\title{
The AFLOW Library of Crystallographic Prototypes: Part 2
}

\author{
David Hicks ${ }^{\mathrm{a}, \mathrm{b}}$, Michael J. Mehlc ${ }^{\mathrm{c}}$, Eric Gossett ${ }^{\mathrm{a}, \mathrm{b}}$, Cormac Toher ${ }^{\mathrm{a}, \mathrm{b}}$, Ohad Levy ${ }^{\mathrm{a}, \mathrm{b}, \mathrm{d}}$, \\ Robert M. Hanson ${ }^{\mathrm{e}}$, Gus Hart ${ }^{\mathrm{f}}$, Stefano Curtarolo ${ }^{\mathrm{a}, \mathrm{g}, \mathrm{h}}$ \\ ${ }^{a}$ Center for Materials Genomics, Duke University, Durham, NC 27708, USA \\ ${ }^{b}$ Department of Mechanical Engineering and Materials Science, Duke University, Durham NC 27708 \\ ${ }^{c}$ Center for Materials Physics and Technology, Code 6390, U.S. Naval Research Laboratory, Washington DC 20375 \\ ${ }^{d}$ Department of Physics, NRCN, P.O. Box 9001, Beer-Sheva 84190, Israel \\ ${ }^{e}$ Department of Chemistry, St. Olaf College, Northfield, Minnesota 55057 \\ ${ }^{f}$ Department of Physics and Astronomy, Brigham Young University, Provo UT 84602 \\ ${ }^{g}$ Materials Science, Electrical Engineering, Physics and Chemistry, Duke University, Durham, North Carolina 27708 \\ ${ }^{h}$ Fritz-Haber-Institut der Max-Planck-Gesellschaft, 14195 Berlin-Dahlem, Germany
}

\begin{abstract}
Materials discovery via high-throughput methods relies on the availability of structural prototypes, which are generally decorated with varying combinations of elements to produce potential new materials. To facilitate the automatic generation of these materials, we developed The AFLOW Library of Crystallographic Prototypes - a collection of crystal prototypes that can be rapidly decorated using the AFLOW software. Part 2 of this work introduces an additional 302 crystal structure prototypes, including at least one from each of the 138 space groups not included in Part 1. Combined with Part 1, the entire library consists of 590 unique crystallographic prototypes covering all 230 space groups. We also present discussions of enantiomorphic space groups, Wigner-Seitz cells, the two-dimensional plane groups, and the various different space group notations used throughout crystallography. All structures - from both Part 1 and Part 2 - are listed in the web version of the library available at http://www . aflow.org/CrystalDatabase.
\end{abstract}

Keywords: Crystal Structure, Space Groups, Wyckoff Positions, Lattice Vectors, Basis Vectors, Database 


\section{Table of Contents}

1. Introduction .......................... 7

2. Enantiomorphic Space Groups $\ldots \ldots \ldots \ldots \ldots \ldots 8$

3. The Wigner-Seitz Primitive Cell ..............9

4. Plane Groups (Two-Dimensional Space Groups) . . 10

4.1. The Parallelogram Crystal System ......... 12

4.1.1. Plane Group \#1: $p 1 \ldots \ldots \ldots \ldots \ldots 12$

4.1.2. Plane Group \#2: $p 2 \ldots \ldots \ldots \ldots \ldots 12$

4.2. The Rectangular Crystal System ...........12

4.2.1. Plane Group \#3: $p 1 m 1 \ldots \ldots \ldots \ldots 12$

4.2.2. Plane Group \#4: $p 1 g 1 \ldots \ldots \ldots \ldots 13$

4.2.3. Plane Group \#5: $c 1 m 1 \ldots \ldots \ldots 13$

4.2.4. Plane Group \#6: $p 2 m m \ldots \ldots \ldots . . . .13$

4.2.5. Plane Group \#7: $p 2 m g$............. 14

4.2.6. Plane Group \#8: $p 2 g g$............ 14

4.2.7. Plane Group \#9: $c 2 m m$.............. 14

4.3. The Square Crystal System ............. 15

4.3.1. Plane Group \#10: $p 4 \ldots \ldots \ldots \ldots \ldots 15$

4.3.2. Plane Group \#11: $p 4 m m \ldots \ldots \ldots \ldots 16$

4.3.3. Plane Group \#12: $p 4 g m \ldots \ldots \ldots \ldots 16$

4.4. The Trigonal Crystal System .............. 16

4.4.1. Plane Group \#13: $p 3 \ldots \ldots \ldots \ldots \ldots 16$

4.4.2. Plane Group \#14: $p 3 m 1 \ldots \ldots \ldots 16$

4.4.3. Plane Group \#15: $p 31 \mathrm{~m} \ldots \ldots \ldots \ldots 17$

4.5. The Hexagonal Crystal System ............ 16

4.5.1. Plane Group \#16: $p 6 \ldots \ldots \ldots \ldots \ldots 17$

4.5.2. Plane Group \#17: $p 6 \mathrm{~mm} \ldots \ldots \ldots \ldots 18$

5. Space Group Notation ..................... 18

6. Conclusion ............................. 18

7. Acknowledgments $\ldots \ldots \ldots \ldots \ldots \ldots \ldots \ldots 24$

8. References ...........................25

\section{Prototypes}

Pī (2)

1. $\mathrm{H}_{2} \mathrm{~S}$ : A2B_aP6_2_aei_i $\ldots \ldots \ldots \ldots \ldots . . . . . . .28$

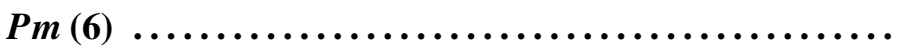

1. $\mathrm{Mo}_{8} \mathrm{P}_{5}$ : A8B5_mP13_6_a7b_3a2b ............. 30

2. FeNi: AB_mP4_6_2b_2a .................. 32

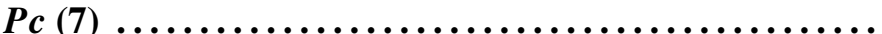

1. $\mathrm{H}_{2} \mathrm{~S}$ IV: A2B_mP12_7_4a_2a $\ldots \ldots \ldots \ldots \ldots \ldots . . . .34$

2. $\mathrm{As}_{2} \mathrm{Ba}: \mathrm{A} 2 \mathrm{~B} \_m P 18 \_7 \_6 \mathrm{a} \_3 \mathrm{a} \ldots \ldots \ldots \ldots \ldots \ldots . . . \ldots 6$

3. $\epsilon-\mathrm{WO}_{3}$ : A3B_mP16_7_6a_2a ............... 38

4. $\mathrm{Rh}_{2} \mathrm{Ga}_{9}:$ A9B2_mP22_7_9a_2a ...............40

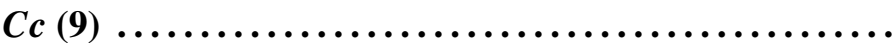

1. $\alpha-\mathrm{P}_{3} \mathrm{~N}_{5}$ : A5B3_mC32_9_5a_3a $\ldots \ldots \ldots \ldots \ldots .42$

2. $\mathrm{H}_{3} \mathrm{Cl}: \mathrm{AB} 3 \_m C 16 \_9 \_a \_3 \mathrm{a} \ldots \ldots . . . \ldots \ldots . . .44$

$P 2 / m(10)$

1. $\delta$-PdCl 2 : A2B_mP6_10_mn_bg ..............46

2. $\mathrm{H}_{3} \mathrm{Cl}: \mathrm{AB} 3 \_\mathrm{mP16} 10 \_\mathrm{mn} \_3 \mathrm{~m} 3 \mathrm{n} \ldots \ldots \ldots \ldots \ldots .48$

3. Muthmannite: ABC2_mP8_10_ac_eh_mn .........50

4. LiSn: AB_mP6_10_en_am ..................52
5. S-carbon: A_mP8_10_2m2n $\ldots \ldots \ldots \ldots \ldots \ldots \ldots . \ldots 54$

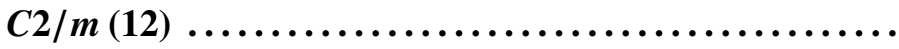

1. Thortveitite: A7B2C2_mC22_12_aij_h_i .........56

2. M-carbon: A_mC16_12_4i .................58

$P 2 / c$ (13)

1. $\mathrm{H}_{2} \mathrm{~S}:$ A2B_mP12_13_2g_ef $\ldots \ldots \ldots \ldots \ldots \ldots \ldots .60$

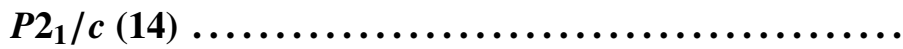

1. $\gamma$-PdCl 2 : A2B_mP6_14_e_a $\ldots \ldots \ldots \ldots \ldots \ldots 62$

2. $\alpha$-Toluene: A7B8_mP120_14_14e_16e .........64 64 C2/c (15)

1. $\mathrm{H}_{3} \mathrm{Cl}: \mathrm{AB} 3 \_m C 16 \_15 \_\mathrm{e} \_c f \ldots \ldots . . . \ldots . . . .71$

2. H-III: A_mC24_15_2e2f .................... 73

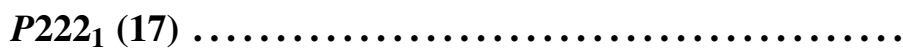

1. $\alpha$-Naumannite: A2B_oP12_17_abe_e ...........75

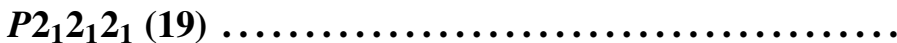

1. $\mathrm{H}_{3} \mathrm{Cl}$ : AB3_oP16_19_a_3a ..................77

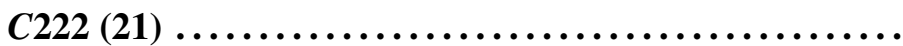

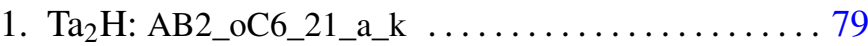

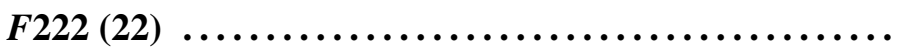

1. $\mathrm{CeRu}_{2} \mathrm{~B}_{2}$ : A2BC2_oF40_22_fi_ad_gh ...........81

2. FeS: AB_oF8_22_a_c ..................... 83

I222 (23)

1. $\mathrm{H}_{3} \mathrm{~S}$ : A3B_ol32_23_ij2k_k ................ 85

2. Stannoidite:

A8B2C12D2E_oI50_23_bcfk_i_3k_j_a . . . . . . . . 87

3. $\mathrm{NaFeS}_{2}$ : ABC2_oI16_23_ab_i_k ..............89

4. BPS 4 : ABC4_oI12_23_a_b_k ...............91

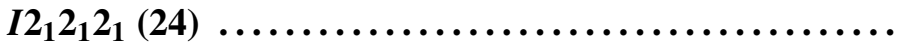

1. Weberite: AB7CD2_oI44_24_a_b3d_c_ac ........993

$\boldsymbol{P m c 2}_{1}$ (26) ...................................

1. $\mathrm{H}_{2} \mathrm{~S}^{*}$ : A2B_oP12_26_abc_ab ............... 95

2. $\beta-\mathrm{SeO}_{2}{ }^{*}$ : A2B_oP12_26_abc_ab .............97

3. TIP 5 : A5B_oP24_26_3a3b2c_ab ............. 99

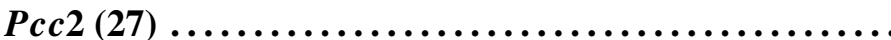

1. $\mathrm{Ca}_{4} \mathrm{Al}_{6} \mathrm{O}_{16} \mathrm{~S}$ :

A6B4C16D_oP108_27_abcd4e_4e_16e_e ..........101

$\operatorname{Pca2}_{1}$ (29)

1. $\mathrm{ZrO}_{2}{ }^{\ddagger}:$ A2B_oP12_29_2a_a $\ldots . . . . . . . . . . . .106$

2. Pyrite ${ }^{\ddagger}$ AB2_oP12_29_a_2a ................ 108

3. Cobaltite: ABC_oP12_29_a_a_a ................110

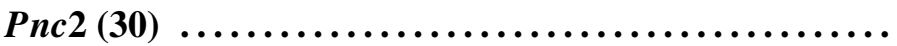

1. $\mathrm{Bi}_{5} \mathrm{Nb}_{3} \mathrm{O}_{15}:$ A5B3C15_oP46_30_a2c_bc_a7c ......112

2. $\mathrm{CuBrSe}_{3}$ : ABC3_oP20_30_2a_c_3c .............115

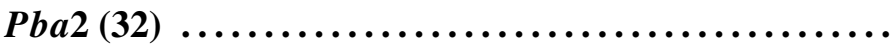

1. $\mathrm{Re}_{2} \mathrm{O}_{5}\left[\mathrm{SO}_{4}\right]_{2}: \mathrm{A} 13 \mathrm{~B} 2 \mathrm{C} 2 \_\mathrm{oP} 34 \_32 \_a 6 c \_c \_c \ldots . .117$

Pna21 (33) $^{2}$

${ }^{*} \mathrm{H}_{2} \mathrm{~S}$ and $\beta-\mathrm{SeO}_{2}$ have the same AFLOW prototype label. They are generated by the same symmetry operations with different sets of parameters.

$\mathrm{ZrO}_{2}$ and Pyrite have similar AFLOW prototype labels (i.e., same symmetry and set of Wyckoff positions with different stoichiometry labels due to alphabetic ordering of atomic species). They are generated by the same symmetry operations with different sets of parameters. 
1. $\kappa$-alumina: A2B3_oP40_33_4a_6a $\ldots \ldots \ldots \ldots \ldots 120$

Pnn2 (34)

1. $\mathrm{TiAl}_{2} \mathrm{Br}_{8}:$ A2B8C_oP22_34_c_4c_a ......... 123

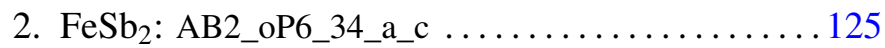

Cmm2 (35)

1. $\mathrm{V}_{2} \mathrm{MoO}_{8}: \mathrm{AB} 8 \mathrm{C} 2 \_$oC22_35_a_ab3e_e ........ 127

Cmc21 (36)

1. HCl: AB_oC8_36_a_a

129

Ccc2 (37)

1. $\mathrm{Li}_{2} \mathrm{Si}_{2} \mathrm{O}_{5}:$ A2B5C2_oC36_37_d_c2d_d

131

Abm2 (39)

1. $\mathrm{Ta}_{3} \mathrm{~S}_{2}$ : A2B3_oC40_39_2d_2c2d ........... 133

2. $\mathrm{VPCl}_{9}$ : A9BC_oC44_39_3c3d_a_c ............. 135

Ama2 (40)

1. $\mathrm{K}_{2} \mathrm{CdPb}$ : AB2C_oC16_40_a_2b_b

137

2. $\mathrm{CeTe}_{3}$ : AB3_oC16_40_b_3b

139

Fmm2 (42)

1. $\mathrm{W}_{3} \mathrm{O}_{10}$ : A10B3_oF52_42_2abce_ab .......... 141

2. BN: AB_oF8_42_a_a .................. 143

Iba2 (45)

1. $\mathrm{MnGa}_{2} \mathrm{Sb}_{2}$ : A2BC2_oI20_45_c_b_c .......... 145

Ima2 (46)

1. TiFeSi: ABC_oI36_46_ac_bc_3b

....

Pnnn (48)

1. $\alpha-\mathrm{RbPr}\left[\mathrm{MoO}_{4}\right]_{2}$ :

A2B8CD_oP24_48_k_2m_d_b

Pccm (49)

1. $\beta$ - $\mathrm{Ta}_{2} \mathrm{O}_{5}$ : A5B2_oP14_49_dehq_ab ......... 151

2. $\mathrm{CsPr}\left[\mathrm{MoO}_{4}\right]_{2}: \mathrm{AB} 2 \mathrm{C} 8 \mathrm{D} \_\mathrm{oP} 24 \_49 \_\mathrm{g} \_\mathrm{q} \_2 \mathrm{qr} \_\mathrm{e} . . .153$

Pban (50)

1. $\mathrm{La}_{2} \mathrm{NiO}_{4}$ : A2BC4_oP28_50_ij_ac_ijm ......... 155

2. $\alpha-\mathrm{Tl}_{2} \mathrm{TeO}_{3}: \mathrm{A} 3 \mathrm{BC} 2 \_\mathrm{oP} 48 \_50 \_3 \mathrm{~m} \_\mathrm{m} \_2 \mathrm{~m} \ldots \ldots . .157$

Pnna (52)

1. $\mathrm{GaCl}_{2}$ : A2B_oP24_52_2e_cd ............ 160

2. $\mathrm{Sr}_{2} \mathrm{Bi}_{3}$ : A3B2_oP20_52_de_cd ............. 162

Pmna (53)

1. $\mathrm{TaNiTe}_{2}$ : ABC2_oP16_53_h_e_gh ............ 164

2. $\mathrm{CuBrSe}_{3}: \mathrm{ABC} 3 \_$oP20_53_e_g_hi ........... 166

Pcca (54)

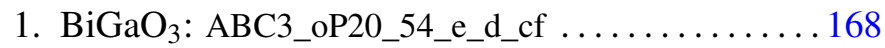

Pbam (55)

1. GeAs 2 : A2B_oP24_55_2g2h_gh ........... 170

2. $\mathrm{Rh}_{5} \mathrm{Ge}_{3}$ : A3B5_oP16_55_ch_agh ........... 172

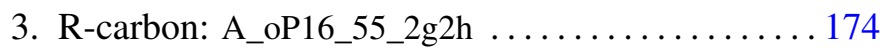

Pnnm (58)

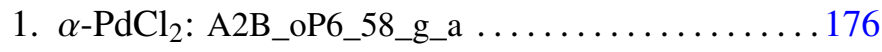

Pmmn (59)

1. FeOCl: ABC_oP6_59_a_b_a .............. 178

Pbcn (60)

1. $\mathrm{Rh}_{2} \mathrm{~S}_{3}:$ A2B3_oP20_60_d_cd ............. 180

2. $\mathrm{WO}_{3}$ : A3B_oP32_60_3d_d .............. 182

3. $\beta$-Toluene: A7B8_oP120_60_7d_8d .......... 185

Pbca (61)
1. Benzene: AB_oP48_61_3c_3c

191

Pnma (62)

1. Tongbaite: A2B3_oP20_62_2c_3c ............ 194

2. Forsterite: A2B4C_oP28_62_ac_2cd_c .......... 196

3. $\mathrm{SrH}_{2}$ : A2B_oP12_62_2c_c .................. 198

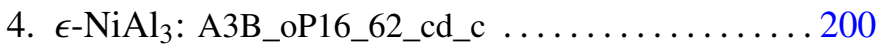

5. Cubanite: AB2C3_oP24_62_c_d_cd .............202

6. Molybdite: AB3_oP16_62_c_3c .............. 204

7. Barite: AB4C_oP24_62_c_2cd_c .............. 206

8. Westerveldite: AB_oP8_62_c_c ............... 208

Cmcm (63)

1. Rasvumite: A2BC3_oC24_63_e_c_cg ..........210

2. $\mathrm{La}_{43} \mathrm{Ni}_{17} \mathrm{Mg}_{5}$ : A43B5C17_oC260_63_c8fg6h_cfg_ce3f2h ........212

3. $\mathrm{MnAl}_{6}$ : A6B_oC28_63_efg_c .............. 218

4. Post-perovskite: AB3C_oC20_63_a_cf_c ........220

5. $\mathrm{MgSO}_{4}$ : AB4C_oC24_63_a_fg_c .............222

6. Anhydrite: AB4C_oC24_63_c_fg_c ............224

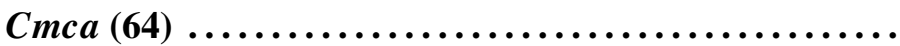

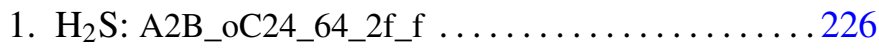

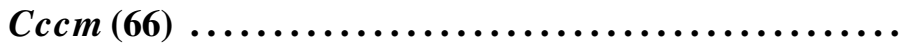

1. $\mathrm{SrAl}_{2} \mathrm{Se}_{4}$ : A2B4C_oC28_66_1_kl_a ........... 228

2. $\mathrm{H}_{3} \mathrm{~S}$ : A3B_oC64_66_gi21m_21 ............230

3. $\beta$-ThI ${ }_{3}$ : A3B_oC64_66_kl2m_bdl ............ 233

Cmma (67)

1. $\mathrm{Al}_{2} \mathrm{CuIr}^{\S}$ : A2BC_oC16_67_ag_b_g ...........236

2. $\mathrm{HoCuP}_{2}{ }^{\S}: \mathrm{ABC} 2 \_\mathrm{oC} 16 \_67 \_b \_g \_a g \ldots \ldots \ldots \ldots 238$

3. $\alpha-\mathrm{FeSe}^{\dagger}$ : AB_oC8_67_a_g ................ 240

4. $\alpha-\mathrm{PbO}^{\dagger}$ : AB_oC8_67_a_g ................... 242

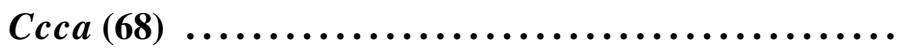

1. PdSn 4 : AB4_oC20_68_a_i ..................244

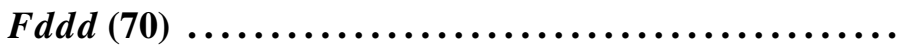

1. $\mathrm{Mn}_{2} \mathrm{~B}: \mathrm{AB} 2 \_\mathrm{oF} 48 \_70 \_f \_f g \ldots \ldots \ldots \ldots \ldots . \ldots \ldots 6$

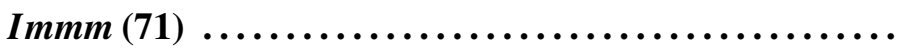

1. $\mathrm{Ta}_{3} \mathrm{~B}_{4}:$ A4B3_oI14_71_gh_cg .............. 248

2. NbPS: ABC_oI12_71_h_j_g .............. 250

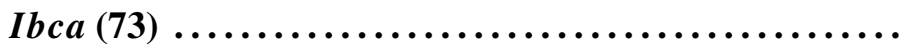

1. $\mathrm{KAg}\left[\mathrm{CO}_{3}\right]$ : ABCD3_oI48_73_d_e_e_ef ......... 252

Imma (74)

1. $\mathrm{KHg}_{2}:$ A2B_oI12_74_h_e ................. 254

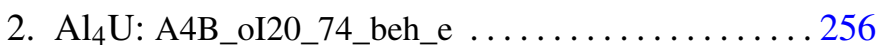

$P 4$ (75)

1. $\mathrm{BaCr}_{2} \mathrm{Ru}_{4} \mathrm{O}_{12}$ :

AB2C12D4_tP76_75_2a2b_2d_12d_4d ..........258

$P 4_{1}$ (76)

1. LaRhC $_{2}$ : A2BC_tP16_76_2a_a_a ............. 262

${ }^{\S} \mathrm{Al}_{2} \mathrm{CuIr}$ and $\mathrm{HoCuP}_{2}$ have similar AFLOW prototype labels (i.e., same symmetry and set of Wyckoff positions with different stoichiometry labels due to alphabetic ordering of atomic species). They are generated by the same symmetry operations with different sets of parameters.

${ }^{\dagger} \alpha$-FeSe and $\alpha$ - $\mathrm{PbO}$ have the same AFLOW prototype label. They are generated by the same symmetry operations with different sets of parameters. 
2. $\mathrm{Cs}_{3} \mathrm{P}_{7}:$ A3B7_tP40_76_3a_7a ............. 264

$\mathrm{P4}_{2}$ (77)

1. Pinnoite: A2B6CD7_tP64_77_2d_6d_d_ab6d ......267

2. $\mathrm{H}_{2}$ S III: A2B_tP48_77_8d_4d ............. 270

$P_{3}$ (78)

1. $\mathrm{Sr}_{2} \mathrm{As}_{2} \mathrm{O}_{7}:$ A2B7C2_tP88_78_4a_14a_4a ....... 273

I4 (79)

1. $\mathrm{TlZn}_{2} \mathrm{Sb}_{2}$ : A2BC2_tI20_79_c_2a_c ........... 277

$I_{1}(80)$

1. $\beta-\mathrm{NbO}_{2}$ : AB2_tI48_80_2b_4b

. 279

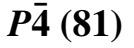

1. $\mathrm{GeSe}_{2}: \mathrm{AB} 2 \_t \mathrm{P} 12 \_81 \_\mathrm{adg} \_2 \mathrm{~h}$

.282 I $\overline{4}$ (82)

1. $\mathrm{Ni}_{3} \mathrm{P}:$ A3B_tI32_82_3g_g

.284

$P 4 / m$ (83)

1. $\mathrm{Ti}_{2} \mathrm{Ge}_{3}: \mathrm{A} 3 \mathrm{~B} 2 \_\mathrm{tP} 10 \_83 \_\mathrm{adk} \_\mathrm{j}$

.286

P4/n (85)

1. $\mathrm{SrBr}_{2}$ : A2B_tP30_85_ab2g_cg ............ 288

$\mathrm{P4}_{2} / \boldsymbol{n}$ (86)

1. $\mathrm{Ti}_{3} \mathrm{P}: \mathrm{AB} 3 \_\mathrm{tP} 32 \_86 \_\mathrm{g} \_3 \mathrm{~g}$

291

$I_{1} / \boldsymbol{a}(88)$

1. $\mathrm{ThCl}_{4}:$ A4B_tI20_88_f_a

294

2. $\alpha-\mathrm{NbO}_{2}: \mathrm{AB} 2 \_\mathrm{tI} 96 \_88 \_2 \mathrm{f} \_4 \mathrm{f}$

296

P422 (89)

1. $\mathrm{C}_{17} \mathrm{FeO}_{4} \mathrm{Pt}$ : A17BC4D_tP184_89_17p_p_4p_io ....300

P4212 (90)

1. $\mathrm{Na}_{4} \mathrm{Ti}_{2} \mathrm{Si}_{8} \mathrm{O}_{22}\left[\mathrm{H}_{2} \mathrm{O}\right]_{4}$ :

A4B2C13D_tP40_90_g_d_cef2g_c

307

2. $\mathrm{BaCu}_{4}[\mathrm{VO}]\left[\mathrm{PO}_{4}\right]_{4}$ :

AB4C17D4E_tP54_90_a_g_c4g_g_c ............310

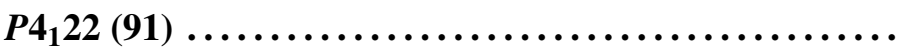

1. ThBC: ABC_tP24_91_d_d_d ....................

$P 4_{2} 22$ (93)

1. $\mathrm{AsPh}_{4} \mathrm{CeS}_{8} \mathrm{P}_{4} \mathrm{Me}_{8}$ :

AB32CD4E8_tP184_93_i_16p_af_2p_4p ........315

$P_{2} 212$ (94)

1. $\mathrm{Na}_{5} \mathrm{Fe}_{3} \mathrm{~F}_{14}: \mathrm{A} 14 \mathrm{~B} 3 \mathrm{C} 5 \_t \mathrm{P} 44 \_94 \_c 3 g \_a d \_b g ~ . . . . .322$

2. $\mathrm{Li}_{2} \mathrm{MoF}_{6}:$ A6B2C_tP18_94_eg_c_a .......... 325

$P_{3} 22$ (95)

1. ThBC: ABC_tP24_95_d_d_d

327

I422 (97)

1. $\mathrm{NaGdCu}_{2} \mathrm{~F}_{8}$ : A2B8CD_tI24_97_d_k_a_b .........329

2. $\mathrm{Ta}_{2} \mathrm{Se}_{8} \mathrm{I}: \mathrm{AB} 8 \mathrm{C} 2 \_\mathrm{tI} 44 \_97 \_\mathrm{e} \_2 \mathrm{k} \_\mathrm{cd}$.

.331 I4, 22 (98)

1. $\mathrm{CdAs}_{2}:$ A2B_tI12_98_f_a

333

P4bm (100)

1. Fresnoite: A2B8C2D_tP26_100_c_abcd_c_a ..... 335

2. $\mathrm{Ce}_{3} \mathrm{Si}_{6} \mathrm{~N}_{11}$ : A3B11C6_tP40_100_ac_bc2d_cd .... 337

$\mathrm{P4}_{2} \mathrm{~cm}$ (101)

1. $\gamma$-MgNiSn: A7B7C2_tP32_101_bde_ade_d ..... 340

$P 4_{2}$ nm (102)

1. $\mathrm{Gd}_{3} \mathrm{Al}_{2}:$ A2B3_tP20_102_2c_b2c

.343

P4cc (103)
1. $\mathrm{NbTe}_{4}: \mathrm{AB} 4 \_\mathrm{tP} 10 \_103 \_\mathrm{a} \_\mathrm{d}$ .345

P4nc (104)

1. $\mathrm{Ba}_{5} \mathrm{In}_{4} \mathrm{Bi}_{5}: \mathrm{A} 5 \mathrm{~B} 5 \mathrm{C} 4$ tP28_104_ac_ac c ....... 347

2. $\mathrm{Tl}_{4} \mathrm{HgI}_{6}:$ AB6C4_tP22_104_a_2ac_c ........... 350

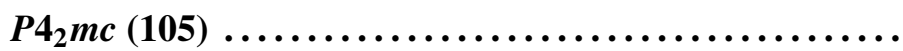

1. $\mathrm{BaGe}_{2} \mathrm{As}_{2}:$ A2BC2_tP20_105_f_ac_2e ........... 352

$P 4_{2} b c$ (106)

1. $\mathrm{NaZn}[\mathrm{OH}]_{3}:$ A3BC3D_tP64_106_3c_c_3c_c ...... 354

I4mm (107)

1. $\mathrm{Co}_{5} \mathrm{Ge}_{7}: \mathrm{A} 5 \mathrm{~B} 7 \_t \mathrm{t} 24 \_107 \_\mathrm{ac} \_\mathrm{abd} . . . \ldots \ldots \ldots . . .358$

2. GeP: AB_tI4_107_a_a ................... 360

I4cm (108)

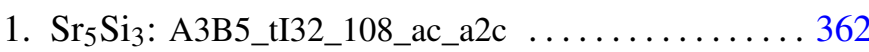

I41 $m$ d (109)

1. LaPtSi: ABC_tI12_109_a_a_a ................. 364

2. NbAs: AB_tI8_109_a_a ...................... 366

$\mathrm{I4}_{1} \boldsymbol{c d}$ (110)

1. $\mathrm{Be}\left[\mathrm{BH}_{4}\right]_{2}:$ A2BC8_tI176_110_2b_b_8b ......... 368

$\boldsymbol{P} \overline{4} 2 m$ (111) .

1. $\mathrm{MnF}_{2}$ : A2B_tP12_111_2n_adf ............. 374

2. NV: AB_tP8_111_n_n ......................... 376

$\boldsymbol{P} \overline{4} 2 c$ (112)

1. $\alpha-\mathrm{CuAlCl}{ }_{4}:$ AB4C_tP12_112_b_n_e ........... 378 $\boldsymbol{P \overline { 4 }}{ }_{1} m$ (113)

1. Akermanite: A2BC7D2_tP24_113_e_a_cef_e ..... 380 $\boldsymbol{P \overline { 4 }}{ }_{1} c$ (114)

1. $\mathrm{SeO}_{3}:$ A3B_tP32_114_3e_e .................... 382

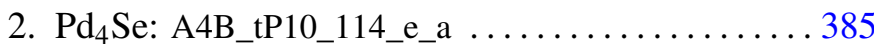

$\boldsymbol{P} \overline{\mathbf{4}} \boldsymbol{m} \mathbf{2}$ (115)

1. $\mathrm{Rh}_{3} \mathrm{P}_{2}:$ A2B3_tP5_115_g_ag ................. 387

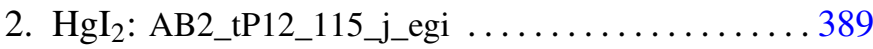

$\boldsymbol{P} \overline{4} \boldsymbol{c} 2$ (116)

1. $\mathrm{Ru}_{2} \mathrm{Sn}_{3}: \mathrm{A} 2 \mathrm{~B} 3 \_\mathrm{tP} 20 \_116 \_b c i \_f j$

391

P $\overline{4} b 2$ (117)

1. $\beta-\mathrm{Bi}_{2} \mathrm{O}_{3}: \mathrm{A} 2 \mathrm{~B} 3 \_t \mathrm{tP} 20 \_117 \_\mathrm{i} \_$adgh $\ldots \ldots \ldots \ldots \ldots 393$

$\boldsymbol{P} \overline{4} n 2$ (118)

1. RuIn 3 : A3B_tP16_118_ei_f

.395

2. $\mathrm{Ir}_{3} \mathrm{Ga}_{5}: \mathrm{A} 5 \mathrm{~B} 3$ _tP32_118_g2i_aceh

.397

I $\overline{4} \mathbf{m} 2$ (119)

1. RbGa 3 : A3B_tI24_119_b2i_af ............. 400

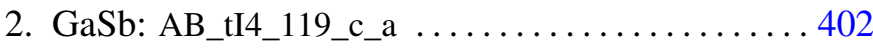

I $\overline{4} c 2$ (120)

1. $\mathrm{KAu}_{4} \mathrm{Sn}_{2}:$ A4BC2_tI28_120_i_d_e ........... 404

$P 4 / \mathbf{m m m}$ (123)

1. $\mathrm{CaRbFe}_{4} \mathrm{As}_{4}$ : A4BC4D_tP10_123_gh_a_i_d ......4406 P4/mcc (124)

1. $\mathrm{Nb}_{4} \mathrm{CoSi}:$ AB4C_tP12_124_a_m_c ............ 408

2. $\mathrm{NbTe}_{4}:$ AB4_tP10_124_a_m ................4 410

$P 4 / n b m$ (125)

1. $\mathrm{PtPb}_{4}:$ A4B_tP10_125_m_a

2. $\mathrm{KCeSe}_{4}: \mathrm{ABC} 4 \_\mathrm{tP} 12 \_125 \_\mathrm{a} \_b \_\mathrm{m} \ldots \ldots \ldots \ldots .414$

$P 4 / n n c$ (126) 
1. $\mathrm{BiAl}_{2} \mathrm{~S}_{4}:$ A2BC4_tP28_126_cd_e_k ..........416

$P 4 / m b m$ (127)

1. ThB 4 : A4B_tP20_127_ehj_g

.418

$P 4 / m n c$ (128)

1. $\mathrm{K}_{2} \mathrm{SnCl}_{6}:$ A6B2C_tP18_128_eh_d_b ..........420

2. $\mathrm{FeCu}_{2} \mathrm{Al}_{7}:$ A7B2C_tP40_128_egi_h_e .........422

P4/ncc (130)

1. $\mathrm{CuBi}_{2} \mathrm{O}_{4}:$ A2BC4_tP28_130_f_c_g ......... 425

2. $\mathrm{Ba}_{5} \mathrm{Si}_{3}: \mathrm{A} 5 \mathrm{~B} 3 \_t \mathrm{P3} 2 \_130 \_c g \_c f \ldots \ldots \ldots \ldots \ldots . \ldots 427$

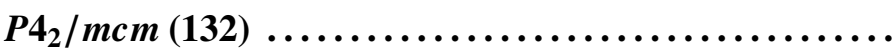

1. $\mathrm{Rb}_{2} \mathrm{TiCu}_{2} \mathrm{~S}_{4}: \mathrm{A} 2 \mathrm{~B} 2 \mathrm{C} 4 \mathrm{D} \_t \mathrm{tP} 18 \_132$ e_i_o_d .......429

2. AgUF6 $:$ AB6C_tP16_132_d_io_a ............441

$\mathrm{P4}_{2} / \boldsymbol{n b c}$ (133)

1. $\beta-\mathrm{V}_{3} \mathrm{~S}:$ AB3_tP32_133_h_i2j .............433

$\mathrm{P4}_{2} / \boldsymbol{m b c}$ (135)

1. Downeyite: A2B_tP24_135_gh_h ...........446

2. $\mathrm{ZnSb}_{2} \mathrm{O}_{4}$ : A4B2C_tP28_135_gh_h_d ........448

$\mathrm{P4}_{2} /$ nmc (137)

1. $\mathrm{Zn}_{3} \mathrm{P}_{2}:$ A2B3_tP40_137_cdf_3g ........... 440

2. $\mathrm{ZrO}_{2}{ }^{\mathrm{I}}:$ A2B_tP6_137_d_a $\ldots . . . \ldots \ldots \ldots . . . .443$

3. $\mathrm{CeCo}_{4} \mathrm{~B}_{4}$ : A4BC4_tP18_137_g_b_g .......... 445

4. $\mathrm{HgI}_{2}{ }^{\mathrm{II}}$ : AB2_tP6_137_a_d ............... 447

$\mathrm{P4}_{2} /$ ncm (138)

1. C: A_tP12_138_bi

449

I4/mmm (139)

1. Calomel: AB_tI8_139_e_e ..............451

I4/mcm (140)

1. $\mathrm{W}_{5} \mathrm{Si}_{3}:$ A3B5_tI32_140_ah_bk

453

2. $\mathrm{Cr}_{5} \mathrm{~B}_{3}$ : A3B5_tI32_140_ah_cl

455

I4, /amd (141)

1. $\alpha$-ThSi ${ }_{2}$ : A2B_tI12_141_e_a .............457

I4, /acd (142)

1. S-III: A_tI16_142_f ..................459

P3 (143)

1. Simpsonite: A4B14C3_hP21_143_bd_ac4d_d .....461

2. $\mathrm{ScRh}_{6} \mathrm{P}_{4}: \mathrm{A} 4 \mathrm{~B} 6 \mathrm{C} \_$hP11_143_bd_2d_a .......... 463

3. $\mathrm{MoS}_{2}$ : AB2_hP12_143_cd_ab2d ............465

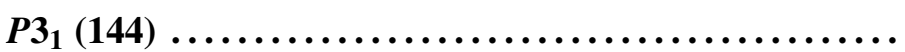

1. $\mathrm{IrGe}_{4}:$ A4B_hP15_144_4a_a ..............467

2. TeZn: AB_hP6_144_a_a ...................4469

$P_{2}$ (145)

1. Sheldrickite:

A2B3C3DE7_hP48_145_2a_3a_3a_a_7a ........471

R3 (146)

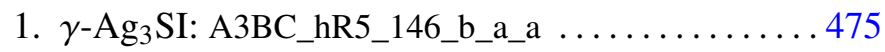

2. $\mathrm{FePSe}_{3}: \mathrm{ABC} 3 \_$hR10_146_2a_2a_2b ...........477 $\boldsymbol{R} \overline{\mathbf{3}}$ (148)

1. Phenakite: A2B4C_hR42_148_2f_4f_f

479

${ }^{\mathrm{I}} \mathrm{ZrO}_{2}$ and $\mathrm{HgI}_{2}$ have similar AFLOW prototype labels (i.e., same symmetry and set of Wyckoff positions with different stoichiometry labels due to alphabetic ordering of atomic species). They are generated by the same symmetry operations with different sets of parameters.
2. $\beta-\mathrm{PdCl}_{2}$ : A2B_hR18_148_2f_f $\ldots \ldots \ldots \ldots \ldots \ldots 483$

P312 (149)

1. $\mathrm{Ti}_{3} \mathrm{O}: \mathrm{AB} 3 \_$hP24_149_acgi_31 .............486

$P_{2} 12$ (153)

1. $\mathrm{CrCl}_{3}$ : A3B_hP24_153_3c_2b ..............488

$P 3_{2} 21$ (154)

1. S-II: A_hP9_154_bc

491

P3m1 (156)

1. $\mathrm{CdI}_{2}: \mathrm{AB} 2$ hP9_156_b2c_3a2bc

.493

2. CuI: AB_hP12_156_2ab3c_2ab3c

.495

3. $\beta$-CuI: AB_hP4_156_ac_ac

P31m (157)

1. $\mathrm{Ag}_{5} \mathrm{~Pb}_{2} \mathrm{O}_{6}: \mathrm{A} 5 \mathrm{~B} 6 \mathrm{C} 2 \_\mathrm{hP} 13 \_157 \_2 \mathrm{ac} \_2 \mathrm{c} \_\mathrm{b}$

499

P3c1 (158)

1. $\beta-\mathrm{RuCl}_{3}$ : A3B_hP8_158_d_a ...............501 $P 31 c$ (159)

1. $\mathrm{Bi}_{2} \mathrm{O}_{3}$ : A2B3_hP20_159_bc_2c ...........503

2. Nierite: A4B3_hP28_159_ab2c_2c ...........506

3. $\mathrm{YbBaCo}_{4} \mathrm{O}_{7}$ : AB4C7D_hP26_159_b_ac_a2c_b .... 509

R3m (160)

1. $\mathrm{H}_{3} \mathrm{~S}:$ A3B_hR4_160_b_a

512

2. $\mathrm{Al}_{8} \mathrm{Cr}_{5}$ : A8B5_hR26_160_a3bc_a3b ......... 514

3. Carbonyl Sulphide: ABC_hR3_160_a_a_a .......5517

4. Moissanite-15R: AB_hR10_160_5a_5a .........519

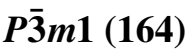

1. $\mathrm{La}_{2} \mathrm{O}_{3}: \mathrm{A} 2 \mathrm{~B} 3$ _hP5_164_d_ad

2. $\delta_{H}^{I I}-\mathrm{NW}_{2}: \mathrm{AB} 2 \_\mathrm{hP} 9 \_164 \_b d \_c 2 \mathrm{~d}$ 523

3. $\mathrm{CuNiSb}_{2}$ : ABC2_hP4_164_a_b_d ............ 525

$P \overline{3} \boldsymbol{c} 1$ (165)

1. $\mathrm{Cu}_{3} \mathrm{P}:$ A3B_hP24_165_bdg_f $\ldots \ldots \ldots \ldots \ldots \ldots . \ldots 27$

$\boldsymbol{R} \overline{\mathbf{3}} \boldsymbol{m}$ (166)

1. $\mathrm{Al}_{4} \mathrm{C}_{3}: \mathrm{A} 4 \mathrm{~B} 3 \_\mathrm{hR7}$ _166_2c_ac

530

2. SmSI: ABC_hR6_166_c_c_c ................ 532

$\boldsymbol{R} \overline{\mathbf{3}} \boldsymbol{c}$ (167)

1. $\mathrm{PrNiO}_{3}: \mathrm{AB} 3 \mathrm{C} \_$hR10_167_b_e_a .............554

2. $\mathrm{KBO}_{2}: \mathrm{ABC} 2 \_\mathrm{hR} 24 \_167 \_\mathrm{e} \_\mathrm{e} \_2 \mathrm{e} . . . \ldots \ldots \ldots . . .536$

$P 6$ (168)

1. $\mathrm{K}_{2} \mathrm{Ta}_{4} \mathrm{O}_{9} \mathrm{~F}_{4}:$ A2B13C4_hP57_168_d_c6d_2d ......539

2. $\mathrm{Al}\left[\mathrm{PO}_{4}\right]: \mathrm{AB} 4 \mathrm{C} \_\mathrm{hP72} \_168 \_2 \mathrm{~d} \_8 \mathrm{~d} \_2 \mathrm{~d} \ldots . . . \ldots . .543$

$P_{1}$ (169)

1. $\alpha-\mathrm{Al}_{2} \mathrm{~S}_{3}$ : A2B3_hP30_169_2a_3a

$P_{5}$ (170)

1. $\mathrm{Al}_{2} \mathrm{~S}_{3}:$ A2B3_hP30_170_2a_3a

550

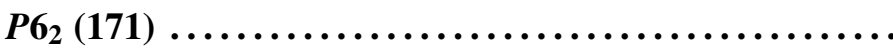

1. $\mathrm{Sr}\left[\mathrm{S}_{2} \mathrm{O}_{6}\right]\left[\mathrm{H}_{2} \mathrm{O}\right]_{4}:$ A10B2C_hP39_171_5c_c_a .....553

$\mathrm{P6}_{4}$ (172)

1. $\mathrm{Sr}\left[\mathrm{S}_{2} \mathrm{O}_{6}\right]\left[\mathrm{H}_{2} \mathrm{O}\right]_{4}:$ A10B2C_hP39_172_5c_c_a .....557

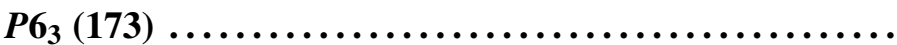

1. $\mathrm{PI}_{3}:$ A3B_hP8_173_c_b .................. 560

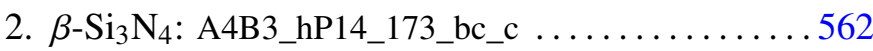

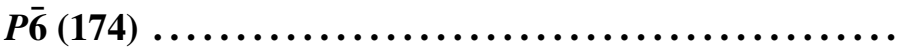

1. $\mathrm{Fe}_{12} \mathrm{Zr}_{2} \mathrm{P}_{7}: \mathrm{A} 12 \mathrm{~B} 7 \mathrm{C} 2 \_$hP21_174_2j2k_ajk_cf .... 564 
2. GdSI: ABC_hP12_174_cj_fk_aj .............. 566 $P 6 / \boldsymbol{m}$ (175)

1. $\mathrm{Nb}_{7} \mathrm{Ru}_{6} \mathrm{~B}_{8}:$ A8B7C6_hP21_175_ck_aj_k ........ 568

2. $\mathrm{Mg}[\mathrm{NH}]:$ ABC_hP36_175_jk_jk_jk ...........5570

$P 6_{3} / \boldsymbol{m}$ (176)

1. $\mathrm{Er}_{3} \mathrm{Ru}_{2}$ : A3B2_hP10_176_h_bd ............. 573

2. $\mathrm{Fe}_{3} \mathrm{Te}_{3} \mathrm{Tl}: \mathrm{A} 3 \mathrm{~B} 3 \mathrm{C} \_\mathrm{hP14} \_176 \_\mathrm{h} \_\mathrm{h} \_\mathrm{d} \ldots . . . . . . .575$

3. $\mathrm{UCl}_{3}$ : A3B_hP8_176_h_d .................. 577

P622 (177)

1. $\mathrm{SiO}_{2}:$ A2B_hP36_177_j21m_n ...............5579

$P 6_{1} 22$ (178)

1. $\mathrm{AuF}_{3}: \mathrm{AB} 3 \_$hP24_178_b_ac ................. 582

2. Sc-V: A_hP6_178_a .....................584

$P_{5} 22$ (179)

1. AuF 3 : AB3_hP24_179_b_ac ................586

$P_{4} 22$ (181)

1. $\beta-\mathrm{SiO}_{2}:$ A2B_hP9_181_j_c $\ldots \ldots \ldots \ldots \ldots . \ldots 58$

P6 mm (183)

1. AuCN: ABC_hP3_183_a_a_a .................590

2. $\mathrm{CrFe}_{3} \mathrm{NiSn}_{5}$ : AB_hP6_183_c_ab ............. 592

P6cc (184)

1. Al[PO 4 ]: AB4C_hP72_184_d_4d_d ............594

P6 3 cm (185)

1. $\mathrm{KNiCl}_{3}$ : A3BC_hP30_185_cd_c_ab ............599

2. $\mathrm{Cu}_{3} \mathrm{P}^{\|}:$A3B_hP24_185_ab2c_c $\ldots . . . \ldots \ldots \ldots . \ldots 62$

3. $\beta$-RuCl ${ }_{3}$ : A3B_hP8_185_c_a $\ldots \ldots \ldots \ldots \ldots \ldots 605$

4. $\mathrm{Na}_{3} \mathrm{As}^{\|}:$: AB3_hP24_185_c_ab2c .............607

$P 6{ }_{3} m c$ (186)

1. $\mathrm{Fe}_{3} \mathrm{Th}_{7}$ : A3B7_hP20_186_c_b2c $\ldots \ldots \ldots \ldots \ldots 610$

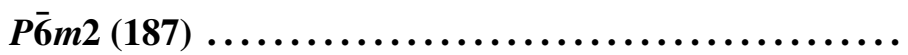

1. $\operatorname{Re}_{3} \mathrm{~N}$ : AB3_hP4_187_e_fh ................6 612

$\boldsymbol{P} \overline{\mathbf{b}} \boldsymbol{c} 2$ (188)

1. LiScI $_{3}:$ A3BC_hP10_188_k_a_e ..............6614

2. $\mathrm{BaSi}_{4} \mathrm{O}_{9}: \mathrm{AB} 9 \mathrm{C} 4 \_\mathrm{hP} 28 \_188$ e_kl_ak ...........616

Pर्62m (189)

1. $\pi-\mathrm{FeMg}_{3} \mathrm{Al}_{8} \mathrm{Si}_{6}$ :

A8BC3D6_hP18_189_bfh_a_g_i

2. $\pi-\mathrm{FeMg}_{3} \mathrm{Al}_{9} \mathrm{Si}_{5}$ :

A9BC3D5_hP18_189_fi_a_g_bh ..............6 622

P $\overline{6} 2 c$ (190)

1. $\mathrm{Li}_{2} \mathrm{Sb}$ : A2B_hP18_190_gh_bf

624

2. $\alpha-\mathrm{Sm}_{3} \mathrm{Ge}_{5}$ : A5B3_hP16_190_bdh_g ..........6 626

3. Troilite: AB_hP24_190_i_afh ...............6628

P6/mcc (192)

1. Beryl: A2B3C18D6_hP58_192_c_f_lm_1 ........631

2. $\mathrm{AlPO}_{4}: \mathrm{AB} 2 \_\mathrm{hP72} \_192 \_\mathrm{m} \_\mathrm{j} 2 \mathrm{kl} \ldots \ldots \ldots \ldots . .635$

$\mathrm{P6}_{3} / \mathrm{mcm}$ (193)

${ }^{\|} \mathrm{Cu}_{3} \mathrm{P}$ and $\mathrm{Na}_{3} \mathrm{As}$ have similar AFLOW prototype labels (i.e., same symmetry and set of Wyckoff positions with different stoichiometry labels due to alphabetic ordering of atomic species). They are generated by the same symmetry operations with different sets of parameters.
1. Mavlyanovite: A5B3_hP16_193_dg_g

$P_{6} / \mathbf{m m c}$ (194)

1. Ni 3 Ti: A3B_hP16_194_gh_ac ...............661

2. $\mathrm{Co}_{2} \mathrm{Al}_{5}:$ A5B2_hP28_194_ahk_ch .............6 643

3. $\mathrm{Al}_{9} \mathrm{Mn}_{3} \mathrm{Si}$ : A9B3C_hP26_194_hk_h_a $\ldots . . . . .646$ $P 23$ (195)

1. $\mathrm{PrRu}_{4} \mathrm{P}_{12}$ : A12BC4_cP34_195_2j_ab_2e

.649

F23 (196)

1. $\mathrm{Cu}_{2} \mathrm{Fe}[\mathrm{CN}]_{6}$ : A12B2C_cF60_196_h_bc_a . ...... 652

2. $\mathrm{MgB}_{12} \mathrm{H}_{12}\left[\mathrm{H}_{2} \mathrm{O}\right]_{12}$ :

A12B36CD12_cF488_196_2h_6h_ac_fgh .........664

$P 2,3$ (198)

1. Sodium Chlorate: ABC3_cP20_198_a_a_b ...... 661 $\boldsymbol{P m} \overline{\mathbf{3}}(\mathbf{2 0 0})$

1. $\mathrm{Mg}_{2} \mathrm{Zn}_{11}:$ A2B11_cP39_200_f_aghij

663

$\boldsymbol{P n} \overline{\mathbf{3}}$ (201)

1. $\mathrm{KSbO}_{3}:$ AB3C_cP60_201_ce_fh_g ............666

$\boldsymbol{F} \boldsymbol{m} \overline{\mathbf{3}}(\mathbf{2 0 2})$

1. $\mathrm{KB}_{6} \mathrm{H}_{6}$ : A6B6C_cF104_202_h_h_c

.669

2. FCC $\mathrm{C}_{60}$ Buckminsterfullerine:

A_cF240_202_h2i

$F d \overline{\mathbf{3}}(\mathbf{2 0 3})$

1. Pyrochlore: A2BCD3E6_cF208_203_e_c_d_f_g . . 675

2. Tychite: A4B2C6D16E_cF232_203_e_d_f_eg_a . .. 679

3. $\mathrm{Rb}_{3} \mathrm{AsSe}_{16}$ : AB3C16_cF160_203_b_ad_eg .......683

$\boldsymbol{P a} \overline{\mathbf{3}}$ (205)

1. $\mathrm{Ca}_{3} \mathrm{Al}_{2} \mathrm{O}_{6}$ : A2B3C6_cP264_205_2d_ab2c2d_6d ...687 687

2. Simple Cubic $\mathrm{C}_{60}$ Buckminsterfullerine: A_cP240_205_10d

.696

Ia $\overline{3}$ (206)

1. AlLi ${ }_{3} \mathrm{~N}_{2}:$ AB3C2_cI96_206_c_e_ad ..............704 P432 (207)

1. $\mathrm{Pd}_{17} \mathrm{Se}_{15}: \mathrm{A} 17 \mathrm{~B} 15 \_c P 64 \_207 \_a c f k \_e i j$

$\mathrm{P4}_{2} 32$ (208)

1. $\mathrm{PH}_{3}:$ A3B_cP16_208_j_b

710

2. $\mathrm{Cs}_{2} \mathrm{ZnFe}[\mathrm{CN}]_{6}$ :

A6B2CD6E_cP64_208_m_ad_b_m_c ...........712

F432 (209)

1. F $F_{6}$ KP: A24BC_cF104_209 j a b $\ldots \ldots \ldots \ldots \ldots .716$

$F 4_{1} 32(210)$

1. $\mathrm{Te}[\mathrm{OH}]_{6}:$ A12B6C_cF608_210_4h_2h_e ........719

I432 (211)

1. $\mathrm{SiO}_{2}$ : A2B_cl72_211_hi_i ..................731

$\mathrm{P4}_{3} 32$ (212)

1. $\mathrm{SrSi}_{2}$ : A2B_cP12_212_c_a

734

$I_{1} 32$ (214)

1. $\mathrm{Ca}_{3} \mathrm{PI}_{3}$ : A3B3C_cI56_214_g_h_a ............736

2. Petzite: A3BC2_cI48_214_f_a_e ............. 739

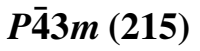

1. $\gamma$-brass: A4B9_cP52_215_ei_3efgi

.741

F $\overline{4} 3 m(216)$ 
1. Quartenary Heusler:

ABCD_cF16_216_c_d_b_a ...................745

$\boldsymbol{P} \overline{43} n$ (218)

1. $\mathrm{Ag}_{3}\left[\mathrm{PO}_{4}\right]:$ A3B4C_cP16_218_c_e_a ..........747

F $\overline{43} \boldsymbol{c}$ (219)

1. Boracite: A7BC3D13_cF192_219_de_b_c_ah

.749

I $\overline{4} 3 d(220)$

1. $\mathrm{Cu}_{15} \mathrm{Si}_{4}: \mathrm{A} 15 \mathrm{~B} 4 \_\mathrm{cI76} \_220 \_$ae_c .............753

2. $\mathrm{Th}_{3} \mathrm{P}_{4}:$ A4B3_cI28_220_c_a .............. 756

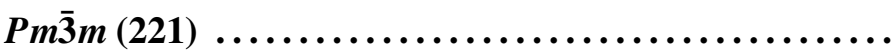

1. $\mathrm{Ca}_{3} \mathrm{Al}_{2} \mathrm{O}_{6}:$ A2B3C6_cP33_221_cd_ag_fh $\ldots \ldots \ldots 758$

$\boldsymbol{P n} \overline{3} n$ (222)

1. $\mathrm{Ce}_{5} \mathrm{Mo}_{3} \mathrm{O}_{16}$ : A5B3C16_cP96_222_ce_d_fi .......761

$\boldsymbol{F} \boldsymbol{m} \overline{3} \boldsymbol{m}$ (225)

1. $\mathrm{Th}_{6} \mathrm{Mn}_{23}:$ A23B6_cF116_225_bd2f_e ..........766

2. $\mathrm{K}_{2} \mathrm{PtCl}_{6}$ : A6B2C_cF36_225_e_c_a .......... 769

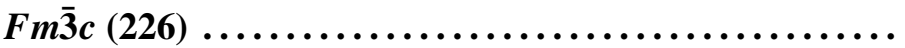

1. $\mathrm{NaZn}_{13}$ : AB13_cF112_226_a_bi ............771

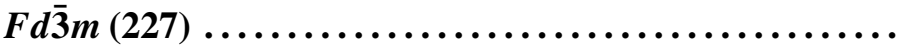

1. Pyrochlore Iridate:

A2B2C7_cF88_227_c_d_af ................. 774

2. Spinel: A3B4_cF56_227_ad_e ............776

$\boldsymbol{F d} \overline{\mathbf{3}} \boldsymbol{c}$ (228)

1. $\mathrm{CuCrCl}_{5}\left[\mathrm{NH}_{3}\right]_{6}: \mathrm{A} 5 \mathrm{BCD6}$ cF416_228_eg_c_b_h ..778

2. $\mathrm{TeO}_{6} \mathrm{H}_{6}:$ A6B_cF224_228_h_c ............. 785

Im $\overline{\mathbf{3}} \boldsymbol{m}$ (229)

1. $\gamma$-brass: A3B10_cI52_229_e_fh ............790

2. $\beta$-Hg H $_{4} \mathrm{Pt}:$ A4B_cI10_229_c_a .............. 793

3. $\mathrm{Ir}_{3} \mathrm{Ge}_{7}$ : A7B3_cI40_229_df_e ............ 795

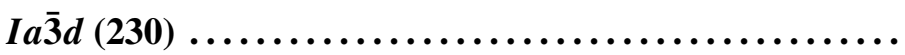

1. Garnet: A2B3C12D3_cI160_230_a_c_h_d ........ 797

\section{Index}

1. Prototype Index ... . . . . . . . . . . . . . . . 988

2. Pearson Symbol Index ................. . 991

3. Strukturbericht Designation Index .......... 996

4. Duplicate AFLOW Label ... . . . . . . . . . . . . . 1000

5. Similar AFLOW Label .... . . . . . . . . . . . . . . 1000

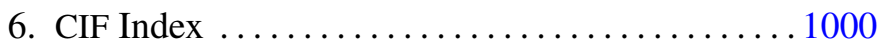

7. POSCAR Index . . . . . . . . . . . . . . . . . 1004

\section{Introduction}

The advent of high-throughput computing has paved the way for efficient and rapid exploration of materials space. Exploiting such computational frameworks has facilitated the predictions of novel materials, including metallic glasses [1], high-entropy oxides [2], candidate photovoltaic absorbers [3], rechargeable battery materials [4], and magnetic Heuslers [5]. Equipped with automated symmetry [6], mechanical [7, 8], and thermal analyses [9, 10, 11], a panoply of material properties can be readily calculated and stored in material-property repositories - such as AFLOW [12, 13, 14, 15, 16, 17, 18], Novel Materials Discovery (NoMaD) [19], Materials Project [20], and the Open Quantum Materials Database (OQMD) [21]. Creating new materials in these computational databases generally entails decorating structural prototypes with various atomic species. To increase the likelihood of synthesizing the conjectured materials, it is advisable to explore previously studied/observed crystal structures as the basis for developing prototype structures.

A variety of resources, such as the Strukturbericht series [22], The Structure of Crystals [23], Pearson's Handbook [24], and The American Mineralogist Crystal Database [25], have cataloged crystal structures over the past century (for a succinct historical recount of crystal structure information, see Ref. [17]). These collections are invaluable for finding crystals of certain symmetries, compositions, Strukturbericht designations, and other structural classifications. Furthermore, they are prime sources for structural prototypes in computational frameworks. However, until recently, much of the data was not easily accessible to the computational materials science community, hindering automatic generation of these prototypes.

A combined effort involving the U.S. Naval Research Laboratory's Crystal Lattice Structures web page and the AFLOW consortium yielded the construction of a new online crystal structure database: The AFLOW Library of Crystallographic Prototypes. The library is the result of a synergistic effort to gather crystal structure prototypes from literature and integrate them into the AFLOW computational framework for automatic generation. The first stage of this venture, Part 1, introduces 288 crystallographic prototypes from 92 different space groups [17]. Each prototype entry contains its symmetry descriptions, lattice and atomic basis vector equations, elements/compounds exhibiting the structure, and citations to the original references. With the AFLOW software, geometry files of the structure can be created in common ab-initio code formats, i.e., VASP [26, 27, 28, 29], QuANTUM ESPRESSO [30], FHIAIMS [31], and ABINIT [32]. Additionally, the infrastructure allows users to tune the internal degree(s) of freedom (lattice and Wyckoff parameters) and alter the atomic species of the structure, providing a robust crystal prototyping tool. The content associated with each prototype entry 
is detailed in Part 1 [17].

The prototype information is also available online at the following URL: http://www.aflow.org/ CrystalDatabase. Along with the structural information listed in the article, the website features additional functionality. An interactive Jmol applet is shown on each prototype entry page, allowing multiple viewing perspectives and differing cell representations (conventional, primitive, supercell, and Wigner-Seitz). Each page is also accompanied by a prototype generator that interfaces with the AFLOW software.

This article presents the continued work and second installment of the AFLOW Library of Crystallographic Prototypes. In Part 2, the crystallographic library is extended by 302 structure prototypes with representatives from the remaining 138 space groups not included in Part 1 . The online version of the library contains all of the prototypes from Part 1 and Part 2.

The outline of this article is as follows: Section 2 highlights the enantiomorphic space groups. Section 3 discusses the Wigner-Seitz cell and showcases the Jmol functionality. Section 4 introduces the two-dimensional plane groups (or "wallpaper" groups). Section 5 describes the different space group symbols, including the Hermann-Mauguin, Hall, International, and Schönflies notations, along with the origin/setting choices used throughout the library.

\section{Enantiomorphic Space Groups}

If we look at space group $P 4_{1}(\# 76)$, we see that it has one Wyckoff position (4a), with operations [33]

$$
(x, y, z)\left(-x,-y, z+\frac{1}{2}\right)\left(-y, x, z+\frac{1}{4}\right)\left(y,-x, z+\frac{3}{4}\right) .
$$

If we then look at space group $P 4_{3}(\# 78)$, we find it also has one (4a) Wyckoff position, with operations

$$
(x, y, z)\left(-x,-y, z+\frac{1}{2}\right)\left(-y, x, z+\frac{3}{4}\right)\left(y,-x, z+\frac{1}{4}\right),
$$

where the only difference is that the $1 / 4$ and $3 / 4$ fractions have swapped positions. We can easily show that space group \#78 is a mirror reflection of \#76 in the $z=0$ plane.

To see this more clearly, consider the $\mathrm{Cs}_{3} \mathrm{P}_{7}$ structure (A3B7_tP40_76_3a_7a $\mathrm{a}^{1}$ ). This structure was found in space group \#76, but if we reflect all of the coordinates through the $z=0$ plane, it transforms into a structure in space group \#78, as shown in the Jmol [34] rendering in Figure 1.

The distance between any pair of atoms is the same in the $P 4_{3}$ structure as it is in the $P 4_{1}$ structure, and the angle between any three atoms is the same in both structures. It

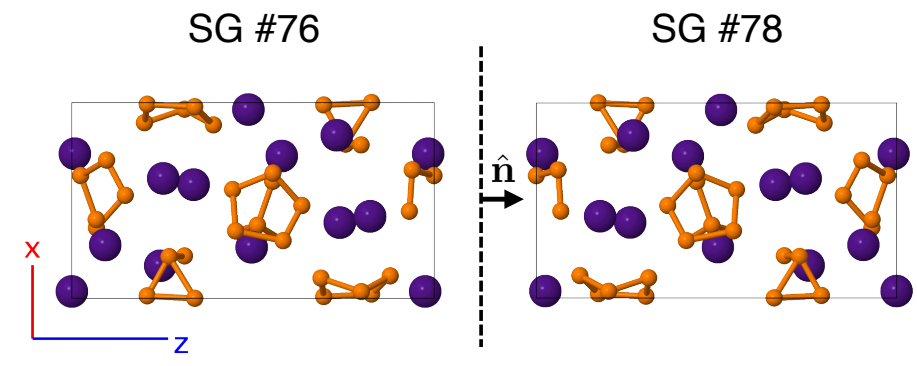

Figure 1: Illustration of enantiomorphic structures. $\mathrm{Cs}_{3} \mathrm{P}_{7}$ in space group $P 4_{1}$ (\#76) (left), and reflected through the $z=0$ plane into space group $\mathrm{P}_{3}$ (\#78) (right). The positive $z$ direction is to the right in both figures, with the mirror plane perpendicular to the page. The figures were produced by Jmol [34].

follows that the structures are degenerate, there is no difference in energy between them, and they should be equally likely to form.

Any structure in space group $P 4_{1}$ can be transformed into $\mathrm{P}_{3}$ by this method. Pairs of space groups which allow these transformations are said to be enantiomorphic [35, 36], or chiral. $^{2}$

There are eleven pairs of enantiomorphic space groups [35, 36]:

- $P 4_{1}(\# 76)$ and $P 4_{3}(\# 78)$,

- $P 4_{1} 22$ (\#91) and $P 4_{3} 22$ (\#95),

- $P 4_{1} 2_{1} 2$ (\#92) and $P 4_{3} 2{ }_{1} 2$ (\#96),

- $P 3_{1}$ (\#144) and $P 3_{2}$ (\#145),

- $P 3_{1} 12$ (\#151) and $P 3_{2} 12$ (\#153),

- $P 3_{1} 21$ (\#152) and $P 3_{2} 21$ (\#154),

- $P 6_{1}(\# 169)$ and $P 6_{5}(\# 170)$,

- $P 6_{2}$ (\#171) and $P 6_{4}$ (\#172),

- $P 6_{1} 22$ (\#178) and $P 6_{5} 22$ (\#179),

- $P 6_{2} 22$ (\#180) and P6422 (\#181), and

- $P 4_{1} 32(\# 213)$ and $P 4_{3} 32(\# 212)$.

In addition, forty-three other space groups allow chiral crystal structures. The complete set of sixty-five space groups are known as the Sohncke groups [35].

\footnotetext{
${ }^{2}$ Formally, any object has chirality if it is not superposable on its mirror image [35]. Chirality is a fundamental aspect of life on Earth. All amino acids found in living organisms are left-handed [37].
}

\footnotetext{
${ }^{1}$ This structure can be found in the Library of Crystallographic Prototypes at http://aflow.org/CrystalDatabase/A3B7_tP40_76_3a_7a.html.
} 


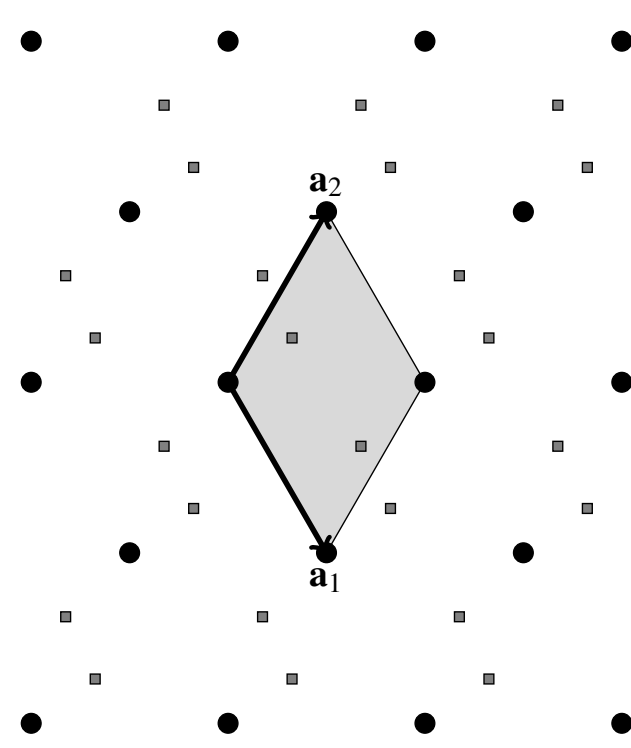

Figure 2: A two-dimensional hexagonal lattice with basis. The black circles show the positions of the lattice vectors, $N_{1} \mathbf{a}_{1}+N_{2} \mathbf{a}_{2}$, where $N_{1}$ and $N_{2}$ are integers and $\mathbf{a}_{1}$ and $\mathbf{a}_{2}$ are given by Equation (2). The gray squares are a two-site basis for this lattice.

\section{The Wigner-Seitz Primitive Cell}

Given a lattice described by a set of primitive lattice vectors, $\mathbf{a}_{1}, \mathbf{a}_{2}$, and $\mathbf{a}_{3}$, we can define a unit cell as a volume which, when translated through every vector of the form $N_{1} \mathbf{a}_{1}+N_{2} \mathbf{a}_{2}+N_{3} \mathbf{a}_{3}$, completely fills space without overlap. Even if we require that this cell have the minimum volume,

$$
V=\mathbf{a}_{1} \cdot\left(\mathbf{a}_{2} \times \mathbf{a}_{3}\right),
$$

this is not a unique definition, and in fact there are an infinite number of choices for the primitive vectors.

As an example, consider the two-dimensional hexagonal lattice with primitive vectors

$$
\begin{aligned}
& \mathbf{a}_{1}=\frac{1}{2} a \hat{\mathbf{x}}-\frac{\sqrt{3}}{2} a \hat{\mathbf{y}} \\
& \mathbf{a}_{2}=\frac{1}{2} a \hat{\mathbf{x}}+\frac{\sqrt{3}}{2} a \hat{\mathbf{y}} .
\end{aligned}
$$

We show this lattice in Figure 2, along with a two-site basis (the gray squares). Perhaps the simplest unit cell we can construct here is a parallelogram, as seen in Figure 2. This is a unit cell, as it has the area of the primitive cell,

$$
A=\frac{\sqrt{3}}{2} a^{2}
$$

each of its replicas contains two basis points, and the cell with its replicas tile the space.

The choice of unit cell is not unique. Consider, for example, an equivalent set of primitive vectors,

$$
\begin{aligned}
& \mathbf{a}_{1}^{\prime}=\frac{3}{2} a \hat{\mathbf{x}}+\frac{\sqrt{3}}{2} a \hat{\mathbf{y}} \\
& \mathbf{a}_{2}^{\prime}=\frac{1}{2} a \hat{\mathbf{x}}+\frac{\sqrt{3}}{2} a \hat{\mathbf{y}},
\end{aligned}
$$

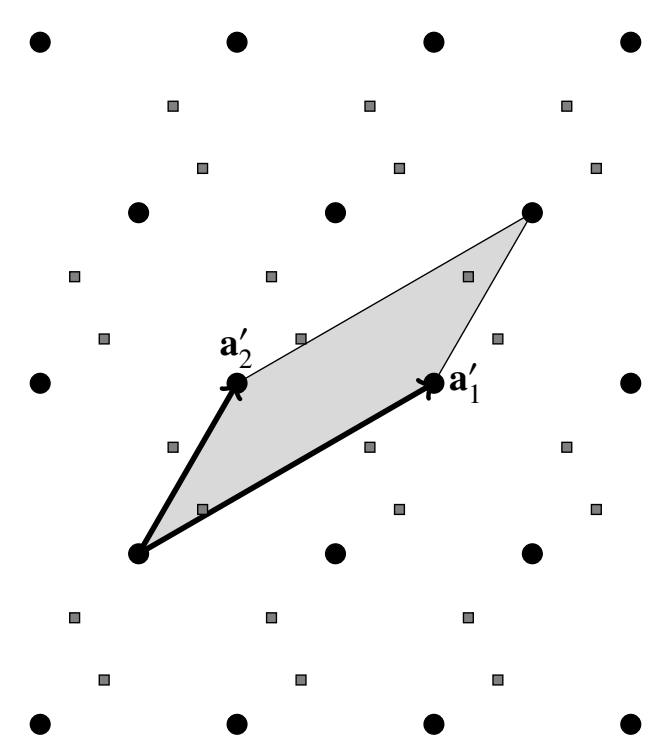

Figure 3: A two-dimensional hexagonal lattice with non-standard primitive vectors. This is the same hexagonal lattice from Figure 2 with primitive vectors given by Equation (4). Each unit cell still contains images of the two sites in the basis.

and the accompanying unit cell shown in Figure 3. This choice of unit cell has the proper area given by Equation (3), contains both basis points, and tiles the space. Both Figures 2 and 3 describe the same lattice plus basis, and so are both unit cells for the lattice.

Though not required, it is frequently useful to have a primitive cell which is uniquely defined and exhibits the symmetry of the lattice. Such a cell, known as the WignerSeitz cell [38], exists for every lattice. The Wigner-Seitz cell is defined as the locus of all points closer to a given lattice point than to any other lattice point. We have constructed the Wigner-Seitz cell for our two-dimensional hexagonal lattice as shown in Figure 4. Like all primitive cells, the Wigner-Seitz cell tiles its space, but unlike other cells it manifests the symmetry of the underlying hexagonal lattice, ${ }^{3}$ in this case exhibiting the six-fold rotation symmetry characteristic of a hexagonal lattice.

By definition, all points in the Wigner-Seitz cell are closer to its origin than to any other lattice point. Since we can center the cell on a point other than the lattice points defined by Equation (1), we can use this fact to determine the nearest neighbors of a given Wyckoff position. Consider the Wigner-Seitz cell in Figure 5, where we center the cell on one of the points in the basis. Only one of the images of the other point in the basis exists in this unit cell, so these two points are nearest neighbors. The only ambiguity in this definition is when a basis point is on the boundary of

\footnotetext{
${ }^{3}$ Note that we say that the Wigner-Seitz cell has the symmetry of the lattice, not the crystal structure. Taken without a basis, the Wigner-Seitz cell in Figure 4 has a six-fold rotation axis about the origin, in agreement with the symmetry of the hexagonal lattice. With the basis, however, the cell shown in Figure 4 does not have full hexagonal symmetry, even though its shape is a hexagon.
} 


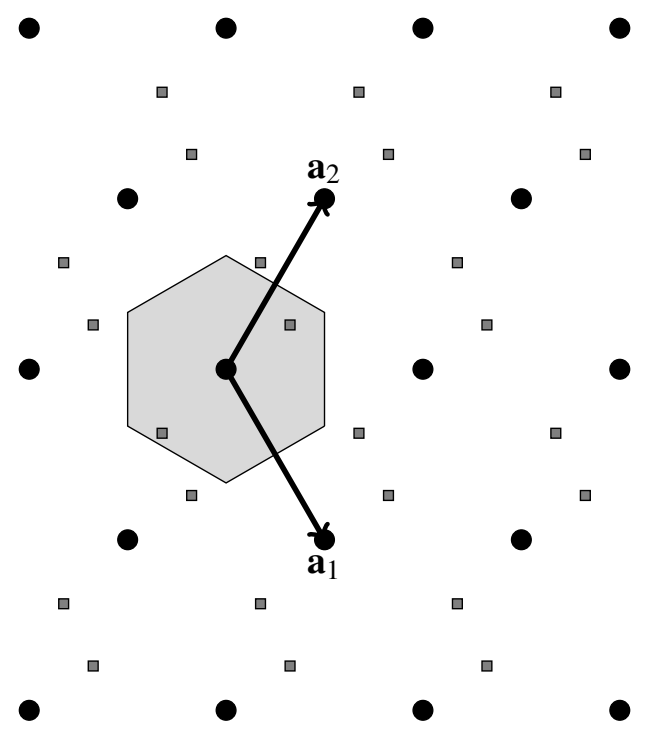

Figure 4: A two-dimensional hexagonal lattice with its corresponding Wigner-Seitz cell centered on a lattice point.

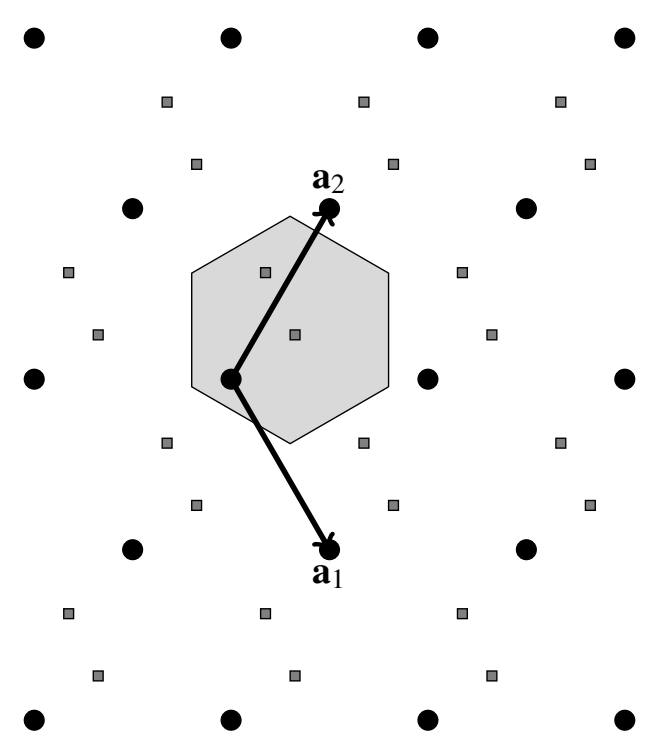

Figure 5: A two-dimensional hexagonal lattice with its corresponding Wigner-Seitz cell centered on one of the atoms.

the Wigner-Seitz zone. In this case there will be multiple copies of the basis point, all of them the same distance from the origin.

Three dimensions complicate the visualization of the Wigner-Seitz cell. We have added the ability to view the Wigner-Seitz cell to every structure in the AFLOW Library of Crystallographic Prototypes, using JSmol [39] to render the cells. As an example, consider the $\mathrm{SiS}_{2}$ structure A2B_oI12_72_j_a. ${ }^{4}$ Figure 6(a) shows the primitive and conventional unit cells of this system, as well as the positions of the silicon and sulfur atoms.

Figure 6(b) shows the Wigner-Seitz cell, centered on the

\footnotetext{
${ }^{4}$ This structure can be found in the Library of Crystallographic Prototypes at http://aflow.org/CrystalDatabase/A2B_oI12_72_j_a.html.
}

origin of the lattice. One can clearly see that the unit cell contains two silicon atoms, with two sulfur atoms in the interior of the zone and two on the boundary.

We can also center the Wigner-Seitz cell on the a particular atom, as shown with an silicon atom in Figure 6(c). This construction unambiguously identifies atoms which are nearest neighbors to the target silicon atom. This procedure can of course be extended to any atom in the primitive cell.

The Wigner-Seitz cell has its analog in reciprocal space. If $\{\mathbf{R}\}$ is the set of all lattice points in three dimensional space, the reciprocal lattice is defined as the set of all vectors $\{\mathbf{K}\}$ such that [40]

$$
e^{i \mathbf{R} \cdot \mathbf{K}}=1
$$

If the real-space lattice is defined by primitive vectors $\mathbf{a}_{1}$, $\mathbf{a}_{2}$, and $\mathbf{a}_{3}$, then the reciprocal lattice is defined by vectors

$$
\mathbf{b}_{i}=\left(\frac{2 \pi}{V}\right) \mathbf{a}_{j} \times \mathbf{a}_{k}
$$

where $(i, j, k)$ are in cyclic order. The reciprocal lattice is dual to the real-space lattice, with

$$
\mathbf{a}_{i} \cdot \mathbf{b}_{j}=2 \pi \delta_{i j}
$$

In matrix notation, we can write this as

$$
2 \pi \mathbf{B}=\left(\mathbf{A}^{T}\right)^{-1},
$$

where $\mathbf{A}, \mathbf{B}$ are matrices whose columns are the lattice vectors $\mathbf{a}$ and reciprocal lattice vectors $\mathbf{b}$, respectively.

This reciprocal lattice has its own Wigner-Seitz cell, the first Brillouin zone. The relationship between real space lattices and their reciprocal space Brillouin zones are discussed in Ref. [14].

\section{Plane Groups (Two-Dimensional Space Groups)}

Even though we live in a world with three physical dimensions, we can often achieve insight into that world by considering corresponding situations in two dimensions [41]. Two-dimensional physics is also important when we consider such materials as graphene [42], $\mathrm{MoS}_{2}$ [43], and similar materials. These systems still form periodic structures, and have symmetry operations associated with translations, rotations and reflections in two dimensions. These form the seventeen "plane groups", often called "wallpaper groups" for obvious reasons.

Since two-dimensional figures readily translate to the page, there are numerous discussions of group properties readily available $[44,45]$, along with some beautiful examples of wallpaper symmetries [46].

At the atomic level, plane groups, like space groups, each possess a set of Wyckoff positions that determine allowed atomic positions in keeping with the symmetry of the group. 
a

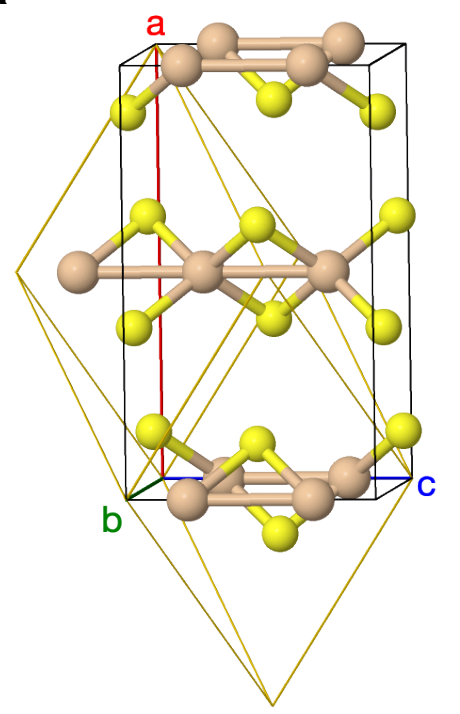

b

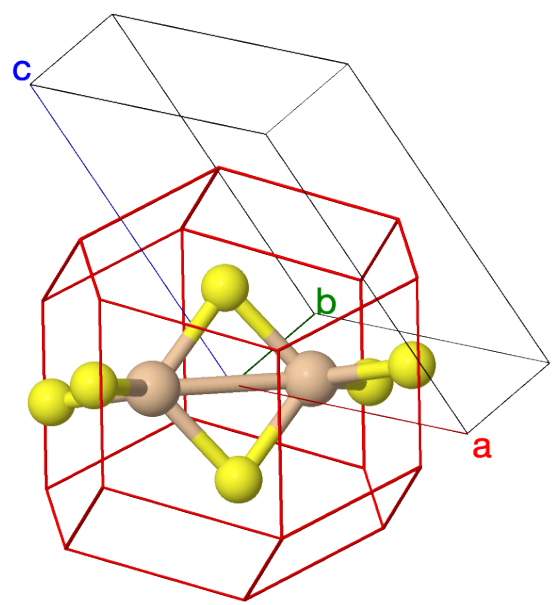

C

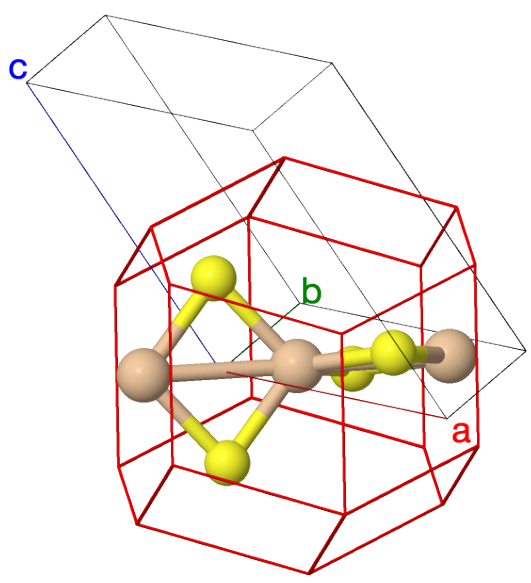

Figure 6: Unit cell representations for body-centered orthorhombic $\mathrm{SiS}_{2}$. The silicon and sulfur atoms are beige and yellow, respectively. (a) The primitive (yellow) and conventional (black) unit cell representations, where the axes are shown with respect to the conventional cell. (b) The WignerSeitz cell (red polyhedron) centered at the origin between two silicon atoms. The axes are shown with respect to the body-centered orthorhombic primitive unit cell (black) defined in Part 1 of the library [17]. (c) The Wigner-Seitz cell (red polyhedron) centered on one of the silicon atoms in the primitive cell. The axes are shown with respect to the body-centered orthorhombic primitive unit cell (black) defined in Part 1 of the library [17].

These have been tabulated in the International Tables [47] and online [33]. Here, we present a graphical illustration of the Wyckoff positions of each of the seventeen space groups, using the notation of the International Tables for each group.

Much as with three-dimensional space groups, the plane groups can be divided into crystal systems, each member of a given system having the same holohedry (rotational symmetry of the point group of the lattice).

There are five crystal systems in two dimensions:

- Simple parallelograms (plane groups \#1-2): the analog to the triclinic system in three dimensions. The primitive vectors for these lattices are given by

$$
\begin{aligned}
& \mathbf{a}_{1}=a \hat{\mathbf{x}} \\
& \mathbf{a}_{2}=b \cos \theta \hat{\mathbf{x}}+b \sin \theta \hat{\mathbf{y}} .
\end{aligned}
$$

The choice of $a, b$, and $\theta$ is completely arbitrary, provided that the choices do not fall into another of the crystal systems.

- Rectangles (plane groups \#3-9): the system is defined by a rectangular lattice

$$
\begin{aligned}
& \mathbf{a}_{1}=a \hat{\mathbf{x}} \\
& \mathbf{a}_{2}=b \hat{\mathbf{y}} .
\end{aligned}
$$

with perpendicular lattice vectors $\left[\theta=90^{\circ}\right.$ in Equation (9)]. The system contains both simple rectangular lattices, where the lattice is defined by Equation (10), and centered rectangular lattices, analogous to basecentered lattices in three dimensions. For the centered lattices (plane groups \#5 and \#9), the conventional cell is defined by Equation (10), and the primitive unit cell is defined by

$$
\begin{aligned}
& \mathbf{a}_{1}=\frac{1}{2} a \hat{\mathbf{x}}-\frac{1}{2} b \hat{\mathbf{y}} \\
& \mathbf{a}_{2}=\frac{1}{2} a \hat{\mathbf{x}}+\frac{1}{2} b \hat{\mathbf{y}} .
\end{aligned}
$$

- Squares (plane groups \#10-12): special cases of the rectangular system given by Equation (10) with $b=a$, so that the primitive lattice is specified by the vectors

$$
\begin{aligned}
& \mathbf{a}_{1}=a \hat{\mathbf{x}} \\
& \mathbf{a}_{2}=a \hat{\mathbf{y}} .
\end{aligned}
$$

The centered rectangular lattice Equation (11) also becomes a square lattice when $a=b$.

- Trigonal (plane groups \#13-15): In this system the primitive vectors are of equal length and separated by an angle of $120^{\circ}$, with a three-fold rotation axis about the origin $\left[b=a\right.$ and $\theta=60^{\circ}$ is given by Equation (9), or $b=\sqrt{3} a$ in Equation (11)]. The lattice vectors are

$$
\begin{aligned}
& \mathbf{a}_{1}=\frac{1}{2} a \hat{\mathbf{x}}-\frac{\sqrt{3}}{2} a \hat{\mathbf{y}} \\
& \mathbf{a}_{2}=\frac{1}{2} a \hat{\mathbf{x}}+\frac{\sqrt{3}}{2} a \hat{\mathbf{y}} .
\end{aligned}
$$

This can be regarded as a special case of the parallelogram in Equation (9) with $b=a$ and $\theta=120^{\circ}$, or of the centered rectangular lattice with $b=\sqrt{3} a$. 
- Hexagonal (plane groups \#16-17): As in the threedimensional case, a distinction is made between the trigonal system, which has a three-fold rotational axis about the origin, and the hexagonal system, which has a six-fold rotational axis. In either case, the primitive vectors of the unit cell are given by Equation (13).

Each section below lists the primitive vectors that describe the lattice associated with the plane group, a table of Wyckoff positions allowed for the space group, and a figure showing possible Wyckoff positions, along with the Wigner-Seitz cell for the lattice.

\subsection{The Parallelogram Crystal System}

\subsubsection{Plane Group \#1: $p 1$}

This plane group has the lowest symmetry possible for a periodic lattice. The primitive lattice vectors are given by Equation (9), and the single Wyckoff position is completely general:

\begin{tabular}{||c|c||}
\hline \hline Label & Lattice Coordinates \\
\hline$(1 \mathrm{a})$ & $(x, y)$ \\
\hline
\end{tabular}

Figure 7 shows a selection of Wyckoff positions for plane group $p 1$. It is worthwhile to note that if there is only one atom in the plane group defined by Equation (9), then we can place it at the origin, which then becomes an inversion site. This immediately promotes the structure to the higher symmetry of plane group $\# 2, p 2$. If the structure contains only two identical atoms, then there is an inversion site between them, and it is once again appropriate to place the structure in space group $p 2$.

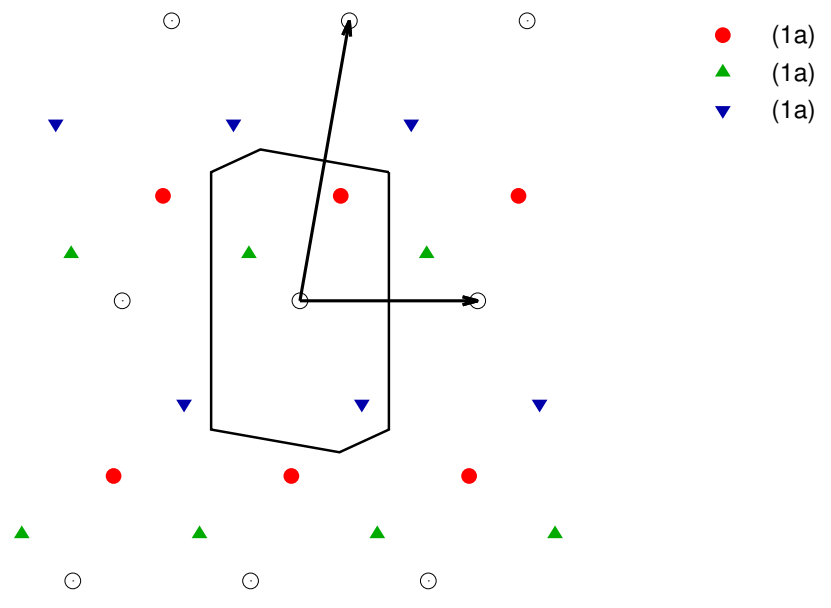

Figure 7: Possible Wyckoff positions for plane group \#1, p1. We show multiple possible Wyckoff positions, as if only one is occupied it can be placed at the origin, and the plane group achieves the higher symmetry of plane group \#2, p2. The black outline represents the boundary of the Wigner-Seitz cell for the lattice in Equation (9). The open circles indicate the lattice points.

\subsubsection{Plane Group \#2: p2}

Plane group \#2, $p 2$, has the same type of primitive lattice as $p 1$, in Equation (9), but now inversion through the origin produces an unchanged structure. This means that any function operating in this crystal system must have the property that

$$
f(x, y)=f(-x,-y) .
$$

This provides several relatively high-symmetry Wyckoff positions for single atoms, in addition to the general Wyckoff position.

\begin{tabular}{||c|c||}
\hline \hline Label & Lattice Coordinates \\
\hline$(2 \mathrm{e})$ & $(x, y)(-x,-y)$ \\
\hline$(1 \mathrm{~d})$ & $(1 / 2,1 / 2)$ \\
\hline$(1 \mathrm{c})$ & $(1 / 2,0)$ \\
\hline$(1 \mathrm{~b})$ & $(0,1 / 2)$ \\
\hline$(1 \mathrm{a})$ & $(0,0)$ \\
\hline \hline
\end{tabular}

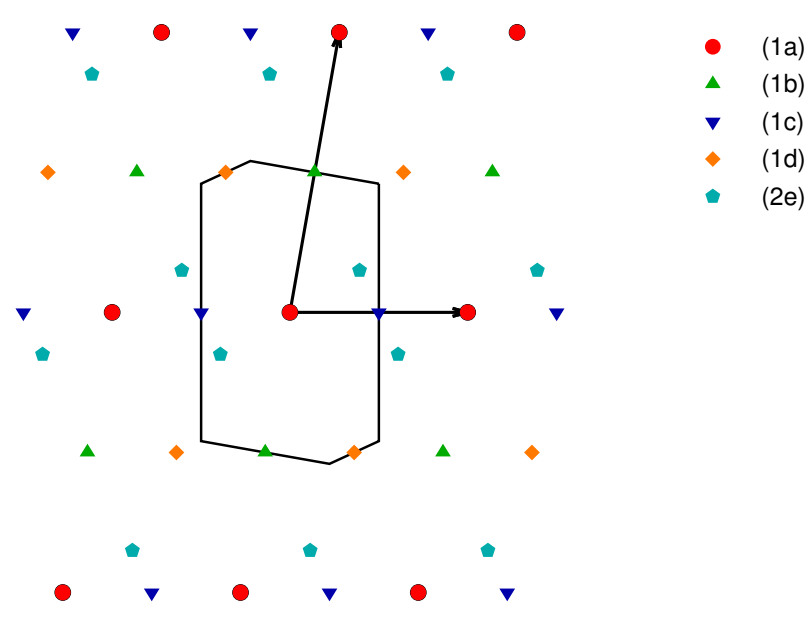

Figure 8: Possible Wyckoff positions for plane group \#2, p2. The positions of the (1a)-(1d) sites are fixed, while the (2e) sites are arbitrary. The black outline represents the boundary of the Wigner-Seitz cell for the lattice in Equation (9).

\subsection{The Rectangular Crystal System}

All of the rectangular plane groups have a conventional cell defined by the primitive vectors in Equation (10). This is also the primitive cell for space groups \#3, \#4, \#6, \#7 and \#8.

\subsubsection{Plane Group \#3: $p 1 m 1$}

This rectangular space group has primitive vectors in Equation (10) and a reflection around $x=0$; that is, any function operating in this space group must have the property that

$$
f(-x, y)=f(x, y)
$$

as well as the periodic properties

$$
f(x+a, y)=f(x, y+b)=f(x, y) .
$$


The Wyckoff positions for space group \#3 are given by

\begin{tabular}{||c|c|}
\hline \hline Label & Lattice Coordinates \\
\hline$(2 \mathrm{c})$ & $(x, y)(-x, y)$ \\
\hline$(1 \mathrm{~b})$ & $(1 / 2, y)$ \\
\hline$(1 \mathrm{a})$ & $(0, y)$ \\
\hline \hline
\end{tabular}

The plane group does not contain the inversion.

A graphical representation of the Wyckoff positions and the Wigner-Seitz cell for plane group \#3 are given in Figure 9 .

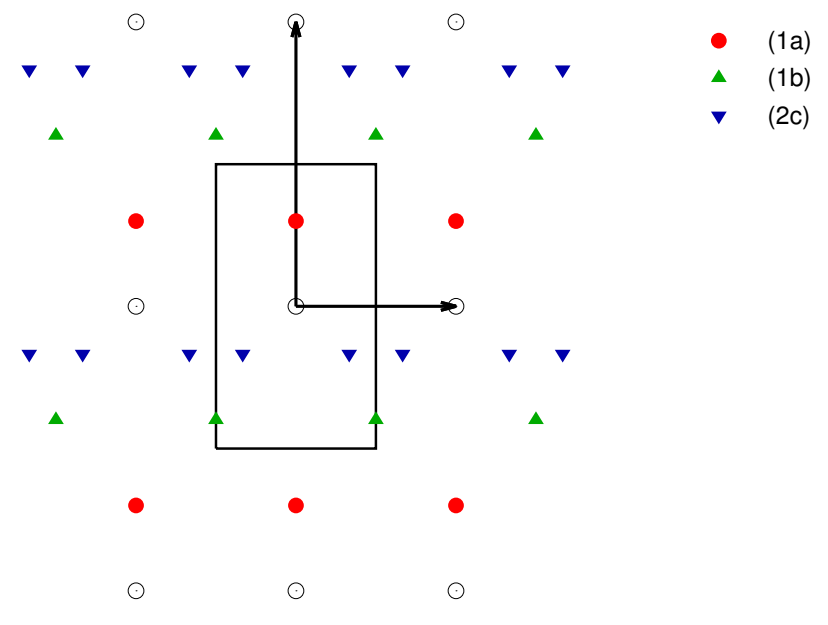

Figure 9: Possible Wyckoff positions for plane group \#3, p1 m1. The black outline represents the boundary of the Wigner-Seitz cell for the lattice in Equation (10). The open circles indicate the lattice points.

\subsubsection{Plane Group \#4: $p 1 g 1$}

This rectangular space group has a glide reflection [44], with a reflection about $x=0$ combined with a translation of $1 / 2 b$ along the $y$ direction. That is, any function showing this symmetry must have the property that

$$
f(x, y)=f(-x, y+1 / 2)
$$

There is only one Wyckoff position:

\begin{tabular}{||c|c||}
\hline \hline Label & Lattice Coordinates \\
\hline$(2 \mathrm{a})$ & $(x, y)(-x, y+1 / 2)$ \\
\hline \hline
\end{tabular}

There is no inversion in this crystal structure.

Figure 10 shows possible occupations of this Wyckoff position. We show two possible occupations. If only one (2a) site is occupied in this crystal system, there is an inversion between the two atoms, and we can place the origin there. In that case $y=1 / 4$, and the system actually has the higher symmetry $p 2 m g(\# 7)$, with atoms on the (2c) sites.

\subsubsection{Plane Group \#5: c1m 1}

This is a centered rectangular space group, with the conventional cell given by Equation (10) and the primitive vectors given by Equation (11). Just as in space group \#4, there

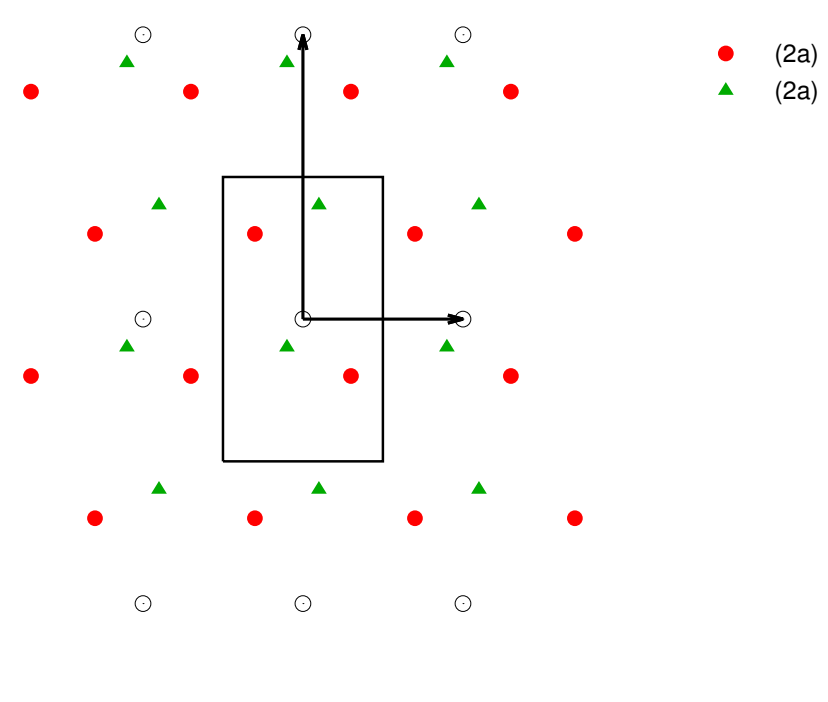

Figure 10: Possible Wyckoff positions for plane group \#4, p1 g1. The black outline represents the boundary of the Wigner-Seitz cell for the lattice in Equation (10). We show two possible occupations of the (2a) Wyckoff position to show that this is not in the higher symmetry plane group p2mg (\#7). The open circles indicate the lattice points.

is a glide reflection in Equation (17). The Wyckoff positions are given by

\begin{tabular}{||c|c||}
\hline \hline Label & Conventional Lattice Coordinates \\
(4b) & $(x, y)(-x, y)$ \\
& $\begin{array}{c}(x+1 / 2, y+1 / 2)(-x+1 / 2, y+1 / 2) \\
(0, y)\end{array}$ \\
\hline (2a) & $\begin{array}{c}(1 / 2, y+1 / 2) \\
\end{array}$ \\
\hline \hline
\end{tabular}

where, following convention, we give the coordinates of the positions in terms of the conventional lattice in Equation (10), but we also explicitly show the translations due to the centered primitive cell below the dashed line. The plane group does not contain the inversion.

The Wyckoff positions for this lattice are sketched in Figure 11.

\subsubsection{Plane Group \#6: $\mathrm{p} 2 \mathrm{~mm}$}

The remaining rectangular plane groups all contain the inversion given by Equation (14). Group \#6 also includes a mirror reflection about $x=0$ in Equation (15). Combining both of these operations show us that there must also be a mirror reflection about $y=0$, as well:

$$
f(x, y)=f(x,-y) .
$$

This leads to several Wyckoff positions, all of which are outlined in Figure 12. 


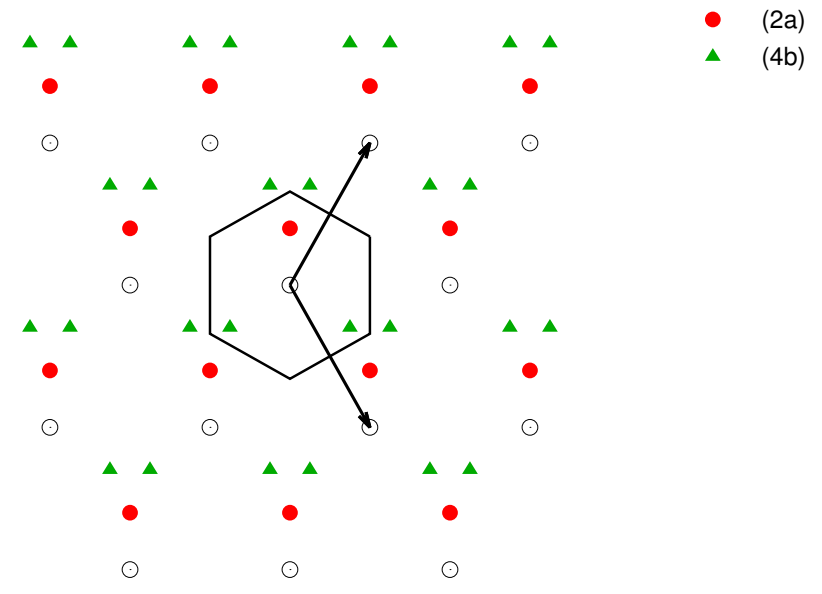

Figure 11: Possible Wyckoff positions for plane group \#5, c1 m1. The black outline represents the boundary of the Wigner-Seitz cell for the primitive cell in Equation (11). The open circles indicate the lattice points.

\begin{tabular}{||c|c||}
\hline \hline Label & Lattice Coordinates \\
\hline$(4 \mathrm{i})$ & $(x, y)(-x,-y)(-x, y)(x,-y)$ \\
\hline$(2 \mathrm{~h})$ & $(1 / 2, y)(1 / 2,-y)$ \\
\hline$(2 \mathrm{~g})$ & $(0, y)(0,-y)$ \\
\hline$(2 \mathrm{f})$ & $(x, 1 / 2)(-x, 1 / 2)$ \\
\hline$(2 \mathrm{e})$ & $(x, 0)(-x, 0)$ \\
\hline$(1 \mathrm{~d})$ & $(1 / 2,1 / 2)$ \\
\hline$(1 \mathrm{c})$ & $(1 / 2,0)$ \\
\hline$(1 \mathrm{~b})$ & $(0,1 / 2)$ \\
\hline$(1 \mathrm{a})$ & $(0,0)$ \\
\hline \hline
\end{tabular}

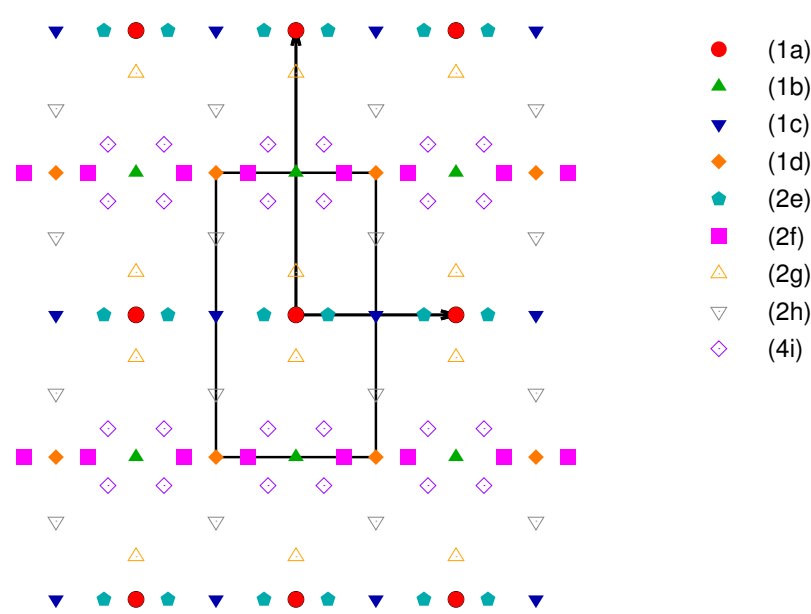

Figure 12: Possible Wyckoff positions for plane group \#6, p2mm. The black outline represents the boundary of the Wigner-Seitz cell for the lattice in Equation (10). Note the mirroring about $x=0$ and $y=0$.

\subsubsection{Plane Group \#7: $p 2 m g$}

In addition to the inversion, this group has a glide reflection about $y=0$ combined with a translation of $1 / 2 a$ along the $x$ direction:

$$
f(x, y)=f(-x+1 / 2, y)
$$

The Wyckoff positions are sketched in Figure 13 and are listed below.

\begin{tabular}{||c|c||}
\hline \hline Label & Lattice Coordinates \\
\hline$(4 d)$ & $(x, y)(-x,-y)(-x+1 / 2, y)(x+1 / 2,-y)$ \\
\hline$(2 \mathrm{c})$ & $(1 / 4, y)(3 / 4,-y)$ \\
\hline$(2 \mathrm{~b})$ & $(0,1 / 2)(1 / 2,1 / 2)$ \\
\hline$(2 \mathrm{a})$ & $(0,0)(1 / 2,0)$ \\
\hline
\end{tabular}

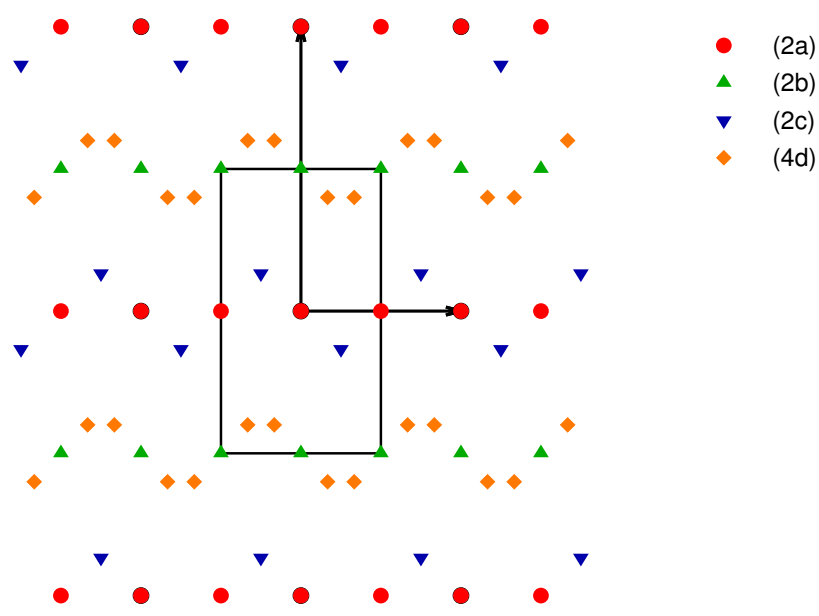

Figure 13: Possible Wyckoff positions for plane group \#7, p2mg. The black outline represents the boundary of the Wigner-Seitz cell for the lattice in Equation (10). Note the mirroring about $x=0$ and $y=0$.

\subsubsection{Plane Group \#8: $p 2 g g$}

This group has two glide reflections as well as the inversion. Its Wyckoff positions are shown in Figure 14 and are listed below.

\begin{tabular}{|c|c||}
\hline \hline Label & Lattice Coordinates \\
\hline$(4 \mathrm{c})$ & $(x, y)(-x,-y)$ \\
& $(-x+1 / 2, y+1 / 2)(x+1 / 2,-y+1 / 2)$ \\
\hline$(2 \mathrm{~b})$ & $(1 / 2,0)(0,1 / 2)$ \\
\hline$(2 \mathrm{a})$ & $(0,0)(1 / 2,1 / 2)$ \\
\hline \hline
\end{tabular}

\subsubsection{Plane Group \#9: c2mm}

Like plane group \#6, $p 2 \mathrm{~mm}$, this group has mirror reflections around both $x=0$ and $y=0$. However, it is a centered lattice, with the primitive cell defined by Equation (11) and the conventional cell defined by Equation (10). The Wyckoff positions are sketched in Figure 15 and listed below. 


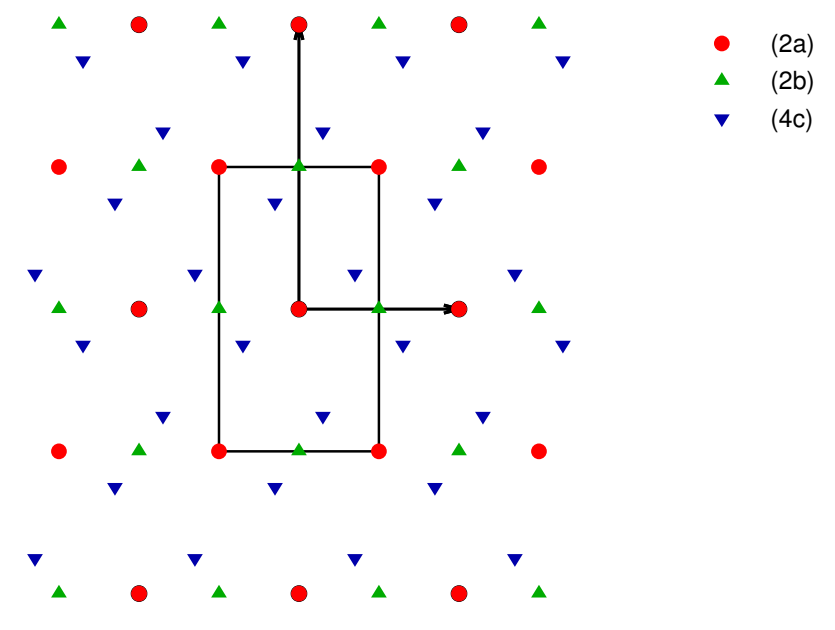

Figure 14: Possible Wyckoff positions for plane group \#8, p2 gg. The black outline represents the boundary of the Wigner-Seitz cell for the lattice in Equation (10).

Note that as with group $c 1 m 1$, \#5, we have listed the coordinates in terms of the conventional unit cell, and included the translations associated with the centered cell in the list.

\begin{tabular}{||c|c||}
\hline \hline Label & Conventional Lattice Coordinates \\
(8f) & $\begin{array}{c}(x, y)(-x,-y)(-x, y)(x,-y) \\
(x+1 / 2, y+1 / 2)(-x+1 / 2,-y+1 / 2) \\
(-x+1 / 2, y+1 / 2)(x+1 / 2,-y+1 / 2)\end{array}$ \\
\hline (4e) & $\begin{array}{c}(0, y)(0,-y) \\
(1 / 2, y+1 / 2)(1 / 2,-y+1 / 2)\end{array}$ \\
\hline (4d) & $\begin{array}{c}(x, 0)(-x, 0) \\
(x+1 / 2,1 / 2)(-x+1 / 2,1 / 2)\end{array}$ \\
\hline (4c) & $\begin{array}{c}(1 / 4,1 / 4)(3 / 4,1 / 4) \\
(3 / 4,3 / 4)(1 / 4,3 / 4)\end{array}$ \\
\hline (2b) & $\begin{array}{r}(0,1 / 2) \\
(1 / 2,0)\end{array}$ \\
\hline (2a) & $\begin{array}{r}(0,0) \\
(1 / 2,1 / 2)\end{array}$ \\
\hline \hline
\end{tabular}

\subsection{The Square Crystal System}

All of the lattices in this system remain unchanged when rotated by $90^{\circ}$ about the origin, so that the crystal obeys the relationship

$$
f(x, y)=f(-y, x)=f(-x,-y)=f(y,-x),
$$

automatically including the inversion from Equation (14). They thus form perfect squares, with the primitive cell given by Equation (12).

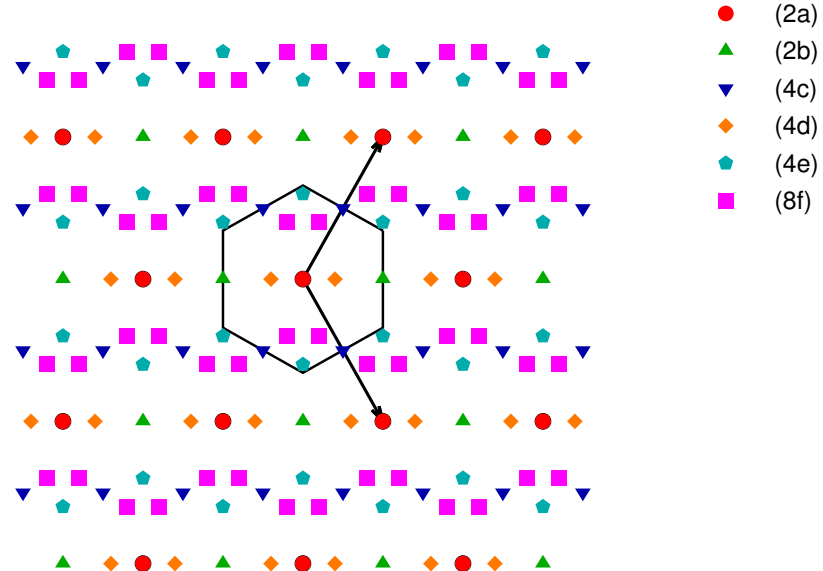

Figure 15: Possible Wyckoff positions for plane group \#9, $\boldsymbol{c 2 m m}$. The black outline represents the boundary of the Wigner-Seitz cell for the primitive cell in Equation (11). The lattice vectors and Wigner-Seitz cell for the conventional cell in Equation (10) are identical to those shown in Figure 12.

\subsubsection{Plane Group \#10: $p 4$}

This is the simplest plane group, with no reflections or glide reflections. The Wyckoff positions are sketched in Figure 16 and listed below.

\begin{tabular}{||c|c||}
\hline \hline Label & Lattice Coordinates \\
\hline$(4 \mathrm{~d})$ & $(x, y)(-x,-y)(-y, x)(y,-x)$ \\
\hline$(2 \mathrm{c})$ & $(1 / 2,0)(0,1 / 2)$ \\
\hline$(1 \mathrm{~b})$ & $(1 / 2,1 / 2)$ \\
\hline$(1 \mathrm{a})$ & $(0,0)$ \\
\hline \hline
\end{tabular}

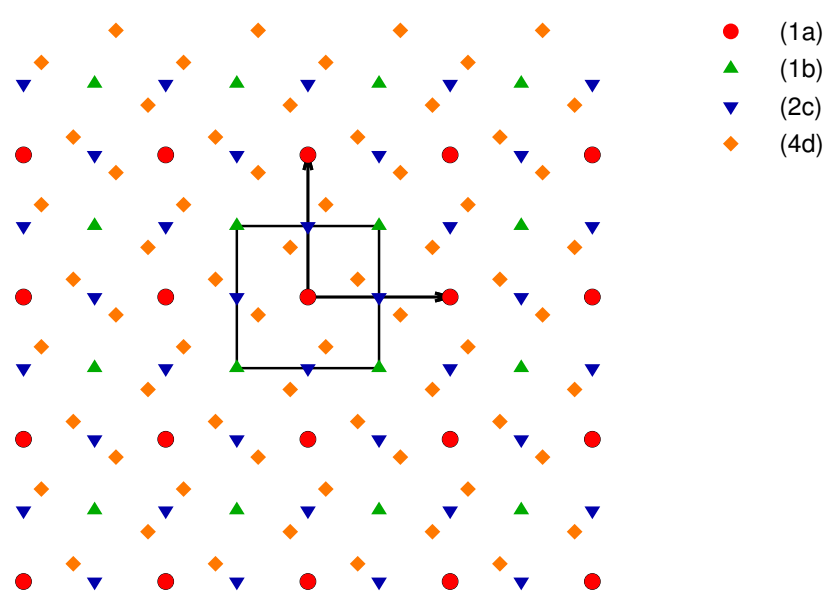

Figure 16: Possible Wyckoff positions for plane group \#10, p4. The black outline represents the boundary of the Wigner-Seitz cell for the lattice in Equation (12). As with all of the square plane groups, this group is invariant with respect to a rotation of $90^{\circ}$ about the origin and contains the inversion. 


\subsubsection{Plane Group \#11: p4mm}

This square plane group includes reflections about $x=0$ and $y=0$. When the $90^{\circ}$ rotations are included, this also generates mirror reflections around the lines $y= \pm x$, so this group admits operations of the form

$$
f(x, y)=f(-x, y)=f(x,-y)=f(y, x)=\cdots .
$$

The Wyckoff positions are sketched in Figure 17 and listed below.

\begin{tabular}{||c|c||}
\hline \hline Label & Lattice Coordinates \\
\hline$(8 \mathrm{~g})$ & $\begin{array}{l}(x, y)(-x,-y)(-y, x)(y,-x) \\
(-x, y)(x,-y)(y, x)(-y,-x)\end{array}$ \\
\hline$(4 \mathrm{f})$ & $(x, x)(-x,-x)(-x, x)(x,-x)$ \\
\hline$(4 \mathrm{e})$ & $(x, 1 / 2)(-x, 1 / 2)(1 / 2, x)(1 / 2,-x)$ \\
\hline$(4 \mathrm{~d})$ & $(x, 0)(-x, 0)(0, x)(0,-x)$ \\
\hline$(2 \mathrm{c})$ & $(1 / 2,0)(0,1 / 2)$ \\
\hline$(1 \mathrm{~b})$ & $(1 / 2,1 / 2)$ \\
\hline$(1 \mathrm{a})$ & $(0,0)$ \\
\hline
\end{tabular}

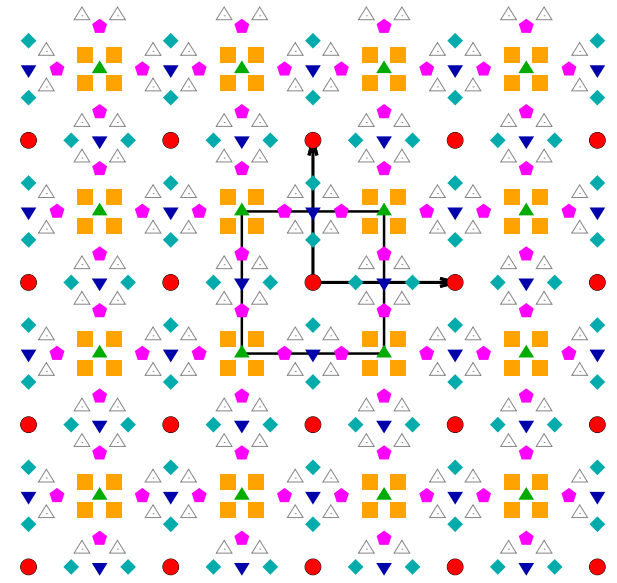

Figure 17: Possible Wyckoff positions for plane group \#11, p4mm. The black outline represents the boundary of the Wigner-Seitz cell for the lattice in Equation (12).

\subsubsection{Plane Group \#12: $p 4 g m$}

This group contains reflections, but they are not centered on the center of rotation, as they are with plane group \#11. The Wyckoff positions are listed below and sketched in Figure 18 .

\begin{tabular}{||c|c||}
\hline \hline Label & Lattice Coordinates \\
\hline$(8 \mathrm{~d})$ & $\begin{array}{r}(x, y)(-x,-y)(-y, x)(y,-x) \\
(-x+1 / 2, y+1 / 2)(x+1 / 2,-y+1 / 2) \\
(y+1 / 2, x+1 / 2)(-y+1 / 2,-x+1 / 2)\end{array}$ \\
\hline$(4 \mathrm{c})$ & $\begin{array}{r}(x, x+1 / 2)(-x,-x+1 / 2) \\
(-x+1 / 2, x)(x+1 / 2,-x)\end{array}$ \\
\hline$(2 \mathrm{~b})$ & $(1 / 2,0)(0,1 / 2)$ \\
\hline$(2 \mathrm{a})$ & $(0,0)(1 / 2,1 / 2)$ \\
\hline \hline
\end{tabular}

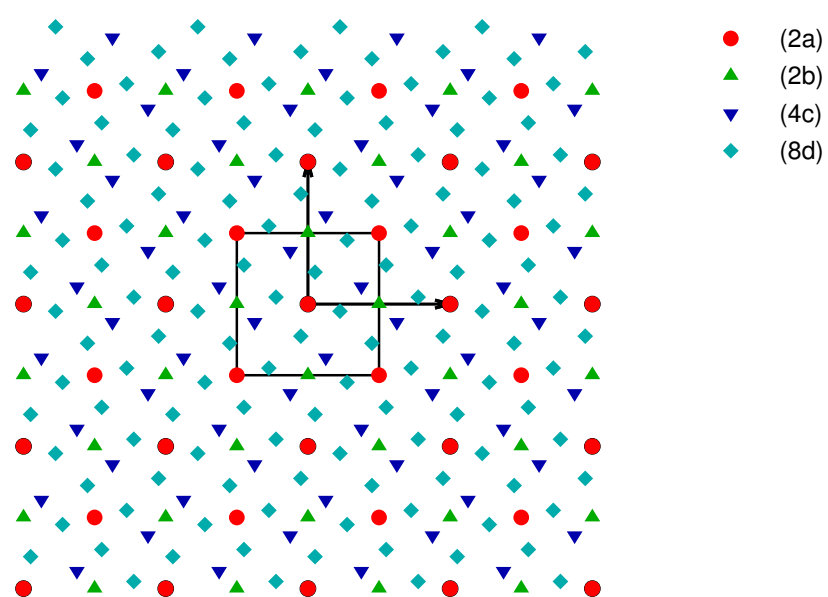

Figure 18: Possible Wyckoff positions for plane group \#12, p4gm. The black outline represents the boundary of the Wigner-Seitz cell for the lattice in Equation (12).

\subsection{The Trigonal Crystal System}

All of the plane groups in the trigonal crystal system are described by the primitive vectors in Equation (13) and are invariant under $120^{\circ}$ rotations about the origin. This system explicitly excludes groups which are invariant with respect to $60^{\circ}$ rotations, and so none of these groups contains the inversion operation in Equation (14).

\subsubsection{Plane Group \#13: p3}

This is the simplest of the trigonal groups, with only the $120^{\circ}$ symmetry operation. The Wyckoff positions are listed below, and sketched in Figure 19.

\begin{tabular}{||c|c||}
\hline \hline Label & Lattice Coordinates \\
\hline$(3 \mathrm{~d})$ & $(x, y)(-y, x-y)(-x+y,-x)$ \\
\hline$(1 \mathrm{c})$ & $(2 / 3,1 / 3)$ \\
\hline$(1 \mathrm{~b})$ & $(1 / 3,2 / 3)$ \\
\hline$(1 \mathrm{a})$ & $(0,0)$ \\
\hline \hline
\end{tabular}

\subsubsection{Plane Group \#14: $p 3 m 1$}

This group includes a reflection about the Cartesian $y$ axis (not the $x$ or $y$ lattice coordinates). The Wyckoff positions are listed below, and sketched in Figure 20.

\begin{tabular}{||c|c||}
\hline \hline Label & Lattice Coordinates \\
\hline$(6 \mathrm{e})$ & $\begin{array}{c}(x, y)(-y, x-y)(-x+y,-x) \\
(-y,-x)(-x+y, y)(x, x-y)\end{array}$ \\
\hline$(3 \mathrm{~d})$ & $(x,-x)(x, 2 x)(-2 x,-x)$ \\
\hline$(1 \mathrm{c})$ & $(2 / 3,1 / 3)$ \\
\hline$(1 \mathrm{~b})$ & $(1 / 3,2 / 3)$ \\
\hline (1a) & $(0,0)$ \\
\hline
\end{tabular}




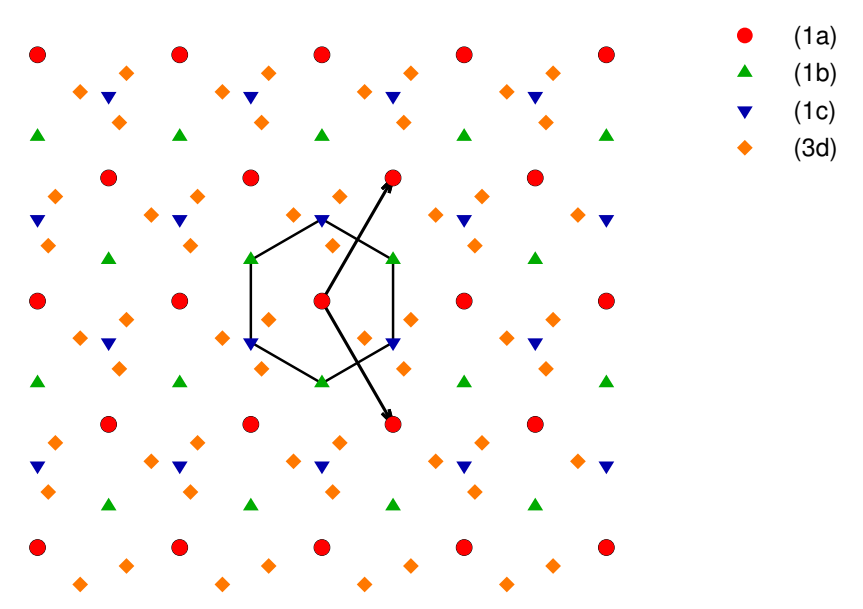

Figure 19: Possible Wyckoff positions for plane group \#13, p3. The black outline represents the boundary of the Wigner-Seitz cell for the lattice in Equation (13). Note the 3-fold rotation axis about the origin.

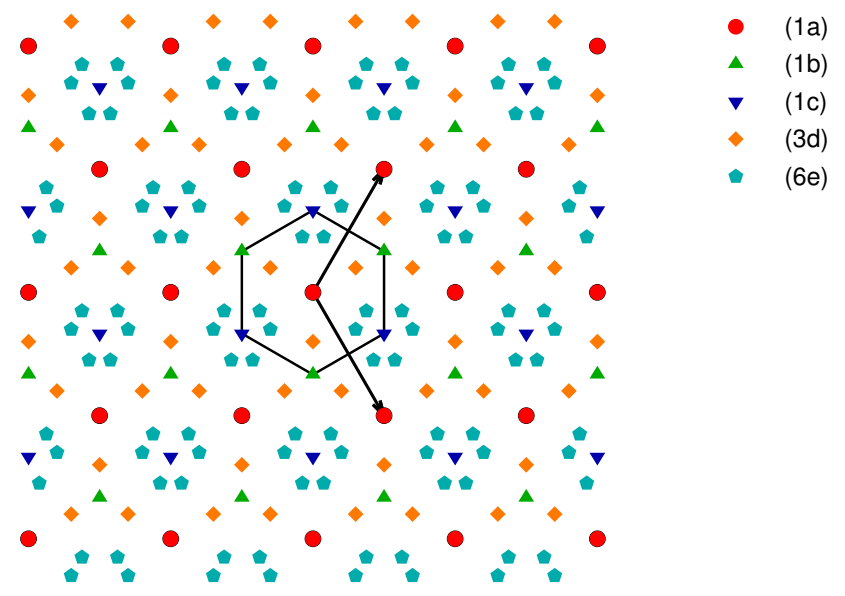

Figure 20: Possible Wyckoff positions for plane group \#14, p3m1. The black outline represents the boundary of the Wigner-Seitz cell for the lattice in Equation (13). Note the reflection about the Cartesian $y$ axis.

\subsubsection{Plane Group \#15: p31m}

This is similar to plane group \#14, but now, instead of a reflection about the $y$-axis, the group includes a reflection about the Cartesian $x$-axis. The Wyckoff positions are listed below, and sketched in Figure 21.

\begin{tabular}{||c|c||}
\hline \hline Label & Lattice Coordinates \\
\hline$(6 \mathrm{~d})$ & $\begin{array}{c}(x, y)(-y, x-y)(-x+y,-x) \\
(y, x)(x-y,-y)(-x,-x+y)\end{array}$ \\
\hline$(3 \mathrm{c})$ & $(x, 0)(0, x)(-x,-x)$ \\
\hline$(2 \mathrm{~b})$ & $(1 / 3,2 / 3)(2 / 3,1 / 3)$ \\
\hline$(1 \mathrm{a})$ & $(0,0)$ \\
\hline
\end{tabular}

\subsection{The Hexagonal Crystal System}

Like the trigonal crystal system, the lattice associated with the hexagonal crystal system is described by the prim-

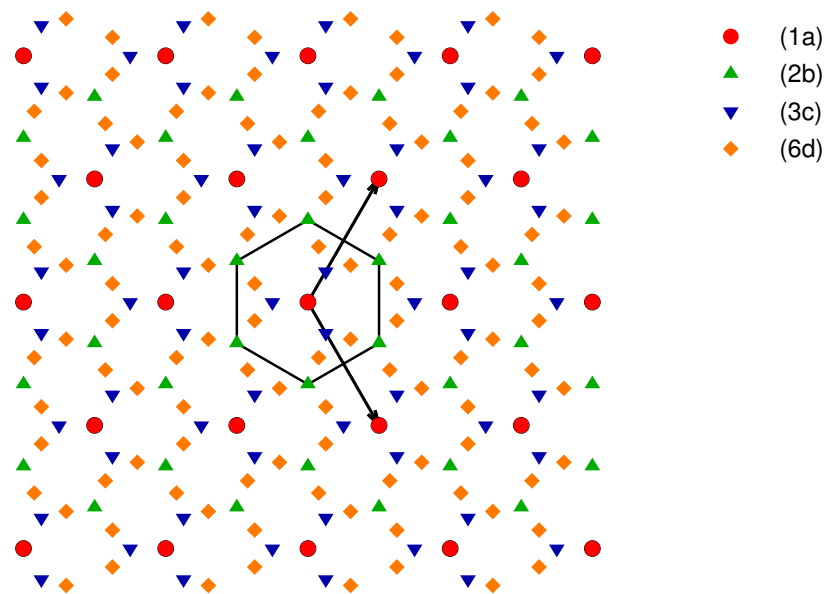

Figure 21: Possible Wyckoff positions for plane group \#15, p31 m. The black outline represents the boundary of the Wigner-Seitz cell for the lattice in Equation (13). Note the reflection about the Cartesian $x$ axis.

itive vectors in Equation (13), but here both groups are invariant under a $60^{\circ}$ degree rotation, and so include the inversion operation in Equation (14).

\subsubsection{Plane Group \#16: $p 6$}

This group includes the $60^{\circ}$ rotation operation, but no reflections. The Wyckoff positions are given below, and described graphically in Figure 22.

\begin{tabular}{||c|c||}
\hline \hline Label & Lattice Coordinates \\
\hline$(6 \mathrm{~d})$ & $\begin{array}{c}(x, y)(-y, x-y)(-x+y,-x) \\
(-x,-y)(y,-x+y)(x-y, x)\end{array}$ \\
\hline$(3 \mathrm{c})$ & $(1 / 2,0)(0,1 / 2)(1 / 2,1 / 2)$ \\
\hline$(2 \mathrm{~b})$ & $(1 / 3,2 / 3)(2 / 3,1 / 3)$ \\
\hline$(1 \mathrm{a})$ & $(0,0)$ \\
\hline
\end{tabular}

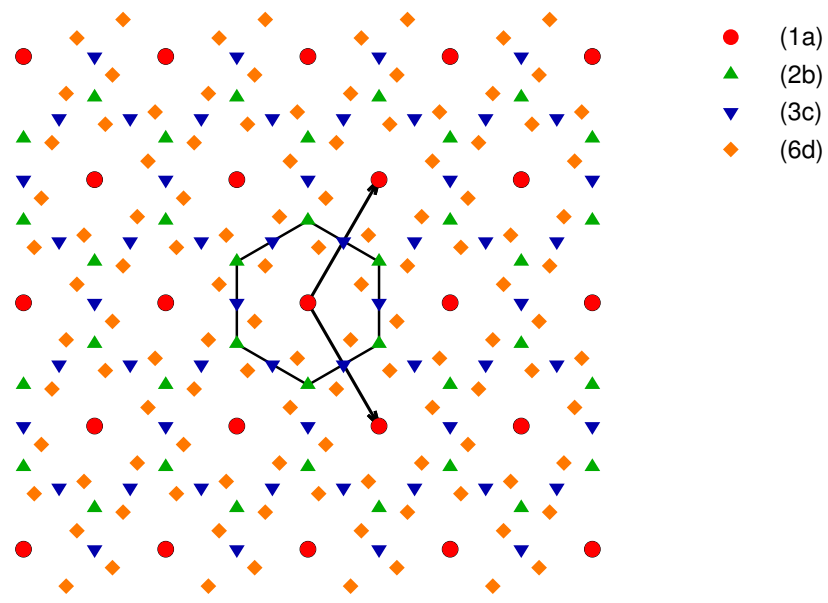

Figure 22: Possible Wyckoff positions for plane group \#16, p6. The black outline represents the boundary of the Wigner-Seitz cell for the lattice in Equation (13). Note the $60^{\circ}$ rotation symmetry about the origin. 


\subsubsection{Plane Group \#17: $p 6 \mathrm{~mm}$}

The final plane group includes the $60^{\circ}$ rotation operation, as well as reflections along both the Cartesian axes. The Wyckoff positions are given below, and described graphically in Figure 23.

\begin{tabular}{||c|c||}
\hline \hline Label & Lattice Coordinates \\
\hline (12f) & $\begin{array}{c}(x, y)(-y, x-y)(-x+y,-x)(-x,-y) \\
(y,-x+y)(x-y, x)(-y,-x)(-x+y, y) \\
(x, x-y)(y, x)(x-y,-y)(-x,-x+y)\end{array}$ \\
\hline (6e) & $\begin{array}{r}(x,-x)(x, 2 x)(-2 x,-x) \\
(-x, x)(-x,-2 x)(2 x, x)\end{array}$ \\
\hline (6d) & $\begin{array}{l}(x, 0)(0, x)(-x,-x) \\
(-x, 0)(0,-x)(x, x)\end{array}$ \\
\hline (3c) & $(1 / 2,0)(0,1 / 2)(1 / 2,1 / 2)$ \\
\hline (2b) & $(1 / 3,2 / 3)(2 / 3,1 / 3)$ \\
\hline (1a) & $(0,0)$ \\
\hline
\end{tabular}

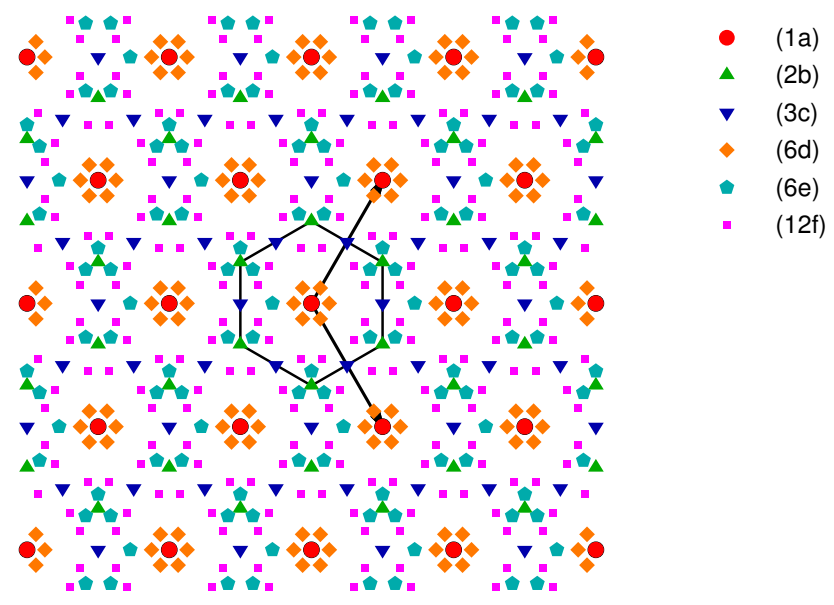

Figure 23: Possible Wyckoff positions for plane group \#17, p6mm. The black outline represents the boundary of the Wigner-Seitz cell for the lattice in Equation (13). Note the $60^{\circ}$ rotation symmetry about the origin as well as the reflections about the Cartesian axes.

\section{Space Group Notation}

There are a variety of space group notation methods. Each structural prototype page includes the space group number and International symbol. The Crystallographic Information Files (CIF) [48] used in the library indicates the number and the Hermann-Mauguin and Hall symbols. Lastly, the VASP POSCAR lists the number and the Hermann-Mauguin and Schönflies symbols.

The notations we use are

- Hermann-Mauguin [49, 50] (in CIF and POSCAR)

- Hall [51] (in CIF)

- "International", a compact form of the HermannMauguin notation used in the International Tables of Crystallography [52] (in entry page).
- Schönflies [53, 54] (in POSCAR)

Note that most notations provide different values for each orientation of a crystal. A complete list of orientations can be found in Hall and Grosse-Kunstleve [55]. In general we use the first space-group orientation listed for a space group on that page. There are two exceptions:

- For some space groups, e.g. \#227, there are two settings, reflecting a choice of origin. We always take the second setting, which places the origin of the realspace coordinate system at an inversion site.

- For rhombohedral unit cells we always use the label for the full hexagonal unit cell $(\mathrm{H})$ rather than the primitive rhombohedral cell (R), which follows our choice to describe the unit cell in terms of the hexagonal lattice parameters $a$ and $c$, rather than the rhombohedral lattice parameters $a$ and $\alpha$.

Complete listings of all space group orientations can be found at

- Concise Space Group Symbols, http://cci.lbl.gov/sginfo/hall_symbols.html

- Elk Spacegroup Manual, http://elk.sourceforge.net/spacegroup.pdf

\section{Conclusion}

Herein, we present the second part of The AFLOW Library of Crystallographic Prototypes. The article includes 302 crystallographic prototypes and provides the corresponding structural information for each entry. The geometry file for each structure can be generated via AFLOW to facilitate high-throughput computation of material properties. This information is also available online at http://www . aflow.org/CrystalDatabase, where it is combined with the prototypes listed in Part 1. 
Table 1: A list of the various space group notations. The space group number, orientation, HermannMauguin symbol, Hall symbol, International symbol, and Schönflies symbol are listed.

\begin{tabular}{|c|c|c|c|c|c|}
\hline Number & Orientation & Hermann-Mauguin & Hall & International & Schönflies \\
\hline 1 & & P 1 & $\mathrm{P} 1$ & $P 1$ & $C_{1}^{1}$ \\
\hline 2 & & $\mathrm{P}-1$ & $-\mathrm{P} 1$ & $P \overline{1}$ & $C_{i}^{1}$ \\
\hline 3 & $\mathrm{~b}$ & P 121 & P 2y & $P 2$ & $C_{2}^{1}$ \\
\hline 4 & $\mathrm{~b}$ & P 1211 & $\mathrm{P} 2 \mathrm{yb}$ & $P 2_{1}$ & $C_{2}^{2}$ \\
\hline 5 & b1 & C 121 & C $2 y$ & $C 2$ & $C_{2}^{3}$ \\
\hline 6 & $\mathrm{~b}$ & $\mathrm{P} 1 \mathrm{~m} 1$ & $P-2 y$ & $P m$ & $C_{s}^{1}$ \\
\hline 7 & b1 & $\mathrm{P} 1 \mathrm{c} 1$ & $\mathrm{P}-2 \mathrm{yc}$ & $P c$ & $C_{s}^{2}$ \\
\hline 8 & b1 & $\mathrm{C} 1 \mathrm{~m} 1$ & $C-2 y$ & $\mathrm{Cm}$ & $C_{s}^{3}$ \\
\hline 9 & b1 & $\mathrm{C} 1 \mathrm{c} 1$ & C $-2 y c$ & $C c$ & $C_{s}^{4}$ \\
\hline 10 & $\mathrm{~b}$ & $\mathrm{P} 12 / \mathrm{m} 1$ & $-\mathrm{P} 2 \mathrm{y}$ & $P 2 / m$ & $C_{2 h}^{1}$ \\
\hline 11 & $\mathrm{~b}$ & P 1 21/m 1 & $-P 2 y b$ & $P 2_{1} / m$ & $C_{2 h}^{2}$ \\
\hline 12 & b1 & $\mathrm{C} 12 / \mathrm{m} 1$ & $-C 2 y$ & $C 2 / m$ & $C_{2 h}^{3}$ \\
\hline 13 & b1 & P $12 / \mathrm{c} 1$ & $-\mathrm{P} 2 \mathrm{yc}$ & $P 2 / c$ & $C_{2 h}^{4}$ \\
\hline 14 & b1 & P 1 21/c 1 & $-\mathrm{P} 2 \mathrm{ybc}$ & $P 2_{1} / c$ & $C_{2 h}^{5}$ \\
\hline 15 & b1 & $\mathrm{C} 12 / \mathrm{c} 1$ & $-\mathrm{C} 2 \mathrm{yc}$ & $C 2 / c$ & $C_{2 h}^{6}$ \\
\hline 16 & & P 222 & P 22 & $P 222$ & $D_{2}^{1}$ \\
\hline 17 & & P 2221 & $\mathrm{P} 2 \mathrm{c} 2$ & $P 222_{1}$ & $D_{2}^{2}$ \\
\hline 18 & & P 21212 & P 2 2ab & $P 2_{1} 2_{1} 2$ & $D_{2}^{3}$ \\
\hline 19 & & P 212121 & P 2ac 2ab & $P 2_{1} 2_{1} 2_{1}$ & $D_{2}^{4}$ \\
\hline 20 & & C 2221 & C 2c 2 & $C 222_{1}$ & $D_{2}^{5}$ \\
\hline 21 & & C 222 & $\mathrm{C} 22$ & $C 222$ & $D_{2}^{6}$ \\
\hline 22 & & F 222 & F 22 & $F 222$ & $D_{2}^{7}$ \\
\hline 23 & & I 222 & I 22 & $I 222$ & $D_{2}^{8}$ \\
\hline 24 & & I 212121 & I $2 b 2 c$ & $I 2_{1} 2_{1} 2_{1}$ & $D_{2}^{9}$ \\
\hline 25 & & $\mathrm{P} \mathrm{m} \mathrm{m} 2$ & P $2-2$ & Pmm2 & $C_{2 v}^{1}$ \\
\hline 26 & & P m c 21 & P $2 c-2$ & $P m c 2_{1}$ & $C_{2 v}^{2}$ \\
\hline 27 & & $\mathrm{Pc}$ c 2 & P $2-2 c$ & $P c c 2$ & $C_{2 v}^{3}$ \\
\hline 28 & & P m a 2 & P $2-2 a$ & Pma2 & $C_{2 v}^{4}$ \\
\hline 29 & & $\mathrm{P} \mathrm{c}$ a 21 & P $2 c-2 a c$ & $\mathrm{Pca}_{1}$ & $C_{2 v}^{5}$ \\
\hline 30 & & P n c 2 & P $2-2 b c$ & Pnc2 & $C_{2 v}^{6}$ \\
\hline 31 & & P m n 21 & P $2 \mathrm{ac}-2$ & $P m n 2_{1}$ & $C_{2 v}^{7}$ \\
\hline 32 & & $\mathrm{~Pb}$ a 2 & P $2-2 a b$ & $\mathrm{Pba} 2$ & $C_{2 v}^{8}$ \\
\hline 33 & & P n a 21 & P $2 c-2 n$ & $P_{n a 2}$ & $C_{2 v}^{9}$ \\
\hline 34 & & P n n 2 & $P 2-2 n$ & Pnn2 & $C_{2 v}^{10}$ \\
\hline 35 & & $\mathrm{C} \mathrm{m} \mathrm{m} 2$ & C $2-2$ & $\mathrm{Cmm} 2$ & $C_{2 v}^{11}$ \\
\hline 36 & & $\mathrm{C} \mathrm{m} \mathrm{c} 21$ & C $2 c-2$ & $C m c 2_{1}$ & $C_{2 v}^{12}$ \\
\hline 37 & & $\mathrm{Cc}$ c 2 & C $2-2 c$ & $C c c 2$ & $C_{2 v}^{13}$ \\
\hline 38 & & A $m$ m 2 & A $2-2$ & Amm2 & $C_{2 v}^{14}$ \\
\hline 39 & & A b m 2 & A $2-2 c$ & Aem2 & $C_{2 v}^{15}$ \\
\hline 40 & & A m a 2 & A $2-2 a$ & Ama2 & $C_{2 v}^{16}$ \\
\hline 41 & & $\mathrm{~A} \mathrm{~b}$ a 2 & A $2-2 \mathrm{ac}$ & Aea 2 & $C_{2 v}^{17}$ \\
\hline 42 & & $\mathrm{~F} \mathrm{~m} \mathrm{~m} 2$ & F $2-2$ & Fmm2 & $C_{2 v}^{18}$ \\
\hline 43 & & $\mathrm{Fd} \mathrm{d} 2$ & F $2-2 d$ & $F d d 2$ & $C_{2 v}^{19}$ \\
\hline 44 & & I m m 2 & I $2-2$ & $\operatorname{Imm} 2$ & $C_{2 v}^{20}$ \\
\hline 45 & & I b a 2 & I $2-2 c$ & $I b a 2$ & $C_{2 v}^{21}$ \\
\hline 46 & & I $\mathrm{m}$ a 2 & I $2-2 a$ & $\operatorname{Ima} 2$ & $C_{2 v}^{22}$ \\
\hline
\end{tabular}


Table 1 (continued): A list of the various space group notations. The space group number, orientation, Hermann-Mauguin symbol, Hall symbol, International symbol, and Schönflies symbol are listed.

\begin{tabular}{|c|c|c|c|c|c|}
\hline Number & Orientation & Hermann-Mauguin & Hall & International & Schönflies \\
\hline 47 & & $\mathrm{P} 2 / \mathrm{m} \mathrm{2} / \mathrm{m} \mathrm{2/m}$ & $-\mathrm{P} 22$ & Pmmm & $D_{2 h}^{1}$ \\
\hline 48 & 2 & $\mathrm{P} 2 / \mathrm{n} 2 / \mathrm{n} 2 / \mathrm{n}: 2$ & $-\mathrm{P} 2 \mathrm{ab} 2 \mathrm{bc}$ & Pnnn & $D_{2 h}^{2}$ \\
\hline 49 & & $\mathrm{P} 2 / \mathrm{c} 2 / \mathrm{c} 2 / \mathrm{m}$ & $-\mathrm{P} 22 \mathrm{c}$ & Pccm & $D_{2 h}^{3}$ \\
\hline 50 & 2 & $\mathrm{P} 2 / \mathrm{b} 2 / \mathrm{a} 2 / \mathrm{n}: 2$ & $-\mathrm{P} 2 \mathrm{ab} 2 \mathrm{~b}$ & Pban & $D_{2 h}^{4}$ \\
\hline 51 & & $\mathrm{P} 21 / \mathrm{m} \mathrm{2} / \mathrm{m} \mathrm{2/a}$ & $-\mathrm{P} 2 \mathrm{a} 2 \mathrm{a}$ & Pmma & $D_{2 h}^{5}$ \\
\hline 52 & & $\mathrm{P} 2 / \mathrm{n} 21 / \mathrm{n} 2 / \mathrm{a}$ & $-\mathrm{P} 2 \mathrm{a} 2 \mathrm{bc}$ & Pnna & $D_{2 h}^{6}$ \\
\hline 53 & & $\mathrm{P} 2 / \mathrm{m} 2 / \mathrm{n} 21 / \mathrm{a}$ & $-\mathrm{P} 2 \mathrm{ac} 2$ & Pmna & $D_{2 h}^{7}$ \\
\hline 54 & & $\mathrm{P} 21 / \mathrm{c} 2 / \mathrm{c} 2 / \mathrm{a}$ & $-\mathrm{P} 2 \mathrm{a} 2 \mathrm{ac}$ & Pcca & $D_{2 h}^{8}$ \\
\hline 55 & & $\mathrm{P} 21 / \mathrm{b} 21 / \mathrm{a} 2 / \mathrm{m}$ & -P $22 a b$ & Pbam & $D_{2 h}^{9}$ \\
\hline 56 & & $\mathrm{P} 21 / \mathrm{c} 21 / \mathrm{c} 2 / \mathrm{n}$ & -P 2ab 2ac & Pccn & $D_{2 h}^{10}$ \\
\hline 57 & & $\mathrm{P} 2 / \mathrm{b} 21 / \mathrm{c} 21 / \mathrm{m}$ & $-P 2 c 2 b$ & $\mathrm{Pbcm}$ & $D_{2 h}^{11}$ \\
\hline 58 & & $\mathrm{P} 21 / \mathrm{n} 21 / \mathrm{n} 2 / \mathrm{m}$ & $-\mathrm{P} 22 \mathrm{n}$ & Pnnm & $D_{2 h}^{12}$ \\
\hline 59 & 2 & P 21/m 21/m 2/n:2 & $-\mathrm{P} 2 \mathrm{ab} 2 \mathrm{a}$ & Pmmn & $D_{2 h}^{13}$ \\
\hline 60 & & $\mathrm{P} 21 / \mathrm{b} 2 / \mathrm{c} 21 / \mathrm{n}$ & $-\mathrm{P} 2 \mathrm{n} 2 \mathrm{ab}$ & $P b c n$ & $D_{2 h}^{14}$ \\
\hline 61 & & $\mathrm{P} 21 / \mathrm{b} 21 / \mathrm{c} 21 / \mathrm{a}$ & $-\mathrm{P} 2 \mathrm{ac} 2 \mathrm{ab}$ & Pbca & $D_{2 h}^{15}$ \\
\hline 62 & & P 21/n 21/m 21/a & $-\mathrm{P} 2 \mathrm{ac} 2 \mathrm{n}$ & Pnma & $D_{2 h}^{16}$ \\
\hline 63 & & $\mathrm{C} 2 / \mathrm{m} 2 / \mathrm{c} 21 / \mathrm{m}$ & $-\mathrm{C} 2 \mathrm{c} 2$ & $\mathrm{Cmcm}$ & $D_{2 h}^{17}$ \\
\hline 64 & & $\mathrm{C} 2 / \mathrm{m} 2 / \mathrm{c} 21 / \mathrm{a}$ & $-\mathrm{C} 2 \mathrm{bc} 2$ & Cmca & $D_{2 h}^{18}$ \\
\hline 65 & & $\mathrm{C} 2 / \mathrm{m} 2 / \mathrm{m} 2 / \mathrm{m}$ & $-\mathrm{C} 22$ & $\mathrm{Cmmm}$ & $D_{2 h}^{19}$ \\
\hline 66 & & $\mathrm{C} 2 / \mathrm{c} 2 / \mathrm{c} 2 / \mathrm{m}$ & $-\mathrm{C} 22 \mathrm{c}$ & Cccm & $D_{2 h}^{20}$ \\
\hline 67 & & $\mathrm{C} 2 / \mathrm{m} 2 / \mathrm{m} 2 / \mathrm{a}$ & $-\mathrm{C} 2 \mathrm{~b} 2$ & Cmma & $D_{2 h}^{21}$ \\
\hline 68 & 2 & $\mathrm{C} 2 / \mathrm{c} 2 / \mathrm{c} 2 / \mathrm{a}: 2$ & $-\mathrm{C} 2 \mathrm{~b} 2 \mathrm{bc}$ & Ccca & $D_{2 h}^{22}$ \\
\hline 69 & & $\mathrm{~F} 2 / \mathrm{m} 2 / \mathrm{m} 2 / \mathrm{m}$ & $-F 22$ & Fmmm & $D_{2 h}^{23}$ \\
\hline 70 & 2 & F $2 / \mathrm{d} 2 / \mathrm{d} 2 / \mathrm{d}: 2$ & $-\mathrm{F} 2 \mathrm{uv} 2 \mathrm{vw}$ & $F d d d$ & $D_{2 h}^{24}$ \\
\hline 71 & & I $2 / \mathrm{m} 2 / \mathrm{m} 2 / \mathrm{m}$ & $-\mathrm{I} 22$ & Immm & $D_{2 h}^{25}$ \\
\hline 72 & & I $2 / \mathrm{b} 2 / \mathrm{a} 2 / \mathrm{m}$ & -I $22 \mathrm{c}$ & Ibam & $D_{2 h}^{26}$ \\
\hline 73 & & I $2 / \mathrm{b} 2 / \mathrm{c} 2 / \mathrm{a}$ & $-\mathrm{I} 2 \mathrm{~b} 2 \mathrm{c}$ & $I b c a$ & $D_{2 h}^{27}$ \\
\hline 74 & & $\mathrm{I} 2 / \mathrm{m} 2 / \mathrm{m} \mathrm{2/a}$ & $-\mathrm{I} 2 \mathrm{~b} 2$ & Imma & $D_{2 h}^{28}$ \\
\hline 75 & & $\mathrm{P} 4$ & $\mathrm{P} 4$ & $P 4$ & $C_{4}^{1}$ \\
\hline 76 & & P 41 & $\mathrm{P} 4 \mathrm{w}$ & $P 4_{1}$ & $C_{4}^{2}$ \\
\hline 77 & & $\mathrm{P} 42$ & $\mathrm{P} 4 \mathrm{c}$ & $P 4_{2}$ & $C_{4}^{3}$ \\
\hline 78 & & P 43 & $\mathrm{P} 4 \mathrm{cw}$ & $P 4_{3}$ & $C_{4}^{4}$ \\
\hline 79 & & I 4 & I 4 & $I 4$ & $C_{4}^{5}$ \\
\hline 80 & & I 41 & I $4 \mathrm{bw}$ & $I 4_{1}$ & $C_{4}^{6}$ \\
\hline 81 & & P -4 & $\mathrm{P}-4$ & $P \overline{4}$ & $S_{4}^{1}$ \\
\hline 82 & & I -4 & I -4 & $I \overline{4}$ & $S_{4}^{2}$ \\
\hline 83 & & $\mathrm{P} 4 / \mathrm{m}$ & $-\mathrm{P} 4$ & $P 4 / m$ & $C_{4 h}^{1}$ \\
\hline 84 & & $\mathrm{P} 42 / \mathrm{m}$ & $-\mathrm{P} 4 \mathrm{c}$ & $P 4_{2} / m$ & $C_{4 h}^{2}$ \\
\hline 85 & 2 & $\mathrm{P} 4 / \mathrm{n}: 2$ & $-\mathrm{P} 4 \mathrm{a}$ & $P 4 / n$ & $C_{4 h}^{3}$ \\
\hline 86 & 2 & $\mathrm{P} 42 / \mathrm{n}: 2$ & $-\mathrm{P} 4 \mathrm{bc}$ & $P 4_{2} / n$ & $C_{4 h}^{4}$ \\
\hline 87 & & $\mathrm{I} 4 / \mathrm{m}$ & $-\mathrm{I} 4$ & $I 4 / m$ & $C_{4 h}^{5}$ \\
\hline 88 & 2 & I $41 / \mathrm{a}: 2$ & -I $4 \mathrm{ad}$ & $I 4_{1} / a$ & $C_{4 h}^{6}$ \\
\hline 89 & & P 422 & P 42 & $P 422$ & $D_{4}^{1}$ \\
\hline 90 & & P 4212 & P 4ab 2ab & $P 42_{1} 2$ & $D_{4}^{2}$ \\
\hline 91 & & P 4122 & $\mathrm{P} 4 \mathrm{w} 2 \mathrm{c}$ & $P 4_{1} 22$ & $D_{4}^{3}$ \\
\hline 92 & & P 41212 & P 4abw 2nw & $P 4_{1} 2_{1} 2$ & $D_{4}^{4}$ \\
\hline
\end{tabular}


Table 1 (continued): A list of the various space group notations. The space group number, orientation, Hermann-Mauguin symbol, Hall symbol, International symbol, and Schönflies symbol are listed.

\begin{tabular}{|c|c|c|c|c|c|}
\hline Number & Orientation & Hermann-Mauguin & Hall & International & Schönflies \\
\hline 93 & & P 4222 & $\mathrm{P} 4 \mathrm{c} 2$ & $P 4_{2} 22$ & $D_{4}^{5}$ \\
\hline 94 & & P 42212 & $\mathrm{P} 4 \mathrm{n} 2 \mathrm{n}$ & $P 4_{2} 2_{1} 2$ & $D_{4}^{6}$ \\
\hline 95 & & P 4322 & $\mathrm{P} 4 \mathrm{cw} 2 \mathrm{c}$ & $\mathrm{P}_{3} 22$ & $D_{4}^{7}$ \\
\hline 96 & & P 43212 & P 4nw 2abw & $P 4_{3} 2_{1} 2$ & $D_{4}^{8}$ \\
\hline 97 & & I 422 & I 42 & $I 422$ & $D_{4}^{9}$ \\
\hline 98 & & I 4122 & I 4bw 2bw & $I 4_{1} 22$ & $D_{4}^{10}$ \\
\hline 99 & & $\mathrm{P} 4 \mathrm{~m} \mathrm{~m}$ & P $4-2$ & $P 4 m m$ & $C_{4 v}^{1}$ \\
\hline 100 & & $\mathrm{P} 4 \mathrm{~b} \mathrm{~m}$ & P $4-2 a b$ & $P 4 b m$ & $C_{4 v}^{2}$ \\
\hline 101 & & $\mathrm{P} 42 \mathrm{c} \mathrm{m}$ & $P 4 c-2 c$ & $\mathrm{P}_{2} \mathrm{~cm}$ & $C_{4 v}^{3}$ \\
\hline 102 & & $\mathrm{P} 42 \mathrm{n} \mathrm{m}$ & $P 4 n-2 n$ & $\mathrm{P4}_{2} \mathrm{~nm}$ & $C_{4 v}^{4}$ \\
\hline 103 & & $\mathrm{P} 4 \mathrm{c} \mathrm{c}$ & P $4-2 c$ & $P 4 c c$ & $C_{4 v}^{5}$ \\
\hline 104 & & $\mathrm{P} 4 \mathrm{n} \mathrm{c}$ & $P 4-2 n$ & $P 4 n c$ & $C_{4 v}^{6}$ \\
\hline 105 & & P $42 \mathrm{~m} \mathrm{c}$ & $P 4 c-2$ & $P 4_{2} m c$ & $C_{4 v}^{7}$ \\
\hline 106 & & $\mathrm{P} 42 \mathrm{~b} \mathrm{c}$ & $P 4 c-2 a b$ & $P_{2} b c$ & $C_{4 v}^{8}$ \\
\hline 107 & & I $4 \mathrm{~m} \mathrm{~m}$ & I $4-2$ & $I 4 m m$ & $C_{4 v}^{9}$ \\
\hline 108 & & $\mathrm{I} 4 \mathrm{c} \mathrm{m}$ & I $4-2 c$ & $I 4 \mathrm{~cm}$ & $C_{4 v}^{10}$ \\
\hline 109 & & $\mathrm{I} 41 \mathrm{~m} \mathrm{~d}$ & I 4bw -2 & $I 4_{1} m d$ & $C_{4 v}^{11}$ \\
\hline 110 & & I $41 \mathrm{c} \mathrm{d}$ & I $4 b w-2 c$ & $I 4_{1} c d$ & $C_{4 v}^{12}$ \\
\hline 111 & & $P-42 m$ & $P-42$ & $P \overline{4} 2 m$ & $D_{2 d}^{1}$ \\
\hline 112 & & $P-42 c$ & $P-42 c$ & $P \overline{4} 2 c$ & $D_{2 d}^{2}$ \\
\hline 113 & & $P-421 m$ & $\mathrm{P}-42 \mathrm{ab}$ & $P \overline{4} 2_{1} m$ & $D_{2 d}^{3}$ \\
\hline 114 & & $P-421 c$ & $P-42 n$ & $P \overline{4} 2_{1} c$ & $D_{2 d}^{4}$ \\
\hline 115 & & $\mathrm{P}-4 \mathrm{~m} 2$ & $P-4-2$ & $P \overline{4} m 2$ & $D_{2 d}^{5}$ \\
\hline 116 & & $P-4$ c 2 & $P-4-2 c$ & $P \overline{4} c 2$ & $D_{2 d}^{6}$ \\
\hline 117 & & $P-4$ b 2 & $P-4-2 a b$ & $P \overline{4} b 2$ & $D_{2 d}^{7}$ \\
\hline 118 & & $P-4$ n 2 & $P-4-2 n$ & $P \overline{4} n 2$ & $D_{2 d}^{8}$ \\
\hline 119 & & $\mathrm{I}-4 \mathrm{~m} 2$ & I $-4-2$ & $I \overline{4} m 2$ & $D_{2 d}^{9}$ \\
\hline 120 & & I - 4 c 2 & I $-4-2 c$ & $I \overline{4} c 2$ & $D_{2 d}^{10}$ \\
\hline 121 & & $\mathrm{I}-42 \mathrm{~m}$ & I -42 & $I \overline{4} 2 m$ & $D_{2 d}^{11}$ \\
\hline 122 & & $\mathrm{I}-42 \mathrm{~d}$ & I -4 2bw & $I \overline{4} 2 d$ & $D_{2 d}^{12}$ \\
\hline 123 & & $\mathrm{P} 4 / \mathrm{m} \mathrm{2/m} \mathrm{2/m}$ & $-\mathrm{P} 42$ & $P 4 / \mathrm{mmm}$ & $D_{4 h}^{1}$ \\
\hline 124 & & $\mathrm{P} 4 / \mathrm{m} 2 / \mathrm{c} 2 / \mathrm{c}$ & $-\mathrm{P} 42 \mathrm{c}$ & $P 4 / m c c$ & $D_{4 h}^{2}$ \\
\hline 125 & 2 & $\mathrm{P} 4 / \mathrm{n} 2 / \mathrm{b} 2 / \mathrm{m}: 2$ & $-P 4 a 2 b$ & $P 4 / n b m$ & $D_{4 h}^{3}$ \\
\hline 126 & 2 & $\mathrm{P} 4 / \mathrm{n} 2 / \mathrm{n} 2 / \mathrm{c}: 2$ & $-\mathrm{P} 4 \mathrm{a} 2 \mathrm{bc}$ & $P 4 / n n c$ & $D_{4 h}^{4}$ \\
\hline 127 & & $\mathrm{P} 4 / \mathrm{m} \mathrm{21/b} \mathrm{2/m}$ & $-\mathrm{P} 42 \mathrm{ab}$ & $P 4 / m b m$ & $D_{4 h}^{5}$ \\
\hline 128 & & $\mathrm{P} 4 / \mathrm{m} \mathrm{21/n} \mathrm{2/c}$ & $-\mathrm{P} 42 \mathrm{n}$ & $P 4 / m n c$ & $D_{4 h}^{6}$ \\
\hline 129 & 2 & $\mathrm{P} 4 / \mathrm{n} \mathrm{21/m} \mathrm{2/m:2}$ & $-\mathrm{P} 4 \mathrm{a} 2 \mathrm{a}$ & P4/nmm & $D_{4 h}^{7}$ \\
\hline 130 & 2 & $\mathrm{P} 4 / \mathrm{n} 21 / \mathrm{c} 2 / \mathrm{c}: 2$ & $-\mathrm{P} 4 \mathrm{a} 2 \mathrm{ac}$ & $P 4 / n c c$ & $D_{4 h}^{8}$ \\
\hline 131 & & $\mathrm{P} 42 / \mathrm{m} \mathrm{2/m} \mathrm{2/c}$ & $-\mathrm{P} 4 \mathrm{c} 2$ & $P 4_{2} / m m c$ & $D_{4 h}^{9}$ \\
\hline 132 & & $\mathrm{P} 42 / \mathrm{m} \mathrm{2/c} \mathrm{2/m}$ & $-\mathrm{P} 4 \mathrm{c} 2 \mathrm{c}$ & $\mathrm{P}_{2} / \mathrm{mcm}$ & $D_{4 h}^{10}$ \\
\hline 133 & 2 & $\mathrm{P} 42 / \mathrm{n} 2 / \mathrm{b} 2 / \mathrm{c}: 2$ & $-\mathrm{P} 4 \mathrm{ac} 2 \mathrm{~b}$ & $P 4_{2} / n b c$ & $D_{4 h}^{11}$ \\
\hline 134 & 2 & $\mathrm{P} 42 / \mathrm{n} \mathrm{2/n} \mathrm{2/m:2}$ & $-\mathrm{P} 4 \mathrm{ac} 2 \mathrm{bc}$ & $P 4_{2} / n n m$ & $D_{4 h}^{12}$ \\
\hline 135 & & $\mathrm{P} 42 / \mathrm{m} \mathrm{21/b} \mathrm{2/c}$ & $-\mathrm{P} 4 \mathrm{c} 2 \mathrm{ab}$ & $P 4_{2} / m b c$ & $D_{4 h}^{13}$ \\
\hline 136 & & $\mathrm{P} 42 / \mathrm{m} 21 / \mathrm{n} 2 / \mathrm{m}$ & $-\mathrm{P} 4 \mathrm{n} 2 \mathrm{n}$ & $P 4_{2} / m n m$ & $D_{4 h}^{14}$ \\
\hline 137 & 2 & $\mathrm{P} 42 / \mathrm{n} \mathrm{21/m} \mathrm{2/c:2}$ & $-\mathrm{P} 4 \mathrm{ac} 2 \mathrm{a}$ & $P 4_{2} / n m c$ & $D_{4 h}^{15}$ \\
\hline 138 & 2 & $\mathrm{P} 42 / \mathrm{n} 21 / \mathrm{c} 2 / \mathrm{m}: 2$ & $-\mathrm{P} 4 \mathrm{ac} 2 \mathrm{ac}$ & $\mathrm{P}_{2} / \mathrm{ncm}$ & $D_{4 h}^{16}$ \\
\hline
\end{tabular}


Table 1 (continued): A list of the various space group notations. The space group number, orientation, Hermann-Mauguin symbol, Hall symbol, International symbol, and Schönflies symbol are listed.

\begin{tabular}{|c|c|c|c|c|c|}
\hline Number & Orientation & Hermann-Mauguin & Hall & International & Schönflies \\
\hline 139 & & $\mathrm{I} 4 / \mathrm{m} \mathrm{2/m} \mathrm{2/m}$ & -I 42 & $I 4 / \mathrm{mmm}$ & $D_{4 h}^{17}$ \\
\hline 140 & & I $4 / \mathrm{m} \mathrm{2/c} 2 / \mathrm{m}$ & $-\mathrm{I} 42 \mathrm{c}$ & $I 4 / \mathrm{mcm}$ & $D_{4 h}^{18}$ \\
\hline 141 & 2 & I $41 / \mathrm{a} 2 / \mathrm{m} 2 / \mathrm{d}: 2$ & $-\mathrm{I} 4 \mathrm{bd} 2$ & $I 4_{1} /$ amd & $D_{4 h}^{19}$ \\
\hline 142 & 2 & I $41 / \mathrm{a} 2 / \mathrm{c} 2 / \mathrm{d}: 2$ & $-\mathrm{I} 4 \mathrm{bd} 2 \mathrm{c}$ & $I 4_{1} /$ acd & $D_{4 h}^{20}$ \\
\hline 143 & & P 3 & P 3 & $P 3$ & $C_{3}^{1}$ \\
\hline 144 & & P 31 & P 31 & $P 3_{1}$ & $C_{3}^{2}$ \\
\hline 145 & & P 32 & P 32 & $P 3_{2}$ & $C_{3}^{3}$ \\
\hline 146 & $\mathrm{H}$ & R 3:H & R 3 & $R 3$ & $C_{3}^{4}$ \\
\hline 147 & & $P-3$ & $-\mathrm{P} 3$ & $P \overline{3}$ & $C_{3 i}^{1}$ \\
\hline 148 & $\mathrm{H}$ & $\mathrm{R}-3: \mathrm{H}$ & $-R 3$ & $R \overline{3}$ & $C_{3 i}^{2}$ \\
\hline 149 & & P 312 & P 32 & $P 312$ & $D_{3}^{1}$ \\
\hline 150 & & P 321 & P 32 " & $P 321$ & $D_{3}^{2}$ \\
\hline 151 & & P 3112 & P 31 2c $\left(\begin{array}{lll}0 & 0 & 1\end{array}\right)$ & $P 3_{1} 12$ & $D_{3}^{3}$ \\
\hline 152 & & P 3121 & P $312 ”$ & $P 3_{1} 21$ & $D_{3}^{4}$ \\
\hline 153 & & P 3212 & P 32 2c $\left(\begin{array}{lll}0 & 0 & -1\end{array}\right)$ & $P 3_{2} 12$ & $D_{3}^{5}$ \\
\hline 154 & & P 3221 & P 32 2" & $P 3_{2} 21$ & $D_{3}^{6}$ \\
\hline 155 & $\mathrm{H}$ & R 32:H & R 32 " & $R 32$ & $D_{3}^{7}$ \\
\hline 156 & & P 3 m 1 & P $3-2 "$ & $P 3 m 1$ & $C_{3 v}^{1}$ \\
\hline 157 & & P $31 \mathrm{~m}$ & P $3-2$ & $P 31 \mathrm{~m}$ & $C_{3 v}^{2}$ \\
\hline 158 & & P 3 c 1 & P $3-2 " c$ & $P 3 c 1$ & $C_{3 v}^{3}$ \\
\hline 159 & & P $31 \mathrm{c}$ & P $3-2 c$ & $P 31 c$ & $C_{3 v}^{4}$ \\
\hline 160 & $\mathrm{H}$ & R 3 m:H & R 3 -2” & $R 3 m$ & $C_{3 v}^{5}$ \\
\hline 161 & $\mathrm{H}$ & R 3 c:H & R $3-2$ "c & $R 3 c$ & $C_{3 v}^{6}$ \\
\hline 162 & & $\mathrm{P}-312 / \mathrm{m}$ & -P 32 & $P \overline{3} 1 \mathrm{~m}$ & $D_{3 d}^{1}$ \\
\hline 163 & & $\mathrm{P}-312 / \mathrm{c}$ & -P $32 c$ & $P \overline{3} 1 c$ & $D_{3 d}^{2}$ \\
\hline 164 & & $\mathrm{P}-32 / \mathrm{m} 1$ & -P $32 ”$ & $P \overline{3} m 1$ & $D_{3 d}^{3}$ \\
\hline 165 & & $\mathrm{P}-32 / \mathrm{c} 1$ & -P 32 "c & $P \overline{3} c 1$ & $D_{3 d}^{4}$ \\
\hline 166 & $\mathrm{H}$ & $\mathrm{R}-3$ 2/m:H & -R $32 ”$ & $R \overline{3} m$ & $D_{3 d}^{5}$ \\
\hline 167 & $\mathrm{H}$ & $\mathrm{R}-3$ 2/c:H & -R 32 "c & $R \overline{3} c$ & $D_{3 d}^{6}$ \\
\hline 168 & & P 6 & P 6 & $P 6$ & $C_{6}^{1}$ \\
\hline 169 & & P 61 & P 61 & $P 6_{1}$ & $C_{6}^{2}$ \\
\hline 170 & & P 65 & P 65 & $P 6_{5}$ & $C_{6}^{3}$ \\
\hline 171 & & P 62 & P 62 & $P 6_{2}$ & $C_{6}^{4}$ \\
\hline 172 & & P 64 & P 64 & $P 6_{4}$ & $C_{6}^{5}$ \\
\hline 173 & & P 63 & P 6c & $P 6_{3}$ & $C_{6}^{6}$ \\
\hline 174 & & P -6 & P -6 & $P \overline{6}$ & $C_{3 h}^{1}$ \\
\hline 175 & & P 6/m & $-\mathrm{P} 6$ & $P 6 / m$ & $C_{6 h}^{1}$ \\
\hline 176 & & $\mathrm{P} 63 / \mathrm{m}$ & $-\mathrm{P} 6 \mathrm{c}$ & $P 6_{3} / m$ & $C_{6 h}^{2}$ \\
\hline 177 & & P 622 & P 62 & $P 622$ & $D_{6}^{1}$ \\
\hline 178 & & P 6122 & P $612\left(\begin{array}{lll}0 & 0 & -1\end{array}\right)$ & $P 6_{1} 22$ & $D_{6}^{2}$ \\
\hline 179 & & P 6522 & P $652\left(\begin{array}{lll}0 & 0 & 1\end{array}\right)$ & $P 6_{5} 22$ & $D_{6}^{3}$ \\
\hline 180 & & P 6222 & P 62 2c $\left(\begin{array}{lll}0 & 0 & 1\end{array}\right)$ & $P 6_{2} 22$ & $D_{6}^{4}$ \\
\hline 181 & & P 6422 & P 64 2c $\left(\begin{array}{lll}0 & 0 & -1\end{array}\right)$ & $P 6_{4} 22$ & $D_{6}^{5}$ \\
\hline 182 & & P 6322 & $\mathrm{P} 6 \mathrm{c} 2 \mathrm{c}$ & $P 6_{3} 22$ & $D_{6}^{6}$ \\
\hline 183 & & P $6 \mathrm{~m} \mathrm{~m}$ & P $6-2$ & P6mm & $C_{6 v}^{1}$ \\
\hline 184 & & P $6 \mathrm{cc}$ & P $6-2 c$ & $P 6 c c$ & $C_{6 v}^{2}$ \\
\hline
\end{tabular}


Table 1 (continued): A list of the various space group notations. The space group number, orientation, Hermann-Mauguin symbol, Hall symbol, International symbol, and Schönflies symbol are listed.

\begin{tabular}{|c|c|c|c|c|c|}
\hline Number & Orientation & Hermann-Mauguin & Hall & International & Schönflies \\
\hline 185 & & P $63 \mathrm{c} \mathrm{m}$ & P 6c -2 & $P 6_{3} \mathrm{~cm}$ & $C_{6 v}^{3}$ \\
\hline 186 & & P $63 \mathrm{~m} \mathrm{c}$ & P $6 c-2 c$ & $P 6_{3} m c$ & $C_{6 v}^{4}$ \\
\hline 187 & & P $-6 \mathrm{~m} 2$ & P -6 2 & $P \overline{6} m 2$ & $D_{3 h}^{1}$ \\
\hline 188 & & $P-6 c 2$ & $P-6 c 2$ & $P \overline{6} c 2$ & $D_{3 h}^{2}$ \\
\hline 189 & & P -6 2 m & $P-6-2$ & $P \overline{6} 2 m$ & $D_{3 h}^{3}$ \\
\hline 190 & & $P-62 c$ & $P-6 c-2 c$ & $P \overline{6} 2 c$ & $D_{3 h}^{4}$ \\
\hline 191 & & $\mathrm{P} 6 / \mathrm{m} \mathrm{2/m} \mathrm{2/m}$ & -P 62 & $P 6 / \mathrm{mmm}$ & $D_{6 h}^{1}$ \\
\hline 192 & & $\mathrm{P} 6 / \mathrm{m} \mathrm{2/c} \mathrm{2/c}$ & $-\mathrm{P} 62 \mathrm{c}$ & $P 6 / m c c$ & $D_{6 h}^{2}$ \\
\hline 193 & & $\mathrm{P} 63 / \mathrm{m} \mathrm{2/c} \mathrm{2/m}$ & $-\mathrm{P} 6 \mathrm{c} 2$ & $\mathrm{PG}_{3} / \mathrm{mcm}$ & $D_{6 h}^{3}$ \\
\hline 194 & & $\mathrm{P} 63 / \mathrm{m} \mathrm{2/m} \mathrm{2/c}$ & $-P$ 6c 2c & $P 6_{3} / m m c$ & $D_{6 h}^{4}$ \\
\hline 195 & & P 23 & P 223 & $P 23$ & $T^{1}$ \\
\hline 196 & & F 23 & F 223 & $F 23$ & $T^{2}$ \\
\hline 197 & & I 23 & I 223 & $I 23$ & $T^{3}$ \\
\hline 198 & & P 213 & P $2 a c 2 a b 3$ & $P 2_{1} 3$ & $T^{4}$ \\
\hline 199 & & I 213 & I $2 b 2 c 3$ & $I 2_{1} 3$ & $T^{5}$ \\
\hline 200 & & $\mathrm{P} 2 / \mathrm{m} \mathrm{-3}$ & $-P 223$ & $P m \overline{3}$ & $T_{h}^{1}$ \\
\hline 201 & 2 & P $2 / n-3: 2$ & $-\mathrm{P} 2 \mathrm{ab} 2 \mathrm{bc} 3$ & $P n \overline{3}$ & $T_{h}^{2}$ \\
\hline 202 & & $\mathrm{~F} 2 / \mathrm{m}-3$ & -F 223 & $F m \overline{3}$ & $T_{h}^{3}$ \\
\hline 203 & 2 & F $2 / d-3: 2$ & -F 2uv 2vw 3 & $F d \overline{3}$ & $T_{h}^{4}$ \\
\hline 204 & & $\mathrm{I} 2 / \mathrm{m}-3$ & -I 223 & $\operatorname{Im} \overline{3}$ & $T_{h}^{5}$ \\
\hline 205 & & P 21/a -3 & -P 2ac 2ab 3 & $P a \overline{3}$ & $T_{h}^{6}$ \\
\hline 206 & & I $21 / a-3$ & $-\mathrm{I} 2 \mathrm{~b} 2 \mathrm{c} 3$ & $I a \overline{3}$ & $T_{h}^{7}$ \\
\hline 207 & & P 432 & P 423 & $P 432$ & $O^{1}$ \\
\hline 208 & & P 4232 & P 4n 23 & $P 4_{2} 32$ & $O^{2}$ \\
\hline 209 & & F 432 & F 423 & $F 432$ & $O^{3}$ \\
\hline 210 & & F 4132 & F $4 \mathrm{~d} 23$ & $F 4_{1} 32$ & $O^{4}$ \\
\hline 211 & & I 432 & I 423 & $I 432$ & $O^{5}$ \\
\hline 212 & & P 4332 & P 4acd 2ab 3 & $P 4_{3} 32$ & $O^{6}$ \\
\hline 213 & & P 4132 & P 4bd 2ab 3 & $P 4_{1} 32$ & $O^{7}$ \\
\hline 214 & & I 4132 & I $4 b d 2 c 3$ & $I 4_{1} 32$ & $O^{8}$ \\
\hline 215 & & P - -4 $3 \mathrm{~m}$ & $P-423$ & $P \overline{4} 3 m$ & $T_{d}^{1}$ \\
\hline 216 & & $\mathrm{~F}-43 \mathrm{~m}$ & F -423 & $F \overline{4} 3 m$ & $T_{d}^{2}$ \\
\hline 217 & & I - $43 \mathrm{~m}$ & I -423 & $I \overline{4} 3 m$ & $T_{d}^{3}$ \\
\hline 218 & & $P-43 n$ & $P-4 n 23$ & $P \overline{4} 3 n$ & $T_{d}^{4}$ \\
\hline 219 & & $F-43 c$ & F $-4 c 23$ & $F \overline{4} 3 c$ & $T_{d}^{5}$ \\
\hline 220 & & $\mathrm{I}-43 \mathrm{~d}$ & I $-4 b d 2 c 3$ & $I \overline{4} 3 d$ & $T_{d}^{6}$ \\
\hline 221 & & $\mathrm{P} 4 / \mathrm{m}-32 / \mathrm{m}$ & -P 423 & $P m \overline{3} m$ & $O_{h}^{1}$ \\
\hline 222 & 2 & P 4/n -3 2/n:2 & $-\mathrm{P} 4 \mathrm{a} 2 \mathrm{bc} 3$ & $P n \overline{3} n$ & $O_{h}^{2}$ \\
\hline 223 & & $\mathrm{P} 42 / \mathrm{m}-32 / n$ & -P 4n 23 & $P m \overline{3} n$ & $O_{h}^{3}$ \\
\hline 224 & 2 & $\mathrm{P} 42 / \mathrm{n}-3$ 2/m:2 & $-P 4 b c 2 b c 3$ & $P n \overline{3} m$ & $O_{h}^{4}$ \\
\hline 225 & & $\mathrm{~F} 4 / \mathrm{m}-32 / \mathrm{m}$ & -F 423 & $F m \overline{3} m$ & $O_{h}^{5}$ \\
\hline 226 & & $\mathrm{~F} 4 / \mathrm{m}-32 / \mathrm{c}$ & $-F 4 c 23$ & $F m \overline{3} c$ & $O_{h}^{6}$ \\
\hline 227 & 2 & $\mathrm{~F} 41 / \mathrm{d}-32 / \mathrm{m}: 2$ & -F 4vw 2vw 3 & $F d \overline{3} m$ & $O_{h}^{7}$ \\
\hline 228 & 2 & $\mathrm{~F} 41 / \mathrm{d}-3 \mathrm{2} / \mathrm{c}: 2$ & $-\mathrm{F} 4 \mathrm{cvw} 2 \mathrm{vw} 3$ & $F d \overline{3} c$ & $O_{h}^{8}$ \\
\hline 229 & & I $4 / \mathrm{m}-32 / \mathrm{m}$ & -I 423 & $\operatorname{Im} \overline{3} m$ & $O_{h}^{9}$ \\
\hline 230 & & I $41 / \mathrm{a}-32 / \mathrm{d}$ & $-\mathrm{I} 4 \mathrm{bd} 2 \mathrm{c} 3$ & $I a \overline{3} d$ & $O_{h}^{10}$ \\
\hline
\end{tabular}




\section{Acknowledgments}

The authors would like to thank D. A. Papaconstantopoulos, who first proposed the Crystal Lattice Structures database, and R. Benjamin Young, who help set up the original web site in the summer of 1995. Special thanks are due to the H. Stokes, for providing updates to the FINDSYM code, and to the librarians at the U.S. Naval Research Laboratory Ruth H. Hooker Research Library, who tracked down many dozens of research articles which are not yet available online. M. J. Mehl is supported by the Kinnear Foundation and under contract from Duke University. We also acknowledge support by the by DOD-ONR (N00014-17-1-2090, N00014-16-1-2326, N00014-15-1-2863, and N00014-161-2781). DH acknowledges support from the Department of Defense through the National Defense Science and Engineering Graduate (NDSEG) Fellowship Program. The AFLOW consortium would like to acknowledge the Duke University Center for Materials Genomics. 


\section{References}

[1] E. Perim, D. Lee, Y. Liu, C. Toher, P. Gong, Y. Li, W. N. Simmons, O. Levy, J. J. Vlassak, J. Schroers, and S. Curtarolo, Spectral descriptors for bulk metallic glasses based on the thermodynamics of competing crystalline phases, Nat. Commun. 7, 12315 (2016), doi:10.1038/ncomms12315.

[2] C. M. Rost, E. Sachet, T. Borman, A. Moballegh, E. C. Dickey, D. Hou, J. L. Jones, S. Curtarolo, and J.-P. Maria, Entropy-stabilized oxides, Nat. Commun. 6, 8485 (2015), doi:10.1038/ncomms9485.

[3] L. Yu and A. Zunger, Identification of Potential Photovoltaic Absorbers Based on First-Principles Spectroscopic Screening of Materials, Phys. Rev. Lett. 108, 068701 (2012), doi:10.1103/PhysRevLett.108. 068701 .

[4] G. Ceder, G. Hauthier, A. Jain, and S. P. Ong, Recharging lithium battery research with firstprinciples methods, MRS Bull. 36, 185-191 (2011), doi:10.1557/mrs.2011.31.

[5] S. Sanvito, C. Oses, J. Xue, A. Tiwari, M. Zic, T. Archer, P. Tozman, M. Venkatesan, J. M. D. Coey, and S. Curtarolo, Accelerated discovery of new magnets in the Heusler alloy family, Sci. Adv. 3, e1602241 (2017), doi:10.1126/sciadv.1602241.

[6] D. Hicks, C. Oses, E. Gossett, G. Gomez, R. H. Taylor, C. Toher, M. J. Mehl, O. Levy, and S. Curtarolo, AFLOW-SYM: platform for the complete, automatic and self-consistent symmetry analysis of crystals, Acta Crystallogr. Sect. A 74, 184-203 (2018), doi:10.1107/S2053273318003066.

[7] C. Toher, C. Oses, J. J. Plata, D. Hicks, F. Rose, O. Levy, M. de Jong, M. D. Asta, M. Fornari, M. Buongiorno Nardelli, and S. Curtarolo, Combining the AFLOW GIBBS and Elastic Libraries to efficiently and robustly screen thermomechanical properties of solids, Phys. Rev. Mater. 1, 015401 (2017), doi:10.1103/PhysRevMaterials.1.015401.

[8] C. Toher, J. J. Plata, O. Levy, M. de Jong, M. D. Asta, M. Buongiorno Nardelli, and S. Curtarolo, High-throughput computational screening of thermal conductivity, Debye temperature, and Grüneisen parameter using a quasiharmonic Debye model, Phys. Rev. B 90, 174107 (2014), doi:10.1103/PhysRevB.90. 174107.

[9] P. Nath, J. J. Plata, D. Usanmaz, R. Al Rahal Al Orabi, M. Fornari, M. Buongiorno Nardelli, C. Toher, and S. Curtarolo, High-Throughput Prediction of Finite-Temperature Properties using the QuasiHarmonic Approximation, Comput. Mater. Sci. 125, 82-91 (2016), doi:10.1016/j.commatsci.2016.07.043.

[10] P. Nath, J. J. Plata, D. Usanmaz, C. Toher, M. Fornari, M. Buongiorno Nardelli, and S. Curtarolo, High throughput combinatorial method for fast and robust prediction of lattice thermal conductivity, Scr. Mater. 129, 88-93 (2017), doi:10.1016/j.scriptamat.2016.09. 034.

[11] J. J. Plata, P. Nath, D. Usanmaz, J. Carrete, C. Toher, M. de Jong, M. D. Asta, M. Fornari, M. Buongiorno Nardelli, and S. Curtarolo, An efficient and accurate framework for calculating lattice thermal conductivity of solids: AFLOW-AAPL Automatic Anharmonic Phonon Library, NPJ Comput. Mater. 3, 45 (2017), doi:10.1038/s41524-017-0046-7.

[12] S. Curtarolo, W. Setyawan, G. L. W. Hart, M. Jahnátek, R. V. Chepulskii, R. H. Taylor, S. Wang, J. Xue, K. Yang, O. Levy, M. J. Mehl, H. T. Stokes, D. O. Demchenko, and D. Morgan, AFLOW: An automatic framework for high-throughput materials discovery, Comput. Mater. Sci. 58, 218-226 (2012), doi:10.1016/ j.commatsci.2012.02.005.

[13] K. Yang, C. Oses, and S. Curtarolo, Modeling OffStoichiometry Materials with a High-Throughput AbInitio Approach, Chem. Mater. 28, 6484-6492 (2016), doi:10.1021/acs.chemmater.6b01449.

[14] W. Setyawan and S. Curtarolo, High-throughput electronic band structure calculations: Challenges and tools, Comput. Mater. Sci. 49, 299-312 (2010), doi: 10.1016/j.commatsci.2010.05.010.

[15] O. Levy, G. L. W. Hart, and S. Curtarolo, Structure maps for hcp metals from first-principles calculations, Phys. Rev. B 81, 174106 (2010), doi:10.1103/ PhysRevB.81.174106.

[16] G. L. W. Hart, S. Curtarolo, T. B. Massalski, and O. Levy, Comprehensive Search for New Phases and Compounds in Binary Alloy Systems Based on Platinum-Group Metals, Using a Computational First-Principles Approach, Phys. Rev. X 3, 041035 (2013), doi:10.1103/PhysRevX.3.041035.

[17] M. J. Mehl, D. Hicks, C. Toher, O. Levy, R. M. Hanson, G. L. W. Hart, and S. Curtarolo, The AFLOW Library of Crystallographic Prototypes: Part 1, Comput. Mater. Sci. 136, S1-S828 (2017), doi:10.1016/j. commatsci.2017.01.017.

[18] A. R. Supka, T. E. Lyons, L. S. I. Liyanage, P. D'Amico, R. Al Rahal Al Orabi, S. Mahatara, 
P. Gopal, C. Toher, D. Ceresoli, A. Calzolari, S. Curtarolo, M. Buongiorno Nardelli, and M. Fornari, AFLOWת: A minimalist approach to high-throughput $a b$ initio calculations including the generation of tight-binding hamiltonians, Comput. Mater. Sci. 136, 76-84 (2017), doi:10.1016/j.commatsci.2017.03.055.

[19] M. Scheffler, C. Draxl, and Computer Center of the Max-Planck Society, Garching, The NoMaD Repository, http://nomad-repository.eu (2014).

[20] A. Jain, G. Hautier, C. J. Moore, S. P. Ong, C. C. Fischer, T. Mueller, K. A. Persson, and G. Ceder, A highthroughput infrastructure for density functional theory calculations, Comput. Mater. Sci. 50, 2295-2310 (2011), doi:10.1016/j.commatsci.2011.02.023.

[21] J. E. Saal, S. Kirklin, M. Aykol, B. Meredig, and C. Wolverton, Materials Design and Discovery with High-Throughput Density Functional Theory: The Open Quantum Materials Database (OQMD), JOM 65, 1501-1509 (2013), doi:10.1007/ s11837-013-0755-4.

[22] P. P. Ewald, K. Herrman, C. Herman, O. Lohrmann, H. Philipp, G. Gottfried, and F. Schossberger, eds., Strukturbericht 1913-1939, vol. I-VII (Akademische Verlagsgesellschaft M. B. H., 1931-1943).

[23] R. W. G. Wyckoff, The Structure of Crystals, vol. IVI (John Wiley \& Sons, New York, London, Sydney, 1963 - 1971), $2^{\text {nd }}$ edn.

[24] W. B. Pearson, A handbook of lattice spacings and structures of metals and alloys (Pergamon Press, New York, 1958).

[25] R. T. Downs and M. Hall-Wallace, The American Mineralogist crystal structure database, Ann. Math. 88, 247-250 (2003).

[26] G. Kresse and J. Hafner, Ab initio molecular dynamics for liquid metals, Phys. Rev. B 47, 558-561 (1993), doi:10.1103/PhysRevB.47.558.

[27] G. Kresse and J. Hafner, Ab initio moleculardynamics simulation of the liquid-metal-amorphoussemiconductor transition in germanium, Phys. Rev. B 49, 14251-14269 (1994), doi:10.1103/PhysRevB.49. 14251.

[28] G. Kresse and J. Furthmüller, Efficiency of ab-initio total energy calculations for metals and semiconductors using a plane-wave basis set, Comput. Mater. Sci. 6, 15-50 (1996), doi:10.1016/0927-0256(96)00008-0.

[29] G. Kresse and J. Furthmüller, Efficient iterative schemes for ab initio total-energy calculations using a plane-wave basis set, Phys. Rev. B 54, 11169-11186 (1996), doi:10.1103/PhysRevB.54.11169.
[30] P. Giannozzi, S. Baroni, N. Bonini, M. Calandra, R. Car, C. Cavazzoni, D. Ceresoli, G. L. Chiarotti, M. Cococcioni, I. Dabo, A. Dal Corso, S. de Gironcoli, S. Fabris, G. Fratesi, R. Gebauer, U. Gerstmann, C. Gougoussis, A. Kokalj, M. Lazzeri, L. Martin-Samos, N. Marzari, F. Mauri, R. Mazzarello, S. Paolini, A. Pasquarello, L. Paulatto, C. Sbraccia, S. Scandolo, G. Sclauzero, A. P. Seitsonen, A. Smogunov, P. Umari, and R. M. Wentzcovitch, QUANTUM ESPRESSO: a modular and open-source software project for quantum simulations of materials, J. Phys.: Condens. Matter 21, 395502 (2009).

[31] V. Blum, R. Gehrke, F. Hanke, P. Havu, V. Havu, $\mathrm{X}$. Ren, K. Reuter, and M. Scheffler, Ab initio molecular simulations with numeric atom-centered orbitals, Comput. Phys. Commun. 180, 2175-2196 (2009).

[32] X. Gonze, J. M. Beuken, R. Caracas, F. Detraux, M. Fuchs, G. M. Rignanese, L. Sindic, M. Verstraete, G. Zerah, F. Jollet, M. Torrent, A. Roy, M. Mikami, P. Ghosez, J. Y. Raty, and D. C. Allan, First-principles computation of material properties: the ABINIT software project, Comput. Mater. Sci. 25, 478-492 (2002), doi:10.1016/S0927-0256(02)00325-7.

[33] M. I. Aroyo, J. M. Perez-Mato, D. Orobengoa, E. Tasci, G. de la Flor, and A. Kirov, Crystallography online: Bilbao crystallographic server, Bulg. Chem. Commun. 43, 183-197 (2011).

[34] Jmol: an open-source Java viewer for chemical structures in $3 D$, http://www.jmol.org/.

[35] Online Dictionary of Crystallography. Maintained by the Commission for Crystallographic Nomenclature of the International Union of Crystallography.

[36] T. Han, ed., International Tables for Crystallography (International Union of Crystallography, 2006), vol. A: Space-group symmetry, chap. 3.1.5, doi:10.1107/ 97809553602060000100 .

[37] D. Sedbrook, Must the Molecules of Life Always be Left-Handed or Right-Handed? Published online July 28, 2016.

[38] N. W. Ashcroft and N. D. Mermin, Solid State Physics (Saunders College Publishing, Orlando, Florida, 1976), chap. 4, pp. 73-75.

[39] JSmol, https://sourceforge.net/projects/jsmol/.

[40] N. W. Ashcroft and N. D. Mermin, Solid State Physics (Saunders College Publishing, Orlando, Florida, 1976), chap. 5.

[41] E. A. Abbott, Flatland: A Romance of Many Dimensions (Project Guttenberg, 1994). 
[42] M. J. Allen, V. C. Tung, and R. B. Kaner, Honeycomb Carbon: A Review of Graphene, Chem. Rev. 110, 132-145 (2010), doi:10.1021/cr900070d.

[43] X. Li and H. Zhu, Two-dimensional MoS 2 : Properties, preparation, and applications, J. Materiomics $\mathbf{1}$, 33-44 (2015), doi:10.1016/j.jmat.2015.03.003.

[44] D. E. Joyce, Wallpaper Groups (1997). (Plane Symmetry Groups).

[45] P. J. Morandi, The Classification of Wallpaper Patterns: From Group Cohomology to Escher's Tessellations (2003). Department of Mathematical Sciences, New Mexico State University, Las Cruces, New Mexico.

[46] D. Eck, Wallpaper Symmetry. Dynamic illustration of symmetries allowed in wallpaper groups.

[47] T. Hahn, ed., International Tables of Crystallography. Volume A: Space-group symmetry (Kluwer Academic publishers, International Union of Crystallography, Chester, England, 2002).

[48] S. R. Hall and B. McMahon, eds., International Tables for Crystallography, vol. G: Definition and exchange of crystallographic data (International Union of Crystallography, Chester, UK, 2006), doi:10.1107/ 97809553602060000107.

[49] C. Hermann, XVI. Zur systematischen Strukturtheorie. I. Eine neue Raumgruppensymbolik, Z. Kristallogr. 68, 257-287 (1928), doi:10.1524/zkri.1928.68.1.257.

[50] C. Mauguin, Sur le symbolisme des groupes de répétition on de symétrie des assemblages cristallins, Z. Kristallogr. 76, 542-558 (1931), doi:10.1524/zkri. 1931.76.1.542.

[51] S. R. Hall, Space-group notation with an explicit origin, Acta Crystallogr. Sect. A 37, 517-525 (1981), doi:10.1107/S0567739481001228.

[52] C. P. Brock, ed., International Tables for Crystallography (International Union of Crystallography, 2016), doi:10.1107/97809553602060000001.

[53] A. Schönflies, Krystallsysteme und Krystalstruktur (B. G. Teabuer, Leipzig, 1891).

[54] A. Schönflies, Theorie der Kristalstruktur (G. Borntraeger, Berlin, 1923).

[55] S. R. Hall and R. W. Grosse-Kunstleve, Concise Space-Group Symbols (2015). 


\section{$\mathrm{H}_{2} \mathrm{~S}$ (90 GPa) Structure: A2B_aP6_2_aei_i}
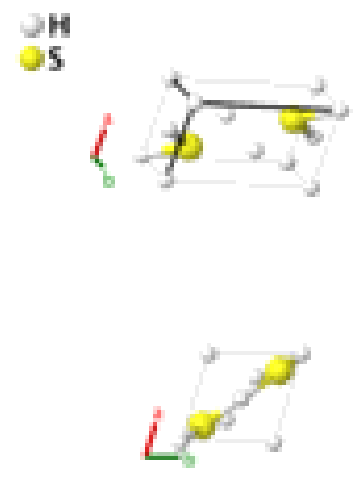

Prototype

AFLOW prototype label

Strukturbericht designation

Pearson symbol

Space group number

Space group symbol

AFLOW prototype command
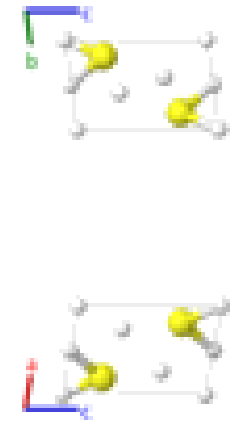

- This structure was found by first-principles electronic structure calculations and is predicted to be the stable structure of $\mathrm{H}_{2} \mathrm{~S}$ in the range $80-140 \mathrm{GPa}$.

- The data presented here was computed at $90 \mathrm{GPa}$.

- The original reference places $\mathrm{H}$ atoms on (1g), (1f) and (2i) sites, with $\mathrm{S}$ atoms on (2i) sites. We have changed the origin so that the $\mathrm{H}$ atoms are now on (1a), (1e) and (2i) sites.

\section{Triclinic primitive vectors:}

$$
\begin{array}{ccc}
\mathbf{a}_{1}= & a \hat{\mathbf{x}} \\
\mathbf{a}_{2}= & b \cos \gamma \hat{\mathbf{x}}+b \sin \gamma \hat{\mathbf{y}} \\
\mathbf{a}_{3}= & c_{x} \hat{\mathbf{x}}+c_{y} \hat{\mathbf{y}}+c_{z} \hat{\mathbf{z}} \\
& & \\
c_{x}= & c \cos \beta \\
c_{y}= & c(\cos \alpha-\cos \beta \cos \gamma) / \sin \gamma \\
c_{z}= & \sqrt{c^{2}-c_{x}^{2}-c_{y}^{2}}
\end{array}
$$
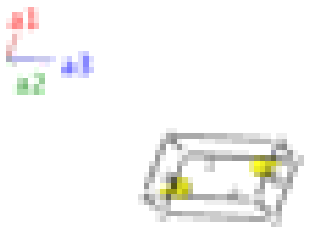

Lattice Coordinates

$\begin{array}{lcl}\mathbf{B}_{1}= & 0 \mathbf{a}_{1}+0 \mathbf{a}_{2}+0 \mathbf{a}_{3}= \\ \mathbf{B}_{2}= & \frac{1}{2} \mathbf{a}_{1}+\frac{1}{2} \mathbf{a}_{2}=\end{array}$
Cartesian Coordinates

$$
0 \hat{\mathbf{x}}+0 \hat{\mathbf{y}}+0 \hat{\mathbf{z}}
$$

$\frac{1}{2}(a+b \cos \gamma) \hat{\mathbf{x}}+\frac{1}{2} b \sin \gamma \hat{\mathbf{y}}$
Wyckoff Position

$(1 a)$

(1e)
Atom Type

H I

H II 


$$
\begin{aligned}
& \mathbf{B}_{3}=x_{3} \mathbf{a}_{1}+y_{3} \mathbf{a}_{2}+z_{3} \mathbf{a}_{3}=\quad\left(x_{3} a+y_{3} b \cos \gamma+z_{3} c_{x}\right) \hat{\mathbf{x}}+ \\
& \left(y_{3} b \sin \gamma+z_{3} c_{y}\right) \hat{\mathbf{y}}+z_{3} c_{z} \hat{\mathbf{z}} \\
& \mathbf{B}_{4}=-x_{3} \mathbf{a}_{1}-y_{3} \mathbf{a}_{2}-z_{3} \mathbf{a}_{3}=\quad\left(-x_{3} a-y_{3} b \cos \gamma-z_{3} c_{x}\right) \hat{\mathbf{x}}+ \\
& \left(-y_{3} b \sin \gamma-z_{3} c_{y}\right) \hat{\mathbf{y}}-z_{3} c_{z} \hat{\mathbf{z}} \\
& \mathbf{B}_{5}=x_{4} \mathbf{a}_{1}+y_{4} \mathbf{a}_{2}+z_{4} \mathbf{a}_{3}=\quad\left(x_{4} a+y_{4} b \cos \gamma+z_{4} c_{x}\right) \hat{\mathbf{x}}+ \\
& \left(y_{4} b \sin \gamma+z_{4} c_{y}\right) \hat{\mathbf{y}}+z_{4} c_{z} \hat{\mathbf{z}} \\
& \mathbf{B}_{6}=-x_{4} \mathbf{a}_{1}-y_{4} \mathbf{a}_{2}-z_{4} \mathbf{a}_{3}=\quad\left(-x_{4} a-y_{4} b \cos \gamma-z_{4} c_{x}\right) \hat{\mathbf{x}}+ \\
& \left(-y_{4} b \sin \gamma-z_{4} c_{y}\right) \hat{\mathbf{y}}-z_{4} c_{z} \hat{\mathbf{z}}
\end{aligned}
$$

\section{References:}

- Y. Li, J. Hao, H. Liu, Y. Li, and Y. Ma, The metallization and superconductivity of dense hydrogen sulfide, J. Chem. Phys. 140, 174712 (2014), doi:10.1063/1.4874158.

\section{Geometry files:}

- CIF: pp. 802

- POSCAR: pp. 802 


\section{$\mathrm{Mo}_{8} \mathrm{P}_{5}$ (High-temperature) Structure: \\ A8B5_mP13_6_a7b_3a2b}
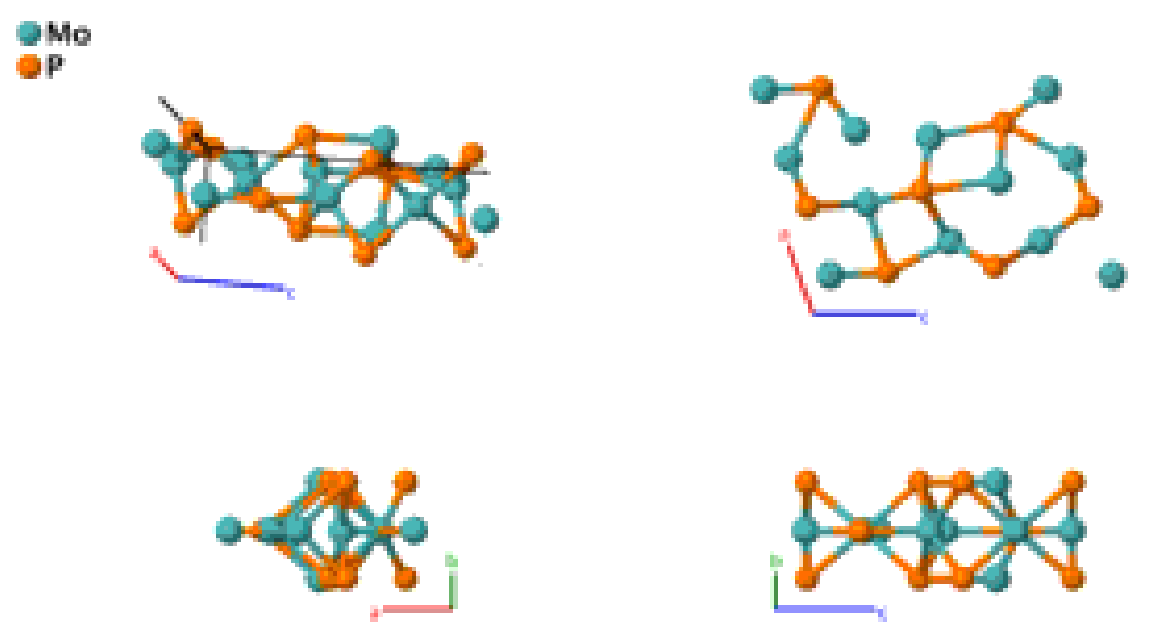

Prototype

: $\quad \mathrm{Mo}_{8} \mathrm{P}_{5}$

AFLOW prototype label

: A8B5_mP13_6_a7b_3a2b

Strukturbericht designation

Pearson symbol

: None

Space group number

: $\mathrm{mP} 13$

Space group symbol

: 6

AFLOW prototype command

$P m$

aflow - -proto=A8B5_mP13_6_a7b_3a2b

- -params $=a, b / a, c / a, \beta, x_{1}, z_{1}, x_{2}, z_{2}, x_{3}, z_{3}, x_{4}, z_{4}, x_{5}, z_{5}, x_{6}, z_{6}, x_{7}, z_{7}, x_{8}, z_{8}$, $x_{9}, z_{9}, x_{10}, z_{10}, x_{11}, z_{11}, x_{12}, z_{12}, x_{13}, z_{13}$

- This high-temperature phase of the Mo-P system is observed between $1580^{\circ} \mathrm{C}-1680^{\circ} \mathrm{C}$ (Johnsson, 1972).

\section{Simple Monoclinic primitive vectors:}

$$
\begin{array}{ccc}
\mathbf{a}_{1} & = & a \hat{\mathbf{x}} \\
\mathbf{a}_{2} & = & b \hat{\mathbf{y}} \\
\mathbf{a}_{3} & = & c \cos \beta \hat{\mathbf{x}}+c \sin \beta \hat{\mathbf{z}}
\end{array}
$$

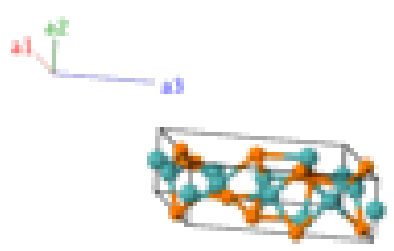

\section{Basis vectors:}

Lattice Coordinates

$\begin{array}{llll}\mathbf{B}_{1}= & x_{1} \mathbf{a}_{1}+z_{1} \mathbf{a}_{3} & = \\ \mathbf{B}_{2}= & x_{2} \mathbf{a}_{1}+z_{2} \mathbf{a}_{3} & = \\ \mathbf{B}_{3}= & x_{3} \mathbf{a}_{1}+z_{3} \mathbf{a}_{3} & = \\ \mathbf{B}_{4}= & x_{4} \mathbf{a}_{1}+z_{4} \mathbf{a}_{3} & =\end{array}$

Cartesian Coordinates

$$
\begin{aligned}
& \left(x_{1} a+z_{1} c \cos \beta\right) \hat{\mathbf{x}}+z_{1} c \sin \beta \hat{\mathbf{z}} \\
& \left(x_{2} a+z_{2} c \cos \beta\right) \hat{\mathbf{x}}+z_{2} c \sin \beta \hat{\mathbf{z}} \\
& \left(x_{3} a+z_{3} c \cos \beta\right) \hat{\mathbf{x}}+z_{3} c \sin \beta \hat{\mathbf{z}} \\
& \left(x_{4} a+z_{4} c \cos \beta\right) \hat{\mathbf{x}}+z_{4} c \sin \beta \hat{\mathbf{z}}
\end{aligned}
$$

Wyckoff Position

Atom Type

(1a)

Mo I

(1a)

P I

(1a)

P II

(1a)

P III 

$\mathbf{B}_{5}=x_{5} \mathbf{a}_{1}+\frac{1}{2} \mathbf{a}_{2}+z_{5} \mathbf{a}_{3}=\left(x_{5} a+z_{5} c \cos \beta\right) \hat{\mathbf{x}}+\frac{1}{2} b \hat{\mathbf{y}}+z_{5} c \sin \beta \hat{\mathbf{z}}$
$\mathbf{B}_{6}=x_{6} \mathbf{a}_{1}+\frac{1}{2} \mathbf{a}_{2}+z_{6} \mathbf{a}_{3}=\left(x_{6} a+z_{6} c \cos \beta\right) \hat{\mathbf{x}}+\frac{1}{2} b \hat{\mathbf{y}}+z_{6} c \sin \beta \hat{\mathbf{z}}$
$\mathbf{B}_{7}=x_{7} \mathbf{a}_{1}+\frac{1}{2} \mathbf{a}_{2}+z_{7} \mathbf{a}_{3}=\left(x_{7} a+z_{7} c \cos \beta\right) \hat{\mathbf{x}}+\frac{1}{2} b \hat{\mathbf{y}}+z_{7} c \sin \beta \hat{\mathbf{z}}$
$\mathbf{B}_{8}=x_{8} \mathbf{a}_{1}+\frac{1}{2} \mathbf{a}_{2}+z_{8} \mathbf{a}_{3}=\left(x_{8} a+z_{8} c \cos \beta\right) \hat{\mathbf{x}}+\frac{1}{2} b \hat{\mathbf{y}}+z_{8} c \sin \beta \hat{\mathbf{z}}$
$\mathbf{B}_{9}=x_{9} \mathbf{a}_{1}+\frac{1}{2} \mathbf{a}_{2}+z_{9} \mathbf{a}_{3}=\left(x_{9} a+z_{9} c \cos \beta\right) \hat{\mathbf{x}}+\frac{1}{2} b \hat{\mathbf{y}}+z_{9} c \sin \beta \hat{\mathbf{z}}$
$\mathbf{B}_{10}=x_{10} \mathbf{a}_{1}+\frac{1}{2} \mathbf{a}_{2}+z_{10} \mathbf{a}_{3}=\left(x_{10} a+z_{10} c \cos \beta\right) \hat{\mathbf{x}}+\frac{1}{2} b \hat{\mathbf{y}}+z_{10} c \sin \beta \hat{\mathbf{z}}$
$\mathbf{B}_{11}=x_{11} \mathbf{a}_{1}+\frac{1}{2} \mathbf{a}_{2}+z_{11} \mathbf{a}_{3}=\left(x_{11} a+z_{11} c \cos \beta\right) \hat{\mathbf{x}}+\frac{1}{2} b \hat{\mathbf{y}}+z_{11} c \sin \beta \hat{\mathbf{z}}$
$\mathbf{B}_{12}=x_{12} \mathbf{a}_{1}+\frac{1}{2} \mathbf{a}_{2}+z_{12} \mathbf{a}_{3}=\left(x_{12} a+z_{12} c \cos \beta\right) \hat{\mathbf{x}}+\frac{1}{2} b \hat{\mathbf{y}}+z_{12} c \sin \beta \hat{\mathbf{z}}$
$\mathbf{B}_{13}=x_{13} \mathbf{a}_{1}+\frac{1}{2} \mathbf{a}_{2}+z_{13} \mathbf{a}_{3}=\left(x_{13} a+z_{13} c \cos \beta\right) \hat{\mathbf{x}}+\frac{1}{2} b \hat{\mathbf{y}}+z_{13} c \sin \beta \hat{\mathbf{z}}$

(1b)

Mo II

Mo III

Mo IV

Mo V

Mo VI

Mo VII

Mo VIII

\section{References:}

- T. Johnsson, The crystal structure of $\mathrm{Mo}_{8} P_{5}$ from twin-crystal data, Acta Chem. Scand. 26, 365-382 (1972), doi:10.3891/acta.chem.scand.26-0365.

\section{Found in:}

- P. Villars and K. Cenzual, Pearson's Crystal Data - Crystal Structure Database for Inorganic Compounds, ASM International (2013).

\section{Geometry files:}

- CIF: pp. 802

- POSCAR: pp. 802 

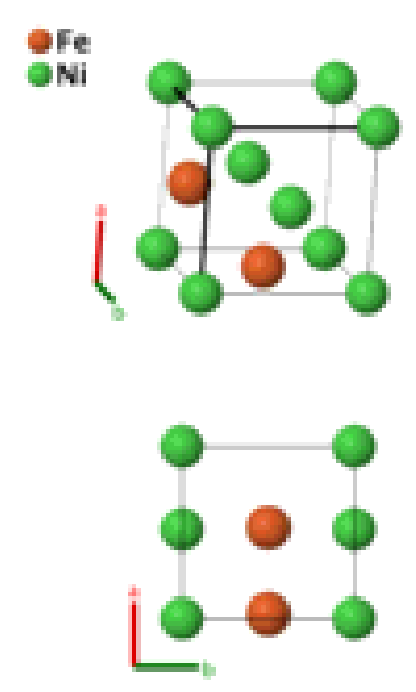
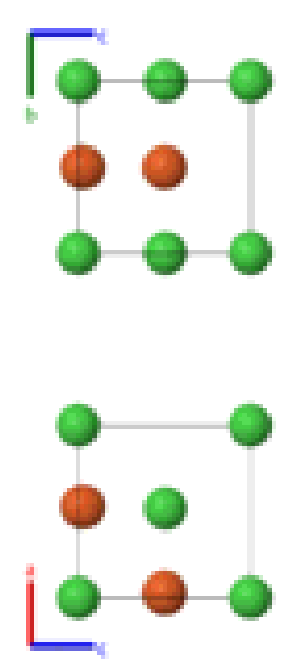

\section{Prototype}

AFLOW prototype label

Strukturbericht designation

Pearson symbol

Space group number

Space group symbol

AFLOW prototype command
: $\mathrm{FeNi}$

: AB_mP4_6_2b_2a

: None

: $\quad \mathrm{mP4}$

$: 6$

: $\quad P m$

\footnotetext{
aflow - -proto $=A B \_m P 4 \_6 \_2 b \_2 a$

- - params $=a, b / a, c / a, \beta, x_{1}, z_{1}, x_{2}, z_{2}, x_{3}, z_{3}, x_{4}, z_{4}$
}

- In the original the site occupations are mixed with Ni majority (0.85) on sites 1a and Fe majority on 1 b.

\section{Simple Monoclinic primitive vectors:}

$$
\begin{array}{ccc}
\mathbf{a}_{1}= & a \hat{\mathbf{x}} \\
\mathbf{a}_{2}= & b \hat{\mathbf{y}} \\
\mathbf{a}_{3}= & c \cos \beta \hat{\mathbf{x}}+c \sin \beta \hat{\mathbf{z}}
\end{array}
$$

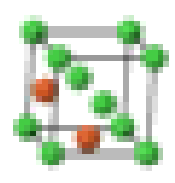

\section{Basis vectors:}

Lattice Coordinates
$\mathbf{B}_{1}=$
$x_{1} \mathbf{a}_{1}+z_{1} \mathbf{a}_{3}$
$=$
$x_{2} \mathbf{a}_{1}+z_{2} \mathbf{a}_{3}$
$=$
$\mathbf{B}_{2}=$
$x_{3} \mathbf{a}_{1}+\frac{1}{2} \mathbf{a}_{2}+z_{3} \mathbf{a}_{3}$
$=$
$\mathbf{B}_{3}=$
$\mathbf{B}_{4}=$
$x_{4} \mathbf{a}_{1}+\frac{1}{2} \mathbf{a}_{2}+z_{4} \mathbf{a}$
$=$
$\left(x_{1} a+z_{1} c \cos \beta\right) \hat{\mathbf{x}}+z_{1} c \sin \beta \hat{\mathbf{z}}$
$\left(x_{2} a+z_{2} c \cos \beta\right) \hat{\mathbf{x}}+z_{2} c \sin \beta \hat{\mathbf{z}}$
$\left(x_{3} a+z_{3} c \cos \beta\right) \hat{\mathbf{x}}+\frac{1}{2} b \hat{\mathbf{y}}+z_{3} c \sin \beta \hat{\mathbf{z}}$
$\left(x_{4} a+z_{4} c \cos \beta\right) \hat{\mathbf{x}}+\frac{1}{2} b \hat{\mathbf{y}}+z_{4} c \sin \beta \hat{\mathbf{z}}$

Cartesian Coordinates
Wyckoff Position

Atom Type

(1a)

$\mathrm{Ni}$ I

(1a)

$\mathrm{Ni}$ II

Fe I

(1b)

Fe II 


\section{References:}

- T. Tagai, H. Takeda, and T. Fukuda, Superstructure of tetrataenite from the Saint Severin meteorite, Zeitschrift für Kristallographie - Crystalline Materials 210, 14-18 (1995), doi:10.1524/zkri.1995.210.1.14.

\section{Found in:}

- P. Villars and K. Cenzual, Pearson's Crystal Data - Crystal Structure Database for Inorganic Compounds, ASM International (2013).

\section{Geometry files:}

- CIF: pp. 802

- POSCAR: pp. 803 


\section{$\mathrm{H}_{2} \mathrm{~S}$ IV Structure: A2B_mP12_7_4a_2a}

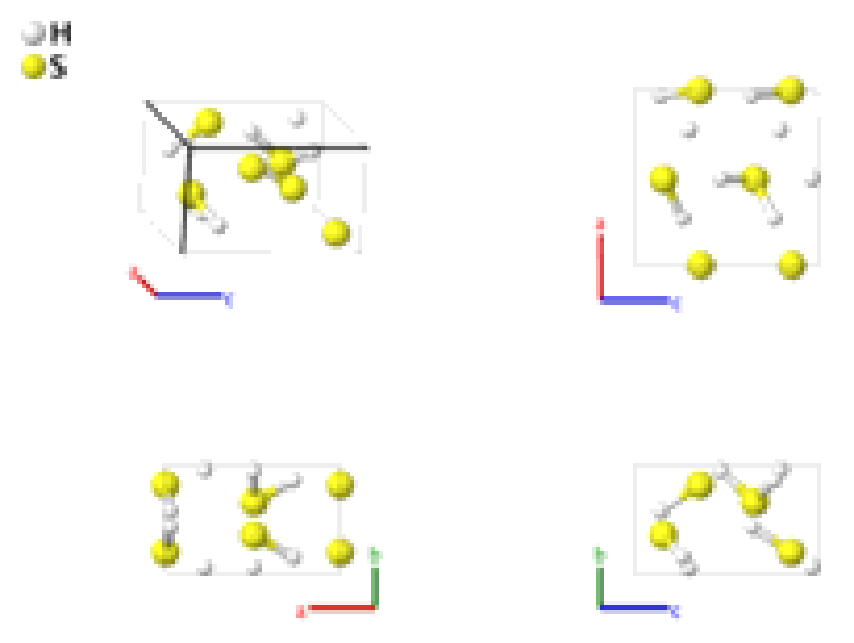

Prototype

AFLOW prototype label

Strukturbericht designation

Pearson symbol

Space group number

Space group symbol

AFLOW prototype command
: $\mathrm{H}_{2} \mathrm{~S}$

: A2B_mP12_7_4a_2a

: None

: $\quad \mathrm{mP} 12$

: 7

: $P c$

- The $\mathrm{H}_{2} \mathrm{~S}$-IV phase is stable at pressures greater than $5 \mathrm{GPa}$ and temperatures below $150 \mathrm{~K}$, and is stable at higher pressures for higher temperatures. Shimizu et. al. (Shimizu, 1995) determined the phase diagram. Endo et al. (Endo, 998) found the crystal structure at $11.4 \mathrm{GPa}$, but could not determine the positions of the hydrogen atoms. Here we use the predicted structure from $\mathrm{Li}$ et al. at $30 \mathrm{GPa}$.

\section{Simple Monoclinic primitive vectors:}
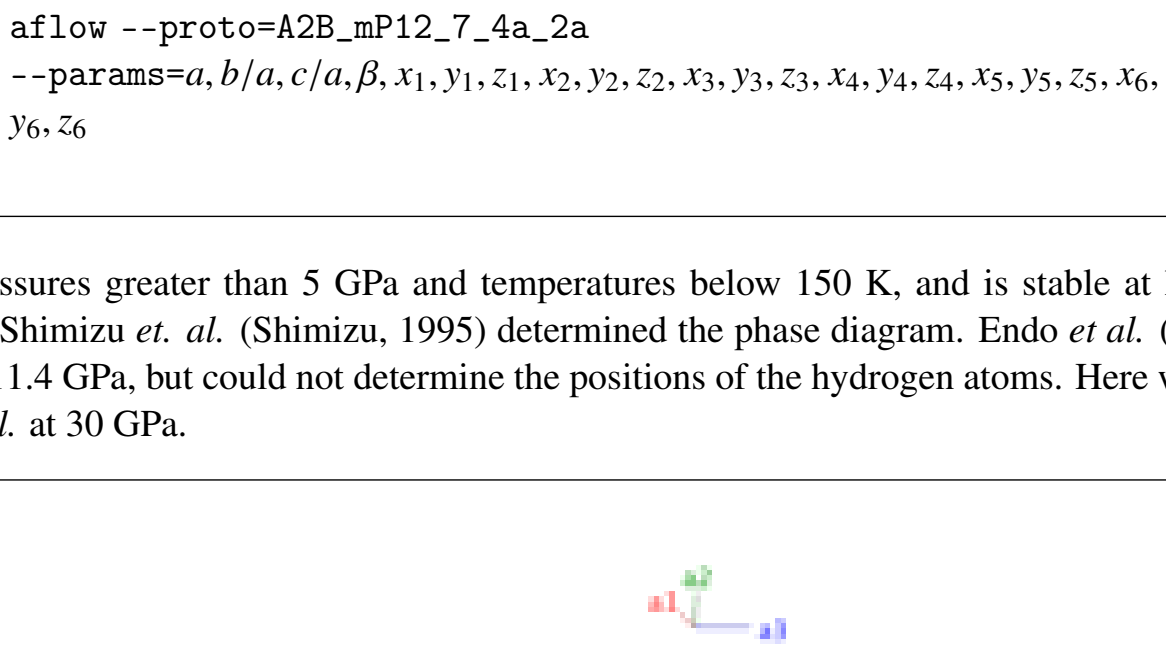

$$
\begin{array}{ccc}
\mathbf{a}_{1} & = & a \hat{\mathbf{x}} \\
\mathbf{a}_{2} & = & b \hat{\mathbf{y}} \\
\mathbf{a}_{3} & = & c \cos \beta \hat{\mathbf{x}}+c \sin \beta \hat{\mathbf{z}}
\end{array}
$$

\begin{tabular}{|c|c|c|c|c|c|c|}
\hline & & Lattice Coordinates & & Cartesian Coordinates & Wyckoff Position & Atom Typ \\
\hline $\mathbf{B}_{1}$ & - & $x_{1} \mathbf{a}_{1}+y_{1} \mathbf{a}_{2}+z_{1} \mathbf{a}_{3}$ & $=$ & $\left(x_{1} a+z_{1} c \cos \beta\right) \hat{\mathbf{x}}+y_{1} b \hat{\mathbf{y}}+z_{1} c \sin \beta \hat{\mathbf{z}}$ & $(2 a)$ & $\mathrm{H} \mathrm{I}$ \\
\hline $\mathbf{B}_{2}$ & $=$ & $x_{1} \mathbf{a}_{1}-y_{1} \mathbf{a}_{2}+\left(\frac{1}{2}+z_{1}\right) \mathbf{a}_{3}$ & $=$ & $\begin{array}{c}\left(\frac{1}{2} c \cos \beta+x_{1} a+z_{1} c \cos \beta\right) \hat{\mathbf{x}}-y_{1} b \hat{\mathbf{y}}+ \\
\left(\frac{1}{2}+z_{1}\right) c \sin \beta \hat{\mathbf{z}}\end{array}$ & $(2 a)$ & $\mathrm{H} \mathrm{I}$ \\
\hline
\end{tabular}

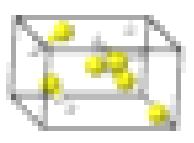

\section{Basis vectors:}




\begin{tabular}{|c|c|c|c|c|c|c|}
\hline $\mathbf{B}_{3}$ & $=$ & $x_{2} \mathbf{a}_{1}+y_{2} \mathbf{a}_{2}+z_{2} \mathbf{a}_{3}$ & $=$ & $\left(x_{2} a+z_{2} c \cos \beta\right) \hat{\mathbf{x}}+y_{2} b \hat{\mathbf{y}}+z_{2} c \sin \beta \hat{\mathbf{z}}$ & $(2 a)$ & H II \\
\hline $\mathbf{B}_{4}$ & $=$ & $x_{2} \mathbf{a}_{1}-y_{2} \mathbf{a}_{2}+\left(\frac{1}{2}+z_{2}\right) \mathbf{a}_{3}$ & $=$ & $\begin{array}{c}\left(\frac{1}{2} c \cos \beta+x_{2} a+z_{2} c \cos \beta\right) \hat{\mathbf{x}}-y_{2} b \hat{\mathbf{y}}+ \\
\left(\frac{1}{2}+z_{2}\right) c \sin \beta \hat{\mathbf{z}}\end{array}$ & $(2 a)$ & H II \\
\hline $\mathbf{B}_{5}$ & $=$ & $x_{3} \mathbf{a}_{1}+y_{3} \mathbf{a}_{2}+z_{3} \mathbf{a}_{3}$ & $=$ & $\left(x_{3} a+z_{3} c \cos \beta\right) \hat{\mathbf{x}}+y_{3} b \hat{\mathbf{y}}+z_{3} c \sin \beta \hat{\mathbf{z}}$ & $(2 a)$ & H III \\
\hline $\mathbf{B}_{6}$ & $=$ & $x_{3} \mathbf{a}_{1}-y_{3} \mathbf{a}_{2}+\left(\frac{1}{2}+z_{3}\right) \mathbf{a}_{3}$ & $=$ & $\begin{array}{c}\left(\frac{1}{2} c \cos \beta+x_{3} a+z_{3} c \cos \beta\right) \hat{\mathbf{x}}-y_{3} b \hat{\mathbf{y}}+ \\
\left(\frac{1}{2}+z_{3}\right) c \sin \beta \hat{\mathbf{z}}\end{array}$ & $(2 a)$ & H III \\
\hline $\mathbf{B}_{7}$ & $=$ & $x_{4} \mathbf{a}_{1}+y_{4} \mathbf{a}_{2}+z_{4} \mathbf{a}_{3}$ & $=$ & $\left(x_{4} a+z_{4} c \cos \beta\right) \hat{\mathbf{x}}+y_{4} b \hat{\mathbf{y}}+z_{4} c \sin \beta \hat{\mathbf{z}}$ & $(2 a)$ & H IV \\
\hline $\mathbf{B}_{8}$ & $=$ & $x_{4} \mathbf{a}_{1}-y_{4} \mathbf{a}_{2}+\left(\frac{1}{2}+z_{4}\right) \mathbf{a}_{3}$ & $=$ & $\begin{array}{c}\left(\frac{1}{2} c \cos \beta+x_{4} a+z_{4} c \cos \beta\right) \hat{\mathbf{x}}-y_{4} b \hat{\mathbf{y}}+ \\
\left(\frac{1}{2}+z_{4}\right) c \sin \beta \hat{\mathbf{z}}\end{array}$ & $(2 a)$ & H IV \\
\hline $\mathbf{B}_{9}$ & $=$ & $x_{5} \mathbf{a}_{1}+y_{5} \mathbf{a}_{2}+z_{5} \mathbf{a}_{3}$ & $=$ & $\left(x_{5} a+z_{5} c \cos \beta\right) \hat{\mathbf{x}}+y_{5} b \hat{\mathbf{y}}+z_{5} c \sin \beta \hat{\mathbf{z}}$ & $(2 a)$ & S I \\
\hline $\mathbf{B}_{10}$ & $=$ & $x_{5} \mathbf{a}_{1}-y_{5} \mathbf{a}_{2}+\left(\frac{1}{2}+z_{5}\right) \mathbf{a}_{3}$ & $=$ & $\begin{array}{c}\left(\frac{1}{2} c \cos \beta+x_{5} a+z_{5} c \cos \beta\right) \hat{\mathbf{x}}-y_{5} b \hat{\mathbf{y}}+ \\
\left(\frac{1}{2}+z_{5}\right) c \sin \beta \hat{\mathbf{z}}\end{array}$ & $(2 a)$ & S I \\
\hline $\mathbf{B}_{11}$ & $=$ & $x_{6} \mathbf{a}_{1}+y_{6} \mathbf{a}_{2}+z_{6} \mathbf{a}_{3}$ & $=$ & $\left(x_{6} a+z_{6} c \cos \beta\right) \hat{\mathbf{x}}+y_{6} b \hat{\mathbf{y}}+z_{6} c \sin \beta \hat{\mathbf{z}}$ & $(2 a)$ & S II \\
\hline $\mathbf{B}_{12}$ & $=$ & $x_{6} \mathbf{a}_{1}-y_{6} \mathbf{a}_{2}+\left(\frac{1}{2}+z_{6}\right) \mathbf{a}_{3}$ & $=$ & $\begin{array}{c}\left(\frac{1}{2} c \cos \beta+x_{6} a+z_{6} c \cos \beta\right) \hat{\mathbf{x}}-y_{6} b \hat{\mathbf{y}}+ \\
\left(\frac{1}{2}+z_{6}\right) c \sin \beta \hat{\mathbf{z}}\end{array}$ & $(2 a)$ & S II \\
\hline
\end{tabular}

\section{References:}

- Y. Li, J. Hao, H. Liu, Y. Li, and Y. Ma, The metallization and superconductivity of dense hydrogen sulfide, J. Chem. Phys. 140, 174712 (2014), doi:10.1063/1.4874158.

- H. Shimizu, H. Yamaguchi, S. Sasaki, A. Honda, S. Endo, and M. Kobayashi, Pressure-temperature phase diagram of solid hydrogen sulfide determined by Raman spectroscopy, Phys. Rev. B 51, 9391-9394 (1995), doi:10.1103/PhysRevB.51.9391.

- S. Endo, A. Honda, K. Koto, O. Shimomura, T. Kikegawa, and N. Hamaya, Crystal structure of high-pressure phase-IV solid hydrogen sulfide, Phys. Rev. B 57, 5699-5703 (1998), doi:10.1103/PhysRevB.57.5699.

\section{Geometry files:}

- CIF: pp. 803

- POSCAR: pp. 803 


\section{$\mathrm{As}_{2} \mathrm{Ba}$ Structure: A2B_mP18_7_6a_3a}
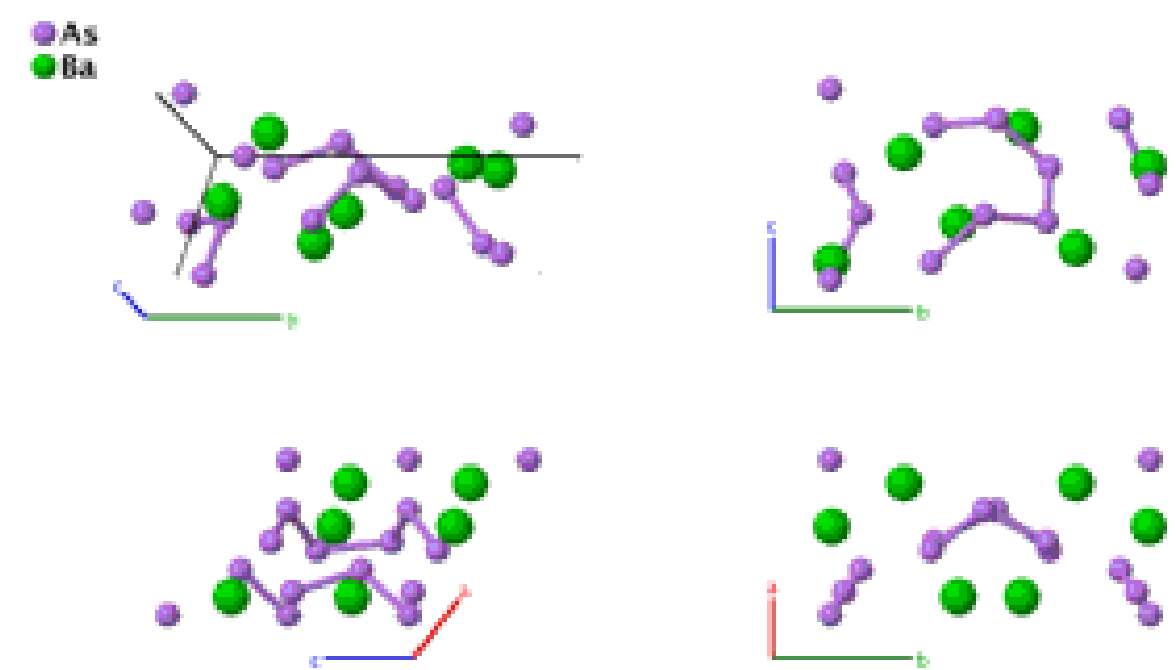

\section{Prototype}

AFLOW prototype label

$: \quad \mathrm{As}_{2} \mathrm{Ba}$

Strukturbericht designation

: A2B_mP18_7_6a_3a

Pearson symbol

None

Space group number

: $\quad \mathrm{mP} 18$

Space group symbol

7

AFLOW prototype command

aflow --proto=A2B_mP18_7_6a_3a

- -params $=a, b / a, c / a, \beta, x_{1}, y_{1}, z_{1}, x_{2}, y_{2}, z_{2}, x_{3}, y_{3}, z_{3}, x_{4}, y_{4}, z_{4}, x_{5}, y_{5}, z_{5}, x_{6}$, $y_{6}, z_{6}, x_{7}, y_{7}, z_{7}, x_{8}, y_{8}, z_{8}, x_{9}, y_{9}, z_{9}$

Simple Monoclinic primitive vectors:

$$
\begin{array}{ccc}
\mathbf{a}_{1} & = & a \hat{\mathbf{x}} \\
\mathbf{a}_{2} & = & b \hat{\mathbf{y}} \\
\mathbf{a}_{3} & = & c \cos \beta \hat{\mathbf{x}}+c \sin \beta \hat{\mathbf{z}}
\end{array}
$$

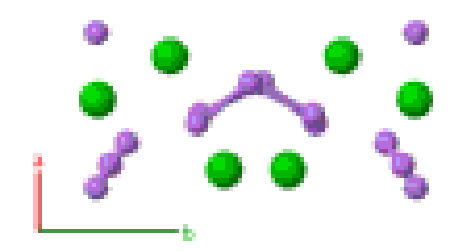




\begin{tabular}{|c|c|c|c|c|c|c|}
\hline $\mathbf{B}_{6}$ & $=$ & $x_{3} \mathbf{a}_{1}-y_{3} \mathbf{a}_{2}+\left(\frac{1}{2}+z_{3}\right) \mathbf{a}_{3}$ & $=$ & $\begin{array}{c}\left(\frac{1}{2} c \cos \beta+x_{3} a+z_{3} c \cos \beta\right) \hat{\mathbf{x}}-y_{3} b \hat{\mathbf{y}}+ \\
\left(\frac{1}{2}+z_{3}\right) c \sin \beta \hat{\mathbf{z}}\end{array}$ & $(2 a)$ & As III \\
\hline $\mathbf{B}_{7}$ & $=$ & $x_{4} \mathbf{a}_{1}+y_{4} \mathbf{a}_{2}+z_{4} \mathbf{a}_{3}$ & $=$ & $\left(x_{4} a+z_{4} c \cos \beta\right) \hat{\mathbf{x}}+y_{4} b \hat{\mathbf{y}}+z_{4} c \sin \beta \hat{\mathbf{z}}$ & $(2 a)$ & As IV \\
\hline $\mathbf{B}_{8}$ & $=$ & $x_{4} \mathbf{a}_{1}-y_{4} \mathbf{a}_{2}+\left(\frac{1}{2}+z_{4}\right) \mathbf{a}_{3}$ & $=$ & $\begin{array}{c}\left(\frac{1}{2} c \cos \beta+x_{4} a+z_{4} c \cos \beta\right) \hat{\mathbf{x}}-y_{4} b \hat{\mathbf{y}}+ \\
\left(\frac{1}{2}+z_{4}\right) c \sin \beta \hat{\mathbf{z}}\end{array}$ & $(2 a)$ & As IV \\
\hline $\mathbf{B}_{9}$ & $=$ & $x_{5} \mathbf{a}_{1}+y_{5} \mathbf{a}_{2}+z_{5} \mathbf{a}_{3}$ & $=$ & $\left(x_{5} a+z_{5} c \cos \beta\right) \hat{\mathbf{x}}+y_{5} b \hat{\mathbf{y}}+z_{5} c \sin \beta \hat{\mathbf{z}}$ & $(2 a)$ & As $\mathrm{V}$ \\
\hline $\mathbf{B}_{10}$ & $=$ & $x_{5} \mathbf{a}_{1}-y_{5} \mathbf{a}_{2}+\left(\frac{1}{2}+z_{5}\right) \mathbf{a}_{3}$ & $=$ & $\begin{array}{c}\left(\frac{1}{2} c \cos \beta+x_{5} a+z_{5} c \cos \beta\right) \hat{\mathbf{x}}-y_{5} b \hat{\mathbf{y}}+ \\
\left(\frac{1}{2}+z_{5}\right) c \sin \beta \hat{\mathbf{z}}\end{array}$ & $(2 a)$ & As $\mathrm{V}$ \\
\hline $\mathbf{B}_{11}$ & $=$ & $x_{6} \mathbf{a}_{1}+y_{6} \mathbf{a}_{2}+z_{6} \mathbf{a}_{3}$ & $=$ & $\left(x_{6} a+z_{6} c \cos \beta\right) \hat{\mathbf{x}}+y_{6} b \hat{\mathbf{y}}+z_{6} c \sin \beta \hat{\mathbf{z}}$ & $(2 a)$ & As VI \\
\hline $\mathbf{B}_{12}$ & $=$ & $x_{6} \mathbf{a}_{1}-y_{6} \mathbf{a}_{2}+\left(\frac{1}{2}+z_{6}\right) \mathbf{a}_{3}$ & $=$ & $\begin{array}{c}\left(\frac{1}{2} c \cos \beta+x_{6} a+z_{6} c \cos \beta\right) \hat{\mathbf{x}}-y_{6} b \hat{\mathbf{y}}+ \\
\left(\frac{1}{2}+z_{6}\right) c \sin \beta \hat{\mathbf{z}}\end{array}$ & $(2 a)$ & As VI \\
\hline $\mathbf{B}_{13}$ & $=$ & $x_{7} \mathbf{a}_{1}+y_{7} \mathbf{a}_{2}+z_{7} \mathbf{a}_{3}$ & $=$ & $\left(x_{7} a+z_{7} c \cos \beta\right) \hat{\mathbf{x}}+y_{7} b \hat{\mathbf{y}}+z_{7} c \sin \beta \hat{\mathbf{z}}$ & $(2 a)$ & $\mathrm{Ba} \mathrm{I}$ \\
\hline $\mathbf{B}_{14}$ & $=$ & $x_{7} \mathbf{a}_{1}-y_{7} \mathbf{a}_{2}+\left(\frac{1}{2}+z_{7}\right) \mathbf{a}_{3}$ & $=$ & $\begin{array}{c}\left(\frac{1}{2} c \cos \beta+x_{7} a+z_{7} c \cos \beta\right) \hat{\mathbf{x}}-y_{7} b \hat{\mathbf{y}}+ \\
\left(\frac{1}{2}+z_{7}\right) c \sin \beta \hat{\mathbf{z}}\end{array}$ & $(2 a)$ & $\mathrm{Ba} \mathrm{I}$ \\
\hline $\mathbf{B}_{15}$ & $=$ & $x_{8} \mathbf{a}_{1}+y_{8} \mathbf{a}_{2}+z_{8} \mathbf{a}_{3}$ & $=$ & $\left(x_{8} a+z_{8} c \cos \beta\right) \hat{\mathbf{x}}+y_{8} b \hat{\mathbf{y}}+z_{8} c \sin \beta \hat{\mathbf{z}}$ & $(2 a)$ & Ba II \\
\hline $\mathbf{B}_{16}$ & $=$ & $x_{8} \mathbf{a}_{1}-y_{8} \mathbf{a}_{2}+\left(\frac{1}{2}+z_{8}\right) \mathbf{a}_{3}$ & $=$ & $\begin{array}{c}\left(\frac{1}{2} c \cos \beta+x_{8} a+z_{8} c \cos \beta\right) \hat{\mathbf{x}}-y_{8} b \hat{\mathbf{y}}+ \\
\left(\frac{1}{2}+z_{8}\right) c \sin \beta \hat{\mathbf{z}}\end{array}$ & $(2 a)$ & Ba II \\
\hline $\mathbf{B}_{17}$ & $=$ & $x_{9} \mathbf{a}_{1}+y_{9} \mathbf{a}_{2}+z_{9} \mathbf{a}_{3}$ & $=$ & $\left(x_{9} a+z_{9} c \cos \beta\right) \hat{\mathbf{x}}+y_{9} b \hat{\mathbf{y}}+z_{9} c \sin \beta \hat{\mathbf{z}}$ & $(2 a)$ & Ba III \\
\hline $\mathbf{B}_{18}$ & $=$ & $x_{9} \mathbf{a}_{1}-y_{9} \mathbf{a}_{2}+\left(\frac{1}{2}+z_{9}\right) \mathbf{a}_{3}$ & $=$ & $\begin{array}{c}\left(\frac{1}{2} c \cos \beta+x_{9} a+z_{9} c \cos \beta\right) \hat{\mathbf{x}}-y_{9} b \hat{\mathbf{y}}+ \\
\left(\frac{1}{2}+z_{9}\right) c \sin \beta \hat{\mathbf{z}}\end{array}$ & $(2 a)$ & Ba III \\
\hline
\end{tabular}

\section{References:}

- F. Emmerling, D. Petri, and C. Röhr, Neue Arsenide mit $A s^{-}$-Ketten und -Ringen: $B a A s_{2}$ und $A^{I} B a_{2} A s_{5}\left(A^{I}=K, R b\right), \mathrm{Z}$. Anorg. Allg. Chem. 630, 2490-2501 (2004), doi:10.1002/zaac.200400257.

\section{Found in:}

- P. Villars and K. Cenzual, Pearson's Crystal Data - Crystal Structure Database for Inorganic Compounds, ASM International (2013).

\section{Geometry files:}

- CIF: pp. 803

- POSCAR: pp. 804 


\section{$\epsilon-\mathrm{WO}_{3}$ (Low-temperature) Structure: A3B_mP16_7_6a_2a}

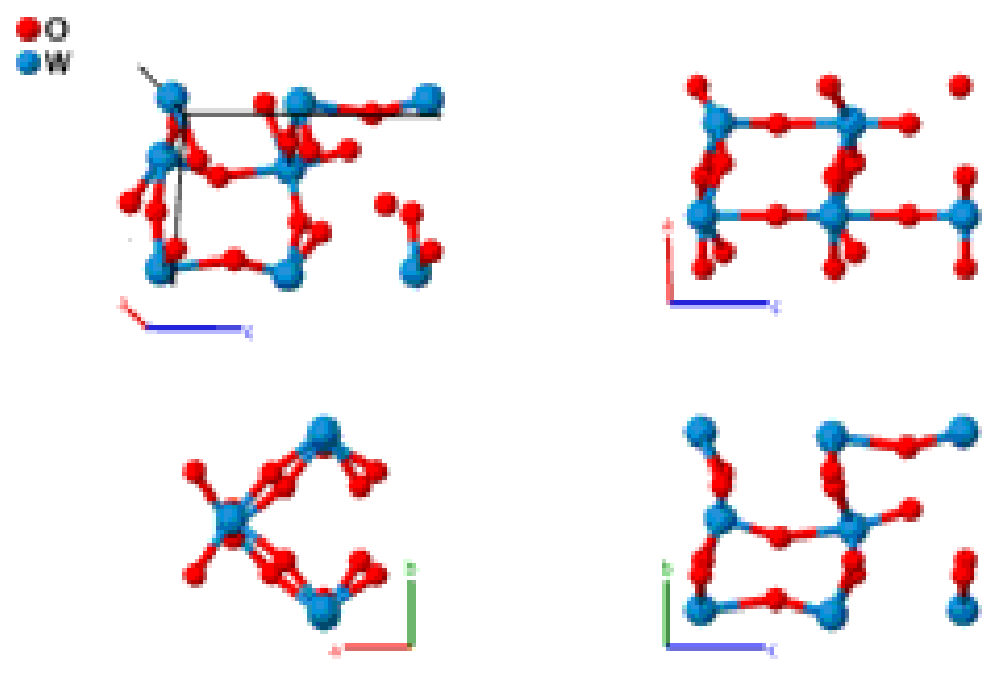

Prototype

AFLOW prototype label

Strukturbericht designation

Pearson symbol

Space group number

Space group symbol

AFLOW prototype command
: $\quad \epsilon-\mathrm{WO}_{3}$

: A3B_mP16_7_6a_2a

: None

: $\quad \mathrm{mP} 16$

: 7

: $\quad P c$ aflow - -proto=A3B_mP16_7_6a_2a

- - params $=a, b / a, c / a, \beta, x_{1}, y_{1}, z_{1}, x_{2}, y_{2}, z_{2}, x_{3}, y_{3}, z_{3}, x_{4}, y_{4}, z_{4}, x_{5}, y_{5}, z_{5}, x_{6}$, $y_{6}, z_{6}, x_{7}, y_{7}, z_{7}, x_{8}, y_{8}, z_{8}$

- This is a low-temperature phase of tungsten trioxide $\left(\mathrm{WO}_{3}\right)$, which was observed at $15 \mathrm{~K}$ (Woodward, 1997).

Simple Monoclinic primitive vectors:
$\mathbf{a}_{1}=\quad a \hat{\mathbf{x}}$
$\mathbf{a}_{2}=\quad b \hat{\mathbf{y}}$
$\mathbf{a}_{3}=c \cos \beta \hat{\mathbf{x}}+c \sin \beta \hat{\mathbf{z}}$

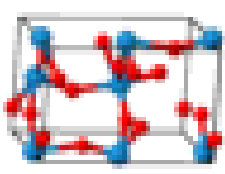

\section{Basis vectors:}

Lattice Coordinates

$$
\begin{aligned}
& \mathbf{B}_{1}=x_{1} \mathbf{a}_{1}+y_{1} \mathbf{a}_{2}+z_{1} \mathbf{a}_{3}=\left(x_{1} a+z_{1} c \cos \beta\right) \hat{\mathbf{x}}+y_{1} b \hat{\mathbf{y}}+z_{1} c \sin \beta \hat{\mathbf{z}} \\
& \mathbf{B}_{2}=x_{1} \mathbf{a}_{1}-y_{1} \mathbf{a}_{2}+\left(\frac{1}{2}+z_{1}\right) \mathbf{a}_{3}=\left(\frac{1}{2} c \cos \beta+x_{1} a+z_{1} c \cos \beta\right) \hat{\mathbf{x}}-y_{1} b \hat{\mathbf{y}}+ \\
& \left(\frac{1}{2}+z_{1}\right) c \sin \beta \hat{\mathbf{z}}
\end{aligned}
$$$$
\mathbf{B}_{3}=x_{2} \mathbf{a}_{1}+y_{2} \mathbf{a}_{2}+z_{2} \mathbf{a}_{3}=\left(x_{2} a+z_{2} c \cos \beta\right) \hat{\mathbf{x}}+y_{2} b \hat{\mathbf{y}}+z_{2} c \sin \beta \hat{\mathbf{z}}
$$$$
\mathbf{B}_{4}=x_{2} \mathbf{a}_{1}-y_{2} \mathbf{a}_{2}+\left(\frac{1}{2}+z_{2}\right) \mathbf{a}_{3}=\left(\frac{1}{2} c \cos \beta+x_{2} a+z_{2} c \cos \beta\right) \hat{\mathbf{x}}-y_{2} b \hat{\mathbf{y}}+
$$$$
\left(\frac{1}{2}+z_{2}\right) c \sin \beta \hat{\mathbf{z}}
$$

Wyckoff Position Atom Type

(2a)

O I

(2a)

O I

(2a)

O II

(2a) 


\begin{tabular}{|c|c|c|c|c|c|c|}
\hline $\mathbf{B}_{5}$ & $=$ & $x_{3} \mathbf{a}_{1}+y_{3} \mathbf{a}_{2}+z_{3} \mathbf{a}_{3}$ & $=$ & $\left(x_{3} a+z_{3} c \cos \beta\right) \hat{\mathbf{x}}+y_{3} b \hat{\mathbf{y}}+z_{3} c \sin \beta \hat{\mathbf{z}}$ & $(2 a)$ & O III \\
\hline $\mathbf{B}_{6}$ & $=$ & $x_{3} \mathbf{a}_{1}-y_{3} \mathbf{a}_{2}+\left(\frac{1}{2}+z_{3}\right) \mathbf{a}_{3}$ & $=$ & $\begin{array}{c}\left(\frac{1}{2} c \cos \beta+x_{3} a+z_{3} c \cos \beta\right) \hat{\mathbf{x}}-y_{3} b \hat{\mathbf{y}}+ \\
\left(\frac{1}{2}+z_{3}\right) c \sin \beta \hat{\mathbf{z}}\end{array}$ & $(2 a)$ & O III \\
\hline $\mathbf{B}_{7}$ & $=$ & $x_{4} \mathbf{a}_{1}+y_{4} \mathbf{a}_{2}+z_{4} \mathbf{a}_{3}$ & $=$ & $\left(x_{4} a+z_{4} c \cos \beta\right) \hat{\mathbf{x}}+y_{4} b \hat{\mathbf{y}}+z_{4} c \sin \beta \hat{\mathbf{z}}$ & $(2 a)$ & O IV \\
\hline $\mathbf{B}_{8}$ & $=$ & $x_{4} \mathbf{a}_{1}-y_{4} \mathbf{a}_{2}+\left(\frac{1}{2}+z_{4}\right) \mathbf{a}_{3}$ & $=$ & $\begin{array}{c}\left(\frac{1}{2} c \cos \beta+x_{4} a+z_{4} c \cos \beta\right) \hat{\mathbf{x}}-y_{4} b \hat{\mathbf{y}}+ \\
\left(\frac{1}{2}+z_{4}\right) c \sin \beta \hat{\mathbf{z}}\end{array}$ & $(2 a)$ & O IV \\
\hline $\mathbf{B}_{9}$ & $=$ & $x_{5} \mathbf{a}_{1}+y_{5} \mathbf{a}_{2}+z_{5} \mathbf{a}_{3}$ & $=$ & $\left(x_{5} a+z_{5} c \cos \beta\right) \hat{\mathbf{x}}+y_{5} b \hat{\mathbf{y}}+z_{5} c \sin \beta \hat{\mathbf{z}}$ & $(2 a)$ & $\mathrm{OV}$ \\
\hline $\mathbf{B}_{10}$ & $=$ & $x_{5} \mathbf{a}_{1}-y_{5} \mathbf{a}_{2}+\left(\frac{1}{2}+z_{5}\right) \mathbf{a}_{3}$ & $=$ & $\begin{array}{c}\left(\frac{1}{2} c \cos \beta+x_{5} a+z_{5} c \cos \beta\right) \hat{\mathbf{x}}-y_{5} b \hat{\mathbf{y}}+ \\
\left(\frac{1}{2}+z_{5}\right) c \sin \beta \hat{\mathbf{z}}\end{array}$ & $(2 a)$ & $\mathrm{OV}$ \\
\hline $\mathbf{B}_{11}$ & $=$ & $x_{6} \mathbf{a}_{1}+y_{6} \mathbf{a}_{2}+z_{6} \mathbf{a}_{3}$ & $=$ & $\left(x_{6} a+z_{6} c \cos \beta\right) \hat{\mathbf{x}}+y_{6} b \hat{\mathbf{y}}+z_{6} c \sin \beta \hat{\mathbf{z}}$ & $(2 a)$ & $\mathrm{O}$ VI \\
\hline $\mathbf{B}_{12}$ & $=$ & $x_{6} \mathbf{a}_{1}-y_{6} \mathbf{a}_{2}+\left(\frac{1}{2}+z_{6}\right) \mathbf{a}_{3}$ & $=$ & $\begin{array}{c}\left(\frac{1}{2} c \cos \beta+x_{6} a+z_{6} c \cos \beta\right) \hat{\mathbf{x}}-y_{6} b \hat{\mathbf{y}}+ \\
\left(\frac{1}{2}+z_{6}\right) c \sin \beta \hat{\mathbf{z}}\end{array}$ & $(2 a)$ & $\mathrm{O} \mathrm{VI}$ \\
\hline $\mathbf{B}_{13}$ & $=$ & $x_{7} \mathbf{a}_{1}+y_{7} \mathbf{a}_{2}+z_{7} \mathbf{a}_{3}$ & $=$ & $\left(x_{7} a+z_{7} c \cos \beta\right) \hat{\mathbf{x}}+y_{7} b \hat{\mathbf{y}}+z_{7} c \sin \beta \hat{\mathbf{z}}$ & $(2 a)$ & W I \\
\hline $\mathbf{B}_{14}$ & $=$ & $x_{7} \mathbf{a}_{1}-y_{7} \mathbf{a}_{2}+\left(\frac{1}{2}+z_{7}\right) \mathbf{a}_{3}$ & $=$ & $\begin{array}{c}\left(\frac{1}{2} c \cos \beta+x_{7} a+z_{7} c \cos \beta\right) \hat{\mathbf{x}}-y_{7} b \hat{\mathbf{y}}+ \\
\left(\frac{1}{2}+z_{7}\right) c \sin \beta \hat{\mathbf{z}}\end{array}$ & $(2 a)$ & W I \\
\hline $\mathbf{B}_{15}$ & $=$ & $x_{8} \mathbf{a}_{1}+y_{8} \mathbf{a}_{2}+z_{8} \mathbf{a}_{3}$ & $=$ & $\left(x_{8} a+z_{8} c \cos \beta\right) \hat{\mathbf{x}}+y_{8} b \hat{\mathbf{y}}+z_{8} c \sin \beta \hat{\mathbf{z}}$ & $(2 a)$ & W II \\
\hline $\mathbf{B}_{16}$ & $=$ & $x_{8} \mathbf{a}_{1}-y_{8} \mathbf{a}_{2}+\left(\frac{1}{2}+z_{8}\right) \mathbf{a}_{3}$ & $=$ & $\begin{array}{c}\left(\frac{1}{2} c \cos \beta+x_{8} a+z_{8} c \cos \beta\right) \hat{\mathbf{x}}-y_{8} b \hat{\mathbf{y}}+ \\
\left(\frac{1}{2}+z_{8}\right) c \sin \beta \hat{\mathbf{z}}\end{array}$ & $(2 a)$ & W II \\
\hline
\end{tabular}

\section{References:}

- P. M. Woodward, A. W. Sleight, and T. Vogt, Ferroelectric tungsten trioxide, J. Solid State Chem. 131, 9-17 (1997), doi:10.1006/jssc.1997.7268.

\section{Found in:}

- P. Villars and K. Cenzual, Pearson's Crystal Data - Crystal Structure Database for Inorganic Compounds, ASM International (2013).

\section{Geometry files:}

- CIF: pp. 804

- POSCAR: pp. 804 


\section{$\mathrm{Rh}_{2} \mathrm{Ga}_{9}$ Structure: A9B2_mP22_7_9a_2a}

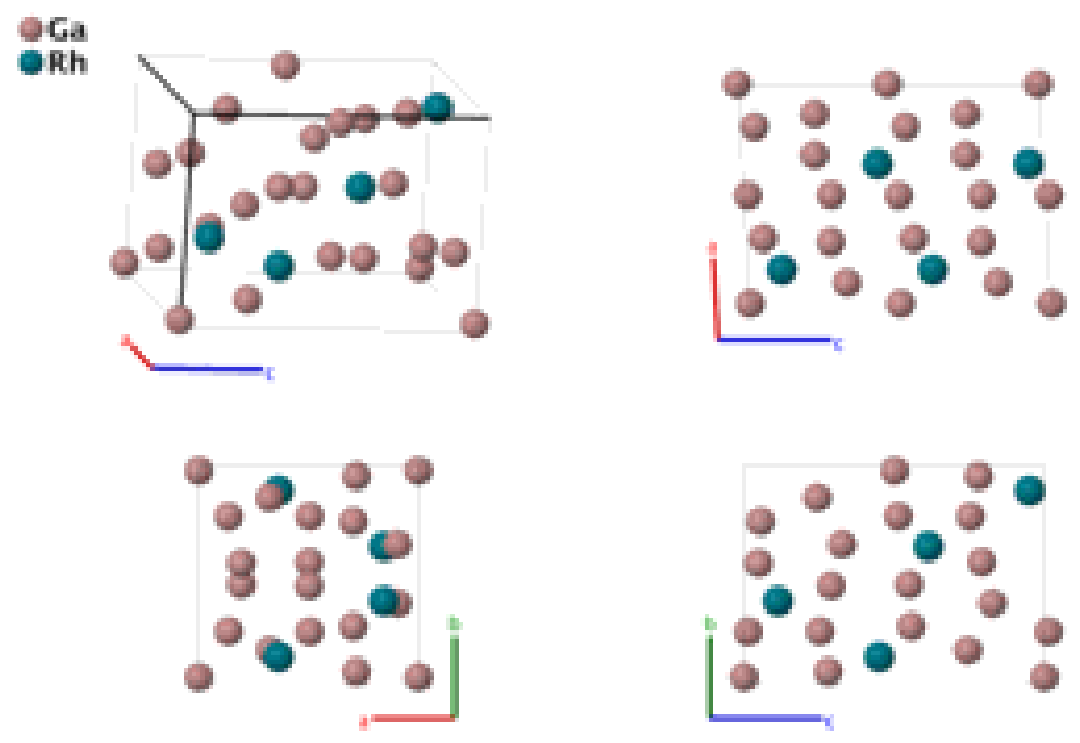

Prototype

AFLOW prototype label

Strukturbericht designation

Pearson symbol

Space group number

Space group symbol

AFLOW prototype command
: $\quad \mathrm{Rh}_{2} \mathrm{Ga} 9$

: A9B2_mP22_7_9a_2a

: None

: $\quad \mathrm{mP} 22$

7

$P c$

Simple Monoclinic primitive vectors:
$\mathbf{a}_{1}=\quad a \hat{\mathbf{x}}$
$\mathbf{a}_{2}=\quad b \hat{\mathbf{y}}$
$\mathbf{a}_{3}=c \cos \beta \hat{\mathbf{x}}+c \sin \beta \hat{\mathbf{z}}$

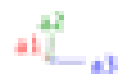

Basis vectors:

Lattice Coordinates

Cartesian Coordinates

$$
\begin{aligned}
& \mathbf{B}_{1}=x_{1} \mathbf{a}_{1}+y_{1} \mathbf{a}_{2}+z_{1} \mathbf{a}_{3}=\left(x_{1} a+z_{1} c \cos \beta\right) \hat{\mathbf{x}}+y_{1} b \hat{\mathbf{y}}+z_{1} c \sin \beta \hat{\mathbf{z}} \\
& \mathbf{B}_{2}=x_{1} \mathbf{a}_{1}-y_{1} \mathbf{a}_{2}+\left(\frac{1}{2}+z_{1}\right) \mathbf{a}_{3}=\left(\frac{1}{2} c \cos \beta+x_{1} a+z_{1} c \cos \beta\right) \hat{\mathbf{x}}-y_{1} b \hat{\mathbf{y}}+ \\
& \left(\frac{1}{2}+z_{1}\right) c \sin \beta \hat{\mathbf{z}} \\
& \mathbf{B}_{3}=x_{2} \mathbf{a}_{1}+y_{2} \mathbf{a}_{2}+z_{2} \mathbf{a}_{3}=\left(x_{2} a+z_{2} c \cos \beta\right) \hat{\mathbf{x}}+y_{2} b \hat{\mathbf{y}}+z_{2} c \sin \beta \hat{\mathbf{z}} \\
& \mathbf{B}_{4}=x_{2} \mathbf{a}_{1}-y_{2} \mathbf{a}_{2}+\left(\frac{1}{2}+z_{2}\right) \mathbf{a}_{3}=\left(\frac{1}{2} c \cos \beta+x_{2} a+z_{2} c \cos \beta\right) \hat{\mathbf{x}}-y_{2} b \hat{\mathbf{y}}+ \\
& \left(\frac{1}{2}+z_{2}\right) c \sin \beta \hat{\mathbf{z}}
\end{aligned}
$$

Wyckoff Position Atom Type

Ga I

$(2 a)$

Ga I

$(2 a)$

Ga II

(2a) 


\begin{tabular}{|c|c|c|c|c|c|c|}
\hline $\mathbf{B}_{5}$ & $=$ & $x_{3} \mathbf{a}_{1}+y_{3} \mathbf{a}_{2}+z_{3} \mathbf{a}_{3}$ & $=$ & $\left(x_{3} a+z_{3} c \cos \beta\right) \hat{\mathbf{x}}+y_{3} b \hat{\mathbf{y}}+z_{3} c \sin \beta \hat{\mathbf{z}}$ & $(2 a)$ & Ga III \\
\hline $\mathbf{B}_{6}$ & $=$ & $x_{3} \mathbf{a}_{1}-y_{3} \mathbf{a}_{2}+\left(\frac{1}{2}+z_{3}\right) \mathbf{a}_{3}$ & $=$ & $\begin{array}{c}\left(\frac{1}{2} c \cos \beta+x_{3} a+z_{3} c \cos \beta\right) \hat{\mathbf{x}}-y_{3} b \hat{\mathbf{y}}+ \\
\left(\frac{1}{2}+z_{3}\right) c \sin \beta \hat{\mathbf{z}}\end{array}$ & $(2 a)$ & Ga III \\
\hline $\mathbf{B}_{7}$ & $=$ & $x_{4} \mathbf{a}_{1}+y_{4} \mathbf{a}_{2}+z_{4} \mathbf{a}_{3}$ & $=$ & $\left(x_{4} a+z_{4} c \cos \beta\right) \hat{\mathbf{x}}+y_{4} b \hat{\mathbf{y}}+z_{4} c \sin \beta \hat{\mathbf{z}}$ & $(2 a)$ & Ga IV \\
\hline $\mathbf{B}_{8}$ & $=$ & $x_{4} \mathbf{a}_{1}-y_{4} \mathbf{a}_{2}+\left(\frac{1}{2}+z_{4}\right) \mathbf{a}_{3}$ & $=$ & $\begin{array}{c}\left(\frac{1}{2} c \cos \beta+x_{4} a+z_{4} c \cos \beta\right) \hat{\mathbf{x}}-y_{4} b \hat{\mathbf{y}}+ \\
\left(\frac{1}{2}+z_{4}\right) c \sin \beta \hat{\mathbf{z}}\end{array}$ & $(2 a)$ & Ga IV \\
\hline $\mathbf{B}_{9}$ & $=$ & $x_{5} \mathbf{a}_{1}+y_{5} \mathbf{a}_{2}+z_{5} \mathbf{a}_{3}$ & $=$ & $\left(x_{5} a+z_{5} c \cos \beta\right) \hat{\mathbf{x}}+y_{5} b \hat{\mathbf{y}}+z_{5} c \sin \beta \hat{\mathbf{z}}$ & $(2 a)$ & $\mathrm{Ga} \mathrm{V}$ \\
\hline $\mathbf{B}_{10}$ & $=$ & $x_{5} \mathbf{a}_{1}-y_{5} \mathbf{a}_{2}+\left(\frac{1}{2}+z_{5}\right) \mathbf{a}_{3}$ & $=$ & $\begin{array}{c}\left(\frac{1}{2} c \cos \beta+x_{5} a+z_{5} c \cos \beta\right) \hat{\mathbf{x}}-y_{5} b \hat{\mathbf{y}}+ \\
\left(\frac{1}{2}+z_{5}\right) c \sin \beta \hat{\mathbf{z}}\end{array}$ & $(2 a)$ & $\mathrm{Ga} \mathrm{V}$ \\
\hline $\mathbf{B}_{11}$ & $=$ & $x_{6} \mathbf{a}_{1}+y_{6} \mathbf{a}_{2}+z_{6} \mathbf{a}_{3}$ & $=$ & $\left(x_{6} a+z_{6} c \cos \beta\right) \hat{\mathbf{x}}+y_{6} b \hat{\mathbf{y}}+z_{6} c \sin \beta \hat{\mathbf{z}}$ & $(2 a)$ & Ga VI \\
\hline $\mathbf{B}_{12}$ & $=$ & $x_{6} \mathbf{a}_{1}-y_{6} \mathbf{a}_{2}+\left(\frac{1}{2}+z_{6}\right) \mathbf{a}_{3}$ & $=$ & $\begin{array}{c}\left(\frac{1}{2} c \cos \beta+x_{6} a+z_{6} c \cos \beta\right) \hat{\mathbf{x}}-y_{6} b \hat{\mathbf{y}}+ \\
\left(\frac{1}{2}+z_{6}\right) c \sin \beta \hat{\mathbf{z}}\end{array}$ & $(2 a)$ & Ga VI \\
\hline $\mathbf{B}_{13}$ & $=$ & $x_{7} \mathbf{a}_{1}+y_{7} \mathbf{a}_{2}+z_{7} \mathbf{a}_{3}$ & $=$ & $\left(x_{7} a+z_{7} c \cos \beta\right) \hat{\mathbf{x}}+y_{7} b \hat{\mathbf{y}}+z_{7} c \sin \beta \hat{\mathbf{z}}$ & $(2 a)$ & Ga VII \\
\hline $\mathbf{B}_{14}$ & $=$ & $x_{7} \mathbf{a}_{1}-y_{7} \mathbf{a}_{2}+\left(\frac{1}{2}+z_{7}\right) \mathbf{a}_{3}$ & $=$ & $\begin{array}{c}\left(\frac{1}{2} c \cos \beta+x_{7} a+z_{7} c \cos \beta\right) \hat{\mathbf{x}}-y_{7} b \hat{\mathbf{y}}+ \\
\left(\frac{1}{2}+z_{7}\right) c \sin \beta \hat{\mathbf{z}}\end{array}$ & $(2 a)$ & Ga VII \\
\hline $\mathbf{B}_{15}$ & $=$ & $x_{8} \mathbf{a}_{1}+y_{8} \mathbf{a}_{2}+z_{8} \mathbf{a}_{3}$ & $=$ & $\left(x_{8} a+z_{8} c \cos \beta\right) \hat{\mathbf{x}}+y_{8} b \hat{\mathbf{y}}+z_{8} c \sin \beta \hat{\mathbf{z}}$ & $(2 a)$ & Ga VIII \\
\hline $\mathbf{B}_{16}$ & $=$ & $x_{8} \mathbf{a}_{1}-y_{8} \mathbf{a}_{2}+\left(\frac{1}{2}+z_{8}\right) \mathbf{a}_{3}$ & $=$ & $\begin{array}{c}\left(\frac{1}{2} c \cos \beta+x_{8} a+z_{8} c \cos \beta\right) \hat{\mathbf{x}}-y_{8} b \hat{\mathbf{y}}+ \\
\left(\frac{1}{2}+z_{8}\right) c \sin \beta \hat{\mathbf{z}}\end{array}$ & $(2 a)$ & Ga VIII \\
\hline $\mathbf{B}_{17}$ & $=$ & $x_{9} \mathbf{a}_{1}+y_{9} \mathbf{a}_{2}+z_{9} \mathbf{a}_{3}$ & $=$ & $\left(x_{9} a+z_{9} c \cos \beta\right) \hat{\mathbf{x}}+y_{9} b \hat{\mathbf{y}}+z_{9} c \sin \beta \hat{\mathbf{z}}$ & $(2 a)$ & Ga IX \\
\hline $\mathbf{B}_{18}$ & $=$ & $x_{9} \mathbf{a}_{1}-y_{9} \mathbf{a}_{2}+\left(\frac{1}{2}+z_{9}\right) \mathbf{a}_{3}$ & $=$ & $\begin{array}{c}\left(\frac{1}{2} c \cos \beta+x_{9} a+z_{9} c \cos \beta\right) \hat{\mathbf{x}}-y_{9} b \hat{\mathbf{y}}+ \\
\left(\frac{1}{2}+z_{9}\right) c \sin \beta \hat{\mathbf{z}}\end{array}$ & $(2 a)$ & Ga IX \\
\hline $\mathbf{B}_{19}$ & $=$ & $x_{10} \mathbf{a}_{1}+y_{10} \mathbf{a}_{2}+z_{10} \mathbf{a}_{3}$ & $=$ & $\begin{array}{c}\left(x_{10} a+z_{10} c \cos \beta\right) \hat{\mathbf{x}}+y_{10} b \hat{\mathbf{y}}+ \\
z_{10} c \sin \beta \hat{\mathbf{z}}\end{array}$ & $(2 a)$ & $\mathrm{Rh} I$ \\
\hline $\mathbf{B}_{20}$ & $=$ & $x_{10} \mathbf{a}_{1}-y_{10} \mathbf{a}_{2}+\left(\frac{1}{2}+z_{10}\right) \mathbf{a}_{3}$ & $=$ & $\begin{array}{c}\left(\frac{1}{2} c \cos \beta+x_{10} a+z_{10} c \cos \beta\right) \hat{\mathbf{x}}- \\
y_{10} b \hat{\mathbf{y}}+\left(\frac{1}{2}+z_{10}\right) c \sin \beta \hat{\mathbf{z}}\end{array}$ & $(2 a)$ & $\mathrm{Rh} I$ \\
\hline $\mathbf{B}_{21}$ & $=$ & $x_{11} \mathbf{a}_{1}+y_{11} \mathbf{a}_{2}+z_{11} \mathbf{a}_{3}$ & $=$ & $\begin{array}{c}\left(x_{11} a+z_{11} c \cos \beta\right) \hat{\mathbf{x}}+y_{11} b \hat{\mathbf{y}}+ \\
z_{11} c \sin \beta \hat{\mathbf{z}}\end{array}$ & $(2 a)$ & $\mathrm{Rh}$ II \\
\hline $\mathbf{B}_{22}$ & $=$ & $x_{11} \mathbf{a}_{1}-y_{11} \mathbf{a}_{2}+\left(\frac{1}{2}+z_{11}\right) \mathbf{a}_{3}$ & $=$ & $\begin{array}{c}\left(\frac{1}{2} c \cos \beta+x_{11} a+z_{11} c \cos \beta\right) \hat{\mathbf{x}}- \\
y_{11} b \hat{\mathbf{y}}+\left(\frac{1}{2}+z_{11}\right) c \sin \beta \hat{\mathbf{z}}\end{array}$ & $(2 a)$ & $\mathrm{Rh}$ II \\
\hline
\end{tabular}

\section{References:}

- M. Boström, H. Rosner, Y. Prots, U. Burkhardt, and Y. Grin, The $\mathrm{Co}_{2} \mathrm{Al}_{9}$ structure type revisited, Z. Anorg. Allg. Chem. 631, 534-541 (2005), doi:10.1002/zaac.200400418.

\section{Found in:}

- P. Villars and K. Cenzual, Pearson's Crystal Data - Crystal Structure Database for Inorganic Compounds, ASM International (2013).

\section{Geometry files:}

- CIF: pp. 804

- POSCAR: pp. 805 


\section{$\alpha-\mathrm{P}_{3} \mathrm{~N}_{5}$ Structure: A5B3_mC32_9_5a_3a}
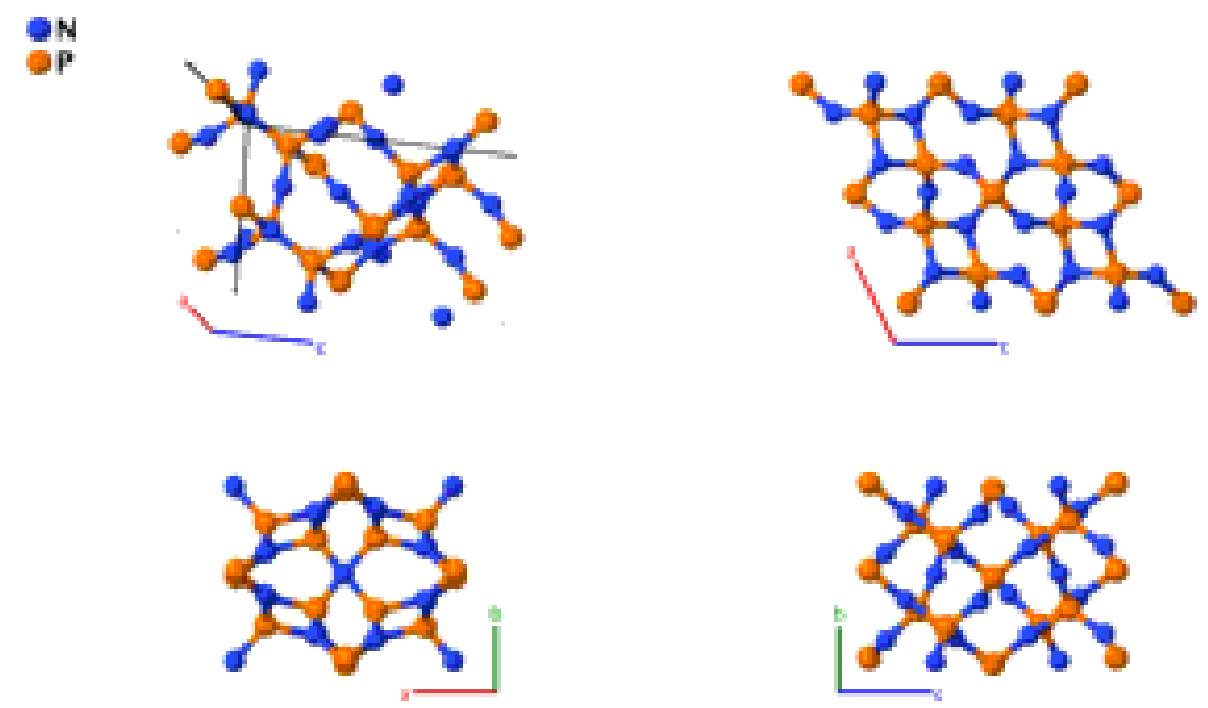

Prototype

$: \quad \alpha-\mathrm{P}_{3} \mathrm{~N}_{5}$

AFLOW prototype label

: A5B3_mC32_9_5a_3a

Strukturbericht designation

: None

Pearson symbol

: $\quad \mathrm{mC} 32$

Space group number

9

Space group symbol

Cc

AFLOW prototype command : aflow--proto=A5B3_mC32_9_5a_3a

- -params $=a, b / a, c / a, \beta, x_{1}, y_{1}, z_{1}, x_{2}, y_{2}, z_{2}, x_{3}, y_{3}, z_{3}, x_{4}, y_{4}, z_{4}, x_{5}, y_{5}, z_{5}, x_{6}$, $y_{6}, z_{6}, x_{7}, y_{7}, z_{7}, x_{8}, y_{8}, z_{8}$

Base-centered Monoclinic primitive vectors:

$$
\begin{array}{llc}
\mathbf{a}_{1}= & \frac{1}{2} a \hat{\mathbf{x}}-\frac{1}{2} b \hat{\mathbf{y}} \\
\mathbf{a}_{2}= & \frac{1}{2} a \hat{\mathbf{x}}+\frac{1}{2} b \hat{\mathbf{y}} \\
\mathbf{a}_{3}= & c \cos \beta \hat{\mathbf{x}}+c \sin \beta \hat{\mathbf{z}}
\end{array}
$$

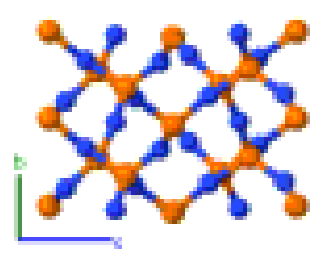

Basis vectors:

Lattice Coordinates

$\mathbf{B}_{1}=\left(x_{1}-y_{1}\right) \mathbf{a}_{1}+\left(x_{1}+y_{1}\right) \mathbf{a}_{2}+z_{1} \mathbf{a}_{3}=$

$=$

$\mathbf{B}_{2}=\left(x_{1}+y_{1}\right) \mathbf{a}_{1}+\left(x_{1}-y_{1}\right) \mathbf{a}_{2}+\quad=\quad\left(\frac{1}{2} c \cos \beta+x_{1} a+z_{1} c \cos \beta\right) \hat{\mathbf{x}}-$ $\left(\frac{1}{2}+z_{1}\right) \mathbf{a}_{3}$

$\mathbf{B}_{3}=\left(x_{2}-y_{2}\right) \mathbf{a}_{1}+\left(x_{2}+y_{2}\right) \mathbf{a}_{2}+z_{2} \mathbf{a}_{3}=\left(x_{2} a+z_{2} c \cos \beta\right) \hat{\mathbf{x}}+y_{2} b \hat{\mathbf{y}}+$
$\mathbf{B}_{4}=\left(x_{2}+y_{2}\right) \mathbf{a}_{1}+\left(x_{2}-y_{2}\right) \mathbf{a}_{2}+=\left(\frac{1}{2} c \cos \beta+x_{2} a+z_{2} c \cos \beta\right) \hat{\mathbf{x}}-$ $\left(\frac{1}{2}+z_{2}\right) \mathbf{a}_{3}$$$
y_{2} b \hat{\mathbf{y}}+\left(\frac{1}{2}+z_{2}\right) c \sin \beta \hat{\mathbf{z}}
$$

$\left(x_{1} a+z_{1} c \cos \beta\right) \hat{\mathbf{x}}+y_{1} b \hat{\mathbf{y}}+$ $z_{1} c \sin \beta \hat{\mathbf{z}}$ $z_{2} c \sin \beta \hat{\mathbf{z}}$

Cartesian Coordinates

$y_{1} b \hat{\mathbf{y}}+\left(\frac{1}{2}+z_{1}\right) c \sin \beta \hat{\mathbf{z}}$

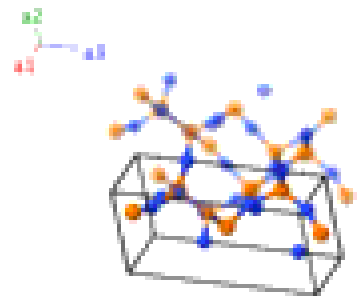

Wyckoff Position Atom Type

(4a)

N I

(4a)

N I

$(4 a)$

N II

(4a)

N II 


\begin{tabular}{|c|c|c|c|c|c|c|}
\hline $\mathbf{B}_{5}$ & $=$ & $\left(x_{3}-y_{3}\right) \mathbf{a}_{1}+\left(x_{3}+y_{3}\right) \mathbf{a}_{2}+z_{3} \mathbf{a}_{3}$ & $=$ & $\begin{array}{c}\left(x_{3} a+z_{3} c \cos \beta\right) \hat{\mathbf{x}}+y_{3} b \hat{\mathbf{y}}+ \\
z_{3} c \sin \beta \hat{\mathbf{z}}\end{array}$ & $(4 a)$ & N III \\
\hline $\mathbf{B}_{6}$ & $=$ & $\begin{array}{c}\left(x_{3}+y_{3}\right) \mathbf{a}_{1}+\left(x_{3}-y_{3}\right) \mathbf{a}_{2}+ \\
\left(\frac{1}{2}+z_{3}\right) \mathbf{a}_{3}\end{array}$ & $=$ & $\begin{array}{c}\left(\frac{1}{2} c \cos \beta+x_{3} a+z_{3} c \cos \beta\right) \hat{\mathbf{x}}- \\
y_{3} b \hat{\mathbf{y}}+\left(\frac{1}{2}+z_{3}\right) c \sin \beta \hat{\mathbf{z}}\end{array}$ & $(4 a)$ & N III \\
\hline $\mathbf{B}_{7}$ & $=$ & $\left(x_{4}-y_{4}\right) \mathbf{a}_{1}+\left(x_{4}+y_{4}\right) \mathbf{a}_{2}+z_{4} \mathbf{a}_{3}$ & $=$ & $\begin{array}{c}\left(x_{4} a+z_{4} c \cos \beta\right) \hat{\mathbf{x}}+y_{4} b \hat{\mathbf{y}}+ \\
z_{4} c \sin \beta \hat{\mathbf{z}}\end{array}$ & $(4 a)$ & N IV \\
\hline $\mathbf{B}_{8}$ & $=$ & $\begin{array}{c}\left(x_{4}+y_{4}\right) \mathbf{a}_{1}+\left(x_{4}-y_{4}\right) \mathbf{a}_{2}+ \\
\left(\frac{1}{2}+z_{4}\right) \mathbf{a}_{3}\end{array}$ & $=$ & $\begin{array}{c}\left(\frac{1}{2} c \cos \beta+x_{4} a+z_{4} c \cos \beta\right) \hat{\mathbf{x}}- \\
y_{4} b \hat{\mathbf{y}}+\left(\frac{1}{2}+z_{4}\right) c \sin \beta \hat{\mathbf{z}}\end{array}$ & $(4 a)$ & N IV \\
\hline $\mathbf{B}_{9}$ & $=$ & $\left(x_{5}-y_{5}\right) \mathbf{a}_{1}+\left(x_{5}+y_{5}\right) \mathbf{a}_{2}+z_{5} \mathbf{a}_{3}$ & $=$ & $\begin{array}{c}\left(x_{5} a+z_{5} c \cos \beta\right) \hat{\mathbf{x}}+y_{5} b \hat{\mathbf{y}}+ \\
z_{5} c \sin \beta \hat{\mathbf{z}}\end{array}$ & $(4 a)$ & N V \\
\hline $\mathbf{B}_{10}$ & $=$ & $\begin{array}{c}\left(x_{5}+y_{5}\right) \mathbf{a}_{1}+\left(x_{5}-y_{5}\right) \mathbf{a}_{2}+ \\
\left(\frac{1}{2}+z_{5}\right) \mathbf{a}_{3}\end{array}$ & $=$ & $\begin{array}{c}\left(\frac{1}{2} c \cos \beta+x_{5} a+z_{5} c \cos \beta\right) \hat{\mathbf{x}}- \\
y_{5} b \hat{\mathbf{y}}+\left(\frac{1}{2}+z_{5}\right) c \sin \beta \hat{\mathbf{z}}\end{array}$ & $(4 a)$ & $\mathrm{N} \mathrm{V}$ \\
\hline $\mathbf{B}_{11}$ & $=$ & $\left(x_{6}-y_{6}\right) \mathbf{a}_{1}+\left(x_{6}+y_{6}\right) \mathbf{a}_{2}+z_{6} \mathbf{a}_{3}$ & $=$ & $\begin{array}{c}\left(x_{6} a+z_{6} c \cos \beta\right) \hat{\mathbf{x}}+y_{6} b \hat{\mathbf{y}}+ \\
z_{6} c \sin \beta \hat{\mathbf{z}}\end{array}$ & $(4 a)$ & P I \\
\hline $\mathbf{B}_{12}$ & $=$ & $\begin{array}{c}\left(x_{6}+y_{6}\right) \mathbf{a}_{1}+\left(x_{6}-y_{6}\right) \mathbf{a}_{2}+ \\
\left(\frac{1}{2}+z_{6}\right) \mathbf{a}_{3}\end{array}$ & $=$ & $\begin{array}{c}\left(\frac{1}{2} c \cos \beta+x_{6} a+z_{6} c \cos \beta\right) \hat{\mathbf{x}}- \\
y_{6} b \hat{\mathbf{y}}+\left(\frac{1}{2}+z_{6}\right) c \sin \beta \hat{\mathbf{z}}\end{array}$ & $(4 a)$ & P I \\
\hline $\mathbf{B}_{13}$ & $=$ & $\left(x_{7}-y_{7}\right) \mathbf{a}_{1}+\left(x_{7}+y_{7}\right) \mathbf{a}_{2}+z_{7} \mathbf{a}_{3}$ & $=$ & $\begin{array}{c}\left(x_{7} a+z_{7} c \cos \beta\right) \hat{\mathbf{x}}+y_{7} b \hat{\mathbf{y}}+ \\
z_{7} c \sin \beta \hat{\mathbf{z}}\end{array}$ & $(4 a)$ & P II \\
\hline $\mathbf{B}_{14}$ & $=$ & $\begin{array}{c}\left(x_{7}+y_{7}\right) \mathbf{a}_{1}+\left(x_{7}-y_{7}\right) \mathbf{a}_{2}+ \\
\left(\frac{1}{2}+z_{7}\right) \mathbf{a}_{3}\end{array}$ & $=$ & $\begin{array}{c}\left(\frac{1}{2} c \cos \beta+x_{7} a+z_{7} c \cos \beta\right) \hat{\mathbf{x}}- \\
y_{7} b \hat{\mathbf{y}}+\left(\frac{1}{2}+z_{7}\right) c \sin \beta \hat{\mathbf{z}}\end{array}$ & $(4 a)$ & P II \\
\hline $\mathbf{B}_{15}$ & $=$ & $\left(x_{8}-y_{8}\right) \mathbf{a}_{1}+\left(x_{8}+y_{8}\right) \mathbf{a}_{2}+z_{8} \mathbf{a}_{3}$ & $=$ & $\begin{array}{c}\left(x_{8} a+z_{8} c \cos \beta\right) \hat{\mathbf{x}}+y_{8} b \hat{\mathbf{y}}+ \\
z_{8} c \sin \beta \hat{\mathbf{z}}\end{array}$ & $(4 a)$ & P III \\
\hline $\mathbf{B}_{16}$ & $=$ & $\begin{array}{c}\left(x_{8}+y_{8}\right) \mathbf{a}_{1}+\left(x_{8}-y_{8}\right) \mathbf{a}_{2}+ \\
\left(\frac{1}{2}+z_{8}\right) \mathbf{a}_{3}\end{array}$ & $=$ & $\begin{array}{c}\left(\frac{1}{2} c \cos \beta+x_{8} a+z_{8} c \cos \beta\right) \hat{\mathbf{x}}- \\
y_{8} b \hat{\mathbf{y}}+\left(\frac{1}{2}+z_{8}\right) c \sin \beta \hat{\mathbf{z}}\end{array}$ & $(4 a)$ & P III \\
\hline
\end{tabular}

\section{References:}

- S. Horstmann, E. Irran, and W. Schnick, Synthesis and Crystal Structure of Phosphorus $(V)$ Nitride $\alpha-P_{3} N_{5}$, Angew. Chem. Int. Ed. 36, 1873-1875 (1997), doi:10.1002/anie.199718731.

\section{Geometry files:}

- CIF: pp. 805

- POSCAR: pp. 805 


\section{$\mathrm{H}_{3} \mathrm{Cl}(20 \mathrm{GPa})$ Structure: AB3_mC16_9_a_3a}
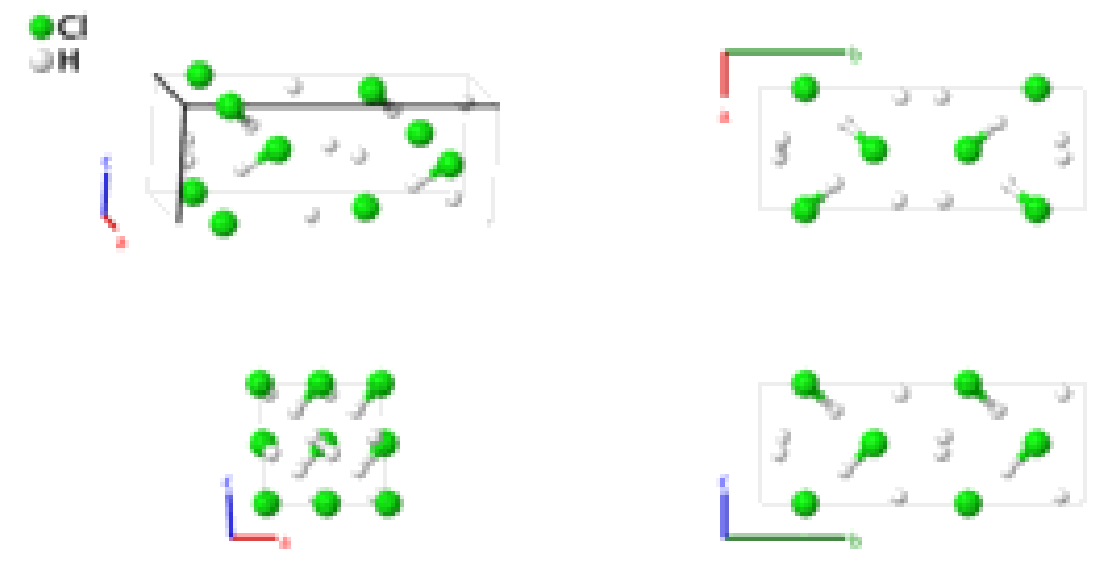

\section{Prototype}

AFLOW prototype label

: $\quad \mathrm{H}_{3} \mathrm{Cl}$

Strukturbericht designation

AB3_mC16_9_a_3a

Pearson symbol

: None

Space group number $\quad: \quad 9$

Space group symbol $\quad: \quad C c$

AFLOW prototype command : aflow--proto=AB3_mC16_9_a_3a

- -params $=a, b / a, c / a, \beta, x_{1}, y_{1}, z_{1}, x_{2}, y_{2}, z_{2}, x_{3}, y_{3}, z_{3}, x_{4}, y_{4}, z_{4}$

- This structure was found via first-principles calculations. The data presented here was computed at a pressure of $20 \mathrm{GPa}$.

Base-centered Monoclinic primitive vectors:

$$
\begin{array}{llc}
\mathbf{a}_{1}= & \frac{1}{2} a \hat{\mathbf{x}}-\frac{1}{2} b \hat{\mathbf{y}} \\
\mathbf{a}_{2}= & \frac{1}{2} a \hat{\mathbf{x}}+\frac{1}{2} b \hat{\mathbf{y}} \\
\mathbf{a}_{3}= & c \cos \beta \hat{\mathbf{x}}+c \sin \beta \hat{\mathbf{z}}
\end{array}
$$
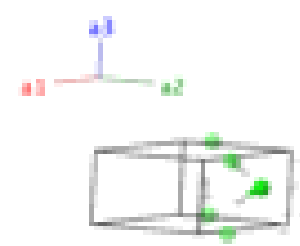

\section{Basis vectors:}

Lattice Coordinates

$$
\begin{aligned}
& \mathbf{B}_{1}=\left(x_{1}-y_{1}\right) \mathbf{a}_{1}+\left(x_{1}+y_{1}\right) \mathbf{a}_{2}+z_{1} \mathbf{a}_{3}=\left(x_{1} a+z_{1} c \cos \beta\right) \hat{\mathbf{x}}+y_{1} b \hat{\mathbf{y}}+ \\
& z_{1} c \sin \beta \hat{\mathbf{z}} \\
& \mathbf{B}_{2}=\left(x_{1}+y_{1}\right) \mathbf{a}_{1}+\left(x_{1}-y_{1}\right) \mathbf{a}_{2}+=\left(\frac{1}{2} c \cos \beta+x_{1} a+z_{1} c \cos \beta\right) \hat{\mathbf{x}}- \\
& \left(\frac{1}{2}+z_{1}\right) \mathbf{a}_{3} \\
& \mathbf{B}_{3}=\left(x_{2}-y_{2}\right) \mathbf{a}_{1}+\left(x_{2}+y_{2}\right) \mathbf{a}_{2}+z_{2} \mathbf{a}_{3}=\left(x_{2} a+z_{2} c \cos \beta\right) \hat{\mathbf{x}}+y_{2} b \hat{\mathbf{y}}+ \\
& z_{2} c \sin \beta \hat{\mathbf{z}} \\
& \mathbf{B}_{4}=\begin{array}{cc}
\left(x_{2}+y_{2}\right) \mathbf{a}_{1}+\left(x_{2}-y_{2}\right) \mathbf{a}_{2}+ & = \\
\left(\frac{1}{2}+z_{2}\right) \mathbf{a}_{3} & \left(\frac{1}{2} c \cos \beta+x_{2} a+z_{2} c \cos \beta\right) \hat{\mathbf{x}}- \\
y_{2} b \hat{\mathbf{y}}+\left(\frac{1}{2}+z_{2}\right) c \sin \beta \hat{\mathbf{z}}
\end{array}
\end{aligned}
$$$$
\mathbf{B}_{5}=\left(x_{3}-y_{3}\right) \mathbf{a}_{1}+\left(x_{3}+y_{3}\right) \mathbf{a}_{2}+z_{3} \mathbf{a}_{3}=\left(x_{3} a+z_{3} c \cos \beta\right) \hat{\mathbf{x}}+y_{3} b \hat{\mathbf{y}}+
$$$$
z_{3} c \sin \beta \hat{\mathbf{z}}
$$

Wyckoff Position Atom Type

H I

(4a) 


\begin{tabular}{|c|c|c|c|c|c|c|}
\hline $\mathbf{B}_{6}$ & $=$ & $\begin{array}{c}\left(x_{3}+y_{3}\right) \mathbf{a}_{1}+\left(x_{3}-y_{3}\right) \mathbf{a}_{2}+ \\
\left(\frac{1}{2}+z_{3}\right) \mathbf{a}_{3}\end{array}$ & $=$ & $\begin{array}{c}\left(\frac{1}{2} c \cos \beta+x_{3} a+z_{3} c \cos \beta\right) \hat{\mathbf{x}}- \\
y_{3} b \hat{\mathbf{y}}+\left(\frac{1}{2}+z_{3}\right) c \sin \beta \hat{\mathbf{z}}\end{array}$ & $(4 a)$ & H II \\
\hline $\mathbf{B}_{7}$ & $=$ & $\left(x_{4}-y_{4}\right) \mathbf{a}_{1}+\left(x_{4}+y_{4}\right) \mathbf{a}_{2}+z_{4} \mathbf{a}_{3}$ & $=$ & $\begin{array}{c}\left(x_{4} a+z_{4} c \cos \beta\right) \hat{\mathbf{x}}+y_{4} b \hat{\mathbf{y}}+ \\
z_{4} c \sin \beta \hat{\mathbf{z}}\end{array}$ & $(4 a)$ & H III \\
\hline $\mathbf{B}_{8}$ & $=$ & $\begin{array}{c}\left(x_{4}+y_{4}\right) \mathbf{a}_{1}+\left(x_{4}-y_{4}\right) \mathbf{a}_{2}+ \\
\left(\frac{1}{2}+z_{4}\right) \mathbf{a}_{3}\end{array}$ & $=$ & $\begin{array}{c}\left(\frac{1}{2} c \cos \beta+x_{4} a+z_{4} c \cos \beta\right) \hat{\mathbf{x}}- \\
y_{4} b \hat{\mathbf{y}}+\left(\frac{1}{2}+z_{4}\right) c \sin \beta \hat{\mathbf{z}}\end{array}$ & $(4 a)$ & H III \\
\hline
\end{tabular}

\section{References:}

- D. Duan, X. Huang, F. Tian, Y. Liu, D. Li, H. Yu, B. Liu, W. Tian, and T. Cui, Predicted Formation of $H_{3}^{+}$in Solid Halogen Polyhydrides at High Pressures, J. Phys. Chem. A 119, 11059-11065 (2015), doi:10.1021/acs.jpca.5b08183.

\section{Geometry files:}

- CIF: pp. 806

- POSCAR: pp. 806 


\section{$\delta$-PdCl 2 Structure: A2B_mP6_10_mn_bg}

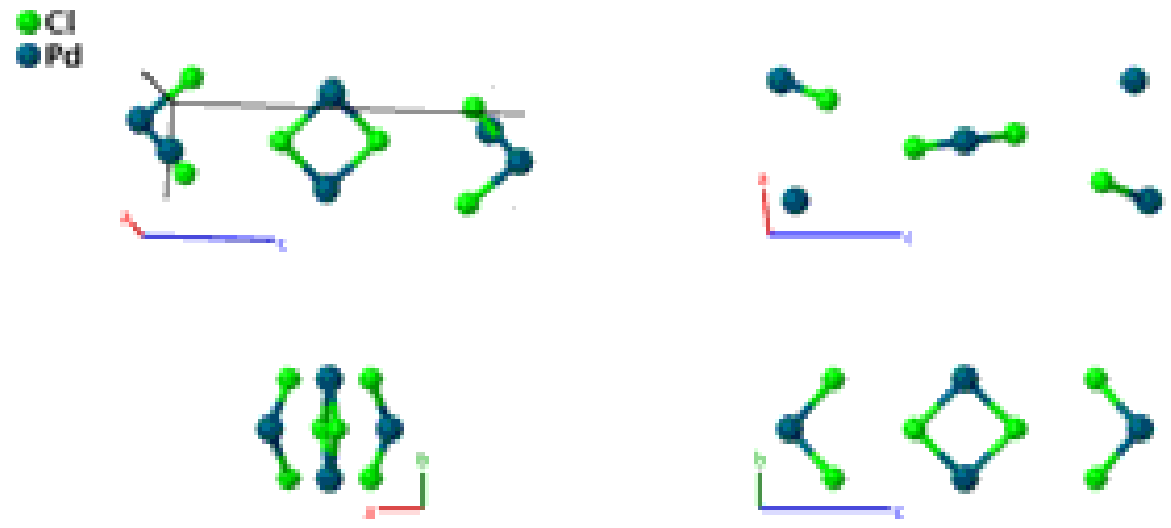

$\begin{array}{lll}\text { Prototype } & : & \delta-\mathrm{PdCl}_{2} \\ \text { AFLOW prototype label } & : & \mathrm{A} 2 \mathrm{~B} \_\mathrm{mP6} 610 \_\mathrm{mn} \_\mathrm{bg} \\ \text { Strukturbericht designation } & : & \text { None } \\ \text { Pearson symbol } & : & \mathrm{mP6} \\ \text { Space group number } & : & 10 \\ \text { Space group symbol } & : & P 2 / m \\ \text { AFLOW prototype command } & : & \text { aflow --proto }=\mathrm{A} 2 \mathrm{~B} \_\mathrm{mP} 6_{-} 10_{-} \mathrm{mn} \_\mathrm{bg} \\ & & -- \text { params }=a, b / a, c / a, \beta, x_{3}, z_{3}, x_{4}, z_{4}\end{array}$

- (Evers, 2010) use the unique-axis $c$ setting of space group $P 2 / \mathrm{m}$. We have switched this to our standard unique-axis $b$ setting. The data was taken at $793 \mathrm{~K}$.

Simple Monoclinic primitive vectors:
$\mathbf{a}_{1}=\quad a \hat{\mathbf{x}}$
$\mathbf{a}_{2}=b \hat{\mathbf{y}}$
$\mathbf{a}_{3}=c \cos \beta \hat{\mathbf{x}}+c \sin \beta \hat{\mathbf{z}}$

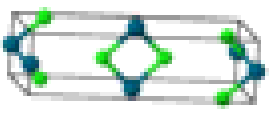

Basis vectors:

\begin{tabular}{|c|c|c|c|c|c|c|}
\hline & & Lattice Coordinates & & Cartesian Coordinates & Wyckoff Position & Atom Type \\
\hline $\mathbf{B}_{1}$ & $=$ & $\frac{1}{2} \mathbf{a}_{2}$ & $=$ & $\frac{1}{2} b \hat{\mathbf{y}}$ & $(1 b)$ & Pd I \\
\hline $\mathbf{B}_{2}$ & $=$ & $\frac{1}{2} \mathbf{a}_{1}+\frac{1}{2} \mathbf{a}_{3}$ & $=$ & $\frac{1}{2}(a+c \cos \beta) \hat{\mathbf{x}}+\frac{1}{2} c \sin \beta \hat{\mathbf{z}}$ & $(1 g)$ & Pd II \\
\hline $\mathbf{B}_{3}$ & $=$ & $x_{3} \mathbf{a}_{1}+z_{3} \mathbf{a}_{3}$ & $=$ & $\left(x_{3} a+z_{3} c \cos \beta\right) \hat{\mathbf{x}}+z_{3} c \sin \beta \hat{\mathbf{z}}$ & $(2 m)$ & $\mathrm{Cl} \mathrm{I}$ \\
\hline $\mathbf{B}_{4}$ & $=$ & $-x_{3} \mathbf{a}_{1}+-z_{3} \mathbf{a}_{3}$ & $=$ & $\left(-x_{3} a-z_{3} c \cos \beta\right) \hat{\mathbf{x}}+-z_{3} c \sin \beta \hat{\mathbf{z}}$ & $(2 m)$ & $\mathrm{Cl} \mathrm{I}$ \\
\hline $\mathbf{B}_{5}$ & $=$ & $x_{4} \mathbf{a}_{1}+\frac{1}{2} \mathbf{a}_{2}+z_{4} \mathbf{a}_{3}$ & $=$ & $\left(x_{4} a+z_{4} c \cos \beta\right) \hat{\mathbf{x}}+\frac{1}{2} b \hat{\mathbf{y}}+z_{4} c \sin \beta \hat{\mathbf{z}}$ & $(2 n)$ & $\mathrm{Cl} \mathrm{II}$ \\
\hline $\mathbf{B}_{6}$ & $=$ & $-x_{4} \mathbf{a}_{1}+\frac{1}{2} \mathbf{a}_{2}-z_{4} \mathbf{a}_{3}$ & $=$ & $\left(-x_{4} a-z_{4} c \cos \beta\right) \hat{\mathbf{x}}+\frac{1}{2} b \hat{\mathbf{y}}-z_{4} c \sin \beta \hat{\mathbf{z}}$ & $(2 n)$ & $\mathrm{Cl} \mathrm{II}$ \\
\hline
\end{tabular}

\section{References:}


- J. Evers, W. Beck, M. Göbel, S. Jakob, P. Mayer, G. Oehlinger, M. Rotter, and T. M. Klapötke, The Structures of $\delta$-PdCl 2 and $\gamma-P_{C C l}$ : Phases with Negative Thermal Expansion in One Direction, Angew. Chem. Int. Ed. 49, 5677-5682 (2010), doi:10.1002/anie.201000680.

\section{Geometry files:}

- CIF: pp. 806

- POSCAR: pp. 806 


\section{$\mathrm{H}_{3} \mathrm{Cl}$ (400 GPa) Structure: AB3_mP16_10_mn_3m3n}

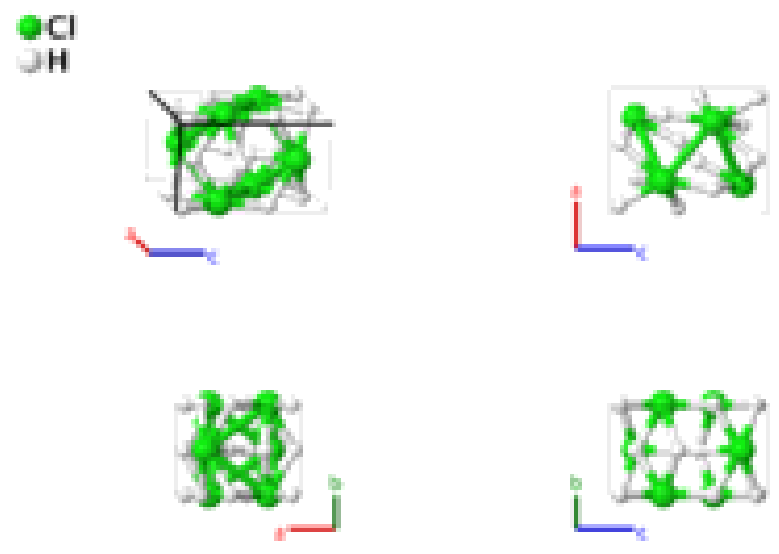

\section{Prototype}

AFLOW prototype label

Strukturbericht designation

Pearson symbol

Space group number

Space group symbol

AFLOW prototype command
: $\quad \mathrm{H}_{3} \mathrm{Cl}$

: AB3_mP16_10_mn_3m3n

: None

: $\quad \mathrm{mP16}$

: 10

: $\quad P 2 / m$ aflow --proto=AB3_mP16_10_mn_3m3n

- params $=a, b / a, c / a, \beta, x_{1}, z_{1}, x_{2}, z_{2}, x_{3}, z_{3}, x_{4}, z_{4}, x_{5}, z_{5}, x_{6}, z_{6}, x_{7}, z_{7}, x_{8}, z_{8}$

- This structure was found via first-principles calculations. The data presented here was computed at a pressure of $400 \mathrm{GPa}$.

\section{Simple Monoclinic primitive vectors:}
$\mathbf{a}_{1}=\quad a \hat{\mathbf{x}}$
$\mathbf{a}_{2}=\quad b \hat{\mathbf{y}}$
$\mathbf{a}_{3}=c \cos \beta \hat{\mathbf{x}}+c \sin \beta \hat{\mathbf{z}}$

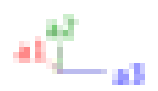

\section{Basis vectors:}

Lattice Coordinates

$\begin{array}{lllll}\mathbf{B}_{1}= & x_{1} \mathbf{a}_{1}+z_{1} \mathbf{a}_{3} & = & & \left(x_{1} a+z_{1} c \cos \beta\right) \hat{\mathbf{x}}+z_{1} c \sin \beta \hat{\mathbf{z}} \\ \mathbf{B}_{2}= & -x_{1} \mathbf{a}_{1}+-z_{1} \mathbf{a}_{3} & = & \left(-x_{1} a-z_{1} c \cos \beta\right) \hat{\mathbf{x}}+-z_{1} c \sin \beta \hat{\mathbf{z}} \\ \mathbf{B}_{3}= & x_{2} \mathbf{a}_{1}+z_{2} \mathbf{a}_{3} & = & \left(x_{2} a+z_{2} c \cos \beta\right) \hat{\mathbf{x}}+z_{2} c \sin \beta \hat{\mathbf{z}} \\ \mathbf{B}_{4}= & -x_{2} \mathbf{a}_{1}+-z_{2} \mathbf{a}_{3}= & = & \left(-x_{2} a-z_{2} c \cos \beta\right) \hat{\mathbf{x}}+-z_{2} c \sin \beta \hat{\mathbf{z}} \\ \mathbf{B}_{5}= & x_{3} \mathbf{a}_{1}+z_{3} \mathbf{a}_{3} & = & \left(x_{3} a+z_{3} c \cos \beta\right) \hat{\mathbf{x}}+z_{3} c \sin \beta \hat{\mathbf{z}} \\ \mathbf{B}_{6}= & -x_{3} \mathbf{a}_{1}+-z_{3} \mathbf{a}_{3}= & & \left(-x_{3} a-z_{3} c \cos \beta\right) \hat{\mathbf{x}}+-z_{3} c \sin \beta \hat{\mathbf{z}}\end{array}$

$\mathbf{B}_{1}=$

$-x_{3} \mathbf{a}_{1}+-z_{3} \mathbf{a}_{3}$
Cartesian Coordinates

$\left(-x_{3} a-z_{3} c \cos \beta\right) \hat{\mathbf{x}}+-z_{3} c \sin \beta \hat{\mathbf{z}}$
Wyckoff Position

(2m)

(2m)

(2m)

(2m)

(2m)

(2m)
Atom Type

Cl I

Cl I

H I

H I

H II

H II 


\begin{tabular}{|c|c|c|c|c|c|c|}
\hline $\mathbf{B}_{7}$ & $=$ & $x_{4} \mathbf{a}_{1}+z_{4} \mathbf{a}_{3}$ & $=$ & $\left(x_{4} a+z_{4} c \cos \beta\right) \hat{\mathbf{x}}+z_{4} c \sin \beta \hat{\mathbf{z}}$ & $(2 m)$ & H III \\
\hline $\mathbf{B}_{8}$ & $=$ & $-x_{4} \mathbf{a}_{1}+-z_{4} \mathbf{a}_{3}$ & $=$ & $\left(-x_{4} a-z_{4} c \cos \beta\right) \hat{\mathbf{x}}+-z_{4} c \sin \beta \hat{\mathbf{z}}$ & $(2 m)$ & H III \\
\hline $\mathbf{B}_{9}$ & $=$ & $x_{5} \mathbf{a}_{1}+\frac{1}{2} \mathbf{a}_{2}+z_{5} \mathbf{a}_{3}$ & $=$ & $\left(x_{5} a+z_{5} c \cos \beta\right) \hat{\mathbf{x}}+\frac{1}{2} b \hat{\mathbf{y}}+z_{5} c \sin \beta \hat{\mathbf{z}}$ & $(2 n)$ & $\mathrm{Cl}$ II \\
\hline $\mathbf{B}_{10}$ & $=$ & $-x_{5} \mathbf{a}_{1}+\frac{1}{2} \mathbf{a}_{2}-z_{5} \mathbf{a}_{3}$ & $=$ & $\left(-x_{5} a-z_{5} c \cos \beta\right) \hat{\mathbf{x}}+\frac{1}{2} b \hat{\mathbf{y}}-z_{5} c \sin \beta \hat{\mathbf{z}}$ & $(2 n)$ & III \\
\hline $\mathbf{B}_{11}$ & $=$ & $x_{6} \mathbf{a}_{1}+\frac{1}{2} \mathbf{a}_{2}+z_{6} \mathbf{a}_{3}$ & $=$ & $\left(x_{6} a+z_{6} c \cos \beta\right) \hat{\mathbf{x}}+\frac{1}{2} b \hat{\mathbf{y}}+z_{6} c \sin \beta \hat{\mathbf{z}}$ & $(2 n)$ & \\
\hline $\mathbf{B}_{12}$ & $=$ & $-x_{6} \mathbf{a}_{1}+\frac{1}{2} \mathbf{a}_{2}-z_{6} \mathbf{a}_{3}$ & $=$ & $\left(-x_{6} a-z_{6} c \cos \beta\right) \hat{\mathbf{x}}+\frac{1}{2} b \hat{\mathbf{y}}-z_{6} c \sin \beta \hat{\mathbf{z}}$ & $(2 n)$ & $\mathrm{V}$ \\
\hline $\mathbf{B}_{13}$ & $=$ & $x_{7} \mathbf{a}_{1}+\frac{1}{2} \mathbf{a}_{2}+z_{7} \mathbf{a}_{3}$ & $=$ & $\left(x_{7} a+z_{7} c \cos \beta\right) \hat{\mathbf{x}}+\frac{1}{2} b \hat{\mathbf{y}}+z_{7} c \sin \beta \hat{\mathbf{z}}$ & $(2 n)$ & 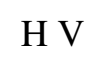 \\
\hline $\mathbf{B}_{14}$ & $=$ & $-x_{7} \mathbf{a}_{1}+\frac{1}{2} \mathbf{a}_{2}-z_{7} \mathbf{a}_{3}$ & $=$ & $\left(-x_{7} a-z_{7} c \cos \beta\right) \hat{\mathbf{x}}+\frac{1}{2} b \hat{\mathbf{y}}-z_{7} c \sin \beta \hat{\mathbf{z}}$ & $(2 n)$ & {$[\mathrm{V}$} \\
\hline $\mathbf{B}_{15}$ & $=$ & $x_{8} \mathbf{a}_{1}+\frac{1}{2} \mathbf{a}_{2}+z_{8} \mathbf{a}_{3}$ & $=$ & $\left(x_{8} a+z_{8} c \cos \beta\right) \hat{\mathbf{x}}+\frac{1}{2} b \hat{\mathbf{y}}+z_{8} c \sin \beta \hat{\mathbf{z}}$ & $(2 n)$ & II \\
\hline $\mathbf{B}_{16}$ & $=$ & $-x_{8} \mathbf{a}_{1}+\frac{1}{2} \mathbf{a}_{2}-z_{8} \mathbf{a}_{3}$ & $=$ & $\left(-x_{8} a-z_{8} c \cos \beta\right) \hat{\mathbf{x}}+\frac{1}{2} b \hat{\mathbf{y}}-z_{8} c \sin \beta \hat{\mathbf{z}}$ & $(2 n)$ & \\
\hline
\end{tabular}

\section{References:}

- Q. Zeng, S. Yu, D. Li, A. R. Oganov, and G. Frapper, Emergence of novel hydrogen chlorides under high pressure, Phys. Chem. Chem. Phys. 19, 8236-8242 (2017), doi:10.1039/C6CP08708F.

\section{Geometry files:}

- CIF: pp. 806

- POSCAR: pp. 807 


\section{Muthmannite (AuAgTe 2 ) Structure: ABC2_mP8_10_ac_eh_mn}

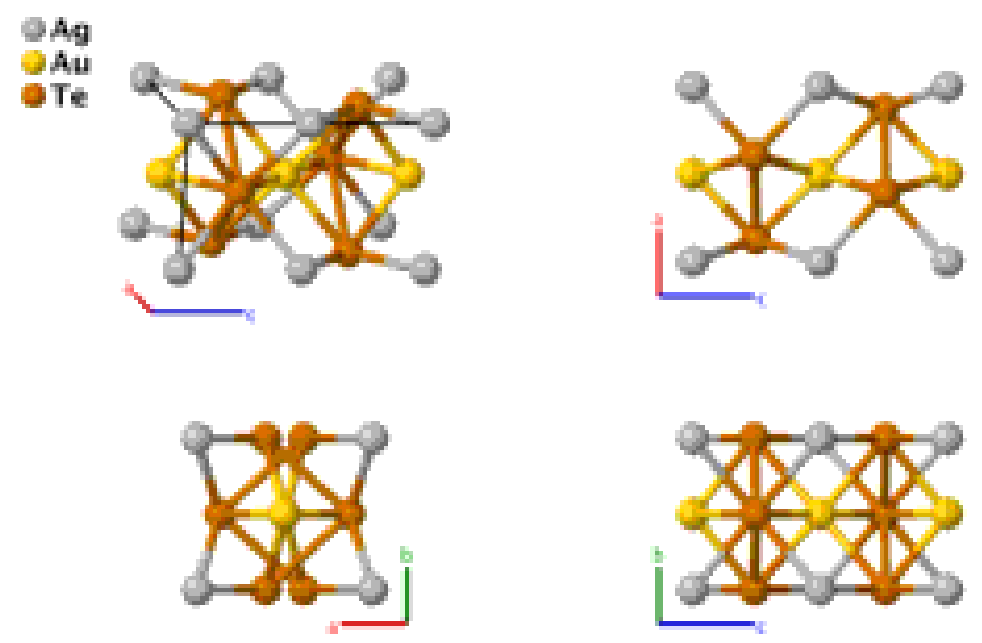

Prototype

AFLOW prototype label

Strukturbericht designation

Pearson symbol

Space group number

Space group symbol

AFLOW prototype command
: $\mathrm{AuAgTe}_{2}$

: ABC2_mP8_10_ac_eh_mn

: None

: $\mathrm{mP8}$

: 10

: $\quad P 2 / m$ aflow --proto $=A B C 2 \_m P 8 \_10 \_a c \_$eh_mn

- -params $=a, b / a, c / a, \beta, x_{5}, z_{5}, x_{6}, z_{6}$

\section{Simple Monoclinic primitive vectors:}
$\mathbf{a}_{1}=\quad a \hat{\mathbf{x}}$
$\mathbf{a}_{2}=\quad b \hat{\mathbf{y}}$
$\mathbf{a}_{3}=c \cos \beta \hat{\mathbf{x}}+c \sin \beta \hat{\mathbf{z}}$

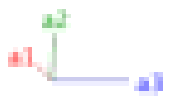

\section{Basis vectors:}

Lattice Coordinates
$\mathbf{B}_{1}=0 \mathbf{a}_{1}+0 \mathbf{a}_{2}+0 \mathbf{a}_{3}=$
$\mathbf{B}_{2}=\quad \frac{1}{2} \mathbf{a}_{3}$
$\mathbf{B}_{3}=\frac{1}{2} \mathbf{a}_{1}+\frac{1}{2} \mathbf{a}_{2}$
$\mathbf{B}_{4}=\frac{1}{2} \mathbf{a}_{1}+\frac{1}{2} \mathbf{a}_{2}+\frac{1}{2} \mathbf{a}_{3}=$
$\mathbf{B}_{5}=$
$x_{5} \mathbf{a}_{1}+z_{5} \mathbf{a}_{3}$
$\mathbf{B}_{6}=$
$=$
$=$
$=$
$=$
$=$

$$
0 \hat{\mathbf{x}}+0 \hat{\mathbf{y}}+0 \hat{\mathbf{z}}
$$$$
\frac{1}{2} c \cos \beta \hat{\mathbf{x}}+\frac{1}{2} c \sin \beta \hat{\mathbf{z}}
$$$$
\frac{1}{2} a \hat{\mathbf{x}}+\frac{1}{2} b \hat{\mathbf{y}}
$$$$
\frac{1}{2}(a+c \cos \beta) \hat{\mathbf{x}}+\frac{1}{2} b \hat{\mathbf{y}}+\frac{1}{2} c \sin \beta \hat{\mathbf{z}}
$$$$
\left(x_{5} a+z_{5} c \cos \beta\right) \hat{\mathbf{x}}+z_{5} c \sin \beta \hat{\mathbf{z}}
$$$$
\left(-x_{5} a-z_{5} c \cos \beta\right) \hat{\mathbf{x}}+-z_{5} c \sin \beta \hat{\mathbf{z}}
$$

Cartesian Coordinates
Wyckoff Position

Atom Type

(1a)

$\mathrm{Ag} I$

(1c)

Ag II

(1e)

$\mathrm{Au} I$

$(1 h)$

Au II

(2m)

Te I

$(2 m)$

Te I 

$\mathbf{B}_{7}=x_{6} \mathbf{a}_{1}+\frac{1}{2} \mathbf{a}_{2}+z_{6} \mathbf{a}_{3}=\left(x_{6} a+z_{6} c \cos \beta\right) \hat{\mathbf{x}}+\frac{1}{2} b \hat{\mathbf{y}}+z_{6} c \sin \beta \hat{\mathbf{z}}$
$(2 n)$
Te II
$\mathbf{B}_{8}=-x_{6} \mathbf{a}_{1}+\frac{1}{2} \mathbf{a}_{2}-z_{6} \mathbf{a}_{3}=\left(-x_{6} a-z_{6} c \cos \beta\right) \hat{\mathbf{x}}+\frac{1}{2} b \hat{\mathbf{y}}-z_{6} c \sin \beta \hat{\mathbf{z}}$
$(2 n)$
Te II

\section{References:}

- L. Bindi, Commensurate-incommensurate phase transition in muthmannite, AuAgTe 2 : first evidence of a modulated structure at low temperature, Philos. Mag. Lett. 88, 533-541 (2008), doi:10.1080/09500830802311072.

\section{Found in:}

- P. Villars and K. Cenzual, Pearson's Crystal Data - Crystal Structure Database for Inorganic Compounds, ASM International (2013).

\section{Geometry files:}

- CIF: pp. 807

- POSCAR: pp. 807 

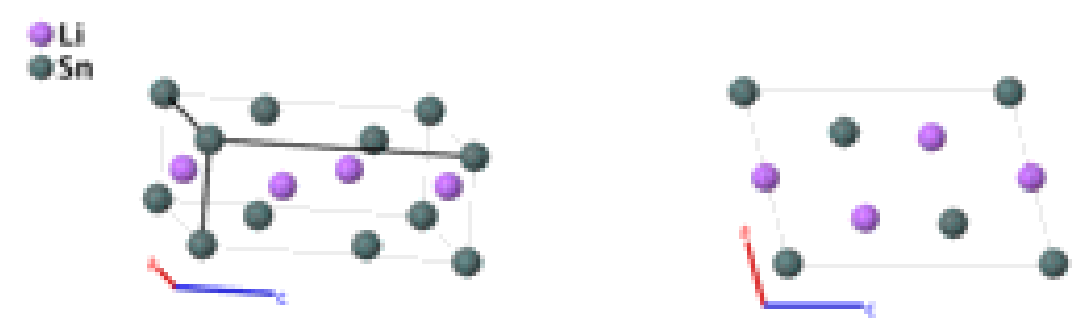

Prototype

: $\quad \operatorname{LiSn}$

AFLOW prototype label

: AB_mP6_10_en_am

Strukturbericht designation

Pearson symbol

: None

Space group number

: $\quad \mathrm{mP6}$

Space group symbol

: 10

AFLOW prototype command : aflow --proto=AB_mP6_10_en_am

- params $=a, b / a, c / a, \beta, x_{3}, z_{3}, x_{4}, z_{4}$

\section{Simple Monoclinic primitive vectors:}

$$
\begin{array}{llc}
\mathbf{a}_{1}= & a \hat{\mathbf{x}} \\
\mathbf{a}_{2}= & b \hat{\mathbf{y}} \\
\mathbf{a}_{3}= & c \cos \beta \hat{\mathbf{x}}+c \sin \beta \hat{\mathbf{z}}
\end{array}
$$
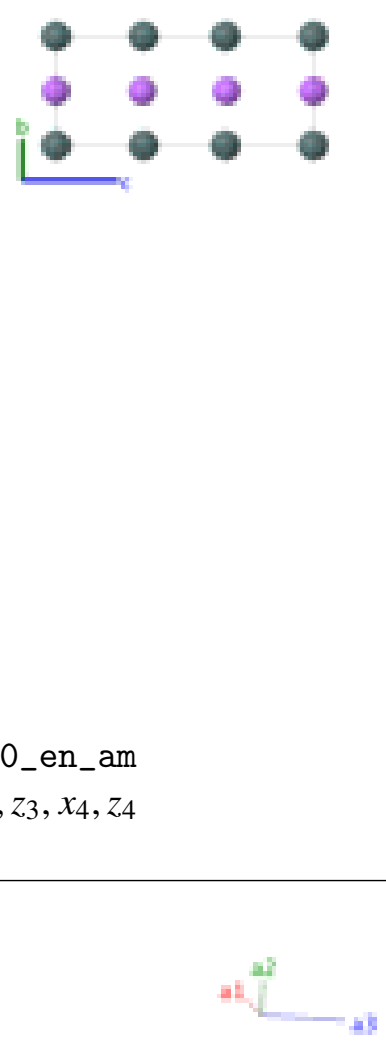

Basis vectors:

Lattice Coordinates

$$
\begin{array}{lcccc}
\mathbf{B}_{1}= & 0 \mathbf{a}_{1}+0 \mathbf{a}_{2}+0 \mathbf{a}_{3} & = & 0 \hat{\mathbf{x}}+0 \hat{\mathbf{y}}+0 \hat{\mathbf{z}} \\
\mathbf{B}_{2}= & \frac{1}{2} \mathbf{a}_{1}+\frac{1}{2} \mathbf{a}_{2} & = & \frac{1}{2} a \hat{\mathbf{x}}+\frac{1}{2} b \hat{\mathbf{y}} \\
\mathbf{B}_{3}= & x_{3} \mathbf{a}_{1}+z_{3} \mathbf{a}_{3} & = & \left(x_{3} a+z_{3} c \cos \beta\right) \hat{\mathbf{x}}+z_{3} c \sin \beta \hat{\mathbf{z}} \\
\mathbf{B}_{4}= & -x_{3} \mathbf{a}_{1}+-z_{3} \mathbf{a}_{3}= & \left(-x_{3} a-z_{3} c \cos \beta\right) \hat{\mathbf{x}}+-z_{3} c \sin \beta \hat{\mathbf{z}} \\
\mathbf{B}_{5}= & x_{4} \mathbf{a}_{1}+\frac{1}{2} \mathbf{a}_{2}+z_{4} \mathbf{a}_{3}= & \left(x_{4} a+z_{4} c \cos \beta\right) \hat{\mathbf{x}}+\frac{1}{2} b \hat{\mathbf{y}}+z_{4} c \sin \beta \hat{\mathbf{z}} \\
\mathbf{B}_{6}= & -x_{4} \mathbf{a}_{1}+\frac{1}{2} \mathbf{a}_{2}-z_{4} \mathbf{a}_{3}= & \left(-x_{4} a-z_{4} c \cos \beta\right) \hat{\mathbf{x}}+\frac{1}{2} b \hat{\mathbf{y}}-z_{4} c \sin \beta \hat{\mathbf{z}}
\end{array}
$$

\section{Cartesian Coordinates}

Wyckoff Position

Atom Type

(1a)

Sn I

(1e)

Li I

(2m)

Sn II

(2m)

Sn II

(2n)

Li II

(2n)

Li II

\section{References:}


- W. Müller and H. Schäfer, Die Kristallstruktur der Phase LiSn, Z. Naturforsch. B 28, 246-248 (1973), doi:10.1515/znb-1973-5-604.

\section{Found in:}

- P. Villars and K. Cenzual, Pearson's Crystal Data - Crystal Structure Database for Inorganic Compounds, ASM International (2013).

\section{Geometry files:}

- CIF: pp. 807

- POSCAR: pp. 808 


\section{S-carbon Structure: A_mP8_10_2m2n}

oc
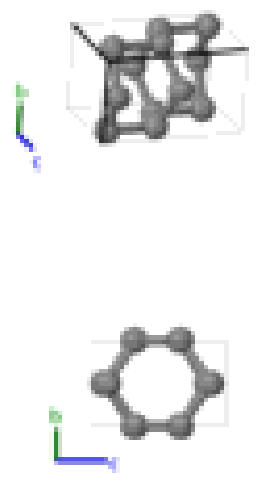
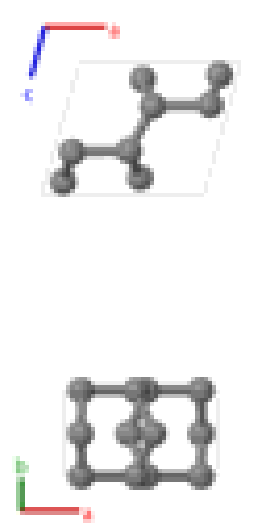

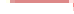

\section{Prototype}

AFLOW prototype label

Strukturbericht designation

Pearson symbol

Space group number

Space group symbol

AFLOW prototype command 

$\mathbf{B}_{4}=-x_{2} \mathbf{a}_{1}+-z_{2} \mathbf{a}_{3}$
$=\left(-x_{2} a-z_{2} c \cos \beta\right) \hat{\mathbf{x}}+-z_{2} c \sin \beta \hat{\mathbf{z}}$
$(2 m)$
C II
$\mathbf{B}_{5}=x_{3} \mathbf{a}_{1}+\frac{1}{2} \mathbf{a}_{2}+z_{3} \mathbf{a}_{3}=\left(x_{3} a+z_{3} c \cos \beta\right) \hat{\mathbf{x}}+\frac{1}{2} b \hat{\mathbf{y}}+z_{3} c \sin \beta \hat{\mathbf{z}}$
$(2 n)$
C III
$\mathbf{B}_{6}=-x_{3} \mathbf{a}_{1}+\frac{1}{2} \mathbf{a}_{2}-z_{3} \mathbf{a}_{3}=\left(-x_{3} a-z_{3} c \cos \beta\right) \hat{\mathbf{x}}+\frac{1}{2} b \hat{\mathbf{y}}-z_{3} c \sin \beta \hat{\mathbf{z}}$
$(2 n)$
C III
$\mathbf{B}_{7}=x_{4} \mathbf{a}_{1}+\frac{1}{2} \mathbf{a}_{2}+z_{4} \mathbf{a}_{3}=\left(x_{4} a+z_{4} c \cos \beta\right) \hat{\mathbf{x}}+\frac{1}{2} b \hat{\mathbf{y}}+z_{4} c \sin \beta \hat{\mathbf{z}}$
C IV
$\mathbf{B}_{8}=-x_{4} \mathbf{a}_{1}+\frac{1}{2} \mathbf{a}_{2}-z_{4} \mathbf{a}_{3}=\left(-x_{4} a-z_{4} c \cos \beta\right) \hat{\mathbf{x}}+\frac{1}{2} b \hat{\mathbf{y}}-z_{4} c \sin \beta \hat{\mathbf{z}}$

\section{References:}

- H. Niu, X.-Q. Chen, S. Wang, D. Li, W. L. Mao, and Y. Li, Families of Superhard Crystalline Carbon Allotropes Constructed via Cold Compression of Graphite and Nanotubes, Phys. Rev. Lett. 108, 135501 (2012), doi:10.1103/PhysRevLett.108.135501.

- F. Tian, X. Dong, Z. Zhao, J. He, and H.-T. Wang, Superhard F-carbon predicted by ab initio particle-swarm optimization methodology, J. Phys.: Condens. Matter 24, 165504 (2012), doi:10.1088/0953-8984/24/16/165504.

- J.-T. Wang, C. Chen, and Y. Kawazoe, Phase conversion from graphite toward a simple monoclinic sp ${ }^{3}$-carbon allotrope, J. Chem. Phys. 137, 024502 (2012), doi:10.1063/1.4732538.

- C. He, L. Sun, C. Zhang, X. Peng, K. Zhang, and J. Zhong, New superhard carbon phases between graphite and diamond, Solid State Commun. 152, 1560-1563 (2012), doi:10.1016/j.ssc.2012.05.022.

- C. He, L. Z. Sun, and J. Zhong, Prediction of superhard carbon allotropes from the segment combination method, J. Superhard Mater. 34, 386-399 (2012), doi:10.3103/S1063457612060123.

\section{Geometry files:}

- CIF: pp. 808

- POSCAR: pp. 808 


\section{Thortveitite $\left(\left[\mathrm{Sc}, \mathrm{Y}_{2} \mathrm{Si}_{2} \mathrm{O}_{7}, S 2_{1}\right)\right.$ Structure: A7B2C2_mC22_12_aij_h_i}
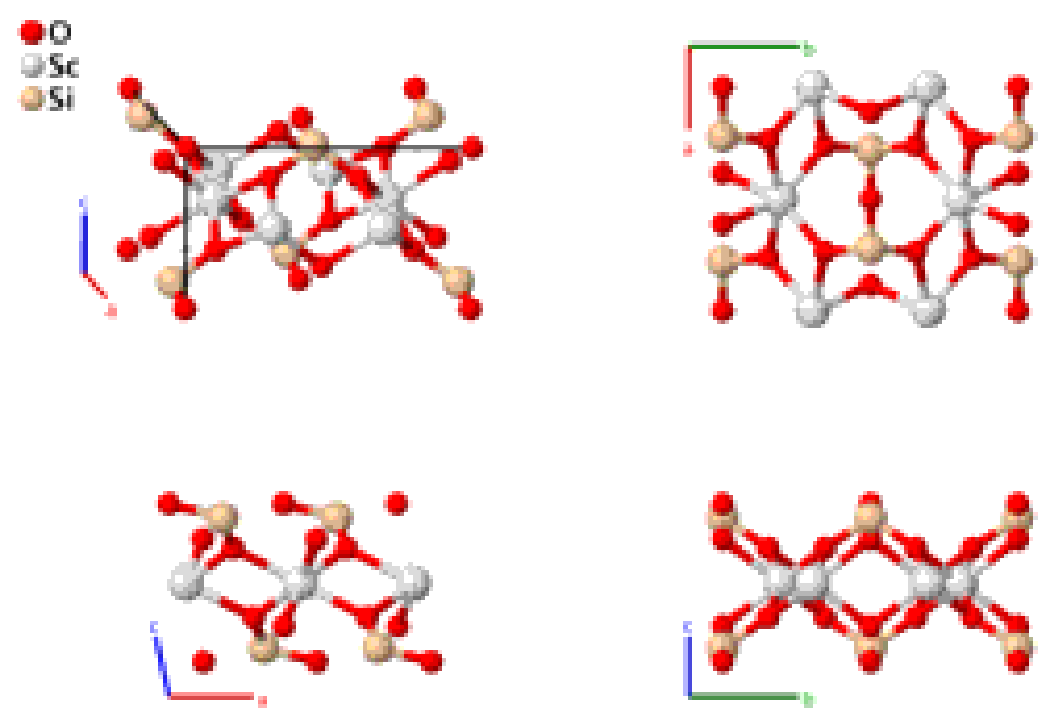

\section{Prototype}

AFLOW prototype label

Strukturbericht designation

Pearson symbol

Space group number

Space group symbol

AFLOW prototype command
: $\quad\left[\mathrm{Sc}, \mathrm{Y}_{2} \mathrm{Si}_{2} \mathrm{O}_{7}\right.$

: A7B2C2_mC22_12_aij_h_i

: $\quad S 21$

: $\quad \mathrm{mC} 22$

$: \quad 12$

: $\quad C 2 / m$ aflow --proto=A7B2C2_mC22_12_aij_h_i

- params $=a, b / a, c / a, \beta, y_{2}, x_{3}, z_{3}, x_{4}, z_{4}, x_{5}, y_{5}, z_{5}$

- Thortveitite is the primary source of scandium, and is one of the simplest sorosilicates, minerals with isolated $\mathrm{Si}_{2} \mathrm{O}_{7}$ groups (Bianchi, 1988).

- Although the (4h) Wyckoff position is randomly occupied by both Sc and Y atoms, we use Sc to represent the site.

- (Bianchi, 1988) gives structural information for several samples of thortveitite. We use the data from sample 1, collected in Iveland, Norway.

Base-centered Monoclinic primitive vectors:

$$
\begin{aligned}
& \mathbf{a}_{1}=\frac{1}{2} a \hat{\mathbf{x}}-\frac{1}{2} b \hat{\mathbf{y}} \\
& \mathbf{a}_{2}=\frac{1}{2} a \hat{\mathbf{x}}+\frac{1}{2} b \hat{\mathbf{y}} \\
& \mathbf{a}_{3}=c \cos \beta \hat{\mathbf{x}}+c \sin \beta \hat{\mathbf{z}}
\end{aligned}
$$

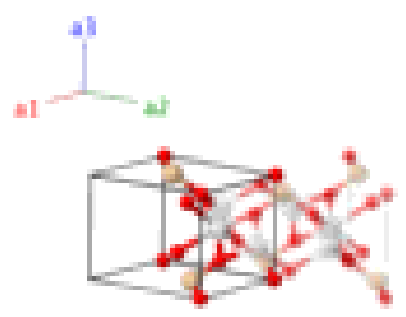

Basis vectors: 


\begin{tabular}{|c|c|c|c|c|c|c|}
\hline $\mathbf{B}_{1}$ & $=$ & $0 \mathbf{a}_{1}+0 \mathbf{a}_{2}+0 \mathbf{a}_{3}$ & $=$ & $0 \hat{\mathbf{x}}+0 \hat{\mathbf{y}}+0 \hat{\mathbf{z}}$ & $(2 a)$ & O I \\
\hline $\mathbf{B}_{2}$ & $=$ & $-y_{2} \mathbf{a}_{1}+y_{2} \mathbf{a}_{2}+\frac{1}{2} \mathbf{a}_{3}$ & $=$ & $\frac{1}{2} c \cos \beta \hat{\mathbf{x}}+y_{2} b \hat{\mathbf{y}}+\frac{1}{2} c \sin \beta \hat{\mathbf{z}}$ & $(4 h)$ & $\mathrm{Sc}$ \\
\hline $\mathbf{B}_{3}$ & $=$ & $y_{2} \mathbf{a}_{1}-y_{2} \mathbf{a}_{2}+\frac{1}{2} \mathbf{a}_{3}$ & $=$ & $\frac{1}{2} c \cos \beta \hat{\mathbf{x}}-y_{2} b \hat{\mathbf{y}}+\frac{1}{2} c \sin \beta \hat{\mathbf{z}}$ & $(4 h)$ & $\mathrm{Sc}$ \\
\hline $\mathbf{B}_{4}$ & $=$ & $x_{3} \mathbf{a}_{1}+x_{3} \mathbf{a}_{2}+z_{3} \mathbf{a}_{3}$ & $=$ & $\left(x_{3} a+z_{3} c \cos \beta\right) \hat{\mathbf{x}}+z_{3} c \sin \beta \hat{\mathbf{z}}$ & $(4 i)$ & $\mathrm{O}$ II \\
\hline $\mathbf{B}_{5}$ & $=$ & $-x_{3} \mathbf{a}_{1}-x_{3} \mathbf{a}_{2}-z_{3} \mathbf{a}_{3}$ & $=$ & $\left(-x_{3} a-z_{3} c \cos \beta\right) \hat{\mathbf{x}}+-z_{3} c \sin \beta \hat{\mathbf{z}}$ & $(4 i)$ & O II \\
\hline $\mathbf{B}_{6}$ & $=$ & $x_{4} \mathbf{a}_{1}+x_{4} \mathbf{a}_{2}+z_{4} \mathbf{a}_{3}$ & $=$ & $\left(x_{4} a+z_{4} c \cos \beta\right) \hat{\mathbf{x}}+z_{4} c \sin \beta \hat{\mathbf{z}}$ & $(4 i)$ & $\mathrm{Si}$ \\
\hline $\mathbf{B}_{7}$ & $=$ & $-x_{4} \mathbf{a}_{1}-x_{4} \mathbf{a}_{2}-z_{4} \mathbf{a}_{3}$ & $=$ & $\left(-x_{4} a-z_{4} c \cos \beta\right) \hat{\mathbf{x}}+-z_{4} c \sin \beta \hat{\mathbf{z}}$ & $(4 i)$ & $\mathrm{Si}$ \\
\hline $\mathbf{B}_{8}$ & $=$ & $\left(x_{5}-y_{5}\right) \mathbf{a}_{1}+\left(x_{5}+y_{5}\right) \mathbf{a}_{2}+z_{5} \mathbf{a}_{3}$ & $=$ & $\begin{array}{c}\left(x_{5} a+z_{5} c \cos \beta\right) \hat{\mathbf{x}}+y_{5} b \hat{\mathbf{y}}+ \\
z_{5} c \sin \beta \hat{\mathbf{z}}\end{array}$ & $(8 j)$ & O III \\
\hline $\mathbf{B}_{9}$ & $=$ & $\begin{array}{c}\left(-x_{5}-y_{5}\right) \mathbf{a}_{1}+\left(-x_{5}+y_{5}\right) \mathbf{a}_{2}- \\
z_{5} \mathbf{a}_{3}\end{array}$ & $=$ & $\begin{array}{c}\left(-x_{5} a-z_{5} c \cos \beta\right) \hat{\mathbf{x}}+y_{5} b \hat{\mathbf{y}}- \\
z_{5} c \sin \beta \hat{\mathbf{z}}\end{array}$ & $(8 j)$ & O III \\
\hline $\mathbf{B}_{10}$ & $=$ & $\begin{array}{c}\left(-x_{5}+y_{5}\right) \mathbf{a}_{1}+\left(-x_{5}-y_{5}\right) \mathbf{a}_{2}- \\
z_{5} \mathbf{a}_{3}\end{array}$ & $=$ & $\begin{array}{c}\left(-x_{5} a-z_{5} c \cos \beta\right) \hat{\mathbf{x}}-y_{5} b \hat{\mathbf{y}}- \\
z_{5} c \sin \beta \hat{\mathbf{z}}\end{array}$ & $(8 j)$ & O III \\
\hline $\mathbf{B}_{11}$ & $=$ & $\left(x_{5}+y_{5}\right) \mathbf{a}_{1}+\left(x_{5}-y_{5}\right) \mathbf{a}_{2}+z_{5} \mathbf{a}_{3}$ & $=$ & $\begin{array}{c}\left(x_{5} a+z_{5} c \cos \beta\right) \hat{\mathbf{x}}-y_{5} b \hat{\mathbf{y}}+ \\
z_{5} c \sin \beta \hat{\mathbf{z}}\end{array}$ & $(8 j)$ & O III \\
\hline
\end{tabular}

\section{References:}

- R. Bianchi, T. Pilati, V. Diella, C. M. Gramaccioli, and G. Mannucci, A re-examination of thortveitite, Am. Mineral. 73, 601-607 (1988).

\section{Found in:}

- R. T. Downs and M. Hall-Wallace, The American Mineralogist Crystal Structure Database, Am. Mineral. 88, 247-250 (2003).

\section{Geometry files:}

- CIF: pp. 808

- POSCAR: pp. 809 


\section{M-carbon Structure: A_mC16_12_4i}

oc
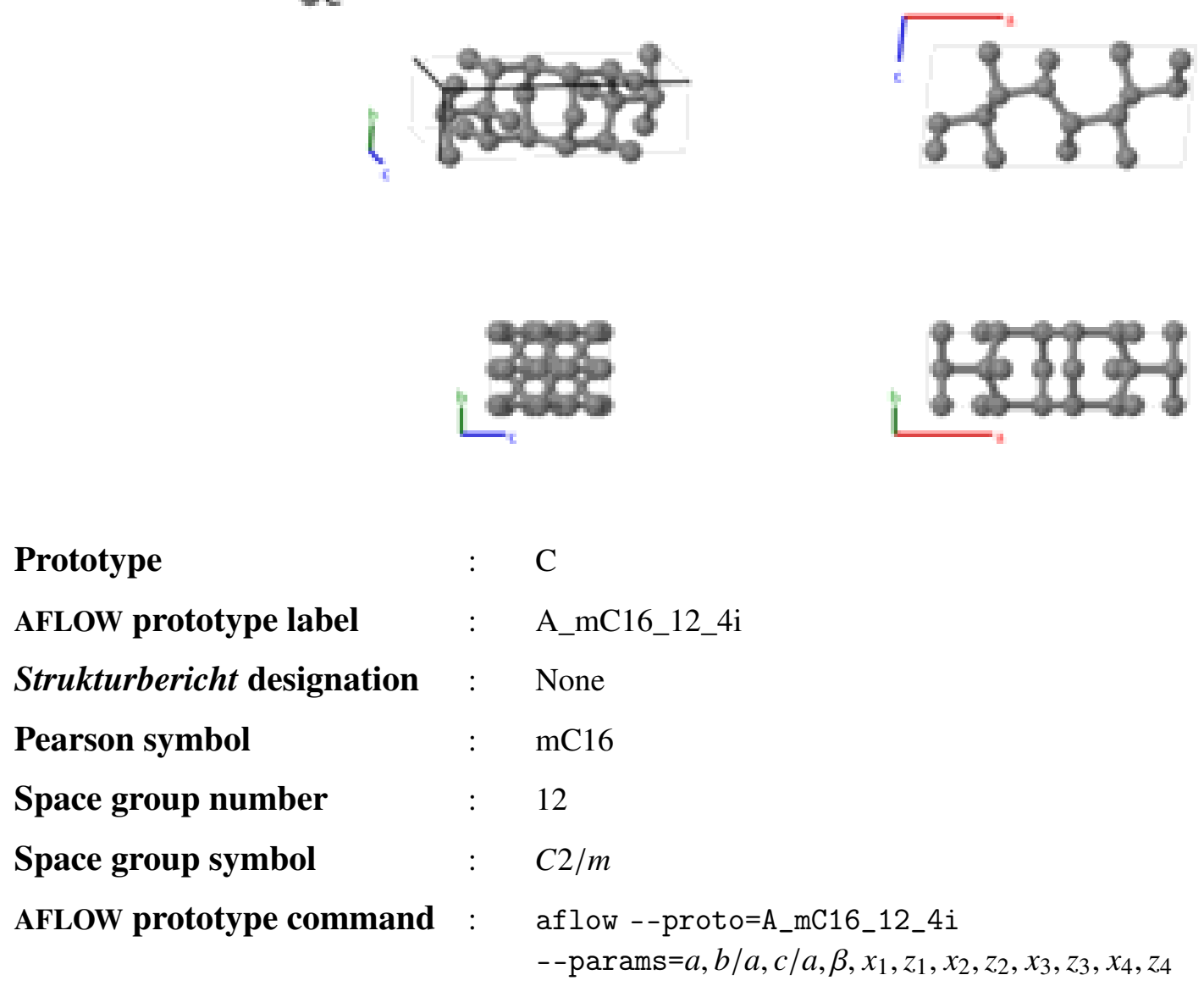

- This structure was originally found by Oganov and Glass, (Oganov, 2006) and was refined and designated M-Carbon by Li et al. (Li, 2009)

Base-centered Monoclinic primitive vectors:

$$
\begin{array}{ll}
\mathbf{a}_{1}= & \frac{1}{2} a \hat{\mathbf{x}}-\frac{1}{2} b \hat{\mathbf{y}} \\
\mathbf{a}_{2}= & \frac{1}{2} a \hat{\mathbf{x}}+\frac{1}{2} b \hat{\mathbf{y}} \\
\mathbf{a}_{3}= & c \cos \beta \hat{\mathbf{x}}+c \sin \beta \hat{\mathbf{z}}
\end{array}
$$

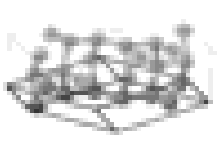

Basis vectors:

Lattice Coordinates

$\mathbf{B}_{1}=x_{1} \mathbf{a}_{1}+x_{1} \mathbf{a}_{2}+z_{1} \mathbf{a}_{3}$

$\mathbf{B}_{2}=-x_{1} \mathbf{a}_{1}-x_{1} \mathbf{a}_{2}-z_{1} \mathbf{a}_{3}$

$\mathbf{B}_{3}=x_{2} \mathbf{a}_{1}+x_{2} \mathbf{a}_{2}+z_{2} \mathbf{a}_{3}$

$\mathbf{B}_{4}=-x_{2} \mathbf{a}_{1}-x_{2} \mathbf{a}_{2}-z_{2} \mathbf{a}_{3}$

$\mathbf{B}_{5}=x_{3} \mathbf{a}_{1}+x_{3} \mathbf{a}_{2}+z_{3} \mathbf{a}_{3}$

$\mathbf{B}_{6}=-x_{3} \mathbf{a}_{1}-x_{3} \mathbf{a}_{2}-z_{3} \mathbf{a}_{3}$

$\mathbf{B}_{8}=-x_{4} \mathbf{a}_{1}-x_{4} \mathbf{a}_{2}-z_{4} \mathbf{a}_{3}$
$\mathbf{B}_{7}=x_{4} \mathbf{a}_{1}+x_{4} \mathbf{a}_{2}+z_{4} \mathbf{a}_{3}$

Cartesian Coordinates

$$
=\quad\left(x_{1} a+z_{1} c \cos \beta\right) \hat{\mathbf{x}}+z_{1} c \sin \beta \hat{\mathbf{z}}
$$$$
=\left(-x_{1} a-z_{1} c \cos \beta\right) \hat{\mathbf{x}}+-z_{1} c \sin \beta \hat{\mathbf{z}}
$$$$
=\quad\left(x_{2} a+z_{2} c \cos \beta\right) \hat{\mathbf{x}}+z_{2} c \sin \beta \hat{\mathbf{z}}
$$$$
=\quad\left(-x_{2} a-z_{2} c \cos \beta\right) \hat{\mathbf{x}}+-z_{2} c \sin \beta \hat{\mathbf{z}}
$$$$
=\quad\left(x_{3} a+z_{3} c \cos \beta\right) \hat{\mathbf{x}}+z_{3} c \sin \beta \hat{\mathbf{z}}
$$$$
=\left(-x_{3} a-z_{3} c \cos \beta\right) \hat{\mathbf{x}}+-z_{3} c \sin \beta \hat{\mathbf{z}}
$$$$
=\quad\left(x_{4} a+z_{4} c \cos \beta\right) \hat{\mathbf{x}}+z_{4} c \sin \beta \hat{\mathbf{z}}
$$$$
=\left(-x_{4} a-z_{4} c \cos \beta\right) \hat{\mathbf{x}}+-z_{4} c \sin \beta \hat{\mathbf{z}}
$$ 


\section{References:}

- Q. Li, Y. Ma, A. R. Oganov, H. Wang, H. Wang, Y. Xu, T. Cui, H.-K. Mao, and G. Zou, Superhard Monoclinic Polymorph of Carbon, Phys. Rev. Lett. 102, 175506 (2009), doi:10.1103/PhysRevLett.102.175506.

- A. R. Oganov and C. W. Glass, Crystal structure prediction using ab initio evolutionary techniques: Principles and applications, J. Chem. Phys. 124, 244704 (2006), doi:10.1063/1.2210932.

\section{Geometry files:}

- CIF: pp. 809

- POSCAR: pp. 809 


\section{$\mathrm{H}_{2} \mathrm{~S}$ (15 GPa) Structure: A2B_mP12_13_2g_ef}
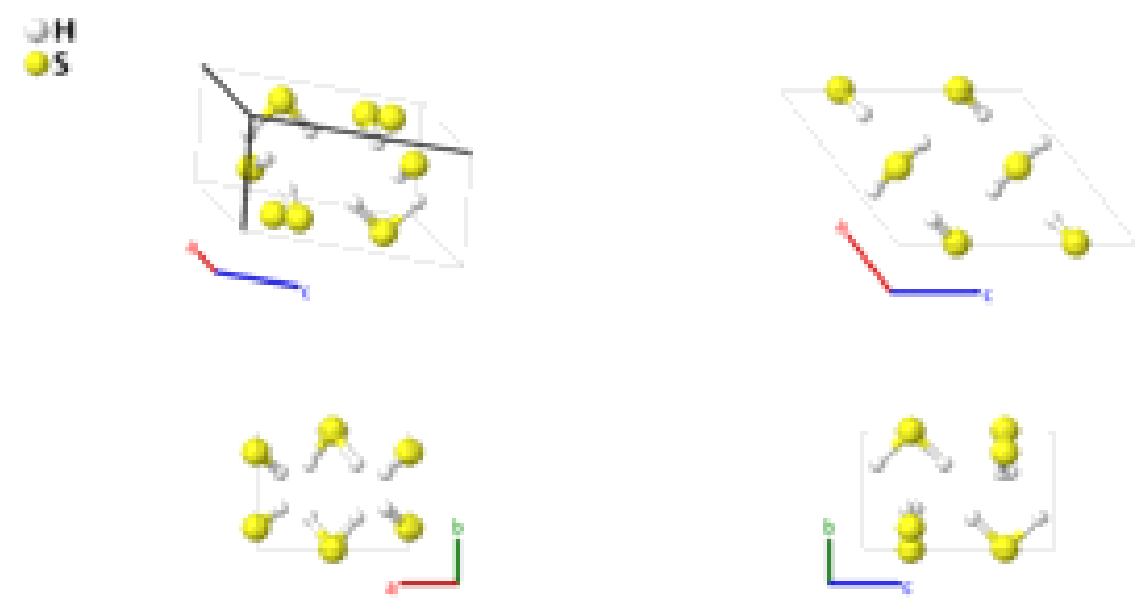

Prototype

: $\mathrm{H}_{2} \mathrm{~S}$

AFLOW prototype label

: A2B_mP12_13_2g_ef

Strukturbericht designation

Pearson symbol

: None

Space group number

: $\mathrm{mP} 12$

Space group symbol

: 13

AFLOW prototype command

: $\quad P 2 / c$

aflow - -proto=A2B_mP12_13_2g_ef

- params $=a, b / a, c / a, \beta, y_{1}, y_{2}, x_{3}, y_{3}, z_{3}, x_{4}, y_{4}, z_{4}$

- This structure was found by first-principles electronic structure calculations and is predicted to be the stable structure of $\mathrm{H}_{2} \mathrm{~S}$ in the range $10-30 \mathrm{GPa}$, which does not agree with the experimental phase diagram. (Shimizu, 1995)

- The data presented here was computed at $15 \mathrm{GPa}$.

\section{Simple Monoclinic primitive vectors:}

$$
\begin{array}{ccc}
\mathbf{a}_{1} & = & a \hat{\mathbf{x}} \\
\mathbf{a}_{2} & = & b \hat{\mathbf{y}} \\
\mathbf{a}_{3} & = & c \cos \beta \hat{\mathbf{x}}+c \sin \beta \hat{\mathbf{z}}
\end{array}
$$

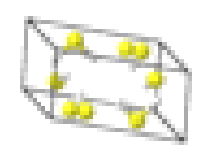

Basis vectors:

Lattice Coordinates

$\begin{array}{llllc}\mathbf{B}_{1}= & y_{1} \mathbf{a}_{2}+\frac{1}{4} \mathbf{a}_{3} & = & \frac{1}{4} c \cos \beta \hat{\mathbf{x}}+y_{1} b \hat{\mathbf{y}}+\frac{1}{4} c \sin \beta \hat{\mathbf{z}} \\ \mathbf{B}_{2}= & -y_{1} \mathbf{a}_{2}+\frac{3}{4} \mathbf{a}_{3} & = & \frac{3}{4} c \cos \beta \hat{\mathbf{x}}-y_{1} b \hat{\mathbf{y}}+\frac{3}{4} c \sin \beta \hat{\mathbf{z}} \\ \mathbf{B}_{3}= & \frac{1}{2} \mathbf{a}_{1}+y_{2} \mathbf{a}_{2}+\frac{1}{4} \mathbf{a}_{3} & = & \left(\frac{1}{2} a+\frac{1}{4} c \cos \beta\right) \hat{\mathbf{x}}+y_{2} b \hat{\mathbf{y}}+\frac{1}{4} c \sin \beta \hat{\mathbf{z}} \\ \mathbf{B}_{4}= & \frac{1}{2} \mathbf{a}_{1}-y_{2} \mathbf{a}_{2}+\frac{3}{4} \mathbf{a}_{3} & = & \left(\frac{1}{2} a+\frac{3}{4} c \cos \beta\right) \hat{\mathbf{x}}-y_{2} b \hat{\mathbf{y}}+\frac{3}{4} c \sin \beta \hat{\mathbf{z}} \\ \mathbf{B}_{5}= & x_{3} \mathbf{a}_{1}+y_{3} \mathbf{a}_{2}+z_{3} \mathbf{a}_{3}= & & \left(x_{3} a+z_{3} c \cos \beta\right) \hat{\mathbf{x}}+y_{3} b \hat{\mathbf{y}}+z_{3} c \sin \beta \hat{\mathbf{z}}\end{array}$

$\mathbf{B}_{1}=$

$=$
Wyckoff Position Atom Type

(2e)

(2e)

(2f)

S II

(2f)

S II

(4g) 


\begin{tabular}{|c|c|c|c|c|c|}
\hline $\mathbf{B}_{6}$ & $=$ & $-x_{3} \mathbf{a}_{1}+y_{3} \mathbf{a}_{2}+\left(\frac{1}{2}-z_{3}\right) \mathbf{a}_{3}$ & $=$ & $\begin{array}{c}\left(\frac{1}{2} c \cos \beta-x_{3} a-z_{3} c \cos \beta\right) \hat{\mathbf{x}}+y_{3} b \hat{\mathbf{y}}+ \\
\left(\frac{1}{2}-z_{3}\right) c \sin \beta \hat{\mathbf{z}}\end{array}$ & $(4 g)$ \\
\hline $\mathbf{B}_{7}$ & $=$ & $-x_{3} \mathbf{a}_{1}-y_{3} \mathbf{a}_{2}-z_{3} \mathbf{a}_{3}$ & $=$ & $\left(-x_{3} a-z_{3} c \cos \beta\right) \hat{\mathbf{x}}-y_{3} b \hat{\mathbf{y}}-z_{3} c \sin \beta \hat{\mathbf{z}}$ & $(4 g)$ \\
\hline $\mathbf{B}_{8}$ & $=$ & $x_{3} \mathbf{a}_{1}-y_{3} \mathbf{a}_{2}+\left(\frac{1}{2}+z_{3}\right) \mathbf{a}_{3}$ & $=$ & $\begin{array}{c}\left(\frac{1}{2} c \cos \beta+x_{3} a+z_{3} c \cos \beta\right) \hat{\mathbf{x}}-y_{3} b \hat{\mathbf{y}}+ \\
\left(\frac{1}{2}+z_{3}\right) c \sin \beta \hat{\mathbf{z}}\end{array}$ & $(4 g)$ \\
\hline $\mathbf{B}_{9}$ & $=$ & $x_{4} \mathbf{a}_{1}+y_{4} \mathbf{a}_{2}+z_{4} \mathbf{a}_{3}$ & $=$ & $\left(x_{4} a+z_{4} c \cos \beta\right) \hat{\mathbf{x}}+y_{4} b \hat{\mathbf{y}}+z_{4} c \sin \beta \hat{\mathbf{z}}$ & $(4 g)$ \\
\hline $\mathbf{B}_{10}$ & $=$ & $-x_{4} \mathbf{a}_{1}+y_{4} \mathbf{a}_{2}+\left(\frac{1}{2}-z_{4}\right) \mathbf{a}_{3}$ & $=$ & $\begin{array}{c}\left(\frac{1}{2} c \cos \beta-x_{4} a-z_{4} c \cos \beta\right) \hat{\mathbf{x}}+y_{4} b \hat{\mathbf{y}}+ \\
\left(\frac{1}{2}-z_{4}\right) c \sin \beta \hat{\mathbf{z}}\end{array}$ & $(4 g)$ \\
\hline $\mathbf{B}_{11}$ & $=$ & $-x_{4} \mathbf{a}_{1}-y_{4} \mathbf{a}_{2}-z_{4} \mathbf{a}_{3}$ & $=$ & $\left(-x_{4} a-z_{4} c \cos \beta\right) \hat{\mathbf{x}}-y_{4} b \hat{\mathbf{y}}-z_{4} c \sin \beta \hat{\mathbf{z}}$ & $(4 g)$ \\
\hline $\mathbf{B}_{12}$ & $=$ & $x_{4} \mathbf{a}_{1}-y_{4} \mathbf{a}_{2}+\left(\frac{1}{2}+z_{4}\right) \mathbf{a}_{3}$ & $=$ & $\begin{array}{c}\left(\frac{1}{2} c \cos \beta+x_{4} a+z_{4} c \cos \beta\right) \hat{\mathbf{x}}-y_{4} b \hat{\mathbf{y}}+ \\
\left(\frac{1}{2}+z_{4}\right) c \sin \beta \hat{\mathbf{z}}\end{array}$ & $(4 g)$ \\
\hline
\end{tabular}

\section{References:}

- Y. Li, J. Hao, H. Liu, Y. Li, and Y. Ma, The metallization and superconductivity of dense hydrogen sulfide, J. Chem. Phys. 140, 174712 (2014), doi:10.1063/1.4874158.

- H. Shimizu, H. Yamaguchi, S. Sasaki, A. Honda, S. Endo, and M. Kobayashi, Pressure-temperature phase diagram of solid hydrogen sulfide determined by Raman spectroscopy, Phys. Rev. B 51, 9391-9394 (1995), doi:10.1103/PhysRevB.51.9391.

\section{Geometry files:}

- CIF: pp. 809

- POSCAR: pp. 810 


\section{$\gamma-\mathrm{PdCl}_{2}$ Structure: A2B_mP6_14_e_a}
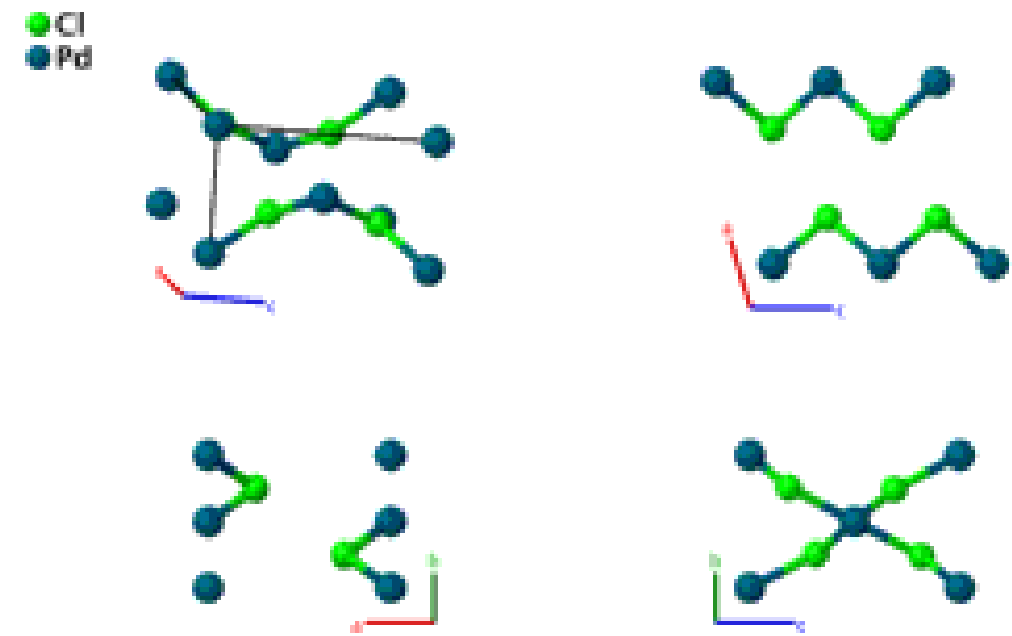

\section{Prototype}

AFLOW prototype label

$: \quad \gamma-\mathrm{PdCl}_{2}$

Strukturbericht designation

: A2B_mP6_14_e_a

Pearson symbol

: None

Space group number

: $\quad \mathrm{mP6}$

Space group symbol

: 14

AFLOW prototype command

: $\quad P 2_{1} / c$

aflow - -proto=A2B_mP6_14_e_a

- params $=a, b / a, c / a, \beta, x_{2}, y_{2}, z_{2}$

- (Evers, 2010) place the Pd atoms on the (2c) Wyckoff position. We have shifted the origin so that the Pd atoms are at the (2a) position.

- Data was taken at $300 \mathrm{~K}$.

\section{Simple Monoclinic primitive vectors:}

$$
\begin{array}{ccc}
\mathbf{a}_{1} & = & a \hat{\mathbf{x}} \\
\mathbf{a}_{2} & = & b \hat{\mathbf{y}} \\
\mathbf{a}_{3} & = & c \cos \beta \hat{\mathbf{x}}+c \sin \beta \hat{\mathbf{z}}
\end{array}
$$
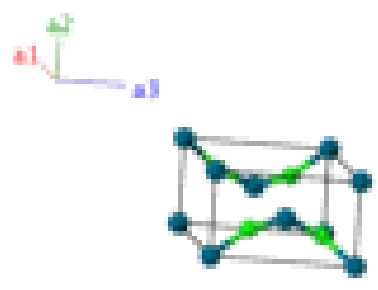

\section{Basis vectors:}

Lattice Coordinates

$\begin{array}{lc}\mathbf{B}_{1}= & 0 \mathbf{a}_{1}+0 \mathbf{a}_{2}+0 \mathbf{a}_{3} \\ \mathbf{B}_{2}= & \frac{1}{2} \mathbf{a}_{2}+\frac{1}{2} \mathbf{a}_{3} \\ \mathbf{B}_{3}= & x_{2} \mathbf{a}_{1}+y_{2} \mathbf{a}_{2}+z_{2} \mathbf{a}_{3}\end{array}$

Cartesian Coordinates

$=$

$=\quad \frac{1}{2} c \cos \beta \hat{\mathbf{x}}+\frac{1}{2} b \hat{\mathbf{y}}+\frac{1}{2} c \sin \beta \hat{\mathbf{z}}$

$=\quad\left(x_{2} a+z_{2} c \cos \beta\right) \hat{\mathbf{x}}+y_{2} b \hat{\mathbf{y}}+$ $z_{2} c \sin \beta \hat{\mathbf{z}}$
Wyckoff Position Atom Type

(2a)

Pd

(2a)

$\mathrm{Pd}$

$(4 e)$
$\mathrm{Cl}$ 


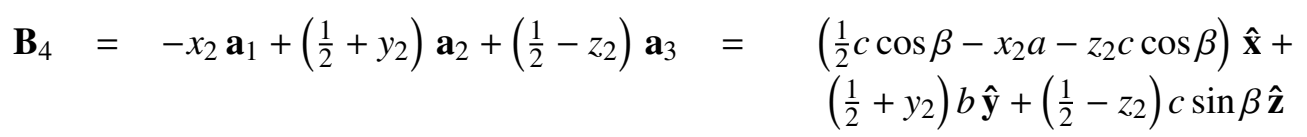

$\mathbf{B}_{5}=-x_{2} \mathbf{a}_{1}-y_{2} \mathbf{a}_{2}-z_{2} \mathbf{a}_{3}=\left(-x_{2} a-z_{2} c \cos \beta\right) \hat{\mathbf{x}}-y_{2} b \hat{\mathbf{y}}-$ $z_{2} c \sin \beta \hat{\mathbf{z}}$

$\mathbf{B}_{6}=x_{2} \mathbf{a}_{1}+\left(\frac{1}{2}-y_{2}\right) \mathbf{a}_{2}+\left(\frac{1}{2}+z_{2}\right) \mathbf{a}_{3}=\left(\frac{1}{2} c \cos \beta+x_{2} a+z_{2} c \cos \beta\right) \hat{\mathbf{x}}+$

$(4 e)$

$\mathrm{Cl}$

\section{References:}

- J. Evers, W. Beck, M. Göbel, S. Jakob, P. Mayer, G. Oehlinger, M. Rotter, and T. M. Klapötke, The Structures of $\delta$ - $P d C l_{2}$ and $\gamma-P_{C l} C_{2}$ : Phases with Negative Thermal Expansion in One Direction, Angew. Chem. Int. Ed. 49, 5677-5682 (2010), doi:10.1002/anie.201000680.

\section{Geometry files:}

- CIF: pp. 810

- POSCAR: pp. 810 


\section{$\alpha$-Toluene Structure: A7B8_mP120_14_14e_16e}
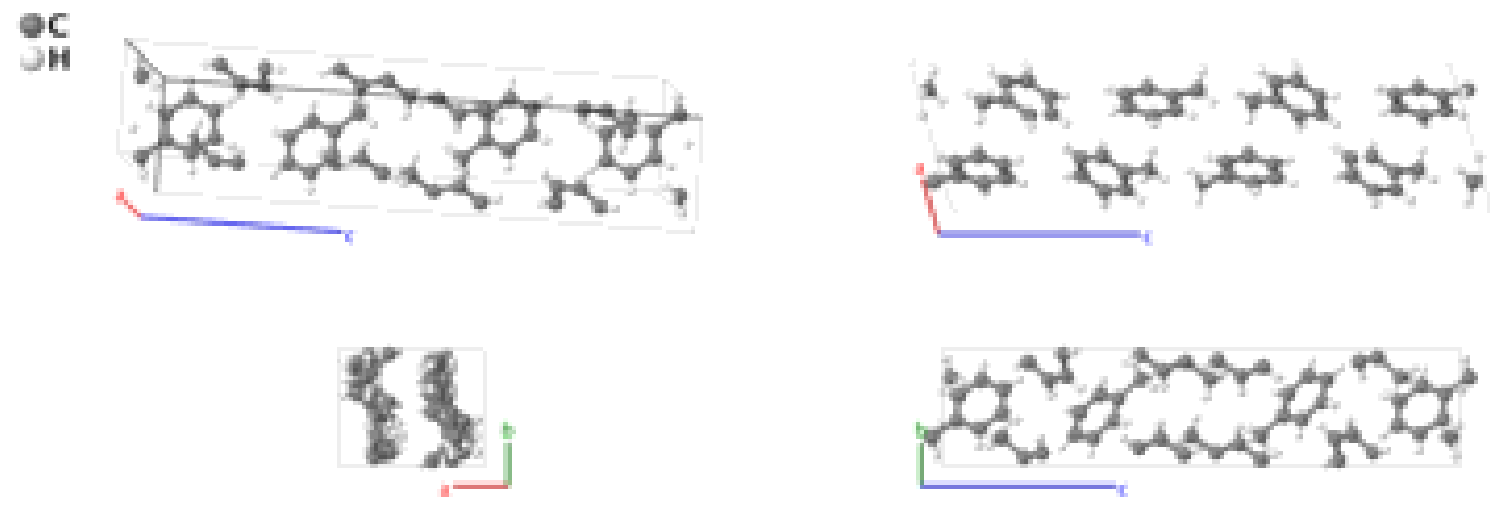

Prototype

AFLOW prototype label

$: \quad \alpha-\mathrm{C}_{7} \mathrm{H}_{8}$

Strukturbericht designation

: A7B8_mP120_14_14e_16e

Pearson symbol

: None

Space group number

$\mathrm{mP} 120$

Space group symbol

AFLOW prototype command

$P 2_{1} / c$

aflow --proto=A7B8_mP120_14_14e_16e

- - params $=a, b / a, c / a, \beta, x_{1}, y_{1}, z_{1}, x_{2}, y_{2}, z_{2}, x_{3}, y_{3}, z_{3}, x_{4}, y_{4}, z_{4}, x_{5}, y_{5}, z_{5}, x_{6}$, $y_{6}, z_{6}, x_{7}, y_{7}, z_{7}, x_{8}, y_{8}, z_{8}, x_{9}, y_{9}, z_{9}, x_{10}, y_{10}, z_{10}, x_{11}, y_{11}, z_{11}, x_{12}, y_{12}, z_{12}, x_{13}, y_{13}$, $z_{13}, x_{14}, y_{14}, z_{14}, x_{15}, y_{15}, z_{15}, x_{16}, y_{16}, z_{16}, x_{17}, y_{17}, z_{17}, x_{18}, y_{18}, z_{18}, x_{19}, y_{19}, z_{19}, x_{20}$, $y_{20}, z_{20}, x_{21}, y_{21}, z_{21}, x_{22}, y_{22}, z_{22}, x_{23}, y_{23}, z_{23}, x_{24}, y_{24}, z_{24}, x_{25}, y_{25}, z_{25}, x_{26}, y_{26}, z_{26}$, $x_{27}, y_{27}, z_{27}, x_{28}, y_{28}, z_{28}, x_{29}, y_{29}, z_{29}, x_{30}, y_{30}, z_{30}$

- $\alpha$-Toluene is the stable low-temperature crystalline structure of the toluene molecule, $\mathrm{C}_{7} \mathrm{H}_{8}$, which crystallizes below $178 \mathrm{~K}$. This data was constructed from experiments at $150 \mathrm{~K}$.

- The hydrogen atomic positions were approximated to agree with the chemistry of the toluene molecule.

\section{Simple Monoclinic primitive vectors:}

$$
\begin{array}{ccc}
\mathbf{a}_{1}= & a \hat{\mathbf{x}} \\
\mathbf{a}_{2}= & b \hat{\mathbf{y}} \\
\mathbf{a}_{3} & = & c \cos \beta \hat{\mathbf{x}}+c \sin \beta \hat{\mathbf{z}}
\end{array}
$$

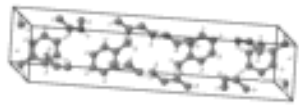

Basis vectors:

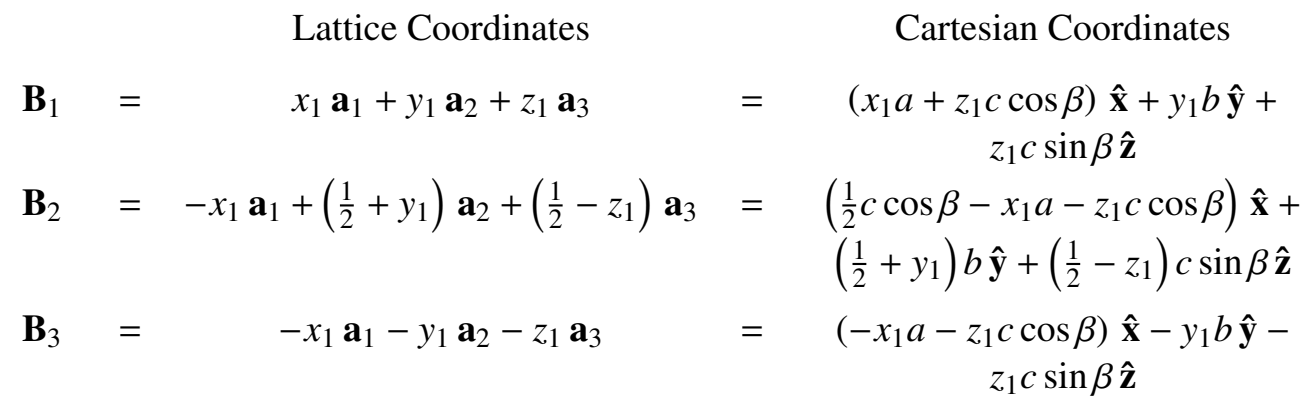

Wyckoff Position Atom Type

$(4 e)$

C I

$(4 e)$

C I 


\begin{tabular}{|c|c|c|c|c|c|c|}
\hline $\mathbf{B}_{4}$ & $=$ & $x_{1} \mathbf{a}_{1}+\left(\frac{1}{2}-y_{1}\right) \mathbf{a}_{2}+\left(\frac{1}{2}+z_{1}\right) \mathbf{a}_{3}$ & $=$ & $\begin{array}{l}\left(\frac{1}{2} c \cos \beta+x_{1} a+z_{1} c \cos \beta\right) \hat{\mathbf{x}}+ \\
\left(\frac{1}{2}-y_{1}\right) b \hat{\mathbf{y}}+\left(\frac{1}{2}+z_{1}\right) c \sin \beta \hat{\mathbf{z}}\end{array}$ & $(4 e)$ & C I \\
\hline $\mathbf{B}_{5}$ & $=$ & $x_{2} \mathbf{a}_{1}+y_{2} \mathbf{a}_{2}+z_{2} \mathbf{a}_{3}$ & $=$ & $\begin{array}{c}\left(x_{2} a+z_{2} c \cos \beta\right) \hat{\mathbf{x}}+y_{2} b \hat{\mathbf{y}}+ \\
z_{2} c \sin \beta \hat{\mathbf{z}}\end{array}$ & $(4 e)$ & C II \\
\hline $\mathbf{B}_{6}$ & $=$ & $-x_{2} \mathbf{a}_{1}+\left(\frac{1}{2}+y_{2}\right) \mathbf{a}_{2}+\left(\frac{1}{2}-z_{2}\right) \mathbf{a}_{3}$ & $=$ & $\begin{array}{c}\left(\frac{1}{2} c \cos \beta-x_{2} a-z_{2} c \cos \beta\right) \hat{\mathbf{x}}+ \\
\left(\frac{1}{2}+y_{2}\right) b \hat{\mathbf{y}}+\left(\frac{1}{2}-z_{2}\right) c \sin \beta \hat{\mathbf{z}}\end{array}$ & $(4 e)$ & C II \\
\hline $\mathbf{B}_{7}$ & $=$ & $-x_{2} \mathbf{a}_{1}-y_{2} \mathbf{a}_{2}-z_{2} \mathbf{a}_{3}$ & $=$ & $\begin{array}{c}\left(-x_{2} a-z_{2} c \cos \beta\right) \hat{\mathbf{x}}-y_{2} b \hat{\mathbf{y}}- \\
z_{2} c \sin \beta \hat{\mathbf{z}}\end{array}$ & $(4 e)$ & C II \\
\hline $\mathbf{B}_{8}$ & $=$ & $x_{2} \mathbf{a}_{1}+\left(\frac{1}{2}-y_{2}\right) \mathbf{a}_{2}+\left(\frac{1}{2}+z_{2}\right) \mathbf{a}_{3}$ & $=$ & $\begin{array}{c}\left(\frac{1}{2} c \cos \beta+x_{2} a+z_{2} c \cos \beta\right) \hat{\mathbf{x}}+ \\
\left(\frac{1}{2}-y_{2}\right) b \hat{\mathbf{y}}+\left(\frac{1}{2}+z_{2}\right) c \sin \beta \hat{\mathbf{z}}\end{array}$ & $(4 e)$ & C II \\
\hline $\mathbf{B}_{9}$ & $=$ & $x_{3} \mathbf{a}_{1}+y_{3} \mathbf{a}_{2}+z_{3} \mathbf{a}_{3}$ & $=$ & $\begin{array}{c}\left(x_{3} a+z_{3} c \cos \beta\right) \hat{\mathbf{x}}+y_{3} b \hat{\mathbf{y}}+ \\
z_{3} c \sin \beta \hat{\mathbf{z}}\end{array}$ & $(4 e)$ & C III \\
\hline $\mathbf{B}_{10}$ & $=$ & $-x_{3} \mathbf{a}_{1}+\left(\frac{1}{2}+y_{3}\right) \mathbf{a}_{2}+\left(\frac{1}{2}-z_{3}\right) \mathbf{a}_{3}$ & $=$ & $\begin{array}{c}\left(\frac{1}{2} c \cos \beta-x_{3} a-z_{3} c \cos \beta\right) \hat{\mathbf{x}}+ \\
\left(\frac{1}{2}+y_{3}\right) b \hat{\mathbf{y}}+\left(\frac{1}{2}-z_{3}\right) c \sin \beta \hat{\mathbf{z}}\end{array}$ & $(4 e)$ & C III \\
\hline $\mathbf{B}_{11}$ & $=$ & $-x_{3} \mathbf{a}_{1}-y_{3} \mathbf{a}_{2}-z_{3} \mathbf{a}_{3}$ & $=$ & $\begin{array}{c}\left(-x_{3} a-z_{3} c \cos \beta\right) \hat{\mathbf{x}}-y_{3} b \hat{\mathbf{y}}- \\
z_{3} c \sin \beta \hat{\mathbf{z}}\end{array}$ & $(4 e)$ & C III \\
\hline $\mathbf{B}_{12}$ & $=$ & $x_{3} \mathbf{a}_{1}+\left(\frac{1}{2}-y_{3}\right) \mathbf{a}_{2}+\left(\frac{1}{2}+z_{3}\right) \mathbf{a}_{3}$ & $=$ & $\begin{array}{c}\left(\frac{1}{2} c \cos \beta+x_{3} a+z_{3} c \cos \beta\right) \hat{\mathbf{x}}+ \\
\left(\frac{1}{2}-y_{3}\right) b \hat{\mathbf{y}}+\left(\frac{1}{2}+z_{3}\right) c \sin \beta \hat{\mathbf{z}}\end{array}$ & $(4 e)$ & C III \\
\hline $\mathbf{B}_{13}$ & $=$ & $x_{4} \mathbf{a}_{1}+y_{4} \mathbf{a}_{2}+z_{4} \mathbf{a}_{3}$ & $=$ & $\begin{array}{c}\left(x_{4} a+z_{4} c \cos \beta\right) \hat{\mathbf{x}}+y_{4} b \hat{\mathbf{y}}+ \\
z_{4} c \sin \beta \hat{\mathbf{z}}\end{array}$ & $(4 e)$ & C IV \\
\hline $\mathbf{B}_{14}$ & $=$ & $-x_{4} \mathbf{a}_{1}+\left(\frac{1}{2}+y_{4}\right) \mathbf{a}_{2}+\left(\frac{1}{2}-z_{4}\right) \mathbf{a}_{3}$ & $=$ & $\begin{array}{c}\left(\frac{1}{2} c \cos \beta-x_{4} a-z_{4} c \cos \beta\right) \hat{\mathbf{x}}+ \\
\left(\frac{1}{2}+y_{4}\right) b \hat{\mathbf{y}}+\left(\frac{1}{2}-z_{4}\right) c \sin \beta \hat{\mathbf{z}}\end{array}$ & $(4 e)$ & C IV \\
\hline $\mathbf{B}_{15}$ & $=$ & $-x_{4} \mathbf{a}_{1}-y_{4} \mathbf{a}_{2}-z_{4} \mathbf{a}_{3}$ & $=$ & $\begin{array}{c}\left(-x_{4} a-z_{4} c \cos \beta\right) \hat{\mathbf{x}}-y_{4} b \hat{\mathbf{y}}- \\
z_{4} c \sin \beta \hat{\mathbf{z}}\end{array}$ & $(4 e)$ & C IV \\
\hline $\mathbf{B}_{16}$ & $=$ & $x_{4} \mathbf{a}_{1}+\left(\frac{1}{2}-y_{4}\right) \mathbf{a}_{2}+\left(\frac{1}{2}+z_{4}\right) \mathbf{a}_{3}$ & $=$ & $\begin{array}{c}\left(\frac{1}{2} c \cos \beta+x_{4} a+z_{4} c \cos \beta\right) \hat{\mathbf{x}}+ \\
\left(\frac{1}{2}-y_{4}\right) b \hat{\mathbf{y}}+\left(\frac{1}{2}+z_{4}\right) c \sin \beta \hat{\mathbf{z}}\end{array}$ & $(4 e)$ & C IV \\
\hline $\mathbf{B}_{17}$ & $=$ & $x_{5} \mathbf{a}_{1}+y_{5} \mathbf{a}_{2}+z_{5} \mathbf{a}_{3}$ & $=$ & $\begin{array}{c}\left(x_{5} a+z_{5} c \cos \beta\right) \hat{\mathbf{x}}+y_{5} b \hat{\mathbf{y}}+ \\
z_{5} c \sin \beta \hat{\mathbf{z}}\end{array}$ & $(4 e)$ & $\mathrm{C} \mathrm{V}$ \\
\hline $\mathbf{B}_{18}$ & $=$ & $-x_{5} \mathbf{a}_{1}+\left(\frac{1}{2}+y_{5}\right) \mathbf{a}_{2}+\left(\frac{1}{2}-z_{5}\right) \mathbf{a}_{3}$ & $=$ & $\begin{array}{c}\left(\frac{1}{2} c \cos \beta-x_{5} a-z_{5} c \cos \beta\right) \hat{\mathbf{x}}+ \\
\left(\frac{1}{2}+y_{5}\right) b \hat{\mathbf{y}}+\left(\frac{1}{2}-z_{5}\right) c \sin \beta \hat{\mathbf{z}}\end{array}$ & $(4 e)$ & $\mathrm{C} \mathrm{V}$ \\
\hline $\mathbf{B}_{19}$ & $=$ & $-x_{5} \mathbf{a}_{1}-y_{5} \mathbf{a}_{2}-z_{5} \mathbf{a}_{3}$ & $=$ & $\begin{array}{c}\left(-x_{5} a-z_{5} c \cos \beta\right) \hat{\mathbf{x}}-y_{5} b \hat{\mathbf{y}}- \\
z_{5} c \sin \beta \hat{\mathbf{z}}\end{array}$ & $(4 e)$ & $\mathrm{C} \mathrm{V}$ \\
\hline $\mathbf{B}_{20}$ & $=$ & $x_{5} \mathbf{a}_{1}+\left(\frac{1}{2}-y_{5}\right) \mathbf{a}_{2}+\left(\frac{1}{2}+z_{5}\right) \mathbf{a}_{3}$ & $=$ & $\begin{array}{c}\left(\frac{1}{2} c \cos \beta+x_{5} a+z_{5} c \cos \beta\right) \hat{\mathbf{x}}+ \\
\left(\frac{1}{2}-y_{5}\right) b \hat{\mathbf{y}}+\left(\frac{1}{2}+z_{5}\right) c \sin \beta \hat{\mathbf{z}}\end{array}$ & $(4 e)$ & $\mathrm{C} \mathrm{V}$ \\
\hline $\mathbf{B}_{21}$ & $=$ & $x_{6} \mathbf{a}_{1}+y_{6} \mathbf{a}_{2}+z_{6} \mathbf{a}_{3}$ & $=$ & $\begin{array}{c}\left(x_{6} a+z_{6} c \cos \beta\right) \hat{\mathbf{x}}+y_{6} b \hat{\mathbf{y}}+ \\
z_{6} c \sin \beta \hat{\mathbf{z}}\end{array}$ & $(4 e)$ & C VI \\
\hline $\mathbf{B}_{22}$ & $=$ & $-x_{6} \mathbf{a}_{1}+\left(\frac{1}{2}+y_{6}\right) \mathbf{a}_{2}+\left(\frac{1}{2}-z_{6}\right) \mathbf{a}_{3}$ & $=$ & $\begin{array}{l}\left(\frac{1}{2} c \cos \beta-x_{6} a-z_{6} c \cos \beta\right) \hat{\mathbf{x}}+ \\
\left(\frac{1}{2}+y_{6}\right) b \hat{\mathbf{y}}+\left(\frac{1}{2}-z_{6}\right) c \sin \beta \hat{\mathbf{z}}\end{array}$ & $(4 e)$ & C VI \\
\hline $\mathbf{B}_{23}$ & $=$ & $-x_{6} \mathbf{a}_{1}-y_{6} \mathbf{a}_{2}-z_{6} \mathbf{a}_{3}$ & $=$ & $\begin{array}{c}\left(-x_{6} a-z_{6} c \cos \beta\right) \hat{\mathbf{x}}-y_{6} b \hat{\mathbf{y}}- \\
z_{6} c \sin \beta \hat{\mathbf{z}}\end{array}$ & $(4 e)$ & C VI \\
\hline $\mathbf{B}_{24}$ & $=$ & $x_{6} \mathbf{a}_{1}+\left(\frac{1}{2}-y_{6}\right) \mathbf{a}_{2}+\left(\frac{1}{2}+z_{6}\right) \mathbf{a}_{3}$ & $=$ & $\begin{array}{c}\left(\frac{1}{2} c \cos \beta+x_{6} a+z_{6} c \cos \beta\right) \hat{\mathbf{x}}+ \\
\left(\frac{1}{2}-y_{6}\right) b \hat{\mathbf{y}}+\left(\frac{1}{2}+z_{6}\right) c \sin \beta \hat{\mathbf{z}}\end{array}$ & $(4 e)$ & C VI \\
\hline $\mathbf{B}_{25}$ & $=$ & $x_{7} \mathbf{a}_{1}+y_{7} \mathbf{a}_{2}+z_{7} \mathbf{a}_{3}$ & $=$ & $\begin{array}{c}\left(x_{7} a+z_{7} c \cos \beta\right) \hat{\mathbf{x}}+y_{7} b \hat{\mathbf{y}}+ \\
z_{7} c \sin \beta \hat{\mathbf{z}}\end{array}$ & $(4 e)$ & C VII \\
\hline
\end{tabular}




\begin{tabular}{|c|c|c|c|c|c|c|}
\hline $\mathbf{B}_{26}$ & $=$ & $-x_{7} \mathbf{a}_{1}+\left(\frac{1}{2}+y_{7}\right) \mathbf{a}_{2}+\left(\frac{1}{2}-z_{7}\right) \mathbf{a}_{3}$ & $=$ & $\begin{array}{r}\left(\frac{1}{2} c \cos \beta-x_{7} a-z_{7} c \cos \beta\right) \hat{\mathbf{x}}+ \\
\left(\frac{1}{2}+y_{7}\right) b \hat{\mathbf{y}}+\left(\frac{1}{2}-z_{7}\right) c \sin \beta \hat{\mathbf{z}}\end{array}$ & $(4 e)$ & C VII \\
\hline $\mathbf{B}_{27}$ & $=$ & $-x_{7} \mathbf{a}_{1}-y_{7} \mathbf{a}_{2}-z_{7} \mathbf{a}_{3}$ & $=$ & $\begin{array}{c}\left(-x_{7} a-z_{7} c \cos \beta\right) \hat{\mathbf{x}}-y_{7} b \hat{\mathbf{y}}- \\
z_{7} c \sin \beta \hat{\mathbf{z}}\end{array}$ & $(4 e)$ & C VII \\
\hline $\mathbf{B}_{28}$ & $=$ & $x_{7} \mathbf{a}_{1}+\left(\frac{1}{2}-y_{7}\right) \mathbf{a}_{2}+\left(\frac{1}{2}+z_{7}\right) \mathbf{a}_{3}$ & $=$ & $\begin{array}{c}\left(\frac{1}{2} c \cos \beta+x_{7} a+z_{7} c \cos \beta\right) \hat{\mathbf{x}}+ \\
\left(\frac{1}{2}-y_{7}\right) b \hat{\mathbf{y}}+\left(\frac{1}{2}+z_{7}\right) c \sin \beta \hat{\mathbf{z}}\end{array}$ & $(4 e)$ & C VII \\
\hline $\mathbf{B}_{29}$ & $=$ & $x_{8} \mathbf{a}_{1}+y_{8} \mathbf{a}_{2}+z_{8} \mathbf{a}_{3}$ & $=$ & $\begin{array}{c}\left(x_{8} a+z_{8} c \cos \beta\right) \hat{\mathbf{x}}+y_{8} b \hat{\mathbf{y}}+ \\
z_{8} c \sin \beta \hat{\mathbf{z}}\end{array}$ & $(4 e)$ & C VIII \\
\hline $\mathbf{B}_{30}$ & $=$ & $-x_{8} \mathbf{a}_{1}+\left(\frac{1}{2}+y_{8}\right) \mathbf{a}_{2}+\left(\frac{1}{2}-z_{8}\right) \mathbf{a}_{3}$ & $=$ & $\begin{array}{c}\left(\frac{1}{2} c \cos \beta-x_{8} a-z_{8} c \cos \beta\right) \hat{\mathbf{x}}+ \\
\left(\frac{1}{2}+y_{8}\right) b \hat{\mathbf{y}}+\left(\frac{1}{2}-z_{8}\right) c \sin \beta \hat{\mathbf{z}}\end{array}$ & $(4 e)$ & C VIII \\
\hline $\mathbf{B}_{31}$ & $=$ & $-x_{8} \mathbf{a}_{1}-y_{8} \mathbf{a}_{2}-z_{8} \mathbf{a}_{3}$ & $=$ & $\begin{array}{c}\left(-x_{8} a-z_{8} c \cos \beta\right) \hat{\mathbf{x}}-y_{8} b \hat{\mathbf{y}}- \\
z_{8} c \sin \beta \hat{\mathbf{z}}\end{array}$ & $(4 e)$ & C VIII \\
\hline $\mathbf{B}_{32}$ & $=$ & $x_{8} \mathbf{a}_{1}+\left(\frac{1}{2}-y_{8}\right) \mathbf{a}_{2}+\left(\frac{1}{2}+z_{8}\right) \mathbf{a}_{3}$ & $=$ & $\begin{array}{c}\left(\frac{1}{2} c \cos \beta+x_{8} a+z_{8} c \cos \beta\right) \hat{\mathbf{x}}+ \\
\left(\frac{1}{2}-y_{8}\right) b \hat{\mathbf{y}}+\left(\frac{1}{2}+z_{8}\right) c \sin \beta \hat{\mathbf{z}}\end{array}$ & $(4 e)$ & C VIII \\
\hline $\mathbf{B}_{33}$ & $=$ & $x_{9} \mathbf{a}_{1}+y_{9} \mathbf{a}_{2}+z_{9} \mathbf{a}_{3}$ & $=$ & $\begin{array}{c}\left(x_{9} a+z_{9} c \cos \beta\right) \hat{\mathbf{x}}+y_{9} b \hat{\mathbf{y}}+ \\
z_{9} c \sin \beta \hat{\mathbf{z}}\end{array}$ & $(4 e)$ & C IX \\
\hline $\mathbf{B}_{34}$ & $=$ & $-x_{9} \mathbf{a}_{1}+\left(\frac{1}{2}+y_{9}\right) \mathbf{a}_{2}+\left(\frac{1}{2}-z_{9}\right) \mathbf{a}_{3}$ & $=$ & $\begin{array}{c}\left(\frac{1}{2} c \cos \beta-x_{9} a-z_{9} c \cos \beta\right) \hat{\mathbf{x}}+ \\
\left(\frac{1}{2}+y_{9}\right) b \hat{\mathbf{y}}+\left(\frac{1}{2}-z_{9}\right) c \sin \beta \hat{\mathbf{z}}\end{array}$ & $(4 e)$ & C IX \\
\hline $\mathbf{B}_{35}$ & $=$ & $-x_{9} \mathbf{a}_{1}-y_{9} \mathbf{a}_{2}-z_{9} \mathbf{a}_{3}$ & $=$ & $\begin{array}{c}\left(-x_{9} a-z_{9} c \cos \beta\right) \hat{\mathbf{x}}-y_{9} b \hat{\mathbf{y}}- \\
z_{9} c \sin \beta \hat{\mathbf{z}}\end{array}$ & $(4 e)$ & C IX \\
\hline $\mathbf{B}_{36}$ & $=$ & $x_{9} \mathbf{a}_{1}+\left(\frac{1}{2}-y_{9}\right) \mathbf{a}_{2}+\left(\frac{1}{2}+z_{9}\right) \mathbf{a}_{3}$ & $=$ & $\begin{array}{c}\left(\frac{1}{2} c \cos \beta+x_{9} a+z_{9} c \cos \beta\right) \hat{\mathbf{x}}+ \\
\left(\frac{1}{2}-y_{9}\right) b \hat{\mathbf{y}}+\left(\frac{1}{2}+z_{9}\right) c \sin \beta \hat{\mathbf{z}}\end{array}$ & $(4 e)$ & C IX \\
\hline $\mathbf{B}_{37}$ & $=$ & $x_{10} \mathbf{a}_{1}+y_{10} \mathbf{a}_{2}+z_{10} \mathbf{a}_{3}$ & $=$ & $\begin{array}{c}\left(x_{10} a+z_{10} c \cos \beta\right) \hat{\mathbf{x}}+y_{10} b \hat{\mathbf{y}}+ \\
z_{10} c \sin \beta \hat{\mathbf{z}}\end{array}$ & $(4 e)$ & $\mathrm{CX}$ \\
\hline $\mathbf{B}_{38}$ & $=$ & $\begin{array}{c}-x_{10} \mathbf{a}_{1}+\left(\frac{1}{2}+y_{10}\right) \mathbf{a}_{2}+ \\
\left(\frac{1}{2}-z_{10}\right) \mathbf{a}_{3}\end{array}$ & $=$ & $\begin{array}{c}\left(\frac{1}{2} c \cos \beta-x_{10} a-z_{10} c \cos \beta\right) \hat{\mathbf{x}}+ \\
\left(\frac{1}{2}+y_{10}\right) b \hat{\mathbf{y}}+\left(\frac{1}{2}-z_{10}\right) c \sin \beta \hat{\mathbf{z}}\end{array}$ & $(4 e)$ & $\mathrm{CX}$ \\
\hline $\mathbf{B}_{39}$ & $=$ & $-x_{10} \mathbf{a}_{1}-y_{10} \mathbf{a}_{2}-z_{10} \mathbf{a}_{3}$ & $=$ & $\begin{array}{c}\left(-x_{10} a-z_{10} c \cos \beta\right) \hat{\mathbf{x}}-y_{10} b \hat{\mathbf{y}}- \\
z_{10} c \sin \beta \hat{\mathbf{z}}\end{array}$ & $(4 e)$ & $\mathrm{CX}$ \\
\hline $\mathbf{B}_{40}$ & $=$ & $x_{10} \mathbf{a}_{1}+\left(\frac{1}{2}-y_{10}\right) \mathbf{a}_{2}+\left(\frac{1}{2}+z_{10}\right) \mathbf{a}_{3}$ & $=$ & $\begin{array}{c}\left(\frac{1}{2} c \cos \beta+x_{10} a+z_{10} c \cos \beta\right) \hat{\mathbf{x}}+ \\
\left(\frac{1}{2}-y_{10}\right) b \hat{\mathbf{y}}+\left(\frac{1}{2}+z_{10}\right) c \sin \beta \hat{\mathbf{z}}\end{array}$ & $(4 e)$ & $\mathrm{CX}$ \\
\hline $\mathbf{B}_{41}$ & $=$ & $x_{11} \mathbf{a}_{1}+y_{11} \mathbf{a}_{2}+z_{11} \mathbf{a}_{3}$ & $=$ & $\begin{array}{c}\left(x_{11} a+z_{11} c \cos \beta\right) \hat{\mathbf{x}}+y_{11} b \hat{\mathbf{y}}+ \\
z_{11} c \sin \beta \hat{\mathbf{z}}\end{array}$ & $(4 e)$ & C XI \\
\hline $\mathbf{B}_{42}$ & $=$ & $\begin{array}{c}-x_{11} \mathbf{a}_{1}+\left(\frac{1}{2}+y_{11}\right) \mathbf{a}_{2}+ \\
\left(\frac{1}{2}-z_{11}\right) \mathbf{a}_{3}\end{array}$ & $=$ & $\begin{array}{c}\left(\frac{1}{2} c \cos \beta-x_{11} a-z_{11} c \cos \beta\right) \hat{\mathbf{x}}+ \\
\left(\frac{1}{2}+y_{11}\right) b \hat{\mathbf{y}}+\left(\frac{1}{2}-z_{11}\right) c \sin \beta \hat{\mathbf{z}}\end{array}$ & $(4 e)$ & C XI \\
\hline $\mathbf{B}_{43}$ & $=$ & $-x_{11} \mathbf{a}_{1}-y_{11} \mathbf{a}_{2}-z_{11} \mathbf{a}_{3}$ & $=$ & $\begin{array}{c}\left(-x_{11} a-z_{11} c \cos \beta\right) \hat{\mathbf{x}}-y_{11} b \hat{\mathbf{y}}- \\
z_{11} c \sin \beta \hat{\mathbf{z}}\end{array}$ & $(4 e)$ & C XI \\
\hline $\mathbf{B}_{44}$ & $=$ & $x_{11} \mathbf{a}_{1}+\left(\frac{1}{2}-y_{11}\right) \mathbf{a}_{2}+\left(\frac{1}{2}+z_{11}\right) \mathbf{a}_{3}$ & $=$ & $\begin{array}{c}\left(\frac{1}{2} c \cos \beta+x_{11} a+z_{11} c \cos \beta\right) \hat{\mathbf{x}}+ \\
\left(\frac{1}{2}-y_{11}\right) b \hat{\mathbf{y}}+\left(\frac{1}{2}+z_{11}\right) c \sin \beta \hat{\mathbf{z}}\end{array}$ & $(4 e)$ & C XI \\
\hline $\mathbf{B}_{45}$ & $=$ & $x_{12} \mathbf{a}_{1}+y_{12} \mathbf{a}_{2}+z_{12} \mathbf{a}_{3}$ & $=$ & $\begin{array}{c}\left(x_{12} a+z_{12} c \cos \beta\right) \hat{\mathbf{x}}+y_{12} b \hat{\mathbf{y}}+ \\
z_{12} c \sin \beta \hat{\mathbf{z}}\end{array}$ & $(4 e)$ & C XII \\
\hline $\mathbf{B}_{46}$ & $=$ & $\begin{array}{c}-x_{12} \mathbf{a}_{1}+\left(\frac{1}{2}+y_{12}\right) \mathbf{a}_{2}+ \\
\left(\frac{1}{2}-z_{12}\right) \mathbf{a}_{3}\end{array}$ & $=$ & $\begin{array}{c}\left(\frac{1}{2} c \cos \beta-x_{12} a-z_{12} c \cos \beta\right) \hat{\mathbf{x}}+ \\
\left(\frac{1}{2}+y_{12}\right) b \hat{\mathbf{y}}+\left(\frac{1}{2}-z_{12}\right) c \sin \beta \hat{\mathbf{z}}\end{array}$ & $(4 e)$ & C XII \\
\hline $\mathbf{B}_{47}$ & $=$ & $-x_{12} \mathbf{a}_{1}-y_{12} \mathbf{a}_{2}-z_{12} \mathbf{a}_{3}$ & $=$ & $\begin{array}{c}\left(-x_{12} a-z_{12} c \cos \beta\right) \hat{\mathbf{x}}-y_{12} b \hat{\mathbf{y}}- \\
z_{12} c \sin \beta \hat{\mathbf{z}}\end{array}$ & $(4 e)$ & C XII \\
\hline
\end{tabular}




$$
\begin{aligned}
& \mathbf{B}_{48}=x_{12} \mathbf{a}_{1}+\left(\frac{1}{2}-y_{12}\right) \mathbf{a}_{2}+\left(\frac{1}{2}+z_{12}\right) \mathbf{a}_{3}=\left(\frac{1}{2} c \cos \beta+x_{12} a+z_{12} c \cos \beta\right) \hat{\mathbf{x}}+ \\
& \left(\frac{1}{2}-y_{12}\right) b \hat{\mathbf{y}}+\left(\frac{1}{2}+z_{12}\right) c \sin \beta \hat{\mathbf{z}} \\
& \mathbf{B}_{49}=x_{13} \mathbf{a}_{1}+y_{13} \mathbf{a}_{2}+z_{13} \mathbf{a}_{3} \quad=\quad\left(x_{13} a+z_{13} c \cos \beta\right) \hat{\mathbf{x}}+y_{13} b \hat{\mathbf{y}}+ \\
& z_{13} c \sin \beta \hat{\mathbf{z}} \\
& \mathbf{B}_{50}=-x_{13} \mathbf{a}_{1}+\left(\frac{1}{2}+y_{13}\right) \mathbf{a}_{2}+\quad=\left(\frac{1}{2} c \cos \beta-x_{13} a-z_{13} c \cos \beta\right) \hat{\mathbf{x}}+ \\
& \left(\frac{1}{2}-z_{13}\right) \mathbf{a}_{3} \quad\left(\frac{1}{2}+y_{13}\right) b \hat{\mathbf{y}}+\left(\frac{1}{2}-z_{13}\right) c \sin \beta \hat{\mathbf{z}} \\
& \mathbf{B}_{51}=-x_{13} \mathbf{a}_{1}-y_{13} \mathbf{a}_{2}-z_{13} \mathbf{a}_{3}=\left(-x_{13} a-z_{13} c \cos \beta\right) \hat{\mathbf{x}}-y_{13} b \hat{\mathbf{y}}- \\
& z_{13} c \sin \beta \hat{\mathbf{z}} \\
& \mathbf{B}_{52}=x_{13} \mathbf{a}_{1}+\left(\frac{1}{2}-y_{13}\right) \mathbf{a}_{2}+\left(\frac{1}{2}+z_{13}\right) \mathbf{a}_{3}=\left(\frac{1}{2} c \cos \beta+x_{13} a+z_{13} c \cos \beta\right) \hat{\mathbf{x}}+ \\
& \left(\frac{1}{2}-y_{13}\right) b \hat{\mathbf{y}}+\left(\frac{1}{2}+z_{13}\right) c \sin \beta \hat{\mathbf{z}} \\
& \mathbf{B}_{53}=x_{14} \mathbf{a}_{1}+y_{14} \mathbf{a}_{2}+z_{14} \mathbf{a}_{3} \quad=\quad\left(x_{14} a+z_{14} c \cos \beta\right) \hat{\mathbf{x}}+y_{14} b \hat{\mathbf{y}}+ \\
& z_{14} c \sin \beta \hat{\mathbf{z}} \\
& \mathbf{B}_{54}=-x_{14} \mathbf{a}_{1}+\left(\frac{1}{2}+y_{14}\right) \mathbf{a}_{2}+\quad=\left(\frac{1}{2} c \cos \beta-x_{14} a-z_{14} c \cos \beta\right) \hat{\mathbf{x}}+ \\
& \left(\frac{1}{2}-z_{14}\right) \mathbf{a}_{3} \quad\left(\frac{1}{2}+y_{14}\right) b \hat{\mathbf{y}}+\left(\frac{1}{2}-z_{14}\right) c \sin \beta \hat{\mathbf{z}} \\
& \mathbf{B}_{55}=-x_{14} \mathbf{a}_{1}-y_{14} \mathbf{a}_{2}-z_{14} \mathbf{a}_{3}=\left(-x_{14} a-z_{14} c \cos \beta\right) \hat{\mathbf{x}}-y_{14} b \hat{\mathbf{y}}- \\
& z_{14} c \sin \beta \hat{\mathbf{z}} \\
& \mathbf{B}_{56}=x_{14} \mathbf{a}_{1}+\left(\frac{1}{2}-y_{14}\right) \mathbf{a}_{2}+\left(\frac{1}{2}+z_{14}\right) \mathbf{a}_{3}=\left(\frac{1}{2} c \cos \beta+x_{14} a+z_{14} c \cos \beta\right) \hat{\mathbf{x}}+ \\
& \left(\frac{1}{2}-y_{14}\right) b \hat{\mathbf{y}}+\left(\frac{1}{2}+z_{14}\right) c \sin \beta \hat{\mathbf{z}} \\
& \mathbf{B}_{57}=x_{15} \mathbf{a}_{1}+y_{15} \mathbf{a}_{2}+z_{15} \mathbf{a}_{3} \quad=\quad\left(x_{15} a+z_{15} c \cos \beta\right) \hat{\mathbf{x}}+y_{15} b \hat{\mathbf{y}}+ \\
& z_{15} c \sin \beta \hat{\mathbf{z}} \\
& \mathbf{B}_{58}=-x_{15} \mathbf{a}_{1}+\left(\frac{1}{2}+y_{15}\right) \mathbf{a}_{2}+\quad=\left(\frac{1}{2} c \cos \beta-x_{15} a-z_{15} c \cos \beta\right) \hat{\mathbf{x}}+ \\
& \left(\frac{1}{2}-z_{15}\right) \mathbf{a}_{3} \\
& \left(\frac{1}{2}+y_{15}\right) b \hat{\mathbf{y}}+\left(\frac{1}{2}-z_{15}\right) c \sin \beta \hat{\mathbf{z}} \\
& \mathbf{B}_{59}=-x_{15} \mathbf{a}_{1}-y_{15} \mathbf{a}_{2}-z_{15} \mathbf{a}_{3}=\left(-x_{15} a-z_{15} c \cos \beta\right) \hat{\mathbf{x}}-y_{15} b \hat{\mathbf{y}}- \\
& z_{15} c \sin \beta \hat{\mathbf{z}} \\
& \mathbf{B}_{60}=x_{15} \mathbf{a}_{1}+\left(\frac{1}{2}-y_{15}\right) \mathbf{a}_{2}+\left(\frac{1}{2}+z_{15}\right) \mathbf{a}_{3}=\left(\frac{1}{2} c \cos \beta+x_{15} a+z_{15} c \cos \beta\right) \hat{\mathbf{x}}+ \\
& \left(\frac{1}{2}-y_{15}\right) b \hat{\mathbf{y}}+\left(\frac{1}{2}+z_{15}\right) c \sin \beta \hat{\mathbf{z}} \\
& \mathbf{B}_{61}=x_{16} \mathbf{a}_{1}+y_{16} \mathbf{a}_{2}+z_{16} \mathbf{a}_{3}=\left(x_{16} a+z_{16} c \cos \beta\right) \hat{\mathbf{x}}+y_{16} b \hat{\mathbf{y}}+ \\
& z_{16} c \sin \beta \hat{\mathbf{z}} \\
& \mathbf{B}_{62}=-x_{16} \mathbf{a}_{1}+\left(\frac{1}{2}+y_{16}\right) \mathbf{a}_{2}+\quad=\left(\frac{1}{2} c \cos \beta-x_{16} a-z_{16} c \cos \beta\right) \hat{\mathbf{x}}+ \\
& \left(\frac{1}{2}-z_{16}\right) \mathbf{a}_{3} \quad\left(\frac{1}{2}+y_{16}\right) b \hat{\mathbf{y}}+\left(\frac{1}{2}-z_{16}\right) c \sin \beta \hat{\mathbf{z}} \\
& \mathbf{B}_{63}=-x_{16} \mathbf{a}_{1}-y_{16} \mathbf{a}_{2}-z_{16} \mathbf{a}_{3}=\left(-x_{16} a-z_{16} c \cos \beta\right) \hat{\mathbf{x}}-y_{16} b \hat{\mathbf{y}}- \\
& z_{16} c \sin \beta \hat{\mathbf{z}} \\
& \mathbf{B}_{64}=x_{16} \mathbf{a}_{1}+\left(\frac{1}{2}-y_{16}\right) \mathbf{a}_{2}+\left(\frac{1}{2}+z_{16}\right) \mathbf{a}_{3}=\left(\frac{1}{2} c \cos \beta+x_{16} a+z_{16} c \cos \beta\right) \hat{\mathbf{x}}+ \\
& \left(\frac{1}{2}-y_{16}\right) b \hat{\mathbf{y}}+\left(\frac{1}{2}+z_{16}\right) c \sin \beta \hat{\mathbf{z}} \\
& \mathbf{B}_{65}=x_{17} \mathbf{a}_{1}+y_{17} \mathbf{a}_{2}+z_{17} \mathbf{a}_{3} \quad=\quad\left(x_{17} a+z_{17} c \cos \beta\right) \hat{\mathbf{x}}+y_{17} b \hat{\mathbf{y}}+ \\
& z_{17} c \sin \beta \hat{\mathbf{z}} \\
& \mathbf{B}_{66}=-x_{17} \mathbf{a}_{1}+\left(\frac{1}{2}+y_{17}\right) \mathbf{a}_{2}+\quad=\left(\frac{1}{2} c \cos \beta-x_{17} a-z_{17} c \cos \beta\right) \hat{\mathbf{x}}+ \\
& \left(\frac{1}{2}-z_{17}\right) \mathbf{a}_{3} \quad\left(\frac{1}{2}+y_{17}\right) b \hat{\mathbf{y}}+\left(\frac{1}{2}-z_{17}\right) c \sin \beta \hat{\mathbf{z}} \\
& \mathbf{B}_{67}=-x_{17} \mathbf{a}_{1}-y_{17} \mathbf{a}_{2}-z_{17} \mathbf{a}_{3}=\left(-x_{17} a-z_{17} c \cos \beta\right) \hat{\mathbf{x}}-y_{17} b \hat{\mathbf{y}}- \\
& z_{17} c \sin \beta \hat{\mathbf{z}} \\
& \mathbf{B}_{68}=x_{17} \mathbf{a}_{1}+\left(\frac{1}{2}-y_{17}\right) \mathbf{a}_{2}+\left(\frac{1}{2}+z_{17}\right) \mathbf{a}_{3}=\left(\frac{1}{2} c \cos \beta+x_{17} a+z_{17} c \cos \beta\right) \hat{\mathbf{x}}+ \\
& \left(\frac{1}{2}-y_{17}\right) b \hat{\mathbf{y}}+\left(\frac{1}{2}+z_{17}\right) c \sin \beta \hat{\mathbf{z}} \\
& \mathbf{B}_{69}=x_{18} \mathbf{a}_{1}+y_{18} \mathbf{a}_{2}+z_{18} \mathbf{a}_{3} \quad=\quad\left(x_{18} a+z_{18} c \cos \beta\right) \hat{\mathbf{x}}+y_{18} b \hat{\mathbf{y}}+ \\
& z_{18} c \sin \beta \hat{\mathbf{z}}
\end{aligned}
$$




\begin{tabular}{|c|c|c|c|c|c|}
\hline $\mathbf{B}_{70}$ & $=$ & $\begin{array}{c}-x_{18} \mathbf{a}_{1}+\left(\frac{1}{2}+y_{18}\right) \mathbf{a}_{2}+ \\
\left(\frac{1}{2}-z_{18}\right) \mathbf{a}_{3}\end{array}$ & $\begin{aligned}= & \left(\frac{1}{2} c \cos \beta-x_{18} a-z_{18} c \cos \beta\right) \hat{\mathbf{x}}+ \\
& \left(\frac{1}{2}+y_{18}\right) b \hat{\mathbf{y}}+\left(\frac{1}{2}-z_{18}\right) c \sin \beta \hat{\mathbf{z}}\end{aligned}$ & $(4 e)$ & H IV \\
\hline $\mathbf{B}_{71}$ & $=$ & $-x_{18} \mathbf{a}_{1}-y_{18} \mathbf{a}_{2}-z_{18} \mathbf{a}_{3}$ & $\begin{array}{c}=\left(-x_{18} a-z_{18} c \cos \beta\right) \hat{\mathbf{x}}-y_{18} b \hat{\mathbf{y}}- \\
z_{18} c \sin \beta \hat{\mathbf{z}}\end{array}$ & $(4 e)$ & H IV \\
\hline $\mathbf{B}_{72}$ & $=$ & $x_{18} \mathbf{a}_{1}+\left(\frac{1}{2}-y_{18}\right) \mathbf{a}_{2}+\left(\frac{1}{2}+z_{18}\right) \mathbf{a}_{3}$ & $\begin{aligned}= & \left(\frac{1}{2} c \cos \beta+x_{18} a+z_{18} c \cos \beta\right) \hat{\mathbf{x}}+ \\
& \left(\frac{1}{2}-y_{18}\right) b \hat{\mathbf{y}}+\left(\frac{1}{2}+z_{18}\right) c \sin \beta \hat{\mathbf{z}}\end{aligned}$ & $(4 e)$ & H IV \\
\hline $\mathbf{B}_{73}$ & $=$ & $x_{19} \mathbf{a}_{1}+y_{19} \mathbf{a}_{2}+z_{19} \mathbf{a}_{3}$ & $\begin{array}{c}=\quad\left(x_{19} a+z_{19} c \cos \beta\right) \hat{\mathbf{x}}+y_{19} b \hat{\mathbf{y}}+ \\
z_{19} c \sin \beta \hat{\mathbf{z}}\end{array}$ & $(4 e)$ & $\mathrm{H} \mathrm{V}$ \\
\hline $\mathbf{B}_{74}$ & $=$ & $\begin{array}{c}-x_{19} \mathbf{a}_{1}+\left(\frac{1}{2}+y_{19}\right) \mathbf{a}_{2}+ \\
\left(\frac{1}{2}-z_{19}\right) \mathbf{a}_{3}\end{array}$ & $\begin{aligned}= & \left(\frac{1}{2} c \cos \beta-x_{19} a-z_{19} c \cos \beta\right) \hat{\mathbf{x}}+ \\
& \left(\frac{1}{2}+y_{19}\right) b \hat{\mathbf{y}}+\left(\frac{1}{2}-z_{19}\right) c \sin \beta \hat{\mathbf{z}}\end{aligned}$ & $(4 e)$ & $\mathrm{H} \mathrm{V}$ \\
\hline $\mathbf{B}_{75}$ & $=$ & $-x_{19} \mathbf{a}_{1}-y_{19} \mathbf{a}_{2}-z_{19} \mathbf{a}_{3}$ & $\begin{array}{c}=\left(-x_{19} a-z_{19} c \cos \beta\right) \hat{\mathbf{x}}-y_{19} b \hat{\mathbf{y}}- \\
z_{19} c \sin \beta \hat{\mathbf{z}}\end{array}$ & $(4 e)$ & $\mathrm{H} \mathrm{V}$ \\
\hline $\mathbf{B}_{76}$ & $=$ & $x_{19} \mathbf{a}_{1}+\left(\frac{1}{2}-y_{19}\right) \mathbf{a}_{2}+\left(\frac{1}{2}+z_{19}\right) \mathbf{a}_{3}$ & $\begin{aligned}= & \left(\frac{1}{2} c \cos \beta+x_{19} a+z_{19} c \cos \beta\right) \hat{\mathbf{x}}+ \\
& \left(\frac{1}{2}-y_{19}\right) b \hat{\mathbf{y}}+\left(\frac{1}{2}+z_{19}\right) c \sin \beta \hat{\mathbf{z}}\end{aligned}$ & $(4 e)$ & $\mathrm{H} \mathrm{V}$ \\
\hline $\mathbf{B}_{77}$ & $=$ & $x_{20} \mathbf{a}_{1}+y_{20} \mathbf{a}_{2}+z_{20} \mathbf{a}_{3}$ & $\begin{array}{c}=\quad\left(x_{20} a+z_{20} c \cos \beta\right) \hat{\mathbf{x}}+y_{20} b \hat{\mathbf{y}}+ \\
z_{20} c \sin \beta \hat{\mathbf{z}}\end{array}$ & $(4 e)$ & H VI \\
\hline $\mathbf{B}_{78}$ & $=$ & $\begin{array}{c}-x_{20} \mathbf{a}_{1}+\left(\frac{1}{2}+y_{20}\right) \mathbf{a}_{2}+ \\
\left(\frac{1}{2}-z_{20}\right) \mathbf{a}_{3}\end{array}$ & $\begin{aligned}= & \left(\frac{1}{2} c \cos \beta-x_{20} a-z_{20} c \cos \beta\right) \hat{\mathbf{x}}+ \\
& \left(\frac{1}{2}+y_{20}\right) b \hat{\mathbf{y}}+\left(\frac{1}{2}-z_{20}\right) c \sin \beta \hat{\mathbf{z}}\end{aligned}$ & $(4 e)$ & H VI \\
\hline $\mathbf{B}_{79}$ & $=$ & $-x_{20} \mathbf{a}_{1}-y_{20} \mathbf{a}_{2}-z_{20} \mathbf{a}_{3}$ & $\begin{array}{c}=\left(-x_{20} a-z_{20} c \cos \beta\right) \hat{\mathbf{x}}-y_{20} b \hat{\mathbf{y}}- \\
z_{20} c \sin \beta \hat{\mathbf{z}}\end{array}$ & $(4 e)$ & H VI \\
\hline $\mathbf{B}_{80}$ & $=$ & $x_{20} \mathbf{a}_{1}+\left(\frac{1}{2}-y_{20}\right) \mathbf{a}_{2}+\left(\frac{1}{2}+z_{20}\right) \mathbf{a}_{3}$ & $\begin{aligned}= & \left(\frac{1}{2} c \cos \beta+x_{20} a+z_{20} c \cos \beta\right) \hat{\mathbf{x}}+ \\
& \left(\frac{1}{2}-y_{20}\right) b \hat{\mathbf{y}}+\left(\frac{1}{2}+z_{20}\right) c \sin \beta \hat{\mathbf{z}}\end{aligned}$ & $(4 e)$ & H VI \\
\hline $\mathbf{B}_{81}$ & $=$ & $x_{21} \mathbf{a}_{1}+y_{21} \mathbf{a}_{2}+z_{21} \mathbf{a}_{3}$ & $\begin{array}{c}=\left(x_{21} a+z_{21} c \cos \beta\right) \hat{\mathbf{x}}+y_{21} b \hat{\mathbf{y}}+ \\
z_{21} c \sin \beta \hat{\mathbf{z}}\end{array}$ & $(4 e)$ & H VII \\
\hline $\mathbf{B}_{82}$ & $=$ & $\begin{array}{c}-x_{21} \mathbf{a}_{1}+\left(\frac{1}{2}+y_{21}\right) \mathbf{a}_{2}+ \\
\left(\frac{1}{2}-z_{21}\right) \mathbf{a}_{3}\end{array}$ & $\begin{aligned}= & \left(\frac{1}{2} c \cos \beta-x_{21} a-z_{21} c \cos \beta\right) \hat{\mathbf{x}}+ \\
& \left(\frac{1}{2}+y_{21}\right) b \hat{\mathbf{y}}+\left(\frac{1}{2}-z_{21}\right) c \sin \beta \hat{\mathbf{z}}\end{aligned}$ & $(4 e)$ & H VII \\
\hline $\mathbf{B}_{83}$ & $=$ & $-x_{21} \mathbf{a}_{1}-y_{21} \mathbf{a}_{2}-z_{21} \mathbf{a}_{3}$ & $\begin{array}{c}=\left(-x_{21} a-z_{21} c \cos \beta\right) \hat{\mathbf{x}}-y_{21} b \hat{\mathbf{y}}- \\
z_{21} c \sin \beta \hat{\mathbf{z}}\end{array}$ & $(4 e)$ & H VII \\
\hline $\mathbf{B}_{84}$ & $=$ & $x_{21} \mathbf{a}_{1}+\left(\frac{1}{2}-y_{21}\right) \mathbf{a}_{2}+\left(\frac{1}{2}+z_{21}\right) \mathbf{a}_{3}$ & $\begin{aligned}= & \left(\frac{1}{2} c \cos \beta+x_{21} a+z_{21} c \cos \beta\right) \hat{\mathbf{x}}+ \\
& \left(\frac{1}{2}-y_{21}\right) b \hat{\mathbf{y}}+\left(\frac{1}{2}+z_{21}\right) c \sin \beta \hat{\mathbf{z}}\end{aligned}$ & $(4 e)$ & H VII \\
\hline $\mathbf{B}_{85}$ & $=$ & $x_{22} \mathbf{a}_{1}+y_{22} \mathbf{a}_{2}+z_{22} \mathbf{a}_{3}$ & $\begin{array}{c}\left(x_{22} a+z_{22} c \cos \beta\right) \hat{\mathbf{x}}+y_{22} b \hat{\mathbf{y}}+ \\
z_{22} c \sin \beta \hat{\mathbf{z}}\end{array}$ & $(4 e)$ & H VIII \\
\hline $\mathbf{B}_{86}$ & $=$ & $\begin{array}{c}-x_{22} \mathbf{a}_{1}+\left(\frac{1}{2}+y_{22}\right) \mathbf{a}_{2}+ \\
\left(\frac{1}{2}-z_{22}\right) \mathbf{a}_{3}\end{array}$ & $\begin{aligned}= & \left(\frac{1}{2} c \cos \beta-x_{22} a-z_{22} c \cos \beta\right) \hat{\mathbf{x}}+ \\
& \left(\frac{1}{2}+y_{22}\right) b \hat{\mathbf{y}}+\left(\frac{1}{2}-z_{22}\right) c \sin \beta \hat{\mathbf{z}}\end{aligned}$ & $(4 e)$ & H VIII \\
\hline $\mathbf{B}_{87}$ & $=$ & $-x_{22} \mathbf{a}_{1}-y_{22} \mathbf{a}_{2}-z_{22} \mathbf{a}_{3}$ & $\begin{array}{c}=\left(-x_{22} a-z_{22} c \cos \beta\right) \hat{\mathbf{x}}-y_{22} b \hat{\mathbf{y}}- \\
z_{22} c \sin \beta \hat{\mathbf{z}}\end{array}$ & $(4 e)$ & H VIII \\
\hline $\mathbf{B}_{88}$ & $=$ & $x_{22} \mathbf{a}_{1}+\left(\frac{1}{2}-y_{22}\right) \mathbf{a}_{2}+\left(\frac{1}{2}+z_{22}\right) \mathbf{a}_{3}$ & $\begin{aligned}= & \left(\frac{1}{2} c \cos \beta+x_{22} a+z_{22} c \cos \beta\right) \hat{\mathbf{x}}+ \\
& \left(\frac{1}{2}-y_{22}\right) b \hat{\mathbf{y}}+\left(\frac{1}{2}+z_{22}\right) c \sin \beta \hat{\mathbf{z}}\end{aligned}$ & $(4 e)$ & H VIII \\
\hline $\mathbf{B}_{89}$ & $=$ & $x_{23} \mathbf{a}_{1}+y_{23} \mathbf{a}_{2}+z_{23} \mathbf{a}_{3}$ & $\begin{array}{c}\left(x_{23} a+z_{23} c \cos \beta\right) \hat{\mathbf{x}}+y_{23} b \hat{\mathbf{y}}+ \\
z_{23} c \sin \beta \hat{\mathbf{z}}\end{array}$ & $(4 e)$ & H IX \\
\hline $\mathbf{B}_{90}$ & $=$ & $\begin{array}{c}-x_{23} \mathbf{a}_{1}+\left(\frac{1}{2}+y_{23}\right) \mathbf{a}_{2}+ \\
\left(\frac{1}{2}-z_{23}\right) \mathbf{a}_{3}\end{array}$ & $\begin{aligned}= & \left(\frac{1}{2} c \cos \beta-x_{23} a-z_{23} c \cos \beta\right) \hat{\mathbf{x}}+ \\
& \left(\frac{1}{2}+y_{23}\right) b \hat{\mathbf{y}}+\left(\frac{1}{2}-z_{23}\right) c \sin \beta \hat{\mathbf{z}}\end{aligned}$ & $(4 e)$ & H IX \\
\hline $\mathbf{B}_{91}$ & $=$ & $-x_{23} \mathbf{a}_{1}-y_{23} \mathbf{a}_{2}-z_{23} \mathbf{a}_{3}$ & $\begin{array}{c}=\left(-x_{23} a-z_{23} c \cos \beta\right) \hat{\mathbf{x}}-y_{23} b \hat{\mathbf{y}}- \\
z_{23} c \sin \beta \hat{\mathbf{z}}\end{array}$ & $(4 e)$ & H IX \\
\hline
\end{tabular}




$$
\begin{aligned}
& \mathbf{B}_{92}=x_{23} \mathbf{a}_{1}+\left(\frac{1}{2}-y_{23}\right) \mathbf{a}_{2}+\left(\frac{1}{2}+z_{23}\right) \mathbf{a}_{3}=\left(\frac{1}{2} c \cos \beta+x_{23} a+z_{23} c \cos \beta\right) \hat{\mathbf{x}}+ \\
& \left(\frac{1}{2}-y_{23}\right) b \hat{\mathbf{y}}+\left(\frac{1}{2}+z_{23}\right) c \sin \beta \hat{\mathbf{z}} \\
& \mathbf{B}_{93}=x_{24} \mathbf{a}_{1}+y_{24} \mathbf{a}_{2}+z_{24} \mathbf{a}_{3} \quad=\quad\left(x_{24} a+z_{24} c \cos \beta\right) \hat{\mathbf{x}}+y_{24} b \hat{\mathbf{y}}+ \\
& z_{24} c \sin \beta \hat{\mathbf{z}} \\
& \mathbf{B}_{94}=-x_{24} \mathbf{a}_{1}+\left(\frac{1}{2}+y_{24}\right) \mathbf{a}_{2}+\quad=\left(\frac{1}{2} c \cos \beta-x_{24} a-z_{24} c \cos \beta\right) \hat{\mathbf{x}}+ \\
& \left(\frac{1}{2}-z_{24}\right) \mathbf{a}_{3} \quad\left(\frac{1}{2}+y_{24}\right) b \hat{\mathbf{y}}+\left(\frac{1}{2}-z_{24}\right) c \sin \beta \hat{\mathbf{z}} \\
& \mathbf{B}_{95}=-x_{24} \mathbf{a}_{1}-y_{24} \mathbf{a}_{2}-z_{24} \mathbf{a}_{3}=\left(-x_{24} a-z_{24} c \cos \beta\right) \hat{\mathbf{x}}-y_{24} b \hat{\mathbf{y}}- \\
& z_{24} c \sin \beta \hat{\mathbf{z}} \\
& \mathbf{B}_{96}=x_{24} \mathbf{a}_{1}+\left(\frac{1}{2}-y_{24}\right) \mathbf{a}_{2}+\left(\frac{1}{2}+z_{24}\right) \mathbf{a}_{3}=\left(\frac{1}{2} c \cos \beta+x_{24} a+z_{24} c \cos \beta\right) \hat{\mathbf{x}}+ \\
& \left(\frac{1}{2}-y_{24}\right) b \hat{\mathbf{y}}+\left(\frac{1}{2}+z_{24}\right) c \sin \beta \hat{\mathbf{z}} \\
& \mathbf{B}_{97}=x_{25} \mathbf{a}_{1}+y_{25} \mathbf{a}_{2}+z_{25} \mathbf{a}_{3} \quad=\quad\left(x_{25} a+z_{25} c \cos \beta\right) \hat{\mathbf{x}}+y_{25} b \hat{\mathbf{y}}+ \\
& z_{25} c \sin \beta \hat{\mathbf{z}} \\
& \mathbf{B}_{98}=-x_{25} \mathbf{a}_{1}+\left(\frac{1}{2}+y_{25}\right) \mathbf{a}_{2}+\quad=\left(\frac{1}{2} c \cos \beta-x_{25} a-z_{25} c \cos \beta\right) \hat{\mathbf{x}}+ \\
& \left(\frac{1}{2}-z_{25}\right) \mathbf{a}_{3} \quad\left(\frac{1}{2}+y_{25}\right) b \hat{\mathbf{y}}+\left(\frac{1}{2}-z_{25}\right) c \sin \beta \hat{\mathbf{z}} \\
& \mathbf{B}_{99}=-x_{25} \mathbf{a}_{1}-y_{25} \mathbf{a}_{2}-z_{25} \mathbf{a}_{3}=\left(-x_{25} a-z_{25} c \cos \beta\right) \hat{\mathbf{x}}-y_{25} b \hat{\mathbf{y}}- \\
& z_{25} c \sin \beta \hat{\mathbf{z}} \\
& \mathbf{B}_{100}=x_{25} \mathbf{a}_{1}+\left(\frac{1}{2}-y_{25}\right) \mathbf{a}_{2}+\left(\frac{1}{2}+z_{25}\right) \mathbf{a}_{3}=\left(\frac{1}{2} c \cos \beta+x_{25} a+z_{25} c \cos \beta\right) \hat{\mathbf{x}}+ \\
& \left(\frac{1}{2}-y_{25}\right) b \hat{\mathbf{y}}+\left(\frac{1}{2}+z_{25}\right) c \sin \beta \hat{\mathbf{z}} \\
& \mathbf{B}_{101}=x_{26} \mathbf{a}_{1}+y_{26} \mathbf{a}_{2}+z_{26} \mathbf{a}_{3}=\left(x_{26} a+z_{26} c \cos \beta\right) \hat{\mathbf{x}}+y_{26} b \hat{\mathbf{y}}+ \\
& z_{26} c \sin \beta \hat{\mathbf{z}} \\
& \mathbf{B}_{102}=-x_{26} \mathbf{a}_{1}+\left(\frac{1}{2}+y_{26}\right) \mathbf{a}_{2}+\quad=\left(\frac{1}{2} c \cos \beta-x_{26} a-z_{26} c \cos \beta\right) \hat{\mathbf{x}}+ \\
& \left(\frac{1}{2}-z_{26}\right) \mathbf{a}_{3} \quad\left(\frac{1}{2}+y_{26}\right) b \hat{\mathbf{y}}+\left(\frac{1}{2}-z_{26}\right) c \sin \beta \hat{\mathbf{z}} \\
& \mathbf{B}_{103}=-x_{26} \mathbf{a}_{1}-y_{26} \mathbf{a}_{2}-z_{26} \mathbf{a}_{3}=\left(-x_{26} a-z_{26} c \cos \beta\right) \hat{\mathbf{x}}-y_{26} b \hat{\mathbf{y}}- \\
& z_{26} c \sin \beta \hat{\mathbf{z}} \\
& \mathbf{B}_{104}=x_{26} \mathbf{a}_{1}+\left(\frac{1}{2}-y_{26}\right) \mathbf{a}_{2}+\left(\frac{1}{2}+z_{26}\right) \mathbf{a}_{3}=\left(\frac{1}{2} c \cos \beta+x_{26} a+z_{26} c \cos \beta\right) \hat{\mathbf{x}}+ \\
& \left(\frac{1}{2}-y_{26}\right) b \hat{\mathbf{y}}+\left(\frac{1}{2}+z_{26}\right) c \sin \beta \hat{\mathbf{z}} \\
& \mathbf{B}_{105}=x_{27} \mathbf{a}_{1}+y_{27} \mathbf{a}_{2}+z_{27} \mathbf{a}_{3} \quad=\quad\left(x_{27} a+z_{27} c \cos \beta\right) \hat{\mathbf{x}}+y_{27} b \hat{\mathbf{y}}+ \\
& z_{27} c \sin \beta \hat{\mathbf{z}} \\
& \mathbf{B}_{106}=-x_{27} \mathbf{a}_{1}+\left(\frac{1}{2}+y_{27}\right) \mathbf{a}_{2}+\quad=\left(\frac{1}{2} c \cos \beta-x_{27} a-z_{27} c \cos \beta\right) \hat{\mathbf{x}}+ \\
& \left(\frac{1}{2}-z_{27}\right) \mathbf{a}_{3} \quad\left(\frac{1}{2}+y_{27}\right) b \hat{\mathbf{y}}+\left(\frac{1}{2}-z_{27}\right) c \sin \beta \hat{\mathbf{z}} \\
& \mathbf{B}_{107}=-x_{27} \mathbf{a}_{1}-y_{27} \mathbf{a}_{2}-z_{27} \mathbf{a}_{3}=\left(-x_{27} a-z_{27} c \cos \beta\right) \hat{\mathbf{x}}-y_{27} b \hat{\mathbf{y}}- \\
& z_{27} c \sin \beta \hat{\mathbf{z}} \\
& \mathbf{B}_{108}=x_{27} \mathbf{a}_{1}+\left(\frac{1}{2}-y_{27}\right) \mathbf{a}_{2}+\left(\frac{1}{2}+z_{27}\right) \mathbf{a}_{3}=\left(\frac{1}{2} c \cos \beta+x_{27} a+z_{27} c \cos \beta\right) \hat{\mathbf{x}}+ \\
& \left(\frac{1}{2}-y_{27}\right) b \hat{\mathbf{y}}+\left(\frac{1}{2}+z_{27}\right) c \sin \beta \hat{\mathbf{z}} \\
& \mathbf{B}_{109}=x_{28} \mathbf{a}_{1}+y_{28} \mathbf{a}_{2}+z_{28} \mathbf{a}_{3}=\left(x_{28} a+z_{28} c \cos \beta\right) \hat{\mathbf{x}}+y_{28} b \hat{\mathbf{y}}+ \\
& z_{28} c \sin \beta \hat{\mathbf{z}} \\
& \mathbf{B}_{110}=-x_{28} \mathbf{a}_{1}+\left(\frac{1}{2}+y_{28}\right) \mathbf{a}_{2}+\quad=\left(\frac{1}{2} c \cos \beta-x_{28} a-z_{28} c \cos \beta\right) \hat{\mathbf{x}}+ \\
& \left(\frac{1}{2}-z_{28}\right) \mathbf{a}_{3} \quad\left(\frac{1}{2}+y_{28}\right) b \hat{\mathbf{y}}+\left(\frac{1}{2}-z_{28}\right) c \sin \beta \hat{\mathbf{z}} \\
& \mathbf{B}_{111}=-x_{28} \mathbf{a}_{1}-y_{28} \mathbf{a}_{2}-z_{28} \mathbf{a}_{3}=\left(-x_{28} a-z_{28} c \cos \beta\right) \hat{\mathbf{x}}-y_{28} b \hat{\mathbf{y}}- \\
& z_{28} c \sin \beta \hat{\mathbf{z}} \\
& \mathbf{B}_{112}=x_{28} \mathbf{a}_{1}+\left(\frac{1}{2}-y_{28}\right) \mathbf{a}_{2}+\left(\frac{1}{2}+z_{28}\right) \mathbf{a}_{3}=\left(\frac{1}{2} c \cos \beta+x_{28} a+z_{28} c \cos \beta\right) \hat{\mathbf{x}}+ \\
& \left(\frac{1}{2}-y_{28}\right) b \hat{\mathbf{y}}+\left(\frac{1}{2}+z_{28}\right) c \sin \beta \hat{\mathbf{z}} \\
& \mathbf{B}_{113}=x_{29} \mathbf{a}_{1}+y_{29} \mathbf{a}_{2}+z_{29} \mathbf{a}_{3} \quad=\quad\left(x_{29} a+z_{29} c \cos \beta\right) \hat{\mathbf{x}}+y_{29} b \hat{\mathbf{y}}+ \\
& z_{29} c \sin \beta \hat{\mathbf{z}}
\end{aligned}
$$




$$
\begin{aligned}
& \mathbf{B}_{114}=-x_{29} \mathbf{a}_{1}+\left(\frac{1}{2}+y_{29}\right) \mathbf{a}_{2}+\quad=\left(\frac{1}{2} c \cos \beta-x_{29} a-z_{29} c \cos \beta\right) \hat{\mathbf{x}}+ \\
& \left(\frac{1}{2}-z_{29}\right) \mathbf{a}_{3} \quad\left(\frac{1}{2}+y_{29}\right) b \hat{\mathbf{y}}+\left(\frac{1}{2}-z_{29}\right) c \sin \beta \hat{\mathbf{z}} \\
& \mathbf{B}_{115}=-x_{29} \mathbf{a}_{1}-y_{29} \mathbf{a}_{2}-z_{29} \mathbf{a}_{3}=\left(-x_{29} a-z_{29} c \cos \beta\right) \hat{\mathbf{x}}-y_{29} b \hat{\mathbf{y}}- \\
& z_{29} c \sin \beta \hat{\mathbf{z}} \\
& \mathbf{B}_{116}=x_{29} \mathbf{a}_{1}+\left(\frac{1}{2}-y_{29}\right) \mathbf{a}_{2}+\left(\frac{1}{2}+z_{29}\right) \mathbf{a}_{3}=\left(\frac{1}{2} c \cos \beta+x_{29} a+z_{29} c \cos \beta\right) \hat{\mathbf{x}}+ \\
& \left(\frac{1}{2}-y_{29}\right) b \hat{\mathbf{y}}+\left(\frac{1}{2}+z_{29}\right) c \sin \beta \hat{\mathbf{z}} \\
& \mathbf{B}_{117}=x_{30} \mathbf{a}_{1}+y_{30} \mathbf{a}_{2}+z_{30} \mathbf{a}_{3} \quad=\quad\left(x_{30} a+z_{30} c \cos \beta\right) \hat{\mathbf{x}}+y_{30} b \hat{\mathbf{y}}+ \\
& z_{30} c \sin \beta \hat{\mathbf{z}} \\
& \mathbf{B}_{118}=-x_{30} \mathbf{a}_{1}+\left(\frac{1}{2}+y_{30}\right) \mathbf{a}_{2}+\quad=\left(\frac{1}{2} c \cos \beta-x_{30} a-z_{30} c \cos \beta\right) \hat{\mathbf{x}}+ \\
& \left(\frac{1}{2}-z_{30}\right) \mathbf{a}_{3} \quad\left(\frac{1}{2}+y_{30}\right) b \hat{\mathbf{y}}+\left(\frac{1}{2}-z_{30}\right) c \sin \beta \hat{\mathbf{z}} \\
& \mathbf{B}_{119}=-x_{30} \mathbf{a}_{1}-y_{30} \mathbf{a}_{2}-z_{30} \mathbf{a}_{3}=\left(-x_{30} a-z_{30} c \cos \beta\right) \hat{\mathbf{x}}-y_{30} b \hat{\mathbf{y}}- \\
& z_{30} c \sin \beta \hat{\mathbf{z}} \\
& \mathbf{B}_{120}=x_{30} \mathbf{a}_{1}+\left(\frac{1}{2}-y_{30}\right) \mathbf{a}_{2}+\left(\frac{1}{2}+z_{30}\right) \mathbf{a}_{3}=\left(\frac{1}{2} c \cos \beta+x_{30} a+z_{30} c \cos \beta\right) \hat{\mathbf{x}}+ \\
& \left(\frac{1}{2}-y_{30}\right) b \hat{\mathbf{y}}+\left(\frac{1}{2}+z_{30}\right) c \sin \beta \hat{\mathbf{z}}
\end{aligned}
$$

\section{References:}

- S. K. Nayak, R. Sathishkumar, and T. N. Guru Row, Directing role of functional groups in selective generation of $C-H-\pi$ interactions: In situ cryo-crystallographic studies on benzyl derivatives, CrystEngComm 12, 3112-3118 (2010), doi:10.1039/C001190H.

\section{Found in:}

- Cambridge Structural Database. CSD Entry: TOLUEN03.

\section{Geometry files:}

- CIF: pp. 810

- POSCAR: pp. 811 


\section{$\mathrm{H}_{3} \mathrm{Cl}$ (50 GPa) Structure: AB3_mC16_15_e_cf}
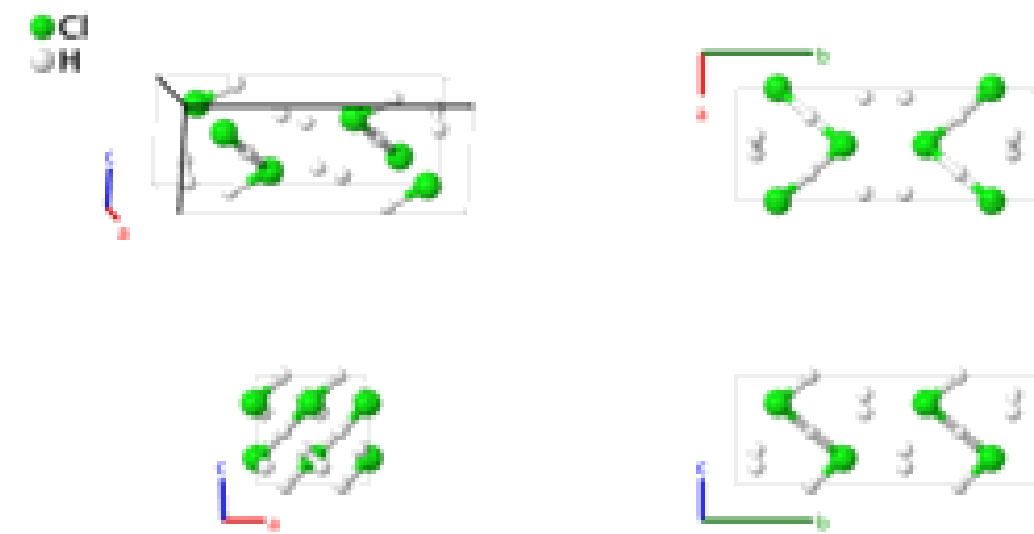

Prototype

AFLOW prototype label

Strukturbericht designation

Pearson symbol

Space group number

Space group symbol

AFLOW prototype command
: $\quad \mathrm{H}_{3} \mathrm{Cl}$

: AB3_mC16_15_e_cf

: None

: $\quad \mathrm{mC16}$

: $\quad 15$

: $\quad C 2 / c$

- This structure was found via first-principles calculations. The data presented here was computed at a pressure of $50 \mathrm{GPa}$.

\section{Base-centered Monoclinic primitive vectors:}

$$
\begin{aligned}
& \mathbf{a}_{1}=\frac{1}{2} a \hat{\mathbf{x}}-\frac{1}{2} b \hat{\mathbf{y}} \\
& \mathbf{a}_{2}=\frac{1}{2} a \hat{\mathbf{x}}+\frac{1}{2} b \hat{\mathbf{y}} \\
& \mathbf{a}_{3}=c \cos \beta \hat{\mathbf{x}}+c \sin \beta \hat{\mathbf{z}}
\end{aligned}
$$
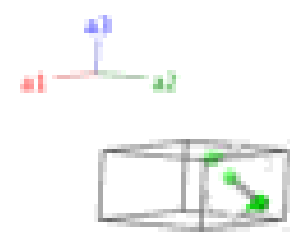

\section{Basis vectors:}

$$
\begin{array}{ccccc}
\mathbf{B}_{1}= & \frac{1}{2} \mathbf{a}_{2} & = & \frac{1}{4} a \hat{\mathbf{x}}+\frac{1}{4} b \hat{\mathbf{y}} \\
\mathbf{B}_{2}= & \frac{1}{2} \mathbf{a}_{1}+\frac{1}{2} \mathbf{a}_{3} & = & \left(\frac{1}{4} a+\frac{1}{2} c \cos \beta\right) \hat{\mathbf{x}}-\frac{1}{4} b \hat{\mathbf{y}}+\frac{1}{2} c \sin \beta \hat{\mathbf{z}} \\
\mathbf{B}_{3}= & -y_{2} \mathbf{a}_{1}+y_{2} \mathbf{a}_{2}+\frac{1}{4} \mathbf{a}_{3} & = & \frac{1}{4} c \cos \beta \hat{\mathbf{x}}+y_{2} b \hat{\mathbf{y}}+\frac{1}{4} c \sin \beta \hat{\mathbf{z}} \\
\mathbf{B}_{4}= & y_{2} \mathbf{a}_{1}-y_{2} \mathbf{a}_{2}+\frac{3}{4} \mathbf{a}_{3} & = & \frac{3}{4} c \cos \beta \hat{\mathbf{x}}-y_{2} b \hat{\mathbf{y}}+\frac{3}{4} c \sin \beta \hat{\mathbf{z}} \\
\mathbf{B}_{5}= & \left(x_{3}-y_{3}\right) \mathbf{a}_{1}+\left(x_{3}+y_{3}\right) \mathbf{a}_{2}+z_{3} \mathbf{a}_{3} & = & \left(x_{3} a+z_{3} c \cos \beta\right) \hat{\mathbf{x}}+y_{3} b \hat{\mathbf{y}}+ \\
& & & & z_{3} c \sin \beta \hat{\mathbf{z}} \\
\mathbf{B}_{6}= & \left(-x_{3}-y_{3}\right) \mathbf{a}_{1}+\left(-x_{3}+y_{3}\right) \mathbf{a}_{2}+ & = & \left(\frac{1}{2} c \cos \beta-x_{3} a-z_{3} c \cos \beta\right) \hat{\mathbf{x}}+ \\
& \left(\frac{1}{2}-z_{3}\right) \mathbf{a}_{3} & & y_{3} b \hat{\mathbf{y}}+\left(\frac{1}{2}-z_{3}\right) c \sin \beta \hat{\mathbf{z}}
\end{array}
$$

Lattice Coordinates

Cartesian Coordinates

\section{Wyckoff Position}

Atom Type

$(4 c)$

H I

H I

$\mathrm{Cl}$

(4e)

$\mathrm{Cl}$

(8f)

H II

(8f)

H II 


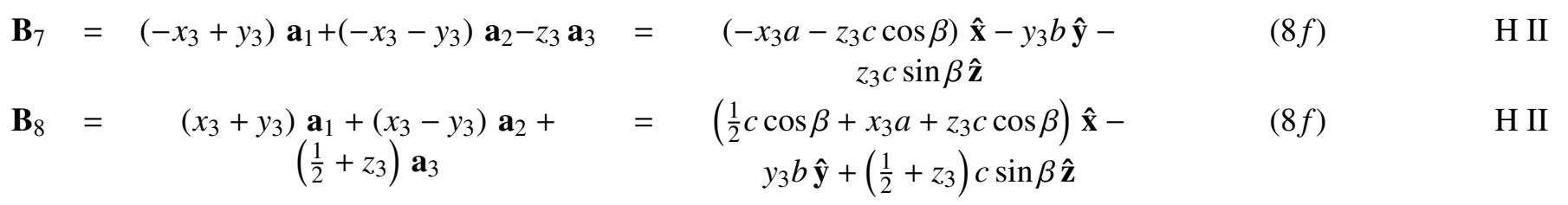

\section{References:}

- D. Duan, X. Huang, F. Tian, Y. Liu, D. Li, H. Yu, B. Liu, W. Tian, and T. Cui, Predicted Formation of $H_{3}^{+}$in Solid Halogen Polyhydrides at High Pressures, J. Phys. Chem. A 119, 11059-11065 (2015), doi:10.1021/acs.jpca.5b08183.

\section{Geometry files:}

- CIF: pp. 811

- POSCAR: pp. 812 


\section{H-III (300 GPa) Structure: A_mC24_15_2e2f}

DH
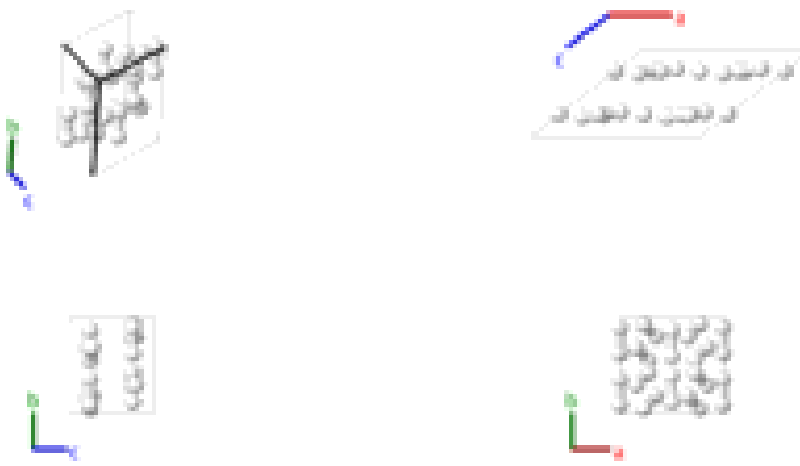

\section{Prototype}

AFLOW prototype label

Strukturbericht designation

Pearson symbol

Space group number

Space group symbol

AFLOW prototype command
$\mathrm{H}$

: A_mC24_15_2e2f

: None

: $\quad \mathrm{mC} 24$

: $\quad 15$

: $\quad C 2 / c$

- This structure was determined by density functional simulations. The authors claim it is in good agreement with experimental data for H-III, and is the lowest energy structure at pressures from approximately 100-250 GPa, including zero-point motion. The data presented here was computed at $300 \mathrm{GPa}$. If we change our description of the unit cell so that

$$
\mathbf{a}_{3} \rightarrow \mathbf{a}_{1}+\mathbf{a}_{2}+\mathbf{a}_{3}
$$

then all of the primitive vectors for the base-centered orthorhombic structure have approximately equal lengths, and the angles between them are approximately $60^{\circ}$. This structure is very close to exhibiting a face-centered cubic lattice.

\section{Base-centered Monoclinic primitive vectors:}

$$
\begin{aligned}
& \mathbf{a}_{1}=\frac{1}{2} a \hat{\mathbf{x}}-\frac{1}{2} b \hat{\mathbf{y}} \\
& \mathbf{a}_{2}=\frac{1}{2} a \hat{\mathbf{x}}+\frac{1}{2} b \hat{\mathbf{y}} \\
& \mathbf{a}_{3}=c \cos \beta \hat{\mathbf{x}}+c \sin \beta \hat{\mathbf{z}}
\end{aligned}
$$

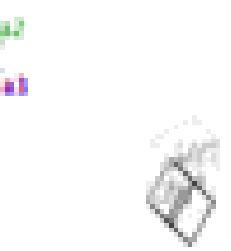

\section{Basis vectors:}

Lattice Coordinates

$\mathbf{B}_{1}=-y_{1} \mathbf{a}_{1}+y_{1} \mathbf{a}_{2}+\frac{1}{4} \mathbf{a}_{3}$

$\mathbf{B}_{2}=$

$\mathbf{B}_{3}=$

$\mathbf{B}_{4}=$
Cartesian Coordinates

$=\quad \frac{1}{4} c \cos \beta \hat{\mathbf{x}}+y_{1} b \hat{\mathbf{y}}+\frac{1}{4} c \sin \beta \hat{\mathbf{z}}$

$=\quad \frac{3}{4} c \cos \beta \hat{\mathbf{x}}-y_{1} b \hat{\mathbf{y}}+\frac{3}{4} c \sin \beta \hat{\mathbf{z}}$

$=\quad \frac{1}{4} c \cos \beta \hat{\mathbf{x}}+y_{2} b \hat{\mathbf{y}}+\frac{1}{4} c \sin \beta \hat{\mathbf{z}}$

$=\quad \frac{3}{4} c \cos \beta \hat{\mathbf{x}}-y_{2} b \hat{\mathbf{y}}+\frac{3}{4} c \sin \beta \hat{\mathbf{z}}$
Wyckoff Position
Atom Type

H I

H I

H II

H II 


\begin{tabular}{|c|c|c|c|c|c|c|}
\hline $\mathbf{B}_{5}$ & $=$ & $\left(x_{3}-y_{3}\right) \mathbf{a}_{1}+\left(x_{3}+y_{3}\right) \mathbf{a}_{2}+z_{3} \mathbf{a}_{3}$ & $=$ & $\begin{array}{c}\left(x_{3} a+z_{3} c \cos \beta\right) \hat{\mathbf{x}}+y_{3} b \hat{\mathbf{y}}+ \\
z_{3} c \sin \beta \hat{\mathbf{z}}\end{array}$ & $(8 f)$ & H III \\
\hline $\mathbf{B}_{6}$ & $=$ & $\begin{array}{c}\left(-x_{3}-y_{3}\right) \mathbf{a}_{1}+\left(-x_{3}+y_{3}\right) \mathbf{a}_{2}+ \\
\left(\frac{1}{2}-z_{3}\right) \mathbf{a}_{3}\end{array}$ & $=$ & $\begin{array}{c}\left(\frac{1}{2} c \cos \beta-x_{3} a-z_{3} c \cos \beta\right) \hat{\mathbf{x}}+ \\
y_{3} b \hat{\mathbf{y}}+\left(\frac{1}{2}-z_{3}\right) c \sin \beta \hat{\mathbf{z}}\end{array}$ & $(8 f)$ & H III \\
\hline $\mathbf{B}_{7}$ & $=$ & $\begin{array}{c}\left(-x_{3}+y_{3}\right) \mathbf{a}_{1}+\left(-x_{3}-y_{3}\right) \mathbf{a}_{2}- \\
z_{3} \mathbf{a}_{3}\end{array}$ & $=$ & $\begin{array}{c}\left(-x_{3} a-z_{3} c \cos \beta\right) \hat{\mathbf{x}}-y_{3} b \hat{\mathbf{y}}- \\
z_{3} c \sin \beta \hat{\mathbf{z}}\end{array}$ & $(8 f)$ & H III \\
\hline $\mathbf{B}_{8}$ & $=$ & $\begin{array}{c}\left(x_{3}+y_{3}\right) \mathbf{a}_{1}+\left(x_{3}-y_{3}\right) \mathbf{a}_{2}+ \\
\left(\frac{1}{2}+z_{3}\right) \mathbf{a}_{3}\end{array}$ & $=$ & $\begin{array}{c}\left(\frac{1}{2} c \cos \beta+x_{3} a+z_{3} c \cos \beta\right) \hat{\mathbf{x}}- \\
y_{3} b \hat{\mathbf{y}}+\left(\frac{1}{2}+z_{3}\right) c \sin \beta \hat{\mathbf{z}}\end{array}$ & $(8 f)$ & H III \\
\hline $\mathbf{B}_{9}$ & $=$ & $\left(x_{4}-y_{4}\right) \mathbf{a}_{1}+\left(x_{4}+y_{4}\right) \mathbf{a}_{2}+z_{4} \mathbf{a}_{3}$ & $=$ & $\begin{array}{c}\left(x_{4} a+z_{4} c \cos \beta\right) \hat{\mathbf{x}}+y_{4} b \hat{\mathbf{y}}+ \\
z_{4} c \sin \beta \hat{\mathbf{z}}\end{array}$ & $(8 f)$ & H IV \\
\hline $\mathbf{B}_{10}$ & $=$ & $\begin{array}{c}\left(-x_{4}-y_{4}\right) \mathbf{a}_{1}+\left(-x_{4}+y_{4}\right) \mathbf{a}_{2}+ \\
\left(\frac{1}{2}-z_{4}\right) \mathbf{a}_{3}\end{array}$ & $=$ & $\begin{array}{c}\left(\frac{1}{2} c \cos \beta-x_{4} a-z_{4} c \cos \beta\right) \hat{\mathbf{x}}+ \\
y_{4} b \hat{\mathbf{y}}+\left(\frac{1}{2}-z_{4}\right) c \sin \beta \hat{\mathbf{z}}\end{array}$ & $(8 f)$ & H IV \\
\hline $\mathbf{B}_{11}$ & $=$ & $\begin{array}{c}\left(-x_{4}+y_{4}\right) \mathbf{a}_{1}+\left(-x_{4}-y_{4}\right) \mathbf{a}_{2}- \\
z_{4} \mathbf{a}_{3}\end{array}$ & $=$ & $\begin{array}{c}\left(-x_{4} a-z_{4} c \cos \beta\right) \hat{\mathbf{x}}-y_{4} b \hat{\mathbf{y}}- \\
z_{4} c \sin \beta \hat{\mathbf{z}}\end{array}$ & $(8 f)$ & H IV \\
\hline $\mathbf{B}_{12}$ & $=$ & $\begin{array}{c}\left(x_{4}+y_{4}\right) \underset{\mathbf{a}_{1}+\left(x_{4}-y_{4}\right) \mathbf{a}_{2}+}{\left(\frac{1}{2}+z_{4}\right) \mathbf{a}_{3}}\end{array}$ & $=$ & $\begin{array}{c}\left(\frac{1}{2} c \cos \beta+x_{4} a+z_{4} c \cos \beta\right) \hat{\mathbf{x}}- \\
y_{4} b \hat{\mathbf{y}}+\left(\frac{1}{2}+z_{4}\right) c \sin \beta \hat{\mathbf{z}}\end{array}$ & $(8 f)$ & H IV \\
\hline
\end{tabular}

\section{References:}

- C. J. Pickard and R. J. Needs, Structure of phase III of solid hydrogen, Nat. Phys. 3, 473-476 (2007), doi:10.1038/nphys625.

\section{Geometry files:}

- CIF: pp. 812

- POSCAR: pp. 812 

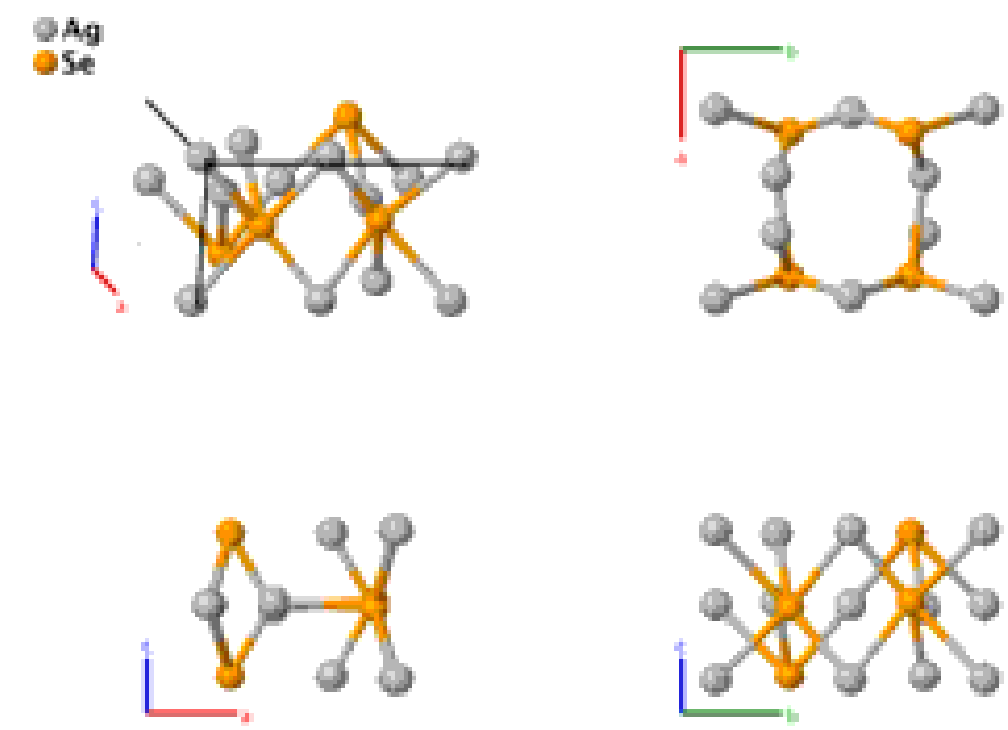

Prototype

AFLOW prototype label

Strukturbericht designation

Pearson symbol

Space group number

Space group symbol

AFLOW prototype command
$: \quad \alpha-\mathrm{Ag}_{2} \mathrm{Se}$

: A2B_oP12_17_abe_e

: None

: $\quad$ oP12

: 17

: $\quad P 222_{1}$

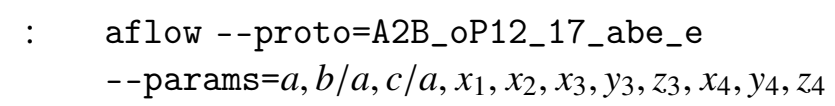

Simple Orthorhombic primitive vectors:

$$
\begin{aligned}
& \mathbf{a}_{1}=a \hat{\mathbf{x}} \\
& \mathbf{a}_{2}=b \hat{\mathbf{y}} \\
& \mathbf{a}_{3}=c \hat{\mathbf{z}}
\end{aligned}
$$

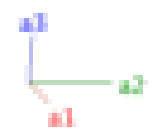

Basis vectors:

Lattice Coordinates

$\mathbf{B}_{1}=$

$\mathbf{B}_{2}=$

$\mathbf{B}_{3}=$

$\mathbf{B}_{4}=$

$\mathbf{B}_{5}=$ $x_{1} \mathbf{a}_{1}$

$$
-x_{1} \mathbf{a}_{1}+\frac{1}{2} \mathbf{a}_{3}
$$$$
x_{2} \mathbf{a}_{1}+\frac{1}{2} \mathbf{a}_{2}
$$

$-x_{2} \mathbf{a}_{1}+\frac{1}{2} \mathbf{a}_{2}+\frac{1}{2} \mathbf{a}_{3}$

$x_{3} \mathbf{a}_{1}+y_{3} \mathbf{a}_{2}+z_{3} \mathbf{a}_{3}$
Cartesian Coordinates

$=$

$=$

$=$

$=$

$=$

$$
x_{1} a \hat{\mathbf{x}}
$$$$
-x_{1} a \hat{\mathbf{x}}+\frac{1}{2} c \hat{\mathbf{z}}
$$$$
x_{2} a \hat{\mathbf{x}}+\frac{1}{2} b \hat{\mathbf{y}}
$$$$
-x_{2} a \hat{\mathbf{x}}+\frac{1}{2} b \hat{\mathbf{y}}+\frac{1}{2} c \hat{\mathbf{z}}
$$$$
x_{3} a \hat{\mathbf{x}}+y_{3} b \hat{\mathbf{y}}+z_{3} c \hat{\mathbf{z}}
$$

Wyckoff Position
Atom Type

Ag I

Ag I

Ag II

Ag II

Ag III 


\begin{tabular}{|c|c|c|c|c|c|c|}
\hline $\mathbf{B}_{6}$ & $=$ & $-x_{3} \mathbf{a}_{1}-y_{3} \mathbf{a}_{2}+\left(\frac{1}{2}+z_{3}\right) \mathbf{a}_{3}$ & $=$ & $-x_{3} a \hat{\mathbf{x}}-y_{3} b \hat{\mathbf{y}}+\left(\frac{1}{2}+z_{3}\right) c \hat{\mathbf{z}}$ & $(4 e)$ & Ag III \\
\hline $\mathbf{B}_{7}$ & $=$ & $-x_{3} \mathbf{a}_{1}+y_{3} \mathbf{a}_{2}+\left(\frac{1}{2}-z_{3}\right) \mathbf{a}_{3}$ & $=$ & $-x_{3} a \hat{\mathbf{x}}+y_{3} b \hat{\mathbf{y}}+\left(\frac{1}{2}-z_{3}\right) c \hat{\mathbf{z}}$ & $(4 e)$ & Ag III \\
\hline $\mathbf{B}_{8}$ & $=$ & $x_{3} \mathbf{a}_{1}-y_{3} \mathbf{a}_{2}-z_{3} \mathbf{a}_{3}$ & $=$ & $x_{3} a \hat{\mathbf{x}}-y_{3} b \hat{\mathbf{y}}-z_{3} c \hat{\mathbf{z}}$ & $(4 e)$ & $\mathrm{Ag}$ III \\
\hline $\mathbf{B}_{9}$ & $=$ & $x_{4} \mathbf{a}_{1}+y_{4} \mathbf{a}_{2}+z_{4} \mathbf{a}_{3}$ & $=$ & $x_{4} a \hat{\mathbf{x}}+y_{4} b \hat{\mathbf{y}}+z_{4} c \hat{\mathbf{z}}$ & $(4 e)$ & $\mathrm{Se}$ \\
\hline $\mathbf{B}_{10}$ & $=$ & $-x_{4} \mathbf{a}_{1}-y_{4} \mathbf{a}_{2}+\left(\frac{1}{2}+z_{4}\right) \mathbf{a}_{3}$ & $=$ & $-x_{4} a \hat{\mathbf{x}}-y_{4} b \hat{\mathbf{y}}+\left(\frac{1}{2}+z_{4}\right) c \hat{\mathbf{z}}$ & $(4 e)$ & $\mathrm{Se}$ \\
\hline $\mathbf{B}_{11}$ & $=$ & $-x_{4} \mathbf{a}_{1}+y_{4} \mathbf{a}_{2}+\left(\frac{1}{2}-z_{4}\right) \mathbf{a}_{3}$ & $=$ & $-x_{4} a \hat{\mathbf{x}}+y_{4} b \hat{\mathbf{y}}+\left(\frac{1}{2}-z_{4}\right) c \hat{\mathbf{z}}$ & $(4 e)$ & $\mathrm{Se}$ \\
\hline $\mathbf{B}_{12}$ & $=$ & $x_{4} \mathbf{a}_{1}-y_{4} \mathbf{a}_{2}-z_{4} \mathbf{a}_{3}$ & $=$ & $x_{4} a \hat{\mathbf{x}}-y_{4} b \hat{\mathbf{y}}-z_{4} c \hat{\mathbf{z}}$ & $(4 e)$ & $\mathrm{Se}$ \\
\hline
\end{tabular}

\section{References:}

- Z. G. Pinsker, C. Ching-liang, R. M. Imamov, and E. L. Lapidus, Determination of the crystal structure of the low-temperature phase $\alpha-\mathrm{Ag}_{2} \mathrm{Se}$, Sov. Phys. Crystallogr. 10, 225-231 (1965).

\section{Found in:}

- P. Villars and K. Cenzual, Pearson's Crystal Data - Crystal Structure Database for Inorganic Compounds, ASM International (2013).

\section{Geometry files:}

- CIF: pp. 812

- POSCAR: pp. 813 


\section{$\mathrm{H}_{3} \mathrm{Cl}(100 \mathrm{GPa})$ Structure: AB3_oP16_19_a_3a}

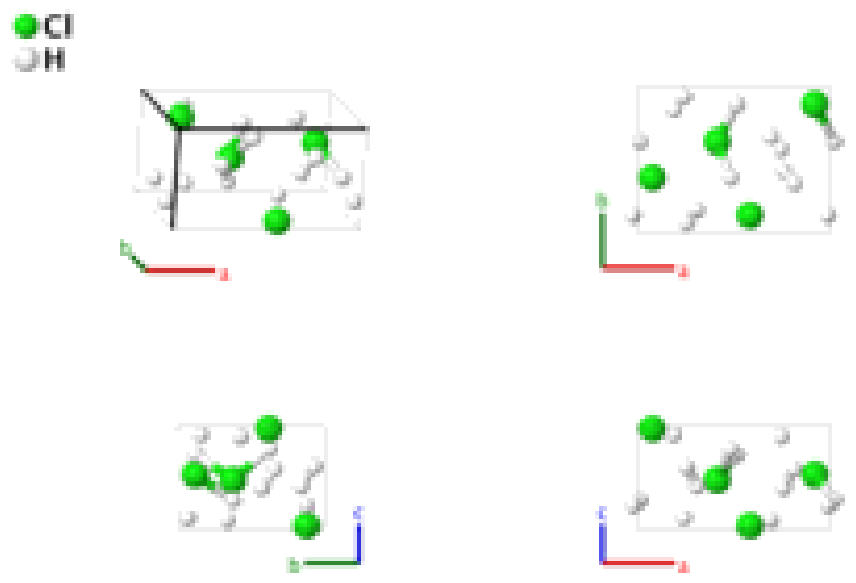

Prototype

AFLOW prototype label

: $\quad \mathrm{H}_{3} \mathrm{Cl}$

Strukturbericht designation

AB3_oP16_19_a_3a

Pearson symbol

None

Space group number

OP16

Space group symbol

$P 2{ }_{1}{ }_{1} 2_{1}$

AFLOW prototype command

aflow --proto=AB3_oP16_19_a_3a

- - params $=a, b / a, c / a, x_{1}, y_{1}, z_{1}, x_{2}, y_{2}, z_{2}, x_{3}, y_{3}, z_{3}, x_{4}, y_{4}, z_{4}$

- This structure was found via first-principles calculations. The data presented here was computed at a pressure of $100 \mathrm{GPa}$.

Simple Orthorhombic primitive vectors:

$$
\begin{aligned}
& \mathbf{a}_{1}=a \hat{\mathbf{x}} \\
& \mathbf{a}_{2}=b \hat{\mathbf{y}} \\
& \mathbf{a}_{3}=c \hat{\mathbf{z}}
\end{aligned}
$$

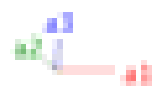

Basis vectors:

Lattice Coordinates

$\mathbf{B}_{1}=x_{1} \mathbf{a}_{1}+y_{1} \mathbf{a}_{2}+z_{1} \mathbf{a}_{3}$

$\mathbf{B}_{2}=\left(\frac{1}{2}-x_{1}\right) \mathbf{a}_{1}-y_{1} \mathbf{a}_{2}+\left(\frac{1}{2}+z_{1}\right) \mathbf{a}_{3}=\left(\frac{1}{2}-x_{1}\right) a \hat{\mathbf{x}}-y_{1} b \hat{\mathbf{y}}+\left(\frac{1}{2}+z_{1}\right) c \hat{\mathbf{z}}$

$\mathbf{B}_{3}=-x_{1} \mathbf{a}_{1}+\left(\frac{1}{2}+y_{1}\right) \mathbf{a}_{2}+\left(\frac{1}{2}-z_{1}\right) \mathbf{a}_{3}=-x_{1} a \hat{\mathbf{x}}+\left(\frac{1}{2}+y_{1}\right) b \hat{\mathbf{y}}+\left(\frac{1}{2}-z_{1}\right) c \hat{\mathbf{z}}$

$\mathbf{B}_{4}=\left(\frac{1}{2}+x_{1}\right) \mathbf{a}_{1}+\left(\frac{1}{2}-y_{1}\right) \mathbf{a}_{2}-z_{1} \mathbf{a}_{3}=\left(\frac{1}{2}+x_{1}\right) a \hat{\mathbf{x}}+\left(\frac{1}{2}-y_{1}\right) b \hat{\mathbf{y}}-z_{1} c \hat{\mathbf{z}}$

$\mathbf{B}_{5}=$
Cartesian Coordinates

$=$

$x_{2} a \hat{\mathbf{x}}+y_{2} b \hat{\mathbf{y}}+z_{2} c \hat{\mathbf{z}}$
Wyckoff Position

(4a)

(4a)

(4a)

(4a)

(4a)
Atom Type

$\mathrm{Cl}$

$\mathrm{Cl}$

$\mathrm{Cl}$

$\mathrm{Cl}$

H I 


\begin{tabular}{|c|c|c|c|c|}
\hline $\mathbf{B}_{6}$ & $=\left(\frac{1}{2}-x_{2}\right) \mathbf{a}_{1}-y_{2} \mathbf{a}_{2}+\left(\frac{1}{2}+z_{2}\right) \mathbf{a}_{3}$ & $=\left(\frac{1}{2}-x_{2}\right) a \hat{\mathbf{x}}-y_{2} b \hat{\mathbf{y}}+\left(\frac{1}{2}+z_{2}\right) c \hat{\mathbf{z}}$ & $(4 a)$ & H I \\
\hline $\mathbf{B}_{7}$ & $=-x_{2} \mathbf{a}_{1}+\left(\frac{1}{2}+y_{2}\right) \mathbf{a}_{2}+\left(\frac{1}{2}-z_{2}\right) \mathbf{a}_{3}$ & $=-x_{2} a \hat{\mathbf{x}}+\left(\frac{1}{2}+y_{2}\right) b \hat{\mathbf{y}}+\left(\frac{1}{2}-z_{2}\right) c \hat{\mathbf{z}}$ & $(4 a)$ & H I \\
\hline $\mathbf{B}_{8}$ & $=\left(\frac{1}{2}+x_{2}\right) \mathbf{a}_{1}+\left(\frac{1}{2}-y_{2}\right) \mathbf{a}_{2}-z_{2} \mathbf{a}_{3}$ & $=\left(\frac{1}{2}+x_{2}\right) a \hat{\mathbf{x}}+\left(\frac{1}{2}-y_{2}\right) b \hat{\mathbf{y}}-z_{2} c \hat{\mathbf{z}}$ & $(4 a)$ & H I \\
\hline $\mathbf{B}_{9}$ & $x_{3} \mathbf{a}_{1}+y_{3} \mathbf{a}_{2}+z_{3} \mathbf{a}_{3}$ & $x_{3} a \hat{\mathbf{x}}+y_{3} b \hat{\mathbf{y}}+z_{3} c \hat{\mathbf{z}}$ & $(4 a)$ & H II \\
\hline $\mathbf{B}_{10}$ & $=\left(\frac{1}{2}-x_{3}\right) \mathbf{a}_{1}-y_{3} \mathbf{a}_{2}+\left(\frac{1}{2}+z_{3}\right) \mathbf{a}_{3}$ & $=\left(\frac{1}{2}-x_{3}\right) a \hat{\mathbf{x}}-y_{3} b \hat{\mathbf{y}}+\left(\frac{1}{2}+z_{3}\right) c \hat{\mathbf{z}}$ & $(4 a)$ & H II \\
\hline $\mathbf{B}_{11}$ & $=-x_{3} \mathbf{a}_{1}+\left(\frac{1}{2}+y_{3}\right) \mathbf{a}_{2}+\left(\frac{1}{2}-z_{3}\right) \mathbf{a}_{3}$ & $=-x_{3} a \hat{\mathbf{x}}+\left(\frac{1}{2}+y_{3}\right) b \hat{\mathbf{y}}+\left(\frac{1}{2}-z_{3}\right) c \hat{\mathbf{z}}$ & $(4 a)$ & H II \\
\hline $\mathbf{B}_{12}$ & $=\left(\frac{1}{2}+x_{3}\right) \mathbf{a}_{1}+\left(\frac{1}{2}-y_{3}\right) \mathbf{a}_{2}-z_{3} \mathbf{a}_{3}$ & $=\left(\frac{1}{2}+x_{3}\right) a \hat{\mathbf{x}}+\left(\frac{1}{2}-y_{3}\right) b \hat{\mathbf{y}}-z_{3} c \hat{\mathbf{z}}$ & $(4 a)$ & H II \\
\hline $\mathbf{B}_{13}$ & $x_{4} \mathbf{a}_{1}+y_{4} \mathbf{a}_{2}+z_{4} \mathbf{a}_{3}$ & $x_{4} a \hat{\mathbf{x}}+y_{4} b \hat{\mathbf{y}}+z_{4} c \hat{\mathbf{z}}$ & $(4 a)$ & H III \\
\hline $\mathbf{B}_{14}$ & $=\left(\frac{1}{2}-x_{4}\right) \mathbf{a}_{1}-y_{4} \mathbf{a}_{2}+\left(\frac{1}{2}+z_{4}\right) \mathbf{a}_{3}$ & $=\left(\frac{1}{2}-x_{4}\right) a \hat{\mathbf{x}}-y_{4} b \hat{\mathbf{y}}+\left(\frac{1}{2}+z_{4}\right) c \hat{\mathbf{z}}$ & $(4 a)$ & H III \\
\hline $\mathbf{B}_{15}$ & $=-x_{4} \mathbf{a}_{1}+\left(\frac{1}{2}+y_{4}\right) \mathbf{a}_{2}+\left(\frac{1}{2}-z_{4}\right) \mathbf{a}_{3}$ & $=-x_{4} a \hat{\mathbf{x}}+\left(\frac{1}{2}+y_{4}\right) b \hat{\mathbf{y}}+\left(\frac{1}{2}-z_{4}\right) c \hat{\mathbf{z}}$ & $(4 a)$ & H III \\
\hline $\mathbf{B}_{16}$ & $=\left(\frac{1}{2}+x_{4}\right) \mathbf{a}_{1}+\left(\frac{1}{2}-y_{4}\right) \mathbf{a}_{2}-z_{4} \mathbf{a}_{3}$ & $=\left(\frac{1}{2}+x_{4}\right) a \hat{\mathbf{x}}+\left(\frac{1}{2}-y_{4}\right) b \hat{\mathbf{y}}-z_{4} c \hat{\mathbf{z}}$ & $(4 a)$ & \\
\hline
\end{tabular}

\section{References:}

- D. Duan, X. Huang, F. Tian, Y. Liu, D. Li, H. Yu, B. Liu, W. Tian, and T. Cui, Predicted Formation of H $H_{3}^{+}$in Solid Halogen Polyhydrides at High Pressures, J. Phys. Chem. A 119, 11059-11065 (2015), doi:10.1021/acs.jpca.5b08183.

\section{Geometry files:}

- CIF: pp. 813

- POSCAR: pp. 813 


\section{$\mathrm{Ta}_{2} \mathrm{H}$ Structure: AB2_oC6_21_a_k}
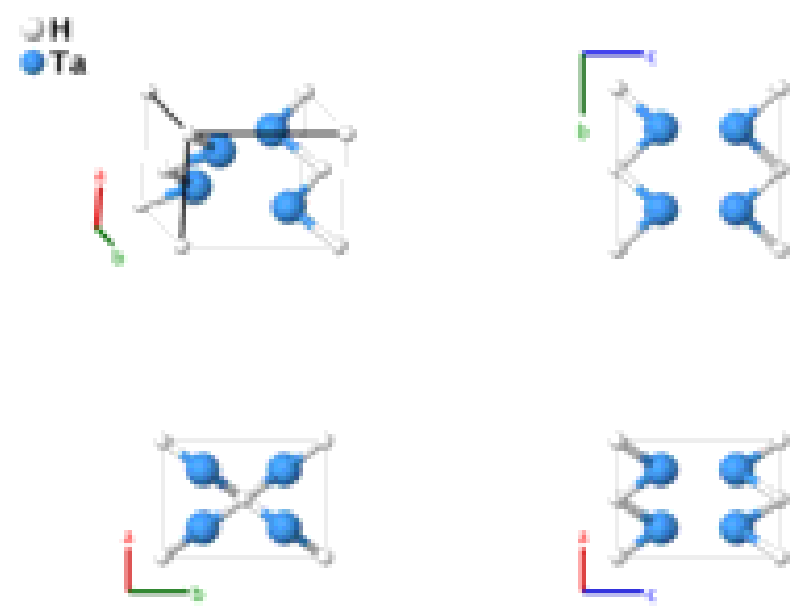

$\begin{array}{lll}\text { Prototype } & : & \mathrm{Ta}_{2} \mathrm{H} \\ \text { AFLOW prototype label } & : & \mathrm{AB} 2 \_\mathrm{C} 6 \_21 \_\mathrm{a} \_\mathrm{k} \\ \text { Strukturbericht designation } & : & \text { None } \\ \text { Pearson symbol } & : & \text { oC6 } \\ \text { Space group number } & : & 21 \\ \text { Space group symbol } & : & C 222 \\ \text { AFLOW prototype command } & : & \text { aflow -proto=AB2_oC6_21_a_k } \\ & & -- \text { params }=a, b / a, c / a, z_{2}\end{array}$

Base-centered Orthorhombic primitive vectors:

$$
\begin{aligned}
& \mathbf{a}_{1}=\frac{1}{2} a \hat{\mathbf{x}}-\frac{1}{2} b \hat{\mathbf{y}} \\
& \mathbf{a}_{2}=\frac{1}{2} a \hat{\mathbf{x}}+\frac{1}{2} b \hat{\mathbf{y}} \\
& \mathbf{a}_{3}=c \quad c \hat{\mathbf{z}}
\end{aligned}
$$

Basis vectors:

Lattice Coordinates

Cartesian Coordinates

$\begin{array}{lcccc}\mathbf{B}_{1}= & 0 \mathbf{a}_{1}+0 \mathbf{a}_{2}+0 \mathbf{a}_{3} & = & 0 \hat{\mathbf{x}}+0 \hat{\mathbf{y}}+0 \hat{\mathbf{z}} \\ \mathbf{B}_{2}= & \frac{1}{2} \mathbf{a}_{2}+z_{2} \mathbf{a}_{3} & = & \frac{1}{4} a \hat{\mathbf{x}}+\frac{1}{4} b \hat{\mathbf{y}}+z_{2} c \hat{\mathbf{z}} \\ \mathbf{B}_{3}= & \frac{1}{2} \mathbf{a}_{1}+-z_{2} \mathbf{a}_{3} & = & \frac{1}{4} a \hat{\mathbf{x}}-\frac{1}{4} b \hat{\mathbf{y}}-z_{2} c \hat{\mathbf{z}}\end{array}$

Wyckoff Position
Atom Type

$\mathrm{H}$

$\mathrm{Ta}$

$\mathrm{Ta}$

\section{References:}

- H. Asano, Y. Ishikawa, and M. Hirabayashi, Single-crystal X-ray diffraction study on the hydrogen ordering in $\mathrm{Ta}_{2} \mathrm{H}, \mathrm{J}$. Appl. Crystallogr. 11, 681-683 (1978), doi:10.1107/S0021889878014260.

Found in: 
- P. Villars and K. Cenzual, Pearson's Crystal Data - Crystal Structure Database for Inorganic Compounds, ASM International (2013).

\section{Geometry files:}

- CIF: pp. 813

- POSCAR: pp. 814 


\section{$\mathrm{CeRu}_{2} \mathrm{~B}_{2}$ Structure: A2BC2_oF40_22_fi_ad_gh}
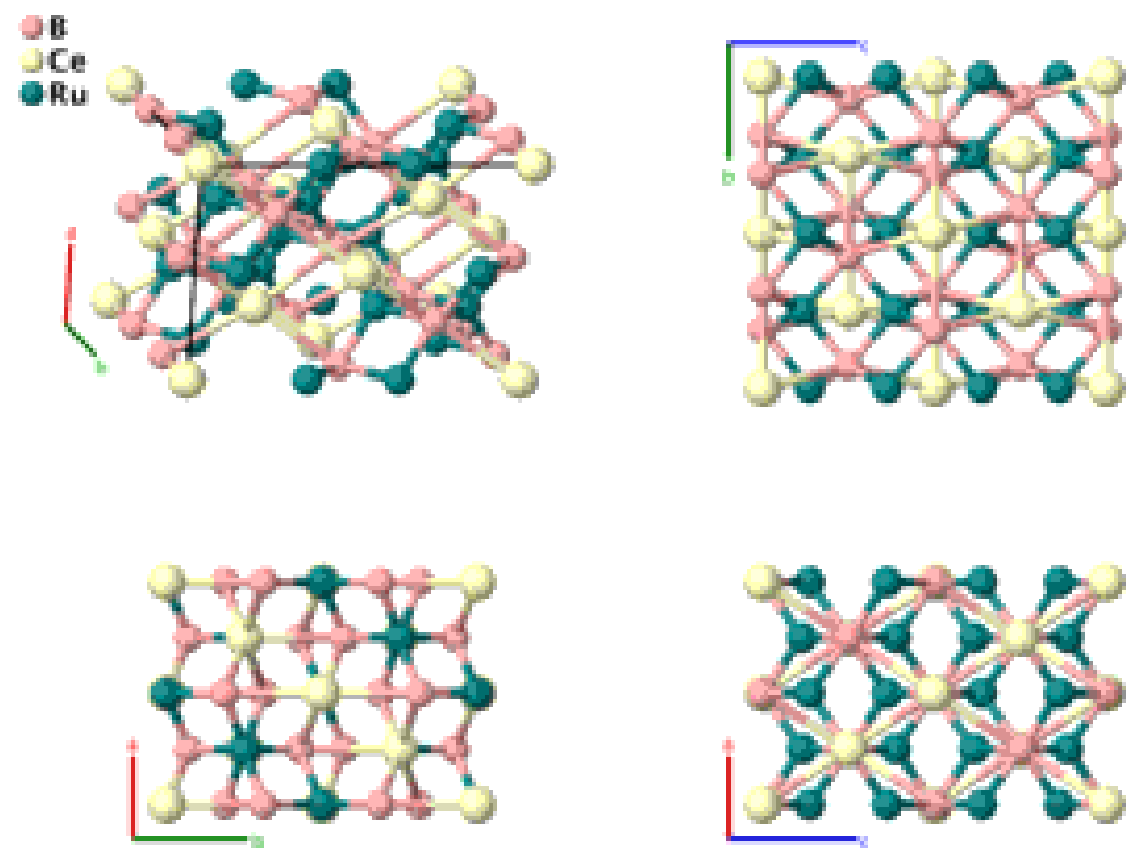

Prototype

AFLOW prototype label

: $\quad \mathrm{CeRu}_{2} \mathrm{~B}_{2}$

Strukturbericht designation : None

Pearson symbol $\quad: \quad \mathrm{oF} 40$

Space group number $\quad: \quad 22$

Space group symbol $\quad: \quad F 222$

AFLOW prototype command : aflow --proto=A2BC2_oF40_22_fi_ad_gh

- params $=a, b / a, c / a, y_{3}, z_{4}, z_{5}, y_{6}$

Face-centered Orthorhombic primitive vectors:

$$
\begin{aligned}
& \mathbf{a}_{1}=\frac{1}{2} b \hat{\mathbf{y}}+\frac{1}{2} c \hat{\mathbf{z}} \\
& \mathbf{a}_{2}=\frac{1}{2} a \hat{\mathbf{x}}+\frac{1}{2} c \hat{\mathbf{z}} \\
& \mathbf{a}_{3}=\frac{1}{2} a \hat{\mathbf{x}}+\frac{1}{2} b \hat{\mathbf{y}}
\end{aligned}
$$

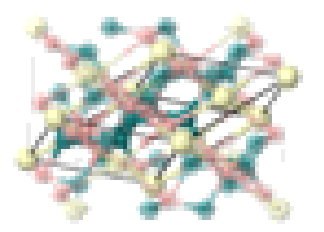

Basis vectors:

Lattice Coordinates

$\begin{array}{llr}\mathbf{B}_{1}= & 0 \mathbf{a}_{1}+0 \mathbf{a}_{2}+0 \mathbf{a}_{3} \\ \mathbf{B}_{2}= & \frac{3}{4} \mathbf{a}_{1}+\frac{3}{4} \mathbf{a}_{2}+\frac{3}{4} \mathbf{a}_{3} \\ \mathbf{B}_{3}= & y_{3} \mathbf{a}_{1}-y_{3} \mathbf{a}_{2}+y_{3} \mathbf{a}_{3}\end{array}$

Cartesian Coordinates

$=$

$=\quad \frac{3}{4} a \hat{\mathbf{x}}+\frac{3}{4} b \hat{\mathbf{y}}+\frac{3}{4} c \hat{\mathbf{z}}$

$=$
Wyckoff Position

(4a)

(4d)

(8f)
Atom Type

Ce I

Ce II

B I 


\begin{tabular}{|c|c|c|c|c|c|c|}
\hline $\mathbf{B}_{4}$ & $=$ & $-y_{3} \mathbf{a}_{1}+y_{3} \mathbf{a}_{2}-y_{3} \mathbf{a}_{3}$ & $=$ & $-y_{3} b \hat{\mathbf{y}}$ & $(8 f)$ & B I \\
\hline $\mathbf{B}_{5}$ & $=$ & $z_{4} \mathbf{a}_{1}+z_{4} \mathbf{a}_{2}-z_{4} \mathbf{a}_{3}$ & $=$ & $z_{4} c \hat{\mathbf{z}}$ & $(8 g)$ & $\mathrm{Ru} I$ \\
\hline $\mathbf{B}_{6}$ & $=$ & $-z_{4} \mathbf{a}_{1}-z_{4} \mathbf{a}_{2}+z_{4} \mathbf{a}_{3}$ & $=$ & $-z_{4} c \hat{\mathbf{z}}$ & $(8 g)$ & $\mathrm{Ru} I$ \\
\hline $\mathbf{B}_{7}$ & $=$ & $z_{5} \mathbf{a}_{1}+z_{5} \mathbf{a}_{2}+\left(\frac{1}{2}-z_{5}\right) \mathbf{a}_{3}$ & $=$ & $\frac{1}{4} a \hat{\mathbf{x}}+\frac{1}{4} b \hat{\mathbf{y}}+z_{5} c \hat{\mathbf{z}}$ & $(8 h)$ & $\mathrm{Ru}$ II \\
\hline $\mathbf{B}_{8}$ & $=$ & $\left(\frac{1}{2}-z_{5}\right) \mathbf{a}_{1}+\left(\frac{1}{2}-z_{5}\right) \mathbf{a}_{2}+z_{5} \mathbf{a}_{3}$ & $=$ & $\frac{1}{4} a \hat{\mathbf{x}}+\frac{1}{4} b \hat{\mathbf{y}}+\left(\frac{1}{2}-z_{5}\right) c \hat{\mathbf{z}}$ & $(8 h)$ & $\mathrm{Ru}$ II \\
\hline $\mathbf{B}_{9}$ & $=$ & $y_{6} \mathbf{a}_{1}+\left(\frac{1}{2}-y_{6}\right) \mathbf{a}_{2}+y_{6} \mathbf{a}_{3}$ & $=$ & $\frac{1}{4} a \hat{\mathbf{x}}+y_{6} b \hat{\mathbf{y}}+\frac{1}{4} c \hat{\mathbf{z}}$ & $(8 i)$ & B II \\
\hline $\mathbf{B}_{10}$ & $=$ & $\left(\frac{1}{2}-y_{6}\right) \mathbf{a}_{1}+y_{6} \mathbf{a}_{2}+\left(\frac{1}{2}-y_{6}\right) \mathbf{a}_{3}$ & $=$ & $\frac{1}{4} a \hat{\mathbf{x}}+\left(\frac{1}{2}-y_{6}\right) b \hat{\mathbf{y}}+\frac{1}{4} c \hat{\mathbf{z}}$ & $(8 i)$ & B II \\
\hline
\end{tabular}

\section{References:}

- P. Rogl, Structural chemistry and phase equilibria of ternary rare earth-platinum metal borides, J. Less-Common Met. 110, 283-294 (1985), doi:10.1016/0022-5088(85)90334-0.

\section{Found in:}

- P. Villars and K. Cenzual, Pearson's Crystal Data - Crystal Structure Database for Inorganic Compounds, ASM International (2013).

\section{Geometry files:}

- CIF: pp. 814

- POSCAR: pp. 814 


\section{FeS (Low-temperature) Structure: AB_oF8_22_a_c}

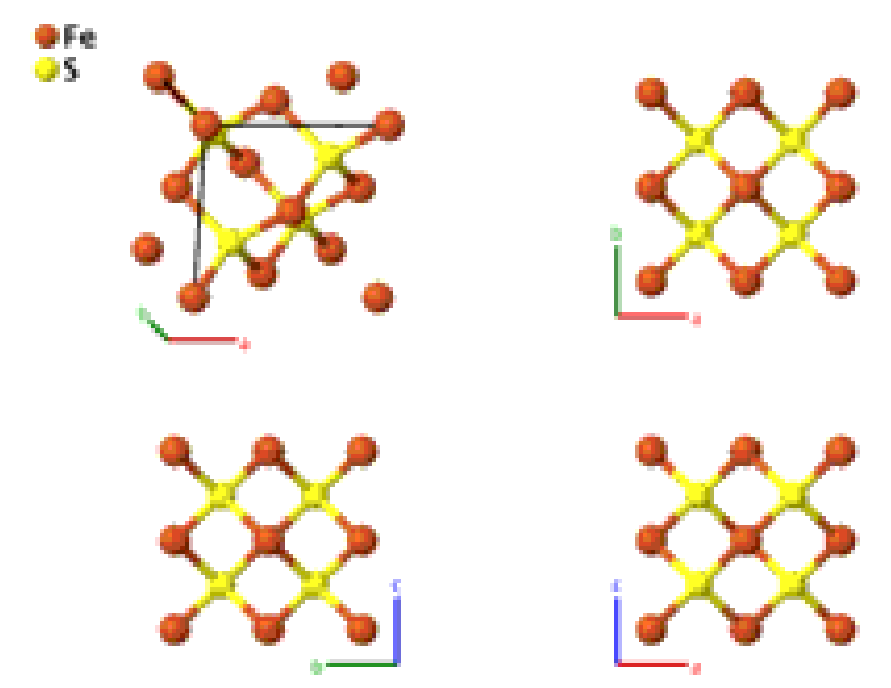

\begin{tabular}{|c|c|c|}
\hline Prototype & & $\mathrm{FeS}$ \\
\hline AFLOW prototype label & : & AB_oF8_22_a_c \\
\hline Strukturbericht designation & & None \\
\hline Pearson symbol & & oF8 \\
\hline Space group number & & 22 \\
\hline Space group symbol & & $F 222$ \\
\hline AFLOW prototype command & $\cdot$ & $\begin{array}{l}\text { aflow }-- \text { proto }=A B_{-} \mathrm{oF} 8 \_22 \_\mathrm{a}_{-} \mathrm{c} \\
- \text {-params }=a, b / a, c / a\end{array}$ \\
\hline
\end{tabular}

Face-centered Orthorhombic primitive vectors:

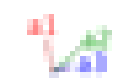

$$
\begin{aligned}
& \mathbf{a}_{1}=\frac{1}{2} b \hat{\mathbf{y}}+\frac{1}{2} c \hat{\mathbf{z}} \\
& \mathbf{a}_{2}=\frac{1}{2} a \hat{\mathbf{x}}+\frac{1}{2} c \hat{\mathbf{z}} \\
& \mathbf{a}_{3}=\frac{1}{2} a \hat{\mathbf{x}}+\frac{1}{2} b \hat{\mathbf{y}}
\end{aligned}
$$

Basis vectors:

Lattice Coordinates

$\mathbf{B}_{1}=0 \mathbf{a}_{1}+0 \mathbf{a}_{2}+0 \mathbf{a}_{3}=$
$\mathbf{B}_{2}=\frac{1}{4} \mathbf{a}_{1}+\frac{1}{4} \mathbf{a}_{2}+\frac{1}{4} \mathbf{a}_{3}=$

$$
\text { Cartesian Coordinates }
$$

$$
\begin{gathered}
0 \hat{\mathbf{x}}+0 \hat{\mathbf{y}}+0 \hat{\mathbf{z}} \\
\frac{1}{4} a \hat{\mathbf{x}}+\frac{1}{4} b \hat{\mathbf{y}}+\frac{1}{4} c \hat{\mathbf{z}}
\end{gathered}
$$

Wyckoff Position

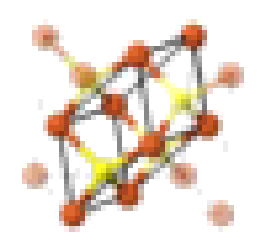

\section{References:}

- M. Wintenberger, B. Srour, C. Meyer, F. Hartmannboutron, Y. Gros, and J. L. Buevoz, First order transitions and magnetic-structure of zincblende-type iron sulfide, Acta Crystallogr. Sect. A 34, S318-S318 (1978).

Found in: 
- P. Villars and K. Cenzual, Pearson's Crystal Data - Crystal Structure Database for Inorganic Compounds, ASM International (2013).

\section{Geometry files:}

- CIF: pp. 814

- POSCAR: pp. 815 


\section{$\mathrm{H}_{3} \mathrm{~S}$ (5 GPa) Structure: A3B_oI32_23_ij2k_k}
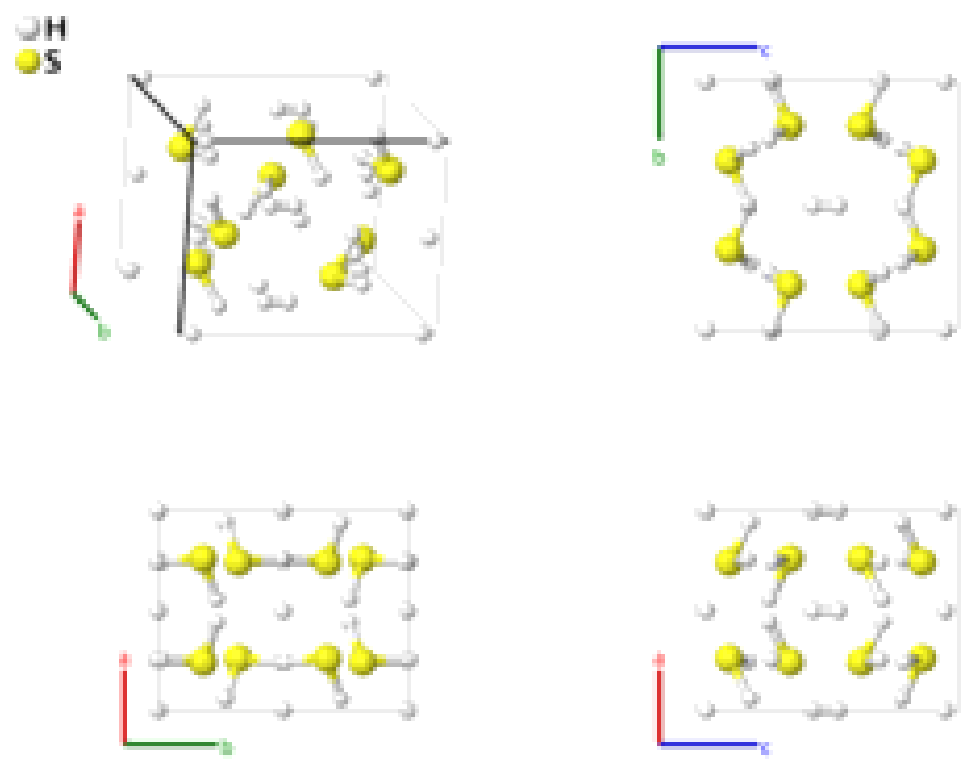

\section{Prototype}

AFLOW prototype label

Strukturbericht designation

Pearson symbol

Space group number

Space group symbol

AFLOW prototype command
: $\mathrm{H}_{3} \mathrm{~S}$

: A3B_OI32_23_ij2k_k

: None

: $\quad$ oI32

: 23

: $\quad I 222$

: aflow--proto=A3B_oI32_23_ij2k_k

--params $=a, b / a, c / a, z_{1}, z_{2}, x_{3}, y_{3}, z_{3}, x_{4}, y_{4}, z_{4}, x_{5}, y_{5}, z_{5}$

- This structure is found in $\mathrm{H}_{3} \mathrm{~S}$ in the pressure range 3.5-17 GPa. The data presented here was taken at $5 \mathrm{GPa}$.

Body-centered Orthorhombic primitive vectors:

$$
\begin{aligned}
& \mathbf{a}_{1}=-\frac{1}{2} a \hat{\mathbf{x}}+\frac{1}{2} b \hat{\mathbf{y}}+\frac{1}{2} c \hat{\mathbf{z}} \\
& \mathbf{a}_{2}=\frac{1}{2} a \hat{\mathbf{x}}-\frac{1}{2} b \hat{\mathbf{y}}+\frac{1}{2} c \hat{\mathbf{z}} \\
& \mathbf{a}_{3}=\frac{1}{2} a \hat{\mathbf{x}}+\frac{1}{2} b \hat{\mathbf{y}}-\frac{1}{2} c \hat{\mathbf{z}}
\end{aligned}
$$

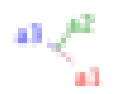

Basis vectors:

Lattice Coordinates
$\mathbf{B}_{1}=$
$\mathbf{B}_{2}=$
$\mathbf{B}_{3}=$
$-z_{1} \mathbf{a}_{1}-z_{1} \mathbf{a}_{2}$
$\left(\frac{1}{2}+z_{2}\right) \mathbf{a}_{1}+z_{2} \mathbf{a}_{2}+\frac{1}{2} \mathbf{a}_{3}$

Cartesian Coordinates

$$
=
$$

$=$

$$
z_{1} c \hat{\mathbf{z}}
$$$$
-z_{1} c \hat{\mathbf{z}}
$$$$
=\quad \frac{1}{2} b \hat{\mathbf{y}}+z_{2} c \hat{\mathbf{z}}
$$

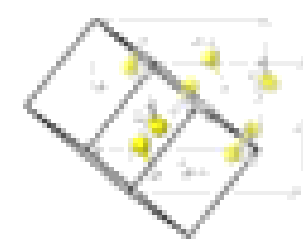




\begin{tabular}{|c|c|c|c|c|c|c|}
\hline $\mathbf{B}_{4}$ & $=$ & $\left(\frac{1}{2}-z_{2}\right) \mathbf{a}_{1}-z_{2} \mathbf{a}_{2}+\frac{1}{2} \mathbf{a}_{3}$ & $=$ & $\frac{1}{2} b \hat{\mathbf{y}}-z_{2} c \hat{\mathbf{z}}$ & $(4 j)$ & H II \\
\hline $\mathbf{B}_{5}$ & $=$ & $\left(y_{3}+z_{3}\right) \mathbf{a}_{1}+\left(x_{3}+z_{3}\right) \mathbf{a}_{2}+\left(x_{3}+y_{3}\right) \mathbf{a}_{3}$ & $=$ & $x_{3} a \hat{\mathbf{x}}+y_{3} b \hat{\mathbf{y}}+z_{3} c \hat{\mathbf{z}}$ & $(8 k)$ & H III \\
\hline $\mathbf{B}_{6}$ & $=$ & $\left(-y_{3}+z_{3}\right) \mathbf{a}_{1}+\left(-x_{3}+z_{3}\right) \mathbf{a}_{2}+\left(-x_{3}-y_{3}\right) \mathbf{a}_{3}$ & $=$ & $-x_{3} a \hat{\mathbf{x}}-y_{3} b \hat{\mathbf{y}}+z_{3} c \hat{\mathbf{z}}$ & $(8 k)$ & H III \\
\hline $\mathbf{B}_{7}$ & $=$ & $\left(y_{3}-z_{3}\right) \mathbf{a}_{1}+\left(-x_{3}-z_{3}\right) \mathbf{a}_{2}+\left(-x_{3}+y_{3}\right) \mathbf{a}_{3}$ & $=$ & $-x_{3} a \hat{\mathbf{x}}+y_{3} b \hat{\mathbf{y}}-z_{3} c \hat{\mathbf{z}}$ & $(8 k)$ & H III \\
\hline $\mathbf{B}_{8}$ & $=$ & $\left(-y_{3}-z_{3}\right) \mathbf{a}_{1}+\left(x_{3}-z_{3}\right) \mathbf{a}_{2}+\left(x_{3}-y_{3}\right) \mathbf{a}_{3}$ & $=$ & $x_{3} a \hat{\mathbf{x}}-y_{3} b \hat{\mathbf{y}}-z_{3} c \hat{\mathbf{z}}$ & $(8 k)$ & H III \\
\hline $\mathbf{B}_{9}$ & $=$ & $\left(y_{4}+z_{4}\right) \mathbf{a}_{1}+\left(x_{4}+z_{4}\right) \mathbf{a}_{2}+\left(x_{4}+y_{4}\right) \mathbf{a}_{3}$ & $=$ & $x_{4} a \hat{\mathbf{x}}+y_{4} b \hat{\mathbf{y}}+z_{4} c \hat{\mathbf{z}}$ & $(8 k)$ & H IV \\
\hline $\mathbf{B}_{10}$ & $=$ & $\left(-y_{4}+z_{4}\right) \mathbf{a}_{1}+\left(-x_{4}+z_{4}\right) \mathbf{a}_{2}+\left(-x_{4}-y_{4}\right) \mathbf{a}_{3}$ & $=$ & $-x_{4} a \hat{\mathbf{x}}-y_{4} b \hat{\mathbf{y}}+z_{4} c \hat{\mathbf{z}}$ & $(8 k)$ & H IV \\
\hline $\mathbf{B}_{11}$ & $=$ & $\left(y_{4}-z_{4}\right) \mathbf{a}_{1}+\left(-x_{4}-z_{4}\right) \mathbf{a}_{2}+\left(-x_{4}+y_{4}\right) \mathbf{a}_{3}$ & $=$ & $-x_{4} a \hat{\mathbf{x}}+y_{4} b \hat{\mathbf{y}}-z_{4} c \hat{\mathbf{z}}$ & $(8 k)$ & H IV \\
\hline $\mathbf{B}_{12}$ & $=$ & $\left(-y_{4}-z_{4}\right) \mathbf{a}_{1}+\left(x_{4}-z_{4}\right) \mathbf{a}_{2}+\left(x_{4}-y_{4}\right) \mathbf{a}_{3}$ & $=$ & $x_{4} a \hat{\mathbf{x}}-y_{4} b \hat{\mathbf{y}}-z_{4} c \hat{\mathbf{z}}$ & $(8 k)$ & H IV \\
\hline $\mathbf{B}_{13}$ & $=$ & $\left(y_{5}+z_{5}\right) \mathbf{a}_{1}+\left(x_{5}+z_{5}\right) \mathbf{a}_{2}+\left(x_{5}+y_{5}\right) \mathbf{a}_{3}$ & $=$ & $x_{5} a \hat{\mathbf{x}}+y_{5} b \hat{\mathbf{y}}+z_{5} c \hat{\mathbf{z}}$ & $(8 k)$ & $\mathrm{S}$ \\
\hline $\mathbf{B}_{14}$ & $=$ & $\left(-y_{5}+z_{5}\right) \mathbf{a}_{1}+\left(-x_{5}+z_{5}\right) \mathbf{a}_{2}+\left(-x_{5}-y_{5}\right) \mathbf{a}_{3}$ & $=$ & $-x_{5} a \hat{\mathbf{x}}-y_{5} b \hat{\mathbf{y}}+z_{5} c \hat{\mathbf{z}}$ & $(8 k)$ & S \\
\hline $\mathbf{B}_{15}$ & $=$ & $\left(y_{5}-z_{5}\right) \mathbf{a}_{1}+\left(-x_{5}-z_{5}\right) \mathbf{a}_{2}+\left(-x_{5}+y_{5}\right) \mathbf{a}_{3}$ & $=$ & $-x_{5} a \hat{\mathbf{x}}+y_{5} b \hat{\mathbf{y}}-z_{5} c \hat{\mathbf{z}}$ & $(8 k)$ & $S$ \\
\hline $\mathbf{B}_{16}$ & $=$ & $\left(-y_{5}-z_{5}\right) \mathbf{a}_{1}+\left(x_{5}-z_{5}\right) \mathbf{a}_{2}+\left(x_{5}-y_{5}\right) \mathbf{a}_{3}$ & $=$ & $x_{5} a \hat{\mathbf{x}}-y_{5} b \hat{\mathbf{y}}-z_{5} c \hat{\mathbf{z}}$ & $(8 k)$ & S \\
\hline
\end{tabular}

\section{References:}

- T. A. Strobel, P. Ganesh, M. Somayazulu, P. R. C. Kent, and R. J. Hemley, Novel Cooperative Interactions and Structural Ordering in $\mathrm{H}_{2} \mathrm{~S}-\mathrm{H}_{2}$, Phys. Rev. Lett. 107, 255503 (2011), doi:10.1103/PhysRevLett.107.255503.

\section{Geometry files:}

- CIF: pp. 815

- POSCAR: pp. 815 
Stannoidite $\left(\mathrm{Cu}_{8}(\mathrm{Fe}, \mathrm{Zn})_{3} \mathrm{Sn}_{2} \mathrm{~S}_{12}\right)$ Structure: A8B2C12D2E_oI50_23_bcfk_i_3k_j_a
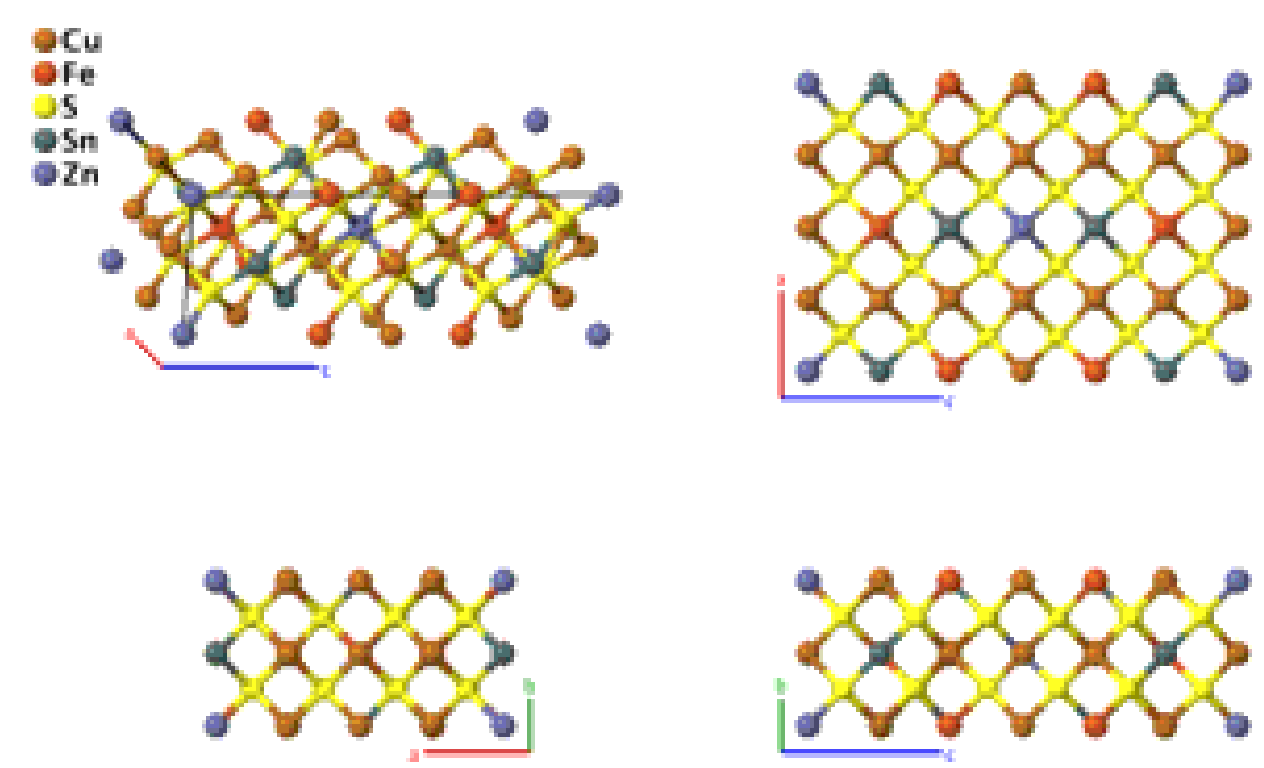

Prototype

: $\quad \mathrm{Cu}_{8}(\mathrm{Fe}, \mathrm{Zn})_{3} \mathrm{Sn}_{2} \mathrm{~S}_{12}$

AFLOW prototype label

: A8B2C12D2E_oI50_23_bcfk_i_3k_j_a

Strukturbericht designation : None

Pearson symbol :

Space group number : $\quad 23$

Space group symbol : $\quad$ I 222

AFLOW prototype command : aflow--proto=A8B2C12D2E_oI50_23_bcfk_i_3k_j_a

- params $=a, b / a, c / a, x_{4}, z_{5}, z_{6}, x_{7}, y_{7}, z_{7}, x_{8}, y_{8}, z_{8}, x_{9}, y_{9}, z_{9}, x_{10}, y_{10}, z_{10}$

- The composition of the $\mathrm{Zn}(2 \mathrm{a})$ site is actually $\mathrm{Zn}_{0.85} \mathrm{Fe}_{0.15}$.

Body-centered Orthorhombic primitive vectors:

$$
\begin{aligned}
& \mathbf{a}_{1}=-\frac{1}{2} a \hat{\mathbf{x}}+\frac{1}{2} b \hat{\mathbf{y}}+\frac{1}{2} c \hat{\mathbf{z}} \\
& \mathbf{a}_{2}=\frac{1}{2} a \hat{\mathbf{x}}-\frac{1}{2} b \hat{\mathbf{y}}+\frac{1}{2} c \hat{\mathbf{z}} \\
& \mathbf{a}_{3}=\frac{1}{2} a \hat{\mathbf{x}}+\frac{1}{2} b \hat{\mathbf{y}}-\frac{1}{2} c \hat{\mathbf{z}}
\end{aligned}
$$
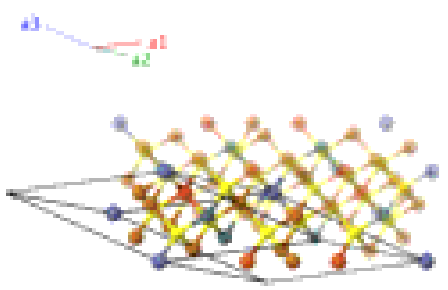

Basis vectors:

Lattice Coordinates
$\mathbf{B}_{1}=$
$0 \mathbf{a}_{1}+0 \mathbf{a}_{2}+0 \mathbf{a}_{3}$
$\mathbf{B}_{2}=$
$\frac{1}{2} \mathbf{a}_{2}+\frac{1}{2} \mathbf{a}_{3}$

Cartesian Coordinates

Wyckoff Position

(2a)

(2b)

Atom Type

$=\quad 0 \hat{\mathbf{x}}+0 \hat{\mathbf{y}}+0 \hat{\mathbf{z}}$

$=\quad \frac{1}{2} a \hat{\mathbf{x}}$ 


\begin{tabular}{|c|c|c|c|c|c|c|}
\hline $\mathbf{B}_{3}$ & $=$ & $\frac{1}{2} \mathbf{a}_{1}+\frac{1}{2} \mathbf{a}_{2}$ & $=$ & $\frac{1}{2} c \hat{\mathbf{z}}$ & $(2 c)$ & $\mathrm{Cu}$ II \\
\hline $\mathbf{B}_{4}$ & $=$ & $\frac{1}{2} \mathbf{a}_{1}+\left(\frac{1}{2}+x_{4}\right) \mathbf{a}_{2}+x_{4} \mathbf{a}_{3}$ & $=$ & $x_{4} a \hat{\mathbf{x}}+\frac{1}{2} c \hat{\mathbf{z}}$ & $(4 f)$ & $\mathrm{Cu}$ III \\
\hline $\mathbf{B}_{5}$ & $=$ & $\frac{1}{2} \mathbf{a}_{1}+\left(\frac{1}{2}-x_{4}\right) \mathbf{a}_{2}-x_{4} \mathbf{a}_{3}$ & $=$ & $-x_{4} a \hat{\mathbf{x}}+\frac{1}{2} c \hat{\mathbf{z}}$ & $(4 f)$ & $\mathrm{Cu}$ III \\
\hline $\mathbf{B}_{6}$ & $=$ & $z_{5} \mathbf{a}_{1}+z_{5} \mathbf{a}_{2}$ & $=$ & $z_{5} c \hat{\mathbf{z}}$ & $(4 i)$ & $\mathrm{Fe}$ \\
\hline $\mathbf{B}_{7}$ & $=$ & $-z_{5} \mathbf{a}_{1}-z_{5} \mathbf{a}_{2}$ & $=$ & $-z_{5} c \hat{\mathbf{z}}$ & $(4 i)$ & $\mathrm{Fe}$ \\
\hline $\mathbf{B}_{8}$ & $=$ & $\left(\frac{1}{2}+z_{6}\right) \mathbf{a}_{1}+z_{6} \mathbf{a}_{2}+\frac{1}{2} \mathbf{a}_{3}$ & $=$ & $\frac{1}{2} b \hat{\mathbf{y}}+z_{6} c \hat{\mathbf{z}}$ & $(4 j)$ & $\mathrm{Sn}$ \\
\hline $\mathbf{B}_{9}$ & $=$ & $\left(\frac{1}{2}-z_{6}\right) \mathbf{a}_{1}-z_{6} \mathbf{a}_{2}+\frac{1}{2} \mathbf{a}_{3}$ & $=$ & $\frac{1}{2} b \hat{\mathbf{y}}-z_{6} c \hat{\mathbf{z}}$ & $(4 j)$ & $\mathrm{Sn}$ \\
\hline $\mathbf{B}_{10}$ & $=$ & $\left(y_{7}+z_{7}\right) \mathbf{a}_{1}+\left(x_{7}+z_{7}\right) \mathbf{a}_{2}+\left(x_{7}+y_{7}\right) \mathbf{a}_{3}$ & $=$ & $x_{7} a \hat{\mathbf{x}}+y_{7} b \hat{\mathbf{y}}+z_{7} c \hat{\mathbf{z}}$ & $(8 k)$ & $\mathrm{Cu} \mathrm{IV}$ \\
\hline $\mathbf{B}_{11}$ & $=$ & $\left(-y_{7}+z_{7}\right) \mathbf{a}_{1}+\left(-x_{7}+z_{7}\right) \mathbf{a}_{2}+\left(-x_{7}-y_{7}\right) \mathbf{a}_{3}$ & $=$ & $-x_{7} a \hat{\mathbf{x}}-y_{7} b \hat{\mathbf{y}}+z_{7} c \hat{\mathbf{z}}$ & $(8 k)$ & $\mathrm{Cu} I \mathrm{~V}$ \\
\hline $\mathbf{B}_{12}$ & $=$ & $\left(y_{7}-z_{7}\right) \mathbf{a}_{1}+\left(-x_{7}-z_{7}\right) \mathbf{a}_{2}+\left(-x_{7}+y_{7}\right) \mathbf{a}_{3}$ & $=$ & $-x_{7} a \hat{\mathbf{x}}+y_{7} b \hat{\mathbf{y}}-z_{7} c \hat{\mathbf{z}}$ & $(8 k)$ & $\mathrm{Cu}$ IV \\
\hline $\mathbf{B}_{13}$ & $=$ & $\left(-y_{7}-z_{7}\right) \mathbf{a}_{1}+\left(x_{7}-z_{7}\right) \mathbf{a}_{2}+\left(x_{7}-y_{7}\right) \mathbf{a}_{3}$ & $=$ & $x_{7} a \hat{\mathbf{x}}-y_{7} b \hat{\mathbf{y}}-z_{7} c \hat{\mathbf{z}}$ & $(8 k)$ & $\mathrm{Cu} \mathrm{IV}$ \\
\hline $\mathbf{B}_{14}$ & $=$ & $\left(y_{8}+z_{8}\right) \mathbf{a}_{1}+\left(x_{8}+z_{8}\right) \mathbf{a}_{2}+\left(x_{8}+y_{8}\right) \mathbf{a}_{3}$ & $=$ & $x_{8} a \hat{\mathbf{x}}+y_{8} b \hat{\mathbf{y}}+z_{8} c \hat{\mathbf{z}}$ & $(8 k)$ & S I \\
\hline $\mathbf{B}_{15}$ & $=$ & $\left(-y_{8}+z_{8}\right) \mathbf{a}_{1}+\left(-x_{8}+z_{8}\right) \mathbf{a}_{2}+\left(-x_{8}-y_{8}\right) \mathbf{a}_{3}$ & $=$ & $-x_{8} a \hat{\mathbf{x}}-y_{8} b \hat{\mathbf{y}}+z_{8} c \hat{\mathbf{z}}$ & $(8 k)$ & S I \\
\hline $\mathbf{B}_{16}$ & $=$ & $\left(y_{8}-z_{8}\right) \mathbf{a}_{1}+\left(-x_{8}-z_{8}\right) \mathbf{a}_{2}+\left(-x_{8}+y_{8}\right) \mathbf{a}_{3}$ & $=$ & $-x_{8} a \hat{\mathbf{x}}+y_{8} b \hat{\mathbf{y}}-z_{8} c \hat{\mathbf{z}}$ & $(8 k)$ & S I \\
\hline $\mathbf{B}_{17}$ & $=$ & $\left(-y_{8}-z_{8}\right) \mathbf{a}_{1}+\left(x_{8}-z_{8}\right) \mathbf{a}_{2}+\left(x_{8}-y_{8}\right) \mathbf{a}_{3}$ & $=$ & $x_{8} a \hat{\mathbf{x}}-y_{8} b \hat{\mathbf{y}}-z_{8} c \hat{\mathbf{z}}$ & $(8 k)$ & S I \\
\hline $\mathbf{B}_{18}$ & $=$ & $\left(y_{9}+z_{9}\right) \mathbf{a}_{1}+\left(x_{9}+z_{9}\right) \mathbf{a}_{2}+\left(x_{9}+y_{9}\right) \mathbf{a}_{3}$ & $=$ & $x_{9} a \hat{\mathbf{x}}+y_{9} b \hat{\mathbf{y}}+z_{9} c \hat{\mathbf{z}}$ & $(8 k)$ & S II \\
\hline $\mathbf{B}_{19}$ & $=$ & $\left(-y_{9}+z_{9}\right) \mathbf{a}_{1}+\left(-x_{9}+z_{9}\right) \mathbf{a}_{2}+\left(-x_{9}-y_{9}\right) \mathbf{a}_{3}$ & $=$ & $-x_{9} a \hat{\mathbf{x}}-y_{9} b \hat{\mathbf{y}}+z_{9} c \hat{\mathbf{z}}$ & $(8 k)$ & S II \\
\hline $\mathbf{B}_{20}$ & $=$ & $\left(y_{9}-z_{9}\right) \mathbf{a}_{1}+\left(-x_{9}-z_{9}\right) \mathbf{a}_{2}+\left(-x_{9}+y_{9}\right) \mathbf{a}_{3}$ & $=$ & $-x_{9} a \hat{\mathbf{x}}+y_{9} b \hat{\mathbf{y}}-z_{9} c \hat{\mathbf{z}}$ & $(8 k)$ & S II \\
\hline $\mathbf{B}_{21}$ & $=$ & $\left(-y_{9}-z_{9}\right) \mathbf{a}_{1}+\left(x_{9}-z_{9}\right) \mathbf{a}_{2}+\left(x_{9}-y_{9}\right) \mathbf{a}_{3}$ & $=$ & $x_{9} a \hat{\mathbf{x}}-y_{9} b \hat{\mathbf{y}}-z_{9} c \hat{\mathbf{z}}$ & $(8 k)$ & S II \\
\hline $\mathbf{B}_{22}$ & $=$ & $\left(y_{10}+z_{10}\right) \mathbf{a}_{1}+\left(x_{10}+z_{10}\right) \mathbf{a}_{2}+\left(x_{10}+y_{10}\right) \mathbf{a}_{3}$ & $=$ & $x_{10} a \hat{\mathbf{x}}+y_{10} b \hat{\mathbf{y}}+z_{10} c \hat{\mathbf{z}}$ & $(8 k)$ & S III \\
\hline $\mathbf{B}_{23}$ & $=$ & $\begin{array}{c}\left(-y_{10}+z_{10}\right) \mathbf{a}_{1}+\left(-x_{10}+z_{10}\right) \mathbf{a}_{2}+ \\
\left(-x_{10}-y_{10}\right) \mathbf{a}_{3}\end{array}$ & $=$ & $-x_{10} a \hat{\mathbf{x}}-y_{10} b \hat{\mathbf{y}}+z_{10} c \hat{\mathbf{z}}$ & $(8 k)$ & S III \\
\hline $\mathbf{B}_{24}$ & $=$ & $\begin{array}{c}\left(y_{10}-z_{10}\right) \mathbf{a}_{1}+\left(-x_{10}-z_{10}\right) \mathbf{a}_{2}+ \\
\left(-x_{10}+y_{10}\right) \mathbf{a}_{3}\end{array}$ & $=$ & $-x_{10} a \hat{\mathbf{x}}+y_{10} b \hat{\mathbf{y}}-z_{10} c \hat{\mathbf{z}}$ & $(8 k)$ & S III \\
\hline $\mathbf{B}_{25}$ & $=$ & $\begin{array}{c}\left(-y_{10}-z_{10}\right) \mathbf{a}_{1}+\left(x_{10}-z_{10}\right) \mathbf{a}_{2}+ \\
\left(x_{10}-y_{10}\right) \mathbf{a}_{3}\end{array}$ & $=$ & $x_{10} a \hat{\mathbf{x}}-y_{10} b \hat{\mathbf{y}}-z_{10} c \hat{\mathbf{z}}$ & $(8 k)$ & S III \\
\hline
\end{tabular}

\section{References:}

- Y. Kudoh and Y. Takéuchi, The superstructure of stannoidite, Zeitschrift für Kristallographie - Crystalline Materials 144, 145-160 (1976), doi:10.1524/zkri.1976.144.16.145.

\section{Geometry files:}

- CIF: pp. 815

- POSCAR: pp. 816 


\section{$\mathrm{NaFeS}_{2}$ Structure: ABC2_oI16_23_ab_i_k}
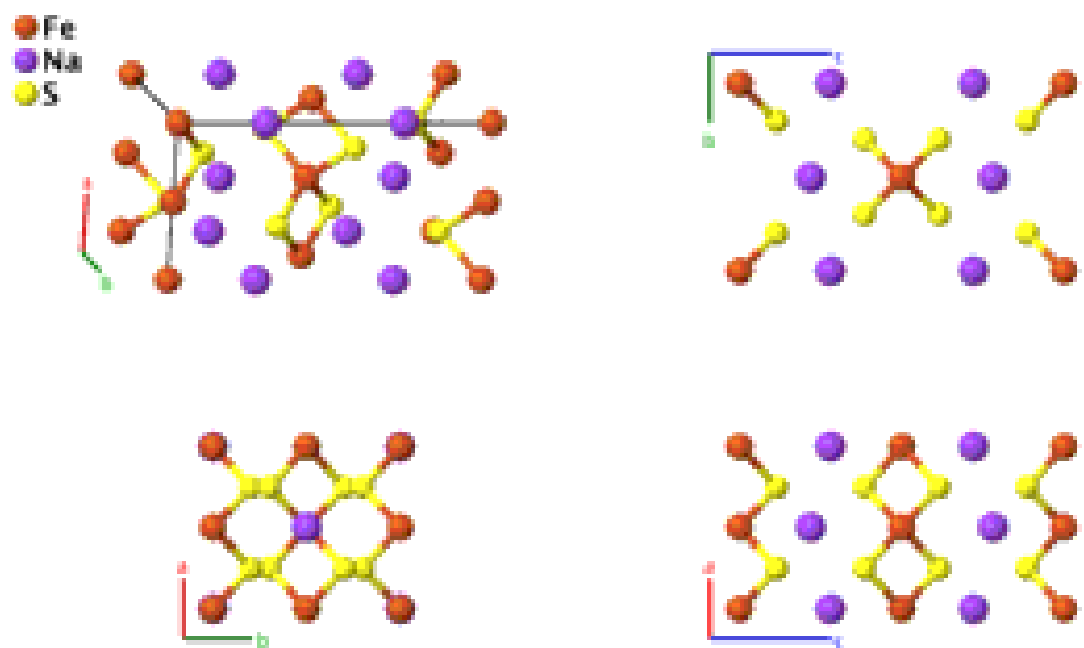

\section{Prototype}

AFLOW prototype label

$: \quad \mathrm{NaFeS}_{2}$

Strukturbericht designation

: ABC2_oI16_23_ab_i_k

Pearson symbol

: None

Space group number $\quad: \quad 23$

Space group symbol $\quad: \quad I 222$

AFLOW prototype command : aflow --proto=ABC2_oI16_23_ab_i_k

- - params $=a, b / a, c / a, z_{3}, x_{4}, y_{4}, z_{4}$

Body-centered Orthorhombic primitive vectors:

$$
\begin{aligned}
& \mathbf{a}_{1}=-\frac{1}{2} a \hat{\mathbf{x}}+\frac{1}{2} b \hat{\mathbf{y}}+\frac{1}{2} c \hat{\mathbf{z}} \\
& \mathbf{a}_{2}=\frac{1}{2} a \hat{\mathbf{x}}-\frac{1}{2} b \hat{\mathbf{y}}+\frac{1}{2} c \hat{\mathbf{z}} \\
& \mathbf{a}_{3}=\frac{1}{2} a \hat{\mathbf{x}}+\frac{1}{2} b \hat{\mathbf{y}}-\frac{1}{2} c \hat{\mathbf{z}}
\end{aligned}
$$

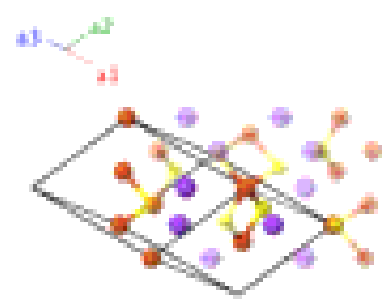

Basis vectors:

\section{Lattice Coordinates}

$\mathbf{B}_{1}=$

$\mathbf{B}_{2}=$

$\mathbf{B}_{3}=$

$\mathbf{B}_{4}=$

$\mathbf{B}_{5}=$

$\mathbf{B}_{6}$

$\mathbf{B}_{7}$

$\mathbf{B}_{8}$

$\frac{1}{2} \mathbf{a}_{2}+\frac{1}{2} \mathbf{a}_{3}$

$z_{3} \mathbf{a}_{1}+z_{3} \mathbf{a}_{2}$

$-z_{3} \mathbf{a}_{1}-z_{3} \mathbf{a}_{2}$
Cartesian Coordinates

$\left(y_{4}+z_{4}\right) \mathbf{a}_{1}+\left(x_{4}+z_{4}\right) \mathbf{a}_{2}+\left(x_{4}+y_{4}\right) \mathbf{a}_{3}$

$\left(-y_{4}+z_{4}\right) \mathbf{a}_{1}+\left(-x_{4}+z_{4}\right) \mathbf{a}_{2}+\left(-x_{4}-y_{4}\right) \mathbf{a}_{3}$

$\left(y_{4}-z_{4}\right) \mathbf{a}_{1}+\left(-x_{4}-z_{4}\right) \mathbf{a}_{2}+\left(-x_{4}+y_{4}\right) \mathbf{a}_{3}$

$\left(-y_{4}-z_{4}\right) \mathbf{a}_{1}+\left(x_{4}-z_{4}\right) \mathbf{a}_{2}+\left(x_{4}-y_{4}\right) \mathbf{a}_{3}$
$=$

$=$

$$
0 \hat{\mathbf{x}}+0 \hat{\mathbf{y}}+0 \hat{\mathbf{z}}
$$$$
\frac{1}{2} a \hat{\mathbf{x}}
$$$$
z_{3} c \hat{\mathbf{z}}
$$$$
-z_{3} c \hat{\mathbf{z}}
$$

$=$

$=\quad x_{4} a \hat{\mathbf{x}}+y_{4} b \hat{\mathbf{y}}+z_{4} c \hat{\mathbf{z}}$

$=-x_{4} a \hat{\mathbf{x}}-y_{4} b \hat{\mathbf{y}}+z_{4} c \hat{\mathbf{z}}$

$=-x_{4} a \hat{\mathbf{x}}+y_{4} b \hat{\mathbf{y}}-z_{4} c \hat{\mathbf{z}}$

$=x_{4} a \hat{\mathbf{x}}-y_{4} b \hat{\mathbf{y}}-z_{4} c \hat{\mathbf{z}}$
Wyckoff Position

(2a)
Atom Type

Fe I

Fe II

$\mathrm{Na}$

$\mathrm{Na}$

S

S

S

S 


\section{References:}

- H. Boller and H. Blaha, Zur Kenntnis des Natriumthioferrates (III), Monatsh. Chem. 114, 145-154 (1983), doi:10.1007/BF00798319.

\section{Found in:}

- P. Villars and K. Cenzual, Pearson's Crystal Data - Crystal Structure Database for Inorganic Compounds, ASM International (2013).

\section{Geometry files:}

- CIF: pp. 816

- POSCAR: pp. 816 


\section{$\mathrm{BPS}_{4}$ Structure: ABC4_oI12_23_a_b_k}
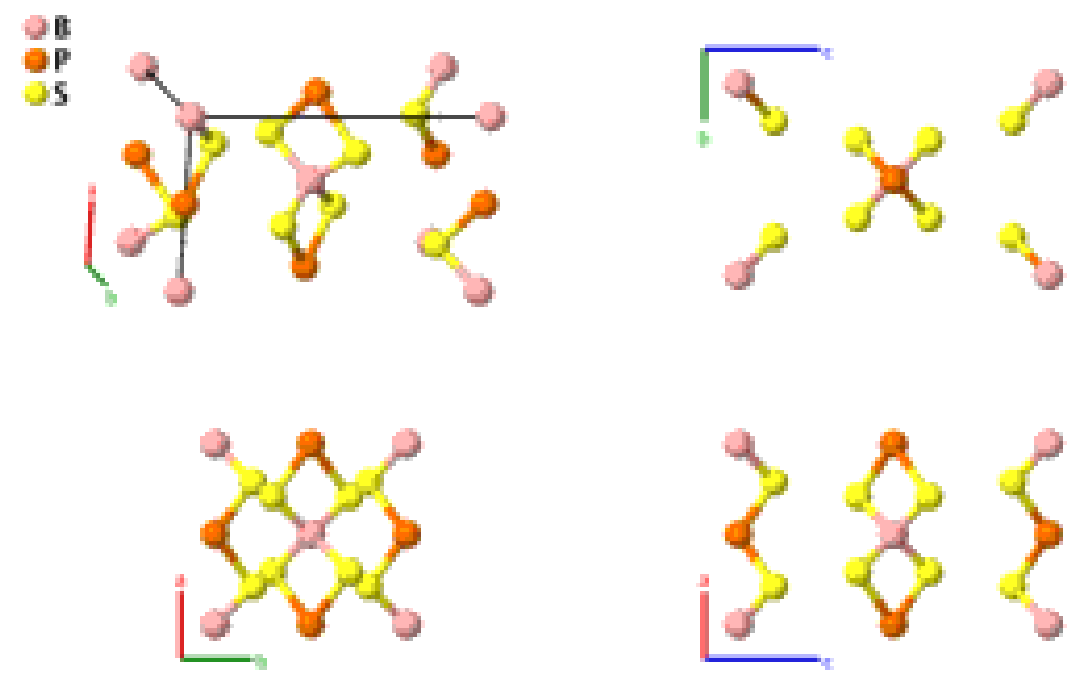

\section{Prototype}

AFLOW prototype label

Strukturbericht designation

Pearson symbol

Space group number

Space group symbol

AFLOW prototype command
$: \quad \mathrm{BPS}_{4}$

: ABC4_oI12_23_a_b_k

: None

: $\quad$ oI12

: 23

: $\quad I 222$ 


\section{References:}

- A. Weiss and H. Schäfer, Zur Kenntnis von Bortetrathiophosphat BPS 4 , Z. Naturforsch. B 18, 81-82 (1963), doi:10.1515/znb-1963-0117.

\section{Found in:}

- P. Villars and K. Cenzual, Pearson's Crystal Data - Crystal Structure Database for Inorganic Compounds, ASM International (2013).

\section{Geometry files:}

- CIF: pp. 816

- POSCAR: pp. 817 


\section{Weberite $\left(\mathrm{Na}_{2} \mathrm{MgAlF}_{7}\right)$ Structure:}

\section{AB7CD2_oI44_24_a_b3d_c_ac}

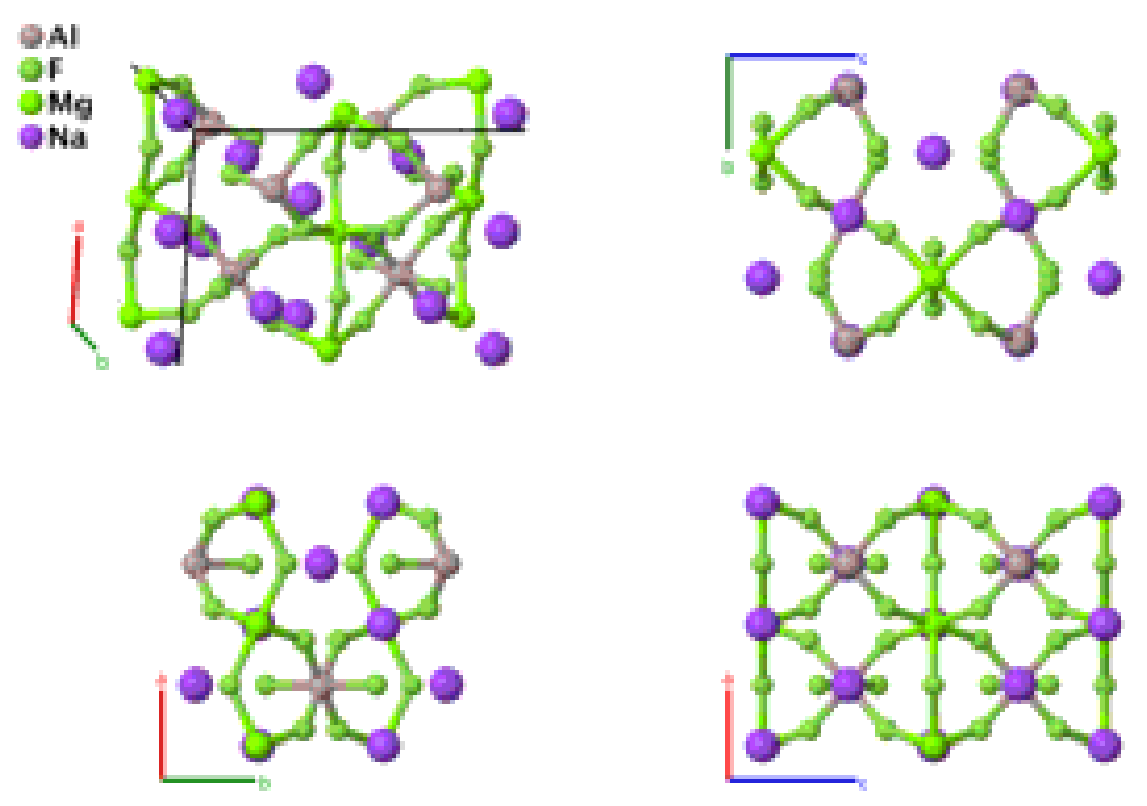

\section{Prototype}

AFLOW prototype label

Strukturbericht designation

Pearson symbol

Space group number

Space group symbol

AFLOW prototype command
: $\quad \mathrm{Na}_{2} \mathrm{MgAlF}_{7}$

: AB7CD2_oI44_24_a_b3d_c_ac

: None

: $\quad$ oI44

$: \quad 24$

: $\quad I 2_{1} 2_{1} 2_{1}$

aflow --proto=AB7CD2_oI44_24_a_b3d_c_ac

- -params $=a, b / a, c / a, x_{1}, x_{2}, y_{3}, z_{4}, z_{5}, x_{6}, y_{6}, z_{6}, x_{7}, y_{7}, z_{7}, x_{8}, y_{8}, z_{8}$

Body-centered Orthorhombic primitive vectors:

$$
\begin{aligned}
& \mathbf{a}_{1}=-\frac{1}{2} a \hat{\mathbf{x}}+\frac{1}{2} b \hat{\mathbf{y}}+\frac{1}{2} c \hat{\mathbf{z}} \\
& \mathbf{a}_{2}=\frac{1}{2} a \hat{\mathbf{x}}-\frac{1}{2} b \hat{\mathbf{y}}+\frac{1}{2} c \hat{\mathbf{z}} \\
& \mathbf{a}_{3}=\frac{1}{2} a \hat{\mathbf{x}}+\frac{1}{2} b \hat{\mathbf{y}}-\frac{1}{2} c \hat{\mathbf{z}}
\end{aligned}
$$

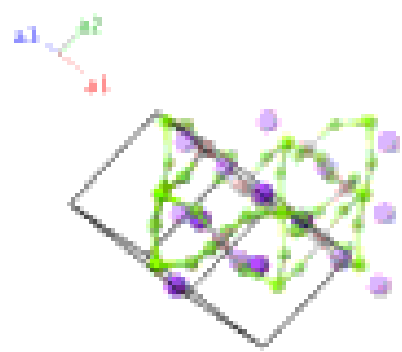

\section{Basis vectors:}

\section{Lattice Coordinates}

$\mathbf{B}_{1}=$

$\mathbf{B}_{2}=$

$\mathbf{B}_{3}=$

$\mathbf{B}_{4}=$ $\frac{1}{4} \mathbf{a}_{1}+\left(\frac{1}{4}+x_{1}\right) \mathbf{a}_{2}+x_{1} \mathbf{a}_{3}$

$\frac{3}{4} \mathbf{a}_{1}+\left(\frac{1}{4}-x_{1}\right) \mathbf{a}_{2}+\left(\frac{1}{2}-x_{1}\right) \mathbf{a}_{3}$

$\frac{1}{4} \mathbf{a}_{1}+\left(\frac{1}{4}+x_{2}\right) \mathbf{a}_{2}+x_{2} \mathbf{a}_{3}$

$\frac{3}{4} \mathbf{a}_{1}+\left(\frac{1}{4}-x_{2}\right) \mathbf{a}_{2}+\left(\frac{1}{2}-x_{2}\right) \mathbf{a}_{3}$
Cartesian Coordinates

$=$

$=$

$=$

$=\quad-x_{2} a \hat{\mathbf{x}}+\frac{1}{2} b \hat{\mathbf{y}}+\frac{1}{4} c \hat{\mathbf{z}}$
Wyckoff Position

(4a)

(4a)

(4a)

(4a)
Atom Type

$\mathrm{Al}$

Al

$\mathrm{Na}$ I

$\mathrm{Na}$ I 


\begin{tabular}{|c|c|c|c|c|c|c|}
\hline $\mathbf{B}_{5}$ & $=$ & $y_{3} \mathbf{a}_{1}+\frac{1}{4} \mathbf{a}_{2}+\left(\frac{1}{4}+y_{3}\right) \mathbf{a}_{3}$ & $=$ & $\frac{1}{4} a \hat{\mathbf{x}}+y_{3} b \hat{\mathbf{y}}$ & $(4 b)$ & F I \\
\hline $\mathbf{B}_{6}$ & $=$ & $\left(\frac{1}{2}-y_{3}\right) \mathbf{a}_{1}+\frac{3}{4} \mathbf{a}_{2}+\left(\frac{1}{4}-y_{3}\right) \mathbf{a}_{3}$ & $=$ & $\frac{1}{4} a \hat{\mathbf{x}}-y_{3} b \hat{\mathbf{y}}+\frac{1}{2} c \hat{\mathbf{z}}$ & $(4 b)$ & F I \\
\hline $\mathbf{B}_{7}$ & $=$ & $\left(\frac{1}{4}+z_{4}\right) \mathbf{a}_{1}+z_{4} \mathbf{a}_{2}+\frac{1}{4} \mathbf{a}_{3}$ & $=$ & $\frac{1}{4} b \hat{\mathbf{y}}+z_{4} c \hat{\mathbf{z}}$ & $(4 c)$ & $\mathrm{Mg}$ \\
\hline $\mathbf{B}_{8}$ & $=$ & $\left(\frac{1}{4}-z_{4}\right) \mathbf{a}_{1}+\left(\frac{1}{2}-z_{4}\right) \mathbf{a}_{2}+\frac{3}{4} \mathbf{a}_{3}$ & $=$ & $\frac{1}{2} a \hat{\mathbf{x}}+\frac{1}{4} b \hat{\mathbf{y}}-z_{4} c \hat{\mathbf{z}}$ & $(4 c)$ & $\mathrm{Mg}$ \\
\hline $\mathbf{B}_{9}$ & $=$ & $\left(\frac{1}{4}+z_{5}\right) \mathbf{a}_{1}+z_{5} \mathbf{a}_{2}+\frac{1}{4} \mathbf{a}_{3}$ & $=$ & $\frac{1}{4} b \hat{\mathbf{y}}+z_{5} c \hat{\mathbf{z}}$ & $(4 c)$ & $\mathrm{Na}$ II \\
\hline $\mathbf{B}_{10}$ & $=$ & $\left(\frac{1}{4}-z_{5}\right) \mathbf{a}_{1}+\left(\frac{1}{2}-z_{5}\right) \mathbf{a}_{2}+\frac{3}{4} \mathbf{a}_{3}$ & $=$ & $\frac{1}{2} a \hat{\mathbf{x}}+\frac{1}{4} b \hat{\mathbf{y}}-z_{5} c \hat{\mathbf{z}}$ & $(4 c)$ & $\mathrm{Na}$ II \\
\hline $\mathbf{B}_{11}$ & $=$ & $\left(y_{6}+z_{6}\right) \mathbf{a}_{1}+\left(x_{6}+z_{6}\right) \mathbf{a}_{2}+\left(x_{6}+y_{6}\right) \mathbf{a}_{3}$ & $=$ & $x_{6} a \hat{\mathbf{x}}+y_{6} b \hat{\mathbf{y}}+z_{6} c \hat{\mathbf{z}}$ & $(8 d)$ & F II \\
\hline $\mathbf{B}_{12}$ & $=$ & $\begin{array}{c}\left(\frac{1}{2}-y_{6}+z_{6}\right) \mathbf{a}_{1}+\left(-x_{6}+z_{6}\right) \mathbf{a}_{2}+ \\
\left(\frac{1}{2}-x_{6}-y_{6}\right) \mathbf{a}_{3}\end{array}$ & $=$ & $-x_{6} a \hat{\mathbf{x}}+\left(\frac{1}{2}-y_{6}\right) b \hat{\mathbf{y}}+z_{6} c \hat{\mathbf{z}}$ & $(8 d)$ & F II \\
\hline $\mathbf{B}_{13}$ & $=$ & $\begin{array}{c}\left(y_{6}-z_{6}\right) \mathbf{a}_{1}+\left(\frac{1}{2}-x_{6}-z_{6}\right) \mathbf{a}_{2}+ \\
\left(\frac{1}{2}-x_{6}+y_{6}\right) \mathbf{a}_{3}\end{array}$ & $=$ & $\left(\frac{1}{2}-x_{6}\right) a \hat{\mathbf{x}}+y_{6} b \hat{\mathbf{y}}-z_{6} c \hat{\mathbf{z}}$ & $(8 d)$ & F II \\
\hline $\mathbf{B}_{14}$ & $=$ & $\begin{array}{c}\left(\frac{1}{2}-y_{6}-z_{6}\right) \mathbf{a}_{1}+\left(\frac{1}{2}+x_{6}-z_{6}\right) \mathbf{a}_{2}+ \\
\left(x_{6}-y_{6}\right) \mathbf{a}_{3}\end{array}$ & $=$ & $x_{6} a \hat{\mathbf{x}}-y_{6} b \hat{\mathbf{y}}+\left(\frac{1}{2}-z_{6}\right) c \hat{\mathbf{z}}$ & $(8 d)$ & F II \\
\hline $\mathbf{B}_{15}$ & $=$ & $\left(y_{7}+z_{7}\right) \mathbf{a}_{1}+\left(x_{7}+z_{7}\right) \mathbf{a}_{2}+\left(x_{7}+y_{7}\right) \mathbf{a}_{3}$ & $=$ & $x_{7} a \hat{\mathbf{x}}+y_{7} b \hat{\mathbf{y}}+z_{7} c \hat{\mathbf{z}}$ & $(8 d)$ & F III \\
\hline $\mathbf{B}_{16}$ & $=$ & $\begin{array}{c}\left(\frac{1}{2}-y_{7}+z_{7}\right) \mathbf{a}_{1}+\left(-x_{7}+z_{7}\right) \mathbf{a}_{2}+ \\
\left(\frac{1}{2}-x_{7}-y_{7}\right) \mathbf{a}_{3}\end{array}$ & $=$ & $-x_{7} a \hat{\mathbf{x}}+\left(\frac{1}{2}-y_{7}\right) b \hat{\mathbf{y}}+z_{7} c \hat{\mathbf{z}}$ & $(8 d)$ & F III \\
\hline $\mathbf{B}_{17}$ & $=$ & $\begin{array}{c}\left(y_{7}-z_{7}\right) \mathbf{a}_{1}+\left(\frac{1}{2}-x_{7}-z_{7}\right) \mathbf{a}_{2}+ \\
\left(\frac{1}{2}-x_{7}+y_{7}\right) \mathbf{a}_{3}\end{array}$ & $=$ & $\left(\frac{1}{2}-x_{7}\right) a \hat{\mathbf{x}}+y_{7} b \hat{\mathbf{y}}-z_{7} c \hat{\mathbf{z}}$ & $(8 d)$ & F III \\
\hline $\mathbf{B}_{18}$ & $=$ & $\begin{array}{c}\left(\frac{1}{2}-y_{7}-z_{7}\right) \mathbf{a}_{1}+\left(\frac{1}{2}+x_{7}-z_{7}\right) \mathbf{a}_{2}+ \\
\left(x_{7}-y_{7}\right) \mathbf{a}_{3}\end{array}$ & $=$ & $x_{7} a \hat{\mathbf{x}}-y_{7} b \hat{\mathbf{y}}+\left(\frac{1}{2}-z_{7}\right) c \hat{\mathbf{z}}$ & $(8 d)$ & F III \\
\hline $\mathbf{B}_{19}$ & $=$ & $\left(y_{8}+z_{8}\right) \mathbf{a}_{1}+\left(x_{8}+z_{8}\right) \mathbf{a}_{2}+\left(x_{8}+y_{8}\right) \mathbf{a}_{3}$ & $=$ & $x_{8} a \hat{\mathbf{x}}+y_{8} b \hat{\mathbf{y}}+z_{8} c \hat{\mathbf{z}}$ & $(8 d)$ & F IV \\
\hline $\mathbf{B}_{20}$ & $=$ & $\begin{array}{c}\left(\frac{1}{2}-y_{8}+z_{8}\right) \mathbf{a}_{1}+\left(-x_{8}+z_{8}\right) \mathbf{a}_{2}+ \\
\left(\frac{1}{2}-x_{8}-y_{8}\right) \mathbf{a}_{3}\end{array}$ & $=$ & $-x_{8} a \hat{\mathbf{x}}+\left(\frac{1}{2}-y_{8}\right) b \hat{\mathbf{y}}+z_{8} c \hat{\mathbf{z}}$ & $(8 d)$ & F IV \\
\hline $\mathbf{B}_{21}$ & $=$ & $\begin{array}{c}\left(y_{8}-z_{8}\right) \mathbf{a}_{1}+\left(\frac{1}{2}-x_{8}-z_{8}\right) \mathbf{a}_{2}+ \\
\left(\frac{1}{2}-x_{8}+y_{8}\right) \mathbf{a}_{3}\end{array}$ & $=$ & $\left(\frac{1}{2}-x_{8}\right) a \hat{\mathbf{x}}+y_{8} b \hat{\mathbf{y}}-z_{8} c \hat{\mathbf{z}}$ & $(8 d)$ & F IV \\
\hline $\mathbf{B}_{22}$ & $=$ & $\begin{array}{c}\left(\frac{1}{2}-y_{8}-z_{8}\right) \mathbf{a}_{1}+\left(\frac{1}{2}+x_{8}-z_{8}\right) \mathbf{a}_{2}+ \\
\left(x_{8}-y_{8}\right) \mathbf{a}_{3}\end{array}$ & $=$ & $x_{8} a \hat{\mathbf{x}}-y_{8} b \hat{\mathbf{y}}+\left(\frac{1}{2}-z_{8}\right) c \hat{\mathbf{z}}$ & $(8 d)$ & F IV \\
\hline
\end{tabular}

\section{References:}

- O. Knop, T. S. Cameron, and K. Jochem, What is the true space group of weberite?, J. Solid State Chem. 43, 213-221 (1982), doi:10.1016/0022-4596(82)90231-6.

\section{Found in:}

- P. Villars and K. Cenzual, Pearson's Crystal Data - Crystal Structure Database for Inorganic Compounds, ASM International (2013).

\section{Geometry files:}

- CIF: pp. 817

- POSCAR: pp. 817 


\section{$\mathrm{H}_{2} \mathrm{~S}$ (70 GPa) Structure: A2B_oP12_26_abc_ab}

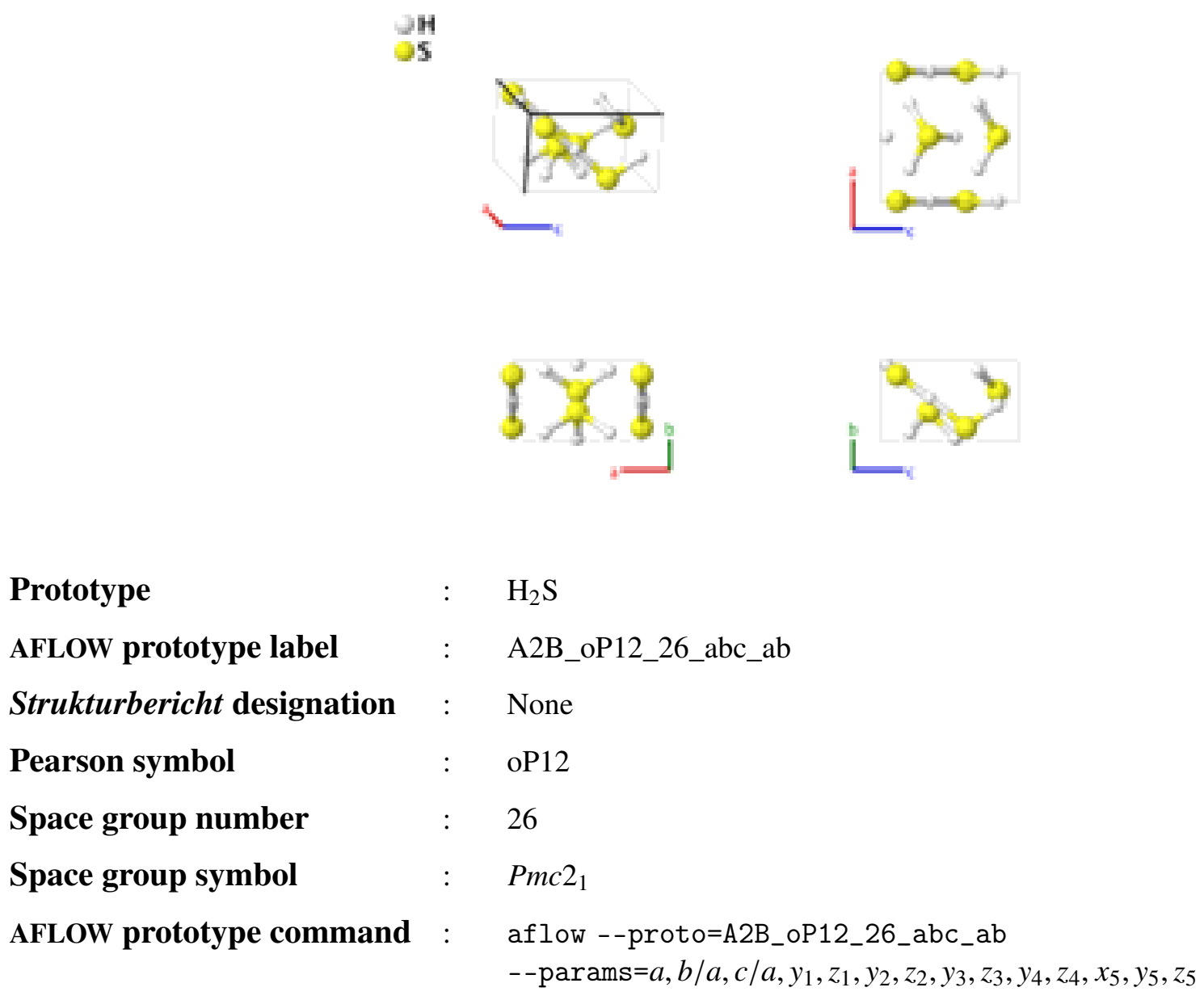

- This structure was found by first-principles electronic structure calculations and is predicted to be the stable structure of $\mathrm{H}_{2} \mathrm{~S}$ in the range $40-80 \mathrm{GPa}$. The data presented here was computed at $70 \mathrm{GPa}$. $\mathrm{H}_{2} \mathrm{~S}$ (pp. 95) and $\beta-\mathrm{SeO}_{2}$ (pp. 97) have the same AFLOW prototype label. They are generated by the same symmetry operations with different sets of parameters (--params) specified in their corresponding CIF files.

\section{Simple Orthorhombic primitive vectors:}

$$
\begin{aligned}
& \mathbf{a}_{1}=a \hat{\mathbf{x}} \\
& \mathbf{a}_{2}=b \hat{\mathbf{y}} \\
& \mathbf{a}_{3}=c \hat{\mathbf{z}}
\end{aligned}
$$

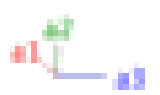

Basis vectors:

$\begin{array}{lccccc} & \text { Lattice Coordinates } & & \text { Cartesian Coordinates } & \text { Wyckoff Position } & \text { Atom Type } \\ \mathbf{B}_{1}= & y_{1} \mathbf{a}_{2}+z_{1} \mathbf{a}_{3} & = & y_{1} b \hat{\mathbf{y}}+z_{1} c \hat{\mathbf{z}} & (2 a) & \text { H I } \\ \mathbf{B}_{2}= & -y_{1} \mathbf{a}_{2}+\left(\frac{1}{2}+z_{1}\right) \mathbf{a}_{3} & = & -y_{1} b \hat{\mathbf{y}}+\left(\frac{1}{2}+z_{1}\right) c \hat{\mathbf{z}} & (2 a) & \text { H I } \\ \mathbf{B}_{3}= & y_{2} \mathbf{a}_{2}+z_{2} \mathbf{a}_{3} & = & y_{2} b \hat{\mathbf{y}}+z_{2} c \hat{\mathbf{z}} & (2 a) & \text { S I }\end{array}$




\begin{tabular}{|c|c|c|c|c|c|c|}
\hline $\mathbf{B}_{4}$ & $=$ & $-y_{2} \mathbf{a}_{2}+\left(\frac{1}{2}+z_{2}\right) \mathbf{a}_{3}$ & $=$ & $-y_{2} b \hat{\mathbf{y}}+\left(\frac{1}{2}+z_{2}\right) c \hat{\mathbf{z}}$ & $(2 a)$ & S I \\
\hline $\mathbf{B}_{5}$ & $=$ & $\frac{1}{2} \mathbf{a}_{1}+y_{3} \mathbf{a}_{2}+z_{3} \mathbf{a}_{3}$ & $=$ & $\frac{1}{2} a \hat{\mathbf{x}}+y_{3} b \hat{\mathbf{y}}+z_{3} c \hat{\mathbf{z}}$ & $(2 b)$ & H II \\
\hline $\mathbf{B}_{6}$ & $=$ & $\frac{1}{2} \mathbf{a}_{1}-y_{3} \mathbf{a}_{2}+\left(\frac{1}{2}+z_{3}\right) \mathbf{a}_{3}$ & $=$ & $\frac{1}{2} a \hat{\mathbf{x}}-y_{3} b \hat{\mathbf{y}}+\left(\frac{1}{2}+z_{3}\right) c \hat{\mathbf{z}}$ & $(2 b)$ & H II \\
\hline $\mathbf{B}_{7}$ & $=$ & $\frac{1}{2} \mathbf{a}_{1}+y_{4} \mathbf{a}_{2}+z_{4} \mathbf{a}_{3}$ & $=$ & $\frac{1}{2} a \hat{\mathbf{x}}+y_{4} b \hat{\mathbf{y}}+z_{4} c \hat{\mathbf{z}}$ & $(2 b)$ & S II \\
\hline $\mathbf{B}_{8}$ & $=$ & $\frac{1}{2} \mathbf{a}_{1}-y_{4} \mathbf{a}_{2}+\left(\frac{1}{2}+z_{4}\right) \mathbf{a}_{3}$ & $=$ & $\frac{1}{2} a \hat{\mathbf{x}}-y_{4} b \hat{\mathbf{y}}+\left(\frac{1}{2}+z_{4}\right) c \hat{\mathbf{z}}$ & $(2 b)$ & S II \\
\hline $\mathbf{B}_{9}$ & $=$ & $x_{5} \mathbf{a}_{1}+y_{5} \mathbf{a}_{2}+z_{5} \mathbf{a}_{3}$ & $=$ & $x_{5} a \hat{\mathbf{x}}+y_{5} b \hat{\mathbf{y}}+z_{5} c \hat{\mathbf{z}}$ & $(4 c)$ & H III \\
\hline $\mathbf{B}_{10}$ & $=$ & $-x_{5} \mathbf{a}_{1}-y_{5} \mathbf{a}_{2}+\left(\frac{1}{2}+z_{5}\right) \mathbf{a}_{3}$ & $=$ & $-x_{5} a \hat{\mathbf{x}}-y_{5} b \hat{\mathbf{y}}+\left(\frac{1}{2}+z_{5}\right) c \hat{\mathbf{z}}$ & $(4 c)$ & H III \\
\hline $\mathbf{B}_{11}$ & $=$ & $x_{5} \mathbf{a}_{1}-y_{5} \mathbf{a}_{2}+\left(\frac{1}{2}+z_{5}\right) \mathbf{a}_{3}$ & $=$ & $x_{5} a \hat{\mathbf{x}}-y_{5} b \hat{\mathbf{y}}+\left(\frac{1}{2}+z_{5}\right) c \hat{\mathbf{z}}$ & $(4 c)$ & H III \\
\hline $\mathbf{B}_{12}$ & $=$ & $-x_{5} \mathbf{a}_{1}+y_{5} \mathbf{a}_{2}+z_{5} \mathbf{a}_{3}$ & $=$ & $-x_{5} a \hat{\mathbf{x}}+y_{5} b \hat{\mathbf{y}}+z_{5} c \hat{\mathbf{z}}$ & $(4 c)$ & H III \\
\hline
\end{tabular}

\section{References:}

- Y. Li, J. Hao, H. Liu, Y. Li, and Y. Ma, The metallization and superconductivity of dense hydrogen sulfide, J. Chem. Phys. 140, 174712 (2014), doi:10.1063/1.4874158.

\section{Geometry files:}

- CIF: pp. 817

- POSCAR: pp. 818 


\section{$\beta-\mathrm{SeO}_{2}$ Structure: A2B_oP12_26_abc_ab}
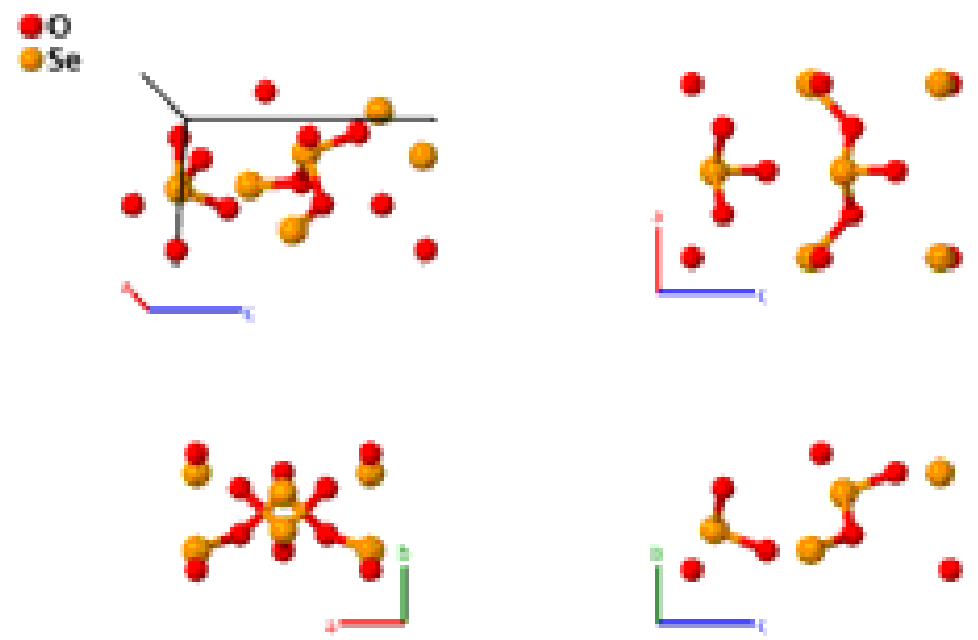

\section{Prototype}

AFLOW prototype label

Strukturbericht designation

Pearson symbol

Space group number

Space group symbol

AFLOW prototype command
: $\quad \beta-\mathrm{SeO}_{2}$

: A2B_oP12_26_abc_ab

: None

: $\quad \mathrm{oP} 12$

: $\quad 26$

: $\quad P m c 2_{1}$

aflow --proto=A2B_oP12_26_abc_ab

- params $=a, b / a, c / a, y_{1}, z_{1}, y_{2}, z_{2}, y_{3}, z_{3}, y_{4}, z_{4}, x_{5}, y_{5}, z_{5}$

- $\mathrm{H}_{2} \mathrm{~S}$ (pp. 95) and $\beta-\mathrm{SeO}_{2}$ (pp. 97) have the same AFLOW prototype label. They are generated by the same symmetry operations with different sets of parameters (--params) specified in their corresponding CIF files.

\section{Simple Orthorhombic primitive vectors:}

$$
\begin{aligned}
& \mathbf{a}_{1}=a \hat{\mathbf{x}} \\
& \mathbf{a}_{2}=b \hat{\mathbf{y}} \\
& \mathbf{a}_{3}=c \hat{\mathbf{z}}
\end{aligned}
$$

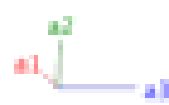

\section{Basis vectors:}

\section{Lattice Coordinates}

$\mathbf{B}_{1}=$

$\mathbf{B}_{2}=$

$\mathbf{B}_{3}=$

$\mathbf{B}_{4}=$

$\mathbf{B}_{5}=$

$$
y_{1} \mathbf{a}_{2}+z_{1} \mathbf{a}_{3}
$$$$
-y_{1} \mathbf{a}_{2}+\left(\frac{1}{2}+z_{1}\right) \mathbf{a}_{3}
$$$$
y_{2} \mathbf{a}_{2}+z_{2} \mathbf{a}_{3}
$$$$
-y_{2} \mathbf{a}_{2}+\left(\frac{1}{2}+z_{2}\right) \mathbf{a}_{3}
$$$$
\frac{1}{2} \mathbf{a}_{1}+y_{3} \mathbf{a}_{2}+z_{3} \mathbf{a}_{3}
$$

$$
=
$$$$
=
$$$$
=
$$$$
=
$$$$
=
$$

\section{Cartesian Coordinates}

$$
\begin{gathered}
y_{1} b \hat{\mathbf{y}}+z_{1} c \hat{\mathbf{z}} \\
-y_{1} b \hat{\mathbf{y}}+\left(\frac{1}{2}+z_{1}\right) c \hat{\mathbf{z}} \\
y_{2} b \hat{\mathbf{y}}+z_{2} c \hat{\mathbf{z}} \\
-y_{2} b \hat{\mathbf{y}}+\left(\frac{1}{2}+z_{2}\right) c \hat{\mathbf{z}} \\
\frac{1}{2} a \hat{\mathbf{x}}+y_{3} b \hat{\mathbf{y}}+z_{3} c \hat{\mathbf{z}}
\end{gathered}
$$

Wyckoff Position
Atom Type

O I

O I

Se I

Se I

O II 


$\begin{array}{rcccccc}\mathbf{B}_{6} & = & \frac{1}{2} \mathbf{a}_{1}-y_{3} \mathbf{a}_{2}+\left(\frac{1}{2}+z_{3}\right) \mathbf{a}_{3} & = & \frac{1}{2} a \hat{\mathbf{x}}-y_{3} b \hat{\mathbf{y}}+\left(\frac{1}{2}+z_{3}\right) c \hat{\mathbf{z}} & (2 b) & \text { O II } \\ \mathbf{B}_{7} & = & \frac{1}{2} \mathbf{a}_{1}+y_{4} \mathbf{a}_{2}+z_{4} \mathbf{a}_{3} & = & \frac{1}{2} a \hat{\mathbf{x}}+y_{4} b \hat{\mathbf{y}}+z_{4} c \hat{\mathbf{z}} & (2 b) & \text { Se II } \\ \mathbf{B}_{8} & = & \frac{1}{2} \mathbf{a}_{1}-y_{4} \mathbf{a}_{2}+\left(\frac{1}{2}+z_{4}\right) \mathbf{a}_{3} & = & \frac{1}{2} a \hat{\mathbf{x}}-y_{4} b \hat{\mathbf{y}}+\left(\frac{1}{2}+z_{4}\right) c \hat{\mathbf{z}} & (2 b) & \text { Se II } \\ \mathbf{B}_{9}= & x_{5} \mathbf{a}_{1}+y_{5} \mathbf{a}_{2}+z_{5} \mathbf{a}_{3} & = & x_{5} a \hat{\mathbf{x}}+y_{5} b \hat{\mathbf{y}}+z_{5} c \hat{\mathbf{z}} & (4 c) & \text { O III } \\ \mathbf{B}_{10}= & -x_{5} \mathbf{a}_{1}-y_{5} \mathbf{a}_{2}+\left(\frac{1}{2}+z_{5}\right) \mathbf{a}_{3} & = & -x_{5} a \hat{\mathbf{x}}-y_{5} b \hat{\mathbf{y}}+\left(\frac{1}{2}+z_{5}\right) c \hat{\mathbf{z}} & (4 c) & \text { O III } \\ \mathbf{B}_{11}= & x_{5} \mathbf{a}_{1}-y_{5} \mathbf{a}_{2}+\left(\frac{1}{2}+z_{5}\right) \mathbf{a}_{3} & = & x_{5} a \hat{\mathbf{x}}-y_{5} b \hat{\mathbf{y}}+\left(\frac{1}{2}+z_{5}\right) c \hat{\mathbf{z}} & (4 c) & \text { O III } \\ \mathbf{B}_{12}= & -x_{5} \mathbf{a}_{1}+y_{5} \mathbf{a}_{2}+z_{5} \mathbf{a}_{3} & = & -x_{5} a \hat{\mathbf{x}}+y_{5} b \hat{\mathbf{y}}+z_{5} c \hat{\mathbf{z}} & (4 c) & \text { O III }\end{array}$

\section{References:}

- D. Orosel, O. Leynaud, P. Balog, and M. Jansen, Pressure-temperature phase diagram of $\mathrm{SeO}_{2}$. Characterization of new phases, J. Solid State Chem. 177, 1631-1638 (2004), doi:10.1016/j.jssc.2003.12.028.

\section{Found in:}

- P. Villars and K. Cenzual, Pearson's Crystal Data - Crystal Structure Database for Inorganic Compounds, ASM International (2013).

\section{Geometry files:}

- CIF: pp. 818

- POSCAR: pp. 818 


\section{TlP 5 Structure: A5B_oP24_26_3a3b2c_ab}
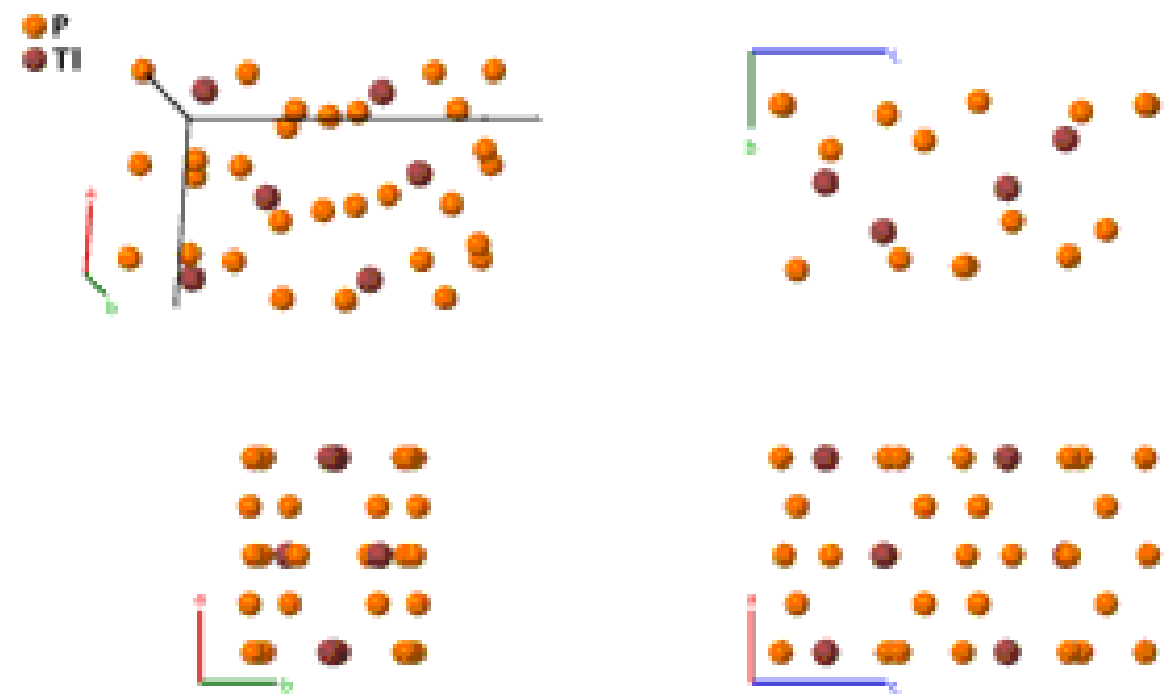

Prototype

$: \quad \mathrm{TlP}_{5}$

AFLOW prototype label

: A5B_oP24_26_3a3b2c_ab

Strukturbericht designation

Pearson symbol

: None

Space group number

: $\quad$ oP24

Space group symbol

: 26

AFLOW prototype command

: $\quad P m c 2_{1}$

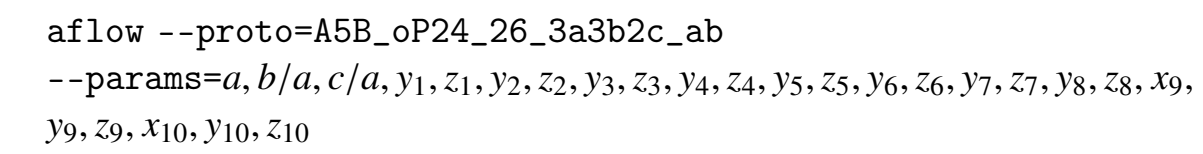

Simple Orthorhombic primitive vectors:

$$
\begin{aligned}
& \mathbf{a}_{1}=a \hat{\mathbf{x}} \\
& \mathbf{a}_{2}=b \hat{\mathbf{y}} \\
& \mathbf{a}_{3}=c \hat{\mathbf{z}}
\end{aligned}
$$

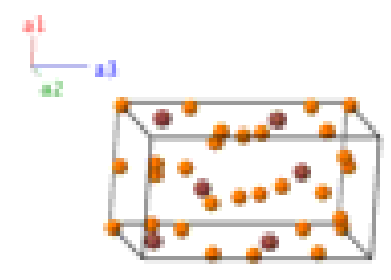

Basis vectors:

Lattice Coordinates
$\mathbf{B}_{1}=$
$y_{1} \mathbf{a}_{2}+z_{1} \mathbf{a}_{3}$
$\mathbf{B}_{2}=$
$-y_{1} \mathbf{a}_{2}+\left(\frac{1}{2}+z_{1}\right) \mathbf{a}_{3}$
$\mathbf{B}_{3}=$
$y_{2} \mathbf{a}_{2}+z_{2} \mathbf{a}_{3}$
$\mathbf{B}_{4}=$
$-y_{2} \mathbf{a}_{2}+\left(\frac{1}{2}+z_{2}\right) \mathbf{a}_{3}$
$\mathbf{B}_{5}=$
$y_{3} \mathbf{a}_{2}+z_{3} \mathbf{a}_{3}$
$\mathbf{B}_{6}=$
$-y_{3} \mathbf{a}_{2}+\left(\frac{1}{2}+z_{3}\right) \mathbf{a}_{3}$
$\mathbf{B}_{7}=$
$y_{4} \mathbf{a}_{2}+z_{4} \mathbf{a}_{3}$
$=$
$=$
$=$
$=$
$=$
$=$
$=$
$=$

$$
\begin{gathered}
y_{1} b \hat{\mathbf{y}}+z_{1} c \hat{\mathbf{z}} \\
-y_{1} b \hat{\mathbf{y}}+\left(\frac{1}{2}+z_{1}\right) c \hat{\mathbf{z}} \\
y_{2} b \hat{\mathbf{y}}+z_{2} c \hat{\mathbf{z}} \\
-y_{2} b \hat{\mathbf{y}}+\left(\frac{1}{2}+z_{2}\right) c \hat{\mathbf{z}} \\
y_{3} b \hat{\mathbf{y}}+z_{3} c \hat{\mathbf{z}} \\
-y_{3} b \hat{\mathbf{y}}+\left(\frac{1}{2}+z_{3}\right) c \hat{\mathbf{z}} \\
y_{4} b \hat{\mathbf{y}}+z_{4} c \hat{\mathbf{z}}
\end{gathered}
$$

Cartesian Coordinates
Wyckoff Position

(2a)
Atom Type

$$
\text { P I }
$$$$
\text { P I }
$$

P II

P II

P III

P III

Tl I 


\begin{tabular}{|c|c|c|c|c|c|c|}
\hline $\mathbf{B}_{8}$ & $=$ & $-y_{4} \mathbf{a}_{2}+\left(\frac{1}{2}+z_{4}\right) \mathbf{a}_{3}$ & $=$ & $-y_{4} b \hat{\mathbf{y}}+\left(\frac{1}{2}+z_{4}\right) c \hat{\mathbf{z}}$ & $(2 a)$ & $\mathrm{Tl} \mathrm{I}$ \\
\hline $\mathbf{B}_{9}$ & $=$ & $\frac{1}{2} \mathbf{a}_{1}+y_{5} \mathbf{a}_{2}+z_{5} \mathbf{a}_{3}$ & $=$ & $\frac{1}{2} a \hat{\mathbf{x}}+y_{5} b \hat{\mathbf{y}}+z_{5} c \hat{\mathbf{z}}$ & $(2 b)$ & P IV \\
\hline $\mathbf{B}_{10}$ & $=$ & $\frac{1}{2} \mathbf{a}_{1}-y_{5} \mathbf{a}_{2}+\left(\frac{1}{2}+z_{5}\right) \mathbf{a}_{3}$ & $=$ & $\frac{1}{2} a \hat{\mathbf{x}}-y_{5} b \hat{\mathbf{y}}+\left(\frac{1}{2}+z_{5}\right) c \hat{\mathbf{z}}$ & $(2 b)$ & P IV \\
\hline $\mathbf{B}_{11}$ & $=$ & $\frac{1}{2} \mathbf{a}_{1}+y_{6} \mathbf{a}_{2}+z_{6} \mathbf{a}_{3}$ & $=$ & $\frac{1}{2} a \hat{\mathbf{x}}+y_{6} b \hat{\mathbf{y}}+z_{6} c \hat{\mathbf{z}}$ & $(2 b)$ & $\mathrm{PV}$ \\
\hline $\mathbf{B}_{12}$ & $=$ & $\frac{1}{2} \mathbf{a}_{1}-y_{6} \mathbf{a}_{2}+\left(\frac{1}{2}+z_{6}\right) \mathbf{a}_{3}$ & $=$ & $\frac{1}{2} a \hat{\mathbf{x}}-y_{6} b \hat{\mathbf{y}}+\left(\frac{1}{2}+z_{6}\right) c \hat{\mathbf{z}}$ & $(2 b)$ & $\mathrm{PV}$ \\
\hline $\mathbf{B}_{13}$ & $=$ & $\frac{1}{2} \mathbf{a}_{1}+y_{7} \mathbf{a}_{2}+z_{7} \mathbf{a}_{3}$ & $=$ & $\frac{1}{2} a \hat{\mathbf{x}}+y_{7} b \hat{\mathbf{y}}+z_{7} c \hat{\mathbf{z}}$ & $(2 b)$ & P VI \\
\hline $\mathbf{B}_{14}$ & $=$ & $\frac{1}{2} \mathbf{a}_{1}-y_{7} \mathbf{a}_{2}+\left(\frac{1}{2}+z_{7}\right) \mathbf{a}_{3}$ & $=$ & $\frac{1}{2} a \hat{\mathbf{x}}-y_{7} b \hat{\mathbf{y}}+\left(\frac{1}{2}+z_{7}\right) c \hat{\mathbf{z}}$ & $(2 b)$ & P VI \\
\hline $\mathbf{B}_{15}$ & $=$ & $\frac{1}{2} \mathbf{a}_{1}+y_{8} \mathbf{a}_{2}+z_{8} \mathbf{a}_{3}$ & $=$ & $\frac{1}{2} a \hat{\mathbf{x}}+y_{8} b \hat{\mathbf{y}}+z_{8} c \hat{\mathbf{z}}$ & $(2 b)$ & Tl II \\
\hline $\mathbf{B}_{16}$ & $=$ & $\frac{1}{2} \mathbf{a}_{1}-y_{8} \mathbf{a}_{2}+\left(\frac{1}{2}+z_{8}\right) \mathbf{a}_{3}$ & $=$ & $\frac{1}{2} a \hat{\mathbf{x}}-y_{8} b \hat{\mathbf{y}}+\left(\frac{1}{2}+z_{8}\right) c \hat{\mathbf{z}}$ & $(2 b)$ & $\mathrm{Tl} \mathrm{II}$ \\
\hline $\mathbf{B}_{17}$ & $=$ & $x_{9} \mathbf{a}_{1}+y_{9} \mathbf{a}_{2}+z_{9} \mathbf{a}_{3}$ & $=$ & $x_{9} a \hat{\mathbf{x}}+y_{9} b \hat{\mathbf{y}}+z_{9} c \hat{\mathbf{z}}$ & $(4 c)$ & P VII \\
\hline $\mathbf{B}_{18}$ & $=$ & $-x_{9} \mathbf{a}_{1}-y_{9} \mathbf{a}_{2}+\left(\frac{1}{2}+z_{9}\right) \mathbf{a}_{3}$ & $=$ & $-x_{9} a \hat{\mathbf{x}}-y_{9} b \hat{\mathbf{y}}+\left(\frac{1}{2}+z_{9}\right) c \hat{\mathbf{z}}$ & $(4 c)$ & P VII \\
\hline $\mathbf{B}_{19}$ & $=$ & $x_{9} \mathbf{a}_{1}-y_{9} \mathbf{a}_{2}+\left(\frac{1}{2}+z_{9}\right) \mathbf{a}_{3}$ & $=$ & $x_{9} a \hat{\mathbf{x}}-y_{9} b \hat{\mathbf{y}}+\left(\frac{1}{2}+z_{9}\right) c \hat{\mathbf{z}}$ & $(4 c)$ & P VII \\
\hline $\mathbf{B}_{20}$ & $=$ & $-x_{9} \mathbf{a}_{1}+y_{9} \mathbf{a}_{2}+z_{9} \mathbf{a}_{3}$ & $=$ & $-x_{9} a \hat{\mathbf{x}}+y_{9} b \hat{\mathbf{y}}+z_{9} c \hat{\mathbf{z}}$ & $(4 c)$ & P VII \\
\hline $\mathbf{B}_{21}$ & $=$ & $x_{10} \mathbf{a}_{1}+y_{10} \mathbf{a}_{2}+z_{10} \mathbf{a}_{3}$ & $=$ & $x_{10} a \hat{\mathbf{x}}+y_{10} b \hat{\mathbf{y}}+z_{10} c \hat{\mathbf{z}}$ & $(4 c)$ & P VIII \\
\hline $\mathbf{B}_{22}$ & $=$ & $-x_{10} \mathbf{a}_{1}-y_{10} \mathbf{a}_{2}+\left(\frac{1}{2}+z_{10}\right) \mathbf{a}_{3}$ & $=$ & $-x_{10} a \hat{\mathbf{x}}-y_{10} b \hat{\mathbf{y}}+\left(\frac{1}{2}+z_{10}\right) c \hat{\mathbf{z}}$ & $(4 c)$ & P VIII \\
\hline $\mathbf{B}_{23}$ & $=$ & $x_{10} \mathbf{a}_{1}-y_{10} \mathbf{a}_{2}+\left(\frac{1}{2}+z_{10}\right) \mathbf{a}_{3}$ & $=$ & $x_{10} a \hat{\mathbf{x}}-y_{10} b \hat{\mathbf{y}}+\left(\frac{1}{2}+z_{10}\right) c \hat{\mathbf{z}}$ & $(4 c)$ & P VIII \\
\hline $\mathbf{B}_{24}$ & $=$ & $-x_{10} \mathbf{a}_{1}+y_{10} \mathbf{a}_{2}+z_{10} \mathbf{a}_{3}$ & $=$ & $-x_{10} a \hat{\mathbf{x}}+y_{10} b \hat{\mathbf{y}}+z_{10} c \hat{\mathbf{z}}$ & $(4 c)$ & P VIII \\
\hline
\end{tabular}

\section{References:}

- O. Olofsson and J. Gullman, The crystal structure of TlP , Acta Chem. Scand. 25, 1327-1337 (1971), doi:10.3891/acta.chem.scand.25-1327.

\section{Found in:}

- P. Villars and K. Cenzual, Pearson's Crystal Data - Crystal Structure Database for Inorganic Compounds, ASM International (2013).

\section{Geometry files:}

- CIF: pp. 818

- POSCAR: pp. 819 


\section{$\mathrm{Ca}_{4} \mathrm{Al}_{6} \mathrm{O}_{16} \mathrm{~S}$ Structure:}

\section{A6B4C16D_oP108_27_abcd4e_4e_16e_e}
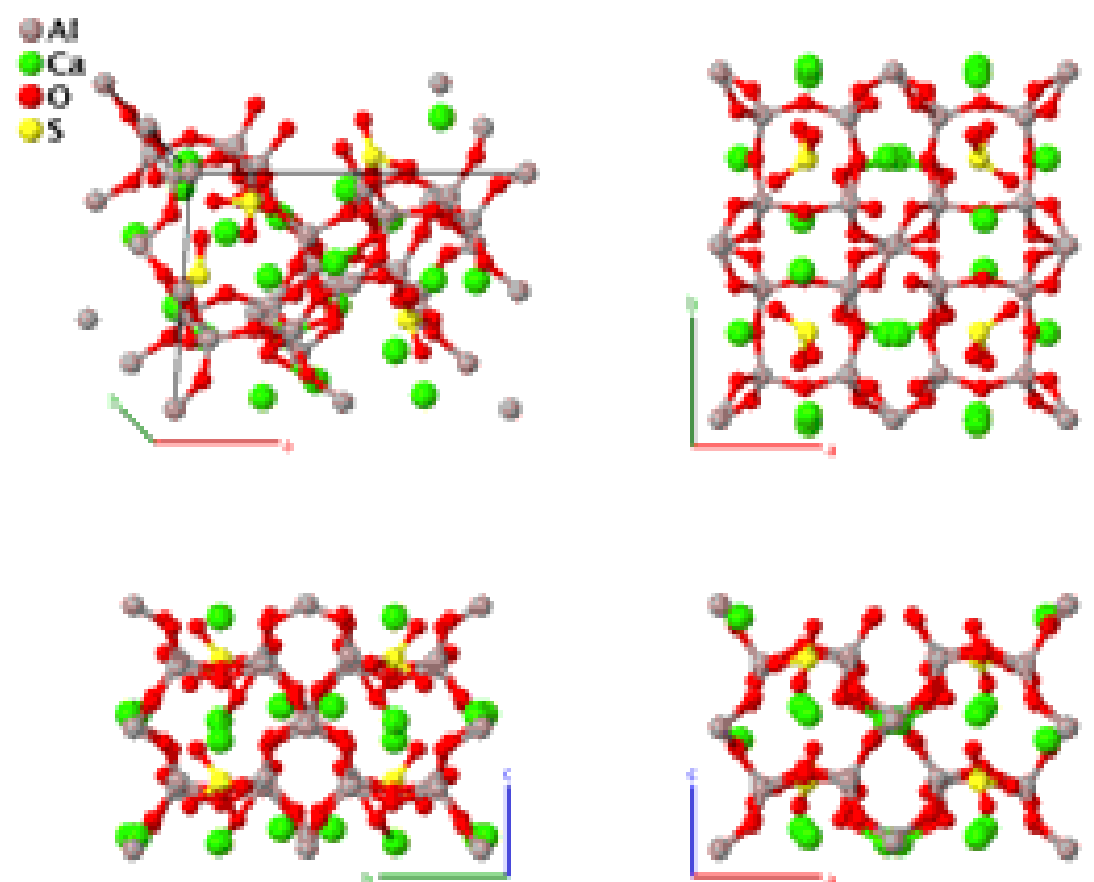

\section{Prototype}

AFLOW prototype label

: $\mathrm{Ca}_{4} \mathrm{Al}_{6} \mathrm{O}_{16} \mathrm{~S}$

Strukturbericht designation

: A6B4C16D_oP108_27_abcd4e_4e_16e_e

Pearson symbol

: None

Space group number

oP108

Space group symbol

: $\quad 27$

AFLOW prototype command

: $\quad P c c 2$
aflow --proto=A6B4C16D_oP108_27_abcd4e_4e_16e_e
- - params $=a, b / a, c / a, z_{1}, z_{2}, z_{3}, z_{4}, x_{5}, y_{5}, z_{5}, x_{6}, y_{6}, z_{6}, x_{7}, y_{7}, z_{7}, x_{8}, y_{8}, z_{8}, x_{9}$, $y_{9}, z_{9}, x_{10}, y_{10}, z_{10}, x_{11}, y_{11}, z_{11}, x_{12}, y_{12}, z_{12}, x_{13}, y_{13}, z_{13}, x_{14}, y_{14}, z_{14}, x_{15}, y_{15}, z_{15}, x_{16}$, $y_{16}, z_{16}, x_{17}, y_{17}, z_{17}, x_{18}, y_{18}, z_{18}, x_{19}, y_{19}, z_{19}, x_{20}, y_{20}, z_{20}, x_{21}, y_{21}, z_{21}, x_{22}, y_{22}, z_{22}$, $x_{23}, y_{23}, z_{23}, x_{24}, y_{24}, z_{24}, x_{25}, y_{25}, z_{25}, x_{26}, y_{26}, z_{26}, x_{27}, y_{27}, z_{27}, x_{28}, y_{28}, z_{28}, x_{29}, y_{29}$, $z_{29}$

Simple Orthorhombic primitive vectors:

$$
\begin{aligned}
& \mathbf{a}_{1}=a \hat{\mathbf{x}} \\
& \mathbf{a}_{2}=b \hat{\mathbf{y}} \\
& \mathbf{a}_{3}=c \hat{\mathbf{z}}
\end{aligned}
$$

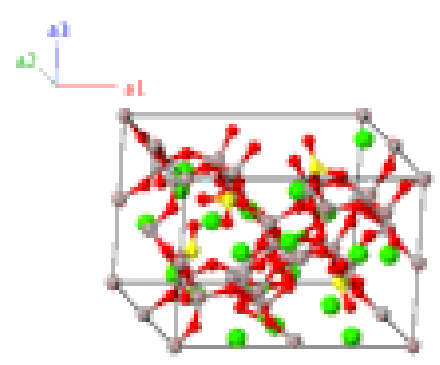


Cartesian Coordinates

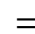

$\mathbf{B}_{1}=$

$z_{1} \mathbf{a}_{3}$

$\mathbf{B}_{2}=$

$\left(\frac{1}{2}+z_{1}\right) \mathbf{a}_{3}$

$\mathbf{B}_{3}=$

$\frac{1}{2} \mathbf{a}_{2}+z_{2} \mathbf{a}_{3}$

$\mathbf{B}_{4}=$

$\mathbf{B}_{5}=$

$\mathbf{B}_{6}=$

$\mathbf{B}_{7}=$

$\mathbf{B}_{8}=$

$\mathbf{B}_{9}=$

$\mathbf{B}_{10}=$

$\mathbf{B}_{11}=$

$\mathbf{B}_{12}=$

$\mathbf{B}_{13}=$

$\mathbf{B}_{14}=$

$\mathbf{B}_{15}=$

$\mathbf{B}_{16}=$

$\mathbf{B}_{17}=$

$\mathbf{B}_{18}=$

$\mathbf{B}_{19}=$

$\mathbf{B}_{20}=$

$\mathbf{B}_{21}=$

$\mathbf{B}_{22}=$

$\mathbf{B}_{23}=$

$\mathbf{B}_{24}=$

$\mathbf{B}_{25}=$

$\mathbf{B}_{26}=$

$\mathbf{B}_{27}=$

$\mathbf{B}_{28}=$

$\mathbf{B}_{29}=$

$\mathbf{B}_{30}=$

$\mathbf{B}_{31}=$

$\mathbf{B}_{32}=$

$\mathbf{B}_{33}=$

$\mathbf{B}_{34}=$

$\mathbf{B}_{35}=$

$\frac{1}{2} \mathbf{a}_{2}+\left(\frac{1}{2}+z_{2}\right) \mathbf{a}_{3}$

$\frac{1}{2} \mathbf{a}_{1}+z_{3} \mathbf{a}_{3}$

$\frac{1}{2} \mathbf{a}_{1}+\left(\frac{1}{2}+z_{3}\right) \mathbf{a}_{3}$

$\frac{1}{2} \mathbf{a}_{1}+\frac{1}{2} \mathbf{a}_{2}+z_{4} \mathbf{a}_{3}$

$\frac{1}{2} \mathbf{a}_{1}+\frac{1}{2} \mathbf{a}_{2}+\left(\frac{1}{2}+z_{4}\right) \mathbf{a}_{3}$

$x_{5} \mathbf{a}_{1}+y_{5} \mathbf{a}_{2}+z_{5} \mathbf{a}_{3}$

$-x_{5} \mathbf{a}_{1}-y_{5} \mathbf{a}_{2}+z_{5} \mathbf{a}_{3}$

$x_{5} \mathbf{a}_{1}-y_{5} \mathbf{a}_{2}+\left(\frac{1}{2}+z_{5}\right) \mathbf{a}_{3}$

$-x_{5} \mathbf{a}_{1}+y_{5} \mathbf{a}_{2}+\left(\frac{1}{2}+z_{5}\right) \mathbf{a}_{3}$

$x_{6} \mathbf{a}_{1}+y_{6} \mathbf{a}_{2}+z_{6} \mathbf{a}_{3}$

$-x_{6} \mathbf{a}_{1}-y_{6} \mathbf{a}_{2}+z_{6} \mathbf{a}_{3}$

$x_{6} \mathbf{a}_{1}-y_{6} \mathbf{a}_{2}+\left(\frac{1}{2}+z_{6}\right) \mathbf{a}_{3}$

$-x_{6} \mathbf{a}_{1}+y_{6} \mathbf{a}_{2}+\left(\frac{1}{2}+z_{6}\right) \mathbf{a}_{3}$

$x_{7} \mathbf{a}_{1}+y_{7} \mathbf{a}_{2}+z_{7} \mathbf{a}_{3}$

$-x_{7} \mathbf{a}_{1}-y_{7} \mathbf{a}_{2}+z_{7} \mathbf{a}_{3}$

$x_{7} \mathbf{a}_{1}-y_{7} \mathbf{a}_{2}+\left(\frac{1}{2}+z_{7}\right) \mathbf{a}_{3}$

$-x_{7} \mathbf{a}_{1}+y_{7} \mathbf{a}_{2}+\left(\frac{1}{2}+z_{7}\right) \mathbf{a}_{3}$

$x_{8} \mathbf{a}_{1}+y_{8} \mathbf{a}_{2}+z_{8} \mathbf{a}_{3}$

$-x_{8} \mathbf{a}_{1}-y_{8} \mathbf{a}_{2}+z_{8} \mathbf{a}_{3}$

$x_{8} \mathbf{a}_{1}-y_{8} \mathbf{a}_{2}+\left(\frac{1}{2}+z_{8}\right) \mathbf{a}_{3}$

$-x_{8} \mathbf{a}_{1}+y_{8} \mathbf{a}_{2}+\left(\frac{1}{2}+z_{8}\right) \mathbf{a}_{3}$

$x_{9} \mathbf{a}_{1}+y_{9} \mathbf{a}_{2}+z_{9} \mathbf{a}_{3}$

$-x_{9} \mathbf{a}_{1}-y_{9} \mathbf{a}_{2}+z_{9} \mathbf{a}_{3}$

$x_{9} \mathbf{a}_{1}-y_{9} \mathbf{a}_{2}+\left(\frac{1}{2}+z_{9}\right) \mathbf{a}_{3}$

$-x_{9} \mathbf{a}_{1}+y_{9} \mathbf{a}_{2}+\left(\frac{1}{2}+z_{9}\right) \mathbf{a}_{3}$

$x_{10} \mathbf{a}_{1}+y_{10} \mathbf{a}_{2}+z_{10} \mathbf{a}_{3}$

$-x_{10} \mathbf{a}_{1}-y_{10} \mathbf{a}_{2}+z_{10} \mathbf{a}_{3}$

$x_{10} \mathbf{a}_{1}-y_{10} \mathbf{a}_{2}+\left(\frac{1}{2}+z_{10}\right) \mathbf{a}_{3}$

$-x_{10} \mathbf{a}_{1}+y_{10} \mathbf{a}_{2}+\left(\frac{1}{2}+z_{10}\right) \mathbf{a}_{3}$

$x_{11} \mathbf{a}_{1}+y_{11} \mathbf{a}_{2}+z_{11} \mathbf{a}_{3}$

$-x_{11} \mathbf{a}_{1}-y_{11} \mathbf{a}_{2}+z_{11} \mathbf{a}_{3}$

$x_{11} \mathbf{a}_{1}-y_{11} \mathbf{a}_{2}+\left(\frac{1}{2}+z_{11}\right) \mathbf{a}_{3}$
$=$

$=$

$=$

$=$

$=$

$=$

$=$

$=$

$=$

$=$

$=$

$=$

$=$

$=$

$=$

$=$

$=$

$=$

$=$

$=$

$=$

$=$

$=$

$=$

$=$

$=$

$=$

$=$

$=$

$=$

$=$

$=$

$=$

$=$

$$
\begin{gathered}
z_{1} c \hat{\mathbf{z}} \\
\left(\frac{1}{2}+z_{1}\right) c \hat{\mathbf{z}} \\
\frac{1}{2} b \hat{\mathbf{y}}+z_{2} c \hat{\mathbf{z}} \\
\frac{1}{2} b \hat{\mathbf{y}}+\left(\frac{1}{2}+z_{2}\right) c \hat{\mathbf{z}} \\
\frac{1}{2} a \hat{\mathbf{x}}+z_{3} c \hat{\mathbf{z}} \\
\frac{1}{2} a \hat{\mathbf{x}}+\left(\frac{1}{2}+z_{3}\right) c \hat{\mathbf{z}} \\
\frac{1}{2} a \hat{\mathbf{x}}+\frac{1}{2} b \hat{\mathbf{y}}+z_{4} c \hat{\mathbf{z}} \\
\frac{1}{2} a \hat{\mathbf{x}}+\frac{1}{2} b \hat{\mathbf{y}}+\left(\frac{1}{2}+z_{4}\right) c \hat{\mathbf{z}} \\
x_{5} a \hat{\mathbf{x}}+y_{5} b \hat{\mathbf{y}}+z_{5} c \hat{\mathbf{z}} \\
-x_{5} a \hat{\mathbf{x}}-y_{5} b \hat{\mathbf{y}}+z_{5} c \hat{\mathbf{z}}
\end{gathered}
$$$$
x_{5} a \hat{\mathbf{x}}-y_{5} b \hat{\mathbf{y}}+\left(\frac{1}{2}+z_{5}\right) c \hat{\mathbf{z}}
$$$$
-x_{5} a \hat{\mathbf{x}}+y_{5} b \hat{\mathbf{y}}+\left(\frac{1}{2}+z_{5}\right) c \hat{\mathbf{z}}
$$$$
x_{6} a \hat{\mathbf{x}}+y_{6} b \hat{\mathbf{y}}+z_{6} c \hat{\mathbf{z}}
$$$$
-x_{6} a \hat{\mathbf{x}}-y_{6} b \hat{\mathbf{y}}+z_{6} c \hat{\mathbf{z}}
$$$$
x_{6} a \hat{\mathbf{x}}-y_{6} b \hat{\mathbf{y}}+\left(\frac{1}{2}+z_{6}\right) c \hat{\mathbf{z}}
$$$$
-x_{6} a \hat{\mathbf{x}}+y_{6} b \hat{\mathbf{y}}+\left(\frac{1}{2}+z_{6}\right) c \hat{\mathbf{z}}
$$$$
x_{7} a \hat{\mathbf{x}}+y_{7} b \hat{\mathbf{y}}+z_{7} c \hat{\mathbf{z}}
$$$$
-x_{7} a \hat{\mathbf{x}}-y_{7} b \hat{\mathbf{y}}+z_{7} c \hat{\mathbf{z}}
$$$$
x_{7} a \hat{\mathbf{x}}-y_{7} b \hat{\mathbf{y}}+\left(\frac{1}{2}+z_{7}\right) c \hat{\mathbf{z}}
$$$$
-x_{7} a \hat{\mathbf{x}}+y_{7} b \hat{\mathbf{y}}+\left(\frac{1}{2}+z_{7}\right) c \hat{\mathbf{z}}
$$$$
x_{8} a \hat{\mathbf{x}}+y_{8} b \hat{\mathbf{y}}+z_{8} c \hat{\mathbf{z}}
$$$$
-x_{8} a \hat{\mathbf{x}}-y_{8} b \hat{\mathbf{y}}+z_{8} c \hat{\mathbf{z}}
$$$$
x_{8} a \hat{\mathbf{x}}-y_{8} b \hat{\mathbf{y}}+\left(\frac{1}{2}+z_{8}\right) c \hat{\mathbf{z}}
$$$$
-x_{8} a \hat{\mathbf{x}}+y_{8} b \hat{\mathbf{y}}+\left(\frac{1}{2}+z_{8}\right) c \hat{\mathbf{z}}
$$$$
x_{9} a \hat{\mathbf{x}}+y_{9} b \hat{\mathbf{y}}+z_{9} c \hat{\mathbf{z}}
$$$$
-x_{9} a \hat{\mathbf{x}}-y_{9} b \hat{\mathbf{y}}+z_{9} c \hat{\mathbf{z}}
$$$$
x_{9} a \hat{\mathbf{x}}-y_{9} b \hat{\mathbf{y}}+\left(\frac{1}{2}+z_{9}\right) c \hat{\mathbf{z}}
$$$$
-x_{9} a \hat{\mathbf{x}}+y_{9} b \hat{\mathbf{y}}+\left(\frac{1}{2}+z_{9}\right) c \hat{\mathbf{z}}
$$$$
x_{10} a \hat{\mathbf{x}}+y_{10} b \hat{\mathbf{y}}+z_{10} c \hat{\mathbf{z}}
$$$$
-x_{10} a \hat{\mathbf{x}}-y_{10} b \hat{\mathbf{y}}+z_{10} c \hat{\mathbf{z}}
$$$$
x_{10} a \hat{\mathbf{x}}-y_{10} b \hat{\mathbf{y}}+\left(\frac{1}{2}+z_{10}\right) c \hat{\mathbf{z}}
$$$$
-x_{10} a \hat{\mathbf{x}}+y_{10} b \hat{\mathbf{y}}+\left(\frac{1}{2}+z_{10}\right) c \hat{\mathbf{z}}
$$$$
x_{11} a \hat{\mathbf{x}}+y_{11} b \hat{\mathbf{y}}+z_{11} c \hat{\mathbf{z}}
$$$$
-x_{11} a \hat{\mathbf{x}}-y_{11} b \hat{\mathbf{y}}+z_{11} c \hat{\mathbf{z}}
$$$$
x_{11} a \hat{\mathbf{x}}-y_{11} b \hat{\mathbf{y}}+\left(\frac{1}{2}+z_{11}\right) c \hat{\mathbf{z}}
$$

Wyckoff Position

Atom Type

(2a)
Al I

Al I

Al II

Al II

Al III

Al III

Al IV

Al IV

Al V

Al V

Al V

Al V

Al VI

Al VI

Al VI

Al VII

Al VII

Al VII

Al VII

Al VIII

Al VIII

Al VIII

Al VIII

Ca I

Ca I

Ca I

Ca I

Ca II

Ca II

Ca II

Ca II

Ca III

Ca III

Ca III
Al VI 


\begin{tabular}{|c|c|c|c|c|c|c|}
\hline $\mathbf{B}_{36}$ & $=$ & $-x_{11} \mathbf{a}_{1}+y_{11} \mathbf{a}_{2}+\left(\frac{1}{2}+z_{11}\right) \mathbf{a}_{3}$ & $=$ & $-x_{11} a \hat{\mathbf{x}}+y_{11} b \hat{\mathbf{y}}+\left(\frac{1}{2}+z_{11}\right) c \hat{\mathbf{z}}$ & $(4 e)$ & Ca III \\
\hline $\mathbf{B}_{37}$ & $=$ & $x_{12} \mathbf{a}_{1}+y_{12} \mathbf{a}_{2}+z_{12} \mathbf{a}_{3}$ & $=$ & $x_{12} a \hat{\mathbf{x}}+y_{12} b \hat{\mathbf{y}}+z_{12} c \hat{\mathbf{z}}$ & $(4 e)$ & $\mathrm{Ca}$ IV \\
\hline $\mathbf{B}_{38}$ & $=$ & $-x_{12} \mathbf{a}_{1}-y_{12} \mathbf{a}_{2}+z_{12} \mathbf{a}_{3}$ & $=$ & $-x_{12} a \hat{\mathbf{x}}-y_{12} b \hat{\mathbf{y}}+z_{12} c \hat{\mathbf{z}}$ & $(4 e)$ & $\mathrm{Ca}$ IV \\
\hline $\mathbf{B}_{39}$ & $=$ & $x_{12} \mathbf{a}_{1}-y_{12} \mathbf{a}_{2}+\left(\frac{1}{2}+z_{12}\right) \mathbf{a}_{3}$ & $=$ & $x_{12} a \hat{\mathbf{x}}-y_{12} b \hat{\mathbf{y}}+\left(\frac{1}{2}+z_{12}\right) c \hat{\mathbf{z}}$ & $(4 e)$ & Ca IV \\
\hline $\mathbf{B}_{40}$ & $=$ & $-x_{12} \mathbf{a}_{1}+y_{12} \mathbf{a}_{2}+\left(\frac{1}{2}+z_{12}\right) \mathbf{a}_{3}$ & $=$ & $-x_{12} a \hat{\mathbf{x}}+y_{12} b \hat{\mathbf{y}}+\left(\frac{1}{2}+z_{12}\right) c \hat{\mathbf{z}}$ & $(4 e)$ & Ca IV \\
\hline $\mathbf{B}_{41}$ & $=$ & $x_{13} \mathbf{a}_{1}+y_{13} \mathbf{a}_{2}+z_{13} \mathbf{a}_{3}$ & $=$ & $x_{13} a \hat{\mathbf{x}}+y_{13} b \hat{\mathbf{y}}+z_{13} c \hat{\mathbf{z}}$ & $(4 e)$ & O I \\
\hline $\mathbf{B}_{42}$ & $=$ & $-x_{13} \mathbf{a}_{1}-y_{13} \mathbf{a}_{2}+z_{13} \mathbf{a}_{3}$ & $=$ & $-x_{13} a \hat{\mathbf{x}}-y_{13} b \hat{\mathbf{y}}+z_{13} c \hat{\mathbf{z}}$ & $(4 e)$ & O I \\
\hline $\mathbf{B}_{43}$ & $=$ & $x_{13} \mathbf{a}_{1}-y_{13} \mathbf{a}_{2}+\left(\frac{1}{2}+z_{13}\right) \mathbf{a}_{3}$ & $=$ & $x_{13} a \hat{\mathbf{x}}-y_{13} b \hat{\mathbf{y}}+\left(\frac{1}{2}+z_{13}\right) c \hat{\mathbf{z}}$ & $(4 e)$ & O I \\
\hline $\mathbf{B}_{44}$ & $=$ & $-x_{13} \mathbf{a}_{1}+y_{13} \mathbf{a}_{2}+\left(\frac{1}{2}+z_{13}\right) \mathbf{a}_{3}$ & $=$ & $-x_{13} a \hat{\mathbf{x}}+y_{13} b \hat{\mathbf{y}}+\left(\frac{1}{2}+z_{13}\right) c \hat{\mathbf{z}}$ & $(4 e)$ & O I \\
\hline $\mathbf{B}_{45}$ & $=$ & $x_{14} \mathbf{a}_{1}+y_{14} \mathbf{a}_{2}+z_{14} \mathbf{a}_{3}$ & $=$ & $x_{14} a \hat{\mathbf{x}}+y_{14} b \hat{\mathbf{y}}+z_{14} c \hat{\mathbf{z}}$ & $(4 e)$ & O II \\
\hline $\mathbf{B}_{46}$ & $=$ & $-x_{14} \mathbf{a}_{1}-y_{14} \mathbf{a}_{2}+z_{14} \mathbf{a}_{3}$ & $=$ & $-x_{14} a \hat{\mathbf{x}}-y_{14} b \hat{\mathbf{y}}+z_{14} c \hat{\mathbf{z}}$ & $(4 e)$ & O II \\
\hline $\mathbf{B}_{47}$ & $=$ & $x_{14} \mathbf{a}_{1}-y_{14} \mathbf{a}_{2}+\left(\frac{1}{2}+z_{14}\right) \mathbf{a}_{3}$ & $=$ & $x_{14} a \hat{\mathbf{x}}-y_{14} b \hat{\mathbf{y}}+\left(\frac{1}{2}+z_{14}\right) c \hat{\mathbf{z}}$ & $(4 e)$ & O II \\
\hline $\mathbf{B}_{48}$ & $=$ & $-x_{14} \mathbf{a}_{1}+y_{14} \mathbf{a}_{2}+\left(\frac{1}{2}+z_{14}\right) \mathbf{a}_{3}$ & $=$ & $-x_{14} a \hat{\mathbf{x}}+y_{14} b \hat{\mathbf{y}}+\left(\frac{1}{2}+z_{14}\right) c \hat{\mathbf{z}}$ & $(4 e)$ & O II \\
\hline $\mathbf{B}_{49}$ & $=$ & $x_{15} \mathbf{a}_{1}+y_{15} \mathbf{a}_{2}+z_{15} \mathbf{a}_{3}$ & $=$ & $x_{15} a \hat{\mathbf{x}}+y_{15} b \hat{\mathbf{y}}+z_{15} c \hat{\mathbf{z}}$ & $(4 e)$ & O III \\
\hline $\mathbf{B}_{50}$ & $=$ & $-x_{15} \mathbf{a}_{1}-y_{15} \mathbf{a}_{2}+z_{15} \mathbf{a}_{3}$ & $=$ & $-x_{15} a \hat{\mathbf{x}}-y_{15} b \hat{\mathbf{y}}+z_{15} c \hat{\mathbf{z}}$ & $(4 e)$ & O III \\
\hline $\mathbf{B}_{51}$ & $=$ & $x_{15} \mathbf{a}_{1}-y_{15} \mathbf{a}_{2}+\left(\frac{1}{2}+z_{15}\right) \mathbf{a}_{3}$ & $=$ & $x_{15} a \hat{\mathbf{x}}-y_{15} b \hat{\mathbf{y}}+\left(\frac{1}{2}+z_{15}\right) c \hat{\mathbf{z}}$ & $(4 e)$ & O III \\
\hline $\mathbf{B}_{52}$ & $=$ & $-x_{15} \mathbf{a}_{1}+y_{15} \mathbf{a}_{2}+\left(\frac{1}{2}+z_{15}\right) \mathbf{a}_{3}$ & $=$ & $-x_{15} a \hat{\mathbf{x}}+y_{15} b \hat{\mathbf{y}}+\left(\frac{1}{2}+z_{15}\right) c \hat{\mathbf{z}}$ & $(4 e)$ & O III \\
\hline $\mathbf{B}_{53}$ & $=$ & $x_{16} \mathbf{a}_{1}+y_{16} \mathbf{a}_{2}+z_{16} \mathbf{a}_{3}$ & $=$ & $x_{16} a \hat{\mathbf{x}}+y_{16} b \hat{\mathbf{y}}+z_{16} c \hat{\mathbf{z}}$ & $(4 e)$ & O IV \\
\hline $\mathbf{B}_{54}$ & $=$ & $-x_{16} \mathbf{a}_{1}-y_{16} \mathbf{a}_{2}+z_{16} \mathbf{a}_{3}$ & $=$ & $-x_{16} a \hat{\mathbf{x}}-y_{16} b \hat{\mathbf{y}}+z_{16} c \hat{\mathbf{z}}$ & $(4 e)$ & O IV \\
\hline $\mathbf{B}_{55}$ & $=$ & $x_{16} \mathbf{a}_{1}-y_{16} \mathbf{a}_{2}+\left(\frac{1}{2}+z_{16}\right) \mathbf{a}_{3}$ & $=$ & $x_{16} a \hat{\mathbf{x}}-y_{16} b \hat{\mathbf{y}}+\left(\frac{1}{2}+z_{16}\right) c \hat{\mathbf{z}}$ & $(4 e)$ & O IV \\
\hline $\mathbf{B}_{56}$ & $=$ & $-x_{16} \mathbf{a}_{1}+y_{16} \mathbf{a}_{2}+\left(\frac{1}{2}+z_{16}\right) \mathbf{a}_{3}$ & $=$ & $-x_{16} a \hat{\mathbf{x}}+y_{16} b \hat{\mathbf{y}}+\left(\frac{1}{2}+z_{16}\right) c \hat{\mathbf{z}}$ & $(4 e)$ & O IV \\
\hline $\mathbf{B}_{57}$ & $=$ & $x_{17} \mathbf{a}_{1}+y_{17} \mathbf{a}_{2}+z_{17} \mathbf{a}_{3}$ & $=$ & $x_{17} a \hat{\mathbf{x}}+y_{17} b \hat{\mathbf{y}}+z_{17} c \hat{\mathbf{z}}$ & $(4 e)$ & $\mathrm{OV}$ \\
\hline $\mathbf{B}_{58}$ & $=$ & $-x_{17} \mathbf{a}_{1}-y_{17} \mathbf{a}_{2}+z_{17} \mathbf{a}_{3}$ & $=$ & $-x_{17} a \hat{\mathbf{x}}-y_{17} b \hat{\mathbf{y}}+z_{17} c \hat{\mathbf{z}}$ & $(4 e)$ & $\mathrm{OV}$ \\
\hline $\mathbf{B}_{59}$ & $=$ & $x_{17} \mathbf{a}_{1}-y_{17} \mathbf{a}_{2}+\left(\frac{1}{2}+z_{17}\right) \mathbf{a}_{3}$ & $=$ & $x_{17} a \hat{\mathbf{x}}-y_{17} b \hat{\mathbf{y}}+\left(\frac{1}{2}+z_{17}\right) c \hat{\mathbf{z}}$ & $(4 e)$ & $\mathrm{OV}$ \\
\hline $\mathbf{B}_{60}$ & $=$ & $-x_{17} \mathbf{a}_{1}+y_{17} \mathbf{a}_{2}+\left(\frac{1}{2}+z_{17}\right) \mathbf{a}_{3}$ & $=$ & $-x_{17} a \hat{\mathbf{x}}+y_{17} b \hat{\mathbf{y}}+\left(\frac{1}{2}+z_{17}\right) c \hat{\mathbf{z}}$ & $(4 e)$ & $\mathrm{OV}$ \\
\hline $\mathbf{B}_{61}$ & $=$ & $x_{18} \mathbf{a}_{1}+y_{18} \mathbf{a}_{2}+z_{18} \mathbf{a}_{3}$ & $=$ & $x_{18} a \hat{\mathbf{x}}+y_{18} b \hat{\mathbf{y}}+z_{18} c \hat{\mathbf{z}}$ & $(4 e)$ & O VI \\
\hline $\mathbf{B}_{62}$ & $=$ & $-x_{18} \mathbf{a}_{1}-y_{18} \mathbf{a}_{2}+z_{18} \mathbf{a}_{3}$ & $=$ & $-x_{18} a \hat{\mathbf{x}}-y_{18} b \hat{\mathbf{y}}+z_{18} c \hat{\mathbf{z}}$ & $(4 e)$ & O VI \\
\hline $\mathbf{B}_{63}$ & $=$ & $x_{18} \mathbf{a}_{1}-y_{18} \mathbf{a}_{2}+\left(\frac{1}{2}+z_{18}\right) \mathbf{a}_{3}$ & $=$ & $x_{18} a \hat{\mathbf{x}}-y_{18} b \hat{\mathbf{y}}+\left(\frac{1}{2}+z_{18}\right) c \hat{\mathbf{z}}$ & $(4 e)$ & O VI \\
\hline $\mathbf{B}_{64}$ & $=$ & $-x_{18} \mathbf{a}_{1}+y_{18} \mathbf{a}_{2}+\left(\frac{1}{2}+z_{18}\right) \mathbf{a}_{3}$ & $=$ & $-x_{18} a \hat{\mathbf{x}}+y_{18} b \hat{\mathbf{y}}+\left(\frac{1}{2}+z_{18}\right) c \hat{\mathbf{z}}$ & $(4 e)$ & O VI \\
\hline $\mathbf{B}_{65}$ & $=$ & $x_{19} \mathbf{a}_{1}+y_{19} \mathbf{a}_{2}+z_{19} \mathbf{a}_{3}$ & $=$ & $x_{19} a \hat{\mathbf{x}}+y_{19} b \hat{\mathbf{y}}+z_{19} c \hat{\mathbf{z}}$ & $(4 e)$ & O VII \\
\hline $\mathbf{B}_{66}$ & $=$ & $-x_{19} \mathbf{a}_{1}-y_{19} \mathbf{a}_{2}+z_{19} \mathbf{a}_{3}$ & $=$ & $-x_{19} a \hat{\mathbf{x}}-y_{19} b \hat{\mathbf{y}}+z_{19} c \hat{\mathbf{z}}$ & $(4 e)$ & O VII \\
\hline $\mathbf{B}_{67}$ & $=$ & $x_{19} \mathbf{a}_{1}-y_{19} \mathbf{a}_{2}+\left(\frac{1}{2}+z_{19}\right) \mathbf{a}_{3}$ & $=$ & $x_{19} a \hat{\mathbf{x}}-y_{19} b \hat{\mathbf{y}}+\left(\frac{1}{2}+z_{19}\right) c \hat{\mathbf{z}}$ & $(4 e)$ & O VII \\
\hline $\mathbf{B}_{68}$ & $=$ & $-x_{19} \mathbf{a}_{1}+y_{19} \mathbf{a}_{2}+\left(\frac{1}{2}+z_{19}\right) \mathbf{a}_{3}$ & $=$ & $-x_{19} a \hat{\mathbf{x}}+y_{19} b \hat{\mathbf{y}}+\left(\frac{1}{2}+z_{19}\right) c \hat{\mathbf{z}}$ & $(4 e)$ & O VII \\
\hline $\mathbf{B}_{69}$ & $=$ & $x_{20} \mathbf{a}_{1}+y_{20} \mathbf{a}_{2}+z_{20} \mathbf{a}_{3}$ & $=$ & $x_{20} a \hat{\mathbf{x}}+y_{20} b \hat{\mathbf{y}}+z_{20} c \hat{\mathbf{z}}$ & $(4 e)$ & O VIII \\
\hline $\mathbf{B}_{70}$ & $=$ & $-x_{20} \mathbf{a}_{1}-y_{20} \mathbf{a}_{2}+z_{20} \mathbf{a}_{3}$ & $=$ & $-x_{20} a \hat{\mathbf{x}}-y_{20} b \hat{\mathbf{y}}+z_{20} c \hat{\mathbf{z}}$ & $(4 e)$ & O VIII \\
\hline $\mathbf{B}_{71}$ & $=$ & $x_{20} \mathbf{a}_{1}-y_{20} \mathbf{a}_{2}+\left(\frac{1}{2}+z_{20}\right) \mathbf{a}_{3}$ & $=$ & $x_{20} a \hat{\mathbf{x}}-y_{20} b \hat{\mathbf{y}}+\left(\frac{1}{2}+z_{20}\right) c \hat{\mathbf{z}}$ & $(4 e)$ & O VIII \\
\hline
\end{tabular}




\begin{tabular}{|c|c|c|c|c|c|c|}
\hline $\mathbf{B}_{72}$ & $=$ & $-x_{20} \mathbf{a}_{1}+y_{20} \mathbf{a}_{2}+\left(\frac{1}{2}+z_{20}\right) \mathbf{a}_{3}$ & $=$ & $-x_{20} a \hat{\mathbf{x}}+y_{20} b \hat{\mathbf{y}}+\left(\frac{1}{2}+z_{20}\right) c \hat{\mathbf{z}}$ & $(4 e)$ & O VIII \\
\hline $\mathbf{B}_{73}$ & $=$ & $x_{21} \mathbf{a}_{1}+y_{21} \mathbf{a}_{2}+z_{21} \mathbf{a}_{3}$ & $=$ & $x_{21} a \hat{\mathbf{x}}+y_{21} b \hat{\mathbf{y}}+z_{21} c \hat{\mathbf{z}}$ & $(4 e)$ & O IX \\
\hline $\mathbf{B}_{74}$ & $=$ & $-x_{21} \mathbf{a}_{1}-y_{21} \mathbf{a}_{2}+z_{21} \mathbf{a}_{3}$ & $=$ & $-x_{21} a \hat{\mathbf{x}}-y_{21} b \hat{\mathbf{y}}+z_{21} c \hat{\mathbf{z}}$ & $(4 e)$ & O IX \\
\hline $\mathbf{B}_{75}$ & $=$ & $x_{21} \mathbf{a}_{1}-y_{21} \mathbf{a}_{2}+\left(\frac{1}{2}+z_{21}\right) \mathbf{a}_{3}$ & $=$ & $x_{21} a \hat{\mathbf{x}}-y_{21} b \hat{\mathbf{y}}+\left(\frac{1}{2}+z_{21}\right) c \hat{\mathbf{z}}$ & $(4 e)$ & O IX \\
\hline $\mathbf{B}_{76}$ & $=$ & $-x_{21} \mathbf{a}_{1}+y_{21} \mathbf{a}_{2}+\left(\frac{1}{2}+z_{21}\right) \mathbf{a}_{3}$ & $=$ & $-x_{21} a \hat{\mathbf{x}}+y_{21} b \hat{\mathbf{y}}+\left(\frac{1}{2}+z_{21}\right) c \hat{\mathbf{z}}$ & $(4 e)$ & O IX \\
\hline $\mathbf{B}_{77}$ & $=$ & $x_{22} \mathbf{a}_{1}+y_{22} \mathbf{a}_{2}+z_{22} \mathbf{a}_{3}$ & $=$ & $x_{22} a \hat{\mathbf{x}}+y_{22} b \hat{\mathbf{y}}+z_{22} c \hat{\mathbf{z}}$ & $(4 e)$ & $\mathrm{OX}$ \\
\hline $\mathbf{B}_{78}$ & $=$ & $-x_{22} \mathbf{a}_{1}-y_{22} \mathbf{a}_{2}+z_{22} \mathbf{a}_{3}$ & $=$ & $-x_{22} a \hat{\mathbf{x}}-y_{22} b \hat{\mathbf{y}}+z_{22} c \hat{\mathbf{z}}$ & $(4 e)$ & $\mathrm{OX}$ \\
\hline $\mathbf{B}_{79}$ & $=$ & $x_{22} \mathbf{a}_{1}-y_{22} \mathbf{a}_{2}+\left(\frac{1}{2}+z_{22}\right) \mathbf{a}_{3}$ & $=$ & $x_{22} a \hat{\mathbf{x}}-y_{22} b \hat{\mathbf{y}}+\left(\frac{1}{2}+z_{22}\right) c \hat{\mathbf{z}}$ & $(4 e)$ & $\mathrm{OX}$ \\
\hline $\mathbf{B}_{80}$ & $=$ & $-x_{22} \mathbf{a}_{1}+y_{22} \mathbf{a}_{2}+\left(\frac{1}{2}+z_{22}\right) \mathbf{a}_{3}$ & $=$ & $-x_{22} a \hat{\mathbf{x}}+y_{22} b \hat{\mathbf{y}}+\left(\frac{1}{2}+z_{22}\right) c \hat{\mathbf{z}}$ & $(4 e)$ & $\mathrm{OX}$ \\
\hline $\mathbf{B}_{81}$ & $=$ & $x_{23} \mathbf{a}_{1}+y_{23} \mathbf{a}_{2}+z_{23} \mathbf{a}_{3}$ & $=$ & $x_{23} a \hat{\mathbf{x}}+y_{23} b \hat{\mathbf{y}}+z_{23} c \hat{\mathbf{z}}$ & $(4 e)$ & O XI \\
\hline $\mathbf{B}_{82}$ & $=$ & $-x_{23} \mathbf{a}_{1}-y_{23} \mathbf{a}_{2}+z_{23} \mathbf{a}_{3}$ & $=$ & $-x_{23} a \hat{\mathbf{x}}-y_{23} b \hat{\mathbf{y}}+z_{23} c \hat{\mathbf{z}}$ & $(4 e)$ & O XI \\
\hline $\mathbf{B}_{83}$ & $=$ & $x_{23} \mathbf{a}_{1}-y_{23} \mathbf{a}_{2}+\left(\frac{1}{2}+z_{23}\right) \mathbf{a}_{3}$ & $=$ & $x_{23} a \hat{\mathbf{x}}-y_{23} b \hat{\mathbf{y}}+\left(\frac{1}{2}+z_{23}\right) c \hat{\mathbf{z}}$ & $(4 e)$ & O XI \\
\hline $\mathbf{B}_{84}$ & $=$ & $-x_{23} \mathbf{a}_{1}+y_{23} \mathbf{a}_{2}+\left(\frac{1}{2}+z_{23}\right) \mathbf{a}_{3}$ & $=$ & $-x_{23} a \hat{\mathbf{x}}+y_{23} b \hat{\mathbf{y}}+\left(\frac{1}{2}+z_{23}\right) c \hat{\mathbf{z}}$ & $(4 e)$ & O XI \\
\hline $\mathbf{B}_{85}$ & $=$ & $x_{24} \mathbf{a}_{1}+y_{24} \mathbf{a}_{2}+z_{24} \mathbf{a}_{3}$ & $=$ & $x_{24} a \hat{\mathbf{x}}+y_{24} b \hat{\mathbf{y}}+z_{24} c \hat{\mathbf{z}}$ & $(4 e)$ & O XII \\
\hline $\mathbf{B}_{86}$ & $=$ & $-x_{24} \mathbf{a}_{1}-y_{24} \mathbf{a}_{2}+z_{24} \mathbf{a}_{3}$ & $=$ & $-x_{24} a \hat{\mathbf{x}}-y_{24} b \hat{\mathbf{y}}+z_{24} c \hat{\mathbf{z}}$ & $(4 e)$ & O XII \\
\hline $\mathbf{B}_{87}$ & $=$ & $x_{24} \mathbf{a}_{1}-y_{24} \mathbf{a}_{2}+\left(\frac{1}{2}+z_{24}\right) \mathbf{a}_{3}$ & $=$ & $x_{24} a \hat{\mathbf{x}}-y_{24} b \hat{\mathbf{y}}+\left(\frac{1}{2}+z_{24}\right) c \hat{\mathbf{z}}$ & $(4 e)$ & O XII \\
\hline $\mathbf{B}_{88}$ & $=$ & $-x_{24} \mathbf{a}_{1}+y_{24} \mathbf{a}_{2}+\left(\frac{1}{2}+z_{24}\right) \mathbf{a}_{3}$ & $=$ & $-x_{24} a \hat{\mathbf{x}}+y_{24} b \hat{\mathbf{y}}+\left(\frac{1}{2}+z_{24}\right) c \hat{\mathbf{z}}$ & $(4 e)$ & O XII \\
\hline $\mathbf{B}_{89}$ & $=$ & $x_{25} \mathbf{a}_{1}+y_{25} \mathbf{a}_{2}+z_{25} \mathbf{a}_{3}$ & $=$ & $x_{25} a \hat{\mathbf{x}}+y_{25} b \hat{\mathbf{y}}+z_{25} c \hat{\mathbf{z}}$ & $(4 e)$ & O XIII \\
\hline $\mathbf{B}_{90}$ & $=$ & $-x_{25} \mathbf{a}_{1}-y_{25} \mathbf{a}_{2}+z_{25} \mathbf{a}_{3}$ & $=$ & $-x_{25} a \hat{\mathbf{x}}-y_{25} b \hat{\mathbf{y}}+z_{25} c \hat{\mathbf{z}}$ & $(4 e)$ & O XIII \\
\hline $\mathbf{B}_{91}$ & $=$ & $x_{25} \mathbf{a}_{1}-y_{25} \mathbf{a}_{2}+\left(\frac{1}{2}+z_{25}\right) \mathbf{a}_{3}$ & $=$ & $x_{25} a \hat{\mathbf{x}}-y_{25} b \hat{\mathbf{y}}+\left(\frac{1}{2}+z_{25}\right) c \hat{\mathbf{z}}$ & $(4 e)$ & O XIII \\
\hline $\mathbf{B}_{92}$ & $=$ & $-x_{25} \mathbf{a}_{1}+y_{25} \mathbf{a}_{2}+\left(\frac{1}{2}+z_{25}\right) \mathbf{a}_{3}$ & $=$ & $-x_{25} a \hat{\mathbf{x}}+y_{25} b \hat{\mathbf{y}}+\left(\frac{1}{2}+z_{25}\right) c \hat{\mathbf{z}}$ & $(4 e)$ & O XIII \\
\hline $\mathbf{B}_{93}$ & $=$ & $x_{26} \mathbf{a}_{1}+y_{26} \mathbf{a}_{2}+z_{26} \mathbf{a}_{3}$ & $=$ & $x_{26} a \hat{\mathbf{x}}+y_{26} b \hat{\mathbf{y}}+z_{26} c \hat{\mathbf{z}}$ & $(4 e)$ & O XIV \\
\hline $\mathbf{B}_{94}$ & $=$ & $-x_{26} \mathbf{a}_{1}-y_{26} \mathbf{a}_{2}+z_{26} \mathbf{a}_{3}$ & $=$ & $-x_{26} a \hat{\mathbf{x}}-y_{26} b \hat{\mathbf{y}}+z_{26} c \hat{\mathbf{z}}$ & $(4 e)$ & O XIV \\
\hline $\mathbf{B}_{95}$ & $=$ & $x_{26} \mathbf{a}_{1}-y_{26} \mathbf{a}_{2}+\left(\frac{1}{2}+z_{26}\right) \mathbf{a}_{3}$ & $=$ & $x_{26} a \hat{\mathbf{x}}-y_{26} b \hat{\mathbf{y}}+\left(\frac{1}{2}+z_{26}\right) c \hat{\mathbf{z}}$ & $(4 e)$ & O XIV \\
\hline $\mathbf{B}_{96}$ & $=$ & $-x_{26} \mathbf{a}_{1}+y_{26} \mathbf{a}_{2}+\left(\frac{1}{2}+z_{26}\right) \mathbf{a}_{3}$ & $=$ & $-x_{26} a \hat{\mathbf{x}}+y_{26} b \hat{\mathbf{y}}+\left(\frac{1}{2}+z_{26}\right) c \hat{\mathbf{z}}$ & $(4 e)$ & O XIV \\
\hline $\mathbf{B}_{97}$ & $=$ & $x_{27} \mathbf{a}_{1}+y_{27} \mathbf{a}_{2}+z_{27} \mathbf{a}_{3}$ & $=$ & $x_{27} a \hat{\mathbf{x}}+y_{27} b \hat{\mathbf{y}}+z_{27} c \hat{\mathbf{z}}$ & $(4 e)$ & $\mathrm{OXV}$ \\
\hline $\mathbf{B}_{98}$ & $=$ & $-x_{27} \mathbf{a}_{1}-y_{27} \mathbf{a}_{2}+z_{27} \mathbf{a}_{3}$ & $=$ & $-x_{27} a \hat{\mathbf{x}}-y_{27} b \hat{\mathbf{y}}+z_{27} c \hat{\mathbf{z}}$ & $(4 e)$ & $\mathrm{OXV}$ \\
\hline $\mathbf{B}_{99}$ & $=$ & $x_{27} \mathbf{a}_{1}-y_{27} \mathbf{a}_{2}+\left(\frac{1}{2}+z_{27}\right) \mathbf{a}_{3}$ & $=$ & $x_{27} a \hat{\mathbf{x}}-y_{27} b \hat{\mathbf{y}}+\left(\frac{1}{2}+z_{27}\right) c \hat{\mathbf{z}}$ & $(4 e)$ & $\mathrm{OXV}$ \\
\hline $\mathbf{B}_{100}$ & $=$ & $-x_{27} \mathbf{a}_{1}+y_{27} \mathbf{a}_{2}+\left(\frac{1}{2}+z_{27}\right) \mathbf{a}_{3}$ & $=$ & $-x_{27} a \hat{\mathbf{x}}+y_{27} b \hat{\mathbf{y}}+\left(\frac{1}{2}+z_{27}\right) c \hat{\mathbf{z}}$ & $(4 e)$ & $\mathrm{O} X \mathrm{~V}$ \\
\hline $\mathbf{B}_{101}$ & $=$ & $x_{28} \mathbf{a}_{1}+y_{28} \mathbf{a}_{2}+z_{28} \mathbf{a}_{3}$ & $=$ & $x_{28} a \hat{\mathbf{x}}+y_{28} b \hat{\mathbf{y}}+z_{28} c \hat{\mathbf{z}}$ & $(4 e)$ & O XVI \\
\hline $\mathbf{B}_{102}$ & $=$ & $-x_{28} \mathbf{a}_{1}-y_{28} \mathbf{a}_{2}+z_{28} \mathbf{a}_{3}$ & $=$ & $-x_{28} a \hat{\mathbf{x}}-y_{28} b \hat{\mathbf{y}}+z_{28} c \hat{\mathbf{z}}$ & $(4 e)$ & O XVI \\
\hline $\mathbf{B}_{103}$ & $=$ & $x_{28} \mathbf{a}_{1}-y_{28} \mathbf{a}_{2}+\left(\frac{1}{2}+z_{28}\right) \mathbf{a}_{3}$ & $=$ & $x_{28} a \hat{\mathbf{x}}-y_{28} b \hat{\mathbf{y}}+\left(\frac{1}{2}+z_{28}\right) c \hat{\mathbf{z}}$ & $(4 e)$ & OXVI \\
\hline $\mathbf{B}_{104}$ & $=$ & $-x_{28} \mathbf{a}_{1}+y_{28} \mathbf{a}_{2}+\left(\frac{1}{2}+z_{28}\right) \mathbf{a}_{3}$ & $=$ & $-x_{28} a \hat{\mathbf{x}}+y_{28} b \hat{\mathbf{y}}+\left(\frac{1}{2}+z_{28}\right) c \hat{\mathbf{z}}$ & $(4 e)$ & OXVI \\
\hline $\mathbf{B}_{105}$ & $=$ & $x_{29} \mathbf{a}_{1}+y_{29} \mathbf{a}_{2}+z_{29} \mathbf{a}_{3}$ & $=$ & $x_{29} a \hat{\mathbf{x}}+y_{29} b \hat{\mathbf{y}}+z_{29} c \hat{\mathbf{z}}$ & $(4 e)$ & $S$ \\
\hline $\mathbf{B}_{106}$ & $=$ & $-x_{29} \mathbf{a}_{1}-y_{29} \mathbf{a}_{2}+z_{29} \mathbf{a}_{3}$ & $=$ & $-x_{29} a \hat{\mathbf{x}}-y_{29} b \hat{\mathbf{y}}+z_{29} c \hat{\mathbf{z}}$ & $(4 e)$ & $S$ \\
\hline $\mathbf{B}_{107}$ & $=$ & $x_{29} \mathbf{a}_{1}-y_{29} \mathbf{a}_{2}+\left(\frac{1}{2}+z_{29}\right) \mathbf{a}_{3}$ & $=$ & $x_{29} a \hat{\mathbf{x}}-y_{29} b \hat{\mathbf{y}}+\left(\frac{1}{2}+z_{29}\right) c \hat{\mathbf{z}}$ & $(4 e)$ & S \\
\hline
\end{tabular}


$\mathbf{B}_{108}=-x_{29} \mathbf{a}_{1}+y_{29} \mathbf{a}_{2}+\left(\frac{1}{2}+z_{29}\right) \mathbf{a}_{3}=-x_{29} a \hat{\mathbf{x}}+y_{29} b \hat{\mathbf{y}}+\left(\frac{1}{2}+z_{29}\right) c \hat{\mathbf{z}}$

$(4 e)$

\section{References:}

- N. J. Calos, C. H. L. Kennard, A. K. Whittaker, and R. L. Davis, Structure of calcium aluminate sulfate $\mathrm{Ca}_{4} A l_{6} \mathrm{O}_{16} S, \mathrm{~J}$. Solid State Chem. 119, 1-7 (1995), doi:10.1016/0022-4596(95)80002-7.

\section{Found in:}

- P. Villars and K. Cenzual, Pearson's Crystal Data - Crystal Structure Database for Inorganic Compounds, ASM International (2013).

\section{Geometry files:}

- CIF: pp. 819

- POSCAR: pp. 819 


\section{$\mathrm{ZrO}_{2}$ Structure: A2B_oP12_29_2a_a}

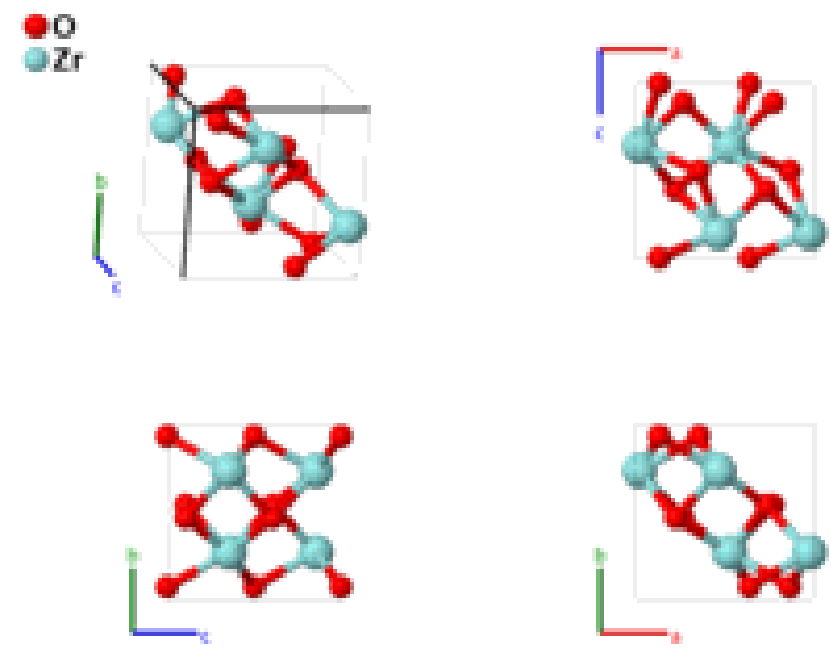

\section{Prototype}

AFLOW prototype label

Strukturbericht designation

Pearson symbol

Space group number

Space group symbol

AFLOW prototype command
$: \quad \mathrm{ZrO}_{2}$

: A2B_oP12_29_2a_a

: None

: $\quad \mathrm{oP} 12$

: 29

: $\quad P c a 2_{1}$

aflow - -proto=A2B_oP12_29_2a_a

- params $=a, b / a, c / a, x_{1}, y_{1}, z_{1}, x_{2}, y_{2}, z_{2}, x_{3}, y_{3}, z_{3}$

- $\mathrm{ZrO}_{2}$ (pp. 106) and Pyrite (pp. 108) have similar AFLOW prototype labels (i.e., same symmetry and set of Wyckoff positions with different stoichiometry labels due to alphabetic ordering of atomic species). They are generated by the same symmetry operations with different sets of parameters (--params) specified in their corresponding CIF files.

\section{Simple Orthorhombic primitive vectors:}

$$
\begin{aligned}
& \mathbf{a}_{1}=a \hat{\mathbf{x}} \\
& \mathbf{a}_{2}=b \hat{\mathbf{y}} \\
& \mathbf{a}_{3}=c \hat{\mathbf{z}}
\end{aligned}
$$
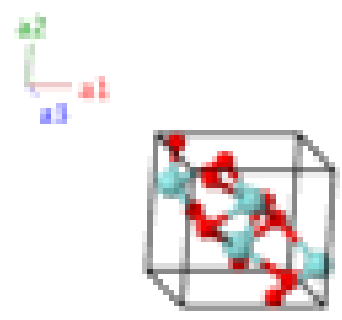

\section{Basis vectors:}

Lattice Coordinates

$\mathbf{B}_{1}=$

$\mathbf{B}_{2}=-x_{1} \mathbf{a}_{1}-y_{1} \mathbf{a}_{2}+\left(\frac{1}{2}+z_{1}\right) \mathbf{a}_{3}$

$\mathbf{B}_{3}=\left(\frac{1}{2}+x_{1}\right) \mathbf{a}_{1}-y_{1} \mathbf{a}_{2}+z_{1} \mathbf{a}_{3}$

$\mathbf{B}_{4}=\left(\frac{1}{2}-x_{1}\right) \mathbf{a}_{1}+y_{1} \mathbf{a}_{2}+\left(\frac{1}{2}+z_{1}\right) \mathbf{a}_{3}=$
Cartesian Coordinates

$=\quad x_{1} a \hat{\mathbf{x}}+y_{1} b \hat{\mathbf{y}}+z_{1} c \hat{\mathbf{z}}$

$=\quad-x_{1} a \hat{\mathbf{x}}-y_{1} b \hat{\mathbf{y}}+\left(\frac{1}{2}+z_{1}\right) c \hat{\mathbf{z}}$

$=\left(\frac{1}{2}+x_{1}\right) a \hat{\mathbf{x}}-y_{1} b \hat{\mathbf{y}}+z_{1} c \hat{\mathbf{z}}$

$=\left(\frac{1}{2}-x_{1}\right) a \hat{\mathbf{x}}+y_{1} b \hat{\mathbf{y}}+\left(\frac{1}{2}+z_{1}\right) c \hat{\mathbf{z}}$
Wyckoff Position Atom Type

$(4 a)$

O I

(4a)

O I

(4a)

O I

(4a)

O I 


\begin{tabular}{|c|c|c|c|c|c|c|}
\hline $\mathbf{B}_{5}$ & $=$ & $x_{2} \mathbf{a}_{1}+y_{2} \mathbf{a}_{2}+z_{2} \mathbf{a}_{3}$ & $=$ & $x_{2} a \hat{\mathbf{x}}+y_{2} b \hat{\mathbf{y}}+z_{2} c \hat{\mathbf{z}}$ & $(4 a)$ & O II \\
\hline $\mathbf{B}_{6}$ & $=$ & $-x_{2} \mathbf{a}_{1}-y_{2} \mathbf{a}_{2}+\left(\frac{1}{2}+z_{2}\right) \mathbf{a}_{3}$ & $=$ & $-x_{2} a \hat{\mathbf{x}}-y_{2} b \hat{\mathbf{y}}+\left(\frac{1}{2}+z_{2}\right) c \hat{\mathbf{z}}$ & $(4 a)$ & O II \\
\hline $\mathbf{B}_{7}$ & $=$ & $\left(\frac{1}{2}+x_{2}\right) \mathbf{a}_{1}-y_{2} \mathbf{a}_{2}+z_{2} \mathbf{a}_{3}$ & $=$ & $\left(\frac{1}{2}+x_{2}\right) a \hat{\mathbf{x}}-y_{2} b \hat{\mathbf{y}}+z_{2} c \hat{\mathbf{z}}$ & $(4 a)$ & O II \\
\hline $\mathbf{B}_{8}$ & $=$ & $\left(\frac{1}{2}-x_{2}\right) \mathbf{a}_{1}+y_{2} \mathbf{a}_{2}+\left(\frac{1}{2}+z_{2}\right) \mathbf{a}_{3}$ & $=$ & $\left(\frac{1}{2}-x_{2}\right) a \hat{\mathbf{x}}+y_{2} b \hat{\mathbf{y}}+\left(\frac{1}{2}+z_{2}\right) c \hat{\mathbf{z}}$ & $(4 a)$ & O II \\
\hline $\mathbf{B}_{9}$ & $=$ & $x_{3} \mathbf{a}_{1}+y_{3} \mathbf{a}_{2}+z_{3} \mathbf{a}_{3}$ & $=$ & $x_{3} a \hat{\mathbf{x}}+y_{3} b \hat{\mathbf{y}}+z_{3} c \hat{\mathbf{z}}$ & $(4 a)$ & $\mathrm{Zr}$ \\
\hline $\mathbf{B}_{10}$ & $=$ & $-x_{3} \mathbf{a}_{1}-y_{3} \mathbf{a}_{2}+\left(\frac{1}{2}+z_{3}\right) \mathbf{a}_{3}$ & $=$ & $-x_{3} a \hat{\mathbf{x}}-y_{3} b \hat{\mathbf{y}}+\left(\frac{1}{2}+z_{3}\right) c \hat{\mathbf{z}}$ & $(4 a)$ & $\mathrm{Zr}$ \\
\hline $\mathbf{B}_{11}$ & $=$ & $\left(\frac{1}{2}+x_{3}\right) \mathbf{a}_{1}-y_{3} \mathbf{a}_{2}+z_{3} \mathbf{a}_{3}$ & $=$ & $\left(\frac{1}{2}+x_{3}\right) a \hat{\mathbf{x}}-y_{3} b \hat{\mathbf{y}}+z_{3} c \hat{\mathbf{z}}$ & $(4 a)$ & $\mathrm{Zr}$ \\
\hline $\mathbf{B}_{12}$ & $=$ & $\left(\frac{1}{2}-x_{3}\right) \mathbf{a}_{1}+y_{3} \mathbf{a}_{2}+\left(\frac{1}{2}+z_{3}\right) \mathbf{a}_{3}$ & $=$ & $\left(\frac{1}{2}-x_{3}\right) a \hat{\mathbf{x}}+y_{3} b \hat{\mathbf{y}}+\left(\frac{1}{2}+z_{3}\right) c \hat{\mathbf{z}}$ & $(4 a)$ & $\mathrm{Zr}$ \\
\hline
\end{tabular}

\section{References:}

- J. Grins, P.-O. Käll, and G. Svensson, Phases in the $Z_{x} \operatorname{Ta}_{1-x}(O, N)_{y}$ system, formed by ammonolysis of Zr-Ta gels: Preparation of a baddeleyite-type solid solution phase $\mathrm{Zr}_{x} \mathrm{Ta}_{1-x} \mathrm{O}_{1+x} N_{1-x}, 0 \leq x \leq 1$, J. Mater. Chem. 4, 1293-1301 (1994), doi:10.1039/JM9940401293.

\section{Found in:}

- P. Villars and K. Cenzual, Pearson's Crystal Data - Crystal Structure Database for Inorganic Compounds, ASM International (2013).

\section{Geometry files:}

- CIF: pp. 820

- POSCAR: pp. 820 


\section{Pyrite $\left(\mathrm{FeS}_{2}\right.$, Low-temperature) Structure:}

\section{AB2 oP12_29_a_2a}
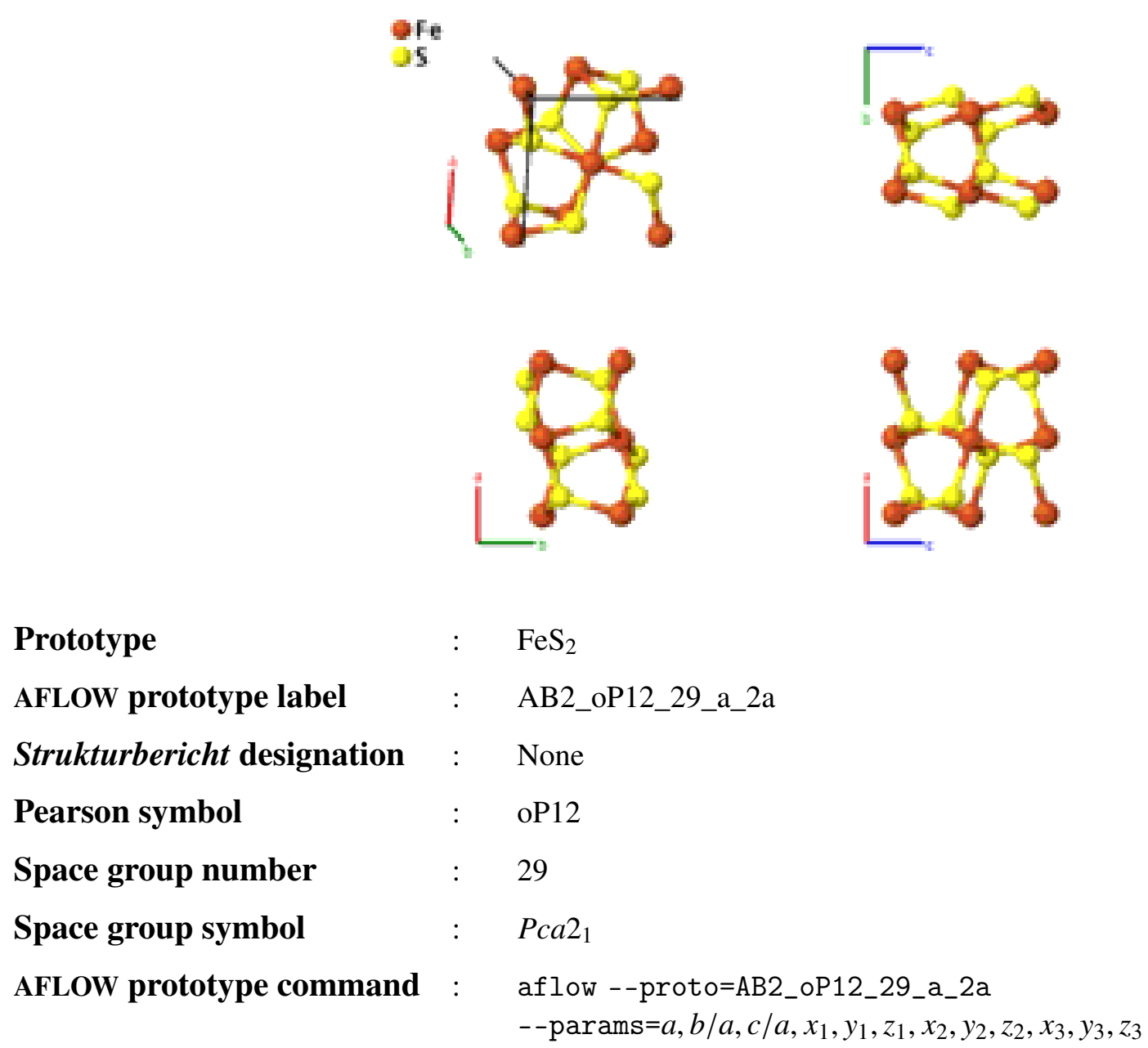

- $\mathrm{ZrO}_{2}$ (pp. 106) and Pyrite (pp. 108) have similar AFLOW prototype labels (i.e., same symmetry and set of Wyckoff positions with different stoichiometry labels due to alphabetic ordering of atomic species). They are generated by the same symmetry operations with different sets of parameters (--params) specified in their corresponding CIF files.

\section{Simple Orthorhombic primitive vectors:}

$$
\begin{aligned}
& \mathbf{a}_{1}=a \hat{\mathbf{x}} \\
& \mathbf{a}_{2}=b \hat{\mathbf{y}} \\
& \mathbf{a}_{3}=c \hat{\mathbf{z}}
\end{aligned}
$$

\begin{tabular}{|c|c|c|c|c|c|}
\hline & Lattice Coordinates & & Cartesian Coordinates & Wyckoff Position & Atom Type \\
\hline $\mathbf{B}_{1}$ & $x_{1} \mathbf{a}_{1}+y_{1} \mathbf{a}_{2}+z_{1} \mathbf{a}_{3}$ & $=$ & $x_{1} a \hat{\mathbf{x}}+y_{1} b \hat{\mathbf{y}}+z_{1} c \hat{\mathbf{z}}$ & $(4 a)$ & $\mathrm{Fe}$ \\
\hline$=$ & $-x_{1} \mathbf{a}_{1}-y_{1} \mathbf{a}_{2}+\left(\frac{1}{2}+z_{1}\right) \mathbf{a}_{3}$ & $=$ & $-x_{1} a \hat{\mathbf{x}}-y_{1} b \hat{\mathbf{y}}+\left(\frac{1}{2}+z_{1}\right) c \hat{\mathbf{z}}$ & $(4 a)$ & $\mathrm{Fe}$ \\
\hline
\end{tabular}

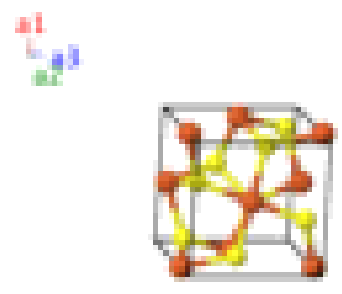

\section{Basis vectors:}




\begin{tabular}{|c|c|c|c|c|c|c|}
\hline $\mathbf{B}_{3}$ & $=$ & $\left(\frac{1}{2}+x_{1}\right) \mathbf{a}_{1}-y_{1} \mathbf{a}_{2}+z_{1} \mathbf{a}_{3}$ & $=$ & $\left(\frac{1}{2}+x_{1}\right) a \hat{\mathbf{x}}-y_{1} b \hat{\mathbf{y}}+z_{1} c \hat{\mathbf{z}}$ & $(4 a)$ & $\mathrm{Fe}$ \\
\hline $\mathbf{B}_{4}$ & $=$ & $\left(\frac{1}{2}-x_{1}\right) \mathbf{a}_{1}+y_{1} \mathbf{a}_{2}+\left(\frac{1}{2}+z_{1}\right) \mathbf{a}_{3}$ & $=$ & $\left(\frac{1}{2}-x_{1}\right) a \hat{\mathbf{x}}+y_{1} b \hat{\mathbf{y}}+\left(\frac{1}{2}+z_{1}\right) c \hat{\mathbf{z}}$ & $(4 a)$ & $\mathrm{Fe}$ \\
\hline $\mathbf{B}_{5}$ & $=$ & $x_{2} \mathbf{a}_{1}+y_{2} \mathbf{a}_{2}+z_{2} \mathbf{a}_{3}$ & $=$ & $x_{2} a \hat{\mathbf{x}}+y_{2} b \hat{\mathbf{y}}+z_{2} c \hat{\mathbf{z}}$ & $(4 a)$ & S I \\
\hline $\mathbf{B}_{6}$ & $=$ & $-x_{2} \mathbf{a}_{1}-y_{2} \mathbf{a}_{2}+\left(\frac{1}{2}+z_{2}\right) \mathbf{a}_{3}$ & $=$ & $-x_{2} a \hat{\mathbf{x}}-y_{2} b \hat{\mathbf{y}}+\left(\frac{1}{2}+z_{2}\right) c \hat{\mathbf{z}}$ & $(4 a)$ & S I \\
\hline $\mathbf{B}_{7}$ & $=$ & $\left(\frac{1}{2}+x_{2}\right) \mathbf{a}_{1}-y_{2} \mathbf{a}_{2}+z_{2} \mathbf{a}_{3}$ & $=$ & $\left(\frac{1}{2}+x_{2}\right) a \hat{\mathbf{x}}-y_{2} b \hat{\mathbf{y}}+z_{2} c \hat{\mathbf{z}}$ & $(4 a)$ & S I \\
\hline $\mathbf{B}_{8}$ & $=$ & $\left(\frac{1}{2}-x_{2}\right) \mathbf{a}_{1}+y_{2} \mathbf{a}_{2}+\left(\frac{1}{2}+z_{2}\right) \mathbf{a}_{3}$ & $=$ & $\left(\frac{1}{2}-x_{2}\right) a \hat{\mathbf{x}}+y_{2} b \hat{\mathbf{y}}+\left(\frac{1}{2}+z_{2}\right) c \hat{\mathbf{z}}$ & $(4 a)$ & S I \\
\hline $\mathbf{B}_{9}$ & $=$ & $x_{3} \mathbf{a}_{1}+y_{3} \mathbf{a}_{2}+z_{3} \mathbf{a}_{3}$ & $=$ & $x_{3} a \hat{\mathbf{x}}+y_{3} b \hat{\mathbf{y}}+z_{3} c \hat{\mathbf{z}}$ & $(4 a)$ & S II \\
\hline $\mathbf{B}_{10}$ & $=$ & $-x_{3} \mathbf{a}_{1}-y_{3} \mathbf{a}_{2}+\left(\frac{1}{2}+z_{3}\right) \mathbf{a}_{3}$ & $=$ & $-x_{3} a \hat{\mathbf{x}}-y_{3} b \hat{\mathbf{y}}+\left(\frac{1}{2}+z_{3}\right) c \hat{\mathbf{z}}$ & $(4 a)$ & S II \\
\hline $\mathbf{B}_{11}$ & $=$ & $\left(\frac{1}{2}+x_{3}\right) \mathbf{a}_{1}-y_{3} \mathbf{a}_{2}+z_{3} \mathbf{a}_{3}$ & $=$ & $\left(\frac{1}{2}+x_{3}\right) a \hat{\mathbf{x}}-y_{3} b \hat{\mathbf{y}}+z_{3} c \hat{\mathbf{z}}$ & $(4 a)$ & S II \\
\hline $\mathbf{B}_{12}$ & $=$ & $\left(\frac{1}{2}-x_{3}\right) \mathbf{a}_{1}+y_{3} \mathbf{a}_{2}+\left(\frac{1}{2}+z_{3}\right) \mathbf{a}_{3}$ & $=$ & $\left(\frac{1}{2}-x_{3}\right) a \hat{\mathbf{x}}+y_{3} b \hat{\mathbf{y}}+\left(\frac{1}{2}+z_{3}\right) c \hat{\mathbf{z}}$ & $(4 a)$ & S II \\
\hline
\end{tabular}

\section{References:}

- P. Bayliss, Crystal chemistry and crystallography of some minerals within the pyrite group, Am. Mineral. 74, 1168-1176 (1989).

\section{Found in:}

- P. Villars and K. Cenzual, Pearson's Crystal Data - Crystal Structure Database for Inorganic Compounds, ASM International (2013).

\section{Geometry files:}

- CIF: pp. 820

- POSCAR: pp. 821 


\section{Cobaltite (CoAsS) Structure: ABC_oP12_29_a_a_a}
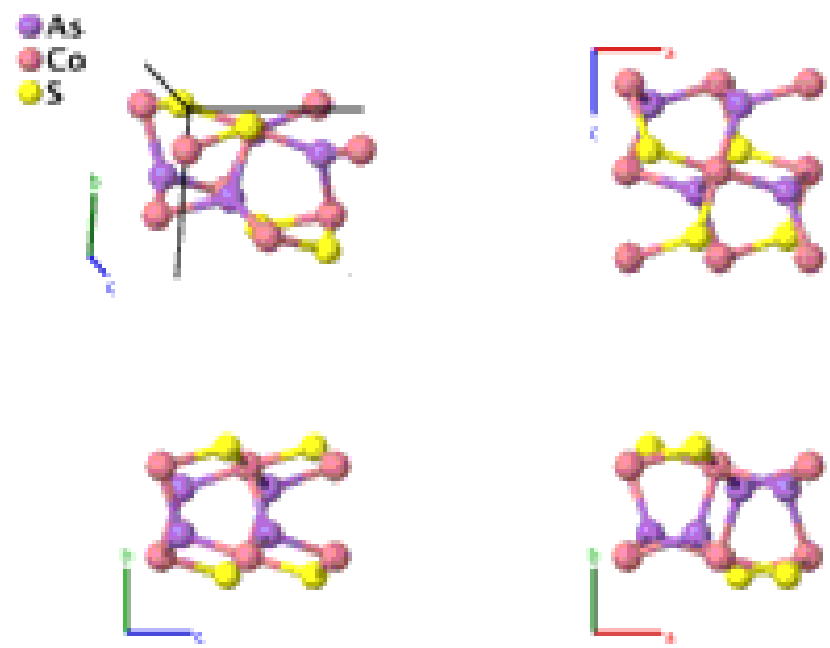

Prototype

AFLOW prototype label

: $\quad$ coAsS

Strukturbericht designation

ABC_oP12_29_a_a_a

Pearson symbol

: None

Space group number

oP12

Space group symbol

: $\quad 29$

AFLOW prototype command

: $\quad P c a 2_{1}$

aflow --proto=ABC_oP12_29_a_a_a

- -params $=a, b / a, c / a, x_{1}, y_{1}, z_{1}, x_{2}, y_{2}, z_{2}, x_{3}, y_{3}, z_{3}$

\section{Simple Orthorhombic primitive vectors:}

$$
\begin{aligned}
& \mathbf{a}_{1}=a \hat{\mathbf{x}} \\
& \mathbf{a}_{2}=b \hat{\mathbf{y}} \\
& \mathbf{a}_{3}=c \hat{\mathbf{z}}
\end{aligned}
$$
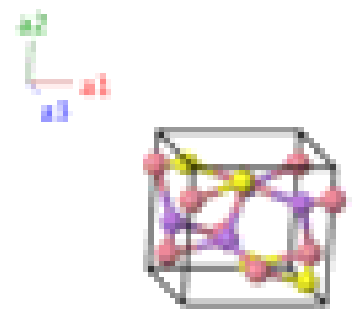

Basis vectors:

Lattice Coordinates

$\mathbf{B}_{1}=x_{1} \mathbf{a}_{1}+y_{1} \mathbf{a}_{2}+z_{1} \mathbf{a}_{3}$

$\mathbf{B}_{2}=-x_{1} \mathbf{a}_{1}-y_{1} \mathbf{a}_{2}+\left(\frac{1}{2}+z_{1}\right) \mathbf{a}_{3}$

$\mathbf{B}_{3}=\left(\frac{1}{2}+x_{1}\right) \mathbf{a}_{1}-y_{1} \mathbf{a}_{2}+z_{1} \mathbf{a}_{3}$

$\mathbf{B}_{4}=\left(\frac{1}{2}-x_{1}\right) \mathbf{a}_{1}+y_{1} \mathbf{a}_{2}+\left(\frac{1}{2}+z_{1}\right)$

$\mathbf{B}_{5}=$

$\mathbf{B}_{6}=$

$\mathbf{B}_{7}=$

$x_{2} \mathbf{a}_{1}+y_{2} \mathbf{a}_{2}+z_{2} \mathbf{a}_{3}$

$-x_{2} \mathbf{a}_{1}-y_{2} \mathbf{a}_{2}+\left(\frac{1}{2}+z_{2}\right) \mathbf{a}_{3}$

$\left(\frac{1}{2}+x_{2}\right) \mathbf{a}_{1}-y_{2} \mathbf{a}_{2}+z_{2} \mathbf{a}_{3}$
Cartesian Coordinates

$=$

$=\quad-x_{1} a \hat{\mathbf{x}}-y_{1} b \hat{\mathbf{y}}+\left(\frac{1}{2}+z_{1}\right) c \hat{\mathbf{z}}$

$=\left(\frac{1}{2}+x_{1}\right) a \hat{\mathbf{x}}-y_{1} b \hat{\mathbf{y}}+z_{1} c \hat{\mathbf{z}}$

$=\left(\frac{1}{2}-x_{1}\right) a \hat{\mathbf{x}}+y_{1} b \hat{\mathbf{y}}+\left(\frac{1}{2}+z_{1}\right) c \hat{\mathbf{z}}$

$=\quad x_{2} a \hat{\mathbf{x}}+y_{2} b \hat{\mathbf{y}}+z_{2} c \hat{\mathbf{z}}$

$=\quad-x_{2} a \hat{\mathbf{x}}-y_{2} b \hat{\mathbf{y}}+\left(\frac{1}{2}+z_{2}\right) c \hat{\mathbf{z}}$

$=\left(\frac{1}{2}+x_{2}\right) a \hat{\mathbf{x}}-y_{2} b \hat{\mathbf{y}}+z_{2} c \hat{\mathbf{z}}$
Wyckoff Position

(4a)

(4a)

(4a)

(4a)

(4a)

(4a)

(4a)
Atom Type

As

As

As

As

Co

Co

Co 


\begin{tabular}{|c|c|c|c|c|c|}
\hline $\mathbf{B}_{8}$ & $=$ & $\left(\frac{1}{2}-x_{2}\right) \mathbf{a}_{1}+y_{2} \mathbf{a}_{2}+\left(\frac{1}{2}+z_{2}\right) \mathbf{a}_{3}$ & $=$ & $\left(\frac{1}{2}-x_{2}\right) a \hat{\mathbf{x}}+y_{2} b \hat{\mathbf{y}}+\left(\frac{1}{2}+z_{2}\right) c \hat{\mathbf{z}}$ & $(4 a)$ \\
\hline $\mathbf{B}_{9}$ & $=$ & $x_{3} \mathbf{a}_{1}+y_{3} \mathbf{a}_{2}+z_{3} \mathbf{a}_{3}$ & $=$ & $x_{3} a \hat{\mathbf{x}}+y_{3} b \hat{\mathbf{y}}+z_{3} c \hat{\mathbf{z}}$ & $(4 a)$ \\
\hline $\mathbf{B}_{10}$ & $=$ & $-x_{3} \mathbf{a}_{1}-y_{3} \mathbf{a}_{2}+\left(\frac{1}{2}+z_{3}\right) \mathbf{a}_{3}$ & $=$ & $-x_{3} a \hat{\mathbf{x}}-y_{3} b \hat{\mathbf{y}}+\left(\frac{1}{2}+z_{3}\right) c \hat{\mathbf{z}}$ & $(4 a)$ \\
\hline $\mathbf{B}_{11}$ & $=$ & $\left(\frac{1}{2}+x_{3}\right) \mathbf{a}_{1}-y_{3} \mathbf{a}_{2}+z_{3} \mathbf{a}_{3}$ & $=$ & $\left(\frac{1}{2}+x_{3}\right) a \hat{\mathbf{x}}-y_{3} b \hat{\mathbf{y}}+z_{3} c \hat{\mathbf{z}}$ & $(4 a)$ \\
\hline $\mathbf{B}_{12}$ & $=$ & $\left(\frac{1}{2}-x_{3}\right) \mathbf{a}_{1}+y_{3} \mathbf{a}_{2}+\left(\frac{1}{2}+z_{3}\right) \mathbf{a}_{3}$ & $=$ & $\left(\frac{1}{2}-x_{3}\right) a \hat{\mathbf{x}}+y_{3} b \hat{\mathbf{y}}+\left(\frac{1}{2}+z_{3}\right) c \hat{\mathbf{z}}$ & $(4 a)$ \\
\hline
\end{tabular}

\section{References:}

- M. E. Fleet and P. C. Burns, Structure and twinning of cobaltite, Can. Mineral. 28, 719-723 (1990).

\section{Found in:}

- P. Villars and K. Cenzual, Pearson's Crystal Data - Crystal Structure Database for Inorganic Compounds, ASM International (2013).

\section{Geometry files:}

- CIF: pp. 821

- POSCAR: pp. 821 


\section{$\mathrm{Bi}_{5} \mathrm{Nb}_{3} \mathrm{O}_{15}$ Structure: A5B3C15_oP46_30_a2c_bc_a7c}
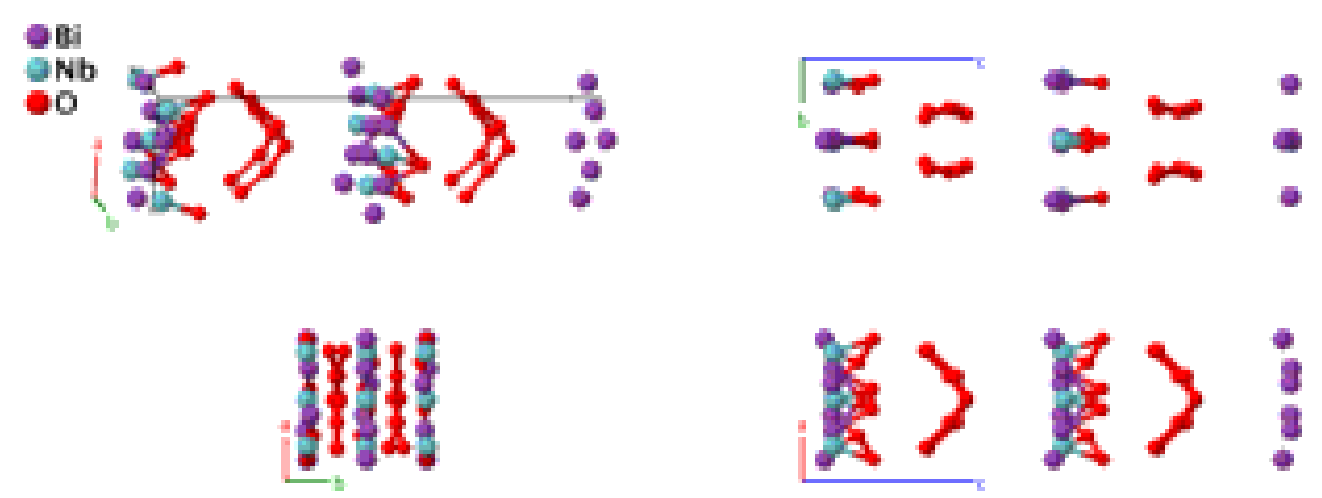

\section{Prototype}

AFLOW prototype label

Strukturbericht designation

Pearson symbol

Space group number

Space group symbol

AFLOW prototype command

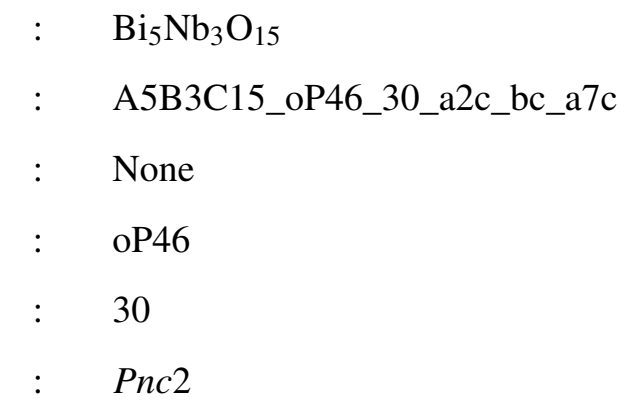

Simple Orthorhombic primitive vectors:

$$
\begin{aligned}
& \mathbf{a}_{1}=a \hat{\mathbf{x}} \\
& \mathbf{a}_{2}=b \hat{\mathbf{y}} \\
& \mathbf{a}_{3}=c \hat{\mathbf{z}}
\end{aligned}
$$

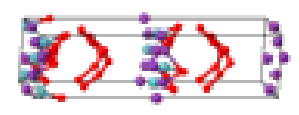

Basis vectors:

Lattice Coordinates
$\mathbf{B}_{1}=$
$z_{1} \mathbf{a}_{3}$
$\mathbf{B}_{2}=\frac{1}{2} \mathbf{a}_{2}+\left(\frac{1}{2}+z_{1}\right) \mathbf{a}_{3}$
$\mathbf{B}_{3}=$
$z_{2} \mathbf{a}_{3}$
$\mathbf{B}_{4}=\frac{1}{2} \mathbf{a}_{2}+\left(\frac{1}{2}+z_{2}\right) \mathbf{a}_{3}$
$\mathbf{B}_{5}=$
$\frac{1}{2} \mathbf{a}_{1}+z_{3} \mathbf{a}_{3}$
$\mathbf{B}_{6}=\frac{1}{2} \mathbf{a}_{1}+\frac{1}{2} \mathbf{a}_{2}+\left(\frac{1}{2}+z_{3}\right) \mathbf{a}_{3}$
$\mathbf{B}_{7}=$
$\mathbf{B}_{8}=$
$x_{4} \mathbf{a}_{1}+y_{4} \mathbf{a}_{2}+z_{4} \mathbf{a}_{3}$
$\mathbf{B}_{9}=x_{4} \mathbf{a}_{1}+\left(\frac{1}{2}-y_{4}\right) \mathbf{a}_{2}+\left(\frac{1}{2}+z_{4}\right) \mathbf{a}_{3}$
$\mathbf{B}_{10}=-x_{4} \mathbf{a}_{1}+\left(\frac{1}{2}+y_{4}\right) \mathbf{a}_{2}+\left(\frac{1}{2}+z_{4}\right) \mathbf{a}_{3}$
$\mathbf{B}_{11}=$
$x_{5} \mathbf{a}_{1}+y_{5} \mathbf{a}_{2}+z_{5} \mathbf{a}_{3}$
$=$
$=\quad \frac{1}{2} b \hat{\mathbf{y}}+\left(\frac{1}{2}+z_{1}\right) c \hat{\mathbf{z}}$
$z_{2} c \hat{\mathbf{z}}$
$=$
$=$
$=$
$=$
$=$
$=$
$=$
$=$
$=$
$z_{1} c \hat{\mathbf{z}}$

Cartesian Coordinates

(2a)

(2a)

(2a)

(2a)
Atom Type

Bi I

Bi I

O I

O I

$\mathrm{Nb}$ I

$\mathrm{Nb} \mathrm{I}$

Bi II

Bi II

Bi II

Bi II

Bi III 


\begin{tabular}{|c|c|c|c|c|c|c|}
\hline $\mathbf{B}_{12}$ & $=$ & $-x_{5} \mathbf{a}_{1}-y_{5} \mathbf{a}_{2}+z 5 \mathbf{a}_{3}$ & $=$ & $-x_{5} a \hat{\mathbf{x}}-y_{5} b \hat{\mathbf{y}}+z_{5} c \hat{\mathbf{z}}$ & $(4 c)$ & Bi III \\
\hline $\mathbf{B}_{13}$ & $=$ & $x_{5} \mathbf{a}_{1}+\left(\frac{1}{2}-y_{5}\right) \mathbf{a}_{2}+\left(\frac{1}{2}+z_{5}\right) \mathbf{a}_{3}$ & $=$ & $x_{5} a \hat{\mathbf{x}}+\left(\frac{1}{2}-y_{5}\right) b \hat{\mathbf{y}}+\left(\frac{1}{2}+z_{5}\right) c \hat{\mathbf{z}}$ & $(4 c)$ & Bi III \\
\hline $\mathbf{B}_{14}$ & $=$ & $-x_{5} \mathbf{a}_{1}+\left(\frac{1}{2}+y_{5}\right) \mathbf{a}_{2}+\left(\frac{1}{2}+z_{5}\right) \mathbf{a}_{3}$ & $=$ & $-x_{5} a \hat{\mathbf{x}}+\left(\frac{1}{2}+y_{5}\right) b \hat{\mathbf{y}}+\left(\frac{1}{2}+z_{5}\right) c \hat{\mathbf{z}}$ & $(4 c)$ & Bi III \\
\hline $\mathbf{B}_{15}$ & $=$ & $x_{6} \mathbf{a}_{1}+y_{6} \mathbf{a}_{2}+z_{6} \mathbf{a}_{3}$ & $=$ & $x_{6} a \hat{\mathbf{x}}+y_{6} b \hat{\mathbf{y}}+z_{6} c \hat{\mathbf{z}}$ & $(4 c)$ & $\mathrm{Nb}$ II \\
\hline $\mathbf{B}_{16}$ & $=$ & $-x_{6} \mathbf{a}_{1}-y_{6} \mathbf{a}_{2}+z_{6} \mathbf{a}_{3}$ & $=$ & $-x_{6} a \hat{\mathbf{x}}-y_{6} b \hat{\mathbf{y}}+z_{6} c \hat{\mathbf{z}}$ & $(4 c)$ & $\mathrm{Nb}$ II \\
\hline $\mathbf{B}_{17}$ & $=$ & $x_{6} \mathbf{a}_{1}+\left(\frac{1}{2}-y_{6}\right) \mathbf{a}_{2}+\left(\frac{1}{2}+z_{6}\right) \mathbf{a}_{3}$ & $=$ & $x_{6} a \hat{\mathbf{x}}+\left(\frac{1}{2}-y_{6}\right) b \hat{\mathbf{y}}+\left(\frac{1}{2}+z_{6}\right) c \hat{\mathbf{z}}$ & $(4 c)$ & $\mathrm{Nb}$ II \\
\hline $\mathbf{B}_{18}$ & $=$ & $-x_{6} \mathbf{a}_{1}+\left(\frac{1}{2}+y_{6}\right) \mathbf{a}_{2}+\left(\frac{1}{2}+z_{6}\right) \mathbf{a}_{3}$ & $=$ & $-x_{6} a \hat{\mathbf{x}}+\left(\frac{1}{2}+y_{6}\right) b \hat{\mathbf{y}}+\left(\frac{1}{2}+z_{6}\right) c \hat{\mathbf{z}}$ & $(4 c)$ & $\mathrm{Nb}$ II \\
\hline $\mathbf{B}_{19}$ & $=$ & $x_{7} \mathbf{a}_{1}+y_{7} \mathbf{a}_{2}+z_{7} \mathbf{a}_{3}$ & $=$ & $x_{7} a \hat{\mathbf{x}}+y_{7} b \hat{\mathbf{y}}+z_{7} c \hat{\mathbf{z}}$ & $(4 c)$ & O II \\
\hline $\mathbf{B}_{20}$ & $=$ & $-x_{7} \mathbf{a}_{1}-y_{7} \mathbf{a}_{2}+z_{7} \mathbf{a}_{3}$ & $=$ & $-x_{7} a \hat{\mathbf{x}}-y_{7} b \hat{\mathbf{y}}+z_{7} c \hat{\mathbf{z}}$ & $(4 c)$ & O II \\
\hline $\mathbf{B}_{21}$ & $=$ & $x_{7} \mathbf{a}_{1}+\left(\frac{1}{2}-y_{7}\right) \mathbf{a}_{2}+\left(\frac{1}{2}+z_{7}\right) \mathbf{a}_{3}$ & $=$ & $x_{7} a \hat{\mathbf{x}}+\left(\frac{1}{2}-y_{7}\right) b \hat{\mathbf{y}}+\left(\frac{1}{2}+z_{7}\right) c \hat{\mathbf{z}}$ & $(4 c)$ & O II \\
\hline $\mathbf{B}_{22}$ & $=$ & $-x_{7} \mathbf{a}_{1}+\left(\frac{1}{2}+y_{7}\right) \mathbf{a}_{2}+\left(\frac{1}{2}+z_{7}\right) \mathbf{a}_{3}$ & $=$ & $-x_{7} a \hat{\mathbf{x}}+\left(\frac{1}{2}+y_{7}\right) b \hat{\mathbf{y}}+\left(\frac{1}{2}+z_{7}\right) c \hat{\mathbf{z}}$ & $(4 c)$ & O II \\
\hline $\mathbf{B}_{23}$ & $=$ & $x_{8} \mathbf{a}_{1}+y_{8} \mathbf{a}_{2}+z_{8} \mathbf{a}_{3}$ & $=$ & $x_{8} a \hat{\mathbf{x}}+y_{8} b \hat{\mathbf{y}}+z_{8} c \hat{\mathbf{z}}$ & $(4 c)$ & O III \\
\hline $\mathbf{B}_{24}$ & $=$ & $-x_{8} \mathbf{a}_{1}-y_{8} \mathbf{a}_{2}+z_{8} \mathbf{a}_{3}$ & $=$ & $-x_{8} a \hat{\mathbf{x}}-y_{8} b \hat{\mathbf{y}}+z_{8} c \hat{\mathbf{z}}$ & $(4 c)$ & O III \\
\hline $\mathbf{B}_{25}$ & $=$ & $x_{8} \mathbf{a}_{1}+\left(\frac{1}{2}-y_{8}\right) \mathbf{a}_{2}+\left(\frac{1}{2}+z_{8}\right) \mathbf{a}_{3}$ & $=$ & $x_{8} a \hat{\mathbf{x}}+\left(\frac{1}{2}-y_{8}\right) b \hat{\mathbf{y}}+\left(\frac{1}{2}+z_{8}\right) c \hat{\mathbf{z}}$ & $(4 c)$ & O III \\
\hline $\mathbf{B}_{26}$ & $=$ & $-x_{8} \mathbf{a}_{1}+\left(\frac{1}{2}+y_{8}\right) \mathbf{a}_{2}+\left(\frac{1}{2}+z_{8}\right) \mathbf{a}_{3}$ & $=$ & $-x_{8} a \hat{\mathbf{x}}+\left(\frac{1}{2}+y_{8}\right) b \hat{\mathbf{y}}+\left(\frac{1}{2}+z_{8}\right) c \hat{\mathbf{z}}$ & $(4 c)$ & O III \\
\hline $\mathbf{B}_{27}$ & $=$ & $x_{9} \mathbf{a}_{1}+y_{9} \mathbf{a}_{2}+z_{9} \mathbf{a}_{3}$ & $=$ & $x_{9} a \hat{\mathbf{x}}+y_{9} b \hat{\mathbf{y}}+z_{9} c \hat{\mathbf{z}}$ & $(4 c)$ & O IV \\
\hline $\mathbf{B}_{28}$ & $=$ & $-x_{9} \mathbf{a}_{1}-y_{9} \mathbf{a}_{2}+z 9 \mathbf{a}_{3}$ & $=$ & $-x_{9} a \hat{\mathbf{x}}-y_{9} b \hat{\mathbf{y}}+z_{9} c \hat{\mathbf{z}}$ & $(4 c)$ & O IV \\
\hline $\mathbf{B}_{29}$ & $=$ & $x_{9} \mathbf{a}_{1}+\left(\frac{1}{2}-y_{9}\right) \mathbf{a}_{2}+\left(\frac{1}{2}+z_{9}\right) \mathbf{a}_{3}$ & $=$ & $x_{9} a \hat{\mathbf{x}}+\left(\frac{1}{2}-y_{9}\right) b \hat{\mathbf{y}}+\left(\frac{1}{2}+z_{9}\right) c \hat{\mathbf{z}}$ & $(4 c)$ & O IV \\
\hline $\mathbf{B}_{30}$ & $=$ & $-x_{9} \mathbf{a}_{1}+\left(\frac{1}{2}+y_{9}\right) \mathbf{a}_{2}+\left(\frac{1}{2}+z 9\right) \mathbf{a}_{3}$ & $=$ & $-x_{9} a \hat{\mathbf{x}}+\left(\frac{1}{2}+y_{9}\right) b \hat{\mathbf{y}}+\left(\frac{1}{2}+z_{9}\right) c \hat{\mathbf{z}}$ & $(4 c)$ & O IV \\
\hline $\mathbf{B}_{31}$ & $=$ & $x_{10} \mathbf{a}_{1}+y_{10} \mathbf{a}_{2}+z_{10} \mathbf{a}_{3}$ & $=$ & $x_{10} a \hat{\mathbf{x}}+y_{10} b \hat{\mathbf{y}}+z_{10} c \hat{\mathbf{z}}$ & $(4 c)$ & $\mathrm{OV}$ \\
\hline $\mathbf{B}_{32}$ & $=$ & $-x_{10} \mathbf{a}_{1}-y_{10} \mathbf{a}_{2}+z_{10} \mathbf{a}_{3}$ & $=$ & $-x_{10} a \hat{\mathbf{x}}-y_{10} b \hat{\mathbf{y}}+z_{10} c \hat{\mathbf{z}}$ & $(4 c)$ & $\mathrm{OV}$ \\
\hline $\mathbf{B}_{33}$ & $=$ & $x_{10} \mathbf{a}_{1}+\left(\frac{1}{2}-y_{10}\right) \mathbf{a}_{2}+\left(\frac{1}{2}+z_{10}\right) \mathbf{a}_{3}$ & $=$ & $x_{10} a \hat{\mathbf{x}}+\left(\frac{1}{2}-y_{10}\right) b \hat{\mathbf{y}}+\left(\frac{1}{2}+z_{10}\right) c \hat{\mathbf{z}}$ & $(4 c)$ & $\mathrm{OV}$ \\
\hline $\mathbf{B}_{34}$ & $=$ & $\begin{array}{c}-x_{10} \mathbf{a}_{1}+\left(\frac{1}{2}+y_{10}\right) \mathbf{a}_{2}+ \\
\left(\frac{1}{2}+z_{10}\right) \mathbf{a}_{3}\end{array}$ & $=$ & $\begin{array}{c}-x_{10} a \hat{\mathbf{x}}+\left(\frac{1}{2}+y_{10}\right) b \hat{\mathbf{y}}+ \\
\left(\frac{1}{2}+z_{10}\right) c \hat{\mathbf{z}}\end{array}$ & $(4 c)$ & $\mathrm{OV}$ \\
\hline $\mathbf{B}_{35}$ & $=$ & $x_{11} \mathbf{a}_{1}+y_{11} \mathbf{a}_{2}+z_{11} \mathbf{a}_{3}$ & $=$ & $x_{11} a \hat{\mathbf{x}}+y_{11} b \hat{\mathbf{y}}+z_{11} c \hat{\mathbf{z}}$ & $(4 c)$ & O VI \\
\hline $\mathbf{B}_{36}$ & $=$ & $-x_{11} \mathbf{a}_{1}-y_{11} \mathbf{a}_{2}+z_{11} \mathbf{a}_{3}$ & $=$ & $-x_{11} a \hat{\mathbf{x}}-y_{11} b \hat{\mathbf{y}}+z_{11} c \hat{\mathbf{z}}$ & $(4 c)$ & O VI \\
\hline $\mathbf{B}_{37}$ & $=$ & $x_{11} \mathbf{a}_{1}+\left(\frac{1}{2}-y_{11}\right) \mathbf{a}_{2}+\left(\frac{1}{2}+z_{11}\right) \mathbf{a}_{3}$ & $=$ & $x_{11} a \hat{\mathbf{x}}+\left(\frac{1}{2}-y_{11}\right) b \hat{\mathbf{y}}+\left(\frac{1}{2}+z_{11}\right) c \hat{\mathbf{z}}$ & $(4 c)$ & O VI \\
\hline $\mathbf{B}_{38}$ & $=$ & $\begin{array}{c}-x_{11} \mathbf{a}_{1}+\left(\frac{1}{2}+y_{11}\right) \mathbf{a}_{2}+ \\
\left(\frac{1}{2}+z_{11}\right) \mathbf{a}_{3}\end{array}$ & $=$ & $\begin{array}{c}-x_{11} a \hat{\mathbf{x}}+\left(\frac{1}{2}+y_{11}\right) b \hat{\mathbf{y}}+ \\
\left(\frac{1}{2}+z_{11}\right) c \hat{\mathbf{z}}\end{array}$ & $(4 c)$ & O VI \\
\hline $\mathbf{B}_{39}$ & $=$ & $x_{12} \mathbf{a}_{1}+y_{12} \mathbf{a}_{2}+z_{12} \mathbf{a}_{3}$ & $=$ & $x_{12} a \hat{\mathbf{x}}+y_{12} b \hat{\mathbf{y}}+z_{12} c \hat{\mathbf{z}}$ & $(4 c)$ & O VII \\
\hline $\mathbf{B}_{40}$ & $=$ & $-x_{12} \mathbf{a}_{1}-y_{12} \mathbf{a}_{2}+z_{12} \mathbf{a}_{3}$ & $=$ & $-x_{12} a \hat{\mathbf{x}}-y_{12} b \hat{\mathbf{y}}+z_{12} c \hat{\mathbf{z}}$ & $(4 c)$ & O VII \\
\hline $\mathbf{B}_{41}$ & $=$ & $x_{12} \mathbf{a}_{1}+\left(\frac{1}{2}-y_{12}\right) \mathbf{a}_{2}+\left(\frac{1}{2}+z_{12}\right) \mathbf{a}_{3}$ & $=$ & $x_{12} a \hat{\mathbf{x}}+\left(\frac{1}{2}-y_{12}\right) b \hat{\mathbf{y}}+\left(\frac{1}{2}+z_{12}\right) c \hat{\mathbf{z}}$ & $(4 c)$ & O VII \\
\hline $\mathbf{B}_{42}$ & $=$ & $\begin{array}{c}-x_{12} \mathbf{a}_{1}+\left(\frac{1}{2}+y_{12}\right) \mathbf{a}_{2}+ \\
\left(\frac{1}{2}+z_{12}\right) \mathbf{a}_{3}\end{array}$ & $=$ & $\begin{array}{c}-x_{12} a \hat{\mathbf{x}}+\left(\frac{1}{2}+y_{12}\right) b \hat{\mathbf{y}}+ \\
\left(\frac{1}{2}+z_{12}\right) c \hat{\mathbf{z}}\end{array}$ & $(4 c)$ & O VII \\
\hline $\mathbf{B}_{43}$ & $=$ & $x_{13} \mathbf{a}_{1}+y_{13} \mathbf{a}_{2}+z_{13} \mathbf{a}_{3}$ & $=$ & $x_{13} a \hat{\mathbf{x}}+y_{13} b \hat{\mathbf{y}}+z_{13} c \hat{\mathbf{z}}$ & $(4 c)$ & O VIII \\
\hline $\mathbf{B}_{44}$ & $=$ & $-x_{13} \mathbf{a}_{1}-y_{13} \mathbf{a}_{2}+z_{13} \mathbf{a}_{3}$ & $=$ & $-x_{13} a \hat{\mathbf{x}}-y_{13} b \hat{\mathbf{y}}+z_{13} c \hat{\mathbf{z}}$ & $(4 c)$ & O VIII \\
\hline $\mathbf{B}_{45}$ & $=$ & $x_{13} \mathbf{a}_{1}+\left(\frac{1}{2}-y_{13}\right) \mathbf{a}_{2}+\left(\frac{1}{2}+z_{13}\right) \mathbf{a}_{3}$ & $=$ & $x_{13} a \hat{\mathbf{x}}+\left(\frac{1}{2}-y_{13}\right) b \hat{\mathbf{y}}+\left(\frac{1}{2}+z_{13}\right) c \hat{\mathbf{z}}$ & $(4 c)$ & O VIII \\
\hline
\end{tabular}




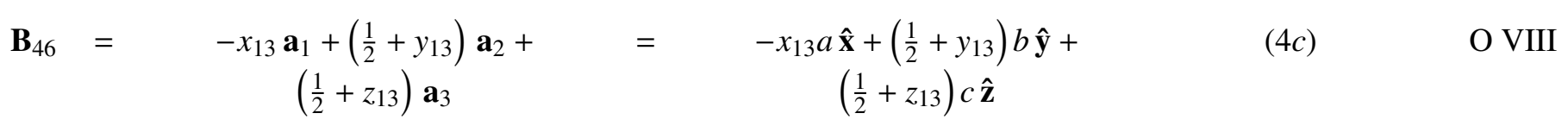

\section{References:}

- S. Tahara, A. Shimada, N. Kumada, and Y. Sugahara, Characterization of $\mathrm{Bi}_{5} \mathrm{Nb}_{3} \mathrm{O}_{15}$ by refinement of neutron diffraction pattern, acid treatment and reaction of the acid-treated product with n-alkylamines, J. Solid State Chem. 180, 2517-2524 (2007), doi:10.1016/j.jssc.2007.05.017.

\section{Found in:}

- P. Villars and K. Cenzual, Pearson's Crystal Data - Crystal Structure Database for Inorganic Compounds, ASM International (2013).

\section{Geometry files:}

- CIF: pp. 821

- POSCAR: pp. 822 


\section{$\mathrm{CuBrSe}_{3}$ Structure: ABC3_oP20_30_2a_c_3c}
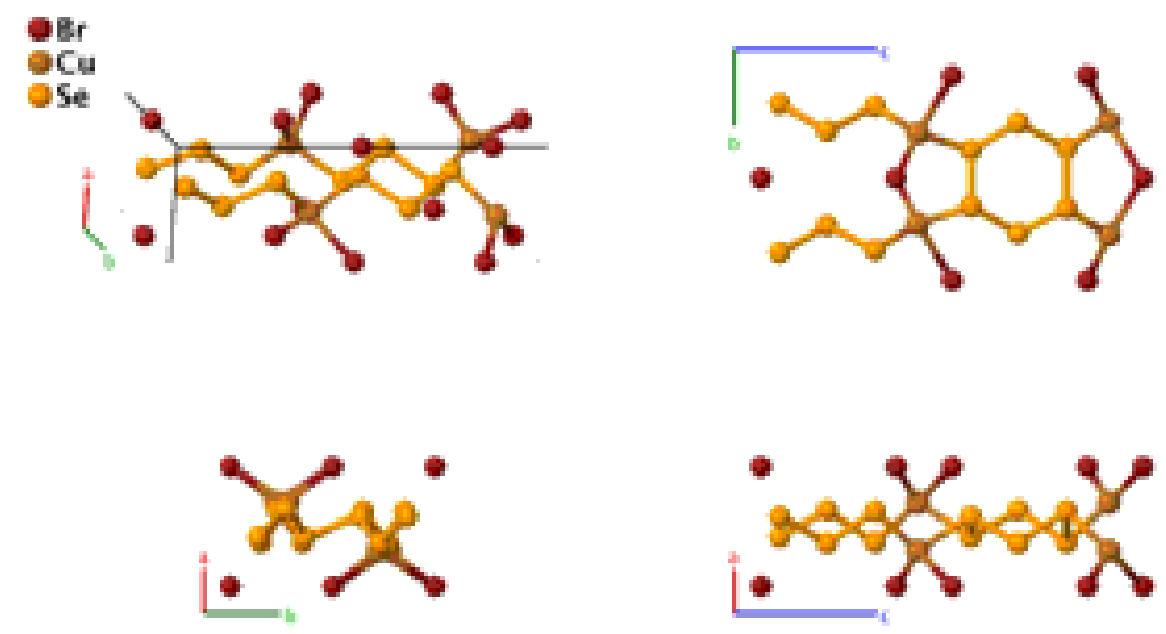

\section{Prototype}

AFLOW prototype label

Strukturbericht designation

Pearson symbol

Space group number

Space group symbol

AFLOW prototype command
: $\quad \mathrm{CuBrSe}_{3}$

: ABC3_oP20_30_2a_c_3c

: None

: $\quad$ oP20

: $\quad 30$

: Pnc2

: aflow --proto=ABC3_oP20_30_2a_c_3c

- - params $=a, b / a, c / a, z_{1}, z_{2}, x_{3}, y_{3}, z_{3}, x_{4}, y_{4}, z_{4}, x_{5}, y_{5}, z_{5}, x_{6}, y_{6}, z_{6}$

Simple Orthorhombic primitive vectors:

$$
\begin{aligned}
& \mathbf{a}_{1}=a \hat{\mathbf{x}} \\
& \mathbf{a}_{2}=b \hat{\mathbf{y}} \\
& \mathbf{a}_{3}=c \hat{\mathbf{z}}
\end{aligned}
$$

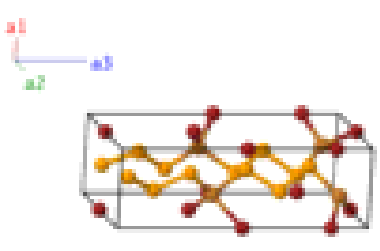

Basis vectors:

Lattice Coordinates

$\begin{array}{lcccc}\mathbf{B}_{1} & = & z_{1} \mathbf{a}_{3} & = & z_{1} c \hat{\mathbf{z}} \\ \mathbf{B}_{2} & = & \frac{1}{2} \mathbf{a}_{2}+\left(\frac{1}{2}+z_{1}\right) \mathbf{a}_{3} & = & \frac{1}{2} b \hat{\mathbf{y}}+\left(\frac{1}{2}+z_{1}\right) c \hat{\mathbf{z}} \\ \mathbf{B}_{3} & z_{2} \mathbf{a}_{3} & = & z_{2} c \hat{\mathbf{z}} \\ \mathbf{B}_{4} & \frac{1}{2} \mathbf{a}_{2}+\left(\frac{1}{2}+z_{2}\right) \mathbf{a}_{3} & = & \frac{1}{2} b \hat{\mathbf{y}}+\left(\frac{1}{2}+z_{2}\right) c \hat{\mathbf{z}} \\ \mathbf{B}_{5} & x_{3} \mathbf{a}_{1}+y_{3} \mathbf{a}_{2}+z_{3} \mathbf{a}_{3} & = & x_{3} a \hat{\mathbf{x}}+y_{3} b \hat{\mathbf{y}}+z_{3} c \hat{\mathbf{z}} \\ \mathbf{B}_{6} & -x_{3} \mathbf{a}_{1}-y_{3} \mathbf{a}_{2}+z_{3} \mathbf{a}_{3} & = & -x_{3} a \hat{\mathbf{x}}-y_{3} b \hat{\mathbf{y}}+z_{3} c \hat{\mathbf{z}} \\ \mathbf{B}_{7} & = & x_{3} \mathbf{a}_{1}+\left(\frac{1}{2}-y_{3}\right) \mathbf{a}_{2}+\left(\frac{1}{2}+z_{3}\right) \mathbf{a}_{3} & = & x_{3} a \hat{\mathbf{x}}+\left(\frac{1}{2}-y_{3}\right) b \hat{\mathbf{y}}+\left(\frac{1}{2}+z_{3}\right) c \hat{\mathbf{z}} \\ \mathbf{B}_{8} & = & -x_{3} \mathbf{a}_{1}+\left(\frac{1}{2}+y_{3}\right) \mathbf{a}_{2}+\left(\frac{1}{2}+z_{3}\right) \mathbf{a}_{3} & = & -x_{3} a \hat{\mathbf{x}}+\left(\frac{1}{2}+y_{3}\right) b \hat{\mathbf{y}}+\left(\frac{1}{2}+z_{3}\right) c \hat{\mathbf{z}}\end{array}$

Wyckoff Position Atom Type

(2a)

$\mathrm{Br} \mathrm{I}$

(2a)

$\mathrm{Br} I$

$(2 a)$

$\mathrm{Br}$ II

(2a)

$\mathrm{Br}$ II

(4c)

$\mathrm{Cu}$

(4c)

$\mathrm{Cu}$

(4c)

$\mathrm{Cu}$

(4c) 


\begin{tabular}{|c|c|c|c|c|c|c|}
\hline $\mathbf{B}_{9}$ & $=$ & $x_{4} \mathbf{a}_{1}+y_{4} \mathbf{a}_{2}+z_{4} \mathbf{a}_{3}$ & $=$ & $x_{4} a \hat{\mathbf{x}}+y_{4} b \hat{\mathbf{y}}+z_{4} c \hat{\mathbf{z}}$ & $(4 c)$ & Se I \\
\hline $\mathbf{B}_{10}$ & $=$ & $-x_{4} \mathbf{a}_{1}-y_{4} \mathbf{a}_{2}+z_{4} \mathbf{a}_{3}$ & $=$ & $-x_{4} a \hat{\mathbf{x}}-y_{4} b \hat{\mathbf{y}}+z_{4} c \hat{\mathbf{z}}$ & $(4 c)$ & Se I \\
\hline $\mathbf{B}_{11}$ & $=$ & $x_{4} \mathbf{a}_{1}+\left(\frac{1}{2}-y_{4}\right) \mathbf{a}_{2}+\left(\frac{1}{2}+z_{4}\right) \mathbf{a}_{3}$ & $=$ & $x_{4} a \hat{\mathbf{x}}+\left(\frac{1}{2}-y_{4}\right) b \hat{\mathbf{y}}+\left(\frac{1}{2}+z_{4}\right) c \hat{\mathbf{z}}$ & $(4 c)$ & Se I \\
\hline $\mathbf{B}_{12}$ & $=$ & $-x_{4} \mathbf{a}_{1}+\left(\frac{1}{2}+y_{4}\right) \mathbf{a}_{2}+\left(\frac{1}{2}+z_{4}\right) \mathbf{a}_{3}$ & $=$ & $-x_{4} a \hat{\mathbf{x}}+\left(\frac{1}{2}+y_{4}\right) b \hat{\mathbf{y}}+\left(\frac{1}{2}+z_{4}\right) c \hat{\mathbf{z}}$ & $(4 c)$ & Se I \\
\hline $\mathbf{B}_{13}$ & $=$ & $x_{5} \mathbf{a}_{1}+y_{5} \mathbf{a}_{2}+z_{5} \mathbf{a}_{3}$ & $=$ & $x_{5} a \hat{\mathbf{x}}+y_{5} b \hat{\mathbf{y}}+z_{5} c \hat{\mathbf{z}}$ & $(4 c)$ & Se II \\
\hline $\mathbf{B}_{14}$ & $=$ & $-x_{5} \mathbf{a}_{1}-y_{5} \mathbf{a}_{2}+z_{5} \mathbf{a}_{3}$ & $=$ & $-x_{5} a \hat{\mathbf{x}}-y_{5} b \hat{\mathbf{y}}+z_{5} c \hat{\mathbf{z}}$ & $(4 c)$ & Se II \\
\hline $\mathbf{B}_{15}$ & $=$ & $x_{5} \mathbf{a}_{1}+\left(\frac{1}{2}-y_{5}\right) \mathbf{a}_{2}+\left(\frac{1}{2}+z_{5}\right) \mathbf{a}_{3}$ & $=$ & $x_{5} a \hat{\mathbf{x}}+\left(\frac{1}{2}-y_{5}\right) b \hat{\mathbf{y}}+\left(\frac{1}{2}+z_{5}\right) c \hat{\mathbf{z}}$ & $(4 c)$ & Se II \\
\hline $\mathbf{B}_{16}$ & $=$ & $-x_{5} \mathbf{a}_{1}+\left(\frac{1}{2}+y_{5}\right) \mathbf{a}_{2}+\left(\frac{1}{2}+z_{5}\right) \mathbf{a}_{3}$ & $=$ & $-x_{5} a \hat{\mathbf{x}}+\left(\frac{1}{2}+y_{5}\right) b \hat{\mathbf{y}}+\left(\frac{1}{2}+z_{5}\right) c \hat{\mathbf{z}}$ & $(4 c)$ & Se II \\
\hline $\mathbf{B}_{17}$ & $=$ & $x_{6} \mathbf{a}_{1}+y_{6} \mathbf{a}_{2}+z_{6} \mathbf{a}_{3}$ & $=$ & $x_{6} a \hat{\mathbf{x}}+y_{6} b \hat{\mathbf{y}}+z_{6} c \hat{\mathbf{z}}$ & $(4 c)$ & Se III \\
\hline $\mathbf{B}_{18}$ & $=$ & $-x_{6} \mathbf{a}_{1}-y_{6} \mathbf{a}_{2}+z_{6} \mathbf{a}_{3}$ & $=$ & $-x_{6} a \hat{\mathbf{x}}-y_{6} b \hat{\mathbf{y}}+z_{6} c \hat{\mathbf{z}}$ & $(4 c)$ & Se III \\
\hline $\mathbf{B}_{19}$ & $=$ & $x_{6} \mathbf{a}_{1}+\left(\frac{1}{2}-y_{6}\right) \mathbf{a}_{2}+\left(\frac{1}{2}+z_{6}\right) \mathbf{a}_{3}$ & $=$ & $x_{6} a \hat{\mathbf{x}}+\left(\frac{1}{2}-y_{6}\right) b \hat{\mathbf{y}}+\left(\frac{1}{2}+z_{6}\right) c \hat{\mathbf{z}}$ & $(4 c)$ & Se III \\
\hline $\mathbf{B}_{20}$ & $=$ & $-x_{6} \mathbf{a}_{1}+\left(\frac{1}{2}+y_{6}\right) \mathbf{a}_{2}+\left(\frac{1}{2}+z_{6}\right) \mathbf{a}_{3}$ & $=$ & $-x_{6} a \hat{\mathbf{x}}+\left(\frac{1}{2}+y_{6}\right) b \hat{\mathbf{y}}+\left(\frac{1}{2}+z_{6}\right) c \hat{\mathbf{z}}$ & $(4 c)$ & Se III \\
\hline
\end{tabular}

\section{References:}

- T. Sakuma, T. Kaneko, T. Kurita, and H. Takahashi, Crystal structure of CuBrSe 3 , J. Phys. Soc. Jpn. 60, 1608-1611 (1991), doi:10.1143/JPSJ.60.1608.

\section{Found in:}

- P. Villars and K. Cenzual, Pearson's Crystal Data - Crystal Structure Database for Inorganic Compounds, ASM International (2013).

\section{Geometry files:}

- CIF: pp. 822

- POSCAR: pp. 822 


\section{$\mathrm{Re}_{2} \mathrm{O}_{5}\left[\mathrm{SO}_{4}\right]_{2}$ Structure: A13B2C2_oP34_32_a6c_c_c}
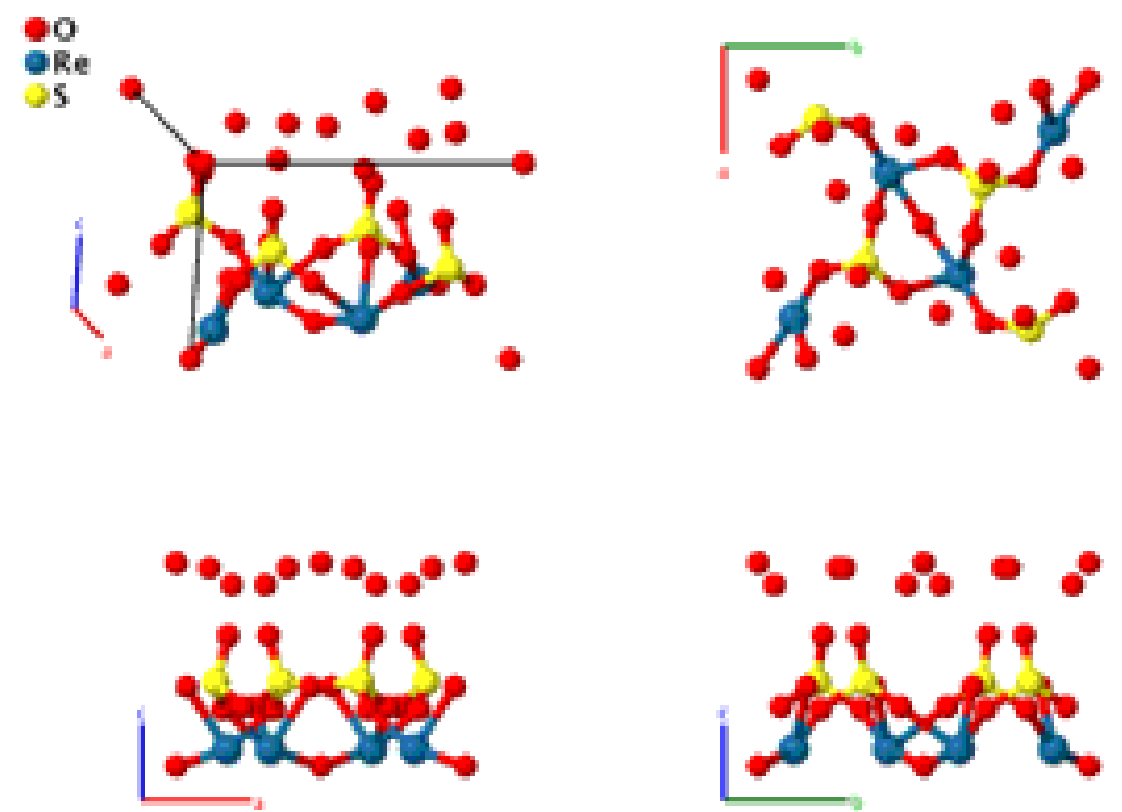

\section{Prototype}

AFLOW prototype label

Strukturbericht designation

Pearson symbol

Space group number

Space group symbol

AFLOW prototype command

$$
\text { : } \quad \mathrm{Re}_{2} \mathrm{O}_{5}\left[\mathrm{SO}_{4}\right]_{2}
$$$$
\text { : A13B2C2_oP34_32_a6c_c_c }
$$

: None

: $\quad \mathrm{oP} 34$

$: \quad 32$

: $\quad P b a 2$

aflow - -proto $=$ A13B2C2_oP34_32_a6c_c_c

- - params $=a, b / a, c / a, z_{1}, x_{2}, y_{2}, z_{2}, x_{3}, y_{3}, z_{3}, x_{4}, y_{4}, z_{4}, x_{5}, y_{5}, z_{5}, x_{6}, y_{6}, z_{6}, x_{7}$, $y_{7}, z_{7}, x_{8}, y_{8}, z_{8}, x_{9}, y_{9}, z_{9}$

Simple Orthorhombic primitive vectors:

$$
\begin{aligned}
& \mathbf{a}_{1}=a \hat{\mathbf{x}} \\
& \mathbf{a}_{2}=b \hat{\mathbf{y}} \\
& \mathbf{a}_{3}=c \hat{\mathbf{z}}
\end{aligned}
$$

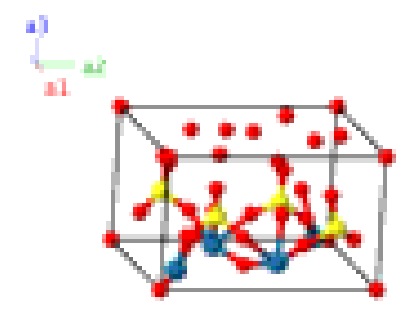

Basis vectors:

Lattice Coordinates

$\begin{array}{lcccc}\mathbf{B}_{1}= & z_{1} \mathbf{a}_{3} & & z_{1} c \hat{\mathbf{z}} \\ \mathbf{B}_{2}= & \frac{1}{2} \mathbf{a}_{1}+\frac{1}{2} \mathbf{a}_{2}+z_{1} \mathbf{a}_{3} & = & \frac{1}{2} a \hat{\mathbf{x}}+\frac{1}{2} b \hat{\mathbf{y}}+z_{1} c \hat{\mathbf{z}} \\ \mathbf{B}_{3}= & x_{2} \mathbf{a}_{1}+y_{2} \mathbf{a}_{2}+z_{2} \mathbf{a}_{3} & = & x_{2} a \hat{\mathbf{x}}+y_{2} b \hat{\mathbf{y}}+z_{2} c \hat{\mathbf{z}} \\ \mathbf{B}_{4}= & -x_{2} \mathbf{a}_{1}-y_{2} \mathbf{a}_{2}+z_{2} \mathbf{a}_{3} & = & -x_{2} a \hat{\mathbf{x}}-y_{2} b \hat{\mathbf{y}}+z_{2} c \hat{\mathbf{z}}\end{array}$

Wyckoff Position Atom Type

(2a)

O I

(2a)

O I

(4c)

O II

$(4 c)$ 


\begin{tabular}{|c|c|c|c|c|c|c|}
\hline $\mathbf{B}_{5}$ & $=$ & $\left(\frac{1}{2}+x_{2}\right) \mathbf{a}_{1}+\left(\frac{1}{2}-y_{2}\right) \mathbf{a}_{2}+z_{2} \mathbf{a}_{3}$ & $=$ & $\left(\frac{1}{2}+x_{2}\right) a \hat{\mathbf{x}}+\left(\frac{1}{2}-y_{2}\right) b \hat{\mathbf{y}}+z_{2} c \hat{\mathbf{z}}$ & $(4 c)$ & O II \\
\hline $\mathbf{B}_{6}$ & $=$ & $\left(\frac{1}{2}-x_{2}\right) \mathbf{a}_{1}+\left(\frac{1}{2}+y_{2}\right) \mathbf{a}_{2}+z_{2} \mathbf{a}_{3}$ & $=$ & $\left(\frac{1}{2}-x_{2}\right) a \hat{\mathbf{x}}+\left(\frac{1}{2}+y_{2}\right) b \hat{\mathbf{y}}+z_{2} c \hat{\mathbf{z}}$ & $(4 c)$ & O II \\
\hline $\mathbf{B}_{7}$ & $=$ & $x_{3} \mathbf{a}_{1}+y_{3} \mathbf{a}_{2}+z_{3} \mathbf{a}_{3}$ & $=$ & $x_{3} a \hat{\mathbf{x}}+y_{3} b \hat{\mathbf{y}}+z_{3} c \hat{\mathbf{z}}$ & $(4 c)$ & O III \\
\hline $\mathbf{B}_{8}$ & $=$ & $-x_{3} \mathbf{a}_{1}-y_{3} \mathbf{a}_{2}+z_{3} \mathbf{a}_{3}$ & $=$ & $-x_{3} a \hat{\mathbf{x}}-y_{3} b \hat{\mathbf{y}}+z_{3} c \hat{\mathbf{z}}$ & $(4 c)$ & O III \\
\hline $\mathbf{B}_{9}$ & $=$ & $\left(\frac{1}{2}+x_{3}\right) \mathbf{a}_{1}+\left(\frac{1}{2}-y_{3}\right) \mathbf{a}_{2}+z_{3} \mathbf{a}_{3}$ & $=$ & $\left(\frac{1}{2}+x_{3}\right) a \hat{\mathbf{x}}+\left(\frac{1}{2}-y_{3}\right) b \hat{\mathbf{y}}+z_{3} c \hat{\mathbf{z}}$ & $(4 c)$ & O III \\
\hline $\mathbf{B}_{10}$ & $=$ & $\left(\frac{1}{2}-x_{3}\right) \mathbf{a}_{1}+\left(\frac{1}{2}+y_{3}\right) \mathbf{a}_{2}+z_{3} \mathbf{a}_{3}$ & $=$ & $\left(\frac{1}{2}-x_{3}\right) a \hat{\mathbf{x}}+\left(\frac{1}{2}+y_{3}\right) b \hat{\mathbf{y}}+z_{3} c \hat{\mathbf{z}}$ & $(4 c)$ & O III \\
\hline $\mathbf{B}_{11}$ & $=$ & $x_{4} \mathbf{a}_{1}+y_{4} \mathbf{a}_{2}+z_{4} \mathbf{a}_{3}$ & $=$ & $x_{4} a \hat{\mathbf{x}}+y_{4} b \hat{\mathbf{y}}+z_{4} c \hat{\mathbf{z}}$ & $(4 c)$ & O IV \\
\hline $\mathbf{B}_{12}$ & $=$ & $-x_{4} \mathbf{a}_{1}-y_{4} \mathbf{a}_{2}+z_{4} \mathbf{a}_{3}$ & $=$ & $-x_{4} a \hat{\mathbf{x}}-y_{4} b \hat{\mathbf{y}}+z_{4} c \hat{\mathbf{z}}$ & $(4 c)$ & O IV \\
\hline $\mathbf{B}_{13}$ & $=$ & $\left(\frac{1}{2}+x_{4}\right) \mathbf{a}_{1}+\left(\frac{1}{2}-y_{4}\right) \mathbf{a}_{2}+z_{4} \mathbf{a}_{3}$ & $=$ & $\left(\frac{1}{2}+x_{4}\right) a \hat{\mathbf{x}}+\left(\frac{1}{2}-y_{4}\right) b \hat{\mathbf{y}}+z_{4} c \hat{\mathbf{z}}$ & $(4 c)$ & O IV \\
\hline $\mathbf{B}_{14}$ & $=$ & $\left(\frac{1}{2}-x_{4}\right) \mathbf{a}_{1}+\left(\frac{1}{2}+y_{4}\right) \mathbf{a}_{2}+z_{4} \mathbf{a}_{3}$ & $=$ & $\left(\frac{1}{2}-x_{4}\right) a \hat{\mathbf{x}}+\left(\frac{1}{2}+y_{4}\right) b \hat{\mathbf{y}}+z_{4} c \hat{\mathbf{z}}$ & $(4 c)$ & O IV \\
\hline $\mathbf{B}_{15}$ & $=$ & $x_{5} \mathbf{a}_{1}+y_{5} \mathbf{a}_{2}+z_{5} \mathbf{a}_{3}$ & $=$ & $x_{5} a \hat{\mathbf{x}}+y_{5} b \hat{\mathbf{y}}+z_{5} c \hat{\mathbf{z}}$ & $(4 c)$ & $\mathrm{OV}$ \\
\hline $\mathbf{B}_{16}$ & $=$ & $-x_{5} \mathbf{a}_{1}-y_{5} \mathbf{a}_{2}+z_{5} \mathbf{a}_{3}$ & $=$ & $-x_{5} a \hat{\mathbf{x}}-y_{5} b \hat{\mathbf{y}}+z_{5} c \hat{\mathbf{z}}$ & $(4 c)$ & $\mathrm{OV}$ \\
\hline $\mathbf{B}_{17}$ & $=$ & $\left(\frac{1}{2}+x_{5}\right) \mathbf{a}_{1}+\left(\frac{1}{2}-y_{5}\right) \mathbf{a}_{2}+z_{5} \mathbf{a}_{3}$ & $=$ & $\left(\frac{1}{2}+x_{5}\right) a \hat{\mathbf{x}}+\left(\frac{1}{2}-y_{5}\right) b \hat{\mathbf{y}}+z_{5} c \hat{\mathbf{z}}$ & $(4 c)$ & $\mathrm{OV}$ \\
\hline $\mathbf{B}_{18}$ & $=$ & $\left(\frac{1}{2}-x_{5}\right) \mathbf{a}_{1}+\left(\frac{1}{2}+y_{5}\right) \mathbf{a}_{2}+z_{5} \mathbf{a}_{3}$ & $=$ & $\left(\frac{1}{2}-x_{5}\right) a \hat{\mathbf{x}}+\left(\frac{1}{2}+y_{5}\right) b \hat{\mathbf{y}}+z_{5} c \hat{\mathbf{z}}$ & $(4 c)$ & $\mathrm{OV}$ \\
\hline $\mathbf{B}_{19}$ & $=$ & $x_{6} \mathbf{a}_{1}+y_{6} \mathbf{a}_{2}+z_{6} \mathbf{a}_{3}$ & $=$ & $x_{6} a \hat{\mathbf{x}}+y_{6} b \hat{\mathbf{y}}+z_{6} c \hat{\mathbf{z}}$ & $(4 c)$ & O VI \\
\hline $\mathbf{B}_{20}$ & $=$ & $-x_{6} \mathbf{a}_{1}-y_{6} \mathbf{a}_{2}+z_{6} \mathbf{a}_{3}$ & $=$ & $-x_{6} a \hat{\mathbf{x}}-y_{6} b \hat{\mathbf{y}}+z_{6} c \hat{\mathbf{z}}$ & $(4 c)$ & O VI \\
\hline $\mathbf{B}_{21}$ & $=$ & $\left(\frac{1}{2}+x_{6}\right) \mathbf{a}_{1}+\left(\frac{1}{2}-y_{6}\right) \mathbf{a}_{2}+z_{6} \mathbf{a}_{3}$ & $=$ & $\left(\frac{1}{2}+x_{6}\right) a \hat{\mathbf{x}}+\left(\frac{1}{2}-y_{6}\right) b \hat{\mathbf{y}}+z_{6} c \hat{\mathbf{z}}$ & $(4 c)$ & $\mathrm{O} \mathrm{VI}$ \\
\hline $\mathbf{B}_{22}$ & $=$ & $\left(\frac{1}{2}-x_{6}\right) \mathbf{a}_{1}+\left(\frac{1}{2}+y_{6}\right) \mathbf{a}_{2}+z_{6} \mathbf{a}_{3}$ & $=$ & $\left(\frac{1}{2}-x_{6}\right) a \hat{\mathbf{x}}+\left(\frac{1}{2}+y_{6}\right) b \hat{\mathbf{y}}+z_{6} c \hat{\mathbf{z}}$ & $(4 c)$ & O VI \\
\hline $\mathbf{B}_{23}$ & $=$ & $x_{7} \mathbf{a}_{1}+y_{7} \mathbf{a}_{2}+z_{7} \mathbf{a}_{3}$ & $=$ & $x_{7} a \hat{\mathbf{x}}+y_{7} b \hat{\mathbf{y}}+z_{7} c \hat{\mathbf{z}}$ & $(4 c)$ & O VII \\
\hline $\mathbf{B}_{24}$ & $=$ & $-x_{7} \mathbf{a}_{1}-y_{7} \mathbf{a}_{2}+z_{7} \mathbf{a}_{3}$ & $=$ & $-x_{7} a \hat{\mathbf{x}}-y_{7} b \hat{\mathbf{y}}+z_{7} c \hat{\mathbf{z}}$ & $(4 c)$ & O VII \\
\hline $\mathbf{B}_{25}$ & $=$ & $\left(\frac{1}{2}+x_{7}\right) \mathbf{a}_{1}+\left(\frac{1}{2}-y_{7}\right) \mathbf{a}_{2}+z_{7} \mathbf{a}_{3}$ & $=$ & $\left(\frac{1}{2}+x_{7}\right) a \hat{\mathbf{x}}+\left(\frac{1}{2}-y_{7}\right) b \hat{\mathbf{y}}+z_{7} c \hat{\mathbf{z}}$ & $(4 c)$ & O VII \\
\hline $\mathbf{B}_{26}$ & $=$ & $\left(\frac{1}{2}-x_{7}\right) \mathbf{a}_{1}+\left(\frac{1}{2}+y_{7}\right) \mathbf{a}_{2}+z_{7} \mathbf{a}_{3}$ & $=$ & $\left(\frac{1}{2}-x_{7}\right) a \hat{\mathbf{x}}+\left(\frac{1}{2}+y_{7}\right) b \hat{\mathbf{y}}+z_{7} c \hat{\mathbf{z}}$ & $(4 c)$ & O VII \\
\hline $\mathbf{B}_{27}$ & $=$ & $x_{8} \mathbf{a}_{1}+y_{8} \mathbf{a}_{2}+z_{8} \mathbf{a}_{3}$ & $=$ & $x_{8} a \hat{\mathbf{x}}+y_{8} b \hat{\mathbf{y}}+z_{8} c \hat{\mathbf{z}}$ & $(4 c)$ & $\operatorname{Re}$ \\
\hline $\mathbf{B}_{28}$ & $=$ & $-x_{8} \mathbf{a}_{1}-y_{8} \mathbf{a}_{2}+z_{8} \mathbf{a}_{3}$ & $=$ & $-x_{8} a \hat{\mathbf{x}}-y_{8} b \hat{\mathbf{y}}+z_{8} c \hat{\mathbf{z}}$ & $(4 c)$ & $\operatorname{Re}$ \\
\hline $\mathbf{B}_{29}$ & $=$ & $\left(\frac{1}{2}+x_{8}\right) \mathbf{a}_{1}+\left(\frac{1}{2}-y_{8}\right) \mathbf{a}_{2}+z_{8} \mathbf{a}_{3}$ & $=$ & $\left(\frac{1}{2}+x_{8}\right) a \hat{\mathbf{x}}+\left(\frac{1}{2}-y_{8}\right) b \hat{\mathbf{y}}+z_{8} c \hat{\mathbf{z}}$ & $(4 c)$ & $\operatorname{Re}$ \\
\hline $\mathbf{B}_{30}$ & $=$ & $\left(\frac{1}{2}-x_{8}\right) \mathbf{a}_{1}+\left(\frac{1}{2}+y_{8}\right) \mathbf{a}_{2}+z_{8} \mathbf{a}_{3}$ & $=$ & $\left(\frac{1}{2}-x_{8}\right) a \hat{\mathbf{x}}+\left(\frac{1}{2}+y_{8}\right) b \hat{\mathbf{y}}+z_{8} c \hat{\mathbf{z}}$ & $(4 c)$ & $\operatorname{Re}$ \\
\hline $\mathbf{B}_{31}$ & $=$ & $x_{9} \mathbf{a}_{1}+y_{9} \mathbf{a}_{2}+z_{9} \mathbf{a}_{3}$ & $=$ & $x_{9} a \hat{\mathbf{x}}+y_{9} b \hat{\mathbf{y}}+z_{9} c \hat{\mathbf{z}}$ & $(4 c)$ & $S$ \\
\hline $\mathbf{B}_{32}$ & $=$ & $-x_{9} \mathbf{a}_{1}-y_{9} \mathbf{a}_{2}+z 9 \mathbf{a}_{3}$ & $=$ & $-x_{9} a \hat{\mathbf{x}}-y_{9} b \hat{\mathbf{y}}+z_{9} c \hat{\mathbf{z}}$ & $(4 c)$ & $\mathrm{S}$ \\
\hline $\mathbf{B}_{33}$ & $=$ & $\left(\frac{1}{2}+x_{9}\right) \mathbf{a}_{1}+\left(\frac{1}{2}-y_{9}\right) \mathbf{a}_{2}+z_{9} \mathbf{a}_{3}$ & $=$ & $\left(\frac{1}{2}+x_{9}\right) a \hat{\mathbf{x}}+\left(\frac{1}{2}-y_{9}\right) b \hat{\mathbf{y}}+z_{9} c \hat{\mathbf{z}}$ & $(4 c)$ & $\mathrm{S}$ \\
\hline $\mathbf{B}_{34}$ & $=$ & $\left(\frac{1}{2}-x_{9}\right) \mathbf{a}_{1}+\left(\frac{1}{2}+y_{9}\right) \mathbf{a}_{2}+z_{9} \mathbf{a}_{3}$ & $=$ & $\left(\frac{1}{2}-x_{9}\right) a \hat{\mathbf{x}}+\left(\frac{1}{2}+y_{9}\right) b \hat{\mathbf{y}}+z_{9} c \hat{\mathbf{z}}$ & $(4 c)$ & S \\
\hline
\end{tabular}

\section{References:}

- U. Betke and M. S. Wickleder, Sulfates of the Refractory Metals: Crystal Structure and Thermal Behavior of $\mathrm{Nb}_{2} \mathrm{O}_{2}\left(\mathrm{SO}_{4}\right)_{3}, \mathrm{MoO}_{2}\left(\mathrm{SO}_{4}\right), \mathrm{WO}\left(\mathrm{SO}_{4}\right)_{2}$, and Two Modifications of $\mathrm{Re}_{2} \mathrm{O}_{5}\left(\mathrm{SO}_{4}\right)_{2}$, Inorg. Chem. 50, 858-872 (2011), doi:10.1021/ic101455z.

\section{Found in:}

- P. Villars and K. Cenzual, Pearson's Crystal Data - Crystal Structure Database for Inorganic Compounds, ASM International (2013). 
Geometry files:

- CIF: pp. 823

- POSCAR: pp. 823 


\section{$\kappa$-alumina $\left(\mathrm{Al}_{2} \mathrm{O}_{3}\right)$ Structure: A2B3_oP40_33_4a_6a}
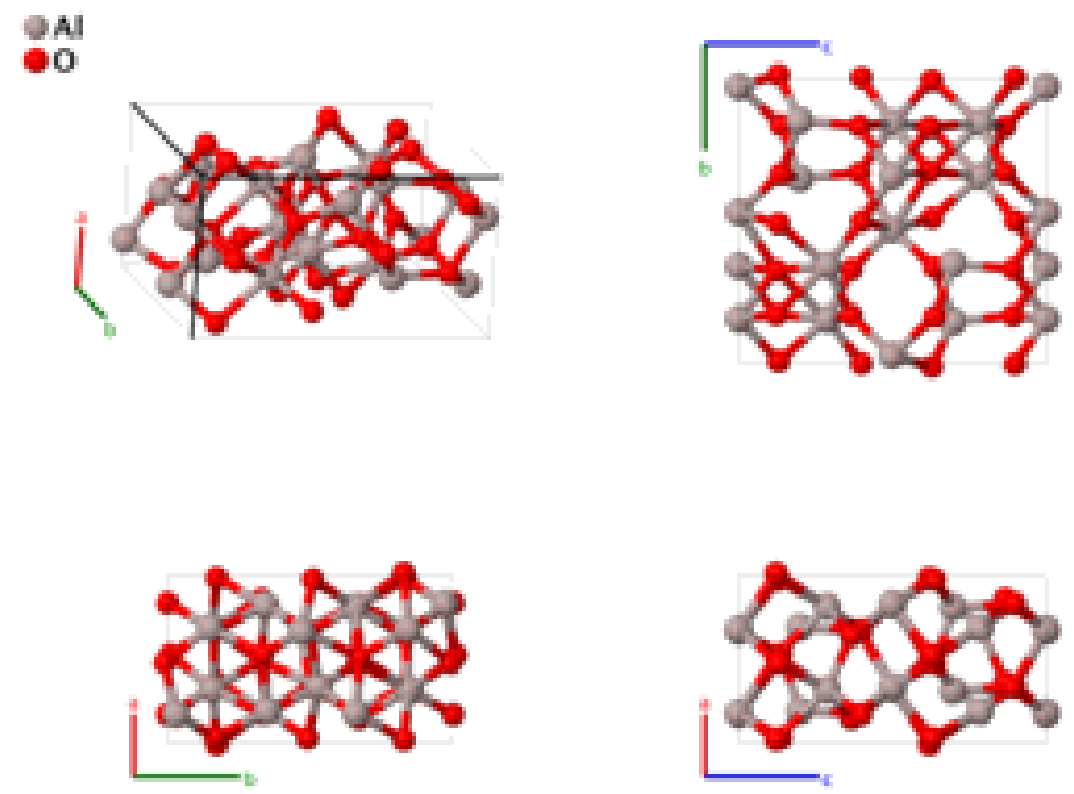

\section{Prototype}

AFLOW prototype label

$: \quad \kappa-\mathrm{Al}_{2} \mathrm{O}_{3}$

Strukturbericht designation

: A2B3_oP40_33_4a_6a

Pearson symbol

: None

Space group number

: $\quad$ oP40

Space group symbol

: $\quad 33$

AFLOW prototype command

: $\quad P_{n a 2}$

aflow --proto $=$ A2B3_oP40_33_4a_6a

- -params $=a, b / a, c / a, x_{1}, y_{1}, z_{1}, x_{2}, y_{2}, z_{2}, x_{3}, y_{3}, z_{3}, x_{4}, y_{4}, z_{4}, x_{5}, y_{5}, z_{5}, x_{6}, y_{6}$,

$z_{6}, x_{7}, y_{7}, z_{7}, x_{8}, y_{8}, z_{8}, x_{9}, y_{9}, z_{9}, x_{10}, y_{10}, z_{10}$

\section{Other compounds with this structure:}

- $(1-x) \mathrm{Fe}_{2} \mathrm{O}_{3} \cdot x \mathrm{Al}_{2} \mathrm{O}_{3},(1-x) \mathrm{Fe}_{2} \mathrm{O}_{3} \cdot x \mathrm{Ga}_{2} \mathrm{O}_{3}$. An approximation to the true crystal structure of $\epsilon-\mathrm{Ga}_{2} \mathrm{O}_{3}$ (Yoshioka, 2007).

\section{Simple Orthorhombic primitive vectors:}

$$
\begin{aligned}
& \mathbf{a}_{1}=a \hat{\mathbf{x}} \\
& \mathbf{a}_{2}=b \hat{\mathbf{y}} \\
& \mathbf{a}_{3}=c \hat{\mathbf{z}}
\end{aligned}
$$

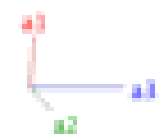

Basis vectors: 


\begin{tabular}{|c|c|c|c|c|c|c|}
\hline $\mathbf{B}_{1}$ & $=$ & $x_{1} \mathbf{a}_{1}+y_{1} \mathbf{a}_{2}+z_{1} \mathbf{a}_{3}$ & $=$ & $x_{1} a \hat{\mathbf{x}}+y_{1} b \hat{\mathbf{y}}+z_{1} c \hat{\mathbf{z}}$ & $(4 a)$ & $\mathrm{Al} \mathrm{I}$ \\
\hline $\mathbf{B}_{2}$ & $=$ & $-x_{1} \mathbf{a}_{1}-y_{1} \mathbf{a}_{2}+\left(\frac{1}{2}+z_{1}\right) \mathbf{a}_{3}$ & $=$ & $-x_{1} a \hat{\mathbf{x}}-y_{1} b \hat{\mathbf{y}}+\left(\frac{1}{2}+z_{1}\right) c \hat{\mathbf{z}}$ & $(4 a)$ & $\mathrm{Al} \mathrm{I}$ \\
\hline $\mathbf{B}_{3}$ & $=$ & $\left(\frac{1}{2}+x_{1}\right) \mathbf{a}_{1}+\left(\frac{1}{2}-y_{1}\right) \mathbf{a}_{2}+z_{1} \mathbf{a}_{3}$ & $=$ & $\left(\frac{1}{2}+x_{1}\right) a \hat{\mathbf{x}}+\left(\frac{1}{2}-y_{1}\right) b \hat{\mathbf{y}}+z_{1} c \hat{\mathbf{z}}$ & $(4 a)$ & $\mathrm{Al} \mathrm{I}$ \\
\hline $\mathbf{B}_{4}$ & $=$ & $\begin{array}{c}\left(\frac{1}{2}-x_{1}\right) \mathbf{a}_{1}+\left(\frac{1}{2}+y_{1}\right) \mathbf{a}_{2}+ \\
\left(\frac{1}{2}+z_{1}\right) \mathbf{a}_{3}\end{array}$ & $=$ & $\begin{array}{c}\left(\frac{1}{2}-x_{1}\right) a \hat{\mathbf{x}}+\left(\frac{1}{2}+y_{1}\right) b \hat{\mathbf{y}}+ \\
\left(\frac{1}{2}+z_{1}\right) c \hat{\mathbf{z}}\end{array}$ & $(4 a)$ & $\mathrm{Al} \mathrm{I}$ \\
\hline $\mathbf{B}_{5}$ & $=$ & $x_{2} \mathbf{a}_{1}+y_{2} \mathbf{a}_{2}+z_{2} \mathbf{a}_{3}$ & $=$ & $x_{2} a \hat{\mathbf{x}}+y_{2} b \hat{\mathbf{y}}+z_{2} c \hat{\mathbf{z}}$ & $(4 a)$ & $\mathrm{Al}$ II \\
\hline $\mathbf{B}_{6}$ & $=$ & $-x_{2} \mathbf{a}_{1}-y_{2} \mathbf{a}_{2}+\left(\frac{1}{2}+z_{2}\right) \mathbf{a}_{3}$ & $=$ & $-x_{2} a \hat{\mathbf{x}}-y_{2} b \hat{\mathbf{y}}+\left(\frac{1}{2}+z_{2}\right) c \hat{\mathbf{z}}$ & $(4 a)$ & Al II \\
\hline $\mathbf{B}_{7}$ & $=$ & $\left(\frac{1}{2}+x_{2}\right) \mathbf{a}_{1}+\left(\frac{1}{2}-y_{2}\right) \mathbf{a}_{2}+z_{2} \mathbf{a}_{3}$ & $=$ & $\left(\frac{1}{2}+x_{2}\right) a \hat{\mathbf{x}}+\left(\frac{1}{2}-y_{2}\right) b \hat{\mathbf{y}}+z_{2} c \hat{\mathbf{z}}$ & $(4 a)$ & Al II \\
\hline $\mathbf{B}_{8}$ & $=$ & $\begin{array}{c}\left(\frac{1}{2}-x_{2}\right) \mathbf{a}_{1}+\left(\frac{1}{2}+y_{2}\right) \mathbf{a}_{2}+ \\
\left(\frac{1}{2}+z_{2}\right) \mathbf{a}_{3}\end{array}$ & $=$ & $\begin{array}{c}\left(\frac{1}{2}-x_{2}\right) a \hat{\mathbf{x}}+\left(\frac{1}{2}+y_{2}\right) b \hat{\mathbf{y}}+ \\
\left(\frac{1}{2}+z_{2}\right) c \hat{\mathbf{z}}\end{array}$ & $(4 a)$ & $\mathrm{Al}$ II \\
\hline $\mathbf{B}_{9}$ & $=$ & $x_{3} \mathbf{a}_{1}+y_{3} \mathbf{a}_{2}+z_{3} \mathbf{a}_{3}$ & $=$ & $x_{3} a \hat{\mathbf{x}}+y_{3} b \hat{\mathbf{y}}+z_{3} c \hat{\mathbf{z}}$ & $(4 a)$ & Al III \\
\hline $\mathbf{B}_{10}$ & $=$ & $-x_{3} \mathbf{a}_{1}-y_{3} \mathbf{a}_{2}+\left(\frac{1}{2}+z_{3}\right) \mathbf{a}_{3}$ & $=$ & $-x_{3} a \hat{\mathbf{x}}-y_{3} b \hat{\mathbf{y}}+\left(\frac{1}{2}+z_{3}\right) c \hat{\mathbf{z}}$ & $(4 a)$ & $\mathrm{Al}$ III \\
\hline $\mathbf{B}_{11}$ & $=$ & $\left(\frac{1}{2}+x_{3}\right) \mathbf{a}_{1}+\left(\frac{1}{2}-y_{3}\right) \mathbf{a}_{2}+z_{3} \mathbf{a}_{3}$ & $=$ & $\left(\frac{1}{2}+x_{3}\right) a \hat{\mathbf{x}}+\left(\frac{1}{2}-y_{3}\right) b \hat{\mathbf{y}}+z_{3} c \hat{\mathbf{z}}$ & $(4 a)$ & $\mathrm{Al} \mathrm{III}$ \\
\hline $\mathbf{B}_{12}$ & $=$ & $\begin{array}{c}\left(\frac{1}{2}-x_{3}\right) \mathbf{a}_{1}+\left(\frac{1}{2}+y_{3}\right) \mathbf{a}_{2}+ \\
\left(\frac{1}{2}+z_{3}\right) \mathbf{a}_{3}\end{array}$ & $=$ & $\begin{array}{c}\left(\frac{1}{2}-x_{3}\right) a \hat{\mathbf{x}}+\left(\frac{1}{2}+y_{3}\right) b \hat{\mathbf{y}}+ \\
\left(\frac{1}{2}+z_{3}\right) c \hat{\mathbf{z}}\end{array}$ & $(4 a)$ & $\mathrm{Al}$ III \\
\hline $\mathbf{B}_{13}$ & $=$ & $x_{4} \mathbf{a}_{1}+y_{4} \mathbf{a}_{2}+z_{4} \mathbf{a}_{3}$ & $=$ & $x_{4} a \hat{\mathbf{x}}+y_{4} b \hat{\mathbf{y}}+z_{4} c \hat{\mathbf{z}}$ & $(4 a)$ & $\mathrm{Al} \mathrm{IV}$ \\
\hline $\mathbf{B}_{14}$ & $=$ & $-x_{4} \mathbf{a}_{1}-y_{4} \mathbf{a}_{2}+\left(\frac{1}{2}+z_{4}\right) \mathbf{a}_{3}$ & $=$ & $-x_{4} a \hat{\mathbf{x}}-y_{4} b \hat{\mathbf{y}}+\left(\frac{1}{2}+z_{4}\right) c \hat{\mathbf{z}}$ & $(4 a)$ & $\mathrm{Al} \mathrm{IV}$ \\
\hline $\mathbf{B}_{15}$ & $=$ & $\left(\frac{1}{2}+x_{4}\right) \mathbf{a}_{1}+\left(\frac{1}{2}-y_{4}\right) \mathbf{a}_{2}+z_{4} \mathbf{a}_{3}$ & $=$ & $\left(\frac{1}{2}+x_{4}\right) a \hat{\mathbf{x}}+\left(\frac{1}{2}-y_{4}\right) b \hat{\mathbf{y}}+z_{4} c \hat{\mathbf{z}}$ & $(4 a)$ & $\mathrm{Al} \mathrm{IV}$ \\
\hline $\mathbf{B}_{16}$ & $=$ & $\begin{array}{c}\left(\frac{1}{2}-x_{4}\right) \mathbf{a}_{1}+\left(\frac{1}{2}+y_{4}\right) \mathbf{a}_{2}+ \\
\left(\frac{1}{2}+z_{4}\right) \mathbf{a}_{3}\end{array}$ & $=$ & $\begin{array}{c}\left(\frac{1}{2}-x_{4}\right) a \hat{\mathbf{x}}+\left(\frac{1}{2}+y_{4}\right) b \hat{\mathbf{y}}+ \\
\left(\frac{1}{2}+z_{4}\right) c \hat{\mathbf{z}}\end{array}$ & $(4 a)$ & $\mathrm{Al} \mathrm{IV}$ \\
\hline $\mathbf{B}_{17}$ & $=$ & $x_{5} \mathbf{a}_{1}+y_{5} \mathbf{a}_{2}+z_{5} \mathbf{a}_{3}$ & $=$ & $x_{5} a \hat{\mathbf{x}}+y_{5} b \hat{\mathbf{y}}+z_{5} c \hat{\mathbf{z}}$ & $(4 a)$ & O I \\
\hline $\mathbf{B}_{18}$ & $=$ & $-x_{5} \mathbf{a}_{1}-y_{5} \mathbf{a}_{2}+\left(\frac{1}{2}+z_{5}\right) \mathbf{a}_{3}$ & $=$ & $-x_{5} a \hat{\mathbf{x}}-y_{5} b \hat{\mathbf{y}}+\left(\frac{1}{2}+z_{5}\right) c \hat{\mathbf{z}}$ & $(4 a)$ & O I \\
\hline $\mathbf{B}_{19}$ & $=$ & $\left(\frac{1}{2}+x_{5}\right) \mathbf{a}_{1}+\left(\frac{1}{2}-y_{5}\right) \mathbf{a}_{2}+z_{5} \mathbf{a}_{3}$ & $=$ & $\left(\frac{1}{2}+x_{5}\right) a \hat{\mathbf{x}}+\left(\frac{1}{2}-y_{5}\right) b \hat{\mathbf{y}}+z_{5} c \hat{\mathbf{z}}$ & $(4 a)$ & $\mathrm{O} \mathrm{I}$ \\
\hline $\mathbf{B}_{20}$ & $=$ & $\begin{array}{c}\left(\frac{1}{2}-x_{5}\right) \mathbf{a}_{1}+\left(\frac{1}{2}+y_{5}\right) \mathbf{a}_{2}+ \\
\left(\frac{1}{2}+z_{5}\right) \mathbf{a}_{3}\end{array}$ & $=$ & $\begin{array}{c}\left(\frac{1}{2}-x_{5}\right) a \hat{\mathbf{x}}+\left(\frac{1}{2}+y_{5}\right) b \hat{\mathbf{y}}+ \\
\left(\frac{1}{2}+z_{5}\right) c \hat{\mathbf{z}}\end{array}$ & $(4 a)$ & O I \\
\hline $\mathbf{B}_{21}$ & $=$ & $x_{6} \mathbf{a}_{1}+y_{6} \mathbf{a}_{2}+z_{6} \mathbf{a}_{3}$ & $=$ & $x_{6} a \hat{\mathbf{x}}+y_{6} b \hat{\mathbf{y}}+z_{6} c \hat{\mathbf{z}}$ & $(4 a)$ & $\mathrm{O}$ II \\
\hline $\mathbf{B}_{22}$ & $=$ & $-x_{6} \mathbf{a}_{1}-y_{6} \mathbf{a}_{2}+\left(\frac{1}{2}+z_{6}\right) \mathbf{a}_{3}$ & $=$ & $-x_{6} a \hat{\mathbf{x}}-y_{6} b \hat{\mathbf{y}}+\left(\frac{1}{2}+z_{6}\right) c \hat{\mathbf{z}}$ & $(4 a)$ & O II \\
\hline $\mathbf{B}_{23}$ & $=$ & $\left(\frac{1}{2}+x_{6}\right) \mathbf{a}_{1}+\left(\frac{1}{2}-y_{6}\right) \mathbf{a}_{2}+z_{6} \mathbf{a}_{3}$ & $=$ & $\left(\frac{1}{2}+x_{6}\right) a \hat{\mathbf{x}}+\left(\frac{1}{2}-y_{6}\right) b \hat{\mathbf{y}}+z_{6} c \hat{\mathbf{z}}$ & $(4 a)$ & O II \\
\hline $\mathbf{B}_{24}$ & $=$ & $\begin{array}{c}\left(\frac{1}{2}-x_{6}\right) \mathbf{a}_{1}+\left(\frac{1}{2}+y_{6}\right) \mathbf{a}_{2}+ \\
\left(\frac{1}{2}+z_{6}\right) \mathbf{a}_{3}\end{array}$ & $=$ & $\begin{array}{c}\left(\frac{1}{2}-x_{6}\right) a \hat{\mathbf{x}}+\left(\frac{1}{2}+y_{6}\right) b \hat{\mathbf{y}}+ \\
\left(\frac{1}{2}+z_{6}\right) c \hat{\mathbf{z}}\end{array}$ & $(4 a)$ & O II \\
\hline $\mathbf{B}_{25}$ & $=$ & $x_{7} \mathbf{a}_{1}+y_{7} \mathbf{a}_{2}+z_{7} \mathbf{a}_{3}$ & $=$ & $x_{7} a \hat{\mathbf{x}}+y_{7} b \hat{\mathbf{y}}+z_{7} c \hat{\mathbf{z}}$ & $(4 a)$ & O III \\
\hline $\mathbf{B}_{26}$ & $=$ & $-x_{7} \mathbf{a}_{1}-y_{7} \mathbf{a}_{2}+\left(\frac{1}{2}+z_{7}\right) \mathbf{a}_{3}$ & $=$ & $-x_{7} a \hat{\mathbf{x}}-y_{7} b \hat{\mathbf{y}}+\left(\frac{1}{2}+z_{7}\right) c \hat{\mathbf{z}}$ & $(4 a)$ & O III \\
\hline $\mathbf{B}_{27}$ & $=$ & $\left(\frac{1}{2}+x_{7}\right) \mathbf{a}_{1}+\left(\frac{1}{2}-y_{7}\right) \mathbf{a}_{2}+z_{7} \mathbf{a}_{3}$ & $=$ & $\left(\frac{1}{2}+x_{7}\right) a \hat{\mathbf{x}}+\left(\frac{1}{2}-y_{7}\right) b \hat{\mathbf{y}}+z_{7} c \hat{\mathbf{z}}$ & $(4 a)$ & O III \\
\hline $\mathbf{B}_{28}$ & $=$ & $\begin{array}{c}\left(\frac{1}{2}-x_{7}\right) \mathbf{a}_{1}+\left(\frac{1}{2}+y_{7}\right) \mathbf{a}_{2}+ \\
\left(\frac{1}{2}+z_{7}\right) \mathbf{a}_{3}\end{array}$ & $=$ & $\begin{array}{c}\left(\frac{1}{2}-x_{7}\right) a \hat{\mathbf{x}}+\left(\frac{1}{2}+y_{7}\right) b \hat{\mathbf{y}}+ \\
\left(\frac{1}{2}+z_{7}\right) c \hat{\mathbf{z}}\end{array}$ & $(4 a)$ & O III \\
\hline $\mathbf{B}_{29}$ & $=$ & $x_{8} \mathbf{a}_{1}+y_{8} \mathbf{a}_{2}+z_{8} \mathbf{a}_{3}$ & $=$ & $x_{8} a \hat{\mathbf{x}}+y_{8} b \hat{\mathbf{y}}+z_{8} c \hat{\mathbf{z}}$ & $(4 a)$ & O IV \\
\hline $\mathbf{B}_{30}$ & $=$ & $-x_{8} \mathbf{a}_{1}-y_{8} \mathbf{a}_{2}+\left(\frac{1}{2}+z_{8}\right) \mathbf{a}_{3}$ & $=$ & $-x_{8} a \hat{\mathbf{x}}-y_{8} b \hat{\mathbf{y}}+\left(\frac{1}{2}+z_{8}\right) c \hat{\mathbf{z}}$ & $(4 a)$ & O IV \\
\hline $\mathbf{B}_{31}$ & $=$ & $\left(\frac{1}{2}+x_{8}\right) \mathbf{a}_{1}+\left(\frac{1}{2}-y_{8}\right) \mathbf{a}_{2}+z_{8} \mathbf{a}_{3}$ & $=$ & $\left(\frac{1}{2}+x_{8}\right) a \hat{\mathbf{x}}+\left(\frac{1}{2}-y_{8}\right) b \hat{\mathbf{y}}+z_{8} c \hat{\mathbf{z}}$ & $(4 a)$ & O IV \\
\hline
\end{tabular}




\begin{tabular}{|c|c|c|c|c|}
\hline $\mathbf{B}_{32}$ & $\begin{array}{c}\left(\frac{1}{2}-x_{8}\right) \mathbf{a}_{1}+\left(\frac{1}{2}+y_{8}\right) \mathbf{a}_{2}+ \\
\left(\frac{1}{2}+z_{8}\right) \mathbf{a}_{3}\end{array}$ & $\begin{array}{c}=\left(\frac{1}{2}-x_{8}\right) a \hat{\mathbf{x}}+\left(\frac{1}{2}+y_{8}\right) b \hat{\mathbf{y}}+ \\
\left(\frac{1}{2}+z_{8}\right) c \hat{\mathbf{z}}\end{array}$ & $(4 a)$ & O IV \\
\hline $\mathbf{B}_{33}$ & $x_{9} \mathbf{a}_{1}+y_{9} \mathbf{a}_{2}+z_{9} \mathbf{a}_{3}$ & $x_{9} a \hat{\mathbf{x}}+y_{9} b \hat{\mathbf{y}}+z_{9} c \hat{\mathbf{z}}$ & $(4 a)$ & $\mathrm{OV}$ \\
\hline $\mathbf{B}_{34}$ & $-x_{9} \mathbf{a}_{1}-y_{9} \mathbf{a}_{2}+\left(\frac{1}{2}+z_{9}\right) \mathbf{a}_{3}$ & $-x_{9} a \hat{\mathbf{x}}-y_{9} b \hat{\mathbf{y}}+\left(\frac{1}{2}+z_{9}\right) c \hat{\mathbf{z}}$ & $(4 a)$ & $\mathrm{OV}$ \\
\hline $\mathbf{B}_{35}$ & $=\left(\frac{1}{2}+x_{9}\right) \mathbf{a}_{1}+\left(\frac{1}{2}-y_{9}\right) \mathbf{a}_{2}+z_{9} \mathbf{a}_{3}$ & $=\left(\frac{1}{2}+x_{9}\right) a \hat{\mathbf{x}}+\left(\frac{1}{2}-y_{9}\right) b \hat{\mathbf{y}}+z_{9} c \hat{\mathbf{z}}$ & $(4 a)$ & $\mathrm{OV}$ \\
\hline $\mathbf{B}_{36}$ & $\begin{array}{c}\left(\frac{1}{2}-x_{9}\right) \mathbf{a}_{1}+\left(\frac{1}{2}+y_{9}\right) \mathbf{a}_{2}+ \\
\left(\frac{1}{2}+z_{9}\right) \mathbf{a}_{3}\end{array}$ & $\begin{array}{c}\left(\frac{1}{2}-x_{9}\right) a \hat{\mathbf{x}}+\left(\frac{1}{2}+y_{9}\right) b \hat{\mathbf{y}}+ \\
\left(\frac{1}{2}+z_{9}\right) c \hat{\mathbf{z}}\end{array}$ & $(4 a)$ & $\mathrm{OV}$ \\
\hline $\mathbf{B}_{37}$ & $x_{10} \mathbf{a}_{1}+y_{10} \mathbf{a}_{2}+z_{10} \mathbf{a}_{3}$ & $x_{10} a \hat{\mathbf{x}}+y_{10} b \hat{\mathbf{y}}+z_{10} c \hat{\mathbf{z}}$ & $(4 a)$ & $\mathrm{O}$ VI \\
\hline $\mathbf{B}_{38}$ & $-x_{10} \mathbf{a}_{1}-y_{10} \mathbf{a}_{2}+\left(\frac{1}{2}+z_{10}\right) \mathbf{a}_{3}$ & $-x_{10} a \hat{\mathbf{x}}-y_{10} b \hat{\mathbf{y}}+\left(\frac{1}{2}+z_{10}\right) c \hat{\mathbf{z}}$ & $(4 a)$ & $\mathrm{O} \mathrm{VI}$ \\
\hline $\mathbf{B}_{39}$ & $=\left(\frac{1}{2}+x_{10}\right) \mathbf{a}_{1}+\left(\frac{1}{2}-y_{10}\right) \mathbf{a}_{2}+z_{10} \mathbf{a}_{3}$ & $=\left(\frac{1}{2}+x_{10}\right) a \hat{\mathbf{x}}+\left(\frac{1}{2}-y_{10}\right) b \hat{\mathbf{y}}+z_{10} c \hat{\mathbf{z}}$ & $(4 a)$ & $\mathrm{O}$ VI \\
\hline $\mathbf{B}_{40}$ & $\begin{array}{c}\left(\frac{1}{2}-x_{10}\right) \mathbf{a}_{1}+\left(\frac{1}{2}+y_{10}\right) \mathbf{a}_{2}+ \\
\left(\frac{1}{2}+z_{10}\right) \mathbf{a}_{3}\end{array}$ & $\begin{array}{c}=\left(\frac{1}{2}-x_{10}\right) a \hat{\mathbf{x}}+\left(\frac{1}{2}+y_{10}\right) b \hat{\mathbf{y}}+ \\
\left(\frac{1}{2}+z_{10}\right) c \hat{\mathbf{z}}\end{array}$ & $(4 a)$ & O VI \\
\hline
\end{tabular}

\section{References:}

- B. Ollivier, R. Retoux, P. Lacorre, D. Massiot, and G. Férey, Crystal structure of $\kappa$-alumina: an X-ray powder diffraction, TEM and NMR study, J. Mater. Chem. 7, 1049-1056 (1997), doi:10.1039/A700054E.

\section{Found in:}

- K. Matsuzaki, H. Yanagi, T. Kamiya, H. Hiramatsu, K. Nomura, M. Hirano, and H. Hosono, Field-induced current modulation in epitaxial film of deep-ultraviolet transparent oxide semiconductor $\mathrm{Ga}_{2} \mathrm{O}_{3}$, Appl. Phys. Lett. 88, 092106 (2006), doi:10.1063/1.2179373.

- S. Yoshioka, H. Hayashi, A. Kuwabara, F. Oba, K. Matsunaga, and I. Tanaka, Structures and energetics of $\mathrm{Ga}_{2} \mathrm{O}_{3}$ polymorphs, J. Phys.: Condens. Matter 19, 346211 (2007), doi:10.1088/0953-8984/19/34/346211.

\section{Geometry files:}

- CIF: pp. 823

- POSCAR: pp. 824 


\section{$\mathrm{TiAl}_{2} \mathrm{Br}_{8}$ Structure: A2B8C_oP22_34_c_4c_a}
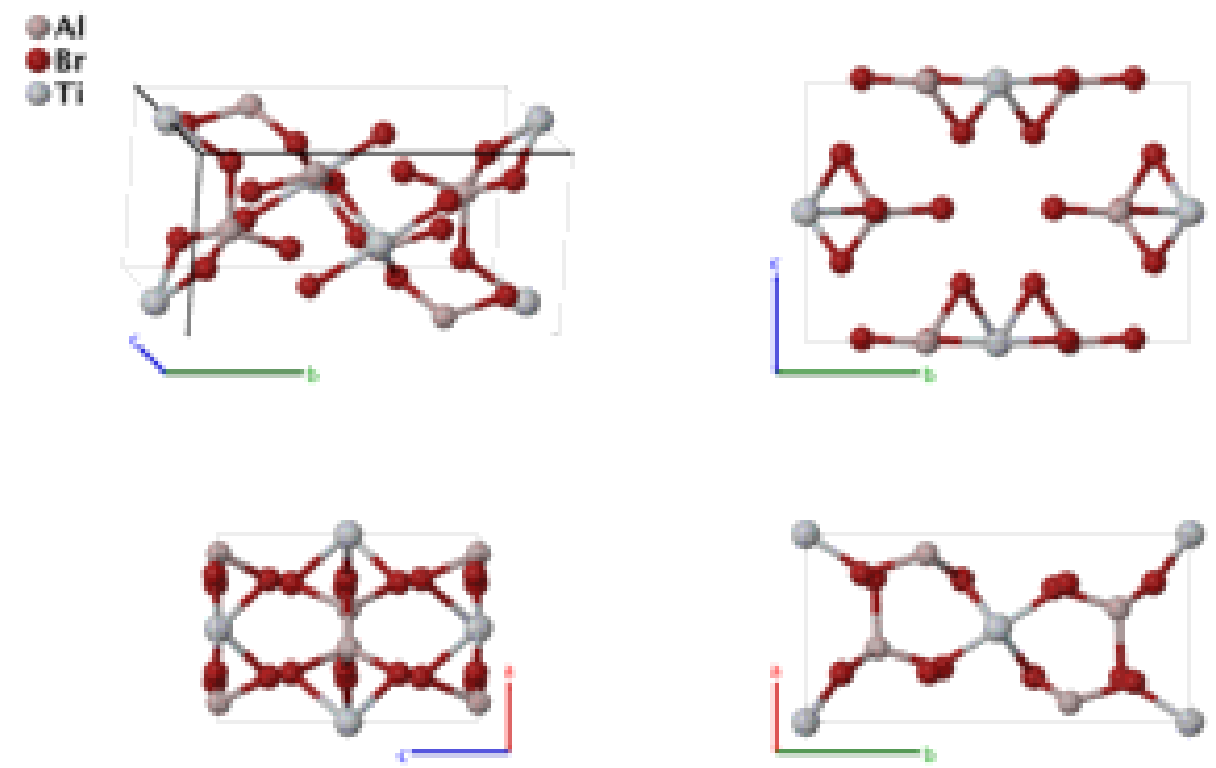

\section{Prototype}

AFLOW prototype label

Strukturbericht designation

Pearson symbol

Space group number

Space group symbol

AFLOW prototype command
: $\quad \mathrm{TiAl}_{2} \mathrm{Br}_{8}$

: A2B8C_oP22_34_c_4c_a

: None

: $\quad$ oP22

: $\quad 34$

: Pnn2

Simple Orthorhombic primitive vectors:

$$
\begin{aligned}
& \mathbf{a}_{1}=a \hat{\mathbf{x}} \\
& \mathbf{a}_{2}=b \hat{\mathbf{y}} \\
& \mathbf{a}_{3}=c \hat{\mathbf{z}}
\end{aligned}
$$

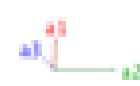

Basis vectors:

Lattice Coordinates
$\mathbf{B}_{1}=$
$z_{1} \mathbf{a}_{3}$
$\mathbf{B}_{2}=$
$\frac{1}{2} \mathbf{a}_{1}+\frac{1}{2} \mathbf{a}_{2}+\left(\frac{1}{2}+z_{1}\right) \mathbf{a}_{3}$
$\mathbf{B}_{3}=$
$x_{2} \mathbf{a}_{1}+y_{2} \mathbf{a}_{2}+z_{2} \mathbf{a}_{3}$
$-x_{2} \mathbf{a}_{1}-y_{2} \mathbf{a}_{2}+z_{2} \mathbf{a}_{3}$
$\mathbf{B}_{4}=$
$\left(\frac{1}{2}+x_{2}\right) \mathbf{a}_{1}+\left(\frac{1}{2}-y_{2}\right) \mathbf{a}_{2}+$ $\left(\frac{1}{2}+z_{2}\right) \mathbf{a}_{3}$

$$
\begin{array}{cc}
= & z_{1} c \hat{\mathbf{z}} \\
= & \frac{1}{2} a \hat{\mathbf{x}}+\frac{1}{2} b \hat{\mathbf{y}}+\left(\frac{1}{2}+z_{1}\right) c \hat{\mathbf{z}} \\
= & x_{2} a \hat{\mathbf{x}}+y_{2} b \hat{\mathbf{y}}+z_{2} c \hat{\mathbf{z}} \\
= & -x_{2} a \hat{\mathbf{x}}-y_{2} b \hat{\mathbf{y}}+z_{2} c \hat{\mathbf{z}} \\
= & \left(\frac{1}{2}+x_{2}\right) a \hat{\mathbf{x}}+\left(\frac{1}{2}-y_{2}\right) b \hat{\mathbf{y}}+ \\
& \left(\frac{1}{2}+z_{2}\right) c \hat{\mathbf{z}}
\end{array}
$$

Cartesian Coordinates
Wyckoff Position Atom Type

(2a)

$\mathrm{Ti}$

(2a)

$\mathrm{Ti}$

$(4 c)$

$\mathrm{Al}$

$(4 c)$

$\mathrm{Al}$

$(4 c)$

$\mathrm{Al}$ 


\begin{tabular}{|c|c|c|c|c|c|c|}
\hline $\mathbf{B}_{6}$ & $=$ & $\begin{array}{c}\left(\frac{1}{2}-x_{2}\right) \mathbf{a}_{1}+\left(\frac{1}{2}+y_{2}\right) \mathbf{a}_{2}+ \\
\left(\frac{1}{2}+z_{2}\right) \mathbf{a}_{3}\end{array}$ & $=$ & $\begin{array}{c}\left(\frac{1}{2}-x_{2}\right) a \hat{\mathbf{x}}+\left(\frac{1}{2}+y_{2}\right) b \hat{\mathbf{y}}+ \\
\left(\frac{1}{2}+z_{2}\right) c \hat{\mathbf{z}}\end{array}$ & $(4 c)$ & $\mathrm{Al}$ \\
\hline $\mathbf{B}_{7}$ & $=$ & $x_{3} \mathbf{a}_{1}+y_{3} \mathbf{a}_{2}+z_{3} \mathbf{a}_{3}$ & $=$ & $x_{3} a \hat{\mathbf{x}}+y_{3} b \hat{\mathbf{y}}+z_{3} c \hat{\mathbf{z}}$ & $(4 c)$ & $\mathrm{Br} \mathrm{I}$ \\
\hline $\mathbf{B}_{8}$ & $=$ & $-x_{3} \mathbf{a}_{1}-y_{3} \mathbf{a}_{2}+z_{3} \mathbf{a}_{3}$ & $=$ & $-x_{3} a \hat{\mathbf{x}}-y_{3} b \hat{\mathbf{y}}+z_{3} c \hat{\mathbf{z}}$ & $(4 c)$ & $\mathrm{Br} \mathrm{I}$ \\
\hline $\mathbf{B}_{9}$ & $=$ & $\begin{array}{c}\left(\frac{1}{2}+x_{3}\right) \mathbf{a}_{1}+\left(\frac{1}{2}-y_{3}\right) \mathbf{a}_{2}+ \\
\left(\frac{1}{2}+z_{3}\right) \mathbf{a}_{3}\end{array}$ & $=$ & $\begin{array}{c}\left(\frac{1}{2}+x_{3}\right) a \hat{\mathbf{x}}+\left(\frac{1}{2}-y_{3}\right) b \hat{\mathbf{y}}+ \\
\left(\frac{1}{2}+z_{3}\right) c \hat{\mathbf{z}}\end{array}$ & $(4 c)$ & $\mathrm{Br} \mathrm{I}$ \\
\hline $\mathbf{B}_{10}$ & $=$ & $\begin{array}{c}\left(\frac{1}{2}-x_{3}\right) \mathbf{a}_{1}+\left(\frac{1}{2}+y_{3}\right) \mathbf{a}_{2}+ \\
\left(\frac{1}{2}+z_{3}\right) \mathbf{a}_{3}\end{array}$ & $=$ & $\begin{array}{c}\left(\frac{1}{2}-x_{3}\right) a \hat{\mathbf{x}}+\left(\frac{1}{2}+y_{3}\right) b \hat{\mathbf{y}}+ \\
\left(\frac{1}{2}+z_{3}\right) c \hat{\mathbf{z}}\end{array}$ & $(4 c)$ & $\mathrm{Br} \mathrm{I}$ \\
\hline $\mathbf{B}_{11}$ & $=$ & $x_{4} \mathbf{a}_{1}+y_{4} \mathbf{a}_{2}+z_{4} \mathbf{a}_{3}$ & $=$ & $x_{4} a \hat{\mathbf{x}}+y_{4} b \hat{\mathbf{y}}+z_{4} c \hat{\mathbf{z}}$ & $(4 c)$ & $\mathrm{Br}$ II \\
\hline $\mathbf{B}_{12}$ & $=$ & $-x_{4} \mathbf{a}_{1}-y_{4} \mathbf{a}_{2}+z_{4} \mathbf{a}_{3}$ & $=$ & $-x_{4} a \hat{\mathbf{x}}-y_{4} b \hat{\mathbf{y}}+z_{4} c \hat{\mathbf{z}}$ & $(4 c)$ & $\mathrm{Br}$ II \\
\hline $\mathbf{B}_{13}$ & $=$ & $\begin{array}{c}\left(\frac{1}{2}+x_{4}\right) \mathbf{a}_{1}+\left(\frac{1}{2}-y_{4}\right) \mathbf{a}_{2}+ \\
\left(\frac{1}{2}+z_{4}\right) \mathbf{a}_{3}\end{array}$ & $=$ & $\begin{array}{c}\left(\frac{1}{2}+x_{4}\right) a \hat{\mathbf{x}}+\left(\frac{1}{2}-y_{4}\right) b \hat{\mathbf{y}}+ \\
\left(\frac{1}{2}+z_{4}\right) c \hat{\mathbf{z}}\end{array}$ & $(4 c)$ & $\mathrm{Br}$ II \\
\hline $\mathbf{B}_{14}$ & $=$ & $\begin{array}{c}\left(\frac{1}{2}-x_{4}\right) \mathbf{a}_{1}+\left(\frac{1}{2}+y_{4}\right) \mathbf{a}_{2}+ \\
\left(\frac{1}{2}+z_{4}\right) \mathbf{a}_{3}\end{array}$ & $=$ & $\begin{array}{c}\left(\frac{1}{2}-x_{4}\right) a \hat{\mathbf{x}}+\left(\frac{1}{2}+y_{4}\right) b \hat{\mathbf{y}}+ \\
\left(\frac{1}{2}+z_{4}\right) c \hat{\mathbf{z}}\end{array}$ & $(4 c)$ & $\mathrm{Br}$ II \\
\hline $\mathbf{B}_{15}$ & $=$ & $x_{5} \mathbf{a}_{1}+y_{5} \mathbf{a}_{2}+z_{5} \mathbf{a}_{3}$ & $=$ & $x_{5} a \hat{\mathbf{x}}+y_{5} b \hat{\mathbf{y}}+z_{5} c \hat{\mathbf{z}}$ & $(4 c)$ & Br III \\
\hline $\mathbf{B}_{16}$ & $=$ & $-x_{5} \mathbf{a}_{1}-y_{5} \mathbf{a}_{2}+z_{5} \mathbf{a}_{3}$ & $=$ & $-x_{5} a \hat{\mathbf{x}}-y_{5} b \hat{\mathbf{y}}+z_{5} c \hat{\mathbf{z}}$ & $(4 c)$ & $\mathrm{Br}$ III \\
\hline $\mathbf{B}_{17}$ & $=$ & $\begin{array}{c}\left(\frac{1}{2}+x_{5}\right) \mathbf{a}_{1}+\left(\frac{1}{2}-y_{5}\right) \mathbf{a}_{2}+ \\
\left(\frac{1}{2}+z_{5}\right) \mathbf{a}_{3}\end{array}$ & $=$ & $\begin{array}{c}\left(\frac{1}{2}+x_{5}\right) a \hat{\mathbf{x}}+\left(\frac{1}{2}-y_{5}\right) b \hat{\mathbf{y}}+ \\
\left(\frac{1}{2}+z_{5}\right) c \hat{\mathbf{z}}\end{array}$ & $(4 c)$ & $\mathrm{Br}$ III \\
\hline $\mathbf{B}_{18}$ & $=$ & $\begin{array}{c}\left(\frac{1}{2}-x_{5}\right) \mathbf{a}_{1}+\left(\frac{1}{2}+y_{5}\right) \mathbf{a}_{2}+ \\
\left(\frac{1}{2}+z_{5}\right) \mathbf{a}_{3}\end{array}$ & $=$ & $\begin{array}{c}\left(\frac{1}{2}-x_{5}\right) a \hat{\mathbf{x}}+\left(\frac{1}{2}+y_{5}\right) b \hat{\mathbf{y}}+ \\
\left(\frac{1}{2}+z_{5}\right) c \hat{\mathbf{z}}\end{array}$ & $(4 c)$ & $\mathrm{Br}$ III \\
\hline $\mathbf{B}_{19}$ & $=$ & $x_{6} \mathbf{a}_{1}+y_{6} \mathbf{a}_{2}+z_{6} \mathbf{a}_{3}$ & $=$ & $x_{6} a \hat{\mathbf{x}}+y_{6} b \hat{\mathbf{y}}+z_{6} c \hat{\mathbf{z}}$ & $(4 c)$ & $\mathrm{Br}$ IV \\
\hline $\mathbf{B}_{20}$ & $=$ & $-x_{6} \mathbf{a}_{1}-y_{6} \mathbf{a}_{2}+z_{6} \mathbf{a}_{3}$ & $=$ & $-x_{6} a \hat{\mathbf{x}}-y_{6} b \hat{\mathbf{y}}+z_{6} c \hat{\mathbf{z}}$ & $(4 c)$ & $\mathrm{Br}$ IV \\
\hline $\mathbf{B}_{21}$ & $=$ & $\begin{array}{c}\left(\frac{1}{2}+x_{6}\right) \mathbf{a}_{1}+\left(\frac{1}{2}-y_{6}\right) \mathbf{a}_{2}+ \\
\left(\frac{1}{2}+z_{6}\right) \mathbf{a}_{3}\end{array}$ & $=$ & $\begin{array}{c}\left(\frac{1}{2}+x_{6}\right) a \hat{\mathbf{x}}+\left(\frac{1}{2}-y_{6}\right) b \hat{\mathbf{y}}+ \\
\left(\frac{1}{2}+z_{6}\right) c \hat{\mathbf{z}}\end{array}$ & $(4 c)$ & $\mathrm{Br}$ IV \\
\hline $\mathbf{B}_{22}$ & $=$ & $\begin{array}{c}\left(\frac{1}{2}-x_{6}\right) \mathbf{a}_{1}+\left(\frac{1}{2}+y_{6}\right) \mathbf{a}_{2}+ \\
\left(\frac{1}{2}+z_{6}\right) \mathbf{a}_{3}\end{array}$ & $=$ & $\begin{array}{c}\left(\frac{1}{2}-x_{6}\right) a \hat{\mathbf{x}}+\left(\frac{1}{2}+y_{6}\right) b \hat{\mathbf{y}}+ \\
\left(\frac{1}{2}+z_{6}\right) c \hat{\mathbf{z}}\end{array}$ & $(4 c)$ & $\mathrm{Br}$ IV \\
\hline
\end{tabular}

\section{References:}

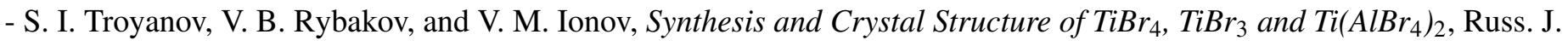
Inorg. Chem. 35, 882-887 (1990).

\section{Found in:}

- P. Villars and K. Cenzual, Pearson's Crystal Data - Crystal Structure Database for Inorganic Compounds, ASM International (2013).

\section{Geometry files:}

- CIF: pp. 824

- POSCAR: pp. 824 

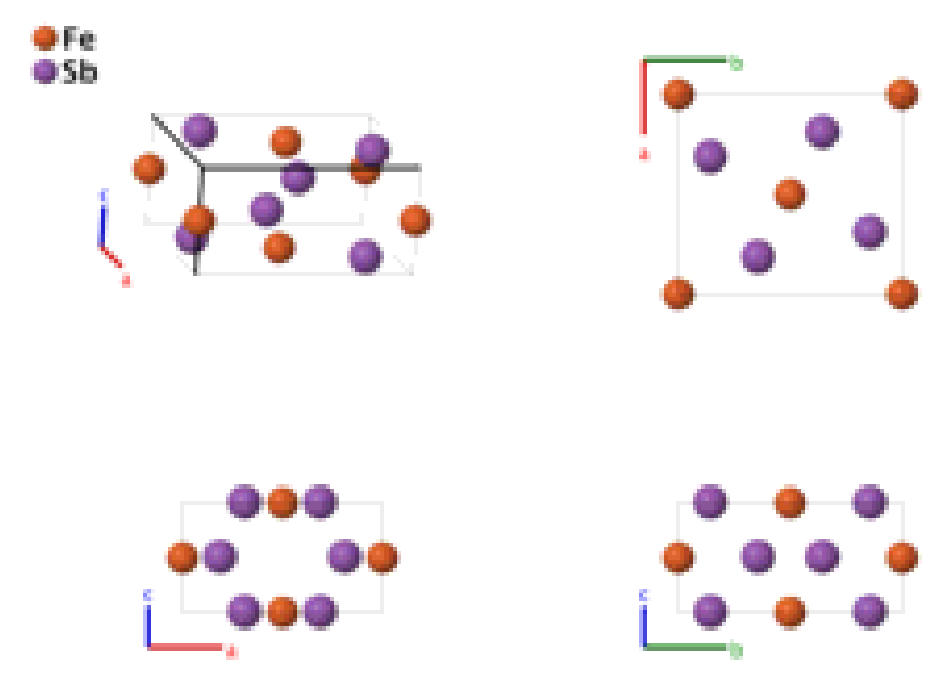

Prototype

: $\quad \mathrm{FeSb}_{2}$

AFLOW prototype label

: AB2_oP6_34_a_c

Strukturbericht designation

: None

Pearson symbol

: $\quad$ oP6

Space group number

: $\quad 34$

Space group symbol

: Pnn2

AFLOW prototype command

aflow --proto=AB2_oP6_34_a_c

- - params $=a, b / a, c / a, z_{1}, x_{2}, y_{2}, z_{2}$

Simple Orthorhombic primitive vectors:
$\mathbf{a}_{1}=a \hat{\mathbf{x}}$
$\mathbf{a}_{2}=b \hat{\mathbf{y}}$
$\mathbf{a}_{3}=c \hat{\mathbf{z}}$

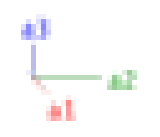

Basis vectors:

Lattice Coordinates
$\mathbf{B}_{1}=$
$z_{1} \mathbf{a}_{3}$
$\mathbf{B}_{2}=\frac{1}{2} \mathbf{a}_{1}+\frac{1}{2} \mathbf{a}_{2}+\left(\frac{1}{2}+z_{1}\right) \mathbf{a}_{3}$
$=$
$=\quad \frac{1}{2} a \hat{\mathbf{x}}+\frac{1}{2} b \hat{\mathbf{y}}+\left(\frac{1}{2}+z_{1}\right) c \hat{\mathbf{z}}$
$z_{1} c \hat{\mathbf{z}}$
$=$
$=$
$x_{2} a \hat{\mathbf{x}}+y_{2} b \hat{\mathbf{y}}+z_{2} c \hat{\mathbf{z}}$
$\mathbf{B}_{4}=-x_{2} \mathbf{a}_{1}-y_{2} \mathbf{a}_{2}+z_{2} \mathbf{a}_{3}$
$-x_{2} a \hat{\mathbf{x}}-y_{2} b \hat{\mathbf{y}}+z_{2} c \hat{\mathbf{z}}$
$\mathbf{B}_{5}=\left(\frac{1}{2}+x_{2}\right) \mathbf{a}_{1}+\left(\frac{1}{2}-y_{2}\right) \mathbf{a}_{2}+$ $\left(\frac{1}{2}+z_{2}\right) \mathbf{a}_{3}$
$\left(\frac{1}{2}+x_{2}\right) a \hat{\mathbf{x}}+\left(\frac{1}{2}-y_{2}\right) b \hat{\mathbf{y}}+$ $\left(\frac{1}{2}+z_{2}\right) c \hat{\mathbf{z}}$

Cartesian Coordinates
Wyckoff Position Atom Type

(2a)

$\mathrm{Fe}$

(2a)

$\mathrm{Fe}$

(4c)

$\mathrm{Sb}$

(4c)

$\mathrm{Sb}$

(4c)

$\mathrm{Sb}$ 
$\mathbf{B}_{6}=\begin{gathered}\left(\frac{1}{2}-x_{2}\right) \mathbf{a}_{1}+\left(\frac{1}{2}+y_{2}\right) \mathbf{a}_{2}+ \\ \left(\frac{1}{2}+z_{2}\right) \mathbf{a}_{3}\end{gathered} \quad \begin{gathered}\left(\frac{1}{2}-x_{2}\right) a \hat{\mathbf{x}}+\left(\frac{1}{2}+y_{2}\right) b \hat{\mathbf{y}}+ \\ \left(\frac{1}{2}+z_{2}\right) c \hat{\mathbf{z}}\end{gathered}$

$(4 c)$

$\mathrm{Sb}$

References:

- H. Holseth and A. Kjekshus, Compounds with the Marcasite Type Crystal Structure. IV. The Crystal Structure of FeSb, Acta Chem. Scand. 23, 3043-3050 (1969), doi:10.3891/acta.chem.scand.23-3043.

\section{Found in:}

- P. Villars and K. Cenzual, Pearson's Crystal Data - Crystal Structure Database for Inorganic Compounds, ASM International (2013).

\section{Geometry files:}

- CIF: pp. 825

- POSCAR: pp. 825 


\section{$\mathrm{V}_{2} \mathrm{MoO}_{8}$ Structure: AB8C2_oC22_35_a_ab3e_e}
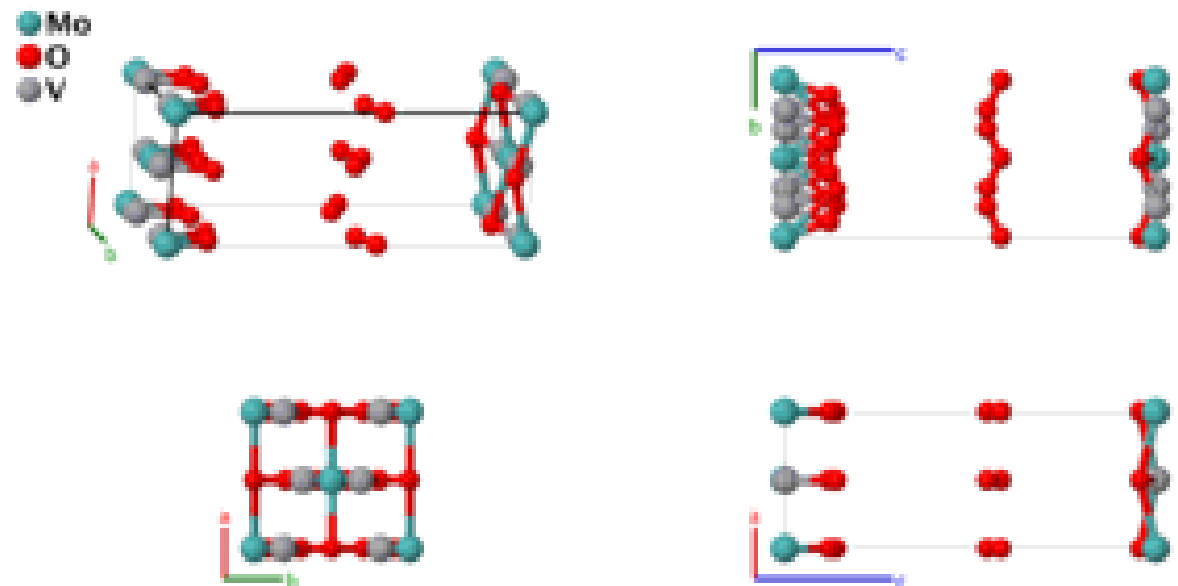

\section{Prototype}

AFLOW prototype label

Strukturbericht designation

Pearson symbol

Space group number

Space group symbol

AFLOW prototype command : aflow--proto=AB8C2_oC22_35_a_ab3e_e

- params $=a, b / a, c / a, z_{1}, z_{2}, z_{3}, y_{4}, z_{4}, y_{5}, z_{5}, y_{6}, z_{6}, y_{7}, z_{7}$

Base-centered Orthorhombic primitive vectors:

$$
\begin{aligned}
& \mathbf{a}_{1}=\frac{1}{2} a \hat{\mathbf{x}}-\frac{1}{2} b \hat{\mathbf{y}} \\
& \mathbf{a}_{2}=\frac{1}{2} a \hat{\mathbf{x}}+\frac{1}{2} b \hat{\mathbf{y}} \\
& \mathbf{a}_{3}=c \quad c \hat{\mathbf{z}}
\end{aligned}
$$

19:

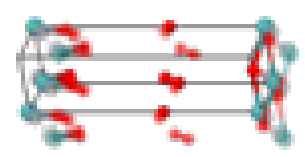

Basis vectors:

Lattice Coordinates

$\begin{array}{lcc}\mathbf{B}_{1}= & z_{1} \mathbf{a}_{3} \\ \mathbf{B}_{2}= & z_{2} \mathbf{a}_{3} \\ \mathbf{B}_{3}= & \frac{1}{2} \mathbf{a}_{1}+\frac{1}{2} \mathbf{a}_{2}+z_{3} \mathbf{a}_{3} \\ \mathbf{B}_{4}= & -y_{4} \mathbf{a}_{1}+y_{4} \mathbf{a}_{2}+z_{4} \mathbf{a}_{3} \\ \mathbf{B}_{5}= & y_{4} \mathbf{a}_{1}-y_{4} \mathbf{a}_{2}+z_{4} \mathbf{a}_{3} \\ \mathbf{B}_{6}= & -y_{5} \mathbf{a}_{1}+y_{5} \mathbf{a}_{2}+z_{5} \mathbf{a}_{3} \\ \mathbf{B}_{7}= & y_{5} \mathbf{a}_{1}-y_{5} \mathbf{a}_{2}+z_{5} \mathbf{a}_{3} \\ \mathbf{B}_{8}= & -y_{6} \mathbf{a}_{1}+y_{6} \mathbf{a}_{2}+z_{6} \mathbf{a}_{3} \\ \mathbf{B}_{9}= & y_{6} \mathbf{a}_{1}-y_{6} \mathbf{a}_{2}+z_{6} \mathbf{a}_{3}\end{array}$

Cartesian Coordinates

$=$

$=$

$=$

$=$

$=$

$=$

$=$

$=$

$=$ $z_{1} c \hat{\mathbf{z}}$

$z_{2} c \hat{\mathbf{z}}$

$\frac{1}{2} a \hat{\mathbf{x}}+z_{3} c \hat{\mathbf{z}}$

$y_{4} b \hat{\mathbf{y}}+z_{4} c \hat{\mathbf{z}}$

$-y_{4} b \hat{\mathbf{y}}+z_{4} c \hat{\mathbf{z}}$

$y_{5} b \hat{\mathbf{y}}+z_{5} c \hat{\mathbf{z}}$

$-y_{5} b \hat{\mathbf{y}}+z_{5} c \hat{\mathbf{z}}$

$y_{6} b \hat{\mathbf{y}}+z_{6} c \hat{\mathbf{z}}$

$-y_{6} b \hat{\mathbf{y}}+z_{6} c \hat{\mathbf{z}}$
Wyckoff Position

(2a)

(2a)

$(4 e)$

$(4 e)$

$(4 e)$

(4e)

$(4 e)$

$(4 e)$
Atom Type

Mo

O I

O II

O III

O III

O IV

O IV

$\mathrm{OV}$

O V 


$\begin{array}{llllll}\mathbf{B}_{10}= & -y_{7} \mathbf{a}_{1}+y_{7} \mathbf{a}_{2}+z_{7} \mathbf{a}_{3} & = & y_{7} b \hat{\mathbf{y}}+z_{7} c \hat{\mathbf{z}} & (4 e) & \mathrm{V} \\ \mathbf{B}_{11}= & y_{7} \mathbf{a}_{1}-y_{7} \mathbf{a}_{2}+z_{7} \mathbf{a}_{3} & = & -y_{7} b \hat{\mathbf{y}}+z_{7} c \hat{\mathbf{z}} & (4 e) & \mathrm{V}\end{array}$

\section{References:}

- P. Mahé-Pailleret, Contribution à l'étude chimique et structurale des composés $\mathrm{AB}_{2} \mathrm{O}$ rencontrés dans les systèmes Mo-VO, UVO et U-Mo-O, Ph.D. thesis, Faculté des sciences de Paris (1970).

\section{Found in:}

- P. Villars and K. Cenzual, Pearson's Crystal Data - Crystal Structure Database for Inorganic Compounds, ASM International (2013).

\section{Geometry files:}

- CIF: pp. 825

- POSCAR: pp. 825 


\section{HCl Structure: AB_oC8_36_a_a}

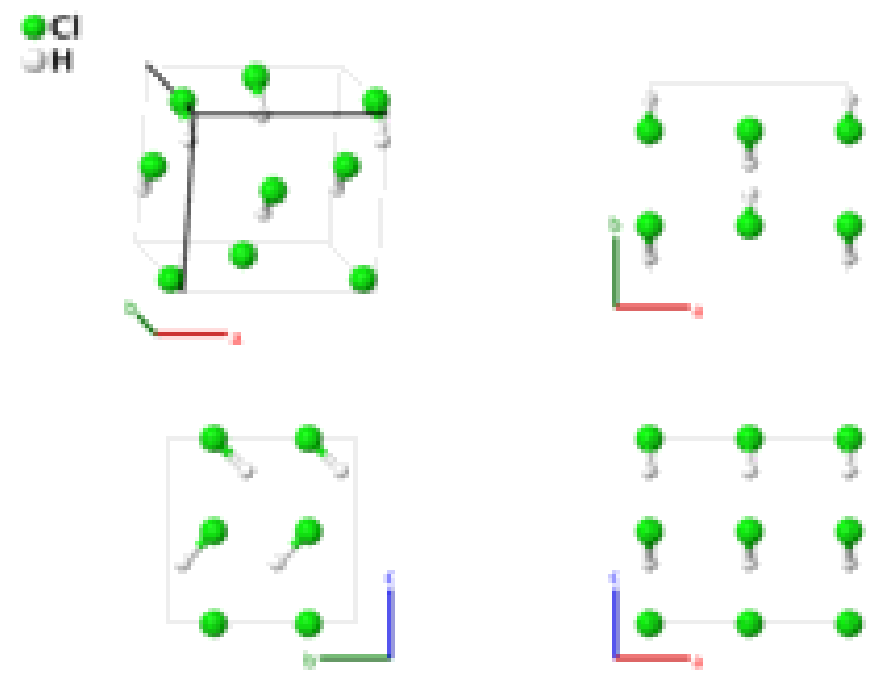

Prototype $\quad: \quad \mathrm{HCl}$

AFLOW prototype label $\quad$ : AB_oC8_36_a_a

Strukturbericht designation : None

Pearson symbol $\quad: \quad \mathrm{oC} 8$

Space group number $\quad: \quad 36$

Space group symbol $\quad: \quad C m c 2_{1}$

AFLOW prototype command : aflow --proto=AB_oC8_36_a_a

- params $=a, b / a, c / a, y_{1}, z_{1}, y_{2}, z_{2}$

- The original reference gives the positions of the atoms in the $B b 2_{1} m$ setting of space group \#36. We have transformed this into the standard $C m c 2_{1}$ setting.

Base-centered Orthorhombic primitive vectors:

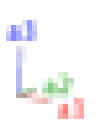

$$
\begin{aligned}
& \mathbf{a}_{1}=\frac{1}{2} a \hat{\mathbf{x}}-\frac{1}{2} b \hat{\mathbf{y}} \\
& \mathbf{a}_{2}=\frac{1}{2} a \hat{\mathbf{x}}+\frac{1}{2} b \hat{\mathbf{y}} \\
& \mathbf{a}_{3}=c \quad c \hat{\mathbf{z}}
\end{aligned}
$$

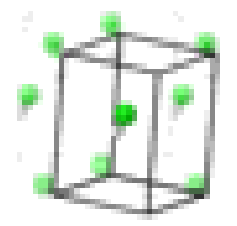

\section{Basis vectors:}

Lattice Coordinates

$\mathbf{B}_{1}=-y_{1} \mathbf{a}_{1}+y_{1} \mathbf{a}_{2}+z_{1} \mathbf{a}_{3}$

$\mathbf{B}_{2}=y_{1} \mathbf{a}_{1}-y_{1} \mathbf{a}_{2}+\left(\frac{1}{2}+z_{1}\right) \mathbf{a}_{3}$

$\mathbf{B}_{3}=-y_{2} \mathbf{a}_{1}+y_{2} \mathbf{a}_{2}+z_{2} \mathbf{a}_{3}$

$\mathbf{B}_{4}=$ 


\section{References:}

- E. Sándor and R. F. C. Farrow, Crystal Structure of Solid Hydrogen Chloride and Deuterium Chloride, Nature 213, 171-172 (1967), doi:10.1038/213171a0.

\section{Geometry files:}

- CIF: pp. 825

- POSCAR: pp. 826 


\section{$\mathrm{Li}_{2} \mathrm{Si}_{2} \mathrm{O}_{5}$ Structure: A2B5C2_oC36_37_d_c2d_d}
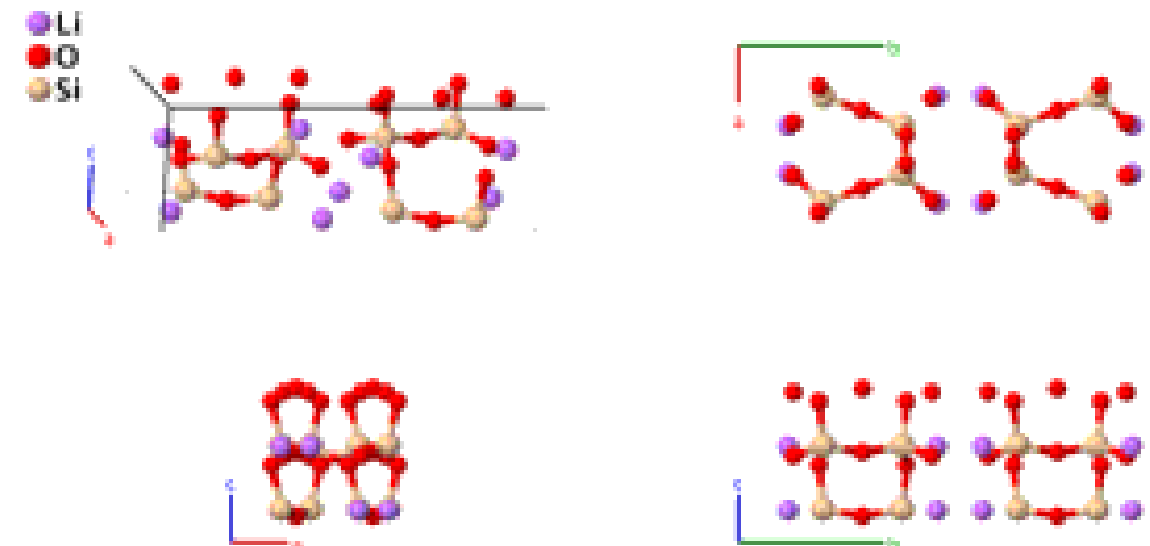

\section{Prototype}

: $\quad \mathrm{Li}_{2} \mathrm{Si}_{2} \mathrm{O}_{5}$

AFLOW prototype label

: A2B5C2_oC36_37_d_c2d_d

Strukturbericht designation : None

Pearson symbol

: $\quad$ oC36

Space group number

$: \quad 37$

Space group symbol

: $\quad C c c 2$

AFLOW prototype command : aflow --proto=A2B5C2_oC36_37_d_c2d_d

- params $=a, b / a, c / a, z_{1}, x_{2}, y_{2}, z_{2}, x_{3}, y_{3}, z_{3}, x_{4}, y_{4}, z_{4}, x_{5}, y_{5}, z_{5}$

\section{Base-centered Orthorhombic primitive vectors:}

$$
\begin{aligned}
& \mathbf{a}_{1}=\frac{1}{2} a \hat{\mathbf{x}}-\frac{1}{2} b \hat{\mathbf{y}} \\
& \mathbf{a}_{2}=\frac{1}{2} a \hat{\mathbf{x}}+\frac{1}{2} b \hat{\mathbf{y}} \\
& \mathbf{a}_{3}=c \quad c \hat{\mathbf{z}}
\end{aligned}
$$

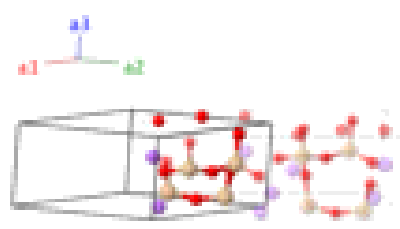

Basis vectors:

\section{Lattice Coordinates}

$\mathbf{B}_{1}=$

$\mathbf{B}_{2}=$

$\mathbf{B}_{3}=$

$\mathbf{B}_{4}=\left(-x_{2}+y_{2}\right) \mathbf{a}_{1}+\left(-x_{2}-y_{2}\right) \mathbf{a}_{2}+z_{2} \mathbf{a}_{3}$

$\mathbf{B}_{5}=\left(x_{2}+y_{2}\right) \mathbf{a}_{1}+\left(x_{2}-y_{2}\right) \mathbf{a}_{2}+\left(\frac{1}{2}+z_{2}\right) \mathbf{a}_{3}$

$\mathbf{B}_{6}=\left(-x_{2}-y_{2}\right) \mathbf{a}_{1}+\left(-x_{2}+y_{2}\right) \mathbf{a}_{2}+$ $\left(\frac{1}{2}+z_{2}\right) \mathbf{a}_{3}$

$\mathbf{B}_{7}=\left(x_{3}-y_{3}\right) \mathbf{a}_{1}+\left(x_{3}+y_{3}\right) \mathbf{a}_{2}+z_{3} \mathbf{a}_{3}$

$\mathbf{B}_{8}=\left(-x_{3}+y_{3}\right) \mathbf{a}_{1}+\left(-x_{3}-y_{3}\right) \mathbf{a}_{2}+z_{3} \mathbf{a}_{3}$

$\mathbf{B}_{9}=\left(x_{3}+y_{3}\right) \mathbf{a}_{1}+\left(x_{3}-y_{3}\right) \mathbf{a}_{2}+\left(\frac{1}{2}+z_{3}\right) \mathbf{a}_{3}$

\section{Cartesian Coordinates}

$=\quad \frac{1}{4} a \hat{\mathbf{x}}+\frac{1}{4} b \hat{\mathbf{y}}+z_{1} c \hat{\mathbf{z}}$

$=\frac{1}{4} a \hat{\mathbf{x}}+\frac{3}{4} b \hat{\mathbf{y}}+\left(\frac{1}{2}+z_{1}\right) c \hat{\mathbf{z}}$

$=\quad x_{2} a \hat{\mathbf{x}}+y_{2} b \hat{\mathbf{y}}+z_{2} c \hat{\mathbf{z}}$

$=\quad-x_{2} a \hat{\mathbf{x}}-y_{2} b \hat{\mathbf{y}}+z_{2} c \hat{\mathbf{z}}$

$=x_{2} a \hat{\mathbf{x}}-y_{2} b \hat{\mathbf{y}}+\left(\frac{1}{2}+z_{2}\right) c \hat{\mathbf{z}}$

$=-x_{2} a \hat{\mathbf{x}}+y_{2} b \hat{\mathbf{y}}+\left(\frac{1}{2}+z_{2}\right) c \hat{\mathbf{z}}$

$=\quad x_{3} a \hat{\mathbf{x}}+y_{3} b \hat{\mathbf{y}}+z_{3} c \hat{\mathbf{z}}$

$=\quad-x_{3} a \hat{\mathbf{x}}-y_{3} b \hat{\mathbf{y}}+z_{3} c \hat{\mathbf{z}}$

$=\quad x_{3} a \hat{\mathbf{x}}-y_{3} b \hat{\mathbf{y}}+\left(\frac{1}{2}+z_{3}\right) c \hat{\mathbf{z}}$
Wyckoff Position Atom Type

O I

O II

O II 


\begin{tabular}{|c|c|c|c|c|c|c|}
\hline $\mathbf{B}_{10}$ & $=$ & $\begin{array}{c}\left(-x_{3}-y_{3}\right) \mathbf{a}_{1}+\left(-x_{3}+y_{3}\right) \mathbf{a}_{2}+ \\
\left(\frac{1}{2}+z_{3}\right) \mathbf{a}_{3}\end{array}$ & $=$ & $-x_{3} a \hat{\mathbf{x}}+y_{3} b \hat{\mathbf{y}}+\left(\frac{1}{2}+z_{3}\right) c \hat{\mathbf{z}}$ & $(8 d)$ & $\mathrm{O}$ II \\
\hline $\mathbf{B}_{11}$ & $=$ & $\left(x_{4}-y_{4}\right) \mathbf{a}_{1}+\left(x_{4}+y_{4}\right) \mathbf{a}_{2}+z_{4} \mathbf{a}_{3}$ & $=$ & $x_{4} a \hat{\mathbf{x}}+y_{4} b \hat{\mathbf{y}}+z_{4} c \hat{\mathbf{z}}$ & $(8 d)$ & O III \\
\hline $\mathbf{B}_{12}$ & $=$ & $\left(-x_{4}+y_{4}\right) \mathbf{a}_{1}+\left(-x_{4}-y_{4}\right) \mathbf{a}_{2}+z_{4} \mathbf{a}_{3}$ & $=$ & $-x_{4} a \hat{\mathbf{x}}-y_{4} b \hat{\mathbf{y}}+z_{4} c \hat{\mathbf{z}}$ & $(8 d)$ & O III \\
\hline $\mathbf{B}_{13}$ & $=$ & $\left(x_{4}+y_{4}\right) \mathbf{a}_{1}+\left(x_{4}-y_{4}\right) \mathbf{a}_{2}+\left(\frac{1}{2}+z_{4}\right) \mathbf{a}_{3}$ & $=$ & $x_{4} a \hat{\mathbf{x}}-y_{4} b \hat{\mathbf{y}}+\left(\frac{1}{2}+z_{4}\right) c \hat{\mathbf{z}}$ & $(8 d)$ & O III \\
\hline $\mathbf{B}_{14}$ & $=$ & $\begin{array}{c}\left(-x_{4}-y_{4}\right) \mathbf{a}_{1}+\left(-x_{4}+y_{4}\right) \mathbf{a}_{2}+ \\
\left(\frac{1}{2}+z_{4}\right) \mathbf{a}_{3}\end{array}$ & $=$ & $-x_{4} a \hat{\mathbf{x}}+y_{4} b \hat{\mathbf{y}}+\left(\frac{1}{2}+z_{4}\right) c \hat{\mathbf{z}}$ & $(8 d)$ & O III \\
\hline $\mathbf{B}_{15}$ & $=$ & $\left(x_{5}-y_{5}\right) \mathbf{a}_{1}+\left(x_{5}+y_{5}\right) \mathbf{a}_{2}+z_{5} \mathbf{a}_{3}$ & $=$ & $x_{5} a \hat{\mathbf{x}}+y_{5} b \hat{\mathbf{y}}+z_{5} c \hat{\mathbf{z}}$ & $(8 d)$ & $\mathrm{Si}$ \\
\hline $\mathbf{B}_{16}$ & $=$ & $\left(-x_{5}+y_{5}\right) \mathbf{a}_{1}+\left(-x_{5}-y_{5}\right) \mathbf{a}_{2}+z_{5} \mathbf{a}_{3}$ & $=$ & $-x_{5} a \hat{\mathbf{x}}-y_{5} b \hat{\mathbf{y}}+z_{5} c \hat{\mathbf{z}}$ & $(8 d)$ & $\mathrm{Si}$ \\
\hline $\mathbf{B}_{17}$ & $=$ & $\left(x_{5}+y_{5}\right) \mathbf{a}_{1}+\left(x_{5}-y_{5}\right) \mathbf{a}_{2}+\left(\frac{1}{2}+z_{5}\right) \mathbf{a}_{3}$ & $=$ & $x_{5} a \hat{\mathbf{x}}-y_{5} b \hat{\mathbf{y}}+\left(\frac{1}{2}+z_{5}\right) c \hat{\mathbf{z}}$ & $(8 d)$ & $\mathrm{Si}$ \\
\hline $\mathbf{B}_{18}$ & $=$ & $\begin{array}{c}\left(-x_{5}-y_{5}\right) \mathbf{a}_{1}+\left(-x_{5}+y_{5}\right) \mathbf{a}_{2}+ \\
\left(\frac{1}{2}+z_{5}\right) \mathbf{a}_{3}\end{array}$ & $=$ & $-x_{5} a \hat{\mathbf{x}}+y_{5} b \hat{\mathbf{y}}+\left(\frac{1}{2}+z_{5}\right) c \hat{\mathbf{z}}$ & $(8 d)$ & $\mathrm{Si}$ \\
\hline
\end{tabular}

\section{References:}

- B. H. W. S. de Jong, P. G. G. Slaats, H. T. J. Supèr, N. Veldman, and A. L. Spek, Extended structures in crystalline phyllosilicates: silica ring systems in lithium, rubidium, cesium, and cesium/lithium phyllosilicate, J. Non Cryst. Solids 176, 164-171 (1994), doi:10.1016/0022-3093(94)90074-4.

\section{Found in:}

- P. Villars and K. Cenzual, Pearson's Crystal Data - Crystal Structure Database for Inorganic Compounds, ASM International (2013).

\section{Geometry files:}

- CIF: pp. 826

- POSCAR: pp. 826 


\section{$\mathrm{Ta}_{3} \mathrm{~S}_{2}$ Structure: A2B3_oC40_39_2d_2c2d}
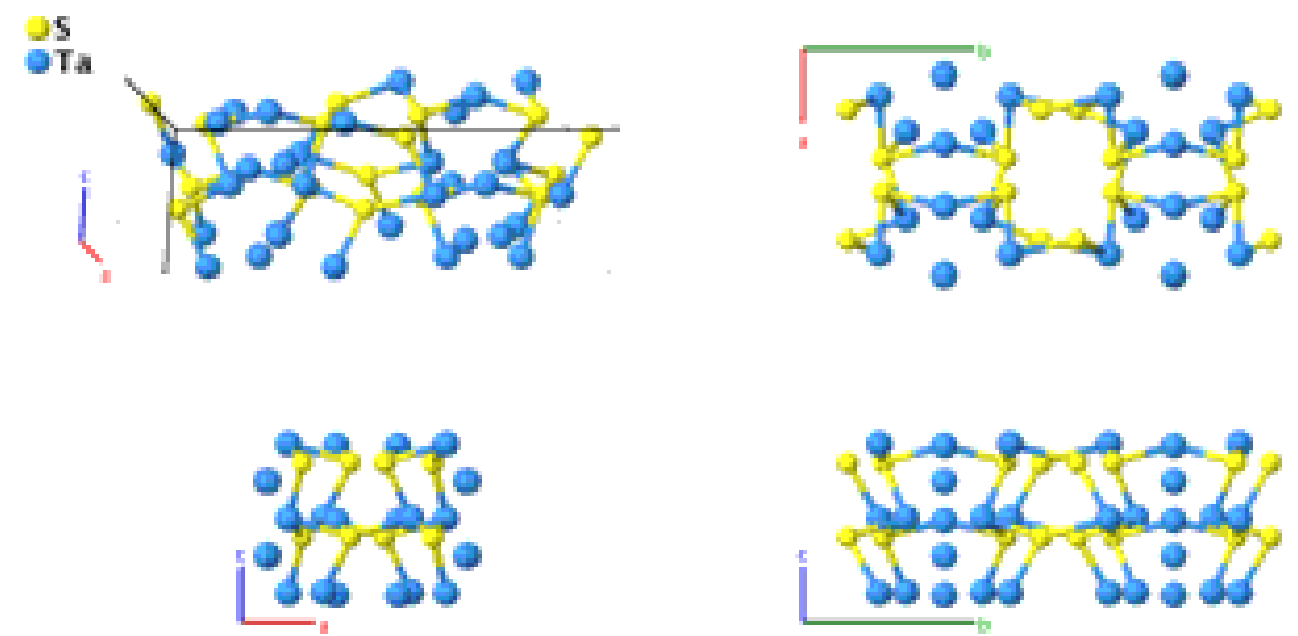

\section{Prototype}

AFLOW prototype label

$: \mathrm{Ta}_{3} \mathrm{~S}_{2}$

Strukturbericht designation : None

Pearson symbol

: $\quad \mathrm{oC} 40$

Space group number $\quad: \quad 39$

Space group symbol : $\quad$ Abm2

AFLOW prototype command : aflow --proto=A2B3_oC40_39_2d_2c2d

- -params $=a, b / a, c / a, x_{1}, z_{1}, x_{2}, z_{2}, x_{3}, y_{3}, z_{3}, x_{4}, y_{4}, z_{4}, x_{5}, y_{5}, z_{5}, x_{6}, y_{6}, z_{6}$

Base-centered Orthorhombic primitive vectors:

$$
\begin{aligned}
& \mathbf{a}_{1}=c \quad a \hat{\mathbf{x}} \\
& \mathbf{a}_{2}=\frac{1}{2} b \hat{\mathbf{y}}-\frac{1}{2} c \hat{\mathbf{z}} \\
& \mathbf{a}_{3}=\frac{1}{2} b \hat{\mathbf{y}}+\frac{1}{2} c \hat{\mathbf{z}}
\end{aligned}
$$

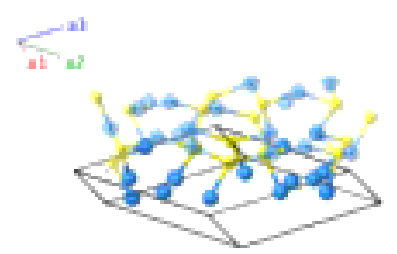

Basis vectors:

Lattice Coordinates

$$
\begin{array}{ccccc}
\mathbf{B}_{1}= & x_{1} \mathbf{a}_{1}+\left(\frac{1}{4}-z_{1}\right) \mathbf{a}_{2}+\left(\frac{1}{4}+z_{1}\right) \mathbf{a}_{3} & = & x_{1} a \hat{\mathbf{x}}+\frac{1}{4} b \hat{\mathbf{y}}+z_{1} c \hat{\mathbf{z}} \\
\mathbf{B}_{2}= & -x_{1} \mathbf{a}_{1}+\left(\frac{3}{4}-z_{1}\right) \mathbf{a}_{2}+\left(\frac{3}{4}+z_{1}\right) \mathbf{a}_{3} & = & -x_{1} a \hat{\mathbf{x}}+\frac{3}{4} b \hat{\mathbf{y}}+z_{1} c \hat{\mathbf{z}} \\
\mathbf{B}_{3}= & x_{2} \mathbf{a}_{1}+\left(\frac{1}{4}-z_{2}\right) \mathbf{a}_{2}+\left(\frac{1}{4}+z_{2}\right) \mathbf{a}_{3} & = & x_{2} a \hat{\mathbf{x}}+\frac{1}{4} b \hat{\mathbf{y}}+z_{2} c \hat{\mathbf{z}} \\
\mathbf{B}_{4}= & -x_{2} \mathbf{a}_{1}+\left(\frac{3}{4}-z_{2}\right) \mathbf{a}_{2}+\left(\frac{3}{4}+z_{2}\right) \mathbf{a}_{3} & = & -x_{2} a \hat{\mathbf{x}}+\frac{3}{4} b \hat{\mathbf{y}}+z_{2} c \hat{\mathbf{z}} \\
\mathbf{B}_{5}= & x_{3} \mathbf{a}_{1}+\left(y_{3}-z_{3}\right) \mathbf{a}_{2}+\left(y_{3}+z_{3}\right) \mathbf{a}_{3} & = & x_{3} a \hat{\mathbf{x}}+y_{3} b \hat{\mathbf{y}}+z_{3} c \hat{\mathbf{z}} \\
\mathbf{B}_{6}= & -x_{3} \mathbf{a}_{1}+\left(-y_{3}-z_{3}\right) \mathbf{a}_{2}+\left(-y_{3}+z_{3}\right) \mathbf{a}_{3} & = & -x_{3} a \hat{\mathbf{x}}-y_{3} b \hat{\mathbf{y}}+z_{3} c \hat{\mathbf{z}} \\
\mathbf{B}_{7}= & x_{3} \mathbf{a}_{1}+\left(\frac{1}{2}-y_{3}-z_{3}\right) \mathbf{a}_{2}+\left(\frac{1}{2}-y_{3}+z_{3}\right) \mathbf{a}_{3} & = & x_{3} a \hat{\mathbf{x}}+\left(\frac{1}{2}-y_{3}\right) b \hat{\mathbf{y}}+z_{3} c \hat{\mathbf{z}} \\
\mathbf{B}_{8}= & -x_{3} \mathbf{a}_{1}+\left(\frac{1}{2}+y_{3}-z_{3}\right) \mathbf{a}_{2}+ & & -x_{3} a \hat{\mathbf{x}}+\left(\frac{1}{2}+y_{3}\right) b \hat{\mathbf{y}}+z_{3} c \hat{\mathbf{z}} \\
& \left(\frac{1}{2}+y_{3}+z_{3}\right) \mathbf{a}_{3} & &
\end{array}
$$

Wyckoff Position Atom Type

Ta I

$(4 c)$

Ta I

$(4 c)$

Ta II

(4c)

Ta II

(8d)

(8d)

(8d)

(8d) 


\begin{tabular}{|c|c|c|c|c|c|c|}
\hline $\mathbf{B}_{9}$ & $=$ & $x_{4} \mathbf{a}_{1}+\left(y_{4}-z_{4}\right) \mathbf{a}_{2}+\left(y_{4}+z_{4}\right) \mathbf{a}_{3}$ & $=$ & $x_{4} a \hat{\mathbf{x}}+y_{4} b \hat{\mathbf{y}}+z_{4} c \hat{\mathbf{z}}$ & $(8 d)$ & S II \\
\hline $\mathbf{B}_{10}$ & $=$ & $-x_{4} \mathbf{a}_{1}+\left(-y_{4}-z_{4}\right) \mathbf{a}_{2}+\left(-y_{4}+z_{4}\right) \mathbf{a}_{3}$ & $=$ & $-x_{4} a \hat{\mathbf{x}}-y_{4} b \hat{\mathbf{y}}+z_{4} c \hat{\mathbf{z}}$ & $(8 d)$ & S II \\
\hline $\mathbf{B}_{11}$ & $=$ & $x_{4} \mathbf{a}_{1}+\left(\frac{1}{2}-y_{4}-z_{4}\right) \mathbf{a}_{2}+\left(\frac{1}{2}-y_{4}+z_{4}\right) \mathbf{a}_{3}$ & $=$ & $x_{4} a \hat{\mathbf{x}}+\left(\frac{1}{2}-y_{4}\right) b \hat{\mathbf{y}}+z_{4} c \hat{\mathbf{z}}$ & $(8 d)$ & S II \\
\hline $\mathbf{B}_{12}$ & $=$ & $\begin{array}{c}-x_{4} \mathbf{a}_{1}+\left(\frac{1}{2}+y_{4}-z_{4}\right) \mathbf{a}_{2}+ \\
\left(\frac{1}{2}+y_{4}+z_{4}\right) \mathbf{a}_{3}\end{array}$ & $=$ & $-x_{4} a \hat{\mathbf{x}}+\left(\frac{1}{2}+y_{4}\right) b \hat{\mathbf{y}}+z_{4} c \hat{\mathbf{z}}$ & $(8 d)$ & S II \\
\hline $\mathbf{B}_{13}$ & $=$ & $x_{5} \mathbf{a}_{1}+\left(y_{5}-z_{5}\right) \mathbf{a}_{2}+\left(y_{5}+z_{5}\right) \mathbf{a}_{3}$ & $=$ & $x_{5} a \hat{\mathbf{x}}+y_{5} b \hat{\mathbf{y}}+z_{5} c \hat{\mathbf{z}}$ & $(8 d)$ & Ta III \\
\hline $\mathbf{B}_{14}$ & $=$ & $-x_{5} \mathbf{a}_{1}+\left(-y_{5}-z_{5}\right) \mathbf{a}_{2}+\left(-y_{5}+z_{5}\right) \mathbf{a}_{3}$ & $=$ & $-x_{5} a \hat{\mathbf{x}}-y_{5} b \hat{\mathbf{y}}+z_{5} c \hat{\mathbf{z}}$ & $(8 d)$ & Ta III \\
\hline $\mathbf{B}_{15}$ & $=$ & $x_{5} \mathbf{a}_{1}+\left(\frac{1}{2}-y_{5}-z_{5}\right) \mathbf{a}_{2}+\left(\frac{1}{2}-y_{5}+z_{5}\right) \mathbf{a}_{3}$ & $=$ & $x_{5} a \hat{\mathbf{x}}+\left(\frac{1}{2}-y_{5}\right) b \hat{\mathbf{y}}+z_{5} c \hat{\mathbf{z}}$ & $(8 d)$ & Ta III \\
\hline $\mathbf{B}_{16}$ & $=$ & $\begin{array}{c}-x_{5} \mathbf{a}_{1}+\left(\frac{1}{2}+y_{5}-z_{5}\right) \mathbf{a}_{2}+ \\
\left(\frac{1}{2}+y_{5}+z_{5}\right) \mathbf{a}_{3}\end{array}$ & $=$ & $-x_{5} a \hat{\mathbf{x}}+\left(\frac{1}{2}+y_{5}\right) b \hat{\mathbf{y}}+z_{5} c \hat{\mathbf{z}}$ & $(8 d)$ & Ta III \\
\hline $\mathbf{B}_{17}$ & $=$ & $x_{6} \mathbf{a}_{1}+\left(y_{6}-z_{6}\right) \mathbf{a}_{2}+\left(y_{6}+z_{6}\right) \mathbf{a}_{3}$ & $=$ & $x_{6} a \hat{\mathbf{x}}+y_{6} b \hat{\mathbf{y}}+z_{6} c \hat{\mathbf{z}}$ & $(8 d)$ & Ta IV \\
\hline $\mathbf{B}_{18}$ & $=$ & $-x_{6} \mathbf{a}_{1}+\left(-y_{6}-z_{6}\right) \mathbf{a}_{2}+\left(-y_{6}+z_{6}\right) \mathbf{a}_{3}$ & $=$ & $-x_{6} a \hat{\mathbf{x}}-y_{6} b \hat{\mathbf{y}}+z_{6} c \hat{\mathbf{z}}$ & $(8 d)$ & Ta IV \\
\hline $\mathbf{B}_{19}$ & $=$ & $x_{6} \mathbf{a}_{1}+\left(\frac{1}{2}-y_{6}-z_{6}\right) \mathbf{a}_{2}+\left(\frac{1}{2}-y_{6}+z_{6}\right) \mathbf{a}_{3}$ & $=$ & $x_{6} a \hat{\mathbf{x}}+\left(\frac{1}{2}-y_{6}\right) b \hat{\mathbf{y}}+z_{6} c \hat{\mathbf{z}}$ & $(8 d)$ & Ta IV \\
\hline $\mathbf{B}_{20}$ & $=$ & $\begin{array}{c}-x_{6} \mathbf{a}_{1}+\left(\frac{1}{2}+y_{6}-z_{6}\right) \mathbf{a}_{2}+ \\
\left(\frac{1}{2}+y_{6}+z_{6}\right) \mathbf{a}_{3}\end{array}$ & $=$ & $-x_{6} a \hat{\mathbf{x}}+\left(\frac{1}{2}+y_{6}\right) b \hat{\mathbf{y}}+z_{6} c \hat{\mathbf{z}}$ & $(8 d)$ & Ta IV \\
\hline
\end{tabular}

\section{References:}

- S. J. Kim, K. S. Nanjundaswamy, and T. Hughbanks, Single-crystal structure of tantalum sulfide $\left(T_{3} S_{2}\right)$. Structure and bonding in the $\operatorname{Ta}_{6} \operatorname{Sn}(n=1,3,4,5$ ?) pentagonal-antiprismatic chain compounds, Inorg. Chem. 30, 159-164 (1991), doi:10.1021/ic00002a004.

\section{Found in:}

- P. Villars and K. Cenzual, Pearson's Crystal Data - Crystal Structure Database for Inorganic Compounds, ASM International (2013).

\section{Geometry files:}

- CIF: pp. 826

- POSCAR: pp. 827 


\section{VPCl 19 Structure: A9BC_oC44_39_3c3d_a_c}
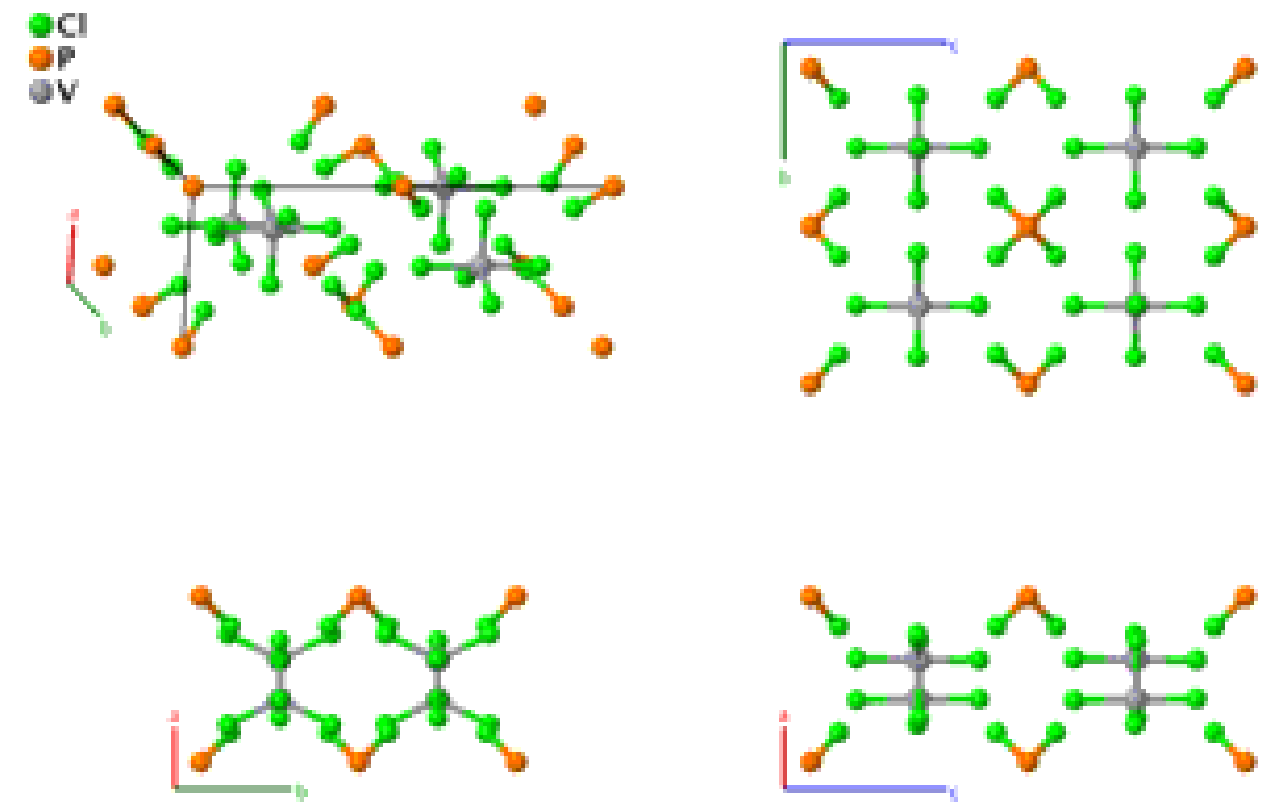

\section{Prototype}

: $\quad \mathrm{VPCl}_{9}$

AFLOW prototype label

: A9BC_oC44_39_3c3d_a_c

Strukturbericht designation : None

Pearson symbol

: $\quad$ oC44

Space group number

: $\quad 39$

Space group symbol

: $\quad A b m 2$

AFLOW prototype command : aflow --proto=A9BC_oC44_39_3c3d_a_c

- params $=a, b / a, c / a, z_{1}, x_{2}, z_{2}, x_{3}, z_{3}, x_{4}, z_{4}, x_{5}, z_{5}, x_{6}, y_{6}, z_{6}, x_{7}, y_{7}, z_{7}, x_{8}, y_{8}$,

Z8

Base-centered Orthorhombic primitive vectors:

$$
\begin{aligned}
& \mathbf{a}_{1}=c \quad a \hat{\mathbf{x}} \\
& \mathbf{a}_{2}=\frac{1}{2} b \hat{\mathbf{y}}-\frac{1}{2} c \hat{\mathbf{z}} \\
& \mathbf{a}_{3}=\frac{1}{2} b \hat{\mathbf{y}}+\frac{1}{2} c \hat{\mathbf{z}}
\end{aligned}
$$

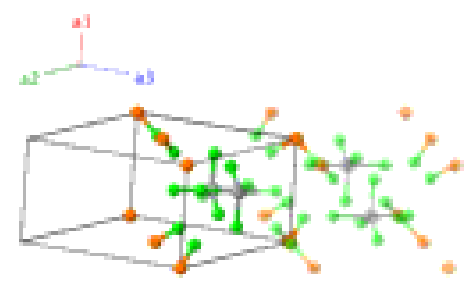

\section{Basis vectors:}

\section{Lattice Coordinates}

$\begin{array}{llclc}\mathbf{B}_{1} & = & -z_{1} \mathbf{a}_{2}+z_{1} \mathbf{a}_{3} & = & z_{1} c \hat{\mathbf{z}} \\ \mathbf{B}_{2} & = & \left(\frac{1}{2}-z_{1}\right) \mathbf{a}_{2}+\left(\frac{1}{2}+z_{1}\right) \mathbf{a}_{3} & = & \frac{1}{2} b \hat{\mathbf{y}}+z_{1} c \hat{\mathbf{z}} \\ \mathbf{B}_{3}= & x_{2} \mathbf{a}_{1}+\left(\frac{1}{4}-z_{2}\right) \mathbf{a}_{2}+\left(\frac{1}{4}+z_{2}\right) \mathbf{a}_{3} & = & x_{2} a \hat{\mathbf{x}}+\frac{1}{4} b \hat{\mathbf{y}}+z_{2} c \hat{\mathbf{z}} \\ \mathbf{B}_{4}= & -x_{2} \mathbf{a}_{1}+\left(\frac{3}{4}-z_{2}\right) \mathbf{a}_{2}+\left(\frac{3}{4}+z_{2}\right) \mathbf{a}_{3} & = & -x_{2} a \hat{\mathbf{x}}+\frac{3}{4} b \hat{\mathbf{y}}+z_{2} c \hat{\mathbf{z}}\end{array}$

Wyckoff Position Atom Type

(4a)

(4a)$$
\mathrm{P}
$$

(4c) 


\begin{tabular}{|c|c|c|c|c|c|c|}
\hline $\mathbf{B}_{5}$ & $=$ & $x_{3} \mathbf{a}_{1}+\left(\frac{1}{4}-z_{3}\right) \mathbf{a}_{2}+\left(\frac{1}{4}+z_{3}\right) \mathbf{a}_{3}$ & $=$ & $x_{3} a \hat{\mathbf{x}}+\frac{1}{4} b \hat{\mathbf{y}}+z_{3} c \hat{\mathbf{z}}$ & $(4 c)$ & $\mathrm{Cl}$ II \\
\hline $\mathbf{B}_{6}$ & $=$ & $-x_{3} \mathbf{a}_{1}+\left(\frac{3}{4}-z_{3}\right) \mathbf{a}_{2}+\left(\frac{3}{4}+z_{3}\right) \mathbf{a}_{3}$ & $=$ & $-x_{3} a \hat{\mathbf{x}}+\frac{3}{4} b \hat{\mathbf{y}}+z_{3} c \hat{\mathbf{z}}$ & $(4 c)$ & $\mathrm{Cl}$ II \\
\hline $\mathbf{B}_{7}$ & $=$ & $x_{4} \mathbf{a}_{1}+\left(\frac{1}{4}-z_{4}\right) \mathbf{a}_{2}+\left(\frac{1}{4}+z_{4}\right) \mathbf{a}_{3}$ & $=$ & $x_{4} a \hat{\mathbf{x}}+\frac{1}{4} b \hat{\mathbf{y}}+z_{4} c \hat{\mathbf{z}}$ & $(4 c)$ & $\mathrm{Cl} \mathrm{III}$ \\
\hline $\mathbf{B}_{8}$ & $=$ & $-x_{4} \mathbf{a}_{1}+\left(\frac{3}{4}-z_{4}\right) \mathbf{a}_{2}+\left(\frac{3}{4}+z_{4}\right) \mathbf{a}_{3}$ & $=$ & $-x_{4} a \hat{\mathbf{x}}+\frac{3}{4} b \hat{\mathbf{y}}+z_{4} c \hat{\mathbf{z}}$ & $(4 c)$ & $\mathrm{Cl} \mathrm{III}$ \\
\hline $\mathbf{B}_{9}$ & $=$ & $x_{5} \mathbf{a}_{1}+\left(\frac{1}{4}-z_{5}\right) \mathbf{a}_{2}+\left(\frac{1}{4}+z_{5}\right) \mathbf{a}_{3}$ & $=$ & $x_{5} a \hat{\mathbf{x}}+\frac{1}{4} b \hat{\mathbf{y}}+z_{5} c \hat{\mathbf{z}}$ & $(4 c)$ & $\mathrm{V}$ \\
\hline $\mathbf{B}_{10}$ & $=$ & $-x_{5} \mathbf{a}_{1}+\left(\frac{3}{4}-z_{5}\right) \mathbf{a}_{2}+\left(\frac{3}{4}+z_{5}\right) \mathbf{a}_{3}$ & $=$ & $-x_{5} a \hat{\mathbf{x}}+\frac{3}{4} b \hat{\mathbf{y}}+z_{5} c \hat{\mathbf{z}}$ & $(4 c)$ & $\mathrm{V}$ \\
\hline $\mathbf{B}_{11}$ & $=$ & $x_{6} \mathbf{a}_{1}+\left(y_{6}-z_{6}\right) \mathbf{a}_{2}+\left(y_{6}+z_{6}\right) \mathbf{a}_{3}$ & $=$ & $x_{6} a \hat{\mathbf{x}}+y_{6} b \hat{\mathbf{y}}+z_{6} c \hat{\mathbf{z}}$ & $(8 d)$ & $\mathrm{Cl} \mathrm{IV}$ \\
\hline $\mathbf{B}_{12}$ & $=$ & $-x_{6} \mathbf{a}_{1}+\left(-y_{6}-z_{6}\right) \mathbf{a}_{2}+\left(-y_{6}+z_{6}\right) \mathbf{a}_{3}$ & $=$ & $-x_{6} a \hat{\mathbf{x}}-y_{6} b \hat{\mathbf{y}}+z_{6} c \hat{\mathbf{z}}$ & $(8 d)$ & $\mathrm{Cl} \mathrm{IV}$ \\
\hline $\mathbf{B}_{13}$ & $=$ & $x_{6} \mathbf{a}_{1}+\left(\frac{1}{2}-y_{6}-z_{6}\right) \mathbf{a}_{2}+\left(\frac{1}{2}-y_{6}+z_{6}\right) \mathbf{a}_{3}$ & $=$ & $x_{6} a \hat{\mathbf{x}}+\left(\frac{1}{2}-y_{6}\right) b \hat{\mathbf{y}}+z_{6} c \hat{\mathbf{z}}$ & $(8 d)$ & $\mathrm{Cl} \mathrm{IV}$ \\
\hline $\mathbf{B}_{14}$ & $=$ & $\begin{array}{c}-x_{6} \mathbf{a}_{1}+\left(\frac{1}{2}+y_{6}-z_{6}\right) \mathbf{a}_{2}+ \\
\left(\frac{1}{2}+y_{6}+z_{6}\right) \mathbf{a}_{3}\end{array}$ & $=$ & $-x_{6} a \hat{\mathbf{x}}+\left(\frac{1}{2}+y_{6}\right) b \hat{\mathbf{y}}+z_{6} c \hat{\mathbf{z}}$ & $(8 d)$ & $\mathrm{Cl} \mathrm{IV}$ \\
\hline $\mathbf{B}_{15}$ & $=$ & $x_{7} \mathbf{a}_{1}+\left(y_{7}-z_{7}\right) \mathbf{a}_{2}+\left(y_{7}+z_{7}\right) \mathbf{a}_{3}$ & $=$ & $x_{7} a \hat{\mathbf{x}}+y_{7} b \hat{\mathbf{y}}+z_{7} c \hat{\mathbf{z}}$ & $(8 d)$ & $\mathrm{Cl} \mathrm{V}$ \\
\hline $\mathbf{B}_{16}$ & $=$ & $-x_{7} \mathbf{a}_{1}+\left(-y_{7}-z_{7}\right) \mathbf{a}_{2}+\left(-y_{7}+z_{7}\right) \mathbf{a}_{3}$ & $=$ & $-x_{7} a \hat{\mathbf{x}}-y_{7} b \hat{\mathbf{y}}+z_{7} c \hat{\mathbf{z}}$ & $(8 d)$ & $\mathrm{Cl} \mathrm{V}$ \\
\hline $\mathbf{B}_{17}$ & $=$ & $x_{7} \mathbf{a}_{1}+\left(\frac{1}{2}-y_{7}-z_{7}\right) \mathbf{a}_{2}+\left(\frac{1}{2}-y_{7}+z_{7}\right) \mathbf{a}_{3}$ & $=$ & $x_{7} a \hat{\mathbf{x}}+\left(\frac{1}{2}-y_{7}\right) b \hat{\mathbf{y}}+z_{7} c \hat{\mathbf{z}}$ & $(8 d)$ & $\mathrm{Cl} \mathrm{V}$ \\
\hline $\mathbf{B}_{18}$ & $=$ & $\begin{array}{c}-x_{7} \mathbf{a}_{1}+\left(\frac{1}{2}+y_{7}-z_{7}\right) \mathbf{a}_{2}+ \\
\left(\frac{1}{2}+y_{7}+z_{7}\right) \mathbf{a}_{3}\end{array}$ & $=$ & $-x_{7} a \hat{\mathbf{x}}+\left(\frac{1}{2}+y_{7}\right) b \hat{\mathbf{y}}+z_{7} c \hat{\mathbf{z}}$ & $(8 d)$ & $\mathrm{Cl} \mathrm{V}$ \\
\hline $\mathbf{B}_{19}$ & $=$ & $x_{8} \mathbf{a}_{1}+\left(y_{8}-z_{8}\right) \mathbf{a}_{2}+\left(y_{8}+z_{8}\right) \mathbf{a}_{3}$ & $=$ & $x_{8} a \hat{\mathbf{x}}+y_{8} b \hat{\mathbf{y}}+z_{8} c \hat{\mathbf{z}}$ & $(8 d)$ & $\mathrm{Cl} \mathrm{VI}$ \\
\hline $\mathbf{B}_{20}$ & $=$ & $-x_{8} \mathbf{a}_{1}+\left(-y_{8}-z_{8}\right) \mathbf{a}_{2}+\left(-y_{8}+z_{8}\right) \mathbf{a}_{3}$ & $=$ & $-x_{8} a \hat{\mathbf{x}}-y_{8} b \hat{\mathbf{y}}+z_{8} c \hat{\mathbf{z}}$ & $(8 d)$ & $\mathrm{Cl} \mathrm{VI}$ \\
\hline $\mathbf{B}_{21}$ & $=$ & $x_{8} \mathbf{a}_{1}+\left(\frac{1}{2}-y_{8}-z_{8}\right) \mathbf{a}_{2}+\left(\frac{1}{2}-y_{8}+z_{8}\right) \mathbf{a}_{3}$ & $=$ & $x_{8} a \hat{\mathbf{x}}+\left(\frac{1}{2}-y_{8}\right) b \hat{\mathbf{y}}+z_{8} c \hat{\mathbf{z}}$ & $(8 d)$ & $\mathrm{Cl} \mathrm{VI}$ \\
\hline $\mathbf{B}_{22}$ & $=$ & $\begin{array}{c}-x_{8} \mathbf{a}_{1}+\left(\frac{1}{2}+y_{8}-z_{8}\right) \mathbf{a}_{2}+ \\
\left(\frac{1}{2}+y_{8}+z_{8}\right) \mathbf{a}_{3}\end{array}$ & $=$ & $-x_{8} a \hat{\mathbf{x}}+\left(\frac{1}{2}+y_{8}\right) b \hat{\mathbf{y}}+z_{8} c \hat{\mathbf{z}}$ & $(8 d)$ & $\mathrm{Cl} \mathrm{VI}$ \\
\hline
\end{tabular}

\section{References:}

- M. L. Ziegler, B. Nuber, K. Weidenhammer, and G. Hoch, Die Molekül-und Kristallstruktur von Tetrachlorophosphoniumpentachlorovanadat (IV), $\left[\mathrm{PCl}_{4}\right]\left[\mathrm{VCl}_{5}\right] /$ The Molecular and Crystal Structure of Tetrachlorophosphoniumpentachlorovanadate (IV), $\left[\mathrm{PCl}_{4}\right]\left[\mathrm{VCl}_{5}\right], \mathrm{Z}$. Naturforsch. B 32, 18-21 (1977), doi:10.1515/znb-1977-0106.

\section{Found in:}

- P. Villars and K. Cenzual, Pearson's Crystal Data - Crystal Structure Database for Inorganic Compounds, ASM International (2013).

\section{Geometry files:}

- CIF: pp. 827

- POSCAR: pp. 827 


\section{$\mathrm{K}_{2} \mathrm{CdPb}$ Structure: AB2C_oC16_40_a_2b_b}
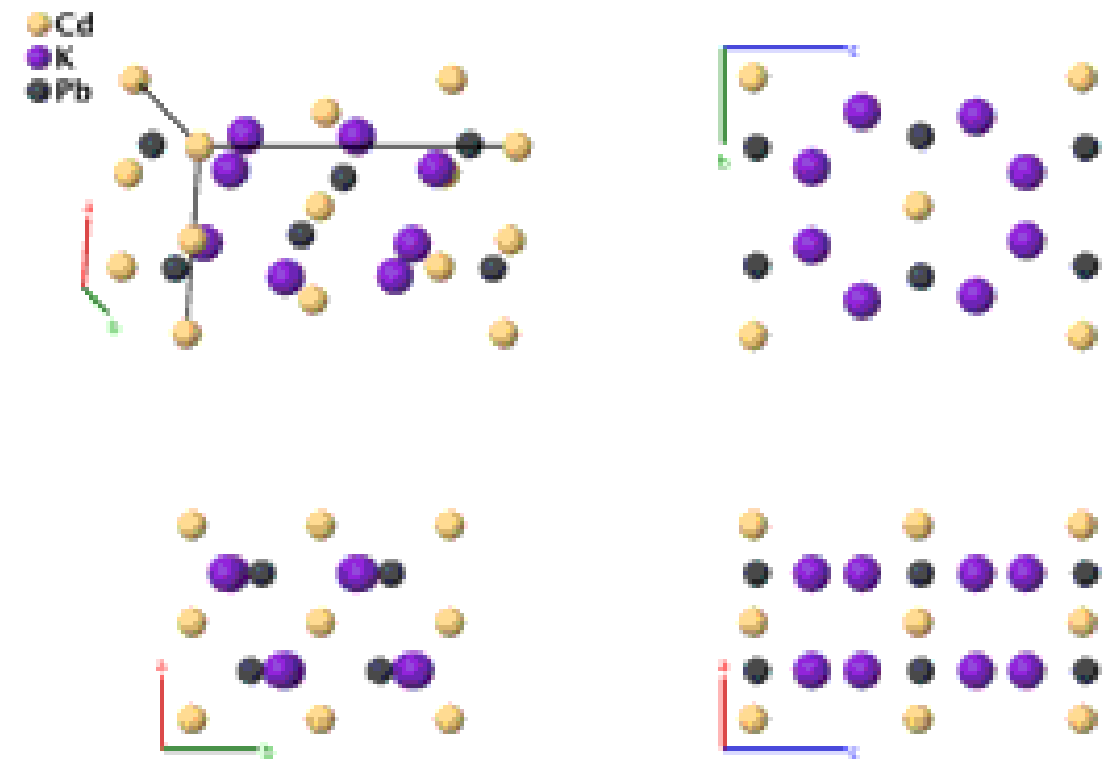

Prototype

$: \quad \mathrm{K}_{2} \mathrm{CdPb}$

AFLOW prototype label

: AB2C_oC16_40_a_2b_b

Strukturbericht designation : None

Pearson symbol :

Space group number $\quad: \quad 40$

Space group symbol $\quad: \quad A m a 2$

AFLOW prototype command : aflow --proto=AB2C_oC16_40_a_2b_b

- -params $=a, b / a, c / a, z_{1}, y_{2}, z_{2}, y_{3}, z_{3}, y_{4}, z_{4}$

Base-centered Orthorhombic primitive vectors:

$$
\begin{aligned}
& \mathbf{a}_{1}=c \quad a \hat{\mathbf{x}} \\
& \mathbf{a}_{2}=\frac{1}{2} b \hat{\mathbf{y}}-\frac{1}{2} c \hat{\mathbf{z}} \\
& \mathbf{a}_{3}=\frac{1}{2} b \hat{\mathbf{y}}+\frac{1}{2} c \hat{\mathbf{z}}
\end{aligned}
$$

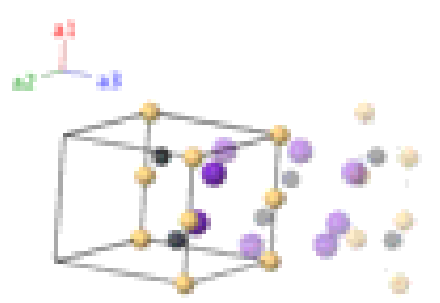

Basis vectors:

Lattice Coordinates
$\mathbf{B}_{1}=$
$-z_{1} \mathbf{a}_{2}+z_{1} \mathbf{a}_{3}$
$\mathbf{B}_{2}=$
$\frac{1}{2} \mathbf{a}_{1}-z_{1} \mathbf{a}_{2}+z_{1} \mathbf{a}_{3}$
$\mathbf{B}_{3}=$
$\mathbf{B}_{4}=$
$\mathbf{B}_{5}=$

$$
\begin{array}{lc}
= & z_{1} c \hat{\mathbf{z}} \\
= & \frac{1}{2} a \hat{\mathbf{x}}+z_{1} c \hat{\mathbf{z}} \\
= & \frac{1}{4} a \hat{\mathbf{x}}+y_{2} b \hat{\mathbf{y}}+z_{2} c \hat{\mathbf{z}} \\
= & \frac{3}{4} a \hat{\mathbf{x}}-y_{2} b \hat{\mathbf{y}}+z_{2} c \hat{\mathbf{z}} \\
= & \frac{1}{4} a \hat{\mathbf{x}}+y_{3} b \hat{\mathbf{y}}+z_{3} c \hat{\mathbf{z}}
\end{array}
$$

Cartesian Coordinates
Wyckoff Position

(4a)
Atom Type

$\mathrm{Cd}$

$\mathrm{Cd}$

K I

K I

K II 


$$
\begin{aligned}
& \mathbf{B}_{6}=\frac{3}{4} \mathbf{a}_{1}+\left(-y_{3}-z_{3}\right) \mathbf{a}_{2}+\left(-y_{3}+z_{3}\right) \mathbf{a}_{3}=\frac{3}{4} a \hat{\mathbf{x}}-y_{3} b \hat{\mathbf{y}}+z_{3} c \hat{\mathbf{z}} \\
& \mathbf{B}_{7}=\frac{1}{4} \mathbf{a}_{1}+\left(y_{4}-z_{4}\right) \mathbf{a}_{2}+\left(y_{4}+z_{4}\right) \mathbf{a}_{3}=\frac{1}{4} a \hat{\mathbf{x}}+y_{4} b \hat{\mathbf{y}}+z_{4} c \hat{\mathbf{z}} \\
& \mathbf{B}_{8}=\frac{3}{4} \mathbf{a}_{1}+\left(-y_{4}-z_{4}\right) \mathbf{a}_{2}+\left(-y_{4}+z_{4}\right) \mathbf{a}_{3}=\frac{3}{4} a \hat{\mathbf{x}}-y_{4} b \hat{\mathbf{y}}+z_{4} c \hat{\mathbf{z}}
\end{aligned}
$$

\section{References:}

- R. Matthes and H.-U. Schuster, Synthese und Struktur der Phasen $K_{2} C d S n$ und $K_{2} C d P b /$ Synthesis and Structure of the Phase $K_{2} C d S n$ and $K_{2} C d P b$, Z. Naturforsch. B 34, 541-543 (1979), doi:10.1515/znb-1979-0403.

\section{Found in:}

- P. Villars and K. Cenzual, Pearson's Crystal Data - Crystal Structure Database for Inorganic Compounds, ASM International (2013).

\section{Geometry files:}

- CIF: pp. 828

- POSCAR: pp. 828 


\section{$\mathrm{CeTe}_{3}$ Structure: AB3_oC16_40_b_3b}
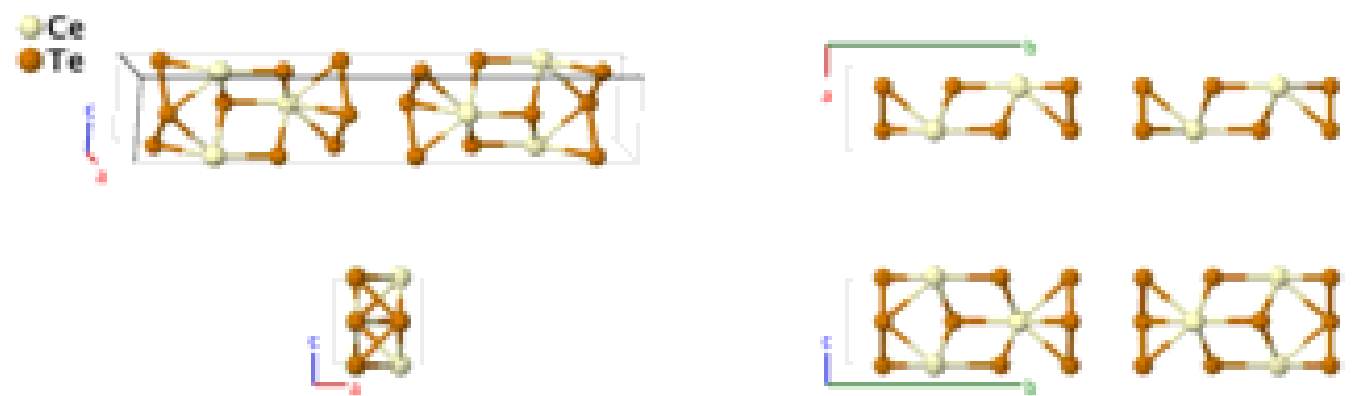

\section{Prototype}

AFLOW prototype label

Strukturbericht designation

Pearson symbol

Space group number

Space group symbol

AFLOW prototype command
$\mathrm{CeTe}_{3}$

: AB3_oC16_40_b_3b

: None

: $\quad$ oC16

: 40

: $\quad A m a 2$

Base-centered Orthorhombic primitive vectors:

$$
\begin{aligned}
& \mathbf{a}_{1}=c \quad a \hat{\mathbf{x}} \\
& \mathbf{a}_{2}=\frac{1}{2} b \hat{\mathbf{y}}-\frac{1}{2} c \hat{\mathbf{z}} \\
& \mathbf{a}_{3}=\frac{1}{2} b \hat{\mathbf{y}}+\frac{1}{2} c \hat{\mathbf{z}}
\end{aligned}
$$

Basis vectors:

Lattice Coordinates

$$
\begin{aligned}
& \mathbf{B}_{1}=\frac{1}{4} \mathbf{a}_{1}+\left(y_{1}-z_{1}\right) \mathbf{a}_{2}+\left(y_{1}+z_{1}\right) \mathbf{a}_{3}=\frac{1}{4} a \hat{\mathbf{x}}+y_{1} b \hat{\mathbf{y}}+z_{1} c \hat{\mathbf{z}} \\
& \mathbf{B}_{2}=\frac{3}{4} \mathbf{a}_{1}+\left(-y_{1}-z_{1}\right) \mathbf{a}_{2}+\left(-y_{1}+z_{1}\right) \mathbf{a}_{3}=\frac{3}{4} a \hat{\mathbf{x}}-y_{1} b \hat{\mathbf{y}}+z_{1} c \hat{\mathbf{z}} \\
& \mathbf{B}_{3}=\frac{1}{4} \mathbf{a}_{1}+\left(y_{2}-z_{2}\right) \mathbf{a}_{2}+\left(y_{2}+z_{2}\right) \mathbf{a}_{3}=\frac{1}{4} a \hat{\mathbf{x}}+y_{2} b \hat{\mathbf{y}}+z_{2} c \hat{\mathbf{z}} \\
& \mathbf{B}_{4}=\frac{3}{4} \mathbf{a}_{1}+\left(-y_{2}-z_{2}\right) \mathbf{a}_{2}+\left(-y_{2}+z_{2}\right) \mathbf{a}_{3}=\frac{3}{4} a \hat{\mathbf{x}}-y_{2} b \hat{\mathbf{y}}+z_{2} c \hat{\mathbf{z}} \\
& \mathbf{B}_{5}=\frac{1}{4} \mathbf{a}_{1}+\left(y_{3}-z_{3}\right) \mathbf{a}_{2}+\left(y_{3}+z_{3}\right) \mathbf{a}_{3}=\frac{1}{4} a \hat{\mathbf{x}}+y_{3} b \hat{\mathbf{y}}+z_{3} c \hat{\mathbf{z}} \\
& \mathbf{B}_{6}=\frac{3}{4} \mathbf{a}_{1}+\left(-y_{3}-z_{3}\right) \mathbf{a}_{2}+\left(-y_{3}+z_{3}\right) \mathbf{a}_{3}=\frac{3}{4} a \hat{\mathbf{x}}-y_{3} b \hat{\mathbf{y}}+z_{3} c \hat{\mathbf{z}} \\
& \mathbf{B}_{7}=\frac{1}{4} \mathbf{a}_{1}+\left(y_{4}-z_{4}\right) \mathbf{a}_{2}+\left(y_{4}+z_{4}\right) \mathbf{a}_{3}=\frac{1}{4} a \hat{\mathbf{x}}+y_{4} b \hat{\mathbf{y}}+z_{4} c \hat{\mathbf{z}} \\
& \mathbf{B}_{8}=\frac{3}{4} \mathbf{a}_{1}+\left(-y_{4}-z_{4}\right) \mathbf{a}_{2}+\left(-y_{4}+z_{4}\right) \mathbf{a}_{3}=\frac{3}{4} a \hat{\mathbf{x}}-y_{4} b \hat{\mathbf{y}}+z_{4} c \hat{\mathbf{z}}
\end{aligned}
$$

Wyckoff Position
Atom Type

$\mathrm{Ce}$

$\mathrm{Ce}$

Te I

Te I

Te II

Te II

Te III

Te III

\section{References:}

- C. Malliakas, S. J. L. Billinge, H. J. Kim, and M. G. Kanatzidis, Square Nets of Tellurium: Rare-Earth Dependent Variation in the Charge-Density Wave of $R_{E T e_{3}}(R E=$ Rare-Earth Element), J. Am. Chem. Soc. 127, 6510-6511 (2005), doi:10.1021/ja0505292.

\section{Found in:}


- P. Villars and K. Cenzual, Pearson's Crystal Data - Crystal Structure Database for Inorganic Compounds, ASM International (2013).

\section{Geometry files:}

- CIF: pp. 828

- POSCAR: pp. 828 


\section{$\mathrm{W}_{3} \mathrm{O}_{10}$ Structure: A10B3_oF52_42_2abce_ab}
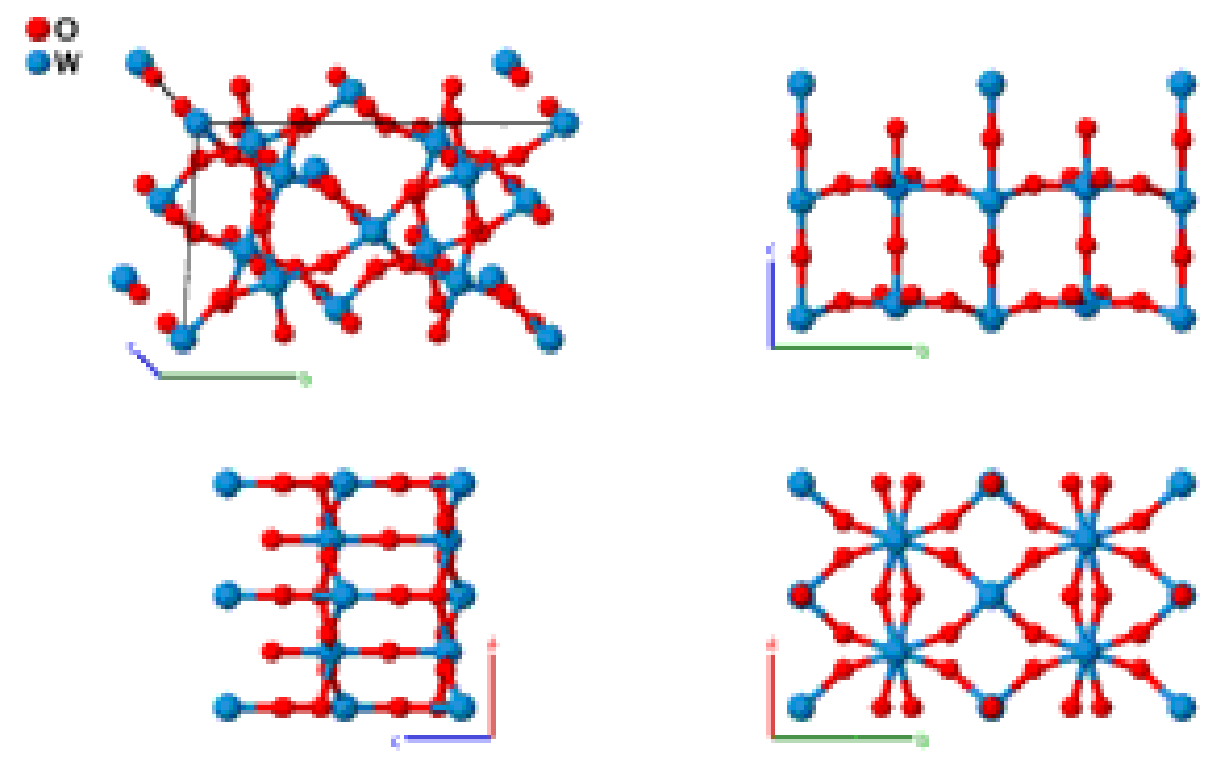

\section{Prototype}

AFLOW prototype label

$: \quad \mathrm{W}_{3} \mathrm{O}_{10}$

Strukturbericht designation

Pearson symbol

: A10B3_oF52_42_2abce_ab

Space group number

: None

Space group symbol

: $\quad 42$

AFLOW prototype command

: $F m m 2$

: aflow --proto=A10B3_oF52_42_2abce_ab

- params $=a, b / a, c / a, z_{1}, z_{2}, z_{3}, z_{4}, z_{5}, y_{6}, z_{6}, x_{7}, y_{7}, z_{7}$

Face-centered Orthorhombic primitive vectors:

$$
\begin{aligned}
& \mathbf{a}_{1}=\frac{1}{2} b \hat{\mathbf{y}}+\frac{1}{2} c \hat{\mathbf{z}} \\
& \mathbf{a}_{2}=\frac{1}{2} a \hat{\mathbf{x}}+\frac{1}{2} c \hat{\mathbf{z}} \\
& \mathbf{a}_{3}=\frac{1}{2} a \hat{\mathbf{x}}+\frac{1}{2} b \hat{\mathbf{y}}
\end{aligned}
$$

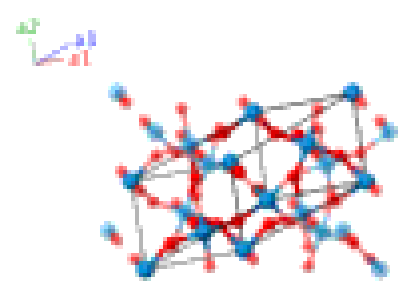

Basis vectors:

Lattice Coordinates

$\begin{array}{lcc}\mathbf{B}_{1}= & z_{1} \mathbf{a}_{1}+z_{1} \mathbf{a}_{2}-z_{1} \mathbf{a}_{3} \\ \mathbf{B}_{2}= & z_{2} \mathbf{a}_{1}+z_{2} \mathbf{a}_{2}-z_{2} \mathbf{a}_{3} \\ \mathbf{B}_{3}= & z_{3} \mathbf{a}_{1}+z_{3} \mathbf{a}_{2}-z_{3} \mathbf{a}_{3} \\ \mathbf{B}_{4}= & z_{4} \mathbf{a}_{1}+z_{4} \mathbf{a}_{2}+\left(\frac{1}{2}-z_{4}\right) \mathbf{a}_{3} \\ \mathbf{B}_{5}= & \left(\frac{1}{2}+z_{4}\right) \mathbf{a}_{1}+\left(\frac{1}{2}+z_{4}\right) \mathbf{a}_{2}-z_{4} \mathbf{a}_{3} \\ \mathbf{B}_{6}= & z_{5} \mathbf{a}_{1}+z_{5} \mathbf{a}_{2}+\left(\frac{1}{2}-z_{5}\right) \mathbf{a}_{3}\end{array}$

Cartesian Coordinates

$=$

$=$

$$
=
$$$$
=\quad \frac{1}{4} a \hat{\mathbf{x}}+\frac{1}{4} b \hat{\mathbf{y}}+z_{4} c \hat{\mathbf{z}}
$$$$
=\frac{1}{4} a \hat{\mathbf{x}}+\frac{1}{4} b \hat{\mathbf{y}}+\left(\frac{1}{2}+z_{4}\right) c \hat{\mathbf{z}}
$$$$
=\quad \frac{1}{4} a \hat{\mathbf{x}}+\frac{1}{4} b \hat{\mathbf{y}}+z_{5} c \hat{\mathbf{z}}
$$

Wyckoff Position Atom Type

(4a)

O I

(4a)

O II

(4a)

W I

(8b)

O III

(8b)

O III

(8b) 


\begin{tabular}{|c|c|c|c|c|c|}
\hline $\mathbf{B}_{7}$ & $=$ & $\left(\frac{1}{2}+z_{5}\right) \mathbf{a}_{1}+\left(\frac{1}{2}+z_{5}\right) \mathbf{a}_{2}-z_{5} \mathbf{a}_{3}$ & $=$ & $\frac{1}{4} a \hat{\mathbf{x}}+\frac{1}{4} b \hat{\mathbf{y}}+\left(\frac{1}{2}+z_{5}\right) c \hat{\mathbf{z}}$ & $(8 b)$ \\
\hline $\mathbf{B}_{8}$ & $=$ & $\left(y_{6}+z_{6}\right) \mathbf{a}_{1}+\left(-y_{6}+z_{6}\right) \mathbf{a}_{2}+\left(y_{6}-z_{6}\right) \mathbf{a}_{3}$ & $=$ & $y_{6} b \hat{\mathbf{y}}+z_{6} c \hat{\mathbf{z}}$ & $(8 c)$ \\
\hline $\mathbf{B}_{9}$ & $=$ & $\left(-y_{6}+z_{6}\right) \mathbf{a}_{1}+\left(y_{6}+z_{6}\right) \mathbf{a}_{2}+\left(-y_{6}-z_{6}\right) \mathbf{a}_{3}$ & $=$ & $-y_{6} b \hat{\mathbf{y}}+z_{6} c \hat{\mathbf{z}}$ & $(8 c)$ \\
\hline $\mathbf{B}_{10}$ & $=$ & $\begin{array}{c}\left(-x_{7}+y_{7}+z_{7}\right) \mathbf{a}_{1}+\left(x_{7}-y_{7}+z_{7}\right) \mathbf{a}_{2}+ \\
\left(x_{7}+y_{7}-z_{7}\right) \mathbf{a}_{3}\end{array}$ & $=$ & $x_{7} a \hat{\mathbf{x}}+y_{7} b \hat{\mathbf{y}}+z_{7} c \hat{\mathbf{z}}$ & $(16 e)$ \\
\hline $\mathbf{B}_{11}$ & $=$ & $\begin{array}{c}\left(x_{7}-y_{7}+z_{7}\right) \mathbf{a}_{1}+\left(-x_{7}+y_{7}+z_{7}\right) \mathbf{a}_{2}+ \\
\left(-x_{7}-y_{7}-z_{7}\right) \mathbf{a}_{3}\end{array}$ & $=$ & $-x_{7} a \hat{\mathbf{x}}-y_{7} b \hat{\mathbf{y}}+z_{7} c \hat{\mathbf{z}}$ & $(16 e)$ \\
\hline $\mathbf{B}_{12}$ & $=$ & $\begin{array}{c}\left(-x_{7}-y_{7}+z_{7}\right) \mathbf{a}_{1}+\left(x_{7}+y_{7}+z_{7}\right) \mathbf{a}_{2}+ \\
\left(x_{7}-y_{7}-z_{7}\right) \mathbf{a}_{3}\end{array}$ & $=$ & $x_{7} a \hat{\mathbf{x}}-y_{7} b \hat{\mathbf{y}}+z_{7} c \hat{\mathbf{z}}$ & $(16 e)$ \\
\hline $\mathbf{B}_{13}$ & $=$ & $\begin{array}{c}\left(x_{7}+y_{7}+z_{7}\right) \mathbf{a}_{1}+\left(-x_{7}-y_{7}+z_{7}\right) \mathbf{a}_{2}+ \\
\left(-x_{7}+y_{7}-z_{7}\right) \mathbf{a}_{3}\end{array}$ & $=$ & $-x_{7} a \hat{\mathbf{x}}+y_{7} b \hat{\mathbf{y}}+z_{7} c \hat{\mathbf{z}}$ & $(16 e)$ \\
\hline
\end{tabular}

\section{References:}

- B. Gerand, G. Nowogrocki, and M. Figlarz, A new tungsten trioxide hydrate, $\mathrm{WO}_{3} \cdot 1 / 3 \mathrm{H}_{2} \mathrm{O}$ : Preparation, characterization, and crystallographic study, J. Solid State Chem. 38, 312-320 (1981), doi:10.1016/0022-4596(81)90062-1.

\section{Found in:}

- P. Villars and K. Cenzual, Pearson's Crystal Data - Crystal Structure Database for Inorganic Compounds, ASM International (2013).

\section{Geometry files:}

- CIF: pp. 828

- POSCAR: pp. 829 


\section{BN (High-pressure, high-temperature) Structure: AB_oF8_42_a_a}

$2 \mathrm{~B}$
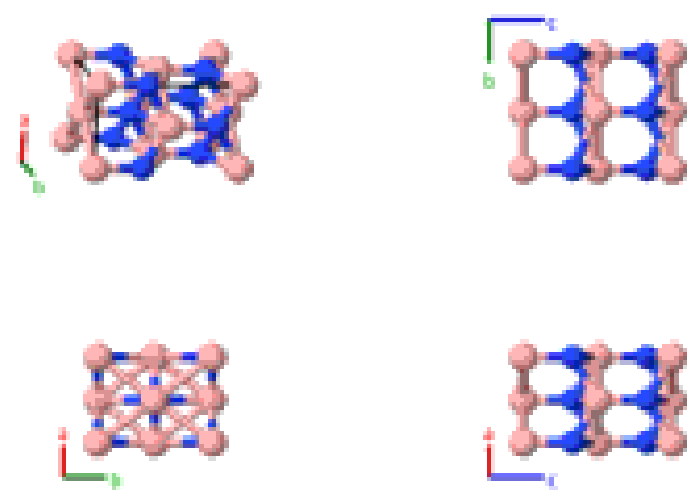

Prototype

: $\mathrm{BN}$

AFLOW prototype label

: AB_oF8_42_a_a

Strukturbericht designation

Pearson symbol

None

Space group number

oF8

Space group symbol

42

AFLOW prototype command

: $\quad F m m 2$

aflow - -proto=AB_oF8_42_a_a

- params $=a, b / a, c / a, z_{1}, z_{2}$

Face-centered Orthorhombic primitive vectors:

$$
\begin{aligned}
& \mathbf{a}_{1}=\frac{1}{2} b \hat{\mathbf{y}}+\frac{1}{2} c \hat{\mathbf{z}} \\
& \mathbf{a}_{2}=\frac{1}{2} a \hat{\mathbf{x}}+\frac{1}{2} c \hat{\mathbf{z}} \\
& \mathbf{a}_{3}=\frac{1}{2} a \hat{\mathbf{x}}+\frac{1}{2} b \hat{\mathbf{y}}
\end{aligned}
$$

\begin{tabular}{|c|c|c|c|c|c|}
\hline & Lattice Coordinates & & Cartesian Coordinates & Wyckoff Position & Atom Type \\
\hline $\mathbf{B}_{1}$ & $z_{1} \mathbf{a}_{1}+z_{1} \mathbf{a}_{2}-z_{1} \mathbf{a}_{3}$ & $=$ & $z_{1} c \hat{\mathbf{z}}$ & $(4 a)$ & B \\
\hline$B_{2}=$ & $z_{2} \mathbf{a}_{1}+z_{2} \mathbf{a}_{2}-z_{2} \mathbf{a}_{3}$ & $=$ & $z_{2} c \hat{\mathbf{z}}$ & $(4 a)$ & $\mathrm{N}$ \\
\hline
\end{tabular}

Basis vectors:

\section{References:}

- A. V. Kurdyumov and G. S. Olejnik, On metastable structures of graphite-like boron nitride, Kristallografiya 29, 792-793 (1984).

Found in:

- P. Villars and K. Cenzual, Pearson's Crystal Data - Crystal Structure Database for Inorganic Compounds, ASM International (2013). 
Geometry files:

- CIF: pp. 829

- POSCAR: pp. 829 


\section{$\mathrm{MnGa}_{2} \mathrm{Sb}_{2}$ Structure: A2BC2_oI20_45_c_b_c}
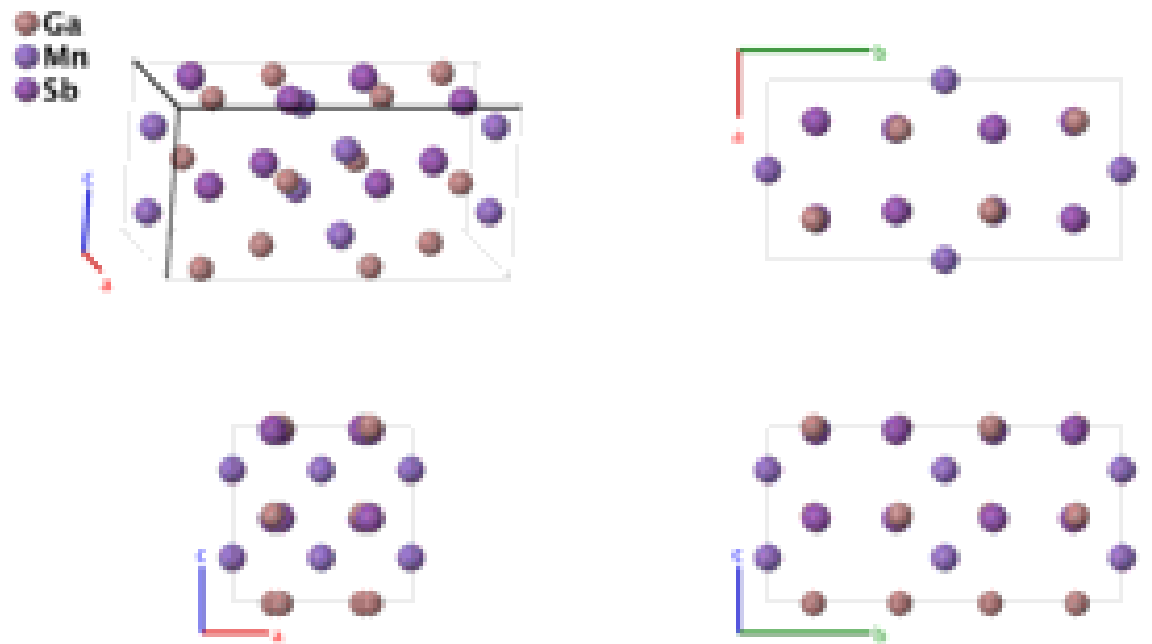

Prototype

AFLOW prototype label

Strukturbericht designation

Pearson symbol

Space group number

Space group symbol

AFLOW prototype command
: $\quad \mathrm{MnGa}_{2} \mathrm{Sb}_{2}$

: A2BC2_oI20_45_c_b_c

: None

: $\quad$ oI20

: $\quad 45$

: $\quad I b a 2$

: aflow --proto=A2BC2_oI20_45_c_b_c

- params $=a, b / a, c / a, z_{1}, x_{2}, y_{2}, z_{2}, x_{3}, y_{3}, z_{3}$

- The Mn site (4b) is reported with an occupation of 0.94 .

Body-centered Orthorhombic primitive vectors:

$$
\begin{aligned}
& \mathbf{a}_{1}=-\frac{1}{2} a \hat{\mathbf{x}}+\frac{1}{2} b \hat{\mathbf{y}}+\frac{1}{2} c \hat{\mathbf{z}} \\
& \mathbf{a}_{2}=\frac{1}{2} a \hat{\mathbf{x}}-\frac{1}{2} b \hat{\mathbf{y}}+\frac{1}{2} c \hat{\mathbf{z}} \\
& \mathbf{a}_{3}=\frac{1}{2} a \hat{\mathbf{x}}+\frac{1}{2} b \hat{\mathbf{y}}-\frac{1}{2} c \hat{\mathbf{z}}
\end{aligned}
$$

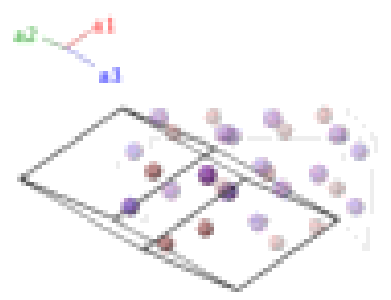

Basis vectors:

Lattice Coordinates

$\mathbf{B}_{1}=\left(\frac{1}{2}+z_{1}\right) \mathbf{a}_{1}+z_{1} \mathbf{a}_{2}+\frac{1}{2} \mathbf{a}_{3}$

$\mathbf{B}_{2}=z_{1} \mathbf{a}_{1}+\left(\frac{1}{2}+z_{1}\right) \mathbf{a}_{2}+\frac{1}{2} \mathbf{a}_{3}$

$\mathbf{B}_{3}=\left(y_{2}+z_{2}\right) \mathbf{a}_{1}+\left(x_{2}+z_{2}\right) \mathbf{a}_{2}+\left(x_{2}+y_{2}\right) \mathbf{a}_{3}=$

$\mathbf{B}_{4}=$

$\left(-x_{2}-y_{2}\right) \mathbf{a}_{3}$
$\mathbf{B}_{4}$

$\mathbf{B}_{5}=\left(\frac{1}{2}-y_{2}+z_{2}\right) \mathbf{a}_{1}+\left(\frac{1}{2}+x_{2}+z_{2}\right) \mathbf{a}_{2}+\quad=\quad x_{2} a \hat{\mathbf{x}}-y_{2} b \hat{\mathbf{y}}+\left(\frac{1}{2}+z_{2}\right) c \hat{\mathbf{z}}$ $\left(x_{2}-y_{2}\right) \mathbf{a}_{3}$
Cartesian Coordinates

Wyckoff Position

Atom Type

$=\quad \frac{1}{2} b \hat{\mathbf{y}}+z_{1} c \hat{\mathbf{z}}$

$(4 b)$

$\mathrm{Mn}$

$=\quad \frac{1}{2} a \hat{\mathbf{x}}+z_{1} c \hat{\mathbf{z}}$

$(4 b)$

$\mathrm{Mn}$

$=\quad x_{2} a \hat{\mathbf{x}}+y_{2} b \hat{\mathbf{y}}+z_{2} c \hat{\mathbf{z}}$

$\mathrm{Ga}$

$(8 c)$

$\mathrm{Ga}$

$(8 c)$

$\mathrm{Ga}$ 


\begin{tabular}{|c|c|c|c|c|}
\hline $\mathbf{B}_{6}$ & $\begin{array}{c}\left(\frac{1}{2}+y_{2}+z_{2}\right) \mathbf{a}_{1}+\left(\frac{1}{2}-x_{2}+z_{2}\right) \mathbf{a}_{2}+ \\
\left(-x_{2}+y_{2}\right) \mathbf{a}_{3}\end{array}$ & $=$ & $-x_{2} a \hat{\mathbf{x}}+y_{2} b \hat{\mathbf{y}}+\left(\frac{1}{2}+z_{2}\right) c \hat{\mathbf{z}}$ & $(8 c)$ \\
\hline $\mathbf{B}_{7}$ & $=\left(y_{3}+z_{3}\right) \mathbf{a}_{1}+\left(x_{3}+z_{3}\right) \mathbf{a}_{2}+\left(x_{3}+y_{3}\right) \mathbf{a}_{3}$ & $=$ & $x_{3} a \hat{\mathbf{x}}+y_{3} b \hat{\mathbf{y}}+z_{3} c \hat{\mathbf{z}}$ & $(8 c)$ \\
\hline $\mathbf{B}_{8}$ & $\begin{array}{c}\left(-y_{3}+z_{3}\right) \mathbf{a}_{1}+\left(-x_{3}+z_{3}\right) \mathbf{a}_{2}+ \\
\left(-x_{3}-y_{3}\right) \mathbf{a}_{3}\end{array}$ & $=$ & $-x_{3} a \hat{\mathbf{x}}-y_{3} b \hat{\mathbf{y}}+z_{3} c \hat{\mathbf{z}}$ & $(8 c)$ \\
\hline $\mathbf{B}_{9}$ & 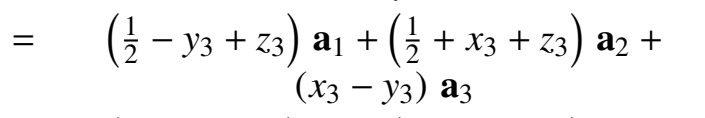 & $=$ & $x_{3} a \hat{\mathbf{x}}-y_{3} b \hat{\mathbf{y}}+\left(\frac{1}{2}+z_{3}\right) c \hat{\mathbf{z}}$ & $(8 c)$ \\
\hline $\mathbf{B}_{10}$ & $\begin{array}{c}\left(\frac{1}{2}+y_{3}+z_{3}\right) \mathbf{a}_{1}+\left(\frac{1}{2}-x_{3}+z_{3}\right) \mathbf{a}_{2}+ \\
\left(-x_{3}+y_{3}\right) \mathbf{a}_{3}\end{array}$ & $=$ & $-x_{3} a \hat{\mathbf{x}}+y_{3} b \hat{\mathbf{y}}+\left(\frac{1}{2}+z_{3}\right) c \hat{\mathbf{z}}$ & $(8 c)$ \\
\hline
\end{tabular}

\section{References:}

- W. Sakakibara, Y. Hayashi, and H. Takizawa, $\mathrm{MnGa}_{2} \mathrm{Sb}_{2}$, a new ferromagnetic compound synthesized under high pressure, J. Ceram. Soc. Jpn. 117, 72-75 (2009), doi:10.2109/jcersj2.117.72.

\section{Found in:}

- P. Villars and K. Cenzual, Pearson's Crystal Data - Crystal Structure Database for Inorganic Compounds, ASM International (2013).

\section{Geometry files:}

- CIF: pp. 829

- POSCAR: pp. 830 


\section{TiFeSi Structure: ABC_oI36_46_ac_bc_3b}
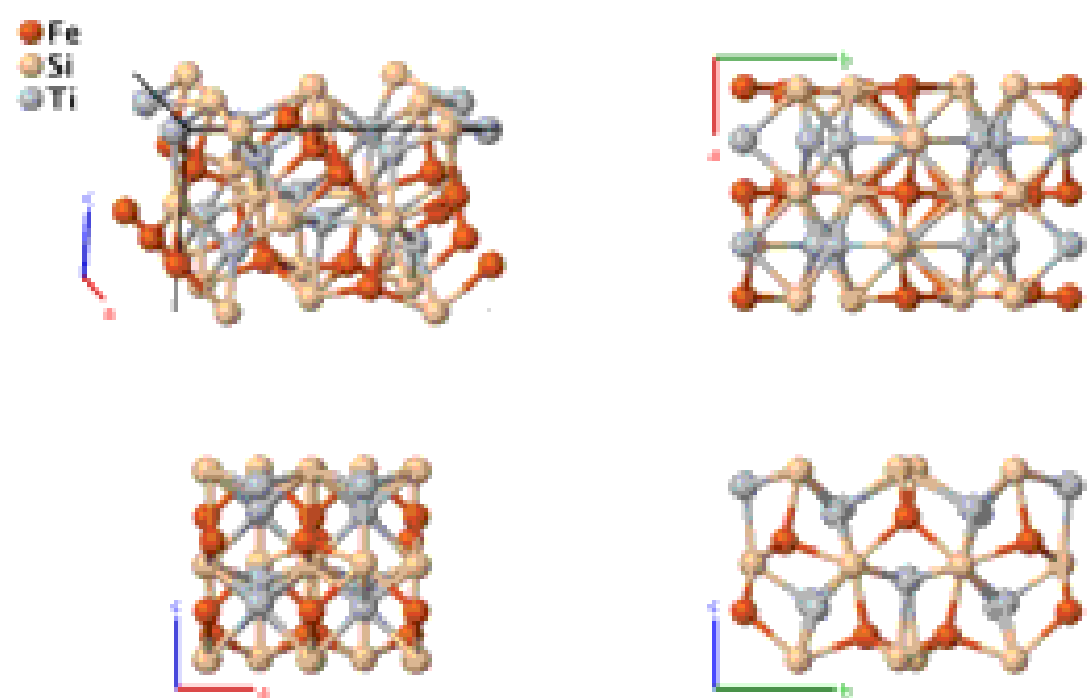

\section{Prototype}

AFLOW prototype label

Strukturbericht designation

Pearson symbol

Space group number

Space group symbol

AFLOW prototype command
: $\quad$ TiFeSi

: ABC_oI36_46_ac_bc_3b

: None

: $\quad$ oI36

$: \quad 46$

: $\quad \operatorname{Ima2}$

Body-centered Orthorhombic primitive vectors:

$$
\begin{aligned}
& \mathbf{a}_{1}=-\frac{1}{2} a \hat{\mathbf{x}}+\frac{1}{2} b \hat{\mathbf{y}}+\frac{1}{2} c \hat{\mathbf{z}} \\
& \mathbf{a}_{2}=\frac{1}{2} a \hat{\mathbf{x}}-\frac{1}{2} b \hat{\mathbf{y}}+\frac{1}{2} c \hat{\mathbf{z}} \\
& \mathbf{a}_{3}=\frac{1}{2} a \hat{\mathbf{x}}+\frac{1}{2} b \hat{\mathbf{y}}-\frac{1}{2} c \hat{\mathbf{z}}
\end{aligned}
$$

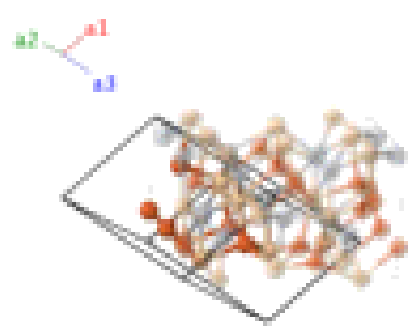

Basis vectors:

\section{Lattice Coordinates}

$\begin{array}{lcc}\mathbf{B}_{1}= & z_{1} \mathbf{a}_{1}+z_{1} \mathbf{a}_{2} \\ \mathbf{B}_{2}= & z_{1} \mathbf{a}_{1}+\left(\frac{1}{2}+z_{1}\right) \mathbf{a}_{2}+\frac{1}{2} \mathbf{a}_{3} \\ \mathbf{B}_{3}= & \left(y_{2}+z_{2}\right) \mathbf{a}_{1}+\left(\frac{1}{4}+z_{2}\right) \mathbf{a}_{2}+\left(\frac{1}{4}+y_{2}\right) \mathbf{a}_{3} \\ \mathbf{B}_{4}= & \left(-y_{2}+z_{2}\right) \mathbf{a}_{1}+\left(\frac{3}{4}+z_{2}\right) \mathbf{a}_{2}+\left(\frac{3}{4}-y_{2}\right) \mathbf{a}_{3} \\ \mathbf{B}_{5}= & \left(y_{3}+z_{3}\right) \mathbf{a}_{1}+\left(\frac{1}{4}+z_{3}\right) \mathbf{a}_{2}+\left(\frac{1}{4}+y_{3}\right) \mathbf{a}_{3} \\ \mathbf{B}_{6}= & \left(-y_{3}+z_{3}\right) \mathbf{a}_{1}+\left(\frac{3}{4}+z_{3}\right) \mathbf{a}_{2}+\left(\frac{3}{4}-y_{3}\right) \mathbf{a}_{3} \\ \mathbf{B}_{7}= & \left(y_{4}+z_{4}\right) \mathbf{a}_{1}+\left(\frac{1}{4}+z_{4}\right) \mathbf{a}_{2}+\left(\frac{1}{4}+y_{4}\right) \mathbf{a}_{3}\end{array}$

Cartesian Coordinates

$=$

$=$

$=$

$=$

$=$

$=$

$=$

$$
\begin{gathered}
z_{1} c \hat{\mathbf{z}} \\
\frac{1}{2} a \hat{\mathbf{x}}+z_{1} c \hat{\mathbf{z}} \\
\frac{1}{4} a \hat{\mathbf{x}}+y_{2} b \hat{\mathbf{y}}+z_{2} c \hat{\mathbf{z}} \\
\frac{3}{4} a \hat{\mathbf{x}}-y_{2} b \hat{\mathbf{y}}+z_{2} c \hat{\mathbf{z}} \\
\frac{1}{4} a \hat{\mathbf{x}}+y_{3} b \hat{\mathbf{y}}+z_{3} c \hat{\mathbf{z}} \\
\frac{3}{4} a \hat{\mathbf{x}}-y_{3} b \hat{\mathbf{y}}+z_{3} c \hat{\mathbf{z}} \\
\frac{1}{4} a \hat{\mathbf{x}}+y_{4} b \hat{\mathbf{y}}+z_{4} c \hat{\mathbf{z}}
\end{gathered}
$$

Wyckoff Position

$(4 a)$
Atom Type

Fe I

$\mathrm{Fe} \mathrm{I}$

Si I

Si I

Ti I

Ti I

Ti II 


\begin{tabular}{|c|c|c|c|c|c|}
\hline $\mathbf{B}_{8}$ & $=$ & $\left(-y_{4}+z_{4}\right) \mathbf{a}_{1}+\left(\frac{3}{4}+z_{4}\right) \mathbf{a}_{2}+\left(\frac{3}{4}-y_{4}\right) \mathbf{a}_{3}$ & $\frac{3}{4} a \hat{\mathbf{x}}-y_{4} b \hat{\mathbf{y}}+z_{4} c \hat{\mathbf{z}}$ & $(4 b)$ & Ti II \\
\hline $\mathbf{B}_{9}$ & $=$ & $\left(y_{5}+z_{5}\right) \mathbf{a}_{1}+\left(\frac{1}{4}+z_{5}\right) \mathbf{a}_{2}+\left(\frac{1}{4}+y_{5}\right) \mathbf{a}_{3}$ & $\frac{1}{4} a \hat{\mathbf{x}}+y_{5} b \hat{\mathbf{y}}+z_{5} c \hat{\mathbf{z}}$ & $(4 b)$ & Ti III \\
\hline $\mathbf{B}_{10}$ & $=$ & $\left(-y_{5}+z_{5}\right) \mathbf{a}_{1}+\left(\frac{3}{4}+z_{5}\right) \mathbf{a}_{2}+\left(\frac{3}{4}-y_{5}\right) \mathbf{a}_{3}$ & $\frac{3}{4} a \hat{\mathbf{x}}-y_{5} b \hat{\mathbf{y}}+z_{5} c \hat{\mathbf{z}}$ & $(4 b)$ & Ti III \\
\hline $\mathbf{B}_{11}$ & $=$ & $\left(y_{6}+z_{6}\right) \mathbf{a}_{1}+\left(x_{6}+z_{6}\right) \mathbf{a}_{2}+\left(x_{6}+y_{6}\right) \mathbf{a}_{3}$ & $x_{6} a \hat{\mathbf{x}}+y_{6} b \hat{\mathbf{y}}+z_{6} c \hat{\mathbf{z}}$ & $(8 c)$ & Fe II \\
\hline $\mathbf{B}_{12}$ & $=$ & $\begin{array}{c}\left(-y_{6}+z_{6}\right) \mathbf{a}_{1}+\left(-x_{6}+z_{6}\right) \mathbf{a}_{2}+ \\
\left(-x_{6}-y_{6}\right) \mathbf{a}_{3}\end{array}$ & $-x_{6} a \hat{\mathbf{x}}-y_{6} b \hat{\mathbf{y}}+z_{6} c \hat{\mathbf{z}}$ & $(8 c)$ & Fe II \\
\hline $\mathbf{B}_{13}$ & $=$ & $\begin{array}{c}\left(-y_{6}+z_{6}\right) \mathbf{a}_{1}+\left(\frac{1}{2}+x_{6}+z_{6}\right) \mathbf{a}_{2}+ \\
\left(\frac{1}{2}+x_{6}-y_{6}\right) \mathbf{a}_{3}\end{array}$ & $=\left(\frac{1}{2}+x_{6}\right) a \hat{\mathbf{x}}-y_{6} b \hat{\mathbf{y}}+z_{6} c \hat{\mathbf{z}}$ & $(8 c)$ & $\mathrm{Fe}$ II \\
\hline $\mathbf{B}_{14}$ & $=$ & $\begin{array}{c}\left(y_{6}+z_{6}\right) \mathbf{a}_{1}+\left(\frac{1}{2}-x_{6}+z_{6}\right) \mathbf{a}_{2}+ \\
\left(\frac{1}{2}-x_{6}+y_{6}\right) \mathbf{a}_{3}\end{array}$ & $=\left(\frac{1}{2}-x_{6}\right) a \hat{\mathbf{x}}+y_{6} b \hat{\mathbf{y}}+z_{6} c \hat{\mathbf{z}}$ & $(8 c)$ & Fe II \\
\hline $\mathbf{B}_{15}$ & $=$ & $\left(y_{7}+z_{7}\right) \mathbf{a}_{1}+\left(x_{7}+z_{7}\right) \mathbf{a}_{2}+\left(x_{7}+y_{7}\right) \mathbf{a}_{3}$ & $x_{7} a \hat{\mathbf{x}}+y_{7} b \hat{\mathbf{y}}+z_{7} c \hat{\mathbf{z}}$ & $(8 c)$ & Si II \\
\hline $\mathbf{B}_{16}$ & $=$ & $\begin{array}{c}\left(-y_{7}+z_{7}\right) \mathbf{a}_{1}+\left(-x_{7}+z_{7}\right) \mathbf{a}_{2}+ \\
\left(-x_{7}-y_{7}\right) \mathbf{a}_{3}\end{array}$ & $-x_{7} a \hat{\mathbf{x}}-y_{7} b \hat{\mathbf{y}}+z_{7} c \hat{\mathbf{z}}$ & $(8 c)$ & Si II \\
\hline $\mathbf{B}_{17}$ & $=$ & $\begin{array}{c}\left(-y_{7}+z_{7}\right) \mathbf{a}_{1}+\left(\frac{1}{2}+x_{7}+z_{7}\right) \mathbf{a}_{2}+ \\
\left(\frac{1}{2}+x_{7}-y_{7}\right) \mathbf{a}_{3}\end{array}$ & $=\left(\frac{1}{2}+x_{7}\right) a \hat{\mathbf{x}}-y_{7} b \hat{\mathbf{y}}+z_{7} c \hat{\mathbf{z}}$ & $(8 c)$ & Si II \\
\hline $\mathbf{B}_{18}$ & $=$ & $\begin{array}{c}\left(y_{7}+z_{7}\right) \mathbf{a}_{1}+\left(\frac{1}{2}-x_{7}+z_{7}\right) \mathbf{a}_{2}+ \\
\left(\frac{1}{2}-x_{7}+y_{7}\right) \mathbf{a}_{3}\end{array}$ & $=\left(\frac{1}{2}-x_{7}\right) a \hat{\mathbf{x}}+y_{7} b \hat{\mathbf{y}}+z_{7} c \hat{\mathbf{z}}$ & $(8 c)$ & Si II \\
\hline
\end{tabular}

\section{References:}

- W. Jeitschko, The crystal structure of TiFeSi and related compounds, Acta Crystallogr. Sect. B Struct. Sci. 26, 815-822 (1970), doi:10.1107/S0567740870003163.

\section{Found in:}

- P. Villars and K. Cenzual, Pearson's Crystal Data - Crystal Structure Database for Inorganic Compounds, ASM International (2013).

\section{Geometry files:}

- CIF: pp. 830

- POSCAR: pp. 830 


\section{$\alpha-\mathrm{RbPr}\left[\mathrm{MoO}_{4}\right]_{2}$ Structure: A2B8CD_oP24_48_k_2m_d_b}
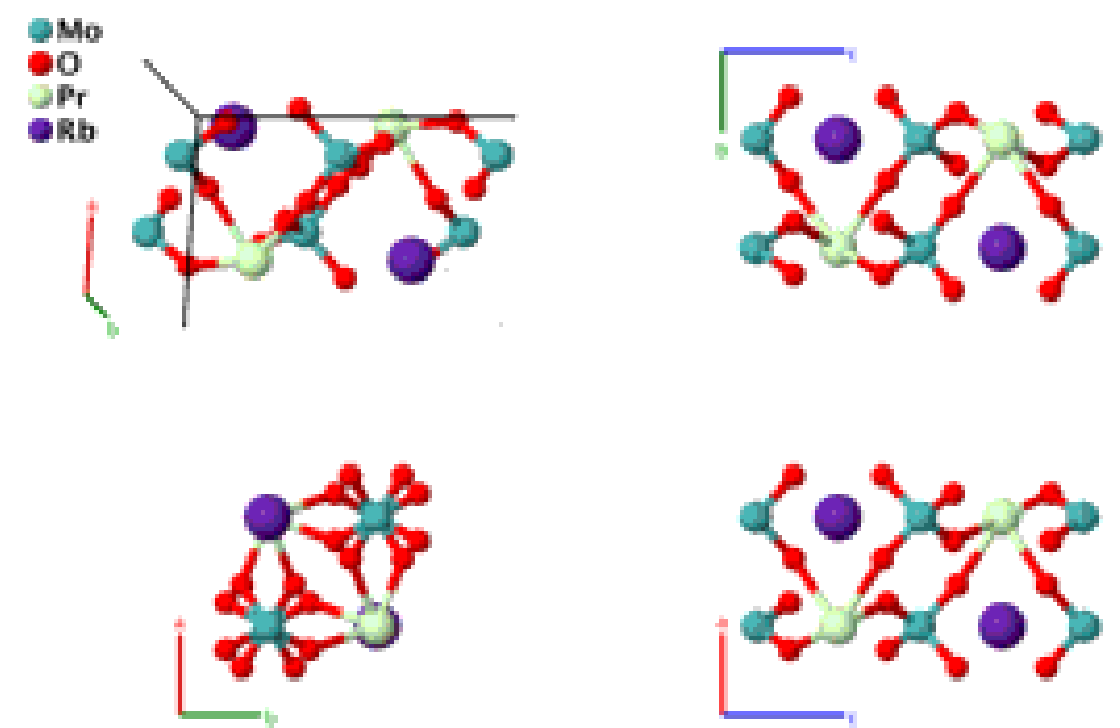

\section{Prototype}

AFLOW prototype label

Strukturbericht designation

Pearson symbol

Space group number

Space group symbol

AFLOW prototype command
: $\quad \alpha-\operatorname{RbPr}\left[\mathrm{MoO}_{4}\right]_{2}$

: A2B8CD_oP24_48_k_2m_d_b

: None

$: \quad$ oP24

: $\quad 48$

: Pnnn

aflow --proto=A2B8CD_oP24_48_k_2m_d_b

- params $=a, b / a, c / a, z_{3}, x_{4}, y_{4}, z_{4}, x_{5}, y_{5}, z_{5}$

Simple Orthorhombic primitive vectors:

$$
\begin{aligned}
& \mathbf{a}_{1}=a \hat{\mathbf{x}} \\
& \mathbf{a}_{2}=b \hat{\mathbf{y}} \\
& \mathbf{a}_{3}=c \hat{\mathbf{z}}
\end{aligned}
$$

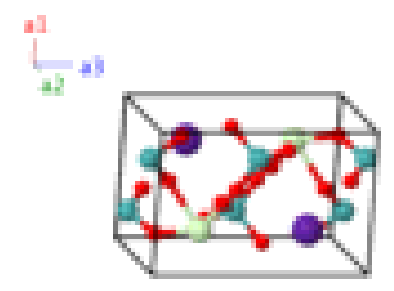

Basis vectors:

Lattice Coordinates

$\begin{array}{lllll}\mathbf{B}_{1}= & \frac{3}{4} \mathbf{a}_{1}+\frac{1}{4} \mathbf{a}_{2}+\frac{1}{4} \mathbf{a}_{3} & = & \frac{3}{4} a \hat{\mathbf{x}}+\frac{1}{4} b \hat{\mathbf{y}}+\frac{1}{4} c \hat{\mathbf{z}} \\ \mathbf{B}_{2}= & \frac{1}{4} \mathbf{a}_{1}+\frac{3}{4} \mathbf{a}_{2}+\frac{3}{4} \mathbf{a}_{3} & = & \frac{1}{4} a \hat{\mathbf{x}}+\frac{3}{4} b \hat{\mathbf{y}}+\frac{3}{4} c \hat{\mathbf{z}} \\ \mathbf{B}_{3}= & \frac{1}{4} \mathbf{a}_{1}+\frac{3}{4} \mathbf{a}_{2}+\frac{1}{4} \mathbf{a}_{3} & = & \frac{1}{4} a \hat{\mathbf{x}}+\frac{3}{4} b \hat{\mathbf{y}}+\frac{1}{4} c \hat{\mathbf{z}} \\ \mathbf{B}_{4}= & \frac{3}{4} \mathbf{a}_{1}+\frac{1}{4} \mathbf{a}_{2}+\frac{3}{4} \mathbf{a}_{3} & = & \frac{3}{4} a \hat{\mathbf{x}}+\frac{1}{4} b \hat{\mathbf{y}}+\frac{3}{4} c \hat{\mathbf{z}} \\ \mathbf{B}_{5}= & \frac{1}{4} \mathbf{a}_{1}+\frac{1}{4} \mathbf{a}_{2}+z_{3} \mathbf{a}_{3} & = & \frac{1}{4} a \hat{\mathbf{x}}+\frac{1}{4} b \hat{\mathbf{y}}+z_{3} c \hat{\mathbf{z}} \\ \mathbf{B}_{6}= & \frac{1}{4} \mathbf{a}_{1}+\frac{1}{4} \mathbf{a}_{2}+\left(\frac{1}{2}-z_{3}\right) \mathbf{a}_{3} & = & \frac{1}{4} a \hat{\mathbf{x}}+\frac{1}{4} b \hat{\mathbf{y}}+\left(\frac{1}{2}-z_{3}\right) c \hat{\mathbf{z}} \\ \mathbf{B}_{7}= & \frac{3}{4} \mathbf{a}_{1}+\frac{3}{4} \mathbf{a}_{2}-z_{3} \mathbf{a}_{3} & = & \frac{3}{4} a \hat{\mathbf{x}}+\frac{3}{4} b \hat{\mathbf{y}}-z_{3} c \hat{\mathbf{z}}\end{array}$

Wyckoff Position Atom Type

(2b)

$\mathrm{Rb}$

(2b)

$\mathrm{Rb}$

$(2 d)$

$(2 d)$

$\operatorname{Pr}$

Pr

(4k)

Mo

(4k)

Mo

(4k)

Mo 


\begin{tabular}{|c|c|c|c|c|c|}
\hline $\mathbf{B}_{8}$ & $=$ & $\frac{3}{4} \mathbf{a}_{1}+\frac{3}{4} \mathbf{a}_{2}+\left(\frac{1}{2}+z_{3}\right) \mathbf{a}_{3}$ & $=$ & $\frac{3}{4} a \hat{\mathbf{x}}+\frac{3}{4} b \hat{\mathbf{y}}+\left(\frac{1}{2}+z_{3}\right) c \hat{\mathbf{z}}$ & $(4 k)$ \\
\hline $\mathbf{B}_{9}$ & $=$ & $x_{4} \mathbf{a}_{1}+y_{4} \mathbf{a}_{2}+z_{4} \mathbf{a}_{3}$ & $=$ & $x_{4} a \hat{\mathbf{x}}+y_{4} b \hat{\mathbf{y}}+z_{4} c \hat{\mathbf{z}}$ & $(8 m)$ \\
\hline $\mathbf{B}_{10}$ & $=$ & $\left(\frac{1}{2}-x_{4}\right) \mathbf{a}_{1}+\left(\frac{1}{2}-y_{4}\right) \mathbf{a}_{2}+z_{4} \mathbf{a}_{3}$ & $=$ & $\left(\frac{1}{2}-x_{4}\right) a \hat{\mathbf{x}}+\left(\frac{1}{2}-y_{4}\right) b \hat{\mathbf{y}}+z_{4} c \hat{\mathbf{z}}$ & $(8 m)$ \\
\hline $\mathbf{B}_{11}$ & $=$ & $\left(\frac{1}{2}-x_{4}\right) \mathbf{a}_{1}+y_{4} \mathbf{a}_{2}+\left(\frac{1}{2}-z_{4}\right) \mathbf{a}_{3}$ & $=$ & $\left(\frac{1}{2}-x_{4}\right) a \hat{\mathbf{x}}+y_{4} b \hat{\mathbf{y}}+\left(\frac{1}{2}-z_{4}\right) c \hat{\mathbf{z}}$ & $(8 m)$ \\
\hline $\mathbf{B}_{12}$ & $=$ & $x_{4} \mathbf{a}_{1}+\left(\frac{1}{2}-y_{4}\right) \mathbf{a}_{2}+\left(\frac{1}{2}-z_{4}\right) \mathbf{a}_{3}$ & $=$ & $x_{4} a \hat{\mathbf{x}}+\left(\frac{1}{2}-y_{4}\right) b \hat{\mathbf{y}}+\left(\frac{1}{2}-z_{4}\right) c \hat{\mathbf{z}}$ & $(8 m)$ \\
\hline $\mathbf{B}_{13}$ & $=$ & $-x_{4} \mathbf{a}_{1}-y_{4} \mathbf{a}_{2}-z_{4} \mathbf{a}_{3}$ & $=$ & $-x_{4} a \hat{\mathbf{x}}-y_{4} b \hat{\mathbf{y}}-z_{4} c \hat{\mathbf{z}}$ & $(8 m)$ \\
\hline $\mathbf{B}_{14}$ & $=$ & $\left(\frac{1}{2}+x_{4}\right) \mathbf{a}_{1}+\left(\frac{1}{2}+y_{4}\right) \mathbf{a}_{2}-z_{4} \mathbf{a}_{3}$ & $=$ & $\left(\frac{1}{2}+x_{4}\right) a \hat{\mathbf{x}}+\left(\frac{1}{2}+y_{4}\right) b \hat{\mathbf{y}}-z_{4} c \hat{\mathbf{z}}$ & $(8 m)$ \\
\hline $\mathbf{B}_{15}$ & $=$ & $\left(\frac{1}{2}+x_{4}\right) \mathbf{a}_{1}-y_{4} \mathbf{a}_{2}+\left(\frac{1}{2}+z_{4}\right) \mathbf{a}_{3}$ & $=$ & $\left(\frac{1}{2}+x_{4}\right) a \hat{\mathbf{x}}-y_{4} b \hat{\mathbf{y}}+\left(\frac{1}{2}+z_{4}\right) c \hat{\mathbf{z}}$ & $(8 m)$ \\
\hline $\mathbf{B}_{16}$ & $=$ & $-x_{4} \mathbf{a}_{1}+\left(\frac{1}{2}+y_{4}\right) \mathbf{a}_{2}+\left(\frac{1}{2}+z_{4}\right) \mathbf{a}_{3}$ & $=$ & $-x_{4} a \hat{\mathbf{x}}+\left(\frac{1}{2}+y_{4}\right) b \hat{\mathbf{y}}+\left(\frac{1}{2}+z_{4}\right) c \hat{\mathbf{z}}$ & $(8 m)$ \\
\hline $\mathbf{B}_{17}$ & $=$ & $x_{5} \mathbf{a}_{1}+y_{5} \mathbf{a}_{2}+z_{5} \mathbf{a}_{3}$ & $=$ & $x_{5} a \hat{\mathbf{x}}+y_{5} b \hat{\mathbf{y}}+z_{5} c \hat{\mathbf{z}}$ & $(8 m)$ \\
\hline $\mathbf{B}_{18}$ & $=$ & $\left(\frac{1}{2}-x_{5}\right) \mathbf{a}_{1}+\left(\frac{1}{2}-y_{5}\right) \mathbf{a}_{2}+z_{5} \mathbf{a}_{3}$ & $=$ & $\left(\frac{1}{2}-x_{5}\right) a \hat{\mathbf{x}}+\left(\frac{1}{2}-y_{5}\right) b \hat{\mathbf{y}}+z_{5} c \hat{\mathbf{z}}$ & $(8 m)$ \\
\hline $\mathbf{B}_{19}$ & $=$ & $\left(\frac{1}{2}-x_{5}\right) \mathbf{a}_{1}+y_{5} \mathbf{a}_{2}+\left(\frac{1}{2}-z_{5}\right) \mathbf{a}_{3}$ & $=$ & $\left(\frac{1}{2}-x_{5}\right) a \hat{\mathbf{x}}+y_{5} b \hat{\mathbf{y}}+\left(\frac{1}{2}-z_{5}\right) c \hat{\mathbf{z}}$ & $(8 m)$ \\
\hline $\mathbf{B}_{20}$ & $=$ & $x_{5} \mathbf{a}_{1}+\left(\frac{1}{2}-y_{5}\right) \mathbf{a}_{2}+\left(\frac{1}{2}-z_{5}\right) \mathbf{a}_{3}$ & $=$ & $x_{5} a \hat{\mathbf{x}}+\left(\frac{1}{2}-y_{5}\right) b \hat{\mathbf{y}}+\left(\frac{1}{2}-z_{5}\right) c \hat{\mathbf{z}}$ & $(8 m)$ \\
\hline $\mathbf{B}_{21}$ & $=$ & $-x_{5} \mathbf{a}_{1}-y_{5} \mathbf{a}_{2}-z_{5} \mathbf{a}_{3}$ & $=$ & $-x_{5} a \hat{\mathbf{x}}-y_{5} b \hat{\mathbf{y}}-z_{5} c \hat{\mathbf{z}}$ & $(8 m)$ \\
\hline $\mathbf{B}_{22}$ & $=$ & $\left(\frac{1}{2}+x_{5}\right) \mathbf{a}_{1}+\left(\frac{1}{2}+y_{5}\right) \mathbf{a}_{2}-z_{5} \mathbf{a}_{3}$ & $=$ & $\left(\frac{1}{2}+x_{5}\right) a \hat{\mathbf{x}}+\left(\frac{1}{2}+y_{5}\right) b \hat{\mathbf{y}}-z_{5} c \hat{\mathbf{z}}$ & $(8 m)$ \\
\hline $\mathbf{B}_{23}$ & $=$ & $\left(\frac{1}{2}+x_{5}\right) \mathbf{a}_{1}-y_{5} \mathbf{a}_{2}+\left(\frac{1}{2}+z_{5}\right) \mathbf{a}_{3}$ & $=$ & $\left(\frac{1}{2}+x_{5}\right) a \hat{\mathbf{x}}-y_{5} b \hat{\mathbf{y}}+\left(\frac{1}{2}+z_{5}\right) c \hat{\mathbf{z}}$ & $(8 m)$ \\
\hline $\mathbf{B}_{24}$ & $=$ & $-x_{5} \mathbf{a}_{1}+\left(\frac{1}{2}+y_{5}\right) \mathbf{a}_{2}+\left(\frac{1}{2}+z_{5}\right) \mathbf{a}_{3}$ & $=$ & $-x_{5} a \hat{\mathbf{x}}+\left(\frac{1}{2}+y_{5}\right) b \hat{\mathbf{y}}+\left(\frac{1}{2}+z_{5}\right) c \hat{\mathbf{z}}$ & $(8 m)$ \\
\hline
\end{tabular}

\section{References:}

- R. F. Klevtsova and P. V. Klevtsov, Polymorphism of rubidium-praseodymium molybdate, $\operatorname{RbPr}\left(\mathrm{MoO}_{4}\right)_{2}$, Kristallografiya 15, 466-470 (1970).

\section{Found in:}

- P. Villars and K. Cenzual, Pearson's Crystal Data - Crystal Structure Database for Inorganic Compounds, ASM International (2013).

\section{Geometry files:}

- CIF: pp. 830

- POSCAR: pp. 831 


\section{$\beta-\mathrm{Ta}_{2} \mathrm{O}_{5}$ Structure: A5B2_oP14_49_dehq_ab}
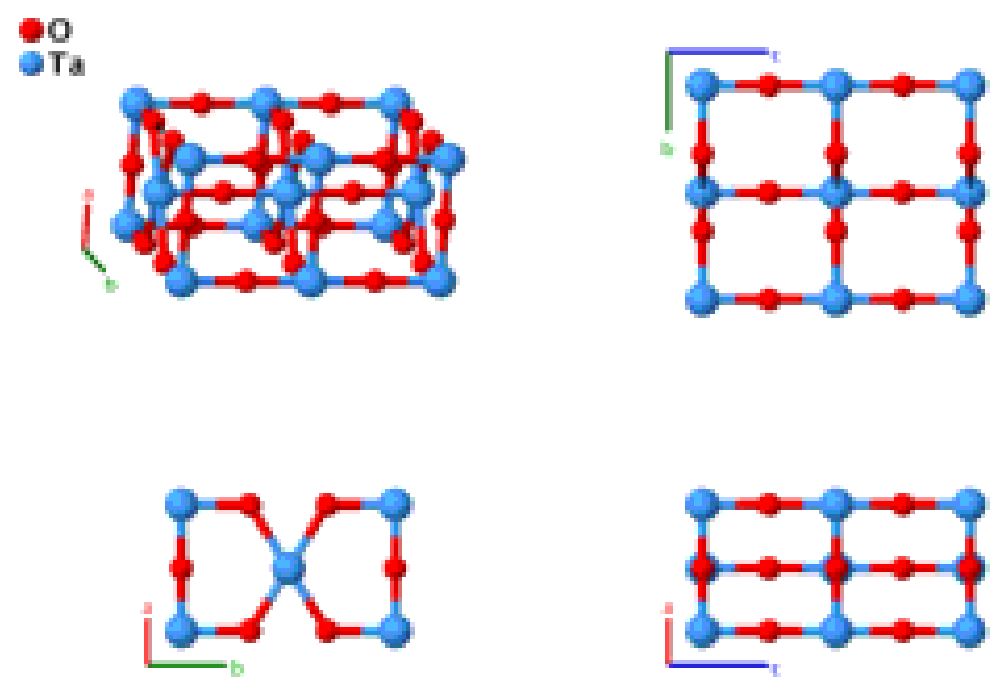

\section{Prototype}

AFLOW prototype label

Strukturbericht designation

Pearson symbol

Space group number

Space group symbol

AFLOW prototype command
: $\quad \beta-\mathrm{Ta}_{2} \mathrm{O}_{5}$

: A5B2_oP14_49_dehq_ab

: None

: $\quad$ oP14

: 49

: $\quad$ Pccm

- - params $=a, b / a, c / a, x_{6}, y_{6}$

- While FINDSYM identifies space group \#49 for this structure (consistent with the reference), AFLOW-SYM and Platon identify \#47. Lowering the tolerance value for AFLOW-SYM resolves the expected space group \#49. Space groups \#47 and \#49 are both reasonable classifications since they are commensurate with subgroup relations.

\section{Simple Orthorhombic primitive vectors:}

$$
\begin{aligned}
& \mathbf{a}_{1}=a \hat{\mathbf{x}} \\
& \mathbf{a}_{2}=b \hat{\mathbf{y}} \\
& \mathbf{a}_{3}=c \hat{\mathbf{z}}
\end{aligned}
$$

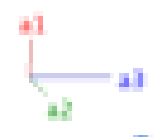

\section{Basis vectors:}

Lattice Coordinates

$\begin{array}{lcc}\mathbf{B}_{1}= & 0 \mathbf{a}_{1}+0 \mathbf{a}_{2}+0 \mathbf{a}_{3} \\ \mathbf{B}_{2}= & \frac{1}{2} \mathbf{a}_{3} \\ \mathbf{B}_{3}= & \frac{1}{2} \mathbf{a}_{1}+\frac{1}{2} \mathbf{a}_{2}\end{array}$

Cartesian Coordinates

$=$

$=$

$=$

$$
\begin{gathered}
0 \hat{\mathbf{x}}+0 \hat{\mathbf{y}}+0 \hat{\mathbf{z}} \\
\frac{1}{2} c \hat{\mathbf{z}} \\
\frac{1}{2} a \hat{\mathbf{x}}+\frac{1}{2} b \hat{\mathbf{y}}
\end{gathered}
$$

Wyckoff Position

$(2 a)$
Atom Type

Ta I

$\mathrm{Ta} \mathrm{I}$

Ta II 


\begin{tabular}{|c|c|c|c|c|c|c|}
\hline $\mathbf{B}_{4}$ & $=$ & $\frac{1}{2} \mathbf{a}_{1}+\frac{1}{2} \mathbf{a}_{2}+\frac{1}{2} \mathbf{a}_{3}$ & $=$ & $\frac{1}{2} a \hat{\mathbf{x}}+\frac{1}{2} b \hat{\mathbf{y}}+\frac{1}{2} c \hat{\mathbf{z}}$ & $(2 b)$ & Ta II \\
\hline $\mathbf{B}_{5}$ & $=$ & $\frac{1}{2} \mathbf{a}_{1}$ & $=$ & $\frac{1}{2} a \hat{\mathbf{x}}$ & $(2 d)$ & O I \\
\hline $\mathbf{B}_{6}$ & $=$ & $\frac{1}{2} \mathbf{a}_{1}+\frac{1}{2} \mathbf{a}_{3}$ & $=$ & $\frac{1}{2} a \hat{\mathbf{x}}+\frac{1}{2} c \hat{\mathbf{z}}$ & $(2 d)$ & O I \\
\hline $\mathbf{B}_{7}$ & $=$ & $\frac{1}{4} \mathbf{a}_{3}$ & $=$ & $\frac{1}{4} c \hat{\mathbf{z}}$ & $(2 e)$ & O II \\
\hline $\mathbf{B}_{8}$ & $=$ & $\frac{3}{4} \mathbf{a}_{3}$ & $=$ & $\frac{3}{4} c \hat{\mathbf{z}}$ & $(2 e)$ & O II \\
\hline $\mathbf{B}_{9}$ & $=$ & $\frac{1}{2} \mathbf{a}_{1}+\frac{1}{2} \mathbf{a}_{2}+\frac{1}{4} \mathbf{a}_{3}$ & $=$ & $\frac{1}{2} a \hat{\mathbf{x}}+\frac{1}{2} b \hat{\mathbf{y}}+\frac{1}{4} c \hat{\mathbf{z}}$ & $(2 h)$ & O III \\
\hline $\mathbf{B}_{10}$ & $=$ & $\frac{1}{2} \mathbf{a}_{1}+\frac{1}{2} \mathbf{a}_{2}+\frac{3}{4} \mathbf{a}_{3}$ & $=$ & $\frac{1}{2} a \hat{\mathbf{x}}+\frac{1}{2} b \hat{\mathbf{y}}+\frac{3}{4} c \hat{\mathbf{z}}$ & $(2 h)$ & O III \\
\hline $\mathbf{B}_{11}$ & $=$ & $x_{6} \mathbf{a}_{1}+y_{6} \mathbf{a}_{2}$ & $=$ & $x_{6} a \hat{\mathbf{x}}+y_{6} b \hat{\mathbf{y}}$ & $(4 q)$ & O IV \\
\hline $\mathbf{B}_{12}$ & $=$ & $-x_{6} \mathbf{a}_{1}-y_{6} \mathbf{a}_{2}$ & $=$ & $-x_{6} a \hat{\mathbf{x}}-y_{6} b \hat{\mathbf{y}}$ & $(4 q)$ & O IV \\
\hline $\mathbf{B}_{13}$ & $=$ & $-x_{6} \mathbf{a}_{1}+y_{6} \mathbf{a}_{2}+\frac{1}{2} \mathbf{a}_{3}$ & $=$ & $-x_{6} a \hat{\mathbf{x}}+y_{6} b \hat{\mathbf{y}}+\frac{1}{2} c \hat{\mathbf{z}}$ & $(4 q)$ & O IV \\
\hline $\mathbf{B}_{14}$ & $=$ & $x_{6} \mathbf{a}_{1}-y_{6} \mathbf{a}_{2}+\frac{1}{2} \mathbf{a}_{3}$ & $=$ & $x_{6} a \hat{\mathbf{x}}-y_{6} b \hat{\mathbf{y}}+\frac{1}{2} c \hat{\mathbf{z}}$ & $(4 q)$ & O IV \\
\hline
\end{tabular}

\section{References:}

- L. A. Aleshina and S. V. Loginova, Rietveld analysis of X-ray diffraction pattern from $\beta-\mathrm{Ta}_{2} \mathrm{O}_{5}$ oxide, Crystallogr. Rep. 47, 415-419 (2002), doi:10.1134/1.1481927.

- H. T. Stokes and D. M. Hatch, FINDSYM: Program for identifying the space group symmetry of a crystal, J. Appl. Crystallogr. 38, 237-238 (2005), doi:10.1107/S0021889804031528.

- D. Hicks, C. Oses, E. Gossett, G. Gomez, R. H. Taylor, C. Toher, M. J. Mehl, O. Levy, and S. Curtarolo, AFLOW-SYM: platform for the complete, automatic and self-consistent symmetry analysis of crystals, Acta Crystallogr. Sect. A 74, 184-203 (2018), doi:10.1107/S2053273318003066.

- A. L. Spek, Single-crystal structure validation with the program PLATON, J. Appl. Crystallogr. 36, 7-13 (2003), doi:10.1107/S0021889802022112.

\section{Found in:}

- P. Villars and K. Cenzual, Pearson's Crystal Data - Crystal Structure Database for Inorganic Compounds, ASM International (2013).

\section{Geometry files:}

- CIF: pp. 831

- POSCAR: pp. 831 


\section{CsPr[$\left[\mathrm{MoO}_{4}\right]_{2}$ Structure: AB2C8D_oP24_49_g_q_2qr_e}
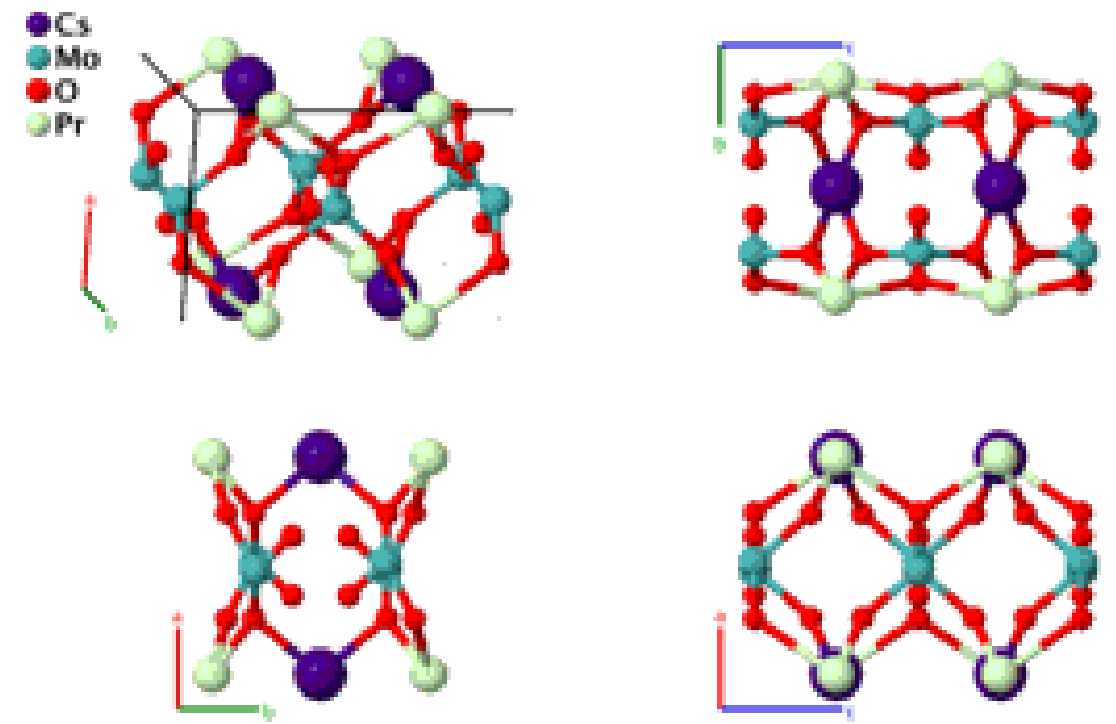

Prototype

AFLOW prototype label

Strukturbericht designation

Pearson symbol

Space group number

Space group symbol

AFLOW prototype command
: $\quad \mathrm{CsPr}\left[\mathrm{MoO}_{4}\right]_{2}$

: AB2C8D_oP24_49_g_q_2qr_e

: None

: $\quad$ oP24

: 49

: $\quad P c c m$

aflow - -proto=AB2C8D_oP24_49_g_q_2qr_e

- -params $=a, b / a, c / a, x_{3}, y_{3}, x_{4}, y_{4}, x_{5}, y_{5}, x_{6}, y_{6}, z_{6}$

Simple Orthorhombic primitive vectors:

$$
\begin{aligned}
& \mathbf{a}_{1}=a \hat{\mathbf{x}} \\
& \mathbf{a}_{2}=b \hat{\mathbf{y}} \\
& \mathbf{a}_{3}=c \hat{\mathbf{z}}
\end{aligned}
$$

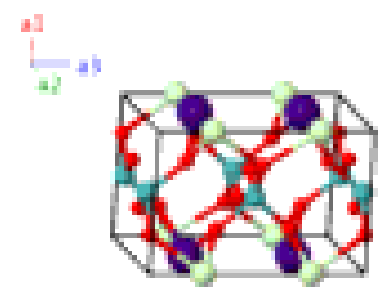

Basis vectors:

Lattice Coordinates

$\begin{array}{lcccc}\mathbf{B}_{1}= & \frac{1}{4} \mathbf{a}_{3} & = & \frac{1}{4} c \hat{\mathbf{z}} \\ \mathbf{B}_{2}= & \frac{3}{4} \mathbf{a}_{3} & = & \frac{3}{4} c \hat{\mathbf{z}} \\ \mathbf{B}_{3}= & \frac{1}{2} \mathbf{a}_{2}+\frac{1}{4} \mathbf{a}_{3} & = & \frac{1}{2} b \hat{\mathbf{y}}+\frac{1}{4} c \hat{\mathbf{z}} \\ \mathbf{B}_{4}= & \frac{1}{2} \mathbf{a}_{2}+\frac{3}{4} \mathbf{a}_{3} & = & \frac{1}{2} b \hat{\mathbf{y}}+\frac{3}{4} c \hat{\mathbf{z}} \\ \mathbf{B}_{5}= & x_{3} \mathbf{a}_{1}+y_{3} \mathbf{a}_{2} & = & x_{3} a \hat{\mathbf{x}}+y_{3} b \hat{\mathbf{y}} \\ \mathbf{B}_{6}= & -x_{3} \mathbf{a}_{1}-y_{3} \mathbf{a}_{2} & = & -x_{3} a \hat{\mathbf{x}}-y_{3} b \hat{\mathbf{y}} \\ \mathbf{B}_{7}= & -x_{3} \mathbf{a}_{1}+y_{3} \mathbf{a}_{2}+\frac{1}{2} \mathbf{a}_{3} & = & -x_{3} a \hat{\mathbf{x}}+y_{3} b \hat{\mathbf{y}}+\frac{1}{2} c \hat{\mathbf{z}}\end{array}$

Wyckoff Position

Atom Type

(2e)

(4q)

(4q)

(4q)
Pr

Pr

Cs

Cs

Mo

Mo

Mo 


\begin{tabular}{|c|c|c|c|c|c|}
\hline $\mathbf{B}_{8}$ & $=$ & $x_{3} \mathbf{a}_{1}-y_{3} \mathbf{a}_{2}+\frac{1}{2} \mathbf{a}_{3}$ & $=$ & $x_{3} a \hat{\mathbf{x}}-y_{3} b \hat{\mathbf{y}}+\frac{1}{2} c \hat{\mathbf{z}}$ & $(4 q)$ \\
\hline $\mathbf{B}_{9}$ & $=$ & $x_{4} \mathbf{a}_{1}+y_{4} \mathbf{a}_{2}$ & $=$ & $x_{4} a \hat{\mathbf{x}}+y_{4} b \hat{\mathbf{y}}$ & $(4 q)$ \\
\hline $\mathbf{B}_{10}$ & $=$ & $-x_{4} \mathbf{a}_{1}-y_{4} \mathbf{a}_{2}$ & $=$ & $-x_{4} a \hat{\mathbf{x}}-y_{4} b \hat{\mathbf{y}}$ & $(4 q)$ \\
\hline $\mathbf{B}_{11}$ & $=$ & $-x_{4} \mathbf{a}_{1}+y_{4} \mathbf{a}_{2}+\frac{1}{2} \mathbf{a}_{3}$ & $=$ & $-x_{4} a \hat{\mathbf{x}}+y_{4} b \hat{\mathbf{y}}+\frac{1}{2} c \hat{\mathbf{z}}$ & $(4 q)$ \\
\hline $\mathbf{B}_{12}$ & $=$ & $x_{4} \mathbf{a}_{1}-y_{4} \mathbf{a}_{2}+\frac{1}{2} \mathbf{a}_{3}$ & $=$ & $x_{4} a \hat{\mathbf{x}}-y_{4} b \hat{\mathbf{y}}+\frac{1}{2} c \hat{\mathbf{z}}$ & $(4 q)$ \\
\hline $\mathbf{B}_{13}$ & $=$ & $x_{5} \mathbf{a}_{1}+y_{5} \mathbf{a}_{2}$ & $=$ & $x_{5} a \hat{\mathbf{x}}+y_{5} b \hat{\mathbf{y}}$ & $(4 q)$ \\
\hline $\mathbf{B}_{14}$ & $=$ & $-x_{5} \mathbf{a}_{1}-y_{5} \mathbf{a}_{2}$ & $=$ & $-x_{5} a \hat{\mathbf{x}}-y_{5} b \hat{\mathbf{y}}$ & $(4 q)$ \\
\hline $\mathbf{B}_{15}$ & $=$ & $-x_{5} \mathbf{a}_{1}+y_{5} \mathbf{a}_{2}+\frac{1}{2} \mathbf{a}_{3}$ & $=$ & $-x_{5} a \hat{\mathbf{x}}+y_{5} b \hat{\mathbf{y}}+\frac{1}{2} c \hat{\mathbf{z}}$ & $(4 q)$ \\
\hline $\mathbf{B}_{16}$ & $=$ & $x_{5} \mathbf{a}_{1}-y_{5} \mathbf{a}_{2}+\frac{1}{2} \mathbf{a}_{3}$ & $=$ & $x_{5} a \hat{\mathbf{x}}-y_{5} b \hat{\mathbf{y}}+\frac{1}{2} c \hat{\mathbf{z}}$ & $(4 q)$ \\
\hline $\mathbf{B}_{17}$ & $=$ & $x_{6} \mathbf{a}_{1}+y_{6} \mathbf{a}_{2}+z_{6} \mathbf{a}_{3}$ & $=$ & $x_{6} a \hat{\mathbf{x}}+y_{6} b \hat{\mathbf{y}}+z_{6} c \hat{\mathbf{z}}$ & $(8 r)$ \\
\hline $\mathbf{B}_{18}$ & $=$ & $-x_{6} \mathbf{a}_{1}-y_{6} \mathbf{a}_{2}+z_{6} \mathbf{a}_{3}$ & $=$ & $-x_{6} a \hat{\mathbf{x}}-y_{6} b \hat{\mathbf{y}}+z_{6} c \hat{\mathbf{z}}$ & $(8 r)$ \\
\hline $\mathbf{B}_{19}$ & $=$ & $-x_{6} \mathbf{a}_{1}+y_{6} \mathbf{a}_{2}+\left(\frac{1}{2}-z_{6}\right) \mathbf{a}_{3}$ & $=$ & $-x_{6} a \hat{\mathbf{x}}+y_{6} b \hat{\mathbf{y}}+\left(\frac{1}{2}-z_{6}\right) c \hat{\mathbf{z}}$ & $(8 r)$ \\
\hline $\mathbf{B}_{20}$ & $=$ & $x_{6} \mathbf{a}_{1}-y_{6} \mathbf{a}_{2}+\left(\frac{1}{2}-z_{6}\right) \mathbf{a}_{3}$ & $=$ & $x_{6} a \hat{\mathbf{x}}-y_{6} b \hat{\mathbf{y}}+\left(\frac{1}{2}-z_{6}\right) c \hat{\mathbf{z}}$ & $(8 r)$ \\
\hline $\mathbf{B}_{21}$ & $=$ & $-x_{6} \mathbf{a}_{1}-y_{6} \mathbf{a}_{2}-z_{6} \mathbf{a}_{3}$ & $=$ & $-x_{6} a \hat{\mathbf{x}}-y_{6} b \hat{\mathbf{y}}-z_{6} c \hat{\mathbf{z}}$ & $(8 r)$ \\
\hline $\mathbf{B}_{22}$ & $=$ & $x_{6} \mathbf{a}_{1}+y_{6} \mathbf{a}_{2}-z_{6} \mathbf{a}_{3}$ & $=$ & $x_{6} a \hat{\mathbf{x}}+y_{6} b \hat{\mathbf{y}}-z_{6} c \hat{\mathbf{z}}$ & $(8 r)$ \\
\hline $\mathbf{B}_{23}$ & $=$ & $x_{6} \mathbf{a}_{1}-y_{6} \mathbf{a}_{2}+\left(\frac{1}{2}+z_{6}\right) \mathbf{a}_{3}$ & $=$ & $x_{6} a \hat{\mathbf{x}}-y_{6} b \hat{\mathbf{y}}+\left(\frac{1}{2}+z_{6}\right) c \hat{\mathbf{z}}$ & $(8 r)$ \\
\hline $\mathbf{B}_{24}$ & $=$ & $-x_{6} \mathbf{a}_{1}+y_{6} \mathbf{a}_{2}+\left(\frac{1}{2}+z_{6}\right) \mathbf{a}_{3}$ & $=$ & $-x_{6} a \hat{\mathbf{x}}+y_{6} b \hat{\mathbf{y}}+\left(\frac{1}{2}+z_{6}\right) c \hat{\mathbf{z}}$ & $(8 r)$ \\
\hline
\end{tabular}

\section{References:}

- V. A. Vinokurov and P. V. Klevtsov, Polymorphism and crystallization of binary cesium-rare earth molybdates CsLn( $\left.\mathrm{MoO}_{4}\right)_{2}$, Sov. Phys. Crystallogr. 17, 102-106 (1972).

\section{Found in:}

- P. Villars and K. Cenzual, Pearson's Crystal Data - Crystal Structure Database for Inorganic Compounds, ASM International (2013).

\section{Geometry files:}

- CIF: pp. 832

- POSCAR: pp. 832 

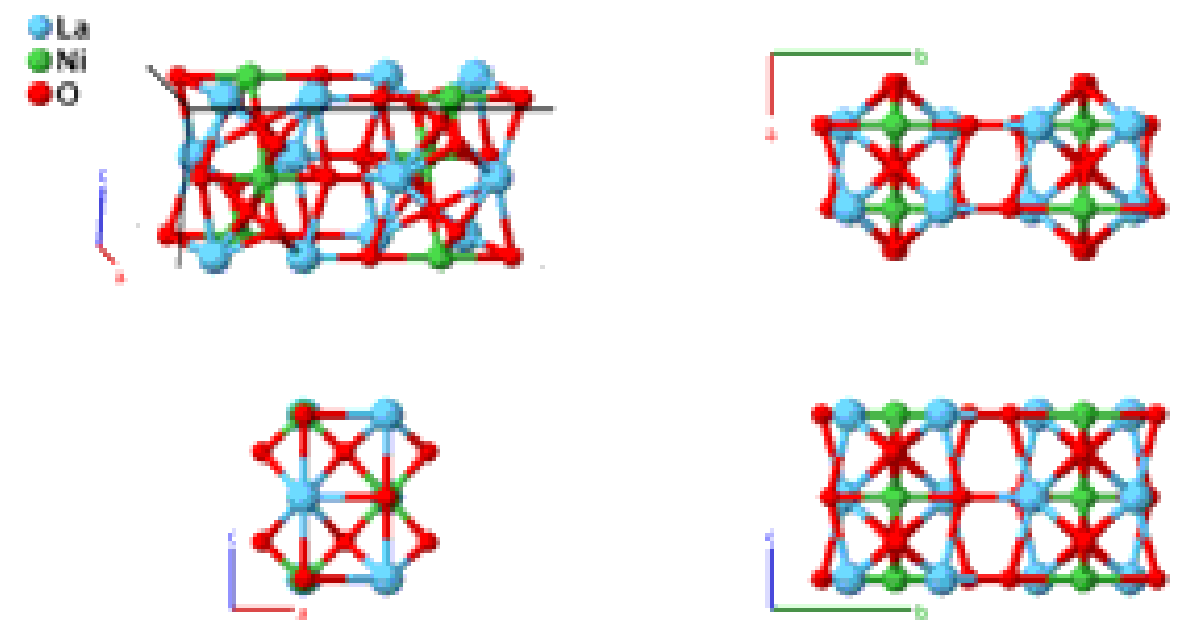

\section{Prototype}

AFLOW prototype label

Strukturbericht designation

Pearson symbol

Space group number

Space group symbol

AFLOW prototype command

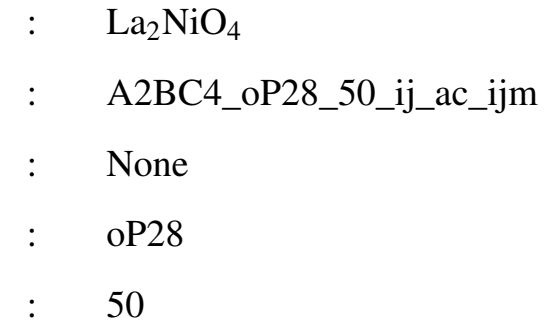

Simple Orthorhombic primitive vectors:

$$
\begin{aligned}
& \mathbf{a}_{1}=a \hat{\mathbf{x}} \\
& \mathbf{a}_{2}=b \hat{\mathbf{y}} \\
& \mathbf{a}_{3}=c \hat{\mathbf{z}}
\end{aligned}
$$

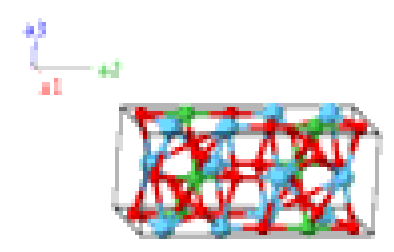

Basis vectors:

Lattice Coordinates

$\begin{array}{ccc}\mathbf{B}_{1}= & \frac{1}{4} \mathbf{a}_{1}+\frac{1}{4} \mathbf{a}_{2} \\ \mathbf{B}_{2}= & \frac{3}{4} \mathbf{a}_{1}+\frac{3}{4} \mathbf{a}_{2} \\ \mathbf{B}_{3}= & \frac{3}{4} \mathbf{a}_{1}+\frac{1}{4} \mathbf{a}_{2}+\frac{1}{2} \mathbf{a}_{3} \\ \mathbf{B}_{4}= & \frac{1}{4} \mathbf{a}_{1}+\frac{3}{4} \mathbf{a}_{2}+\frac{1}{2} \mathbf{a}_{3} \\ \mathbf{B}_{5}= & \frac{1}{4} \mathbf{a}_{1}+y_{3} \mathbf{a}_{2} \\ \mathbf{B}_{6}= & \frac{1}{4} \mathbf{a}_{1}+\left(\frac{1}{2}-y_{3}\right) \mathbf{a}_{2} \\ \mathbf{B}_{7}= & \frac{3}{4} \mathbf{a}_{1}-y_{3} \mathbf{a}_{2} \\ \mathbf{B}_{8}= & \frac{3}{4} \mathbf{a}_{1}+\left(\frac{1}{2}+y_{3}\right) \mathbf{a}_{2} \\ \mathbf{B}_{9}= & \frac{1}{4} \mathbf{a}_{1}+y_{4} \mathbf{a}_{2}\end{array}$

Cartesian Coordinates

$=$

$=$

$=$

$=$

$=$

$=$

$=$
$=$

$=$

$=$

$=$

$$
\begin{gathered}
\frac{1}{4} a \hat{\mathbf{x}}+\frac{1}{4} b \hat{\mathbf{y}} \\
\frac{3}{4} a \hat{\mathbf{x}}+\frac{3}{4} b \hat{\mathbf{y}} \\
\frac{3}{4} a \hat{\mathbf{x}}+\frac{1}{4} b \hat{\mathbf{y}}+\frac{1}{2} c \hat{\mathbf{z}} \\
\frac{1}{4} a \hat{\mathbf{x}}+\frac{3}{4} b \hat{\mathbf{y}}+\frac{1}{2} c \hat{\mathbf{z}} \\
\frac{1}{4} a \hat{\mathbf{x}}+y_{3} b \hat{\mathbf{y}} \\
\frac{1}{4} a \hat{\mathbf{x}}+\left(\frac{1}{2}-y_{3}\right) b \hat{\mathbf{y}} \\
\frac{3}{4} a \hat{\mathbf{x}}-y_{3} b \hat{\mathbf{y}} \\
\frac{3}{4} a \hat{\mathbf{x}}+\left(\frac{1}{2}+y_{3}\right) b \hat{\mathbf{y}} \\
\frac{1}{4} a \hat{\mathbf{x}}+y_{4} b \hat{\mathbf{y}}
\end{gathered}
$$

Wyckoff Position

(2a)

(2a)
Atom Type

Ni I

Ni I

Ni II

Ni II

La I

La I

La I

La I

O I 


\begin{tabular}{|c|c|c|c|c|c|c|}
\hline $\mathbf{B}_{10}$ & $=$ & $\frac{1}{4} \mathbf{a}_{1}+\left(\frac{1}{2}-y_{4}\right) \mathbf{a}_{2}$ & $=$ & $\frac{1}{4} a \hat{\mathbf{x}}+\left(\frac{1}{2}-y_{4}\right) b \hat{\mathbf{y}}$ & $(4 i)$ & O I \\
\hline $\mathbf{B}_{11}$ & $=$ & $\frac{3}{4} \mathbf{a}_{1}-y_{4} \mathbf{a}_{2}$ & $=$ & $\frac{3}{4} a \hat{\mathbf{x}}-y_{4} b \hat{\mathbf{y}}$ & $(4 i)$ & O I \\
\hline $\mathbf{B}_{12}$ & $=$ & $\frac{3}{4} \mathbf{a}_{1}+\left(\frac{1}{2}+y_{4}\right) \mathbf{a}_{2}$ & $=$ & $\frac{3}{4} a \hat{\mathbf{x}}+\left(\frac{1}{2}+y_{4}\right) b \hat{\mathbf{y}}$ & $(4 i)$ & $\mathrm{O} \mathrm{I}$ \\
\hline $\mathbf{B}_{13}$ & $=$ & $\frac{1}{4} \mathbf{a}_{1}+y_{5} \mathbf{a}_{2}+\frac{1}{2} \mathbf{a}_{3}$ & $=$ & $\frac{1}{4} a \hat{\mathbf{x}}+y_{5} b \hat{\mathbf{y}}+\frac{1}{2} c \hat{\mathbf{z}}$ & $(4 j)$ & La II \\
\hline $\mathbf{B}_{14}$ & $=$ & $\frac{1}{4} \mathbf{a}_{1}+\left(\frac{1}{2}-y_{5}\right) \mathbf{a}_{2}+\frac{1}{2} \mathbf{a}_{3}$ & $=$ & $\frac{1}{4} a \hat{\mathbf{x}}+\left(\frac{1}{2}-y_{5}\right) b \hat{\mathbf{y}}+\frac{1}{2} c \hat{\mathbf{z}}$ & $(4 j)$ & La II \\
\hline $\mathbf{B}_{15}$ & $=$ & $\frac{3}{4} \mathbf{a}_{1}-y_{5} \mathbf{a}_{2}+\frac{1}{2} \mathbf{a}_{3}$ & $=$ & $\frac{3}{4} a \hat{\mathbf{x}}-y_{5} b \hat{\mathbf{y}}+\frac{1}{2} c \hat{\mathbf{z}}$ & $(4 j)$ & La II \\
\hline $\mathbf{B}_{16}$ & $=$ & $\frac{3}{4} \mathbf{a}_{1}+\left(\frac{1}{2}+y_{5}\right) \mathbf{a}_{2}+\frac{1}{2} \mathbf{a}_{3}$ & $=$ & $\frac{3}{4} a \hat{\mathbf{x}}+\left(\frac{1}{2}+y_{5}\right) b \hat{\mathbf{y}}+\frac{1}{2} c \hat{\mathbf{z}}$ & $(4 j)$ & La II \\
\hline $\mathbf{B}_{17}$ & $=$ & $\frac{1}{4} \mathbf{a}_{1}+y_{6} \mathbf{a}_{2}+\frac{1}{2} \mathbf{a}_{3}$ & $=$ & $\frac{1}{4} a \hat{\mathbf{x}}+y_{6} b \hat{\mathbf{y}}+\frac{1}{2} c \hat{\mathbf{z}}$ & $(4 j)$ & O II \\
\hline $\mathbf{B}_{18}$ & $=$ & $\frac{1}{4} \mathbf{a}_{1}+\left(\frac{1}{2}-y_{6}\right) \mathbf{a}_{2}+\frac{1}{2} \mathbf{a}_{3}$ & $=$ & $\frac{1}{4} a \hat{\mathbf{x}}+\left(\frac{1}{2}-y_{6}\right) b \hat{\mathbf{y}}+\frac{1}{2} c \hat{\mathbf{z}}$ & $(4 j)$ & O II \\
\hline $\mathbf{B}_{19}$ & $=$ & $\frac{3}{4} \mathbf{a}_{1}-y_{6} \mathbf{a}_{2}+\frac{1}{2} \mathbf{a}_{3}$ & $=$ & $\frac{3}{4} a \hat{\mathbf{x}}-y_{6} b \hat{\mathbf{y}}+\frac{1}{2} c \hat{\mathbf{z}}$ & $(4 j)$ & O II \\
\hline $\mathbf{B}_{20}$ & $=$ & $\frac{3}{4} \mathbf{a}_{1}+\left(\frac{1}{2}+y_{6}\right) \mathbf{a}_{2}+\frac{1}{2} \mathbf{a}_{3}$ & $=$ & $\frac{3}{4} a \hat{\mathbf{x}}+\left(\frac{1}{2}+y_{6}\right) b \hat{\mathbf{y}}+\frac{1}{2} c \hat{\mathbf{z}}$ & $(4 j)$ & O II \\
\hline $\mathbf{B}_{21}$ & $=$ & $x_{7} \mathbf{a}_{1}+y_{7} \mathbf{a}_{2}+z_{7} \mathbf{a}_{3}$ & $=$ & $x_{7} a \hat{\mathbf{x}}+y_{7} b \hat{\mathbf{y}}+z_{7} c \hat{\mathbf{z}}$ & $(8 m)$ & O III \\
\hline $\mathbf{B}_{22}$ & $=$ & $\left(\frac{1}{2}-x_{7}\right) \mathbf{a}_{1}+\left(\frac{1}{2}-y_{7}\right) \mathbf{a}_{2}+z_{7} \mathbf{a}_{3}$ & $=$ & $\left(\frac{1}{2}-x_{7}\right) a \hat{\mathbf{x}}+\left(\frac{1}{2}-y_{7}\right) b \hat{\mathbf{y}}+z_{7} c \hat{\mathbf{z}}$ & $(8 m)$ & O III \\
\hline $\mathbf{B}_{23}$ & $=$ & $\left(\frac{1}{2}-x_{7}\right) \mathbf{a}_{1}+y_{7} \mathbf{a}_{2}-z_{7} \mathbf{a}_{3}$ & $=$ & $\left(\frac{1}{2}-x_{7}\right) a \hat{\mathbf{x}}+y_{7} b \hat{\mathbf{y}}-z_{7} c \hat{\mathbf{z}}$ & $(8 m)$ & O III \\
\hline $\mathbf{B}_{24}$ & $=$ & $x_{7} \mathbf{a}_{1}+\left(\frac{1}{2}-y_{7}\right) \mathbf{a}_{2}-z_{7} \mathbf{a}_{3}$ & $=$ & $x_{7} a \hat{\mathbf{x}}+\left(\frac{1}{2}-y_{7}\right) b \hat{\mathbf{y}}-z_{7} c \hat{\mathbf{z}}$ & $(8 m)$ & O III \\
\hline $\mathbf{B}_{25}$ & $=$ & $-x_{7} \mathbf{a}_{1}-y_{7} \mathbf{a}_{2}-z_{7} \mathbf{a}_{3}$ & $=$ & $-x_{7} a \hat{\mathbf{x}}-y_{7} b \hat{\mathbf{y}}-z_{7} c \hat{\mathbf{z}}$ & $(8 m)$ & O III \\
\hline $\mathbf{B}_{26}$ & $=$ & $\left(\frac{1}{2}+x_{7}\right) \mathbf{a}_{1}+\left(\frac{1}{2}+y_{7}\right) \mathbf{a}_{2}-z_{7} \mathbf{a}_{3}$ & $=$ & $\left(\frac{1}{2}+x_{7}\right) a \hat{\mathbf{x}}+\left(\frac{1}{2}+y_{7}\right) b \hat{\mathbf{y}}-z_{7} c \hat{\mathbf{z}}$ & $(8 m)$ & O III \\
\hline $\mathbf{B}_{27}$ & $=$ & $\left(\frac{1}{2}+x_{7}\right) \mathbf{a}_{1}-y_{7} \mathbf{a}_{2}+z_{7} \mathbf{a}_{3}$ & $=$ & $\left(\frac{1}{2}+x_{7}\right) a \hat{\mathbf{x}}-y_{7} b \hat{\mathbf{y}}+z_{7} c \hat{\mathbf{z}}$ & $(8 m)$ & O III \\
\hline $\mathbf{B}_{28}$ & $=$ & $-x_{7} \mathbf{a}_{1}+\left(\frac{1}{2}+y_{7}\right) \mathbf{a}_{2}+z_{7} \mathbf{a}_{3}$ & $=$ & $-x_{7} a \hat{\mathbf{x}}+\left(\frac{1}{2}+y_{7}\right) b \hat{\mathbf{y}}+z_{7} c \hat{\mathbf{z}}$ & $(8 m)$ & O III \\
\hline
\end{tabular}

\section{References:}

- P. Odier, M. Leblanc, and J. Choisnet, Structural characterization of an orthorhombic form of $\mathrm{La}_{2} \mathrm{NiO}_{4}$, Mater. Res. Bull. 21, 787-796 (1986), doi:10.1016/0025-5408(86)90163-7.

\section{Found in:}

- P. Villars and K. Cenzual, Pearson's Crystal Data - Crystal Structure Database for Inorganic Compounds, ASM International (2013).

\section{Geometry files:}

- CIF: pp. 832

- POSCAR: pp. 832 


\section{$\alpha-\mathrm{Tl}_{2} \mathrm{TeO}_{3}$ Structure: A3BC2_oP48_50_3m_m_2m}
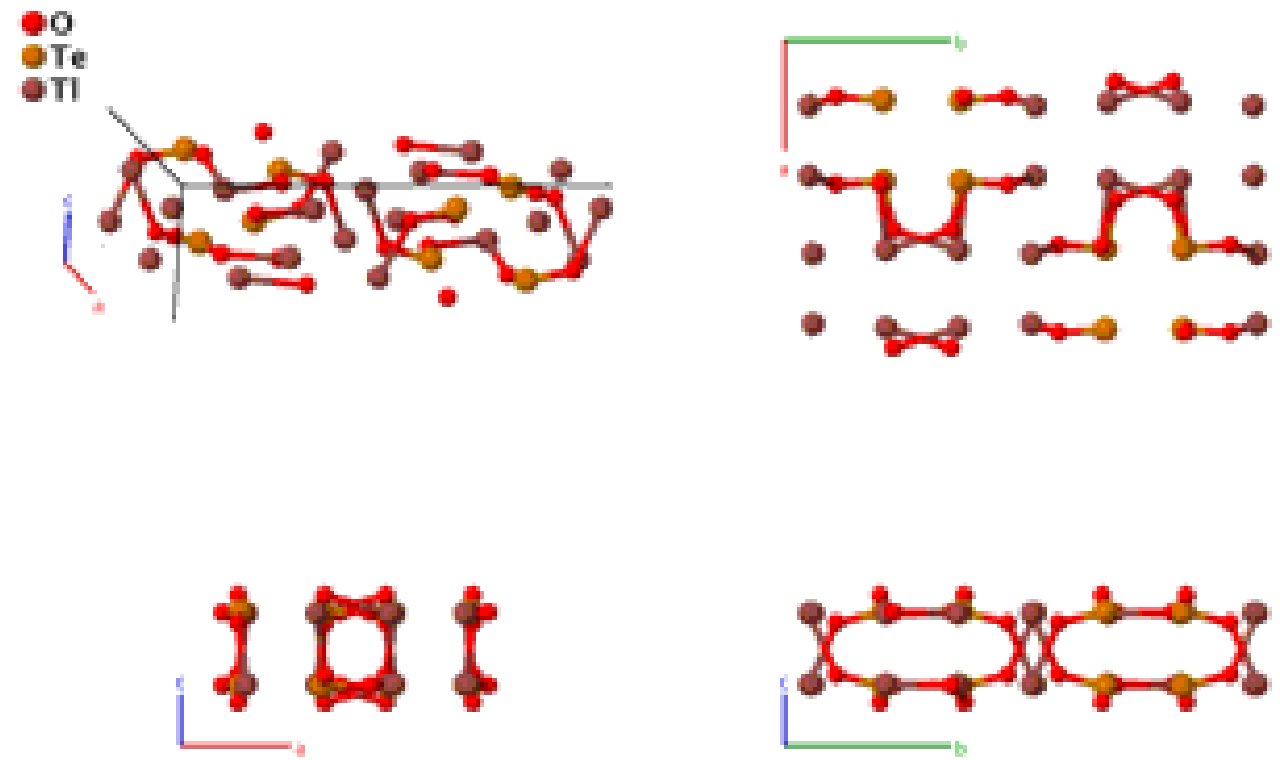

Prototype

: $\quad \alpha-\mathrm{Tl}_{2} \mathrm{TeO}_{3}$

AFLOW prototype label

: A3BC2_oP48_50_3m_m_2m

Strukturbericht designation : None

Pearson symbol $\quad: \quad$ oP48

Space group number $\quad: \quad 50$

Space group symbol : Pban

AFLOW prototype command : aflow --proto=A3BC2_oP48_50_3m_m_2m

- -params $=a, b / a, c / a, x_{1}, y_{1}, z_{1}, x_{2}, y_{2}, z_{2}, x_{3}, y_{3}, z_{3}, x_{4}, y_{4}, z_{4}, x_{5}, y_{5}, z_{5}, x_{6}, y_{6}$, $z_{6}$

Simple Orthorhombic primitive vectors:

$$
\begin{aligned}
& \mathbf{a}_{1}=a \hat{\mathbf{x}} \\
& \mathbf{a}_{2}=b \hat{\mathbf{y}} \\
& \mathbf{a}_{3}=c \hat{\mathbf{z}}
\end{aligned}
$$

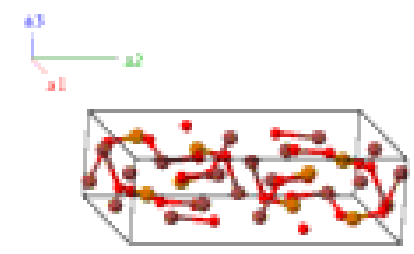

Basis vectors:

Lattice Coordinates

$\mathbf{B}_{1}=\quad x_{1} \mathbf{a}_{1}+y_{1} \mathbf{a}_{2}+z_{1} \mathbf{a}_{3}$

$=$

$\mathbf{B}_{2}=$

$\mathbf{B}_{3}=$

$\mathbf{B}_{4}=$

$x_{1} a \hat{\mathbf{x}}+y_{1} b \hat{\mathbf{y}}+z_{1} c \hat{\mathbf{z}}$

$=\left(\frac{1}{2}-x_{1}\right) a \hat{\mathbf{x}}+\left(\frac{1}{2}-y_{1}\right) b \hat{\mathbf{y}}+z_{1} c \hat{\mathbf{z}}$

$=\left(\frac{1}{2}-x_{1}\right) a \hat{\mathbf{x}}+y_{1} b \hat{\mathbf{y}}-z_{1} c \hat{\mathbf{z}}$

$=$

$x_{1} a \hat{\mathbf{x}}+\left(\frac{1}{2}-y_{1}\right) b \hat{\mathbf{y}}-z_{1} c \hat{\mathbf{z}}$
Wyckoff Position

Atom Type

$(8 m)$

O I

$(8 m)$

O I

$(8 m)$

O I

(8m)

O I 


\begin{tabular}{|c|c|c|c|c|c|c|}
\hline $\mathbf{B}_{5}$ & $=$ & $-x_{1} \mathbf{a}_{1}-y_{1} \mathbf{a}_{2}-z_{1} \mathbf{a}_{3}$ & $=$ & $-x_{1} a \hat{\mathbf{x}}-y_{1} b \hat{\mathbf{y}}-z_{1} c \hat{\mathbf{z}}$ & $(8 m)$ & O I \\
\hline $\mathbf{B}_{6}$ & $=$ & $\left(\frac{1}{2}+x_{1}\right) \mathbf{a}_{1}+\left(\frac{1}{2}+y_{1}\right) \mathbf{a}_{2}-z_{1} \mathbf{a}_{3}$ & $=$ & $\left(\frac{1}{2}+x_{1}\right) a \hat{\mathbf{x}}+\left(\frac{1}{2}+y_{1}\right) b \hat{\mathbf{y}}-z_{1} c \hat{\mathbf{z}}$ & $(8 m)$ & $\mathrm{O} I$ \\
\hline $\mathbf{B}_{7}$ & $=$ & $\left(\frac{1}{2}+x_{1}\right) \mathbf{a}_{1}-y_{1} \mathbf{a}_{2}+z_{1} \mathbf{a}_{3}$ & $=$ & $\left(\frac{1}{2}+x_{1}\right) a \hat{\mathbf{x}}-y_{1} b \hat{\mathbf{y}}+z_{1} c \hat{\mathbf{z}}$ & $(8 m)$ & $\mathrm{O} \mathrm{I}$ \\
\hline $\mathbf{B}_{8}$ & $=$ & $-x_{1} \mathbf{a}_{1}+\left(\frac{1}{2}+y_{1}\right) \mathbf{a}_{2}+z_{1} \mathbf{a}_{3}$ & $=$ & $-x_{1} a \hat{\mathbf{x}}+\left(\frac{1}{2}+y_{1}\right) b \hat{\mathbf{y}}+z_{1} c \hat{\mathbf{z}}$ & $(8 m)$ & O I \\
\hline $\mathbf{B}_{9}$ & $=$ & $x_{2} \mathbf{a}_{1}+y_{2} \mathbf{a}_{2}+z_{2} \mathbf{a}_{3}$ & $=$ & $x_{2} a \hat{\mathbf{x}}+y_{2} b \hat{\mathbf{y}}+z_{2} c \hat{\mathbf{z}}$ & $(8 m)$ & $\mathrm{O}$ II \\
\hline $\mathbf{B}_{10}$ & $=$ & $\left(\frac{1}{2}-x_{2}\right) \mathbf{a}_{1}+\left(\frac{1}{2}-y_{2}\right) \mathbf{a}_{2}+z_{2} \mathbf{a}_{3}$ & $=$ & $\left(\frac{1}{2}-x_{2}\right) a \hat{\mathbf{x}}+\left(\frac{1}{2}-y_{2}\right) b \hat{\mathbf{y}}+z_{2} c \hat{\mathbf{z}}$ & $(8 m)$ & $\mathrm{O}$ II \\
\hline $\mathbf{B}_{11}$ & $=$ & $\left(\frac{1}{2}-x_{2}\right) \mathbf{a}_{1}+y_{2} \mathbf{a}_{2}-z_{2} \mathbf{a}_{3}$ & $=$ & $\left(\frac{1}{2}-x_{2}\right) a \hat{\mathbf{x}}+y_{2} b \hat{\mathbf{y}}-z_{2} c \hat{\mathbf{z}}$ & $(8 m)$ & O II \\
\hline $\mathbf{B}_{12}$ & $=$ & $x_{2} \mathbf{a}_{1}+\left(\frac{1}{2}-y_{2}\right) \mathbf{a}_{2}-z_{2} \mathbf{a}_{3}$ & $=$ & $x_{2} a \hat{\mathbf{x}}+\left(\frac{1}{2}-y_{2}\right) b \hat{\mathbf{y}}-z_{2} c \hat{\mathbf{z}}$ & $(8 m)$ & O II \\
\hline $\mathbf{B}_{13}$ & $=$ & $-x_{2} \mathbf{a}_{1}-y_{2} \mathbf{a}_{2}-z_{2} \mathbf{a}_{3}$ & $=$ & $-x_{2} a \hat{\mathbf{x}}-y_{2} b \hat{\mathbf{y}}-z_{2} c \hat{\mathbf{z}}$ & $(8 m)$ & $\mathrm{O}$ II \\
\hline $\mathbf{B}_{14}$ & $=$ & $\left(\frac{1}{2}+x_{2}\right) \mathbf{a}_{1}+\left(\frac{1}{2}+y_{2}\right) \mathbf{a}_{2}-z_{2} \mathbf{a}_{3}$ & $=$ & $\left(\frac{1}{2}+x_{2}\right) a \hat{\mathbf{x}}+\left(\frac{1}{2}+y_{2}\right) b \hat{\mathbf{y}}-z_{2} c \hat{\mathbf{z}}$ & $(8 m)$ & O II \\
\hline $\mathbf{B}_{15}$ & $=$ & $\left(\frac{1}{2}+x_{2}\right) \mathbf{a}_{1}-y_{2} \mathbf{a}_{2}+z_{2} \mathbf{a}_{3}$ & $=$ & $\left(\frac{1}{2}+x_{2}\right) a \hat{\mathbf{x}}-y_{2} b \hat{\mathbf{y}}+z_{2} c \hat{\mathbf{z}}$ & $(8 m)$ & O II \\
\hline $\mathbf{B}_{16}$ & $=$ & $-x_{2} \mathbf{a}_{1}+\left(\frac{1}{2}+y_{2}\right) \mathbf{a}_{2}+z_{2} \mathbf{a}_{3}$ & $=$ & $-x_{2} a \hat{\mathbf{x}}+\left(\frac{1}{2}+y_{2}\right) b \hat{\mathbf{y}}+z_{2} c \hat{\mathbf{z}}$ & $(8 m)$ & O II \\
\hline $\mathbf{B}_{17}$ & $=$ & $x_{3} \mathbf{a}_{1}+y_{3} \mathbf{a}_{2}+z_{3} \mathbf{a}_{3}$ & $=$ & $x_{3} a \hat{\mathbf{x}}+y_{3} b \hat{\mathbf{y}}+z_{3} c \hat{\mathbf{z}}$ & $(8 m)$ & O III \\
\hline $\mathbf{B}_{18}$ & $=$ & $\left(\frac{1}{2}-x_{3}\right) \mathbf{a}_{1}+\left(\frac{1}{2}-y_{3}\right) \mathbf{a}_{2}+z_{3} \mathbf{a}_{3}$ & $=$ & $\left(\frac{1}{2}-x_{3}\right) a \hat{\mathbf{x}}+\left(\frac{1}{2}-y_{3}\right) b \hat{\mathbf{y}}+z_{3} c \hat{\mathbf{z}}$ & $(8 m)$ & O III \\
\hline $\mathbf{B}_{19}$ & $=$ & $\left(\frac{1}{2}-x_{3}\right) \mathbf{a}_{1}+y_{3} \mathbf{a}_{2}-z_{3} \mathbf{a}_{3}$ & $=$ & $\left(\frac{1}{2}-x_{3}\right) a \hat{\mathbf{x}}+y_{3} b \hat{\mathbf{y}}-z_{3} c \hat{\mathbf{z}}$ & $(8 m)$ & O III \\
\hline $\mathbf{B}_{20}$ & $=$ & $x_{3} \mathbf{a}_{1}+\left(\frac{1}{2}-y_{3}\right) \mathbf{a}_{2}-z_{3} \mathbf{a}_{3}$ & $=$ & $x_{3} a \hat{\mathbf{x}}+\left(\frac{1}{2}-y_{3}\right) b \hat{\mathbf{y}}-z_{3} c \hat{\mathbf{z}}$ & $(8 m)$ & O III \\
\hline $\mathbf{B}_{21}$ & $=$ & $-x_{3} \mathbf{a}_{1}-y_{3} \mathbf{a}_{2}-z_{3} \mathbf{a}_{3}$ & $=$ & $-x_{3} a \hat{\mathbf{x}}-y_{3} b \hat{\mathbf{y}}-z_{3} c \hat{\mathbf{z}}$ & $(8 m)$ & O III \\
\hline $\mathbf{B}_{22}$ & $=$ & $\left(\frac{1}{2}+x_{3}\right) \mathbf{a}_{1}+\left(\frac{1}{2}+y_{3}\right) \mathbf{a}_{2}-z_{3} \mathbf{a}_{3}$ & $=$ & $\left(\frac{1}{2}+x_{3}\right) a \hat{\mathbf{x}}+\left(\frac{1}{2}+y_{3}\right) b \hat{\mathbf{y}}-z_{3} c \hat{\mathbf{z}}$ & $(8 m)$ & O III \\
\hline $\mathbf{B}_{23}$ & $=$ & $\left(\frac{1}{2}+x_{3}\right) \mathbf{a}_{1}-y_{3} \mathbf{a}_{2}+z_{3} \mathbf{a}_{3}$ & $=$ & $\left(\frac{1}{2}+x_{3}\right) a \hat{\mathbf{x}}-y_{3} b \hat{\mathbf{y}}+z_{3} c \hat{\mathbf{z}}$ & $(8 m)$ & O III \\
\hline $\mathbf{B}_{24}$ & $=$ & $-x_{3} \mathbf{a}_{1}+\left(\frac{1}{2}+y_{3}\right) \mathbf{a}_{2}+z_{3} \mathbf{a}_{3}$ & $=$ & $-x_{3} a \hat{\mathbf{x}}+\left(\frac{1}{2}+y_{3}\right) b \hat{\mathbf{y}}+z_{3} c \hat{\mathbf{z}}$ & $(8 m)$ & O III \\
\hline $\mathbf{B}_{25}$ & $=$ & $x_{4} \mathbf{a}_{1}+y_{4} \mathbf{a}_{2}+z_{4} \mathbf{a}_{3}$ & $=$ & $x_{4} a \hat{\mathbf{x}}+y_{4} b \hat{\mathbf{y}}+z_{4} c \hat{\mathbf{z}}$ & $(8 m)$ & $\mathrm{Te}$ \\
\hline $\mathbf{B}_{26}$ & $=$ & $\left(\frac{1}{2}-x_{4}\right) \mathbf{a}_{1}+\left(\frac{1}{2}-y_{4}\right) \mathbf{a}_{2}+z_{4} \mathbf{a}_{3}$ & $=$ & $\left(\frac{1}{2}-x_{4}\right) a \hat{\mathbf{x}}+\left(\frac{1}{2}-y_{4}\right) b \hat{\mathbf{y}}+z_{4} c \hat{\mathbf{z}}$ & $(8 m)$ & $\mathrm{Te}$ \\
\hline $\mathbf{B}_{27}$ & $=$ & $\left(\frac{1}{2}-x_{4}\right) \mathbf{a}_{1}+y_{4} \mathbf{a}_{2}-z_{4} \mathbf{a}_{3}$ & $=$ & $\left(\frac{1}{2}-x_{4}\right) a \hat{\mathbf{x}}+y_{4} b \hat{\mathbf{y}}-z_{4} c \hat{\mathbf{z}}$ & $(8 m)$ & $\mathrm{Te}$ \\
\hline $\mathbf{B}_{28}$ & $=$ & $x_{4} \mathbf{a}_{1}+\left(\frac{1}{2}-y_{4}\right) \mathbf{a}_{2}-z_{4} \mathbf{a}_{3}$ & $=$ & $x_{4} a \hat{\mathbf{x}}+\left(\frac{1}{2}-y_{4}\right) b \hat{\mathbf{y}}-z_{4} c \hat{\mathbf{z}}$ & $(8 m)$ & $\mathrm{Te}$ \\
\hline $\mathbf{B}_{29}$ & $=$ & $-x_{4} \mathbf{a}_{1}-y_{4} \mathbf{a}_{2}-z_{4} \mathbf{a}_{3}$ & $=$ & $-x_{4} a \hat{\mathbf{x}}-y_{4} b \hat{\mathbf{y}}-z_{4} c \hat{\mathbf{z}}$ & $(8 m)$ & $\mathrm{Te}$ \\
\hline $\mathbf{B}_{30}$ & $=$ & $\left(\frac{1}{2}+x_{4}\right) \mathbf{a}_{1}+\left(\frac{1}{2}+y_{4}\right) \mathbf{a}_{2}-z_{4} \mathbf{a}_{3}$ & $=$ & $\left(\frac{1}{2}+x_{4}\right) a \hat{\mathbf{x}}+\left(\frac{1}{2}+y_{4}\right) b \hat{\mathbf{y}}-z_{4} c \hat{\mathbf{z}}$ & $(8 m)$ & $\mathrm{Te}$ \\
\hline $\mathbf{B}_{31}$ & $=$ & $\left(\frac{1}{2}+x_{4}\right) \mathbf{a}_{1}-y_{4} \mathbf{a}_{2}+z_{4} \mathbf{a}_{3}$ & $=$ & $\left(\frac{1}{2}+x_{4}\right) a \hat{\mathbf{x}}-y_{4} b \hat{\mathbf{y}}+z_{4} c \hat{\mathbf{z}}$ & $(8 m)$ & $\mathrm{Te}$ \\
\hline $\mathbf{B}_{32}$ & $=$ & $-x_{4} \mathbf{a}_{1}+\left(\frac{1}{2}+y_{4}\right) \mathbf{a}_{2}+z_{4} \mathbf{a}_{3}$ & $=$ & $-x_{4} a \hat{\mathbf{x}}+\left(\frac{1}{2}+y_{4}\right) b \hat{\mathbf{y}}+z_{4} c \hat{\mathbf{z}}$ & $(8 m)$ & $\mathrm{Te}$ \\
\hline $\mathbf{B}_{33}$ & $=$ & $x_{5} \mathbf{a}_{1}+y_{5} \mathbf{a}_{2}+z_{5} \mathbf{a}_{3}$ & $=$ & $x_{5} a \hat{\mathbf{x}}+y_{5} b \hat{\mathbf{y}}+z_{5} c \hat{\mathbf{z}}$ & $(8 m)$ & $\mathrm{Tl} \mathrm{I}$ \\
\hline $\mathbf{B}_{34}$ & $=$ & $\left(\frac{1}{2}-x_{5}\right) \mathbf{a}_{1}+\left(\frac{1}{2}-y_{5}\right) \mathbf{a}_{2}+z_{5} \mathbf{a}_{3}$ & $=$ & $\left(\frac{1}{2}-x_{5}\right) a \hat{\mathbf{x}}+\left(\frac{1}{2}-y_{5}\right) b \hat{\mathbf{y}}+z_{5} c \hat{\mathbf{z}}$ & $(8 m)$ & $\mathrm{Tl} \mathrm{I}$ \\
\hline $\mathbf{B}_{35}$ & $=$ & $\left(\frac{1}{2}-x_{5}\right) \mathbf{a}_{1}+y_{5} \mathbf{a}_{2}-z_{5} \mathbf{a}_{3}$ & $=$ & $\left(\frac{1}{2}-x_{5}\right) a \hat{\mathbf{x}}+y_{5} b \hat{\mathbf{y}}-z_{5} c \hat{\mathbf{z}}$ & $(8 m)$ & $\mathrm{Tl} \mathrm{I}$ \\
\hline $\mathbf{B}_{36}$ & $=$ & $x_{5} \mathbf{a}_{1}+\left(\frac{1}{2}-y_{5}\right) \mathbf{a}_{2}-z_{5} \mathbf{a}_{3}$ & $=$ & $x_{5} a \hat{\mathbf{x}}+\left(\frac{1}{2}-y_{5}\right) b \hat{\mathbf{y}}-z_{5} c \hat{\mathbf{z}}$ & $(8 m)$ & $\mathrm{Tl} \mathrm{I}$ \\
\hline $\mathbf{B}_{37}$ & $=$ & $-x_{5} \mathbf{a}_{1}-y_{5} \mathbf{a}_{2}-z_{5} \mathbf{a}_{3}$ & $=$ & $-x_{5} a \hat{\mathbf{x}}-y_{5} b \hat{\mathbf{y}}-z_{5} c \hat{\mathbf{z}}$ & $(8 m)$ & $\mathrm{Tl} \mathrm{I}$ \\
\hline $\mathbf{B}_{38}$ & $=$ & $\left(\frac{1}{2}+x_{5}\right) \mathbf{a}_{1}+\left(\frac{1}{2}+y_{5}\right) \mathbf{a}_{2}-z_{5} \mathbf{a}_{3}$ & $=$ & $\left(\frac{1}{2}+x_{5}\right) a \hat{\mathbf{x}}+\left(\frac{1}{2}+y_{5}\right) b \hat{\mathbf{y}}-z_{5} c \hat{\mathbf{z}}$ & $(8 m)$ & $\mathrm{Tl} \mathrm{I}$ \\
\hline $\mathbf{B}_{39}$ & $=$ & $\left(\frac{1}{2}+x_{5}\right) \mathbf{a}_{1}-y_{5} \mathbf{a}_{2}+z_{5} \mathbf{a}_{3}$ & $=$ & $\left(\frac{1}{2}+x_{5}\right) a \hat{\mathbf{x}}-y_{5} b \hat{\mathbf{y}}+z_{5} c \hat{\mathbf{z}}$ & $(8 m)$ & $\mathrm{Tl} \mathrm{I}$ \\
\hline $\mathbf{B}_{40}$ & $=$ & $-x_{5} \mathbf{a}_{1}+\left(\frac{1}{2}+y_{5}\right) \mathbf{a}_{2}+z_{5} \mathbf{a}_{3}$ & $=$ & $-x_{5} a \hat{\mathbf{x}}+\left(\frac{1}{2}+y_{5}\right) b \hat{\mathbf{y}}+z_{5} c \hat{\mathbf{z}}$ & $(8 m)$ & $\mathrm{Tl} \mathrm{I}$ \\
\hline
\end{tabular}




\begin{tabular}{|c|c|c|c|c|c|}
\hline $\mathbf{B}_{41}$ & $=$ & $x_{6} \mathbf{a}_{1}+y_{6} \mathbf{a}_{2}+z_{6} \mathbf{a}_{3}$ & $=$ & $x_{6} a \hat{\mathbf{x}}+y_{6} b \hat{\mathbf{y}}+z_{6} c \hat{\mathbf{z}}$ & $(8 m)$ \\
\hline $\mathbf{B}_{42}$ & $=$ & $\left(\frac{1}{2}-x_{6}\right) \mathbf{a}_{1}+\left(\frac{1}{2}-y_{6}\right) \mathbf{a}_{2}+z_{6} \mathbf{a}_{3}$ & $=$ & $\left(\frac{1}{2}-x_{6}\right) a \hat{\mathbf{x}}+\left(\frac{1}{2}-y_{6}\right) b \hat{\mathbf{y}}+z_{6} c \hat{\mathbf{z}}$ & $(8 m)$ \\
\hline $\mathbf{B}_{43}$ & $=$ & $\left(\frac{1}{2}-x_{6}\right) \mathbf{a}_{1}+y_{6} \mathbf{a}_{2}-z_{6} \mathbf{a}_{3}$ & $=$ & $\left(\frac{1}{2}-x_{6}\right) a \hat{\mathbf{x}}+y_{6} b \hat{\mathbf{y}}-z_{6} c \hat{\mathbf{z}}$ & $(8 m)$ \\
\hline $\mathbf{B}_{44}$ & $=$ & $x_{6} \mathbf{a}_{1}+\left(\frac{1}{2}-y_{6}\right) \mathbf{a}_{2}-z_{6} \mathbf{a}_{3}$ & $=$ & $x_{6} a \hat{\mathbf{x}}+\left(\frac{1}{2}-y_{6}\right) b \hat{\mathbf{y}}-z_{6} c \hat{\mathbf{z}}$ & $(8 m)$ \\
\hline $\mathbf{B}_{45}$ & $=$ & $-x_{6} \mathbf{a}_{1}-y_{6} \mathbf{a}_{2}-z_{6} \mathbf{a}_{3}$ & $=$ & $-x_{6} a \hat{\mathbf{x}}-y_{6} b \hat{\mathbf{y}}-z_{6} c \hat{\mathbf{z}}$ & $(8 m)$ \\
\hline $\mathbf{B}_{46}$ & $=$ & $\left(\frac{1}{2}+x_{6}\right) \mathbf{a}_{1}+\left(\frac{1}{2}+y_{6}\right) \mathbf{a}_{2}-z_{6} \mathbf{a}_{3}$ & $=$ & $\left(\frac{1}{2}+x_{6}\right) a \hat{\mathbf{x}}+\left(\frac{1}{2}+y_{6}\right) b \hat{\mathbf{y}}-z_{6} c \hat{\mathbf{z}}$ & $(8 m)$ \\
\hline $\mathbf{B}_{47}$ & $=$ & $\left(\frac{1}{2}+x_{6}\right) \mathbf{a}_{1}-y_{6} \mathbf{a}_{2}+z_{6} \mathbf{a}_{3}$ & $=$ & $\left(\frac{1}{2}+x_{6}\right) a \hat{\mathbf{x}}-y_{6} b \hat{\mathbf{y}}+z_{6} c \hat{\mathbf{z}}$ & $(8 m)$ \\
\hline $\mathbf{B}_{48}$ & $=$ & $-x_{6} \mathbf{a}_{1}+\left(\frac{1}{2}+y_{6}\right) \mathbf{a}_{2}+z_{6} \mathbf{a}_{3}$ & $=$ & $-x_{6} a \hat{\mathbf{x}}+\left(\frac{1}{2}+y_{6}\right) b \hat{\mathbf{y}}+z_{6} c \hat{\mathbf{z}}$ & $(8 m)$ \\
\hline
\end{tabular}

\section{References:}

- F. Rieger and A.-V. Mudring, Phase transition in $\mathrm{Tl}_{2} \mathrm{TeO}_{3}$ : Influence and origin of the thallium lone pair distortion, Inorg. Chem. 46, 446-452 (2007), doi:10.1021/ic061273j.

\section{Found in:}

- P. Villars and K. Cenzual, Pearson's Crystal Data - Crystal Structure Database for Inorganic Compounds, ASM International (2013).

\section{Geometry files:}

- CIF: pp. 833

- POSCAR: pp. 833 
$\mathrm{GaCl}_{2}$ (High-temperature) Structure:

A2B_oP24_52_2e_cd
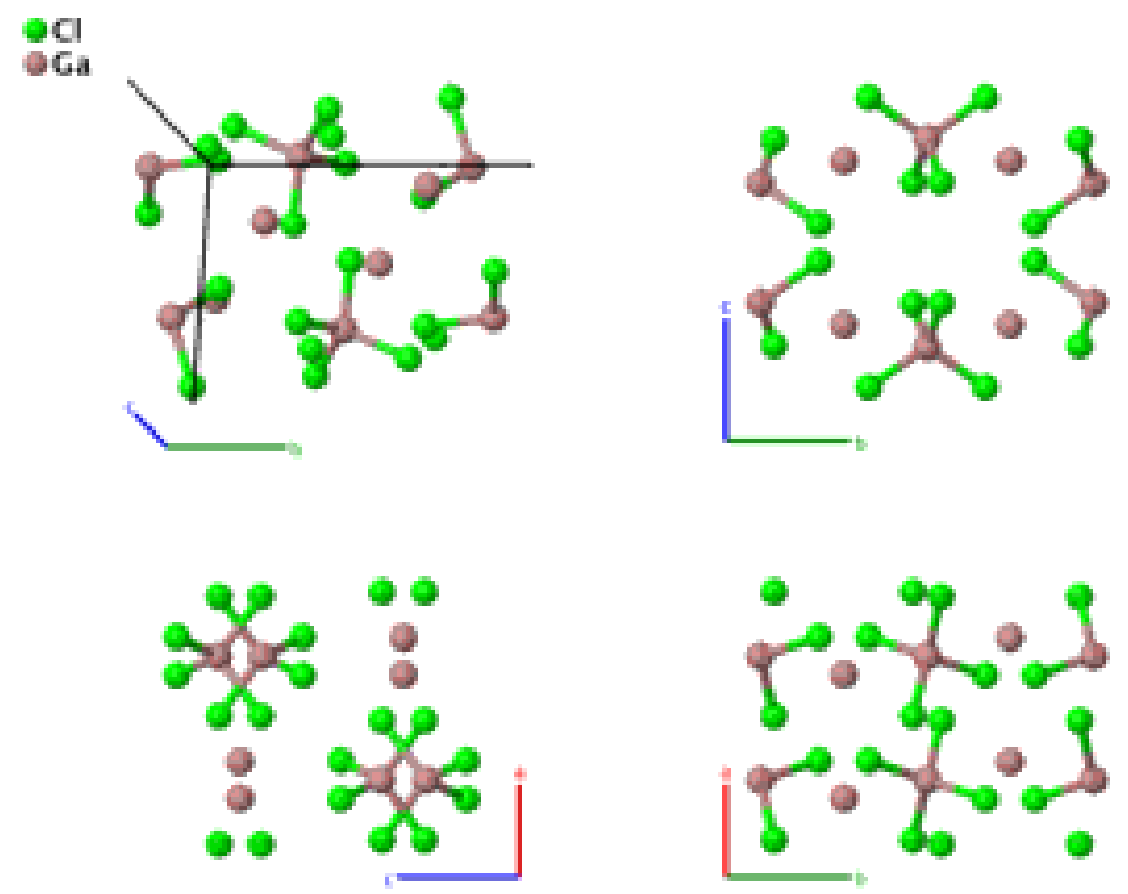

\section{Prototype}

: $\quad \mathrm{GaCl}_{2}$

AFLOW prototype label

: A2B_oP24_52_2e_cd

Strukturbericht designation

Pearson symbol

: None

Space group number

: $\quad$ oP24

Space group symbol

: 52

AFLOW prototype command

: Pnna

: aflow --proto=A2B_oP24_52_2e_cd

- -params $=a, b / a, c / a, z_{1}, x_{2}, x_{3}, y_{3}, z_{3}, x_{4}, y_{4}, z_{4}$

Simple Orthorhombic primitive vectors:

$$
\begin{aligned}
& \mathbf{a}_{1}=a \hat{\mathbf{x}} \\
& \mathbf{a}_{2}=b \hat{\mathbf{y}} \\
& \mathbf{a}_{3}=c \hat{\mathbf{z}}
\end{aligned}
$$

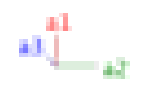

Basis vectors:

Lattice Coordinates

$$
\begin{aligned}
& \mathbf{B}_{1}= \\
& \mathbf{B}_{2}= \\
& \frac{1}{4} \mathbf{a}_{1}+z_{1} \mathbf{a}_{3} \\
& = \\
& =\quad \frac{1}{4} a \hat{\mathbf{x}}+\frac{1}{2} b \hat{\mathbf{y}}+\left(\frac{1}{2}-z_{1}\right) c \hat{\mathbf{z}} \\
& \frac{1}{4} a \hat{\mathbf{x}}+z_{1} c \hat{\mathbf{z}} \\
& \frac{1}{4} \mathbf{a}_{1}+\frac{1}{2} \mathbf{a}_{2}+\left(\frac{1}{2}-z_{1}\right) \mathbf{a}_{3}
\end{aligned}
$$$$
\text { Cartesian Coordinates }
$$

Wyckoff Position

Atom Type

$(4 c)$

Ga I

$(4 c)$ 


\begin{tabular}{|c|c|c|c|c|c|c|}
\hline $\mathbf{B}_{3}$ & $=$ & $\frac{3}{4} \mathbf{a}_{1}+-z_{1} \mathbf{a}_{3}$ & $=$ & $\frac{3}{4} a \hat{\mathbf{x}}+-z_{1} c \hat{\mathbf{z}}$ & $(4 c)$ & Ga I \\
\hline $\mathbf{B}_{4}$ & $=$ & $\frac{3}{4} \mathbf{a}_{1}+\frac{1}{2} \mathbf{a}_{2}+\left(\frac{1}{2}+z_{1}\right) \mathbf{a}_{3}$ & $=$ & $\frac{3}{4} a \hat{\mathbf{x}}+\frac{1}{2} b \hat{\mathbf{y}}+\left(\frac{1}{2}+z_{1}\right) c \hat{\mathbf{z}}$ & $(4 c)$ & Ga I \\
\hline $\mathbf{B}_{5}$ & $=$ & $x_{2} \mathbf{a}_{1}+\frac{1}{4} \mathbf{a}_{2}+\frac{1}{4} \mathbf{a}_{3}$ & $=$ & $x_{2} a \hat{\mathbf{x}}+\frac{1}{4} b \hat{\mathbf{y}}+\frac{1}{4} c \hat{\mathbf{z}}$ & $(4 d)$ & Ga II \\
\hline $\mathbf{B}_{6}$ & $=$ & $\left(\frac{1}{2}-x_{2}\right) \mathbf{a}_{1}+\frac{3}{4} \mathbf{a}_{2}+\frac{1}{4} \mathbf{a}_{3}$ & $=$ & $\left(\frac{1}{2}-x_{2}\right) a \hat{\mathbf{x}}+\frac{3}{4} b \hat{\mathbf{y}}+\frac{1}{4} c \hat{\mathbf{z}}$ & $(4 d)$ & Ga II \\
\hline $\mathbf{B}_{7}$ & $=$ & $-x_{2} \mathbf{a}_{1}+\frac{3}{4} \mathbf{a}_{2}+\frac{3}{4} \mathbf{a}_{3}$ & $=$ & $-x_{2} a \hat{\mathbf{x}}+\frac{3}{4} b \hat{\mathbf{y}}+\frac{3}{4} c \hat{\mathbf{z}}$ & $(4 d)$ & Ga II \\
\hline $\mathbf{B}_{8}$ & $=$ & $\left(\frac{1}{2}+x_{2}\right) \mathbf{a}_{1}+\frac{1}{4} \mathbf{a}_{2}+\frac{3}{4} \mathbf{a}_{3}$ & $=$ & $\left(\frac{1}{2}+x_{2}\right) a \hat{\mathbf{x}}+\frac{1}{4} b \hat{\mathbf{y}}+\frac{3}{4} c \hat{\mathbf{z}}$ & $(4 d)$ & Ga II \\
\hline $\mathbf{B}_{9}$ & $=$ & $x_{3} \mathbf{a}_{1}+y_{3} \mathbf{a}_{2}+z_{3} \mathbf{a}_{3}$ & $=$ & $x_{3} a \hat{\mathbf{x}}+y_{3} b \hat{\mathbf{y}}+z_{3} c \hat{\mathbf{z}}$ & $(8 e)$ & $\mathrm{Cl} \mathrm{I}$ \\
\hline $\mathbf{B}_{10}$ & $=$ & $\left(\frac{1}{2}-x_{3}\right) \mathbf{a}_{1}-y_{3} \mathbf{a}_{2}+z_{3} \mathbf{a}_{3}$ & $=$ & $\left(\frac{1}{2}-x_{3}\right) a \hat{\mathbf{x}}-y_{3} b \hat{\mathbf{y}}+z_{3} c \hat{\mathbf{z}}$ & $(8 e)$ & $\mathrm{Cl} \mathrm{I}$ \\
\hline $\mathbf{B}_{11}$ & $=$ & $\begin{array}{c}\left(\frac{1}{2}-x_{3}\right) \mathbf{a}_{1}+\left(\frac{1}{2}+y_{3}\right) \mathbf{a}_{2}+ \\
\left(\frac{1}{2}-z_{3}\right) \mathbf{a}_{3}\end{array}$ & $=$ & $\begin{array}{c}\left(\frac{1}{2}-x_{3}\right) a \hat{\mathbf{x}}+\left(\frac{1}{2}+y_{3}\right) b \hat{\mathbf{y}}+ \\
\left(\frac{1}{2}-z_{3}\right) c \hat{\mathbf{z}}\end{array}$ & $(8 e)$ & $\mathrm{Cl} \mathrm{I}$ \\
\hline $\mathbf{B}_{12}$ & $=$ & $x_{3} \mathbf{a}_{1}+\left(\frac{1}{2}-y_{3}\right) \mathbf{a}_{2}+\left(\frac{1}{2}-z_{3}\right) \mathbf{a}_{3}$ & $=$ & $x_{3} a \hat{\mathbf{x}}+\left(\frac{1}{2}-y_{3}\right) b \hat{\mathbf{y}}+\left(\frac{1}{2}-z_{3}\right) c \hat{\mathbf{z}}$ & $(8 e)$ & $\mathrm{Cl} \mathrm{I}$ \\
\hline $\mathbf{B}_{13}$ & $=$ & $-x_{3} \mathbf{a}_{1}-y_{3} \mathbf{a}_{2}-z_{3} \mathbf{a}_{3}$ & $=$ & $-x_{3} a \hat{\mathbf{x}}-y_{3} b \hat{\mathbf{y}}-z_{3} c \hat{\mathbf{z}}$ & $(8 e)$ & $\mathrm{Cl} \mathrm{I}$ \\
\hline $\mathbf{B}_{14}$ & $=$ & $\left(\frac{1}{2}+x_{3}\right) \mathbf{a}_{1}+y_{3} \mathbf{a}_{2}-z_{3} \mathbf{a}_{3}$ & $=$ & $\left(\frac{1}{2}+x_{3}\right) a \hat{\mathbf{x}}+y_{3} b \hat{\mathbf{y}}-z_{3} c \hat{\mathbf{z}}$ & $(8 e)$ & $\mathrm{Cl} \mathrm{I}$ \\
\hline $\mathbf{B}_{15}$ & $=$ & $\begin{array}{c}\left(\frac{1}{2}+x_{3}\right) \mathbf{a}_{1}+\left(\frac{1}{2}-y_{3}\right) \mathbf{a}_{2}+ \\
\left(\frac{1}{2}+z_{3}\right) \mathbf{a}_{3}\end{array}$ & $=$ & $\begin{array}{c}\left(\frac{1}{2}+x_{3}\right) a \hat{\mathbf{x}}+\left(\frac{1}{2}-y_{3}\right) b \hat{\mathbf{y}}+ \\
\left(\frac{1}{2}+z_{3}\right) c \hat{\mathbf{z}}\end{array}$ & $(8 e)$ & $\mathrm{Cl} \mathrm{I}$ \\
\hline $\mathbf{B}_{16}$ & $=$ & $-x_{3} \mathbf{a}_{1}+\left(\frac{1}{2}+y_{3}\right) \mathbf{a}_{2}+\left(\frac{1}{2}+z_{3}\right) \mathbf{a}_{3}$ & $=$ & $-x_{3} a \hat{\mathbf{x}}+\left(\frac{1}{2}+y_{3}\right) b \hat{\mathbf{y}}+\left(\frac{1}{2}+z_{3}\right) c \hat{\mathbf{z}}$ & $(8 e)$ & $\mathrm{Cl} \mathrm{I}$ \\
\hline $\mathbf{B}_{17}$ & $=$ & $x_{4} \mathbf{a}_{1}+y_{4} \mathbf{a}_{2}+z_{4} \mathbf{a}_{3}$ & $=$ & $x_{4} a \hat{\mathbf{x}}+y_{4} b \hat{\mathbf{y}}+z_{4} c \hat{\mathbf{z}}$ & $(8 e)$ & $\mathrm{Cl}$ II \\
\hline $\mathbf{B}_{18}$ & $=$ & $\left(\frac{1}{2}-x_{4}\right) \mathbf{a}_{1}-y_{4} \mathbf{a}_{2}+z_{4} \mathbf{a}_{3}$ & $=$ & $\left(\frac{1}{2}-x_{4}\right) a \hat{\mathbf{x}}-y_{4} b \hat{\mathbf{y}}+z_{4} c \hat{\mathbf{z}}$ & $(8 e)$ & $\mathrm{Cl}$ II \\
\hline $\mathbf{B}_{19}$ & $=$ & $\begin{array}{c}\left(\frac{1}{2}-x_{4}\right) \mathbf{a}_{1}+\left(\frac{1}{2}+y_{4}\right) \mathbf{a}_{2}+ \\
\left(\frac{1}{2}-z_{4}\right) \mathbf{a}_{3}\end{array}$ & $=$ & $\begin{array}{c}\left(\frac{1}{2}-x_{4}\right) a \hat{\mathbf{x}}+\left(\frac{1}{2}+y_{4}\right) b \hat{\mathbf{y}}+ \\
\left(\frac{1}{2}-z_{4}\right) c \hat{\mathbf{z}}\end{array}$ & $(8 e)$ & $\mathrm{Cl}$ II \\
\hline $\mathbf{B}_{20}$ & $=$ & $x_{4} \mathbf{a}_{1}+\left(\frac{1}{2}-y_{4}\right) \mathbf{a}_{2}+\left(\frac{1}{2}-z_{4}\right) \mathbf{a}_{3}$ & $=$ & $x_{4} a \hat{\mathbf{x}}+\left(\frac{1}{2}-y_{4}\right) b \hat{\mathbf{y}}+\left(\frac{1}{2}-z_{4}\right) c \hat{\mathbf{z}}$ & $(8 e)$ & $\mathrm{Cl}$ II \\
\hline $\mathbf{B}_{21}$ & $=$ & $-x_{4} \mathbf{a}_{1}-y_{4} \mathbf{a}_{2}-z_{4} \mathbf{a}_{3}$ & $=$ & $-x_{4} a \hat{\mathbf{x}}-y_{4} b \hat{\mathbf{y}}-z_{4} c \hat{\mathbf{z}}$ & $(8 e)$ & $\mathrm{Cl}$ II \\
\hline $\mathbf{B}_{22}$ & $=$ & $\left(\frac{1}{2}+x_{4}\right) \mathbf{a}_{1}+y_{4} \mathbf{a}_{2}-z_{4} \mathbf{a}_{3}$ & $=$ & $\left(\frac{1}{2}+x_{4}\right) a \hat{\mathbf{x}}+y_{4} b \hat{\mathbf{y}}-z_{4} c \hat{\mathbf{z}}$ & $(8 e)$ & $\mathrm{Cl}$ II \\
\hline $\mathbf{B}_{23}$ & $=$ & $\begin{array}{c}\left(\frac{1}{2}+x_{4}\right) \mathbf{a}_{1}+\left(\frac{1}{2}-y_{4}\right) \mathbf{a}_{2}+ \\
\left(\frac{1}{2}+z_{4}\right) \mathbf{a}_{3}\end{array}$ & $=$ & $\begin{array}{c}\left(\frac{1}{2}+x_{4}\right) a \hat{\mathbf{x}}+\left(\frac{1}{2}-y_{4}\right) b \hat{\mathbf{y}}+ \\
\left(\frac{1}{2}+z_{4}\right) c \hat{\mathbf{z}}\end{array}$ & $(8 e)$ & $\mathrm{Cl}$ II \\
\hline $\mathbf{B}_{24}$ & $=$ & $-x_{4} \mathbf{a}_{1}+\left(\frac{1}{2}+y_{4}\right) \mathbf{a}_{2}+\left(\frac{1}{2}+z_{4}\right) \mathbf{a}_{3}$ & $=$ & $-x_{4} a \hat{\mathbf{x}}+\left(\frac{1}{2}+y_{4}\right) b \hat{\mathbf{y}}+\left(\frac{1}{2}+z_{4}\right) c \hat{\mathbf{z}}$ & $(8 e)$ & $\mathrm{Cl}$ II \\
\hline
\end{tabular}

\section{References:}

- A. P. Wilkinson, A. K. Cheetham, and D. E. Cox, Study of oxidation-state contrast in gallium dichloride by synchrotron X-ray anomalous scattering, Acta Crystallogr. Sect. B Struct. Sci. 47, 155-161 (1991), doi:10.1107/S0108768190010485.

\section{Found in:}

- P. Villars and K. Cenzual, Pearson's Crystal Data - Crystal Structure Database for Inorganic Compounds, ASM International (2013).

\section{Geometry files:}

- CIF: pp. 833

- POSCAR: pp. 834 


\section{$\mathrm{Sr}_{2} \mathrm{Bi}_{3}$ Structure: A3B2_oP20_52_de_cd}
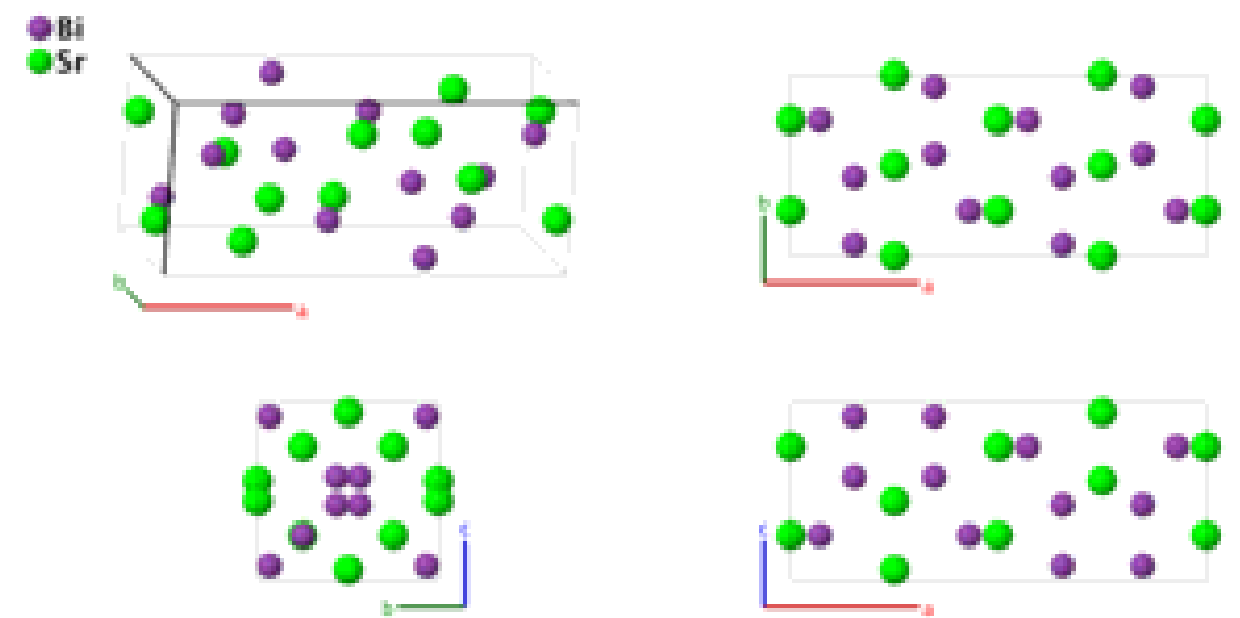

Prototype

$: \mathrm{Sr}_{2} \mathrm{Bi}_{3}$

AFLOW prototype label

: A3B2_oP20_52_de_cd

Strukturbericht designation : None

Pearson symbol

: $\quad$ oP20

Space group number

: 52

Space group symbol

: Pnna

AFLOW prototype command : aflow --proto=A3B2_oP20_52_de_cd

- -params $=a, b / a, c / a, z_{1}, x_{2}, x_{3}, x_{4}, y_{4}, z_{4}$

Simple Orthorhombic primitive vectors:

$$
\begin{aligned}
& \mathbf{a}_{1}=a \hat{\mathbf{x}} \\
& \mathbf{a}_{2}=b \hat{\mathbf{y}} \\
& \mathbf{a}_{3}=c \hat{\mathbf{z}}
\end{aligned}
$$

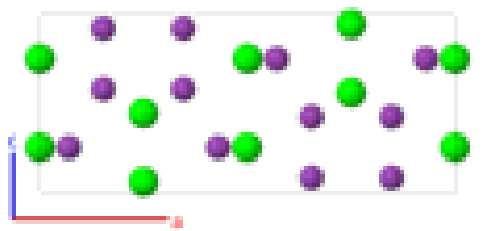

\section{Basis vectors:}

Lattice Coordinates

$\mathbf{B}_{1}=\quad \frac{1}{4} \mathbf{a}_{1}+z_{1} \mathbf{a}_{3}$

$\mathbf{B}_{2}=\frac{1}{4} \mathbf{a}_{1}+\frac{1}{2} \mathbf{a}_{2}+\left(\frac{1}{2}-z_{1}\right) \mathbf{a}_{3}$

$\mathbf{B}_{3}=\quad \frac{3}{4} \mathbf{a}_{1}+-z_{1} \mathbf{a}_{3}$

$\mathbf{B}_{4}=\frac{3}{4} \mathbf{a}_{1}+\frac{1}{2} \mathbf{a}_{2}+\left(\frac{1}{2}+z_{1}\right) \mathbf{a}_{3}$

$\mathbf{B}_{5}=$

$\mathbf{B}_{6}=$

$\mathbf{B}_{7}=$

$\mathbf{B}_{8}=$

$\mathbf{B}_{9}=$ $x_{2} \mathbf{a}_{1}+\frac{1}{4} \mathbf{a}_{2}+\frac{1}{4} \mathbf{a}_{3}$

$\left(\frac{1}{2}-x_{2}\right) \mathbf{a}_{1}+\frac{3}{4} \mathbf{a}_{2}+\frac{1}{4} \mathbf{a}_{3}$

$-x_{2} \mathbf{a}_{1}+\frac{3}{4} \mathbf{a}_{2}+\frac{3}{4} \mathbf{a}_{3}$

$\left(\frac{1}{2}+x_{2}\right) \mathbf{a}_{1}+\frac{1}{4} \mathbf{a}_{2}+\frac{3}{4} \mathbf{a}_{3}$

$x_{3} \mathbf{a}_{1}+\frac{1}{4} \mathbf{a}_{2}+\frac{1}{4} \mathbf{a}_{3}$
Cartesian Coordinates

$=\quad \frac{1}{4} a \hat{\mathbf{x}}+z_{1} c \hat{\mathbf{z}}$

$=\quad \frac{1}{4} a \hat{\mathbf{x}}+\frac{1}{2} b \hat{\mathbf{y}}+\left(\frac{1}{2}-z_{1}\right) c \hat{\mathbf{z}}$

$=\quad \frac{3}{4} a \hat{\mathbf{x}}+-z_{1} c \hat{\mathbf{z}}$

$=\quad \frac{3}{4} a \hat{\mathbf{x}}+\frac{1}{2} b \hat{\mathbf{y}}+\left(\frac{1}{2}+z_{1}\right) c \hat{\mathbf{z}}$

$=\quad x_{2} a \hat{\mathbf{x}}+\frac{1}{4} b \hat{\mathbf{y}}+\frac{1}{4} c \hat{\mathbf{z}}$

$=\left(\frac{1}{2}-x_{2}\right) a \hat{\mathbf{x}}+\frac{3}{4} b \hat{\mathbf{y}}+\frac{1}{4} c \hat{\mathbf{z}}$

$=\quad-x_{2} a \hat{\mathbf{x}}+\frac{3}{4} b \hat{\mathbf{y}}+\frac{3}{4} c \hat{\mathbf{z}}$

$=\quad\left(\frac{1}{2}+x_{2}\right) a \hat{\mathbf{x}}+\frac{1}{4} b \hat{\mathbf{y}}+\frac{3}{4} c \hat{\mathbf{z}}$

$=\quad x_{3} a \hat{\mathbf{x}}+\frac{1}{4} b \hat{\mathbf{y}}+\frac{1}{4} c \hat{\mathbf{z}}$

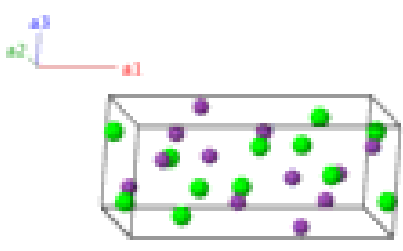




\begin{tabular}{|c|c|c|c|c|c|c|}
\hline $\mathbf{B}_{10}$ & $=$ & $\left(\frac{1}{2}-x_{3}\right) \mathbf{a}_{1}+\frac{3}{4} \mathbf{a}_{2}+\frac{1}{4} \mathbf{a}_{3}$ & $=$ & $\left(\frac{1}{2}-x_{3}\right) a \hat{\mathbf{x}}+\frac{3}{4} b \hat{\mathbf{y}}+\frac{1}{4} c \hat{\mathbf{z}}$ & $(4 d)$ & Sr II \\
\hline $\mathbf{B}_{11}$ & $=$ & $-x_{3} \mathbf{a}_{1}+\frac{3}{4} \mathbf{a}_{2}+\frac{3}{4} \mathbf{a}_{3}$ & $=$ & $-x_{3} a \hat{\mathbf{x}}+\frac{3}{4} b \hat{\mathbf{y}}+\frac{3}{4} c \hat{\mathbf{z}}$ & $(4 d)$ & Sr II \\
\hline $\mathbf{B}_{12}$ & $=$ & $\left(\frac{1}{2}+x_{3}\right) \mathbf{a}_{1}+\frac{1}{4} \mathbf{a}_{2}+\frac{3}{4} \mathbf{a}_{3}$ & $=$ & $\left(\frac{1}{2}+x_{3}\right) a \hat{\mathbf{x}}+\frac{1}{4} b \hat{\mathbf{y}}+\frac{3}{4} c \hat{\mathbf{z}}$ & $(4 d)$ & Sr II \\
\hline $\mathbf{B}_{13}$ & $=$ & $x_{4} \mathbf{a}_{1}+y_{4} \mathbf{a}_{2}+z_{4} \mathbf{a}_{3}$ & $=$ & $x_{4} a \hat{\mathbf{x}}+y_{4} b \hat{\mathbf{y}}+z_{4} c \hat{\mathbf{z}}$ & $(8 e)$ & $\mathrm{Bi}$ II \\
\hline $\mathbf{B}_{14}$ & $=$ & $\left(\frac{1}{2}-x_{4}\right) \mathbf{a}_{1}-y_{4} \mathbf{a}_{2}+z_{4} \mathbf{a}_{3}$ & $=$ & $\left(\frac{1}{2}-x_{4}\right) a \hat{\mathbf{x}}-y_{4} b \hat{\mathbf{y}}+z_{4} c \hat{\mathbf{z}}$ & $(8 e)$ & Bi II \\
\hline $\mathbf{B}_{15}$ & $=$ & $\begin{array}{c}\left(\frac{1}{2}-x_{4}\right) \mathbf{a}_{1}+\left(\frac{1}{2}+y_{4}\right) \mathbf{a}_{2}+ \\
\left(\frac{1}{2}-z_{4}\right) \mathbf{a}_{3}\end{array}$ & $=$ & $\begin{array}{c}\left(\frac{1}{2}-x_{4}\right) a \hat{\mathbf{x}}+\left(\frac{1}{2}+y_{4}\right) b \hat{\mathbf{y}}+ \\
\left(\frac{1}{2}-z_{4}\right) c \hat{\mathbf{z}}\end{array}$ & $(8 e)$ & Bi II \\
\hline $\mathbf{B}_{16}$ & $=$ & $x_{4} \mathbf{a}_{1}+\left(\frac{1}{2}-y_{4}\right) \mathbf{a}_{2}+\left(\frac{1}{2}-z_{4}\right) \mathbf{a}_{3}$ & $=$ & $x_{4} a \hat{\mathbf{x}}+\left(\frac{1}{2}-y_{4}\right) b \hat{\mathbf{y}}+\left(\frac{1}{2}-z_{4}\right) c \hat{\mathbf{z}}$ & $(8 e)$ & $\mathrm{Bi}$ II \\
\hline $\mathbf{B}_{17}$ & $=$ & $-x_{4} \mathbf{a}_{1}-y_{4} \mathbf{a}_{2}-z_{4} \mathbf{a}_{3}$ & $=$ & $-x_{4} a \hat{\mathbf{x}}-y_{4} b \hat{\mathbf{y}}-z_{4} c \hat{\mathbf{z}}$ & $(8 e)$ & Bi II \\
\hline $\mathbf{B}_{18}$ & $=$ & $\left(\frac{1}{2}+x_{4}\right) \mathbf{a}_{1}+y_{4} \mathbf{a}_{2}-z_{4} \mathbf{a}_{3}$ & $=$ & $\left(\frac{1}{2}+x_{4}\right) a \hat{\mathbf{x}}+y_{4} b \hat{\mathbf{y}}-z_{4} c \hat{\mathbf{z}}$ & $(8 e)$ & Bi II \\
\hline $\mathbf{B}_{19}$ & $=$ & $\begin{array}{c}\left(\frac{1}{2}+x_{4}\right) \mathbf{a}_{1}+\left(\frac{1}{2}-y_{4}\right) \mathbf{a}_{2}+ \\
\left(\frac{1}{2}+z_{4}\right) \mathbf{a}_{3}\end{array}$ & $=$ & $\begin{array}{c}\left(\frac{1}{2}+x_{4}\right) a \hat{\mathbf{x}}+\left(\frac{1}{2}-y_{4}\right) b \hat{\mathbf{y}}+ \\
\left(\frac{1}{2}+z_{4}\right) c \hat{\mathbf{z}}\end{array}$ & $(8 e)$ & Bi II \\
\hline $\mathbf{B}_{20}$ & $=$ & $-x_{4} \mathbf{a}_{1}+\left(\frac{1}{2}+y_{4}\right) \mathbf{a}_{2}+\left(\frac{1}{2}+z_{4}\right) \mathbf{a}_{3}$ & $=$ & $-x_{4} a \hat{\mathbf{x}}+\left(\frac{1}{2}+y_{4}\right) b \hat{\mathbf{y}}+\left(\frac{1}{2}+z_{4}\right) c \hat{\mathbf{z}}$ & $(8 e)$ & $\mathrm{Bi}$ II \\
\hline
\end{tabular}

\section{References:}

- F. Merlo and M. L. Fornasini, Crystal structure of some phases and alloying behaviour in alkaline earths, europium and ytterbium pnictides, Mater. Res. Bull. 29, 149-154 (1994), doi:10.1016/0025-5408(94)90135-X.

\section{Found in:}

- P. Villars and K. Cenzual, Pearson's Crystal Data - Crystal Structure Database for Inorganic Compounds, ASM International (2013).

\section{Geometry files:}

- CIF: pp. 834

- POSCAR: pp. 834 


\section{$\mathrm{TaNiTe}_{2}$ Structure: ABC2_oP16_53_h_e_gh}
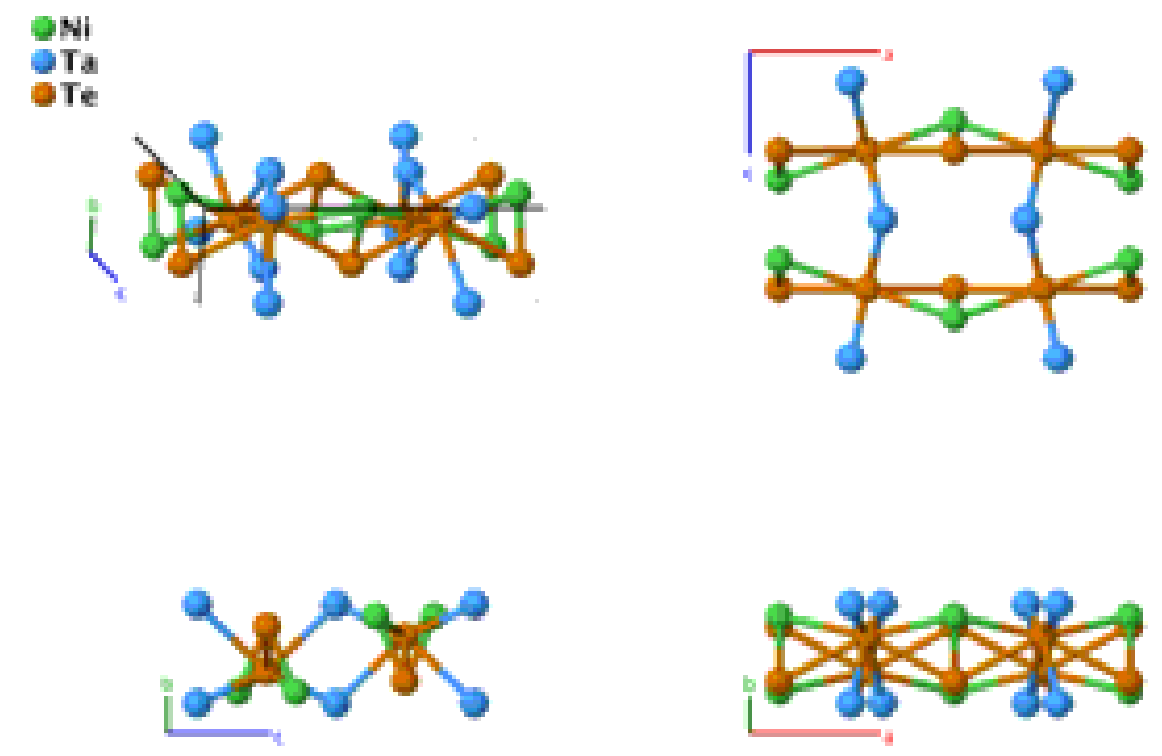

Prototype

$: \quad \mathrm{TaNiTe}_{2}$

AFLOW prototype label

: ABC2_oP16_53_h_e_gh

Strukturbericht designation : None

Pearson symbol

: $\quad$ oP16

Space group number $\quad: \quad 53$

Space group symbol : Pmna

AFLOW prototype command : aflow --proto=ABC2_oP16_53_h_e_gh

- -params $=a, b / a, c / a, x_{1}, y_{2}, y_{3}, z_{3}, y_{4}, z_{4}$

\section{Simple Orthorhombic primitive vectors:}

$$
\begin{aligned}
& \mathbf{a}_{1}=a \hat{\mathbf{x}} \\
& \mathbf{a}_{2}=b \hat{\mathbf{y}} \\
& \mathbf{a}_{3}=c \hat{\mathbf{z}}
\end{aligned}
$$

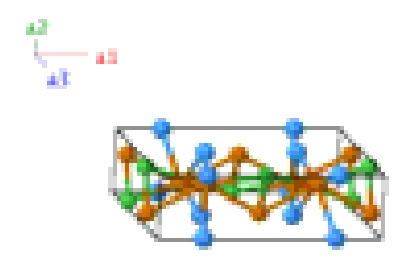

\section{Basis vectors:}

Lattice Coordinates

$\begin{array}{llclc}\mathbf{B}_{1}= & x_{1} \mathbf{a}_{1} & & x_{1} a \hat{\mathbf{x}} \\ \mathbf{B}_{2}= & \left(\frac{1}{2}-x_{1}\right) \mathbf{a}_{1}+\frac{1}{2} \mathbf{a}_{3} & = & \left(\frac{1}{2}-x_{1}\right) a \hat{\mathbf{x}}+\frac{1}{2} c \hat{\mathbf{z}} \\ \mathbf{B}_{3}= & -x_{1} \mathbf{a}_{1} & = & -x_{1} a \hat{\mathbf{x}} \\ \mathbf{B}_{4}= & \left(\frac{1}{2}+x_{1}\right) \mathbf{a}_{1}+\frac{1}{2} \mathbf{a}_{3} & = & \left(\frac{1}{2}+x_{1}\right) a \hat{\mathbf{x}}+\frac{1}{2} c \hat{\mathbf{z}} \\ \mathbf{B}_{5}= & \frac{1}{4} \mathbf{a}_{1}+y_{2} \mathbf{a}_{2}+\frac{1}{4} \mathbf{a}_{3} & & = & \frac{1}{4} a \hat{\mathbf{x}}+y_{2} b \hat{\mathbf{y}}+\frac{1}{4} c \hat{\mathbf{z}}\end{array}$

Wyckoff Position

Atom Type

(4e)

$\mathrm{Ta}$

(4e)

$\mathrm{Ta}$

$(4 e)$

Ta

(4e)

$\mathrm{Ta}$

$(4 g)$ 


\begin{tabular}{|c|c|c|c|c|c|c|}
\hline $\mathbf{B}_{6}$ & $=$ & $\frac{1}{4} \mathbf{a}_{1}-y_{2} \mathbf{a}_{2}+\frac{3}{4} \mathbf{a}_{3}$ & $=$ & $\frac{1}{4} a \hat{\mathbf{x}}-y_{2} b \hat{\mathbf{y}}+\frac{3}{4} c \hat{\mathbf{z}}$ & $(4 g)$ & Te I \\
\hline $\mathbf{B}_{7}$ & $=$ & $\frac{3}{4} \mathbf{a}_{1}-y_{2} \mathbf{a}_{2}+\frac{3}{4} \mathbf{a}_{3}$ & $=$ & $\frac{3}{4} a \hat{\mathbf{x}}-y_{2} b \hat{\mathbf{y}}+\frac{3}{4} c \hat{\mathbf{z}}$ & $(4 g)$ & $\mathrm{Te} \mathrm{I}$ \\
\hline $\mathbf{B}_{8}$ & $=$ & $\frac{3}{4} \mathbf{a}_{1}+y_{2} \mathbf{a}_{2}+\frac{1}{4} \mathbf{a}_{3}$ & $=$ & $\frac{3}{4} a \hat{\mathbf{x}}+y_{2} b \hat{\mathbf{y}}+\frac{1}{4} c \hat{\mathbf{z}}$ & $(4 g)$ & Te I \\
\hline $\mathbf{B}_{9}$ & $=$ & $y_{3} \mathbf{a}_{2}+z_{3} \mathbf{a}_{3}$ & $=$ & $y_{3} b \hat{\mathbf{y}}+z_{3} c \hat{\mathbf{z}}$ & $(4 h)$ & $\mathrm{Ni}$ \\
\hline $\mathbf{B}_{10}$ & $=$ & $\frac{1}{2} \mathbf{a}_{1}-y_{3} \mathbf{a}_{2}+\left(\frac{1}{2}+z_{3}\right) \mathbf{a}_{3}$ & $=$ & $\frac{1}{2} a \hat{\mathbf{x}}-y_{3} b \hat{\mathbf{y}}+\left(\frac{1}{2}+z_{3}\right) c \hat{\mathbf{z}}$ & $(4 h)$ & $\mathrm{Ni}$ \\
\hline $\mathbf{B}_{11}$ & $=$ & $\frac{1}{2} \mathbf{a}_{1}+y_{3} \mathbf{a}_{2}+\left(\frac{1}{2}-z_{3}\right) \mathbf{a}_{3}$ & $=$ & $\frac{1}{2} a \hat{\mathbf{x}}+y_{3} b \hat{\mathbf{y}}+\left(\frac{1}{2}-z_{3}\right) c \hat{\mathbf{z}}$ & $(4 h)$ & $\mathrm{Ni}$ \\
\hline $\mathbf{B}_{12}$ & $=$ & $-y_{3} \mathbf{a}_{2}-z_{3} \mathbf{a}_{3}$ & $=$ & $-y_{3} b \hat{\mathbf{y}}-z_{3} c \hat{\mathbf{z}}$ & $(4 h)$ & $\mathrm{Ni}$ \\
\hline $\mathbf{B}_{13}$ & $=$ & $y_{4} \mathbf{a}_{2}+z_{4} \mathbf{a}_{3}$ & $=$ & $y_{4} b \hat{\mathbf{y}}+z_{4} c \hat{\mathbf{z}}$ & $(4 h)$ & Te II \\
\hline $\mathbf{B}_{14}$ & $=$ & $\frac{1}{2} \mathbf{a}_{1}-y_{4} \mathbf{a}_{2}+\left(\frac{1}{2}+z_{4}\right) \mathbf{a}_{3}$ & $=$ & $\frac{1}{2} a \hat{\mathbf{x}}-y_{4} b \hat{\mathbf{y}}+\left(\frac{1}{2}+z_{4}\right) c \hat{\mathbf{z}}$ & $(4 h)$ & Te II \\
\hline $\mathbf{B}_{15}$ & $=$ & $\frac{1}{2} \mathbf{a}_{1}+y_{4} \mathbf{a}_{2}+\left(\frac{1}{2}-z_{4}\right) \mathbf{a}_{3}$ & $=$ & $\frac{1}{2} a \hat{\mathbf{x}}+y_{4} b \hat{\mathbf{y}}+\left(\frac{1}{2}-z_{4}\right) c \hat{\mathbf{z}}$ & $(4 h)$ & Te II \\
\hline $\mathbf{B}_{16}$ & $=$ & $-y_{4} \mathbf{a}_{2}-z_{4} \mathbf{a}_{3}$ & $=$ & $-y_{4} b \hat{\mathbf{y}}-z_{4} c \hat{\mathbf{z}}$ & $(4 h)$ & Te II \\
\hline
\end{tabular}

\section{References:}

- W. Tremel, Isolated and Condensed $\mathrm{Ta}_{2} \mathrm{Ni}_{2}$ Clusters in the Layered Tellurides $\mathrm{Ta}_{2} \mathrm{Ni}_{2} \mathrm{Te}_{4}$ and $\mathrm{Ta}_{2} \mathrm{Ni}_{3} \mathrm{Te}_{5}$, Angew. Chem. Int. Ed. 30, 840-843 (1991), doi:10.1002/anie.199108401.

\section{Found in:}

- P. Villars and K. Cenzual, Pearson's Crystal Data - Crystal Structure Database for Inorganic Compounds, ASM International (2013).

\section{Geometry files:}

- CIF: pp. 834

- POSCAR: pp. 835 


\section{$\mathrm{CuBrSe}{ }_{3}$ Structure: ABC3_oP20_53_e_g_hi}
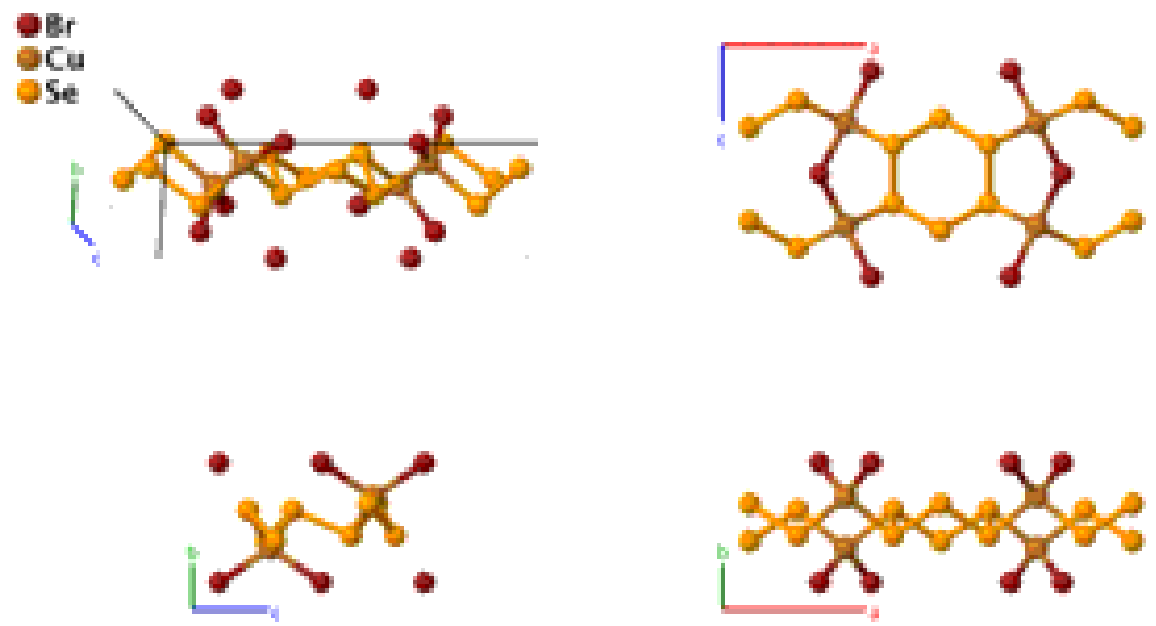

\section{Prototype}

AFLOW prototype label

Strukturbericht designation

Pearson symbol

Space group number

Space group symbol

AFLOW prototype command
: $\mathrm{CuBrSe}_{3}$

: ABC3_oP20_53_e_g_hi

: None

: $\quad$ oP20

: 53

\section{: Pmna}

Simple Orthorhombic primitive vectors:

$$
\begin{aligned}
& \mathbf{a}_{1}=a \hat{\mathbf{x}} \\
& \mathbf{a}_{2}=b \hat{\mathbf{y}} \\
& \mathbf{a}_{3}=c \hat{\mathbf{z}}
\end{aligned}
$$

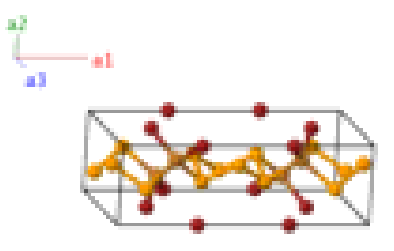

Basis vectors:

Lattice Coordinates

$\begin{array}{llc}\mathbf{B}_{1}= & x_{1} \mathbf{a}_{1} \\ \mathbf{B}_{2}= & \left(\frac{1}{2}-x_{1}\right) \mathbf{a}_{1}+\frac{1}{2} \mathbf{a}_{3} \\ \mathbf{B}_{3}= & -x_{1} \mathbf{a}_{1} \\ \mathbf{B}_{4}= & \left(\frac{1}{2}+x_{1}\right) \mathbf{a}_{1}+\frac{1}{2} \mathbf{a}_{3} \\ \mathbf{B}_{5}= & \frac{1}{4} \mathbf{a}_{1}+y_{2} \mathbf{a}_{2}+\frac{1}{4} \mathbf{a}_{3} \\ \mathbf{B}_{6}= & \frac{1}{4} \mathbf{a}_{1}-y_{2} \mathbf{a}_{2}+\frac{3}{4} \mathbf{a}_{3} \\ \mathbf{B}_{7}= & \frac{3}{4} \mathbf{a}_{1}-y_{2} \mathbf{a}_{2}+\frac{3}{4} \mathbf{a}_{3} \\ \mathbf{B}_{8}= & \frac{3}{4} \mathbf{a}_{1}+y_{2} \mathbf{a}_{2}+\frac{1}{4} \mathbf{a}_{3}\end{array}$

Cartesian Coordinates

$$
=
$$$$
=
$$$$
=
$$$$
=
$$$$
=
$$$$
=
$$$$
=
$$$$
=
$$$$
=
$$

$$
\begin{gathered}
x_{1} a \hat{\mathbf{x}} \\
\left(\frac{1}{2}-x_{1}\right) a \hat{\mathbf{x}}+\frac{1}{2} c \hat{\mathbf{z}} \\
-x_{1} a \hat{\mathbf{x}} \\
\left(\frac{1}{2}+x_{1}\right) a \hat{\mathbf{x}}+\frac{1}{2} c \hat{\mathbf{z}} \\
\frac{1}{4} a \hat{\mathbf{x}}+y_{2} b \hat{\mathbf{y}}+\frac{1}{4} c \hat{\mathbf{z}} \\
\frac{1}{4} a \hat{\mathbf{x}}-y_{2} b \hat{\mathbf{y}}+\frac{3}{4} c \hat{\mathbf{z}} \\
\frac{3}{4} a \hat{\mathbf{x}}-y_{2} b \hat{\mathbf{y}}+\frac{3}{4} c \hat{\mathbf{z}} \\
\frac{3}{4} a \hat{\mathbf{x}}+y_{2} b \hat{\mathbf{y}}+\frac{1}{4} c \hat{\mathbf{z}}
\end{gathered}
$$

Wyckoff Position

Atom Type

$(4 e)$

$\mathrm{Br}$

(4e)

$\mathrm{Br}$

(4e)

$\mathrm{Br}$

(4e)

$\mathrm{Br}$

(4g)

$\mathrm{Cu}$

(4g)

$\mathrm{Cu}$

(4g)

$\mathrm{Cu}$

(4g)

$\mathrm{Cu}$ 


\begin{tabular}{|c|c|c|c|c|c|c|}
\hline $\mathbf{B}_{9}$ & $=$ & $y_{3} \mathbf{a}_{2}+z_{3} \mathbf{a}_{3}$ & $=$ & $y_{3} b \hat{\mathbf{y}}+z_{3} c \hat{\mathbf{z}}$ & $(4 h)$ & Se I \\
\hline $\mathbf{B}_{10}$ & $=$ & $\frac{1}{2} \mathbf{a}_{1}-y_{3} \mathbf{a}_{2}+\left(\frac{1}{2}+z_{3}\right) \mathbf{a}_{3}$ & $=$ & $\frac{1}{2} a \hat{\mathbf{x}}-y_{3} b \hat{\mathbf{y}}+\left(\frac{1}{2}+z_{3}\right) c \hat{\mathbf{z}}$ & $(4 h)$ & Se I \\
\hline $\mathbf{B}_{11}$ & $=$ & $\frac{1}{2} \mathbf{a}_{1}+y_{3} \mathbf{a}_{2}+\left(\frac{1}{2}-z_{3}\right) \mathbf{a}_{3}$ & $=$ & $\frac{1}{2} a \hat{\mathbf{x}}+y_{3} b \hat{\mathbf{y}}+\left(\frac{1}{2}-z_{3}\right) c \hat{\mathbf{z}}$ & $(4 h)$ & Se I \\
\hline $\mathbf{B}_{12}$ & $=$ & $-y_{3} \mathbf{a}_{2}-z_{3} \mathbf{a}_{3}$ & $=$ & $-y_{3} b \hat{\mathbf{y}}-z_{3} c \hat{\mathbf{z}}$ & $(4 h)$ & Se I \\
\hline $\mathbf{B}_{13}$ & $=$ & $x_{4} \mathbf{a}_{1}+y_{4} \mathbf{a}_{2}+z_{4} \mathbf{a}_{3}$ & $=$ & $x_{4} a \hat{\mathbf{x}}+y_{4} b \hat{\mathbf{y}}+z_{4} c \hat{\mathbf{z}}$ & $(8 i)$ & Se II \\
\hline $\mathbf{B}_{14}$ & $=$ & $\left(\frac{1}{2}-x_{4}\right) \mathbf{a}_{1}-y_{4} \mathbf{a}_{2}+\left(\frac{1}{2}+z_{4}\right) \mathbf{a}_{3}$ & $=$ & $\left(\frac{1}{2}-x_{4}\right) a \hat{\mathbf{x}}-y_{4} b \hat{\mathbf{y}}+\left(\frac{1}{2}+z_{4}\right) c \hat{\mathbf{z}}$ & $(8 i)$ & Se II \\
\hline $\mathbf{B}_{15}$ & $=$ & $\left(\frac{1}{2}-x_{4}\right) \mathbf{a}_{1}+y_{4} \mathbf{a}_{2}+\left(\frac{1}{2}-z_{4}\right) \mathbf{a}_{3}$ & $=$ & $\left(\frac{1}{2}-x_{4}\right) a \hat{\mathbf{x}}+y_{4} b \hat{\mathbf{y}}+\left(\frac{1}{2}-z_{4}\right) c \hat{\mathbf{z}}$ & $(8 i)$ & Se II \\
\hline $\mathbf{B}_{16}$ & $=$ & $x_{4} \mathbf{a}_{1}-y_{4} \mathbf{a}_{2}-z_{4} \mathbf{a}_{3}$ & $=$ & $x_{4} a \hat{\mathbf{x}}-y_{4} b \hat{\mathbf{y}}-z_{4} c \hat{\mathbf{z}}$ & $(8 i)$ & Se II \\
\hline $\mathbf{B}_{17}$ & $=$ & $-x_{4} \mathbf{a}_{1}-y_{4} \mathbf{a}_{2}-z_{4} \mathbf{a}_{3}$ & $=$ & $-x_{4} a \hat{\mathbf{x}}-y_{4} b \hat{\mathbf{y}}-z_{4} c \hat{\mathbf{z}}$ & $(8 i)$ & Se II \\
\hline $\mathbf{B}_{18}$ & $=$ & $\left(\frac{1}{2}+x_{4}\right) \mathbf{a}_{1}+y_{4} \mathbf{a}_{2}+\left(\frac{1}{2}-z_{4}\right) \mathbf{a}_{3}$ & $=$ & $\left(\frac{1}{2}+x_{4}\right) a \hat{\mathbf{x}}+y_{4} b \hat{\mathbf{y}}+\left(\frac{1}{2}-z_{4}\right) c \hat{\mathbf{z}}$ & $(8 i)$ & Se II \\
\hline $\mathbf{B}_{19}$ & $=$ & $\left(\frac{1}{2}+x_{4}\right) \mathbf{a}_{1}-y_{4} \mathbf{a}_{2}+\left(\frac{1}{2}+z_{4}\right) \mathbf{a}_{3}$ & $=$ & $\left(\frac{1}{2}+x_{4}\right) a \hat{\mathbf{x}}-y_{4} b \hat{\mathbf{y}}+\left(\frac{1}{2}+z_{4}\right) c \hat{\mathbf{z}}$ & $(8 i)$ & Se II \\
\hline $\mathbf{B}_{20}$ & $=$ & $-x_{4} \mathbf{a}_{1}+y_{4} \mathbf{a}_{2}+z_{4} \mathbf{a}_{3}$ & $=$ & $-x_{4} a \hat{\mathbf{x}}+y_{4} b \hat{\mathbf{y}}+z_{4} c \hat{\mathbf{z}}$ & $(8 i)$ & Se II \\
\hline
\end{tabular}

\section{References:}

- H. M. Haendler and P. M. Carkner, The crystal structure of copper bromide triselenide, $\mathrm{CuBrSe}$, J. Solid State Chem. 29, 35-39 (1979), doi:10.1016/0022-4596(79)90206-8.

\section{Found in:}

- P. Villars and K. Cenzual, Pearson's Crystal Data - Crystal Structure Database for Inorganic Compounds, ASM International (2013).

\section{Geometry files:}

- CIF: pp. 835

- POSCAR: pp. 835 


\section{$\mathrm{BiGaO}_{3}$ Structure: ABC3_oP20_54_e_d_cf}

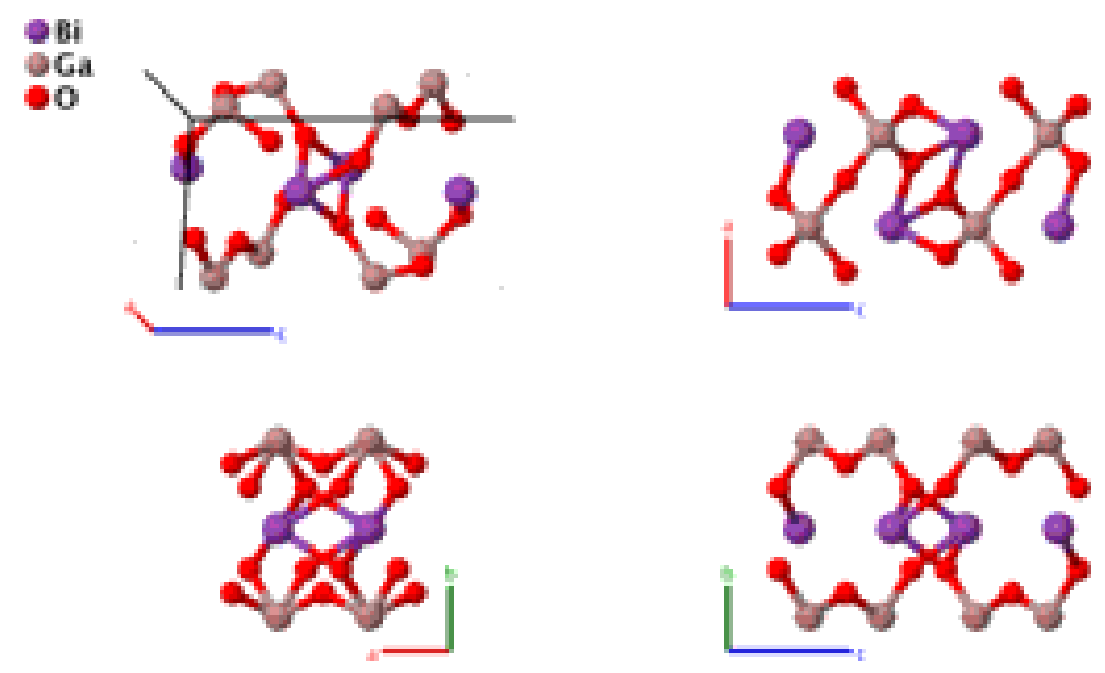

Prototype

AFLOW prototype label

Strukturbericht designation

Pearson symbol

Space group number

Space group symbol

AFLOW prototype command
: $\quad \mathrm{BiGaO}_{3}$

: ABC3_oP20_54_e_d_cf

: None

: $\quad$ oP20

: 54

: $\quad$ Pcca

: aflow --proto=ABC3_oP20_54_e_d_cf

- -params $=a, b / a, c / a, y_{1}, z_{2}, z_{3}, x_{4}, y_{4}, z_{4}$

Simple Orthorhombic primitive vectors:

$$
\begin{aligned}
& \mathbf{a}_{1}=a \hat{\mathbf{x}} \\
& \mathbf{a}_{2}=b \hat{\mathbf{y}} \\
& \mathbf{a}_{3}=c \hat{\mathbf{z}}
\end{aligned}
$$

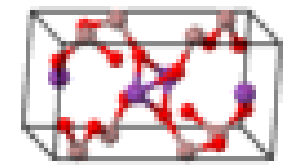

Basis vectors:

Lattice Coordinates

$\begin{array}{lcccc}\mathbf{B}_{1}= & y_{1} \mathbf{a}_{2}+\frac{1}{4} \mathbf{a}_{3} & = & y_{1} b \hat{\mathbf{y}}+\frac{1}{4} c \hat{\mathbf{z}} \\ \mathbf{B}_{2}= & \frac{1}{2} \mathbf{a}_{1}-y_{1} \mathbf{a}_{2}+\frac{1}{4} \mathbf{a}_{3} & = & \frac{1}{2} a \hat{\mathbf{x}}-y_{1} b \hat{\mathbf{y}}+\frac{1}{4} c \hat{\mathbf{z}} \\ \mathbf{B}_{3}= & -y_{1} \mathbf{a}_{2}+\frac{3}{4} \mathbf{a}_{3} & = & -y_{1} b \hat{\mathbf{y}}+\frac{3}{4} c \hat{\mathbf{z}} \\ \mathbf{B}_{4}= & \frac{1}{2} \mathbf{a}_{1}+y_{1} \mathbf{a}_{2}+\frac{3}{4} \mathbf{a}_{3} & = & \frac{1}{2} a \hat{\mathbf{x}}+y_{1} b \hat{\mathbf{y}}+\frac{3}{4} c \hat{\mathbf{z}} \\ \mathbf{B}_{5}= & \frac{1}{4} \mathbf{a}_{1}+z_{2} \mathbf{a}_{3} & = & \frac{1}{4} a \hat{\mathbf{x}}+z_{2} c \hat{\mathbf{z}} \\ \mathbf{B}_{6}= & \frac{3}{4} \mathbf{a}_{1}+\left(\frac{1}{2}-z_{2}\right) \mathbf{a}_{3} & = & \frac{3}{4} a \hat{\mathbf{x}}+\left(\frac{1}{2}-z_{2}\right) c \hat{\mathbf{z}} \\ \mathbf{B}_{7}= & \frac{3}{4} \mathbf{a}_{1}+-z_{2} \mathbf{a}_{3} & = & \frac{3}{4} a \hat{\mathbf{x}}+-z_{2} c \hat{\mathbf{z}} \\ \mathbf{B}_{8}= & \frac{1}{4} \mathbf{a}_{1}+\left(\frac{1}{2}+z_{2}\right) \mathbf{a}_{3} & = & \frac{1}{4} a \hat{\mathbf{x}}+\left(\frac{1}{2}+z_{2}\right) c \hat{\mathbf{z}}\end{array}$

Wyckoff Position Atom Type

O I

O I

O I

(4c)

O I

(4d)

$\mathrm{Ga}$

(4d)

Ga

(4d)

Ga

(4d)

Ga 


\begin{tabular}{|c|c|c|c|c|c|}
\hline $\mathbf{B}_{9}$ & $=$ & $\frac{1}{4} \mathbf{a}_{1}+\frac{1}{2} \mathbf{a}_{2}+z_{3} \mathbf{a}_{3}$ & $=$ & $\frac{1}{4} a \hat{\mathbf{x}}+\frac{1}{2} b \hat{\mathbf{y}}+z_{3} c \hat{\mathbf{z}}$ & $(4 e)$ \\
\hline $\mathbf{B}_{10}$ & $=$ & $\frac{3}{4} \mathbf{a}_{1}+\frac{1}{2} \mathbf{a}_{2}+\left(\frac{1}{2}-z_{3}\right) \mathbf{a}_{3}$ & $=$ & $\frac{3}{4} a \hat{\mathbf{x}}+\frac{1}{2} b \hat{\mathbf{y}}+\left(\frac{1}{2}-z_{3}\right) c \hat{\mathbf{z}}$ & $(4 e)$ \\
\hline $\mathbf{B}_{11}$ & $=$ & $\frac{3}{4} \mathbf{a}_{1}+\frac{1}{2} \mathbf{a}_{2}-z_{3} \mathbf{a}_{3}$ & $=$ & $\frac{3}{4} a \hat{\mathbf{x}}+\frac{1}{2} b \hat{\mathbf{y}}-z_{3} c \hat{\mathbf{z}}$ & $(4 e)$ \\
\hline $\mathbf{B}_{12}$ & $=$ & $\frac{1}{4} \mathbf{a}_{1}+\frac{1}{2} \mathbf{a}_{2}+\left(\frac{1}{2}+z_{3}\right) \mathbf{a}_{3}$ & $=$ & $\frac{1}{4} a \hat{\mathbf{x}}+\frac{1}{2} b \hat{\mathbf{y}}+\left(\frac{1}{2}+z_{3}\right) c \hat{\mathbf{z}}$ & $(4 e)$ \\
\hline $\mathbf{B}_{13}$ & $=$ & $x_{4} \mathbf{a}_{1}+y_{4} \mathbf{a}_{2}+z_{4} \mathbf{a}_{3}$ & $=$ & $x_{4} a \hat{\mathbf{x}}+y_{4} b \hat{\mathbf{y}}+z_{4} c \hat{\mathbf{z}}$ & $(8 f)$ \\
\hline $\mathbf{B}_{14}$ & $=$ & $\left(\frac{1}{2}-x_{4}\right) \mathbf{a}_{1}-y_{4} \mathbf{a}_{2}+z_{4} \mathbf{a}_{3}$ & $=$ & $\left(\frac{1}{2}-x_{4}\right) a \hat{\mathbf{x}}-y_{4} b \hat{\mathbf{y}}+z_{4} c \hat{\mathbf{z}}$ & $(8 f)$ \\
\hline $\mathbf{B}_{15}$ & $=$ & $-x_{4} \mathbf{a}_{1}+y_{4} \mathbf{a}_{2}+\left(\frac{1}{2}-z_{4}\right) \mathbf{a}_{3}$ & $=$ & $-x_{4} a \hat{\mathbf{x}}+y_{4} b \hat{\mathbf{y}}+\left(\frac{1}{2}-z_{4}\right) c \hat{\mathbf{z}}$ & $(8 f)$ \\
\hline $\mathbf{B}_{16}$ & $=$ & $\left(\frac{1}{2}+x_{4}\right) \mathbf{a}_{1}-y_{4} \mathbf{a}_{2}+\left(\frac{1}{2}-z_{4}\right) \mathbf{a}_{3}$ & $=$ & $\left(\frac{1}{2}+x_{4}\right) a \hat{\mathbf{x}}-y_{4} b \hat{\mathbf{y}}+\left(\frac{1}{2}-z_{4}\right) c \hat{\mathbf{z}}$ & $(8 f)$ \\
\hline $\mathbf{B}_{17}$ & $=$ & $-x_{4} \mathbf{a}_{1}-y_{4} \mathbf{a}_{2}-z_{4} \mathbf{a}_{3}$ & $=$ & $-x_{4} a \hat{\mathbf{x}}-y_{4} b \hat{\mathbf{y}}-z_{4} c \hat{\mathbf{z}}$ & $(8 f)$ \\
\hline $\mathbf{B}_{18}$ & $=$ & $\left(\frac{1}{2}+x_{4}\right) \mathbf{a}_{1}+y_{4} \mathbf{a}_{2}-z_{4} \mathbf{a}_{3}$ & $=$ & $\left(\frac{1}{2}+x_{4}\right) a \hat{\mathbf{x}}+y_{4} b \hat{\mathbf{y}}-z_{4} c \hat{\mathbf{z}}$ & $(8 f)$ \\
\hline $\mathbf{B}_{19}$ & $=$ & $x_{4} \mathbf{a}_{1}-y_{4} \mathbf{a}_{2}+\left(\frac{1}{2}+z_{4}\right) \mathbf{a}_{3}$ & $=$ & $x_{4} a \hat{\mathbf{x}}-y_{4} b \hat{\mathbf{y}}+\left(\frac{1}{2}+z_{4}\right) c \hat{\mathbf{z}}$ & $(8 f)$ \\
\hline $\mathbf{B}_{20}$ & $=$ & $\left(\frac{1}{2}-x_{4}\right) \mathbf{a}_{1}+y_{4} \mathbf{a}_{2}+\left(\frac{1}{2}+z_{4}\right) \mathbf{a}_{3}$ & $=$ & $\left(\frac{1}{2}-x_{4}\right) a \hat{\mathbf{x}}+y_{4} b \hat{\mathbf{y}}+\left(\frac{1}{2}+z_{4}\right) c \hat{\mathbf{z}}$ & $(8 f)$ \\
\hline
\end{tabular}

\section{References:}

- H. Yusa, A. A. Belik, E. Takayama-Muromachi, N. Hirao, and Y. Ohishi, High-pressure phase transitions in BiMO $3(M=$ $A l, G a$, and In): In situ x-ray diffraction and Raman scattering experiments, Phys. Rev. B 80, 214103 (2009), doi:10.1103/PhysRevB.80.214103.

\section{Found in:}

- P. Villars and K. Cenzual, Pearson's Crystal Data - Crystal Structure Database for Inorganic Compounds, ASM International (2013).

\section{Geometry files:}

- CIF: pp. 835

- POSCAR: pp. 836 


\section{$\mathrm{GeAs}_{2}$ Structure: A2B_oP24_55_2g2h_gh}
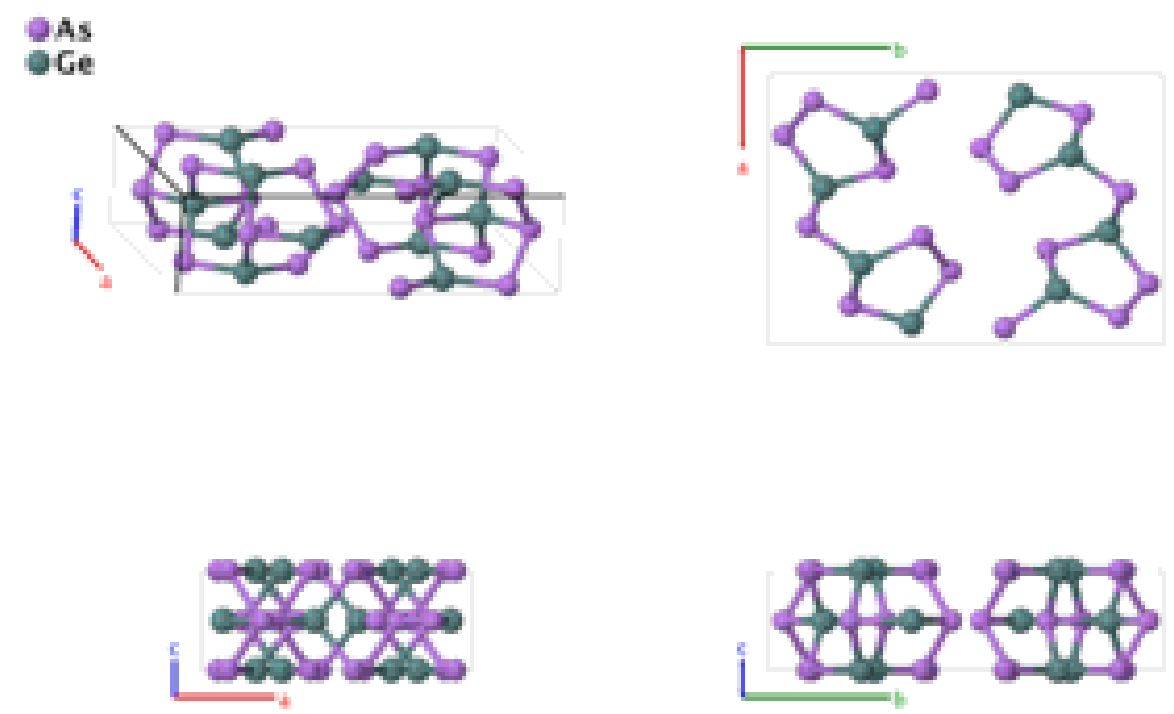

Prototype

: $\mathrm{GeAs}_{2}$

AFLOW prototype label

A2B_oP24_55_2g2h_gh

Strukturbericht designation : None

$\begin{array}{lll}\text { Pearson symbol } & \text { oP24 }\end{array}$

Space group number $\quad$ : 55

Space group symbol $\quad: \quad$ Pbam

AFLOW prototype command : aflow --proto=A2B_oP24_55_2g2h_gh

- params $=a, b / a, c / a, x_{1}, y_{1}, x_{2}, y_{2}, x_{3}, y_{3}, x_{4}, y_{4}, x_{5}, y_{5}, x_{6}, y_{6}$

Simple Orthorhombic primitive vectors:

$$
\begin{aligned}
& \mathbf{a}_{1}=a \hat{\mathbf{x}} \\
& \mathbf{a}_{2}=b \hat{\mathbf{y}} \\
& \mathbf{a}_{3}=c \hat{\mathbf{z}}
\end{aligned}
$$

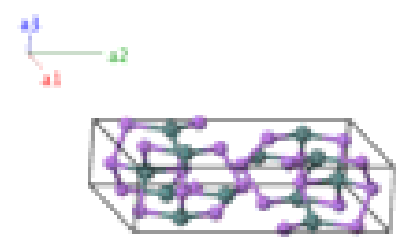

\section{Basis vectors:}

Lattice Coordinates

$\begin{array}{lcccc}\mathbf{B}_{1}= & x_{1} \mathbf{a}_{1}+y_{1} \mathbf{a}_{2} & = & x_{1} a \hat{\mathbf{x}}+y_{1} b \hat{\mathbf{y}} \\ \mathbf{B}_{2}= & -x_{1} \mathbf{a}_{1}-y_{1} \mathbf{a}_{2} & = & -x_{1} a \hat{\mathbf{x}}-y_{1} b \hat{\mathbf{y}} \\ \mathbf{B}_{3}= & \left(\frac{1}{2}-x_{1}\right) \mathbf{a}_{1}+\left(\frac{1}{2}+y_{1}\right) \mathbf{a}_{2} & = & \left(\frac{1}{2}-x_{1}\right) a \hat{\mathbf{x}}+\left(\frac{1}{2}+y_{1}\right) b \hat{\mathbf{y}} \\ \mathbf{B}_{4}= & \left(\frac{1}{2}+x_{1}\right) \mathbf{a}_{1}+\left(\frac{1}{2}-y_{1}\right) \mathbf{a}_{2} & = & \left(\frac{1}{2}+x_{1}\right) a \hat{\mathbf{x}}+\left(\frac{1}{2}-y_{1}\right) b \hat{\mathbf{y}} \\ \mathbf{B}_{5}= & x_{2} \mathbf{a}_{1}+y_{2} \mathbf{a}_{2} & = & x_{2} a \hat{\mathbf{x}}+y_{2} b \hat{\mathbf{y}} \\ \mathbf{B}_{6}= & -x_{2} \mathbf{a}_{1}-y_{2} \mathbf{a}_{2} & = & -x_{2} a \hat{\mathbf{x}}-y_{2} b \hat{\mathbf{y}}\end{array}$

Wyckoff Position Atom Type

$(4 g)$

As I

$(4 g)$

As I

$(4 g)$

As I

$(4 g)$

As I

$(4 g)$

As II

$(4 g)$ 


\begin{tabular}{|c|c|c|c|c|c|c|}
\hline $\mathbf{B}_{7}$ & $=$ & $\left(\frac{1}{2}-x_{2}\right) \mathbf{a}_{1}+\left(\frac{1}{2}+y_{2}\right) \mathbf{a}_{2}$ & $=$ & $\left(\frac{1}{2}-x_{2}\right) a \hat{\mathbf{x}}+\left(\frac{1}{2}+y_{2}\right) b \hat{\mathbf{y}}$ & $(4 g)$ & As II \\
\hline $\mathbf{B}_{8}$ & $=$ & $\left(\frac{1}{2}+x_{2}\right) \mathbf{a}_{1}+\left(\frac{1}{2}-y_{2}\right) \mathbf{a}_{2}$ & $=$ & $\left(\frac{1}{2}+x_{2}\right) a \hat{\mathbf{x}}+\left(\frac{1}{2}-y_{2}\right) b \hat{\mathbf{y}}$ & $(4 g)$ & As II \\
\hline $\mathbf{B}_{9}$ & $=$ & $x_{3} \mathbf{a}_{1}+y_{3} \mathbf{a}_{2}$ & $=$ & $x_{3} a \hat{\mathbf{x}}+y_{3} b \hat{\mathbf{y}}$ & $(4 g)$ & Ge I \\
\hline $\mathbf{B}_{10}$ & $=$ & $-x_{3} \mathbf{a}_{1}-y_{3} \mathbf{a}_{2}$ & $=$ & $-x_{3} a \hat{\mathbf{x}}-y_{3} b \hat{\mathbf{y}}$ & $(4 g)$ & Ge I \\
\hline $\mathbf{B}_{11}$ & $=$ & $\left(\frac{1}{2}-x_{3}\right) \mathbf{a}_{1}+\left(\frac{1}{2}+y_{3}\right) \mathbf{a}_{2}$ & $=$ & $\left(\frac{1}{2}-x_{3}\right) a \hat{\mathbf{x}}+\left(\frac{1}{2}+y_{3}\right) b \hat{\mathbf{y}}$ & $(4 g)$ & Ge I \\
\hline $\mathbf{B}_{12}$ & $=$ & $\left(\frac{1}{2}+x_{3}\right) \mathbf{a}_{1}+\left(\frac{1}{2}-y_{3}\right) \mathbf{a}_{2}$ & $=$ & $\left(\frac{1}{2}+x_{3}\right) a \hat{\mathbf{x}}+\left(\frac{1}{2}-y_{3}\right) b \hat{\mathbf{y}}$ & $(4 g)$ & Ge I \\
\hline $\mathbf{B}_{13}$ & $=$ & $x_{4} \mathbf{a}_{1}+y_{4} \mathbf{a}_{2}+\frac{1}{2} \mathbf{a}_{3}$ & $=$ & $x_{4} a \hat{\mathbf{x}}+y_{4} b \hat{\mathbf{y}}+\frac{1}{2} c \hat{\mathbf{z}}$ & $(4 h)$ & As III \\
\hline $\mathbf{B}_{14}$ & $=$ & $-x_{4} \mathbf{a}_{1}-y_{4} \mathbf{a}_{2}+\frac{1}{2} \mathbf{a}_{3}$ & $=$ & $-x_{4} a \hat{\mathbf{x}}-y_{4} b \hat{\mathbf{y}}+\frac{1}{2} c \hat{\mathbf{z}}$ & $(4 h)$ & As III \\
\hline $\mathbf{B}_{15}$ & $=$ & $\left(\frac{1}{2}-x_{4}\right) \mathbf{a}_{1}+\left(\frac{1}{2}+y_{4}\right) \mathbf{a}_{2}+\frac{1}{2} \mathbf{a}_{3}$ & $=$ & $\left(\frac{1}{2}-x_{4}\right) a \hat{\mathbf{x}}+\left(\frac{1}{2}+y_{4}\right) b \hat{\mathbf{y}}+\frac{1}{2} c \hat{\mathbf{z}}$ & $(4 h)$ & As III \\
\hline $\mathbf{B}_{16}$ & $=$ & $\left(\frac{1}{2}+x_{4}\right) \mathbf{a}_{1}+\left(\frac{1}{2}-y_{4}\right) \mathbf{a}_{2}+\frac{1}{2} \mathbf{a}_{3}$ & $=$ & $\left(\frac{1}{2}+x_{4}\right) a \hat{\mathbf{x}}+\left(\frac{1}{2}-y_{4}\right) b \hat{\mathbf{y}}+\frac{1}{2} c \hat{\mathbf{z}}$ & $(4 h)$ & As III \\
\hline $\mathbf{B}_{17}$ & $=$ & $x_{5} \mathbf{a}_{1}+y_{5} \mathbf{a}_{2}+\frac{1}{2} \mathbf{a}_{3}$ & $=$ & $x_{5} a \hat{\mathbf{x}}+y_{5} b \hat{\mathbf{y}}+\frac{1}{2} c \hat{\mathbf{z}}$ & $(4 h)$ & As IV \\
\hline $\mathbf{B}_{18}$ & $=$ & $-x_{5} \mathbf{a}_{1}-y_{5} \mathbf{a}_{2}+\frac{1}{2} \mathbf{a}_{3}$ & $=$ & $-x_{5} a \hat{\mathbf{x}}-y_{5} b \hat{\mathbf{y}}+\frac{1}{2} c \hat{\mathbf{z}}$ & $(4 h)$ & As IV \\
\hline $\mathbf{B}_{19}$ & $=$ & $\left(\frac{1}{2}-x_{5}\right) \mathbf{a}_{1}+\left(\frac{1}{2}+y_{5}\right) \mathbf{a}_{2}+\frac{1}{2} \mathbf{a}_{3}$ & $=$ & $\left(\frac{1}{2}-x_{5}\right) a \hat{\mathbf{x}}+\left(\frac{1}{2}+y_{5}\right) b \hat{\mathbf{y}}+\frac{1}{2} c \hat{\mathbf{z}}$ & $(4 h)$ & As IV \\
\hline $\mathbf{B}_{20}$ & $=$ & $\left(\frac{1}{2}+x_{5}\right) \mathbf{a}_{1}+\left(\frac{1}{2}-y_{5}\right) \mathbf{a}_{2}+\frac{1}{2} \mathbf{a}_{3}$ & $=$ & $\left(\frac{1}{2}+x_{5}\right) a \hat{\mathbf{x}}+\left(\frac{1}{2}-y_{5}\right) b \hat{\mathbf{y}}+\frac{1}{2} c \hat{\mathbf{z}}$ & $(4 h)$ & As IV \\
\hline $\mathbf{B}_{21}$ & $=$ & $x_{6} \mathbf{a}_{1}+y_{6} \mathbf{a}_{2}+\frac{1}{2} \mathbf{a}_{3}$ & $=$ & $x_{6} a \hat{\mathbf{x}}+y_{6} b \hat{\mathbf{y}}+\frac{1}{2} c \hat{\mathbf{z}}$ & $(4 h)$ & Ge II \\
\hline $\mathbf{B}_{22}$ & $=$ & $-x_{6} \mathbf{a}_{1}-y_{6} \mathbf{a}_{2}+\frac{1}{2} \mathbf{a}_{3}$ & $=$ & $-x_{6} a \hat{\mathbf{x}}-y_{6} b \hat{\mathbf{y}}+\frac{1}{2} c \hat{\mathbf{z}}$ & $(4 h)$ & Ge II \\
\hline $\mathbf{B}_{23}$ & $=$ & $\left(\frac{1}{2}-x_{6}\right) \mathbf{a}_{1}+\left(\frac{1}{2}+y_{6}\right) \mathbf{a}_{2}+\frac{1}{2} \mathbf{a}_{3}$ & $=$ & $\left(\frac{1}{2}-x_{6}\right) a \hat{\mathbf{x}}+\left(\frac{1}{2}+y_{6}\right) b \hat{\mathbf{y}}+\frac{1}{2} c \hat{\mathbf{z}}$ & $(4 h)$ & Ge II \\
\hline $\mathbf{B}_{24}$ & $=$ & $\left(\frac{1}{2}+x_{6}\right) \mathbf{a}_{1}+\left(\frac{1}{2}-y_{6}\right) \mathbf{a}_{2}+\frac{1}{2} \mathbf{a}_{3}$ & $=$ & $\left(\frac{1}{2}+x_{6}\right) a \hat{\mathbf{x}}+\left(\frac{1}{2}-y_{6}\right) b \hat{\mathbf{y}}+\frac{1}{2} c \hat{\mathbf{z}}$ & $(4 h)$ & Ge II \\
\hline
\end{tabular}

\section{References:}

- T. Wadsten, Crystal structures of $\mathrm{SiP}_{2}$, SiAs 2 , and GeP, Acta Chem. Scand. 21, 593-594 (1967), doi:10.3891/acta.chem.scand.21-0593.

\section{Found in:}

- P. Villars and K. Cenzual, Pearson's Crystal Data - Crystal Structure Database for Inorganic Compounds, ASM International (2013).

\section{Geometry files:}

- CIF: pp. 836

- POSCAR: pp. 836 
$\mathrm{Rh}_{5} \mathrm{Ge}_{3}$ Structure: A3B5_oP16_55_ch_agh
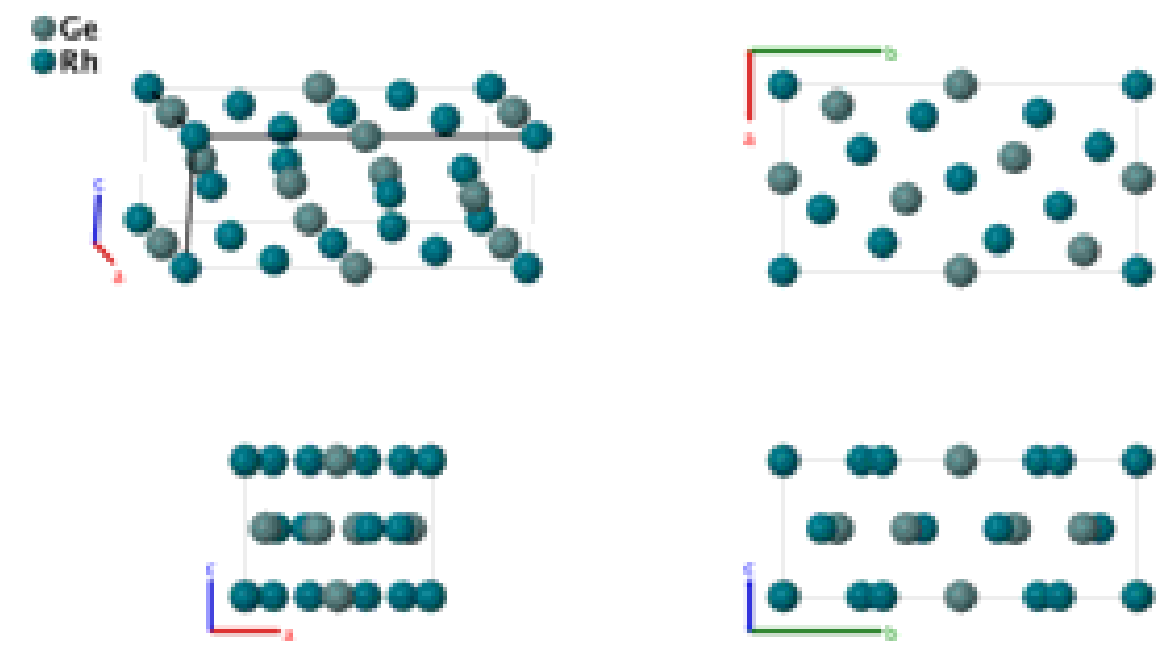

\section{Prototype}

AFLOW prototype label

Strukturbericht designation

Pearson symbol

Space group number

Space group symbol

AFLOW prototype command
: $\quad \mathrm{Rh}_{5} \mathrm{Ge}_{3}$

: A3B5_oP16_55_ch_agh

: None

: $\quad$ oP16

: 55

: Pbam

\section{Simple Orthorhombic primitive vectors:}

$$
\begin{aligned}
& \mathbf{a}_{1}=a \hat{\mathbf{x}} \\
& \mathbf{a}_{2}=b \hat{\mathbf{y}} \\
& \mathbf{a}_{3}=c \hat{\mathbf{z}}
\end{aligned}
$$

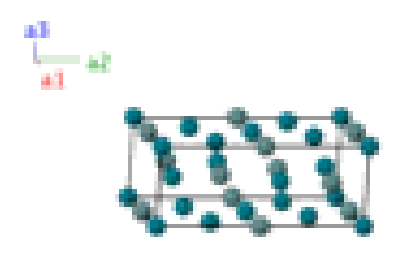

Basis vectors:

\section{Lattice Coordinates}

$\mathbf{B}_{1}=$

$\mathbf{B}_{2}=$

$\mathbf{B}_{3}=$

$\mathbf{B}_{4}=$

$\mathbf{B}_{5}=$

$\mathbf{B}_{6}=$

$$
0 \mathbf{a}_{1}+0 \mathbf{a}_{2}+0 \mathbf{a}_{3}
$$$$
\frac{1}{2} \mathbf{a}_{1}+\frac{1}{2} \mathbf{a}_{2}
$$

$\frac{1}{2} \mathbf{a}_{2}$

$\frac{1}{2} \mathbf{a}_{1}$

$$
x_{3} \mathbf{a}_{1}+y_{3} \mathbf{a}_{2}
$$

$-x_{3} \mathbf{a}_{1}-y_{3} \mathbf{a}_{2}$

$\mathbf{B}_{7}=$

$\mathbf{B}_{8}=$ aflow - -proto=A3B5_oP16_55_ch_agh

- params $=a, b / a, c / a, x_{3}, y_{3}, x_{4}, y_{4}, x_{5}, y_{5}$ 


\begin{tabular}{|c|c|c|c|c|c|}
\hline $\mathbf{B}_{9}$ & $=$ & $x_{4} \mathbf{a}_{1}+y_{4} \mathbf{a}_{2}+\frac{1}{2} \mathbf{a}_{3}$ & $=$ & $x_{4} a \hat{\mathbf{x}}+y_{4} b \hat{\mathbf{y}}+\frac{1}{2} c \hat{\mathbf{z}}$ & $(4 h)$ \\
\hline $\mathbf{B}_{10}$ & $=$ & $-x_{4} \mathbf{a}_{1}-y_{4} \mathbf{a}_{2}+\frac{1}{2} \mathbf{a}_{3}$ & $=$ & $-x_{4} a \hat{\mathbf{x}}-y_{4} b \hat{\mathbf{y}}+\frac{1}{2} c \hat{\mathbf{z}}$ & $(4 h)$ \\
\hline $\mathbf{B}_{11}$ & $=$ & $\left(\frac{1}{2}-x_{4}\right) \mathbf{a}_{1}+\left(\frac{1}{2}+y_{4}\right) \mathbf{a}_{2}+\frac{1}{2} \mathbf{a}_{3}$ & $=$ & $\left(\frac{1}{2}-x_{4}\right) a \hat{\mathbf{x}}+\left(\frac{1}{2}+y_{4}\right) b \hat{\mathbf{y}}+\frac{1}{2} c \hat{\mathbf{z}}$ & $(4 h)$ \\
\hline $\mathbf{B}_{12}$ & $=$ & $\left(\frac{1}{2}+x_{4}\right) \mathbf{a}_{1}+\left(\frac{1}{2}-y_{4}\right) \mathbf{a}_{2}+\frac{1}{2} \mathbf{a}_{3}$ & $=$ & $\left(\frac{1}{2}+x_{4}\right) a \hat{\mathbf{x}}+\left(\frac{1}{2}-y_{4}\right) b \hat{\mathbf{y}}+\frac{1}{2} c \hat{\mathbf{z}}$ & $(4 h)$ \\
\hline $\mathbf{B}_{13}$ & $=$ & $x_{5} \mathbf{a}_{1}+y_{5} \mathbf{a}_{2}+\frac{1}{2} \mathbf{a}_{3}$ & $=$ & $x_{5} a \hat{\mathbf{x}}+y_{5} b \hat{\mathbf{y}}+\frac{1}{2} c \hat{\mathbf{z}}$ & $(4 h)$ \\
\hline $\mathbf{B}_{14}$ & $=$ & $-x_{5} \mathbf{a}_{1}-y_{5} \mathbf{a}_{2}+\frac{1}{2} \mathbf{a}_{3}$ & $=$ & $-x_{5} a \hat{\mathbf{x}}-y_{5} b \hat{\mathbf{y}}+\frac{1}{2} c \hat{\mathbf{z}}$ & $(4 h)$ \\
\hline $\mathbf{B}_{15}$ & $=$ & $\left(\frac{1}{2}-x_{5}\right) \mathbf{a}_{1}+\left(\frac{1}{2}+y_{5}\right) \mathbf{a}_{2}+\frac{1}{2} \mathbf{a}_{3}$ & $=$ & $\left(\frac{1}{2}-x_{5}\right) a \hat{\mathbf{x}}+\left(\frac{1}{2}+y_{5}\right) b \hat{\mathbf{y}}+\frac{1}{2} c \hat{\mathbf{z}}$ & $(4 h)$ \\
\hline $\mathbf{B}_{16}$ & $=$ & $\left(\frac{1}{2}+x_{5}\right) \mathbf{a}_{1}+\left(\frac{1}{2}-y_{5}\right) \mathbf{a}_{2}+\frac{1}{2} \mathbf{a}_{3}$ & $=$ & $\left(\frac{1}{2}+x_{5}\right) a \hat{\mathbf{x}}+\left(\frac{1}{2}-y_{5}\right) b \hat{\mathbf{y}}+\frac{1}{2} c \hat{\mathbf{z}}$ & $(4 h)$ \\
\hline
\end{tabular}

\section{References:}

- S. Geller, The rhodium-germanium system. I. The crystal structures of $R h_{2} G e, R h_{5} G e_{3}$ and $R h G e$, Acta Cryst. 8, 15-21 (1955), doi:10.1107/S0365110X55000030.

\section{Found in:}

- P. Villars and K. Cenzual, Pearson's Crystal Data - Crystal Structure Database for Inorganic Compounds, ASM International (2013).

\section{Geometry files:}

- CIF: pp. 836

- POSCAR: pp. 837 


\section{R-carbon Structure: A_oP16_55_2g2h}

$\phi c$
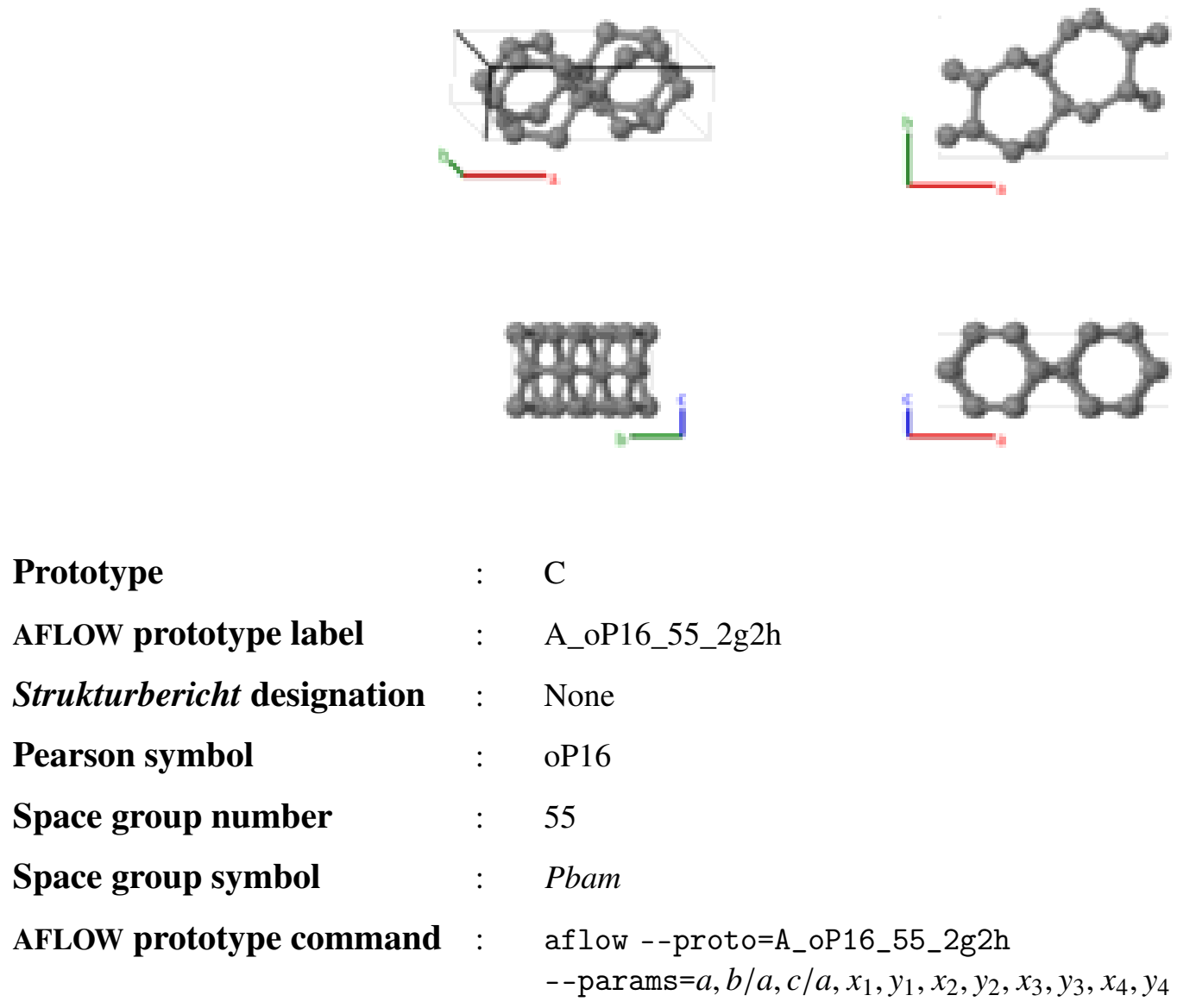

- This is a predicted "superhard" allotrope of carbon. Shortly after this paper was published, another paper predicted a similar phase, called "H-carbon" (He, 2012). The similarity between the two structures can be seen by shifting the origin by (1/2) $\mathbf{a}_{1}$. Other sources (Zhao, 2012) refer to this structure as "O-carbon."

\section{Simple Orthorhombic primitive vectors:}

$$
\begin{aligned}
& \mathbf{a}_{1}=a \hat{\mathbf{x}} \\
& \mathbf{a}_{2}=b \hat{\mathbf{y}} \\
& \mathbf{a}_{3}=c \hat{\mathbf{z}}
\end{aligned}
$$

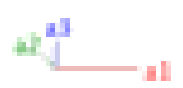

Basis vectors:

Lattice Coordinates

$\begin{array}{lccl}\mathbf{B}_{1} & & x_{1} \mathbf{a}_{1}+y_{1} \mathbf{a}_{2} & = \\ \mathbf{B}_{2} & & -x_{1} \mathbf{a}_{1}-y_{1} \mathbf{a}_{2} & = \\ \mathbf{B}_{3} & = & \left(\frac{1}{2}-x_{1}\right) \mathbf{a}_{1}+\left(\frac{1}{2}+y_{1}\right) \mathbf{a}_{2} & = \\ \mathbf{B}_{4}= & \left(\frac{1}{2}+x_{1}\right) \mathbf{a}_{1}+\left(\frac{1}{2}-y_{1}\right) \mathbf{a}_{2} & = \\ \mathbf{B}_{5} & & x_{2} \mathbf{a}_{1}+y_{2} \mathbf{a}_{2} & =\end{array}$

Cartesian Coordinates

$=$

$=$

$=\left(\frac{1}{2}-x_{1}\right) a \hat{\mathbf{x}}+\left(\frac{1}{2}+y_{1}\right) b \hat{\mathbf{y}}$

$=\left(\frac{1}{2}+x_{1}\right) a \hat{\mathbf{x}}+\left(\frac{1}{2}-y_{1}\right) b \hat{\mathbf{y}}$

$=\quad x_{2} a \hat{\mathbf{x}}+y_{2} b \hat{\mathbf{y}}$
Wyckoff Position

Atom Type

(4g)

C I

$(4 g)$

C I

$(4 g)$

C I

(4g)

C I

(4g) 


\begin{tabular}{|c|c|c|c|c|c|c|}
\hline $\mathbf{B}_{6}$ & $=$ & $-x_{2} \mathbf{a}_{1}-y_{2} \mathbf{a}_{2}$ & $=$ & $-x_{2} a \hat{\mathbf{x}}-y_{2} b \hat{\mathbf{y}}$ & $(4 g)$ & C II \\
\hline $\mathbf{B}_{7}$ & $=$ & $\left(\frac{1}{2}-x_{2}\right) \mathbf{a}_{1}+\left(\frac{1}{2}+y_{2}\right) \mathbf{a}_{2}$ & $=$ & $\left(\frac{1}{2}-x_{2}\right) a \hat{\mathbf{x}}+\left(\frac{1}{2}+y_{2}\right) b \hat{\mathbf{y}}$ & $(4 g)$ & C II \\
\hline $\mathbf{B}_{8}$ & $=$ & $\left(\frac{1}{2}+x_{2}\right) \mathbf{a}_{1}+\left(\frac{1}{2}-y_{2}\right) \mathbf{a}_{2}$ & $=$ & $\left(\frac{1}{2}+x_{2}\right) a \hat{\mathbf{x}}+\left(\frac{1}{2}-y_{2}\right) b \hat{\mathbf{y}}$ & $(4 g)$ & C II \\
\hline $\mathbf{B}_{9}$ & $=$ & $x_{3} \mathbf{a}_{1}+y_{3} \mathbf{a}_{2}+\frac{1}{2} \mathbf{a}_{3}$ & $=$ & $x_{3} a \hat{\mathbf{x}}+y_{3} b \hat{\mathbf{y}}+\frac{1}{2} c \hat{\mathbf{z}}$ & $(4 h)$ & III \\
\hline $\mathbf{B}_{10}$ & $=$ & $-x_{3} \mathbf{a}_{1}-y_{3} \mathbf{a}_{2}+\frac{1}{2} \mathbf{a}_{3}$ & $=$ & $-x_{3} a \hat{\mathbf{x}}-y_{3} b \hat{\mathbf{y}}+\frac{1}{2} c \hat{\mathbf{z}}$ & $(4 h)$ & III \\
\hline $\mathbf{B}_{11}$ & $=$ & $\left(\frac{1}{2}-x_{3}\right) \mathbf{a}_{1}+\left(\frac{1}{2}+y_{3}\right) \mathbf{a}_{2}+\frac{1}{2} \mathbf{a}_{3}$ & $=$ & $\left(\frac{1}{2}-x_{3}\right) a \hat{\mathbf{x}}+\left(\frac{1}{2}+y_{3}\right) b \hat{\mathbf{y}}+\frac{1}{2} c \hat{\mathbf{z}}$ & $(4 h)$ & C III \\
\hline $\mathbf{B}_{12}$ & $=$ & $\left(\frac{1}{2}+x_{3}\right) \mathbf{a}_{1}+\left(\frac{1}{2}-y_{3}\right) \mathbf{a}_{2}+\frac{1}{2} \mathbf{a}_{3}$ & $=$ & $\left(\frac{1}{2}+x_{3}\right) a \hat{\mathbf{x}}+\left(\frac{1}{2}-y_{3}\right) b \hat{\mathbf{y}}+\frac{1}{2} c \hat{\mathbf{z}}$ & $(4 h)$ & C III \\
\hline $\mathbf{B}_{13}$ & $=$ & $x_{4} \mathbf{a}_{1}+y_{4} \mathbf{a}_{2}+\frac{1}{2} \mathbf{a}_{3}$ & $=$ & $x_{4} a \hat{\mathbf{x}}+y_{4} b \hat{\mathbf{y}}+\frac{1}{2} c \hat{\mathbf{z}}$ & $(4 h)$ & IV \\
\hline $\mathbf{B}_{14}$ & $=$ & $-x_{4} \mathbf{a}_{1}-y_{4} \mathbf{a}_{2}+\frac{1}{2} \mathbf{a}_{3}$ & $=$ & $-x_{4} a \hat{\mathbf{x}}-y_{4} b \hat{\mathbf{y}}+\frac{1}{2} c \hat{\mathbf{z}}$ & $(4 h)$ & C IV \\
\hline $\mathbf{B}_{15}$ & $=$ & $\left(\frac{1}{2}-x_{4}\right) \mathbf{a}_{1}+\left(\frac{1}{2}+y_{4}\right) \mathbf{a}_{2}+\frac{1}{2} \mathbf{a}_{3}$ & $=$ & $\left(\frac{1}{2}-x_{4}\right) a \hat{\mathbf{x}}+\left(\frac{1}{2}+y_{4}\right) b \hat{\mathbf{y}}+\frac{1}{2} c \hat{\mathbf{z}}$ & $(4 h)$ & \\
\hline $\mathbf{B}_{16}$ & $=$ & $\left(\frac{1}{2}+x_{4}\right) \mathbf{a}_{1}+\left(\frac{1}{2}-y_{4}\right) \mathbf{a}_{2}+\frac{1}{2} \mathbf{a}_{3}$ & $=$ & $\left(\frac{1}{2}+x_{4}\right) a \hat{\mathbf{x}}+\left(\frac{1}{2}-y_{4}\right) b \hat{\mathbf{y}}+\frac{1}{2} c \hat{\mathbf{z}}$ & $(4 h)$ & \\
\hline
\end{tabular}

\section{References:}

- H. Niu, X.-Q. Chen, S. Wang, D. Li, W. L. Mao, and Y. Li, Families of Superhard Crystalline Carbon Allotropes Constructed via Cold Compression of Graphite and Nanotubes, Phys. Rev. Lett. 108, 135501 (2012), doi:10.1103/PhysRevLett.108.135501.

- C. He, L. Sun, C. Zhang, X. Peng, K. Zhang, and J. Zhong, New superhard carbon phases between graphite and diamond, Solid State Commun. 152, 1560-1563 (2012), doi:10.1016/j.ssc.2012.05.022.

- C. He, L. Z. Sun, and J. Zhong, Prediction of superhard carbon allotropes from the segment combination method, J. Superhard Mater. 34, 386-399 (2012), doi:10.3103/S1063457612060123.

- Z. Zhao, F. Tian, X. Dong, Q. Li, Q. Wang, H. Wang, X. Zhong, B. Xu, D. Yu, J. He, H.-T. Wang, Y. Ma, and Y. Tian, Tetragonal Allotrope of Group 14 Elements, J. Am. Chem. Soc. 134, 12362-12365 (2012), doi:10.1021/ja304380p.

\section{Geometry files:}

- CIF: pp. 837

- POSCAR: pp. 837 


\section{$\alpha-\mathrm{PdCl}_{2}(C 50)$ Structure: A2B_oP6_58_g_a}
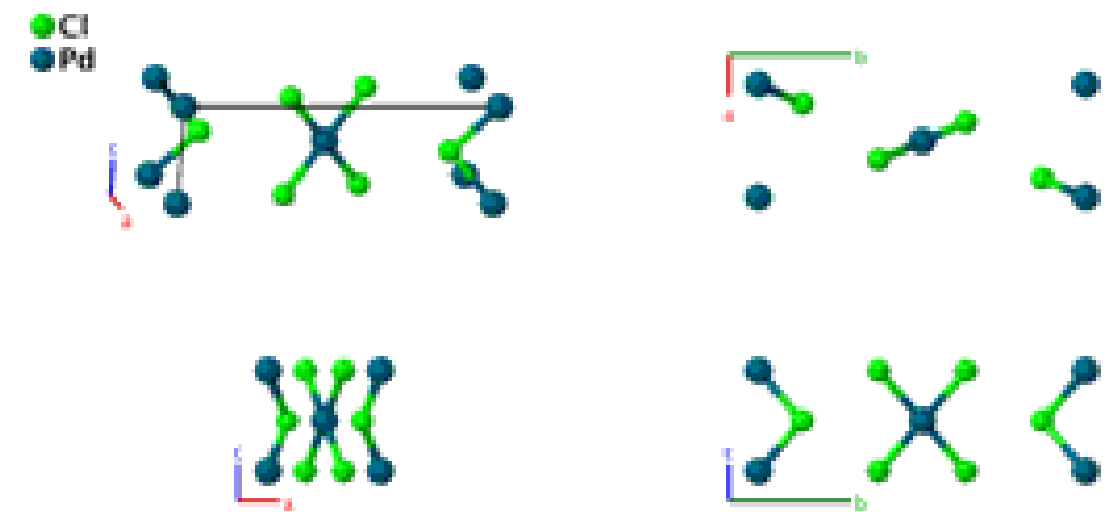

\section{Prototype}

: $\quad \alpha-\mathrm{PdCl}_{2}$

AFLOW prototype label

: A2B_oP6_58_g_a

Strukturbericht designation

: $\quad$ 550

Pearson symbol

: oP6

Space group number

: 58

Space group symbol

: Pnnm

AFLOW prototype command : aflow --proto=A2B_oP6_58_g_a

- params $=a, b / a, c / a, x_{2}, y_{2}$

- (Evers, 2010) implicitly places the Pd atoms at the (2b) Wyckoff position. We have shifted the Pd atoms to the (2a) site.

\section{Simple Orthorhombic primitive vectors:}

$$
\begin{aligned}
& \mathbf{a}_{1}=a \hat{\mathbf{x}} \\
& \mathbf{a}_{2}=b \hat{\mathbf{y}} \\
& \mathbf{a}_{3}=c \hat{\mathbf{z}}
\end{aligned}
$$

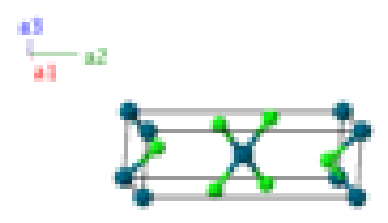

\section{Basis vectors:}

Lattice Coordinates

$\mathbf{B}_{1}=\quad 0 \mathbf{a}_{1}+0 \mathbf{a}_{2}+0 \mathbf{a}_{3}$

$\mathbf{B}_{2}=\frac{1}{2} \mathbf{a}_{1}+\frac{1}{2} \mathbf{a}_{2}+\frac{1}{2} \mathbf{a}_{3}$

$\mathbf{B}_{3}=$

$\mathbf{B}_{4}=$

$x_{2} \mathbf{a}_{1}+y_{2} \mathbf{a}_{2}$

$-x_{2} \mathbf{a}_{1}-y_{2} \mathbf{a}_{2}$

$\mathbf{B}_{5}=\left(\frac{1}{2}-x_{2}\right) \mathbf{a}_{1}+\left(\frac{1}{2}+y_{2}\right) \mathbf{a}_{2}+\frac{1}{2} \mathbf{a}_{3}$

$\mathbf{B}_{6}=\left(\frac{1}{2}+x_{2}\right) \mathbf{a}_{1}+\left(\frac{1}{2}-y_{2}\right) \mathbf{a}_{2}+\frac{1}{2} \mathbf{a}_{3}$

\section{Cartesian Coordinates}

$=$

$=$

$$
0 \hat{\mathbf{x}}+0 \hat{\mathbf{y}}+0 \hat{\mathbf{z}}
$$$$
\frac{1}{2} a \hat{\mathbf{x}}+\frac{1}{2} b \hat{\mathbf{y}}+\frac{1}{2} c \hat{\mathbf{z}}
$$

$=$

$$
x_{2} a \hat{\mathbf{x}}+y_{2} b \hat{\mathbf{y}}
$$$$
-x_{2} a \hat{\mathbf{x}}-y_{2} b \hat{\mathbf{y}}
$$

$=\left(\frac{1}{2}-x_{2}\right) a \hat{\mathbf{x}}+\left(\frac{1}{2}+y_{2}\right) b \hat{\mathbf{y}}+\frac{1}{2} c \hat{\mathbf{z}}$

$=\left(\frac{1}{2}+x_{2}\right) a \hat{\mathbf{x}}+\left(\frac{1}{2}-y_{2}\right) b \hat{\mathbf{y}}+\frac{1}{2} c \hat{\mathbf{z}}$
Wyckoff Position

(2a)
Atom Type

Pd

Pd

$\mathrm{Cl}$

$\mathrm{Cl}$

$\mathrm{Cl}$

$\mathrm{Cl}$

\section{References:}


- J. Evers, W. Beck, M. Göbel, S. Jakob, P. Mayer, G. Oehlinger, M. Rotter, and T. M. Klapötke, The Structures of $\delta$-PdCl 2 and $\gamma-P_{C C l}$ : Phases with Negative Thermal Expansion in One Direction, Angew. Chem. Int. Ed. 49, 5677-5682 (2010), doi:10.1002/anie.201000680.

\section{Geometry files:}

- CIF: pp. 837

- POSCAR: pp. 838 


\section{FeOCl Structure: ABC_oP6_59_a_b_a}
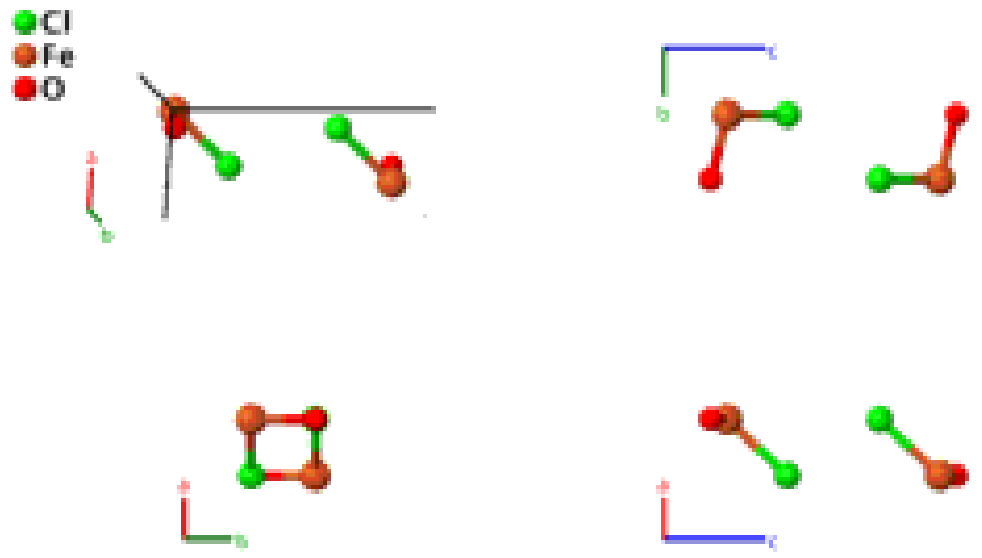

\section{Prototype}

: $\mathrm{FeOCl}$

AFLOW prototype label

: ABC_oP6_59_a_b_a

Strukturbericht designation

Pearson symbol

: None

Space group number

: oP6

Space group symbol

: 59

AFLOW prototype command

: Pmmn

aflow --proto=ABC_oP6_59_a_b_a

- params $=a, b / a, c / a, z_{1}, z_{2}, z_{3}$

\section{Other compounds with this structure:}

- $M \mathrm{OCl},(M=\mathrm{Ti}, \mathrm{V}, \mathrm{Cr}, \mathrm{Fe})$, the superconducting alkali metal intercalcates $\alpha-M \mathrm{~N} X(M=\mathrm{Ti}, \mathrm{Zr}, \mathrm{Hf} ; X=\mathrm{Cl}, \mathrm{Br}, \mathrm{I})$.

\section{Simple Orthorhombic primitive vectors:}

$$
\begin{aligned}
& \mathbf{a}_{1}=a \hat{\mathbf{x}} \\
& \mathbf{a}_{2}=b \hat{\mathbf{y}} \\
& \mathbf{a}_{3}=c \hat{\mathbf{z}}
\end{aligned}
$$

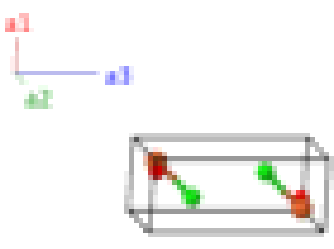

\section{Basis vectors:}

Lattice Coordinates

$\mathbf{B}_{1}=\frac{1}{4} \mathbf{a}_{1}+\frac{1}{4} \mathbf{a}_{2}+z_{1} \mathbf{a}_{3}$

$\mathbf{B}_{2}=\frac{3}{4} \mathbf{a}_{1}+\frac{3}{4} \mathbf{a}_{2}-z_{1} \mathbf{a}_{3}$

$\mathbf{B}_{3}=\frac{1}{4} \mathbf{a}_{1}+\frac{1}{4} \mathbf{a}_{2}+z_{2} \mathbf{a}_{3}$

$\mathbf{B}_{4}=\frac{3}{4} \mathbf{a}_{1}+\frac{3}{4} \mathbf{a}_{2}-z_{2} \mathbf{a}_{3}$

$\mathbf{B}_{5}=\frac{1}{4} \mathbf{a}_{1}+\frac{3}{4} \mathbf{a}_{2}+z_{3} \mathbf{a}_{3}$

$\mathbf{B}_{6}=\frac{3}{4} \mathbf{a}_{1}+\frac{1}{4} \mathbf{a}_{2}-z_{3} \mathbf{a}_{3}$
Cartesian Coordinates

$=$

$=$

$=$

$=$

$=$

$=$

$$
\begin{aligned}
& \frac{1}{4} a \hat{\mathbf{x}}+\frac{1}{4} b \hat{\mathbf{y}}+z_{1} c \hat{\mathbf{z}} \\
& \frac{3}{4} a \hat{\mathbf{x}}+\frac{3}{4} b \hat{\mathbf{y}}-z_{1} c \hat{\mathbf{z}} \\
& \frac{1}{4} a \hat{\mathbf{x}}+\frac{1}{4} b \hat{\mathbf{y}}+z_{2} c \hat{\mathbf{z}} \\
& \frac{3}{4} a \hat{\mathbf{x}}+\frac{3}{4} b \hat{\mathbf{y}}-z_{2} c \hat{\mathbf{z}} \\
& \frac{1}{4} a \hat{\mathbf{x}}+\frac{3}{4} b \hat{\mathbf{y}}+z_{3} c \hat{\mathbf{z}} \\
& \frac{3}{4} a \hat{\mathbf{x}}+\frac{1}{4} b \hat{\mathbf{y}}-z_{3} c \hat{\mathbf{z}}
\end{aligned}
$$

Wyckoff Position
Atom Type

$\mathrm{Cl}$

$\mathrm{Cl}$

$\mathrm{O}$

$\mathrm{O}$

$\mathrm{Fe}$

$\mathrm{Fe}$ 


\section{References:}

- S. M. Kauzlarich, J. L. Stanton, J. Faber, and B. A. Averill, Neutron profile refinement of the structure of FeOCl and FeOCl(TTF $)_{1 / 8.5}$, J. Am. Chem. Soc. 108, 7946-7951 (1986), doi:10.1021/ja00285a011.

\section{Geometry files:}

- CIF: pp. 838

- POSCAR: pp. 838 


\section{$\mathrm{Rh}_{2} \mathrm{~S}_{3}$ Structure: A2B3_oP20_60_d_cd}
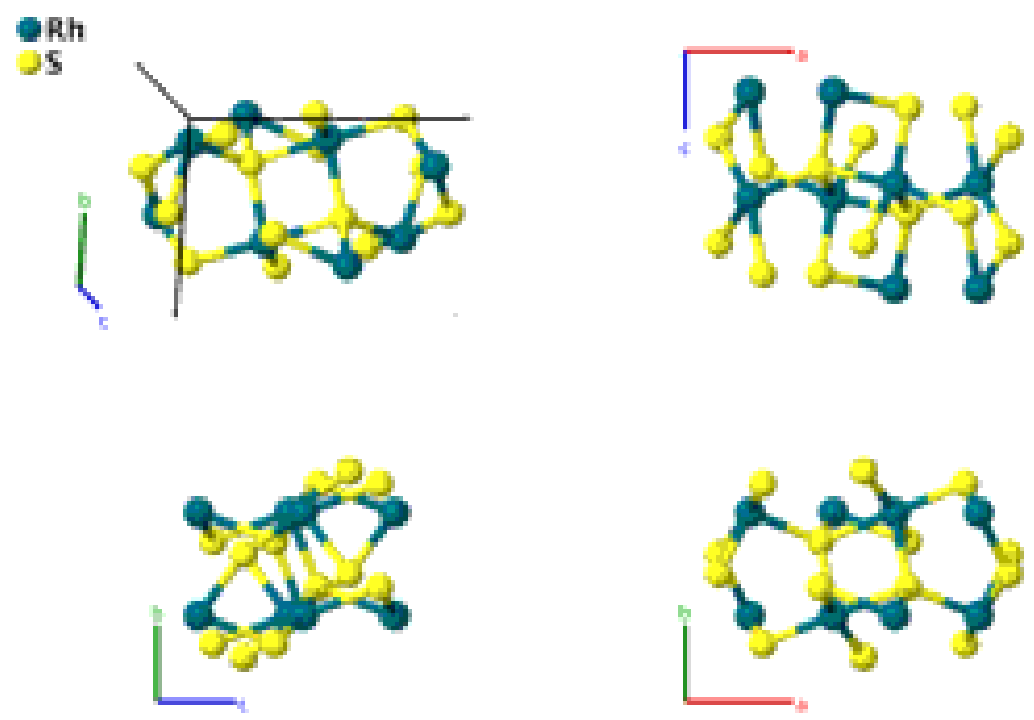

\section{Prototype}

AFLOW prototype label

$: \quad \mathrm{Rh}_{2} \mathrm{~S}_{3}$

Strukturbericht designation

: A2B3_oP20_60_d_cd

Pearson symbol

: None

Space group number

: $\quad$ oP20

Space group symbol

: 60

AFLOW prototype command : aflow --proto=A2B3_oP20_60_d_cd

: Pbcn

- -params $=a, b / a, c / a, y_{1}, x_{2}, y_{2}, z_{2}, x_{3}, y_{3}, z_{3}$

Simple Orthorhombic primitive vectors:

$$
\begin{aligned}
& \mathbf{a}_{1}=a \hat{\mathbf{x}} \\
& \mathbf{a}_{2}=b \hat{\mathbf{y}} \\
& \mathbf{a}_{3}=c \hat{\mathbf{z}}
\end{aligned}
$$

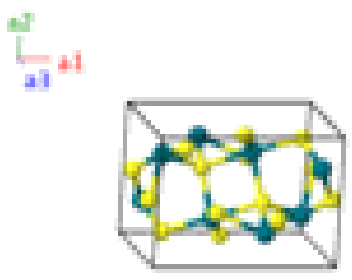

\section{Basis vectors:}

Lattice Coordinates

$\begin{array}{lcccc}\mathbf{B}_{1} & = & y_{1} \mathbf{a}_{2}+\frac{1}{4} \mathbf{a}_{3} & & \\ \mathbf{B}_{2} & = & \frac{1}{2} \mathbf{a}_{1}+\left(\frac{1}{2}-y_{1}\right) \mathbf{a}_{2}+\frac{3}{4} \mathbf{a}_{3} & = & \frac{1}{2} a \hat{\mathbf{x}}+\left(\frac{1}{2}-y_{1}\right) b \hat{\mathbf{y}}+\frac{3}{4} c \hat{\mathbf{z}} \\ \mathbf{B}_{3}= & -y_{1} \mathbf{a}_{2}+\frac{3}{4} \mathbf{a}_{3} & = & -y_{1} b \hat{\mathbf{y}}+\frac{3}{4} c \hat{\mathbf{z}} \\ \mathbf{B}_{4}= & \frac{1}{2} \mathbf{a}_{1}+\left(\frac{1}{2}+y_{1}\right) \mathbf{a}_{2}+\frac{1}{4} \mathbf{a}_{3} & = & \frac{1}{2} a \hat{\mathbf{x}}+\left(\frac{1}{2}+y_{1}\right) b \hat{\mathbf{y}}+\frac{1}{4} c \hat{\mathbf{z}} \\ \mathbf{B}_{5}= & x_{2} \mathbf{a}_{1}+y_{2} \mathbf{a}_{2}+z_{2} \mathbf{a}_{3} & = & x_{2} a \hat{\mathbf{x}}+y_{2} b \hat{\mathbf{y}}+z_{2} c \hat{\mathbf{z}} \\ \mathbf{B}_{6}= & \left(\frac{1}{2}-x_{2}\right) \mathbf{a}_{1}+\left(\frac{1}{2}-y_{2}\right) \mathbf{a}_{2}+ & & & \left(\frac{1}{2}-x_{2}\right) a \hat{\mathbf{x}}+\left(\frac{1}{2}-y_{2}\right) b \hat{\mathbf{y}}+ \\ & \left(\frac{1}{2}+z_{2}\right) \mathbf{a}_{3} & & & \left(\frac{1}{2}+z_{2}\right) c \hat{\mathbf{z}}\end{array}$

Wyckoff Position Atom Type

$(4 c)$ 


\begin{tabular}{|c|c|c|c|c|c|c|}
\hline $\mathbf{B}_{7}$ & $=$ & $-x_{2} \mathbf{a}_{1}+y_{2} \mathbf{a}_{2}+\left(\frac{1}{2}-z_{2}\right) \mathbf{a}_{3}$ & $=$ & $-x_{2} a \hat{\mathbf{x}}+y_{2} b \hat{\mathbf{y}}+\left(\frac{1}{2}-z_{2}\right) c \hat{\mathbf{z}}$ & $(8 d)$ & $\mathrm{Rh}$ \\
\hline $\mathbf{B}_{8}$ & $=$ & $\left(\frac{1}{2}+x_{2}\right) \mathbf{a}_{1}+\left(\frac{1}{2}-y_{2}\right) \mathbf{a}_{2}-z_{2} \mathbf{a}_{3}$ & $=$ & $\left(\frac{1}{2}+x_{2}\right) a \hat{\mathbf{x}}+\left(\frac{1}{2}-y_{2}\right) b \hat{\mathbf{y}}-z_{2} c \hat{\mathbf{z}}$ & $(8 d)$ & $\mathrm{Rh}$ \\
\hline $\mathbf{B}_{9}$ & $=$ & $-x_{2} \mathbf{a}_{1}-y_{2} \mathbf{a}_{2}-z_{2} \mathbf{a}_{3}$ & $=$ & $-x_{2} a \hat{\mathbf{x}}-y_{2} b \hat{\mathbf{y}}-z_{2} c \hat{\mathbf{z}}$ & $(8 d)$ & $\mathrm{Rh}$ \\
\hline $\mathbf{B}_{10}$ & $=$ & $\begin{array}{c}\left(\frac{1}{2}+x_{2}\right) \mathbf{a}_{1}+\left(\frac{1}{2}+y_{2}\right) \mathbf{a}_{2}+ \\
\left(\frac{1}{2}-z_{2}\right) \mathbf{a}_{3}\end{array}$ & $=$ & $\begin{array}{c}\left(\frac{1}{2}+x_{2}\right) a \hat{\mathbf{x}}+\left(\frac{1}{2}+y_{2}\right) b \hat{\mathbf{y}}+ \\
\left(\frac{1}{2}-z_{2}\right) c \hat{\mathbf{z}}\end{array}$ & $(8 d)$ & $\mathrm{Rh}$ \\
\hline $\mathbf{B}_{11}$ & $=$ & $x_{2} \mathbf{a}_{1}-y_{2} \mathbf{a}_{2}+\left(\frac{1}{2}+z_{2}\right) \mathbf{a}_{3}$ & $=$ & $x_{2} a \hat{\mathbf{x}}-y_{2} b \hat{\mathbf{y}}+\left(\frac{1}{2}+z_{2}\right) c \hat{\mathbf{z}}$ & $(8 d)$ & $\mathrm{Rh}$ \\
\hline $\mathbf{B}_{12}$ & $=$ & $\left(\frac{1}{2}-x_{2}\right) \mathbf{a}_{1}+\left(\frac{1}{2}+y_{2}\right) \mathbf{a}_{2}+z_{2} \mathbf{a}_{3}$ & $=$ & $\left(\frac{1}{2}-x_{2}\right) a \hat{\mathbf{x}}+\left(\frac{1}{2}+y_{2}\right) b \hat{\mathbf{y}}+z_{2} c \hat{\mathbf{z}}$ & $(8 d)$ & $\mathrm{Rh}$ \\
\hline $\mathbf{B}_{13}$ & $=$ & $x_{3} \mathbf{a}_{1}+y_{3} \mathbf{a}_{2}+z_{3} \mathbf{a}_{3}$ & $=$ & $x_{3} a \hat{\mathbf{x}}+y_{3} b \hat{\mathbf{y}}+z_{3} c \hat{\mathbf{z}}$ & $(8 d)$ & S II \\
\hline $\mathbf{B}_{14}$ & $=$ & $\begin{array}{c}\left(\frac{1}{2}-x_{3}\right) \mathbf{a}_{1}+\left(\frac{1}{2}-y_{3}\right) \mathbf{a}_{2}+ \\
\left(\frac{1}{2}+z_{3}\right) \mathbf{a}_{3}\end{array}$ & $=$ & $\begin{array}{c}\left(\frac{1}{2}-x_{3}\right) a \hat{\mathbf{x}}+\left(\frac{1}{2}-y_{3}\right) b \hat{\mathbf{y}}+ \\
\left(\frac{1}{2}+z_{3}\right) c \hat{\mathbf{z}}\end{array}$ & $(8 d)$ & S II \\
\hline $\mathbf{B}_{15}$ & $=$ & $-x_{3} \mathbf{a}_{1}+y_{3} \mathbf{a}_{2}+\left(\frac{1}{2}-z_{3}\right) \mathbf{a}_{3}$ & $=$ & $-x_{3} a \hat{\mathbf{x}}+y_{3} b \hat{\mathbf{y}}+\left(\frac{1}{2}-z_{3}\right) c \hat{\mathbf{z}}$ & $(8 d)$ & S II \\
\hline $\mathbf{B}_{16}$ & $=$ & $\left(\frac{1}{2}+x_{3}\right) \mathbf{a}_{1}+\left(\frac{1}{2}-y_{3}\right) \mathbf{a}_{2}-z_{3} \mathbf{a}_{3}$ & $=$ & $\left(\frac{1}{2}+x_{3}\right) a \hat{\mathbf{x}}+\left(\frac{1}{2}-y_{3}\right) b \hat{\mathbf{y}}-z_{3} c \hat{\mathbf{z}}$ & $(8 d)$ & S II \\
\hline $\mathbf{B}_{17}$ & $=$ & $-x_{3} \mathbf{a}_{1}-y_{3} \mathbf{a}_{2}-z_{3} \mathbf{a}_{3}$ & $=$ & $-x_{3} a \hat{\mathbf{x}}-y_{3} b \hat{\mathbf{y}}-z_{3} c \hat{\mathbf{z}}$ & $(8 d)$ & S II \\
\hline $\mathbf{B}_{18}$ & $=$ & $\begin{array}{c}\left(\frac{1}{2}+x_{3}\right) \mathbf{a}_{1}+\left(\frac{1}{2}+y_{3}\right) \mathbf{a}_{2}+ \\
\left(\frac{1}{2}-z_{3}\right) \mathbf{a}_{3}\end{array}$ & $=$ & $\begin{array}{c}\left(\frac{1}{2}+x_{3}\right) a \hat{\mathbf{x}}+\left(\frac{1}{2}+y_{3}\right) b \hat{\mathbf{y}}+ \\
\left(\frac{1}{2}-z_{3}\right) c \hat{\mathbf{z}}\end{array}$ & $(8 d)$ & S II \\
\hline $\mathbf{B}_{19}$ & $=$ & $x_{3} \mathbf{a}_{1}-y_{3} \mathbf{a}_{2}+\left(\frac{1}{2}+z_{3}\right) \mathbf{a}_{3}$ & $=$ & $x_{3} a \hat{\mathbf{x}}-y_{3} b \hat{\mathbf{y}}+\left(\frac{1}{2}+z_{3}\right) c \hat{\mathbf{z}}$ & $(8 d)$ & S II \\
\hline $\mathbf{B}_{20}$ & $=$ & $\left(\frac{1}{2}-x_{3}\right) \mathbf{a}_{1}+\left(\frac{1}{2}+y_{3}\right) \mathbf{a}_{2}+z_{3} \mathbf{a}_{3}$ & $=$ & $\left(\frac{1}{2}-x_{3}\right) a \hat{\mathbf{x}}+\left(\frac{1}{2}+y_{3}\right) b \hat{\mathbf{y}}+z_{3} c \hat{\mathbf{z}}$ & $(8 d)$ & S II \\
\hline
\end{tabular}

\section{References:}

- E. P. F. and Hulliger, The crystal structure of $R h_{2} S_{3}$, in Acta Cryst. (Munksgaard Int Publ LTD 35 Norre Sogade, PO Box 2148, DK-1016 Copenhagen, Denmark, 1966), p. A66.

\section{Found in:}

- P. Villars and K. Cenzual, Pearson's Crystal Data - Crystal Structure Database for Inorganic Compounds, ASM International (2013).

\section{Geometry files:}

- CIF: pp. 838

- POSCAR: pp. 839 


\section{$\mathrm{WO}_{3}$ Structure: A3B_oP32_60_3d_d}
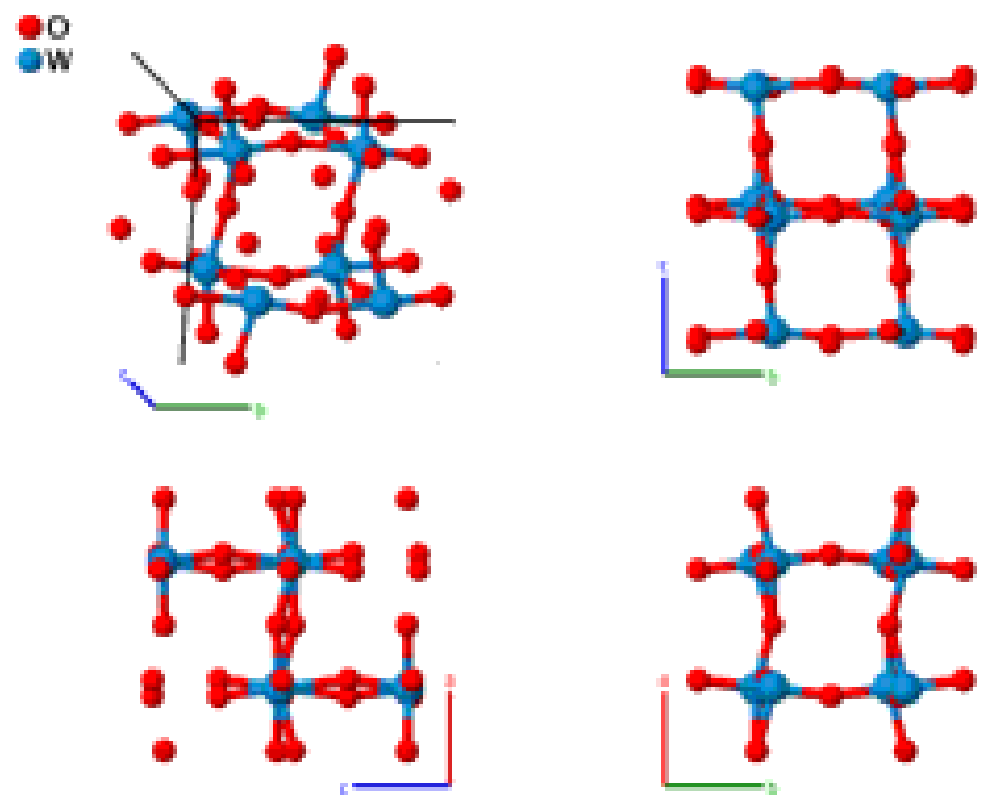

\section{Prototype}

AFLOW prototype label

$: \quad \mathrm{WO}_{3}$

Strukturbericht designation

Pearson symbol

: A3B_oP32_60_3d_d

Space group number

: None

Space group symbol

: $\quad$ oP32

AFLOW prototype command

: 60

: $\quad$ Pbcn

\section{Simple Orthorhombic primitive vectors:}

$$
\begin{aligned}
& \mathbf{a}_{1}=a \hat{\mathbf{x}} \\
& \mathbf{a}_{2}=b \hat{\mathbf{y}} \\
& \mathbf{a}_{3}=c \hat{\mathbf{z}}
\end{aligned}
$$

\begin{tabular}{|c|c|c|c|c|c|c|}
\hline & & Lattice Coordinates & & Cartesian Coordinates & Wyckoff Position & Atom Type \\
\hline $\mathbf{B}_{1}$ & $=$ & $x_{1} \mathbf{a}_{1}+y_{1} \mathbf{a}_{2}+z_{1} \mathbf{a}_{3}$ & $=$ & $x_{1} a \hat{\mathbf{x}}+y_{1} b \hat{\mathbf{y}}+z_{1} c \hat{\mathbf{z}}$ & $(8 d)$ & O I \\
\hline $\mathbf{B}_{2}$ & $=$ & $\begin{array}{c}\left(\frac{1}{2}-x_{1}\right) \mathbf{a}_{1}+\left(\frac{1}{2}-y_{1}\right) \mathbf{a}_{2}+ \\
\left(\frac{1}{2}+z_{1}\right) \mathbf{a}_{3}\end{array}$ & $=$ & $\begin{array}{c}\left(\frac{1}{2}-x_{1}\right) a \hat{\mathbf{x}}+\left(\frac{1}{2}-y_{1}\right) b \hat{\mathbf{y}}+ \\
\left(\frac{1}{2}+z_{1}\right) c \hat{\mathbf{z}}\end{array}$ & $(8 d)$ & O I \\
\hline $\mathbf{B}_{3}$ & $=$ & $-x_{1} \mathbf{a}_{1}+y_{1} \mathbf{a}_{2}+\left(\frac{1}{2}-z_{1}\right) \mathbf{a}_{3}$ & $=$ & $-x_{1} a \hat{\mathbf{x}}+y_{1} b \hat{\mathbf{y}}+\left(\frac{1}{2}-z_{1}\right) c \hat{\mathbf{z}}$ & $(8 d)$ & O I \\
\hline $\mathbf{B}_{4}$ & $=$ & $\left(\frac{1}{2}+x_{1}\right) \mathbf{a}_{1}+\left(\frac{1}{2}-y_{1}\right) \mathbf{a}_{2}-z_{1} \mathbf{a}_{3}$ & $=$ & $\left(\frac{1}{2}+x_{1}\right) a \hat{\mathbf{x}}+\left(\frac{1}{2}-y_{1}\right) b \hat{\mathbf{y}}-z_{1} c \hat{\mathbf{z}}$ & $(8 d)$ & O I \\
\hline
\end{tabular}

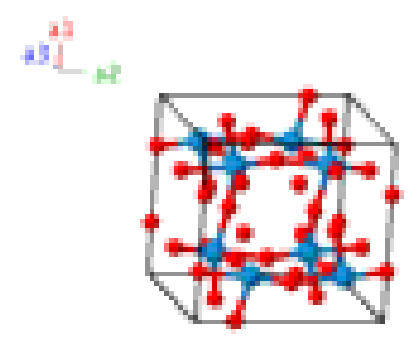

\section{Basis vectors:}




\begin{tabular}{|c|c|c|c|c|c|c|}
\hline $\mathbf{B}_{5}$ & $=$ & $-x_{1} \mathbf{a}_{1}-y_{1} \mathbf{a}_{2}-z_{1} \mathbf{a}_{3}$ & $=$ & $-x_{1} a \hat{\mathbf{x}}-y_{1} b \hat{\mathbf{y}}-z_{1} c \hat{\mathbf{z}}$ & $(8 d)$ & O I \\
\hline $\mathbf{B}_{6}$ & $=$ & $\begin{array}{c}\left(\frac{1}{2}+x_{1}\right) \mathbf{a}_{1}+\left(\frac{1}{2}+y_{1}\right) \mathbf{a}_{2}+ \\
\left(\frac{1}{2}-z_{1}\right) \mathbf{a}_{3}\end{array}$ & $=$ & $\begin{array}{c}\left(\frac{1}{2}+x_{1}\right) a \hat{\mathbf{x}}+\left(\frac{1}{2}+y_{1}\right) b \hat{\mathbf{y}}+ \\
\left(\frac{1}{2}-z_{1}\right) c \hat{\mathbf{z}}\end{array}$ & $(8 d)$ & O I \\
\hline $\mathbf{B}_{7}$ & $=$ & $x_{1} \mathbf{a}_{1}-y_{1} \mathbf{a}_{2}+\left(\frac{1}{2}+z_{1}\right) \mathbf{a}_{3}$ & $=$ & $x_{1} a \hat{\mathbf{x}}-y_{1} b \hat{\mathbf{y}}+\left(\frac{1}{2}+z_{1}\right) c \hat{\mathbf{z}}$ & $(8 d)$ & O I \\
\hline $\mathbf{B}_{8}$ & $=$ & $\left(\frac{1}{2}-x_{1}\right) \mathbf{a}_{1}+\left(\frac{1}{2}+y_{1}\right) \mathbf{a}_{2}+z_{1} \mathbf{a}_{3}$ & $=$ & $\left(\frac{1}{2}-x_{1}\right) a \hat{\mathbf{x}}+\left(\frac{1}{2}+y_{1}\right) b \hat{\mathbf{y}}+z_{1} c \hat{\mathbf{z}}$ & $(8 d)$ & O I \\
\hline $\mathbf{B}_{9}$ & $=$ & $x_{2} \mathbf{a}_{1}+y_{2} \mathbf{a}_{2}+z_{2} \mathbf{a}_{3}$ & $=$ & $x_{2} a \hat{\mathbf{x}}+y_{2} b \hat{\mathbf{y}}+z_{2} c \hat{\mathbf{z}}$ & $(8 d)$ & O II \\
\hline $\mathbf{B}_{10}$ & $=$ & $\begin{array}{c}\left(\frac{1}{2}-x_{2}\right) \mathbf{a}_{1}+\left(\frac{1}{2}-y_{2}\right) \mathbf{a}_{2}+ \\
\left(\frac{1}{2}+z_{2}\right) \mathbf{a}_{3}\end{array}$ & $=$ & $\begin{array}{c}\left(\frac{1}{2}-x_{2}\right) a \hat{\mathbf{x}}+\left(\frac{1}{2}-y_{2}\right) b \hat{\mathbf{y}}+ \\
\left(\frac{1}{2}+z_{2}\right) c \hat{\mathbf{z}}\end{array}$ & $(8 d)$ & O II \\
\hline $\mathbf{B}_{11}$ & $=$ & $-x_{2} \mathbf{a}_{1}+y_{2} \mathbf{a}_{2}+\left(\frac{1}{2}-z_{2}\right) \mathbf{a}_{3}$ & $=$ & $-x_{2} a \hat{\mathbf{x}}+y_{2} b \hat{\mathbf{y}}+\left(\frac{1}{2}-z_{2}\right) c \hat{\mathbf{z}}$ & $(8 d)$ & O II \\
\hline $\mathbf{B}_{12}$ & $=$ & $\left(\frac{1}{2}+x_{2}\right) \mathbf{a}_{1}+\left(\frac{1}{2}-y_{2}\right) \mathbf{a}_{2}-z_{2} \mathbf{a}_{3}$ & $=$ & $\left(\frac{1}{2}+x_{2}\right) a \hat{\mathbf{x}}+\left(\frac{1}{2}-y_{2}\right) b \hat{\mathbf{y}}-z_{2} c \hat{\mathbf{z}}$ & $(8 d)$ & O II \\
\hline $\mathbf{B}_{13}$ & $=$ & $-x_{2} \mathbf{a}_{1}-y_{2} \mathbf{a}_{2}-z_{2} \mathbf{a}_{3}$ & $=$ & $-x_{2} a \hat{\mathbf{x}}-y_{2} b \hat{\mathbf{y}}-z_{2} c \hat{\mathbf{z}}$ & $(8 d)$ & O II \\
\hline $\mathbf{B}_{14}$ & $=$ & $\begin{array}{c}\left(\frac{1}{2}+x_{2}\right) \mathbf{a}_{1}+\left(\frac{1}{2}+y_{2}\right) \mathbf{a}_{2}+ \\
\left(\frac{1}{2}-z_{2}\right) \mathbf{a}_{3}\end{array}$ & $=$ & $\begin{array}{c}\left(\frac{1}{2}+x_{2}\right) a \hat{\mathbf{x}}+\left(\frac{1}{2}+y_{2}\right) b \hat{\mathbf{y}}+ \\
\left(\frac{1}{2}-z_{2}\right) c \hat{\mathbf{z}}\end{array}$ & $(8 d)$ & O II \\
\hline $\mathbf{B}_{15}$ & $=$ & $x_{2} \mathbf{a}_{1}-y_{2} \mathbf{a}_{2}+\left(\frac{1}{2}+z_{2}\right) \mathbf{a}_{3}$ & $=$ & $x_{2} a \hat{\mathbf{x}}-y_{2} b \hat{\mathbf{y}}+\left(\frac{1}{2}+z_{2}\right) c \hat{\mathbf{z}}$ & $(8 d)$ & O II \\
\hline $\mathbf{B}_{16}$ & $=$ & $\left(\frac{1}{2}-x_{2}\right) \mathbf{a}_{1}+\left(\frac{1}{2}+y_{2}\right) \mathbf{a}_{2}+z_{2} \mathbf{a}_{3}$ & $=$ & $\left(\frac{1}{2}-x_{2}\right) a \hat{\mathbf{x}}+\left(\frac{1}{2}+y_{2}\right) b \hat{\mathbf{y}}+z_{2} c \hat{\mathbf{z}}$ & $(8 d)$ & O II \\
\hline $\mathbf{B}_{17}$ & $=$ & $x_{3} \mathbf{a}_{1}+y_{3} \mathbf{a}_{2}+z_{3} \mathbf{a}_{3}$ & $=$ & $x_{3} a \hat{\mathbf{x}}+y_{3} b \hat{\mathbf{y}}+z_{3} c \hat{\mathbf{z}}$ & $(8 d)$ & O III \\
\hline $\mathbf{B}_{18}$ & $=$ & $\begin{array}{c}\left(\frac{1}{2}-x_{3}\right) \mathbf{a}_{1}+\left(\frac{1}{2}-y_{3}\right) \mathbf{a}_{2}+ \\
\left(\frac{1}{2}+z_{3}\right) \mathbf{a}_{3}\end{array}$ & $=$ & $\begin{array}{c}\left(\frac{1}{2}-x_{3}\right) a \hat{\mathbf{x}}+\left(\frac{1}{2}-y_{3}\right) b \hat{\mathbf{y}}+ \\
\left(\frac{1}{2}+z_{3}\right) c \hat{\mathbf{z}}\end{array}$ & $(8 d)$ & O III \\
\hline $\mathbf{B}_{19}$ & $=$ & $-x_{3} \mathbf{a}_{1}+y_{3} \mathbf{a}_{2}+\left(\frac{1}{2}-z_{3}\right) \mathbf{a}_{3}$ & $=$ & $-x_{3} a \hat{\mathbf{x}}+y_{3} b \hat{\mathbf{y}}+\left(\frac{1}{2}-z_{3}\right) c \hat{\mathbf{z}}$ & $(8 d)$ & O III \\
\hline $\mathbf{B}_{20}$ & $=$ & $\left(\frac{1}{2}+x_{3}\right) \mathbf{a}_{1}+\left(\frac{1}{2}-y_{3}\right) \mathbf{a}_{2}-z_{3} \mathbf{a}_{3}$ & $=$ & $\left(\frac{1}{2}+x_{3}\right) a \hat{\mathbf{x}}+\left(\frac{1}{2}-y_{3}\right) b \hat{\mathbf{y}}-z_{3} c \hat{\mathbf{z}}$ & $(8 d)$ & O III \\
\hline $\mathbf{B}_{21}$ & $=$ & $-x_{3} \mathbf{a}_{1}-y_{3} \mathbf{a}_{2}-z_{3} \mathbf{a}_{3}$ & $=$ & $-x_{3} a \hat{\mathbf{x}}-y_{3} b \hat{\mathbf{y}}-z_{3} c \hat{\mathbf{z}}$ & $(8 d)$ & O III \\
\hline $\mathbf{B}_{22}$ & $=$ & $\begin{array}{c}\left(\frac{1}{2}+x_{3}\right) \mathbf{a}_{1}+\left(\frac{1}{2}+y_{3}\right) \mathbf{a}_{2}+ \\
\left(\frac{1}{2}-z_{3}\right) \mathbf{a}_{3}\end{array}$ & $=$ & $\begin{array}{c}\left(\frac{1}{2}+x_{3}\right) a \hat{\mathbf{x}}+\left(\frac{1}{2}+y_{3}\right) b \hat{\mathbf{y}}+ \\
\left(\frac{1}{2}-z_{3}\right) c \hat{\mathbf{z}}\end{array}$ & $(8 d)$ & O III \\
\hline $\mathbf{B}_{23}$ & $=$ & $x_{3} \mathbf{a}_{1}-y_{3} \mathbf{a}_{2}+\left(\frac{1}{2}+z_{3}\right) \mathbf{a}_{3}$ & $=$ & $x_{3} a \hat{\mathbf{x}}-y_{3} b \hat{\mathbf{y}}+\left(\frac{1}{2}+z_{3}\right) c \hat{\mathbf{z}}$ & $(8 d)$ & O III \\
\hline $\mathbf{B}_{24}$ & $=$ & $\left(\frac{1}{2}-x_{3}\right) \mathbf{a}_{1}+\left(\frac{1}{2}+y_{3}\right) \mathbf{a}_{2}+z_{3} \mathbf{a}_{3}$ & $=$ & $\left(\frac{1}{2}-x_{3}\right) a \hat{\mathbf{x}}+\left(\frac{1}{2}+y_{3}\right) b \hat{\mathbf{y}}+z_{3} c \hat{\mathbf{z}}$ & $(8 d)$ & O III \\
\hline $\mathbf{B}_{25}$ & $=$ & $x_{4} \mathbf{a}_{1}+y_{4} \mathbf{a}_{2}+z_{4} \mathbf{a}_{3}$ & $=$ & $x_{4} a \hat{\mathbf{x}}+y_{4} b \hat{\mathbf{y}}+z_{4} c \hat{\mathbf{z}}$ & $(8 d)$ & $\mathrm{W}$ \\
\hline $\mathbf{B}_{26}$ & $=$ & $\begin{array}{c}\left(\frac{1}{2}-x_{4}\right) \mathbf{a}_{1}+\left(\frac{1}{2}-y_{4}\right) \mathbf{a}_{2}+ \\
\left(\frac{1}{2}+z_{4}\right) \mathbf{a}_{3}\end{array}$ & $=$ & $\begin{array}{c}\left(\frac{1}{2}-x_{4}\right) a \hat{\mathbf{x}}+\left(\frac{1}{2}-y_{4}\right) b \hat{\mathbf{y}}+ \\
\left(\frac{1}{2}+z_{4}\right) c \hat{\mathbf{z}}\end{array}$ & $(8 d)$ & $\mathrm{W}$ \\
\hline $\mathbf{B}_{27}$ & $=$ & $-x_{4} \mathbf{a}_{1}+y_{4} \mathbf{a}_{2}+\left(\frac{1}{2}-z_{4}\right) \mathbf{a}_{3}$ & $=$ & $-x_{4} a \hat{\mathbf{x}}+y_{4} b \hat{\mathbf{y}}+\left(\frac{1}{2}-z_{4}\right) c \hat{\mathbf{z}}$ & $(8 d)$ & $\mathrm{W}$ \\
\hline $\mathbf{B}_{28}$ & $=$ & $\left(\frac{1}{2}+x_{4}\right) \mathbf{a}_{1}+\left(\frac{1}{2}-y_{4}\right) \mathbf{a}_{2}-z_{4} \mathbf{a}_{3}$ & $=$ & $\left(\frac{1}{2}+x_{4}\right) a \hat{\mathbf{x}}+\left(\frac{1}{2}-y_{4}\right) b \hat{\mathbf{y}}-z_{4} c \hat{\mathbf{z}}$ & $(8 d)$ & $\mathrm{W}$ \\
\hline $\mathbf{B}_{29}$ & $=$ & $-x_{4} \mathbf{a}_{1}-y_{4} \mathbf{a}_{2}-z_{4} \mathbf{a}_{3}$ & $=$ & $-x_{4} a \hat{\mathbf{x}}-y_{4} b \hat{\mathbf{y}}-z_{4} c \hat{\mathbf{z}}$ & $(8 d)$ & $\mathrm{W}$ \\
\hline $\mathbf{B}_{30}$ & $=$ & $\begin{array}{c}\left(\frac{1}{2}+x_{4}\right) \mathbf{a}_{1}+\left(\frac{1}{2}+y_{4}\right) \mathbf{a}_{2}+ \\
\left(\frac{1}{2}-z_{4}\right) \mathbf{a}_{3}\end{array}$ & $=$ & $\begin{array}{c}\left(\frac{1}{2}+x_{4}\right) a \hat{\mathbf{x}}+\left(\frac{1}{2}+y_{4}\right) b \hat{\mathbf{y}}+ \\
\left(\frac{1}{2}-z_{4}\right) c \hat{\mathbf{z}}\end{array}$ & $(8 d)$ & $\mathrm{W}$ \\
\hline $\mathbf{B}_{31}$ & $=$ & $x_{4} \mathbf{a}_{1}-y_{4} \mathbf{a}_{2}+\left(\frac{1}{2}+z_{4}\right) \mathbf{a}_{3}$ & $=$ & $x_{4} a \hat{\mathbf{x}}-y_{4} b \hat{\mathbf{y}}+\left(\frac{1}{2}+z_{4}\right) c \hat{\mathbf{z}}$ & $(8 d)$ & $\mathrm{W}$ \\
\hline $\mathbf{B}_{32}$ & $=$ & $\left(\frac{1}{2}-x_{4}\right) \mathbf{a}_{1}+\left(\frac{1}{2}+y_{4}\right) \mathbf{a}_{2}+z_{4} \mathbf{a}_{3}$ & $=$ & $\left(\frac{1}{2}-x_{4}\right) a \hat{\mathbf{x}}+\left(\frac{1}{2}+y_{4}\right) b \hat{\mathbf{y}}+z_{4} c \hat{\mathbf{z}}$ & $(8 d)$ & $\mathrm{W}$ \\
\hline
\end{tabular}

\section{References:}

- T. Vogt, P. M. Woodward, and B. A. Hunter, The high-temperature phases of WO3, J. Solid State Chem. 144, 209-215 (1999), doi:10.1006/jssc.1999.8173. 


\section{Found in:}

- P. Villars and K. Cenzual, Pearson's Crystal Data - Crystal Structure Database for Inorganic Compounds, ASM International (2013).

\section{Geometry files:}

- CIF: pp. 839

- POSCAR: pp. 839 


\section{$\beta$-Toluene Structure: A7B8_oP120_60_7d_8d}
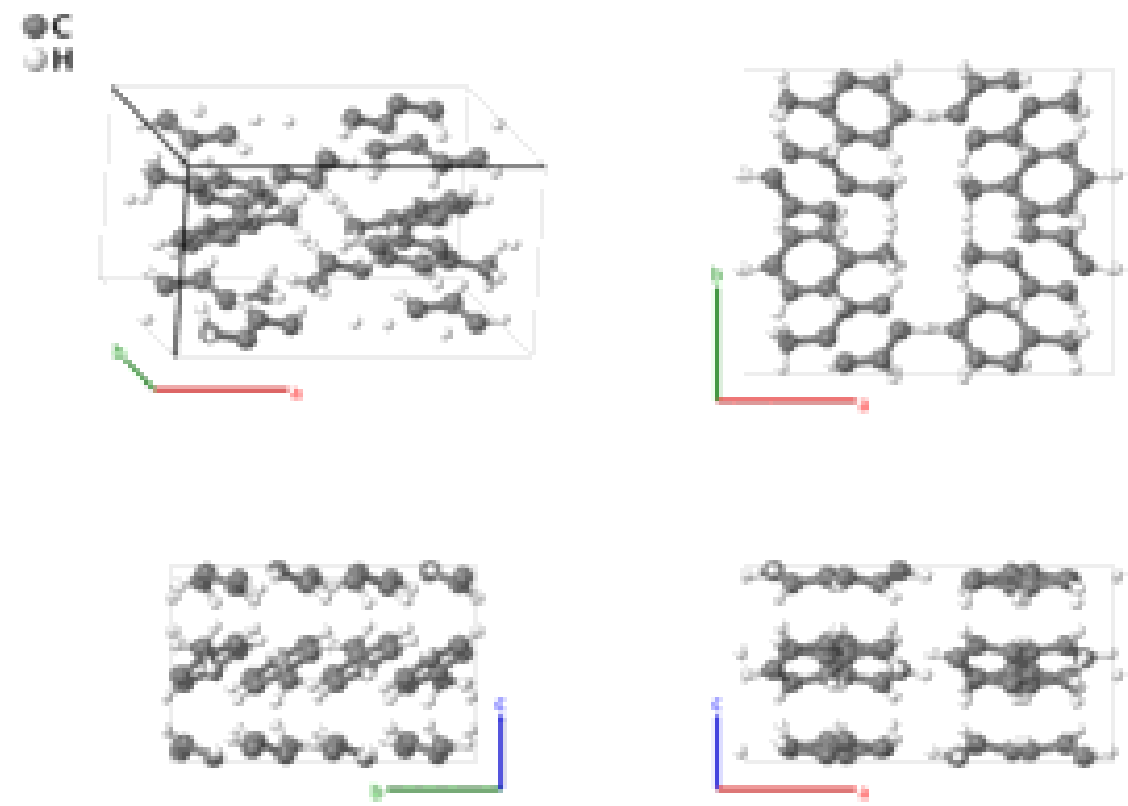

\section{Prototype}

AFLOW prototype label

Strukturbericht designation

Pearson symbol

Space group number

Space group symbol

AFLOW prototype command
: $\quad \beta-\mathrm{C}_{7} \mathrm{H}_{8}$

: A7B8_oP120_60_7d_8d

: None

: $\quad$ oP120

$: 60$

: $P b c n$

- $\beta$-Toluene is a metastable crystalline structure of the toluene molecule, $\mathrm{C}_{7} \mathrm{H}_{8}$, which crystallizes below $178 \mathrm{~K}$. This data was constructed from experiments at $105 \mathrm{~K}$.

- The hydrogen atomic positions were approximated to agree with the chemistry of the toluene molecule.

\section{Simple Orthorhombic primitive vectors:}

$$
\begin{aligned}
& \mathbf{a}_{1}=a \hat{\mathbf{x}} \\
& \mathbf{a}_{2}=b \hat{\mathbf{y}} \\
& \mathbf{a}_{3}=c \hat{\mathbf{z}}
\end{aligned}
$$

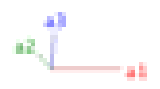


Lattice Coordinates

$\begin{array}{ccc}\mathbf{B}_{1}= & x_{1} \mathbf{a}_{1}+y_{1} \mathbf{a}_{2}+z_{1} \mathbf{a}_{3} \\ \mathbf{B}_{2}= & \left(\frac{1}{2}-x_{1}\right) \mathbf{a}_{1}+\left(\frac{1}{2}-y_{1}\right) \mathbf{a}_{2} \\ \left(\frac{1}{2}+z_{1}\right) \mathbf{a}_{3}\end{array}$

$\mathbf{B}_{3}=-x_{1} \mathbf{a}_{1}+y_{1} \mathbf{a}_{2}+\left(\frac{1}{2}-z_{1}\right) \mathbf{a}_{3}$

$\mathbf{B}_{4}=\left(\frac{1}{2}+x_{1}\right) \mathbf{a}_{1}+\left(\frac{1}{2}-y_{1}\right) \mathbf{a}_{2}-z_{1} \mathbf{a}_{3}$

$\mathbf{B}_{5}=-x_{1} \mathbf{a}_{1}-y_{1} \mathbf{a}_{2}-z_{1} \mathbf{a}_{3}$

$\mathbf{B}_{6}=\left(\frac{1}{2}+x_{1}\right) \mathbf{a}_{1}+\left(\frac{1}{2}+y_{1}\right) \mathbf{a}_{2}+$ $\left(\frac{1}{2}-z_{1}\right) \mathbf{a}_{3}$

$\mathbf{B}_{7}=x_{1} \mathbf{a}_{1}-y_{1} \mathbf{a}_{2}+\left(\frac{1}{2}+z_{1}\right) \mathbf{a}_{3}$

$\mathbf{B}_{8}=\left(\frac{1}{2}-x_{1}\right) \mathbf{a}_{1}+\left(\frac{1}{2}+y_{1}\right) \mathbf{a}_{2}+z_{1} \mathbf{a}_{3}$

$\mathbf{B}_{9}=x_{2} \mathbf{a}_{1}+y_{2} \mathbf{a}_{2}+z_{2} \mathbf{a}_{3}$

$\mathbf{B}_{10}=\left(\frac{1}{2}-x_{2}\right) \mathbf{a}_{1}+\left(\frac{1}{2}-y_{2}\right) \mathbf{a}_{2}+$

$$
\left(\frac{1}{2}+z_{2}\right) \mathbf{a}_{3}
$$

$\mathbf{B}_{11}=-x_{2} \mathbf{a}_{1}+y_{2} \mathbf{a}_{2}+\left(\frac{1}{2}-z_{2}\right) \mathbf{a}_{3}$

$\mathbf{B}_{12}=\left(\frac{1}{2}+x_{2}\right) \mathbf{a}_{1}+\left(\frac{1}{2}-y_{2}\right) \mathbf{a}_{2}-z_{2} \mathbf{a}_{3}$

$\mathbf{B}_{13}=-x_{2} \mathbf{a}_{1}-y_{2} \mathbf{a}_{2}-z_{2} \mathbf{a}_{3}$

$\mathbf{B}_{14}=\left(\frac{1}{2}+x_{2}\right) \mathbf{a}_{1}+\left(\frac{1}{2}+y_{2}\right) \mathbf{a}_{2}+$ $\left(\frac{1}{2}-z_{2}\right) \mathbf{a}_{3}$

$\mathbf{B}_{15}=x_{2} \mathbf{a}_{1}-y_{2} \mathbf{a}_{2}+\left(\frac{1}{2}+z_{2}\right) \mathbf{a}_{3}$

$\mathbf{B}_{16}=\left(\frac{1}{2}-x_{2}\right) \mathbf{a}_{1}+\left(\frac{1}{2}+y_{2}\right) \mathbf{a}_{2}+z_{2} \mathbf{a}_{3}$

$\mathbf{B}_{17}=x_{3} \mathbf{a}_{1}+y_{3} \mathbf{a}_{2}+z_{3} \mathbf{a}_{3}$

$\mathbf{B}_{18}=\left(\frac{1}{2}-x_{3}\right) \mathbf{a}_{1}+\left(\frac{1}{2}-y_{3}\right) \mathbf{a}_{2}+$ $\left(\frac{1}{2}+z_{3}\right) \mathbf{a}_{3}$

$\mathbf{B}_{19}=-x_{3} \mathbf{a}_{1}+y_{3} \mathbf{a}_{2}+\left(\frac{1}{2}-z_{3}\right) \mathbf{a}_{3}$

$\mathbf{B}_{20}=\left(\frac{1}{2}+x_{3}\right) \mathbf{a}_{1}+\left(\frac{1}{2}-y_{3}\right) \mathbf{a}_{2}-z_{3} \mathbf{a}_{3}$

$\mathbf{B}_{21}=-x_{3} \mathbf{a}_{1}-y_{3} \mathbf{a}_{2}-z_{3} \mathbf{a}_{3}$

$\mathbf{B}_{22}=\left(\frac{1}{2}+x_{3}\right) \mathbf{a}_{1}+\left(\frac{1}{2}+y_{3}\right) \mathbf{a}_{2}+$ $\left(\frac{1}{2}-z_{3}\right) \mathbf{a}_{3}$

$\mathbf{B}_{23}=x_{3} \mathbf{a}_{1}-y_{3} \mathbf{a}_{2}+\left(\frac{1}{2}+z_{3}\right) \mathbf{a}_{3}$

$\mathbf{B}_{24}=\left(\frac{1}{2}-x_{3}\right) \mathbf{a}_{1}+\left(\frac{1}{2}+y_{3}\right) \mathbf{a}_{2}+z_{3} \mathbf{a}_{3}$

$\mathbf{B}_{25}=x_{4} \mathbf{a}_{1}+y_{4} \mathbf{a}_{2}+z_{4} \mathbf{a}_{3}$

$\mathbf{B}_{26}=\left(\frac{1}{2}-x_{4}\right) \mathbf{a}_{1}+\left(\frac{1}{2}-y_{4}\right) \mathbf{a}_{2}+$ $\left(\frac{1}{2}+z_{4}\right) \mathbf{a}_{3}$

$\mathbf{B}_{27}=-x_{4} \mathbf{a}_{1}+y_{4} \mathbf{a}_{2}+\left(\frac{1}{2}-z_{4}\right) \mathbf{a}_{3}$

$\mathbf{B}_{28}=\left(\frac{1}{2}+x_{4}\right) \mathbf{a}_{1}+\left(\frac{1}{2}-y_{4}\right) \mathbf{a}_{2}-z_{4} \mathbf{a}_{3}$

$\mathbf{B}_{29}=\quad-x_{4} \mathbf{a}_{1}-y_{4} \mathbf{a}_{2}-z_{4} \mathbf{a}_{3}$
Cartesian Coordinates

$$
=
$$$$
x_{1} a \hat{\mathbf{x}}+y_{1} b \hat{\mathbf{y}}+z_{1} c \hat{\mathbf{z}}
$$$$
\left(\frac{1}{2}-x_{1}\right) a \hat{\mathbf{x}}+\left(\frac{1}{2}-y_{1}\right) b \hat{\mathbf{y}}+
$$$$
\left(\frac{1}{2}+z_{1}\right) c \hat{\mathbf{z}}
$$$$
=\quad-x_{1} a \hat{\mathbf{x}}+y_{1} b \hat{\mathbf{y}}+\left(\frac{1}{2}-z_{1}\right) c \hat{\mathbf{z}}
$$$$
=\left(\frac{1}{2}+x_{1}\right) a \hat{\mathbf{x}}+\left(\frac{1}{2}-y_{1}\right) b \hat{\mathbf{y}}-z_{1} c \hat{\mathbf{z}}
$$$$
=\quad-x_{1} a \hat{\mathbf{x}}-y_{1} b \hat{\mathbf{y}}-z_{1} c \hat{\mathbf{z}}
$$$$
=\left(\frac{1}{2}+x_{1}\right) a \hat{\mathbf{x}}+\left(\frac{1}{2}+y_{1}\right) b \hat{\mathbf{y}}+
$$$$
\left(\frac{1}{2}-z_{1}\right) c \hat{\mathbf{z}}
$$$$
=\quad x_{1} a \hat{\mathbf{x}}-y_{1} b \hat{\mathbf{y}}+\left(\frac{1}{2}+z_{1}\right) c \hat{\mathbf{z}}
$$$$
=\left(\frac{1}{2}-x_{1}\right) a \hat{\mathbf{x}}+\left(\frac{1}{2}+y_{1}\right) b \hat{\mathbf{y}}+z_{1} c \hat{\mathbf{z}}
$$$$
=\quad x_{2} a \hat{\mathbf{x}}+y_{2} b \hat{\mathbf{y}}+z_{2} c \hat{\mathbf{z}}
$$$$
=\left(\frac{1}{2}-x_{2}\right) a \hat{\mathbf{x}}+\left(\frac{1}{2}-y_{2}\right) b \hat{\mathbf{y}}+
$$$$
\left(\frac{1}{2}+z_{2}\right) c \hat{\mathbf{z}}
$$$$
=\quad-x_{2} a \hat{\mathbf{x}}+y_{2} b \hat{\mathbf{y}}+\left(\frac{1}{2}-z_{2}\right) c \hat{\mathbf{z}}
$$$$
=\left(\frac{1}{2}+x_{2}\right) a \hat{\mathbf{x}}+\left(\frac{1}{2}-y_{2}\right) b \hat{\mathbf{y}}-z_{2} c \hat{\mathbf{z}}
$$$$
=\quad-x_{2} a \hat{\mathbf{x}}-y_{2} b \hat{\mathbf{y}}-z_{2} c \hat{\mathbf{z}}
$$$$
=\left(\frac{1}{2}+x_{2}\right) a \hat{\mathbf{x}}+\left(\frac{1}{2}+y_{2}\right) b \hat{\mathbf{y}}+
$$$$
\left(\frac{1}{2}-z_{2}\right) c \hat{\mathbf{z}}
$$$$
=\quad x_{2} a \hat{\mathbf{x}}-y_{2} b \hat{\mathbf{y}}+\left(\frac{1}{2}+z_{2}\right) c \hat{\mathbf{z}}
$$$$
=\left(\frac{1}{2}-x_{2}\right) a \hat{\mathbf{x}}+\left(\frac{1}{2}+y_{2}\right) b \hat{\mathbf{y}}+z_{2} c \hat{\mathbf{z}}
$$$$
=\quad x_{3} a \hat{\mathbf{x}}+y_{3} b \hat{\mathbf{y}}+z_{3} c \hat{\mathbf{z}}
$$$$
=\left(\frac{1}{2}-x_{3}\right) a \hat{\mathbf{x}}+\left(\frac{1}{2}-y_{3}\right) b \hat{\mathbf{y}}+
$$$$
\left(\frac{1}{2}+z_{3}\right) c \hat{\mathbf{z}}
$$$$
=\quad-x_{3} a \hat{\mathbf{x}}+y_{3} b \hat{\mathbf{y}}+\left(\frac{1}{2}-z_{3}\right) c \hat{\mathbf{z}}
$$$$
=\left(\frac{1}{2}+x_{3}\right) a \hat{\mathbf{x}}+\left(\frac{1}{2}-y_{3}\right) b \hat{\mathbf{y}}-z_{3} c \hat{\mathbf{z}}
$$$$
=\quad-x_{3} a \hat{\mathbf{x}}-y_{3} b \hat{\mathbf{y}}-z_{3} c \hat{\mathbf{z}}
$$$$
=\left(\frac{1}{2}+x_{3}\right) a \hat{\mathbf{x}}+\left(\frac{1}{2}+y_{3}\right) b \hat{\mathbf{y}}+
$$$$
\left(\frac{1}{2}-z_{3}\right) c \hat{\mathbf{z}}
$$$$
=\quad x_{3} a \hat{\mathbf{x}}-y_{3} b \hat{\mathbf{y}}+\left(\frac{1}{2}+z_{3}\right) c \hat{\mathbf{z}}
$$$$
=\left(\frac{1}{2}-x_{3}\right) a \hat{\mathbf{x}}+\left(\frac{1}{2}+y_{3}\right) b \hat{\mathbf{y}}+z_{3} c \hat{\mathbf{z}}
$$$$
=\quad x_{4} a \hat{\mathbf{x}}+y_{4} b \hat{\mathbf{y}}+z_{4} c \hat{\mathbf{z}}
$$$$
=\left(\frac{1}{2}-x_{4}\right) a \hat{\mathbf{x}}+\left(\frac{1}{2}-y_{4}\right) b \hat{\mathbf{y}}+
$$$$
\left(\frac{1}{2}+z_{4}\right) c \hat{\mathbf{z}}
$$

C I

C I

C I

C I

C I

C I

C I

C I

C II

C II

C II

C II

C II

C II

C II

C II

C III

C III

C III

C III

C III

C III

C III

C III

C IV

C IV

C IV

C IV

C IV 


\begin{tabular}{|c|c|c|c|c|c|c|}
\hline $\mathbf{B}_{30}$ & $=$ & $\begin{array}{c}\left(\frac{1}{2}+x_{4}\right) \mathbf{a}_{1}+\left(\frac{1}{2}+y_{4}\right) \mathbf{a}_{2}+ \\
\left(\frac{1}{2}-z_{4}\right) \mathbf{a}_{3}\end{array}$ & $=$ & $\begin{array}{c}\left(\frac{1}{2}+x_{4}\right) a \hat{\mathbf{x}}+\left(\frac{1}{2}+y_{4}\right) b \hat{\mathbf{y}}+ \\
\left(\frac{1}{2}-z_{4}\right) c \hat{\mathbf{z}}\end{array}$ & $(8 d)$ & C IV \\
\hline $\mathbf{B}_{31}$ & $=$ & $x_{4} \mathbf{a}_{1}-y_{4} \mathbf{a}_{2}+\left(\frac{1}{2}+z_{4}\right) \mathbf{a}_{3}$ & $=$ & $x_{4} a \hat{\mathbf{x}}-y_{4} b \hat{\mathbf{y}}+\left(\frac{1}{2}+z_{4}\right) c \hat{\mathbf{z}}$ & $(8 d)$ & C IV \\
\hline $\mathbf{B}_{32}$ & $=$ & $\left(\frac{1}{2}-x_{4}\right) \mathbf{a}_{1}+\left(\frac{1}{2}+y_{4}\right) \mathbf{a}_{2}+z_{4} \mathbf{a}_{3}$ & $=$ & $\left(\frac{1}{2}-x_{4}\right) a \hat{\mathbf{x}}+\left(\frac{1}{2}+y_{4}\right) b \hat{\mathbf{y}}+z_{4} c \hat{\mathbf{z}}$ & $(8 d)$ & C IV \\
\hline $\mathbf{B}_{33}$ & $=$ & $x_{5} \mathbf{a}_{1}+y_{5} \mathbf{a}_{2}+z_{5} \mathbf{a}_{3}$ & $=$ & $x_{5} a \hat{\mathbf{x}}+y_{5} b \hat{\mathbf{y}}+z_{5} c \hat{\mathbf{z}}$ & $(8 d)$ & $\mathrm{CV}$ \\
\hline $\mathbf{B}_{34}$ & $=$ & $\begin{array}{c}\left(\frac{1}{2}-x_{5}\right) \mathbf{a}_{1}+\left(\frac{1}{2}-y_{5}\right) \mathbf{a}_{2}+ \\
\left(\frac{1}{2}+z_{5}\right) \mathbf{a}_{3}\end{array}$ & $=$ & $\begin{array}{c}\left(\frac{1}{2}-x_{5}\right) a \hat{\mathbf{x}}+\left(\frac{1}{2}-y_{5}\right) b \hat{\mathbf{y}}+ \\
\left(\frac{1}{2}+z_{5}\right) c \hat{\mathbf{z}}\end{array}$ & $(8 d)$ & $\mathrm{C} \mathrm{V}$ \\
\hline $\mathbf{B}_{35}$ & $=$ & $-x_{5} \mathbf{a}_{1}+y_{5} \mathbf{a}_{2}+\left(\frac{1}{2}-z_{5}\right) \mathbf{a}_{3}$ & $=$ & $-x_{5} a \hat{\mathbf{x}}+y_{5} b \hat{\mathbf{y}}+\left(\frac{1}{2}-z_{5}\right) c \hat{\mathbf{z}}$ & $(8 d)$ & $\mathrm{C} \mathrm{V}$ \\
\hline $\mathbf{B}_{36}$ & $=$ & $\left(\frac{1}{2}+x_{5}\right) \mathbf{a}_{1}+\left(\frac{1}{2}-y_{5}\right) \mathbf{a}_{2}-z_{5} \mathbf{a}_{3}$ & $=$ & $\left(\frac{1}{2}+x_{5}\right) a \hat{\mathbf{x}}+\left(\frac{1}{2}-y_{5}\right) b \hat{\mathbf{y}}-z_{5} c \hat{\mathbf{z}}$ & $(8 d)$ & $\mathrm{CV}$ \\
\hline $\mathbf{B}_{37}$ & $=$ & $-x_{5} \mathbf{a}_{1}-y_{5} \mathbf{a}_{2}-z_{5} \mathbf{a}_{3}$ & $=$ & $-x_{5} a \hat{\mathbf{x}}-y_{5} b \hat{\mathbf{y}}-z_{5} c \hat{\mathbf{z}}$ & $(8 d)$ & $\mathrm{C} \mathrm{V}$ \\
\hline $\mathbf{B}_{38}$ & $=$ & $\begin{array}{c}\left(\frac{1}{2}+x_{5}\right) \mathbf{a}_{1}+\left(\frac{1}{2}+y_{5}\right) \mathbf{a}_{2}+ \\
\left(\frac{1}{2}-z_{5}\right) \mathbf{a}_{3}\end{array}$ & $=$ & $\begin{array}{c}\left(\frac{1}{2}+x_{5}\right) a \hat{\mathbf{x}}+\left(\frac{1}{2}+y_{5}\right) b \hat{\mathbf{y}}+ \\
\left(\frac{1}{2}-z_{5}\right) c \hat{\mathbf{z}}\end{array}$ & $(8 d)$ & $\mathrm{CV}$ \\
\hline $\mathbf{B}_{39}$ & $=$ & $x_{5} \mathbf{a}_{1}-y_{5} \mathbf{a}_{2}+\left(\frac{1}{2}+z_{5}\right) \mathbf{a}_{3}$ & $=$ & $x_{5} a \hat{\mathbf{x}}-y_{5} b \hat{\mathbf{y}}+\left(\frac{1}{2}+z_{5}\right) c \hat{\mathbf{z}}$ & $(8 d)$ & $\mathrm{C} \mathrm{V}$ \\
\hline $\mathbf{B}_{40}$ & $=$ & $\left(\frac{1}{2}-x_{5}\right) \mathbf{a}_{1}+\left(\frac{1}{2}+y_{5}\right) \mathbf{a}_{2}+z_{5} \mathbf{a}_{3}$ & $=$ & $\left(\frac{1}{2}-x_{5}\right) a \hat{\mathbf{x}}+\left(\frac{1}{2}+y_{5}\right) b \hat{\mathbf{y}}+z_{5} c \hat{\mathbf{z}}$ & $(8 d)$ & $\mathrm{C} \mathrm{V}$ \\
\hline $\mathbf{B}_{41}$ & $=$ & $x_{6} \mathbf{a}_{1}+y_{6} \mathbf{a}_{2}+z_{6} \mathbf{a}_{3}$ & $=$ & $x_{6} a \hat{\mathbf{x}}+y_{6} b \hat{\mathbf{y}}+z_{6} c \hat{\mathbf{z}}$ & $(8 d)$ & $\mathrm{C} \mathrm{VI}$ \\
\hline $\mathbf{B}_{42}$ & $=$ & $\begin{array}{c}\left(\frac{1}{2}-x_{6}\right) \mathbf{a}_{1}+\left(\frac{1}{2}-y_{6}\right) \mathbf{a}_{2}+ \\
\left(\frac{1}{2}+z_{6}\right) \mathbf{a}_{3}\end{array}$ & $=$ & $\begin{array}{c}\left(\frac{1}{2}-x_{6}\right) a \hat{\mathbf{x}}+\left(\frac{1}{2}-y_{6}\right) b \hat{\mathbf{y}}+ \\
\left(\frac{1}{2}+z_{6}\right) c \hat{\mathbf{z}}\end{array}$ & $(8 d)$ & C VI \\
\hline $\mathbf{B}_{43}$ & $=$ & $-x_{6} \mathbf{a}_{1}+y_{6} \mathbf{a}_{2}+\left(\frac{1}{2}-z_{6}\right) \mathbf{a}_{3}$ & $=$ & $-x_{6} a \hat{\mathbf{x}}+y_{6} b \hat{\mathbf{y}}+\left(\frac{1}{2}-z_{6}\right) c \hat{\mathbf{z}}$ & $(8 d)$ & C VI \\
\hline $\mathbf{B}_{44}$ & $=$ & $\left(\frac{1}{2}+x_{6}\right) \mathbf{a}_{1}+\left(\frac{1}{2}-y_{6}\right) \mathbf{a}_{2}-z_{6} \mathbf{a}_{3}$ & $=$ & $\left(\frac{1}{2}+x_{6}\right) a \hat{\mathbf{x}}+\left(\frac{1}{2}-y_{6}\right) b \hat{\mathbf{y}}-z_{6} c \hat{\mathbf{z}}$ & $(8 d)$ & $\mathrm{C} \mathrm{VI}$ \\
\hline $\mathbf{B}_{45}$ & $=$ & $-x_{6} \mathbf{a}_{1}-y_{6} \mathbf{a}_{2}-z_{6} \mathbf{a}_{3}$ & $=$ & $-x_{6} a \hat{\mathbf{x}}-y_{6} b \hat{\mathbf{y}}-z_{6} c \hat{\mathbf{z}}$ & $(8 d)$ & $\mathrm{C} \mathrm{VI}$ \\
\hline $\mathbf{B}_{46}$ & $=$ & $\begin{array}{c}\left(\frac{1}{2}+x_{6}\right) \mathbf{a}_{1}+\left(\frac{1}{2}+y_{6}\right) \mathbf{a}_{2}+ \\
\left(\frac{1}{2}-z_{6}\right) \mathbf{a}_{3}\end{array}$ & $=$ & $\begin{array}{c}\left(\frac{1}{2}+x_{6}\right) a \hat{\mathbf{x}}+\left(\frac{1}{2}+y_{6}\right) b \hat{\mathbf{y}}+ \\
\left(\frac{1}{2}-z_{6}\right) c \hat{\mathbf{z}}\end{array}$ & $(8 d)$ & C VI \\
\hline $\mathbf{B}_{47}$ & $=$ & $x_{6} \mathbf{a}_{1}-y_{6} \mathbf{a}_{2}+\left(\frac{1}{2}+z_{6}\right) \mathbf{a}_{3}$ & $=$ & $x_{6} a \hat{\mathbf{x}}-y_{6} b \hat{\mathbf{y}}+\left(\frac{1}{2}+z_{6}\right) c \hat{\mathbf{z}}$ & $(8 d)$ & C VI \\
\hline $\mathbf{B}_{48}$ & $=$ & $\left(\frac{1}{2}-x_{6}\right) \mathbf{a}_{1}+\left(\frac{1}{2}+y_{6}\right) \mathbf{a}_{2}+z_{6} \mathbf{a}_{3}$ & $=$ & $\left(\frac{1}{2}-x_{6}\right) a \hat{\mathbf{x}}+\left(\frac{1}{2}+y_{6}\right) b \hat{\mathbf{y}}+z_{6} c \hat{\mathbf{z}}$ & $(8 d)$ & $\mathrm{C}$ VI \\
\hline $\mathbf{B}_{49}$ & $=$ & $x_{7} \mathbf{a}_{1}+y_{7} \mathbf{a}_{2}+z_{7} \mathbf{a}_{3}$ & $=$ & $x_{7} a \hat{\mathbf{x}}+y_{7} b \hat{\mathbf{y}}+z_{7} c \hat{\mathbf{z}}$ & $(8 d)$ & C VII \\
\hline $\mathbf{B}_{50}$ & $=$ & $\begin{array}{c}\left(\frac{1}{2}-x_{7}\right) \mathbf{a}_{1}+\left(\frac{1}{2}-y_{7}\right) \mathbf{a}_{2}+ \\
\left(\frac{1}{2}+z_{7}\right) \mathbf{a}_{3}\end{array}$ & $=$ & $\begin{array}{c}\left(\frac{1}{2}-x_{7}\right) a \hat{\mathbf{x}}+\left(\frac{1}{2}-y_{7}\right) b \hat{\mathbf{y}}+ \\
\left(\frac{1}{2}+z_{7}\right) c \hat{\mathbf{z}}\end{array}$ & $(8 d)$ & C VII \\
\hline $\mathbf{B}_{51}$ & $=$ & $-x_{7} \mathbf{a}_{1}+y_{7} \mathbf{a}_{2}+\left(\frac{1}{2}-z_{7}\right) \mathbf{a}_{3}$ & $=$ & $-x_{7} a \hat{\mathbf{x}}+y_{7} b \hat{\mathbf{y}}+\left(\frac{1}{2}-z_{7}\right) c \hat{\mathbf{z}}$ & $(8 d)$ & C VII \\
\hline $\mathbf{B}_{52}$ & $=$ & $\left(\frac{1}{2}+x_{7}\right) \mathbf{a}_{1}+\left(\frac{1}{2}-y_{7}\right) \mathbf{a}_{2}-z_{7} \mathbf{a}_{3}$ & $=$ & $\left(\frac{1}{2}+x_{7}\right) a \hat{\mathbf{x}}+\left(\frac{1}{2}-y_{7}\right) b \hat{\mathbf{y}}-z_{7} c \hat{\mathbf{z}}$ & $(8 d)$ & C VII \\
\hline $\mathbf{B}_{53}$ & $=$ & $-x_{7} \mathbf{a}_{1}-y_{7} \mathbf{a}_{2}-z_{7} \mathbf{a}_{3}$ & $=$ & $-x_{7} a \hat{\mathbf{x}}-y_{7} b \hat{\mathbf{y}}-z_{7} c \hat{\mathbf{z}}$ & $(8 d)$ & C VII \\
\hline $\mathbf{B}_{54}$ & $=$ & $\begin{array}{c}\left(\frac{1}{2}+x_{7}\right) \mathbf{a}_{1}+\left(\frac{1}{2}+y_{7}\right) \mathbf{a}_{2}+ \\
\left(\frac{1}{2}-z_{7}\right) \mathbf{a}_{3}\end{array}$ & $=$ & $\begin{array}{c}\left(\frac{1}{2}+x_{7}\right) a \hat{\mathbf{x}}+\left(\frac{1}{2}+y_{7}\right) b \hat{\mathbf{y}}+ \\
\left(\frac{1}{2}-z_{7}\right) c \hat{\mathbf{z}}\end{array}$ & $(8 d)$ & C VII \\
\hline $\mathbf{B}_{55}$ & $=$ & $x_{7} \mathbf{a}_{1}-y_{7} \mathbf{a}_{2}+\left(\frac{1}{2}+z_{7}\right) \mathbf{a}_{3}$ & $=$ & $x_{7} a \hat{\mathbf{x}}-y_{7} b \hat{\mathbf{y}}+\left(\frac{1}{2}+z_{7}\right) c \hat{\mathbf{z}}$ & $(8 d)$ & C VII \\
\hline $\mathbf{B}_{56}$ & $=$ & $\left(\frac{1}{2}-x_{7}\right) \mathbf{a}_{1}+\left(\frac{1}{2}+y_{7}\right) \mathbf{a}_{2}+z_{7} \mathbf{a}_{3}$ & $=$ & $\left(\frac{1}{2}-x_{7}\right) a \hat{\mathbf{x}}+\left(\frac{1}{2}+y_{7}\right) b \hat{\mathbf{y}}+z_{7} c \hat{\mathbf{z}}$ & $(8 d)$ & C VII \\
\hline $\mathbf{B}_{57}$ & $=$ & $x_{8} \mathbf{a}_{1}+y_{8} \mathbf{a}_{2}+z_{8} \mathbf{a}_{3}$ & $=$ & $x_{8} a \hat{\mathbf{x}}+y_{8} b \hat{\mathbf{y}}+z_{8} c \hat{\mathbf{z}}$ & $(8 d)$ & $\mathrm{H} \mathrm{I}$ \\
\hline $\mathbf{B}_{58}$ & $=$ & $\begin{array}{c}\left(\frac{1}{2}-x_{8}\right) \mathbf{a}_{1}+\left(\frac{1}{2}-y_{8}\right) \mathbf{a}_{2}+ \\
\left(\frac{1}{2}+z_{8}\right) \mathbf{a}_{3}\end{array}$ & $=$ & $\begin{array}{c}\left(\frac{1}{2}-x_{8}\right) a \hat{\mathbf{x}}+\left(\frac{1}{2}-y_{8}\right) b \hat{\mathbf{y}}+ \\
\left(\frac{1}{2}+z_{8}\right) c \hat{\mathbf{z}}\end{array}$ & $(8 d)$ & $\mathrm{H} \mathrm{I}$ \\
\hline $\mathbf{B}_{59}$ & $=$ & $-x_{8} \mathbf{a}_{1}+y_{8} \mathbf{a}_{2}+\left(\frac{1}{2}-z_{8}\right) \mathbf{a}_{3}$ & $=$ & $-x_{8} a \hat{\mathbf{x}}+y_{8} b \hat{\mathbf{y}}+\left(\frac{1}{2}-z_{8}\right) c \hat{\mathbf{z}}$ & $(8 d)$ & $\mathrm{H} \mathrm{I}$ \\
\hline
\end{tabular}




\begin{tabular}{|c|c|c|c|c|c|c|}
\hline $\mathbf{B}_{60}$ & $=$ & $\left(\frac{1}{2}+x_{8}\right) \mathbf{a}_{1}+\left(\frac{1}{2}-y_{8}\right) \mathbf{a}_{2}-z_{8} \mathbf{a}_{3}$ & $=$ & $\left(\frac{1}{2}+x_{8}\right) a \hat{\mathbf{x}}+\left(\frac{1}{2}-y_{8}\right) b \hat{\mathbf{y}}-z_{8} c \hat{\mathbf{z}}$ & $(8 d)$ & $\mathrm{H} \mathrm{I}$ \\
\hline $\mathbf{B}_{61}$ & $=$ & $-x_{8} \mathbf{a}_{1}-y_{8} \mathbf{a}_{2}-z_{8} \mathbf{a}_{3}$ & $=$ & $-x_{8} a \hat{\mathbf{x}}-y_{8} b \hat{\mathbf{y}}-z_{8} c \hat{\mathbf{z}}$ & $(8 d)$ & H I \\
\hline $\mathbf{B}_{62}$ & $=$ & $\begin{array}{c}\left(\frac{1}{2}+x_{8}\right) \mathbf{a}_{1}+\left(\frac{1}{2}+y_{8}\right) \mathbf{a}_{2}+ \\
\left(\frac{1}{2}-z_{8}\right) \mathbf{a}_{3}\end{array}$ & $=$ & $\begin{array}{c}\left(\frac{1}{2}+x_{8}\right) a \hat{\mathbf{x}}+\left(\frac{1}{2}+y_{8}\right) b \hat{\mathbf{y}}+ \\
\left(\frac{1}{2}-z_{8}\right) c \hat{\mathbf{z}}\end{array}$ & $(8 d)$ & H I \\
\hline $\mathbf{B}_{63}$ & $=$ & $x_{8} \mathbf{a}_{1}-y_{8} \mathbf{a}_{2}+\left(\frac{1}{2}+z_{8}\right) \mathbf{a}_{3}$ & $=$ & $x_{8} a \hat{\mathbf{x}}-y_{8} b \hat{\mathbf{y}}+\left(\frac{1}{2}+z_{8}\right) c \hat{\mathbf{z}}$ & $(8 d)$ & $\mathrm{H} \mathrm{I}$ \\
\hline $\mathbf{B}_{64}$ & $=$ & $\left(\frac{1}{2}-x_{8}\right) \mathbf{a}_{1}+\left(\frac{1}{2}+y_{8}\right) \mathbf{a}_{2}+z_{8} \mathbf{a}_{3}$ & $=$ & $\left(\frac{1}{2}-x_{8}\right) a \hat{\mathbf{x}}+\left(\frac{1}{2}+y_{8}\right) b \hat{\mathbf{y}}+z_{8} c \hat{\mathbf{z}}$ & $(8 d)$ & H I \\
\hline $\mathbf{B}_{65}$ & $=$ & $x_{9} \mathbf{a}_{1}+y_{9} \mathbf{a}_{2}+z_{9} \mathbf{a}_{3}$ & $=$ & $x_{9} a \hat{\mathbf{x}}+y_{9} b \hat{\mathbf{y}}+z_{9} c \hat{\mathbf{z}}$ & $(8 d)$ & H II \\
\hline $\mathbf{B}_{66}$ & $=$ & $\begin{array}{c}\left(\frac{1}{2}-x_{9}\right) \mathbf{a}_{1}+\left(\frac{1}{2}-y_{9}\right) \mathbf{a}_{2}+ \\
\left(\frac{1}{2}+z_{9}\right) \mathbf{a}_{3}\end{array}$ & $=$ & $\begin{array}{c}\left(\frac{1}{2}-x_{9}\right) a \hat{\mathbf{x}}+\left(\frac{1}{2}-y_{9}\right) b \hat{\mathbf{y}}+ \\
\left(\frac{1}{2}+z_{9}\right) c \hat{\mathbf{z}}\end{array}$ & $(8 d)$ & H II \\
\hline $\mathbf{B}_{67}$ & $=$ & $-x_{9} \mathbf{a}_{1}+y_{9} \mathbf{a}_{2}+\left(\frac{1}{2}-z_{9}\right) \mathbf{a}_{3}$ & $=$ & $-x_{9} a \hat{\mathbf{x}}+y_{9} b \hat{\mathbf{y}}+\left(\frac{1}{2}-z_{9}\right) c \hat{\mathbf{z}}$ & $(8 d)$ & H II \\
\hline $\mathbf{B}_{68}$ & $=$ & $\left(\frac{1}{2}+x_{9}\right) \mathbf{a}_{1}+\left(\frac{1}{2}-y_{9}\right) \mathbf{a}_{2}-z_{9} \mathbf{a}_{3}$ & $=$ & $\left(\frac{1}{2}+x_{9}\right) a \hat{\mathbf{x}}+\left(\frac{1}{2}-y_{9}\right) b \hat{\mathbf{y}}-z_{9} c \hat{\mathbf{z}}$ & $(8 d)$ & H II \\
\hline $\mathbf{B}_{69}$ & $=$ & $-x_{9} \mathbf{a}_{1}-y_{9} \mathbf{a}_{2}-z_{9} \mathbf{a}_{3}$ & $=$ & $-x_{9} a \hat{\mathbf{x}}-y_{9} b \hat{\mathbf{y}}-z_{9} c \hat{\mathbf{z}}$ & $(8 d)$ & H II \\
\hline $\mathbf{B}_{70}$ & $=$ & $\begin{array}{c}\left(\frac{1}{2}+x_{9}\right) \mathbf{a}_{1}+\left(\frac{1}{2}+y_{9}\right) \mathbf{a}_{2}+ \\
\left(\frac{1}{2}-z_{9}\right) \mathbf{a}_{3}\end{array}$ & $=$ & $\begin{array}{c}\left(\frac{1}{2}+x_{9}\right) a \hat{\mathbf{x}}+\left(\frac{1}{2}+y_{9}\right) b \hat{\mathbf{y}}+ \\
\left(\frac{1}{2}-z_{9}\right) c \hat{\mathbf{z}}\end{array}$ & $(8 d)$ & H II \\
\hline $\mathbf{B}_{71}$ & $=$ & $x_{9} \mathbf{a}_{1}-y_{9} \mathbf{a}_{2}+\left(\frac{1}{2}+z_{9}\right) \mathbf{a}_{3}$ & $=$ & $x_{9} a \hat{\mathbf{x}}-y_{9} b \hat{\mathbf{y}}+\left(\frac{1}{2}+z_{9}\right) c \hat{\mathbf{z}}$ & $(8 d)$ & $\mathrm{H}$ II \\
\hline $\mathbf{B}_{72}$ & $=$ & $\left(\frac{1}{2}-x_{9}\right) \mathbf{a}_{1}+\left(\frac{1}{2}+y_{9}\right) \mathbf{a}_{2}+z_{9} \mathbf{a}_{3}$ & $=$ & $\left(\frac{1}{2}-x_{9}\right) a \hat{\mathbf{x}}+\left(\frac{1}{2}+y_{9}\right) b \hat{\mathbf{y}}+z_{9} c \hat{\mathbf{z}}$ & $(8 d)$ & H II \\
\hline $\mathbf{B}_{73}$ & $=$ & $x_{10} \mathbf{a}_{1}+y_{10} \mathbf{a}_{2}+z_{10} \mathbf{a}_{3}$ & $=$ & $x_{10} a \hat{\mathbf{x}}+y_{10} b \hat{\mathbf{y}}+z_{10} c \hat{\mathbf{z}}$ & $(8 d)$ & H III \\
\hline $\mathbf{B}_{74}$ & $=$ & $\begin{array}{c}\left(\frac{1}{2}-x_{10}\right) \mathbf{a}_{1}+\left(\frac{1}{2}-y_{10}\right) \mathbf{a}_{2}+ \\
\left(\frac{1}{2}+z_{10}\right) \mathbf{a}_{3}\end{array}$ & $=$ & $\begin{array}{c}\left(\frac{1}{2}-x_{10}\right) a \hat{\mathbf{x}}+\left(\frac{1}{2}-y_{10}\right) b \hat{\mathbf{y}}+ \\
\left(\frac{1}{2}+z_{10}\right) c \hat{\mathbf{z}}\end{array}$ & $(8 d)$ & H III \\
\hline $\mathbf{B}_{75}$ & $=$ & $-x_{10} \mathbf{a}_{1}+y_{10} \mathbf{a}_{2}+\left(\frac{1}{2}-z_{10}\right) \mathbf{a}_{3}$ & $=$ & $-x_{10} a \hat{\mathbf{x}}+y_{10} b \hat{\mathbf{y}}+\left(\frac{1}{2}-z_{10}\right) c \hat{\mathbf{z}}$ & $(8 d)$ & H III \\
\hline $\mathbf{B}_{76}$ & $=$ & $\left(\frac{1}{2}+x_{10}\right) \mathbf{a}_{1}+\left(\frac{1}{2}-y_{10}\right) \mathbf{a}_{2}-z_{10} \mathbf{a}_{3}$ & $=$ & $\left(\frac{1}{2}+x_{10}\right) a \hat{\mathbf{x}}+\left(\frac{1}{2}-y_{10}\right) b \hat{\mathbf{y}}-z_{10} c \hat{\mathbf{z}}$ & $(8 d)$ & H III \\
\hline $\mathbf{B}_{77}$ & $=$ & $-x_{10} \mathbf{a}_{1}-y_{10} \mathbf{a}_{2}-z_{10} \mathbf{a}_{3}$ & $=$ & $-x_{10} a \hat{\mathbf{x}}-y_{10} b \hat{\mathbf{y}}-z_{10} c \hat{\mathbf{z}}$ & $(8 d)$ & H III \\
\hline $\mathbf{B}_{78}$ & $=$ & $\begin{array}{c}\left(\frac{1}{2}+x_{10}\right) \mathbf{a}_{1}+\left(\frac{1}{2}+y_{10}\right) \mathbf{a}_{2}+ \\
\left(\frac{1}{2}-z_{10}\right) \mathbf{a}_{3}\end{array}$ & $=$ & $\begin{array}{c}\left(\frac{1}{2}+x_{10}\right) a \hat{\mathbf{x}}+\left(\frac{1}{2}+y_{10}\right) b \hat{\mathbf{y}}+ \\
\left(\frac{1}{2}-z_{10}\right) c \hat{\mathbf{z}}\end{array}$ & $(8 d)$ & H III \\
\hline $\mathbf{B}_{79}$ & $=$ & $x_{10} \mathbf{a}_{1}-y_{10} \mathbf{a}_{2}+\left(\frac{1}{2}+z_{10}\right) \mathbf{a}_{3}$ & $=$ & $x_{10} a \hat{\mathbf{x}}-y_{10} b \hat{\mathbf{y}}+\left(\frac{1}{2}+z_{10}\right) c \hat{\mathbf{z}}$ & $(8 d)$ & H III \\
\hline $\mathbf{B}_{80}$ & $=$ & $\left(\frac{1}{2}-x_{10}\right) \mathbf{a}_{1}+\left(\frac{1}{2}+y_{10}\right) \mathbf{a}_{2}+z_{10} \mathbf{a}_{3}$ & $=$ & $\left(\frac{1}{2}-x_{10}\right) a \hat{\mathbf{x}}+\left(\frac{1}{2}+y_{10}\right) b \hat{\mathbf{y}}+z_{10} c \hat{\mathbf{z}}$ & $(8 d)$ & H III \\
\hline $\mathbf{B}_{81}$ & $=$ & $x_{11} \mathbf{a}_{1}+y_{11} \mathbf{a}_{2}+z_{11} \mathbf{a}_{3}$ & $=$ & $x_{11} a \hat{\mathbf{x}}+y_{11} b \hat{\mathbf{y}}+z_{11} c \hat{\mathbf{z}}$ & $(8 d)$ & H IV \\
\hline $\mathbf{B}_{82}$ & $=$ & $\begin{array}{c}\left(\frac{1}{2}-x_{11}\right) \mathbf{a}_{1}+\left(\frac{1}{2}-y_{11}\right) \mathbf{a}_{2}+ \\
\left(\frac{1}{2}+z_{11}\right) \mathbf{a}_{3}\end{array}$ & $=$ & $\begin{array}{c}\left(\frac{1}{2}-x_{11}\right) a \hat{\mathbf{x}}+\left(\frac{1}{2}-y_{11}\right) b \hat{\mathbf{y}}+ \\
\left(\frac{1}{2}+z_{11}\right) c \hat{\mathbf{z}}\end{array}$ & $(8 d)$ & H IV \\
\hline $\mathbf{B}_{83}$ & $=$ & $-x_{11} \mathbf{a}_{1}+y_{11} \mathbf{a}_{2}+\left(\frac{1}{2}-z_{11}\right) \mathbf{a}_{3}$ & $=$ & $-x_{11} a \hat{\mathbf{x}}+y_{11} b \hat{\mathbf{y}}+\left(\frac{1}{2}-z_{11}\right) c \hat{\mathbf{z}}$ & $(8 d)$ & H IV \\
\hline $\mathbf{B}_{84}$ & $=$ & $\left(\frac{1}{2}+x_{11}\right) \mathbf{a}_{1}+\left(\frac{1}{2}-y_{11}\right) \mathbf{a}_{2}-z_{11} \mathbf{a}_{3}$ & $=$ & $\left(\frac{1}{2}+x_{11}\right) a \hat{\mathbf{x}}+\left(\frac{1}{2}-y_{11}\right) b \hat{\mathbf{y}}-z_{11} c \hat{\mathbf{z}}$ & $(8 d)$ & H IV \\
\hline $\mathbf{B}_{85}$ & $=$ & $-x_{11} \mathbf{a}_{1}-y_{11} \mathbf{a}_{2}-z_{11} \mathbf{a}_{3}$ & $=$ & $-x_{11} a \hat{\mathbf{x}}-y_{11} b \hat{\mathbf{y}}-z_{11} c \hat{\mathbf{z}}$ & $(8 d)$ & H IV \\
\hline $\mathbf{B}_{86}$ & $=$ & $\begin{array}{c}\left(\frac{1}{2}+x_{11}\right) \mathbf{a}_{1}+\left(\frac{1}{2}+y_{11}\right) \mathbf{a}_{2}+ \\
\left(\frac{1}{2}-z_{11}\right) \mathbf{a}_{3}\end{array}$ & $=$ & $\begin{array}{c}\left(\frac{1}{2}+x_{11}\right) a \hat{\mathbf{x}}+\left(\frac{1}{2}+y_{11}\right) b \hat{\mathbf{y}}+ \\
\left(\frac{1}{2}-z_{11}\right) c \hat{\mathbf{z}}\end{array}$ & $(8 d)$ & H IV \\
\hline $\mathbf{B}_{87}$ & $=$ & $x_{11} \mathbf{a}_{1}-y_{11} \mathbf{a}_{2}+\left(\frac{1}{2}+z_{11}\right) \mathbf{a}_{3}$ & $=$ & $x_{11} a \hat{\mathbf{x}}-y_{11} b \hat{\mathbf{y}}+\left(\frac{1}{2}+z_{11}\right) c \hat{\mathbf{z}}$ & $(8 d)$ & H IV \\
\hline $\mathbf{B}_{88}$ & $=$ & $\left(\frac{1}{2}-x_{11}\right) \mathbf{a}_{1}+\left(\frac{1}{2}+y_{11}\right) \mathbf{a}_{2}+z_{11} \mathbf{a}_{3}$ & $=$ & $\left(\frac{1}{2}-x_{11}\right) a \hat{\mathbf{x}}+\left(\frac{1}{2}+y_{11}\right) b \hat{\mathbf{y}}+z_{11} c \hat{\mathbf{z}}$ & $(8 d)$ & H IV \\
\hline $\mathbf{B}_{89}$ & $=$ & $x_{12} \mathbf{a}_{1}+y_{12} \mathbf{a}_{2}+z_{12} \mathbf{a}_{3}$ & $=$ & $x_{12} a \hat{\mathbf{x}}+y_{12} b \hat{\mathbf{y}}+z_{12} c \hat{\mathbf{z}}$ & $(8 d)$ & $\mathrm{H} \mathrm{V}$ \\
\hline
\end{tabular}




\begin{tabular}{|c|c|c|c|c|}
\hline $\mathbf{B}_{90}$ & $\begin{array}{c}=\left(\frac{1}{2}-x_{12}\right) \mathbf{a}_{1}+\left(\frac{1}{2}-y_{12}\right) \mathbf{a}_{2}+ \\
\left(\frac{1}{2}+z_{12}\right) \mathbf{a}_{3}\end{array}$ & $\begin{array}{c}=\left(\frac{1}{2}-x_{12}\right) a \hat{\mathbf{x}}+\left(\frac{1}{2}-y_{12}\right) b \hat{\mathbf{y}}+ \\
\left(\frac{1}{2}+z_{12}\right) c \hat{\mathbf{z}}\end{array}$ & $(8 d)$ & $\mathrm{H} \mathrm{V}$ \\
\hline $\mathbf{B}_{91}$ & $-x_{12} \mathbf{a}_{1}+y_{12} \mathbf{a}_{2}+\left(\frac{1}{2}-z_{12}\right) \mathbf{a}_{3}$ & $-x_{12} a \hat{\mathbf{x}}+y_{12} b \hat{\mathbf{y}}+\left(\frac{1}{2}-z_{12}\right) c \hat{\mathbf{z}}$ & $(8 d)$ & $\mathrm{H} \mathrm{V}$ \\
\hline $\mathbf{B}_{92}$ & $=\left(\frac{1}{2}+x_{12}\right) \mathbf{a}_{1}+\left(\frac{1}{2}-y_{12}\right) \mathbf{a}_{2}-z_{12} \mathbf{a}_{3}$ & $=\left(\frac{1}{2}+x_{12}\right) a \hat{\mathbf{x}}+\left(\frac{1}{2}-y_{12}\right) b \hat{\mathbf{y}}-z_{12} c \hat{\mathbf{z}}$ & $(8 d)$ & H V \\
\hline $\mathbf{B}_{93}$ & $-x_{12} \mathbf{a}_{1}-y_{12} \mathbf{a}_{2}-z_{12} \mathbf{a}_{3}$ & $-x_{12} a \hat{\mathbf{x}}-y_{12} b \hat{\mathbf{y}}-z_{12} c \hat{\mathbf{z}}$ & $(8 d)$ & $\mathrm{H} \mathrm{V}$ \\
\hline $\mathbf{B}_{94}$ & $\begin{array}{c}\left(\frac{1}{2}+x_{12}\right) \mathbf{a}_{1}+\left(\frac{1}{2}+y_{12}\right) \mathbf{a}_{2}+ \\
\left(\frac{1}{2}-z_{12}\right) \mathbf{a}_{3}\end{array}$ & $\begin{array}{c}=\left(\frac{1}{2}+x_{12}\right) a \hat{\mathbf{x}}+\left(\frac{1}{2}+y_{12}\right) b \hat{\mathbf{y}}+ \\
\left(\frac{1}{2}-z_{12}\right) c \hat{\mathbf{z}}\end{array}$ & $(8 d)$ & $\mathrm{H} \mathrm{V}$ \\
\hline $\mathbf{B}_{95}$ & $x_{12} \mathbf{a}_{1}-y_{12} \mathbf{a}_{2}+\left(\frac{1}{2}+z_{12}\right) \mathbf{a}_{3}$ & $x_{12} a \hat{\mathbf{x}}-y_{12} b \hat{\mathbf{y}}+\left(\frac{1}{2}+z_{12}\right) c \hat{\mathbf{z}}$ & $(8 d)$ & $\mathrm{H} \mathrm{V}$ \\
\hline $\mathbf{B}_{96}$ & $=\left(\frac{1}{2}-x_{12}\right) \mathbf{a}_{1}+\left(\frac{1}{2}+y_{12}\right) \mathbf{a}_{2}+z_{12} \mathbf{a}_{3}$ & $=\left(\frac{1}{2}-x_{12}\right) a \hat{\mathbf{x}}+\left(\frac{1}{2}+y_{12}\right) b \hat{\mathbf{y}}+z_{12} c \hat{\mathbf{z}}$ & $(8 d)$ & $\mathrm{H} \mathrm{V}$ \\
\hline $\mathbf{B}_{97}$ & $x_{13} \mathbf{a}_{1}+y_{13} \mathbf{a}_{2}+z_{13} \mathbf{a}_{3}$ & $x_{13} a \hat{\mathbf{x}}+y_{13} b \hat{\mathbf{y}}+z_{13} c \hat{\mathbf{z}}$ & $(8 d)$ & H VI \\
\hline $\mathbf{B}_{98}$ & $\begin{array}{c}\left(\frac{1}{2}-x_{13}\right) \mathbf{a}_{1}+\left(\frac{1}{2}-y_{13}\right) \mathbf{a}_{2}+ \\
\left(\frac{1}{2}+z_{13}\right) \mathbf{a}_{3}\end{array}$ & $\begin{array}{c}\left(\frac{1}{2}-x_{13}\right) a \hat{\mathbf{x}}+\left(\frac{1}{2}-y_{13}\right) b \hat{\mathbf{y}}+ \\
\left(\frac{1}{2}+z_{13}\right) c \hat{\mathbf{z}}\end{array}$ & $(8 d)$ & H VI \\
\hline $\mathbf{B}_{99}$ & $-x_{13} \mathbf{a}_{1}+y_{13} \mathbf{a}_{2}+\left(\frac{1}{2}-z_{13}\right) \mathbf{a}_{3}$ & $-x_{13} a \hat{\mathbf{x}}+y_{13} b \hat{\mathbf{y}}+\left(\frac{1}{2}-z_{13}\right) c \hat{\mathbf{z}}$ & $(8 d)$ & H VI \\
\hline $\mathbf{B}_{100}$ & $=\left(\frac{1}{2}+x_{13}\right) \mathbf{a}_{1}+\left(\frac{1}{2}-y_{13}\right) \mathbf{a}_{2}-z_{13} \mathbf{a}_{3}$ & $=\left(\frac{1}{2}+x_{13}\right) a \hat{\mathbf{x}}+\left(\frac{1}{2}-y_{13}\right) b \hat{\mathbf{y}}-z_{13} c \hat{\mathbf{z}}$ & $(8 d)$ & H VI \\
\hline $\mathbf{B}_{101}$ & $-x_{13} \mathbf{a}_{1}-y_{13} \mathbf{a}_{2}-z_{13} \mathbf{a}_{3}$ & $-x_{13} a \hat{\mathbf{x}}-y_{13} b \hat{\mathbf{y}}-z_{13} c \hat{\mathbf{z}}$ & $(8 d)$ & H VI \\
\hline $\mathbf{B}_{102}$ & $\begin{array}{c}=\left(\frac{1}{2}+x_{13}\right) \mathbf{a}_{1}+\left(\frac{1}{2}+y_{13}\right) \mathbf{a}_{2}+ \\
\left(\frac{1}{2}-z_{13}\right) \mathbf{a}_{3}\end{array}$ & $\begin{array}{c}\left(\frac{1}{2}+x_{13}\right) a \hat{\mathbf{x}}+\left(\frac{1}{2}+y_{13}\right) b \hat{\mathbf{y}}+ \\
\left(\frac{1}{2}-z_{13}\right) c \hat{\mathbf{z}}\end{array}$ & $(8 d)$ & H VI \\
\hline $\mathbf{B}_{103}$ & $x_{13} \mathbf{a}_{1}-y_{13} \mathbf{a}_{2}+\left(\frac{1}{2}+z_{13}\right) \mathbf{a}_{3}$ & $x_{13} a \hat{\mathbf{x}}-y_{13} b \hat{\mathbf{y}}+\left(\frac{1}{2}+z_{13}\right) c \hat{\mathbf{z}}$ & $(8 d)$ & H VI \\
\hline $\mathbf{B}_{104}$ & $=\left(\frac{1}{2}-x_{13}\right) \mathbf{a}_{1}+\left(\frac{1}{2}+y_{13}\right) \mathbf{a}_{2}+z_{13} \mathbf{a}_{3}$ & $=\left(\frac{1}{2}-x_{13}\right) a \hat{\mathbf{x}}+\left(\frac{1}{2}+y_{13}\right) b \hat{\mathbf{y}}+z_{13} c \hat{\mathbf{z}}$ & $(8 d)$ & H VI \\
\hline $\mathbf{B}_{105}$ & $x_{14} \mathbf{a}_{1}+y_{14} \mathbf{a}_{2}+z_{14} \mathbf{a}_{3}$ & $x_{14} a \hat{\mathbf{x}}+y_{14} b \hat{\mathbf{y}}+z_{14} c \hat{\mathbf{z}}$ & $(8 d)$ & H VII \\
\hline $\mathbf{B}_{106}$ & $\begin{array}{c}\left(\frac{1}{2}-x_{14}\right) \mathbf{a}_{1}+\left(\frac{1}{2}-y_{14}\right) \mathbf{a}_{2}+ \\
\left(\frac{1}{2}+z_{14}\right) \mathbf{a}_{3}\end{array}$ & $\begin{array}{c}\left(\frac{1}{2}-x_{14}\right) a \hat{\mathbf{x}}+\left(\frac{1}{2}-y_{14}\right) b \hat{\mathbf{y}}+ \\
\left(\frac{1}{2}+z_{14}\right) c \hat{\mathbf{z}}\end{array}$ & $(8 d)$ & H VII \\
\hline $\mathbf{B}_{107}$ & $-x_{14} \mathbf{a}_{1}+y_{14} \mathbf{a}_{2}+\left(\frac{1}{2}-z_{14}\right) \mathbf{a}_{3}$ & $-x_{14} a \hat{\mathbf{x}}+y_{14} b \hat{\mathbf{y}}+\left(\frac{1}{2}-z_{14}\right) c \hat{\mathbf{z}}$ & $(8 d)$ & H VII \\
\hline $\mathbf{B}_{108}$ & $=\left(\frac{1}{2}+x_{14}\right) \mathbf{a}_{1}+\left(\frac{1}{2}-y_{14}\right) \mathbf{a}_{2}-z_{14} \mathbf{a}_{3}$ & $=\left(\frac{1}{2}+x_{14}\right) a \hat{\mathbf{x}}+\left(\frac{1}{2}-y_{14}\right) b \hat{\mathbf{y}}-z_{14} c \hat{\mathbf{z}}$ & $(8 d)$ & H VII \\
\hline $\mathbf{B}_{109}$ & $-x_{14} \mathbf{a}_{1}-y_{14} \mathbf{a}_{2}-z_{14} \mathbf{a}_{3}$ & $-x_{14} a \hat{\mathbf{x}}-y_{14} b \hat{\mathbf{y}}-z_{14} c \hat{\mathbf{z}}$ & $(8 d)$ & H VII \\
\hline $\mathbf{B}_{110}$ & $\begin{array}{c}\left(\frac{1}{2}+x_{14}\right) \mathbf{a}_{1}+\left(\frac{1}{2}+y_{14}\right) \mathbf{a}_{2}+ \\
\left(\frac{1}{2}-z_{14}\right) \mathbf{a}_{3}\end{array}$ & $\begin{array}{c}\left(\frac{1}{2}+x_{14}\right) a \hat{\mathbf{x}}+\left(\frac{1}{2}+y_{14}\right) b \hat{\mathbf{y}}+ \\
\left(\frac{1}{2}-z_{14}\right) c \hat{\mathbf{z}}\end{array}$ & $(8 d)$ & H VII \\
\hline $\mathbf{B}_{111}$ & $x_{14} \mathbf{a}_{1}-y_{14} \mathbf{a}_{2}+\left(\frac{1}{2}+z_{14}\right) \mathbf{a}_{3}$ & $x_{14} a \hat{\mathbf{x}}-y_{14} b \hat{\mathbf{y}}+\left(\frac{1}{2}+z_{14}\right) c \hat{\mathbf{z}}$ & $(8 d)$ & H VII \\
\hline $\mathbf{B}_{112}$ & $=\left(\frac{1}{2}-x_{14}\right) \mathbf{a}_{1}+\left(\frac{1}{2}+y_{14}\right) \mathbf{a}_{2}+z_{14} \mathbf{a}_{3}$ & $=\left(\frac{1}{2}-x_{14}\right) a \hat{\mathbf{x}}+\left(\frac{1}{2}+y_{14}\right) b \hat{\mathbf{y}}+z_{14} c \hat{\mathbf{z}}$ & $(8 d)$ & H VII \\
\hline $\mathbf{B}_{113}$ & $x_{15} \mathbf{a}_{1}+y_{15} \mathbf{a}_{2}+z_{15} \mathbf{a}_{3}$ & $x_{15} a \hat{\mathbf{x}}+y_{15} b \hat{\mathbf{y}}+z_{15} c \hat{\mathbf{z}}$ & $(8 d)$ & H VIII \\
\hline $\mathbf{B}_{114}$ & $\begin{array}{c}\left(\frac{1}{2}-x_{15}\right) \mathbf{a}_{1}+\left(\frac{1}{2}-y_{15}\right) \mathbf{a}_{2}+ \\
\left(\frac{1}{2}+z_{15}\right) \mathbf{a}_{3}\end{array}$ & $\begin{array}{c}\left(\frac{1}{2}-x_{15}\right) a \hat{\mathbf{x}}+\left(\frac{1}{2}-y_{15}\right) b \hat{\mathbf{y}}+ \\
\left(\frac{1}{2}+z_{15}\right) c \hat{\mathbf{z}}\end{array}$ & $(8 d)$ & H VIII \\
\hline $\mathbf{B}_{115}$ & $-x_{15} \mathbf{a}_{1}+y_{15} \mathbf{a}_{2}+\left(\frac{1}{2}-z_{15}\right) \mathbf{a}_{3}$ & $-x_{15} a \hat{\mathbf{x}}+y_{15} b \hat{\mathbf{y}}+\left(\frac{1}{2}-z_{15}\right) c \hat{\mathbf{z}}$ & $(8 d)$ & H VIII \\
\hline $\mathbf{B}_{116}$ & $=\left(\frac{1}{2}+x_{15}\right) \mathbf{a}_{1}+\left(\frac{1}{2}-y_{15}\right) \mathbf{a}_{2}-z_{15} \mathbf{a}_{3}$ & $=\left(\frac{1}{2}+x_{15}\right) a \hat{\mathbf{x}}+\left(\frac{1}{2}-y_{15}\right) b \hat{\mathbf{y}}-z_{15} c \hat{\mathbf{z}}$ & $(8 d)$ & H VIII \\
\hline $\mathbf{B}_{117}$ & $-x_{15} \mathbf{a}_{1}-y_{15} \mathbf{a}_{2}-z_{15} \mathbf{a}_{3}$ & $-x_{15} a \hat{\mathbf{x}}-y_{15} b \hat{\mathbf{y}}-z_{15} c \hat{\mathbf{z}}$ & $(8 d)$ & H VIII \\
\hline $\mathbf{B}_{118}$ & $\begin{array}{c}\left(\frac{1}{2}+x_{15}\right) \mathbf{a}_{1}+\left(\frac{1}{2}+y_{15}\right) \mathbf{a}_{2}+ \\
\left(\frac{1}{2}-z_{15}\right) \mathbf{a}_{3}\end{array}$ & $\begin{array}{c}\left(\frac{1}{2}+x_{15}\right) a \hat{\mathbf{x}}+\left(\frac{1}{2}+y_{15}\right) b \hat{\mathbf{y}}+ \\
\left(\frac{1}{2}-z_{15}\right) c \hat{\mathbf{z}}\end{array}$ & $(8 d)$ & H VIII \\
\hline $\mathbf{B}_{119}$ & $x_{15} \mathbf{a}_{1}-y_{15} \mathbf{a}_{2}+\left(\frac{1}{2}+z_{15}\right) \mathbf{a}_{3}$ & $x_{15} a \hat{\mathbf{x}}-y_{15} b \hat{\mathbf{y}}+\left(\frac{1}{2}+z_{15}\right) c \hat{\mathbf{z}}$ & $(8 d)$ & H VIII \\
\hline
\end{tabular}




$$
\mathbf{B}_{120}=\left(\frac{1}{2}-x_{15}\right) \mathbf{a}_{1}+\left(\frac{1}{2}+y_{15}\right) \mathbf{a}_{2}+z_{15} \mathbf{a}_{3}=\left(\frac{1}{2}-x_{15}\right) a \hat{\mathbf{x}}+\left(\frac{1}{2}+y_{15}\right) b \hat{\mathbf{y}}+z_{15} c \hat{\mathbf{z}}
$$

\section{References:}

- D. Andre, R. Fourme, J. Bruneaux-Poulle, and L. Bosio, Crystal structure of the metastable $\beta$-phase of toluene, J. Mol. Struct. 81, 253-259 (1982), doi:10.1016/0022-2860(82)85338-6.

\section{Geometry files:}

- CIF: pp. 839

- POSCAR: pp. 840 


\section{Benzene Structure: AB_oP48_61_3c_3c}
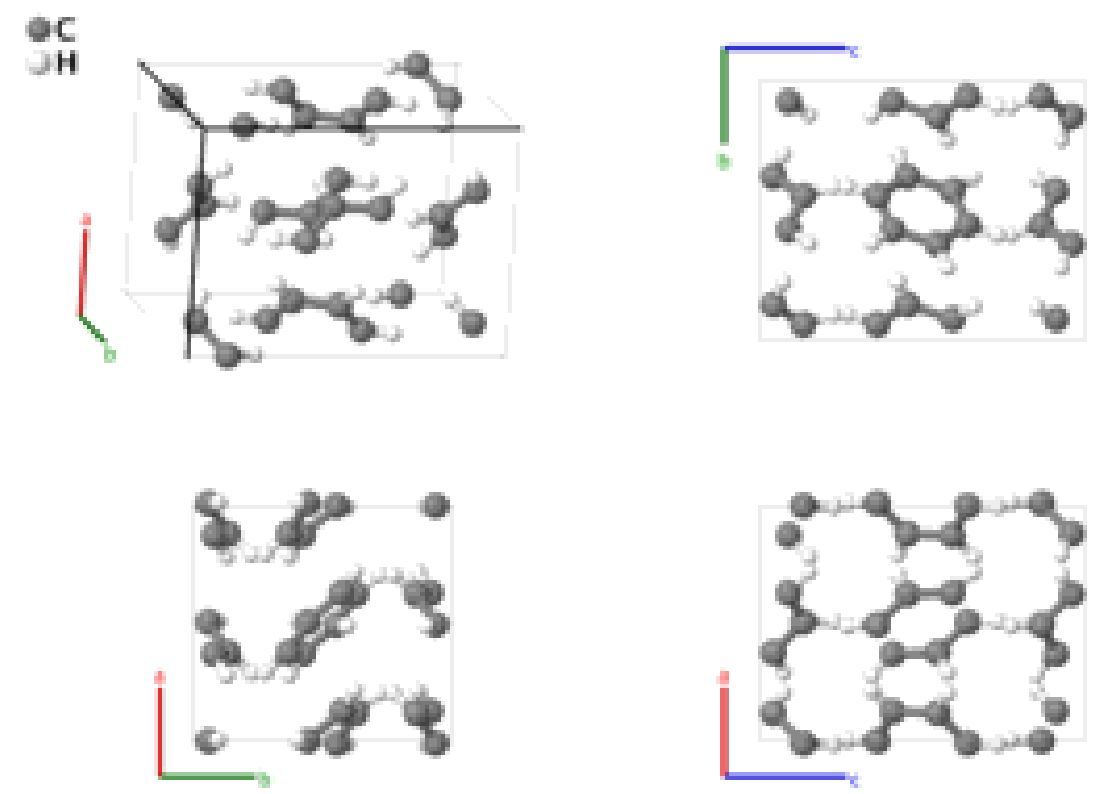

\section{Prototype}

AFLOW prototype label

Strukturbericht designation

Pearson symbol

Space group number

Space group symbol

AFLOW prototype command
: Benzene

: $\quad$ AB_oP48_61_3c_3c

: None

: $\quad$ oP48

: 61

: $\quad P b c a$

- Benzene is a liquid at temperatures above $6^{\circ} \mathrm{C}(279 \mathrm{~K})$. This data was constructed from experiments at $150 \mathrm{~K}$.

- The hydrogen atomic positions were approximated to agree with the chemistry of the benzene molecule.

\section{Simple Orthorhombic primitive vectors:}

$$
\begin{aligned}
& \mathbf{a}_{1}=a \hat{\mathbf{x}} \\
& \mathbf{a}_{2}=b \hat{\mathbf{y}} \\
& \mathbf{a}_{3}=c \hat{\mathbf{z}}
\end{aligned}
$$

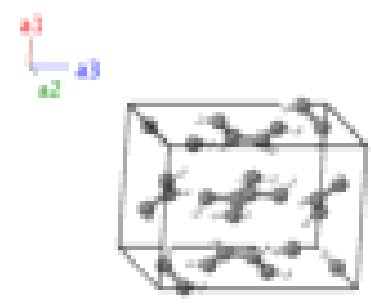

Basis vectors:

Lattice Coordinates

$\mathbf{B}_{1}=$

$x_{1} \mathbf{a}_{1}+y_{1} \mathbf{a}_{2}+z_{1} \mathbf{a}_{3}$
Cartesian Coordinates

$=$

$x_{1} a \hat{\mathbf{x}}+y_{1} b \hat{\mathbf{y}}+z_{1} c \hat{\mathbf{z}}$
Wyckoff Position

(8c)
Atom Type

C I 


\begin{tabular}{|c|c|c|c|c|c|}
\hline $\mathbf{B}_{2}$ & $=$ & $\left(\frac{1}{2}-x_{1}\right) \mathbf{a}_{1}-y_{1} \mathbf{a}_{2}+\left(\frac{1}{2}+z_{1}\right) \mathbf{a}_{3}$ & $=\left(\frac{1}{2}-x_{1}\right) a \hat{\mathbf{x}}-y_{1} b \hat{\mathbf{y}}+\left(\frac{1}{2}+z_{1}\right) c \hat{\mathbf{z}}$ & $(8 c)$ & $\mathrm{C} \mathrm{I}$ \\
\hline $\mathbf{B}_{3}$ & $=$ & $-x_{1} \mathbf{a}_{1}+\left(\frac{1}{2}+y_{1}\right) \mathbf{a}_{2}+\left(\frac{1}{2}-z_{1}\right) \mathbf{a}_{3}$ & $=-x_{1} a \hat{\mathbf{x}}+\left(\frac{1}{2}+y_{1}\right) b \hat{\mathbf{y}}+\left(\frac{1}{2}-z_{1}\right) c \hat{\mathbf{z}}$ & $(8 c)$ & C I \\
\hline $\mathbf{B}_{4}$ & $=$ & $\left(\frac{1}{2}+x_{1}\right) \mathbf{a}_{1}+\left(\frac{1}{2}-y_{1}\right) \mathbf{a}_{2}-z_{1} \mathbf{a}_{3}$ & $=\left(\frac{1}{2}+x_{1}\right) a \hat{\mathbf{x}}+\left(\frac{1}{2}-y_{1}\right) b \hat{\mathbf{y}}-z_{1} c \hat{\mathbf{z}}$ & $(8 c)$ & C I \\
\hline $\mathbf{B}_{5}$ & $=$ & $-x_{1} \mathbf{a}_{1}-y_{1} \mathbf{a}_{2}-z_{1} \mathbf{a}_{3}$ & $-x_{1} a \hat{\mathbf{x}}-y_{1} b \hat{\mathbf{y}}-z_{1} c \hat{\mathbf{z}}$ & $(8 c)$ & C I \\
\hline $\mathbf{B}_{6}$ & $=$ & $\left(\frac{1}{2}+x_{1}\right) \mathbf{a}_{1}+y_{1} \mathbf{a}_{2}+\left(\frac{1}{2}-z_{1}\right) \mathbf{a}_{3}$ & $=\left(\frac{1}{2}+x_{1}\right) a \hat{\mathbf{x}}+y_{1} b \hat{\mathbf{y}}+\left(\frac{1}{2}-z_{1}\right) c \hat{\mathbf{z}}$ & $(8 c)$ & $\mathrm{C} \mathrm{I}$ \\
\hline $\mathbf{B}_{7}$ & $=$ & $x_{1} \mathbf{a}_{1}+\left(\frac{1}{2}-y_{1}\right) \mathbf{a}_{2}+\left(\frac{1}{2}+z_{1}\right) \mathbf{a}_{3}$ & $=\quad x_{1} a \hat{\mathbf{x}}+\left(\frac{1}{2}-y_{1}\right) b \hat{\mathbf{y}}+\left(\frac{1}{2}+z_{1}\right) c \hat{\mathbf{z}}$ & $(8 c)$ & C I \\
\hline $\mathbf{B}_{8}$ & $=$ & $\left(\frac{1}{2}-x_{1}\right) \mathbf{a}_{1}+\left(\frac{1}{2}+y_{1}\right) \mathbf{a}_{2}+z_{1} \mathbf{a}_{3}$ & $=\left(\frac{1}{2}-x_{1}\right) a \hat{\mathbf{x}}+\left(\frac{1}{2}+y_{1}\right) b \hat{\mathbf{y}}+z_{1} c \hat{\mathbf{z}}$ & $(8 c)$ & C I \\
\hline $\mathbf{B}_{9}$ & $=$ & $x_{2} \mathbf{a}_{1}+y_{2} \mathbf{a}_{2}+z_{2} \mathbf{a}_{3}$ & $x_{2} a \hat{\mathbf{x}}+y_{2} b \hat{\mathbf{y}}+z_{2} c \hat{\mathbf{z}}$ & $(8 c)$ & C II \\
\hline $\mathbf{B}_{10}$ & $=$ & $\left(\frac{1}{2}-x_{2}\right) \mathbf{a}_{1}-y_{2} \mathbf{a}_{2}+\left(\frac{1}{2}+z_{2}\right) \mathbf{a}_{3}$ & $=\left(\frac{1}{2}-x_{2}\right) a \hat{\mathbf{x}}-y_{2} b \hat{\mathbf{y}}+\left(\frac{1}{2}+z_{2}\right) c \hat{\mathbf{z}}$ & $(8 c)$ & C II \\
\hline $\mathbf{B}_{11}$ & $=$ & $-x_{2} \mathbf{a}_{1}+\left(\frac{1}{2}+y_{2}\right) \mathbf{a}_{2}+\left(\frac{1}{2}-z_{2}\right) \mathbf{a}_{3}$ & $=-x_{2} a \hat{\mathbf{x}}+\left(\frac{1}{2}+y_{2}\right) b \hat{\mathbf{y}}+\left(\frac{1}{2}-z_{2}\right) c \hat{\mathbf{z}}$ & $(8 c)$ & C II \\
\hline $\mathbf{B}_{12}$ & $=$ & $\left(\frac{1}{2}+x_{2}\right) \mathbf{a}_{1}+\left(\frac{1}{2}-y_{2}\right) \mathbf{a}_{2}-z_{2} \mathbf{a}_{3}$ & $=\left(\frac{1}{2}+x_{2}\right) a \hat{\mathbf{x}}+\left(\frac{1}{2}-y_{2}\right) b \hat{\mathbf{y}}-z_{2} c \hat{\mathbf{z}}$ & $(8 c)$ & C II \\
\hline $\mathbf{B}_{13}$ & $=$ & $-x_{2} \mathbf{a}_{1}-y_{2} \mathbf{a}_{2}-z_{2} \mathbf{a}_{3}$ & $-x_{2} a \hat{\mathbf{x}}-y_{2} b \hat{\mathbf{y}}-z_{2} c \hat{\mathbf{z}}$ & $(8 c)$ & C II \\
\hline $\mathbf{B}_{14}$ & $=$ & $\left(\frac{1}{2}+x_{2}\right) \mathbf{a}_{1}+y_{2} \mathbf{a}_{2}+\left(\frac{1}{2}-z_{2}\right) \mathbf{a}_{3}$ & $=\left(\frac{1}{2}+x_{2}\right) a \hat{\mathbf{x}}+y_{2} b \hat{\mathbf{y}}+\left(\frac{1}{2}-z_{2}\right) c \hat{\mathbf{z}}$ & $(8 c)$ & C II \\
\hline $\mathbf{B}_{15}$ & $=$ & $x_{2} \mathbf{a}_{1}+\left(\frac{1}{2}-y_{2}\right) \mathbf{a}_{2}+\left(\frac{1}{2}+z_{2}\right) \mathbf{a}_{3}$ & $=\quad x_{2} a \hat{\mathbf{x}}+\left(\frac{1}{2}-y_{2}\right) b \hat{\mathbf{y}}+\left(\frac{1}{2}+z_{2}\right) c \hat{\mathbf{z}}$ & $(8 c)$ & C II \\
\hline $\mathbf{B}_{16}$ & $=$ & $\left(\frac{1}{2}-x_{2}\right) \mathbf{a}_{1}+\left(\frac{1}{2}+y_{2}\right) \mathbf{a}_{2}+z_{2} \mathbf{a}_{3}$ & $=\left(\frac{1}{2}-x_{2}\right) a \hat{\mathbf{x}}+\left(\frac{1}{2}+y_{2}\right) b \hat{\mathbf{y}}+z_{2} c \hat{\mathbf{z}}$ & $(8 c)$ & C II \\
\hline $\mathbf{B}_{17}$ & $=$ & $x_{3} \mathbf{a}_{1}+y_{3} \mathbf{a}_{2}+z_{3} \mathbf{a}_{3}$ & $x_{3} a \hat{\mathbf{x}}+y_{3} b \hat{\mathbf{y}}+z_{3} c \hat{\mathbf{z}}$ & $(8 c)$ & C III \\
\hline $\mathbf{B}_{18}$ & $=$ & $\left(\frac{1}{2}-x_{3}\right) \mathbf{a}_{1}-y_{3} \mathbf{a}_{2}+\left(\frac{1}{2}+z_{3}\right) \mathbf{a}_{3}$ & $=\left(\frac{1}{2}-x_{3}\right) a \hat{\mathbf{x}}-y_{3} b \hat{\mathbf{y}}+\left(\frac{1}{2}+z_{3}\right) c \hat{\mathbf{z}}$ & $(8 c)$ & C III \\
\hline $\mathbf{B}_{19}$ & $=$ & $-x_{3} \mathbf{a}_{1}+\left(\frac{1}{2}+y_{3}\right) \mathbf{a}_{2}+\left(\frac{1}{2}-z_{3}\right) \mathbf{a}_{3}$ & $=-x_{3} a \hat{\mathbf{x}}+\left(\frac{1}{2}+y_{3}\right) b \hat{\mathbf{y}}+\left(\frac{1}{2}-z_{3}\right) c \hat{\mathbf{z}}$ & $(8 c)$ & C III \\
\hline $\mathbf{B}_{20}$ & $=$ & $\left(\frac{1}{2}+x_{3}\right) \mathbf{a}_{1}+\left(\frac{1}{2}-y_{3}\right) \mathbf{a}_{2}-z_{3} \mathbf{a}_{3}$ & $=\left(\frac{1}{2}+x_{3}\right) a \hat{\mathbf{x}}+\left(\frac{1}{2}-y_{3}\right) b \hat{\mathbf{y}}-z_{3} c \hat{\mathbf{z}}$ & $(8 c)$ & C III \\
\hline $\mathbf{B}_{21}$ & $=$ & $-x_{3} \mathbf{a}_{1}-y_{3} \mathbf{a}_{2}-z_{3} \mathbf{a}_{3}$ & $-x_{3} a \hat{\mathbf{x}}-y_{3} b \hat{\mathbf{y}}-z_{3} c \hat{\mathbf{z}}$ & $(8 c)$ & C III \\
\hline $\mathbf{B}_{22}$ & $=$ & $\left(\frac{1}{2}+x_{3}\right) \mathbf{a}_{1}+y_{3} \mathbf{a}_{2}+\left(\frac{1}{2}-z_{3}\right) \mathbf{a}_{3}$ & $=\left(\frac{1}{2}+x_{3}\right) a \hat{\mathbf{x}}+y_{3} b \hat{\mathbf{y}}+\left(\frac{1}{2}-z_{3}\right) c \hat{\mathbf{z}}$ & $(8 c)$ & C III \\
\hline $\mathbf{B}_{23}$ & $=$ & $x_{3} \mathbf{a}_{1}+\left(\frac{1}{2}-y_{3}\right) \mathbf{a}_{2}+\left(\frac{1}{2}+z_{3}\right) \mathbf{a}_{3}$ & $=\quad x_{3} a \hat{\mathbf{x}}+\left(\frac{1}{2}-y_{3}\right) b \hat{\mathbf{y}}+\left(\frac{1}{2}+z_{3}\right) c \hat{\mathbf{z}}$ & $(8 c)$ & C III \\
\hline $\mathbf{B}_{24}$ & $=$ & $\left(\frac{1}{2}-x_{3}\right) \mathbf{a}_{1}+\left(\frac{1}{2}+y_{3}\right) \mathbf{a}_{2}+z_{3} \mathbf{a}_{3}$ & $=\left(\frac{1}{2}-x_{3}\right) a \hat{\mathbf{x}}+\left(\frac{1}{2}+y_{3}\right) b \hat{\mathbf{y}}+z_{3} c \hat{\mathbf{z}}$ & $(8 c)$ & C III \\
\hline $\mathbf{B}_{25}$ & $=$ & $x_{4} \mathbf{a}_{1}+y_{4} \mathbf{a}_{2}+z_{4} \mathbf{a}_{3}$ & $x_{4} a \hat{\mathbf{x}}+y_{4} b \hat{\mathbf{y}}+z_{4} c \hat{\mathbf{z}}$ & $(8 c)$ & H I \\
\hline $\mathbf{B}_{26}$ & $=$ & $\left(\frac{1}{2}-x_{4}\right) \mathbf{a}_{1}-y_{4} \mathbf{a}_{2}+\left(\frac{1}{2}+z_{4}\right) \mathbf{a}_{3}$ & $=\left(\frac{1}{2}-x_{4}\right) a \hat{\mathbf{x}}-y_{4} b \hat{\mathbf{y}}+\left(\frac{1}{2}+z_{4}\right) c \hat{\mathbf{z}}$ & $(8 c)$ & H I \\
\hline $\mathbf{B}_{27}$ & $=$ & $-x_{4} \mathbf{a}_{1}+\left(\frac{1}{2}+y_{4}\right) \mathbf{a}_{2}+\left(\frac{1}{2}-z_{4}\right) \mathbf{a}_{3}$ & $=-x_{4} a \hat{\mathbf{x}}+\left(\frac{1}{2}+y_{4}\right) b \hat{\mathbf{y}}+\left(\frac{1}{2}-z_{4}\right) c \hat{\mathbf{z}}$ & $(8 c)$ & H I \\
\hline $\mathbf{B}_{28}$ & $=$ & $\left(\frac{1}{2}+x_{4}\right) \mathbf{a}_{1}+\left(\frac{1}{2}-y_{4}\right) \mathbf{a}_{2}-z_{4} \mathbf{a}_{3}$ & $=\left(\frac{1}{2}+x_{4}\right) a \hat{\mathbf{x}}+\left(\frac{1}{2}-y_{4}\right) b \hat{\mathbf{y}}-z_{4} c \hat{\mathbf{z}}$ & $(8 c)$ & H I \\
\hline $\mathbf{B}_{29}$ & $=$ & $-x_{4} \mathbf{a}_{1}-y_{4} \mathbf{a}_{2}-z_{4} \mathbf{a}_{3}$ & $-x_{4} a \hat{\mathbf{x}}-y_{4} b \hat{\mathbf{y}}-z_{4} c \hat{\mathbf{z}}$ & $(8 c)$ & H I \\
\hline $\mathbf{B}_{30}$ & $=$ & $\left(\frac{1}{2}+x_{4}\right) \mathbf{a}_{1}+y_{4} \mathbf{a}_{2}+\left(\frac{1}{2}-z_{4}\right) \mathbf{a}_{3}$ & $=\left(\frac{1}{2}+x_{4}\right) a \hat{\mathbf{x}}+y_{4} b \hat{\mathbf{y}}+\left(\frac{1}{2}-z_{4}\right) c \hat{\mathbf{z}}$ & $(8 c)$ & H I \\
\hline $\mathbf{B}_{31}$ & $=$ & $x_{4} \mathbf{a}_{1}+\left(\frac{1}{2}-y_{4}\right) \mathbf{a}_{2}+\left(\frac{1}{2}+z_{4}\right) \mathbf{a}_{3}$ & $=\quad x_{4} a \hat{\mathbf{x}}+\left(\frac{1}{2}-y_{4}\right) b \hat{\mathbf{y}}+\left(\frac{1}{2}+z_{4}\right) c \hat{\mathbf{z}}$ & $(8 c)$ & H I \\
\hline $\mathbf{B}_{32}$ & $=$ & $\left(\frac{1}{2}-x_{4}\right) \mathbf{a}_{1}+\left(\frac{1}{2}+y_{4}\right) \mathbf{a}_{2}+z_{4} \mathbf{a}_{3}$ & $=\left(\frac{1}{2}-x_{4}\right) a \hat{\mathbf{x}}+\left(\frac{1}{2}+y_{4}\right) b \hat{\mathbf{y}}+z_{4} c \hat{\mathbf{z}}$ & $(8 c)$ & $\mathrm{H} \mathrm{I}$ \\
\hline $\mathbf{B}_{33}$ & $=$ & $x_{5} \mathbf{a}_{1}+y_{5} \mathbf{a}_{2}+z_{5} \mathbf{a}_{3}$ & $x_{5} a \hat{\mathbf{x}}+y_{5} b \hat{\mathbf{y}}+z_{5} c \hat{\mathbf{z}}$ & $(8 c)$ & $\mathrm{H}$ II \\
\hline $\mathbf{B}_{34}$ & $=$ & $\left(\frac{1}{2}-x_{5}\right) \mathbf{a}_{1}-y_{5} \mathbf{a}_{2}+\left(\frac{1}{2}+z_{5}\right) \mathbf{a}_{3}$ & $=\left(\frac{1}{2}-x_{5}\right) a \hat{\mathbf{x}}-y_{5} b \hat{\mathbf{y}}+\left(\frac{1}{2}+z_{5}\right) c \hat{\mathbf{z}}$ & $(8 c)$ & H II \\
\hline $\mathbf{B}_{35}$ & $=$ & $-x_{5} \mathbf{a}_{1}+\left(\frac{1}{2}+y_{5}\right) \mathbf{a}_{2}+\left(\frac{1}{2}-z_{5}\right) \mathbf{a}_{3}$ & $=-x_{5} a \hat{\mathbf{x}}+\left(\frac{1}{2}+y_{5}\right) b \hat{\mathbf{y}}+\left(\frac{1}{2}-z_{5}\right) c \hat{\mathbf{z}}$ & $(8 c)$ & H II \\
\hline $\mathbf{B}_{36}$ & $=$ & $\left(\frac{1}{2}+x_{5}\right) \mathbf{a}_{1}+\left(\frac{1}{2}-y_{5}\right) \mathbf{a}_{2}-z_{5} \mathbf{a}_{3}$ & $=\left(\frac{1}{2}+x_{5}\right) a \hat{\mathbf{x}}+\left(\frac{1}{2}-y_{5}\right) b \hat{\mathbf{y}}-z_{5} c \hat{\mathbf{z}}$ & $(8 c)$ & H II \\
\hline $\mathbf{B}_{37}$ & $=$ & $-x_{5} \mathbf{a}_{1}-y_{5} \mathbf{a}_{2}-z_{5} \mathbf{a}_{3}$ & $-x_{5} a \hat{\mathbf{x}}-y_{5} b \hat{\mathbf{y}}-z_{5} c \hat{\mathbf{z}}$ & $(8 c)$ & H II \\
\hline
\end{tabular}




\begin{tabular}{|c|c|c|c|}
\hline $\mathbf{B}_{38}$ & $=\left(\frac{1}{2}+x_{5}\right) \mathbf{a}_{1}+y_{5} \mathbf{a}_{2}+\left(\frac{1}{2}-z_{5}\right) \mathbf{a}_{3}$ & $=\left(\frac{1}{2}+x_{5}\right) a \hat{\mathbf{x}}+y_{5} b \hat{\mathbf{y}}+\left(\frac{1}{2}-z_{5}\right) c \hat{\mathbf{z}}$ & $(8 c)$ \\
\hline $\mathbf{B}_{39}$ & $=\quad x_{5} \mathbf{a}_{1}+\left(\frac{1}{2}-y_{5}\right) \mathbf{a}_{2}+\left(\frac{1}{2}+z_{5}\right) \mathbf{a}_{3}$ & $=\quad x_{5} a \hat{\mathbf{x}}+\left(\frac{1}{2}-y_{5}\right) b \hat{\mathbf{y}}+\left(\frac{1}{2}+z_{5}\right) c \hat{\mathbf{z}}$ & $(8 c)$ \\
\hline $\mathbf{B}_{40}$ & $=\left(\frac{1}{2}-x_{5}\right) \mathbf{a}_{1}+\left(\frac{1}{2}+y_{5}\right) \mathbf{a}_{2}+z_{5} \mathbf{a}_{3}$ & $=\left(\frac{1}{2}-x_{5}\right) a \hat{\mathbf{x}}+\left(\frac{1}{2}+y_{5}\right) b \hat{\mathbf{y}}+z_{5} c \hat{\mathbf{z}}$ & $(8 c)$ \\
\hline $\mathbf{B}_{41}$ & $x_{6} \mathbf{a}_{1}+y_{6} \mathbf{a}_{2}+z_{6} \mathbf{a}_{3}$ & $x_{6} a \hat{\mathbf{x}}+y_{6} b \hat{\mathbf{y}}+z_{6} c \hat{\mathbf{z}}$ & $(8 c)$ \\
\hline $\mathbf{B}_{42}$ & $=\left(\frac{1}{2}-x_{6}\right) \mathbf{a}_{1}-y_{6} \mathbf{a}_{2}+\left(\frac{1}{2}+z_{6}\right) \mathbf{a}_{3}$ & $=\left(\frac{1}{2}-x_{6}\right) a \hat{\mathbf{x}}-y_{6} b \hat{\mathbf{y}}+\left(\frac{1}{2}+z_{6}\right) c \hat{\mathbf{z}}$ & $(8 c)$ \\
\hline $\mathbf{B}_{43}$ & $=-x_{6} \mathbf{a}_{1}+\left(\frac{1}{2}+y_{6}\right) \mathbf{a}_{2}+\left(\frac{1}{2}-z_{6}\right) \mathbf{a}_{3}$ & $=-x_{6} a \hat{\mathbf{x}}+\left(\frac{1}{2}+y_{6}\right) b \hat{\mathbf{y}}+\left(\frac{1}{2}-z_{6}\right) c \hat{\mathbf{z}}$ & $(8 c)$ \\
\hline $\mathbf{B}_{44}$ & $=\left(\frac{1}{2}+x_{6}\right) \mathbf{a}_{1}+\left(\frac{1}{2}-y_{6}\right) \mathbf{a}_{2}-z_{6} \mathbf{a}_{3}$ & $=\left(\frac{1}{2}+x_{6}\right) a \hat{\mathbf{x}}+\left(\frac{1}{2}-y_{6}\right) b \hat{\mathbf{y}}-z_{6} c \hat{\mathbf{z}}$ & $(8 c)$ \\
\hline $\mathbf{B}_{45}$ & $-x_{6} \mathbf{a}_{1}-y_{6} \mathbf{a}_{2}-z_{6} \mathbf{a}_{3}$ & $-x_{6} a \hat{\mathbf{x}}-y_{6} b \hat{\mathbf{y}}-z_{6} c \hat{\mathbf{z}}$ & $(8 c)$ \\
\hline $\mathbf{B}_{46}$ & $=\left(\frac{1}{2}+x_{6}\right) \mathbf{a}_{1}+y_{6} \mathbf{a}_{2}+\left(\frac{1}{2}-z_{6}\right) \mathbf{a}_{3}$ & $=\left(\frac{1}{2}+x_{6}\right) a \hat{\mathbf{x}}+y_{6} b \hat{\mathbf{y}}+\left(\frac{1}{2}-z_{6}\right) c \hat{\mathbf{z}}$ & $(8 c)$ \\
\hline $\mathbf{B}_{47}$ & $=\quad x_{6} \mathbf{a}_{1}+\left(\frac{1}{2}-y_{6}\right) \mathbf{a}_{2}+\left(\frac{1}{2}+z_{6}\right) \mathbf{a}_{3}$ & $=\quad x_{6} a \hat{\mathbf{x}}+\left(\frac{1}{2}-y_{6}\right) b \hat{\mathbf{y}}+\left(\frac{1}{2}+z_{6}\right) c \hat{\mathbf{z}}$ & $(8 c)$ \\
\hline $3_{48}$ & $=\left(\frac{1}{2}-x_{6}\right) \mathbf{a}_{1}+\left(\frac{1}{2}+y_{6}\right) \mathbf{a}_{2}+z_{6} \mathbf{a}_{3}$ & $=\left(\frac{1}{2}-x_{6}\right) a \hat{\mathbf{x}}+\left(\frac{1}{2}+y_{6}\right) b \hat{\mathbf{y}}+z_{6} c \hat{\mathbf{z}}$ & $(8 c)$ \\
\hline
\end{tabular}

\section{References:}

- S. K. Nayak, R. Sathishkumar, and T. N. Guru Row, Directing role of functional groups in selective generation of C-H- $\pi$ interactions: In situ cryo-crystallographic studies on benzyl derivatives, CrystEngComm 12, 3112-3118 (2010), doi:10.1039/C001190H.

\section{Geometry files:}

- CIF: pp. 841

- POSCAR: pp. 841 


\section{Tongbaite $\left(\mathrm{Cr}_{3} \mathrm{C}_{2}, D 5_{10}\right)$ Structure: A2B3_oP20_62_2c_3c}
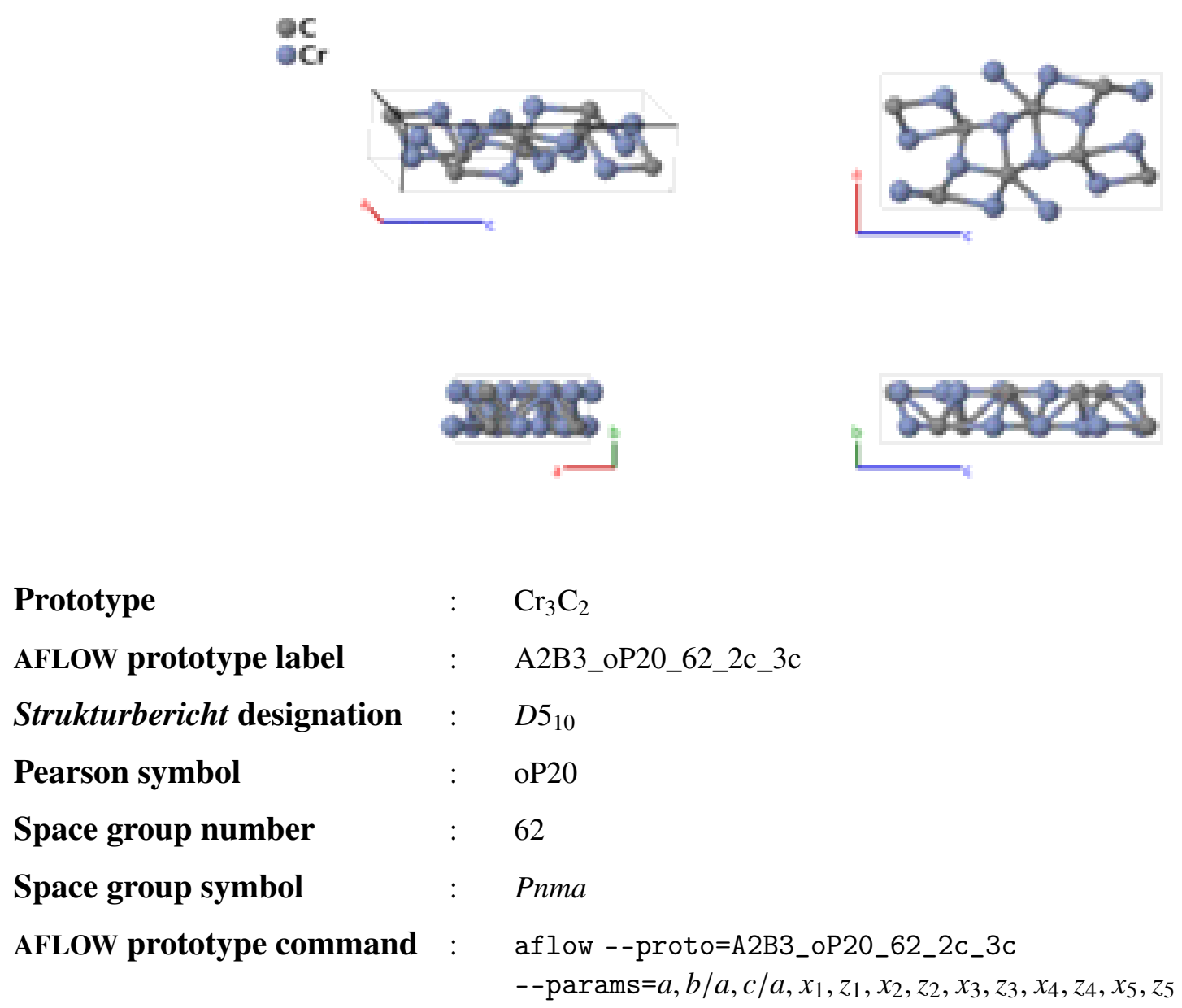

\section{Other compounds with this structure:}

- $\mathrm{Hf}_{3} \mathrm{P}_{2}$

- Several authors remark that this is the anti-type of Stibnite $\left(\mathrm{Sb}_{2} \mathrm{~S}_{3}, D 5_{8}, \mathrm{~A} 3 \mathrm{~B} 2 \_\mathrm{oP} 20 \_62 \_3 \mathrm{c} \_2 \mathrm{c}\right)$.

\section{Simple Orthorhombic primitive vectors:}

$$
\begin{aligned}
& \mathbf{a}_{1}=a \hat{\mathbf{x}} \\
& \mathbf{a}_{2}=b \hat{\mathbf{y}} \\
& \mathbf{a}_{3}=c \hat{\mathbf{z}}
\end{aligned}
$$

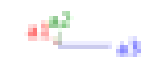

Basis vectors:
Lattice Coordinates
Cartesian Coordinates
Wyckoff Position
Atom Type
$\mathbf{B}_{1}=x_{1} \mathbf{a}_{1}+\frac{1}{4} \mathbf{a}_{2}+z_{1} \mathbf{a}_{3}$
$=\quad x_{1} a \hat{\mathbf{x}}+\frac{1}{4} b \hat{\mathbf{y}}+z_{1} c \hat{\mathbf{z}}$
$\mathbf{B}_{2}=\left(\frac{1}{2}-x_{1}\right) \mathbf{a}_{1}+\frac{3}{4} \mathbf{a}_{2}+\left(\frac{1}{2}+z_{1}\right) \mathbf{a}_{3}=\left(\frac{1}{2}-x_{1}\right) a \hat{\mathbf{x}}+\frac{3}{4} b \hat{\mathbf{y}}+\left(\frac{1}{2}+z_{1}\right) c \hat{\mathbf{z}}$
$\mathbf{B}_{3}=$
$-x_{1} \mathbf{a}_{1}+\frac{3}{4} \mathbf{a}_{2}-z_{1} \mathbf{a}_{3}$
$=\quad-x_{1} a \hat{\mathbf{x}}+\frac{3}{4} b \hat{\mathbf{y}}-z_{1} c \hat{\mathbf{z}}$
C I 


\begin{tabular}{|c|c|c|c|c|c|c|}
\hline $\mathbf{B}_{4}$ & $=$ & $\left(\frac{1}{2}+x_{1}\right) \mathbf{a}_{1}+\frac{1}{4} \mathbf{a}_{2}+\left(\frac{1}{2}-z_{1}\right) \mathbf{a}_{3}$ & $=$ & $\left(\frac{1}{2}+x_{1}\right) a \hat{\mathbf{x}}+\frac{1}{4} b \hat{\mathbf{y}}+\left(\frac{1}{2}-z_{1}\right) c \hat{\mathbf{z}}$ & $(4 c)$ & C I \\
\hline $\mathbf{B}_{5}$ & $=$ & $x_{2} \mathbf{a}_{1}+\frac{1}{4} \mathbf{a}_{2}+z_{2} \mathbf{a}_{3}$ & $=$ & $x_{2} a \hat{\mathbf{x}}+\frac{1}{4} b \hat{\mathbf{y}}+z_{2} c \hat{\mathbf{z}}$ & $(4 c)$ & C II \\
\hline $\mathbf{B}_{6}$ & $=$ & $\left(\frac{1}{2}-x_{2}\right) \mathbf{a}_{1}+\frac{3}{4} \mathbf{a}_{2}+\left(\frac{1}{2}+z_{2}\right) \mathbf{a}_{3}$ & $=$ & $\left(\frac{1}{2}-x_{2}\right) a \hat{\mathbf{x}}+\frac{3}{4} b \hat{\mathbf{y}}+\left(\frac{1}{2}+z_{2}\right) c \hat{\mathbf{z}}$ & $(4 c)$ & C II \\
\hline $\mathbf{B}_{7}$ & $=$ & $-x_{2} \mathbf{a}_{1}+\frac{3}{4} \mathbf{a}_{2}-z_{2} \mathbf{a}_{3}$ & $=$ & $-x_{2} a \hat{\mathbf{x}}+\frac{3}{4} b \hat{\mathbf{y}}-z_{2} c \hat{\mathbf{z}}$ & $(4 c)$ & C II \\
\hline $\mathbf{B}_{8}$ & $=$ & $\left(\frac{1}{2}+x_{2}\right) \mathbf{a}_{1}+\frac{1}{4} \mathbf{a}_{2}+\left(\frac{1}{2}-z_{2}\right) \mathbf{a}_{3}$ & $=$ & $\left(\frac{1}{2}+x_{2}\right) a \hat{\mathbf{x}}+\frac{1}{4} b \hat{\mathbf{y}}+\left(\frac{1}{2}-z_{2}\right) c \hat{\mathbf{z}}$ & $(4 c)$ & C II \\
\hline $\mathbf{B}_{9}$ & $=$ & $x_{3} \mathbf{a}_{1}+\frac{1}{4} \mathbf{a}_{2}+z_{3} \mathbf{a}_{3}$ & $=$ & $x_{3} a \hat{\mathbf{x}}+\frac{1}{4} b \hat{\mathbf{y}}+z_{3} c \hat{\mathbf{z}}$ & $(4 c)$ & $\mathrm{Cr} \mathrm{I}$ \\
\hline $\mathbf{B}_{10}$ & $=$ & $\left(\frac{1}{2}-x_{3}\right) \mathbf{a}_{1}+\frac{3}{4} \mathbf{a}_{2}+\left(\frac{1}{2}+z_{3}\right) \mathbf{a}_{3}$ & $=$ & $\left(\frac{1}{2}-x_{3}\right) a \hat{\mathbf{x}}+\frac{3}{4} b \hat{\mathbf{y}}+\left(\frac{1}{2}+z_{3}\right) c \hat{\mathbf{z}}$ & $(4 c)$ & Cr I \\
\hline $\mathbf{B}_{11}$ & $=$ & $-x_{3} \mathbf{a}_{1}+\frac{3}{4} \mathbf{a}_{2}-z_{3} \mathbf{a}_{3}$ & $=$ & $-x_{3} a \hat{\mathbf{x}}+\frac{3}{4} b \hat{\mathbf{y}}-z_{3} c \hat{\mathbf{z}}$ & $(4 c)$ & $\mathrm{Cr} \mathrm{I}$ \\
\hline $\mathbf{B}_{12}$ & $=$ & $\left(\frac{1}{2}+x_{3}\right) \mathbf{a}_{1}+\frac{1}{4} \mathbf{a}_{2}+\left(\frac{1}{2}-z_{3}\right) \mathbf{a}_{3}$ & $=$ & $\left(\frac{1}{2}+x_{3}\right) a \hat{\mathbf{x}}+\frac{1}{4} b \hat{\mathbf{y}}+\left(\frac{1}{2}-z_{3}\right) c \hat{\mathbf{z}}$ & $(4 c)$ & $\mathrm{Cr} \mathrm{I}$ \\
\hline $\mathbf{B}_{13}$ & $=$ & $x_{4} \mathbf{a}_{1}+\frac{1}{4} \mathbf{a}_{2}+z_{4} \mathbf{a}_{3}$ & $=$ & $x_{4} a \hat{\mathbf{x}}+\frac{1}{4} b \hat{\mathbf{y}}+z_{4} c \hat{\mathbf{z}}$ & $(4 c)$ & Cr II \\
\hline $\mathbf{B}_{14}$ & $=$ & $\left(\frac{1}{2}-x_{4}\right) \mathbf{a}_{1}+\frac{3}{4} \mathbf{a}_{2}+\left(\frac{1}{2}+z_{4}\right) \mathbf{a}_{3}$ & $=$ & $\left(\frac{1}{2}-x_{4}\right) a \hat{\mathbf{x}}+\frac{3}{4} b \hat{\mathbf{y}}+\left(\frac{1}{2}+z_{4}\right) c \hat{\mathbf{z}}$ & $(4 c)$ & Cr II \\
\hline $\mathbf{B}_{15}$ & $=$ & $-x_{4} \mathbf{a}_{1}+\frac{3}{4} \mathbf{a}_{2}-z_{4} \mathbf{a}_{3}$ & $=$ & $-x_{4} a \hat{\mathbf{x}}+\frac{3}{4} b \hat{\mathbf{y}}-z_{4} c \hat{\mathbf{z}}$ & $(4 c)$ & Cr II \\
\hline $\mathbf{B}_{16}$ & $=$ & $\left(\frac{1}{2}+x_{4}\right) \mathbf{a}_{1}+\frac{1}{4} \mathbf{a}_{2}+\left(\frac{1}{2}-z_{4}\right) \mathbf{a}_{3}$ & $=$ & $\left(\frac{1}{2}+x_{4}\right) a \hat{\mathbf{x}}+\frac{1}{4} b \hat{\mathbf{y}}+\left(\frac{1}{2}-z_{4}\right) c \hat{\mathbf{z}}$ & $(4 c)$ & Cr II \\
\hline $\mathbf{B}_{17}$ & $=$ & $x_{5} \mathbf{a}_{1}+\frac{1}{4} \mathbf{a}_{2}+z_{5} \mathbf{a}_{3}$ & $=$ & $x_{5} a \hat{\mathbf{x}}+\frac{1}{4} b \hat{\mathbf{y}}+z_{5} c \hat{\mathbf{z}}$ & $(4 c)$ & Cr III \\
\hline $\mathbf{B}_{18}$ & $=$ & $\left(\frac{1}{2}-x_{5}\right) \mathbf{a}_{1}+\frac{3}{4} \mathbf{a}_{2}+\left(\frac{1}{2}+z_{5}\right) \mathbf{a}_{3}$ & $=$ & $\left(\frac{1}{2}-x_{5}\right) a \hat{\mathbf{x}}+\frac{3}{4} b \hat{\mathbf{y}}+\left(\frac{1}{2}+z_{5}\right) c \hat{\mathbf{z}}$ & $(4 c)$ & Cr III \\
\hline $\mathbf{B}_{19}$ & $=$ & $-x_{5} \mathbf{a}_{1}+\frac{3}{4} \mathbf{a}_{2}-z_{5} \mathbf{a}_{3}$ & $=$ & $-x_{5} a \hat{\mathbf{x}}+\frac{3}{4} b \hat{\mathbf{y}}-z_{5} c \hat{\mathbf{z}}$ & $(4 c)$ & Cr III \\
\hline $\mathbf{B}_{20}$ & $=$ & $\left(\frac{1}{2}+x_{5}\right) \mathbf{a}_{1}+\frac{1}{4} \mathbf{a}_{2}+\left(\frac{1}{2}-z_{5}\right) \mathbf{a}_{3}$ & $=$ & $\left(\frac{1}{2}+x_{5}\right) a \hat{\mathbf{x}}+\frac{1}{4} b \hat{\mathbf{y}}+\left(\frac{1}{2}-z_{5}\right) c \hat{\mathbf{z}}$ & $(4 c)$ & Cr III \\
\hline
\end{tabular}

\section{References:}

- S. Rundqvist and G. Runnsjö, Crystal Structure Refinement of $\mathrm{Cr}_{3} C_{2}$, Acta Chem. Scand. 23, 1191-1199 (1969), doi:10.3891/acta.chem.scand.23-1191.

\section{Geometry files:}

- CIF: pp. 841

- POSCAR: pp. 842 
Forsterite $\left(\mathrm{Mg}_{2} \mathrm{SiO}_{4}, \mathrm{S1}_{2}\right)$ Structure:

A2B4C_oP28_62_ac_2cd_c

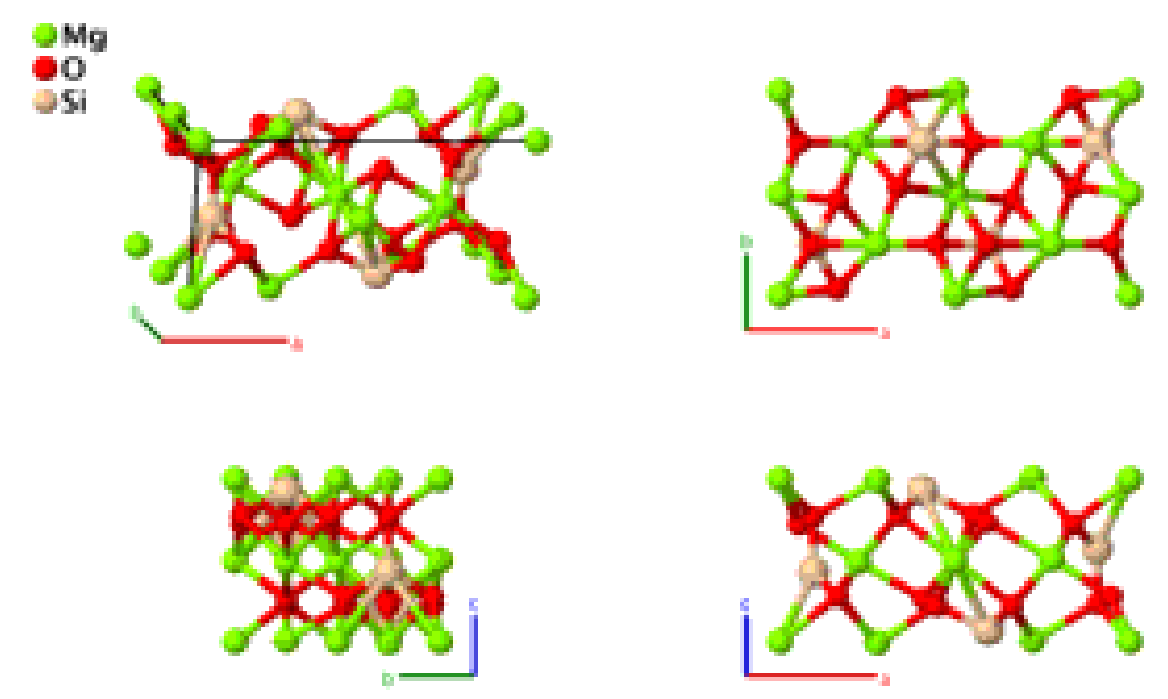

\section{Prototype}

AFLOW prototype label

Strukturbericht designation

Pearson symbol

Space group number

Space group symbol

AFLOW prototype command
: $\quad \mathrm{Mg}_{2} \mathrm{SiO}_{4}$

: A2B4C_oP28_62_ac_2cd_c

: $\quad S 1_{2}$

: $\quad$ oP28

: 62

: Pnma

: $\quad$ aflow --proto=A2B4C_oP28_62_ac_2cd_c

- params $=a, b / a, c / a, x_{2}, z_{2}, x_{3}, z_{3}, x_{4}, z_{4}, x_{5}, z_{5}, x_{6}, y_{6}, z_{6}$

\section{Other compounds with this structure:}

- $\mathrm{Fe}_{2} \mathrm{SiO}_{4}$ (fayalite), (Mg,Fe)CaSiO $4,(\mathrm{Mg}, \mathrm{Mn}) \mathrm{SiO}_{4}, \mathrm{Al}_{2} \mathrm{BeO}_{4}, \mathrm{Fe}_{2} \mathrm{SiS}_{4}, \mathrm{Mg}_{2} \mathrm{GeS}_{4}, \mathrm{Mg}_{2} \mathrm{GeS}_{4}, \mathrm{Mn}_{2} \mathrm{GeS}_{4}, \mathrm{Tm}_{2} \mathrm{ZnS}_{4}$

- This structure is the magnesium end-point of olivine, $(\mathrm{Mg}, \mathrm{Fe})_{2} \mathrm{SiO}_{4}$. (Hazen, 1976) reports the structure in the Pbnm setting of space group \#62. We have transformed this into the standard Pnma setting. We use the structural data at $23^{\circ} \mathrm{C}$.

\section{Simple Orthorhombic primitive vectors:}

$$
\begin{aligned}
& \mathbf{a}_{1}=a \hat{\mathbf{x}} \\
& \mathbf{a}_{2}=b \hat{\mathbf{y}} \\
& \mathbf{a}_{3}=c \hat{\mathbf{z}}
\end{aligned}
$$
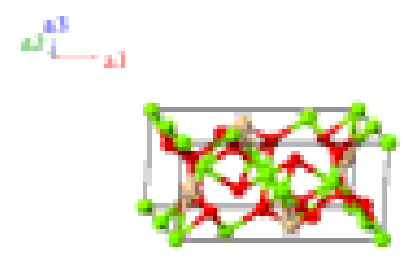

Basis vectors: 


\begin{tabular}{|c|c|c|c|c|c|c|}
\hline $\mathbf{B}_{1}$ & $=$ & $0 \mathbf{a}_{1}+0 \mathbf{a}_{2}+0 \mathbf{a}_{3}$ & $=$ & $0 \hat{\mathbf{x}}+0 \hat{\mathbf{y}}+0 \hat{\mathbf{z}}$ & $(4 a)$ & $\mathrm{Mg} \mathrm{I}$ \\
\hline $\mathbf{B}_{2}$ & $=$ & $\frac{1}{2} \mathbf{a}_{1}+\frac{1}{2} \mathbf{a}_{3}$ & $=$ & $\frac{1}{2} a \hat{\mathbf{x}}+\frac{1}{2} c \hat{\mathbf{z}}$ & $(4 a)$ & $\mathrm{Mg} \mathrm{I}$ \\
\hline $\mathbf{B}_{3}$ & $=$ & $\frac{1}{2} \mathbf{a}_{2}$ & $=$ & $\frac{1}{2} b \hat{\mathbf{y}}$ & $(4 a)$ & $\mathrm{Mg} \mathrm{I}$ \\
\hline $\mathbf{B}_{4}$ & $=$ & $\frac{1}{2} \mathbf{a}_{1}+\frac{1}{2} \mathbf{a}_{2}+\frac{1}{2} \mathbf{a}_{3}$ & $=$ & $\frac{1}{2} a \hat{\mathbf{x}}+\frac{1}{2} b \hat{\mathbf{y}}+\frac{1}{2} c \hat{\mathbf{z}}$ & $(4 a)$ & $\mathrm{Mg} \mathrm{I}$ \\
\hline $\mathbf{B}_{5}$ & $=$ & $x_{2} \mathbf{a}_{1}+\frac{1}{4} \mathbf{a}_{2}+z_{2} \mathbf{a}_{3}$ & $=$ & $x_{2} a \hat{\mathbf{x}}+\frac{1}{4} b \hat{\mathbf{y}}+z_{2} c \hat{\mathbf{z}}$ & $(4 c)$ & Mg II \\
\hline $\mathbf{B}_{6}$ & $=$ & $\left(\frac{1}{2}-x_{2}\right) \mathbf{a}_{1}+\frac{3}{4} \mathbf{a}_{2}+\left(\frac{1}{2}+z_{2}\right) \mathbf{a}_{3}$ & $=$ & $\left(\frac{1}{2}-x_{2}\right) a \hat{\mathbf{x}}+\frac{3}{4} b \hat{\mathbf{y}}+\left(\frac{1}{2}+z_{2}\right) c \hat{\mathbf{z}}$ & $(4 c)$ & Mg II \\
\hline $\mathbf{B}_{7}$ & $=$ & $-x_{2} \mathbf{a}_{1}+\frac{3}{4} \mathbf{a}_{2}-z_{2} \mathbf{a}_{3}$ & $=$ & $-x_{2} a \hat{\mathbf{x}}+\frac{3}{4} b \hat{\mathbf{y}}-z_{2} c \hat{\mathbf{z}}$ & $(4 c)$ & $\mathrm{Mg}$ II \\
\hline $\mathbf{B}_{8}$ & $=$ & $\left(\frac{1}{2}+x_{2}\right) \mathbf{a}_{1}+\frac{1}{4} \mathbf{a}_{2}+\left(\frac{1}{2}-z_{2}\right) \mathbf{a}_{3}$ & $=$ & $\left(\frac{1}{2}+x_{2}\right) a \hat{\mathbf{x}}+\frac{1}{4} b \hat{\mathbf{y}}+\left(\frac{1}{2}-z_{2}\right) c \hat{\mathbf{z}}$ & $(4 c)$ & Mg II \\
\hline $\mathbf{B}_{9}$ & $=$ & $x_{3} \mathbf{a}_{1}+\frac{1}{4} \mathbf{a}_{2}+z_{3} \mathbf{a}_{3}$ & $=$ & $x_{3} a \hat{\mathbf{x}}+\frac{1}{4} b \hat{\mathbf{y}}+z_{3} c \hat{\mathbf{z}}$ & $(4 c)$ & O I \\
\hline $\mathbf{B}_{10}$ & $=$ & $\left(\frac{1}{2}-x_{3}\right) \mathbf{a}_{1}+\frac{3}{4} \mathbf{a}_{2}+\left(\frac{1}{2}+z_{3}\right) \mathbf{a}_{3}$ & $=$ & $\left(\frac{1}{2}-x_{3}\right) a \hat{\mathbf{x}}+\frac{3}{4} b \hat{\mathbf{y}}+\left(\frac{1}{2}+z_{3}\right) c \hat{\mathbf{z}}$ & $(4 c)$ & O I \\
\hline $\mathbf{B}_{11}$ & $=$ & $-x_{3} \mathbf{a}_{1}+\frac{3}{4} \mathbf{a}_{2}-z_{3} \mathbf{a}_{3}$ & $=$ & $-x_{3} a \hat{\mathbf{x}}+\frac{3}{4} b \hat{\mathbf{y}}-z_{3} c \hat{\mathbf{z}}$ & $(4 c)$ & O I \\
\hline $\mathbf{B}_{12}$ & $=$ & $\left(\frac{1}{2}+x_{3}\right) \mathbf{a}_{1}+\frac{1}{4} \mathbf{a}_{2}+\left(\frac{1}{2}-z_{3}\right) \mathbf{a}_{3}$ & $=$ & $\left(\frac{1}{2}+x_{3}\right) a \hat{\mathbf{x}}+\frac{1}{4} b \hat{\mathbf{y}}+\left(\frac{1}{2}-z_{3}\right) c \hat{\mathbf{z}}$ & $(4 c)$ & O I \\
\hline $\mathbf{B}_{13}$ & $=$ & $x_{4} \mathbf{a}_{1}+\frac{1}{4} \mathbf{a}_{2}+z_{4} \mathbf{a}_{3}$ & $=$ & $x_{4} a \hat{\mathbf{x}}+\frac{1}{4} b \hat{\mathbf{y}}+z_{4} c \hat{\mathbf{z}}$ & $(4 c)$ & O II \\
\hline $\mathbf{B}_{14}$ & $=$ & $\left(\frac{1}{2}-x_{4}\right) \mathbf{a}_{1}+\frac{3}{4} \mathbf{a}_{2}+\left(\frac{1}{2}+z_{4}\right) \mathbf{a}_{3}$ & $=$ & $\left(\frac{1}{2}-x_{4}\right) a \hat{\mathbf{x}}+\frac{3}{4} b \hat{\mathbf{y}}+\left(\frac{1}{2}+z_{4}\right) c \hat{\mathbf{z}}$ & $(4 c)$ & O II \\
\hline $\mathbf{B}_{15}$ & $=$ & $-x_{4} \mathbf{a}_{1}+\frac{3}{4} \mathbf{a}_{2}-z_{4} \mathbf{a}_{3}$ & $=$ & $-x_{4} a \hat{\mathbf{x}}+\frac{3}{4} b \hat{\mathbf{y}}-z_{4} c \hat{\mathbf{z}}$ & $(4 c)$ & O II \\
\hline $\mathbf{B}_{16}$ & $=$ & $\left(\frac{1}{2}+x_{4}\right) \mathbf{a}_{1}+\frac{1}{4} \mathbf{a}_{2}+\left(\frac{1}{2}-z_{4}\right) \mathbf{a}_{3}$ & $=$ & $\left(\frac{1}{2}+x_{4}\right) a \hat{\mathbf{x}}+\frac{1}{4} b \hat{\mathbf{y}}+\left(\frac{1}{2}-z_{4}\right) c \hat{\mathbf{z}}$ & $(4 c)$ & O II \\
\hline $\mathbf{B}_{17}$ & $=$ & $x_{5} \mathbf{a}_{1}+\frac{1}{4} \mathbf{a}_{2}+z_{5} \mathbf{a}_{3}$ & $=$ & $x_{5} a \hat{\mathbf{x}}+\frac{1}{4} b \hat{\mathbf{y}}+z_{5} c \hat{\mathbf{z}}$ & $(4 c)$ & $\mathrm{Si}$ \\
\hline $\mathbf{B}_{18}$ & $=$ & $\left(\frac{1}{2}-x_{5}\right) \mathbf{a}_{1}+\frac{3}{4} \mathbf{a}_{2}+\left(\frac{1}{2}+z_{5}\right) \mathbf{a}_{3}$ & $=$ & $\left(\frac{1}{2}-x_{5}\right) a \hat{\mathbf{x}}+\frac{3}{4} b \hat{\mathbf{y}}+\left(\frac{1}{2}+z_{5}\right) c \hat{\mathbf{z}}$ & $(4 c)$ & $\mathrm{Si}$ \\
\hline $\mathbf{B}_{19}$ & $=$ & $-x_{5} \mathbf{a}_{1}+\frac{3}{4} \mathbf{a}_{2}-z_{5} \mathbf{a}_{3}$ & $=$ & $-x_{5} a \hat{\mathbf{x}}+\frac{3}{4} b \hat{\mathbf{y}}-z_{5} c \hat{\mathbf{z}}$ & $(4 c)$ & $\mathrm{Si}$ \\
\hline $\mathbf{B}_{20}$ & $=$ & $\left(\frac{1}{2}+x_{5}\right) \mathbf{a}_{1}+\frac{1}{4} \mathbf{a}_{2}+\left(\frac{1}{2}-z_{5}\right) \mathbf{a}_{3}$ & $=$ & $\left(\frac{1}{2}+x_{5}\right) a \hat{\mathbf{x}}+\frac{1}{4} b \hat{\mathbf{y}}+\left(\frac{1}{2}-z_{5}\right) c \hat{\mathbf{z}}$ & $(4 c)$ & $\mathrm{Si}$ \\
\hline $\mathbf{B}_{21}$ & $=$ & $x_{6} \mathbf{a}_{1}+y_{6} \mathbf{a}_{2}+z_{6} \mathbf{a}_{3}$ & $=$ & $x_{6} a \hat{\mathbf{x}}+y_{6} b \hat{\mathbf{y}}+z_{6} c \hat{\mathbf{z}}$ & $(8 d)$ & O III \\
\hline $\mathbf{B}_{22}$ & $=$ & $\left(\frac{1}{2}-x_{6}\right) \mathbf{a}_{1}-y_{6} \mathbf{a}_{2}+\left(\frac{1}{2}+z_{6}\right) \mathbf{a}_{3}$ & $=$ & $\left(\frac{1}{2}-x_{6}\right) a \hat{\mathbf{x}}-y_{6} b \hat{\mathbf{y}}+\left(\frac{1}{2}+z_{6}\right) c \hat{\mathbf{z}}$ & $(8 d)$ & O III \\
\hline $\mathbf{B}_{23}$ & $=$ & $-x_{6} \mathbf{a}_{1}+\left(\frac{1}{2}+y_{6}\right) \mathbf{a}_{2}-z_{6} \mathbf{a}_{3}$ & $=$ & $-x_{6} a \hat{\mathbf{x}}+\left(\frac{1}{2}+y_{6}\right) b \hat{\mathbf{y}}-z_{6} c \hat{\mathbf{z}}$ & $(8 d)$ & O III \\
\hline $\mathbf{B}_{24}$ & $=$ & $\begin{array}{c}\left(\frac{1}{2}+x_{6}\right) \mathbf{a}_{1}+\left(\frac{1}{2}-y_{6}\right) \mathbf{a}_{2}+ \\
\left(\frac{1}{2}-z_{6}\right) \mathbf{a}_{3}\end{array}$ & $=$ & $\begin{array}{c}\left(\frac{1}{2}+x_{6}\right) a \hat{\mathbf{x}}+\left(\frac{1}{2}-y_{6}\right) b \hat{\mathbf{y}}+ \\
\left(\frac{1}{2}-z_{6}\right) c \hat{\mathbf{z}}\end{array}$ & $(8 d)$ & O III \\
\hline $\mathbf{B}_{25}$ & $=$ & $-x_{6} \mathbf{a}_{1}-y_{6} \mathbf{a}_{2}-z_{6} \mathbf{a}_{3}$ & $=$ & $-x_{6} a \hat{\mathbf{x}}-y_{6} b \hat{\mathbf{y}}-z_{6} c \hat{\mathbf{z}}$ & $(8 d)$ & O III \\
\hline $\mathbf{B}_{26}$ & $=$ & $\left(\frac{1}{2}+x_{6}\right) \mathbf{a}_{1}+y_{6} \mathbf{a}_{2}+\left(\frac{1}{2}-z_{6}\right) \mathbf{a}_{3}$ & $=$ & $\left(\frac{1}{2}+x_{6}\right) a \hat{\mathbf{x}}+y_{6} b \hat{\mathbf{y}}+\left(\frac{1}{2}-z_{6}\right) c \hat{\mathbf{z}}$ & $(8 d)$ & O III \\
\hline $\mathbf{B}_{27}$ & $=$ & $x_{6} \mathbf{a}_{1}+\left(\frac{1}{2}-y_{6}\right) \mathbf{a}_{2}+z_{6} \mathbf{a}_{3}$ & $=$ & $x_{6} a \hat{\mathbf{x}}+\left(\frac{1}{2}-y_{6}\right) b \hat{\mathbf{y}}+z_{6} c \hat{\mathbf{z}}$ & $(8 d)$ & O III \\
\hline $\mathbf{B}_{28}$ & $=$ & $\begin{array}{c}\left(\frac{1}{2}-x_{6}\right) \mathbf{a}_{1}+\left(\frac{1}{2}+y_{6}\right) \mathbf{a}_{2}+ \\
\left(\frac{1}{2}+z_{6}\right) \mathbf{a}_{3}\end{array}$ & $=$ & $\begin{array}{c}\left(\frac{1}{2}-x_{6}\right) a \hat{\mathbf{x}}+\left(\frac{1}{2}+y_{6}\right) b \hat{\mathbf{y}}+ \\
\left(\frac{1}{2}+z_{6}\right) c \hat{\mathbf{z}}\end{array}$ & $(8 d)$ & O III \\
\hline
\end{tabular}

\section{References:}

- R. M. Hazen, Effects of temperature and pressure on the crystal structure of forsterite, Am. Mineral. 61, 1280-1293 (1976).

\section{Geometry files:}

- CIF: pp. 842

- POSCAR: pp. 842 


\section{$\mathrm{SrH}_{2}(C 29)$ Structure: A2B_oP12_62_2c_c}
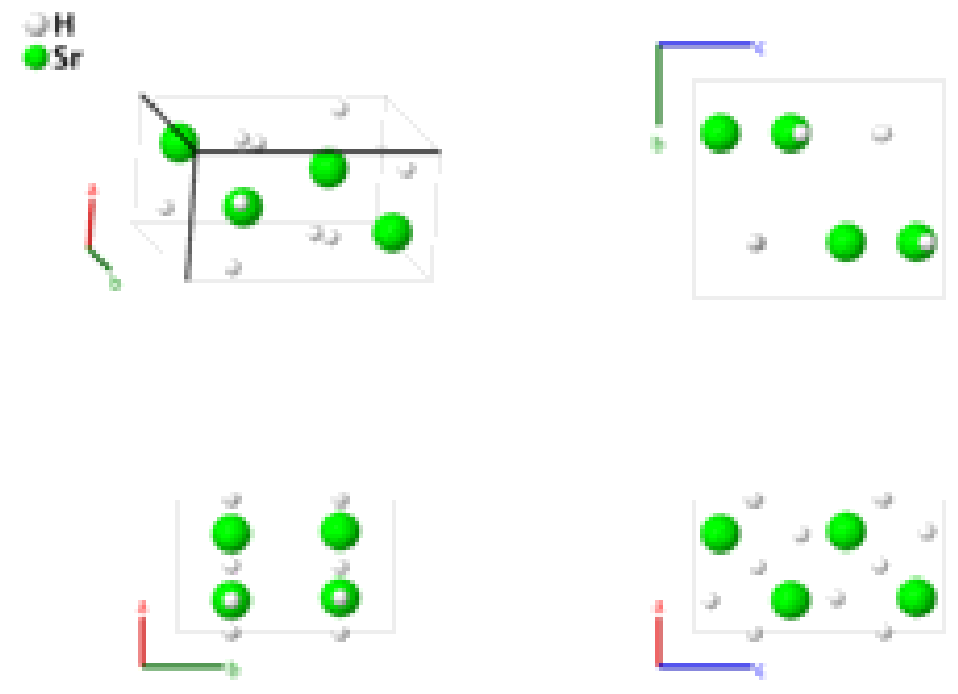

Prototype

AFLOW prototype label

Strukturbericht designation

Pearson symbol

Space group number

Space group symbol

AFLOW prototype command
: $\quad \mathrm{SrH}_{2}$

: A2B_oP12_62_2c_c

: $\quad C 29$

: $\quad \mathrm{oP} 12$

: 62

: Pnma

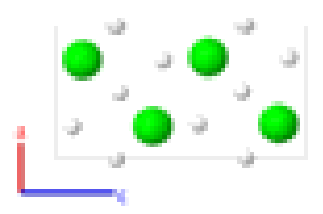

Other compounds with this structure:

- $\mathrm{CaH}_{2}$

Simple Orthorhombic primitive vectors:

$$
\begin{aligned}
& \mathbf{a}_{1}=a \hat{\mathbf{x}} \\
& \mathbf{a}_{2}=b \hat{\mathbf{y}} \\
& \mathbf{a}_{3}=c \hat{\mathbf{z}}
\end{aligned}
$$

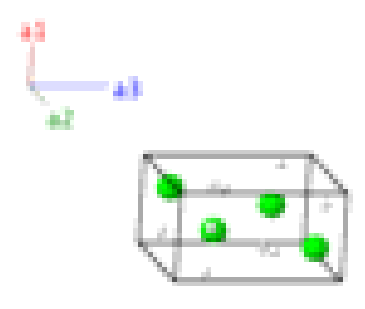

Basis vectors:

$\begin{array}{llcccc} & \text { Lattice Coordinates } & & \text { Cartesian Coordinates } & \text { Wyckoff Position } & \text { Atom Type } \\ \mathbf{B}_{1}= & x_{1} \mathbf{a}_{1}+\frac{1}{4} \mathbf{a}_{2}+z_{1} \mathbf{a}_{3} & = & x_{1} a \hat{\mathbf{x}}+\frac{1}{4} b \hat{\mathbf{y}}+z_{1} c \hat{\mathbf{z}} & (4 c) & \text { H I } \\ \mathbf{B}_{2}= & \left(\frac{1}{2}-x_{1}\right) \mathbf{a}_{1}+\frac{3}{4} \mathbf{a}_{2}+\left(\frac{1}{2}+z_{1}\right) \mathbf{a}_{3} & = & \left(\frac{1}{2}-x_{1}\right) a \hat{\mathbf{x}}+\frac{3}{4} b \hat{\mathbf{y}}+\left(\frac{1}{2}+z_{1}\right) c \hat{\mathbf{z}} & (4 c) & \text { H I } \\ \mathbf{B}_{3}= & -x_{1} \mathbf{a}_{1}+\frac{3}{4} \mathbf{a}_{2}-z_{1} \mathbf{a}_{3} & = & -x_{1} a \hat{\mathbf{x}}+\frac{3}{4} b \hat{\mathbf{y}}-z_{1} c \hat{\mathbf{z}} & (4 c) & \text { H I }\end{array}$




\begin{tabular}{|c|c|c|c|c|c|c|}
\hline $\mathbf{B}_{4}$ & $=$ & $\left(\frac{1}{2}+x_{1}\right) \mathbf{a}_{1}+\frac{1}{4} \mathbf{a}_{2}+\left(\frac{1}{2}-z_{1}\right) \mathbf{a}_{3}$ & $=$ & $\left(\frac{1}{2}+x_{1}\right) a \hat{\mathbf{x}}+\frac{1}{4} b \hat{\mathbf{y}}+\left(\frac{1}{2}-z_{1}\right) c \hat{\mathbf{z}}$ & $(4 c)$ & H I \\
\hline $\mathbf{B}_{5}$ & $=$ & $x_{2} \mathbf{a}_{1}+\frac{1}{4} \mathbf{a}_{2}+z_{2} \mathbf{a}_{3}$ & $=$ & $x_{2} a \hat{\mathbf{x}}+\frac{1}{4} b \hat{\mathbf{y}}+z_{2} c \hat{\mathbf{z}}$ & $(4 c)$ & H II \\
\hline $\mathbf{B}_{6}$ & $=$ & $\left(\frac{1}{2}-x_{2}\right) \mathbf{a}_{1}+\frac{3}{4} \mathbf{a}_{2}+\left(\frac{1}{2}+z_{2}\right) \mathbf{a}_{3}$ & $=$ & $\left(\frac{1}{2}-x_{2}\right) a \hat{\mathbf{x}}+\frac{3}{4} b \hat{\mathbf{y}}+\left(\frac{1}{2}+z_{2}\right) c \hat{\mathbf{z}}$ & $(4 c)$ & H II \\
\hline $\mathbf{B}_{7}$ & $=$ & $-x_{2} \mathbf{a}_{1}+\frac{3}{4} \mathbf{a}_{2}-z_{2} \mathbf{a}_{3}$ & $=$ & $-x_{2} a \hat{\mathbf{x}}+\frac{3}{4} b \hat{\mathbf{y}}-z_{2} c \hat{\mathbf{z}}$ & $(4 c)$ & H II \\
\hline $\mathbf{B}_{8}$ & $=$ & $\left(\frac{1}{2}+x_{2}\right) \mathbf{a}_{1}+\frac{1}{4} \mathbf{a}_{2}+\left(\frac{1}{2}-z_{2}\right) \mathbf{a}_{3}$ & $=$ & $\left(\frac{1}{2}+x_{2}\right) a \hat{\mathbf{x}}+\frac{1}{4} b \hat{\mathbf{y}}+\left(\frac{1}{2}-z_{2}\right) c \hat{\mathbf{z}}$ & $(4 c)$ & H II \\
\hline $\mathbf{B}_{9}$ & $=$ & $x_{3} \mathbf{a}_{1}+\frac{1}{4} \mathbf{a}_{2}+z_{3} \mathbf{a}_{3}$ & $=$ & $x_{3} a \hat{\mathbf{x}}+\frac{1}{4} b \hat{\mathbf{y}}+z_{3} c \hat{\mathbf{z}}$ & $(4 c)$ & $\mathrm{Sr}$ \\
\hline $\mathbf{B}_{10}$ & $=$ & $\left(\frac{1}{2}-x_{3}\right) \mathbf{a}_{1}+\frac{3}{4} \mathbf{a}_{2}+\left(\frac{1}{2}+z_{3}\right) \mathbf{a}_{3}$ & $=$ & $\left(\frac{1}{2}-x_{3}\right) a \hat{\mathbf{x}}+\frac{3}{4} b \hat{\mathbf{y}}+\left(\frac{1}{2}+z_{3}\right) c \hat{\mathbf{z}}$ & $(4 c)$ & $\mathrm{Sr}$ \\
\hline $\mathbf{B}_{11}$ & $=$ & $-x_{3} \mathbf{a}_{1}+\frac{3}{4} \mathbf{a}_{2}-z_{3} \mathbf{a}_{3}$ & $=$ & $-x_{3} a \hat{\mathbf{x}}+\frac{3}{4} b \hat{\mathbf{y}}-z_{3} c \hat{\mathbf{z}}$ & $(4 c)$ & $\mathrm{Sr}$ \\
\hline $\mathbf{B}_{12}$ & $=$ & $\left(\frac{1}{2}+x_{3}\right) \mathbf{a}_{1}+\frac{1}{4} \mathbf{a}_{2}+\left(\frac{1}{2}-z_{3}\right) \mathbf{a}_{3}$ & $=$ & $\left(\frac{1}{2}+x_{3}\right) a \hat{\mathbf{x}}+\frac{1}{4} b \hat{\mathbf{y}}+\left(\frac{1}{2}-z_{3}\right) c \hat{\mathbf{z}}$ & $(4 c)$ & $\mathrm{Sr}$ \\
\hline
\end{tabular}

\section{References:}

- R. C. Ropp, Encyclopedia of the Alkaline Earth Compounds (Elsevier, Oxford, 2013), chap. 2, pp. 30-31.

\section{Geometry files:}

- CIF: pp. 842

- POSCAR: pp. 843 


\section{$\epsilon-\mathrm{NiAl}_{3}\left(D 0_{20}\right)$ Structure: A3B_oP16_62_cd_c}

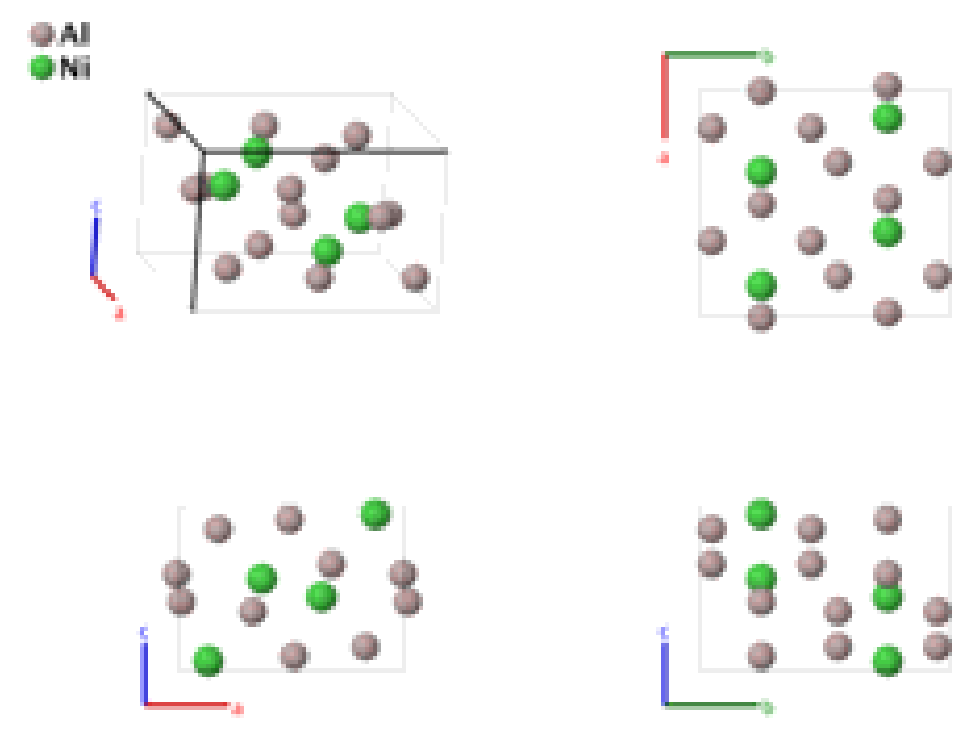

Prototype

AFLOW prototype label

Strukturbericht designation

Pearson symbol

Space group number

Space group symbol

AFLOW prototype command
: $\quad \epsilon-\mathrm{NiAl}_{3}$

: A3B_oP16_62_cd_c

: $\quad D 0_{20}$

: $\quad$ oP16

: 62

: Pnma

\section{Simple Orthorhombic primitive vectors:}

$$
\begin{aligned}
& \mathbf{a}_{1}=a \hat{\mathbf{x}} \\
& \mathbf{a}_{2}=b \hat{\mathbf{y}} \\
& \mathbf{a}_{3}=c \hat{\mathbf{z}}
\end{aligned}
$$
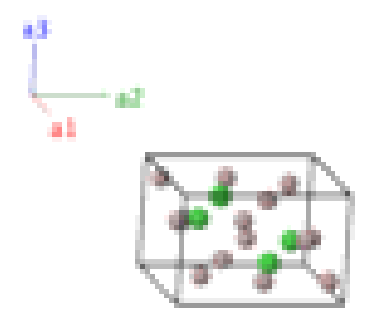

Basis vectors:

Lattice Coordinates

$$
\begin{array}{llccc}
\mathbf{B}_{1}= & x_{1} \mathbf{a}_{1}+\frac{1}{4} \mathbf{a}_{2}+z_{1} \mathbf{a}_{3} & = & x_{1} a \hat{\mathbf{x}}+\frac{1}{4} b \hat{\mathbf{y}}+z_{1} c \hat{\mathbf{z}} \\
\mathbf{B}_{2}= & \left(\frac{1}{2}-x_{1}\right) \mathbf{a}_{1}+\frac{3}{4} \mathbf{a}_{2}+\left(\frac{1}{2}+z_{1}\right) \mathbf{a}_{3} & = & \left(\frac{1}{2}-x_{1}\right) a \hat{\mathbf{x}}+\frac{3}{4} b \hat{\mathbf{y}}+\left(\frac{1}{2}+z_{1}\right) c \hat{\mathbf{z}} \\
\mathbf{B}_{3}= & -x_{1} \mathbf{a}_{1}+\frac{3}{4} \mathbf{a}_{2}-z_{1} \mathbf{a}_{3} & = & -x_{1} a \hat{\mathbf{x}}+\frac{3}{4} b \hat{\mathbf{y}}-z_{1} c \hat{\mathbf{z}} \\
\mathbf{B}_{4}= & \left(\frac{1}{2}+x_{1}\right) \mathbf{a}_{1}+\frac{1}{4} \mathbf{a}_{2}+\left(\frac{1}{2}-z_{1}\right) \mathbf{a}_{3} & = & \left(\frac{1}{2}+x_{1}\right) a \hat{\mathbf{x}}+\frac{1}{4} b \hat{\mathbf{y}}+\left(\frac{1}{2}-z_{1}\right) c \hat{\mathbf{z}} \\
\mathbf{B}_{5}= & x_{2} \mathbf{a}_{1}+\frac{1}{4} \mathbf{a}_{2}+z_{2} \mathbf{a}_{3} & & & x_{2} a \hat{\mathbf{x}}+\frac{1}{4} b \hat{\mathbf{y}}+z_{2} c \hat{\mathbf{z}} \\
\mathbf{B}_{6} & = & \left(\frac{1}{2}-x_{2}\right) \mathbf{a}_{1}+\frac{3}{4} \mathbf{a}_{2}+\left(\frac{1}{2}+z_{2}\right) \mathbf{a}_{3} & = & \left(\frac{1}{2}-x_{2}\right) a \hat{\mathbf{x}}+\frac{3}{4} b \hat{\mathbf{y}}+\left(\frac{1}{2}+z_{2}\right) c \hat{\mathbf{z}}
\end{array}
$$

Wyckoff Position

$(4 c)$
Atom Type

Al I

Al I

Al I

Al I

$\mathrm{Ni}$

$\mathrm{Ni}$ 


\begin{tabular}{|c|c|c|c|c|c|c|}
\hline $\mathbf{B}_{7}$ & $=$ & $-x_{2} \mathbf{a}_{1}+\frac{3}{4} \mathbf{a}_{2}-z_{2} \mathbf{a}_{3}$ & $=$ & $-x_{2} a \hat{\mathbf{x}}+\frac{3}{4} b \hat{\mathbf{y}}-z_{2} c \hat{\mathbf{z}}$ & $(4 c)$ & $\mathrm{Ni}$ \\
\hline $\mathbf{B}_{8}$ & $=$ & $\left(\frac{1}{2}+x_{2}\right) \mathbf{a}_{1}+\frac{1}{4} \mathbf{a}_{2}+\left(\frac{1}{2}-z_{2}\right) \mathbf{a}_{3}$ & $=$ & $\left(\frac{1}{2}+x_{2}\right) a \hat{\mathbf{x}}+\frac{1}{4} b \hat{\mathbf{y}}+\left(\frac{1}{2}-z_{2}\right) c \hat{\mathbf{z}}$ & $(4 c)$ & $\mathrm{Ni}$ \\
\hline $\mathbf{B}_{9}$ & $=$ & $x_{3} \mathbf{a}_{1}+y_{3} \mathbf{a}_{2}+z_{3} \mathbf{a}_{3}$ & $=$ & $x_{3} a \hat{\mathbf{x}}+y_{3} b \hat{\mathbf{y}}+z_{3} c \hat{\mathbf{z}}$ & $(8 d)$ & $\mathrm{Al}$ II \\
\hline $\mathbf{B}_{10}$ & $=$ & $\left(\frac{1}{2}-x_{3}\right) \mathbf{a}_{1}-y_{3} \mathbf{a}_{2}+\left(\frac{1}{2}+z_{3}\right) \mathbf{a}_{3}$ & $=$ & $\left(\frac{1}{2}-x_{3}\right) a \hat{\mathbf{x}}-y_{3} b \hat{\mathbf{y}}+\left(\frac{1}{2}+z_{3}\right) c \hat{\mathbf{z}}$ & $(8 d)$ & Al II \\
\hline $\mathbf{B}_{11}$ & $=$ & $-x_{3} \mathbf{a}_{1}+\left(\frac{1}{2}+y_{3}\right) \mathbf{a}_{2}-z_{3} \mathbf{a}_{3}$ & $=$ & $-x_{3} a \hat{\mathbf{x}}+\left(\frac{1}{2}+y_{3}\right) b \hat{\mathbf{y}}-z_{3} c \hat{\mathbf{z}}$ & $(8 d)$ & Al II \\
\hline $\mathbf{B}_{12}$ & $=$ & $\begin{array}{c}\left(\frac{1}{2}+x_{3}\right) \mathbf{a}_{1}+\left(\frac{1}{2}-y_{3}\right) \mathbf{a}_{2}+ \\
\left(\frac{1}{2}-z_{3}\right) \mathbf{a}_{3}\end{array}$ & $=$ & $\begin{array}{c}\left(\frac{1}{2}+x_{3}\right) a \hat{\mathbf{x}}+\left(\frac{1}{2}-y_{3}\right) b \hat{\mathbf{y}}+ \\
\left(\frac{1}{2}-z_{3}\right) c \hat{\mathbf{z}}\end{array}$ & $(8 d)$ & $\mathrm{Al}$ II \\
\hline $\mathbf{B}_{13}$ & $=$ & $-x_{3} \mathbf{a}_{1}-y_{3} \mathbf{a}_{2}-z_{3} \mathbf{a}_{3}$ & $=$ & $-x_{3} a \hat{\mathbf{x}}-y_{3} b \hat{\mathbf{y}}-z_{3} c \hat{\mathbf{z}}$ & $(8 d)$ & $\mathrm{Al} \mathrm{II}$ \\
\hline $\mathbf{B}_{14}$ & $=$ & $\left(\frac{1}{2}+x_{3}\right) \mathbf{a}_{1}+y_{3} \mathbf{a}_{2}+\left(\frac{1}{2}-z_{3}\right) \mathbf{a}_{3}$ & $=$ & $\left(\frac{1}{2}+x_{3}\right) a \hat{\mathbf{x}}+y_{3} b \hat{\mathbf{y}}+\left(\frac{1}{2}-z_{3}\right) c \hat{\mathbf{z}}$ & $(8 d)$ & Al II \\
\hline $\mathbf{B}_{15}$ & $=$ & $x_{3} \mathbf{a}_{1}+\left(\frac{1}{2}-y_{3}\right) \mathbf{a}_{2}+z_{3} \mathbf{a}_{3}$ & $=$ & $x_{3} a \hat{\mathbf{x}}+\left(\frac{1}{2}-y_{3}\right) b \hat{\mathbf{y}}+z_{3} c \hat{\mathbf{z}}$ & $(8 d)$ & $\mathrm{Al} \mathrm{II}$ \\
\hline $\mathbf{B}_{16}$ & $=$ & $\begin{array}{c}\left(\frac{1}{2}-x_{3}\right) \mathbf{a}_{1}+\left(\frac{1}{2}+y_{3}\right) \mathbf{a}_{2}+ \\
\left(\frac{1}{2}+z_{3}\right) \mathbf{a}_{3}\end{array}$ & $=$ & $\begin{array}{c}\left(\frac{1}{2}-x_{3}\right) a \hat{\mathbf{x}}+\left(\frac{1}{2}+y_{3}\right) b \hat{\mathbf{y}}+ \\
\left(\frac{1}{2}+z_{3}\right) c \hat{\mathbf{z}}\end{array}$ & $(8 d)$ & $\mathrm{Al}$ II \\
\hline
\end{tabular}

\section{References:}

- A. J. Bradley and A. Taylor, The crystal structures of $\mathrm{Ni}_{2} \mathrm{Al}_{3}$ and $\mathrm{NiAl}_{3}$, Philos. Mag. 23, 1049-1067 (1937), doi:10.1080/14786443708561875.

\section{Found in:}

- W. B. Pearson, A Handbook of Lattice Spacings and Structures of Metals and Alloys, no. N.R.C. No. 4303 in International Series of Monographs on Metal Physics and Physical Metallurgy (Pergamon Press, Oxford, London, Edinburgh, New York, Paris, Frankfort, 1958), 1964 reprint with corrections edn.

\section{Geometry files:}

- CIF: pp. 843

- POSCAR: pp. 843 
Cubanite $\left(\mathrm{CuFe}_{2} \mathrm{~S}_{3}, E 9_{e}\right)$ Structure:

AB2C3_oP24_62_c_d_cd
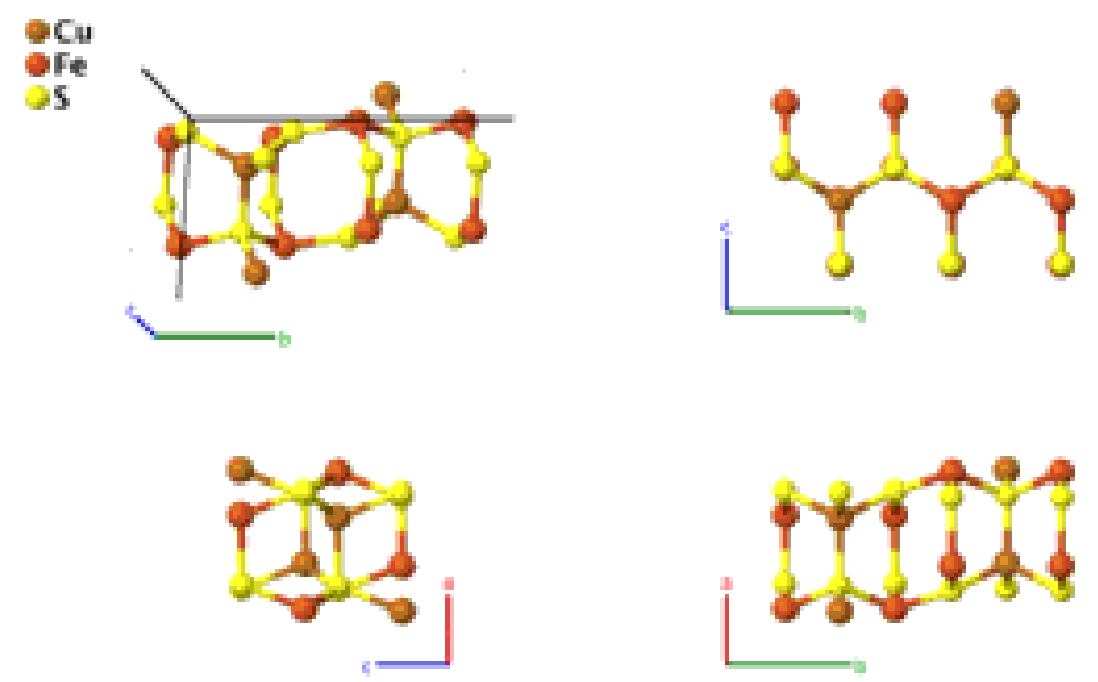

Prototype

AFLOW prototype label

Strukturbericht designation

Pearson symbol

Space group number

Space group symbol

AFLOW prototype command
: $\quad \mathrm{CuFe}_{2} \mathrm{~S}_{3}$

: AB2C3_oP24_62_c_d_cd

: $\quad E 9_{e}$

: $\quad$ oP24

: 62

: Pnma

: aflow --proto=AB2C3_oP24_62_c_d_cd

- params $=a, b / a, c / a, x_{1}, z_{1}, x_{2}, z_{2}, x_{3}, y_{3}, z_{3}, x_{4}, y_{4}, z_{4}$

- (Szymański, 1974) uses the Pcmn orientation of space group \#62 to describe this structure. We have swapped the $\hat{\mathbf{x}}$ and $\hat{\mathbf{z}}$ axis to transform this into the standard Pnma orientation.

Simple Orthorhombic primitive vectors:

$$
\begin{aligned}
& \mathbf{a}_{1}=a \hat{\mathbf{x}} \\
& \mathbf{a}_{2}=b \hat{\mathbf{y}} \\
& \mathbf{a}_{3}=c \hat{\mathbf{z}}
\end{aligned}
$$
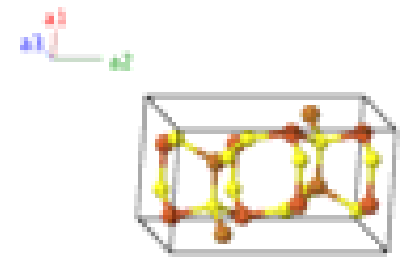

Basis vectors:

$$
\text { Lattice Coordinates }
$$

$$
\begin{array}{llccc}
\mathbf{B}_{1}= & x_{1} \mathbf{a}_{1}+\frac{1}{4} \mathbf{a}_{2}+z_{1} \mathbf{a}_{3} & = & x_{1} a \hat{\mathbf{x}}+\frac{1}{4} b \hat{\mathbf{y}}+z_{1} c \hat{\mathbf{z}} \\
\mathbf{B}_{2}= & \left(\frac{1}{2}-x_{1}\right) \mathbf{a}_{1}+\frac{3}{4} \mathbf{a}_{2}+\left(\frac{1}{2}+z_{1}\right) \mathbf{a}_{3} & = & \left(\frac{1}{2}-x_{1}\right) a \hat{\mathbf{x}}+\frac{3}{4} b \hat{\mathbf{y}}+\left(\frac{1}{2}+z_{1}\right) c \hat{\mathbf{z}} \\
\mathbf{B}_{3}= & -x_{1} \mathbf{a}_{1}+\frac{3}{4} \mathbf{a}_{2}-z_{1} \mathbf{a}_{3} & = & -x_{1} a \hat{\mathbf{x}}+\frac{3}{4} b \hat{\mathbf{y}}-z_{1} c \hat{\mathbf{z}} \\
\mathbf{B}_{4}= & \left(\frac{1}{2}+x_{1}\right) \mathbf{a}_{1}+\frac{1}{4} \mathbf{a}_{2}+\left(\frac{1}{2}-z_{1}\right) \mathbf{a}_{3} & = & \left(\frac{1}{2}+x_{1}\right) a \hat{\mathbf{x}}+\frac{1}{4} b \hat{\mathbf{y}}+\left(\frac{1}{2}-z_{1}\right) c \hat{\mathbf{z}}
\end{array}
$$$$
\text { Cartesian Coordinates }
$$

Wyckoff Position Atom Type

$\mathrm{Cu}$

$\mathrm{Cu}$

$\mathrm{Cu}$

$\mathrm{Cu}$ 


\begin{tabular}{|c|c|c|c|c|c|c|}
\hline $\mathbf{B}_{5}$ & $=$ & $x_{2} \mathbf{a}_{1}+\frac{1}{4} \mathbf{a}_{2}+z_{2} \mathbf{a}_{3}$ & $=$ & $x_{2} a \hat{\mathbf{x}}+\frac{1}{4} b \hat{\mathbf{y}}+z_{2} c \hat{\mathbf{z}}$ & $(4 c)$ & S I \\
\hline $\mathbf{B}_{6}$ & $=$ & $\left(\frac{1}{2}-x_{2}\right) \mathbf{a}_{1}+\frac{3}{4} \mathbf{a}_{2}+\left(\frac{1}{2}+z_{2}\right) \mathbf{a}_{3}$ & $=$ & $\left(\frac{1}{2}-x_{2}\right) a \hat{\mathbf{x}}+\frac{3}{4} b \hat{\mathbf{y}}+\left(\frac{1}{2}+z_{2}\right) c \hat{\mathbf{z}}$ & $(4 c)$ & S I \\
\hline $\mathbf{B}_{7}$ & $=$ & $-x_{2} \mathbf{a}_{1}+\frac{3}{4} \mathbf{a}_{2}-z_{2} \mathbf{a}_{3}$ & $=$ & $-x_{2} a \hat{\mathbf{x}}+\frac{3}{4} b \hat{\mathbf{y}}-z_{2} c \hat{\mathbf{z}}$ & $(4 c)$ & S I \\
\hline $\mathbf{B}_{8}$ & $=$ & $\left(\frac{1}{2}+x_{2}\right) \mathbf{a}_{1}+\frac{1}{4} \mathbf{a}_{2}+\left(\frac{1}{2}-z_{2}\right) \mathbf{a}_{3}$ & $=$ & $\left(\frac{1}{2}+x_{2}\right) a \hat{\mathbf{x}}+\frac{1}{4} b \hat{\mathbf{y}}+\left(\frac{1}{2}-z_{2}\right) c \hat{\mathbf{z}}$ & $(4 c)$ & S I \\
\hline $\mathbf{B}_{9}$ & $=$ & $x_{3} \mathbf{a}_{1}+y_{3} \mathbf{a}_{2}+z_{3} \mathbf{a}_{3}$ & $=$ & $x_{3} a \hat{\mathbf{x}}+y_{3} b \hat{\mathbf{y}}+z_{3} c \hat{\mathbf{z}}$ & $(8 d)$ & $\mathrm{Fe}$ \\
\hline $\mathbf{B}_{10}$ & $=$ & $\left(\frac{1}{2}-x_{3}\right) \mathbf{a}_{1}-y_{3} \mathbf{a}_{2}+\left(\frac{1}{2}+z_{3}\right) \mathbf{a}_{3}$ & $=$ & $\left(\frac{1}{2}-x_{3}\right) a \hat{\mathbf{x}}-y_{3} b \hat{\mathbf{y}}+\left(\frac{1}{2}+z_{3}\right) c \hat{\mathbf{z}}$ & $(8 d)$ & $\mathrm{Fe}$ \\
\hline $\mathbf{B}_{11}$ & $=$ & $-x_{3} \mathbf{a}_{1}+\left(\frac{1}{2}+y_{3}\right) \mathbf{a}_{2}-z_{3} \mathbf{a}_{3}$ & $=$ & $-x_{3} a \hat{\mathbf{x}}+\left(\frac{1}{2}+y_{3}\right) b \hat{\mathbf{y}}-z_{3} c \hat{\mathbf{z}}$ & $(8 d)$ & $\mathrm{Fe}$ \\
\hline $\mathbf{B}_{12}$ & $=$ & $\begin{array}{c}\left(\frac{1}{2}+x_{3}\right) \mathbf{a}_{1}+\left(\frac{1}{2}-y_{3}\right) \mathbf{a}_{2}+ \\
\left(\frac{1}{2}-z_{3}\right) \mathbf{a}_{3}\end{array}$ & $=$ & $\begin{array}{c}\left(\frac{1}{2}+x_{3}\right) a \hat{\mathbf{x}}+\left(\frac{1}{2}-y_{3}\right) b \hat{\mathbf{y}}+ \\
\left(\frac{1}{2}-z_{3}\right) c \hat{\mathbf{z}}\end{array}$ & $(8 d)$ & $\mathrm{Fe}$ \\
\hline $\mathbf{B}_{13}$ & $=$ & $-x_{3} \mathbf{a}_{1}-y_{3} \mathbf{a}_{2}-z_{3} \mathbf{a}_{3}$ & $=$ & $-x_{3} a \hat{\mathbf{x}}-y_{3} b \hat{\mathbf{y}}-z_{3} c \hat{\mathbf{z}}$ & $(8 d)$ & $\mathrm{Fe}$ \\
\hline $\mathbf{B}_{14}$ & $=$ & $\left(\frac{1}{2}+x_{3}\right) \mathbf{a}_{1}+y_{3} \mathbf{a}_{2}+\left(\frac{1}{2}-z_{3}\right) \mathbf{a}_{3}$ & $=$ & $\left(\frac{1}{2}+x_{3}\right) a \hat{\mathbf{x}}+y_{3} b \hat{\mathbf{y}}+\left(\frac{1}{2}-z_{3}\right) c \hat{\mathbf{z}}$ & $(8 d)$ & $\mathrm{Fe}$ \\
\hline $\mathbf{B}_{15}$ & $=$ & $x_{3} \mathbf{a}_{1}+\left(\frac{1}{2}-y_{3}\right) \mathbf{a}_{2}+z_{3} \mathbf{a}_{3}$ & $=$ & $x_{3} a \hat{\mathbf{x}}+\left(\frac{1}{2}-y_{3}\right) b \hat{\mathbf{y}}+z_{3} c \hat{\mathbf{z}}$ & $(8 d)$ & $\mathrm{Fe}$ \\
\hline $\mathbf{B}_{16}$ & $=$ & $\begin{array}{c}\left(\frac{1}{2}-x_{3}\right) \mathbf{a}_{1}+\left(\frac{1}{2}+y_{3}\right) \mathbf{a}_{2}+ \\
\left(\frac{1}{2}+z_{3}\right) \mathbf{a}_{3}\end{array}$ & $=$ & $\begin{array}{c}\left(\frac{1}{2}-x_{3}\right) a \hat{\mathbf{x}}+\left(\frac{1}{2}+y_{3}\right) b \hat{\mathbf{y}}+ \\
\left(\frac{1}{2}+z_{3}\right) c \hat{\mathbf{z}}\end{array}$ & $(8 d)$ & $\mathrm{Fe}$ \\
\hline $\mathbf{B}_{17}$ & $=$ & $x_{4} \mathbf{a}_{1}+y_{4} \mathbf{a}_{2}+z_{4} \mathbf{a}_{3}$ & $=$ & $x_{4} a \hat{\mathbf{x}}+y_{4} b \hat{\mathbf{y}}+z_{4} c \hat{\mathbf{z}}$ & $(8 d)$ & S II \\
\hline $\mathbf{B}_{18}$ & $=$ & $\left(\frac{1}{2}-x_{4}\right) \mathbf{a}_{1}-y_{4} \mathbf{a}_{2}+\left(\frac{1}{2}+z_{4}\right) \mathbf{a}_{3}$ & $=$ & $\left(\frac{1}{2}-x_{4}\right) a \hat{\mathbf{x}}-y_{4} b \hat{\mathbf{y}}+\left(\frac{1}{2}+z_{4}\right) c \hat{\mathbf{z}}$ & $(8 d)$ & S II \\
\hline $\mathbf{B}_{19}$ & $=$ & $-x_{4} \mathbf{a}_{1}+\left(\frac{1}{2}+y_{4}\right) \mathbf{a}_{2}-z_{4} \mathbf{a}_{3}$ & $=$ & $-x_{4} a \hat{\mathbf{x}}+\left(\frac{1}{2}+y_{4}\right) b \hat{\mathbf{y}}-z_{4} c \hat{\mathbf{z}}$ & $(8 d)$ & S II \\
\hline $\mathbf{B}_{20}$ & $=$ & $\begin{array}{c}\left(\frac{1}{2}+x_{4}\right) \mathbf{a}_{1}+\left(\frac{1}{2}-y_{4}\right) \mathbf{a}_{2}+ \\
\left(\frac{1}{2}-z_{4}\right) \mathbf{a}_{3}\end{array}$ & $=$ & $\begin{array}{c}\left(\frac{1}{2}+x_{4}\right) a \hat{\mathbf{x}}+\left(\frac{1}{2}-y_{4}\right) b \hat{\mathbf{y}}+ \\
\left(\frac{1}{2}-z_{4}\right) c \hat{\mathbf{z}}\end{array}$ & $(8 d)$ & S II \\
\hline $\mathbf{B}_{21}$ & $=$ & $-x_{4} \mathbf{a}_{1}-y_{4} \mathbf{a}_{2}-z_{4} \mathbf{a}_{3}$ & $=$ & $-x_{4} a \hat{\mathbf{x}}-y_{4} b \hat{\mathbf{y}}-z_{4} c \hat{\mathbf{z}}$ & $(8 d)$ & S II \\
\hline $\mathbf{B}_{22}$ & $=$ & $\left(\frac{1}{2}+x_{4}\right) \mathbf{a}_{1}+y_{4} \mathbf{a}_{2}+\left(\frac{1}{2}-z_{4}\right) \mathbf{a}_{3}$ & $=$ & $\left(\frac{1}{2}+x_{4}\right) a \hat{\mathbf{x}}+y_{4} b \hat{\mathbf{y}}+\left(\frac{1}{2}-z_{4}\right) c \hat{\mathbf{z}}$ & $(8 d)$ & S II \\
\hline $\mathbf{B}_{23}$ & $=$ & $x_{4} \mathbf{a}_{1}+\left(\frac{1}{2}-y_{4}\right) \mathbf{a}_{2}+z_{4} \mathbf{a}_{3}$ & $=$ & $x_{4} a \hat{\mathbf{x}}+\left(\frac{1}{2}-y_{4}\right) b \hat{\mathbf{y}}+z_{4} c \hat{\mathbf{z}}$ & $(8 d)$ & S II \\
\hline $\mathbf{B}_{24}$ & $=$ & $\begin{array}{c}\left(\frac{1}{2}-x_{4}\right) \mathbf{a}_{1}+\left(\frac{1}{2}+y_{4}\right) \mathbf{a}_{2}+ \\
\left(\frac{1}{2}+z_{4}\right) \mathbf{a}_{3}\end{array}$ & $=$ & $\begin{array}{c}\left(\frac{1}{2}-x_{4}\right) a \hat{\mathbf{x}}+\left(\frac{1}{2}+y_{4}\right) b \hat{\mathbf{y}}+ \\
\left(\frac{1}{2}+z_{4}\right) c \hat{\mathbf{z}}\end{array}$ & $(8 d)$ & S II \\
\hline
\end{tabular}

\section{References:}

- T. Szymański, A refinement of the structure of cubanite, $\mathrm{CuFe}_{2} \mathrm{~S}_{3}$, Zeitschrift für Kristallographie - Crystalline Materials 140, 218-239 (1974), doi:10.1524/zkri-1974-3-407.

\section{Geometry files:}

- CIF: pp. 843

- POSCAR: pp. 844 


\section{Molybdite $\left(\mathrm{MoO}_{3}, \mathrm{DO}_{8}\right)$ Structure: AB3_oP16_62_c_3c}
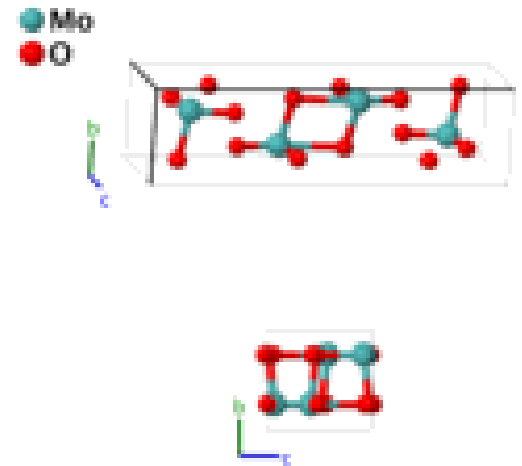
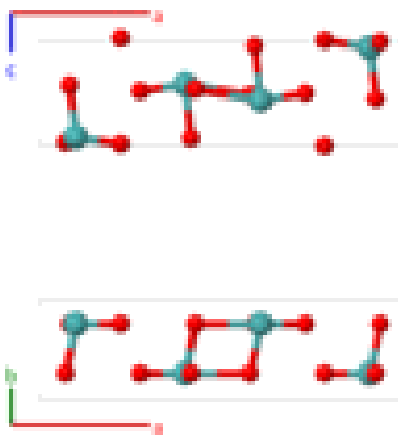

Prototype

AFLOW prototype label

Strukturbericht designation

Pearson symbol

Space group number

Space group symbol

AFLOW prototype command
: $\quad \mathrm{MoO}_{3}$

: AB3_oP16_62_c_3c

: $\quad \mathrm{DO}_{8}$

: $\quad$ oP16

: 62

Pnma

aflow --proto $=$ AB3_oP16_62_C_3c

- params $=a, b / a, c / a, x_{1}, z_{1}, x_{2}, z_{2}, x_{3}, z_{3}, x_{4}, z_{4}$

- The unit cell and atomic positions were originally given in the Pbnm orientation of space group \#62. We have rotated the crystal axis so that $\hat{\mathbf{y}} \rightarrow \hat{\mathbf{x}} \rightarrow \hat{\mathbf{z}}$ to put the system in the standard Pnma representation.

Simple Orthorhombic primitive vectors:

$$
\begin{aligned}
& \mathbf{a}_{1}=a \hat{\mathbf{x}} \\
& \mathbf{a}_{2}=b \hat{\mathbf{y}} \\
& \mathbf{a}_{3}=c \hat{\mathbf{z}}
\end{aligned}
$$

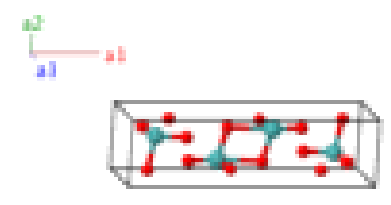

\section{Basis vectors:}

Lattice Coordinates

$$
\begin{array}{llccc}
\mathbf{B}_{1}= & x_{1} \mathbf{a}_{1}+\frac{1}{4} \mathbf{a}_{2}+z_{1} \mathbf{a}_{3} & = & x_{1} a \hat{\mathbf{x}}+\frac{1}{4} b \hat{\mathbf{y}}+z_{1} c \hat{\mathbf{z}} \\
\mathbf{B}_{2}= & \left(\frac{1}{2}-x_{1}\right) \mathbf{a}_{1}+\frac{3}{4} \mathbf{a}_{2}+\left(\frac{1}{2}+z_{1}\right) \mathbf{a}_{3} & = & \left(\frac{1}{2}-x_{1}\right) a \hat{\mathbf{x}}+\frac{3}{4} b \hat{\mathbf{y}}+\left(\frac{1}{2}+z_{1}\right) c \hat{\mathbf{z}} \\
\mathbf{B}_{3}= & -x_{1} \mathbf{a}_{1}+\frac{3}{4} \mathbf{a}_{2}-z_{1} \mathbf{a}_{3} & = & -x_{1} a \hat{\mathbf{x}}+\frac{3}{4} b \hat{\mathbf{y}}-z_{1} c \hat{\mathbf{z}} \\
\mathbf{B}_{4}= & \left(\frac{1}{2}+x_{1}\right) \mathbf{a}_{1}+\frac{1}{4} \mathbf{a}_{2}+\left(\frac{1}{2}-z_{1}\right) \mathbf{a}_{3} & = & \left(\frac{1}{2}+x_{1}\right) a \hat{\mathbf{x}}+\frac{1}{4} b \hat{\mathbf{y}}+\left(\frac{1}{2}-z_{1}\right) c \hat{\mathbf{z}} \\
\mathbf{B}_{5}= & x_{2} \mathbf{a}_{1}+\frac{1}{4} \mathbf{a}_{2}+z_{2} \mathbf{a}_{3} & = & x_{2} a \hat{\mathbf{x}}+\frac{1}{4} b \hat{\mathbf{y}}+z_{2} c \hat{\mathbf{z}} \\
\mathbf{B}_{6}= & \left(\frac{1}{2}-x_{2}\right) \mathbf{a}_{1}+\frac{3}{4} \mathbf{a}_{2}+\left(\frac{1}{2}+z_{2}\right) \mathbf{a}_{3} & = & \left(\frac{1}{2}-x_{2}\right) a \hat{\mathbf{x}}+\frac{3}{4} b \hat{\mathbf{y}}+\left(\frac{1}{2}+z_{2}\right) c \hat{\mathbf{z}} \\
\mathbf{B}_{7}= & -x_{2} \mathbf{a}_{1}+\frac{3}{4} \mathbf{a}_{2}-z_{2} \mathbf{a}_{3} & = & -x_{2} a \hat{\mathbf{x}}+\frac{3}{4} b \hat{\mathbf{y}}-z_{2} c \hat{\mathbf{z}} \\
\mathbf{B}_{8}= & \left(\frac{1}{2}+x_{2}\right) \mathbf{a}_{1}+\frac{1}{4} \mathbf{a}_{2}+\left(\frac{1}{2}-z_{2}\right) \mathbf{a}_{3}= & \left(\frac{1}{2}+x_{2}\right) a \hat{\mathbf{x}}+\frac{1}{4} b \hat{\mathbf{y}}+\left(\frac{1}{2}-z_{2}\right) c \hat{\mathbf{z}}
\end{array}
$$

Cartesian Coordinates
Wyckoff Position Atom Type
Mo

Mo

Mo

Mo

O I

O I

O I

O I 


\begin{tabular}{|c|c|c|c|c|c|}
\hline $\mathbf{B}_{9}$ & $=$ & $x_{3} \mathbf{a}_{1}+\frac{1}{4} \mathbf{a}_{2}+z_{3} \mathbf{a}_{3}$ & $=$ & $x_{3} a \hat{\mathbf{x}}+\frac{1}{4} b \hat{\mathbf{y}}+z_{3} c \hat{\mathbf{z}}$ & $(4 c)$ \\
\hline $\mathbf{B}_{10}$ & $=$ & $\left(\frac{1}{2}-x_{3}\right) \mathbf{a}_{1}+\frac{3}{4} \mathbf{a}_{2}+\left(\frac{1}{2}+z_{3}\right) \mathbf{a}_{3}$ & $=$ & $\left(\frac{1}{2}-x_{3}\right) a \hat{\mathbf{x}}+\frac{3}{4} b \hat{\mathbf{y}}+\left(\frac{1}{2}+z_{3}\right) c \hat{\mathbf{z}}$ & $(4 c)$ \\
\hline $\mathbf{B}_{11}$ & $=$ & $-x_{3} \mathbf{a}_{1}+\frac{3}{4} \mathbf{a}_{2}-z_{3} \mathbf{a}_{3}$ & $=$ & $-x_{3} a \hat{\mathbf{x}}+\frac{3}{4} b \hat{\mathbf{y}}-z_{3} c \hat{\mathbf{z}}$ & $(4 c)$ \\
\hline $\mathbf{B}_{12}$ & $=$ & $\left(\frac{1}{2}+x_{3}\right) \mathbf{a}_{1}+\frac{1}{4} \mathbf{a}_{2}+\left(\frac{1}{2}-z_{3}\right) \mathbf{a}_{3}$ & $=$ & $\left(\frac{1}{2}+x_{3}\right) a \hat{\mathbf{x}}+\frac{1}{4} b \hat{\mathbf{y}}+\left(\frac{1}{2}-z_{3}\right) c \hat{\mathbf{z}}$ & $(4 c)$ \\
\hline $\mathbf{B}_{13}$ & $=$ & $x_{4} \mathbf{a}_{1}+\frac{1}{4} \mathbf{a}_{2}+z_{4} \mathbf{a}_{3}$ & $=$ & $x_{4} a \hat{\mathbf{x}}+\frac{1}{4} b \hat{\mathbf{y}}+z_{4} c \hat{\mathbf{z}}$ & $(4 c)$ \\
\hline $\mathbf{B}_{14}$ & $=$ & $\left(\frac{1}{2}-x_{4}\right) \mathbf{a}_{1}+\frac{3}{4} \mathbf{a}_{2}+\left(\frac{1}{2}+z_{4}\right) \mathbf{a}_{3}$ & $=$ & $\left(\frac{1}{2}-x_{4}\right) a \hat{\mathbf{x}}+\frac{3}{4} b \hat{\mathbf{y}}+\left(\frac{1}{2}+z_{4}\right) c \hat{\mathbf{z}}$ & $(4 c)$ \\
\hline $\mathbf{B}_{15}$ & $=$ & $-x_{4} \mathbf{a}_{1}+\frac{3}{4} \mathbf{a}_{2}-z_{4} \mathbf{a}_{3}$ & $=$ & $-x_{4} a \hat{\mathbf{x}}+\frac{3}{4} b \hat{\mathbf{y}}-z_{4} c \hat{\mathbf{z}}$ & $(4 c)$ \\
\hline $\mathbf{B}_{16}$ & $=$ & $\left(\frac{1}{2}+x_{4}\right) \mathbf{a}_{1}+\frac{1}{4} \mathbf{a}_{2}+\left(\frac{1}{2}-z_{4}\right) \mathbf{a}_{3}$ & $=$ & $\left(\frac{1}{2}+x_{4}\right) a \hat{\mathbf{x}}+\frac{1}{4} b \hat{\mathbf{y}}+\left(\frac{1}{2}-z_{4}\right) c \hat{\mathbf{z}}$ & $(4 c)$ \\
\hline
\end{tabular}

\section{References:}

- H. Sitepu, B. H. O'Connor, and D. Li, Comparative evaluation of the March and generalized spherical harmonic preferred orientation models using X-ray diffraction data for molybdite and calcite powders, J. Appl. Crystallogr. 38, 158-167 (2005), doi:10.1107/S0021889804031231.

\section{Geometry files:}

- CIF: pp. 844

- POSCAR: pp. 844 


\section{Barite $\left(\mathrm{BaSO}_{4}, \mathrm{HO}_{2}\right)$ Structure:}

\section{AB4C_oP24_62_c_2cd_c}

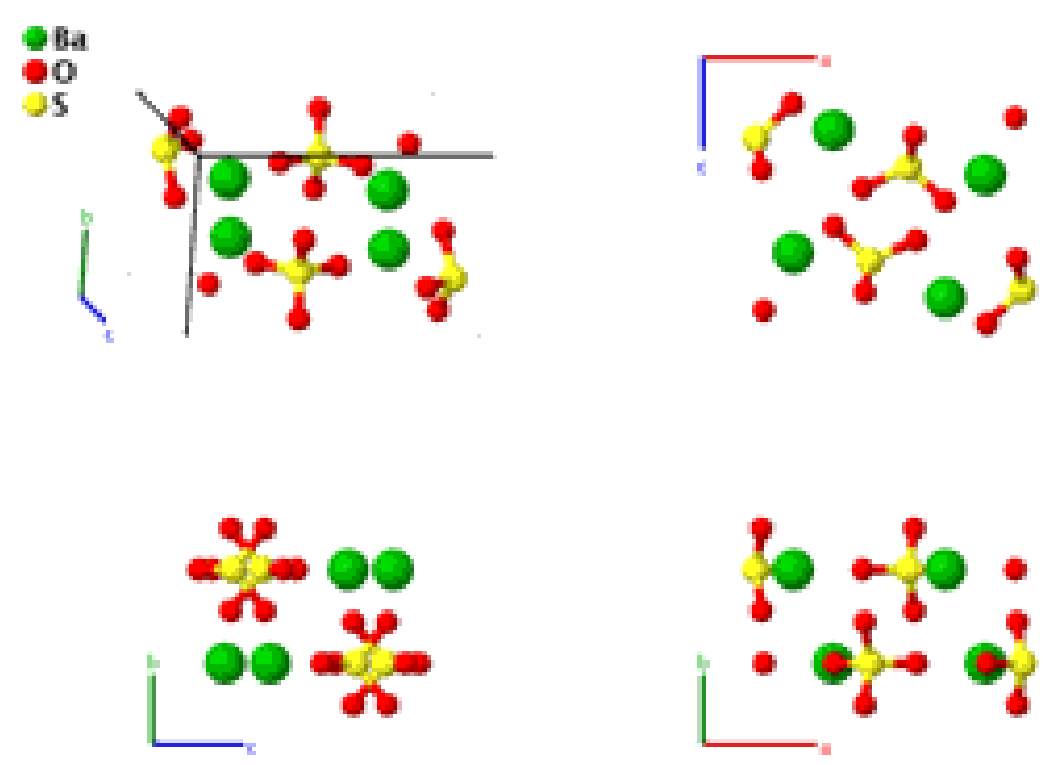

\section{Prototype}

AFLOW prototype label

Strukturbericht designation

Pearson symbol

Space group number

Space group symbol

AFLOW prototype command
$: \quad \mathrm{BaSO}_{4}$

: AB4C_oP24_62_c_2cd_c

: $\quad \mathrm{HO}_{2}$

: $\quad$ oP24

: 62

: Pnma

aflow --proto=AB4C_oP24_62_c_2cd_c

- params $=a, b / a, c / a, x_{1}, z_{1}, x_{2}, z_{2}, x_{3}, z_{3}, x_{4}, z_{4}, x_{5}, y_{5}, z_{5}$

Other compounds with this structure:

- $\mathrm{SrSO}_{4}$ (celestite), $\mathrm{PbSO}_{4}$ (anglesite), $\mathrm{KGaH}_{4}$

Simple Orthorhombic primitive vectors:

$$
\begin{aligned}
& \mathbf{a}_{1}=a \hat{\mathbf{x}} \\
& \mathbf{a}_{2}=b \hat{\mathbf{y}} \\
& \mathbf{a}_{3}=c \hat{\mathbf{z}}
\end{aligned}
$$

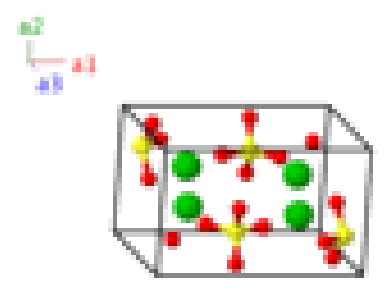

Basis vectors:

Lattice Coordinates

$\mathbf{B}_{1}=\quad x_{1} \mathbf{a}_{1}+\frac{1}{4} \mathbf{a}_{2}+z_{1} \mathbf{a}_{3}$

$\mathbf{B}_{2}=$

$\left(\frac{1}{2}-x_{1}\right) \mathbf{a}_{1}+\frac{3}{4} \mathbf{a}_{2}+\left(\frac{1}{2}+z_{1}\right) \mathbf{a}_{3}$

$=$

$=$

Cartesian Coordinates

Wyckoff Position

Atom Type

$x_{1} a \hat{\mathbf{x}}+\frac{1}{4} b \hat{\mathbf{y}}+z_{1} c \hat{\mathbf{z}}$

(4c)

$\mathrm{Ba}$

(4c)

$\mathrm{Ba}$ 


\begin{tabular}{|c|c|c|c|c|c|c|}
\hline $\mathbf{B}_{3}$ & $=$ & $-x_{1} \mathbf{a}_{1}+\frac{3}{4} \mathbf{a}_{2}-z_{1} \mathbf{a}_{3}$ & $=$ & $-x_{1} a \hat{\mathbf{x}}+\frac{3}{4} b \hat{\mathbf{y}}-z_{1} c \hat{\mathbf{z}}$ & $(4 c)$ & $\mathrm{Ba}$ \\
\hline $\mathbf{B}_{4}$ & $=$ & $\left(\frac{1}{2}+x_{1}\right) \mathbf{a}_{1}+\frac{1}{4} \mathbf{a}_{2}+\left(\frac{1}{2}-z_{1}\right) \mathbf{a}_{3}$ & $=$ & $\left(\frac{1}{2}+x_{1}\right) a \hat{\mathbf{x}}+\frac{1}{4} b \hat{\mathbf{y}}+\left(\frac{1}{2}-z_{1}\right) c \hat{\mathbf{z}}$ & $(4 c)$ & $\mathrm{Ba}$ \\
\hline $\mathbf{B}_{5}$ & $=$ & $x_{2} \mathbf{a}_{1}+\frac{1}{4} \mathbf{a}_{2}+z_{2} \mathbf{a}_{3}$ & $=$ & $x_{2} a \hat{\mathbf{x}}+\frac{1}{4} b \hat{\mathbf{y}}+z_{2} c \hat{\mathbf{z}}$ & $(4 c)$ & O I \\
\hline $\mathbf{B}_{6}$ & $=$ & $\left(\frac{1}{2}-x_{2}\right) \mathbf{a}_{1}+\frac{3}{4} \mathbf{a}_{2}+\left(\frac{1}{2}+z_{2}\right) \mathbf{a}_{3}$ & $=$ & $\left(\frac{1}{2}-x_{2}\right) a \hat{\mathbf{x}}+\frac{3}{4} b \hat{\mathbf{y}}+\left(\frac{1}{2}+z_{2}\right) c \hat{\mathbf{z}}$ & $(4 c)$ & O I \\
\hline $\mathbf{B}_{7}$ & $=$ & $-x_{2} \mathbf{a}_{1}+\frac{3}{4} \mathbf{a}_{2}-z_{2} \mathbf{a}_{3}$ & $=$ & $-x_{2} a \hat{\mathbf{x}}+\frac{3}{4} b \hat{\mathbf{y}}-z_{2} c \hat{\mathbf{z}}$ & $(4 c)$ & O I \\
\hline $\mathbf{B}_{8}$ & $=$ & $\left(\frac{1}{2}+x_{2}\right) \mathbf{a}_{1}+\frac{1}{4} \mathbf{a}_{2}+\left(\frac{1}{2}-z_{2}\right) \mathbf{a}_{3}$ & $=$ & $\left(\frac{1}{2}+x_{2}\right) a \hat{\mathbf{x}}+\frac{1}{4} b \hat{\mathbf{y}}+\left(\frac{1}{2}-z_{2}\right) c \hat{\mathbf{z}}$ & $(4 c)$ & O I \\
\hline $\mathbf{B}_{9}$ & $=$ & $x_{3} \mathbf{a}_{1}+\frac{1}{4} \mathbf{a}_{2}+z_{3} \mathbf{a}_{3}$ & $=$ & $x_{3} a \hat{\mathbf{x}}+\frac{1}{4} b \hat{\mathbf{y}}+z_{3} c \hat{\mathbf{z}}$ & $(4 c)$ & O II \\
\hline $\mathbf{B}_{10}$ & $=$ & $\left(\frac{1}{2}-x_{3}\right) \mathbf{a}_{1}+\frac{3}{4} \mathbf{a}_{2}+\left(\frac{1}{2}+z_{3}\right) \mathbf{a}_{3}$ & $=$ & $\left(\frac{1}{2}-x_{3}\right) a \hat{\mathbf{x}}+\frac{3}{4} b \hat{\mathbf{y}}+\left(\frac{1}{2}+z_{3}\right) c \hat{\mathbf{z}}$ & $(4 c)$ & O II \\
\hline $\mathbf{B}_{11}$ & $=$ & $-x_{3} \mathbf{a}_{1}+\frac{3}{4} \mathbf{a}_{2}-z_{3} \mathbf{a}_{3}$ & $=$ & $-x_{3} a \hat{\mathbf{x}}+\frac{3}{4} b \hat{\mathbf{y}}-z_{3} c \hat{\mathbf{z}}$ & $(4 c)$ & O II \\
\hline $\mathbf{B}_{12}$ & $=$ & $\left(\frac{1}{2}+x_{3}\right) \mathbf{a}_{1}+\frac{1}{4} \mathbf{a}_{2}+\left(\frac{1}{2}-z_{3}\right) \mathbf{a}_{3}$ & $=$ & $\left(\frac{1}{2}+x_{3}\right) a \hat{\mathbf{x}}+\frac{1}{4} b \hat{\mathbf{y}}+\left(\frac{1}{2}-z_{3}\right) c \hat{\mathbf{z}}$ & $(4 c)$ & O II \\
\hline $\mathbf{B}_{13}$ & $=$ & $x_{4} \mathbf{a}_{1}+\frac{1}{4} \mathbf{a}_{2}+z_{4} \mathbf{a}_{3}$ & $=$ & $x_{4} a \hat{\mathbf{x}}+\frac{1}{4} b \hat{\mathbf{y}}+z_{4} c \hat{\mathbf{z}}$ & $(4 c)$ & $\mathrm{S}$ \\
\hline $\mathbf{B}_{14}$ & $=$ & $\left(\frac{1}{2}-x_{4}\right) \mathbf{a}_{1}+\frac{3}{4} \mathbf{a}_{2}+\left(\frac{1}{2}+z_{4}\right) \mathbf{a}_{3}$ & $=$ & $\left(\frac{1}{2}-x_{4}\right) a \hat{\mathbf{x}}+\frac{3}{4} b \hat{\mathbf{y}}+\left(\frac{1}{2}+z_{4}\right) c \hat{\mathbf{z}}$ & $(4 c)$ & $\mathrm{S}$ \\
\hline $\mathbf{B}_{15}$ & $=$ & $-x_{4} \mathbf{a}_{1}+\frac{3}{4} \mathbf{a}_{2}-z_{4} \mathbf{a}_{3}$ & $=$ & $-x_{4} a \hat{\mathbf{x}}+\frac{3}{4} b \hat{\mathbf{y}}-z_{4} c \hat{\mathbf{z}}$ & $(4 c)$ & $\mathrm{S}$ \\
\hline $\mathbf{B}_{16}$ & $=$ & $\left(\frac{1}{2}+x_{4}\right) \mathbf{a}_{1}+\frac{1}{4} \mathbf{a}_{2}+\left(\frac{1}{2}-z_{4}\right) \mathbf{a}_{3}$ & $=$ & $\left(\frac{1}{2}+x_{4}\right) a \hat{\mathbf{x}}+\frac{1}{4} b \hat{\mathbf{y}}+\left(\frac{1}{2}-z_{4}\right) c \hat{\mathbf{z}}$ & $(4 c)$ & $\mathrm{S}$ \\
\hline $\mathbf{B}_{17}$ & $=$ & $x_{5} \mathbf{a}_{1}+y_{5} \mathbf{a}_{2}+z_{5} \mathbf{a}_{3}$ & $=$ & $x_{5} a \hat{\mathbf{x}}+y_{5} b \hat{\mathbf{y}}+z_{5} c \hat{\mathbf{z}}$ & $(8 d)$ & O III \\
\hline $\mathbf{B}_{18}$ & $=$ & $\left(\frac{1}{2}-x_{5}\right) \mathbf{a}_{1}-y_{5} \mathbf{a}_{2}+\left(\frac{1}{2}+z_{5}\right) \mathbf{a}_{3}$ & $=$ & $\left(\frac{1}{2}-x_{5}\right) a \hat{\mathbf{x}}-y_{5} b \hat{\mathbf{y}}+\left(\frac{1}{2}+z_{5}\right) c \hat{\mathbf{z}}$ & $(8 d)$ & O III \\
\hline $\mathbf{B}_{19}$ & $=$ & $-x_{5} \mathbf{a}_{1}+\left(\frac{1}{2}+y_{5}\right) \mathbf{a}_{2}-z_{5} \mathbf{a}_{3}$ & $=$ & $-x_{5} a \hat{\mathbf{x}}+\left(\frac{1}{2}+y_{5}\right) b \hat{\mathbf{y}}-z_{5} c \hat{\mathbf{z}}$ & $(8 d)$ & O III \\
\hline $\mathbf{B}_{20}$ & $=$ & $\begin{array}{c}\left(\frac{1}{2}+x_{5}\right) \mathbf{a}_{1}+\left(\frac{1}{2}-y_{5}\right) \mathbf{a}_{2}+ \\
\left(\frac{1}{2}-z_{5}\right) \mathbf{a}_{3}\end{array}$ & $=$ & $\begin{array}{c}\left(\frac{1}{2}+x_{5}\right) a \hat{\mathbf{x}}+\left(\frac{1}{2}-y_{5}\right) b \hat{\mathbf{y}}+ \\
\left(\frac{1}{2}-z_{5}\right) c \hat{\mathbf{z}}\end{array}$ & $(8 d)$ & O III \\
\hline $\mathbf{B}_{21}$ & $=$ & $-x_{5} \mathbf{a}_{1}-y_{5} \mathbf{a}_{2}-z_{5} \mathbf{a}_{3}$ & $=$ & $-x_{5} a \hat{\mathbf{x}}-y_{5} b \hat{\mathbf{y}}-z_{5} c \hat{\mathbf{z}}$ & $(8 d)$ & O III \\
\hline $\mathbf{B}_{22}$ & $=$ & $\left(\frac{1}{2}+x_{5}\right) \mathbf{a}_{1}+y_{5} \mathbf{a}_{2}+\left(\frac{1}{2}-z_{5}\right) \mathbf{a}_{3}$ & $=$ & $\left(\frac{1}{2}+x_{5}\right) a \hat{\mathbf{x}}+y_{5} b \hat{\mathbf{y}}+\left(\frac{1}{2}-z_{5}\right) c \hat{\mathbf{z}}$ & $(8 d)$ & O III \\
\hline $\mathbf{B}_{23}$ & $=$ & $x_{5} \mathbf{a}_{1}+\left(\frac{1}{2}-y_{5}\right) \mathbf{a}_{2}+z_{5} \mathbf{a}_{3}$ & $=$ & $x_{5} a \hat{\mathbf{x}}+\left(\frac{1}{2}-y_{5}\right) b \hat{\mathbf{y}}+z_{5} c \hat{\mathbf{z}}$ & $(8 d)$ & O III \\
\hline $\mathbf{B}_{24}$ & $=$ & $\begin{array}{c}\left(\frac{1}{2}-x_{5}\right) \mathbf{a}_{1}+\left(\frac{1}{2}+y_{5}\right) \mathbf{a}_{2}+ \\
\left(\frac{1}{2}+z_{5}\right) \mathbf{a}_{3}\end{array}$ & $=$ & $\begin{array}{c}\left(\frac{1}{2}-x_{5}\right) a \hat{\mathbf{x}}+\left(\frac{1}{2}+y_{5}\right) b \hat{\mathbf{y}}+ \\
\left(\frac{1}{2}+z_{5}\right) c \hat{\mathbf{z}}\end{array}$ & $(8 d)$ & O III \\
\hline
\end{tabular}

\section{References:}

- A. A. Colville and K. Staudhammer, A refinement of the structure of barite, Am. Mineral. 52, 1877-1880 (1967).

\section{Found in:}

- D. Barthelmy, Mineralogy Database (2012). Barite.

\section{Geometry files:}

- CIF: pp. 844

- POSCAR: pp. 845 


\section{Westerveldite (FeAs, B14) Structure:}

AB_oP8_62_c_c

As
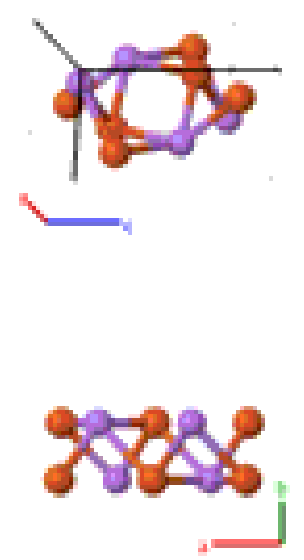

Prototype : FeAs

AFLOW prototype label $\quad$ : $\quad$ AB_oP8_62_c_c

Strukturbericht designation : $\quad B 14$

Pearson symbol $\quad: \quad$ oP8

Space group number $\quad: \quad 62$

Space group symbol $\quad: \quad$ Pnma

AFLOW prototype command : aflow--proto=AB_oP8_62_c_c

- params $=a, b / a, c / a, x_{1}, z_{1}, x_{2}, z_{2}$

\section{Other compounds with this structure:}

- CoAs

- The B31 (MnP, AB_oP8_62_c_c) structure is similar to this one. (Brandes, 1992) lists B31 as the primary structure, but we include FeAs here for completeness. We use the data (Selte, 1972) reported at $14 \mathrm{~K}$.

\section{Simple Orthorhombic primitive vectors:}

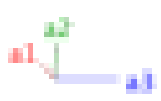
$\mathbf{a}_{1}=a \hat{\mathbf{x}}$
$\mathbf{a}_{2}=b \hat{\mathbf{y}}$
$\mathbf{a}_{3}=c \hat{\mathbf{z}}$

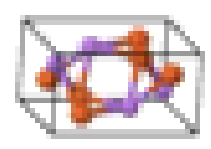

Basis vectors: 


\begin{tabular}{|c|c|c|c|c|c|}
\hline $\mathbf{B}_{1}$ & $=$ & $x_{1} \mathbf{a}_{1}+\frac{1}{4} \mathbf{a}_{2}+z_{1} \mathbf{a}_{3}$ & $=$ & $x_{1} a \hat{\mathbf{x}}+\frac{1}{4} b \hat{\mathbf{y}}+z_{1} c \hat{\mathbf{z}}$ & $(4 c)$ \\
\hline $\mathbf{B}_{2}$ & $=$ & $\left(\frac{1}{2}-x_{1}\right) \mathbf{a}_{1}+\frac{3}{4} \mathbf{a}_{2}+\left(\frac{1}{2}+z_{1}\right) \mathbf{a}_{3}$ & $=$ & $\left(\frac{1}{2}-x_{1}\right) a \hat{\mathbf{x}}+\frac{3}{4} b \hat{\mathbf{y}}+\left(\frac{1}{2}+z_{1}\right) c \hat{\mathbf{z}}$ & $(4 c)$ \\
\hline $\mathbf{B}_{3}$ & $=$ & $-x_{1} \mathbf{a}_{1}+\frac{3}{4} \mathbf{a}_{2}-z_{1} \mathbf{a}_{3}$ & $=$ & $-x_{1} a \hat{\mathbf{x}}+\frac{3}{4} b \hat{\mathbf{y}}-z_{1} c \hat{\mathbf{z}}$ & $(4 c)$ \\
\hline $\mathbf{B}_{4}$ & $=$ & $\left(\frac{1}{2}+x_{1}\right) \mathbf{a}_{1}+\frac{1}{4} \mathbf{a}_{2}+\left(\frac{1}{2}-z_{1}\right) \mathbf{a}_{3}$ & $=$ & $\left(\frac{1}{2}+x_{1}\right) a \hat{\mathbf{x}}+\frac{1}{4} b \hat{\mathbf{y}}+\left(\frac{1}{2}-z_{1}\right) c \hat{\mathbf{z}}$ & $(4 c)$ \\
\hline $\mathbf{B}_{5}$ & $=$ & $x_{2} \mathbf{a}_{1}+\frac{1}{4} \mathbf{a}_{2}+z_{2} \mathbf{a}_{3}$ & $=$ & $x_{2} a \hat{\mathbf{x}}+\frac{1}{4} b \hat{\mathbf{y}}+z_{2} c \hat{\mathbf{z}}$ & $(4 c)$ \\
\hline $\mathbf{B}_{6}$ & $=$ & $\left(\frac{1}{2}-x_{2}\right) \mathbf{a}_{1}+\frac{3}{4} \mathbf{a}_{2}+\left(\frac{1}{2}+z_{2}\right) \mathbf{a}_{3}$ & $=$ & $\left(\frac{1}{2}-x_{2}\right) a \hat{\mathbf{x}}+\frac{3}{4} b \hat{\mathbf{y}}+\left(\frac{1}{2}+z_{2}\right) c \hat{\mathbf{z}}$ & $(4 c)$ \\
\hline $\mathbf{B}_{7}$ & $=$ & $-x_{2} \mathbf{a}_{1}+\frac{3}{4} \mathbf{a}_{2}-z_{2} \mathbf{a}_{3}$ & $=$ & $-x_{2} a \hat{\mathbf{x}}+\frac{3}{4} b \hat{\mathbf{y}}-z_{2} c \hat{\mathbf{z}}$ & $(4 c)$ \\
\hline $\mathbf{B}_{8}$ & $=$ & $\left(\frac{1}{2}+x_{2}\right) \mathbf{a}_{1}+\frac{1}{4} \mathbf{a}_{2}+\left(\frac{1}{2}-z_{2}\right) \mathbf{a}_{3}$ & $=$ & $\left(\frac{1}{2}+x_{2}\right) a \hat{\mathbf{x}}+\frac{1}{4} b \hat{\mathbf{y}}+\left(\frac{1}{2}-z_{2}\right) c \hat{\mathbf{z}}$ & $(4 c)$ \\
\hline
\end{tabular}

\section{References:}

- K. Selte, A. Kjekshus, and A. F. Andresen, Magnetic Structure and Properties of FeAs, Acta Chem. Scand. 26, 3101-3113 (1972), doi:10.3891/acta.chem.scand.26-3101.

- E. Parthé, L. Gelato, B. Chabot, M. Penso, K. Cenzula, and R. Gladyshevskii, Standardized Data and Crystal Chemical Characterization of Inorganic Structure Types, Gmelin Handbook of Inorganic and Organometallic Chemistry, vol. 2 (Springer-Verlag, Berlin, Heidelberg, 1993), 8 edn., doi:10.1007/978-3-662-02909-1_3.

\section{Found in:}

- J. R. Jeffries, N. P. Butch, H. Cynn, S. R. Saha, K. Kirshenbaum, S. T. Weir, Y. K. Vohra, and J. Paglione, Interplay between magnetism, structure, and strong electron-phonon coupling in binary FeAs under pressure, Phys. Rev. B 83, 134520 (2011), doi:10.1103/PhysRevB.83.134520.

\section{Geometry files:}

- CIF: pp. 845

- POSCAR: pp. 845 


\section{Rasvumite $\left(\mathrm{KFe}_{2} \mathrm{~S}_{3}\right)$ Structure: A2BC3_oC24_63_e_c_cg}
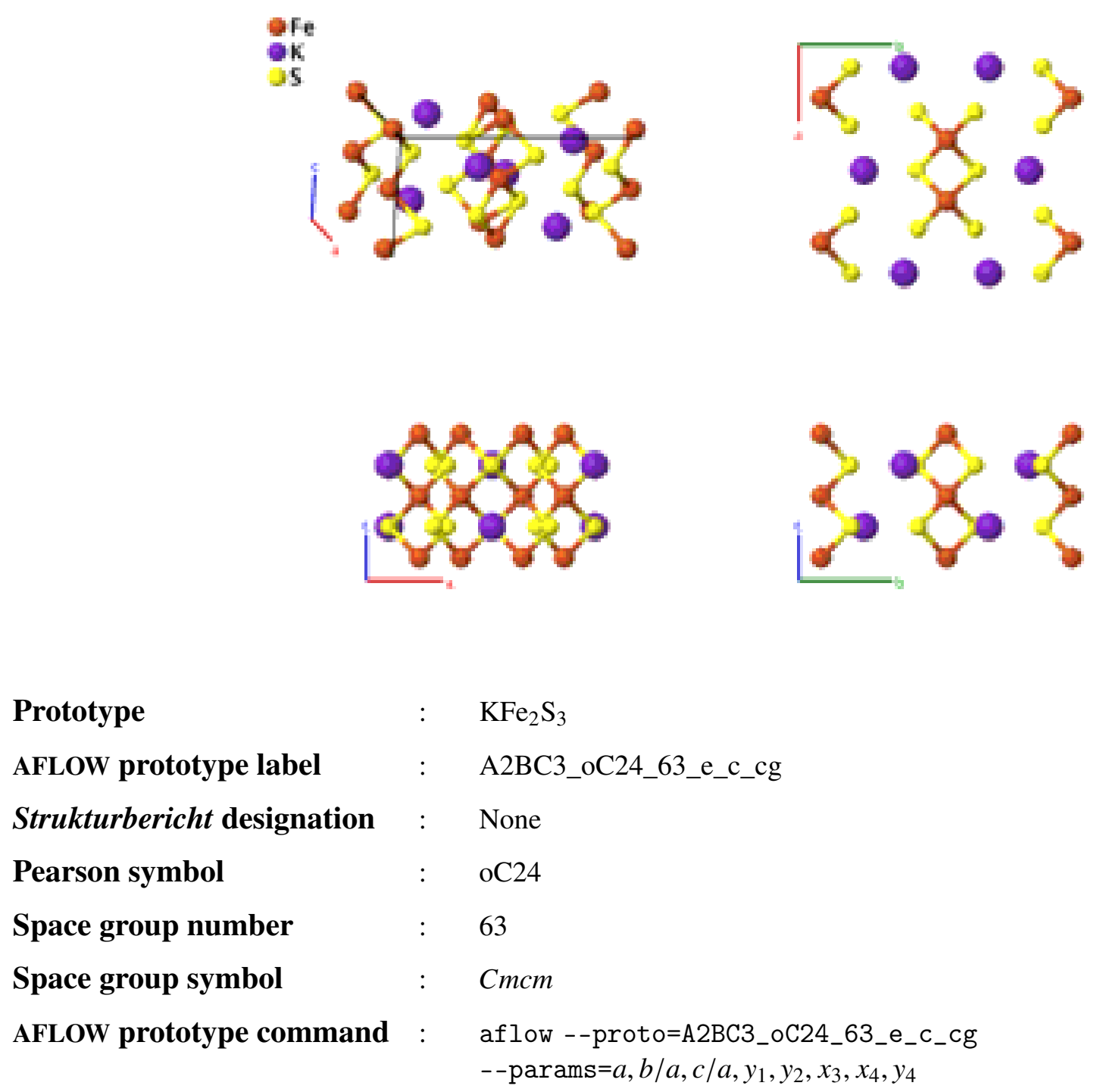

Other compounds with this structure:

- $\mathrm{BaFe}_{2} \mathrm{~S}_{3}, \mathrm{RbFe}_{2} \mathrm{~S}_{3}, \mathrm{CsFe}_{2} \mathrm{~S}_{3}$

Base-centered Orthorhombic primitive vectors:

$$
\begin{aligned}
& \mathbf{a}_{1}=\frac{1}{2} a \hat{\mathbf{x}}-\frac{1}{2} b \hat{\mathbf{y}} \\
& \mathbf{a}_{2}=\frac{1}{2} a \hat{\mathbf{x}}+\frac{1}{2} b \hat{\mathbf{y}} \\
& \mathbf{a}_{3}=c \quad c \hat{\mathbf{z}}
\end{aligned}
$$
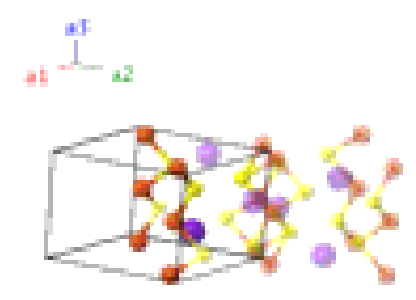

Basis vectors:

\section{Lattice Coordinates}

$\begin{array}{lll}\mathbf{B}_{1}= & -y_{1} \mathbf{a}_{1}+y_{1} \mathbf{a}_{2}+\frac{1}{4} \mathbf{a}_{3} \\ \mathbf{B}_{2}= & y_{1} \mathbf{a}_{1}-y_{1} \mathbf{a}_{2}+\frac{3}{4} \mathbf{a}_{3}\end{array}$

$\mathbf{B}_{1}=$
$\mathbf{B}_{2}=$

$y_{1} \mathbf{a}_{1}-y_{1} \mathbf{a}_{2}+\frac{3}{4} \mathbf{a}_{3}$ 


\begin{tabular}{|c|c|c|c|c|c|}
\hline $\mathbf{B}_{3}$ & $=$ & $-y_{2} \mathbf{a}_{1}+y_{2} \mathbf{a}_{2}+\frac{1}{4} \mathbf{a}_{3}$ & $=$ & $y_{2} b \hat{\mathbf{y}}+\frac{1}{4} c \hat{\mathbf{z}}$ & $(4 c)$ \\
\hline $\mathbf{B}_{4}$ & $=$ & $y_{2} \mathbf{a}_{1}-y_{2} \mathbf{a}_{2}+\frac{3}{4} \mathbf{a}_{3}$ & $=$ & $-y_{2} b \hat{\mathbf{y}}+\frac{3}{4} c \hat{\mathbf{z}}$ & $(4 c)$ \\
\hline $\mathbf{B}_{5}$ & $=$ & $x_{3} \mathbf{a}_{1}+x_{3} \mathbf{a}_{2}$ & $=$ & $x_{3} a \hat{\mathbf{x}}$ & $(8 e)$ \\
\hline $\mathbf{B}_{6}$ & $=$ & $-x_{3} \mathbf{a}_{1}-x_{3} \mathbf{a}_{2}+\frac{1}{2} \mathbf{a}_{3}$ & $=$ & $-x_{3} a \hat{\mathbf{x}}+\frac{1}{2} c \hat{\mathbf{z}}$ & $(8 e)$ \\
\hline $\mathbf{B}_{7}$ & $=$ & $-x_{3} \mathbf{a}_{1}-x_{3} \mathbf{a}_{2}$ & $=$ & $-x_{3} a \hat{\mathbf{x}}$ & $(8 e)$ \\
\hline $\mathbf{B}_{8}$ & $=$ & $x_{3} \mathbf{a}_{1}+x_{3} \mathbf{a}_{2}+\frac{1}{2} \mathbf{a}_{3}$ & $=$ & $x_{3} a \hat{\mathbf{x}}+\frac{1}{2} c \hat{\mathbf{z}}$ & $(8 e)$ \\
\hline $\mathbf{B}_{9}$ & $=$ & $\left(x_{4}-y_{4}\right) \mathbf{a}_{1}+\left(x_{4}+y_{4}\right) \mathbf{a}_{2}+\frac{1}{4} \mathbf{a}_{3}$ & $=$ & $x_{4} a \hat{\mathbf{x}}+y_{4} b \hat{\mathbf{y}}+\frac{1}{4} c \hat{\mathbf{z}}$ & $(8 g)$ \\
\hline $\mathbf{B}_{10}$ & $=$ & $\left(-x_{4}+y_{4}\right) \mathbf{a}_{1}+\left(-x_{4}-y_{4}\right) \mathbf{a}_{2}+\frac{3}{4} \mathbf{a}_{3}$ & $=$ & $-x_{4} a \hat{\mathbf{x}}-y_{4} b \hat{\mathbf{y}}+\frac{3}{4} c \hat{\mathbf{z}}$ & $(8 g)$ \\
\hline $\mathbf{B}_{11}$ & $=$ & $\left(-x_{4}-y_{4}\right) \mathbf{a}_{1}+\left(-x_{4}+y_{4}\right) \mathbf{a}_{2}+\frac{1}{4} \mathbf{a}_{3}$ & $=$ & $-x_{4} a \hat{\mathbf{x}}+y_{4} b \hat{\mathbf{y}}+\frac{1}{4} c \hat{\mathbf{z}}$ & $(8 g)$ \\
\hline $\mathbf{B}_{12}$ & $=$ & $\left(x_{4}+y_{4}\right) \mathbf{a}_{1}+\left(x_{4}-y_{4}\right) \mathbf{a}_{2}+\frac{3}{4} \mathbf{a}_{3}$ & $=$ & $x_{4} a \hat{\mathbf{x}}-y_{4} b \hat{\mathbf{y}}+\frac{3}{4} c \hat{\mathbf{z}}$ & $(8 g)$ \\
\hline
\end{tabular}

\section{References:}

- J. R. Clark and G. E. Brown, Jr., Crystal structure of rasvumite, $\mathrm{KFe}_{2} \mathrm{~S}_{3}$, Am. Mineral. 65, 477-482 (1980).

\section{Found in:}

- R. T. Downs and M. Hall-Wallace, The American Mineralogist Crystal Structure Database, Am. Mineral. 88, 247-250 (2003).

\section{Geometry files:}

- CIF: pp. 845

- POSCAR: pp. 846 


\section{$\mathrm{La}_{43} \mathrm{Ni}_{17} \mathrm{Mg}_{5}$ Structure:}

\section{A43B5C17_oC260_63_c8fg6h_cfg_ce3f2h}
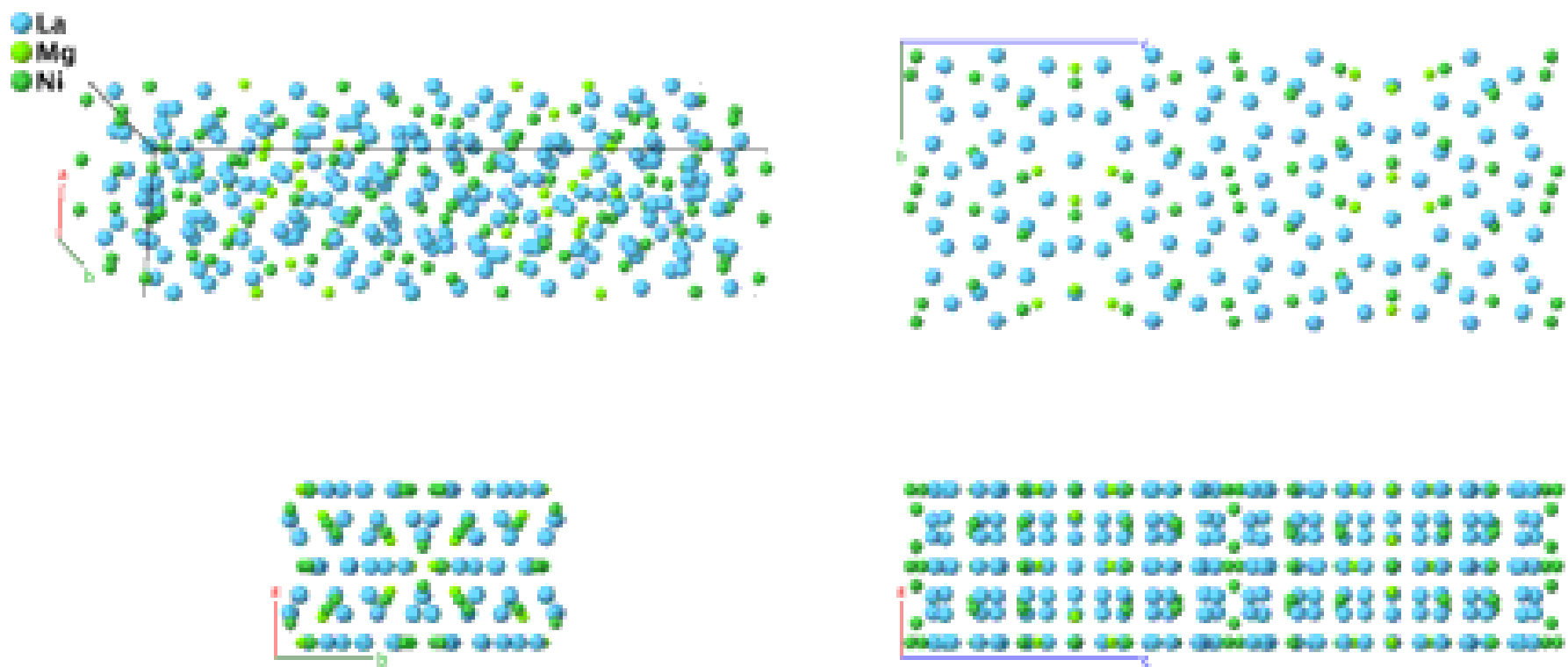

Prototype

AFLOW prototype label

Strukturbericht designation

Pearson symbol

Space group number

Space group symbol

AFLOW prototype command
$: \quad \mathrm{La}_{43} \mathrm{Ni}_{17} \mathrm{Mg}_{5}$

: A43B5C17_oC260_63_c8fg6h_cfg_ce3f2h

: None

: $\quad$ oC260

: 63

: $\mathrm{Cmcm}$

aflow --proto=A43B5C17_oC260_63_c8fg6h_cfg_ce $3 \mathrm{f} 2 \mathrm{~h}$

- -params $=a, b / a, c / a, y_{1}, y_{2}, y_{3}, x_{4}, y_{5}, z_{5}, y_{6}, z_{6}, y_{7}, z_{7}, y_{8}, z_{8}, y_{9}, z_{9}, y_{10}, z_{10}, y_{11}$, $z_{11}, y_{12}, z_{12}, y_{13}, z_{13}, y_{14}, z_{14}, y_{15}, z_{15}, y_{16}, z_{16}, x_{17}, y_{17}, x_{18}, y_{18}, x_{19}, y_{19}, z_{19}, x_{20}, y_{20}$, $z_{20}, x_{21}, y_{21}, z_{21}, x_{22}, y_{22}, z_{22}, x_{23}, y_{23}, z_{23}, x_{24}, y_{24}, z_{24}, x_{25}, y_{25}, z_{25}, x_{26}, y_{26}, z_{26}$

Base-centered Orthorhombic primitive vectors:

$$
\begin{aligned}
& \mathbf{a}_{1}=\frac{1}{2} a \hat{\mathbf{x}}-\frac{1}{2} b \hat{\mathbf{y}} \\
& \mathbf{a}_{2}=\frac{1}{2} a \hat{\mathbf{x}}+\frac{1}{2} b \hat{\mathbf{y}} \\
& \mathbf{a}_{3}=c \quad c \hat{\mathbf{z}}
\end{aligned}
$$

Basis vectors:

Lattice Coordinates

$\begin{array}{lll}\mathbf{B}_{1} & = & -y_{1} \mathbf{a}_{1}+y_{1} \mathbf{a}_{2}+\frac{1}{4} \mathbf{a}_{3} \\ \mathbf{B}_{2} & = & y_{1} \mathbf{a}_{1}-y_{1} \mathbf{a}_{2}+\frac{3}{4} \mathbf{a}_{3} \\ \mathbf{B}_{3}= & -y_{2} \mathbf{a}_{1}+y_{2} \mathbf{a}_{2}+\frac{1}{4} \mathbf{a}_{3} \\ \mathbf{B}_{4}= & y_{2} \mathbf{a}_{1}-y_{2} \mathbf{a}_{2}+\frac{3}{4} \mathbf{a}_{3}\end{array}$

$\mathbf{B}_{1}=$

$\mathbf{B}_{2}=$

$y_{2} \mathbf{a}_{1}-y_{2} \mathbf{a}_{2}+\frac{3}{4} \mathbf{a}_{3}$
Cartesian Coordinates

$=$

$=$

$=$

$=$

$$
\begin{gathered}
y_{1} b \hat{\mathbf{y}}+\frac{1}{4} c \hat{\mathbf{z}} \\
-y_{1} b \hat{\mathbf{y}}+\frac{3}{4} c \hat{\mathbf{z}} \\
y_{2} b \hat{\mathbf{y}}+\frac{1}{4} c \hat{\mathbf{z}} \\
-y_{2} b \hat{\mathbf{y}}+\frac{3}{4} c \hat{\mathbf{z}}
\end{gathered}
$$

Wyckoff Position
Atom Type

La I

La I

Mg I

Mg I 


\begin{tabular}{|c|c|c|c|c|c|c|}
\hline $\mathbf{B}_{5}$ & $=$ & $-y_{3} \mathbf{a}_{1}+y_{3} \mathbf{a}_{2}+\frac{1}{4} \mathbf{a}_{3}$ & $=$ & $y_{3} b \hat{\mathbf{y}}+\frac{1}{4} c \hat{\mathbf{z}}$ & $(4 c)$ & Ni I \\
\hline $\mathbf{B}_{6}$ & $=$ & $y_{3} \mathbf{a}_{1}-y_{3} \mathbf{a}_{2}+\frac{3}{4} \mathbf{a}_{3}$ & $=$ & $-y_{3} b \hat{\mathbf{y}}+\frac{3}{4} c \hat{\mathbf{z}}$ & $(4 c)$ & $\mathrm{Ni} I$ \\
\hline $\mathbf{B}_{7}$ & $=$ & $x_{4} \mathbf{a}_{1}+x_{4} \mathbf{a}_{2}$ & $=$ & $x_{4} a \hat{\mathbf{x}}$ & $(8 e)$ & Ni II \\
\hline $\mathbf{B}_{8}$ & $=$ & $-x_{4} \mathbf{a}_{1}-x_{4} \mathbf{a}_{2}+\frac{1}{2} \mathbf{a}_{3}$ & $=$ & $-x_{4} a \hat{\mathbf{x}}+\frac{1}{2} c \hat{\mathbf{z}}$ & $(8 e)$ & Ni II \\
\hline $\mathbf{B}_{9}$ & $=$ & $-x_{4} \mathbf{a}_{1}-x_{4} \mathbf{a}_{2}$ & $=$ & $-x_{4} a \hat{\mathbf{x}}$ & $(8 e)$ & Ni II \\
\hline $\mathbf{B}_{10}$ & $=$ & $x_{4} \mathbf{a}_{1}+x_{4} \mathbf{a}_{2}+\frac{1}{2} \mathbf{a}_{3}$ & $=$ & $x_{4} a \hat{\mathbf{x}}+\frac{1}{2} c \hat{\mathbf{z}}$ & $(8 e)$ & Ni II \\
\hline $\mathbf{B}_{11}$ & $=$ & $-y_{5} \mathbf{a}_{1}+y_{5} \mathbf{a}_{2}+z_{5} \mathbf{a}_{3}$ & $=$ & $y_{5} b \hat{\mathbf{y}}+z_{5} c \hat{\mathbf{z}}$ & $(8 f)$ & La II \\
\hline $\mathbf{B}_{12}$ & $=$ & $y_{5} \mathbf{a}_{1}-y_{5} \mathbf{a}_{2}+\left(\frac{1}{2}+z_{5}\right) \mathbf{a}_{3}$ & $=$ & $-y_{5} b \hat{\mathbf{y}}+\left(\frac{1}{2}+z_{5}\right) c \hat{\mathbf{z}}$ & $(8 f)$ & La II \\
\hline $\mathbf{B}_{13}$ & $=$ & $-y_{5} \mathbf{a}_{1}+y_{5} \mathbf{a}_{2}+\left(\frac{1}{2}-z_{5}\right) \mathbf{a}_{3}$ & $=$ & $y_{5} b \hat{\mathbf{y}}+\left(\frac{1}{2}-z_{5}\right) c \hat{\mathbf{z}}$ & $(8 f)$ & La II \\
\hline $\mathbf{B}_{14}$ & $=$ & $y_{5} \mathbf{a}_{1}-y_{5} \mathbf{a}_{2}-z_{5} \mathbf{a}_{3}$ & $=$ & $-y_{5} b \hat{\mathbf{y}}-z_{5} c \hat{\mathbf{z}}$ & $(8 f)$ & La II \\
\hline $\mathbf{B}_{15}$ & $=$ & $-y_{6} \mathbf{a}_{1}+y_{6} \mathbf{a}_{2}+z_{6} \mathbf{a}_{3}$ & $=$ & $y_{6} b \hat{\mathbf{y}}+z_{6} c \hat{\mathbf{z}}$ & $(8 f)$ & La III \\
\hline $\mathbf{B}_{16}$ & $=$ & $y_{6} \mathbf{a}_{1}-y_{6} \mathbf{a}_{2}+\left(\frac{1}{2}+z_{6}\right) \mathbf{a}_{3}$ & $=$ & $-y_{6} b \hat{\mathbf{y}}+\left(\frac{1}{2}+z_{6}\right) c \hat{\mathbf{z}}$ & $(8 f)$ & La III \\
\hline $\mathbf{B}_{17}$ & $=$ & $-y_{6} \mathbf{a}_{1}+y_{6} \mathbf{a}_{2}+\left(\frac{1}{2}-z_{6}\right) \mathbf{a}_{3}$ & $=$ & $y_{6} b \hat{\mathbf{y}}+\left(\frac{1}{2}-z_{6}\right) c \hat{\mathbf{z}}$ & $(8 f)$ & La III \\
\hline $\mathbf{B}_{18}$ & $=$ & $y_{6} \mathbf{a}_{1}-y_{6} \mathbf{a}_{2}-z_{6} \mathbf{a}_{3}$ & $=$ & $-y_{6} b \hat{\mathbf{y}}-z_{6} c \hat{\mathbf{z}}$ & $(8 f)$ & La III \\
\hline $\mathbf{B}_{19}$ & $=$ & $-y_{7} \mathbf{a}_{1}+y_{7} \mathbf{a}_{2}+z_{7} \mathbf{a}_{3}$ & $=$ & $y_{7} b \hat{\mathbf{y}}+z_{7} c \hat{\mathbf{z}}$ & $(8 f)$ & La IV \\
\hline $\mathbf{B}_{20}$ & $=$ & $y_{7} \mathbf{a}_{1}-y_{7} \mathbf{a}_{2}+\left(\frac{1}{2}+z_{7}\right) \mathbf{a}_{3}$ & $=$ & $-y_{7} b \hat{\mathbf{y}}+\left(\frac{1}{2}+z_{7}\right) c \hat{\mathbf{z}}$ & $(8 f)$ & La IV \\
\hline $\mathbf{B}_{21}$ & $=$ & $-y_{7} \mathbf{a}_{1}+y_{7} \mathbf{a}_{2}+\left(\frac{1}{2}-z_{7}\right) \mathbf{a}_{3}$ & $=$ & $y_{7} b \hat{\mathbf{y}}+\left(\frac{1}{2}-z_{7}\right) c \hat{\mathbf{z}}$ & $(8 f)$ & La IV \\
\hline $\mathbf{B}_{22}$ & $=$ & $y_{7} \mathbf{a}_{1}-y_{7} \mathbf{a}_{2}-z_{7} \mathbf{a}_{3}$ & $=$ & $-y_{7} b \hat{\mathbf{y}}-z_{7} c \hat{\mathbf{z}}$ & $(8 f)$ & La IV \\
\hline $\mathbf{B}_{23}$ & $=$ & $-y_{8} \mathbf{a}_{1}+y_{8} \mathbf{a}_{2}+z_{8} \mathbf{a}_{3}$ & $=$ & $y_{8} b \hat{\mathbf{y}}+z_{8} c \hat{\mathbf{z}}$ & $(8 f)$ & $\mathrm{La} \mathrm{V}$ \\
\hline $\mathbf{B}_{24}$ & $=$ & $y_{8} \mathbf{a}_{1}-y_{8} \mathbf{a}_{2}+\left(\frac{1}{2}+z_{8}\right) \mathbf{a}_{3}$ & $=$ & $-y_{8} b \hat{\mathbf{y}}+\left(\frac{1}{2}+z_{8}\right) c \hat{\mathbf{z}}$ & $(8 f)$ & La V \\
\hline $\mathbf{B}_{25}$ & $=$ & $-y_{8} \mathbf{a}_{1}+y_{8} \mathbf{a}_{2}+\left(\frac{1}{2}-z_{8}\right) \mathbf{a}_{3}$ & $=$ & $y_{8} b \hat{\mathbf{y}}+\left(\frac{1}{2}-z_{8}\right) c \hat{\mathbf{z}}$ & $(8 f)$ & La V \\
\hline $\mathbf{B}_{26}$ & $=$ & $y_{8} \mathbf{a}_{1}-y_{8} \mathbf{a}_{2}-z_{8} \mathbf{a}_{3}$ & $=$ & $-y_{8} b \hat{\mathbf{y}}-z_{8} c \hat{\mathbf{z}}$ & $(8 f)$ & $\mathrm{La} \mathrm{V}$ \\
\hline $\mathbf{B}_{27}$ & $=$ & $-y_{9} \mathbf{a}_{1}+y_{9} \mathbf{a}_{2}+z_{9} \mathbf{a}_{3}$ & $=$ & $y_{9} b \hat{\mathbf{y}}+z_{9} c \hat{\mathbf{z}}$ & $(8 f)$ & La VI \\
\hline $\mathbf{B}_{28}$ & $=$ & $y_{9} \mathbf{a}_{1}-y_{9} \mathbf{a}_{2}+\left(\frac{1}{2}+z_{9}\right) \mathbf{a}_{3}$ & $=$ & $-y_{9} b \hat{\mathbf{y}}+\left(\frac{1}{2}+z_{9}\right) c \hat{\mathbf{z}}$ & $(8 f)$ & La VI \\
\hline $\mathbf{B}_{29}$ & $=$ & $-y_{9} \mathbf{a}_{1}+y_{9} \mathbf{a}_{2}+\left(\frac{1}{2}-z_{9}\right) \mathbf{a}_{3}$ & $=$ & $y_{9} b \hat{\mathbf{y}}+\left(\frac{1}{2}-z_{9}\right) c \hat{\mathbf{z}}$ & $(8 f)$ & La VI \\
\hline $\mathbf{B}_{30}$ & $=$ & $y_{9} \mathbf{a}_{1}-y_{9} \mathbf{a}_{2}-z_{9} \mathbf{a}_{3}$ & $=$ & $-y_{9} b \hat{\mathbf{y}}-z_{9} c \hat{\mathbf{z}}$ & $(8 f)$ & La VI \\
\hline $\mathbf{B}_{31}$ & $=$ & $-y_{10} \mathbf{a}_{1}+y_{10} \mathbf{a}_{2}+z_{10} \mathbf{a}_{3}$ & $=$ & $y_{10} b \hat{\mathbf{y}}+z_{10} c \hat{\mathbf{z}}$ & $(8 f)$ & La VII \\
\hline $\mathbf{B}_{32}$ & $=$ & $y_{10} \mathbf{a}_{1}-y_{10} \mathbf{a}_{2}+\left(\frac{1}{2}+z_{10}\right) \mathbf{a}_{3}$ & $=$ & $-y_{10} b \hat{\mathbf{y}}+\left(\frac{1}{2}+z_{10}\right) c \hat{\mathbf{z}}$ & $(8 f)$ & La VII \\
\hline $\mathbf{B}_{33}$ & $=$ & $-y_{10} \mathbf{a}_{1}+y_{10} \mathbf{a}_{2}+\left(\frac{1}{2}-z_{10}\right) \mathbf{a}_{3}$ & $=$ & $y_{10} b \hat{\mathbf{y}}+\left(\frac{1}{2}-z_{10}\right) c \hat{\mathbf{z}}$ & $(8 f)$ & La VII \\
\hline $\mathbf{B}_{34}$ & $=$ & $y_{10} \mathbf{a}_{1}-y_{10} \mathbf{a}_{2}-z_{10} \mathbf{a}_{3}$ & $=$ & $-y_{10} b \hat{\mathbf{y}}-z_{10} c \hat{\mathbf{z}}$ & $(8 f)$ & La VII \\
\hline $\mathbf{B}_{35}$ & $=$ & $-y_{11} \mathbf{a}_{1}+y_{11} \mathbf{a}_{2}+z_{11} \mathbf{a}_{3}$ & $=$ & $y_{11} b \hat{\mathbf{y}}+z_{11} c \hat{\mathbf{z}}$ & $(8 f)$ & La VIII \\
\hline $\mathbf{B}_{36}$ & $=$ & $y_{11} \mathbf{a}_{1}-y_{11} \mathbf{a}_{2}+\left(\frac{1}{2}+z_{11}\right) \mathbf{a}_{3}$ & $=$ & $-y_{11} b \hat{\mathbf{y}}+\left(\frac{1}{2}+z_{11}\right) c \hat{\mathbf{z}}$ & $(8 f)$ & La VIII \\
\hline $\mathbf{B}_{37}$ & $=$ & $-y_{11} \mathbf{a}_{1}+y_{11} \mathbf{a}_{2}+\left(\frac{1}{2}-z_{11}\right) \mathbf{a}_{3}$ & $=$ & $y_{11} b \hat{\mathbf{y}}+\left(\frac{1}{2}-z_{11}\right) c \hat{\mathbf{z}}$ & $(8 f)$ & La VIII \\
\hline $\mathbf{B}_{38}$ & $=$ & $y_{11} \mathbf{a}_{1}-y_{11} \mathbf{a}_{2}-z_{11} \mathbf{a}_{3}$ & $=$ & $-y_{11} b \hat{\mathbf{y}}-z_{11} c \hat{\mathbf{z}}$ & $(8 f)$ & La VIII \\
\hline $\mathbf{B}_{39}$ & $=$ & $-y_{12} \mathbf{a}_{1}+y_{12} \mathbf{a}_{2}+z_{12} \mathbf{a}_{3}$ & $=$ & $y_{12} b \hat{\mathbf{y}}+z_{12} c \hat{\mathbf{z}}$ & $(8 f)$ & La IX \\
\hline $\mathbf{B}_{40}$ & $=$ & $y_{12} \mathbf{a}_{1}-y_{12} \mathbf{a}_{2}+\left(\frac{1}{2}+z_{12}\right) \mathbf{a}_{3}$ & $=$ & $-y_{12} b \hat{\mathbf{y}}+\left(\frac{1}{2}+z_{12}\right) c \hat{\mathbf{z}}$ & $(8 f)$ & La IX \\
\hline
\end{tabular}




\begin{tabular}{|c|c|c|c|c|c|c|}
\hline $\mathbf{B}_{41}$ & $=$ & $-y_{12} \mathbf{a}_{1}+y_{12} \mathbf{a}_{2}+\left(\frac{1}{2}-z_{12}\right) \mathbf{a}_{3}$ & $=$ & $y_{12} b \hat{\mathbf{y}}+\left(\frac{1}{2}-z_{12}\right) c \hat{\mathbf{z}}$ & $(8 f)$ & La IX \\
\hline $\mathbf{B}_{42}$ & $=$ & $y_{12} \mathbf{a}_{1}-y_{12} \mathbf{a}_{2}-z_{12} \mathbf{a}_{3}$ & $=$ & $-y_{12} b \hat{\mathbf{y}}-z_{12} c \hat{\mathbf{z}}$ & $(8 f)$ & La IX \\
\hline $\mathbf{B}_{43}$ & $=$ & $-y_{13} \mathbf{a}_{1}+y_{13} \mathbf{a}_{2}+z_{13} \mathbf{a}_{3}$ & $=$ & $y_{13} b \hat{\mathbf{y}}+z_{13} c \hat{\mathbf{z}}$ & $(8 f)$ & Mg II \\
\hline $\mathbf{B}_{44}$ & $=$ & $y_{13} \mathbf{a}_{1}-y_{13} \mathbf{a}_{2}+\left(\frac{1}{2}+z_{13}\right) \mathbf{a}_{3}$ & $=$ & $-y_{13} b \hat{\mathbf{y}}+\left(\frac{1}{2}+z_{13}\right) c \hat{\mathbf{z}}$ & $(8 f)$ & $\mathrm{Mg}$ II \\
\hline $\mathbf{B}_{45}$ & $=$ & $-y_{13} \mathbf{a}_{1}+y_{13} \mathbf{a}_{2}+\left(\frac{1}{2}-z_{13}\right) \mathbf{a}_{3}$ & $=$ & $y_{13} b \hat{\mathbf{y}}+\left(\frac{1}{2}-z_{13}\right) c \hat{\mathbf{z}}$ & $(8 f)$ & Mg II \\
\hline $\mathbf{B}_{46}$ & $=$ & $y_{13} \mathbf{a}_{1}-y_{13} \mathbf{a}_{2}-z_{13} \mathbf{a}_{3}$ & $=$ & $-y_{13} b \hat{\mathbf{y}}-z_{13} c \hat{\mathbf{z}}$ & $(8 f)$ & $\mathrm{Mg}$ II \\
\hline $\mathbf{B}_{47}$ & $=$ & $-y_{14} \mathbf{a}_{1}+y_{14} \mathbf{a}_{2}+z_{14} \mathbf{a}_{3}$ & $=$ & $y_{14} b \hat{\mathbf{y}}+z_{14} c \hat{\mathbf{z}}$ & $(8 f)$ & Ni III \\
\hline $\mathbf{B}_{48}$ & $=$ & $y_{14} \mathbf{a}_{1}-y_{14} \mathbf{a}_{2}+\left(\frac{1}{2}+z_{14}\right) \mathbf{a}_{3}$ & $=$ & $-y_{14} b \hat{\mathbf{y}}+\left(\frac{1}{2}+z_{14}\right) c \hat{\mathbf{z}}$ & $(8 f)$ & Ni III \\
\hline $\mathbf{B}_{49}$ & $=$ & $-y_{14} \mathbf{a}_{1}+y_{14} \mathbf{a}_{2}+\left(\frac{1}{2}-z_{14}\right) \mathbf{a}_{3}$ & $=$ & $y_{14} b \hat{\mathbf{y}}+\left(\frac{1}{2}-z_{14}\right) c \hat{\mathbf{z}}$ & $(8 f)$ & Ni III \\
\hline $\mathbf{B}_{50}$ & $=$ & $y_{14} \mathbf{a}_{1}-y_{14} \mathbf{a}_{2}-z_{14} \mathbf{a}_{3}$ & $=$ & $-y_{14} b \hat{\mathbf{y}}-z_{14} c \hat{\mathbf{z}}$ & $(8 f)$ & Ni III \\
\hline $\mathbf{B}_{51}$ & $=$ & $-y_{15} \mathbf{a}_{1}+y_{15} \mathbf{a}_{2}+z_{15} \mathbf{a}_{3}$ & $=$ & $y_{15} b \hat{\mathbf{y}}+z_{15} c \hat{\mathbf{z}}$ & $(8 f)$ & Ni IV \\
\hline $\mathbf{B}_{52}$ & $=$ & $y_{15} \mathbf{a}_{1}-y_{15} \mathbf{a}_{2}+\left(\frac{1}{2}+z_{15}\right) \mathbf{a}_{3}$ & $=$ & $-y_{15} b \hat{\mathbf{y}}+\left(\frac{1}{2}+z_{15}\right) c \hat{\mathbf{z}}$ & $(8 f)$ & Ni IV \\
\hline $\mathbf{B}_{53}$ & $=$ & $-y_{15} \mathbf{a}_{1}+y_{15} \mathbf{a}_{2}+\left(\frac{1}{2}-z_{15}\right) \mathbf{a}_{3}$ & $=$ & $y_{15} b \hat{\mathbf{y}}+\left(\frac{1}{2}-z_{15}\right) c \hat{\mathbf{z}}$ & $(8 f)$ & Ni IV \\
\hline $\mathbf{B}_{54}$ & $=$ & $y_{15} \mathbf{a}_{1}-y_{15} \mathbf{a}_{2}-z_{15} \mathbf{a}_{3}$ & $=$ & $-y_{15} b \hat{\mathbf{y}}-z_{15} c \hat{\mathbf{z}}$ & $(8 f)$ & Ni IV \\
\hline $\mathbf{B}_{55}$ & $=$ & $-y_{16} \mathbf{a}_{1}+y_{16} \mathbf{a}_{2}+z_{16} \mathbf{a}_{3}$ & $=$ & $y_{16} b \hat{\mathbf{y}}+z_{16} c \hat{\mathbf{z}}$ & $(8 f)$ & $\mathrm{Ni} \mathrm{V}$ \\
\hline $\mathbf{B}_{56}$ & $=$ & $y_{16} \mathbf{a}_{1}-y_{16} \mathbf{a}_{2}+\left(\frac{1}{2}+z_{16}\right) \mathbf{a}_{3}$ & $=$ & $-y_{16} b \hat{\mathbf{y}}+\left(\frac{1}{2}+z_{16}\right) c \hat{\mathbf{z}}$ & $(8 f)$ & $\mathrm{Ni} \mathrm{V}$ \\
\hline $\mathbf{B}_{57}$ & $=$ & $-y_{16} \mathbf{a}_{1}+y_{16} \mathbf{a}_{2}+\left(\frac{1}{2}-z_{16}\right) \mathbf{a}_{3}$ & $=$ & $y_{16} b \hat{\mathbf{y}}+\left(\frac{1}{2}-z_{16}\right) c \hat{\mathbf{z}}$ & $(8 f)$ & Ni V \\
\hline $\mathbf{B}_{58}$ & $=$ & $y_{16} \mathbf{a}_{1}-y_{16} \mathbf{a}_{2}-z_{16} \mathbf{a}_{3}$ & $=$ & $-y_{16} b \hat{\mathbf{y}}-z_{16} c \hat{\mathbf{z}}$ & $(8 f)$ & Ni V \\
\hline $\mathbf{B}_{59}$ & $=$ & $\left(x_{17}-y_{17}\right) \mathbf{a}_{1}+\left(x_{17}+y_{17}\right) \mathbf{a}_{2}+\frac{1}{4} \mathbf{a}_{3}$ & $=$ & $x_{17} a \hat{\mathbf{x}}+y_{17} b \hat{\mathbf{y}}+\frac{1}{4} c \hat{\mathbf{z}}$ & $(8 g)$ & La X \\
\hline $\mathbf{B}_{60}$ & $=$ & $\begin{array}{c}\left(-x_{17}+y_{17}\right) \mathbf{a}_{1}+\left(-x_{17}-y_{17}\right) \mathbf{a}_{2}+ \\
\frac{3}{4} \mathbf{a}_{3}\end{array}$ & $=$ & $-x_{17} a \hat{\mathbf{x}}-y_{17} b \hat{\mathbf{y}}+\frac{3}{4} c \hat{\mathbf{z}}$ & $(8 g)$ & La X \\
\hline $\mathbf{B}_{61}$ & $=$ & $\begin{array}{c}\left(-x_{17}-y_{17}\right) \mathbf{a}_{1}+\left(-x_{17}+y_{17}\right) \mathbf{a}_{2}+ \\
\frac{1}{4} \mathbf{a}_{3}\end{array}$ & $=$ & $-x_{17} a \hat{\mathbf{x}}+y_{17} b \hat{\mathbf{y}}+\frac{1}{4} c \hat{\mathbf{z}}$ & $(8 g)$ & La X \\
\hline $\mathbf{B}_{62}$ & $=$ & $\left(x_{17}+y_{17}\right) \mathbf{a}_{1}+\left(x_{17}-y_{17}\right) \mathbf{a}_{2}+\frac{3}{4} \mathbf{a}_{3}$ & $=$ & $x_{17} a \hat{\mathbf{x}}-y_{17} b \hat{\mathbf{y}}+\frac{3}{4} c \hat{\mathbf{z}}$ & $(8 g)$ & La X \\
\hline $\mathbf{B}_{63}$ & $=$ & $\left(x_{18}-y_{18}\right) \mathbf{a}_{1}+\left(x_{18}+y_{18}\right) \mathbf{a}_{2}+\frac{1}{4} \mathbf{a}_{3}$ & $=$ & $x_{18} a \hat{\mathbf{x}}+y_{18} b \hat{\mathbf{y}}+\frac{1}{4} c \hat{\mathbf{z}}$ & $(8 g)$ & $\mathrm{Mg}$ III \\
\hline $\mathbf{B}_{64}$ & $=$ & $\begin{array}{c}\left(-x_{18}+y_{18}\right) \mathbf{a}_{1}+\left(-x_{18}-y_{18}\right) \mathbf{a}_{2}+ \\
\frac{3}{4} \mathbf{a}_{3}\end{array}$ & $=$ & $-x_{18} a \hat{\mathbf{x}}-y_{18} b \hat{\mathbf{y}}+\frac{3}{4} c \hat{\mathbf{z}}$ & $(8 g)$ & Mg III \\
\hline $\mathbf{B}_{65}$ & $=$ & $\begin{array}{c}\left(-x_{18}-y_{18}\right) \mathbf{a}_{1}+\left(-x_{18}+y_{18}\right) \mathbf{a}_{2}+ \\
\frac{1}{4} \mathbf{a}_{3}\end{array}$ & $=$ & $-x_{18} a \hat{\mathbf{x}}+y_{18} b \hat{\mathbf{y}}+\frac{1}{4} c \hat{\mathbf{z}}$ & $(8 g)$ & Mg III \\
\hline $\mathbf{B}_{66}$ & $=$ & $\left(x_{18}+y_{18}\right) \mathbf{a}_{1}+\left(x_{18}-y_{18}\right) \mathbf{a}_{2}+\frac{3}{4} \mathbf{a}_{3}$ & $=$ & $x_{18} a \hat{\mathbf{x}}-y_{18} b \hat{\mathbf{y}}+\frac{3}{4} c \hat{\mathbf{z}}$ & $(8 g)$ & $\mathrm{Mg}$ III \\
\hline $\mathbf{B}_{67}$ & $=$ & $\left(x_{19}-y_{19}\right) \mathbf{a}_{1}+\left(x_{19}+y_{19}\right) \mathbf{a}_{2}+z_{19} \mathbf{a}_{3}$ & $=$ & $x_{19} a \hat{\mathbf{x}}+y_{19} b \hat{\mathbf{y}}+z_{19} c \hat{\mathbf{z}}$ & $(16 h)$ & La XI \\
\hline $\mathbf{B}_{68}$ & $=$ & $\begin{array}{c}\left(-x_{19}+y_{19}\right) \mathbf{a}_{1}+\left(-x_{19}-y_{19}\right) \mathbf{a}_{2}+ \\
\left(\frac{1}{2}+z_{19}\right) \mathbf{a}_{3}\end{array}$ & $=$ & $-x_{19} a \hat{\mathbf{x}}-y_{19} b \hat{\mathbf{y}}+\left(\frac{1}{2}+z_{19}\right) c \hat{\mathbf{z}}$ & $(16 h)$ & La XI \\
\hline $\mathbf{B}_{69}$ & $=$ & $\begin{array}{c}\left(-x_{19}-y_{19}\right) \mathbf{a}_{1}+\left(-x_{19}+y_{19}\right) \mathbf{a}_{2}+ \\
\left(\frac{1}{2}-z_{19}\right) \mathbf{a}_{3}\end{array}$ & $=$ & $-x_{19} a \hat{\mathbf{x}}+y_{19} b \hat{\mathbf{y}}+\left(\frac{1}{2}-z_{19}\right) c \hat{\mathbf{z}}$ & $(16 h)$ & La XI \\
\hline $\mathbf{B}_{70}$ & $=$ & $\left(x_{19}+y_{19}\right) \mathbf{a}_{1}+\left(x_{19}-y_{19}\right) \mathbf{a}_{2}-z_{19} \mathbf{a}_{3}$ & $=$ & $x_{19} a \hat{\mathbf{x}}-y_{19} b \hat{\mathbf{y}}-z_{19} c \hat{\mathbf{z}}$ & $(16 h)$ & La XI \\
\hline $\mathbf{B}_{71}$ & $=$ & $\begin{array}{c}\left(-x_{19}+y_{19}\right) \mathbf{a}_{1}+\left(-x_{19}-y_{19}\right) \mathbf{a}_{2}- \\
z_{19} \mathbf{a}_{3}\end{array}$ & $=$ & $-x_{19} a \hat{\mathbf{x}}-y_{19} b \hat{\mathbf{y}}-z_{19} c \hat{\mathbf{z}}$ & $(16 h)$ & La XI \\
\hline $\mathbf{B}_{72}$ & $=$ & $\begin{array}{c}\left(x_{19}-y_{19}\right) \mathbf{a}_{1}+\left(x_{19}+y_{19}\right) \mathbf{a}_{2}+ \\
\left(\frac{1}{2}-z_{19}\right) \mathbf{a}_{3}\end{array}$ & $=$ & $x_{19} a \hat{\mathbf{x}}+y_{19} b \hat{\mathbf{y}}+\left(\frac{1}{2}-z_{19}\right) c \hat{\mathbf{z}}$ & $(16 h)$ & La XI \\
\hline
\end{tabular}




\begin{tabular}{|c|c|c|c|c|c|c|}
\hline $\mathbf{B}_{73}$ & $=$ & $\begin{array}{c}\left(x_{19}+y_{19}\right) \mathbf{a}_{1}+\left(x_{19}-y_{19}\right) \mathbf{a}_{2}+ \\
\left(\frac{1}{2}+z_{19}\right) \mathbf{a}_{3}\end{array}$ & $=$ & $x_{19} a \hat{\mathbf{x}}-y_{19} b \hat{\mathbf{y}}+\left(\frac{1}{2}+z_{19}\right) c \hat{\mathbf{z}}$ & $(16 h)$ & La XI \\
\hline $\mathbf{B}_{74}$ & $=$ & $\begin{array}{c}\left(-x_{19}-y_{19}\right) \mathbf{a}_{1}+\left(-x_{19}+y_{19}\right) \mathbf{a}_{2}+ \\
z_{19} \mathbf{a}_{3}\end{array}$ & $=$ & $-x_{19} a \hat{\mathbf{x}}+y_{19} b \hat{\mathbf{y}}+z_{19} c \hat{\mathbf{z}}$ & $(16 h)$ & La XI \\
\hline $\mathbf{B}_{75}$ & $=$ & $\left(x_{20}-y_{20}\right) \mathbf{a}_{1}+\left(x_{20}+y_{20}\right) \mathbf{a}_{2}+z_{20} \mathbf{a}_{3}$ & $=$ & $x_{20} a \hat{\mathbf{x}}+y_{20} b \hat{\mathbf{y}}+z_{20} c \hat{\mathbf{z}}$ & $(16 h)$ & La XII \\
\hline $\mathbf{B}_{76}$ & $=$ & $\begin{array}{c}\left(-x_{20}+y_{20}\right) \mathbf{a}_{1}+\left(-x_{20}-y_{20}\right) \mathbf{a}_{2}+ \\
\left(\frac{1}{2}+z_{20}\right) \mathbf{a}_{3}\end{array}$ & $=$ & $-x_{20} a \hat{\mathbf{x}}-y_{20} b \hat{\mathbf{y}}+\left(\frac{1}{2}+z_{20}\right) c \hat{\mathbf{z}}$ & $(16 h)$ & La XII \\
\hline $\mathbf{B}_{77}$ & $=$ & $\begin{array}{c}\left(-x_{20}-y_{20}\right) \mathbf{a}_{1}+\left(-x_{20}+y_{20}\right) \mathbf{a}_{2}+ \\
\left(\frac{1}{2}-z_{20}\right) \mathbf{a}_{3}\end{array}$ & $=$ & $-x_{20} a \hat{\mathbf{x}}+y_{20} b \hat{\mathbf{y}}+\left(\frac{1}{2}-z_{20}\right) c \hat{\mathbf{z}}$ & $(16 h)$ & La XII \\
\hline $\mathbf{B}_{78}$ & $=$ & $\left(x_{20}+y_{20}\right) \mathbf{a}_{1}+\left(x_{20}-y_{20}\right) \mathbf{a}_{2}-z_{20} \mathbf{a}_{3}$ & $=$ & $x_{20} a \hat{\mathbf{x}}-y_{20} b \hat{\mathbf{y}}-z_{20} c \hat{\mathbf{z}}$ & $(16 h)$ & La XII \\
\hline $\mathbf{B}_{79}$ & $=$ & $\begin{array}{c}\left(-x_{20}+y_{20}\right) \mathbf{a}_{1}+\left(-x_{20}-y_{20}\right) \mathbf{a}_{2}- \\
z_{20} \mathbf{a}_{3}\end{array}$ & $=$ & $-x_{20} a \hat{\mathbf{x}}-y_{20} b \hat{\mathbf{y}}-z_{20} c \hat{\mathbf{z}}$ & $(16 h)$ & La XII \\
\hline $\mathbf{B}_{80}$ & $=$ & $\begin{array}{c}\left(x_{20}-y_{20}\right) \mathbf{a}_{1}+\left(x_{20}+y_{20}\right) \mathbf{a}_{2}+ \\
\left(\frac{1}{2}-z_{20}\right) \mathbf{a}_{3}\end{array}$ & $=$ & $x_{20} a \hat{\mathbf{x}}+y_{20} b \hat{\mathbf{y}}+\left(\frac{1}{2}-z_{20}\right) c \hat{\mathbf{z}}$ & $(16 h)$ & La XII \\
\hline $\mathbf{B}_{81}$ & $=$ & $\begin{array}{c}\left(x_{20}+y_{20}\right) \mathbf{a}_{1}+\left(x_{20}-y_{20}\right) \mathbf{a}_{2}+ \\
\left(\frac{1}{2}+z_{20}\right) \mathbf{a}_{3}\end{array}$ & $=$ & $x_{20} a \hat{\mathbf{x}}-y_{20} b \hat{\mathbf{y}}+\left(\frac{1}{2}+z_{20}\right) c \hat{\mathbf{z}}$ & $(16 h)$ & La XII \\
\hline $\mathbf{B}_{82}$ & $=$ & $\begin{array}{c}\left(-x_{20}-y_{20}\right) \mathbf{a}_{1}+\left(-x_{20}+y_{20}\right) \mathbf{a}_{2}+ \\
z_{20} \mathbf{a}_{3}\end{array}$ & $=$ & $-x_{20} a \hat{\mathbf{x}}+y_{20} b \hat{\mathbf{y}}+z_{20} c \hat{\mathbf{z}}$ & $(16 h)$ & La XII \\
\hline $\mathbf{B}_{83}$ & $=$ & $\left(x_{21}-y_{21}\right) \mathbf{a}_{1}+\left(x_{21}+y_{21}\right) \mathbf{a}_{2}+z_{21} \mathbf{a}_{3}$ & $=$ & $x_{21} a \hat{\mathbf{x}}+y_{21} b \hat{\mathbf{y}}+z_{21} c \hat{\mathbf{z}}$ & $(16 h)$ & La XIII \\
\hline $\mathbf{B}_{84}$ & $=$ & $\begin{array}{c}\left(-x_{21}+y_{21}\right) \mathbf{a}_{1}+\left(-x_{21}-y_{21}\right) \mathbf{a}_{2}+ \\
\left(\frac{1}{2}+z_{21}\right) \mathbf{a}_{3}\end{array}$ & $=$ & $-x_{21} a \hat{\mathbf{x}}-y_{21} b \hat{\mathbf{y}}+\left(\frac{1}{2}+z_{21}\right) c \hat{\mathbf{z}}$ & $(16 h)$ & La XIII \\
\hline $\mathbf{B}_{85}$ & $=$ & $\begin{array}{c}\left(-x_{21}-y_{21}\right) \mathbf{a}_{1}+\left(-x_{21}+y_{21}\right) \mathbf{a}_{2}+ \\
\left(\frac{1}{2}-z_{21}\right) \mathbf{a}_{3}\end{array}$ & $=$ & $-x_{21} a \hat{\mathbf{x}}+y_{21} b \hat{\mathbf{y}}+\left(\frac{1}{2}-z_{21}\right) c \hat{\mathbf{z}}$ & $(16 h)$ & La XIII \\
\hline $\mathbf{B}_{86}$ & $=$ & $\left(x_{21}+y_{21}\right) \mathbf{a}_{1}+\left(x_{21}-y_{21}\right) \mathbf{a}_{2}-z_{21} \mathbf{a}_{3}$ & $=$ & $x_{21} a \hat{\mathbf{x}}-y_{21} b \hat{\mathbf{y}}-z_{21} c \hat{\mathbf{z}}$ & $(16 h)$ & La XIII \\
\hline $\mathbf{B}_{87}$ & $=$ & $\begin{array}{c}\left(-x_{21}+y_{21}\right) \mathbf{a}_{1}+\left(-x_{21}-y_{21}\right) \mathbf{a}_{2}- \\
z_{21} \mathbf{a}_{3}\end{array}$ & $=$ & $-x_{21} a \hat{\mathbf{x}}-y_{21} b \hat{\mathbf{y}}-z_{21} c \hat{\mathbf{z}}$ & $(16 h)$ & La XIII \\
\hline $\mathbf{B}_{88}$ & $=$ & $\begin{array}{c}\left(x_{21}-y_{21}\right) \mathbf{a}_{1}+\left(x_{21}+y_{21}\right) \mathbf{a}_{2}+ \\
\left(\frac{1}{2}-z_{21}\right) \mathbf{a}_{3}\end{array}$ & $=$ & $x_{21} a \hat{\mathbf{x}}+y_{21} b \hat{\mathbf{y}}+\left(\frac{1}{2}-z_{21}\right) c \hat{\mathbf{z}}$ & $(16 h)$ & La XIII \\
\hline $\mathbf{B}_{89}$ & $=$ & $\begin{array}{c}\left(x_{21}+y_{21}\right) \mathbf{a}_{1}+\left(x_{21}-y_{21}\right) \mathbf{a}_{2}+ \\
\left(\frac{1}{2}+z_{21}\right) \mathbf{a}_{3}\end{array}$ & $=$ & $x_{21} a \hat{\mathbf{x}}-y_{21} b \hat{\mathbf{y}}+\left(\frac{1}{2}+z_{21}\right) c \hat{\mathbf{z}}$ & $(16 h)$ & La XIII \\
\hline $\mathbf{B}_{90}$ & $=$ & $\begin{array}{c}\left(-x_{21}-y_{21}\right) \mathbf{a}_{1}+\left(-x_{21}+y_{21}\right) \mathbf{a}_{2}+ \\
z_{21} \mathbf{a}_{3}\end{array}$ & $=$ & $-x_{21} a \hat{\mathbf{x}}+y_{21} b \hat{\mathbf{y}}+z_{21} c \hat{\mathbf{z}}$ & $(16 h)$ & La XIII \\
\hline $\mathbf{B}_{91}$ & $=$ & $\left(x_{22}-y_{22}\right) \mathbf{a}_{1}+\left(x_{22}+y_{22}\right) \mathbf{a}_{2}+z_{22} \mathbf{a}_{3}$ & $=$ & $x_{22} a \hat{\mathbf{x}}+y_{22} b \hat{\mathbf{y}}+z_{22} c \hat{\mathbf{z}}$ & $(16 h)$ & La XIV \\
\hline $\mathbf{B}_{92}$ & $=$ & $\begin{array}{c}\left(-x_{22}+y_{22}\right) \mathbf{a}_{1}+\left(-x_{22}-y_{22}\right) \mathbf{a}_{2}+ \\
\left(\frac{1}{2}+z_{22}\right) \mathbf{a}_{3}\end{array}$ & $=$ & $-x_{22} a \hat{\mathbf{x}}-y_{22} b \hat{\mathbf{y}}+\left(\frac{1}{2}+z_{22}\right) c \hat{\mathbf{z}}$ & $(16 h)$ & La XIV \\
\hline $\mathbf{B}_{93}$ & $=$ & $\begin{array}{c}\left(-x_{22}-y_{22}\right) \mathbf{a}_{1}+\left(-x_{22}+y_{22}\right) \mathbf{a}_{2}+ \\
\left(\frac{1}{2}-z_{22}\right) \mathbf{a}_{3}\end{array}$ & $=$ & $-x_{22} a \hat{\mathbf{x}}+y_{22} b \hat{\mathbf{y}}+\left(\frac{1}{2}-z_{22}\right) c \hat{\mathbf{z}}$ & $(16 h)$ & La XIV \\
\hline $\mathbf{B}_{94}$ & $=$ & $\left(x_{22}+y_{22}\right) \mathbf{a}_{1}+\left(x_{22}-y_{22}\right) \mathbf{a}_{2}-z_{22} \mathbf{a}_{3}$ & $=$ & $x_{22} a \hat{\mathbf{x}}-y_{22} b \hat{\mathbf{y}}-z_{22} c \hat{\mathbf{z}}$ & $(16 h)$ & La XIV \\
\hline $\mathbf{B}_{95}$ & $=$ & $\begin{array}{c}\left(-x_{22}+y_{22}\right) \mathbf{a}_{1}+\left(-x_{22}-y_{22}\right) \mathbf{a}_{2}- \\
z_{22} \mathbf{a}_{3}\end{array}$ & $=$ & $-x_{22} a \hat{\mathbf{x}}-y_{22} b \hat{\mathbf{y}}-z_{22} c \hat{\mathbf{z}}$ & $(16 h)$ & La XIV \\
\hline $\mathbf{B}_{96}$ & $=$ & $\begin{array}{c}\left(x_{22}-y_{22}\right) \mathbf{a}_{1}+\left(x_{22}+y_{22}\right) \mathbf{a}_{2}+ \\
\left(\frac{1}{2}-z_{22}\right) \mathbf{a}_{3}\end{array}$ & $=$ & $x_{22} a \hat{\mathbf{x}}+y_{22} b \hat{\mathbf{y}}+\left(\frac{1}{2}-z_{22}\right) c \hat{\mathbf{z}}$ & $(16 h)$ & La XIV \\
\hline $\mathbf{B}_{97}$ & $=$ & $\begin{array}{c}\left(x_{22}+y_{22}\right) \mathbf{a}_{1}+\left(x_{22}-y_{22}\right) \mathbf{a}_{2}+ \\
\left(\frac{1}{2}+z_{22}\right) \mathbf{a}_{3}\end{array}$ & $=$ & $x_{22} a \hat{\mathbf{x}}-y_{22} b \hat{\mathbf{y}}+\left(\frac{1}{2}+z_{22}\right) c \hat{\mathbf{z}}$ & $(16 h)$ & La XIV \\
\hline
\end{tabular}




\begin{tabular}{|c|c|c|c|c|c|c|}
\hline $\mathbf{B}_{98}$ & $=$ & $\begin{array}{c}\left(-x_{22}-y_{22}\right) \mathbf{a}_{1}+\left(-x_{22}+y_{22}\right) \mathbf{a}_{2}+ \\
z_{22} \mathbf{a}_{3}\end{array}$ & $=$ & $-x_{22} a \hat{\mathbf{x}}+y_{22} b \hat{\mathbf{y}}+z_{22} c \hat{\mathbf{z}}$ & $(16 h)$ & La XIV \\
\hline $\mathbf{B}_{99}$ & $=$ & $\left(x_{23}-y_{23}\right) \mathbf{a}_{1}+\left(x_{23}+y_{23}\right) \mathbf{a}_{2}+z_{23} \mathbf{a}_{3}$ & $=$ & $x_{23} a \hat{\mathbf{x}}+y_{23} b \hat{\mathbf{y}}+z_{23} c \hat{\mathbf{z}}$ & $(16 h)$ & La XV \\
\hline $\mathbf{B}_{100}$ & $=$ & $\begin{array}{c}\left(-x_{23}+y_{23}\right) \mathbf{a}_{1}+\left(-x_{23}-y_{23}\right) \mathbf{a}_{2}+ \\
\left(\frac{1}{2}+z_{23}\right) \mathbf{a}_{3}\end{array}$ & $=$ & $-x_{23} a \hat{\mathbf{x}}-y_{23} b \hat{\mathbf{y}}+\left(\frac{1}{2}+z_{23}\right) c \hat{\mathbf{z}}$ & $(16 h)$ & La XV \\
\hline $\mathbf{B}_{101}$ & $=$ & $\begin{array}{c}\left(-x_{23}-y_{23}\right) \mathbf{a}_{1}+\left(-x_{23}+y_{23}\right) \mathbf{a}_{2}+ \\
\left(\frac{1}{2}-z_{23}\right) \mathbf{a}_{3}\end{array}$ & $=$ & $-x_{23} a \hat{\mathbf{x}}+y_{23} b \hat{\mathbf{y}}+\left(\frac{1}{2}-z_{23}\right) c \hat{\mathbf{z}}$ & $(16 h)$ & La XV \\
\hline $\mathbf{B}_{102}$ & $=$ & $\left(x_{23}+y_{23}\right) \mathbf{a}_{1}+\left(x_{23}-y_{23}\right) \mathbf{a}_{2}-z_{23} \mathbf{a}_{3}$ & $=$ & $x_{23} a \hat{\mathbf{x}}-y_{23} b \hat{\mathbf{y}}-z_{23} c \hat{\mathbf{z}}$ & $(16 h)$ & La XV \\
\hline $\mathbf{B}_{103}$ & $=$ & $\begin{array}{c}\left(-x_{23}+y_{23}\right) \mathbf{a}_{1}+\left(-x_{23}-y_{23}\right) \mathbf{a}_{2}- \\
z_{23} \mathbf{a}_{3}\end{array}$ & $=$ & $-x_{23} a \hat{\mathbf{x}}-y_{23} b \hat{\mathbf{y}}-z_{23} c \hat{\mathbf{z}}$ & $(16 h)$ & La XV \\
\hline $\mathbf{B}_{104}$ & $=$ & $\begin{array}{c}\left(x_{23}-y_{23}\right) \mathbf{a}_{1}+\left(x_{23}+y_{23}\right) \mathbf{a}_{2}+ \\
\left(\frac{1}{2}-z_{23}\right) \mathbf{a}_{3}\end{array}$ & $=$ & $x_{23} a \hat{\mathbf{x}}+y_{23} b \hat{\mathbf{y}}+\left(\frac{1}{2}-z_{23}\right) c \hat{\mathbf{z}}$ & $(16 h)$ & La XV \\
\hline $\mathbf{B}_{105}$ & $=$ & $\begin{array}{c}\left(x_{23}+y_{23}\right) \mathbf{a}_{1}+\left(x_{23}-y_{23}\right) \mathbf{a}_{2}+ \\
\left(\frac{1}{2}+z_{23}\right) \mathbf{a}_{3}\end{array}$ & $=$ & $x_{23} a \hat{\mathbf{x}}-y_{23} b \hat{\mathbf{y}}+\left(\frac{1}{2}+z_{23}\right) c \hat{\mathbf{z}}$ & $(16 h)$ & La XV \\
\hline $\mathbf{B}_{106}$ & $=$ & $\begin{array}{c}\left(-x_{23}-y_{23}\right) \mathbf{a}_{1}+\left(-x_{23}+y_{23}\right) \mathbf{a}_{2}+ \\
z_{23} \mathbf{a}_{3}\end{array}$ & $=$ & $-x_{23} a \hat{\mathbf{x}}+y_{23} b \hat{\mathbf{y}}+z_{23} c \hat{\mathbf{z}}$ & $(16 h)$ & La XV \\
\hline $\mathbf{B}_{107}$ & $=$ & $\left(x_{24}-y_{24}\right) \mathbf{a}_{1}+\left(x_{24}+y_{24}\right) \mathbf{a}_{2}+z_{24} \mathbf{a}_{3}$ & $=$ & $x_{24} a \hat{\mathbf{x}}+y_{24} b \hat{\mathbf{y}}+z_{24} c \hat{\mathbf{z}}$ & $(16 h)$ & La XVI \\
\hline $\mathbf{B}_{108}$ & $=$ & $\begin{array}{c}\left(-x_{24}+y_{24}\right) \mathbf{a}_{1}+\left(-x_{24}-y_{24}\right) \mathbf{a}_{2}+ \\
\left(\frac{1}{2}+z_{24}\right) \mathbf{a}_{3}\end{array}$ & $=$ & $-x_{24} a \hat{\mathbf{x}}-y_{24} b \hat{\mathbf{y}}+\left(\frac{1}{2}+z_{24}\right) c \hat{\mathbf{z}}$ & $(16 h)$ & La XVI \\
\hline $\mathbf{B}_{109}$ & $=$ & $\begin{array}{c}\left(-x_{24}-y_{24}\right) \mathbf{a}_{1}+\left(-x_{24}+y_{24}\right) \mathbf{a}_{2}+ \\
\left(\frac{1}{2}-z_{24}\right) \mathbf{a}_{3}\end{array}$ & $=$ & $-x_{24} a \hat{\mathbf{x}}+y_{24} b \hat{\mathbf{y}}+\left(\frac{1}{2}-z_{24}\right) c \hat{\mathbf{z}}$ & $(16 h)$ & La XVI \\
\hline $\mathbf{B}_{110}$ & $=$ & $\left(x_{24}+y_{24}\right) \mathbf{a}_{1}+\left(x_{24}-y_{24}\right) \mathbf{a}_{2}-z_{24} \mathbf{a}_{3}$ & $=$ & $x_{24} a \hat{\mathbf{x}}-y_{24} b \hat{\mathbf{y}}-z_{24} c \hat{\mathbf{z}}$ & $(16 h)$ & La XVI \\
\hline $\mathbf{B}_{111}$ & $=$ & $\begin{array}{c}\left(-x_{24}+y_{24}\right) \mathbf{a}_{1}+\left(-x_{24}-y_{24}\right) \mathbf{a}_{2}- \\
z_{24} \mathbf{a}_{3}\end{array}$ & $=$ & $-x_{24} a \hat{\mathbf{x}}-y_{24} b \hat{\mathbf{y}}-z_{24} c \hat{\mathbf{z}}$ & $(16 h)$ & La XVI \\
\hline $\mathbf{B}_{112}$ & $=$ & $\begin{array}{c}\left(x_{24}-y_{24}\right) \mathbf{a}_{1}+\left(x_{24}+y_{24}\right) \mathbf{a}_{2}+ \\
\left(\frac{1}{2}-z_{24}\right) \mathbf{a}_{3}\end{array}$ & $=$ & $x_{24} a \hat{\mathbf{x}}+y_{24} b \hat{\mathbf{y}}+\left(\frac{1}{2}-z_{24}\right) c \hat{\mathbf{z}}$ & $(16 h)$ & La XVI \\
\hline $\mathbf{B}_{113}$ & $=$ & $\begin{array}{c}\left(x_{24}+y_{24}\right) \mathbf{a}_{1}+\left(x_{24}-y_{24}\right) \mathbf{a}_{2}+ \\
\left(\frac{1}{2}+z_{24}\right) \mathbf{a}_{3}\end{array}$ & $=$ & $x_{24} a \hat{\mathbf{x}}-y_{24} b \hat{\mathbf{y}}+\left(\frac{1}{2}+z_{24}\right) c \hat{\mathbf{z}}$ & $(16 h)$ & La XVI \\
\hline $\mathbf{B}_{114}$ & $=$ & $\begin{array}{c}\left(-x_{24}-y_{24}\right) \mathbf{a}_{1}+\left(-x_{24}+y_{24}\right) \mathbf{a}_{2}+ \\
z_{24} \mathbf{a}_{3}\end{array}$ & $=$ & $-x_{24} a \hat{\mathbf{x}}+y_{24} b \hat{\mathbf{y}}+z_{24} c \hat{\mathbf{z}}$ & $(16 h)$ & La XVI \\
\hline $\mathbf{B}_{115}$ & $=$ & $\left(x_{25}-y_{25}\right) \mathbf{a}_{1}+\left(x_{25}+y_{25}\right) \mathbf{a}_{2}+z_{25} \mathbf{a}_{3}$ & $=$ & $x_{25} a \hat{\mathbf{x}}+y_{25} b \hat{\mathbf{y}}+z_{25} c \hat{\mathbf{z}}$ & $(16 h)$ & $\mathrm{Ni}$ VI \\
\hline $\mathbf{B}_{116}$ & $=$ & $\begin{array}{c}\left(-x_{25}+y_{25}\right) \mathbf{a}_{1}+\left(-x_{25}-y_{25}\right) \mathbf{a}_{2}+ \\
\left(\frac{1}{2}+z_{25}\right) \mathbf{a}_{3}\end{array}$ & $=$ & $-x_{25} a \hat{\mathbf{x}}-y_{25} b \hat{\mathbf{y}}+\left(\frac{1}{2}+z_{25}\right) c \hat{\mathbf{z}}$ & $(16 h)$ & $\mathrm{Ni}$ VI \\
\hline $\mathbf{B}_{117}$ & $=$ & $\begin{array}{c}\left(-x_{25}-y_{25}\right) \mathbf{a}_{1}+\left(-x_{25}+y_{25}\right) \mathbf{a}_{2}+ \\
\left(\frac{1}{2}-z_{25}\right) \mathbf{a}_{3}\end{array}$ & $=$ & $-x_{25} a \hat{\mathbf{x}}+y_{25} b \hat{\mathbf{y}}+\left(\frac{1}{2}-z_{25}\right) c \hat{\mathbf{z}}$ & $(16 h)$ & $\mathrm{Ni}$ VI \\
\hline $\mathbf{B}_{118}$ & $=$ & $\left(x_{25}+y_{25}\right) \mathbf{a}_{1}+\left(x_{25}-y_{25}\right) \mathbf{a}_{2}-z_{25} \mathbf{a}_{3}$ & $=$ & $x_{25} a \hat{\mathbf{x}}-y_{25} b \hat{\mathbf{y}}-z_{25} c \hat{\mathbf{z}}$ & $(16 h)$ & $\mathrm{Ni}$ VI \\
\hline $\mathbf{B}_{119}$ & $=$ & $\begin{array}{c}\left(-x_{25}+y_{25}\right) \mathbf{a}_{1}+\left(-x_{25}-y_{25}\right) \mathbf{a}_{2}- \\
z_{25} \mathbf{a}_{3}\end{array}$ & $=$ & $-x_{25} a \hat{\mathbf{x}}-y_{25} b \hat{\mathbf{y}}-z_{25} c \hat{\mathbf{z}}$ & $(16 h)$ & $\mathrm{Ni}$ VI \\
\hline $\mathbf{B}_{120}$ & $=$ & $\begin{array}{c}\left(x_{25}-y_{25}\right) \mathbf{a}_{1}+\left(x_{25}+y_{25}\right) \mathbf{a}_{2}+ \\
\left(\frac{1}{2}-z_{25}\right) \mathbf{a}_{3}\end{array}$ & $=$ & $x_{25} a \hat{\mathbf{x}}+y_{25} b \hat{\mathbf{y}}+\left(\frac{1}{2}-z_{25}\right) c \hat{\mathbf{z}}$ & $(16 h)$ & $\mathrm{Ni}$ VI \\
\hline $\mathbf{B}_{121}$ & $=$ & $\begin{array}{c}\left(x_{25}+y_{25}\right) \mathbf{a}_{1}+\left(x_{25}-y_{25}\right) \mathbf{a}_{2}+ \\
\left(\frac{1}{2}+z_{25}\right) \mathbf{a}_{3}\end{array}$ & $=$ & $x_{25} a \hat{\mathbf{x}}-y_{25} b \hat{\mathbf{y}}+\left(\frac{1}{2}+z_{25}\right) c \hat{\mathbf{z}}$ & $(16 h)$ & $\mathrm{Ni}$ VI \\
\hline $\mathbf{B}_{122}$ & $=$ & $\begin{array}{c}\left(-x_{25}-y_{25}\right) \mathbf{a}_{1}+\left(-x_{25}+y_{25}\right) \mathbf{a}_{2}+ \\
z_{25} \mathbf{a}_{3}\end{array}$ & $=$ & $-x_{25} a \hat{\mathbf{x}}+y_{25} b \hat{\mathbf{y}}+z_{25} c \hat{\mathbf{z}}$ & $(16 h)$ & $\mathrm{Ni}$ VI \\
\hline $\mathbf{B}_{123}$ & $=$ & $\left(x_{26}-y_{26}\right) \mathbf{a}_{1}+\left(x_{26}+y_{26}\right) \mathbf{a}_{2}+z_{26} \mathbf{a}_{3}$ & $=$ & $x_{26} a \hat{\mathbf{x}}+y_{26} b \hat{\mathbf{y}}+z_{26} c \hat{\mathbf{z}}$ & $(16 h)$ & Ni VII \\
\hline
\end{tabular}




$$
\begin{aligned}
& \mathbf{B}_{124}=\left(-x_{26}+y_{26}\right) \mathbf{a}_{1}+\left(-x_{26}-y_{26}\right) \mathbf{a}_{2}+=-x_{26} a \hat{\mathbf{x}}-y_{26} b \hat{\mathbf{y}}+\left(\frac{1}{2}+z_{26}\right) c \hat{\mathbf{z}} \\
& \left(\frac{1}{2}+z_{26}\right) \mathbf{a}_{3} \\
& \mathbf{B}_{125}=\left(-x_{26}-y_{26}\right) \mathbf{a}_{1}+\left(-x_{26}+y_{26}\right) \mathbf{a}_{2}+=-x_{26} a \hat{\mathbf{x}}+y_{26} b \hat{\mathbf{y}}+\left(\frac{1}{2}-z_{26}\right) c \hat{\mathbf{z}} \quad \text { (16h) } \quad \text { Ni VII } \\
& \left(\frac{1}{2}-z_{26}\right) \mathbf{a}_{3} \\
& \mathbf{B}_{126}=\left(x_{26}+y_{26}\right) \mathbf{a}_{1}+\left(x_{26}-y_{26}\right) \mathbf{a}_{2}-z_{26} \mathbf{a}_{3}=x_{26} a \hat{\mathbf{x}}-y_{26} b \hat{\mathbf{y}}-z_{26} c \hat{\mathbf{z}} \\
& \mathbf{B}_{127}=\left(-x_{26}+y_{26}\right) \mathbf{a}_{1}+\left(-x_{26}-y_{26}\right) \mathbf{a}_{2}-=-x_{26} a \hat{\mathbf{x}}-y_{26} b \hat{\mathbf{y}}-z_{26} c \hat{\mathbf{z}} \\
& \mathbf{B}_{128}=\left(x_{26}-y_{26}\right) \mathbf{a}_{1}+\left(x_{26}+y_{26}\right) \mathbf{a}_{2}+\quad=\quad x_{26} a \hat{\mathbf{x}}+y_{26} b \hat{\mathbf{y}}+\left(\frac{1}{2}-z_{26}\right) c \hat{\mathbf{z}} \quad \text { (16h) Ni VII } \\
& \left(\frac{1}{2}-z_{26}\right) \mathbf{a}_{3} \\
& \mathbf{B}_{129}=\left(x_{26}+y_{26}\right) \mathbf{a}_{1}+\left(x_{26}-y_{26}\right) \mathbf{a}_{2}+\quad=\quad x_{26} a \hat{\mathbf{x}}-y_{26} b \hat{\mathbf{y}}+\left(\frac{1}{2}+z_{26}\right) c \hat{\mathbf{z}} \\
& \left(\frac{1}{2}+z_{26}\right) \mathbf{a}_{3} \\
& \mathbf{B}_{130}=\left(-x_{26}-y_{26}\right) \mathbf{a}_{1}+\left(-x_{26}+y_{26}\right) \mathbf{a}_{2}+=-x_{26} a \hat{\mathbf{x}}+y_{26} b \hat{\mathbf{y}}+z_{26} c \hat{\mathbf{z}} \\
& z_{26} \mathbf{a}_{3}
\end{aligned}
$$

\section{References:}

- P. Solokha, S. De Negri, V. Pavlyuk, and A. Saccone, Anti-Mackay Polyicosahedral Clusters in La-Ni-Mg Ternary Compounds: Synthesis and Crystal Structure of the $\mathrm{La}_{43} \mathrm{Ni}_{17} \mathrm{Mg}_{5}$ New Intermetallic Phase, Inorg. Chem. 48, 11586-11593 (2009), doi:10.1021/ic901422v.

\section{Geometry files:}

- CIF: pp. 846

- POSCAR: pp. 846 


\section{$\operatorname{MnAl}_{6}\left(D 2_{h}\right)$ Structure: A6B_oC28_63_efg_c}

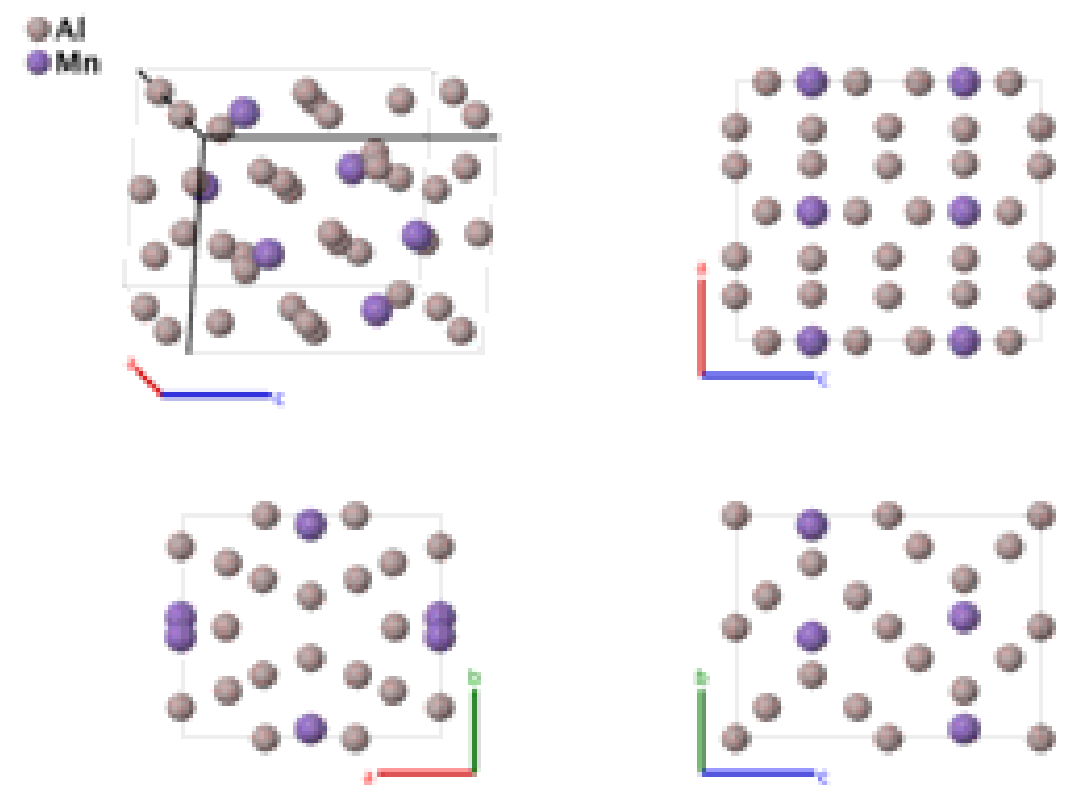

Prototype : $\mathrm{MnAl}_{6}$

AFLOW prototype label : : A6B_oC28_63_efg_c

Strukturbericht designation : $D 2_{h}$

Pearson symbol $\quad: \quad{ }_{0} C 28$

Space group number $\quad: \quad 63$

Space group symbol $\quad: \quad \mathrm{Cmcm}$

AFLOW prototype command : aflow--proto=A6B_oC28_63_efg_c

- params $=a, b / a, c / a, y_{1}, x_{2}, y_{3}, z_{3}, x_{4}, y_{4}$

Base-centered Orthorhombic primitive vectors:

$$
\begin{aligned}
& \mathbf{a}_{1}=\frac{1}{2} a \hat{\mathbf{x}}-\frac{1}{2} b \hat{\mathbf{y}} \\
& \mathbf{a}_{2}=\frac{1}{2} a \hat{\mathbf{x}}+\frac{1}{2} b \hat{\mathbf{y}} \\
& \mathbf{a}_{3}=c \quad c \hat{\mathbf{z}}
\end{aligned}
$$

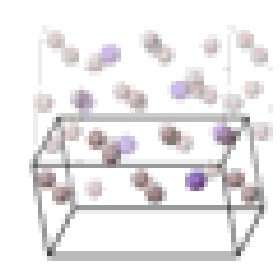

Basis vectors:

Lattice Coordinates

$\mathbf{B}_{1}=-y_{1} \mathbf{a}_{1}+y_{1} \mathbf{a}_{2}+\frac{1}{4} \mathbf{a}_{3}$

$\mathbf{B}_{2}=$

$\mathbf{B}_{3}=$

$\mathbf{B}_{4}=$

$\mathbf{B}_{5}=$

$$
-y_{1} \mathbf{a}_{1}+y_{1} \mathbf{a}_{2}+\frac{1}{4} \mathbf{a}_{3}
$$$$
y_{1} \mathbf{a}_{1}-y_{1} \mathbf{a}_{2}+\frac{3}{4} \mathbf{a}_{3}
$$$$
x_{2} \mathbf{a}_{1}+x_{2} \mathbf{a}_{2}
$$$$
-x_{2} \mathbf{a}_{1}-x_{2} \mathbf{a}_{2}+\frac{1}{2} \mathbf{a}_{3}
$$$$
-x_{2} \mathbf{a}_{1}-x_{2} \mathbf{a}_{2}
$$

$-x_{2} \mathbf{a}_{1}-x_{2} \mathbf{a}_{2}$
Cartesian Coordinates

$=\quad y_{1} b \hat{\mathbf{y}}+\frac{1}{4} c \hat{\mathbf{z}}$

$=\quad-y_{1} b \hat{\mathbf{y}}+\frac{3}{4} c \hat{\mathbf{z}}$

$=\quad x_{2} a \hat{\mathbf{x}}$

$=\quad-x_{2} a \hat{\mathbf{x}}+\frac{1}{2} c \hat{\mathbf{z}}$

$=\quad-x_{2} a \hat{\mathbf{x}}$
Wyckoff Position

(4c)
Atom Type

$\mathrm{Mn}$

$\mathrm{Mn}$

Al I

Al I

Al I 


\begin{tabular}{|c|c|c|c|c|c|c|}
\hline $\mathbf{B}_{6}$ & $=$ & $x_{2} \mathbf{a}_{1}+x_{2} \mathbf{a}_{2}+\frac{1}{2} \mathbf{a}_{3}$ & $=$ & $x_{2} a \hat{\mathbf{x}}+\frac{1}{2} c \hat{\mathbf{z}}$ & $(8 e)$ & $\mathrm{Al} \mathrm{I}$ \\
\hline $\mathbf{B}_{7}$ & $=$ & $-y_{3} \mathbf{a}_{1}+y_{3} \mathbf{a}_{2}+z_{3} \mathbf{a}_{3}$ & $=$ & $y_{3} b \hat{\mathbf{y}}+z_{3} c \hat{\mathbf{z}}$ & $(8 f)$ & Al II \\
\hline $\mathbf{B}_{8}$ & $=$ & $y_{3} \mathbf{a}_{1}-y_{3} \mathbf{a}_{2}+\left(\frac{1}{2}+z_{3}\right) \mathbf{a}_{3}$ & $=$ & $-y_{3} b \hat{\mathbf{y}}+\left(\frac{1}{2}+z_{3}\right) c \hat{\mathbf{z}}$ & $(8 f)$ & Al II \\
\hline $\mathbf{B}_{9}$ & $=$ & $-y_{3} \mathbf{a}_{1}+y_{3} \mathbf{a}_{2}+\left(\frac{1}{2}-z_{3}\right) \mathbf{a}_{3}$ & $=$ & $y_{3} b \hat{\mathbf{y}}+\left(\frac{1}{2}-z_{3}\right) c \hat{\mathbf{z}}$ & $(8 f)$ & Al II \\
\hline $\mathbf{B}_{10}$ & $=$ & $y_{3} \mathbf{a}_{1}-y_{3} \mathbf{a}_{2}-z_{3} \mathbf{a}_{3}$ & $=$ & $-y_{3} b \hat{\mathbf{y}}-z_{3} c \hat{\mathbf{z}}$ & $(8 f)$ & Al II \\
\hline $\mathbf{B}_{11}$ & $=$ & $\left(x_{4}-y_{4}\right) \mathbf{a}_{1}+\left(x_{4}+y_{4}\right) \mathbf{a}_{2}+\frac{1}{4} \mathbf{a}_{3}$ & $=$ & $x_{4} a \hat{\mathbf{x}}+y_{4} b \hat{\mathbf{y}}+\frac{1}{4} c \hat{\mathbf{z}}$ & $(8 g)$ & $\mathrm{Al}$ III \\
\hline $\mathbf{B}_{12}$ & $=$ & $\left(-x_{4}+y_{4}\right) \mathbf{a}_{1}+\left(-x_{4}-y_{4}\right) \mathbf{a}_{2}+\frac{3}{4} \mathbf{a}_{3}$ & $=$ & $-x_{4} a \hat{\mathbf{x}}-y_{4} b \hat{\mathbf{y}}+\frac{3}{4} c \hat{\mathbf{z}}$ & $(8 g)$ & $\mathrm{Al}$ III \\
\hline $\mathbf{B}_{13}$ & $=$ & $\left(-x_{4}-y_{4}\right) \mathbf{a}_{1}+\left(-x_{4}+y_{4}\right) \mathbf{a}_{2}+\frac{1}{4} \mathbf{a}_{3}$ & $=$ & $-x_{4} a \hat{\mathbf{x}}+y_{4} b \hat{\mathbf{y}}+\frac{1}{4} c \hat{\mathbf{z}}$ & $(8 g)$ & $\mathrm{Al} \mathrm{III}$ \\
\hline $\mathbf{B}_{14}$ & $=$ & $\left(x_{4}+y_{4}\right) \mathbf{a}_{1}+\left(x_{4}-y_{4}\right) \mathbf{a}_{2}+\frac{3}{4} \mathbf{a}_{3}$ & $=$ & $x_{4} a \hat{\mathbf{x}}-y_{4} b \hat{\mathbf{y}}+\frac{3}{4} c \hat{\mathbf{z}}$ & $(8 g)$ & Al III \\
\hline
\end{tabular}

\section{References:}

- A. Kontio and P. Coppens, New study of the structure of MnAl , Acta Crystallogr. Sect. B Struct. Sci. 37, 433-435 (1981), doi:10.1107/S0567740881003191.

\section{Geometry files:}

- CIF: pp. 847

- POSCAR: pp. 847 


\section{Post-perovskite $\left(\mathrm{MgSiO}_{3}\right)$ Structure: AB3C_oC20_63_a_cf_c}
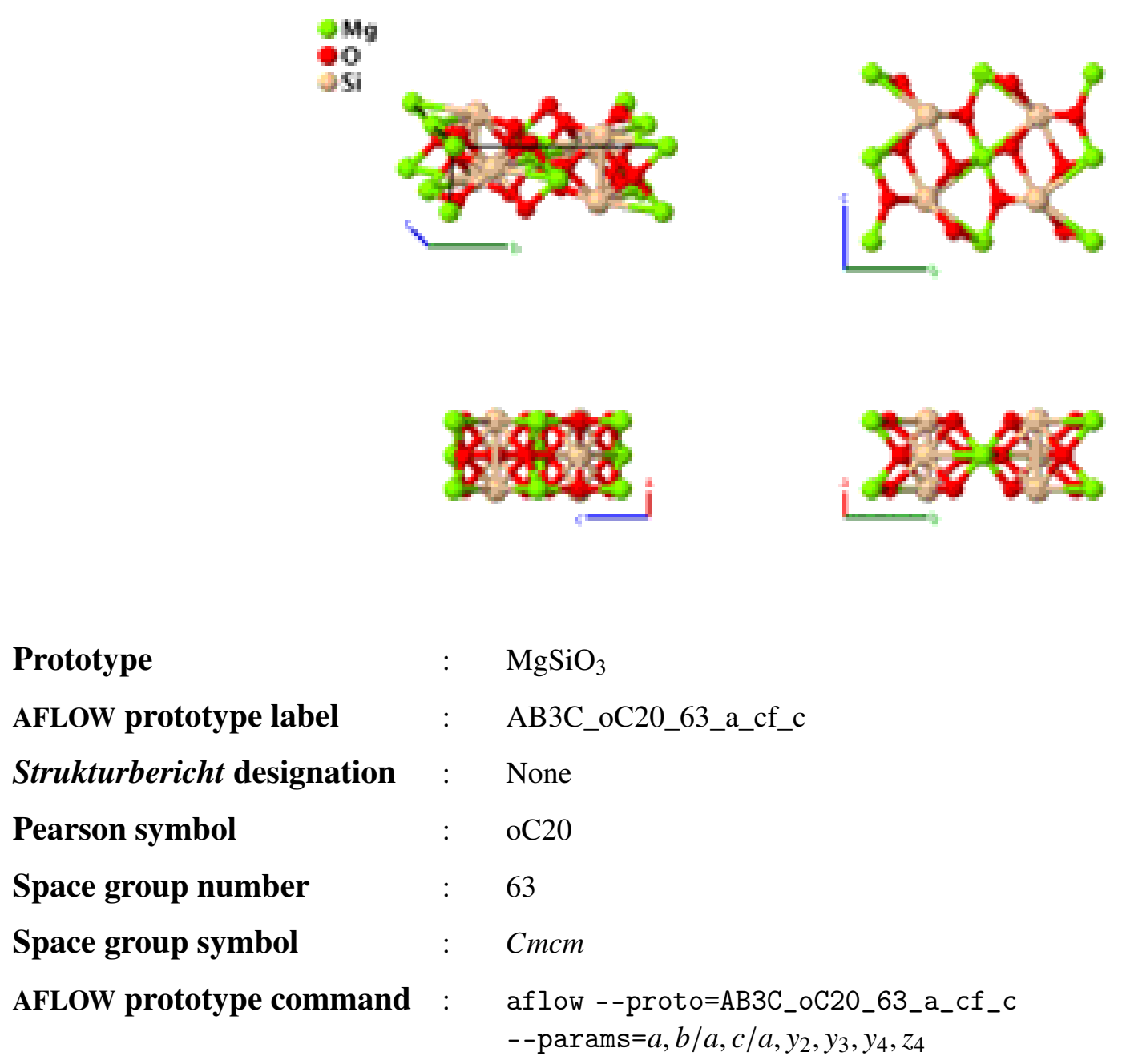

Other compounds with this structure:

- $\mathrm{CaIrO}_{3}, \mathrm{MgGeO}_{3}, \mathrm{NaMgF}_{3}$

- This structure was determined by a combination of x-ray diffraction measurements and atomistic simulations of $\mathrm{MgSiO}_{3}$ at a pressure of $121 \mathrm{GPa}$ and a temperature of $300 \mathrm{~K}$. This approximates the conditions at the Earth's core-mantle boundary.

Base-centered Orthorhombic primitive vectors:

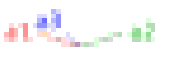

$$
\begin{aligned}
& \mathbf{a}_{1}=\frac{1}{2} a \hat{\mathbf{x}}-\frac{1}{2} b \hat{\mathbf{y}} \\
& \mathbf{a}_{2}=\frac{1}{2} a \hat{\mathbf{x}}+\frac{1}{2} b \hat{\mathbf{y}} \\
& \mathbf{a}_{3}=c \quad c \hat{\mathbf{z}}
\end{aligned}
$$

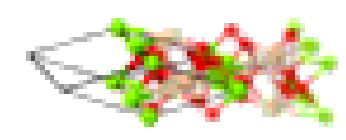


Lattice Coordinates

$\begin{array}{lccr}\mathbf{B}_{1} & & 0 \mathbf{a}_{1}+0 \mathbf{a}_{2}+0 \mathbf{a}_{3} & = \\ \mathbf{B}_{2} & = & \frac{1}{2} \mathbf{a}_{3} & = \\ \mathbf{B}_{3} & & -y_{2} \mathbf{a}_{1}+y_{2} \mathbf{a}_{2}+\frac{1}{4} \mathbf{a}_{3} & = \\ \mathbf{B}_{4}= & y_{2} \mathbf{a}_{1}-y_{2} \mathbf{a}_{2}+\frac{3}{4} \mathbf{a}_{3} & = \\ \mathbf{B}_{5}= & -y_{3} \mathbf{a}_{1}+y_{3} \mathbf{a}_{2}+\frac{1}{4} \mathbf{a}_{3} & = \\ \mathbf{B}_{6}= & y_{3} \mathbf{a}_{1}-y_{3} \mathbf{a}_{2}+\frac{3}{4} \mathbf{a}_{3} & = \\ \mathbf{B}_{7}= & -y_{4} \mathbf{a}_{1}+y_{4} \mathbf{a}_{2}+z_{4} \mathbf{a}_{3} & = \\ \mathbf{B}_{8}= & y_{4} \mathbf{a}_{1}-y_{4} \mathbf{a}_{2}+\left(\frac{1}{2}+z_{4}\right) \mathbf{a}_{3} & = \\ \mathbf{B}_{9}= & -y_{4} \mathbf{a}_{1}+y_{4} \mathbf{a}_{2}+\left(\frac{1}{2}-z_{4}\right) \mathbf{a}_{3} & = \\ \mathbf{B}_{10}= & y_{4} \mathbf{a}_{1}-y_{4} \mathbf{a}_{2}-z_{4} \mathbf{a}_{3} & =\end{array}$

Cartesian Coordinates

$=$

$=$

$=$

$=$

$=$

$=$

$=$

$=$

$=$

$=$

$$
0 \hat{\mathbf{x}}+0 \hat{\mathbf{y}}+0 \hat{\mathbf{z}}
$$$$
\frac{1}{2} c \hat{\mathbf{z}}
$$$$
y_{2} b \hat{\mathbf{y}}+\frac{1}{4} c \hat{\mathbf{z}}
$$$$
-y_{2} b \hat{\mathbf{y}}+\frac{3}{4} c \hat{\mathbf{z}}
$$$$
y_{3} b \hat{\mathbf{y}}+\frac{1}{4} c \hat{\mathbf{z}}
$$$$
-y_{3} b \hat{\mathbf{y}}+\frac{3}{4} c \hat{\mathbf{z}}
$$$$
y_{4} b \hat{\mathbf{y}}+z_{4} c \hat{\mathbf{z}}
$$$$
-y_{4} b \hat{\mathbf{y}}+\left(\frac{1}{2}+z_{4}\right) c \hat{\mathbf{z}}
$$$$
y_{4} b \hat{\mathbf{y}}+\left(\frac{1}{2}-z_{4}\right) c \hat{\mathbf{z}}
$$

$-y_{4} b \hat{\mathbf{y}}-z_{4} c \hat{\mathbf{z}}$
Wyckoff Position

(4a)

(4a)

$(8 f)$
Atom Type

$\mathrm{Mg}$

$\mathrm{Mg}$

O I

O I

$\mathrm{Si}$

$\mathrm{Si}$

O II

O II

O II

O II

\section{References:}

- M. Murakami, K. Hirose, K. Kawamura, N. Sata, and Y. Ohishi, Post-Perovskite Phase Transition in MgSiO${ }_{3}$, Science 304, 855-858 (2004), doi:10.1126/science.1095932.

\section{Geometry files:}

- CIF: pp. 848

- POSCAR: pp. 848 


\section{$\mathrm{MgSO}_{4}$ Structure: AB4C_oC24_63_a_fg_c}
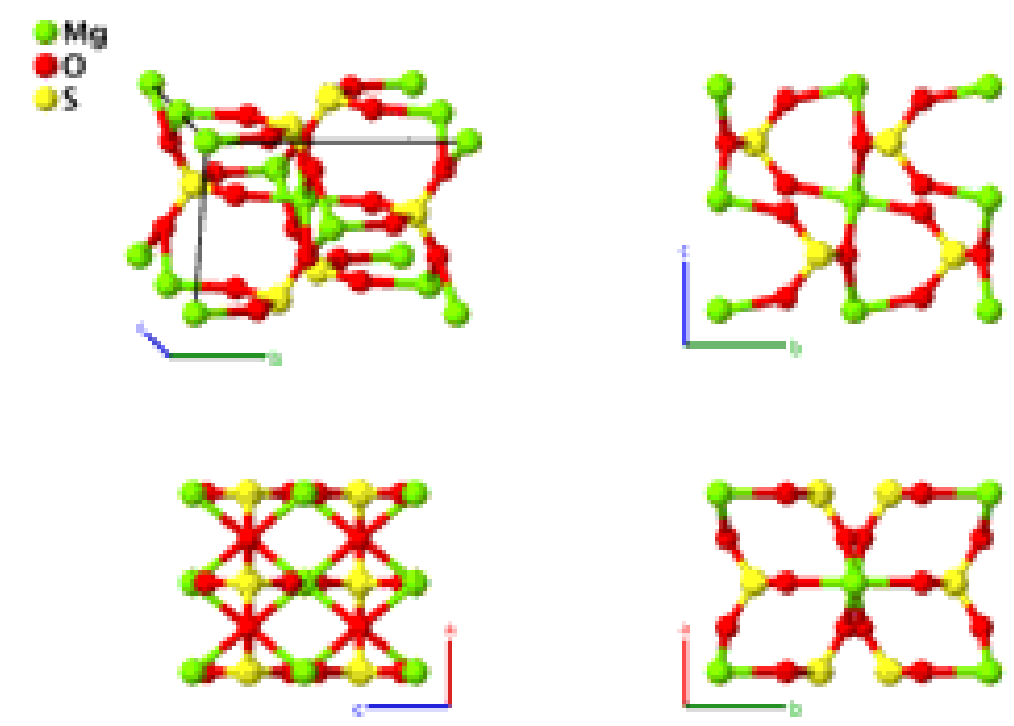

\section{Prototype}

AFLOW prototype label

: $\mathrm{MgO}_{4} \mathrm{~S}$

Strukturbericht designation

: AB4C_oC24_63_a_fg_c

Pearson symbol

: None

Space group number

: $\quad$ oC24

Space group symbol

$: \quad 63$

AFLOW prototype command

: $\mathrm{Cmcm}$

aflow --proto=AB4C_oC24_63_a_fg_c

- params $=a, b / a, c / a, y_{2}, y_{3}, z_{3}, x_{4}, y_{4}$

Other compounds with this structure:

- $\mathrm{CdCrO}_{4}, \mathrm{CoCrO}_{4}, \mathrm{MgCrO}_{4}, \mathrm{NiCrO}_{4}, \mathrm{NiSO}_{4}$

Base-centered Orthorhombic primitive vectors:

$4 y_{12}$
$\mathbf{a}_{1}=\frac{1}{2} a \hat{\mathbf{x}}-\frac{1}{2} b \hat{\mathbf{y}}$
$\mathbf{a}_{2}=\frac{1}{2} a \hat{\mathbf{x}}+\frac{1}{2} b \hat{\mathbf{y}}$
$\mathbf{a}_{3}=c \hat{\mathbf{z}}$

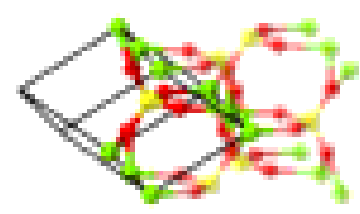

Basis vectors:

Lattice Coordinates

$\begin{array}{ccc}\mathbf{B}_{1}= & 0 \mathbf{a}_{1}+0 \mathbf{a}_{2}+0 \mathbf{a}_{3} \\ \mathbf{B}_{2}= & \frac{1}{2} \mathbf{a}_{3} \\ \mathbf{B}_{3}= & -y_{2} \mathbf{a}_{1}+y_{2} \mathbf{a}_{2}+\frac{1}{4} \mathbf{a}_{3}\end{array}$

Cartesian Coordinates

$=$

$=$

$=$
$0 \hat{\mathbf{x}}+0 \hat{\mathbf{y}}+0 \hat{\mathbf{z}}$

$\frac{1}{2} c \hat{\mathbf{z}}$

$y_{2} b \hat{\mathbf{y}}+\frac{1}{4} c \hat{\mathbf{z}}$
Wyckoff Position

Atom Type

(4a)

(4a)

(4c)
$\mathrm{Mg}$

$\mathrm{Mg}$

S 


\begin{tabular}{|c|c|c|c|c|c|c|}
\hline $\mathbf{B}_{4}$ & $=$ & $y_{2} \mathbf{a}_{1}-y_{2} \mathbf{a}_{2}+\frac{3}{4} \mathbf{a}_{3}$ & $=$ & $-y_{2} b \hat{\mathbf{y}}+\frac{3}{4} c \hat{\mathbf{z}}$ & $(4 c)$ & $\mathrm{S}$ \\
\hline $\mathbf{B}_{5}$ & $=$ & $-y_{3} \mathbf{a}_{1}+y_{3} \mathbf{a}_{2}+z_{3} \mathbf{a}_{3}$ & $=$ & $y_{3} b \hat{\mathbf{y}}+z_{3} c \hat{\mathbf{z}}$ & $(8 f)$ & $\mathrm{O} \mathrm{I}$ \\
\hline $\mathbf{B}_{6}$ & $=$ & $y_{3} \mathbf{a}_{1}-y_{3} \mathbf{a}_{2}+\left(\frac{1}{2}+z_{3}\right) \mathbf{a}_{3}$ & $=$ & $-y_{3} b \hat{\mathbf{y}}+\left(\frac{1}{2}+z_{3}\right) c \hat{\mathbf{z}}$ & $(8 f)$ & O I \\
\hline $\mathbf{B}_{7}$ & $=$ & $-y_{3} \mathbf{a}_{1}+y_{3} \mathbf{a}_{2}+\left(\frac{1}{2}-z_{3}\right) \mathbf{a}_{3}$ & $=$ & $y_{3} b \hat{\mathbf{y}}+\left(\frac{1}{2}-z_{3}\right) c \hat{\mathbf{z}}$ & $(8 f)$ & O I \\
\hline $\mathbf{B}_{8}$ & $=$ & $y_{3} \mathbf{a}_{1}-y_{3} \mathbf{a}_{2}-z_{3} \mathbf{a}_{3}$ & $=$ & $-y_{3} b \hat{\mathbf{y}}-z_{3} c \hat{\mathbf{z}}$ & $(8 f)$ & O I \\
\hline $\mathbf{B}_{9}$ & $=$ & $\left(x_{4}-y_{4}\right) \mathbf{a}_{1}+\left(x_{4}+y_{4}\right) \mathbf{a}_{2}+\frac{1}{4} \mathbf{a}_{3}$ & $=$ & $x_{4} a \hat{\mathbf{x}}+y_{4} b \hat{\mathbf{y}}+\frac{1}{4} c \hat{\mathbf{z}}$ & $(8 g)$ & O II \\
\hline $\mathbf{B}_{10}$ & $=$ & $\left(-x_{4}+y_{4}\right) \mathbf{a}_{1}+\left(-x_{4}-y_{4}\right) \mathbf{a}_{2}+\frac{3}{4} \mathbf{a}_{3}$ & $=$ & $-x_{4} a \hat{\mathbf{x}}-y_{4} b \hat{\mathbf{y}}+\frac{3}{4} c \hat{\mathbf{z}}$ & $(8 g)$ & O II \\
\hline $\mathbf{B}_{11}$ & $=$ & $\left(-x_{4}-y_{4}\right) \mathbf{a}_{1}+\left(-x_{4}+y_{4}\right) \mathbf{a}_{2}+\frac{1}{4} \mathbf{a}_{3}$ & $=$ & $-x_{4} a \hat{\mathbf{x}}+y_{4} b \hat{\mathbf{y}}+\frac{1}{4} c \hat{\mathbf{z}}$ & $(8 g)$ & O II \\
\hline $\mathbf{B}_{12}$ & $=$ & $\left(x_{4}+y_{4}\right) \mathbf{a}_{1}+\left(x_{4}-y_{4}\right) \mathbf{a}_{2}+\frac{3}{4} \mathbf{a}_{3}$ & $=$ & $x_{4} a \hat{\mathbf{x}}-y_{4} b \hat{\mathbf{y}}+\frac{3}{4} c \hat{\mathbf{z}}$ & $(8 g)$ & O II \\
\hline
\end{tabular}

\section{References:}

- P. J. Rentzeperis and C. T. Soldatos, The crystal structure of the anhydrous magnesium sulphate, Acta Cryst. 11, 686-688 (1958), doi:10.1107/S0365110X58001857.

\section{Found in:}

- R. T. Downs and M. Hall-Wallace, The American Mineralogist Crystal Structure Database, Am. Mineral. 88, 247-250 (2003).

\section{Geometry files:}

- CIF: pp. 848

- POSCAR: pp. 848 


\section{Anhydrite $\left(\mathrm{CaSO}_{4}, \mathrm{HO}_{1}\right)$ Structure: AB4C_oC24_63_c_fg_c}
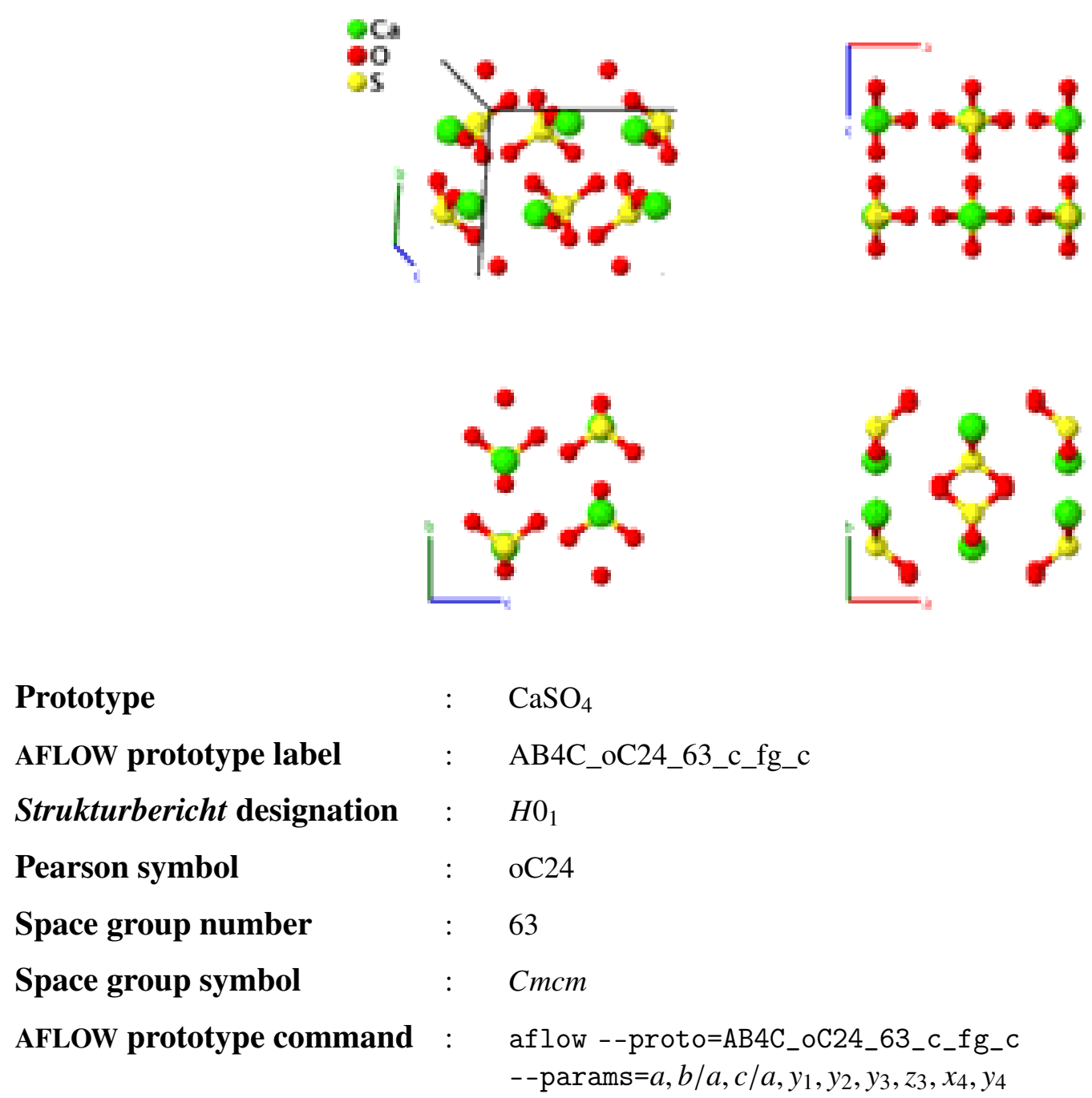

\section{Other compounds with this structure:}

- $\mathrm{CdCrO}_{4}, \mathrm{MgCrO}_{4}, \mathrm{MgSO}_{4}, \mathrm{NaBF}_{4}, \mathrm{NaCrO}_{4}, \mathrm{NiSO}_{4}$

- (Hawthorne, 1975) give the structure in the Amma setting of space group \#63. We have transformed this to the standard $\mathrm{Cmcm}$ setting. The addition of water into the anhydrite crystal transforms it into gypsum.

Base-centered Orthorhombic primitive vectors:

$$
\begin{array}{ll}
\mathbf{a}_{1}= & \frac{1}{2} a \hat{\mathbf{x}}-\frac{1}{2} b \hat{\mathbf{y}} \\
\mathbf{a}_{2}= & \frac{1}{2} a \hat{\mathbf{x}}+\frac{1}{2} b \hat{\mathbf{y}} \\
\mathbf{a}_{3}= & c \hat{\mathbf{z}}
\end{array}
$$

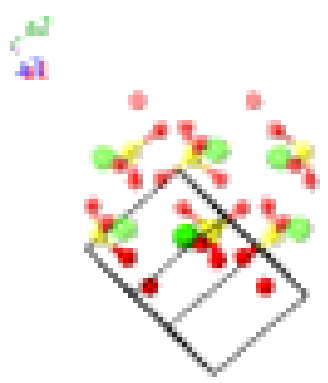

Basis vectors: 
Lattice Coordinates

$\begin{array}{lcc}\mathbf{B}_{1}= & -y_{1} \mathbf{a}_{1}+y_{1} \mathbf{a}_{2}+\frac{1}{4} \mathbf{a}_{3} \\ \mathbf{B}_{2}= & y_{1} \mathbf{a}_{1}-y_{1} \mathbf{a}_{2}+\frac{3}{4} \mathbf{a}_{3} \\ \mathbf{B}_{3}= & -y_{2} \mathbf{a}_{1}+y_{2} \mathbf{a}_{2}+\frac{1}{4} \mathbf{a}_{3} \\ \mathbf{B}_{4}= & y_{2} \mathbf{a}_{1}-y_{2} \mathbf{a}_{2}+\frac{3}{4} \mathbf{a}_{3} \\ \mathbf{B}_{5}= & -y_{3} \mathbf{a}_{1}+y_{3} \mathbf{a}_{2}+z_{3} \mathbf{a}_{3} \\ \mathbf{B}_{6}= & y_{3} \mathbf{a}_{1}-y_{3} \mathbf{a}_{2}+\left(\frac{1}{2}+z_{3}\right) \mathbf{a}_{3} \\ \mathbf{B}_{7}= & -y_{3} \mathbf{a}_{1}+y_{3} \mathbf{a}_{2}+\left(\frac{1}{2}-z_{3}\right) \mathbf{a}_{3} \\ \mathbf{B}_{8}= & y_{3} \mathbf{a}_{1}-y_{3} \mathbf{a}_{2}-z_{3} \mathbf{a}_{3} \\ \mathbf{B}_{9}= & \left(x_{4}-y_{4}\right) \mathbf{a}_{1}+\left(x_{4}+y_{4}\right) \mathbf{a}_{2}+\frac{1}{4} \mathbf{a}_{3} \\ \mathbf{B}_{10}= & \left(-x_{4}+y_{4}\right) \mathbf{a}_{1}+\left(-x_{4}-y_{4}\right) \mathbf{a}_{2}+\frac{3}{4} \mathbf{a}_{3} \\ \mathbf{B}_{11}= & \left(-x_{4}-y_{4}\right) \mathbf{a}_{1}+\left(-x_{4}+y_{4}\right) \mathbf{a}_{2}+\frac{1}{4} \mathbf{a}_{3} \\ \mathbf{B}_{12}= & \left(x_{4}+y_{4}\right) \mathbf{a}_{1}+\left(x_{4}-y_{4}\right) \mathbf{a}_{2}+\frac{3}{4} \mathbf{a}_{3}\end{array}$

Cartesian Coordinates

$=$

$=\quad-y_{1} b \hat{\mathbf{y}}+\frac{3}{4} c \hat{\mathbf{z}}$

$=\quad y_{2} b \hat{\mathbf{y}}+\frac{1}{4} c \hat{\mathbf{z}}$

$=\quad-y_{2} b \hat{\mathbf{y}}+\frac{3}{4} c \hat{\mathbf{z}}$

$=\quad y_{3} b \hat{\mathbf{y}}+z_{3} c \hat{\mathbf{z}}$

$=-y_{3} b \hat{\mathbf{y}}+\left(\frac{1}{2}+z_{3}\right) c \hat{\mathbf{z}}$

$=\quad y_{3} b \hat{\mathbf{y}}+\left(\frac{1}{2}-z_{3}\right) c \hat{\mathbf{z}}$

$=\quad-y_{3} b \hat{\mathbf{y}}-z_{3} c \hat{\mathbf{z}}$

$=\quad x_{4} a \hat{\mathbf{x}}+y_{4} b \hat{\mathbf{y}}+\frac{1}{4} c \hat{\mathbf{z}}$

$=-x_{4} a \hat{\mathbf{x}}-y_{4} b \hat{\mathbf{y}}+\frac{3}{4} c \hat{\mathbf{z}}$

$=-x_{4} a \hat{\mathbf{x}}+y_{4} b \hat{\mathbf{y}}+\frac{1}{4} c \hat{\mathbf{z}}$

$=\quad x_{4} a \hat{\mathbf{x}}-y_{4} b \hat{\mathbf{y}}+\frac{3}{4} c \hat{\mathbf{z}}$
Wyckoff Position
Atom Type

$\mathrm{Ca}$

$\mathrm{Ca}$

$\mathrm{S}$

$\mathrm{S}$

O I

O I

O I

O I

O II

O II

O II

O II

\section{References:}

- F. C. Hawthorne and R. B. Ferguson, Anhydrous sulphates. II. Refinement of the crystal structure of anhydrite, Can. Mineral. 13, 289-292 (1975).

\section{Geometry files:}

- CIF: pp. 849

- POSCAR: pp. 849 


\section{$\mathrm{H}_{2} \mathrm{~S}$ (170 GPa) Structure: A2B_oC24_64_2f_f}
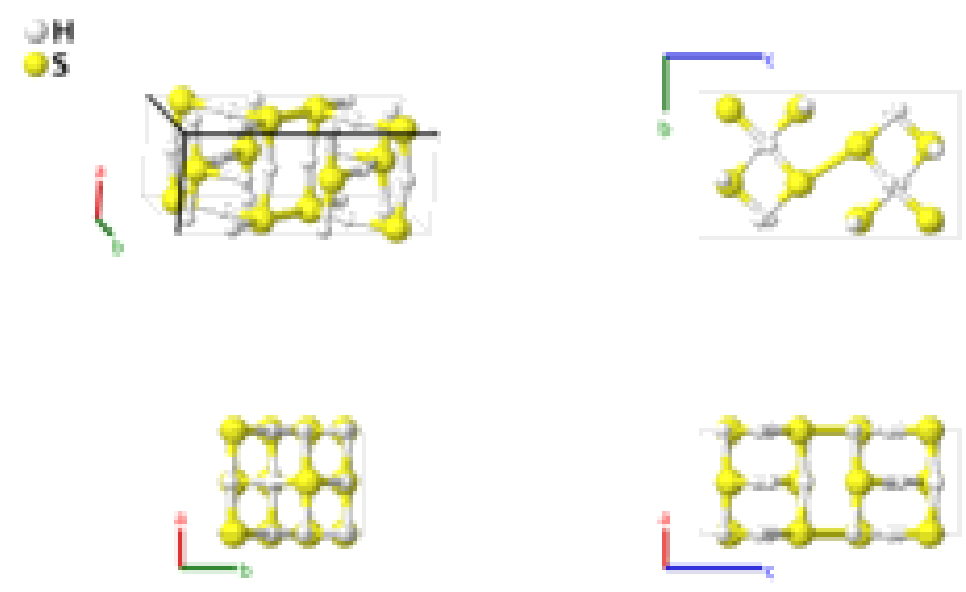

Prototype

AFLOW prototype label

: $\mathrm{H}_{2} \mathrm{~S}$

Strukturbericht designation

: A2B_oC24_64_2f_f

Pearson symbol

: None

Space group number

: $\quad$ oC24

Space group symbol

: 64

AFLOW prototype command

: $\quad$ Cmca

aflow --proto=A2B_oC24_64_2f_f

- - params $=a, b / a, c / a, y_{1}, z_{1}, y_{2}, z_{2}, y_{3}, z_{3}$

- This structure was found by first-principles electronic structure calculations and is predicted to be the stable structure of $\mathrm{H}_{2} \mathrm{~S}$ for pressures $>140 \mathrm{GPa}$. At $160 \mathrm{GPa}$ it is predicted to be a conventional superconductor with an approximate transition temperature of $80 \mathrm{~K}$, however it is unlikely that this is the crystal structure of the $190 \mathrm{~K}$ superconductor, which is likely the A3B_cI8_229_b_a phase of $\mathrm{H}_{3} \mathrm{~S}$ (Bernstein, 2015). The data presented here was computed at $170 \mathrm{GPa}$.

\section{Base-centered Orthorhombic primitive vectors:}

$$
\begin{aligned}
& \mathbf{a}_{1}=\frac{1}{2} a \hat{\mathbf{x}}-\frac{1}{2} b \hat{\mathbf{y}} \\
& \mathbf{a}_{2}=\frac{1}{2} a \hat{\mathbf{x}}+\frac{1}{2} b \hat{\mathbf{y}} \\
& \mathbf{a}_{3}=c \quad c \hat{\mathbf{z}}
\end{aligned}
$$

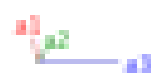

\section{Basis vectors:}

$\begin{array}{llllll} & \text { Lattice Coordinates } & & \text { Cartesian Coordinates } & \text { Wyckoff Position } & \text { Atom Type } \\ \mathbf{B}_{1}= & -y_{1} \mathbf{a}_{1}+y_{1} \mathbf{a}_{2}+z_{1} \mathbf{a}_{3} & = & y_{1} b \hat{\mathbf{y}}+z_{1} c \hat{\mathbf{z}} & (8 f) & \text { H I } \\ \mathbf{B}_{2}= & \left(\frac{1}{2}+y_{1}\right) \mathbf{a}_{1}+\left(\frac{1}{2}-y_{1}\right) \mathbf{a}_{2}+\left(\frac{1}{2}+z_{1}\right) \mathbf{a}_{3} & = & \frac{1}{2} a \hat{\mathbf{x}}-y_{1} b \hat{\mathbf{y}}+\left(\frac{1}{2}+z_{1}\right) c \hat{\mathbf{z}} & (8 f) & \text { H I } \\ \mathbf{B}_{3}= & \left(\frac{1}{2}-y_{1}\right) \mathbf{a}_{1}+\left(\frac{1}{2}+y_{1}\right) \mathbf{a}_{2}+\left(\frac{1}{2}-z_{1}\right) \mathbf{a}_{3} & = & \frac{1}{2} a \hat{\mathbf{x}}+y_{1} b \hat{\mathbf{y}}+\left(\frac{1}{2}-z_{1}\right) c \hat{\mathbf{z}} & (8 f) & \text { H I } \\ \mathbf{B}_{4}= & y_{1} \mathbf{a}_{1}-y_{1} \mathbf{a}_{2}-z_{1} \mathbf{a}_{3} & = & -y_{1} b \hat{\mathbf{y}}-z_{1} c \hat{\mathbf{z}} & (8 f) & \text { H I } \\ \mathbf{B}_{5}= & -y_{2} \mathbf{a}_{1}+y_{2} \mathbf{a}_{2}+z_{2} \mathbf{a}_{3} & = & y_{2} b \hat{\mathbf{y}}+z_{2} c \hat{\mathbf{z}} & (8 f) & \text { H II }\end{array}$




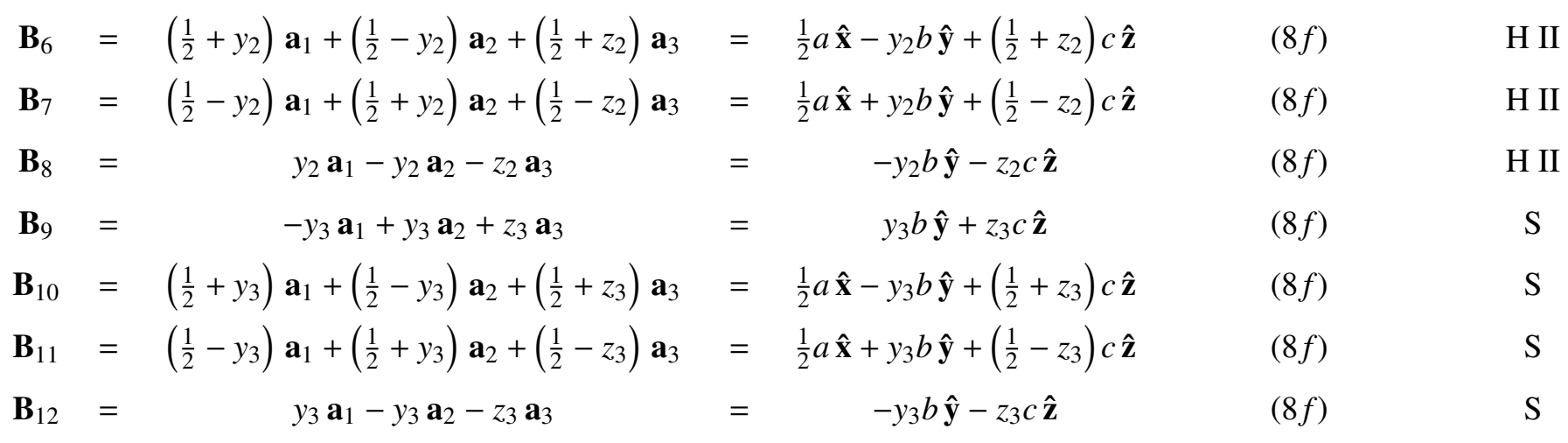

\section{References:}

- Y. Li, J. Hao, H. Liu, Y. Li, and Y. Ma, The metallization and superconductivity of dense hydrogen sulfide, J. Chem. Phys. 140, 174712 (2014), doi:10.1063/1.4874158.

\section{Geometry files:}

- CIF: pp. 849

- POSCAR: pp. 849 


\section{$\mathrm{SrAl}_{2} \mathrm{Se}_{4}$ Structure: A2B4C_oC28_66_1_kl_a}
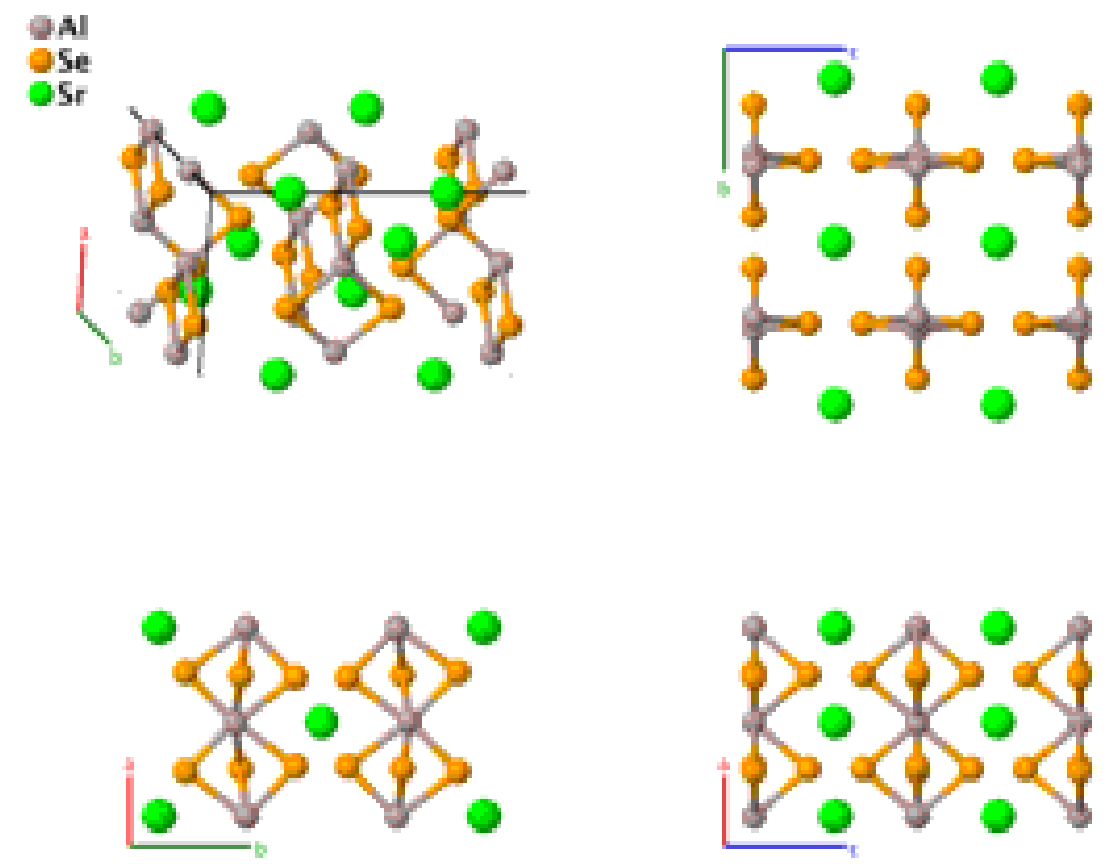

\section{Prototype}

AFLOW prototype label

Strukturbericht designation

Pearson symbol

Space group number

Space group symbol

AFLOW prototype command
: $\quad \mathrm{SrAl}_{2} \mathrm{Se}_{4}$

: A2B4C_oC28_66_1_kl_a

: None

: $\quad$ oC28

: 66

: $\quad$ Cccm

\section{Base-centered Orthorhombic primitive vectors:}

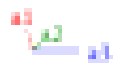

$$
\begin{aligned}
& \mathbf{a}_{1}=\frac{1}{2} a \hat{\mathbf{x}}-\frac{1}{2} b \hat{\mathbf{y}} \\
& \mathbf{a}_{2}=\frac{1}{2} a \hat{\mathbf{x}}+\frac{1}{2} b \hat{\mathbf{y}} \\
& \mathbf{a}_{3}=c \quad c \hat{\mathbf{z}}
\end{aligned}
$$

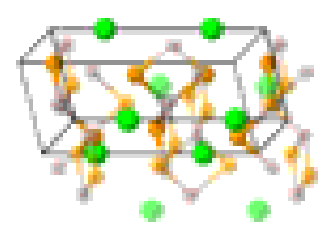

Basis vectors:

\section{Lattice Coordinates}

$\begin{array}{ccc}\mathbf{B}_{1}= & \frac{1}{4} \mathbf{a}_{3} \\ \mathbf{B}_{2}= & \frac{3}{4} \mathbf{a}_{3} \\ \mathbf{B}_{3}= & \frac{1}{2} \mathbf{a}_{2}+z_{2} \mathbf{a}_{3}\end{array}$

Cartesian Coordinates

$=$

$=$

$=\quad \frac{1}{4} a \hat{\mathbf{x}}+\frac{1}{4} b \hat{\mathbf{y}}+z_{2} c \hat{\mathbf{z}}$
Wyckoff Position

$(4 a)$

(4a)

(8k)
Atom Type

$\mathrm{Sr}$ $\mathrm{Sr}$

Se I 


\begin{tabular}{|c|c|c|c|c|c|c|}
\hline $\mathbf{B}_{4}$ & $=$ & $\frac{1}{2} \mathbf{a}_{1}+\left(\frac{1}{2}-z_{2}\right) \mathbf{a}_{3}$ & $=$ & $\frac{1}{4} a \hat{\mathbf{x}}-\frac{1}{4} b \hat{\mathbf{y}}+\left(\frac{1}{2}-z_{2}\right) c \hat{\mathbf{z}}$ & $(8 k)$ & Se I \\
\hline $\mathbf{B}_{5}$ & $=$ & $\frac{1}{2} \mathbf{a}_{2}-z_{2} \mathbf{a}_{3}$ & $=$ & $\frac{1}{4} a \hat{\mathbf{x}}+\frac{1}{4} b \hat{\mathbf{y}}-z_{2} c \hat{\mathbf{z}}$ & $(8 k)$ & Se I \\
\hline $\mathbf{B}_{6}$ & $=$ & $\frac{1}{2} \mathbf{a}_{1}+\left(\frac{1}{2}+z_{2}\right) \mathbf{a}_{3}$ & $=$ & $\frac{1}{4} a \hat{\mathbf{x}}+\frac{3}{4} b \hat{\mathbf{y}}+\left(\frac{1}{2}+z_{2}\right) c \hat{\mathbf{z}}$ & $(8 k)$ & Se I \\
\hline $\mathbf{B}_{7}$ & $=$ & $\left(x_{3}-y_{3}\right) \mathbf{a}_{1}+\left(x_{3}+y_{3}\right) \mathbf{a}_{2}$ & $=$ & $x_{3} a \hat{\mathbf{x}}+y_{3} b \hat{\mathbf{y}}$ & $(8 l)$ & $\mathrm{Al}$ \\
\hline $\mathbf{B}_{8}$ & $=$ & $\left(-x_{3}+y_{3}\right) \mathbf{a}_{1}+\left(-x_{3}-y_{3}\right) \mathbf{a}_{2}$ & $=$ & $-x_{3} a \hat{\mathbf{x}}-y_{3} b \hat{\mathbf{y}}$ & $(8 l)$ & $\mathrm{Al}$ \\
\hline $\mathbf{B}_{9}$ & $=$ & $\left(-x_{3}-y_{3}\right) \mathbf{a}_{1}+\left(-x_{3}+y_{3}\right) \mathbf{a}_{2}+\frac{1}{2} \mathbf{a}_{3}$ & $=$ & $-x_{3} a \hat{\mathbf{x}}+y_{3} b \hat{\mathbf{y}}+\frac{1}{2} c \hat{\mathbf{z}}$ & $(8 l)$ & $\mathrm{Al}$ \\
\hline $\mathbf{B}_{10}$ & $=$ & $\left(x_{3}+y_{3}\right) \mathbf{a}_{1}+\left(x_{3}-y_{3}\right) \mathbf{a}_{2}+\frac{1}{2} \mathbf{a}_{3}$ & $=$ & $x_{3} a \hat{\mathbf{x}}-y_{3} b \hat{\mathbf{y}}+\frac{1}{2} c \hat{\mathbf{z}}$ & $(8 l)$ & $\mathrm{Al}$ \\
\hline $\mathbf{B}_{11}$ & $=$ & $\left(x_{4}-y_{4}\right) \mathbf{a}_{1}+\left(x_{4}+y_{4}\right) \mathbf{a}_{2}$ & $=$ & $x_{4} a \hat{\mathbf{x}}+y_{4} b \hat{\mathbf{y}}$ & $(8 l)$ & Se II \\
\hline $\mathbf{B}_{12}$ & $=$ & $\left(-x_{4}+y_{4}\right) \mathbf{a}_{1}+\left(-x_{4}-y_{4}\right) \mathbf{a}_{2}$ & $=$ & $-x_{4} a \hat{\mathbf{x}}-y_{4} b \hat{\mathbf{y}}$ & $(8 l)$ & Se II \\
\hline $\mathbf{B}_{13}$ & $=$ & $\left(-x_{4}-y_{4}\right) \mathbf{a}_{1}+\left(-x_{4}+y_{4}\right) \mathbf{a}_{2}+\frac{1}{2} \mathbf{a}_{3}$ & $=$ & $-x_{4} a \hat{\mathbf{x}}+y_{4} b \hat{\mathbf{y}}+\frac{1}{2} c \hat{\mathbf{z}}$ & $(8 l)$ & Se II \\
\hline $\mathbf{B}_{14}$ & $=$ & $\left(x_{4}+y_{4}\right) \mathbf{a}_{1}+\left(x_{4}-y_{4}\right) \mathbf{a}_{2}+\frac{1}{2} \mathbf{a}_{3}$ & $=$ & $x_{4} a \hat{\mathbf{x}}-y_{4} b \hat{\mathbf{y}}+\frac{1}{2} c \hat{\mathbf{z}}$ & $(8 l)$ & Se II \\
\hline
\end{tabular}

\section{References:}

- W. Klee and H. Schäfer, $\mathrm{CaAl}_{2} \mathrm{Se}_{4}$ und $\mathrm{SrAl}_{2} \mathrm{Se}_{4}$-Strukturvarianten des TlSe-Typs $/ \mathrm{CaAl}_{2} \mathrm{Se}_{4}$ and $\mathrm{SrAl}_{2} \mathrm{Se}_{4}$-Variants of the TlSe-Structure, Z. Naturforsch. B 33, 829-833 (1978), doi:10.1515/znb-1978-0803.

\section{Found in:}

- P. Villars and K. Cenzual, Pearson's Crystal Data - Crystal Structure Database for Inorganic Compounds, ASM International (2013).

\section{Geometry files:}

- CIF: pp. 850

- POSCAR: pp. 850 


\section{H3S (60 GPa) Structure: A3B_oC64_66_gi2lm_21}
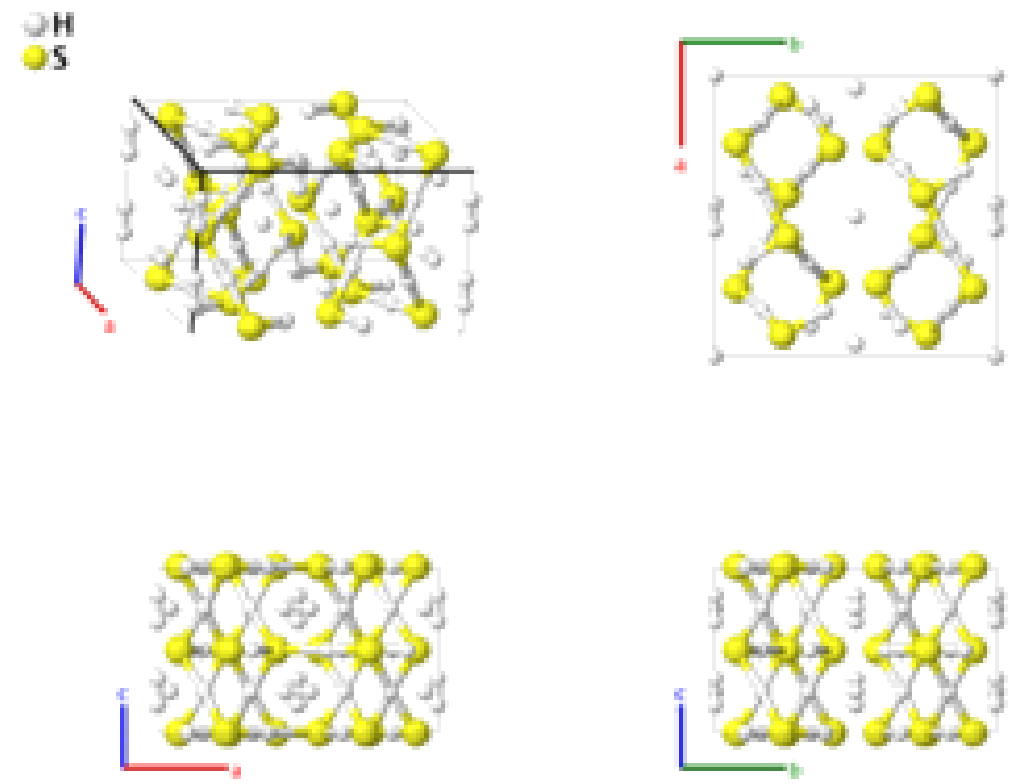

Prototype

AFLOW prototype label

Strukturbericht designation

Pearson symbol

Space group number

Space group symbol

AFLOW prototype command
: $\quad \mathrm{H}_{3} \mathrm{~S}$

: A3B_oC64_66_gi21m_21

: None

: $\quad$ oC64

: 66

: $\quad \mathrm{Cccm}$

- params $=a, b / a, c / a, x_{1}, z_{2}, x_{3}, y_{3}, x_{4}, y_{4}, x_{5}, y_{5}, x_{6}, y_{6}, x_{7}, y_{7}, z_{7}$

- This structure was found by first-principles electronic structure calculations and is predicted to be the stable structure of $\mathrm{H}_{3} \mathrm{~S}$ for pressures between 40 and $90 \mathrm{GPa}$.

- The data presented here was computed at $60 \mathrm{GPa}$.

Base-centered Orthorhombic primitive vectors:

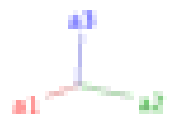

$$
\begin{aligned}
& \mathbf{a}_{1}=\frac{1}{2} a \hat{\mathbf{x}}-\frac{1}{2} b \hat{\mathbf{y}} \\
& \mathbf{a}_{2}=\frac{1}{2} a \hat{\mathbf{x}}+\frac{1}{2} b \hat{\mathbf{y}} \\
& \mathbf{a}_{3}=c \quad c \hat{\mathbf{z}}
\end{aligned}
$$

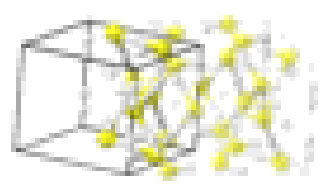

\section{Basis vectors:}




\begin{tabular}{|c|c|c|c|c|c|c|}
\hline $\mathbf{B}_{1}$ & $=$ & $x_{1} \mathbf{a}_{1}+x_{1} \mathbf{a}_{2}+\frac{1}{4} \mathbf{a}_{3}$ & $=$ & $x_{1} a \hat{\mathbf{x}}+\frac{1}{4} c \hat{\mathbf{z}}$ & $(8 g)$ & $\mathrm{H} \mathrm{I}$ \\
\hline $\mathbf{B}_{2}$ & $=$ & $-x_{1} \mathbf{a}_{1}-x_{1} \mathbf{a}_{2}+\frac{1}{4} \mathbf{a}_{3}$ & $=$ & $-x_{1} a \hat{\mathbf{x}}+\frac{1}{4} c \hat{\mathbf{z}}$ & $(8 g)$ & $\mathrm{H} \mathrm{I}$ \\
\hline $\mathbf{B}_{3}$ & $=$ & $-x_{1} \mathbf{a}_{1}-x_{1} \mathbf{a}_{2}+\frac{3}{4} \mathbf{a}_{3}$ & $=$ & $-x_{1} a \hat{\mathbf{x}}+\frac{3}{4} c \hat{\mathbf{z}}$ & $(8 g)$ & $\mathrm{H} \mathrm{I}$ \\
\hline $\mathbf{B}_{4}$ & $=$ & $x_{1} \mathbf{a}_{1}+x_{1} \mathbf{a}_{2}+\frac{3}{4} \mathbf{a}_{3}$ & $=$ & $x_{1} a \hat{\mathbf{x}}+\frac{3}{4} c \hat{\mathbf{z}}$ & $(8 g)$ & $\mathrm{H} \mathrm{I}$ \\
\hline $\mathbf{B}_{5}$ & $=$ & $z_{2} \mathbf{a}_{3}$ & $=$ & $z_{2} c \hat{\mathbf{z}}$ & $(8 i)$ & H II \\
\hline $\mathbf{B}_{6}$ & $=$ & $\left(\frac{1}{2}-z_{2}\right) \mathbf{a}_{3}$ & $=$ & $\left(\frac{1}{2}-z_{2}\right) c \hat{\mathbf{z}}$ & $(8 i)$ & H II \\
\hline $\mathbf{B}_{7}$ & $=$ & $-z_{2} \mathbf{a}_{3}$ & $=$ & $-z_{2} c \hat{\mathbf{z}}$ & $(8 i)$ & H II \\
\hline $\mathbf{B}_{8}$ & $=$ & $\left(\frac{1}{2}+z_{2}\right) \mathbf{a}_{3}$ & $=$ & $\left(\frac{1}{2}+z_{2}\right) c \hat{\mathbf{z}}$ & $(8 i)$ & H II \\
\hline $\mathbf{B}_{9}$ & $=$ & $\left(x_{3}-y_{3}\right) \mathbf{a}_{1}+\left(x_{3}+y_{3}\right) \mathbf{a}_{2}$ & $=$ & $x_{3} a \hat{\mathbf{x}}+y_{3} b \hat{\mathbf{y}}$ & $(8 l)$ & H III \\
\hline $\mathbf{B}_{10}$ & $=$ & $\left(-x_{3}+y_{3}\right) \mathbf{a}_{1}+\left(-x_{3}-y_{3}\right) \mathbf{a}_{2}$ & $=$ & $-x_{3} a \hat{\mathbf{x}}-y_{3} b \hat{\mathbf{y}}$ & $(8 l)$ & H III \\
\hline $\mathbf{B}_{11}$ & $=$ & $\left(-x_{3}-y_{3}\right) \mathbf{a}_{1}+\left(-x_{3}+y_{3}\right) \mathbf{a}_{2}+\frac{1}{2} \mathbf{a}_{3}$ & $=$ & $-x_{3} a \hat{\mathbf{x}}+y_{3} b \hat{\mathbf{y}}+\frac{1}{2} c \hat{\mathbf{z}}$ & $(8 l)$ & H III \\
\hline $\mathbf{B}_{12}$ & $=$ & $\left(x_{3}+y_{3}\right) \mathbf{a}_{1}+\left(x_{3}-y_{3}\right) \mathbf{a}_{2}+\frac{1}{2} \mathbf{a}_{3}$ & $=$ & $x_{3} a \hat{\mathbf{x}}-y_{3} b \hat{\mathbf{y}}+\frac{1}{2} c \hat{\mathbf{z}}$ & $(8 l)$ & H III \\
\hline $\mathbf{B}_{13}$ & $=$ & $\left(x_{4}-y_{4}\right) \mathbf{a}_{1}+\left(x_{4}+y_{4}\right) \mathbf{a}_{2}$ & $=$ & $x_{4} a \hat{\mathbf{x}}+y_{4} b \hat{\mathbf{y}}$ & $(8 l)$ & H IV \\
\hline $\mathbf{B}_{14}$ & $=$ & $\left(-x_{4}+y_{4}\right) \mathbf{a}_{1}+\left(-x_{4}-y_{4}\right) \mathbf{a}_{2}$ & $=$ & $-x_{4} a \hat{\mathbf{x}}-y_{4} b \hat{\mathbf{y}}$ & $(8 l)$ & H IV \\
\hline $\mathbf{B}_{15}$ & $=$ & $\left(-x_{4}-y_{4}\right) \mathbf{a}_{1}+\left(-x_{4}+y_{4}\right) \mathbf{a}_{2}+\frac{1}{2} \mathbf{a}_{3}$ & $=$ & $-x_{4} a \hat{\mathbf{x}}+y_{4} b \hat{\mathbf{y}}+\frac{1}{2} c \hat{\mathbf{z}}$ & $(8 l)$ & H IV \\
\hline $\mathbf{B}_{16}$ & $=$ & $\left(x_{4}+y_{4}\right) \mathbf{a}_{1}+\left(x_{4}-y_{4}\right) \mathbf{a}_{2}+\frac{1}{2} \mathbf{a}_{3}$ & $=$ & $x_{4} a \hat{\mathbf{x}}-y_{4} b \hat{\mathbf{y}}+\frac{1}{2} c \hat{\mathbf{z}}$ & $(8 l)$ & H IV \\
\hline $\mathbf{B}_{17}$ & $=$ & $\left(x_{5}-y_{5}\right) \mathbf{a}_{1}+\left(x_{5}+y_{5}\right) \mathbf{a}_{2}$ & $=$ & $x_{5} a \hat{\mathbf{x}}+y_{5} b \hat{\mathbf{y}}$ & $(8 l)$ & S I \\
\hline $\mathbf{B}_{18}$ & $=$ & $\left(-x_{5}+y_{5}\right) \mathbf{a}_{1}+\left(-x_{5}-y_{5}\right) \mathbf{a}_{2}$ & $=$ & $-x_{5} a \hat{\mathbf{x}}-y_{5} b \hat{\mathbf{y}}$ & $(8 l)$ & S I \\
\hline $\mathbf{B}_{19}$ & $=$ & $\left(-x_{5}-y_{5}\right) \mathbf{a}_{1}+\left(-x_{5}+y_{5}\right) \mathbf{a}_{2}+\frac{1}{2} \mathbf{a}_{3}$ & $=$ & $-x_{5} a \hat{\mathbf{x}}+y_{5} b \hat{\mathbf{y}}+\frac{1}{2} c \hat{\mathbf{z}}$ & $(8 l)$ & S I \\
\hline $\mathbf{B}_{20}$ & $=$ & $\left(x_{5}+y_{5}\right) \mathbf{a}_{1}+\left(x_{5}-y_{5}\right) \mathbf{a}_{2}+\frac{1}{2} \mathbf{a}_{3}$ & $=$ & $x_{5} a \hat{\mathbf{x}}-y_{5} b \hat{\mathbf{y}}+\frac{1}{2} c \hat{\mathbf{z}}$ & $(8 l)$ & S I \\
\hline $\mathbf{B}_{21}$ & $=$ & $\left(x_{6}-y_{6}\right) \mathbf{a}_{1}+\left(x_{6}+y_{6}\right) \mathbf{a}_{2}$ & $=$ & $x_{6} a \hat{\mathbf{x}}+y_{6} b \hat{\mathbf{y}}$ & $(8 l)$ & S II \\
\hline $\mathbf{B}_{22}$ & $=$ & $\left(-x_{6}+y_{6}\right) \mathbf{a}_{1}+\left(-x_{6}-y_{6}\right) \mathbf{a}_{2}$ & $=$ & $-x_{6} a \hat{\mathbf{x}}-y_{6} b \hat{\mathbf{y}}$ & $(8 l)$ & S II \\
\hline $\mathbf{B}_{23}$ & $=$ & $\left(-x_{6}-y_{6}\right) \mathbf{a}_{1}+\left(-x_{6}+y_{6}\right) \mathbf{a}_{2}+\frac{1}{2} \mathbf{a}_{3}$ & $=$ & $-x_{6} a \hat{\mathbf{x}}+y_{6} b \hat{\mathbf{y}}+\frac{1}{2} c \hat{\mathbf{z}}$ & $(8 l)$ & S II \\
\hline $\mathbf{B}_{24}$ & $=$ & $\left(x_{6}+y_{6}\right) \mathbf{a}_{1}+\left(x_{6}-y_{6}\right) \mathbf{a}_{2}+\frac{1}{2} \mathbf{a}_{3}$ & $=$ & $x_{6} a \hat{\mathbf{x}}-y_{6} b \hat{\mathbf{y}}+\frac{1}{2} c \hat{\mathbf{z}}$ & $(8 l)$ & S II \\
\hline $\mathbf{B}_{25}$ & $=$ & $\left(x_{7}-y_{7}\right) \mathbf{a}_{1}+\left(x_{7}+y_{7}\right) \mathbf{a}_{2}+z_{7} \mathbf{a}_{3}$ & $=$ & $x_{7} a \hat{\mathbf{x}}+y_{7} b \hat{\mathbf{y}}+z_{7} c \hat{\mathbf{z}}$ & $(16 m)$ & H V \\
\hline $\mathbf{B}_{26}$ & $=$ & $\left(-x_{7}+y_{7}\right) \mathbf{a}_{1}+\left(-x_{7}-y_{7}\right) \mathbf{a}_{2}+z_{7} \mathbf{a}_{3}$ & $=$ & $-x_{7} a \hat{\mathbf{x}}-y_{7} b \hat{\mathbf{y}}+z_{7} c \hat{\mathbf{z}}$ & $(16 m)$ & $\mathrm{H} \mathrm{V}$ \\
\hline $\mathbf{B}_{27}$ & $=$ & $\begin{array}{c}\left(-x_{7}-y_{7}\right) \mathbf{a}_{1}+\left(-x_{7}+y_{7}\right) \mathbf{a}_{2}+ \\
\left(\frac{1}{2}-z_{7}\right) \mathbf{a}_{3}\end{array}$ & $=$ & $-x_{7} a \hat{\mathbf{x}}+y_{7} b \hat{\mathbf{y}}+\left(\frac{1}{2}-z_{7}\right) c \hat{\mathbf{z}}$ & $(16 m)$ & H V \\
\hline $\mathbf{B}_{28}$ & $=$ & $\left(x_{7}+y_{7}\right) \mathbf{a}_{1}+\left(x_{7}-y_{7}\right) \mathbf{a}_{2}+\left(\frac{1}{2}-z_{7}\right) \mathbf{a}_{3}$ & $=$ & $x_{7} a \hat{\mathbf{x}}-y_{7} b \hat{\mathbf{y}}+\left(\frac{1}{2}-z_{7}\right) c \hat{\mathbf{z}}$ & $(16 m)$ & $\mathrm{H} \mathrm{V}$ \\
\hline $\mathbf{B}_{29}$ & $=$ & $\left(-x_{7}+y_{7}\right) \mathbf{a}_{1}+\left(-x_{7}-y_{7}\right) \mathbf{a}_{2}-z_{7} \mathbf{a}_{3}$ & $=$ & $-x_{7} a \hat{\mathbf{x}}-y_{7} b \hat{\mathbf{y}}-z_{7} c \hat{\mathbf{z}}$ & $(16 m)$ & $\mathrm{H} \mathrm{V}$ \\
\hline $\mathbf{B}_{30}$ & $=$ & $\left(x_{7}-y_{7}\right) \mathbf{a}_{1}+\left(x_{7}+y_{7}\right) \mathbf{a}_{2}-z_{7} \mathbf{a}_{3}$ & $=$ & $x_{7} a \hat{\mathbf{x}}+y_{7} b \hat{\mathbf{y}}-z_{7} c \hat{\mathbf{z}}$ & $(16 m)$ & $\mathrm{H} \mathrm{V}$ \\
\hline $\mathbf{B}_{31}$ & $=$ & $\left(x_{7}+y_{7}\right) \mathbf{a}_{1}+\left(x_{7}-y_{7}\right) \mathbf{a}_{2}+\left(\frac{1}{2}+z_{7}\right) \mathbf{a}_{3}$ & $=$ & $x_{7} a \hat{\mathbf{x}}-y_{7} b \hat{\mathbf{y}}+\left(\frac{1}{2}+z_{7}\right) c \hat{\mathbf{z}}$ & $(16 m)$ & H V \\
\hline $\mathbf{B}_{32}$ & $=$ & $\begin{array}{c}\left(-x_{7}-y_{7}\right) \mathbf{a}_{1}+\left(-x_{7}+y_{7}\right) \mathbf{a}_{2}+ \\
\left(\frac{1}{2}+z_{7}\right) \mathbf{a}_{3}\end{array}$ & $=$ & $-x_{7} a \hat{\mathbf{x}}+y_{7} b \hat{\mathbf{y}}+\left(\frac{1}{2}+z_{7}\right) c \hat{\mathbf{z}}$ & $(16 m)$ & H V \\
\hline
\end{tabular}

\section{References:}

- D. Duan, Y. Liu, F. Tian, D. Li, X. Huang, Z. Zhao, H. Yu, B. Liu, W. Tian, and T. Cui, Pressure-induced metallization of dense $\left(H_{2} S_{2}\right)_{2} H_{2}$ with high- $T_{c}$ superconductivity, Sci. Rep. 4, 6968 (2014), doi:10.1038/srep06968. 
Geometry files:

- CIF: pp. 850

- POSCAR: pp. 850 


\section{$\beta$-ThI 3 Structure: A3B_oC64_66_k12m_bdl}
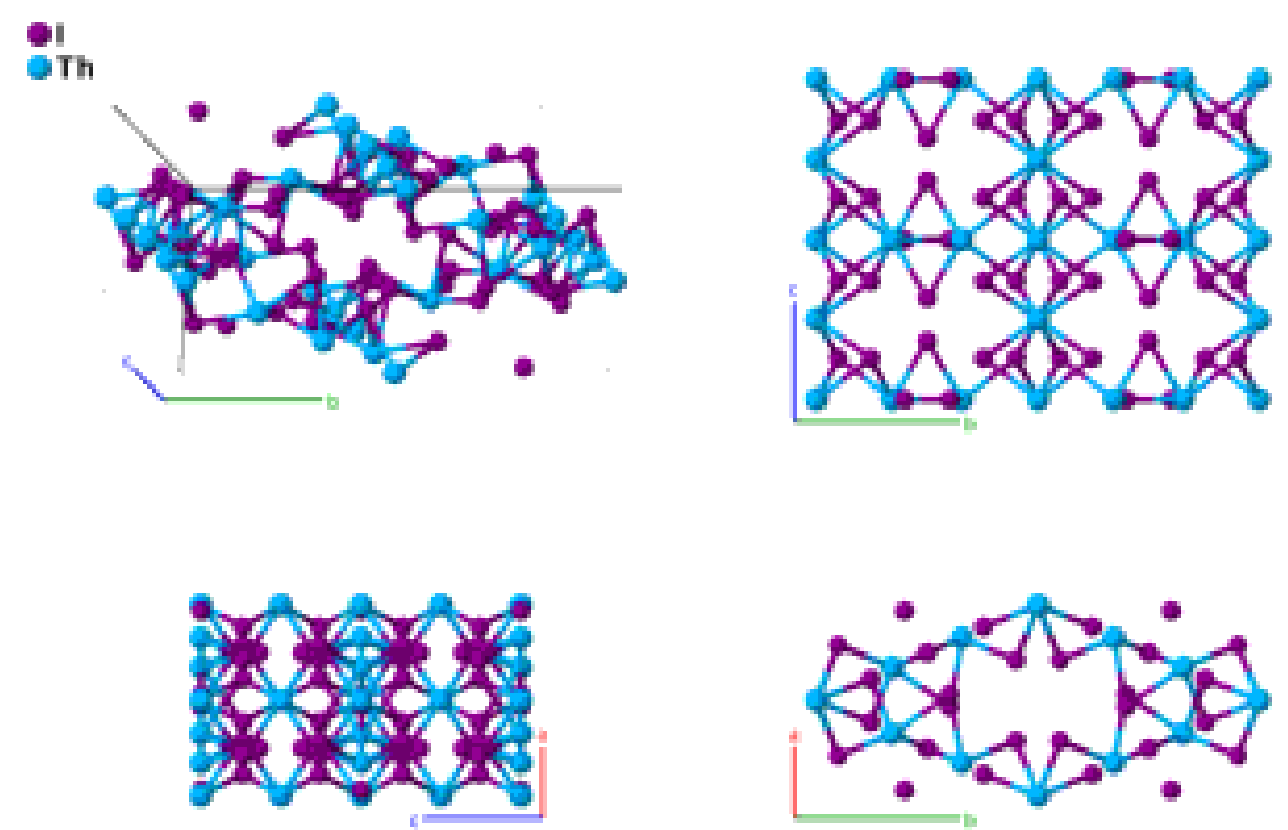

Prototype

AFLOW prototype label

$: \quad \beta-\mathrm{ThI}_{3}$

Strukturbericht designation : None

Pearson symbol : oC64

Space group number $\quad: \quad 66$

Space group symbol $\quad: \quad \mathrm{Cccm}$

AFLOW prototype command : aflow --proto=A3B_oC64_66_kl2m_bdl

- params $=a, b / a, c / a, z_{3}, x_{4}, y_{4}, x_{5}, y_{5}, x_{6}, y_{6}, z_{6}, x_{7}, y_{7}, z_{7}$

Base-centered Orthorhombic primitive vectors:

$$
\begin{array}{ll}
\mathbf{a}_{1}= & \frac{1}{2} a \hat{\mathbf{x}}-\frac{1}{2} b \hat{\mathbf{y}} \\
\mathbf{a}_{2}= & \frac{1}{2} a \hat{\mathbf{x}}+\frac{1}{2} b \hat{\mathbf{y}} \\
\mathbf{a}_{3}= & c \hat{\mathbf{z}}
\end{array}
$$

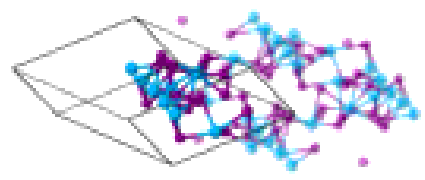

Basis vectors:

Lattice Coordinates

$\begin{array}{lc}\mathbf{B}_{1}= & \frac{1}{2} \mathbf{a}_{1}+\frac{1}{2} \mathbf{a}_{2}+\frac{1}{4} \mathbf{a}_{3} \\ \mathbf{B}_{2}= & \frac{1}{2} \mathbf{a}_{1}+\frac{1}{2} \mathbf{a}_{2}+\frac{3}{4} \mathbf{a}_{3} \\ \mathbf{B}_{3}= & \frac{1}{2} \mathbf{a}_{1}+\frac{1}{2} \mathbf{a}_{2} \\ \mathbf{B}_{4}= & \frac{1}{2} \mathbf{a}_{1}+\frac{1}{2} \mathbf{a}_{2}+\frac{1}{2} \mathbf{a}_{3}\end{array}$

Cartesian Coordinates

$=$

$=\quad \frac{1}{2} a \hat{\mathbf{x}}+\frac{3}{4} c \hat{\mathbf{z}}$

$=\quad \frac{1}{2} a \hat{\mathbf{x}}$

$=\quad \frac{1}{2} a \hat{\mathbf{x}}+\frac{1}{2} c \hat{\mathbf{z}}$
Wyckoff Position

$(4 b)$

$(4 b)$

(4d)

(4d)
Atom Type

Th I

Th I

Th II

Th II 


\begin{tabular}{|c|c|c|c|c|c|c|}
\hline $\mathbf{B}_{5}$ & $=$ & $\frac{1}{2} \mathbf{a}_{2}+z_{3} \mathbf{a}_{3}$ & $=$ & $\frac{1}{4} a \hat{\mathbf{x}}+\frac{1}{4} b \hat{\mathbf{y}}+z_{3} c \hat{\mathbf{z}}$ & $(8 k)$ & I I \\
\hline $\mathbf{B}_{6}$ & $=$ & $\frac{1}{2} \mathbf{a}_{1}+\left(\frac{1}{2}-z_{3}\right) \mathbf{a}_{3}$ & $=$ & $\frac{1}{4} a \hat{\mathbf{x}}-\frac{1}{4} b \hat{\mathbf{y}}+\left(\frac{1}{2}-z_{3}\right) c \hat{\mathbf{z}}$ & $(8 k)$ & I I \\
\hline $\mathbf{B}_{7}$ & $=$ & $\frac{1}{2} \mathbf{a}_{2}-z_{3} \mathbf{a}_{3}$ & $=$ & $\frac{1}{4} a \hat{\mathbf{x}}+\frac{1}{4} b \hat{\mathbf{y}}-z_{3} c \hat{\mathbf{z}}$ & $(8 k)$ & I I \\
\hline $\mathbf{B}_{8}$ & $=$ & $\frac{1}{2} \mathbf{a}_{1}+\left(\frac{1}{2}+z_{3}\right) \mathbf{a}_{3}$ & $=$ & $\frac{1}{4} a \hat{\mathbf{x}}+\frac{3}{4} b \hat{\mathbf{y}}+\left(\frac{1}{2}+z_{3}\right) c \hat{\mathbf{z}}$ & $(8 k)$ & I I \\
\hline $\mathbf{B}_{9}$ & $=$ & $\left(x_{4}-y_{4}\right) \mathbf{a}_{1}+\left(x_{4}+y_{4}\right) \mathbf{a}_{2}$ & $=$ & $x_{4} a \hat{\mathbf{x}}+y_{4} b \hat{\mathbf{y}}$ & $(8 l)$ & I II \\
\hline $\mathbf{B}_{10}$ & $=$ & $\left(-x_{4}+y_{4}\right) \mathbf{a}_{1}+\left(-x_{4}-y_{4}\right) \mathbf{a}_{2}$ & $=$ & $-x_{4} a \hat{\mathbf{x}}-y_{4} b \hat{\mathbf{y}}$ & $(8 l)$ & I II \\
\hline $\mathbf{B}_{11}$ & $=$ & $\left(-x_{4}-y_{4}\right) \mathbf{a}_{1}+\left(-x_{4}+y_{4}\right) \mathbf{a}_{2}+\frac{1}{2} \mathbf{a}_{3}$ & $=$ & $-x_{4} a \hat{\mathbf{x}}+y_{4} b \hat{\mathbf{y}}+\frac{1}{2} c \hat{\mathbf{z}}$ & $(8 l)$ & I II \\
\hline $\mathbf{B}_{12}$ & $=$ & $\left(x_{4}+y_{4}\right) \mathbf{a}_{1}+\left(x_{4}-y_{4}\right) \mathbf{a}_{2}+\frac{1}{2} \mathbf{a}_{3}$ & $=$ & $x_{4} a \hat{\mathbf{x}}-y_{4} b \hat{\mathbf{y}}+\frac{1}{2} c \hat{\mathbf{z}}$ & $(8 l)$ & I II \\
\hline $\mathbf{B}_{13}$ & $=$ & $\left(x_{5}-y_{5}\right) \mathbf{a}_{1}+\left(x_{5}+y_{5}\right) \mathbf{a}_{2}$ & $=$ & $x_{5} a \hat{\mathbf{x}}+y_{5} b \hat{\mathbf{y}}$ & $(8 l)$ & Th III \\
\hline $\mathbf{B}_{14}$ & $=$ & $\left(-x_{5}+y_{5}\right) \mathbf{a}_{1}+\left(-x_{5}-y_{5}\right) \mathbf{a}_{2}$ & $=$ & $-x_{5} a \hat{\mathbf{x}}-y_{5} b \hat{\mathbf{y}}$ & $(8 l)$ & Th III \\
\hline $\mathbf{B}_{15}$ & $=$ & $\left(-x_{5}-y_{5}\right) \mathbf{a}_{1}+\left(-x_{5}+y_{5}\right) \mathbf{a}_{2}+\frac{1}{2} \mathbf{a}_{3}$ & $=$ & $-x_{5} a \hat{\mathbf{x}}+y_{5} b \hat{\mathbf{y}}+\frac{1}{2} c \hat{\mathbf{z}}$ & $(8 l)$ & Th III \\
\hline $\mathbf{B}_{16}$ & $=$ & $\left(x_{5}+y_{5}\right) \mathbf{a}_{1}+\left(x_{5}-y_{5}\right) \mathbf{a}_{2}+\frac{1}{2} \mathbf{a}_{3}$ & $=$ & $x_{5} a \hat{\mathbf{x}}-y_{5} b \hat{\mathbf{y}}+\frac{1}{2} c \hat{\mathbf{z}}$ & $(8 l)$ & Th III \\
\hline $\mathbf{B}_{17}$ & $=$ & $\left(x_{6}-y_{6}\right) \mathbf{a}_{1}+\left(x_{6}+y_{6}\right) \mathbf{a}_{2}+z_{6} \mathbf{a}_{3}$ & $=$ & $x_{6} a \hat{\mathbf{x}}+y_{6} b \hat{\mathbf{y}}+z_{6} c \hat{\mathbf{z}}$ & $(16 m)$ & I III \\
\hline $\mathbf{B}_{18}$ & $=$ & $\left(-x_{6}+y_{6}\right) \mathbf{a}_{1}+\left(-x_{6}-y_{6}\right) \mathbf{a}_{2}+z_{6} \mathbf{a}_{3}$ & $=$ & $-x_{6} a \hat{\mathbf{x}}-y_{6} b \hat{\mathbf{y}}+z_{6} c \hat{\mathbf{z}}$ & $(16 m)$ & I III \\
\hline $\mathbf{B}_{19}$ & $=$ & $\begin{array}{c}\left(-x_{6}-y_{6}\right) \underset{\mathbf{a}_{1}+\left(-x_{6}+y_{6}\right) \mathbf{a}_{2}+}{\left(\frac{1}{2}-z_{6}\right) \mathbf{a}_{3}}\end{array}$ & $=$ & $-x_{6} a \hat{\mathbf{x}}+y_{6} b \hat{\mathbf{y}}+\left(\frac{1}{2}-z_{6}\right) c \hat{\mathbf{z}}$ & $(16 m)$ & I III \\
\hline $\mathbf{B}_{20}$ & $=$ & $\left(x_{6}+y_{6}\right) \mathbf{a}_{1}+\left(x_{6}-y_{6}\right) \mathbf{a}_{2}+\left(\frac{1}{2}-z_{6}\right) \mathbf{a}_{3}$ & $=$ & $x_{6} a \hat{\mathbf{x}}-y_{6} b \hat{\mathbf{y}}+\left(\frac{1}{2}-z_{6}\right) c \hat{\mathbf{z}}$ & $(16 m)$ & I III \\
\hline $\mathbf{B}_{21}$ & $=$ & $\left(-x_{6}+y_{6}\right) \mathbf{a}_{1}+\left(-x_{6}-y_{6}\right) \mathbf{a}_{2}-z_{6} \mathbf{a}_{3}$ & $=$ & $-x_{6} a \hat{\mathbf{x}}-y_{6} b \hat{\mathbf{y}}-z_{6} c \hat{\mathbf{z}}$ & $(16 m)$ & I III \\
\hline $\mathbf{B}_{22}$ & $=$ & $\left(x_{6}-y_{6}\right) \mathbf{a}_{1}+\left(x_{6}+y_{6}\right) \mathbf{a}_{2}-z_{6} \mathbf{a}_{3}$ & $=$ & $x_{6} a \hat{\mathbf{x}}+y_{6} b \hat{\mathbf{y}}-z_{6} c \hat{\mathbf{z}}$ & $(16 m)$ & I III \\
\hline $\mathbf{B}_{23}$ & $=$ & $\left(x_{6}+y_{6}\right) \mathbf{a}_{1}+\left(x_{6}-y_{6}\right) \mathbf{a}_{2}+\left(\frac{1}{2}+z_{6}\right) \mathbf{a}_{3}$ & $=$ & $x_{6} a \hat{\mathbf{x}}-y_{6} b \hat{\mathbf{y}}+\left(\frac{1}{2}+z_{6}\right) c \hat{\mathbf{z}}$ & $(16 m)$ & I III \\
\hline $\mathbf{B}_{24}$ & $=$ & $\begin{array}{c}\left(-x_{6}-y_{6}\right) \mathbf{a}_{1}+\left(-x_{6}+y_{6}\right) \mathbf{a}_{2}+ \\
\left(\frac{1}{2}+z_{6}\right) \mathbf{a}_{3}\end{array}$ & $=$ & $-x_{6} a \hat{\mathbf{x}}+y_{6} b \hat{\mathbf{y}}+\left(\frac{1}{2}+z_{6}\right) c \hat{\mathbf{z}}$ & $(16 m)$ & I III \\
\hline $\mathbf{B}_{25}$ & $=$ & $\left(x_{7}-y_{7}\right) \mathbf{a}_{1}+\left(x_{7}+y_{7}\right) \mathbf{a}_{2}+z_{7} \mathbf{a}_{3}$ & $=$ & $x_{7} a \hat{\mathbf{x}}+y_{7} b \hat{\mathbf{y}}+z_{7} c \hat{\mathbf{z}}$ & $(16 m)$ & I IV \\
\hline $\mathbf{B}_{26}$ & $=$ & $\left(-x_{7}+y_{7}\right) \mathbf{a}_{1}+\left(-x_{7}-y_{7}\right) \mathbf{a}_{2}+z_{7} \mathbf{a}_{3}$ & $=$ & $-x_{7} a \hat{\mathbf{x}}-y_{7} b \hat{\mathbf{y}}+z_{7} c \hat{\mathbf{z}}$ & $(16 m)$ & I IV \\
\hline $\mathbf{B}_{27}$ & $=$ & $\begin{array}{c}\left(-x_{7}-y_{7}\right) \mathbf{a}_{1}+\left(-x_{7}+y_{7}\right) \mathbf{a}_{2}+ \\
\left(\frac{1}{2}-z_{7}\right) \mathbf{a}_{3}\end{array}$ & $=$ & $-x_{7} a \hat{\mathbf{x}}+y_{7} b \hat{\mathbf{y}}+\left(\frac{1}{2}-z_{7}\right) c \hat{\mathbf{z}}$ & $(16 m)$ & I IV \\
\hline $\mathbf{B}_{28}$ & $=$ & $\left(x_{7}+y_{7}\right) \mathbf{a}_{1}+\left(x_{7}-y_{7}\right) \mathbf{a}_{2}+\left(\frac{1}{2}-z_{7}\right) \mathbf{a}_{3}$ & $=$ & $x_{7} a \hat{\mathbf{x}}-y_{7} b \hat{\mathbf{y}}+\left(\frac{1}{2}-z_{7}\right) c \hat{\mathbf{z}}$ & $(16 m)$ & I IV \\
\hline $\mathbf{B}_{29}$ & $=$ & $\left(-x_{7}+y_{7}\right) \mathbf{a}_{1}+\left(-x_{7}-y_{7}\right) \mathbf{a}_{2}-z_{7} \mathbf{a}_{3}$ & $=$ & $-x_{7} a \hat{\mathbf{x}}-y_{7} b \hat{\mathbf{y}}-z_{7} c \hat{\mathbf{z}}$ & $(16 m)$ & I IV \\
\hline $\mathbf{B}_{30}$ & $=$ & $\left(x_{7}-y_{7}\right) \mathbf{a}_{1}+\left(x_{7}+y_{7}\right) \mathbf{a}_{2}-z_{7} \mathbf{a}_{3}$ & $=$ & $x_{7} a \hat{\mathbf{x}}+y_{7} b \hat{\mathbf{y}}-z_{7} c \hat{\mathbf{z}}$ & $(16 m)$ & I IV \\
\hline $\mathbf{B}_{31}$ & $=$ & $\left(x_{7}+y_{7}\right) \mathbf{a}_{1}+\left(x_{7}-y_{7}\right) \mathbf{a}_{2}+\left(\frac{1}{2}+z_{7}\right) \mathbf{a}_{3}$ & $=$ & $x_{7} a \hat{\mathbf{x}}-y_{7} b \hat{\mathbf{y}}+\left(\frac{1}{2}+z_{7}\right) c \hat{\mathbf{z}}$ & $(16 m)$ & I IV \\
\hline $\mathbf{B}_{32}$ & $=$ & $\begin{array}{c}\left(-x_{7}-y_{7}\right) \mathbf{a}_{1}+\left(-x_{7}+y_{7}\right) \mathbf{a}_{2}+ \\
\left(\frac{1}{2}+z_{7}\right) \mathbf{a}_{3}\end{array}$ & $=$ & $-x_{7} a \hat{\mathbf{x}}+y_{7} b \hat{\mathbf{y}}+\left(\frac{1}{2}+z_{7}\right) c \hat{\mathbf{z}}$ & $(16 m)$ & I IV \\
\hline
\end{tabular}

\section{References:}

- H. P. Beck and C. Strobel, $\mathrm{ThI}_{3}$, ein Janus unter den Verbindungen mit Metall-Metall-Wechselwirkungen, Angew. Chem. 94, 558-559 (1982), doi:10.1002/ange.19820940731.

\section{Found in:}

- R. T. Downs and M. Hall-Wallace, The American Mineralogist Crystal Structure Database, Am. Mineral. 88, 247-250 (2003). 
Geometry files:

- CIF: pp. 851

- POSCAR: pp. 851 


\section{Al 2 CuIr Structure: A2BC_oC16_67_ag_b_g}
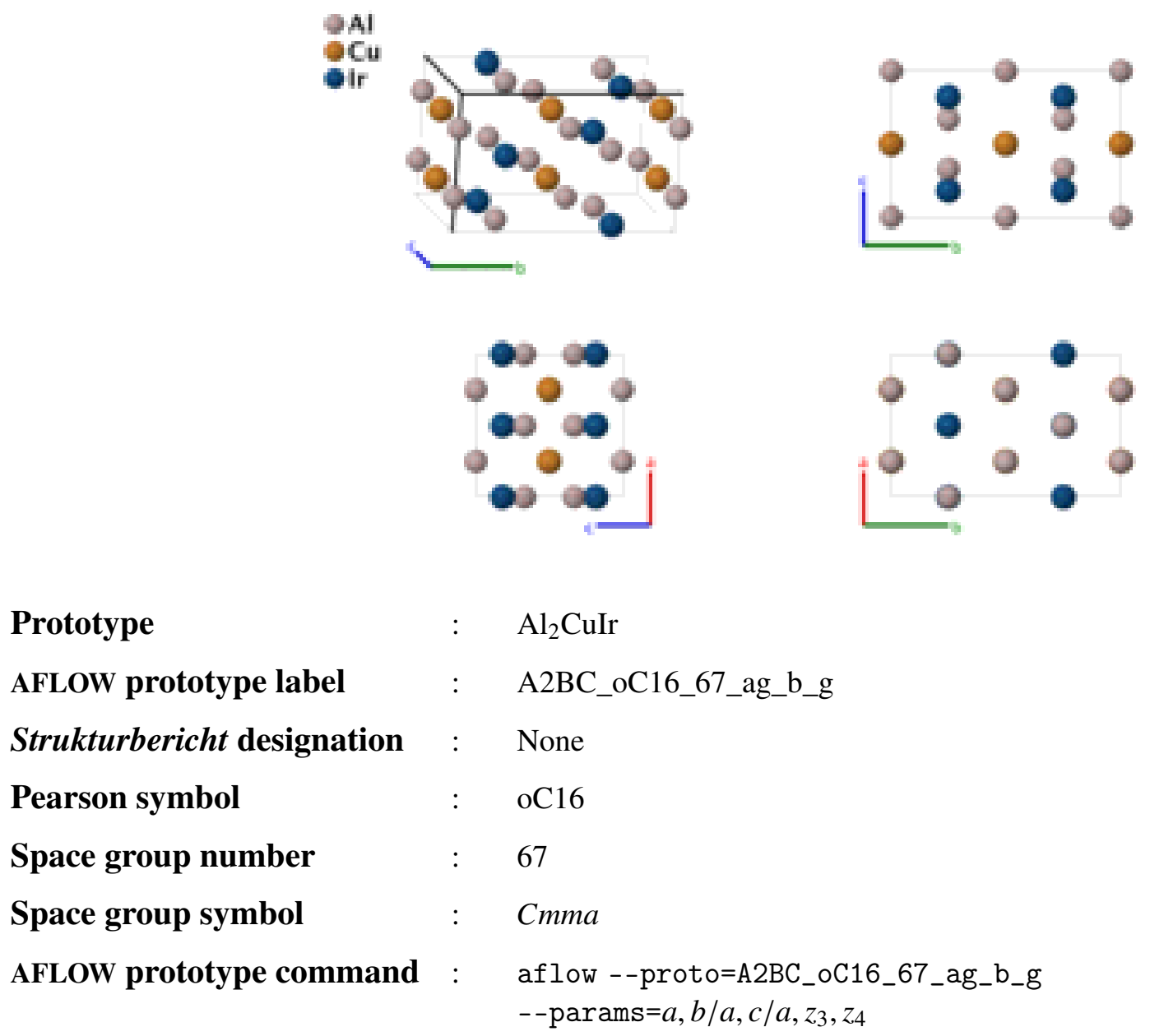

- $\mathrm{Al}_{2} \mathrm{CuIr}$ (pp. 236) and $\mathrm{CuHoP}_{2}$ (pp. 238) have similar AFLOW prototype labels (i.e., same symmetry and set of Wyckoff positions with different stoichiometry labels due to alphabetic ordering of atomic species). They are generated by the same symmetry operations with different sets of parameters (--params) specified in their corresponding CIF files.

\section{Base-centered Orthorhombic primitive vectors:}

$$
\begin{array}{ll}
\mathbf{a}_{1}= & \frac{1}{2} a \hat{\mathbf{x}}-\frac{1}{2} b \hat{\mathbf{y}} \\
\mathbf{a}_{2}= & \frac{1}{2} a \hat{\mathbf{x}}+\frac{1}{2} b \hat{\mathbf{y}} \\
\mathbf{a}_{3}= & c \hat{\mathbf{z}}
\end{array}
$$

\section{Basis vectors:}

Lattice Coordinates

$\begin{array}{llcl}\mathbf{B}_{1}= & \frac{1}{4} \mathbf{a}_{1}+\frac{1}{4} \mathbf{a}_{2} & = \\ \mathbf{B}_{2}= & \frac{3}{4} \mathbf{a}_{1}+\frac{3}{4} \mathbf{a}_{2} & = \\ \mathbf{B}_{3}= & \frac{1}{4} \mathbf{a}_{1}+\frac{1}{4} \mathbf{a}_{2}+\frac{1}{2} \mathbf{a}_{3} & = \\ \mathbf{B}_{4}= & \frac{3}{4} \mathbf{a}_{1}+\frac{3}{4} \mathbf{a}_{2}+\frac{1}{2} \mathbf{a}_{3} & =\end{array}$

Cartesian Coordinates

$$
\begin{gathered}
\frac{1}{4} a \hat{\mathbf{x}} \\
\frac{3}{4} a \hat{\mathbf{x}} \\
\frac{1}{4} a \hat{\mathbf{x}}+\frac{1}{2} c \hat{\mathbf{z}} \\
\frac{3}{4} a \hat{\mathbf{x}}+\frac{1}{2} c \hat{\mathbf{z}}
\end{gathered}
$$

Wyckoff Position
Atom Type

$\mathrm{Al} \mathrm{I}$

Al I

$\mathrm{Cu}$

$\mathrm{Cu}$ 

$\mathbf{B}_{5}=\frac{3}{4} \mathbf{a}_{1}+\frac{1}{4} \mathbf{a}_{2}+z_{3} \mathbf{a}_{3}=$
$\frac{1}{2} a \hat{\mathbf{x}}-\frac{1}{4} b \hat{\mathbf{y}}+z_{3} c \hat{\mathbf{z}}$
$(4 g)$
Al II
$\mathbf{B}_{6}=\frac{1}{4} \mathbf{a}_{1}+\frac{3}{4} \mathbf{a}_{2}-z_{3} \mathbf{a}_{3}=\frac{1}{2} a \hat{\mathbf{x}}+\frac{1}{4} b \hat{\mathbf{y}}-z_{3} c \hat{\mathbf{z}}$
$(4 g)$
Al II
$\mathbf{B}_{7}=\frac{3}{4} \mathbf{a}_{1}+\frac{1}{4} \mathbf{a}_{2}+z_{4} \mathbf{a}_{3}=\frac{1}{2} a \hat{\mathbf{x}}-\frac{1}{4} b \hat{\mathbf{y}}+z_{4} c \hat{\mathbf{z}}$
$(4 g)$
Ir
$\mathbf{B}_{8}=\frac{1}{4} \mathbf{a}_{1}+\frac{3}{4} \mathbf{a}_{2}-z_{4} \mathbf{a}_{3}=\frac{1}{2} a \hat{\mathbf{x}}+\frac{1}{4} b \hat{\mathbf{y}}-z_{4} c \hat{\mathbf{z}}$
$(4 g)$
Ir

\section{References:}

- L. Meshi, V. Ezersky, D. Kapush, and B. Grushko, Crystal structure of the Al ${ }_{2}$ CuIr phase, J. Alloys Compd. 496, 208-211 (2010), doi:10.1016/j.jallcom.2010.02.129.

\section{Found in:}

- P. Villars and K. Cenzual, Pearson's Crystal Data - Crystal Structure Database for Inorganic Compounds, ASM International (2013).

\section{Geometry files:}

- CIF: pp. 851

- POSCAR: pp. 852 


\section{HoCuP 2 Structure: ABC2_oC16_67_b_g_ag}
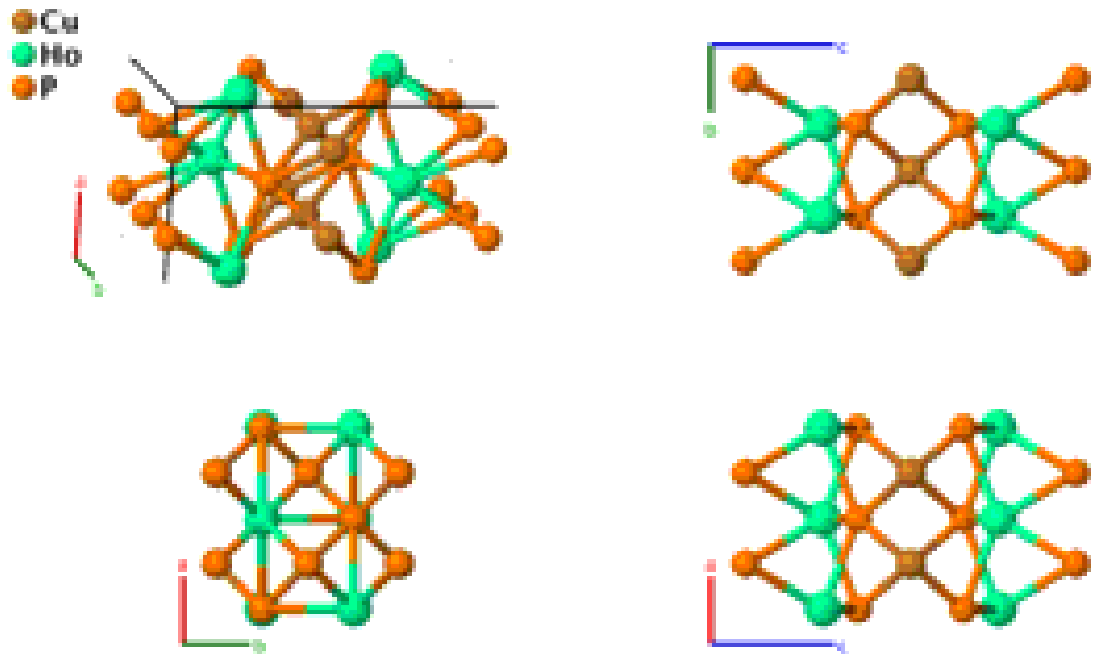

\section{Prototype}

AFLOW prototype label

: $\quad \mathrm{HoCuP}_{2}$

Strukturbericht designation

Pearson symbol

: ABC2_oC16_67_b_g_ag

Space group number

: None

Space group symbol

: $\quad \mathrm{oC} 16$

AFLOW prototype command

: 67

: Cmma

aflow --proto=ABC2_oC16_67_b_g_ag

- - params $=a, b / a, c / a, z_{3}, z_{4}$

- $\mathrm{Al}_{2} \mathrm{CuIr}$ (pp. 236) and $\mathrm{CuHoP}_{2}$ (pp. 238) have similar AFLOW prototype labels (i.e., same symmetry and set of Wyckoff positions with different stoichiometry labels due to alphabetic ordering of atomic species). They are generated by the same symmetry operations with different sets of parameters (--params) specified in their corresponding CIF files.

\section{Base-centered Orthorhombic primitive vectors:}

$$
\begin{aligned}
& \mathbf{a}_{1}=\frac{1}{2} a \hat{\mathbf{x}}-\frac{1}{2} b \hat{\mathbf{y}} \\
& \mathbf{a}_{2}=\frac{1}{2} a \hat{\mathbf{x}}+\frac{1}{2} b \hat{\mathbf{y}} \\
& \mathbf{a}_{3}=c \quad c \hat{\mathbf{z}}
\end{aligned}
$$

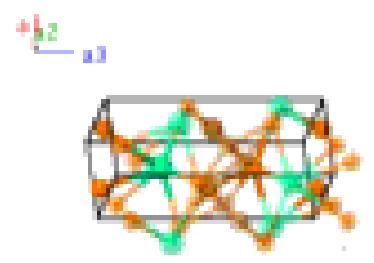

Basis vectors:

Lattice Coordinates

$\begin{array}{llclc}\mathbf{B}_{1}= & \frac{1}{4} \mathbf{a}_{1}+\frac{1}{4} \mathbf{a}_{2} & = & \frac{1}{4} a \hat{\mathbf{x}} \\ \mathbf{B}_{2}= & \frac{3}{4} \mathbf{a}_{1}+\frac{3}{4} \mathbf{a}_{2} & = & \frac{3}{4} a \hat{\mathbf{x}} \\ \mathbf{B}_{3}= & \frac{1}{4} \mathbf{a}_{1}+\frac{1}{4} \mathbf{a}_{2}+\frac{1}{2} \mathbf{a}_{3} & = & \frac{1}{4} a \hat{\mathbf{x}}+\frac{1}{2} c \hat{\mathbf{z}} \\ \mathbf{B}_{4}= & \frac{3}{4} \mathbf{a}_{1}+\frac{3}{4} \mathbf{a}_{2}+\frac{1}{2} \mathbf{a}_{3} & = & \frac{3}{4} a \hat{\mathbf{x}}+\frac{1}{2} c \hat{\mathbf{z}}\end{array}$

Wyckoff Position
Atom Type

P I

P I

$\mathrm{Cu}$

$\mathrm{Cu}$ 


$\begin{array}{rlllll}\mathbf{B}_{5}= & \frac{3}{4} \mathbf{a}_{1}+\frac{1}{4} \mathbf{a}_{2}+z_{3} \mathbf{a}_{3} & = & \frac{1}{2} a \hat{\mathbf{x}}-\frac{1}{4} b \hat{\mathbf{y}}+z_{3} c \hat{\mathbf{z}} & \text { (4g) } & \text { Ho } \\ \mathbf{B}_{6}= & \frac{1}{4} \mathbf{a}_{1}+\frac{3}{4} \mathbf{a}_{2}-z_{3} \mathbf{a}_{3} & = & \frac{1}{2} a \hat{\mathbf{x}}+\frac{1}{4} b \hat{\mathbf{y}}-z_{3} c \hat{\mathbf{z}} & (4 g) & \text { Ho } \\ \mathbf{B}_{7}= & \frac{3}{4} \mathbf{a}_{1}+\frac{1}{4} \mathbf{a}_{2}+z_{4} \mathbf{a}_{3} & = & \frac{1}{2} a \hat{\mathbf{x}}-\frac{1}{4} b \hat{\mathbf{y}}+z_{4} c \hat{\mathbf{z}} & (4 g) & \text { P II } \\ \mathbf{B}_{8}= & \frac{1}{4} \mathbf{a}_{1}+\frac{3}{4} \mathbf{a}_{2}-z_{4} \mathbf{a}_{3} & = & \frac{1}{2} a \hat{\mathbf{x}}+\frac{1}{4} b \hat{\mathbf{y}}-z_{4} c \hat{\mathbf{z}} & \text { P II }\end{array}$

\section{References:}

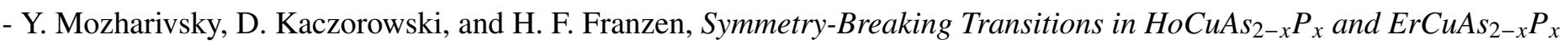
$(x=0-2)$ : Crystal Structure, Application of Landau Theory, Magnetic and Electrical Properties, Z. Anorg. Allg. Chem. 627, 2163-2172 (2001), doi:10.1002/1521-3749(200109)627:9<2163::AID-ZAAC2163>3.0.CO;2-N.

\section{Found in:}

- P. Villars and K. Cenzual, Pearson's Crystal Data - Crystal Structure Database for Inorganic Compounds, ASM International (2013).

\section{Geometry files:}

- CIF: pp. 852

- POSCAR: pp. 852 


\section{$\alpha$-FeSe Structure: AB_oC8_67_a_g}
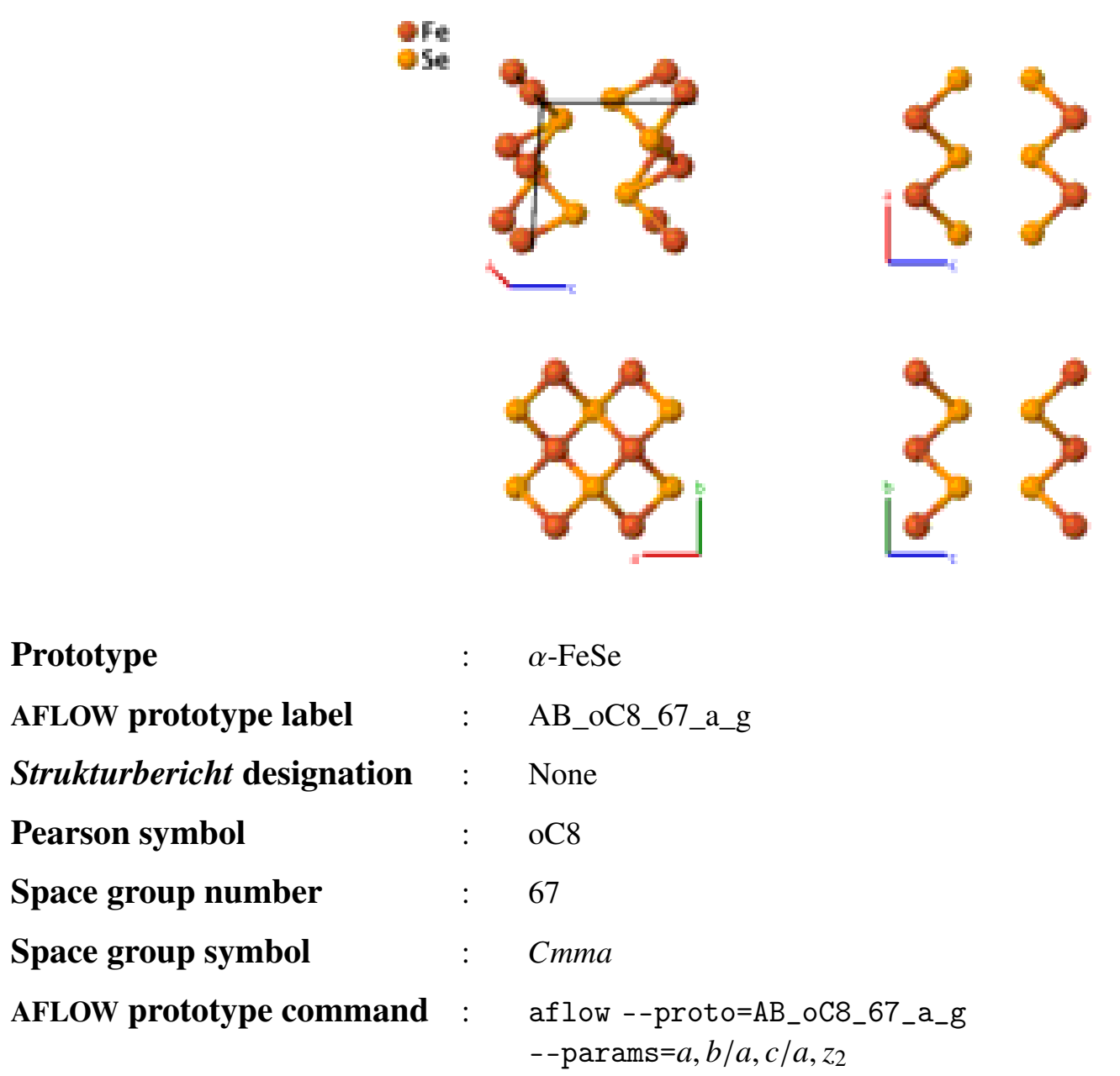

- We follow the reference in calling this $\alpha$-FeSe. Some other authorities refer to the $B 10(\mathrm{PbO})$-like phase of FeSe as $\alpha$ $\mathrm{FeSe}$, calling this phase $\beta$-FeSe. The authors note that "the Se ion concentration is close to 1." The data is presented for the structure at 7 K. $\alpha$-FeSe (pp. 240) and $\alpha$-PbO (pp. 242)) have the same AFLOW prototype label. They are generated by the same symmetry operations with different sets of parameters (--params) specified in their corresponding CIF files.

\section{Base-centered Orthorhombic primitive vectors:}

$$
\begin{aligned}
& \mathbf{a}_{1}=\frac{1}{2} a \hat{\mathbf{x}}-\frac{1}{2} b \hat{\mathbf{y}} \\
& \mathbf{a}_{2}=\frac{1}{2} a \hat{\mathbf{x}}+\frac{1}{2} b \hat{\mathbf{y}} \\
& \mathbf{a}_{3}=c \quad c \hat{\mathbf{z}}
\end{aligned}
$$
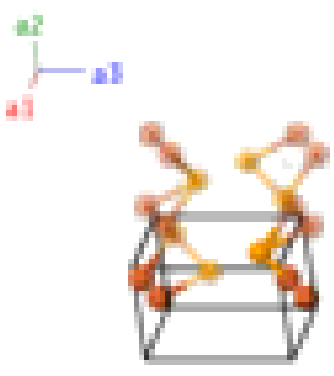

Basis vectors:

$\begin{array}{cccccc} & \text { Lattice Coordinates } & & \text { Cartesian Coordinates } & \text { Wyckoff Position } & \text { Atom Type } \\ \mathbf{B}_{1}= & \frac{1}{4} \mathbf{a}_{1}+\frac{1}{4} \mathbf{a}_{2} & = & \frac{1}{4} a \hat{\mathbf{x}} & (4 a) & \mathrm{Fe} \\ \mathbf{B}_{2}= & \frac{3}{4} \mathbf{a}_{1}+\frac{3}{4} \mathbf{a}_{2} & = & \frac{3}{4} a \hat{\mathbf{x}} & (4 a) & \mathrm{Fe}\end{array}$




\begin{tabular}{|c|c|c|c|c|}
\hline $\mathbf{B}_{3}$ & $\frac{3}{4} \mathbf{a}_{1}+\frac{1}{4} \mathbf{a}_{2}+z_{2} \mathbf{a}_{3}$ & $=$ & $\frac{1}{2} a \hat{\mathbf{x}}-\frac{1}{4} b \hat{\mathbf{y}}+z_{2} c \hat{\mathbf{z}}$ & $(4 g)$ \\
\hline $\mathbf{B}_{4}=$ & $\frac{1}{4} \mathbf{a}_{1}+\frac{3}{4} \mathbf{a}_{2}-z_{2} \mathbf{a}_{3}$ & $=$ & $\frac{1}{2} a \hat{\mathbf{x}}+\frac{1}{4} b \hat{\mathbf{y}}-z_{2} c \hat{\mathbf{z}}$ & $(4 g)$ \\
\hline
\end{tabular}

\section{References:}

- D. Louca, K. Horigane, A. Llobet, R. Arita, S. Ji, N. Katayama, S. Konbu, K. Nakamura, T.-Y. Koo, P. Tong, and K. Yamada, Local atomic structure of superconducting $\mathrm{FeSe}_{1-x} \mathrm{Te}_{x}$, Phys. Rev. B 81, 134524 (2010), doi:10.1103/PhysRevB.81.134524.

\section{Geometry files:}

- CIF: pp. 852

- POSCAR: pp. 853 


\section{$\alpha$-PbO Structure: AB_oC8_67_a_g}

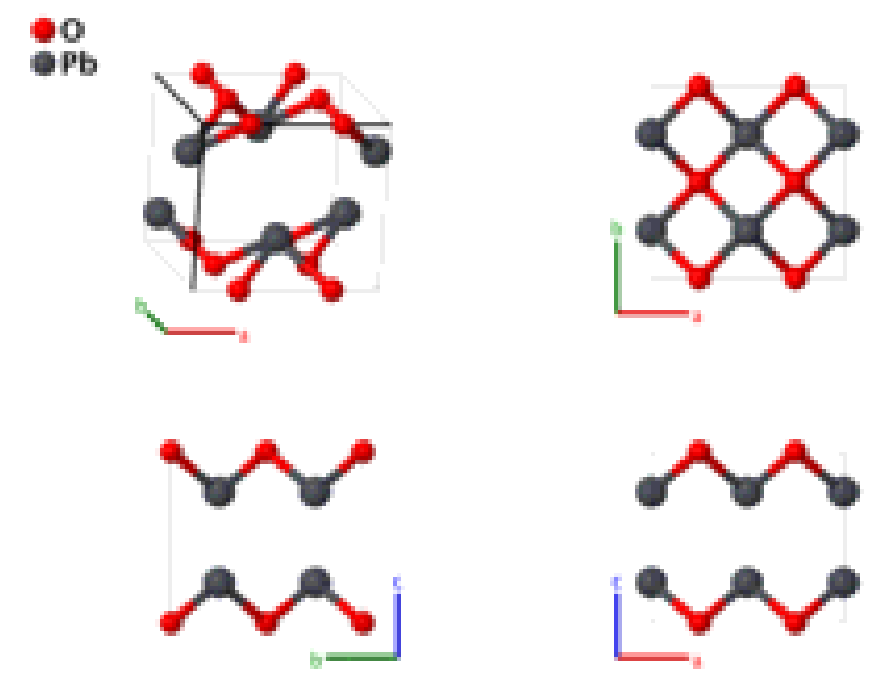

$\begin{array}{lll}\text { Prototype } & : & \alpha-\mathrm{PbO} \\ \text { AFLOW prototype label } & : & \text { AB_oC8_67_a_g } \\ \text { Strukturbericht designation } & : & \text { None } \\ \text { Pearson symbol } & : & \mathrm{oC} 8 \\ \text { Space group number } & : & 67 \\ \text { Space group symbol } & : & \text { Cmma } \\ \text { AFLOW prototype command } & : & \text { aflow --proto }=\mathrm{AB} \_ \text {oC8_67_a_g } \\ & & -- \text { params }=a, b / a, c / a, z_{2}\end{array}$

- FINDSYM identifies space group \#67 for this structure (consistent with the reference); however, since $b / a \approx 1$, AFLOWSYM and Platon identify \#129. Lowering the tolerance value for AFLOW-SYM resolves the expected space group \#67. Space groups \#67 and \#129 are both reasonable classifications since they are commensurate with subgroup relations. $\alpha$-FeSe (pp. 240) and $\alpha$-PbO (pp. 242)) have the same AFLOW prototype label. They are generated by the same symmetry operations with different sets of parameters (--params) specified in their corresponding CIF files.

\section{Base-centered Orthorhombic primitive vectors:}

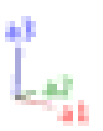

$$
\begin{aligned}
& \mathbf{a}_{1}=\frac{1}{2} a \hat{\mathbf{x}}-\frac{1}{2} b \hat{\mathbf{y}} \\
& \mathbf{a}_{2}=\frac{1}{2} a \hat{\mathbf{x}}+\frac{1}{2} b \hat{\mathbf{y}} \\
& \mathbf{a}_{3}=c \quad c \hat{\mathbf{z}}
\end{aligned}
$$

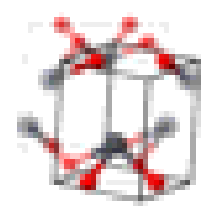

Basis vectors:

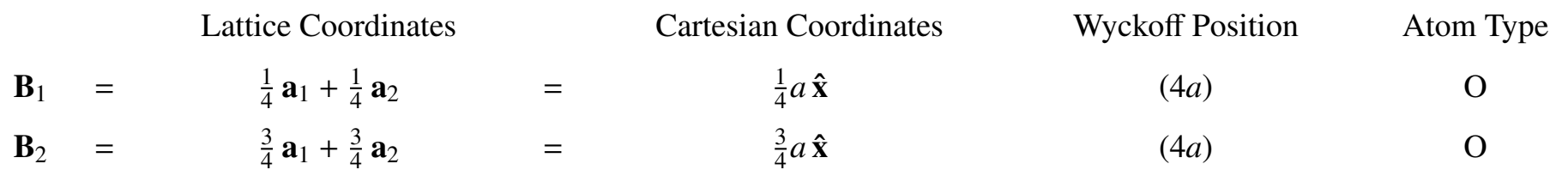



$\mathbf{B}_{3}=$
$\frac{3}{4} \mathbf{a}_{1}+\frac{1}{4} \mathbf{a}_{2}+z_{2} \mathbf{a}_{3}$
$=$
$\frac{1}{2} a \hat{\mathbf{x}}-\frac{1}{4} b \hat{\mathbf{y}}+z_{2} c \hat{\mathbf{z}}$
(4g)
$\mathrm{Pb}$
$\mathbf{B}_{4}=$
$\frac{1}{4} \mathbf{a}_{1}+\frac{3}{4} \mathbf{a}_{2}-z_{2} \mathbf{a}_{3}$
$\frac{1}{2} a \hat{\mathbf{x}}+\frac{1}{4} b \hat{\mathbf{y}}-z_{2} c \hat{\mathbf{z}}$
$(4 g)$
$\mathrm{Pb}$

\section{References:}

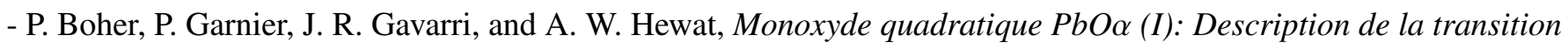
structurale ferroélastique, J. Solid State Chem. 57, 343-350 (1985), doi:10.1016/0022-4596(85)90197-5.

- H. T. Stokes and D. M. Hatch, FINDSYM: Program for identifying the space group symmetry of a crystal, J. Appl. Crystallogr. 38, 237-238 (2005), doi:10.1107/S0021889804031528.

- D. Hicks, C. Oses, E. Gossett, G. Gomez, R. H. Taylor, C. Toher, M. J. Mehl, O. Levy, and S. Curtarolo, AFLOW-SYM: platform for the complete, automatic and self-consistent symmetry analysis of crystals, Acta Crystallogr. Sect. A 74, 184-203 (2018), doi:10.1107/S2053273318003066.

- A. L. Spek, Single-crystal structure validation with the program PLATON, J. Appl. Crystallogr. 36, 7-13 (2003), doi:10.1107/S0021889802022112.

\section{Found in:}

- P. Villars and K. Cenzual, Pearson's Crystal Data - Crystal Structure Database for Inorganic Compounds, ASM International (2013).

\section{Geometry files:}

- CIF: pp. 853

- POSCAR: pp. 853 


\section{$\mathrm{PdSn}_{4}$ Structure: AB4_oC20_68_a_i}
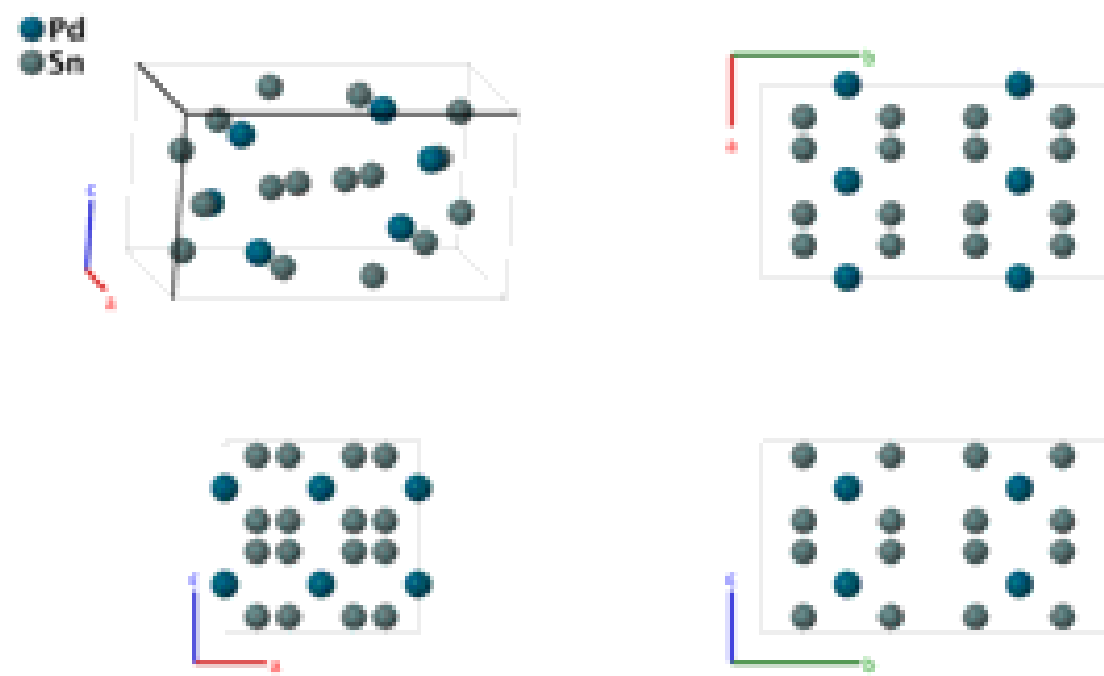

Prototype

AFLOW prototype label

Strukturbericht designation

Pearson symbol

Space group number

Space group symbol

AFLOW prototype command
: $\quad \mathrm{PdSn}_{4}$

: AB4_oC20_68_a_i

: None

: $\quad$ oC20

: 68

: $\quad$ ccca

: $\quad$ aflow --proto=AB4_oC20_68_a_i

- params $=a, b / a, c / a, x_{2}, y_{2}, z_{2}$

Base-centered Orthorhombic primitive vectors:

$$
\begin{aligned}
& \mathbf{a}_{1}=\frac{1}{2} a \hat{\mathbf{x}}-\frac{1}{2} b \hat{\mathbf{y}} \\
& \mathbf{a}_{2}=\frac{1}{2} a \hat{\mathbf{x}}+\frac{1}{2} b \hat{\mathbf{y}} \\
& \mathbf{a}_{3}=c \quad c \hat{\mathbf{z}}
\end{aligned}
$$

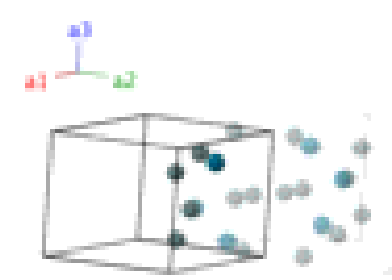

Basis vectors:

\section{Lattice Coordinates}

$$
\begin{aligned}
& \mathbf{B}_{1}=\quad \frac{3}{4} \mathbf{a}_{1}+\frac{1}{4} \mathbf{a}_{2}+\frac{1}{4} \mathbf{a}_{3} \\
& \mathbf{B}_{2}=\quad \frac{1}{4} \mathbf{a}_{1}+\frac{3}{4} \mathbf{a}_{2}+\frac{3}{4} \mathbf{a}_{3} \\
& \mathbf{B}_{3}=\left(x_{2}-y_{2}\right) \mathbf{a}_{1}+\left(x_{2}+y_{2}\right) \mathbf{a}_{2}+z_{2} \mathbf{a}_{3} \\
& \mathbf{B}_{4}= \\
& \left(\frac{1}{2}-x_{2}+y_{2}\right) \mathbf{a}_{1}+ \\
& \left(\frac{1}{2}-x_{2}-y_{2}\right) \mathbf{a}_{2}+z_{2} \mathbf{a}_{3} \\
& \mathbf{B}_{5}=\left(-x_{2}-y_{2}\right) \mathbf{a}_{1}+\left(-x_{2}+y_{2}\right) \mathbf{a}_{2}+\quad=\quad-x_{2} a \hat{\mathbf{x}}+y_{2} b \hat{\mathbf{y}}+\left(\frac{1}{2}-z_{2}\right) c \hat{\mathbf{z}} \\
& \left(\frac{1}{2}-z_{2}\right) \mathbf{a}_{3} \\
& \mathbf{B}_{6}=\begin{array}{c}
\left(\frac{1}{2}+x_{2}+y_{2}\right) \mathbf{a}_{1}+ \\
\left(\frac{1}{2}+x_{2}-y_{2}\right) \mathbf{a}_{2}+\left(\frac{1}{2}-z_{2}\right) \mathbf{a}_{3}
\end{array} \quad=\left(\frac{1}{2}+x_{2}\right) a \hat{\mathbf{x}}-y_{2} b \hat{\mathbf{y}}+\left(\frac{1}{2}-z_{2}\right) c \hat{\mathbf{z}}
\end{aligned}
$$

Cartesian Coordinates 


\begin{tabular}{|c|c|c|c|c|c|}
\hline $\mathbf{B}_{7}$ & $=$ & $\left(-x_{2}+y_{2}\right) \mathbf{a}_{1}+\left(-x_{2}-y_{2}\right) \mathbf{a}_{2}-z_{2} \mathbf{a}_{3}$ & $=$ & $-x_{2} a \hat{\mathbf{x}}-y_{2} b \hat{\mathbf{y}}-z_{2} c \hat{\mathbf{z}}$ & $(16 i)$ \\
\hline $\mathbf{B}_{8}$ & $=$ & $\begin{array}{c}\left(\frac{1}{2}+x_{2}-y_{2}\right) \mathbf{a}_{1}+ \\
\left(\frac{1}{2}+x_{2}+y_{2}\right) \mathbf{a}_{2}-z_{2} \mathbf{a}_{3}\end{array}$ & $=$ & $\left(\frac{1}{2}+x_{2}\right) a \hat{\mathbf{x}}+y_{2} b \hat{\mathbf{y}}-z_{2} c \hat{\mathbf{z}}$ & $(16 i)$ \\
\hline $\mathbf{B}_{9}$ & $=$ & $\begin{array}{c}\left(x_{2}+y_{2}\right) \mathbf{a}_{1}+\left(x_{2}-y_{2}\right) \mathbf{a}_{2}+ \\
\left(\frac{1}{2}+z_{2}\right) \mathbf{a}_{3}\end{array}$ & $=$ & $x_{2} a \hat{\mathbf{x}}-y_{2} b \hat{\mathbf{y}}+\left(\frac{1}{2}+z_{2}\right) c \hat{\mathbf{z}}$ & $(16 i)$ \\
\hline $\mathbf{B}_{10}$ & $=$ & $\begin{array}{c}\left(\frac{1}{2}-x_{2}-y_{2}\right) \mathbf{a}_{1}+ \\
\left(\frac{1}{2}-x_{2}+y_{2}\right) \mathbf{a}_{2}+\left(\frac{1}{2}+z_{2}\right) \mathbf{a}_{3}\end{array}$ & & $\left(\frac{1}{2}-x_{2}\right) a \hat{\mathbf{x}}+y_{2} b \hat{\mathbf{y}}+\left(\frac{1}{2}+z_{2}\right) c \hat{\mathbf{z}}$ & $(16 i)$ \\
\hline
\end{tabular}

\section{References:}

- J. Nylén, F. J. García García, B. D. Mosel, R. Pöttgen, and U. Häussermann, Structural relationships, phase stability and bonding of compounds $P d S n_{n}(n=2$, 3, 4), Solid State Sci. 6, 147-155 (2004),

doi:10.1016/j.solidstatesciences.2003.09.011.

\section{Found in:}

- P. Villars and K. Cenzual, Pearson's Crystal Data - Crystal Structure Database for Inorganic Compounds, ASM International (2013).

\section{Geometry files:}

- CIF: pp. 853

- POSCAR: pp. 854 


\section{$\mathrm{Mn}_{2} \mathrm{~B}\left(D 1_{f}\right)$ Structure: AB2_oF48_70_f_fg}
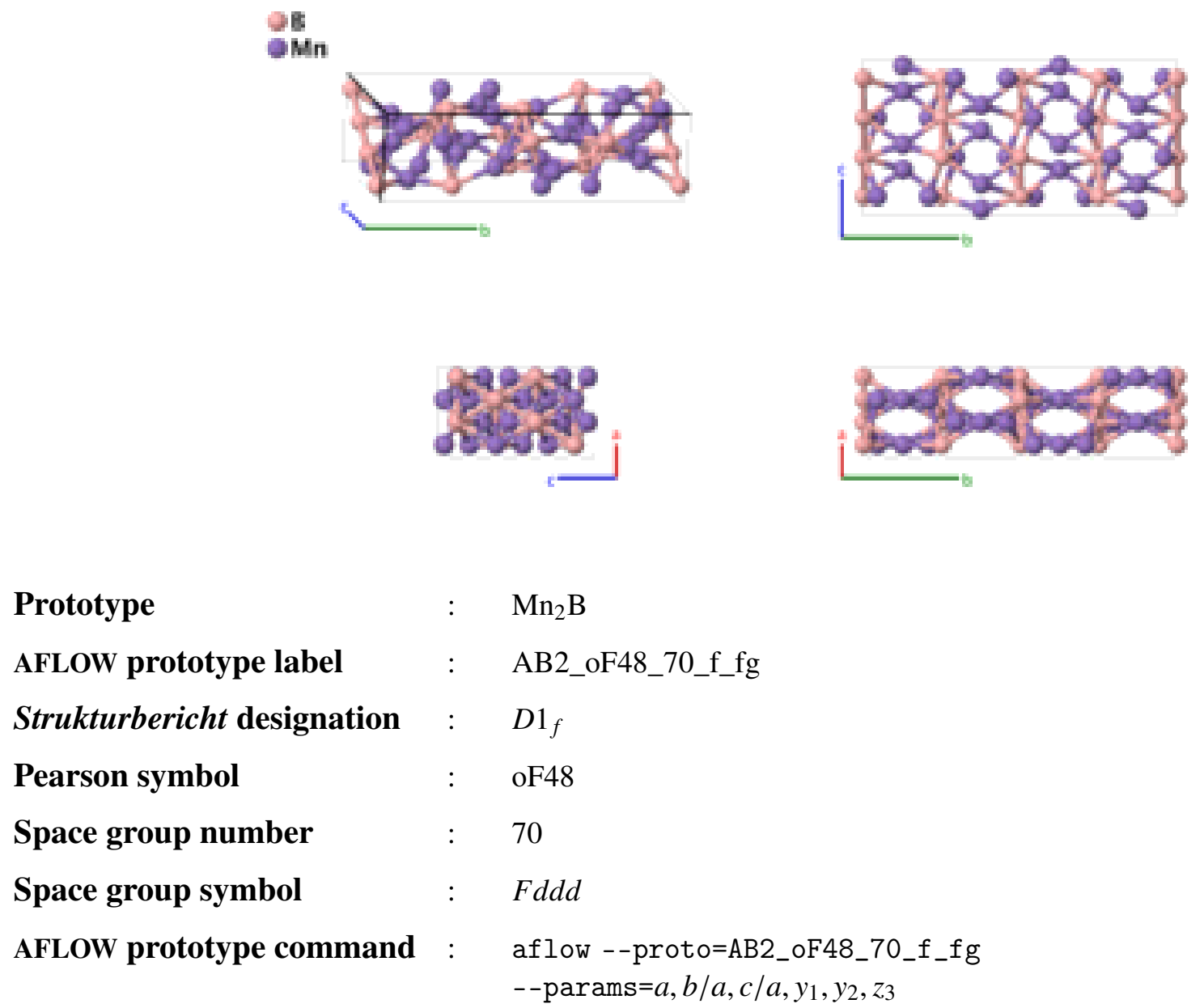

\section{Other compounds with this structure:}

- $\mathrm{Cr}_{2} \mathrm{~B}$

- Early works, e.g. (Pearson, 1958) referred to this structure as $\mathrm{Mn}_{4} \mathrm{~B}$, with the same space group and Wyckoff positions. The stoichiometry was fixed by assuming that the (16e) Boron positions were only half-occupied. Tergenius's 1981 refinement of the structure showed that the (16e) sites were totally filled, fixing the stoichiometry to $\mathrm{Mn}_{2} \mathrm{~B}$. A similar reanalysis showed that the similar structure known has $\mathrm{Cr}_{4} \mathrm{~B}$ also had composition $\mathrm{Cr}_{2} \mathrm{~B}$. Tergenius gives the atomic positions using the first setting of space group $F d d d \# 70$. We have translated this into the second setting, where the origin is on an inversion site. As a part of this process the primitive axes were also rotated compared to Tergenius.

\section{Face-centered Orthorhombic primitive vectors:}

$$
\begin{aligned}
& \mathbf{a}_{1}=\frac{1}{2} b \hat{\mathbf{y}}+\frac{1}{2} c \hat{\mathbf{z}} \\
& \mathbf{a}_{2}=\frac{1}{2} a \hat{\mathbf{x}}+\frac{1}{2} c \hat{\mathbf{z}} \\
& \mathbf{a}_{3}=\frac{1}{2} a \hat{\mathbf{x}}+\frac{1}{2} b \hat{\mathbf{y}}
\end{aligned}
$$

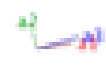

\begin{tabular}{|c|c|c|c|c|}
\hline & Lattice Coordinates & Cartesian Coordinates & Wyckoff Position & Atom Type \\
\hline $\mathbf{B}_{1}=$ & $y_{1} \mathbf{a}_{1}+\left(\frac{1}{4}-y_{1}\right) \mathbf{a}_{2}+y_{1} \mathbf{a}_{3}$ & $\frac{1}{8} a \hat{\mathbf{x}}+y_{1} b \hat{\mathbf{y}}+\frac{1}{8} c \hat{\mathbf{z}}$ & $(16 f)$ & B \\
\hline
\end{tabular}

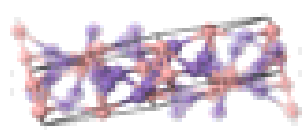

Basis vectors: 


\begin{tabular}{|c|c|c|c|c|c|c|}
\hline $\mathbf{B}_{2}$ & $=$ & $\left(\frac{1}{4}-y_{1}\right) \mathbf{a}_{1}+y_{1} \mathbf{a}_{2}+\left(\frac{1}{4}-y_{1}\right) \mathbf{a}_{3}$ & $=$ & $\frac{1}{8} a \hat{\mathbf{x}}+\left(\frac{1}{4}-y_{1}\right) b \hat{\mathbf{y}}+\frac{1}{8} c \hat{\mathbf{z}}$ & $(16 f)$ & B \\
\hline $\mathbf{B}_{3}$ & $=$ & $-y_{1} \mathbf{a}_{1}+\left(\frac{3}{4}+y_{1}\right) \mathbf{a}_{2}-y_{1} \mathbf{a}_{3}$ & $=$ & $\frac{3}{8} a \hat{\mathbf{x}}-y_{1} b \hat{\mathbf{y}}+\frac{3}{8} c \hat{\mathbf{z}}$ & $(16 f)$ & B \\
\hline $\mathbf{B}_{4}$ & $=$ & $\left(\frac{3}{4}+y_{1}\right) \mathbf{a}_{1}-y_{1} \mathbf{a}_{2}+\left(\frac{3}{4}+y_{1}\right) \mathbf{a}_{3}$ & $=$ & $\frac{3}{8} a \hat{\mathbf{x}}+\left(\frac{3}{4}+y_{1}\right) b \hat{\mathbf{y}}+\frac{3}{8} c \hat{\mathbf{z}}$ & $(16 f)$ & B \\
\hline $\mathbf{B}_{5}$ & $=$ & $y_{2} \mathbf{a}_{1}+\left(\frac{1}{4}-y_{2}\right) \mathbf{a}_{2}+y_{2} \mathbf{a}_{3}$ & $=$ & $\frac{1}{8} a \hat{\mathbf{x}}+y_{2} b \hat{\mathbf{y}}+\frac{1}{8} c \hat{\mathbf{z}}$ & $(16 f)$ & Mn I \\
\hline $\mathbf{B}_{6}$ & $=$ & $\left(\frac{1}{4}-y_{2}\right) \mathbf{a}_{1}+y_{2} \mathbf{a}_{2}+\left(\frac{1}{4}-y_{2}\right) \mathbf{a}_{3}$ & $=$ & $\frac{1}{8} a \hat{\mathbf{x}}+\left(\frac{1}{4}-y_{2}\right) b \hat{\mathbf{y}}+\frac{1}{8} c \hat{\mathbf{z}}$ & $(16 f)$ & Mn I \\
\hline $\mathbf{B}_{7}$ & $=$ & $-y_{2} \mathbf{a}_{1}+\left(\frac{3}{4}+y_{2}\right) \mathbf{a}_{2}-y_{2} \mathbf{a}_{3}$ & $=$ & $\frac{3}{8} a \hat{\mathbf{x}}-y_{2} b \hat{\mathbf{y}}+\frac{3}{8} c \hat{\mathbf{z}}$ & $(16 f)$ & Mn I \\
\hline $\mathbf{B}_{8}$ & $=$ & $\left(\frac{3}{4}+y_{2}\right) \mathbf{a}_{1}-y_{2} \mathbf{a}_{2}+\left(\frac{3}{4}+y_{2}\right) \mathbf{a}_{3}$ & $=$ & $\frac{3}{8} a \hat{\mathbf{x}}+\left(\frac{3}{4}+y_{2}\right) b \hat{\mathbf{y}}+\frac{3}{8} c \hat{\mathbf{z}}$ & $(16 f)$ & Mn I \\
\hline $\mathbf{B}_{9}$ & $=$ & $z_{3} \mathbf{a}_{1}+z_{3} \mathbf{a}_{2}+\left(\frac{1}{4}-z_{3}\right) \mathbf{a}_{3}$ & $=$ & $\frac{1}{8} a \hat{\mathbf{x}}+\frac{1}{8} b \hat{\mathbf{y}}+z_{3} c \hat{\mathbf{z}}$ & $(16 g)$ & Mn II \\
\hline $\mathbf{B}_{10}$ & $=$ & $\left(\frac{1}{4}-z_{3}\right) \mathbf{a}_{1}+\left(\frac{1}{4}-z_{3}\right) \mathbf{a}_{2}+z_{3} \mathbf{a}_{3}$ & $=$ & $\frac{1}{8} a \hat{\mathbf{x}}+\frac{1}{8} b \hat{\mathbf{y}}+\left(\frac{1}{4}-z_{3}\right) c \hat{\mathbf{z}}$ & $(16 g)$ & Mn II \\
\hline $\mathbf{B}_{11}$ & $=$ & $-z_{3} \mathbf{a}_{1}-z_{3} \mathbf{a}_{2}+\left(\frac{3}{4}+z_{3}\right) \mathbf{a}_{3}$ & $=$ & $\frac{3}{8} a \hat{\mathbf{x}}+\frac{3}{8} b \hat{\mathbf{y}}-z_{3} c \hat{\mathbf{z}}$ & $(16 g)$ & Mn II \\
\hline $\mathbf{B}_{12}$ & $=$ & $\left(\frac{3}{4}+z_{3}\right) \mathbf{a}_{1}+\left(\frac{3}{4}+z_{3}\right) \mathbf{a}_{2}-z_{3} \mathbf{a}_{3}$ & $=$ & $\frac{3}{8} a \hat{\mathbf{x}}+\frac{3}{8} b \hat{\mathbf{y}}+\left(\frac{3}{4}+z_{3}\right) c \hat{\mathbf{z}}$ & $(16 g)$ & Mn II \\
\hline
\end{tabular}

\section{References:}

- L.-E. Tergenius, Refinement of the crystal structure of orthorhombic $M n_{2} B$ (formerly denoted $M n_{4} B$ ), J. Less-Common Met. 82, 335-340 (1981), doi:10.1016/0022-5088(81)90236-8.

- W. B. Pearson, A Handbook of Lattice Spacings and Structures of Metals and Alloys, no. N.R.C. No. 4303 in International Series of Monographs on Metal Physics and Physical Metallurgy (Pergamon Press, Oxford, London, Edinburgh, New York, Paris, Frankfort, 1958), 1964 reprint with corrections edn.

\section{Geometry files:}

- CIF: pp. 854

- POSCAR: pp. 854 


\section{$\mathrm{Ta}_{3} \mathrm{~B}_{4}\left(D 7_{b}\right)$ Structure: A4B3_oI14_71_gh_cg}
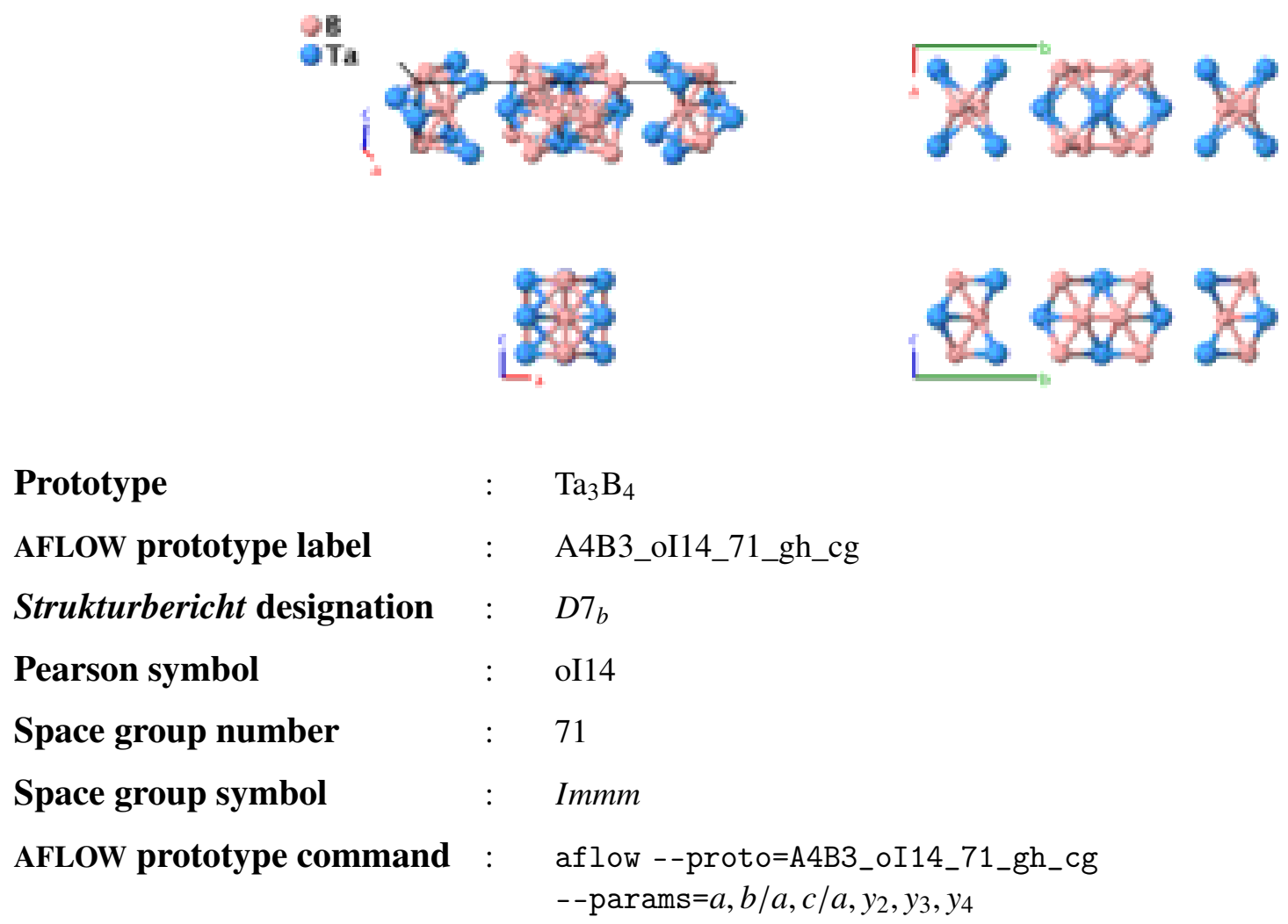

\section{Other compounds with this structure:}

- $\mathrm{B}_{4} \mathrm{CoMo}_{2}, \mathrm{~B}_{4} \mathrm{Cr}_{3}, \mathrm{~B}_{4} \mathrm{FeMo}_{2}, \mathrm{~B}_{4} \mathrm{Mn}_{3}, \mathrm{~B}_{4} \mathrm{Mo}_{2} \mathrm{Ni}, \mathrm{B}_{4} \mathrm{Nb}_{3}, \mathrm{~B}_{4} \mathrm{Ta}_{3}, \mathrm{~B}_{4} \mathrm{~V}_{3}$

Body-centered Orthorhombic primitive vectors:

$$
\begin{aligned}
& \mathbf{a}_{1}=-\frac{1}{2} a \hat{\mathbf{x}}+\frac{1}{2} b \hat{\mathbf{y}}+\frac{1}{2} c \hat{\mathbf{z}} \\
& \mathbf{a}_{2}=\frac{1}{2} a \hat{\mathbf{x}}-\frac{1}{2} b \hat{\mathbf{y}}+\frac{1}{2} c \hat{\mathbf{z}} \\
& \mathbf{a}_{3}=\frac{1}{2} a \hat{\mathbf{x}}+\frac{1}{2} b \hat{\mathbf{y}}-\frac{1}{2} c \hat{\mathbf{z}}
\end{aligned}
$$

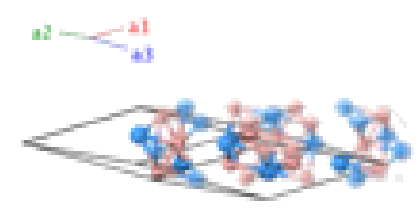

Basis vectors:

Lattice Coordinates

$\begin{array}{lcccc}\mathbf{B}_{1} & = & \frac{1}{2} \mathbf{a}_{1}+\frac{1}{2} \mathbf{a}_{2} & = & \frac{1}{2} c \hat{\mathbf{z}} \\ \mathbf{B}_{2} & y_{2} \mathbf{a}_{1}+y_{2} \mathbf{a}_{3} & = & y_{2} b \hat{\mathbf{y}} \\ \mathbf{B}_{3} & -y_{2} \mathbf{a}_{1}+-y_{2} \mathbf{a}_{3} & = & -y_{2} b \hat{\mathbf{y}} \\ \mathbf{B}_{4}= & y_{3} \mathbf{a}_{1}+y_{3} \mathbf{a}_{3} & = & y_{3} b \hat{\mathbf{y}} \\ \mathbf{B}_{5}= & -y_{3} \mathbf{a}_{1}+-y_{3} \mathbf{a}_{3} & = & -y_{3} b \hat{\mathbf{y}} \\ \mathbf{B}_{6}= & \left(\frac{1}{2}+y_{4}\right) \mathbf{a}_{1}+\frac{1}{2} \mathbf{a}_{2}+y_{4} \mathbf{a}_{3} & = & y_{4} b \hat{\mathbf{y}}+\frac{1}{2} c \hat{\mathbf{z}} \\ \mathbf{B}_{7} & = & \left(\frac{1}{2}-y_{4}\right) \mathbf{a}_{1}+\frac{1}{2} \mathbf{a}_{2}-y_{4} \mathbf{a}_{3} & = & -y_{4} b \hat{\mathbf{y}}+\frac{1}{2} c \hat{\mathbf{z}}\end{array}$

Wyckoff Position

(2c)
Atom Type

Ta I

B I

B I

Ta II

Ta II

B II

B II

\section{References:}

- R. Kiessling, The Borides of Tantalum, Acta Chem. Scand. 3, 603-615 (1949), doi:10.3891/acta.chem.scand.03-0603. 


\section{Found in:}

- R. M. Minyaev and R. Hoffmann, Transition-metal borides with the tantalum boride $\left(\mathrm{Ta}_{3} B_{4}\right)$ crystal structure: their electronic and bonding properties, Chem. Mater. 3, 547-557 (1991), doi:10.1021/cm00015a035.

\section{Geometry files:}

- CIF: pp. 854

- POSCAR: pp. 855 

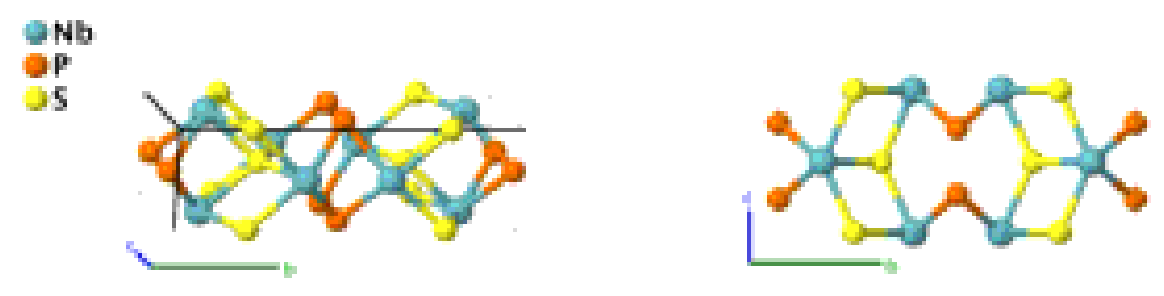

Prototype

: $\quad$ NbPS

AFLOW prototype label

: $\quad$ ABC_oI12_71_h_j_g

Strukturbericht designation

: None

Pearson symbol

: $\quad$ I12

Space group number

71

Space group symbol

Immm

AFLOW prototype command : aflow --proto=ABC_oI12_71_h_j_g

- params $=a, b / a, c / a, y_{1}, y_{2}, z_{3}$

\section{Other compounds with this structure:}

- TaPS, NbPSe, HfSbTe, ZrSbTe

Body-centered Orthorhombic primitive vectors:

$$
\begin{aligned}
& \mathbf{a}_{1}=-\frac{1}{2} a \hat{\mathbf{x}}+\frac{1}{2} b \hat{\mathbf{y}}+\frac{1}{2} c \hat{\mathbf{z}} \\
& \mathbf{a}_{2}=\frac{1}{2} a \hat{\mathbf{x}}-\frac{1}{2} b \hat{\mathbf{y}}+\frac{1}{2} c \hat{\mathbf{z}} \\
& \mathbf{a}_{3}=\frac{1}{2} a \hat{\mathbf{x}}+\frac{1}{2} b \hat{\mathbf{y}}-\frac{1}{2} c \hat{\mathbf{z}}
\end{aligned}
$$

Basis vectors:

Lattice Coordinates

$\begin{array}{lccl}\mathbf{B}_{1}= & y_{1} \mathbf{a}_{1}+y_{1} \mathbf{a}_{3} & = \\ \mathbf{B}_{2}= & -y_{1} \mathbf{a}_{1}+-y_{1} \mathbf{a}_{3} & = \\ \mathbf{B}_{3}= & \left(\frac{1}{2}+y_{2}\right) \mathbf{a}_{1}+\frac{1}{2} \mathbf{a}_{2}+y_{2} \mathbf{a}_{3} & = \\ \mathbf{B}_{4}= & \left(\frac{1}{2}-y_{2}\right) \mathbf{a}_{1}+\frac{1}{2} \mathbf{a}_{2}-y_{2} \mathbf{a}_{3} & = \\ \mathbf{B}_{5}= & z_{3} \mathbf{a}_{1}+\left(\frac{1}{2}+z_{3}\right) \mathbf{a}_{2}+\frac{1}{2} \mathbf{a}_{3} & = \\ \mathbf{B}_{6}= & -z_{3} \mathbf{a}_{1}+\left(\frac{1}{2}-z_{3}\right) \mathbf{a}_{2}+\frac{1}{2} \mathbf{a}_{3} & =\end{array}$

Cartesian Coordinates

$=$

$=$

$=$

$=$

$=$

$=$

$$
\begin{gathered}
y_{1} b \hat{\mathbf{y}} \\
-y_{1} b \hat{\mathbf{y}} \\
y_{2} b \hat{\mathbf{y}}+\frac{1}{2} c \hat{\mathbf{z}} \\
-y_{2} b \hat{\mathbf{y}}+\frac{1}{2} c \hat{\mathbf{z}} \\
\frac{1}{2} a \hat{\mathbf{x}}+z_{3} c \hat{\mathbf{z}} \\
\frac{1}{2} a \hat{\mathbf{x}}+-z_{3} c \hat{\mathbf{z}}
\end{gathered}
$$

Wyckoff Position

(4g)

(4g)

(4h)

(4h)

(4j)

(4j)
Atom Type

S

S

$\mathrm{Nb}$

$\mathrm{Nb}$

$\mathrm{P}$

P 


\section{References:}

- P. C. Donohue and P. E. Bierstedt, Synthesis, crystal structure, and superconducting properties of niobium phosphorus sulfide, niobium phosphorus selenide and tantalum phosphorus sulfide, Inorg. Chem. 8, 2690-2694 (1969), doi:10.1021/ic50082a031.

\section{Geometry files:}

- CIF: pp. 855

- POSCAR: pp. 855 


\section{$\mathrm{KAg}\left[\mathrm{CO}_{3}\right]$ Structure: ABCD3_oI48_73_d_e_e_ef}
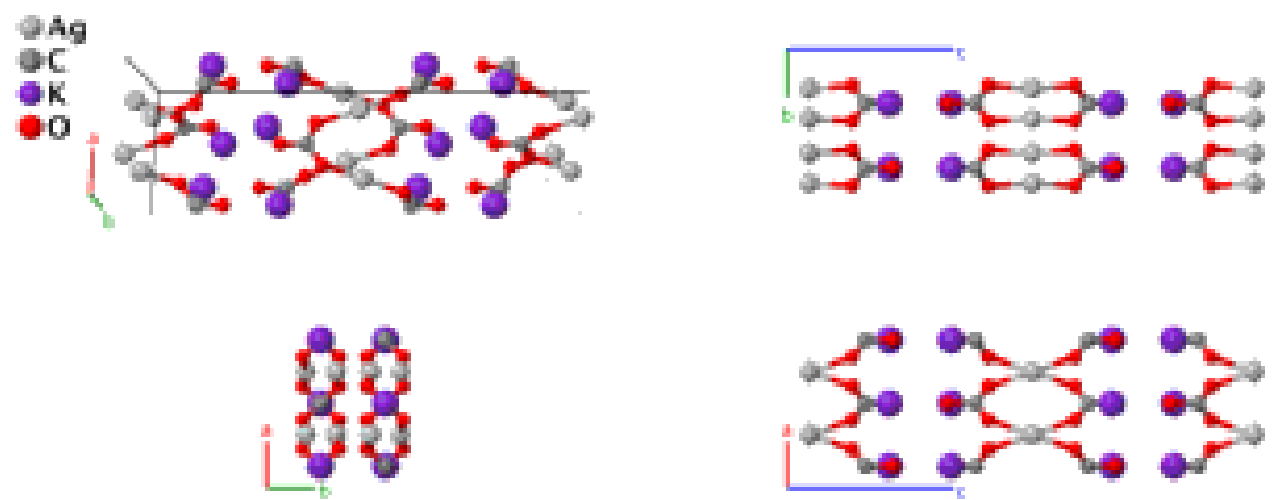

Prototype

AFLOW prototype label

Strukturbericht designation

Pearson symbol

Space group number

Space group symbol

AFLOW prototype command
: $\mathrm{KAg}\left[\mathrm{CO}_{3}\right]$

: ABCD3_oI48_73_d_e_e_ef

: None

: $\quad$ oI48

: 73

: $\quad$ Ibca

aflow --proto=ABCD3_oI48_73_d_e_e_ef

- params $=a, b / a, c / a, y_{1}, z_{2}, z_{3}, z_{4}, x_{5}, y_{5}, z_{5}$

Body-centered Orthorhombic primitive vectors:

$$
\begin{aligned}
& \mathbf{a}_{1}=-\frac{1}{2} a \hat{\mathbf{x}}+\frac{1}{2} b \hat{\mathbf{y}}+\frac{1}{2} c \hat{\mathbf{z}} \\
& \mathbf{a}_{2}=\frac{1}{2} a \hat{\mathbf{x}}-\frac{1}{2} b \hat{\mathbf{y}}+\frac{1}{2} c \hat{\mathbf{z}} \\
& \mathbf{a}_{3}=\frac{1}{2} a \hat{\mathbf{x}}+\frac{1}{2} b \hat{\mathbf{y}}-\frac{1}{2} c \hat{\mathbf{z}}
\end{aligned}
$$

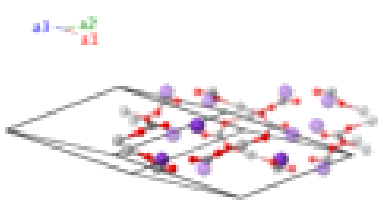

Basis vectors:

Lattice Coordinates

$\begin{array}{llclc}\mathbf{B}_{1}= & y_{1} \mathbf{a}_{1}+\frac{1}{4} \mathbf{a}_{2}+\left(\frac{1}{4}+y_{1}\right) \mathbf{a}_{3} & = & \frac{1}{4} a \hat{\mathbf{x}}+y_{1} b \hat{\mathbf{y}} \\ \mathbf{B}_{2} & = & \left(\frac{1}{2}-y_{1}\right) \mathbf{a}_{1}+\frac{3}{4} \mathbf{a}_{2}+\left(\frac{1}{4}-y_{1}\right) \mathbf{a}_{3} & = & \frac{1}{4} a \hat{\mathbf{x}}-y_{1} b \hat{\mathbf{y}}+\frac{1}{2} c \hat{\mathbf{z}} \\ \mathbf{B}_{3} & = & -y_{1} \mathbf{a}_{1}+\frac{3}{4} \mathbf{a}_{2}+\left(\frac{3}{4}-y_{1}\right) \mathbf{a}_{3} & = & \frac{3}{4} a \hat{\mathbf{x}}-y_{1} b \hat{\mathbf{y}} \\ \mathbf{B}_{4}= & \left(\frac{1}{2}+y_{1}\right) \mathbf{a}_{1}+\frac{1}{4} \mathbf{a}_{2}+\left(\frac{3}{4}+y_{1}\right) \mathbf{a}_{3} & = & \frac{1}{4} a \hat{\mathbf{x}}+\left(\frac{1}{2}+y_{1}\right) b \hat{\mathbf{y}} \\ \mathbf{B}_{5} & = & \left(\frac{1}{4}+z_{2}\right) \mathbf{a}_{1}+z_{2} \mathbf{a}_{2}+\frac{1}{4} \mathbf{a}_{3} & = & \frac{1}{4} b \hat{\mathbf{y}}+z_{2} c \hat{\mathbf{z}} \\ \mathbf{B}_{6} & = & \left(\frac{1}{4}-z_{2}\right) \mathbf{a}_{1}+\left(\frac{1}{2}-z_{2}\right) \mathbf{a}_{2}+\frac{3}{4} \mathbf{a}_{3} & = & \frac{1}{2} a \hat{\mathbf{x}}+\frac{1}{4} b \hat{\mathbf{y}}-z_{2} c \hat{\mathbf{z}} \\ \mathbf{B}_{7} & = & \left(\frac{3}{4}-z_{2}\right) \mathbf{a}_{1}-z_{2} \mathbf{a}_{2}+\frac{3}{4} \mathbf{a}_{3} & = & \frac{3}{4} b \hat{\mathbf{y}}-z_{2} c \hat{\mathbf{z}} \\ \mathbf{B}_{8}= & \left(\frac{3}{4}+z_{2}\right) \mathbf{a}_{1}+\left(\frac{1}{2}+z_{2}\right) \mathbf{a}_{2}+\frac{1}{4} \mathbf{a}_{3} & = & \frac{1}{4} b \hat{\mathbf{y}}+\left(\frac{1}{2}+z_{2}\right) c \hat{\mathbf{z}} \\ \mathbf{B}_{9}= & \left(\frac{1}{4}+z_{3}\right) \mathbf{a}_{1}+z_{3} \mathbf{a}_{2}+\frac{1}{4} \mathbf{a}_{3} & = & \frac{1}{4} b \hat{\mathbf{y}}+z_{3} c \hat{\mathbf{z}} \\ \mathbf{B}_{10}= & \left(\frac{1}{4}-z_{3}\right) \mathbf{a}_{1}+\left(\frac{1}{2}-z_{3}\right) \mathbf{a}_{2}+\frac{3}{4} \mathbf{a}_{3} & = & \frac{1}{2} a \hat{\mathbf{x}}+\frac{1}{4} b \hat{\mathbf{y}}-z_{3} c \hat{\mathbf{z}} \\ \mathbf{B}_{11}= & \left(\frac{3}{4}-z_{3}\right) \mathbf{a}_{1}-z_{3} \mathbf{a}_{2}+\frac{3}{4} \mathbf{a}_{3} & = & \frac{3}{4} b \hat{\mathbf{y}}-z_{3} c \hat{\mathbf{z}}\end{array}$

Wyckoff Position Atom Type

$\mathrm{Ag}$

Ag

$\mathrm{Ag}$

$\mathrm{Ag}$
C

C

C

C

K

K

K 


\begin{tabular}{|c|c|c|c|c|c|}
\hline $\mathbf{B}_{12}$ & $=$ & $\left(\frac{3}{4}+z_{3}\right) \mathbf{a}_{1}+\left(\frac{1}{2}+z_{3}\right) \mathbf{a}_{2}+\frac{1}{4} \mathbf{a}_{3}$ & $=$ & $\frac{1}{4} b \hat{\mathbf{y}}+\left(\frac{1}{2}+z_{3}\right) c \hat{\mathbf{z}}$ & $(8 e)$ \\
\hline $\mathbf{B}_{13}$ & $=$ & $\left(\frac{1}{4}+z_{4}\right) \mathbf{a}_{1}+z_{4} \mathbf{a}_{2}+\frac{1}{4} \mathbf{a}_{3}$ & $=$ & $\frac{1}{4} b \hat{\mathbf{y}}+z_{4} c \hat{\mathbf{z}}$ & $(8 e)$ \\
\hline $\mathbf{B}_{14}$ & $=$ & $\left(\frac{1}{4}-z_{4}\right) \mathbf{a}_{1}+\left(\frac{1}{2}-z_{4}\right) \mathbf{a}_{2}+\frac{3}{4} \mathbf{a}_{3}$ & $=$ & $\frac{1}{2} a \hat{\mathbf{x}}+\frac{1}{4} b \hat{\mathbf{y}}-z_{4} c \hat{\mathbf{z}}$ & $(8 e)$ \\
\hline $\mathbf{B}_{15}$ & $=$ & $\left(\frac{3}{4}-z_{4}\right) \mathbf{a}_{1}-z_{4} \mathbf{a}_{2}+\frac{3}{4} \mathbf{a}_{3}$ & $=$ & $\frac{3}{4} b \hat{\mathbf{y}}-z_{4} c \hat{\mathbf{z}}$ & $(8 e)$ \\
\hline $\mathbf{B}_{16}$ & $=$ & $\left(\frac{3}{4}+z_{4}\right) \mathbf{a}_{1}+\left(\frac{1}{2}+z_{4}\right) \mathbf{a}_{2}+\frac{1}{4} \mathbf{a}_{3}$ & $=$ & $\frac{1}{4} b \hat{\mathbf{y}}+\left(\frac{1}{2}+z_{4}\right) c \hat{\mathbf{z}}$ & $(8 e)$ \\
\hline $\mathbf{B}_{17}$ & $=$ & $\left(y_{5}+z_{5}\right) \mathbf{a}_{1}+\left(x_{5}+z_{5}\right) \mathbf{a}_{2}+\left(x_{5}+y_{5}\right) \mathbf{a}_{3}$ & $=$ & $x_{5} a \hat{\mathbf{x}}+y_{5} b \hat{\mathbf{y}}+z_{5} c \hat{\mathbf{z}}$ & $(16 f)$ \\
\hline $\mathbf{B}_{18}$ & $=$ & $\begin{array}{c}\left(\frac{1}{2}-y_{5}+z_{5}\right) \mathbf{a}_{1}+\left(-x_{5}+z_{5}\right) \mathbf{a}_{2}+ \\
\left(\frac{1}{2}-x_{5}-y_{5}\right) \mathbf{a}_{3}\end{array}$ & $=$ & $-x_{5} a \hat{\mathbf{x}}+\left(\frac{1}{2}-y_{5}\right) b \hat{\mathbf{y}}+z_{5} c \hat{\mathbf{z}}$ & $(16 f)$ \\
\hline $\mathbf{B}_{19}$ & $=$ & $\begin{array}{c}\left(y_{5}-z_{5}\right) \mathbf{a}_{1}+\left(\frac{1}{2}-x_{5}-z_{5}\right) \mathbf{a}_{2}+ \\
\left(\frac{1}{2}-x_{5}+y_{5}\right) \mathbf{a}_{3}\end{array}$ & $=$ & $\left(\frac{1}{2}-x_{5}\right) a \hat{\mathbf{x}}+y_{5} b \hat{\mathbf{y}}-z_{5} c \hat{\mathbf{z}}$ & $(16 f)$ \\
\hline $\mathbf{B}_{20}$ & $=$ & $\begin{array}{c}\left(\frac{1}{2}-y_{5}-z_{5}\right) \mathbf{a}_{1}+\left(\frac{1}{2}+x_{5}-z_{5}\right) \mathbf{a}_{2}+ \\
\left(x_{5}-y_{5}\right) \mathbf{a}_{3}\end{array}$ & $=$ & $x_{5} a \hat{\mathbf{x}}-y_{5} b \hat{\mathbf{y}}+\left(\frac{1}{2}-z_{5}\right) c \hat{\mathbf{z}}$ & $(16 f)$ \\
\hline $\mathbf{B}_{21}$ & $=$ & $\begin{array}{c}\left(-y_{5}-z_{5}\right) \mathbf{a}_{1}+\left(-x_{5}-z_{5}\right) \mathbf{a}_{2}+ \\
\left(-x_{5}-y_{5}\right) \mathbf{a}_{3}\end{array}$ & $=$ & $-x_{5} a \hat{\mathbf{x}}-y_{5} b \hat{\mathbf{y}}-z_{5} c \hat{\mathbf{z}}$ & $(16 f)$ \\
\hline $\mathbf{B}_{22}$ & $=$ & $\begin{array}{c}\left(\frac{1}{2}+y_{5}-z_{5}\right) \mathbf{a}_{1}+\left(x_{5}-z_{5}\right) \mathbf{a}_{2}+ \\
\left(\frac{1}{2}+x_{5}+y_{5}\right) \mathbf{a}_{3}\end{array}$ & $=$ & $x_{5} a \hat{\mathbf{x}}+\left(\frac{1}{2}+y_{5}\right) b \hat{\mathbf{y}}-z_{5} c \hat{\mathbf{z}}$ & $(16 f)$ \\
\hline $\mathbf{B}_{23}$ & $=$ & $\begin{array}{c}\left(-y_{5}+z_{5}\right) \mathbf{a}_{1}+\left(\frac{1}{2}+x_{5}+z_{5}\right) \mathbf{a}_{2}+ \\
\left(\frac{1}{2}+x_{5}-y_{5}\right) \mathbf{a}_{3}\end{array}$ & $=$ & $\left(\frac{1}{2}+x_{5}\right) a \hat{\mathbf{x}}-y_{5} b \hat{\mathbf{y}}+z_{5} c \hat{\mathbf{z}}$ & $(16 f)$ \\
\hline $\mathbf{B}_{24}$ & $=$ & $\begin{array}{c}\left(\frac{1}{2}+y_{5}+z_{5}\right) \mathbf{a}_{1}+\left(\frac{1}{2}-x_{5}+z_{5}\right) \mathbf{a}_{2}+ \\
\left(-x_{5}+y_{5}\right) \mathbf{a}_{3}\end{array}$ & $=$ & $-x_{5} a \hat{\mathbf{x}}+y_{5} b \hat{\mathbf{y}}+\left(\frac{1}{2}+z_{5}\right) c \hat{\mathbf{z}}$ & $(16 f)$ \\
\hline
\end{tabular}

\section{References:}

- Y.-Q. Zheng, L.-X. Zhou, J.-L. Lin, and S.-W. Zhang, Refinement of the crystal structure of potassium catena-carbonato-argentate(I), $\mathrm{K}\left[\mathrm{Ag}\left(\mathrm{CO}_{3}\right)\right]$, Zeitschrift für Kristallographie - New Crystal Structures 215, 467-468 (2000), doi:10.1515/ncrs-2000-0405.

\section{Found in:}

- P. Villars and K. Cenzual, Pearson's Crystal Data - Crystal Structure Database for Inorganic Compounds, ASM International (2013).

\section{Geometry files:}

- CIF: pp. 855

- POSCAR: pp. 856 

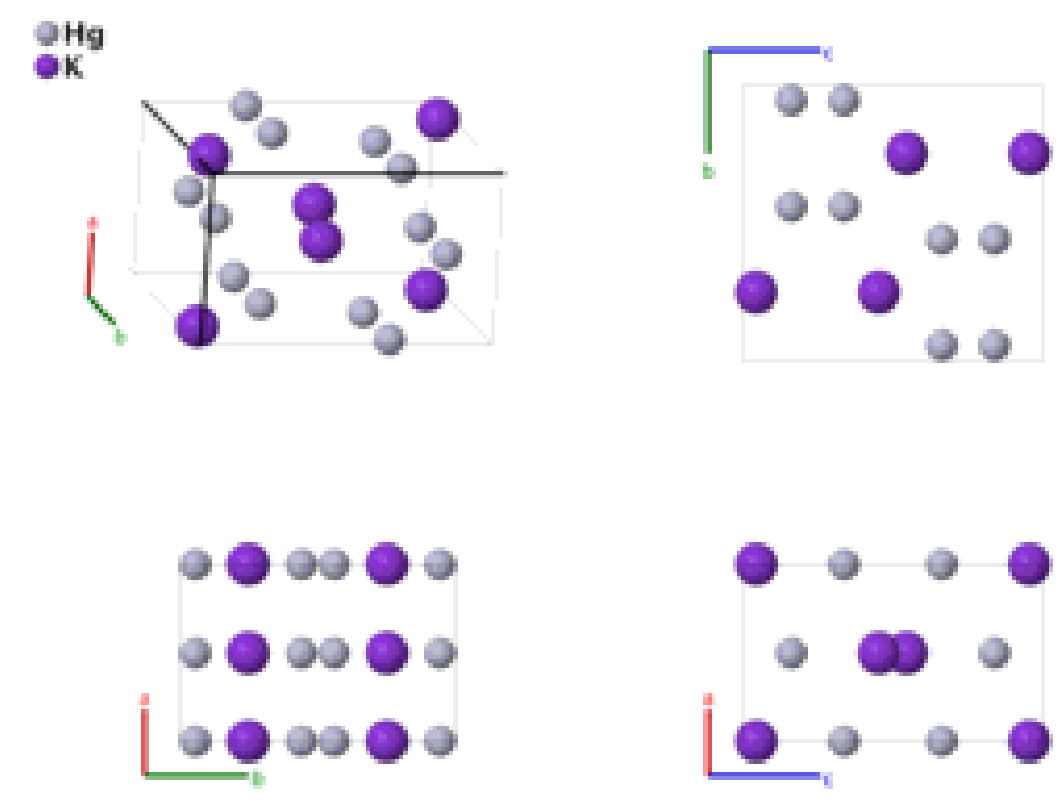

Prototype

AFLOW prototype label

: $\mathrm{KHg}_{2}$

Strukturbericht designation

: A2B_oI12_74_h_e

Pearson symbol

: None

Space group number

: $\mathrm{oI} 12$

Space group symbol

: 74

AFLOW prototype command

: Imma

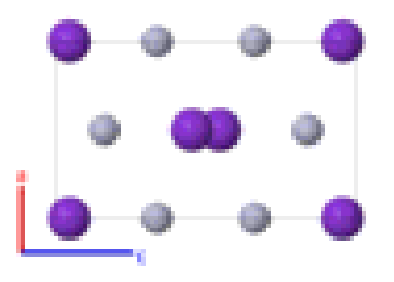

aflow --proto=A2B_oI12_74_h_e

- params $=a, b / a, c / a, z_{1}, y_{2}, z_{2}$

Body-centered Orthorhombic primitive vectors:

$$
\begin{aligned}
& \mathbf{a}_{1}=-\frac{1}{2} a \hat{\mathbf{x}}+\frac{1}{2} b \hat{\mathbf{y}}+\frac{1}{2} c \hat{\mathbf{z}} \\
& \mathbf{a}_{2}=\frac{1}{2} a \hat{\mathbf{x}}-\frac{1}{2} b \hat{\mathbf{y}}+\frac{1}{2} c \hat{\mathbf{z}} \\
& \mathbf{a}_{3}=\frac{1}{2} a \hat{\mathbf{x}}+\frac{1}{2} b \hat{\mathbf{y}}-\frac{1}{2} c \hat{\mathbf{z}}
\end{aligned}
$$

$$
\text { i1) }
$$

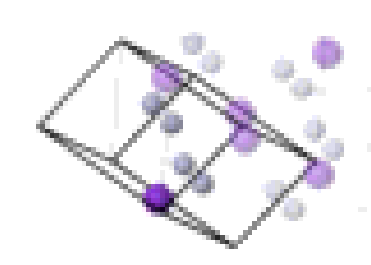

\section{Basis vectors:}

Lattice Coordinates

$\begin{array}{llcll}\mathbf{B}_{1}= & \left(\frac{1}{4}+z_{1}\right) \mathbf{a}_{1}+z_{1} \mathbf{a}_{2}+\frac{1}{4} \mathbf{a}_{3} & = & \frac{1}{4} b \hat{\mathbf{y}}+z_{1} c \hat{\mathbf{z}} \\ \mathbf{B}_{2}= & \left(\frac{3}{4}-z_{1}\right) \mathbf{a}_{1}-z_{1} \mathbf{a}_{2}+\frac{3}{4} \mathbf{a}_{3} & = & \frac{3}{4} b \hat{\mathbf{y}}-z_{1} c \hat{\mathbf{z}} \\ \mathbf{B}_{3}= & \left(y_{2}+z_{2}\right) \mathbf{a}_{1}+z_{2} \mathbf{a}_{2}+y_{2} \mathbf{a}_{3} & = & y_{2} b \hat{\mathbf{y}}+z_{2} c \hat{\mathbf{z}} \\ \mathbf{B}_{4}= & \left(\frac{1}{2}-y_{2}+z_{2}\right) \mathbf{a}_{1}+z_{2} \mathbf{a}_{2}+\left(\frac{1}{2}-y_{2}\right) \mathbf{a}_{3} & = & \left(\frac{1}{2}-y_{2}\right) b \hat{\mathbf{y}}+z_{2} c \hat{\mathbf{z}}\end{array}$

$\mathbf{B}_{1}=$

Ba

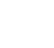



$\mathbf{B}_{5}=$
$\left(\frac{1}{2}+y_{2}-z_{2}\right) \mathbf{a}_{1}-z_{2} \mathbf{a}_{2}+\left(\frac{1}{2}+y_{2}\right) \mathbf{a}_{3}$
$=$
$\left(\frac{1}{2}+y_{2}\right) b \hat{\mathbf{y}}-z_{2} c \hat{\mathbf{z}}$
(8h)
$\mathrm{Hg}$
$\mathbf{B}_{6}=$
$\left(-y_{2}-z_{2}\right) \mathbf{a}_{1}-z_{2} \mathbf{a}_{2}-y_{2} \mathbf{a}_{3}$
$=$
$-y_{2} b \hat{\mathbf{y}}-z_{2} c \hat{\mathbf{z}}$
$(8 h)$
$\mathrm{Hg}$

\section{References:}

- E. J. Duwell and N. C. Baenziger, The crystal structures of $\mathrm{KHg}$ and $\mathrm{KHg}_{2}$, Acta Cryst. 8, 705-710 (1955), doi:10.1107/S0365110X55002168.

\section{Found in:}

- P. Villars and K. Cenzual, Pearson's Crystal Data - Crystal Structure Database for Inorganic Compounds, ASM International (2013).

\section{Geometry files:}

- CIF: pp. 856

- POSCAR: pp. 856 


\section{$\mathrm{Al}_{4} \mathrm{U}\left(D 1_{b}\right)$ Structure: A4B_oI20_74_beh_e}
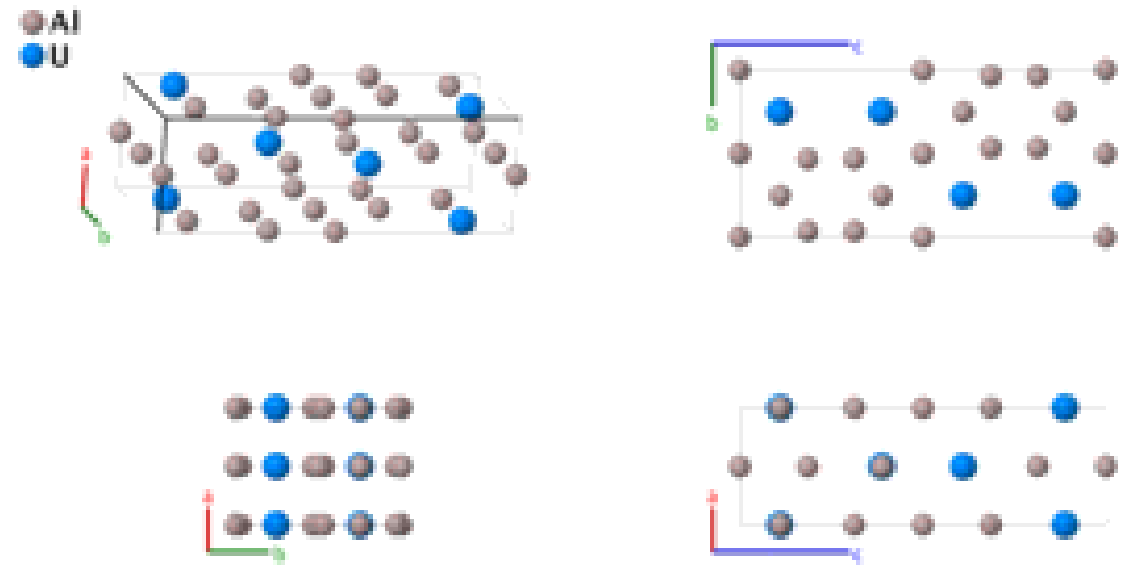

Prototype

AFLOW prototype label

Strukturbericht designation

Pearson symbol

Space group number

Space group symbol

AFLOW prototype command
: $\quad \mathrm{Al}_{4} \mathrm{U}$

: A4B_oI20_74_beh_e

: $D 1_{b}$

: $\quad$ oI20

: 74

: Imma

aflow --proto=A4B_oI20_74_beh_e

- params $=a, b / a, c / a, z_{2}, z_{3}, y_{4}, z_{4}$

\section{Other compounds with this structure:}

- $\mathrm{Al}_{4} \mathrm{Gd}, \mathrm{Al}_{4} \mathrm{~Np}, \mathrm{Al}_{4} \mathrm{Pu}$

Body-centered Orthorhombic primitive vectors:

$$
\begin{aligned}
& \mathbf{a}_{1}=-\frac{1}{2} a \hat{\mathbf{x}}+\frac{1}{2} b \hat{\mathbf{y}}+\frac{1}{2} c \hat{\mathbf{z}} \\
& \mathbf{a}_{2}=\frac{1}{2} a \hat{\mathbf{x}}-\frac{1}{2} b \hat{\mathbf{y}}+\frac{1}{2} c \hat{\mathbf{z}} \\
& \mathbf{a}_{3}=\frac{1}{2} a \hat{\mathbf{x}}+\frac{1}{2} b \hat{\mathbf{y}}-\frac{1}{2} c \hat{\mathbf{z}}
\end{aligned}
$$

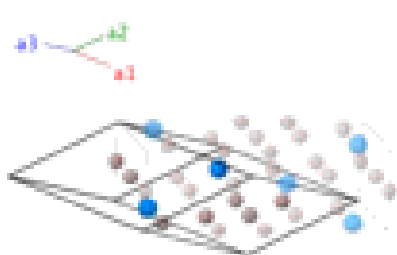

Basis vectors:

\section{Lattice Coordinates}

$\mathbf{B}_{1}=$

$\mathbf{B}_{2}=$

$\mathbf{B}_{3}=$

$\mathbf{B}_{4}=$

$\mathbf{B}_{5}=$

$\mathbf{B}_{6}=$

$\mathbf{B}_{7}=$ $\frac{1}{2} \mathbf{a}_{1}+\frac{1}{2} \mathbf{a}_{2}$

$\frac{1}{2} \mathbf{a}_{2}+\frac{1}{2} \mathbf{a}_{3}$

$\left(\frac{1}{4}+z_{2}\right) \mathbf{a}_{1}+z_{2} \mathbf{a}_{2}+\frac{1}{4} \mathbf{a}_{3}$

$\left(\frac{3}{4}-z_{2}\right) \mathbf{a}_{1}-z_{2} \mathbf{a}_{2}+\frac{3}{4} \mathbf{a}_{3}$

$\left(\frac{1}{4}+z_{3}\right) \mathbf{a}_{1}+z_{3} \mathbf{a}_{2}+\frac{1}{4} \mathbf{a}_{3}$

$\left(\frac{3}{4}-z_{3}\right) \mathbf{a}_{1}-z_{3} \mathbf{a}_{2}+\frac{3}{4} \mathbf{a}_{3}$

$\left(y_{4}+z_{4}\right) \mathbf{a}_{1}+z_{4} \mathbf{a}_{2}+y_{4} \mathbf{a}_{3}$
Cartesian Coordinates

$=$

$=$

$=$

$=$

$=$

$=$

$=$
Wyckoff Position

(4b)

(4b)

(4e)

(4e)

$(4 e)$

$(4 e)$

(8h)
Atom Type

Al I

Al I

Al II

Al II

U

Al III 

$\mathbf{B}_{8}=\left(\frac{1}{2}-y_{4}+z_{4}\right) \mathbf{a}_{1}+z_{4} \mathbf{a}_{2}+\left(\frac{1}{2}-y_{4}\right) \mathbf{a}_{3}$
$=\left(\frac{1}{2}-y_{4}\right) b \hat{\mathbf{y}}+z_{4} c \hat{\mathbf{z}}$
(8h)
Al III
$\mathbf{B}_{9}=\left(\frac{1}{2}+y_{4}-z_{4}\right) \mathbf{a}_{1}-z_{4} \mathbf{a}_{2}+\left(\frac{1}{2}+y_{4}\right) \mathbf{a}_{3}=\left(\frac{1}{2}+y_{4}\right) b \hat{\mathbf{y}}-z_{4} c \hat{\mathbf{z}}$
(8h)
Al III
$\mathbf{B}_{10}=$
$\left(-y_{4}-z_{4}\right) \mathbf{a}_{1}-z_{4} \mathbf{a}_{2}-y_{4} \mathbf{a}_{3}$
$=\quad-y_{4} b \hat{\mathbf{y}}-z_{4} c \hat{\mathbf{z}}$
(8h)
Al III

\section{References:}

- H. U. Borgstedt and H. Wedemeyer, Gmelin Handbook of Inorganic Chemistry (Springer-Verlag, Berlin Heidelberg, 1989), vol. Supplement B 2, chap. 3, p. 144.

\section{Geometry files:}

- CIF: pp. 856

- POSCAR: pp. 857 


\section{$\mathrm{BaCr}_{2} \mathrm{Ru}_{4} \mathrm{O}_{12}$ Structure:}

\section{AB2C12D4_tP76_75_2a2b_2d_12d_4d}
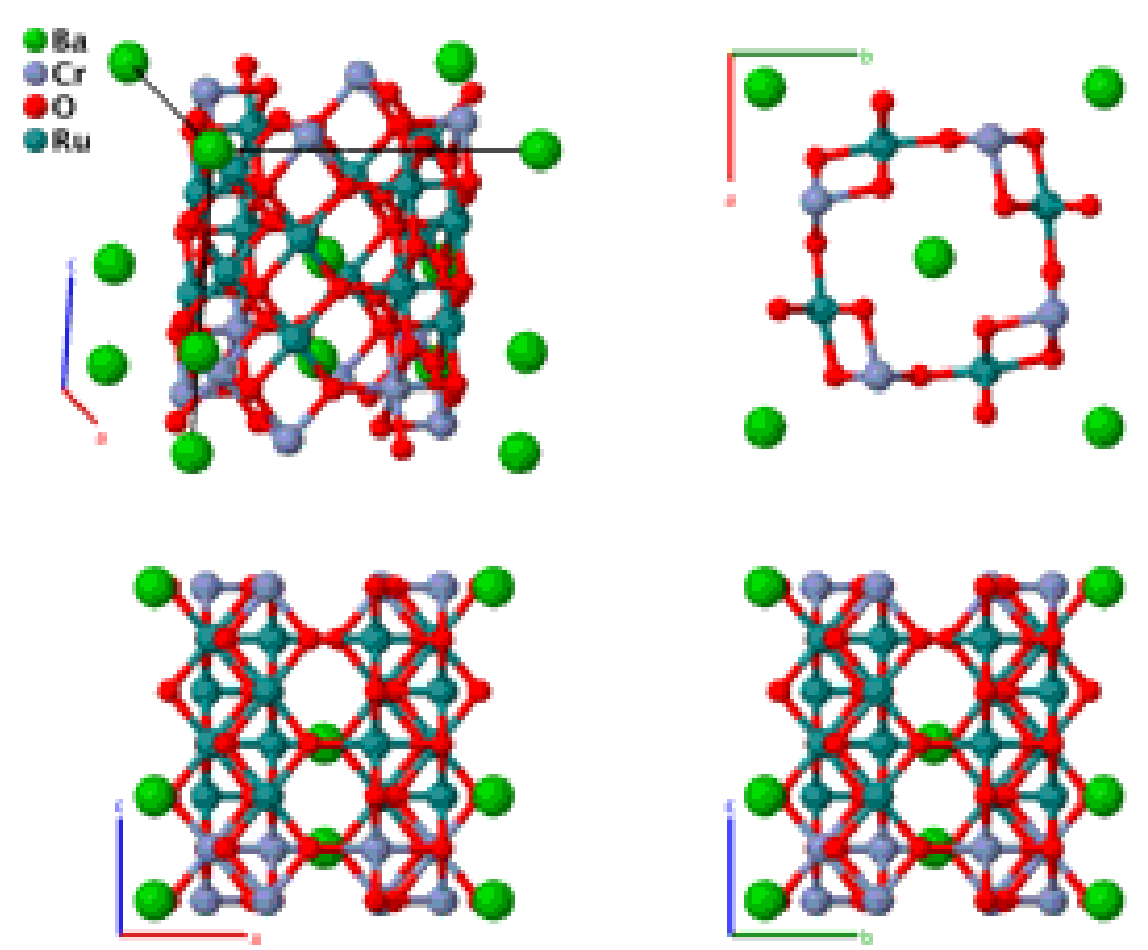

\section{Prototype}

AFLOW prototype label

: $\quad \mathrm{BaCr}_{2} \mathrm{Ru}_{4} \mathrm{O}_{12}$

Strukturbericht designation

Pearson symbol

: AB2C12D4_tP76_75_2a2b_2d_12d_4d

Space group number

: None

Space group symbol

$: \quad \mathrm{tP76}$

AFLOW prototype command

: 75

: $\quad P 4$

aflow --proto=AB2C12D4_tP76_75_2a2b_2d_12d_4d

- -params $=a, c / a, z_{1}, z_{2}, z_{3}, z_{4}, x_{5}, y_{5}, z_{5}, x_{6}, y_{6}, z_{6}, x_{7}, y_{7}, z_{7}, x_{8}, y_{8}, z_{8}, x_{9}, y_{9}, z_{9}$, $x_{10}, y_{10}, z_{10}, x_{11}, y_{11}, z_{11}, x_{12}, y_{12}, z_{12}, x_{13}, y_{13}, z_{13}, x_{14}, y_{14}, z_{14}, x_{15}, y_{15}, z_{15}, x_{16}, y_{16}$, $z_{16}, x_{17}, y_{17}, z_{17}, x_{18}, y_{18}, z_{18}, x_{19}, y_{19}, z_{19}, x_{20}, y_{20}, z_{20}, x_{21}, y_{21}, z_{21}, x_{22}, y_{22}, z_{22}$

Simple Tetragonal primitive vectors:

$$
\begin{aligned}
& \mathbf{a}_{1}=a \hat{\mathbf{x}} \\
& \mathbf{a}_{2}=a \hat{\mathbf{y}} \\
& \mathbf{a}_{3}=c \hat{\mathbf{z}}
\end{aligned}
$$

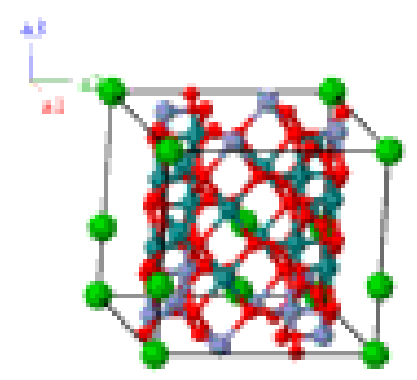

Basis vectors: 


\begin{tabular}{|c|c|c|c|c|c|c|}
\hline $\mathbf{B}_{1}$ & $=$ & $z_{1} \mathbf{a}_{3}$ & $=$ & $z_{1} c \hat{\mathbf{z}}$ & $(1 a)$ & $\mathrm{Ba} \mathrm{I}$ \\
\hline $\mathbf{B}_{2}$ & $=$ & $z_{2} \mathbf{a}_{3}$ & $=$ & $z_{2} c \hat{\mathbf{z}}$ & $(1 a)$ & Ba II \\
\hline $\mathbf{B}_{3}$ & $=$ & $\frac{1}{2} \mathbf{a}_{1}+\frac{1}{2} \mathbf{a}_{2}+z_{3} \mathbf{a}_{3}$ & $=$ & $\frac{1}{2} a \hat{\mathbf{x}}+\frac{1}{2} a \hat{\mathbf{y}}+z_{3} c \hat{\mathbf{z}}$ & $(1 b)$ & Ba III \\
\hline $\mathbf{B}_{4}$ & $=$ & $\frac{1}{2} \mathbf{a}_{1}+\frac{1}{2} \mathbf{a}_{2}+z_{4} \mathbf{a}_{3}$ & $=$ & $\frac{1}{2} a \hat{\mathbf{x}}+\frac{1}{2} a \hat{\mathbf{y}}+z_{4} c \hat{\mathbf{z}}$ & $(1 b)$ & $\mathrm{Ba} I V$ \\
\hline $\mathbf{B}_{5}$ & $=$ & $x_{5} \mathbf{a}_{1}+y_{5} \mathbf{a}_{2}+z_{5} \mathbf{a}_{3}$ & $=$ & $x_{5} a \hat{\mathbf{x}}+y_{5} a \hat{\mathbf{y}}+z_{5} c \hat{\mathbf{z}}$ & $(4 d)$ & $\mathrm{Cr} \mathrm{I}$ \\
\hline $\mathbf{B}_{6}$ & $=$ & $-x_{5} \mathbf{a}_{1}-y_{5} \mathbf{a}_{2}+z_{5} \mathbf{a}_{3}$ & $=$ & $-x_{5} a \hat{\mathbf{x}}-y_{5} a \hat{\mathbf{y}}+z_{5} c \hat{\mathbf{z}}$ & $(4 d)$ & $\mathrm{Cr} \mathrm{I}$ \\
\hline $\mathbf{B}_{7}$ & $=$ & $-y_{5} \mathbf{a}_{1}+x_{5} \mathbf{a}_{2}+z_{5} \mathbf{a}_{3}$ & $=$ & $-y_{5} a \hat{\mathbf{x}}+x_{5} a \hat{\mathbf{y}}+z_{5} c \hat{\mathbf{z}}$ & $(4 d)$ & $\mathrm{Cr} \mathrm{I}$ \\
\hline $\mathbf{B}_{8}$ & $=$ & $y_{5} \mathbf{a}_{1}-x_{5} \mathbf{a}_{2}+z_{5} \mathbf{a}_{3}$ & $=$ & $y_{5} a \hat{\mathbf{x}}-x_{5} a \hat{\mathbf{y}}+z_{5} c \hat{\mathbf{z}}$ & $(4 d)$ & $\mathrm{Cr} \mathrm{I}$ \\
\hline $\mathbf{B}_{9}$ & $=$ & $x_{6} \mathbf{a}_{1}+y_{6} \mathbf{a}_{2}+z_{6} \mathbf{a}_{3}$ & $=$ & $x_{6} a \hat{\mathbf{x}}+y_{6} a \hat{\mathbf{y}}+z_{6} c \hat{\mathbf{z}}$ & $(4 d)$ & Cr II \\
\hline $\mathbf{B}_{10}$ & $=$ & $-x_{6} \mathbf{a}_{1}-y_{6} \mathbf{a}_{2}+z_{6} \mathbf{a}_{3}$ & $=$ & $-x_{6} a \hat{\mathbf{x}}-y_{6} a \hat{\mathbf{y}}+z_{6} c \hat{\mathbf{z}}$ & $(4 d)$ & Cr II \\
\hline $\mathbf{B}_{11}$ & $=$ & $-y_{6} \mathbf{a}_{1}+x_{6} \mathbf{a}_{2}+z_{6} \mathbf{a}_{3}$ & $=$ & $-y_{6} a \hat{\mathbf{x}}+x_{6} a \hat{\mathbf{y}}+z_{6} c \hat{\mathbf{z}}$ & $(4 d)$ & Cr II \\
\hline $\mathbf{B}_{12}$ & $=$ & $y_{6} \mathbf{a}_{1}-x_{6} \mathbf{a}_{2}+z_{6} \mathbf{a}_{3}$ & $=$ & $y_{6} a \hat{\mathbf{x}}-x_{6} a \hat{\mathbf{y}}+z_{6} c \hat{\mathbf{z}}$ & $(4 d)$ & Cr II \\
\hline $\mathbf{B}_{13}$ & $=$ & $x_{7} \mathbf{a}_{1}+y_{7} \mathbf{a}_{2}+z_{7} \mathbf{a}_{3}$ & $=$ & $x_{7} a \hat{\mathbf{x}}+y_{7} a \hat{\mathbf{y}}+z_{7} c \hat{\mathbf{z}}$ & $(4 d)$ & $\mathrm{O} \mathrm{I}$ \\
\hline $\mathbf{B}_{14}$ & $=$ & $-x_{7} \mathbf{a}_{1}-y_{7} \mathbf{a}_{2}+z_{7} \mathbf{a}_{3}$ & $=$ & $-x_{7} a \hat{\mathbf{x}}-y_{7} a \hat{\mathbf{y}}+z_{7} c \hat{\mathbf{z}}$ & $(4 d)$ & O I \\
\hline $\mathbf{B}_{15}$ & $=$ & $-y_{7} \mathbf{a}_{1}+x_{7} \mathbf{a}_{2}+z_{7} \mathbf{a}_{3}$ & $=$ & $-y_{7} a \hat{\mathbf{x}}+x_{7} a \hat{\mathbf{y}}+z_{7} c \hat{\mathbf{z}}$ & $(4 d)$ & O I \\
\hline $\mathbf{B}_{16}$ & $=$ & $y_{7} \mathbf{a}_{1}-x_{7} \mathbf{a}_{2}+z_{7} \mathbf{a}_{3}$ & $=$ & $y_{7} a \hat{\mathbf{x}}-x_{7} a \hat{\mathbf{y}}+z_{7} c \hat{\mathbf{z}}$ & $(4 d)$ & $\mathrm{O} \mathrm{I}$ \\
\hline $\mathbf{B}_{17}$ & $=$ & $x_{8} \mathbf{a}_{1}+y_{8} \mathbf{a}_{2}+z_{8} \mathbf{a}_{3}$ & $=$ & $x_{8} a \hat{\mathbf{x}}+y_{8} a \hat{\mathbf{y}}+z_{8} c \hat{\mathbf{z}}$ & $(4 d)$ & O II \\
\hline $\mathbf{B}_{18}$ & $=$ & $-x_{8} \mathbf{a}_{1}-y_{8} \mathbf{a}_{2}+z_{8} \mathbf{a}_{3}$ & $=$ & $-x_{8} a \hat{\mathbf{x}}-y_{8} a \hat{\mathbf{y}}+z_{8} c \hat{\mathbf{z}}$ & $(4 d)$ & O II \\
\hline $\mathbf{B}_{19}$ & $=$ & $-y_{8} \mathbf{a}_{1}+x_{8} \mathbf{a}_{2}+z_{8} \mathbf{a}_{3}$ & $=$ & $-y_{8} a \hat{\mathbf{x}}+x_{8} a \hat{\mathbf{y}}+z_{8} c \hat{\mathbf{z}}$ & $(4 d)$ & $\mathrm{O}$ II \\
\hline $\mathbf{B}_{20}$ & $=$ & $y_{8} \mathbf{a}_{1}-x_{8} \mathbf{a}_{2}+z_{8} \mathbf{a}_{3}$ & $=$ & $y_{8} a \hat{\mathbf{x}}-x_{8} a \hat{\mathbf{y}}+z_{8} c \hat{\mathbf{z}}$ & $(4 d)$ & O II \\
\hline $\mathbf{B}_{21}$ & $=$ & $x_{9} \mathbf{a}_{1}+y_{9} \mathbf{a}_{2}+z_{9} \mathbf{a}_{3}$ & $=$ & $x_{9} a \hat{\mathbf{x}}+y_{9} a \hat{\mathbf{y}}+z_{9} c \hat{\mathbf{z}}$ & $(4 d)$ & O III \\
\hline $\mathbf{B}_{22}$ & $=$ & $-x_{9} \mathbf{a}_{1}-y_{9} \mathbf{a}_{2}+z_{9} \mathbf{a}_{3}$ & $=$ & $-x_{9} a \hat{\mathbf{x}}-y_{9} a \hat{\mathbf{y}}+z_{9} c \hat{\mathbf{z}}$ & $(4 d)$ & O III \\
\hline $\mathbf{B}_{23}$ & $=$ & $-y_{9} \mathbf{a}_{1}+x_{9} \mathbf{a}_{2}+z_{9} \mathbf{a}_{3}$ & $=$ & $-y_{9} a \hat{\mathbf{x}}+x_{9} a \hat{\mathbf{y}}+z_{9} c \hat{\mathbf{z}}$ & $(4 d)$ & O III \\
\hline $\mathbf{B}_{24}$ & $=$ & $y_{9} \mathbf{a}_{1}-x_{9} \mathbf{a}_{2}+z_{9} \mathbf{a}_{3}$ & $=$ & $y_{9} a \hat{\mathbf{x}}-x_{9} a \hat{\mathbf{y}}+z_{9} c \hat{\mathbf{z}}$ & $(4 d)$ & O III \\
\hline $\mathbf{B}_{25}$ & $=$ & $x_{10} \mathbf{a}_{1}+y_{10} \mathbf{a}_{2}+z_{10} \mathbf{a}_{3}$ & $=$ & $x_{10} a \hat{\mathbf{x}}+y_{10} a \hat{\mathbf{y}}+z_{10} c \hat{\mathbf{z}}$ & $(4 d)$ & O IV \\
\hline $\mathbf{B}_{26}$ & $=$ & $-x_{10} \mathbf{a}_{1}-y_{10} \mathbf{a}_{2}+z_{10} \mathbf{a}_{3}$ & $=$ & $-x_{10} a \hat{\mathbf{x}}-y_{10} a \hat{\mathbf{y}}+z_{10} c \hat{\mathbf{z}}$ & $(4 d)$ & O IV \\
\hline $\mathbf{B}_{27}$ & $=$ & $-y_{10} \mathbf{a}_{1}+x_{10} \mathbf{a}_{2}+z_{10} \mathbf{a}_{3}$ & $=$ & $-y_{10} a \hat{\mathbf{x}}+x_{10} a \hat{\mathbf{y}}+z_{10} c \hat{\mathbf{z}}$ & $(4 d)$ & O IV \\
\hline $\mathbf{B}_{28}$ & $=$ & $y_{10} \mathbf{a}_{1}-x_{10} \mathbf{a}_{2}+z_{10} \mathbf{a}_{3}$ & $=$ & $y_{10} a \hat{\mathbf{x}}-x_{10} a \hat{\mathbf{y}}+z_{10} c \hat{\mathbf{z}}$ & $(4 d)$ & O IV \\
\hline $\mathbf{B}_{29}$ & $=$ & $x_{11} \mathbf{a}_{1}+y_{11} \mathbf{a}_{2}+z_{11} \mathbf{a}_{3}$ & $=$ & $x_{11} a \hat{\mathbf{x}}+y_{11} a \hat{\mathbf{y}}+z_{11} c \hat{\mathbf{z}}$ & $(4 d)$ & $\mathrm{OV}$ \\
\hline $\mathbf{B}_{30}$ & $=$ & $-x_{11} \mathbf{a}_{1}-y_{11} \mathbf{a}_{2}+z_{11} \mathbf{a}_{3}$ & $=$ & $-x_{11} a \hat{\mathbf{x}}-y_{11} a \hat{\mathbf{y}}+z_{11} c \hat{\mathbf{z}}$ & $(4 d)$ & $\mathrm{OV}$ \\
\hline $\mathbf{B}_{31}$ & $=$ & $-y_{11} \mathbf{a}_{1}+x_{11} \mathbf{a}_{2}+z_{11} \mathbf{a}_{3}$ & $=$ & $-y_{11} a \hat{\mathbf{x}}+x_{11} a \hat{\mathbf{y}}+z_{11} c \hat{\mathbf{z}}$ & $(4 d)$ & $\mathrm{OV}$ \\
\hline $\mathbf{B}_{32}$ & $=$ & $y_{11} \mathbf{a}_{1}-x_{11} \mathbf{a}_{2}+z_{11} \mathbf{a}_{3}$ & $=$ & $y_{11} a \hat{\mathbf{x}}-x_{11} a \hat{\mathbf{y}}+z_{11} c \hat{\mathbf{z}}$ & $(4 d)$ & $\mathrm{OV}$ \\
\hline $\mathbf{B}_{33}$ & $=$ & $x_{12} \mathbf{a}_{1}+y_{12} \mathbf{a}_{2}+z_{12} \mathbf{a}_{3}$ & $=$ & $x_{12} a \hat{\mathbf{x}}+y_{12} a \hat{\mathbf{y}}+z_{12} c \hat{\mathbf{z}}$ & $(4 d)$ & $\mathrm{O}$ VI \\
\hline $\mathbf{B}_{34}$ & $=$ & $-x_{12} \mathbf{a}_{1}-y_{12} \mathbf{a}_{2}+z_{12} \mathbf{a}_{3}$ & $=$ & $-x_{12} a \hat{\mathbf{x}}-y_{12} a \hat{\mathbf{y}}+z_{12} c \hat{\mathbf{z}}$ & $(4 d)$ & $\mathrm{O}$ VI \\
\hline $\mathbf{B}_{35}$ & $=$ & $-y_{12} \mathbf{a}_{1}+x_{12} \mathbf{a}_{2}+z_{12} \mathbf{a}_{3}$ & $=$ & $-y_{12} a \hat{\mathbf{x}}+x_{12} a \hat{\mathbf{y}}+z_{12} c \hat{\mathbf{z}}$ & $(4 d)$ & O VI \\
\hline $\mathbf{B}_{36}$ & $=$ & $y_{12} \mathbf{a}_{1}-x_{12} \mathbf{a}_{2}+z_{12} \mathbf{a}_{3}$ & $=$ & $y_{12} a \hat{\mathbf{x}}-x_{12} a \hat{\mathbf{y}}+z_{12} c \hat{\mathbf{z}}$ & $(4 d)$ & $\mathrm{O} \mathrm{VI}$ \\
\hline
\end{tabular}




\begin{tabular}{|c|c|c|c|c|c|c|}
\hline $\mathbf{B}_{37}$ & $=$ & $x_{13} \mathbf{a}_{1}+y_{13} \mathbf{a}_{2}+z_{13} \mathbf{a}_{3}$ & $=$ & $x_{13} a \hat{\mathbf{x}}+y_{13} a \hat{\mathbf{y}}+z_{13} c \hat{\mathbf{z}}$ & $(4 d)$ & O VII \\
\hline $\mathbf{B}_{38}$ & $=$ & $-x_{13} \mathbf{a}_{1}-y_{13} \mathbf{a}_{2}+z_{13} \mathbf{a}_{3}$ & $=$ & $-x_{13} a \hat{\mathbf{x}}-y_{13} a \hat{\mathbf{y}}+z_{13} c \hat{\mathbf{z}}$ & $(4 d)$ & O VII \\
\hline $\mathbf{B}_{39}$ & $=$ & $-y_{13} \mathbf{a}_{1}+x_{13} \mathbf{a}_{2}+z_{13} \mathbf{a}_{3}$ & $=$ & $-y_{13} a \hat{\mathbf{x}}+x_{13} a \hat{\mathbf{y}}+z_{13} c \hat{\mathbf{z}}$ & $(4 d)$ & O VII \\
\hline $\mathbf{B}_{40}$ & $=$ & $y_{13} \mathbf{a}_{1}-x_{13} \mathbf{a}_{2}+z_{13} \mathbf{a}_{3}$ & $=$ & $y_{13} a \hat{\mathbf{x}}-x_{13} a \hat{\mathbf{y}}+z_{13} c \hat{\mathbf{z}}$ & $(4 d)$ & O VII \\
\hline $\mathbf{B}_{41}$ & $=$ & $x_{14} \mathbf{a}_{1}+y_{14} \mathbf{a}_{2}+z_{14} \mathbf{a}_{3}$ & $=$ & $x_{14} a \hat{\mathbf{x}}+y_{14} a \hat{\mathbf{y}}+z_{14} c \hat{\mathbf{z}}$ & $(4 d)$ & O VIII \\
\hline $\mathbf{B}_{42}$ & $=$ & $-x_{14} \mathbf{a}_{1}-y_{14} \mathbf{a}_{2}+z_{14} \mathbf{a}_{3}$ & $=$ & $-x_{14} a \hat{\mathbf{x}}-y_{14} a \hat{\mathbf{y}}+z_{14} c \hat{\mathbf{z}}$ & $(4 d)$ & O VIII \\
\hline $\mathbf{B}_{43}$ & $=$ & $-y_{14} \mathbf{a}_{1}+x_{14} \mathbf{a}_{2}+z_{14} \mathbf{a}_{3}$ & $=$ & $-y_{14} a \hat{\mathbf{x}}+x_{14} a \hat{\mathbf{y}}+z_{14} c \hat{\mathbf{z}}$ & $(4 d)$ & O VIII \\
\hline $\mathbf{B}_{44}$ & $=$ & $y_{14} \mathbf{a}_{1}-x_{14} \mathbf{a}_{2}+z_{14} \mathbf{a}_{3}$ & $=$ & $y_{14} a \hat{\mathbf{x}}-x_{14} a \hat{\mathbf{y}}+z_{14} c \hat{\mathbf{z}}$ & $(4 d)$ & O VIII \\
\hline $\mathbf{B}_{45}$ & $=$ & $x_{15} \mathbf{a}_{1}+y_{15} \mathbf{a}_{2}+z_{15} \mathbf{a}_{3}$ & $=$ & $x_{15} a \hat{\mathbf{x}}+y_{15} a \hat{\mathbf{y}}+z_{15} c \hat{\mathbf{z}}$ & $(4 d)$ & O IX \\
\hline $\mathbf{B}_{46}$ & $=$ & $-x_{15} \mathbf{a}_{1}-y_{15} \mathbf{a}_{2}+z_{15} \mathbf{a}_{3}$ & $=$ & $-x_{15} a \hat{\mathbf{x}}-y_{15} a \hat{\mathbf{y}}+z_{15} c \hat{\mathbf{z}}$ & $(4 d)$ & O IX \\
\hline $\mathbf{B}_{47}$ & $=$ & $-y_{15} \mathbf{a}_{1}+x_{15} \mathbf{a}_{2}+z_{15} \mathbf{a}_{3}$ & $=$ & $-y_{15} a \hat{\mathbf{x}}+x_{15} a \hat{\mathbf{y}}+z_{15} c \hat{\mathbf{z}}$ & $(4 d)$ & O IX \\
\hline $\mathbf{B}_{48}$ & $=$ & $y_{15} \mathbf{a}_{1}-x_{15} \mathbf{a}_{2}+z_{15} \mathbf{a}_{3}$ & $=$ & $y_{15} a \hat{\mathbf{x}}-x_{15} a \hat{\mathbf{y}}+z_{15} c \hat{\mathbf{z}}$ & $(4 d)$ & O IX \\
\hline $\mathbf{B}_{49}$ & $=$ & $x_{16} \mathbf{a}_{1}+y_{16} \mathbf{a}_{2}+z_{16} \mathbf{a}_{3}$ & $=$ & $x_{16} a \hat{\mathbf{x}}+y_{16} a \hat{\mathbf{y}}+z_{16} c \hat{\mathbf{z}}$ & $(4 d)$ & $\mathrm{OX}$ \\
\hline $\mathbf{B}_{50}$ & $=$ & $-x_{16} \mathbf{a}_{1}-y_{16} \mathbf{a}_{2}+z_{16} \mathbf{a}_{3}$ & $=$ & $-x_{16} a \hat{\mathbf{x}}-y_{16} a \hat{\mathbf{y}}+z_{16} c \hat{\mathbf{z}}$ & $(4 d)$ & $\mathrm{OX}$ \\
\hline $\mathbf{B}_{51}$ & $=$ & $-y_{16} \mathbf{a}_{1}+x_{16} \mathbf{a}_{2}+z_{16} \mathbf{a}_{3}$ & $=$ & $-y_{16} a \hat{\mathbf{x}}+x_{16} a \hat{\mathbf{y}}+z_{16} c \hat{\mathbf{z}}$ & $(4 d)$ & $\mathrm{OX}$ \\
\hline $\mathbf{B}_{52}$ & $=$ & $y_{16} \mathbf{a}_{1}-x_{16} \mathbf{a}_{2}+z_{16} \mathbf{a}_{3}$ & $=$ & $y_{16} a \hat{\mathbf{x}}-x_{16} a \hat{\mathbf{y}}+z_{16} c \hat{\mathbf{z}}$ & $(4 d)$ & $\mathrm{OX}$ \\
\hline $\mathbf{B}_{53}$ & $=$ & $x_{17} \mathbf{a}_{1}+y_{17} \mathbf{a}_{2}+z_{17} \mathbf{a}_{3}$ & $=$ & $x_{17} a \hat{\mathbf{x}}+y_{17} a \hat{\mathbf{y}}+z_{17} c \hat{\mathbf{z}}$ & $(4 d)$ & $\mathrm{O} X \mathrm{XI}$ \\
\hline $\mathbf{B}_{54}$ & $=$ & $-x_{17} \mathbf{a}_{1}-y_{17} \mathbf{a}_{2}+z_{17} \mathbf{a}_{3}$ & $=$ & $-x_{17} a \hat{\mathbf{x}}-y_{17} a \hat{\mathbf{y}}+z_{17} c \hat{\mathbf{z}}$ & $(4 d)$ & $\mathrm{O} X \mathrm{XI}$ \\
\hline $\mathbf{B}_{55}$ & $=$ & $-y_{17} \mathbf{a}_{1}+x_{17} \mathbf{a}_{2}+z_{17} \mathbf{a}_{3}$ & $=$ & $-y_{17} a \hat{\mathbf{x}}+x_{17} a \hat{\mathbf{y}}+z_{17} c \hat{\mathbf{z}}$ & $(4 d)$ & O XI \\
\hline $\mathbf{B}_{56}$ & $=$ & $y_{17} \mathbf{a}_{1}-x_{17} \mathbf{a}_{2}+z_{17} \mathbf{a}_{3}$ & $=$ & $y_{17} a \hat{\mathbf{x}}-x_{17} a \hat{\mathbf{y}}+z_{17} c \hat{\mathbf{z}}$ & $(4 d)$ & $\mathrm{O} X \mathrm{XI}$ \\
\hline $\mathbf{B}_{57}$ & $=$ & $x_{18} \mathbf{a}_{1}+y_{18} \mathbf{a}_{2}+z_{18} \mathbf{a}_{3}$ & $=$ & $x_{18} a \hat{\mathbf{x}}+y_{18} a \hat{\mathbf{y}}+z_{18} c \hat{\mathbf{z}}$ & $(4 d)$ & O XII \\
\hline $\mathbf{B}_{58}$ & $=$ & $-x_{18} \mathbf{a}_{1}-y_{18} \mathbf{a}_{2}+z_{18} \mathbf{a}_{3}$ & $=$ & $-x_{18} a \hat{\mathbf{x}}-y_{18} a \hat{\mathbf{y}}+z_{18} c \hat{\mathbf{z}}$ & $(4 d)$ & O XII \\
\hline $\mathbf{B}_{59}$ & $=$ & $-y_{18} \mathbf{a}_{1}+x_{18} \mathbf{a}_{2}+z_{18} \mathbf{a}_{3}$ & $=$ & $-y_{18} a \hat{\mathbf{x}}+x_{18} a \hat{\mathbf{y}}+z_{18} c \hat{\mathbf{z}}$ & $(4 d)$ & O XII \\
\hline $\mathbf{B}_{60}$ & $=$ & $y_{18} \mathbf{a}_{1}-x_{18} \mathbf{a}_{2}+z_{18} \mathbf{a}_{3}$ & $=$ & $y_{18} a \hat{\mathbf{x}}-x_{18} a \hat{\mathbf{y}}+z_{18} c \hat{\mathbf{z}}$ & $(4 d)$ & O XII \\
\hline $\mathbf{B}_{61}$ & $=$ & $x_{19} \mathbf{a}_{1}+y_{19} \mathbf{a}_{2}+z_{19} \mathbf{a}_{3}$ & $=$ & $x_{19} a \hat{\mathbf{x}}+y_{19} a \hat{\mathbf{y}}+z_{19} c \hat{\mathbf{z}}$ & $(4 d)$ & $\mathrm{Ru} I$ \\
\hline $\mathbf{B}_{62}$ & $=$ & $-x_{19} \mathbf{a}_{1}-y_{19} \mathbf{a}_{2}+z_{19} \mathbf{a}_{3}$ & $=$ & $-x_{19} a \hat{\mathbf{x}}-y_{19} a \hat{\mathbf{y}}+z_{19} c \hat{\mathbf{z}}$ & $(4 d)$ & $\mathrm{Ru} \mathrm{I}$ \\
\hline $\mathbf{B}_{63}$ & $=$ & $-y_{19} \mathbf{a}_{1}+x_{19} \mathbf{a}_{2}+z_{19} \mathbf{a}_{3}$ & $=$ & $-y_{19} a \hat{\mathbf{x}}+x_{19} a \hat{\mathbf{y}}+z_{19} c \hat{\mathbf{z}}$ & $(4 d)$ & $\mathrm{Ru} I$ \\
\hline $\mathbf{B}_{64}$ & $=$ & $y_{19} \mathbf{a}_{1}-x_{19} \mathbf{a}_{2}+z_{19} \mathbf{a}_{3}$ & $=$ & $y_{19} a \hat{\mathbf{x}}-x_{19} a \hat{\mathbf{y}}+z_{19} c \hat{\mathbf{z}}$ & $(4 d)$ & $\mathrm{Ru} I$ \\
\hline $\mathbf{B}_{65}$ & $=$ & $x_{20} \mathbf{a}_{1}+y_{20} \mathbf{a}_{2}+z_{20} \mathbf{a}_{3}$ & $=$ & $x_{20} a \hat{\mathbf{x}}+y_{20} a \hat{\mathbf{y}}+z_{20} c \hat{\mathbf{z}}$ & $(4 d)$ & $\mathrm{Ru}$ II \\
\hline $\mathbf{B}_{66}$ & $=$ & $-x_{20} \mathbf{a}_{1}-y_{20} \mathbf{a}_{2}+z_{20} \mathbf{a}_{3}$ & $=$ & $-x_{20} a \hat{\mathbf{x}}-y_{20} a \hat{\mathbf{y}}+z_{20} c \hat{\mathbf{z}}$ & $(4 d)$ & $\mathrm{Ru} I I$ \\
\hline $\mathbf{B}_{67}$ & $=$ & $-y_{20} \mathbf{a}_{1}+x_{20} \mathbf{a}_{2}+z_{20} \mathbf{a}_{3}$ & $=$ & $-y_{20} a \hat{\mathbf{x}}+x_{20} a \hat{\mathbf{y}}+z_{20} c \hat{\mathbf{z}}$ & $(4 d)$ & $\mathrm{Ru}$ II \\
\hline $\mathbf{B}_{68}$ & $=$ & $y_{20} \mathbf{a}_{1}-x_{20} \mathbf{a}_{2}+z_{20} \mathbf{a}_{3}$ & $=$ & $y_{20} a \hat{\mathbf{x}}-x_{20} a \hat{\mathbf{y}}+z_{20} c \hat{\mathbf{z}}$ & $(4 d)$ & $\mathrm{Ru}$ II \\
\hline $\mathbf{B}_{69}$ & $=$ & $x_{21} \mathbf{a}_{1}+y_{21} \mathbf{a}_{2}+z_{21} \mathbf{a}_{3}$ & $=$ & $x_{21} a \hat{\mathbf{x}}+y_{21} a \hat{\mathbf{y}}+z_{21} c \hat{\mathbf{z}}$ & $(4 d)$ & $\mathrm{Ru}$ III \\
\hline $\mathbf{B}_{70}$ & $=$ & $-x_{21} \mathbf{a}_{1}-y_{21} \mathbf{a}_{2}+z_{21} \mathbf{a}_{3}$ & $=$ & $-x_{21} a \hat{\mathbf{x}}-y_{21} a \hat{\mathbf{y}}+z_{21} c \hat{\mathbf{z}}$ & $(4 d)$ & $\mathrm{Ru}$ III \\
\hline $\mathbf{B}_{71}$ & $=$ & $-y_{21} \mathbf{a}_{1}+x_{21} \mathbf{a}_{2}+z_{21} \mathbf{a}_{3}$ & $=$ & $-y_{21} a \hat{\mathbf{x}}+x_{21} a \hat{\mathbf{y}}+z_{21} c \hat{\mathbf{z}}$ & $(4 d)$ & $\mathrm{Ru}$ III \\
\hline $\mathbf{B}_{72}$ & $=$ & $y_{21} \mathbf{a}_{1}-x_{21} \mathbf{a}_{2}+z_{21} \mathbf{a}_{3}$ & $=$ & $y_{21} a \hat{\mathbf{x}}-x_{21} a \hat{\mathbf{y}}+z_{21} c \hat{\mathbf{z}}$ & $(4 d)$ & $\mathrm{Ru}$ III \\
\hline
\end{tabular}




\begin{tabular}{|c|c|c|c|c|c|c|}
\hline $\mathbf{B}_{73}$ & $=$ & $x_{22} \mathbf{a}_{1}+y_{22} \mathbf{a}_{2}+z_{22} \mathbf{a}_{3}$ & $=$ & $x_{22} a \hat{\mathbf{x}}+y_{22} a \hat{\mathbf{y}}+z_{22} c \hat{\mathbf{z}}$ & $(4 d)$ & $\mathrm{Ru} I V$ \\
\hline $\mathbf{B}_{74}$ & $=$ & $-x_{22} \mathbf{a}_{1}-y_{22} \mathbf{a}_{2}+z_{22} \mathbf{a}_{3}$ & $=$ & $-x_{22} a \hat{\mathbf{x}}-y_{22} a \hat{\mathbf{y}}+z_{22} c \hat{\mathbf{z}}$ & $(4 d)$ & $\mathrm{Ru} I V$ \\
\hline $\mathbf{B}_{75}$ & $=$ & $-y_{22} \mathbf{a}_{1}+x_{22} \mathbf{a}_{2}+z_{22} \mathbf{a}_{3}$ & $=$ & $-y_{22} a \hat{\mathbf{x}}+x_{22} a \hat{\mathbf{y}}+z_{22} c \hat{\mathbf{z}}$ & $(4 d)$ & $\mathrm{Ru} I V$ \\
\hline $\mathbf{B}_{76}$ & $=$ & $y_{22} \mathbf{a}_{1}-x_{22} \mathbf{a}_{2}+z_{22} \mathbf{a}_{3}$ & $=$ & $y_{22} a \hat{\mathbf{x}}-x_{22} a \hat{\mathbf{y}}+z_{22} c \hat{\mathbf{z}}$ & $(4 d)$ & $\mathrm{Ru}$ IV \\
\hline
\end{tabular}

\section{References:}

- M. C. Cadée and A. Prodan, Tripling of the short axis in the hollandite structure, Mater. Res. Bull. 14, 613-618 (1979), doi:10.1016/0025-5408(79)90043-6.

\section{Found in:}

- P. Villars and K. Cenzual, Pearson's Crystal Data - Crystal Structure Database for Inorganic Compounds, ASM International (2013).

\section{Geometry files:}

- CIF: pp. 857

- POSCAR: pp. 857 


\section{LaRhC 2 Structure: A2BC_tP16_76_2a_a_a}
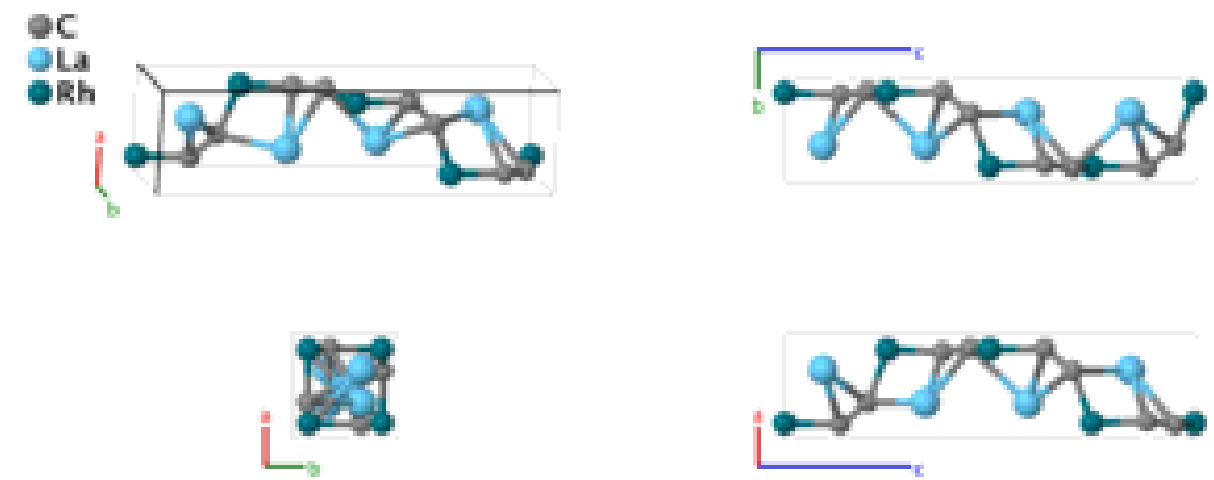

\section{Prototype}

AFLOW prototype label

Strukturbericht designation

Pearson symbol

Space group number

Space group symbol

AFLOW prototype command
: $\quad \mathrm{LaRhC}_{2}$

: A2BC_tP16_76_2a_a_a

: None

$: \quad \mathrm{tP} 16$

: $\quad 76$

: $\quad P 4_{1}$

aflow --proto=A2BC_tP16_76_2a_a_a

- -params $=a, c / a, x_{1}, y_{1}, z_{1}, x_{2}, y_{2}, z_{2}, x_{3}, y_{3}, z_{3}, x_{4}, y_{4}, z_{4}$

\section{Simple Tetragonal primitive vectors:}

$$
\begin{aligned}
& \mathbf{a}_{1}=a \hat{\mathbf{x}} \\
& \mathbf{a}_{2}=a \hat{\mathbf{y}} \\
& \mathbf{a}_{3}=c \hat{\mathbf{z}}
\end{aligned}
$$

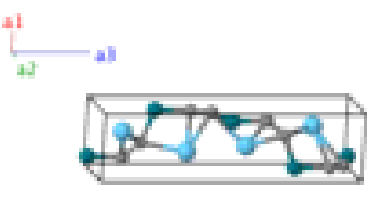

Basis vectors:

\section{Lattice Coordinates}

$\mathbf{B}_{1}=$

$\mathbf{B}_{2}=$

$\mathbf{B}_{3}$

$\mathbf{B}_{4}$

$\mathbf{B}_{5}=$

$\mathbf{B}_{6}=-x_{2} \mathbf{a}_{1}-y_{2} \mathbf{a}_{2}+\left(\frac{1}{2}+z_{2}\right) \mathbf{a}_{3}$

$\mathbf{B}_{7}=-y_{2} \mathbf{a}_{1}+x_{2} \mathbf{a}_{2}+\left(\frac{1}{4}+z_{2}\right) \mathbf{a}_{3}$

$\mathbf{B}_{8}=y_{2} \mathbf{a}_{1}-x_{2} \mathbf{a}_{2}+\left(\frac{3}{4}+z_{2}\right) \mathbf{a}_{3}$

$\mathbf{B}_{9}=$

$\mathbf{B}_{10}=$

$\mathbf{B}_{11}=$

$\mathbf{B}_{12}=$ $x_{1} \mathbf{a}_{1}+y_{1} \mathbf{a}_{2}+z_{1} \mathbf{a}_{3}$

$x_{3} \mathbf{a}_{1}+y_{3} \mathbf{a}_{2}+z_{3} \mathbf{a}_{3}$

$-x_{3} \mathbf{a}_{1}-y_{3} \mathbf{a}_{2}+\left(\frac{1}{2}+z_{3}\right) \mathbf{a}_{3}$

$-y_{3} \mathbf{a}_{1}+x_{3} \mathbf{a}_{2}+\left(\frac{1}{4}+z_{3}\right) \mathbf{a}_{3}$

$y_{3} \mathbf{a}_{1}-x_{3} \mathbf{a}_{2}+\left(\frac{3}{4}+z_{3}\right) \mathbf{a}_{3}$
Cartesian Coordinates

$=\quad x_{1} a \hat{\mathbf{x}}+y_{1} a \hat{\mathbf{y}}+z_{1} c \hat{\mathbf{z}}$

$=\quad-x_{1} a \hat{\mathbf{x}}-y_{1} a \hat{\mathbf{y}}+\left(\frac{1}{2}+z_{1}\right) c \hat{\mathbf{z}}$

$=-y_{1} a \hat{\mathbf{x}}+x_{1} a \hat{\mathbf{y}}+\left(\frac{1}{4}+z_{1}\right) c \hat{\mathbf{z}}$

$=\quad y_{1} a \hat{\mathbf{x}}-x_{1} a \hat{\mathbf{y}}+\left(\frac{3}{4}+z_{1}\right) c \hat{\mathbf{z}}$

$=\quad x_{2} a \hat{\mathbf{x}}+y_{2} a \hat{\mathbf{y}}+z_{2} c \hat{\mathbf{z}}$

$=-x_{2} a \hat{\mathbf{x}}-y_{2} a \hat{\mathbf{y}}+\left(\frac{1}{2}+z_{2}\right) c \hat{\mathbf{z}}$

$=-y_{2} a \hat{\mathbf{x}}+x_{2} a \hat{\mathbf{y}}+\left(\frac{1}{4}+z_{2}\right) c \hat{\mathbf{z}}$

$=\quad y_{2} a \hat{\mathbf{x}}-x_{2} a \hat{\mathbf{y}}+\left(\frac{3}{4}+z_{2}\right) c \hat{\mathbf{z}}$

$=\quad x_{3} a \hat{\mathbf{x}}+y_{3} a \hat{\mathbf{y}}+z_{3} c \hat{\mathbf{z}}$

$=-x_{3} a \hat{\mathbf{x}}-y_{3} a \hat{\mathbf{y}}+\left(\frac{1}{2}+z_{3}\right) c \hat{\mathbf{z}}$

$=-y_{3} a \hat{\mathbf{x}}+x_{3} a \hat{\mathbf{y}}+\left(\frac{1}{4}+z_{3}\right) c \hat{\mathbf{z}}$

$=\quad y_{3} a \hat{\mathbf{x}}-x_{3} a \hat{\mathbf{y}}+\left(\frac{3}{4}+z_{3}\right) c \hat{\mathbf{z}}$
Wyckoff Position
Atom Type

C I

C I

C I

C I

C II

C II

C II

C II

$\mathrm{La}$

$\mathrm{La}$

$\mathrm{La}$

$\mathrm{La}$ 


\begin{tabular}{|c|c|c|c|c|c|c|}
\hline $\mathbf{B}_{13}$ & $=$ & $x_{4} \mathbf{a}_{1}+y_{4} \mathbf{a}_{2}+z_{4} \mathbf{a}_{3}$ & $=$ & $x_{4} a \hat{\mathbf{x}}+y_{4} a \hat{\mathbf{y}}+z_{4} c \hat{\mathbf{z}}$ & $(4 a)$ & $\mathrm{Rh}$ \\
\hline $\mathbf{B}_{14}$ & $=$ & $-x_{4} \mathbf{a}_{1}-y_{4} \mathbf{a}_{2}+\left(\frac{1}{2}+z_{4}\right) \mathbf{a}_{3}$ & $=$ & $-x_{4} a \hat{\mathbf{x}}-y_{4} a \hat{\mathbf{y}}+\left(\frac{1}{2}+z_{4}\right) c \hat{\mathbf{z}}$ & $(4 a)$ & $\mathrm{Rh}$ \\
\hline $\mathbf{B}_{15}$ & $=$ & $-y_{4} \mathbf{a}_{1}+x_{4} \mathbf{a}_{2}+\left(\frac{1}{4}+z_{4}\right) \mathbf{a}_{3}$ & $=$ & $-y_{4} a \hat{\mathbf{x}}+x_{4} a \hat{\mathbf{y}}+\left(\frac{1}{4}+z_{4}\right) c \hat{\mathbf{z}}$ & $(4 a)$ & $\mathrm{Rh}$ \\
\hline $\mathbf{B}_{16}$ & $=$ & $y_{4} \mathbf{a}_{1}-x_{4} \mathbf{a}_{2}+\left(\frac{3}{4}+z_{4}\right) \mathbf{a}_{3}$ & $=$ & $y_{4} a \hat{\mathbf{x}}-x_{4} a \hat{\mathbf{y}}+\left(\frac{3}{4}+z_{4}\right) c \hat{\mathbf{z}}$ & $(4 a)$ & $\mathrm{Rh}$ \\
\hline
\end{tabular}

\section{References:}

- A. O. Tsokol', O. I. Bodak, E. P. Marusin, and V. E. Zavodnik, X-ray diffraction studies of ternary $R R h C_{2}(R=L a, C e, P r$, Nd, Sm) compounds, Kristallografiya 33, 345-348 (1988).

\section{Found in:}

- P. Villars and K. Cenzual, Pearson's Crystal Data - Crystal Structure Database for Inorganic Compounds, ASM International (2013).

\section{Geometry files:}

- CIF: pp. 858

- POSCAR: pp. 858 


\section{$\mathrm{Cs}_{3} \mathrm{P}_{7}$ Structure: A3B7_tP40_76_3a_7a}
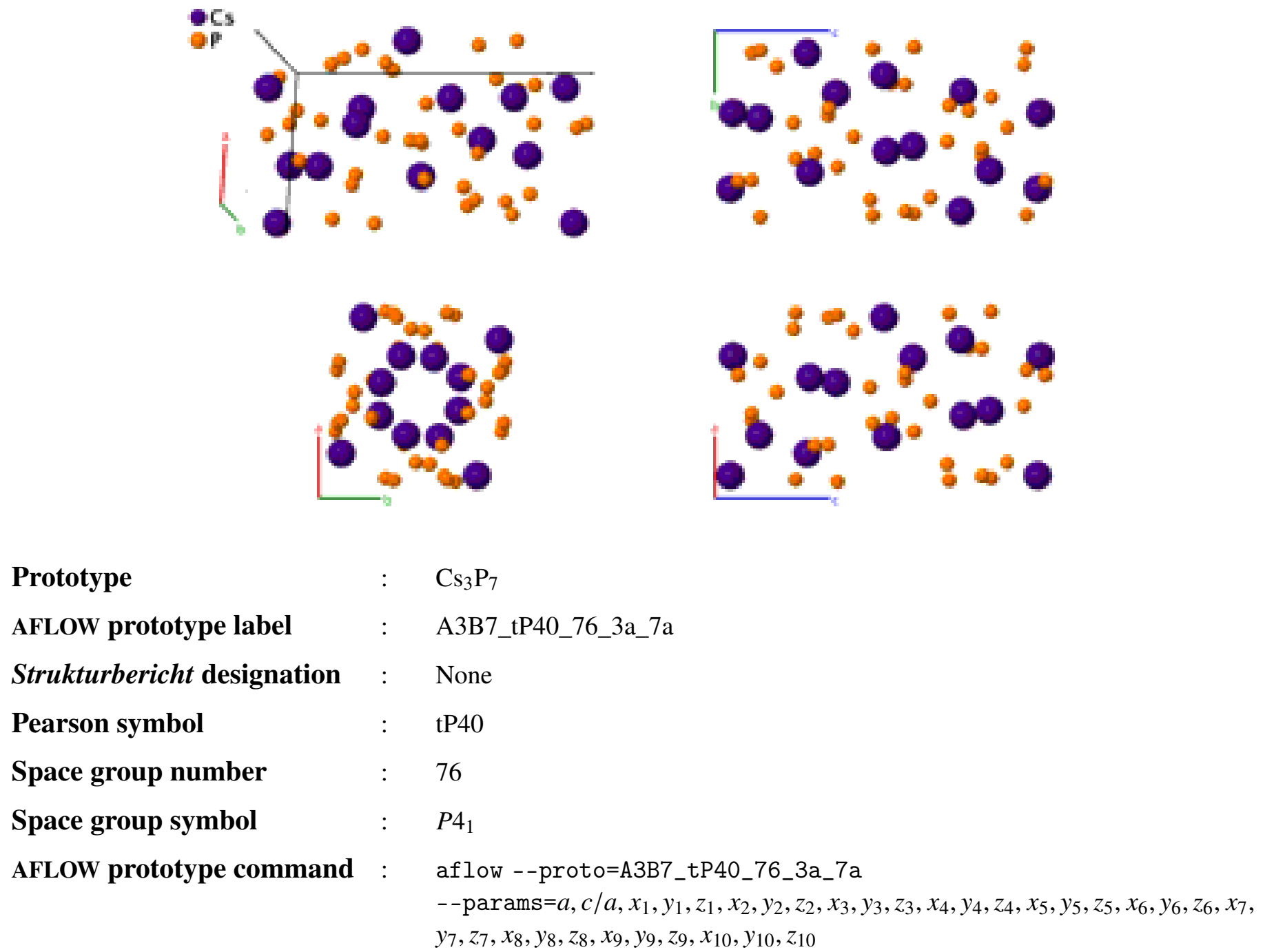

Simple Tetragonal primitive vectors:

$$
\begin{aligned}
& \mathbf{a}_{1}=a \hat{\mathbf{x}} \\
& \mathbf{a}_{2}=a \hat{\mathbf{y}} \\
& \mathbf{a}_{3}=c \hat{\mathbf{z}}
\end{aligned}
$$

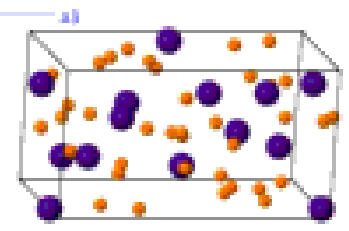

Basis vectors:

Lattice Coordinates

$\mathbf{B}_{1}=x_{1} \mathbf{a}_{1}+y_{1} \mathbf{a}_{2}+z_{1} \mathbf{a}_{3}$

$\mathbf{B}_{2}=-x_{1} \mathbf{a}_{1}-y_{1} \mathbf{a}_{2}+\left(\frac{1}{2}+z_{1}\right) \mathbf{a}_{3}$

$\mathbf{B}_{3}=-y_{1} \mathbf{a}_{1}+x_{1} \mathbf{a}_{2}+\left(\frac{1}{4}+z_{1}\right) \mathbf{a}_{3}$

$\mathbf{B}_{4}=y_{1} \mathbf{a}_{1}-x_{1} \mathbf{a}_{2}+\left(\frac{3}{4}+z_{1}\right) \mathbf{a}_{3}$

$\mathbf{B}_{5}=$

$x_{2} \mathbf{a}_{1}+y_{2} \mathbf{a}_{2}+z_{2} \mathbf{a}_{3}$

$\mathbf{B}_{6}=$

$-x_{2} \mathbf{a}_{1}-y_{2} \mathbf{a}_{2}+\left(\frac{1}{2}+z_{2}\right) \mathbf{a}_{3}$
$=$

Cartesian Coordinates

$$
=
$$$$
=
$$

$=$

$=$

$=$

$$
x_{1} a \hat{\mathbf{x}}+y_{1} a \hat{\mathbf{y}}+z_{1} c \hat{\mathbf{z}}
$$$$
-x_{1} a \hat{\mathbf{x}}-y_{1} a \hat{\mathbf{y}}+\left(\frac{1}{2}+z_{1}\right) c \hat{\mathbf{z}}
$$$$
-y_{1} a \hat{\mathbf{x}}+x_{1} a \hat{\mathbf{y}}+\left(\frac{1}{4}+z_{1}\right) c \hat{\mathbf{z}}
$$$$
y_{1} a \hat{\mathbf{x}}-x_{1} a \hat{\mathbf{y}}+\left(\frac{3}{4}+z_{1}\right) c \hat{\mathbf{z}}
$$$$
x_{2} a \hat{\mathbf{x}}+y_{2} a \hat{\mathbf{y}}+z_{2} c \hat{\mathbf{z}}
$$$$
-x_{2} a \hat{\mathbf{x}}-y_{2} a \hat{\mathbf{y}}+\left(\frac{1}{2}+z_{2}\right) c \hat{\mathbf{z}}
$$

Wyckoff Position

Atom Type

(4a)

Cs I

(4a)

Cs I

(4a)

Cs I

(4a)

Cs I

(4a)

Cs II

(4a) 


\begin{tabular}{|c|c|c|c|c|c|c|}
\hline $\mathbf{B}_{7}$ & $=$ & $-y_{2} \mathbf{a}_{1}+x_{2} \mathbf{a}_{2}+\left(\frac{1}{4}+z_{2}\right) \mathbf{a}_{3}$ & $=$ & $-y_{2} a \hat{\mathbf{x}}+x_{2} a \hat{\mathbf{y}}+\left(\frac{1}{4}+z_{2}\right) c \hat{\mathbf{z}}$ & $(4 a)$ & Cs II \\
\hline $\mathbf{B}_{8}$ & $=$ & $y_{2} \mathbf{a}_{1}-x_{2} \mathbf{a}_{2}+\left(\frac{3}{4}+z_{2}\right) \mathbf{a}_{3}$ & $=$ & $y_{2} a \hat{\mathbf{x}}-x_{2} a \hat{\mathbf{y}}+\left(\frac{3}{4}+z_{2}\right) c \hat{\mathbf{z}}$ & $(4 a)$ & Cs II \\
\hline $\mathbf{B}_{9}$ & $=$ & $x_{3} \mathbf{a}_{1}+y_{3} \mathbf{a}_{2}+z_{3} \mathbf{a}_{3}$ & $=$ & $x_{3} a \hat{\mathbf{x}}+y_{3} a \hat{\mathbf{y}}+z_{3} c \hat{\mathbf{z}}$ & $(4 a)$ & Cs III \\
\hline $\mathbf{B}_{10}$ & $=$ & $-x_{3} \mathbf{a}_{1}-y_{3} \mathbf{a}_{2}+\left(\frac{1}{2}+z_{3}\right) \mathbf{a}_{3}$ & $=$ & $-x_{3} a \hat{\mathbf{x}}-y_{3} a \hat{\mathbf{y}}+\left(\frac{1}{2}+z_{3}\right) c \hat{\mathbf{z}}$ & $(4 a)$ & Cs III \\
\hline $\mathbf{B}_{11}$ & $=$ & $-y_{3} \mathbf{a}_{1}+x_{3} \mathbf{a}_{2}+\left(\frac{1}{4}+z_{3}\right) \mathbf{a}_{3}$ & $=$ & $-y_{3} a \hat{\mathbf{x}}+x_{3} a \hat{\mathbf{y}}+\left(\frac{1}{4}+z_{3}\right) c \hat{\mathbf{z}}$ & $(4 a)$ & Cs III \\
\hline $\mathbf{B}_{12}$ & $=$ & $y_{3} \mathbf{a}_{1}-x_{3} \mathbf{a}_{2}+\left(\frac{3}{4}+z_{3}\right) \mathbf{a}_{3}$ & $=$ & $y_{3} a \hat{\mathbf{x}}-x_{3} a \hat{\mathbf{y}}+\left(\frac{3}{4}+z_{3}\right) c \hat{\mathbf{z}}$ & $(4 a)$ & Cs III \\
\hline $\mathbf{B}_{13}$ & $=$ & $x_{4} \mathbf{a}_{1}+y_{4} \mathbf{a}_{2}+z_{4} \mathbf{a}_{3}$ & $=$ & $x_{4} a \hat{\mathbf{x}}+y_{4} a \hat{\mathbf{y}}+z_{4} c \hat{\mathbf{z}}$ & $(4 a)$ & P I \\
\hline $\mathbf{B}_{14}$ & $=$ & $-x_{4} \mathbf{a}_{1}-y_{4} \mathbf{a}_{2}+\left(\frac{1}{2}+z_{4}\right) \mathbf{a}_{3}$ & $=$ & $-x_{4} a \hat{\mathbf{x}}-y_{4} a \hat{\mathbf{y}}+\left(\frac{1}{2}+z_{4}\right) c \hat{\mathbf{z}}$ & $(4 a)$ & P I \\
\hline $\mathbf{B}_{15}$ & $=$ & $-y_{4} \mathbf{a}_{1}+x_{4} \mathbf{a}_{2}+\left(\frac{1}{4}+z_{4}\right) \mathbf{a}_{3}$ & $=$ & $-y_{4} a \hat{\mathbf{x}}+x_{4} a \hat{\mathbf{y}}+\left(\frac{1}{4}+z_{4}\right) c \hat{\mathbf{z}}$ & $(4 a)$ & P I \\
\hline $\mathbf{B}_{16}$ & $=$ & $y_{4} \mathbf{a}_{1}-x_{4} \mathbf{a}_{2}+\left(\frac{3}{4}+z_{4}\right) \mathbf{a}_{3}$ & $=$ & $y_{4} a \hat{\mathbf{x}}-x_{4} a \hat{\mathbf{y}}+\left(\frac{3}{4}+z_{4}\right) c \hat{\mathbf{z}}$ & $(4 a)$ & P I \\
\hline $\mathbf{B}_{17}$ & $=$ & $x_{5} \mathbf{a}_{1}+y_{5} \mathbf{a}_{2}+z_{5} \mathbf{a}_{3}$ & $=$ & $x_{5} a \hat{\mathbf{x}}+y_{5} a \hat{\mathbf{y}}+z_{5} c \hat{\mathbf{z}}$ & $(4 a)$ & P II \\
\hline $\mathbf{B}_{18}$ & $=$ & $-x_{5} \mathbf{a}_{1}-y_{5} \mathbf{a}_{2}+\left(\frac{1}{2}+z_{5}\right) \mathbf{a}_{3}$ & $=$ & $-x_{5} a \hat{\mathbf{x}}-y_{5} a \hat{\mathbf{y}}+\left(\frac{1}{2}+z_{5}\right) c \hat{\mathbf{z}}$ & $(4 a)$ & P II \\
\hline $\mathbf{B}_{19}$ & $=$ & $-y_{5} \mathbf{a}_{1}+x_{5} \mathbf{a}_{2}+\left(\frac{1}{4}+z_{5}\right) \mathbf{a}_{3}$ & $=$ & $-y_{5} a \hat{\mathbf{x}}+x_{5} a \hat{\mathbf{y}}+\left(\frac{1}{4}+z_{5}\right) c \hat{\mathbf{z}}$ & $(4 a)$ & P II \\
\hline $\mathbf{B}_{20}$ & $=$ & $y_{5} \mathbf{a}_{1}-x_{5} \mathbf{a}_{2}+\left(\frac{3}{4}+z_{5}\right) \mathbf{a}_{3}$ & $=$ & $y_{5} a \hat{\mathbf{x}}-x_{5} a \hat{\mathbf{y}}+\left(\frac{3}{4}+z_{5}\right) c \hat{\mathbf{z}}$ & $(4 a)$ & P II \\
\hline $\mathbf{B}_{21}$ & $=$ & $x_{6} \mathbf{a}_{1}+y_{6} \mathbf{a}_{2}+z_{6} \mathbf{a}_{3}$ & $=$ & $x_{6} a \hat{\mathbf{x}}+y_{6} a \hat{\mathbf{y}}+z_{6} c \hat{\mathbf{z}}$ & $(4 a)$ & P III \\
\hline $\mathbf{B}_{22}$ & $=$ & $-x_{6} \mathbf{a}_{1}-y_{6} \mathbf{a}_{2}+\left(\frac{1}{2}+z_{6}\right) \mathbf{a}_{3}$ & $=$ & $-x_{6} a \hat{\mathbf{x}}-y_{6} a \hat{\mathbf{y}}+\left(\frac{1}{2}+z_{6}\right) c \hat{\mathbf{z}}$ & $(4 a)$ & P III \\
\hline $\mathbf{B}_{23}$ & $=$ & $-y_{6} \mathbf{a}_{1}+x_{6} \mathbf{a}_{2}+\left(\frac{1}{4}+z_{6}\right) \mathbf{a}_{3}$ & $=$ & $-y_{6} a \hat{\mathbf{x}}+x_{6} a \hat{\mathbf{y}}+\left(\frac{1}{4}+z_{6}\right) c \hat{\mathbf{z}}$ & $(4 a)$ & P III \\
\hline $\mathbf{B}_{24}$ & $=$ & $y_{6} \mathbf{a}_{1}-x_{6} \mathbf{a}_{2}+\left(\frac{3}{4}+z_{6}\right) \mathbf{a}_{3}$ & $=$ & $y_{6} a \hat{\mathbf{x}}-x_{6} a \hat{\mathbf{y}}+\left(\frac{3}{4}+z_{6}\right) c \hat{\mathbf{z}}$ & $(4 a)$ & P III \\
\hline $\mathbf{B}_{25}$ & $=$ & $x_{7} \mathbf{a}_{1}+y_{7} \mathbf{a}_{2}+z_{7} \mathbf{a}_{3}$ & $=$ & $x_{7} a \hat{\mathbf{x}}+y_{7} a \hat{\mathbf{y}}+z_{7} c \hat{\mathbf{z}}$ & $(4 a)$ & P IV \\
\hline $\mathbf{B}_{26}$ & $=$ & $-x_{7} \mathbf{a}_{1}-y_{7} \mathbf{a}_{2}+\left(\frac{1}{2}+z_{7}\right) \mathbf{a}_{3}$ & $=$ & $-x_{7} a \hat{\mathbf{x}}-y_{7} a \hat{\mathbf{y}}+\left(\frac{1}{2}+z_{7}\right) c \hat{\mathbf{z}}$ & $(4 a)$ & P IV \\
\hline $\mathbf{B}_{27}$ & $=$ & $-y_{7} \mathbf{a}_{1}+x_{7} \mathbf{a}_{2}+\left(\frac{1}{4}+z_{7}\right) \mathbf{a}_{3}$ & $=$ & $-y_{7} a \hat{\mathbf{x}}+x_{7} a \hat{\mathbf{y}}+\left(\frac{1}{4}+z_{7}\right) c \hat{\mathbf{z}}$ & $(4 a)$ & P IV \\
\hline $\mathbf{B}_{28}$ & $=$ & $y_{7} \mathbf{a}_{1}-x_{7} \mathbf{a}_{2}+\left(\frac{3}{4}+z_{7}\right) \mathbf{a}_{3}$ & $=$ & $y_{7} a \hat{\mathbf{x}}-x_{7} a \hat{\mathbf{y}}+\left(\frac{3}{4}+z_{7}\right) c \hat{\mathbf{z}}$ & $(4 a)$ & P IV \\
\hline $\mathbf{B}_{29}$ & $=$ & $x_{8} \mathbf{a}_{1}+y_{8} \mathbf{a}_{2}+z_{8} \mathbf{a}_{3}$ & $=$ & $x_{8} a \hat{\mathbf{x}}+y_{8} a \hat{\mathbf{y}}+z_{8} c \hat{\mathbf{z}}$ & $(4 a)$ & $\mathrm{PV}$ \\
\hline $\mathbf{B}_{30}$ & $=$ & $-x_{8} \mathbf{a}_{1}-y_{8} \mathbf{a}_{2}+\left(\frac{1}{2}+z_{8}\right) \mathbf{a}_{3}$ & $=$ & $-x_{8} a \hat{\mathbf{x}}-y_{8} a \hat{\mathbf{y}}+\left(\frac{1}{2}+z_{8}\right) c \hat{\mathbf{z}}$ & $(4 a)$ & P V \\
\hline $\mathbf{B}_{31}$ & $=$ & $-y_{8} \mathbf{a}_{1}+x_{8} \mathbf{a}_{2}+\left(\frac{1}{4}+z_{8}\right) \mathbf{a}_{3}$ & $=$ & $-y_{8} a \hat{\mathbf{x}}+x_{8} a \hat{\mathbf{y}}+\left(\frac{1}{4}+z_{8}\right) c \hat{\mathbf{z}}$ & $(4 a)$ & P V \\
\hline $\mathbf{B}_{32}$ & $=$ & $y_{8} \mathbf{a}_{1}-x_{8} \mathbf{a}_{2}+\left(\frac{3}{4}+z_{8}\right) \mathbf{a}_{3}$ & $=$ & $y_{8} a \hat{\mathbf{x}}-x_{8} a \hat{\mathbf{y}}+\left(\frac{3}{4}+z_{8}\right) c \hat{\mathbf{z}}$ & $(4 a)$ & $\mathrm{P} \mathrm{V}$ \\
\hline $\mathbf{B}_{33}$ & $=$ & $x_{9} \mathbf{a}_{1}+y_{9} \mathbf{a}_{2}+z_{9} \mathbf{a}_{3}$ & $=$ & $x_{9} a \hat{\mathbf{x}}+y_{9} a \hat{\mathbf{y}}+z_{9} c \hat{\mathbf{z}}$ & $(4 a)$ & P VI \\
\hline $\mathbf{B}_{34}$ & $=$ & $-x_{9} \mathbf{a}_{1}-y_{9} \mathbf{a}_{2}+\left(\frac{1}{2}+z_{9}\right) \mathbf{a}_{3}$ & $=$ & $-x_{9} a \hat{\mathbf{x}}-y_{9} a \hat{\mathbf{y}}+\left(\frac{1}{2}+z_{9}\right) c \hat{\mathbf{z}}$ & $(4 a)$ & P VI \\
\hline $\mathbf{B}_{35}$ & $=$ & $-y_{9} \mathbf{a}_{1}+x_{9} \mathbf{a}_{2}+\left(\frac{1}{4}+z_{9}\right) \mathbf{a}_{3}$ & $=$ & $-y_{9} a \hat{\mathbf{x}}+x_{9} a \hat{\mathbf{y}}+\left(\frac{1}{4}+z_{9}\right) c \hat{\mathbf{z}}$ & $(4 a)$ & P VI \\
\hline $\mathbf{B}_{36}$ & $=$ & $y_{9} \mathbf{a}_{1}-x_{9} \mathbf{a}_{2}+\left(\frac{3}{4}+z_{9}\right) \mathbf{a}_{3}$ & $=$ & $y_{9} a \hat{\mathbf{x}}-x_{9} a \hat{\mathbf{y}}+\left(\frac{3}{4}+z_{9}\right) c \hat{\mathbf{z}}$ & $(4 a)$ & P VI \\
\hline $\mathbf{B}_{37}$ & $=$ & $x_{10} \mathbf{a}_{1}+y_{10} \mathbf{a}_{2}+z_{10} \mathbf{a}_{3}$ & $=$ & $x_{10} a \hat{\mathbf{x}}+y_{10} a \hat{\mathbf{y}}+z_{10} c \hat{\mathbf{z}}$ & $(4 a)$ & P VII \\
\hline $\mathbf{B}_{38}$ & $=$ & $-x_{10} \mathbf{a}_{1}-y_{10} \mathbf{a}_{2}+\left(\frac{1}{2}+z_{10}\right) \mathbf{a}_{3}$ & $=$ & $-x_{10} a \hat{\mathbf{x}}-y_{10} a \hat{\mathbf{y}}+\left(\frac{1}{2}+z_{10}\right) c \hat{\mathbf{z}}$ & $(4 a)$ & P VII \\
\hline $\mathbf{B}_{39}$ & $=$ & $-y_{10} \mathbf{a}_{1}+x_{10} \mathbf{a}_{2}+\left(\frac{1}{4}+z_{10}\right) \mathbf{a}_{3}$ & $=$ & $-y_{10} a \hat{\mathbf{x}}+x_{10} a \hat{\mathbf{y}}+\left(\frac{1}{4}+z_{10}\right) c \hat{\mathbf{z}}$ & $(4 a)$ & P VII \\
\hline $\mathbf{B}_{40}$ & $=$ & $y_{10} \mathbf{a}_{1}-x_{10} \mathbf{a}_{2}+\left(\frac{3}{4}+z_{10}\right) \mathbf{a}_{3}$ & $=$ & $y_{10} a \hat{\mathbf{x}}-x_{10} a \hat{\mathbf{y}}+\left(\frac{3}{4}+z_{10}\right) c \hat{\mathbf{z}}$ & $(4 a)$ & P VII \\
\hline
\end{tabular}

\section{References:}

- T. Meyer, W. Hönle, and H. G. von Schnering, Zur Chemie und Strukturchemie von Phosphiden und Polyphosphiden. 44. 
Tricäsiumheptaphosphid $\mathrm{Cs}_{3} P_{7}$ : Darstellung, Struktur und Eigenschaften, Z. Anorg. Allg. Chem. 552, 69-80 (1987), doi:10.1002/zaac.19875520907.

\section{Found in:}

- R. J. D. Tilley, Crystals and Crystal Structures (Wiley, Chichester, England, 2006), chap. 5, p. 102.

\section{Geometry files:}

- CIF: pp. 858

- POSCAR: pp. 859 


\section{Pinnoite $\left(\mathrm{MgB}_{2} \mathrm{O}(\mathrm{OH})_{6}\right)$ Structure:}

\section{A2B6CD7_tP64_77_2d_6d_d_ab6d}
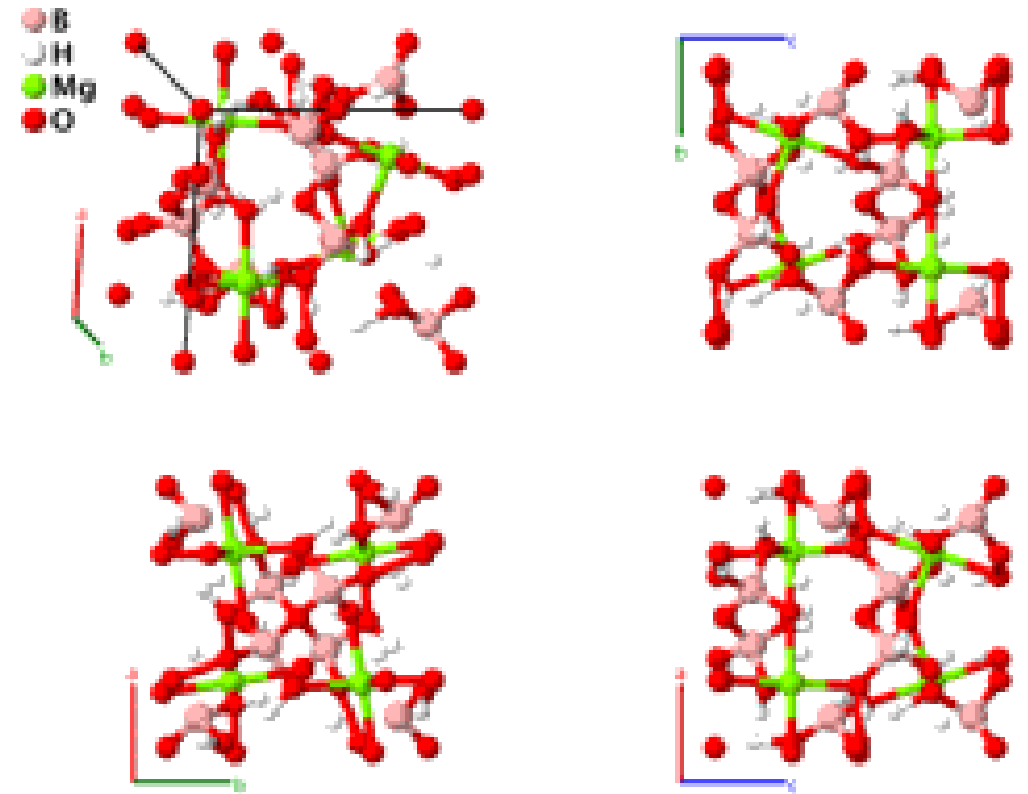

Prototype

AFLOW prototype label

Strukturbericht designation

Pearson symbol

Space group number

Space group symbol

AFLOW prototype command
: $\quad \mathrm{MgB}_{2} \mathrm{O}(\mathrm{OH})_{6}$

: A2B6CD7_tP64_77_2d_6d_d_ab6d

: None

: $\quad$ tP64

: 77

: $\quad P 4_{2}$

aflow - -proto=A2B6CD7_tP64_77_2d_6d_d_ab6d

- - params $=a, c / a, z_{1}, z_{2}, x_{3}, y_{3}, z_{3}, x_{4}, y_{4}, z_{4}, x_{5}, y_{5}, z_{5}, x_{6}, y_{6}, z_{6}, x_{7}, y_{7}, z_{7}, x_{8}, y_{8}$,

$z_{8}, x_{9}, y_{9}, z_{9}, x_{10}, y_{10}, z_{10}, x_{11}, y_{11}, z_{11}, x_{12}, y_{12}, z_{12}, x_{13}, y_{13}, z_{13}, x_{14}, y_{14}, z_{14}, x_{15}, y_{15}, z_{15}$,

$x_{16}, y_{16}, z_{16}, x_{17}, y_{17}, z_{17}$

Simple Tetragonal primitive vectors:

$$
\begin{aligned}
& \mathbf{a}_{1}=a \hat{\mathbf{x}} \\
& \mathbf{a}_{2}=a \hat{\mathbf{y}} \\
& \mathbf{a}_{3}=c \hat{\mathbf{z}}
\end{aligned}
$$

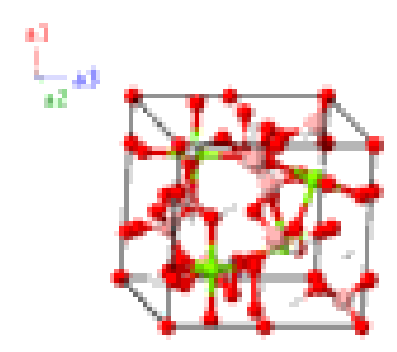

Basis vectors:

Lattice Coordinates
$\mathbf{B}_{1}=$
$z_{1} \mathbf{a}_{3}$
$\mathbf{B}_{2}=$
$\left(\frac{1}{2}+z_{1}\right) \mathbf{a}_{3}$
$=$
$=$

$$
z_{1} c \hat{\mathbf{z}}
$$$$
\left(\frac{1}{2}+z_{1}\right) c \hat{\mathbf{z}}
$$

Cartesian Coordinates
Wyckoff Position

Atom Type

(2a)

O I

(2a)

O I 


\begin{tabular}{|c|c|c|c|c|c|c|}
\hline $\mathbf{B}_{3}$ & $=$ & $\frac{1}{2} \mathbf{a}_{1}+\frac{1}{2} \mathbf{a}_{2}+z_{2} \mathbf{a}_{3}$ & $=$ & $\frac{1}{2} a \hat{\mathbf{x}}+\frac{1}{2} a \hat{\mathbf{y}}+z_{2} c \hat{\mathbf{z}}$ & $(2 b)$ & $\mathrm{O}$ II \\
\hline $\mathbf{B}_{4}$ & $=$ & $\frac{1}{2} \mathbf{a}_{1}+\frac{1}{2} \mathbf{a}_{2}+\left(\frac{1}{2}+z_{2}\right) \mathbf{a}_{3}$ & $=$ & $\frac{1}{2} a \hat{\mathbf{x}}+\frac{1}{2} a \hat{\mathbf{y}}+\left(\frac{1}{2}+z_{2}\right) c \hat{\mathbf{z}}$ & $(2 b)$ & $\mathrm{O}$ II \\
\hline $\mathbf{B}_{5}$ & $=$ & $x_{3} \mathbf{a}_{1}+y_{3} \mathbf{a}_{2}+z_{3} \mathbf{a}_{3}$ & $=$ & $x_{3} a \hat{\mathbf{x}}+y_{3} a \hat{\mathbf{y}}+z_{3} c \hat{\mathbf{z}}$ & $(4 d)$ & B I \\
\hline $\mathbf{B}_{6}$ & $=$ & $-x_{3} \mathbf{a}_{1}-y_{3} \mathbf{a}_{2}+z_{3} \mathbf{a}_{3}$ & $=$ & $-x_{3} a \hat{\mathbf{x}}-y_{3} a \hat{\mathbf{y}}+z_{3} c \hat{\mathbf{z}}$ & $(4 d)$ & B I \\
\hline $\mathbf{B}_{7}$ & $=$ & $-y_{3} \mathbf{a}_{1}+x_{3} \mathbf{a}_{2}+\left(\frac{1}{2}+z_{3}\right) \mathbf{a}_{3}$ & $=$ & $-y_{3} a \hat{\mathbf{x}}+x_{3} a \hat{\mathbf{y}}+\left(\frac{1}{2}+z_{3}\right) c \hat{\mathbf{z}}$ & $(4 d)$ & B I \\
\hline $\mathbf{B}_{8}$ & $=$ & $y_{3} \mathbf{a}_{1}-x_{3} \mathbf{a}_{2}+\left(\frac{1}{2}+z_{3}\right) \mathbf{a}_{3}$ & $=$ & $y_{3} a \hat{\mathbf{x}}-x_{3} a \hat{\mathbf{y}}+\left(\frac{1}{2}+z_{3}\right) c \hat{\mathbf{z}}$ & $(4 d)$ & B I \\
\hline $\mathbf{B}_{9}$ & $=$ & $x_{4} \mathbf{a}_{1}+y_{4} \mathbf{a}_{2}+z_{4} \mathbf{a}_{3}$ & $=$ & $x_{4} a \hat{\mathbf{x}}+y_{4} a \hat{\mathbf{y}}+z_{4} c \hat{\mathbf{z}}$ & $(4 d)$ & B II \\
\hline $\mathbf{B}_{10}$ & $=$ & $-x_{4} \mathbf{a}_{1}-y_{4} \mathbf{a}_{2}+z_{4} \mathbf{a}_{3}$ & $=$ & $-x_{4} a \hat{\mathbf{x}}-y_{4} a \hat{\mathbf{y}}+z_{4} c \hat{\mathbf{z}}$ & $(4 d)$ & B II \\
\hline $\mathbf{B}_{11}$ & $=$ & $-y_{4} \mathbf{a}_{1}+x_{4} \mathbf{a}_{2}+\left(\frac{1}{2}+z_{4}\right) \mathbf{a}_{3}$ & $=$ & $-y_{4} a \hat{\mathbf{x}}+x_{4} a \hat{\mathbf{y}}+\left(\frac{1}{2}+z_{4}\right) c \hat{\mathbf{z}}$ & $(4 d)$ & B II \\
\hline $\mathbf{B}_{12}$ & $=$ & $y_{4} \mathbf{a}_{1}-x_{4} \mathbf{a}_{2}+\left(\frac{1}{2}+z_{4}\right) \mathbf{a}_{3}$ & $=$ & $y_{4} a \hat{\mathbf{x}}-x_{4} a \hat{\mathbf{y}}+\left(\frac{1}{2}+z_{4}\right) c \hat{\mathbf{z}}$ & $(4 d)$ & B II \\
\hline $\mathbf{B}_{13}$ & $=$ & $x_{5} \mathbf{a}_{1}+y_{5} \mathbf{a}_{2}+z_{5} \mathbf{a}_{3}$ & $=$ & $x_{5} a \hat{\mathbf{x}}+y_{5} a \hat{\mathbf{y}}+z_{5} c \hat{\mathbf{z}}$ & $(4 d)$ & $\mathrm{H} \mathrm{I}$ \\
\hline $\mathbf{B}_{14}$ & $=$ & $-x_{5} \mathbf{a}_{1}-y_{5} \mathbf{a}_{2}+z_{5} \mathbf{a}_{3}$ & $=$ & $-x_{5} a \hat{\mathbf{x}}-y_{5} a \hat{\mathbf{y}}+z_{5} c \hat{\mathbf{z}}$ & $(4 d)$ & $\mathrm{H} \mathrm{I}$ \\
\hline $\mathbf{B}_{15}$ & $=$ & $-y_{5} \mathbf{a}_{1}+x_{5} \mathbf{a}_{2}+\left(\frac{1}{2}+z_{5}\right) \mathbf{a}_{3}$ & $=$ & $-y_{5} a \hat{\mathbf{x}}+x_{5} a \hat{\mathbf{y}}+\left(\frac{1}{2}+z_{5}\right) c \hat{\mathbf{z}}$ & $(4 d)$ & H I \\
\hline $\mathbf{B}_{16}$ & $=$ & $y_{5} \mathbf{a}_{1}-x_{5} \mathbf{a}_{2}+\left(\frac{1}{2}+z_{5}\right) \mathbf{a}_{3}$ & $=$ & $y_{5} a \hat{\mathbf{x}}-x_{5} a \hat{\mathbf{y}}+\left(\frac{1}{2}+z_{5}\right) c \hat{\mathbf{z}}$ & $(4 d)$ & $\mathrm{H} \mathrm{I}$ \\
\hline $\mathbf{B}_{17}$ & $=$ & $x_{6} \mathbf{a}_{1}+y_{6} \mathbf{a}_{2}+z_{6} \mathbf{a}_{3}$ & $=$ & $x_{6} a \hat{\mathbf{x}}+y_{6} a \hat{\mathbf{y}}+z_{6} c \hat{\mathbf{z}}$ & $(4 d)$ & H II \\
\hline $\mathbf{B}_{18}$ & $=$ & $-x_{6} \mathbf{a}_{1}-y_{6} \mathbf{a}_{2}+z_{6} \mathbf{a}_{3}$ & $=$ & $-x_{6} a \hat{\mathbf{x}}-y_{6} a \hat{\mathbf{y}}+z_{6} c \hat{\mathbf{z}}$ & $(4 d)$ & H II \\
\hline $\mathbf{B}_{19}$ & $=$ & $-y_{6} \mathbf{a}_{1}+x_{6} \mathbf{a}_{2}+\left(\frac{1}{2}+z_{6}\right) \mathbf{a}_{3}$ & $=$ & $-y_{6} a \hat{\mathbf{x}}+x_{6} a \hat{\mathbf{y}}+\left(\frac{1}{2}+z_{6}\right) c \hat{\mathbf{z}}$ & $(4 d)$ & H II \\
\hline $\mathbf{B}_{20}$ & $=$ & $y_{6} \mathbf{a}_{1}-x_{6} \mathbf{a}_{2}+\left(\frac{1}{2}+z_{6}\right) \mathbf{a}_{3}$ & $=$ & $y_{6} a \hat{\mathbf{x}}-x_{6} a \hat{\mathbf{y}}+\left(\frac{1}{2}+z_{6}\right) c \hat{\mathbf{z}}$ & $(4 d)$ & H II \\
\hline $\mathbf{B}_{21}$ & $=$ & $x_{7} \mathbf{a}_{1}+y_{7} \mathbf{a}_{2}+z_{7} \mathbf{a}_{3}$ & $=$ & $x_{7} a \hat{\mathbf{x}}+y_{7} a \hat{\mathbf{y}}+z_{7} c \hat{\mathbf{z}}$ & $(4 d)$ & H III \\
\hline $\mathbf{B}_{22}$ & $=$ & $-x_{7} \mathbf{a}_{1}-y_{7} \mathbf{a}_{2}+z_{7} \mathbf{a}_{3}$ & $=$ & $-x_{7} a \hat{\mathbf{x}}-y_{7} a \hat{\mathbf{y}}+z_{7} c \hat{\mathbf{z}}$ & $(4 d)$ & H III \\
\hline $\mathbf{B}_{23}$ & $=$ & $-y_{7} \mathbf{a}_{1}+x_{7} \mathbf{a}_{2}+\left(\frac{1}{2}+z_{7}\right) \mathbf{a}_{3}$ & $=$ & $-y_{7} a \hat{\mathbf{x}}+x_{7} a \hat{\mathbf{y}}+\left(\frac{1}{2}+z_{7}\right) c \hat{\mathbf{z}}$ & $(4 d)$ & H III \\
\hline $\mathbf{B}_{24}$ & $=$ & $y_{7} \mathbf{a}_{1}-x_{7} \mathbf{a}_{2}+\left(\frac{1}{2}+z_{7}\right) \mathbf{a}_{3}$ & $=$ & $y_{7} a \hat{\mathbf{x}}-x_{7} a \hat{\mathbf{y}}+\left(\frac{1}{2}+z_{7}\right) c \hat{\mathbf{z}}$ & $(4 d)$ & H III \\
\hline $\mathbf{B}_{25}$ & $=$ & $x_{8} \mathbf{a}_{1}+y_{8} \mathbf{a}_{2}+z_{8} \mathbf{a}_{3}$ & $=$ & $x_{8} a \hat{\mathbf{x}}+y_{8} a \hat{\mathbf{y}}+z_{8} c \hat{\mathbf{z}}$ & $(4 d)$ & H IV \\
\hline $\mathbf{B}_{26}$ & $=$ & $-x_{8} \mathbf{a}_{1}-y_{8} \mathbf{a}_{2}+z_{8} \mathbf{a}_{3}$ & $=$ & $-x_{8} a \hat{\mathbf{x}}-y_{8} a \hat{\mathbf{y}}+z_{8} c \hat{\mathbf{z}}$ & $(4 d)$ & H IV \\
\hline $\mathbf{B}_{27}$ & $=$ & $-y_{8} \mathbf{a}_{1}+x_{8} \mathbf{a}_{2}+\left(\frac{1}{2}+z_{8}\right) \mathbf{a}_{3}$ & $=$ & $-y_{8} a \hat{\mathbf{x}}+x_{8} a \hat{\mathbf{y}}+\left(\frac{1}{2}+z_{8}\right) c \hat{\mathbf{z}}$ & $(4 d)$ & H IV \\
\hline $\mathbf{B}_{28}$ & $=$ & $y_{8} \mathbf{a}_{1}-x_{8} \mathbf{a}_{2}+\left(\frac{1}{2}+z_{8}\right) \mathbf{a}_{3}$ & $=$ & $y_{8} a \hat{\mathbf{x}}-x_{8} a \hat{\mathbf{y}}+\left(\frac{1}{2}+z_{8}\right) c \hat{\mathbf{z}}$ & $(4 d)$ & H IV \\
\hline $\mathbf{B}_{29}$ & $=$ & $x_{9} \mathbf{a}_{1}+y_{9} \mathbf{a}_{2}+z_{9} \mathbf{a}_{3}$ & $=$ & $x_{9} a \hat{\mathbf{x}}+y_{9} a \hat{\mathbf{y}}+z_{9} c \hat{\mathbf{z}}$ & $(4 d)$ & $\mathrm{H} \mathrm{V}$ \\
\hline $\mathbf{B}_{30}$ & $=$ & $-x_{9} \mathbf{a}_{1}-y_{9} \mathbf{a}_{2}+z 9 \mathbf{a}_{3}$ & $=$ & $-x_{9} a \hat{\mathbf{x}}-y_{9} a \hat{\mathbf{y}}+z_{9} c \hat{\mathbf{z}}$ & $(4 d)$ & $\mathrm{H} \mathrm{V}$ \\
\hline $\mathbf{B}_{31}$ & $=$ & $-y_{9} \mathbf{a}_{1}+x_{9} \mathbf{a}_{2}+\left(\frac{1}{2}+z_{9}\right) \mathbf{a}_{3}$ & $=$ & $-y_{9} a \hat{\mathbf{x}}+x_{9} a \hat{\mathbf{y}}+\left(\frac{1}{2}+z_{9}\right) c \hat{\mathbf{z}}$ & $(4 d)$ & H V \\
\hline $\mathbf{B}_{32}$ & $=$ & $y_{9} \mathbf{a}_{1}-x_{9} \mathbf{a}_{2}+\left(\frac{1}{2}+z_{9}\right) \mathbf{a}_{3}$ & $=$ & $y_{9} a \hat{\mathbf{x}}-x_{9} a \hat{\mathbf{y}}+\left(\frac{1}{2}+z_{9}\right) c \hat{\mathbf{z}}$ & $(4 d)$ & H V \\
\hline $\mathbf{B}_{33}$ & $=$ & $x_{10} \mathbf{a}_{1}+y_{10} \mathbf{a}_{2}+z_{10} \mathbf{a}_{3}$ & $=$ & $x_{10} a \hat{\mathbf{x}}+y_{10} a \hat{\mathbf{y}}+z_{10} c \hat{\mathbf{z}}$ & $(4 d)$ & $\mathrm{H}$ VI \\
\hline $\mathbf{B}_{34}$ & $=$ & $-x_{10} \mathbf{a}_{1}-y_{10} \mathbf{a}_{2}+z_{10} \mathbf{a}_{3}$ & $=$ & $-x_{10} a \hat{\mathbf{x}}-y_{10} a \hat{\mathbf{y}}+z_{10} c \hat{\mathbf{z}}$ & $(4 d)$ & H VI \\
\hline $\mathbf{B}_{35}$ & $=$ & $-y_{10} \mathbf{a}_{1}+x_{10} \mathbf{a}_{2}+\left(\frac{1}{2}+z_{10}\right) \mathbf{a}_{3}$ & $=$ & $-y_{10} a \hat{\mathbf{x}}+x_{10} a \hat{\mathbf{y}}+\left(\frac{1}{2}+z_{10}\right) c \hat{\mathbf{z}}$ & $(4 d)$ & H VI \\
\hline $\mathbf{B}_{36}$ & $=$ & $y_{10} \mathbf{a}_{1}-x_{10} \mathbf{a}_{2}+\left(\frac{1}{2}+z_{10}\right) \mathbf{a}_{3}$ & $=$ & $y_{10} a \hat{\mathbf{x}}-x_{10} a \hat{\mathbf{y}}+\left(\frac{1}{2}+z_{10}\right) c \hat{\mathbf{z}}$ & $(4 d)$ & H VI \\
\hline $\mathbf{B}_{37}$ & $=$ & $x_{11} \mathbf{a}_{1}+y_{11} \mathbf{a}_{2}+z_{11} \mathbf{a}_{3}$ & $=$ & $x_{11} a \hat{\mathbf{x}}+y_{11} a \hat{\mathbf{y}}+z_{11} c \hat{\mathbf{z}}$ & $(4 d)$ & $\mathrm{Mg}$ \\
\hline $\mathbf{B}_{38}$ & $=$ & $-x_{11} \mathbf{a}_{1}-y_{11} \mathbf{a}_{2}+z_{11} \mathbf{a}_{3}$ & $=$ & $-x_{11} a \hat{\mathbf{x}}-y_{11} a \hat{\mathbf{y}}+z_{11} c \hat{\mathbf{z}}$ & $(4 d)$ & $\mathrm{Mg}$ \\
\hline
\end{tabular}




\begin{tabular}{|c|c|c|c|c|c|c|}
\hline $\mathbf{B}_{39}$ & $=$ & $-y_{11} \mathbf{a}_{1}+x_{11} \mathbf{a}_{2}+\left(\frac{1}{2}+z_{11}\right) \mathbf{a}_{3}$ & $=$ & $-y_{11} a \hat{\mathbf{x}}+x_{11} a \hat{\mathbf{y}}+\left(\frac{1}{2}+z_{11}\right) c \hat{\mathbf{z}}$ & $(4 d)$ & $\mathrm{Mg}$ \\
\hline $\mathbf{B}_{40}$ & $=$ & $y_{11} \mathbf{a}_{1}-x_{11} \mathbf{a}_{2}+\left(\frac{1}{2}+z_{11}\right) \mathbf{a}_{3}$ & $=$ & $y_{11} a \hat{\mathbf{x}}-x_{11} a \hat{\mathbf{y}}+\left(\frac{1}{2}+z_{11}\right) c \hat{\mathbf{z}}$ & $(4 d)$ & $\mathrm{Mg}$ \\
\hline $\mathbf{B}_{41}$ & $=$ & $x_{12} \mathbf{a}_{1}+y_{12} \mathbf{a}_{2}+z_{12} \mathbf{a}_{3}$ & $=$ & $x_{12} a \hat{\mathbf{x}}+y_{12} a \hat{\mathbf{y}}+z_{12} c \hat{\mathbf{z}}$ & $(4 d)$ & O III \\
\hline $\mathbf{B}_{42}$ & $=$ & $-x_{12} \mathbf{a}_{1}-y_{12} \mathbf{a}_{2}+z_{12} \mathbf{a}_{3}$ & $=$ & $-x_{12} a \hat{\mathbf{x}}-y_{12} a \hat{\mathbf{y}}+z_{12} c \hat{\mathbf{z}}$ & $(4 d)$ & O III \\
\hline $\mathbf{B}_{43}$ & $=$ & $-y_{12} \mathbf{a}_{1}+x_{12} \mathbf{a}_{2}+\left(\frac{1}{2}+z_{12}\right) \mathbf{a}_{3}$ & $=$ & $-y_{12} a \hat{\mathbf{x}}+x_{12} a \hat{\mathbf{y}}+\left(\frac{1}{2}+z_{12}\right) c \hat{\mathbf{z}}$ & $(4 d)$ & O III \\
\hline $\mathbf{B}_{44}$ & $=$ & $y_{12} \mathbf{a}_{1}-x_{12} \mathbf{a}_{2}+\left(\frac{1}{2}+z_{12}\right) \mathbf{a}_{3}$ & $=$ & $y_{12} a \hat{\mathbf{x}}-x_{12} a \hat{\mathbf{y}}+\left(\frac{1}{2}+z_{12}\right) c \hat{\mathbf{z}}$ & $(4 d)$ & O III \\
\hline $\mathbf{B}_{45}$ & $=$ & $x_{13} \mathbf{a}_{1}+y_{13} \mathbf{a}_{2}+z_{13} \mathbf{a}_{3}$ & $=$ & $x_{13} a \hat{\mathbf{x}}+y_{13} a \hat{\mathbf{y}}+z_{13} c \hat{\mathbf{z}}$ & $(4 d)$ & O IV \\
\hline $\mathbf{B}_{46}$ & $=$ & $-x_{13} \mathbf{a}_{1}-y_{13} \mathbf{a}_{2}+z_{13} \mathbf{a}_{3}$ & $=$ & $-x_{13} a \hat{\mathbf{x}}-y_{13} a \hat{\mathbf{y}}+z_{13} c \hat{\mathbf{z}}$ & $(4 d)$ & O IV \\
\hline $\mathbf{B}_{47}$ & $=$ & $-y_{13} \mathbf{a}_{1}+x_{13} \mathbf{a}_{2}+\left(\frac{1}{2}+z_{13}\right) \mathbf{a}_{3}$ & $=$ & $-y_{13} a \hat{\mathbf{x}}+x_{13} a \hat{\mathbf{y}}+\left(\frac{1}{2}+z_{13}\right) c \hat{\mathbf{z}}$ & $(4 d)$ & O IV \\
\hline $\mathbf{B}_{48}$ & $=$ & $y_{13} \mathbf{a}_{1}-x_{13} \mathbf{a}_{2}+\left(\frac{1}{2}+z_{13}\right) \mathbf{a}_{3}$ & $=$ & $y_{13} a \hat{\mathbf{x}}-x_{13} a \hat{\mathbf{y}}+\left(\frac{1}{2}+z_{13}\right) c \hat{\mathbf{z}}$ & $(4 d)$ & O IV \\
\hline $\mathbf{B}_{49}$ & $=$ & $x_{14} \mathbf{a}_{1}+y_{14} \mathbf{a}_{2}+z_{14} \mathbf{a}_{3}$ & $=$ & $x_{14} a \hat{\mathbf{x}}+y_{14} a \hat{\mathbf{y}}+z_{14} c \hat{\mathbf{z}}$ & $(4 d)$ & $\mathrm{OV}$ \\
\hline $\mathbf{B}_{50}$ & $=$ & $-x_{14} \mathbf{a}_{1}-y_{14} \mathbf{a}_{2}+z_{14} \mathbf{a}_{3}$ & $=$ & $-x_{14} a \hat{\mathbf{x}}-y_{14} a \hat{\mathbf{y}}+z_{14} c \hat{\mathbf{z}}$ & $(4 d)$ & $\mathrm{OV}$ \\
\hline $\mathbf{B}_{51}$ & $=$ & $-y_{14} \mathbf{a}_{1}+x_{14} \mathbf{a}_{2}+\left(\frac{1}{2}+z_{14}\right) \mathbf{a}_{3}$ & $=$ & $-y_{14} a \hat{\mathbf{x}}+x_{14} a \hat{\mathbf{y}}+\left(\frac{1}{2}+z_{14}\right) c \hat{\mathbf{z}}$ & $(4 d)$ & $\mathrm{OV}$ \\
\hline $\mathbf{B}_{52}$ & $=$ & $y_{14} \mathbf{a}_{1}-x_{14} \mathbf{a}_{2}+\left(\frac{1}{2}+z_{14}\right) \mathbf{a}_{3}$ & $=$ & $y_{14} a \hat{\mathbf{x}}-x_{14} a \hat{\mathbf{y}}+\left(\frac{1}{2}+z_{14}\right) c \hat{\mathbf{z}}$ & $(4 d)$ & $\mathrm{OV}$ \\
\hline $\mathbf{B}_{53}$ & $=$ & $x_{15} \mathbf{a}_{1}+y_{15} \mathbf{a}_{2}+z_{15} \mathbf{a}_{3}$ & $=$ & $x_{15} a \hat{\mathbf{x}}+y_{15} a \hat{\mathbf{y}}+z_{15} c \hat{\mathbf{z}}$ & $(4 d)$ & O VI \\
\hline $\mathbf{B}_{54}$ & $=$ & $-x_{15} \mathbf{a}_{1}-y_{15} \mathbf{a}_{2}+z_{15} \mathbf{a}_{3}$ & $=$ & $-x_{15} a \hat{\mathbf{x}}-y_{15} a \hat{\mathbf{y}}+z_{15} c \hat{\mathbf{z}}$ & $(4 d)$ & O VI \\
\hline $\mathbf{B}_{55}$ & $=$ & $-y_{15} \mathbf{a}_{1}+x_{15} \mathbf{a}_{2}+\left(\frac{1}{2}+z_{15}\right) \mathbf{a}_{3}$ & $=$ & $-y_{15} a \hat{\mathbf{x}}+x_{15} a \hat{\mathbf{y}}+\left(\frac{1}{2}+z_{15}\right) c \hat{\mathbf{z}}$ & $(4 d)$ & $\mathrm{O} \mathrm{VI}$ \\
\hline $\mathbf{B}_{56}$ & $=$ & $y_{15} \mathbf{a}_{1}-x_{15} \mathbf{a}_{2}+\left(\frac{1}{2}+z_{15}\right) \mathbf{a}_{3}$ & $=$ & $y_{15} a \hat{\mathbf{x}}-x_{15} a \hat{\mathbf{y}}+\left(\frac{1}{2}+z_{15}\right) c \hat{\mathbf{z}}$ & $(4 d)$ & O VI \\
\hline $\mathbf{B}_{57}$ & $=$ & $x_{16} \mathbf{a}_{1}+y_{16} \mathbf{a}_{2}+z_{16} \mathbf{a}_{3}$ & $=$ & $x_{16} a \hat{\mathbf{x}}+y_{16} a \hat{\mathbf{y}}+z_{16} c \hat{\mathbf{z}}$ & $(4 d)$ & O VII \\
\hline $\mathbf{B}_{58}$ & $=$ & $-x_{16} \mathbf{a}_{1}-y_{16} \mathbf{a}_{2}+z_{16} \mathbf{a}_{3}$ & $=$ & $-x_{16} a \hat{\mathbf{x}}-y_{16} a \hat{\mathbf{y}}+z_{16} c \hat{\mathbf{z}}$ & $(4 d)$ & O VII \\
\hline $\mathbf{B}_{59}$ & $=$ & $-y_{16} \mathbf{a}_{1}+x_{16} \mathbf{a}_{2}+\left(\frac{1}{2}+z_{16}\right) \mathbf{a}_{3}$ & $=$ & $-y_{16} a \hat{\mathbf{x}}+x_{16} a \hat{\mathbf{y}}+\left(\frac{1}{2}+z_{16}\right) c \hat{\mathbf{z}}$ & $(4 d)$ & O VII \\
\hline $\mathbf{B}_{60}$ & $=$ & $y_{16} \mathbf{a}_{1}-x_{16} \mathbf{a}_{2}+\left(\frac{1}{2}+z_{16}\right) \mathbf{a}_{3}$ & $=$ & $y_{16} a \hat{\mathbf{x}}-x_{16} a \hat{\mathbf{y}}+\left(\frac{1}{2}+z_{16}\right) c \hat{\mathbf{z}}$ & $(4 d)$ & O VII \\
\hline $\mathbf{B}_{61}$ & $=$ & $x_{17} \mathbf{a}_{1}+y_{17} \mathbf{a}_{2}+z_{17} \mathbf{a}_{3}$ & $=$ & $x_{17} a \hat{\mathbf{x}}+y_{17} a \hat{\mathbf{y}}+z_{17} c \hat{\mathbf{z}}$ & $(4 d)$ & O VIII \\
\hline $\mathbf{B}_{62}$ & $=$ & $-x_{17} \mathbf{a}_{1}-y_{17} \mathbf{a}_{2}+z_{17} \mathbf{a}_{3}$ & $=$ & $-x_{17} a \hat{\mathbf{x}}-y_{17} a \hat{\mathbf{y}}+z_{17} c \hat{\mathbf{z}}$ & $(4 d)$ & O VIII \\
\hline $\mathbf{B}_{63}$ & $=$ & $-y_{17} \mathbf{a}_{1}+x_{17} \mathbf{a}_{2}+\left(\frac{1}{2}+z_{17}\right) \mathbf{a}_{3}$ & $=$ & $-y_{17} a \hat{\mathbf{x}}+x_{17} a \hat{\mathbf{y}}+\left(\frac{1}{2}+z_{17}\right) c \hat{\mathbf{z}}$ & $(4 d)$ & O VIII \\
\hline $\mathbf{B}_{64}$ & $=$ & $y_{17} \mathbf{a}_{1}-x_{17} \mathbf{a}_{2}+\left(\frac{1}{2}+z_{17}\right) \mathbf{a}_{3}$ & $=$ & $y_{17} a \hat{\mathbf{x}}-x_{17} a \hat{\mathbf{y}}+\left(\frac{1}{2}+z_{17}\right) c \hat{\mathbf{z}}$ & $(4 d)$ & O VIII \\
\hline
\end{tabular}

\section{References:}

- E. A. Genkina and Y. A. Malinovskii, Refinement of the structure of pinnoite: Location of hydrogen atoms, Sov. Phys. Crystallogr. 28, 475-477 (1983).

\section{Found in:}

- P. Villars and K. Cenzual, Pearson's Crystal Data - Crystal Structure Database for Inorganic Compounds, ASM International (2013).

\section{Geometry files:}

- CIF: pp. 859

- POSCAR: pp. 859 


\section{$\mathrm{H}_{2} \mathrm{~S}$ III Structure: A2B_tP48_77_8d_4d}
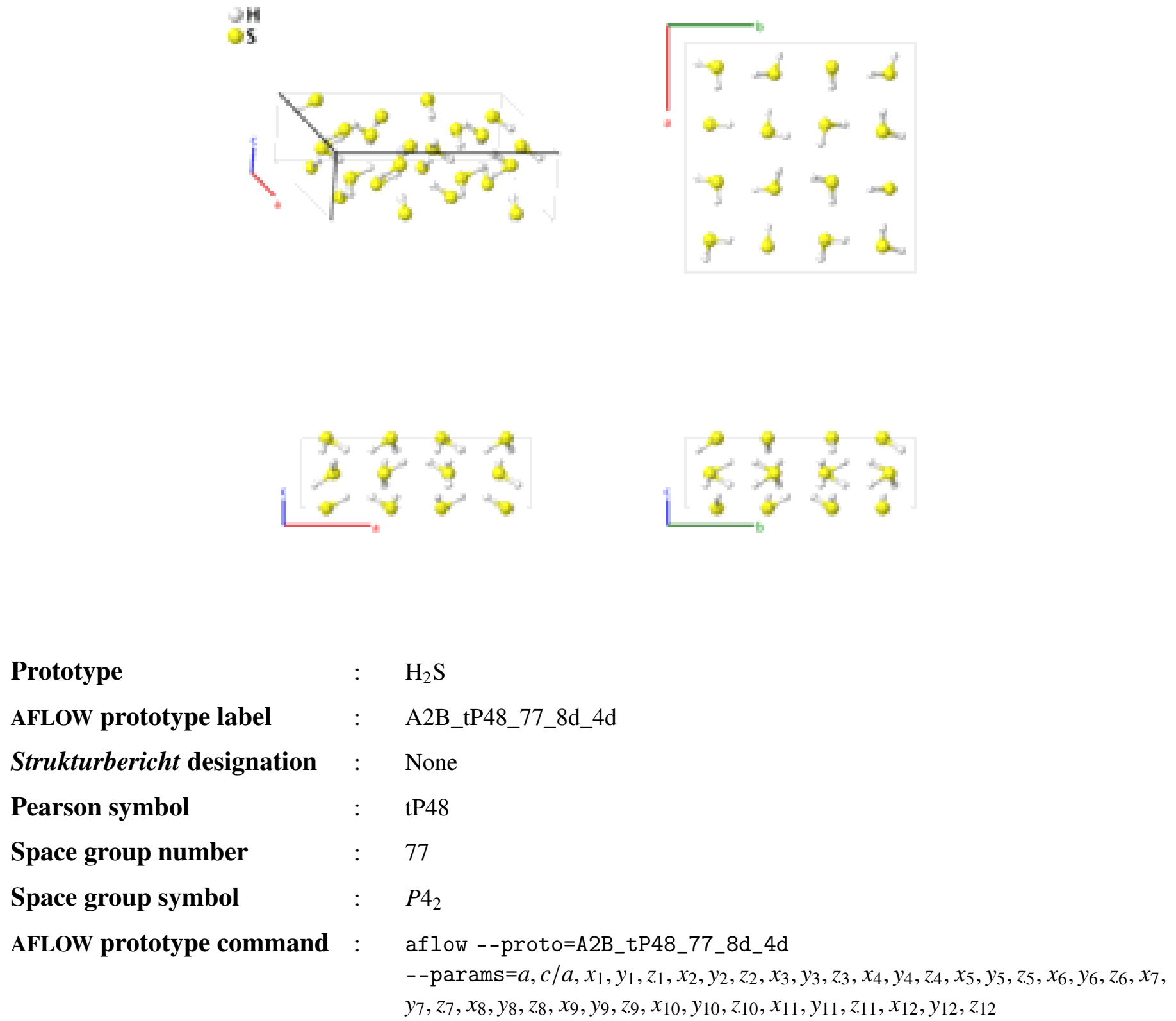

- This is one candidate structure for the $\mathrm{H}_{2} \mathrm{~S}$ III structure, which is stable at pressures under $4 \mathrm{GPa}$ and temperatures less than $\approx 100 \mathrm{~K}$ (Shimizu, 1995). The data presented here was for $\mathrm{D}_{2} \mathrm{~S}$ at $102 \mathrm{~K}$ and ambient pressure.

\section{Simple Tetragonal primitive vectors:}

$$
\begin{aligned}
& \mathbf{a}_{1}=a \hat{\mathbf{x}} \\
& \mathbf{a}_{2}=a \hat{\mathbf{y}} \\
& \mathbf{a}_{3}=c \hat{\mathbf{z}}
\end{aligned}
$$

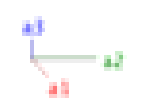


Lattice Coordinates

$$
\begin{aligned}
& \mathbf{B}_{1}= \\
& x_{1} \mathbf{a}_{1}+y_{1} \mathbf{a}_{2}+z_{1} \mathbf{a}_{3} \\
& -x_{1} \mathbf{a}_{1}-y_{1} \mathbf{a}_{2}+z_{1} \mathbf{a}_{3}
\end{aligned}
$$

$\mathbf{B}_{3}=$

$\mathbf{B}_{4}=$

$\mathbf{B}_{5}=$

$\mathbf{B}_{6}=$

$\mathbf{B}_{7}=$

$\mathbf{B}_{8}=$

$\mathbf{B}_{9}=$

$\mathbf{B}_{10}=$

$\mathbf{B}_{11}=$

$\mathbf{B}_{12}=$

$\mathbf{B}_{13}=$

$\mathbf{B}_{14}=$

$\mathbf{B}_{15}=$

$\mathbf{B}_{16}=$

$\mathbf{B}_{17}=$

$\mathbf{B}_{18}=$

$\mathbf{B}_{19}=$

$\mathbf{B}_{20}=$

$\mathbf{B}_{21}=$

$\mathbf{B}_{22}=$

$\mathbf{B}_{23}=$

$\mathbf{B}_{24}=$

$\mathbf{B}_{25}=$

$\mathbf{B}_{26}=$

$\mathbf{B}_{27}=$

$\mathbf{B}_{28}=$

$\mathbf{B}_{29}=$

$\mathbf{B}_{30}=$

$\mathbf{B}_{31}=$

$\mathbf{B}_{32}=$

$\mathbf{B}_{33}=$

$\mathbf{B}_{34}=$

$\mathbf{B}_{35}=$

$$
-y_{1} \mathbf{a}_{1}+x_{1} \mathbf{a}_{2}+\left(\frac{1}{2}+z_{1}\right) \mathbf{a}_{3}
$$$$
y_{1} \mathbf{a}_{1}-x_{1} \mathbf{a}_{2}+\left(\frac{1}{2}+z_{1}\right) \mathbf{a}_{3}
$$$$
x_{2} \mathbf{a}_{1}+y_{2} \mathbf{a}_{2}+z_{2} \mathbf{a}_{3}
$$$$
-x_{2} \mathbf{a}_{1}-y_{2} \mathbf{a}_{2}+z_{2} \mathbf{a}_{3}
$$

$-y_{2} \mathbf{a}_{1}+x_{2} \mathbf{a}_{2}+\left(\frac{1}{2}+z_{2}\right) \mathbf{a}_{3}$

$y_{2} \mathbf{a}_{1}-x_{2} \mathbf{a}_{2}+\left(\frac{1}{2}+z_{2}\right) \mathbf{a}_{3}$

$x_{3} \mathbf{a}_{1}+y_{3} \mathbf{a}_{2}+z_{3} \mathbf{a}_{3}$

$-x_{3} \mathbf{a}_{1}-y_{3} \mathbf{a}_{2}+z_{3} \mathbf{a}_{3}$

$-y_{3} \mathbf{a}_{1}+x_{3} \mathbf{a}_{2}+\left(\frac{1}{2}+z_{3}\right) \mathbf{a}_{3}$

$y_{3} \mathbf{a}_{1}-x_{3} \mathbf{a}_{2}+\left(\frac{1}{2}+z_{3}\right) \mathbf{a}_{3}$

$$
x_{4} \mathbf{a}_{1}+y_{4} \mathbf{a}_{2}+z_{4} \mathbf{a}_{3}
$$$$
-x_{4} \mathbf{a}_{1}-y_{4} \mathbf{a}_{2}+z_{4} \mathbf{a}_{3}
$$

$-y_{4} \mathbf{a}_{1}+x_{4} \mathbf{a}_{2}+\left(\frac{1}{2}+z_{4}\right) \mathbf{a}_{3}$

$$
y_{4} \mathbf{a}_{1}-x_{4} \mathbf{a}_{2}+\left(\frac{1}{2}+z_{4}\right) \mathbf{a}_{3}
$$

$x_{5} \mathbf{a}_{1}+y_{5} \mathbf{a}_{2}+z_{5} \mathbf{a}_{3}$

$-x_{5} \mathbf{a}_{1}-y_{5} \mathbf{a}_{2}+z_{5} \mathbf{a}_{3}$

$-y_{5} \mathbf{a}_{1}+x_{5} \mathbf{a}_{2}+\left(\frac{1}{2}+z_{5}\right) \mathbf{a}_{3}$

$y_{5} \mathbf{a}_{1}-x_{5} \mathbf{a}_{2}+\left(\frac{1}{2}+z_{5}\right) \mathbf{a}_{3}$

$x_{6} \mathbf{a}_{1}+y_{6} \mathbf{a}_{2}+z_{6} \mathbf{a}_{3}$

$-x_{6} \mathbf{a}_{1}-y_{6} \mathbf{a}_{2}+z_{6} \mathbf{a}_{3}$

$-y_{6} \mathbf{a}_{1}+x_{6} \mathbf{a}_{2}+\left(\frac{1}{2}+z_{6}\right) \mathbf{a}_{3}$

$y_{6} \mathbf{a}_{1}-x_{6} \mathbf{a}_{2}+\left(\frac{1}{2}+z_{6}\right) \mathbf{a}_{3}$

$$
x_{7} \mathbf{a}_{1}+y_{7} \mathbf{a}_{2}+z_{7} \mathbf{a}_{3}
$$

$-x_{7} \mathbf{a}_{1}-y_{7} \mathbf{a}_{2}+z_{7} \mathbf{a}_{3}$

$-y_{7} \mathbf{a}_{1}+x_{7} \mathbf{a}_{2}+\left(\frac{1}{2}+z_{7}\right) \mathbf{a}_{3}$

$y_{7} \mathbf{a}_{1}-x_{7} \mathbf{a}_{2}+\left(\frac{1}{2}+z_{7}\right) \mathbf{a}_{3}$

$x_{8} \mathbf{a}_{1}+y_{8} \mathbf{a}_{2}+z_{8} \mathbf{a}_{3}$

$-x_{8} \mathbf{a}_{1}-y_{8} \mathbf{a}_{2}+z_{8} \mathbf{a}_{3}$

$-y_{8} \mathbf{a}_{1}+x_{8} \mathbf{a}_{2}+\left(\frac{1}{2}+z_{8}\right) \mathbf{a}_{3}$

$y_{8} \mathbf{a}_{1}-x_{8} \mathbf{a}_{2}+\left(\frac{1}{2}+z_{8}\right) \mathbf{a}_{3}$

$x_{9} \mathbf{a}_{1}+y_{9} \mathbf{a}_{2}+z_{9} \mathbf{a}_{3}$

$-x_{9} \mathbf{a}_{1}-y_{9} \mathbf{a}_{2}+z_{9} \mathbf{a}_{3}$

$-y_{9} \mathbf{a}_{1}+x_{9} \mathbf{a}_{2}+\left(\frac{1}{2}+z_{9}\right) \mathbf{a}_{3}$
Cartesian Coordinates$$
=
$$

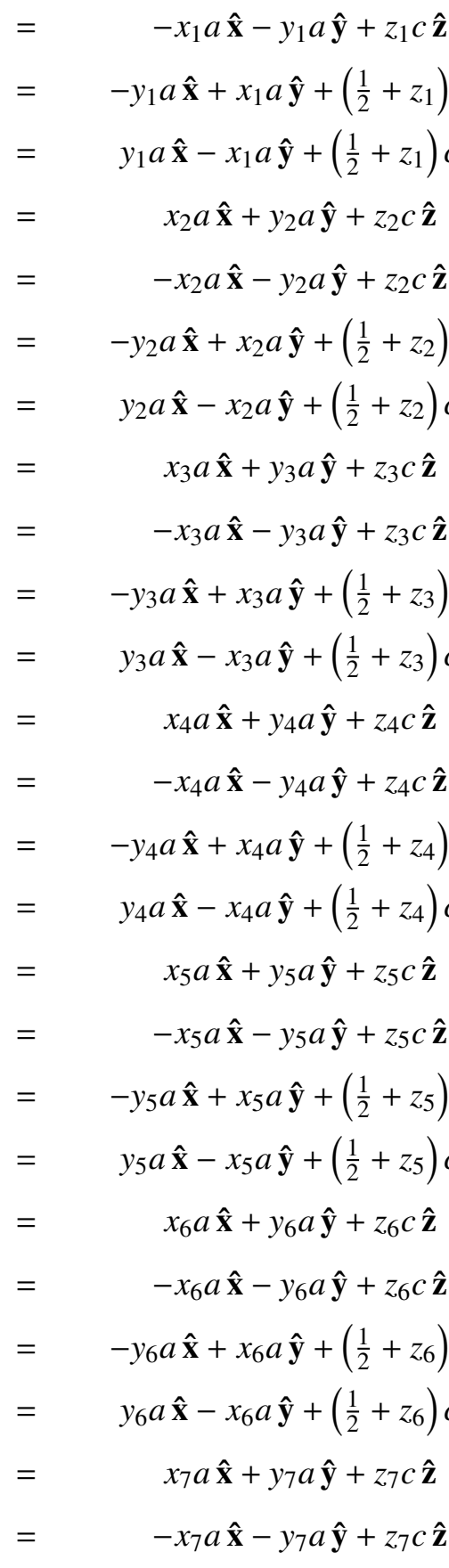

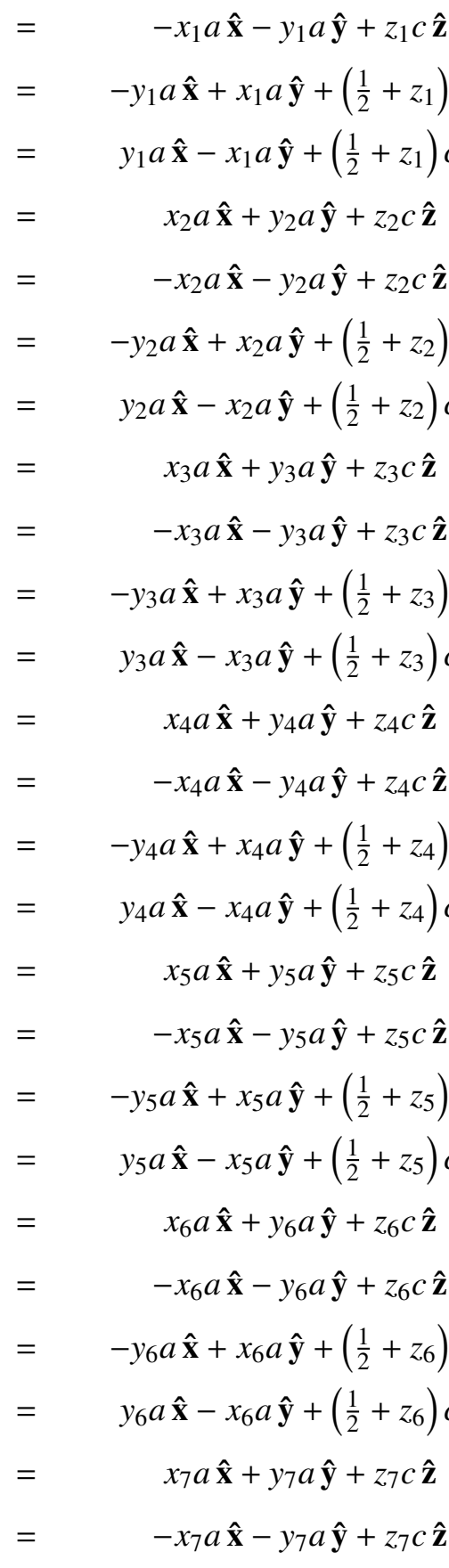

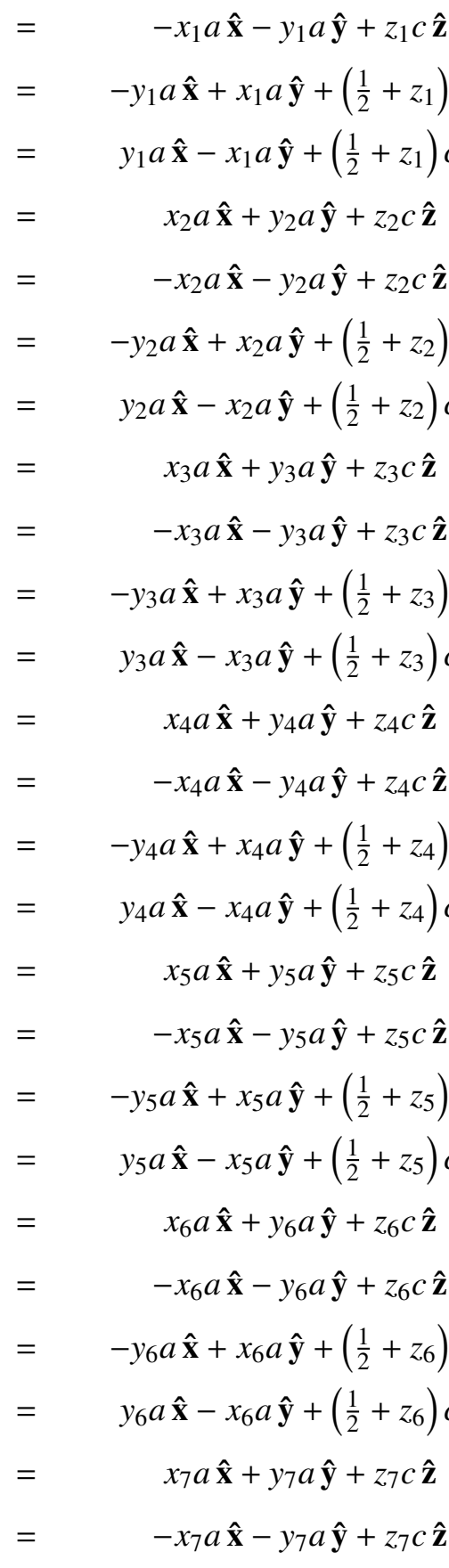

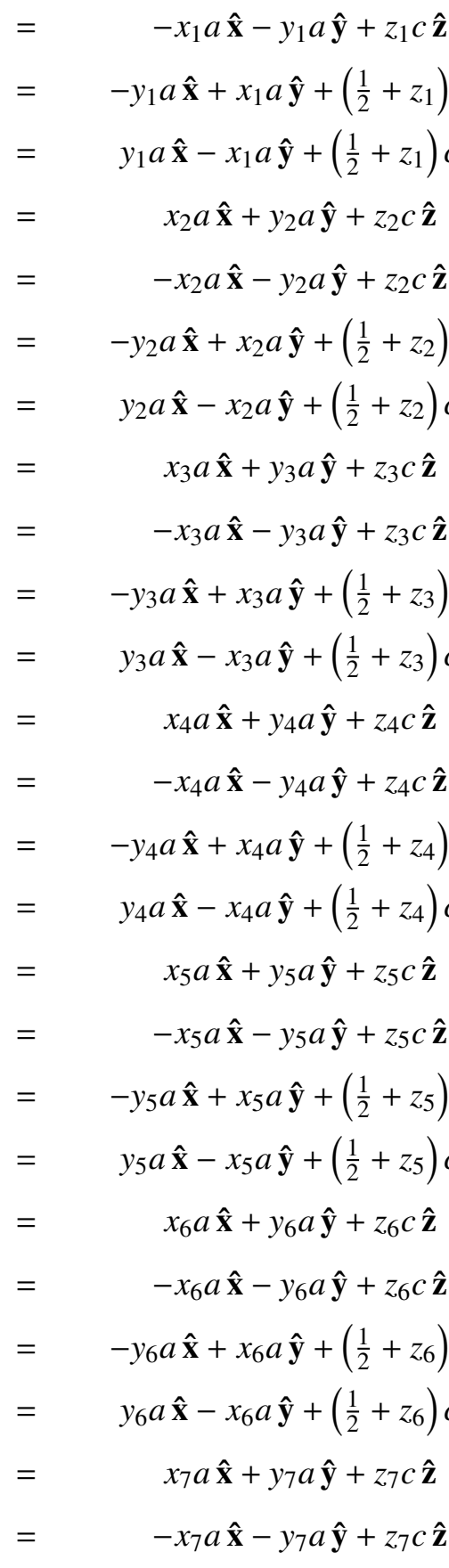

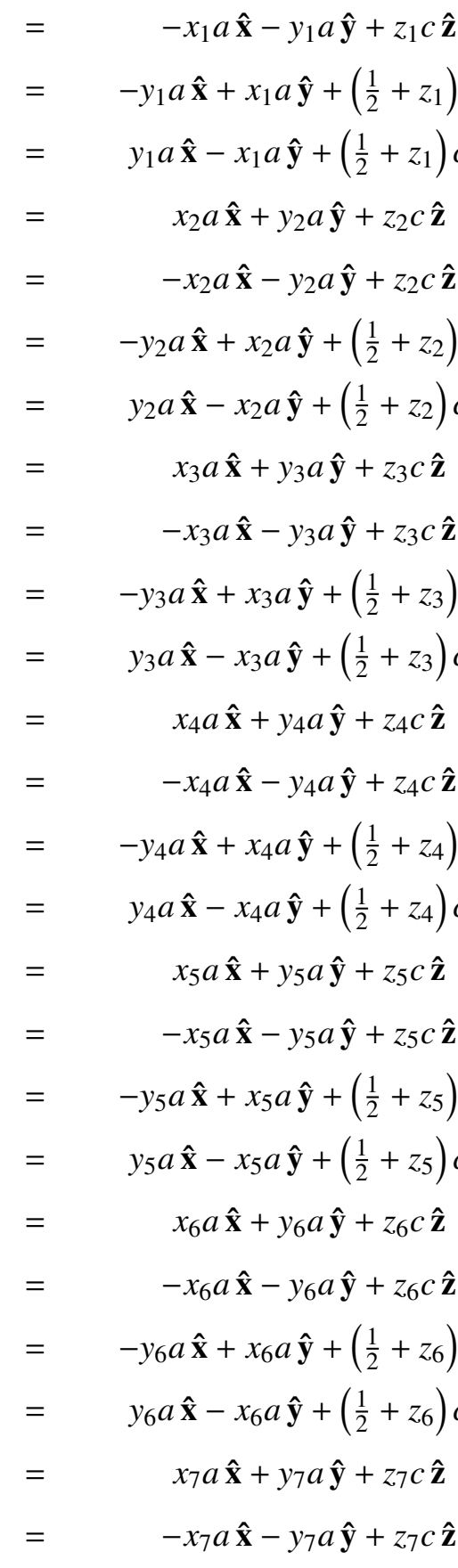

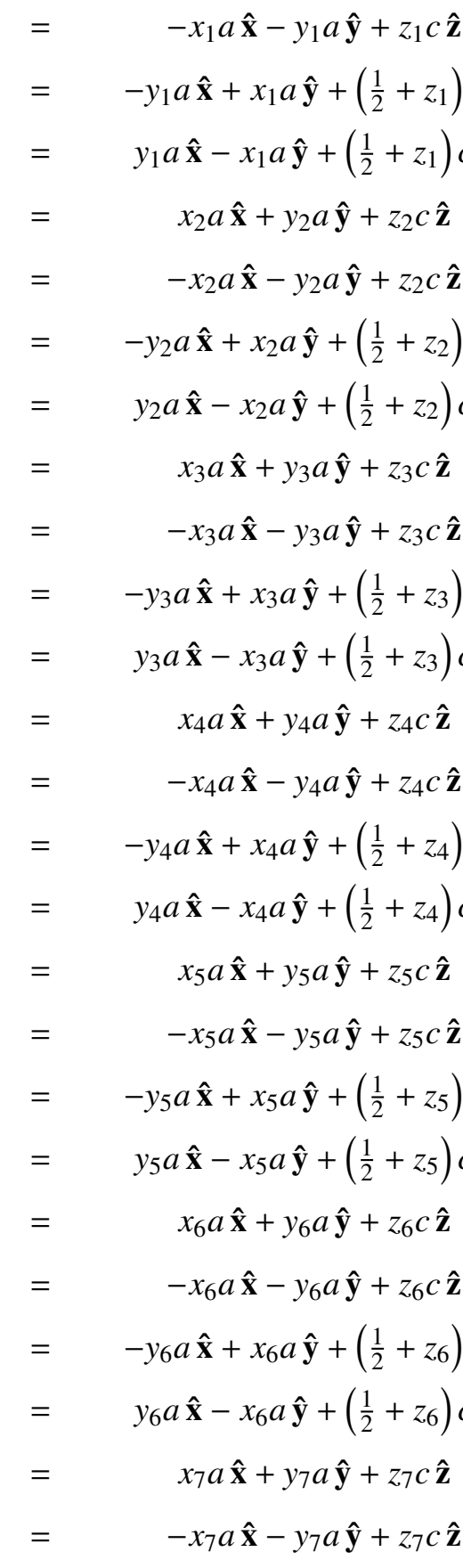

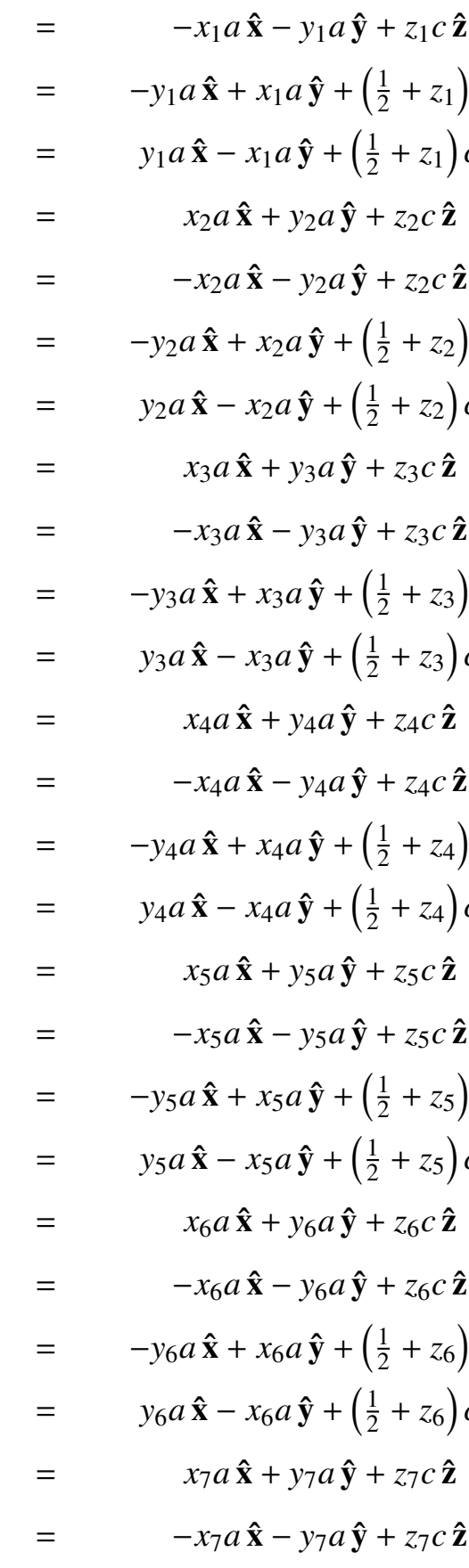

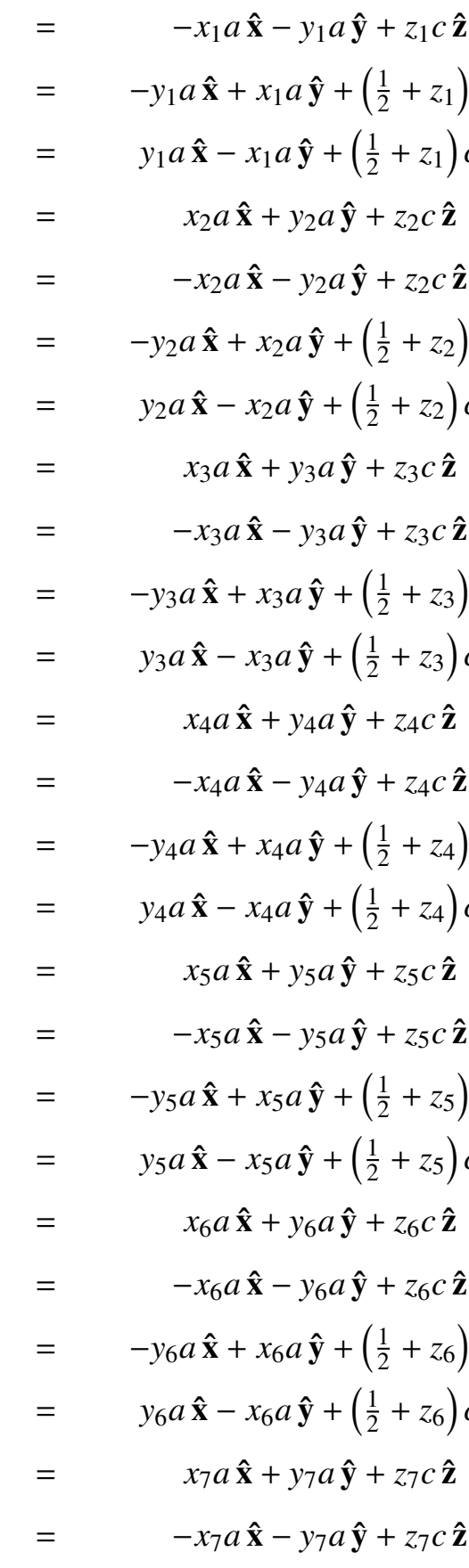

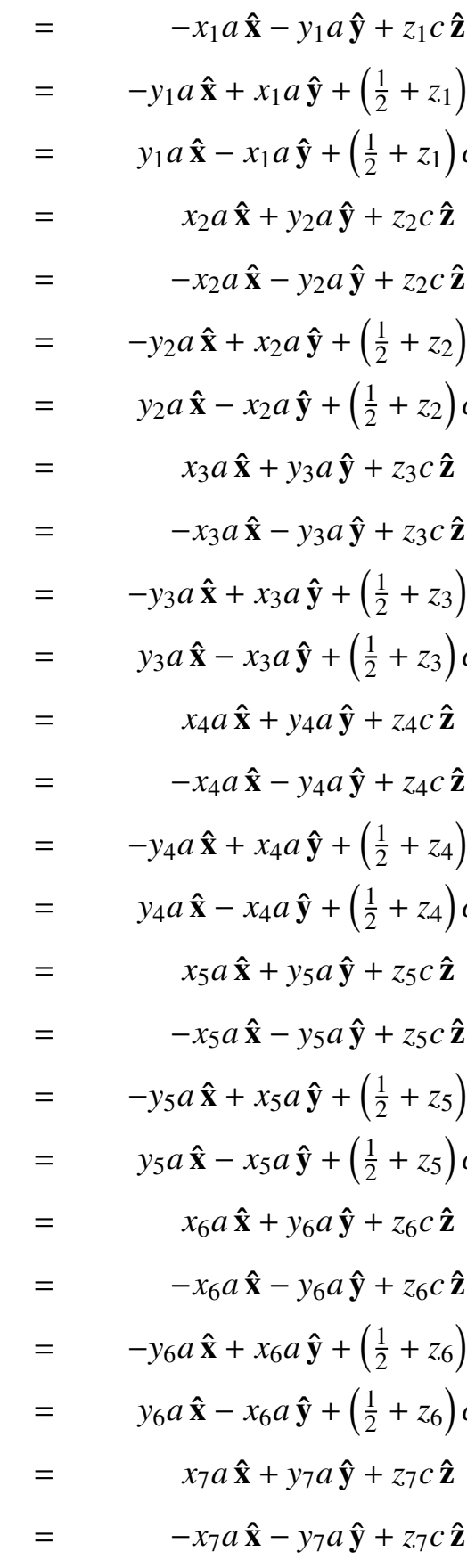

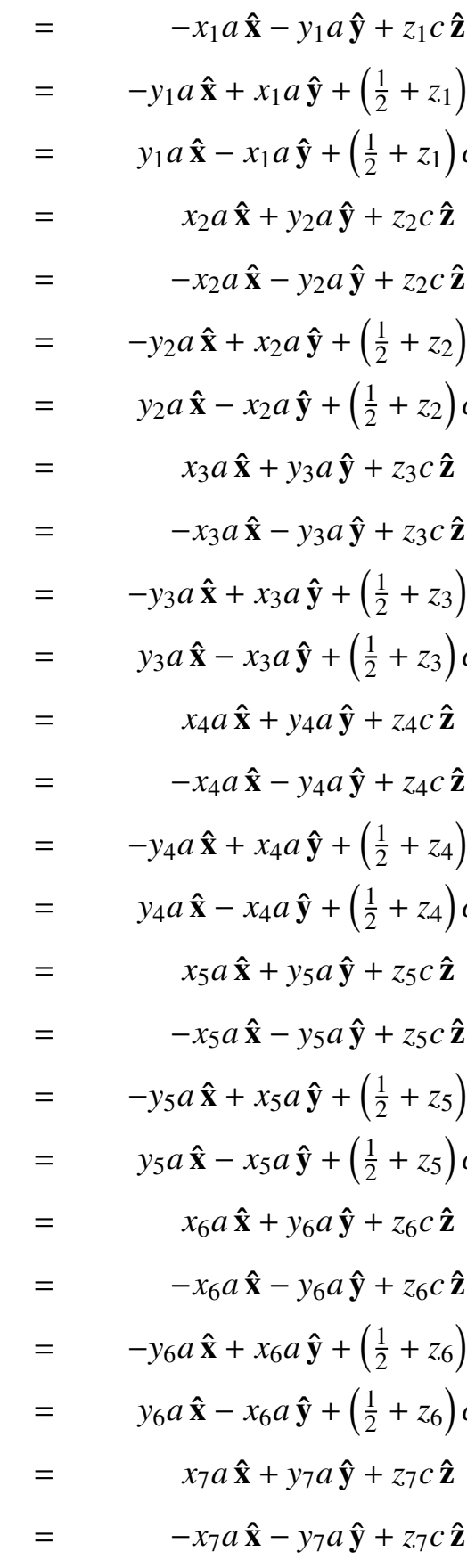

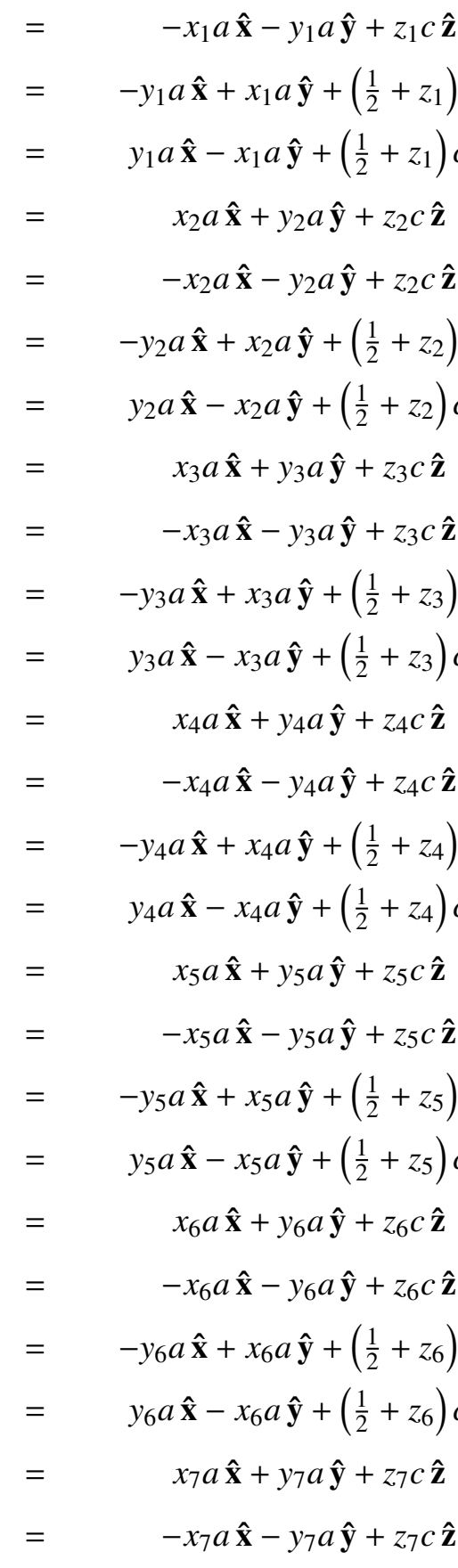

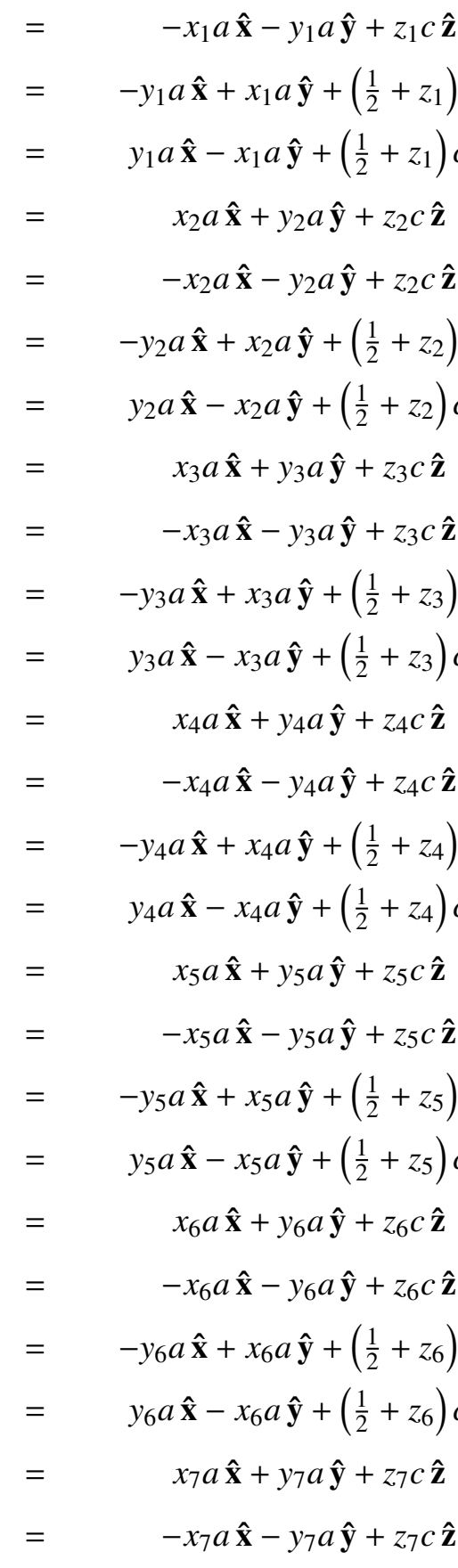$$
=
$$

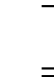

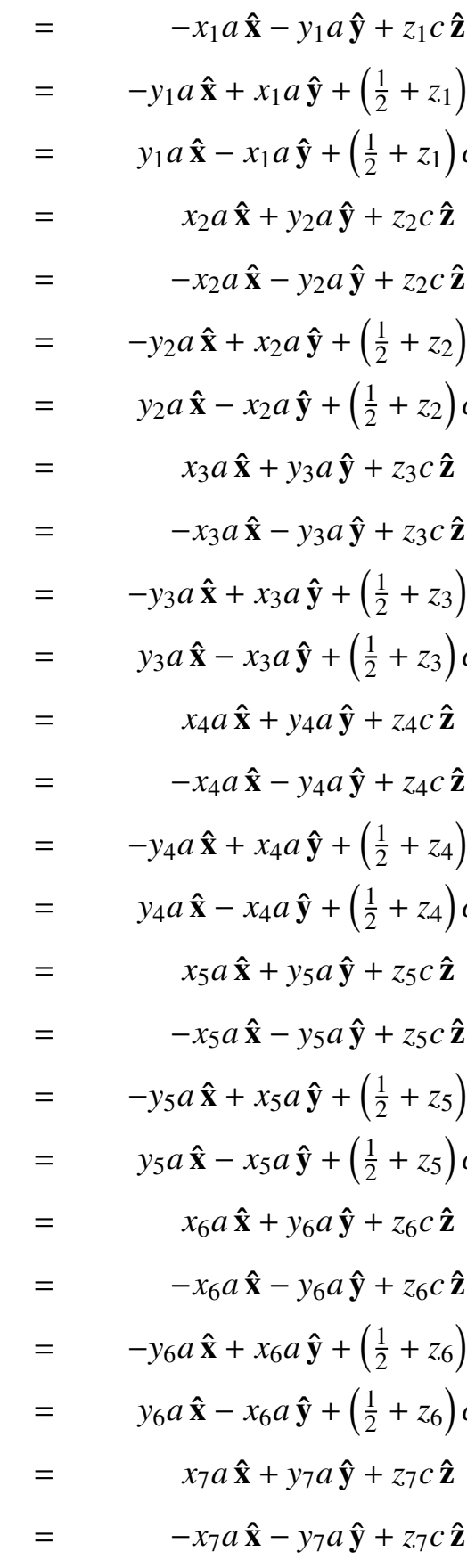$$
=
$$$$
=
$$$$
=
$$

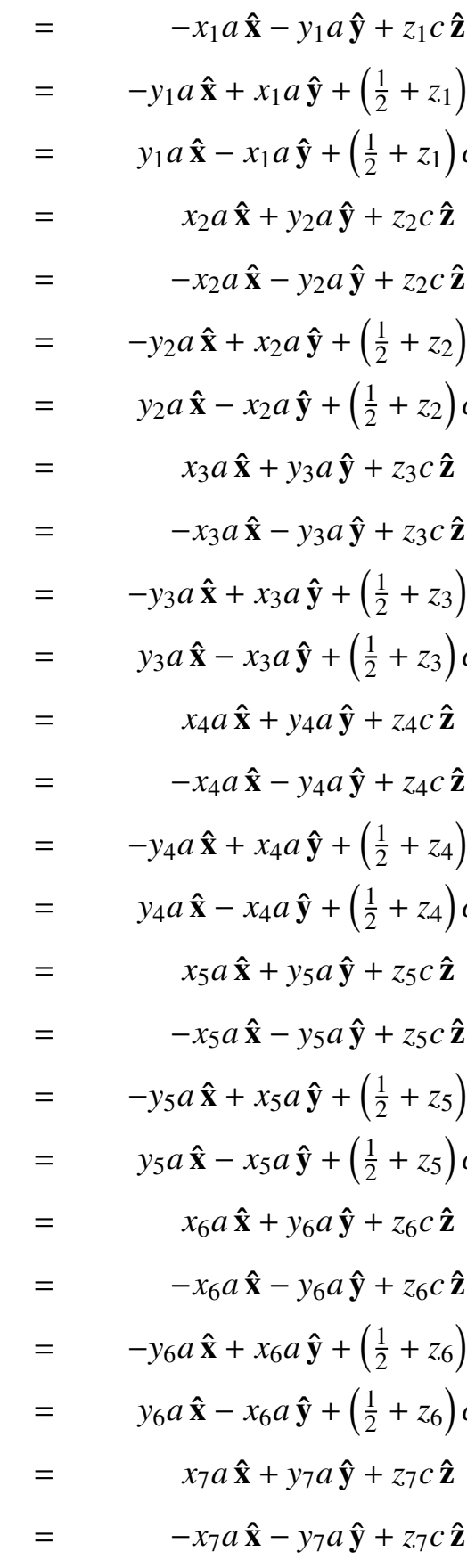

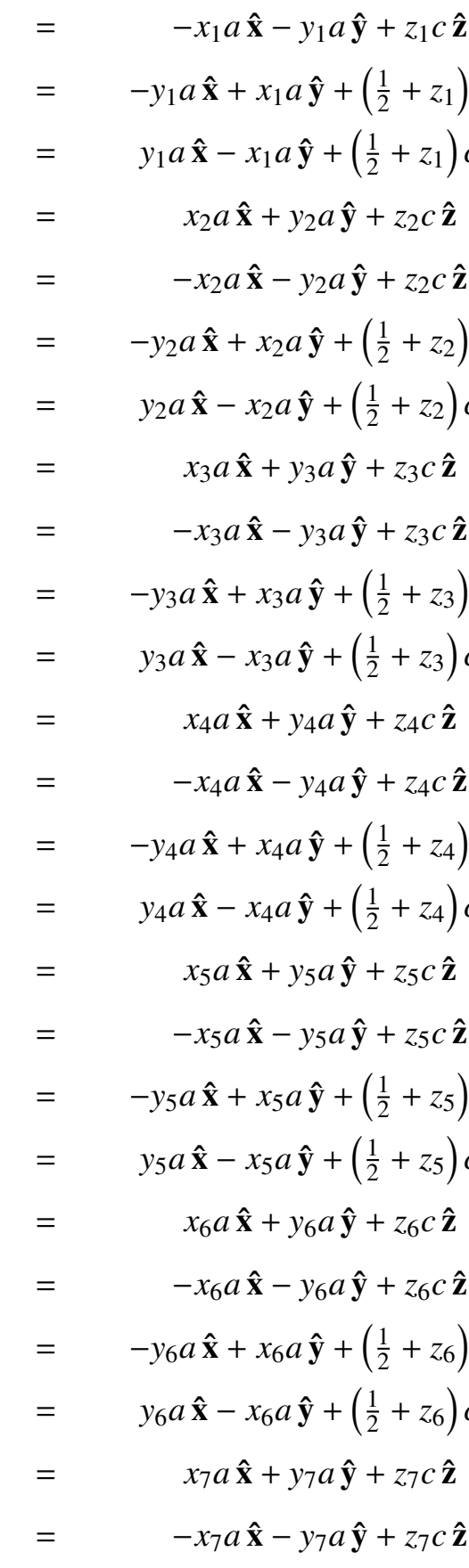

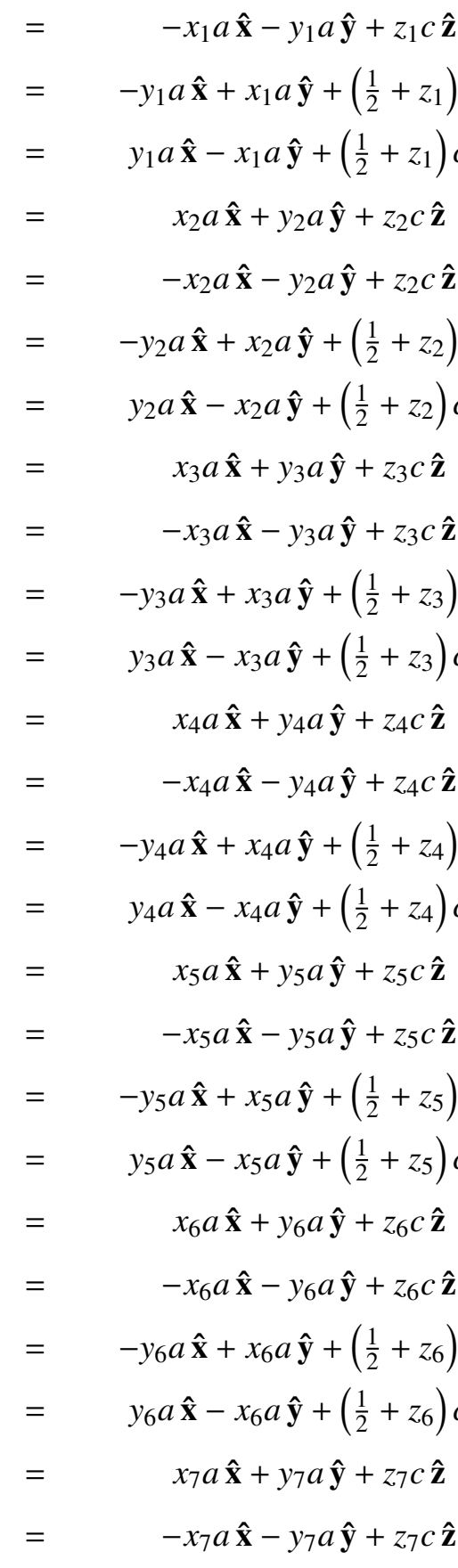

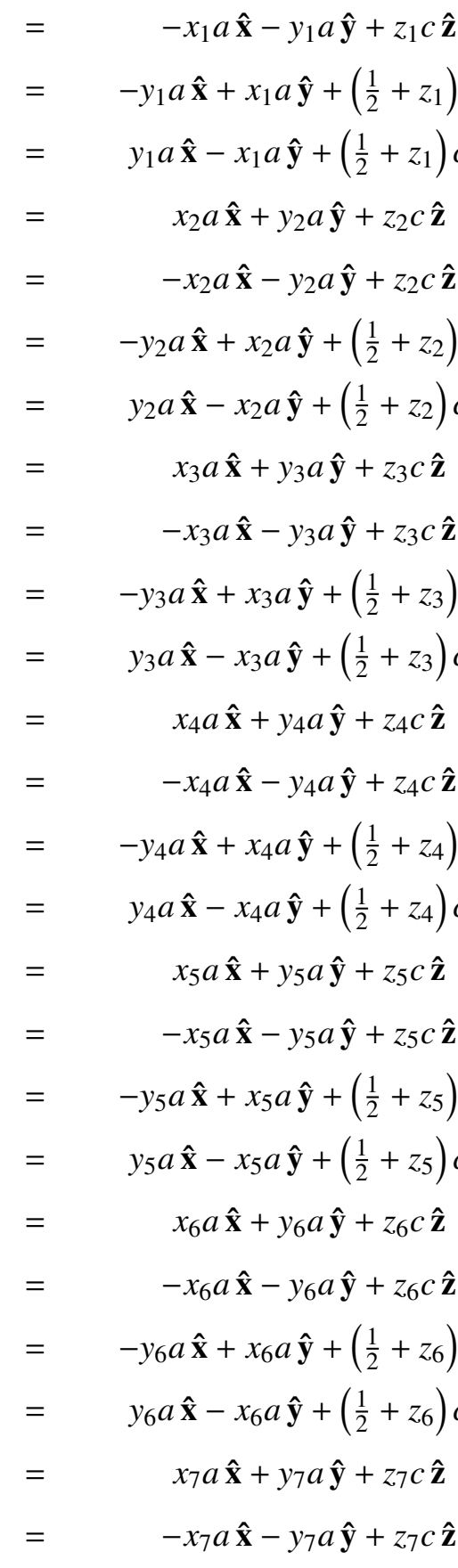

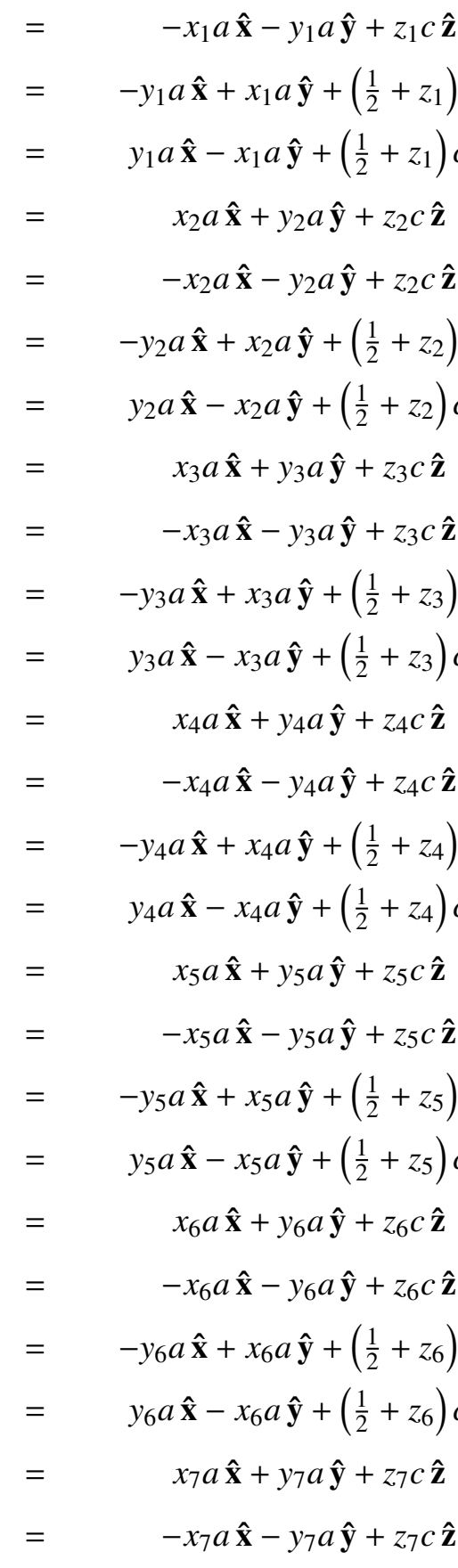

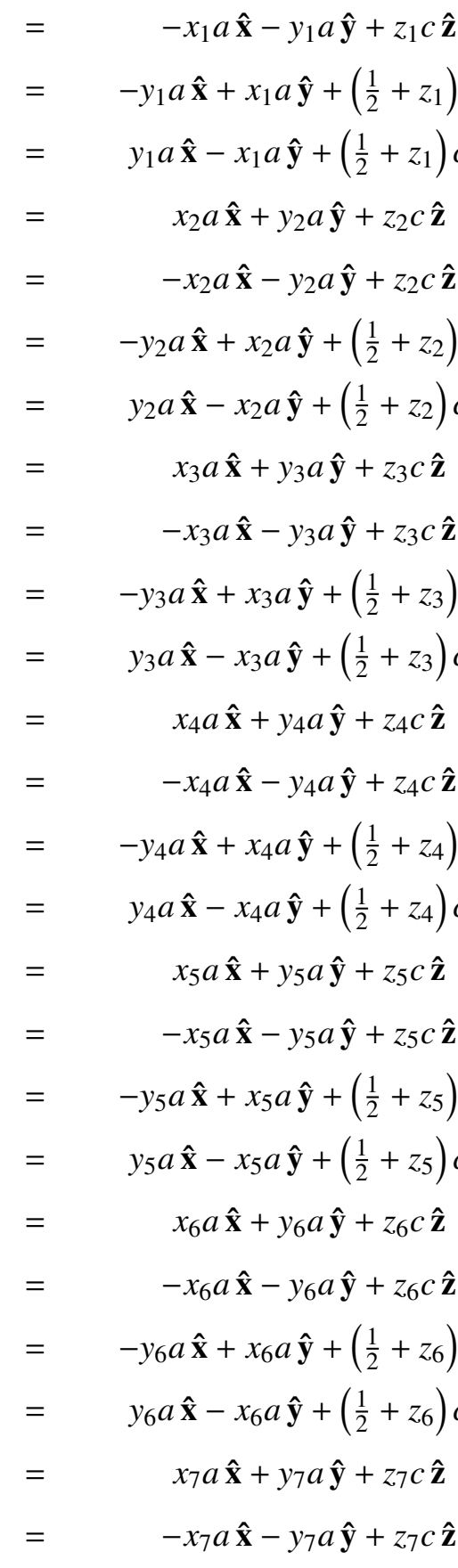

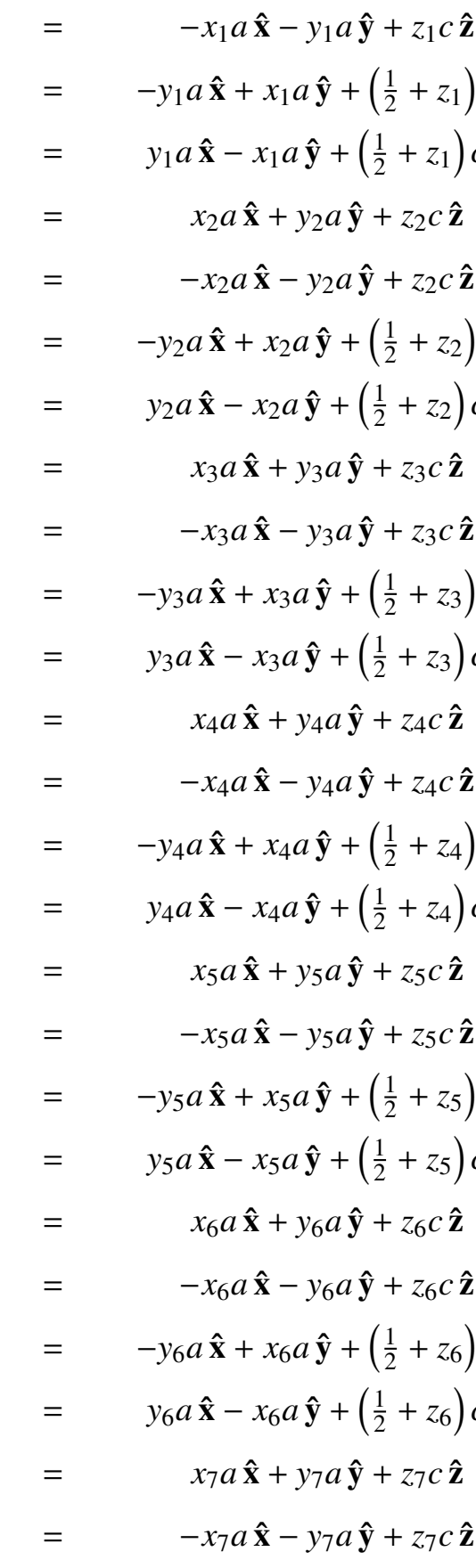

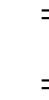

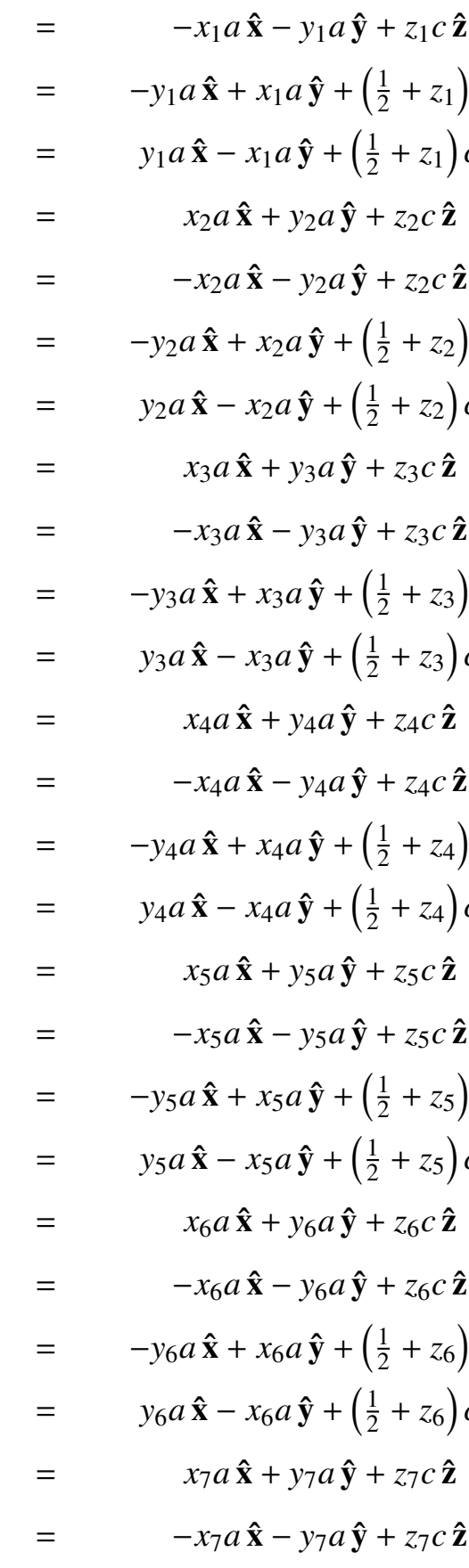

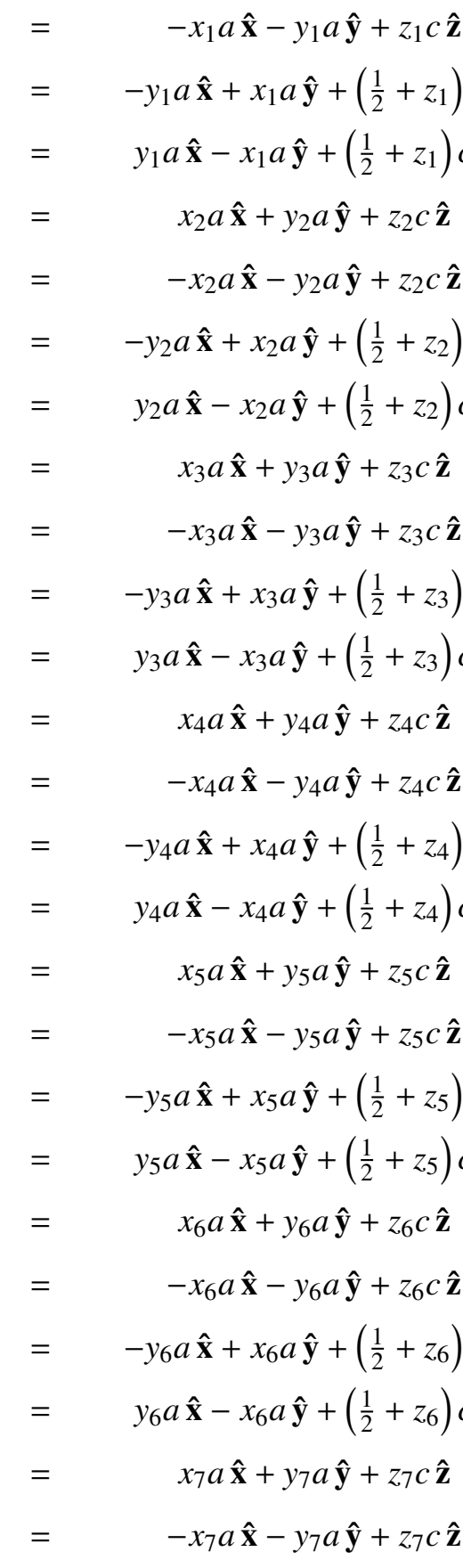

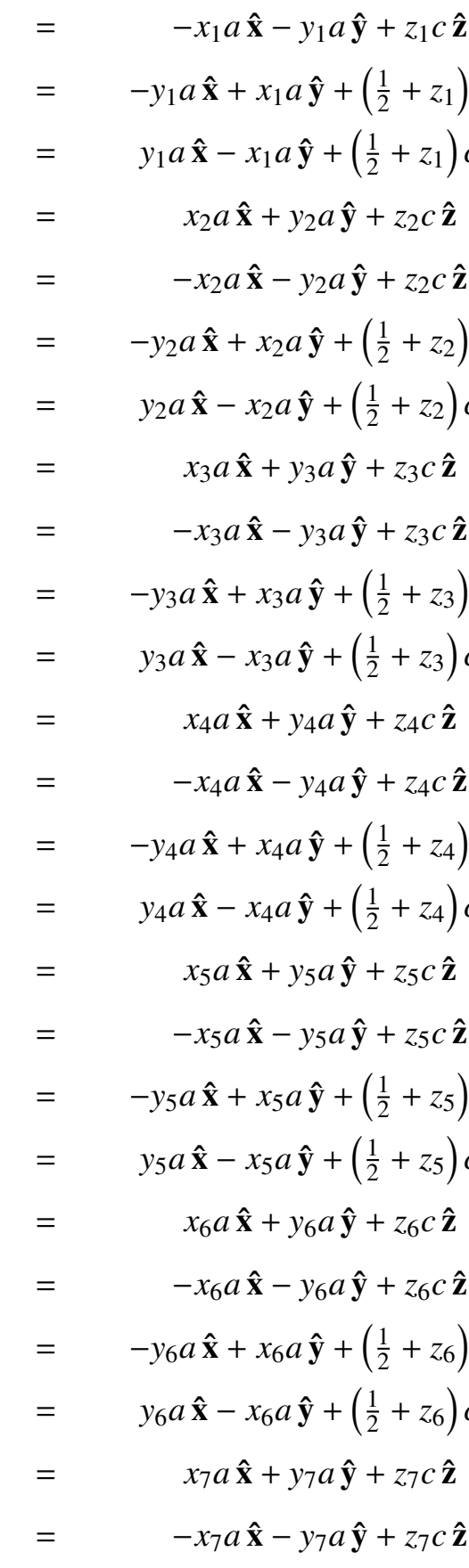$$
=-y_{7} a \hat{\mathbf{x}}+x_{7} a \hat{\mathbf{y}}+\left(\frac{1}{2}+z_{7}\right) c \hat{\mathbf{z}}
$$$$
=\quad y_{7} a \hat{\mathbf{x}}-x_{7} a \hat{\mathbf{y}}+\left(\frac{1}{2}+z_{7}\right) c \hat{\mathbf{z}}
$$$$
=\quad x_{8} a \hat{\mathbf{x}}+y_{8} a \hat{\mathbf{y}}+z_{8} c \hat{\mathbf{z}}
$$$$
=\quad-x_{8} a \hat{\mathbf{x}}-y_{8} a \hat{\mathbf{y}}+z_{8} c \hat{\mathbf{z}}
$$$$
=\quad-y_{8} a \hat{\mathbf{x}}+x_{8} a \hat{\mathbf{y}}+\left(\frac{1}{2}+z_{8}\right) c \hat{\mathbf{z}}
$$$$
=\quad y_{8} a \hat{\mathbf{x}}-x_{8} a \hat{\mathbf{y}}+\left(\frac{1}{2}+z_{8}\right) c \hat{\mathbf{z}}
$$$$
=\quad x_{9} a \hat{\mathbf{x}}+y_{9} a \hat{\mathbf{y}}+z_{9} c \hat{\mathbf{z}}
$$$$
=\quad-x_{9} a \hat{\mathbf{x}}-y_{9} a \hat{\mathbf{y}}+z_{9} c \hat{\mathbf{z}}
$$$$
=\quad-y_{9} a \hat{\mathbf{x}}+x_{9} a \hat{\mathbf{y}}+\left(\frac{1}{2}+z_{9}\right) c \hat{\mathbf{z}}
$$

Wyckoff Position
Atom Type

$$
\text { H I }
$$

H I

H I

H I

H II

H II

H II

H II

H III

H III

H III

H III

H IV

H IV

H IV

H IV

H V

H V

H V

H V

H VI

H VI

H VI

H VI

H VII

H VII

H VII

H VII

H VIII

H VIII

H VIII

H VIII

S I

S I

S I 


\begin{tabular}{|c|c|c|c|c|c|c|}
\hline $\mathbf{B}_{36}$ & $=$ & $y_{9} \mathbf{a}_{1}-x_{9} \mathbf{a}_{2}+\left(\frac{1}{2}+z_{9}\right) \mathbf{a}_{3}$ & $=$ & $y_{9} a \hat{\mathbf{x}}-x_{9} a \hat{\mathbf{y}}+\left(\frac{1}{2}+z_{9}\right) c \hat{\mathbf{z}}$ & $(4 d)$ & S I \\
\hline $\mathbf{B}_{37}$ & $=$ & $x_{10} \mathbf{a}_{1}+y_{10} \mathbf{a}_{2}+z_{10} \mathbf{a}_{3}$ & $=$ & $x_{10} a \hat{\mathbf{x}}+y_{10} a \hat{\mathbf{y}}+z_{10} c \hat{\mathbf{z}}$ & $(4 d)$ & S II \\
\hline $\mathbf{B}_{38}$ & $=$ & $-x_{10} \mathbf{a}_{1}-y_{10} \mathbf{a}_{2}+z_{10} \mathbf{a}_{3}$ & $=$ & $-x_{10} a \hat{\mathbf{x}}-y_{10} a \hat{\mathbf{y}}+z_{10} c \hat{\mathbf{z}}$ & $(4 d)$ & S II \\
\hline $\mathbf{B}_{39}$ & $=$ & $-y_{10} \mathbf{a}_{1}+x_{10} \mathbf{a}_{2}+\left(\frac{1}{2}+z_{10}\right) \mathbf{a}_{3}$ & $=$ & $-y_{10} a \hat{\mathbf{x}}+x_{10} a \hat{\mathbf{y}}+\left(\frac{1}{2}+z_{10}\right) c \hat{\mathbf{z}}$ & $(4 d)$ & S II \\
\hline $\mathbf{B}_{40}$ & $=$ & $y_{10} \mathbf{a}_{1}-x_{10} \mathbf{a}_{2}+\left(\frac{1}{2}+z_{10}\right) \mathbf{a}_{3}$ & $=$ & $y_{10} a \hat{\mathbf{x}}-x_{10} a \hat{\mathbf{y}}+\left(\frac{1}{2}+z_{10}\right) c \hat{\mathbf{z}}$ & $(4 d)$ & S II \\
\hline $\mathbf{B}_{41}$ & $=$ & $x_{11} \mathbf{a}_{1}+y_{11} \mathbf{a}_{2}+z_{11} \mathbf{a}_{3}$ & $=$ & $x_{11} a \hat{\mathbf{x}}+y_{11} a \hat{\mathbf{y}}+z_{11} c \hat{\mathbf{z}}$ & $(4 d)$ & S III \\
\hline $\mathbf{B}_{42}$ & $=$ & $-x_{11} \mathbf{a}_{1}-y_{11} \mathbf{a}_{2}+z_{11} \mathbf{a}_{3}$ & $=$ & $-x_{11} a \hat{\mathbf{x}}-y_{11} a \hat{\mathbf{y}}+z_{11} c \hat{\mathbf{z}}$ & $(4 d)$ & S III \\
\hline $\mathbf{B}_{43}$ & $=$ & $-y_{11} \mathbf{a}_{1}+x_{11} \mathbf{a}_{2}+\left(\frac{1}{2}+z_{11}\right) \mathbf{a}_{3}$ & $=$ & $-y_{11} a \hat{\mathbf{x}}+x_{11} a \hat{\mathbf{y}}+\left(\frac{1}{2}+z_{11}\right) c \hat{\mathbf{z}}$ & $(4 d)$ & S III \\
\hline $\mathbf{B}_{44}$ & $=$ & $y_{11} \mathbf{a}_{1}-x_{11} \mathbf{a}_{2}+\left(\frac{1}{2}+z_{11}\right) \mathbf{a}_{3}$ & $=$ & $y_{11} a \hat{\mathbf{x}}-x_{11} a \hat{\mathbf{y}}+\left(\frac{1}{2}+z_{11}\right) c \hat{\mathbf{z}}$ & $(4 d)$ & S III \\
\hline $\mathbf{B}_{45}$ & $=$ & $x_{12} \mathbf{a}_{1}+y_{12} \mathbf{a}_{2}+z_{12} \mathbf{a}_{3}$ & $=$ & $x_{12} a \hat{\mathbf{x}}+y_{12} a \hat{\mathbf{y}}+z_{12} c \hat{\mathbf{z}}$ & $(4 d)$ & S IV \\
\hline $\mathbf{B}_{46}$ & $=$ & $-x_{12} \mathbf{a}_{1}-y_{12} \mathbf{a}_{2}+z_{12} \mathbf{a}_{3}$ & $=$ & $-x_{12} a \hat{\mathbf{x}}-y_{12} a \hat{\mathbf{y}}+z_{12} c \hat{\mathbf{z}}$ & $(4 d)$ & S IV \\
\hline $\mathbf{B}_{47}$ & $=$ & $-y_{12} \mathbf{a}_{1}+x_{12} \mathbf{a}_{2}+\left(\frac{1}{2}+z_{12}\right) \mathbf{a}_{3}$ & $=$ & $-y_{12} a \hat{\mathbf{x}}+x_{12} a \hat{\mathbf{y}}+\left(\frac{1}{2}+z_{12}\right) c \hat{\mathbf{z}}$ & $(4 d)$ & S IV \\
\hline $\mathbf{B}_{48}$ & $=$ & $y_{12} \mathbf{a}_{1}-x_{12} \mathbf{a}_{2}+\left(\frac{1}{2}+z_{12}\right) \mathbf{a}_{3}$ & $=$ & $y_{12} a \hat{\mathbf{x}}-x_{12} a \hat{\mathbf{y}}+\left(\frac{1}{2}+z_{12}\right) c \hat{\mathbf{z}}$ & $(4 d)$ & S IV \\
\hline
\end{tabular}

\section{References:}

- E. Sándor and S. O. Ogunade, Structure and Phase Transition in Solid Hydrogen and Deuterium Sulphides, Nature 224, 905-907 (1969), doi:10.1038/224905b0.

\section{Found in:}

- H. Shimizu, H. Yamaguchi, S. Sasaki, A. Honda, S. Endo, and M. Kobayashi, Pressure-temperature phase diagram of solid hydrogen sulfide determined by Raman spectroscopy, Phys. Rev. B 51, 9391-9394 (1995), doi:10.1103/PhysRevB.51.9391.

\section{Geometry files:}

- CIF: pp. 860

- POSCAR: pp. 860 


\section{$\mathrm{Sr}_{2} \mathrm{As}_{2} \mathrm{O}_{7}$ Structure: A2B7C2_tP88_78_4a_14a_4a}
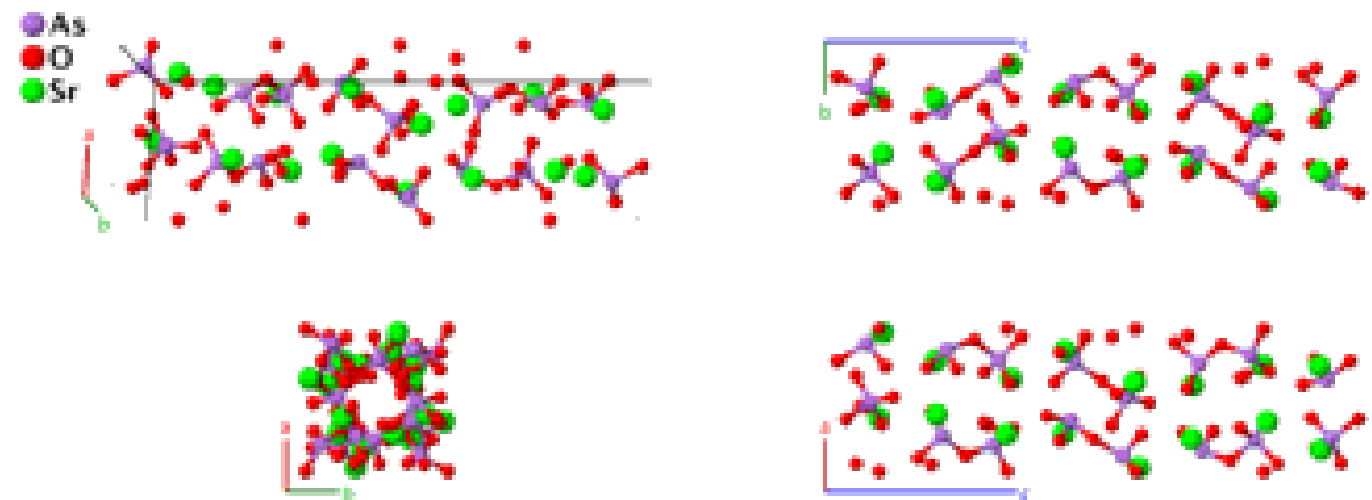

\section{Prototype}

AFLOW prototype label

Strukturbericht designation

Pearson symbol

Space group number

Space group symbol

AFLOW prototype command
: $\quad \mathrm{Sr}_{2} \mathrm{As}_{2} \mathrm{O}_{7}$

: A2B7C2_tP88_78_4a_14a_4a

: None

: $\quad$ tP88

$: \quad 78$

: $\quad P_{3}$

aflow --proto $=$ A2B7C2_tP88_78_4a_14a_4a

- -params $=a, c / a, x_{1}, y_{1}, z_{1}, x_{2}, y_{2}, z_{2}, x_{3}, y_{3}, z_{3}, x_{4}, y_{4}, z_{4}, x_{5}, y_{5}, z_{5}, x_{6}, y_{6}, z_{6}, x_{7}$,

$y_{7}, z_{7}, x_{8}, y_{8}, z_{8}, x_{9}, y_{9}, z_{9}, x_{10}, y_{10}, z_{10}, x_{11}, y_{11}, z_{11}, x_{12}, y_{12}, z_{12}, x_{13}, y_{13}, z_{13}, x_{14}, y_{14}$,

$z_{14}, x_{15}, y_{15}, z_{15}, x_{16}, y_{16}, z_{16}, x_{17}, y_{17}, z_{17}, x_{18}, y_{18}, z_{18}, x_{19}, y_{19}, z_{19}, x_{20}, y_{20}, z_{20}, x_{21}$,

$y_{21}, z_{21}, x_{22}, y_{22}, z_{22}$

\section{Simple Tetragonal primitive vectors:}

$$
\begin{aligned}
& \mathbf{a}_{1}=a \hat{\mathbf{x}} \\
& \mathbf{a}_{2}=a \hat{\mathbf{y}} \\
& \mathbf{a}_{3}=c \hat{\mathbf{z}}
\end{aligned}
$$

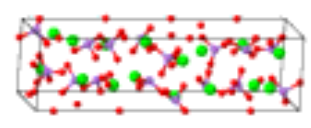

Basis vectors:

\section{Lattice Coordinates}

$\mathbf{B}_{1}=x_{1} \mathbf{a}_{1}+y_{1} \mathbf{a}_{2}+z_{1} \mathbf{a}_{3}$

$\mathbf{B}_{2}=$

$\mathbf{B}_{3}=$

$\mathbf{B}_{4}=$

$\mathbf{B}_{5}=$

$\mathbf{B}_{6}=$

$\mathbf{B}_{7}=$

$\mathbf{B}_{8}=$

$\mathbf{B}_{9}=$

$\mathbf{B}_{10}=$ $-x_{1} \mathbf{a}_{1}-y_{1} \mathbf{a}_{2}+\left(\frac{1}{2}+z_{1}\right) \mathbf{a}_{3}$

$-y_{1} \mathbf{a}_{1}+x_{1} \mathbf{a}_{2}+\left(\frac{3}{4}+z_{1}\right) \mathbf{a}_{3}$

$y_{1} \mathbf{a}_{1}-x_{1} \mathbf{a}_{2}+\left(\frac{1}{4}+z_{1}\right) \mathbf{a}_{3}$

$x_{2} \mathbf{a}_{1}+y_{2} \mathbf{a}_{2}+z_{2} \mathbf{a}_{3}$

$-x_{2} \mathbf{a}_{1}-y_{2} \mathbf{a}_{2}+\left(\frac{1}{2}+z_{2}\right) \mathbf{a}$

$-y_{2} \mathbf{a}_{1}+x_{2} \mathbf{a}_{2}+\left(\frac{3}{4}+z_{2}\right) \mathbf{a}_{3}$

$y_{2} \mathbf{a}_{1}-x_{2} \mathbf{a}_{2}+\left(\frac{1}{4}+z_{2}\right) \mathbf{a}_{3}$

$x_{3} \mathbf{a}_{1}+y_{3} \mathbf{a}_{2}+z_{3} \mathbf{a}_{3}$

$-x_{3} \mathbf{a}_{1}-y_{3} \mathbf{a}_{2}+\left(\frac{1}{2}+z_{3}\right) \mathbf{a}_{3}$

\section{Cartesian Coordinates}

$=$

$=$

$=$

$=$

$=$

$=$

$=$

$=$

$=$

$=$

$=$

$$
x_{1} a \hat{\mathbf{x}}+y_{1} a \hat{\mathbf{y}}+z_{1} c \hat{\mathbf{z}}
$$$$
-x_{1} a \hat{\mathbf{x}}-y_{1} a \hat{\mathbf{y}}+\left(\frac{1}{2}+z_{1}\right) c \hat{\mathbf{z}}
$$$$
-y_{1} a \hat{\mathbf{x}}+x_{1} a \hat{\mathbf{y}}+\left(\frac{3}{4}+z_{1}\right) c \hat{\mathbf{z}}
$$$$
y_{1} a \hat{\mathbf{x}}-x_{1} a \hat{\mathbf{y}}+\left(\frac{1}{4}+z_{1}\right) c \hat{\mathbf{z}}
$$$$
x_{2} a \hat{\mathbf{x}}+y_{2} a \hat{\mathbf{y}}+z_{2} c \hat{\mathbf{z}}
$$$$
-x_{2} a \hat{\mathbf{x}}-y_{2} a \hat{\mathbf{y}}+\left(\frac{1}{2}+z_{2}\right) c \hat{\mathbf{z}}
$$$$
-y_{2} a \hat{\mathbf{x}}+x_{2} a \hat{\mathbf{y}}+\left(\frac{3}{4}+z_{2}\right) c \hat{\mathbf{z}}
$$$$
y_{2} a \hat{\mathbf{x}}-x_{2} a \hat{\mathbf{y}}+\left(\frac{1}{4}+z_{2}\right) c \hat{\mathbf{z}}
$$$$
x_{3} a \hat{\mathbf{x}}+y_{3} a \hat{\mathbf{y}}+z_{3} c \hat{\mathbf{z}}
$$$$
-x_{3} a \hat{\mathbf{x}}-y_{3} a \hat{\mathbf{y}}+\left(\frac{1}{2}+z_{3}\right) c \hat{\mathbf{z}}
$$

Wyckoff Position

(4a)
Atom Type

As I

As I

As I

As I

As II

As II

As II

As II

As III

As III 


\begin{tabular}{|c|c|c|c|c|c|c|}
\hline $\mathbf{B}_{11}$ & $=$ & $-y_{3} \mathbf{a}_{1}+x_{3} \mathbf{a}_{2}+\left(\frac{3}{4}+z_{3}\right) \mathbf{a}_{3}$ & $=$ & $-y_{3} a \hat{\mathbf{x}}+x_{3} a \hat{\mathbf{y}}+\left(\frac{3}{4}+z_{3}\right) c \hat{\mathbf{z}}$ & $(4 a)$ & As III \\
\hline $\mathbf{B}_{12}$ & $=$ & $y_{3} \mathbf{a}_{1}-x_{3} \mathbf{a}_{2}+\left(\frac{1}{4}+z_{3}\right) \mathbf{a}_{3}$ & $=$ & $y_{3} a \hat{\mathbf{x}}-x_{3} a \hat{\mathbf{y}}+\left(\frac{1}{4}+z_{3}\right) c \hat{\mathbf{z}}$ & $(4 a)$ & As III \\
\hline $\mathbf{B}_{13}$ & $=$ & $x_{4} \mathbf{a}_{1}+y_{4} \mathbf{a}_{2}+z_{4} \mathbf{a}_{3}$ & $=$ & $x_{4} a \hat{\mathbf{x}}+y_{4} a \hat{\mathbf{y}}+z_{4} c \hat{\mathbf{z}}$ & $(4 a)$ & As IV \\
\hline $\mathbf{B}_{14}$ & $=$ & $-x_{4} \mathbf{a}_{1}-y_{4} \mathbf{a}_{2}+\left(\frac{1}{2}+z_{4}\right) \mathbf{a}_{3}$ & $=$ & $-x_{4} a \hat{\mathbf{x}}-y_{4} a \hat{\mathbf{y}}+\left(\frac{1}{2}+z_{4}\right) c \hat{\mathbf{z}}$ & $(4 a)$ & As IV \\
\hline $\mathbf{B}_{15}$ & $=$ & $-y_{4} \mathbf{a}_{1}+x_{4} \mathbf{a}_{2}+\left(\frac{3}{4}+z_{4}\right) \mathbf{a}_{3}$ & $=$ & $-y_{4} a \hat{\mathbf{x}}+x_{4} a \hat{\mathbf{y}}+\left(\frac{3}{4}+z_{4}\right) c \hat{\mathbf{z}}$ & $(4 a)$ & As IV \\
\hline $\mathbf{B}_{16}$ & $=$ & $y_{4} \mathbf{a}_{1}-x_{4} \mathbf{a}_{2}+\left(\frac{1}{4}+z_{4}\right) \mathbf{a}_{3}$ & $=$ & $y_{4} a \hat{\mathbf{x}}-x_{4} a \hat{\mathbf{y}}+\left(\frac{1}{4}+z_{4}\right) c \hat{\mathbf{z}}$ & $(4 a)$ & As IV \\
\hline $\mathbf{B}_{17}$ & $=$ & $x_{5} \mathbf{a}_{1}+y_{5} \mathbf{a}_{2}+z_{5} \mathbf{a}_{3}$ & $=$ & $x_{5} a \hat{\mathbf{x}}+y_{5} a \hat{\mathbf{y}}+z_{5} c \hat{\mathbf{z}}$ & $(4 a)$ & O I \\
\hline $\mathbf{B}_{18}$ & $=$ & $-x_{5} \mathbf{a}_{1}-y_{5} \mathbf{a}_{2}+\left(\frac{1}{2}+z_{5}\right) \mathbf{a}_{3}$ & $=$ & $-x_{5} a \hat{\mathbf{x}}-y_{5} a \hat{\mathbf{y}}+\left(\frac{1}{2}+z_{5}\right) c \hat{\mathbf{z}}$ & $(4 a)$ & O I \\
\hline $\mathbf{B}_{19}$ & $=$ & $-y_{5} \mathbf{a}_{1}+x_{5} \mathbf{a}_{2}+\left(\frac{3}{4}+z_{5}\right) \mathbf{a}_{3}$ & $=$ & $-y_{5} a \hat{\mathbf{x}}+x_{5} a \hat{\mathbf{y}}+\left(\frac{3}{4}+z_{5}\right) c \hat{\mathbf{z}}$ & $(4 a)$ & O I \\
\hline $\mathbf{B}_{20}$ & $=$ & $y_{5} \mathbf{a}_{1}-x_{5} \mathbf{a}_{2}+\left(\frac{1}{4}+z_{5}\right) \mathbf{a}_{3}$ & $=$ & $y_{5} a \hat{\mathbf{x}}-x_{5} a \hat{\mathbf{y}}+\left(\frac{1}{4}+z_{5}\right) c \hat{\mathbf{z}}$ & $(4 a)$ & O I \\
\hline $\mathbf{B}_{21}$ & $=$ & $x_{6} \mathbf{a}_{1}+y_{6} \mathbf{a}_{2}+z_{6} \mathbf{a}_{3}$ & $=$ & $x_{6} a \hat{\mathbf{x}}+y_{6} a \hat{\mathbf{y}}+z_{6} c \hat{\mathbf{z}}$ & $(4 a)$ & O II \\
\hline $\mathbf{B}_{22}$ & $=$ & $-x_{6} \mathbf{a}_{1}-y_{6} \mathbf{a}_{2}+\left(\frac{1}{2}+z_{6}\right) \mathbf{a}_{3}$ & $=$ & $-x_{6} a \hat{\mathbf{x}}-y_{6} a \hat{\mathbf{y}}+\left(\frac{1}{2}+z_{6}\right) c \hat{\mathbf{z}}$ & $(4 a)$ & O II \\
\hline $\mathbf{B}_{23}$ & $=$ & $-y_{6} \mathbf{a}_{1}+x_{6} \mathbf{a}_{2}+\left(\frac{3}{4}+z_{6}\right) \mathbf{a}_{3}$ & $=$ & $-y_{6} a \hat{\mathbf{x}}+x_{6} a \hat{\mathbf{y}}+\left(\frac{3}{4}+z_{6}\right) c \hat{\mathbf{z}}$ & $(4 a)$ & O II \\
\hline $\mathbf{B}_{24}$ & $=$ & $y_{6} \mathbf{a}_{1}-x_{6} \mathbf{a}_{2}+\left(\frac{1}{4}+z_{6}\right) \mathbf{a}_{3}$ & $=$ & $y_{6} a \hat{\mathbf{x}}-x_{6} a \hat{\mathbf{y}}+\left(\frac{1}{4}+z_{6}\right) c \hat{\mathbf{z}}$ & $(4 a)$ & O II \\
\hline $\mathbf{B}_{25}$ & $=$ & $x_{7} \mathbf{a}_{1}+y_{7} \mathbf{a}_{2}+z_{7} \mathbf{a}_{3}$ & $=$ & $x_{7} a \hat{\mathbf{x}}+y_{7} a \hat{\mathbf{y}}+z_{7} c \hat{\mathbf{z}}$ & $(4 a)$ & O III \\
\hline $\mathbf{B}_{26}$ & $=$ & $-x_{7} \mathbf{a}_{1}-y_{7} \mathbf{a}_{2}+\left(\frac{1}{2}+z_{7}\right) \mathbf{a}_{3}$ & $=$ & $-x_{7} a \hat{\mathbf{x}}-y_{7} a \hat{\mathbf{y}}+\left(\frac{1}{2}+z_{7}\right) c \hat{\mathbf{z}}$ & $(4 a)$ & O III \\
\hline $\mathbf{B}_{27}$ & $=$ & $-y_{7} \mathbf{a}_{1}+x_{7} \mathbf{a}_{2}+\left(\frac{3}{4}+z_{7}\right) \mathbf{a}_{3}$ & $=$ & $-y_{7} a \hat{\mathbf{x}}+x_{7} a \hat{\mathbf{y}}+\left(\frac{3}{4}+z_{7}\right) c \hat{\mathbf{z}}$ & $(4 a)$ & O III \\
\hline $\mathbf{B}_{28}$ & $=$ & $y_{7} \mathbf{a}_{1}-x_{7} \mathbf{a}_{2}+\left(\frac{1}{4}+z_{7}\right) \mathbf{a}_{3}$ & $=$ & $y_{7} a \hat{\mathbf{x}}-x_{7} a \hat{\mathbf{y}}+\left(\frac{1}{4}+z_{7}\right) c \hat{\mathbf{z}}$ & $(4 a)$ & O III \\
\hline $\mathbf{B}_{29}$ & $=$ & $x_{8} \mathbf{a}_{1}+y_{8} \mathbf{a}_{2}+z_{8} \mathbf{a}_{3}$ & $=$ & $x_{8} a \hat{\mathbf{x}}+y_{8} a \hat{\mathbf{y}}+z_{8} c \hat{\mathbf{z}}$ & $(4 a)$ & O IV \\
\hline $\mathbf{B}_{30}$ & $=$ & $-x_{8} \mathbf{a}_{1}-y_{8} \mathbf{a}_{2}+\left(\frac{1}{2}+z_{8}\right) \mathbf{a}_{3}$ & $=$ & $-x_{8} a \hat{\mathbf{x}}-y_{8} a \hat{\mathbf{y}}+\left(\frac{1}{2}+z_{8}\right) c \hat{\mathbf{z}}$ & $(4 a)$ & O IV \\
\hline $\mathbf{B}_{31}$ & $=$ & $-y_{8} \mathbf{a}_{1}+x_{8} \mathbf{a}_{2}+\left(\frac{3}{4}+z_{8}\right) \mathbf{a}_{3}$ & $=$ & $-y_{8} a \hat{\mathbf{x}}+x_{8} a \hat{\mathbf{y}}+\left(\frac{3}{4}+z_{8}\right) c \hat{\mathbf{z}}$ & $(4 a)$ & O IV \\
\hline $\mathbf{B}_{32}$ & $=$ & $y_{8} \mathbf{a}_{1}-x_{8} \mathbf{a}_{2}+\left(\frac{1}{4}+z_{8}\right) \mathbf{a}_{3}$ & $=$ & $y_{8} a \hat{\mathbf{x}}-x_{8} a \hat{\mathbf{y}}+\left(\frac{1}{4}+z_{8}\right) c \hat{\mathbf{z}}$ & $(4 a)$ & O IV \\
\hline $\mathbf{B}_{33}$ & $=$ & $x_{9} \mathbf{a}_{1}+y_{9} \mathbf{a}_{2}+z_{9} \mathbf{a}_{3}$ & $=$ & $x_{9} a \hat{\mathbf{x}}+y_{9} a \hat{\mathbf{y}}+z_{9} c \hat{\mathbf{z}}$ & $(4 a)$ & $\mathrm{OV}$ \\
\hline $\mathbf{B}_{34}$ & $=$ & $-x_{9} \mathbf{a}_{1}-y_{9} \mathbf{a}_{2}+\left(\frac{1}{2}+z_{9}\right) \mathbf{a}_{3}$ & $=$ & $-x_{9} a \hat{\mathbf{x}}-y_{9} a \hat{\mathbf{y}}+\left(\frac{1}{2}+z_{9}\right) c \hat{\mathbf{z}}$ & $(4 a)$ & $\mathrm{OV}$ \\
\hline $\mathbf{B}_{35}$ & $=$ & $-y_{9} \mathbf{a}_{1}+x_{9} \mathbf{a}_{2}+\left(\frac{3}{4}+z_{9}\right) \mathbf{a}_{3}$ & $=$ & $-y_{9} a \hat{\mathbf{x}}+x_{9} a \hat{\mathbf{y}}+\left(\frac{3}{4}+z_{9}\right) c \hat{\mathbf{z}}$ & $(4 a)$ & $\mathrm{OV}$ \\
\hline $\mathbf{B}_{36}$ & $=$ & $y_{9} \mathbf{a}_{1}-x_{9} \mathbf{a}_{2}+\left(\frac{1}{4}+z_{9}\right) \mathbf{a}_{3}$ & $=$ & $y_{9} a \hat{\mathbf{x}}-x_{9} a \hat{\mathbf{y}}+\left(\frac{1}{4}+z_{9}\right) c \hat{\mathbf{z}}$ & $(4 a)$ & $\mathrm{OV}$ \\
\hline $\mathbf{B}_{37}$ & $=$ & $x_{10} \mathbf{a}_{1}+y_{10} \mathbf{a}_{2}+z_{10} \mathbf{a}_{3}$ & $=$ & $x_{10} a \hat{\mathbf{x}}+y_{10} a \hat{\mathbf{y}}+z_{10} c \hat{\mathbf{z}}$ & $(4 a)$ & O VI \\
\hline $\mathbf{B}_{38}$ & $=$ & $-x_{10} \mathbf{a}_{1}-y_{10} \mathbf{a}_{2}+\left(\frac{1}{2}+z_{10}\right) \mathbf{a}_{3}$ & $=$ & $-x_{10} a \hat{\mathbf{x}}-y_{10} a \hat{\mathbf{y}}+\left(\frac{1}{2}+z_{10}\right) c \hat{\mathbf{z}}$ & $(4 a)$ & O VI \\
\hline $\mathbf{B}_{39}$ & $=$ & $-y_{10} \mathbf{a}_{1}+x_{10} \mathbf{a}_{2}+\left(\frac{3}{4}+z_{10}\right) \mathbf{a}_{3}$ & $=$ & $-y_{10} a \hat{\mathbf{x}}+x_{10} a \hat{\mathbf{y}}+\left(\frac{3}{4}+z_{10}\right) c \hat{\mathbf{z}}$ & $(4 a)$ & O VI \\
\hline $\mathbf{B}_{40}$ & $=$ & $y_{10} \mathbf{a}_{1}-x_{10} \mathbf{a}_{2}+\left(\frac{1}{4}+z_{10}\right) \mathbf{a}_{3}$ & $=$ & $y_{10} a \hat{\mathbf{x}}-x_{10} a \hat{\mathbf{y}}+\left(\frac{1}{4}+z_{10}\right) c \hat{\mathbf{z}}$ & $(4 a)$ & O VI \\
\hline $\mathbf{B}_{41}$ & $=$ & $x_{11} \mathbf{a}_{1}+y_{11} \mathbf{a}_{2}+z_{11} \mathbf{a}_{3}$ & $=$ & $x_{11} a \hat{\mathbf{x}}+y_{11} a \hat{\mathbf{y}}+z_{11} c \hat{\mathbf{z}}$ & $(4 a)$ & O VII \\
\hline $\mathbf{B}_{42}$ & $=$ & $-x_{11} \mathbf{a}_{1}-y_{11} \mathbf{a}_{2}+\left(\frac{1}{2}+z_{11}\right) \mathbf{a}_{3}$ & $=$ & $-x_{11} a \hat{\mathbf{x}}-y_{11} a \hat{\mathbf{y}}+\left(\frac{1}{2}+z_{11}\right) c \hat{\mathbf{z}}$ & $(4 a)$ & O VII \\
\hline $\mathbf{B}_{43}$ & $=$ & $-y_{11} \mathbf{a}_{1}+x_{11} \mathbf{a}_{2}+\left(\frac{3}{4}+z_{11}\right) \mathbf{a}_{3}$ & $=$ & $-y_{11} a \hat{\mathbf{x}}+x_{11} a \hat{\mathbf{y}}+\left(\frac{3}{4}+z_{11}\right) c \hat{\mathbf{z}}$ & $(4 a)$ & O VII \\
\hline $\mathbf{B}_{44}$ & $=$ & $y_{11} \mathbf{a}_{1}-x_{11} \mathbf{a}_{2}+\left(\frac{1}{4}+z_{11}\right) \mathbf{a}_{3}$ & $=$ & $y_{11} a \hat{\mathbf{x}}-x_{11} a \hat{\mathbf{y}}+\left(\frac{1}{4}+z_{11}\right) c \hat{\mathbf{z}}$ & $(4 a)$ & O VII \\
\hline $\mathbf{B}_{45}$ & $=$ & $x_{12} \mathbf{a}_{1}+y_{12} \mathbf{a}_{2}+z_{12} \mathbf{a}_{3}$ & $=$ & $x_{12} a \hat{\mathbf{x}}+y_{12} a \hat{\mathbf{y}}+z_{12} c \hat{\mathbf{z}}$ & $(4 a)$ & O VIII \\
\hline $\mathbf{B}_{46}$ & $=$ & $-x_{12} \mathbf{a}_{1}-y_{12} \mathbf{a}_{2}+\left(\frac{1}{2}+z_{12}\right) \mathbf{a}_{3}$ & $=$ & $-x_{12} a \hat{\mathbf{x}}-y_{12} a \hat{\mathbf{y}}+\left(\frac{1}{2}+z_{12}\right) c \hat{\mathbf{z}}$ & $(4 a)$ & O VIII \\
\hline
\end{tabular}




\begin{tabular}{|c|c|c|c|c|c|c|}
\hline $\mathbf{B}_{47}$ & $=$ & $-y_{12} \mathbf{a}_{1}+x_{12} \mathbf{a}_{2}+\left(\frac{3}{4}+z_{12}\right) \mathbf{a}_{3}$ & $=$ & $-y_{12} a \hat{\mathbf{x}}+x_{12} a \hat{\mathbf{y}}+\left(\frac{3}{4}+z_{12}\right) c \hat{\mathbf{z}}$ & $(4 a)$ & O VIII \\
\hline $\mathbf{B}_{48}$ & $=$ & $y_{12} \mathbf{a}_{1}-x_{12} \mathbf{a}_{2}+\left(\frac{1}{4}+z_{12}\right) \mathbf{a}_{3}$ & $=$ & $y_{12} a \hat{\mathbf{x}}-x_{12} a \hat{\mathbf{y}}+\left(\frac{1}{4}+z_{12}\right) c \hat{\mathbf{z}}$ & $(4 a)$ & O VIII \\
\hline $\mathbf{B}_{49}$ & $=$ & $x_{13} \mathbf{a}_{1}+y_{13} \mathbf{a}_{2}+z_{13} \mathbf{a}_{3}$ & $=$ & $x_{13} a \hat{\mathbf{x}}+y_{13} a \hat{\mathbf{y}}+z_{13} c \hat{\mathbf{z}}$ & $(4 a)$ & O IX \\
\hline $\mathbf{B}_{50}$ & $=$ & $-x_{13} \mathbf{a}_{1}-y_{13} \mathbf{a}_{2}+\left(\frac{1}{2}+z_{13}\right) \mathbf{a}_{3}$ & $=$ & $-x_{13} a \hat{\mathbf{x}}-y_{13} a \hat{\mathbf{y}}+\left(\frac{1}{2}+z_{13}\right) c \hat{\mathbf{z}}$ & $(4 a)$ & O IX \\
\hline $\mathbf{B}_{51}$ & $=$ & $-y_{13} \mathbf{a}_{1}+x_{13} \mathbf{a}_{2}+\left(\frac{3}{4}+z_{13}\right) \mathbf{a}_{3}$ & $=$ & $-y_{13} a \hat{\mathbf{x}}+x_{13} a \hat{\mathbf{y}}+\left(\frac{3}{4}+z_{13}\right) c \hat{\mathbf{z}}$ & $(4 a)$ & O IX \\
\hline $\mathbf{B}_{52}$ & $=$ & $y_{13} \mathbf{a}_{1}-x_{13} \mathbf{a}_{2}+\left(\frac{1}{4}+z_{13}\right) \mathbf{a}_{3}$ & $=$ & $y_{13} a \hat{\mathbf{x}}-x_{13} a \hat{\mathbf{y}}+\left(\frac{1}{4}+z_{13}\right) c \hat{\mathbf{z}}$ & $(4 a)$ & O IX \\
\hline $\mathbf{B}_{53}$ & $=$ & $x_{14} \mathbf{a}_{1}+y_{14} \mathbf{a}_{2}+z_{14} \mathbf{a}_{3}$ & $=$ & $x_{14} a \hat{\mathbf{x}}+y_{14} a \hat{\mathbf{y}}+z_{14} c \hat{\mathbf{z}}$ & $(4 a)$ & $\mathrm{OX}$ \\
\hline $\mathbf{B}_{54}$ & $=$ & $-x_{14} \mathbf{a}_{1}-y_{14} \mathbf{a}_{2}+\left(\frac{1}{2}+z_{14}\right) \mathbf{a}_{3}$ & $=$ & $-x_{14} a \hat{\mathbf{x}}-y_{14} a \hat{\mathbf{y}}+\left(\frac{1}{2}+z_{14}\right) c \hat{\mathbf{z}}$ & $(4 a)$ & $\mathrm{OX}$ \\
\hline $\mathbf{B}_{55}$ & $=$ & $-y_{14} \mathbf{a}_{1}+x_{14} \mathbf{a}_{2}+\left(\frac{3}{4}+z_{14}\right) \mathbf{a}_{3}$ & $=$ & $-y_{14} a \hat{\mathbf{x}}+x_{14} a \hat{\mathbf{y}}+\left(\frac{3}{4}+z_{14}\right) c \hat{\mathbf{z}}$ & $(4 a)$ & $\mathrm{OX}$ \\
\hline $\mathbf{B}_{56}$ & $=$ & $y_{14} \mathbf{a}_{1}-x_{14} \mathbf{a}_{2}+\left(\frac{1}{4}+z_{14}\right) \mathbf{a}_{3}$ & $=$ & $y_{14} a \hat{\mathbf{x}}-x_{14} a \hat{\mathbf{y}}+\left(\frac{1}{4}+z_{14}\right) c \hat{\mathbf{z}}$ & $(4 a)$ & $\mathrm{OX}$ \\
\hline $\mathbf{B}_{57}$ & $=$ & $x_{15} \mathbf{a}_{1}+y_{15} \mathbf{a}_{2}+z_{15} \mathbf{a}_{3}$ & $=$ & $x_{15} a \hat{\mathbf{x}}+y_{15} a \hat{\mathbf{y}}+z_{15} c \hat{\mathbf{z}}$ & $(4 a)$ & O XI \\
\hline $\mathbf{B}_{58}$ & $=$ & $-x_{15} \mathbf{a}_{1}-y_{15} \mathbf{a}_{2}+\left(\frac{1}{2}+z_{15}\right) \mathbf{a}_{3}$ & $=$ & $-x_{15} a \hat{\mathbf{x}}-y_{15} a \hat{\mathbf{y}}+\left(\frac{1}{2}+z_{15}\right) c \hat{\mathbf{z}}$ & $(4 a)$ & O XI \\
\hline $\mathbf{B}_{59}$ & $=$ & $-y_{15} \mathbf{a}_{1}+x_{15} \mathbf{a}_{2}+\left(\frac{3}{4}+z_{15}\right) \mathbf{a}_{3}$ & $=$ & $-y_{15} a \hat{\mathbf{x}}+x_{15} a \hat{\mathbf{y}}+\left(\frac{3}{4}+z_{15}\right) c \hat{\mathbf{z}}$ & $(4 a)$ & O XI \\
\hline $\mathbf{B}_{60}$ & $=$ & $y_{15} \mathbf{a}_{1}-x_{15} \mathbf{a}_{2}+\left(\frac{1}{4}+z_{15}\right) \mathbf{a}_{3}$ & $=$ & $y_{15} a \hat{\mathbf{x}}-x_{15} a \hat{\mathbf{y}}+\left(\frac{1}{4}+z_{15}\right) c \hat{\mathbf{z}}$ & $(4 a)$ & O XI \\
\hline $\mathbf{B}_{61}$ & $=$ & $x_{16} \mathbf{a}_{1}+y_{16} \mathbf{a}_{2}+z_{16} \mathbf{a}_{3}$ & $=$ & $x_{16} a \hat{\mathbf{x}}+y_{16} a \hat{\mathbf{y}}+z_{16} c \hat{\mathbf{z}}$ & $(4 a)$ & O XII \\
\hline $\mathbf{B}_{62}$ & $=$ & $-x_{16} \mathbf{a}_{1}-y_{16} \mathbf{a}_{2}+\left(\frac{1}{2}+z_{16}\right) \mathbf{a}_{3}$ & $=$ & $-x_{16} a \hat{\mathbf{x}}-y_{16} a \hat{\mathbf{y}}+\left(\frac{1}{2}+z_{16}\right) c \hat{\mathbf{z}}$ & $(4 a)$ & O XII \\
\hline $\mathbf{B}_{63}$ & $=$ & $-y_{16} \mathbf{a}_{1}+x_{16} \mathbf{a}_{2}+\left(\frac{3}{4}+z_{16}\right) \mathbf{a}_{3}$ & $=$ & $-y_{16} a \hat{\mathbf{x}}+x_{16} a \hat{\mathbf{y}}+\left(\frac{3}{4}+z_{16}\right) c \hat{\mathbf{z}}$ & $(4 a)$ & O XII \\
\hline $\mathbf{B}_{64}$ & $=$ & $y_{16} \mathbf{a}_{1}-x_{16} \mathbf{a}_{2}+\left(\frac{1}{4}+z_{16}\right) \mathbf{a}_{3}$ & $=$ & $y_{16} a \hat{\mathbf{x}}-x_{16} a \hat{\mathbf{y}}+\left(\frac{1}{4}+z_{16}\right) c \hat{\mathbf{z}}$ & $(4 a)$ & O XII \\
\hline $\mathbf{B}_{65}$ & $=$ & $x_{17} \mathbf{a}_{1}+y_{17} \mathbf{a}_{2}+z_{17} \mathbf{a}_{3}$ & $=$ & $x_{17} a \hat{\mathbf{x}}+y_{17} a \hat{\mathbf{y}}+z_{17} c \hat{\mathbf{z}}$ & $(4 a)$ & O XIII \\
\hline $\mathbf{B}_{66}$ & $=$ & $-x_{17} \mathbf{a}_{1}-y_{17} \mathbf{a}_{2}+\left(\frac{1}{2}+z_{17}\right) \mathbf{a}_{3}$ & $=$ & $-x_{17} a \hat{\mathbf{x}}-y_{17} a \hat{\mathbf{y}}+\left(\frac{1}{2}+z_{17}\right) c \hat{\mathbf{z}}$ & $(4 a)$ & O XIII \\
\hline $\mathbf{B}_{67}$ & $=$ & $-y_{17} \mathbf{a}_{1}+x_{17} \mathbf{a}_{2}+\left(\frac{3}{4}+z_{17}\right) \mathbf{a}_{3}$ & $=$ & $-y_{17} a \hat{\mathbf{x}}+x_{17} a \hat{\mathbf{y}}+\left(\frac{3}{4}+z_{17}\right) c \hat{\mathbf{z}}$ & $(4 a)$ & O XIII \\
\hline $\mathbf{B}_{68}$ & $=$ & $y_{17} \mathbf{a}_{1}-x_{17} \mathbf{a}_{2}+\left(\frac{1}{4}+z_{17}\right) \mathbf{a}_{3}$ & $=$ & $y_{17} a \hat{\mathbf{x}}-x_{17} a \hat{\mathbf{y}}+\left(\frac{1}{4}+z_{17}\right) c \hat{\mathbf{z}}$ & $(4 a)$ & O XIII \\
\hline $\mathbf{B}_{69}$ & $=$ & $x_{18} \mathbf{a}_{1}+y_{18} \mathbf{a}_{2}+z_{18} \mathbf{a}_{3}$ & $=$ & $x_{18} a \hat{\mathbf{x}}+y_{18} a \hat{\mathbf{y}}+z_{18} c \hat{\mathbf{z}}$ & $(4 a)$ & O XIV \\
\hline $\mathbf{B}_{70}$ & $=$ & $-x_{18} \mathbf{a}_{1}-y_{18} \mathbf{a}_{2}+\left(\frac{1}{2}+z_{18}\right) \mathbf{a}_{3}$ & $=$ & $-x_{18} a \hat{\mathbf{x}}-y_{18} a \hat{\mathbf{y}}+\left(\frac{1}{2}+z_{18}\right) c \hat{\mathbf{z}}$ & $(4 a)$ & O XIV \\
\hline $\mathbf{B}_{71}$ & $=$ & $-y_{18} \mathbf{a}_{1}+x_{18} \mathbf{a}_{2}+\left(\frac{3}{4}+z_{18}\right) \mathbf{a}_{3}$ & $=$ & $-y_{18} a \hat{\mathbf{x}}+x_{18} a \hat{\mathbf{y}}+\left(\frac{3}{4}+z_{18}\right) c \hat{\mathbf{z}}$ & $(4 a)$ & O XIV \\
\hline $\mathbf{B}_{72}$ & $=$ & $y_{18} \mathbf{a}_{1}-x_{18} \mathbf{a}_{2}+\left(\frac{1}{4}+z_{18}\right) \mathbf{a}_{3}$ & $=$ & $y_{18} a \hat{\mathbf{x}}-x_{18} a \hat{\mathbf{y}}+\left(\frac{1}{4}+z_{18}\right) c \hat{\mathbf{z}}$ & $(4 a)$ & O XIV \\
\hline $\mathbf{B}_{73}$ & $=$ & $x_{19} \mathbf{a}_{1}+y_{19} \mathbf{a}_{2}+z_{19} \mathbf{a}_{3}$ & $=$ & $x_{19} a \hat{\mathbf{x}}+y_{19} a \hat{\mathbf{y}}+z_{19} c \hat{\mathbf{z}}$ & $(4 a)$ & Sr I \\
\hline $\mathbf{B}_{74}$ & $=$ & $-x_{19} \mathbf{a}_{1}-y_{19} \mathbf{a}_{2}+\left(\frac{1}{2}+z_{19}\right) \mathbf{a}_{3}$ & $=$ & $-x_{19} a \hat{\mathbf{x}}-y_{19} a \hat{\mathbf{y}}+\left(\frac{1}{2}+z_{19}\right) c \hat{\mathbf{z}}$ & $(4 a)$ & Sr I \\
\hline $\mathbf{B}_{75}$ & $=$ & $-y_{19} \mathbf{a}_{1}+x_{19} \mathbf{a}_{2}+\left(\frac{3}{4}+z_{19}\right) \mathbf{a}_{3}$ & $=$ & $-y_{19} a \hat{\mathbf{x}}+x_{19} a \hat{\mathbf{y}}+\left(\frac{3}{4}+z_{19}\right) c \hat{\mathbf{z}}$ & $(4 a)$ & Sr I \\
\hline $\mathbf{B}_{76}$ & $=$ & $y_{19} \mathbf{a}_{1}-x_{19} \mathbf{a}_{2}+\left(\frac{1}{4}+z_{19}\right) \mathbf{a}_{3}$ & $=$ & $y_{19} a \hat{\mathbf{x}}-x_{19} a \hat{\mathbf{y}}+\left(\frac{1}{4}+z_{19}\right) c \hat{\mathbf{z}}$ & $(4 a)$ & Sr I \\
\hline $\mathbf{B}_{77}$ & $=$ & $x_{20} \mathbf{a}_{1}+y_{20} \mathbf{a}_{2}+z_{20} \mathbf{a}_{3}$ & $=$ & $x_{20} a \hat{\mathbf{x}}+y_{20} a \hat{\mathbf{y}}+z_{20} c \hat{\mathbf{z}}$ & $(4 a)$ & Sr II \\
\hline $\mathbf{B}_{78}$ & $=$ & $-x_{20} \mathbf{a}_{1}-y_{20} \mathbf{a}_{2}+\left(\frac{1}{2}+z_{20}\right) \mathbf{a}_{3}$ & $=$ & $-x_{20} a \hat{\mathbf{x}}-y_{20} a \hat{\mathbf{y}}+\left(\frac{1}{2}+z_{20}\right) c \hat{\mathbf{z}}$ & $(4 a)$ & Sr II \\
\hline $\mathbf{B}_{79}$ & $=$ & $-y_{20} \mathbf{a}_{1}+x_{20} \mathbf{a}_{2}+\left(\frac{3}{4}+z_{20}\right) \mathbf{a}_{3}$ & $=$ & $-y_{20} a \hat{\mathbf{x}}+x_{20} a \hat{\mathbf{y}}+\left(\frac{3}{4}+z_{20}\right) c \hat{\mathbf{z}}$ & $(4 a)$ & Sr II \\
\hline $\mathbf{B}_{80}$ & $=$ & $y_{20} \mathbf{a}_{1}-x_{20} \mathbf{a}_{2}+\left(\frac{1}{4}+z_{20}\right) \mathbf{a}_{3}$ & $=$ & $y_{20} a \hat{\mathbf{x}}-x_{20} a \hat{\mathbf{y}}+\left(\frac{1}{4}+z_{20}\right) c \hat{\mathbf{z}}$ & $(4 a)$ & Sr II \\
\hline $\mathbf{B}_{81}$ & $=$ & $x_{21} \mathbf{a}_{1}+y_{21} \mathbf{a}_{2}+z_{21} \mathbf{a}_{3}$ & $=$ & $x_{21} a \hat{\mathbf{x}}+y_{21} a \hat{\mathbf{y}}+z_{21} c \hat{\mathbf{z}}$ & $(4 a)$ & Sr III \\
\hline $\mathbf{B}_{82}$ & $=$ & $-x_{21} \mathbf{a}_{1}-y_{21} \mathbf{a}_{2}+\left(\frac{1}{2}+z_{21}\right) \mathbf{a}_{3}$ & $=$ & $-x_{21} a \hat{\mathbf{x}}-y_{21} a \hat{\mathbf{y}}+\left(\frac{1}{2}+z_{21}\right) c \hat{\mathbf{z}}$ & $(4 a)$ & Sr III \\
\hline
\end{tabular}




\begin{tabular}{|c|c|c|c|c|c|c|}
\hline $\mathbf{B}_{83}$ & $=$ & $-y_{21} \mathbf{a}_{1}+x_{21} \mathbf{a}_{2}+\left(\frac{3}{4}+z_{21}\right) \mathbf{a}_{3}$ & $=$ & $-y_{21} a \hat{\mathbf{x}}+x_{21} a \hat{\mathbf{y}}+\left(\frac{3}{4}+z_{21}\right) c \hat{\mathbf{z}}$ & $(4 a)$ & Sr III \\
\hline $\mathbf{B}_{84}$ & $=$ & $y_{21} \mathbf{a}_{1}-x_{21} \mathbf{a}_{2}+\left(\frac{1}{4}+z_{21}\right) \mathbf{a}_{3}$ & $=$ & $y_{21} a \hat{\mathbf{x}}-x_{21} a \hat{\mathbf{y}}+\left(\frac{1}{4}+z_{21}\right) c \hat{\mathbf{z}}$ & $(4 a)$ & Sr III \\
\hline $\mathbf{B}_{85}$ & $=$ & $x_{22} \mathbf{a}_{1}+y_{22} \mathbf{a}_{2}+z_{22} \mathbf{a}_{3}$ & $=$ & $x_{22} a \hat{\mathbf{x}}+y_{22} a \hat{\mathbf{y}}+z_{22} c \hat{\mathbf{z}}$ & $(4 a)$ & Sr IV \\
\hline $\mathbf{B}_{86}$ & $=$ & $-x_{22} \mathbf{a}_{1}-y_{22} \mathbf{a}_{2}+\left(\frac{1}{2}+z_{22}\right) \mathbf{a}_{3}$ & $=$ & $-x_{22} a \hat{\mathbf{x}}-y_{22} a \hat{\mathbf{y}}+\left(\frac{1}{2}+z_{22}\right) c \hat{\mathbf{z}}$ & $(4 a)$ & Sr IV \\
\hline $\mathbf{B}_{87}$ & $=$ & $-y_{22} \mathbf{a}_{1}+x_{22} \mathbf{a}_{2}+\left(\frac{3}{4}+z_{22}\right) \mathbf{a}_{3}$ & $=$ & $-y_{22} a \hat{\mathbf{x}}+x_{22} a \hat{\mathbf{y}}+\left(\frac{3}{4}+z_{22}\right) c \hat{\mathbf{z}}$ & $(4 a)$ & Sr IV \\
\hline $\mathbf{B}_{88}$ & $=$ & $y_{22} \mathbf{a}_{1}-x_{22} \mathbf{a}_{2}+\left(\frac{1}{4}+z_{22}\right) \mathbf{a}_{3}$ & $=$ & $y_{22} a \hat{\mathbf{x}}-x_{22} a \hat{\mathbf{y}}+\left(\frac{1}{4}+z_{22}\right) c \hat{\mathbf{z}}$ & $(4 a)$ & Sr IV \\
\hline
\end{tabular}

\section{References:}

- A. Mbarek and F. Edhokkar, The P4 $4_{3}$ enantiomorph of $\mathrm{Sr}_{2} \mathrm{As}_{2} \mathrm{O}_{7}$, Acta Crystallogr. E 69, i84-i84 (2013), doi: $10.1107 /$ S1600536813031619.

\section{Found in:}

- P. Villars and K. Cenzual, Pearson's Crystal Data - Crystal Structure Database for Inorganic Compounds, ASM International (2013).

\section{Geometry files:}

- CIF: pp. 860

- POSCAR: pp. 861 


\section{$\mathrm{TlZn}_{2} \mathrm{Sb}_{2}$ Structure: A2BC2_tI20_79_c_2a_c}
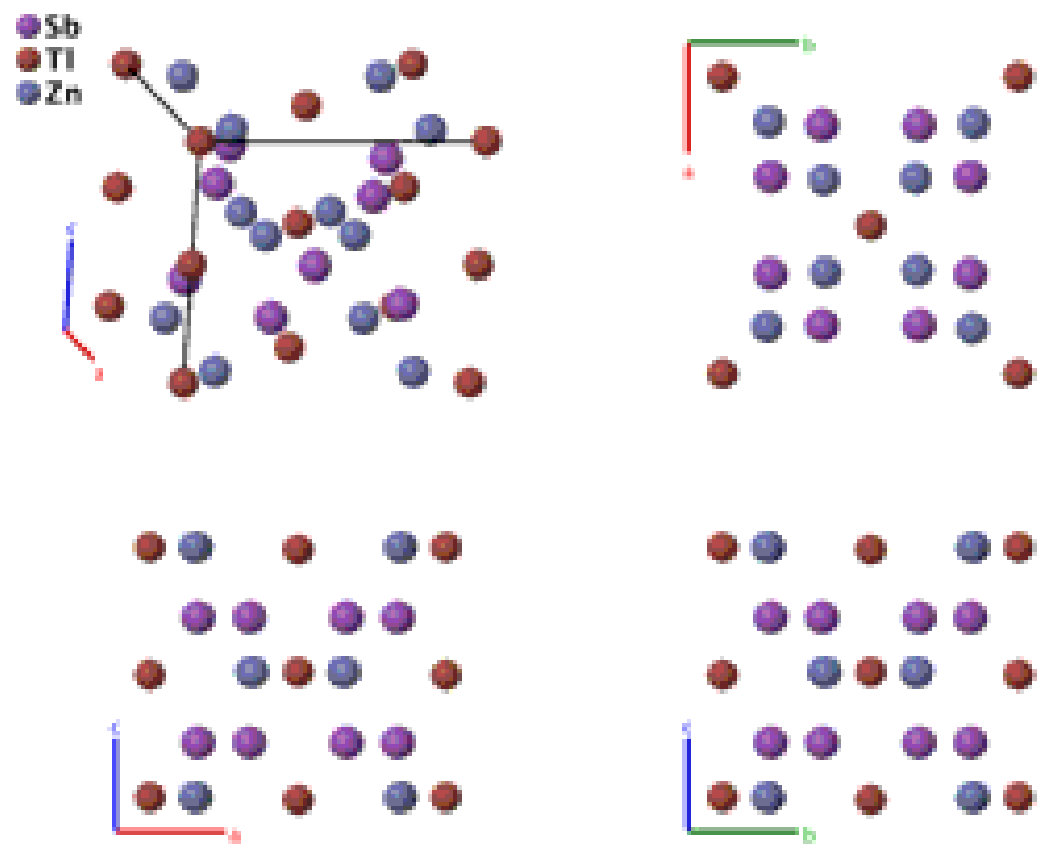

Prototype

: $\quad \mathrm{TlZn}_{2} \mathrm{Sb}_{2}$

AFLOW prototype label

A2BC2_tI20_79_c_2a_c

Strukturbericht designation

None

Pearson symbol

$\mathrm{I} 20$

Space group number

79

Space group symbol

I4

AFLOW prototype command

aflow --proto=A2BC2_tI20_79_c_2a_c

- -params $=a, c / a, z_{1}, z_{2}, x_{3}, y_{3}, z_{3}, x_{4}, y_{4}, z_{4}$

Body-centered Tetragonal primitive vectors:

$$
\begin{aligned}
& \mathbf{a}_{1}=-\frac{1}{2} a \hat{\mathbf{x}}+\frac{1}{2} a \hat{\mathbf{y}}+\frac{1}{2} c \hat{\mathbf{z}} \\
& \mathbf{a}_{2}=\frac{1}{2} a \hat{\mathbf{x}}-\frac{1}{2} a \hat{\mathbf{y}}+\frac{1}{2} c \hat{\mathbf{z}} \\
& \mathbf{a}_{3}=\frac{1}{2} a \hat{\mathbf{x}}+\frac{1}{2} a \hat{\mathbf{y}}-\frac{1}{2} c \hat{\mathbf{z}}
\end{aligned}
$$

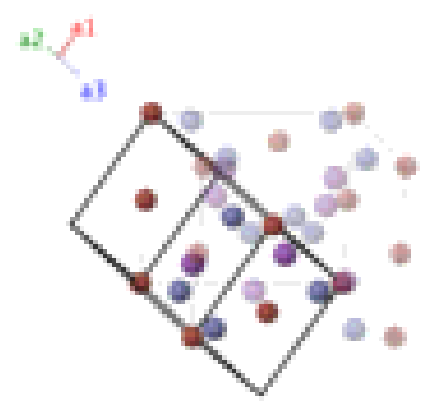

\section{Basis vectors:}

Lattice Coordinates

$\begin{array}{lccc}\mathbf{B}_{1}= & z_{1} \mathbf{a}_{1}+z_{1} \mathbf{a}_{2} & = & z_{1} c \hat{\mathbf{z}} \\ \mathbf{B}_{2}= & z_{2} \mathbf{a}_{1}+z_{2} \mathbf{a}_{2} & = & z_{2} c \hat{\mathbf{z}} \\ \mathbf{B}_{3}= & \left(y_{3}+z_{3}\right) \mathbf{a}_{1}+\left(x_{3}+z_{3}\right) \mathbf{a}_{2}+\left(x_{3}+y_{3}\right) \mathbf{a}_{3} & = & x_{3} a \hat{\mathbf{x}}+y_{3} a \hat{\mathbf{y}}+z_{3} c \hat{\mathbf{z}} \\ \mathbf{B}_{4}= & \left(-y_{3}+z_{3}\right) \mathbf{a}_{1}+\left(-x_{3}+z_{3}\right) \mathbf{a}_{2}+\left(-x_{3}-y_{3}\right) \mathbf{a}_{3} & = & -x_{3} a \hat{\mathbf{x}}-y_{3} a \hat{\mathbf{y}}+z_{3} c \hat{\mathbf{z}}\end{array}$

$\mathbf{B}_{1}=$
Wyckoff Position Atom Type

(2a)

Tl I

(2a)

Tl II

(8c)

$\mathrm{Sb}$

(8c) 

$\mathbf{B}_{5}=\left(x_{3}+z_{3}\right) \mathbf{a}_{1}+\left(-y_{3}+z_{3}\right) \mathbf{a}_{2}+\left(x_{3}-y_{3}\right) \mathbf{a}_{3}=-y_{3} a \hat{\mathbf{x}}+x_{3} a \hat{\mathbf{y}}+z_{3} c \hat{\mathbf{z}}$
$(8 c)$
$\mathrm{Sb}$
$\mathbf{B}_{6}=\left(-x_{3}+z_{3}\right) \mathbf{a}_{1}+\left(y_{3}+z_{3}\right) \mathbf{a}_{2}+\left(-x_{3}+y_{3}\right) \mathbf{a}_{3}=y_{3} a \hat{\mathbf{x}}-x_{3} a \hat{\mathbf{y}}+z_{3} c \hat{\mathbf{z}}$
$(8 c)$
$\mathrm{Sb}$
$\mathbf{B}_{7}=\left(y_{4}+z_{4}\right) \mathbf{a}_{1}+\left(x_{4}+z_{4}\right) \mathbf{a}_{2}+\left(x_{4}+y_{4}\right) \mathbf{a}_{3}=x_{4} a \hat{\mathbf{x}}+y_{4} a \hat{\mathbf{y}}+z_{4} c \hat{\mathbf{z}}$
$\mathrm{Zn}$
$\mathbf{B}_{8}=\left(-y_{4}+z_{4}\right) \mathbf{a}_{1}+\left(-x_{4}+z_{4}\right) \mathbf{a}_{2}+\left(-x_{4}-y_{4}\right) \mathbf{a}_{3}=$
$=-x_{4} a \hat{\mathbf{x}}-y_{4} a \hat{\mathbf{y}}+z_{4} c \hat{\mathbf{z}}$
$(8 c)$
$\mathrm{Zn}$
$\mathbf{B}_{9}=\left(x_{4}+z_{4}\right) \mathbf{a}_{1}+\left(-y_{4}+z_{4}\right) \mathbf{a}_{2}+\left(x_{4}-y_{4}\right) \mathbf{a}_{3}=-y_{4} a \hat{\mathbf{x}}+x_{4} a \hat{\mathbf{y}}+z_{4} c \hat{\mathbf{z}}$
$\mathrm{Zn}$
$\mathbf{B}_{10}=\left(-x_{4}+z_{4}\right) \mathbf{a}_{1}+\left(y_{4}+z_{4}\right) \mathbf{a}_{2}+\left(-x_{4}+y_{4}\right) \mathbf{a}_{3}=y_{4} a \hat{\mathbf{x}}-x_{4} a \hat{\mathbf{y}}+z_{4} c \hat{\mathbf{z}}$
$(8 c)$
$\mathrm{Zn}$

\section{References:}

- A. Czybulka, B. Krenkel, and H.-U. Schuster, Ternäre zintl-Verbindungen mit thallium als elektronendonator, J. Less-Common Met. 137, 311-322 (1988), doi:10.1016/0022-5088(88)90096-3.

\section{Found in:}

- P. Villars and K. Cenzual, Pearson's Crystal Data - Crystal Structure Database for Inorganic Compounds, ASM International (2013).

\section{Geometry files:}

- CIF: pp. 861

- POSCAR: pp. 862 


\section{$\beta-\mathrm{NbO}_{2}$ Structure: AB2_tI48_80_2b_4b}
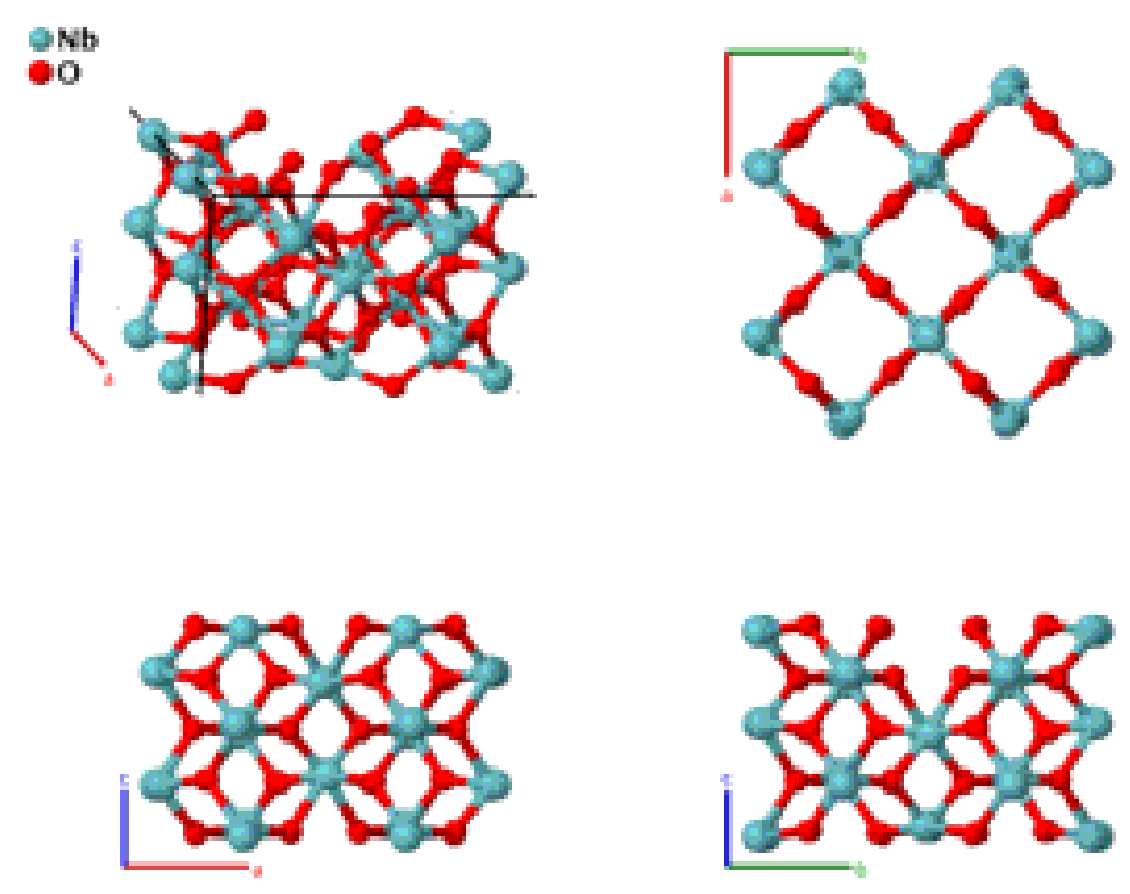

\section{Prototype}

AFLOW prototype label

$: \quad \beta-\mathrm{NbO}_{2}$

\section{Strukturbericht designation}

: AB2_tI48_80_2b_4b

Pearson symbol

: None

Space group number

$\mathrm{tI} 48$

Space group symbol

: $\quad 80$

AFLOW prototype command

$: \quad I 4_{1}$

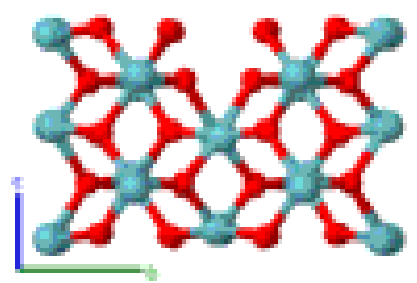

- This crystal is not quite stoichiometric. The actual composition was found to be $\mathrm{NbO}_{2-x}$, where $0.002 \leq x \leq 0.01$.

Body-centered Tetragonal primitive vectors:

$$
\begin{aligned}
& \mathbf{a}_{1}=-\frac{1}{2} a \hat{\mathbf{x}}+\frac{1}{2} a \hat{\mathbf{y}}+\frac{1}{2} c \hat{\mathbf{z}} \\
& \mathbf{a}_{2}=\frac{1}{2} a \hat{\mathbf{x}}-\frac{1}{2} a \hat{\mathbf{y}}+\frac{1}{2} c \hat{\mathbf{z}} \\
& \mathbf{a}_{3}=\frac{1}{2} a \hat{\mathbf{x}}+\frac{1}{2} a \hat{\mathbf{y}}-\frac{1}{2} c \hat{\mathbf{z}}
\end{aligned}
$$

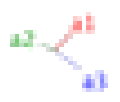

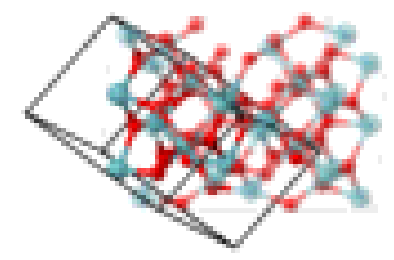

Basis vectors:

Lattice Coordinates

$\mathbf{B}_{1}=\quad \begin{gathered}\left(y_{1}+z_{1}\right) \mathbf{a}_{1}+\left(x_{1}+z_{1}\right) \mathbf{a}_{2}+ \\ \left(x_{1}+y_{1}\right) \mathbf{a}_{3}\end{gathered}=$
Cartesian Coordinates

$x_{1} a \hat{\mathbf{x}}+y_{1} a \hat{\mathbf{y}}+z_{1} c \hat{\mathbf{z}}$

Wyckoff Position

$(8 b)$
Atom Type

$\mathrm{Nb} \mathrm{I}$ 


\begin{tabular}{|c|c|c|c|c|c|c|}
\hline $\mathbf{B}_{2}$ & $=$ & $\begin{array}{c}\left(-y_{1}+z_{1}\right) \mathbf{a}_{1}+\left(-x_{1}+z_{1}\right) \mathbf{a}_{2}+ \\
\left(-x_{1}-y_{1}\right) \mathbf{a}_{3}\end{array}$ & $=$ & $-x_{1} a \hat{\mathbf{x}}-y_{1} a \hat{\mathbf{y}}+z_{1} c \hat{\mathbf{z}}$ & $(8 b)$ & $\mathrm{Nb} \mathrm{I}$ \\
\hline $\mathbf{B}_{3}$ & $=$ & $\begin{array}{c}\left(\frac{3}{4}+x_{1}+z_{1}\right) \mathbf{a}_{1}+ \\
\left(\frac{1}{4}-y_{1}+z_{1}\right) \mathbf{a}_{2}+\left(\frac{1}{2}+x_{1}-y_{1}\right) \mathbf{a}_{3}\end{array}$ & $=$ & $-y_{1} a \hat{\mathbf{x}}+\left(\frac{1}{2}+x_{1}\right) a \hat{\mathbf{y}}+\left(\frac{1}{4}+z_{1}\right) c \hat{\mathbf{z}}$ & $(8 b)$ & $\mathrm{Nb} \mathrm{I}$ \\
\hline $\mathbf{B}_{4}$ & $=$ & $\begin{array}{c}\left(\frac{3}{4}-x_{1}+z_{1}\right) \mathbf{a}_{1}+ \\
\left(\frac{1}{4}+y_{1}+z_{1}\right) \mathbf{a}_{2}+\left(\frac{1}{2}-x_{1}+y_{1}\right) \mathbf{a}_{3}\end{array}$ & $=$ & $y_{1} a \hat{\mathbf{x}}+\left(\frac{1}{2}-x_{1}\right) a \hat{\mathbf{y}}+\left(\frac{1}{4}+z_{1}\right) c \hat{\mathbf{z}}$ & $(8 b)$ & $\mathrm{Nb} \mathrm{I}$ \\
\hline $\mathbf{B}_{5}$ & $=$ & $\begin{array}{c}\left(y_{2}+z_{2}\right) \mathbf{a}_{1}+\left(x_{2}+z_{2}\right) \mathbf{a}_{2}+ \\
\left(x_{2}+y_{2}\right) \mathbf{a}_{3}\end{array}$ & $=$ & $x_{2} a \hat{\mathbf{x}}+y_{2} a \hat{\mathbf{y}}+z_{2} c \hat{\mathbf{z}}$ & $(8 b)$ & $\mathrm{Nb}$ II \\
\hline $\mathbf{B}_{6}$ & $=$ & $\begin{array}{c}\left(-y_{2}+z_{2}\right) \mathbf{a}_{1}+\left(-x_{2}+z_{2}\right) \mathbf{a}_{2}+ \\
\left(-x_{2}-y_{2}\right) \mathbf{a}_{3}\end{array}$ & $=$ & $-x_{2} a \hat{\mathbf{x}}-y_{2} a \hat{\mathbf{y}}+z_{2} c \hat{\mathbf{z}}$ & $(8 b)$ & $\mathrm{Nb}$ II \\
\hline $\mathbf{B}_{7}$ & $=$ & $\begin{array}{c}\left(\frac{3}{4}+x_{2}+z_{2}\right) \mathbf{a}_{1}+ \\
\left(\frac{1}{4}-y_{2}+z_{2}\right) \mathbf{a}_{2}+\left(\frac{1}{2}+x_{2}-y_{2}\right) \mathbf{a}_{3}\end{array}$ & $=$ & $-y_{2} a \hat{\mathbf{x}}+\left(\frac{1}{2}+x_{2}\right) a \hat{\mathbf{y}}+\left(\frac{1}{4}+z_{2}\right) c \hat{\mathbf{z}}$ & $(8 b)$ & $\mathrm{Nb}$ II \\
\hline $\mathbf{B}_{8}$ & $=$ & $\begin{array}{c}\left(\frac{3}{4}-x_{2}+z_{2}\right) \mathbf{a}_{1}+ \\
\left(\frac{1}{4}+y_{2}+z_{2}\right) \mathbf{a}_{2}+\left(\frac{1}{2}-x_{2}+y_{2}\right) \mathbf{a}_{3}\end{array}$ & $=$ & $y_{2} a \hat{\mathbf{x}}+\left(\frac{1}{2}-x_{2}\right) a \hat{\mathbf{y}}+\left(\frac{1}{4}+z_{2}\right) c \hat{\mathbf{z}}$ & $(8 b)$ & $\mathrm{Nb}$ II \\
\hline $\mathbf{B}_{9}$ & $=$ & $\begin{array}{c}\left(y_{3}+z_{3}\right) \mathbf{a}_{1}+\left(x_{3}+z_{3}\right) \mathbf{a}_{2}+ \\
\left(x_{3}+y_{3}\right) \mathbf{a}_{3}\end{array}$ & $=$ & $x_{3} a \hat{\mathbf{x}}+y_{3} a \hat{\mathbf{y}}+z_{3} c \hat{\mathbf{z}}$ & $(8 b)$ & O I \\
\hline $\mathbf{B}_{10}$ & $=$ & $\begin{array}{c}\left(-y_{3}+z_{3}\right) \mathbf{a}_{1}+\left(-x_{3}+z_{3}\right) \mathbf{a}_{2}+ \\
\left(-x_{3}-y_{3}\right) \mathbf{a}_{3}\end{array}$ & $=$ & $-x_{3} a \hat{\mathbf{x}}-y_{3} a \hat{\mathbf{y}}+z_{3} c \hat{\mathbf{z}}$ & $(8 b)$ & O I \\
\hline $\mathbf{B}_{11}$ & $=$ & $\begin{array}{c}\left(\frac{3}{4}+x_{3}+z_{3}\right) \mathbf{a}_{1}+ \\
\left(\frac{1}{4}-y_{3}+z_{3}\right) \mathbf{a}_{2}+\left(\frac{1}{2}+x_{3}-y_{3}\right) \mathbf{a}_{3}\end{array}$ & $=$ & $-y_{3} a \hat{\mathbf{x}}+\left(\frac{1}{2}+x_{3}\right) a \hat{\mathbf{y}}+\left(\frac{1}{4}+z_{3}\right) c \hat{\mathbf{z}}$ & $(8 b)$ & O I \\
\hline $\mathbf{B}_{12}$ & $=$ & $\begin{array}{c}\left(\frac{3}{4}-x_{3}+z_{3}\right) \mathbf{a}_{1}+ \\
\left(\frac{1}{4}+y_{3}+z_{3}\right) \mathbf{a}_{2}+\left(\frac{1}{2}-x_{3}+y_{3}\right) \mathbf{a}_{3}\end{array}$ & $=$ & $y_{3} a \hat{\mathbf{x}}+\left(\frac{1}{2}-x_{3}\right) a \hat{\mathbf{y}}+\left(\frac{1}{4}+z_{3}\right) c \hat{\mathbf{z}}$ & $(8 b)$ & O I \\
\hline $\mathbf{B}_{13}$ & $=$ & $\begin{array}{c}\left(y_{4}+z_{4}\right) \mathbf{a}_{1}+\left(x_{4}+z_{4}\right) \mathbf{a}_{2}+ \\
\left(x_{4}+y_{4}\right) \mathbf{a}_{3}\end{array}$ & $=$ & $x_{4} a \hat{\mathbf{x}}+y_{4} a \hat{\mathbf{y}}+z_{4} c \hat{\mathbf{z}}$ & $(8 b)$ & O II \\
\hline $\mathbf{B}_{14}$ & $=$ & $\begin{array}{c}\left(-y_{4}+z_{4}\right) \mathbf{a}_{1}+\left(-x_{4}+z_{4}\right) \mathbf{a}_{2}+ \\
\left(-x_{4}-y_{4}\right) \mathbf{a}_{3}\end{array}$ & $=$ & $-x_{4} a \hat{\mathbf{x}}-y_{4} a \hat{\mathbf{y}}+z_{4} c \hat{\mathbf{z}}$ & $(8 b)$ & $\mathrm{O}$ II \\
\hline $\mathbf{B}_{15}$ & $=$ & $\begin{array}{c}\left(\frac{3}{4}+x_{4}+z_{4}\right) \mathbf{a}_{1}+ \\
\left(\frac{1}{4}-y_{4}+z_{4}\right) \mathbf{a}_{2}+\left(\frac{1}{2}+x_{4}-y_{4}\right) \mathbf{a}_{3}\end{array}$ & $=$ & $-y_{4} a \hat{\mathbf{x}}+\left(\frac{1}{2}+x_{4}\right) a \hat{\mathbf{y}}+\left(\frac{1}{4}+z_{4}\right) c \hat{\mathbf{z}}$ & $(8 b)$ & O II \\
\hline $\mathbf{B}_{16}$ & $=$ & $\begin{array}{c}\left(\frac{3}{4}-x_{4}+z_{4}\right) \mathbf{a}_{1}+ \\
\left(\frac{1}{4}+y_{4}+z_{4}\right) \mathbf{a}_{2}+\left(\frac{1}{2}-x_{4}+y_{4}\right) \mathbf{a}_{3}\end{array}$ & $=$ & $y_{4} a \hat{\mathbf{x}}+\left(\frac{1}{2}-x_{4}\right) a \hat{\mathbf{y}}+\left(\frac{1}{4}+z_{4}\right) c \hat{\mathbf{z}}$ & $(8 b)$ & O II \\
\hline $\mathbf{B}_{17}$ & $=$ & $\begin{array}{c}\left(y_{5}+z_{5}\right) \mathbf{a}_{1}+\left(x_{5}+z_{5}\right) \mathbf{a}_{2}+ \\
\left(x_{5}+y_{5}\right) \mathbf{a}_{3}\end{array}$ & $=$ & $x_{5} a \hat{\mathbf{x}}+y_{5} a \hat{\mathbf{y}}+z_{5} c \hat{\mathbf{z}}$ & $(8 b)$ & O III \\
\hline $\mathbf{B}_{18}$ & $=$ & $\begin{array}{c}\left(-y_{5}+z_{5}\right) \mathbf{a}_{1}+\left(-x_{5}+z_{5}\right) \mathbf{a}_{2}+ \\
\left(-x_{5}-y_{5}\right) \mathbf{a}_{3}\end{array}$ & $=$ & $-x_{5} a \hat{\mathbf{x}}-y_{5} a \hat{\mathbf{y}}+z_{5} c \hat{\mathbf{z}}$ & $(8 b)$ & O III \\
\hline $\mathbf{B}_{19}$ & $=$ & $\begin{array}{c}\left(\frac{3}{4}+x_{5}+z_{5}\right) \mathbf{a}_{1}+ \\
\left(\frac{1}{4}-y_{5}+z_{5}\right) \mathbf{a}_{2}+\left(\frac{1}{2}+x_{5}-y_{5}\right) \mathbf{a}_{3}\end{array}$ & $=$ & $-y_{5} a \hat{\mathbf{x}}+\left(\frac{1}{2}+x_{5}\right) a \hat{\mathbf{y}}+\left(\frac{1}{4}+z_{5}\right) c \hat{\mathbf{z}}$ & $(8 b)$ & O III \\
\hline $\mathbf{B}_{20}$ & $=$ & $\begin{array}{c}\left(\frac{3}{4}-x_{5}+z_{5}\right) \mathbf{a}_{1}+ \\
\left(\frac{1}{4}+y_{5}+z_{5}\right) \mathbf{a}_{2}+\left(\frac{1}{2}-x_{5}+y_{5}\right) \mathbf{a}_{3}\end{array}$ & $=$ & $y_{5} a \hat{\mathbf{x}}+\left(\frac{1}{2}-x_{5}\right) a \hat{\mathbf{y}}+\left(\frac{1}{4}+z_{5}\right) c \hat{\mathbf{z}}$ & $(8 b)$ & O III \\
\hline $\mathbf{B}_{21}$ & $=$ & $\begin{array}{c}\left(y_{6}+z_{6}\right) \mathbf{a}_{1}+\left(x_{6}+z_{6}\right) \mathbf{a}_{2}+ \\
\left(x_{6}+y_{6}\right) \mathbf{a}_{3}\end{array}$ & $=$ & $x_{6} a \hat{\mathbf{x}}+y_{6} a \hat{\mathbf{y}}+z_{6} c \hat{\mathbf{z}}$ & $(8 b)$ & O IV \\
\hline $\mathbf{B}_{22}$ & $=$ & $\begin{array}{c}\left(-y_{6}+z_{6}\right) \mathbf{a}_{1}+\left(-x_{6}+z_{6}\right) \mathbf{a}_{2}+ \\
\left(-x_{6}-y_{6}\right) \mathbf{a}_{3}\end{array}$ & $=$ & $-x_{6} a \hat{\mathbf{x}}-y_{6} a \hat{\mathbf{y}}+z_{6} c \hat{\mathbf{z}}$ & $(8 b)$ & O IV \\
\hline $\mathbf{B}_{23}$ & $=$ & $\begin{array}{c}\left(\frac{3}{4}+x_{6}+z_{6}\right) \mathbf{a}_{1}+ \\
\left(\frac{1}{4}-y_{6}+z_{6}\right) \mathbf{a}_{2}+\left(\frac{1}{2}+x_{6}-y_{6}\right) \mathbf{a}_{3}\end{array}$ & $=$ & $-y_{6} a \hat{\mathbf{x}}+\left(\frac{1}{2}+x_{6}\right) a \hat{\mathbf{y}}+\left(\frac{1}{4}+z_{6}\right) c \hat{\mathbf{z}}$ & $(8 b)$ & O IV \\
\hline
\end{tabular}


$\mathbf{B}_{24}=\begin{gathered}\left(\frac{3}{4}-x_{6}+z_{6}\right) \mathbf{a}_{1}+ \\ \left(\frac{1}{4}+y_{6}+z_{6}\right) \mathbf{a}_{2}+\left(\frac{1}{2}-x_{6}+y_{6}\right) \mathbf{a}_{3}\end{gathered}=y_{6} a \hat{\mathbf{x}}+\left(\frac{1}{2}-x_{6}\right) a \hat{\mathbf{y}}+\left(\frac{1}{4}+z_{6}\right) c \hat{\mathbf{z}}$

\section{References:}

- H.-J. Schweizer and R. Gruehn, Zur Darstellung und Kristallstruktur von $\beta-\mathrm{NbO}_{2}$ / Synthesis and Crystal Structure of $\beta$-NbO $\mathrm{O}_{2}$, Z. Naturforsch. B 37, 1361-1368 (1982), doi:10.1515/znb-1982-1101.

\section{Found in:}

- P. Villars and L. D. Calvert, eds., Pearson's Handbook of Crystallographic Data (ASM International, Materials Park OH, 1991), vol. IV, chap. , p. 4535.

\section{Geometry files:}

- CIF: pp. 862

- POSCAR: pp. 862 


\section{$\mathrm{GeSe}_{2}$ (High-pressure) Structure: AB2_tP12_81_adg_2h}
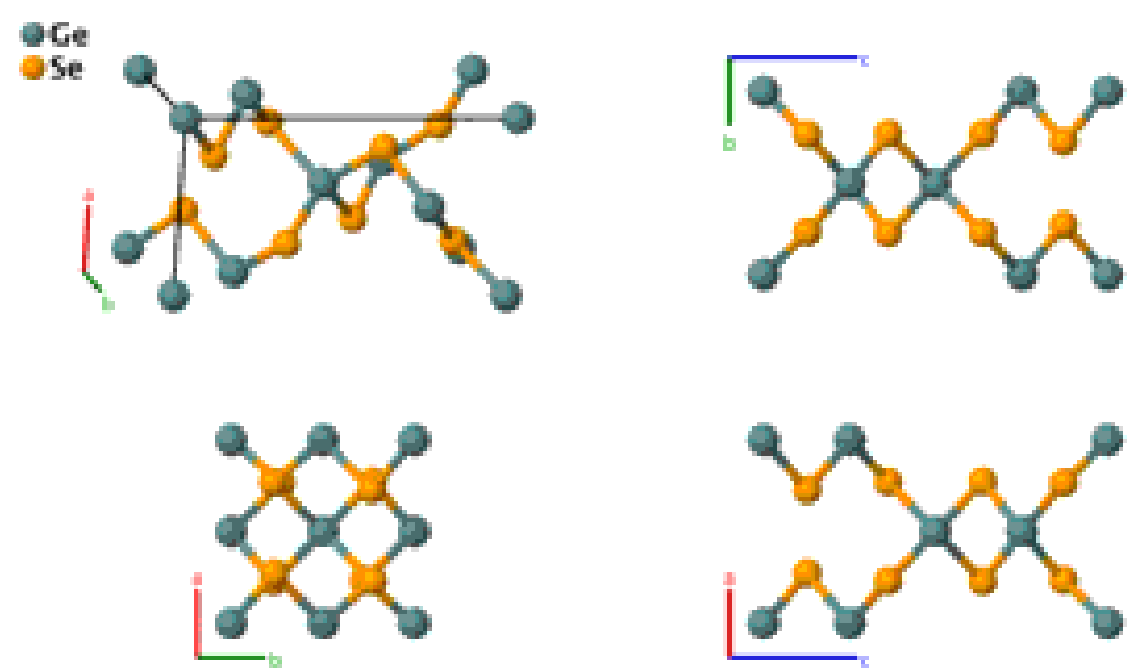

\section{Prototype}

AFLOW prototype label

: $\mathrm{GeSe}_{2}$

Strukturbericht designation

Pearson symbol

: AB2_tP12_81_adg_2h

Space group number

$: \quad \mathrm{tP} 12$

Space group symbol

: 81

AFLOW prototype command

$$
: \quad P \overline{4}
$$

- -params $=a, c / a, z_{3}, x_{4}, y_{4}, z_{4}, x_{5}, y_{5}, z_{5}$

\section{Simple Tetragonal primitive vectors:}

$$
\begin{aligned}
& \mathbf{a}_{1}=a \hat{\mathbf{x}} \\
& \mathbf{a}_{2}=a \hat{\mathbf{y}} \\
& \mathbf{a}_{3}=c \hat{\mathbf{z}}
\end{aligned}
$$

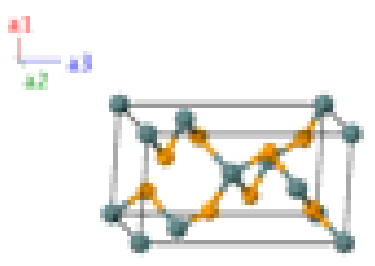

Basis vectors:

Lattice Coordinates

$\begin{array}{lcccc}\mathbf{B}_{1}= & 0 \mathbf{a}_{1}+0 \mathbf{a}_{2}+0 \mathbf{a}_{3} & = & 0 \hat{\mathbf{x}}+0 \hat{\mathbf{y}}+0 \hat{\mathbf{z}} \\ \mathbf{B}_{2}= & \frac{1}{2} \mathbf{a}_{1}+\frac{1}{2} \mathbf{a}_{2}+\frac{1}{2} \mathbf{a}_{3} & = & \frac{1}{2} a \hat{\mathbf{x}}+\frac{1}{2} a \hat{\mathbf{y}}+\frac{1}{2} c \hat{\mathbf{z}} \\ \mathbf{B}_{3}= & \frac{1}{2} \mathbf{a}_{2}+z_{3} \mathbf{a}_{3} & = & \frac{1}{2} a \hat{\mathbf{y}}+z_{3} c \hat{\mathbf{z}} \\ \mathbf{B}_{4}= & \frac{1}{2} \mathbf{a}_{1}+-z_{3} \mathbf{a}_{3} & = & \frac{1}{2} a \hat{\mathbf{x}}+-z_{3} c \hat{\mathbf{z}} \\ \mathbf{B}_{5}= & x_{4} \mathbf{a}_{1}+y_{4} \mathbf{a}_{2}+z_{4} \mathbf{a}_{3} & = & x_{4} a \hat{\mathbf{x}}+y_{4} a \hat{\mathbf{y}}+z_{4} c \hat{\mathbf{z}} \\ \mathbf{B}_{6}= & -x_{4} \mathbf{a}_{1}-y_{4} \mathbf{a}_{2}+z_{4} \mathbf{a}_{3} & = & -x_{4} a \hat{\mathbf{x}}-y_{4} a \hat{\mathbf{y}}+z_{4} c \hat{\mathbf{z}} \\ \mathbf{B}_{7}= & y_{4} \mathbf{a}_{1}-x_{4} \mathbf{a}_{2}-z_{4} \mathbf{a}_{3} & = & y_{4} a \hat{\mathbf{x}}-x_{4} a \hat{\mathbf{y}}-z_{4} c \hat{\mathbf{z}} \\ \mathbf{B}_{8}= & -y_{4} \mathbf{a}_{1}+x_{4} \mathbf{a}_{2}-z_{4} \mathbf{a}_{3} & = & -y_{4} a \hat{\mathbf{x}}+x_{4} a \hat{\mathbf{y}}-z_{4} c \hat{\mathbf{z}}\end{array}$

Wyckoff Position
Atom Type

Ge I

Ge II

Ge III

Ge III

Se I

Se I

Se I

Se I 


$\begin{array}{rlllll}\mathbf{B}_{9}= & x_{5} \mathbf{a}_{1}+y_{5} \mathbf{a}_{2}+z_{5} \mathbf{a}_{3} & = & x_{5} a \hat{\mathbf{x}}+y_{5} a \hat{\mathbf{y}}+z_{5} c \hat{\mathbf{z}} & (4 h) & \text { Se II } \\ \mathbf{B}_{10}= & -x_{5} \mathbf{a}_{1}-y_{5} \mathbf{a}_{2}+z_{5} \mathbf{a}_{3}= & -x_{5} a \hat{\mathbf{x}}-y_{5} a \hat{\mathbf{y}}+z_{5} c \hat{\mathbf{z}} & (4 h) & \text { Se II } \\ \mathbf{B}_{11}= & y_{5} \mathbf{a}_{1}-x_{5} \mathbf{a}_{2}-z_{5} \mathbf{a}_{3}= & y_{5} a \hat{\mathbf{x}}-x_{5} a \hat{\mathbf{y}}-z_{5} c \hat{\mathbf{z}} & \text { Se II } \\ \mathbf{B}_{12}= & -y_{5} \mathbf{a}_{1}+x_{5} \mathbf{a}_{2}-z_{5} \mathbf{a}_{3}= & -y_{5} a \hat{\mathbf{x}}+x_{5} a \hat{\mathbf{y}}-z_{5} c \hat{\mathbf{z}} & \text { Se II }\end{array}$

\section{References:}

- A. Grzechnik, S. Stølen, E. Bakken, T. Grande, and M. Mezouar, Structural transformations in three-dimensional crystalline GeSe $e_{2}$ at high pressures and high temperatures, J. Solid State Chem. 150, 121-127 (2000), doi:10.1006/jssc.1999.8557.

\section{Found in:}

- P. Villars and K. Cenzual, Pearson's Crystal Data - Crystal Structure Database for Inorganic Compounds, ASM International (2013).

\section{Geometry files:}

- CIF: pp. 862

- POSCAR: pp. 863 


\section{$\mathrm{Ni}_{3} \mathrm{P}\left(D 0_{e}\right)$ Structure: A3B_tI32_82_3g_g}
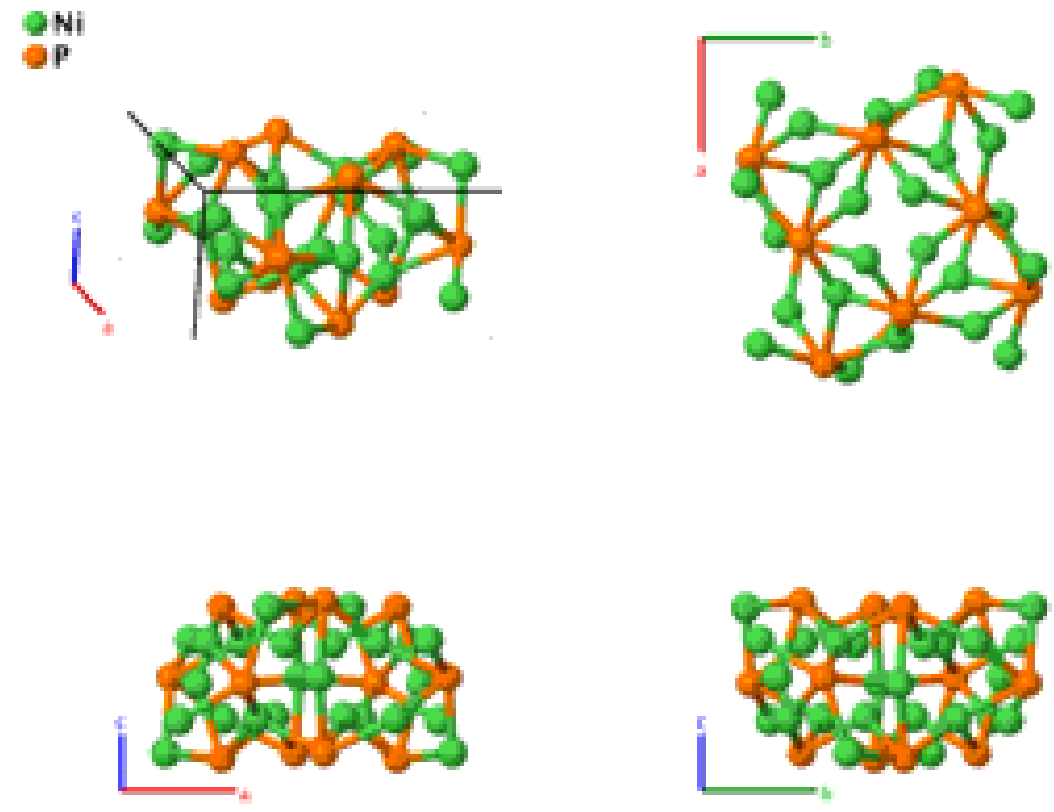

\section{Prototype}

AFLOW prototype label

$: \quad \mathrm{Ni}_{3} \mathrm{P}$

Strukturbericht designation

: A3B_tI32_82_3g_g

Pearson symbol

$0_{e}$

Space group number

$\mathrm{tI} 32$

Space group symbol

82

AFLOW prototype command

$: \quad I \overline{4}$

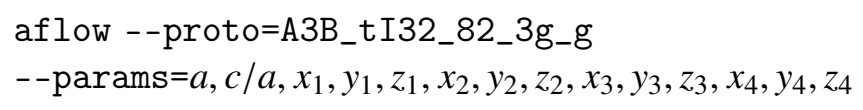

Other compounds with this structure:

- $\mathrm{Cr}_{3} \mathrm{P}, \mathrm{Fe}_{3} \mathrm{P}, \mathrm{Mn}_{3} \mathrm{P}, \mathrm{Mo}_{3} \mathrm{P}, \mathrm{Ti}_{3} \mathrm{P}, \mathrm{V}_{3} \mathrm{P}, \mathrm{BFe}_{3}$

Body-centered Tetragonal primitive vectors:

$$
\begin{aligned}
& \mathbf{a}_{1}=-\frac{1}{2} a \hat{\mathbf{x}}+\frac{1}{2} a \hat{\mathbf{y}}+\frac{1}{2} c \hat{\mathbf{z}} \\
& \mathbf{a}_{2}=\frac{1}{2} a \hat{\mathbf{x}}-\frac{1}{2} a \hat{\mathbf{y}}+\frac{1}{2} c \hat{\mathbf{z}} \\
& \mathbf{a}_{3}=\frac{1}{2} a \hat{\mathbf{x}}+\frac{1}{2} a \hat{\mathbf{y}}-\frac{1}{2} c \hat{\mathbf{z}}
\end{aligned}
$$

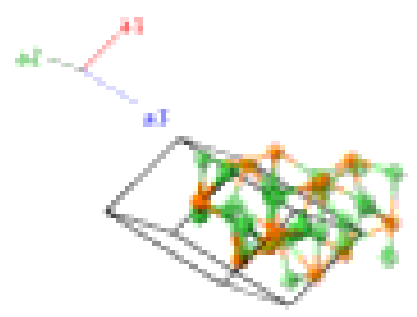

Basis vectors: 


\begin{tabular}{|c|c|c|c|c|c|c|}
\hline $\mathbf{B}_{2}$ & $=$ & $\left(-y_{1}+z_{1}\right) \mathbf{a}_{1}+\left(-x_{1}+z_{1}\right) \mathbf{a}_{2}+\left(-x_{1}-y_{1}\right) \mathbf{a}_{3}$ & $=$ & $-x_{1} a \hat{\mathbf{x}}-y_{1} a \hat{\mathbf{y}}+z_{1} c \hat{\mathbf{z}}$ & $(8 g)$ & Ni I \\
\hline $\mathbf{B}_{3}$ & $=$ & $\left(-x_{1}-z_{1}\right) \mathbf{a}_{1}+\left(y_{1}-z_{1}\right) \mathbf{a}_{2}+\left(-x_{1}+y_{1}\right) \mathbf{a}_{3}$ & $=$ & $y_{1} a \hat{\mathbf{x}}-x_{1} a \hat{\mathbf{y}}-z_{1} c \hat{\mathbf{z}}$ & $(8 g)$ & Ni I \\
\hline $\mathbf{B}_{4}$ & $=$ & $\left(x_{1}-z_{1}\right) \mathbf{a}_{1}+\left(-y_{1}-z_{1}\right) \mathbf{a}_{2}+\left(x_{1}-y_{1}\right) \mathbf{a}_{3}$ & $=$ & $-y_{1} a \hat{\mathbf{x}}+x_{1} a \hat{\mathbf{y}}-z_{1} c \hat{\mathbf{z}}$ & $(8 g)$ & Ni I \\
\hline $\mathbf{B}_{5}$ & $=$ & $\left(y_{2}+z_{2}\right) \mathbf{a}_{1}+\left(x_{2}+z_{2}\right) \mathbf{a}_{2}+\left(x_{2}+y_{2}\right) \mathbf{a}_{3}$ & $=$ & $x_{2} a \hat{\mathbf{x}}+y_{2} a \hat{\mathbf{y}}+z_{2} c \hat{\mathbf{z}}$ & $(8 g)$ & Ni II \\
\hline $\mathbf{B}_{6}$ & $=$ & $\left(-y_{2}+z_{2}\right) \mathbf{a}_{1}+\left(-x_{2}+z_{2}\right) \mathbf{a}_{2}+\left(-x_{2}-y_{2}\right) \mathbf{a}_{3}$ & $=$ & $-x_{2} a \hat{\mathbf{x}}-y_{2} a \hat{\mathbf{y}}+z_{2} c \hat{\mathbf{z}}$ & $(8 g)$ & Ni II \\
\hline $\mathbf{B}_{7}$ & $=$ & $\left(-x_{2}-z_{2}\right) \mathbf{a}_{1}+\left(y_{2}-z_{2}\right) \mathbf{a}_{2}+\left(-x_{2}+y_{2}\right) \mathbf{a}_{3}$ & $=$ & $y_{2} a \hat{\mathbf{x}}-x_{2} a \hat{\mathbf{y}}-z_{2} c \hat{\mathbf{z}}$ & $(8 g)$ & Ni II \\
\hline $\mathbf{B}_{8}$ & $=$ & $\left(x_{2}-z_{2}\right) \mathbf{a}_{1}+\left(-y_{2}-z_{2}\right) \mathbf{a}_{2}+\left(x_{2}-y_{2}\right) \mathbf{a}_{3}$ & $=$ & $-y_{2} a \hat{\mathbf{x}}+x_{2} a \hat{\mathbf{y}}-z_{2} c \hat{\mathbf{z}}$ & $(8 g)$ & Ni II \\
\hline $\mathbf{B}_{9}$ & $=$ & $\left(y_{3}+z_{3}\right) \mathbf{a}_{1}+\left(x_{3}+z_{3}\right) \mathbf{a}_{2}+\left(x_{3}+y_{3}\right) \mathbf{a}_{3}$ & $=$ & $x_{3} a \hat{\mathbf{x}}+y_{3} a \hat{\mathbf{y}}+z_{3} c \hat{\mathbf{z}}$ & $(8 g)$ & Ni III \\
\hline $\mathbf{B}_{10}$ & $=$ & $\left(-y_{3}+z_{3}\right) \mathbf{a}_{1}+\left(-x_{3}+z_{3}\right) \mathbf{a}_{2}+\left(-x_{3}-y_{3}\right) \mathbf{a}_{3}$ & $=$ & $-x_{3} a \hat{\mathbf{x}}-y_{3} a \hat{\mathbf{y}}+z_{3} c \hat{\mathbf{z}}$ & $(8 g)$ & Ni III \\
\hline $\mathbf{B}_{11}$ & $=$ & $\left(-x_{3}-z_{3}\right) \mathbf{a}_{1}+\left(y_{3}-z_{3}\right) \mathbf{a}_{2}+\left(-x_{3}+y_{3}\right) \mathbf{a}_{3}$ & $=$ & $y_{3} a \hat{\mathbf{x}}-x_{3} a \hat{\mathbf{y}}-z_{3} c \hat{\mathbf{z}}$ & $(8 g)$ & Ni III \\
\hline $\mathbf{B}_{12}$ & $=$ & $\left(x_{3}-z_{3}\right) \mathbf{a}_{1}+\left(-y_{3}-z_{3}\right) \mathbf{a}_{2}+\left(x_{3}-y_{3}\right) \mathbf{a}_{3}$ & $=$ & $-y_{3} a \hat{\mathbf{x}}+x_{3} a \hat{\mathbf{y}}-z_{3} c \hat{\mathbf{z}}$ & $(8 g)$ & Ni III \\
\hline $\mathbf{B}_{13}$ & $=$ & $\left(y_{4}+z_{4}\right) \mathbf{a}_{1}+\left(x_{4}+z_{4}\right) \mathbf{a}_{2}+\left(x_{4}+y_{4}\right) \mathbf{a}_{3}$ & $=$ & $x_{4} a \hat{\mathbf{x}}+y_{4} a \hat{\mathbf{y}}+z_{4} c \hat{\mathbf{z}}$ & $(8 g)$ & $\mathrm{P}$ \\
\hline $\mathbf{B}_{14}$ & $=$ & $\left(-y_{4}+z_{4}\right) \mathbf{a}_{1}+\left(-x_{4}+z_{4}\right) \mathbf{a}_{2}+\left(-x_{4}-y_{4}\right) \mathbf{a}_{3}$ & $=$ & $-x_{4} a \hat{\mathbf{x}}-y_{4} a \hat{\mathbf{y}}+z_{4} c \hat{\mathbf{z}}$ & $(8 g)$ & $\mathrm{P}$ \\
\hline $\mathbf{B}_{15}$ & $=$ & $\left(-x_{4}-z_{4}\right) \mathbf{a}_{1}+\left(y_{4}-z_{4}\right) \mathbf{a}_{2}+\left(-x_{4}+y_{4}\right) \mathbf{a}_{3}$ & $=$ & $y_{4} a \hat{\mathbf{x}}-x_{4} a \hat{\mathbf{y}}-z_{4} c \hat{\mathbf{z}}$ & $(8 g)$ & $\mathrm{P}$ \\
\hline $\mathbf{B}_{16}$ & $=$ & $\left(x_{4}-z_{4}\right) \mathbf{a}_{1}+\left(-y_{4}-z_{4}\right) \mathbf{a}_{2}+\left(x_{4}-y_{4}\right) \mathbf{a}_{3}$ & $=$ & $-y_{4} a \hat{\mathbf{x}}+x_{4} a \hat{\mathbf{y}}-z_{4} c \hat{\mathbf{z}}$ & $(8 g)$ & $\mathrm{P}$ \\
\hline
\end{tabular}

\section{References:}

- S. Rundqvist, E. Hassler, and L. Lundvik, Refinement of the Ni $i_{3} P$ Structure, Acta Chem. Scand. 16, 242-243 (1962), doi:10.3891/acta.chem.scand.16-0242.

\section{Geometry files:}

- CIF: pp. 863

- POSCAR: pp. 863 


\section{$\mathrm{Ti}_{2} \mathrm{Ge}_{3}$ Structure: A3B2_tP10_83_adk_j}
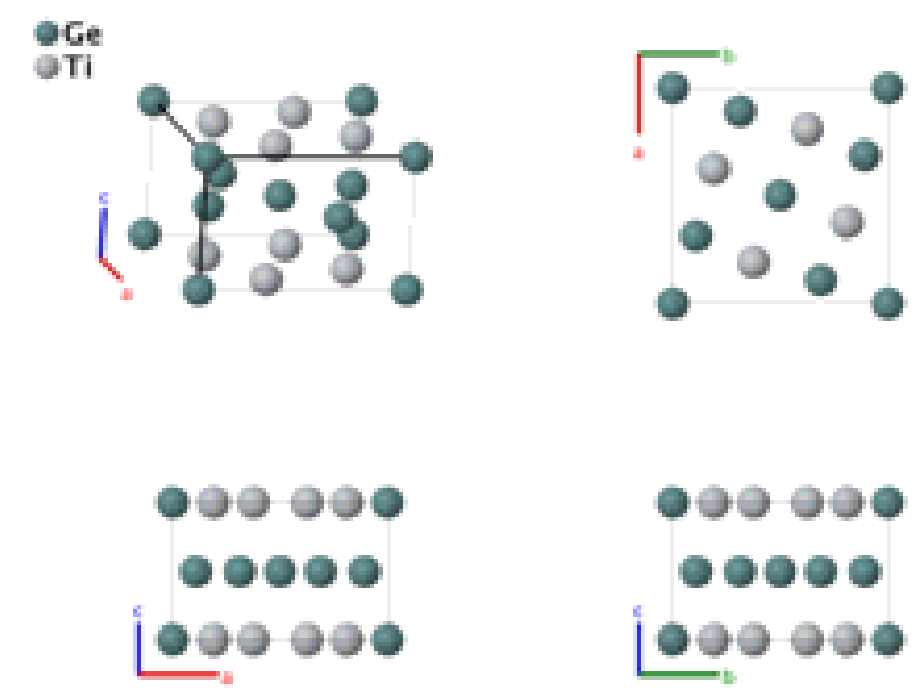

Prototype

: $\quad \mathrm{Ti}_{2} \mathrm{Ge}_{3}$

AFLOW prototype label

: A3B2_tP10_83_adk_j

Strukturbericht designation

: None

Pearson symbol

: $\quad \mathrm{tP} 10$

Space group number

: 83

Space group symbol

: $\quad P 4 / m$

AFLOW prototype command

aflow - -proto $=A 3 B 2 \_t P 10 \_83 \_a d k \_j$

- params $=a, c / a, x_{3}, y_{3}, x_{4}, y_{4}$

Simple Tetragonal primitive vectors:

$$
\begin{aligned}
& \mathbf{a}_{1}=a \hat{\mathbf{x}} \\
& \mathbf{a}_{2}=a \hat{\mathbf{y}} \\
& \mathbf{a}_{3}=c \hat{\mathbf{z}}
\end{aligned}
$$

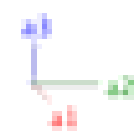

Basis vectors:

Lattice Coordinates

$\begin{array}{llc}\mathbf{B}_{1} & = & 0 \mathbf{a}_{1}+0 \mathbf{a}_{2}+0 \mathbf{a}_{3} \\ \mathbf{B}_{2} & = & \frac{1}{2} \mathbf{a}_{1}+\frac{1}{2} \mathbf{a}_{2}+\frac{1}{2} \mathbf{a}_{3} \\ \mathbf{B}_{3}= & x_{3} \mathbf{a}_{1}+y_{3} \mathbf{a}_{2} \\ \mathbf{B}_{4}= & -x_{3} \mathbf{a}_{1}-y_{3} \mathbf{a}_{2} \\ \mathbf{B}_{5}= & -y_{3} \mathbf{a}_{1}+x_{3} \mathbf{a}_{2} \\ \mathbf{B}_{6}= & y_{3} \mathbf{a}_{1}-x_{3} \mathbf{a}_{2}\end{array}$

Cartesian Coordinates

$=$

$=$

$=$

$=$

$=$

$=$

$$
\begin{gathered}
0 \hat{\mathbf{x}}+0 \hat{\mathbf{y}}+0 \hat{\mathbf{z}} \\
\frac{1}{2} a \hat{\mathbf{x}}+\frac{1}{2} a \hat{\mathbf{y}}+\frac{1}{2} c \hat{\mathbf{z}} \\
x_{3} a \hat{\mathbf{x}}+y_{3} a \hat{\mathbf{y}} \\
-x_{3} a \hat{\mathbf{x}}-y_{3} a \hat{\mathbf{y}} \\
-y_{3} a \hat{\mathbf{x}}+x_{3} a \hat{\mathbf{y}} \\
y_{3} a \hat{\mathbf{x}}-x_{3} a \hat{\mathbf{y}}
\end{gathered}
$$

Wyckoff Position

(1a)

(4j)

(4j)

(4j)

(4j)
Atom Type

Ge I

Ge II

$\mathrm{Ti}$

$\mathrm{Ti}$

$\mathrm{Ti}$

$\mathrm{Ti}$ 


$\begin{array}{rlrlrl}\mathbf{B}_{7}= & x_{4} \mathbf{a}_{1}+y_{4} \mathbf{a}_{2}+\frac{1}{2} \mathbf{a}_{3} & = & x_{4} a \hat{\mathbf{x}}+y_{4} a \hat{\mathbf{y}}+\frac{1}{2} c \hat{\mathbf{z}} & (4 k) & \text { Ge III } \\ \mathbf{B}_{8}= & -x_{4} \mathbf{a}_{1}-y_{4} \mathbf{a}_{2}+\frac{1}{2} \mathbf{a}_{3}= & -x_{4} a \hat{\mathbf{x}}-y_{4} a \hat{\mathbf{y}}+\frac{1}{2} c \hat{\mathbf{z}} & (4 k) & \text { Ge III } \\ \mathbf{B}_{9}= & -y_{4} \mathbf{a}_{1}+x_{4} \mathbf{a}_{2}+\frac{1}{2} \mathbf{a}_{3}= & -y_{4} a \hat{\mathbf{x}}+x_{4} a \hat{\mathbf{y}}+\frac{1}{2} c \hat{\mathbf{z}} & (4 k) & \text { Ge III } \\ \mathbf{B}_{10}= & y_{4} \mathbf{a}_{1}-x_{4} \mathbf{a}_{2}+\frac{1}{2} \mathbf{a}_{3}= & y_{4} a \hat{\mathbf{x}}-x_{4} a \hat{\mathbf{y}}+\frac{1}{2} c \hat{\mathbf{z}} & \text { Ge III }\end{array}$

\section{References:}

- K. Schubert, H. G. Meissner, M. Pötzschke, W. Rossteutscher, and E. Stolz, Einige Strukturdaten metallischer Phasen (7), Naturwissenschaften 49, 57-57 (1962), doi:10.1007/BF00595382.

\section{Found in:}

- P. Villars and K. Cenzual, Pearson's Crystal Data - Crystal Structure Database for Inorganic Compounds, ASM International (2013).

\section{Geometry files:}

- CIF: pp. 863

- POSCAR: pp. 864 


\section{$\mathrm{SrBr}_{2}$ Structure: A2B_tP30_85_ab2g_cg}
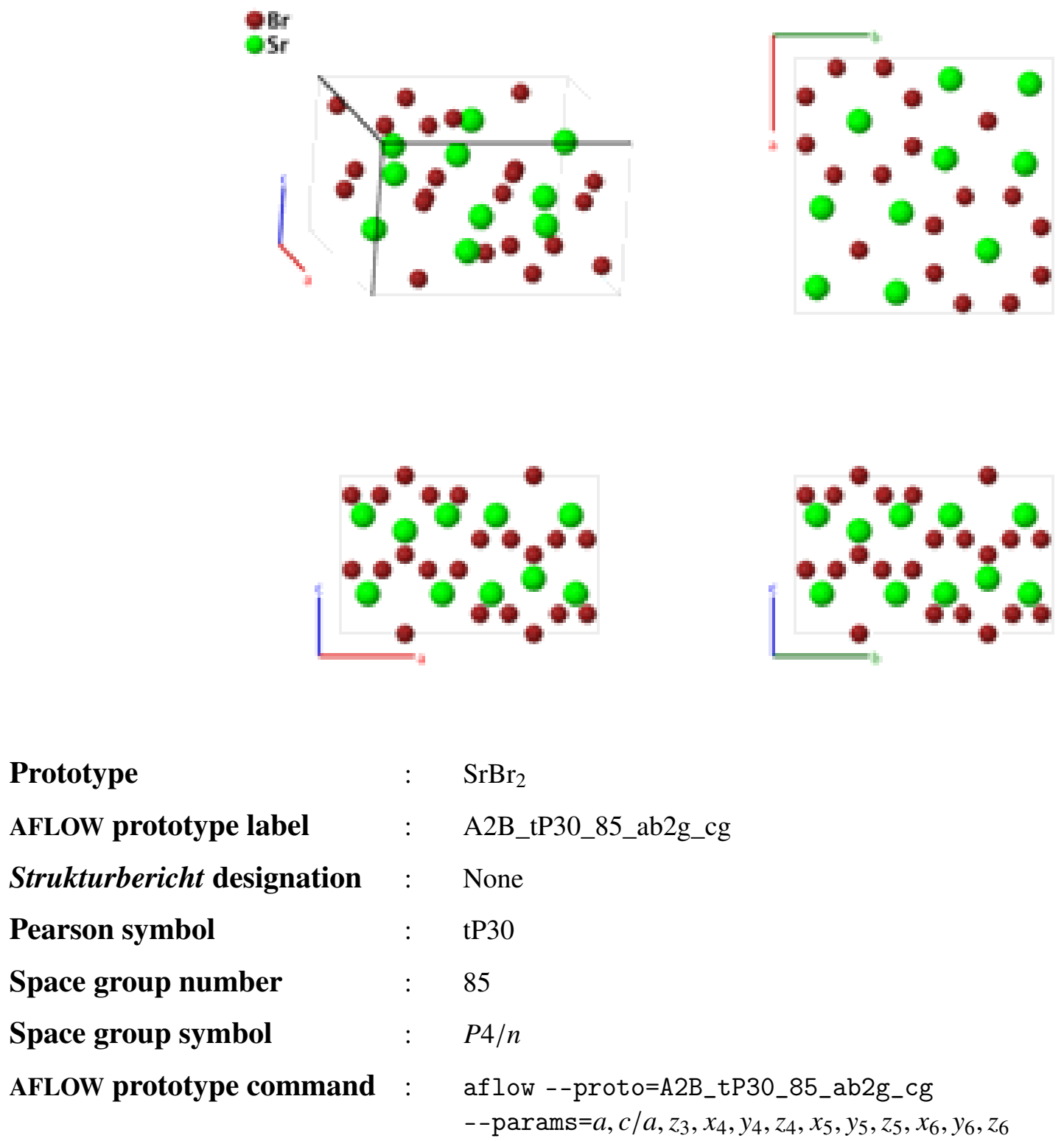

- Using the work of (Kamermans, 1939), (Herrmann, 1943) designated the structure $\mathrm{SrBr}_{2}$ as Strukturbericht C53, and placed it in space group Pnma \#62. (Sass, 1963) pointed out that the structure proposed by Kamermans did not agree with powder diffraction data, and proposed this structure, also found by (Frit, 1969). (Parthé, 1993) gives the current structure the $C 53$ designation. We will follow the original Strukturbericht, and give the C53 designation to the Pnma structure.

\section{Simple Tetragonal primitive vectors:}

$$
\begin{aligned}
& \mathbf{a}_{1}=a \hat{\mathbf{x}} \\
& \mathbf{a}_{2}=a \hat{\mathbf{y}} \\
& \mathbf{a}_{3}=c \hat{\mathbf{z}}
\end{aligned}
$$

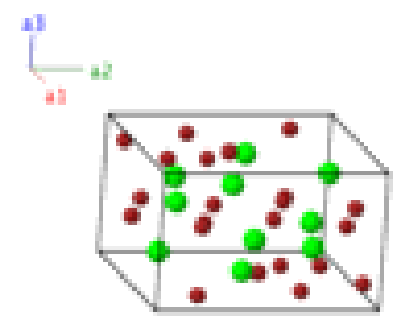

\section{Basis vectors:}


Lattice Coordinates

\begin{tabular}{|c|c|c|}
\hline $\mathbf{B}_{1}$ & $=$ & $\frac{1}{4} \mathbf{a}_{1}+\frac{3}{4} \mathbf{a}_{2}$ \\
\hline $\mathbf{B}_{2}$ & $=$ & $\frac{3}{4} \mathbf{a}_{1}+\frac{1}{4} \mathbf{a}_{2}$ \\
\hline $\mathbf{B}_{3}$ & $=$ & $\frac{1}{4} \mathbf{a}_{1}+\frac{3}{4} \mathbf{a}_{2}+\frac{1}{2} \mathbf{a}_{3}$ \\
\hline $\mathbf{B}_{4}$ & $=$ & $\frac{3}{4} \mathbf{a}_{1}+\frac{1}{4} \mathbf{a}_{2}+\frac{1}{2} \mathbf{a}_{3}$ \\
\hline $\mathbf{B}_{5}$ & $=$ & $\frac{1}{4} \mathbf{a}_{1}+\frac{1}{4} \mathbf{a}_{2}+z_{3} \mathbf{a}_{3}$ \\
\hline $\mathbf{B}_{6}$ & $=$ & $\frac{3}{4} \mathbf{a}_{1}+\frac{3}{4} \mathbf{a}_{2}-z_{3} \mathbf{a}_{3}$ \\
\hline $\mathbf{B}_{7}$ & $=$ & $x_{4} \mathbf{a}_{1}+y_{4} \mathbf{a}_{2}+z_{4} \mathbf{a}_{3}$ \\
\hline $\mathbf{B}_{8}$ & $=$ & $\left(\frac{1}{2}-x_{4}\right) \mathbf{a}_{1}+\left(\frac{1}{2}-y_{4}\right) \mathbf{a}_{2}+z_{4} \mathbf{a}_{3}$ \\
\hline $\mathbf{B}_{9}$ & $=$ & $\left(\frac{1}{2}-y_{4}\right) \mathbf{a}_{1}+x_{4} \mathbf{a}_{2}+z_{4} \mathbf{a}_{3}$ \\
\hline $\mathbf{B}_{10}$ & $=$ & $y_{4} \mathbf{a}_{1}+\left(\frac{1}{2}-x_{4}\right) \mathbf{a}_{2}+z_{4} \mathbf{a}_{3}$ \\
\hline $\mathbf{B}_{11}$ & $=$ & $-x_{4} \mathbf{a}_{1}-y_{4} \mathbf{a}_{2}-z_{4} \mathbf{a}_{3}$ \\
\hline $\mathbf{B}_{12}$ & $=$ & $\left(\frac{1}{2}+x_{4}\right) \mathbf{a}_{1}+\left(\frac{1}{2}+y_{4}\right) \mathbf{a}_{2}-z_{4} \mathbf{a}_{3}$ \\
\hline $\mathbf{B}_{13}$ & $=$ & $\left(\frac{1}{2}+y_{4}\right) \mathbf{a}_{1}-x_{4} \mathbf{a}_{2}-z_{4} \mathbf{a}_{3}$ \\
\hline $\mathbf{B}_{14}$ & $=$ & $-y_{4} \mathbf{a}_{1}+\left(\frac{1}{2}+x_{4}\right) \mathbf{a}_{2}-z_{4} \mathbf{a}_{3}$ \\
\hline $\mathbf{B}_{15}$ & $=$ & $x_{5} \mathbf{a}_{1}+y_{5} \mathbf{a}_{2}+z_{5} \mathbf{a}_{3}$ \\
\hline $\mathbf{B}_{16}$ & $=$ & $\left(\frac{1}{2}-x_{5}\right) \mathbf{a}_{1}+\left(\frac{1}{2}-y_{5}\right) \mathbf{a}_{2}+z_{5} \mathbf{a}_{3}$ \\
\hline $\mathbf{B}_{17}$ & $=$ & $\left(\frac{1}{2}-y_{5}\right) \mathbf{a}_{1}+x_{5} \mathbf{a}_{2}+z_{5} \mathbf{a}_{3}$ \\
\hline $\mathbf{B}_{18}$ & $=$ & $y_{5} \mathbf{a}_{1}+\left(\frac{1}{2}-x_{5}\right) \mathbf{a}_{2}+z_{5} \mathbf{a}_{3}$ \\
\hline $\mathbf{B}_{19}$ & $=$ & $-x_{5} \mathbf{a}_{1}-y_{5} \mathbf{a}_{2}-z_{5} \mathbf{a}_{3}$ \\
\hline $\mathbf{B}_{20}$ & $=$ & $\left(\frac{1}{2}+x_{5}\right) \mathbf{a}_{1}+\left(\frac{1}{2}+y_{5}\right) \mathbf{a}_{2}-z_{5} \mathbf{a}_{3}$ \\
\hline $\mathbf{B}_{21}$ & $=$ & $\left(\frac{1}{2}+y_{5}\right) \mathbf{a}_{1}-x_{5} \mathbf{a}_{2}-z_{5} \mathbf{a}_{3}$ \\
\hline $\mathbf{B}_{22}$ & $=$ & $-y_{5} \mathbf{a}_{1}+\left(\frac{1}{2}+x_{5}\right) \mathbf{a}_{2}-z_{5} \mathbf{a}_{3}$ \\
\hline $\mathbf{B}_{23}$ & $=$ & $x_{6} \mathbf{a}_{1}+y_{6} \mathbf{a}_{2}+z_{6} \mathbf{a}_{3}$ \\
\hline $\mathbf{B}_{24}$ & $=$ & $\left(\frac{1}{2}-x_{6}\right) \mathbf{a}_{1}+\left(\frac{1}{2}-y_{6}\right) \mathbf{a}_{2}+z_{6} \mathbf{a}_{3}$ \\
\hline $\mathbf{B}_{25}$ & $=$ & $\left(\frac{1}{2}-y_{6}\right) \mathbf{a}_{1}+x_{6} \mathbf{a}_{2}+z_{6} \mathbf{a}_{3}$ \\
\hline $\mathbf{B}_{26}$ & $=$ & $y_{6} \mathbf{a}_{1}+\left(\frac{1}{2}-x_{6}\right) \mathbf{a}_{2}+z_{6} \mathbf{a}_{3}$ \\
\hline $\mathbf{B}_{27}$ & $=$ & $-x_{6} \mathbf{a}_{1}-y_{6} \mathbf{a}_{2}-z_{6} \mathbf{a}_{3}$ \\
\hline $\mathbf{B}_{28}$ & $=$ & $\left(\frac{1}{2}+x_{6}\right) \mathbf{a}_{1}+\left(\frac{1}{2}+y_{6}\right) \mathbf{a}_{2}-z_{6} \mathbf{a}_{3}$ \\
\hline $\mathbf{B}_{29}$ & $=$ & $\left(\frac{1}{2}+y_{6}\right) \mathbf{a}_{1}-x_{6} \mathbf{a}_{2}-z_{6} \mathbf{a}_{3}$ \\
\hline $\mathbf{B}_{30}$ & $=$ & $-y_{6} \mathbf{a}_{1}+\left(\frac{1}{2}+x_{6}\right) \mathbf{a}_{2}-z_{6} \mathbf{a}_{3}$ \\
\hline
\end{tabular}

Cartesian Coordinates

$=$

$=$

$=$

$=$

$=$

$=$

$=$

$=$

$=$

$=$

$=$

$=$

$=$

$=$

$=$

$=$

$=$

$=$

$=$

$=$

$=$

$=$

$=$

$=$

$=$

$=$

$=$

$=$

$=$

$=$

$=$

$=$

$=$

$$
\frac{1}{4} a \hat{\mathbf{x}}+\frac{3}{4} a \hat{\mathbf{y}}
$$$$
\frac{3}{4} a \hat{\mathbf{x}}+\frac{1}{4} a \hat{\mathbf{y}}
$$$$
\frac{1}{4} a \hat{\mathbf{x}}+\frac{3}{4} a \hat{\mathbf{y}}+\frac{1}{2} c \hat{\mathbf{z}}
$$$$
\frac{3}{4} a \hat{\mathbf{x}}+\frac{1}{4} a \hat{\mathbf{y}}+\frac{1}{2} c \hat{\mathbf{z}}
$$$$
\frac{1}{4} a \hat{\mathbf{x}}+\frac{1}{4} a \hat{\mathbf{y}}+z_{3} c \hat{\mathbf{z}}
$$$$
\frac{3}{4} a \hat{\mathbf{x}}+\frac{3}{4} a \hat{\mathbf{y}}-z_{3} c \hat{\mathbf{z}}
$$$$
x_{4} a \hat{\mathbf{x}}+y_{4} a \hat{\mathbf{y}}+z_{4} c \hat{\mathbf{z}}
$$$$
\left(\frac{1}{2}-x_{4}\right) a \hat{\mathbf{x}}+\left(\frac{1}{2}-y_{4}\right) a \hat{\mathbf{y}}+z_{4} c \hat{\mathbf{z}}
$$$$
\left(\frac{1}{2}-y_{4}\right) a \hat{\mathbf{x}}+x_{4} a \hat{\mathbf{y}}+z_{4} c \hat{\mathbf{z}}
$$$$
y_{4} a \hat{\mathbf{x}}+\left(\frac{1}{2}-x_{4}\right) a \hat{\mathbf{y}}+z_{4} c \hat{\mathbf{z}}
$$$$
-x_{4} a \hat{\mathbf{x}}-y_{4} a \hat{\mathbf{y}}-z_{4} c \hat{\mathbf{z}}
$$$$
\left(\frac{1}{2}+x_{4}\right) a \hat{\mathbf{x}}+\left(\frac{1}{2}+y_{4}\right) a \hat{\mathbf{y}}-z_{4} c \hat{\mathbf{z}}
$$$$
\left(\frac{1}{2}+y_{4}\right) a \hat{\mathbf{x}}-x_{4} a \hat{\mathbf{y}}-z_{4} c \hat{\mathbf{z}}
$$$$
-y_{4} a \hat{\mathbf{x}}+\left(\frac{1}{2}+x_{4}\right) a \hat{\mathbf{y}}-z_{4} c \hat{\mathbf{z}}
$$$$
x_{5} a \hat{\mathbf{x}}+y_{5} a \hat{\mathbf{y}}+z_{5} c \hat{\mathbf{z}}
$$$$
\left(\frac{1}{2}-x_{5}\right) a \hat{\mathbf{x}}+\left(\frac{1}{2}-y_{5}\right) a \hat{\mathbf{y}}+z_{5} c \hat{\mathbf{z}}
$$$$
\left(\frac{1}{2}-y_{5}\right) a \hat{\mathbf{x}}+x_{5} a \hat{\mathbf{y}}+z_{5} c \hat{\mathbf{z}}
$$$$
y_{5} a \hat{\mathbf{x}}+\left(\frac{1}{2}-x_{5}\right) a \hat{\mathbf{y}}+z_{5} c \hat{\mathbf{z}}
$$$$
-x_{5} a \hat{\mathbf{x}}-y_{5} a \hat{\mathbf{y}}-z_{5} c \hat{\mathbf{z}}
$$$$
\left(\frac{1}{2}+x_{5}\right) a \hat{\mathbf{x}}+\left(\frac{1}{2}+y_{5}\right) a \hat{\mathbf{y}}-z_{5} c \hat{\mathbf{z}}
$$$$
\left(\frac{1}{2}+y_{5}\right) a \hat{\mathbf{x}}-x_{5} a \hat{\mathbf{y}}-z_{5} c \hat{\mathbf{z}}
$$$$
-y_{5} a \hat{\mathbf{x}}+\left(\frac{1}{2}+x_{5}\right) a \hat{\mathbf{y}}-z_{5} c \hat{\mathbf{z}}
$$$$
x_{6} a \hat{\mathbf{x}}+y_{6} a \hat{\mathbf{y}}+z_{6} c \hat{\mathbf{z}}
$$$$
\left(\frac{1}{2}-x_{6}\right) a \hat{\mathbf{x}}+\left(\frac{1}{2}-y_{6}\right) a \hat{\mathbf{y}}+z_{6} c \hat{\mathbf{z}}
$$$$
\left(\frac{1}{2}-y_{6}\right) a \hat{\mathbf{x}}+x_{6} a \hat{\mathbf{y}}+z_{6} c \hat{\mathbf{z}}
$$$$
y_{6} a \hat{\mathbf{x}}+\left(\frac{1}{2}-x_{6}\right) a \hat{\mathbf{y}}+z_{6} c \hat{\mathbf{z}}
$$$$
-x_{6} a \hat{\mathbf{x}}-y_{6} a \hat{\mathbf{y}}-z_{6} c \hat{\mathbf{z}}
$$$$
\left(\frac{1}{2}+x_{6}\right) a \hat{\mathbf{x}}+\left(\frac{1}{2}+y_{6}\right) a \hat{\mathbf{y}}-z_{6} c \hat{\mathbf{z}}
$$$$
\left(\frac{1}{2}+y_{6}\right) a \hat{\mathbf{x}}-x_{6} a \hat{\mathbf{y}}-z_{6} c \hat{\mathbf{z}}
$$$$
-y_{6} a \hat{\mathbf{x}}+\left(\frac{1}{2}+x_{6}\right) a \hat{\mathbf{y}}-z_{6} c \hat{\mathbf{z}}
$$

Wyckoff Position

Atom Type

(2a)

Br I

(2a)
Br I

Br II

Br II

Sr I

Sr I

Br III

Br III

$\mathrm{Br}$ III

Br III

Br III

Br III

Br III

Br III

Br IV

Br IV

Br IV

Br IV

Br IV

Br IV

Br IV

Br IV

Sr II

Sr II

Sr II

Sr II

Sr II

Sr II

Sr II

Sr II

\section{References:}

- B. Frit and M. M. Chbany, Les halogeno-carbonates de strontium, J. Inorg. Nucl. Chem. 31, 2685-2693 (1969), doi:10.1016/0022-1902(69)80182-X.

- K. Herrmann, ed., Strukturbericht Band VII 1939 (Akademische Verlagsgesellschaft M. B. H., Leipzig, 1943).

- M. A. Kamermans, The Crystal Structure of $\mathrm{SrBr}_{2}$, Zeitschrift für Kristallographie - Crystalline Materials 101, 406-411

(1939), doi:10.1524/zkri.1939.101.1.406. 
- R. L. Sass, T. Brackett, and E. Brackett, The Crystal Structure of Strontium Bromide, J. Phys. Chem. 67, 2862-2863 (1963), doi:10.1021/j100806a516.

- E. Parthé, L. Gelato, B. Chabot, M. Penso, K. Cenzula, and R. Gladyshevskii, Standardized Data and Crystal Chemical Characterization of Inorganic Structure Types, Gmelin Handbook of Inorganic and Organometallic Chemistry, vol. 2 (Springer-Verlag, Berlin, Heidelberg, 1993), 8 edn., doi:10.1007/978-3-662-02909-1_3.

\section{Found in:}

- P. Villars and K. Cenzual, Pearson's Crystal Data - Crystal Structure Database for Inorganic Compounds, ASM International (2013).

\section{Geometry files:}

- CIF: pp. 864

- POSCAR: pp. 864 


\section{Ti 3 P Structure: AB3_tP32_86_g_3g}
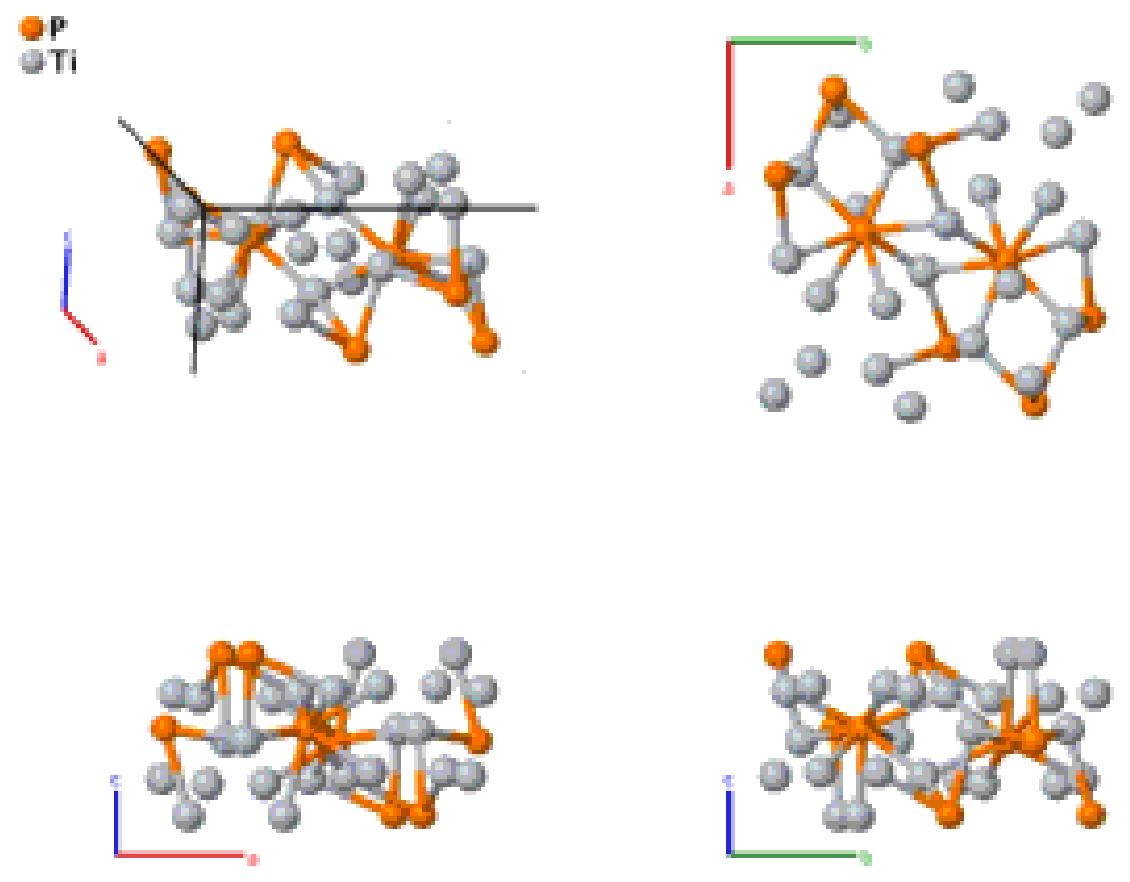

\section{Prototype}

AFLOW prototype label

Strukturbericht designation

Pearson symbol

$: \quad \mathrm{Ti}_{3} \mathrm{P}$

Space group number

tP32

Space group symbol

86

AFLOW prototype command

: $\quad P 4_{2} / n$

$$
\text { - - params }=a, c / a, x_{1}, y_{1}, z_{1}, x_{2}, y_{2}, z_{2}, x_{3}, y_{3}, z_{3}, x_{4}, y_{4}, z_{4}
$$

Simple Tetragonal primitive vectors:

$$
\begin{aligned}
& \mathbf{a}_{1}=a \hat{\mathbf{x}} \\
& \mathbf{a}_{2}=a \hat{\mathbf{y}} \\
& \mathbf{a}_{3}=c \hat{\mathbf{z}}
\end{aligned}
$$

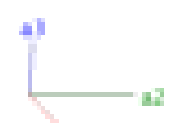

-11

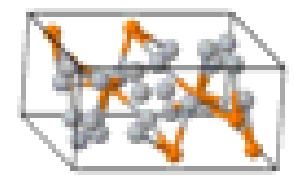

Basis vectors:

Lattice Coordinates

Cartesian Coordinates

$\mathbf{B}_{1}=x_{1} \mathbf{a}_{1}+y_{1} \mathbf{a}_{2}+z_{1} \mathbf{a}_{3}$

$\mathbf{B}_{2}=\left(\frac{1}{2}-x_{1}\right) \mathbf{a}_{1}+\left(\frac{1}{2}-y_{1}\right) \mathbf{a}_{2}+z_{1} \mathbf{a}_{3}$
$=$

$x_{1} a \hat{\mathbf{x}}+y_{1} a \hat{\mathbf{y}}+z_{1} c \hat{\mathbf{z}}$

$=\left(\frac{1}{2}-x_{1}\right) a \hat{\mathbf{x}}+\left(\frac{1}{2}-y_{1}\right) a \hat{\mathbf{y}}+z_{1} c \hat{\mathbf{z}}$
Wyckoff Position

Atom Type

$(8 g)$

$\mathrm{P}$

$(8 g)$ 


\begin{tabular}{|c|c|c|c|c|c|c|}
\hline $\mathbf{B}_{3}$ & $=$ & $-y_{1} \mathbf{a}_{1}+\left(\frac{1}{2}+x_{1}\right) \mathbf{a}_{2}+\left(\frac{1}{2}+z_{1}\right) \mathbf{a}_{3}$ & $=$ & $-y_{1} a \hat{\mathbf{x}}+\left(\frac{1}{2}+x_{1}\right) a \hat{\mathbf{y}}+\left(\frac{1}{2}+z_{1}\right) c \hat{\mathbf{z}}$ & $(8 g)$ & $\mathrm{P}$ \\
\hline $\mathbf{B}_{4}$ & $=$ & $\left(\frac{1}{2}+y_{1}\right) \mathbf{a}_{1}-x_{1} \mathbf{a}_{2}+\left(\frac{1}{2}+z_{1}\right) \mathbf{a}_{3}$ & $=$ & $\left(\frac{1}{2}+y_{1}\right) a \hat{\mathbf{x}}-x_{1} a \hat{\mathbf{y}}+\left(\frac{1}{2}+z_{1}\right) c \hat{\mathbf{z}}$ & $(8 g)$ & $\mathrm{P}$ \\
\hline $\mathbf{B}_{5}$ & $=$ & $-x_{1} \mathbf{a}_{1}-y_{1} \mathbf{a}_{2}-z_{1} \mathbf{a}_{3}$ & $=$ & $-x_{1} a \hat{\mathbf{x}}-y_{1} a \hat{\mathbf{y}}-z_{1} c \hat{\mathbf{z}}$ & $(8 g)$ & $\mathrm{P}$ \\
\hline $\mathbf{B}_{6}$ & $=$ & $\left(\frac{1}{2}+x_{1}\right) \mathbf{a}_{1}+\left(\frac{1}{2}+y_{1}\right) \mathbf{a}_{2}-z_{1} \mathbf{a}_{3}$ & $=$ & $\left(\frac{1}{2}+x_{1}\right) a \hat{\mathbf{x}}+\left(\frac{1}{2}+y_{1}\right) a \hat{\mathbf{y}}-z_{1} c \hat{\mathbf{z}}$ & $(8 g)$ & $\mathrm{P}$ \\
\hline $\mathbf{B}_{7}$ & $=$ & $y_{1} \mathbf{a}_{1}+\left(\frac{1}{2}-x_{1}\right) \mathbf{a}_{2}+\left(\frac{1}{2}-z_{1}\right) \mathbf{a}_{3}$ & $=$ & $y_{1} a \hat{\mathbf{x}}+\left(\frac{1}{2}-x_{1}\right) a \hat{\mathbf{y}}+\left(\frac{1}{2}-z_{1}\right) c \hat{\mathbf{z}}$ & $(8 g)$ & $\mathrm{P}$ \\
\hline $\mathbf{B}_{8}$ & $=$ & $\left(\frac{1}{2}-y_{1}\right) \mathbf{a}_{1}+x_{1} \mathbf{a}_{2}+\left(\frac{1}{2}-z_{1}\right) \mathbf{a}_{3}$ & $=$ & $\left(\frac{1}{2}-y_{1}\right) a \hat{\mathbf{x}}+x_{1} a \hat{\mathbf{y}}+\left(\frac{1}{2}-z_{1}\right) c \hat{\mathbf{z}}$ & $(8 g)$ & $\mathrm{P}$ \\
\hline $\mathbf{B}_{9}$ & $=$ & $x_{2} \mathbf{a}_{1}+y_{2} \mathbf{a}_{2}+z_{2} \mathbf{a}_{3}$ & $=$ & $x_{2} a \hat{\mathbf{x}}+y_{2} a \hat{\mathbf{y}}+z_{2} c \hat{\mathbf{z}}$ & $(8 g)$ & Ti I \\
\hline $\mathbf{B}_{10}$ & $=$ & $\left(\frac{1}{2}-x_{2}\right) \mathbf{a}_{1}+\left(\frac{1}{2}-y_{2}\right) \mathbf{a}_{2}+z_{2} \mathbf{a}_{3}$ & $=$ & $\left(\frac{1}{2}-x_{2}\right) a \hat{\mathbf{x}}+\left(\frac{1}{2}-y_{2}\right) a \hat{\mathbf{y}}+z_{2} c \hat{\mathbf{z}}$ & $(8 g)$ & Ti I \\
\hline $\mathbf{B}_{11}$ & $=$ & $-y_{2} \mathbf{a}_{1}+\left(\frac{1}{2}+x_{2}\right) \mathbf{a}_{2}+\left(\frac{1}{2}+z_{2}\right) \mathbf{a}_{3}$ & $=$ & $-y_{2} a \hat{\mathbf{x}}+\left(\frac{1}{2}+x_{2}\right) a \hat{\mathbf{y}}+\left(\frac{1}{2}+z_{2}\right) c \hat{\mathbf{z}}$ & $(8 g)$ & Ti I \\
\hline $\mathbf{B}_{12}$ & $=$ & $\left(\frac{1}{2}+y_{2}\right) \mathbf{a}_{1}-x_{2} \mathbf{a}_{2}+\left(\frac{1}{2}+z_{2}\right) \mathbf{a}_{3}$ & $=$ & $\left(\frac{1}{2}+y_{2}\right) a \hat{\mathbf{x}}-x_{2} a \hat{\mathbf{y}}+\left(\frac{1}{2}+z_{2}\right) c \hat{\mathbf{z}}$ & $(8 g)$ & Ti I \\
\hline $\mathbf{B}_{13}$ & $=$ & $-x_{2} \mathbf{a}_{1}-y_{2} \mathbf{a}_{2}-z_{2} \mathbf{a}_{3}$ & $=$ & $-x_{2} a \hat{\mathbf{x}}-y_{2} a \hat{\mathbf{y}}-z_{2} c \hat{\mathbf{z}}$ & $(8 g)$ & Ti I \\
\hline $\mathbf{B}_{14}$ & $=$ & $\left(\frac{1}{2}+x_{2}\right) \mathbf{a}_{1}+\left(\frac{1}{2}+y_{2}\right) \mathbf{a}_{2}-z_{2} \mathbf{a}_{3}$ & $=$ & $\left(\frac{1}{2}+x_{2}\right) a \hat{\mathbf{x}}+\left(\frac{1}{2}+y_{2}\right) a \hat{\mathbf{y}}-z_{2} c \hat{\mathbf{z}}$ & $(8 g)$ & Ti I \\
\hline $\mathbf{B}_{15}$ & $=$ & $y_{2} \mathbf{a}_{1}+\left(\frac{1}{2}-x_{2}\right) \mathbf{a}_{2}+\left(\frac{1}{2}-z_{2}\right) \mathbf{a}_{3}$ & $=$ & $y_{2} a \hat{\mathbf{x}}+\left(\frac{1}{2}-x_{2}\right) a \hat{\mathbf{y}}+\left(\frac{1}{2}-z_{2}\right) c \hat{\mathbf{z}}$ & $(8 g)$ & Ti I \\
\hline $\mathbf{B}_{16}$ & $=$ & $\left(\frac{1}{2}-y_{2}\right) \mathbf{a}_{1}+x_{2} \mathbf{a}_{2}+\left(\frac{1}{2}-z_{2}\right) \mathbf{a}_{3}$ & $=$ & $\left(\frac{1}{2}-y_{2}\right) a \hat{\mathbf{x}}+x_{2} a \hat{\mathbf{y}}+\left(\frac{1}{2}-z_{2}\right) c \hat{\mathbf{z}}$ & $(8 g)$ & Ti I \\
\hline $\mathbf{B}_{17}$ & $=$ & $x_{3} \mathbf{a}_{1}+y_{3} \mathbf{a}_{2}+z_{3} \mathbf{a}_{3}$ & $=$ & $x_{3} a \hat{\mathbf{x}}+y_{3} a \hat{\mathbf{y}}+z_{3} c \hat{\mathbf{z}}$ & $(8 g)$ & Ti II \\
\hline $\mathbf{B}_{18}$ & $=$ & $\left(\frac{1}{2}-x_{3}\right) \mathbf{a}_{1}+\left(\frac{1}{2}-y_{3}\right) \mathbf{a}_{2}+z_{3} \mathbf{a}_{3}$ & $=$ & $\left(\frac{1}{2}-x_{3}\right) a \hat{\mathbf{x}}+\left(\frac{1}{2}-y_{3}\right) a \hat{\mathbf{y}}+z_{3} c \hat{\mathbf{z}}$ & $(8 g)$ & Ti II \\
\hline $\mathbf{B}_{19}$ & $=$ & $-y_{3} \mathbf{a}_{1}+\left(\frac{1}{2}+x_{3}\right) \mathbf{a}_{2}+\left(\frac{1}{2}+z_{3}\right) \mathbf{a}_{3}$ & $=$ & $-y_{3} a \hat{\mathbf{x}}+\left(\frac{1}{2}+x_{3}\right) a \hat{\mathbf{y}}+\left(\frac{1}{2}+z_{3}\right) c \hat{\mathbf{z}}$ & $(8 g)$ & Ti II \\
\hline $\mathbf{B}_{20}$ & $=$ & $\left(\frac{1}{2}+y_{3}\right) \mathbf{a}_{1}-x_{3} \mathbf{a}_{2}+\left(\frac{1}{2}+z_{3}\right) \mathbf{a}_{3}$ & $=$ & $\left(\frac{1}{2}+y_{3}\right) a \hat{\mathbf{x}}-x_{3} a \hat{\mathbf{y}}+\left(\frac{1}{2}+z_{3}\right) c \hat{\mathbf{z}}$ & $(8 g)$ & Ti II \\
\hline $\mathbf{B}_{21}$ & $=$ & $-x_{3} \mathbf{a}_{1}-y_{3} \mathbf{a}_{2}-z_{3} \mathbf{a}_{3}$ & $=$ & $-x_{3} a \hat{\mathbf{x}}-y_{3} a \hat{\mathbf{y}}-z_{3} c \hat{\mathbf{z}}$ & $(8 g)$ & Ti II \\
\hline $\mathbf{B}_{22}$ & $=$ & $\left(\frac{1}{2}+x_{3}\right) \mathbf{a}_{1}+\left(\frac{1}{2}+y_{3}\right) \mathbf{a}_{2}-z_{3} \mathbf{a}_{3}$ & $=$ & $\left(\frac{1}{2}+x_{3}\right) a \hat{\mathbf{x}}+\left(\frac{1}{2}+y_{3}\right) a \hat{\mathbf{y}}-z_{3} c \hat{\mathbf{z}}$ & $(8 g)$ & Ti II \\
\hline $\mathbf{B}_{23}$ & $=$ & $y_{3} \mathbf{a}_{1}+\left(\frac{1}{2}-x_{3}\right) \mathbf{a}_{2}+\left(\frac{1}{2}-z_{3}\right) \mathbf{a}_{3}$ & $=$ & $y_{3} a \hat{\mathbf{x}}+\left(\frac{1}{2}-x_{3}\right) a \hat{\mathbf{y}}+\left(\frac{1}{2}-z_{3}\right) c \hat{\mathbf{z}}$ & $(8 g)$ & Ti II \\
\hline $\mathbf{B}_{24}$ & $=$ & $\left(\frac{1}{2}-y_{3}\right) \mathbf{a}_{1}+x_{3} \mathbf{a}_{2}+\left(\frac{1}{2}-z_{3}\right) \mathbf{a}_{3}$ & $=$ & $\left(\frac{1}{2}-y_{3}\right) a \hat{\mathbf{x}}+x_{3} a \hat{\mathbf{y}}+\left(\frac{1}{2}-z_{3}\right) c \hat{\mathbf{z}}$ & $(8 g)$ & $\mathrm{Ti}$ II \\
\hline $\mathbf{B}_{25}$ & $=$ & $x_{4} \mathbf{a}_{1}+y_{4} \mathbf{a}_{2}+z_{4} \mathbf{a}_{3}$ & $=$ & $x_{4} a \hat{\mathbf{x}}+y_{4} a \hat{\mathbf{y}}+z_{4} c \hat{\mathbf{z}}$ & $(8 g)$ & Ti III \\
\hline $\mathbf{B}_{26}$ & $=$ & $\left(\frac{1}{2}-x_{4}\right) \mathbf{a}_{1}+\left(\frac{1}{2}-y_{4}\right) \mathbf{a}_{2}+z_{4} \mathbf{a}_{3}$ & $=$ & $\left(\frac{1}{2}-x_{4}\right) a \hat{\mathbf{x}}+\left(\frac{1}{2}-y_{4}\right) a \hat{\mathbf{y}}+z_{4} c \hat{\mathbf{z}}$ & $(8 g)$ & Ti III \\
\hline $\mathbf{B}_{27}$ & $=$ & $-y_{4} \mathbf{a}_{1}+\left(\frac{1}{2}+x_{4}\right) \mathbf{a}_{2}+\left(\frac{1}{2}+z_{4}\right) \mathbf{a}_{3}$ & $=$ & $-y_{4} a \hat{\mathbf{x}}+\left(\frac{1}{2}+x_{4}\right) a \hat{\mathbf{y}}+\left(\frac{1}{2}+z_{4}\right) c \hat{\mathbf{z}}$ & $(8 g)$ & Ti III \\
\hline $\mathbf{B}_{28}$ & $=$ & $\left(\frac{1}{2}+y_{4}\right) \mathbf{a}_{1}-x_{4} \mathbf{a}_{2}+\left(\frac{1}{2}+z_{4}\right) \mathbf{a}_{3}$ & $=$ & $\left(\frac{1}{2}+y_{4}\right) a \hat{\mathbf{x}}-x_{4} a \hat{\mathbf{y}}+\left(\frac{1}{2}+z_{4}\right) c \hat{\mathbf{z}}$ & $(8 g)$ & Ti III \\
\hline $\mathbf{B}_{29}$ & $=$ & $-x_{4} \mathbf{a}_{1}-y_{4} \mathbf{a}_{2}-z_{4} \mathbf{a}_{3}$ & $=$ & $-x_{4} a \hat{\mathbf{x}}-y_{4} a \hat{\mathbf{y}}-z_{4} c \hat{\mathbf{z}}$ & $(8 g)$ & Ti III \\
\hline $\mathbf{B}_{30}$ & $=$ & $\left(\frac{1}{2}+x_{4}\right) \mathbf{a}_{1}+\left(\frac{1}{2}+y_{4}\right) \mathbf{a}_{2}-z_{4} \mathbf{a}_{3}$ & $=$ & $\left(\frac{1}{2}+x_{4}\right) a \hat{\mathbf{x}}+\left(\frac{1}{2}+y_{4}\right) a \hat{\mathbf{y}}-z_{4} c \hat{\mathbf{z}}$ & $(8 g)$ & Ti III \\
\hline $\mathbf{B}_{31}$ & $=$ & $y_{4} \mathbf{a}_{1}+\left(\frac{1}{2}-x_{4}\right) \mathbf{a}_{2}+\left(\frac{1}{2}-z_{4}\right) \mathbf{a}_{3}$ & $=$ & $y_{4} a \hat{\mathbf{x}}+\left(\frac{1}{2}-x_{4}\right) a \hat{\mathbf{y}}+\left(\frac{1}{2}-z_{4}\right) c \hat{\mathbf{z}}$ & $(8 g)$ & Ti III \\
\hline $\mathbf{B}_{32}$ & $=$ & $\left(\frac{1}{2}-y_{4}\right) \mathbf{a}_{1}+x_{4} \mathbf{a}_{2}+\left(\frac{1}{2}-z_{4}\right) \mathbf{a}_{3}$ & $=$ & $\left(\frac{1}{2}-y_{4}\right) a \hat{\mathbf{x}}+x_{4} a \hat{\mathbf{y}}+\left(\frac{1}{2}-z_{4}\right) c \hat{\mathbf{z}}$ & $(8 g)$ & Ti III \\
\hline
\end{tabular}

\section{References:}

- V. N. Eremenko and V. E. Listovnichii, State diagram of the Ti-P system, Dopov. Akad. Nauk Ukr. RSR pp. 1176-1179 (1965).

\section{Found in:}

- P. Villars and K. Cenzual, Pearson's Crystal Data - Crystal Structure Database for Inorganic Compounds, ASM International (2013). 
Geometry files:

- CIF: pp. 865

- POSCAR: pp. 865 


\section{$\mathrm{ThCl}_{4}$ Structure: A4B_tI20_88_f_a}
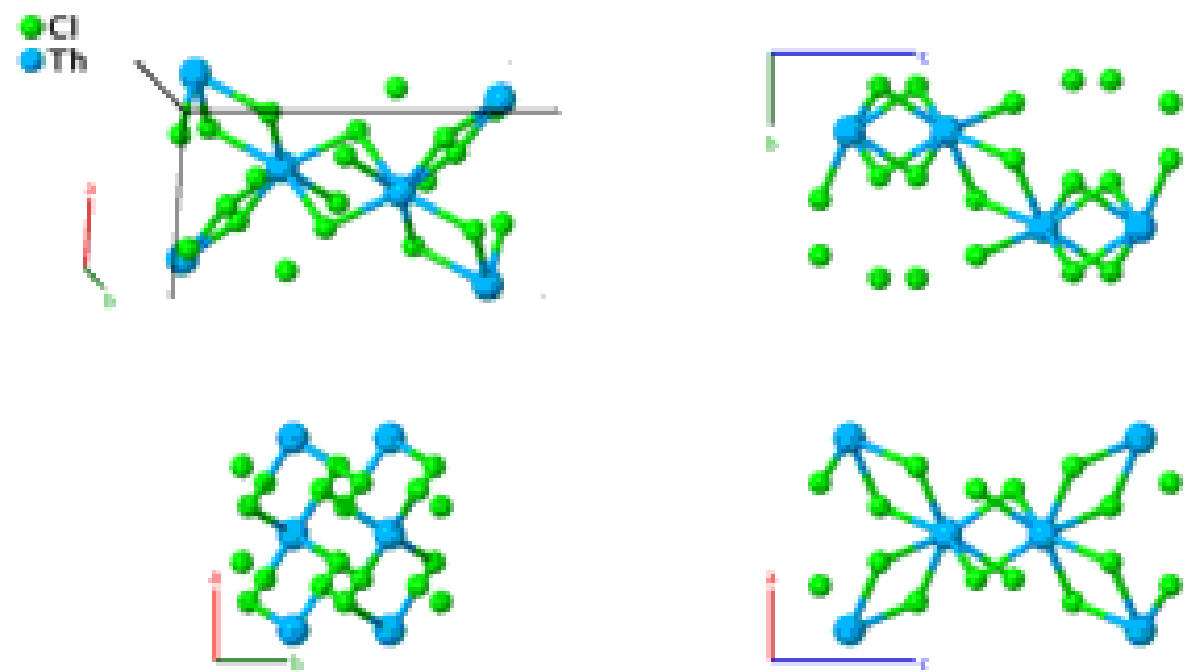

\section{Prototype}

AFLOW prototype label

Strukturbericht designation

Pearson symbol

Space group number

Space group symbol

AFLOW prototype command
: $\quad \mathrm{ThCl}_{4}$

: A4B_tI20_88_f_a

: None

: $\quad$ tI20

$: \quad 88$

: $\quad I 4_{1} / a$

Body-centered Tetragonal primitive vectors:

$$
\begin{aligned}
& \mathbf{a}_{1}=-\frac{1}{2} a \hat{\mathbf{x}}+\frac{1}{2} a \hat{\mathbf{y}}+\frac{1}{2} c \hat{\mathbf{z}} \\
& \mathbf{a}_{2}=\frac{1}{2} a \hat{\mathbf{x}}-\frac{1}{2} a \hat{\mathbf{y}}+\frac{1}{2} c \hat{\mathbf{z}} \\
& \mathbf{a}_{3}=\frac{1}{2} a \hat{\mathbf{x}}+\frac{1}{2} a \hat{\mathbf{y}}-\frac{1}{2} c \hat{\mathbf{z}}
\end{aligned}
$$

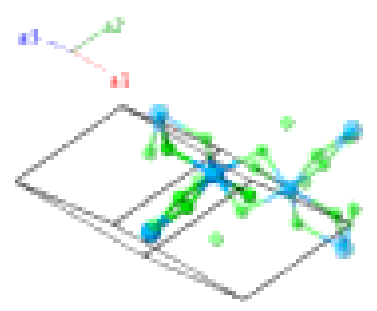

\section{Basis vectors:}

\section{Lattice Coordinates}

$\begin{array}{ccc}\mathbf{B}_{1}= & \frac{3}{8} \mathbf{a}_{1}+\frac{1}{8} \mathbf{a}_{2}+\frac{1}{4} \mathbf{a}_{3} \\ \mathbf{B}_{2}= & \frac{5}{8} \mathbf{a}_{1}+\frac{7}{8} \mathbf{a}_{2}+\frac{3}{4} \mathbf{a}_{3} \\ \mathbf{B}_{3}= & \begin{array}{c}\left(y_{2}+z_{2}\right) \mathbf{a}_{1}+\left(x_{2}+z_{2}\right) \mathbf{a}_{2}+ \\ \left(x_{2}+y_{2}\right) \mathbf{a}_{3}\end{array} \\ \mathbf{B}_{4}= & \begin{array}{c}\left(\frac{1}{2}-y_{2}+z_{2}\right) \mathbf{a}_{1}+\left(-x_{2}+z_{2}\right) \mathbf{a}_{2}+= \\ \left(\frac{1}{2}-x_{2}-y_{2}\right) \mathbf{a}_{3}\end{array} \\ \mathbf{B}_{5}= & \left(\frac{1}{2}+x_{2}+z_{2}\right) \mathbf{a}_{1}+\left(-y_{2}+z_{2}\right) \mathbf{a}_{2}+= \\ \left(x_{2}-y_{2}\right) \mathbf{a}_{3}\end{array}$
Cartesian Coordinates

$=$

$=$

$=$

$=$

$$
-x_{2} a \hat{\mathbf{x}}+\left(\frac{1}{2}-y_{2}\right) a \hat{\mathbf{y}}+z_{2} c \hat{\mathbf{z}}
$$

$\left(\frac{3}{4}-y_{2}\right) a \hat{\mathbf{x}}+\left(\frac{1}{4}+x_{2}\right) a \hat{\mathbf{y}}+$ $\left(\frac{1}{4}+z_{2}\right) c \hat{\mathbf{z}}$
Wyckoff Position Atom Type

$(4 a)$

Th

(4a)

Th

(16f)

$\mathrm{Cl}$

(16f)

Cl

(16f)

$\mathrm{Cl}$ 


\begin{tabular}{|c|c|c|c|c|c|}
\hline $\mathbf{B}_{6}$ & $=$ & $\begin{array}{c}\left(\frac{1}{2}-x_{2}+z_{2}\right) \mathbf{a}_{1}+ \\
\left(\frac{1}{2}+y_{2}+z_{2}\right) \mathbf{a}_{2}+\left(\frac{1}{2}-x_{2}+y_{2}\right) \mathbf{a}_{3}\end{array}$ & $=$ & $\begin{array}{c}\left(\frac{1}{4}+y_{2}\right) a \hat{\mathbf{x}}+\left(\frac{1}{4}-x_{2}\right) a \hat{\mathbf{y}}+ \\
\left(\frac{1}{4}+z_{2}\right) c \hat{\mathbf{z}}\end{array}$ & $(16 f)$ \\
\hline $\mathbf{B}_{7}$ & $=$ & $\begin{array}{c}\left(-y_{2}-z_{2}\right) \mathbf{a}_{1}+\left(-x_{2}-z_{2}\right) \mathbf{a}_{2}+ \\
\left(-x_{2}-y_{2}\right) \mathbf{a}_{3}\end{array}$ & $=$ & $-x_{2} a \hat{\mathbf{x}}-y_{2} a \hat{\mathbf{y}}-z_{2} c \hat{\mathbf{z}}$ & $(16 f)$ \\
\hline $\mathbf{B}_{8}$ & $=$ & $\begin{array}{c}\left(\frac{1}{2}+y_{2}-z_{2}\right) \mathbf{a}_{1}+\left(x_{2}-z_{2}\right) \mathbf{a}_{2}+ \\
\left(\frac{1}{2}+x_{2}+y_{2}\right) \mathbf{a}_{3}\end{array}$ & $=$ & $x_{2} a \hat{\mathbf{x}}+\left(\frac{1}{2}+y_{2}\right) a \hat{\mathbf{y}}-z_{2} c \hat{\mathbf{z}}$ & $(16 f)$ \\
\hline $\mathbf{B}_{9}$ & $=$ & $\begin{array}{c}\left(\frac{1}{2}-x_{2}-z_{2}\right) \mathbf{a}_{1}+\left(y_{2}-z_{2}\right) \mathbf{a}_{2}+ \\
\left(-x_{2}+y_{2}\right) \mathbf{a}_{3}\end{array}$ & $=$ & $\begin{array}{c}\left(-\frac{1}{4}+y_{2}\right) a \hat{\mathbf{x}}+\left(\frac{1}{4}-x_{2}\right) a \hat{\mathbf{y}}+ \\
\left(\frac{1}{4}-z_{2}\right) c \hat{\mathbf{z}}\end{array}$ & $(16 f)$ \\
\hline $\mathbf{B}_{10}$ & $=$ & $\begin{array}{c}\left(\frac{1}{2}+x_{2}-z_{2}\right) \mathbf{a}_{1}+ \\
\left(\frac{1}{2}-y_{2}-z_{2}\right) \mathbf{a}_{2}+\left(\frac{1}{2}+x_{2}-y_{2}\right) \mathbf{a}_{3}\end{array}$ & $=$ & $\begin{array}{c}\left(\frac{1}{4}-y_{2}\right) a \hat{\mathbf{x}}+\left(\frac{1}{4}+x_{2}\right) a \hat{\mathbf{y}}+ \\
\left(\frac{1}{4}-z_{2}\right) c \hat{\mathbf{z}}\end{array}$ & $(16 f)$ \\
\hline
\end{tabular}

\section{References:}

- J. T. Mason, M. C. Jha, and P. Chiotti, Crystal structures of ThCl 4 polymorphs, J. Less-Common Met. 34, 143-151 (1974), doi:10.1016/0022-5088(74)90224-0.

\section{Found in:}

- P. Villars and K. Cenzual, Pearson's Crystal Data - Crystal Structure Database for Inorganic Compounds, ASM International (2013).

\section{Geometry files:}

- CIF: pp. 865

- POSCAR: pp. 865 


\section{$\alpha-\mathrm{NbO}_{2}$ Structure: AB2_tI96_88_2f_4f}
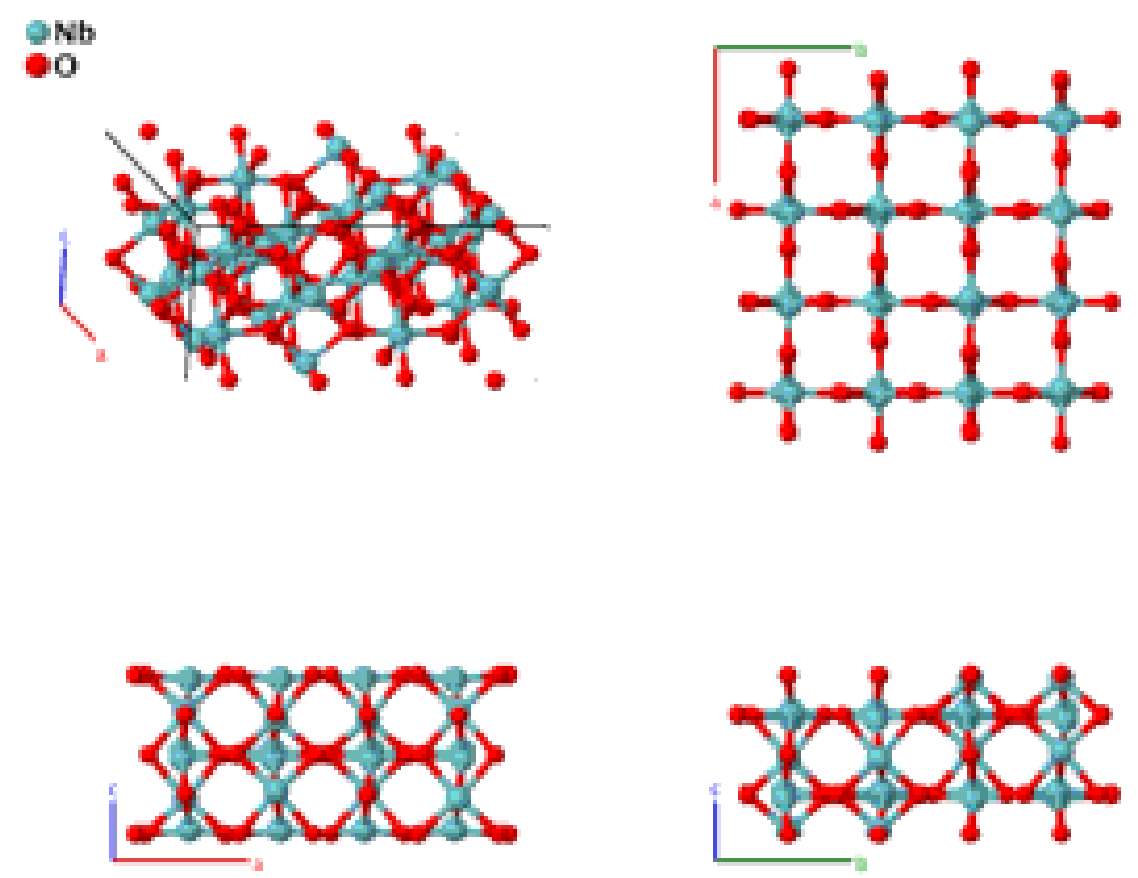

\section{Prototype}

AFLOW prototype label

$\quad \alpha-\mathrm{NbO}_{2}$

Strukturbericht designation

: AB2_tI96_88_2f_4f

Pearson symbol

: None

Space group number

: $\quad$ tI96

Space group symbol

: $\quad 88$

AFLOW prototype command

$: \quad I 4_{1} / a$

- Although Bolzan et al. (Bolzan, 1994) also gives structural information $\alpha-\mathrm{NbO}_{2}$, Pynn et al. (Pynn, 1996) is the only reference we found which unambiguously states that this structure is reported in setting 2 of space group \#88.

Body-centered Tetragonal primitive vectors:

$$
\begin{aligned}
& \mathbf{a}_{1}=-\frac{1}{2} a \hat{\mathbf{x}}+\frac{1}{2} a \hat{\mathbf{y}}+\frac{1}{2} c \hat{\mathbf{z}} \\
& \mathbf{a}_{2}=\frac{1}{2} a \hat{\mathbf{x}}-\frac{1}{2} a \hat{\mathbf{y}}+\frac{1}{2} c \hat{\mathbf{z}} \\
& \mathbf{a}_{3}=\frac{1}{2} a \hat{\mathbf{x}}+\frac{1}{2} a \hat{\mathbf{y}}-\frac{1}{2} c \hat{\mathbf{z}}
\end{aligned}
$$
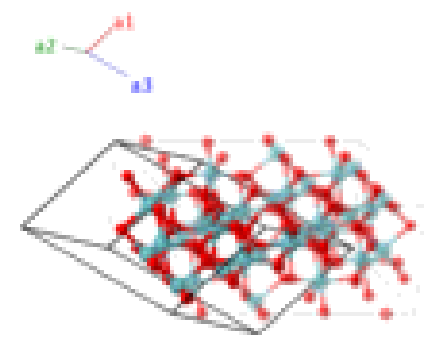

Basis vectors: 
Lattice Coordinates

$\mathbf{B}_{1}=\begin{gathered}\left(y_{1}+z_{1}\right) \mathbf{a}_{1}+\left(x_{1}+z_{1}\right) \mathbf{a}_{2}+ \\ \left(x_{1}+y_{1}\right) \mathbf{a}_{3}\end{gathered}$

$\mathbf{B}_{2}=\left(\frac{1}{2}-y_{1}+z_{1}\right) \mathbf{a}_{1}+\left(-x_{1}+z_{1}\right) \mathbf{a}_{2}+=$ $\left(\frac{1}{2}-x_{1}-y_{1}\right) \mathbf{a}_{3}$

$\mathbf{B}_{3}=\left(\frac{1}{2}+x_{1}+z_{1}\right) \mathbf{a}_{1}+\left(-y_{1}+z_{1}\right) \mathbf{a}_{2}+=$ $\left(x_{1}-y_{1}\right) \mathbf{a}_{3}$

$\mathbf{B}_{4}=\begin{gathered}\left(\frac{1}{2}-x_{1}+z_{1}\right) \mathbf{a}_{1}+ \\ \left(\frac{1}{2}+y_{1}+z_{1}\right) \mathbf{a}_{2}+\left(\frac{1}{2}-x_{1}+y_{1}\right) \mathbf{a}_{3}\end{gathered}$

$\mathbf{B}_{5}=\left(-y_{1}-z_{1}\right) \mathbf{a}_{1}+\left(-x_{1}-z_{1}\right) \mathbf{a}_{2}+=$ $\left(-x_{1}-y_{1}\right) \mathbf{a}_{3}$

$\mathbf{B}_{6}=\left(\frac{1}{2}+y_{1}-z_{1}\right) \mathbf{a}_{1}+\left(x_{1}-z_{1}\right) \mathbf{a}_{2}+=$ $\left(\frac{1}{2}+x_{1}+y_{1}\right) \mathbf{a}_{3}$

$\mathbf{B}_{7}=\left(\frac{1}{2}-x_{1}-z_{1}\right) \mathbf{a}_{1}+\left(y_{1}-z_{1}\right) \mathbf{a}_{2}+=\left(-\frac{1}{4}+y_{1}\right) a \hat{\mathbf{x}}+\left(\frac{1}{4}-x_{1}\right) a \hat{\mathbf{y}}+$ $\left(-x_{1}+y_{1}\right) \mathbf{a}_{3}$

$\mathbf{B}_{8}=\begin{gathered}\left(\frac{1}{2}+x_{1}-z_{1}\right) \mathbf{a}_{1}+ \\ \left(\frac{1}{2}-y_{1}-z_{1}\right) \mathbf{a}_{2}+\left(\frac{1}{2}+x_{1}-y_{1}\right) \mathbf{a}_{3}\end{gathered}$

$\mathbf{B}_{9}=\left(y_{2}+z_{2}\right) \mathbf{a}_{1}+\left(x_{2}+z_{2}\right) \mathbf{a}_{2}+=$ $\left(x_{2}+y_{2}\right) \mathbf{a}_{3}$

$\mathbf{B}_{10}=\left(\frac{1}{2}-y_{2}+z_{2}\right) \mathbf{a}_{1}+\left(-x_{2}+z_{2}\right) \mathbf{a}_{2}+=$ $\left(\frac{1}{2}-x_{2}-y_{2}\right) \mathbf{a}_{3}$

$\mathbf{B}_{11}=\left(\frac{1}{2}+x_{2}+z_{2}\right) \mathbf{a}_{1}+\left(-y_{2}+z_{2}\right) \mathbf{a}_{2}+=$ $\left(x_{2}-y_{2}\right) \mathbf{a}_{3}$

$\mathbf{B}_{12}=\begin{gathered}\left(\frac{1}{2}-x_{2}+z_{2}\right) \mathbf{a}_{1}+ \\ \left(\frac{1}{2}+y_{2}+z_{2}\right) \mathbf{a}_{2}+\left(\frac{1}{2}-x_{2}+y_{2}\right) \mathbf{a}_{3}\end{gathered}$

$\mathbf{B}_{13}=\left(-y_{2}-z_{2}\right) \mathbf{a}_{1}+\left(-x_{2}-z_{2}\right) \mathbf{a}_{2}+=$ $\left(-x_{2}-y_{2}\right) \mathbf{a}_{3}$

$\mathbf{B}_{14}=\left(\frac{1}{2}+y_{2}-z_{2}\right) \mathbf{a}_{1}+\left(x_{2}-z_{2}\right) \mathbf{a}_{2}+=$ $\left(\frac{1}{2}+x_{2}+y_{2}\right) \mathbf{a}_{3}$

$\mathbf{B}_{15}=\left(\frac{1}{2}-x_{2}-z_{2}\right) \mathbf{a}_{1}+\left(y_{2}-z_{2}\right) \mathbf{a}_{2}+$ $\left(-x_{2}+y_{2}\right) \mathbf{a}_{3}$

$\mathbf{B}_{16}=\begin{gathered}\left(\frac{1}{2}+x_{2}-z_{2}\right) \mathbf{a}_{1}+ \\ \left(\frac{1}{2}-y_{2}-z_{2}\right) \mathbf{a}_{2}+\left(\frac{1}{2}+x_{2}-y_{2}\right) \mathbf{a}_{3}\end{gathered}$

$\mathbf{B}_{17}=\left(y_{3}+z_{3}\right) \mathbf{a}_{1}+\left(x_{3}+z_{3}\right) \mathbf{a}_{2}+=$ $\left(x_{3}+y_{3}\right) \mathbf{a}_{3}$

$\mathbf{B}_{18}=\left(\frac{1}{2}-y_{3}+z_{3}\right) \mathbf{a}_{1}+\left(-x_{3}+z_{3}\right) \mathbf{a}_{2}+=$ $\left(\frac{1}{2}-x_{3}-y_{3}\right) \mathbf{a}_{3}$

$\mathbf{B}_{19}=\left(\frac{1}{2}+x_{3}+z_{3}\right) \mathbf{a}_{1}+\left(-y_{3}+z_{3}\right) \mathbf{a}_{2}+$ $\left(x_{3}-y_{3}\right) \mathbf{a}_{3}$

$\mathbf{B}_{20}=\begin{gathered}\left(\frac{1}{2}-x_{3}+z_{3}\right) \mathbf{a}_{1}+ \\ \left(\frac{1}{2}+y_{3}+z_{3}\right) \mathbf{a}_{2}+\left(\frac{1}{2}-x_{3}+y_{3}\right) \mathbf{a}_{3}\end{gathered}$

$\mathbf{B}_{21}=\left(-y_{3}-z_{3}\right) \mathbf{a}_{1}+\left(-x_{3}-z_{3}\right) \mathbf{a}_{2}+=$ $\left(-x_{3}-y_{3}\right) \mathbf{a}_{3}$
Cartesian Coordinates

$x_{1} a \hat{\mathbf{x}}+y_{1} a \hat{\mathbf{y}}+z_{1} c \hat{\mathbf{z}}$

$(16 f)$

$(16 f)$

$(16 f)$

(16f)

$\mathrm{Nb}$ II

$$
\left(\frac{1}{4}-z_{2}\right) c \hat{\mathbf{z}}
$$

$\mathrm{Nb}$ II

$$
\left(\frac{1}{4}-z_{2}\right) c \hat{\mathbf{z}}
$$

O I

O I

O I

O I

$$
\left(\frac{1}{4}+z_{3}\right) c \hat{\mathbf{z}}
$$

$=\left(\frac{1}{4}+y_{3}\right) a \hat{\mathbf{x}}+\left(\frac{1}{4}-x_{3}\right) a \hat{\mathbf{y}}+$

$$
\left(\frac{1}{4}+z_{3}\right) c \hat{\mathbf{z}}
$$

$\mathrm{Nb} I$

$\mathrm{Nb}$ I

$\mathrm{Nb} \mathrm{I}$

$\mathrm{Nb} \mathrm{I}$

$\mathrm{Nb} \mathrm{I}$

$\mathrm{Nb} \mathrm{I}$

$\mathrm{Nb}$ I

$\mathrm{Nb}$ I

$\mathrm{Nb}$ II

$\mathrm{Nb}$ II

$\mathrm{Nb}$ II

$\mathrm{Nb}$ II

$\mathrm{Nb}$ II

$\mathrm{Nb}$ II

$$
-x_{3} a \hat{\mathbf{x}}-y_{3} a \hat{\mathbf{y}}-z_{3} c \hat{\mathbf{z}}
$$




\begin{tabular}{|c|c|c|c|c|c|}
\hline $\mathbf{B}_{22}$ & $\begin{array}{c}=\left(\frac{1}{2}+y_{3}-z_{3}\right) \mathbf{a}_{1}+\left(x_{3}-z_{3}\right) \mathbf{a}_{2}+ \\
\left(\frac{1}{2}+x_{3}+y_{3}\right) \mathbf{a}_{3}\end{array}$ & $=$ & $x_{3} a \hat{\mathbf{x}}+\left(\frac{1}{2}+y_{3}\right) a \hat{\mathbf{y}}-z_{3} c \hat{\mathbf{z}}$ & $(16 f)$ & $\mathrm{O} I$ \\
\hline $\mathbf{B}_{23}$ & $\begin{array}{c}=\left(\frac{1}{2}-x_{3}-z_{3}\right) \mathbf{a}_{1}+\left(y_{3}-z_{3}\right) \mathbf{a}_{2}+ \\
\left(-x_{3}+y_{3}\right) \mathbf{a}_{3}\end{array}$ & $=$ & $\begin{array}{c}\left(-\frac{1}{4}+y_{3}\right) a \hat{\mathbf{x}}+\left(\frac{1}{4}-x_{3}\right) a \hat{\mathbf{y}}+ \\
\left(\frac{1}{4}-z_{3}\right) c \hat{\mathbf{z}}\end{array}$ & $(16 f)$ & O I \\
\hline $\mathbf{B}_{24}$ & $=\begin{array}{c}\left(\frac{1}{2}+x_{3}-z_{3}\right) \mathbf{a}_{1}+ \\
\left(\frac{1}{2}-y_{3}-z_{3}\right) \mathbf{a}_{2}+\left(\frac{1}{2}+x_{3}-y_{3}\right) \mathbf{a}_{3}\end{array}$ & $=$ & $\begin{array}{c}\left(\frac{1}{4}-y_{3}\right) a \hat{\mathbf{x}}+\left(\frac{1}{4}+x_{3}\right) a \hat{\mathbf{y}}+ \\
\left(\frac{1}{4}-z_{3}\right) c \hat{\mathbf{z}}\end{array}$ & $(16 f)$ & O I \\
\hline $\mathbf{B}_{25}$ & $\begin{array}{c}=\left(y_{4}+z_{4}\right) \mathbf{a}_{1}+\left(x_{4}+z_{4}\right) \mathbf{a}_{2}+ \\
\left(x_{4}+y_{4}\right) \mathbf{a}_{3}\end{array}$ & $=$ & $x_{4} a \hat{\mathbf{x}}+y_{4} a \hat{\mathbf{y}}+z_{4} c \hat{\mathbf{z}}$ & $(16 f)$ & O II \\
\hline $\mathbf{B}_{26}$ & $\begin{array}{c}=\left(\frac{1}{2}-y_{4}+z_{4}\right) \mathbf{a}_{1}+\left(-x_{4}+z_{4}\right) \mathbf{a}_{2}+ \\
\left(\frac{1}{2}-x_{4}-y_{4}\right) \mathbf{a}_{3}\end{array}$ & $=$ & $-x_{4} a \hat{\mathbf{x}}+\left(\frac{1}{2}-y_{4}\right) a \hat{\mathbf{y}}+z_{4} c \hat{\mathbf{z}}$ & $(16 f)$ & O II \\
\hline $\mathbf{B}_{27}$ & $\begin{array}{c}=\left(\frac{1}{2}+x_{4}+z_{4}\right) \mathbf{a}_{1}+\left(-y_{4}+z_{4}\right) \mathbf{a}_{2}+ \\
\left(x_{4}-y_{4}\right) \mathbf{a}_{3}\end{array}$ & $=$ & $\begin{array}{c}\left(\frac{3}{4}-y_{4}\right) a \hat{\mathbf{x}}+\left(\frac{1}{4}+x_{4}\right) a \hat{\mathbf{y}}+ \\
\left(\frac{1}{4}+z_{4}\right) c \hat{\mathbf{z}}\end{array}$ & $(16 f)$ & O II \\
\hline $\mathbf{B}_{28}$ & $\begin{array}{c}=\quad\left(\frac{1}{2}-x_{4}+z_{4}\right) \mathbf{a}_{1}+ \\
\left(\frac{1}{2}+y_{4}+z_{4}\right) \mathbf{a}_{2}+\left(\frac{1}{2}-x_{4}+y_{4}\right) \mathbf{a}_{3}\end{array}$ & $=$ & $\begin{array}{c}\left(\frac{1}{4}+y_{4}\right) a \hat{\mathbf{x}}+\left(\frac{1}{4}-x_{4}\right) a \hat{\mathbf{y}}+ \\
\left(\frac{1}{4}+z_{4}\right) c \hat{\mathbf{z}}\end{array}$ & $(16 f)$ & O II \\
\hline $\mathbf{B}_{29}$ & $\begin{array}{c}=\left(-y_{4}-z_{4}\right) \mathbf{a}_{1}+\left(-x_{4}-z_{4}\right) \mathbf{a}_{2}+ \\
\left(-x_{4}-y_{4}\right) \mathbf{a}_{3}\end{array}$ & $=$ & $-x_{4} a \hat{\mathbf{x}}-y_{4} a \hat{\mathbf{y}}-z_{4} c \hat{\mathbf{z}}$ & $(16 f)$ & O II \\
\hline $\mathbf{B}_{30}$ & $\begin{array}{c}=\left(\frac{1}{2}+y_{4}-z_{4}\right) \mathbf{a}_{1}+\left(x_{4}-z_{4}\right) \mathbf{a}_{2}+ \\
\left(\frac{1}{2}+x_{4}+y_{4}\right) \mathbf{a}_{3}\end{array}$ & $=$ & $x_{4} a \hat{\mathbf{x}}+\left(\frac{1}{2}+y_{4}\right) a \hat{\mathbf{y}}-z_{4} c \hat{\mathbf{z}}$ & $(16 f)$ & O II \\
\hline $\mathbf{B}_{31}$ & $\begin{array}{c}=\left(\frac{1}{2}-x_{4}-z_{4}\right) \mathbf{a}_{1}+\left(y_{4}-z_{4}\right) \mathbf{a}_{2}+ \\
\left(-x_{4}+y_{4}\right) \mathbf{a}_{3}\end{array}$ & $=$ & $\begin{array}{c}\left(-\frac{1}{4}+y_{4}\right) a \hat{\mathbf{x}}+\left(\frac{1}{4}-x_{4}\right) a \hat{\mathbf{y}}+ \\
\left(\frac{1}{4}-z_{4}\right) c \hat{\mathbf{z}}\end{array}$ & $(16 f)$ & O II \\
\hline $\mathbf{B}_{32}$ & $=\begin{array}{c}\left(\frac{1}{2}+x_{4}-z_{4}\right) \mathbf{a}_{1}+ \\
\left(\frac{1}{2}-y_{4}-z_{4}\right) \mathbf{a}_{2}+\left(\frac{1}{2}+x_{4}-y_{4}\right) \mathbf{a}_{3}\end{array}$ & $=$ & $\begin{array}{c}\left(\frac{1}{4}-y_{4}\right) a \hat{\mathbf{x}}+\left(\frac{1}{4}+x_{4}\right) a \hat{\mathbf{y}}+ \\
\left(\frac{1}{4}-z_{4}\right) c \hat{\mathbf{z}}\end{array}$ & $(16 f)$ & O II \\
\hline $\mathbf{B}_{33}$ & $\begin{array}{c}=\left(y_{5}+z_{5}\right) \mathbf{a}_{1}+\left(x_{5}+z_{5}\right) \mathbf{a}_{2}+ \\
\left(x_{5}+y_{5}\right) \mathbf{a}_{3}\end{array}$ & $=$ & $x_{5} a \hat{\mathbf{x}}+y_{5} a \hat{\mathbf{y}}+z_{5} c \hat{\mathbf{z}}$ & $(16 f)$ & O III \\
\hline $\mathbf{B}_{34}$ & $\begin{array}{c}=\left(\frac{1}{2}-y_{5}+z_{5}\right) \mathbf{a}_{1}+\left(-x_{5}+z_{5}\right) \mathbf{a}_{2}+ \\
\left(\frac{1}{2}-x_{5}-y_{5}\right) \mathbf{a}_{3}\end{array}$ & $=$ & $-x_{5} a \hat{\mathbf{x}}+\left(\frac{1}{2}-y_{5}\right) a \hat{\mathbf{y}}+z_{5} c \hat{\mathbf{z}}$ & $(16 f)$ & O III \\
\hline $\mathbf{B}_{35}$ & $\begin{array}{c}=\left(\frac{1}{2}+x_{5}+z_{5}\right) \mathbf{a}_{1}+\left(-y_{5}+z_{5}\right) \mathbf{a}_{2}+ \\
\left(x_{5}-y_{5}\right) \mathbf{a}_{3}\end{array}$ & $=$ & $\begin{array}{c}\left(\frac{3}{4}-y_{5}\right) a \hat{\mathbf{x}}+\left(\frac{1}{4}+x_{5}\right) a \hat{\mathbf{y}}+ \\
\left(\frac{1}{4}+z_{5}\right) c \hat{\mathbf{z}}\end{array}$ & $(16 f)$ & O III \\
\hline $\mathbf{B}_{36}$ & $=\begin{array}{c}\left(\frac{1}{2}-x_{5}+z_{5}\right) \mathbf{a}_{1}+ \\
\left(\frac{1}{2}+y_{5}+z_{5}\right) \mathbf{a}_{2}+\left(\frac{1}{2}-x_{5}+y_{5}\right) \mathbf{a}_{3}\end{array}$ & $=$ & $\begin{array}{c}\left(\frac{1}{4}+y_{5}\right) a \hat{\mathbf{x}}+\left(\frac{1}{4}-x_{5}\right) a \hat{\mathbf{y}}+ \\
\left(\frac{1}{4}+z_{5}\right) c \hat{\mathbf{z}}\end{array}$ & $(16 f)$ & O III \\
\hline $\mathbf{B}_{37}$ & $\begin{array}{c}=\left(-y_{5}-z_{5}\right) \mathbf{a}_{1}+\left(-x_{5}-z_{5}\right) \mathbf{a}_{2}+ \\
\left(-x_{5}-y_{5}\right) \mathbf{a}_{3}\end{array}$ & $=$ & $-x_{5} a \hat{\mathbf{x}}-y_{5} a \hat{\mathbf{y}}-z_{5} c \hat{\mathbf{z}}$ & $(16 f)$ & O III \\
\hline $\mathbf{B}_{38}$ & $\begin{array}{c}=\left(\frac{1}{2}+y_{5}-z_{5}\right) \mathbf{a}_{1}+\left(x_{5}-z_{5}\right) \mathbf{a}_{2}+ \\
\left(\frac{1}{2}+x_{5}+y_{5}\right) \mathbf{a}_{3}\end{array}$ & $=$ & $x_{5} a \hat{\mathbf{x}}+\left(\frac{1}{2}+y_{5}\right) a \hat{\mathbf{y}}-z_{5} c \hat{\mathbf{z}}$ & $(16 f)$ & O III \\
\hline $\mathbf{B}_{39}$ & $\begin{array}{c}=\left(\frac{1}{2}-x_{5}-z_{5}\right) \mathbf{a}_{1}+\left(y_{5}-z_{5}\right) \mathbf{a}_{2}+ \\
\left(-x_{5}+y_{5}\right) \mathbf{a}_{3}\end{array}$ & $=$ & $\begin{array}{c}\left(-\frac{1}{4}+y_{5}\right) a \hat{\mathbf{x}}+\left(\frac{1}{4}-x_{5}\right) a \hat{\mathbf{y}}+ \\
\left(\frac{1}{4}-z_{5}\right) c \hat{\mathbf{z}}\end{array}$ & $(16 f)$ & O III \\
\hline $\mathbf{B}_{40}$ & $=\begin{array}{c}\left(\frac{1}{2}+x_{5}-z_{5}\right) \mathbf{a}_{1}+ \\
\left(\frac{1}{2}-y_{5}-z_{5}\right) \mathbf{a}_{2}+\left(\frac{1}{2}+x_{5}-y_{5}\right) \mathbf{a}_{3}\end{array}$ & $=$ & $\begin{array}{c}\left(\frac{1}{4}-y_{5}\right) a \hat{\mathbf{x}}+\left(\frac{1}{4}+x_{5}\right) a \hat{\mathbf{y}}+ \\
\left(\frac{1}{4}-z_{5}\right) c \hat{\mathbf{z}}\end{array}$ & $(16 f)$ & O III \\
\hline $\mathbf{B}_{41}$ & $\begin{array}{c}\left(y_{6}+z_{6}\right) \mathbf{a}_{1}+\left(x_{6}+z_{6}\right) \mathbf{a}_{2}+ \\
\left(x_{6}+y_{6}\right) \mathbf{a}_{3}\end{array}$ & $=$ & $x_{6} a \hat{\mathbf{x}}+y_{6} a \hat{\mathbf{y}}+z_{6} c \hat{\mathbf{z}}$ & $(16 f)$ & O IV \\
\hline $\mathbf{B}_{42}$ & $\begin{array}{c}=\left(\frac{1}{2}-y_{6}+z_{6}\right) \mathbf{a}_{1}+\left(-x_{6}+z_{6}\right) \mathbf{a}_{2}+ \\
\left(\frac{1}{2}-x_{6}-y_{6}\right) \mathbf{a}_{3}\end{array}$ & $=$ & $-x_{6} a \hat{\mathbf{x}}+\left(\frac{1}{2}-y_{6}\right) a \hat{\mathbf{y}}+z_{6} c \hat{\mathbf{z}}$ & $(16 f)$ & O IV \\
\hline
\end{tabular}




\begin{tabular}{|c|c|c|c|c|c|c|}
\hline $\mathbf{B}_{43}$ & $=$ & $\begin{array}{c}\left(\frac{1}{2}+x_{6}+z_{6}\right) \mathbf{a}_{1}+\left(-y_{6}+z_{6}\right) \mathbf{a}_{2}+ \\
\left(x_{6}-y_{6}\right) \mathbf{a}_{3}\end{array}$ & $=$ & $\begin{array}{c}\left(\frac{3}{4}-y_{6}\right) a \hat{\mathbf{x}}+\left(\frac{1}{4}+x_{6}\right) a \hat{\mathbf{y}}+ \\
\left(\frac{1}{4}+z_{6}\right) c \hat{\mathbf{z}}\end{array}$ & $(16 f)$ & O IV \\
\hline $\mathbf{B}_{44}$ & $=$ & $\begin{array}{c}\left(\frac{1}{2}-x_{6}+z_{6}\right) \mathbf{a}_{1}+ \\
\left(\frac{1}{2}+y_{6}+z_{6}\right) \mathbf{a}_{2}+\left(\frac{1}{2}-x_{6}+y_{6}\right) \mathbf{a}_{3}\end{array}$ & $=$ & $\begin{array}{c}\left(\frac{1}{4}+y_{6}\right) a \hat{\mathbf{x}}+\left(\frac{1}{4}-x_{6}\right) a \hat{\mathbf{y}}+ \\
\left(\frac{1}{4}+z_{6}\right) c \hat{\mathbf{z}}\end{array}$ & $(16 f)$ & O IV \\
\hline $\mathbf{B}_{45}$ & $=$ & $\begin{array}{c}\left(-y_{6}-z_{6}\right) \mathbf{a}_{1}+\left(-x_{6}-z_{6}\right) \mathbf{a}_{2}+ \\
\left(-x_{6}-y_{6}\right) \mathbf{a}_{3}\end{array}$ & $=$ & $-x_{6} a \hat{\mathbf{x}}-y_{6} a \hat{\mathbf{y}}-z_{6} c \hat{\mathbf{z}}$ & $(16 f)$ & O IV \\
\hline $\mathbf{B}_{46}$ & $=$ & $\begin{array}{c}\left(\frac{1}{2}+y_{6}-z_{6}\right) \mathbf{a}_{1}+\left(x_{6}-z_{6}\right) \mathbf{a}_{2}+ \\
\left(\frac{1}{2}+x_{6}+y_{6}\right) \mathbf{a}_{3}\end{array}$ & $=$ & $x_{6} a \hat{\mathbf{x}}+\left(\frac{1}{2}+y_{6}\right) a \hat{\mathbf{y}}-z_{6} c \hat{\mathbf{z}}$ & $(16 f)$ & O IV \\
\hline $\mathbf{B}_{47}$ & $=$ & $\begin{array}{c}\left(\frac{1}{2}-x_{6}-z_{6}\right) \mathbf{a}_{1}+\left(y_{6}-z_{6}\right) \mathbf{a}_{2}+ \\
\left(-x_{6}+y_{6}\right) \mathbf{a}_{3}\end{array}$ & $=$ & $\begin{array}{c}\left(-\frac{1}{4}+y_{6}\right) a \hat{\mathbf{x}}+\left(\frac{1}{4}-x_{6}\right) a \hat{\mathbf{y}}+ \\
\left(\frac{1}{4}-z_{6}\right) c \hat{\mathbf{z}}\end{array}$ & $(16 f)$ & O IV \\
\hline $\mathbf{B}_{48}$ & $=$ & $\begin{array}{c}\left(\frac{1}{2}+x_{6}-z_{6}\right) \mathbf{a}_{1}+ \\
\left(\frac{1}{2}-y_{6}-z_{6}\right) \mathbf{a}_{2}+\left(\frac{1}{2}+x_{6}-y_{6}\right) \mathbf{a}_{3}\end{array}$ & $=$ & $\begin{array}{c}\left(\frac{1}{4}-y_{6}\right) a \hat{\mathbf{x}}+\left(\frac{1}{4}+x_{6}\right) a \hat{\mathbf{y}}+ \\
\left(\frac{1}{4}-z_{6}\right) c \hat{\mathbf{z}}\end{array}$ & $(16 f)$ & O IV \\
\hline
\end{tabular}

\section{References:}

- R. Pynn, J. D. Axe, and R. Thomas, Structural distortions in the low-temperature phase of $\mathrm{NbO}_{2}$, Phys. Rev. B 13, 2965-2975 (1976), doi:10.1103/PhysRevB.13.2965.

\section{Found in:}

- A. A. Bolzan, C. Fong, B. J. Kennedy, and C. J. Howard, A Powder Neutron Diffraction Study of Semiconducting and Metallic Niobium Dioxide, J. Solid State Chem. 113, 9-14 (1994), doi:10.1006/jssc.1994.1334.

\section{Geometry files:}

- CIF: pp. 866

- POSCAR: pp. 866 


\section{$\mathrm{C}_{17} \mathrm{FeO}_{4} \mathrm{Pt}$ Structure: A17BC4D_tP184_89_17p_p_4p_io}
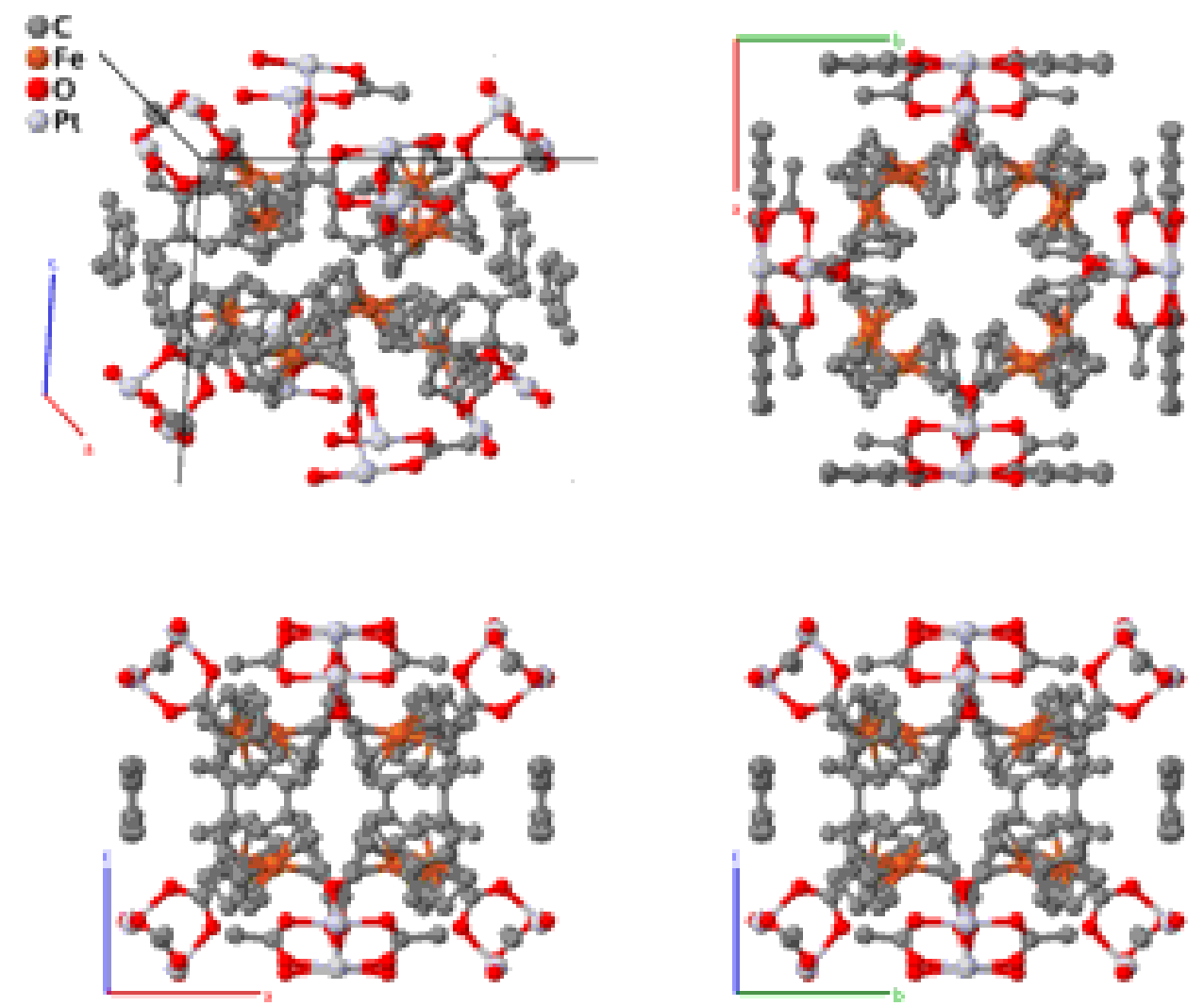

Prototype

AFLOW prototype label

Strukturbericht designation

Pearson symbol

Space group number

Space group symbol

AFLOW prototype command
: $\quad \mathrm{C}_{17} \mathrm{FeO}_{4} \mathrm{Pt}$

: $\quad$ A17BC4D_tP184_89_17p_p_4p_io

: None

: $\quad$ tP184

: 89

: $\quad P 422$

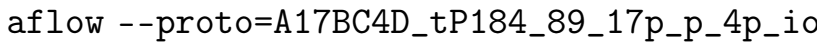

- params $=a, c / a, z_{1}, x_{2}, x_{3}, y_{3}, z_{3}, x_{4}, y_{4}, z_{4}, x_{5}, y_{5}, z_{5}, x_{6}, y_{6}, z_{6}, x_{7}, y_{7}, z_{7}, x_{8}, y_{8}$, $z_{8}, x_{9}, y_{9}, z_{9}, x_{10}, y_{10}, z_{10}, x_{11}, y_{11}, z_{11}, x_{12}, y_{12}, z_{12}, x_{13}, y_{13}, z_{13}, x_{14}, y_{14}, z_{14}, x_{15}, y_{15}, z_{15}$, $x_{16}, y_{16}, z_{16}, x_{17}, y_{17}, z_{17}, x_{18}, y_{18}, z_{18}, x_{19}, y_{19}, z_{19}, x_{20}, y_{20}, z_{20}, x_{21}, y_{21}, z_{21}, x_{22}, y_{22}$, $z_{22}, x_{23}, y_{23}, z_{23}, x_{24}, y_{24}, z_{24}$

- Structures exhibiting space group \#89 are quite rare. According to (Hoffmann, 2014), there are only two entries in the Inorganic Crystal Structure Database with space group \#89; however, they are incorrectly classified. This structure is listed in the Cambridge Structure Database (ID=863010). Only the non-hydrogen atoms are listed. 


$$
\begin{aligned}
& \mathbf{a}_{1}=a \hat{\mathbf{x}} \\
& \mathbf{a}_{2}=a \hat{\mathbf{y}} \\
& \mathbf{a}_{3}=c \hat{\mathbf{z}}
\end{aligned}
$$

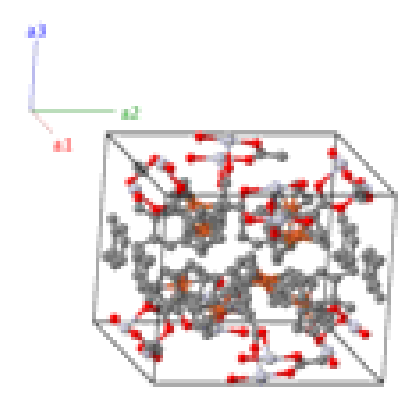

\section{Basis vectors:}

\section{Lattice Coordinates}

$\mathbf{B}_{1}=$

$\mathbf{B}_{2}=$

$\mathbf{B}_{3}=$

$\mathbf{B}_{4}=$

$\mathbf{B}_{5}=$

$\mathbf{B}_{6}=$

$\mathbf{B}_{7}=$

$\mathbf{B}_{8}=$

$\mathbf{B}_{9}=$

$\mathbf{B}_{10}=$

$\mathbf{B}_{11}=$

$\mathbf{B}_{12}=$

$\mathbf{B}_{13}=$

$\mathbf{B}_{14}=$

$\mathbf{B}_{15}=$

$\mathbf{B}_{16}=$

$\mathbf{B}_{17}=$

$\mathbf{B}_{18}=$

$\mathbf{B}_{19}=$

$\mathbf{B}_{20}=$

$\mathbf{B}_{21}=$

$\mathbf{B}_{22}=$

$\mathbf{B}_{23}=$

$\mathbf{B}_{24}=$

$\mathbf{B}_{25}=$

$\mathbf{B}_{26}=$

$\mathbf{B}_{27}=$

$$
\frac{1}{2} \mathbf{a}_{2}+z_{1} \mathbf{a}_{3}
$$$$
\frac{1}{2} \mathbf{a}_{1}+z_{1} \mathbf{a}_{3}
$$$$
\frac{1}{2} \mathbf{a}_{2}-z_{1} \mathbf{a}_{3}
$$$$
\frac{1}{2} \mathbf{a}_{1}+-z_{1} \mathbf{a}_{3}
$$$$
x_{2} \mathbf{a}_{1}+\frac{1}{2} \mathbf{a}_{2}
$$$$
-x_{2} \mathbf{a}_{1}+\frac{1}{2} \mathbf{a}_{2}
$$$$
\frac{1}{2} \mathbf{a}_{1}+x_{2} \mathbf{a}_{2}
$$$$
\frac{1}{2} \mathbf{a}_{1}-x_{2} \mathbf{a}_{2}
$$

$$
x_{3} \mathbf{a}_{1}+y_{3} \mathbf{a}_{2}+z_{3} \mathbf{a}_{3}
$$$$
-x_{3} \mathbf{a}_{1}-y_{3} \mathbf{a}_{2}+z_{3} \mathbf{a}_{3}
$$$$
-y_{3} \mathbf{a}_{1}+x_{3} \mathbf{a}_{2}+z_{3} \mathbf{a}_{3}
$$$$
y_{3} \mathbf{a}_{1}-x_{3} \mathbf{a}_{2}+z_{3} \mathbf{a}_{3}
$$$$
-x_{3} \mathbf{a}_{1}+y_{3} \mathbf{a}_{2}-z_{3} \mathbf{a}_{3}
$$$$
x_{3} \mathbf{a}_{1}-y_{3} \mathbf{a}_{2}-z_{3} \mathbf{a}_{3}
$$$$
y_{3} \mathbf{a}_{1}+x_{3} \mathbf{a}_{2}-z_{3} \mathbf{a}_{3}
$$$$
-y_{3} \mathbf{a}_{1}-x_{3} \mathbf{a}_{2}-z_{3} \mathbf{a}_{3}
$$$$
x_{4} \mathbf{a}_{1}+y_{4} \mathbf{a}_{2}+z_{4} \mathbf{a}_{3}
$$$$
-x_{4} \mathbf{a}_{1}-y_{4} \mathbf{a}_{2}+z_{4} \mathbf{a}_{3}
$$$$
-y_{4} \mathbf{a}_{1}+x_{4} \mathbf{a}_{2}+z_{4} \mathbf{a}_{3}
$$$$
y_{4} \mathbf{a}_{1}-x_{4} \mathbf{a}_{2}+z_{4} \mathbf{a}_{3}
$$$$
-x_{4} \mathbf{a}_{1}+y_{4} \mathbf{a}_{2}-z_{4} \mathbf{a}_{3}
$$$$
x_{4} \mathbf{a}_{1}-y_{4} \mathbf{a}_{2}-z_{4} \mathbf{a}_{3}
$$$$
y_{4} \mathbf{a}_{1}+x_{4} \mathbf{a}_{2}-z_{4} \mathbf{a}_{3}
$$$$
-y_{4} \mathbf{a}_{1}-x_{4} \mathbf{a}_{2}-z_{4} \mathbf{a}_{3}
$$$$
x_{5} \mathbf{a}_{1}+y_{5} \mathbf{a}_{2}+z_{5} \mathbf{a}_{3}
$$$$
-x_{5} \mathbf{a}_{1}-y_{5} \mathbf{a}_{2}+z_{5} \mathbf{a}_{3}
$$$$
-y_{5} \mathbf{a}_{1}+x_{5} \mathbf{a}_{2}+z_{5} \mathbf{a}_{3}
$$

$=$

$=$

$=$

$=$

$=$

$=$

$=$

$=$
$=$

$=$

$=$

$=$

$=$

$=$

$=$

$=$

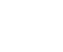

$=$

$=$

$=$

$-$

$=$

$=$

$=$

$=$

$=$

$=$

$=$

$=$

$=$

$=$

$=$

$=$

$=$

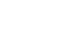

$=$

$=$
Cartesian Coordinates

$$
\begin{array}{r}
\frac{1}{2} a \hat{\mathbf{y}}+z_{1} c \hat{\mathbf{z}} \\
\frac{1}{2} a \hat{\mathbf{x}}+z_{1} c \hat{\mathbf{z}} \\
\frac{1}{2} a \hat{\mathbf{y}}-z_{1} c \hat{\mathbf{z}} \\
\frac{1}{2} a \hat{\mathbf{x}}+-z_{1} c \hat{\mathbf{z}} \\
x_{2} a \hat{\mathbf{x}}+\frac{1}{2} a \hat{\mathbf{y}} \\
-x_{2} a \hat{\mathbf{x}}+\frac{1}{2} a \hat{\mathbf{y}} \\
\frac{1}{2} a \hat{\mathbf{x}}+x_{2} a \hat{\mathbf{y}} \\
\frac{1}{2} a \hat{\mathbf{x}}-x_{2} a \hat{\mathbf{y}}
\end{array}
$$$$
x_{3} a \hat{\mathbf{x}}+y_{3} a \hat{\mathbf{y}}+z_{3} c \hat{\mathbf{z}}
$$$$
-x_{3} a \hat{\mathbf{x}}-y_{3} a \hat{\mathbf{y}}+z_{3} c \hat{\mathbf{z}}
$$$$
-y_{3} a \hat{\mathbf{x}}+x_{3} a \hat{\mathbf{y}}+z_{3} c \hat{\mathbf{z}}
$$$$
y_{3} a \hat{\mathbf{x}}-x_{3} a \hat{\mathbf{y}}+z_{3} c \hat{\mathbf{z}}
$$$$
-x_{3} a \hat{\mathbf{x}}+y_{3} a \hat{\mathbf{y}}-z_{3} c \hat{\mathbf{z}}
$$$$
x_{3} a \hat{\mathbf{x}}-y_{3} a \hat{\mathbf{y}}-z_{3} c \hat{\mathbf{z}}
$$$$
y_{3} a \hat{\mathbf{x}}+x_{3} a \hat{\mathbf{y}}-z_{3} c \hat{\mathbf{z}}
$$$$
-y_{3} a \hat{\mathbf{x}}-x_{3} a \hat{\mathbf{y}}-z_{3} c \hat{\mathbf{z}}
$$$$
x_{4} a \hat{\mathbf{x}}+y_{4} a \hat{\mathbf{y}}+z_{4} c \hat{\mathbf{z}}
$$$$
-x_{4} a \hat{\mathbf{x}}-y_{4} a \hat{\mathbf{y}}+z_{4} c \hat{\mathbf{z}}
$$$$
-y_{4} a \hat{\mathbf{x}}+x_{4} a \hat{\mathbf{y}}+z_{4} c \hat{\mathbf{z}}
$$$$
y_{4} a \hat{\mathbf{x}}-x_{4} a \hat{\mathbf{y}}+z_{4} c \hat{\mathbf{z}}
$$$$
-x_{4} a \hat{\mathbf{x}}+y_{4} a \hat{\mathbf{y}}-z_{4} c \hat{\mathbf{z}}
$$$$
x_{4} a \hat{\mathbf{x}}-y_{4} a \hat{\mathbf{y}}-z_{4} c \hat{\mathbf{z}}
$$$$
y_{4} a \hat{\mathbf{x}}+x_{4} a \hat{\mathbf{y}}-z_{4} c \hat{\mathbf{z}}
$$$$
-y_{4} a \hat{\mathbf{x}}-x_{4} a \hat{\mathbf{y}}-z_{4} c \hat{\mathbf{z}}
$$$$
x_{5} a \hat{\mathbf{x}}+y_{5} a \hat{\mathbf{y}}+z_{5} c \hat{\mathbf{z}}
$$$$
-x_{5} a \hat{\mathbf{x}}-y_{5} a \hat{\mathbf{y}}+z_{5} c \hat{\mathbf{z}}
$$$$
-y_{5} a \hat{\mathbf{x}}+x_{5} a \hat{\mathbf{y}}+z_{5} c \hat{\mathbf{z}}
$$

Wyckoff Position
Atom Type

Pt I

Pt I

Pt I

Pt I

Pt II

Pt II

Pt II

Pt II

C I

C I

C I

C I

C I

C I

C I

C I

C II

C II

C II

C II

C II

C II

C II

C II

C III

C III

C III 


\begin{tabular}{|c|c|c|c|c|c|c|}
\hline $\mathbf{B}_{28}$ & $=$ & $y_{5} \mathbf{a}_{1}-x_{5} \mathbf{a}_{2}+z_{5} \mathbf{a}_{3}$ & $=$ & $y_{5} a \hat{\mathbf{x}}-x_{5} a \hat{\mathbf{y}}+z_{5} c \hat{\mathbf{z}}$ & $(8 p)$ & C III \\
\hline $\mathbf{B}_{29}$ & $=$ & $-x_{5} \mathbf{a}_{1}+y_{5} \mathbf{a}_{2}-z_{5} \mathbf{a}_{3}$ & $=$ & $-x_{5} a \hat{\mathbf{x}}+y_{5} a \hat{\mathbf{y}}-z_{5} c \hat{\mathbf{z}}$ & $(8 p)$ & C III \\
\hline $\mathbf{B}_{30}$ & $=$ & $x_{5} \mathbf{a}_{1}-y_{5} \mathbf{a}_{2}-z_{5} \mathbf{a}_{3}$ & $=$ & $x_{5} a \hat{\mathbf{x}}-y_{5} a \hat{\mathbf{y}}-z_{5} c \hat{\mathbf{z}}$ & $(8 p)$ & C III \\
\hline $\mathbf{B}_{31}$ & $=$ & $y_{5} \mathbf{a}_{1}+x_{5} \mathbf{a}_{2}-z_{5} \mathbf{a}_{3}$ & $=$ & $y_{5} a \hat{\mathbf{x}}+x_{5} a \hat{\mathbf{y}}-z_{5} c \hat{\mathbf{z}}$ & $(8 p)$ & C III \\
\hline $\mathbf{B}_{32}$ & $=$ & $-y_{5} \mathbf{a}_{1}-x_{5} \mathbf{a}_{2}-z_{5} \mathbf{a}_{3}$ & $=$ & $-y_{5} a \hat{\mathbf{x}}-x_{5} a \hat{\mathbf{y}}-z_{5} c \hat{\mathbf{z}}$ & $(8 p)$ & C III \\
\hline $\mathbf{B}_{33}$ & $=$ & $x_{6} \mathbf{a}_{1}+y_{6} \mathbf{a}_{2}+z_{6} \mathbf{a}_{3}$ & $=$ & $x_{6} a \hat{\mathbf{x}}+y_{6} a \hat{\mathbf{y}}+z_{6} c \hat{\mathbf{z}}$ & $(8 p)$ & C IV \\
\hline $\mathbf{B}_{34}$ & $=$ & $-x_{6} \mathbf{a}_{1}-y_{6} \mathbf{a}_{2}+z_{6} \mathbf{a}_{3}$ & $=$ & $-x_{6} a \hat{\mathbf{x}}-y_{6} a \hat{\mathbf{y}}+z_{6} c \hat{\mathbf{z}}$ & $(8 p)$ & C IV \\
\hline $\mathbf{B}_{35}$ & $=$ & $-y_{6} \mathbf{a}_{1}+x_{6} \mathbf{a}_{2}+z_{6} \mathbf{a}_{3}$ & $=$ & $-y_{6} a \hat{\mathbf{x}}+x_{6} a \hat{\mathbf{y}}+z_{6} c \hat{\mathbf{z}}$ & $(8 p)$ & C IV \\
\hline $\mathbf{B}_{36}$ & $=$ & $y_{6} \mathbf{a}_{1}-x_{6} \mathbf{a}_{2}+z_{6} \mathbf{a}_{3}$ & $=$ & $y_{6} a \hat{\mathbf{x}}-x_{6} a \hat{\mathbf{y}}+z_{6} c \hat{\mathbf{z}}$ & $(8 p)$ & C IV \\
\hline $\mathbf{B}_{37}$ & $=$ & $-x_{6} \mathbf{a}_{1}+y_{6} \mathbf{a}_{2}-z_{6} \mathbf{a}_{3}$ & $=$ & $-x_{6} a \hat{\mathbf{x}}+y_{6} a \hat{\mathbf{y}}-z_{6} c \hat{\mathbf{z}}$ & $(8 p)$ & C IV \\
\hline $\mathbf{B}_{38}$ & $=$ & $x_{6} \mathbf{a}_{1}-y_{6} \mathbf{a}_{2}-z_{6} \mathbf{a}_{3}$ & $=$ & $x_{6} a \hat{\mathbf{x}}-y_{6} a \hat{\mathbf{y}}-z_{6} c \hat{\mathbf{z}}$ & $(8 p)$ & C IV \\
\hline $\mathbf{B}_{39}$ & $=$ & $y_{6} \mathbf{a}_{1}+x_{6} \mathbf{a}_{2}-z_{6} \mathbf{a}_{3}$ & $=$ & $y_{6} a \hat{\mathbf{x}}+x_{6} a \hat{\mathbf{y}}-z_{6} c \hat{\mathbf{z}}$ & $(8 p)$ & C IV \\
\hline $\mathbf{B}_{40}$ & $=$ & $-y_{6} \mathbf{a}_{1}-x_{6} \mathbf{a}_{2}-z_{6} \mathbf{a}_{3}$ & $=$ & $-y_{6} a \hat{\mathbf{x}}-x_{6} a \hat{\mathbf{y}}-z_{6} c \hat{\mathbf{z}}$ & $(8 p)$ & C IV \\
\hline $\mathbf{B}_{41}$ & $=$ & $x_{7} \mathbf{a}_{1}+y_{7} \mathbf{a}_{2}+z_{7} \mathbf{a}_{3}$ & $=$ & $x_{7} a \hat{\mathbf{x}}+y_{7} a \hat{\mathbf{y}}+z_{7} c \hat{\mathbf{z}}$ & $(8 p)$ & $\mathrm{C} \mathrm{V}$ \\
\hline $\mathbf{B}_{42}$ & $=$ & $-x_{7} \mathbf{a}_{1}-y_{7} \mathbf{a}_{2}+z_{7} \mathbf{a}_{3}$ & $=$ & $-x_{7} a \hat{\mathbf{x}}-y_{7} a \hat{\mathbf{y}}+z_{7} c \hat{\mathbf{z}}$ & $(8 p)$ & $\mathrm{C} \mathrm{V}$ \\
\hline $\mathbf{B}_{43}$ & $=$ & $-y_{7} \mathbf{a}_{1}+x_{7} \mathbf{a}_{2}+z_{7} \mathbf{a}_{3}$ & $=$ & $-y_{7} a \hat{\mathbf{x}}+x_{7} a \hat{\mathbf{y}}+z_{7} c \hat{\mathbf{z}}$ & $(8 p)$ & $\mathrm{C} \mathrm{V}$ \\
\hline $\mathbf{B}_{44}$ & $=$ & $y_{7} \mathbf{a}_{1}-x_{7} \mathbf{a}_{2}+z_{7} \mathbf{a}_{3}$ & $=$ & $y_{7} a \hat{\mathbf{x}}-x_{7} a \hat{\mathbf{y}}+z_{7} c \hat{\mathbf{z}}$ & $(8 p)$ & $\mathrm{C} \mathrm{V}$ \\
\hline $\mathbf{B}_{45}$ & $=$ & $-x_{7} \mathbf{a}_{1}+y_{7} \mathbf{a}_{2}-z_{7} \mathbf{a}_{3}$ & $=$ & $-x_{7} a \hat{\mathbf{x}}+y_{7} a \hat{\mathbf{y}}-z_{7} c \hat{\mathbf{z}}$ & $(8 p)$ & $\mathrm{C} \mathrm{V}$ \\
\hline $\mathbf{B}_{46}$ & $=$ & $x_{7} \mathbf{a}_{1}-y_{7} \mathbf{a}_{2}-z_{7} \mathbf{a}_{3}$ & $=$ & $x_{7} a \hat{\mathbf{x}}-y_{7} a \hat{\mathbf{y}}-z_{7} c \hat{\mathbf{z}}$ & $(8 p)$ & $\mathrm{C} \mathrm{V}$ \\
\hline $\mathbf{B}_{47}$ & $=$ & $y_{7} \mathbf{a}_{1}+x_{7} \mathbf{a}_{2}-z_{7} \mathbf{a}_{3}$ & $=$ & $y_{7} a \hat{\mathbf{x}}+x_{7} a \hat{\mathbf{y}}-z_{7} c \hat{\mathbf{z}}$ & $(8 p)$ & $\mathrm{C} \mathrm{V}$ \\
\hline $\mathbf{B}_{48}$ & $=$ & $-y_{7} \mathbf{a}_{1}-x_{7} \mathbf{a}_{2}-z_{7} \mathbf{a}_{3}$ & $=$ & $-y_{7} a \hat{\mathbf{x}}-x_{7} a \hat{\mathbf{y}}-z_{7} c \hat{\mathbf{z}}$ & $(8 p)$ & $\mathrm{C} \mathrm{V}$ \\
\hline $\mathbf{B}_{49}$ & $=$ & $x_{8} \mathbf{a}_{1}+y_{8} \mathbf{a}_{2}+z_{8} \mathbf{a}_{3}$ & $=$ & $x_{8} a \hat{\mathbf{x}}+y_{8} a \hat{\mathbf{y}}+z_{8} c \hat{\mathbf{z}}$ & $(8 p)$ & C VI \\
\hline $\mathbf{B}_{50}$ & $=$ & $-x_{8} \mathbf{a}_{1}-y_{8} \mathbf{a}_{2}+z_{8} \mathbf{a}_{3}$ & $=$ & $-x_{8} a \hat{\mathbf{x}}-y_{8} a \hat{\mathbf{y}}+z_{8} c \hat{\mathbf{z}}$ & $(8 p)$ & C VI \\
\hline $\mathbf{B}_{51}$ & $=$ & $-y_{8} \mathbf{a}_{1}+x_{8} \mathbf{a}_{2}+z_{8} \mathbf{a}_{3}$ & $=$ & $-y_{8} a \hat{\mathbf{x}}+x_{8} a \hat{\mathbf{y}}+z_{8} c \hat{\mathbf{z}}$ & $(8 p)$ & $\mathrm{C}$ VI \\
\hline $\mathbf{B}_{52}$ & $=$ & $y_{8} \mathbf{a}_{1}-x_{8} \mathbf{a}_{2}+z_{8} \mathbf{a}_{3}$ & $=$ & $y_{8} a \hat{\mathbf{x}}-x_{8} a \hat{\mathbf{y}}+z_{8} c \hat{\mathbf{z}}$ & $(8 p)$ & C VI \\
\hline $\mathbf{B}_{53}$ & $=$ & $-x_{8} \mathbf{a}_{1}+y_{8} \mathbf{a}_{2}-z_{8} \mathbf{a}_{3}$ & $=$ & $-x_{8} a \hat{\mathbf{x}}+y_{8} a \hat{\mathbf{y}}-z_{8} c \hat{\mathbf{z}}$ & $(8 p)$ & C VI \\
\hline $\mathbf{B}_{54}$ & $=$ & $x_{8} \mathbf{a}_{1}-y_{8} \mathbf{a}_{2}-z_{8} \mathbf{a}_{3}$ & $=$ & $x_{8} a \hat{\mathbf{x}}-y_{8} a \hat{\mathbf{y}}-z_{8} c \hat{\mathbf{z}}$ & $(8 p)$ & C VI \\
\hline $\mathbf{B}_{55}$ & $=$ & $y_{8} \mathbf{a}_{1}+x_{8} \mathbf{a}_{2}-z_{8} \mathbf{a}_{3}$ & $=$ & $y_{8} a \hat{\mathbf{x}}+x_{8} a \hat{\mathbf{y}}-z_{8} c \hat{\mathbf{z}}$ & $(8 p)$ & C VI \\
\hline $\mathbf{B}_{56}$ & $=$ & $-y_{8} \mathbf{a}_{1}-x_{8} \mathbf{a}_{2}-z_{8} \mathbf{a}_{3}$ & $=$ & $-y_{8} a \hat{\mathbf{x}}-x_{8} a \hat{\mathbf{y}}-z_{8} c \hat{\mathbf{z}}$ & $(8 p)$ & $\mathrm{C}$ VI \\
\hline $\mathbf{B}_{57}$ & $=$ & $x_{9} \mathbf{a}_{1}+y_{9} \mathbf{a}_{2}+z_{9} \mathbf{a}_{3}$ & $=$ & $x_{9} a \hat{\mathbf{x}}+y_{9} a \hat{\mathbf{y}}+z_{9} c \hat{\mathbf{z}}$ & $(8 p)$ & C VII \\
\hline $\mathbf{B}_{58}$ & $=$ & $-x_{9} \mathbf{a}_{1}-y_{9} \mathbf{a}_{2}+z_{9} \mathbf{a}_{3}$ & $=$ & $-x_{9} a \hat{\mathbf{x}}-y_{9} a \hat{\mathbf{y}}+z_{9} c \hat{\mathbf{z}}$ & $(8 p)$ & C VII \\
\hline $\mathbf{B}_{59}$ & $=$ & $-y_{9} \mathbf{a}_{1}+x_{9} \mathbf{a}_{2}+z_{9} \mathbf{a}_{3}$ & $=$ & $-y_{9} a \hat{\mathbf{x}}+x_{9} a \hat{\mathbf{y}}+z_{9} c \hat{\mathbf{z}}$ & $(8 p)$ & C VII \\
\hline $\mathbf{B}_{60}$ & $=$ & $y_{9} \mathbf{a}_{1}-x_{9} \mathbf{a}_{2}+z_{9} \mathbf{a}_{3}$ & $=$ & $y_{9} a \hat{\mathbf{x}}-x_{9} a \hat{\mathbf{y}}+z_{9} c \hat{\mathbf{z}}$ & $(8 p)$ & C VII \\
\hline $\mathbf{B}_{61}$ & $=$ & $-x_{9} \mathbf{a}_{1}+y_{9} \mathbf{a}_{2}-z_{9} \mathbf{a}_{3}$ & $=$ & $-x_{9} a \hat{\mathbf{x}}+y_{9} a \hat{\mathbf{y}}-z_{9} c \hat{\mathbf{z}}$ & $(8 p)$ & C VII \\
\hline $\mathbf{B}_{62}$ & $=$ & $x_{9} \mathbf{a}_{1}-y_{9} \mathbf{a}_{2}-z_{9} \mathbf{a}_{3}$ & $=$ & $x_{9} a \hat{\mathbf{x}}-y_{9} a \hat{\mathbf{y}}-z_{9} c \hat{\mathbf{z}}$ & $(8 p)$ & C VII \\
\hline $\mathbf{B}_{63}$ & $=$ & $y_{9} \mathbf{a}_{1}+x_{9} \mathbf{a}_{2}-z_{9} \mathbf{a}_{3}$ & $=$ & $y_{9} a \hat{\mathbf{x}}+x_{9} a \hat{\mathbf{y}}-z_{9} c \hat{\mathbf{z}}$ & $(8 p)$ & C VII \\
\hline
\end{tabular}




\begin{tabular}{|c|c|c|c|c|c|c|}
\hline $\mathbf{B}_{64}$ & $=$ & $-y_{9} \mathbf{a}_{1}-x_{9} \mathbf{a}_{2}-z 9 \mathbf{a}_{3}$ & $=$ & $-y_{9} a \hat{\mathbf{x}}-x_{9} a \hat{\mathbf{y}}-z_{9} c \hat{\mathbf{z}}$ & $(8 p)$ & C VII \\
\hline $\mathbf{B}_{65}$ & $=$ & $x_{10} \mathbf{a}_{1}+y_{10} \mathbf{a}_{2}+z_{10} \mathbf{a}_{3}$ & $=$ & $x_{10} a \hat{\mathbf{x}}+y_{10} a \hat{\mathbf{y}}+z_{10} c \hat{\mathbf{z}}$ & $(8 p)$ & C VIII \\
\hline $\mathbf{B}_{66}$ & $=$ & $-x_{10} \mathbf{a}_{1}-y_{10} \mathbf{a}_{2}+z_{10} \mathbf{a}_{3}$ & $=$ & $-x_{10} a \hat{\mathbf{x}}-y_{10} a \hat{\mathbf{y}}+z_{10} c \hat{\mathbf{z}}$ & $(8 p)$ & C VIII \\
\hline $\mathbf{B}_{67}$ & $=$ & $-y_{10} \mathbf{a}_{1}+x_{10} \mathbf{a}_{2}+z_{10} \mathbf{a}_{3}$ & $=$ & $-y_{10} a \hat{\mathbf{x}}+x_{10} a \hat{\mathbf{y}}+z_{10} c \hat{\mathbf{z}}$ & $(8 p)$ & C VIII \\
\hline $\mathbf{B}_{68}$ & $=$ & $y_{10} \mathbf{a}_{1}-x_{10} \mathbf{a}_{2}+z_{10} \mathbf{a}_{3}$ & $=$ & $y_{10} a \hat{\mathbf{x}}-x_{10} a \hat{\mathbf{y}}+z_{10} c \hat{\mathbf{z}}$ & $(8 p)$ & C VIII \\
\hline $\mathbf{B}_{69}$ & $=$ & $-x_{10} \mathbf{a}_{1}+y_{10} \mathbf{a}_{2}-z_{10} \mathbf{a}_{3}$ & $=$ & $-x_{10} a \hat{\mathbf{x}}+y_{10} a \hat{\mathbf{y}}-z_{10} c \hat{\mathbf{z}}$ & $(8 p)$ & C VIII \\
\hline $\mathbf{B}_{70}$ & $=$ & $x_{10} \mathbf{a}_{1}-y_{10} \mathbf{a}_{2}-z_{10} \mathbf{a}_{3}$ & $=$ & $x_{10} a \hat{\mathbf{x}}-y_{10} a \hat{\mathbf{y}}-z_{10} c \hat{\mathbf{z}}$ & $(8 p)$ & C VIII \\
\hline $\mathbf{B}_{71}$ & $=$ & $y_{10} \mathbf{a}_{1}+x_{10} \mathbf{a}_{2}-z_{10} \mathbf{a}_{3}$ & $=$ & $y_{10} a \hat{\mathbf{x}}+x_{10} a \hat{\mathbf{y}}-z_{10} c \hat{\mathbf{z}}$ & $(8 p)$ & C VIII \\
\hline $\mathbf{B}_{72}$ & $=$ & $-y_{10} \mathbf{a}_{1}-x_{10} \mathbf{a}_{2}-z_{10} \mathbf{a}_{3}$ & $=$ & $-y_{10} a \hat{\mathbf{x}}-x_{10} a \hat{\mathbf{y}}-z_{10} c \hat{\mathbf{z}}$ & $(8 p)$ & C VIII \\
\hline $\mathbf{B}_{73}$ & $=$ & $x_{11} \mathbf{a}_{1}+y_{11} \mathbf{a}_{2}+z_{11} \mathbf{a}_{3}$ & $=$ & $x_{11} a \hat{\mathbf{x}}+y_{11} a \hat{\mathbf{y}}+z_{11} c \hat{\mathbf{z}}$ & $(8 p)$ & C IX \\
\hline $\mathbf{B}_{74}$ & $=$ & $-x_{11} \mathbf{a}_{1}-y_{11} \mathbf{a}_{2}+z_{11} \mathbf{a}_{3}$ & $=$ & $-x_{11} a \hat{\mathbf{x}}-y_{11} a \hat{\mathbf{y}}+z_{11} c \hat{\mathbf{z}}$ & $(8 p)$ & C IX \\
\hline $\mathbf{B}_{75}$ & $=$ & $-y_{11} \mathbf{a}_{1}+x_{11} \mathbf{a}_{2}+z_{11} \mathbf{a}_{3}$ & $=$ & $-y_{11} a \hat{\mathbf{x}}+x_{11} a \hat{\mathbf{y}}+z_{11} c \hat{\mathbf{z}}$ & $(8 p)$ & C IX \\
\hline $\mathbf{B}_{76}$ & $=$ & $y_{11} \mathbf{a}_{1}-x_{11} \mathbf{a}_{2}+z_{11} \mathbf{a}_{3}$ & $=$ & $y_{11} a \hat{\mathbf{x}}-x_{11} a \hat{\mathbf{y}}+z_{11} c \hat{\mathbf{z}}$ & $(8 p)$ & C IX \\
\hline $\mathbf{B}_{77}$ & $=$ & $-x_{11} \mathbf{a}_{1}+y_{11} \mathbf{a}_{2}-z_{11} \mathbf{a}_{3}$ & $=$ & $-x_{11} a \hat{\mathbf{x}}+y_{11} a \hat{\mathbf{y}}-z_{11} c \hat{\mathbf{z}}$ & $(8 p)$ & C IX \\
\hline $\mathbf{B}_{78}$ & $=$ & $x_{11} \mathbf{a}_{1}-y_{11} \mathbf{a}_{2}-z_{11} \mathbf{a}_{3}$ & $=$ & $x_{11} a \hat{\mathbf{x}}-y_{11} a \hat{\mathbf{y}}-z_{11} c \hat{\mathbf{z}}$ & $(8 p)$ & C IX \\
\hline $\mathbf{B}_{79}$ & $=$ & $y_{11} \mathbf{a}_{1}+x_{11} \mathbf{a}_{2}-z_{11} \mathbf{a}_{3}$ & $=$ & $y_{11} a \hat{\mathbf{x}}+x_{11} a \hat{\mathbf{y}}-z_{11} c \hat{\mathbf{z}}$ & $(8 p)$ & C IX \\
\hline $\mathbf{B}_{80}$ & $=$ & $-y_{11} \mathbf{a}_{1}-x_{11} \mathbf{a}_{2}-z_{11} \mathbf{a}_{3}$ & $=$ & $-y_{11} a \hat{\mathbf{x}}-x_{11} a \hat{\mathbf{y}}-z_{11} c \hat{\mathbf{z}}$ & $(8 p)$ & C IX \\
\hline $\mathbf{B}_{81}$ & $=$ & $x_{12} \mathbf{a}_{1}+y_{12} \mathbf{a}_{2}+z_{12} \mathbf{a}_{3}$ & $=$ & $x_{12} a \hat{\mathbf{x}}+y_{12} a \hat{\mathbf{y}}+z_{12} c \hat{\mathbf{z}}$ & $(8 p)$ & $\mathrm{CX}$ \\
\hline $\mathbf{B}_{82}$ & $=$ & $-x_{12} \mathbf{a}_{1}-y_{12} \mathbf{a}_{2}+z_{12} \mathbf{a}_{3}$ & $=$ & $-x_{12} a \hat{\mathbf{x}}-y_{12} a \hat{\mathbf{y}}+z_{12} c \hat{\mathbf{z}}$ & $(8 p)$ & $\mathrm{CX}$ \\
\hline $\mathbf{B}_{83}$ & $=$ & $-y_{12} \mathbf{a}_{1}+x_{12} \mathbf{a}_{2}+z_{12} \mathbf{a}_{3}$ & $=$ & $-y_{12} a \hat{\mathbf{x}}+x_{12} a \hat{\mathbf{y}}+z_{12} c \hat{\mathbf{z}}$ & $(8 p)$ & $\mathrm{CX}$ \\
\hline $\mathbf{B}_{84}$ & $=$ & $y_{12} \mathbf{a}_{1}-x_{12} \mathbf{a}_{2}+z_{12} \mathbf{a}_{3}$ & $=$ & $y_{12} a \hat{\mathbf{x}}-x_{12} a \hat{\mathbf{y}}+z_{12} c \hat{\mathbf{z}}$ & $(8 p)$ & $\mathrm{CX}$ \\
\hline $\mathbf{B}_{85}$ & $=$ & $-x_{12} \mathbf{a}_{1}+y_{12} \mathbf{a}_{2}-z_{12} \mathbf{a}_{3}$ & $=$ & $-x_{12} a \hat{\mathbf{x}}+y_{12} a \hat{\mathbf{y}}-z_{12} c \hat{\mathbf{z}}$ & $(8 p)$ & $\mathrm{CX}$ \\
\hline $\mathbf{B}_{86}$ & $=$ & $x_{12} \mathbf{a}_{1}-y_{12} \mathbf{a}_{2}-z_{12} \mathbf{a}_{3}$ & $=$ & $x_{12} a \hat{\mathbf{x}}-y_{12} a \hat{\mathbf{y}}-z_{12} c \hat{\mathbf{z}}$ & $(8 p)$ & $\mathrm{CX}$ \\
\hline $\mathbf{B}_{87}$ & $=$ & $y_{12} \mathbf{a}_{1}+x_{12} \mathbf{a}_{2}-z_{12} \mathbf{a}_{3}$ & $=$ & $y_{12} a \hat{\mathbf{x}}+x_{12} a \hat{\mathbf{y}}-z_{12} c \hat{\mathbf{z}}$ & $(8 p)$ & $\mathrm{CX}$ \\
\hline $\mathbf{B}_{88}$ & $=$ & $-y_{12} \mathbf{a}_{1}-x_{12} \mathbf{a}_{2}-z_{12} \mathbf{a}_{3}$ & $=$ & $-y_{12} a \hat{\mathbf{x}}-x_{12} a \hat{\mathbf{y}}-z_{12} c \hat{\mathbf{z}}$ & $(8 p)$ & $\mathrm{CX}$ \\
\hline $\mathbf{B}_{89}$ & $=$ & $x_{13} \mathbf{a}_{1}+y_{13} \mathbf{a}_{2}+z_{13} \mathbf{a}_{3}$ & $=$ & $x_{13} a \hat{\mathbf{x}}+y_{13} a \hat{\mathbf{y}}+z_{13} c \hat{\mathbf{z}}$ & $(8 p)$ & C XI \\
\hline $\mathbf{B}_{90}$ & $=$ & $-x_{13} \mathbf{a}_{1}-y_{13} \mathbf{a}_{2}+z_{13} \mathbf{a}_{3}$ & $=$ & $-x_{13} a \hat{\mathbf{x}}-y_{13} a \hat{\mathbf{y}}+z_{13} c \hat{\mathbf{z}}$ & $(8 p)$ & C XI \\
\hline $\mathbf{B}_{91}$ & $=$ & $-y_{13} \mathbf{a}_{1}+x_{13} \mathbf{a}_{2}+z_{13} \mathbf{a}_{3}$ & $=$ & $-y_{13} a \hat{\mathbf{x}}+x_{13} a \hat{\mathbf{y}}+z_{13} c \hat{\mathbf{z}}$ & $(8 p)$ & C XI \\
\hline $\mathbf{B}_{92}$ & $=$ & $y_{13} \mathbf{a}_{1}-x_{13} \mathbf{a}_{2}+z_{13} \mathbf{a}_{3}$ & $=$ & $y_{13} a \hat{\mathbf{x}}-x_{13} a \hat{\mathbf{y}}+z_{13} c \hat{\mathbf{z}}$ & $(8 p)$ & C XI \\
\hline $\mathbf{B}_{93}$ & $=$ & $-x_{13} \mathbf{a}_{1}+y_{13} \mathbf{a}_{2}-z_{13} \mathbf{a}_{3}$ & $=$ & $-x_{13} a \hat{\mathbf{x}}+y_{13} a \hat{\mathbf{y}}-z_{13} c \hat{\mathbf{z}}$ & $(8 p)$ & C XI \\
\hline $\mathbf{B}_{94}$ & $=$ & $x_{13} \mathbf{a}_{1}-y_{13} \mathbf{a}_{2}-z_{13} \mathbf{a}_{3}$ & $=$ & $x_{13} a \hat{\mathbf{x}}-y_{13} a \hat{\mathbf{y}}-z_{13} c \hat{\mathbf{z}}$ & $(8 p)$ & C XI \\
\hline $\mathbf{B}_{95}$ & $=$ & $y_{13} \mathbf{a}_{1}+x_{13} \mathbf{a}_{2}-z_{13} \mathbf{a}_{3}$ & $=$ & $y_{13} a \hat{\mathbf{x}}+x_{13} a \hat{\mathbf{y}}-z_{13} c \hat{\mathbf{z}}$ & $(8 p)$ & C XI \\
\hline $\mathbf{B}_{96}$ & $=$ & $-y_{13} \mathbf{a}_{1}-x_{13} \mathbf{a}_{2}-z_{13} \mathbf{a}_{3}$ & $=$ & $-y_{13} a \hat{\mathbf{x}}-x_{13} a \hat{\mathbf{y}}-z_{13} c \hat{\mathbf{z}}$ & $(8 p)$ & C XI \\
\hline $\mathbf{B}_{97}$ & $=$ & $x_{14} \mathbf{a}_{1}+y_{14} \mathbf{a}_{2}+z_{14} \mathbf{a}_{3}$ & $=$ & $x_{14} a \hat{\mathbf{x}}+y_{14} a \hat{\mathbf{y}}+z_{14} c \hat{\mathbf{z}}$ & $(8 p)$ & C XII \\
\hline $\mathbf{B}_{98}$ & $=$ & $-x_{14} \mathbf{a}_{1}-y_{14} \mathbf{a}_{2}+z_{14} \mathbf{a}_{3}$ & $=$ & $-x_{14} a \hat{\mathbf{x}}-y_{14} a \hat{\mathbf{y}}+z_{14} c \hat{\mathbf{z}}$ & $(8 p)$ & C XII \\
\hline $\mathbf{B}_{99}$ & $=$ & $-y_{14} \mathbf{a}_{1}+x_{14} \mathbf{a}_{2}+z_{14} \mathbf{a}_{3}$ & $=$ & $-y_{14} a \hat{\mathbf{x}}+x_{14} a \hat{\mathbf{y}}+z_{14} c \hat{\mathbf{z}}$ & $(8 p)$ & C XII \\
\hline
\end{tabular}




\begin{tabular}{|c|c|c|c|c|c|c|}
\hline $\mathbf{B}_{100}$ & $=$ & $y_{14} \mathbf{a}_{1}-x_{14} \mathbf{a}_{2}+z_{14} \mathbf{a}_{3}$ & $=$ & $y_{14} a \hat{\mathbf{x}}-x_{14} a \hat{\mathbf{y}}+z_{14} c \hat{\mathbf{z}}$ & $(8 p)$ & C XII \\
\hline $\mathbf{B}_{101}$ & $=$ & $-x_{14} \mathbf{a}_{1}+y_{14} \mathbf{a}_{2}-z_{14} \mathbf{a}_{3}$ & $=$ & $-x_{14} a \hat{\mathbf{x}}+y_{14} a \hat{\mathbf{y}}-z_{14} c \hat{\mathbf{z}}$ & $(8 p)$ & C XII \\
\hline $\mathbf{B}_{102}$ & $=$ & $x_{14} \mathbf{a}_{1}-y_{14} \mathbf{a}_{2}-z_{14} \mathbf{a}_{3}$ & $=$ & $x_{14} a \hat{\mathbf{x}}-y_{14} a \hat{\mathbf{y}}-z_{14} c \hat{\mathbf{z}}$ & $(8 p)$ & C XII \\
\hline $\mathbf{B}_{103}$ & $=$ & $y_{14} \mathbf{a}_{1}+x_{14} \mathbf{a}_{2}-z_{14} \mathbf{a}_{3}$ & $=$ & $y_{14} a \hat{\mathbf{x}}+x_{14} a \hat{\mathbf{y}}-z_{14} c \hat{\mathbf{z}}$ & $(8 p)$ & C XII \\
\hline $\mathbf{B}_{104}$ & $=$ & $-y_{14} \mathbf{a}_{1}-x_{14} \mathbf{a}_{2}-z_{14} \mathbf{a}_{3}$ & $=$ & $-y_{14} a \hat{\mathbf{x}}-x_{14} a \hat{\mathbf{y}}-z_{14} c \hat{\mathbf{z}}$ & $(8 p)$ & C XII \\
\hline $\mathbf{B}_{105}$ & $=$ & $x_{15} \mathbf{a}_{1}+y_{15} \mathbf{a}_{2}+z_{15} \mathbf{a}_{3}$ & $=$ & $x_{15} a \hat{\mathbf{x}}+y_{15} a \hat{\mathbf{y}}+z_{15} c \hat{\mathbf{z}}$ & $(8 p)$ & C XIII \\
\hline $\mathbf{B}_{106}$ & $=$ & $-x_{15} \mathbf{a}_{1}-y_{15} \mathbf{a}_{2}+z_{15} \mathbf{a}_{3}$ & $=$ & $-x_{15} a \hat{\mathbf{x}}-y_{15} a \hat{\mathbf{y}}+z_{15} c \hat{\mathbf{z}}$ & $(8 p)$ & C XIII \\
\hline $\mathbf{B}_{107}$ & $=$ & $-y_{15} \mathbf{a}_{1}+x_{15} \mathbf{a}_{2}+z_{15} \mathbf{a}_{3}$ & $=$ & $-y_{15} a \hat{\mathbf{x}}+x_{15} a \hat{\mathbf{y}}+z_{15} c \hat{\mathbf{z}}$ & $(8 p)$ & C XIII \\
\hline $\mathbf{B}_{108}$ & $=$ & $y_{15} \mathbf{a}_{1}-x_{15} \mathbf{a}_{2}+z_{15} \mathbf{a}_{3}$ & $=$ & $y_{15} a \hat{\mathbf{x}}-x_{15} a \hat{\mathbf{y}}+z_{15} c \hat{\mathbf{z}}$ & $(8 p)$ & C XIII \\
\hline $\mathbf{B}_{109}$ & $=$ & $-x_{15} \mathbf{a}_{1}+y_{15} \mathbf{a}_{2}-z_{15} \mathbf{a}_{3}$ & $=$ & $-x_{15} a \hat{\mathbf{x}}+y_{15} a \hat{\mathbf{y}}-z_{15} c \hat{\mathbf{z}}$ & $(8 p)$ & C XIII \\
\hline $\mathbf{B}_{110}$ & $=$ & $x_{15} \mathbf{a}_{1}-y_{15} \mathbf{a}_{2}-z_{15} \mathbf{a}_{3}$ & $=$ & $x_{15} a \hat{\mathbf{x}}-y_{15} a \hat{\mathbf{y}}-z_{15} c \hat{\mathbf{z}}$ & $(8 p)$ & C XIII \\
\hline $\mathbf{B}_{111}$ & $=$ & $y_{15} \mathbf{a}_{1}+x_{15} \mathbf{a}_{2}-z_{15} \mathbf{a}_{3}$ & $=$ & $y_{15} a \hat{\mathbf{x}}+x_{15} a \hat{\mathbf{y}}-z_{15} c \hat{\mathbf{z}}$ & $(8 p)$ & C XIII \\
\hline $\mathbf{B}_{112}$ & $=$ & $-y_{15} \mathbf{a}_{1}-x_{15} \mathbf{a}_{2}-z_{15} \mathbf{a}_{3}$ & $=$ & $-y_{15} a \hat{\mathbf{x}}-x_{15} a \hat{\mathbf{y}}-z_{15} c \hat{\mathbf{z}}$ & $(8 p)$ & C XIII \\
\hline $\mathbf{B}_{113}$ & $=$ & $x_{16} \mathbf{a}_{1}+y_{16} \mathbf{a}_{2}+z_{16} \mathbf{a}_{3}$ & $=$ & $x_{16} a \hat{\mathbf{x}}+y_{16} a \hat{\mathbf{y}}+z_{16} c \hat{\mathbf{z}}$ & $(8 p)$ & C XIV \\
\hline $\mathbf{B}_{114}$ & $=$ & $-x_{16} \mathbf{a}_{1}-y_{16} \mathbf{a}_{2}+z_{16} \mathbf{a}_{3}$ & $=$ & $-x_{16} a \hat{\mathbf{x}}-y_{16} a \hat{\mathbf{y}}+z_{16} c \hat{\mathbf{z}}$ & $(8 p)$ & C XIV \\
\hline $\mathbf{B}_{115}$ & $=$ & $-y_{16} \mathbf{a}_{1}+x_{16} \mathbf{a}_{2}+z_{16} \mathbf{a}_{3}$ & $=$ & $-y_{16} a \hat{\mathbf{x}}+x_{16} a \hat{\mathbf{y}}+z_{16} c \hat{\mathbf{z}}$ & $(8 p)$ & C XIV \\
\hline $\mathbf{B}_{116}$ & $=$ & $y_{16} \mathbf{a}_{1}-x_{16} \mathbf{a}_{2}+z_{16} \mathbf{a}_{3}$ & $=$ & $y_{16} a \hat{\mathbf{x}}-x_{16} a \hat{\mathbf{y}}+z_{16} c \hat{\mathbf{z}}$ & $(8 p)$ & C XIV \\
\hline $\mathbf{B}_{117}$ & $=$ & $-x_{16} \mathbf{a}_{1}+y_{16} \mathbf{a}_{2}-z_{16} \mathbf{a}_{3}$ & $=$ & $-x_{16} a \hat{\mathbf{x}}+y_{16} a \hat{\mathbf{y}}-z_{16} c \hat{\mathbf{z}}$ & $(8 p)$ & C XIV \\
\hline $\mathbf{B}_{118}$ & $=$ & $x_{16} \mathbf{a}_{1}-y_{16} \mathbf{a}_{2}-z_{16} \mathbf{a}_{3}$ & $=$ & $x_{16} a \hat{\mathbf{x}}-y_{16} a \hat{\mathbf{y}}-z_{16} c \hat{\mathbf{z}}$ & $(8 p)$ & C XIV \\
\hline $\mathbf{B}_{119}$ & $=$ & $y_{16} \mathbf{a}_{1}+x_{16} \mathbf{a}_{2}-z_{16} \mathbf{a}_{3}$ & $=$ & $y_{16} a \hat{\mathbf{x}}+x_{16} a \hat{\mathbf{y}}-z_{16} c \hat{\mathbf{z}}$ & $(8 p)$ & C XIV \\
\hline $\mathbf{B}_{120}$ & $=$ & $-y_{16} \mathbf{a}_{1}-x_{16} \mathbf{a}_{2}-z_{16} \mathbf{a}_{3}$ & $=$ & $-y_{16} a \hat{\mathbf{x}}-x_{16} a \hat{\mathbf{y}}-z_{16} c \hat{\mathbf{z}}$ & $(8 p)$ & C XIV \\
\hline $\mathbf{B}_{121}$ & $=$ & $x_{17} \mathbf{a}_{1}+y_{17} \mathbf{a}_{2}+z_{17} \mathbf{a}_{3}$ & $=$ & $x_{17} a \hat{\mathbf{x}}+y_{17} a \hat{\mathbf{y}}+z_{17} c \hat{\mathbf{z}}$ & $(8 p)$ & $\mathrm{CXV}$ \\
\hline $\mathbf{B}_{122}$ & $=$ & $-x_{17} \mathbf{a}_{1}-y_{17} \mathbf{a}_{2}+z_{17} \mathbf{a}_{3}$ & $=$ & $-x_{17} a \hat{\mathbf{x}}-y_{17} a \hat{\mathbf{y}}+z_{17} c \hat{\mathbf{z}}$ & $(8 p)$ & $\mathrm{CXV}$ \\
\hline $\mathbf{B}_{123}$ & $=$ & $-y_{17} \mathbf{a}_{1}+x_{17} \mathbf{a}_{2}+z_{17} \mathbf{a}_{3}$ & $=$ & $-y_{17} a \hat{\mathbf{x}}+x_{17} a \hat{\mathbf{y}}+z_{17} c \hat{\mathbf{z}}$ & $(8 p)$ & $\mathrm{CXV}$ \\
\hline $\mathbf{B}_{124}$ & $=$ & $y_{17} \mathbf{a}_{1}-x_{17} \mathbf{a}_{2}+z_{17} \mathbf{a}_{3}$ & $=$ & $y_{17} a \hat{\mathbf{x}}-x_{17} a \hat{\mathbf{y}}+z_{17} c \hat{\mathbf{z}}$ & $(8 p)$ & $\mathrm{CXV}$ \\
\hline $\mathbf{B}_{125}$ & $=$ & $-x_{17} \mathbf{a}_{1}+y_{17} \mathbf{a}_{2}-z_{17} \mathbf{a}_{3}$ & $=$ & $-x_{17} a \hat{\mathbf{x}}+y_{17} a \hat{\mathbf{y}}-z_{17} c \hat{\mathbf{z}}$ & $(8 p)$ & $\mathrm{CXV}$ \\
\hline $\mathbf{B}_{126}$ & $=$ & $x_{17} \mathbf{a}_{1}-y_{17} \mathbf{a}_{2}-z_{17} \mathbf{a}_{3}$ & $=$ & $x_{17} a \hat{\mathbf{x}}-y_{17} a \hat{\mathbf{y}}-z_{17} c \hat{\mathbf{z}}$ & $(8 p)$ & $\mathrm{CXV}$ \\
\hline $\mathbf{B}_{127}$ & $=$ & $y_{17} \mathbf{a}_{1}+x_{17} \mathbf{a}_{2}-z_{17} \mathbf{a}_{3}$ & $=$ & $y_{17} a \hat{\mathbf{x}}+x_{17} a \hat{\mathbf{y}}-z_{17} c \hat{\mathbf{z}}$ & $(8 p)$ & $\mathrm{CXV}$ \\
\hline $\mathbf{B}_{128}$ & $=$ & $-y_{17} \mathbf{a}_{1}-x_{17} \mathbf{a}_{2}-z_{17} \mathbf{a}_{3}$ & $=$ & $-y_{17} a \hat{\mathbf{x}}-x_{17} a \hat{\mathbf{y}}-z_{17} c \hat{\mathbf{z}}$ & $(8 p)$ & $\mathrm{CXV}$ \\
\hline $\mathbf{B}_{129}$ & $=$ & $x_{18} \mathbf{a}_{1}+y_{18} \mathbf{a}_{2}+z_{18} \mathbf{a}_{3}$ & $=$ & $x_{18} a \hat{\mathbf{x}}+y_{18} a \hat{\mathbf{y}}+z_{18} c \hat{\mathbf{z}}$ & $(8 p)$ & C XVI \\
\hline $\mathbf{B}_{130}$ & $=$ & $-x_{18} \mathbf{a}_{1}-y_{18} \mathbf{a}_{2}+z_{18} \mathbf{a}_{3}$ & $=$ & $-x_{18} a \hat{\mathbf{x}}-y_{18} a \hat{\mathbf{y}}+z_{18} c \hat{\mathbf{z}}$ & $(8 p)$ & C XVI \\
\hline $\mathbf{B}_{131}$ & $=$ & $-y_{18} \mathbf{a}_{1}+x_{18} \mathbf{a}_{2}+z_{18} \mathbf{a}_{3}$ & $=$ & $-y_{18} a \hat{\mathbf{x}}+x_{18} a \hat{\mathbf{y}}+z_{18} c \hat{\mathbf{z}}$ & $(8 p)$ & C XVI \\
\hline $\mathbf{B}_{132}$ & $=$ & $y_{18} \mathbf{a}_{1}-x_{18} \mathbf{a}_{2}+z_{18} \mathbf{a}_{3}$ & $=$ & $y_{18} a \hat{\mathbf{x}}-x_{18} a \hat{\mathbf{y}}+z_{18} c \hat{\mathbf{z}}$ & $(8 p)$ & C XVI \\
\hline $\mathbf{B}_{133}$ & $=$ & $-x_{18} \mathbf{a}_{1}+y_{18} \mathbf{a}_{2}-z_{18} \mathbf{a}_{3}$ & $=$ & $-x_{18} a \hat{\mathbf{x}}+y_{18} a \hat{\mathbf{y}}-z_{18} c \hat{\mathbf{z}}$ & $(8 p)$ & C XVI \\
\hline $\mathbf{B}_{134}$ & $=$ & $x_{18} \mathbf{a}_{1}-y_{18} \mathbf{a}_{2}-z_{18} \mathbf{a}_{3}$ & $=$ & $x_{18} a \hat{\mathbf{x}}-y_{18} a \hat{\mathbf{y}}-z_{18} c \hat{\mathbf{z}}$ & $(8 p)$ & \\
\hline $\mathbf{B}_{135}$ & $=$ & $y_{18} \mathbf{a}_{1}+x_{18} \mathbf{a}_{2}-z_{18} \mathbf{a}_{3}$ & $=$ & $y_{18} a \hat{\mathbf{x}}+x_{18} a \hat{\mathbf{y}}-z_{18} c \hat{\mathbf{z}}$ & $(8 p)$ & \\
\hline
\end{tabular}




\begin{tabular}{|c|c|c|c|c|c|c|}
\hline $\mathbf{B}_{136}$ & $=$ & $-y_{18} \mathbf{a}_{1}-x_{18} \mathbf{a}_{2}-z_{18} \mathbf{a}_{3}$ & $=$ & $-y_{18} a \hat{\mathbf{x}}-x_{18} a \hat{\mathbf{y}}-z_{18} c \hat{\mathbf{z}}$ & $(8 p)$ & C XVI \\
\hline $\mathbf{B}_{137}$ & $=$ & $x_{19} \mathbf{a}_{1}+y_{19} \mathbf{a}_{2}+z_{19} \mathbf{a}_{3}$ & $=$ & $x_{19} a \hat{\mathbf{x}}+y_{19} a \hat{\mathbf{y}}+z_{19} c \hat{\mathbf{z}}$ & $(8 p)$ & C XVII \\
\hline $\mathbf{B}_{138}$ & $=$ & $-x_{19} \mathbf{a}_{1}-y_{19} \mathbf{a}_{2}+z_{19} \mathbf{a}_{3}$ & $=$ & $-x_{19} a \hat{\mathbf{x}}-y_{19} a \hat{\mathbf{y}}+z_{19} c \hat{\mathbf{z}}$ & $(8 p)$ & C XVII \\
\hline $\mathbf{B}_{139}$ & $=$ & $-y_{19} \mathbf{a}_{1}+x_{19} \mathbf{a}_{2}+z_{19} \mathbf{a}_{3}$ & $=$ & $-y_{19} a \hat{\mathbf{x}}+x_{19} a \hat{\mathbf{y}}+z_{19} c \hat{\mathbf{z}}$ & $(8 p)$ & C XVII \\
\hline $\mathbf{B}_{140}$ & $=$ & $y_{19} \mathbf{a}_{1}-x_{19} \mathbf{a}_{2}+z_{19} \mathbf{a}_{3}$ & $=$ & $y_{19} a \hat{\mathbf{x}}-x_{19} a \hat{\mathbf{y}}+z_{19} c \hat{\mathbf{z}}$ & $(8 p)$ & C XVII \\
\hline $\mathbf{B}_{141}$ & $=$ & $-x_{19} \mathbf{a}_{1}+y_{19} \mathbf{a}_{2}-z_{19} \mathbf{a}_{3}$ & $=$ & $-x_{19} a \hat{\mathbf{x}}+y_{19} a \hat{\mathbf{y}}-z_{19} c \hat{\mathbf{z}}$ & $(8 p)$ & C XVII \\
\hline $\mathbf{B}_{142}$ & $=$ & $x_{19} \mathbf{a}_{1}-y_{19} \mathbf{a}_{2}-z_{19} \mathbf{a}_{3}$ & $=$ & $x_{19} a \hat{\mathbf{x}}-y_{19} a \hat{\mathbf{y}}-z_{19} c \hat{\mathbf{z}}$ & $(8 p)$ & C XVII \\
\hline $\mathbf{B}_{143}$ & $=$ & $y_{19} \mathbf{a}_{1}+x_{19} \mathbf{a}_{2}-z_{19} \mathbf{a}_{3}$ & $=$ & $y_{19} a \hat{\mathbf{x}}+x_{19} a \hat{\mathbf{y}}-z_{19} c \hat{\mathbf{z}}$ & $(8 p)$ & C XVII \\
\hline $\mathbf{B}_{144}$ & $=$ & $-y_{19} \mathbf{a}_{1}-x_{19} \mathbf{a}_{2}-z_{19} \mathbf{a}_{3}$ & $=$ & $-y_{19} a \hat{\mathbf{x}}-x_{19} a \hat{\mathbf{y}}-z_{19} c \hat{\mathbf{z}}$ & $(8 p)$ & C XVII \\
\hline $\mathbf{B}_{145}$ & $=$ & $x_{20} \mathbf{a}_{1}+y_{20} \mathbf{a}_{2}+z_{20} \mathbf{a}_{3}$ & $=$ & $x_{20} a \hat{\mathbf{x}}+y_{20} a \hat{\mathbf{y}}+z_{20} c \hat{\mathbf{z}}$ & $(8 p)$ & $\mathrm{Fe}$ \\
\hline $\mathbf{B}_{146}$ & $=$ & $-x_{20} \mathbf{a}_{1}-y_{20} \mathbf{a}_{2}+z_{20} \mathbf{a}_{3}$ & $=$ & $-x_{20} a \hat{\mathbf{x}}-y_{20} a \hat{\mathbf{y}}+z_{20} c \hat{\mathbf{z}}$ & $(8 p)$ & $\mathrm{Fe}$ \\
\hline $\mathbf{B}_{147}$ & $=$ & $-y_{20} \mathbf{a}_{1}+x_{20} \mathbf{a}_{2}+z_{20} \mathbf{a}_{3}$ & $=$ & $-y_{20} a \hat{\mathbf{x}}+x_{20} a \hat{\mathbf{y}}+z_{20} c \hat{\mathbf{z}}$ & $(8 p)$ & $\mathrm{Fe}$ \\
\hline $\mathbf{B}_{148}$ & $=$ & $y_{20} \mathbf{a}_{1}-x_{20} \mathbf{a}_{2}+z_{20} \mathbf{a}_{3}$ & $=$ & $y_{20} a \hat{\mathbf{x}}-x_{20} a \hat{\mathbf{y}}+z_{20} c \hat{\mathbf{z}}$ & $(8 p)$ & $\mathrm{Fe}$ \\
\hline $\mathbf{B}_{149}$ & $=$ & $-x_{20} \mathbf{a}_{1}+y_{20} \mathbf{a}_{2}-z_{20} \mathbf{a}_{3}$ & $=$ & $-x_{20} a \hat{\mathbf{x}}+y_{20} a \hat{\mathbf{y}}-z_{20} c \hat{\mathbf{z}}$ & $(8 p)$ & $\mathrm{Fe}$ \\
\hline $\mathbf{B}_{150}$ & $=$ & $x_{20} \mathbf{a}_{1}-y_{20} \mathbf{a}_{2}-z_{20} \mathbf{a}_{3}$ & $=$ & $x_{20} a \hat{\mathbf{x}}-y_{20} a \hat{\mathbf{y}}-z_{20} c \hat{\mathbf{z}}$ & $(8 p)$ & $\mathrm{Fe}$ \\
\hline $\mathbf{B}_{151}$ & $=$ & $y_{20} \mathbf{a}_{1}+x_{20} \mathbf{a}_{2}-z_{20} \mathbf{a}_{3}$ & $=$ & $y_{20} a \hat{\mathbf{x}}+x_{20} a \hat{\mathbf{y}}-z_{20} c \hat{\mathbf{z}}$ & $(8 p)$ & $\mathrm{Fe}$ \\
\hline $\mathbf{B}_{152}$ & $=$ & $-y_{20} \mathbf{a}_{1}-x_{20} \mathbf{a}_{2}-z_{20} \mathbf{a}_{3}$ & $=$ & $-y_{20} a \hat{\mathbf{x}}-x_{20} a \hat{\mathbf{y}}-z_{20} c \hat{\mathbf{z}}$ & $(8 p)$ & $\mathrm{Fe}$ \\
\hline $\mathbf{B}_{153}$ & $=$ & $x_{21} \mathbf{a}_{1}+y_{21} \mathbf{a}_{2}+z_{21} \mathbf{a}_{3}$ & $=$ & $x_{21} a \hat{\mathbf{x}}+y_{21} a \hat{\mathbf{y}}+z_{21} c \hat{\mathbf{z}}$ & $(8 p)$ & O I \\
\hline $\mathbf{B}_{154}$ & $=$ & $-x_{21} \mathbf{a}_{1}-y_{21} \mathbf{a}_{2}+z_{21} \mathbf{a}_{3}$ & $=$ & $-x_{21} a \hat{\mathbf{x}}-y_{21} a \hat{\mathbf{y}}+z_{21} c \hat{\mathbf{z}}$ & $(8 p)$ & O I \\
\hline $\mathbf{B}_{155}$ & $=$ & $-y_{21} \mathbf{a}_{1}+x_{21} \mathbf{a}_{2}+z_{21} \mathbf{a}_{3}$ & $=$ & $-y_{21} a \hat{\mathbf{x}}+x_{21} a \hat{\mathbf{y}}+z_{21} c \hat{\mathbf{z}}$ & $(8 p)$ & O I \\
\hline $\mathbf{B}_{156}$ & $=$ & $y_{21} \mathbf{a}_{1}-x_{21} \mathbf{a}_{2}+z_{21} \mathbf{a}_{3}$ & $=$ & $y_{21} a \hat{\mathbf{x}}-x_{21} a \hat{\mathbf{y}}+z_{21} c \hat{\mathbf{z}}$ & $(8 p)$ & O I \\
\hline $\mathbf{B}_{157}$ & $=$ & $-x_{21} \mathbf{a}_{1}+y_{21} \mathbf{a}_{2}-z_{21} \mathbf{a}_{3}$ & $=$ & $-x_{21} a \hat{\mathbf{x}}+y_{21} a \hat{\mathbf{y}}-z_{21} c \hat{\mathbf{z}}$ & $(8 p)$ & O I \\
\hline $\mathbf{B}_{158}$ & $=$ & $x_{21} \mathbf{a}_{1}-y_{21} \mathbf{a}_{2}-z_{21} \mathbf{a}_{3}$ & $=$ & $x_{21} a \hat{\mathbf{x}}-y_{21} a \hat{\mathbf{y}}-z_{21} c \hat{\mathbf{z}}$ & $(8 p)$ & O I \\
\hline $\mathbf{B}_{159}$ & $=$ & $y_{21} \mathbf{a}_{1}+x_{21} \mathbf{a}_{2}-z_{21} \mathbf{a}_{3}$ & $=$ & $y_{21} a \hat{\mathbf{x}}+x_{21} a \hat{\mathbf{y}}-z_{21} c \hat{\mathbf{z}}$ & $(8 p)$ & O I \\
\hline $\mathbf{B}_{160}$ & $=$ & $-y_{21} \mathbf{a}_{1}-x_{21} \mathbf{a}_{2}-z_{21} \mathbf{a}_{3}$ & $=$ & $-y_{21} a \hat{\mathbf{x}}-x_{21} a \hat{\mathbf{y}}-z_{21} c \hat{\mathbf{z}}$ & $(8 p)$ & O I \\
\hline $\mathbf{B}_{161}$ & $=$ & $x_{22} \mathbf{a}_{1}+y_{22} \mathbf{a}_{2}+z_{22} \mathbf{a}_{3}$ & $=$ & $x_{22} a \hat{\mathbf{x}}+y_{22} a \hat{\mathbf{y}}+z_{22} c \hat{\mathbf{z}}$ & $(8 p)$ & O II \\
\hline $\mathbf{B}_{162}$ & $=$ & $-x_{22} \mathbf{a}_{1}-y_{22} \mathbf{a}_{2}+z_{22} \mathbf{a}_{3}$ & $=$ & $-x_{22} a \hat{\mathbf{x}}-y_{22} a \hat{\mathbf{y}}+z_{22} c \hat{\mathbf{z}}$ & $(8 p)$ & O II \\
\hline $\mathbf{B}_{163}$ & $=$ & $-y_{22} \mathbf{a}_{1}+x_{22} \mathbf{a}_{2}+z_{22} \mathbf{a}_{3}$ & $=$ & $-y_{22} a \hat{\mathbf{x}}+x_{22} a \hat{\mathbf{y}}+z_{22} c \hat{\mathbf{z}}$ & $(8 p)$ & O II \\
\hline $\mathbf{B}_{164}$ & $=$ & $y_{22} \mathbf{a}_{1}-x_{22} \mathbf{a}_{2}+z_{22} \mathbf{a}_{3}$ & $=$ & $y_{22} a \hat{\mathbf{x}}-x_{22} a \hat{\mathbf{y}}+z_{22} c \hat{\mathbf{z}}$ & $(8 p)$ & O II \\
\hline $\mathbf{B}_{165}$ & $=$ & $-x_{22} \mathbf{a}_{1}+y_{22} \mathbf{a}_{2}-z_{22} \mathbf{a}_{3}$ & $=$ & $-x_{22} a \hat{\mathbf{x}}+y_{22} a \hat{\mathbf{y}}-z_{22} c \hat{\mathbf{z}}$ & $(8 p)$ & O II \\
\hline $\mathbf{B}_{166}$ & $=$ & $x_{22} \mathbf{a}_{1}-y_{22} \mathbf{a}_{2}-z_{22} \mathbf{a}_{3}$ & $=$ & $x_{22} a \hat{\mathbf{x}}-y_{22} a \hat{\mathbf{y}}-z_{22} c \hat{\mathbf{z}}$ & $(8 p)$ & O II \\
\hline $\mathbf{B}_{167}$ & $=$ & $y_{22} \mathbf{a}_{1}+x_{22} \mathbf{a}_{2}-z_{22} \mathbf{a}_{3}$ & $=$ & $y_{22} a \hat{\mathbf{x}}+x_{22} a \hat{\mathbf{y}}-z_{22} c \hat{\mathbf{z}}$ & $(8 p)$ & O II \\
\hline $\mathbf{B}_{168}$ & $=$ & $-y_{22} \mathbf{a}_{1}-x_{22} \mathbf{a}_{2}-z_{22} \mathbf{a}_{3}$ & $=$ & $-y_{22} a \hat{\mathbf{x}}-x_{22} a \hat{\mathbf{y}}-z_{22} c \hat{\mathbf{z}}$ & $(8 p)$ & O II \\
\hline $\mathbf{B}_{169}$ & $=$ & $x_{23} \mathbf{a}_{1}+y_{23} \mathbf{a}_{2}+z_{23} \mathbf{a}_{3}$ & $=$ & $x_{23} a \hat{\mathbf{x}}+y_{23} a \hat{\mathbf{y}}+z_{23} c \hat{\mathbf{z}}$ & $(8 p)$ & O III \\
\hline $\mathbf{B}_{170}$ & $=$ & $-x_{23} \mathbf{a}_{1}-y_{23} \mathbf{a}_{2}+z_{23} \mathbf{a}_{3}$ & $=$ & $-x_{23} a \hat{\mathbf{x}}-y_{23} a \hat{\mathbf{y}}+z_{23} c \hat{\mathbf{z}}$ & $(8 p)$ & O III \\
\hline $\mathbf{B}_{171}$ & $=$ & $-y_{23} \mathbf{a}_{1}+x_{23} \mathbf{a}_{2}+z_{23} \mathbf{a}_{3}$ & $=$ & $-y_{23} a \hat{\mathbf{x}}+x_{23} a \hat{\mathbf{y}}+z_{23} c \hat{\mathbf{z}}$ & $(8 p)$ & O III \\
\hline
\end{tabular}




\begin{tabular}{|c|c|c|c|c|c|c|}
\hline $\mathbf{B}_{172}$ & $=$ & $y_{23} \mathbf{a}_{1}-x_{23} \mathbf{a}_{2}+z_{23} \mathbf{a}_{3}$ & $=$ & $y_{23} a \hat{\mathbf{x}}-x_{23} a \hat{\mathbf{y}}+z_{23} c \hat{\mathbf{z}}$ & $(8 p)$ & O III \\
\hline $\mathbf{B}_{173}$ & $=$ & $-x_{23} \mathbf{a}_{1}+y_{23} \mathbf{a}_{2}-z_{23} \mathbf{a}_{3}$ & $=$ & $-x_{23} a \hat{\mathbf{x}}+y_{23} a \hat{\mathbf{y}}-z_{23} c \hat{\mathbf{z}}$ & $(8 p)$ & O III \\
\hline $\mathbf{B}_{174}$ & $=$ & $x_{23} \mathbf{a}_{1}-y_{23} \mathbf{a}_{2}-z_{23} \mathbf{a}_{3}$ & $=$ & $x_{23} a \hat{\mathbf{x}}-y_{23} a \hat{\mathbf{y}}-z_{23} c \hat{\mathbf{z}}$ & $(8 p)$ & O III \\
\hline $\mathbf{B}_{175}$ & $=$ & $y_{23} \mathbf{a}_{1}+x_{23} \mathbf{a}_{2}-z_{23} \mathbf{a}_{3}$ & $=$ & $y_{23} a \hat{\mathbf{x}}+x_{23} a \hat{\mathbf{y}}-z_{23} c \hat{\mathbf{z}}$ & $(8 p)$ & O III \\
\hline $\mathbf{B}_{176}$ & $=$ & $-y_{23} \mathbf{a}_{1}-x_{23} \mathbf{a}_{2}-z_{23} \mathbf{a}_{3}$ & $=$ & $-y_{23} a \hat{\mathbf{x}}-x_{23} a \hat{\mathbf{y}}-z_{23} c \hat{\mathbf{z}}$ & $(8 p)$ & O III \\
\hline $\mathbf{B}_{177}$ & $=$ & $x_{24} \mathbf{a}_{1}+y_{24} \mathbf{a}_{2}+z_{24} \mathbf{a}_{3}$ & $=$ & $x_{24} a \hat{\mathbf{x}}+y_{24} a \hat{\mathbf{y}}+z_{24} c \hat{\mathbf{z}}$ & $(8 p)$ & O IV \\
\hline $\mathbf{B}_{178}$ & $=$ & $-x_{24} \mathbf{a}_{1}-y_{24} \mathbf{a}_{2}+z_{24} \mathbf{a}_{3}$ & $=$ & $-x_{24} a \hat{\mathbf{x}}-y_{24} a \hat{\mathbf{y}}+z_{24} c \hat{\mathbf{z}}$ & $(8 p)$ & O IV \\
\hline $\mathbf{B}_{179}$ & $=$ & $-y_{24} \mathbf{a}_{1}+x_{24} \mathbf{a}_{2}+z_{24} \mathbf{a}_{3}$ & $=$ & $-y_{24} a \hat{\mathbf{x}}+x_{24} a \hat{\mathbf{y}}+z_{24} c \hat{\mathbf{z}}$ & $(8 p)$ & O IV \\
\hline $\mathbf{B}_{180}$ & $=$ & $y_{24} \mathbf{a}_{1}-x_{24} \mathbf{a}_{2}+z_{24} \mathbf{a}_{3}$ & $=$ & $y_{24} a \hat{\mathbf{x}}-x_{24} a \hat{\mathbf{y}}+z_{24} c \hat{\mathbf{z}}$ & $(8 p)$ & O IV \\
\hline $\mathbf{B}_{181}$ & $=$ & $-x_{24} \mathbf{a}_{1}+y_{24} \mathbf{a}_{2}-z_{24} \mathbf{a}_{3}$ & $=$ & $-x_{24} a \hat{\mathbf{x}}+y_{24} a \hat{\mathbf{y}}-z_{24} c \hat{\mathbf{z}}$ & $(8 p)$ & O IV \\
\hline $\mathbf{B}_{182}$ & $=$ & $x_{24} \mathbf{a}_{1}-y_{24} \mathbf{a}_{2}-z_{24} \mathbf{a}_{3}$ & $=$ & $x_{24} a \hat{\mathbf{x}}-y_{24} a \hat{\mathbf{y}}-z_{24} c \hat{\mathbf{z}}$ & $(8 p)$ & O IV \\
\hline $\mathbf{B}_{183}$ & $=$ & $y_{24} \mathbf{a}_{1}+x_{24} \mathbf{a}_{2}-z_{24} \mathbf{a}_{3}$ & $=$ & $y_{24} a \hat{\mathbf{x}}+x_{24} a \hat{\mathbf{y}}-z_{24} c \hat{\mathbf{z}}$ & $(8 p)$ & O IV \\
\hline $\mathbf{B}_{184}$ & $=$ & $-y_{24} \mathbf{a}_{1}-x_{24} \mathbf{a}_{2}-z_{24} \mathbf{a}_{3}$ & $=$ & $-y_{24} a \hat{\mathbf{x}}-x_{24} a \hat{\mathbf{y}}-z_{24} c \hat{\mathbf{z}}$ & $(8 p)$ & O IV \\
\hline
\end{tabular}

\section{References:}

- S. Tanaka and K. Mashima, Interaction of Ferrocene Moieties Across a Square Pt $t_{4}$ Unit: Synthesis, Characterization, and Electrochemical Properties of Carboxylate-Bridged Bimetallic $\mathrm{Pt}_{4} \mathrm{Fe}_{n}(n=2,3$, and 4) Complexes, Inorg. Chem. 50, 11384-11393 (2011), doi:10.1021/ic201012m.

\section{Found in:}

- C. R. Groom, I. J. Bruno, M. P. Lightfoot, and S. C. Ward, The Cambridge Structural Database, Acta Crystallogr. Sect. B Struct. Sci. 72, 171-179 (2016), doi:10.1107/S2052520616003954.

- F. Hoffmann, The Fascination of Crystals and Symmetry (2014). 230 - The space group list project.

\section{Geometry files:}

- CIF: pp. 866

- POSCAR: pp. 867 
$\mathrm{Na}_{4} \mathrm{Ti}_{2} \mathrm{Si}_{8} \mathrm{O}_{22}\left[\mathrm{H}_{2} \mathrm{O}\right]_{4}$ Structure:

A4B2C13D_tP40_90_g_d_cef2g_c
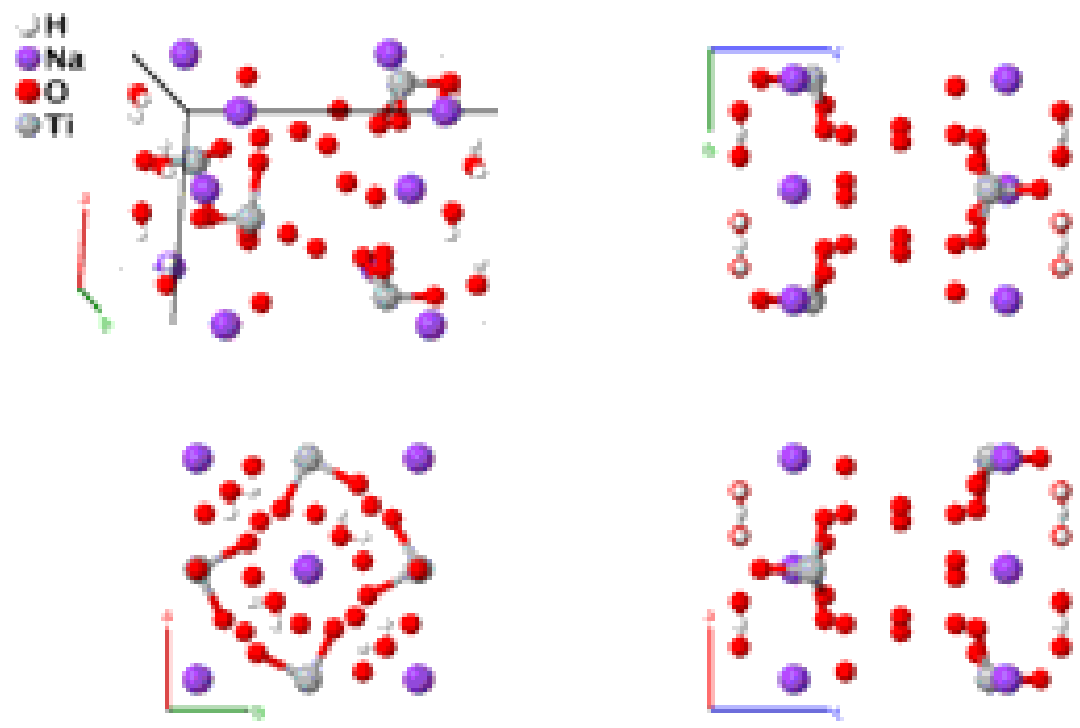

Prototype

AFLOW prototype label

Strukturbericht designation

Pearson symbol

Space group number

Space group symbol

AFLOW prototype command
$: \quad \mathrm{Na}_{4} \mathrm{Ti}_{2} \mathrm{Si}_{8} \mathrm{O}_{22}\left[\mathrm{H}_{2} \mathrm{O}\right]_{4}$

: A4B2C13D_tP40_90_g_d_cef2g_c

: None

: $\quad$ PP40

: $\quad 90$

: $\quad P 42_{1} 2$

Simple Tetragonal primitive vectors:

$$
\begin{aligned}
& \mathbf{a}_{1}=a \hat{\mathbf{x}} \\
& \mathbf{a}_{2}=a \hat{\mathbf{y}} \\
& \mathbf{a}_{3}=c \hat{\mathbf{z}}
\end{aligned}
$$

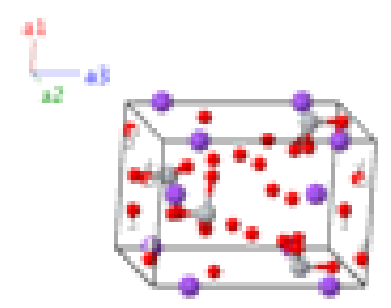

Basis vectors:

Lattice Coordinates

$\begin{array}{llc}\mathbf{B}_{1}= & \frac{1}{2} \mathbf{a}_{2}+z_{1} \mathbf{a}_{3} \\ \mathbf{B}_{2}= & \frac{1}{2} \mathbf{a}_{1}+-z_{1} \mathbf{a}_{3} \\ \mathbf{B}_{3}= & \frac{1}{2} \mathbf{a}_{2}+z_{2} \mathbf{a}_{3} \\ \mathbf{B}_{4}= & \frac{1}{2} \mathbf{a}_{1}+-z_{2} \mathbf{a}_{3} \\ \mathbf{B}_{5}= & z_{3} \mathbf{a}_{3}\end{array}$

Cartesian Coordinates

$=$

$=\quad \frac{1}{2} a \hat{\mathbf{x}}+-z 1 c \hat{\mathbf{z}}$

$=\quad \frac{1}{2} a \hat{\mathbf{y}}+z_{2} c \hat{\mathbf{z}}$

$=\quad \frac{1}{2} a \hat{\mathbf{x}}+-z_{2} c \hat{\mathbf{z}}$

$=\quad z_{3} c \hat{\mathbf{z}}$
Wyckoff Position

Atom Type

(2c)

(4d)
O I

O I

$\mathrm{Ti}$

$\mathrm{Ti}$

$\mathrm{Na}$ 


\begin{tabular}{|c|c|c|c|c|c|c|}
\hline $\mathbf{B}_{6}$ & $=$ & $\frac{1}{2} \mathbf{a}_{1}+\frac{1}{2} \mathbf{a}_{2}+z_{3} \mathbf{a}_{3}$ & $=$ & $\frac{1}{2} a \hat{\mathbf{x}}+\frac{1}{2} a \hat{\mathbf{y}}+z_{3} c \hat{\mathbf{z}}$ & $(4 d)$ & $\mathrm{Na}$ \\
\hline $\mathbf{B}_{7}$ & $=$ & $\frac{1}{2} \mathbf{a}_{1}+\frac{1}{2} \mathbf{a}_{2}-z_{3} \mathbf{a}_{3}$ & $=$ & $\frac{1}{2} a \hat{\mathbf{x}}+\frac{1}{2} a \hat{\mathbf{y}}-z_{3} c \hat{\mathbf{z}}$ & $(4 d)$ & $\mathrm{Na}$ \\
\hline $\mathbf{B}_{8}$ & $=$ & $-z_{3} \mathbf{a}_{3}$ & $=$ & $-z_{3} c \hat{\mathbf{z}}$ & $(4 d)$ & $\mathrm{Na}$ \\
\hline $\mathbf{B}_{9}$ & $=$ & $x_{4} \mathbf{a}_{1}+x_{4} \mathbf{a}_{2}$ & $=$ & $x_{4} a \hat{\mathbf{x}}+x_{4} a \hat{\mathbf{y}}$ & $(4 e)$ & O II \\
\hline $\mathbf{B}_{10}$ & $=$ & $-x_{4} \mathbf{a}_{1}-x_{4} \mathbf{a}_{2}$ & $=$ & $-x_{4} a \hat{\mathbf{x}}-x_{4} a \hat{\mathbf{y}}$ & $(4 e)$ & O II \\
\hline $\mathbf{B}_{11}$ & $=$ & $\left(\frac{1}{2}-x_{4}\right) \mathbf{a}_{1}+\left(\frac{1}{2}+x_{4}\right) \mathbf{a}_{2}$ & $=$ & $\left(\frac{1}{2}-x_{4}\right) a \hat{\mathbf{x}}+\left(\frac{1}{2}+x_{4}\right) a \hat{\mathbf{y}}$ & $(4 e)$ & O II \\
\hline $\mathbf{B}_{12}$ & $=$ & $\left(\frac{1}{2}+x_{4}\right) \mathbf{a}_{1}+\left(\frac{1}{2}-x_{4}\right) \mathbf{a}_{2}$ & $=$ & $\left(\frac{1}{2}+x_{4}\right) a \hat{\mathbf{x}}+\left(\frac{1}{2}-x_{4}\right) a \hat{\mathbf{y}}$ & $(4 e)$ & O II \\
\hline $\mathbf{B}_{13}$ & $=$ & $x_{5} \mathbf{a}_{1}+x_{5} \mathbf{a}_{2}+\frac{1}{2} \mathbf{a}_{3}$ & $=$ & $x_{5} a \hat{\mathbf{x}}+x_{5} a \hat{\mathbf{y}}+\frac{1}{2} c \hat{\mathbf{z}}$ & $(4 f)$ & O III \\
\hline $\mathbf{B}_{14}$ & $=$ & $-x_{5} \mathbf{a}_{1}-x_{5} \mathbf{a}_{2}+\frac{1}{2} \mathbf{a}_{3}$ & $=$ & $-x_{5} a \hat{\mathbf{x}}-x_{5} a \hat{\mathbf{y}}+\frac{1}{2} c \hat{\mathbf{z}}$ & $(4 f)$ & O III \\
\hline $\mathbf{B}_{15}$ & $=$ & $\left(\frac{1}{2}-x_{5}\right) \mathbf{a}_{1}+\left(\frac{1}{2}+x_{5}\right) \mathbf{a}_{2}+\frac{1}{2} \mathbf{a}_{3}$ & $=$ & $\left(\frac{1}{2}-x_{5}\right) a \hat{\mathbf{x}}+\left(\frac{1}{2}+x_{5}\right) a \hat{\mathbf{y}}+\frac{1}{2} c \hat{\mathbf{z}}$ & $(4 f)$ & O III \\
\hline $\mathbf{B}_{16}$ & $=$ & $\left(\frac{1}{2}+x_{5}\right) \mathbf{a}_{1}+\left(\frac{1}{2}-x_{5}\right) \mathbf{a}_{2}+\frac{1}{2} \mathbf{a}_{3}$ & $=$ & $\left(\frac{1}{2}+x_{5}\right) a \hat{\mathbf{x}}+\left(\frac{1}{2}-x_{5}\right) a \hat{\mathbf{y}}+\frac{1}{2} c \hat{\mathbf{z}}$ & $(4 f)$ & O III \\
\hline $\mathbf{B}_{17}$ & $=$ & $x_{6} \mathbf{a}_{1}+y_{6} \mathbf{a}_{2}+z_{6} \mathbf{a}_{3}$ & $=$ & $x_{6} a \hat{\mathbf{x}}+y_{6} a \hat{\mathbf{y}}+z_{6} c \hat{\mathbf{z}}$ & $(8 g)$ & $\mathrm{H}$ \\
\hline $\mathbf{B}_{18}$ & $=$ & $-x_{6} \mathbf{a}_{1}-y_{6} \mathbf{a}_{2}+z_{6} \mathbf{a}_{3}$ & $=$ & $-x_{6} a \hat{\mathbf{x}}-y_{6} a \hat{\mathbf{y}}+z_{6} c \hat{\mathbf{z}}$ & $(8 g)$ & $\mathrm{H}$ \\
\hline $\mathbf{B}_{19}$ & $=$ & $\left(\frac{1}{2}-y_{6}\right) \mathbf{a}_{1}+\left(\frac{1}{2}+x_{6}\right) \mathbf{a}_{2}+z_{6} \mathbf{a}_{3}$ & $=$ & $\left(\frac{1}{2}-y_{6}\right) a \hat{\mathbf{x}}+\left(\frac{1}{2}+x_{6}\right) a \hat{\mathbf{y}}+z_{6} c \hat{\mathbf{z}}$ & $(8 g)$ & $\mathrm{H}$ \\
\hline $\mathbf{B}_{20}$ & $=$ & $\left(\frac{1}{2}+y_{6}\right) \mathbf{a}_{1}+\left(\frac{1}{2}-x_{6}\right) \mathbf{a}_{2}+z_{6} \mathbf{a}_{3}$ & $=$ & $\left(\frac{1}{2}+y_{6}\right) a \hat{\mathbf{x}}+\left(\frac{1}{2}-x_{6}\right) a \hat{\mathbf{y}}+z_{6} c \hat{\mathbf{z}}$ & $(8 g)$ & $\mathrm{H}$ \\
\hline $\mathbf{B}_{21}$ & $=$ & $\left(\frac{1}{2}-x_{6}\right) \mathbf{a}_{1}+\left(\frac{1}{2}+y_{6}\right) \mathbf{a}_{2}-z_{6} \mathbf{a}_{3}$ & $=$ & $\left(\frac{1}{2}-x_{6}\right) a \hat{\mathbf{x}}+\left(\frac{1}{2}+y_{6}\right) a \hat{\mathbf{y}}-z_{6} c \hat{\mathbf{z}}$ & $(8 g)$ & $\mathrm{H}$ \\
\hline $\mathbf{B}_{22}$ & $=$ & $\left(\frac{1}{2}+x_{6}\right) \mathbf{a}_{1}+\left(\frac{1}{2}-y_{6}\right) \mathbf{a}_{2}-z_{6} \mathbf{a}_{3}$ & $=$ & $\left(\frac{1}{2}+x_{6}\right) a \hat{\mathbf{x}}+\left(\frac{1}{2}-y_{6}\right) a \hat{\mathbf{y}}-z_{6} c \hat{\mathbf{z}}$ & $(8 g)$ & $\mathrm{H}$ \\
\hline $\mathbf{B}_{23}$ & $=$ & $y_{6} \mathbf{a}_{1}+x_{6} \mathbf{a}_{2}-z_{6} \mathbf{a}_{3}$ & $=$ & $y_{6} a \hat{\mathbf{x}}+x_{6} a \hat{\mathbf{y}}-z_{6} c \hat{\mathbf{z}}$ & $(8 g)$ & $\mathrm{H}$ \\
\hline $\mathbf{B}_{24}$ & $=$ & $-y_{6} \mathbf{a}_{1}-x_{6} \mathbf{a}_{2}-z_{6} \mathbf{a}_{3}$ & $=$ & $-y_{6} a \hat{\mathbf{x}}-x_{6} a \hat{\mathbf{y}}-z_{6} c \hat{\mathbf{z}}$ & $(8 g)$ & $\mathrm{H}$ \\
\hline $\mathbf{B}_{25}$ & $=$ & $x_{7} \mathbf{a}_{1}+y_{7} \mathbf{a}_{2}+z_{7} \mathbf{a}_{3}$ & $=$ & $x_{7} a \hat{\mathbf{x}}+y_{7} a \hat{\mathbf{y}}+z_{7} c \hat{\mathbf{z}}$ & $(8 g)$ & O IV \\
\hline $\mathbf{B}_{26}$ & $=$ & $-x_{7} \mathbf{a}_{1}-y_{7} \mathbf{a}_{2}+z_{7} \mathbf{a}_{3}$ & $=$ & $-x_{7} a \hat{\mathbf{x}}-y_{7} a \hat{\mathbf{y}}+z_{7} c \hat{\mathbf{z}}$ & $(8 g)$ & O IV \\
\hline $\mathbf{B}_{27}$ & $=$ & $\left(\frac{1}{2}-y_{7}\right) \mathbf{a}_{1}+\left(\frac{1}{2}+x_{7}\right) \mathbf{a}_{2}+z_{7} \mathbf{a}_{3}$ & $=$ & $\left(\frac{1}{2}-y_{7}\right) a \hat{\mathbf{x}}+\left(\frac{1}{2}+x_{7}\right) a \hat{\mathbf{y}}+z_{7} c \hat{\mathbf{z}}$ & $(8 g)$ & O IV \\
\hline $\mathbf{B}_{28}$ & $=$ & $\left(\frac{1}{2}+y_{7}\right) \mathbf{a}_{1}+\left(\frac{1}{2}-x_{7}\right) \mathbf{a}_{2}+z_{7} \mathbf{a}_{3}$ & $=$ & $\left(\frac{1}{2}+y_{7}\right) a \hat{\mathbf{x}}+\left(\frac{1}{2}-x_{7}\right) a \hat{\mathbf{y}}+z_{7} c \hat{\mathbf{z}}$ & $(8 g)$ & O IV \\
\hline $\mathbf{B}_{29}$ & $=$ & $\left(\frac{1}{2}-x_{7}\right) \mathbf{a}_{1}+\left(\frac{1}{2}+y_{7}\right) \mathbf{a}_{2}-z_{7} \mathbf{a}_{3}$ & $=$ & $\left(\frac{1}{2}-x_{7}\right) a \hat{\mathbf{x}}+\left(\frac{1}{2}+y_{7}\right) a \hat{\mathbf{y}}-z_{7} c \hat{\mathbf{z}}$ & $(8 g)$ & O IV \\
\hline $\mathbf{B}_{30}$ & $=$ & $\left(\frac{1}{2}+x_{7}\right) \mathbf{a}_{1}+\left(\frac{1}{2}-y_{7}\right) \mathbf{a}_{2}-z_{7} \mathbf{a}_{3}$ & $=$ & $\left(\frac{1}{2}+x_{7}\right) a \hat{\mathbf{x}}+\left(\frac{1}{2}-y_{7}\right) a \hat{\mathbf{y}}-z_{7} c \hat{\mathbf{z}}$ & $(8 g)$ & O IV \\
\hline $\mathbf{B}_{31}$ & $=$ & $y_{7} \mathbf{a}_{1}+x_{7} \mathbf{a}_{2}-z_{7} \mathbf{a}_{3}$ & $=$ & $y_{7} a \hat{\mathbf{x}}+x_{7} a \hat{\mathbf{y}}-z_{7} c \hat{\mathbf{z}}$ & $(8 g)$ & O IV \\
\hline $\mathbf{B}_{32}$ & $=$ & $-y_{7} \mathbf{a}_{1}-x_{7} \mathbf{a}_{2}-z_{7} \mathbf{a}_{3}$ & $=$ & $-y_{7} a \hat{\mathbf{x}}-x_{7} a \hat{\mathbf{y}}-z_{7} c \hat{\mathbf{z}}$ & $(8 g)$ & O IV \\
\hline $\mathbf{B}_{33}$ & $=$ & $x_{8} \mathbf{a}_{1}+y_{8} \mathbf{a}_{2}+z_{8} \mathbf{a}_{3}$ & $=$ & $x_{8} a \hat{\mathbf{x}}+y_{8} a \hat{\mathbf{y}}+z_{8} c \hat{\mathbf{z}}$ & $(8 g)$ & $\mathrm{OV}$ \\
\hline $\mathbf{B}_{34}$ & $=$ & $-x_{8} \mathbf{a}_{1}-y_{8} \mathbf{a}_{2}+z_{8} \mathbf{a}_{3}$ & $=$ & $-x_{8} a \hat{\mathbf{x}}-y_{8} a \hat{\mathbf{y}}+z_{8} c \hat{\mathbf{z}}$ & $(8 g)$ & $\mathrm{OV}$ \\
\hline $\mathbf{B}_{35}$ & $=$ & $\left(\frac{1}{2}-y_{8}\right) \mathbf{a}_{1}+\left(\frac{1}{2}+x_{8}\right) \mathbf{a}_{2}+z_{8} \mathbf{a}_{3}$ & $=$ & $\left(\frac{1}{2}-y_{8}\right) a \hat{\mathbf{x}}+\left(\frac{1}{2}+x_{8}\right) a \hat{\mathbf{y}}+z_{8} c \hat{\mathbf{z}}$ & $(8 g)$ & $\mathrm{OV}$ \\
\hline $\mathbf{B}_{36}$ & $=$ & $\left(\frac{1}{2}+y_{8}\right) \mathbf{a}_{1}+\left(\frac{1}{2}-x_{8}\right) \mathbf{a}_{2}+z_{8} \mathbf{a}_{3}$ & $=$ & $\left(\frac{1}{2}+y_{8}\right) a \hat{\mathbf{x}}+\left(\frac{1}{2}-x_{8}\right) a \hat{\mathbf{y}}+z_{8} c \hat{\mathbf{z}}$ & $(8 g)$ & $\mathrm{O} \mathrm{V}$ \\
\hline $\mathbf{B}_{37}$ & $=$ & $\left(\frac{1}{2}-x_{8}\right) \mathbf{a}_{1}+\left(\frac{1}{2}+y_{8}\right) \mathbf{a}_{2}-z_{8} \mathbf{a}_{3}$ & $=$ & $\left(\frac{1}{2}-x_{8}\right) a \hat{\mathbf{x}}+\left(\frac{1}{2}+y_{8}\right) a \hat{\mathbf{y}}-z_{8} c \hat{\mathbf{z}}$ & $(8 g)$ & $\mathrm{O} \mathrm{V}$ \\
\hline $\mathbf{B}_{38}$ & $=$ & $\left(\frac{1}{2}+x_{8}\right) \mathbf{a}_{1}+\left(\frac{1}{2}-y_{8}\right) \mathbf{a}_{2}-z_{8} \mathbf{a}_{3}$ & $=$ & $\left(\frac{1}{2}+x_{8}\right) a \hat{\mathbf{x}}+\left(\frac{1}{2}-y_{8}\right) a \hat{\mathbf{y}}-z_{8} c \hat{\mathbf{z}}$ & $(8 g)$ & $\mathrm{OV}$ \\
\hline $\mathbf{B}_{39}$ & $=$ & $y_{8} \mathbf{a}_{1}+x_{8} \mathbf{a}_{2}-z_{8} \mathbf{a}_{3}$ & $=$ & $y_{8} a \hat{\mathbf{x}}+x_{8} a \hat{\mathbf{y}}-z_{8} c \hat{\mathbf{z}}$ & $(8 g)$ & $\mathrm{O} \mathrm{V}$ \\
\hline $\mathbf{B}_{40}$ & $=$ & $-y_{8} \mathbf{a}_{1}-x_{8} \mathbf{a}_{2}-z_{8} \mathbf{a}_{3}$ & $=$ & $-y_{8} a \hat{\mathbf{x}}-x_{8} a \hat{\mathbf{y}}-z_{8} c \hat{\mathbf{z}}$ & $(8 g)$ & $\mathrm{OV}$ \\
\hline
\end{tabular}

\section{References:}


- S. Ferdov, U. Kolitsch, C. Lengauer, E. Tillmanns, Z. Lin, and R. A. Sá Ferreira, Refinement of the layered titanosilicate AM-1 from single-crystal X-ray diffraction data, Acta Crystallogr. E 63, i186-i186 (2007), doi:10.1107/S160053680704812X.

\section{Found in:}

- P. Villars and K. Cenzual, Pearson's Crystal Data - Crystal Structure Database for Inorganic Compounds, ASM International (2013).

\section{Geometry files:}

- CIF: pp. 868

- POSCAR: pp. 868 


\section{$\mathrm{BaCu}_{4}[\mathrm{VO}]\left[\mathrm{PO}_{4}\right]_{4}$ Structure:}

\section{AB4C17D4E_tP54_90_a_g_c4g_g_c}
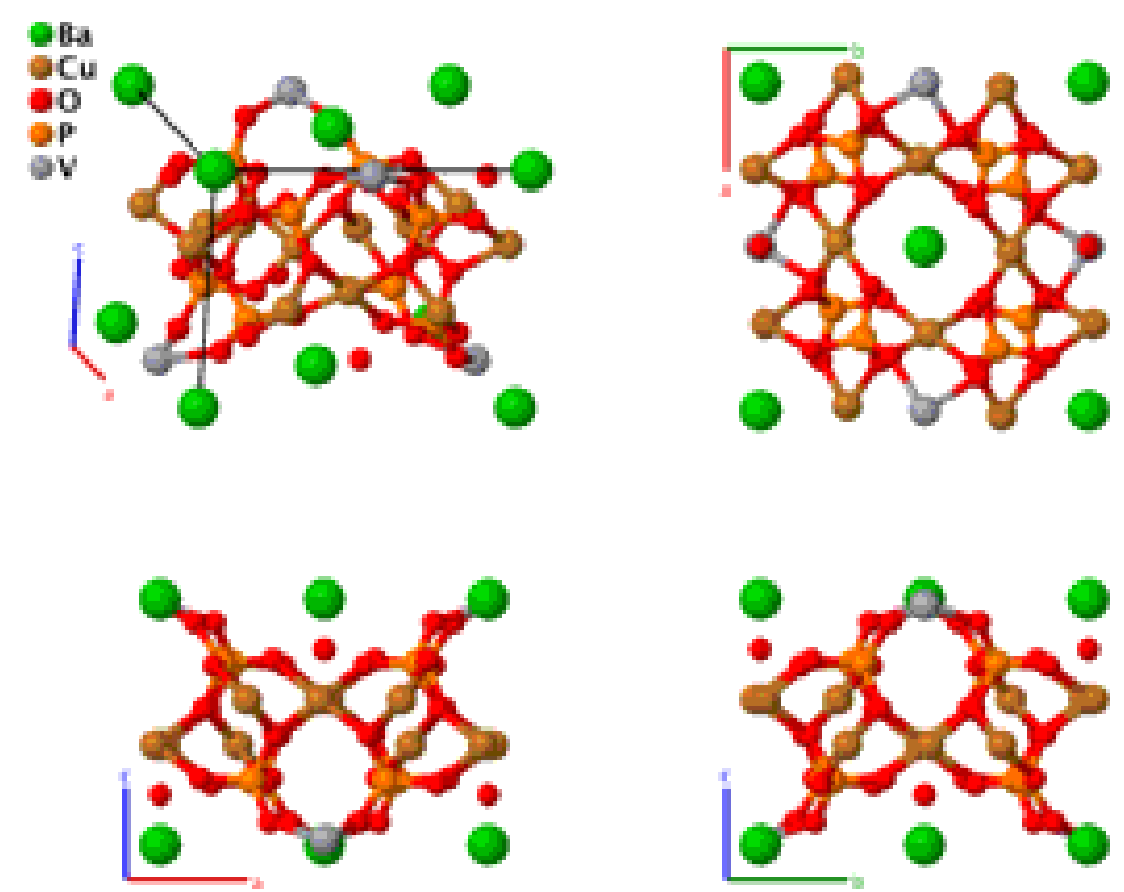

\section{Prototype}

AFLOW prototype label

: $\quad \mathrm{BaCu}_{4}[\mathrm{VO}]\left[\mathrm{PO}_{4}\right]_{4}$

Strukturbericht designation : None

Pearson symbol

: $\quad$ PP54

Space group number

: $\quad 90$

Space group symbol

: $\quad P 42_{12}$

AFLOW prototype command

aflow --proto=AB4C17D4E_tP54_90_a_g_c4g_g_c

- - params $=a, c / a, z_{2}, z_{3}, x_{4}, y_{4}, z_{4}, x_{5}, y_{5}, z_{5}, x_{6}, y_{6}, z_{6}, x_{7}, y_{7}, z_{7}, x_{8}, y_{8}, z_{8}, x_{9}, y_{9}$,

$z_{9}$

Simple Tetragonal primitive vectors:

$$
\begin{aligned}
& \mathbf{a}_{1}=a \hat{\mathbf{x}} \\
& \mathbf{a}_{2}=a \hat{\mathbf{y}} \\
& \mathbf{a}_{3}=c \hat{\mathbf{z}}
\end{aligned}
$$

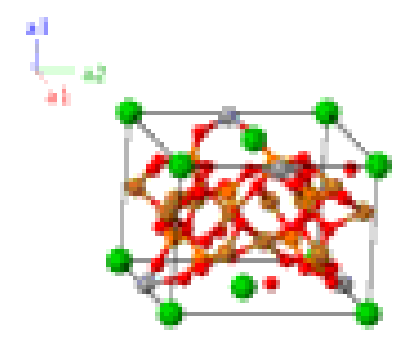

Basis vectors:

Lattice Coordinates

$\mathbf{B}_{1}=0 \mathbf{a}_{1}+0 \mathbf{a}_{2}+0 \mathbf{a}_{3}$
Cartesian Coordinates

$=$

$$
0 \hat{\mathbf{x}}+0 \hat{\mathbf{y}}+0 \hat{\mathbf{z}}
$$

Wyckoff Position

(2a)
Atom Type

$\mathrm{Ba}$ 


\begin{tabular}{|c|c|c|c|c|}
\hline $\mathbf{B}_{2}$ & $\frac{1}{2} \mathbf{a}_{1}+\frac{1}{2} \mathbf{a}_{2}$ & $\frac{1}{2} a \hat{\mathbf{x}}+\frac{1}{2} a \hat{\mathbf{y}}$ & $(2 a)$ & $\mathrm{Ba}$ \\
\hline $\mathbf{B}_{3}$ & $\frac{1}{2} \mathbf{a}_{2}+z_{2} \mathbf{a}_{3}$ & $\frac{1}{2} a \hat{\mathbf{y}}+z_{2} c \hat{\mathbf{z}}$ & $(2 c)$ & O I \\
\hline $\mathbf{B}_{4}$ & $\frac{1}{2} \mathbf{a}_{1}+-z_{2} \mathbf{a}_{3}$ & $\frac{1}{2} a \hat{\mathbf{x}}+-z_{2} c \hat{\mathbf{z}}$ & $(2 c)$ & $\mathrm{O}$ I \\
\hline $\mathbf{B}_{5}$ & $\frac{1}{2} \mathbf{a}_{2}+z_{3} \mathbf{a}_{3}$ & $\frac{1}{2} a \hat{\mathbf{y}}+z_{3} c \hat{\mathbf{z}}$ & $(2 c)$ & $\mathrm{V}$ \\
\hline $\mathbf{B}_{6}$ & $\frac{1}{2} \mathbf{a}_{1}+-z_{3} \mathbf{a}_{3}$ & $\frac{1}{2} a \hat{\mathbf{x}}+-z_{3} c \hat{\mathbf{z}}$ & $(2 c)$ & $\mathrm{V}$ \\
\hline $\mathbf{B}_{7}$ & $x_{4} \mathbf{a}_{1}+y_{4} \mathbf{a}_{2}+z_{4} \mathbf{a}_{3}$ & $x_{4} a \hat{\mathbf{x}}+y_{4} a \hat{\mathbf{y}}+z_{4} c \hat{\mathbf{z}}$ & $(8 g)$ & $\mathrm{Cu}$ \\
\hline $\mathbf{B}_{8}$ & $-x_{4} \mathbf{a}_{1}-y_{4} \mathbf{a}_{2}+z_{4} \mathbf{a}_{3}$ & $-x_{4} a \hat{\mathbf{x}}-y_{4} a \hat{\mathbf{y}}+z_{4} c \hat{\mathbf{z}}$ & $(8 g)$ & $\mathrm{Cu}$ \\
\hline $\mathbf{B}_{9}$ & $=\left(\frac{1}{2}-y_{4}\right) \mathbf{a}_{1}+\left(\frac{1}{2}+x_{4}\right) \mathbf{a}_{2}+z_{4} \mathbf{a}_{3}$ & $=\left(\frac{1}{2}-y_{4}\right) a \hat{\mathbf{x}}+\left(\frac{1}{2}+x_{4}\right) a \hat{\mathbf{y}}+z_{4} c \hat{\mathbf{z}}$ & $(8 g)$ & $\mathrm{Cu}$ \\
\hline $\mathbf{B}_{10}$ & $=\left(\frac{1}{2}+y_{4}\right) \mathbf{a}_{1}+\left(\frac{1}{2}-x_{4}\right) \mathbf{a}_{2}+z_{4} \mathbf{a}_{3}$ & $=\left(\frac{1}{2}+y_{4}\right) a \hat{\mathbf{x}}+\left(\frac{1}{2}-x_{4}\right) a \hat{\mathbf{y}}+z_{4} c \hat{\mathbf{z}}$ & $(8 g)$ & $\mathrm{Cu}$ \\
\hline $\mathbf{B}_{11}$ & $=\left(\frac{1}{2}-x_{4}\right) \mathbf{a}_{1}+\left(\frac{1}{2}+y_{4}\right) \mathbf{a}_{2}-z_{4} \mathbf{a}_{3}$ & $=\left(\frac{1}{2}-x_{4}\right) a \hat{\mathbf{x}}+\left(\frac{1}{2}+y_{4}\right) a \hat{\mathbf{y}}-z_{4} c \hat{\mathbf{z}}$ & $(8 g)$ & $\mathrm{Cu}$ \\
\hline $\mathbf{B}_{12}$ & $=\left(\frac{1}{2}+x_{4}\right) \mathbf{a}_{1}+\left(\frac{1}{2}-y_{4}\right) \mathbf{a}_{2}-z_{4} \mathbf{a}_{3}$ & $=\left(\frac{1}{2}+x_{4}\right) a \hat{\mathbf{x}}+\left(\frac{1}{2}-y_{4}\right) a \hat{\mathbf{y}}-z_{4} c \hat{\mathbf{z}}$ & $(8 g)$ & $\mathrm{Cu}$ \\
\hline $\mathbf{B}_{13}$ & $y_{4} \mathbf{a}_{1}+x_{4} \mathbf{a}_{2}-z_{4} \mathbf{a}_{3}$ & $y_{4} a \hat{\mathbf{x}}+x_{4} a \hat{\mathbf{y}}-z_{4} c \hat{\mathbf{z}}$ & $(8 g)$ & $\mathrm{Cu}$ \\
\hline $\mathbf{B}_{14}$ & $-y_{4} \mathbf{a}_{1}-x_{4} \mathbf{a}_{2}-z_{4} \mathbf{a}_{3}$ & $-y_{4} a \hat{\mathbf{x}}-x_{4} a \hat{\mathbf{y}}-z_{4} c \hat{\mathbf{z}}$ & $(8 g)$ & $\mathrm{Cu}$ \\
\hline $\mathbf{B}_{15}$ & $x_{5} \mathbf{a}_{1}+y_{5} \mathbf{a}_{2}+z_{5} \mathbf{a}_{3}$ & $x_{5} a \hat{\mathbf{x}}+y_{5} a \hat{\mathbf{y}}+z_{5} c \hat{\mathbf{z}}$ & $(8 g)$ & O II \\
\hline $\mathbf{B}_{16}$ & $-x_{5} \mathbf{a}_{1}-y_{5} \mathbf{a}_{2}+z_{5} \mathbf{a}_{3}$ & $-x_{5} a \hat{\mathbf{x}}-y_{5} a \hat{\mathbf{y}}+z_{5} c \hat{\mathbf{z}}$ & $(8 g)$ & O II \\
\hline $\mathbf{B}_{17}$ & $=\left(\frac{1}{2}-y_{5}\right) \mathbf{a}_{1}+\left(\frac{1}{2}+x_{5}\right) \mathbf{a}_{2}+z_{5} \mathbf{a}_{3}$ & $=\left(\frac{1}{2}-y_{5}\right) a \hat{\mathbf{x}}+\left(\frac{1}{2}+x_{5}\right) a \hat{\mathbf{y}}+z_{5} c \hat{\mathbf{z}}$ & $(8 g)$ & $\mathrm{O}$ II \\
\hline $\mathbf{B}_{18}$ & $=\left(\frac{1}{2}+y_{5}\right) \mathbf{a}_{1}+\left(\frac{1}{2}-x_{5}\right) \mathbf{a}_{2}+z_{5} \mathbf{a}_{3}$ & $=\left(\frac{1}{2}+y_{5}\right) a \hat{\mathbf{x}}+\left(\frac{1}{2}-x_{5}\right) a \hat{\mathbf{y}}+z_{5} c \hat{\mathbf{z}}$ & $(8 g)$ & O II \\
\hline $\mathbf{B}_{19}$ & $=\left(\frac{1}{2}-x_{5}\right) \mathbf{a}_{1}+\left(\frac{1}{2}+y_{5}\right) \mathbf{a}_{2}-z_{5} \mathbf{a}_{3}$ & $=\left(\frac{1}{2}-x_{5}\right) a \hat{\mathbf{x}}+\left(\frac{1}{2}+y_{5}\right) a \hat{\mathbf{y}}-z_{5} c \hat{\mathbf{z}}$ & $(8 g)$ & O II \\
\hline $\mathbf{B}_{20}$ & $=\left(\frac{1}{2}+x_{5}\right) \mathbf{a}_{1}+\left(\frac{1}{2}-y_{5}\right) \mathbf{a}_{2}-z_{5} \mathbf{a}_{3}$ & $=\left(\frac{1}{2}+x_{5}\right) a \hat{\mathbf{x}}+\left(\frac{1}{2}-y_{5}\right) a \hat{\mathbf{y}}-z_{5} c \hat{\mathbf{z}}$ & $(8 g)$ & O II \\
\hline $\mathbf{B}_{21}$ & $y_{5} \mathbf{a}_{1}+x_{5} \mathbf{a}_{2}-z_{5} \mathbf{a}_{3}$ & $y_{5} a \hat{\mathbf{x}}+x_{5} a \hat{\mathbf{y}}-z_{5} c \hat{\mathbf{z}}$ & $(8 g)$ & O II \\
\hline $\mathbf{B}_{22}$ & $-y_{5} \mathbf{a}_{1}-x_{5} \mathbf{a}_{2}-z_{5} \mathbf{a}_{3}$ & $-y_{5} a \hat{\mathbf{x}}-x_{5} a \hat{\mathbf{y}}-z_{5} c \hat{\mathbf{z}}$ & $(8 g)$ & O II \\
\hline $\mathbf{B}_{23}$ & $x_{6} \mathbf{a}_{1}+y_{6} \mathbf{a}_{2}+z_{6} \mathbf{a}_{3}$ & $x_{6} a \hat{\mathbf{x}}+y_{6} a \hat{\mathbf{y}}+z_{6} c \hat{\mathbf{z}}$ & $(8 g)$ & O III \\
\hline $\mathbf{B}_{24}$ & $-x_{6} \mathbf{a}_{1}-y_{6} \mathbf{a}_{2}+z_{6} \mathbf{a}_{3}$ & $-x_{6} a \hat{\mathbf{x}}-y_{6} a \hat{\mathbf{y}}+z_{6} c \hat{\mathbf{z}}$ & $(8 g)$ & O III \\
\hline $\mathbf{B}_{25}$ & $=\left(\frac{1}{2}-y_{6}\right) \mathbf{a}_{1}+\left(\frac{1}{2}+x_{6}\right) \mathbf{a}_{2}+z_{6} \mathbf{a}_{3}$ & $=\left(\frac{1}{2}-y_{6}\right) a \hat{\mathbf{x}}+\left(\frac{1}{2}+x_{6}\right) a \hat{\mathbf{y}}+z_{6} c \hat{\mathbf{z}}$ & $(8 g)$ & O III \\
\hline $\mathbf{B}_{26}$ & $=\left(\frac{1}{2}+y_{6}\right) \mathbf{a}_{1}+\left(\frac{1}{2}-x_{6}\right) \mathbf{a}_{2}+z_{6} \mathbf{a}_{3}$ & $=\left(\frac{1}{2}+y_{6}\right) a \hat{\mathbf{x}}+\left(\frac{1}{2}-x_{6}\right) a \hat{\mathbf{y}}+z_{6} c \hat{\mathbf{z}}$ & $(8 g)$ & O III \\
\hline $\mathbf{B}_{27}$ & $=\left(\frac{1}{2}-x_{6}\right) \mathbf{a}_{1}+\left(\frac{1}{2}+y_{6}\right) \mathbf{a}_{2}-z_{6} \mathbf{a}_{3}$ & $=\left(\frac{1}{2}-x_{6}\right) a \hat{\mathbf{x}}+\left(\frac{1}{2}+y_{6}\right) a \hat{\mathbf{y}}-z_{6} c \hat{\mathbf{z}}$ & $(8 g)$ & O III \\
\hline $\mathbf{B}_{28}$ & $=\left(\frac{1}{2}+x_{6}\right) \mathbf{a}_{1}+\left(\frac{1}{2}-y_{6}\right) \mathbf{a}_{2}-z_{6} \mathbf{a}_{3}$ & $=\left(\frac{1}{2}+x_{6}\right) a \hat{\mathbf{x}}+\left(\frac{1}{2}-y_{6}\right) a \hat{\mathbf{y}}-z_{6} c \hat{\mathbf{z}}$ & $(8 g)$ & O III \\
\hline $\mathbf{B}_{29}$ & $y_{6} \mathbf{a}_{1}+x_{6} \mathbf{a}_{2}-z_{6} \mathbf{a}_{3}$ & $y_{6} a \hat{\mathbf{x}}+x_{6} a \hat{\mathbf{y}}-z_{6} c \hat{\mathbf{z}}$ & $(8 g)$ & O III \\
\hline $\mathbf{B}_{30}$ & $-y_{6} \mathbf{a}_{1}-x_{6} \mathbf{a}_{2}-z_{6} \mathbf{a}_{3}$ & $-y_{6} a \hat{\mathbf{x}}-x_{6} a \hat{\mathbf{y}}-z_{6} c \hat{\mathbf{z}}$ & $(8 g)$ & O III \\
\hline $\mathbf{B}_{31}$ & $x_{7} \mathbf{a}_{1}+y_{7} \mathbf{a}_{2}+z_{7} \mathbf{a}_{3}$ & $x_{7} a \hat{\mathbf{x}}+y_{7} a \hat{\mathbf{y}}+z_{7} c \hat{\mathbf{z}}$ & $(8 g)$ & O IV \\
\hline $\mathbf{B}_{32}$ & $-x_{7} \mathbf{a}_{1}-y_{7} \mathbf{a}_{2}+z_{7} \mathbf{a}_{3}$ & $-x_{7} a \hat{\mathbf{x}}-y_{7} a \hat{\mathbf{y}}+z_{7} c \hat{\mathbf{z}}$ & $(8 g)$ & O IV \\
\hline $\mathbf{B}_{33}$ & $=\left(\frac{1}{2}-y_{7}\right) \mathbf{a}_{1}+\left(\frac{1}{2}+x_{7}\right) \mathbf{a}_{2}+z_{7} \mathbf{a}_{3}$ & $=\left(\frac{1}{2}-y_{7}\right) a \hat{\mathbf{x}}+\left(\frac{1}{2}+x_{7}\right) a \hat{\mathbf{y}}+z_{7} c \hat{\mathbf{z}}$ & $(8 g)$ & O IV \\
\hline $\mathbf{B}_{34}$ & $=\left(\frac{1}{2}+y_{7}\right) \mathbf{a}_{1}+\left(\frac{1}{2}-x_{7}\right) \mathbf{a}_{2}+z_{7} \mathbf{a}_{3}$ & $=\left(\frac{1}{2}+y_{7}\right) a \hat{\mathbf{x}}+\left(\frac{1}{2}-x_{7}\right) a \hat{\mathbf{y}}+z_{7} c \hat{\mathbf{z}}$ & $(8 g)$ & O IV \\
\hline $\mathbf{B}_{35}$ & $=\left(\frac{1}{2}-x_{7}\right) \mathbf{a}_{1}+\left(\frac{1}{2}+y_{7}\right) \mathbf{a}_{2}-z_{7} \mathbf{a}_{3}$ & $=\left(\frac{1}{2}-x_{7}\right) a \hat{\mathbf{x}}+\left(\frac{1}{2}+y_{7}\right) a \hat{\mathbf{y}}-z_{7} c \hat{\mathbf{z}}$ & $(8 g)$ & O IV \\
\hline $\mathbf{B}_{36}$ & $=\left(\frac{1}{2}+x_{7}\right) \mathbf{a}_{1}+\left(\frac{1}{2}-y_{7}\right) \mathbf{a}_{2}-z_{7} \mathbf{a}_{3}$ & $=\left(\frac{1}{2}+x_{7}\right) a \hat{\mathbf{x}}+\left(\frac{1}{2}-y_{7}\right) a \hat{\mathbf{y}}-z_{7} c \hat{\mathbf{z}}$ & $(8 g)$ & O IV \\
\hline $\mathbf{B}_{37}$ & $y_{7} \mathbf{a}_{1}+x_{7} \mathbf{a}_{2}-z_{7} \mathbf{a}_{3}$ & $y_{7} a \hat{\mathbf{x}}+x_{7} a \hat{\mathbf{y}}-z_{7} c \hat{\mathbf{z}}$ & $(8 g)$ & O IV \\
\hline
\end{tabular}




\begin{tabular}{|c|c|c|c|c|c|c|}
\hline $\mathbf{B}_{38}$ & $=$ & $-y_{7} \mathbf{a}_{1}-x_{7} \mathbf{a}_{2}-z_{7} \mathbf{a}_{3}$ & $=$ & $-y_{7} a \hat{\mathbf{x}}-x_{7} a \hat{\mathbf{y}}-z_{7} c \hat{\mathbf{z}}$ & $(8 g)$ & O IV \\
\hline $\mathbf{B}_{39}$ & $=$ & $x_{8} \mathbf{a}_{1}+y_{8} \mathbf{a}_{2}+z_{8} \mathbf{a}_{3}$ & $=$ & $x_{8} a \hat{\mathbf{x}}+y_{8} a \hat{\mathbf{y}}+z_{8} c \hat{\mathbf{z}}$ & $(8 g)$ & $\mathrm{OV}$ \\
\hline $\mathbf{B}_{40}$ & $=$ & $-x_{8} \mathbf{a}_{1}-y_{8} \mathbf{a}_{2}+z_{8} \mathbf{a}_{3}$ & $=$ & $-x_{8} a \hat{\mathbf{x}}-y_{8} a \hat{\mathbf{y}}+z_{8} c \hat{\mathbf{z}}$ & $(8 g)$ & $\mathrm{OV}$ \\
\hline $\mathbf{B}_{41}$ & $=$ & $\left(\frac{1}{2}-y_{8}\right) \mathbf{a}_{1}+\left(\frac{1}{2}+x_{8}\right) \mathbf{a}_{2}+z_{8} \mathbf{a}_{3}$ & $=$ & $\left(\frac{1}{2}-y_{8}\right) a \hat{\mathbf{x}}+\left(\frac{1}{2}+x_{8}\right) a \hat{\mathbf{y}}+z_{8} c \hat{\mathbf{z}}$ & $(8 g)$ & $\mathrm{OV}$ \\
\hline $\mathbf{B}_{42}$ & $=$ & $\left(\frac{1}{2}+y_{8}\right) \mathbf{a}_{1}+\left(\frac{1}{2}-x_{8}\right) \mathbf{a}_{2}+z_{8} \mathbf{a}_{3}$ & $=$ & $\left(\frac{1}{2}+y_{8}\right) a \hat{\mathbf{x}}+\left(\frac{1}{2}-x_{8}\right) a \hat{\mathbf{y}}+z_{8} c \hat{\mathbf{z}}$ & $(8 g)$ & $\mathrm{OV}$ \\
\hline $\mathbf{B}_{43}$ & $=$ & $\left(\frac{1}{2}-x_{8}\right) \mathbf{a}_{1}+\left(\frac{1}{2}+y_{8}\right) \mathbf{a}_{2}-z_{8} \mathbf{a}_{3}$ & $=$ & $\left(\frac{1}{2}-x_{8}\right) a \hat{\mathbf{x}}+\left(\frac{1}{2}+y_{8}\right) a \hat{\mathbf{y}}-z_{8} c \hat{\mathbf{z}}$ & $(8 g)$ & $\mathrm{OV}$ \\
\hline $\mathbf{B}_{44}$ & $=$ & $\left(\frac{1}{2}+x_{8}\right) \mathbf{a}_{1}+\left(\frac{1}{2}-y_{8}\right) \mathbf{a}_{2}-z_{8} \mathbf{a}_{3}$ & $=$ & $\left(\frac{1}{2}+x_{8}\right) a \hat{\mathbf{x}}+\left(\frac{1}{2}-y_{8}\right) a \hat{\mathbf{y}}-z_{8} c \hat{\mathbf{z}}$ & $(8 g)$ & $\mathrm{OV}$ \\
\hline $\mathbf{B}_{45}$ & $=$ & $y_{8} \mathbf{a}_{1}+x_{8} \mathbf{a}_{2}-z_{8} \mathbf{a}_{3}$ & $=$ & $y_{8} a \hat{\mathbf{x}}+x_{8} a \hat{\mathbf{y}}-z_{8} c \hat{\mathbf{z}}$ & $(8 g)$ & $\mathrm{OV}$ \\
\hline $\mathbf{B}_{46}$ & $=$ & $-y_{8} \mathbf{a}_{1}-x_{8} \mathbf{a}_{2}-z_{8} \mathbf{a}_{3}$ & $=$ & $-y_{8} a \hat{\mathbf{x}}-x_{8} a \hat{\mathbf{y}}-z_{8} c \hat{\mathbf{z}}$ & $(8 g)$ & $\mathrm{OV}$ \\
\hline $\mathbf{B}_{47}$ & $=$ & $x_{9} \mathbf{a}_{1}+y_{9} \mathbf{a}_{2}+z_{9} \mathbf{a}_{3}$ & $=$ & $x_{9} a \hat{\mathbf{x}}+y_{9} a \hat{\mathbf{y}}+z_{9} c \hat{\mathbf{z}}$ & $(8 g)$ & $\mathrm{P}$ \\
\hline $\mathbf{B}_{48}$ & $=$ & $-x_{9} \mathbf{a}_{1}-y_{9} \mathbf{a}_{2}+z_{9} \mathbf{a}_{3}$ & $=$ & $-x_{9} a \hat{\mathbf{x}}-y_{9} a \hat{\mathbf{y}}+z_{9} c \hat{\mathbf{z}}$ & $(8 g)$ & $\mathrm{P}$ \\
\hline $\mathbf{B}_{49}$ & $=$ & $\left(\frac{1}{2}-y_{9}\right) \mathbf{a}_{1}+\left(\frac{1}{2}+x_{9}\right) \mathbf{a}_{2}+z_{9} \mathbf{a}_{3}$ & $=$ & $\left(\frac{1}{2}-y_{9}\right) a \hat{\mathbf{x}}+\left(\frac{1}{2}+x_{9}\right) a \hat{\mathbf{y}}+z_{9} c \hat{\mathbf{z}}$ & $(8 g)$ & $\mathrm{P}$ \\
\hline $\mathbf{B}_{50}$ & $=$ & $\left(\frac{1}{2}+y_{9}\right) \mathbf{a}_{1}+\left(\frac{1}{2}-x_{9}\right) \mathbf{a}_{2}+z_{9} \mathbf{a}_{3}$ & $=$ & $\left(\frac{1}{2}+y_{9}\right) a \hat{\mathbf{x}}+\left(\frac{1}{2}-x_{9}\right) a \hat{\mathbf{y}}+z_{9} c \hat{\mathbf{z}}$ & $(8 g)$ & $\mathrm{P}$ \\
\hline $\mathbf{B}_{51}$ & $=$ & $\left(\frac{1}{2}-x_{9}\right) \mathbf{a}_{1}+\left(\frac{1}{2}+y_{9}\right) \mathbf{a}_{2}-z_{9} \mathbf{a}_{3}$ & $=$ & $\left(\frac{1}{2}-x_{9}\right) a \hat{\mathbf{x}}+\left(\frac{1}{2}+y_{9}\right) a \hat{\mathbf{y}}-z_{9} c \hat{\mathbf{z}}$ & $(8 g)$ & $\mathrm{P}$ \\
\hline $\mathbf{B}_{52}$ & $=$ & $\left(\frac{1}{2}+x_{9}\right) \mathbf{a}_{1}+\left(\frac{1}{2}-y_{9}\right) \mathbf{a}_{2}-z_{9} \mathbf{a}_{3}$ & $=$ & $\left(\frac{1}{2}+x_{9}\right) a \hat{\mathbf{x}}+\left(\frac{1}{2}-y_{9}\right) a \hat{\mathbf{y}}-z_{9} c \hat{\mathbf{z}}$ & $(8 g)$ & $\mathrm{P}$ \\
\hline $\mathbf{B}_{53}$ & $=$ & $y_{9} \mathbf{a}_{1}+x_{9} \mathbf{a}_{2}-z_{9} \mathbf{a}_{3}$ & $=$ & $y_{9} a \hat{\mathbf{x}}+x_{9} a \hat{\mathbf{y}}-z_{9} c \hat{\mathbf{z}}$ & $(8 g)$ & $\mathrm{P}$ \\
\hline $\mathbf{B}_{54}$ & $=$ & $-y_{9} \mathbf{a}_{1}-x_{9} \mathbf{a}_{2}-z 9 \mathbf{a}_{3}$ & $=$ & $-y_{9} a \hat{\mathbf{x}}-x_{9} a \hat{\mathbf{y}}-z_{9} c \hat{\mathbf{z}}$ & $(8 g)$ & $\mathrm{P}$ \\
\hline
\end{tabular}

\section{References:}

- S. Meyer and $\mathrm{H}$. Müller-Buschbaum, $\mathrm{Cu}_{4} \mathrm{O}_{12}$-Baugruppen aus planaren $\mathrm{CuO}_{4}$-Polygonen im Barium-Vanadyl-Oxocuprat(II)-phosphat $\mathrm{Ba}(\mathrm{VO}) \mathrm{Cu}_{4}\left(\mathrm{PO}_{4}\right)_{4}$, Z. Anorg. Allg. Chem. 623, 1693-1698 (1997), doi:10.1002/zaac.19976231103.

\section{Found in:}

- P. Villars and K. Cenzual, Pearson's Crystal Data - Crystal Structure Database for Inorganic Compounds, ASM International (2013).

\section{Geometry files:}

- CIF: pp. 868

- POSCAR: pp. 869 


\section{ThBC Structure: ABC_tP24_91_d_d_d}
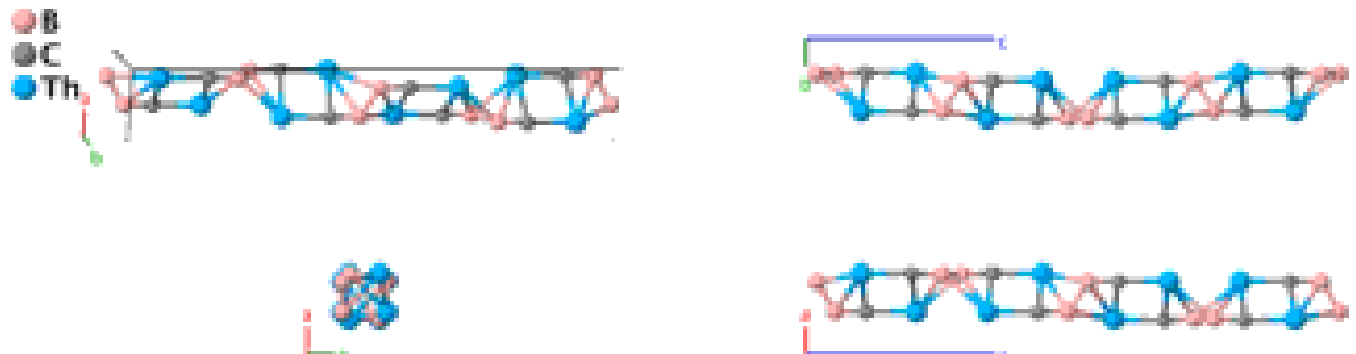

\section{Prototype}

: $\quad$ ThBC

AFLOW prototype label

: $\quad$ ABC_tP24_91_d_d_d

Strukturbericht designation

Pearson symbol

: None

Space group number $\quad: \quad 91$

Space group symbol $\quad: \quad P 4_{1} 22$

AFLOW prototype command : aflow --proto=ABC_tP24_91_d_d_d

- params $=a, c / a, x_{1}, y_{1}, z_{1}, x_{2}, y_{2}, z_{2}, x_{3}, y_{3}, z_{3}$

\section{Simple Tetragonal primitive vectors:}

$$
\begin{aligned}
& \mathbf{a}_{1}=a \hat{\mathbf{x}} \\
& \mathbf{a}_{2}=a \hat{\mathbf{y}} \\
& \mathbf{a}_{3}=c \hat{\mathbf{z}}
\end{aligned}
$$

\begin{tabular}{|c|c|c|c|c|c|c|}
\hline & & Lattice Coordinates & & Cartesian Coordinates & Wyckoff Position & Atom Type \\
\hline $\mathbf{B}_{1}$ & $=$ & $x_{1} \mathbf{a}_{1}+y_{1} \mathbf{a}_{2}+z_{1} \mathbf{a}_{3}$ & $=$ & $x_{1} a \hat{\mathbf{x}}+y_{1} a \hat{\mathbf{y}}+z_{1} c \hat{\mathbf{z}}$ & $(8 d)$ & $\mathrm{B}$ \\
\hline $\mathbf{B}_{2}$ & $=$ & $-x_{1} \mathbf{a}_{1}-y_{1} \mathbf{a}_{2}+\left(\frac{1}{2}+z_{1}\right) \mathbf{a}_{3}$ & $=$ & $-x_{1} a \hat{\mathbf{x}}-y_{1} a \hat{\mathbf{y}}+\left(\frac{1}{2}+z_{1}\right) c \hat{\mathbf{z}}$ & $(8 d)$ & $\mathrm{B}$ \\
\hline $\mathbf{B}_{3}$ & $=$ & $-y_{1} \mathbf{a}_{1}+x_{1} \mathbf{a}_{2}+\left(\frac{1}{4}+z_{1}\right) \mathbf{a}_{3}$ & $=$ & $-y_{1} a \hat{\mathbf{x}}+x_{1} a \hat{\mathbf{y}}+\left(\frac{1}{4}+z_{1}\right) c \hat{\mathbf{z}}$ & $(8 d)$ & $\mathrm{B}$ \\
\hline $\mathbf{B}_{4}$ & $=$ & $y_{1} \mathbf{a}_{1}-x_{1} \mathbf{a}_{2}+\left(\frac{3}{4}+z_{1}\right) \mathbf{a}_{3}$ & $=$ & $y_{1} a \hat{\mathbf{x}}-x_{1} a \hat{\mathbf{y}}+\left(\frac{3}{4}+z_{1}\right) c \hat{\mathbf{z}}$ & $(8 d)$ & $\mathrm{B}$ \\
\hline $\mathbf{B}_{5}$ & $=$ & $-x_{1} \mathbf{a}_{1}+y_{1} \mathbf{a}_{2}-z_{1} \mathbf{a}_{3}$ & $=$ & $-x_{1} a \hat{\mathbf{x}}+y_{1} a \hat{\mathbf{y}}-z_{1} c \hat{\mathbf{z}}$ & $(8 d)$ & B \\
\hline $\mathbf{B}_{6}$ & $=$ & $x_{1} \mathbf{a}_{1}-y_{1} \mathbf{a}_{2}+\left(\frac{1}{2}-z_{1}\right) \mathbf{a}_{3}$ & $=$ & $x_{1} a \hat{\mathbf{x}}-y_{1} a \hat{\mathbf{y}}+\left(\frac{1}{2}-z_{1}\right) c \hat{\mathbf{z}}$ & $(8 d)$ & $\mathrm{B}$ \\
\hline $\mathbf{B}_{7}$ & $=$ & $y_{1} \mathbf{a}_{1}+x_{1} \mathbf{a}_{2}+\left(\frac{3}{4}-z_{1}\right) \mathbf{a}_{3}$ & $=$ & $y_{1} a \hat{\mathbf{x}}+x_{1} a \hat{\mathbf{y}}+\left(\frac{3}{4}-z_{1}\right) c \hat{\mathbf{z}}$ & $(8 d)$ & $\mathrm{B}$ \\
\hline $\mathbf{B}_{8}$ & $=$ & $-y_{1} \mathbf{a}_{1}-x_{1} \mathbf{a}_{2}+\left(\frac{1}{4}-z_{1}\right) \mathbf{a}_{3}$ & $=$ & $-y_{1} a \hat{\mathbf{x}}-x_{1} a \hat{\mathbf{y}}+\left(\frac{1}{4}-z_{1}\right) c \hat{\mathbf{z}}$ & $(8 d)$ & $\mathrm{B}$ \\
\hline $\mathbf{B}_{9}$ & $=$ & $x_{2} \mathbf{a}_{1}+y_{2} \mathbf{a}_{2}+z_{2} \mathbf{a}_{3}$ & $=$ & $x_{2} a \hat{\mathbf{x}}+y_{2} a \hat{\mathbf{y}}+z_{2} c \hat{\mathbf{z}}$ & $(8 d)$ & $\mathrm{C}$ \\
\hline $\mathbf{B}_{10}$ & $=$ & $-x_{2} \mathbf{a}_{1}-y_{2} \mathbf{a}_{2}+\left(\frac{1}{2}+z_{2}\right) \mathbf{a}_{3}$ & $=$ & $-x_{2} a \hat{\mathbf{x}}-y_{2} a \hat{\mathbf{y}}+\left(\frac{1}{2}+z_{2}\right) c \hat{\mathbf{z}}$ & $(8 d)$ & $\mathrm{C}$ \\
\hline $\mathbf{B}_{11}$ & $=$ & $-y_{2} \mathbf{a}_{1}+x_{2} \mathbf{a}_{2}+\left(\frac{1}{4}+z_{2}\right) \mathbf{a}_{3}$ & $=$ & $-y_{2} a \hat{\mathbf{x}}+x_{2} a \hat{\mathbf{y}}+\left(\frac{1}{4}+z_{2}\right) c \hat{\mathbf{z}}$ & $(8 d)$ & $\mathrm{C}$ \\
\hline $\mathbf{B}_{12}$ & $=$ & $y_{2} \mathbf{a}_{1}-x_{2} \mathbf{a}_{2}+\left(\frac{3}{4}+z_{2}\right) \mathbf{a}_{3}$ & $=$ & $y_{2} a \hat{\mathbf{x}}-x_{2} a \hat{\mathbf{y}}+\left(\frac{3}{4}+z_{2}\right) c \hat{\mathbf{z}}$ & $(8 d)$ & $\mathrm{C}$ \\
\hline $\mathbf{B}_{13}$ & $=$ & $-x_{2} \mathbf{a}_{1}+y_{2} \mathbf{a}_{2}-z_{2} \mathbf{a}_{3}$ & $=$ & $-x_{2} a \hat{\mathbf{x}}+y_{2} a \hat{\mathbf{y}}-z_{2} c \hat{\mathbf{z}}$ & $(8 d)$ & $\mathrm{C}$ \\
\hline $\mathbf{B}_{14}$ & $=$ & $x_{2} \mathbf{a}_{1}-y_{2} \mathbf{a}_{2}+\left(\frac{1}{2}-z_{2}\right) \mathbf{a}_{3}$ & $=$ & $x_{2} a \hat{\mathbf{x}}-y_{2} a \hat{\mathbf{y}}+\left(\frac{1}{2}-z_{2}\right) c \hat{\mathbf{z}}$ & $(8 d)$ & $\mathrm{C}$ \\
\hline
\end{tabular}

\section{काष्तिक}

Basis vectors: 


\begin{tabular}{|c|c|c|c|c|c|c|}
\hline $\mathbf{B}_{15}$ & $=$ & $y_{2} \mathbf{a}_{1}+x_{2} \mathbf{a}_{2}+\left(\frac{3}{4}-z_{2}\right) \mathbf{a}_{3}$ & $=$ & $y_{2} a \hat{\mathbf{x}}+x_{2} a \hat{\mathbf{y}}+\left(\frac{3}{4}-z_{2}\right) c \hat{\mathbf{z}}$ & $(8 d)$ & $\mathrm{C}$ \\
\hline $\mathbf{B}_{16}$ & $=$ & $-y_{2} \mathbf{a}_{1}-x_{2} \mathbf{a}_{2}+\left(\frac{1}{4}-z_{2}\right) \mathbf{a}_{3}$ & $=$ & $-y_{2} a \hat{\mathbf{x}}-x_{2} a \hat{\mathbf{y}}+\left(\frac{1}{4}-z_{2}\right) c \hat{\mathbf{z}}$ & $(8 d)$ & $\mathrm{C}$ \\
\hline $\mathbf{B}_{17}$ & $=$ & $x_{3} \mathbf{a}_{1}+y_{3} \mathbf{a}_{2}+z_{3} \mathbf{a}_{3}$ & $=$ & $x_{3} a \hat{\mathbf{x}}+y_{3} a \hat{\mathbf{y}}+z_{3} c \hat{\mathbf{z}}$ & $(8 d)$ & $\mathrm{Th}$ \\
\hline $\mathbf{B}_{18}$ & $=$ & $-x_{3} \mathbf{a}_{1}-y_{3} \mathbf{a}_{2}+\left(\frac{1}{2}+z_{3}\right) \mathbf{a}_{3}$ & $=$ & $-x_{3} a \hat{\mathbf{x}}-y_{3} a \hat{\mathbf{y}}+\left(\frac{1}{2}+z_{3}\right) c \hat{\mathbf{z}}$ & $(8 d)$ & $\mathrm{Th}$ \\
\hline $\mathbf{B}_{19}$ & $=$ & $-y_{3} \mathbf{a}_{1}+x_{3} \mathbf{a}_{2}+\left(\frac{1}{4}+z_{3}\right) \mathbf{a}_{3}$ & $=$ & $-y_{3} a \hat{\mathbf{x}}+x_{3} a \hat{\mathbf{y}}+\left(\frac{1}{4}+z_{3}\right) c \hat{\mathbf{z}}$ & $(8 d)$ & $\mathrm{Th}$ \\
\hline $\mathbf{B}_{20}$ & $=$ & $y_{3} \mathbf{a}_{1}-x_{3} \mathbf{a}_{2}+\left(\frac{3}{4}+z_{3}\right) \mathbf{a}_{3}$ & $=$ & $y_{3} a \hat{\mathbf{x}}-x_{3} a \hat{\mathbf{y}}+\left(\frac{3}{4}+z_{3}\right) c \hat{\mathbf{z}}$ & $(8 d)$ & Th \\
\hline $\mathbf{B}_{21}$ & $=$ & $-x_{3} \mathbf{a}_{1}+y_{3} \mathbf{a}_{2}-z_{3} \mathbf{a}_{3}$ & $=$ & $-x_{3} a \hat{\mathbf{x}}+y_{3} a \hat{\mathbf{y}}-z_{3} c \hat{\mathbf{z}}$ & $(8 d)$ & Th \\
\hline $\mathbf{B}_{22}$ & $=$ & $x_{3} \mathbf{a}_{1}-y_{3} \mathbf{a}_{2}+\left(\frac{1}{2}-z_{3}\right) \mathbf{a}_{3}$ & $=$ & $x_{3} a \hat{\mathbf{x}}-y_{3} a \hat{\mathbf{y}}+\left(\frac{1}{2}-z_{3}\right) c \hat{\mathbf{z}}$ & $(8 d)$ & Th \\
\hline $\mathbf{B}_{23}$ & $=$ & $y_{3} \mathbf{a}_{1}+x_{3} \mathbf{a}_{2}+\left(\frac{3}{4}-z_{3}\right) \mathbf{a}_{3}$ & $=$ & $y_{3} a \hat{\mathbf{x}}+x_{3} a \hat{\mathbf{y}}+\left(\frac{3}{4}-z_{3}\right) c \hat{\mathbf{z}}$ & $(8 d)$ & $\mathrm{Th}$ \\
\hline $\mathbf{B}_{24}$ & $=$ & $-y_{3} \mathbf{a}_{1}-x_{3} \mathbf{a}_{2}+\left(\frac{1}{4}-z_{3}\right) \mathbf{a}_{3}$ & $=$ & $-y_{3} a \hat{\mathbf{x}}-x_{3} a \hat{\mathbf{y}}+\left(\frac{1}{4}-z_{3}\right) c \hat{\mathbf{z}}$ & $(8 d)$ & Th \\
\hline
\end{tabular}

\section{References:}

- P. Rogl, The crystal structure of ThBC, J. Nucl. Mat. 73, 198-203 (1978), doi:10.1016/0022-3115(78)90560-3.

\section{Found in:}

- P. Villars and K. Cenzual, Pearson's Crystal Data - Crystal Structure Database for Inorganic Compounds, ASM International (2013).

\section{Geometry files:}

- CIF: pp. 869

- POSCAR: pp. 869 


\section{$\mathrm{AsPh}_{4} \mathrm{CeS}_{8} \mathrm{P}_{4} \mathrm{Me}_{8}$ Structure: AB32CD4E8_tP184_93_i_16p_af_2p_4p}
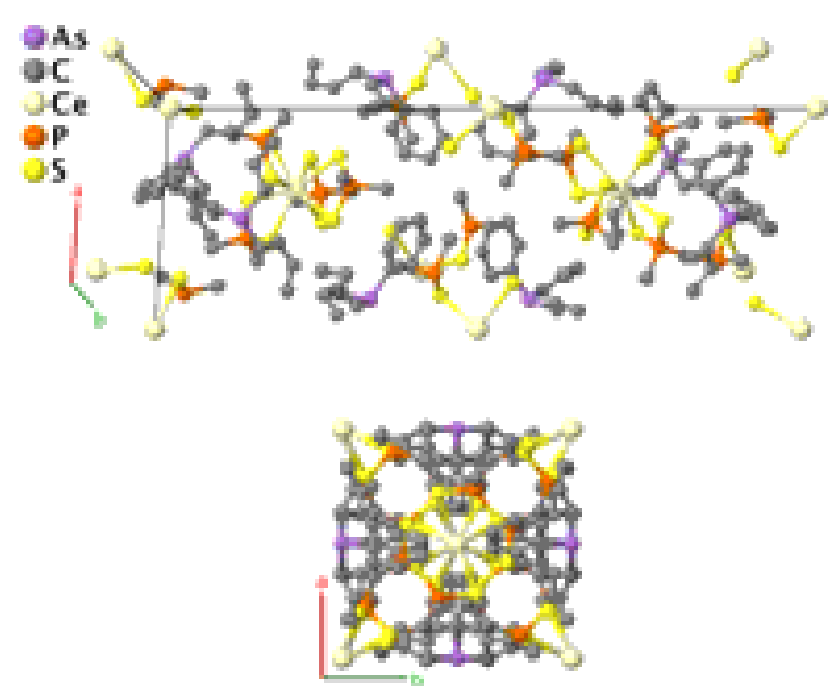
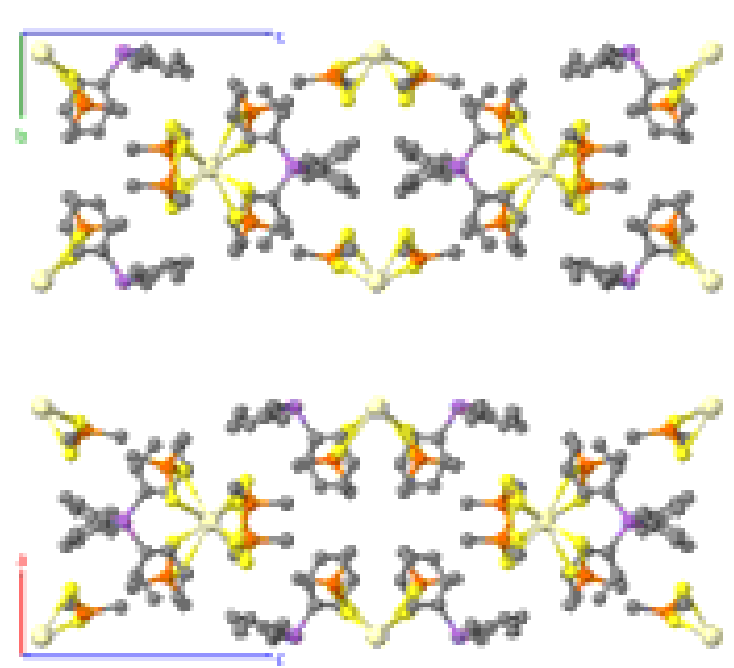

\section{Prototype}

AFLOW prototype label

Strukturbericht designation

Pearson symbol

Space group number

Space group symbol

AFLOW prototype command
$\mathrm{AsPh}_{4} \mathrm{CeS}_{8} \mathrm{P}_{4} \mathrm{Me}_{8}$

AB32CD4E8_tP184_93_i_16p_af_2p_4p

: None

: $\quad$ tP184

: $\quad 93$

: $\quad P 4_{2} 22$

- Structures exhibiting space group \#93 are quite rare. According to (Hoffmann, 2014), there are no entries in the Inorganic Crystal Structure Database with space group \#93. The hydrogen atoms are not included in this prototype. $(\mathrm{Ph}=$ Phenyl and $\mathrm{Me}=$ Methyl $)$

\section{Simple Tetragonal primitive vectors:}

$$
\begin{aligned}
& \mathbf{a}_{1}=a \hat{\mathbf{x}} \\
& \mathbf{a}_{2}=a \hat{\mathbf{y}} \\
& \mathbf{a}_{3}=c \hat{\mathbf{z}}
\end{aligned}
$$

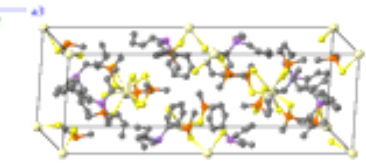

Basis vectors:

\section{Lattice Coordinates}

$\begin{array}{ccc}\mathbf{B}_{1}= & 0 \mathbf{a}_{1}+0 \mathbf{a}_{2}+0 \mathbf{a}_{3} \\ \mathbf{B}_{2}= & \frac{1}{2} \mathbf{a}_{3} \\ \mathbf{B}_{3}= & \frac{1}{2} \mathbf{a}_{1}+\frac{1}{2} \mathbf{a}_{2}+\frac{1}{4} \mathbf{a}_{3}\end{array}$

Cartesian Coordinates

$=$

$=$

$=$

$$
\begin{gathered}
0 \hat{\mathbf{x}}+0 \hat{\mathbf{y}}+0 \hat{\mathbf{z}} \\
\frac{1}{2} c \hat{\mathbf{z}} \\
\frac{1}{2} a \hat{\mathbf{x}}+\frac{1}{2} a \hat{\mathbf{y}}+\frac{1}{4} c \hat{\mathbf{z}}
\end{gathered}
$$

Wyckoff Position

$(2 a)$

$(2 f)$
Atom Type

Ce I

Ce I

Ce II 


\begin{tabular}{|c|c|c|c|c|c|c|}
\hline $\mathbf{B}_{4}$ & $=$ & $\frac{1}{2} \mathbf{a}_{1}+\frac{1}{2} \mathbf{a}_{2}+\frac{3}{4} \mathbf{a}_{3}$ & $=$ & $\frac{1}{2} a \hat{\mathbf{x}}+\frac{1}{2} a \hat{\mathbf{y}}+\frac{3}{4} c \hat{\mathbf{z}}$ & $(2 f)$ & Ce II \\
\hline $\mathbf{B}_{5}$ & $=$ & $\frac{1}{2} \mathbf{a}_{2}+z_{3} \mathbf{a}_{3}$ & $=$ & $\frac{1}{2} a \hat{\mathbf{y}}+z_{3} c \hat{\mathbf{z}}$ & $(4 i)$ & As \\
\hline $\mathbf{B}_{6}$ & $=$ & $\frac{1}{2} \mathbf{a}_{1}+\left(\frac{1}{2}+z_{3}\right) \mathbf{a}_{3}$ & $=$ & $\frac{1}{2} a \hat{\mathbf{x}}+\left(\frac{1}{2}+z_{3}\right) c \hat{\mathbf{z}}$ & $(4 i)$ & As \\
\hline $\mathbf{B}_{7}$ & $=$ & $\frac{1}{2} \mathbf{a}_{2}-z_{3} \mathbf{a}_{3}$ & $=$ & $\frac{1}{2} a \hat{\mathbf{y}}-z_{3} c \hat{\mathbf{z}}$ & $(4 i)$ & As \\
\hline $\mathbf{B}_{8}$ & $=$ & $\frac{1}{2} \mathbf{a}_{1}+\left(\frac{1}{2}-z_{3}\right) \mathbf{a}_{3}$ & $=$ & $\frac{1}{2} a \hat{\mathbf{x}}+\left(\frac{1}{2}-z_{3}\right) c \hat{\mathbf{z}}$ & $(4 i)$ & As \\
\hline $\mathbf{B}_{9}$ & $=$ & $x_{4} \mathbf{a}_{1}+y_{4} \mathbf{a}_{2}+z_{4} \mathbf{a}_{3}$ & $=$ & $x_{4} a \hat{\mathbf{x}}+y_{4} a \hat{\mathbf{y}}+z_{4} c \hat{\mathbf{z}}$ & $(8 p)$ & C I \\
\hline $\mathbf{B}_{10}$ & $=$ & $-x_{4} \mathbf{a}_{1}-y_{4} \mathbf{a}_{2}+z_{4} \mathbf{a}_{3}$ & $=$ & $-x_{4} a \hat{\mathbf{x}}-y_{4} a \hat{\mathbf{y}}+z_{4} c \hat{\mathbf{z}}$ & $(8 p)$ & C I \\
\hline $\mathbf{B}_{11}$ & $=$ & $-y_{4} \mathbf{a}_{1}+x_{4} \mathbf{a}_{2}+\left(\frac{1}{2}+z_{4}\right) \mathbf{a}_{3}$ & $=$ & $-y_{4} a \hat{\mathbf{x}}+x_{4} a \hat{\mathbf{y}}+\left(\frac{1}{2}+z_{4}\right) c \hat{\mathbf{z}}$ & $(8 p)$ & C I \\
\hline $\mathbf{B}_{12}$ & $=$ & $y_{4} \mathbf{a}_{1}-x_{4} \mathbf{a}_{2}+\left(\frac{1}{2}+z_{4}\right) \mathbf{a}_{3}$ & $=$ & $y_{4} a \hat{\mathbf{x}}-x_{4} a \hat{\mathbf{y}}+\left(\frac{1}{2}+z_{4}\right) c \hat{\mathbf{z}}$ & $(8 p)$ & $\mathrm{C} \mathrm{I}$ \\
\hline $\mathbf{B}_{13}$ & $=$ & $-x_{4} \mathbf{a}_{1}+y_{4} \mathbf{a}_{2}-z_{4} \mathbf{a}_{3}$ & $=$ & $-x_{4} a \hat{\mathbf{x}}+y_{4} a \hat{\mathbf{y}}-z_{4} c \hat{\mathbf{z}}$ & $(8 p)$ & $\mathrm{C} \mathrm{I}$ \\
\hline $\mathbf{B}_{14}$ & $=$ & $x_{4} \mathbf{a}_{1}-y_{4} \mathbf{a}_{2}-z_{4} \mathbf{a}_{3}$ & $=$ & $x_{4} a \hat{\mathbf{x}}-y_{4} a \hat{\mathbf{y}}-z_{4} c \hat{\mathbf{z}}$ & $(8 p)$ & C I \\
\hline $\mathbf{B}_{15}$ & $=$ & $y_{4} \mathbf{a}_{1}+x_{4} \mathbf{a}_{2}+\left(\frac{1}{2}-z_{4}\right) \mathbf{a}_{3}$ & $=$ & $y_{4} a \hat{\mathbf{x}}+x_{4} a \hat{\mathbf{y}}+\left(\frac{1}{2}-z_{4}\right) c \hat{\mathbf{z}}$ & $(8 p)$ & C I \\
\hline $\mathbf{B}_{16}$ & $=$ & $-y_{4} \mathbf{a}_{1}-x_{4} \mathbf{a}_{2}+\left(\frac{1}{2}-z_{4}\right) \mathbf{a}_{3}$ & $=$ & $-y_{4} a \hat{\mathbf{x}}-x_{4} a \hat{\mathbf{y}}+\left(\frac{1}{2}-z_{4}\right) c \hat{\mathbf{z}}$ & $(8 p)$ & C I \\
\hline $\mathbf{B}_{17}$ & $=$ & $x_{5} \mathbf{a}_{1}+y_{5} \mathbf{a}_{2}+z_{5} \mathbf{a}_{3}$ & $=$ & $x_{5} a \hat{\mathbf{x}}+y_{5} a \hat{\mathbf{y}}+z_{5} c \hat{\mathbf{z}}$ & $(8 p)$ & C II \\
\hline $\mathbf{B}_{18}$ & $=$ & $-x_{5} \mathbf{a}_{1}-y_{5} \mathbf{a}_{2}+z_{5} \mathbf{a}_{3}$ & $=$ & $-x_{5} a \hat{\mathbf{x}}-y_{5} a \hat{\mathbf{y}}+z_{5} c \hat{\mathbf{z}}$ & $(8 p)$ & C II \\
\hline $\mathbf{B}_{19}$ & $=$ & $-y_{5} \mathbf{a}_{1}+x_{5} \mathbf{a}_{2}+\left(\frac{1}{2}+z_{5}\right) \mathbf{a}_{3}$ & $=$ & $-y_{5} a \hat{\mathbf{x}}+x_{5} a \hat{\mathbf{y}}+\left(\frac{1}{2}+z_{5}\right) c \hat{\mathbf{z}}$ & $(8 p)$ & C II \\
\hline $\mathbf{B}_{20}$ & $=$ & $y_{5} \mathbf{a}_{1}-x_{5} \mathbf{a}_{2}+\left(\frac{1}{2}+z_{5}\right) \mathbf{a}_{3}$ & $=$ & $y_{5} a \hat{\mathbf{x}}-x_{5} a \hat{\mathbf{y}}+\left(\frac{1}{2}+z_{5}\right) c \hat{\mathbf{z}}$ & $(8 p)$ & C II \\
\hline $\mathbf{B}_{21}$ & $=$ & $-x_{5} \mathbf{a}_{1}+y_{5} \mathbf{a}_{2}-z_{5} \mathbf{a}_{3}$ & $=$ & $-x_{5} a \hat{\mathbf{x}}+y_{5} a \hat{\mathbf{y}}-z_{5} c \hat{\mathbf{z}}$ & $(8 p)$ & C II \\
\hline $\mathbf{B}_{22}$ & $=$ & $x_{5} \mathbf{a}_{1}-y_{5} \mathbf{a}_{2}-z_{5} \mathbf{a}_{3}$ & $=$ & $x_{5} a \hat{\mathbf{x}}-y_{5} a \hat{\mathbf{y}}-z_{5} c \hat{\mathbf{z}}$ & $(8 p)$ & C II \\
\hline $\mathbf{B}_{23}$ & $=$ & $y_{5} \mathbf{a}_{1}+x_{5} \mathbf{a}_{2}+\left(\frac{1}{2}-z_{5}\right) \mathbf{a}_{3}$ & $=$ & $y_{5} a \hat{\mathbf{x}}+x_{5} a \hat{\mathbf{y}}+\left(\frac{1}{2}-z_{5}\right) c \hat{\mathbf{z}}$ & $(8 p)$ & C II \\
\hline $\mathbf{B}_{24}$ & $=$ & $-y_{5} \mathbf{a}_{1}-x_{5} \mathbf{a}_{2}+\left(\frac{1}{2}-z_{5}\right) \mathbf{a}_{3}$ & $=$ & $-y_{5} a \hat{\mathbf{x}}-x_{5} a \hat{\mathbf{y}}+\left(\frac{1}{2}-z_{5}\right) c \hat{\mathbf{z}}$ & $(8 p)$ & C II \\
\hline $\mathbf{B}_{25}$ & $=$ & $x_{6} \mathbf{a}_{1}+y_{6} \mathbf{a}_{2}+z_{6} \mathbf{a}_{3}$ & $=$ & $x_{6} a \hat{\mathbf{x}}+y_{6} a \hat{\mathbf{y}}+z_{6} c \hat{\mathbf{z}}$ & $(8 p)$ & C III \\
\hline $\mathbf{B}_{26}$ & $=$ & $-x_{6} \mathbf{a}_{1}-y_{6} \mathbf{a}_{2}+z_{6} \mathbf{a}_{3}$ & $=$ & $-x_{6} a \hat{\mathbf{x}}-y_{6} a \hat{\mathbf{y}}+z_{6} c \hat{\mathbf{z}}$ & $(8 p)$ & C III \\
\hline $\mathbf{B}_{27}$ & $=$ & $-y_{6} \mathbf{a}_{1}+x_{6} \mathbf{a}_{2}+\left(\frac{1}{2}+z_{6}\right) \mathbf{a}_{3}$ & $=$ & $-y_{6} a \hat{\mathbf{x}}+x_{6} a \hat{\mathbf{y}}+\left(\frac{1}{2}+z_{6}\right) c \hat{\mathbf{z}}$ & $(8 p)$ & C III \\
\hline $\mathbf{B}_{28}$ & $=$ & $y_{6} \mathbf{a}_{1}-x_{6} \mathbf{a}_{2}+\left(\frac{1}{2}+z_{6}\right) \mathbf{a}_{3}$ & $=$ & $y_{6} a \hat{\mathbf{x}}-x_{6} a \hat{\mathbf{y}}+\left(\frac{1}{2}+z_{6}\right) c \hat{\mathbf{z}}$ & $(8 p)$ & C III \\
\hline $\mathbf{B}_{29}$ & $=$ & $-x_{6} \mathbf{a}_{1}+y_{6} \mathbf{a}_{2}-z_{6} \mathbf{a}_{3}$ & $=$ & $-x_{6} a \hat{\mathbf{x}}+y_{6} a \hat{\mathbf{y}}-z_{6} c \hat{\mathbf{z}}$ & $(8 p)$ & C III \\
\hline $\mathbf{B}_{30}$ & $=$ & $x_{6} \mathbf{a}_{1}-y_{6} \mathbf{a}_{2}-z_{6} \mathbf{a}_{3}$ & $=$ & $x_{6} a \hat{\mathbf{x}}-y_{6} a \hat{\mathbf{y}}-z_{6} c \hat{\mathbf{z}}$ & $(8 p)$ & C III \\
\hline $\mathbf{B}_{31}$ & $=$ & $y_{6} \mathbf{a}_{1}+x_{6} \mathbf{a}_{2}+\left(\frac{1}{2}-z_{6}\right) \mathbf{a}_{3}$ & $=$ & $y_{6} a \hat{\mathbf{x}}+x_{6} a \hat{\mathbf{y}}+\left(\frac{1}{2}-z_{6}\right) c \hat{\mathbf{z}}$ & $(8 p)$ & III \\
\hline $\mathbf{B}_{32}$ & $=$ & $-y_{6} \mathbf{a}_{1}-x_{6} \mathbf{a}_{2}+\left(\frac{1}{2}-z_{6}\right) \mathbf{a}_{3}$ & $=$ & $-y_{6} a \hat{\mathbf{x}}-x_{6} a \hat{\mathbf{y}}+\left(\frac{1}{2}-z_{6}\right) c \hat{\mathbf{z}}$ & $(8 p)$ & C III \\
\hline $\mathbf{B}_{33}$ & $=$ & $x_{7} \mathbf{a}_{1}+y_{7} \mathbf{a}_{2}+z_{7} \mathbf{a}_{3}$ & $=$ & $x_{7} a \hat{\mathbf{x}}+y_{7} a \hat{\mathbf{y}}+z_{7} c \hat{\mathbf{z}}$ & $(8 p)$ & C IV \\
\hline $\mathbf{B}_{34}$ & $=$ & $-x_{7} \mathbf{a}_{1}-y_{7} \mathbf{a}_{2}+z_{7} \mathbf{a}_{3}$ & $=$ & $-x_{7} a \hat{\mathbf{x}}-y_{7} a \hat{\mathbf{y}}+z_{7} c \hat{\mathbf{z}}$ & $(8 p)$ & C IV \\
\hline $\mathbf{B}_{35}$ & $=$ & $-y_{7} \mathbf{a}_{1}+x_{7} \mathbf{a}_{2}+\left(\frac{1}{2}+z_{7}\right) \mathbf{a}_{3}$ & $=$ & $-y_{7} a \hat{\mathbf{x}}+x_{7} a \hat{\mathbf{y}}+\left(\frac{1}{2}+z_{7}\right) c \hat{\mathbf{z}}$ & $(8 p)$ & IV \\
\hline $\mathbf{B}_{36}$ & $=$ & $y_{7} \mathbf{a}_{1}-x_{7} \mathbf{a}_{2}+\left(\frac{1}{2}+z_{7}\right) \mathbf{a}_{3}$ & $=$ & $y_{7} a \hat{\mathbf{x}}-x_{7} a \hat{\mathbf{y}}+\left(\frac{1}{2}+z_{7}\right) c \hat{\mathbf{z}}$ & $(8 p)$ & C IV \\
\hline $\mathbf{B}_{37}$ & $=$ & $-x_{7} \mathbf{a}_{1}+y_{7} \mathbf{a}_{2}-z_{7} \mathbf{a}_{3}$ & $=$ & $-x_{7} a \hat{\mathbf{x}}+y_{7} a \hat{\mathbf{y}}-z_{7} c \hat{\mathbf{z}}$ & $(8 p)$ & C IV \\
\hline $\mathbf{B}_{38}$ & $=$ & $x_{7} \mathbf{a}_{1}-y_{7} \mathbf{a}_{2}-z_{7} \mathbf{a}_{3}$ & $=$ & $x_{7} a \hat{\mathbf{x}}-y_{7} a \hat{\mathbf{y}}-z_{7} c \hat{\mathbf{z}}$ & $(8 p)$ & C IV \\
\hline $\mathbf{B}_{39}$ & $=$ & $y_{7} \mathbf{a}_{1}+x_{7} \mathbf{a}_{2}+\left(\frac{1}{2}-z_{7}\right) \mathbf{a}_{3}$ & $=$ & $y_{7} a \hat{\mathbf{x}}+x_{7} a \hat{\mathbf{y}}+\left(\frac{1}{2}-z_{7}\right) c \hat{\mathbf{z}}$ & $(8 p)$ & \\
\hline
\end{tabular}




\begin{tabular}{|c|c|c|c|c|c|c|}
\hline $\mathbf{B}_{40}$ & $=$ & $-y_{7} \mathbf{a}_{1}-x_{7} \mathbf{a}_{2}+\left(\frac{1}{2}-z_{7}\right) \mathbf{a}_{3}$ & $=$ & $-y_{7} a \hat{\mathbf{x}}-x_{7} a \hat{\mathbf{y}}+\left(\frac{1}{2}-z_{7}\right) c \hat{\mathbf{z}}$ & $(8 p)$ & C IV \\
\hline $\mathbf{B}_{41}$ & $=$ & $x_{8} \mathbf{a}_{1}+y_{8} \mathbf{a}_{2}+z_{8} \mathbf{a}_{3}$ & $=$ & $x_{8} a \hat{\mathbf{x}}+y_{8} a \hat{\mathbf{y}}+z_{8} c \hat{\mathbf{z}}$ & $(8 p)$ & $\mathrm{C} \mathrm{V}$ \\
\hline $\mathbf{B}_{42}$ & $=$ & $-x_{8} \mathbf{a}_{1}-y_{8} \mathbf{a}_{2}+z_{8} \mathbf{a}_{3}$ & $=$ & $-x_{8} a \hat{\mathbf{x}}-y_{8} a \hat{\mathbf{y}}+z_{8} c \hat{\mathbf{z}}$ & $(8 p)$ & $\mathrm{CV}$ \\
\hline $\mathbf{B}_{43}$ & $=$ & $-y_{8} \mathbf{a}_{1}+x_{8} \mathbf{a}_{2}+\left(\frac{1}{2}+z_{8}\right) \mathbf{a}_{3}$ & $=$ & $-y_{8} a \hat{\mathbf{x}}+x_{8} a \hat{\mathbf{y}}+\left(\frac{1}{2}+z_{8}\right) c \hat{\mathbf{z}}$ & $(8 p)$ & $\mathrm{C} \mathrm{V}$ \\
\hline $\mathbf{B}_{44}$ & $=$ & $y_{8} \mathbf{a}_{1}-x_{8} \mathbf{a}_{2}+\left(\frac{1}{2}+z_{8}\right) \mathbf{a}_{3}$ & $=$ & $y_{8} a \hat{\mathbf{x}}-x_{8} a \hat{\mathbf{y}}+\left(\frac{1}{2}+z_{8}\right) c \hat{\mathbf{z}}$ & $(8 p)$ & $\mathrm{C} \mathrm{V}$ \\
\hline $\mathbf{B}_{45}$ & $=$ & $-x_{8} \mathbf{a}_{1}+y_{8} \mathbf{a}_{2}-z_{8} \mathbf{a}_{3}$ & $=$ & $-x_{8} a \hat{\mathbf{x}}+y_{8} a \hat{\mathbf{y}}-z_{8} c \hat{\mathbf{z}}$ & $(8 p)$ & $\mathrm{C} \mathrm{V}$ \\
\hline $\mathbf{B}_{46}$ & $=$ & $x_{8} \mathbf{a}_{1}-y_{8} \mathbf{a}_{2}-z_{8} \mathbf{a}_{3}$ & $=$ & $x_{8} a \hat{\mathbf{x}}-y_{8} a \hat{\mathbf{y}}-z_{8} c \hat{\mathbf{z}}$ & $(8 p)$ & $\mathrm{C} \mathrm{V}$ \\
\hline $\mathbf{B}_{47}$ & $=$ & $y_{8} \mathbf{a}_{1}+x_{8} \mathbf{a}_{2}+\left(\frac{1}{2}-z_{8}\right) \mathbf{a}_{3}$ & $=$ & $y_{8} a \hat{\mathbf{x}}+x_{8} a \hat{\mathbf{y}}+\left(\frac{1}{2}-z_{8}\right) c \hat{\mathbf{z}}$ & $(8 p)$ & $\mathrm{C} \mathrm{V}$ \\
\hline $\mathbf{B}_{48}$ & $=$ & $-y_{8} \mathbf{a}_{1}-x_{8} \mathbf{a}_{2}+\left(\frac{1}{2}-z_{8}\right) \mathbf{a}_{3}$ & $=$ & $-y_{8} a \hat{\mathbf{x}}-x_{8} a \hat{\mathbf{y}}+\left(\frac{1}{2}-z_{8}\right) c \hat{\mathbf{z}}$ & $(8 p)$ & $\mathrm{C} \mathrm{V}$ \\
\hline $\mathbf{B}_{49}$ & $=$ & $x_{9} \mathbf{a}_{1}+y_{9} \mathbf{a}_{2}+z_{9} \mathbf{a}_{3}$ & $=$ & $x_{9} a \hat{\mathbf{x}}+y_{9} a \hat{\mathbf{y}}+z_{9} c \hat{\mathbf{z}}$ & $(8 p)$ & C VI \\
\hline $\mathbf{B}_{50}$ & $=$ & $-x_{9} \mathbf{a}_{1}-y_{9} \mathbf{a}_{2}+z_{9} \mathbf{a}_{3}$ & $=$ & $-x_{9} a \hat{\mathbf{x}}-y_{9} a \hat{\mathbf{y}}+z_{9} c \hat{\mathbf{z}}$ & $(8 p)$ & $\mathrm{C}$ VI \\
\hline $\mathbf{B}_{51}$ & $=$ & $-y_{9} \mathbf{a}_{1}+x_{9} \mathbf{a}_{2}+\left(\frac{1}{2}+z_{9}\right) \mathbf{a}_{3}$ & $=$ & $-y_{9} a \hat{\mathbf{x}}+x_{9} a \hat{\mathbf{y}}+\left(\frac{1}{2}+z_{9}\right) c \hat{\mathbf{z}}$ & $(8 p)$ & C VI \\
\hline $\mathbf{B}_{52}$ & $=$ & $y_{9} \mathbf{a}_{1}-x_{9} \mathbf{a}_{2}+\left(\frac{1}{2}+z_{9}\right) \mathbf{a}_{3}$ & $=$ & $y_{9} a \hat{\mathbf{x}}-x_{9} a \hat{\mathbf{y}}+\left(\frac{1}{2}+z_{9}\right) c \hat{\mathbf{z}}$ & $(8 p)$ & C VI \\
\hline $\mathbf{B}_{53}$ & $=$ & $-x_{9} \mathbf{a}_{1}+y_{9} \mathbf{a}_{2}-z_{9} \mathbf{a}_{3}$ & $=$ & $-x_{9} a \hat{\mathbf{x}}+y_{9} a \hat{\mathbf{y}}-z_{9} c \hat{\mathbf{z}}$ & $(8 p)$ & C VI \\
\hline $\mathbf{B}_{54}$ & $=$ & $x_{9} \mathbf{a}_{1}-y_{9} \mathbf{a}_{2}-z_{9} \mathbf{a}_{3}$ & $=$ & $x_{9} a \hat{\mathbf{x}}-y_{9} a \hat{\mathbf{y}}-z_{9} c \hat{\mathbf{z}}$ & $(8 p)$ & C VI \\
\hline $\mathbf{B}_{55}$ & $=$ & $y_{9} \mathbf{a}_{1}+x_{9} \mathbf{a}_{2}+\left(\frac{1}{2}-z_{9}\right) \mathbf{a}_{3}$ & $=$ & $y_{9} a \hat{\mathbf{x}}+x_{9} a \hat{\mathbf{y}}+\left(\frac{1}{2}-z_{9}\right) c \hat{\mathbf{z}}$ & $(8 p)$ & C VI \\
\hline $\mathbf{B}_{56}$ & $=$ & $-y_{9} \mathbf{a}_{1}-x_{9} \mathbf{a}_{2}+\left(\frac{1}{2}-z_{9}\right) \mathbf{a}_{3}$ & $=$ & $-y_{9} a \hat{\mathbf{x}}-x_{9} a \hat{\mathbf{y}}+\left(\frac{1}{2}-z_{9}\right) c \hat{\mathbf{z}}$ & $(8 p)$ & C VI \\
\hline $\mathbf{B}_{57}$ & $=$ & $x_{10} \mathbf{a}_{1}+y_{10} \mathbf{a}_{2}+z_{10} \mathbf{a}_{3}$ & $=$ & $x_{10} a \hat{\mathbf{x}}+y_{10} a \hat{\mathbf{y}}+z_{10} c \hat{\mathbf{z}}$ & $(8 p)$ & C VII \\
\hline $\mathbf{B}_{58}$ & $=$ & $-x_{10} \mathbf{a}_{1}-y_{10} \mathbf{a}_{2}+z_{10} \mathbf{a}_{3}$ & $=$ & $-x_{10} a \hat{\mathbf{x}}-y_{10} a \hat{\mathbf{y}}+z_{10} c \hat{\mathbf{z}}$ & $(8 p)$ & C VII \\
\hline $\mathbf{B}_{59}$ & $=$ & $-y_{10} \mathbf{a}_{1}+x_{10} \mathbf{a}_{2}+\left(\frac{1}{2}+z_{10}\right) \mathbf{a}_{3}$ & $=$ & $-y_{10} a \hat{\mathbf{x}}+x_{10} a \hat{\mathbf{y}}+\left(\frac{1}{2}+z_{10}\right) c \hat{\mathbf{z}}$ & $(8 p)$ & C VII \\
\hline $\mathbf{B}_{60}$ & $=$ & $y_{10} \mathbf{a}_{1}-x_{10} \mathbf{a}_{2}+\left(\frac{1}{2}+z_{10}\right) \mathbf{a}_{3}$ & $=$ & $y_{10} a \hat{\mathbf{x}}-x_{10} a \hat{\mathbf{y}}+\left(\frac{1}{2}+z_{10}\right) c \hat{\mathbf{z}}$ & $(8 p)$ & C VII \\
\hline $\mathbf{B}_{61}$ & $=$ & $-x_{10} \mathbf{a}_{1}+y_{10} \mathbf{a}_{2}-z_{10} \mathbf{a}_{3}$ & $=$ & $-x_{10} a \hat{\mathbf{x}}+y_{10} a \hat{\mathbf{y}}-z_{10} c \hat{\mathbf{z}}$ & $(8 p)$ & C VII \\
\hline $\mathbf{B}_{62}$ & $=$ & $x_{10} \mathbf{a}_{1}-y_{10} \mathbf{a}_{2}-z_{10} \mathbf{a}_{3}$ & $=$ & $x_{10} a \hat{\mathbf{x}}-y_{10} a \hat{\mathbf{y}}-z_{10} c \hat{\mathbf{z}}$ & $(8 p)$ & C VII \\
\hline $\mathbf{B}_{63}$ & $=$ & $y_{10} \mathbf{a}_{1}+x_{10} \mathbf{a}_{2}+\left(\frac{1}{2}-z_{10}\right) \mathbf{a}_{3}$ & $=$ & $y_{10} a \hat{\mathbf{x}}+x_{10} a \hat{\mathbf{y}}+\left(\frac{1}{2}-z_{10}\right) c \hat{\mathbf{z}}$ & $(8 p)$ & C VII \\
\hline $\mathbf{B}_{64}$ & $=$ & $-y_{10} \mathbf{a}_{1}-x_{10} \mathbf{a}_{2}+\left(\frac{1}{2}-z_{10}\right) \mathbf{a}_{3}$ & $=$ & $-y_{10} a \hat{\mathbf{x}}-x_{10} a \hat{\mathbf{y}}+\left(\frac{1}{2}-z_{10}\right) c \hat{\mathbf{z}}$ & $(8 p)$ & C VII \\
\hline $\mathbf{B}_{65}$ & $=$ & $x_{11} \mathbf{a}_{1}+y_{11} \mathbf{a}_{2}+z_{11} \mathbf{a}_{3}$ & $=$ & $x_{11} a \hat{\mathbf{x}}+y_{11} a \hat{\mathbf{y}}+z_{11} c \hat{\mathbf{z}}$ & $(8 p)$ & C VIII \\
\hline $\mathbf{B}_{66}$ & $=$ & $-x_{11} \mathbf{a}_{1}-y_{11} \mathbf{a}_{2}+z_{11} \mathbf{a}_{3}$ & $=$ & $-x_{11} a \hat{\mathbf{x}}-y_{11} a \hat{\mathbf{y}}+z_{11} c \hat{\mathbf{z}}$ & $(8 p)$ & C VIII \\
\hline $\mathbf{B}_{67}$ & $=$ & $-y_{11} \mathbf{a}_{1}+x_{11} \mathbf{a}_{2}+\left(\frac{1}{2}+z_{11}\right) \mathbf{a}_{3}$ & $=$ & $-y_{11} a \hat{\mathbf{x}}+x_{11} a \hat{\mathbf{y}}+\left(\frac{1}{2}+z_{11}\right) c \hat{\mathbf{z}}$ & $(8 p)$ & C VIII \\
\hline $\mathbf{B}_{68}$ & $=$ & $y_{11} \mathbf{a}_{1}-x_{11} \mathbf{a}_{2}+\left(\frac{1}{2}+z_{11}\right) \mathbf{a}_{3}$ & $=$ & $y_{11} a \hat{\mathbf{x}}-x_{11} a \hat{\mathbf{y}}+\left(\frac{1}{2}+z_{11}\right) c \hat{\mathbf{z}}$ & $(8 p)$ & C VIII \\
\hline $\mathbf{B}_{69}$ & $=$ & $-x_{11} \mathbf{a}_{1}+y_{11} \mathbf{a}_{2}-z_{11} \mathbf{a}_{3}$ & $=$ & $-x_{11} a \hat{\mathbf{x}}+y_{11} a \hat{\mathbf{y}}-z_{11} c \hat{\mathbf{z}}$ & $(8 p)$ & C VIII \\
\hline $\mathbf{B}_{70}$ & $=$ & $x_{11} \mathbf{a}_{1}-y_{11} \mathbf{a}_{2}-z_{11} \mathbf{a}_{3}$ & $=$ & $x_{11} a \hat{\mathbf{x}}-y_{11} a \hat{\mathbf{y}}-z_{11} c \hat{\mathbf{z}}$ & $(8 p)$ & C VIII \\
\hline $\mathbf{B}_{71}$ & $=$ & $y_{11} \mathbf{a}_{1}+x_{11} \mathbf{a}_{2}+\left(\frac{1}{2}-z_{11}\right) \mathbf{a}_{3}$ & $=$ & $y_{11} a \hat{\mathbf{x}}+x_{11} a \hat{\mathbf{y}}+\left(\frac{1}{2}-z_{11}\right) c \hat{\mathbf{z}}$ & $(8 p)$ & C VIII \\
\hline $\mathbf{B}_{72}$ & $=$ & $-y_{11} \mathbf{a}_{1}-x_{11} \mathbf{a}_{2}+\left(\frac{1}{2}-z_{11}\right) \mathbf{a}_{3}$ & $=$ & $-y_{11} a \hat{\mathbf{x}}-x_{11} a \hat{\mathbf{y}}+\left(\frac{1}{2}-z_{11}\right) c \hat{\mathbf{z}}$ & $(8 p)$ & C VIII \\
\hline $\mathbf{B}_{73}$ & $=$ & $x_{12} \mathbf{a}_{1}+y_{12} \mathbf{a}_{2}+z_{12} \mathbf{a}_{3}$ & $=$ & $x_{12} a \hat{\mathbf{x}}+y_{12} a \hat{\mathbf{y}}+z_{12} c \hat{\mathbf{z}}$ & $(8 p)$ & C IX \\
\hline $\mathbf{B}_{74}$ & $=$ & $-x_{12} \mathbf{a}_{1}-y_{12} \mathbf{a}_{2}+z_{12} \mathbf{a}_{3}$ & $=$ & $-x_{12} a \hat{\mathbf{x}}-y_{12} a \hat{\mathbf{y}}+z_{12} c \hat{\mathbf{z}}$ & $(8 p)$ & C IX \\
\hline $\mathbf{B}_{75}$ & $=$ & $-y_{12} \mathbf{a}_{1}+x_{12} \mathbf{a}_{2}+\left(\frac{1}{2}+z_{12}\right) \mathbf{a}_{3}$ & $=$ & $-y_{12} a \hat{\mathbf{x}}+x_{12} a \hat{\mathbf{y}}+\left(\frac{1}{2}+z_{12}\right) c \hat{\mathbf{z}}$ & $(8 p)$ & C IX \\
\hline
\end{tabular}




\begin{tabular}{|c|c|c|c|c|c|c|}
\hline $\mathbf{B}_{76}$ & $=$ & $y_{12} \mathbf{a}_{1}-x_{12} \mathbf{a}_{2}+\left(\frac{1}{2}+z_{12}\right) \mathbf{a}_{3}$ & $=$ & $y_{12} a \hat{\mathbf{x}}-x_{12} a \hat{\mathbf{y}}+\left(\frac{1}{2}+z_{12}\right) c \hat{\mathbf{z}}$ & $(8 p)$ & C IX \\
\hline $\mathbf{B}_{77}$ & $=$ & $-x_{12} \mathbf{a}_{1}+y_{12} \mathbf{a}_{2}-z_{12} \mathbf{a}_{3}$ & $=$ & $-x_{12} a \hat{\mathbf{x}}+y_{12} a \hat{\mathbf{y}}-z_{12} c \hat{\mathbf{z}}$ & $(8 p)$ & C IX \\
\hline $\mathbf{B}_{78}$ & $=$ & $x_{12} \mathbf{a}_{1}-y_{12} \mathbf{a}_{2}-z_{12} \mathbf{a}_{3}$ & $=$ & $x_{12} a \hat{\mathbf{x}}-y_{12} a \hat{\mathbf{y}}-z_{12} c \hat{\mathbf{z}}$ & $(8 p)$ & C IX \\
\hline $\mathbf{B}_{79}$ & $=$ & $y_{12} \mathbf{a}_{1}+x_{12} \mathbf{a}_{2}+\left(\frac{1}{2}-z_{12}\right) \mathbf{a}_{3}$ & $=$ & $y_{12} a \hat{\mathbf{x}}+x_{12} a \hat{\mathbf{y}}+\left(\frac{1}{2}-z_{12}\right) c \hat{\mathbf{z}}$ & $(8 p)$ & C IX \\
\hline $\mathbf{B}_{80}$ & $=$ & $-y_{12} \mathbf{a}_{1}-x_{12} \mathbf{a}_{2}+\left(\frac{1}{2}-z_{12}\right) \mathbf{a}_{3}$ & $=$ & $-y_{12} a \hat{\mathbf{x}}-x_{12} a \hat{\mathbf{y}}+\left(\frac{1}{2}-z_{12}\right) c \hat{\mathbf{z}}$ & $(8 p)$ & C IX \\
\hline $\mathbf{B}_{81}$ & $=$ & $x_{13} \mathbf{a}_{1}+y_{13} \mathbf{a}_{2}+z_{13} \mathbf{a}_{3}$ & $=$ & $x_{13} a \hat{\mathbf{x}}+y_{13} a \hat{\mathbf{y}}+z_{13} c \hat{\mathbf{z}}$ & $(8 p)$ & $\mathrm{CX}$ \\
\hline $\mathbf{B}_{82}$ & $=$ & $-x_{13} \mathbf{a}_{1}-y_{13} \mathbf{a}_{2}+z_{13} \mathbf{a}_{3}$ & $=$ & $-x_{13} a \hat{\mathbf{x}}-y_{13} a \hat{\mathbf{y}}+z_{13} c \hat{\mathbf{z}}$ & $(8 p)$ & $\mathrm{CX}$ \\
\hline $\mathbf{B}_{83}$ & $=$ & $-y_{13} \mathbf{a}_{1}+x_{13} \mathbf{a}_{2}+\left(\frac{1}{2}+z_{13}\right) \mathbf{a}_{3}$ & $=$ & $-y_{13} a \hat{\mathbf{x}}+x_{13} a \hat{\mathbf{y}}+\left(\frac{1}{2}+z_{13}\right) c \hat{\mathbf{z}}$ & $(8 p)$ & $\mathrm{CX}$ \\
\hline $\mathbf{B}_{84}$ & $=$ & $y_{13} \mathbf{a}_{1}-x_{13} \mathbf{a}_{2}+\left(\frac{1}{2}+z_{13}\right) \mathbf{a}_{3}$ & $=$ & $y_{13} a \hat{\mathbf{x}}-x_{13} a \hat{\mathbf{y}}+\left(\frac{1}{2}+z_{13}\right) c \hat{\mathbf{z}}$ & $(8 p)$ & $\mathrm{CX}$ \\
\hline $\mathbf{B}_{85}$ & $=$ & $-x_{13} \mathbf{a}_{1}+y_{13} \mathbf{a}_{2}-z_{13} \mathbf{a}_{3}$ & $=$ & $-x_{13} a \hat{\mathbf{x}}+y_{13} a \hat{\mathbf{y}}-z_{13} c \hat{\mathbf{z}}$ & $(8 p)$ & $\mathrm{CX}$ \\
\hline $\mathbf{B}_{86}$ & $=$ & $x_{13} \mathbf{a}_{1}-y_{13} \mathbf{a}_{2}-z_{13} \mathbf{a}_{3}$ & $=$ & $x_{13} a \hat{\mathbf{x}}-y_{13} a \hat{\mathbf{y}}-z_{13} c \hat{\mathbf{z}}$ & $(8 p)$ & $\mathrm{CX}$ \\
\hline $\mathbf{B}_{87}$ & $=$ & $y_{13} \mathbf{a}_{1}+x_{13} \mathbf{a}_{2}+\left(\frac{1}{2}-z_{13}\right) \mathbf{a}_{3}$ & $=$ & $y_{13} a \hat{\mathbf{x}}+x_{13} a \hat{\mathbf{y}}+\left(\frac{1}{2}-z_{13}\right) c \hat{\mathbf{z}}$ & $(8 p)$ & $\mathrm{CX}$ \\
\hline $\mathbf{B}_{88}$ & $=$ & $-y_{13} \mathbf{a}_{1}-x_{13} \mathbf{a}_{2}+\left(\frac{1}{2}-z_{13}\right) \mathbf{a}_{3}$ & $=$ & $-y_{13} a \hat{\mathbf{x}}-x_{13} a \hat{\mathbf{y}}+\left(\frac{1}{2}-z_{13}\right) c \hat{\mathbf{z}}$ & $(8 p)$ & $\mathrm{CX}$ \\
\hline $\mathbf{B}_{89}$ & $=$ & $x_{14} \mathbf{a}_{1}+y_{14} \mathbf{a}_{2}+z_{14} \mathbf{a}_{3}$ & $=$ & $x_{14} a \hat{\mathbf{x}}+y_{14} a \hat{\mathbf{y}}+z_{14} c \hat{\mathbf{z}}$ & $(8 p)$ & $\mathrm{C} \mathrm{XI}$ \\
\hline $\mathbf{B}_{90}$ & $=$ & $-x_{14} \mathbf{a}_{1}-y_{14} \mathbf{a}_{2}+z_{14} \mathbf{a}_{3}$ & $=$ & $-x_{14} a \hat{\mathbf{x}}-y_{14} a \hat{\mathbf{y}}+z_{14} c \hat{\mathbf{z}}$ & $(8 p)$ & C XI \\
\hline $\mathbf{B}_{91}$ & $=$ & $-y_{14} \mathbf{a}_{1}+x_{14} \mathbf{a}_{2}+\left(\frac{1}{2}+z_{14}\right) \mathbf{a}_{3}$ & $=$ & $-y_{14} a \hat{\mathbf{x}}+x_{14} a \hat{\mathbf{y}}+\left(\frac{1}{2}+z_{14}\right) c \hat{\mathbf{z}}$ & $(8 p)$ & C XI \\
\hline $\mathbf{B}_{92}$ & $=$ & $y_{14} \mathbf{a}_{1}-x_{14} \mathbf{a}_{2}+\left(\frac{1}{2}+z_{14}\right) \mathbf{a}_{3}$ & $=$ & $y_{14} a \hat{\mathbf{x}}-x_{14} a \hat{\mathbf{y}}+\left(\frac{1}{2}+z_{14}\right) c \hat{\mathbf{z}}$ & $(8 p)$ & $\mathrm{C} \mathrm{XI}$ \\
\hline $\mathbf{B}_{93}$ & $=$ & $-x_{14} \mathbf{a}_{1}+y_{14} \mathbf{a}_{2}-z_{14} \mathbf{a}_{3}$ & $=$ & $-x_{14} a \hat{\mathbf{x}}+y_{14} a \hat{\mathbf{y}}-z_{14} c \hat{\mathbf{z}}$ & $(8 p)$ & C XI \\
\hline $\mathbf{B}_{94}$ & $=$ & $x_{14} \mathbf{a}_{1}-y_{14} \mathbf{a}_{2}-z_{14} \mathbf{a}_{3}$ & $=$ & $x_{14} a \hat{\mathbf{x}}-y_{14} a \hat{\mathbf{y}}-z_{14} c \hat{\mathbf{z}}$ & $(8 p)$ & C XI \\
\hline $\mathbf{B}_{95}$ & $=$ & $y_{14} \mathbf{a}_{1}+x_{14} \mathbf{a}_{2}+\left(\frac{1}{2}-z_{14}\right) \mathbf{a}_{3}$ & $=$ & $y_{14} a \hat{\mathbf{x}}+x_{14} a \hat{\mathbf{y}}+\left(\frac{1}{2}-z_{14}\right) c \hat{\mathbf{z}}$ & $(8 p)$ & C XI \\
\hline $\mathbf{B}_{96}$ & $=$ & $-y_{14} \mathbf{a}_{1}-x_{14} \mathbf{a}_{2}+\left(\frac{1}{2}-z_{14}\right) \mathbf{a}_{3}$ & $=$ & $-y_{14} a \hat{\mathbf{x}}-x_{14} a \hat{\mathbf{y}}+\left(\frac{1}{2}-z_{14}\right) c \hat{\mathbf{z}}$ & $(8 p)$ & $\mathrm{C} \mathrm{XI}$ \\
\hline $\mathbf{B}_{97}$ & $=$ & $x_{15} \mathbf{a}_{1}+y_{15} \mathbf{a}_{2}+z_{15} \mathbf{a}_{3}$ & $=$ & $x_{15} a \hat{\mathbf{x}}+y_{15} a \hat{\mathbf{y}}+z_{15} c \hat{\mathbf{z}}$ & $(8 p)$ & C XII \\
\hline $\mathbf{B}_{98}$ & $=$ & $-x_{15} \mathbf{a}_{1}-y_{15} \mathbf{a}_{2}+z_{15} \mathbf{a}_{3}$ & $=$ & $-x_{15} a \hat{\mathbf{x}}-y_{15} a \hat{\mathbf{y}}+z_{15} c \hat{\mathbf{z}}$ & $(8 p)$ & C XII \\
\hline $\mathbf{B}_{99}$ & $=$ & $-y_{15} \mathbf{a}_{1}+x_{15} \mathbf{a}_{2}+\left(\frac{1}{2}+z_{15}\right) \mathbf{a}_{3}$ & $=$ & $-y_{15} a \hat{\mathbf{x}}+x_{15} a \hat{\mathbf{y}}+\left(\frac{1}{2}+z_{15}\right) c \hat{\mathbf{z}}$ & $(8 p)$ & C XII \\
\hline $\mathbf{B}_{100}$ & $=$ & $y_{15} \mathbf{a}_{1}-x_{15} \mathbf{a}_{2}+\left(\frac{1}{2}+z_{15}\right) \mathbf{a}_{3}$ & $=$ & $y_{15} a \hat{\mathbf{x}}-x_{15} a \hat{\mathbf{y}}+\left(\frac{1}{2}+z_{15}\right) c \hat{\mathbf{z}}$ & $(8 p)$ & C XII \\
\hline $\mathbf{B}_{101}$ & $=$ & $-x_{15} \mathbf{a}_{1}+y_{15} \mathbf{a}_{2}-z_{15} \mathbf{a}_{3}$ & $=$ & $-x_{15} a \hat{\mathbf{x}}+y_{15} a \hat{\mathbf{y}}-z_{15} c \hat{\mathbf{z}}$ & $(8 p)$ & C XII \\
\hline $\mathbf{B}_{102}$ & $=$ & $x_{15} \mathbf{a}_{1}-y_{15} \mathbf{a}_{2}-z_{15} \mathbf{a}_{3}$ & $=$ & $x_{15} a \hat{\mathbf{x}}-y_{15} a \hat{\mathbf{y}}-z_{15} c \hat{\mathbf{z}}$ & $(8 p)$ & C XII \\
\hline $\mathbf{B}_{103}$ & $=$ & $y_{15} \mathbf{a}_{1}+x_{15} \mathbf{a}_{2}+\left(\frac{1}{2}-z_{15}\right) \mathbf{a}_{3}$ & $=$ & $y_{15} a \hat{\mathbf{x}}+x_{15} a \hat{\mathbf{y}}+\left(\frac{1}{2}-z_{15}\right) c \hat{\mathbf{z}}$ & $(8 p)$ & C XII \\
\hline $\mathbf{B}_{104}$ & $=$ & $-y_{15} \mathbf{a}_{1}-x_{15} \mathbf{a}_{2}+\left(\frac{1}{2}-z_{15}\right) \mathbf{a}_{3}$ & $=$ & $-y_{15} a \hat{\mathbf{x}}-x_{15} a \hat{\mathbf{y}}+\left(\frac{1}{2}-z_{15}\right) c \hat{\mathbf{z}}$ & $(8 p)$ & C XII \\
\hline $\mathbf{B}_{105}$ & $=$ & $x_{16} \mathbf{a}_{1}+y_{16} \mathbf{a}_{2}+z_{16} \mathbf{a}_{3}$ & $=$ & $x_{16} a \hat{\mathbf{x}}+y_{16} a \hat{\mathbf{y}}+z_{16} c \hat{\mathbf{z}}$ & $(8 p)$ & C XIII \\
\hline $\mathbf{B}_{106}$ & $=$ & $-x_{16} \mathbf{a}_{1}-y_{16} \mathbf{a}_{2}+z_{16} \mathbf{a}_{3}$ & $=$ & $-x_{16} a \hat{\mathbf{x}}-y_{16} a \hat{\mathbf{y}}+z_{16} c \hat{\mathbf{z}}$ & $(8 p)$ & C XIII \\
\hline $\mathbf{B}_{107}$ & $=$ & $-y_{16} \mathbf{a}_{1}+x_{16} \mathbf{a}_{2}+\left(\frac{1}{2}+z_{16}\right) \mathbf{a}_{3}$ & $=$ & $-y_{16} a \hat{\mathbf{x}}+x_{16} a \hat{\mathbf{y}}+\left(\frac{1}{2}+z_{16}\right) c \hat{\mathbf{z}}$ & $(8 p)$ & C XIII \\
\hline $\mathbf{B}_{108}$ & $=$ & $y_{16} \mathbf{a}_{1}-x_{16} \mathbf{a}_{2}+\left(\frac{1}{2}+z_{16}\right) \mathbf{a}_{3}$ & $=$ & $y_{16} a \hat{\mathbf{x}}-x_{16} a \hat{\mathbf{y}}+\left(\frac{1}{2}+z_{16}\right) c \hat{\mathbf{z}}$ & $(8 p)$ & C XIII \\
\hline $\mathbf{B}_{109}$ & $=$ & $-x_{16} \mathbf{a}_{1}+y_{16} \mathbf{a}_{2}-z_{16} \mathbf{a}_{3}$ & $=$ & $-x_{16} a \hat{\mathbf{x}}+y_{16} a \hat{\mathbf{y}}-z_{16} c \hat{\mathbf{z}}$ & $(8 p)$ & C XIII \\
\hline $\mathbf{B}_{110}$ & $=$ & $x_{16} \mathbf{a}_{1}-y_{16} \mathbf{a}_{2}-z_{16} \mathbf{a}_{3}$ & $=$ & $x_{16} a \hat{\mathbf{x}}-y_{16} a \hat{\mathbf{y}}-z_{16} c \hat{\mathbf{z}}$ & $(8 p)$ & C XIII \\
\hline $\mathbf{B}_{111}$ & $=$ & $y_{16} \mathbf{a}_{1}+x_{16} \mathbf{a}_{2}+\left(\frac{1}{2}-z_{16}\right) \mathbf{a}_{3}$ & $=$ & $y_{16} a \hat{\mathbf{x}}+x_{16} a \hat{\mathbf{y}}+\left(\frac{1}{2}-z_{16}\right) c \hat{\mathbf{z}}$ & $(8 p)$ & C XIII \\
\hline
\end{tabular}




\begin{tabular}{|c|c|c|c|c|c|c|}
\hline $\mathbf{B}_{112}$ & $=$ & $-y_{16} \mathbf{a}_{1}-x_{16} \mathbf{a}_{2}+\left(\frac{1}{2}-z_{16}\right) \mathbf{a}_{3}$ & $=$ & $-y_{16} a \hat{\mathbf{x}}-x_{16} a \hat{\mathbf{y}}+\left(\frac{1}{2}-z_{16}\right) c \hat{\mathbf{z}}$ & $(8 p)$ & C XIII \\
\hline $\mathbf{B}_{113}$ & $=$ & $x_{17} \mathbf{a}_{1}+y_{17} \mathbf{a}_{2}+z_{17} \mathbf{a}_{3}$ & $=$ & $x_{17} a \hat{\mathbf{x}}+y_{17} a \hat{\mathbf{y}}+z_{17} c \hat{\mathbf{z}}$ & $(8 p)$ & C XIV \\
\hline $\mathbf{B}_{114}$ & $=$ & $-x_{17} \mathbf{a}_{1}-y_{17} \mathbf{a}_{2}+z_{17} \mathbf{a}_{3}$ & $=$ & $-x_{17} a \hat{\mathbf{x}}-y_{17} a \hat{\mathbf{y}}+z_{17} c \hat{\mathbf{z}}$ & $(8 p)$ & C XIV \\
\hline $\mathbf{B}_{115}$ & $=$ & $-y_{17} \mathbf{a}_{1}+x_{17} \mathbf{a}_{2}+\left(\frac{1}{2}+z_{17}\right) \mathbf{a}_{3}$ & $=$ & $-y_{17} a \hat{\mathbf{x}}+x_{17} a \hat{\mathbf{y}}+\left(\frac{1}{2}+z_{17}\right) c \hat{\mathbf{z}}$ & $(8 p)$ & C XIV \\
\hline $\mathbf{B}_{116}$ & $=$ & $y_{17} \mathbf{a}_{1}-x_{17} \mathbf{a}_{2}+\left(\frac{1}{2}+z_{17}\right) \mathbf{a}_{3}$ & $=$ & $y_{17} a \hat{\mathbf{x}}-x_{17} a \hat{\mathbf{y}}+\left(\frac{1}{2}+z_{17}\right) c \hat{\mathbf{z}}$ & $(8 p)$ & C XIV \\
\hline $\mathbf{B}_{117}$ & $=$ & $-x_{17} \mathbf{a}_{1}+y_{17} \mathbf{a}_{2}-z_{17} \mathbf{a}_{3}$ & $=$ & $-x_{17} a \hat{\mathbf{x}}+y_{17} a \hat{\mathbf{y}}-z_{17} c \hat{\mathbf{z}}$ & $(8 p)$ & C XIV \\
\hline $\mathbf{B}_{118}$ & $=$ & $x_{17} \mathbf{a}_{1}-y_{17} \mathbf{a}_{2}-z_{17} \mathbf{a}_{3}$ & $=$ & $x_{17} a \hat{\mathbf{x}}-y_{17} a \hat{\mathbf{y}}-z_{17} c \hat{\mathbf{z}}$ & $(8 p)$ & C XIV \\
\hline $\mathbf{B}_{119}$ & $=$ & $y_{17} \mathbf{a}_{1}+x_{17} \mathbf{a}_{2}+\left(\frac{1}{2}-z_{17}\right) \mathbf{a}_{3}$ & $=$ & $y_{17} a \hat{\mathbf{x}}+x_{17} a \hat{\mathbf{y}}+\left(\frac{1}{2}-z_{17}\right) c \hat{\mathbf{z}}$ & $(8 p)$ & C XIV \\
\hline $\mathbf{B}_{120}$ & $=$ & $-y_{17} \mathbf{a}_{1}-x_{17} \mathbf{a}_{2}+\left(\frac{1}{2}-z_{17}\right) \mathbf{a}_{3}$ & $=$ & $-y_{17} a \hat{\mathbf{x}}-x_{17} a \hat{\mathbf{y}}+\left(\frac{1}{2}-z_{17}\right) c \hat{\mathbf{z}}$ & $(8 p)$ & C XIV \\
\hline $\mathbf{B}_{121}$ & $=$ & $x_{18} \mathbf{a}_{1}+y_{18} \mathbf{a}_{2}+z_{18} \mathbf{a}_{3}$ & $=$ & $x_{18} a \hat{\mathbf{x}}+y_{18} a \hat{\mathbf{y}}+z_{18} c \hat{\mathbf{z}}$ & $(8 p)$ & $\mathrm{CXV}$ \\
\hline $\mathbf{B}_{122}$ & $=$ & $-x_{18} \mathbf{a}_{1}-y_{18} \mathbf{a}_{2}+z_{18} \mathbf{a}_{3}$ & $=$ & $-x_{18} a \hat{\mathbf{x}}-y_{18} a \hat{\mathbf{y}}+z_{18} c \hat{\mathbf{z}}$ & $(8 p)$ & $\mathrm{CXV}$ \\
\hline $\mathbf{B}_{123}$ & $=$ & $-y_{18} \mathbf{a}_{1}+x_{18} \mathbf{a}_{2}+\left(\frac{1}{2}+z_{18}\right) \mathbf{a}_{3}$ & $=$ & $-y_{18} a \hat{\mathbf{x}}+x_{18} a \hat{\mathbf{y}}+\left(\frac{1}{2}+z_{18}\right) c \hat{\mathbf{z}}$ & $(8 p)$ & $\mathrm{CXV}$ \\
\hline $\mathbf{B}_{124}$ & $=$ & $y_{18} \mathbf{a}_{1}-x_{18} \mathbf{a}_{2}+\left(\frac{1}{2}+z_{18}\right) \mathbf{a}_{3}$ & $=$ & $y_{18} a \hat{\mathbf{x}}-x_{18} a \hat{\mathbf{y}}+\left(\frac{1}{2}+z_{18}\right) c \hat{\mathbf{z}}$ & $(8 p)$ & $\mathrm{CXV}$ \\
\hline $\mathbf{B}_{125}$ & $=$ & $-x_{18} \mathbf{a}_{1}+y_{18} \mathbf{a}_{2}-z_{18} \mathbf{a}_{3}$ & $=$ & $-x_{18} a \hat{\mathbf{x}}+y_{18} a \hat{\mathbf{y}}-z_{18} c \hat{\mathbf{z}}$ & $(8 p)$ & $\mathrm{CXV}$ \\
\hline $\mathbf{B}_{126}$ & $=$ & $x_{18} \mathbf{a}_{1}-y_{18} \mathbf{a}_{2}-z_{18} \mathbf{a}_{3}$ & $=$ & $x_{18} a \hat{\mathbf{x}}-y_{18} a \hat{\mathbf{y}}-z_{18} c \hat{\mathbf{z}}$ & $(8 p)$ & $\mathrm{CXV}$ \\
\hline $\mathbf{B}_{127}$ & $=$ & $y_{18} \mathbf{a}_{1}+x_{18} \mathbf{a}_{2}+\left(\frac{1}{2}-z_{18}\right) \mathbf{a}_{3}$ & $=$ & $y_{18} a \hat{\mathbf{x}}+x_{18} a \hat{\mathbf{y}}+\left(\frac{1}{2}-z_{18}\right) c \hat{\mathbf{z}}$ & $(8 p)$ & $\mathrm{CXV}$ \\
\hline $\mathbf{B}_{128}$ & $=$ & $-y_{18} \mathbf{a}_{1}-x_{18} \mathbf{a}_{2}+\left(\frac{1}{2}-z_{18}\right) \mathbf{a}_{3}$ & $=$ & $-y_{18} a \hat{\mathbf{x}}-x_{18} a \hat{\mathbf{y}}+\left(\frac{1}{2}-z_{18}\right) c \hat{\mathbf{z}}$ & $(8 p)$ & $\mathrm{CXV}$ \\
\hline $\mathbf{B}_{129}$ & $=$ & $x_{19} \mathbf{a}_{1}+y_{19} \mathbf{a}_{2}+z_{19} \mathbf{a}_{3}$ & $=$ & $x_{19} a \hat{\mathbf{x}}+y_{19} a \hat{\mathbf{y}}+z_{19} c \hat{\mathbf{z}}$ & $(8 p)$ & C XVI \\
\hline $\mathbf{B}_{130}$ & $=$ & $-x_{19} \mathbf{a}_{1}-y_{19} \mathbf{a}_{2}+z_{19} \mathbf{a}_{3}$ & $=$ & $-x_{19} a \hat{\mathbf{x}}-y_{19} a \hat{\mathbf{y}}+z_{19} c \hat{\mathbf{z}}$ & $(8 p)$ & C XVI \\
\hline $\mathbf{B}_{131}$ & $=$ & $-y_{19} \mathbf{a}_{1}+x_{19} \mathbf{a}_{2}+\left(\frac{1}{2}+z_{19}\right) \mathbf{a}_{3}$ & $=$ & $-y_{19} a \hat{\mathbf{x}}+x_{19} a \hat{\mathbf{y}}+\left(\frac{1}{2}+z_{19}\right) c \hat{\mathbf{z}}$ & $(8 p)$ & C XVI \\
\hline $\mathbf{B}_{132}$ & $=$ & $y_{19} \mathbf{a}_{1}-x_{19} \mathbf{a}_{2}+\left(\frac{1}{2}+z_{19}\right) \mathbf{a}_{3}$ & $=$ & $y_{19} a \hat{\mathbf{x}}-x_{19} a \hat{\mathbf{y}}+\left(\frac{1}{2}+z_{19}\right) c \hat{\mathbf{z}}$ & $(8 p)$ & C XVI \\
\hline $\mathbf{B}_{133}$ & $=$ & $-x_{19} \mathbf{a}_{1}+y_{19} \mathbf{a}_{2}-z_{19} \mathbf{a}_{3}$ & $=$ & $-x_{19} a \hat{\mathbf{x}}+y_{19} a \hat{\mathbf{y}}-z_{19} c \hat{\mathbf{z}}$ & $(8 p)$ & C XVI \\
\hline $\mathbf{B}_{134}$ & $=$ & $x_{19} \mathbf{a}_{1}-y_{19} \mathbf{a}_{2}-z_{19} \mathbf{a}_{3}$ & $=$ & $x_{19} a \hat{\mathbf{x}}-y_{19} a \hat{\mathbf{y}}-z_{19} c \hat{\mathbf{z}}$ & $(8 p)$ & C XVI \\
\hline $\mathbf{B}_{135}$ & $=$ & $y_{19} \mathbf{a}_{1}+x_{19} \mathbf{a}_{2}+\left(\frac{1}{2}-z_{19}\right) \mathbf{a}_{3}$ & $=$ & $y_{19} a \hat{\mathbf{x}}+x_{19} a \hat{\mathbf{y}}+\left(\frac{1}{2}-z_{19}\right) c \hat{\mathbf{z}}$ & $(8 p)$ & C XVI \\
\hline $\mathbf{B}_{136}$ & $=$ & $-y_{19} \mathbf{a}_{1}-x_{19} \mathbf{a}_{2}+\left(\frac{1}{2}-z_{19}\right) \mathbf{a}_{3}$ & $=$ & $-y_{19} a \hat{\mathbf{x}}-x_{19} a \hat{\mathbf{y}}+\left(\frac{1}{2}-z_{19}\right) c \hat{\mathbf{z}}$ & $(8 p)$ & C XVI \\
\hline $\mathbf{B}_{137}$ & $=$ & $x_{20} \mathbf{a}_{1}+y_{20} \mathbf{a}_{2}+z_{20} \mathbf{a}_{3}$ & $=$ & $x_{20} a \hat{\mathbf{x}}+y_{20} a \hat{\mathbf{y}}+z_{20} c \hat{\mathbf{z}}$ & $(8 p)$ & P I \\
\hline $\mathbf{B}_{138}$ & $=$ & $-x_{20} \mathbf{a}_{1}-y_{20} \mathbf{a}_{2}+z_{20} \mathbf{a}_{3}$ & $=$ & $-x_{20} a \hat{\mathbf{x}}-y_{20} a \hat{\mathbf{y}}+z_{20} c \hat{\mathbf{z}}$ & $(8 p)$ & P I \\
\hline $\mathbf{B}_{139}$ & $=$ & $-y_{20} \mathbf{a}_{1}+x_{20} \mathbf{a}_{2}+\left(\frac{1}{2}+z_{20}\right) \mathbf{a}_{3}$ & $=$ & $-y_{20} a \hat{\mathbf{x}}+x_{20} a \hat{\mathbf{y}}+\left(\frac{1}{2}+z_{20}\right) c \hat{\mathbf{z}}$ & $(8 p)$ & P I \\
\hline $\mathbf{B}_{140}$ & $=$ & $y_{20} \mathbf{a}_{1}-x_{20} \mathbf{a}_{2}+\left(\frac{1}{2}+z_{20}\right) \mathbf{a}_{3}$ & $=$ & $y_{20} a \hat{\mathbf{x}}-x_{20} a \hat{\mathbf{y}}+\left(\frac{1}{2}+z_{20}\right) c \hat{\mathbf{z}}$ & $(8 p)$ & P I \\
\hline $\mathbf{B}_{141}$ & $=$ & $-x_{20} \mathbf{a}_{1}+y_{20} \mathbf{a}_{2}-z_{20} \mathbf{a}_{3}$ & $=$ & $-x_{20} a \hat{\mathbf{x}}+y_{20} a \hat{\mathbf{y}}-z_{20} c \hat{\mathbf{z}}$ & $(8 p)$ & P I \\
\hline $\mathbf{B}_{142}$ & $=$ & $x_{20} \mathbf{a}_{1}-y_{20} \mathbf{a}_{2}-z_{20} \mathbf{a}_{3}$ & $=$ & $x_{20} a \hat{\mathbf{x}}-y_{20} a \hat{\mathbf{y}}-z_{20} c \hat{\mathbf{z}}$ & $(8 p)$ & P I \\
\hline $\mathbf{B}_{143}$ & $=$ & $y_{20} \mathbf{a}_{1}+x_{20} \mathbf{a}_{2}+\left(\frac{1}{2}-z_{20}\right) \mathbf{a}_{3}$ & $=$ & $y_{20} a \hat{\mathbf{x}}+x_{20} a \hat{\mathbf{y}}+\left(\frac{1}{2}-z_{20}\right) c \hat{\mathbf{z}}$ & $(8 p)$ & P I \\
\hline $\mathbf{B}_{144}$ & $=$ & $-y_{20} \mathbf{a}_{1}-x_{20} \mathbf{a}_{2}+\left(\frac{1}{2}-z_{20}\right) \mathbf{a}_{3}$ & $=$ & $-y_{20} a \hat{\mathbf{x}}-x_{20} a \hat{\mathbf{y}}+\left(\frac{1}{2}-z_{20}\right) c \hat{\mathbf{z}}$ & $(8 p)$ & P I \\
\hline $\mathbf{B}_{145}$ & $=$ & $x_{21} \mathbf{a}_{1}+y_{21} \mathbf{a}_{2}+z_{21} \mathbf{a}_{3}$ & $=$ & $x_{21} a \hat{\mathbf{x}}+y_{21} a \hat{\mathbf{y}}+z_{21} c \hat{\mathbf{z}}$ & $(8 p)$ & P II \\
\hline $\mathbf{B}_{146}$ & $=$ & $-x_{21} \mathbf{a}_{1}-y_{21} \mathbf{a}_{2}+z_{21} \mathbf{a}_{3}$ & $=$ & $-x_{21} a \hat{\mathbf{x}}-y_{21} a \hat{\mathbf{y}}+z_{21} c \hat{\mathbf{z}}$ & $(8 p)$ & P II \\
\hline $\mathbf{B}_{147}$ & $=$ & $-y_{21} \mathbf{a}_{1}+x_{21} \mathbf{a}_{2}+\left(\frac{1}{2}+z_{21}\right) \mathbf{a}_{3}$ & $=$ & $-y_{21} a \hat{\mathbf{x}}+x_{21} a \hat{\mathbf{y}}+\left(\frac{1}{2}+z_{21}\right) c \hat{\mathbf{z}}$ & $(8 p)$ & P II \\
\hline
\end{tabular}




\begin{tabular}{|c|c|c|c|c|c|}
\hline $\mathbf{B}_{148}$ & $=$ & $y_{21} \mathbf{a}_{1}-x_{21} \mathbf{a}_{2}+\left(\frac{1}{2}+z_{21}\right) \mathbf{a}_{3}$ & $=$ & $y_{21} a \hat{\mathbf{x}}-x_{21} a \hat{\mathbf{y}}+\left(\frac{1}{2}+z_{21}\right) c \hat{\mathbf{z}}$ & $(8 p)$ \\
\hline $\mathbf{B}_{149}$ & $=$ & $-x_{21} \mathbf{a}_{1}+y_{21} \mathbf{a}_{2}-z_{21} \mathbf{a}_{3}$ & $=$ & $-x_{21} a \hat{\mathbf{x}}+y_{21} a \hat{\mathbf{y}}-z_{21} c \hat{\mathbf{z}}$ & $(8 p)$ \\
\hline $\mathbf{B}_{150}$ & $=$ & $x_{21} \mathbf{a}_{1}-y_{21} \mathbf{a}_{2}-z_{21} \mathbf{a}_{3}$ & $=$ & $x_{21} a \hat{\mathbf{x}}-y_{21} a \hat{\mathbf{y}}-z_{21} c \hat{\mathbf{z}}$ & $(8 p)$ \\
\hline $\mathbf{B}_{151}$ & $=$ & $y_{21} \mathbf{a}_{1}+x_{21} \mathbf{a}_{2}+\left(\frac{1}{2}-z_{21}\right) \mathbf{a}_{3}$ & $=$ & $y_{21} a \hat{\mathbf{x}}+x_{21} a \hat{\mathbf{y}}+\left(\frac{1}{2}-z_{21}\right) c \hat{\mathbf{z}}$ & $(8 p)$ \\
\hline $\mathbf{B}_{152}$ & $=$ & $-y_{21} \mathbf{a}_{1}-x_{21} \mathbf{a}_{2}+\left(\frac{1}{2}-z_{21}\right) \mathbf{a}_{3}$ & $=$ & $-y_{21} a \hat{\mathbf{x}}-x_{21} a \hat{\mathbf{y}}+\left(\frac{1}{2}-z_{21}\right) c \hat{\mathbf{z}}$ & $(8 p)$ \\
\hline $\mathbf{B}_{153}$ & $=$ & $x_{22} \mathbf{a}_{1}+y_{22} \mathbf{a}_{2}+z_{22} \mathbf{a}_{3}$ & $=$ & $x_{22} a \hat{\mathbf{x}}+y_{22} a \hat{\mathbf{y}}+z_{22} c \hat{\mathbf{z}}$ & $(8 p)$ \\
\hline $\mathbf{B}_{154}$ & $=$ & $-x_{22} \mathbf{a}_{1}-y_{22} \mathbf{a}_{2}+z_{22} \mathbf{a}_{3}$ & $=$ & $-x_{22} a \hat{\mathbf{x}}-y_{22} a \hat{\mathbf{y}}+z_{22} c \hat{\mathbf{z}}$ & $(8 p)$ \\
\hline $\mathbf{B}_{155}$ & $=$ & $-y_{22} \mathbf{a}_{1}+x_{22} \mathbf{a}_{2}+\left(\frac{1}{2}+z_{22}\right) \mathbf{a}_{3}$ & $=$ & $-y_{22} a \hat{\mathbf{x}}+x_{22} a \hat{\mathbf{y}}+\left(\frac{1}{2}+z_{22}\right) c \hat{\mathbf{z}}$ & $(8 p)$ \\
\hline $\mathbf{B}_{156}$ & $=$ & $y_{22} \mathbf{a}_{1}-x_{22} \mathbf{a}_{2}+\left(\frac{1}{2}+z_{22}\right) \mathbf{a}_{3}$ & $=$ & $y_{22} a \hat{\mathbf{x}}-x_{22} a \hat{\mathbf{y}}+\left(\frac{1}{2}+z_{22}\right) c \hat{\mathbf{z}}$ & $(8 p)$ \\
\hline $\mathbf{B}_{157}$ & $=$ & $-x_{22} \mathbf{a}_{1}+y_{22} \mathbf{a}_{2}-z_{22} \mathbf{a}_{3}$ & $=$ & $-x_{22} a \hat{\mathbf{x}}+y_{22} a \hat{\mathbf{y}}-z_{22} c \hat{\mathbf{z}}$ & $(8 p)$ \\
\hline $\mathbf{B}_{158}$ & $=$ & $x_{22} \mathbf{a}_{1}-y_{22} \mathbf{a}_{2}-z_{22} \mathbf{a}_{3}$ & $=$ & $x_{22} a \hat{\mathbf{x}}-y_{22} a \hat{\mathbf{y}}-z_{22} c \hat{\mathbf{z}}$ & $(8 p)$ \\
\hline $\mathbf{B}_{159}$ & $=$ & $y_{22} \mathbf{a}_{1}+x_{22} \mathbf{a}_{2}+\left(\frac{1}{2}-z_{22}\right) \mathbf{a}_{3}$ & $=$ & $y_{22} a \hat{\mathbf{x}}+x_{22} a \hat{\mathbf{y}}+\left(\frac{1}{2}-z_{22}\right) c \hat{\mathbf{z}}$ & $(8 p)$ \\
\hline $\mathbf{B}_{160}$ & $=$ & $-y_{22} \mathbf{a}_{1}-x_{22} \mathbf{a}_{2}+\left(\frac{1}{2}-z_{22}\right) \mathbf{a}_{3}$ & $=$ & $-y_{22} a \hat{\mathbf{x}}-x_{22} a \hat{\mathbf{y}}+\left(\frac{1}{2}-z_{22}\right) c \hat{\mathbf{z}}$ & $(8 p)$ \\
\hline $\mathbf{B}_{161}$ & $=$ & $x_{23} \mathbf{a}_{1}+y_{23} \mathbf{a}_{2}+z_{23} \mathbf{a}_{3}$ & $=$ & $x_{23} a \hat{\mathbf{x}}+y_{23} a \hat{\mathbf{y}}+z_{23} c \hat{\mathbf{z}}$ & $(8 p)$ \\
\hline $\mathbf{B}_{162}$ & $=$ & $-x_{23} \mathbf{a}_{1}-y_{23} \mathbf{a}_{2}+z_{23} \mathbf{a}_{3}$ & $=$ & $-x_{23} a \hat{\mathbf{x}}-y_{23} a \hat{\mathbf{y}}+z_{23} c \hat{\mathbf{z}}$ & $(8 p)$ \\
\hline $\mathbf{B}_{163}$ & $=$ & $-y_{23} \mathbf{a}_{1}+x_{23} \mathbf{a}_{2}+\left(\frac{1}{2}+z_{23}\right) \mathbf{a}_{3}$ & $=$ & $-y_{23} a \hat{\mathbf{x}}+x_{23} a \hat{\mathbf{y}}+\left(\frac{1}{2}+z_{23}\right) c \hat{\mathbf{z}}$ & $(8 p)$ \\
\hline $\mathbf{B}_{164}$ & $=$ & $y_{23} \mathbf{a}_{1}-x_{23} \mathbf{a}_{2}+\left(\frac{1}{2}+z_{23}\right) \mathbf{a}_{3}$ & $=$ & $y_{23} a \hat{\mathbf{x}}-x_{23} a \hat{\mathbf{y}}+\left(\frac{1}{2}+z_{23}\right) c \hat{\mathbf{z}}$ & $(8 p)$ \\
\hline $\mathbf{B}_{165}$ & $=$ & $-x_{23} \mathbf{a}_{1}+y_{23} \mathbf{a}_{2}-z_{23} \mathbf{a}_{3}$ & $=$ & $-x_{23} a \hat{\mathbf{x}}+y_{23} a \hat{\mathbf{y}}-z_{23} c \hat{\mathbf{z}}$ & $(8 p)$ \\
\hline $\mathbf{B}_{166}$ & $=$ & $x_{23} \mathbf{a}_{1}-y_{23} \mathbf{a}_{2}-z_{23} \mathbf{a}_{3}$ & $=$ & $x_{23} a \hat{\mathbf{x}}-y_{23} a \hat{\mathbf{y}}-z_{23} c \hat{\mathbf{z}}$ & $(8 p)$ \\
\hline $\mathbf{B}_{167}$ & $=$ & $y_{23} \mathbf{a}_{1}+x_{23} \mathbf{a}_{2}+\left(\frac{1}{2}-z_{23}\right) \mathbf{a}_{3}$ & $=$ & $y_{23} a \hat{\mathbf{x}}+x_{23} a \hat{\mathbf{y}}+\left(\frac{1}{2}-z_{23}\right) c \hat{\mathbf{z}}$ & $(8 p)$ \\
\hline $\mathbf{B}_{168}$ & $=$ & $-y_{23} \mathbf{a}_{1}-x_{23} \mathbf{a}_{2}+\left(\frac{1}{2}-z_{23}\right) \mathbf{a}_{3}$ & $=$ & $-y_{23} a \hat{\mathbf{x}}-x_{23} a \hat{\mathbf{y}}+\left(\frac{1}{2}-z_{23}\right) c \hat{\mathbf{z}}$ & $(8 p)$ \\
\hline $\mathbf{B}_{169}$ & $=$ & $x_{24} \mathbf{a}_{1}+y_{24} \mathbf{a}_{2}+z_{24} \mathbf{a}_{3}$ & $=$ & $x_{24} a \hat{\mathbf{x}}+y_{24} a \hat{\mathbf{y}}+z_{24} c \hat{\mathbf{z}}$ & $(8 p)$ \\
\hline $\mathbf{B}_{170}$ & $=$ & $-x_{24} \mathbf{a}_{1}-y_{24} \mathbf{a}_{2}+z_{24} \mathbf{a}_{3}$ & $=$ & $-x_{24} a \hat{\mathbf{x}}-y_{24} a \hat{\mathbf{y}}+z_{24} c \hat{\mathbf{z}}$ & $(8 p)$ \\
\hline $\mathbf{B}_{171}$ & $=$ & $-y_{24} \mathbf{a}_{1}+x_{24} \mathbf{a}_{2}+\left(\frac{1}{2}+z_{24}\right) \mathbf{a}_{3}$ & $=$ & $-y_{24} a \hat{\mathbf{x}}+x_{24} a \hat{\mathbf{y}}+\left(\frac{1}{2}+z_{24}\right) c \hat{\mathbf{z}}$ & $(8 p)$ \\
\hline $\mathbf{B}_{172}$ & $=$ & $y_{24} \mathbf{a}_{1}-x_{24} \mathbf{a}_{2}+\left(\frac{1}{2}+z_{24}\right) \mathbf{a}_{3}$ & $=$ & $y_{24} a \hat{\mathbf{x}}-x_{24} a \hat{\mathbf{y}}+\left(\frac{1}{2}+z_{24}\right) c \hat{\mathbf{z}}$ & $(8 p)$ \\
\hline $\mathbf{B}_{173}$ & $=$ & $-x_{24} \mathbf{a}_{1}+y_{24} \mathbf{a}_{2}-z_{24} \mathbf{a}_{3}$ & $=$ & $-x_{24} a \hat{\mathbf{x}}+y_{24} a \hat{\mathbf{y}}-z_{24} c \hat{\mathbf{z}}$ & $(8 p)$ \\
\hline $\mathbf{B}_{174}$ & $=$ & $x_{24} \mathbf{a}_{1}-y_{24} \mathbf{a}_{2}-z_{24} \mathbf{a}_{3}$ & $=$ & $x_{24} a \hat{\mathbf{x}}-y_{24} a \hat{\mathbf{y}}-z_{24} c \hat{\mathbf{z}}$ & $(8 p)$ \\
\hline $\mathbf{B}_{175}$ & $=$ & $y_{24} \mathbf{a}_{1}+x_{24} \mathbf{a}_{2}+\left(\frac{1}{2}-z_{24}\right) \mathbf{a}_{3}$ & $=$ & $y_{24} a \hat{\mathbf{x}}+x_{24} a \hat{\mathbf{y}}+\left(\frac{1}{2}-z_{24}\right) c \hat{\mathbf{z}}$ & $(8 p)$ \\
\hline $\mathbf{B}_{176}$ & $=$ & $-y_{24} \mathbf{a}_{1}-x_{24} \mathbf{a}_{2}+\left(\frac{1}{2}-z_{24}\right) \mathbf{a}_{3}$ & $=$ & $-y_{24} a \hat{\mathbf{x}}-x_{24} a \hat{\mathbf{y}}+\left(\frac{1}{2}-z_{24}\right) c \hat{\mathbf{z}}$ & $(8 p)$ \\
\hline $\mathbf{B}_{177}$ & $=$ & $x_{25} \mathbf{a}_{1}+y_{25} \mathbf{a}_{2}+z_{25} \mathbf{a}_{3}$ & $=$ & $x_{25} a \hat{\mathbf{x}}+y_{25} a \hat{\mathbf{y}}+z_{25} c \hat{\mathbf{z}}$ & $(8 p)$ \\
\hline $\mathbf{B}_{178}$ & $=$ & $-x_{25} \mathbf{a}_{1}-y_{25} \mathbf{a}_{2}+z_{25} \mathbf{a}_{3}$ & $=$ & $-x_{25} a \hat{\mathbf{x}}-y_{25} a \hat{\mathbf{y}}+z_{25} c \hat{\mathbf{z}}$ & $(8 p)$ \\
\hline $\mathbf{B}_{179}$ & $=$ & $-y_{25} \mathbf{a}_{1}+x_{25} \mathbf{a}_{2}+\left(\frac{1}{2}+z_{25}\right) \mathbf{a}_{3}$ & $=$ & $-y_{25} a \hat{\mathbf{x}}+x_{25} a \hat{\mathbf{y}}+\left(\frac{1}{2}+z_{25}\right) c \hat{\mathbf{z}}$ & $(8 p)$ \\
\hline $\mathbf{B}_{180}$ & $=$ & $y_{25} \mathbf{a}_{1}-x_{25} \mathbf{a}_{2}+\left(\frac{1}{2}+z_{25}\right) \mathbf{a}_{3}$ & $=$ & $y_{25} a \hat{\mathbf{x}}-x_{25} a \hat{\mathbf{y}}+\left(\frac{1}{2}+z_{25}\right) c \hat{\mathbf{z}}$ & $(8 p)$ \\
\hline $\mathbf{B}_{181}$ & $=$ & $-x_{25} \mathbf{a}_{1}+y_{25} \mathbf{a}_{2}-z_{25} \mathbf{a}_{3}$ & $=$ & $-x_{25} a \hat{\mathbf{x}}+y_{25} a \hat{\mathbf{y}}-z_{25} c \hat{\mathbf{z}}$ & $(8 p)$ \\
\hline $\mathbf{B}_{182}$ & $=$ & $x_{25} \mathbf{a}_{1}-y_{25} \mathbf{a}_{2}-z_{25} \mathbf{a}_{3}$ & $=$ & $x_{25} a \hat{\mathbf{x}}-y_{25} a \hat{\mathbf{y}}-z_{25} c \hat{\mathbf{z}}$ & $(8 p)$ \\
\hline $\mathbf{B}_{183}$ & $=$ & $y_{25} \mathbf{a}_{1}+x_{25} \mathbf{a}_{2}+\left(\frac{1}{2}-z_{25}\right) \mathbf{a}_{3}$ & $=$ & $y_{25} a \hat{\mathbf{x}}+x_{25} a \hat{\mathbf{y}}+\left(\frac{1}{2}-z_{25}\right) c \hat{\mathbf{z}}$ & $(8 p)$ \\
\hline
\end{tabular}




$$
\mathbf{B}_{184}=-y_{25} \mathbf{a}_{1}-x_{25} \mathbf{a}_{2}+\left(\frac{1}{2}-z_{25}\right) \mathbf{a}_{3}=-y_{25} a \hat{\mathbf{x}}-x_{25} a \hat{\mathbf{y}}+\left(\frac{1}{2}-z_{25}\right) c \hat{\mathbf{z}}
$$

\section{References:}

- S. Spiliadis, A. A. Pinkerton, and D. Schwarzenbach, Crystal and molecular structures of $\left[A s P h_{4}\right]\left[L n\left(S_{2} P M e_{2}\right)_{4}\right](L n=$ Ce or $\mathrm{Tm}$ ) and their comparison with results obtained from paramagnetic nuclear magnetic resonance data in solution, $\mathrm{J}$. Chem. Soc. Dalton Trans. pp. 1809-1813 (1982), doi:10.1039/DT9820001809.

\section{Found in:}

- F. Hoffmann, The Fascination of Crystals and Symmetry (2014). 230 - The space group list project.

\section{Geometry files:}

- CIF: pp. 870

- POSCAR: pp. 870 
$\mathrm{Na}_{5} \mathrm{Fe}_{3} \mathrm{~F}_{14}$ (High-temperature) Structure:

\section{A14B3C5_tP44_94_c3g_ad_bg}
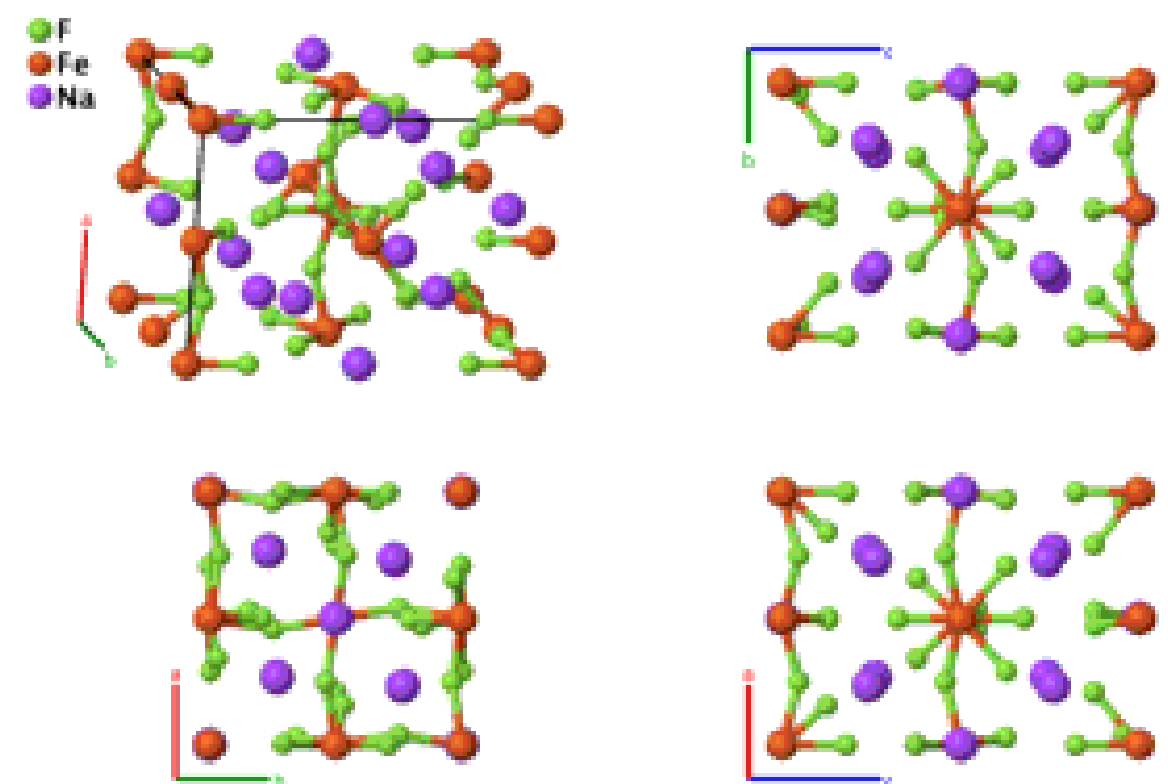

\section{Prototype}

AFLOW prototype label

: $\quad \mathrm{Na}_{5} \mathrm{Fe}_{3} \mathrm{~F}_{14}$

Strukturbericht designation

Pearson symbol

: A14B3C5_tP44_94_c3g_ad_bg

Space group number

: None

Space group symbol

: $\quad$ tP44

AFLOW prototype command

: 94

: $\quad P 4_{2} 2_{1} 2$

aflow --proto=A14B3C5_tP44_94_c3g_ad_bg

- -params $=a, c / a, z_{3}, z_{4}, x_{5}, y_{5}, z_{5}, x_{6}, y_{6}, z_{6}, x_{7}, y_{7}, z_{7}, x_{8}, y_{8}, z_{8}$

\section{Simple Tetragonal primitive vectors:}

$$
\begin{aligned}
& \mathbf{a}_{1}=a \hat{\mathbf{x}} \\
& \mathbf{a}_{2}=a \hat{\mathbf{y}} \\
& \mathbf{a}_{3}=c \hat{\mathbf{z}}
\end{aligned}
$$

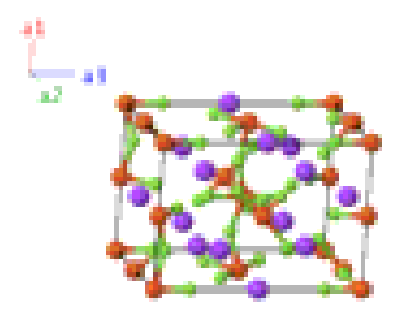

Basis vectors:

Lattice Coordinates

$\begin{array}{lcc}\mathbf{B}_{1}= & 0 \mathbf{a}_{1}+0 \mathbf{a}_{2}+0 \mathbf{a}_{3} \\ \mathbf{B}_{2}= & \frac{1}{2} \mathbf{a}_{1}+\frac{1}{2} \mathbf{a}_{2}+\frac{1}{2} \mathbf{a}_{3} \\ \mathbf{B}_{3}= & \frac{1}{2} \mathbf{a}_{3} \\ \mathbf{B}_{4}= & \frac{1}{2} \mathbf{a}_{1}+\frac{1}{2} \mathbf{a}_{2}\end{array}$

Cartesian Coordinates

$\begin{array}{lcr}= & 0 \hat{\mathbf{x}}+0 \hat{\mathbf{y}}+0 \hat{\mathbf{z}} \\ = & \frac{1}{2} a \hat{\mathbf{x}}+\frac{1}{2} a \hat{\mathbf{y}}+\frac{1}{2} c \hat{\mathbf{z}} \\ = & \frac{1}{2} c \hat{\mathbf{z}} \\ = & \frac{1}{2} a \hat{\mathbf{x}}+\frac{1}{2} a \hat{\mathbf{y}}\end{array}$

Wyckoff Position

$(2 a)$

(2a)

(2b)

(2b)
Atom Type

Fe I

Fe I

$\mathrm{Na}$ I

$\mathrm{Na}$ I 


\begin{tabular}{|c|c|c|c|c|c|c|}
\hline $\mathbf{B}_{5}$ & $=$ & $z_{3} \mathbf{a}_{3}$ & $=$ & $z_{3} c \hat{\mathbf{z}}$ & $(4 c)$ & F I \\
\hline $\mathbf{B}_{6}$ & $=$ & $\frac{1}{2} \mathbf{a}_{1}+\frac{1}{2} \mathbf{a}_{2}+\left(\frac{1}{2}+z_{3}\right) \mathbf{a}_{3}$ & $=$ & $\frac{1}{2} a \hat{\mathbf{x}}+\frac{1}{2} a \hat{\mathbf{y}}+\left(\frac{1}{2}+z_{3}\right) c \hat{\mathbf{z}}$ & $(4 c)$ & F I \\
\hline $\mathbf{B}_{7}$ & $=$ & $\frac{1}{2} \mathbf{a}_{1}+\frac{1}{2} \mathbf{a}_{2}+\left(\frac{1}{2}-z_{3}\right) \mathbf{a}_{3}$ & $=$ & $\frac{1}{2} a \hat{\mathbf{x}}+\frac{1}{2} a \hat{\mathbf{y}}+\left(\frac{1}{2}-z_{3}\right) c \hat{\mathbf{z}}$ & $(4 c)$ & F I \\
\hline $\mathbf{B}_{8}$ & $=$ & $-z_{3} \mathbf{a}_{3}$ & $=$ & $-z_{3} c \hat{\mathbf{z}}$ & $(4 c)$ & F I \\
\hline $\mathbf{B}_{9}$ & $=$ & $\frac{1}{2} \mathbf{a}_{2}+z_{4} \mathbf{a}_{3}$ & $=$ & $\frac{1}{2} a \hat{\mathbf{y}}+z_{4} c \hat{\mathbf{z}}$ & $(4 d)$ & Fe II \\
\hline $\mathbf{B}_{10}$ & $=$ & $\frac{1}{2} \mathbf{a}_{2}+\left(\frac{1}{2}+z_{4}\right) \mathbf{a}_{3}$ & $=$ & $\frac{1}{2} a \hat{\mathbf{y}}+\left(\frac{1}{2}+z_{4}\right) c \hat{\mathbf{z}}$ & $(4 d)$ & Fe II \\
\hline $\mathbf{B}_{11}$ & $=$ & $\frac{1}{2} \mathbf{a}_{1}+\left(\frac{1}{2}-z_{4}\right) \mathbf{a}_{3}$ & $=$ & $\frac{1}{2} a \hat{\mathbf{x}}+\left(\frac{1}{2}-z_{4}\right) c \hat{\mathbf{z}}$ & $(4 d)$ & Fe II \\
\hline $\mathbf{B}_{12}$ & $=$ & $\frac{1}{2} \mathbf{a}_{1}+-z_{4} \mathbf{a}_{3}$ & $=$ & $\frac{1}{2} a \hat{\mathbf{x}}+-z_{4} c \hat{\mathbf{z}}$ & $(4 d)$ & Fe II \\
\hline $\mathbf{B}_{13}$ & $=$ & $x_{5} \mathbf{a}_{1}+y_{5} \mathbf{a}_{2}+z_{5} \mathbf{a}_{3}$ & $=$ & $x_{5} a \hat{\mathbf{x}}+y_{5} a \hat{\mathbf{y}}+z_{5} c \hat{\mathbf{z}}$ & $(8 g)$ & F II \\
\hline $\mathbf{B}_{14}$ & $=$ & $-x_{5} \mathbf{a}_{1}-y_{5} \mathbf{a}_{2}+z_{5} \mathbf{a}_{3}$ & $=$ & $-x_{5} a \hat{\mathbf{x}}-y_{5} a \hat{\mathbf{y}}+z_{5} c \hat{\mathbf{z}}$ & $(8 g)$ & F II \\
\hline $\mathbf{B}_{15}$ & $=$ & $\begin{array}{c}\left(\frac{1}{2}-y_{5}\right) \mathbf{a}_{1}+\left(\frac{1}{2}+x_{5}\right) \mathbf{a}_{2}+ \\
\left(\frac{1}{2}+z_{5}\right) \mathbf{a}_{3}\end{array}$ & $=$ & $\begin{array}{c}\left(\frac{1}{2}-y_{5}\right) a \hat{\mathbf{x}}+\left(\frac{1}{2}+x_{5}\right) a \hat{\mathbf{y}}+ \\
\left(\frac{1}{2}+z_{5}\right) c \hat{\mathbf{z}}\end{array}$ & $(8 g)$ & F II \\
\hline $\mathbf{B}_{16}$ & $=$ & $\begin{array}{c}\left(\frac{1}{2}+y_{5}\right) \mathbf{a}_{1}+\left(\frac{1}{2}-x_{5}\right) \mathbf{a}_{2}+ \\
\left(\frac{1}{2}+z_{5}\right) \mathbf{a}_{3}\end{array}$ & $=$ & $\begin{array}{c}\left(\frac{1}{2}+y_{5}\right) a \hat{\mathbf{x}}+\left(\frac{1}{2}-x_{5}\right) a \hat{\mathbf{y}}+ \\
\left(\frac{1}{2}+z_{5}\right) c \hat{\mathbf{z}}\end{array}$ & $(8 g)$ & F II \\
\hline $\mathbf{B}_{17}$ & $=$ & $\begin{array}{c}\left(\frac{1}{2}-x_{5}\right) \mathbf{a}_{1}+\left(\frac{1}{2}+y_{5}\right) \mathbf{a}_{2}+ \\
\left(\frac{1}{2}-z_{5}\right) \mathbf{a}_{3}\end{array}$ & $=$ & $\begin{array}{c}\left(\frac{1}{2}-x_{5}\right) a \hat{\mathbf{x}}+\left(\frac{1}{2}+y_{5}\right) a \hat{\mathbf{y}}+ \\
\left(\frac{1}{2}-z_{5}\right) c \hat{\mathbf{z}}\end{array}$ & $(8 g)$ & F II \\
\hline $\mathbf{B}_{18}$ & $=$ & $\begin{array}{c}\left(\frac{1}{2}+x_{5}\right) \mathbf{a}_{1}+\left(\frac{1}{2}-y_{5}\right) \mathbf{a}_{2}+ \\
\left(\frac{1}{2}-z_{5}\right) \mathbf{a}_{3}\end{array}$ & $=$ & $\begin{array}{c}\left(\frac{1}{2}+x_{5}\right) a \hat{\mathbf{x}}+\left(\frac{1}{2}-y_{5}\right) a \hat{\mathbf{y}}+ \\
\left(\frac{1}{2}-z_{5}\right) c \hat{\mathbf{z}}\end{array}$ & $(8 g)$ & F II \\
\hline $\mathbf{B}_{19}$ & $=$ & $y_{5} \mathbf{a}_{1}+x_{5} \mathbf{a}_{2}-z_{5} \mathbf{a}_{3}$ & $=$ & $y_{5} a \hat{\mathbf{x}}+x_{5} a \hat{\mathbf{y}}-z_{5} c \hat{\mathbf{z}}$ & $(8 g)$ & F II \\
\hline $\mathbf{B}_{20}$ & $=$ & $-y_{5} \mathbf{a}_{1}-x_{5} \mathbf{a}_{2}-z_{5} \mathbf{a}_{3}$ & $=$ & $-y_{5} a \hat{\mathbf{x}}-x_{5} a \hat{\mathbf{y}}-z_{5} c \hat{\mathbf{z}}$ & $(8 g)$ & F II \\
\hline $\mathbf{B}_{21}$ & $=$ & $x_{6} \mathbf{a}_{1}+y_{6} \mathbf{a}_{2}+z_{6} \mathbf{a}_{3}$ & $=$ & $x_{6} a \hat{\mathbf{x}}+y_{6} a \hat{\mathbf{y}}+z_{6} c \hat{\mathbf{z}}$ & $(8 g)$ & F III \\
\hline $\mathbf{B}_{22}$ & $=$ & $-x_{6} \mathbf{a}_{1}-y_{6} \mathbf{a}_{2}+z_{6} \mathbf{a}_{3}$ & $=$ & $-x_{6} a \hat{\mathbf{x}}-y_{6} a \hat{\mathbf{y}}+z_{6} c \hat{\mathbf{z}}$ & $(8 g)$ & F III \\
\hline $\mathbf{B}_{23}$ & $=$ & $\begin{array}{c}\left(\frac{1}{2}-y_{6}\right) \mathbf{a}_{1}+\left(\frac{1}{2}+x_{6}\right) \mathbf{a}_{2}+ \\
\left(\frac{1}{2}+z_{6}\right) \mathbf{a}_{3}\end{array}$ & $=$ & $\begin{array}{c}\left(\frac{1}{2}-y_{6}\right) a \hat{\mathbf{x}}+\left(\frac{1}{2}+x_{6}\right) a \hat{\mathbf{y}}+ \\
\left(\frac{1}{2}+z_{6}\right) c \hat{\mathbf{z}}\end{array}$ & $(8 g)$ & F III \\
\hline $\mathbf{B}_{24}$ & $=$ & $\begin{array}{c}\left(\frac{1}{2}+y_{6}\right) \mathbf{a}_{1}+\left(\frac{1}{2}-x_{6}\right) \mathbf{a}_{2}+ \\
\left(\frac{1}{2}+z_{6}\right) \mathbf{a}_{3}\end{array}$ & $=$ & $\begin{array}{c}\left(\frac{1}{2}+y_{6}\right) a \hat{\mathbf{x}}+\left(\frac{1}{2}-x_{6}\right) a \hat{\mathbf{y}}+ \\
\left(\frac{1}{2}+z_{6}\right) c \hat{\mathbf{z}}\end{array}$ & $(8 g)$ & F III \\
\hline $\mathbf{B}_{25}$ & $=$ & $\begin{array}{c}\left(\frac{1}{2}-x_{6}\right) \mathbf{a}_{1}+\left(\frac{1}{2}+y_{6}\right) \mathbf{a}_{2}+ \\
\left(\frac{1}{2}-z_{6}\right) \mathbf{a}_{3}\end{array}$ & $=$ & $\begin{array}{c}\left(\frac{1}{2}-x_{6}\right) a \hat{\mathbf{x}}+\left(\frac{1}{2}+y_{6}\right) a \hat{\mathbf{y}}+ \\
\left(\frac{1}{2}-z_{6}\right) c \hat{\mathbf{z}}\end{array}$ & $(8 g)$ & F III \\
\hline $\mathbf{B}_{26}$ & $=$ & $\begin{array}{c}\left(\frac{1}{2}+x_{6}\right) \mathbf{a}_{1}+\left(\frac{1}{2}-y_{6}\right) \mathbf{a}_{2}+ \\
\left(\frac{1}{2}-z_{6}\right) \mathbf{a}_{3}\end{array}$ & $=$ & $\begin{array}{c}\left(\frac{1}{2}+x_{6}\right) a \hat{\mathbf{x}}+\left(\frac{1}{2}-y_{6}\right) a \hat{\mathbf{y}}+ \\
\left(\frac{1}{2}-z_{6}\right) c \hat{\mathbf{z}}\end{array}$ & $(8 g)$ & F III \\
\hline $\mathbf{B}_{27}$ & $=$ & $y_{6} \mathbf{a}_{1}+x_{6} \mathbf{a}_{2}-z_{6} \mathbf{a}_{3}$ & $=$ & $y_{6} a \hat{\mathbf{x}}+x_{6} a \hat{\mathbf{y}}-z_{6} c \hat{\mathbf{z}}$ & $(8 g)$ & F III \\
\hline $\mathbf{B}_{28}$ & $=$ & $-y_{6} \mathbf{a}_{1}-x_{6} \mathbf{a}_{2}-z_{6} \mathbf{a}_{3}$ & $=$ & $-y_{6} a \hat{\mathbf{x}}-x_{6} a \hat{\mathbf{y}}-z_{6} c \hat{\mathbf{z}}$ & $(8 g)$ & F III \\
\hline $\mathbf{B}_{29}$ & $=$ & $x_{7} \mathbf{a}_{1}+y_{7} \mathbf{a}_{2}+z_{7} \mathbf{a}_{3}$ & $=$ & $x_{7} a \hat{\mathbf{x}}+y_{7} a \hat{\mathbf{y}}+z_{7} c \hat{\mathbf{z}}$ & $(8 g)$ & F IV \\
\hline $\mathbf{B}_{30}$ & $=$ & $-x_{7} \mathbf{a}_{1}-y_{7} \mathbf{a}_{2}+z_{7} \mathbf{a}_{3}$ & $=$ & $-x_{7} a \hat{\mathbf{x}}-y_{7} a \hat{\mathbf{y}}+z_{7} c \hat{\mathbf{z}}$ & $(8 g)$ & F IV \\
\hline $\mathbf{B}_{31}$ & $=$ & $\begin{array}{c}\left(\frac{1}{2}-y_{7}\right) \mathbf{a}_{1}+\left(\frac{1}{2}+x_{7}\right) \mathbf{a}_{2}+ \\
\left(\frac{1}{2}+z_{7}\right) \mathbf{a}_{3}\end{array}$ & $=$ & $\begin{array}{c}\left(\frac{1}{2}-y_{7}\right) a \hat{\mathbf{x}}+\left(\frac{1}{2}+x_{7}\right) a \hat{\mathbf{y}}+ \\
\left(\frac{1}{2}+z_{7}\right) c \hat{\mathbf{z}}\end{array}$ & $(8 g)$ & F IV \\
\hline $\mathbf{B}_{32}$ & $=$ & $\begin{array}{c}\left(\frac{1}{2}+y_{7}\right) \mathbf{a}_{1}+\left(\frac{1}{2}-x_{7}\right) \mathbf{a}_{2}+ \\
\left(\frac{1}{2}+z_{7}\right) \mathbf{a}_{3}\end{array}$ & $=$ & $\begin{array}{c}\left(\frac{1}{2}+y_{7}\right) a \hat{\mathbf{x}}+\left(\frac{1}{2}-x_{7}\right) a \hat{\mathbf{y}}+ \\
\left(\frac{1}{2}+z_{7}\right) c \hat{\mathbf{z}}\end{array}$ & $(8 g)$ & F IV \\
\hline
\end{tabular}




\begin{tabular}{|c|c|c|c|c|c|c|}
\hline $\mathbf{B}_{33}$ & $=$ & $\begin{array}{c}\left(\frac{1}{2}-x_{7}\right) \mathbf{a}_{1}+\left(\frac{1}{2}+y_{7}\right) \mathbf{a}_{2}+ \\
\left(\frac{1}{2}-z_{7}\right) \mathbf{a}_{3}\end{array}$ & $=$ & $\begin{array}{c}\left(\frac{1}{2}-x_{7}\right) a \hat{\mathbf{x}}+\left(\frac{1}{2}+y_{7}\right) a \hat{\mathbf{y}}+ \\
\left(\frac{1}{2}-z_{7}\right) c \hat{\mathbf{z}}\end{array}$ & $(8 g)$ & F IV \\
\hline $\mathbf{B}_{34}$ & $=$ & $\begin{array}{c}\left(\frac{1}{2}+x_{7}\right) \mathbf{a}_{1}+\left(\frac{1}{2}-y_{7}\right) \mathbf{a}_{2}+ \\
\left(\frac{1}{2}-z_{7}\right) \mathbf{a}_{3}\end{array}$ & $=$ & $\begin{array}{c}\left(\frac{1}{2}+x_{7}\right) a \hat{\mathbf{x}}+\left(\frac{1}{2}-y_{7}\right) a \hat{\mathbf{y}}+ \\
\left(\frac{1}{2}-z_{7}\right) c \hat{\mathbf{z}}\end{array}$ & $(8 g)$ & F IV \\
\hline $\mathbf{B}_{35}$ & $=$ & $y_{7} \mathbf{a}_{1}+x_{7} \mathbf{a}_{2}-z_{7} \mathbf{a}_{3}$ & $=$ & $y_{7} a \hat{\mathbf{x}}+x_{7} a \hat{\mathbf{y}}-z_{7} c \hat{\mathbf{z}}$ & $(8 g)$ & F IV \\
\hline $\mathbf{B}_{36}$ & $=$ & $-y_{7} \mathbf{a}_{1}-x_{7} \mathbf{a}_{2}-z_{7} \mathbf{a}_{3}$ & $=$ & $-y_{7} a \hat{\mathbf{x}}-x_{7} a \hat{\mathbf{y}}-z_{7} c \hat{\mathbf{z}}$ & $(8 g)$ & F IV \\
\hline $\mathbf{B}_{37}$ & $=$ & $x_{8} \mathbf{a}_{1}+y_{8} \mathbf{a}_{2}+z_{8} \mathbf{a}_{3}$ & $=$ & $x_{8} a \hat{\mathbf{x}}+y_{8} a \hat{\mathbf{y}}+z_{8} c \hat{\mathbf{z}}$ & $(8 g)$ & $\mathrm{Na}$ II \\
\hline $\mathbf{B}_{38}$ & $=$ & $-x_{8} \mathbf{a}_{1}-y_{8} \mathbf{a}_{2}+z_{8} \mathbf{a}_{3}$ & $=$ & $-x_{8} a \hat{\mathbf{x}}-y_{8} a \hat{\mathbf{y}}+z_{8} c \hat{\mathbf{z}}$ & $(8 g)$ & $\mathrm{Na}$ II \\
\hline $\mathbf{B}_{39}$ & $=$ & $\begin{array}{c}\left(\frac{1}{2}-y_{8}\right) \mathbf{a}_{1}+\left(\frac{1}{2}+x_{8}\right) \mathbf{a}_{2}+ \\
\left(\frac{1}{2}+z_{8}\right) \mathbf{a}_{3}\end{array}$ & $=$ & $\begin{array}{c}\left(\frac{1}{2}-y_{8}\right) a \hat{\mathbf{x}}+\left(\frac{1}{2}+x_{8}\right) a \hat{\mathbf{y}}+ \\
\left(\frac{1}{2}+z_{8}\right) c \hat{\mathbf{z}}\end{array}$ & $(8 g)$ & $\mathrm{Na} I I$ \\
\hline $\mathbf{B}_{40}$ & $=$ & $\begin{array}{c}\left(\frac{1}{2}+y_{8}\right) \mathbf{a}_{1}+\left(\frac{1}{2}-x_{8}\right) \mathbf{a}_{2}+ \\
\left(\frac{1}{2}+z_{8}\right) \mathbf{a}_{3}\end{array}$ & $=$ & $\begin{array}{c}\left(\frac{1}{2}+y_{8}\right) a \hat{\mathbf{x}}+\left(\frac{1}{2}-x_{8}\right) a \hat{\mathbf{y}}+ \\
\left(\frac{1}{2}+z_{8}\right) c \hat{\mathbf{z}}\end{array}$ & $(8 g)$ & $\mathrm{Na} I I$ \\
\hline $\mathbf{B}_{41}$ & $=$ & $\begin{array}{c}\left(\frac{1}{2}-x_{8}\right) \mathbf{a}_{1}+\left(\frac{1}{2}+y_{8}\right) \mathbf{a}_{2}+ \\
\left(\frac{1}{2}-z_{8}\right) \mathbf{a}_{3}\end{array}$ & $=$ & $\begin{array}{c}\left(\frac{1}{2}-x_{8}\right) a \hat{\mathbf{x}}+\left(\frac{1}{2}+y_{8}\right) a \hat{\mathbf{y}}+ \\
\left(\frac{1}{2}-z_{8}\right) c \hat{\mathbf{z}}\end{array}$ & $(8 g)$ & $\mathrm{Na} I I$ \\
\hline $\mathbf{B}_{42}$ & $=$ & $\begin{array}{c}\left(\frac{1}{2}+x_{8}\right) \mathbf{a}_{1}+\left(\frac{1}{2}-y_{8}\right) \mathbf{a}_{2}+ \\
\left(\frac{1}{2}-z_{8}\right) \mathbf{a}_{3}\end{array}$ & $=$ & $\begin{array}{c}\left(\frac{1}{2}+x_{8}\right) a \hat{\mathbf{x}}+\left(\frac{1}{2}-y_{8}\right) a \hat{\mathbf{y}}+ \\
\left(\frac{1}{2}-z_{8}\right) c \hat{\mathbf{z}}\end{array}$ & $(8 g)$ & $\mathrm{Na} I I$ \\
\hline $\mathbf{B}_{43}$ & $=$ & $y_{8} \mathbf{a}_{1}+x_{8} \mathbf{a}_{2}-z_{8} \mathbf{a}_{3}$ & $=$ & $y_{8} a \hat{\mathbf{x}}+x_{8} a \hat{\mathbf{y}}-z_{8} c \hat{\mathbf{z}}$ & $(8 g)$ & $\mathrm{Na} I I$ \\
\hline $\mathbf{B}_{44}$ & $=$ & $-y_{8} \mathbf{a}_{1}-x_{8} \mathbf{a}_{2}-z_{8} \mathbf{a}_{3}$ & $=$ & $-y_{8} a \hat{\mathbf{x}}-x_{8} a \hat{\mathbf{y}}-z_{8} c \hat{\mathbf{z}}$ & $(8 g)$ & $\mathrm{Na}$ II \\
\hline
\end{tabular}

\section{References:}

- M. Vlasse, F. Menil, C. Moriliere, J. M. Dance, A. Tressaud, and J. Portier, Etude cristallographique et par effet Mössbauer du fluorure ferrimagnétique $\mathrm{Na}_{5} \mathrm{Fe}_{3} \mathrm{~F}_{14} \gamma$, J. Solid State Chem. 17, 291-298 (1976), doi:10.1016/0022-4596(76)90134-1.

\section{Found in:}

- P. Villars and K. Cenzual, Pearson's Crystal Data - Crystal Structure Database for Inorganic Compounds, ASM International (2013).

\section{Geometry files:}

- CIF: pp. 871

- POSCAR: pp. 871 

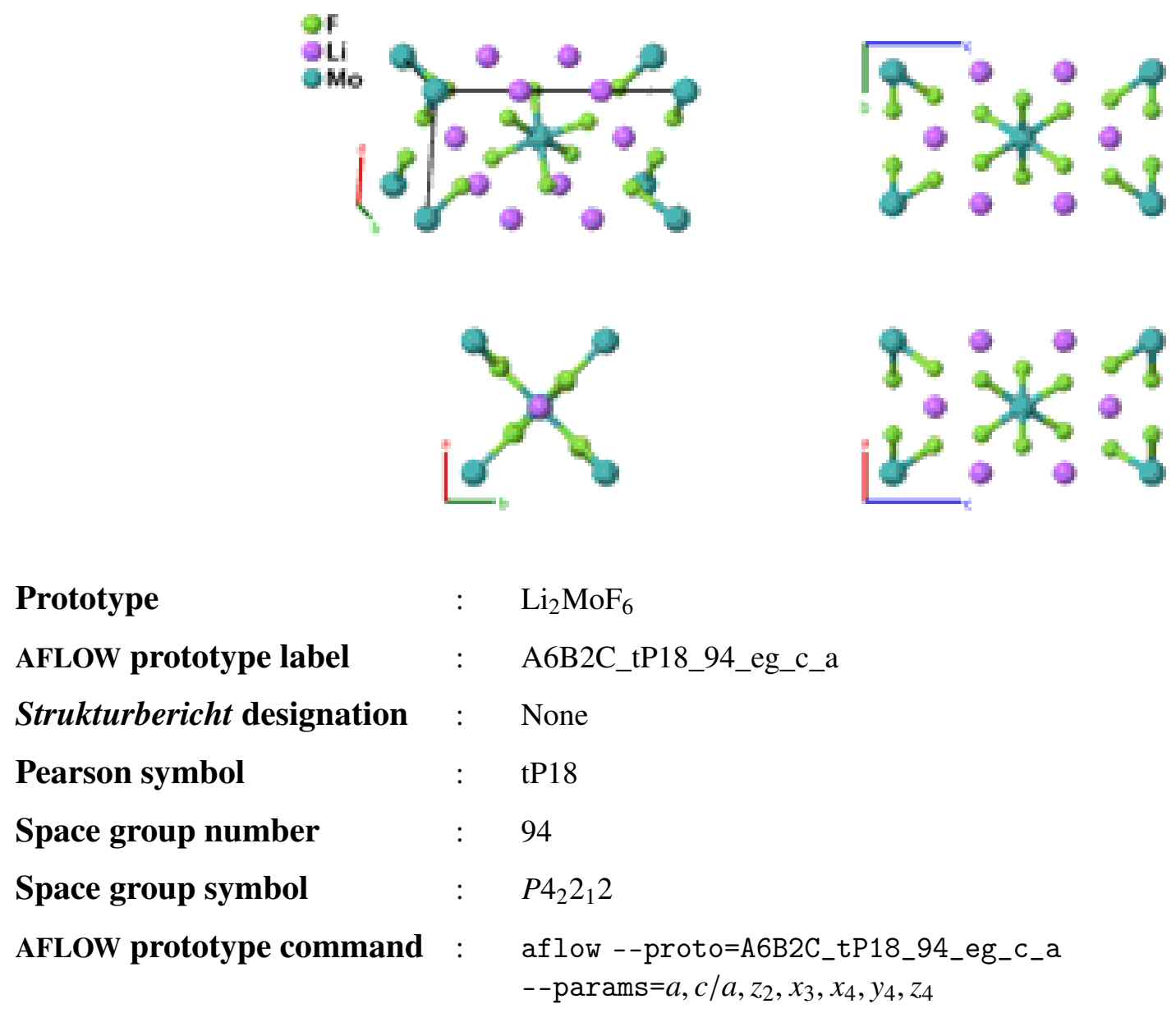

Prototype

AFLOW prototype label

: $\quad \mathrm{Li}_{2} \mathrm{MoF}_{6}$

Strukturbericht designation

: A6B2C_tP18_94_eg_c_a

Pearson symbol

: None

Space group number

: $\quad$ tP18

Space group symbol

: 94

AFLOW prototype command

$$
\text { : } \quad P 4_{2} 2{ }_{1} 2
$$

aflow --proto=A6B2C_tP18_94_eg_c_a

--params $=a, c / a, z_{2}, x_{3}, x_{4}, y_{4}, z_{4}$

\section{Simple Tetragonal primitive vectors:}

$$
\begin{aligned}
& \mathbf{a}_{1}=a \hat{\mathbf{x}} \\
& \mathbf{a}_{2}=a \hat{\mathbf{y}} \\
& \mathbf{a}_{3}=c \hat{\mathbf{z}}
\end{aligned}
$$

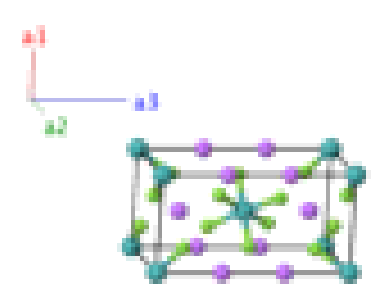

Basis vectors:

Lattice Coordinates

$\begin{array}{ccc}\mathbf{B}_{1}= & 0 \mathbf{a}_{1}+0 \mathbf{a}_{2}+0 \mathbf{a}_{3} \\ \mathbf{B}_{2}= & \frac{1}{2} \mathbf{a}_{1}+\frac{1}{2} \mathbf{a}_{2}+\frac{1}{2} \mathbf{a}_{3} \\ \mathbf{B}_{3}= & z_{2} \mathbf{a}_{3} \\ \mathbf{B}_{4}= & \frac{1}{2} \mathbf{a}_{1}+\frac{1}{2} \mathbf{a}_{2}+\left(\frac{1}{2}+z_{2}\right) \mathbf{a}_{3} \\ \mathbf{B}_{5}= & \frac{1}{2} \mathbf{a}_{1}+\frac{1}{2} \mathbf{a}_{2}+\left(\frac{1}{2}-z_{2}\right) \mathbf{a}_{3} \\ \mathbf{B}_{6}= & -z_{2} \mathbf{a}_{3} \\ \mathbf{B}_{7}= & x_{3} \mathbf{a}_{1}+x_{3} \mathbf{a}_{2} \\ \mathbf{B}_{8}= & -x_{3} \mathbf{a}_{1}-x_{3} \mathbf{a}_{2}\end{array}$

Cartesian Coordinates

$=$

$=$

$=$

$=$

$=$

$=$

$=$

$=$

$$
0 \hat{\mathbf{x}}+0 \hat{\mathbf{y}}+0 \hat{\mathbf{z}}
$$$$
\frac{1}{2} a \hat{\mathbf{X}}+\frac{1}{2} a \hat{\mathbf{y}}+\frac{1}{2} c \hat{\mathbf{z}}
$$$$
z_{2} c \hat{\mathbf{z}}
$$$$
\frac{1}{2} a \hat{\mathbf{x}}+\frac{1}{2} a \hat{\mathbf{y}}+\left(\frac{1}{2}+z_{2}\right) c \hat{\mathbf{z}}
$$$$
\frac{1}{2} a \hat{\mathbf{x}}+\frac{1}{2} a \hat{\mathbf{y}}+\left(\frac{1}{2}-z_{2}\right) c \hat{\mathbf{z}}
$$$$
-z_{2} c \hat{\mathbf{z}}
$$

$$
x_{3} a \hat{\mathbf{x}}+x_{3} a \hat{\mathbf{y}}
$$$$
-x_{3} a \hat{\mathbf{x}}-x_{3} a \hat{\mathbf{y}}
$$

Wyckoff Position Atom Type

(2a) Mo

(2a) Mo

F I

$(4 e)$ 


\begin{tabular}{|c|c|c|c|c|c|c|}
\hline $\mathbf{B}_{9}$ & $=$ & $\left(\frac{1}{2}-x_{3}\right) \mathbf{a}_{1}+\left(\frac{1}{2}+x_{3}\right) \mathbf{a}_{2}+\frac{1}{2} \mathbf{a}_{3}$ & $=$ & $\left(\frac{1}{2}-x_{3}\right) a \hat{\mathbf{x}}+\left(\frac{1}{2}+x_{3}\right) a \hat{\mathbf{y}}+\frac{1}{2} c \hat{\mathbf{z}}$ & $(4 e)$ & F I \\
\hline $\mathbf{B}_{10}$ & $=$ & $\left(\frac{1}{2}+x_{3}\right) \mathbf{a}_{1}+\left(\frac{1}{2}-x_{3}\right) \mathbf{a}_{2}+\frac{1}{2} \mathbf{a}_{3}$ & $=$ & $\left(\frac{1}{2}+x_{3}\right) a \hat{\mathbf{x}}+\left(\frac{1}{2}-x_{3}\right) a \hat{\mathbf{y}}+\frac{1}{2} c \hat{\mathbf{z}}$ & $(4 e)$ & F I \\
\hline $\mathbf{B}_{11}$ & $=$ & $x_{4} \mathbf{a}_{1}+y_{4} \mathbf{a}_{2}+z_{4} \mathbf{a}_{3}$ & $=$ & $x_{4} a \hat{\mathbf{x}}+y_{4} a \hat{\mathbf{y}}+z_{4} c \hat{\mathbf{z}}$ & $(8 g)$ & F II \\
\hline $\mathbf{B}_{12}$ & $=$ & $-x_{4} \mathbf{a}_{1}-y_{4} \mathbf{a}_{2}+z_{4} \mathbf{a}_{3}$ & $=$ & $-x_{4} a \hat{\mathbf{x}}-y_{4} a \hat{\mathbf{y}}+z_{4} c \hat{\mathbf{z}}$ & $(8 g)$ & F II \\
\hline $\mathbf{B}_{13}$ & $=$ & $\begin{array}{c}\left(\frac{1}{2}-y_{4}\right) \mathbf{a}_{1}+\left(\frac{1}{2}+x_{4}\right) \mathbf{a}_{2}+ \\
\left(\frac{1}{2}+z_{4}\right) \mathbf{a}_{3}\end{array}$ & $=$ & $\begin{array}{c}\left(\frac{1}{2}-y_{4}\right) a \hat{\mathbf{x}}+\left(\frac{1}{2}+x_{4}\right) a \hat{\mathbf{y}}+ \\
\left(\frac{1}{2}+z_{4}\right) c \hat{\mathbf{z}}\end{array}$ & $(8 g)$ & F II \\
\hline $\mathbf{B}_{14}$ & $=$ & $\begin{array}{c}\left(\frac{1}{2}+y_{4}\right) \mathbf{a}_{1}+\left(\frac{1}{2}-x_{4}\right) \mathbf{a}_{2}+ \\
\left(\frac{1}{2}+z_{4}\right) \mathbf{a}_{3}\end{array}$ & $=$ & $\begin{array}{c}\left(\frac{1}{2}+y_{4}\right) a \hat{\mathbf{x}}+\left(\frac{1}{2}-x_{4}\right) a \hat{\mathbf{y}}+ \\
\left(\frac{1}{2}+z_{4}\right) c \hat{\mathbf{z}}\end{array}$ & $(8 g)$ & F II \\
\hline $\mathbf{B}_{15}$ & $=$ & $\begin{array}{c}\left(\frac{1}{2}-x_{4}\right) \mathbf{a}_{1}+\left(\frac{1}{2}+y_{4}\right) \mathbf{a}_{2}+ \\
\left(\frac{1}{2}-z_{4}\right) \mathbf{a}_{3}\end{array}$ & $=$ & $\begin{array}{c}\left(\frac{1}{2}-x_{4}\right) a \hat{\mathbf{x}}+\left(\frac{1}{2}+y_{4}\right) a \hat{\mathbf{y}}+ \\
\left(\frac{1}{2}-z_{4}\right) c \hat{\mathbf{z}}\end{array}$ & $(8 g)$ & F II \\
\hline $\mathbf{B}_{16}$ & $=$ & $\begin{array}{c}\left(\frac{1}{2}+x_{4}\right) \mathbf{a}_{1}+\left(\frac{1}{2}-y_{4}\right) \mathbf{a}_{2}+ \\
\left(\frac{1}{2}-z_{4}\right) \mathbf{a}_{3}\end{array}$ & $=$ & $\begin{array}{c}\left(\frac{1}{2}+x_{4}\right) a \hat{\mathbf{x}}+\left(\frac{1}{2}-y_{4}\right) a \hat{\mathbf{y}}+ \\
\left(\frac{1}{2}-z_{4}\right) c \hat{\mathbf{z}}\end{array}$ & $(8 g)$ & F II \\
\hline $\mathbf{B}_{17}$ & $=$ & $y_{4} \mathbf{a}_{1}+x_{4} \mathbf{a}_{2}-z_{4} \mathbf{a}_{3}$ & $=$ & $y_{4} a \hat{\mathbf{x}}+x_{4} a \hat{\mathbf{y}}-z_{4} c \hat{\mathbf{z}}$ & $(8 g)$ & F II \\
\hline $\mathbf{B}_{18}$ & $=$ & $-y_{4} \mathbf{a}_{1}-x_{4} \mathbf{a}_{2}-z_{4} \mathbf{a}_{3}$ & $=$ & $-y_{4} a \hat{\mathbf{x}}-x_{4} a \hat{\mathbf{y}}-z_{4} c \hat{\mathbf{z}}$ & $(8 g)$ & F II \\
\hline
\end{tabular}

\section{References:}

- G. Brunton, The crystal structure of $\mathrm{Li}_{2} \mathrm{MoF}_{6}$, Mater. Res. Bull. 6, 555-560 (1971), doi:10.1016/0025-5408(71)90004-3.

\section{Found in:}

- P. Villars and K. Cenzual, Pearson's Crystal Data - Crystal Structure Database for Inorganic Compounds, ASM International (2013).

\section{Geometry files:}

- CIF: pp. 872

- POSCAR: pp. 872 


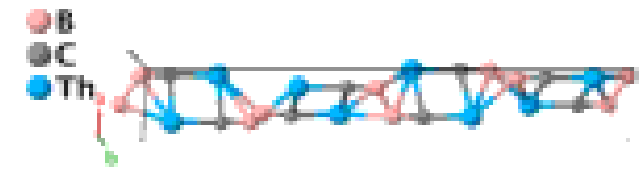

\section{Prototype}

AFLOW prototype label

Strukturbericht designation

Pearson symbol

Space group number

Space group symbol

AFLOW prototype command
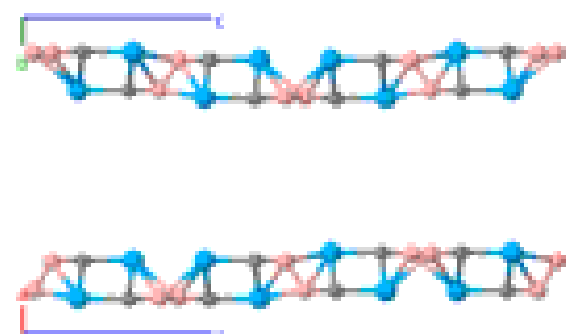

- This structure is the enantiomorph of the ThBC (ABC_tP24_91_d_d_d) structure, and was generated by reflecting the coordinates of the space group \#91 structure through the $z=0$ plane.

Simple Tetragonal primitive vectors:

$$
\begin{aligned}
& \mathbf{a}_{1}=a \hat{\mathbf{x}} \\
& \mathbf{a}_{2}=a \hat{\mathbf{y}} \\
& \mathbf{a}_{3}=c \hat{\mathbf{z}}
\end{aligned}
$$

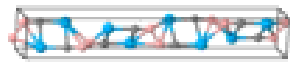

Basis vectors:

Lattice Coordinates

$\mathbf{B}_{1}=x_{1} \mathbf{a}_{1}+y_{1} \mathbf{a}_{2}+z_{1} \mathbf{a}_{3}$

$\mathbf{B}_{2}=-x_{1} \mathbf{a}_{1}-y_{1} \mathbf{a}_{2}+\left(\frac{1}{2}+z_{1}\right) \mathbf{a}_{3}$

$\mathbf{B}_{3}=-y_{1} \mathbf{a}_{1}+x_{1} \mathbf{a}_{2}+\left(\frac{3}{4}+z_{1}\right) \mathbf{a}_{3}$

$\mathbf{B}_{4}=y_{1} \mathbf{a}_{1}-x_{1} \mathbf{a}_{2}+\left(\frac{1}{4}+z_{1}\right) \mathbf{a}_{3}$

$\mathbf{B}_{5}=-x_{1} \mathbf{a}_{1}+y_{1} \mathbf{a}_{2}-z_{1} \mathbf{a}_{3}$

$\mathbf{B}_{6}=x_{1} \mathbf{a}_{1}-y_{1} \mathbf{a}_{2}+\left(\frac{1}{2}-z_{1}\right) \mathbf{a}_{3}$

$\mathbf{B}_{7}=y_{1} \mathbf{a}_{1}+x_{1} \mathbf{a}_{2}+\left(\frac{1}{4}-z_{1}\right) \mathbf{a}_{3}$

$\mathbf{B}_{8}=-y_{1} \mathbf{a}_{1}-x_{1} \mathbf{a}_{2}+\left(\frac{3}{4}-z_{1}\right) \mathbf{a}_{3}$

$\mathbf{B}_{9}=x_{2} \mathbf{a}_{1}+y_{2} \mathbf{a}_{2}+z_{2} \mathbf{a}_{3}$

$\mathbf{B}_{10}=-x_{2} \mathbf{a}_{1}-y_{2} \mathbf{a}_{2}+\left(\frac{1}{2}+z_{2}\right) \mathbf{a}_{3}$

$\mathbf{B}_{11}=-y_{2} \mathbf{a}_{1}+x_{2} \mathbf{a}_{2}+\left(\frac{3}{4}+z_{2}\right) \mathbf{a}_{3}$

$\mathbf{B}_{12}=y_{2} \mathbf{a}_{1}-x_{2} \mathbf{a}_{2}+\left(\frac{1}{4}+z_{2}\right) \mathbf{a}_{3}$
Cartesian Coordinates

$=$

$=\quad-x_{1} a \hat{\mathbf{x}}-y_{1} a \hat{\mathbf{y}}+\left(\frac{1}{2}+z_{1}\right) c \hat{\mathbf{z}}$

$=\quad-y_{1} a \hat{\mathbf{x}}+x_{1} a \hat{\mathbf{y}}+\left(\frac{3}{4}+z_{1}\right) c \hat{\mathbf{z}}$

$=\quad y_{1} a \hat{\mathbf{x}}-x_{1} a \hat{\mathbf{y}}+\left(\frac{1}{4}+z_{1}\right) c \hat{\mathbf{z}}$

$=\quad-x_{1} a \hat{\mathbf{x}}+y_{1} a \hat{\mathbf{y}}-z_{1} c \hat{\mathbf{z}}$

$=\quad x_{1} a \hat{\mathbf{x}}-y_{1} a \hat{\mathbf{y}}+\left(\frac{1}{2}-z_{1}\right) c \hat{\mathbf{z}}$

$=y_{1} a \hat{\mathbf{x}}+x_{1} a \hat{\mathbf{y}}+\left(\frac{1}{4}-z_{1}\right) c \hat{\mathbf{z}}$

$=\quad-y_{1} a \hat{\mathbf{x}}-x_{1} a \hat{\mathbf{y}}+\left(\frac{3}{4}-z_{1}\right) c \hat{\mathbf{z}}$

$=\quad x_{2} a \hat{\mathbf{x}}+y_{2} a \hat{\mathbf{y}}+z_{2} c \hat{\mathbf{z}}$

$=-x_{2} a \hat{\mathbf{x}}-y_{2} a \hat{\mathbf{y}}+\left(\frac{1}{2}+z_{2}\right) c \hat{\mathbf{z}}$

$=\quad-y_{2} a \hat{\mathbf{x}}+x_{2} a \hat{\mathbf{y}}+\left(\frac{3}{4}+z_{2}\right) c \hat{\mathbf{z}}$

$=\quad y_{2} a \hat{\mathbf{x}}-x_{2} a \hat{\mathbf{y}}+\left(\frac{1}{4}+z_{2}\right) c \hat{\mathbf{z}}$
Wyckoff Position
Atom Type

B

B

B

B

B

B

C

C

C

C 


\begin{tabular}{|c|c|c|c|c|c|c|}
\hline $\mathbf{B}_{13}$ & $=$ & $-x_{2} \mathbf{a}_{1}+y_{2} \mathbf{a}_{2}-z_{2} \mathbf{a}_{3}$ & $=$ & $-x_{2} a \hat{\mathbf{x}}+y_{2} a \hat{\mathbf{y}}-z_{2} c \hat{\mathbf{z}}$ & $(8 d)$ & $\mathrm{C}$ \\
\hline $\mathbf{B}_{14}$ & $=$ & $x_{2} \mathbf{a}_{1}-y_{2} \mathbf{a}_{2}+\left(\frac{1}{2}-z_{2}\right) \mathbf{a}_{3}$ & $=$ & $x_{2} a \hat{\mathbf{x}}-y_{2} a \hat{\mathbf{y}}+\left(\frac{1}{2}-z_{2}\right) c \hat{\mathbf{z}}$ & $(8 d)$ & $\mathrm{C}$ \\
\hline $\mathbf{B}_{15}$ & $=$ & $y_{2} \mathbf{a}_{1}+x_{2} \mathbf{a}_{2}+\left(\frac{1}{4}-z_{2}\right) \mathbf{a}_{3}$ & $=$ & $y_{2} a \hat{\mathbf{x}}+x_{2} a \hat{\mathbf{y}}+\left(\frac{1}{4}-z_{2}\right) c \hat{\mathbf{z}}$ & $(8 d)$ & $\mathrm{C}$ \\
\hline $\mathbf{B}_{16}$ & $=$ & $-y_{2} \mathbf{a}_{1}-x_{2} \mathbf{a}_{2}+\left(\frac{3}{4}-z_{2}\right) \mathbf{a}_{3}$ & $=$ & $-y_{2} a \hat{\mathbf{x}}-x_{2} a \hat{\mathbf{y}}+\left(\frac{3}{4}-z_{2}\right) c \hat{\mathbf{z}}$ & $(8 d)$ & $\mathrm{C}$ \\
\hline $\mathbf{B}_{17}$ & $=$ & $x_{3} \mathbf{a}_{1}+y_{3} \mathbf{a}_{2}+z_{3} \mathbf{a}_{3}$ & $=$ & $x_{3} a \hat{\mathbf{x}}+y_{3} a \hat{\mathbf{y}}+z_{3} c \hat{\mathbf{z}}$ & $(8 d)$ & Th \\
\hline $\mathbf{B}_{18}$ & $=$ & $-x_{3} \mathbf{a}_{1}-y_{3} \mathbf{a}_{2}+\left(\frac{1}{2}+z_{3}\right) \mathbf{a}_{3}$ & $=$ & $-x_{3} a \hat{\mathbf{x}}-y_{3} a \hat{\mathbf{y}}+\left(\frac{1}{2}+z_{3}\right) c \hat{\mathbf{z}}$ & $(8 d)$ & Th \\
\hline $\mathbf{B}_{19}$ & $=$ & $-y_{3} \mathbf{a}_{1}+x_{3} \mathbf{a}_{2}+\left(\frac{3}{4}+z_{3}\right) \mathbf{a}_{3}$ & $=$ & $-y_{3} a \hat{\mathbf{x}}+x_{3} a \hat{\mathbf{y}}+\left(\frac{3}{4}+z_{3}\right) c \hat{\mathbf{z}}$ & $(8 d)$ & Th \\
\hline $\mathbf{B}_{20}$ & $=$ & $y_{3} \mathbf{a}_{1}-x_{3} \mathbf{a}_{2}+\left(\frac{1}{4}+z_{3}\right) \mathbf{a}_{3}$ & $=$ & $y_{3} a \hat{\mathbf{x}}-x_{3} a \hat{\mathbf{y}}+\left(\frac{1}{4}+z_{3}\right) c \hat{\mathbf{z}}$ & $(8 d)$ & Th \\
\hline $\mathbf{B}_{21}$ & $=$ & $-x_{3} \mathbf{a}_{1}+y_{3} \mathbf{a}_{2}-z_{3} \mathbf{a}_{3}$ & $=$ & $-x_{3} a \hat{\mathbf{x}}+y_{3} a \hat{\mathbf{y}}-z_{3} c \hat{\mathbf{z}}$ & $(8 d)$ & Th \\
\hline $\mathbf{B}_{22}$ & $=$ & $x_{3} \mathbf{a}_{1}-y_{3} \mathbf{a}_{2}+\left(\frac{1}{2}-z_{3}\right) \mathbf{a}_{3}$ & $=$ & $x_{3} a \hat{\mathbf{x}}-y_{3} a \hat{\mathbf{y}}+\left(\frac{1}{2}-z_{3}\right) c \hat{\mathbf{z}}$ & $(8 d)$ & Th \\
\hline $\mathbf{B}_{23}$ & $=$ & $y_{3} \mathbf{a}_{1}+x_{3} \mathbf{a}_{2}+\left(\frac{1}{4}-z_{3}\right) \mathbf{a}_{3}$ & $=$ & $y_{3} a \hat{\mathbf{x}}+x_{3} a \hat{\mathbf{y}}+\left(\frac{1}{4}-z_{3}\right) c \hat{\mathbf{z}}$ & $(8 d)$ & Th \\
\hline $\mathbf{B}_{24}$ & $=$ & $-y_{3} \mathbf{a}_{1}-x_{3} \mathbf{a}_{2}+\left(\frac{3}{4}-z_{3}\right) \mathbf{a}_{3}$ & $=$ & $-y_{3} a \hat{\mathbf{x}}-x_{3} a \hat{\mathbf{y}}+\left(\frac{3}{4}-z_{3}\right) c \hat{\mathbf{z}}$ & $(8 d)$ & Th \\
\hline
\end{tabular}

\section{References:}

- P. Rogl, The crystal structure of ThBC, J. Nucl. Mat. 73, 198-203 (1978), doi:10.1016/0022-3115(78)90560-3.

\section{Geometry files:}

- CIF: pp. 872

- POSCAR: pp. 872 


\section{$\mathrm{NaGdCu} \mathrm{F}_{8}$ Structure: A2B8CD_tI24_97_d_k_a_b}
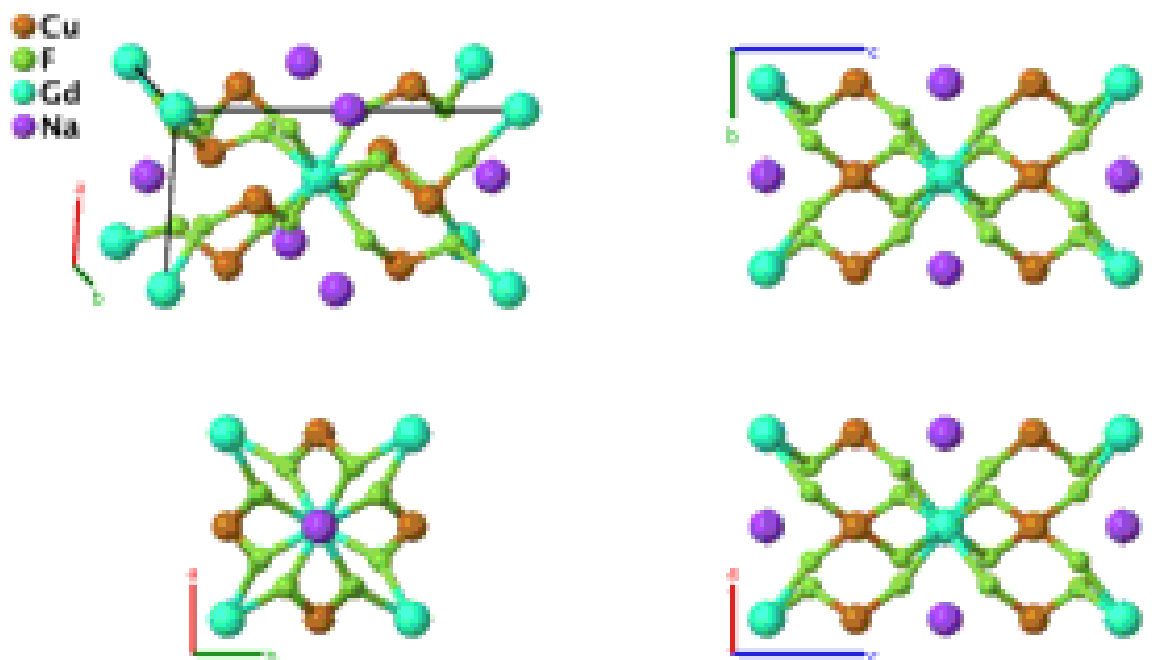

Prototype

AFLOW prototype label

Strukturbericht designation

Pearson symbol

Space group number

Space group symbol

AFLOW prototype command
: $\quad \mathrm{NaGdCu}_{2} \mathrm{~F}_{8}$

: A2B8CD_tI24_97_d_k_a_b

: None

: $\quad$ tI24

: $\quad 97$

: $\quad I 422$

\section{- params $=a, c / a, x_{4}, y_{4}, z_{4}$}

Body-centered Tetragonal primitive vectors:

$$
\begin{aligned}
& \mathbf{a}_{1}=-\frac{1}{2} a \hat{\mathbf{x}}+\frac{1}{2} a \hat{\mathbf{y}}+\frac{1}{2} c \hat{\mathbf{z}} \\
& \mathbf{a}_{2}=\frac{1}{2} a \hat{\mathbf{x}}-\frac{1}{2} a \hat{\mathbf{y}}+\frac{1}{2} c \hat{\mathbf{z}} \\
& \mathbf{a}_{3}=\frac{1}{2} a \hat{\mathbf{x}}+\frac{1}{2} a \hat{\mathbf{y}}-\frac{1}{2} c \hat{\mathbf{z}}
\end{aligned}
$$

\section{Basis vectors:}

Lattice Coordinates

$\begin{array}{lcc}\mathbf{B}_{1}= & 0 \mathbf{a}_{1}+0 \mathbf{a}_{2}+0 \mathbf{a}_{3} \\ \mathbf{B}_{2}= & \frac{1}{2} \mathbf{a}_{1}+\frac{1}{2} \mathbf{a}_{2} \\ \mathbf{B}_{3}= & \frac{3}{4} \mathbf{a}_{1}+\frac{1}{4} \mathbf{a}_{2}+\frac{1}{2} \mathbf{a}_{3} \\ \mathbf{B}_{4}= & \frac{1}{4} \mathbf{a}_{1}+\frac{3}{4} \mathbf{a}_{2}+\frac{1}{2} \mathbf{a}_{3}\end{array}$

$\mathbf{B}_{5}=\left(y_{4}+z_{4}\right) \mathbf{a}_{1}+\left(x_{4}+z_{4}\right) \mathbf{a}_{2}+\left(x_{4}+y_{4}\right) \mathbf{a}_{3}$

$\mathbf{B}_{6}=\left(-y_{4}+z_{4}\right) \mathbf{a}_{1}+\left(-x_{4}+z_{4}\right) \mathbf{a}_{2}+\left(-x_{4}-y_{4}\right) \mathbf{a}_{3}=$

$\mathbf{B}_{7}=\left(x_{4}+z_{4}\right) \mathbf{a}_{1}+\left(-y_{4}+z_{4}\right) \mathbf{a}_{2}+\left(x_{4}-y_{4}\right) \mathbf{a}_{3}=$

$\mathbf{B}_{8}=\left(-x_{4}+z_{4}\right) \mathbf{a}_{1}+\left(y_{4}+z_{4}\right) \mathbf{a}_{2}+\left(-x_{4}+y_{4}\right) \mathbf{a}_{3}=$
Cartesian Coordinates

$$
=
$$$$
=
$$$$
=
$$$$
=
$$$$
=
$$$$
=
$$$$
=-x_{4} a \hat{\mathbf{x}}-y_{4} a \hat{\mathbf{y}}+z_{4} c \hat{\mathbf{z}}
$$

$=-y_{4} a \hat{\mathbf{x}}+x_{4} a \hat{\mathbf{y}}+z_{4} c \hat{\mathbf{z}}$

$y_{4} a \hat{\mathbf{x}}-x_{4} a \hat{\mathbf{y}}+z_{4} c \hat{\mathbf{z}}$
Wyckoff Position Atom Type

(2a)

$\mathrm{Gd}$

$\mathrm{Na}$

$(4 d)$

$\mathrm{Cu}$

$(4 d)$

$\mathrm{Cu}$

\section{F}

F

F

F 
$\mathbf{B}_{9}=\left(y_{4}-z_{4}\right) \mathbf{a}_{1}+\left(-x_{4}-z_{4}\right) \mathbf{a}_{2}+\left(-x_{4}+y_{4}\right) \mathbf{a}_{3}=-x_{4} a \hat{\mathbf{x}}+y_{4} a \hat{\mathbf{y}}-z_{4} c \hat{\mathbf{z}}$
$\mathbf{B}_{10}=\left(-y_{4}-z_{4}\right) \mathbf{a}_{1}+\left(x_{4}-z_{4}\right) \mathbf{a}_{2}+\left(x_{4}-y_{4}\right) \mathbf{a}_{3}=x_{4} a \hat{\mathbf{x}}-y_{4} a \hat{\mathbf{y}}-z_{4} c \hat{\mathbf{z}}$
$\mathbf{B}_{11}=\left(x_{4}-z_{4}\right) \mathbf{a}_{1}+\left(y_{4}-z_{4}\right) \mathbf{a}_{2}+\left(x_{4}+y_{4}\right) \mathbf{a}_{3}=y_{4} a \hat{\mathbf{x}}+x_{4} a \hat{\mathbf{y}}-z_{4} c \hat{\mathbf{z}}$
$\mathbf{B}_{12}=\left(-x_{4}-z_{4}\right) \mathbf{a}_{1}+\left(-y_{4}-z_{4}\right) \mathbf{a}_{2}+\left(-x_{4}-y_{4}\right) \mathbf{a}_{3}=-y_{4} a \hat{\mathbf{x}}-x_{4} a \hat{\mathbf{y}}-z_{4} c \hat{\mathbf{z}}$

\section{References:}

- C. De Nadaï, A. Demourgues, L. Lozano, P. Gravereau, and J. Grannec, Structural investigations of new copper fluorides $\mathrm{NaRECu}_{2} \mathrm{~F}_{8}\left(\mathrm{RE}^{3+}=\mathrm{Sm}^{3+}, \mathrm{Eu}^{3+}, \mathrm{Gd}^{3+}, Y^{3+}, \mathrm{Er}^{3+}, \mathrm{Yb}^{3+}\right)$, J. Mater. Chem. 8, 2487-2491 (1998), doi:10.1039/A803015D.

\section{Found in:}

- P. Villars and K. Cenzual, Pearson's Crystal Data - Crystal Structure Database for Inorganic Compounds, ASM International (2013).

\section{Geometry files:}

- CIF: pp. 873

- POSCAR: pp. 873 


\section{$\mathrm{Ta}_{2} \mathrm{Se}_{8} \mathrm{I}$ Structure: AB8C2_tI44_97_e_2k_cd}
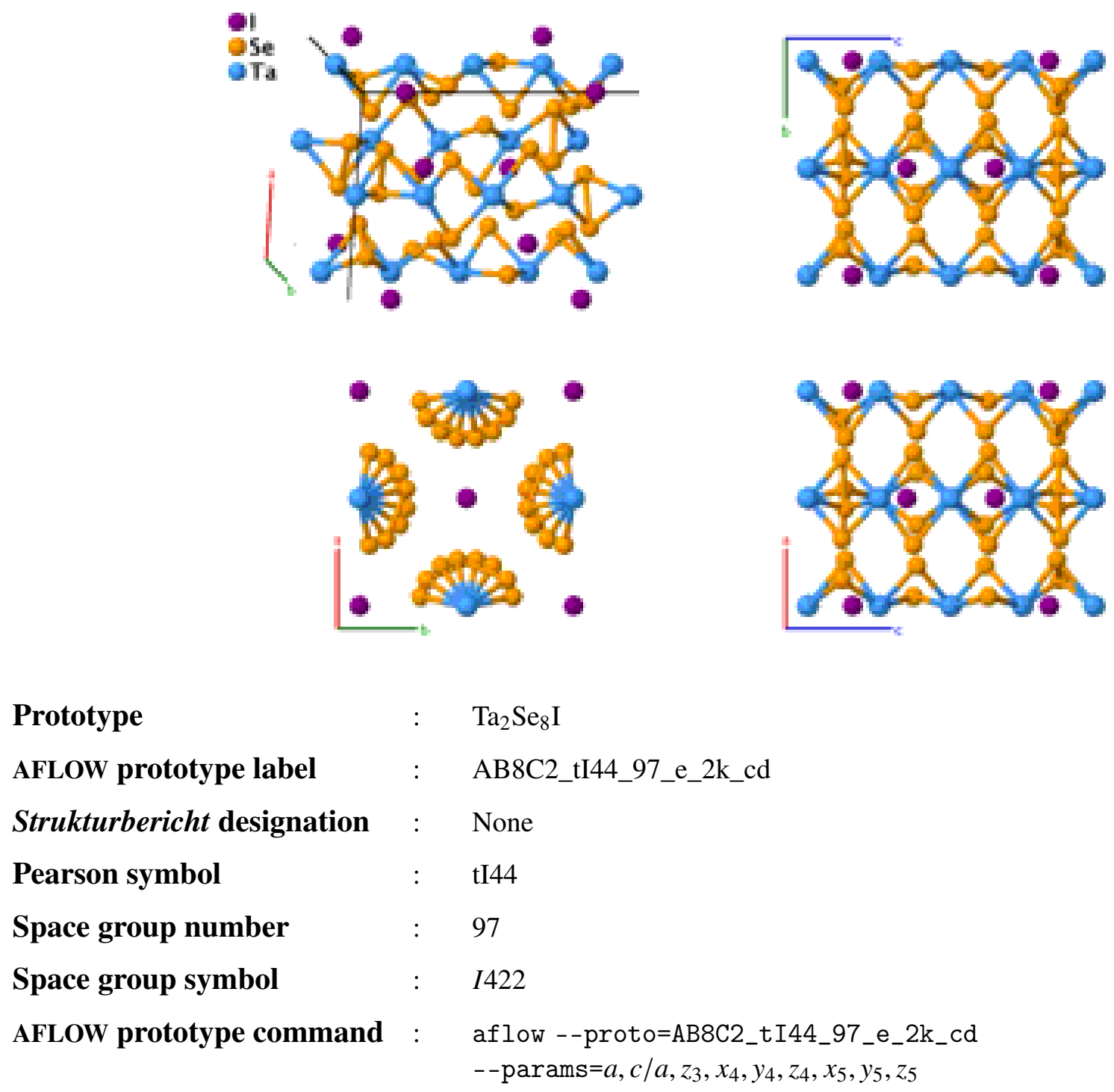

Prototype

: $\quad \mathrm{Ta}_{2} \mathrm{Se}_{8} \mathrm{I}$

AFLOW prototype label

: AB8C2_tI44_97_e_2k_cd

Strukturbericht designation : None

Pearson symbol $\quad: \quad t I 44$

Space group number $\quad: \quad 97$

Space group symbol $\quad: \quad I 422$

AFLOW prototype command : aflow --proto=AB8C2_tI44_97_e_2k_cd

- params $=a, c / a, z_{3}, x_{4}, y_{4}, z_{4}, x_{5}, y_{5}, z_{5}$

Body-centered Tetragonal primitive vectors:

$$
\begin{aligned}
& \mathbf{a}_{1}=-\frac{1}{2} a \hat{\mathbf{x}}+\frac{1}{2} a \hat{\mathbf{y}}+\frac{1}{2} c \hat{\mathbf{z}} \\
& \mathbf{a}_{2}=\frac{1}{2} a \hat{\mathbf{x}}-\frac{1}{2} a \hat{\mathbf{y}}+\frac{1}{2} c \hat{\mathbf{z}} \\
& \mathbf{a}_{3}=\frac{1}{2} a \hat{\mathbf{x}}+\frac{1}{2} a \hat{\mathbf{y}}-\frac{1}{2} c \hat{\mathbf{z}}
\end{aligned}
$$

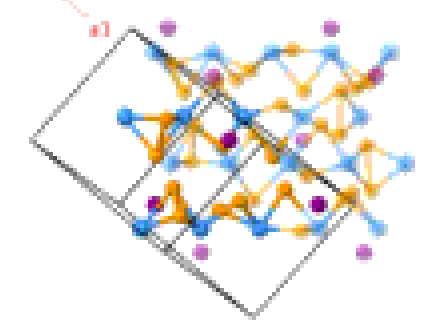

Basis vectors:

Lattice Coordinates

$\begin{array}{rlc}\mathbf{B}_{1}= & \frac{1}{2} \mathbf{a}_{1}+\frac{1}{2} \mathbf{a}_{3} \\ \mathbf{B}_{2}= & \frac{1}{2} \mathbf{a}_{2}+\frac{1}{2} \mathbf{a}_{3} \\ \mathbf{B}_{3}= & \frac{3}{4} \mathbf{a}_{1}+\frac{1}{4} \mathbf{a}_{2}+\frac{1}{2} \mathbf{a}_{3} \\ \mathbf{B}_{4}= & \frac{1}{4} \mathbf{a}_{1}+\frac{3}{4} \mathbf{a}_{2}+\frac{1}{2} \mathbf{a}_{3}\end{array}$

Cartesian Coordinates

$=\quad \frac{1}{2} a \hat{\mathbf{y}}$

$=\quad \frac{1}{2} a \hat{\mathbf{x}}$

$=\quad \frac{1}{2} a \hat{\mathbf{y}}+\frac{1}{4} c \hat{\mathbf{z}}$

$=\quad \frac{1}{2} a \hat{\mathbf{x}}+\frac{1}{4} c \hat{\mathbf{z}}$
Wyckoff Position

(4c)

$(4 c)$

(4d)

(4d)
Atom Type

Ta I

Ta I

Ta II

Ta II 


\begin{tabular}{|c|c|c|c|c|c|c|}
\hline $\mathbf{B}_{5}$ & $=$ & $z_{3} \mathbf{a}_{1}+z_{3} \mathbf{a}_{2}$ & $=$ & $z_{3} c \hat{\mathbf{z}}$ & $(4 e)$ & I \\
\hline $\mathbf{B}_{6}$ & $=$ & $-z_{3} \mathbf{a}_{1}-z_{3} \mathbf{a}_{2}$ & $=$ & $-z_{3} c \hat{\mathbf{z}}$ & $(4 e)$ & I \\
\hline $\mathbf{B}_{7}$ & $=$ & $\left(y_{4}+z_{4}\right) \mathbf{a}_{1}+\left(x_{4}+z_{4}\right) \mathbf{a}_{2}+\left(x_{4}+y_{4}\right) \mathbf{a}_{3}$ & $=$ & $x_{4} a \hat{\mathbf{x}}+y_{4} a \hat{\mathbf{y}}+z_{4} c \hat{\mathbf{z}}$ & $(16 k)$ & Se I \\
\hline $\mathbf{B}_{8}$ & $=$ & $\left(-y_{4}+z_{4}\right) \mathbf{a}_{1}+\left(-x_{4}+z_{4}\right) \mathbf{a}_{2}+\left(-x_{4}-y_{4}\right) \mathbf{a}_{3}$ & $=$ & $-x_{4} a \hat{\mathbf{x}}-y_{4} a \hat{\mathbf{y}}+z_{4} c \hat{\mathbf{z}}$ & $(16 k)$ & Se I \\
\hline $\mathbf{B}_{9}$ & $=$ & $\left(x_{4}+z_{4}\right) \mathbf{a}_{1}+\left(-y_{4}+z_{4}\right) \mathbf{a}_{2}+\left(x_{4}-y_{4}\right) \mathbf{a}_{3}$ & $=$ & $-y_{4} a \hat{\mathbf{x}}+x_{4} a \hat{\mathbf{y}}+z_{4} c \hat{\mathbf{z}}$ & $(16 k)$ & Se I \\
\hline $\mathbf{B}_{10}$ & $=$ & $\left(-x_{4}+z_{4}\right) \mathbf{a}_{1}+\left(y_{4}+z_{4}\right) \mathbf{a}_{2}+\left(-x_{4}+y_{4}\right) \mathbf{a}_{3}$ & $=$ & $y_{4} a \hat{\mathbf{x}}-x_{4} a \hat{\mathbf{y}}+z_{4} c \hat{\mathbf{z}}$ & $(16 k)$ & Se I \\
\hline $\mathbf{B}_{11}$ & $=$ & $\left(y_{4}-z_{4}\right) \mathbf{a}_{1}+\left(-x_{4}-z_{4}\right) \mathbf{a}_{2}+\left(-x_{4}+y_{4}\right) \mathbf{a}_{3}$ & $=$ & $-x_{4} a \hat{\mathbf{x}}+y_{4} a \hat{\mathbf{y}}-z_{4} c \hat{\mathbf{z}}$ & $(16 k)$ & Se I \\
\hline $\mathbf{B}_{12}$ & $=$ & $\left(-y_{4}-z_{4}\right) \mathbf{a}_{1}+\left(x_{4}-z_{4}\right) \mathbf{a}_{2}+\left(x_{4}-y_{4}\right) \mathbf{a}_{3}$ & $=$ & $x_{4} a \hat{\mathbf{x}}-y_{4} a \hat{\mathbf{y}}-z_{4} c \hat{\mathbf{z}}$ & $(16 k)$ & Se I \\
\hline $\mathbf{B}_{13}$ & $=$ & $\left(x_{4}-z_{4}\right) \mathbf{a}_{1}+\left(y_{4}-z_{4}\right) \mathbf{a}_{2}+\left(x_{4}+y_{4}\right) \mathbf{a}_{3}$ & $=$ & $y_{4} a \hat{\mathbf{x}}+x_{4} a \hat{\mathbf{y}}-z_{4} c \hat{\mathbf{z}}$ & $(16 k)$ & Se I \\
\hline $\mathbf{B}_{14}$ & $=$ & $\left(-x_{4}-z_{4}\right) \mathbf{a}_{1}+\left(-y_{4}-z_{4}\right) \mathbf{a}_{2}+\left(-x_{4}-y_{4}\right) \mathbf{a}_{3}$ & $=$ & $-y_{4} a \hat{\mathbf{x}}-x_{4} a \hat{\mathbf{y}}-z_{4} c \hat{\mathbf{z}}$ & $(16 k)$ & Se I \\
\hline $\mathbf{B}_{15}$ & $=$ & $\left(y_{5}+z_{5}\right) \mathbf{a}_{1}+\left(x_{5}+z_{5}\right) \mathbf{a}_{2}+\left(x_{5}+y_{5}\right) \mathbf{a}_{3}$ & $=$ & $x_{5} a \hat{\mathbf{x}}+y_{5} a \hat{\mathbf{y}}+z_{5} c \hat{\mathbf{z}}$ & $(16 k)$ & Se II \\
\hline $\mathbf{B}_{16}$ & $=$ & $\left(-y_{5}+z_{5}\right) \mathbf{a}_{1}+\left(-x_{5}+z_{5}\right) \mathbf{a}_{2}+\left(-x_{5}-y_{5}\right) \mathbf{a}_{3}$ & $=$ & $-x_{5} a \hat{\mathbf{x}}-y_{5} a \hat{\mathbf{y}}+z_{5} c \hat{\mathbf{z}}$ & $(16 k)$ & Se II \\
\hline $\mathbf{B}_{17}$ & $=$ & $\left(x_{5}+z_{5}\right) \mathbf{a}_{1}+\left(-y_{5}+z_{5}\right) \mathbf{a}_{2}+\left(x_{5}-y_{5}\right) \mathbf{a}_{3}$ & $=$ & $-y_{5} a \hat{\mathbf{x}}+x_{5} a \hat{\mathbf{y}}+z_{5} c \hat{\mathbf{z}}$ & $(16 k)$ & Se II \\
\hline $\mathbf{B}_{18}$ & $=$ & $\left(-x_{5}+z_{5}\right) \mathbf{a}_{1}+\left(y_{5}+z_{5}\right) \mathbf{a}_{2}+\left(-x_{5}+y_{5}\right) \mathbf{a}_{3}$ & $=$ & $y_{5} a \hat{\mathbf{x}}-x_{5} a \hat{\mathbf{y}}+z_{5} c \hat{\mathbf{z}}$ & $(16 k)$ & Se II \\
\hline $\mathbf{B}_{19}$ & $=$ & $\left(y_{5}-z_{5}\right) \mathbf{a}_{1}+\left(-x_{5}-z_{5}\right) \mathbf{a}_{2}+\left(-x_{5}+y_{5}\right) \mathbf{a}_{3}$ & $=$ & $-x_{5} a \hat{\mathbf{x}}+y_{5} a \hat{\mathbf{y}}-z_{5} c \hat{\mathbf{z}}$ & $(16 k)$ & Se II \\
\hline $\mathbf{B}_{20}$ & $=$ & $\left(-y_{5}-z_{5}\right) \mathbf{a}_{1}+\left(x_{5}-z_{5}\right) \mathbf{a}_{2}+\left(x_{5}-y_{5}\right) \mathbf{a}_{3}$ & $=$ & $x_{5} a \hat{\mathbf{x}}-y_{5} a \hat{\mathbf{y}}-z_{5} c \hat{\mathbf{z}}$ & $(16 k)$ & Se II \\
\hline $\mathbf{B}_{21}$ & $=$ & $\left(x_{5}-z_{5}\right) \mathbf{a}_{1}+\left(y_{5}-z_{5}\right) \mathbf{a}_{2}+\left(x_{5}+y_{5}\right) \mathbf{a}_{3}$ & $=$ & $y_{5} a \hat{\mathbf{x}}+x_{5} a \hat{\mathbf{y}}-z_{5} c \hat{\mathbf{z}}$ & $(16 k)$ & Se II \\
\hline $\mathbf{B}_{22}$ & $=$ & $\left(-x_{5}-z_{5}\right) \mathbf{a}_{1}+\left(-y_{5}-z_{5}\right) \mathbf{a}_{2}+\left(-x_{5}-y_{5}\right) \mathbf{a}_{3}$ & $=$ & $-y_{5} a \hat{\mathbf{x}}-x_{5} a \hat{\mathbf{y}}-z_{5} c \hat{\mathbf{z}}$ & $(16 k)$ & Se II \\
\hline
\end{tabular}

\section{References:}

- P. Gressier, A. Meerschaut, L. Guemas, J. Rouxel, and P. Monceau, Characterization of the new series of quasi one-dimensional compounds $\left(M X_{4}\right)_{n} Y(M=N b, T a ; X=S$, Se; $Y=B r, I)$, J. Solid State Chem. 51, 141-151 (1984), doi:10.1016/0022-4596(84)90327-X.

\section{Found in:}

- P. Villars and K. Cenzual, Pearson's Crystal Data - Crystal Structure Database for Inorganic Compounds, ASM International (2013).

\section{Geometry files:}

- CIF: pp. 873

- POSCAR: pp. 874 


\section{$\mathrm{CdAs}_{2}$ Structure: A2B_tI12_98_f_a}
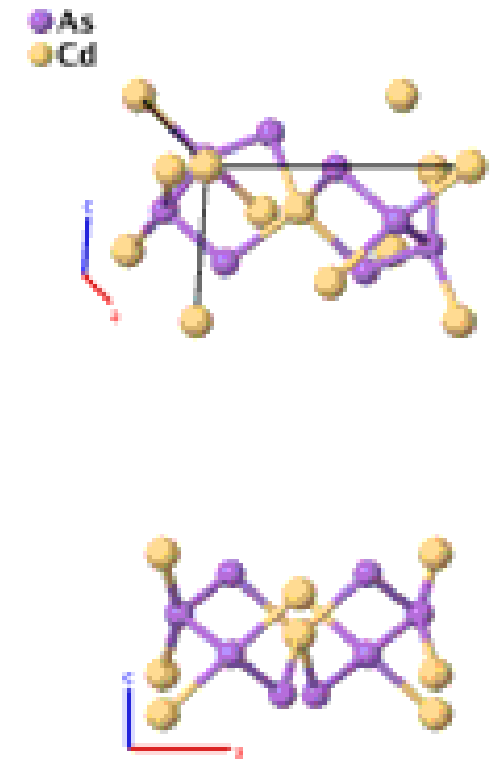
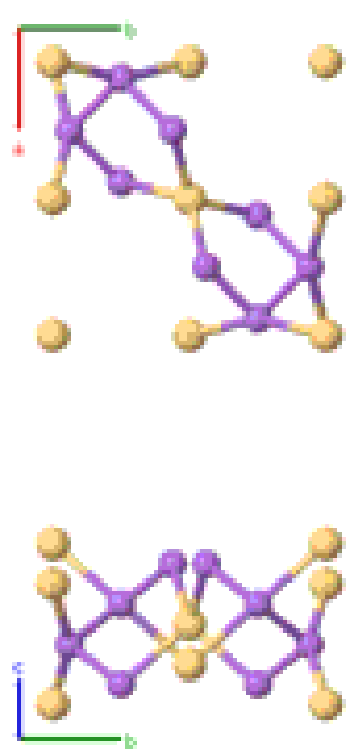

Prototype

AFLOW prototype label

Strukturbericht designation

Pearson symbol

Space group number

Space group symbol

AFLOW prototype command
: $\quad \mathrm{CdAs}_{2}$

: A2B_tI12_98_f_a

: None

: $\quad \mathrm{tI} 12$

: $\quad 98$

: $\quad I 4_{1} 22$

Body-centered Tetragonal primitive vectors:

$$
\begin{aligned}
& \mathbf{a}_{1}=-\frac{1}{2} a \hat{\mathbf{x}}+\frac{1}{2} a \hat{\mathbf{y}}+\frac{1}{2} c \hat{\mathbf{z}} \\
& \mathbf{a}_{2}=\frac{1}{2} a \hat{\mathbf{x}}-\frac{1}{2} a \hat{\mathbf{y}}+\frac{1}{2} c \hat{\mathbf{z}} \\
& \mathbf{a}_{3}=\frac{1}{2} a \hat{\mathbf{x}}+\frac{1}{2} a \hat{\mathbf{y}}-\frac{1}{2} c \hat{\mathbf{z}}
\end{aligned}
$$

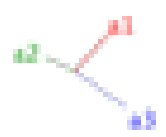

Basis vectors:

Lattice Coordinates
$\mathbf{B}_{1}=$
$0 \mathbf{a}_{1}+0 \mathbf{a}_{2}+0 \mathbf{a}_{3}$
$\mathbf{B}_{2}=$
$\frac{3}{4} \mathbf{a}_{1}+\frac{1}{4} \mathbf{a}_{2}+\frac{1}{2} \mathbf{a}_{3}$
$\mathbf{B}_{3}=$
$\mathbf{B}_{4}=$
$\frac{3}{8} \mathbf{a}_{1}+\left(\frac{1}{8}+x_{2}\right) \mathbf{a}_{2}+\left(\frac{1}{4}+x_{2}\right) \mathbf{a}_{3}$
$\frac{7}{8} \mathbf{a}_{1}+\left(\frac{1}{8}-x_{2}\right) \mathbf{a}_{2}+\left(\frac{3}{4}-x_{2}\right) \mathbf{a}_{3}$
$=$
$=\quad \frac{1}{2} a \hat{\mathbf{y}}+\frac{1}{4} c \hat{\mathbf{z}}$
$=\quad x_{2} a \hat{\mathbf{x}}+\frac{1}{4} a \hat{\mathbf{y}}+\frac{1}{8} c \hat{\mathbf{z}}$
$=\quad-x_{2} a \hat{\mathbf{x}}+\frac{3}{4} a \hat{\mathbf{y}}+\frac{1}{8} c \hat{\mathbf{z}}$

Cartesian Coordinates

Wyckoff Position

Atom Type

$(4 a)$

$\mathrm{Cd}$

(4a)

Cd

$(8 f)$

As

(8f) 

$\mathbf{B}_{5}=\left(\frac{7}{8}+x_{2}\right)$
$\mathbf{a}_{1}+\frac{1}{8} \mathbf{a}_{2}+\left(\frac{1}{4}+x_{2}\right) \mathbf{a}_{3}$
$=\frac{3}{4} a \hat{\mathbf{x}}+\left(\frac{1}{2}+x_{2}\right) a \hat{\mathbf{y}}+\frac{3}{8} c \hat{\mathbf{z}}$
$(8 f)$
As
$\mathbf{B}_{6}=\left(\frac{7}{8}-x_{2}\right) \mathbf{a}_{1}+\frac{5}{8} \mathbf{a}_{2}+\left(\frac{3}{4}-x_{2}\right) \mathbf{a}_{3}=\frac{1}{4} a \hat{\mathbf{x}}+\left(\frac{1}{2}-x_{2}\right) a \hat{\mathbf{y}}+\frac{3}{8} c \hat{\mathbf{z}}$
$(8 f)$
As

\section{References:}

- V. N. Yakimovich, V. A. Rubtsov, and V. M. Trukhan, Phase Relationships in the $C d P_{4}-Z n P_{2}-C d A s_{2}-Z n A s_{2}$ System, Inorg. Mat. 32, 579-582 (1996).

\section{Found in:}

- P. Villars and K. Cenzual, Pearson's Crystal Data - Crystal Structure Database for Inorganic Compounds, ASM International (2013).

\section{Geometry files:}

- CIF: pp. 874

- POSCAR: pp. 874 


\section{Fresnoite $\left(\mathrm{Ba}_{2} \mathrm{TiSi}_{2} \mathrm{O}_{8}\right)$ Structure:}

\section{A2B8C2D_tP26_100_c_abcd_c_a}

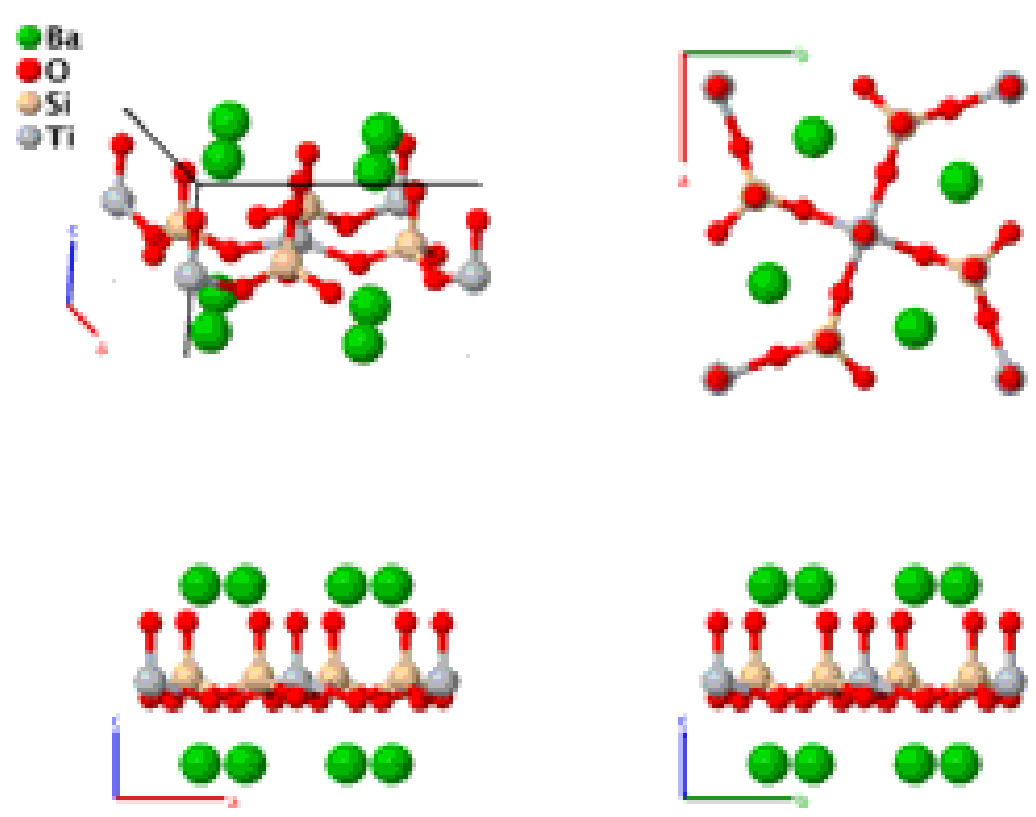

Prototype $\quad: \mathrm{Ba}_{2} \mathrm{TiSi}_{2} \mathrm{O}_{8}$

AFLOW prototype label $\quad$ : A2B8C2D_tP26_100_c_abcd_c_a

Strukturbericht designation : None

Pearson symbol $\quad: \quad$ tP26

Space group number $\quad: \quad 100$

Space group symbol $\quad: \quad P 4 b m$

AFLOW prototype command : aflow --proto=A2B8C2D_tP26_100_c_abcd_c_a

- - params $=a, c / a, z_{1}, z_{2}, z_{3}, x_{4}, z_{4}, x_{5}, z_{5}, x_{6}, z_{6}, x_{7}, y_{7}, z_{7}$

- Found in the Big Creek-Rush Creek sanbornite deposit, 5 miles NE of Trimmer, Fresno Co. California.

Simple Tetragonal primitive vectors:

$$
\begin{aligned}
& \mathbf{a}_{1}=a \hat{\mathbf{x}} \\
& \mathbf{a}_{2}=a \hat{\mathbf{y}} \\
& \mathbf{a}_{3}=c \hat{\mathbf{z}}
\end{aligned}
$$

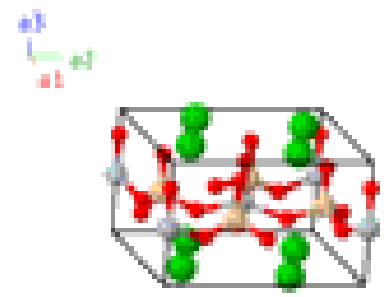

Basis vectors:

Lattice Coordinates

$\mathbf{B}_{1}=$
Cartesian Coordinates

$z_{1} c \hat{\mathbf{z}}$
Wyckoff Position
Atom Type

$=$ (2a)

O I 


\begin{tabular}{|c|c|c|c|c|c|c|}
\hline $\mathbf{B}_{2}$ & $=$ & $\frac{1}{2} \mathbf{a}_{1}+\frac{1}{2} \mathbf{a}_{2}+z_{1} \mathbf{a}_{3}$ & $=$ & $\frac{1}{2} a \hat{\mathbf{x}}+\frac{1}{2} a \hat{\mathbf{y}}+z_{1} c \hat{\mathbf{z}}$ & $(2 a)$ & O I \\
\hline $\mathbf{B}_{3}$ & $=$ & $z_{2} \mathbf{a}_{3}$ & $=$ & $z_{2} c \hat{\mathbf{z}}$ & $(2 a)$ & $\mathrm{Ti}$ \\
\hline $\mathbf{B}_{4}$ & $=$ & $\frac{1}{2} \mathbf{a}_{1}+\frac{1}{2} \mathbf{a}_{2}+z_{2} \mathbf{a}_{3}$ & $=$ & $\frac{1}{2} a \hat{\mathbf{x}}+\frac{1}{2} a \hat{\mathbf{y}}+z_{2} c \hat{\mathbf{z}}$ & $(2 a)$ & $\mathrm{Ti}$ \\
\hline $\mathbf{B}_{5}$ & $=$ & $\frac{1}{2} \mathbf{a}_{1}+z_{3} \mathbf{a}_{3}$ & $=$ & $\frac{1}{2} a \hat{\mathbf{x}}+z_{3} c \hat{\mathbf{z}}$ & $(2 b)$ & O II \\
\hline $\mathbf{B}_{6}$ & $=$ & $\frac{1}{2} \mathbf{a}_{2}+z_{3} \mathbf{a}_{3}$ & $=$ & $\frac{1}{2} a \hat{\mathbf{y}}+z_{3} c \hat{\mathbf{z}}$ & $(2 b)$ & O II \\
\hline $\mathbf{B}_{7}$ & $=$ & $x_{4} \mathbf{a}_{1}+\left(\frac{1}{2}+x_{4}\right) \mathbf{a}_{2}+z_{4} \mathbf{a}_{3}$ & $=$ & $x_{4} a \hat{\mathbf{x}}+\left(\frac{1}{2}+x_{4}\right) a \hat{\mathbf{y}}+z_{4} c \hat{\mathbf{z}}$ & $(4 c)$ & $\mathrm{Ba}$ \\
\hline $\mathbf{B}_{8}$ & $=$ & $-x_{4} \mathbf{a}_{1}+\left(\frac{1}{2}-x_{4}\right) \mathbf{a}_{2}+z_{4} \mathbf{a}_{3}$ & $=$ & $-x_{4} a \hat{\mathbf{x}}+\left(\frac{1}{2}-x_{4}\right) a \hat{\mathbf{y}}+z_{4} c \hat{\mathbf{z}}$ & $(4 c)$ & $\mathrm{Ba}$ \\
\hline $\mathbf{B}_{9}$ & $=$ & $\left(\frac{1}{2}-x_{4}\right) \mathbf{a}_{1}+x_{4} \mathbf{a}_{2}+z_{4} \mathbf{a}_{3}$ & $=$ & $\left(\frac{1}{2}-x_{4}\right) a \hat{\mathbf{x}}+x_{4} a \hat{\mathbf{y}}+z_{4} c \hat{\mathbf{z}}$ & $(4 c)$ & $\mathrm{Ba}$ \\
\hline $\mathbf{B}_{10}$ & $=$ & $\left(\frac{1}{2}+x_{4}\right) \mathbf{a}_{1}-x_{4} \mathbf{a}_{2}+z_{4} \mathbf{a}_{3}$ & $=$ & $\left(\frac{1}{2}+x_{4}\right) a \hat{\mathbf{x}}-x_{4} a \hat{\mathbf{y}}+z_{4} c \hat{\mathbf{z}}$ & $(4 c)$ & $\mathrm{Ba}$ \\
\hline $\mathbf{B}_{11}$ & $=$ & $x_{5} \mathbf{a}_{1}+\left(\frac{1}{2}+x_{5}\right) \mathbf{a}_{2}+z_{5} \mathbf{a}_{3}$ & $=$ & $x_{5} a \hat{\mathbf{x}}+\left(\frac{1}{2}+x_{5}\right) a \hat{\mathbf{y}}+z_{5} c \hat{\mathbf{z}}$ & $(4 c)$ & O III \\
\hline $\mathbf{B}_{12}$ & $=$ & $-x_{5} \mathbf{a}_{1}+\left(\frac{1}{2}-x_{5}\right) \mathbf{a}_{2}+z_{5} \mathbf{a}_{3}$ & $=$ & $-x_{5} a \hat{\mathbf{x}}+\left(\frac{1}{2}-x_{5}\right) a \hat{\mathbf{y}}+z_{5} c \hat{\mathbf{z}}$ & $(4 c)$ & O III \\
\hline $\mathbf{B}_{13}$ & $=$ & $\left(\frac{1}{2}-x_{5}\right) \mathbf{a}_{1}+x_{5} \mathbf{a}_{2}+z_{5} \mathbf{a}_{3}$ & $=$ & $\left(\frac{1}{2}-x_{5}\right) a \hat{\mathbf{x}}+x_{5} a \hat{\mathbf{y}}+z_{5} c \hat{\mathbf{z}}$ & $(4 c)$ & O III \\
\hline $\mathbf{B}_{14}$ & $=$ & $\left(\frac{1}{2}+x_{5}\right) \mathbf{a}_{1}-x_{5} \mathbf{a}_{2}+z_{5} \mathbf{a}_{3}$ & $=$ & $\left(\frac{1}{2}+x_{5}\right) a \hat{\mathbf{x}}-x_{5} a \hat{\mathbf{y}}+z_{5} c \hat{\mathbf{z}}$ & $(4 c)$ & O III \\
\hline $\mathbf{B}_{15}$ & $=$ & $x_{6} \mathbf{a}_{1}+\left(\frac{1}{2}+x_{6}\right) \mathbf{a}_{2}+z_{6} \mathbf{a}_{3}$ & $=$ & $x_{6} a \hat{\mathbf{x}}+\left(\frac{1}{2}+x_{6}\right) a \hat{\mathbf{y}}+z_{6} c \hat{\mathbf{z}}$ & $(4 c)$ & $\mathrm{Si}$ \\
\hline $\mathbf{B}_{16}$ & $=$ & $-x_{6} \mathbf{a}_{1}+\left(\frac{1}{2}-x_{6}\right) \mathbf{a}_{2}+z_{6} \mathbf{a}_{3}$ & $=$ & $-x_{6} a \hat{\mathbf{x}}+\left(\frac{1}{2}-x_{6}\right) a \hat{\mathbf{y}}+z_{6} c \hat{\mathbf{z}}$ & $(4 c)$ & $\mathrm{Si}$ \\
\hline $\mathbf{B}_{17}$ & $=$ & $\left(\frac{1}{2}-x_{6}\right) \mathbf{a}_{1}+x_{6} \mathbf{a}_{2}+z_{6} \mathbf{a}_{3}$ & $=$ & $\left(\frac{1}{2}-x_{6}\right) a \hat{\mathbf{x}}+x_{6} a \hat{\mathbf{y}}+z_{6} c \hat{\mathbf{z}}$ & $(4 c)$ & $\mathrm{Si}$ \\
\hline $\mathbf{B}_{18}$ & $=$ & $\left(\frac{1}{2}+x_{6}\right) \mathbf{a}_{1}-x_{6} \mathbf{a}_{2}+z_{6} \mathbf{a}_{3}$ & $=$ & $\left(\frac{1}{2}+x_{6}\right) a \hat{\mathbf{x}}-x_{6} a \hat{\mathbf{y}}+z_{6} c \hat{\mathbf{z}}$ & $(4 c)$ & $\mathrm{Si}$ \\
\hline $\mathbf{B}_{19}$ & $=$ & $x_{7} \mathbf{a}_{1}+y_{7} \mathbf{a}_{2}+z_{7} \mathbf{a}_{3}$ & $=$ & $x_{7} a \hat{\mathbf{x}}+y_{7} a \hat{\mathbf{y}}+z_{7} c \hat{\mathbf{z}}$ & $(8 d)$ & O IV \\
\hline $\mathbf{B}_{20}$ & $=$ & $-x_{7} \mathbf{a}_{1}-y_{7} \mathbf{a}_{2}+z_{7} \mathbf{a}_{3}$ & $=$ & $-x_{7} a \hat{\mathbf{x}}-y_{7} a \hat{\mathbf{y}}+z_{7} c \hat{\mathbf{z}}$ & $(8 d)$ & O IV \\
\hline $\mathbf{B}_{21}$ & $=$ & $-y_{7} \mathbf{a}_{1}+x_{7} \mathbf{a}_{2}+z_{7} \mathbf{a}_{3}$ & $=$ & $-y_{7} a \hat{\mathbf{x}}+x_{7} a \hat{\mathbf{y}}+z_{7} c \hat{\mathbf{z}}$ & $(8 d)$ & O IV \\
\hline $\mathbf{B}_{22}$ & $=$ & $y_{7} \mathbf{a}_{1}-x_{7} \mathbf{a}_{2}+z_{7} \mathbf{a}_{3}$ & $=$ & $y_{7} a \hat{\mathbf{x}}-x_{7} a \hat{\mathbf{y}}+z_{7} c \hat{\mathbf{z}}$ & $(8 d)$ & O IV \\
\hline $\mathbf{B}_{23}$ & $=$ & $\left(\frac{1}{2}+x_{7}\right) \mathbf{a}_{1}+\left(\frac{1}{2}-y_{7}\right) \mathbf{a}_{2}+z_{7} \mathbf{a}_{3}$ & $=$ & $\left(\frac{1}{2}+x_{7}\right) a \hat{\mathbf{x}}+\left(\frac{1}{2}-y_{7}\right) a \hat{\mathbf{y}}+z_{7} c \hat{\mathbf{z}}$ & $(8 d)$ & O IV \\
\hline $\mathbf{B}_{24}$ & $=$ & $\left(\frac{1}{2}-x_{7}\right) \mathbf{a}_{1}+\left(\frac{1}{2}+y_{7}\right) \mathbf{a}_{2}+z_{7} \mathbf{a}_{3}$ & $=$ & $\left(\frac{1}{2}-x_{7}\right) a \hat{\mathbf{x}}+\left(\frac{1}{2}+y_{7}\right) a \hat{\mathbf{y}}+z_{7} c \hat{\mathbf{z}}$ & $(8 d)$ & O IV \\
\hline $\mathbf{B}_{25}$ & $=$ & $\left(\frac{1}{2}-y_{7}\right) \mathbf{a}_{1}+\left(\frac{1}{2}-x_{7}\right) \mathbf{a}_{2}+z_{7} \mathbf{a}_{3}$ & $=$ & $\left(\frac{1}{2}-y_{7}\right) a \hat{\mathbf{x}}+\left(\frac{1}{2}-x_{7}\right) a \hat{\mathbf{y}}+z_{7} c \hat{\mathbf{z}}$ & $(8 d)$ & O IV \\
\hline $\mathbf{B}_{26}$ & $=$ & $\left(\frac{1}{2}+y_{7}\right) \mathbf{a}_{1}+\left(\frac{1}{2}+x_{7}\right) \mathbf{a}_{2}+z_{7} \mathbf{a}_{3}$ & $=$ & $\left(\frac{1}{2}+y_{7}\right) a \hat{\mathbf{x}}+\left(\frac{1}{2}+x_{7}\right) a \hat{\mathbf{y}}+z_{7} c \hat{\mathbf{z}}$ & $(8 d)$ & O IV \\
\hline
\end{tabular}

\section{References:}

- S. A. Markgraf, A. Halliya, A. S. Bhalla, R. E. Newnham, and C. T. Prewitt, X-ray structure refinement and pyroelectric investigation of fresnoite, $\mathrm{Ba}_{2} \mathrm{TiSi}_{2} \mathrm{O}_{8}$, Ferroelectrics 62, 17-26 (1985), doi:10.1080/00150198508017914.

\section{Geometry files:}

- CIF: pp. 874

- POSCAR: pp. 875 


\section{$\mathrm{Ce}_{3} \mathrm{Si}_{6} \mathrm{~N}_{11}$ Structure: A3B11C6_tP40_100_ac_bc2d_cd}
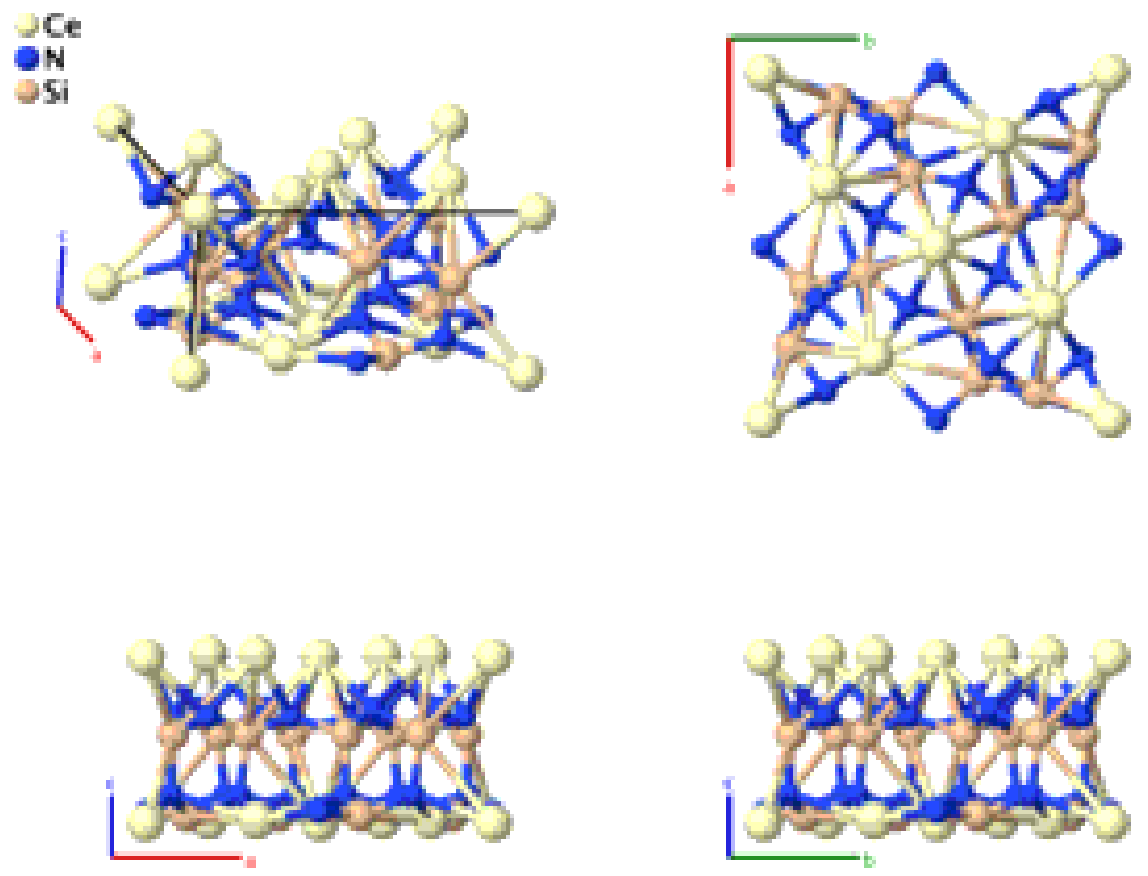

\section{Prototype}

AFLOW prototype label

Strukturbericht designation

Pearson symbol

Space group number

Space group symbol

AFLOW prototype command : aflow --proto=A3B11C6_tP40_100_ac_bc2d_cd

- params $=a, c / a, z_{1}, z_{2}, x_{3}, z_{3}, x_{4}, z_{4}, x_{5}, z_{5}, x_{6}, y_{6}, z_{6}, x_{7}, y_{7}, z_{7}, x_{8}, y_{8}, z_{8}$

Simple Tetragonal primitive vectors:

$$
\begin{aligned}
& \mathbf{a}_{1}=a \hat{\mathbf{x}} \\
& \mathbf{a}_{2}=a \hat{\mathbf{y}} \\
& \mathbf{a}_{3}=c \hat{\mathbf{z}}
\end{aligned}
$$

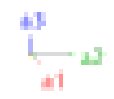

Basis vectors:

Lattice Coordinates
$\mathbf{B}_{1}=$ $z_{1} \mathbf{a}_{3}$
$\mathbf{B}_{2}=$
$\frac{1}{2} \mathbf{a}_{1}+\frac{1}{2} \mathbf{a}_{2}+z_{1} \mathbf{a}_{3}$
$=$
$=$
$z_{1} c \hat{\mathbf{z}}$
$\frac{1}{2} a \hat{\mathbf{x}}+\frac{1}{2} a \hat{\mathbf{y}}+z_{1} c \hat{\mathbf{z}}$

Cartesian Coordinates
Wyckoff Position Atom Type

(2a)

Ce I

(2a)

Ce I 


\begin{tabular}{|c|c|c|c|c|c|c|}
\hline $\mathbf{B}_{3}$ & $=$ & $\frac{1}{2} \mathbf{a}_{1}+z_{2} \mathbf{a}_{3}$ & $=$ & $\frac{1}{2} a \hat{\mathbf{x}}+z_{2} c \hat{\mathbf{z}}$ & $(2 b)$ & $\mathrm{N}$ I \\
\hline $\mathbf{B}_{4}$ & $=$ & $\frac{1}{2} \mathbf{a}_{2}+z_{2} \mathbf{a}_{3}$ & $=$ & $\frac{1}{2} a \hat{\mathbf{y}}+z_{2} c \hat{\mathbf{z}}$ & $(2 b)$ & $\mathrm{N} \mathrm{I}$ \\
\hline $\mathbf{B}_{5}$ & $=$ & $x_{3} \mathbf{a}_{1}+\left(\frac{1}{2}+x_{3}\right) \mathbf{a}_{2}+z_{3} \mathbf{a}_{3}$ & $=$ & $x_{3} a \hat{\mathbf{x}}+\left(\frac{1}{2}+x_{3}\right) a \hat{\mathbf{y}}+z_{3} c \hat{\mathbf{z}}$ & $(4 c)$ & Ce II \\
\hline $\mathbf{B}_{6}$ & $=$ & $-x_{3} \mathbf{a}_{1}+\left(\frac{1}{2}-x_{3}\right) \mathbf{a}_{2}+z_{3} \mathbf{a}_{3}$ & $=$ & $-x_{3} a \hat{\mathbf{x}}+\left(\frac{1}{2}-x_{3}\right) a \hat{\mathbf{y}}+z_{3} c \hat{\mathbf{z}}$ & $(4 c)$ & Ce II \\
\hline $\mathbf{B}_{7}$ & $=$ & $\left(\frac{1}{2}-x_{3}\right) \mathbf{a}_{1}+x_{3} \mathbf{a}_{2}+z_{3} \mathbf{a}_{3}$ & $=$ & $\left(\frac{1}{2}-x_{3}\right) a \hat{\mathbf{x}}+x_{3} a \hat{\mathbf{y}}+z_{3} c \hat{\mathbf{z}}$ & $(4 c)$ & Ce II \\
\hline $\mathbf{B}_{8}$ & $=$ & $\left(\frac{1}{2}+x_{3}\right) \mathbf{a}_{1}-x_{3} \mathbf{a}_{2}+z_{3} \mathbf{a}_{3}$ & $=$ & $\left(\frac{1}{2}+x_{3}\right) a \hat{\mathbf{x}}-x_{3} a \hat{\mathbf{y}}+z_{3} c \hat{\mathbf{z}}$ & $(4 c)$ & Ce II \\
\hline $\mathbf{B}_{9}$ & $=$ & $x_{4} \mathbf{a}_{1}+\left(\frac{1}{2}+x_{4}\right) \mathbf{a}_{2}+z_{4} \mathbf{a}_{3}$ & $=$ & $x_{4} a \hat{\mathbf{x}}+\left(\frac{1}{2}+x_{4}\right) a \hat{\mathbf{y}}+z_{4} c \hat{\mathbf{z}}$ & $(4 c)$ & $\mathrm{N}$ II \\
\hline $\mathbf{B}_{10}$ & $=$ & $-x_{4} \mathbf{a}_{1}+\left(\frac{1}{2}-x_{4}\right) \mathbf{a}_{2}+z_{4} \mathbf{a}_{3}$ & $=$ & $-x_{4} a \hat{\mathbf{x}}+\left(\frac{1}{2}-x_{4}\right) a \hat{\mathbf{y}}+z_{4} c \hat{\mathbf{z}}$ & $(4 c)$ & $\mathrm{N}$ II \\
\hline $\mathbf{B}_{11}$ & $=$ & $\left(\frac{1}{2}-x_{4}\right) \mathbf{a}_{1}+x_{4} \mathbf{a}_{2}+z_{4} \mathbf{a}_{3}$ & $=$ & $\left(\frac{1}{2}-x_{4}\right) a \hat{\mathbf{x}}+x_{4} a \hat{\mathbf{y}}+z_{4} c \hat{\mathbf{z}}$ & $(4 c)$ & $\mathrm{N}$ II \\
\hline $\mathbf{B}_{12}$ & $=$ & $\left(\frac{1}{2}+x_{4}\right) \mathbf{a}_{1}-x_{4} \mathbf{a}_{2}+z_{4} \mathbf{a}_{3}$ & $=$ & $\left(\frac{1}{2}+x_{4}\right) a \hat{\mathbf{x}}-x_{4} a \hat{\mathbf{y}}+z_{4} c \hat{\mathbf{z}}$ & $(4 c)$ & $\mathrm{N}$ II \\
\hline $\mathbf{B}_{13}$ & $=$ & $x_{5} \mathbf{a}_{1}+\left(\frac{1}{2}+x_{5}\right) \mathbf{a}_{2}+z_{5} \mathbf{a}_{3}$ & $=$ & $x_{5} a \hat{\mathbf{x}}+\left(\frac{1}{2}+x_{5}\right) a \hat{\mathbf{y}}+z_{5} c \hat{\mathbf{z}}$ & $(4 c)$ & Si I \\
\hline $\mathbf{B}_{14}$ & $=$ & $-x_{5} \mathbf{a}_{1}+\left(\frac{1}{2}-x_{5}\right) \mathbf{a}_{2}+z_{5} \mathbf{a}_{3}$ & $=$ & $-x_{5} a \hat{\mathbf{x}}+\left(\frac{1}{2}-x_{5}\right) a \hat{\mathbf{y}}+z_{5} c \hat{\mathbf{z}}$ & $(4 c)$ & Si I \\
\hline $\mathbf{B}_{15}$ & $=$ & $\left(\frac{1}{2}-x_{5}\right) \mathbf{a}_{1}+x_{5} \mathbf{a}_{2}+z_{5} \mathbf{a}_{3}$ & $=$ & $\left(\frac{1}{2}-x_{5}\right) a \hat{\mathbf{x}}+x_{5} a \hat{\mathbf{y}}+z_{5} c \hat{\mathbf{z}}$ & $(4 c)$ & Si I \\
\hline $\mathbf{B}_{16}$ & $=$ & $\left(\frac{1}{2}+x_{5}\right) \mathbf{a}_{1}-x_{5} \mathbf{a}_{2}+z_{5} \mathbf{a}_{3}$ & $=$ & $\left(\frac{1}{2}+x_{5}\right) a \hat{\mathbf{x}}-x_{5} a \hat{\mathbf{y}}+z_{5} c \hat{\mathbf{z}}$ & $(4 c)$ & Si I \\
\hline $\mathbf{B}_{17}$ & $=$ & $x_{6} \mathbf{a}_{1}+y_{6} \mathbf{a}_{2}+z_{6} \mathbf{a}_{3}$ & $=$ & $x_{6} a \hat{\mathbf{x}}+y_{6} a \hat{\mathbf{y}}+z_{6} c \hat{\mathbf{z}}$ & $(8 d)$ & N III \\
\hline $\mathbf{B}_{18}$ & $=$ & $-x_{6} \mathbf{a}_{1}-y_{6} \mathbf{a}_{2}+z_{6} \mathbf{a}_{3}$ & $=$ & $-x_{6} a \hat{\mathbf{x}}-y_{6} a \hat{\mathbf{y}}+z_{6} c \hat{\mathbf{z}}$ & $(8 d)$ & N III \\
\hline $\mathbf{B}_{19}$ & $=$ & $-y_{6} \mathbf{a}_{1}+x_{6} \mathbf{a}_{2}+z_{6} \mathbf{a}_{3}$ & $=$ & $-y_{6} a \hat{\mathbf{x}}+x_{6} a \hat{\mathbf{y}}+z_{6} c \hat{\mathbf{z}}$ & $(8 d)$ & N III \\
\hline $\mathbf{B}_{20}$ & $=$ & $y_{6} \mathbf{a}_{1}-x_{6} \mathbf{a}_{2}+z_{6} \mathbf{a}_{3}$ & $=$ & $y_{6} a \hat{\mathbf{x}}-x_{6} a \hat{\mathbf{y}}+z_{6} c \hat{\mathbf{z}}$ & $(8 d)$ & N III \\
\hline $\mathbf{B}_{21}$ & $=$ & $\left(\frac{1}{2}+x_{6}\right) \mathbf{a}_{1}+\left(\frac{1}{2}-y_{6}\right) \mathbf{a}_{2}+z_{6} \mathbf{a}_{3}$ & $=$ & $\left(\frac{1}{2}+x_{6}\right) a \hat{\mathbf{x}}+\left(\frac{1}{2}-y_{6}\right) a \hat{\mathbf{y}}+z_{6} c \hat{\mathbf{z}}$ & $(8 d)$ & N III \\
\hline $\mathbf{B}_{22}$ & $=$ & $\left(\frac{1}{2}-x_{6}\right) \mathbf{a}_{1}+\left(\frac{1}{2}+y_{6}\right) \mathbf{a}_{2}+z_{6} \mathbf{a}_{3}$ & $=$ & $\left(\frac{1}{2}-x_{6}\right) a \hat{\mathbf{x}}+\left(\frac{1}{2}+y_{6}\right) a \hat{\mathbf{y}}+z_{6} c \hat{\mathbf{z}}$ & $(8 d)$ & N III \\
\hline $\mathbf{B}_{23}$ & $=$ & $\left(\frac{1}{2}-y_{6}\right) \mathbf{a}_{1}+\left(\frac{1}{2}-x_{6}\right) \mathbf{a}_{2}+z_{6} \mathbf{a}_{3}$ & $=$ & $\left(\frac{1}{2}-y_{6}\right) a \hat{\mathbf{x}}+\left(\frac{1}{2}-x_{6}\right) a \hat{\mathbf{y}}+z_{6} c \hat{\mathbf{z}}$ & $(8 d)$ & N III \\
\hline $\mathbf{B}_{24}$ & $=$ & $\left(\frac{1}{2}+y_{6}\right) \mathbf{a}_{1}+\left(\frac{1}{2}+x_{6}\right) \mathbf{a}_{2}+z_{6} \mathbf{a}_{3}$ & $=$ & $\left(\frac{1}{2}+y_{6}\right) a \hat{\mathbf{x}}+\left(\frac{1}{2}+x_{6}\right) a \hat{\mathbf{y}}+z_{6} c \hat{\mathbf{z}}$ & $(8 d)$ & N III \\
\hline $\mathbf{B}_{25}$ & $=$ & $x_{7} \mathbf{a}_{1}+y_{7} \mathbf{a}_{2}+z_{7} \mathbf{a}_{3}$ & $=$ & $x_{7} a \hat{\mathbf{x}}+y_{7} a \hat{\mathbf{y}}+z_{7} c \hat{\mathbf{z}}$ & $(8 d)$ & N IV \\
\hline $\mathbf{B}_{26}$ & $=$ & $-x_{7} \mathbf{a}_{1}-y_{7} \mathbf{a}_{2}+z_{7} \mathbf{a}_{3}$ & $=$ & $-x_{7} a \hat{\mathbf{x}}-y_{7} a \hat{\mathbf{y}}+z_{7} c \hat{\mathbf{z}}$ & $(8 d)$ & N IV \\
\hline $\mathbf{B}_{27}$ & $=$ & $-y_{7} \mathbf{a}_{1}+x_{7} \mathbf{a}_{2}+z_{7} \mathbf{a}_{3}$ & $=$ & $-y_{7} a \hat{\mathbf{x}}+x_{7} a \hat{\mathbf{y}}+z_{7} c \hat{\mathbf{z}}$ & $(8 d)$ & N IV \\
\hline $\mathbf{B}_{28}$ & $=$ & $y_{7} \mathbf{a}_{1}-x_{7} \mathbf{a}_{2}+z_{7} \mathbf{a}_{3}$ & $=$ & $y_{7} a \hat{\mathbf{x}}-x_{7} a \hat{\mathbf{y}}+z_{7} c \hat{\mathbf{z}}$ & $(8 d)$ & N IV \\
\hline $\mathbf{B}_{29}$ & $=$ & $\left(\frac{1}{2}+x_{7}\right) \mathbf{a}_{1}+\left(\frac{1}{2}-y_{7}\right) \mathbf{a}_{2}+z_{7} \mathbf{a}_{3}$ & $=$ & $\left(\frac{1}{2}+x_{7}\right) a \hat{\mathbf{x}}+\left(\frac{1}{2}-y_{7}\right) a \hat{\mathbf{y}}+z_{7} c \hat{\mathbf{z}}$ & $(8 d)$ & N IV \\
\hline $\mathbf{B}_{30}$ & $=$ & $\left(\frac{1}{2}-x_{7}\right) \mathbf{a}_{1}+\left(\frac{1}{2}+y_{7}\right) \mathbf{a}_{2}+z_{7} \mathbf{a}_{3}$ & $=$ & $\left(\frac{1}{2}-x_{7}\right) a \hat{\mathbf{x}}+\left(\frac{1}{2}+y_{7}\right) a \hat{\mathbf{y}}+z_{7} c \hat{\mathbf{z}}$ & $(8 d)$ & N IV \\
\hline $\mathbf{B}_{31}$ & $=$ & $\left(\frac{1}{2}-y_{7}\right) \mathbf{a}_{1}+\left(\frac{1}{2}-x_{7}\right) \mathbf{a}_{2}+z_{7} \mathbf{a}_{3}$ & $=$ & $\left(\frac{1}{2}-y_{7}\right) a \hat{\mathbf{x}}+\left(\frac{1}{2}-x_{7}\right) a \hat{\mathbf{y}}+z_{7} c \hat{\mathbf{z}}$ & $(8 d)$ & N IV \\
\hline $\mathbf{B}_{32}$ & $=$ & $\left(\frac{1}{2}+y_{7}\right) \mathbf{a}_{1}+\left(\frac{1}{2}+x_{7}\right) \mathbf{a}_{2}+z_{7} \mathbf{a}_{3}$ & $=$ & $\left(\frac{1}{2}+y_{7}\right) a \hat{\mathbf{x}}+\left(\frac{1}{2}+x_{7}\right) a \hat{\mathbf{y}}+z_{7} c \hat{\mathbf{z}}$ & $(8 d)$ & N IV \\
\hline $\mathbf{B}_{33}$ & $=$ & $x_{8} \mathbf{a}_{1}+y_{8} \mathbf{a}_{2}+z_{8} \mathbf{a}_{3}$ & $=$ & $x_{8} a \hat{\mathbf{x}}+y_{8} a \hat{\mathbf{y}}+z_{8} c \hat{\mathbf{z}}$ & $(8 d)$ & Si II \\
\hline $\mathbf{B}_{34}$ & $=$ & $-x_{8} \mathbf{a}_{1}-y_{8} \mathbf{a}_{2}+z_{8} \mathbf{a}_{3}$ & $=$ & $-x_{8} a \hat{\mathbf{x}}-y_{8} a \hat{\mathbf{y}}+z_{8} c \hat{\mathbf{z}}$ & $(8 d)$ & Si II \\
\hline $\mathbf{B}_{35}$ & $=$ & $-y_{8} \mathbf{a}_{1}+x_{8} \mathbf{a}_{2}+z_{8} \mathbf{a}_{3}$ & $=$ & $-y_{8} a \hat{\mathbf{x}}+x_{8} a \hat{\mathbf{y}}+z_{8} c \hat{\mathbf{z}}$ & $(8 d)$ & Si II \\
\hline $\mathbf{B}_{36}$ & $=$ & $y_{8} \mathbf{a}_{1}-x_{8} \mathbf{a}_{2}+z_{8} \mathbf{a}_{3}$ & $=$ & $y_{8} a \hat{\mathbf{x}}-x_{8} a \hat{\mathbf{y}}+z_{8} c \hat{\mathbf{z}}$ & $(8 d)$ & Si II \\
\hline $\mathbf{B}_{37}$ & $=$ & $\left(\frac{1}{2}+x_{8}\right) \mathbf{a}_{1}+\left(\frac{1}{2}-y_{8}\right) \mathbf{a}_{2}+z_{8} \mathbf{a}_{3}$ & $=$ & $\left(\frac{1}{2}+x_{8}\right) a \hat{\mathbf{x}}+\left(\frac{1}{2}-y_{8}\right) a \hat{\mathbf{y}}+z_{8} c \hat{\mathbf{z}}$ & $(8 d)$ & Si II \\
\hline $\mathbf{B}_{38}$ & $=$ & $\left(\frac{1}{2}-x_{8}\right) \mathbf{a}_{1}+\left(\frac{1}{2}+y_{8}\right) \mathbf{a}_{2}+z_{8} \mathbf{a}_{3}$ & $=$ & $\left(\frac{1}{2}-x_{8}\right) a \hat{\mathbf{x}}+\left(\frac{1}{2}+y_{8}\right) a \hat{\mathbf{y}}+z_{8} c \hat{\mathbf{z}}$ & $(8 d)$ & Si II \\
\hline
\end{tabular}




$$
\begin{aligned}
& \mathbf{B}_{39}=\left(\frac{1}{2}-y_{8}\right) \mathbf{a}_{1}+\left(\frac{1}{2}-x_{8}\right) \mathbf{a}_{2}+z_{8} \mathbf{a}_{3}=\left(\frac{1}{2}-y_{8}\right) a \hat{\mathbf{x}}+\left(\frac{1}{2}-x_{8}\right) a \hat{\mathbf{y}}+z_{8} c \hat{\mathbf{z}} \\
& \mathbf{B}_{40}=\left(\frac{1}{2}+y_{8}\right) \mathbf{a}_{1}+\left(\frac{1}{2}+x_{8}\right) \mathbf{a}_{2}+z_{8} \mathbf{a}_{3}=\left(\frac{1}{2}+y_{8}\right) a \hat{\mathbf{x}}+\left(\frac{1}{2}+x_{8}\right) a \hat{\mathbf{y}}+z_{8} c \hat{\mathbf{z}}
\end{aligned}
$$

\section{References:}

- M. Woike and W. Jeitschko, Preparation and Crystal Structure of the Nitridosilicates $\operatorname{Ln}_{3} \operatorname{Si}_{6} N_{11}$ (Ln=La, Ce, $\mathrm{Pr}, \mathrm{Nd}$, $\mathrm{Sm})$ and $\mathrm{LnSi}_{3} \mathrm{~N}_{5}(\mathrm{Ln}=\mathrm{Ce}, \mathrm{Pr}$, Nd), Inorg. Chem. 34, 5105-5108 (1995), doi:10.1021/ic00125a005.

\section{Found in:}

- P. Villars and K. Cenzual, Pearson's Crystal Data - Crystal Structure Database for Inorganic Compounds, ASM International (2013).

\section{Geometry files:}

- CIF: pp. 875

- POSCAR: pp. 875 


\section{$\gamma$-MgNiSn Structure: A7B7C2_tP32_101_bde_ade_d}
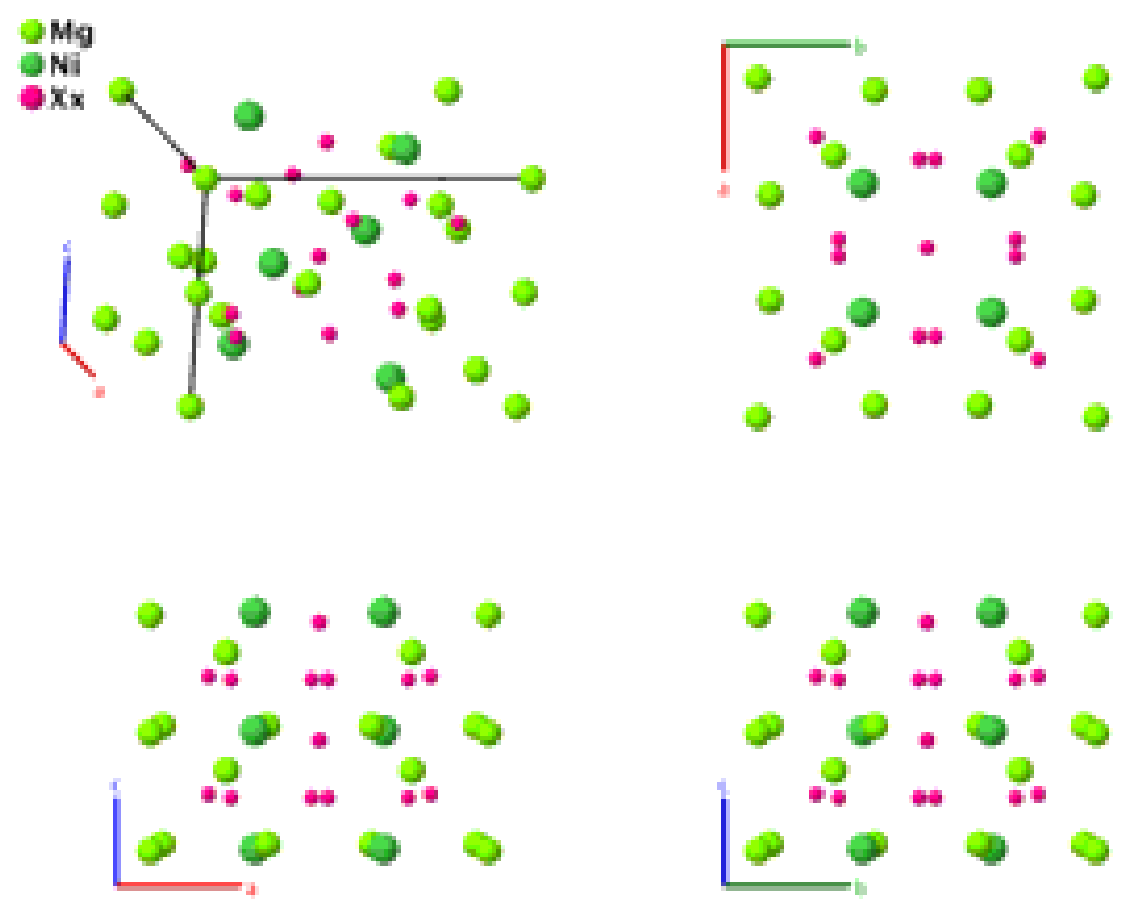

\section{Prototype}

AFLOW prototype label

Strukturbericht designation

Pearson symbol

Space group number

Space group symbol

AFLOW prototype command
: $\quad \gamma$-MgNiSn

: A7B7C2_tP32_101_bde_ade_d

: None

: $\quad$ tP32

: $\quad 101$

: $\quad \mathrm{P}_{2} \mathrm{~cm}$

aflow --proto=A7B7C2_tP32_101_bde_ade_d

- params $=a, c / a, z_{1}, z_{2}, x_{3}, z_{3}, x_{4}, z_{4}, x_{5}, z_{5}, x_{6}, y_{6}, z_{6}, x_{7}, y_{7}, z_{7}$

- This is the $\gamma$ phase of the Mg-Ni-Sn ternary system. The (2b), (8e) and (4d) Wyckoff positions are partially occupied and are represented by the labels M I, M II, and M III, respectively. Here, M I is $0.88 \mathrm{Mg}+0.12 \mathrm{Ni}, \mathrm{M} \mathrm{II}$ is $0.96 \mathrm{Mg}+0.05 \mathrm{Ni}$, and $\mathrm{M}$ III is $0.88 \mathrm{Sn}+0.12 \mathrm{Mg}$. The Jmol image does not distinguish between the different M labels and is represented by the "Xx" atoms.

\section{Simple Tetragonal primitive vectors:}

$$
\begin{aligned}
& \mathbf{a}_{1}=a \hat{\mathbf{x}} \\
& \mathbf{a}_{2}=a \hat{\mathbf{y}} \\
& \mathbf{a}_{3}=c \hat{\mathbf{z}}
\end{aligned}
$$

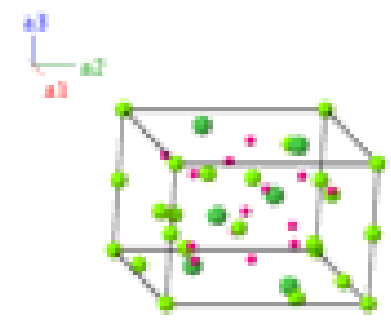

Basis vectors: 
Lattice Coordinates

$\mathbf{B}_{1}=z_{1} \mathbf{a}_{3}=$

$\mathbf{B}_{2}=$

$\left(\frac{1}{2}+z_{1}\right) \mathbf{a}_{3}$

$\mathbf{B}_{3}=$

$\frac{1}{2} \mathbf{a}_{1}+\frac{1}{2} \mathbf{a}_{2}+z_{2} \mathbf{a}_{3}$

$\mathbf{B}_{4}=$

$\mathbf{B}_{5}=$

$\mathbf{B}_{6}=$

$\mathbf{B}_{7}=$

$\mathbf{B}_{8}=$

$\mathbf{B}_{9}=$

$\mathbf{B}_{10}=$

$\mathbf{B}_{11}=$

$\mathbf{B}_{12}=$

$\mathbf{B}_{13}=$

$\mathbf{B}_{14}=$

$\mathbf{B}_{15}=$

$\mathbf{B}_{16}=$

$\mathbf{B}_{17}=$

$\mathbf{B}_{18}=$

$\mathbf{B}_{19}=$

$\mathbf{B}_{20}=$

$\mathbf{B}_{21}=$

$\mathbf{B}_{22}=$

$\mathbf{B}_{23}=$

$\mathbf{B}_{24}=$

$\mathbf{B}_{25}=$

$\mathbf{B}_{26}=$

$\mathbf{B}_{27}=$

$\mathbf{B}_{28}=$

$\mathbf{B}_{29}=$

$\mathbf{B}_{30}=$

$\mathbf{B}_{31}=$

$\mathbf{B}_{32}=$
Cartesian Coordinates

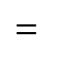

$=$

$=$

$=$

$=$

$=$

$=$

$=$

$=$

$=$

$=$

$=$

$=$

$=$

$=$

$=$

$=$

$=$

$=$

$=$

$$
=
$$

$=$

$=$

$=$

$=$

$=$

$=$

$=$

$=$

$=$

$=$

$=$
Wyckoff Position

Atom Type

(2a)
Mg I

Mg I

M I

M I

M II

M II

M II

M II

Mg II

Mg II

Mg II

Mg II

$\mathrm{Ni}$

$\mathrm{Ni}$

$\mathrm{Ni}$

$\mathrm{Ni}$

M III

M III

M III

M III

M III

M III

M III

M III

Mg III

Mg III

Mg III

Mg III

Mg III

Mg III

Mg III

Mg III

\section{References:}

- M. Boudard, P. Bordet, H. Vincent, and F. Audebert, The structure of the Y-phase in the Mg-Ni-Sn system, J. Alloys Compd. 372, 121-128 (2004), doi:10.1016/j.jallcom.2003.09.142. 


\section{Found in:}

- P. Villars and K. Cenzual, Pearson's Crystal Data - Crystal Structure Database for Inorganic Compounds, ASM International (2013).

\section{Geometry files:}

- CIF: pp. 875

- POSCAR: pp. 876 


\section{$\mathrm{Gd}_{3} \mathrm{Al}_{2}$ Structure: A2B3_tP20_102_2c_b2c}
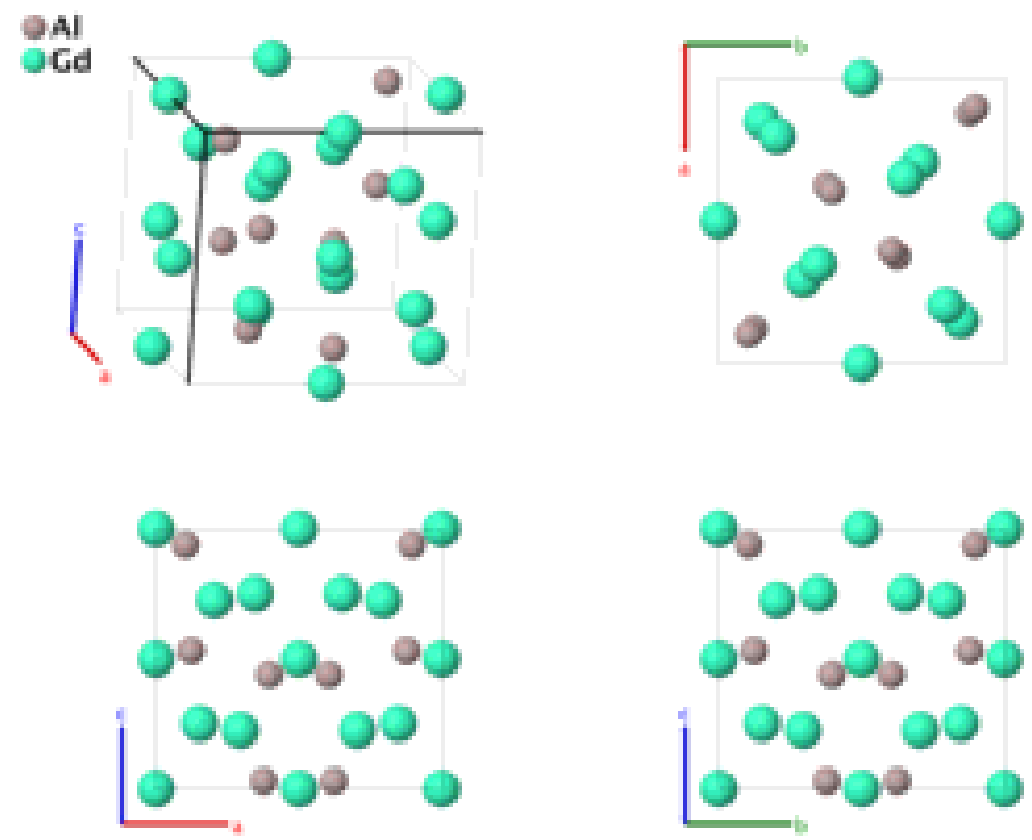

Prototype

AFLOW prototype label

: $\quad \mathrm{Gd}_{3} \mathrm{Al}_{2}$

Strukturbericht designation

: A2B3_tP20_102_2c_b2c

Pearson symbol

: None

Space group number

: $\quad$ tP20

Space group symbol

: 102

AFLOW prototype command

$\mathrm{P}_{2} \mathrm{~nm}$

aflow - -proto $=$ A2B3_tP20_102_2c_b2c

- params $=a, c / a, z_{1}, x_{2}, z_{2}, x_{3}, z_{3}, x_{4}, z_{4}, x_{5}, z_{5}$

Simple Tetragonal primitive vectors:

$$
\begin{aligned}
& \mathbf{a}_{1}=a \hat{\mathbf{x}} \\
& \mathbf{a}_{2}=a \hat{\mathbf{y}} \\
& \mathbf{a}_{3}=c \hat{\mathbf{z}}
\end{aligned}
$$

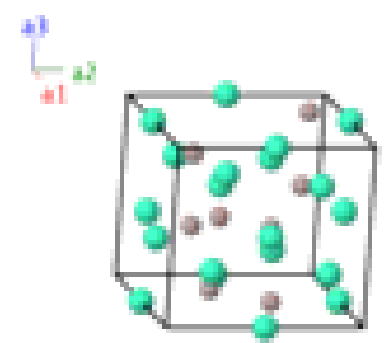

\section{Basis vectors:}

$$
\text { Lattice Coordinates }
$$

$\begin{array}{llc}\mathbf{B}_{1}= & \frac{1}{2} \mathbf{a}_{2}+z_{1} \mathbf{a}_{3} \\ \mathbf{B}_{2}= & \frac{1}{2} \mathbf{a}_{2}+\left(\frac{1}{2}+z_{1}\right) \mathbf{a}_{3} \\ \mathbf{B}_{3}= & \frac{1}{2} \mathbf{a}_{1}+\left(\frac{1}{2}+z_{1}\right) \mathbf{a}_{3} \\ \mathbf{B}_{4}= & \frac{1}{2} \mathbf{a}_{1}+z_{1} \mathbf{a}_{3}\end{array}$

$\mathbf{B}_{1}=$ $\frac{1}{2} \mathbf{a}_{1}+z_{1} \mathbf{a}_{3}$
Cartesian Coordinates

$=$

$=\quad \frac{1}{2} a \hat{\mathbf{y}}+\left(\frac{1}{2}+z_{1}\right) c \hat{\mathbf{z}}$

$=\quad \frac{1}{2} a \hat{\mathbf{x}}+\left(\frac{1}{2}+z_{1}\right) c \hat{\mathbf{z}}$

$=\quad \frac{1}{2} a \hat{\mathbf{x}}+z_{1} c \hat{\mathbf{z}}$
Wyckoff Position

$(4 b)$

(4b)

(4b)

(4b)
Atom Type

Gd I

Gd I

Gd I

Gd I 


\begin{tabular}{|c|c|c|c|c|c|c|}
\hline $\mathbf{B}_{5}$ & $=$ & $x_{2} \mathbf{a}_{1}+x_{2} \mathbf{a}_{2}+z_{2} \mathbf{a}_{3}$ & $=$ & $x_{2} a \hat{\mathbf{x}}+x_{2} a \hat{\mathbf{y}}+z_{2} c \hat{\mathbf{z}}$ & $(4 c)$ & $\mathrm{Al} \mathrm{I}$ \\
\hline $\mathbf{B}_{6}$ & $=$ & $-x_{2} \mathbf{a}_{1}-x_{2} \mathbf{a}_{2}+z_{2} \mathbf{a}_{3}$ & $=$ & $-x_{2} a \hat{\mathbf{x}}-x_{2} a \hat{\mathbf{y}}+z_{2} c \hat{\mathbf{z}}$ & $(4 c)$ & $\mathrm{Al} \mathrm{I}$ \\
\hline $\mathbf{B}_{7}$ & $=$ & $\begin{array}{c}\left(\frac{1}{2}-x_{2}\right) \mathbf{a}_{1}+\left(\frac{1}{2}+x_{2}\right) \mathbf{a}_{2}+ \\
\left(\frac{1}{2}+z_{2}\right) \mathbf{a}_{3}\end{array}$ & $=$ & $\begin{array}{c}\left(\frac{1}{2}-x_{2}\right) a \hat{\mathbf{x}}+\left(\frac{1}{2}+x_{2}\right) a \hat{\mathbf{y}}+ \\
\left(\frac{1}{2}+z_{2}\right) c \hat{\mathbf{z}}\end{array}$ & $(4 c)$ & $\mathrm{Al} \mathrm{I}$ \\
\hline $\mathbf{B}_{8}$ & $=$ & $\begin{array}{c}\left(\frac{1}{2}+x_{2}\right) \mathbf{a}_{1}+\left(\frac{1}{2}-x_{2}\right) \mathbf{a}_{2}+ \\
\left(\frac{1}{2}+z_{2}\right) \mathbf{a}_{3}\end{array}$ & $=$ & $\begin{array}{c}\left(\frac{1}{2}+x_{2}\right) a \hat{\mathbf{x}}+\left(\frac{1}{2}-x_{2}\right) a \hat{\mathbf{y}}+ \\
\left(\frac{1}{2}+z_{2}\right) c \hat{\mathbf{z}}\end{array}$ & $(4 c)$ & $\mathrm{Al} \mathrm{I}$ \\
\hline $\mathbf{B}_{9}$ & $=$ & $x_{3} \mathbf{a}_{1}+x_{3} \mathbf{a}_{2}+z_{3} \mathbf{a}_{3}$ & $=$ & $x_{3} a \hat{\mathbf{x}}+x_{3} a \hat{\mathbf{y}}+z_{3} c \hat{\mathbf{z}}$ & $(4 c)$ & Al II \\
\hline $\mathbf{B}_{10}$ & $=$ & $-x_{3} \mathbf{a}_{1}-x_{3} \mathbf{a}_{2}+z_{3} \mathbf{a}_{3}$ & $=$ & $-x_{3} a \hat{\mathbf{x}}-x_{3} a \hat{\mathbf{y}}+z_{3} c \hat{\mathbf{z}}$ & $(4 c)$ & Al II \\
\hline $\mathbf{B}_{11}$ & $=$ & $\begin{array}{c}\left(\frac{1}{2}-x_{3}\right) \mathbf{a}_{1}+\left(\frac{1}{2}+x_{3}\right) \mathbf{a}_{2}+ \\
\left(\frac{1}{2}+z_{3}\right) \mathbf{a}_{3}\end{array}$ & $=$ & $\begin{array}{c}\left(\frac{1}{2}-x_{3}\right) a \hat{\mathbf{x}}+\left(\frac{1}{2}+x_{3}\right) a \hat{\mathbf{y}}+ \\
\left(\frac{1}{2}+z_{3}\right) c \hat{\mathbf{z}}\end{array}$ & $(4 c)$ & Al II \\
\hline $\mathbf{B}_{12}$ & $=$ & $\begin{array}{c}\left(\frac{1}{2}+x_{3}\right) \mathbf{a}_{1}+\left(\frac{1}{2}-x_{3}\right) \mathbf{a}_{2}+ \\
\left(\frac{1}{2}+z_{3}\right) \mathbf{a}_{3}\end{array}$ & $=$ & $\begin{array}{c}\left(\frac{1}{2}+x_{3}\right) a \hat{\mathbf{x}}+\left(\frac{1}{2}-x_{3}\right) a \hat{\mathbf{y}}+ \\
\left(\frac{1}{2}+z_{3}\right) c \hat{\mathbf{z}}\end{array}$ & $(4 c)$ & Al II \\
\hline $\mathbf{B}_{13}$ & $=$ & $x_{4} \mathbf{a}_{1}+x_{4} \mathbf{a}_{2}+z_{4} \mathbf{a}_{3}$ & $=$ & $x_{4} a \hat{\mathbf{x}}+x_{4} a \hat{\mathbf{y}}+z_{4} c \hat{\mathbf{z}}$ & $(4 c)$ & Gd II \\
\hline $\mathbf{B}_{14}$ & $=$ & $-x_{4} \mathbf{a}_{1}-x_{4} \mathbf{a}_{2}+z_{4} \mathbf{a}_{3}$ & $=$ & $-x_{4} a \hat{\mathbf{x}}-x_{4} a \hat{\mathbf{y}}+z_{4} c \hat{\mathbf{z}}$ & $(4 c)$ & Gd II \\
\hline $\mathbf{B}_{15}$ & $=$ & $\begin{array}{c}\left(\frac{1}{2}-x_{4}\right) \mathbf{a}_{1}+\left(\frac{1}{2}+x_{4}\right) \mathbf{a}_{2}+ \\
\left(\frac{1}{2}+z_{4}\right) \mathbf{a}_{3}\end{array}$ & $=$ & $\begin{array}{c}\left(\frac{1}{2}-x_{4}\right) a \hat{\mathbf{x}}+\left(\frac{1}{2}+x_{4}\right) a \hat{\mathbf{y}}+ \\
\left(\frac{1}{2}+z_{4}\right) c \hat{\mathbf{z}}\end{array}$ & $(4 c)$ & Gd II \\
\hline $\mathbf{B}_{16}$ & $=$ & $\begin{array}{c}\left(\frac{1}{2}+x_{4}\right) \mathbf{a}_{1}+\left(\frac{1}{2}-x_{4}\right) \mathbf{a}_{2}+ \\
\left(\frac{1}{2}+z_{4}\right) \mathbf{a}_{3}\end{array}$ & $=$ & $\begin{array}{c}\left(\frac{1}{2}+x_{4}\right) a \hat{\mathbf{x}}+\left(\frac{1}{2}-x_{4}\right) a \hat{\mathbf{y}}+ \\
\left(\frac{1}{2}+z_{4}\right) c \hat{\mathbf{z}}\end{array}$ & $(4 c)$ & Gd II \\
\hline $\mathbf{B}_{17}$ & $=$ & $x_{5} \mathbf{a}_{1}+x_{5} \mathbf{a}_{2}+z_{5} \mathbf{a}_{3}$ & $=$ & $x_{5} a \hat{\mathbf{x}}+x_{5} a \hat{\mathbf{y}}+z_{5} c \hat{\mathbf{z}}$ & $(4 c)$ & Gd III \\
\hline $\mathbf{B}_{18}$ & $=$ & $-x_{5} \mathbf{a}_{1}-x_{5} \mathbf{a}_{2}+z_{5} \mathbf{a}_{3}$ & $=$ & $-x_{5} a \hat{\mathbf{x}}-x_{5} a \hat{\mathbf{y}}+z_{5} c \hat{\mathbf{z}}$ & $(4 c)$ & Gd III \\
\hline $\mathbf{B}_{19}$ & $=$ & $\begin{array}{c}\left(\frac{1}{2}-x_{5}\right) \mathbf{a}_{1}+\left(\frac{1}{2}+x_{5}\right) \mathbf{a}_{2}+ \\
\left(\frac{1}{2}+z_{5}\right) \mathbf{a}_{3}\end{array}$ & $=$ & $\begin{array}{c}\left(\frac{1}{2}-x_{5}\right) a \hat{\mathbf{x}}+\left(\frac{1}{2}+x_{5}\right) a \hat{\mathbf{y}}+ \\
\left(\frac{1}{2}+z_{5}\right) c \hat{\mathbf{z}}\end{array}$ & $(4 c)$ & Gd III \\
\hline $\mathbf{B}_{20}$ & $=$ & $\begin{array}{c}\left(\frac{1}{2}+x_{5}\right) \mathbf{a}_{1}+\left(\frac{1}{2}-x_{5}\right) \mathbf{a}_{2}+ \\
\left(\frac{1}{2}+z_{5}\right) \mathbf{a}_{3}\end{array}$ & $=$ & $\begin{array}{c}\left(\frac{1}{2}+x_{5}\right) a \hat{\mathbf{x}}+\left(\frac{1}{2}-x_{5}\right) a \hat{\mathbf{y}}+ \\
\left(\frac{1}{2}+z_{5}\right) c \hat{\mathbf{z}}\end{array}$ & $(4 c)$ & Gd III \\
\hline
\end{tabular}

\section{References:}

- K. H. J. Buschow, Rare earth-aluminium intermetallic compounds of the form RAl and $R_{3} A l_{2}$, J. Less-Common Met. 8 , 209-212 (1965), doi:10.1016/0022-5088(65)90047-0.

\section{Found in:}

- P. Villars and K. Cenzual, Pearson's Crystal Data - Crystal Structure Database for Inorganic Compounds, ASM International (2013).

\section{Geometry files:}

- CIF: pp. 876

- POSCAR: pp. 876 

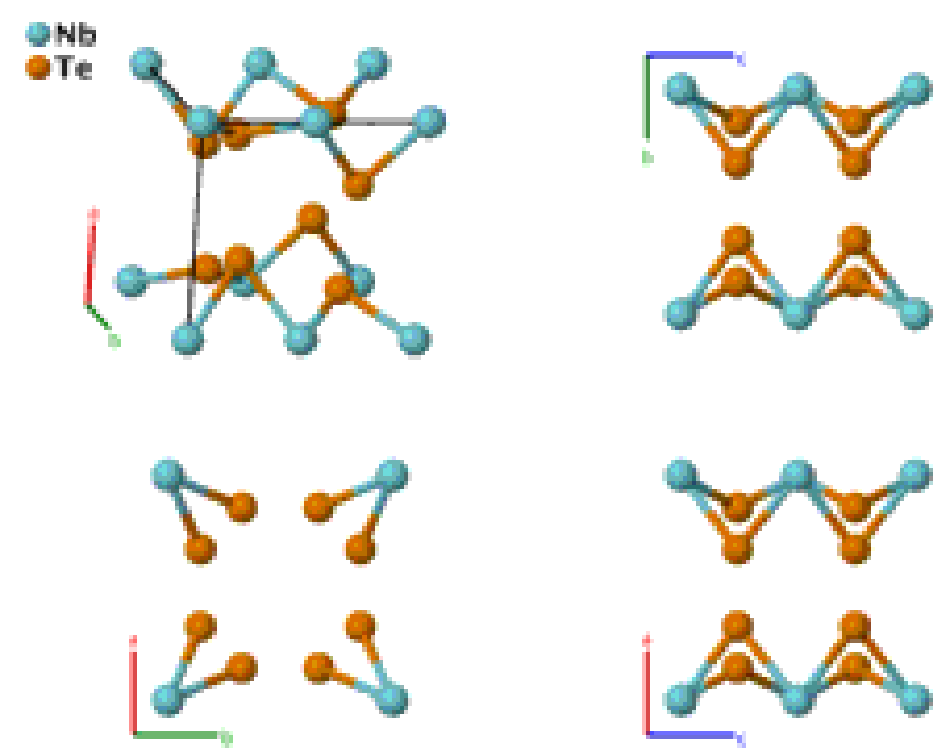

\section{Prototype}

AFLOW prototype label

Strukturbericht designation

Pearson symbol

Space group number

Space group symbol

AFLOW prototype command
: $\quad \mathrm{NbTe}_{4}$

: AB4_tP10_103_a_d

: None

: $\quad$ PP10

: 103

: $\quad P 4 c c$

\section{Simple Tetragonal primitive vectors:}

$$
\begin{aligned}
& \mathbf{a}_{1}=a \hat{\mathbf{x}} \\
& \mathbf{a}_{2}=a \hat{\mathbf{y}} \\
& \mathbf{a}_{3}=c \hat{\mathbf{z}}
\end{aligned}
$$

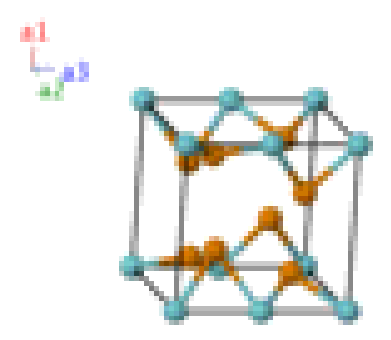

Basis vectors:

Lattice Coordinates

$\mathbf{B}_{1}=$

$\mathbf{B}_{2}=$

$\mathbf{B}_{3}=$

$\mathbf{B}_{4}=$

$\mathbf{B}_{5}=$

$\mathbf{B}_{6}=$ $z_{1} \mathbf{a}_{3}$

$\left(\frac{1}{2}+z_{1}\right) \mathbf{a}_{3}$

$x_{2} \mathbf{a}_{1}+y_{2} \mathbf{a}_{2}+z_{2} \mathbf{a}_{3}$

$-x_{2} \mathbf{a}_{1}-y_{2} \mathbf{a}_{2}+z_{2} \mathbf{a}_{3}$

$-y_{2} \mathbf{a}_{1}+x_{2} \mathbf{a}_{2}+z_{2} \mathbf{a}_{3}$

$y_{2} \mathbf{a}_{1}-x_{2} \mathbf{a}_{2}+z_{2} \mathbf{a}_{3}$
Cartesian Coordinates

$=$

$=$

$=$

$=$

$=$

$=$
Wyckoff Position

(2a)

(2a)

$(8 d)$

$(8 d)$
Atom Type

$\mathrm{Nb}$

$\mathrm{Nb}$

$\mathrm{Te}$

$\mathrm{Te}$

Te

$\mathrm{Te}$ 

$\mathbf{B}_{7}=x_{2} \mathbf{a}_{1}-y_{2} \mathbf{a}_{2}+\left(\frac{1}{2}+z_{2}\right) \mathbf{a}_{3}=x_{2} a \hat{\mathbf{x}}-y_{2} a \hat{\mathbf{y}}+\left(\frac{1}{2}+z_{2}\right) c \hat{\mathbf{z}}$
$(8 d)$
$\mathbf{B}_{8}=-x_{2} \mathbf{a}_{1}+y_{2} \mathbf{a}_{2}+\left(\frac{1}{2}+z_{2}\right) \mathbf{a}_{3}=-x_{2} a \hat{\mathbf{x}}+y_{2} a \hat{\mathbf{y}}+\left(\frac{1}{2}+z_{2}\right) c \hat{\mathbf{z}}$
$(8 d)$
$\mathrm{Te}$
$\mathbf{B}_{9}=-y_{2} \mathbf{a}_{1}-x_{2} \mathbf{a}_{2}+\left(\frac{1}{2}+z_{2}\right) \mathbf{a}_{3}=-y_{2} a \hat{\mathbf{x}}-x_{2} a \hat{\mathbf{y}}+\left(\frac{1}{2}+z_{2}\right) c \hat{\mathbf{z}}$
$\mathrm{Te}$
$\mathbf{B}_{10}=y_{2} \mathbf{a}_{1}+x_{2} \mathbf{a}_{2}+\left(\frac{1}{2}+z_{2}\right) \mathbf{a}_{3}=y_{2} a \hat{\mathbf{x}}+x_{2} a \hat{\mathbf{y}}+\left(\frac{1}{2}+z_{2}\right) c \hat{\mathbf{z}}$
$\mathrm{Te}$

\section{References:}

- H. Böhm, The high temperature modification of niobium tetratelluride $\mathrm{NbTe}_{4}$, Zeitschrift für Kristallographie - Crystalline Materials 180, 113-122 (1987), doi:10.1524/zkri.1987.180.1-4.113.

\section{Found in:}

- P. Villars and K. Cenzual, Pearson's Crystal Data - Crystal Structure Database for Inorganic Compounds, ASM International (2013).

\section{Geometry files:}

- CIF: pp. 877

- POSCAR: pp. 877 
Ba $\mathrm{In}_{4} \mathrm{Bi}_{5}$ Structure: A5B5C4_tP28_104_ac_ac_c
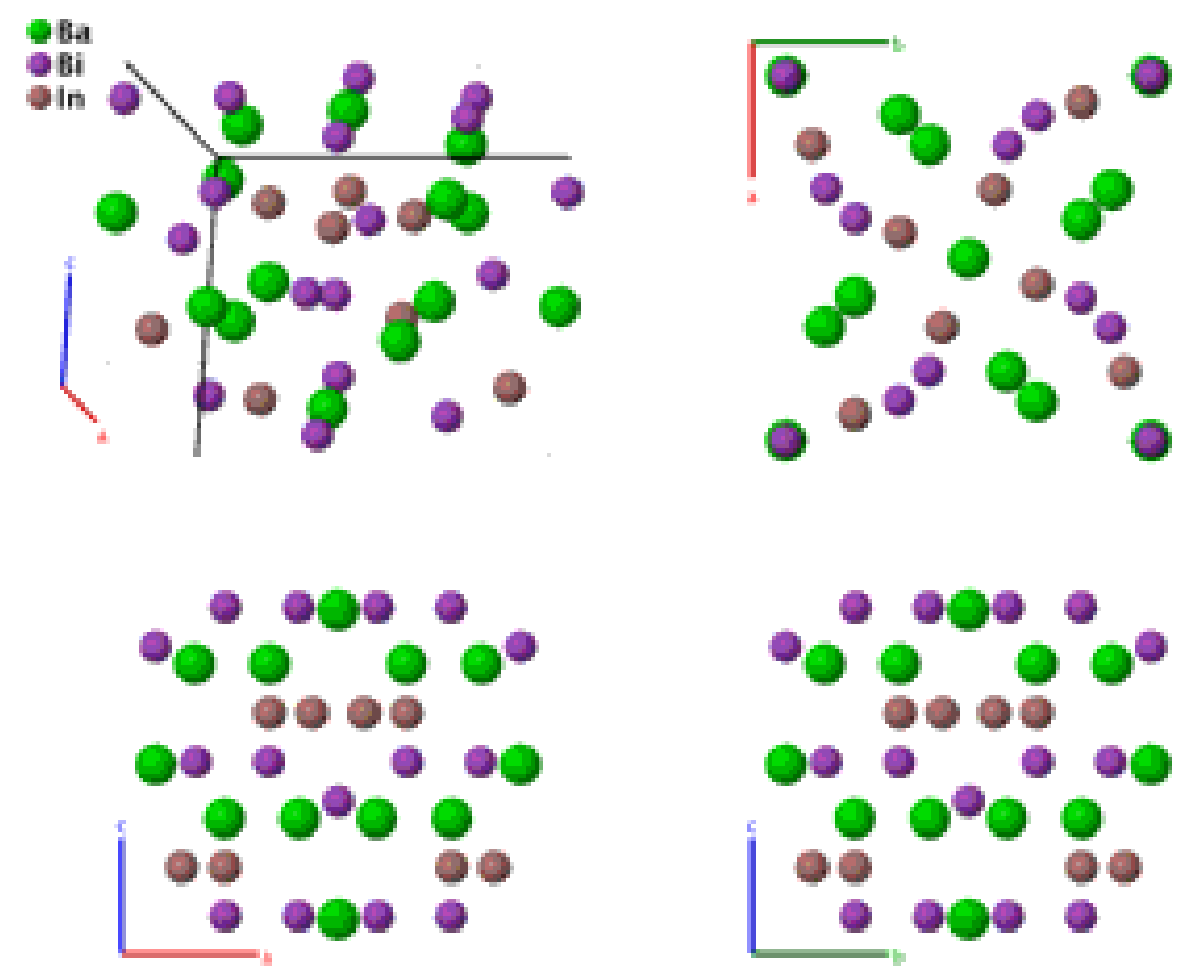

\section{Prototype}

AFLOW prototype label

: $\quad \mathrm{Ba}_{5} \mathrm{In}_{4} \mathrm{Bi}_{5}$

Strukturbericht designation

Pearson symbol

A5B5C4_tP28_104_ac_ac_c

Space group number

None

Space group symbol

AFLOW prototype command

$$
\text { : } \quad \text { tP28 }
$$

: 104

: $\quad P 4 n c$

\section{Simple Tetragonal primitive vectors:}

$$
\begin{aligned}
& \mathbf{a}_{1}=a \hat{\mathbf{x}} \\
& \mathbf{a}_{2}=a \hat{\mathbf{y}} \\
& \mathbf{a}_{3}=c \hat{\mathbf{z}}
\end{aligned}
$$

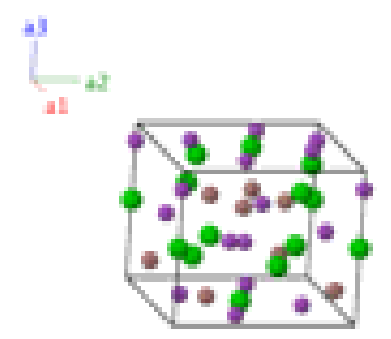

Basis vectors:

Lattice Coordinates

$$
\begin{aligned}
& \mathbf{B}_{1}= \\
& \mathbf{B}_{2}= \\
& z_{1} \mathbf{a}_{3} \\
& \frac{1}{2} \mathbf{a}_{1}+\frac{1}{2} \mathbf{a}_{2}+\left(\frac{1}{2}+z_{1}\right) \mathbf{a}_{3} \\
& = \\
& =\quad \frac{1}{2} a \hat{\mathbf{x}}+\frac{1}{2} a \hat{\mathbf{y}}+\left(\frac{1}{2}+z_{1}\right) c \hat{\mathbf{z}} \\
& z_{1} c \hat{\mathbf{z}}
\end{aligned}
$$

Cartesian Coordinates

Wyckoff Position

$(2 a)$

(2a)
Atom Type

$\mathrm{Ba}$ I

Ba I 


\begin{tabular}{|c|c|c|c|c|c|c|}
\hline $\mathbf{B}_{3}$ & $=$ & $z_{2} \mathbf{a}_{3}$ & $=$ & $z_{2} c \hat{\mathbf{z}}$ & $(2 a)$ & Bi I \\
\hline $\mathbf{B}_{4}$ & $=$ & $\frac{1}{2} \mathbf{a}_{1}+\frac{1}{2} \mathbf{a}_{2}+\left(\frac{1}{2}+z_{2}\right) \mathbf{a}_{3}$ & $=$ & $\frac{1}{2} a \hat{\mathbf{x}}+\frac{1}{2} a \hat{\mathbf{y}}+\left(\frac{1}{2}+z_{2}\right) c \hat{\mathbf{z}}$ & $(2 a)$ & Bi I \\
\hline $\mathbf{B}_{5}$ & $=$ & $x_{3} \mathbf{a}_{1}+y_{3} \mathbf{a}_{2}+z_{3} \mathbf{a}_{3}$ & $=$ & $x_{3} a \hat{\mathbf{x}}+y_{3} a \hat{\mathbf{y}}+z_{3} c \hat{\mathbf{z}}$ & $(8 c)$ & Ba II \\
\hline $\mathbf{B}_{6}$ & $=$ & $-x_{3} \mathbf{a}_{1}-y_{3} \mathbf{a}_{2}+z_{3} \mathbf{a}_{3}$ & $=$ & $-x_{3} a \hat{\mathbf{x}}-y_{3} a \hat{\mathbf{y}}+z_{3} c \hat{\mathbf{z}}$ & $(8 c)$ & Ba II \\
\hline $\mathbf{B}_{7}$ & $=$ & $-y_{3} \mathbf{a}_{1}+x_{3} \mathbf{a}_{2}+z_{3} \mathbf{a}_{3}$ & $=$ & $-y_{3} a \hat{\mathbf{x}}+x_{3} a \hat{\mathbf{y}}+z_{3} c \hat{\mathbf{z}}$ & $(8 c)$ & Ba II \\
\hline $\mathbf{B}_{8}$ & $=$ & $y_{3} \mathbf{a}_{1}-x_{3} \mathbf{a}_{2}+z_{3} \mathbf{a}_{3}$ & $=$ & $y_{3} a \hat{\mathbf{x}}-x_{3} a \hat{\mathbf{y}}+z_{3} c \hat{\mathbf{z}}$ & $(8 c)$ & Ba II \\
\hline $\mathbf{B}_{9}$ & $=$ & $\begin{array}{c}\left(\frac{1}{2}+x_{3}\right) \mathbf{a}_{1}+\left(\frac{1}{2}-y_{3}\right) \mathbf{a}_{2}+ \\
\left(\frac{1}{2}+z_{3}\right) \mathbf{a}_{3}\end{array}$ & $=$ & $\begin{array}{c}\left(\frac{1}{2}+x_{3}\right) a \hat{\mathbf{x}}+\left(\frac{1}{2}-y_{3}\right) a \hat{\mathbf{y}}+ \\
\left(\frac{1}{2}+z_{3}\right) c \hat{\mathbf{z}}\end{array}$ & $(8 c)$ & Ba II \\
\hline $\mathbf{B}_{10}$ & $=$ & $\begin{array}{c}\left(\frac{1}{2}-x_{3}\right) \mathbf{a}_{1}+\left(\frac{1}{2}+y_{3}\right) \mathbf{a}_{2}+ \\
\left(\frac{1}{2}+z_{3}\right) \mathbf{a}_{3}\end{array}$ & $=$ & $\begin{array}{c}\left(\frac{1}{2}-x_{3}\right) a \hat{\mathbf{x}}+\left(\frac{1}{2}+y_{3}\right) a \hat{\mathbf{y}}+ \\
\left(\frac{1}{2}+z_{3}\right) c \hat{\mathbf{z}}\end{array}$ & $(8 c)$ & Ba II \\
\hline $\mathbf{B}_{11}$ & $=$ & $\begin{array}{c}\left(\frac{1}{2}-y_{3}\right) \mathbf{a}_{1}+\left(\frac{1}{2}-x_{3}\right) \mathbf{a}_{2}+ \\
\left(\frac{1}{2}+z_{3}\right) \mathbf{a}_{3}\end{array}$ & $=$ & $\begin{array}{c}\left(\frac{1}{2}-y_{3}\right) a \hat{\mathbf{x}}+\left(\frac{1}{2}-x_{3}\right) a \hat{\mathbf{y}}+ \\
\left(\frac{1}{2}+z_{3}\right) c \hat{\mathbf{z}}\end{array}$ & $(8 c)$ & Ba II \\
\hline $\mathbf{B}_{12}$ & $=$ & $\begin{array}{c}\left(\frac{1}{2}+y_{3}\right) \mathbf{a}_{1}+\left(\frac{1}{2}+x_{3}\right) \mathbf{a}_{2}+ \\
\left(\frac{1}{2}+z_{3}\right) \mathbf{a}_{3}\end{array}$ & $=$ & $\begin{array}{c}\left(\frac{1}{2}+y_{3}\right) a \hat{\mathbf{x}}+\left(\frac{1}{2}+x_{3}\right) a \hat{\mathbf{y}}+ \\
\left(\frac{1}{2}+z_{3}\right) c \hat{\mathbf{z}}\end{array}$ & $(8 c)$ & Ba II \\
\hline $\mathbf{B}_{13}$ & $=$ & $x_{4} \mathbf{a}_{1}+y_{4} \mathbf{a}_{2}+z_{4} \mathbf{a}_{3}$ & $=$ & $x_{4} a \hat{\mathbf{x}}+y_{4} a \hat{\mathbf{y}}+z_{4} c \hat{\mathbf{z}}$ & $(8 c)$ & Bi II \\
\hline $\mathbf{B}_{14}$ & $=$ & $-x_{4} \mathbf{a}_{1}-y_{4} \mathbf{a}_{2}+z_{4} \mathbf{a}_{3}$ & $=$ & $-x_{4} a \hat{\mathbf{x}}-y_{4} a \hat{\mathbf{y}}+z_{4} c \hat{\mathbf{z}}$ & $(8 c)$ & Bi II \\
\hline $\mathbf{B}_{15}$ & $=$ & $-y_{4} \mathbf{a}_{1}+x_{4} \mathbf{a}_{2}+z_{4} \mathbf{a}_{3}$ & $=$ & $-y_{4} a \hat{\mathbf{x}}+x_{4} a \hat{\mathbf{y}}+z_{4} c \hat{\mathbf{z}}$ & $(8 c)$ & Bi II \\
\hline $\mathbf{B}_{16}$ & $=$ & $y_{4} \mathbf{a}_{1}-x_{4} \mathbf{a}_{2}+z_{4} \mathbf{a}_{3}$ & $=$ & $y_{4} a \hat{\mathbf{x}}-x_{4} a \hat{\mathbf{y}}+z_{4} c \hat{\mathbf{z}}$ & $(8 c)$ & $\mathrm{Bi}$ II \\
\hline $\mathbf{B}_{17}$ & $=$ & $\begin{array}{c}\left(\frac{1}{2}+x_{4}\right) \mathbf{a}_{1}+\left(\frac{1}{2}-y_{4}\right) \mathbf{a}_{2}+ \\
\left(\frac{1}{2}+z_{4}\right) \mathbf{a}_{3}\end{array}$ & $=$ & $\begin{array}{c}\left(\frac{1}{2}+x_{4}\right) a \hat{\mathbf{x}}+\left(\frac{1}{2}-y_{4}\right) a \hat{\mathbf{y}}+ \\
\left(\frac{1}{2}+z_{4}\right) c \hat{\mathbf{z}}\end{array}$ & $(8 c)$ & Bi II \\
\hline $\mathbf{B}_{18}$ & $=$ & $\begin{array}{c}\left(\frac{1}{2}-x_{4}\right) \mathbf{a}_{1}+\left(\frac{1}{2}+y_{4}\right) \mathbf{a}_{2}+ \\
\left(\frac{1}{2}+z_{4}\right) \mathbf{a}_{3}\end{array}$ & $=$ & $\begin{array}{c}\left(\frac{1}{2}-x_{4}\right) a \hat{\mathbf{x}}+\left(\frac{1}{2}+y_{4}\right) a \hat{\mathbf{y}}+ \\
\left(\frac{1}{2}+z_{4}\right) c \hat{\mathbf{z}}\end{array}$ & $(8 c)$ & Bi II \\
\hline $\mathbf{B}_{19}$ & $=$ & $\begin{array}{c}\left(\frac{1}{2}-y_{4}\right) \mathbf{a}_{1}+\left(\frac{1}{2}-x_{4}\right) \mathbf{a}_{2}+ \\
\left(\frac{1}{2}+z_{4}\right) \mathbf{a}_{3}\end{array}$ & $=$ & $\begin{array}{c}\left(\frac{1}{2}-y_{4}\right) a \hat{\mathbf{x}}+\left(\frac{1}{2}-x_{4}\right) a \hat{\mathbf{y}}+ \\
\left(\frac{1}{2}+z_{4}\right) c \hat{\mathbf{z}}\end{array}$ & $(8 c)$ & Bi II \\
\hline $\mathbf{B}_{20}$ & $=$ & $\begin{array}{c}\left(\frac{1}{2}+y_{4}\right) \mathbf{a}_{1}+\left(\frac{1}{2}+x_{4}\right) \mathbf{a}_{2}+ \\
\left(\frac{1}{2}+z_{4}\right) \mathbf{a}_{3}\end{array}$ & $=$ & $\begin{array}{c}\left(\frac{1}{2}+y_{4}\right) a \hat{\mathbf{x}}+\left(\frac{1}{2}+x_{4}\right) a \hat{\mathbf{y}}+ \\
\left(\frac{1}{2}+z_{4}\right) c \hat{\mathbf{z}}\end{array}$ & $(8 c)$ & Bi II \\
\hline $\mathbf{B}_{21}$ & $=$ & $x_{5} \mathbf{a}_{1}+y_{5} \mathbf{a}_{2}+z_{5} \mathbf{a}_{3}$ & $=$ & $x_{5} a \hat{\mathbf{x}}+y_{5} a \hat{\mathbf{y}}+z_{5} c \hat{\mathbf{z}}$ & $(8 c)$ & In \\
\hline $\mathbf{B}_{22}$ & $=$ & $-x_{5} \mathbf{a}_{1}-y_{5} \mathbf{a}_{2}+z_{5} \mathbf{a}_{3}$ & $=$ & $-x_{5} a \hat{\mathbf{x}}-y_{5} a \hat{\mathbf{y}}+z_{5} c \hat{\mathbf{z}}$ & $(8 c)$ & In \\
\hline $\mathbf{B}_{23}$ & $=$ & $-y_{5} \mathbf{a}_{1}+x_{5} \mathbf{a}_{2}+z_{5} \mathbf{a}_{3}$ & $=$ & $-y_{5} a \hat{\mathbf{x}}+x_{5} a \hat{\mathbf{y}}+z_{5} c \hat{\mathbf{z}}$ & $(8 c)$ & In \\
\hline $\mathbf{B}_{24}$ & $=$ & $y_{5} \mathbf{a}_{1}-x_{5} \mathbf{a}_{2}+z_{5} \mathbf{a}_{3}$ & $=$ & $y_{5} a \hat{\mathbf{x}}-x_{5} a \hat{\mathbf{y}}+z_{5} c \hat{\mathbf{z}}$ & $(8 c)$ & In \\
\hline $\mathbf{B}_{25}$ & $=$ & $\begin{array}{c}\left(\frac{1}{2}+x_{5}\right) \mathbf{a}_{1}+\left(\frac{1}{2}-y_{5}\right) \mathbf{a}_{2}+ \\
\left(\frac{1}{2}+z_{5}\right) \mathbf{a}_{3}\end{array}$ & $=$ & $\begin{array}{c}\left(\frac{1}{2}+x_{5}\right) a \hat{\mathbf{x}}+\left(\frac{1}{2}-y_{5}\right) a \hat{\mathbf{y}}+ \\
\left(\frac{1}{2}+z_{5}\right) c \hat{\mathbf{z}}\end{array}$ & $(8 c)$ & In \\
\hline $\mathbf{B}_{26}$ & $=$ & $\begin{array}{c}\left(\frac{1}{2}-x_{5}\right) \mathbf{a}_{1}+\left(\frac{1}{2}+y_{5}\right) \mathbf{a}_{2}+ \\
\left(\frac{1}{2}+z_{5}\right) \mathbf{a}_{3}\end{array}$ & $=$ & $\begin{array}{c}\left(\frac{1}{2}-x_{5}\right) a \hat{\mathbf{x}}+\left(\frac{1}{2}+y_{5}\right) a \hat{\mathbf{y}}+ \\
\left(\frac{1}{2}+z_{5}\right) c \hat{\mathbf{z}}\end{array}$ & $(8 c)$ & In \\
\hline $\mathbf{B}_{27}$ & $=$ & $\begin{array}{c}\left(\frac{1}{2}-y_{5}\right) \mathbf{a}_{1}+\left(\frac{1}{2}-x_{5}\right) \mathbf{a}_{2}+ \\
\left(\frac{1}{2}+z_{5}\right) \mathbf{a}_{3}\end{array}$ & $=$ & $\begin{array}{c}\left(\frac{1}{2}-y_{5}\right) a \hat{\mathbf{x}}+\left(\frac{1}{2}-x_{5}\right) a \hat{\mathbf{y}}+ \\
\left(\frac{1}{2}+z_{5}\right) c \hat{\mathbf{z}}\end{array}$ & $(8 c)$ & In \\
\hline $\mathbf{B}_{28}$ & $=$ & $\begin{array}{c}\left(\frac{1}{2}+y_{5}\right) \mathbf{a}_{1}+\left(\frac{1}{2}+x_{5}\right) \mathbf{a}_{2}+ \\
\left(\frac{1}{2}+z_{5}\right) \mathbf{a}_{3}\end{array}$ & $=$ & $\begin{array}{c}\left(\frac{1}{2}+y_{5}\right) a \hat{\mathbf{x}}+\left(\frac{1}{2}+x_{5}\right) a \hat{\mathbf{y}}+ \\
\left(\frac{1}{2}+z_{5}\right) c \hat{\mathbf{z}}\end{array}$ & $(8 c)$ & In \\
\hline
\end{tabular}




\section{References:}

- S. Ponou, T. F. Fässler, G. Tobías, E. Canadell, A. Cho, and S. C. Sevov, Synthesis, Characterization, and Electronic Structure of Ba $I_{5}{ }_{4} B i_{5}:$ An Acentric and One-Electron Deficient Phase, Chem. Euro. J. 10, 3615-3621 (2004), doi:10.1002/chem.200306061.

\section{Found in:}

- P. Villars and K. Cenzual, Pearson's Crystal Data - Crystal Structure Database for Inorganic Compounds, ASM International (2013).

\section{Geometry files:}

- CIF: pp. 877

- POSCAR: pp. 877 


\section{$\mathrm{Tl}_{4} \mathrm{HgI}_{6}$ Structure: AB6C4_tP22_104_a_2ac_c}
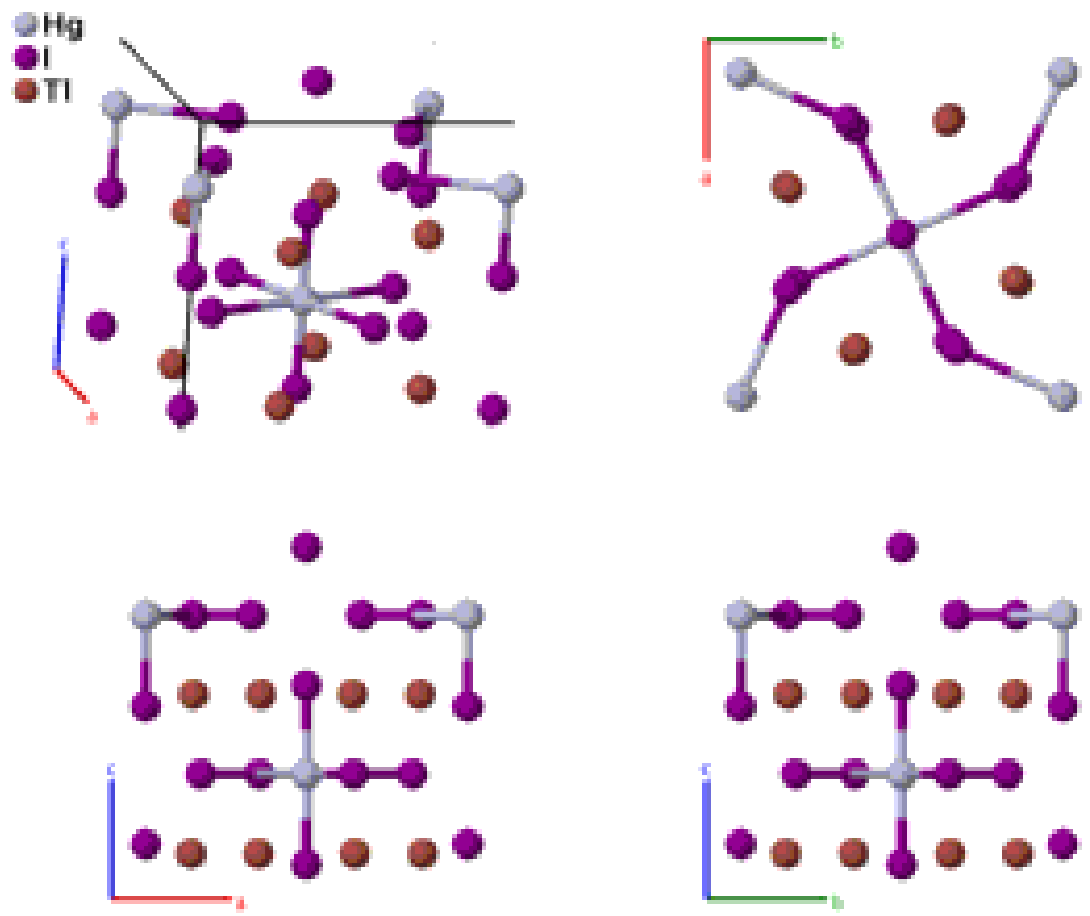

Prototype

AFLOW prototype label

: $\quad \mathrm{Tl}_{4} \mathrm{HgI}_{6}$

\section{Strukturbericht designation}

Pearson symbol AB6C4_tP22_104_a_2ac_c

Space group number None

Space group symbol

$\begin{aligned} \text { AFLOW prototype command : } & \text { aflow --proto }=\mathrm{AB} 6 \mathrm{C} 4_{-} \mathrm{tP} 22_{-} 104_{-} \mathrm{a}_{-} 2 \mathrm{ac} \_\mathrm{c} \\ & - \text {-params }=a, c / a, z_{1}, z_{2}, z_{3}, x_{4}, y_{4}, z_{4}, x_{5}, y_{5}, z_{5}\end{aligned}$

tP22

: 104

: $\quad P 4 n c$

- The second I site is reported with an occupancy 0.92 .

Simple Tetragonal primitive vectors:

$$
\begin{aligned}
& \mathbf{a}_{1}=a \hat{\mathbf{x}} \\
& \mathbf{a}_{2}=a \hat{\mathbf{y}} \\
& \mathbf{a}_{3}=c \hat{\mathbf{z}}
\end{aligned}
$$

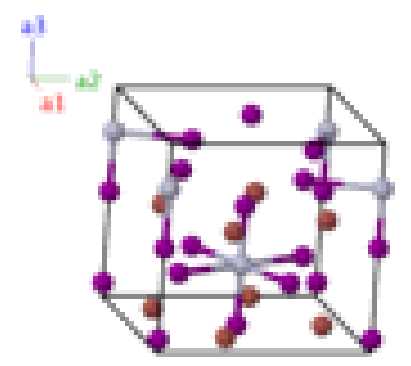

Basis vectors:

Lattice Coordinates

$\mathbf{B}_{1}=$

$z_{1} \mathbf{a}_{3}$

$=$
Cartesian Coordinates

$z_{1} c \hat{\mathbf{z}}$
Wyckoff Position

(2a)
Atom Type

$\mathrm{Hg}$ 


\begin{tabular}{|c|c|c|c|c|c|c|}
\hline $\mathbf{B}_{2}$ & $=$ & $\frac{1}{2} \mathbf{a}_{1}+\frac{1}{2} \mathbf{a}_{2}+\left(\frac{1}{2}+z_{1}\right) \mathbf{a}_{3}$ & $=$ & $\frac{1}{2} a \hat{\mathbf{x}}+\frac{1}{2} a \hat{\mathbf{y}}+\left(\frac{1}{2}+z_{1}\right) c \hat{\mathbf{z}}$ & $(2 a)$ & $\mathrm{Hg}$ \\
\hline $\mathbf{B}_{3}$ & $=$ & $z_{2} \mathbf{a}_{3}$ & $=$ & $z_{2} c \hat{\mathbf{z}}$ & $(2 a)$ & I I \\
\hline $\mathbf{B}_{4}$ & $=$ & $\frac{1}{2} \mathbf{a}_{1}+\frac{1}{2} \mathbf{a}_{2}+\left(\frac{1}{2}+z_{2}\right) \mathbf{a}_{3}$ & $=$ & $\frac{1}{2} a \hat{\mathbf{x}}+\frac{1}{2} a \hat{\mathbf{y}}+\left(\frac{1}{2}+z_{2}\right) c \hat{\mathbf{z}}$ & $(2 a)$ & I I \\
\hline $\mathbf{B}_{5}$ & $=$ & $z_{3} \mathbf{a}_{3}$ & $=$ & $z_{3} c \hat{\mathbf{z}}$ & $(2 a)$ & I II \\
\hline $\mathbf{B}_{6}$ & $=$ & $\frac{1}{2} \mathbf{a}_{1}+\frac{1}{2} \mathbf{a}_{2}+\left(\frac{1}{2}+z_{3}\right) \mathbf{a}_{3}$ & $=$ & $\frac{1}{2} a \hat{\mathbf{x}}+\frac{1}{2} a \hat{\mathbf{y}}+\left(\frac{1}{2}+z_{3}\right) c \hat{\mathbf{z}}$ & $(2 a)$ & I II \\
\hline $\mathbf{B}_{7}$ & $=$ & $x_{4} \mathbf{a}_{1}+y_{4} \mathbf{a}_{2}+z_{4} \mathbf{a}_{3}$ & $=$ & $x_{4} a \hat{\mathbf{x}}+y_{4} a \hat{\mathbf{y}}+z_{4} c \hat{\mathbf{z}}$ & $(8 c)$ & I III \\
\hline $\mathbf{B}_{8}$ & $=$ & $-x_{4} \mathbf{a}_{1}-y_{4} \mathbf{a}_{2}+z_{4} \mathbf{a}_{3}$ & $=$ & $-x_{4} a \hat{\mathbf{x}}-y_{4} a \hat{\mathbf{y}}+z_{4} c \hat{\mathbf{z}}$ & $(8 c)$ & I III \\
\hline $\mathbf{B}_{9}$ & $=$ & $-y_{4} \mathbf{a}_{1}+x_{4} \mathbf{a}_{2}+z_{4} \mathbf{a}_{3}$ & $=$ & $-y_{4} a \hat{\mathbf{x}}+x_{4} a \hat{\mathbf{y}}+z_{4} c \hat{\mathbf{z}}$ & $(8 c)$ & I III \\
\hline $\mathbf{B}_{10}$ & $=$ & $y_{4} \mathbf{a}_{1}-x_{4} \mathbf{a}_{2}+z_{4} \mathbf{a}_{3}$ & $=$ & $y_{4} a \hat{\mathbf{x}}-x_{4} a \hat{\mathbf{y}}+z_{4} c \hat{\mathbf{z}}$ & $(8 c)$ & I III \\
\hline $\mathbf{B}_{11}$ & $=$ & $\begin{array}{c}\left(\frac{1}{2}+x_{4}\right) \mathbf{a}_{1}+\left(\frac{1}{2}-y_{4}\right) \mathbf{a}_{2}+ \\
\left(\frac{1}{2}+z_{4}\right) \mathbf{a}_{3}\end{array}$ & $=$ & $\begin{array}{c}\left(\frac{1}{2}+x_{4}\right) a \hat{\mathbf{x}}+\left(\frac{1}{2}-y_{4}\right) a \hat{\mathbf{y}}+ \\
\left(\frac{1}{2}+z_{4}\right) c \hat{\mathbf{z}}\end{array}$ & $(8 c)$ & I III \\
\hline $\mathbf{B}_{12}$ & $=$ & $\begin{array}{c}\left(\frac{1}{2}-x_{4}\right) \mathbf{a}_{1}+\left(\frac{1}{2}+y_{4}\right) \mathbf{a}_{2}+ \\
\left(\frac{1}{2}+z_{4}\right) \mathbf{a}_{3}\end{array}$ & $=$ & $\begin{array}{c}\left(\frac{1}{2}-x_{4}\right) a \hat{\mathbf{x}}+\left(\frac{1}{2}+y_{4}\right) a \hat{\mathbf{y}}+ \\
\left(\frac{1}{2}+z_{4}\right) c \hat{\mathbf{z}}\end{array}$ & $(8 c)$ & I III \\
\hline $\mathbf{B}_{13}$ & $=$ & $\begin{array}{c}\left(\frac{1}{2}-y_{4}\right) \mathbf{a}_{1}+\left(\frac{1}{2}-x_{4}\right) \mathbf{a}_{2}+ \\
\left(\frac{1}{2}+z_{4}\right) \mathbf{a}_{3}\end{array}$ & $=$ & $\begin{array}{c}\left(\frac{1}{2}-y_{4}\right) a \hat{\mathbf{x}}+\left(\frac{1}{2}-x_{4}\right) a \hat{\mathbf{y}}+ \\
\left(\frac{1}{2}+z_{4}\right) c \hat{\mathbf{z}}\end{array}$ & $(8 c)$ & I III \\
\hline $\mathbf{B}_{14}$ & $=$ & $\begin{array}{c}\left(\frac{1}{2}+y_{4}\right) \mathbf{a}_{1}+\left(\frac{1}{2}+x_{4}\right) \mathbf{a}_{2}+ \\
\left(\frac{1}{2}+z_{4}\right) \mathbf{a}_{3}\end{array}$ & $=$ & $\begin{array}{c}\left(\frac{1}{2}+y_{4}\right) a \hat{\mathbf{x}}+\left(\frac{1}{2}+x_{4}\right) a \hat{\mathbf{y}}+ \\
\left(\frac{1}{2}+z_{4}\right) c \hat{\mathbf{z}}\end{array}$ & $(8 c)$ & I III \\
\hline $\mathbf{B}_{15}$ & $=$ & $x_{5} \mathbf{a}_{1}+y_{5} \mathbf{a}_{2}+z_{5} \mathbf{a}_{3}$ & $=$ & $x_{5} a \hat{\mathbf{x}}+y_{5} a \hat{\mathbf{y}}+z_{5} c \hat{\mathbf{z}}$ & $(8 c)$ & $\mathrm{Tl}$ \\
\hline $\mathbf{B}_{16}$ & $=$ & $-x_{5} \mathbf{a}_{1}-y_{5} \mathbf{a}_{2}+z_{5} \mathbf{a}_{3}$ & $=$ & $-x_{5} a \hat{\mathbf{x}}-y_{5} a \hat{\mathbf{y}}+z_{5} c \hat{\mathbf{z}}$ & $(8 c)$ & $\mathrm{Tl}$ \\
\hline $\mathbf{B}_{17}$ & $=$ & $-y_{5} \mathbf{a}_{1}+x_{5} \mathbf{a}_{2}+z_{5} \mathbf{a}_{3}$ & $=$ & $-y_{5} a \hat{\mathbf{x}}+x_{5} a \hat{\mathbf{y}}+z_{5} c \hat{\mathbf{z}}$ & $(8 c)$ & $\mathrm{Tl}$ \\
\hline $\mathbf{B}_{18}$ & $=$ & $y_{5} \mathbf{a}_{1}-x_{5} \mathbf{a}_{2}+z_{5} \mathbf{a}_{3}$ & $=$ & $y_{5} a \hat{\mathbf{x}}-x_{5} a \hat{\mathbf{y}}+z_{5} c \hat{\mathbf{z}}$ & $(8 c)$ & $\mathrm{Tl}$ \\
\hline $\mathbf{B}_{19}$ & $=$ & $\begin{array}{c}\left(\frac{1}{2}+x_{5}\right) \mathbf{a}_{1}+\left(\frac{1}{2}-y_{5}\right) \mathbf{a}_{2}+ \\
\left(\frac{1}{2}+z_{5}\right) \mathbf{a}_{3}\end{array}$ & $=$ & $\begin{array}{c}\left(\frac{1}{2}+x_{5}\right) a \hat{\mathbf{x}}+\left(\frac{1}{2}-y_{5}\right) a \hat{\mathbf{y}}+ \\
\left(\frac{1}{2}+z_{5}\right) c \hat{\mathbf{z}}\end{array}$ & $(8 c)$ & $\mathrm{Tl}$ \\
\hline $\mathbf{B}_{20}$ & $=$ & $\begin{array}{c}\left(\frac{1}{2}-x_{5}\right) \mathbf{a}_{1}+\left(\frac{1}{2}+y_{5}\right) \mathbf{a}_{2}+ \\
\left(\frac{1}{2}+z_{5}\right) \mathbf{a}_{3}\end{array}$ & $=$ & $\begin{array}{c}\left(\frac{1}{2}-x_{5}\right) a \hat{\mathbf{x}}+\left(\frac{1}{2}+y_{5}\right) a \hat{\mathbf{y}}+ \\
\left(\frac{1}{2}+z_{5}\right) c \hat{\mathbf{z}}\end{array}$ & $(8 c)$ & $\mathrm{Tl}$ \\
\hline $\mathbf{B}_{21}$ & $=$ & $\begin{array}{c}\left(\frac{1}{2}-y_{5}\right) \mathbf{a}_{1}+\left(\frac{1}{2}-x_{5}\right) \mathbf{a}_{2}+ \\
\left(\frac{1}{2}+z_{5}\right) \mathbf{a}_{3}\end{array}$ & $=$ & $\begin{array}{c}\left(\frac{1}{2}-y_{5}\right) a \hat{\mathbf{x}}+\left(\frac{1}{2}-x_{5}\right) a \hat{\mathbf{y}}+ \\
\left(\frac{1}{2}+z_{5}\right) c \hat{\mathbf{z}}\end{array}$ & $(8 c)$ & $\mathrm{Tl}$ \\
\hline $\mathbf{B}_{22}$ & $=$ & $\begin{array}{c}\left(\frac{1}{2}+y_{5}\right) \mathbf{a}_{1}+\left(\frac{1}{2}+x_{5}\right) \mathbf{a}_{2}+ \\
\left(\frac{1}{2}+z_{5}\right) \mathbf{a}_{3}\end{array}$ & $=$ & $\begin{array}{c}\left(\frac{1}{2}+y_{5}\right) a \hat{\mathbf{x}}+\left(\frac{1}{2}+x_{5}\right) a \hat{\mathbf{y}}+ \\
\left(\frac{1}{2}+z_{5}\right) c \hat{\mathbf{z}}\end{array}$ & $(8 c)$ & $\mathrm{Tl}$ \\
\hline
\end{tabular}

\section{References:}

- D. V. Badikov, V. V. Badikov, G. M. Kuz'micheva, V. L. Panyutin, V. B. Rybakov, V. I. Chizhikov, G. S. Shevyrdyaeva, and E. S. Shcherbakova, Growth and X-ray diffraction study of $\mathrm{Tl}_{4} \mathrm{HgI}_{6}$ crystals, Inorg. Mat. 40, 314-320 (2004), doi:10.1023/B:INMA.0000020535.59699.ff.

\section{Found in:}

- P. Villars and K. Cenzual, Pearson's Crystal Data - Crystal Structure Database for Inorganic Compounds, ASM International (2013).

\section{Geometry files:}

- CIF: pp. 878

- POSCAR: pp. 878 


\section{$\mathrm{BaGe}_{2} \mathrm{As}_{2}$ Structure: A2BC2_tP20_105_f_ac_2e}

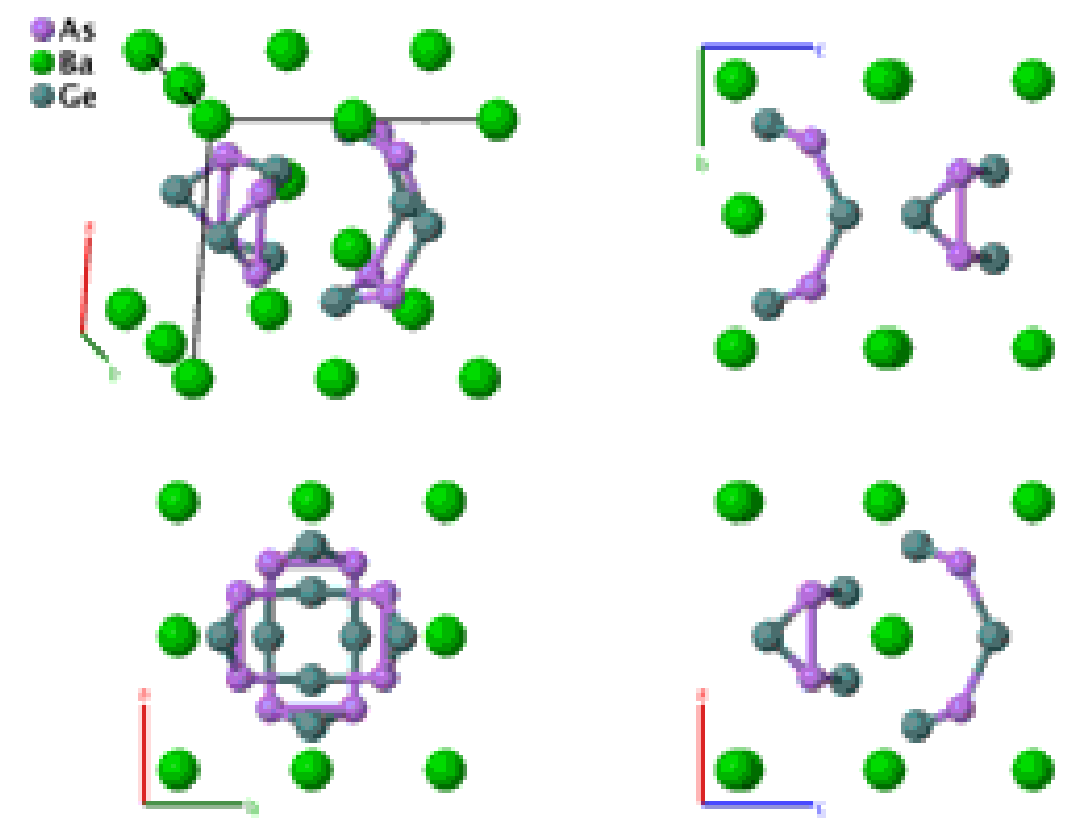

\section{Prototype}

AFLOW prototype label

Strukturbericht designation

Pearson symbol

Space group number

Space group symbol

AFLOW prototype command
: $\quad \mathrm{BaGe}_{2} \mathrm{As}_{2}$

: A2BC2_tP20_105_f_ac_2e

: None

: $\quad$ tP20

: 105

: $\quad P 4_{2} m c$

aflow --proto=A2BC2_tP20_105_f_ac_2e

- -params $=a, c / a, z_{1}, z_{2}, x_{3}, z_{3}, x_{4}, z_{4}, x_{5}, y_{5}, z_{5}$

\section{Simple Tetragonal primitive vectors:}

$$
\begin{aligned}
& \mathbf{a}_{1}=a \hat{\mathbf{x}} \\
& \mathbf{a}_{2}=a \hat{\mathbf{y}} \\
& \mathbf{a}_{3}=c \hat{\mathbf{z}}
\end{aligned}
$$

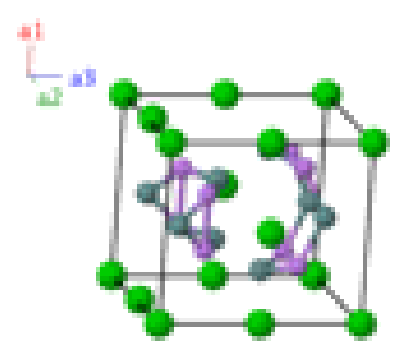

Basis vectors:

Lattice Coordinates

$\begin{array}{lcc}\mathbf{B}_{1}= & z_{1} \mathbf{a}_{3} \\ \mathbf{B}_{2}= & \left(\frac{1}{2}+z_{1}\right) \mathbf{a}_{3} \\ \mathbf{B}_{3}= & \frac{1}{2} \mathbf{a}_{2}+z_{2} \mathbf{a}_{3} \\ \mathbf{B}_{4}= & \frac{1}{2} \mathbf{a}_{1}+\left(\frac{1}{2}+z_{2}\right) \mathbf{a}_{3} \\ \mathbf{B}_{5}= & x_{3} \mathbf{a}_{1}+\frac{1}{2} \mathbf{a}_{2}+z_{3} \mathbf{a}_{3}\end{array}$

$\mathbf{B}_{1}=$

$x_{3} \mathbf{a}_{1}+\frac{1}{2} \mathbf{a}_{2}+z_{3} \mathbf{a}_{3}$
Cartesian Coordinates

$$
=
$$

$$
=
$$$$
=
$$$$
=
$$$$
=
$$$$
=
$$

$z_{1} c \hat{\mathbf{z}}$

$$
\left(\frac{1}{2}+z_{1}\right) c \hat{\mathbf{z}}
$$$$
\frac{1}{2} a \hat{\mathbf{y}}+z_{2} c \hat{\mathbf{z}}
$$$$
\frac{1}{2} a \hat{\mathbf{x}}+\left(\frac{1}{2}+z_{2}\right) c \hat{\mathbf{z}}
$$$$
x_{3} a \hat{\mathbf{x}}+\frac{1}{2} a \hat{\mathbf{y}}+z_{3} c \hat{\mathbf{z}}
$$

Wyckoff Position

(2a)
Atom Type

Ba I

Ba I

Ba II

Ba II

Ge I 


\begin{tabular}{|c|c|c|c|c|c|c|}
\hline $\mathbf{B}_{6}$ & $=$ & $-x_{3} \mathbf{a}_{1}+\frac{1}{2} \mathbf{a}_{2}+z_{3} \mathbf{a}_{3}$ & $=$ & $-x_{3} a \hat{\mathbf{x}}+\frac{1}{2} a \hat{\mathbf{y}}+z_{3} c \hat{\mathbf{z}}$ & $(4 e)$ & Ge I \\
\hline $\mathbf{B}_{7}$ & $=$ & $\frac{1}{2} \mathbf{a}_{1}+x_{3} \mathbf{a}_{2}+\left(\frac{1}{2}+z_{3}\right) \mathbf{a}_{3}$ & $=$ & $\frac{1}{2} a \hat{\mathbf{x}}+x_{3} a \hat{\mathbf{y}}+\left(\frac{1}{2}+z_{3}\right) c \hat{\mathbf{z}}$ & $(4 e)$ & Ge I \\
\hline $\mathbf{B}_{8}$ & $=$ & $\frac{1}{2} \mathbf{a}_{1}-x_{3} \mathbf{a}_{2}+\left(\frac{1}{2}+z_{3}\right) \mathbf{a}_{3}$ & $=$ & $\frac{1}{2} a \hat{\mathbf{x}}-x_{3} a \hat{\mathbf{y}}+\left(\frac{1}{2}+z_{3}\right) c \hat{\mathbf{z}}$ & $(4 e)$ & Ge I \\
\hline $\mathbf{B}_{9}$ & $=$ & $x_{4} \mathbf{a}_{1}+\frac{1}{2} \mathbf{a}_{2}+z_{4} \mathbf{a}_{3}$ & $=$ & $x_{4} a \hat{\mathbf{x}}+\frac{1}{2} a \hat{\mathbf{y}}+z_{4} c \hat{\mathbf{z}}$ & $(4 e)$ & Ge II \\
\hline $\mathbf{B}_{10}$ & $=$ & $-x_{4} \mathbf{a}_{1}+\frac{1}{2} \mathbf{a}_{2}+z_{4} \mathbf{a}_{3}$ & $=$ & $-x_{4} a \hat{\mathbf{x}}+\frac{1}{2} a \hat{\mathbf{y}}+z_{4} c \hat{\mathbf{z}}$ & $(4 e)$ & Ge II \\
\hline $\mathbf{B}_{11}$ & $=$ & $\frac{1}{2} \mathbf{a}_{1}+x_{4} \mathbf{a}_{2}+\left(\frac{1}{2}+z_{4}\right) \mathbf{a}_{3}$ & $=$ & $\frac{1}{2} a \hat{\mathbf{x}}+x_{4} a \hat{\mathbf{y}}+\left(\frac{1}{2}+z_{4}\right) c \hat{\mathbf{z}}$ & $(4 e)$ & Ge II \\
\hline $\mathbf{B}_{12}$ & $=$ & $\frac{1}{2} \mathbf{a}_{1}-x_{4} \mathbf{a}_{2}+\left(\frac{1}{2}+z_{4}\right) \mathbf{a}_{3}$ & $=$ & $\frac{1}{2} a \hat{\mathbf{x}}-x_{4} a \hat{\mathbf{y}}+\left(\frac{1}{2}+z_{4}\right) c \hat{\mathbf{z}}$ & $(4 e)$ & Ge II \\
\hline $\mathbf{B}_{13}$ & $=$ & $x_{5} \mathbf{a}_{1}+y_{5} \mathbf{a}_{2}+z_{5} \mathbf{a}_{3}$ & $=$ & $x_{5} a \hat{\mathbf{x}}+y_{5} a \hat{\mathbf{y}}+z_{5} c \hat{\mathbf{z}}$ & $(8 f)$ & As \\
\hline $\mathbf{B}_{14}$ & $=$ & $-x_{5} \mathbf{a}_{1}-y_{5} \mathbf{a}_{2}+z_{5} \mathbf{a}_{3}$ & $=$ & $-x_{5} a \hat{\mathbf{x}}-y_{5} a \hat{\mathbf{y}}+z_{5} c \hat{\mathbf{z}}$ & $(8 f)$ & As \\
\hline $\mathbf{B}_{15}$ & $=$ & $-y_{5} \mathbf{a}_{1}+x_{5} \mathbf{a}_{2}+\left(\frac{1}{2}+z_{5}\right) \mathbf{a}_{3}$ & $=$ & $-y_{5} a \hat{\mathbf{x}}+x_{5} a \hat{\mathbf{y}}+\left(\frac{1}{2}+z_{5}\right) c \hat{\mathbf{z}}$ & $(8 f)$ & As \\
\hline $\mathbf{B}_{16}$ & $=$ & $y_{5} \mathbf{a}_{1}-x_{5} \mathbf{a}_{2}+\left(\frac{1}{2}+z_{5}\right) \mathbf{a}_{3}$ & $=$ & $y_{5} a \hat{\mathbf{x}}-x_{5} a \hat{\mathbf{y}}+\left(\frac{1}{2}+z_{5}\right) c \hat{\mathbf{z}}$ & $(8 f)$ & As \\
\hline $\mathbf{B}_{17}$ & $=$ & $x_{5} \mathbf{a}_{1}-y_{5} \mathbf{a}_{2}+z_{5} \mathbf{a}_{3}$ & $=$ & $x_{5} a \hat{\mathbf{x}}-y_{5} a \hat{\mathbf{y}}+z_{5} c \hat{\mathbf{z}}$ & $(8 f)$ & As \\
\hline $\mathbf{B}_{18}$ & $=$ & $-x_{5} \mathbf{a}_{1}+y_{5} \mathbf{a}_{2}+z_{5} \mathbf{a}_{3}$ & $=$ & $-x_{5} a \hat{\mathbf{x}}+y_{5} a \hat{\mathbf{y}}+z_{5} c \hat{\mathbf{z}}$ & $(8 f)$ & As \\
\hline $\mathbf{B}_{19}$ & $=$ & $-y_{5} \mathbf{a}_{1}-x_{5} \mathbf{a}_{2}+\left(\frac{1}{2}+z_{5}\right) \mathbf{a}_{3}$ & $=$ & $-y_{5} a \hat{\mathbf{x}}-x_{5} a \hat{\mathbf{y}}+\left(\frac{1}{2}+z_{5}\right) c \hat{\mathbf{z}}$ & $(8 f)$ & As \\
\hline $\mathbf{B}_{20}$ & $=$ & $y_{5} \mathbf{a}_{1}+x_{5} \mathbf{a}_{2}+\left(\frac{1}{2}+z_{5}\right) \mathbf{a}_{3}$ & $=$ & $y_{5} a \hat{\mathbf{x}}+x_{5} a \hat{\mathbf{y}}+\left(\frac{1}{2}+z_{5}\right) c \hat{\mathbf{z}}$ & $(8 f)$ & As \\
\hline
\end{tabular}

\section{References:}

- B. Eisenmann and H. Schäfer, Zintlphasen mit binären Anionen: Zur Kenntnis von BaGe $\mathrm{P}_{2}$ und $\mathrm{BaGe}_{2} \mathrm{As}_{2} /$ Zintl Phases with Binary Anions: $\mathrm{BaGe}_{2} \mathrm{P}_{2}$ and $\mathrm{BaGe}_{2} \mathrm{As}_{2}$, Z. Naturforsch. B 36, $415-419$ (1981), doi:10.1515/znb-1981-0403.

\section{Found in:}

- P. Villars and K. Cenzual, Pearson's Crystal Data - Crystal Structure Database for Inorganic Compounds, ASM International (2013).

\section{Geometry files:}

- CIF: pp. 878

- POSCAR: pp. 878 


\section{$\mathrm{NaZn}[\mathrm{OH}]_{3}$ Structure: A3BC3D_tP64_106_3c_c_3c_c}
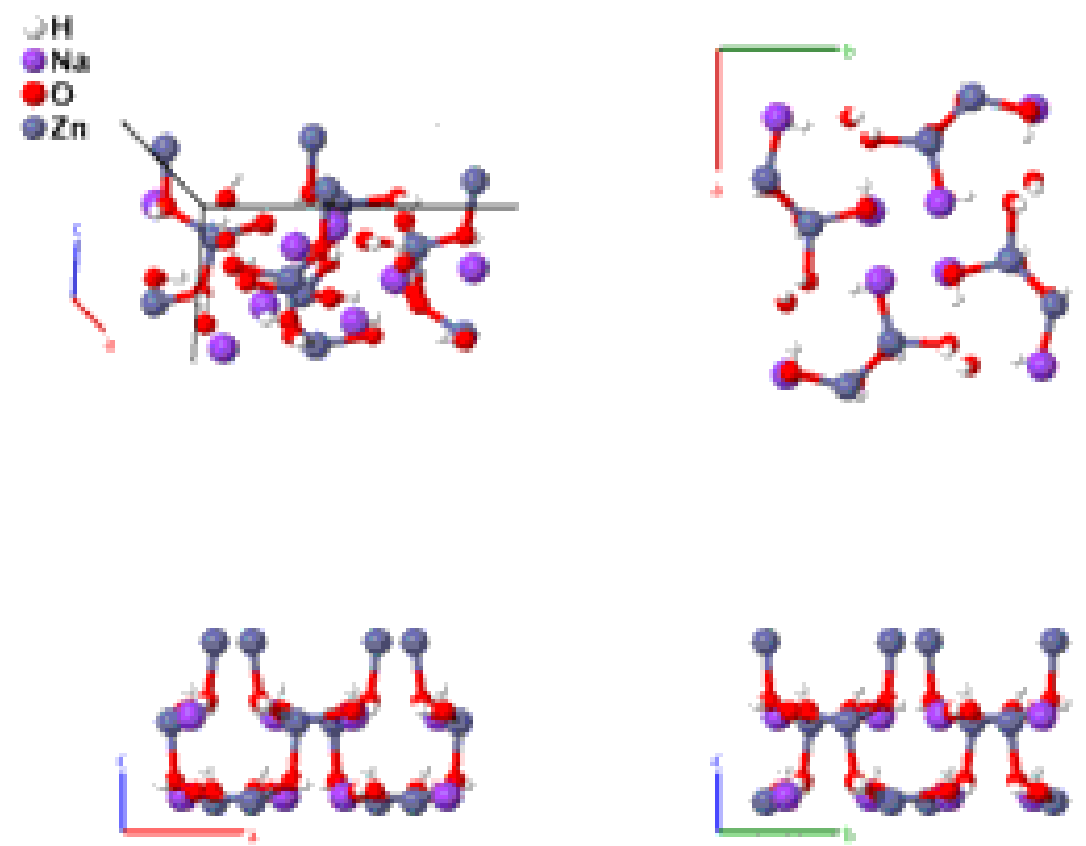

Prototype

AFLOW prototype label

Strukturbericht designation

Pearson symbol

Space group number

Space group symbol

AFLOW prototype command
: $\quad \mathrm{NaZn}[\mathrm{OH}]_{3}$

: A3BC3D_tP64_106_3c_c_3c_c

: None

: $\quad$ tP64

: 106

: $\quad P 4_{2} b c$

: $\quad$ aflow --proto=A3BC3D_tP64_106_3c_c_3c_c

- -params $=a, c / a, x_{1}, y_{1}, z_{1}, x_{2}, y_{2}, z_{2}, x_{3}, y_{3}, z_{3}, x_{4}, y_{4}, z_{4}, x_{5}, y_{5}, z_{5}, x_{6}, y_{6}, z_{6}, x_{7}$, $y_{7}, z_{7}, x_{8}, y_{8}, z_{8}$

Simple Tetragonal primitive vectors:

$$
\begin{aligned}
& \mathbf{a}_{1}=a \hat{\mathbf{x}} \\
& \mathbf{a}_{2}=a \hat{\mathbf{y}} \\
& \mathbf{a}_{3}=c \hat{\mathbf{z}}
\end{aligned}
$$

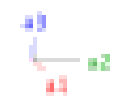

Basis vectors:

\section{Lattice Coordinates}

$\mathbf{B}_{1}=$

$\mathbf{B}_{2}=$

$x_{1} \mathbf{a}_{1}+y_{1} \mathbf{a}_{2}+z_{1} \mathbf{a}_{3}$

$-x_{1} \mathbf{a}_{1}-y_{1} \mathbf{a}_{2}+z_{1} \mathbf{a}_{3}$
Cartesian Coordinates

$=$

$=$

$x_{1} a \hat{\mathbf{x}}+y_{1} a \hat{\mathbf{y}}+z_{1} c \hat{\mathbf{z}}$
Wyckoff Position

$(8 c)$

$(8 c)$
Atom Type

H I

H I 


\begin{tabular}{|c|c|c|c|c|c|c|}
\hline $\mathbf{B}_{3}$ & $=$ & $-y_{1} \mathbf{a}_{1}+x_{1} \mathbf{a}_{2}+\left(\frac{1}{2}+z_{1}\right) \mathbf{a}_{3}$ & $=$ & $-y_{1} a \hat{\mathbf{x}}+x_{1} a \hat{\mathbf{y}}+\left(\frac{1}{2}+z_{1}\right) c \hat{\mathbf{z}}$ & $(8 c)$ & H I \\
\hline $\mathbf{B}_{4}$ & $=$ & $y_{1} \mathbf{a}_{1}-x_{1} \mathbf{a}_{2}+\left(\frac{1}{2}+z_{1}\right) \mathbf{a}_{3}$ & $=$ & $y_{1} a \hat{\mathbf{x}}-x_{1} a \hat{\mathbf{y}}+\left(\frac{1}{2}+z_{1}\right) c \hat{\mathbf{z}}$ & $(8 c)$ & H I \\
\hline $\mathbf{B}_{5}$ & $=$ & $\left(\frac{1}{2}+x_{1}\right) \mathbf{a}_{1}+\left(\frac{1}{2}-y_{1}\right) \mathbf{a}_{2}+z_{1} \mathbf{a}_{3}$ & $=$ & $\left(\frac{1}{2}+x_{1}\right) a \hat{\mathbf{x}}+\left(\frac{1}{2}-y_{1}\right) a \hat{\mathbf{y}}+z_{1} c \hat{\mathbf{z}}$ & $(8 c)$ & H I \\
\hline $\mathbf{B}_{6}$ & $=$ & $\left(\frac{1}{2}-x_{1}\right) \mathbf{a}_{1}+\left(\frac{1}{2}+y_{1}\right) \mathbf{a}_{2}+z_{1} \mathbf{a}_{3}$ & $=$ & $\left(\frac{1}{2}-x_{1}\right) a \hat{\mathbf{x}}+\left(\frac{1}{2}+y_{1}\right) a \hat{\mathbf{y}}+z_{1} c \hat{\mathbf{z}}$ & $(8 c)$ & H I \\
\hline $\mathbf{B}_{7}$ & $=$ & $\begin{array}{c}\left(\frac{1}{2}-y_{1}\right) \mathbf{a}_{1}+\left(\frac{1}{2}-x_{1}\right) \mathbf{a}_{2}+ \\
\left(\frac{1}{2}+z_{1}\right) \mathbf{a}_{3}\end{array}$ & $=$ & $\begin{array}{c}\left(\frac{1}{2}-y_{1}\right) a \hat{\mathbf{x}}+\left(\frac{1}{2}-x_{1}\right) a \hat{\mathbf{y}}+ \\
\left(\frac{1}{2}+z_{1}\right) c \hat{\mathbf{z}}\end{array}$ & $(8 c)$ & $\mathrm{H} \mathrm{I}$ \\
\hline $\mathbf{B}_{8}$ & $=$ & $\begin{array}{c}\left(\frac{1}{2}+y_{1}\right) \mathbf{a}_{1}+\left(\frac{1}{2}+x_{1}\right) \mathbf{a}_{2}+ \\
\left(\frac{1}{2}+z_{1}\right) \mathbf{a}_{3}\end{array}$ & $=$ & $\begin{array}{c}\left(\frac{1}{2}+y_{1}\right) a \hat{\mathbf{x}}+\left(\frac{1}{2}+x_{1}\right) a \hat{\mathbf{y}}+ \\
\left(\frac{1}{2}+z_{1}\right) c \hat{\mathbf{z}}\end{array}$ & $(8 c)$ & H I \\
\hline $\mathbf{B}_{9}$ & $=$ & $x_{2} \mathbf{a}_{1}+y_{2} \mathbf{a}_{2}+z_{2} \mathbf{a}_{3}$ & $=$ & $x_{2} a \hat{\mathbf{x}}+y_{2} a \hat{\mathbf{y}}+z_{2} c \hat{\mathbf{z}}$ & $(8 c)$ & H II \\
\hline $\mathbf{B}_{10}$ & $=$ & $-x_{2} \mathbf{a}_{1}-y_{2} \mathbf{a}_{2}+z_{2} \mathbf{a}_{3}$ & $=$ & $-x_{2} a \hat{\mathbf{x}}-y_{2} a \hat{\mathbf{y}}+z_{2} c \hat{\mathbf{z}}$ & $(8 c)$ & H II \\
\hline $\mathbf{B}_{11}$ & $=$ & $-y_{2} \mathbf{a}_{1}+x_{2} \mathbf{a}_{2}+\left(\frac{1}{2}+z_{2}\right) \mathbf{a}_{3}$ & $=$ & $-y_{2} a \hat{\mathbf{x}}+x_{2} a \hat{\mathbf{y}}+\left(\frac{1}{2}+z_{2}\right) c \hat{\mathbf{z}}$ & $(8 c)$ & $\mathrm{H}$ II \\
\hline $\mathbf{B}_{12}$ & $=$ & $y_{2} \mathbf{a}_{1}-x_{2} \mathbf{a}_{2}+\left(\frac{1}{2}+z_{2}\right) \mathbf{a}_{3}$ & $=$ & $y_{2} a \hat{\mathbf{x}}-x_{2} a \hat{\mathbf{y}}+\left(\frac{1}{2}+z_{2}\right) c \hat{\mathbf{z}}$ & $(8 c)$ & $\mathrm{H}$ II \\
\hline $\mathbf{B}_{13}$ & $=$ & $\left(\frac{1}{2}+x_{2}\right) \mathbf{a}_{1}+\left(\frac{1}{2}-y_{2}\right) \mathbf{a}_{2}+z_{2} \mathbf{a}_{3}$ & $=$ & $\left(\frac{1}{2}+x_{2}\right) a \hat{\mathbf{x}}+\left(\frac{1}{2}-y_{2}\right) a \hat{\mathbf{y}}+z_{2} c \hat{\mathbf{z}}$ & $(8 c)$ & H II \\
\hline $\mathbf{B}_{14}$ & $=$ & $\left(\frac{1}{2}-x_{2}\right) \mathbf{a}_{1}+\left(\frac{1}{2}+y_{2}\right) \mathbf{a}_{2}+z_{2} \mathbf{a}_{3}$ & $=$ & $\left(\frac{1}{2}-x_{2}\right) a \hat{\mathbf{x}}+\left(\frac{1}{2}+y_{2}\right) a \hat{\mathbf{y}}+z_{2} c \hat{\mathbf{z}}$ & $(8 c)$ & H II \\
\hline $\mathbf{B}_{15}$ & $=$ & $\begin{array}{c}\left(\frac{1}{2}-y_{2}\right) \mathbf{a}_{1}+\left(\frac{1}{2}-x_{2}\right) \mathbf{a}_{2}+ \\
\left(\frac{1}{2}+z_{2}\right) \mathbf{a}_{3}\end{array}$ & $=$ & $\begin{array}{c}\left(\frac{1}{2}-y_{2}\right) a \hat{\mathbf{x}}+\left(\frac{1}{2}-x_{2}\right) a \hat{\mathbf{y}}+ \\
\left(\frac{1}{2}+z_{2}\right) c \hat{\mathbf{z}}\end{array}$ & $(8 c)$ & H II \\
\hline $\mathbf{B}_{16}$ & $=$ & $\begin{array}{c}\left(\frac{1}{2}+y_{2}\right) \mathbf{a}_{1}+\left(\frac{1}{2}+x_{2}\right) \mathbf{a}_{2}+ \\
\left(\frac{1}{2}+z_{2}\right) \mathbf{a}_{3}\end{array}$ & $=$ & $\begin{array}{c}\left(\frac{1}{2}+y_{2}\right) a \hat{\mathbf{x}}+\left(\frac{1}{2}+x_{2}\right) a \hat{\mathbf{y}}+ \\
\left(\frac{1}{2}+z_{2}\right) c \hat{\mathbf{z}}\end{array}$ & $(8 c)$ & H II \\
\hline $\mathbf{B}_{17}$ & $=$ & $x_{3} \mathbf{a}_{1}+y_{3} \mathbf{a}_{2}+z_{3} \mathbf{a}_{3}$ & $=$ & $x_{3} a \hat{\mathbf{x}}+y_{3} a \hat{\mathbf{y}}+z_{3} c \hat{\mathbf{z}}$ & $(8 c)$ & H III \\
\hline $\mathbf{B}_{18}$ & $=$ & $-x_{3} \mathbf{a}_{1}-y_{3} \mathbf{a}_{2}+z_{3} \mathbf{a}_{3}$ & $=$ & $-x_{3} a \hat{\mathbf{x}}-y_{3} a \hat{\mathbf{y}}+z_{3} c \hat{\mathbf{z}}$ & $(8 c)$ & H III \\
\hline $\mathbf{B}_{19}$ & $=$ & $-y_{3} \mathbf{a}_{1}+x_{3} \mathbf{a}_{2}+\left(\frac{1}{2}+z_{3}\right) \mathbf{a}_{3}$ & $=$ & $-y_{3} a \hat{\mathbf{x}}+x_{3} a \hat{\mathbf{y}}+\left(\frac{1}{2}+z_{3}\right) c \hat{\mathbf{z}}$ & $(8 c)$ & H III \\
\hline $\mathbf{B}_{20}$ & $=$ & $y_{3} \mathbf{a}_{1}-x_{3} \mathbf{a}_{2}+\left(\frac{1}{2}+z_{3}\right) \mathbf{a}_{3}$ & $=$ & $y_{3} a \hat{\mathbf{x}}-x_{3} a \hat{\mathbf{y}}+\left(\frac{1}{2}+z_{3}\right) c \hat{\mathbf{z}}$ & $(8 c)$ & H III \\
\hline $\mathbf{B}_{21}$ & $=$ & $\left(\frac{1}{2}+x_{3}\right) \mathbf{a}_{1}+\left(\frac{1}{2}-y_{3}\right) \mathbf{a}_{2}+z_{3} \mathbf{a}_{3}$ & $=$ & $\left(\frac{1}{2}+x_{3}\right) a \hat{\mathbf{x}}+\left(\frac{1}{2}-y_{3}\right) a \hat{\mathbf{y}}+z_{3} c \hat{\mathbf{z}}$ & $(8 c)$ & H III \\
\hline $\mathbf{B}_{22}$ & $=$ & $\left(\frac{1}{2}-x_{3}\right) \mathbf{a}_{1}+\left(\frac{1}{2}+y_{3}\right) \mathbf{a}_{2}+z_{3} \mathbf{a}_{3}$ & $=$ & $\left(\frac{1}{2}-x_{3}\right) a \hat{\mathbf{x}}+\left(\frac{1}{2}+y_{3}\right) a \hat{\mathbf{y}}+z_{3} c \hat{\mathbf{z}}$ & $(8 c)$ & H III \\
\hline $\mathbf{B}_{23}$ & $=$ & $\begin{array}{c}\left(\frac{1}{2}-y_{3}\right) \mathbf{a}_{1}+\left(\frac{1}{2}-x_{3}\right) \mathbf{a}_{2}+ \\
\left(\frac{1}{2}+z_{3}\right) \mathbf{a}_{3}\end{array}$ & $=$ & $\begin{array}{c}\left(\frac{1}{2}-y_{3}\right) a \hat{\mathbf{x}}+\left(\frac{1}{2}-x_{3}\right) a \hat{\mathbf{y}}+ \\
\left(\frac{1}{2}+z_{3}\right) c \hat{\mathbf{z}}\end{array}$ & $(8 c)$ & H III \\
\hline $\mathbf{B}_{24}$ & $=$ & $\begin{array}{c}\left(\frac{1}{2}+y_{3}\right) \mathbf{a}_{1}+\left(\frac{1}{2}+x_{3}\right) \mathbf{a}_{2}+ \\
\left(\frac{1}{2}+z_{3}\right) \mathbf{a}_{3}\end{array}$ & $=$ & $\begin{array}{c}\left(\frac{1}{2}+y_{3}\right) a \hat{\mathbf{x}}+\left(\frac{1}{2}+x_{3}\right) a \hat{\mathbf{y}}+ \\
\left(\frac{1}{2}+z_{3}\right) c \hat{\mathbf{z}}\end{array}$ & $(8 c)$ & H III \\
\hline $\mathbf{B}_{25}$ & $=$ & $x_{4} \mathbf{a}_{1}+y_{4} \mathbf{a}_{2}+z_{4} \mathbf{a}_{3}$ & $=$ & $x_{4} a \hat{\mathbf{x}}+y_{4} a \hat{\mathbf{y}}+z_{4} c \hat{\mathbf{z}}$ & $(8 c)$ & $\mathrm{Na}$ \\
\hline $\mathbf{B}_{26}$ & $=$ & $-x_{4} \mathbf{a}_{1}-y_{4} \mathbf{a}_{2}+z_{4} \mathbf{a}_{3}$ & $=$ & $-x_{4} a \hat{\mathbf{x}}-y_{4} a \hat{\mathbf{y}}+z_{4} c \hat{\mathbf{z}}$ & $(8 c)$ & $\mathrm{Na}$ \\
\hline $\mathbf{B}_{27}$ & $=$ & $-y_{4} \mathbf{a}_{1}+x_{4} \mathbf{a}_{2}+\left(\frac{1}{2}+z_{4}\right) \mathbf{a}_{3}$ & $=$ & $-y_{4} a \hat{\mathbf{x}}+x_{4} a \hat{\mathbf{y}}+\left(\frac{1}{2}+z_{4}\right) c \hat{\mathbf{z}}$ & $(8 c)$ & $\mathrm{Na}$ \\
\hline $\mathbf{B}_{28}$ & $=$ & $y_{4} \mathbf{a}_{1}-x_{4} \mathbf{a}_{2}+\left(\frac{1}{2}+z_{4}\right) \mathbf{a}_{3}$ & $=$ & $y_{4} a \hat{\mathbf{x}}-x_{4} a \hat{\mathbf{y}}+\left(\frac{1}{2}+z_{4}\right) c \hat{\mathbf{z}}$ & $(8 c)$ & $\mathrm{Na}$ \\
\hline $\mathbf{B}_{29}$ & $=$ & $\left(\frac{1}{2}+x_{4}\right) \mathbf{a}_{1}+\left(\frac{1}{2}-y_{4}\right) \mathbf{a}_{2}+z_{4} \mathbf{a}_{3}$ & $=$ & $\left(\frac{1}{2}+x_{4}\right) a \hat{\mathbf{x}}+\left(\frac{1}{2}-y_{4}\right) a \hat{\mathbf{y}}+z_{4} c \hat{\mathbf{z}}$ & $(8 c)$ & $\mathrm{Na}$ \\
\hline $\mathbf{B}_{30}$ & $=$ & $\left(\frac{1}{2}-x_{4}\right) \mathbf{a}_{1}+\left(\frac{1}{2}+y_{4}\right) \mathbf{a}_{2}+z_{4} \mathbf{a}_{3}$ & $=$ & $\left(\frac{1}{2}-x_{4}\right) a \hat{\mathbf{x}}+\left(\frac{1}{2}+y_{4}\right) a \hat{\mathbf{y}}+z_{4} c \hat{\mathbf{z}}$ & $(8 c)$ & $\mathrm{Na}$ \\
\hline $\mathbf{B}_{31}$ & $=$ & $\begin{array}{c}\left(\frac{1}{2}-y_{4}\right) \mathbf{a}_{1}+\left(\frac{1}{2}-x_{4}\right) \mathbf{a}_{2}+ \\
\left(\frac{1}{2}+z_{4}\right) \mathbf{a}_{3}\end{array}$ & $=$ & $\begin{array}{c}\left(\frac{1}{2}-y_{4}\right) a \hat{\mathbf{x}}+\left(\frac{1}{2}-x_{4}\right) a \hat{\mathbf{y}}+ \\
\left(\frac{1}{2}+z_{4}\right) c \hat{\mathbf{z}}\end{array}$ & $(8 c)$ & $\mathrm{Na}$ \\
\hline $\mathbf{B}_{32}$ & $=$ & $\begin{array}{c}\left(\frac{1}{2}+y_{4}\right) \mathbf{a}_{1}+\left(\frac{1}{2}+x_{4}\right) \mathbf{a}_{2}+ \\
\left(\frac{1}{2}+z_{4}\right) \mathbf{a}_{3}\end{array}$ & $=$ & $\begin{array}{c}\left(\frac{1}{2}+y_{4}\right) a \hat{\mathbf{x}}+\left(\frac{1}{2}+x_{4}\right) a \hat{\mathbf{y}}+ \\
\left(\frac{1}{2}+z_{4}\right) c \hat{\mathbf{z}}\end{array}$ & $(8 c)$ & $\mathrm{Na}$ \\
\hline
\end{tabular}




\begin{tabular}{|c|c|c|c|c|c|c|}
\hline $\mathbf{B}_{33}$ & $=$ & $x_{5} \mathbf{a}_{1}+y_{5} \mathbf{a}_{2}+z_{5} \mathbf{a}_{3}$ & $=$ & $x_{5} a \hat{\mathbf{x}}+y_{5} a \hat{\mathbf{y}}+z_{5} c \hat{\mathbf{z}}$ & $(8 c)$ & O I \\
\hline $\mathbf{B}_{34}$ & $=$ & $-x_{5} \mathbf{a}_{1}-y_{5} \mathbf{a}_{2}+z_{5} \mathbf{a}_{3}$ & $=$ & $-x_{5} a \hat{\mathbf{x}}-y_{5} a \hat{\mathbf{y}}+z_{5} c \hat{\mathbf{z}}$ & $(8 c)$ & $\mathrm{O} I$ \\
\hline $\mathbf{B}_{35}$ & $=$ & $-y_{5} \mathbf{a}_{1}+x_{5} \mathbf{a}_{2}+\left(\frac{1}{2}+z_{5}\right) \mathbf{a}_{3}$ & $=$ & $-y_{5} a \hat{\mathbf{x}}+x_{5} a \hat{\mathbf{y}}+\left(\frac{1}{2}+z_{5}\right) c \hat{\mathbf{z}}$ & $(8 c)$ & O I \\
\hline $\mathbf{B}_{36}$ & $=$ & $y_{5} \mathbf{a}_{1}-x_{5} \mathbf{a}_{2}+\left(\frac{1}{2}+z_{5}\right) \mathbf{a}_{3}$ & $=$ & $y_{5} a \hat{\mathbf{x}}-x_{5} a \hat{\mathbf{y}}+\left(\frac{1}{2}+z_{5}\right) c \hat{\mathbf{z}}$ & $(8 c)$ & O I \\
\hline $\mathbf{B}_{37}$ & $=$ & $\left(\frac{1}{2}+x_{5}\right) \mathbf{a}_{1}+\left(\frac{1}{2}-y_{5}\right) \mathbf{a}_{2}+z_{5} \mathbf{a}_{3}$ & $=$ & $\left(\frac{1}{2}+x_{5}\right) a \hat{\mathbf{x}}+\left(\frac{1}{2}-y_{5}\right) a \hat{\mathbf{y}}+z_{5} c \hat{\mathbf{z}}$ & $(8 c)$ & $\mathrm{O} \mathrm{I}$ \\
\hline $\mathbf{B}_{38}$ & $=$ & $\left(\frac{1}{2}-x_{5}\right) \mathbf{a}_{1}+\left(\frac{1}{2}+y_{5}\right) \mathbf{a}_{2}+z_{5} \mathbf{a}_{3}$ & $=$ & $\left(\frac{1}{2}-x_{5}\right) a \hat{\mathbf{x}}+\left(\frac{1}{2}+y_{5}\right) a \hat{\mathbf{y}}+z_{5} c \hat{\mathbf{z}}$ & $(8 c)$ & O I \\
\hline $\mathbf{B}_{39}$ & $=$ & $\begin{array}{c}\left(\frac{1}{2}-y_{5}\right) \mathbf{a}_{1}+\left(\frac{1}{2}-x_{5}\right) \mathbf{a}_{2}+ \\
\left(\frac{1}{2}+z_{5}\right) \mathbf{a}_{3}\end{array}$ & $=$ & $\begin{array}{c}\left(\frac{1}{2}-y_{5}\right) a \hat{\mathbf{x}}+\left(\frac{1}{2}-x_{5}\right) a \hat{\mathbf{y}}+ \\
\left(\frac{1}{2}+z_{5}\right) c \hat{\mathbf{z}}\end{array}$ & $(8 c)$ & O I \\
\hline $\mathbf{B}_{40}$ & $=$ & $\begin{array}{c}\left(\frac{1}{2}+y_{5}\right) \mathbf{a}_{1}+\left(\frac{1}{2}+x_{5}\right) \mathbf{a}_{2}+ \\
\left(\frac{1}{2}+z_{5}\right) \mathbf{a}_{3}\end{array}$ & $=$ & $\begin{array}{c}\left(\frac{1}{2}+y_{5}\right) a \hat{\mathbf{x}}+\left(\frac{1}{2}+x_{5}\right) a \hat{\mathbf{y}}+ \\
\left(\frac{1}{2}+z_{5}\right) c \hat{\mathbf{z}}\end{array}$ & $(8 c)$ & O I \\
\hline $\mathbf{B}_{41}$ & $=$ & $x_{6} \mathbf{a}_{1}+y_{6} \mathbf{a}_{2}+z_{6} \mathbf{a}_{3}$ & $=$ & $x_{6} a \hat{\mathbf{x}}+y_{6} a \hat{\mathbf{y}}+z_{6} c \hat{\mathbf{z}}$ & $(8 c)$ & O II \\
\hline $\mathbf{B}_{42}$ & $=$ & $-x_{6} \mathbf{a}_{1}-y_{6} \mathbf{a}_{2}+z_{6} \mathbf{a}_{3}$ & $=$ & $-x_{6} a \hat{\mathbf{x}}-y_{6} a \hat{\mathbf{y}}+z_{6} c \hat{\mathbf{z}}$ & $(8 c)$ & O II \\
\hline $\mathbf{B}_{43}$ & $=$ & $-y_{6} \mathbf{a}_{1}+x_{6} \mathbf{a}_{2}+\left(\frac{1}{2}+z_{6}\right) \mathbf{a}_{3}$ & $=$ & $-y_{6} a \hat{\mathbf{x}}+x_{6} a \hat{\mathbf{y}}+\left(\frac{1}{2}+z_{6}\right) c \hat{\mathbf{z}}$ & $(8 c)$ & O II \\
\hline $\mathbf{B}_{44}$ & $=$ & $y_{6} \mathbf{a}_{1}-x_{6} \mathbf{a}_{2}+\left(\frac{1}{2}+z_{6}\right) \mathbf{a}_{3}$ & $=$ & $y_{6} a \hat{\mathbf{x}}-x_{6} a \hat{\mathbf{y}}+\left(\frac{1}{2}+z_{6}\right) c \hat{\mathbf{z}}$ & $(8 c)$ & O II \\
\hline $\mathbf{B}_{45}$ & $=$ & $\left(\frac{1}{2}+x_{6}\right) \mathbf{a}_{1}+\left(\frac{1}{2}-y_{6}\right) \mathbf{a}_{2}+z_{6} \mathbf{a}_{3}$ & $=$ & $\left(\frac{1}{2}+x_{6}\right) a \hat{\mathbf{x}}+\left(\frac{1}{2}-y_{6}\right) a \hat{\mathbf{y}}+z_{6} c \hat{\mathbf{z}}$ & $(8 c)$ & O II \\
\hline $\mathbf{B}_{46}$ & $=$ & $\left(\frac{1}{2}-x_{6}\right) \mathbf{a}_{1}+\left(\frac{1}{2}+y_{6}\right) \mathbf{a}_{2}+z_{6} \mathbf{a}_{3}$ & $=$ & $\left(\frac{1}{2}-x_{6}\right) a \hat{\mathbf{x}}+\left(\frac{1}{2}+y_{6}\right) a \hat{\mathbf{y}}+z_{6} c \hat{\mathbf{z}}$ & $(8 c)$ & O II \\
\hline $\mathbf{B}_{47}$ & $=$ & $\begin{array}{c}\left(\frac{1}{2}-y_{6}\right) \mathbf{a}_{1}+\left(\frac{1}{2}-x_{6}\right) \mathbf{a}_{2}+ \\
\left(\frac{1}{2}+z_{6}\right) \mathbf{a}_{3}\end{array}$ & $=$ & $\begin{array}{c}\left(\frac{1}{2}-y_{6}\right) a \hat{\mathbf{x}}+\left(\frac{1}{2}-x_{6}\right) a \hat{\mathbf{y}}+ \\
\left(\frac{1}{2}+z_{6}\right) c \hat{\mathbf{z}}\end{array}$ & $(8 c)$ & O II \\
\hline $\mathbf{B}_{48}$ & $=$ & $\begin{array}{c}\left(\frac{1}{2}+y_{6}\right) \mathbf{a}_{1}+\left(\frac{1}{2}+x_{6}\right) \mathbf{a}_{2}+ \\
\left(\frac{1}{2}+z_{6}\right) \mathbf{a}_{3}\end{array}$ & $=$ & $\begin{array}{c}\left(\frac{1}{2}+y_{6}\right) a \hat{\mathbf{x}}+\left(\frac{1}{2}+x_{6}\right) a \hat{\mathbf{y}}+ \\
\left(\frac{1}{2}+z_{6}\right) c \hat{\mathbf{z}}\end{array}$ & $(8 c)$ & O II \\
\hline $\mathbf{B}_{49}$ & $=$ & $x_{7} \mathbf{a}_{1}+y_{7} \mathbf{a}_{2}+z_{7} \mathbf{a}_{3}$ & $=$ & $x_{7} a \hat{\mathbf{x}}+y_{7} a \hat{\mathbf{y}}+z_{7} c \hat{\mathbf{z}}$ & $(8 c)$ & O III \\
\hline $\mathbf{B}_{50}$ & $=$ & $-x_{7} \mathbf{a}_{1}-y_{7} \mathbf{a}_{2}+z_{7} \mathbf{a}_{3}$ & $=$ & $-x_{7} a \hat{\mathbf{x}}-y_{7} a \hat{\mathbf{y}}+z_{7} c \hat{\mathbf{z}}$ & $(8 c)$ & O III \\
\hline $\mathbf{B}_{51}$ & $=$ & $-y_{7} \mathbf{a}_{1}+x_{7} \mathbf{a}_{2}+\left(\frac{1}{2}+z_{7}\right) \mathbf{a}_{3}$ & $=$ & $-y_{7} a \hat{\mathbf{x}}+x_{7} a \hat{\mathbf{y}}+\left(\frac{1}{2}+z_{7}\right) c \hat{\mathbf{z}}$ & $(8 c)$ & O III \\
\hline $\mathbf{B}_{52}$ & $=$ & $y_{7} \mathbf{a}_{1}-x_{7} \mathbf{a}_{2}+\left(\frac{1}{2}+z_{7}\right) \mathbf{a}_{3}$ & $=$ & $y_{7} a \hat{\mathbf{x}}-x_{7} a \hat{\mathbf{y}}+\left(\frac{1}{2}+z_{7}\right) c \hat{\mathbf{z}}$ & $(8 c)$ & O III \\
\hline $\mathbf{B}_{53}$ & $=$ & $\left(\frac{1}{2}+x_{7}\right) \mathbf{a}_{1}+\left(\frac{1}{2}-y_{7}\right) \mathbf{a}_{2}+z_{7} \mathbf{a}_{3}$ & $=$ & $\left(\frac{1}{2}+x_{7}\right) a \hat{\mathbf{x}}+\left(\frac{1}{2}-y_{7}\right) a \hat{\mathbf{y}}+z_{7} c \hat{\mathbf{z}}$ & $(8 c)$ & O III \\
\hline $\mathbf{B}_{54}$ & $=$ & $\left(\frac{1}{2}-x_{7}\right) \mathbf{a}_{1}+\left(\frac{1}{2}+y_{7}\right) \mathbf{a}_{2}+z_{7} \mathbf{a}_{3}$ & $=$ & $\left(\frac{1}{2}-x_{7}\right) a \hat{\mathbf{x}}+\left(\frac{1}{2}+y_{7}\right) a \hat{\mathbf{y}}+z_{7} c \hat{\mathbf{z}}$ & $(8 c)$ & O III \\
\hline $\mathbf{B}_{55}$ & $=$ & $\begin{array}{c}\left(\frac{1}{2}-y_{7}\right) \mathbf{a}_{1}+\left(\frac{1}{2}-x_{7}\right) \mathbf{a}_{2}+ \\
\left(\frac{1}{2}+z_{7}\right) \mathbf{a}_{3}\end{array}$ & $=$ & $\begin{array}{c}\left(\frac{1}{2}-y_{7}\right) a \hat{\mathbf{x}}+\left(\frac{1}{2}-x_{7}\right) a \hat{\mathbf{y}}+ \\
\left(\frac{1}{2}+z_{7}\right) c \hat{\mathbf{z}}\end{array}$ & $(8 c)$ & O III \\
\hline $\mathbf{B}_{56}$ & $=$ & $\begin{array}{c}\left(\frac{1}{2}+y_{7}\right) \mathbf{a}_{1}+\left(\frac{1}{2}+x_{7}\right) \mathbf{a}_{2}+ \\
\left(\frac{1}{2}+z_{7}\right) \mathbf{a}_{3}\end{array}$ & $=$ & $\begin{array}{c}\left(\frac{1}{2}+y_{7}\right) a \hat{\mathbf{x}}+\left(\frac{1}{2}+x_{7}\right) a \hat{\mathbf{y}}+ \\
\left(\frac{1}{2}+z_{7}\right) c \hat{\mathbf{z}}\end{array}$ & $(8 c)$ & O III \\
\hline $\mathbf{B}_{57}$ & $=$ & $x_{8} \mathbf{a}_{1}+y_{8} \mathbf{a}_{2}+z_{8} \mathbf{a}_{3}$ & $=$ & $x_{8} a \hat{\mathbf{x}}+y_{8} a \hat{\mathbf{y}}+z_{8} c \hat{\mathbf{z}}$ & $(8 c)$ & $\mathrm{Zn}$ \\
\hline $\mathbf{B}_{58}$ & $=$ & $-x_{8} \mathbf{a}_{1}-y_{8} \mathbf{a}_{2}+z_{8} \mathbf{a}_{3}$ & $=$ & $-x_{8} a \hat{\mathbf{x}}-y_{8} a \hat{\mathbf{y}}+z_{8} c \hat{\mathbf{z}}$ & $(8 c)$ & $\mathrm{Zn}$ \\
\hline $\mathbf{B}_{59}$ & $=$ & $-y_{8} \mathbf{a}_{1}+x_{8} \mathbf{a}_{2}+\left(\frac{1}{2}+z_{8}\right) \mathbf{a}_{3}$ & $=$ & $-y_{8} a \hat{\mathbf{x}}+x_{8} a \hat{\mathbf{y}}+\left(\frac{1}{2}+z_{8}\right) c \hat{\mathbf{z}}$ & $(8 c)$ & $\mathrm{Zn}$ \\
\hline $\mathbf{B}_{60}$ & $=$ & $y_{8} \mathbf{a}_{1}-x_{8} \mathbf{a}_{2}+\left(\frac{1}{2}+z_{8}\right) \mathbf{a}_{3}$ & $=$ & $y_{8} a \hat{\mathbf{x}}-x_{8} a \hat{\mathbf{y}}+\left(\frac{1}{2}+z_{8}\right) c \hat{\mathbf{z}}$ & $(8 c)$ & $\mathrm{Zn}$ \\
\hline $\mathbf{B}_{61}$ & $=$ & $\left(\frac{1}{2}+x_{8}\right) \mathbf{a}_{1}+\left(\frac{1}{2}-y_{8}\right) \mathbf{a}_{2}+z_{8} \mathbf{a}_{3}$ & $=$ & $\left(\frac{1}{2}+x_{8}\right) a \hat{\mathbf{x}}+\left(\frac{1}{2}-y_{8}\right) a \hat{\mathbf{y}}+z_{8} c \hat{\mathbf{z}}$ & $(8 c)$ & $\mathrm{Zn}$ \\
\hline $\mathbf{B}_{62}$ & $=$ & $\left(\frac{1}{2}-x_{8}\right) \mathbf{a}_{1}+\left(\frac{1}{2}+y_{8}\right) \mathbf{a}_{2}+z_{8} \mathbf{a}_{3}$ & $=$ & $\left(\frac{1}{2}-x_{8}\right) a \hat{\mathbf{x}}+\left(\frac{1}{2}+y_{8}\right) a \hat{\mathbf{y}}+z_{8} c \hat{\mathbf{z}}$ & $(8 c)$ & $\mathrm{Zn}$ \\
\hline $\mathbf{B}_{63}$ & $=$ & $\begin{array}{c}\left(\frac{1}{2}-y_{8}\right) \mathbf{a}_{1}+\left(\frac{1}{2}-x_{8}\right) \mathbf{a}_{2}+ \\
\left(\frac{1}{2}+z_{8}\right) \mathbf{a}_{3}\end{array}$ & $=$ & $\begin{array}{c}\left(\frac{1}{2}-y_{8}\right) a \hat{\mathbf{x}}+\left(\frac{1}{2}-x_{8}\right) a \hat{\mathbf{y}}+ \\
\left(\frac{1}{2}+z_{8}\right) c \hat{\mathbf{z}}\end{array}$ & $(8 c)$ & $\mathrm{Zn}$ \\
\hline
\end{tabular}


$\mathbf{B}_{64}=\begin{array}{cc}\left(\frac{1}{2}+y_{8}\right) \mathbf{a}_{1}+\left(\frac{1}{2}+x_{8}\right) \mathbf{a}_{2}+ \\ \left(\frac{1}{2}+z_{8}\right) \mathbf{a}_{3}\end{array} \quad \begin{gathered}\left(\frac{1}{2}+y_{8}\right) a \hat{\mathbf{x}}+\left(\frac{1}{2}+x_{8}\right) a \hat{\mathbf{y}}+ \\ \left(\frac{1}{2}+z_{8}\right) c \hat{\mathbf{z}}\end{gathered}$

$(8 c)$

$\mathrm{Zn}$

References:

- R. Stahl and H. Jacobs, Synthese und Kristallstruktur von $\mathrm{NaZn}(\mathrm{OH})_{3} \cdot 3 \mathrm{H}_{2} \mathrm{O}$ und $\mathrm{NaZn}(\mathrm{OH})_{3}$, Z. Anorg. Allg. Chem. 624, 25-29 (1998), doi:10.1002/(SICI)1521-3749(199801)624:1<25::AID-ZAAC25>3.0.CO;2-8.

\section{Found in:}

- P. Villars and K. Cenzual, Pearson's Crystal Data - Crystal Structure Database for Inorganic Compounds, ASM International (2013).

\section{Geometry files:}

- CIF: pp. 879

- POSCAR: pp. 879 


\section{$\mathrm{Co}_{5} \mathrm{Ge}_{7}$ Structure: A5B7_tI24_107_ac_abd}
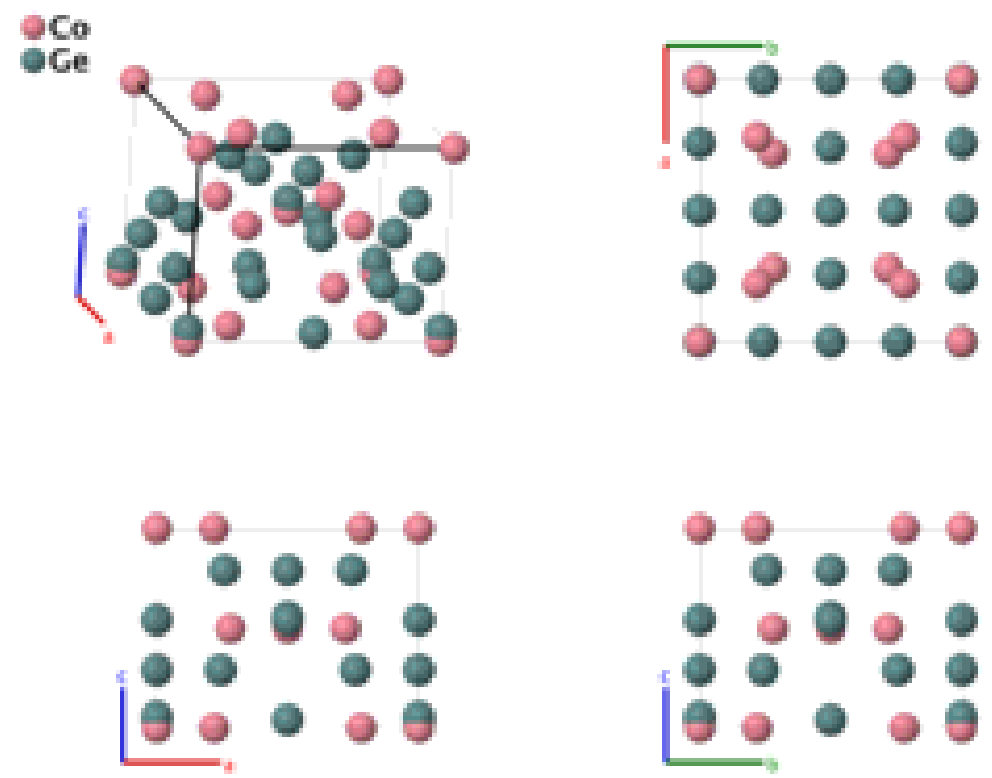

\section{Prototype}

$: \mathrm{Co}_{5} \mathrm{Ge}_{7}$

AFLOW prototype label

: A5B7_tI24_107_ac_abd

Strukturbericht designation

Pearson symbol

: None

Space group number

$: \quad \mathrm{tI} 24$

Space group symbol

: $\quad 107$

AFLOW prototype command

: $\quad I 4 \mathrm{~mm}$

aflow --proto=A5B7_tI24_107_ac_abd
--params $=a, c / a, z_{1}, z_{2}, z_{3}, x_{4}, z_{4}, x_{5}, z_{5}$

Body-centered Tetragonal primitive vectors:

$$
\begin{aligned}
& \mathbf{a}_{1}=-\frac{1}{2} a \hat{\mathbf{x}}+\frac{1}{2} a \hat{\mathbf{y}}+\frac{1}{2} c \hat{\mathbf{z}} \\
& \mathbf{a}_{2}=\frac{1}{2} a \hat{\mathbf{x}}-\frac{1}{2} a \hat{\mathbf{y}}+\frac{1}{2} c \hat{\mathbf{z}} \\
& \mathbf{a}_{3}=\frac{1}{2} a \hat{\mathbf{x}}+\frac{1}{2} a \hat{\mathbf{y}}-\frac{1}{2} c \hat{\mathbf{z}}
\end{aligned}
$$

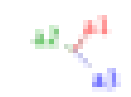

Basis vectors:

\section{Lattice Coordinates}

$\mathbf{B}_{1}=$

$\mathbf{B}_{2}=$

$\mathbf{B}_{3}=$

$\mathbf{B}_{4}=$

$\mathbf{B}_{5}=$

$$
z_{1} \mathbf{a}_{1}+z_{1} \mathbf{a}_{2}
$$$$
z_{2} \mathbf{a}_{1}+z_{2} \mathbf{a}_{2}
$$$$
\left(\frac{1}{2}+z_{3}\right) \mathbf{a}_{1}+z_{3} \mathbf{a}_{2}+\frac{1}{2} \mathbf{a}_{3}
$$$$
z_{3} \mathbf{a}_{1}+\left(\frac{1}{2}+z_{3}\right) \mathbf{a}_{2}+\frac{1}{2} \mathbf{a}_{3}
$$

$\left(x_{4}+z_{4}\right) \mathbf{a}_{1}+\left(x_{4}+z_{4}\right) \mathbf{a}_{2}+2 x_{4} \mathbf{a}_{3}$
Cartesian Coordinates

$=$

$=$

$=\quad \frac{1}{2} a \hat{\mathbf{y}}+z_{3} c \hat{\mathbf{z}}$

$=\quad \frac{1}{2} a \hat{\mathbf{x}}+z_{3} c \hat{\mathbf{z}}$

$=\quad x_{4} a \hat{\mathbf{x}}+x_{4} a \hat{\mathbf{y}}+z_{4} c \hat{\mathbf{z}}$
Wyckoff Position

(2a)

(2a)
Atom Type

Co I

Ge I

Ge II

Ge II

Co II 


$\begin{array}{rlrlrrr}\mathbf{B}_{6}= & \left(-x_{4}+z_{4}\right) \mathbf{a}_{1}+\left(-x_{4}+z_{4}\right) \mathbf{a}_{2}-2 x_{4} \mathbf{a}_{3} & = & -x_{4} a \hat{\mathbf{x}}-x_{4} a \hat{\mathbf{y}}+z_{4} c \hat{\mathbf{z}} & (8 c) & \text { Co II } \\ \mathbf{B}_{7}= & \left(x_{4}+z_{4}\right) \mathbf{a}_{1}+\left(-x_{4}+z_{4}\right) \mathbf{a}_{2} & = & -x_{4} a \hat{\mathbf{x}}+x_{4} a \hat{\mathbf{y}}+z_{4} c \hat{\mathbf{z}} & (8 c) & \text { Co II } \\ \mathbf{B}_{8} & = & \left(-x_{4}+z_{4}\right) \mathbf{a}_{1}+\left(x_{4}+z_{4}\right) \mathbf{a}_{2} & = & x_{4} a \hat{\mathbf{x}}-x_{4} a \hat{\mathbf{y}}+z_{4} c \hat{\mathbf{z}} & (8 c) & \text { Co II } \\ \mathbf{B}_{9}= & z_{5} \mathbf{a}_{1}+\left(x_{5}+z_{5}\right) \mathbf{a}_{2}+x_{5} \mathbf{a}_{3} & = & x_{5} a \hat{\mathbf{x}}+z_{5} c \hat{\mathbf{z}} & (8 d) & \text { Ge III } \\ \mathbf{B}_{10} & = & z_{5} \mathbf{a}_{1}+\left(-x_{5}+z_{5}\right) \mathbf{a}_{2}-x_{5} \mathbf{a}_{3} & = & -x_{5} a \hat{\mathbf{x}}+z_{5} c \hat{\mathbf{z}} & (8 d) & \text { Ge III } \\ \mathbf{B}_{11}= & \left(x_{5}+z_{5}\right) \mathbf{a}_{1}+z_{5} \mathbf{a}_{2}+x_{5} \mathbf{a}_{3} & = & x_{5} a \hat{\mathbf{y}}+z_{5} c \hat{\mathbf{z}} & (8 d) & \text { Ge III } \\ \mathbf{B}_{12}= & \left(-x_{5}+z_{5}\right) \mathbf{a}_{1}+z_{5} \mathbf{a}_{2}-x_{5} \mathbf{a}_{3} & = & -x_{5} a \hat{\mathbf{y}}+z_{5} c \hat{\mathbf{z}} & (8 d) & \text { Ge III }\end{array}$

\section{References:}

- K. Schubert, T. R. Anantharaman, H. O. K. Ata, H. G. Meissner, M. Pötzschke, W. Rossteutscher, and E. Stolz, Einige strukturelle Ergebnisse an metallischen Phasen (6), Naturwissenschaften 47, 512 (1960), doi:10.1007/BF00641115.

\section{Found in:}

- P. Villars and K. Cenzual, Pearson's Crystal Data - Crystal Structure Database for Inorganic Compounds, ASM International (2013).

\section{Geometry files:}

- CIF: pp. 879

- POSCAR: pp. 880 


\section{GeP (High-pressure, superconducting) Structure:} AB_tI4_107_a_a
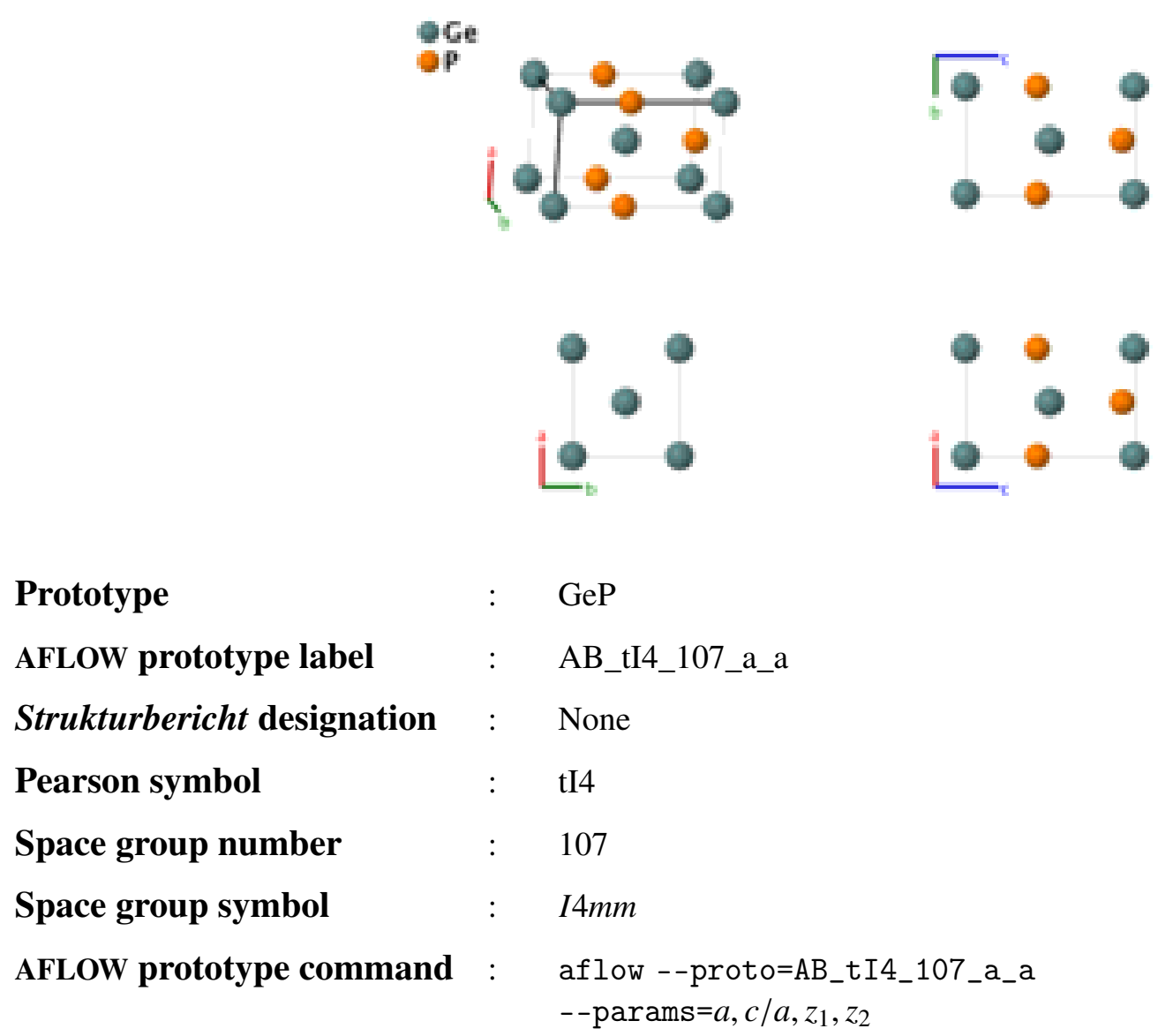

Body-centered Tetragonal primitive vectors:

$$
\begin{aligned}
& \mathbf{a}_{1}=-\frac{1}{2} a \hat{\mathbf{x}}+\frac{1}{2} a \hat{\mathbf{y}}+\frac{1}{2} c \hat{\mathbf{z}} \\
& \mathbf{a}_{2}=\frac{1}{2} a \hat{\mathbf{x}}-\frac{1}{2} a \hat{\mathbf{y}}+\frac{1}{2} c \hat{\mathbf{z}} \\
& \mathbf{a}_{3}=\frac{1}{2} a \hat{\mathbf{x}}+\frac{1}{2} a \hat{\mathbf{y}}-\frac{1}{2} c \hat{\mathbf{z}}
\end{aligned}
$$

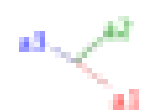

Basis vectors:

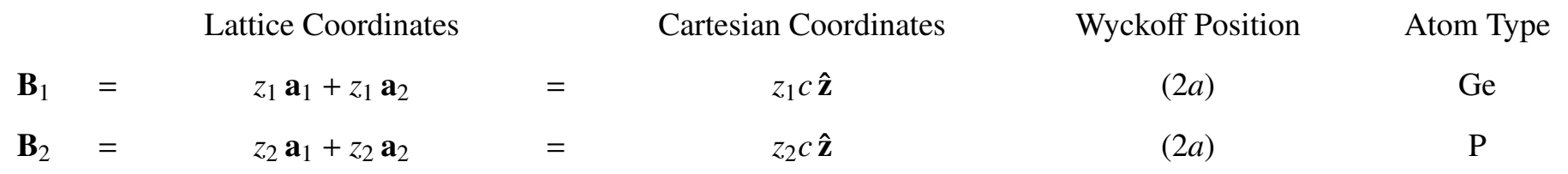

\section{References:}

- P. C. Donohue and H. S. Young, Synthesis, structure, and superconductivity of new high pressure phases in the systems Ge-P and Ge-As, J. Solid State Chem. 1, 143-149 (1970), doi:10.1016/0022-4596(70)90005-8.

\section{Found in:}

- P. Villars and K. Cenzual, Pearson's Crystal Data - Crystal Structure Database for Inorganic Compounds, ASM International (2013). 
Geometry files:

- CIF: pp. 880

- POSCAR: pp. 880 


\section{$\mathrm{Sr}_{5} \mathrm{Si}_{3}$ Structure: A3B5_tI32_108_ac_a2c}
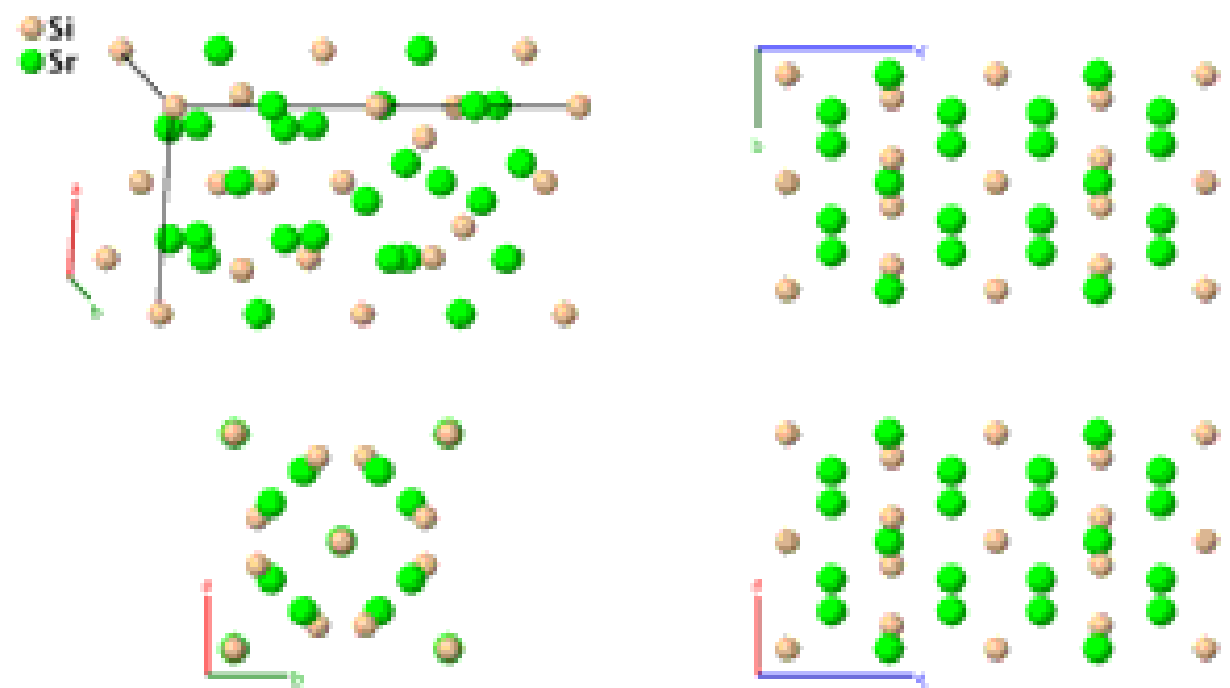

\section{Prototype}

$: \quad \mathrm{Sr}_{5} \mathrm{Si}_{3}$

AFLOW prototype label

: A3B5_tI32_108_ac_a2c

Strukturbericht designation : None

Pearson symbol $\quad: \quad t \mathrm{tI} 32$

Space group number $\quad: 108$

Space group symbol $\quad: \quad I 4 \mathrm{~cm}$

AFLOW prototype command : aflow --proto=A3B5_tI32_108_ac_a2c

- params $=a, c / a, z_{1}, z_{2}, x_{3}, z_{3}, x_{4}, z_{4}, x_{5}, z_{5}$

Body-centered Tetragonal primitive vectors:

$$
\begin{aligned}
& \mathbf{a}_{1}=-\frac{1}{2} a \hat{\mathbf{x}}+\frac{1}{2} a \hat{\mathbf{y}}+\frac{1}{2} c \hat{\mathbf{z}} \\
& \mathbf{a}_{2}=\frac{1}{2} a \hat{\mathbf{x}}-\frac{1}{2} a \hat{\mathbf{y}}+\frac{1}{2} c \hat{\mathbf{z}} \\
& \mathbf{a}_{3}=\frac{1}{2} a \hat{\mathbf{x}}+\frac{1}{2} a \hat{\mathbf{y}}-\frac{1}{2} c \hat{\mathbf{z}}
\end{aligned}
$$

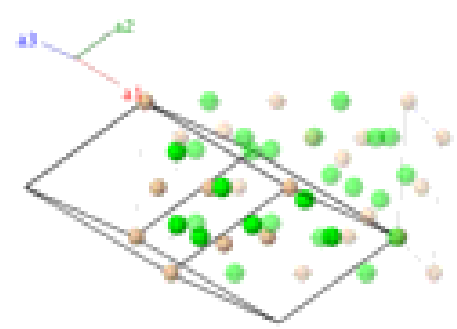

\section{Basis vectors:}

Lattice Coordinates

$$
\begin{aligned}
& \mathbf{B}_{1}=\quad z_{1} \mathbf{a}_{1}+z_{1} \mathbf{a}_{2} \\
& \mathbf{B}_{2}= \\
& \mathbf{B}_{3}= \\
& \left(\frac{1}{2}+z_{1}\right) \mathbf{a}_{1}+\left(\frac{1}{2}+z_{1}\right) \mathbf{a}_{2} \\
& \mathbf{B}_{4}=\left(\frac{1}{2}+z_{2}\right) \mathbf{a}_{1}+\left(\frac{1}{2}+z_{2}\right) \mathbf{a}_{2} \\
& \mathbf{B}_{5}= \\
& \left(\frac{1}{2}+x_{3}+z_{3}\right) \mathbf{a}_{1}+\left(x_{3}+z_{3}\right) \mathbf{a}_{2}+ \\
& \left(\frac{1}{2}+2 x_{3}\right) \mathbf{a}_{3} \\
& \mathbf{B}_{6}=\left(\frac{1}{2}-x_{3}+z_{3}\right) \mathbf{a}_{1}+\left(-x_{3}+z_{3}\right) \mathbf{a}_{2}+\quad=-x_{3} a \hat{\mathbf{x}}+\left(\frac{1}{2}-x_{3}\right) a \hat{\mathbf{y}}+z_{3} c \hat{\mathbf{z}} \\
& \left(\frac{1}{2}-2 x_{3}\right) \mathbf{a}_{3} \\
& = \\
& z_{1} c \hat{\mathbf{z}} \\
& =\quad\left(\frac{1}{2}+z_{1}\right) c \hat{\mathbf{z}} \\
& z_{2} c \hat{\mathbf{z}} \\
& \left(\frac{1}{2}+z_{2}\right) c \hat{\mathbf{z}} \\
& =\quad x_{3} a \hat{\mathbf{x}}+\left(\frac{1}{2}+x_{3}\right) a \hat{\mathbf{y}}+z_{3} c \hat{\mathbf{z}}
\end{aligned}
$$

Wyckoff Position Atom Type Si I Si I Sr I Sr I Si II 


\begin{tabular}{|c|c|c|c|c|c|c|}
\hline $\mathbf{B}_{7}$ & $=$ & $\left(x_{3}+z_{3}\right) \mathbf{a}_{1}+\left(\frac{1}{2}-x_{3}+z_{3}\right) \mathbf{a}_{2}+\frac{1}{2} \mathbf{a}_{3}$ & $=$ & $\left(\frac{1}{2}-x_{3}\right) a \hat{\mathbf{x}}+x_{3} a \hat{\mathbf{y}}+z_{3} c \hat{\mathbf{z}}$ & $(8 c)$ & Si II \\
\hline $\mathbf{B}_{8}$ & $=$ & $\left(-x_{3}+z_{3}\right) \mathbf{a}_{1}+\left(\frac{1}{2}+x_{3}+z_{3}\right) \mathbf{a}_{2}+\frac{1}{2} \mathbf{a}_{3}$ & $=$ & $\left(\frac{1}{2}+x_{3}\right) a \hat{\mathbf{x}}-x_{3} a \hat{\mathbf{y}}+z_{3} c \hat{\mathbf{z}}$ & $(8 c)$ & Si II \\
\hline $\mathbf{B}_{9}$ & $=$ & $\begin{array}{c}\left(\frac{1}{2}+x_{4}+z_{4}\right) \mathbf{a}_{1}+\left(x_{4}+z_{4}\right) \mathbf{a}_{2}+ \\
\left(\frac{1}{2}+2 x_{4}\right) \mathbf{a}_{3}\end{array}$ & $=$ & $x_{4} a \hat{\mathbf{x}}+\left(\frac{1}{2}+x_{4}\right) a \hat{\mathbf{y}}+z_{4} c \hat{\mathbf{z}}$ & $(8 c)$ & Sr II \\
\hline $\mathbf{B}_{10}$ & $=$ & $\begin{array}{c}\left(\frac{1}{2}-x_{4}+z_{4}\right) \mathbf{a}_{1}+\left(-x_{4}+z_{4}\right) \mathbf{a}_{2}+ \\
\left(\frac{1}{2}-2 x_{4}\right) \mathbf{a}_{3}\end{array}$ & $=$ & $-x_{4} a \hat{\mathbf{x}}+\left(\frac{1}{2}-x_{4}\right) a \hat{\mathbf{y}}+z_{4} c \hat{\mathbf{z}}$ & $(8 c)$ & Sr II \\
\hline $\mathbf{B}_{11}$ & $=$ & $\left(x_{4}+z_{4}\right) \mathbf{a}_{1}+\left(\frac{1}{2}-x_{4}+z_{4}\right) \mathbf{a}_{2}+\frac{1}{2} \mathbf{a}_{3}$ & $=$ & $\left(\frac{1}{2}-x_{4}\right) a \hat{\mathbf{x}}+x_{4} a \hat{\mathbf{y}}+z_{4} c \hat{\mathbf{z}}$ & $(8 c)$ & Sr II \\
\hline $\mathbf{B}_{12}$ & $=$ & $\left(-x_{4}+z_{4}\right) \mathbf{a}_{1}+\left(\frac{1}{2}+x_{4}+z_{4}\right) \mathbf{a}_{2}+\frac{1}{2} \mathbf{a}_{3}$ & $=$ & $\left(\frac{1}{2}+x_{4}\right) a \hat{\mathbf{x}}-x_{4} a \hat{\mathbf{y}}+z_{4} c \hat{\mathbf{z}}$ & $(8 c)$ & Sr II \\
\hline $\mathbf{B}_{13}$ & $=$ & $\begin{array}{c}\left(\frac{1}{2}+x_{5}+z_{5}\right) \mathbf{a}_{1}+\left(x_{5}+z_{5}\right) \mathbf{a}_{2}+ \\
\left(\frac{1}{2}+2 x_{5}\right) \mathbf{a}_{3}\end{array}$ & $=$ & $x_{5} a \hat{\mathbf{x}}+\left(\frac{1}{2}+x_{5}\right) a \hat{\mathbf{y}}+z_{5} c \hat{\mathbf{z}}$ & $(8 c)$ & Sr III \\
\hline $\mathbf{B}_{14}$ & $=$ & $\begin{array}{c}\left(\frac{1}{2}-x_{5}+z_{5}\right) \mathbf{a}_{1}+\left(-x_{5}+z_{5}\right) \mathbf{a}_{2}+ \\
\left(\frac{1}{2}-2 x_{5}\right) \mathbf{a}_{3}\end{array}$ & $=$ & $-x_{5} a \hat{\mathbf{x}}+\left(\frac{1}{2}-x_{5}\right) a \hat{\mathbf{y}}+z_{5} c \hat{\mathbf{z}}$ & $(8 c)$ & Sr III \\
\hline $\mathbf{B}_{15}$ & $=$ & $\left(x_{5}+z_{5}\right) \mathbf{a}_{1}+\left(\frac{1}{2}-x_{5}+z_{5}\right) \mathbf{a}_{2}+\frac{1}{2} \mathbf{a}_{3}$ & $=$ & $\left(\frac{1}{2}-x_{5}\right) a \hat{\mathbf{x}}+x_{5} a \hat{\mathbf{y}}+z_{5} c \hat{\mathbf{z}}$ & $(8 c)$ & Sr III \\
\hline $\mathbf{B}_{16}$ & $=$ & $\left(-x_{5}+z_{5}\right) \mathbf{a}_{1}+\left(\frac{1}{2}+x_{5}+z_{5}\right) \mathbf{a}_{2}+\frac{1}{2} \mathbf{a}_{3}$ & $=$ & $\left(\frac{1}{2}+x_{5}\right) a \hat{\mathbf{x}}-x_{5} a \hat{\mathbf{y}}+z_{5} c \hat{\mathbf{z}}$ & $(8 c)$ & Sr III \\
\hline
\end{tabular}

\section{References:}

- G. Nagorsen, G. Rocktäschel, H. Schäfer, and A. Weiss, Die Kristallstruktur der Phase $\mathrm{Sr}_{5} \mathrm{Si}_{3}$, Z. Naturforsch. B 22, 101-102 (1967), doi:10.1515/znb-1967-0122.

\section{Found in:}

- P. Villars and K. Cenzual, Pearson's Crystal Data - Crystal Structure Database for Inorganic Compounds, ASM International (2013).

\section{Geometry files:}

- CIF: pp. 880

- POSCAR: pp. 881 


\section{LaPtSi Structure: ABC_tI12_109_a_a_a}
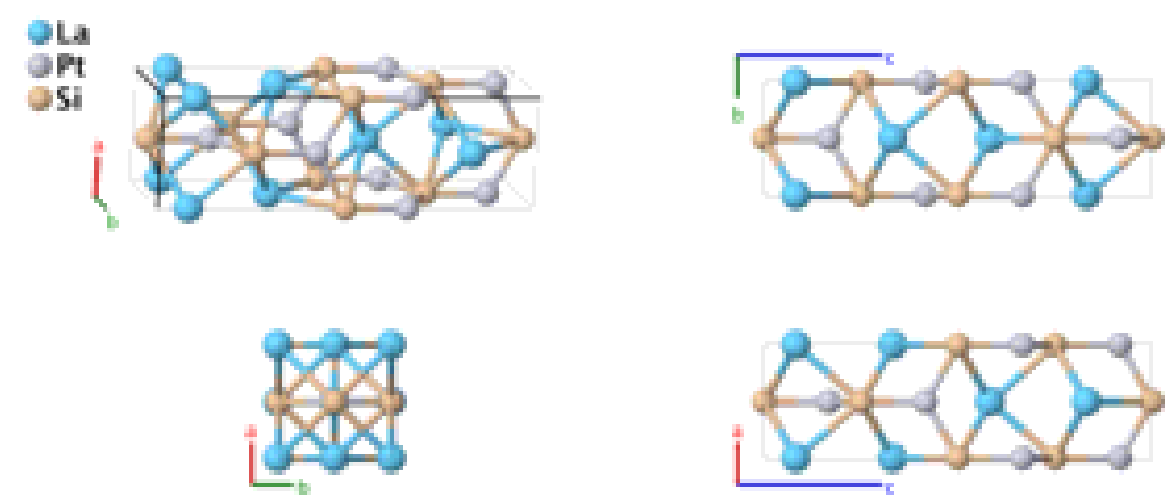

$\begin{array}{lll}\text { Prototype } & : & \text { LaPtSi } \\ \text { AFLOW prototype label } & : & \text { ABC_tI12_109_a_a_a } \\ \text { Strukturbericht designation } & : & \text { None } \\ \text { Pearson symbol } & : & \mathrm{tI} 12 \\ \text { Space group number } & : & 109 \\ \text { Space group symbol } & : & I 4_{1} m d \\ \text { AFLOW prototype command } & : & \text { aflow - proto }=\mathrm{ABC} \_t I 12_{-} 109 \_a \_a \_a \\ & & -- \text { params }=a, c / a, z_{1}, z_{2}, z_{3}\end{array}$

Body-centered Tetragonal primitive vectors:

$$
\begin{aligned}
& \mathbf{a}_{1}=-\frac{1}{2} a \hat{\mathbf{x}}+\frac{1}{2} a \hat{\mathbf{y}}+\frac{1}{2} c \hat{\mathbf{z}} \\
& \mathbf{a}_{2}=\frac{1}{2} a \hat{\mathbf{x}}-\frac{1}{2} a \hat{\mathbf{y}}+\frac{1}{2} c \hat{\mathbf{z}} \\
& \mathbf{a}_{3}=\frac{1}{2} a \hat{\mathbf{x}}+\frac{1}{2} a \hat{\mathbf{y}}-\frac{1}{2} c \hat{\mathbf{z}}
\end{aligned}
$$

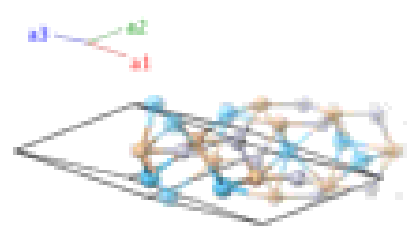

Basis vectors:

Lattice Coordinates
$\mathbf{B}_{1}=$
$z_{1} \mathbf{a}_{1}+z_{1} \mathbf{a}_{2}$
$\mathbf{B}_{2}=$
$\left(\frac{3}{4}+z_{1}\right) \mathbf{a}_{1}+\left(\frac{1}{4}+z_{1}\right) \mathbf{a}_{2}+\frac{1}{2} \mathbf{a}_{3}$
$z_{2} \mathbf{a}_{1}+z_{2} \mathbf{a}_{2}$
$\mathbf{B}_{3}=$$$
a_{2} a_{1}+z_{2} a_{2}
$$
$\mathbf{B}_{4}=\left(\frac{3}{4}+z_{2}\right) \mathbf{a}_{1}+\left(\frac{1}{4}+z_{2}\right) \mathbf{a}_{2}+\frac{1}{2} \mathbf{a}_{3}$
$\mathbf{B}_{5}=$
$\mathbf{B}_{6}=$

$$
\begin{gathered}
z_{3} \mathbf{a}_{1}+z_{3} \mathbf{a}_{2} \\
\left(\frac{3}{4}+z_{3}\right) \mathbf{a}_{1}+\left(\frac{1}{4}+z_{3}\right) \mathbf{a}_{2}+\frac{1}{2} \mathbf{a}_{3}
\end{gathered}
$$$$
=
$$
$=\quad \frac{1}{2} a \hat{\mathbf{y}}+\left(\frac{1}{4}+z_{1}\right) c \hat{\mathbf{z}}$
$=$
$z_{2} c \hat{\mathbf{z}}$
$=\quad \frac{1}{2} a \hat{\mathbf{y}}+\left(\frac{1}{4}+z_{2}\right) c \hat{\mathbf{z}}$
$=$
$z_{3} c \hat{\mathbf{z}}$
$=\quad \frac{1}{2} a \hat{\mathbf{y}}+\left(\frac{1}{4}+z_{3}\right) c \hat{\mathbf{z}}$

Cartesian Coordinates

Wyckoff Position

Atom Type

(4a)

$\mathrm{La}$

$(4 a)$

$\mathrm{La}$

(4a)

$\mathrm{Pt}$

(4a)

$\mathrm{Pt}$

$(4 a)$

$\mathrm{Si}$

\section{References:}

- K. Klepp and E. Parthé, RPtSi phases $(R=L a, C e, P r, N d$, Sm and $G d)$ with an ordered $\mathrm{ThSi}_{2}$ derivative structure, Acta Crystallogr. Sect. B Struct. Sci. 38, 1105-1108 (1982), doi:10.1107/S056774088200507X.

\section{Found in:}

- P. Villars and K. Cenzual, Pearson's Crystal Data - Crystal Structure Database for Inorganic Compounds, ASM International (2013). 
Geometry files:

- CIF: pp. 881

- POSCAR: pp. 881 


\section{NbAs Structure: AB_tI8_109_a_a}
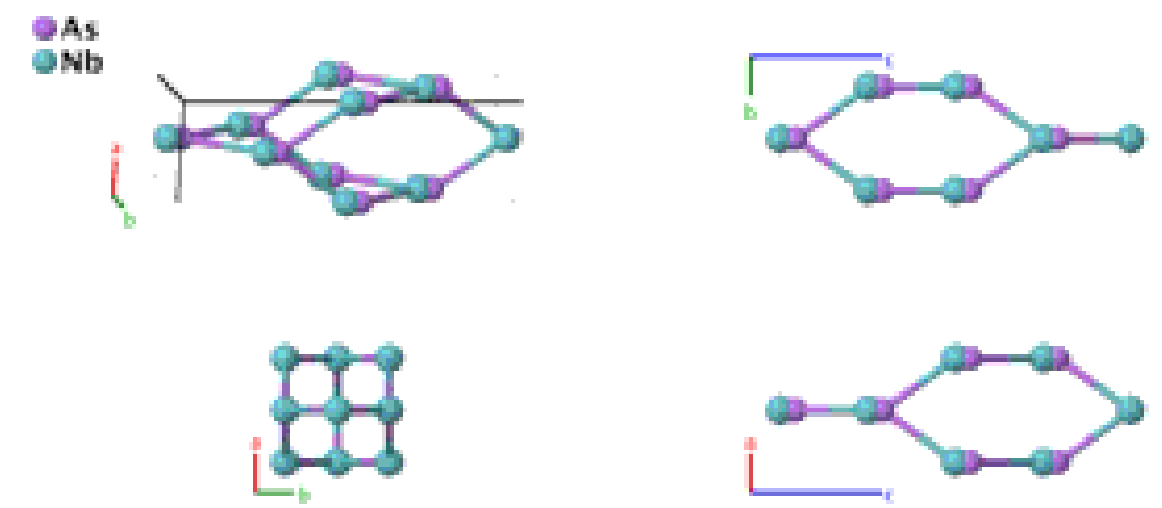

\section{Prototype}

: $\quad \mathrm{NbAs}$

AFLOW prototype label

: AB_tI8_109_a_a

Strukturbericht designation

: None

Pearson symbol

: $\quad$ tI8

Space group number $\quad: \quad 109$

Space group symbol $\quad: \quad I 4_{1} m d$

AFLOW prototype command : aflow --proto=AB_tI8_109_a_a

- params $=a, c / a, z_{1}, z_{2}$

Body-centered Tetragonal primitive vectors:

$$
\begin{aligned}
& \mathbf{a}_{1}=-\frac{1}{2} a \hat{\mathbf{x}}+\frac{1}{2} a \hat{\mathbf{y}}+\frac{1}{2} c \hat{\mathbf{z}} \\
& \mathbf{a}_{2}=\frac{1}{2} a \hat{\mathbf{x}}-\frac{1}{2} a \hat{\mathbf{y}}+\frac{1}{2} c \hat{\mathbf{z}} \\
& \mathbf{a}_{3}=\frac{1}{2} a \hat{\mathbf{x}}+\frac{1}{2} a \hat{\mathbf{y}}-\frac{1}{2} c \hat{\mathbf{z}}
\end{aligned}
$$

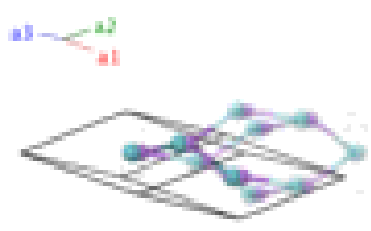

Basis vectors:

Lattice Coordinates

Cartesian Coordinates

Wyckoff Position

Atom Type

$\begin{array}{llccc}\mathbf{B}_{1} & & z_{1} \mathbf{a}_{1}+z_{1} \mathbf{a}_{2} & & z_{1} c \hat{\mathbf{z}} \\ \mathbf{B}_{2} & = & \left(\frac{3}{4}+z_{1}\right) \mathbf{a}_{1}+\left(\frac{1}{4}+z_{1}\right) \mathbf{a}_{2}+\frac{1}{2} \mathbf{a}_{3} & = & \frac{1}{2} a \hat{\mathbf{y}}+\left(\frac{1}{4}+z_{1}\right) c \hat{\mathbf{z}} \\ \mathbf{B}_{3}= & z_{2} \mathbf{a}_{1}+z_{2} \mathbf{a}_{2} & = & z_{2} c \hat{\mathbf{z}} \\ \mathbf{B}_{4}= & \left(\frac{3}{4}+z_{2}\right) \mathbf{a}_{1}+\left(\frac{1}{4}+z_{2}\right) \mathbf{a}_{2}+\frac{1}{2} \mathbf{a}_{3} & = & \frac{1}{2} a \hat{\mathbf{y}}+\left(\frac{1}{4}+z_{2}\right) c \hat{\mathbf{z}}\end{array}$

$(4 a)$

As

(4a)

As

$(4 a)$

$\mathrm{Nb}$

$(4 a)$

$\mathrm{Nb}$

\section{References:}

- S. Furuseth and A. Kjekshus, On the Arsenides and Antimonides of Niobium, Acta Chem. Scand. 18, 1180-1195 (1964), doi:10.3891/acta.chem.scand.18-1180.

Found in:

- P. Villars and K. Cenzual, Pearson's Crystal Data - Crystal Structure Database for Inorganic Compounds, ASM International (2013). 
Geometry files:

- CIF: pp. 881

- POSCAR: pp. 882 


\section{$\mathrm{Be}\left[\mathrm{BH}_{4}\right]_{2}$ Structure: A2BC8_tI176_110_2b_b_8b}
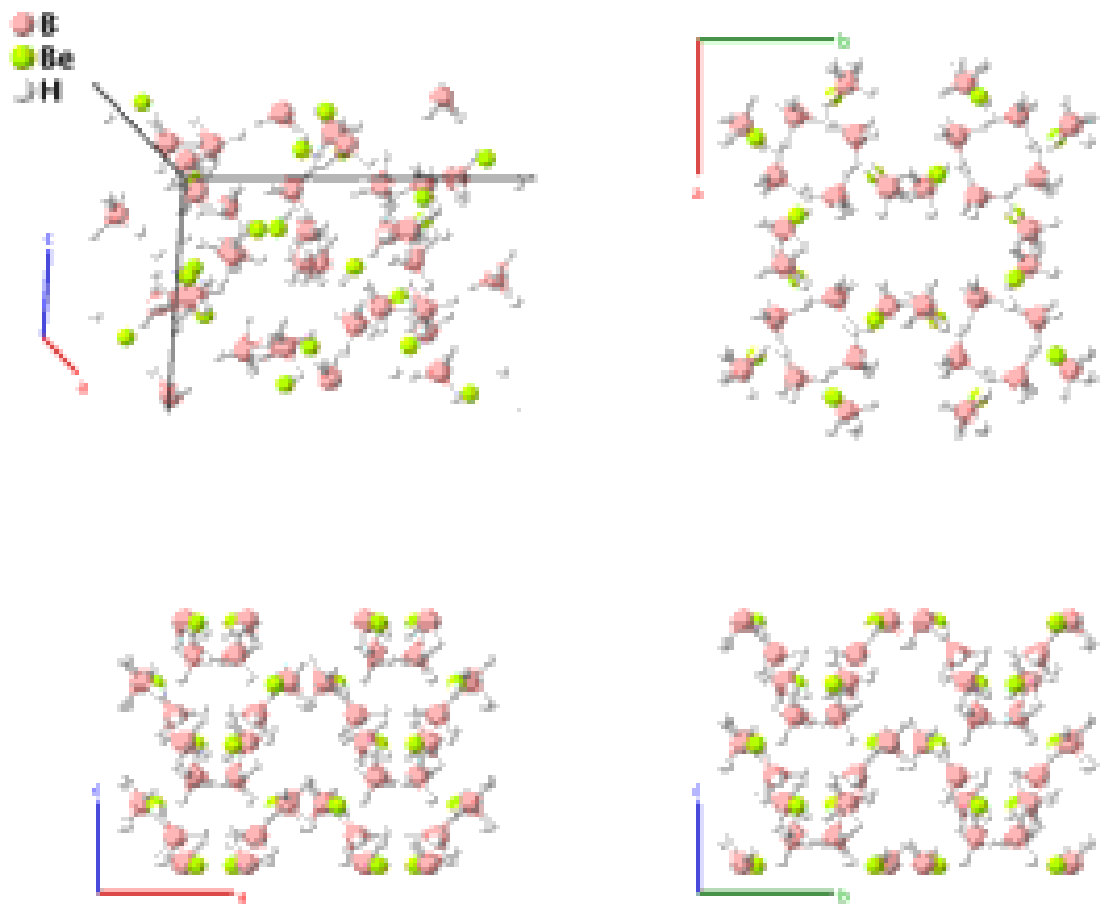

Prototype

AFLOW prototype label

Strukturbericht designation

Pearson symbol

Space group number

Space group symbol

AFLOW prototype command
: $\quad \mathrm{Be}\left[\mathrm{BH}_{4}\right]_{2}$

: A2BC8_tI176_110_2b_b_8b

: None

: $\quad$ tI176

: 110

: $\quad I 4_{1} c d$

- -params $=a, c / a, x_{1}, y_{1}, z_{1}, x_{2}, y_{2}, z_{2}, x_{3}, y_{3}, z_{3}, x_{4}, y_{4}, z_{4}, x_{5}, y_{5}, z_{5}, x_{6}, y_{6}, z_{6}, x_{7}$, $y_{7}, z_{7}, x_{8}, y_{8}, z_{8}, x_{9}, y_{9}, z_{9}, x_{10}, y_{10}, z_{10}, x_{11}, y_{11}, z_{11}$

Body-centered Tetragonal primitive vectors:

$$
\begin{aligned}
& \mathbf{a}_{1}=-\frac{1}{2} a \hat{\mathbf{x}}+\frac{1}{2} a \hat{\mathbf{y}}+\frac{1}{2} c \hat{\mathbf{z}} \\
& \mathbf{a}_{2}=\frac{1}{2} a \hat{\mathbf{x}}-\frac{1}{2} a \hat{\mathbf{y}}+\frac{1}{2} c \hat{\mathbf{z}} \\
& \mathbf{a}_{3}=\frac{1}{2} a \hat{\mathbf{x}}+\frac{1}{2} a \hat{\mathbf{y}}-\frac{1}{2} c \hat{\mathbf{z}}
\end{aligned}
$$

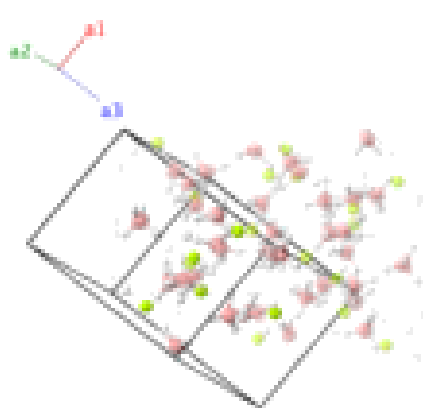

Basis vectors:

Lattice Coordinates

$\mathbf{B}_{1}=$

$$
\left(x_{1}+y_{1}\right) \mathbf{a}_{3}
$$

Cartesian Coordinates

$x_{1} a \hat{\mathbf{x}}+y_{1} a \hat{\mathbf{y}}+z_{1} c \hat{\mathbf{z}}$

$=$ 


$$
\begin{aligned}
& \mathbf{B}_{2}=\left(-y_{1}+z_{1}\right) \mathbf{a}_{1}+\left(-x_{1}+z_{1}\right) \mathbf{a}_{2}+\quad=\quad-x_{1} a \hat{\mathbf{x}}-y_{1} a \hat{\mathbf{y}}+z_{1} c \hat{\mathbf{z}} \\
& \mathbf{B}_{3}=\left(\frac{3}{4}+x_{1}+z_{1}\right) \mathbf{a}_{1}+\quad=-y_{1} a \hat{\mathbf{x}}+\left(\frac{1}{2}+x_{1}\right) a \hat{\mathbf{y}}+\left(\frac{1}{4}+z_{1}\right) c \hat{\mathbf{z}} \\
& \left(\frac{1}{4}-y_{1}+z_{1}\right) \mathbf{a}_{2}+\left(\frac{1}{2}+x_{1}-y_{1}\right) \mathbf{a}_{3} \\
& \mathbf{B}_{4}=\begin{array}{c}
\left(\frac{3}{4}-x_{1}+z_{1}\right) \mathbf{a}_{1}+ \\
\left(\frac{1}{4}+y_{1}+z_{1}\right) \mathbf{a}_{2}+\left(\frac{1}{2}-x_{1}+y_{1}\right) \mathbf{a}_{3}
\end{array} \\
& \mathbf{B}_{5}=\left(\frac{1}{2}-y_{1}+z_{1}\right) \mathbf{a}_{1}+\quad=\quad x_{1} a \hat{\mathbf{x}}-y_{1} a \hat{\mathbf{y}}+\left(\frac{1}{2}+z_{1}\right) c \hat{\mathbf{z}} \\
& \left(\frac{1}{2}+x_{1}+z_{1}\right) \mathbf{a}_{2}+\left(x_{1}-y_{1}\right) \mathbf{a}_{3} \\
& \mathbf{B}_{6}=\begin{array}{c}
\left(\frac{1}{2}+y_{1}+z_{1}\right) \mathbf{a}_{1}+ \\
\left(\frac{1}{2}-x_{1}+z_{1}\right) \mathbf{a}_{2}+\left(-x_{1}+y_{1}\right) \mathbf{a}_{3}
\end{array} \\
& \mathbf{B}_{7}=\begin{array}{c}
\left(\frac{1}{4}-x_{1}+z_{1}\right) \mathbf{a}_{1}+ \\
\left(\frac{3}{4}-y_{1}+z_{1}\right) \mathbf{a}_{2}+\left(\frac{1}{2}-x_{1}-y_{1}\right) \mathbf{a}_{3}
\end{array} \\
& \mathbf{B}_{8}=\begin{array}{c}
\left(\frac{1}{4}+x_{1}+z_{1}\right) \mathbf{a}_{1}+ \\
\left(\frac{3}{4}+y_{1}+z_{1}\right) \mathbf{a}_{2}+\left(\frac{1}{2}+x_{1}+y_{1}\right) \mathbf{a}_{3}
\end{array} \\
& \mathbf{B}_{9}=\left(y_{2}+z_{2}\right) \mathbf{a}_{1}+\left(x_{2}+z_{2}\right) \mathbf{a}_{2}+\quad=\quad x_{2} a \hat{\mathbf{x}}+y_{2} a \hat{\mathbf{y}}+z_{2} c \hat{\mathbf{z}} \\
& \left(x_{2}+y_{2}\right) \mathbf{a}_{3} \\
& \begin{array}{cc}
\mathbf{B}_{10}= & \begin{array}{c}
\left(-y_{2}+z_{2}\right) \mathbf{a}_{1}+\left(-x_{2}+z_{2}\right) \mathbf{a}_{2}+ \\
\left(-x_{2}-y_{2}\right) \mathbf{a}_{3}
\end{array} \\
\mathbf{B}_{11}= & \left(\frac{3}{4}+x_{2}+z_{2}\right) \mathbf{a}_{1}+ \\
& \left(\frac{1}{4}-y_{2}+z_{2}\right) \mathbf{a}_{2}+\left(\frac{1}{2}+x_{2}-y_{2}\right) \mathbf{a}_{3}
\end{array} \\
& \mathbf{B}_{12}=\begin{array}{c}
\left(\frac{3}{4}-x_{2}+z_{2}\right) \mathbf{a}_{1}+ \\
\left(\frac{1}{4}+y_{2}+z_{2}\right) \mathbf{a}_{2}+\left(\frac{1}{2}-x_{2}+y_{2}\right) \mathbf{a}_{3}
\end{array} \\
& \mathbf{B}_{13}=\begin{array}{c}
\left(\frac{1}{2}-y_{2}+z_{2}\right) \mathbf{a}_{1}+ \\
\left(\frac{1}{2}+x_{2}+z_{2}\right) \mathbf{a}_{2}+\left(x_{2}-y_{2}\right) \mathbf{a}_{3}
\end{array} \\
& \mathbf{B}_{14}=\begin{array}{c}
\left(\frac{1}{2}+y_{2}+z_{2}\right) \mathbf{a}_{1}+ \\
\left(\frac{1}{2}-x_{2}+z_{2}\right) \mathbf{a}_{2}+\left(-x_{2}+y_{2}\right) \mathbf{a}_{3}
\end{array} \\
& \mathbf{B}_{15}=\begin{array}{c}
\left(\frac{1}{4}-x_{2}+z_{2}\right) \mathbf{a}_{1}+ \\
\left(\frac{3}{4}-y_{2}+z_{2}\right) \mathbf{a}_{2}+\left(\frac{1}{2}-x_{2}-y_{2}\right) \mathbf{a}_{3}
\end{array} \\
& \mathbf{B}_{16}=\begin{array}{c}
\left(\frac{1}{4}+x_{2}+z_{2}\right) \mathbf{a}_{1}+ \\
\left(\frac{3}{4}+y_{2}+z_{2}\right) \mathbf{a}_{2}+\left(\frac{1}{2}+x_{2}+y_{2}\right) \mathbf{a}_{3}
\end{array} \\
& \mathbf{B}_{17}=\left(y_{3}+z_{3}\right) \mathbf{a}_{1}+\left(x_{3}+z_{3}\right) \mathbf{a}_{2}+\quad=\quad x_{3} a \hat{\mathbf{x}}+y_{3} a \hat{\mathbf{y}}+z_{3} c \hat{\mathbf{z}} \\
& \left(x_{3}+y_{3}\right) \mathbf{a}_{3} \\
& =\quad-x_{1} a \hat{\mathbf{x}}+y_{1} a \hat{\mathbf{y}}+\left(\frac{1}{2}+z_{1}\right) c \hat{\mathbf{z}} \\
& =\left(\frac{1}{2}-y_{1}\right) a \hat{\mathbf{x}}-x_{1} a \hat{\mathbf{y}}+\left(\frac{1}{4}+z_{1}\right) c \hat{\mathbf{z}} \quad(16 b) \\
& =\left(\frac{1}{2}+y_{1}\right) a \hat{\mathbf{x}}+x_{1} a \hat{\mathbf{y}}+\left(\frac{1}{4}+z_{1}\right) c \hat{\mathbf{z}} \\
& -x_{2} a \hat{\mathbf{x}}-y_{2} a \hat{\mathbf{y}}+z_{2} c \hat{\mathbf{z}} \\
& =-y_{2} a \hat{\mathbf{x}}+\left(\frac{1}{2}+x_{2}\right) a \hat{\mathbf{y}}+\left(\frac{1}{4}+z_{2}\right) c \hat{\mathbf{z}} \\
& =y_{2} a \hat{\mathbf{x}}+\left(\frac{1}{2}-x_{2}\right) a \hat{\mathbf{y}}+\left(\frac{1}{4}+z_{2}\right) c \hat{\mathbf{z}} \\
& =\quad x_{2} a \hat{\mathbf{x}}-y_{2} a \hat{\mathbf{y}}+\left(\frac{1}{2}+z_{2}\right) c \hat{\mathbf{z}} \\
& =\quad-x_{2} a \hat{\mathbf{x}}+y_{2} a \hat{\mathbf{y}}+\left(\frac{1}{2}+z_{2}\right) c \hat{\mathbf{z}} \quad(16 b) \\
& =\left(\frac{1}{2}-y_{2}\right) a \hat{\mathbf{x}}-x_{2} a \hat{\mathbf{y}}+\left(\frac{1}{4}+z_{2}\right) c \hat{\mathbf{z}} \\
& =\left(\frac{1}{2}+y_{2}\right) a \hat{\mathbf{x}}+x_{2} a \hat{\mathbf{y}}+\left(\frac{1}{4}+z_{2}\right) c \hat{\mathbf{z}} \\
& \mathbf{B}_{19}=\begin{array}{c}
\left(\frac{3}{4}+x_{3}+z_{3}\right) \mathbf{a}_{1}+ \\
\left(\frac{1}{4}-y_{3}+z_{3}\right) \mathbf{a}_{2}+\left(\frac{1}{2}+x_{3}-y_{3}\right) \mathbf{a}_{3}
\end{array} \\
& \left(-x_{3}-y_{3}\right) \mathbf{a}_{3} \\
& \begin{aligned}
& \mathbf{B}_{20}=\left(\frac{3}{4}-x_{3}+z_{3}\right) \mathbf{a}_{1}+ \\
&\left(\frac{1}{4}+y_{3}+z_{3}\right) \mathbf{a}_{2}+\left(\frac{1}{2}-x_{3}+y_{3}\right) \mathbf{a}_{3}
\end{aligned} \\
& \begin{array}{r}
\mathbf{B}_{21}= \\
\left(\frac{1}{2}+x_{3}+z_{3}\right) \mathbf{a}_{2}+\left(x_{3}-y_{3}\right) \mathbf{a}_{3}
\end{array} \\
& \mathbf{B}_{22}=\begin{array}{c}
\left(\frac{1}{2}+y_{3}+z_{3}\right) \mathbf{a}_{1}+ \\
\left(\frac{1}{2}-x_{3}+z_{3}\right) \mathbf{a}_{2}+\left(-x_{3}+y_{3}\right) \mathbf{a}_{3}
\end{array}=-x_{3} a \hat{\mathbf{x}}+y_{3} a \hat{\mathbf{y}}+\left(\frac{1}{2}+z_{3}\right) c \hat{\mathbf{z}}
\end{aligned}
$$




\begin{tabular}{|c|c|c|c|c|c|c|}
\hline $\mathbf{B}_{23}$ & $=$ & $\begin{array}{c}\left(\frac{1}{4}-x_{3}+z_{3}\right) \mathbf{a}_{1}+ \\
\left(\frac{3}{4}-y_{3}+z_{3}\right) \mathbf{a}_{2}+\left(\frac{1}{2}-x_{3}-y_{3}\right) \mathbf{a}_{3}\end{array}$ & $=$ & $\left(\frac{1}{2}-y_{3}\right) a \hat{\mathbf{x}}-x_{3} a \hat{\mathbf{y}}+\left(\frac{1}{4}+z_{3}\right) c \hat{\mathbf{z}}$ & $(16 b)$ & $\mathrm{Be}$ \\
\hline $\mathbf{B}_{24}$ & $=$ & $\begin{array}{c}\left(\frac{1}{4}+x_{3}+z_{3}\right) \mathbf{a}_{1}+ \\
\left(\frac{3}{4}+y_{3}+z_{3}\right) \mathbf{a}_{2}+\left(\frac{1}{2}+x_{3}+y_{3}\right) \mathbf{a}_{3}\end{array}$ & $=$ & $\left(\frac{1}{2}+y_{3}\right) a \hat{\mathbf{x}}+x_{3} a \hat{\mathbf{y}}+\left(\frac{1}{4}+z_{3}\right) c \hat{\mathbf{z}}$ & $(16 b)$ & $\mathrm{Be}$ \\
\hline $\mathbf{B}_{25}$ & $=$ & $\begin{array}{c}\left(y_{4}+z_{4}\right) \mathbf{a}_{1}+\left(x_{4}+z_{4}\right) \mathbf{a}_{2}+ \\
\left(x_{4}+y_{4}\right) \mathbf{a}_{3}\end{array}$ & $=$ & $x_{4} a \hat{\mathbf{x}}+y_{4} a \hat{\mathbf{y}}+z_{4} c \hat{\mathbf{z}}$ & $(16 b)$ & H I \\
\hline $\mathbf{B}_{26}$ & $=$ & $\begin{array}{c}\left(-y_{4}+z_{4}\right) \mathbf{a}_{1}+\left(-x_{4}+z_{4}\right) \mathbf{a}_{2}+ \\
\left(-x_{4}-y_{4}\right) \mathbf{a}_{3}\end{array}$ & $=$ & $-x_{4} a \hat{\mathbf{x}}-y_{4} a \hat{\mathbf{y}}+z_{4} c \hat{\mathbf{z}}$ & $(16 b)$ & H I \\
\hline $\mathbf{B}_{27}$ & $=$ & $\begin{array}{c}\left(\frac{3}{4}+x_{4}+z_{4}\right) \mathbf{a}_{1}+ \\
\left(\frac{1}{4}-y_{4}+z_{4}\right) \mathbf{a}_{2}+\left(\frac{1}{2}+x_{4}-y_{4}\right) \mathbf{a}_{3}\end{array}$ & $=$ & $-y_{4} a \hat{\mathbf{x}}+\left(\frac{1}{2}+x_{4}\right) a \hat{\mathbf{y}}+\left(\frac{1}{4}+z_{4}\right) c \hat{\mathbf{z}}$ & $(16 b)$ & H I \\
\hline $\mathbf{B}_{28}$ & $=$ & $\begin{array}{c}\left(\frac{3}{4}-x_{4}+z_{4}\right) \mathbf{a}_{1}+ \\
\left(\frac{1}{4}+y_{4}+z_{4}\right) \mathbf{a}_{2}+\left(\frac{1}{2}-x_{4}+y_{4}\right) \mathbf{a}_{3}\end{array}$ & $=$ & $y_{4} a \hat{\mathbf{x}}+\left(\frac{1}{2}-x_{4}\right) a \hat{\mathbf{y}}+\left(\frac{1}{4}+z_{4}\right) c \hat{\mathbf{z}}$ & $(16 b)$ & H I \\
\hline $\mathbf{B}_{29}$ & $=$ & $\begin{array}{c}\left(\frac{1}{2}-y_{4}+z_{4}\right) \mathbf{a}_{1}+ \\
\left(\frac{1}{2}+x_{4}+z_{4}\right) \mathbf{a}_{2}+\left(x_{4}-y_{4}\right) \mathbf{a}_{3}\end{array}$ & $=$ & $x_{4} a \hat{\mathbf{x}}-y_{4} a \hat{\mathbf{y}}+\left(\frac{1}{2}+z_{4}\right) c \hat{\mathbf{z}}$ & $(16 b)$ & H I \\
\hline $\mathbf{B}_{30}$ & $=$ & $\begin{array}{c}\left(\frac{1}{2}+y_{4}+z_{4}\right) \mathbf{a}_{1}+ \\
\left(\frac{1}{2}-x_{4}+z_{4}\right) \mathbf{a}_{2}+\left(-x_{4}+y_{4}\right) \mathbf{a}_{3}\end{array}$ & $=$ & $-x_{4} a \hat{\mathbf{x}}+y_{4} a \hat{\mathbf{y}}+\left(\frac{1}{2}+z_{4}\right) c \hat{\mathbf{z}}$ & $(16 b)$ & H I \\
\hline $\mathbf{B}_{31}$ & $=$ & $\begin{array}{c}\left(\frac{1}{4}-x_{4}+z_{4}\right) \mathbf{a}_{1}+ \\
\left(\frac{3}{4}-y_{4}+z_{4}\right) \mathbf{a}_{2}+\left(\frac{1}{2}-x_{4}-y_{4}\right) \mathbf{a}_{3}\end{array}$ & $=$ & $\left(\frac{1}{2}-y_{4}\right) a \hat{\mathbf{x}}-x_{4} a \hat{\mathbf{y}}+\left(\frac{1}{4}+z_{4}\right) c \hat{\mathbf{z}}$ & $(16 b)$ & H I \\
\hline $\mathbf{B}_{32}$ & $=$ & $\begin{array}{c}\left(\frac{1}{4}+x_{4}+z_{4}\right) \mathbf{a}_{1}+ \\
\left(\frac{3}{4}+y_{4}+z_{4}\right) \mathbf{a}_{2}+\left(\frac{1}{2}+x_{4}+y_{4}\right) \mathbf{a}_{3}\end{array}$ & $=$ & $\left(\frac{1}{2}+y_{4}\right) a \hat{\mathbf{x}}+x_{4} a \hat{\mathbf{y}}+\left(\frac{1}{4}+z_{4}\right) c \hat{\mathbf{z}}$ & $(16 b)$ & H I \\
\hline $\mathbf{B}_{33}$ & $=$ & $\begin{array}{c}\left(y_{5}+z_{5}\right) \mathbf{a}_{1}+\left(x_{5}+z_{5}\right) \mathbf{a}_{2}+ \\
\left(x_{5}+y_{5}\right) \mathbf{a}_{3}\end{array}$ & $=$ & $x_{5} a \hat{\mathbf{x}}+y_{5} a \hat{\mathbf{y}}+z_{5} c \hat{\mathbf{z}}$ & $(16 b)$ & H II \\
\hline $\mathbf{B}_{34}$ & $=$ & $\begin{array}{c}\left(-y_{5}+z_{5}\right) \mathbf{a}_{1}+\left(-x_{5}+z_{5}\right) \mathbf{a}_{2}+ \\
\left(-x_{5}-y_{5}\right) \mathbf{a}_{3}\end{array}$ & $=$ & $-x_{5} a \hat{\mathbf{x}}-y_{5} a \hat{\mathbf{y}}+z_{5} c \hat{\mathbf{z}}$ & $(16 b)$ & H II \\
\hline $\mathbf{B}_{35}$ & $=$ & $\begin{array}{c}\left(\frac{3}{4}+x_{5}+z_{5}\right) \mathbf{a}_{1}+ \\
\left(\frac{1}{4}-y_{5}+z_{5}\right) \mathbf{a}_{2}+\left(\frac{1}{2}+x_{5}-y_{5}\right) \mathbf{a}_{3}\end{array}$ & $=$ & $-y_{5} a \hat{\mathbf{x}}+\left(\frac{1}{2}+x_{5}\right) a \hat{\mathbf{y}}+\left(\frac{1}{4}+z_{5}\right) c \hat{\mathbf{z}}$ & $(16 b)$ & H II \\
\hline $\mathbf{B}_{36}$ & $=$ & $\begin{array}{c}\left(\frac{3}{4}-x_{5}+z_{5}\right) \mathbf{a}_{1}+ \\
\left(\frac{1}{4}+y_{5}+z_{5}\right) \mathbf{a}_{2}+\left(\frac{1}{2}-x_{5}+y_{5}\right) \mathbf{a}_{3}\end{array}$ & $=$ & $y_{5} a \hat{\mathbf{x}}+\left(\frac{1}{2}-x_{5}\right) a \hat{\mathbf{y}}+\left(\frac{1}{4}+z_{5}\right) c \hat{\mathbf{z}}$ & $(16 b)$ & H II \\
\hline $\mathbf{B}_{37}$ & $=$ & $\begin{array}{c}\left(\frac{1}{2}-y_{5}+z_{5}\right) \mathbf{a}_{1}+ \\
\left(\frac{1}{2}+x_{5}+z_{5}\right) \mathbf{a}_{2}+\left(x_{5}-y_{5}\right) \mathbf{a}_{3}\end{array}$ & $=$ & $x_{5} a \hat{\mathbf{x}}-y_{5} a \hat{\mathbf{y}}+\left(\frac{1}{2}+z_{5}\right) c \hat{\mathbf{z}}$ & $(16 b)$ & H II \\
\hline $\mathbf{B}_{38}$ & $=$ & $\begin{array}{c}\left(\frac{1}{2}+y_{5}+z_{5}\right) \mathbf{a}_{1}+ \\
\left(\frac{1}{2}-x_{5}+z_{5}\right) \mathbf{a}_{2}+\left(-x_{5}+y_{5}\right) \mathbf{a}_{3}\end{array}$ & $=$ & $-x_{5} a \hat{\mathbf{x}}+y_{5} a \hat{\mathbf{y}}+\left(\frac{1}{2}+z_{5}\right) c \hat{\mathbf{z}}$ & $(16 b)$ & H II \\
\hline $\mathbf{B}_{39}$ & $=$ & $\begin{array}{c}\left(\frac{1}{4}-x_{5}+z_{5}\right) \mathbf{a}_{1}+ \\
\left(\frac{3}{4}-y_{5}+z_{5}\right) \mathbf{a}_{2}+\left(\frac{1}{2}-x_{5}-y_{5}\right) \mathbf{a}_{3}\end{array}$ & $=$ & $\left(\frac{1}{2}-y_{5}\right) a \hat{\mathbf{x}}-x_{5} a \hat{\mathbf{y}}+\left(\frac{1}{4}+z_{5}\right) c \hat{\mathbf{z}}$ & $(16 b)$ & H II \\
\hline $\mathbf{B}_{40}$ & $=$ & $\begin{array}{c}\left(\frac{1}{4}+x_{5}+z_{5}\right) \mathbf{a}_{1}+ \\
\left(\frac{3}{4}+y_{5}+z_{5}\right) \mathbf{a}_{2}+\left(\frac{1}{2}+x_{5}+y_{5}\right) \mathbf{a}_{3}\end{array}$ & $=$ & $\left(\frac{1}{2}+y_{5}\right) a \hat{\mathbf{x}}+x_{5} a \hat{\mathbf{y}}+\left(\frac{1}{4}+z_{5}\right) c \hat{\mathbf{z}}$ & $(16 b)$ & H II \\
\hline $\mathbf{B}_{41}$ & $=$ & $\begin{array}{c}\left(y_{6}+z_{6}\right) \mathbf{a}_{1}+\left(x_{6}+z_{6}\right) \mathbf{a}_{2}+ \\
\left(x_{6}+y_{6}\right) \mathbf{a}_{3}\end{array}$ & $=$ & $x_{6} a \hat{\mathbf{x}}+y_{6} a \hat{\mathbf{y}}+z_{6} c \hat{\mathbf{z}}$ & $(16 b)$ & H III \\
\hline $\mathbf{B}_{42}$ & $=$ & $\begin{array}{c}\left(-y_{6}+z_{6}\right) \mathbf{a}_{1}+\left(-x_{6}+z_{6}\right) \mathbf{a}_{2}+ \\
\left(-x_{6}-y_{6}\right) \mathbf{a}_{3}\end{array}$ & $=$ & $-x_{6} a \hat{\mathbf{x}}-y_{6} a \hat{\mathbf{y}}+z_{6} c \hat{\mathbf{z}}$ & $(16 b)$ & H III \\
\hline $\mathbf{B}_{43}$ & $=$ & $\begin{array}{c}\left(\frac{3}{4}+x_{6}+z_{6}\right) \mathbf{a}_{1}+ \\
\left(\frac{1}{4}-y_{6}+z_{6}\right) \mathbf{a}_{2}+\left(\frac{1}{2}+x_{6}-y_{6}\right) \mathbf{a}_{3}\end{array}$ & $=$ & $-y_{6} a \hat{\mathbf{x}}+\left(\frac{1}{2}+x_{6}\right) a \hat{\mathbf{y}}+\left(\frac{1}{4}+z_{6}\right) c \hat{\mathbf{z}}$ & $(16 b)$ & H III \\
\hline
\end{tabular}




$$
\begin{aligned}
& \mathbf{B}_{44}=\begin{array}{c}
\left(\frac{3}{4}-x_{6}+z_{6}\right) \mathbf{a}_{1}+ \\
\left(\frac{1}{4}+y_{6}+z_{6}\right) \mathbf{a}_{2}+\left(\frac{1}{2}-x_{6}+y_{6}\right) \mathbf{a}_{3}
\end{array} \\
& \mathbf{B}_{45}=\left(\frac{1}{2}-y_{6}+z_{6}\right) \mathbf{a}_{1}+\quad=\quad x_{6} a \hat{\mathbf{x}}-y_{6} a \hat{\mathbf{y}}+\left(\frac{1}{2}+z_{6}\right) c \hat{\mathbf{z}} \\
& \left(\frac{1}{2}+x_{6}+z_{6}\right) \mathbf{a}_{2}+\left(x_{6}-y_{6}\right) \mathbf{a}_{3} \\
& \mathbf{B}_{46}=\begin{array}{c}
\left(\frac{1}{2}+y_{6}+z_{6}\right) \mathbf{a}_{1}+ \\
\left(\frac{1}{2}-x_{6}+z_{6}\right) \mathbf{a}_{2}+\left(-x_{6}+y_{6}\right) \mathbf{a}_{3}
\end{array} \\
& \mathbf{B}_{47}=\begin{array}{c}
\left(\frac{1}{4}-x_{6}+z_{6}\right) \mathbf{a}_{1}+ \\
\left(\frac{3}{4}-y_{6}+z_{6}\right) \mathbf{a}_{2}+\left(\frac{1}{2}-x_{6}-y_{6}\right) \mathbf{a}_{3}
\end{array} \\
& \mathbf{B}_{48}=\left(\frac{1}{4}+x_{6}+z_{6}\right) \mathbf{a}_{1}+\quad=\left(\frac{1}{2}+y_{6}\right) a \hat{\mathbf{x}}+x_{6} a \hat{\mathbf{y}}+\left(\frac{1}{4}+z_{6}\right) c \hat{\mathbf{z}} \\
& \left(\frac{3}{4}+y_{6}+z_{6}\right) \mathbf{a}_{2}+\left(\frac{1}{2}+x_{6}+y_{6}\right) \mathbf{a}_{3} \\
& \mathbf{B}_{49}=\left(y_{7}+z_{7}\right) \mathbf{a}_{1}+\left(x_{7}+z_{7}\right) \mathbf{a}_{2}+\quad=\quad x_{7} a \hat{\mathbf{x}}+y_{7} a \hat{\mathbf{y}}+z_{7} c \hat{\mathbf{z}} \\
& \left(x_{7}+y_{7}\right) \mathbf{a}_{3} \\
& =\quad-x_{6} a \hat{\mathbf{x}}+y_{6} a \hat{\mathbf{y}}+\left(\frac{1}{2}+z_{6}\right) c \hat{\mathbf{z}} \\
& =\left(\frac{1}{2}-y_{6}\right) a \hat{\mathbf{x}}-x_{6} a \hat{\mathbf{y}}+\left(\frac{1}{4}+z_{6}\right) c \hat{\mathbf{z}} \quad(16 b) \\
& \mathbf{B}_{50}=\left(-y_{7}+z_{7}\right) \mathbf{a}_{1}+\left(-x_{7}+z_{7}\right) \mathbf{a}_{2}+\quad=\quad-x_{7} a \hat{\mathbf{x}}-y_{7} a \hat{\mathbf{y}}+z_{7} c \hat{\mathbf{z}} \\
& \mathbf{B}_{51}=\begin{array}{c}
\left(\frac{3}{4}+x_{7}+z_{7}\right) \mathbf{a}_{1}+ \\
\left(\frac{1}{4}-y_{7}+z_{7}\right) \mathbf{a}_{2}+\left(\frac{1}{2}+x_{7}-y_{7}\right) \mathbf{a}_{3}
\end{array} \\
& \mathbf{B}_{52}=\begin{array}{c}
\left(\frac{3}{4}-x_{7}+z_{7}\right) \mathbf{a}_{1}+ \\
\left(\frac{1}{4}+y_{7}+z_{7}\right) \mathbf{a}_{2}+\left(\frac{1}{2}-x_{7}+y_{7}\right) \mathbf{a}_{3}
\end{array} \\
& \mathbf{B}_{53}=\begin{array}{c}
\left(\frac{1}{2}-y_{7}+z_{7}\right) \mathbf{a}_{1}+ \\
\left(\frac{1}{2}+x_{7}+z_{7}\right) \mathbf{a}_{2}+\left(x_{7}-y_{7}\right) \mathbf{a}_{3}
\end{array} \\
& \mathbf{B}_{54}=\begin{array}{c}
\left(\frac{1}{2}+y_{7}+z_{7}\right) \mathbf{a}_{1}+ \\
\left(\frac{1}{2}-x_{7}+z_{7}\right) \mathbf{a}_{2}+\left(-x_{7}+y_{7}\right) \mathbf{a}_{3}
\end{array} \\
& \mathbf{B}_{55}=\begin{array}{c}
\left(\frac{1}{4}-x_{7}+z_{7}\right) \mathbf{a}_{1}+ \\
\left(\frac{3}{4}-y_{7}+z_{7}\right) \mathbf{a}_{2}+\left(\frac{1}{2}-x_{7}-y_{7}\right) \mathbf{a}_{3}
\end{array} \\
& \mathbf{B}_{56}=\begin{array}{c}
\left(\frac{1}{4}+x_{7}+z_{7}\right) \mathbf{a}_{1}+ \\
\left(\frac{3}{4}+y_{7}+z_{7}\right) \mathbf{a}_{2}+\left(\frac{1}{2}+x_{7}+y_{7}\right) \mathbf{a}_{3}
\end{array} \\
& \mathbf{B}_{57}=\left(y_{8}+z_{8}\right) \mathbf{a}_{1}+\left(x_{8}+z_{8}\right) \mathbf{a}_{2}+\quad=\quad x_{8} a \hat{\mathbf{x}}+y_{8} a \hat{\mathbf{y}}+z_{8} c \hat{\mathbf{z}} \\
& \left(x_{8}+y_{8}\right) \mathbf{a}_{3} \\
& =-y_{7} a \hat{\mathbf{x}}+\left(\frac{1}{2}+x_{7}\right) a \hat{\mathbf{y}}+\left(\frac{1}{4}+z_{7}\right) c \hat{\mathbf{z}} \\
& =y_{7} a \hat{\mathbf{x}}+\left(\frac{1}{2}-x_{7}\right) a \hat{\mathbf{y}}+\left(\frac{1}{4}+z_{7}\right) c \hat{\mathbf{z}} \\
& =\quad x_{7} a \hat{\mathbf{x}}-y_{7} a \hat{\mathbf{y}}+\left(\frac{1}{2}+z_{7}\right) c \hat{\mathbf{z}} \\
& =\quad-x_{7} a \hat{\mathbf{x}}+y_{7} a \hat{\mathbf{y}}+\left(\frac{1}{2}+z_{7}\right) c \hat{\mathbf{z}} \\
& =\left(\frac{1}{2}-y_{7}\right) a \hat{\mathbf{x}}-x_{7} a \hat{\mathbf{y}}+\left(\frac{1}{4}+z_{7}\right) c \hat{\mathbf{z}} \\
& =\left(\frac{1}{2}+y_{7}\right) a \hat{\mathbf{x}}+x_{7} a \hat{\mathbf{y}}+\left(\frac{1}{4}+z_{7}\right) c \hat{\mathbf{z}} \\
& -x_{8} a \hat{\mathbf{x}}-y_{8} a \hat{\mathbf{y}}+z_{8} c \hat{\mathbf{z}} \\
& \begin{array}{cc}
\mathbf{B}_{58}= & \begin{array}{c}
\left(-y_{8}+z_{8}\right) \mathbf{a}_{1}+\left(-x_{8}+z_{8}\right) \mathbf{a}_{2}+ \\
\left(-x_{8}-y_{8}\right) \mathbf{a}_{3}
\end{array} \\
\mathbf{B}_{59}= & \left(\frac{3}{4}+x_{8}+z_{8}\right) \mathbf{a}_{1}+ \\
& \left(\frac{1}{4}-y_{8}+z_{8}\right) \mathbf{a}_{2}+\left(\frac{1}{2}+x_{8}-y_{8}\right) \mathbf{a}_{3}
\end{array} \\
& \mathbf{B}_{60}=\begin{array}{c}
\left(\frac{3}{4}-x_{8}+z_{8}\right) \mathbf{a}_{1}+ \\
\left(\frac{1}{4}+y_{8}+z_{8}\right) \mathbf{a}_{2}+\left(\frac{1}{2}-x_{8}+y_{8}\right) \mathbf{a}_{3}
\end{array} \\
& \mathbf{B}_{61}=\begin{array}{c}
\left(\frac{1}{2}-y_{8}+z_{8}\right) \mathbf{a}_{1}+ \\
\left(\frac{1}{2}+x_{8}+z_{8}\right) \mathbf{a}_{2}+\left(x_{8}-y_{8}\right) \mathbf{a}_{3}
\end{array} \\
& \mathbf{B}_{62}=\begin{array}{c}
\left(\frac{1}{2}+y_{8}+z_{8}\right) \mathbf{a}_{1}+ \\
\left(\frac{1}{2}-x_{8}+z_{8}\right) \mathbf{a}_{2}+\left(-x_{8}+y_{8}\right) \mathbf{a}_{3}
\end{array} \\
& \mathbf{B}_{63}=\begin{array}{c}
\left(\frac{1}{4}-x_{8}+z_{8}\right) \mathbf{a}_{1}+ \\
\left(\frac{3}{4}-y_{8}+z_{8}\right) \mathbf{a}_{2}+\left(\frac{1}{2}-x_{8}-y_{8}\right) \mathbf{a}_{3}
\end{array} \\
& \mathbf{B}_{64}=\left(\frac{1}{4}+x_{8}+z_{8}\right) \mathbf{a}_{1}+=\left(\frac{1}{2}+y_{8}\right) a \hat{\mathbf{x}}+x_{8} a \hat{\mathbf{y}}+\left(\frac{1}{4}+z_{8}\right) c \hat{\mathbf{z}} \\
& \left(\frac{3}{4}+y_{8}+z_{8}\right) \mathbf{a}_{2}+\left(\frac{1}{2}+x_{8}+y_{8}\right) \mathbf{a}_{3} \\
& =y_{8} a \hat{\mathbf{x}}+\left(\frac{1}{2}-x_{8}\right) a \hat{\mathbf{y}}+\left(\frac{1}{4}+z_{8}\right) c \hat{\mathbf{z}}
\end{aligned}
$$




\begin{tabular}{|c|c|c|c|c|c|c|}
\hline $\mathbf{B}_{65}$ & $=$ & $\begin{array}{c}\left(y_{9}+z_{9}\right) \mathbf{a}_{1}+\left(x_{9}+z_{9}\right) \mathbf{a}_{2}+ \\
\left(x_{9}+y_{9}\right) \mathbf{a}_{3}\end{array}$ & $=$ & $x_{9} a \hat{\mathbf{x}}+y_{9} a \hat{\mathbf{y}}+z_{9} c \hat{\mathbf{z}}$ & $(16 b)$ & H VI \\
\hline $\mathbf{B}_{66}$ & $=$ & $\begin{array}{c}\left(-y_{9}+z_{9}\right) \mathbf{a}_{1}+\left(-x_{9}+z_{9}\right) \mathbf{a}_{2}+ \\
\left(-x_{9}-y_{9}\right) \mathbf{a}_{3}\end{array}$ & $=$ & $-x_{9} a \hat{\mathbf{x}}-y_{9} a \hat{\mathbf{y}}+z_{9} c \hat{\mathbf{z}}$ & $(16 b)$ & H VI \\
\hline $\mathbf{B}_{67}$ & $=$ & $\begin{array}{c}\left(\frac{3}{4}+x_{9}+z_{9}\right) \mathbf{a}_{1}+ \\
\left(\frac{1}{4}-y_{9}+z_{9}\right) \mathbf{a}_{2}+\left(\frac{1}{2}+x_{9}-y_{9}\right) \mathbf{a}_{3}\end{array}$ & $=$ & $-y_{9} a \hat{\mathbf{x}}+\left(\frac{1}{2}+x_{9}\right) a \hat{\mathbf{y}}+\left(\frac{1}{4}+z_{9}\right) c \hat{\mathbf{z}}$ & $(16 b)$ & H VI \\
\hline $\mathbf{B}_{68}$ & $=$ & $\begin{array}{c}\left(\frac{3}{4}-x_{9}+z_{9}\right) \mathbf{a}_{1}+ \\
\left(\frac{1}{4}+y_{9}+z_{9}\right) \mathbf{a}_{2}+\left(\frac{1}{2}-x_{9}+y_{9}\right) \mathbf{a}_{3}\end{array}$ & $=$ & $y_{9} a \hat{\mathbf{x}}+\left(\frac{1}{2}-x_{9}\right) a \hat{\mathbf{y}}+\left(\frac{1}{4}+z_{9}\right) c \hat{\mathbf{z}}$ & $(16 b)$ & H VI \\
\hline $\mathbf{B}_{69}$ & $=$ & $\begin{array}{c}\left(\frac{1}{2}-y_{9}+z_{9}\right) \mathbf{a}_{1}+ \\
\left(\frac{1}{2}+x_{9}+z_{9}\right) \mathbf{a}_{2}+\left(x_{9}-y_{9}\right) \mathbf{a}_{3}\end{array}$ & $=$ & $x_{9} a \hat{\mathbf{x}}-y_{9} a \hat{\mathbf{y}}+\left(\frac{1}{2}+z_{9}\right) c \hat{\mathbf{z}}$ & $(16 b)$ & H VI \\
\hline $\mathbf{B}_{70}$ & $=$ & $\begin{array}{c}\left(\frac{1}{2}+y_{9}+z_{9}\right) \mathbf{a}_{1}+ \\
\left(\frac{1}{2}-x_{9}+z_{9}\right) \mathbf{a}_{2}+\left(-x_{9}+y_{9}\right) \mathbf{a}_{3}\end{array}$ & $=$ & $-x_{9} a \hat{\mathbf{x}}+y_{9} a \hat{\mathbf{y}}+\left(\frac{1}{2}+z_{9}\right) c \hat{\mathbf{z}}$ & $(16 b)$ & H VI \\
\hline $\mathbf{B}_{71}$ & $=$ & $\begin{array}{c}\left(\frac{1}{4}-x_{9}+z_{9}\right) \mathbf{a}_{1}+ \\
\left(\frac{3}{4}-y_{9}+z 9\right) \mathbf{a}_{2}+\left(\frac{1}{2}-x_{9}-y_{9}\right) \mathbf{a}_{3}\end{array}$ & $=$ & $\left(\frac{1}{2}-y_{9}\right) a \hat{\mathbf{x}}-x_{9} a \hat{\mathbf{y}}+\left(\frac{1}{4}+z_{9}\right) c \hat{\mathbf{z}}$ & $(16 b)$ & H VI \\
\hline $\mathbf{B}_{72}$ & $=$ & $\begin{array}{c}\left(\frac{1}{4}+x_{9}+z_{9}\right) \mathbf{a}_{1}+ \\
\left(\frac{3}{4}+y_{9}+z_{9}\right) \mathbf{a}_{2}+\left(\frac{1}{2}+x_{9}+y_{9}\right) \mathbf{a}_{3}\end{array}$ & $=$ & $\left(\frac{1}{2}+y_{9}\right) a \hat{\mathbf{x}}+x_{9} a \hat{\mathbf{y}}+\left(\frac{1}{4}+z_{9}\right) c \hat{\mathbf{z}}$ & $(16 b)$ & H VI \\
\hline $\mathbf{B}_{73}$ & $=$ & $\begin{array}{c}\left(y_{10}+z_{10}\right) \mathbf{a}_{1}+\left(x_{10}+z_{10}\right) \mathbf{a}_{2}+ \\
\left(x_{10}+y_{10}\right) \mathbf{a}_{3}\end{array}$ & $=$ & $x_{10} a \hat{\mathbf{x}}+y_{10} a \hat{\mathbf{y}}+z_{10} c \hat{\mathbf{z}}$ & $(16 b)$ & H VII \\
\hline $\mathbf{B}_{74}$ & $=$ & $\begin{array}{c}\left(-y_{10}+z_{10}\right) \mathbf{a}_{1}+ \\
\left(-x_{10}+z_{10}\right) \mathbf{a}_{2}+\left(-x_{10}-y_{10}\right) \mathbf{a}_{3}\end{array}$ & $=$ & $-x_{10} a \hat{\mathbf{x}}-y_{10} a \hat{\mathbf{y}}+z_{10} c \hat{\mathbf{z}}$ & $(16 b)$ & H VII \\
\hline $\mathbf{B}_{75}$ & $=$ & $\begin{array}{c}\left(\frac{3}{4}+x_{10}+z_{10}\right) \mathbf{a}_{1}+ \\
\left(\frac{1}{4}-y_{10}+z_{10}\right) \mathbf{a}_{2}+ \\
\left(\frac{1}{2}+x_{10}-y_{10}\right) \mathbf{a}_{3}\end{array}$ & $=$ & $\begin{array}{c}-y_{10} a \hat{\mathbf{x}}+\left(\frac{1}{2}+x_{10}\right) a \hat{\mathbf{y}}+ \\
\left(\frac{1}{4}+z_{10}\right) c \hat{\mathbf{z}}\end{array}$ & $(16 b)$ & H VII \\
\hline $\mathbf{B}_{76}$ & $=$ & $\begin{array}{l}\left(\frac{3}{4}-x_{10}+z_{10}\right) \mathbf{a}_{1}+ \\
\left(\frac{1}{4}+y_{10}+z_{10}\right) \mathbf{a}_{2}+ \\
\left(\frac{1}{2}-x_{10}+y_{10}\right) \mathbf{a}_{3}\end{array}$ & $=$ & $y_{10} a \hat{\mathbf{x}}+\left(\frac{1}{2}-x_{10}\right) a \hat{\mathbf{y}}+\left(\frac{1}{4}+z_{10}\right) c \hat{\mathbf{z}}$ & $(16 b)$ & H VII \\
\hline $\mathbf{B}_{77}$ & $=$ & $\begin{array}{c}\left(\frac{1}{2}-y_{10}+z_{10}\right) \mathbf{a}_{1}+ \\
\left(\frac{1}{2}+x_{10}+z_{10}\right) \mathbf{a}_{2}+\left(x_{10}-y_{10}\right) \mathbf{a}_{3}\end{array}$ & $=$ & $x_{10} a \hat{\mathbf{x}}-y_{10} a \hat{\mathbf{y}}+\left(\frac{1}{2}+z_{10}\right) c \hat{\mathbf{z}}$ & $(16 b)$ & H VII \\
\hline $\mathbf{B}_{78}$ & $=$ & $\begin{array}{c}\left(\frac{1}{2}+y_{10}+z_{10}\right) \mathbf{a}_{1}+ \\
\left(\frac{1}{2}-x_{10}+z_{10}\right) \mathbf{a}_{2}+ \\
\left(-x_{10}+y_{10}\right) \mathbf{a}_{3}\end{array}$ & $=$ & $-x_{10} a \hat{\mathbf{x}}+y_{10} a \hat{\mathbf{y}}+\left(\frac{1}{2}+z_{10}\right) c \hat{\mathbf{z}}$ & $(16 b)$ & H VII \\
\hline $\mathbf{B}_{79}$ & $=$ & $\begin{array}{c}\left(\frac{1}{4}-x_{10}+z_{10}\right) \mathbf{a}_{1}+ \\
\left(\frac{3}{4}-y_{10}+z_{10}\right) \mathbf{a}_{2}+ \\
\left(\frac{1}{2}-x_{10}-y_{10}\right) \mathbf{a}_{3}\end{array}$ & $=$ & $\left(\frac{1}{2}-y_{10}\right) a \hat{\mathbf{x}}-x_{10} a \hat{\mathbf{y}}+\left(\frac{1}{4}+z_{10}\right) c \hat{\mathbf{z}}$ & $(16 b)$ & H VII \\
\hline $\mathbf{B}_{80}$ & $=$ & $\begin{array}{c}\left(\frac{1}{4}+x_{10}+z_{10}\right) \mathbf{a}_{1}+ \\
\left(\frac{3}{4}+y_{10}+z_{10}\right) \mathbf{a}_{2}+ \\
\left(\frac{1}{2}+x_{10}+y_{10}\right) \mathbf{a}_{3}\end{array}$ & $=$ & $\left(\frac{1}{2}+y_{10}\right) a \hat{\mathbf{x}}+x_{10} a \hat{\mathbf{y}}+\left(\frac{1}{4}+z_{10}\right) c \hat{\mathbf{z}}$ & $(16 b)$ & H VII \\
\hline $\mathbf{B}_{81}$ & $=$ & $\begin{array}{c}\left(y_{11}+z_{11}\right) \mathbf{a}_{1}+\left(x_{11}+z_{11}\right) \mathbf{a}_{2}+ \\
\left(x_{11}+y_{11}\right) \mathbf{a}_{3}\end{array}$ & $=$ & $x_{11} a \hat{\mathbf{x}}+y_{11} a \hat{\mathbf{y}}+z_{11} c \hat{\mathbf{z}}$ & $(16 b)$ & H VIII \\
\hline $\mathbf{B}_{82}$ & $=$ & $\begin{array}{c}\left(-y_{11}+z_{11}\right) \mathbf{a}_{1}+ \\
\left(-x_{11}+z_{11}\right) \mathbf{a}_{2}+\left(-x_{11}-y_{11}\right) \mathbf{a}_{3}\end{array}$ & $=$ & $-x_{11} a \hat{\mathbf{x}}-y_{11} a \hat{\mathbf{y}}+z_{11} c \hat{\mathbf{z}}$ & $(16 b)$ & H VIII \\
\hline $\mathbf{B}_{83}$ & $=$ & $\begin{array}{c}\left(\frac{3}{4}+x_{11}+z_{11}\right) \mathbf{a}_{1}+ \\
\left(\frac{1}{4}-y_{11}+z_{11}\right) \mathbf{a}_{2}+ \\
\left(\frac{1}{2}+x_{11}-y_{11}\right) \mathbf{a}_{3}\end{array}$ & $=$ & $\begin{array}{c}-y_{11} a \hat{\mathbf{x}}+\left(\frac{1}{2}+x_{11}\right) a \hat{\mathbf{y}}+ \\
\left(\frac{1}{4}+z_{11}\right) c \hat{\mathbf{z}}\end{array}$ & $(16 b)$ & H VIII \\
\hline
\end{tabular}




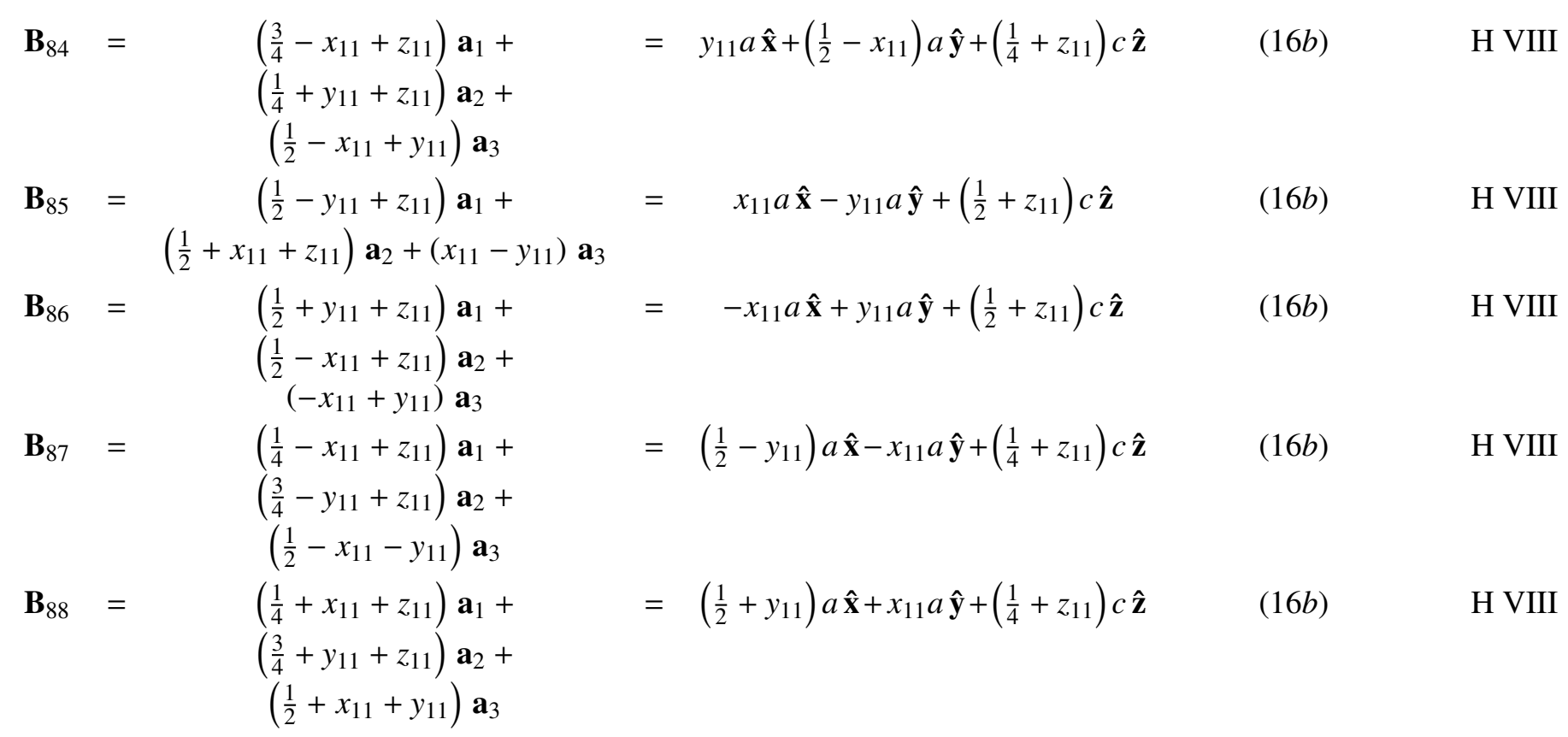

\section{References:}

- D. S. Marynick and W. N. Lipscomb, Crystal structure of beryllium borohydride, Inorg. Chem. 11, 820-823 (1972), doi:10.1021/ic50110a033.

\section{Found in:}

- P. Villars and K. Cenzual, Pearson's Crystal Data - Crystal Structure Database for Inorganic Compounds, ASM International (2013).

\section{Geometry files:}

- CIF: pp. 882

- POSCAR: pp. 882 


\section{$\mathrm{MnF}_{2}$ Structure: A2B_tP12_111_2n_adf}
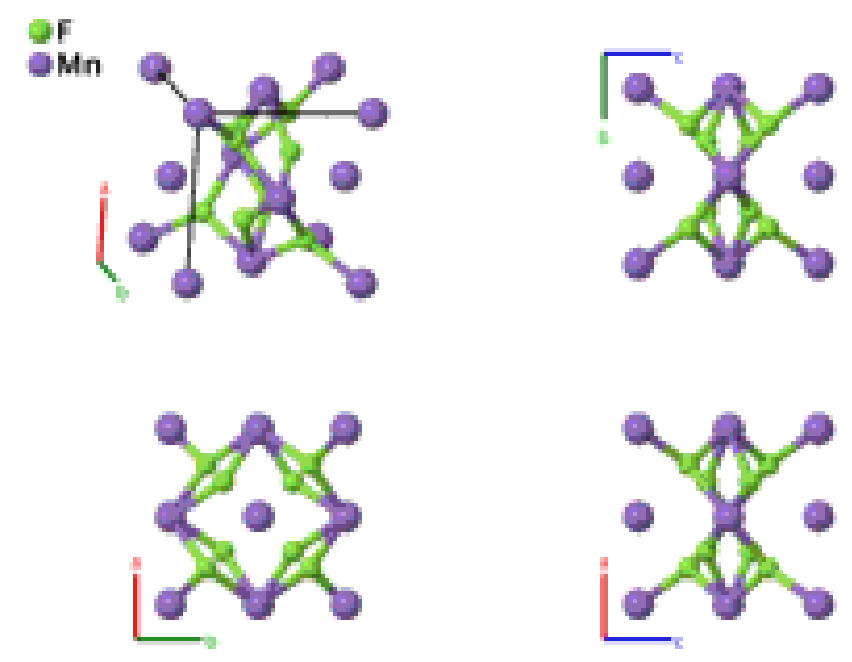

\section{Prototype}

AFLOW prototype label

: $\quad \mathrm{MnF}_{2}$

Strukturbericht designation

: A2B_tP12_111_2n_adf

Pearson symbol

: None

Space group number

: $\quad \mathrm{tP} 12$

Space group symbol

: $\quad 111$

AFLOW prototype command

: $\quad P \overline{4} 2 m$

aflow - -proto $=$ A2B_tP12_111_2n_adf
- -params $=a, c / a, x_{4}, z_{4}, x_{5}, z_{5}$

Simple Tetragonal primitive vectors:
$\mathbf{a}_{1}=a \hat{\mathbf{x}}$
$\mathbf{a}_{2}=a \hat{\mathbf{y}}$
$\mathbf{a}_{3}=c \hat{\mathbf{z}}$
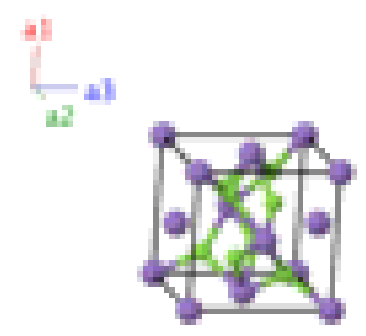

Basis vectors:

Lattice Coordinates

$\begin{array}{lcccc}\mathbf{B}_{1}= & 0 \mathbf{a}_{1}+0 \mathbf{a}_{2}+0 \mathbf{a}_{3} & = & 0 \hat{\mathbf{x}}+0 \hat{\mathbf{y}}+0 \hat{\mathbf{z}} \\ \mathbf{B}_{2}= & \frac{1}{2} \mathbf{a}_{1}+\frac{1}{2} \mathbf{a}_{2} & = & \frac{1}{2} a \hat{\mathbf{x}}+\frac{1}{2} a \hat{\mathbf{y}} \\ \mathbf{B}_{3}= & \frac{1}{2} \mathbf{a}_{1}+\frac{1}{2} \mathbf{a}_{3} & = & \frac{1}{2} a \hat{\mathbf{x}}+\frac{1}{2} c \hat{\mathbf{z}} \\ \mathbf{B}_{4}= & \frac{1}{2} \mathbf{a}_{2}+\frac{1}{2} \mathbf{a}_{3} & = & \frac{1}{2} a \hat{\mathbf{y}}+\frac{1}{2} c \hat{\mathbf{z}} \\ \mathbf{B}_{5}= & x_{4} \mathbf{a}_{1}+x_{4} \mathbf{a}_{2}+z_{4} \mathbf{a}_{3} & = & x_{4} a \hat{\mathbf{x}}+x_{4} a \hat{\mathbf{y}}+z_{4} c \hat{\mathbf{z}} \\ \mathbf{B}_{6}= & -x_{4} \mathbf{a}_{1}-x_{4} \mathbf{a}_{2}+z_{4} \mathbf{a}_{3}= & -x_{4} a \hat{\mathbf{x}}-x_{4} a \hat{\mathbf{y}}+z_{4} c \hat{\mathbf{z}} \\ \mathbf{B}_{7}= & x_{4} \mathbf{a}_{1}-x_{4} \mathbf{a}_{2}-z_{4} \mathbf{a}_{3} & = & x_{4} a \hat{\mathbf{x}}-x_{4} a \hat{\mathbf{y}}-z_{4} c \hat{\mathbf{z}}\end{array}$

Wyckoff Position

(1a)

(4n)

(4n)

$(4 n)$
Atom Type

Mn I

Mn II

Mn III

Mn III

F I

F I

F I 


$\begin{array}{rlllll}\mathbf{B}_{8}=-x_{4} \mathbf{a}_{1}+x_{4} \mathbf{a}_{2}-z_{4} \mathbf{a}_{3} & = & -x_{4} a \hat{\mathbf{x}}+x_{4} a \hat{\mathbf{y}}-z_{4} c \hat{\mathbf{z}} & (4 n) & \text { F I } \\ \mathbf{B}_{9}= & x_{5} \mathbf{a}_{1}+x_{5} \mathbf{a}_{2}+z_{5} \mathbf{a}_{3}=x_{5} a \hat{\mathbf{x}}+x_{5} a \hat{\mathbf{y}}+z_{5} c \hat{\mathbf{z}} & (4 n) & \text { F II } \\ \mathbf{B}_{10}=-x_{5} \mathbf{a}_{1}-x_{5} \mathbf{a}_{2}+z_{5} \mathbf{a}_{3}= & -x_{5} a \hat{\mathbf{x}}-x_{5} a \hat{\mathbf{y}}+z_{5} c \hat{\mathbf{z}} & (4 n) & \text { F II } \\ \mathbf{B}_{11}= & x_{5} \mathbf{a}_{1}-x_{5} \mathbf{a}_{2}-z_{5} \mathbf{a}_{3}= & x_{5} a \hat{\mathbf{x}}-x_{5} a \hat{\mathbf{y}}-z_{5} c \hat{\mathbf{z}} & (4 n) & \text { F II } \\ \mathbf{B}_{12}= & -x_{5} \mathbf{a}_{1}+x_{5} \mathbf{a}_{2}-z_{5} \mathbf{a}_{3}= & -x_{5} a \hat{\mathbf{x}}+x_{5} a \hat{\mathbf{y}}-z_{5} c \hat{\mathbf{z}} & \text { F II }\end{array}$

\section{References:}

- T. Yagi, J. C. Jamieson, and P. B. Moore, Polymorphism in MnF (rutile type) at high pressures, J. Geophys. Res. 84, 1113-1115 (1979), doi:10.1029/JB084iB03p01113.

\section{Found in:}

- P. Villars and K. Cenzual, Pearson's Crystal Data - Crystal Structure Database for Inorganic Compounds, ASM International (2013).

\section{Geometry files:}

- CIF: pp. 883

- POSCAR: pp. 883 

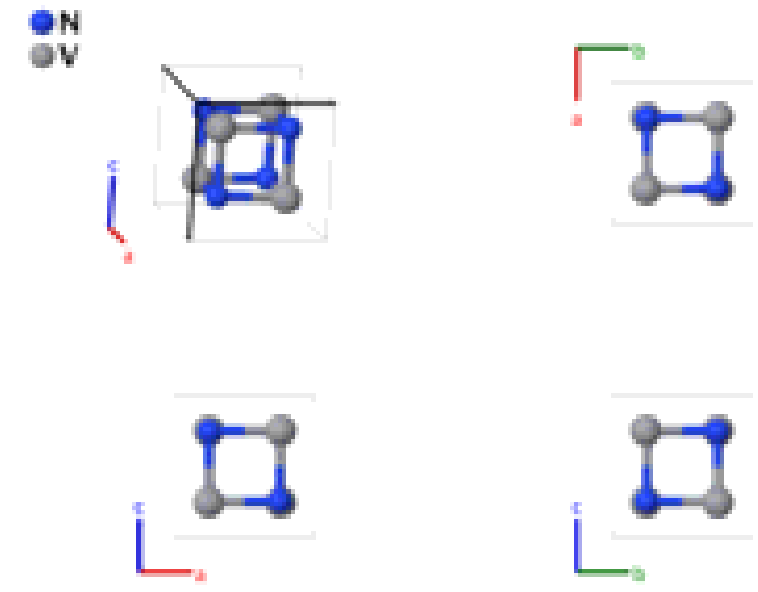

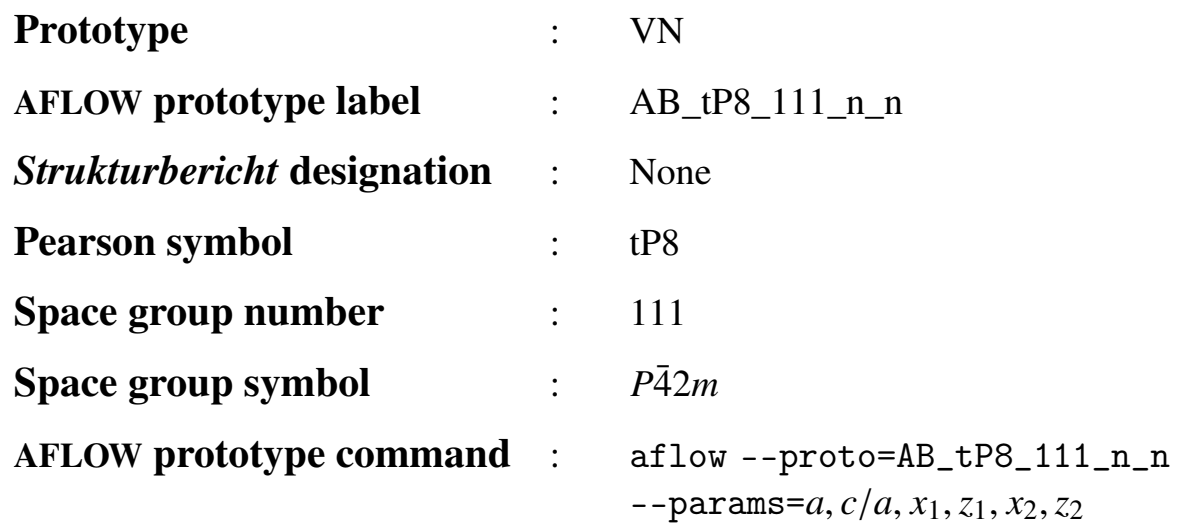

- FINDSYM identifies space group \#111 for this structure (consistent with the reference); however, since $c / a \approx 1$, AFLOW-SYM and Platon identify \#215. Lowering the tolerance value for AFLOW-SYM resolves the expected space group \#111. Space groups \#111 and \#215 are both reasonable classifications since they are commensurate with subgroup relations.

\section{Simple Tetragonal primitive vectors:}

$$
\begin{aligned}
& \mathbf{a}_{1}=a \hat{\mathbf{x}} \\
& \mathbf{a}_{2}=a \hat{\mathbf{y}} \\
& \mathbf{a}_{3}=c \hat{\mathbf{z}}
\end{aligned}
$$

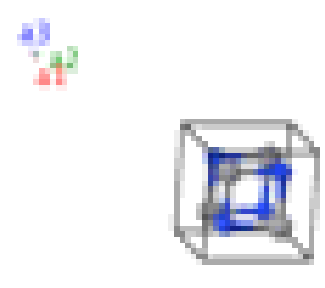

\section{Basis vectors:}

$\begin{array}{llllll} & \text { Lattice Coordinates } & & \text { Cartesian Coordinates } & \text { Wyckoff Position } & \text { Atom Type } \\ \mathbf{B}_{1}= & x_{1} \mathbf{a}_{1}+x_{1} \mathbf{a}_{2}+z_{1} \mathbf{a}_{3} & = & x_{1} a \hat{\mathbf{x}}+x_{1} a \hat{\mathbf{y}}+z_{1} c \hat{\mathbf{z}} & (4 n) & \mathrm{N} \\ \mathbf{B}_{2}= & -x_{1} \mathbf{a}_{1}-x_{1} \mathbf{a}_{2}+z_{1} \mathbf{a}_{3} & = & -x_{1} a \hat{\mathbf{x}}-x_{1} a \hat{\mathbf{y}}+z_{1} c \hat{\mathbf{z}} & (4 n) & \mathrm{N} \\ \mathbf{B}_{3}= & x_{1} \mathbf{a}_{1}-x_{1} \mathbf{a}_{2}-z_{1} \mathbf{a}_{3} & = & x_{1} a \hat{\mathbf{x}}-x_{1} a \hat{\mathbf{y}}-z_{1} c \hat{\mathbf{z}} & (4 n) & \mathrm{N} \\ \mathbf{B}_{4}= & -x_{1} \mathbf{a}_{1}+x_{1} \mathbf{a}_{2}-z_{1} \mathbf{a}_{3} & = & -x_{1} a \hat{\mathbf{x}}+x_{1} a \hat{\mathbf{y}}-z_{1} c \hat{\mathbf{z}} & (4 n) & \mathrm{N}\end{array}$




$\begin{array}{llllll}\mathbf{B}_{5}= & x_{2} \mathbf{a}_{1}+x_{2} \mathbf{a}_{2}+z_{2} \mathbf{a}_{3} & = & x_{2} a \hat{\mathbf{x}}+x_{2} a \hat{\mathbf{y}}+z_{2} c \hat{\mathbf{z}} & (4 n) & \mathrm{V} \\ \mathbf{B}_{6}= & -x_{2} \mathbf{a}_{1}-x_{2} \mathbf{a}_{2}+z_{2} \mathbf{a}_{3}= & -x_{2} a \hat{\mathbf{x}}-x_{2} a \hat{\mathbf{y}}+z_{2} c \hat{\mathbf{z}} & (4 n) & \mathrm{V} \\ \mathbf{B}_{7}= & x_{2} \mathbf{a}_{1}-x_{2} \mathbf{a}_{2}-z_{2} \mathbf{a}_{3}= & x_{2} a \hat{\mathbf{x}}-x_{2} a \hat{\mathbf{y}}-z_{2} c \hat{\mathbf{z}} & (4 n) & \mathrm{V} \\ \mathbf{B}_{8}= & -x_{2} \mathbf{a}_{1}+x_{2} \mathbf{a}_{2}-z_{2} \mathbf{a}_{3}= & -x_{2} a \hat{\mathbf{x}}+x_{2} a \hat{\mathbf{y}}-z_{2} c \hat{\mathbf{z}} & (4 n) & \mathrm{V}\end{array}$

\section{References:}

- F. Kubel, W. Lengauer, K. Yvon, K. Knorr, and A. Junod, Structural phase transition at $205 \mathrm{~K}$ in stoichiometric vanadium nitride, Phys. Rev. B 38, 12908 (1988), doi:10.1103/PhysRevB.38.12908.

- H. T. Stokes and D. M. Hatch, FINDSYM: Program for identifying the space group symmetry of a crystal, J. Appl. Crystallogr. 38, 237-238 (2005), doi:10.1107/S0021889804031528.

- D. Hicks, C. Oses, E. Gossett, G. Gomez, R. H. Taylor, C. Toher, M. J. Mehl, O. Levy, and S. Curtarolo, AFLOW-SYM: platform for the complete, automatic and self-consistent symmetry analysis of crystals, Acta Crystallogr. Sect. A 74, 184-203 (2018), doi:10.1107/S2053273318003066.

- A. L. Spek, Single-crystal structure validation with the program PLATON, J. Appl. Crystallogr. 36, 7-13 (2003), doi:10.1107/S0021889802022112.

\section{Found in:}

- P. Villars and K. Cenzual, Pearson's Crystal Data - Crystal Structure Database for Inorganic Compounds, ASM International (2013).

\section{Geometry files:}

- CIF: pp. 883

- POSCAR: pp. 884 

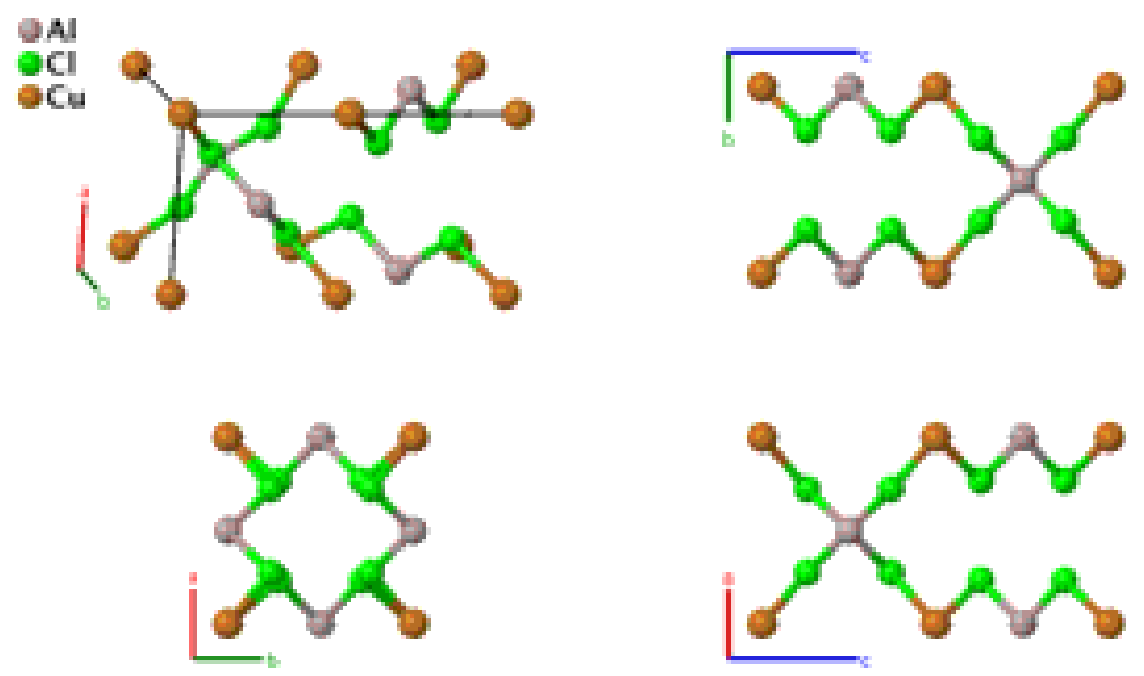

\section{Prototype}

AFLOW prototype label

$: \quad \alpha-\mathrm{CuAlCl}_{4}$

Strukturbericht designation

: AB4C_tP12_112_b_n_e

Pearson symbol

: None

Space group number

: $\quad$ tP12

Space group symbol

$: \quad 112$

AFLOW prototype command

$$
\text { : } \quad P \overline{4} 2 c
$$

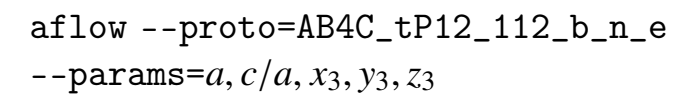

\section{Simple Tetragonal primitive vectors:}

$$
\begin{aligned}
& \mathbf{a}_{1}=a \hat{\mathbf{x}} \\
& \mathbf{a}_{2}=a \hat{\mathbf{y}} \\
& \mathbf{a}_{3}=c \hat{\mathbf{z}}
\end{aligned}
$$

\begin{tabular}{|c|c|c|c|c|c|c|}
\hline & & Lattice Coordinates & & Cartesian Coordinates & Wyckoff Position & Atom Type \\
\hline $\mathbf{B}_{1}$ & $=$ & $\frac{1}{2} \mathbf{a}_{1}+\frac{1}{4} \mathbf{a}_{3}$ & $=$ & $\frac{1}{2} a \hat{\mathbf{x}}+\frac{1}{4} c \hat{\mathbf{z}}$ & $(2 b)$ & $\mathrm{Al}$ \\
\hline $\mathbf{B}_{2}$ & $=$ & $\frac{1}{2} \mathbf{a}_{2}+\frac{3}{4} \mathbf{a}_{3}$ & $=$ & $\frac{1}{2} a \hat{\mathbf{y}}+\frac{3}{4} c \hat{\mathbf{z}}$ & $(2 b)$ & $\mathrm{Al}$ \\
\hline $\mathbf{B}_{3}$ & $=$ & $0 \mathbf{a}_{1}+0 \mathbf{a}_{2}+0 \mathbf{a}_{3}$ & $=$ & $0 \hat{\mathbf{x}}+0 \hat{\mathbf{y}}+0 \hat{\mathbf{z}}$ & $(2 e)$ & $\mathrm{Cu}$ \\
\hline $\mathbf{B}_{4}$ & $=$ & $\frac{1}{2} \mathbf{a}_{3}$ & $=$ & $\frac{1}{2} c \hat{\mathbf{z}}$ & $(2 e)$ & $\mathrm{Cu}$ \\
\hline $\mathbf{B}_{5}$ & $=$ & $x_{3} \mathbf{a}_{1}+y_{3} \mathbf{a}_{2}+z_{3} \mathbf{a}_{3}$ & $=$ & $x_{3} a \hat{\mathbf{x}}+y_{3} a \hat{\mathbf{y}}+z_{3} c \hat{\mathbf{z}}$ & $(8 n)$ & $\mathrm{Cl}$ \\
\hline $\mathbf{B}_{6}$ & $=$ & $-x_{3} \mathbf{a}_{1}-y_{3} \mathbf{a}_{2}+z_{3} \mathbf{a}_{3}$ & $=$ & $-x_{3} a \hat{\mathbf{x}}-y_{3} a \hat{\mathbf{y}}+z_{3} c \hat{\mathbf{z}}$ & $(8 n)$ & $\mathrm{Cl}$ \\
\hline $\mathbf{B}_{7}$ & $=$ & $y_{3} \mathbf{a}_{1}-x_{3} \mathbf{a}_{2}-z_{3} \mathbf{a}_{3}$ & $=$ & $y_{3} a \hat{\mathbf{x}}-x_{3} a \hat{\mathbf{y}}-z_{3} c \hat{\mathbf{z}}$ & $(8 n)$ & $\mathrm{Cl}$ \\
\hline $\mathbf{B}_{8}$ & $=$ & $-y_{3} \mathbf{a}_{1}+x_{3} \mathbf{a}_{2}-z_{3} \mathbf{a}_{3}$ & $=$ & $-y_{3} a \hat{\mathbf{x}}+x_{3} a \hat{\mathbf{y}}-z_{3} c \hat{\mathbf{z}}$ & $(8 n)$ & $\mathrm{Cl}$ \\
\hline
\end{tabular}

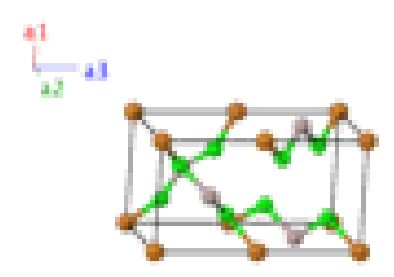

Basis vectors: 


$\mathbf{B}_{9}=-x_{3} \mathbf{a}_{1}+y_{3} \mathbf{a}_{2}+\left(\frac{1}{2}-z_{3}\right) \mathbf{a}_{3}=-x_{3} a \hat{\mathbf{x}}+y_{3} a \hat{\mathbf{y}}+\left(\frac{1}{2}-z_{3}\right) c \hat{\mathbf{z}}$
$\mathbf{B}_{10}=x_{3} \mathbf{a}_{1}-y_{3} \mathbf{a}_{2}+\left(\frac{1}{2}-z_{3}\right) \mathbf{a}_{3}=x_{3} a \hat{\mathbf{x}}-y_{3} a \hat{\mathbf{y}}+\left(\frac{1}{2}-z_{3}\right) c \hat{\mathbf{z}}$
$\mathbf{B}_{11}=-y_{3} \mathbf{a}_{1}-x_{3} \mathbf{a}_{2}+\left(\frac{1}{2}+z_{3}\right) \mathbf{a}_{3}=-y_{3} a \hat{\mathbf{x}}-x_{3} a \hat{\mathbf{y}}+\left(\frac{1}{2}+z_{3}\right) c \hat{\mathbf{z}}$
$\mathbf{B}_{12}=y_{3} \mathbf{a}_{1}+x_{3} \mathbf{a}_{2}+\left(\frac{1}{2}+z_{3}\right) \mathbf{a}_{3}=y_{3} a \hat{\mathbf{x}}+x_{3} a \hat{\mathbf{y}}+\left(\frac{1}{2}+z_{3}\right) c \hat{\mathbf{z}}$

\section{References:}

- J. D. Martin, B. R. Leafblad, R. M. Sullivan, and P. D. Boyle, $\alpha$-and $\beta$-CuAlCl 4 : Framework Construction Using Corner-Shared Tetrahedral Metal-Halide Building Blocks, Inorg. Chem. 37, 1341-1346 (1998), doi:10.1021/ic971148v.

\section{Found in:}

- P. Villars and K. Cenzual, Pearson's Crystal Data - Crystal Structure Database for Inorganic Compounds, ASM International (2013).

\section{Geometry files:}

- CIF: pp. 884

- POSCAR: pp. 884 


\section{Akermanite $\left(\mathrm{Ca}_{2} \mathrm{MgSi}_{2} \mathrm{O}_{7}, S 5_{3}\right)$ Structure: A2BC7D2_tP24_113_e_a_cef_e}
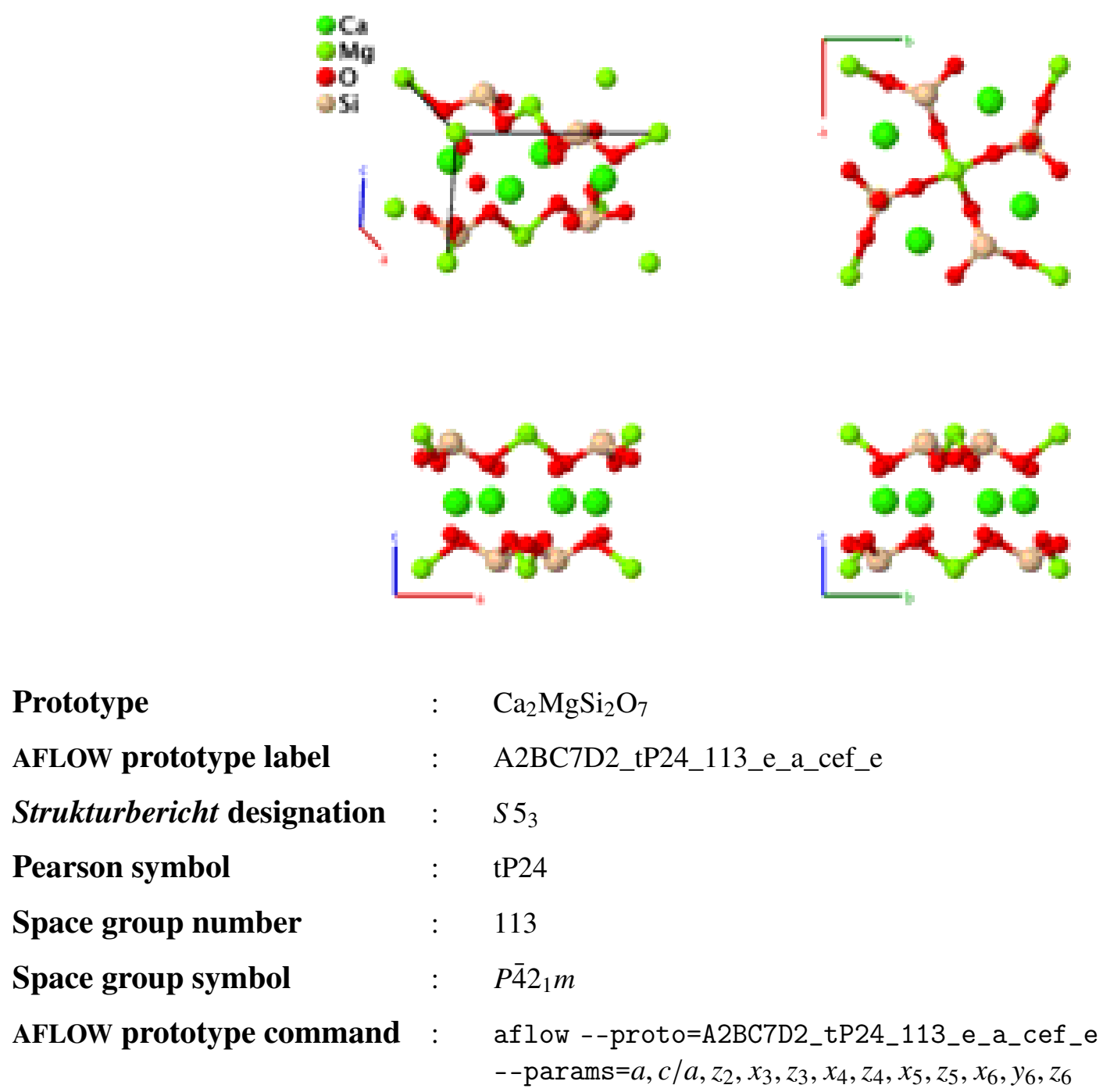

AFLOW prototype label

: $\quad \mathrm{Ca}_{2} \mathrm{MgSi}_{2} \mathrm{O}_{7}$

Strukturbericht designation : A2BC7D2_tP24_113_e_a_cef_e

Pearson symbol $: \quad S 5_{3}$

Space group number

: $\quad$ tP24

Space group symbol

: 113

AFLOW prototype command

: $\quad P \overline{4} 2_{1} m$

\section{Other compounds with this structure:}

- $(\mathrm{Ca}, \mathrm{Na})_{2}(\mathrm{Al}, \mathrm{Mg}, \mathrm{Fe})(\mathrm{Si}, \mathrm{Al})_{2} \mathrm{O}_{7}$ (melilite), $(\mathrm{Ca}, \mathrm{Na})_{2}(\mathrm{Al}, \mathrm{Mg}, \mathrm{Fe})\left(\mathrm{Si}_{2} \mathrm{O}_{7}\right)$ (alumoakermanite), $\mathrm{Ca}_{2} \mathrm{Al}_{2} \mathrm{SiO}_{7}$ (gehlenite), $\mathrm{Ca}_{2} \mathrm{BeSi}_{2} \mathrm{O}_{7}$ (gugiaite), $\mathrm{Ca}_{2} \mathrm{ZnSi}_{2} \mathrm{O}_{7}$ (hardystonite), $\mathrm{Ca}_{2} \mathrm{~B}_{2} \mathrm{SiO}_{7}$ (okayamalite), $(\mathrm{Ca}, \mathrm{Na})_{2}\left(\mathrm{Mg}, \mathrm{Al}, \mathrm{Si}_{3} \mathrm{O}_{7}, \mathrm{Sr}_{2} \mathrm{ZrSi}_{2} \mathrm{O}_{7}\right.$, $\mathrm{Sr}_{2} \mathrm{MnGe}_{2} \mathrm{O}_{7}, \mathrm{Sr}_{2} \mathrm{MnGe}_{2} \mathrm{~S}_{6} \mathrm{O}$

- Akermanite is an end point of the mineral melilite, which, like thortveitite $\left(S 2_{1}\right)$, is a sorosilicate, a mineral containing isolated $\mathrm{Si}_{2} \mathrm{O}_{7}$ or related groups. We have followed (Parthé, 1997) and use akermanite to represent the entire class of materials. We use the ambient pressure data from (Yang, 1997) to describe the structure.

\section{Simple Tetragonal primitive vectors:}

$$
\begin{aligned}
& \mathbf{a}_{1}=a \hat{\mathbf{x}} \\
& \mathbf{a}_{2}=a \hat{\mathbf{y}} \\
& \mathbf{a}_{3}=c \hat{\mathbf{z}}
\end{aligned}
$$

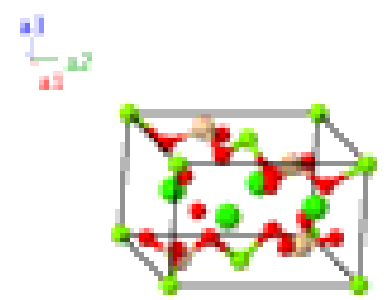


Lattice Coordinates

\begin{tabular}{|c|c|c|}
\hline $\mathbf{B}_{1}$ & $=$ & $0 \mathbf{a}_{1}+0 \mathbf{a}_{2}+0 \mathbf{a}_{3}$ \\
\hline $\mathbf{B}_{2}$ & $=$ & $\frac{1}{2} \mathbf{a}_{1}+\frac{1}{2} \mathbf{a}_{2}$ \\
\hline $\mathbf{B}_{3}$ & $=$ & $\frac{1}{2} \mathbf{a}_{2}+z_{2} \mathbf{a}_{3}$ \\
\hline $\mathbf{B}_{4}$ & $=$ & $\frac{1}{2} \mathbf{a}_{1}+-z_{2} \mathbf{a}_{3}$ \\
\hline $\mathbf{B}_{5}$ & $=$ & $x_{3} \mathbf{a}_{1}+\left(\frac{1}{2}+x_{3}\right) \mathbf{a}_{2}+z_{3} \mathbf{a}_{3}$ \\
\hline $\mathbf{B}_{6}$ & $=$ & $-x_{3} \mathbf{a}_{1}+\left(\frac{1}{2}-x_{3}\right) \mathbf{a}_{2}+z_{3} \mathbf{a}_{3}$ \\
\hline $\mathbf{B}_{7}$ & $=$ & $\left(\frac{1}{2}+x_{3}\right) \mathbf{a}_{1}-x_{3} \mathbf{a}_{2}-z_{3} \mathbf{a}_{3}$ \\
\hline $\mathbf{B}_{8}$ & $=$ & $\left(\frac{1}{2}-x_{3}\right) \mathbf{a}_{1}+x_{3} \mathbf{a}_{2}-z_{3} \mathbf{a}_{3}$ \\
\hline $\mathbf{B}_{9}$ & $=$ & $x_{4} \mathbf{a}_{1}+\left(\frac{1}{2}+x_{4}\right) \mathbf{a}_{2}+z_{4} \mathbf{a}_{3}$ \\
\hline $\mathbf{B}_{10}$ & $=$ & $-x_{4} \mathbf{a}_{1}+\left(\frac{1}{2}-x_{4}\right) \mathbf{a}_{2}+z_{4} \mathbf{a}_{3}$ \\
\hline $\mathbf{B}_{11}$ & $=$ & $\left(\frac{1}{2}+x_{4}\right) \mathbf{a}_{1}-x_{4} \mathbf{a}_{2}-z_{4} \mathbf{a}_{3}$ \\
\hline $\mathbf{B}_{12}$ & $=$ & $\left(\frac{1}{2}-x_{4}\right) \mathbf{a}_{1}+x_{4} \mathbf{a}_{2}-z_{4} \mathbf{a}_{3}$ \\
\hline $\mathbf{B}_{13}$ & $=$ & $x_{5} \mathbf{a}_{1}+\left(\frac{1}{2}+x_{5}\right) \mathbf{a}_{2}+z_{5} \mathbf{a}_{3}$ \\
\hline $\mathbf{B}_{14}$ & $=$ & $-x_{5} \mathbf{a}_{1}+\left(\frac{1}{2}-x_{5}\right) \mathbf{a}_{2}+z_{5} \mathbf{a}_{3}$ \\
\hline $\mathbf{B}_{15}$ & $=$ & $\left(\frac{1}{2}+x_{5}\right) \mathbf{a}_{1}-x_{5} \mathbf{a}_{2}-z_{5} \mathbf{a}_{3}$ \\
\hline $\mathbf{B}_{16}$ & $=$ & $\left(\frac{1}{2}-x_{5}\right) \mathbf{a}_{1}+x_{5} \mathbf{a}_{2}-z_{5} \mathbf{a}_{3}$ \\
\hline $\mathbf{B}_{17}$ & $=$ & $x_{6} \mathbf{a}_{1}+y_{6} \mathbf{a}_{2}+z_{6} \mathbf{a}_{3}$ \\
\hline $\mathbf{B}_{18}$ & $=$ & $-x_{6} \mathbf{a}_{1}-y_{6} \mathbf{a}_{2}+z_{6} \mathbf{a}_{3}$ \\
\hline $\mathbf{B}_{19}$ & $=$ & $y_{6} \mathbf{a}_{1}-x_{6} \mathbf{a}_{2}-z_{6} \mathbf{a}_{3}$ \\
\hline $\mathbf{B}_{20}$ & $=$ & $-y_{6} \mathbf{a}_{1}+x_{6} \mathbf{a}_{2}-z_{6} \mathbf{a}_{3}$ \\
\hline $\mathbf{B}_{21}$ & $=$ & $\left(\frac{1}{2}-x_{6}\right) \mathbf{a}_{1}+\left(\frac{1}{2}+y_{6}\right) \mathbf{a}_{2}-z_{6} \mathbf{a}_{3}$ \\
\hline $\mathbf{B}_{22}$ & $=$ & $\left(\frac{1}{2}+x_{6}\right) \mathbf{a}_{1}+\left(\frac{1}{2}-y_{6}\right) \mathbf{a}_{2}-z_{6} \mathbf{a}_{3}$ \\
\hline $\mathbf{B}_{23}$ & $=$ & $\left(\frac{1}{2}-y_{6}\right) \mathbf{a}_{1}+\left(\frac{1}{2}-x_{6}\right) \mathbf{a}_{2}+z_{6} \mathbf{a}_{3}$ \\
\hline $\mathbf{B}_{24}$ & $=$ & $\left(\frac{1}{2}+y_{6}\right) \mathbf{a}_{1}+\left(\frac{1}{2}+x_{6}\right) \mathbf{a}_{2}+z_{6} \mathbf{a}_{3}$ \\
\hline
\end{tabular}

Cartesian Coordinates

$$
\begin{array}{ll}
= & 0 \hat{\mathbf{x}}+0 \hat{\mathbf{y}}+0 \hat{\mathbf{z}} \\
= & \frac{1}{2} a \hat{\mathbf{x}}+\frac{1}{2} a \hat{\mathbf{y}} \\
= & \frac{1}{2} a \hat{\mathbf{y}}+z_{2} c \hat{\mathbf{z}} \\
= & \frac{1}{2} a \hat{\mathbf{x}}+-z_{2} c \hat{\mathbf{z}}
\end{array}
$$$$
=\quad x_{3} a \hat{\mathbf{x}}+\left(\frac{1}{2}+x_{3}\right) a \hat{\mathbf{y}}+z_{3} c \hat{\mathbf{z}}
$$$$
=\quad-x_{3} a \hat{\mathbf{x}}+\left(\frac{1}{2}-x_{3}\right) a \hat{\mathbf{y}}+z_{3} c \hat{\mathbf{z}}
$$$$
=\left(\frac{1}{2}+x_{3}\right) a \hat{\mathbf{x}}-x_{3} a \hat{\mathbf{y}}-z_{3} c \hat{\mathbf{z}}
$$$$
=\left(\frac{1}{2}-x_{3}\right) a \hat{\mathbf{x}}+x_{3} a \hat{\mathbf{y}}-z_{3} c \hat{\mathbf{z}}
$$$$
=\quad x_{4} a \hat{\mathbf{x}}+\left(\frac{1}{2}+x_{4}\right) a \hat{\mathbf{y}}+z_{4} c \hat{\mathbf{z}}
$$$$
=\quad-x_{4} a \hat{\mathbf{x}}+\left(\frac{1}{2}-x_{4}\right) a \hat{\mathbf{y}}+z_{4} c \hat{\mathbf{z}}
$$$$
=\left(\frac{1}{2}+x_{4}\right) a \hat{\mathbf{x}}-x_{4} a \hat{\mathbf{y}}-z_{4} c \hat{\mathbf{z}}
$$$$
=\left(\frac{1}{2}-x_{4}\right) a \hat{\mathbf{x}}+x_{4} a \hat{\mathbf{y}}-z_{4} c \hat{\mathbf{z}}
$$$$
=\quad x_{5} a \hat{\mathbf{x}}+\left(\frac{1}{2}+x_{5}\right) a \hat{\mathbf{y}}+z_{5} c \hat{\mathbf{z}}
$$$$
=\quad-x_{5} a \hat{\mathbf{x}}+\left(\frac{1}{2}-x_{5}\right) a \hat{\mathbf{y}}+z_{5} c \hat{\mathbf{z}}
$$$$
=\left(\frac{1}{2}+x_{5}\right) a \hat{\mathbf{x}}-x_{5} a \hat{\mathbf{y}}-z_{5} c \hat{\mathbf{z}}
$$$$
=\left(\frac{1}{2}-x_{5}\right) a \hat{\mathbf{x}}+x_{5} a \hat{\mathbf{y}}-z_{5} c \hat{\mathbf{z}}
$$$$
=\quad x_{6} a \hat{\mathbf{x}}+y_{6} a \hat{\mathbf{y}}+z_{6} c \hat{\mathbf{z}}
$$$$
=\quad-x_{6} a \hat{\mathbf{x}}-y_{6} a \hat{\mathbf{y}}+z_{6} c \hat{\mathbf{z}}
$$$$
=\quad y_{6} a \hat{\mathbf{x}}-x_{6} a \hat{\mathbf{y}}-z_{6} c \hat{\mathbf{z}}
$$$$
=\quad-y_{6} a \hat{\mathbf{x}}+x_{6} a \hat{\mathbf{y}}-z_{6} c \hat{\mathbf{z}}
$$$$
=\left(\frac{1}{2}-x_{6}\right) a \hat{\mathbf{x}}+\left(\frac{1}{2}+y_{6}\right) a \hat{\mathbf{y}}-z_{6} c \hat{\mathbf{z}}
$$$$
=\left(\frac{1}{2}+x_{6}\right) a \hat{\mathbf{x}}+\left(\frac{1}{2}-y_{6}\right) a \hat{\mathbf{y}}-z_{6} c \hat{\mathbf{z}}
$$$$
=\left(\frac{1}{2}-y_{6}\right) a \hat{\mathbf{x}}+\left(\frac{1}{2}-x_{6}\right) a \hat{\mathbf{y}}+z_{6} c \hat{\mathbf{z}}
$$$$
=\left(\frac{1}{2}+y_{6}\right) a \hat{\mathbf{x}}+\left(\frac{1}{2}+x_{6}\right) a \hat{\mathbf{y}}+z_{6} c \hat{\mathbf{z}}
$$

Wyckoff Position
Atom Type

$\mathrm{Mg}$

$\mathrm{Mg}$

O I

O I

$\mathrm{Ca}$

$\mathrm{Ca}$

$\mathrm{Ca}$

$\mathrm{Ca}$

O II

O II

O II

O II

$\mathrm{Si}$

$\mathrm{Si}$

$\mathrm{Si}$

$\mathrm{Si}$

O III

O III

O III

O III

O III

O III

O III

O III

\section{References:}

- H. Yang, R. M. Hazen, R. T. Downs, and L. W. Finger, Structural change associated with the incommensurate-normal phase transition in akermanite, $\mathrm{Ca}_{2} \mathrm{MgSi}_{2} \mathrm{O}_{7}$, at high pressure, Phys. Chem. Miner. 24, 510-519 (1997), doi:10.1007/s002690050066.

- E. Parthé, L. Gelato, B. Chabot, M. Penso, K. Cenzula, and R. Gladyshevskii, Standardized Data and Crystal Chemical Characterization of Inorganic Structure Types, Gmelin Handbook of Inorganic and Organometallic Chemistry, vol. 2 (Springer-Verlag, Berlin, Heidelberg, 1993), 8 edn., doi:10.1007/978-3-662-02909-1_3.

\section{Geometry files:}

- CIF: pp. 884

- POSCAR: pp. 885 


\section{$\mathrm{SeO}_{3}$ Structure: A3B_tP32_114_3e_e}
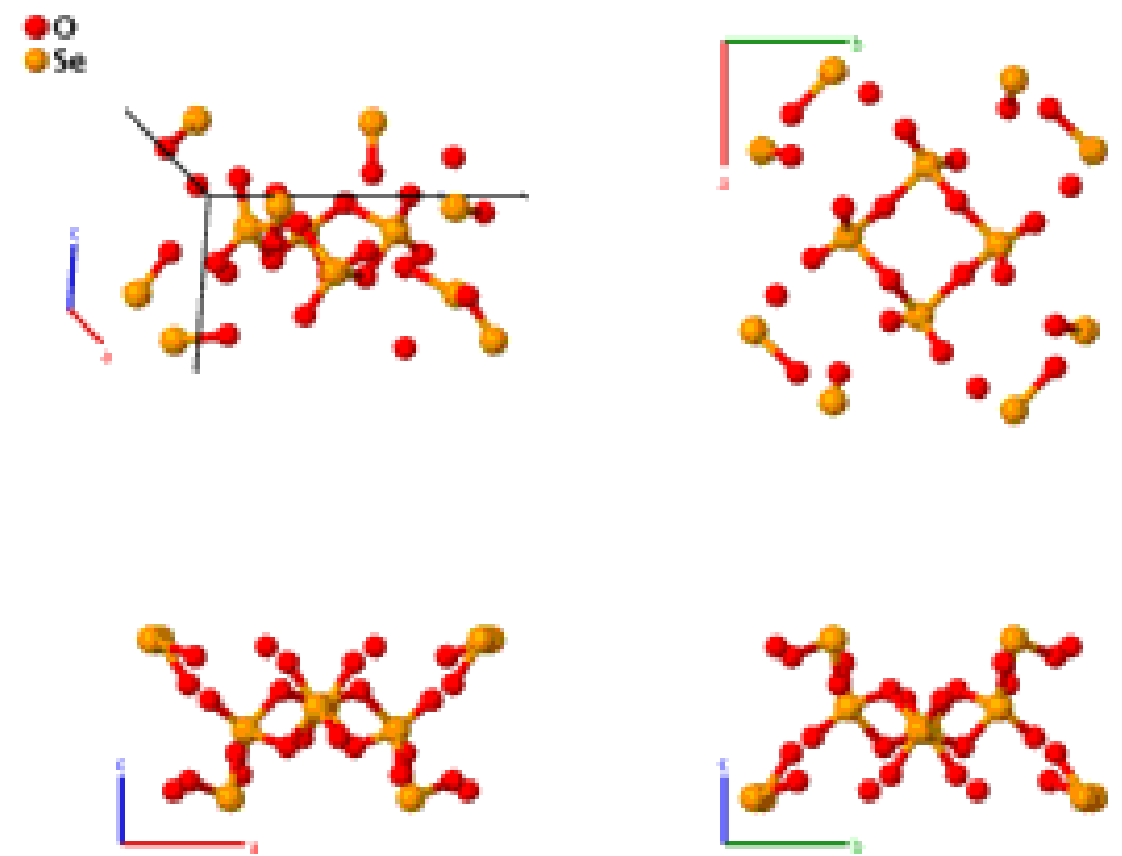

Prototype

AFLOW prototype label

Strukturbericht designation

Pearson symbol

Space group number

Space group symbol

AFLOW prototype command
$: \quad \mathrm{SeO}_{3}$

: A3B_tP32_114_3e_e

: None

: $\quad$ tP32

: $\quad 114$

: $\quad P \overline{4} 2{ }_{1} c$

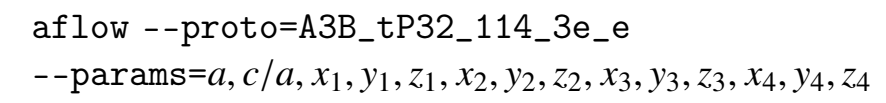

\section{Simple Tetragonal primitive vectors:}

$$
\begin{aligned}
& \mathbf{a}_{1}=a \hat{\mathbf{x}} \\
& \mathbf{a}_{2}=a \hat{\mathbf{y}} \\
& \mathbf{a}_{3}=c \hat{\mathbf{z}}
\end{aligned}
$$
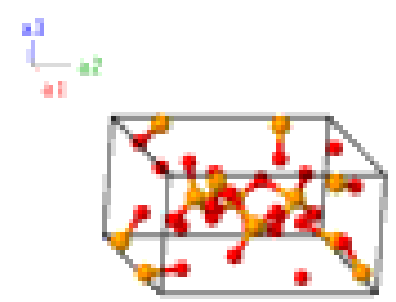

Basis vectors:

Lattice Coordinates

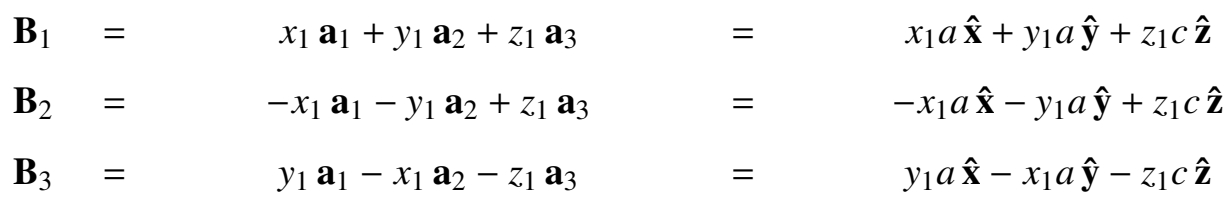

$\mathbf{B}_{1}=x_{1} \mathbf{a}_{1}+y_{1} \mathbf{a}_{2}+z_{1} \mathbf{a}_{3}$

$=$
Cartesian Coordinates

Wyckoff Position

Atom Type

$\begin{array}{ll}(8 e) & \text { O I } \\ (8 e) & \text { O I } \\ (8 e) & \text { O I }\end{array}$




\begin{tabular}{|c|c|c|c|c|c|c|}
\hline $\mathbf{B}_{4}$ & $=$ & $-y_{1} \mathbf{a}_{1}+x_{1} \mathbf{a}_{2}-z_{1} \mathbf{a}_{3}$ & $=$ & $-y_{1} a \hat{\mathbf{x}}+x_{1} a \hat{\mathbf{y}}-z_{1} c \hat{\mathbf{z}}$ & $(8 e)$ & O I \\
\hline $\mathbf{B}_{5}$ & $=$ & $\begin{array}{c}\left(\frac{1}{2}-x_{1}\right) \mathbf{a}_{1}+\left(\frac{1}{2}+y_{1}\right) \mathbf{a}_{2}+ \\
\left(\frac{1}{2}-z_{1}\right) \mathbf{a}_{3}\end{array}$ & $=$ & $\begin{array}{c}\left(\frac{1}{2}-x_{1}\right) a \hat{\mathbf{x}}+\left(\frac{1}{2}+y_{1}\right) a \hat{\mathbf{y}}+ \\
\left(\frac{1}{2}-z_{1}\right) c \hat{\mathbf{z}}\end{array}$ & $(8 e)$ & $\mathrm{O} \mathrm{I}$ \\
\hline $\mathbf{B}_{6}$ & $=$ & $\begin{array}{c}\left(\frac{1}{2}+x_{1}\right) \mathbf{a}_{1}+\left(\frac{1}{2}-y_{1}\right) \mathbf{a}_{2}+ \\
\left(\frac{1}{2}-z_{1}\right) \mathbf{a}_{3}\end{array}$ & $=$ & $\begin{array}{c}\left(\frac{1}{2}+x_{1}\right) a \hat{\mathbf{x}}+\left(\frac{1}{2}-y_{1}\right) a \hat{\mathbf{y}}+ \\
\left(\frac{1}{2}-z_{1}\right) c \hat{\mathbf{z}}\end{array}$ & $(8 e)$ & $\mathrm{O} \mathrm{I}$ \\
\hline $\mathbf{B}_{7}$ & $=$ & $\begin{array}{c}\left(\frac{1}{2}-y_{1}\right) \mathbf{a}_{1}+\left(\frac{1}{2}-x_{1}\right) \mathbf{a}_{2}+ \\
\left(\frac{1}{2}+z_{1}\right) \mathbf{a}_{3}\end{array}$ & $=$ & $\begin{array}{c}\left(\frac{1}{2}-y_{1}\right) a \hat{\mathbf{x}}+\left(\frac{1}{2}-x_{1}\right) a \hat{\mathbf{y}}+ \\
\left(\frac{1}{2}+z_{1}\right) c \hat{\mathbf{z}}\end{array}$ & $(8 e)$ & $\mathrm{O} \mathrm{I}$ \\
\hline $\mathbf{B}_{8}$ & $=$ & $\begin{array}{c}\left(\frac{1}{2}+y_{1}\right) \mathbf{a}_{1}+\left(\frac{1}{2}+x_{1}\right) \mathbf{a}_{2}+ \\
\left(\frac{1}{2}+z_{1}\right) \mathbf{a}_{3}\end{array}$ & $=$ & $\begin{array}{c}\left(\frac{1}{2}+y_{1}\right) a \hat{\mathbf{x}}+\left(\frac{1}{2}+x_{1}\right) a \hat{\mathbf{y}}+ \\
\left(\frac{1}{2}+z_{1}\right) c \hat{\mathbf{z}}\end{array}$ & $(8 e)$ & O I \\
\hline $\mathbf{B}_{9}$ & $=$ & $x_{2} \mathbf{a}_{1}+y_{2} \mathbf{a}_{2}+z_{2} \mathbf{a}_{3}$ & $=$ & $x_{2} a \hat{\mathbf{x}}+y_{2} a \hat{\mathbf{y}}+z_{2} c \hat{\mathbf{z}}$ & $(8 e)$ & $\mathrm{O}$ II \\
\hline $\mathbf{B}_{10}$ & $=$ & $-x_{2} \mathbf{a}_{1}-y_{2} \mathbf{a}_{2}+z_{2} \mathbf{a}_{3}$ & $=$ & $-x_{2} a \hat{\mathbf{x}}-y_{2} a \hat{\mathbf{y}}+z_{2} c \hat{\mathbf{z}}$ & $(8 e)$ & O II \\
\hline $\mathbf{B}_{11}$ & $=$ & $y_{2} \mathbf{a}_{1}-x_{2} \mathbf{a}_{2}-z_{2} \mathbf{a}_{3}$ & $=$ & $y_{2} a \hat{\mathbf{x}}-x_{2} a \hat{\mathbf{y}}-z_{2} c \hat{\mathbf{z}}$ & $(8 e)$ & O II \\
\hline $\mathbf{B}_{12}$ & $=$ & $-y_{2} \mathbf{a}_{1}+x_{2} \mathbf{a}_{2}-z_{2} \mathbf{a}_{3}$ & $=$ & $-y_{2} a \hat{\mathbf{x}}+x_{2} a \hat{\mathbf{y}}-z_{2} c \hat{\mathbf{z}}$ & $(8 e)$ & O II \\
\hline $\mathbf{B}_{13}$ & $=$ & $\begin{array}{c}\left(\frac{1}{2}-x_{2}\right) \mathbf{a}_{1}+\left(\frac{1}{2}+y_{2}\right) \mathbf{a}_{2}+ \\
\left(\frac{1}{2}-z_{2}\right) \mathbf{a}_{3}\end{array}$ & $=$ & $\begin{array}{c}\left(\frac{1}{2}-x_{2}\right) a \hat{\mathbf{x}}+\left(\frac{1}{2}+y_{2}\right) a \hat{\mathbf{y}}+ \\
\left(\frac{1}{2}-z_{2}\right) c \hat{\mathbf{z}}\end{array}$ & $(8 e)$ & O II \\
\hline $\mathbf{B}_{14}$ & $=$ & $\begin{array}{c}\left(\frac{1}{2}+x_{2}\right) \mathbf{a}_{1}+\left(\frac{1}{2}-y_{2}\right) \mathbf{a}_{2}+ \\
\left(\frac{1}{2}-z_{2}\right) \mathbf{a}_{3}\end{array}$ & $=$ & $\begin{array}{c}\left(\frac{1}{2}+x_{2}\right) a \hat{\mathbf{x}}+\left(\frac{1}{2}-y_{2}\right) a \hat{\mathbf{y}}+ \\
\left(\frac{1}{2}-z_{2}\right) c \hat{\mathbf{z}}\end{array}$ & $(8 e)$ & O II \\
\hline $\mathbf{B}_{15}$ & $=$ & $\begin{array}{c}\left(\frac{1}{2}-y_{2}\right) \mathbf{a}_{1}+\left(\frac{1}{2}-x_{2}\right) \mathbf{a}_{2}+ \\
\left(\frac{1}{2}+z_{2}\right) \mathbf{a}_{3}\end{array}$ & $=$ & $\begin{array}{c}\left(\frac{1}{2}-y_{2}\right) a \hat{\mathbf{x}}+\left(\frac{1}{2}-x_{2}\right) a \hat{\mathbf{y}}+ \\
\left(\frac{1}{2}+z_{2}\right) c \hat{\mathbf{z}}\end{array}$ & $(8 e)$ & O II \\
\hline $\mathbf{B}_{16}$ & $=$ & $\begin{array}{c}\left(\frac{1}{2}+y_{2}\right) \mathbf{a}_{1}+\left(\frac{1}{2}+x_{2}\right) \mathbf{a}_{2}+ \\
\left(\frac{1}{2}+z_{2}\right) \mathbf{a}_{3}\end{array}$ & $=$ & $\begin{array}{c}\left(\frac{1}{2}+y_{2}\right) a \hat{\mathbf{x}}+\left(\frac{1}{2}+x_{2}\right) a \hat{\mathbf{y}}+ \\
\left(\frac{1}{2}+z_{2}\right) c \hat{\mathbf{z}}\end{array}$ & $(8 e)$ & O II \\
\hline $\mathbf{B}_{17}$ & $=$ & $x_{3} \mathbf{a}_{1}+y_{3} \mathbf{a}_{2}+z_{3} \mathbf{a}_{3}$ & $=$ & $x_{3} a \hat{\mathbf{x}}+y_{3} a \hat{\mathbf{y}}+z_{3} c \hat{\mathbf{z}}$ & $(8 e)$ & O III \\
\hline $\mathbf{B}_{18}$ & $=$ & $-x_{3} \mathbf{a}_{1}-y_{3} \mathbf{a}_{2}+z_{3} \mathbf{a}_{3}$ & $=$ & $-x_{3} a \hat{\mathbf{x}}-y_{3} a \hat{\mathbf{y}}+z_{3} c \hat{\mathbf{z}}$ & $(8 e)$ & O III \\
\hline $\mathbf{B}_{19}$ & $=$ & $y_{3} \mathbf{a}_{1}-x_{3} \mathbf{a}_{2}-z_{3} \mathbf{a}_{3}$ & $=$ & $y_{3} a \hat{\mathbf{x}}-x_{3} a \hat{\mathbf{y}}-z_{3} c \hat{\mathbf{z}}$ & $(8 e)$ & O III \\
\hline $\mathbf{B}_{20}$ & $=$ & $-y_{3} \mathbf{a}_{1}+x_{3} \mathbf{a}_{2}-z_{3} \mathbf{a}_{3}$ & $=$ & $-y_{3} a \hat{\mathbf{x}}+x_{3} a \hat{\mathbf{y}}-z_{3} c \hat{\mathbf{z}}$ & $(8 e)$ & O III \\
\hline $\mathbf{B}_{21}$ & $=$ & $\begin{array}{c}\left(\frac{1}{2}-x_{3}\right) \mathbf{a}_{1}+\left(\frac{1}{2}+y_{3}\right) \mathbf{a}_{2}+ \\
\left(\frac{1}{2}-z_{3}\right) \mathbf{a}_{3}\end{array}$ & $=$ & $\begin{array}{c}\left(\frac{1}{2}-x_{3}\right) a \hat{\mathbf{x}}+\left(\frac{1}{2}+y_{3}\right) a \hat{\mathbf{y}}+ \\
\left(\frac{1}{2}-z_{3}\right) c \hat{\mathbf{z}}\end{array}$ & $(8 e)$ & O III \\
\hline $\mathbf{B}_{22}$ & $=$ & $\begin{array}{c}\left(\frac{1}{2}+x_{3}\right) \mathbf{a}_{1}+\left(\frac{1}{2}-y_{3}\right) \mathbf{a}_{2}+ \\
\left(\frac{1}{2}-z_{3}\right) \mathbf{a}_{3}\end{array}$ & $=$ & $\begin{array}{c}\left(\frac{1}{2}+x_{3}\right) a \hat{\mathbf{x}}+\left(\frac{1}{2}-y_{3}\right) a \hat{\mathbf{y}}+ \\
\left(\frac{1}{2}-z_{3}\right) c \hat{\mathbf{z}}\end{array}$ & $(8 e)$ & O III \\
\hline $\mathbf{B}_{23}$ & $=$ & $\begin{array}{c}\left(\frac{1}{2}-y_{3}\right) \mathbf{a}_{1}+\left(\frac{1}{2}-x_{3}\right) \mathbf{a}_{2}+ \\
\left(\frac{1}{2}+z_{3}\right) \mathbf{a}_{3}\end{array}$ & $=$ & $\begin{array}{c}\left(\frac{1}{2}-y_{3}\right) a \hat{\mathbf{x}}+\left(\frac{1}{2}-x_{3}\right) a \hat{\mathbf{y}}+ \\
\left(\frac{1}{2}+z_{3}\right) c \hat{\mathbf{z}}\end{array}$ & $(8 e)$ & O III \\
\hline $\mathbf{B}_{24}$ & $=$ & $\begin{array}{c}\left(\frac{1}{2}+y_{3}\right) \mathbf{a}_{1}+\left(\frac{1}{2}+x_{3}\right) \mathbf{a}_{2}+ \\
\left(\frac{1}{2}+z_{3}\right) \mathbf{a}_{3}\end{array}$ & $=$ & $\begin{array}{c}\left(\frac{1}{2}+y_{3}\right) a \hat{\mathbf{x}}+\left(\frac{1}{2}+x_{3}\right) a \hat{\mathbf{y}}+ \\
\left(\frac{1}{2}+z_{3}\right) c \hat{\mathbf{z}}\end{array}$ & $(8 e)$ & O III \\
\hline $\mathbf{B}_{25}$ & $=$ & $x_{4} \mathbf{a}_{1}+y_{4} \mathbf{a}_{2}+z_{4} \mathbf{a}_{3}$ & $=$ & $x_{4} a \hat{\mathbf{x}}+y_{4} a \hat{\mathbf{y}}+z_{4} c \hat{\mathbf{z}}$ & $(8 e)$ & $\mathrm{Se}$ \\
\hline $\mathbf{B}_{26}$ & $=$ & $-x_{4} \mathbf{a}_{1}-y_{4} \mathbf{a}_{2}+z_{4} \mathbf{a}_{3}$ & $=$ & $-x_{4} a \hat{\mathbf{x}}-y_{4} a \hat{\mathbf{y}}+z_{4} c \hat{\mathbf{z}}$ & $(8 e)$ & $\mathrm{Se}$ \\
\hline $\mathbf{B}_{27}$ & $=$ & $y_{4} \mathbf{a}_{1}-x_{4} \mathbf{a}_{2}-z_{4} \mathbf{a}_{3}$ & $=$ & $y_{4} a \hat{\mathbf{x}}-x_{4} a \hat{\mathbf{y}}-z_{4} c \hat{\mathbf{z}}$ & $(8 e)$ & $\mathrm{Se}$ \\
\hline $\mathbf{B}_{28}$ & $=$ & $-y_{4} \mathbf{a}_{1}+x_{4} \mathbf{a}_{2}-z_{4} \mathbf{a}_{3}$ & $=$ & $-y_{4} a \hat{\mathbf{x}}+x_{4} a \hat{\mathbf{y}}-z_{4} c \hat{\mathbf{z}}$ & $(8 e)$ & $\mathrm{Se}$ \\
\hline $\mathbf{B}_{29}$ & $=$ & $\begin{array}{c}\left(\frac{1}{2}-x_{4}\right) \mathbf{a}_{1}+\left(\frac{1}{2}+y_{4}\right) \mathbf{a}_{2}+ \\
\left(\frac{1}{2}-z_{4}\right) \mathbf{a}_{3}\end{array}$ & $=$ & $\begin{array}{c}\left(\frac{1}{2}-x_{4}\right) a \hat{\mathbf{x}}+\left(\frac{1}{2}+y_{4}\right) a \hat{\mathbf{y}}+ \\
\left(\frac{1}{2}-z_{4}\right) c \hat{\mathbf{z}}\end{array}$ & $(8 e)$ & $\mathrm{Se}$ \\
\hline
\end{tabular}




\begin{tabular}{|c|c|c|c|c|c|}
\hline $\mathbf{B}_{30}$ & $=$ & $\begin{array}{c}\left(\frac{1}{2}+x_{4}\right) \mathbf{a}_{1}+\left(\frac{1}{2}-y_{4}\right) \mathbf{a}_{2}+ \\
\left(\frac{1}{2}-z_{4}\right) \mathbf{a}_{3}\end{array}$ & $=$ & $\begin{array}{c}\left(\frac{1}{2}+x_{4}\right) a \hat{\mathbf{x}}+\left(\frac{1}{2}-y_{4}\right) a \hat{\mathbf{y}}+ \\
\left(\frac{1}{2}-z_{4}\right) c \hat{\mathbf{z}}\end{array}$ & $(8 e)$ \\
\hline $\mathbf{B}_{31}$ & $=$ & $\begin{array}{c}\left(\frac{1}{2}-y_{4}\right) \mathbf{a}_{1}+\left(\frac{1}{2}-x_{4}\right) \mathbf{a}_{2}+ \\
\left(\frac{1}{2}+z_{4}\right) \mathbf{a}_{3}\end{array}$ & $=$ & $\begin{array}{c}\left(\frac{1}{2}-y_{4}\right) a \hat{\mathbf{x}}+\left(\frac{1}{2}-x_{4}\right) a \hat{\mathbf{y}}+ \\
\left(\frac{1}{2}+z_{4}\right) c \hat{\mathbf{z}}\end{array}$ & $(8 e)$ \\
\hline $\mathbf{B}_{32}$ & $=$ & $\begin{array}{c}\left(\frac{1}{2}+y_{4}\right) \mathbf{a}_{1}+\left(\frac{1}{2}+x_{4}\right) \mathbf{a}_{2}+ \\
\left(\frac{1}{2}+z_{4}\right) \mathbf{a}_{3}\end{array}$ & $=$ & $\begin{array}{c}\left(\frac{1}{2}+y_{4}\right) a \hat{\mathbf{x}}+\left(\frac{1}{2}+x_{4}\right) a \hat{\mathbf{y}}+ \\
\left(\frac{1}{2}+z_{4}\right) c \hat{\mathbf{z}}\end{array}$ & $(8 e)$ \\
\hline
\end{tabular}

\section{References:}

- F. C. Mijlhoff and C. H. MacGillavry, Symmetry and unit-cell dimensions of selenium trioxide, Acta Cryst. 15, 620-620 (1962), doi:10.1107/S0365110X62001644.

\section{Found in:}

- P. Villars and K. Cenzual, Pearson's Crystal Data - Crystal Structure Database for Inorganic Compounds, ASM International (2013).

\section{Geometry files:}

- CIF: pp. 885

- POSCAR: pp. 885 


\section{$\mathrm{Pd}_{4}$ Se Structure: A4B_tP10_114_e_a}
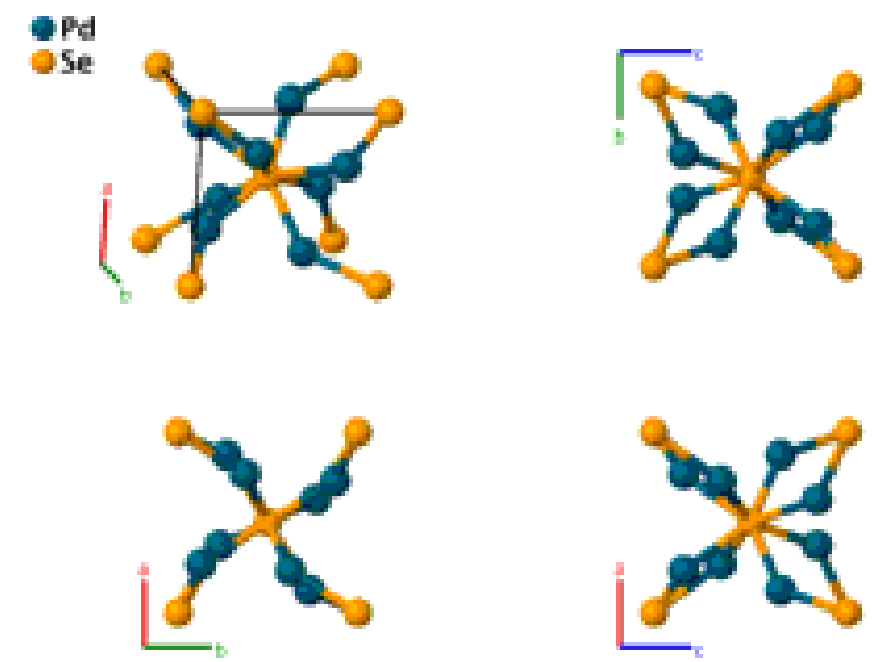

Prototype

$\mathrm{Pd}_{4} \mathrm{Se}$

AFLOW prototype label

: A4B_tP10_114_e_a

Strukturbericht designation

Pearson symbol

: None

Space group number

: $\quad$ P10

Space group symbol

: 114

AFLOW prototype command

: $\quad P \overline{4} 2_{1} c$

aflow - -proto $=A 4 B \_t P 10 \_114 \_$__a

- params $=a, c / a, x_{2}, y_{2}, z_{2}$

\section{Simple Tetragonal primitive vectors:}

$$
\begin{aligned}
& \mathbf{a}_{1}=a \hat{\mathbf{x}} \\
& \mathbf{a}_{2}=a \hat{\mathbf{y}} \\
& \mathbf{a}_{3}=c \hat{\mathbf{z}}
\end{aligned}
$$

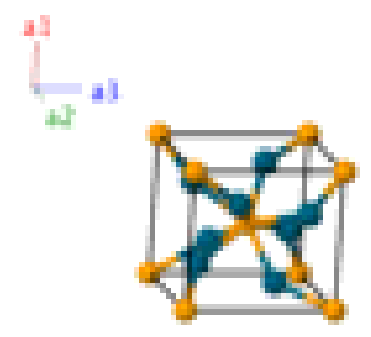

Basis vectors:

Lattice Coordinates

$\begin{array}{llc}\mathbf{B}_{1}= & 0 \mathbf{a}_{1}+0 \mathbf{a}_{2}+0 \mathbf{a}_{3} \\ \mathbf{B}_{2}= & \frac{1}{2} \mathbf{a}_{1}+\frac{1}{2} \mathbf{a}_{2}+\frac{1}{2} \mathbf{a}_{3} \\ \mathbf{B}_{3}= & x_{2} \mathbf{a}_{1}+y_{2} \mathbf{a}_{2}+z_{2} \mathbf{a}_{3} \\ \mathbf{B}_{4}= & -x_{2} \mathbf{a}_{1}-y_{2} \mathbf{a}_{2}+z_{2} \mathbf{a}_{3} \\ \mathbf{B}_{5}= & y_{2} \mathbf{a}_{1}-x_{2} \mathbf{a}_{2}-z_{2} \mathbf{a}_{3} \\ \mathbf{B}_{6}= & -y_{2} \mathbf{a}_{1}+x_{2} \mathbf{a}_{2}-z_{2} \mathbf{a}_{3}\end{array}$

$\mathbf{B}_{1}=$

$-y_{2} \mathbf{a}_{1}+x_{2} \mathbf{a}_{2}-z_{2} \mathbf{a}_{3}$

$\mathbf{B}_{6}=$
Cartesian Coordinates

$$
0 \hat{\mathbf{x}}+0 \hat{\mathbf{y}}+0 \hat{\mathbf{z}}
$$$$
\frac{1}{2} a \hat{\mathbf{x}}+\frac{1}{2} a \hat{\mathbf{y}}+\frac{1}{2} c \hat{\mathbf{z}}
$$$$
x_{2} a \hat{\mathbf{x}}+y_{2} a \hat{\mathbf{y}}+z_{2} c \hat{\mathbf{z}}
$$$$
-x_{2} a \hat{\mathbf{x}}-y_{2} a \hat{\mathbf{y}}+z_{2} c \hat{\mathbf{z}}
$$$$
y_{2} a \hat{\mathbf{x}}-x_{2} a \hat{\mathbf{y}}-z_{2} c \hat{\mathbf{z}}
$$$$
-y_{2} a \hat{\mathbf{x}}+x_{2} a \hat{\mathbf{y}}-z_{2} c \hat{\mathbf{z}}
$$

Wyckoff Position Atom Type

(2a) $\mathrm{Se}$

(2a) $\mathrm{Se}$ $\mathrm{Pd}$ Pd Pd

$(8 e)$ 


\begin{tabular}{|c|c|c|c|c|c|}
\hline $\mathbf{B}_{7}$ & $=$ & $\begin{array}{c}\left(\frac{1}{2}-x_{2}\right) \mathbf{a}_{1}+\left(\frac{1}{2}+y_{2}\right) \mathbf{a}_{2}+ \\
\left(\frac{1}{2}-z_{2}\right) \mathbf{a}_{3}\end{array}$ & $=$ & $\begin{array}{c}\left(\frac{1}{2}-x_{2}\right) a \hat{\mathbf{x}}+\left(\frac{1}{2}+y_{2}\right) a \hat{\mathbf{y}}+ \\
\left(\frac{1}{2}-z_{2}\right) c \hat{\mathbf{z}}\end{array}$ & $(8 e)$ \\
\hline $\mathbf{B}_{8}$ & $=$ & $\begin{array}{c}\left(\frac{1}{2}+x_{2}\right) \mathbf{a}_{1}+\left(\frac{1}{2}-y_{2}\right) \mathbf{a}_{2}+ \\
\left(\frac{1}{2}-z_{2}\right) \mathbf{a}_{3}\end{array}$ & $=$ & $\begin{array}{c}\left(\frac{1}{2}+x_{2}\right) a \hat{\mathbf{x}}+\left(\frac{1}{2}-y_{2}\right) a \hat{\mathbf{y}}+ \\
\left(\frac{1}{2}-z_{2}\right) c \hat{\mathbf{z}}\end{array}$ & $(8 e)$ \\
\hline $\mathbf{B}_{9}$ & $=$ & $\begin{array}{c}\left(\frac{1}{2}-y_{2}\right) \mathbf{a}_{1}+\left(\frac{1}{2}-x_{2}\right) \mathbf{a}_{2}+ \\
\left(\frac{1}{2}+z_{2}\right) \mathbf{a}_{3}\end{array}$ & $=$ & $\begin{array}{c}\left(\frac{1}{2}-y_{2}\right) a \hat{\mathbf{x}}+\left(\frac{1}{2}-x_{2}\right) a \hat{\mathbf{y}}+ \\
\left(\frac{1}{2}+z_{2}\right) c \hat{\mathbf{z}}\end{array}$ & $(8 e)$ \\
\hline $\mathbf{B}_{10}$ & $=$ & $\begin{array}{c}\left(\frac{1}{2}+y_{2}\right) \mathbf{a}_{1}+\left(\frac{1}{2}+x_{2}\right) \mathbf{a}_{2}+ \\
\left(\frac{1}{2}+z_{2}\right) \mathbf{a}_{3}\end{array}$ & $=$ & $\begin{array}{c}\left(\frac{1}{2}+y_{2}\right) a \hat{\mathbf{x}}+\left(\frac{1}{2}+x_{2}\right) a \hat{\mathbf{y}}+ \\
\left(\frac{1}{2}+z_{2}\right) c \hat{\mathbf{z}}\end{array}$ & $(8 e)$ \\
\hline
\end{tabular}

\section{References:}

- F. Grønvold and E. Røst, On the sulfides, selenides and tellurides of palladium, Acta Chem. Scand. 10, 1620-1634 (1956), doi:10.3891/acta.chem.scand.10-1620.

\section{Found in:}

- P. Villars and K. Cenzual, Pearson's Crystal Data - Crystal Structure Database for Inorganic Compounds, ASM International (2013).

\section{Geometry files:}

- CIF: pp. 885

- POSCAR: pp. 886 


\section{$\mathrm{Rh}_{3} \mathrm{P}_{2}$ Structure: A2B3_tP5_115_g_ag}
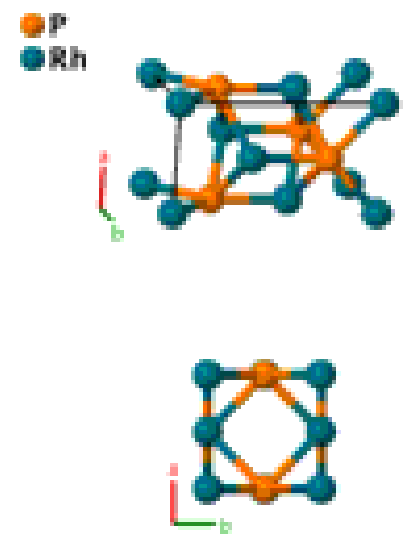
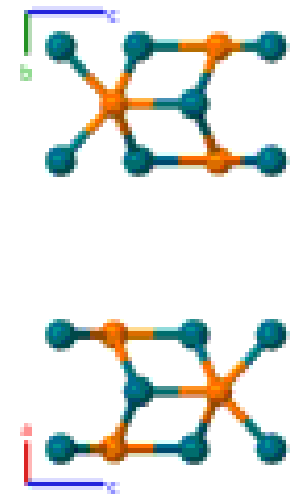

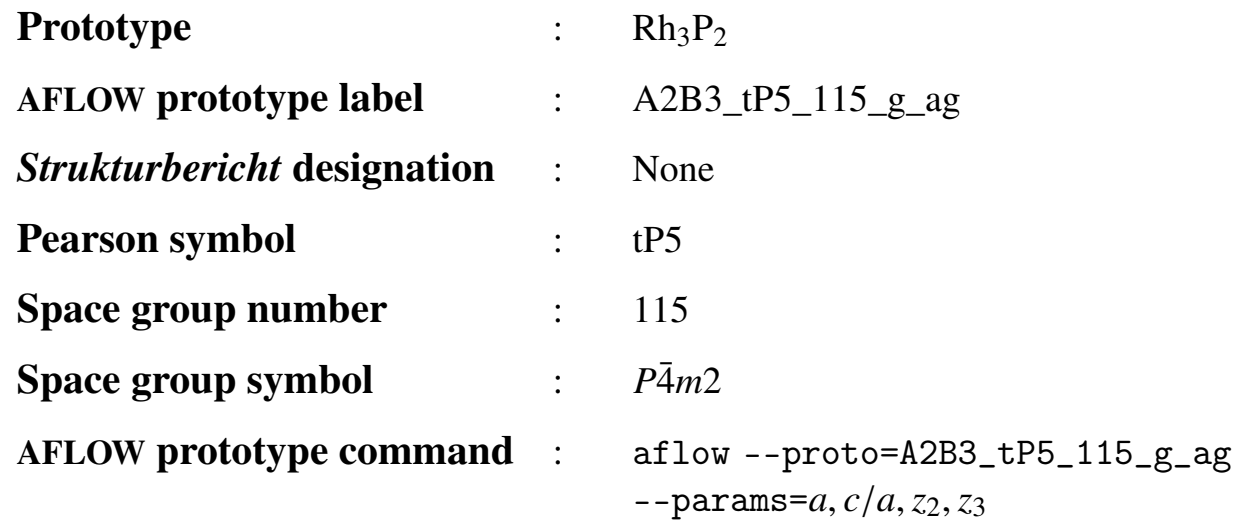

Simple Tetragonal primitive vectors:

$$
\begin{aligned}
& \mathbf{a}_{1}=a \hat{\mathbf{x}} \\
& \mathbf{a}_{2}=a \hat{\mathbf{y}} \\
& \mathbf{a}_{3}=c \hat{\mathbf{z}}
\end{aligned}
$$

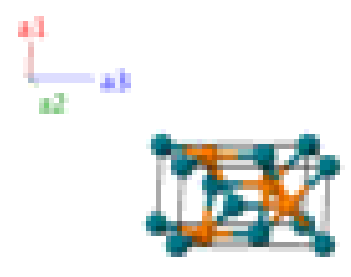

Basis vectors:

Lattice Coordinates

$\mathbf{B}_{1}=0 \mathbf{a}_{1}+0 \mathbf{a}_{2}+0 \mathbf{a}_{3}$

$\mathbf{B}_{2}=$

$\frac{1}{2} \mathbf{a}_{2}+z_{2} \mathbf{a}_{3}$

$\mathbf{B}_{3}=$

$\mathbf{B}_{4}=$

$\mathbf{B}_{5}=$ $\frac{1}{2} \mathbf{a}_{1}+-z_{2} \mathbf{a}_{3}$

$\frac{1}{2} \mathbf{a}_{2}+z_{3} \mathbf{a}_{3}$

$\frac{1}{2} \mathbf{a}_{1}+-z_{3} \mathbf{a}_{3}$
Cartesian Coordinates

$=$

$0 \hat{\mathbf{x}}+0 \hat{\mathbf{y}}+0 \hat{\mathbf{z}}$

$\frac{1}{2} a \hat{\mathbf{y}}+z_{2} c \hat{\mathbf{z}}$

$\frac{1}{2} a \hat{\mathbf{x}}+-z_{2} c \hat{\mathbf{z}}$

$\frac{1}{2} a \hat{\mathbf{y}}+z_{3} c \hat{\mathbf{z}}$

$\frac{1}{2} a \hat{\mathbf{x}}+-z_{3} c \hat{\mathbf{z}}$
Wyckoff Position

(1a)
Atom Type

Rh I

$\mathrm{P}$

$\mathrm{P}$

$\mathrm{Rh}$ II

$\mathrm{Rh}$ II

\section{References:}

- E. H. El Ghadraoui, R. Guerin, and M. Sergent, Diphosphure de trirhodium, $R h_{3} P_{2}$ : premier exemple d'une structure lacunaire ordonnée de type anti-PbFCl, Acta Crystallogr. C 39, 1493-1494 (1983), doi:10.1107/S0108270183009002.

Found in: 
- P. Villars and K. Cenzual, Pearson's Crystal Data - Crystal Structure Database for Inorganic Compounds, ASM International (2013).

\section{Geometry files:}

- CIF: pp. 886

- POSCAR: pp. 886 


\section{$\mathrm{HgI}_{2}$ Structure: AB2_tP12_115_j_egi}
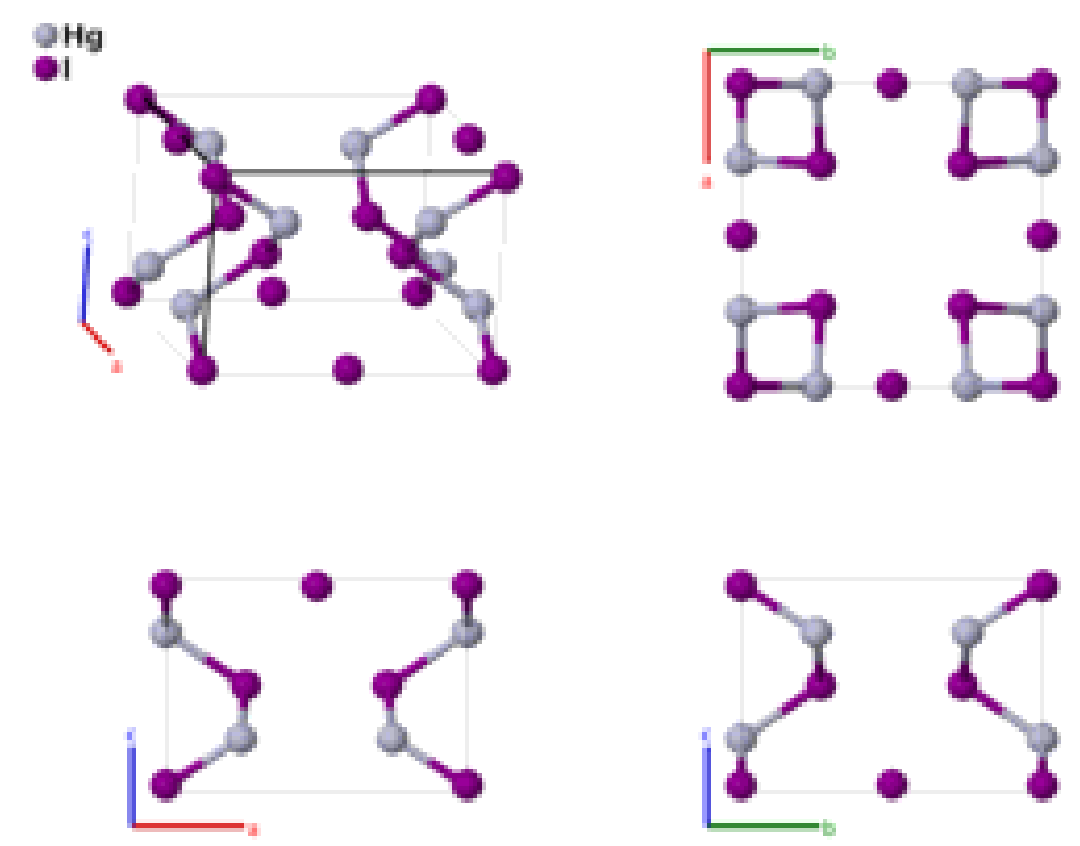

\section{Prototype}

AFLOW prototype label

Strukturbericht designation

Pearson symbol

Space group number

Space group symbol

AFLOW prototype command
: $\quad \mathrm{HgI}_{2}$

$$
\text { : AB2_tP12_115_j_egi }
$$

: None

: $\quad$ tP12

: 115

: $\quad P \overline{4} m 2$

aflow - -proto=AB2_tP12_115_j_egi

- -params $=a, c / a, z_{1}, z_{2}, x_{3}, x_{4}, z_{4}$

Simple Tetragonal primitive vectors:

$$
\begin{aligned}
& \mathbf{a}_{1}=a \hat{\mathbf{x}} \\
& \mathbf{a}_{2}=a \hat{\mathbf{y}} \\
& \mathbf{a}_{3}=c \hat{\mathbf{z}}
\end{aligned}
$$

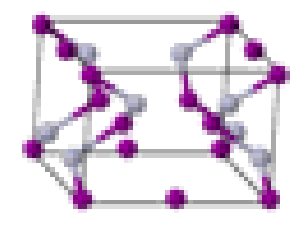

Basis vectors:

Lattice Coordinates

$\begin{array}{ccc}\mathbf{B}_{1} & = & z_{1} \mathbf{a}_{3} \\ \mathbf{B}_{2} & = & -z_{1} \mathbf{a}_{3} \\ \mathbf{B}_{3} & = & \frac{1}{2} \mathbf{a}_{2}+z_{2} \mathbf{a}_{3} \\ \mathbf{B}_{4} & = & \frac{1}{2} \mathbf{a}_{1}+-z_{2} \mathbf{a}_{3}\end{array}$

$\mathbf{B}_{1}=$

$\mathbf{B}_{2}=$

$=$

$=$

$=$

$=$
Wyckoff Position

$(2 e)$
Atom Type 


\begin{tabular}{|c|c|c|c|c|c|c|}
\hline $\mathbf{B}_{5}$ & $=$ & $x_{3} \mathbf{a}_{1}+x_{3} \mathbf{a}_{2}+\frac{1}{2} \mathbf{a}_{3}$ & $=$ & $x_{3} a \hat{\mathbf{x}}+x_{3} a \hat{\mathbf{y}}+\frac{1}{2} c \hat{\mathbf{z}}$ & $(4 i)$ & I III \\
\hline $\mathbf{B}_{6}$ & $=$ & $-x_{3} \mathbf{a}_{1}-x_{3} \mathbf{a}_{2}+\frac{1}{2} \mathbf{a}_{3}$ & $=$ & $-x_{3} a \hat{\mathbf{x}}-x_{3} a \hat{\mathbf{y}}+\frac{1}{2} c \hat{\mathbf{z}}$ & $(4 i)$ & I III \\
\hline $\mathbf{B}_{7}$ & $=$ & $x_{3} \mathbf{a}_{1}-x_{3} \mathbf{a}_{2}+\frac{1}{2} \mathbf{a}_{3}$ & $=$ & $x_{3} a \hat{\mathbf{x}}-x_{3} a \hat{\mathbf{y}}+\frac{1}{2} c \hat{\mathbf{z}}$ & $(4 i)$ & I III \\
\hline $\mathbf{B}_{8}$ & $=$ & $-x_{3} \mathbf{a}_{1}+x_{3} \mathbf{a}_{2}+\frac{1}{2} \mathbf{a}_{3}$ & $=$ & $-x_{3} a \hat{\mathbf{x}}+x_{3} a \hat{\mathbf{y}}+\frac{1}{2} c \hat{\mathbf{z}}$ & $(4 i)$ & I III \\
\hline $\mathbf{B}_{9}$ & $=$ & $x_{4} \mathbf{a}_{1}+z_{4} \mathbf{a}_{3}$ & $=$ & $x_{4} a \hat{\mathbf{x}}+z_{4} c \hat{\mathbf{z}}$ & $(4 j)$ & $\mathrm{Hg}$ \\
\hline $\mathbf{B}_{10}$ & $=$ & $-x_{4} \mathbf{a}_{1}+z_{4} \mathbf{a}_{3}$ & $=$ & $-x_{4} a \hat{\mathbf{x}}+z_{4} c \hat{\mathbf{z}}$ & $(4 j)$ & $\mathrm{Hg}$ \\
\hline $\mathbf{B}_{11}$ & $=$ & $-x_{4} \mathbf{a}_{2}-z_{4} \mathbf{a}_{3}$ & $=$ & $-x_{4} a \hat{\mathbf{y}}-z_{4} c \hat{\mathbf{z}}$ & $(4 j)$ & $\mathrm{Hg}$ \\
\hline $\mathbf{B}_{12}$ & $=$ & $x_{4} \mathbf{a}_{2}-z_{4} \mathbf{a}_{3}$ & $=$ & $x_{4} a \hat{\mathbf{y}}-z_{4} c \hat{\mathbf{z}}$ & $(4 j)$ & $\mathrm{Hg}$ \\
\hline
\end{tabular}

\section{References:}

- M. Hostettler, H. Birkedal, and D. Schwarzenbach, The structure of orange HgI 2 . I. Polytypic layer structure, Acta Crystallogr. Sect. B Struct. Sci. 58, 903-913 (2002), doi:10.1107/S010876810201618X.

\section{Found in:}

- P. Villars and K. Cenzual, Pearson's Crystal Data - Crystal Structure Database for Inorganic Compounds, ASM International (2013).

\section{Geometry files:}

- CIF: pp. 886

- POSCAR: pp. 887 


\section{$\mathrm{Ru}_{2} \mathrm{Sn}_{3}$ Structure: A2B3_tP20_116_bci_fj}
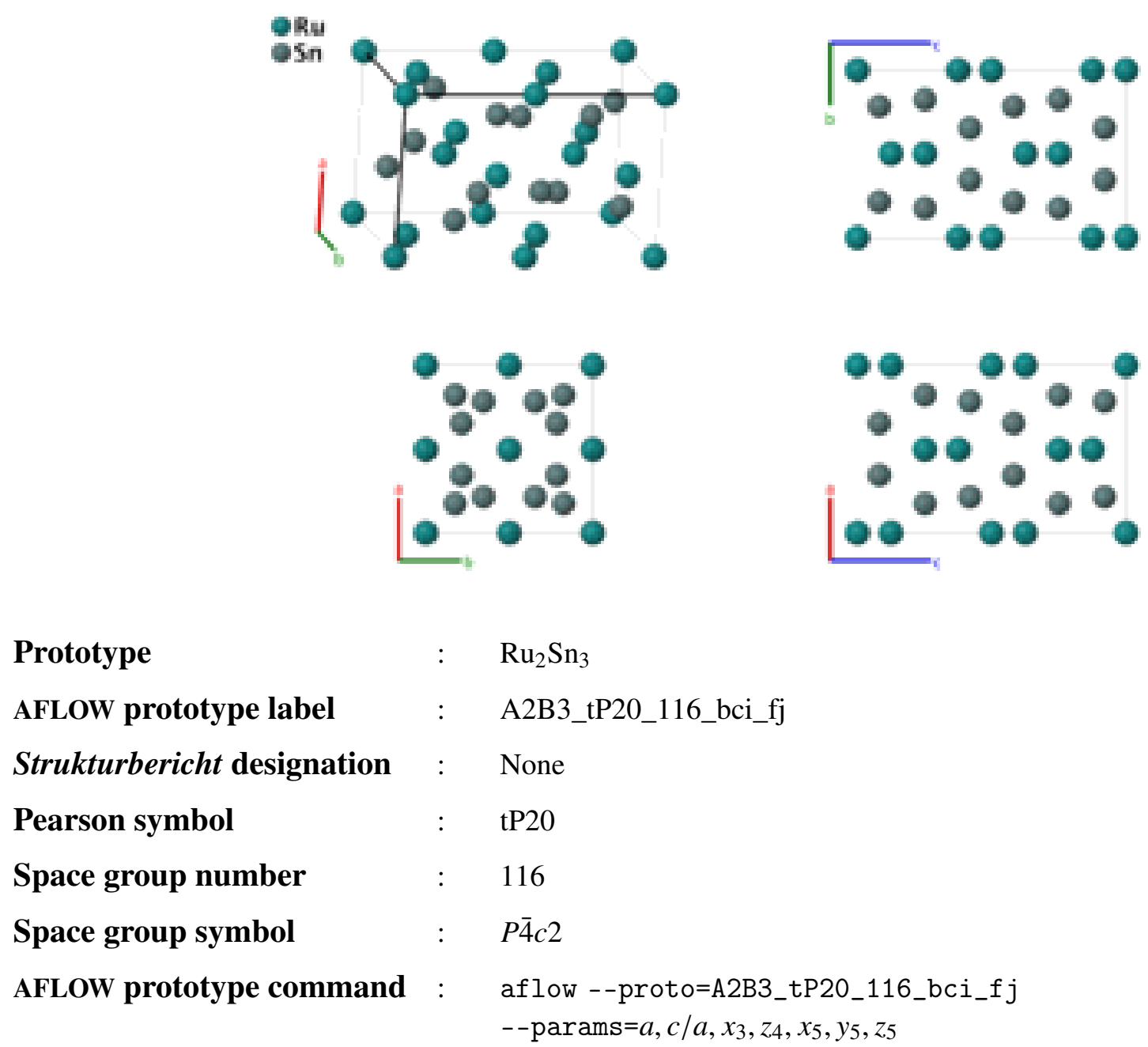

Simple Tetragonal primitive vectors:

$$
\begin{aligned}
& \mathbf{a}_{1}=a \hat{\mathbf{x}} \\
& \mathbf{a}_{2}=a \hat{\mathbf{y}} \\
& \mathbf{a}_{3}=c \hat{\mathbf{z}}
\end{aligned}
$$

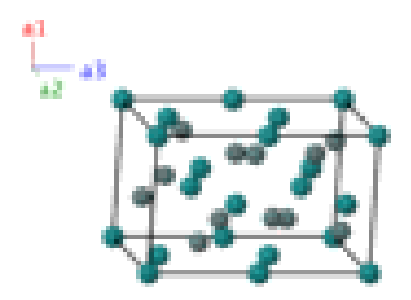

Basis vectors:

Lattice Coordinates

$\mathbf{B}_{1}=\quad \frac{1}{2} \mathbf{a}_{1}+\frac{1}{2} \mathbf{a}_{2}+\frac{1}{4} \mathbf{a}_{3}$

$\mathbf{B}_{2}=\frac{1}{2} \mathbf{a}_{1}+\frac{1}{2} \mathbf{a}_{2}+\frac{3}{4} \mathbf{a}_{3}$

$\mathbf{B}_{3}=$

$\mathbf{B}_{4}=$

$\mathbf{B}_{5}=$

$\mathbf{B}_{6}=$

$\mathbf{B}_{7}=$

$$
0 \mathbf{a}_{1}+0 \mathbf{a}_{2}+0 \mathbf{a}_{3}
$$$$
\frac{1}{2} \mathbf{a}_{3}
$$

$x_{3} \mathbf{a}_{1}+x_{3} \mathbf{a}_{2}+\frac{3}{4} \mathbf{a}_{3}$

$-x_{3} \mathbf{a}_{1}-x_{3} \mathbf{a}_{2}+\frac{3}{4} \mathbf{a}_{3}$

$x_{3} \mathbf{a}_{1}-x_{3} \mathbf{a}_{2}+\frac{1}{4} \mathbf{a}_{3}$
Cartesian Coordinates

$=$

$=$

$=$

$=$

$=$

$=$

$=$

$$
\frac{1}{2} a \hat{\mathbf{x}}+\frac{1}{2} a \hat{\mathbf{y}}+\frac{1}{4} c \hat{\mathbf{z}}
$$$$
\frac{1}{2} a \hat{\mathbf{x}}+\frac{1}{2} a \hat{\mathbf{y}}+\frac{3}{4} c \hat{\mathbf{z}}
$$$$
0 \hat{\mathbf{x}}+0 \hat{\mathbf{y}}+0 \hat{\mathbf{z}}
$$$$
\frac{1}{2} c \hat{\mathbf{z}}
$$$$
x_{3} a \hat{\mathbf{x}}+x_{3} a \hat{\mathbf{y}}+\frac{3}{4} c \hat{\mathbf{z}}
$$$$
-x_{3} a \hat{\mathbf{x}}-x_{3} a \hat{\mathbf{y}}+\frac{3}{4} c \hat{\mathbf{z}}
$$$$
x_{3} a \hat{\mathbf{x}}-x_{3} a \hat{\mathbf{y}}+\frac{1}{4} c \hat{\mathbf{z}}
$$

Wyckoff Position

$(2 b)$

$(4 f)$
Atom Type

$\mathrm{Ru} I$

$\mathrm{Ru} I$

$\mathrm{Ru}$ II

Ru II

Sn I

Sn I

Sn I 


\begin{tabular}{|c|c|c|c|c|c|c|}
\hline $\mathbf{B}_{8}$ & $=$ & $-x_{3} \mathbf{a}_{1}+x_{3} \mathbf{a}_{2}+\frac{1}{4} \mathbf{a}_{3}$ & $=$ & $-x_{3} a \hat{\mathbf{x}}+x_{3} a \hat{\mathbf{y}}+\frac{1}{4} c \hat{\mathbf{z}}$ & $(4 f)$ & Sn I \\
\hline $\mathbf{B}_{9}$ & $=$ & $\frac{1}{2} \mathbf{a}_{2}+z_{4} \mathbf{a}_{3}$ & $=$ & $\frac{1}{2} a \hat{\mathbf{y}}+z_{4} c \hat{\mathbf{z}}$ & $(4 i)$ & $\mathrm{Ru}$ III \\
\hline $\mathbf{B}_{10}$ & $=$ & $\frac{1}{2} \mathbf{a}_{1}+-z_{4} \mathbf{a}_{3}$ & $=$ & $\frac{1}{2} a \hat{\mathbf{x}}+-z_{4} c \hat{\mathbf{z}}$ & $(4 i)$ & $\mathrm{Ru}$ III \\
\hline $\mathbf{B}_{11}$ & $=$ & $\frac{1}{2} \mathbf{a}_{2}+\left(\frac{1}{2}+z_{4}\right) \mathbf{a}_{3}$ & $=$ & $\frac{1}{2} a \hat{\mathbf{y}}+\left(\frac{1}{2}+z_{4}\right) c \hat{\mathbf{z}}$ & $(4 i)$ & $\mathrm{Ru}$ III \\
\hline $\mathbf{B}_{12}$ & $=$ & $\frac{1}{2} \mathbf{a}_{1}+\left(\frac{1}{2}-z_{4}\right) \mathbf{a}_{3}$ & $=$ & $\frac{1}{2} a \hat{\mathbf{x}}+\left(\frac{1}{2}-z_{4}\right) c \hat{\mathbf{z}}$ & $(4 i)$ & $\mathrm{Ru}$ III \\
\hline $\mathbf{B}_{13}$ & $=$ & $x_{5} \mathbf{a}_{1}+y_{5} \mathbf{a}_{2}+z_{5} \mathbf{a}_{3}$ & $=$ & $x_{5} a \hat{\mathbf{x}}+y_{5} a \hat{\mathbf{y}}+z_{5} c \hat{\mathbf{z}}$ & $(8 j)$ & Sn II \\
\hline $\mathbf{B}_{14}$ & $=$ & $-x_{5} \mathbf{a}_{1}-y_{5} \mathbf{a}_{2}+z_{5} \mathbf{a}_{3}$ & $=$ & $-x_{5} a \hat{\mathbf{x}}-y_{5} a \hat{\mathbf{y}}+z_{5} c \hat{\mathbf{z}}$ & $(8 j)$ & Sn II \\
\hline $\mathbf{B}_{15}$ & $=$ & $y_{5} \mathbf{a}_{1}-x_{5} \mathbf{a}_{2}-z_{5} \mathbf{a}_{3}$ & $=$ & $y_{5} a \hat{\mathbf{x}}-x_{5} a \hat{\mathbf{y}}-z_{5} c \hat{\mathbf{z}}$ & $(8 j)$ & Sn II \\
\hline $\mathbf{B}_{16}$ & $=$ & $-y_{5} \mathbf{a}_{1}+x_{5} \mathbf{a}_{2}-z_{5} \mathbf{a}_{3}$ & $=$ & $-y_{5} a \hat{\mathbf{x}}+x_{5} a \hat{\mathbf{y}}-z_{5} c \hat{\mathbf{z}}$ & $(8 j)$ & Sn II \\
\hline $\mathbf{B}_{17}$ & $=$ & $x_{5} \mathbf{a}_{1}-y_{5} \mathbf{a}_{2}+\left(\frac{1}{2}+z_{5}\right) \mathbf{a}_{3}$ & $=$ & $x_{5} a \hat{\mathbf{x}}-y_{5} a \hat{\mathbf{y}}+\left(\frac{1}{2}+z_{5}\right) c \hat{\mathbf{z}}$ & $(8 j)$ & Sn II \\
\hline $\mathbf{B}_{18}$ & $=$ & $-x_{5} \mathbf{a}_{1}+y_{5} \mathbf{a}_{2}+\left(\frac{1}{2}+z_{5}\right) \mathbf{a}_{3}$ & $=$ & $-x_{5} a \hat{\mathbf{x}}+y_{5} a \hat{\mathbf{y}}+\left(\frac{1}{2}+z_{5}\right) c \hat{\mathbf{z}}$ & $(8 j)$ & Sn II \\
\hline $\mathbf{B}_{19}$ & $=$ & $y_{5} \mathbf{a}_{1}+x_{5} \mathbf{a}_{2}+\left(\frac{1}{2}-z_{5}\right) \mathbf{a}_{3}$ & $=$ & $y_{5} a \hat{\mathbf{x}}+x_{5} a \hat{\mathbf{y}}+\left(\frac{1}{2}-z_{5}\right) c \hat{\mathbf{z}}$ & $(8 j)$ & Sn II \\
\hline $\mathbf{B}_{20}$ & $=$ & $-y_{5} \mathbf{a}_{1}-x_{5} \mathbf{a}_{2}+\left(\frac{1}{2}-z_{5}\right) \mathbf{a}_{3}$ & $=$ & $-y_{5} a \hat{\mathbf{x}}-x_{5} a \hat{\mathbf{y}}+\left(\frac{1}{2}-z_{5}\right) c \hat{\mathbf{z}}$ & $(8 j)$ & Sn II \\
\hline
\end{tabular}

\section{References:}

- O. Schwomma, H. Nowotny, and A. Wittmann, Untersuchungen im System: Ru-Sn, Monatsh. Chem. 95, 1538-1543 (1964), doi:10.1007/BF00901709.

\section{Found in:}

- P. Villars and K. Cenzual, Pearson's Crystal Data - Crystal Structure Database for Inorganic Compounds, ASM International (2013).

\section{Geometry files:}

- CIF: pp. 887

- POSCAR: pp. 887 
$\beta-\mathrm{Bi}_{2} \mathrm{O}_{3}$ (High-temperature) Structure: A2B3_tP20_117_i_adgh
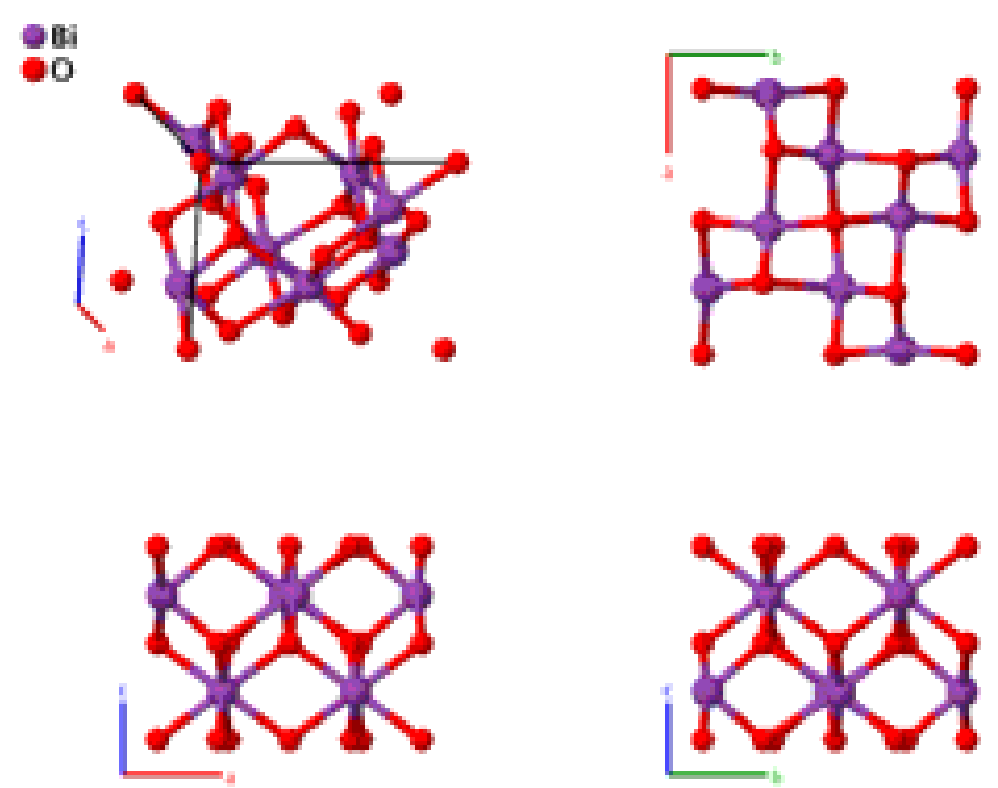

\section{Prototype}

AFLOW prototype label

Strukturbericht designation

Pearson symbol

Space group number

Space group symbol

AFLOW prototype command
: $\quad \beta-\mathrm{Bi}_{2} \mathrm{O}_{3}$

: A2B3_tP20_117_i_adgh

: None

: $\quad$ tP20

: $\quad 117$

: $\quad P \overline{4} b 2$

Simple Tetragonal primitive vectors:

$$
\begin{aligned}
& \mathbf{a}_{1}=a \hat{\mathbf{x}} \\
& \mathbf{a}_{2}=a \hat{\mathbf{y}} \\
& \mathbf{a}_{3}=c \hat{\mathbf{z}}
\end{aligned}
$$

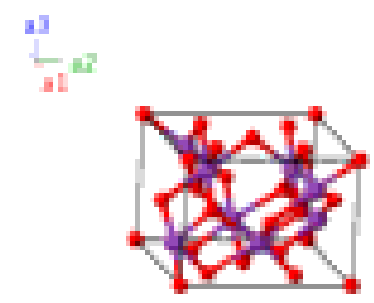

Basis vectors:

Lattice Coordinates

$\begin{array}{lcc}\mathbf{B}_{1}= & 0 \mathbf{a}_{1}+0 \mathbf{a}_{2}+0 \mathbf{a}_{3} \\ \mathbf{B}_{2}= & \frac{1}{2} \mathbf{a}_{1}+\frac{1}{2} \mathbf{a}_{2} \\ \mathbf{B}_{3}= & \frac{1}{2} \mathbf{a}_{2}+\frac{1}{2} \mathbf{a}_{3}\end{array}$

$\mathbf{B}_{1}=$

$\mathbf{B}_{2}=$

$\mathbf{B}_{3}$
Cartesian Coordinates

$=$

$=$

$=$

$$
\begin{gathered}
0 \hat{\mathbf{x}}+0 \hat{\mathbf{y}}+0 \hat{\mathbf{z}} \\
\frac{1}{2} a \hat{\mathbf{x}}+\frac{1}{2} a \hat{\mathbf{y}} \\
\frac{1}{2} a \hat{\mathbf{y}}+\frac{1}{2} c \hat{\mathbf{z}}
\end{gathered}
$$

Wyckoff Position

Atom Type

(2a)

O I

(2a)

O I

(2d) 


\begin{tabular}{|c|c|c|c|c|c|c|}
\hline $\mathbf{B}_{4}$ & $=$ & $\frac{1}{2} \mathbf{a}_{1}+\frac{1}{2} \mathbf{a}_{3}$ & $=$ & $\frac{1}{2} a \hat{\mathbf{x}}+\frac{1}{2} c \hat{\mathbf{z}}$ & $(2 d)$ & $\mathrm{O}$ II \\
\hline $\mathbf{B}_{5}$ & $=$ & $x_{3} \mathbf{a}_{1}+\left(\frac{1}{2}+x_{3}\right) \mathbf{a}_{2}$ & $=$ & $x_{3} a \hat{\mathbf{x}}+\left(\frac{1}{2}+x_{3}\right) a \hat{\mathbf{y}}$ & $(4 g)$ & O III \\
\hline $\mathbf{B}_{6}$ & $=$ & $-x_{3} \mathbf{a}_{1}+\left(\frac{1}{2}-x_{3}\right) \mathbf{a}_{2}$ & $=$ & $-x_{3} a \hat{\mathbf{x}}+\left(\frac{1}{2}-x_{3}\right) a \hat{\mathbf{y}}$ & $(4 g)$ & O III \\
\hline $\mathbf{B}_{7}$ & $=$ & $\left(\frac{1}{2}+x_{3}\right) \mathbf{a}_{1}-x_{3} \mathbf{a}_{2}$ & $=$ & $\left(\frac{1}{2}+x_{3}\right) a \hat{\mathbf{x}}-x_{3} a \hat{\mathbf{y}}$ & $(4 g)$ & O III \\
\hline $\mathbf{B}_{8}$ & $=$ & $\left(\frac{1}{2}-x_{3}\right) \mathbf{a}_{1}+x_{3} \mathbf{a}_{2}$ & $=$ & $\left(\frac{1}{2}-x_{3}\right) a \hat{\mathbf{x}}+x_{3} a \hat{\mathbf{y}}$ & $(4 g)$ & O III \\
\hline $\mathbf{B}_{9}$ & $=$ & $x_{4} \mathbf{a}_{1}+\left(\frac{1}{2}+x_{4}\right) \mathbf{a}_{2}+\frac{1}{2} \mathbf{a}_{3}$ & $=$ & $x_{4} a \hat{\mathbf{x}}+\left(\frac{1}{2}+x_{4}\right) a \hat{\mathbf{y}}+\frac{1}{2} c \hat{\mathbf{z}}$ & $(4 h)$ & O IV \\
\hline $\mathbf{B}_{10}$ & $=$ & $-x_{4} \mathbf{a}_{1}+\left(\frac{1}{2}-x_{4}\right) \mathbf{a}_{2}+\frac{1}{2} \mathbf{a}_{3}$ & $=$ & $-x_{4} a \hat{\mathbf{x}}+\left(\frac{1}{2}-x_{4}\right) a \hat{\mathbf{y}}+\frac{1}{2} c \hat{\mathbf{z}}$ & $(4 h)$ & O IV \\
\hline $\mathbf{B}_{11}$ & $=$ & $\left(\frac{1}{2}+x_{4}\right) \mathbf{a}_{1}-x_{4} \mathbf{a}_{2}+\frac{1}{2} \mathbf{a}_{3}$ & $=$ & $\left(\frac{1}{2}+x_{4}\right) a \hat{\mathbf{x}}-x_{4} a \hat{\mathbf{y}}+\frac{1}{2} c \hat{\mathbf{z}}$ & $(4 h)$ & O IV \\
\hline $\mathbf{B}_{12}$ & $=$ & $\left(\frac{1}{2}-x_{4}\right) \mathbf{a}_{1}+x_{4} \mathbf{a}_{2}+\frac{1}{2} \mathbf{a}_{3}$ & $=$ & $\left(\frac{1}{2}-x_{4}\right) a \hat{\mathbf{x}}+x_{4} a \hat{\mathbf{y}}+\frac{1}{2} c \hat{\mathbf{z}}$ & $(4 h)$ & O IV \\
\hline $\mathbf{B}_{13}$ & $=$ & $x_{5} \mathbf{a}_{1}+y_{5} \mathbf{a}_{2}+z_{5} \mathbf{a}_{3}$ & $=$ & $x_{5} a \hat{\mathbf{x}}+y_{5} a \hat{\mathbf{y}}+z_{5} c \hat{\mathbf{z}}$ & $(8 i)$ & $\mathrm{Bi}$ \\
\hline $\mathbf{B}_{14}$ & $=$ & $-x_{5} \mathbf{a}_{1}-y_{5} \mathbf{a}_{2}+z_{5} \mathbf{a}_{3}$ & $=$ & $-x_{5} a \hat{\mathbf{x}}-y_{5} a \hat{\mathbf{y}}+z_{5} c \hat{\mathbf{z}}$ & $(8 i)$ & $\mathrm{Bi}$ \\
\hline $\mathbf{B}_{15}$ & $=$ & $y_{5} \mathbf{a}_{1}-x_{5} \mathbf{a}_{2}-z_{5} \mathbf{a}_{3}$ & $=$ & $y_{5} a \hat{\mathbf{x}}-x_{5} a \hat{\mathbf{y}}-z_{5} c \hat{\mathbf{z}}$ & $(8 i)$ & $\mathrm{Bi}$ \\
\hline $\mathbf{B}_{16}$ & $=$ & $-y_{5} \mathbf{a}_{1}+x_{5} \mathbf{a}_{2}-z_{5} \mathbf{a}_{3}$ & $=$ & $-y_{5} a \hat{\mathbf{x}}+x_{5} a \hat{\mathbf{y}}-z_{5} c \hat{\mathbf{z}}$ & $(8 i)$ & $\mathrm{Bi}$ \\
\hline $\mathbf{B}_{17}$ & $=$ & $\left(\frac{1}{2}+x_{5}\right) \mathbf{a}_{1}+\left(\frac{1}{2}-y_{5}\right) \mathbf{a}_{2}+z_{5} \mathbf{a}_{3}$ & $=$ & $\left(\frac{1}{2}+x_{5}\right) a \hat{\mathbf{x}}+\left(\frac{1}{2}-y_{5}\right) a \hat{\mathbf{y}}+z_{5} c \hat{\mathbf{z}}$ & $(8 i)$ & $\mathrm{Bi}$ \\
\hline $\mathbf{B}_{18}$ & $=$ & $\left(\frac{1}{2}-x_{5}\right) \mathbf{a}_{1}+\left(\frac{1}{2}+y_{5}\right) \mathbf{a}_{2}+z_{5} \mathbf{a}_{3}$ & $=$ & $\left(\frac{1}{2}-x_{5}\right) a \hat{\mathbf{x}}+\left(\frac{1}{2}+y_{5}\right) a \hat{\mathbf{y}}+z_{5} c \hat{\mathbf{z}}$ & $(8 i)$ & $\mathrm{Bi}$ \\
\hline $\mathbf{B}_{19}$ & $=$ & $\left(\frac{1}{2}+y_{5}\right) \mathbf{a}_{1}+\left(\frac{1}{2}+x_{5}\right) \mathbf{a}_{2}-z_{5} \mathbf{a}_{3}$ & $=$ & $\left(\frac{1}{2}+y_{5}\right) a \hat{\mathbf{x}}+\left(\frac{1}{2}+x_{5}\right) a \hat{\mathbf{y}}-z_{5} c \hat{\mathbf{z}}$ & $(8 i)$ & $\mathrm{Bi}$ \\
\hline $\mathbf{B}_{20}$ & $=$ & $\left(\frac{1}{2}-y_{5}\right) \mathbf{a}_{1}+\left(\frac{1}{2}-x_{5}\right) \mathbf{a}_{2}-z_{5} \mathbf{a}_{3}$ & $=$ & $\left(\frac{1}{2}-y_{5}\right) a \hat{\mathbf{x}}+\left(\frac{1}{2}-x_{5}\right) a \hat{\mathbf{y}}-z_{5} c \hat{\mathbf{z}}$ & $(8 i)$ & $\mathrm{Bi}$ \\
\hline
\end{tabular}

\section{References:}

- L. G. Sillén, X-ray studies on bismuth trioxide, Ark. Kem. Mineral. Geol. 12A, 1-15 (1937).

\section{Found in:}

- P. Villars and K. Cenzual, Pearson's Crystal Data - Crystal Structure Database for Inorganic Compounds, ASM International (2013).

\section{Geometry files:}

- CIF: pp. 887

- POSCAR: pp. 888 


\section{RuIn ${ }_{3}$ Structure: A3B_tP16_118_ei_f}

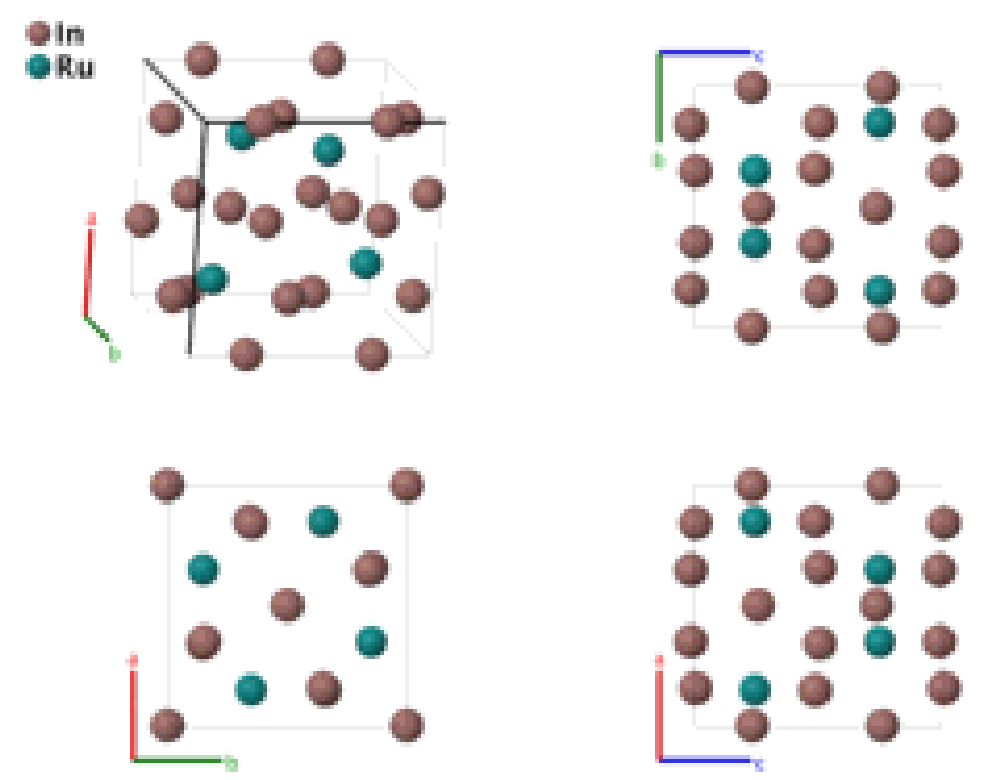

Prototype

AFLOW prototype label

Strukturbericht designation

Pearson symbol

Space group number

Space group symbol

AFLOW prototype command
: $\quad \mathrm{RuIn}_{3}$

: A3B_tP16_118_ei_f

: None

: $\quad$ PP16

: $\quad 118$

: $\quad P \overline{4} n 2$

\section{Simple Tetragonal primitive vectors:}

$$
\begin{aligned}
& \mathbf{a}_{1}=a \hat{\mathbf{x}} \\
& \mathbf{a}_{2}=a \hat{\mathbf{y}} \\
& \mathbf{a}_{3}=c \hat{\mathbf{z}}
\end{aligned}
$$

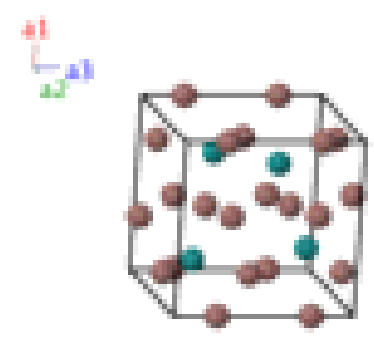

\begin{tabular}{|c|c|c|c|c|}
\hline $\mathbf{B}_{1}$ & $=$ & $z_{1} \mathbf{a}_{3}$ & $=$ & $z_{1} c \hat{\mathbf{z}}$ \\
\hline $\mathbf{B}_{2}$ & $=$ & $-z_{1} \mathbf{a}_{3}$ & $=$ & $-z_{1} c \hat{\mathbf{z}}$ \\
\hline $\mathbf{B}_{3}$ & $=$ & $\frac{1}{2} \mathbf{a}_{1}+\frac{1}{2} \mathbf{a}_{2}+\left(\frac{1}{2}+z_{1}\right) \mathbf{a}_{3}$ & $=$ & $\frac{1}{2} a \hat{\mathbf{x}}+\frac{1}{2} a \hat{\mathbf{y}}+\left(\frac{1}{2}+z_{1}\right) c \hat{\mathbf{z}}$ \\
\hline $\mathbf{B}_{4}$ & $=$ & $\frac{1}{2} \mathbf{a}_{1}+\frac{1}{2} \mathbf{a}_{2}+\left(\frac{1}{2}-z_{1}\right) \mathbf{a}_{3}$ & $=$ & $\frac{1}{2} a \hat{\mathbf{x}}+\frac{1}{2} a \hat{\mathbf{y}}+\left(\frac{1}{2}-z_{1}\right) c \hat{\mathbf{z}}$ \\
\hline $\mathbf{B}_{5}$ & $=$ & $x_{2} \mathbf{a}_{1}+\left(\frac{1}{2}-x_{2}\right) \mathbf{a}_{2}+\frac{1}{4} \mathbf{a}_{3}$ & $=$ & $x_{2} a \hat{\mathbf{x}}+\left(\frac{1}{2}-x_{2}\right) a \hat{\mathbf{y}}+\frac{1}{4} c \hat{\mathbf{z}}$ \\
\hline
\end{tabular}

\section{Basis vectors:}

Lattice Coordinates 


\begin{tabular}{|c|c|c|c|c|c|c|}
\hline $\mathbf{B}_{6}$ & $=$ & $-x_{2} \mathbf{a}_{1}+\left(\frac{1}{2}+x_{2}\right) \mathbf{a}_{2}+\frac{1}{4} \mathbf{a}_{3}$ & $=$ & $-x_{2} a \hat{\mathbf{x}}+\left(\frac{1}{2}+x_{2}\right) a \hat{\mathbf{y}}+\frac{1}{4} c \hat{\mathbf{z}}$ & $(4 f)$ & $\mathrm{Ru}$ \\
\hline $\mathbf{B}_{7}$ & $=$ & $\left(\frac{1}{2}-x_{2}\right) \mathbf{a}_{1}-x_{2} \mathbf{a}_{2}+\frac{3}{4} \mathbf{a}_{3}$ & $=$ & $\left(\frac{1}{2}-x_{2}\right) a \hat{\mathbf{x}}-x_{2} a \hat{\mathbf{y}}+\frac{3}{4} c \hat{\mathbf{z}}$ & $(4 f)$ & $\mathrm{Ru}$ \\
\hline $\mathbf{B}_{8}$ & $=$ & $\left(\frac{1}{2}+x_{2}\right) \mathbf{a}_{1}+x_{2} \mathbf{a}_{2}+\frac{3}{4} \mathbf{a}_{3}$ & $=$ & $\left(\frac{1}{2}+x_{2}\right) a \hat{\mathbf{x}}+x_{2} a \hat{\mathbf{y}}+\frac{3}{4} c \hat{\mathbf{z}}$ & $(4 f)$ & $\mathrm{Ru}$ \\
\hline $\mathbf{B}_{9}$ & $=$ & $x_{3} \mathbf{a}_{1}+y_{3} \mathbf{a}_{2}+z_{3} \mathbf{a}_{3}$ & $=$ & $x_{3} a \hat{\mathbf{x}}+y_{3} a \hat{\mathbf{y}}+z_{3} c \hat{\mathbf{z}}$ & $(8 i)$ & In II \\
\hline $\mathbf{B}_{10}$ & $=$ & $-x_{3} \mathbf{a}_{1}-y_{3} \mathbf{a}_{2}+z_{3} \mathbf{a}_{3}$ & $=$ & $-x_{3} a \hat{\mathbf{x}}-y_{3} a \hat{\mathbf{y}}+z_{3} c \hat{\mathbf{z}}$ & $(8 i)$ & In II \\
\hline $\mathbf{B}_{11}$ & $=$ & $y_{3} \mathbf{a}_{1}-x_{3} \mathbf{a}_{2}-z_{3} \mathbf{a}_{3}$ & $=$ & $y_{3} a \hat{\mathbf{x}}-x_{3} a \hat{\mathbf{y}}-z_{3} c \hat{\mathbf{z}}$ & $(8 i)$ & In II \\
\hline $\mathbf{B}_{12}$ & $=$ & $-y_{3} \mathbf{a}_{1}+x_{3} \mathbf{a}_{2}-z_{3} \mathbf{a}_{3}$ & $=$ & $-y_{3} a \hat{\mathbf{x}}+x_{3} a \hat{\mathbf{y}}-z_{3} c \hat{\mathbf{z}}$ & $(8 i)$ & In II \\
\hline $\mathbf{B}_{13}$ & $=$ & $\begin{array}{c}\left(\frac{1}{2}+x_{3}\right) \mathbf{a}_{1}+\left(\frac{1}{2}-y_{3}\right) \mathbf{a}_{2}+ \\
\left(\frac{1}{2}+z_{3}\right) \mathbf{a}_{3}\end{array}$ & $=$ & $\begin{array}{c}\left(\frac{1}{2}+x_{3}\right) a \hat{\mathbf{x}}+\left(\frac{1}{2}-y_{3}\right) a \hat{\mathbf{y}}+ \\
\left(\frac{1}{2}+z_{3}\right) c \hat{\mathbf{z}}\end{array}$ & $(8 i)$ & In II \\
\hline $\mathbf{B}_{14}$ & $=$ & $\begin{array}{c}\left(\frac{1}{2}-x_{3}\right) \mathbf{a}_{1}+\left(\frac{1}{2}+y_{3}\right) \mathbf{a}_{2}+ \\
\left(\frac{1}{2}+z_{3}\right) \mathbf{a}_{3}\end{array}$ & $=$ & $\begin{array}{c}\left(\frac{1}{2}-x_{3}\right) a \hat{\mathbf{x}}+\left(\frac{1}{2}+y_{3}\right) a \hat{\mathbf{y}}+ \\
\left(\frac{1}{2}+z_{3}\right) c \hat{\mathbf{z}}\end{array}$ & $(8 i)$ & In II \\
\hline $\mathbf{B}_{15}$ & $=$ & $\begin{array}{c}\left(\frac{1}{2}+y_{3}\right) \mathbf{a}_{1}+\left(\frac{1}{2}+x_{3}\right) \mathbf{a}_{2}+ \\
\left(\frac{1}{2}-z_{3}\right) \mathbf{a}_{3}\end{array}$ & $=$ & $\begin{array}{c}\left(\frac{1}{2}+y_{3}\right) a \hat{\mathbf{x}}+\left(\frac{1}{2}+x_{3}\right) a \hat{\mathbf{y}}+ \\
\left(\frac{1}{2}-z_{3}\right) c \hat{\mathbf{z}}\end{array}$ & $(8 i)$ & In II \\
\hline $\mathbf{B}_{16}$ & $=$ & $\begin{array}{c}\left(\frac{1}{2}-y_{3}\right) \mathbf{a}_{1}+\left(\frac{1}{2}-x_{3}\right) \mathbf{a}_{2}+ \\
\left(\frac{1}{2}-z_{3}\right) \mathbf{a}_{3}\end{array}$ & $=$ & $\begin{array}{c}\left(\frac{1}{2}-y_{3}\right) a \hat{\mathbf{x}}+\left(\frac{1}{2}-x_{3}\right) a \hat{\mathbf{y}}+ \\
\left(\frac{1}{2}-z_{3}\right) c \hat{\mathbf{z}}\end{array}$ & $(8 i)$ & In II \\
\hline
\end{tabular}

\section{References:}

- R. B. Roof, Z. Fisk, and J. L. Smith, Crystal data for RuIn, Powder Diffraction 1, 20-21 (1986), doi: $10.1017 /$ S0885715600011234.

\section{Found in:}

- P. Villars and K. Cenzual, Pearson's Crystal Data - Crystal Structure Database for Inorganic Compounds, ASM International (2013).

\section{Geometry files:}

- CIF: pp. 888

- POSCAR: pp. 888 


\section{$\mathrm{Ir}_{3} \mathrm{Ga}_{5}$ Structure: A5B3_tP32_118_g2i_aceh}
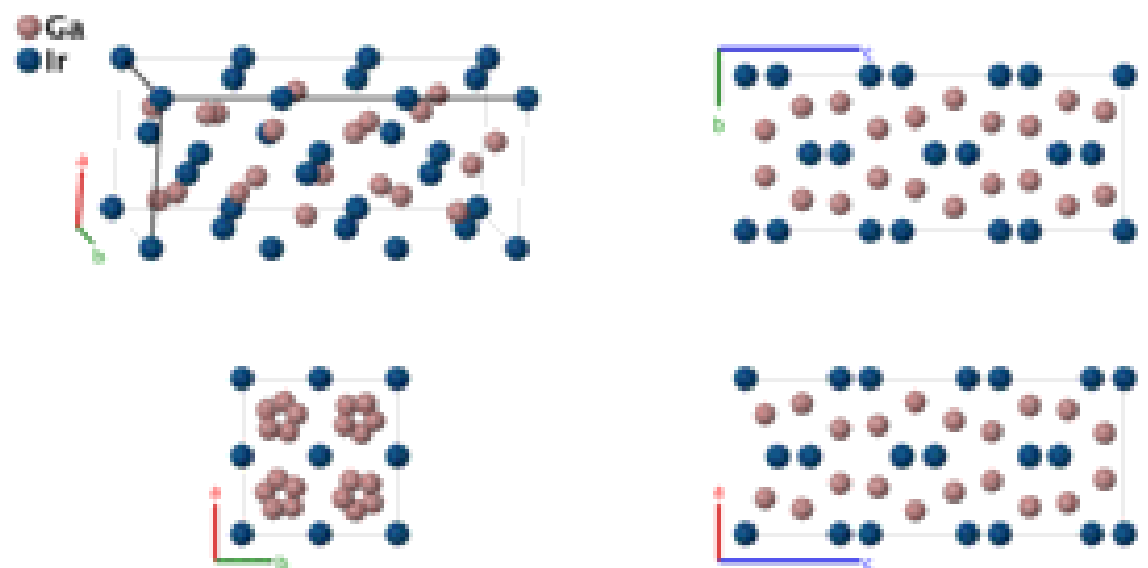

\section{Prototype}

AFLOW prototype label

: $\quad \mathrm{Ir}_{3} \mathrm{Ga}_{5}$

Strukturbericht designation

Pearson symbol

: A5B3_tP32_118_g2i_aceh

Space group number

: None

Space group symbol

: $\quad$ tP32

AFLOW prototype command

: $\quad 118$

: $\quad P \overline{4} n 2$

aflow --proto=A5B3_tP32_118_g2i_aceh

- params $=a, c / a, z_{3}, x_{4}, z_{5}, x_{6}, y_{6}, z_{6}, x_{7}, y_{7}, z_{7}$

\section{Simple Tetragonal primitive vectors:}

$$
\begin{aligned}
& \mathbf{a}_{1}=a \hat{\mathbf{x}} \\
& \mathbf{a}_{2}=a \hat{\mathbf{y}} \\
& \mathbf{a}_{3}=c \hat{\mathbf{z}}
\end{aligned}
$$

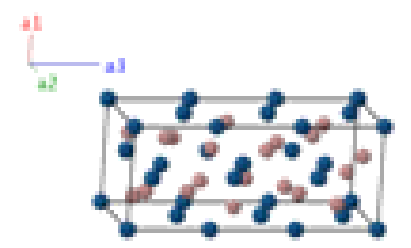

Basis vectors:

Lattice Coordinates

$\begin{array}{lcccc}\mathbf{B}_{1}= & 0 \mathbf{a}_{1}+0 \mathbf{a}_{2}+0 \mathbf{a}_{3} & = & 0 \hat{\mathbf{x}}+0 \hat{\mathbf{y}}+0 \hat{\mathbf{z}} \\ \mathbf{B}_{2}= & \frac{1}{2} \mathbf{a}_{1}+\frac{1}{2} \mathbf{a}_{2}+\frac{1}{2} \mathbf{a}_{3} & = & \frac{1}{2} a \hat{\mathbf{x}}+\frac{1}{2} a \hat{\mathbf{y}}+\frac{1}{2} c \hat{\mathbf{z}} \\ \mathbf{B}_{3}= & \frac{1}{2} \mathbf{a}_{2}+\frac{1}{4} \mathbf{a}_{3} & = & \frac{1}{2} a \hat{\mathbf{y}}+\frac{1}{4} c \hat{\mathbf{z}} \\ \mathbf{B}_{4}= & \frac{1}{2} \mathbf{a}_{1}+\frac{3}{4} \mathbf{a}_{3} & = & \frac{1}{2} a \hat{\mathbf{x}}+\frac{3}{4} c \hat{\mathbf{z}} \\ \mathbf{B}_{5}= & z_{3} \mathbf{a}_{3} & -z_{3} \mathbf{a}_{3} & = & z_{3} c \hat{\mathbf{z}} \\ \mathbf{B}_{6}= & \frac{1}{2} \mathbf{a}_{1}+\frac{1}{2} \mathbf{a}_{2}+\left(\frac{1}{2}+z_{3}\right) \mathbf{a}_{3} & = & \frac{1}{2} a \hat{\mathbf{x}}+\frac{1}{2} a \hat{\mathbf{y}}+\left(\frac{1}{2}+z_{3}\right) c \hat{\mathbf{z}} \\ \mathbf{B}_{7}= & \frac{1}{2} \mathbf{a}_{1}+\frac{1}{2} \mathbf{a}_{2}+\left(\frac{1}{2}-z_{3}\right) \mathbf{a}_{3} & = & \frac{1}{2} a \hat{\mathbf{x}}+\frac{1}{2} a \hat{\mathbf{y}}+\left(\frac{1}{2}-z_{3}\right) c \hat{\mathbf{z}} \\ \mathbf{B}_{8}= & x_{4} \mathbf{a}_{1}+\left(\frac{1}{2}+x_{4}\right) \mathbf{a}_{2}+\frac{1}{4} \mathbf{a}_{3} & = & x_{4} a \hat{\mathbf{x}}+\left(\frac{1}{2}+x_{4}\right) a \hat{\mathbf{y}}+\frac{1}{4} c \hat{\mathbf{z}} \\ \mathbf{B}_{9}= & -x_{4} \mathbf{a}_{1}+\left(\frac{1}{2}-x_{4}\right) \mathbf{a}_{2}+\frac{1}{4} \mathbf{a}_{3} & = & -x_{4} a \hat{\mathbf{x}}+\left(\frac{1}{2}-x_{4}\right) a \hat{\mathbf{y}}+\frac{1}{4} c \hat{\mathbf{z}} \\ \mathbf{B}_{10}= & & \end{array}$

Wyckoff Position Atom Type

(2a)

Ir I

(2a)

Ir I

(2c)

Ir II

(2c)

Ir II

(4e)

Ir III

(4e)

Ir III

(4e)

Ir III

(4e)

Ir III

(4g)

Ga I

(4g)

Ga I 


\begin{tabular}{|c|c|c|c|c|c|c|}
\hline $\mathbf{B}_{11}$ & $=$ & $\left(\frac{1}{2}+x_{4}\right) \mathbf{a}_{1}-x_{4} \mathbf{a}_{2}+\frac{3}{4} \mathbf{a}_{3}$ & $=$ & $\left(\frac{1}{2}+x_{4}\right) a \hat{\mathbf{x}}-x_{4} a \hat{\mathbf{y}}+\frac{3}{4} c \hat{\mathbf{z}}$ & $(4 g)$ & $\mathrm{Ga} \mathrm{I}$ \\
\hline $\mathbf{B}_{12}$ & $=$ & $\left(\frac{1}{2}-x_{4}\right) \mathbf{a}_{1}+x_{4} \mathbf{a}_{2}+\frac{3}{4} \mathbf{a}_{3}$ & $=$ & $\left(\frac{1}{2}-x_{4}\right) a \hat{\mathbf{x}}+x_{4} a \hat{\mathbf{y}}+\frac{3}{4} c \hat{\mathbf{z}}$ & $(4 g)$ & $\mathrm{Ga} I$ \\
\hline $\mathbf{B}_{13}$ & $=$ & $\frac{1}{2} \mathbf{a}_{2}+z_{5} \mathbf{a}_{3}$ & $=$ & $\frac{1}{2} a \hat{\mathbf{y}}+z_{5} c \hat{\mathbf{z}}$ & $(4 h)$ & Ir IV \\
\hline $\mathbf{B}_{14}$ & $=$ & $\frac{1}{2} \mathbf{a}_{1}+-z_{5} \mathbf{a}_{3}$ & $=$ & $\frac{1}{2} a \hat{\mathbf{x}}+-z_{5} c \hat{\mathbf{z}}$ & $(4 h)$ & Ir IV \\
\hline $\mathbf{B}_{15}$ & $=$ & $\frac{1}{2} \mathbf{a}_{1}+\left(\frac{1}{2}+z_{5}\right) \mathbf{a}_{3}$ & $=$ & $\frac{1}{2} a \hat{\mathbf{x}}+\left(\frac{1}{2}+z_{5}\right) c \hat{\mathbf{z}}$ & $(4 h)$ & Ir IV \\
\hline $\mathbf{B}_{16}$ & $=$ & $\frac{1}{2} \mathbf{a}_{2}+\left(\frac{1}{2}-z_{5}\right) \mathbf{a}_{3}$ & $=$ & $\frac{1}{2} a \hat{\mathbf{y}}+\left(\frac{1}{2}-z_{5}\right) c \hat{\mathbf{z}}$ & $(4 h)$ & Ir IV \\
\hline $\mathbf{B}_{17}$ & $=$ & $x_{6} \mathbf{a}_{1}+y_{6} \mathbf{a}_{2}+z_{6} \mathbf{a}_{3}$ & $=$ & $x_{6} a \hat{\mathbf{x}}+y_{6} a \hat{\mathbf{y}}+z_{6} c \hat{\mathbf{z}}$ & $(8 i)$ & Ga II \\
\hline $\mathbf{B}_{18}$ & $=$ & $-x_{6} \mathbf{a}_{1}-y_{6} \mathbf{a}_{2}+z_{6} \mathbf{a}_{3}$ & $=$ & $-x_{6} a \hat{\mathbf{x}}-y_{6} a \hat{\mathbf{y}}+z_{6} c \hat{\mathbf{z}}$ & $(8 i)$ & Ga II \\
\hline $\mathbf{B}_{19}$ & $=$ & $y_{6} \mathbf{a}_{1}-x_{6} \mathbf{a}_{2}-z_{6} \mathbf{a}_{3}$ & $=$ & $y_{6} a \hat{\mathbf{x}}-x_{6} a \hat{\mathbf{y}}-z_{6} c \hat{\mathbf{z}}$ & $(8 i)$ & Ga II \\
\hline $\mathbf{B}_{20}$ & $=$ & $-y_{6} \mathbf{a}_{1}+x_{6} \mathbf{a}_{2}-z_{6} \mathbf{a}_{3}$ & $=$ & $-y_{6} a \hat{\mathbf{x}}+x_{6} a \hat{\mathbf{y}}-z_{6} c \hat{\mathbf{z}}$ & $(8 i)$ & Ga II \\
\hline $\mathbf{B}_{21}$ & $=$ & $\begin{array}{c}\left(\frac{1}{2}+x_{6}\right) \mathbf{a}_{1}+\left(\frac{1}{2}-y_{6}\right) \mathbf{a}_{2}+ \\
\left(\frac{1}{2}+z_{6}\right) \mathbf{a}_{3}\end{array}$ & $=$ & $\begin{array}{c}\left(\frac{1}{2}+x_{6}\right) a \hat{\mathbf{x}}+\left(\frac{1}{2}-y_{6}\right) a \hat{\mathbf{y}}+ \\
\left(\frac{1}{2}+z_{6}\right) c \hat{\mathbf{z}}\end{array}$ & $(8 i)$ & Ga II \\
\hline $\mathbf{B}_{22}$ & $=$ & $\begin{array}{c}\left(\frac{1}{2}-x_{6}\right) \mathbf{a}_{1}+\left(\frac{1}{2}+y_{6}\right) \mathbf{a}_{2}+ \\
\left(\frac{1}{2}+z_{6}\right) \mathbf{a}_{3}\end{array}$ & $=$ & $\begin{array}{c}\left(\frac{1}{2}-x_{6}\right) a \hat{\mathbf{x}}+\left(\frac{1}{2}+y_{6}\right) a \hat{\mathbf{y}}+ \\
\left(\frac{1}{2}+z_{6}\right) c \hat{\mathbf{z}}\end{array}$ & $(8 i)$ & Ga II \\
\hline $\mathbf{B}_{23}$ & $=$ & $\begin{array}{c}\left(\frac{1}{2}+y_{6}\right) \mathbf{a}_{1}+\left(\frac{1}{2}+x_{6}\right) \mathbf{a}_{2}+ \\
\left(\frac{1}{2}-z_{6}\right) \mathbf{a}_{3}\end{array}$ & $=$ & $\begin{array}{c}\left(\frac{1}{2}+y_{6}\right) a \hat{\mathbf{x}}+\left(\frac{1}{2}+x_{6}\right) a \hat{\mathbf{y}}+ \\
\left(\frac{1}{2}-z_{6}\right) c \hat{\mathbf{z}}\end{array}$ & $(8 i)$ & Ga II \\
\hline $\mathbf{B}_{24}$ & $=$ & $\begin{array}{c}\left(\frac{1}{2}-y_{6}\right) \mathbf{a}_{1}+\left(\frac{1}{2}-x_{6}\right) \mathbf{a}_{2}+ \\
\left(\frac{1}{2}-z_{6}\right) \mathbf{a}_{3}\end{array}$ & $=$ & $\begin{array}{c}\left(\frac{1}{2}-y_{6}\right) a \hat{\mathbf{x}}+\left(\frac{1}{2}-x_{6}\right) a \hat{\mathbf{y}}+ \\
\left(\frac{1}{2}-z_{6}\right) c \hat{\mathbf{z}}\end{array}$ & $(8 i)$ & Ga II \\
\hline $\mathbf{B}_{25}$ & $=$ & $x_{7} \mathbf{a}_{1}+y_{7} \mathbf{a}_{2}+z_{7} \mathbf{a}_{3}$ & $=$ & $x_{7} a \hat{\mathbf{x}}+y_{7} a \hat{\mathbf{y}}+z_{7} c \hat{\mathbf{z}}$ & $(8 i)$ & Ga III \\
\hline $\mathbf{B}_{26}$ & $=$ & $-x_{7} \mathbf{a}_{1}-y_{7} \mathbf{a}_{2}+z_{7} \mathbf{a}_{3}$ & $=$ & $-x_{7} a \hat{\mathbf{x}}-y_{7} a \hat{\mathbf{y}}+z_{7} c \hat{\mathbf{z}}$ & $(8 i)$ & Ga III \\
\hline $\mathbf{B}_{27}$ & $=$ & $y_{7} \mathbf{a}_{1}-x_{7} \mathbf{a}_{2}-z_{7} \mathbf{a}_{3}$ & $=$ & $y_{7} a \hat{\mathbf{x}}-x_{7} a \hat{\mathbf{y}}-z_{7} c \hat{\mathbf{z}}$ & $(8 i)$ & Ga III \\
\hline $\mathbf{B}_{28}$ & $=$ & $-y_{7} \mathbf{a}_{1}+x_{7} \mathbf{a}_{2}-z_{7} \mathbf{a}_{3}$ & $=$ & $-y_{7} a \hat{\mathbf{x}}+x_{7} a \hat{\mathbf{y}}-z_{7} c \hat{\mathbf{z}}$ & $(8 i)$ & Ga III \\
\hline $\mathbf{B}_{29}$ & $=$ & $\begin{array}{c}\left(\frac{1}{2}+x_{7}\right) \mathbf{a}_{1}+\left(\frac{1}{2}-y_{7}\right) \mathbf{a}_{2}+ \\
\left(\frac{1}{2}+z_{7}\right) \mathbf{a}_{3}\end{array}$ & $=$ & $\begin{array}{c}\left(\frac{1}{2}+x_{7}\right) a \hat{\mathbf{x}}+\left(\frac{1}{2}-y_{7}\right) a \hat{\mathbf{y}}+ \\
\left(\frac{1}{2}+z_{7}\right) c \hat{\mathbf{z}}\end{array}$ & $(8 i)$ & Ga III \\
\hline $\mathbf{B}_{30}$ & $=$ & $\begin{array}{c}\left(\frac{1}{2}-x_{7}\right) \mathbf{a}_{1}+\left(\frac{1}{2}+y_{7}\right) \mathbf{a}_{2}+ \\
\left(\frac{1}{2}+z_{7}\right) \mathbf{a}_{3}\end{array}$ & $=$ & $\begin{array}{c}\left(\frac{1}{2}-x_{7}\right) a \hat{\mathbf{x}}+\left(\frac{1}{2}+y_{7}\right) a \hat{\mathbf{y}}+ \\
\left(\frac{1}{2}+z_{7}\right) c \hat{\mathbf{z}}\end{array}$ & $(8 i)$ & Ga III \\
\hline $\mathbf{B}_{31}$ & $=$ & $\begin{array}{c}\left(\frac{1}{2}+y_{7}\right) \mathbf{a}_{1}+\left(\frac{1}{2}+x_{7}\right) \mathbf{a}_{2}+ \\
\left(\frac{1}{2}-z_{7}\right) \mathbf{a}_{3}\end{array}$ & $=$ & $\begin{array}{c}\left(\frac{1}{2}+y_{7}\right) a \hat{\mathbf{x}}+\left(\frac{1}{2}+x_{7}\right) a \hat{\mathbf{y}}+ \\
\left(\frac{1}{2}-z_{7}\right) c \hat{\mathbf{z}}\end{array}$ & $(8 i)$ & Ga III \\
\hline $\mathbf{B}_{32}$ & $=$ & $\begin{array}{c}\left(\frac{1}{2}-y_{7}\right) \mathbf{a}_{1}+\left(\frac{1}{2}-x_{7}\right) \mathbf{a}_{2}+ \\
\left(\frac{1}{2}-z_{7}\right) \mathbf{a}_{3}\end{array}$ & $=$ & $\begin{array}{c}\left(\frac{1}{2}-y_{7}\right) a \hat{\mathbf{x}}+\left(\frac{1}{2}-x_{7}\right) a \hat{\mathbf{y}}+ \\
\left(\frac{1}{2}-z_{7}\right) c \hat{\mathbf{z}}\end{array}$ & $(8 i)$ & Ga III \\
\hline
\end{tabular}

\section{References:}

- H. Völlenkle, A. Wittmann, and H. Nowotny, Die Kristallstrukturen von $R h_{10} G a_{17}$ und $\operatorname{Ir}_{3} G a_{5}$, Monatsh. Chem. 98, 176-183 (1967), doi:10.1007/BF00901115.

\section{Found in:}

- P. Villars and K. Cenzual, Pearson's Crystal Data - Crystal Structure Database for Inorganic Compounds, ASM International (2013).

\section{Geometry files:}

- CIF: pp. 888 
- POSCAR: pp. 889 


\section{$\mathrm{RbGa}_{3}$ Structure: A3B_tI24_119_b2i_af}
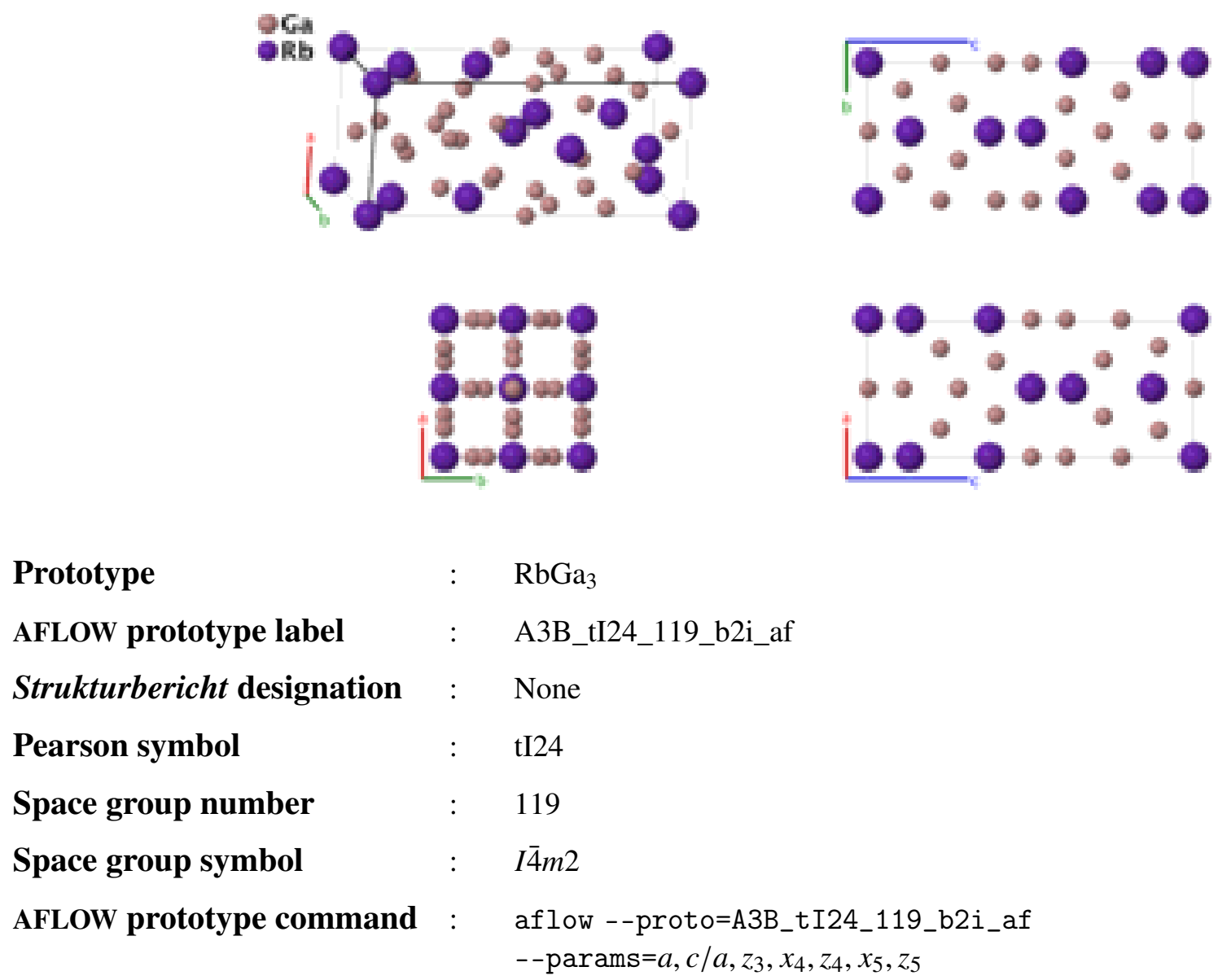

Other compounds with this structure:

- $\mathrm{CsGa}_{3}, \mathrm{KGa}_{3}$

Body-centered Tetragonal primitive vectors:

$$
\begin{aligned}
& \mathbf{a}_{1}=-\frac{1}{2} a \hat{\mathbf{x}}+\frac{1}{2} a \hat{\mathbf{y}}+\frac{1}{2} c \hat{\mathbf{z}} \\
& \mathbf{a}_{2}=\frac{1}{2} a \hat{\mathbf{x}}-\frac{1}{2} a \hat{\mathbf{y}}+\frac{1}{2} c \hat{\mathbf{z}} \\
& \mathbf{a}_{3}=\frac{1}{2} a \hat{\mathbf{x}}+\frac{1}{2} a \hat{\mathbf{y}}-\frac{1}{2} c \hat{\mathbf{z}}
\end{aligned}
$$

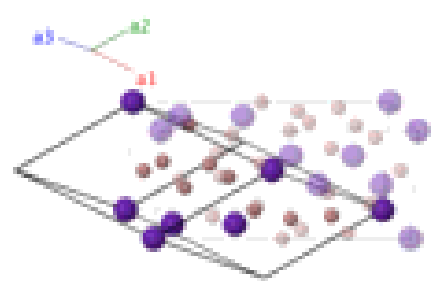

Basis vectors:

Lattice Coordinates

$\begin{array}{lccl}\mathbf{B}_{1} & & 0 \mathbf{a}_{1}+0 \mathbf{a}_{2}+0 \mathbf{a}_{3} & = \\ \mathbf{B}_{2} & = & \frac{1}{2} \mathbf{a}_{1}+\frac{1}{2} \mathbf{a}_{2} & = \\ \mathbf{B}_{3}= & \left(\frac{1}{2}+z_{3}\right) \mathbf{a}_{1}+z_{3} \mathbf{a}_{2}+\frac{1}{2} \mathbf{a}_{3} & = \\ \mathbf{B}_{4}= & -z_{3} \mathbf{a}_{1}+\left(\frac{1}{2}-z_{3}\right) \mathbf{a}_{2}+\frac{1}{2} \mathbf{a}_{3} & = \\ \mathbf{B}_{5} & = & z_{4} \mathbf{a}_{1}+\left(x_{4}+z_{4}\right) \mathbf{a}_{2}+x_{4} \mathbf{a}_{3} & = \\ \mathbf{B}_{6} & = & z_{4} \mathbf{a}_{1}+\left(-x_{4}+z_{4}\right) \mathbf{a}_{2}-x_{4} \mathbf{a}_{3} & = \\ \mathbf{B}_{7} & = & \left(-x_{4}-z_{4}\right) \mathbf{a}_{1}-z_{4} \mathbf{a}_{2}-x_{4} \mathbf{a}_{3} & =\end{array}$

Cartesian Coordinates

$=$

$=$

$=$

$=$

$=$

$=$

$=$

$$
\begin{gathered}
0 \hat{\mathbf{x}}+0 \hat{\mathbf{y}}+0 \hat{\mathbf{z}} \\
\frac{1}{2} c \hat{\mathbf{z}} \\
\frac{1}{2} a \hat{\mathbf{y}}+z_{3} c \hat{\mathbf{z}} \\
\frac{1}{2} a \hat{\mathbf{x}}+-z_{3} c \hat{\mathbf{z}} \\
x_{4} a \hat{\mathbf{x}}+z_{4} c \hat{\mathbf{z}} \\
-x_{4} a \hat{\mathbf{x}}+z_{4} c \hat{\mathbf{z}} \\
-x_{4} a \hat{\mathbf{y}}-z_{4} c \hat{\mathbf{z}}
\end{gathered}
$$

Wyckoff Position
Atom Type

$\mathrm{Rb} \mathrm{I}$

Ga I

$\mathrm{Rb}$ II

$\mathrm{Rb}$ II

Ga II

Ga II

Ga II 


$\begin{array}{rlllll}\mathbf{B}_{8}= & \left(x_{4}-z_{4}\right) \mathbf{a}_{1}-z_{4} \mathbf{a}_{2}+x_{4} \mathbf{a}_{3} & = & x_{4} a \hat{\mathbf{y}}-z_{4} c \hat{\mathbf{z}} \\ \mathbf{B}_{9}= & z_{5} \mathbf{a}_{1}+\left(x_{5}+z_{5}\right) \mathbf{a}_{2}+x_{5} \mathbf{a}_{3} & = & x_{5} a \hat{\mathbf{x}}+z_{5} c \hat{\mathbf{z}} \\ \mathbf{B}_{10}= & z_{5} \mathbf{a}_{1}+\left(-x_{5}+z_{5}\right) \mathbf{a}_{2}-x_{5} \mathbf{a}_{3}= & -x_{5} a \hat{\mathbf{x}}+z_{5} c \hat{\mathbf{z}} \\ \mathbf{B}_{11}=\left(-x_{5}-z_{5}\right) \mathbf{a}_{1}-z_{5} \mathbf{a}_{2}-x_{5} \mathbf{a}_{3}= & -x_{5} a \hat{\mathbf{y}}-z_{5} c \hat{\mathbf{z}} \\ \mathbf{B}_{12}=\left(x_{5}-z_{5}\right) \mathbf{a}_{1}-z_{5} \mathbf{a}_{2}+x_{5} \mathbf{a}_{3}= & x_{5} a \hat{\mathbf{y}}-z_{5} c \hat{\mathbf{z}} & \text { Ga II }\end{array}$

\section{References:}

- R. G. Ling and C. Belin, Preparation and Crystal Structure Determination of the New Intermetallic Compound RbGa 3 , Z. Anorg. Allg. Chem. 480, 181-185 (1981), doi:10.1002/zaac.19814800923.

\section{Found in:}

- P. Villars and L. Calvert, Pearson's Handbook of Crystallographic Data for Intermetallic Phases (ASM International, Materials Park, OK, 1991), vol. III, chap. , p. 3545.

\section{Geometry files:}

- CIF: pp. 889

- POSCAR: pp. 889 


\section{GaSb Structure: AB_tI4_119_c_a}

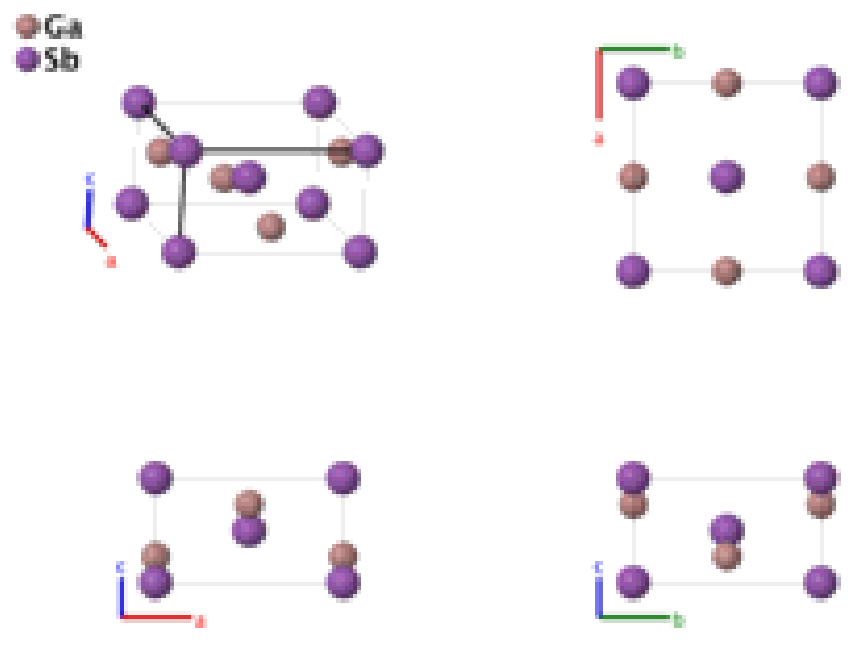

Prototype

: $\mathrm{GaSb}$

AFLOW prototype label

: AB_tI4_119_c_a

Strukturbericht designation

: None

Pearson symbol $\quad$ : $\quad$ tI4

Space group number $\quad$ : 119

Space group symbol $\quad: \quad I \overline{4} m 2$

AFLOW prototype command : aflow--proto=AB_tI4_119_c_a

- params $=a, c / a$

Body-centered Tetragonal primitive vectors:

$$
\begin{aligned}
& \mathbf{a}_{1}=-\frac{1}{2} a \hat{\mathbf{x}}+\frac{1}{2} a \hat{\mathbf{y}}+\frac{1}{2} c \hat{\mathbf{z}} \\
& \mathbf{a}_{2}=\frac{1}{2} a \hat{\mathbf{x}}-\frac{1}{2} a \hat{\mathbf{y}}+\frac{1}{2} c \hat{\mathbf{z}} \\
& \mathbf{a}_{3}=\frac{1}{2} a \hat{\mathbf{x}}+\frac{1}{2} a \hat{\mathbf{y}}-\frac{1}{2} c \hat{\mathbf{z}}
\end{aligned}
$$

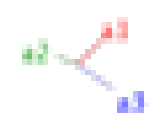

\section{Basis vectors:}

Lattice Coordinates

$\mathbf{B}_{1}=0 \mathbf{a}_{1}+0 \mathbf{a}_{2}+0 \mathbf{a}_{3}=$
$\mathbf{B}_{2}=\frac{3}{4} \mathbf{a}_{1}+\frac{1}{4} \mathbf{a}_{2}+\frac{1}{2} \mathbf{a}_{3}=$

Cartesian Coordinates

$$
\begin{gathered}
0 \hat{\mathbf{x}}+0 \hat{\mathbf{y}}+0 \hat{\mathbf{z}} \\
\frac{1}{2} a \hat{\mathbf{y}}+\frac{1}{4} c \hat{\mathbf{z}}
\end{gathered}
$$

Wyckoff Position

(2a)
Atom Type

$\mathrm{Sb}$

$\mathrm{Ga}$

\section{References:}

- T. R. R. McDonald, R. Sard, and E. Gregory, Retention of GaSb (II) at low temperatures and one atmosphere pressure, J. Appl. Phys. 36, 1498-1499 (1965), doi:10.1063/1.1714346.

Found in: 
- P. Villars and K. Cenzual, Pearson's Crystal Data - Crystal Structure Database for Inorganic Compounds, ASM International (2013).

\section{Geometry files:}

- CIF: pp. 889

- POSCAR: pp. 890 

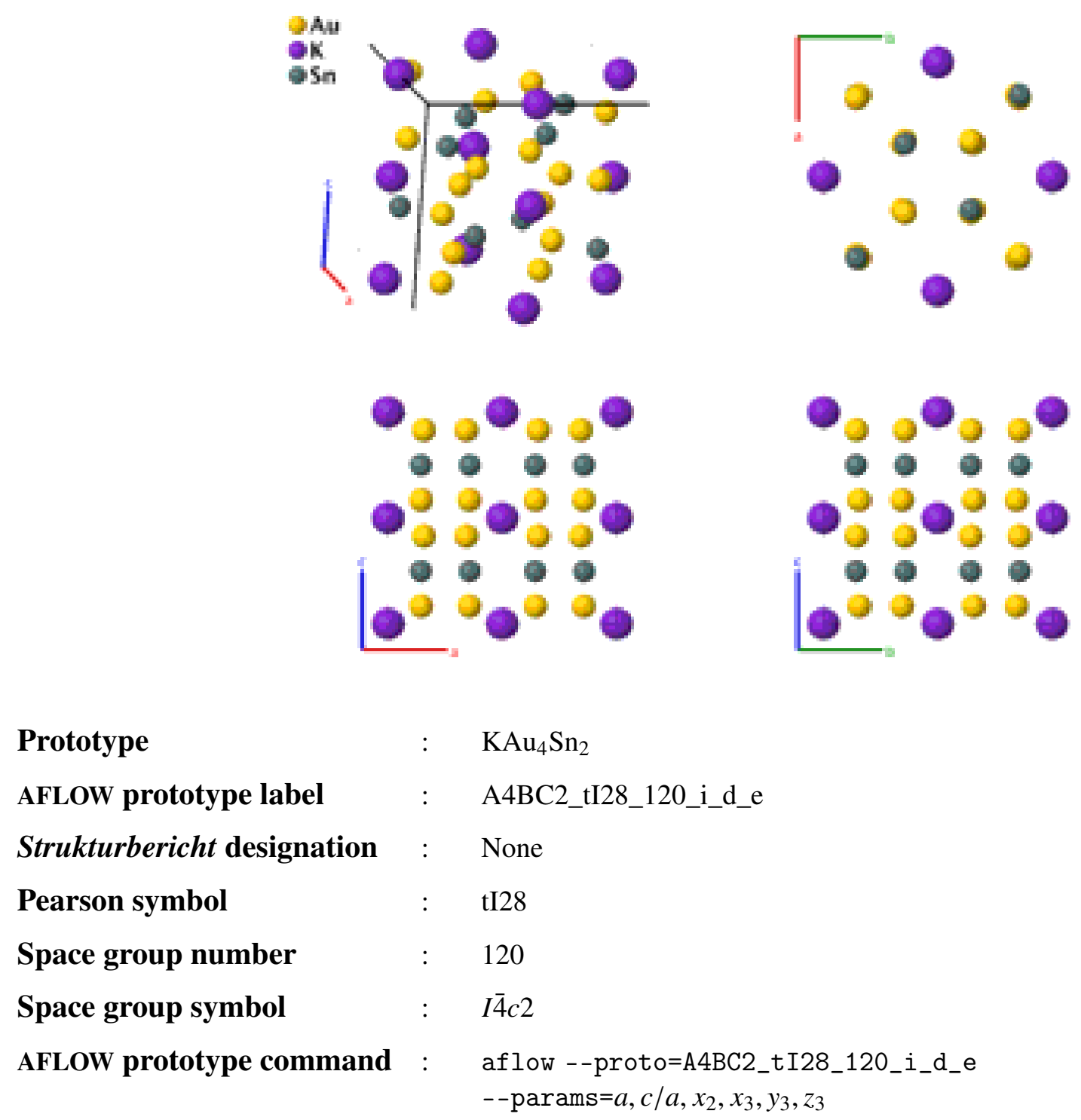

Body-centered Tetragonal primitive vectors:

$$
\begin{aligned}
& \mathbf{a}_{1}=-\frac{1}{2} a \hat{\mathbf{x}}+\frac{1}{2} a \hat{\mathbf{y}}+\frac{1}{2} c \hat{\mathbf{z}} \\
& \mathbf{a}_{2}=\frac{1}{2} a \hat{\mathbf{x}}-\frac{1}{2} a \hat{\mathbf{y}}+\frac{1}{2} c \hat{\mathbf{z}} \\
& \mathbf{a}_{3}=\frac{1}{2} a \hat{\mathbf{x}}+\frac{1}{2} a \hat{\mathbf{y}}-\frac{1}{2} c \hat{\mathbf{z}}
\end{aligned}
$$

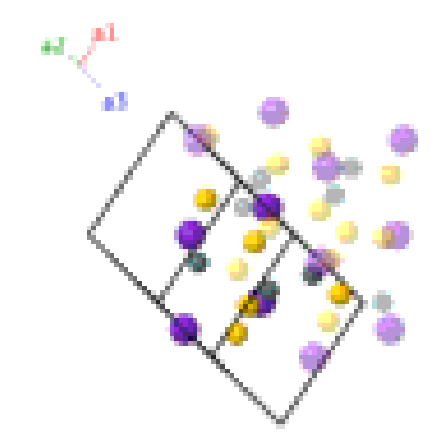

Basis vectors:

Lattice Coordinates

$\begin{array}{lcccc}\mathbf{B}_{1}= & \frac{1}{2} \mathbf{a}_{1}+\frac{1}{2} \mathbf{a}_{3} & = & \frac{1}{2} a \hat{\mathbf{y}} \\ \mathbf{B}_{2}= & \frac{1}{2} \mathbf{a}_{2}+\frac{1}{2} \mathbf{a}_{3} & = & \frac{1}{2} a \hat{\mathbf{x}} \\ \mathbf{B}_{3}= & \left(\frac{1}{4}+x_{2}\right) \mathbf{a}_{1}+\left(\frac{1}{4}+x_{2}\right) \mathbf{a}_{2}+2 x_{2} \mathbf{a}_{3} & = & x_{2} a \hat{\mathbf{x}}+x_{2} a \hat{\mathbf{y}}+\frac{1}{4} c \hat{\mathbf{z}} \\ \mathbf{B}_{4}= & \left(\frac{1}{4}-x_{2}\right) \mathbf{a}_{1}+\left(\frac{1}{4}-x_{2}\right) \mathbf{a}_{2}-2 x_{2} \mathbf{a}_{3} & = & -x_{2} a \hat{\mathbf{x}}-x_{2} a \hat{\mathbf{y}}+\frac{1}{4} c \hat{\mathbf{z}}\end{array}$




\begin{tabular}{|c|c|c|c|c|c|c|}
\hline $\mathbf{B}_{5}$ & $=$ & $\left(\frac{3}{4}-x_{2}\right) \mathbf{a}_{1}+\left(\frac{3}{4}+x_{2}\right) \mathbf{a}_{2}$ & $=$ & $x_{2} a \hat{\mathbf{x}}-x_{2} a \hat{\mathbf{y}}+\frac{3}{4} c \hat{\mathbf{z}}$ & $(8 e)$ & $\mathrm{Sn}$ \\
\hline $\mathbf{B}_{6}$ & $=$ & $\left(\frac{3}{4}+x_{2}\right) \mathbf{a}_{1}+\left(\frac{3}{4}-x_{2}\right) \mathbf{a}_{2}$ & $=$ & $-x_{2} a \hat{\mathbf{x}}+x_{2} a \hat{\mathbf{y}}+\frac{3}{4} c \hat{\mathbf{z}}$ & $(8 e)$ & $\mathrm{Sn}$ \\
\hline $\mathbf{B}_{7}$ & $=$ & $\left(y_{3}+z_{3}\right) \mathbf{a}_{1}+\left(x_{3}+z_{3}\right) \mathbf{a}_{2}+\left(x_{3}+y_{3}\right) \mathbf{a}_{3}$ & $=$ & $x_{3} a \hat{\mathbf{x}}+y_{3} a \hat{\mathbf{y}}+z_{3} c \hat{\mathbf{z}}$ & $(16 i)$ & $\mathrm{Au}$ \\
\hline $\mathbf{B}_{8}$ & $=$ & $\begin{array}{c}\left(-y_{3}+z_{3}\right) \mathbf{a}_{1}+\left(-x_{3}+z_{3}\right) \mathbf{a}_{2}+ \\
\left(-x_{3}-y_{3}\right) \mathbf{a}_{3}\end{array}$ & $=$ & $-x_{3} a \hat{\mathbf{x}}-y_{3} a \hat{\mathbf{y}}+z_{3} c \hat{\mathbf{z}}$ & $(16 i)$ & $\mathrm{Au}$ \\
\hline $\mathbf{B}_{9}$ & $=$ & $\begin{array}{c}\left(-x_{3}-z_{3}\right) \mathbf{a}_{1}+\left(y_{3}-z_{3}\right) \mathbf{a}_{2}+ \\
\left(-x_{3}+y_{3}\right) \mathbf{a}_{3}\end{array}$ & $=$ & $y_{3} a \hat{\mathbf{x}}-x_{3} a \hat{\mathbf{y}}-z_{3} c \hat{\mathbf{z}}$ & $(16 i)$ & $\mathrm{Au}$ \\
\hline $\mathbf{B}_{10}$ & $=$ & $\left(x_{3}-z_{3}\right) \mathbf{a}_{1}+\left(-y_{3}-z_{3}\right) \mathbf{a}_{2}+\left(x_{3}-y_{3}\right) \mathbf{a}_{3}$ & $=$ & $-y_{3} a \hat{\mathbf{x}}+x_{3} a \hat{\mathbf{y}}-z_{3} c \hat{\mathbf{z}}$ & $(16 i)$ & $\mathrm{Au}$ \\
\hline $\mathbf{B}_{11}$ & $=$ & $\begin{array}{c}\left(\frac{1}{2}-y_{3}+z_{3}\right) \mathbf{a}_{1}+\left(\frac{1}{2}+x_{3}+z_{3}\right) \mathbf{a}_{2}+ \\
\left(x_{3}-y_{3}\right) \mathbf{a}_{3}\end{array}$ & $=$ & $x_{3} a \hat{\mathbf{x}}-y_{3} a \hat{\mathbf{y}}+\left(\frac{1}{2}+z_{3}\right) c \hat{\mathbf{z}}$ & $(16 i)$ & $\mathrm{Au}$ \\
\hline $\mathbf{B}_{12}$ & $=$ & $\begin{array}{c}\left(\frac{1}{2}+y_{3}+z_{3}\right) \mathbf{a}_{1}+\left(\frac{1}{2}-x_{3}+z_{3}\right) \mathbf{a}_{2}+ \\
\left(-x_{3}+y_{3}\right) \mathbf{a}_{3}\end{array}$ & $=$ & $-x_{3} a \hat{\mathbf{x}}+y_{3} a \hat{\mathbf{y}}+\left(\frac{1}{2}+z_{3}\right) c \hat{\mathbf{z}}$ & $(16 i)$ & $\mathrm{Au}$ \\
\hline $\mathbf{B}_{13}$ & $=$ & $\begin{array}{c}\left(\frac{1}{2}+x_{3}-z_{3}\right) \mathbf{a}_{1}+\left(\frac{1}{2}+y_{3}-z_{3}\right) \mathbf{a}_{2}+ \\
\left(x_{3}+y_{3}\right) \mathbf{a}_{3}\end{array}$ & $=$ & $y_{3} a \hat{\mathbf{x}}+x_{3} a \hat{\mathbf{y}}+\left(\frac{1}{2}-z_{3}\right) c \hat{\mathbf{z}}$ & $(16 i)$ & $\mathrm{Au}$ \\
\hline $\mathbf{B}_{14}$ & $=$ & $\begin{array}{c}\left(\frac{1}{2}-x_{3}-z_{3}\right) \mathbf{a}_{1}+\left(\frac{1}{2}-y_{3}-z_{3}\right) \mathbf{a}_{2}+ \\
\left(-x_{3}-y_{3}\right) \mathbf{a}_{3}\end{array}$ & $=$ & $-y_{3} a \hat{\mathbf{x}}-x_{3} a \hat{\mathbf{y}}+\left(\frac{1}{2}-z_{3}\right) c \hat{\mathbf{z}}$ & $(16 i)$ & $\mathrm{Au}$ \\
\hline
\end{tabular}

\section{References:}

- H.-D. Sinnen and H.-U. Schuster, Darstellung und Struktur des KAu $\mathrm{Sn}_{2}$ / Preparation and Crystal Structure of $\mathrm{KAu}_{4} \mathrm{Sn}_{2}$, Z. Naturforsch. B 33, 1077-1079 (1978), doi:10.1515/znb-1978-1004.

\section{Found in:}

- P. Villars and K. Cenzual, Pearson's Crystal Data - Crystal Structure Database for Inorganic Compounds, ASM International (2013).

\section{Geometry files:}

- CIF: pp. 890

- POSCAR: pp. 890 


\section{$\mathrm{CaRbFe}_{4} \mathrm{As}_{4}$ (Superconducting) Structure: A4BC4D_tP10_123_gh_a_i_d}
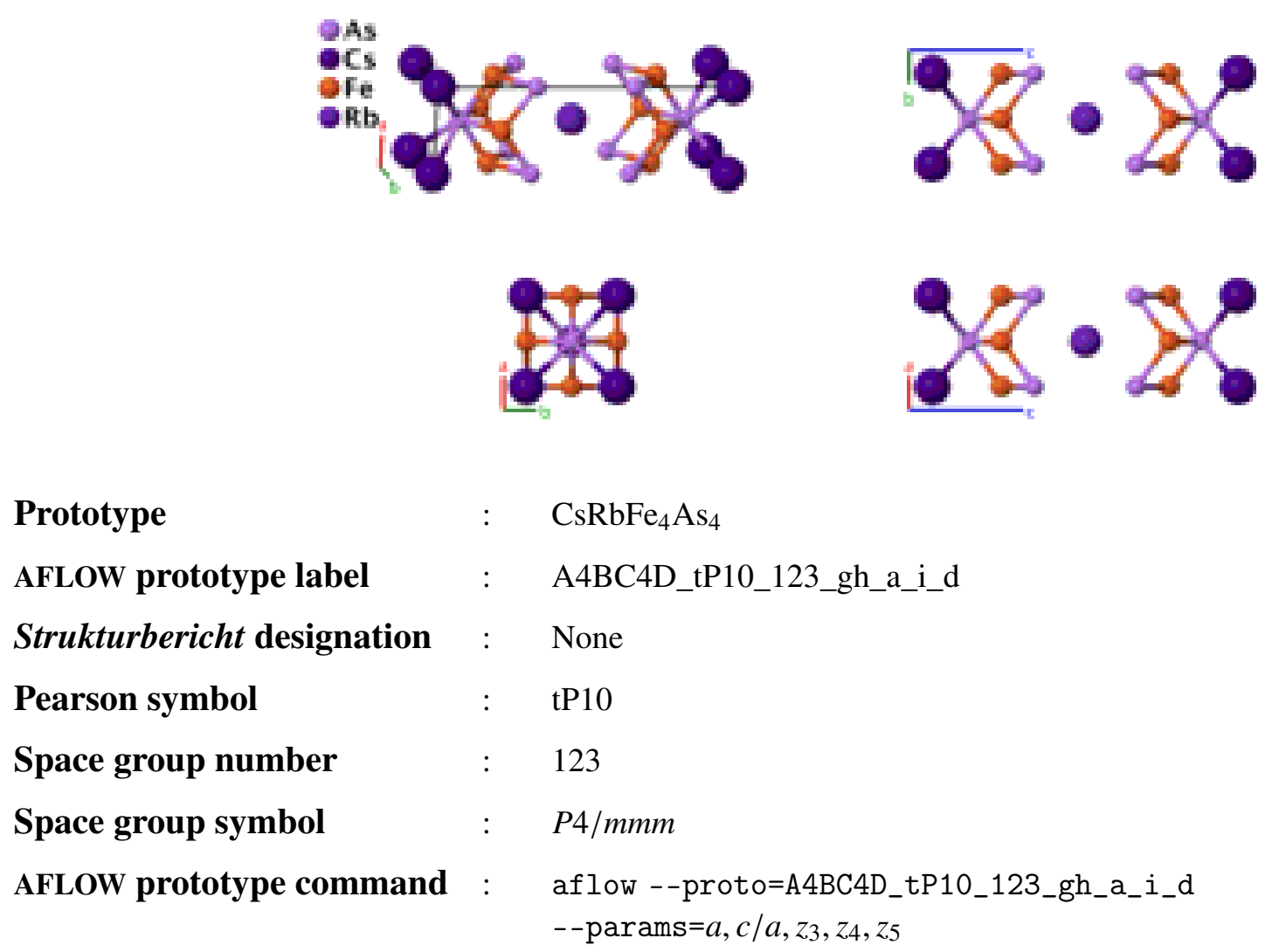

Prototype

AFLOW prototype label

$: \quad \mathrm{CsRbFe}_{4} \mathrm{As}_{4}$

Strukturbericht designation

: A4BC4D_tP10_123_gh_a_i_d

Pearson symbol

: None

Space group number

tP10

Space group symbol

123

AFLOW prototype command

: $\quad P 4 / \mathrm{mmm}$

\section{Other compounds with this structure:}

- $\mathrm{CaKFe}_{4} \mathrm{As}_{4}, \mathrm{CaCsFe}_{4} \mathrm{As}_{4}, \mathrm{SrRbFe}_{4} \mathrm{As}_{4}, \mathrm{SrCsFe}_{4} \mathrm{As}_{4}, \mathrm{BaCsFe}_{4} \mathrm{As}_{4}$

- These compounds form a family of stoichiometric superconductors with transition temperatures $T_{\mathrm{c}}$ ranging from $26-$ $37 \mathrm{~K}$.

Simple Tetragonal primitive vectors:

$$
\begin{aligned}
& \mathbf{a}_{1}=a \hat{\mathbf{x}} \\
& \mathbf{a}_{2}=a \hat{\mathbf{y}} \\
& \mathbf{a}_{3}=c \hat{\mathbf{z}}
\end{aligned}
$$

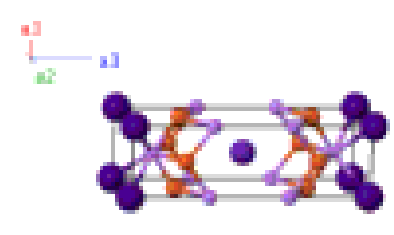

Basis vectors:

Lattice Coordinates

$\begin{array}{lcccc}\mathbf{B}_{1} & = & 0 \mathbf{a}_{1}+0 \mathbf{a}_{2}+0 \mathbf{a}_{3} & = & 0 \hat{\mathbf{x}}+0 \hat{\mathbf{y}}+0 \hat{\mathbf{z}} \\ \mathbf{B}_{2}= & \frac{1}{2} \mathbf{a}_{1}+\frac{1}{2} \mathbf{a}_{2}+\frac{1}{2} \mathbf{a}_{3} & = & \frac{1}{2} a \hat{\mathbf{x}}+\frac{1}{2} a \hat{\mathbf{y}}+\frac{1}{2} c \hat{\mathbf{z}} \\ \mathbf{B}_{3}= & z_{3} \mathbf{a}_{3} & = & z_{3} c \hat{\mathbf{z}} \\ \mathbf{B}_{4}= & -z_{3} \mathbf{a}_{3} & = & -z_{3} c \hat{\mathbf{z}} \\ \mathbf{B}_{5} & = & \frac{1}{2} \mathbf{a}_{1}+\frac{1}{2} \mathbf{a}_{2}+z_{4} \mathbf{a}_{3} & = & \frac{1}{2} a \hat{\mathbf{x}}+\frac{1}{2} a \hat{\mathbf{y}}+z_{4} c \hat{\mathbf{z}}\end{array}$

Wyckoff Position
Atom Type

Cs

$\mathrm{Rb}$

As I

As I

As II 


\begin{tabular}{|c|c|c|c|c|c|c|}
\hline $\mathbf{B}_{6}$ & $=$ & $\frac{1}{2} \mathbf{a}_{1}+\frac{1}{2} \mathbf{a}_{2}-z_{4} \mathbf{a}_{3}$ & $=$ & $\frac{1}{2} a \hat{\mathbf{x}}+\frac{1}{2} a \hat{\mathbf{y}}-z_{4} c \hat{\mathbf{z}}$ & $(2 h)$ & As II \\
\hline $\mathbf{B}_{7}$ & $=$ & $\frac{1}{2} \mathbf{a}_{2}+z_{5} \mathbf{a}_{3}$ & $=$ & $\frac{1}{2} a \hat{\mathbf{y}}+z_{5} c \hat{\mathbf{z}}$ & $(4 i)$ & $\mathrm{Fe}$ \\
\hline $\mathbf{B}_{8}$ & $=$ & $\frac{1}{2} \mathbf{a}_{1}+z_{5} \mathbf{a}_{3}$ & $=$ & $\frac{1}{2} a \hat{\mathbf{x}}+z_{5} c \hat{\mathbf{z}}$ & $(4 i)$ & $\mathrm{Fe}$ \\
\hline $\mathbf{B}_{9}$ & $=$ & $\frac{1}{2} \mathbf{a}_{2}-z_{5} \mathbf{a}_{3}$ & $=$ & $\frac{1}{2} a \hat{\mathbf{y}}-z_{5} c \hat{\mathbf{z}}$ & $(4 i)$ & $\mathrm{Fe}$ \\
\hline $\mathbf{B}_{10}$ & $=$ & $\frac{1}{2} \mathbf{a}_{1}+-z_{5} \mathbf{a}_{3}$ & $=$ & $\frac{1}{2} a \hat{\mathbf{x}}+-z_{5} c \hat{\mathbf{z}}$ & $(4 i)$ & $\mathrm{Fe}$ \\
\hline
\end{tabular}

\section{References:}

- A. Iyo, K. Kawashima, T. Kinjo, T. Nishio, S. Ishida, H. Fujihisa, Y. Gotoh, K. Kihou, H. Eisaki, and Y. Yoshida, New-Structure-Type Fe-Based Superconductors: $\mathrm{CaAFe}_{4} A s_{4}(A=K, \mathrm{Rb}, \mathrm{Cs})$ and $\mathrm{SrAFe}_{4} A s_{4}(A=R b, C s)$, J. Am. Chem. Soc. 138, 3410-3415 (2016), doi:10.1021/jacs.5b12571.

\section{Geometry files:}

- CIF: pp. 890

- POSCAR: pp. 891 


\section{$\mathrm{Nb}_{4} \mathrm{CoSi}$ Structure: AB4C_tP12_124_a_m_c}

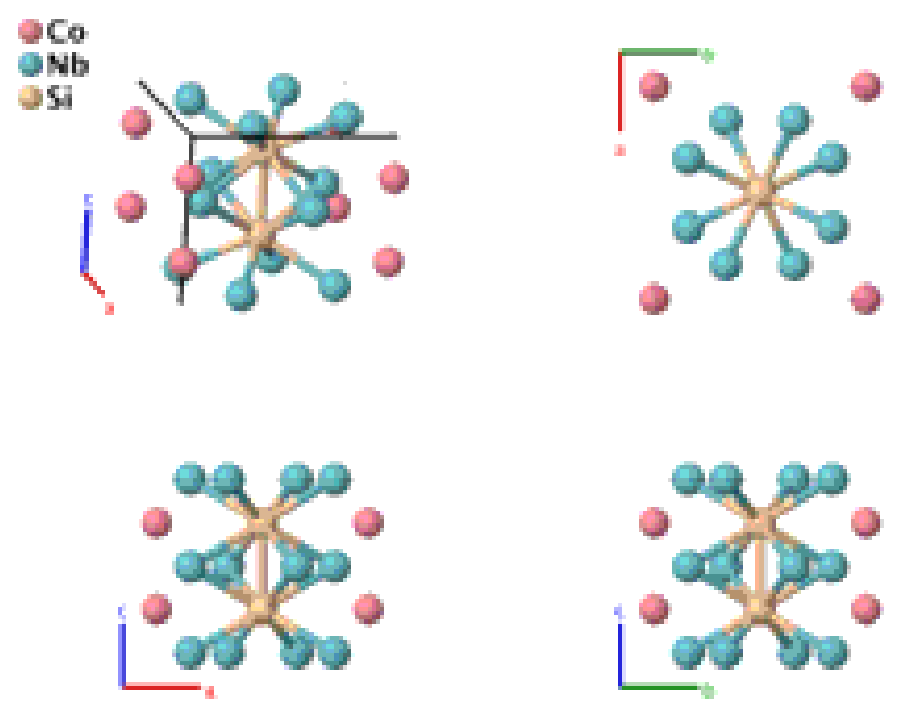

\section{Prototype}

AFLOW prototype label

Strukturbericht designation

Pearson symbol

Space group number

Space group symbol

AFLOW prototype command
: $\quad \mathrm{Nb}_{4} \mathrm{CoSi}$

: $\quad$ AB4C_tP12_124_a_m_c

: None

: $\quad \mathrm{tP} 12$

: $\quad 124$

: $\quad P 4 / m c c$

Simple Tetragonal primitive vectors:

$$
\begin{aligned}
& \mathbf{a}_{1}=a \hat{\mathbf{x}} \\
& \mathbf{a}_{2}=a \hat{\mathbf{y}} \\
& \mathbf{a}_{3}=c \hat{\mathbf{z}}
\end{aligned}
$$
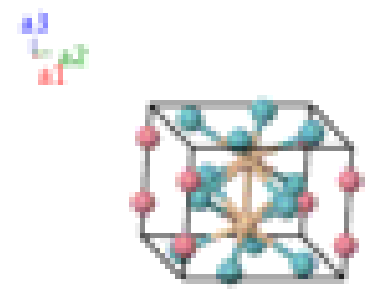

\section{Basis vectors:}

Lattice Coordinates

$\begin{array}{lcc}\mathbf{B}_{1}= & \frac{1}{4} \mathbf{a}_{3} \\ \mathbf{B}_{2}= & \frac{3}{4} \mathbf{a}_{3} \\ \mathbf{B}_{3}= & \frac{1}{2} \mathbf{a}_{1}+\frac{1}{2} \mathbf{a}_{2}+\frac{1}{4} \mathbf{a}_{3} \\ \mathbf{B}_{4}= & \frac{1}{2} \mathbf{a}_{1}+\frac{1}{2} \mathbf{a}_{2}+\frac{3}{4} \mathbf{a}_{3} \\ \mathbf{B}_{5}= & x_{3} \mathbf{a}_{1}+y_{3} \mathbf{a}_{2} \\ \mathbf{B}_{6}= & -x_{3} \mathbf{a}_{1}-y_{3} \mathbf{a}_{2}\end{array}$

$$
=
$$

$=$

$=$

$=$

$=$

$=$
Cartesian Coordinates

$\frac{1}{4} c \hat{\mathbf{z}}$

$\frac{3}{4} c \hat{\mathbf{Z}}$

$\frac{1}{2} a \hat{\mathbf{X}}+\frac{1}{2} a \hat{\mathbf{y}}+\frac{1}{4} c \hat{\mathbf{z}}$

$\frac{1}{2} a \hat{\mathbf{X}}+\frac{1}{2} a \hat{\mathbf{y}}+\frac{3}{4} c \hat{\mathbf{z}}$

$x_{3} a \hat{\mathbf{x}}+y_{3} a \hat{\mathbf{y}}$

$-x_{3} a \hat{\mathbf{x}}-y_{3} a \hat{\mathbf{y}}$
Wyckoff Position

(2a)

(2a)

(2c)

(2c)

$(8 m)$

$(8 m)$
Atom Type

Co

Co

$\mathrm{Si}$

$\mathrm{Si}$

$\mathrm{Nb}$

$\mathrm{Nb}$ 


\begin{tabular}{|c|c|c|c|c|c|c|}
\hline $\mathbf{B}_{7}$ & $=$ & $-y_{3} \mathbf{a}_{1}+x_{3} \mathbf{a}_{2}$ & $=$ & $-y_{3} a \hat{\mathbf{x}}+x_{3} a \hat{\mathbf{y}}$ & $(8 m)$ & $\mathrm{Nb}$ \\
\hline $\mathbf{B}_{8}$ & $=$ & $y_{3} \mathbf{a}_{1}-x_{3} \mathbf{a}_{2}$ & $=$ & $y_{3} a \hat{\mathbf{x}}-x_{3} a \hat{\mathbf{y}}$ & $(8 m)$ & $\mathrm{Nb}$ \\
\hline $\mathbf{B}_{9}$ & $=$ & $-x_{3} \mathbf{a}_{1}+y_{3} \mathbf{a}_{2}+\frac{1}{2} \mathbf{a}_{3}$ & $=$ & $-x_{3} a \hat{\mathbf{x}}+y_{3} a \hat{\mathbf{y}}+\frac{1}{2} c \hat{\mathbf{z}}$ & $(8 m)$ & $\mathrm{Nb}$ \\
\hline $\mathbf{B}_{10}$ & $=$ & $x_{3} \mathbf{a}_{1}-y_{3} \mathbf{a}_{2}+\frac{1}{2} \mathbf{a}_{3}$ & $=$ & $x_{3} a \hat{\mathbf{x}}-y_{3} a \hat{\mathbf{y}}+\frac{1}{2} c \hat{\mathbf{z}}$ & $(8 m)$ & $\mathrm{Nb}$ \\
\hline $\mathbf{B}_{11}$ & $=$ & $y_{3} \mathbf{a}_{1}+x_{3} \mathbf{a}_{2}+\frac{1}{2} \mathbf{a}_{3}$ & $=$ & $y_{3} a \hat{\mathbf{x}}+x_{3} a \hat{\mathbf{y}}+\frac{1}{2} c \hat{\mathbf{z}}$ & $(8 m)$ & $\mathrm{Nb}$ \\
\hline $\mathbf{B}_{12}$ & $=$ & $-y_{3} \mathbf{a}_{1}-x_{3} \mathbf{a}_{2}+\frac{1}{2} \mathbf{a}_{3}$ & $=$ & $-y_{3} a \hat{\mathbf{x}}-x_{3} a \hat{\mathbf{y}}+\frac{1}{2} c \hat{\mathbf{z}}$ & $(8 m)$ & $\mathrm{Nb}$ \\
\hline
\end{tabular}

\section{References:}

- E. I. Gladyshevskii and Y. B. Kuz'ma, The compounds $\mathrm{Nb}_{4} \mathrm{FeSi}, \mathrm{Nb}_{4} \mathrm{CoSi}, \mathrm{Nb}{ }_{4} \mathrm{NiSi}$ and their crystal structures, J. Struct. Chem. 6, 60-63 (1965), doi:10.1007/BF00743870.

\section{Found in:}

- P. Villars and K. Cenzual, Pearson's Crystal Data - Crystal Structure Database for Inorganic Compounds, ASM International (2013).

\section{Geometry files:}

- CIF: pp. 891

- POSCAR: pp. 891 

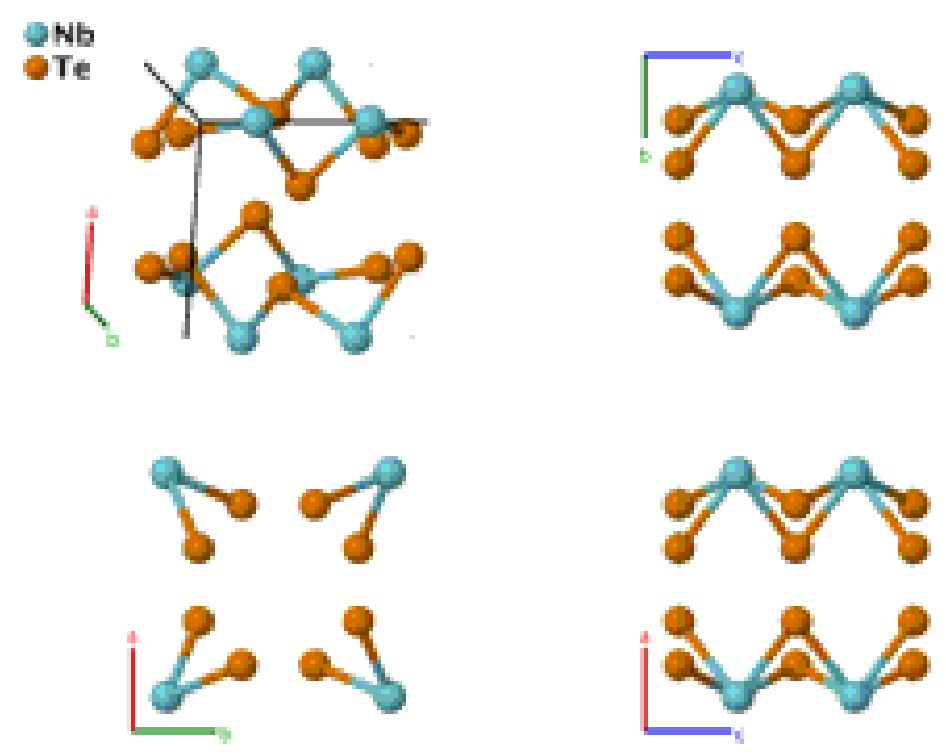

\section{Prototype}

AFLOW prototype label

: $\quad \mathrm{NbTe}_{4}$

Strukturbericht designation

Pearson symbol

: AB4_tP10_124_a_m

Space group number

: None

Space group symbol

: $\quad$ PP10

AFLOW prototype command

: 124

: $P 4 / m c c$

: aflow--proto=AB4_tP10_124_a_m

- params $=a, c / a, x_{2}, y_{2}$

\section{Simple Tetragonal primitive vectors:}

$$
\begin{aligned}
& \mathbf{a}_{1}=a \hat{\mathbf{x}} \\
& \mathbf{a}_{2}=a \hat{\mathbf{y}} \\
& \mathbf{a}_{3}=c \hat{\mathbf{z}}
\end{aligned}
$$

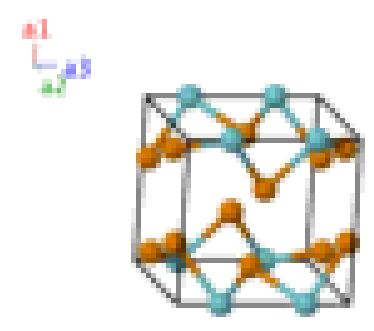

\section{Basis vectors:}

$\begin{array}{lccccc} & \text { Lattice Coordinates } & & \text { Cartesian Coordinates } & \text { Wyckoff Position } & \text { Atom Type } \\ \mathbf{B}_{1}= & \frac{1}{4} \mathbf{a}_{3} & = & \frac{1}{4} c \hat{\mathbf{z}} & (2 a) & \mathrm{Nb} \\ \mathbf{B}_{2}= & \frac{3}{4} \mathbf{a}_{3} & = & \frac{3}{4} c \hat{\mathbf{z}} & (2 a) & \mathrm{Nb} \\ \mathbf{B}_{3}= & x_{2} \mathbf{a}_{1}+y_{2} \mathbf{a}_{2} & = & x_{2} a \hat{\mathbf{x}}+y_{2} a \hat{\mathbf{y}} & (8 m) & \mathrm{Te} \\ \mathbf{B}_{4}= & -x_{2} \mathbf{a}_{1}-y_{2} \mathbf{a}_{2} & = & -x_{2} a \hat{\mathbf{x}}-y_{2} a \hat{\mathbf{y}} & (8 m) & \mathrm{Te} \\ \mathbf{B}_{5}= & -y_{2} \mathbf{a}_{1}+x_{2} \mathbf{a}_{2} & = & -y_{2} a \hat{\mathbf{x}}+x_{2} a \hat{\mathbf{y}} & (8 m) & \mathrm{Te} \\ \mathbf{B}_{6}= & y_{2} \mathbf{a}_{1}-x_{2} \mathbf{a}_{2} & = & y_{2} a \hat{\mathbf{x}}-x_{2} a \hat{\mathbf{y}} & (8 m) & \mathrm{Te}\end{array}$




$\begin{array}{rlllll}\mathbf{B}_{7}= & -x_{2} \mathbf{a}_{1}+y_{2} \mathbf{a}_{2}+\frac{1}{2} \mathbf{a}_{3} & = & -x_{2} a \hat{\mathbf{x}}+y_{2} a \hat{\mathbf{y}}+\frac{1}{2} c \hat{\mathbf{z}} & (8 m) & \mathrm{Te} \\ \mathbf{B}_{8}= & x_{2} \mathbf{a}_{1}-y_{2} \mathbf{a}_{2}+\frac{1}{2} \mathbf{a}_{3} & = & x_{2} a \hat{\mathbf{x}}-y_{2} a \hat{\mathbf{y}}+\frac{1}{2} c \hat{\mathbf{z}} & (8 m) & \mathrm{Te} \\ \mathbf{B}_{9}= & y_{2} \mathbf{a}_{1}+x_{2} \mathbf{a}_{2}+\frac{1}{2} \mathbf{a}_{3}= & y_{2} a \hat{\mathbf{x}}+x_{2} a \hat{\mathbf{y}}+\frac{1}{2} c \hat{\mathbf{z}} & (8 m) & \mathrm{Te} \\ \mathbf{B}_{10}= & -y_{2} \mathbf{a}_{1}-x_{2} \mathbf{a}_{2}+\frac{1}{2} \mathbf{a}_{3}= & -y_{2} a \hat{\mathbf{x}}-x_{2} a \hat{\mathbf{y}}+\frac{1}{2} c \hat{\mathbf{z}} & (8 m) & \mathrm{Te}\end{array}$

\section{References:}

- K. Selte and A. Kjekshus, On the crystal structure of NbTe 4 , Acta Chem. Scand. 18, 690-696 (1964), doi:10.3891/acta.chem.scand.18-0690.

\section{Found in:}

- P. Villars and K. Cenzual, Pearson's Crystal Data - Crystal Structure Database for Inorganic Compounds, ASM International (2013).

\section{Geometry files:}

- CIF: pp. 891

- POSCAR: pp. 892 


\section{$\mathrm{PtPb}_{4}$ Structure: A4B_tP10_125_m_a}

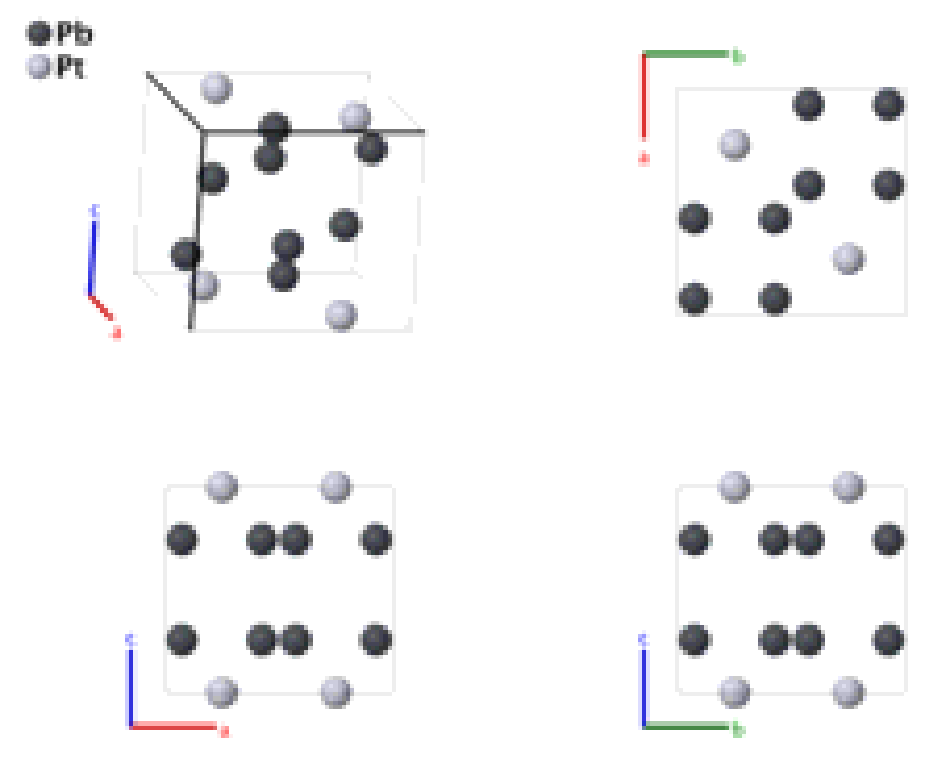

Prototype

AFLOW prototype label

Strukturbericht designation

Pearson symbol

Space group number

Space group symbol

AFLOW prototype command
: $\quad \mathrm{PtPb}_{4}$

: A4B_tP10_125_m_a

: None

: $\quad$ PP10

: $\quad 125$

: $\quad P 4 / n b m$

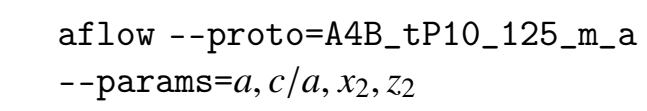

Simple Tetragonal primitive vectors:

$$
\begin{aligned}
& \mathbf{a}_{1}=a \hat{\mathbf{x}} \\
& \mathbf{a}_{2}=a \hat{\mathbf{y}} \\
& \mathbf{a}_{3}=c \hat{\mathbf{z}}
\end{aligned}
$$
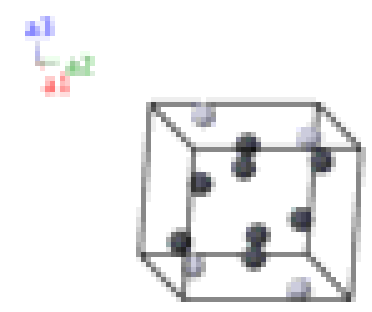

\section{Basis vectors:}

Lattice Coordinates

$\begin{array}{lcccc}\mathbf{B}_{1}= & \frac{1}{4} \mathbf{a}_{1}+\frac{1}{4} \mathbf{a}_{2} & = & \frac{1}{4} a \hat{\mathbf{x}}+\frac{1}{4} a \hat{\mathbf{y}} \\ \mathbf{B}_{2}= & \frac{3}{4} \mathbf{a}_{1}+\frac{3}{4} \mathbf{a}_{2} & = & \frac{3}{4} a \hat{\mathbf{x}}+\frac{3}{4} a \hat{\mathbf{y}} \\ \mathbf{B}_{3}= & x_{2} \mathbf{a}_{1}-x_{2} \mathbf{a}_{2}+z_{2} \mathbf{a}_{3} & = & x_{2} a \hat{\mathbf{x}}-x_{2} a \hat{\mathbf{y}}+z_{2} c \hat{\mathbf{z}} \\ \mathbf{B}_{4}= & \left(\frac{1}{2}-x_{2}\right) \mathbf{a}_{1}+\left(\frac{1}{2}+x_{2}\right) \mathbf{a}_{2}+z_{2} \mathbf{a}_{3} & = & \left(\frac{1}{2}-x_{2}\right) a \hat{\mathbf{x}}+\left(\frac{1}{2}+x_{2}\right) a \hat{\mathbf{y}}+z_{2} c \hat{\mathbf{z}} \\ \mathbf{B}_{5}= & \left(\frac{1}{2}+x_{2}\right) \mathbf{a}_{1}+x_{2} \mathbf{a}_{2}+z_{2} \mathbf{a}_{3} & & & \left(\frac{1}{2}+x_{2}\right) a \hat{\mathbf{x}}+x_{2} a \hat{\mathbf{y}}+z_{2} c \hat{\mathbf{z}}\end{array}$

$\mathbf{B}_{1}=$
Cartesian Coordinates

Wyckoff Position

(2a)

(2a)

$(8 m)$

$(8 m)$

(8m)
Atom Type

$\mathrm{Pt}$

Pt

$\mathrm{Pb}$

$\mathrm{Pb}$

$\mathrm{Pb}$ 


\begin{tabular}{|c|c|c|c|c|c|}
\hline $\mathbf{B}_{6}$ & $=$ & $-x_{2} \mathbf{a}_{1}+\left(\frac{1}{2}-x_{2}\right) \mathbf{a}_{2}+z_{2} \mathbf{a}_{3}$ & $=$ & $-x_{2} a \hat{\mathbf{x}}+\left(\frac{1}{2}-x_{2}\right) a \hat{\mathbf{y}}+z_{2} c \hat{\mathbf{z}}$ & $(8 m)$ \\
\hline $\mathbf{B}_{7}$ & $=$ & $\left(\frac{1}{2}-x_{2}\right) \mathbf{a}_{1}-x_{2} \mathbf{a}_{2}-z_{2} \mathbf{a}_{3}$ & $=$ & $\left(\frac{1}{2}-x_{2}\right) a \hat{\mathbf{x}}-x_{2} a \hat{\mathbf{y}}-z_{2} c \hat{\mathbf{z}}$ & $(8 m)$ \\
\hline $\mathbf{B}_{8}$ & $=$ & $x_{2} \mathbf{a}_{1}+\left(\frac{1}{2}+x_{2}\right) \mathbf{a}_{2}-z_{2} \mathbf{a}_{3}$ & $=$ & $x_{2} a \hat{\mathbf{x}}+\left(\frac{1}{2}+x_{2}\right) a \hat{\mathbf{y}}-z_{2} c \hat{\mathbf{z}}$ & $(8 m)$ \\
\hline $\mathbf{B}_{9}$ & $=$ & $-x_{2} \mathbf{a}_{1}+x_{2} \mathbf{a}_{2}-z_{2} \mathbf{a}_{3}$ & $=$ & $-x_{2} a \hat{\mathbf{x}}+x_{2} a \hat{\mathbf{y}}-z_{2} c \hat{\mathbf{z}}$ & $(8 m)$ \\
\hline $\mathbf{B}_{10}$ & $=$ & $\left(\frac{1}{2}+x_{2}\right) \mathbf{a}_{1}+\left(\frac{1}{2}-x_{2}\right) \mathbf{a}_{2}-z_{2} \mathbf{a}_{3}$ & $=$ & $\left(\frac{1}{2}+x_{2}\right) a \hat{\mathbf{x}}+\left(\frac{1}{2}-x_{2}\right) a \hat{\mathbf{y}}-z_{2} c \hat{\mathbf{z}}$ & $(8 m)$ \\
\hline
\end{tabular}

\section{References:}

- R. Graham, G. C. S. Waghorn, and P. T. Davies, An X-ray investigation of the lead-platinum system, Acta Cryst. 7, 634-635 (1954).

\section{Found in:}

- P. Villars and K. Cenzual, Pearson's Crystal Data - Crystal Structure Database for Inorganic Compounds, ASM International (2013).

\section{Geometry files:}

- CIF: pp. 892

- POSCAR: pp. 892 

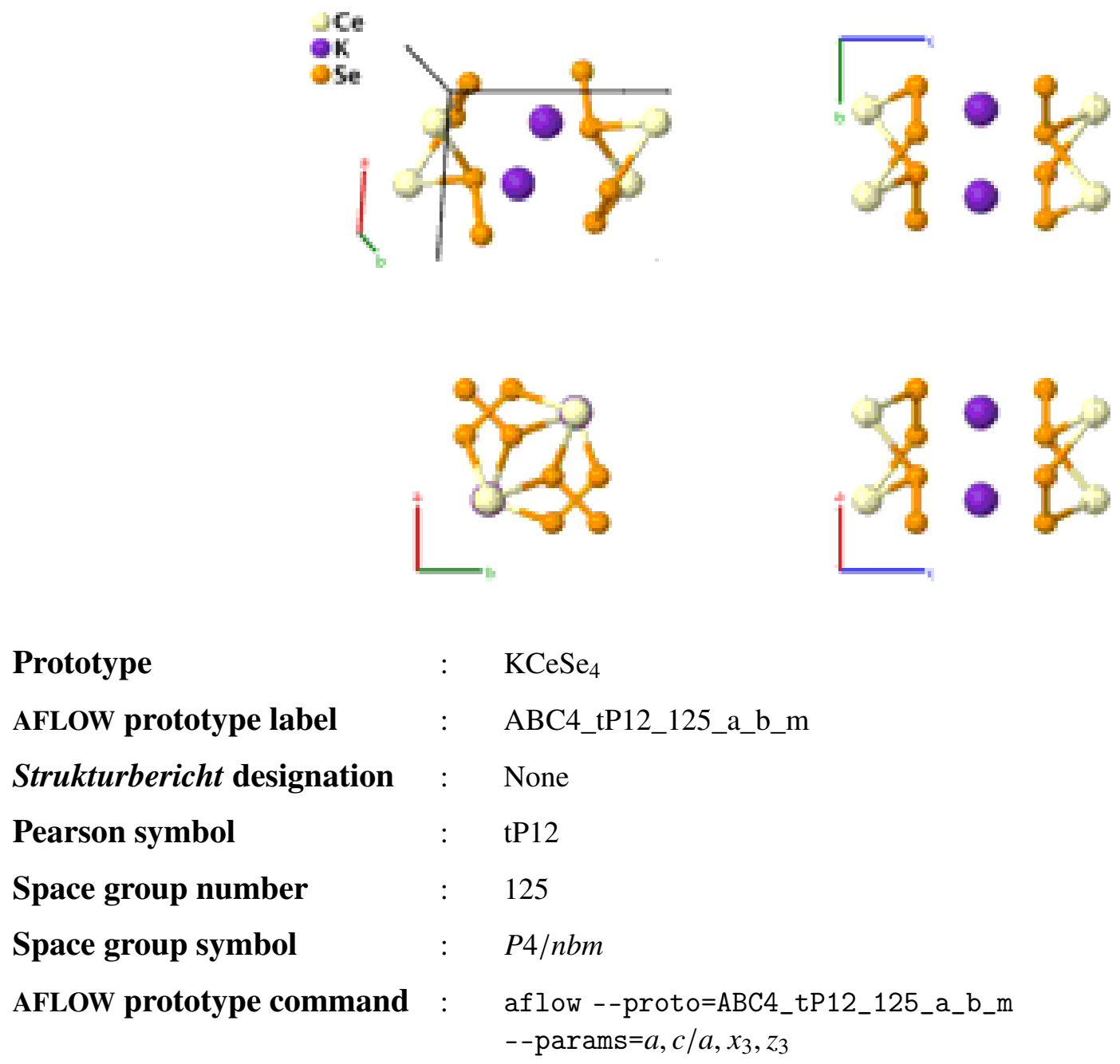

\section{Simple Tetragonal primitive vectors:}

$$
\begin{aligned}
& \mathbf{a}_{1}=a \hat{\mathbf{x}} \\
& \mathbf{a}_{2}=a \hat{\mathbf{y}} \\
& \mathbf{a}_{3}=c \hat{\mathbf{z}}
\end{aligned}
$$

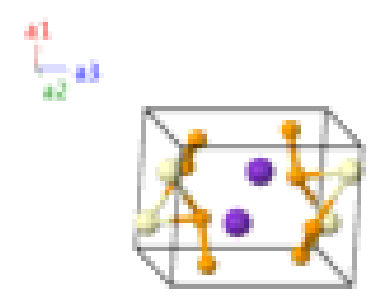

\section{Basis vectors:}

\section{Lattice Coordinates}

$\begin{array}{lcccc}\mathbf{B}_{1}= & \frac{1}{4} \mathbf{a}_{1}+\frac{1}{4} \mathbf{a}_{2} & = & \frac{1}{4} a \hat{\mathbf{x}}+\frac{1}{4} a \hat{\mathbf{y}} \\ \mathbf{B}_{2}= & \frac{3}{4} \mathbf{a}_{1}+\frac{3}{4} \mathbf{a}_{2} & = & \frac{3}{4} a \hat{\mathbf{x}}+\frac{3}{4} a \hat{\mathbf{y}} \\ \mathbf{B}_{3}= & \frac{1}{4} \mathbf{a}_{1}+\frac{1}{4} \mathbf{a}_{2}+\frac{1}{2} \mathbf{a}_{3} & = & \frac{1}{4} a \hat{\mathbf{x}}+\frac{1}{4} a \hat{\mathbf{y}}+\frac{1}{2} c \hat{\mathbf{z}} \\ \mathbf{B}_{4}= & \frac{3}{4} \mathbf{a}_{1}+\frac{3}{4} \mathbf{a}_{2}+\frac{1}{2} \mathbf{a}_{3} & = & \frac{3}{4} a \hat{\mathbf{x}}+\frac{3}{4} a \hat{\mathbf{y}}+\frac{1}{2} c \hat{\mathbf{z}} \\ \mathbf{B}_{5}= & x_{3} \mathbf{a}_{1}-x_{3} \mathbf{a}_{2}+z_{3} \mathbf{a}_{3} & = & x_{3} a \hat{\mathbf{x}}-x_{3} a \hat{\mathbf{y}}+z_{3} c \hat{\mathbf{z}} \\ \mathbf{B}_{6}= & \left(\frac{1}{2}-x_{3}\right) \mathbf{a}_{1}+\left(\frac{1}{2}+x_{3}\right) \mathbf{a}_{2}+z_{3} \mathbf{a}_{3} & = & \left(\frac{1}{2}-x_{3}\right) a \hat{\mathbf{x}}+\left(\frac{1}{2}+x_{3}\right) a \hat{\mathbf{y}}+z_{3} c \hat{\mathbf{z}}\end{array}$

$\mathbf{B}_{1}=$

$\mathbf{B}_{2}=$

$\mathbf{B}_{3}=$
Cartesian Coordinates

Wyckoff Position Atom Type

(2a)

(2a)

$(2 b)$

(2b)

$(8 m)$

$(8 m)$
$\mathrm{Ce}$

$\mathrm{Ce}$

K

K

$\mathrm{Se}$

$\mathrm{Se}$ 


\begin{tabular}{|c|c|c|c|c|c|}
\hline $\mathbf{B}_{7}$ & $=$ & $\left(\frac{1}{2}+x_{3}\right) \mathbf{a}_{1}+x_{3} \mathbf{a}_{2}+z_{3} \mathbf{a}_{3}$ & $=$ & $\left(\frac{1}{2}+x_{3}\right) a \hat{\mathbf{x}}+x_{3} a \hat{\mathbf{y}}+z_{3} c \hat{\mathbf{z}}$ & $(8 m)$ \\
\hline $\mathbf{B}_{8}$ & $=$ & $-x_{3} \mathbf{a}_{1}+\left(\frac{1}{2}-x_{3}\right) \mathbf{a}_{2}+z_{3} \mathbf{a}_{3}$ & $=$ & $-x_{3} a \hat{\mathbf{x}}+\left(\frac{1}{2}-x_{3}\right) a \hat{\mathbf{y}}+z_{3} c \hat{\mathbf{z}}$ & $(8 m)$ \\
\hline $\mathbf{B}_{9}$ & $=$ & $\left(\frac{1}{2}-x_{3}\right) \mathbf{a}_{1}-x_{3} \mathbf{a}_{2}-z_{3} \mathbf{a}_{3}$ & $=$ & $\left(\frac{1}{2}-x_{3}\right) a \hat{\mathbf{x}}-x_{3} a \hat{\mathbf{y}}-z_{3} c \hat{\mathbf{z}}$ & $(8 m)$ \\
\hline $\mathbf{B}_{10}$ & $=$ & $x_{3} \mathbf{a}_{1}+\left(\frac{1}{2}+x_{3}\right) \mathbf{a}_{2}-z_{3} \mathbf{a}_{3}$ & $=$ & $x_{3} a \hat{\mathbf{x}}+\left(\frac{1}{2}+x_{3}\right) a \hat{\mathbf{y}}-z_{3} c \hat{\mathbf{z}}$ & $(8 m)$ \\
\hline $\mathbf{B}_{11}$ & $=$ & $-x_{3} \mathbf{a}_{1}+x_{3} \mathbf{a}_{2}-z_{3} \mathbf{a}_{3}$ & $=$ & $-x_{3} a \hat{\mathbf{x}}+x_{3} a \hat{\mathbf{y}}-z_{3} c \hat{\mathbf{z}}$ & $(8 m)$ \\
\hline $\mathbf{B}_{12}$ & $=$ & $\left.\frac{1}{2}+x_{3}\right) \mathbf{a}_{1}+\left(\frac{1}{2}-x_{3}\right) \mathbf{a}_{2}-z_{3} \mathbf{a}_{3}$ & $=$ & $\left(\frac{1}{2}+x_{3}\right) a \hat{\mathbf{x}}+\left(\frac{1}{2}-x_{3}\right) a \hat{\mathbf{y}}-z_{3} c \hat{\mathbf{z}}$ & $(8 m)$ \\
\hline
\end{tabular}

\section{References:}

- A. C. Sutorik and M. G. Kanatzidis, $\mathrm{KCeSe}_{4}$ : A New Solid-State Lanthanide Polychalcogenide, Angew. Chem. Int. Ed. 31, 1594-1596 (1992), doi:10.1002/anie.199215941.

\section{Found in:}

- P. Villars and K. Cenzual, Pearson's Crystal Data - Crystal Structure Database for Inorganic Compounds, ASM International (2013).

\section{Geometry files:}

- CIF: pp. 892

- POSCAR: pp. 893 


\section{$\mathrm{BiAl}_{2} \mathrm{~S}_{4}$ Structure: A2BC4_tP28_126_cd_e_k}
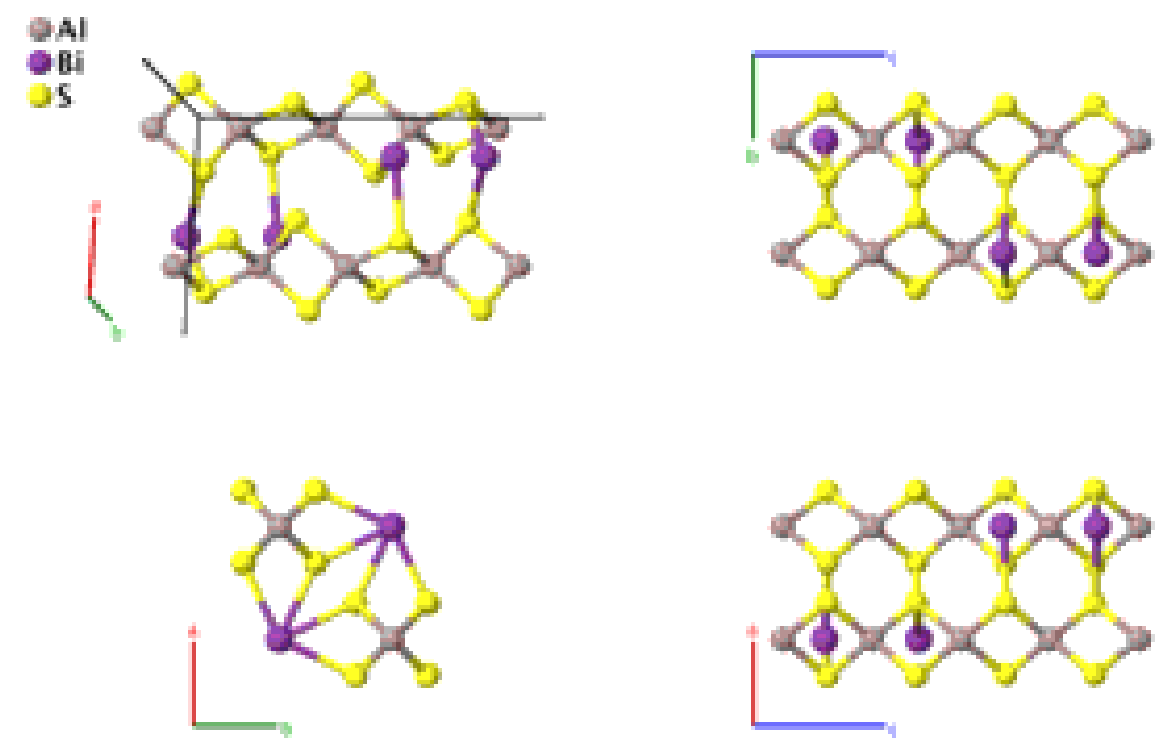

\section{Prototype}

AFLOW prototype label

Strukturbericht designation

Pearson symbol

Space group number

Space group symbol

AFLOW prototype command
$: \quad \mathrm{BiAl}_{2} \mathrm{~S}_{4}$

: A2BC4_tP28_126_cd_e_k

: None

: $\quad$ tP28

: $\quad 126$

: $\quad P 4 / n n c$

aflow - -proto=A2BC4_tP28_126_cd_e_k

- params $=a, c / a, z_{3}, x_{4}, y_{4}, z_{4}$

\section{Simple Tetragonal primitive vectors:}

$$
\begin{aligned}
& \mathbf{a}_{1}=a \hat{\mathbf{x}} \\
& \mathbf{a}_{2}=a \hat{\mathbf{y}} \\
& \mathbf{a}_{3}=c \hat{\mathbf{z}}
\end{aligned}
$$

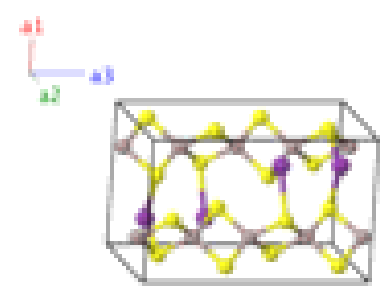

\section{Basis vectors:}

Lattice Coordinates

$\mathbf{B}_{1}=\quad \frac{1}{4} \mathbf{a}_{1}+\frac{3}{4} \mathbf{a}_{2}+\frac{3}{4} \mathbf{a}_{3}$

$\mathbf{B}_{2}=\frac{3}{4} \mathbf{a}_{1}+\frac{1}{4} \mathbf{a}_{2}+\frac{3}{4} \mathbf{a}_{3}$

$\mathbf{B}_{3}=\quad \frac{3}{4} \mathbf{a}_{1}+\frac{1}{4} \mathbf{a}_{2}+\frac{1}{4} \mathbf{a}_{3}$

$\mathbf{B}_{4}=\frac{1}{4} \mathbf{a}_{1}+\frac{3}{4} \mathbf{a}_{2}+\frac{1}{4} \mathbf{a}_{3}$

$\mathbf{B}_{5}=$

$\mathbf{B}_{6}=$

$\frac{1}{4} \mathbf{a}_{1}+\frac{3}{4} \mathbf{a}_{2}$

$\frac{3}{4} \mathbf{a}_{1}+\frac{1}{4} \mathbf{a}_{2}$
Cartesian Coordinates

$=\quad \frac{1}{4} a \hat{\mathbf{x}}+\frac{3}{4} a \hat{\mathbf{y}}+\frac{3}{4} c \hat{\mathbf{z}}$

$=\quad \frac{3}{4} a \hat{\mathbf{x}}+\frac{1}{4} a \hat{\mathbf{y}}+\frac{3}{4} c \hat{\mathbf{z}}$

$=\quad \frac{3}{4} a \hat{\mathbf{x}}+\frac{1}{4} a \hat{\mathbf{y}}+\frac{1}{4} c \hat{\mathbf{z}}$

$=\quad \frac{1}{4} a \hat{\mathbf{x}}+\frac{3}{4} a \hat{\mathbf{y}}+\frac{1}{4} c \hat{\mathbf{z}}$

$=\quad \frac{1}{4} a \hat{\mathbf{x}}+\frac{3}{4} a \hat{\mathbf{y}}$

$=\quad \frac{3}{4} a \hat{\mathbf{x}}+\frac{1}{4} a \hat{\mathbf{y}}$
Wyckoff Position

(4c)

(4d)
Atom Type

Al I

Al I

Al I

Al I

Al II

Al II 


\begin{tabular}{|c|c|c|c|c|c|}
\hline $\mathbf{B}_{7}$ & $=$ & $\frac{1}{4} \mathbf{a}_{1}+\frac{3}{4} \mathbf{a}_{2}+\frac{1}{2} \mathbf{a}_{3}$ & $=$ & $\frac{1}{4} a \hat{\mathbf{x}}+\frac{3}{4} a \hat{\mathbf{y}}+\frac{1}{2} c \hat{\mathbf{z}}$ & $(4 d)$ \\
\hline $\mathbf{B}_{8}$ & $=$ & $\frac{3}{4} \mathbf{a}_{1}+\frac{1}{4} \mathbf{a}_{2}+\frac{1}{2} \mathbf{a}_{3}$ & $=$ & $\frac{3}{4} a \hat{\mathbf{x}}+\frac{1}{4} a \hat{\mathbf{y}}+\frac{1}{2} c \hat{\mathbf{z}}$ & $(4 d)$ \\
\hline $\mathbf{B}_{9}$ & $=$ & $\frac{1}{4} \mathbf{a}_{1}+\frac{1}{4} \mathbf{a}_{2}+z_{3} \mathbf{a}_{3}$ & $=$ & $\frac{1}{4} a \hat{\mathbf{x}}+\frac{1}{4} a \hat{\mathbf{y}}+z_{3} c \hat{\mathbf{z}}$ & $(4 e)$ \\
\hline $\mathbf{B}_{10}$ & $=$ & $\frac{1}{4} \mathbf{a}_{1}+\frac{1}{4} \mathbf{a}_{2}+\left(\frac{1}{2}-z_{3}\right) \mathbf{a}_{3}$ & $=$ & $\frac{1}{4} a \hat{\mathbf{x}}+\frac{1}{4} a \hat{\mathbf{y}}+\left(\frac{1}{2}-z_{3}\right) c \hat{\mathbf{z}}$ & $(4 e)$ \\
\hline $\mathbf{B}_{11}$ & $=$ & $\frac{3}{4} \mathbf{a}_{1}+\frac{3}{4} \mathbf{a}_{2}-z_{3} \mathbf{a}_{3}$ & $=$ & $\frac{3}{4} a \hat{\mathbf{x}}+\frac{3}{4} a \hat{\mathbf{y}}-z_{3} c \hat{\mathbf{z}}$ & $(4 e)$ \\
\hline $\mathbf{B}_{12}$ & $=$ & $\frac{3}{4} \mathbf{a}_{1}+\frac{3}{4} \mathbf{a}_{2}+\left(\frac{1}{2}+z_{3}\right) \mathbf{a}_{3}$ & $=$ & $\frac{3}{4} a \hat{\mathbf{x}}+\frac{3}{4} a \hat{\mathbf{y}}+\left(\frac{1}{2}+z_{3}\right) c \hat{\mathbf{z}}$ & $(4 e)$ \\
\hline $\mathbf{B}_{13}$ & $=$ & $x_{4} \mathbf{a}_{1}+y_{4} \mathbf{a}_{2}+z_{4} \mathbf{a}_{3}$ & $=$ & $x_{4} a \hat{\mathbf{x}}+y_{4} a \hat{\mathbf{y}}+z_{4} c \hat{\mathbf{z}}$ & $(16 k)$ \\
\hline $\mathbf{B}_{14}$ & $=$ & $\left(\frac{1}{2}-x_{4}\right) \mathbf{a}_{1}+\left(\frac{1}{2}-y_{4}\right) \mathbf{a}_{2}+z_{4} \mathbf{a}_{3}$ & $=$ & $\left(\frac{1}{2}-x_{4}\right) a \hat{\mathbf{x}}+\left(\frac{1}{2}-y_{4}\right) a \hat{\mathbf{y}}+z_{4} c \hat{\mathbf{z}}$ & $(16 k)$ \\
\hline $\mathbf{B}_{15}$ & $=$ & $\left(\frac{1}{2}-y_{4}\right) \mathbf{a}_{1}+x_{4} \mathbf{a}_{2}+z_{4} \mathbf{a}_{3}$ & $=$ & $\left(\frac{1}{2}-y_{4}\right) a \hat{\mathbf{x}}+x_{4} a \hat{\mathbf{y}}+z_{4} c \hat{\mathbf{z}}$ & $(16 k)$ \\
\hline $\mathbf{B}_{16}$ & $=$ & $y_{4} \mathbf{a}_{1}+\left(\frac{1}{2}-x_{4}\right) \mathbf{a}_{2}+z_{4} \mathbf{a}_{3}$ & $=$ & $y_{4} a \hat{\mathbf{x}}+\left(\frac{1}{2}-x_{4}\right) a \hat{\mathbf{y}}+z_{4} c \hat{\mathbf{z}}$ & $(16 k)$ \\
\hline $\mathbf{B}_{17}$ & $=$ & $\left(\frac{1}{2}-x_{4}\right) \mathbf{a}_{1}+y_{4} \mathbf{a}_{2}+\left(\frac{1}{2}-z_{4}\right) \mathbf{a}_{3}$ & $=$ & $\left(\frac{1}{2}-x_{4}\right) a \hat{\mathbf{x}}+y_{4} a \hat{\mathbf{y}}+\left(\frac{1}{2}-z_{4}\right) c \hat{\mathbf{z}}$ & $(16 k)$ \\
\hline $\mathbf{B}_{18}$ & $=$ & $x_{4} \mathbf{a}_{1}+\left(\frac{1}{2}-y_{4}\right) \mathbf{a}_{2}+\left(\frac{1}{2}-z_{4}\right) \mathbf{a}_{3}$ & $=$ & $x_{4} a \hat{\mathbf{x}}+\left(\frac{1}{2}-y_{4}\right) a \hat{\mathbf{y}}+\left(\frac{1}{2}-z_{4}\right) c \hat{\mathbf{z}}$ & $(16 k)$ \\
\hline $\mathbf{B}_{19}$ & $=$ & $y_{4} \mathbf{a}_{1}+x_{4} \mathbf{a}_{2}+\left(\frac{1}{2}-z_{4}\right) \mathbf{a}_{3}$ & $=$ & $y_{4} a \hat{\mathbf{x}}+x_{4} a \hat{\mathbf{y}}+\left(\frac{1}{2}-z_{4}\right) c \hat{\mathbf{z}}$ & $(16 k)$ \\
\hline $\mathbf{B}_{20}$ & $=$ & $\begin{array}{c}\left(\frac{1}{2}-y_{4}\right) \mathbf{a}_{1}+\left(\frac{1}{2}-x_{4}\right) \mathbf{a}_{2}+ \\
\left(\frac{1}{2}-z_{4}\right) \mathbf{a}_{3}\end{array}$ & $=$ & $\begin{array}{c}\left(\frac{1}{2}-y_{4}\right) a \hat{\mathbf{x}}+\left(\frac{1}{2}-x_{4}\right) a \hat{\mathbf{y}}+ \\
\left(\frac{1}{2}-z_{4}\right) c \hat{\mathbf{z}}\end{array}$ & $(16 k)$ \\
\hline $\mathbf{B}_{21}$ & $=$ & $-x_{4} \mathbf{a}_{1}-y_{4} \mathbf{a}_{2}-z_{4} \mathbf{a}_{3}$ & $=$ & $-x_{4} a \hat{\mathbf{x}}-y_{4} a \hat{\mathbf{y}}-z_{4} c \hat{\mathbf{z}}$ & $(16 k)$ \\
\hline $\mathbf{B}_{22}$ & $=$ & $\left(\frac{1}{2}+x_{4}\right) \mathbf{a}_{1}+\left(\frac{1}{2}+y_{4}\right) \mathbf{a}_{2}-z_{4} \mathbf{a}_{3}$ & $=$ & $\left(\frac{1}{2}+x_{4}\right) a \hat{\mathbf{x}}+\left(\frac{1}{2}+y_{4}\right) a \hat{\mathbf{y}}-z_{4} c \hat{\mathbf{z}}$ & $(16 k)$ \\
\hline $\mathbf{B}_{23}$ & $=$ & $\left(\frac{1}{2}+y_{4}\right) \mathbf{a}_{1}-x_{4} \mathbf{a}_{2}-z_{4} \mathbf{a}_{3}$ & $=$ & $\left(\frac{1}{2}+y_{4}\right) a \hat{\mathbf{x}}-x_{4} a \hat{\mathbf{y}}-z_{4} c \hat{\mathbf{z}}$ & $(16 k)$ \\
\hline $\mathbf{B}_{24}$ & $=$ & $-y_{4} \mathbf{a}_{1}+\left(\frac{1}{2}+x_{4}\right) \mathbf{a}_{2}-z_{4} \mathbf{a}_{3}$ & $=$ & $-y_{4} a \hat{\mathbf{x}}+\left(\frac{1}{2}+x_{4}\right) a \hat{\mathbf{y}}-z_{4} c \hat{\mathbf{z}}$ & $(16 k)$ \\
\hline $\mathbf{B}_{25}$ & $=$ & $\left(\frac{1}{2}+x_{4}\right) \mathbf{a}_{1}-y_{4} \mathbf{a}_{2}+\left(\frac{1}{2}+z_{4}\right) \mathbf{a}_{3}$ & $=$ & $\left(\frac{1}{2}+x_{4}\right) a \hat{\mathbf{x}}-y_{4} a \hat{\mathbf{y}}+\left(\frac{1}{2}+z_{4}\right) c \hat{\mathbf{z}}$ & $(16 k)$ \\
\hline $\mathbf{B}_{26}$ & $=$ & $-x_{4} \mathbf{a}_{1}+\left(\frac{1}{2}+y_{4}\right) \mathbf{a}_{2}+\left(\frac{1}{2}+z_{4}\right) \mathbf{a}_{3}$ & $=$ & $-x_{4} a \hat{\mathbf{x}}+\left(\frac{1}{2}+y_{4}\right) a \hat{\mathbf{y}}+\left(\frac{1}{2}+z_{4}\right) c \hat{\mathbf{z}}$ & $(16 k)$ \\
\hline $\mathbf{B}_{27}$ & $=$ & $-y_{4} \mathbf{a}_{1}-x_{4} \mathbf{a}_{2}+\left(\frac{1}{2}+z_{4}\right) \mathbf{a}_{3}$ & $=$ & $-y_{4} a \hat{\mathbf{x}}-x_{4} a \hat{\mathbf{y}}+\left(\frac{1}{2}+z_{4}\right) c \hat{\mathbf{z}}$ & $(16 k)$ \\
\hline $\mathbf{B}_{28}$ & $=$ & $\begin{array}{c}\left(\frac{1}{2}+y_{4}\right) \mathbf{a}_{1}+\left(\frac{1}{2}+x_{4}\right) \mathbf{a}_{2}+ \\
\left(\frac{1}{2}+z_{4}\right) \mathbf{a}_{3}\end{array}$ & $=$ & $\begin{array}{c}\left(\frac{1}{2}+y_{4}\right) a \hat{\mathbf{x}}+\left(\frac{1}{2}+x_{4}\right) a \hat{\mathbf{y}}+ \\
\left(\frac{1}{2}+z_{4}\right) c \hat{\mathbf{z}}\end{array}$ & $(16 k)$ \\
\hline
\end{tabular}

\section{References:}

- H. Kalpen, W. Hönle, M. Somer, U. Schwarz, K. Peters, H. G. von Schnering, and R. Blachnik,

Bismut(II)-chalkogenometallate(III) Bi $\mathrm{i}_{2} \mathrm{M}_{4} \mathrm{X}_{8}$, Verbindungen mit Bi ${ }_{2}^{4+}$-Hanteln $(M=A l, G a ; X=S, S e)$, Z. Anorg. Allg.

Chem. 624, 1137-1147 (1998), doi:10.1002/(SICI)1521-3749(199807)624:7<1137::AID-ZAAC1137>3.0.CO;2-B.

\section{Found in:}

- P. Villars and K. Cenzual, Pearson's Crystal Data - Crystal Structure Database for Inorganic Compounds, ASM International (2013).

\section{Geometry files:}

- CIF: pp. 893

- POSCAR: pp. 893 


\section{$\mathrm{ThB}_{4}\left(D 1_{e}\right)$ Structure: A4B_tP20_127_ehj_g}
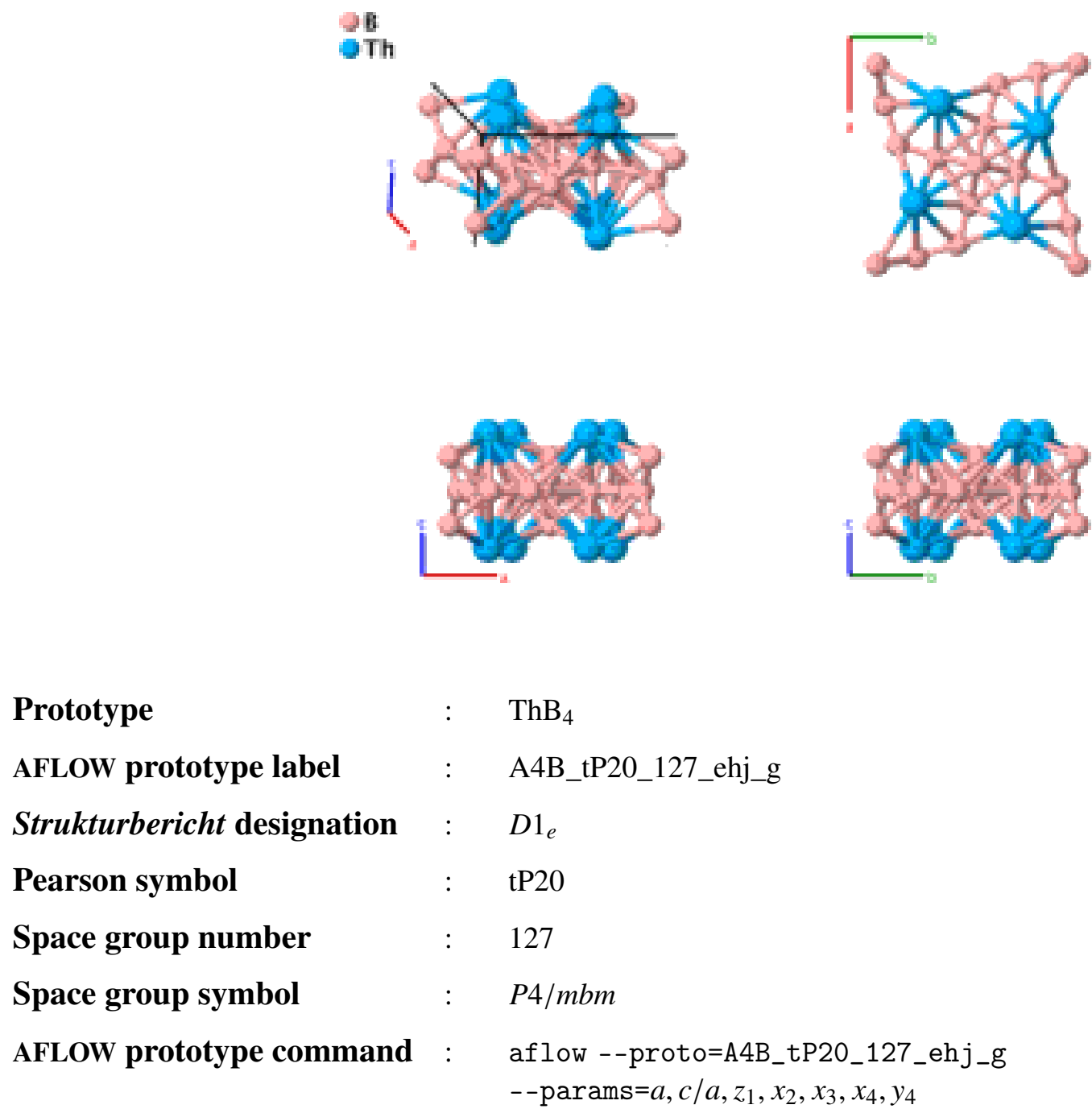

Other compounds with this structure:

- $\mathrm{B}_{4} \mathrm{Ce}, \mathrm{B}_{4} \mathrm{U}, \mathrm{B}_{4} \mathrm{Y}$

\section{Simple Tetragonal primitive vectors:}

$$
\begin{aligned}
& \mathbf{a}_{1}=a \hat{\mathbf{x}} \\
& \mathbf{a}_{2}=a \hat{\mathbf{y}} \\
& \mathbf{a}_{3}=c \hat{\mathbf{z}}
\end{aligned}
$$

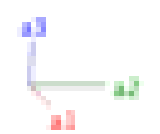

Basis vectors:

Lattice Coordinates

$\begin{array}{ccccc}\mathbf{B}_{1}= & z_{1} \mathbf{a}_{3} & & & z_{1} c \hat{\mathbf{z}} \\ \mathbf{B}_{2}= & \frac{1}{2} \mathbf{a}_{1}+\frac{1}{2} \mathbf{a}_{2}-z_{1} \mathbf{a}_{3} & & & \frac{1}{2} a \hat{\mathbf{x}}+\frac{1}{2} a \hat{\mathbf{y}}-z_{1} c \hat{\mathbf{z}}\end{array}$

Wyckoff Position

Atom Type

$(4 e)$

B I

(4e) 


\begin{tabular}{|c|c|c|c|c|c|c|}
\hline $\mathbf{B}_{3}$ & $=$ & $-z_{1} \mathbf{a}_{3}$ & $=$ & $-z_{1} c \hat{\mathbf{z}}$ & $(4 e)$ & B I \\
\hline $\mathbf{B}_{4}$ & $=$ & $\frac{1}{2} \mathbf{a}_{1}+\frac{1}{2} \mathbf{a}_{2}+z_{1} \mathbf{a}_{3}$ & $=$ & $\frac{1}{2} a \hat{\mathbf{x}}+\frac{1}{2} a \hat{\mathbf{y}}+z_{1} c \hat{\mathbf{z}}$ & $(4 e)$ & B I \\
\hline $\mathbf{B}_{5}$ & $=$ & $x_{2} \mathbf{a}_{1}+\left(\frac{1}{2}+x_{2}\right) \mathbf{a}_{2}$ & $=$ & $x_{2} a \hat{\mathbf{x}}+\left(\frac{1}{2}+x_{2}\right) a \hat{\mathbf{y}}$ & $(4 g)$ & Th \\
\hline $\mathbf{B}_{6}$ & $=$ & $-x_{2} \mathbf{a}_{1}+\left(\frac{1}{2}-x_{2}\right) \mathbf{a}_{2}$ & $=$ & $-x_{2} a \hat{\mathbf{x}}+\left(\frac{1}{2}-x_{2}\right) a \hat{\mathbf{y}}$ & $(4 g)$ & Th \\
\hline $\mathbf{B}_{7}$ & $=$ & $\left(\frac{1}{2}-x_{2}\right) \mathbf{a}_{1}+x_{2} \mathbf{a}_{2}$ & $=$ & $\left(\frac{1}{2}-x_{2}\right) a \hat{\mathbf{x}}+x_{2} a \hat{\mathbf{y}}$ & $(4 g)$ & Th \\
\hline $\mathbf{B}_{8}$ & $=$ & $\left(\frac{1}{2}+x_{2}\right) \mathbf{a}_{1}-x_{2} \mathbf{a}_{2}$ & $=$ & $\left(\frac{1}{2}+x_{2}\right) a \hat{\mathbf{x}}-x_{2} a \hat{\mathbf{y}}$ & $(4 g)$ & Th \\
\hline $\mathbf{B}_{9}$ & $=$ & $x_{3} \mathbf{a}_{1}+\left(\frac{1}{2}+x_{3}\right) \mathbf{a}_{2}+\frac{1}{2} \mathbf{a}_{3}$ & $=$ & $x_{3} a \hat{\mathbf{x}}+\left(\frac{1}{2}+x_{3}\right) a \hat{\mathbf{y}}+\frac{1}{2} c \hat{\mathbf{z}}$ & $(4 h)$ & B II \\
\hline $\mathbf{B}_{10}$ & $=$ & $-x_{3} \mathbf{a}_{1}+\left(\frac{1}{2}-x_{3}\right) \mathbf{a}_{2}+\frac{1}{2} \mathbf{a}_{3}$ & $=$ & $-x_{3} a \hat{\mathbf{x}}+\left(\frac{1}{2}-x_{3}\right) a \hat{\mathbf{y}}+\frac{1}{2} c \hat{\mathbf{z}}$ & $(4 h)$ & B II \\
\hline $\mathbf{B}_{11}$ & $=$ & $\left(\frac{1}{2}-x_{3}\right) \mathbf{a}_{1}+x_{3} \mathbf{a}_{2}+\frac{1}{2} \mathbf{a}_{3}$ & $=$ & $\left(\frac{1}{2}-x_{3}\right) a \hat{\mathbf{x}}+x_{3} a \hat{\mathbf{y}}+\frac{1}{2} c \hat{\mathbf{z}}$ & $(4 h)$ & B II \\
\hline $\mathbf{B}_{12}$ & $=$ & $\left(\frac{1}{2}+x_{3}\right) \mathbf{a}_{1}-x_{3} \mathbf{a}_{2}+\frac{1}{2} \mathbf{a}_{3}$ & $=$ & $\left(\frac{1}{2}+x_{3}\right) a \hat{\mathbf{x}}-x_{3} a \hat{\mathbf{y}}+\frac{1}{2} c \hat{\mathbf{z}}$ & $(4 h)$ & B II \\
\hline $\mathbf{B}_{13}$ & $=$ & $x_{4} \mathbf{a}_{1}+y_{4} \mathbf{a}_{2}+\frac{1}{2} \mathbf{a}_{3}$ & $=$ & $x_{4} a \hat{\mathbf{x}}+y_{4} a \hat{\mathbf{y}}+\frac{1}{2} c \hat{\mathbf{z}}$ & $(8 j)$ & B III \\
\hline $\mathbf{B}_{14}$ & $=$ & $-x_{4} \mathbf{a}_{1}-y_{4} \mathbf{a}_{2}+\frac{1}{2} \mathbf{a}_{3}$ & $=$ & $-x_{4} a \hat{\mathbf{x}}-y_{4} a \hat{\mathbf{y}}+\frac{1}{2} c \hat{\mathbf{z}}$ & $(8 j)$ & B III \\
\hline $\mathbf{B}_{15}$ & $=$ & $-y_{4} \mathbf{a}_{1}+x_{4} \mathbf{a}_{2}+\frac{1}{2} \mathbf{a}_{3}$ & $=$ & $-y_{4} a \hat{\mathbf{x}}+x_{4} a \hat{\mathbf{y}}+\frac{1}{2} c \hat{\mathbf{z}}$ & $(8 j)$ & B III \\
\hline $\mathbf{B}_{16}$ & $=$ & $y_{4} \mathbf{a}_{1}-x_{4} \mathbf{a}_{2}+\frac{1}{2} \mathbf{a}_{3}$ & $=$ & $y_{4} a \hat{\mathbf{x}}-x_{4} a \hat{\mathbf{y}}+\frac{1}{2} c \hat{\mathbf{z}}$ & $(8 j)$ & B III \\
\hline $\mathbf{B}_{17}$ & $=$ & $\left(\frac{1}{2}-x_{4}\right) \mathbf{a}_{1}+\left(\frac{1}{2}+y_{4}\right) \mathbf{a}_{2}+\frac{1}{2} \mathbf{a}_{3}$ & $=$ & $\left(\frac{1}{2}-x_{4}\right) a \hat{\mathbf{x}}+\left(\frac{1}{2}+y_{4}\right) a \hat{\mathbf{y}}+\frac{1}{2} c \hat{\mathbf{z}}$ & $(8 j)$ & B III \\
\hline $\mathbf{B}_{18}$ & $=$ & $\left(\frac{1}{2}+x_{4}\right) \mathbf{a}_{1}+\left(\frac{1}{2}-y_{4}\right) \mathbf{a}_{2}+\frac{1}{2} \mathbf{a}_{3}$ & $=$ & $\left(\frac{1}{2}+x_{4}\right) a \hat{\mathbf{x}}+\left(\frac{1}{2}-y_{4}\right) a \hat{\mathbf{y}}+\frac{1}{2} c \hat{\mathbf{z}}$ & $(8 j)$ & B III \\
\hline $\mathbf{B}_{19}$ & $=$ & $\left(\frac{1}{2}+y_{4}\right) \mathbf{a}_{1}+\left(\frac{1}{2}+x_{4}\right) \mathbf{a}_{2}+\frac{1}{2} \mathbf{a}_{3}$ & $=$ & $\left(\frac{1}{2}+y_{4}\right) a \hat{\mathbf{x}}+\left(\frac{1}{2}+x_{4}\right) a \hat{\mathbf{y}}+\frac{1}{2} c \hat{\mathbf{z}}$ & $(8 j)$ & B III \\
\hline $\mathbf{B}_{20}$ & $=$ & $\left(\frac{1}{2}-y_{4}\right) \mathbf{a}_{1}+\left(\frac{1}{2}-x_{4}\right) \mathbf{a}_{2}+\frac{1}{2} \mathbf{a}_{3}$ & $=$ & $\left(\frac{1}{2}-y_{4}\right) a \hat{\mathbf{x}}+\left(\frac{1}{2}-x_{4}\right) a \hat{\mathbf{y}}+\frac{1}{2} c \hat{\mathbf{z}}$ & $(8 j)$ & B III \\
\hline
\end{tabular}

\section{References:}

- A. Zalkin and D. H. Templeton, The Crystal Structures of $\mathrm{CeB}_{4}, T h B_{4}$, and $U B_{4}$, J. Chem. Phys. 18, 391 (1950), doi:10.1063/1.1747637.

\section{Geometry files:}

- CIF: pp. 893

- POSCAR: pp. 894 
$\mathrm{K}_{2} \mathrm{SnCl}_{6}$ (Low-temperature) Structure:

A6B2C_tP18_128_eh_d_b
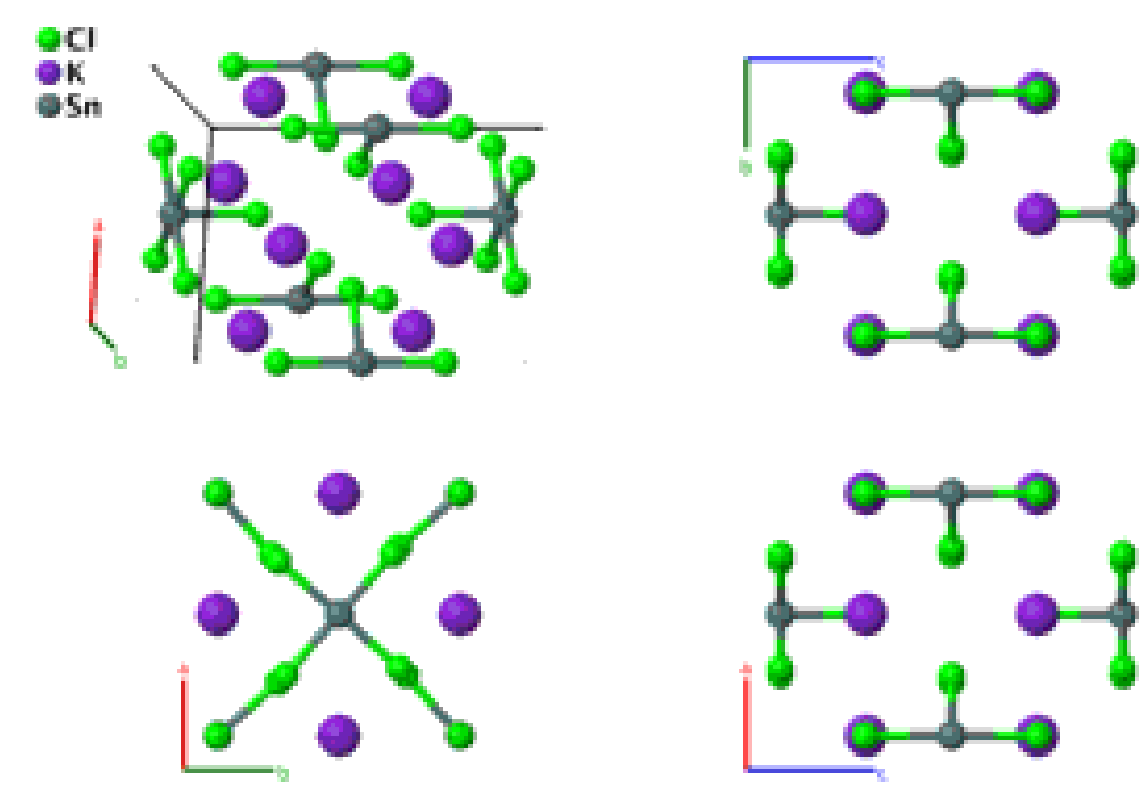

\section{Prototype}

AFLOW prototype label

Strukturbericht designation

Pearson symbol

Space group number

Space group symbol

AFLOW prototype command
$: \quad \mathrm{K}_{2} \mathrm{SnCl}_{6}$

: A6B2C_tP18_128_eh_d_b

: None

: $\quad$ tP18

: 128

: $\quad P 4 / m n c$

aflow - -proto=A6B2C_tP18_128_eh_d_b

- params $=a, c / a, z_{3}, x_{4}, y_{4}$

Simple Tetragonal primitive vectors:

$$
\begin{aligned}
& \mathbf{a}_{1}=a \hat{\mathbf{x}} \\
& \mathbf{a}_{2}=a \hat{\mathbf{y}} \\
& \mathbf{a}_{3}=c \hat{\mathbf{z}}
\end{aligned}
$$

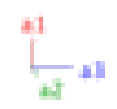

Basis vectors:

Lattice Coordinates
$\mathbf{B}_{1}=$
$\mathbf{B}_{2}=$
$\mathbf{B}_{3}=$
$\mathbf{B}_{4}=$
$\mathbf{B}_{5}=$

$\frac{1}{2} \mathbf{a}_{3}$

$\frac{1}{2} \mathbf{a}_{1}+\frac{1}{2} \mathbf{a}_{2}$

$\frac{1}{2} \mathbf{a}_{2}+\frac{1}{4} \mathbf{a}_{3}$

$\frac{1}{2} \mathbf{a}_{1}+\frac{1}{4} \mathbf{a}_{3}$

$\frac{1}{2} \mathbf{a}_{2}+\frac{3}{4} \mathbf{a}_{3}$
Cartesian Coordinates

$=$

$=$

$=$

$=$

$=$

$$
\begin{gathered}
\frac{1}{2} c \hat{\mathbf{z}} \\
\frac{1}{2} a \hat{\mathbf{x}}+\frac{1}{2} a \hat{\mathbf{y}} \\
\frac{1}{2} a \hat{\mathbf{y}}+\frac{1}{4} c \hat{\mathbf{z}} \\
\frac{1}{2} a \hat{\mathbf{x}}+\frac{1}{4} c \hat{\mathbf{z}} \\
\frac{1}{2} a \hat{\mathbf{y}}+\frac{3}{4} c \hat{\mathbf{z}}
\end{gathered}
$$

Wyckoff Position

Atom Type

$(2 b)$
Sn

Sn

K

K

K 


\begin{tabular}{|c|c|c|c|c|c|c|}
\hline $\mathbf{B}_{6}$ & $=$ & $\frac{1}{2} \mathbf{a}_{1}+\frac{3}{4} \mathbf{a}_{3}$ & $=$ & $\frac{1}{2} a \hat{\mathbf{x}}+\frac{3}{4} c \hat{\mathbf{z}}$ & $(4 d)$ & $\mathrm{K}$ \\
\hline $\mathbf{B}_{7}$ & $=$ & $z_{3} \mathbf{a}_{3}$ & $=$ & $z_{3} c \hat{\mathbf{z}}$ & $(4 e)$ & $\mathrm{Cl} \mathrm{I}$ \\
\hline $\mathbf{B}_{8}$ & $=$ & $\frac{1}{2} \mathbf{a}_{1}+\frac{1}{2} \mathbf{a}_{2}+\left(\frac{1}{2}-z_{3}\right) \mathbf{a}_{3}$ & $=$ & $\frac{1}{2} a \hat{\mathbf{x}}+\frac{1}{2} a \hat{\mathbf{y}}+\left(\frac{1}{2}-z_{3}\right) c \hat{\mathbf{z}}$ & $(4 e)$ & $\mathrm{Cl} \mathrm{I}$ \\
\hline $\mathbf{B}_{9}$ & $=$ & $-z_{3} \mathbf{a}_{3}$ & $=$ & $-z_{3} c \hat{\mathbf{z}}$ & $(4 e)$ & $\mathrm{Cl} \mathrm{I}$ \\
\hline $\mathbf{B}_{10}$ & $=$ & $\frac{1}{2} \mathbf{a}_{1}+\frac{1}{2} \mathbf{a}_{2}+\left(\frac{1}{2}+z_{3}\right) \mathbf{a}_{3}$ & $=$ & $\frac{1}{2} a \hat{\mathbf{x}}+\frac{1}{2} a \hat{\mathbf{y}}+\left(\frac{1}{2}+z_{3}\right) c \hat{\mathbf{z}}$ & $(4 e)$ & $\mathrm{Cl} \mathrm{I}$ \\
\hline $\mathbf{B}_{11}$ & $=$ & $x_{4} \mathbf{a}_{1}+y_{4} \mathbf{a}_{2}$ & $=$ & $x_{4} a \hat{\mathbf{x}}+y_{4} a \hat{\mathbf{y}}$ & $(8 h)$ & $\mathrm{Cl}$ II \\
\hline $\mathbf{B}_{12}$ & $=$ & $-x_{4} \mathbf{a}_{1}-y_{4} \mathbf{a}_{2}$ & $=$ & $-x_{4} a \hat{\mathbf{x}}-y_{4} a \hat{\mathbf{y}}$ & $(8 h)$ & $\mathrm{Cl}$ II \\
\hline $\mathbf{B}_{13}$ & $=$ & $-y_{4} \mathbf{a}_{1}+x_{4} \mathbf{a}_{2}$ & $=$ & $-y_{4} a \hat{\mathbf{x}}+x_{4} a \hat{\mathbf{y}}$ & $(8 h)$ & $\mathrm{Cl}$ II \\
\hline $\mathbf{B}_{14}$ & $=$ & $y_{4} \mathbf{a}_{1}-x_{4} \mathbf{a}_{2}$ & $=$ & $y_{4} a \hat{\mathbf{x}}-x_{4} a \hat{\mathbf{y}}$ & $(8 h)$ & $\mathrm{Cl}$ II \\
\hline $\mathbf{B}_{15}$ & $=$ & $\left(\frac{1}{2}-x_{4}\right) \mathbf{a}_{1}+\left(\frac{1}{2}+y_{4}\right) \mathbf{a}_{2}+\frac{1}{2} \mathbf{a}_{3}$ & $=$ & $\left(\frac{1}{2}-x_{4}\right) a \hat{\mathbf{x}}+\left(\frac{1}{2}+y_{4}\right) a \hat{\mathbf{y}}+\frac{1}{2} c \hat{\mathbf{z}}$ & $(8 h)$ & $\mathrm{Cl}$ II \\
\hline $\mathbf{B}_{16}$ & $=$ & $\left(\frac{1}{2}+x_{4}\right) \mathbf{a}_{1}+\left(\frac{1}{2}-y_{4}\right) \mathbf{a}_{2}+\frac{1}{2} \mathbf{a}_{3}$ & $=$ & $\left(\frac{1}{2}+x_{4}\right) a \hat{\mathbf{x}}+\left(\frac{1}{2}-y_{4}\right) a \hat{\mathbf{y}}+\frac{1}{2} c \hat{\mathbf{z}}$ & $(8 h)$ & $\mathrm{Cl}$ II \\
\hline $\mathbf{B}_{17}$ & $=$ & $\left(\frac{1}{2}+y_{4}\right) \mathbf{a}_{1}+\left(\frac{1}{2}+x_{4}\right) \mathbf{a}_{2}+\frac{1}{2} \mathbf{a}_{3}$ & $=$ & $\left(\frac{1}{2}+y_{4}\right) a \hat{\mathbf{x}}+\left(\frac{1}{2}+x_{4}\right) a \hat{\mathbf{y}}+\frac{1}{2} c \hat{\mathbf{z}}$ & $(8 h)$ & $\mathrm{Cl}$ II \\
\hline $\mathbf{B}_{18}$ & $=$ & $\left(\frac{1}{2}-y_{4}\right) \mathbf{a}_{1}+\left(\frac{1}{2}-x_{4}\right) \mathbf{a}_{2}+\frac{1}{2} \mathbf{a}_{3}$ & $=$ & $\left(\frac{1}{2}-y_{4}\right) a \hat{\mathbf{x}}+\left(\frac{1}{2}-x_{4}\right) a \hat{\mathbf{y}}+\frac{1}{2} c \hat{\mathbf{z}}$ & $(8 h)$ & $\mathrm{Cl}$ II \\
\hline
\end{tabular}

\section{References:}

- H. Boysen and A. W. Hewat, A neutron powder investigation of the structural changes in $\mathrm{K}_{2} \mathrm{SnCl}_{6}$, Acta Crystallogr. Sect. B Struct. Sci. 34, 1412-1418 (1978), doi:10.1107/S0567740878005816.

\section{Found in:}

- P. Villars and K. Cenzual, Pearson's Crystal Data - Crystal Structure Database for Inorganic Compounds, ASM International (2013).

\section{Geometry files:}

- CIF: pp. 894

- POSCAR: pp. 894 


\section{$\mathrm{FeCu}_{2} \mathrm{Al}_{7}\left(E 9_{a}\right)$ Structure: A7B2C_tP40_128_egi_h_e}
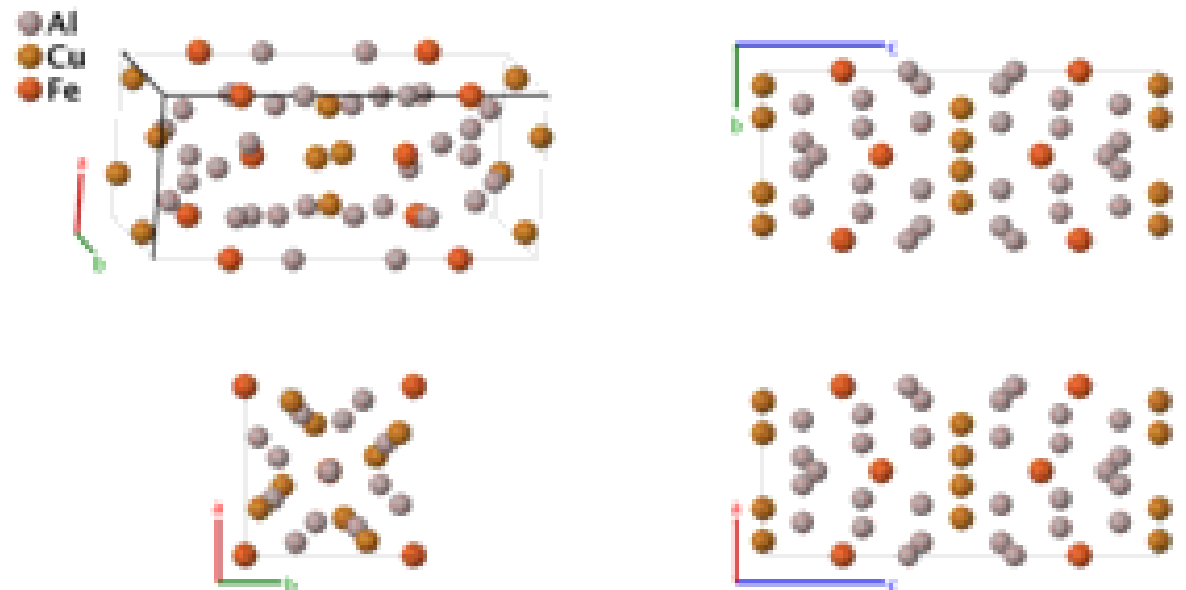

\section{Prototype}

AFLOW prototype label

Strukturbericht designation

Pearson symbol

Space group number

Space group symbol

AFLOW prototype command
$: \quad \mathrm{FeCu}_{2} \mathrm{Al}_{7}$

: A7B2C_tP40_128_egi_h_e

: $E 9_{a}$

: $\quad$ tP40

: $\quad 128$

: $\quad P 4 / m n c$

aflow --proto=A7B2C_tP40_128_egi_h_e

- -params $=a, c / a, z_{1}, z_{2}, x_{3}, x_{4}, y_{4}, x_{5}, y_{5}, z_{5}$

\section{Other compounds with this structure:}

- $\mathrm{T}(\mathrm{CoCuAl})$, an alloy with an approximate composition of $\mathrm{Co}_{2} \mathrm{Cu}_{4.9} \mathrm{Al}_{17.7}, \mathrm{NiCu}_{3} \mathrm{Al}_{6}$

\section{Simple Tetragonal primitive vectors:}

$$
\begin{aligned}
& \mathbf{a}_{1}=a \hat{\mathbf{x}} \\
& \mathbf{a}_{2}=a \hat{\mathbf{y}} \\
& \mathbf{a}_{3}=c \hat{\mathbf{z}}
\end{aligned}
$$

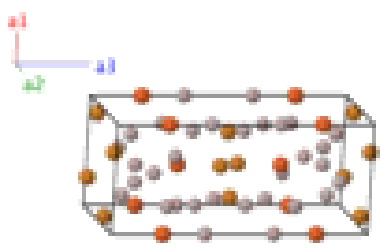

Basis vectors:

Lattice Coordinates

$\begin{array}{llclc}\mathbf{B}_{1} & & z_{1} \mathbf{a}_{3} & & z_{1} c \hat{\mathbf{z}} \\ \mathbf{B}_{2} & & \frac{1}{2} \mathbf{a}_{1}+\frac{1}{2} \mathbf{a}_{2}+\left(\frac{1}{2}-z_{1}\right) \mathbf{a}_{3} & = & \frac{1}{2} a \hat{\mathbf{x}}+\frac{1}{2} a \hat{\mathbf{y}}+\left(\frac{1}{2}-z_{1}\right) c \hat{\mathbf{z}} \\ \mathbf{B}_{3}= & -z_{1} \mathbf{a}_{3} & = & -z_{1} c \hat{\mathbf{z}} \\ \mathbf{B}_{4}= & \frac{1}{2} \mathbf{a}_{1}+\frac{1}{2} \mathbf{a}_{2}+\left(\frac{1}{2}+z_{1}\right) \mathbf{a}_{3} & = & \frac{1}{2} a \hat{\mathbf{x}}+\frac{1}{2} a \hat{\mathbf{y}}+\left(\frac{1}{2}+z_{1}\right) c \hat{\mathbf{z}} \\ \mathbf{B}_{5}= & z_{2} \mathbf{a}_{3} & = & z_{2} c \hat{\mathbf{z}} \\ \mathbf{B}_{6} & & \frac{1}{2} \mathbf{a}_{1}+\frac{1}{2} \mathbf{a}_{2}+\left(\frac{1}{2}-z_{2}\right) \mathbf{a}_{3} & = & \frac{1}{2} a \hat{\mathbf{x}}+\frac{1}{2} a \hat{\mathbf{y}}+\left(\frac{1}{2}-z_{2}\right) c \hat{\mathbf{z}} \\ \mathbf{B}_{7} & -z_{2} \mathbf{a}_{3} & & & -z_{2} c \hat{\mathbf{z}}\end{array}$

Wyckoff Position Atom Type

$(4 e)$

Al I

$(4 e)$

Al I

$(4 e)$

Al I

$(4 e)$

Al I

(4e)

$\mathrm{Fe}$

(4e)

$\mathrm{Fe}$

(4e)

$\mathrm{Fe}$ 


\begin{tabular}{|c|c|c|c|c|c|c|}
\hline $\mathbf{B}_{8}$ & $=$ & $\frac{1}{2} \mathbf{a}_{1}+\frac{1}{2} \mathbf{a}_{2}+\left(\frac{1}{2}+z_{2}\right) \mathbf{a}_{3}$ & $=$ & $\frac{1}{2} a \hat{\mathbf{x}}+\frac{1}{2} a \hat{\mathbf{y}}+\left(\frac{1}{2}+z_{2}\right) c \hat{\mathbf{z}}$ & $(4 e)$ & $\mathrm{Fe}$ \\
\hline $\mathbf{B}_{9}$ & $=$ & $x_{3} \mathbf{a}_{1}+\left(\frac{1}{2}+x_{3}\right) \mathbf{a}_{2}+\frac{1}{4} \mathbf{a}_{3}$ & $=$ & $x_{3} a \hat{\mathbf{x}}+\left(\frac{1}{2}+x_{3}\right) a \hat{\mathbf{y}}+\frac{1}{4} c \hat{\mathbf{z}}$ & $(8 g)$ & $\mathrm{Al}$ II \\
\hline $\mathbf{B}_{10}$ & $=$ & $-x_{3} \mathbf{a}_{1}+\left(\frac{1}{2}-x_{3}\right) \mathbf{a}_{2}+\frac{1}{4} \mathbf{a}_{3}$ & $=$ & $-x_{3} a \hat{\mathbf{x}}+\left(\frac{1}{2}-x_{3}\right) a \hat{\mathbf{y}}+\frac{1}{4} c \hat{\mathbf{z}}$ & $(8 g)$ & Al II \\
\hline $\mathbf{B}_{11}$ & $=$ & $\left(\frac{1}{2}-x_{3}\right) \mathbf{a}_{1}+x_{3} \mathbf{a}_{2}+\frac{1}{4} \mathbf{a}_{3}$ & $=$ & $\left(\frac{1}{2}-x_{3}\right) a \hat{\mathbf{x}}+x_{3} a \hat{\mathbf{y}}+\frac{1}{4} c \hat{\mathbf{z}}$ & $(8 g)$ & $\mathrm{Al} \mathrm{II}$ \\
\hline $\mathbf{B}_{12}$ & $=$ & $\left(\frac{1}{2}+x_{3}\right) \mathbf{a}_{1}-x_{3} \mathbf{a}_{2}+\frac{1}{4} \mathbf{a}_{3}$ & $=$ & $\left(\frac{1}{2}+x_{3}\right) a \hat{\mathbf{x}}-x_{3} a \hat{\mathbf{y}}+\frac{1}{4} c \hat{\mathbf{z}}$ & $(8 g)$ & $\mathrm{Al}$ II \\
\hline $\mathbf{B}_{13}$ & $=$ & $-x_{3} \mathbf{a}_{1}+\left(\frac{1}{2}-x_{3}\right) \mathbf{a}_{2}+\frac{3}{4} \mathbf{a}_{3}$ & $=$ & $-x_{3} a \hat{\mathbf{x}}+\left(\frac{1}{2}-x_{3}\right) a \hat{\mathbf{y}}+\frac{3}{4} c \hat{\mathbf{z}}$ & $(8 g)$ & $\mathrm{Al}$ II \\
\hline $\mathbf{B}_{14}$ & $=$ & $x_{3} \mathbf{a}_{1}+\left(\frac{1}{2}+x_{3}\right) \mathbf{a}_{2}+\frac{3}{4} \mathbf{a}_{3}$ & $=$ & $x_{3} a \hat{\mathbf{x}}+\left(\frac{1}{2}+x_{3}\right) a \hat{\mathbf{y}}+\frac{3}{4} c \hat{\mathbf{z}}$ & $(8 g)$ & $\mathrm{Al}$ II \\
\hline $\mathbf{B}_{15}$ & $=$ & $\left(\frac{1}{2}+x_{3}\right) \mathbf{a}_{1}-x_{3} \mathbf{a}_{2}+\frac{3}{4} \mathbf{a}_{3}$ & $=$ & $\left(\frac{1}{2}+x_{3}\right) a \hat{\mathbf{x}}-x_{3} a \hat{\mathbf{y}}+\frac{3}{4} c \hat{\mathbf{z}}$ & $(8 g)$ & $\mathrm{Al}$ II \\
\hline $\mathbf{B}_{16}$ & $=$ & $\left(\frac{1}{2}-x_{3}\right) \mathbf{a}_{1}+x_{3} \mathbf{a}_{2}+\frac{3}{4} \mathbf{a}_{3}$ & $=$ & $\left(\frac{1}{2}-x_{3}\right) a \hat{\mathbf{x}}+x_{3} a \hat{\mathbf{y}}+\frac{3}{4} c \hat{\mathbf{z}}$ & $(8 g)$ & Al II \\
\hline $\mathbf{B}_{17}$ & $=$ & $x_{4} \mathbf{a}_{1}+y_{4} \mathbf{a}_{2}$ & $=$ & $x_{4} a \hat{\mathbf{x}}+y_{4} a \hat{\mathbf{y}}$ & $(8 h)$ & $\mathrm{Cu}$ \\
\hline $\mathbf{B}_{18}$ & $=$ & $-x_{4} \mathbf{a}_{1}-y_{4} \mathbf{a}_{2}$ & $=$ & $-x_{4} a \hat{\mathbf{x}}-y_{4} a \hat{\mathbf{y}}$ & $(8 h)$ & $\mathrm{Cu}$ \\
\hline $\mathbf{B}_{19}$ & $=$ & $-y_{4} \mathbf{a}_{1}+x_{4} \mathbf{a}_{2}$ & $=$ & $-y_{4} a \hat{\mathbf{x}}+x_{4} a \hat{\mathbf{y}}$ & $(8 h)$ & $\mathrm{Cu}$ \\
\hline $\mathbf{B}_{20}$ & $=$ & $y_{4} \mathbf{a}_{1}-x_{4} \mathbf{a}_{2}$ & $=$ & $y_{4} a \hat{\mathbf{x}}-x_{4} a \hat{\mathbf{y}}$ & $(8 h)$ & $\mathrm{Cu}$ \\
\hline $\mathbf{B}_{21}$ & $=$ & $\left(\frac{1}{2}-x_{4}\right) \mathbf{a}_{1}+\left(\frac{1}{2}+y_{4}\right) \mathbf{a}_{2}+\frac{1}{2} \mathbf{a}_{3}$ & $=$ & $\left(\frac{1}{2}-x_{4}\right) a \hat{\mathbf{x}}+\left(\frac{1}{2}+y_{4}\right) a \hat{\mathbf{y}}+\frac{1}{2} c \hat{\mathbf{z}}$ & $(8 h)$ & $\mathrm{Cu}$ \\
\hline $\mathbf{B}_{22}$ & $=$ & $\left(\frac{1}{2}+x_{4}\right) \mathbf{a}_{1}+\left(\frac{1}{2}-y_{4}\right) \mathbf{a}_{2}+\frac{1}{2} \mathbf{a}_{3}$ & $=$ & $\left(\frac{1}{2}+x_{4}\right) a \hat{\mathbf{x}}+\left(\frac{1}{2}-y_{4}\right) a \hat{\mathbf{y}}+\frac{1}{2} c \hat{\mathbf{z}}$ & $(8 h)$ & $\mathrm{Cu}$ \\
\hline $\mathbf{B}_{23}$ & $=$ & $\left(\frac{1}{2}+y_{4}\right) \mathbf{a}_{1}+\left(\frac{1}{2}+x_{4}\right) \mathbf{a}_{2}+\frac{1}{2} \mathbf{a}_{3}$ & $=$ & $\left(\frac{1}{2}+y_{4}\right) a \hat{\mathbf{x}}+\left(\frac{1}{2}+x_{4}\right) a \hat{\mathbf{y}}+\frac{1}{2} c \hat{\mathbf{z}}$ & $(8 h)$ & $\mathrm{Cu}$ \\
\hline $\mathbf{B}_{24}$ & $=$ & $\left(\frac{1}{2}-y_{4}\right) \mathbf{a}_{1}+\left(\frac{1}{2}-x_{4}\right) \mathbf{a}_{2}+\frac{1}{2} \mathbf{a}_{3}$ & $=$ & $\left(\frac{1}{2}-y_{4}\right) a \hat{\mathbf{x}}+\left(\frac{1}{2}-x_{4}\right) a \hat{\mathbf{y}}+\frac{1}{2} c \hat{\mathbf{z}}$ & $(8 h)$ & $\mathrm{Cu}$ \\
\hline $\mathbf{B}_{25}$ & $=$ & $x_{5} \mathbf{a}_{1}+y_{5} \mathbf{a}_{2}+z_{5} \mathbf{a}_{3}$ & $=$ & $x_{5} a \hat{\mathbf{x}}+y_{5} a \hat{\mathbf{y}}+z_{5} c \hat{\mathbf{z}}$ & $(16 i)$ & Al III \\
\hline $\mathbf{B}_{26}$ & $=$ & $-x_{5} \mathbf{a}_{1}-y_{5} \mathbf{a}_{2}+z_{5} \mathbf{a}_{3}$ & $=$ & $-x_{5} a \hat{\mathbf{x}}-y_{5} a \hat{\mathbf{y}}+z_{5} c \hat{\mathbf{z}}$ & $(16 i)$ & $\mathrm{Al}$ III \\
\hline $\mathbf{B}_{27}$ & $=$ & $-y_{5} \mathbf{a}_{1}+x_{5} \mathbf{a}_{2}+z_{5} \mathbf{a}_{3}$ & $=$ & $-y_{5} a \hat{\mathbf{x}}+x_{5} a \hat{\mathbf{y}}+z_{5} c \hat{\mathbf{z}}$ & $(16 i)$ & $\mathrm{Al}$ III \\
\hline $\mathbf{B}_{28}$ & $=$ & $y_{5} \mathbf{a}_{1}-x_{5} \mathbf{a}_{2}+z_{5} \mathbf{a}_{3}$ & $=$ & $y_{5} a \hat{\mathbf{x}}-x_{5} a \hat{\mathbf{y}}+z_{5} c \hat{\mathbf{z}}$ & $(16 i)$ & Al III \\
\hline $\mathbf{B}_{29}$ & $=$ & $\begin{array}{c}\left(\frac{1}{2}-x_{5}\right) \mathbf{a}_{1}+\left(\frac{1}{2}+y_{5}\right) \mathbf{a}_{2}+ \\
\left(\frac{1}{2}-z_{5}\right) \mathbf{a}_{3}\end{array}$ & $=$ & $\begin{array}{c}\left(\frac{1}{2}-x_{5}\right) a \hat{\mathbf{x}}+\left(\frac{1}{2}+y_{5}\right) a \hat{\mathbf{y}}+ \\
\left(\frac{1}{2}-z_{5}\right) c \hat{\mathbf{z}}\end{array}$ & $(16 i)$ & $\mathrm{Al}$ III \\
\hline $\mathbf{B}_{30}$ & $=$ & $\begin{array}{c}\left(\frac{1}{2}+x_{5}\right) \mathbf{a}_{1}+\left(\frac{1}{2}-y_{5}\right) \mathbf{a}_{2}+ \\
\left(\frac{1}{2}-z_{5}\right) \mathbf{a}_{3}\end{array}$ & $=$ & $\begin{array}{c}\left(\frac{1}{2}+x_{5}\right) a \hat{\mathbf{x}}+\left(\frac{1}{2}-y_{5}\right) a \hat{\mathbf{y}}+ \\
\left(\frac{1}{2}-z_{5}\right) c \hat{\mathbf{z}}\end{array}$ & $(16 i)$ & $\mathrm{Al} \mathrm{III}$ \\
\hline $\mathbf{B}_{31}$ & $=$ & $\begin{array}{c}\left(\frac{1}{2}+y_{5}\right) \mathbf{a}_{1}+\left(\frac{1}{2}+x_{5}\right) \mathbf{a}_{2}+ \\
\left(\frac{1}{2}-z_{5}\right) \mathbf{a}_{3}\end{array}$ & $=$ & $\begin{array}{c}\left(\frac{1}{2}+y_{5}\right) a \hat{\mathbf{x}}+\left(\frac{1}{2}+x_{5}\right) a \hat{\mathbf{y}}+ \\
\left(\frac{1}{2}-z_{5}\right) c \hat{\mathbf{z}}\end{array}$ & $(16 i)$ & Al III \\
\hline $\mathbf{B}_{32}$ & $=$ & $\begin{array}{c}\left(\frac{1}{2}-y_{5}\right) \mathbf{a}_{1}+\left(\frac{1}{2}-x_{5}\right) \mathbf{a}_{2}+ \\
\left(\frac{1}{2}-z_{5}\right) \mathbf{a}_{3}\end{array}$ & $=$ & $\begin{array}{c}\left(\frac{1}{2}-y_{5}\right) a \hat{\mathbf{x}}+\left(\frac{1}{2}-x_{5}\right) a \hat{\mathbf{y}}+ \\
\left(\frac{1}{2}-z_{5}\right) c \hat{\mathbf{z}}\end{array}$ & $(16 i)$ & Al III \\
\hline $\mathbf{B}_{33}$ & $=$ & $-x_{5} \mathbf{a}_{1}-y_{5} \mathbf{a}_{2}-z_{5} \mathbf{a}_{3}$ & $=$ & $-x_{5} a \hat{\mathbf{x}}-y_{5} a \hat{\mathbf{y}}-z_{5} c \hat{\mathbf{z}}$ & $(16 i)$ & $\mathrm{Al} \mathrm{III}$ \\
\hline $\mathbf{B}_{34}$ & $=$ & $x_{5} \mathbf{a}_{1}+y_{5} \mathbf{a}_{2}-z_{5} \mathbf{a}_{3}$ & $=$ & $x_{5} a \hat{\mathbf{x}}+y_{5} a \hat{\mathbf{y}}-z_{5} c \hat{\mathbf{z}}$ & $(16 i)$ & $\mathrm{Al}$ III \\
\hline $\mathbf{B}_{35}$ & $=$ & $y_{5} \mathbf{a}_{1}-x_{5} \mathbf{a}_{2}-z_{5} \mathbf{a}_{3}$ & $=$ & $y_{5} a \hat{\mathbf{x}}-x_{5} a \hat{\mathbf{y}}-z_{5} c \hat{\mathbf{z}}$ & $(16 i)$ & $\mathrm{Al}$ III \\
\hline $\mathbf{B}_{36}$ & $=$ & $-y_{5} \mathbf{a}_{1}+x_{5} \mathbf{a}_{2}-z_{5} \mathbf{a}_{3}$ & $=$ & $-y_{5} a \hat{\mathbf{x}}+x_{5} a \hat{\mathbf{y}}-z_{5} c \hat{\mathbf{z}}$ & $(16 i)$ & $\mathrm{Al} \mathrm{III}$ \\
\hline $\mathbf{B}_{37}$ & $=$ & $\begin{array}{c}\left(\frac{1}{2}+x_{5}\right) \mathbf{a}_{1}+\left(\frac{1}{2}-y_{5}\right) \mathbf{a}_{2}+ \\
\left(\frac{1}{2}+z_{5}\right) \mathbf{a}_{3}\end{array}$ & $=$ & $\begin{array}{c}\left(\frac{1}{2}+x_{5}\right) a \hat{\mathbf{x}}+\left(\frac{1}{2}-y_{5}\right) a \hat{\mathbf{y}}+ \\
\left(\frac{1}{2}+z_{5}\right) c \hat{\mathbf{z}}\end{array}$ & $(16 i)$ & Al III \\
\hline $\mathbf{B}_{38}$ & $=$ & $\begin{array}{c}\left(\frac{1}{2}-x_{5}\right) \mathbf{a}_{1}+\left(\frac{1}{2}+y_{5}\right) \mathbf{a}_{2}+ \\
\left(\frac{1}{2}+z_{5}\right) \mathbf{a}_{3}\end{array}$ & $=$ & $\begin{array}{c}\left(\frac{1}{2}-x_{5}\right) a \hat{\mathbf{x}}+\left(\frac{1}{2}+y_{5}\right) a \hat{\mathbf{y}}+ \\
\left(\frac{1}{2}+z_{5}\right) c \hat{\mathbf{z}}\end{array}$ & $(16 i)$ & $\mathrm{Al}$ III \\
\hline
\end{tabular}




$\begin{array}{cccc}\mathbf{B}_{39}= & \left(\frac{1}{2}-y_{5}\right) \mathbf{a}_{1}+\left(\frac{1}{2}-x_{5}\right) \mathbf{a}_{2}+ & & \left(\frac{1}{2}-y_{5}\right) a \hat{\mathbf{x}}+\left(\frac{1}{2}-x_{5}\right) a \hat{\mathbf{y}}+ \\ \left(\frac{1}{2}+z_{5}\right) \mathbf{a}_{3} & \left(\frac{1}{2}+z_{5}\right) c \hat{\mathbf{z}} & \text { (16i) } \\ \mathbf{B}_{40}= & \left(\frac{1}{2}+y_{5}\right) \mathbf{a}_{1}+\left(\frac{1}{2}+x_{5}\right) \mathbf{a}_{2}+ & = & \left(\frac{1}{2}+y_{5}\right) a \hat{\mathbf{x}}+\left(\frac{1}{2}+x_{5}\right) a \hat{\mathbf{y}}+ \\ \left(\frac{1}{2}+z_{5}\right) \mathbf{a}_{3} & & \left(\frac{1}{2}+z_{5}\right) c \hat{\mathbf{z}}\end{array}$

\section{References:}

- M. G. Bown and P. J. Brown, The structure of $\mathrm{FeCu}_{2} \mathrm{Al}_{7}$ and $T(\mathrm{CoCuAl})$, Acta Cryst. 9, 911-914 (1956), doi:10.1107/S0365110X56002576.

\section{Geometry files:}

- CIF: pp. 895

- POSCAR: pp. 895 


\section{$\mathrm{CuBi}_{2} \mathrm{O}_{4}$ Structure: A2BC4_tP28_130_f_c_g}
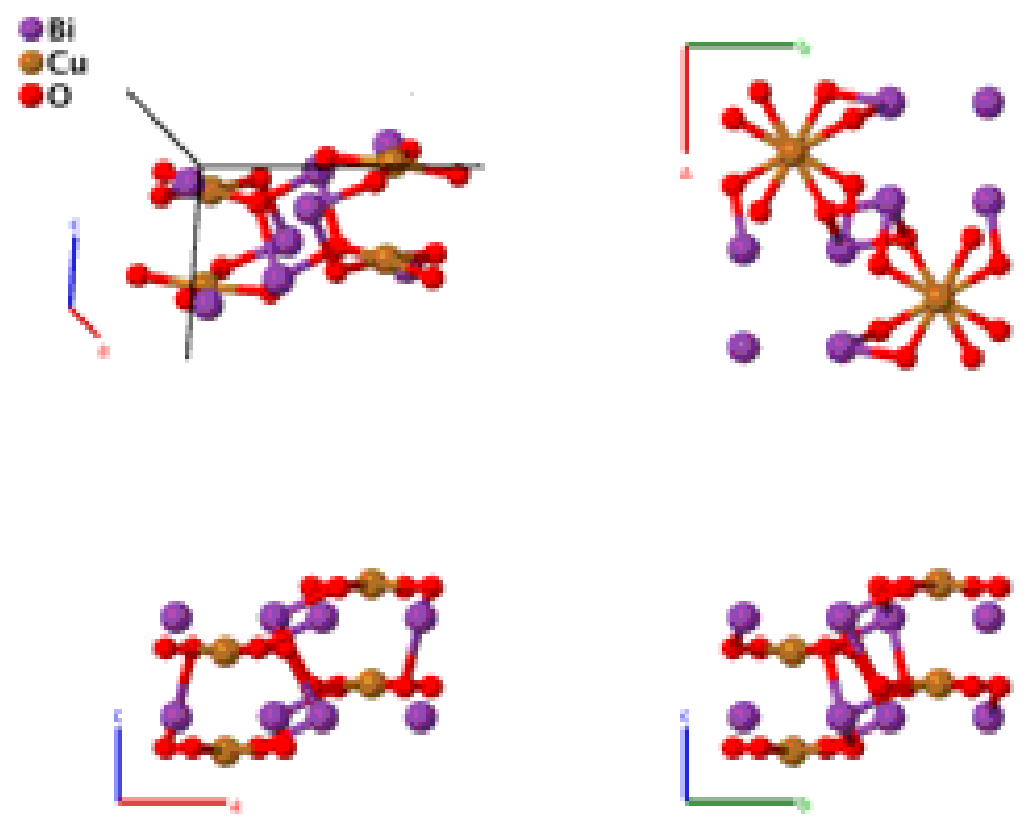

\section{Prototype}

AFLOW prototype label

Strukturbericht designation

Pearson symbol

Space group number

Space group symbol

AFLOW prototype command
: $\quad \mathrm{CuBi}_{2} \mathrm{O}_{4}$

: A2BC4_tP28_130_f_c_g

: None

$: \quad$ tP28

: 130

: $\quad P 4 / n c c$

Simple Tetragonal primitive vectors:

$$
\begin{aligned}
& \mathbf{a}_{1}=a \hat{\mathbf{x}} \\
& \mathbf{a}_{2}=a \hat{\mathbf{y}} \\
& \mathbf{a}_{3}=c \hat{\mathbf{z}}
\end{aligned}
$$

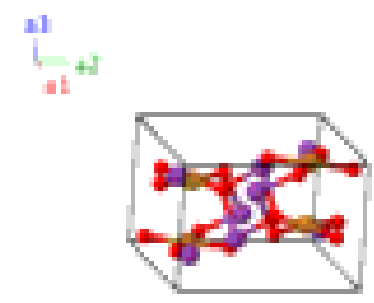

\section{Basis vectors:}

Lattice Coordinates

$\begin{array}{lcccc}\mathbf{B}_{1}= & \frac{1}{4} \mathbf{a}_{1}+\frac{1}{4} \mathbf{a}_{2}+z_{1} \mathbf{a}_{3} & = & \frac{1}{4} a \hat{\mathbf{x}}+\frac{1}{4} a \hat{\mathbf{y}}+z_{1} c \hat{\mathbf{z}} \\ \mathbf{B}_{2}= & \frac{3}{4} \mathbf{a}_{1}+\frac{3}{4} \mathbf{a}_{2}+\left(\frac{1}{2}-z_{1}\right) \mathbf{a}_{3} & = & \frac{3}{4} a \hat{\mathbf{x}}+\frac{3}{4} a \hat{\mathbf{y}}+\left(\frac{1}{2}-z_{1}\right) c \hat{\mathbf{z}} \\ \mathbf{B}_{3}= & \frac{3}{4} \mathbf{a}_{1}+\frac{3}{4} \mathbf{a}_{2}-z_{1} \mathbf{a}_{3} & = & \frac{3}{4} a \hat{\mathbf{x}}+\frac{3}{4} a \hat{\mathbf{y}}-z_{1} c \hat{\mathbf{z}} \\ \mathbf{B}_{4}= & \frac{1}{4} \mathbf{a}_{1}+\frac{1}{4} \mathbf{a}_{2}+\left(\frac{1}{2}+z_{1}\right) \mathbf{a}_{3} & = & \frac{1}{4} a \hat{\mathbf{x}}+\frac{1}{4} a \hat{\mathbf{y}}+\left(\frac{1}{2}+z_{1}\right) c \hat{\mathbf{z}}\end{array}$

Wyckoff Position

Atom Type

$\begin{array}{ll}(4 c) & \mathrm{Cu} \\ (4 c) & \mathrm{Cu} \\ (4 c) & \mathrm{Cu} \\ (4 c) & \mathrm{Cu}\end{array}$




\begin{tabular}{|c|c|c|c|c|c|}
\hline $\mathbf{B}_{5}$ & $=$ & $x_{2} \mathbf{a}_{1}-x_{2} \mathbf{a}_{2}+\frac{1}{4} \mathbf{a}_{3}$ & $=$ & $x_{2} a \hat{\mathbf{x}}-x_{2} a \hat{\mathbf{y}}+\frac{1}{4} c \hat{\mathbf{z}}$ & $(8 f)$ \\
\hline $\mathbf{B}_{6}$ & $=$ & $\left(\frac{1}{2}-x_{2}\right) \mathbf{a}_{1}+\left(\frac{1}{2}-x_{2}\right) \mathbf{a}_{2}+\frac{1}{4} \mathbf{a}_{3}$ & $=$ & $\left(\frac{1}{2}-x_{2}\right) a \hat{\mathbf{x}}+\left(\frac{1}{2}-x_{2}\right) a \hat{\mathbf{y}}+\frac{1}{4} c \hat{\mathbf{z}}$ & $(8 f)$ \\
\hline $\mathbf{B}_{7}$ & $=$ & $\left(\frac{1}{2}+x_{2}\right) \mathbf{a}_{1}+x_{2} \mathbf{a}_{2}+\frac{1}{4} \mathbf{a}_{3}$ & $=$ & $\left(\frac{1}{2}+x_{2}\right) a \hat{\mathbf{x}}+x_{2} a \hat{\mathbf{y}}+\frac{1}{4} c \hat{\mathbf{z}}$ & $(8 f)$ \\
\hline $\mathbf{B}_{8}$ & $=$ & $-x_{2} \mathbf{a}_{1}+\left(\frac{1}{2}-x_{2}\right) \mathbf{a}_{2}+\frac{1}{4} \mathbf{a}_{3}$ & $=$ & $-x_{2} a \hat{\mathbf{x}}+\left(\frac{1}{2}-x_{2}\right) a \hat{\mathbf{y}}+\frac{1}{4} c \hat{\mathbf{z}}$ & $(8 f)$ \\
\hline $\mathbf{B}_{9}$ & $=$ & $-x_{2} \mathbf{a}_{1}+x_{2} \mathbf{a}_{2}+\frac{3}{4} \mathbf{a}_{3}$ & $=$ & $-x_{2} a \hat{\mathbf{x}}+x_{2} a \hat{\mathbf{y}}+\frac{3}{4} c \hat{\mathbf{z}}$ & $(8 f)$ \\
\hline $\mathbf{B}_{10}$ & $=$ & $\left(\frac{1}{2}+x_{2}\right) \mathbf{a}_{1}+\left(\frac{1}{2}-x_{2}\right) \mathbf{a}_{2}+\frac{3}{4} \mathbf{a}_{3}$ & $=$ & $\left(\frac{1}{2}+x_{2}\right) a \hat{\mathbf{x}}+\left(\frac{1}{2}-x_{2}\right) a \hat{\mathbf{y}}+\frac{3}{4} c \hat{\mathbf{z}}$ & $(8 f)$ \\
\hline $\mathbf{B}_{11}$ & $=$ & $\left(\frac{1}{2}-x_{2}\right) \mathbf{a}_{1}-x_{2} \mathbf{a}_{2}+\frac{3}{4} \mathbf{a}_{3}$ & $=$ & $\left(\frac{1}{2}-x_{2}\right) a \hat{\mathbf{x}}-x_{2} a \hat{\mathbf{y}}+\frac{3}{4} c \hat{\mathbf{z}}$ & $(8 f)$ \\
\hline $\mathbf{B}_{12}$ & $=$ & $x_{2} \mathbf{a}_{1}+\left(\frac{1}{2}+x_{2}\right) \mathbf{a}_{2}+\frac{3}{4} \mathbf{a}_{3}$ & $=$ & $x_{2} a \hat{\mathbf{x}}+\left(\frac{1}{2}+x_{2}\right) a \hat{\mathbf{y}}+\frac{3}{4} c \hat{\mathbf{z}}$ & $(8 f)$ \\
\hline $\mathbf{B}_{13}$ & $=$ & $x_{3} \mathbf{a}_{1}+y_{3} \mathbf{a}_{2}+z_{3} \mathbf{a}_{3}$ & $=$ & $x_{3} a \hat{\mathbf{x}}+y_{3} a \hat{\mathbf{y}}+z_{3} c \hat{\mathbf{z}}$ & $(16 g)$ \\
\hline $\mathbf{B}_{14}$ & $=$ & $\left(\frac{1}{2}-x_{3}\right) \mathbf{a}_{1}+\left(\frac{1}{2}-y_{3}\right) \mathbf{a}_{2}+z_{3} \mathbf{a}_{3}$ & $=$ & $\left(\frac{1}{2}-x_{3}\right) a \hat{\mathbf{x}}+\left(\frac{1}{2}-y_{3}\right) a \hat{\mathbf{y}}+z_{3} c \hat{\mathbf{z}}$ & $(16 g)$ \\
\hline $\mathbf{B}_{15}$ & $=$ & $\left(\frac{1}{2}-y_{3}\right) \mathbf{a}_{1}+x_{3} \mathbf{a}_{2}+z_{3} \mathbf{a}_{3}$ & $=$ & $\left(\frac{1}{2}-y_{3}\right) a \hat{\mathbf{x}}+x_{3} a \hat{\mathbf{y}}+z_{3} c \hat{\mathbf{z}}$ & $(16 g)$ \\
\hline $\mathbf{B}_{16}$ & $=$ & $y_{3} \mathbf{a}_{1}+\left(\frac{1}{2}-x_{3}\right) \mathbf{a}_{2}+z_{3} \mathbf{a}_{3}$ & $=$ & $y_{3} a \hat{\mathbf{x}}+\left(\frac{1}{2}-x_{3}\right) a \hat{\mathbf{y}}+z_{3} c \hat{\mathbf{z}}$ & $(16 g)$ \\
\hline $\mathbf{B}_{17}$ & $=$ & $-x_{3} \mathbf{a}_{1}+\left(\frac{1}{2}+y_{3}\right) \mathbf{a}_{2}+\left(\frac{1}{2}-z_{3}\right) \mathbf{a}_{3}$ & $=$ & $-x_{3} a \hat{\mathbf{x}}+\left(\frac{1}{2}+y_{3}\right) a \hat{\mathbf{y}}+\left(\frac{1}{2}-z_{3}\right) c \hat{\mathbf{z}}$ & $(16 g)$ \\
\hline $\mathbf{B}_{18}$ & $=$ & $\left(\frac{1}{2}+x_{3}\right) \mathbf{a}_{1}-y_{3} \mathbf{a}_{2}+\left(\frac{1}{2}-z_{3}\right) \mathbf{a}_{3}$ & $=$ & $\left(\frac{1}{2}+x_{3}\right) a \hat{\mathbf{x}}-y_{3} a \hat{\mathbf{y}}+\left(\frac{1}{2}-z_{3}\right) c \hat{\mathbf{z}}$ & $(16 g)$ \\
\hline $\mathbf{B}_{19}$ & $=$ & $\begin{array}{c}\left(\frac{1}{2}+y_{3}\right) \mathbf{a}_{1}+\left(\frac{1}{2}+x_{3}\right) \mathbf{a}_{2}+ \\
\left(\frac{1}{2}-z_{3}\right) \mathbf{a}_{3}\end{array}$ & $=$ & $\begin{array}{c}\left(\frac{1}{2}+y_{3}\right) a \hat{\mathbf{x}}+\left(\frac{1}{2}+x_{3}\right) a \hat{\mathbf{y}}+ \\
\left(\frac{1}{2}-z_{3}\right) c \hat{\mathbf{z}}\end{array}$ & $(16 g)$ \\
\hline $\mathbf{B}_{20}$ & $=$ & $-y_{3} \mathbf{a}_{1}-x_{3} \mathbf{a}_{2}+\left(\frac{1}{2}-z_{3}\right) \mathbf{a}_{3}$ & $=$ & $-y_{3} a \hat{\mathbf{x}}-x_{3} a \hat{\mathbf{y}}+\left(\frac{1}{2}-z_{3}\right) c \hat{\mathbf{z}}$ & $(16 g)$ \\
\hline $\mathbf{B}_{21}$ & $=$ & $-x_{3} \mathbf{a}_{1}-y_{3} \mathbf{a}_{2}-z_{3} \mathbf{a}_{3}$ & $=$ & $-x_{3} a \hat{\mathbf{x}}-y_{3} a \hat{\mathbf{y}}-z_{3} c \hat{\mathbf{z}}$ & $(16 g)$ \\
\hline $\mathbf{B}_{22}$ & $=$ & $\left(\frac{1}{2}+x_{3}\right) \mathbf{a}_{1}+\left(\frac{1}{2}+y_{3}\right) \mathbf{a}_{2}-z_{3} \mathbf{a}_{3}$ & $=$ & $\left(\frac{1}{2}+x_{3}\right) a \hat{\mathbf{x}}+\left(\frac{1}{2}+y_{3}\right) a \hat{\mathbf{y}}-z_{3} c \hat{\mathbf{z}}$ & $(16 g)$ \\
\hline $\mathbf{B}_{23}$ & $=$ & $\left(\frac{1}{2}+y_{3}\right) \mathbf{a}_{1}-x_{3} \mathbf{a}_{2}-z_{3} \mathbf{a}_{3}$ & $=$ & $\left(\frac{1}{2}+y_{3}\right) a \hat{\mathbf{x}}-x_{3} a \hat{\mathbf{y}}-z_{3} c \hat{\mathbf{z}}$ & $(16 g)$ \\
\hline $\mathbf{B}_{24}$ & $=$ & $-y_{3} \mathbf{a}_{1}+\left(\frac{1}{2}+x_{3}\right) \mathbf{a}_{2}-z_{3} \mathbf{a}_{3}$ & $=$ & $-y_{3} a \hat{\mathbf{x}}+\left(\frac{1}{2}+x_{3}\right) a \hat{\mathbf{y}}-z_{3} c \hat{\mathbf{z}}$ & $(16 g)$ \\
\hline $\mathbf{B}_{25}$ & $=$ & $x_{3} \mathbf{a}_{1}+\left(\frac{1}{2}-y_{3}\right) \mathbf{a}_{2}+\left(\frac{1}{2}+z_{3}\right) \mathbf{a}_{3}$ & $=$ & $x_{3} a \hat{\mathbf{x}}+\left(\frac{1}{2}-y_{3}\right) a \hat{\mathbf{y}}+\left(\frac{1}{2}+z_{3}\right) c \hat{\mathbf{z}}$ & $(16 g)$ \\
\hline $\mathbf{B}_{26}$ & $=$ & $\left(\frac{1}{2}-x_{3}\right) \mathbf{a}_{1}+y_{3} \mathbf{a}_{2}+\left(\frac{1}{2}+z_{3}\right) \mathbf{a}_{3}$ & $=$ & $\left(\frac{1}{2}-x_{3}\right) a \hat{\mathbf{x}}+y_{3} a \hat{\mathbf{y}}+\left(\frac{1}{2}+z_{3}\right) c \hat{\mathbf{z}}$ & $(16 g)$ \\
\hline $\mathbf{B}_{27}$ & $=$ & $\begin{array}{c}\left(\frac{1}{2}-y_{3}\right) \mathbf{a}_{1}+\left(\frac{1}{2}-x_{3}\right) \mathbf{a}_{2}+ \\
\left(\frac{1}{2}+z_{3}\right) \mathbf{a}_{3}\end{array}$ & $=$ & $\begin{array}{c}\left(\frac{1}{2}-y_{3}\right) a \hat{\mathbf{x}}+\left(\frac{1}{2}-x_{3}\right) a \hat{\mathbf{y}}+ \\
\left(\frac{1}{2}+z_{3}\right) c \hat{\mathbf{z}}\end{array}$ & $(16 g)$ \\
\hline $\mathbf{B}_{28}$ & $=$ & $y_{3} \mathbf{a}_{1}+x_{3} \mathbf{a}_{2}+\left(\frac{1}{2}+z_{3}\right) \mathbf{a}_{3}$ & $=$ & $y_{3} a \hat{\mathbf{x}}+x_{3} a \hat{\mathbf{y}}+\left(\frac{1}{2}+z_{3}\right) c \hat{\mathbf{z}}$ & $(16 g)$ \\
\hline
\end{tabular}

\section{References:}

- J.-C. Boivin, J. Trehoux, and D. Thomas, Étude structurale de $\mathrm{CuBi}_{2} \mathrm{O}_{4}$, Bull. Soc. fr. Mineral. Crystallogr. 99, 193-196 (1976).

\section{Found in:}

- P. Villars and K. Cenzual, Pearson's Crystal Data - Crystal Structure Database for Inorganic Compounds, ASM International (2013).

\section{Geometry files:}

- CIF: pp. 895

- POSCAR: pp. 896 


\section{$\mathrm{Ba}_{5} \mathrm{Si}_{3}$ Structure: A5B3_tP32_130_cg_cf}
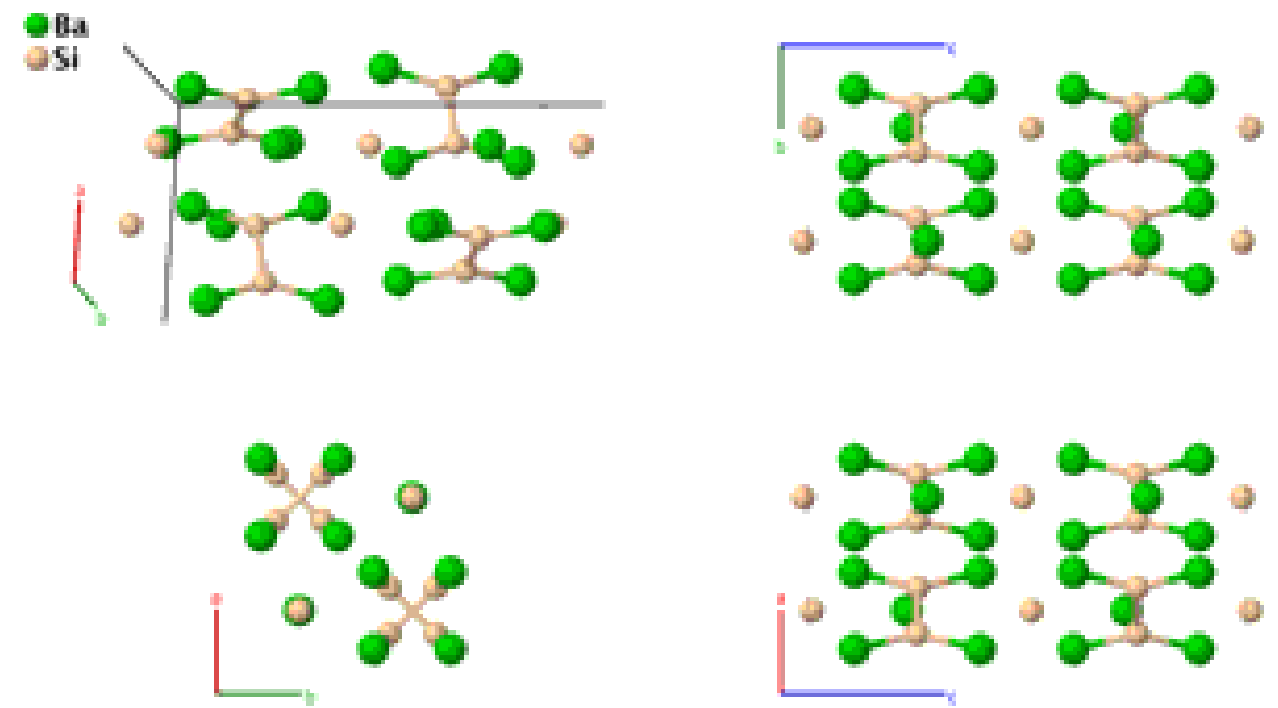

Prototype

$\mathrm{Ba}_{5} \mathrm{Si}_{3}$

AFLOW prototype label

: A5B3_tP32_130_cg_cf

Strukturbericht designation : None

Pearson symbol : $\quad$ tP32

Space group number $\quad: \quad 130$

Space group symbol $\quad: \quad P 4 / n c c$

AFLOW prototype command : aflow --proto=A5B3_tP32_130_cg_cf

- params $=a, c / a, z_{1}, z_{2}, x_{3}, x_{4}, y_{4}, z_{4}$

Simple Tetragonal primitive vectors:

$$
\begin{aligned}
& \mathbf{a}_{1}=a \hat{\mathbf{x}} \\
& \mathbf{a}_{2}=a \hat{\mathbf{y}} \\
& \mathbf{a}_{3}=c \hat{\mathbf{z}}
\end{aligned}
$$

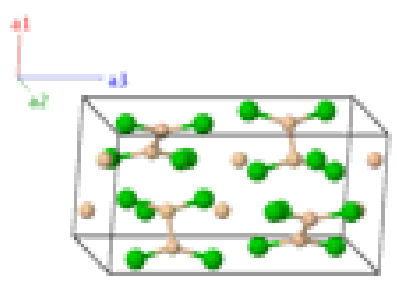

Basis vectors:

Lattice Coordinates

$\mathbf{B}_{1}=\frac{1}{4} \mathbf{a}_{1}+\frac{1}{4} \mathbf{a}_{2}+z_{1} \mathbf{a}_{3}$

$\mathbf{B}_{2}=\frac{3}{4} \mathbf{a}_{1}+\frac{3}{4} \mathbf{a}_{2}+\left(\frac{1}{2}-z_{1}\right) \mathbf{a}_{3}$

$\mathbf{B}_{3}=\quad \frac{3}{4} \mathbf{a}_{1}+\frac{3}{4} \mathbf{a}_{2}-z_{1} \mathbf{a}_{3}$

$\mathbf{B}_{4}=\frac{1}{4} \mathbf{a}_{1}+\frac{1}{4} \mathbf{a}_{2}+\left(\frac{1}{2}+z_{1}\right) \mathbf{a}_{3}$

$\mathbf{B}_{5}=$

$\mathbf{B}_{6}=$

$\mathbf{B}_{7}=$

$$
\frac{1}{4} \mathbf{a}_{1}+\frac{1}{4} \mathbf{a}_{2}+z_{2} \mathbf{a}_{3}
$$

$$
\frac{3}{4} \mathbf{a}_{1}+\frac{3}{4} \mathbf{a}_{2}+\left(\frac{1}{2}-z_{2}\right) \mathbf{a}_{3}
$$

$\frac{3}{4} \mathbf{a}_{1}+\frac{3}{4} \mathbf{a}_{2}-z_{2} \mathbf{a}_{3}$
Cartesian Coordinates

$=$

$=$

$=$

$=$

$=$

$=$

$=$

$$
\frac{1}{4} a \hat{\mathbf{x}}+\frac{1}{4} a \hat{\mathbf{y}}+z_{1} c \hat{\mathbf{z}}
$$$$
\frac{3}{4} a \hat{\mathbf{x}}+\frac{3}{4} a \hat{\mathbf{y}}+\left(\frac{1}{2}-z_{1}\right) c \hat{\mathbf{z}}
$$$$
\frac{3}{4} a \hat{\mathbf{x}}+\frac{3}{4} a \hat{\mathbf{y}}-z_{1} c \hat{\mathbf{z}}
$$$$
\frac{1}{4} a \hat{\mathbf{x}}+\frac{1}{4} a \hat{\mathbf{y}}+\left(\frac{1}{2}+z_{1}\right) c \hat{\mathbf{z}}
$$$$
\frac{1}{4} a \hat{\mathbf{x}}+\frac{1}{4} a \hat{\mathbf{y}}+z_{2} c \hat{\mathbf{z}}
$$$$
\frac{3}{4} a \hat{\mathbf{x}}+\frac{3}{4} a \hat{\mathbf{y}}+\left(\frac{1}{2}-z_{2}\right) c \hat{\mathbf{z}}
$$$$
\frac{3}{4} a \hat{\mathbf{x}}+\frac{3}{4} a \hat{\mathbf{y}}-z_{2} c \hat{\mathbf{z}}
$$

Wyckoff Position Atom Type

$(4 c)$

$\mathrm{Ba} I$

Ba I

Ba I

Ba I

(4c)

Si I

Si I

(4c)

Si I 


\begin{tabular}{|c|c|c|c|c|c|c|}
\hline $\mathbf{B}_{8}$ & $=$ & $\frac{1}{4} \mathbf{a}_{1}+\frac{1}{4} \mathbf{a}_{2}+\left(\frac{1}{2}+z_{2}\right) \mathbf{a}_{3}$ & $=$ & $\frac{1}{4} a \hat{\mathbf{x}}+\frac{1}{4} a \hat{\mathbf{y}}+\left(\frac{1}{2}+z_{2}\right) c \hat{\mathbf{z}}$ & $(4 c)$ & Si I \\
\hline $\mathbf{B}_{9}$ & $=$ & $x_{3} \mathbf{a}_{1}-x_{3} \mathbf{a}_{2}+\frac{1}{4} \mathbf{a}_{3}$ & $=$ & $x_{3} a \hat{\mathbf{x}}-x_{3} a \hat{\mathbf{y}}+\frac{1}{4} c \hat{\mathbf{z}}$ & $(8 f)$ & Si II \\
\hline $\mathbf{B}_{10}$ & $=$ & $\left(\frac{1}{2}-x_{3}\right) \mathbf{a}_{1}+\left(\frac{1}{2}-x_{3}\right) \mathbf{a}_{2}+\frac{1}{4} \mathbf{a}_{3}$ & $=$ & $\left(\frac{1}{2}-x_{3}\right) a \hat{\mathbf{x}}+\left(\frac{1}{2}-x_{3}\right) a \hat{\mathbf{y}}+\frac{1}{4} c \hat{\mathbf{z}}$ & $(8 f)$ & Si II \\
\hline $\mathbf{B}_{11}$ & $=$ & $\left(\frac{1}{2}+x_{3}\right) \mathbf{a}_{1}+x_{3} \mathbf{a}_{2}+\frac{1}{4} \mathbf{a}_{3}$ & $=$ & $\left(\frac{1}{2}+x_{3}\right) a \hat{\mathbf{x}}+x_{3} a \hat{\mathbf{y}}+\frac{1}{4} c \hat{\mathbf{z}}$ & $(8 f)$ & Si II \\
\hline $\mathbf{B}_{12}$ & $=$ & $-x_{3} \mathbf{a}_{1}+\left(\frac{1}{2}-x_{3}\right) \mathbf{a}_{2}+\frac{1}{4} \mathbf{a}_{3}$ & $=$ & $-x_{3} a \hat{\mathbf{x}}+\left(\frac{1}{2}-x_{3}\right) a \hat{\mathbf{y}}+\frac{1}{4} c \hat{\mathbf{z}}$ & $(8 f)$ & Si II \\
\hline $\mathbf{B}_{13}$ & $=$ & $-x_{3} \mathbf{a}_{1}+x_{3} \mathbf{a}_{2}+\frac{3}{4} \mathbf{a}_{3}$ & $=$ & $-x_{3} a \hat{\mathbf{x}}+x_{3} a \hat{\mathbf{y}}+\frac{3}{4} c \hat{\mathbf{z}}$ & $(8 f)$ & Si II \\
\hline $\mathbf{B}_{14}$ & $=$ & $\left(\frac{1}{2}+x_{3}\right) \mathbf{a}_{1}+\left(\frac{1}{2}-x_{3}\right) \mathbf{a}_{2}+\frac{3}{4} \mathbf{a}_{3}$ & $=$ & $\left(\frac{1}{2}+x_{3}\right) a \hat{\mathbf{x}}+\left(\frac{1}{2}-x_{3}\right) a \hat{\mathbf{y}}+\frac{3}{4} c \hat{\mathbf{z}}$ & $(8 f)$ & Si II \\
\hline $\mathbf{B}_{15}$ & $=$ & $\left(\frac{1}{2}-x_{3}\right) \mathbf{a}_{1}-x_{3} \mathbf{a}_{2}+\frac{3}{4} \mathbf{a}_{3}$ & $=$ & $\left(\frac{1}{2}-x_{3}\right) a \hat{\mathbf{x}}-x_{3} a \hat{\mathbf{y}}+\frac{3}{4} c \hat{\mathbf{z}}$ & $(8 f)$ & Si II \\
\hline $\mathbf{B}_{16}$ & $=$ & $x_{3} \mathbf{a}_{1}+\left(\frac{1}{2}+x_{3}\right) \mathbf{a}_{2}+\frac{3}{4} \mathbf{a}_{3}$ & $=$ & $x_{3} a \hat{\mathbf{x}}+\left(\frac{1}{2}+x_{3}\right) a \hat{\mathbf{y}}+\frac{3}{4} c \hat{\mathbf{z}}$ & $(8 f)$ & Si II \\
\hline $\mathbf{B}_{17}$ & $=$ & $x_{4} \mathbf{a}_{1}+y_{4} \mathbf{a}_{2}+z_{4} \mathbf{a}_{3}$ & $=$ & $x_{4} a \hat{\mathbf{x}}+y_{4} a \hat{\mathbf{y}}+z_{4} c \hat{\mathbf{z}}$ & $(16 g)$ & Ba II \\
\hline $\mathbf{B}_{18}$ & $=$ & $\left(\frac{1}{2}-x_{4}\right) \mathbf{a}_{1}+\left(\frac{1}{2}-y_{4}\right) \mathbf{a}_{2}+z_{4} \mathbf{a}_{3}$ & $=$ & $\left(\frac{1}{2}-x_{4}\right) a \hat{\mathbf{x}}+\left(\frac{1}{2}-y_{4}\right) a \hat{\mathbf{y}}+z_{4} c \hat{\mathbf{z}}$ & $(16 g)$ & Ba II \\
\hline $\mathbf{B}_{19}$ & $=$ & $\left(\frac{1}{2}-y_{4}\right) \mathbf{a}_{1}+x_{4} \mathbf{a}_{2}+z_{4} \mathbf{a}_{3}$ & $=$ & $\left(\frac{1}{2}-y_{4}\right) a \hat{\mathbf{x}}+x_{4} a \hat{\mathbf{y}}+z_{4} c \hat{\mathbf{z}}$ & $(16 g)$ & $\mathrm{Ba}$ II \\
\hline $\mathbf{B}_{20}$ & $=$ & $y_{4} \mathbf{a}_{1}+\left(\frac{1}{2}-x_{4}\right) \mathbf{a}_{2}+z_{4} \mathbf{a}_{3}$ & $=$ & $y_{4} a \hat{\mathbf{x}}+\left(\frac{1}{2}-x_{4}\right) a \hat{\mathbf{y}}+z_{4} c \hat{\mathbf{z}}$ & $(16 g)$ & Ba II \\
\hline $\mathbf{B}_{21}$ & $=$ & $-x_{4} \mathbf{a}_{1}+\left(\frac{1}{2}+y_{4}\right) \mathbf{a}_{2}+\left(\frac{1}{2}-z_{4}\right) \mathbf{a}_{3}$ & $=$ & $-x_{4} a \hat{\mathbf{x}}+\left(\frac{1}{2}+y_{4}\right) a \hat{\mathbf{y}}+\left(\frac{1}{2}-z_{4}\right) c \hat{\mathbf{z}}$ & $(16 g)$ & Ba II \\
\hline $\mathbf{B}_{22}$ & $=$ & $\left(\frac{1}{2}+x_{4}\right) \mathbf{a}_{1}-y_{4} \mathbf{a}_{2}+\left(\frac{1}{2}-z_{4}\right) \mathbf{a}_{3}$ & $=$ & $\left(\frac{1}{2}+x_{4}\right) a \hat{\mathbf{x}}-y_{4} a \hat{\mathbf{y}}+\left(\frac{1}{2}-z_{4}\right) c \hat{\mathbf{z}}$ & $(16 g)$ & Ba II \\
\hline $\mathbf{B}_{23}$ & $=$ & $\begin{array}{c}\left(\frac{1}{2}+y_{4}\right) \mathbf{a}_{1}+\left(\frac{1}{2}+x_{4}\right) \mathbf{a}_{2}+ \\
\left(\frac{1}{2}-z_{4}\right) \mathbf{a}_{3}\end{array}$ & $=$ & $\begin{array}{c}\left(\frac{1}{2}+y_{4}\right) a \hat{\mathbf{x}}+\left(\frac{1}{2}+x_{4}\right) a \hat{\mathbf{y}}+ \\
\left(\frac{1}{2}-z_{4}\right) c \hat{\mathbf{z}}\end{array}$ & $(16 g)$ & Ba II \\
\hline $\mathbf{B}_{24}$ & $=$ & $-y_{4} \mathbf{a}_{1}-x_{4} \mathbf{a}_{2}+\left(\frac{1}{2}-z_{4}\right) \mathbf{a}_{3}$ & $=$ & $-y_{4} a \hat{\mathbf{x}}-x_{4} a \hat{\mathbf{y}}+\left(\frac{1}{2}-z_{4}\right) c \hat{\mathbf{z}}$ & $(16 g)$ & Ba II \\
\hline $\mathbf{B}_{25}$ & $=$ & $-x_{4} \mathbf{a}_{1}-y_{4} \mathbf{a}_{2}-z_{4} \mathbf{a}_{3}$ & $=$ & $-x_{4} a \hat{\mathbf{x}}-y_{4} a \hat{\mathbf{y}}-z_{4} c \hat{\mathbf{z}}$ & $(16 g)$ & Ba II \\
\hline $\mathbf{B}_{26}$ & $=$ & $\left(\frac{1}{2}+x_{4}\right) \mathbf{a}_{1}+\left(\frac{1}{2}+y_{4}\right) \mathbf{a}_{2}-z_{4} \mathbf{a}_{3}$ & $=$ & $\left(\frac{1}{2}+x_{4}\right) a \hat{\mathbf{x}}+\left(\frac{1}{2}+y_{4}\right) a \hat{\mathbf{y}}-z_{4} c \hat{\mathbf{z}}$ & $(16 g)$ & Ba II \\
\hline $\mathbf{B}_{27}$ & $=$ & $\left(\frac{1}{2}+y_{4}\right) \mathbf{a}_{1}-x_{4} \mathbf{a}_{2}-z_{4} \mathbf{a}_{3}$ & $=$ & $\left(\frac{1}{2}+y_{4}\right) a \hat{\mathbf{x}}-x_{4} a \hat{\mathbf{y}}-z_{4} c \hat{\mathbf{z}}$ & $(16 g)$ & Ba II \\
\hline $\mathbf{B}_{28}$ & $=$ & $-y_{4} \mathbf{a}_{1}+\left(\frac{1}{2}+x_{4}\right) \mathbf{a}_{2}-z_{4} \mathbf{a}_{3}$ & $=$ & $-y_{4} a \hat{\mathbf{x}}+\left(\frac{1}{2}+x_{4}\right) a \hat{\mathbf{y}}-z_{4} c \hat{\mathbf{z}}$ & $(16 g)$ & Ba II \\
\hline $\mathbf{B}_{29}$ & $=$ & $x_{4} \mathbf{a}_{1}+\left(\frac{1}{2}-y_{4}\right) \mathbf{a}_{2}+\left(\frac{1}{2}+z_{4}\right) \mathbf{a}_{3}$ & $=$ & $x_{4} a \hat{\mathbf{x}}+\left(\frac{1}{2}-y_{4}\right) a \hat{\mathbf{y}}+\left(\frac{1}{2}+z_{4}\right) c \hat{\mathbf{z}}$ & $(16 g)$ & Ba II \\
\hline $\mathbf{B}_{30}$ & $=$ & $\left(\frac{1}{2}-x_{4}\right) \mathbf{a}_{1}+y_{4} \mathbf{a}_{2}+\left(\frac{1}{2}+z_{4}\right) \mathbf{a}_{3}$ & $=$ & $\left(\frac{1}{2}-x_{4}\right) a \hat{\mathbf{x}}+y_{4} a \hat{\mathbf{y}}+\left(\frac{1}{2}+z_{4}\right) c \hat{\mathbf{z}}$ & $(16 g)$ & Ba II \\
\hline $\mathbf{B}_{31}$ & $=$ & $\begin{array}{c}\left(\frac{1}{2}-y_{4}\right) \mathbf{a}_{1}+\left(\frac{1}{2}-x_{4}\right) \mathbf{a}_{2}+ \\
\left(\frac{1}{2}+z_{4}\right) \mathbf{a}_{3}\end{array}$ & $=$ & $\begin{array}{c}\left(\frac{1}{2}-y_{4}\right) a \hat{\mathbf{x}}+\left(\frac{1}{2}-x_{4}\right) a \hat{\mathbf{y}}+ \\
\left(\frac{1}{2}+z_{4}\right) c \hat{\mathbf{z}}\end{array}$ & $(16 g)$ & $\mathrm{Ba}$ II \\
\hline $\mathbf{B}_{32}$ & $=$ & $y_{4} \mathbf{a}_{1}+x_{4} \mathbf{a}_{2}+\left(\frac{1}{2}+z_{4}\right) \mathbf{a}_{3}$ & $=$ & $y_{4} a \hat{\mathbf{x}}+x_{4} a \hat{\mathbf{y}}+\left(\frac{1}{2}+z_{4}\right) c \hat{\mathbf{z}}$ & $(16 g)$ & Ba II \\
\hline
\end{tabular}

\section{References:}

- R. Nesper and F. Zürcher, Refinement of the crystal structure of pentabarium trisilicide, $B a_{5} \mathrm{Si}_{3}$, Z. Kristallogr. B 214, 20 (1966), doi:10.1515/ncrs-1999-0113.

\section{Geometry files:}

- CIF: pp. 896

- POSCAR: pp. 896 


\section{$\mathrm{Rb}_{2} \mathrm{TiCu}_{2} \mathrm{~S}_{4}$ Structure: A2B2C4D_tP18_132_e_i_o_d}
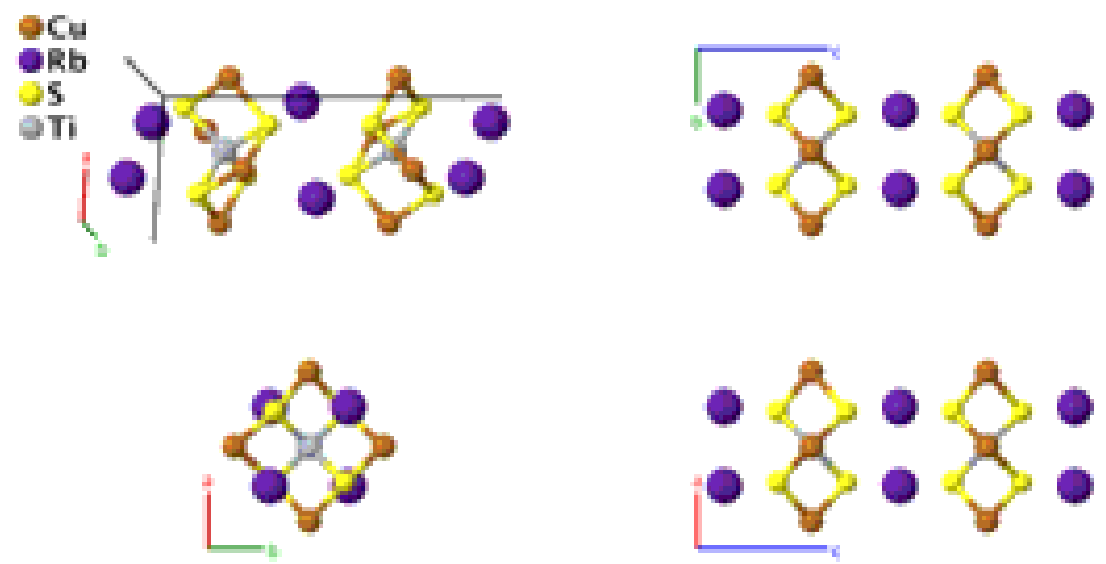

Prototype

AFLOW prototype label

Strukturbericht designation

Pearson symbol

Space group number

Space group symbol

AFLOW prototype command
: $\quad \mathrm{Rb}_{2} \mathrm{TiCu}_{2} \mathrm{Se}_{4}$

: A2B2C4D_tP18_132_e_i_o_d

: None

: $\quad$ tP18

: 132

: $\quad P 4_{2} / \mathrm{mcm}$

aflow --proto=A2B2C4D_tP18_132_e_i_o_d

- params $=a, c / a, x_{3}, x_{4}, z_{4}$

\section{Other compounds with this structure:}

- $\mathrm{Cs}_{2} \mathrm{TiAg}_{2} \mathrm{~S}_{4}, \mathrm{Cs}_{2} \mathrm{TiCu}_{2} \mathrm{Se}_{4}$

- The atomic positions for this structure are not given in the main text, but are provided as a Crystallographic Information File (CIF), ic001346d.cif.

\section{Simple Tetragonal primitive vectors:}

$$
\begin{aligned}
& \mathbf{a}_{1}=a \hat{\mathbf{x}} \\
& \mathbf{a}_{2}=a \hat{\mathbf{y}} \\
& \mathbf{a}_{3}=c \hat{\mathbf{z}}
\end{aligned}
$$

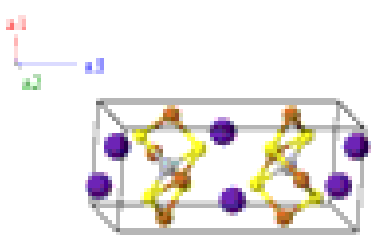

Basis vectors:

Lattice Coordinates

$\begin{array}{llc}\mathbf{B}_{1}= & \frac{1}{2} \mathbf{a}_{1}+\frac{1}{2} \mathbf{a}_{2}+\frac{1}{4} \mathbf{a}_{3} \\ \mathbf{B}_{2}= & \frac{1}{2} \mathbf{a}_{1}+\frac{1}{2} \mathbf{a}_{2}+\frac{3}{4} \mathbf{a}_{3} \\ \mathbf{B}_{3}= & \frac{1}{2} \mathbf{a}_{2}+\frac{1}{4} \mathbf{a}_{3} \\ \mathbf{B}_{4}= & \frac{1}{2} \mathbf{a}_{1}+\frac{3}{4} \mathbf{a}_{3} \\ \mathbf{B}_{5}= & \frac{1}{2} \mathbf{a}_{2}+\frac{3}{4} \mathbf{a}_{3}\end{array}$

$\mathbf{B}_{1}=\frac{1}{2} \mathbf{a}_{1}+\frac{1}{2} \mathbf{a}_{2}+\frac{1}{4} \mathbf{a}_{3}$

$=$

$=$

$=$

$=$

$=$
Cartesian Coordinates

$\frac{1}{2} a \hat{\mathbf{x}}+\frac{1}{2} a \hat{\mathbf{y}}+\frac{1}{4} c \hat{\mathbf{z}}$

$\frac{1}{2} a \hat{\mathbf{x}}+\frac{1}{2} a \hat{\mathbf{y}}+\frac{3}{4} c \hat{\mathbf{z}}$

$\frac{1}{2} a \hat{\mathbf{y}}+\frac{1}{4} c \hat{\mathbf{z}}$

$\frac{1}{2} a \hat{\mathbf{x}}+\frac{3}{4} c \hat{\mathbf{z}}$

$\frac{1}{2} a \hat{\mathbf{y}}+\frac{3}{4} c \hat{\mathbf{z}}$
Wyckoff Position

$(2 d)$

$(2 d)$

$(4 e)$

$(4 e)$
Atom Type

$\mathrm{Ti}$

Ti

$\mathrm{Cu}$

$\mathrm{Cu}$

$\mathrm{Cu}$ 


\begin{tabular}{|c|c|c|c|c|c|c|}
\hline $\mathbf{B}_{6}$ & $=$ & $\frac{1}{2} \mathbf{a}_{1}+\frac{1}{4} \mathbf{a}_{3}$ & $=$ & $\frac{1}{2} a \hat{\mathbf{x}}+\frac{1}{4} c \hat{\mathbf{z}}$ & $(4 e)$ & $\mathrm{Cu}$ \\
\hline $\mathbf{B}_{7}$ & $=$ & $x_{3} \mathbf{a}_{1}+x_{3} \mathbf{a}_{2}$ & $=$ & $x_{3} a \hat{\mathbf{x}}+x_{3} a \hat{\mathbf{y}}$ & $(4 i)$ & $\mathrm{Rb}$ \\
\hline $\mathbf{B}_{8}$ & $=$ & $-x_{3} \mathbf{a}_{1}-x_{3} \mathbf{a}_{2}$ & $=$ & $-x_{3} a \hat{\mathbf{x}}-x_{3} a \hat{\mathbf{y}}$ & $(4 i)$ & $\mathrm{Rb}$ \\
\hline $\mathbf{B}_{9}$ & $=$ & $-x_{3} \mathbf{a}_{1}+x_{3} \mathbf{a}_{2}+\frac{1}{2} \mathbf{a}_{3}$ & $=$ & $-x_{3} a \hat{\mathbf{x}}+x_{3} a \hat{\mathbf{y}}+\frac{1}{2} c \hat{\mathbf{z}}$ & $(4 i)$ & $\mathrm{Rb}$ \\
\hline $\mathbf{B}_{10}$ & $=$ & $x_{3} \mathbf{a}_{1}-x_{3} \mathbf{a}_{2}+\frac{1}{2} \mathbf{a}_{3}$ & $=$ & $x_{3} a \hat{\mathbf{x}}-x_{3} a \hat{\mathbf{y}}+\frac{1}{2} c \hat{\mathbf{z}}$ & $(4 i)$ & $\mathrm{Rb}$ \\
\hline $\mathbf{B}_{11}$ & $=$ & $x_{4} \mathbf{a}_{1}+x_{4} \mathbf{a}_{2}+z_{4} \mathbf{a}_{3}$ & $=$ & $x_{4} a \hat{\mathbf{x}}+x_{4} a \hat{\mathbf{y}}+z_{4} c \hat{\mathbf{z}}$ & $(8 o)$ & $\mathrm{S}$ \\
\hline $\mathbf{B}_{12}$ & $=$ & $-x_{4} \mathbf{a}_{1}-x_{4} \mathbf{a}_{2}+z_{4} \mathbf{a}_{3}$ & $=$ & $-x_{4} a \hat{\mathbf{x}}-x_{4} a \hat{\mathbf{y}}+z_{4} c \hat{\mathbf{z}}$ & $(8 o)$ & S \\
\hline $\mathbf{B}_{13}$ & $=$ & $-x_{4} \mathbf{a}_{1}+x_{4} \mathbf{a}_{2}+\left(\frac{1}{2}+z_{4}\right) \mathbf{a}_{3}$ & $=$ & $-x_{4} a \hat{\mathbf{x}}+x_{4} a \hat{\mathbf{y}}+\left(\frac{1}{2}+z_{4}\right) c \hat{\mathbf{z}}$ & $(8 o)$ & $\mathrm{S}$ \\
\hline $\mathbf{B}_{14}$ & $=$ & $x_{4} \mathbf{a}_{1}-x_{4} \mathbf{a}_{2}+\left(\frac{1}{2}+z_{4}\right) \mathbf{a}_{3}$ & $=$ & $x_{4} a \hat{\mathbf{x}}-x_{4} a \hat{\mathbf{y}}+\left(\frac{1}{2}+z_{4}\right) c \hat{\mathbf{z}}$ & $(8 o)$ & $\mathrm{S}$ \\
\hline $\mathbf{B}_{15}$ & $=$ & $-x_{4} \mathbf{a}_{1}+x_{4} \mathbf{a}_{2}+\left(\frac{1}{2}-z_{4}\right) \mathbf{a}_{3}$ & $=$ & $-x_{4} a \hat{\mathbf{x}}+x_{4} a \hat{\mathbf{y}}+\left(\frac{1}{2}-z_{4}\right) c \hat{\mathbf{z}}$ & $(8 o)$ & $\mathrm{S}$ \\
\hline $\mathbf{B}_{16}$ & $=$ & $x_{4} \mathbf{a}_{1}-x_{4} \mathbf{a}_{2}+\left(\frac{1}{2}-z_{4}\right) \mathbf{a}_{3}$ & $=$ & $x_{4} a \hat{\mathbf{x}}-x_{4} a \hat{\mathbf{y}}+\left(\frac{1}{2}-z_{4}\right) c \hat{\mathbf{z}}$ & $(8 o)$ & $\mathrm{S}$ \\
\hline $\mathbf{B}_{17}$ & $=$ & $x_{4} \mathbf{a}_{1}+x_{4} \mathbf{a}_{2}-z_{4} \mathbf{a}_{3}$ & $=$ & $x_{4} a \hat{\mathbf{x}}+x_{4} a \hat{\mathbf{y}}-z_{4} c \hat{\mathbf{z}}$ & $(8 o)$ & $S$ \\
\hline $\mathbf{B}_{18}$ & $=$ & $-x_{4} \mathbf{a}_{1}-x_{4} \mathbf{a}_{2}-z_{4} \mathbf{a}_{3}$ & $=$ & $-x_{4} a \hat{\mathbf{x}}-x_{4} a \hat{\mathbf{y}}-z_{4} c \hat{\mathbf{z}}$ & $(8 o)$ & $\mathrm{S}$ \\
\hline
\end{tabular}

\section{References:}

- F. Q. Huang and J. A. Ibers, New Layered Materials: Syntheses, Structures, and Optical Properties of $\mathrm{K}_{2} \mathrm{TiCu}_{2} \mathrm{~S}_{4}$, $\mathrm{Rb}_{2} \mathrm{TiCu}_{2} \mathrm{~S}_{4}, \mathrm{Rb}_{2} \mathrm{TiAg}_{2} \mathrm{~S}_{4}, \mathrm{Cs}_{2} \mathrm{TiAg}_{2} \mathrm{~S}_{4}$, and $\mathrm{Cs}_{2} \mathrm{TiCu}_{2} \mathrm{Se}_{4}$, Inorg. Chem. 40, 2602-2607 (2001), doi:10.1021/ic001346d.

\section{Geometry files:}

- CIF: pp. 896

- POSCAR: pp. 897 


\section{AgUF 6 Structure: AB6C_tP16_132_d_io_a}
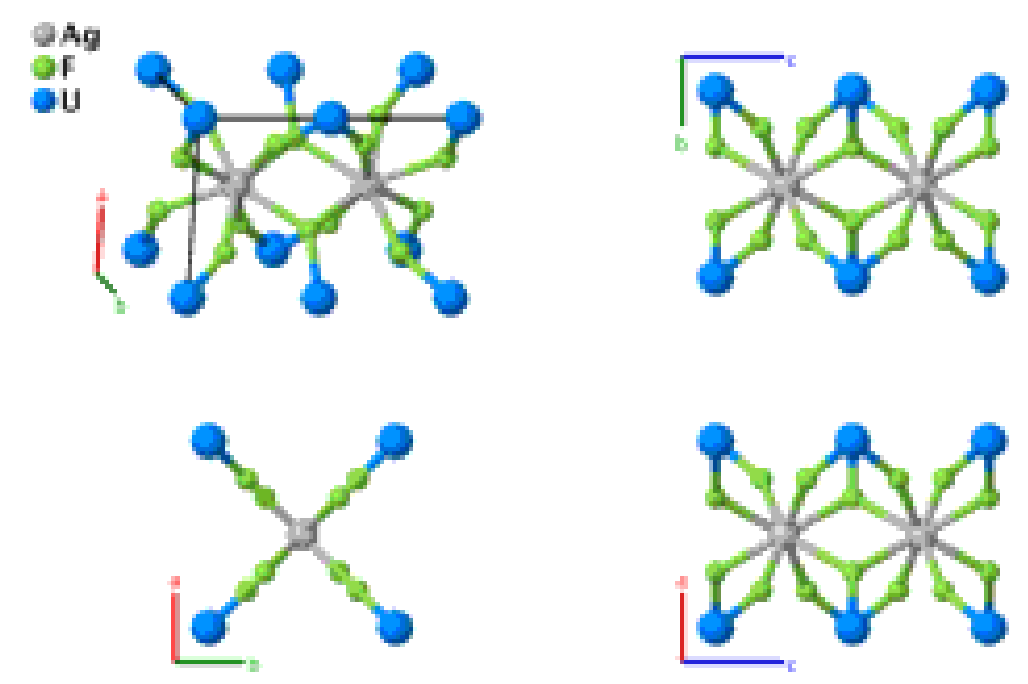

\section{Prototype}

AFLOW prototype label

$: \quad \mathrm{AgUF}_{6}$

Strukturbericht designation

Pearson symbol

: AB6C_tP16_132_d_io_a

Space group number

: None

Space group symbol

: $\quad$ tP16

AFLOW prototype command

$$
\text { : } \quad P 4_{2} / \mathrm{mcm}
$$

: aflow --proto=AB6C_tP16_132_d_io_a

- params $=a, c / a, x_{3}, x_{4}, z_{4}$

Simple Tetragonal primitive vectors:

$$
\begin{aligned}
& \mathbf{a}_{1}=a \hat{\mathbf{x}} \\
& \mathbf{a}_{2}=a \hat{\mathbf{y}} \\
& \mathbf{a}_{3}=c \hat{\mathbf{z}}
\end{aligned}
$$

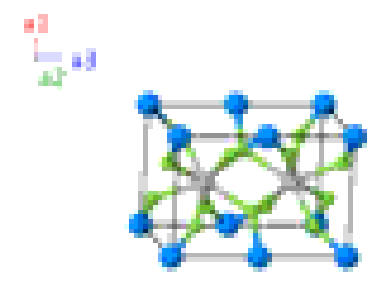

Basis vectors:

Lattice Coordinates
$\mathbf{B}_{1}=$
$0 \mathbf{a}_{1}+0 \mathbf{a}_{2}+0 \mathbf{a}_{3}$
$\mathbf{B}_{2}=$
$\frac{1}{2} \mathbf{a}_{3}$
$\mathbf{B}_{3}=$
$\mathbf{B}_{4}=$
$\mathbf{B}_{5}=$
$\mathbf{B}_{6}=$
$\mathbf{B}_{7}=$
$=$
$=$
$=$
$=$
$=$
$=$
$=$

$$
\begin{gathered}
0 \hat{\mathbf{x}}+0 \hat{\mathbf{y}}+0 \hat{\mathbf{z}} \\
\frac{1}{2} c \hat{\mathbf{z}} \\
\frac{1}{2} a \hat{\mathbf{x}}+\frac{1}{2} a \hat{\mathbf{y}}+\frac{1}{4} c \hat{\mathbf{z}} \\
\frac{1}{2} a \hat{\mathbf{x}}+\frac{1}{2} a \hat{\mathbf{y}}+\frac{3}{4} c \hat{\mathbf{z}} \\
x_{3} a \hat{\mathbf{x}}+x_{3} a \hat{\mathbf{y}}
\end{gathered}
$$$$
\frac{1}{2} a \hat{\mathbf{x}}+\frac{1}{2} a \hat{\mathbf{y}}+\frac{1}{4} c \hat{\mathbf{z}}
$$$$
\frac{1}{2} a \hat{\mathbf{x}}+\frac{1}{2} a \hat{\mathbf{y}}+\frac{3}{4} c \hat{\mathbf{z}}
$$$$
-x_{3} a \hat{\mathbf{x}}-x_{3} a \hat{\mathbf{y}}
$$$$
-x_{3} a \hat{\mathbf{x}}+x_{3} a \hat{\mathbf{y}}+\frac{1}{2} c \hat{\mathbf{z}}
$$

Cartesian Coordinates
Wyckoff Position

Atom Type

(2a)

(2a)

(2d)

(2d)

(4i)

(4i)

(4i)
$\mathrm{U}$ $\mathrm{Ag}$ $\mathrm{Ag}$ F I F I F I 


\begin{tabular}{|c|c|c|c|c|c|c|}
\hline $\mathbf{B}_{8}$ & $=$ & $x_{3} \mathbf{a}_{1}-x_{3} \mathbf{a}_{2}+\frac{1}{2} \mathbf{a}_{3}$ & $=$ & $x_{3} a \hat{\mathbf{x}}-x_{3} a \hat{\mathbf{y}}+\frac{1}{2} c \hat{\mathbf{z}}$ & $(4 i)$ & F I \\
\hline $\mathbf{B}_{9}$ & $=$ & $x_{4} \mathbf{a}_{1}+x_{4} \mathbf{a}_{2}+z_{4} \mathbf{a}_{3}$ & $=$ & $x_{4} a \hat{\mathbf{x}}+x_{4} a \hat{\mathbf{y}}+z_{4} c \hat{\mathbf{z}}$ & $(8 o)$ & F II \\
\hline $\mathbf{B}_{10}$ & $=$ & $-x_{4} \mathbf{a}_{1}-x_{4} \mathbf{a}_{2}+z_{4} \mathbf{a}_{3}$ & $=$ & $-x_{4} a \hat{\mathbf{x}}-x_{4} a \hat{\mathbf{y}}+z_{4} c \hat{\mathbf{z}}$ & $(8 o)$ & F II \\
\hline $\mathbf{B}_{11}$ & $=$ & $-x_{4} \mathbf{a}_{1}+x_{4} \mathbf{a}_{2}+\left(\frac{1}{2}+z_{4}\right) \mathbf{a}_{3}$ & $=$ & $-x_{4} a \hat{\mathbf{x}}+x_{4} a \hat{\mathbf{y}}+\left(\frac{1}{2}+z_{4}\right) c \hat{\mathbf{z}}$ & $(8 o)$ & F II \\
\hline $\mathbf{B}_{12}$ & $=$ & $x_{4} \mathbf{a}_{1}-x_{4} \mathbf{a}_{2}+\left(\frac{1}{2}+z_{4}\right) \mathbf{a}_{3}$ & $=$ & $x_{4} a \hat{\mathbf{x}}-x_{4} a \hat{\mathbf{y}}+\left(\frac{1}{2}+z_{4}\right) c \hat{\mathbf{z}}$ & $(8 o)$ & F II \\
\hline $\mathbf{B}_{13}$ & $=$ & $-x_{4} \mathbf{a}_{1}+x_{4} \mathbf{a}_{2}+\left(\frac{1}{2}-z_{4}\right) \mathbf{a}_{3}$ & $=$ & $-x_{4} a \hat{\mathbf{x}}+x_{4} a \hat{\mathbf{y}}+\left(\frac{1}{2}-z_{4}\right) c \hat{\mathbf{z}}$ & $(8 o)$ & F II \\
\hline $\mathbf{B}_{14}$ & $=$ & $x_{4} \mathbf{a}_{1}-x_{4} \mathbf{a}_{2}+\left(\frac{1}{2}-z_{4}\right) \mathbf{a}_{3}$ & $=$ & $x_{4} a \hat{\mathbf{x}}-x_{4} a \hat{\mathbf{y}}+\left(\frac{1}{2}-z_{4}\right) c \hat{\mathbf{z}}$ & $(8 o)$ & F II \\
\hline $\mathbf{B}_{15}$ & $=$ & $x_{4} \mathbf{a}_{1}+x_{4} \mathbf{a}_{2}-z_{4} \mathbf{a}_{3}$ & $=$ & $x_{4} a \hat{\mathbf{x}}+x_{4} a \hat{\mathbf{y}}-z_{4} c \hat{\mathbf{z}}$ & $(8 o)$ & F II \\
\hline $\mathbf{B}_{16}$ & $=$ & $-x_{4} \mathbf{a}_{1}-x_{4} \mathbf{a}_{2}-z_{4} \mathbf{a}_{3}$ & $=$ & $-x_{4} a \hat{\mathbf{x}}-x_{4} a \hat{\mathbf{y}}-z_{4} c \hat{\mathbf{z}}$ & $(8 o)$ & F II \\
\hline
\end{tabular}

\section{References:}

- P. Charpin, Structure cristalline des hexafluorures complexes d'uranium V et d'argent de potassium d'ammonium de rubidium ou de thallium, C. R. Hebd. Séances Acad. Sci. 260, 1914-1916 (1965).

\section{Found in:}

- P. Villars and K. Cenzual, Pearson's Crystal Data - Crystal Structure Database for Inorganic Compounds, ASM International (2013).

\section{Geometry files:}

- CIF: pp. 897

- POSCAR: pp. 897 


\section{$\beta-\mathrm{V}_{3} \mathrm{~S}$ Structure: AB3_tP32_133_h_i2j}
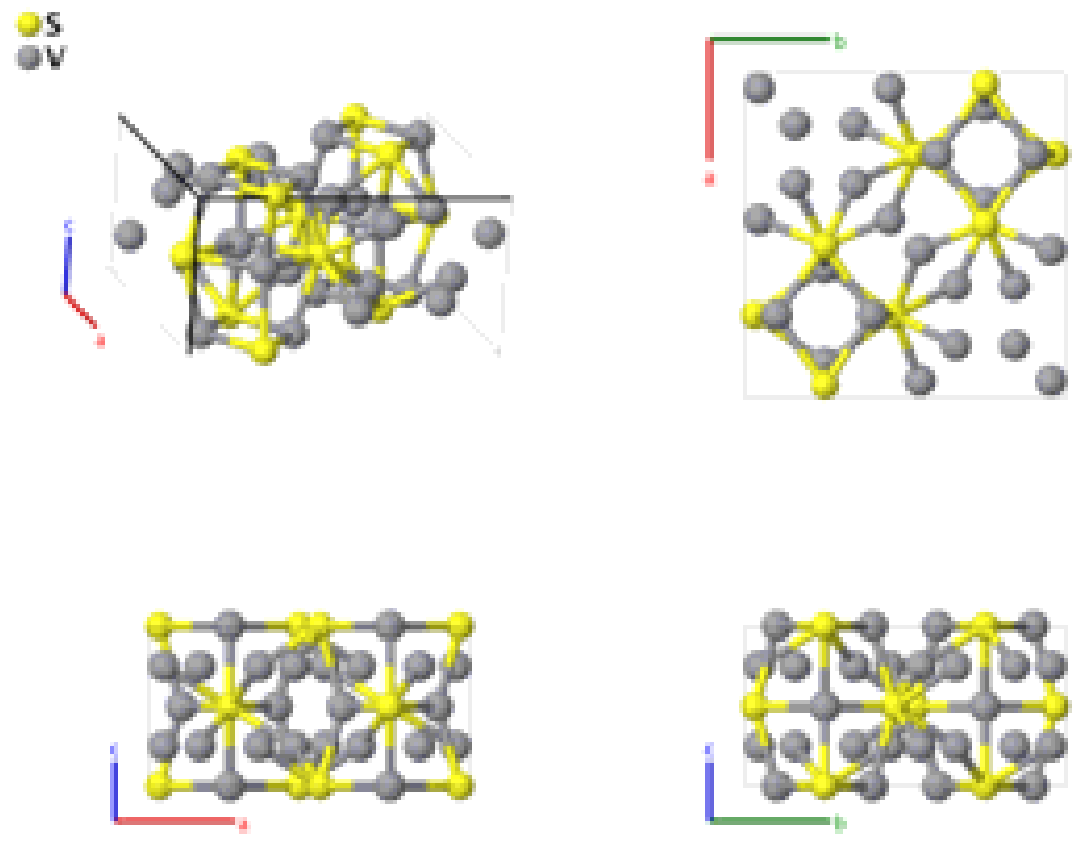

Prototype

: $\quad \beta-\mathrm{V}_{3} \mathrm{~S}$

AFLOW prototype label

: AB3_tP32_133_h_i2j

Strukturbericht designation

: None

Pearson symbol

: $\quad$ PP32

Space group number

133

Space group symbol

$\mathrm{P}_{2} / n b c$

AFLOW prototype command

aflow --proto=AB3_tP32_133_h_i2j

- -params $=a, c / a, x_{1}, x_{2}, x_{3}, x_{4}$

\section{Simple Tetragonal primitive vectors:}

$$
\begin{aligned}
& \mathbf{a}_{1}=a \hat{\mathbf{x}} \\
& \mathbf{a}_{2}=a \hat{\mathbf{y}} \\
& \mathbf{a}_{3}=c \hat{\mathbf{z}}
\end{aligned}
$$

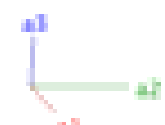

inII

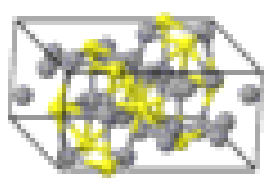

Basis vectors:

Lattice Coordinates

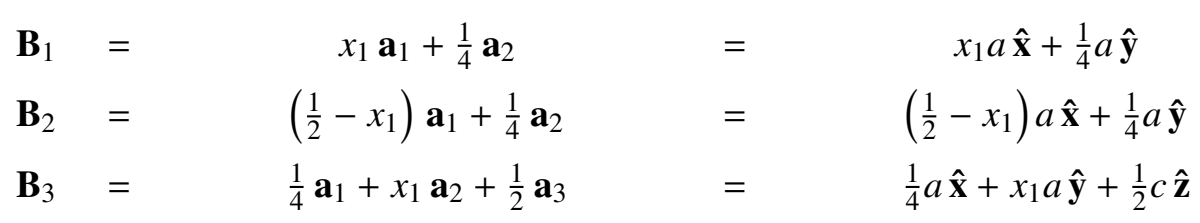

$\mathbf{B}_{1}=$

$\mathbf{B}_{2}=$

$\frac{1}{4} \mathbf{a}_{1}+x_{1} \mathbf{a}_{2}+\frac{1}{2} \mathbf{a}_{3}$

$=$
Cartesian Coordinates

Wyckoff Position

Atom Type

$(8 h)$

$(8 h)$

$(8 h)$
$\mathrm{S}$ 


\begin{tabular}{|c|c|c|c|c|c|c|}
\hline $\mathbf{B}_{4}$ & $=$ & $\frac{1}{4} \mathbf{a}_{1}+\left(\frac{1}{2}-x_{1}\right) \mathbf{a}_{2}+\frac{1}{2} \mathbf{a}_{3}$ & $=$ & $\frac{1}{4} a \hat{\mathbf{x}}+\left(\frac{1}{2}-x_{1}\right) a \hat{\mathbf{y}}+\frac{1}{2} c \hat{\mathbf{z}}$ & $(8 h)$ & $S$ \\
\hline $\mathbf{B}_{5}$ & $=$ & $-x_{1} \mathbf{a}_{1}+\frac{3}{4} \mathbf{a}_{2}$ & $=$ & $-x_{1} a \hat{\mathbf{x}}+\frac{3}{4} a \hat{\mathbf{y}}$ & $(8 h)$ & $S$ \\
\hline $\mathbf{B}_{6}$ & $=$ & $\left(\frac{1}{2}+x_{1}\right) \mathbf{a}_{1}+\frac{3}{4} \mathbf{a}_{2}$ & $=$ & $\left(\frac{1}{2}+x_{1}\right) a \hat{\mathbf{x}}+\frac{3}{4} a \hat{\mathbf{y}}$ & $(8 h)$ & $S$ \\
\hline $\mathbf{B}_{7}$ & $=$ & $\frac{3}{4} \mathbf{a}_{1}-x_{1} \mathbf{a}_{2}+\frac{1}{2} \mathbf{a}_{3}$ & $=$ & $\frac{3}{4} a \hat{\mathbf{x}}-x_{1} a \hat{\mathbf{y}}+\frac{1}{2} c \hat{\mathbf{z}}$ & $(8 h)$ & $S$ \\
\hline $\mathbf{B}_{8}$ & $=$ & $\frac{3}{4} \mathbf{a}_{1}+\left(\frac{1}{2}+x_{1}\right) \mathbf{a}_{2}+\frac{1}{2} \mathbf{a}_{3}$ & $=$ & $\frac{3}{4} a \hat{\mathbf{x}}+\left(\frac{1}{2}+x_{1}\right) a \hat{\mathbf{y}}+\frac{1}{2} c \hat{\mathbf{z}}$ & $(8 h)$ & S \\
\hline $\mathbf{B}_{9}$ & $=$ & $x_{2} \mathbf{a}_{1}+\frac{1}{4} \mathbf{a}_{2}+\frac{1}{2} \mathbf{a}_{3}$ & $=$ & $x_{2} a \hat{\mathbf{x}}+\frac{1}{4} a \hat{\mathbf{y}}+\frac{1}{2} c \hat{\mathbf{z}}$ & $(8 i)$ & V I \\
\hline $\mathbf{B}_{10}$ & $=$ & $\left(\frac{1}{2}-x_{2}\right) \mathbf{a}_{1}+\frac{1}{4} \mathbf{a}_{2}+\frac{1}{2} \mathbf{a}_{3}$ & $=$ & $\left(\frac{1}{2}-x_{2}\right) a \hat{\mathbf{x}}+\frac{1}{4} a \hat{\mathbf{y}}+\frac{1}{2} c \hat{\mathbf{z}}$ & $(8 i)$ & V I \\
\hline $\mathbf{B}_{11}$ & $=$ & $\frac{1}{4} \mathbf{a}_{1}+x_{2} \mathbf{a}_{2}$ & $=$ & $\frac{1}{4} a \hat{\mathbf{x}}+x_{2} a \hat{\mathbf{y}}$ & $(8 i)$ & V I \\
\hline $\mathbf{B}_{12}$ & $=$ & $\frac{1}{4} \mathbf{a}_{1}+\left(\frac{1}{2}-x_{2}\right) \mathbf{a}_{2}$ & $=$ & $\frac{1}{4} a \hat{\mathbf{x}}+\left(\frac{1}{2}-x_{2}\right) a \hat{\mathbf{y}}$ & $(8 i)$ & V I \\
\hline $\mathbf{B}_{13}$ & $=$ & $-x_{2} \mathbf{a}_{1}+\frac{3}{4} \mathbf{a}_{2}+\frac{1}{2} \mathbf{a}_{3}$ & $=$ & $-x_{2} a \hat{\mathbf{x}}+\frac{3}{4} a \hat{\mathbf{y}}+\frac{1}{2} c \hat{\mathbf{z}}$ & $(8 i)$ & V I \\
\hline $\mathbf{B}_{14}$ & $=$ & $\left(\frac{1}{2}+x_{2}\right) \mathbf{a}_{1}+\frac{3}{4} \mathbf{a}_{2}+\frac{1}{2} \mathbf{a}_{3}$ & $=$ & $\left(\frac{1}{2}+x_{2}\right) a \hat{\mathbf{x}}+\frac{3}{4} a \hat{\mathbf{y}}+\frac{1}{2} c \hat{\mathbf{z}}$ & $(8 i)$ & V I \\
\hline $\mathbf{B}_{15}$ & $=$ & $\frac{3}{4} \mathbf{a}_{1}-x_{2} \mathbf{a}_{2}$ & $=$ & $\frac{3}{4} a \hat{\mathbf{x}}-x_{2} a \hat{\mathbf{y}}$ & $(8 i)$ & V I \\
\hline $\mathbf{B}_{16}$ & $=$ & $\frac{3}{4} \mathbf{a}_{1}+\left(\frac{1}{2}+x_{2}\right) \mathbf{a}_{2}$ & $=$ & $\frac{3}{4} a \hat{\mathbf{x}}+\left(\frac{1}{2}+x_{2}\right) a \hat{\mathbf{y}}$ & $(8 i)$ & V I \\
\hline $\mathbf{B}_{17}$ & $=$ & $x_{3} \mathbf{a}_{1}+x_{3} \mathbf{a}_{2}+\frac{1}{4} \mathbf{a}_{3}$ & $=$ & $x_{3} a \hat{\mathbf{x}}+x_{3} a \hat{\mathbf{y}}+\frac{1}{4} c \hat{\mathbf{z}}$ & $(8 j)$ & V II \\
\hline $\mathbf{B}_{18}$ & $=$ & $\left(\frac{1}{2}-x_{3}\right) \mathbf{a}_{1}+\left(\frac{1}{2}-x_{3}\right) \mathbf{a}_{2}+\frac{1}{4} \mathbf{a}_{3}$ & $=$ & $\left(\frac{1}{2}-x_{3}\right) a \hat{\mathbf{x}}+\left(\frac{1}{2}-x_{3}\right) a \hat{\mathbf{y}}+\frac{1}{4} c \hat{\mathbf{z}}$ & $(8 j)$ & V II \\
\hline $\mathbf{B}_{19}$ & $=$ & $\left(\frac{1}{2}-x_{3}\right) \mathbf{a}_{1}+x_{3} \mathbf{a}_{2}+\frac{3}{4} \mathbf{a}_{3}$ & $=$ & $\left(\frac{1}{2}-x_{3}\right) a \hat{\mathbf{x}}+x_{3} a \hat{\mathbf{y}}+\frac{3}{4} c \hat{\mathbf{z}}$ & $(8 j)$ & V II \\
\hline $\mathbf{B}_{20}$ & $=$ & $x_{3} \mathbf{a}_{1}+\left(\frac{1}{2}-x_{3}\right) \mathbf{a}_{2}+\frac{3}{4} \mathbf{a}_{3}$ & $=$ & $x_{3} a \hat{\mathbf{x}}+\left(\frac{1}{2}-x_{3}\right) a \hat{\mathbf{y}}+\frac{3}{4} c \hat{\mathbf{z}}$ & $(8 j)$ & V II \\
\hline $\mathbf{B}_{21}$ & $=$ & $-x_{3} \mathbf{a}_{1}-x_{3} \mathbf{a}_{2}+\frac{3}{4} \mathbf{a}_{3}$ & $=$ & $-x_{3} a \hat{\mathbf{x}}-x_{3} a \hat{\mathbf{y}}+\frac{3}{4} c \hat{\mathbf{z}}$ & $(8 j)$ & V II \\
\hline $\mathbf{B}_{22}$ & $=$ & $\left(\frac{1}{2}+x_{3}\right) \mathbf{a}_{1}+\left(\frac{1}{2}+x_{3}\right) \mathbf{a}_{2}+\frac{3}{4} \mathbf{a}_{3}$ & $=$ & $\left(\frac{1}{2}+x_{3}\right) a \hat{\mathbf{x}}+\left(\frac{1}{2}+x_{3}\right) a \hat{\mathbf{y}}+\frac{3}{4} c \hat{\mathbf{z}}$ & $(8 j)$ & V II \\
\hline $\mathbf{B}_{23}$ & $=$ & $\left(\frac{1}{2}+x_{3}\right) \mathbf{a}_{1}-x_{3} \mathbf{a}_{2}+\frac{1}{4} \mathbf{a}_{3}$ & $=$ & $\left(\frac{1}{2}+x_{3}\right) a \hat{\mathbf{x}}-x_{3} a \hat{\mathbf{y}}+\frac{1}{4} c \hat{\mathbf{z}}$ & $(8 j)$ & V II \\
\hline $\mathbf{B}_{24}$ & $=$ & $-x_{3} \mathbf{a}_{1}+\left(\frac{1}{2}+x_{3}\right) \mathbf{a}_{2}+\frac{1}{4} \mathbf{a}_{3}$ & $=$ & $-x_{3} a \hat{\mathbf{x}}+\left(\frac{1}{2}+x_{3}\right) a \hat{\mathbf{y}}+\frac{1}{4} c \hat{\mathbf{z}}$ & $(8 j)$ & V II \\
\hline $\mathbf{B}_{25}$ & $=$ & $x_{4} \mathbf{a}_{1}+x_{4} \mathbf{a}_{2}+\frac{1}{4} \mathbf{a}_{3}$ & $=$ & $x_{4} a \hat{\mathbf{x}}+x_{4} a \hat{\mathbf{y}}+\frac{1}{4} c \hat{\mathbf{z}}$ & $(8 j)$ & V III \\
\hline $\mathbf{B}_{26}$ & $=$ & $\left(\frac{1}{2}-x_{4}\right) \mathbf{a}_{1}+\left(\frac{1}{2}-x_{4}\right) \mathbf{a}_{2}+\frac{1}{4} \mathbf{a}_{3}$ & $=$ & $\left(\frac{1}{2}-x_{4}\right) a \hat{\mathbf{x}}+\left(\frac{1}{2}-x_{4}\right) a \hat{\mathbf{y}}+\frac{1}{4} c \hat{\mathbf{z}}$ & $(8 j)$ & V III \\
\hline $\mathbf{B}_{27}$ & $=$ & $\left(\frac{1}{2}-x_{4}\right) \mathbf{a}_{1}+x_{4} \mathbf{a}_{2}+\frac{3}{4} \mathbf{a}_{3}$ & $=$ & $\left(\frac{1}{2}-x_{4}\right) a \hat{\mathbf{x}}+x_{4} a \hat{\mathbf{y}}+\frac{3}{4} c \hat{\mathbf{z}}$ & $(8 j)$ & V III \\
\hline $\mathbf{B}_{28}$ & $=$ & $x_{4} \mathbf{a}_{1}+\left(\frac{1}{2}-x_{4}\right) \mathbf{a}_{2}+\frac{3}{4} \mathbf{a}_{3}$ & $=$ & $x_{4} a \hat{\mathbf{x}}+\left(\frac{1}{2}-x_{4}\right) a \hat{\mathbf{y}}+\frac{3}{4} c \hat{\mathbf{z}}$ & $(8 j)$ & V III \\
\hline $\mathbf{B}_{29}$ & $=$ & $-x_{4} \mathbf{a}_{1}-x_{4} \mathbf{a}_{2}+\frac{3}{4} \mathbf{a}_{3}$ & $=$ & $-x_{4} a \hat{\mathbf{x}}-x_{4} a \hat{\mathbf{y}}+\frac{3}{4} c \hat{\mathbf{z}}$ & $(8 j)$ & V III \\
\hline $\mathbf{B}_{30}$ & $=$ & $\left(\frac{1}{2}+x_{4}\right) \mathbf{a}_{1}+\left(\frac{1}{2}+x_{4}\right) \mathbf{a}_{2}+\frac{3}{4} \mathbf{a}_{3}$ & $=$ & $\left(\frac{1}{2}+x_{4}\right) a \hat{\mathbf{x}}+\left(\frac{1}{2}+x_{4}\right) a \hat{\mathbf{y}}+\frac{3}{4} c \hat{\mathbf{z}}$ & $(8 j)$ & V III \\
\hline $\mathbf{B}_{31}$ & $=$ & $\left(\frac{1}{2}+x_{4}\right) \mathbf{a}_{1}-x_{4} \mathbf{a}_{2}+\frac{1}{4} \mathbf{a}_{3}$ & $=$ & $\left(\frac{1}{2}+x_{4}\right) a \hat{\mathbf{x}}-x_{4} a \hat{\mathbf{y}}+\frac{1}{4} c \hat{\mathbf{z}}$ & $(8 j)$ & V III \\
\hline $\mathbf{B}_{32}$ & $=$ & $-x_{4} \mathbf{a}_{1}+\left(\frac{1}{2}+x_{4}\right) \mathbf{a}_{2}+\frac{1}{4} \mathbf{a}_{3}$ & $=$ & $-x_{4} a \hat{\mathbf{x}}+\left(\frac{1}{2}+x_{4}\right) a \hat{\mathbf{y}}+\frac{1}{4} c \hat{\mathbf{z}}$ & $(8 j)$ & V III \\
\hline
\end{tabular}

\section{References:}

- B. Pedersen and F. Grønvold, The crystal structures of $\alpha-V_{3} S$ and $\beta-V_{3} S$, Acta Cryst. 12, 1022-1027 (1959), doi:10.1107/S0365110X59002869.

\section{Found in:}

- P. Villars and K. Cenzual, Pearson's Crystal Data - Crystal Structure Database for Inorganic Compounds, ASM International (2013).

\section{Geometry files:}


- CIF: pp. 897

- POSCAR: pp. 898 


\section{Downeyite $\left(\mathrm{SeO}_{2}, \mathrm{C} 47\right)$ Structure:}

\section{A2B_tP24_135_gh_h}
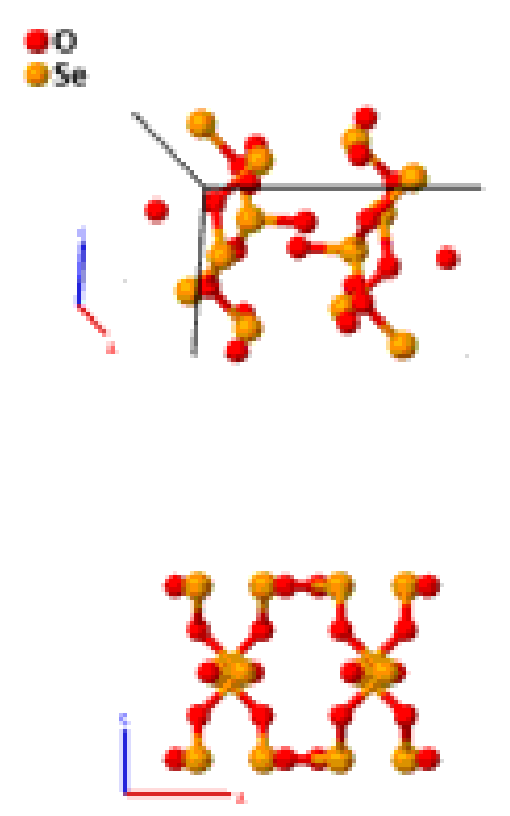
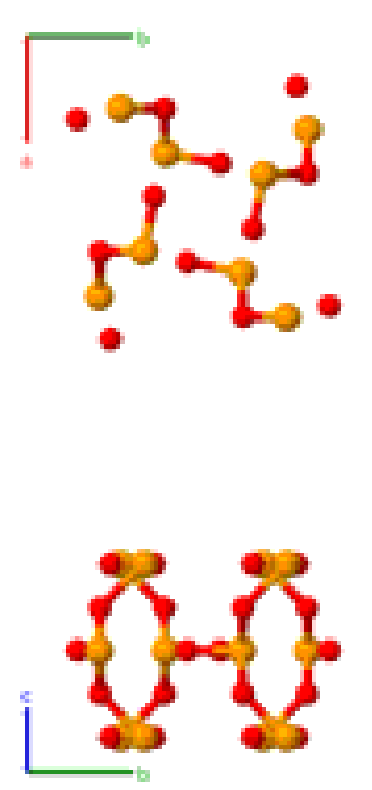

\section{Prototype}

AFLOW prototype label

Strukturbericht designation

Pearson symbol

Space group number

Space group symbol

AFLOW prototype command
: $\quad \mathrm{SeO}_{2}$

: A2B_tP24_135_gh_h

: $\quad$ C47

: $\quad \mathrm{tP} 24$

: $\quad 135$

: $\quad P 4_{2} / m b c$

aflow --proto=A2B_tP24_135_gh_h

- - params $=a, c / a, x_{1}, x_{2}, y_{2}, x_{3}, y_{3}$

- Data for this structure was taken at $139 \mathrm{~K}$.

Simple Tetragonal primitive vectors:

$$
\begin{aligned}
& \mathbf{a}_{1}=a \hat{\mathbf{x}} \\
& \mathbf{a}_{2}=a \hat{\mathbf{y}} \\
& \mathbf{a}_{3}=c \hat{\mathbf{z}}
\end{aligned}
$$
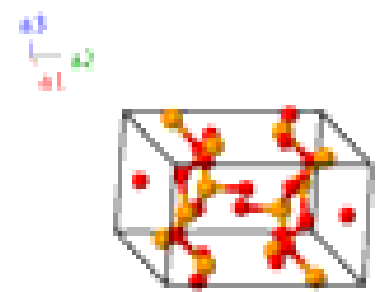

Basis vectors:

Lattice Coordinates

$\mathbf{B}_{1}=x_{1} \mathbf{a}_{1}+\left(\frac{1}{2}+x_{1}\right) \mathbf{a}_{2}+\frac{1}{4} \mathbf{a}_{3}=x_{1} a \hat{\mathbf{x}}+\left(\frac{1}{2}+x_{1}\right) a \hat{\mathbf{y}}+\frac{1}{4} c \hat{\mathbf{z}}$
Wyckoff Position

Atom Type

$(8 g)$

O I 


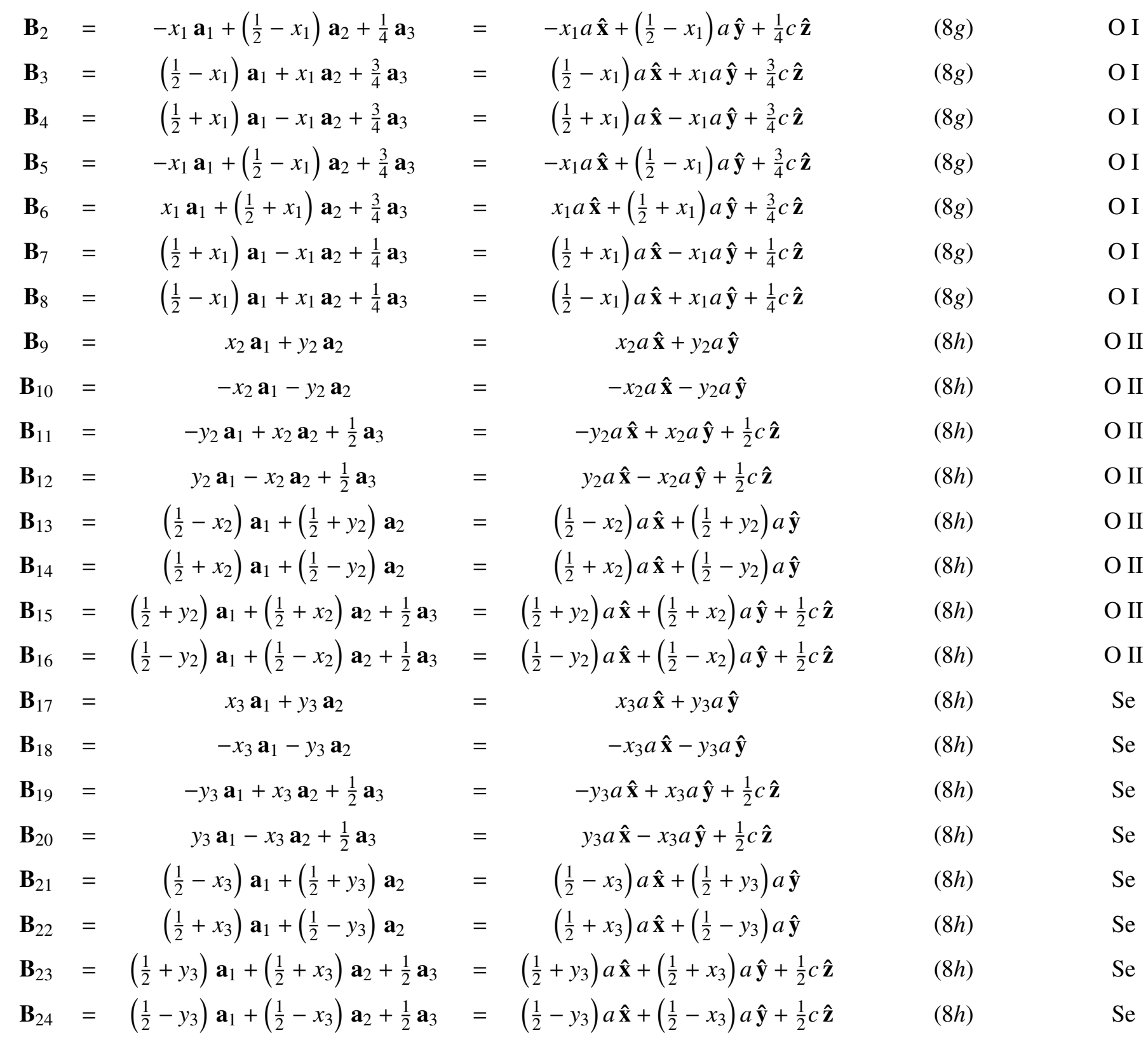

\section{References:}

- K. Ståhl, J. P. Legros, and J. Galy, The crystal structure of $\mathrm{SeO}_{2}$ at 139 and 286 K, Kristallografiya 202, 99-107 (1992), doi:10.1524/zkri.1992.202.14.99.

\section{Found in:}

- R. T. Downs and M. Hall-Wallace, The American Mineralogist Crystal Structure Database, Am. Mineral. 88, 247-250 (2003).

\section{Geometry files:}

- CIF: pp. 898

- POSCAR: pp. 898 


\section{$\mathrm{ZnSb}_{2} \mathrm{O}_{4}$ Structure: A4B2C_tP28_135_gh_h_d}
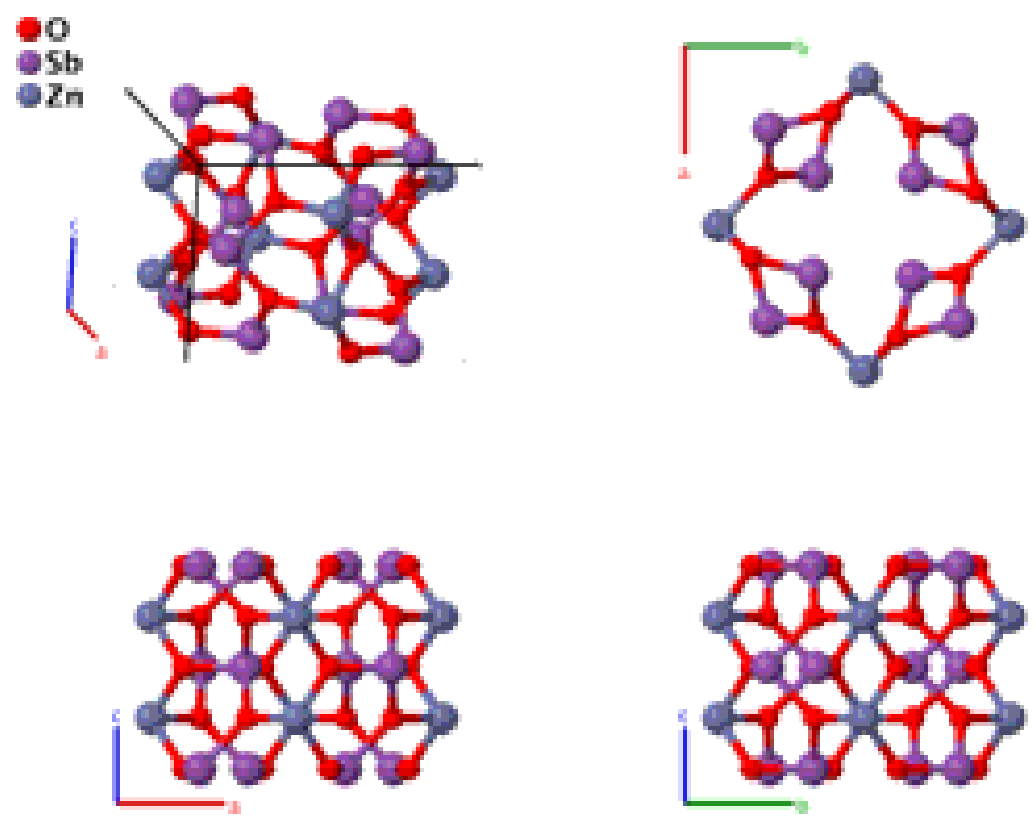

\section{Prototype}

AFLOW prototype label

: $\quad \mathrm{ZnSb}_{2} \mathrm{O}_{4}$

Strukturbericht designation

: A4B2C_tP28_135_gh_h_d

Pearson symbol

: None

Space group number

: $\quad$ tP28

Space group symbol

: 135

AFLOW prototype command

: $\quad P 4_{2} / m b c$

aflow - -proto=A4B2C_tP28_135_gh_h_d
- params $=a, c / a, x_{2}, x_{3}, y_{3}, x_{4}, y_{4}$

Simple Tetragonal primitive vectors:

$$
\begin{aligned}
& \mathbf{a}_{1}=a \hat{\mathbf{x}} \\
& \mathbf{a}_{2}=a \hat{\mathbf{y}} \\
& \mathbf{a}_{3}=c \hat{\mathbf{z}}
\end{aligned}
$$

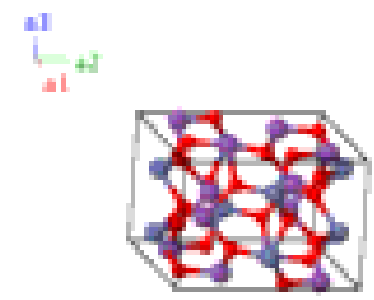

Basis vectors:

Lattice Coordinates

$\begin{array}{lll}\mathbf{B}_{1}= & \frac{1}{2} \mathbf{a}_{2}+\frac{1}{4} \mathbf{a}_{3} \\ \mathbf{B}_{2}= & \frac{1}{2} \mathbf{a}_{1}+\frac{3}{4} \mathbf{a}_{3} \\ \mathbf{B}_{3}= & \frac{1}{2} \mathbf{a}_{2}+\frac{3}{4} \mathbf{a}_{3} \\ \mathbf{B}_{4}= & \frac{1}{2} \mathbf{a}_{1}+\frac{1}{4} \mathbf{a}_{3}\end{array}$

Cartesian Coordinates

$=$

$=$

$=$

$=$

$$
\begin{aligned}
& \frac{1}{2} a \hat{\mathbf{y}}+\frac{1}{4} c \hat{\mathbf{z}} \\
& \frac{1}{2} a \hat{\mathbf{x}}+\frac{3}{4} c \hat{\mathbf{z}} \\
& \frac{1}{2} a \hat{\mathbf{y}}+\frac{3}{4} c \hat{\mathbf{z}} \\
& \frac{1}{2} a \hat{\mathbf{x}}+\frac{1}{4} c \hat{\mathbf{z}}
\end{aligned}
$$

Wyckoff Position

Atom Type

(4d)

$\mathrm{Zn}$

(4d)

$\mathrm{Zn}$

(4d)

$\mathrm{Zn}$

(4d)

Zn 


\begin{tabular}{|c|c|c|c|c|c|c|}
\hline $\mathbf{B}_{5}$ & $=$ & $x_{2} \mathbf{a}_{1}+\left(\frac{1}{2}+x_{2}\right) \mathbf{a}_{2}+\frac{1}{4} \mathbf{a}_{3}$ & $=$ & $x_{2} a \hat{\mathbf{x}}+\left(\frac{1}{2}+x_{2}\right) a \hat{\mathbf{y}}+\frac{1}{4} c \hat{\mathbf{z}}$ & $(8 g)$ & O I \\
\hline $\mathbf{B}_{6}$ & $=$ & $-x_{2} \mathbf{a}_{1}+\left(\frac{1}{2}-x_{2}\right) \mathbf{a}_{2}+\frac{1}{4} \mathbf{a}_{3}$ & $=$ & $-x_{2} a \hat{\mathbf{x}}+\left(\frac{1}{2}-x_{2}\right) a \hat{\mathbf{y}}+\frac{1}{4} c \hat{\mathbf{z}}$ & $(8 g)$ & O I \\
\hline $\mathbf{B}_{7}$ & $=$ & $\left(\frac{1}{2}-x_{2}\right) \mathbf{a}_{1}+x_{2} \mathbf{a}_{2}+\frac{3}{4} \mathbf{a}_{3}$ & $=$ & $\left(\frac{1}{2}-x_{2}\right) a \hat{\mathbf{x}}+x_{2} a \hat{\mathbf{y}}+\frac{3}{4} c \hat{\mathbf{z}}$ & $(8 g)$ & $\mathrm{O} \mathrm{I}$ \\
\hline $\mathbf{B}_{8}$ & $=$ & $\left(\frac{1}{2}+x_{2}\right) \mathbf{a}_{1}-x_{2} \mathbf{a}_{2}+\frac{3}{4} \mathbf{a}_{3}$ & $=$ & $\left(\frac{1}{2}+x_{2}\right) a \hat{\mathbf{x}}-x_{2} a \hat{\mathbf{y}}+\frac{3}{4} c \hat{\mathbf{z}}$ & $(8 g)$ & O I \\
\hline $\mathbf{B}_{9}$ & $=$ & $-x_{2} \mathbf{a}_{1}+\left(\frac{1}{2}-x_{2}\right) \mathbf{a}_{2}+\frac{3}{4} \mathbf{a}_{3}$ & $=$ & $-x_{2} a \hat{\mathbf{x}}+\left(\frac{1}{2}-x_{2}\right) a \hat{\mathbf{y}}+\frac{3}{4} c \hat{\mathbf{z}}$ & $(8 g)$ & O I \\
\hline $\mathbf{B}_{10}$ & $=$ & $x_{2} \mathbf{a}_{1}+\left(\frac{1}{2}+x_{2}\right) \mathbf{a}_{2}+\frac{3}{4} \mathbf{a}_{3}$ & $=$ & $x_{2} a \hat{\mathbf{x}}+\left(\frac{1}{2}+x_{2}\right) a \hat{\mathbf{y}}+\frac{3}{4} c \hat{\mathbf{z}}$ & $(8 g)$ & O I \\
\hline $\mathbf{B}_{11}$ & $=$ & $\left(\frac{1}{2}+x_{2}\right) \mathbf{a}_{1}-x_{2} \mathbf{a}_{2}+\frac{1}{4} \mathbf{a}_{3}$ & $=$ & $\left(\frac{1}{2}+x_{2}\right) a \hat{\mathbf{x}}-x_{2} a \hat{\mathbf{y}}+\frac{1}{4} c \hat{\mathbf{z}}$ & $(8 g)$ & O I \\
\hline $\mathbf{B}_{12}$ & $=$ & $\left(\frac{1}{2}-x_{2}\right) \mathbf{a}_{1}+x_{2} \mathbf{a}_{2}+\frac{1}{4} \mathbf{a}_{3}$ & $=$ & $\left(\frac{1}{2}-x_{2}\right) a \hat{\mathbf{x}}+x_{2} a \hat{\mathbf{y}}+\frac{1}{4} c \hat{\mathbf{z}}$ & $(8 g)$ & O I \\
\hline $\mathbf{B}_{13}$ & $=$ & $x_{3} \mathbf{a}_{1}+y_{3} \mathbf{a}_{2}$ & $=$ & $x_{3} a \hat{\mathbf{x}}+y_{3} a \hat{\mathbf{y}}$ & $(8 h)$ & O II \\
\hline $\mathbf{B}_{14}$ & $=$ & $-x_{3} \mathbf{a}_{1}-y_{3} \mathbf{a}_{2}$ & $=$ & $-x_{3} a \hat{\mathbf{x}}-y_{3} a \hat{\mathbf{y}}$ & $(8 h)$ & O II \\
\hline $\mathbf{B}_{15}$ & $=$ & $-y_{3} \mathbf{a}_{1}+x_{3} \mathbf{a}_{2}+\frac{1}{2} \mathbf{a}_{3}$ & $=$ & $-y_{3} a \hat{\mathbf{x}}+x_{3} a \hat{\mathbf{y}}+\frac{1}{2} c \hat{\mathbf{z}}$ & $(8 h)$ & O II \\
\hline $\mathbf{B}_{16}$ & $=$ & $y_{3} \mathbf{a}_{1}-x_{3} \mathbf{a}_{2}+\frac{1}{2} \mathbf{a}_{3}$ & $=$ & $y_{3} a \hat{\mathbf{x}}-x_{3} a \hat{\mathbf{y}}+\frac{1}{2} c \hat{\mathbf{z}}$ & $(8 h)$ & O II \\
\hline $\mathbf{B}_{17}$ & $=$ & $\left(\frac{1}{2}-x_{3}\right) \mathbf{a}_{1}+\left(\frac{1}{2}+y_{3}\right) \mathbf{a}_{2}$ & $=$ & $\left(\frac{1}{2}-x_{3}\right) a \hat{\mathbf{x}}+\left(\frac{1}{2}+y_{3}\right) a \hat{\mathbf{y}}$ & $(8 h)$ & O II \\
\hline $\mathbf{B}_{18}$ & $=$ & $\left(\frac{1}{2}+x_{3}\right) \mathbf{a}_{1}+\left(\frac{1}{2}-y_{3}\right) \mathbf{a}_{2}$ & $=$ & $\left(\frac{1}{2}+x_{3}\right) a \hat{\mathbf{x}}+\left(\frac{1}{2}-y_{3}\right) a \hat{\mathbf{y}}$ & $(8 h)$ & O II \\
\hline $\mathbf{B}_{19}$ & $=$ & $\left(\frac{1}{2}+y_{3}\right) \mathbf{a}_{1}+\left(\frac{1}{2}+x_{3}\right) \mathbf{a}_{2}+\frac{1}{2} \mathbf{a}_{3}$ & $=$ & $\left(\frac{1}{2}+y_{3}\right) a \hat{\mathbf{x}}+\left(\frac{1}{2}+x_{3}\right) a \hat{\mathbf{y}}+\frac{1}{2} c \hat{\mathbf{z}}$ & $(8 h)$ & O II \\
\hline $\mathbf{B}_{20}$ & $=$ & $\left(\frac{1}{2}-y_{3}\right) \mathbf{a}_{1}+\left(\frac{1}{2}-x_{3}\right) \mathbf{a}_{2}+\frac{1}{2} \mathbf{a}_{3}$ & $=$ & $\left(\frac{1}{2}-y_{3}\right) a \hat{\mathbf{x}}+\left(\frac{1}{2}-x_{3}\right) a \hat{\mathbf{y}}+\frac{1}{2} c \hat{\mathbf{z}}$ & $(8 h)$ & O II \\
\hline $\mathbf{B}_{21}$ & $=$ & $x_{4} \mathbf{a}_{1}+y_{4} \mathbf{a}_{2}$ & $=$ & $x_{4} a \hat{\mathbf{x}}+y_{4} a \hat{\mathbf{y}}$ & $(8 h)$ & $\mathrm{Sb}$ \\
\hline $\mathbf{B}_{22}$ & $=$ & $-x_{4} \mathbf{a}_{1}-y_{4} \mathbf{a}_{2}$ & $=$ & $-x_{4} a \hat{\mathbf{x}}-y_{4} a \hat{\mathbf{y}}$ & $(8 h)$ & $\mathrm{Sb}$ \\
\hline $\mathbf{B}_{23}$ & $=$ & $-y_{4} \mathbf{a}_{1}+x_{4} \mathbf{a}_{2}+\frac{1}{2} \mathbf{a}_{3}$ & $=$ & $-y_{4} a \hat{\mathbf{x}}+x_{4} a \hat{\mathbf{y}}+\frac{1}{2} c \hat{\mathbf{z}}$ & $(8 h)$ & $\mathrm{Sb}$ \\
\hline $\mathbf{B}_{24}$ & $=$ & $y_{4} \mathbf{a}_{1}-x_{4} \mathbf{a}_{2}+\frac{1}{2} \mathbf{a}_{3}$ & $=$ & $y_{4} a \hat{\mathbf{x}}-x_{4} a \hat{\mathbf{y}}+\frac{1}{2} c \hat{\mathbf{z}}$ & $(8 h)$ & $\mathrm{Sb}$ \\
\hline $\mathbf{B}_{25}$ & $=$ & $\left(\frac{1}{2}-x_{4}\right) \mathbf{a}_{1}+\left(\frac{1}{2}+y_{4}\right) \mathbf{a}_{2}$ & $=$ & $\left(\frac{1}{2}-x_{4}\right) a \hat{\mathbf{x}}+\left(\frac{1}{2}+y_{4}\right) a \hat{\mathbf{y}}$ & $(8 h)$ & $\mathrm{Sb}$ \\
\hline $\mathbf{B}_{26}$ & $=$ & $\left(\frac{1}{2}+x_{4}\right) \mathbf{a}_{1}+\left(\frac{1}{2}-y_{4}\right) \mathbf{a}_{2}$ & $=$ & $\left(\frac{1}{2}+x_{4}\right) a \hat{\mathbf{x}}+\left(\frac{1}{2}-y_{4}\right) a \hat{\mathbf{y}}$ & $(8 h)$ & $\mathrm{Sb}$ \\
\hline $\mathbf{B}_{27}$ & $=$ & $\left(\frac{1}{2}+y_{4}\right) \mathbf{a}_{1}+\left(\frac{1}{2}+x_{4}\right) \mathbf{a}_{2}+\frac{1}{2} \mathbf{a}_{3}$ & $=$ & $\left(\frac{1}{2}+y_{4}\right) a \hat{\mathbf{x}}+\left(\frac{1}{2}+x_{4}\right) a \hat{\mathbf{y}}+\frac{1}{2} c \hat{\mathbf{z}}$ & $(8 h)$ & $\mathrm{Sb}$ \\
\hline $\mathbf{B}_{28}$ & $=$ & $\left(\frac{1}{2}-y_{4}\right) \mathbf{a}_{1}+\left(\frac{1}{2}-x_{4}\right) \mathbf{a}_{2}+\frac{1}{2} \mathbf{a}_{3}$ & $=$ & $\left(\frac{1}{2}-y_{4}\right) a \hat{\mathbf{x}}+\left(\frac{1}{2}-x_{4}\right) a \hat{\mathbf{y}}+\frac{1}{2} c \hat{\mathbf{z}}$ & $(8 h)$ & $\mathrm{Sb}$ \\
\hline
\end{tabular}

\section{References:}

- S. Ståhl, The crystal structure of $\mathrm{ZnSb}_{2} \mathrm{O}_{4}$ and isomorphous compounds, Ark. Kem. Mineral. Geol. 17B, 1-7 (1943).

\section{Found in:}

- P. Villars and K. Cenzual, Pearson's Crystal Data - Crystal Structure Database for Inorganic Compounds, ASM International (2013).

\section{Geometry files:}

- CIF: pp. 899

- POSCAR: pp. 899 


\section{$\mathrm{Zn}_{3} \mathrm{P}_{2}\left(D 5_{9}\right)$ Structure: A2B3_tP40_137_cdf_3g}
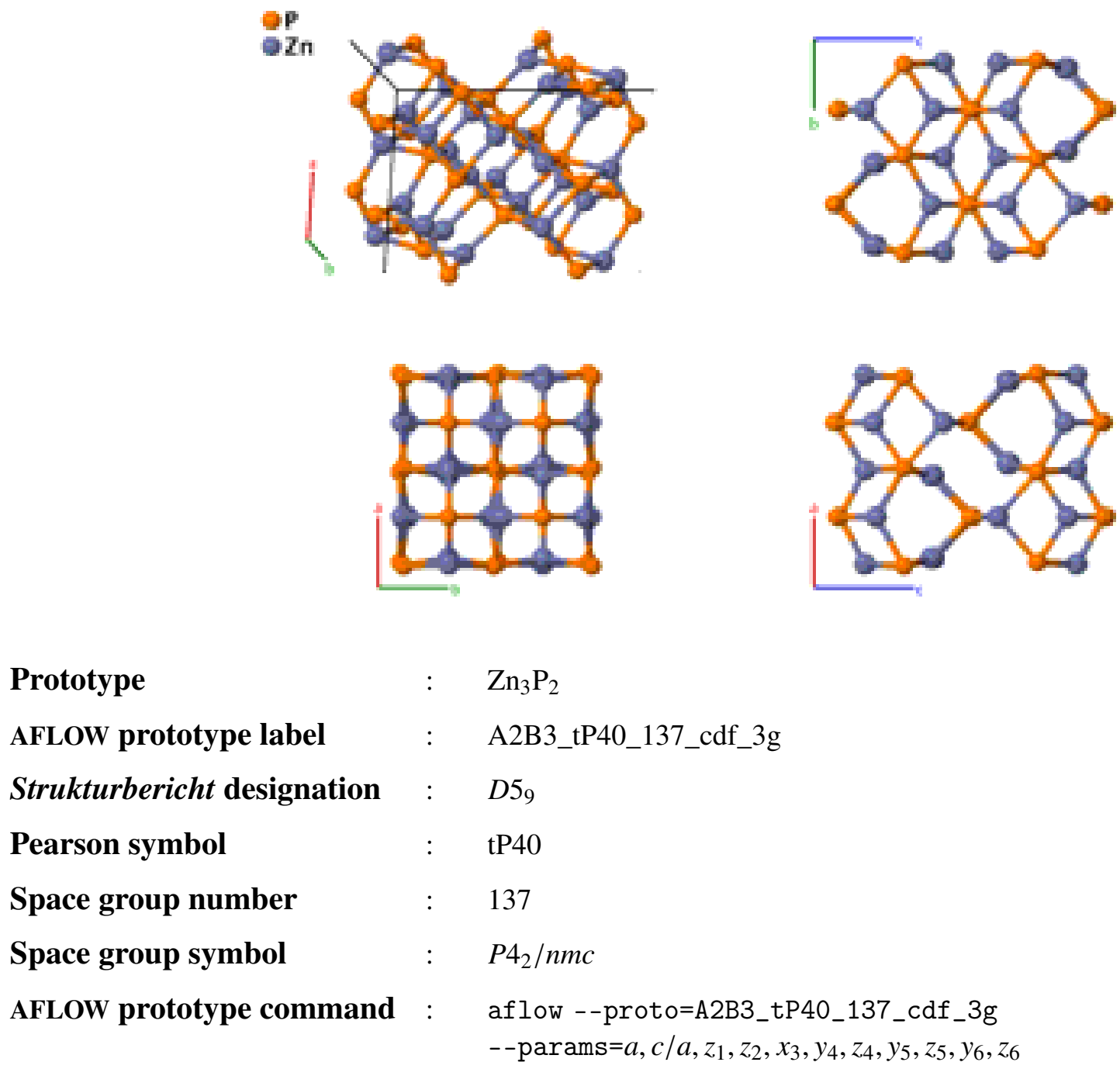

\section{Other compounds with this structure:}

- $\alpha-\mathrm{As}_{2} \mathrm{Cd}_{3}, \alpha-\mathrm{As}_{2} \mathrm{Zn}_{3}, \mathrm{Cd}_{3} \mathrm{P}_{2}$

- (Stackelberg, 1935) gives the atomic positions in the first setting of space group $P 4_{2} / n m c \# 137$. We have changed this to the second setting, placing the origin of the system at the inversion site. On page 803, (Pearson, 1958) gives the space group as $P 4_{2} / m m c \# 131$, but it is correctly given as $P 4_{2} / n m c$ on page 111 .

\section{Simple Tetragonal primitive vectors:}

$$
\begin{aligned}
& \mathbf{a}_{1}=a \hat{\mathbf{x}} \\
& \mathbf{a}_{2}=a \hat{\mathbf{y}} \\
& \mathbf{a}_{3}=c \hat{\mathbf{z}}
\end{aligned}
$$

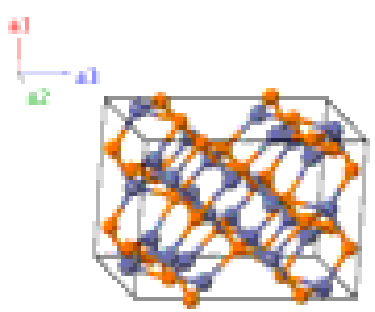

Basis vectors: 


\begin{tabular}{|c|c|c|c|c|c|c|}
\hline $\mathbf{B}_{1}$ & $=$ & $\frac{3}{4} \mathbf{a}_{1}+\frac{1}{4} \mathbf{a}_{2}+z_{1} \mathbf{a}_{3}$ & $=$ & $\frac{3}{4} a \hat{\mathbf{x}}+\frac{1}{4} a \hat{\mathbf{y}}+z_{1} c \hat{\mathbf{z}}$ & $(4 c)$ & P I \\
\hline $\mathbf{B}_{2}$ & $=$ & $\frac{1}{4} \mathbf{a}_{1}+\frac{3}{4} \mathbf{a}_{2}+\left(\frac{1}{2}+z_{1}\right) \mathbf{a}_{3}$ & $=$ & $\frac{1}{4} a \hat{\mathbf{x}}+\frac{3}{4} a \hat{\mathbf{y}}+\left(\frac{1}{2}+z_{1}\right) c \hat{\mathbf{z}}$ & $(4 c)$ & P I \\
\hline $\mathbf{B}_{3}$ & $=$ & $\frac{1}{4} \mathbf{a}_{1}+\frac{3}{4} \mathbf{a}_{2}-z_{1} \mathbf{a}_{3}$ & $=$ & $\frac{1}{4} a \hat{\mathbf{x}}+\frac{3}{4} a \hat{\mathbf{y}}-z_{1} c \hat{\mathbf{z}}$ & $(4 c)$ & P I \\
\hline $\mathbf{B}_{4}$ & $=$ & $\frac{3}{4} \mathbf{a}_{1}+\frac{1}{4} \mathbf{a}_{2}+\left(\frac{1}{2}-z_{1}\right) \mathbf{a}_{3}$ & $=$ & $\frac{3}{4} a \hat{\mathbf{x}}+\frac{1}{4} a \hat{\mathbf{y}}+\left(\frac{1}{2}-z_{1}\right) c \hat{\mathbf{z}}$ & $(4 c)$ & P I \\
\hline $\mathbf{B}_{5}$ & $=$ & $\frac{1}{4} \mathbf{a}_{1}+\frac{1}{4} \mathbf{a}_{2}+z_{2} \mathbf{a}_{3}$ & $=$ & $\frac{1}{4} a \hat{\mathbf{x}}+\frac{1}{4} a \hat{\mathbf{y}}+z_{2} c \hat{\mathbf{z}}$ & $(4 d)$ & P II \\
\hline $\mathbf{B}_{6}$ & $=$ & $\frac{1}{4} \mathbf{a}_{1}+\frac{1}{4} \mathbf{a}_{2}+\left(\frac{1}{2}+z_{2}\right) \mathbf{a}_{3}$ & $=$ & $\frac{1}{4} a \hat{\mathbf{x}}+\frac{1}{4} a \hat{\mathbf{y}}+\left(\frac{1}{2}+z_{2}\right) c \hat{\mathbf{z}}$ & $(4 d)$ & P II \\
\hline $\mathbf{B}_{7}$ & $=$ & $\frac{3}{4} \mathbf{a}_{1}+\frac{3}{4} \mathbf{a}_{2}-z_{2} \mathbf{a}_{3}$ & $=$ & $\frac{3}{4} a \hat{\mathbf{x}}+\frac{3}{4} a \hat{\mathbf{y}}-z_{2} c \hat{\mathbf{z}}$ & $(4 d)$ & P II \\
\hline $\mathbf{B}_{8}$ & $=$ & $\frac{3}{4} \mathbf{a}_{1}+\frac{3}{4} \mathbf{a}_{2}+\left(\frac{1}{2}-z_{2}\right) \mathbf{a}_{3}$ & $=$ & $\frac{3}{4} a \hat{\mathbf{x}}+\frac{3}{4} a \hat{\mathbf{y}}+\left(\frac{1}{2}-z_{2}\right) c \hat{\mathbf{z}}$ & $(4 d)$ & P II \\
\hline $\mathbf{B}_{9}$ & $=$ & $x_{3} \mathbf{a}_{1}-x_{3} \mathbf{a}_{2}+\frac{1}{4} \mathbf{a}_{3}$ & $=$ & $x_{3} a \hat{\mathbf{x}}-x_{3} a \hat{\mathbf{y}}+\frac{1}{4} c \hat{\mathbf{z}}$ & $(8 f)$ & P III \\
\hline $\mathbf{B}_{10}$ & $=$ & $\left(\frac{1}{2}-x_{3}\right) \mathbf{a}_{1}+\left(\frac{1}{2}+x_{3}\right) \mathbf{a}_{2}+\frac{1}{4} \mathbf{a}_{3}$ & $=$ & $\left(\frac{1}{2}-x_{3}\right) a \hat{\mathbf{x}}+\left(\frac{1}{2}+x_{3}\right) a \hat{\mathbf{y}}+\frac{1}{4} c \hat{\mathbf{z}}$ & $(8 f)$ & P III \\
\hline $\mathbf{B}_{11}$ & $=$ & $\left(\frac{1}{2}+x_{3}\right) \mathbf{a}_{1}+x_{3} \mathbf{a}_{2}+\frac{3}{4} \mathbf{a}_{3}$ & $=$ & $\left(\frac{1}{2}+x_{3}\right) a \hat{\mathbf{x}}+x_{3} a \hat{\mathbf{y}}+\frac{3}{4} c \hat{\mathbf{z}}$ & $(8 f)$ & P III \\
\hline $\mathbf{B}_{12}$ & $=$ & $-x_{3} \mathbf{a}_{1}+\left(\frac{1}{2}-x_{3}\right) \mathbf{a}_{2}+\frac{3}{4} \mathbf{a}_{3}$ & $=$ & $-x_{3} a \hat{\mathbf{x}}+\left(\frac{1}{2}-x_{3}\right) a \hat{\mathbf{y}}+\frac{3}{4} c \hat{\mathbf{z}}$ & $(8 f)$ & P III \\
\hline $\mathbf{B}_{13}$ & $=$ & $-x_{3} \mathbf{a}_{1}+x_{3} \mathbf{a}_{2}+\frac{3}{4} \mathbf{a}_{3}$ & $=$ & $-x_{3} a \hat{\mathbf{x}}+x_{3} a \hat{\mathbf{y}}+\frac{3}{4} c \hat{\mathbf{z}}$ & $(8 f)$ & P III \\
\hline $\mathbf{B}_{14}$ & $=$ & $\left(\frac{1}{2}+x_{3}\right) \mathbf{a}_{1}+\left(\frac{1}{2}-x_{3}\right) \mathbf{a}_{2}+\frac{3}{4} \mathbf{a}_{3}$ & $=$ & $\left(\frac{1}{2}+x_{3}\right) a \hat{\mathbf{x}}+\left(\frac{1}{2}-x_{3}\right) a \hat{\mathbf{y}}+\frac{3}{4} c \hat{\mathbf{z}}$ & $(8 f)$ & P III \\
\hline $\mathbf{B}_{15}$ & $=$ & $\left(\frac{1}{2}-x_{3}\right) \mathbf{a}_{1}-x_{3} \mathbf{a}_{2}+\frac{1}{4} \mathbf{a}_{3}$ & $=$ & $\left(\frac{1}{2}-x_{3}\right) a \hat{\mathbf{x}}-x_{3} a \hat{\mathbf{y}}+\frac{1}{4} c \hat{\mathbf{z}}$ & $(8 f)$ & P III \\
\hline $\mathbf{B}_{16}$ & $=$ & $x_{3} \mathbf{a}_{1}+\left(\frac{1}{2}+x_{3}\right) \mathbf{a}_{2}+\frac{1}{4} \mathbf{a}_{3}$ & $=$ & $x_{3} a \hat{\mathbf{x}}+\left(\frac{1}{2}+x_{3}\right) a \hat{\mathbf{y}}+\frac{1}{4} c \hat{\mathbf{z}}$ & $(8 f)$ & P III \\
\hline $\mathbf{B}_{17}$ & $=$ & $\frac{1}{4} \mathbf{a}_{1}+y_{4} \mathbf{a}_{2}+z_{4} \mathbf{a}_{3}$ & $=$ & $\frac{1}{4} a \hat{\mathbf{x}}+y_{4} a \hat{\mathbf{y}}+z_{4} c \hat{\mathbf{z}}$ & $(8 g)$ & Zn I \\
\hline $\mathbf{B}_{18}$ & $=$ & $\frac{1}{4} \mathbf{a}_{1}+\left(\frac{1}{2}-y_{4}\right) \mathbf{a}_{2}+z_{4} \mathbf{a}_{3}$ & $=$ & $\frac{1}{4} a \hat{\mathbf{x}}+\left(\frac{1}{2}-y_{4}\right) a \hat{\mathbf{y}}+z_{4} c \hat{\mathbf{z}}$ & $(8 g)$ & $\mathrm{Zn} \mathrm{I}$ \\
\hline $\mathbf{B}_{19}$ & $=$ & $\left(\frac{1}{2}-y_{4}\right) \mathbf{a}_{1}+\frac{1}{4} \mathbf{a}_{2}+\left(\frac{1}{2}+z_{4}\right) \mathbf{a}_{3}$ & $=$ & $\left(\frac{1}{2}-y_{4}\right) a \hat{\mathbf{x}}+\frac{1}{4} a \hat{\mathbf{y}}+\left(\frac{1}{2}+z_{4}\right) c \hat{\mathbf{z}}$ & $(8 g)$ & Zn I \\
\hline $\mathbf{B}_{20}$ & $=$ & $y_{4} \mathbf{a}_{1}+\frac{1}{4} \mathbf{a}_{2}+\left(\frac{1}{2}+z_{4}\right) \mathbf{a}_{3}$ & $=$ & $y_{4} a \hat{\mathbf{x}}+\frac{1}{4} a \hat{\mathbf{y}}+\left(\frac{1}{2}+z_{4}\right) c \hat{\mathbf{z}}$ & $(8 g)$ & Zn I \\
\hline $\mathbf{B}_{21}$ & $=$ & $\frac{3}{4} \mathbf{a}_{1}+\left(\frac{1}{2}+y_{4}\right) \mathbf{a}_{2}-z_{4} \mathbf{a}_{3}$ & $=$ & $\frac{3}{4} a \hat{\mathbf{x}}+\left(\frac{1}{2}+y_{4}\right) a \hat{\mathbf{y}}-z_{4} c \hat{\mathbf{z}}$ & $(8 g)$ & Zn I \\
\hline $\mathbf{B}_{22}$ & $=$ & $\frac{3}{4} \mathbf{a}_{1}-y_{4} \mathbf{a}_{2}-z_{4} \mathbf{a}_{3}$ & $=$ & $\frac{3}{4} a \hat{\mathbf{x}}-y_{4} a \hat{\mathbf{y}}-z_{4} c \hat{\mathbf{z}}$ & $(8 g)$ & Zn I \\
\hline $\mathbf{B}_{23}$ & $=$ & $\left(\frac{1}{2}+y_{4}\right) \mathbf{a}_{1}+\frac{3}{4} \mathbf{a}_{2}+\left(\frac{1}{2}-z_{4}\right) \mathbf{a}_{3}$ & $=$ & $\left(\frac{1}{2}+y_{4}\right) a \hat{\mathbf{x}}+\frac{3}{4} a \hat{\mathbf{y}}+\left(\frac{1}{2}-z_{4}\right) c \hat{\mathbf{z}}$ & $(8 g)$ & Zn I \\
\hline $\mathbf{B}_{24}$ & $=$ & $-y_{4} \mathbf{a}_{1}+\frac{3}{4} \mathbf{a}_{2}+\left(\frac{1}{2}-z_{4}\right) \mathbf{a}_{3}$ & $=$ & $-y_{4} a \hat{\mathbf{x}}+\frac{3}{4} a \hat{\mathbf{y}}+\left(\frac{1}{2}-z_{4}\right) c \hat{\mathbf{z}}$ & $(8 g)$ & Zn I \\
\hline $\mathbf{B}_{25}$ & $=$ & $\frac{1}{4} \mathbf{a}_{1}+y_{5} \mathbf{a}_{2}+z_{5} \mathbf{a}_{3}$ & $=$ & $\frac{1}{4} a \hat{\mathbf{x}}+y_{5} a \hat{\mathbf{y}}+z_{5} c \hat{\mathbf{z}}$ & $(8 g)$ & Zn II \\
\hline $\mathbf{B}_{26}$ & $=$ & $\frac{1}{4} \mathbf{a}_{1}+\left(\frac{1}{2}-y_{5}\right) \mathbf{a}_{2}+z_{5} \mathbf{a}_{3}$ & $=$ & $\frac{1}{4} a \hat{\mathbf{x}}+\left(\frac{1}{2}-y_{5}\right) a \hat{\mathbf{y}}+z_{5} c \hat{\mathbf{z}}$ & $(8 g)$ & Zn II \\
\hline $\mathbf{B}_{27}$ & $=$ & $\left(\frac{1}{2}-y_{5}\right) \mathbf{a}_{1}+\frac{1}{4} \mathbf{a}_{2}+\left(\frac{1}{2}+z_{5}\right) \mathbf{a}_{3}$ & $=$ & $\left(\frac{1}{2}-y_{5}\right) a \hat{\mathbf{x}}+\frac{1}{4} a \hat{\mathbf{y}}+\left(\frac{1}{2}+z_{5}\right) c \hat{\mathbf{z}}$ & $(8 g)$ & $\mathrm{Zn} \mathrm{II}$ \\
\hline $\mathbf{B}_{28}$ & $=$ & $y_{5} \mathbf{a}_{1}+\frac{1}{4} \mathbf{a}_{2}+\left(\frac{1}{2}+z_{5}\right) \mathbf{a}_{3}$ & $=$ & $y_{5} a \hat{\mathbf{x}}+\frac{1}{4} a \hat{\mathbf{y}}+\left(\frac{1}{2}+z_{5}\right) c \hat{\mathbf{z}}$ & $(8 g)$ & $\mathrm{Zn}$ II \\
\hline $\mathbf{B}_{29}$ & $=$ & $\frac{3}{4} \mathbf{a}_{1}+\left(\frac{1}{2}+y_{5}\right) \mathbf{a}_{2}-z_{5} \mathbf{a}_{3}$ & $=$ & $\frac{3}{4} a \hat{\mathbf{x}}+\left(\frac{1}{2}+y_{5}\right) a \hat{\mathbf{y}}-z_{5} c \hat{\mathbf{z}}$ & $(8 g)$ & Zn II \\
\hline $\mathbf{B}_{30}$ & $=$ & $\frac{3}{4} \mathbf{a}_{1}-y_{5} \mathbf{a}_{2}-z_{5} \mathbf{a}_{3}$ & $=$ & $\frac{3}{4} a \hat{\mathbf{x}}-y_{5} a \hat{\mathbf{y}}-z_{5} c \hat{\mathbf{z}}$ & $(8 g)$ & Zn II \\
\hline $\mathbf{B}_{31}$ & $=$ & $\left(\frac{1}{2}+y_{5}\right) \mathbf{a}_{1}+\frac{3}{4} \mathbf{a}_{2}+\left(\frac{1}{2}-z_{5}\right) \mathbf{a}_{3}$ & $=$ & $\left(\frac{1}{2}+y_{5}\right) a \hat{\mathbf{x}}+\frac{3}{4} a \hat{\mathbf{y}}+\left(\frac{1}{2}-z_{5}\right) c \hat{\mathbf{z}}$ & $(8 g)$ & Zn II \\
\hline $\mathbf{B}_{32}$ & $=$ & $-y_{5} \mathbf{a}_{1}+\frac{3}{4} \mathbf{a}_{2}+\left(\frac{1}{2}-z_{5}\right) \mathbf{a}_{3}$ & $=$ & $-y_{5} a \hat{\mathbf{x}}+\frac{3}{4} a \hat{\mathbf{y}}+\left(\frac{1}{2}-z_{5}\right) c \hat{\mathbf{z}}$ & $(8 g)$ & Zn II \\
\hline $\mathbf{B}_{33}$ & $=$ & $\frac{1}{4} \mathbf{a}_{1}+y_{6} \mathbf{a}_{2}+z_{6} \mathbf{a}_{3}$ & $=$ & $\frac{1}{4} a \hat{\mathbf{x}}+y_{6} a \hat{\mathbf{y}}+z_{6} c \hat{\mathbf{z}}$ & $(8 g)$ & $\mathrm{Zn} \mathrm{III}$ \\
\hline $\mathbf{B}_{34}$ & $=$ & $\frac{1}{4} \mathbf{a}_{1}+\left(\frac{1}{2}-y_{6}\right) \mathbf{a}_{2}+z_{6} \mathbf{a}_{3}$ & $=$ & $\frac{1}{4} a \hat{\mathbf{x}}+\left(\frac{1}{2}-y_{6}\right) a \hat{\mathbf{y}}+z_{6} c \hat{\mathbf{z}}$ & $(8 g)$ & Zn III \\
\hline $\mathbf{B}_{35}$ & $=$ & $\left(\frac{1}{2}-y_{6}\right) \mathbf{a}_{1}+\frac{1}{4} \mathbf{a}_{2}+\left(\frac{1}{2}+z_{6}\right) \mathbf{a}_{3}$ & $=$ & $\left(\frac{1}{2}-y_{6}\right) a \hat{\mathbf{x}}+\frac{1}{4} a \hat{\mathbf{y}}+\left(\frac{1}{2}+z_{6}\right) c \hat{\mathbf{z}}$ & $(8 g)$ & Zn III \\
\hline $\mathbf{B}_{36}$ & $=$ & $y_{6} \mathbf{a}_{1}+\frac{1}{4} \mathbf{a}_{2}+\left(\frac{1}{2}+z_{6}\right) \mathbf{a}_{3}$ & $=$ & $y_{6} a \hat{\mathbf{x}}+\frac{1}{4} a \hat{\mathbf{y}}+\left(\frac{1}{2}+z_{6}\right) c \hat{\mathbf{z}}$ & $(8 g)$ & Zn III \\
\hline
\end{tabular}




$\begin{array}{llllll}\mathbf{B}_{37}= & \frac{3}{4} \mathbf{a}_{1}+\left(\frac{1}{2}+y_{6}\right) \mathbf{a}_{2}-z_{6} \mathbf{a}_{3} & = & \frac{3}{4} a \hat{\mathbf{x}}+\left(\frac{1}{2}+y_{6}\right) a \hat{\mathbf{y}}-z_{6} c \hat{\mathbf{z}} \\ \mathbf{B}_{38}= & \frac{3}{4} \mathbf{a}_{1}-y_{6} \mathbf{a}_{2}-z_{6} \mathbf{a}_{3} & = & \frac{3}{4} a \hat{\mathbf{x}}-y_{6} a \hat{\mathbf{y}}-z_{6} c \hat{\mathbf{z}} & (8 g) & \mathrm{Zn} \text { III } \\ \mathbf{B}_{39}= & \left(\frac{1}{2}+y_{6}\right) \mathbf{a}_{1}+\frac{3}{4} \mathbf{a}_{2}+\left(\frac{1}{2}-z_{6}\right) \mathbf{a}_{3}= & \left(\frac{1}{2}+y_{6}\right) a \hat{\mathbf{x}}+\frac{3}{4} a \hat{\mathbf{y}}+\left(\frac{1}{2}-z_{6}\right) c \hat{\mathbf{z}} \\ \mathbf{B}_{40}= & -y_{6} \mathbf{a}_{1}+\frac{3}{4} \mathbf{a}_{2}+\left(\frac{1}{2}-z_{6}\right) \mathbf{a}_{3}= & -y_{6} a \hat{\mathbf{x}}+\frac{3}{4} a \hat{\mathbf{y}}+\left(\frac{1}{2}-z_{6}\right) c \hat{\mathbf{z}}\end{array}$

\section{References:}

- M. v. Stackelberg and R. Paulu, Untersuchungen an den Phosphiden und Arseniden des Zinks und Cadmiums. Das $Z_{3} P_{2}$-Gitter, Z. Phys. Chem. B 28, 427-460 (1935), doi:10.1515/zpch-1935-2841.

\section{Found in:}

- R. T. Downs and M. Hall-Wallace, The American Mineralogist Crystal Structure Database, Am. Mineral. 88, 247-250 (2003).

- W. B. Pearson, A Handbook of Lattice Spacings and Structures of Metals and Alloys, no. N.R.C. No. 4303 in International Series of Monographs on Metal Physics and Physical Metallurgy (Pergamon Press, Oxford, London, Edinburgh, New York, Paris, Frankfort, 1958), 1964 reprint with corrections edn.

\section{Geometry files:}

- CIF: pp. 899

- POSCAR: pp. 900 


\section{$\mathrm{ZrO}_{2}$ (High-temperature) Structure: A2B_tP6_137_d_a}

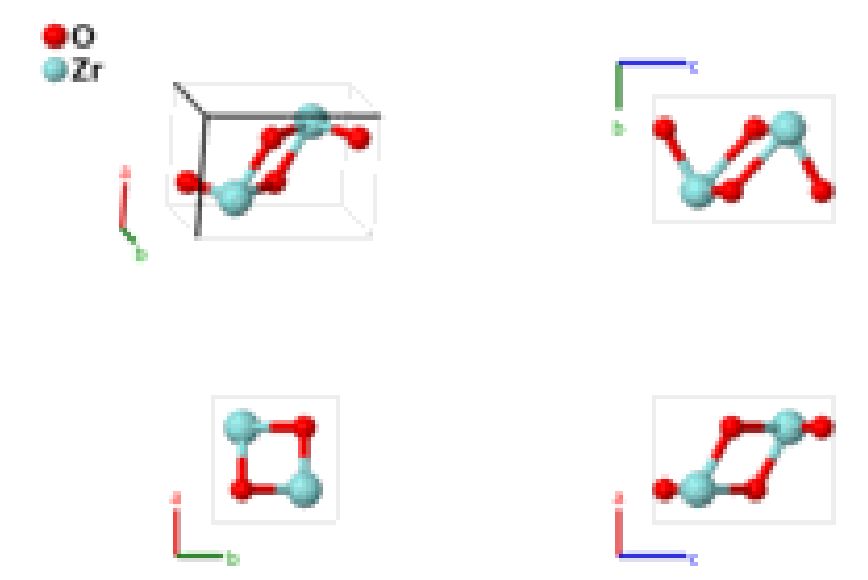

\section{Prototype}

AFLOW prototype label

Strukturbericht designation

Pearson symbol

Space group number

Space group symbol

AFLOW prototype command
$: \quad \mathrm{ZrO}_{2}$

: A2B_tP6_137_d_a

: None

: tP6

: $\quad 137$

: $\quad P 4_{2} / n m c$ aflow --proto=A2B_tP6_137_d_a

- -params $=a, c / a, z_{2}$

- $\mathrm{ZrO}_{2}$ (pp. 443) and $\mathrm{HgI}_{2}$ (pp. 447) have similar AFLOW prototype labels (i.e., same symmetry and set of Wyckoff positions with different stoichiometry labels due to alphabetic ordering of atomic species). They are generated by the same symmetry operations with different sets of parameters (--params) specified in their corresponding CIF files.

\section{Simple Tetragonal primitive vectors:}

$$
\begin{aligned}
& \mathbf{a}_{1}=a \hat{\mathbf{x}} \\
& \mathbf{a}_{2}=a \hat{\mathbf{y}} \\
& \mathbf{a}_{3}=c \hat{\mathbf{z}}
\end{aligned}
$$

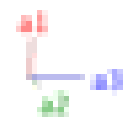

\section{Basis vectors:}

\section{Lattice Coordinates}

$$
\mathbf{B}_{1}=\quad \frac{3}{4} \mathbf{a}_{1}+\frac{1}{4} \mathbf{a}_{2}+\frac{3}{4} \mathbf{a}_{3}
$$

$\mathbf{B}_{2}=$

$\mathbf{B}_{3}=$

$\mathbf{B}_{4}=\frac{1}{4} \mathbf{a}_{1}+\frac{1}{4} \mathbf{a}_{2}+\left(\frac{1}{2}+z_{2}\right) \mathbf{a}_{3}$

$\mathbf{B}_{5}=\frac{3}{4} \mathbf{a}_{1}+\frac{3}{4} \mathbf{a}_{2}-z_{2} \mathbf{a}_{3}$

$\mathbf{B}_{6}=\frac{3}{4} \mathbf{a}_{1}+\frac{3}{4} \mathbf{a}_{2}+\left(\frac{1}{2}-z_{2}\right) \mathbf{a}_{3}$
Cartesian Coordinates

$=$

$=$

$=$

$=$

$=$

$=$

$$
\frac{3}{4} a \hat{\mathbf{x}}+\frac{1}{4} a \hat{\mathbf{y}}+\frac{3}{4} c \hat{\mathbf{z}}
$$$$
\frac{1}{4} a \hat{\mathbf{x}}+\frac{3}{4} a \hat{\mathbf{y}}+\frac{1}{4} c \hat{\mathbf{z}}
$$$$
\frac{1}{4} a \hat{\mathbf{x}}+\frac{1}{4} a \hat{\mathbf{y}}+z_{2} c \hat{\mathbf{z}}
$$$$
\frac{1}{4} a \hat{\mathbf{x}}+\frac{1}{4} a \hat{\mathbf{y}}+\left(\frac{1}{2}+z_{2}\right) c \hat{\mathbf{z}}
$$$$
\frac{3}{4} a \hat{\mathbf{x}}+\frac{3}{4} a \hat{\mathbf{y}}-z_{2} c \hat{\mathbf{z}}
$$$$
\frac{3}{4} a \hat{\mathbf{x}}+\frac{3}{4} a \hat{\mathbf{y}}+\left(\frac{1}{2}-z_{2}\right) c \hat{\mathbf{z}}
$$

Wyckoff Position
Atom Type

$\mathrm{Zr}$

$\mathrm{Zr}$

O

O

O

O 


\section{References:}

- G. Teufer, The crystal structure of tetragonal $\mathrm{ZrO}_{2}$, Acta Cryst. 15, 1187-1187 (1962), doi:10.1107/S0365110X62003114.

\section{Found in:}

- P. Villars and K. Cenzual, Pearson's Crystal Data - Crystal Structure Database for Inorganic Compounds, ASM International (2013).

\section{Geometry files:}

- CIF: pp. 900

- POSCAR: pp. 900 


\section{$\mathrm{CeCo}_{4} \mathrm{~B}_{4}$ Structure: A4BC4_tP18_137_g_b_g}
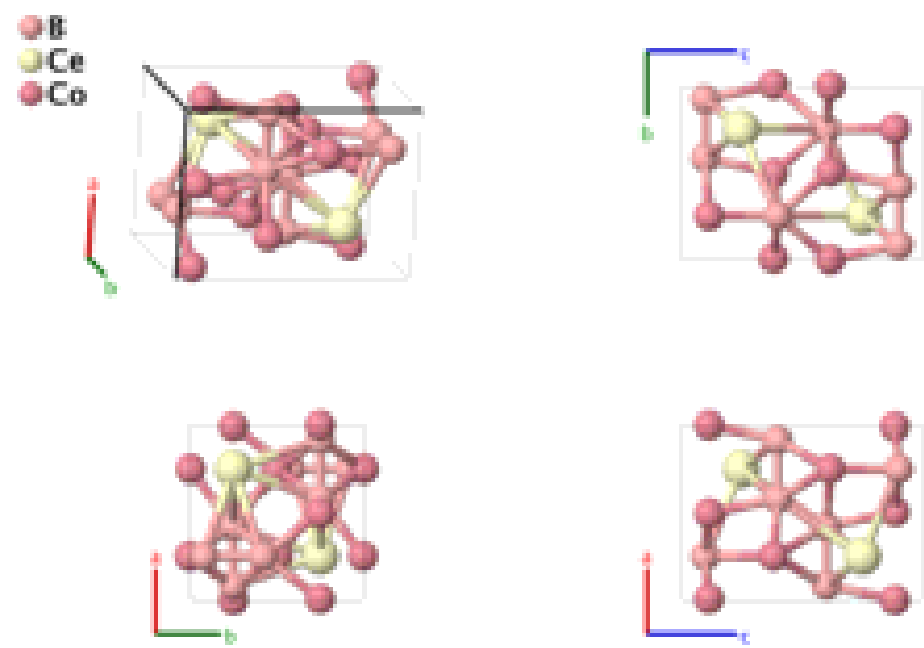

\section{Prototype}

AFLOW prototype label

: $\quad \mathrm{CeCo}_{4} \mathrm{~B}_{4}$

Strukturbericht designation

: A4BC4_tP18_137_g_b_g

Pearson symbol

: None

Space group number

: $\quad$ tP18

Space group symbol

AFLOW prototype command

$\mathrm{P}_{2} /$ nmc

aflow --proto=A4BC4_tP18_137_g_b_g

- params $=a, c / a, y_{2}, z_{2}, y_{3}, z_{3}$

Simple Tetragonal primitive vectors:

$$
\begin{aligned}
& \mathbf{a}_{1}=a \hat{\mathbf{x}} \\
& \mathbf{a}_{2}=a \hat{\mathbf{y}} \\
& \mathbf{a}_{3}=c \hat{\mathbf{z}}
\end{aligned}
$$

Basis vectors:

\section{Lattice Coordinates}

$\mathbf{B}_{1}=\frac{3}{4} \mathbf{a}_{1}+\frac{1}{4} \mathbf{a}_{2}+\frac{1}{4} \mathbf{a}_{3}$

$\mathbf{B}_{2}=\frac{1}{4} \mathbf{a}_{1}+\frac{3}{4} \mathbf{a}_{2}+\frac{3}{4} \mathbf{a}_{3}$

$\mathbf{B}_{3}=\frac{1}{4} \mathbf{a}_{1}+y_{2} \mathbf{a}_{2}+z_{2} \mathbf{a}_{3}$

$\mathbf{B}_{4}=\frac{1}{4} \mathbf{a}_{1}+\left(\frac{1}{2}-y_{2}\right) \mathbf{a}_{2}+z_{2} \mathbf{a}_{3}$

$\mathbf{B}_{5}=\left(\frac{1}{2}-y_{2}\right) \mathbf{a}_{1}+\frac{1}{4} \mathbf{a}_{2}+\left(\frac{1}{2}+z_{2}\right) \mathbf{a}_{3}$

$\mathbf{B}_{6}=$

$\mathbf{B}_{7}=$

$\mathbf{B}_{8}=$ $y_{2} \mathbf{a}_{1}+\frac{1}{4} \mathbf{a}_{2}+\left(\frac{1}{2}+z_{2}\right) \mathbf{a}_{3}$

$$
\frac{3}{4} \mathbf{a}_{1}+\left(\frac{1}{2}+y_{2}\right) \mathbf{a}_{2}-z_{2} \mathbf{a}_{3}
$$

$\frac{3}{4} \mathbf{a}_{1}-y_{2} \mathbf{a}_{2}-z_{2} \mathbf{a}_{3}$

\section{Cartesian Coordinates}

$=$

$=$

$=$

$=$

$=$

$=$

$=$

$=$

$$
\frac{3}{4} a \hat{\mathbf{x}}+\frac{1}{4} a \hat{\mathbf{y}}+\frac{1}{4} c \hat{\mathbf{z}}
$$$$
\frac{1}{4} a \hat{\mathbf{X}}+\frac{3}{4} a \hat{\mathbf{y}}+\frac{3}{4} c \hat{\mathbf{z}}
$$$$
\frac{1}{4} a \hat{\mathbf{x}}+y_{2} a \hat{\mathbf{y}}+z_{2} c \hat{\mathbf{z}}
$$$$
\frac{1}{4} a \hat{\mathbf{x}}+\left(\frac{1}{2}-y_{2}\right) a \hat{\mathbf{y}}+z_{2} c \hat{\mathbf{z}}
$$$$
\left(\frac{1}{2}-y_{2}\right) a \hat{\mathbf{x}}+\frac{1}{4} a \hat{\mathbf{y}}+\left(\frac{1}{2}+z_{2}\right) c \hat{\mathbf{z}}
$$$$
y_{2} a \hat{\mathbf{x}}+\frac{1}{4} a \hat{\mathbf{y}}+\left(\frac{1}{2}+z_{2}\right) c \hat{\mathbf{z}}
$$$$
\frac{3}{4} a \hat{\mathbf{x}}+\left(\frac{1}{2}+y_{2}\right) a \hat{\mathbf{y}}-z_{2} c \hat{\mathbf{z}}
$$$$
\frac{3}{4} a \hat{\mathbf{x}}-y_{2} a \hat{\mathbf{y}}-z_{2} c \hat{\mathbf{z}}
$$

Wyckoff Position
Atom Type

$\mathrm{Ce}$

$\mathrm{Ce}$

B

B

B

B

B

B 


\begin{tabular}{|c|c|c|c|c|c|c|}
\hline $\mathbf{B}_{9}$ & $=$ & $\left(\frac{1}{2}+y_{2}\right) \mathbf{a}_{1}+\frac{3}{4} \mathbf{a}_{2}+\left(\frac{1}{2}-z_{2}\right) \mathbf{a}_{3}$ & $=$ & $\left(\frac{1}{2}+y_{2}\right) a \hat{\mathbf{x}}+\frac{3}{4} a \hat{\mathbf{y}}+\left(\frac{1}{2}-z_{2}\right) c \hat{\mathbf{z}}$ & $(8 g)$ & B \\
\hline $\mathbf{B}_{10}$ & $=$ & $-y_{2} \mathbf{a}_{1}+\frac{3}{4} \mathbf{a}_{2}+\left(\frac{1}{2}-z_{2}\right) \mathbf{a}_{3}$ & $=$ & $-y_{2} a \hat{\mathbf{x}}+\frac{3}{4} a \hat{\mathbf{y}}+\left(\frac{1}{2}-z_{2}\right) c \hat{\mathbf{z}}$ & $(8 g)$ & $\mathrm{B}$ \\
\hline $\mathbf{B}_{11}$ & $=$ & $\frac{1}{4} \mathbf{a}_{1}+y_{3} \mathbf{a}_{2}+z_{3} \mathbf{a}_{3}$ & $=$ & $\frac{1}{4} a \hat{\mathbf{x}}+y_{3} a \hat{\mathbf{y}}+z_{3} c \hat{\mathbf{z}}$ & $(8 g)$ & $\mathrm{Co}$ \\
\hline $\mathbf{B}_{12}$ & $=$ & $\frac{1}{4} \mathbf{a}_{1}+\left(\frac{1}{2}-y_{3}\right) \mathbf{a}_{2}+z_{3} \mathbf{a}_{3}$ & $=$ & $\frac{1}{4} a \hat{\mathbf{x}}+\left(\frac{1}{2}-y_{3}\right) a \hat{\mathbf{y}}+z_{3} c \hat{\mathbf{z}}$ & $(8 g)$ & $\mathrm{Co}$ \\
\hline $\mathbf{B}_{13}$ & $=$ & $\left(\frac{1}{2}-y_{3}\right) \mathbf{a}_{1}+\frac{1}{4} \mathbf{a}_{2}+\left(\frac{1}{2}+z_{3}\right) \mathbf{a}_{3}$ & $=$ & $\left(\frac{1}{2}-y_{3}\right) a \hat{\mathbf{x}}+\frac{1}{4} a \hat{\mathbf{y}}+\left(\frac{1}{2}+z_{3}\right) c \hat{\mathbf{z}}$ & $(8 g)$ & $\mathrm{Co}$ \\
\hline $\mathbf{B}_{14}$ & $=$ & $y_{3} \mathbf{a}_{1}+\frac{1}{4} \mathbf{a}_{2}+\left(\frac{1}{2}+z_{3}\right) \mathbf{a}_{3}$ & $=$ & $y_{3} a \hat{\mathbf{x}}+\frac{1}{4} a \hat{\mathbf{y}}+\left(\frac{1}{2}+z_{3}\right) c \hat{\mathbf{z}}$ & $(8 g)$ & $\mathrm{Co}$ \\
\hline $\mathbf{B}_{15}$ & $=$ & $\frac{3}{4} \mathbf{a}_{1}+\left(\frac{1}{2}+y_{3}\right) \mathbf{a}_{2}-z_{3} \mathbf{a}_{3}$ & $=$ & $\frac{3}{4} a \hat{\mathbf{x}}+\left(\frac{1}{2}+y_{3}\right) a \hat{\mathbf{y}}-z_{3} c \hat{\mathbf{z}}$ & $(8 g)$ & $\mathrm{Co}$ \\
\hline $\mathbf{B}_{16}$ & $=$ & $\frac{3}{4} \mathbf{a}_{1}-y_{3} \mathbf{a}_{2}-z_{3} \mathbf{a}_{3}$ & $=$ & $\frac{3}{4} a \hat{\mathbf{x}}-y_{3} a \hat{\mathbf{y}}-z_{3} c \hat{\mathbf{z}}$ & $(8 g)$ & $\mathrm{Co}$ \\
\hline $\mathbf{B}_{17}$ & $=$ & $\left(\frac{1}{2}+y_{3}\right) \mathbf{a}_{1}+\frac{3}{4} \mathbf{a}_{2}+\left(\frac{1}{2}-z_{3}\right) \mathbf{a}_{3}$ & $=$ & $\left(\frac{1}{2}+y_{3}\right) a \hat{\mathbf{x}}+\frac{3}{4} a \hat{\mathbf{y}}+\left(\frac{1}{2}-z_{3}\right) c \hat{\mathbf{z}}$ & $(8 g)$ & $\mathrm{Co}$ \\
\hline $\mathbf{B}_{18}$ & $=$ & $-y_{3} \mathbf{a}_{1}+\frac{3}{4} \mathbf{a}_{2}+\left(\frac{1}{2}-z_{3}\right) \mathbf{a}_{3}$ & $=$ & $-y_{3} a \hat{\mathbf{x}}+\frac{3}{4} a \hat{\mathbf{y}}+\left(\frac{1}{2}-z_{3}\right) c \hat{\mathbf{z}}$ & $(8 g)$ & $\mathrm{Co}$ \\
\hline
\end{tabular}

\section{References:}

- Y. B. Kuzma and N. S. Bilonizhko, Crystal structure of the compounds $\mathrm{CeCo}_{4} B_{4}$ and its analogs, Sov. Phys. Crystallogr.

16, 897-898 (1972).

\section{Found in:}

- P. Villars and K. Cenzual, Pearson's Crystal Data - Crystal Structure Database for Inorganic Compounds, ASM International (2013).

\section{Geometry files:}

- CIF: pp. 900

- POSCAR: pp. 901 

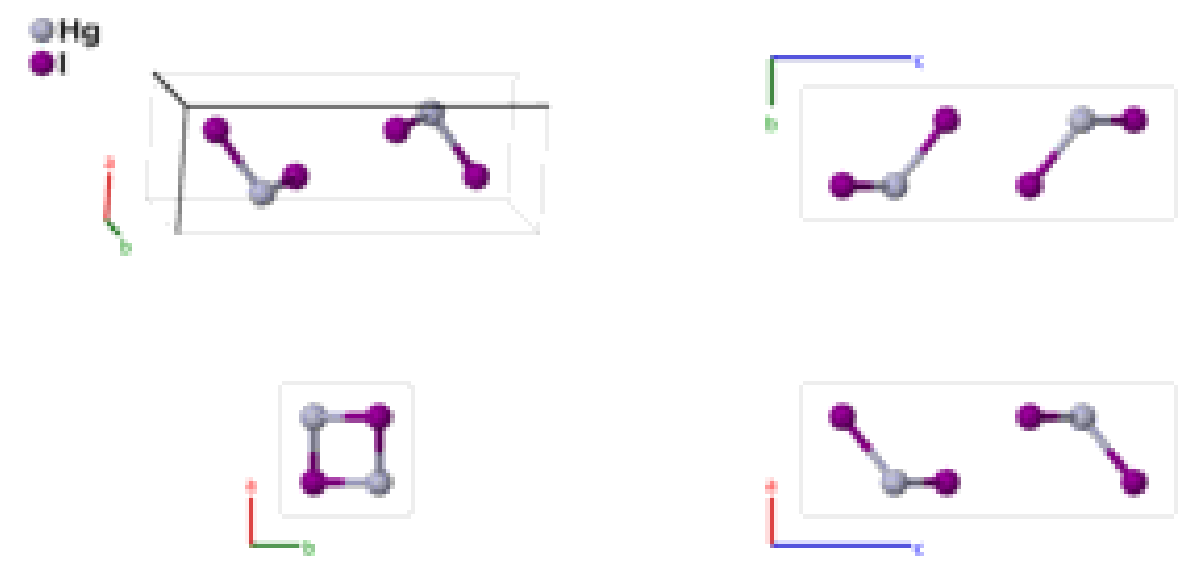

$\begin{array}{lll}\text { Prototype } & : & \mathrm{HgI}_{2} \\ \text { AFLOW prototype label } & : & \mathrm{AB} 2 \_t \mathrm{P} 6 \_137 \_\mathrm{a} \_\mathrm{d} \\ \text { Strukturbericht } \text { designation } & : & \mathrm{C} 13 \\ \text { Pearson symbol } & : & \mathrm{tP6} \\ \text { Space group number } & : & 137 \\ \text { Space group symbol } & : & P 4_{2} / n m c \\ \text { AFLOW prototype command } & : & \text { aflow --proto }=\mathrm{AB} 2 \_t \mathrm{tP} 6_{-} 137_{-} \mathrm{a} \_\mathrm{d} \\ & & - \text {-params }=a, c / a, z_{2}\end{array}$

- The CIF and POSCAR files contain the data at room temperature, $293 \mathrm{~K}$. $\mathrm{ZrO}_{2}$ (pp. 443) and $\mathrm{HgI}_{2}$ (pp. 447) have similar AFLOW prototype labels (i.e., same symmetry and set of Wyckoff positions with different stoichiometry labels due to alphabetic ordering of atomic species). They are generated by the same symmetry operations with different sets of parameters (--params) specified in their corresponding CIF files.

\section{Simple Tetragonal primitive vectors:}

$$
\begin{aligned}
& \mathbf{a}_{1}=a \hat{\mathbf{x}} \\
& \mathbf{a}_{2}=a \hat{\mathbf{y}} \\
& \mathbf{a}_{3}=c \hat{\mathbf{z}}
\end{aligned}
$$

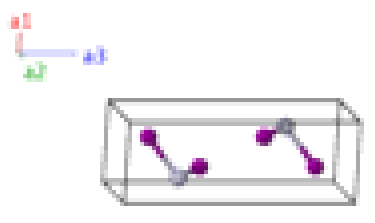

\section{Basis vectors:}

Lattice Coordinates

$\begin{array}{llclc}\mathbf{B}_{1}= & \frac{3}{4} \mathbf{a}_{1}+\frac{1}{4} \mathbf{a}_{2}+\frac{3}{4} \mathbf{a}_{3} & = & \frac{3}{4} a \hat{\mathbf{x}}+\frac{1}{4} a \hat{\mathbf{y}}+\frac{3}{4} c \hat{\mathbf{z}} \\ \mathbf{B}_{2}= & \frac{1}{4} \mathbf{a}_{1}+\frac{3}{4} \mathbf{a}_{2}+\frac{1}{4} \mathbf{a}_{3} & = & \frac{1}{4} a \hat{\mathbf{x}}+\frac{3}{4} a \hat{\mathbf{y}}+\frac{1}{4} c \hat{\mathbf{z}} \\ \mathbf{B}_{3}= & \frac{1}{4} \mathbf{a}_{1}+\frac{1}{4} \mathbf{a}_{2}+z_{2} \mathbf{a}_{3} & = & \frac{1}{4} a \hat{\mathbf{x}}+\frac{1}{4} a \hat{\mathbf{y}}+z_{2} c \hat{\mathbf{z}} \\ \mathbf{B}_{4}= & \frac{1}{4} \mathbf{a}_{1}+\frac{1}{4} \mathbf{a}_{2}+\left(\frac{1}{2}+z_{2}\right) \mathbf{a}_{3} & = & \frac{1}{4} a \hat{\mathbf{x}}+\frac{1}{4} a \hat{\mathbf{y}}+\left(\frac{1}{2}+z_{2}\right) c \hat{\mathbf{z}} \\ \mathbf{B}_{5}= & \frac{3}{4} \mathbf{a}_{1}+\frac{3}{4} \mathbf{a}_{2}-z_{2} \mathbf{a}_{3} & = & \frac{3}{4} a \hat{\mathbf{x}}+\frac{3}{4} a \hat{\mathbf{y}}-z_{2} c \hat{\mathbf{z}} \\ \mathbf{B}_{6}= & \frac{3}{4} \mathbf{a}_{1}+\frac{3}{4} \mathbf{a}_{2}+\left(\frac{1}{2}-z_{2}\right) \mathbf{a}_{3} & = & \frac{3}{4} a \hat{\mathbf{x}}+\frac{3}{4} a \hat{\mathbf{y}}+\left(\frac{1}{2}-z_{2}\right) c \hat{\mathbf{z}}\end{array}$

Wyckoff Position

Atom Type

(2a)

$\mathrm{Hg}$

(2a)

$\mathrm{Hg}$

(4d)

(4d)

(4d)

$\mathrm{Hg}$
$\mathrm{Hg}$
I
I
I
I




\section{References:}

- D. Schwarzenbach, H. Birkedal, M. Hostettler, and P. Fischer, Neutron diffraction investigation of the temperature dependence of crystal structure and thermal motions of red $\mathrm{HgI}_{2}$, Acta Crystallogr. Sect. B Struct. Sci. 63, 826-835 (2007), doi:10.1107/S0108768107043327.

\section{Found in:}

- R. T. Downs and M. Hall-Wallace, The American Mineralogist Crystal Structure Database, Am. Mineral. 88, 247-250 (2003).

\section{Geometry files:}

- CIF: pp. 901

- POSCAR: pp. 901 


\section{C (T12 Group IV) Structure: A_tP12_138_bi}

\section{QC}
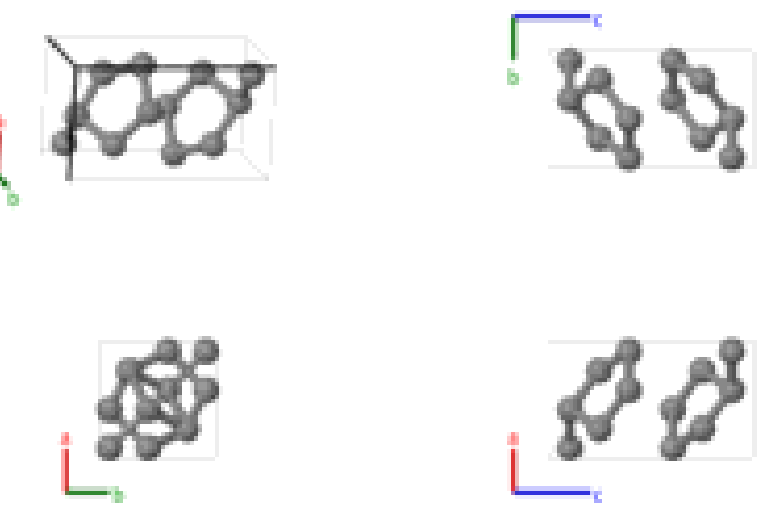

$\begin{array}{lll}\text { Prototype } & : & \text { C } \\ \text { AFLOW prototype label } & : & \text { A_tP12_138_bi } \\ \text { Strukturbericht designation } & : & \text { None } \\ \text { Pearson symbol } & : & \mathrm{tP} 12 \\ \text { Space group number } & : & 138 \\ \text { Space group symbol } & : & P 4_{2} / \mathrm{ncm} \\ \text { AFLOW prototype command } & : & \begin{array}{l}\text { aflow --proto }=A_{-} \mathrm{tP} 12_{-} 138 \_b i \\ \end{array} \\ & & - \text {-params }=a, c / a, x_{2}, z_{2}\end{array}$

Other elements with this structure:

- $\mathrm{Si}, \mathrm{Ge}$

- This is a tetragonal allotrope of the diamond structure found computationally in $\mathrm{C}, \mathrm{Si}$, and $\mathrm{Ge}$. The authors state "The T12 polymorph naturally accounts for the experimental $d$ spacings and Raman spectra of synthesized metastable Ge and Si-XIII phases with long-puzzling unknown structures, respectively."

\section{Simple Tetragonal primitive vectors:}

$$
\begin{aligned}
& \mathbf{a}_{1}=a \hat{\mathbf{x}} \\
& \mathbf{a}_{2}=a \hat{\mathbf{y}} \\
& \mathbf{a}_{3}=c \hat{\mathbf{z}}
\end{aligned}
$$
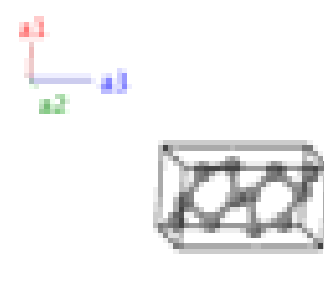

Basis vectors:

Lattice Coordinates

$\begin{array}{lll}\mathbf{B}_{1}= & \frac{3}{4} \mathbf{a}_{1}+\frac{1}{4} \mathbf{a}_{2}+\frac{3}{4} \mathbf{a}_{3} \\ \mathbf{B}_{2}= & \frac{1}{4} \mathbf{a}_{1}+\frac{3}{4} \mathbf{a}_{2}+\frac{1}{4} \mathbf{a}_{3} \\ \mathbf{B}_{3}= & \frac{1}{4} \mathbf{a}_{1}+\frac{3}{4} \mathbf{a}_{2}+\frac{3}{4} \mathbf{a}_{3}\end{array}$

$\mathbf{B}_{1}=\quad \frac{3}{4} \mathbf{a}_{1}+\frac{1}{4} \mathbf{a}_{2}+\frac{3}{4} \mathbf{a}_{3}$

$\mathbf{B}_{3}=$
Cartesian Coordinates

$=$

$=$

$=$

$$
\begin{aligned}
& \frac{3}{4} a \hat{\mathbf{x}}+\frac{1}{4} a \hat{\mathbf{y}}+\frac{3}{4} c \hat{\mathbf{z}} \\
& \frac{1}{4} a \hat{\mathbf{x}}+\frac{3}{4} a \hat{\mathbf{y}}+\frac{1}{4} c \hat{\mathbf{z}} \\
& \frac{1}{4} a \hat{\mathbf{x}}+\frac{3}{4} a \hat{\mathbf{y}}+\frac{3}{4} c \hat{\mathbf{z}}
\end{aligned}
$$

Wyckoff Position

(4b)
Atom Type

C I

C I

C I 


\begin{tabular}{|c|c|c|c|c|c|c|}
\hline $\mathbf{B}_{4}$ & $=$ & $\frac{3}{4} \mathbf{a}_{1}+\frac{1}{4} \mathbf{a}_{2}+\frac{1}{4} \mathbf{a}_{3}$ & $=$ & $\frac{3}{4} a \hat{\mathbf{x}}+\frac{1}{4} a \hat{\mathbf{y}}+\frac{1}{4} c \hat{\mathbf{z}}$ & $(4 b)$ & $\mathrm{CI}$ \\
\hline $\mathbf{B}_{5}$ & $=$ & $x_{2} \mathbf{a}_{1}+x_{2} \mathbf{a}_{2}+z_{2} \mathbf{a}_{3}$ & $=$ & $x_{2} a \hat{\mathbf{x}}+x_{2} a \hat{\mathbf{y}}+z_{2} c \hat{\mathbf{z}}$ & $(8 i)$ & C II \\
\hline $\mathbf{B}_{6}$ & $=$ & $\left(\frac{1}{2}-x_{2}\right) \mathbf{a}_{1}+\left(\frac{1}{2}-x_{2}\right) \mathbf{a}_{2}+z_{2} \mathbf{a}_{3}$ & $=$ & $\left(\frac{1}{2}-x_{2}\right) a \hat{\mathbf{x}}+\left(\frac{1}{2}-x_{2}\right) a \hat{\mathbf{y}}+z_{2} c \hat{\mathbf{z}}$ & $(8 i)$ & C I \\
\hline $\mathbf{B}_{7}$ & $=$ & $\left(\frac{1}{2}-x_{2}\right) \mathbf{a}_{1}+x_{2} \mathbf{a}_{2}+\left(\frac{1}{2}+z_{2}\right) \mathbf{a}_{3}$ & $=$ & $\left(\frac{1}{2}-x_{2}\right) a \hat{\mathbf{x}}+x_{2} a \hat{\mathbf{y}}+\left(\frac{1}{2}+z_{2}\right) c \hat{\mathbf{z}}$ & $(8 i)$ & C II \\
\hline $\mathbf{B}_{8}$ & $=$ & $x_{2} \mathbf{a}_{1}+\left(\frac{1}{2}-x_{2}\right) \mathbf{a}_{2}+\left(\frac{1}{2}+z_{2}\right) \mathbf{a}_{3}$ & $=$ & $x_{2} a \hat{\mathbf{x}}+\left(\frac{1}{2}-x_{2}\right) a \hat{\mathbf{y}}+\left(\frac{1}{2}+z_{2}\right) c \hat{\mathbf{z}}$ & $(8 i)$ & C II \\
\hline $\mathbf{B}_{9}$ & $=$ & $-x_{2} \mathbf{a}_{1}+\left(\frac{1}{2}+x_{2}\right) \mathbf{a}_{2}+\left(\frac{1}{2}-z_{2}\right) \mathbf{a}_{3}$ & $=$ & $-x_{2} a \hat{\mathbf{x}}+\left(\frac{1}{2}+x_{2}\right) a \hat{\mathbf{y}}+\left(\frac{1}{2}-z_{2}\right) c \hat{\mathbf{z}}$ & $(8 i)$ & C II \\
\hline $\mathbf{B}_{10}$ & $=$ & $\left(\frac{1}{2}+x_{2}\right) \mathbf{a}_{1}-x_{2} \mathbf{a}_{2}+\left(\frac{1}{2}-z_{2}\right) \mathbf{a}_{3}$ & $=$ & $\left(\frac{1}{2}+x_{2}\right) a \hat{\mathbf{x}}-x_{2} a \hat{\mathbf{y}}+\left(\frac{1}{2}-z_{2}\right) c \hat{\mathbf{z}}$ & $(8 i)$ & C II \\
\hline $\mathbf{B}_{11}$ & $=$ & $\left(\frac{1}{2}+x_{2}\right) \mathbf{a}_{1}+\left(\frac{1}{2}+x_{2}\right) \mathbf{a}_{2}-z_{2} \mathbf{a}_{3}$ & $=$ & $\left(\frac{1}{2}+x_{2}\right) a \hat{\mathbf{x}}+\left(\frac{1}{2}+x_{2}\right) a \hat{\mathbf{y}}-z_{2} c \hat{\mathbf{z}}$ & $(8 i)$ & C II \\
\hline $\mathbf{B}_{12}$ & $=$ & $-x_{2} \mathbf{a}_{1}-x_{2} \mathbf{a}_{2}-z_{2} \mathbf{a}_{3}$ & $=$ & $-x_{2} a \hat{\mathbf{x}}-x_{2} a \hat{\mathbf{y}}-z_{2} c \hat{\mathbf{z}}$ & $(8 i)$ & \\
\hline
\end{tabular}

\section{References:}

- Z. Zhao, F. Tian, X. Dong, Q. Li, Q. Wang, H. Wang, X. Zhong, B. Xu, D. Yu, J. He, H.-T. Wang, Y. Ma, and Y. Tian, Tetragonal Allotrope of Group 14 Elements, J. Am. Chem. Soc. 134, 12362-12365 (2012), doi:10.1021/ja304380p.

\section{Geometry files:}

- CIF: pp. 901

- POSCAR: pp. 902 


\section{Calomel $\left(\mathrm{Hg}_{2} \mathrm{Cl}_{2}, D 3_{1}\right)$ Structure: AB_tI8_139_e_e}
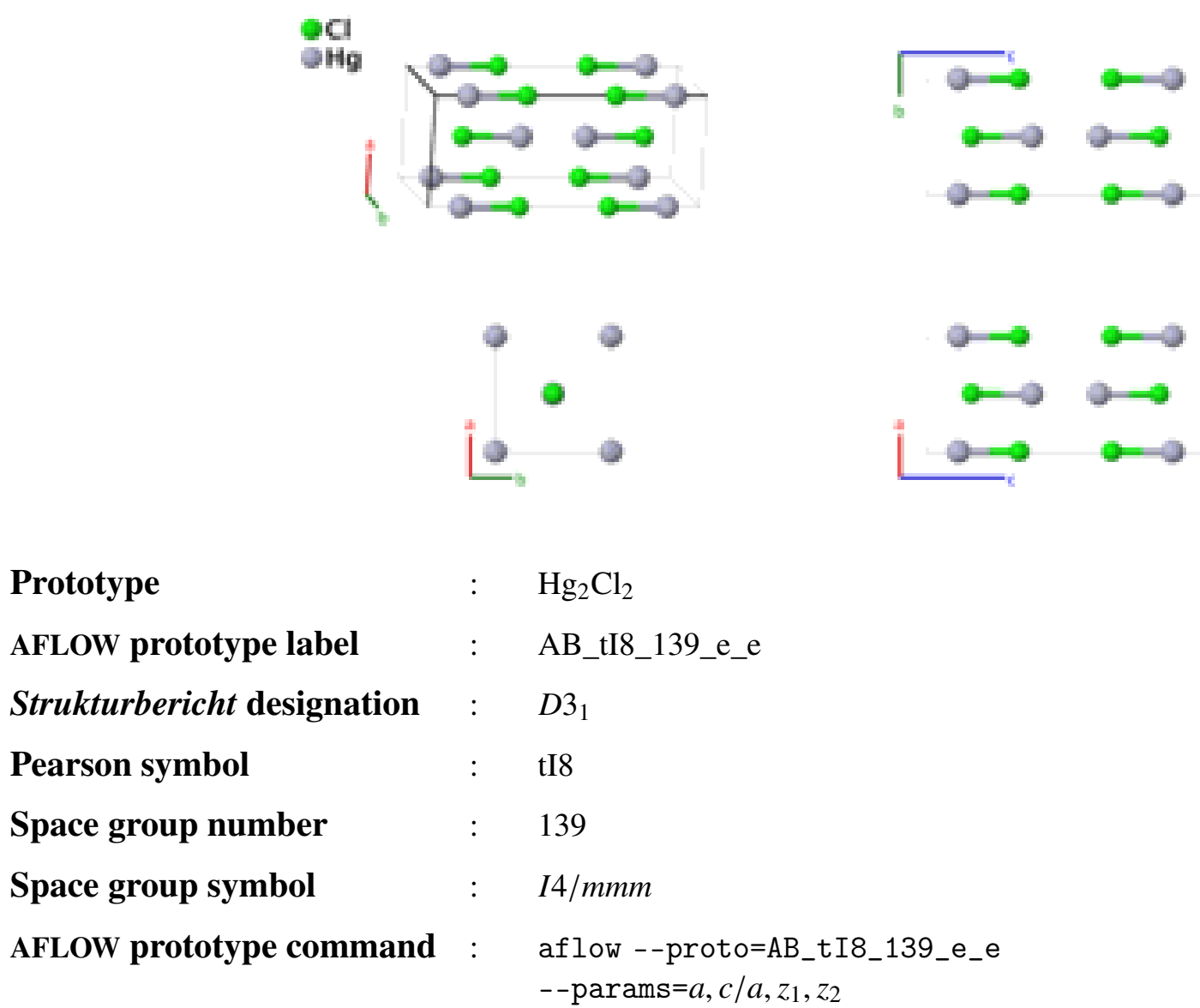

Body-centered Tetragonal primitive vectors:

$$
\begin{aligned}
& \mathbf{a}_{1}=-\frac{1}{2} a \hat{\mathbf{x}}+\frac{1}{2} a \hat{\mathbf{y}}+\frac{1}{2} c \hat{\mathbf{z}} \\
& \mathbf{a}_{2}=\frac{1}{2} a \hat{\mathbf{x}}-\frac{1}{2} a \hat{\mathbf{y}}+\frac{1}{2} c \hat{\mathbf{z}} \\
& \mathbf{a}_{3}=\frac{1}{2} a \hat{\mathbf{x}}+\frac{1}{2} a \hat{\mathbf{y}}-\frac{1}{2} c \hat{\mathbf{z}}
\end{aligned}
$$

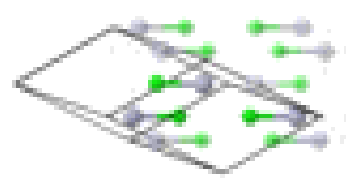

Basis vectors:

Lattice Coordinates

$\begin{array}{llll}\mathbf{B}_{1}= & z_{1} \mathbf{a}_{1}+z_{1} \mathbf{a}_{2} & = \\ \mathbf{B}_{2}= & -z_{1} \mathbf{a}_{1}-z_{1} \mathbf{a}_{2} & = \\ \mathbf{B}_{3}= & z_{2} \mathbf{a}_{1}+z_{2} \mathbf{a}_{2} & = \\ \mathbf{B}_{4}= & -z_{2} \mathbf{a}_{1}-z_{2} \mathbf{a}_{2} & =\end{array}$

\section{References:}

- N. J. Calos, C. H. L. Kennard, and R. L. Davis, The structure of calomel, $\mathrm{Hg}_{2} \mathrm{Cl}_{2}$, derived from neutron powder data, $\mathrm{Z}$. Kristallogr. 187, 305-307 (1989), doi:10.1524/zkri.1989.187.14.305.

Found in:

- R. T. Downs and M. Hall-Wallace, The American Mineralogist Crystal Structure Database, Am. Mineral. 88, 247-250 (2003). 
Geometry files:

- CIF: pp. 902

- POSCAR: pp. 902 


\section{$\mathrm{W}_{5} \mathrm{Si}_{3}\left(D 8_{m}\right)$ Structure: A3B5_tI32_140_ah_bk}
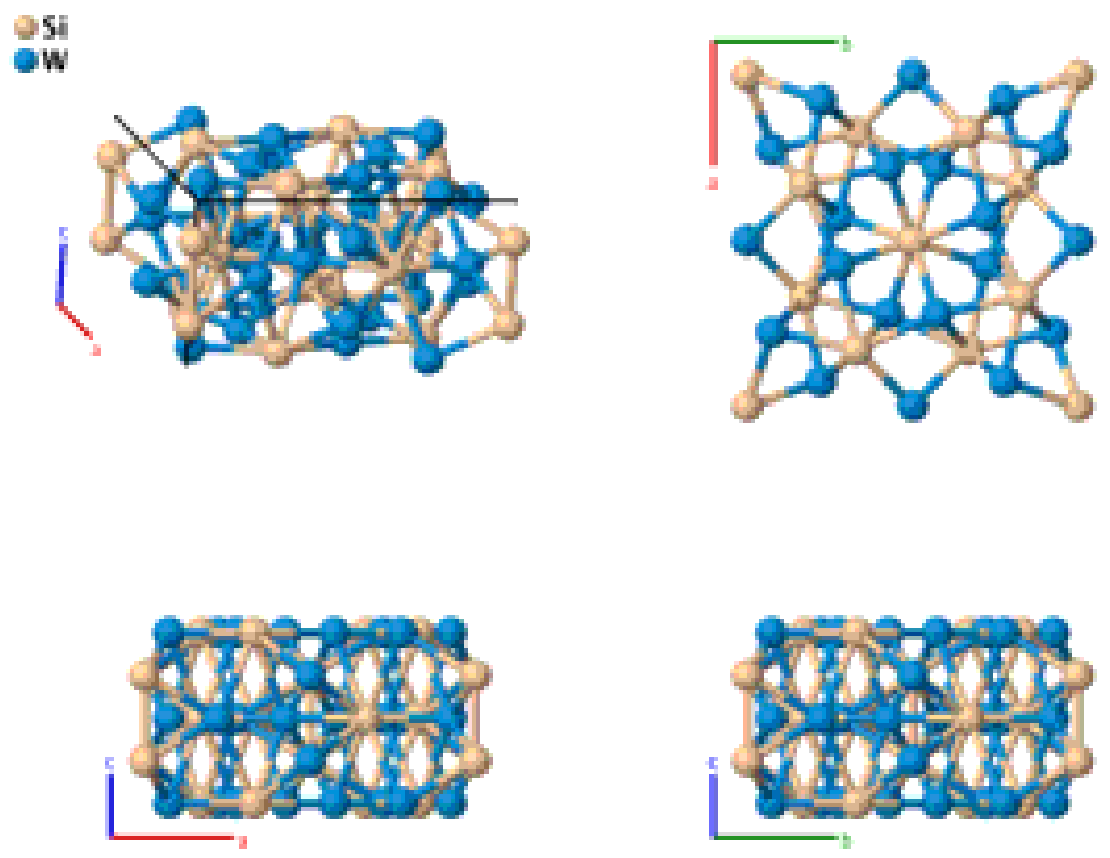

\begin{tabular}{|c|c|c|}
\hline Prototype & $\cdot$ & $\mathrm{W}_{5} \mathrm{Si}_{3}$ \\
\hline AFLOW prototype label & & A3B5_tI32_140_ah_bk \\
\hline Strukturbericht designation & 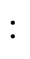 & $D 8_{m}$ \\
\hline Pearson symbol & & $\mathrm{tI} 32$ \\
\hline Space group number & $\cdot$ & 140 \\
\hline Space group symbol & 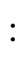 & $I 4 / \mathrm{mcm}$ \\
\hline AFLOW prototype command & & $\begin{array}{l}\text { aflow --proto=A3B5_tI32_140_ah_bk } \\
\text {--params }=a, c / a, x_{3}, x_{4}, y_{4}\end{array}$ \\
\hline
\end{tabular}

Other compounds with this structure:

- $\mathrm{Cr}_{5} \mathrm{Ge}_{3}, \mathrm{Cr}_{5} \mathrm{Si}_{3}, \mathrm{Mo}_{5} \mathrm{Si}_{3}, \mathrm{Nb}_{5} \mathrm{Si}_{3}, \mathrm{Ta}_{5} \mathrm{Si}_{3}, \mathrm{~V}_{5} \mathrm{Si}_{3}$

- (Pearson, 1958) refers to this as the "T1 phase."

Body-centered Tetragonal primitive vectors:

$$
\begin{aligned}
& \mathbf{a}_{1}=-\frac{1}{2} a \hat{\mathbf{x}}+\frac{1}{2} a \hat{\mathbf{y}}+\frac{1}{2} c \hat{\mathbf{z}} \\
& \mathbf{a}_{2}=\frac{1}{2} a \hat{\mathbf{x}}-\frac{1}{2} a \hat{\mathbf{y}}+\frac{1}{2} c \hat{\mathbf{z}} \\
& \mathbf{a}_{3}=\frac{1}{2} a \hat{\mathbf{x}}+\frac{1}{2} a \hat{\mathbf{y}}-\frac{1}{2} c \hat{\mathbf{z}}
\end{aligned}
$$

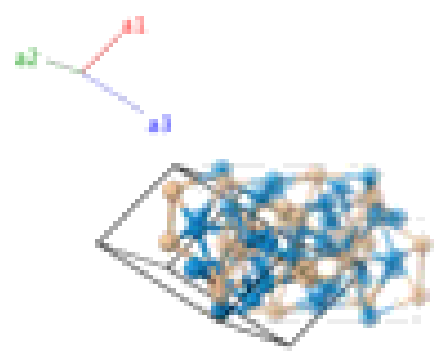


Lattice Coordinates

\begin{tabular}{|c|c|c|c|c|}
\hline $\mathbf{B}_{1}$ & $=$ & $\frac{1}{4} \mathbf{a}_{1}+\frac{1}{4} \mathbf{a}_{2}$ & $=$ & $\frac{1}{4} c \hat{\mathbf{z}}$ \\
\hline $\mathbf{B}_{2}$ & $=$ & $\frac{3}{4} \mathbf{a}_{1}+\frac{3}{4} \mathbf{a}_{2}$ & $=$ & $\frac{3}{4} c \hat{\mathbf{z}}$ \\
\hline $\mathbf{B}_{3}$ & $=$ & $\frac{3}{4} \mathbf{a}_{1}+\frac{1}{4} \mathbf{a}_{2}+\frac{1}{2} \mathbf{a}_{3}$ & $=$ & $\frac{1}{2} a \hat{\mathbf{y}}+\frac{1}{4} c \hat{\mathbf{z}}$ \\
\hline $\mathbf{B}_{4}$ & $=$ & $\frac{1}{4} \mathbf{a}_{1}+\frac{3}{4} \mathbf{a}_{2}+\frac{1}{2} \mathbf{a}_{3}$ & $=$ & $\frac{1}{2} a \hat{\mathbf{x}}+\frac{1}{4} c \hat{\mathbf{z}}$ \\
\hline $\mathbf{B}_{5}$ & $=$ & $\left(\frac{1}{2}+x_{3}\right) \mathbf{a}_{1}+x_{3} \mathbf{a}_{2}+\left(\frac{1}{2}+2 x_{3}\right) \mathbf{a}_{3}$ & $=$ & $x_{3} a \hat{\mathbf{x}}+\left(\frac{1}{2}+x_{3}\right) a \hat{\mathbf{y}}$ \\
\hline $\mathbf{B}_{6}$ & $=$ & $\left(\frac{1}{2}-x_{3}\right) \mathbf{a}_{1}-x_{3} \mathbf{a}_{2}+\left(\frac{1}{2}-2 x_{3}\right) \mathbf{a}_{3}$ & $=$ & $-x_{3} a \hat{\mathbf{x}}+\left(\frac{1}{2}-x_{3}\right) a \hat{\mathbf{y}}$ \\
\hline $\mathbf{B}_{7}$ & $=$ & $x_{3} \mathbf{a}_{1}+\left(\frac{1}{2}-x_{3}\right) \mathbf{a}_{2}+\frac{1}{2} \mathbf{a}_{3}$ & $=$ & $\left(\frac{1}{2}-x_{3}\right) a \hat{\mathbf{x}}+x_{3} a \hat{\mathbf{y}}$ \\
\hline $\mathbf{B}_{8}$ & $=$ & $-x_{3} \mathbf{a}_{1}+\left(\frac{1}{2}+x_{3}\right) \mathbf{a}_{2}+\frac{1}{2} \mathbf{a}_{3}$ & $=$ & $\left(\frac{1}{2}+x_{3}\right) a \hat{\mathbf{x}}-x_{3} a \hat{\mathbf{y}}$ \\
\hline $\mathbf{B}_{9}$ & $=$ & $y_{4} \mathbf{a}_{1}+x_{4} \mathbf{a}_{2}+\left(x_{4}+y_{4}\right) \mathbf{a}_{3}$ & $=$ & $x_{4} a \hat{\mathbf{x}}+y_{4} a \hat{\mathbf{y}}$ \\
\hline $\mathbf{B}_{10}$ & $=$ & $-y_{4} \mathbf{a}_{1}-x_{4} \mathbf{a}_{2}+\left(-x_{4}-y_{4}\right) \mathbf{a}_{3}$ & $=$ & $-x_{4} a \hat{\mathbf{x}}-y_{4} a \hat{\mathbf{y}}$ \\
\hline $\mathbf{B}_{11}$ & $=$ & $x_{4} \mathbf{a}_{1}-y_{4} \mathbf{a}_{2}+\left(x_{4}-y_{4}\right) \mathbf{a}_{3}$ & $=$ & $-y_{4} a \hat{\mathbf{x}}+x_{4} a \hat{\mathbf{y}}$ \\
\hline $\mathbf{B}_{12}$ & $=$ & $-x_{4} \mathbf{a}_{1}+y_{4} \mathbf{a}_{2}+\left(-x_{4}+y_{4}\right) \mathbf{a}_{3}$ & $=$ & $y_{4} a \hat{\mathbf{x}}-x_{4} a \hat{\mathbf{y}}$ \\
\hline $\mathbf{B}_{13}$ & $=$ & $\left(\frac{1}{2}+y_{4}\right) \mathbf{a}_{1}+\left(\frac{1}{2}-x_{4}\right) \mathbf{a}_{2}+\left(-x_{4}+y_{4}\right) \mathbf{a}_{3}$ & $=$ & $-x_{4} a \hat{\mathbf{x}}+y_{4} a \hat{\mathbf{y}}+\frac{1}{2} c \hat{\mathbf{z}}$ \\
\hline $\mathbf{B}_{14}$ & $=$ & $\left(\frac{1}{2}-y_{4}\right) \mathbf{a}_{1}+\left(\frac{1}{2}+x_{4}\right) \mathbf{a}_{2}+\left(x_{4}-y_{4}\right) \mathbf{a}_{3}$ & $=$ & $x_{4} a \hat{\mathbf{x}}-y_{4} a \hat{\mathbf{y}}+\frac{1}{2} c \hat{\mathbf{z}}$ \\
\hline $\mathbf{B}_{15}$ & $=$ & $\left(\frac{1}{2}+x_{4}\right) \mathbf{a}_{1}+\left(\frac{1}{2}+y_{4}\right) \mathbf{a}_{2}+\left(x_{4}+y_{4}\right) \mathbf{a}_{3}$ & $=$ & $y_{4} a \hat{\mathbf{x}}+x_{4} a \hat{\mathbf{y}}+\frac{1}{2} c \hat{\mathbf{z}}$ \\
\hline $\mathbf{B}_{16}$ & $=$ & $\left(\frac{1}{2}-x_{4}\right) \mathbf{a}_{1}+\left(\frac{1}{2}-y_{4}\right) \mathbf{a}_{2}+\left(-x_{4}-y_{4}\right) \mathbf{a}_{3}$ & $=$ & $-y_{4} a \hat{\mathbf{x}}-x_{4} a \hat{\mathbf{y}}+\frac{1}{2} c \hat{\mathbf{z}}$ \\
\hline
\end{tabular}

Wyckoff Position

(4a)

(4a)
Atom Type

Si I

Si I

W I

W I

Si II

Si II

Si II

Si II

W II

W II

W II

W II

W II

W II

W II

W II

\section{References:}

- B. Aronsson, The Crystal Structure of $\mathrm{Mo}_{5} \mathrm{Si}_{3}$ and $\mathrm{W}_{5} \mathrm{Si}_{3}$, Acta Chem. Scand. 9, 1107-1110 (1955), doi:10.3891/acta.chem.scand.09-1107.

\section{Geometry files:}

- CIF: pp. 902

- POSCAR: pp. 903 


\section{$\mathrm{Cr}_{5} \mathrm{~B}_{3}\left(D 8_{l}\right)$ Structure: A3B5_tI32_140_ah_cl}
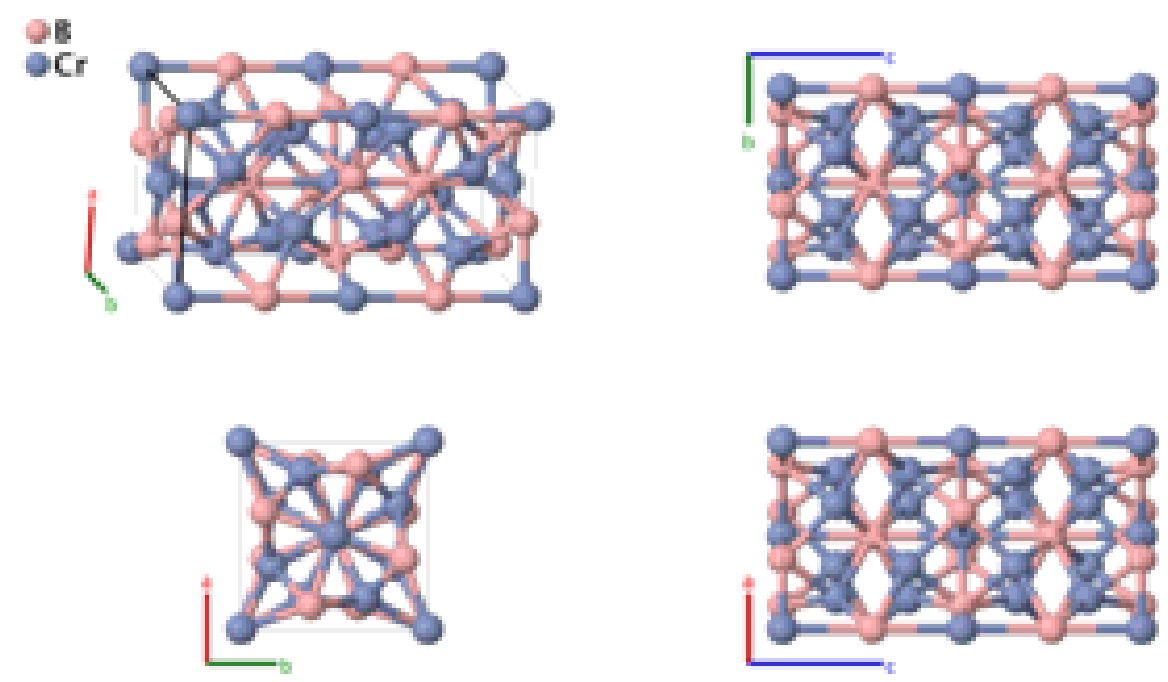

$\begin{array}{lll}\text { Prototype } & : & \mathrm{Cr}_{5} \mathrm{~B}_{3} \\ \text { AFLOW prototype label } & : & \mathrm{A} 3 \mathrm{~B} 5 \_\mathrm{tI} 32_{-} 140 \_\mathrm{ah} \_\mathrm{cl} \\ \text { Strukturbericht designation } & : & \mathrm{D} 8_{l} \\ \text { Pearson symbol } & : & \mathrm{tI} 32 \\ \text { Space group number } & : & 140 \\ \text { Space group symbol } & : & I 4 / \mathrm{mcm} \\ \text { AFLOW prototype command } & : & \begin{array}{l}\text { aflow --proto=A3B5_tI32_140_ah_cl } \\ \text {--params }=a, c / a, x_{3}, x_{4}, z_{4}\end{array}\end{array}$

\section{Other compounds with this structure:}

- $\mathrm{Eu}_{5} \mathrm{Ge}_{3}, \mathrm{Pr}_{5} \mathrm{Si}_{3}, \mathrm{Ta}_{5} \mathrm{Ge}_{3}, \mathrm{Nb}_{5} \mathrm{Si}_{3}, \mathrm{Ta}_{5} \mathrm{Si}_{3}, \mathrm{EuIrGe}_{2}, \mathrm{Fe}_{5} \mathrm{~PB}_{2}, \mathrm{Fe}_{5} \mathrm{SiB}_{2}, \mathrm{Fe}_{4} \mathrm{CoPB}_{2}, \mathrm{Fe}_{4} \mathrm{MnPB}_{2}, \mathrm{Fe}_{4} \mathrm{CoSiB}_{2}, \mathrm{Fe}_{4} \mathrm{MnSiB}_{2}$, $\mathrm{Mo}_{5} \mathrm{SiB}_{2}, \mathrm{Sr}_{5} \mathrm{In}_{3}, \mathrm{Gd}_{5} \mathrm{CoSi}_{2}, \mathrm{Ca}_{5} \mathrm{Si}_{3}$,

- We have been unable to obtain a copy of (Bertaut, 1953), so we use the data found online from (Downs, 2003). Although $\mathrm{Cr}_{5} \mathrm{~B}_{3}$ is universally regarded as the prototype for $D 8_{l}$, it appears that no determination of the internal parameters has ever been made. The values found in (Bertaut, 1953) seem to be resonable choices.

Body-centered Tetragonal primitive vectors:

$$
\begin{aligned}
& \mathbf{a}_{1}=-\frac{1}{2} a \hat{\mathbf{x}}+\frac{1}{2} a \hat{\mathbf{y}}+\frac{1}{2} c \hat{\mathbf{z}} \\
& \mathbf{a}_{2}=\frac{1}{2} a \hat{\mathbf{x}}-\frac{1}{2} a \hat{\mathbf{y}}+\frac{1}{2} c \hat{\mathbf{z}} \\
& \mathbf{a}_{3}=\frac{1}{2} a \hat{\mathbf{x}}+\frac{1}{2} a \hat{\mathbf{y}}-\frac{1}{2} c \hat{\mathbf{z}}
\end{aligned}
$$

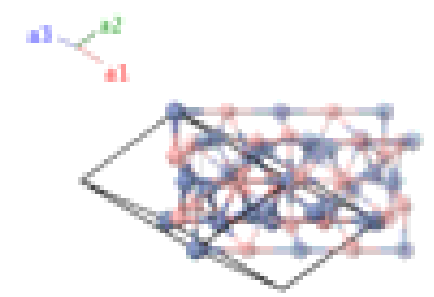

\section{Basis vectors:}

Lattice Coordinates

$\mathbf{B}_{1}=$
Cartesian Coordinates

$=$
Wyckoff Position

(4a)
Atom Type

B I 


\begin{tabular}{|c|c|c|c|c|c|c|}
\hline $\mathbf{B}_{2}$ & $=$ & $\frac{3}{4} \mathbf{a}_{1}+\frac{3}{4} \mathbf{a}_{2}$ & $=$ & $\frac{3}{4} c \hat{\mathbf{z}}$ & $(4 a)$ & B I \\
\hline $\mathbf{B}_{3}$ & $=$ & $0 \mathbf{a}_{1}+0 \mathbf{a}_{2}+0 \mathbf{a}_{3}$ & $=$ & $0 \hat{\mathbf{x}}+0 \hat{\mathbf{y}}+0 \hat{\mathbf{z}}$ & $(4 c)$ & Cr I \\
\hline $\mathbf{B}_{4}$ & $=$ & $\frac{1}{2} \mathbf{a}_{1}+\frac{1}{2} \mathbf{a}_{2}$ & $=$ & $\frac{1}{2} c \hat{\mathbf{z}}$ & $(4 c)$ & $\mathrm{Cr} \mathrm{I}$ \\
\hline $\mathbf{B}_{5}$ & $=$ & $\left(\frac{1}{2}+x_{3}\right) \mathbf{a}_{1}+x_{3} \mathbf{a}_{2}+\left(\frac{1}{2}+2 x_{3}\right) \mathbf{a}_{3}$ & $=$ & $x_{3} a \hat{\mathbf{x}}+\left(\frac{1}{2}+x_{3}\right) a \hat{\mathbf{y}}$ & $(8 h)$ & B II \\
\hline $\mathbf{B}_{6}$ & $=$ & $\left(\frac{1}{2}-x_{3}\right) \mathbf{a}_{1}-x_{3} \mathbf{a}_{2}+\left(\frac{1}{2}-2 x_{3}\right) \mathbf{a}_{3}$ & $=$ & $-x_{3} a \hat{\mathbf{x}}+\left(\frac{1}{2}-x_{3}\right) a \hat{\mathbf{y}}$ & $(8 h)$ & B II \\
\hline $\mathbf{B}_{7}$ & $=$ & $x_{3} \mathbf{a}_{1}+\left(\frac{1}{2}-x_{3}\right) \mathbf{a}_{2}+\frac{1}{2} \mathbf{a}_{3}$ & $=$ & $\left(\frac{1}{2}-x_{3}\right) a \hat{\mathbf{x}}+x_{3} a \hat{\mathbf{y}}$ & $(8 h)$ & B II \\
\hline $\mathbf{B}_{8}$ & $=$ & $-x_{3} \mathbf{a}_{1}+\left(\frac{1}{2}+x_{3}\right) \mathbf{a}_{2}+\frac{1}{2} \mathbf{a}_{3}$ & $=$ & $\left(\frac{1}{2}+x_{3}\right) a \hat{\mathbf{x}}-x_{3} a \hat{\mathbf{y}}$ & $(8 h)$ & B II \\
\hline $\mathbf{B}_{9}$ & $=$ & $\begin{array}{c}\left(\frac{1}{2}+x_{4}+z_{4}\right) \mathbf{a}_{1}+\left(x_{4}+z_{4}\right) \mathbf{a}_{2}+ \\
\left(\frac{1}{2}+2 x_{4}\right) \mathbf{a}_{3}\end{array}$ & $=$ & $x_{4} a \hat{\mathbf{x}}+\left(\frac{1}{2}+x_{4}\right) a \hat{\mathbf{y}}+z_{4} c \hat{\mathbf{z}}$ & $(16 l)$ & Cr II \\
\hline $\mathbf{B}_{10}$ & $=$ & $\begin{array}{c}\left(\frac{1}{2}-x_{4}+z_{4}\right) \mathbf{a}_{1}+\left(-x_{4}+z_{4}\right) \mathbf{a}_{2}+ \\
\left(\frac{1}{2}-2 x_{4}\right) \mathbf{a}_{3}\end{array}$ & $=$ & $-x_{4} a \hat{\mathbf{x}}+\left(\frac{1}{2}-x_{4}\right) a \hat{\mathbf{y}}+z_{4} c \hat{\mathbf{z}}$ & $(16 l)$ & Cr II \\
\hline $\mathbf{B}_{11}$ & $=$ & $\left(x_{4}+z_{4}\right) \mathbf{a}_{1}+\left(\frac{1}{2}-x_{4}+z_{4}\right) \mathbf{a}_{2}+\frac{1}{2} \mathbf{a}_{3}$ & $=$ & $\left(\frac{1}{2}-x_{4}\right) a \hat{\mathbf{x}}+x_{4} a \hat{\mathbf{y}}+z_{4} c \hat{\mathbf{z}}$ & $(16 l)$ & Cr II \\
\hline $\mathbf{B}_{12}$ & $=$ & $\left(-x_{4}+z_{4}\right) \mathbf{a}_{1}+\left(\frac{1}{2}+x_{4}+z_{4}\right) \mathbf{a}_{2}+\frac{1}{2} \mathbf{a}_{3}$ & $=$ & $\left(\frac{1}{2}+x_{4}\right) a \hat{\mathbf{x}}-x_{4} a \hat{\mathbf{y}}+z_{4} c \hat{\mathbf{z}}$ & $(16 l)$ & Cr II \\
\hline $\mathbf{B}_{13}$ & $=$ & $\left(x_{4}-z_{4}\right) \mathbf{a}_{1}+\left(\frac{1}{2}-x_{4}-z_{4}\right) \mathbf{a}_{2}+\frac{1}{2} \mathbf{a}_{3}$ & $=$ & $\left(\frac{1}{2}-x_{4}\right) a \hat{\mathbf{x}}+x_{4} a \hat{\mathbf{y}}-z_{4} c \hat{\mathbf{z}}$ & $(16 l)$ & Cr II \\
\hline $\mathbf{B}_{14}$ & $=$ & $\left(-x_{4}-z_{4}\right) \mathbf{a}_{1}+\left(\frac{1}{2}+x_{4}-z_{4}\right) \mathbf{a}_{2}+\frac{1}{2} \mathbf{a}_{3}$ & $=$ & $\left(\frac{1}{2}+x_{4}\right) a \hat{\mathbf{x}}-x_{4} a \hat{\mathbf{y}}-z_{4} c \hat{\mathbf{z}}$ & $(16 l)$ & Cr II \\
\hline $\mathbf{B}_{15}$ & $=$ & $\begin{array}{c}\left(\frac{1}{2}+x_{4}-z_{4}\right) \mathbf{a}_{1}+\left(x_{4}-z_{4}\right) \mathbf{a}_{2}+ \\
\left(\frac{1}{2}+2 x_{4}\right) \mathbf{a}_{3}\end{array}$ & $=$ & $x_{4} a \hat{\mathbf{x}}+\left(\frac{1}{2}+x_{4}\right) a \hat{\mathbf{y}}-z_{4} c \hat{\mathbf{z}}$ & $(16 l)$ & Cr II \\
\hline $\mathbf{B}_{16}$ & $=$ & $\begin{array}{c}\left(\frac{1}{2}-x_{4}-z_{4}\right) \mathbf{a}_{1}+\left(-x_{4}-z_{4}\right) \mathbf{a}_{2}+ \\
\left(\frac{1}{2}-2 x_{4}\right) \mathbf{a}_{3}\end{array}$ & $=$ & $-x_{4} a \hat{\mathbf{x}}+\left(\frac{1}{2}-x_{4}\right) a \hat{\mathbf{y}}-z_{4} c \hat{\mathbf{z}}$ & $(16 l)$ & $\mathrm{Cr}$ II \\
\hline
\end{tabular}

\section{References:}

- F. Bertaut and P. Blum, Etude des borures de chrome, C. R. Hebd. Séances Acad. Sci. 236, 1055-1056 (1953).

\section{Found in:}

- R. T. Downs and M. Hall-Wallace, The American Mineralogist Crystal Structure Database, Am. Mineral. 88, 247-250 (2003).

\section{Geometry files:}

- CIF: pp. 903

- POSCAR: pp. 903 


\section{$\alpha-\mathrm{ThSi}_{2}\left(C_{c}\right)$ Structure: A2B_tI12_141_e_a}
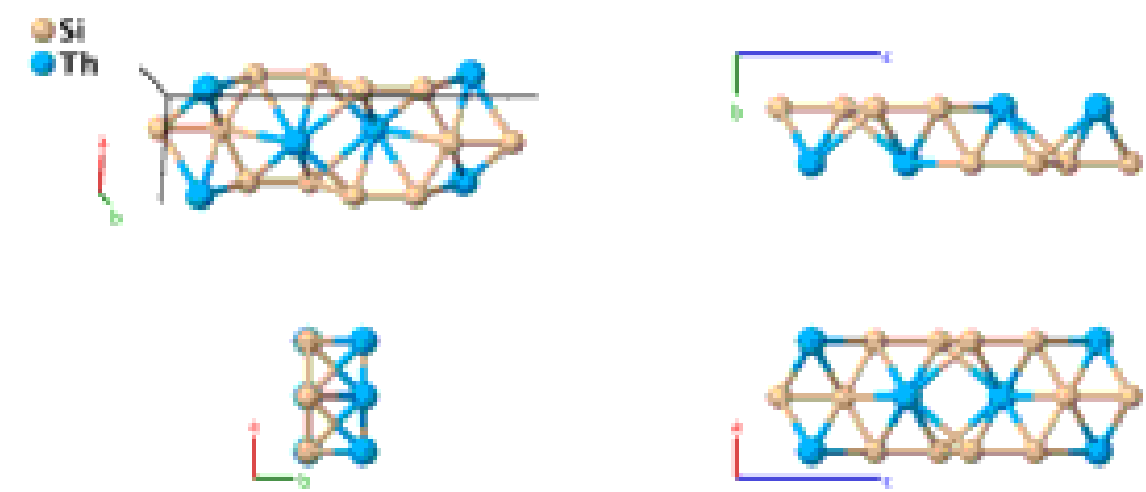

\section{Prototype}

AFLOW prototype label

: $\quad \alpha-\mathrm{ThSi}_{2}$

Strukturbericht designation

: A2B_tI12_141_e_a

Pearson symbol

$C_{c}$

Space group number

Space group symbol

141

AFLOW prototype command

I4 $4_{1} /$ amd

aflow - -proto=A2B_tI12_141_e_a

- params $=a, c / a, z_{2}$

Body-centered Tetragonal primitive vectors:

$$
\begin{aligned}
& \mathbf{a}_{1}=-\frac{1}{2} a \hat{\mathbf{x}}+\frac{1}{2} a \hat{\mathbf{y}}+\frac{1}{2} c \hat{\mathbf{z}} \\
& \mathbf{a}_{2}=\frac{1}{2} a \hat{\mathbf{x}}-\frac{1}{2} a \hat{\mathbf{y}}+\frac{1}{2} c \hat{\mathbf{z}} \\
& \mathbf{a}_{3}=\frac{1}{2} a \hat{\mathbf{x}}+\frac{1}{2} a \hat{\mathbf{y}}-\frac{1}{2} c \hat{\mathbf{z}}
\end{aligned}
$$

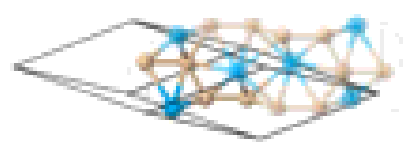

Basis vectors:

Lattice Coordinates

$\mathbf{B}_{1}=$

$\frac{7}{8} \mathbf{a}_{1}+\frac{1}{8} \mathbf{a}_{2}+\frac{3}{4} \mathbf{a}_{3}$

$\mathbf{B}_{2}=\frac{1}{8} \mathbf{a}_{1}+\frac{7}{8} \mathbf{a}_{2}+\frac{1}{4} \mathbf{a}_{3}$

$\mathbf{B}_{3}=\left(\frac{1}{4}+z_{2}\right) \mathbf{a}_{1}+z_{2} \mathbf{a}_{2}+\frac{1}{4} \mathbf{a}_{3}$

$\mathbf{B}_{4}=z_{2} \mathbf{a}_{1}+\left(\frac{1}{4}+z_{2}\right) \mathbf{a}_{2}+\frac{3}{4} \mathbf{a}_{3}$

$\mathbf{B}_{5}=\left(\frac{3}{4}-z_{2}\right) \mathbf{a}_{1}-z_{2} \mathbf{a}_{2}+\frac{3}{4} \mathbf{a}_{3}$

$\mathbf{B}_{6}=-z_{2} \mathbf{a}_{1}+\left(\frac{3}{4}-z_{2}\right) \mathbf{a}_{2}+\frac{1}{4} \mathbf{a}_{3}$

$\begin{array}{cc} & \text { Cartesian Coordinates } \\ = & \frac{3}{4} a \hat{\mathbf{y}}+\frac{1}{8} c \hat{\mathbf{z}} \\ = & \frac{1}{2} a \hat{\mathbf{x}}+\frac{3}{4} a \hat{\mathbf{y}}+\frac{3}{8} c \hat{\mathbf{z}} \\ = & \frac{1}{4} a \hat{\mathbf{y}}+z_{2} c \hat{\mathbf{z}} \\ = & \frac{1}{2} a \hat{\mathbf{x}}+\frac{1}{4} a \hat{\mathbf{y}}+\left(-\frac{1}{4}+z_{2}\right) c \hat{\mathbf{z}} \\ = & \frac{3}{4} a \hat{\mathbf{y}}-z_{2} c \hat{\mathbf{z}} \\ = & \frac{1}{2} a \hat{\mathbf{x}}+\frac{3}{4} a \hat{\mathbf{y}}+\left(\frac{1}{4}-z_{2}\right) c \hat{\mathbf{z}}\end{array}$

Cartesian Coordinates

Wyckoff Position

Atom Type

$(4 a)$

Th

(4a)

Th

(8e)

$\mathrm{Si}$

(8e)

Si

(8e)

Si

(8e)

$\mathrm{Si}$

\section{References:}

- G. Brauer and A. Mitius, Die Kristallstruktur des Thoriumsilicids ThSi, Z. Anorg. Allg. Chem. 249, 325-339 (1942), doi:10.1002/zaac.19422490401.

\section{Found in:}

- W. B. Pearson, The Crystal Chemistry and Physics of Metals and Alloys (Wiley Interscience, New York, London, Sydney, Tornoto, 1972). 
Geometry files:

- CIF: pp. 904

- POSCAR: pp. 904 


\section{S-III Structure: A_tI16_142_f}

05
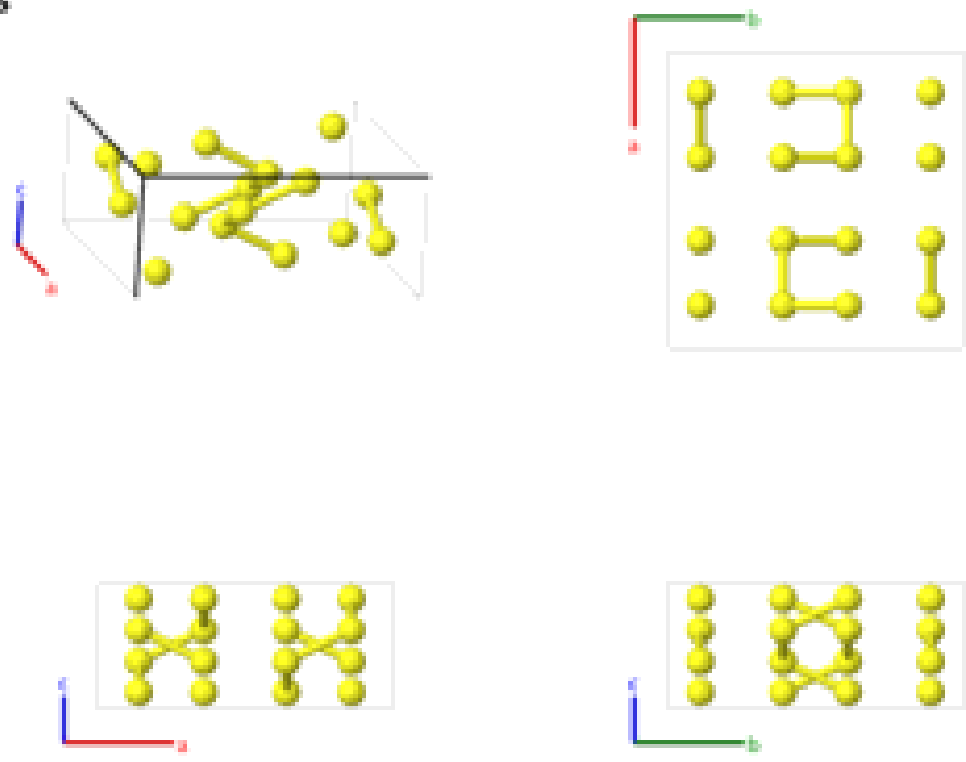

Prototype

AFLOW prototype label

Strukturbericht designation

Pearson symbol

Space group number

Space group symbol

AFLOW prototype command : aflow --proto=A_tI16_142_f

- - params $=a, c / a, x_{1}$

\section{Other elements with this structure:}

- Se (Se-VII, prepared at $450 \mathrm{~K}$ and $20 \mathrm{GPa}$ )

- The S-III phase is found when sulfur is pressurized above $36 \mathrm{GPa}$ at $300 \mathrm{~K}$. At $300 \mathrm{~K}$ it is stable up to $83 \mathrm{GPa}$.

- This data was taken at $12 \mathrm{GPa}$ and $300 \mathrm{~K}$.

Body-centered Tetragonal primitive vectors:

$$
\begin{aligned}
& \mathbf{a}_{1}=-\frac{1}{2} a \hat{\mathbf{x}}+\frac{1}{2} a \hat{\mathbf{y}}+\frac{1}{2} c \hat{\mathbf{z}} \\
& \mathbf{a}_{2}=\frac{1}{2} a \hat{\mathbf{x}}-\frac{1}{2} a \hat{\mathbf{y}}+\frac{1}{2} c \hat{\mathbf{z}} \\
& \mathbf{a}_{3}=\frac{1}{2} a \hat{\mathbf{x}}+\frac{1}{2} a \hat{\mathbf{y}}-\frac{1}{2} c \hat{\mathbf{z}}
\end{aligned}
$$
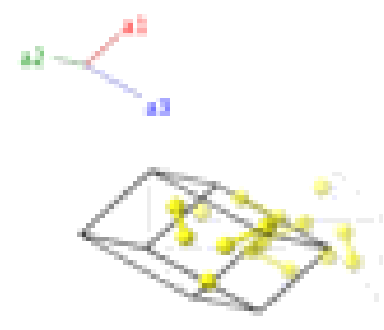
Lattice Coordinates

$$
\begin{gathered}
\mathbf{B}_{1}=\quad\left(\frac{3}{8}+x_{1}\right) \mathbf{a}_{1}+\left(\frac{1}{8}+x_{1}\right) \mathbf{a}_{2} \\
\left(\frac{1}{4}+2 x_{1}\right) \mathbf{a}_{3}
\end{gathered}
$$

$\mathbf{B}_{2}=\left(\frac{3}{8}-x_{1}\right) \mathbf{a}_{1}+\left(\frac{1}{8}-x_{1}\right)$ $\left(\frac{1}{4}-2 x_{1}\right) \mathbf{a}_{3}$

$\mathbf{B}_{3}=\left(\frac{1}{8}+x_{1}\right) \mathbf{a}_{1}+\left(\frac{3}{8}-x_{1}\right) \mathbf{a}_{2}+\frac{3}{4} \mathbf{a}_{3}$

$\mathbf{B}_{4}=\left(\frac{1}{8}-x_{1}\right) \mathbf{a}_{1}+\left(\frac{3}{8}+x_{1}\right) \mathbf{a}_{2}+\frac{3}{4} \mathbf{a}_{3}$

$\mathbf{B}_{5}=$

$\left(\frac{5}{8}-x_{1}\right) \mathbf{a}_{1}+\left(\frac{7}{8}-x_{1}\right) \mathbf{a}_{2}+$ $\left(\frac{3}{4}-2 x_{1}\right) \mathbf{a}_{3}$

$\mathbf{B}_{6}=$

$$
\left(\frac{5}{8}+x_{1}\right) \mathbf{a}_{1}+\left(\frac{7}{8}+x_{1}\right) \mathbf{a}_{2}+
$$
$\left(\frac{3}{4}+2 x_{1}\right) \mathbf{a}_{3}$

$\mathbf{B}_{7}=\left(\frac{7}{8}-x_{1}\right) \mathbf{a}_{1}+\left(\frac{5}{8}+x_{1}\right) \mathbf{a}_{2}+\frac{1}{4} \mathbf{a}_{3}=x_{1} a \hat{\mathbf{x}}+\left(\frac{1}{4}-x_{1}\right) a \hat{\mathbf{y}}+\frac{5}{8} c \hat{\mathbf{z}}$

$\mathbf{B}_{8}=\left(\frac{7}{8}+x_{1}\right) \mathbf{a}_{1}+\left(\frac{5}{8}-x_{1}\right) \mathbf{a}_{2}+\frac{1}{4} \mathbf{a}_{3}=-x_{1} a \hat{\mathbf{x}}+\left(\frac{1}{4}+x_{1}\right) a \hat{\mathbf{y}}+\frac{5}{8} c \hat{\mathbf{z}}$
Wyckoff Position Atom Type

$(16 f)$

(16f)

S

(16f)

S

$(16 f)$

S

S

S

S

S

\section{References:}

- O. Degtyareva, E. Gregoryanz, M. Somayazulu, P. Dera, H. Mao, and R. J. Hemley, Novel chain structures in group VI elements, Nat. Mater. 4, 152-155 (2005), doi:10.1038/nmat1294.

\section{Geometry files:}

- CIF: pp. 904

- POSCAR: pp. 905 


\section{Simpsonite $\left(\mathrm{Ta}_{3} \mathrm{Al}_{4} \mathrm{O}_{13}[\mathrm{OH}]\right)$ Structure: A4B14C3_hP21_143_bd_ac4d_d}
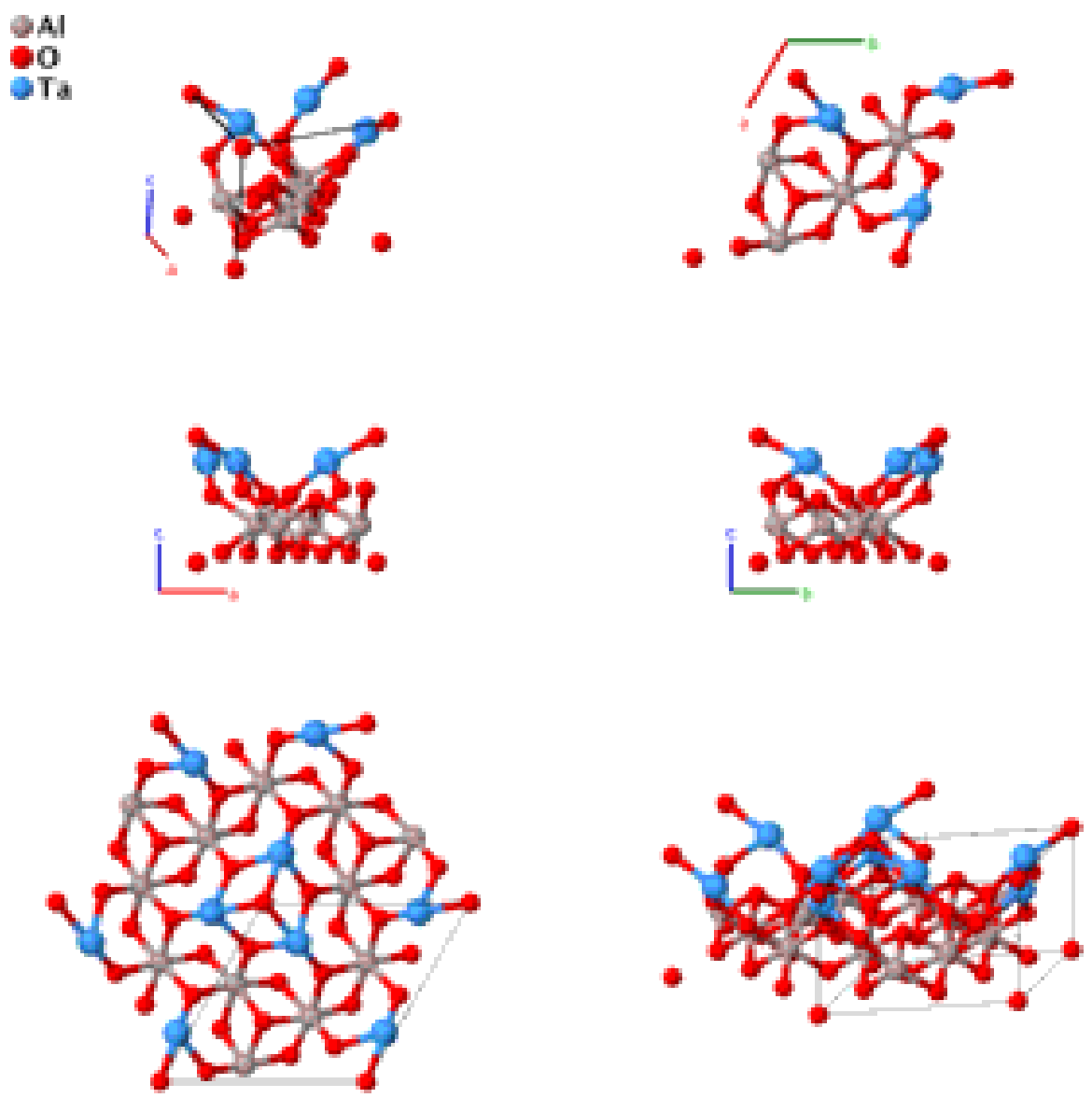

Prototype

AFLOW prototype label

: $\quad \mathrm{Ta}_{3} \mathrm{Al}_{4} \mathrm{O}_{13}[\mathrm{OH}]$

Strukturbericht designation : None

Pearson symbol $\quad: \quad \mathrm{hP} 21$

Space group number : $\quad 143$

Space group symbol $\quad: \quad P 3$

AFLOW prototype command : aflow --proto=A4B14C3_hP21_143_bd_ac4d_d

- params $=a, c / a, z_{1}, z_{2}, z_{3}, x_{4}, y_{4}, z_{4}, x_{5}, y_{5}, z_{5}, x_{6}, y_{6}, z_{6}, x_{7}, y_{7}, z_{7}, x_{8}, y_{8}, z_{8}, x_{9}$, $y_{9}, z_{9}$

- The $\mathrm{OH}$ molecule is centered on the (1c) site; however, it is only listed as $\mathrm{O}$ in this prototype.

\section{Trigonal Hexagonal primitive vectors:}

$$
\begin{array}{ll}
\mathbf{a}_{1}= & \frac{1}{2} a \hat{\mathbf{x}}-\frac{\sqrt{3}}{2} a \hat{\mathbf{y}} \\
\mathbf{a}_{2}= & \frac{1}{2} a \hat{\mathbf{x}}+\frac{\sqrt{3}}{2} a \hat{\mathbf{y}} \\
\mathbf{a}_{3}= & c \hat{\mathbf{z}}
\end{array}
$$
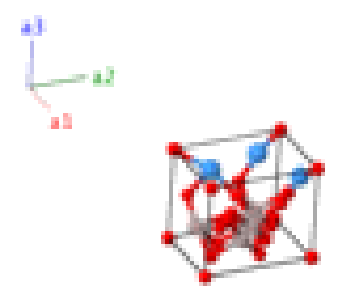


\section{Basis vectors:}

Lattice Coordinates

$\mathbf{B}_{1}=z_{1} \mathbf{a}_{3}=$

$\mathbf{B}_{2}=\frac{1}{3} \mathbf{a}_{1}+\frac{2}{3} \mathbf{a}_{2}+z_{2} \mathbf{a}_{3}$

$\mathbf{B}_{3}=\frac{2}{3} \mathbf{a}_{1}+\frac{1}{3} \mathbf{a}_{2}+z_{3} \mathbf{a}_{3}$

$\mathbf{B}_{4}=x_{4} \mathbf{a}_{1}+y_{4} \mathbf{a}_{2}+z_{4} \mathbf{a}_{3}$

$\mathbf{B}_{5}=-y_{4} \mathbf{a}_{1}+\left(x_{4}-y_{4}\right) \mathbf{a}_{2}+z_{4} \mathbf{a}_{3}=$

$\mathbf{B}_{6}=\left(-x_{4}+y_{4}\right) \mathbf{a}_{1}-x_{4} \mathbf{a}_{2}+z_{4} \mathbf{a}_{3}=$

$\mathbf{B}_{7}=x_{5} \mathbf{a}_{1}+y_{5} \mathbf{a}_{2}+z_{5} \mathbf{a}_{3}=$

$\mathbf{B}_{8}=-y_{5} \mathbf{a}_{1}+\left(x_{5}-y_{5}\right) \mathbf{a}_{2}+z_{5} \mathbf{a}_{3}=$

$\mathbf{B}_{9}=\left(-x_{5}+y_{5}\right) \mathbf{a}_{1}-x_{5} \mathbf{a}_{2}+z_{5} \mathbf{a}_{3}=$

$\mathbf{B}_{10}=x_{6} \mathbf{a}_{1}+y_{6} \mathbf{a}_{2}+z_{6} \mathbf{a}_{3}$

$\mathbf{B}_{11}=-y_{6} \mathbf{a}_{1}+\left(x_{6}-y_{6}\right) \mathbf{a}_{2}+z_{6} \mathbf{a}_{3}=$

$\mathbf{B}_{12}=\left(-x_{6}+y_{6}\right) \mathbf{a}_{1}-x_{6} \mathbf{a}_{2}+z_{6} \mathbf{a}_{3}=$

$\mathbf{B}_{13}=x_{7} \mathbf{a}_{1}+y_{7} \mathbf{a}_{2}+z_{7} \mathbf{a}_{3}$

$\mathbf{B}_{14}=-y_{7} \mathbf{a}_{1}+\left(x_{7}-y_{7}\right) \mathbf{a}_{2}+z_{7} \mathbf{a}_{3}=$

$\mathbf{B}_{15}=\left(-x_{7}+y_{7}\right) \mathbf{a}_{1}-x_{7} \mathbf{a}_{2}+z_{7} \mathbf{a}_{3}=$

$\mathbf{B}_{16}=x_{8} \mathbf{a}_{1}+y_{8} \mathbf{a}_{2}+z_{8} \mathbf{a}_{3}$

$\mathbf{B}_{17}=-y_{8} \mathbf{a}_{1}+\left(x_{8}-y_{8}\right) \mathbf{a}_{2}+z_{8} \mathbf{a}_{3}=$

$\mathbf{B}_{18}=\left(-x_{8}+y_{8}\right) \mathbf{a}_{1}-x_{8} \mathbf{a}_{2}+z_{8} \mathbf{a}_{3}=$

$\mathbf{B}_{19}=x_{9} \mathbf{a}_{1}+y_{9} \mathbf{a}_{2}+z_{9} \mathbf{a}_{3}=$

$\mathbf{B}_{20}=-y_{9} \mathbf{a}_{1}+\left(x_{9}-y_{9}\right) \mathbf{a}_{2}+z_{9} \mathbf{a}_{3}=$

$\mathbf{B}_{21}=\left(-x_{9}+y_{9}\right) \mathbf{a}_{1}-x_{9} \mathbf{a}_{2}+z_{9} \mathbf{a}_{3}=$
Cartesian Coordinates

$=$

$=$

$z_{1} c \hat{\mathbf{z}}$

$=\quad \frac{1}{2} a \hat{\mathbf{x}}-\frac{1}{2 \sqrt{3}} a \hat{\mathbf{y}}+z_{3} c \hat{\mathbf{z}}$

$=\frac{1}{2}\left(x_{4}+y_{4}\right) a \hat{\mathbf{x}}+\frac{\sqrt{3}}{2}\left(-x_{4}+y_{4}\right) a \hat{\mathbf{y}}+z_{4} c \hat{\mathbf{z}}$

$=\left(\frac{1}{2} x_{4}-y_{4}\right) a \hat{\mathbf{x}}+\frac{\sqrt{3}}{2} x_{4} a \hat{\mathbf{y}}+z_{4} c \hat{\mathbf{z}}$

$=\left(-x_{4}+\frac{1}{2} y_{4}\right) a \hat{\mathbf{x}}-\frac{\sqrt{3}}{2} y_{4} a \hat{\mathbf{y}}+z_{4} c \hat{\mathbf{z}}$

$=\frac{1}{2}\left(x_{5}+y_{5}\right) a \hat{\mathbf{x}}+\frac{\sqrt{3}}{2}\left(-x_{5}+y_{5}\right) a \hat{\mathbf{y}}+z_{5} c \hat{\mathbf{z}}$

$=\left(\frac{1}{2} x_{5}-y_{5}\right) a \hat{\mathbf{x}}+\frac{\sqrt{3}}{2} x_{5} a \hat{\mathbf{y}}+z_{5} c \hat{\mathbf{z}}$

$=\left(-x_{5}+\frac{1}{2} y_{5}\right) a \hat{\mathbf{x}}-\frac{\sqrt{3}}{2} y_{5} a \hat{\mathbf{y}}+z_{5} c \hat{\mathbf{z}}$

$=\frac{1}{2}\left(x_{6}+y_{6}\right) a \hat{\mathbf{x}}+\frac{\sqrt{3}}{2}\left(-x_{6}+y_{6}\right) a \hat{\mathbf{y}}+z_{6} c \hat{\mathbf{z}}$

$=\left(\frac{1}{2} x_{6}-y_{6}\right) a \hat{\mathbf{x}}+\frac{\sqrt{3}}{2} x_{6} a \hat{\mathbf{y}}+z_{6} c \hat{\mathbf{z}}$

$=\left(-x_{6}+\frac{1}{2} y_{6}\right) a \hat{\mathbf{x}}-\frac{\sqrt{3}}{2} y_{6} a \hat{\mathbf{y}}+z_{6} c \hat{\mathbf{z}}$

$=\frac{1}{2}\left(x_{7}+y_{7}\right) a \hat{\mathbf{x}}+\frac{\sqrt{3}}{2}\left(-x_{7}+y_{7}\right) a \hat{\mathbf{y}}+z_{7} c \hat{\mathbf{z}}$

$=\left(\frac{1}{2} x_{7}-y_{7}\right) a \hat{\mathbf{x}}+\frac{\sqrt{3}}{2} x_{7} a \hat{\mathbf{y}}+z_{7} c \hat{\mathbf{z}}$

$=\left(-x_{7}+\frac{1}{2} y_{7}\right) a \hat{\mathbf{x}}-\frac{\sqrt{3}}{2} y_{7} a \hat{\mathbf{y}}+z_{7} c \hat{\mathbf{z}}$

$=\frac{1}{2}\left(x_{8}+y_{8}\right) a \hat{\mathbf{x}}+\frac{\sqrt{3}}{2}\left(-x_{8}+y_{8}\right) a \hat{\mathbf{y}}+z_{8} c \hat{\mathbf{z}}$

$=\left(\frac{1}{2} x_{8}-y_{8}\right) a \hat{\mathbf{x}}+\frac{\sqrt{3}}{2} x_{8} a \hat{\mathbf{y}}+z_{8} c \hat{\mathbf{z}}$

$=\left(-x_{8}+\frac{1}{2} y_{8}\right) a \hat{\mathbf{x}}-\frac{\sqrt{3}}{2} y_{8} a \hat{\mathbf{y}}+z_{8} c \hat{\mathbf{z}}$

$=\frac{1}{2}\left(x_{9}+y_{9}\right) a \hat{\mathbf{x}}+\frac{\sqrt{3}}{2}\left(-x_{9}+y_{9}\right) a \hat{\mathbf{y}}+z_{9} c \hat{\mathbf{z}}$

$\left(\frac{1}{2} x_{9}-y_{9}\right) a \hat{\mathbf{x}}+\frac{\sqrt{3}}{2} x_{9} a \hat{\mathbf{y}}+z_{9} c \hat{\mathbf{z}}$

$\left(-x_{9}+\frac{1}{2} y_{9}\right) a \hat{\mathbf{x}}-\frac{\sqrt{3}}{2} y_{9} a \hat{\mathbf{y}}+z_{9} c \hat{\mathbf{z}}$
Wyckoff Position Atom Type

(1a)

O I

Al I

O II
Al II

Al II

Al II

O III

O III

O III

O IV

O IV

O IV

O V

O V

O V

O VI

O VI

O VI

$\mathrm{Ta}$

$\mathrm{Ta}$

$\mathrm{Ta}$

\section{References:}

- T. S. Ercit, F. C. Hawthorne, and P. Cerny, The crystal structure of alumotantite; its relation to the structures of simpsonite and the $(\mathrm{Al}, \mathrm{Ga})(\mathrm{Ta}, \mathrm{Nb}) \mathrm{O}_{4}$ compounds, Can. Mineral. 30, 653-662 (1992).

\section{Found in:}

- P. Villars and K. Cenzual, Pearson's Crystal Data - Crystal Structure Database for Inorganic Compounds, ASM International (2013).

\section{Geometry files:}

- CIF: pp. 905

- POSCAR: pp. 905 


\section{$\mathrm{ScRh}_{6} \mathrm{P}_{4}$ Structure: A4B6C_hP11_143_bd_2d_a}
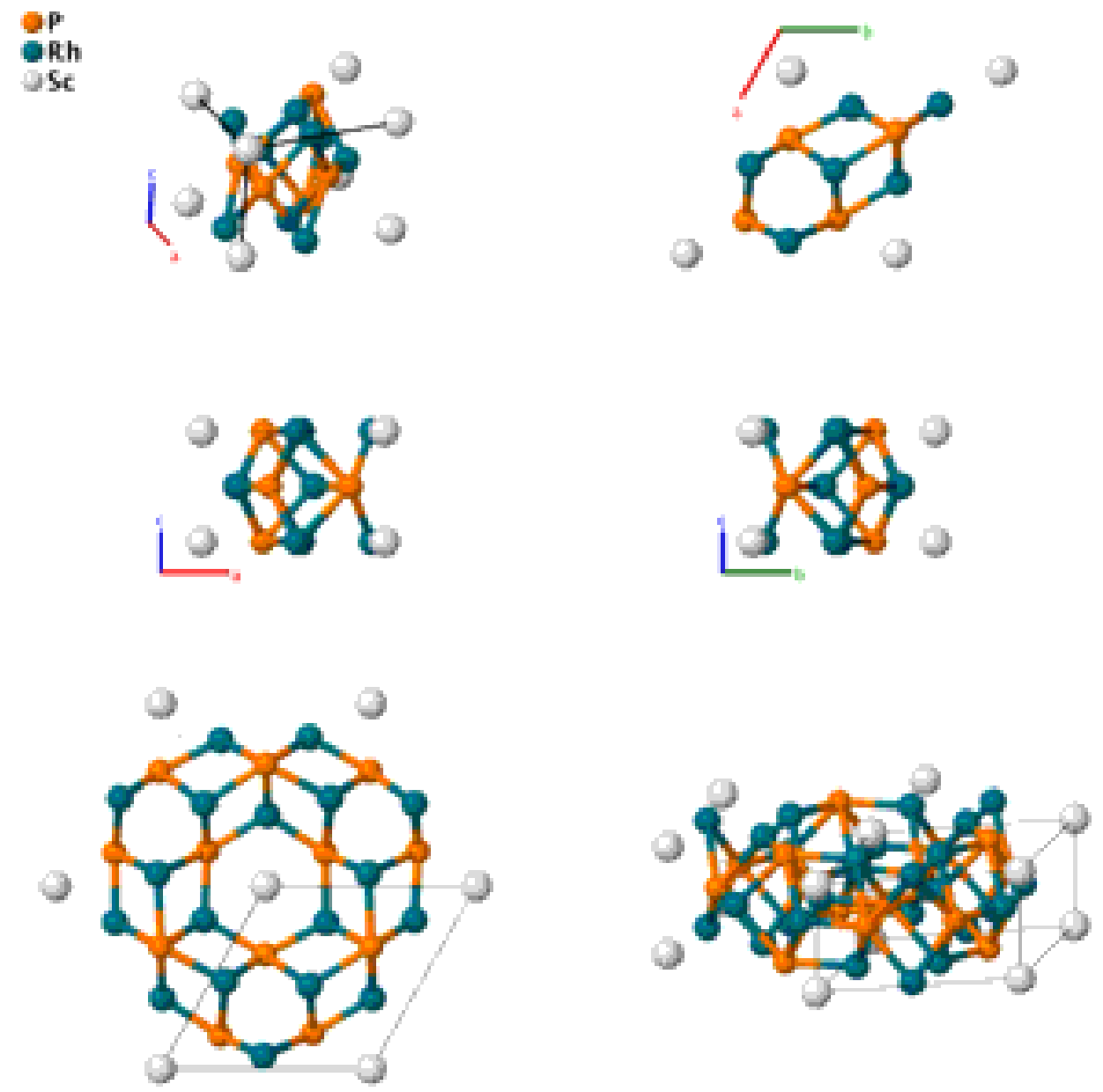

\section{Prototype}

AFLOW prototype label

$: \quad \mathrm{ScRh}_{6} \mathrm{P}_{4}$

Strukturbericht designation

: A4B6C_hP11_143_bd_2d_a

Pearson symbol

: None

Space group number

: $\quad \mathrm{hP} 11$

Space group symbol

: 143

AFLOW prototype command

: $\quad P 3$

\footnotetext{
aflow - -proto=A4B6C_hP11_143_bd_2d_a

- params $=a, c / a, z_{1}, z_{2}, x_{3}, y_{3}, z_{3}, x_{4}, y_{4}, z_{4}, x_{5}, y_{5}, z_{5}$
}

- While FINDSYM identifies space group \#143 for this structure (consistent with the reference), AFLOW-SYM and Platon identify \#174 and \#187, respectively. Lowering the tolerance value for AFLOW-SYM resolves the expected space group \#143. Space groups \#143, \#174, and \#187 are reasonable classifications since they are commensurate with subgroup relations. 


\section{Trigonal Hexagonal primitive vectors:}

$$
\begin{array}{ll}
\mathbf{a}_{1}= & \frac{1}{2} a \hat{\mathbf{x}}-\frac{\sqrt{3}}{2} a \hat{\mathbf{y}} \\
\mathbf{a}_{2}= & \frac{1}{2} a \hat{\mathbf{x}}+\frac{\sqrt{3}}{2} a \hat{\mathbf{y}} \\
\mathbf{a}_{3}= & c \hat{\mathbf{z}}
\end{array}
$$

\begin{tabular}{|c|c|c|c|c|c|c|}
\hline & & Lattice Coordinates & & Cartesian Coordinates & Wyckoff Position & Atom Type \\
\hline $\mathbf{B}_{1}$ & $=$ & $z_{1} \mathbf{a}_{3}$ & $=$ & $z_{1} c \hat{\mathbf{z}}$ & $(1 a)$ & $\mathrm{Sc}$ \\
\hline $\mathbf{B}_{2}$ & $=$ & $\frac{1}{3} \mathbf{a}_{1}+\frac{2}{3} \mathbf{a}_{2}+z_{2} \mathbf{a}_{3}$ & $=$ & $\frac{1}{2} a \hat{\mathbf{x}}+\frac{1}{2 \sqrt{3}} a \hat{\mathbf{y}}+z_{2} c \hat{\mathbf{z}}$ & $(1 b)$ & P I \\
\hline $\mathbf{B}_{3}$ & $=$ & $x_{3} \mathbf{a}_{1}+y_{3} \mathbf{a}_{2}+z_{3} \mathbf{a}_{3}$ & $=$ & $\frac{1}{2}\left(x_{3}+y_{3}\right) a \hat{\mathbf{x}}+\frac{\sqrt{3}}{2}\left(-x_{3}+y_{3}\right) a \hat{\mathbf{y}}+z_{3} c \hat{\mathbf{z}}$ & $(3 d)$ & P II \\
\hline $\mathbf{B}_{4}$ & $=$ & $-y_{3} \mathbf{a}_{1}+\left(x_{3}-y_{3}\right) \mathbf{a}_{2}+z_{3} \mathbf{a}_{3}$ & $=$ & $\left(\frac{1}{2} x_{3}-y_{3}\right) a \hat{\mathbf{x}}+\frac{\sqrt{3}}{2} x_{3} a \hat{\mathbf{y}}+z_{3} c \hat{\mathbf{z}}$ & $(3 d)$ & P II \\
\hline $\mathbf{B}_{5}$ & $=$ & $\left(-x_{3}+y_{3}\right) \mathbf{a}_{1}-x_{3} \mathbf{a}_{2}+z_{3} \mathbf{a}_{3}$ & $=$ & $\left(-x_{3}+\frac{1}{2} y_{3}\right) a \hat{\mathbf{x}}-\frac{\sqrt{3}}{2} y_{3} a \hat{\mathbf{y}}+z_{3} c \hat{\mathbf{z}}$ & $(3 d)$ & P II \\
\hline $\mathbf{B}_{6}$ & $=$ & $x_{4} \mathbf{a}_{1}+y_{4} \mathbf{a}_{2}+z_{4} \mathbf{a}_{3}$ & $=$ & $\frac{1}{2}\left(x_{4}+y_{4}\right) a \hat{\mathbf{x}}+\frac{\sqrt{3}}{2}\left(-x_{4}+y_{4}\right) a \hat{\mathbf{y}}+z_{4} c \hat{\mathbf{z}}$ & $(3 d)$ & $\mathrm{Rh} I$ \\
\hline $\mathbf{B}_{7}$ & $=$ & $-y_{4} \mathbf{a}_{1}+\left(x_{4}-y_{4}\right) \mathbf{a}_{2}+z_{4} \mathbf{a}_{3}$ & $=$ & $\left(\frac{1}{2} x_{4}-y_{4}\right) a \hat{\mathbf{x}}+\frac{\sqrt{3}}{2} x_{4} a \hat{\mathbf{y}}+z_{4} c \hat{\mathbf{z}}$ & $(3 d)$ & $\mathrm{Rh} I$ \\
\hline $\mathbf{B}_{8}$ & $=$ & $\left(-x_{4}+y_{4}\right) \mathbf{a}_{1}-x_{4} \mathbf{a}_{2}+z_{4} \mathbf{a}_{3}$ & $=$ & $\left(-x_{4}+\frac{1}{2} y_{4}\right) a \hat{\mathbf{x}}-\frac{\sqrt{3}}{2} y_{4} a \hat{\mathbf{y}}+z_{4} c \hat{\mathbf{z}}$ & $(3 d)$ & $\mathrm{Rh} I$ \\
\hline $\mathbf{B}_{9}$ & $=$ & $x_{5} \mathbf{a}_{1}+y_{5} \mathbf{a}_{2}+z_{5} \mathbf{a}_{3}$ & $=$ & $\frac{1}{2}\left(x_{5}+y_{5}\right) a \hat{\mathbf{x}}+\frac{\sqrt{3}}{2}\left(-x_{5}+y_{5}\right) a \hat{\mathbf{y}}+z_{5} c \hat{\mathbf{z}}$ & $(3 d)$ & $\mathrm{Rh}$ II \\
\hline $\mathbf{B}_{10}$ & $=$ & $-y_{5} \mathbf{a}_{1}+\left(x_{5}-y_{5}\right) \mathbf{a}_{2}+z_{5} \mathbf{a}_{3}$ & $=$ & $\left(\frac{1}{2} x_{5}-y_{5}\right) a \hat{\mathbf{x}}+\frac{\sqrt{3}}{2} x_{5} a \hat{\mathbf{y}}+z_{5} c \hat{\mathbf{z}}$ & $(3 d)$ & $\mathrm{Rh}$ II \\
\hline $\mathbf{B}_{11}$ & $=$ & $\left(-x_{5}+y_{5}\right) \mathbf{a}_{1}-x_{5} \mathbf{a}_{2}+z_{5} \mathbf{a}_{3}$ & $=$ & $\left(-x_{5}+\frac{1}{2} y_{5}\right) a \hat{\mathbf{x}}-\frac{\sqrt{3}}{2} y_{5} a \hat{\mathbf{y}}+z_{5} c \hat{\mathbf{z}}$ & $(3 d)$ & $\mathrm{Rh}$ II \\
\hline
\end{tabular}

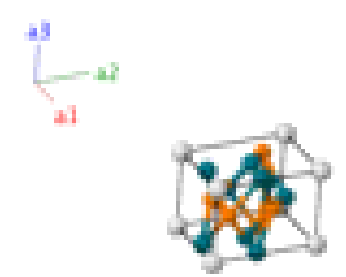

\section{Basis vectors:}

\section{References:}

- U. Pfannenschmidt, U. C. Rodewald, and R. Pöttgen, Bismuth flux crystal growth of RERh $P_{4}(R E=S c, Y b, L u):$ new phosphides with a superstructure of the $\mathrm{LiCo}_{6} \mathrm{P}_{4}$ type, Monatsh. Chem. 142, 219-224 (2011), doi:10.1007/s00706-011-0450-5.

- H. T. Stokes and D. M. Hatch, FINDSYM: Program for identifying the space group symmetry of a crystal, J. Appl. Crystallogr. 38, 237-238 (2005), doi:10.1107/S0021889804031528.

- D. Hicks, C. Oses, E. Gossett, G. Gomez, R. H. Taylor, C. Toher, M. J. Mehl, O. Levy, and S. Curtarolo, AFLOW-SYM: platform for the complete, automatic and self-consistent symmetry analysis of crystals, Acta Crystallogr. Sect. A 74, 184-203 (2018), doi:10.1107/S2053273318003066.

- A. L. Spek, Single-crystal structure validation with the program PLATON, J. Appl. Crystallogr. 36, 7-13 (2003), doi:10.1107/S0021889802022112.

\section{Found in:}

- P. Villars and K. Cenzual, Pearson's Crystal Data - Crystal Structure Database for Inorganic Compounds, ASM International (2013).

\section{Geometry files:}

- CIF: pp. 905

- POSCAR: pp. 906 


\section{$\mathrm{MoS}_{2}$ Structure: AB2_hP12_143_cd_ab2d}
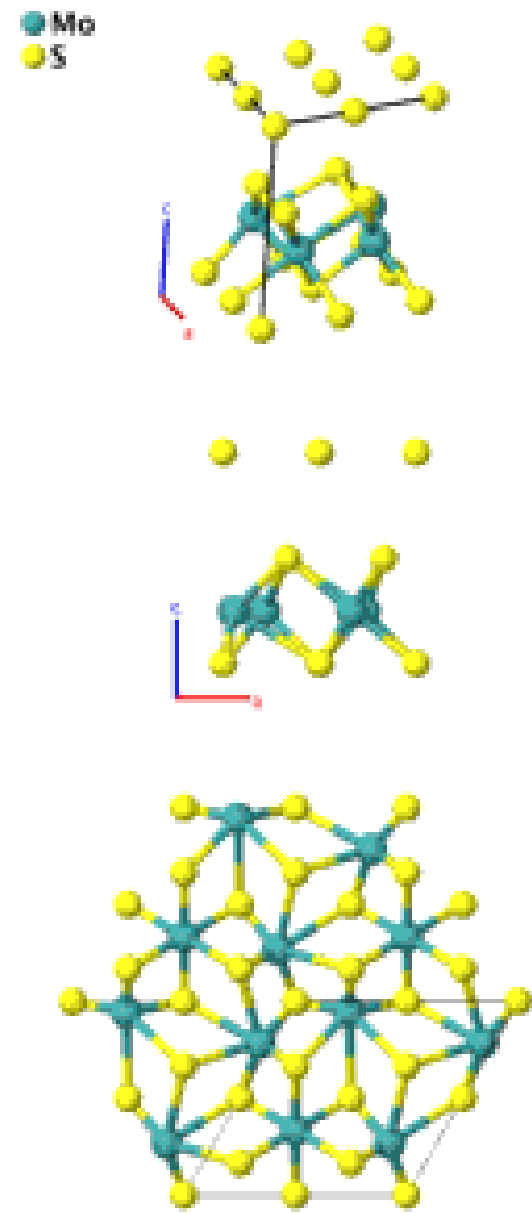
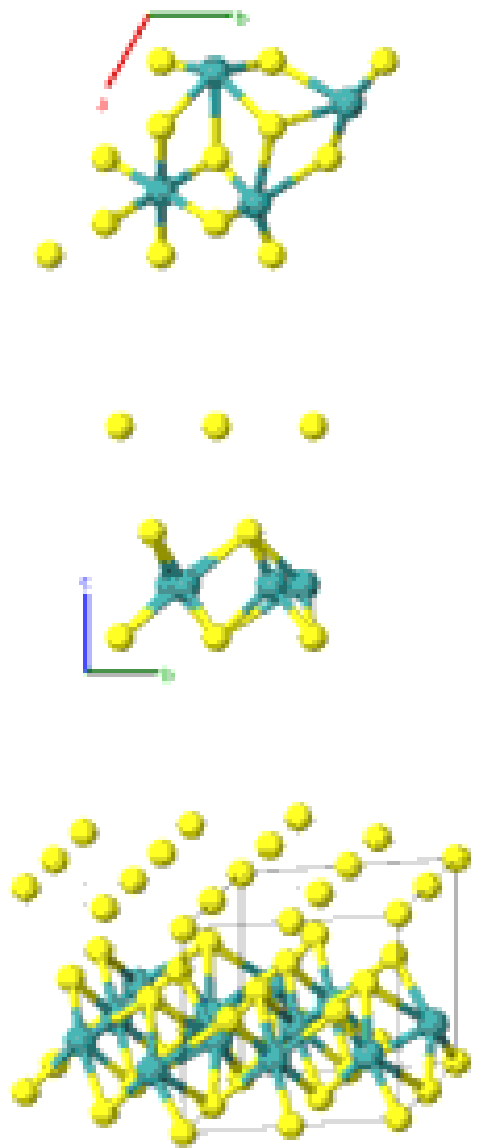

Prototype

AFLOW prototype label

Strukturbericht designation

Pearson symbol

Space group number

Space group symbol

AFLOW prototype command : aflow --proto=AB2_hP12_143_cd_ab2d

- -params $=a, c / a, z_{1}, z_{2}, z_{3}, x_{4}, y_{4}, z_{4}, x_{5}, y_{5}, z_{5}, x_{6}, y_{6}, z_{6}$

Trigonal Hexagonal primitive vectors:

$$
\begin{array}{ll}
\mathbf{a}_{1}= & \frac{1}{2} a \hat{\mathbf{x}}-\frac{\sqrt{3}}{2} a \hat{\mathbf{y}} \\
\mathbf{a}_{2}= & \frac{1}{2} a \hat{\mathbf{x}}+\frac{\sqrt{3}}{2} a \hat{\mathbf{y}} \\
\mathbf{a}_{3}= & c \hat{\mathbf{z}}
\end{array}
$$

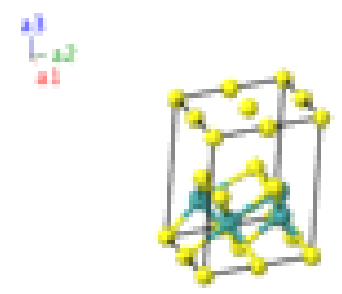

Basis vectors: 


$$
\begin{array}{llclc}
\mathbf{B}_{1}= & z_{1} \mathbf{a}_{3} & = & z_{1} c \hat{\mathbf{z}} \\
\mathbf{B}_{2}= & \frac{1}{3} \mathbf{a}_{1}+\frac{2}{3} \mathbf{a}_{2}+z_{2} \mathbf{a}_{3} & = & \frac{1}{2} a \hat{\mathbf{x}}+\frac{1}{2 \sqrt{3}} a \hat{\mathbf{y}}+z_{2} c \hat{\mathbf{z}} \\
\mathbf{B}_{3}= & \frac{2}{3} \mathbf{a}_{1}+\frac{1}{3} \mathbf{a}_{2}+z_{3} \mathbf{a}_{3} & = & \frac{1}{2} a \hat{\mathbf{x}}-\frac{1}{2 \sqrt{3}} a \hat{\mathbf{y}}+z_{3} c \hat{\mathbf{z}} \\
\mathbf{B}_{4}= & x_{4} \mathbf{a}_{1}+y_{4} \mathbf{a}_{2}+z_{4} \mathbf{a}_{3} & = & \frac{1}{2}\left(x_{4}+y_{4}\right) a \hat{\mathbf{x}}+\frac{\sqrt{3}}{2}\left(-x_{4}+y_{4}\right) a \hat{\mathbf{y}}+z_{4} c \hat{\mathbf{z}} \\
\mathbf{B}_{5}= & -y_{4} \mathbf{a}_{1}+\left(x_{4}-y_{4}\right) \mathbf{a}_{2}+z_{4} \mathbf{a}_{3} & = & \left(\frac{1}{2} x_{4}-y_{4}\right) a \hat{\mathbf{x}}+\frac{\sqrt{3}}{2} x_{4} a \hat{\mathbf{y}}+z_{4} c \hat{\mathbf{z}} \\
\mathbf{B}_{6}= & \left(-x_{4}+y_{4}\right) \mathbf{a}_{1}-x_{4} \mathbf{a}_{2}+z_{4} \mathbf{a}_{3} & = & \left(-x_{4}+\frac{1}{2} y_{4}\right) a \hat{\mathbf{x}}-\frac{\sqrt{3}}{2} y_{4} a \hat{\mathbf{y}}+z_{4} c \hat{\mathbf{z}} \\
\mathbf{B}_{7}= & x_{5} \mathbf{a}_{1}+y_{5} \mathbf{a}_{2}+z_{5} \mathbf{a}_{3} & = & \frac{1}{2}\left(x_{5}+y_{5}\right) a \hat{\mathbf{x}}+\frac{\sqrt{3}}{2}\left(-x_{5}+y_{5}\right) a \hat{\mathbf{y}}+z_{5} c \hat{\mathbf{z}} \\
\mathbf{B}_{8}= & -y_{5} \mathbf{a}_{1}+\left(x_{5}-y_{5}\right) \mathbf{a}_{2}+z_{5} \mathbf{a}_{3}= & \left(\frac{1}{2} x_{5}-y_{5}\right) a \hat{\mathbf{x}}+\frac{\sqrt{3}}{2} x_{5} a \hat{\mathbf{y}}+z_{5} c \hat{\mathbf{z}} \\
\mathbf{B}_{9}= & \left(-x_{5}+y_{5}\right) \mathbf{a}_{1}-x_{5} \mathbf{a}_{2}+z_{5} \mathbf{a}_{3}= & \left(-x_{5}+\frac{1}{2} y_{5}\right) a \hat{\mathbf{x}}-\frac{\sqrt{3}}{2} y_{5} a \hat{\mathbf{y}}+z_{5} c \hat{\mathbf{z}} \\
\mathbf{B}_{10}= & x_{6} \mathbf{a}_{1}+y_{6} \mathbf{a}_{2}+z_{6} \mathbf{a}_{3} & = & \frac{1}{2}\left(x_{6}+y_{6}\right) a \hat{\mathbf{x}}+\frac{\sqrt{3}}{2}\left(-x_{6}+y_{6}\right) a \hat{\mathbf{y}}+z_{6} c \hat{\mathbf{z}} \\
\mathbf{B}_{11}= & -y_{6} \mathbf{a}_{1}+\left(x_{6}-y_{6}\right) \mathbf{a}_{2}+z_{6} \mathbf{a}_{3}= & & \left(\frac{1}{2} x_{6}-y_{6}\right) a \hat{\mathbf{x}}+\frac{\sqrt{3}}{2} x_{6} a \hat{\mathbf{y}}+z_{6} c \hat{\mathbf{z}} \\
\mathbf{B}_{12}= & \left(-x_{6}+y_{6}\right) \mathbf{a}_{1}-x_{6} \mathbf{a}_{2}+z_{6} \mathbf{a}_{3} & = & \left(-x_{6}+\frac{1}{2} y_{6}\right) a \hat{\mathbf{x}}-\frac{\sqrt{3}}{2} y_{6} a \hat{\mathbf{y}}+z_{6} c \hat{\mathbf{z}}
\end{array}
$$

Mo I

Mo II

Mo II

Mo II

S III

S III

S III

S IV

S IV

S IV

\section{References:}

- K. E. Dungey, M. D. Curtis, and J. E. Penner-Hahn, Structural characterization and thermal stability of $\mathrm{MoS}_{2}$ intercalation compounds, Chem. Mater. 10, 2152-2161 (1998), doi:10.1021/cm980034u.

\section{Found in:}

- P. Villars and K. Cenzual, Pearson's Crystal Data - Crystal Structure Database for Inorganic Compounds, ASM International (2013).

\section{Geometry files:}

- CIF: pp. 906

- POSCAR: pp. 906 
$\mathrm{IrGe}_{4}$ Structure: A4B_hP15_144_4a_a
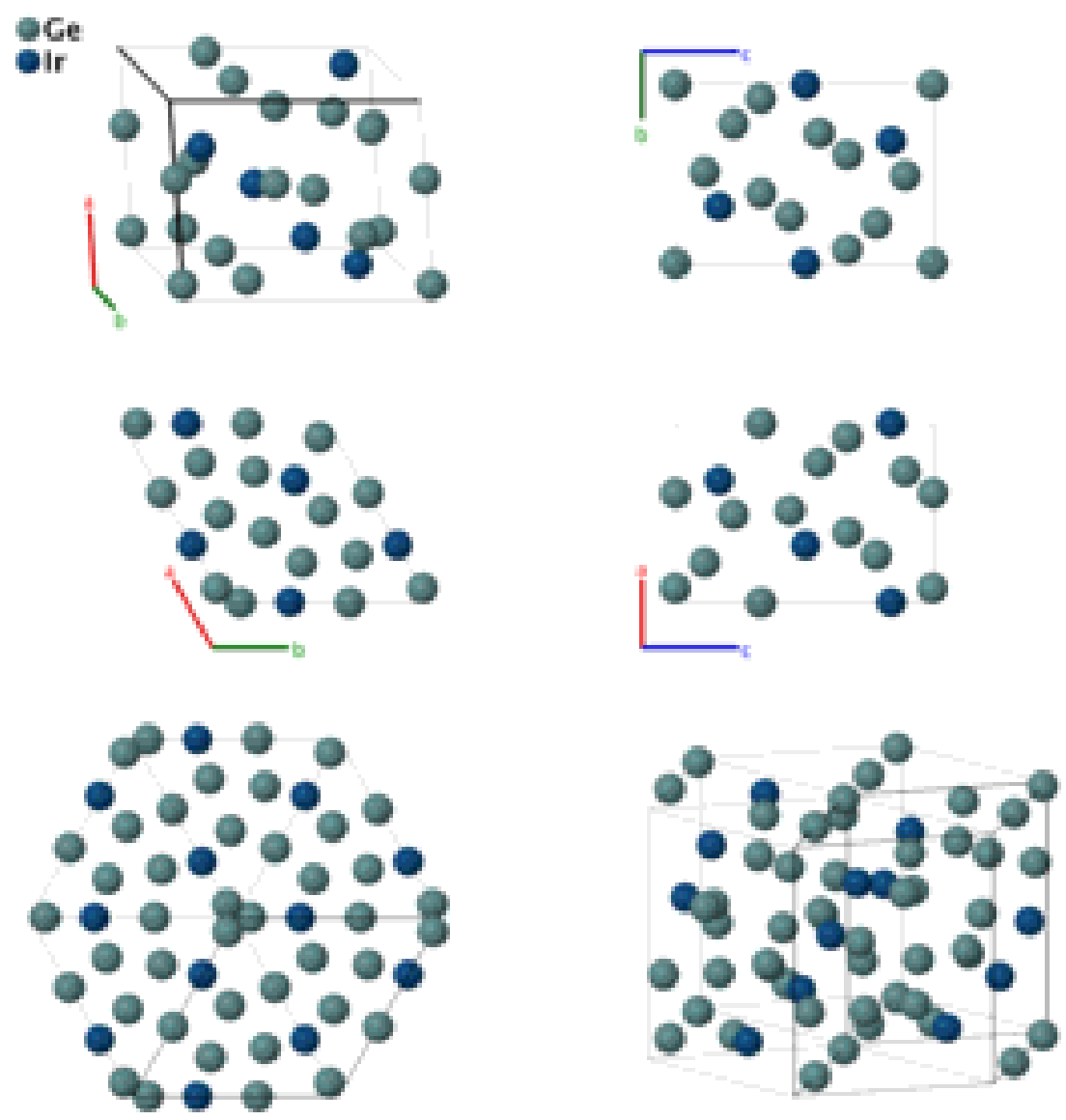

Prototype

$: \quad \mathrm{IrGe}_{4}$

AFLOW prototype label

: A4B_hP15_144_4a_a

Strukturbericht designation

Pearson symbol

: None

Space group number

: $\mathrm{hP} 15$

Space group symbol

: 144

AFLOW prototype command

: $P 3_{1}$

aflow --proto $=A 4 B_{-}$hP15_144_4a_a

--params $=a, c / a, x_{1}, y_{1}, z_{1}, x_{2}, y_{2}, z_{2}, x_{3}, y_{3}, z_{3}, x_{4}, y_{4}, z_{4}, x_{5}, y_{5}, z_{5}$

Trigonal Hexagonal primitive vectors:

$$
\begin{array}{ll}
\mathbf{a}_{1}= & \frac{1}{2} a \hat{\mathbf{x}}-\frac{\sqrt{3}}{2} a \hat{\mathbf{y}} \\
\mathbf{a}_{2}= & \frac{1}{2} a \hat{\mathbf{x}}+\frac{\sqrt{3}}{2} a \hat{\mathbf{y}} \\
\mathbf{a}_{3}= & c \hat{\mathbf{z}}
\end{array}
$$

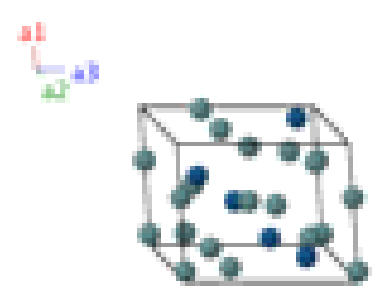

Basis vectors: 


\begin{tabular}{|c|c|c|c|c|c|c|}
\hline $\mathbf{B}_{1}$ & $=$ & $x_{1} \mathbf{a}_{1}+y_{1} \mathbf{a}_{2}+z_{1} \mathbf{a}_{3}$ & $=$ & $\begin{array}{c}\frac{1}{2}\left(x_{1}+y_{1}\right) a \hat{\mathbf{x}}+ \\
\sqrt{3}\left(-x_{1}+y_{1}\right) a \hat{\mathbf{v}}+71 \hat{\mathbf{z}}\end{array}$ & $(3 a)$ & Ge I \\
\hline $\mathbf{B}_{2}$ & $=$ & $-y_{1} \mathbf{a}_{1}+\left(x_{1}-y_{1}\right) \mathbf{a}_{2}+\left(\frac{1}{3}+z_{1}\right) \mathbf{a}_{3}$ & $=$ & $\begin{array}{c}\left(\frac{1}{2} x_{1}-y_{1}\right) a \hat{\mathbf{x}}+\frac{\sqrt{3}}{2} x_{1} a \hat{\mathbf{y}}+ \\
\left(\frac{1}{3}+z_{1}\right) c \hat{\mathbf{z}}\end{array}$ & $(3 a)$ & Ge I \\
\hline $\mathbf{B}_{3}$ & $=$ & $\left(-x_{1}+y_{1}\right) \mathbf{a}_{1}-x_{1} \mathbf{a}_{2}+\left(\frac{2}{3}+z_{1}\right) \mathbf{a}_{3}$ & $=$ & $\begin{array}{c}\left(-x_{1}+\frac{1}{2} y_{1}\right) a \hat{\mathbf{x}}-\frac{\sqrt{3}}{2} y_{1} a \hat{\mathbf{y}}+ \\
\left(\frac{2}{3}+z_{1}\right) c \hat{\mathbf{z}}\end{array}$ & $(3 a)$ & Ge I \\
\hline $\mathbf{B}_{4}$ & $=$ & $x_{2} \mathbf{a}_{1}+y_{2} \mathbf{a}_{2}+z_{2} \mathbf{a}_{3}$ & $=$ & $\begin{array}{c}\frac{1}{2}\left(x_{2}+y_{2}\right) a \hat{\mathbf{x}}+ \\
\frac{\sqrt{3}}{2}\left(-x_{2}+y_{2}\right) a \hat{\mathbf{y}}+z_{2} c \hat{\mathbf{z}}\end{array}$ & $(3 a)$ & Ge II \\
\hline $\mathbf{B}_{5}$ & $=$ & $-y_{2} \mathbf{a}_{1}+\left(x_{2}-y_{2}\right) \mathbf{a}_{2}+\left(\frac{1}{3}+z_{2}\right) \mathbf{a}_{3}$ & $=$ & $\begin{array}{c}\left(\frac{1}{2} x_{2}-y_{2}\right) a \hat{\mathbf{x}}+\frac{\sqrt{3}}{2} x_{2} a \hat{\mathbf{y}}+ \\
\left(\frac{1}{3}+z_{2}\right) c \hat{\mathbf{z}}\end{array}$ & $(3 a)$ & Ge II \\
\hline $\mathbf{B}_{6}$ & $=$ & $\left(-x_{2}+y_{2}\right) \mathbf{a}_{1}-x_{2} \mathbf{a}_{2}+\left(\frac{2}{3}+z_{2}\right) \mathbf{a}_{3}$ & $=$ & $\begin{array}{c}\left(-x_{2}+\frac{1}{2} y_{2}\right) a \hat{\mathbf{x}}-\frac{\sqrt{3}}{2} y_{2} a \hat{\mathbf{y}}+ \\
\left(\frac{2}{3}+z_{2}\right) c \hat{\mathbf{z}}\end{array}$ & $(3 a)$ & Ge II \\
\hline $\mathbf{B}_{7}$ & $=$ & $x_{3} \mathbf{a}_{1}+y_{3} \mathbf{a}_{2}+z_{3} \mathbf{a}_{3}$ & $=$ & $\begin{array}{c}\frac{1}{2}\left(x_{3}+y_{3}\right) a \hat{\mathbf{x}}+ \\
\frac{\sqrt{3}}{2}\left(-x_{3}+y_{3}\right) a \hat{\mathbf{y}}+z_{3} c \hat{\mathbf{z}}\end{array}$ & $(3 a)$ & Ge III \\
\hline $\mathbf{B}_{8}$ & $=$ & $-y_{3} \mathbf{a}_{1}+\left(x_{3}-y_{3}\right) \mathbf{a}_{2}+\left(\frac{1}{3}+z_{3}\right) \mathbf{a}_{3}$ & $=$ & $\begin{array}{c}\left(\frac{1}{2} x_{3}-y_{3}\right) a \hat{\mathbf{x}}+\frac{\sqrt{3}}{2} x_{3} a \hat{\mathbf{y}}+ \\
\left(\frac{1}{3}+z_{3}\right) c \hat{\mathbf{z}}\end{array}$ & $(3 a)$ & Ge III \\
\hline $\mathbf{B}_{9}$ & $=$ & $\left(-x_{3}+y_{3}\right) \mathbf{a}_{1}-x_{3} \mathbf{a}_{2}+\left(\frac{2}{3}+z_{3}\right) \mathbf{a}_{3}$ & $=$ & $\begin{array}{c}\left(-x_{3}+\frac{1}{2} y_{3}\right) a \hat{\mathbf{x}}-\frac{\sqrt{3}}{2} y_{3} a \hat{\mathbf{y}}+ \\
\left(\frac{2}{3}+z_{3}\right) c \hat{\mathbf{z}}\end{array}$ & $(3 a)$ & Ge III \\
\hline $\mathbf{B}_{10}$ & $=$ & $x_{4} \mathbf{a}_{1}+y_{4} \mathbf{a}_{2}+z_{4} \mathbf{a}_{3}$ & $=$ & $\begin{array}{c}\frac{1}{2}\left(x_{4}+y_{4}\right) a \hat{\mathbf{x}}+ \\
\frac{\sqrt{3}}{2}\left(-x_{4}+y_{4}\right) a \hat{\mathbf{y}}+z_{4} c \hat{\mathbf{z}}\end{array}$ & $(3 a)$ & Ge IV \\
\hline $\mathbf{B}_{11}$ & $=$ & $-y_{4} \mathbf{a}_{1}+\left(x_{4}-y_{4}\right) \mathbf{a}_{2}+\left(\frac{1}{3}+z_{4}\right) \mathbf{a}_{3}$ & $=$ & $\begin{array}{c}\left(\frac{1}{2} x_{4}-y_{4}\right) a \hat{\mathbf{x}}+\frac{\sqrt{3}}{2} x_{4} a \hat{\mathbf{y}}+ \\
\left(\frac{1}{3}+z_{4}\right) c \hat{\mathbf{z}}\end{array}$ & $(3 a)$ & Ge IV \\
\hline $\mathbf{B}_{12}$ & $=$ & $\left(-x_{4}+y_{4}\right) \mathbf{a}_{1}-x_{4} \mathbf{a}_{2}+\left(\frac{2}{3}+z_{4}\right) \mathbf{a}_{3}$ & $=$ & $\begin{array}{c}\left(-x_{4}+\frac{1}{2} y_{4}\right) a \hat{\mathbf{x}}-\frac{\sqrt{3}}{2} y_{4} a \hat{\mathbf{y}}+ \\
\left(\frac{2}{3}+z_{4}\right) c \hat{\mathbf{z}}\end{array}$ & $(3 a)$ & Ge IV \\
\hline $\mathbf{B}_{13}$ & $=$ & $x_{5} \mathbf{a}_{1}+y_{5} \mathbf{a}_{2}+z_{5} \mathbf{a}_{3}$ & $=$ & $\begin{array}{c}\frac{1}{2}\left(x_{5}+y_{5}\right) a \hat{\mathbf{x}}+ \\
\frac{\sqrt{3}}{2}\left(-x_{5}+y_{5}\right) a \hat{\mathbf{y}}+z_{5} c \hat{\mathbf{z}}\end{array}$ & $(3 a)$ & Ir \\
\hline $\mathbf{B}_{14}$ & $=$ & $-y_{5} \mathbf{a}_{1}+\left(x_{5}-y_{5}\right) \mathbf{a}_{2}+\left(\frac{1}{3}+z_{5}\right) \mathbf{a}_{3}$ & $=$ & $\begin{array}{c}\left(\frac{1}{2} x_{5}-y_{5}\right) a \hat{\mathbf{x}}+\frac{\sqrt{3}}{2} x_{5} a \hat{\mathbf{y}}+ \\
\left(\frac{1}{3}+z_{5}\right) c \hat{\mathbf{z}}\end{array}$ & $(3 a)$ & $\mathrm{Ir}$ \\
\hline $\mathbf{B}_{15}$ & $=$ & $\left(-x_{5}+y_{5}\right) \mathbf{a}_{1}-x_{5} \mathbf{a}_{2}+\left(\frac{2}{3}+z_{5}\right) \mathbf{a}_{3}$ & $=$ & $\begin{array}{c}\left(-x_{5}+\frac{1}{2} y_{5}\right) a \hat{\mathbf{x}}-\frac{\sqrt{3}}{2} y_{5} a \hat{\mathbf{y}}+ \\
\left(\frac{2}{3}+z_{5}\right) c \hat{\mathbf{z}}\end{array}$ & $(3 a)$ & Ir \\
\hline
\end{tabular}

\section{References:}

- K. Schubert, S. Bhan, T. K. Biswas, K. Frank, and P. K. Panday, Einige Strukturdaten metallischer Phasen, Naturwissenschaften 55, 542-543 (1968), doi:10.1007/BF00660131.

\section{Found in:}

- P. Villars and K. Cenzual, Pearson's Crystal Data - Crystal Structure Database for Inorganic Compounds, ASM International (2013).

\section{Geometry files:}

- CIF: pp. 906

- POSCAR: pp. 907 


\section{TeZn (High-pressure) Structure: AB_hP6_144_a_a}
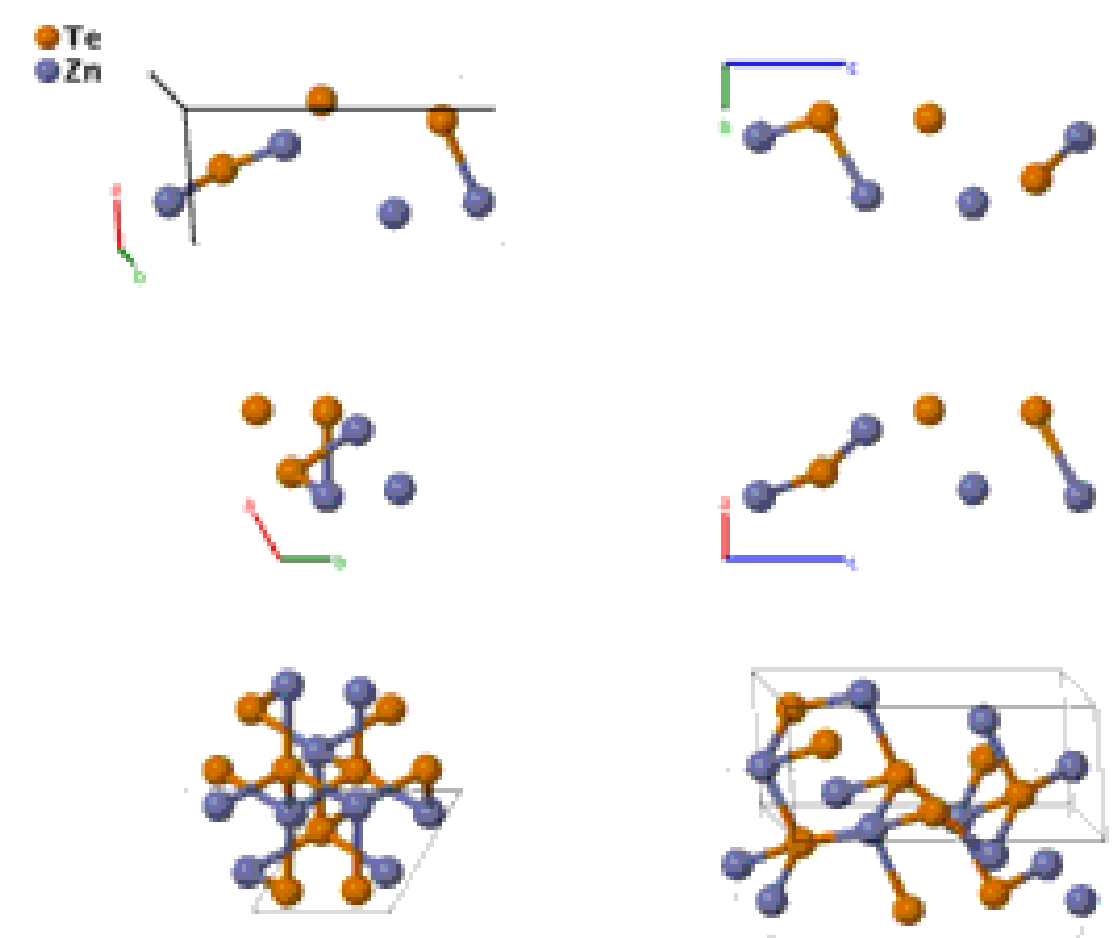

\section{Prototype}

AFLOW prototype label

: $\quad \mathrm{ZnTe}$

Strukturbericht designation

: AB_hP6_144_a_a

Pearson symbol

: None

Space group number

: $\quad \mathrm{hP6}$

Space group symbol

: $\quad 144$

AFLOW prototype command

: $\quad P 3_{1}$

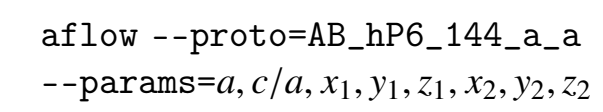

Trigonal Hexagonal primitive vectors:

$$
\begin{array}{ll}
\mathbf{a}_{1}= & \frac{1}{2} a \hat{\mathbf{x}}-\frac{\sqrt{3}}{2} a \hat{\mathbf{y}} \\
\mathbf{a}_{2}= & \frac{1}{2} a \hat{\mathbf{x}}+\frac{\sqrt{3}}{2} a \hat{\mathbf{y}} \\
\mathbf{a}_{3}= & c \hat{\mathbf{z}}
\end{array}
$$

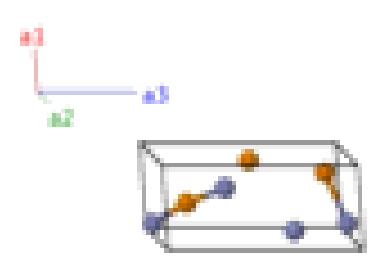

Basis vectors:

\section{Lattice Coordinates}

$\mathbf{B}_{1}=$

$x_{1} \mathbf{a}_{1}+y_{1} \mathbf{a}_{2}+z_{1} \mathbf{a}_{3}$

$=$

Cartesian Coordinates

$\frac{1}{2}\left(x_{1}+y_{1}\right) a \hat{\mathbf{x}}+$

$\frac{\sqrt{3}}{2}\left(-x_{1}+y_{1}\right) a \hat{\mathbf{y}}+z_{1} c \hat{\mathbf{z}}$

$\mathbf{B}_{2}=-y_{1} \mathbf{a}_{1}+\left(x_{1}-y_{1}\right) \mathbf{a}_{2}+\left(\frac{1}{3}+z_{1}\right) \mathbf{a}_{3}=\left(\frac{1}{2} x_{1}-y_{1}\right) a \hat{\mathbf{x}}+\frac{\sqrt{3}}{2} x_{1} a \hat{\mathbf{y}}+$

$\left(\frac{1}{3}+z_{1}\right) c \hat{\mathbf{z}}$
Wyckoff Position Atom Type

(3a)

$\mathrm{Te}$

$(3 a)$

$\mathrm{Te}$ 


\begin{tabular}{|c|c|c|c|c|c|}
\hline $\mathbf{B}_{3}$ & $=$ & $\left(-x_{1}+y_{1}\right) \mathbf{a}_{1}-x_{1} \mathbf{a}_{2}+\left(\frac{2}{3}+z_{1}\right) \mathbf{a}_{3}$ & $=$ & $\begin{array}{c}\left(-x_{1}+\frac{1}{2} y_{1}\right) a \hat{\mathbf{x}}-\frac{\sqrt{3}}{2} y_{1} a \hat{\mathbf{y}}+ \\
\left(\frac{2}{3}+z_{1}\right) c \hat{\mathbf{z}}\end{array}$ & $(3 a)$ \\
\hline $\mathbf{B}_{4}$ & $=$ & $x_{2} \mathbf{a}_{1}+y_{2} \mathbf{a}_{2}+z_{2} \mathbf{a}_{3}$ & $=$ & $\begin{array}{c}\frac{1}{2}\left(x_{2}+y_{2}\right) a \hat{\mathbf{x}}+ \\
\frac{\sqrt{3}}{2}\left(-x_{2}+y_{2}\right) a \hat{\mathbf{y}}+z_{2} c \hat{\mathbf{z}}\end{array}$ & $(3 a)$ \\
\hline $\mathbf{B}_{5}$ & $=$ & $-y_{2} \mathbf{a}_{1}+\left(x_{2}-y_{2}\right) \mathbf{a}_{2}+\left(\frac{1}{3}+z_{2}\right) \mathbf{a}_{3}$ & $=$ & $\begin{array}{c}\left(\frac{1}{2} x_{2}-y_{2}\right) a \hat{\mathbf{x}}+\frac{\sqrt{3}}{2} x_{2} a \hat{\mathbf{y}}+ \\
\left(\frac{1}{3}+z_{2}\right) c \hat{\mathbf{z}}\end{array}$ & $(3 a)$ \\
\hline $\mathbf{B}_{6}$ & $=$ & $\left(-x_{2}+y_{2}\right) \mathbf{a}_{1}-x_{2} \mathbf{a}_{2}+\left(\frac{2}{3}+z_{2}\right) \mathbf{a}_{3}$ & $=$ & $\begin{array}{c}\left(-x_{2}+\frac{1}{2} y_{2}\right) a \hat{\mathbf{x}}-\frac{\sqrt{3}}{2} y_{2} a \hat{\mathbf{y}}+ \\
\left(\frac{2}{3}+z_{2}\right) c \hat{\mathbf{z}}\end{array}$ & $(3 a)$ \\
\hline
\end{tabular}

\section{References:}

- K. Kusaba and D. J. Weidner, Structure of high pressure phase I in ZnTe, in AIP Conference Proceedings (1994), vol. 309, pp. 553-556, doi:10.1063/1.46096.

\section{Found in:}

- P. Villars and K. Cenzual, Pearson's Crystal Data - Crystal Structure Database for Inorganic Compounds, ASM International (2013).

\section{Geometry files:}

- CIF: pp. 907

- POSCAR: pp. 907 


\section{Sheldrickite $\left(\mathrm{NaCa}_{3}\left[\mathrm{CO}_{3}\right]_{2} \mathrm{~F}_{3}\left[\mathrm{H}_{2} \mathrm{O}\right]\right)$ Structure: A2B3C3DE7_hP48_145_2a_3a_3a_a_7a}
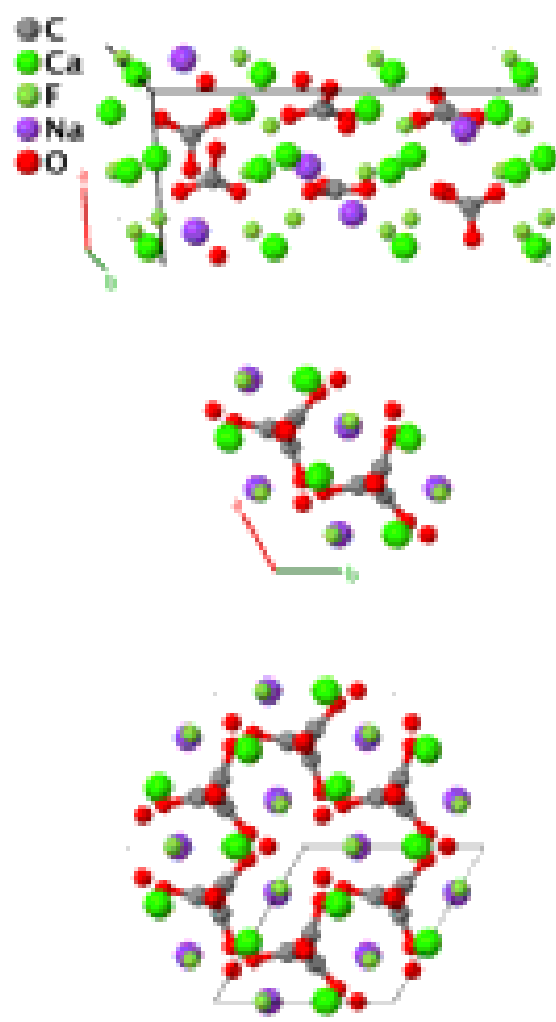

Prototype

AFLOW prototype label

Strukturbericht designation

Pearson symbol

Space group number

Space group symbol

AFLOW prototype command
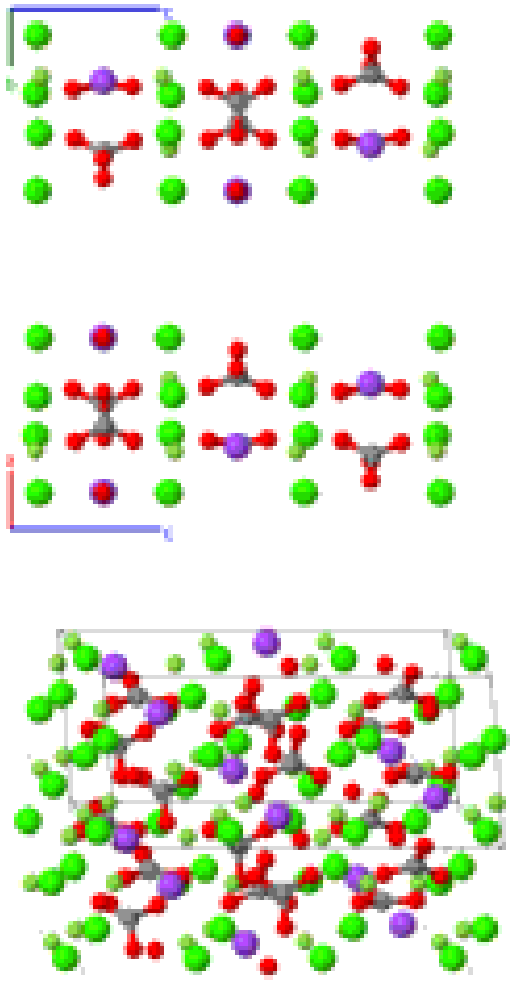

- The $\mathrm{H}_{2} \mathrm{O}$ molecule is centered on one of the (3a) sites; however, it is only listed as $\mathrm{O}$ in this prototype.

\section{Trigonal Hexagonal primitive vectors:}

$$
\begin{array}{ll}
\mathbf{a}_{1}= & \frac{1}{2} a \hat{\mathbf{x}}-\frac{\sqrt{3}}{2} a \hat{\mathbf{y}} \\
\mathbf{a}_{2}= & \frac{1}{2} a \hat{\mathbf{x}}+\frac{\sqrt{3}}{2} a \hat{\mathbf{y}} \\
\mathbf{a}_{3}= & c \hat{\mathbf{z}}
\end{array}
$$

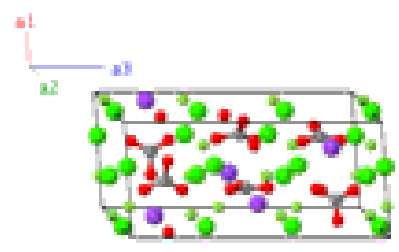

Basis vectors: 
Lattice Coordinates

Cartesian Coordinates

$$
\begin{aligned}
& \mathbf{B}_{1}=x_{1} \mathbf{a}_{1}+y_{1} \mathbf{a}_{2}+z_{1} \mathbf{a}_{3} \\
& = \\
& \frac{1}{2}\left(x_{1}+y_{1}\right) a \hat{\mathbf{x}}+ \\
& \frac{\sqrt{3}}{2}\left(-x_{1}+y_{1}\right) a \hat{\mathbf{y}}+z_{1} c \hat{\mathbf{z}} \\
& \mathbf{B}_{2}=-y_{1} \mathbf{a}_{1}+\left(x_{1}-y_{1}\right) \mathbf{a}_{2}+\left(\frac{2}{3}+z_{1}\right) \mathbf{a}_{3}=\left(\frac{1}{2} x_{1}-y_{1}\right) a \hat{\mathbf{x}}+\frac{\sqrt{3}}{2} x_{1} a \hat{\mathbf{y}}+ \\
& \left(\frac{2}{3}+z_{1}\right) c \hat{\mathbf{z}} \\
& \mathbf{B}_{3}=\left(-x_{1}+y_{1}\right) \mathbf{a}_{1}-x_{1} \mathbf{a}_{2}+\left(\frac{1}{3}+z_{1}\right) \mathbf{a}_{3}=\left(-x_{1}+\frac{1}{2} y_{1}\right) a \hat{\mathbf{x}}-\frac{\sqrt{3}}{2} y_{1} a \hat{\mathbf{y}}+ \\
& \left(\frac{1}{3}+z_{1}\right) c \hat{\mathbf{z}} \\
& \mathbf{B}_{4}=x_{2} \mathbf{a}_{1}+y_{2} \mathbf{a}_{2}+z_{2} \mathbf{a}_{3} \quad=\quad \frac{1}{2}\left(x_{2}+y_{2}\right) a \hat{\mathbf{x}}+ \\
& \frac{\sqrt{3}}{2}\left(-x_{2}+y_{2}\right) a \hat{\mathbf{y}}+z_{2} c \hat{\mathbf{z}} \\
& \mathbf{B}_{5}=-y_{2} \mathbf{a}_{1}+\left(x_{2}-y_{2}\right) \mathbf{a}_{2}+\left(\frac{2}{3}+z_{2}\right) \mathbf{a}_{3}=\left(\frac{1}{2} x_{2}-y_{2}\right) a \hat{\mathbf{x}}+\frac{\sqrt{3}}{2} x_{2} a \hat{\mathbf{y}}+ \\
& \left(\frac{2}{3}+z_{2}\right) c \hat{\mathbf{z}} \\
& \mathbf{B}_{6}=\left(-x_{2}+y_{2}\right) \mathbf{a}_{1}-x_{2} \mathbf{a}_{2}+\left(\frac{1}{3}+z_{2}\right) \mathbf{a}_{3}=\left(-x_{2}+\frac{1}{2} y_{2}\right) a \hat{\mathbf{x}}-\frac{\sqrt{3}}{2} y_{2} a \hat{\mathbf{y}}+ \\
& \left(\frac{1}{3}+z_{2}\right) c \hat{\mathbf{z}} \\
& \mathbf{B}_{7}=x_{3} \mathbf{a}_{1}+y_{3} \mathbf{a}_{2}+z_{3} \mathbf{a}_{3} \quad=\quad \frac{1}{2}\left(x_{3}+y_{3}\right) a \hat{\mathbf{x}}+ \\
& \frac{\sqrt{3}}{2}\left(-x_{3}+y_{3}\right) a \hat{\mathbf{y}}+z_{3} c \hat{\mathbf{z}} \\
& \mathbf{B}_{8}=-y_{3} \mathbf{a}_{1}+\left(x_{3}-y_{3}\right) \mathbf{a}_{2}+\left(\frac{2}{3}+z_{3}\right) \mathbf{a}_{3}=\left(\frac{1}{2} x_{3}-y_{3}\right) a \hat{\mathbf{x}}+\frac{\sqrt{3}}{2} x_{3} a \hat{\mathbf{y}}+ \\
& \left(\frac{2}{3}+z_{3}\right) c \hat{\mathbf{z}} \\
& \mathbf{B}_{9}=\left(-x_{3}+y_{3}\right) \mathbf{a}_{1}-x_{3} \mathbf{a}_{2}+\left(\frac{1}{3}+z_{3}\right) \mathbf{a}_{3}=\left(-x_{3}+\frac{1}{2} y_{3}\right) a \hat{\mathbf{x}}-\frac{\sqrt{3}}{2} y_{3} a \hat{\mathbf{y}}+ \\
& \left(\frac{1}{3}+z_{3}\right) c \hat{\mathbf{z}} \\
& \mathbf{B}_{10}=x_{4} \mathbf{a}_{1}+y_{4} \mathbf{a}_{2}+z_{4} \mathbf{a}_{3} \quad=\quad \frac{1}{2}\left(x_{4}+y_{4}\right) a \hat{\mathbf{x}}+ \\
& \frac{\sqrt{3}}{2}\left(-x_{4}+y_{4}\right) a \hat{\mathbf{y}}+z_{4} c \hat{\mathbf{z}} \\
& \mathbf{B}_{11}=-y_{4} \mathbf{a}_{1}+\left(x_{4}-y_{4}\right) \mathbf{a}_{2}+\left(\frac{2}{3}+z_{4}\right) \mathbf{a}_{3}=\left(\frac{1}{2} x_{4}-y_{4}\right) a \hat{\mathbf{x}}+\frac{\sqrt{3}}{2} x_{4} a \hat{\mathbf{y}}+ \\
& \left(\frac{2}{3}+z_{4}\right) c \hat{\mathbf{z}} \\
& \mathbf{B}_{12}=\left(-x_{4}+y_{4}\right) \mathbf{a}_{1}-x_{4} \mathbf{a}_{2}+\left(\frac{1}{3}+z_{4}\right) \mathbf{a}_{3}=\left(-x_{4}+\frac{1}{2} y_{4}\right) a \hat{\mathbf{x}}-\frac{\sqrt{3}}{2} y_{4} a \hat{\mathbf{y}}+ \\
& \left(\frac{1}{3}+z_{4}\right) c \hat{\mathbf{z}} \\
& \mathbf{B}_{13}=x_{5} \mathbf{a}_{1}+y_{5} \mathbf{a}_{2}+z_{5} \mathbf{a}_{3} \quad=\quad \frac{1}{2}\left(x_{5}+y_{5}\right) a \hat{\mathbf{x}}+ \\
& \frac{\sqrt{3}}{2}\left(-x_{5}+y_{5}\right) a \hat{\mathbf{y}}+z_{5} c \hat{\mathbf{z}} \\
& \mathbf{B}_{14}=-y_{5} \mathbf{a}_{1}+\left(x_{5}-y_{5}\right) \mathbf{a}_{2}+\left(\frac{2}{3}+z_{5}\right) \mathbf{a}_{3}=\left(\frac{1}{2} x_{5}-y_{5}\right) a \hat{\mathbf{x}}+\frac{\sqrt{3}}{2} x_{5} a \hat{\mathbf{y}}+ \\
& \left(\frac{2}{3}+z_{5}\right) c \hat{\mathbf{z}} \\
& \mathbf{B}_{15}=\left(-x_{5}+y_{5}\right) \mathbf{a}_{1}-x_{5} \mathbf{a}_{2}+\left(\frac{1}{3}+z_{5}\right) \mathbf{a}_{3}=\left(-x_{5}+\frac{1}{2} y_{5}\right) a \hat{\mathbf{x}}-\frac{\sqrt{3}}{2} y_{5} a \hat{\mathbf{y}}+ \\
& \left(\frac{1}{3}+z_{5}\right) c \hat{\mathbf{z}} \\
& \mathbf{B}_{16}=x_{6} \mathbf{a}_{1}+y_{6} \mathbf{a}_{2}+z_{6} \mathbf{a}_{3} \quad=\quad \frac{1}{2}\left(x_{6}+y_{6}\right) a \hat{\mathbf{x}}+ \\
& \frac{\sqrt{3}}{2}\left(-x_{6}+y_{6}\right) a \hat{\mathbf{y}}+z_{6} c \hat{\mathbf{z}} \\
& \mathbf{B}_{17}=-y_{6} \mathbf{a}_{1}+\left(x_{6}-y_{6}\right) \mathbf{a}_{2}+\left(\frac{2}{3}+z_{6}\right) \mathbf{a}_{3}=\left(\frac{1}{2} x_{6}-y_{6}\right) a \hat{\mathbf{x}}+\frac{\sqrt{3}}{2} x_{6} a \hat{\mathbf{y}}+ \\
& \left(\frac{2}{3}+z_{6}\right) c \hat{\mathbf{z}} \\
& \mathbf{B}_{18}=\left(-x_{6}+y_{6}\right) \mathbf{a}_{1}-x_{6} \mathbf{a}_{2}+\left(\frac{1}{3}+z_{6}\right) \mathbf{a}_{3}=\left(-x_{6}+\frac{1}{2} y_{6}\right) a \hat{\mathbf{x}}-\frac{\sqrt{3}}{2} y_{6} a \hat{\mathbf{y}}+ \\
& \left(\frac{1}{3}+z_{6}\right) c \hat{\mathbf{z}} \\
& \mathbf{B}_{19}=x_{7} \mathbf{a}_{1}+y_{7} \mathbf{a}_{2}+z_{7} \mathbf{a}_{3} \quad=\quad \frac{1}{2}\left(x_{7}+y_{7}\right) a \hat{\mathbf{x}}+ \\
& \frac{\sqrt{3}}{2}\left(-x_{7}+y_{7}\right) a \hat{\mathbf{y}}+z_{7} c \hat{\mathbf{z}} \\
& \mathbf{B}_{20}=-y_{7} \mathbf{a}_{1}+\left(x_{7}-y_{7}\right) \mathbf{a}_{2}+\left(\frac{2}{3}+z_{7}\right) \mathbf{a}_{3}=\left(\frac{1}{2} x_{7}-y_{7}\right) a \hat{\mathbf{x}}+\frac{\sqrt{3}}{2} x_{7} a \hat{\mathbf{y}}+ \\
& \left(\frac{2}{3}+z_{7}\right) c \hat{\mathbf{z}}
\end{aligned}
$$

C I

C II

C II

Ca I

Ca I

Ca I

Ca II

Ca II

Ca II

Ca III

Ca III

Ca III

F I

F I

F I

F II 


\begin{tabular}{|c|c|c|c|c|c|c|}
\hline $\mathbf{B}_{21}$ & $=$ & $\left(-x_{7}+y_{7}\right) \mathbf{a}_{1}-x_{7} \mathbf{a}_{2}+\left(\frac{1}{3}+z_{7}\right) \mathbf{a}_{3}$ & $=$ & $\begin{array}{c}\left(-x_{7}+\frac{1}{2} y_{7}\right) a \hat{\mathbf{x}}-\frac{\sqrt{3}}{2} y_{7} a \hat{\mathbf{y}}+ \\
\left(\frac{1}{3}+z_{7}\right) c \hat{\mathbf{z}}\end{array}$ & $(3 a)$ & F II \\
\hline $\mathbf{B}_{22}$ & $=$ & $x_{8} \mathbf{a}_{1}+y_{8} \mathbf{a}_{2}+z_{8} \mathbf{a}_{3}$ & $=$ & $\begin{array}{c}\frac{1}{2}\left(x_{8}+y_{8}\right) a \hat{\mathbf{x}}+ \\
\frac{\sqrt{3}}{2}\left(-x_{8}+y_{8}\right) a \hat{\mathbf{y}}+z_{8} c \hat{\mathbf{z}}\end{array}$ & $(3 a)$ & F III \\
\hline $\mathbf{B}_{23}$ & $=$ & $-y_{8} \mathbf{a}_{1}+\left(x_{8}-y_{8}\right) \mathbf{a}_{2}+\left(\frac{2}{3}+z_{8}\right) \mathbf{a}_{3}$ & $=$ & $\begin{array}{c}\left(\frac{1}{2} x_{8}-y_{8}\right) a \hat{\mathbf{x}}+\frac{\sqrt{3}}{2} x_{8} a \hat{\mathbf{y}}+ \\
\left(\frac{2}{3}+z_{8}\right) c \hat{\mathbf{z}}\end{array}$ & $(3 a)$ & F III \\
\hline $\mathbf{B}_{24}$ & $=$ & $\left(-x_{8}+y_{8}\right) \mathbf{a}_{1}-x_{8} \mathbf{a}_{2}+\left(\frac{1}{3}+z_{8}\right) \mathbf{a}_{3}$ & $=$ & $\begin{array}{c}\left(-x_{8}+\frac{1}{2} y_{8}\right) a \hat{\mathbf{x}}-\frac{\sqrt{3}}{2} y_{8} a \hat{\mathbf{y}}+ \\
\left(\frac{1}{3}+z_{8}\right) c \hat{\mathbf{z}}\end{array}$ & $(3 a)$ & F III \\
\hline $\mathbf{B}_{25}$ & $=$ & $x_{9} \mathbf{a}_{1}+y_{9} \mathbf{a}_{2}+z_{9} \mathbf{a}_{3}$ & $=$ & $\begin{array}{c}\frac{1}{2}\left(x_{9}+y_{9}\right) a \hat{\mathbf{x}}+ \\
\frac{\sqrt{3}}{2}\left(-x_{9}+y_{9}\right) a \hat{\mathbf{y}}+z_{9} c \hat{\mathbf{z}}\end{array}$ & $(3 a)$ & $\mathrm{Na}$ \\
\hline $\mathbf{B}_{26}$ & $=$ & $-y_{9} \mathbf{a}_{1}+\left(x_{9}-y_{9}\right) \mathbf{a}_{2}+\left(\frac{2}{3}+z_{9}\right) \mathbf{a}_{3}$ & $=$ & $\begin{array}{c}\left(\frac{1}{2} x_{9}-y_{9}\right) a \hat{\mathbf{x}}+\frac{\sqrt{3}}{2} x_{9} a \hat{\mathbf{y}}+ \\
\left(\frac{2}{3}+z_{9}\right) c \hat{\mathbf{z}}\end{array}$ & $(3 a)$ & $\mathrm{Na}$ \\
\hline $\mathbf{B}_{27}$ & $=$ & $\left(-x_{9}+y_{9}\right) \mathbf{a}_{1}-x_{9} \mathbf{a}_{2}+\left(\frac{1}{3}+z_{9}\right) \mathbf{a}_{3}$ & $=$ & $\begin{array}{c}\left(-x_{9}+\frac{1}{2} y_{9}\right) a \hat{\mathbf{x}}-\frac{\sqrt{3}}{2} y_{9} a \hat{\mathbf{y}}+ \\
\left(\frac{1}{3}+z_{9}\right) c \hat{\mathbf{z}}\end{array}$ & $(3 a)$ & $\mathrm{Na}$ \\
\hline $\mathbf{B}_{28}$ & $=$ & $x_{10} \mathbf{a}_{1}+y_{10} \mathbf{a}_{2}+z_{10} \mathbf{a}_{3}$ & $=$ & $\begin{array}{c}\frac{1}{2}\left(x_{10}+y_{10}\right) a \hat{\mathbf{x}}+ \\
\frac{\sqrt{3}}{2}\left(-x_{10}+y_{10}\right) a \hat{\mathbf{y}}+z_{10} c \hat{\mathbf{z}}\end{array}$ & $(3 a)$ & O I \\
\hline $\mathbf{B}_{29}$ & $=$ & $\begin{array}{c}-y_{10} \mathbf{a}_{1}+\left(x_{10}-y_{10}\right) \mathbf{a}_{2}+ \\
\left(\frac{2}{3}+z_{10}\right) \mathbf{a}_{3}\end{array}$ & $=$ & $\begin{array}{c}\left(\frac{1}{2} x_{10}-y_{10}\right) a \hat{\mathbf{x}}+\frac{\sqrt{3}}{2} x_{10} a \hat{\mathbf{y}}+ \\
\left(\frac{2}{3}+z_{10}\right) c \hat{\mathbf{z}}\end{array}$ & $(3 a)$ & O I \\
\hline $\mathbf{B}_{30}$ & $=$ & $\begin{array}{c}\left(-x_{10}+y_{10}\right) \mathbf{a}_{1}-x_{10} \mathbf{a}_{2}+ \\
\left(\frac{1}{3}+z_{10}\right) \mathbf{a}_{3}\end{array}$ & $=$ & $\begin{array}{c}\left(-x_{10}+\frac{1}{2} y_{10}\right) a \hat{\mathbf{x}}-\frac{\sqrt{3}}{2} y_{10} a \hat{\mathbf{y}}+ \\
\left(\frac{1}{3}+z_{10}\right) c \hat{\mathbf{z}}\end{array}$ & $(3 a)$ & O I \\
\hline $\mathbf{B}_{31}$ & $=$ & $x_{11} \mathbf{a}_{1}+y_{11} \mathbf{a}_{2}+z_{11} \mathbf{a}_{3}$ & $=$ & $\begin{array}{c}\frac{1}{2}\left(x_{11}+y_{11}\right) a \hat{\mathbf{x}}+ \\
\frac{\sqrt{3}}{2}\left(-x_{11}+y_{11}\right) a \hat{\mathbf{y}}+z_{11} c \hat{\mathbf{z}}\end{array}$ & $(3 a)$ & O II \\
\hline $\mathbf{B}_{32}$ & $=$ & $\begin{array}{c}-y_{11} \mathbf{a}_{1}+\left(x_{11}-y_{11}\right) \mathbf{a}_{2}+ \\
\left(\frac{2}{3}+z_{11}\right) \mathbf{a}_{3}\end{array}$ & $=$ & $\begin{array}{c}\left(\frac{1}{2} x_{11}-y_{11}\right) a \hat{\mathbf{x}}+\frac{\sqrt{3}}{2} x_{11} a \hat{\mathbf{y}}+ \\
\left(\frac{2}{3}+z_{11}\right) c \hat{\mathbf{z}}\end{array}$ & $(3 a)$ & O II \\
\hline $\mathbf{B}_{33}$ & $=$ & $\begin{array}{c}\left(-x_{11}+y_{11}\right) \mathbf{a}_{1}-x_{11} \mathbf{a}_{2}+ \\
\left(\frac{1}{3}+z_{11}\right) \mathbf{a}_{3}\end{array}$ & $=$ & $\begin{array}{c}\left(-x_{11}+\frac{1}{2} y_{11}\right) a \hat{\mathbf{x}}-\frac{\sqrt{3}}{2} y_{11} a \hat{\mathbf{y}}+ \\
\left(\frac{1}{3}+z_{11}\right) c \hat{\mathbf{z}}\end{array}$ & $(3 a)$ & O II \\
\hline $\mathbf{B}_{34}$ & $=$ & $x_{12} \mathbf{a}_{1}+y_{12} \mathbf{a}_{2}+z_{12} \mathbf{a}_{3}$ & $=$ & $\begin{array}{c}\frac{1}{2}\left(x_{12}+y_{12}\right) a \hat{\mathbf{x}}+ \\
\frac{\sqrt{3}}{2}\left(-x_{12}+y_{12}\right) a \hat{\mathbf{y}}+z_{12} c \hat{\mathbf{z}}\end{array}$ & $(3 a)$ & O III \\
\hline $\mathbf{B}_{35}$ & $=$ & $\begin{array}{c}-y_{12} \mathbf{a}_{1}+\left(x_{12}-y_{12}\right) \mathbf{a}_{2}+ \\
\left(\frac{2}{3}+z_{12}\right) \mathbf{a}_{3}\end{array}$ & $=$ & $\begin{array}{c}\left(\frac{1}{2} x_{12}-y_{12}\right) a \hat{\mathbf{x}}+\frac{\sqrt{3}}{2} x_{12} a \hat{\mathbf{y}}+ \\
\left(\frac{2}{3}+z_{12}\right) c \hat{\mathbf{z}}\end{array}$ & $(3 a)$ & O III \\
\hline $\mathbf{B}_{36}$ & $=$ & $\begin{array}{c}\left(-x_{12}+y_{12}\right) \mathbf{a}_{1}-x_{12} \mathbf{a}_{2}+ \\
\left(\frac{1}{3}+z_{12}\right) \mathbf{a}_{3}\end{array}$ & $=$ & $\begin{array}{c}\left(-x_{12}+\frac{1}{2} y_{12}\right) a \hat{\mathbf{x}}-\frac{\sqrt{3}}{2} y_{12} a \hat{\mathbf{y}}+ \\
\left(\frac{1}{3}+z_{12}\right) c \hat{\mathbf{z}}\end{array}$ & $(3 a)$ & O III \\
\hline $\mathbf{B}_{37}$ & $=$ & $x_{13} \mathbf{a}_{1}+y_{13} \mathbf{a}_{2}+z_{13} \mathbf{a}_{3}$ & $=$ & $\begin{array}{c}\frac{1}{2}\left(x_{13}+y_{13}\right) a \hat{\mathbf{x}}+ \\
\frac{\sqrt{3}}{2}\left(-x_{13}+y_{13}\right) a \hat{\mathbf{y}}+z_{13} c \hat{\mathbf{z}}\end{array}$ & $(3 a)$ & O IV \\
\hline $\mathbf{B}_{38}$ & $=$ & $\begin{array}{c}-y_{13} \mathbf{a}_{1}+\left(x_{13}-y_{13}\right) \mathbf{a}_{2}+ \\
\left(\frac{2}{3}+z_{13}\right) \mathbf{a}_{3}\end{array}$ & $=$ & $\begin{array}{c}\left(\frac{1}{2} x_{13}-y_{13}\right) a \hat{\mathbf{x}}+\frac{\sqrt{3}}{2} x_{13} a \hat{\mathbf{y}}+ \\
\left(\frac{2}{3}+z_{13}\right) c \hat{\mathbf{z}}\end{array}$ & $(3 a)$ & O IV \\
\hline $\mathbf{B}_{39}$ & $=$ & $\begin{array}{c}\left(-x_{13}+y_{13}\right) \mathbf{a}_{1}-x_{13} \mathbf{a}_{2}+ \\
\left(\frac{1}{3}+z_{13}\right) \mathbf{a}_{3}\end{array}$ & $=$ & $\begin{array}{c}\left(-x_{13}+\frac{1}{2} y_{13}\right) a \hat{\mathbf{x}}-\frac{\sqrt{3}}{2} y_{13} a \hat{\mathbf{y}}+ \\
\left(\frac{1}{3}+z_{13}\right) c \hat{\mathbf{z}}\end{array}$ & $(3 a)$ & O IV \\
\hline $\mathbf{B}_{40}$ & $=$ & $x_{14} \mathbf{a}_{1}+y_{14} \mathbf{a}_{2}+z_{14} \mathbf{a}_{3}$ & $=$ & $\begin{array}{c}\frac{1}{2}\left(x_{14}+y_{14}\right) a \hat{\mathbf{x}}+ \\
\frac{\sqrt{3}}{2}\left(-x_{14}+y_{14}\right) a \hat{\mathbf{y}}+z_{14} c \hat{\mathbf{z}}\end{array}$ & $(3 a)$ & $\mathrm{OV}$ \\
\hline
\end{tabular}




\begin{tabular}{|c|c|c|c|c|c|c|}
\hline $\mathbf{B}_{41}$ & $=$ & $\begin{array}{c}-y_{14} \mathbf{a}_{1}+\left(x_{14}-y_{14}\right) \mathbf{a}_{2}+ \\
\left(\frac{2}{3}+z_{14}\right) \mathbf{a}_{3}\end{array}$ & $=$ & $\begin{array}{c}\left(\frac{1}{2} x_{14}-y_{14}\right) a \hat{\mathbf{x}}+\frac{\sqrt{3}}{2} x_{14} a \hat{\mathbf{y}}+ \\
\left(\frac{2}{3}+z_{14}\right) c \hat{\mathbf{z}}\end{array}$ & $(3 a)$ & $\mathrm{O} \mathrm{V}$ \\
\hline $\mathbf{B}_{42}$ & $=$ & $\begin{array}{c}\left(-x_{14}+y_{14}\right) \mathbf{a}_{1}-x_{14} \mathbf{a}_{2}+ \\
\left(\frac{1}{3}+z_{14}\right) \mathbf{a}_{3}\end{array}$ & $=$ & $\begin{array}{c}\left(-x_{14}+\frac{1}{2} y_{14}\right) a \hat{\mathbf{x}}-\frac{\sqrt{3}}{2} y_{14} a \hat{\mathbf{y}}+ \\
\left(\frac{1}{3}+z_{14}\right) c \hat{\mathbf{z}}\end{array}$ & $(3 a)$ & $\mathrm{OV}$ \\
\hline $\mathbf{B}_{43}$ & $=$ & $x_{15} \mathbf{a}_{1}+y_{15} \mathbf{a}_{2}+z_{15} \mathbf{a}_{3}$ & $=$ & $\begin{array}{c}\frac{1}{2}\left(x_{15}+y_{15}\right) a \hat{\mathbf{x}}+ \\
\frac{\sqrt{3}}{2}\left(-x_{15}+y_{15}\right) a \hat{\mathbf{y}}+z_{15} c \hat{\mathbf{z}}\end{array}$ & $(3 a)$ & O VI \\
\hline $\mathbf{B}_{44}$ & $=$ & $\begin{array}{c}-y_{15} \mathbf{a}_{1}+\left(x_{15}-y_{15}\right) \mathbf{a}_{2}+ \\
\left(\frac{2}{3}+z_{15}\right) \mathbf{a}_{3}\end{array}$ & $=$ & $\begin{array}{c}\left(\frac{1}{2} x_{15}-y_{15}\right) a \hat{\mathbf{x}}+\frac{\sqrt{3}}{2} x_{15} a \hat{\mathbf{y}}+ \\
\left(\frac{2}{3}+z_{15}\right) c \hat{\mathbf{z}}\end{array}$ & $(3 a)$ & O VI \\
\hline $\mathbf{B}_{45}$ & $=$ & $\begin{array}{c}\left(-x_{15}+y_{15}\right) \mathbf{a}_{1}-x_{15} \mathbf{a}_{2}+ \\
\left(\frac{1}{3}+z_{15}\right) \mathbf{a}_{3}\end{array}$ & $=$ & $\begin{array}{c}\left(-x_{15}+\frac{1}{2} y_{15}\right) a \hat{\mathbf{x}}-\frac{\sqrt{3}}{2} y_{15} a \hat{\mathbf{y}}+ \\
\left(\frac{1}{3}+z_{15}\right) c \hat{\mathbf{z}}\end{array}$ & $(3 a)$ & O VI \\
\hline $\mathbf{B}_{46}$ & $=$ & $x_{16} \mathbf{a}_{1}+y_{16} \mathbf{a}_{2}+z_{16} \mathbf{a}_{3}$ & $=$ & $\begin{array}{c}\frac{1}{2}\left(x_{16}+y_{16}\right) a \hat{\mathbf{x}}+ \\
\frac{\sqrt{3}}{2}\left(-x_{16}+y_{16}\right) a \hat{\mathbf{y}}+z_{16} c \hat{\mathbf{z}}\end{array}$ & $(3 a)$ & O VII \\
\hline $\mathbf{B}_{47}$ & $=$ & $\begin{array}{c}-y_{16} \mathbf{a}_{1}+\left(x_{16}-y_{16}\right) \mathbf{a}_{2}+ \\
\left(\frac{2}{3}+z_{16}\right) \mathbf{a}_{3}\end{array}$ & $=$ & $\begin{array}{c}\left(\frac{1}{2} x_{16}-y_{16}\right) a \hat{\mathbf{x}}+\frac{\sqrt{3}}{2} x_{16} a \hat{\mathbf{y}}+ \\
\left(\frac{2}{3}+z_{16}\right) c \hat{\mathbf{z}}\end{array}$ & $(3 a)$ & O VII \\
\hline $\mathbf{B}_{48}$ & $=$ & $\begin{array}{c}\left(-x_{16}+y_{16}\right) \mathbf{a}_{1}-x_{16} \mathbf{a}_{2}+ \\
\left(\frac{1}{3}+z_{16}\right) \mathbf{a}_{3}\end{array}$ & $=$ & $\begin{array}{c}\left(-x_{16}+\frac{1}{2} y_{16}\right) a \hat{\mathbf{x}}-\frac{\sqrt{3}}{2} y_{16} a \hat{\mathbf{y}}+ \\
\left(\frac{1}{3}+z_{16}\right) c \hat{\mathbf{z}}\end{array}$ & $(3 a)$ & O VII \\
\hline
\end{tabular}

\section{References:}

- J. D. Grice, R. A. Gault, and J. Van Velthuizen, Sheldrickite, a new sodium-calcium-fluorocarbonate mineral species from Mont Saint-Hilaire, Quebec, Can. Mineral. 35, 181-187 (1997).

\section{Found in:}

- P. Villars and K. Cenzual, Pearson's Crystal Data - Crystal Structure Database for Inorganic Compounds, ASM International (2013).

\section{Geometry files:}

- CIF: pp. 907

- POSCAR: pp. 907 


\section{$\gamma-\mathrm{Ag}_{3} \mathrm{SI}$ (Low-temperature) Structure:}

\section{A3BC_hR5_146_b_a_a}
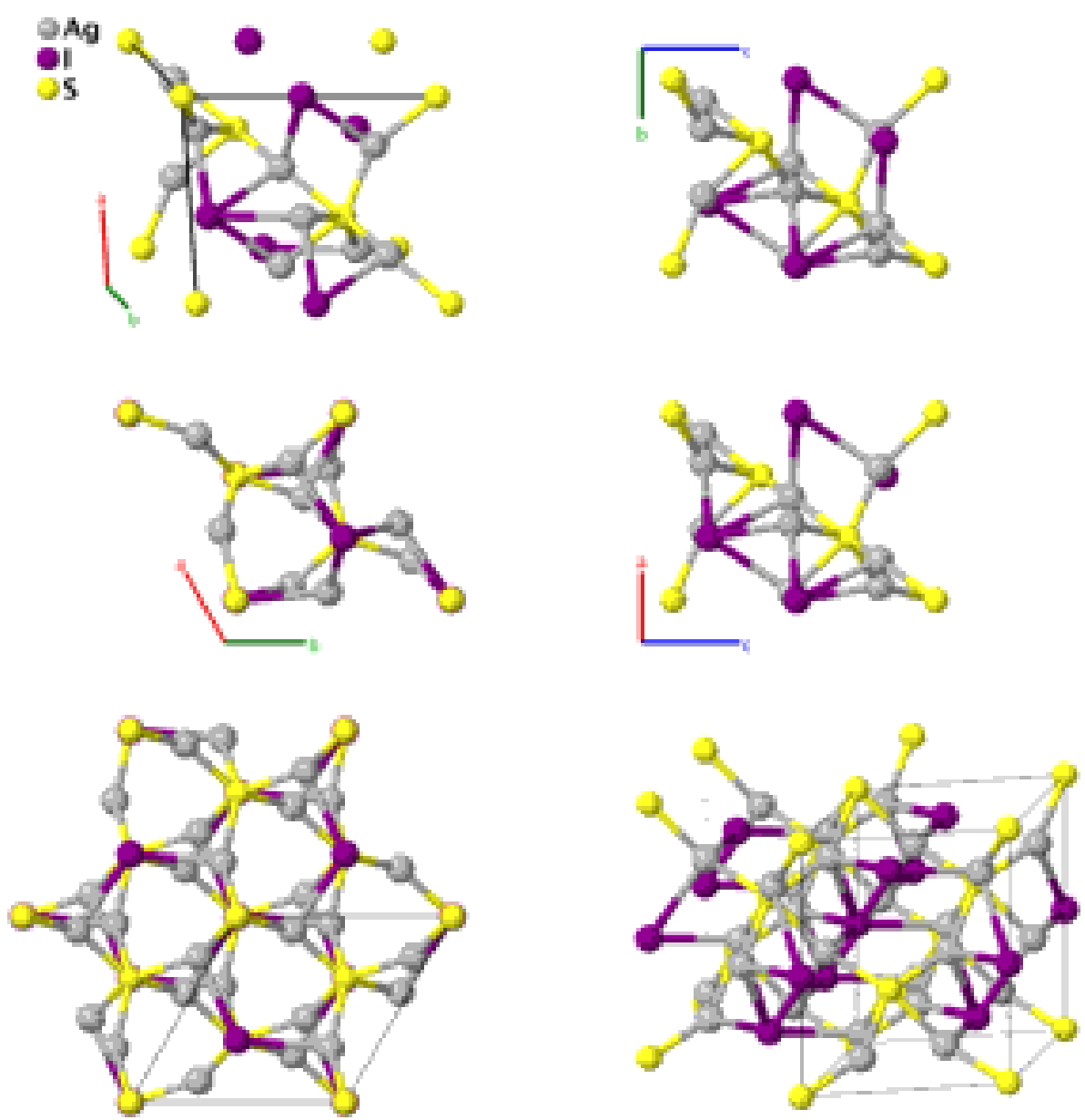

\section{Prototype}

AFLOW prototype label

$: \quad \gamma-\mathrm{Ag}_{3} \mathrm{SI}$

Strukturbericht designation

Pearson symbol

Space group number

Space group symbol

: $\quad R 3$
AFLOW prototype command
aflow --proto $=A 3 B C \_$hR5_146_b_a_a [--hex]
- -params $=a, c / a, x_{1}, x_{2}, x_{3}, y_{3}, z_{3}$

Rhombohedral primitive vectors:

$$
\begin{array}{llc}
\mathbf{a}_{1}= & \frac{1}{2} a \hat{\mathbf{x}}-\frac{1}{2 \sqrt{3}} a \hat{\mathbf{y}}+\frac{1}{3} c \hat{\mathbf{z}} \\
\mathbf{a}_{2}= & \frac{1}{\sqrt{3}} a \hat{\mathbf{y}}+\frac{1}{3} c \hat{\mathbf{z}} \\
\mathbf{a}_{3}= & -\frac{1}{2} a \hat{\mathbf{x}}-\frac{1}{2 \sqrt{3}} a \hat{\mathbf{y}}+\frac{1}{3} c \hat{\mathbf{z}}
\end{array}
$$

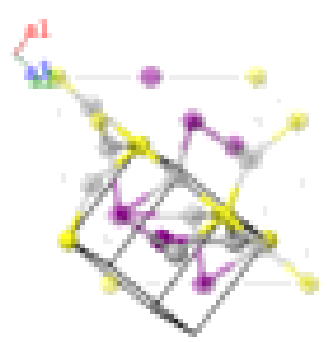




$$
\begin{array}{cccc}
\mathbf{B}_{1}= & x_{1} \mathbf{a}_{1}+x_{1} \mathbf{a}_{2}+x_{1} \mathbf{a}_{3}= & x_{1} c \hat{\mathbf{z}} \\
\mathbf{B}_{2}= & x_{2} \mathbf{a}_{1}+x_{2} \mathbf{a}_{2}+x_{2} \mathbf{a}_{3}= & x_{2} c \hat{\mathbf{z}} \\
\mathbf{B}_{3}= & x_{3} \mathbf{a}_{1}+y_{3} \mathbf{a}_{2}+z_{3} \mathbf{a}_{3}= & \frac{1}{2}\left(x_{3}-z_{3}\right) a \hat{\mathbf{x}}+\left(-\frac{1}{2 \sqrt{3}} x_{3}+\frac{1}{\sqrt{3}} y_{3}-\frac{1}{2 \sqrt{3}} z_{3}\right) a \hat{\mathbf{y}}+ \\
& & \frac{1}{3}\left(x_{3}+y_{3}+z_{3}\right) c \hat{\mathbf{z}} \\
\mathbf{B}_{4}= & z_{3} \mathbf{a}_{1}+x_{3} \mathbf{a}_{2}+y_{3} \mathbf{a}_{3}= & \frac{1}{2}\left(-y_{3}+z_{3}\right) a \hat{\mathbf{x}}+\left(\frac{1}{\sqrt{3}} x_{3}-\frac{1}{2 \sqrt{3}} y_{3}-\frac{1}{2 \sqrt{3}} z_{3}\right) a \hat{\mathbf{y}}+ \\
& & \frac{1}{3}\left(x_{3}+y_{3}+z_{3}\right) c \hat{\mathbf{z}} \\
\mathbf{B}_{5}= & y_{3} \mathbf{a}_{1}+z_{3} \mathbf{a}_{2}+x_{3} \mathbf{a}_{3}= & \frac{1}{2}\left(-x_{3}+y_{3}\right) a \hat{\mathbf{x}}+\left(-\frac{1}{2 \sqrt{3}} x_{3}-\frac{1}{2 \sqrt{3}} y_{3}+\frac{1}{\sqrt{3}} z_{3}\right) a \hat{\mathbf{y}}+ \\
& & \frac{1}{3}\left(x_{3}+y_{3}+z_{3}\right) c \hat{\mathbf{z}}
\end{array}
$$

I S

$\mathrm{Ag}$

Ag

$\mathrm{Ag}$

\section{References:}

- S. Hoshino, T. Sakuma, and Y. Fujii, A Structural Phase Transition in Superionic Conductor $\mathrm{Ag}_{3}$ SI, J. Phys. Soc. Jpn. 47, 1252-1259 (1979), doi:10.1143/JPSJ.47.1252.

\section{Found in:}

- P. Villars and K. Cenzual, Pearson's Crystal Data - Crystal Structure Database for Inorganic Compounds, ASM International (2013).

\section{Geometry files:}

- CIF: pp. 908

- POSCAR: pp. 908 


\section{$\mathrm{FePSe}_{3}$ Structure: ABC3_hR10_146_2a_2a_2b}
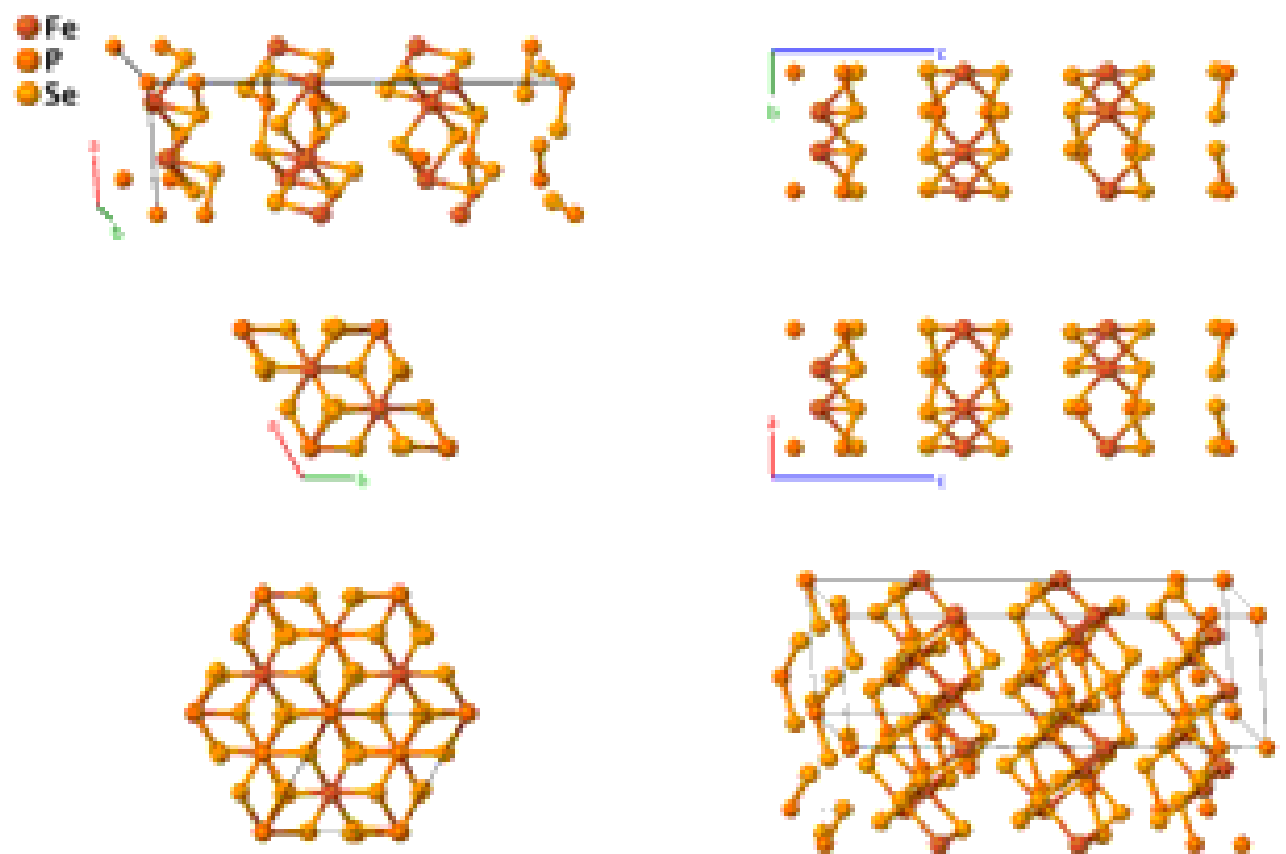

Prototype

AFLOW prototype label

Strukturbericht designation

Pearson symbol

Space group number

Space group symbol

AFLOW prototype command
: $\quad \mathrm{FePSe}_{3}$

: ABC3_hR10_146_2a_2a_2b

: None

: $\quad \mathrm{hR} 10$

: 146

$: \quad R 3$ aflow --proto=ABC3_hR10_146_2a_2a_2b [--hex]

- - params $=a, c / a, x_{1}, x_{2}, x_{3}, x_{4}, x_{5}, y_{5}, z_{5}, x_{6}, y_{6}, z_{6}$

Rhombohedral primitive vectors:

$$
\begin{array}{llc}
\mathbf{a}_{1}= & \frac{1}{2} a \hat{\mathbf{x}}-\frac{1}{2 \sqrt{3}} a \hat{\mathbf{y}}+\frac{1}{3} c \hat{\mathbf{z}} \\
\mathbf{a}_{2}= & \frac{1}{\sqrt{3}} a \hat{\mathbf{y}}+\frac{1}{3} c \hat{\mathbf{z}} \\
\mathbf{a}_{3}= & -\frac{1}{2} a \hat{\mathbf{x}}-\frac{1}{2 \sqrt{3}} a \hat{\mathbf{y}}+\frac{1}{3} c \hat{\mathbf{z}}
\end{array}
$$

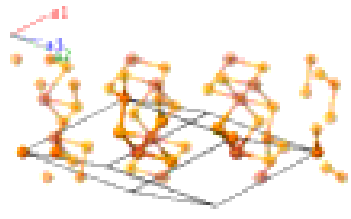

\section{Basis vectors:}

Lattice Coordinates

$$
\begin{array}{cccc}
\mathbf{B}_{1}=x_{1} \mathbf{a}_{1}+x_{1} \mathbf{a}_{2}+x_{1} \mathbf{a}_{3}= & x_{1} c \hat{\mathbf{z}} \\
\mathbf{B}_{2}=x_{2} \mathbf{a}_{1}+x_{2} \mathbf{a}_{2}+x_{2} \mathbf{a}_{3}= & x_{2} c \hat{\mathbf{z}} \\
\mathbf{B}_{3}=x_{3} \mathbf{a}_{1}+x_{3} \mathbf{a}_{2}+x_{3} \mathbf{a}_{3}= & x_{3} c \hat{\mathbf{z}} \\
\mathbf{B}_{4}=x_{4} \mathbf{a}_{1}+x_{4} \mathbf{a}_{2}+x_{4} \mathbf{a}_{3}= & x_{4} c \hat{\mathbf{z}} \\
\mathbf{B}_{5}=x_{5} \mathbf{a}_{1}+y_{5} \mathbf{a}_{2}+z_{5} \mathbf{a}_{3}= & \frac{1}{2}\left(x_{5}-z_{5}\right) a \hat{\mathbf{x}}+\left(-\frac{1}{2 \sqrt{3}} x_{5}+\frac{1}{\sqrt{3}} y_{5}-\frac{1}{2 \sqrt{3}} z_{5}\right) a \hat{\mathbf{y}}+ \\
& & \frac{1}{3}\left(x_{5}+y_{5}+z_{5}\right) c \hat{\mathbf{z}}
\end{array}
$$

Cartesian Coordinates
Wyckoff Position Atom Type

(1a)

Fe I

(1a)

Fe II

(1a)

P I

(1a)

P II

(3b)

Se I 


$$
\begin{aligned}
& \mathbf{B}_{6}=z_{5} \mathbf{a}_{1}+x_{5} \mathbf{a}_{2}+y_{5} \mathbf{a}_{3}=\frac{1}{2}\left(-y_{5}+z_{5}\right) a \hat{\mathbf{x}}+\left(\frac{1}{\sqrt{3}} x_{5}-\frac{1}{2 \sqrt{3}} y_{5}-\frac{1}{2 \sqrt{3}} z_{5}\right) a \hat{\mathbf{y}}+ \\
& \frac{1}{3}\left(x_{5}+y_{5}+z_{5}\right) c \hat{\mathbf{z}} \\
& \mathbf{B}_{7}=y_{5} \mathbf{a}_{1}+z_{5} \mathbf{a}_{2}+x_{5} \mathbf{a}_{3}=\frac{1}{2}\left(-x_{5}+y_{5}\right) a \hat{\mathbf{x}}+\left(-\frac{1}{2 \sqrt{3}} x_{5}-\frac{1}{2 \sqrt{3}} y_{5}+\frac{1}{\sqrt{3}} z_{5}\right) a \hat{\mathbf{y}}+ \\
& \frac{1}{3}\left(x_{5}+y_{5}+z_{5}\right) c \hat{\mathbf{z}} \\
& \mathbf{B}_{8}=x_{6} \mathbf{a}_{1}+y_{6} \mathbf{a}_{2}+z_{6} \mathbf{a}_{3}=\frac{1}{2}\left(x_{6}-z_{6}\right) a \hat{\mathbf{x}}+\left(-\frac{1}{2 \sqrt{3}} x_{6}+\frac{1}{\sqrt{3}} y_{6}-\frac{1}{2 \sqrt{3}} z_{6}\right) a \hat{\mathbf{y}}+ \\
& \frac{1}{3}\left(x_{6}+y_{6}+z_{6}\right) c \hat{\mathbf{z}} \\
& \mathbf{B}_{9}=z_{6} \mathbf{a}_{1}+x_{6} \mathbf{a}_{2}+y_{6} \mathbf{a}_{3}=\frac{1}{2}\left(-y_{6}+z_{6}\right) a \hat{\mathbf{x}}+\left(\frac{1}{\sqrt{3}} x_{6}-\frac{1}{2 \sqrt{3}} y_{6}-\frac{1}{2 \sqrt{3}} z_{6}\right) a \hat{\mathbf{y}}+ \\
& \frac{1}{3}\left(x_{6}+y_{6}+z_{6}\right) c \hat{\mathbf{z}} \\
& \mathbf{B}_{10}=y_{6} \mathbf{a}_{1}+z_{6} \mathbf{a}_{2}+x_{6} \mathbf{a}_{3}=\frac{1}{2}\left(-x_{6}+y_{6}\right) a \hat{\mathbf{x}}+\left(-\frac{1}{2 \sqrt{3}} x_{6}-\frac{1}{2 \sqrt{3}} y_{6}+\frac{1}{\sqrt{3}} z_{6}\right) a \hat{\mathbf{y}}+ \\
& \frac{1}{3}\left(x_{6}+y_{6}+z_{6}\right) c \hat{\mathbf{z}}
\end{aligned}
$$

\section{References:}

- W. Klingen, G. Eulenberger, and H. Hahn, Uber die Kristallstrukturen von $\mathrm{Fe}_{2} \mathrm{P}_{2} \mathrm{Se}_{6}$ und $\mathrm{Fe}_{2} \mathrm{P}_{2} \mathrm{~S}_{6}$, Z. Anorg. Allg. Chem. 401, 97-112 (1973), doi:10.1002/zaac.19734010113.

\section{Found in:}

- P. Villars and K. Cenzual, Pearson's Crystal Data - Crystal Structure Database for Inorganic Compounds, ASM International (2013).

\section{Geometry files:}

- CIF: pp. 908

- POSCAR: pp. 909 


\section{Phenakite $\left(\mathrm{Be}_{2} \mathrm{SiO}_{4}, S 1_{3}\right)$ Structure:}

\section{A2B4C_hR42_148_2f_4f_f}
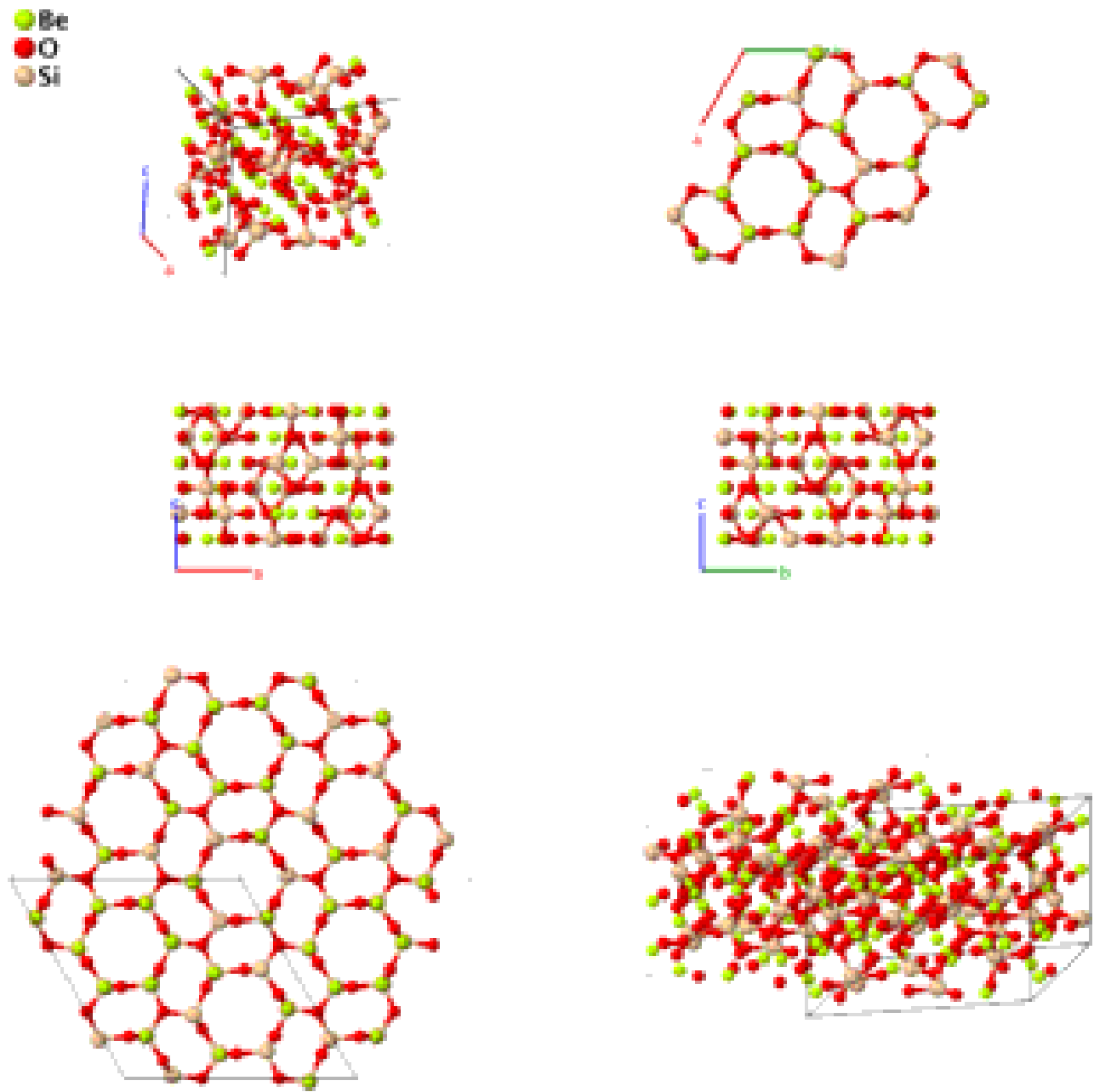

Prototype

: $\quad \mathrm{Be}_{2} \mathrm{SiO}_{4}$

AFLOW prototype label

: A2B4C_hR42_148_2f_4f_f

Strukturbericht designation : $\quad S 1_{3}$

Pearson symbol

: $\quad \mathrm{hR} 42$

Space group number $\quad: \quad 148$

Space group symbol $\quad: \quad R \overline{3}$

AFLOW prototype command : $\quad$ aflow --proto=A2B4C_hR42_148_2f_4f_f $[--h e x]$

- - params $=a, c / a, x_{1}, y_{1}, z_{1}, x_{2}, y_{2}, z_{2}, x_{3}, y_{3}, z_{3}, x_{4}, y_{4}, z_{4}, x_{5}, y_{5}, z_{5}, x_{6}, y_{6}, z_{6}, x_{7}$, $y_{7}, z_{7}$

Other compounds with this structure:

- $\mathrm{Zn}_{2} \mathrm{SiO}_{4}$ (willemite), $(\mathrm{Zn}, \mathrm{Mn})_{2} \mathrm{SiO}_{4}$ (troostite), $\mathrm{LiZnPO}_{4}$ 


\section{Rhombohedral primitive vectors:}

$$
\begin{array}{llc}
\mathbf{a}_{1}= & \frac{1}{2} a \hat{\mathbf{x}}-\frac{1}{2 \sqrt{3}} a \hat{\mathbf{y}}+\frac{1}{3} c \hat{\mathbf{z}} \\
\mathbf{a}_{2}= & \frac{1}{\sqrt{3}} a \hat{\mathbf{y}}+\frac{1}{3} c \hat{\mathbf{z}} \\
\mathbf{a}_{3}= & -\frac{1}{2} a \hat{\mathbf{x}}-\frac{1}{2 \sqrt{3}} a \hat{\mathbf{y}}+\frac{1}{3} c \hat{\mathbf{z}}
\end{array}
$$

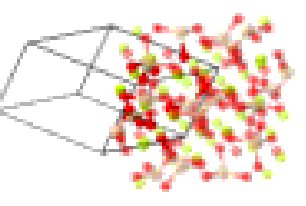

\section{Basis vectors:}

Lattice Coordinates

Cartesian Coordinates

$$
\begin{aligned}
& \mathbf{B}_{1}=x_{1} \mathbf{a}_{1}+y_{1} \mathbf{a}_{2}+z_{1} \mathbf{a}_{3}=\frac{1}{2}\left(x_{1}-z_{1}\right) a \hat{\mathbf{x}}+\left(-\frac{1}{2 \sqrt{3}} x_{1}+\frac{1}{\sqrt{3}} y_{1}-\frac{1}{2 \sqrt{3}} z_{1}\right) a \hat{\mathbf{y}}+ \\
& \frac{1}{3}\left(x_{1}+y_{1}+z_{1}\right) c \hat{\mathbf{z}} \\
& \mathbf{B}_{2}=z_{1} \mathbf{a}_{1}+x_{1} \mathbf{a}_{2}+y_{1} \mathbf{a}_{3}=\frac{1}{2}\left(-y_{1}+z_{1}\right) a \hat{\mathbf{x}}+\left(\frac{1}{\sqrt{3}} x_{1}-\frac{1}{2 \sqrt{3}} y_{1}-\frac{1}{2 \sqrt{3}} z_{1}\right) a \hat{\mathbf{y}}+ \\
& \frac{1}{3}\left(x_{1}+y_{1}+z_{1}\right) c \hat{\mathbf{z}} \\
& \mathbf{B}_{3}=y_{1} \mathbf{a}_{1}+z_{1} \mathbf{a}_{2}+x_{1} \mathbf{a}_{3}= \\
& \frac{1}{2}\left(-x_{1}+y_{1}\right) a \hat{\mathbf{x}}+ \\
& \left(-\frac{1}{2 \sqrt{3}} x_{1}-\frac{1}{2 \sqrt{3}} y_{1}+\frac{1}{\sqrt{3}} z_{1}\right) a \hat{\mathbf{y}}+ \\
& \frac{1}{3}\left(x_{1}+y_{1}+z_{1}\right) c \hat{\mathbf{z}} \\
& \mathbf{B}_{4}=-x_{1} \mathbf{a}_{1}-y_{1} \mathbf{a}_{2}-z_{1} \mathbf{a}_{3}=\frac{1}{2}\left(-x_{1}+z_{1}\right) a \hat{\mathbf{x}}+\left(\frac{1}{2 \sqrt{3}} x_{1}-\frac{1}{\sqrt{3}} y_{1}+\frac{1}{2 \sqrt{3}} z_{1}\right) a \hat{\mathbf{y}}- \\
& \frac{1}{3}\left(x_{1}+y_{1}+z_{1}\right) c \hat{\mathbf{z}}
\end{aligned}
$$$$
\mathbf{B}_{5}=-z_{1} \mathbf{a}_{1}-x_{1} \mathbf{a}_{2}-y_{1} \mathbf{a}_{3}=\frac{1}{2}\left(y_{1}-z_{1}\right) a \hat{\mathbf{x}}+\left(-\frac{1}{\sqrt{3}} x_{1}+\frac{1}{2 \sqrt{3}} y_{1}+\frac{1}{2 \sqrt{3}} z_{1}\right) a \hat{\mathbf{y}}-
$$$$
\frac{1}{3}\left(x_{1}+y_{1}+z_{1}\right) c \hat{\mathbf{z}}
$$$$
\mathbf{B}_{6}=-y_{1} \mathbf{a}_{1}-z_{1} \mathbf{a}_{2}-x_{1} \mathbf{a}_{3}=\frac{1}{2}\left(x_{1}-y_{1}\right) a \hat{\mathbf{x}}+\left(\frac{1}{2 \sqrt{3}} x_{1}+\frac{1}{2 \sqrt{3}} y_{1}-\frac{1}{\sqrt{3}} z_{1}\right) a \hat{\mathbf{y}}-
$$$$
\frac{1}{3}\left(x_{1}+y_{1}+z_{1}\right) c \hat{\mathbf{z}}
$$$$
\mathbf{B}_{7}=x_{2} \mathbf{a}_{1}+y_{2} \mathbf{a}_{2}+z_{2} \mathbf{a}_{3}=\frac{1}{2}\left(x_{2}-z_{2}\right) a \hat{\mathbf{x}}+\left(-\frac{1}{2 \sqrt{3}} x_{2}+\frac{1}{\sqrt{3}} y_{2}-\frac{1}{2 \sqrt{3}} z_{2}\right) a \hat{\mathbf{y}}+
$$$$
\frac{1}{3}\left(x_{2}+y_{2}+z_{2}\right) c \hat{\mathbf{z}}
$$$$
\mathbf{B}_{8}=z_{2} \mathbf{a}_{1}+x_{2} \mathbf{a}_{2}+y_{2} \mathbf{a}_{3}=\frac{1}{2}\left(-y_{2}+z_{2}\right) a \hat{\mathbf{x}}+\left(\frac{1}{\sqrt{3}} x_{2}-\frac{1}{2 \sqrt{3}} y_{2}-\frac{1}{2 \sqrt{3}} z_{2}\right) a \hat{\mathbf{y}}+
$$$$
\frac{1}{3}\left(x_{2}+y_{2}+z_{2}\right) c \hat{\mathbf{z}}
$$$$
\mathbf{B}_{9}=y_{2} \mathbf{a}_{1}+z_{2} \mathbf{a}_{2}+x_{2} \mathbf{a}_{3}=
$$$$
\frac{1}{2}\left(-x_{2}+y_{2}\right) a \hat{\mathbf{x}}+
$$$$
\left(-\frac{1}{2 \sqrt{3}} x_{2}-\frac{1}{2 \sqrt{3}} y_{2}+\frac{1}{\sqrt{3}} z_{2}\right) a \hat{\mathbf{y}}+
$$$$
\frac{1}{3}\left(x_{2}+y_{2}+z_{2}\right) c \hat{\mathbf{z}}
$$

$\mathbf{B}_{11}=-z_{2} \mathbf{a}_{1}-x_{2} \mathbf{a}_{2}-y_{2} \mathbf{a}_{3}=\frac{1}{2}\left(y_{2}-z_{2}\right) a \hat{\mathbf{x}}+\left(-\frac{1}{\sqrt{3}} x_{2}+\frac{1}{2 \sqrt{3}} y_{2}+\frac{1}{2 \sqrt{3}} z_{2}\right) a \hat{\mathbf{y}}-$ $\frac{1}{3}\left(x_{2}+y_{2}+z_{2}\right) c \hat{\mathbf{z}}$

$\mathbf{B}_{12}=-y_{2} \mathbf{a}_{1}-z_{2} \mathbf{a}_{2}-x_{2} \mathbf{a}_{3}=\frac{1}{2}\left(x_{2}-y_{2}\right) a \hat{\mathbf{x}}+\left(\frac{1}{2 \sqrt{3}} x_{2}+\frac{1}{2 \sqrt{3}} y_{2}-\frac{1}{\sqrt{3}} z_{2}\right) a \hat{\mathbf{y}}-$

$$
\frac{1}{3}\left(x_{2}+y_{2}+z_{2}\right) c \hat{\mathbf{z}}
$$

$\mathbf{B}_{13}=x_{3} \mathbf{a}_{1}+y_{3} \mathbf{a}_{2}+z_{3} \mathbf{a}_{3}=\frac{1}{2}\left(x_{3}-z_{3}\right) a \hat{\mathbf{x}}+\left(-\frac{1}{2 \sqrt{3}} x_{3}+\frac{1}{\sqrt{3}} y_{3}-\frac{1}{2 \sqrt{3}} z_{3}\right) a \hat{\mathbf{y}}+$ $\frac{1}{3}\left(x_{3}+y_{3}+z_{3}\right) c \hat{\mathbf{z}}$

$\mathbf{B}_{14}=z_{3} \mathbf{a}_{1}+x_{3} \mathbf{a}_{2}+y_{3} \mathbf{a}_{3}=\frac{1}{2}\left(-y_{3}+z_{3}\right) a \hat{\mathbf{x}}+\left(\frac{1}{\sqrt{3}} x_{3}-\frac{1}{2 \sqrt{3}} y_{3}-\frac{1}{2 \sqrt{3}} z_{3}\right) a \hat{\mathbf{y}}+$ $\frac{1}{3}\left(x_{3}+y_{3}+z_{3}\right) c \hat{\mathbf{z}}$
Be II

Be II

Be II

Be I

Be I

Be I

Be I

Be I

Be I

Be II

Be II

Be II

O I 


$$
\mathbf{B}_{15}=y_{3} \mathbf{a}_{1}+z_{3} \mathbf{a}_{2}+x_{3} \mathbf{a}_{3}=\begin{gathered}
\frac{1}{2}\left(-x_{3}+y_{3}\right) a \hat{\mathbf{x}}+ \\
\left(-\frac{1}{2 \sqrt{3}} x_{3}-\frac{1}{2 \sqrt{3}} y_{3}+\frac{1}{\sqrt{3}} z_{3}\right) a \hat{\mathbf{y}}+ \\
\frac{1}{3}\left(x_{3}+y_{3}+z_{3}\right) c \hat{\mathbf{z}}
\end{gathered}
$$

$\mathbf{B}_{16}=-x_{3} \mathbf{a}_{1}-y_{3} \mathbf{a}_{2}-z_{3} \mathbf{a}_{3}=\frac{1}{2}\left(-x_{3}+z_{3}\right) a \hat{\mathbf{x}}+\left(\frac{1}{2 \sqrt{3}} x_{3}-\frac{1}{\sqrt{3}} y_{3}+\frac{1}{2 \sqrt{3}} z_{3}\right) a \hat{\mathbf{y}}-$

$\mathbf{B}_{17}=-z_{3} \mathbf{a}_{1}-x_{3} \mathbf{a}_{2}-y_{3} \mathbf{a}_{3}=\frac{1}{2}\left(y_{3}-z_{3}\right) a \hat{\mathbf{x}}+\left(-\frac{1}{\sqrt{3}} x_{3}+\frac{1}{2 \sqrt{3}} y_{3}+\frac{1}{2 \sqrt{3}} z_{3}\right) a \hat{\mathbf{y}}-$

$\mathbf{B}_{18}=-y_{3} \mathbf{a}_{1}-z_{3} \mathbf{a}_{2}-x_{3} \mathbf{a}_{3}=\frac{1}{2}\left(x_{3}-y_{3}\right) a \hat{\mathbf{x}}+\left(\frac{1}{2 \sqrt{3}} x_{3}+\frac{1}{2 \sqrt{3}} y_{3}-\frac{1}{\sqrt{3}} z_{3}\right) a \hat{\mathbf{y}}-$

$\mathbf{B}_{19}=x_{4} \mathbf{a}_{1}+y_{4} \mathbf{a}_{2}+z_{4} \mathbf{a}_{3}=\begin{gathered}\frac{1}{2}\left(x_{4}-z_{4}\right) a \hat{\mathbf{x}}+\left(-\frac{1}{2 \sqrt{3}} x_{4}+\frac{1}{\sqrt{3}} y_{4}-\frac{1}{2 \sqrt{3}} z_{4}\right) a \hat{\mathbf{y}}+ \\ \frac{1}{3}\left(x_{4}+y_{4}+z_{4}\right) c \hat{\mathbf{z}}\end{gathered}$

O II

$\mathbf{B}_{20}=z_{4} \mathbf{a}_{1}+x_{4} \mathbf{a}_{2}+y_{4} \mathbf{a}_{3}=\frac{1}{2}\left(-y_{4}+z_{4}\right) a \hat{\mathbf{x}}+\left(\frac{1}{\sqrt{3}} x_{4}-\frac{1}{2 \sqrt{3}} y_{4}-\frac{1}{2 \sqrt{3}} z_{4}\right) a \hat{\mathbf{y}}+$

O II

$\mathbf{B}_{21}=y_{4} \mathbf{a}_{1}+z_{4} \mathbf{a}_{2}+x_{4} \mathbf{a}_{3}=$

$$
\frac{1}{3}\left(x_{4}+y_{4}+z_{4}\right) c \hat{\mathbf{z}}
$$

O II

$$
\begin{gathered}
\left(-\frac{1}{2 \sqrt{3}} x_{4}-\frac{1}{2 \sqrt{3}} y_{4}+\frac{1}{\sqrt{3}} z_{4}\right) a \hat{\mathbf{y}}+ \\
\frac{1}{3}\left(x_{4}+y_{4}+z_{4}\right) c \hat{\mathbf{z}}
\end{gathered}
$$

$\mathbf{B}_{22}=-x_{4} \mathbf{a}_{1}-y_{4} \mathbf{a}_{2}-z_{4} \mathbf{a}_{3}=\frac{1}{2}\left(-x_{4}+z_{4}\right) a \hat{\mathbf{x}}+\left(\frac{1}{2 \sqrt{3}} x_{4}-\frac{1}{\sqrt{3}} y_{4}+\frac{1}{2 \sqrt{3}} z_{4}\right) a \hat{\mathbf{y}}-$

O II

$\mathbf{B}_{23}=-z_{4} \mathbf{a}_{1}-x_{4} \mathbf{a}_{2}-y_{4} \mathbf{a}_{3}=\begin{gathered}\frac{1}{3}\left(x_{4}+y_{4}+z_{4}\right) c \hat{\mathbf{z}} \\ \frac{1}{2}\left(y_{4}-z_{4}\right) a \hat{\mathbf{x}}+\left(-\frac{1}{\sqrt{3}} x_{4}+\frac{1}{2 \sqrt{3}} y_{4}+\frac{1}{2 \sqrt{3}} z_{4}\right) a \hat{\mathbf{y}}- \\ \frac{1}{3}\left(x_{4}+y_{4}+z_{4}\right) c \hat{\mathbf{z}}\end{gathered}$

O II

$\mathbf{B}_{24}=-y_{4} \mathbf{a}_{1}-z_{4} \mathbf{a}_{2}-x_{4} \mathbf{a}_{3}=\frac{1}{2}\left(x_{4}-y_{4}\right) a \hat{\mathbf{x}}+\left(\frac{1}{2 \sqrt{3}} x_{4}+\frac{1}{2 \sqrt{3}} y_{4}-\frac{1}{\sqrt{3}} z_{4}\right) a \hat{\mathbf{y}}-$

O II

$\mathbf{B}_{25}=x_{5} \mathbf{a}_{1}+y_{5} \mathbf{a}_{2}+z_{5} \mathbf{a}_{3}=\frac{1}{2}\left(x_{5}-z_{5}\right) a \hat{\mathbf{x}}+\left(-\frac{1}{2 \sqrt{3}} x_{5}+\frac{1}{\sqrt{3}} y_{5}-\frac{1}{2 \sqrt{3}} z_{5}\right) a \hat{\mathbf{y}}+$

O III

$\mathbf{B}_{26}=z_{5} \mathbf{a}_{1}+x_{5} \mathbf{a}_{2}+y_{5} \mathbf{a}_{3}=\frac{1}{2}\left(-y_{5}+z_{5}\right) a \hat{\mathbf{x}}+\left(\frac{1}{\sqrt{3}} x_{5}-\frac{1}{2 \sqrt{3}} y_{5}-\frac{1}{2 \sqrt{3}} z_{5}\right) a \hat{\mathbf{y}}+$

O III

$\mathbf{B}_{27}=y_{5} \mathbf{a}_{1}+z_{5} \mathbf{a}_{2}+x_{5} \mathbf{a}_{3}=$

$$
\frac{1}{3}\left(x_{5}+y_{5}+z_{5}\right) c \hat{\mathbf{z}}
$$

$$
\begin{gathered}
\frac{1}{2}\left(-x_{5}+y_{5}\right) a \hat{\mathbf{x}}+ \\
\left(-\frac{1}{2 \sqrt{3}} x_{5}-\frac{1}{2 \sqrt{3}} y_{5}+\frac{1}{\sqrt{3}} z_{5}\right) a \hat{\mathbf{y}}+ \\
\frac{1}{3}\left(x_{5}+y_{5}+z_{5}\right) c \hat{\mathbf{z}}
\end{gathered}
$$

$\mathbf{B}_{28}=-x_{5} \mathbf{a}_{1}-y_{5} \mathbf{a}_{2}-z_{5} \mathbf{a}_{3}=\frac{1}{2}\left(-x_{5}+z_{5}\right) a \hat{\mathbf{x}}+\left(\frac{1}{2 \sqrt{3}} x_{5}-\frac{1}{\sqrt{3}} y_{5}+\frac{1}{2 \sqrt{3}} z_{5}\right) a \hat{\mathbf{y}}-$

O III

$\mathbf{B}_{29}=-z_{5} \mathbf{a}_{1}-x_{5} \mathbf{a}_{2}-y_{5} \mathbf{a}_{3}=\frac{1}{2}\left(y_{5}-z_{5}\right) a \hat{\mathbf{x}}+\left(-\frac{1}{\sqrt{3}} x_{5}+\frac{1}{2 \sqrt{3}} y_{5}+\frac{1}{2 \sqrt{3}} z_{5}\right) a \hat{\mathbf{y}}-$

O III

$\mathbf{B}_{30}=-y_{5} \mathbf{a}_{1}-z_{5} \mathbf{a}_{2}-x_{5} \mathbf{a}_{3}=\frac{1}{2}\left(x_{5}-y_{5}\right) a \hat{\mathbf{x}}+\left(\frac{1}{2 \sqrt{3}} x_{5}+\frac{1}{2 \sqrt{3}} y_{5}-\frac{1}{\sqrt{3}} z_{5}\right) a \hat{\mathbf{y}}-$

O III

$\mathbf{B}_{31}=x_{6} \mathbf{a}_{1}+y_{6} \mathbf{a}_{2}+z_{6} \mathbf{a}_{3}=\frac{1}{2}\left(x_{6}-z_{6}\right) a \hat{\mathbf{x}}+\left(-\frac{1}{2 \sqrt{3}} x_{6}+\frac{1}{\sqrt{3}} y_{6}-\frac{1}{2 \sqrt{3}} z_{6}\right) a \hat{\mathbf{y}}+$

O IV

$\mathbf{B}_{32}=z_{6} \mathbf{a}_{1}+x_{6} \mathbf{a}_{2}+y_{6} \mathbf{a}_{3}=\frac{1}{2}\left(-y_{6}+z_{6}\right) a \hat{\mathbf{x}}+\left(\frac{1}{\sqrt{3}} x_{6}-\frac{1}{2 \sqrt{3}} y_{6}-\frac{1}{2 \sqrt{3}} z_{6}\right) a \hat{\mathbf{y}}+$

O IV 


$$
\begin{aligned}
& \mathbf{B}_{33}=y_{6} \mathbf{a}_{1}+z_{6} \mathbf{a}_{2}+x_{6} \mathbf{a}_{3}=\begin{array}{c}
\frac{1}{2}\left(-x_{6}+y_{6}\right) a \hat{\mathbf{x}}+ \\
\left(-\frac{1}{2 \sqrt{3}} x_{6}-\frac{1}{2 \sqrt{3}} y_{6}+\frac{1}{\sqrt{3}} z_{6}\right) a \hat{\mathbf{y}}+ \\
\frac{1}{3}\left(x_{6}+y_{6}+z_{6}\right) c \hat{\mathbf{z}}
\end{array} \\
& \mathbf{B}_{34}=-x_{6} \mathbf{a}_{1}-y_{6} \mathbf{a}_{2}-z_{6} \mathbf{a}_{3}=\frac{1}{2}\left(-x_{6}+z_{6}\right) a \hat{\mathbf{x}}+\left(\frac{1}{2 \sqrt{3}} x_{6}-\frac{1}{\sqrt{3}} y_{6}+\frac{1}{2 \sqrt{3}} z_{6}\right) a \hat{\mathbf{y}}- \\
& \frac{1}{3}\left(x_{6}+y_{6}+z_{6}\right) c \hat{\mathbf{z}} \\
& \mathbf{B}_{35}=-z_{6} \mathbf{a}_{1}-x_{6} \mathbf{a}_{2}-y_{6} \mathbf{a}_{3}=\frac{1}{2}\left(y_{6}-z_{6}\right) a \hat{\mathbf{x}}+\left(-\frac{1}{\sqrt{3}} x_{6}+\frac{1}{2 \sqrt{3}} y_{6}+\frac{1}{2 \sqrt{3}} z_{6}\right) a \hat{\mathbf{y}}- \\
& \frac{1}{3}\left(x_{6}+y_{6}+z_{6}\right) c \hat{\mathbf{z}} \\
& \mathbf{B}_{36}=-y_{6} \mathbf{a}_{1}-z_{6} \mathbf{a}_{2}-x_{6} \mathbf{a}_{3}=\frac{1}{2}\left(x_{6}-y_{6}\right) a \hat{\mathbf{x}}+\left(\frac{1}{2 \sqrt{3}} x_{6}+\frac{1}{2 \sqrt{3}} y_{6}-\frac{1}{\sqrt{3}} z_{6}\right) a \hat{\mathbf{y}}- \\
& \frac{1}{3}\left(x_{6}+y_{6}+z_{6}\right) c \hat{\mathbf{z}} \\
& \mathbf{B}_{37}=x_{7} \mathbf{a}_{1}+y_{7} \mathbf{a}_{2}+z_{7} \mathbf{a}_{3}=\frac{1}{2}\left(x_{7}-z_{7}\right) a \hat{\mathbf{x}}+\left(-\frac{1}{2 \sqrt{3}} x_{7}+\frac{1}{\sqrt{3}} y_{7}-\frac{1}{2 \sqrt{3}} z_{7}\right) a \hat{\mathbf{y}}+ \\
& \frac{1}{3}\left(x_{7}+y_{7}+z_{7}\right) c \hat{\mathbf{z}} \\
& \mathbf{B}_{38}=z_{7} \mathbf{a}_{1}+x_{7} \mathbf{a}_{2}+y_{7} \mathbf{a}_{3}=\frac{1}{2}\left(-y_{7}+z_{7}\right) a \hat{\mathbf{x}}+\left(\frac{1}{\sqrt{3}} x_{7}-\frac{1}{2 \sqrt{3}} y_{7}-\frac{1}{2 \sqrt{3}} z_{7}\right) a \hat{\mathbf{y}}+ \\
& \frac{1}{3}\left(x_{7}+y_{7}+z_{7}\right) c \hat{\mathbf{z}} \\
& \mathbf{B}_{39}=y_{7} \mathbf{a}_{1}+z_{7} \mathbf{a}_{2}+x_{7} \mathbf{a}_{3}=\frac{1}{2}\left(-x_{7}+y_{7}\right) a \hat{\mathbf{x}}+ \\
& \left(-\frac{1}{2 \sqrt{3}} x_{7}-\frac{1}{2 \sqrt{3}} y_{7}+\frac{1}{\sqrt{3}} z_{7}\right) a \hat{\mathbf{y}}+ \\
& \frac{1}{3}\left(x_{7}+y_{7}+z_{7}\right) c \hat{\mathbf{z}} \\
& \mathbf{B}_{40}=-x_{7} \mathbf{a}_{1}-y_{7} \mathbf{a}_{2}-z_{7} \mathbf{a}_{3}=\frac{1}{2}\left(-x_{7}+z_{7}\right) a \hat{\mathbf{x}}+\left(\frac{1}{2 \sqrt{3}} x_{7}-\frac{1}{\sqrt{3}} y_{7}+\frac{1}{2 \sqrt{3}} z_{7}\right) a \hat{\mathbf{y}}- \\
& \frac{1}{3}\left(x_{7}+y_{7}+z_{7}\right) c \hat{\mathbf{z}} \\
& \mathbf{B}_{41}=-z_{7} \mathbf{a}_{1}-x_{7} \mathbf{a}_{2}-y_{7} \mathbf{a}_{3}=\frac{1}{2}\left(y_{7}-z_{7}\right) a \hat{\mathbf{x}}+\left(-\frac{1}{\sqrt{3}} x_{7}+\frac{1}{2 \sqrt{3}} y_{7}+\frac{1}{2 \sqrt{3}} z_{7}\right) a \hat{\mathbf{y}}- \\
& \frac{1}{3}\left(x_{7}+y_{7}+z_{7}\right) c \hat{\mathbf{z}} \\
& \mathbf{B}_{42}=-y_{7} \mathbf{a}_{1}-z_{7} \mathbf{a}_{2}-x_{7} \mathbf{a}_{3}=\frac{1}{2}\left(x_{7}-y_{7}\right) a \hat{\mathbf{x}}+\left(\frac{1}{2 \sqrt{3}} x_{7}+\frac{1}{2 \sqrt{3}} y_{7}-\frac{1}{\sqrt{3}} z_{7}\right) a \hat{\mathbf{y}}- \\
& \frac{1}{3}\left(x_{7}+y_{7}+z_{7}\right) c \hat{\mathbf{z}}
\end{aligned}
$$

\section{References:}

- R. M. Hazen and L. W. Finger, High-Temperature Crystal Chemistry of Phenakite $\left(\mathrm{Be}_{2} \mathrm{SiO}_{4}\right)$ and Chrysoberyl $\left(\mathrm{BeAl}_{2} \mathrm{O}_{4}\right)$, Phys. Chem. Miner. 14, 426-434 (1987), doi:10.1007/BF00628819.

\section{Geometry files:}

- CIF: pp. 909

- POSCAR: pp. 909 


\section{$\beta-\mathrm{PdCl}_{2}$ Structure: A2B_hR18_148_2f_f}
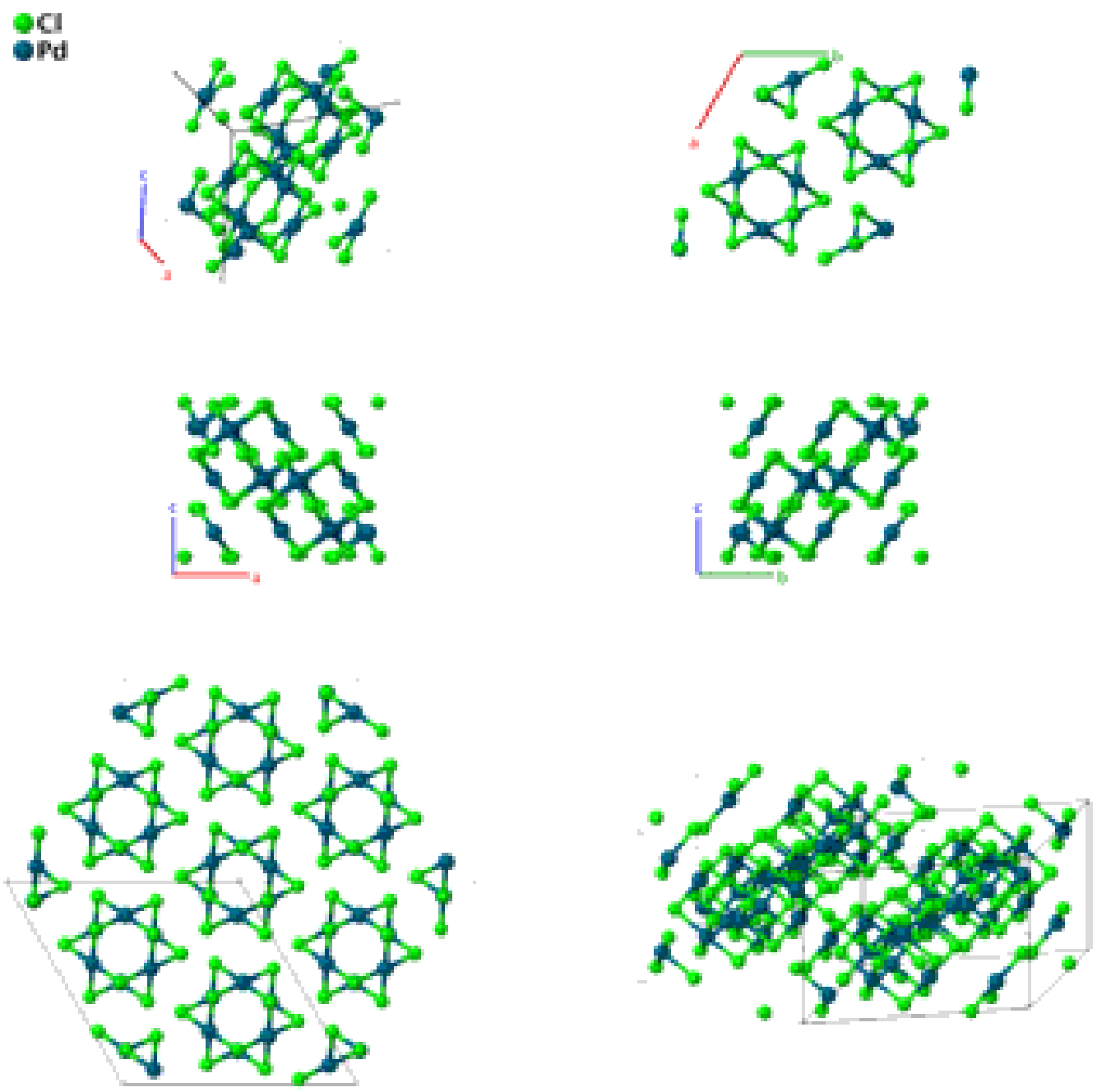

Prototype

AFLOW prototype label

Strukturbericht designation

Pearson symbol

Space group number

Space group symbol

AFLOW prototype command
: $\quad \beta-\mathrm{PdCl}_{2}$

: A2B_hR18_148_2f_f

: None

: $\quad$ hR18

: $\quad 148$

: $R \overline{3}$ 


\section{Rhombohedral primitive vectors:}

$$
\begin{array}{llc}
\mathbf{a}_{1}= & \frac{1}{2} a \hat{\mathbf{x}}-\frac{1}{2 \sqrt{3}} a \hat{\mathbf{y}}+\frac{1}{3} c \hat{\mathbf{z}} \\
\mathbf{a}_{2}= & \frac{1}{\sqrt{3}} a \hat{\mathbf{y}}+\frac{1}{3} c \hat{\mathbf{z}} \\
\mathbf{a}_{3}= & -\frac{1}{2} a \hat{\mathbf{x}}-\frac{1}{2 \sqrt{3}} a \hat{\mathbf{y}}+\frac{1}{3} c \hat{\mathbf{z}}
\end{array}
$$

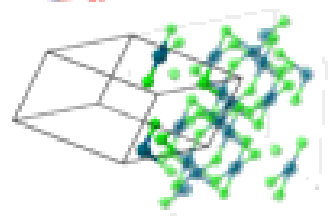

\section{Basis vectors:}

Lattice Coordinates

Cartesian Coordinates

$$
\begin{aligned}
& \mathbf{B}_{1}=x_{1} \mathbf{a}_{1}+y_{1} \mathbf{a}_{2}+z_{1} \mathbf{a}_{3}=\frac{1}{2}\left(x_{1}-z_{1}\right) a \hat{\mathbf{x}}+\left(-\frac{1}{2 \sqrt{3}} x_{1}+\frac{1}{\sqrt{3}} y_{1}-\frac{1}{2 \sqrt{3}} z_{1}\right) a \hat{\mathbf{y}}+ \\
& \frac{1}{3}\left(x_{1}+y_{1}+z_{1}\right) c \hat{\mathbf{z}} \\
& \mathbf{B}_{2}=z_{1} \mathbf{a}_{1}+x_{1} \mathbf{a}_{2}+y_{1} \mathbf{a}_{3}=\frac{1}{2}\left(-y_{1}+z_{1}\right) a \hat{\mathbf{x}}+\left(\frac{1}{\sqrt{3}} x_{1}-\frac{1}{2 \sqrt{3}} y_{1}-\frac{1}{2 \sqrt{3}} z_{1}\right) a \hat{\mathbf{y}}+ \\
& \frac{1}{3}\left(x_{1}+y_{1}+z_{1}\right) c \hat{\mathbf{z}} \\
& \mathbf{B}_{3}=y_{1} \mathbf{a}_{1}+z_{1} \mathbf{a}_{2}+x_{1} \mathbf{a}_{3}= \\
& \frac{1}{2}\left(-x_{1}+y_{1}\right) a \hat{\mathbf{x}}+ \\
& \left(-\frac{1}{2 \sqrt{3}} x_{1}-\frac{1}{2 \sqrt{3}} y_{1}+\frac{1}{\sqrt{3}} z_{1}\right) a \hat{\mathbf{y}}+ \\
& \frac{1}{3}\left(x_{1}+y_{1}+z_{1}\right) c \hat{\mathbf{z}}
\end{aligned}
$$$$
\mathbf{B}_{4}=-x_{1} \mathbf{a}_{1}-y_{1} \mathbf{a}_{2}-z_{1} \mathbf{a}_{3}=\frac{1}{2}\left(-x_{1}+z_{1}\right) a \hat{\mathbf{x}}+\left(\frac{1}{2 \sqrt{3}} x_{1}-\frac{1}{\sqrt{3}} y_{1}+\frac{1}{2 \sqrt{3}} z_{1}\right) a \hat{\mathbf{y}}-
$$$$
\frac{1}{3}\left(x_{1}+y_{1}+z_{1}\right) c \hat{\mathbf{z}}
$$$$
\mathbf{B}_{5}=-z_{1} \mathbf{a}_{1}-x_{1} \mathbf{a}_{2}-y_{1} \mathbf{a}_{3}=\frac{1}{2}\left(y_{1}-z_{1}\right) a \hat{\mathbf{x}}+\left(-\frac{1}{\sqrt{3}} x_{1}+\frac{1}{2 \sqrt{3}} y_{1}+\frac{1}{2 \sqrt{3}} z_{1}\right) a \hat{\mathbf{y}}-
$$$$
\frac{1}{3}\left(x_{1}+y_{1}+z_{1}\right) c \hat{\mathbf{z}}
$$$$
\mathbf{B}_{6}=-y_{1} \mathbf{a}_{1}-z_{1} \mathbf{a}_{2}-x_{1} \mathbf{a}_{3}=\frac{1}{2}\left(x_{1}-y_{1}\right) a \hat{\mathbf{x}}+\left(\frac{1}{2 \sqrt{3}} x_{1}+\frac{1}{2 \sqrt{3}} y_{1}-\frac{1}{\sqrt{3}} z_{1}\right) a \hat{\mathbf{y}}-
$$$$
\frac{1}{3}\left(x_{1}+y_{1}+z_{1}\right) c \hat{\mathbf{z}}
$$$$
\mathbf{B}_{7}=x_{2} \mathbf{a}_{1}+y_{2} \mathbf{a}_{2}+z_{2} \mathbf{a}_{3}=\frac{1}{2}\left(x_{2}-z_{2}\right) a \hat{\mathbf{x}}+\left(-\frac{1}{2 \sqrt{3}} x_{2}+\frac{1}{\sqrt{3}} y_{2}-\frac{1}{2 \sqrt{3}} z_{2}\right) a \hat{\mathbf{y}}+
$$$$
\frac{1}{3}\left(x_{2}+y_{2}+z_{2}\right) c \hat{\mathbf{z}}
$$$$
\mathbf{B}_{8}=z_{2} \mathbf{a}_{1}+x_{2} \mathbf{a}_{2}+y_{2} \mathbf{a}_{3}=\frac{1}{2}\left(-y_{2}+z_{2}\right) a \hat{\mathbf{x}}+\left(\frac{1}{\sqrt{3}} x_{2}-\frac{1}{2 \sqrt{3}} y_{2}-\frac{1}{2 \sqrt{3}} z_{2}\right) a \hat{\mathbf{y}}+
$$$$
\frac{1}{3}\left(x_{2}+y_{2}+z_{2}\right) c \hat{\mathbf{z}}
$$$$
\mathbf{B}_{9}=y_{2} \mathbf{a}_{1}+z_{2} \mathbf{a}_{2}+x_{2} \mathbf{a}_{3}=
$$$$
\frac{1}{2}\left(-x_{2}+y_{2}\right) a \hat{\mathbf{x}}+
$$$$
\left(-\frac{1}{2 \sqrt{3}} x_{2}-\frac{1}{2 \sqrt{3}} y_{2}+\frac{1}{\sqrt{3}} z_{2}\right) a \hat{\mathbf{y}}+
$$$$
\frac{1}{3}\left(x_{2}+y_{2}+z_{2}\right) c \hat{\mathbf{z}}
$$$$
\mathbf{B}_{10}=-x_{2} \mathbf{a}_{1}-y_{2} \mathbf{a}_{2}-z_{2} \mathbf{a}_{3}=\frac{1}{2}\left(-x_{2}+z_{2}\right) a \hat{\mathbf{x}}+\left(\frac{1}{2 \sqrt{3}} x_{2}-\frac{1}{\sqrt{3}} y_{2}+\frac{1}{2 \sqrt{3}} z_{2}\right) a \hat{\mathbf{y}}-
$$$$
\frac{1}{3}\left(x_{2}+y_{2}+z_{2}\right) c \hat{\mathbf{z}}
$$$$
\mathbf{B}_{11}=-z_{2} \mathbf{a}_{1}-x_{2} \mathbf{a}_{2}-y_{2} \mathbf{a}_{3}=\frac{1}{2}\left(y_{2}-z_{2}\right) a \hat{\mathbf{x}}+\left(-\frac{1}{\sqrt{3}} x_{2}+\frac{1}{2 \sqrt{3}} y_{2}+\frac{1}{2 \sqrt{3}} z_{2}\right) a \hat{\mathbf{y}}-
$$$$
\frac{1}{3}\left(x_{2}+y_{2}+z_{2}\right) c \hat{\mathbf{z}}
$$$$
\mathbf{B}_{12}=-y_{2} \mathbf{a}_{1}-z_{2} \mathbf{a}_{2}-x_{2} \mathbf{a}_{3}=\frac{1}{2}\left(x_{2}-y_{2}\right) a \hat{\mathbf{x}}+\left(\frac{1}{2 \sqrt{3}} x_{2}+\frac{1}{2 \sqrt{3}} y_{2}-\frac{1}{\sqrt{3}} z_{2}\right) a \hat{\mathbf{y}}-
$$$$
\frac{1}{3}\left(x_{2}+y_{2}+z_{2}\right) c \hat{\mathbf{z}}
$$$$
\mathbf{B}_{13}=x_{3} \mathbf{a}_{1}+y_{3} \mathbf{a}_{2}+z_{3} \mathbf{a}_{3}=\frac{1}{2}\left(x_{3}-z_{3}\right) a \hat{\mathbf{x}}+\left(-\frac{1}{2 \sqrt{3}} x_{3}+\frac{1}{\sqrt{3}} y_{3}-\frac{1}{2 \sqrt{3}} z_{3}\right) a \hat{\mathbf{y}}+
$$$$
\frac{1}{3}\left(x_{3}+y_{3}+z_{3}\right) c \hat{\mathbf{z}}
$$$$
\mathbf{B}_{14}=z_{3} \mathbf{a}_{1}+x_{3} \mathbf{a}_{2}+y_{3} \mathbf{a}_{3}=\frac{1}{2}\left(-y_{3}+z_{3}\right) a \hat{\mathbf{x}}+\left(\frac{1}{\sqrt{3}} x_{3}-\frac{1}{2 \sqrt{3}} y_{3}-\frac{1}{2 \sqrt{3}} z_{3}\right) a \hat{\mathbf{y}}+
$$$$
\frac{1}{3}\left(x_{3}+y_{3}+z_{3}\right) c \hat{\mathbf{z}}
$$

Wyckoff Position Atom Type

Cl I

Cl I

Cl II

Cl II

Cl II

Cl II

Cl II

Pd 


$$
\begin{aligned}
& \mathbf{B}_{15}=y_{3} \mathbf{a}_{1}+z_{3} \mathbf{a}_{2}+x_{3} \mathbf{a}_{3}=\quad \frac{1}{2}\left(-x_{3}+y_{3}\right) a \hat{\mathbf{x}}+\quad \quad \quad \quad \text { Pd } \\
& \left(-\frac{1}{2 \sqrt{3}} x_{3}-\frac{1}{2 \sqrt{3}} y_{3}+\frac{1}{\sqrt{3}} z_{3}\right) a \hat{\mathbf{y}}+ \\
& \frac{1}{3}\left(x_{3}+y_{3}+z_{3}\right) c \hat{\mathbf{z}} \\
& \mathbf{B}_{16}=-x_{3} \mathbf{a}_{1}-y_{3} \mathbf{a}_{2}-z_{3} \mathbf{a}_{3}=\frac{1}{2}\left(-x_{3}+z_{3}\right) a \hat{\mathbf{x}}+\left(\frac{1}{2 \sqrt{3}} x_{3}-\frac{1}{\sqrt{3}} y_{3}+\frac{1}{2 \sqrt{3}} z_{3}\right) a \hat{\mathbf{y}}-\quad \text { (6f) } \quad \mathrm{Pd} \\
& \frac{1}{3}\left(x_{3}+y_{3}+z_{3}\right) c \hat{\mathbf{z}} \\
& \mathbf{B}_{17}=-z_{3} \mathbf{a}_{1}-x_{3} \mathbf{a}_{2}-y_{3} \mathbf{a}_{3}=\frac{1}{2}\left(y_{3}-z_{3}\right) a \hat{\mathbf{x}}+\left(-\frac{1}{\sqrt{3}} x_{3}+\frac{1}{2 \sqrt{3}} y_{3}+\frac{1}{2 \sqrt{3}} z_{3}\right) a \hat{\mathbf{y}}-\quad \frac{1}{3}\left(x_{3}+y_{3}+z_{3}\right) c \hat{\mathbf{z}} \\
& \mathbf{B}_{18}=-y_{3} \mathbf{a}_{1}-z_{3} \mathbf{a}_{2}-x_{3} \mathbf{a}_{3}=\frac{1}{2}\left(x_{3}-y_{3}\right) a \hat{\mathbf{x}}+\left(\frac{1}{2 \sqrt{3}} x_{3}+\frac{1}{2 \sqrt{3}} y_{3}-\frac{1}{\sqrt{3}} z_{3}\right) a \hat{\mathbf{y}}-\quad \text { (6f) } \\
& \frac{1}{3}\left(x_{3}+y_{3}+z_{3}\right) c \hat{\mathbf{z}}
\end{aligned}
$$

\section{References:}

- D. B. Dell'Amico, F. Calderazzo, F. Marchetti, and S. Ramello, Molecular Structure of $\left[\mathrm{Pd}_{6} \mathrm{Cl}_{12}\right]$ in Single Crystals Chemically Grown at Room Temperature, Angew. Chem. Int. Ed. 35, 1331-1333 (1996), doi:10.1002/anie.199613311. - P. Villars and K. Cenzual, eds., Structure Types (Springer, Berlin, Heidelberg, 2010), Landolt-Börnstein - Group III Condensed Matter, vol. 43A8, chap. Part 8: SpaceGroups (156) P3m1 - (148) R-3, doi:10.1007/978-3-540-70892-6_423.

\section{Geometry files:}

- CIF: pp. 909

- POSCAR: pp. 910 


\section{$\mathrm{Ti}_{3} \mathrm{O}$ (Room-temperature) Structure:}

\section{AB3_hP24_149_acgi_31}
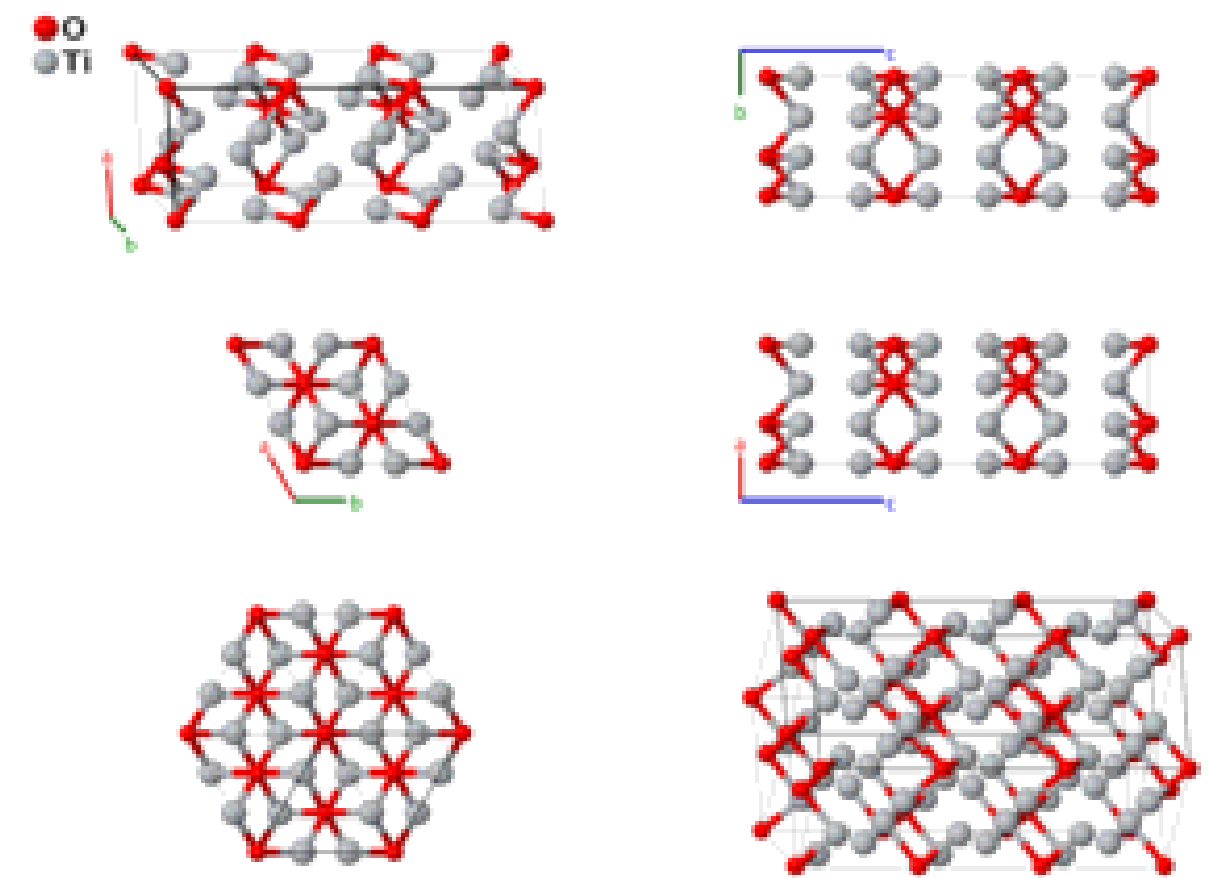

Prototype

AFLOW prototype label

$: \quad \mathrm{Ti}_{3} \mathrm{O}$

Strukturbericht designation : None

Pearson symbol : $\quad \mathrm{hP} 24$

Space group number : $\quad 149$

Space group symbol $\quad: \quad P 312$

AFLOW prototype command : aflow --proto=AB3_hP24_149_acgi_31

- - params $=a, c / a, z_{3}, z_{4}, x_{5}, y_{5}, z_{5}, x_{6}, y_{6}, z_{6}, x_{7}, y_{7}, z_{7}$

Trigonal Hexagonal primitive vectors:

$$
\begin{array}{ll}
\mathbf{a}_{1}= & \frac{1}{2} a \hat{\mathbf{x}}-\frac{\sqrt{3}}{2} a \hat{\mathbf{y}} \\
\mathbf{a}_{2}= & \frac{1}{2} a \hat{\mathbf{x}}+\frac{\sqrt{3}}{2} a \hat{\mathbf{y}} \\
\mathbf{a}_{3}= & c \hat{\mathbf{z}}
\end{array}
$$

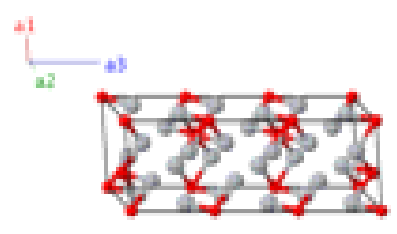

Basis vectors:

Lattice Coordinates

$\begin{array}{lcc}\mathbf{B}_{1}= & 0 \mathbf{a}_{1}+0 \mathbf{a}_{2}+0 \mathbf{a}_{3} \\ \mathbf{B}_{2}= & \frac{1}{3} \mathbf{a}_{1}+\frac{2}{3} \mathbf{a}_{2} \\ \mathbf{B}_{3}= & z_{3} \mathbf{a}_{3} \\ \mathbf{B}_{4}= & -z_{3} \mathbf{a}_{3}\end{array}$

Cartesian Coordinates

$=$

$=$

$=$

$=$

$$
\begin{gathered}
0 \hat{\mathbf{x}}+0 \hat{\mathbf{y}}+0 \hat{\mathbf{z}} \\
\frac{1}{2} a \hat{\mathbf{x}}+\frac{1}{2 \sqrt{3}} a \hat{\mathbf{y}} \\
z_{3} c \hat{\mathbf{z}}
\end{gathered}
$$$$
-z_{3} c \hat{\mathbf{z}}
$$

Wyckoff Position

Atom Type

(1a)

O I

(1c)

O II

(2g)

O III

(2g)

O III 


\begin{tabular}{|c|c|c|c|c|c|}
\hline $\mathbf{B}_{5}$ & $=$ & $\frac{2}{3} \mathbf{a}_{1}+\frac{1}{3} \mathbf{a}_{2}+z_{4} \mathbf{a}_{3}$ & $\frac{1}{2} a \hat{\mathbf{x}}-\frac{1}{2 \sqrt{3}} a \hat{\mathbf{y}}+z_{4} c \hat{\mathbf{z}}$ & $(2 i)$ & O IV \\
\hline $\mathbf{B}_{6}$ & $=$ & $\frac{2}{3} \mathbf{a}_{1}+\frac{1}{3} \mathbf{a}_{2}-z_{4} \mathbf{a}_{3}$ & $\frac{1}{2} a \hat{\mathbf{x}}-\frac{1}{2 \sqrt{3}} a \hat{\mathbf{y}}-z_{4} c \hat{\mathbf{z}}$ & $(2 i)$ & O IV \\
\hline $\mathbf{B}_{7}$ & $=$ & $x_{5} \mathbf{a}_{1}+y_{5} \mathbf{a}_{2}+z_{5} \mathbf{a}_{3}$ & $=\frac{1}{2}\left(x_{5}+y_{5}\right) a \hat{\mathbf{x}}+\frac{\sqrt{3}}{2}\left(-x_{5}+y_{5}\right) a \hat{\mathbf{y}}+z_{5} c \hat{\mathbf{z}}$ & $(6 l)$ & Ti I \\
\hline $\mathbf{B}_{8}$ & $=$ & $-y_{5} \mathbf{a}_{1}+\left(x_{5}-y_{5}\right) \mathbf{a}_{2}+z_{5} \mathbf{a}_{3}$ & $\left(\frac{1}{2} x_{5}-y_{5}\right) a \hat{\mathbf{x}}+\frac{\sqrt{3}}{2} x_{5} a \hat{\mathbf{y}}+z_{5} c \hat{\mathbf{z}}$ & $(6 l)$ & Ti I \\
\hline $\mathbf{B}_{9}$ & $=$ & $\left(-x_{5}+y_{5}\right) \mathbf{a}_{1}-x_{5} \mathbf{a}_{2}+z_{5} \mathbf{a}_{3}$ & $\left(-x_{5}+\frac{1}{2} y_{5}\right) a \hat{\mathbf{x}}-\frac{\sqrt{3}}{2} y_{5} a \hat{\mathbf{y}}+z_{5} c \hat{\mathbf{z}}$ & $(6 l)$ & Ti I \\
\hline $\mathbf{B}_{10}$ & $=$ & $-y_{5} \mathbf{a}_{1}-x_{5} \mathbf{a}_{2}-z_{5} \mathbf{a}_{3}$ & $\begin{array}{c}-\frac{1}{2}\left(x_{5}+y_{5}\right) a \hat{\mathbf{x}}+\frac{\sqrt{3}}{2}\left(-x_{5}+y_{5}\right) a \hat{\mathbf{y}}- \\
z_{5} c \hat{\mathbf{z}}\end{array}$ & $(6 l)$ & Ti I \\
\hline $\mathbf{B}_{11}$ & $=$ & $\left(-x_{5}+y_{5}\right) \mathbf{a}_{1}+y_{5} \mathbf{a}_{2}-z_{5} \mathbf{a}_{3}$ & $\left(-\frac{1}{2} x_{5}+y_{5}\right) a \hat{\mathbf{x}}+\frac{\sqrt{3}}{2} x_{5} a \hat{\mathbf{y}}-z_{5} c \hat{\mathbf{z}}$ & $(6 l)$ & Ti I \\
\hline $\mathbf{B}_{12}$ & $=$ & $x_{5} \mathbf{a}_{1}+\left(x_{5}-y_{5}\right) \mathbf{a}_{2}-z_{5} \mathbf{a}_{3}$ & $\left(x_{5}-\frac{1}{2} y_{5}\right) a \hat{\mathbf{x}}-\frac{\sqrt{3}}{2} y_{5} a \hat{\mathbf{y}}-z_{5} c \hat{\mathbf{z}}$ & $(6 l)$ & Ti I \\
\hline $\mathbf{B}_{13}$ & $=$ & $x_{6} \mathbf{a}_{1}+y_{6} \mathbf{a}_{2}+z_{6} \mathbf{a}_{3}$ & $=\frac{1}{2}\left(x_{6}+y_{6}\right) a \hat{\mathbf{x}}+\frac{\sqrt{3}}{2}\left(-x_{6}+y_{6}\right) a \hat{\mathbf{y}}+z_{6} c \hat{\mathbf{z}}$ & $(6 l)$ & Ti II \\
\hline $\mathbf{B}_{14}$ & $=$ & $-y_{6} \mathbf{a}_{1}+\left(x_{6}-y_{6}\right) \mathbf{a}_{2}+z_{6} \mathbf{a}_{3}$ & $\left(\frac{1}{2} x_{6}-y_{6}\right) a \hat{\mathbf{x}}+\frac{\sqrt{3}}{2} x_{6} a \hat{\mathbf{y}}+z_{6} c \hat{\mathbf{z}}$ & $(6 l)$ & Ti II \\
\hline $\mathbf{B}_{15}$ & $=$ & $\left(-x_{6}+y_{6}\right) \mathbf{a}_{1}-x_{6} \mathbf{a}_{2}+z_{6} \mathbf{a}_{3}$ & $\left(-x_{6}+\frac{1}{2} y_{6}\right) a \hat{\mathbf{x}}-\frac{\sqrt{3}}{2} y_{6} a \hat{\mathbf{y}}+z_{6} c \hat{\mathbf{z}}$ & $(6 l)$ & Ti II \\
\hline $\mathbf{B}_{16}$ & $=$ & $-y_{6} \mathbf{a}_{1}-x_{6} \mathbf{a}_{2}-z_{6} \mathbf{a}_{3}$ & $\begin{array}{c}-\frac{1}{2}\left(x_{6}+y_{6}\right) a \hat{\mathbf{x}}+\frac{\sqrt{3}}{2}\left(-x_{6}+y_{6}\right) a \hat{\mathbf{y}}- \\
z_{6} c \hat{\mathbf{z}}\end{array}$ & $(6 l)$ & Ti II \\
\hline $\mathbf{B}_{17}$ & $=$ & $\left(-x_{6}+y_{6}\right) \mathbf{a}_{1}+y_{6} \mathbf{a}_{2}-z_{6} \mathbf{a}_{3}$ & $\left(-\frac{1}{2} x_{6}+y_{6}\right) a \hat{\mathbf{x}}+\frac{\sqrt{3}}{2} x_{6} a \hat{\mathbf{y}}-z_{6} c \hat{\mathbf{z}}$ & $(6 l)$ & Ti II \\
\hline $\mathbf{B}_{18}$ & $=$ & $x_{6} \mathbf{a}_{1}+\left(x_{6}-y_{6}\right) \mathbf{a}_{2}-z_{6} \mathbf{a}_{3}$ & $\left(x_{6}-\frac{1}{2} y_{6}\right) a \hat{\mathbf{x}}-\frac{\sqrt{3}}{2} y_{6} a \hat{\mathbf{y}}-z_{6} c \hat{\mathbf{z}}$ & $(6 l)$ & Ti II \\
\hline $\mathbf{B}_{19}$ & $=$ & $x_{7} \mathbf{a}_{1}+y_{7} \mathbf{a}_{2}+z_{7} \mathbf{a}_{3}$ & $=\frac{1}{2}\left(x_{7}+y_{7}\right) a \hat{\mathbf{x}}+\frac{\sqrt{3}}{2}\left(-x_{7}+y_{7}\right) a \hat{\mathbf{y}}+z_{7} c \hat{\mathbf{z}}$ & $(6 l)$ & Ti III \\
\hline $\mathbf{B}_{20}$ & $=$ & $-y_{7} \mathbf{a}_{1}+\left(x_{7}-y_{7}\right) \mathbf{a}_{2}+z_{7} \mathbf{a}_{3}$ & $\left(\frac{1}{2} x_{7}-y_{7}\right) a \hat{\mathbf{x}}+\frac{\sqrt{3}}{2} x_{7} a \hat{\mathbf{y}}+z_{7} c \hat{\mathbf{z}}$ & $(6 l)$ & Ti III \\
\hline $\mathbf{B}_{21}$ & $=$ & $\left(-x_{7}+y_{7}\right) \mathbf{a}_{1}-x_{7} \mathbf{a}_{2}+z_{7} \mathbf{a}_{3}$ & $\left(-x_{7}+\frac{1}{2} y_{7}\right) a \hat{\mathbf{x}}-\frac{\sqrt{3}}{2} y_{7} a \hat{\mathbf{y}}+z_{7} c \hat{\mathbf{z}}$ & $(6 l)$ & Ti III \\
\hline $\mathbf{B}_{22}$ & $=$ & $-y_{7} \mathbf{a}_{1}-x_{7} \mathbf{a}_{2}-z_{7} \mathbf{a}_{3}$ & $\begin{array}{c}-\frac{1}{2}\left(x_{7}+y_{7}\right) a \hat{\mathbf{x}}+\frac{\sqrt{3}}{2}\left(-x_{7}+y_{7}\right) a \hat{\mathbf{y}}- \\
z_{7} c \hat{\mathbf{z}}\end{array}$ & $(6 l)$ & Ti III \\
\hline $\mathbf{B}_{23}$ & $=$ & $\left(-x_{7}+y_{7}\right) \mathbf{a}_{1}+y_{7} \mathbf{a}_{2}-z_{7} \mathbf{a}_{3}$ & $\left(-\frac{1}{2} x_{7}+y_{7}\right) a \hat{\mathbf{x}}+\frac{\sqrt{3}}{2} x_{7} a \hat{\mathbf{y}}-z_{7} c \hat{\mathbf{z}}$ & $(6 l)$ & Ti III \\
\hline $\mathbf{B}_{24}$ & $=$ & $x_{7} \mathbf{a}_{1}+\left(x_{7}-y_{7}\right) \mathbf{a}_{2}-z_{7} \mathbf{a}_{3}$ & $\left(x_{7}-\frac{1}{2} y_{7}\right) a \hat{\mathbf{x}}-\frac{\sqrt{3}}{2} y_{7} a \hat{\mathbf{y}}-z_{7} c \hat{\mathbf{z}}$ & $(6 l)$ & Ti III \\
\hline
\end{tabular}

\section{References:}

- A. Jostsons and A. S. Malin, The ordered structure of Ti ${ }_{3} O$, Acta Crystallogr. Sect. B Struct. Sci. 24, 211-213 (1968), doi:10.1107/S0567740868001974.

\section{Found in:}

- P. Villars and K. Cenzual, Pearson's Crystal Data - Crystal Structure Database for Inorganic Compounds, ASM International (2013).

\section{Geometry files:}

- CIF: pp. 910

- POSCAR: pp. 910 


\section{$\mathrm{CrCl}_{3}$ Structure: A3B_hP24_153_3c_2b}
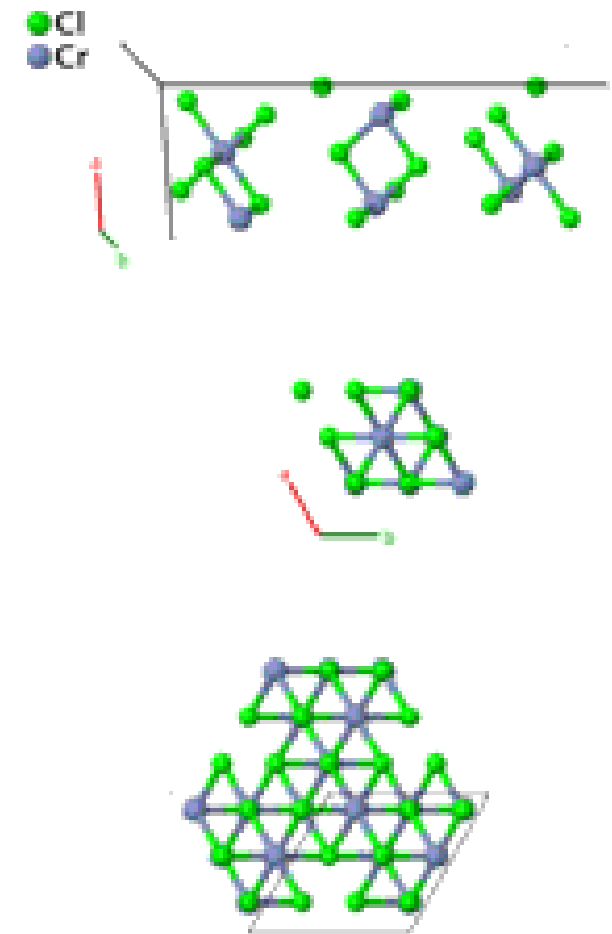
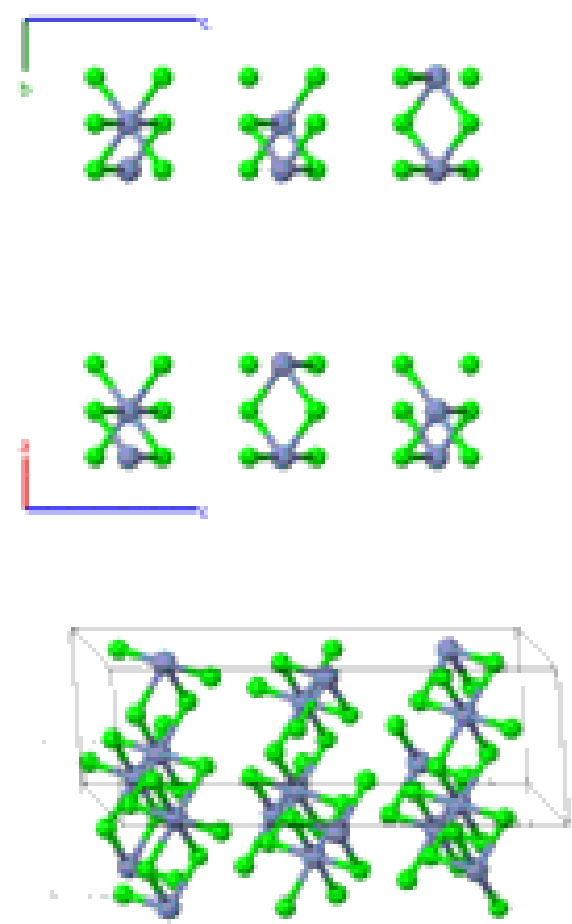

Prototype

AFLOW prototype label

Strukturbericht designation

Pearson symbol

Space group number

Space group symbol

AFLOW prototype command
: $\quad \mathrm{CrCl}_{3}$

: A3B_hP24_153_3c_2b

: None

: $\mathrm{hP} 24$

153

$P 3_{2} 12$

- This structure is the enantiomorph of the $\mathrm{CrCl}_{3}\left(\mathrm{D0}_{4}, \mathrm{~A} 3 \mathrm{~B} \_\mathrm{hP} 24 \_151 \_3 \mathrm{c} \_2 \mathrm{a}\right)$ structure, and was generated by reflecting the coordinates of the space group \#151 structure through the $z=0$ plane.

\section{Trigonal Hexagonal primitive vectors:}

$$
\begin{array}{ll}
\mathbf{a}_{1}= & \frac{1}{2} a \hat{\mathbf{x}}-\frac{\sqrt{3}}{2} a \hat{\mathbf{y}} \\
\mathbf{a}_{2}= & \frac{1}{2} a \hat{\mathbf{x}}+\frac{\sqrt{3}}{2} a \hat{\mathbf{y}} \\
\mathbf{a}_{3}= & c \hat{\mathbf{z}}
\end{array}
$$

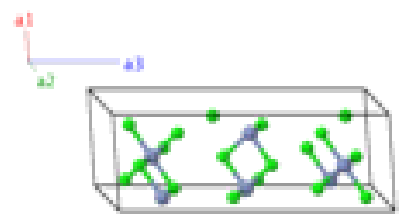

\section{Basis vectors:}

Lattice Coordinates

$\begin{array}{lllll}\mathbf{B}_{1}= & x_{1} \mathbf{a}_{1}-x_{1} \mathbf{a}_{2}+\frac{1}{6} \mathbf{a}_{3} & = & -\sqrt{3} x_{1} a \hat{\mathbf{y}}+\frac{1}{6} c \hat{\mathbf{z}} \\ \mathbf{B}_{2}= & x_{1} \mathbf{a}_{1}+2 x_{1} \mathbf{a}_{2}+\frac{5}{6} \mathbf{a}_{3} & = & \frac{3}{2} x_{1} a \hat{\mathbf{x}}+\frac{\sqrt{3}}{2} x_{1} a \hat{\mathbf{y}}+\frac{5}{6} c \hat{\mathbf{z}}\end{array}$

$x_{1} \mathbf{a}_{1}-x_{1} \mathbf{a}_{2}+\frac{1}{6} \mathbf{a}_{3}$

$=\quad \frac{3}{2} x_{1} a \hat{\mathbf{x}}+\frac{\sqrt{3}}{2} x_{1} a \hat{\mathbf{y}}+\frac{5}{6} c \hat{\mathbf{z}}$
Wyckoff Position

Atom Type

(3b)

Cr I

(3b)
Cr I 


\begin{tabular}{|c|c|c|c|c|c|c|}
\hline $\mathbf{B}_{3}$ & $=$ & $-2 x_{1} \mathbf{a}_{1}-x_{1} \mathbf{a}_{2}+\frac{1}{2} \mathbf{a}_{3}$ & $=$ & $-\frac{3}{2} x_{1} a \hat{\mathbf{x}}+\frac{\sqrt{3}}{2} x_{1} a \hat{\mathbf{y}}+\frac{1}{2} c \hat{\mathbf{z}}$ & $(3 b)$ & $\mathrm{Cr} \mathrm{I}$ \\
\hline $\mathbf{B}_{4}$ & $=$ & $x_{2} \mathbf{a}_{1}-x_{2} \mathbf{a}_{2}+\frac{1}{6} \mathbf{a}_{3}$ & $=$ & $-\sqrt{3} x_{2} a \hat{\mathbf{y}}+\frac{1}{6} c \hat{\mathbf{z}}$ & $(3 b)$ & Cr II \\
\hline $\mathbf{B}_{5}$ & $=$ & $x_{2} \mathbf{a}_{1}+2 x_{2} \mathbf{a}_{2}+\frac{5}{6} \mathbf{a}_{3}$ & $=$ & $\frac{3}{2} x_{2} a \hat{\mathbf{x}}+\frac{\sqrt{3}}{2} x_{2} a \hat{\mathbf{y}}+\frac{5}{6} c \hat{\mathbf{z}}$ & $(3 b)$ & Cr II \\
\hline $\mathbf{B}_{6}$ & $=$ & $-2 x_{2} \mathbf{a}_{1}-x_{2} \mathbf{a}_{2}+\frac{1}{2} \mathbf{a}_{3}$ & $=$ & $-\frac{3}{2} x_{2} a \hat{\mathbf{x}}+\frac{\sqrt{3}}{2} x_{2} a \hat{\mathbf{y}}+\frac{1}{2} c \hat{\mathbf{z}}$ & $(3 b)$ & Cr II \\
\hline $\mathbf{B}_{7}$ & $=$ & $x_{3} \mathbf{a}_{1}+y_{3} \mathbf{a}_{2}+z_{3} \mathbf{a}_{3}$ & $=$ & $\begin{array}{c}\frac{1}{2}\left(x_{3}+y_{3}\right) a \hat{\mathbf{x}}+ \\
\frac{\sqrt{3}}{2}\left(-x_{3}+y_{3}\right) a \hat{\mathbf{y}}+z_{3} c \hat{\mathbf{z}}\end{array}$ & $(6 c)$ & $\mathrm{Cl} \mathrm{I}$ \\
\hline $\mathbf{B}_{8}$ & $=$ & $-y_{3} \mathbf{a}_{1}+\left(x_{3}-y_{3}\right) \mathbf{a}_{2}+\left(\frac{2}{3}+z_{3}\right) \mathbf{a}_{3}$ & $=$ & $\begin{array}{c}\left(\frac{1}{2} x_{3}-y_{3}\right) a \hat{\mathbf{x}}+\frac{\sqrt{3}}{2} x_{3} a \hat{\mathbf{y}}+ \\
\left(\frac{2}{3}+z_{3}\right) c \hat{\mathbf{z}}\end{array}$ & $(6 c)$ & $\mathrm{Cl} \mathrm{I}$ \\
\hline $\mathbf{B}_{9}$ & $=$ & $\left(-x_{3}+y_{3}\right) \mathbf{a}_{1}-x_{3} \mathbf{a}_{2}+\left(\frac{1}{3}+z_{3}\right) \mathbf{a}_{3}$ & $=$ & $\begin{array}{c}\left(-x_{3}+\frac{1}{2} y_{3}\right) a \hat{\mathbf{x}}-\frac{\sqrt{3}}{2} y_{3} a \hat{\mathbf{y}}+ \\
\left(\frac{1}{3}+z_{3}\right) c \hat{\mathbf{z}}\end{array}$ & $(6 c)$ & $\mathrm{Cl} \mathrm{I}$ \\
\hline $\mathbf{B}_{10}$ & $=$ & $-y_{3} \mathbf{a}_{1}-x_{3} \mathbf{a}_{2}+\left(\frac{1}{3}-z_{3}\right) \mathbf{a}_{3}$ & $=$ & $\begin{array}{c}-\frac{1}{2}\left(x_{3}+y_{3}\right) a \hat{\mathbf{x}}+ \\
\frac{\sqrt{3}}{2}\left(-x_{3}+y_{3}\right) a \hat{\mathbf{y}}+\left(\frac{1}{3}-z_{3}\right) c \hat{\mathbf{z}}\end{array}$ & $(6 c)$ & $\mathrm{Cl} \mathrm{I}$ \\
\hline $\mathbf{B}_{11}$ & $=$ & $\left(-x_{3}+y_{3}\right) \mathbf{a}_{1}+y_{3} \mathbf{a}_{2}+\left(\frac{2}{3}-z_{3}\right) \mathbf{a}_{3}$ & $=$ & $\begin{array}{c}\left(-\frac{1}{2} x_{3}+y_{3}\right) a \hat{\mathbf{x}}+\frac{\sqrt{3}}{2} x_{3} a \hat{\mathbf{y}}+ \\
\left(\frac{2}{3}-z_{3}\right) c \hat{\mathbf{z}}\end{array}$ & $(6 c)$ & $\mathrm{Cl} \mathrm{I}$ \\
\hline $\mathbf{B}_{12}$ & $=$ & $x_{3} \mathbf{a}_{1}+\left(x_{3}-y_{3}\right) \mathbf{a}_{2}-z_{3} \mathbf{a}_{3}$ & $=$ & $\left(x_{3}-\frac{1}{2} y_{3}\right) a \hat{\mathbf{x}}-\frac{\sqrt{3}}{2} y_{3} a \hat{\mathbf{y}}-z_{3} c \hat{\mathbf{z}}$ & $(6 c)$ & $\mathrm{Cl} \mathrm{I}$ \\
\hline $\mathbf{B}_{13}$ & $=$ & $x_{4} \mathbf{a}_{1}+y_{4} \mathbf{a}_{2}+z_{4} \mathbf{a}_{3}$ & $=$ & $\begin{array}{c}\frac{1}{2}\left(x_{4}+y_{4}\right) a \hat{\mathbf{x}}+ \\
\frac{\sqrt{3}}{2}\left(-x_{4}+y_{4}\right) a \hat{\mathbf{y}}+z_{4} c \hat{\mathbf{z}}\end{array}$ & $(6 c)$ & $\mathrm{Cl} \mathrm{II}$ \\
\hline $\mathbf{B}_{14}$ & $=$ & $-y_{4} \mathbf{a}_{1}+\left(x_{4}-y_{4}\right) \mathbf{a}_{2}+\left(\frac{2}{3}+z_{4}\right) \mathbf{a}_{3}$ & $=$ & $\begin{array}{c}\left(\frac{1}{2} x_{4}-y_{4}\right) a \hat{\mathbf{x}}+\frac{\sqrt{3}}{2} x_{4} a \hat{\mathbf{y}}+ \\
\left(\frac{2}{3}+z_{4}\right) c \hat{\mathbf{z}}\end{array}$ & $(6 c)$ & $\mathrm{Cl} \mathrm{II}$ \\
\hline $\mathbf{B}_{15}$ & $=$ & $\left(-x_{4}+y_{4}\right) \mathbf{a}_{1}-x_{4} \mathbf{a}_{2}+\left(\frac{1}{3}+z_{4}\right) \mathbf{a}_{3}$ & $=$ & $\begin{array}{c}\left(-x_{4}+\frac{1}{2} y_{4}\right) a \hat{\mathbf{x}}-\frac{\sqrt{3}}{2} y_{4} a \hat{\mathbf{y}}+ \\
\left(\frac{1}{3}+z_{4}\right) c \hat{\mathbf{z}}\end{array}$ & $(6 c)$ & $\mathrm{Cl}$ II \\
\hline $\mathbf{B}_{16}$ & $=$ & $-y_{4} \mathbf{a}_{1}-x_{4} \mathbf{a}_{2}+\left(\frac{1}{3}-z_{4}\right) \mathbf{a}_{3}$ & $=$ & $\begin{array}{c}-\frac{1}{2}\left(x_{4}+y_{4}\right) a \hat{\mathbf{x}}+ \\
\frac{\sqrt{3}}{2}\left(-x_{4}+y_{4}\right) a \hat{\mathbf{y}}+\left(\frac{1}{3}-z_{4}\right) c \hat{\mathbf{z}}\end{array}$ & $(6 c)$ & $\mathrm{Cl}$ II \\
\hline $\mathbf{B}_{17}$ & $=$ & $\left(-x_{4}+y_{4}\right) \mathbf{a}_{1}+y_{4} \mathbf{a}_{2}+\left(\frac{2}{3}-z_{4}\right) \mathbf{a}_{3}$ & $=$ & $\begin{array}{c}\left(-\frac{1}{2} x_{4}+y_{4}\right) a \hat{\mathbf{x}}+\frac{\sqrt{3}}{2} x_{4} a \hat{\mathbf{y}}+ \\
\left(\frac{2}{3}-z_{4}\right) c \hat{\mathbf{z}}\end{array}$ & $(6 c)$ & $\mathrm{Cl} \mathrm{II}$ \\
\hline $\mathbf{B}_{18}$ & $=$ & $x_{4} \mathbf{a}_{1}+\left(x_{4}-y_{4}\right) \mathbf{a}_{2}-z_{4} \mathbf{a}_{3}$ & $=$ & $\left(x_{4}-\frac{1}{2} y_{4}\right) a \hat{\mathbf{x}}-\frac{\sqrt{3}}{2} y_{4} a \hat{\mathbf{y}}-z_{4} c \hat{\mathbf{z}}$ & $(6 c)$ & $\mathrm{Cl}$ II \\
\hline $\mathbf{B}_{19}$ & $=$ & $x_{5} \mathbf{a}_{1}+y_{5} \mathbf{a}_{2}+z_{5} \mathbf{a}_{3}$ & $=$ & $\begin{array}{c}\frac{1}{2}\left(x_{5}+y_{5}\right) a \hat{\mathbf{x}}+ \\
\frac{\sqrt{3}}{2}\left(-x_{5}+y_{5}\right) a \hat{\mathbf{y}}+z_{5} c \hat{\mathbf{z}}\end{array}$ & $(6 c)$ & $\mathrm{Cl}$ III \\
\hline $\mathbf{B}_{20}$ & $=$ & $-y_{5} \mathbf{a}_{1}+\left(x_{5}-y_{5}\right) \mathbf{a}_{2}+\left(\frac{2}{3}+z_{5}\right) \mathbf{a}_{3}$ & $=$ & $\begin{array}{c}\left(\frac{1}{2} x_{5}-y_{5}\right) a \hat{\mathbf{x}}+\frac{\sqrt{3}}{2} x_{5} a \hat{\mathbf{y}}+ \\
\left(\frac{2}{3}+z_{5}\right) c \hat{\mathbf{z}}\end{array}$ & $(6 c)$ & $\mathrm{Cl}$ III \\
\hline $\mathbf{B}_{21}$ & $=$ & $\left(-x_{5}+y_{5}\right) \mathbf{a}_{1}-x_{5} \mathbf{a}_{2}+\left(\frac{1}{3}+z_{5}\right) \mathbf{a}_{3}$ & $=$ & $\begin{array}{c}\left(-x_{5}+\frac{1}{2} y_{5}\right) a \hat{\mathbf{x}}-\frac{\sqrt{3}}{2} y_{5} a \hat{\mathbf{y}}+ \\
\left(\frac{1}{3}+z_{5}\right) c \hat{\mathbf{z}}\end{array}$ & $(6 c)$ & $\mathrm{Cl}$ III \\
\hline $\mathbf{B}_{22}$ & $=$ & $-y_{5} \mathbf{a}_{1}-x_{5} \mathbf{a}_{2}+\left(\frac{1}{3}-z_{5}\right) \mathbf{a}_{3}$ & $=$ & $\begin{array}{c}-\frac{1}{2}\left(x_{5}+y_{5}\right) a \hat{\mathbf{x}}+ \\
\frac{\sqrt{3}}{2}\left(-x_{5}+y_{5}\right) a \hat{\mathbf{y}}+\left(\frac{1}{3}-z_{5}\right) c \hat{\mathbf{z}}\end{array}$ & $(6 c)$ & $\mathrm{Cl}$ III \\
\hline $\mathbf{B}_{23}$ & $=$ & $\left(-x_{5}+y_{5}\right) \mathbf{a}_{1}+y_{5} \mathbf{a}_{2}+\left(\frac{2}{3}-z_{5}\right) \mathbf{a}_{3}$ & $=$ & $\begin{array}{c}\left(-\frac{1}{2} x_{5}+y_{5}\right) a \hat{\mathbf{x}}+\frac{\sqrt{3}}{2} x_{5} a \hat{\mathbf{y}}+ \\
\left(\frac{2}{3}-z_{5}\right) c \hat{\mathbf{z}}\end{array}$ & $(6 c)$ & $\mathrm{Cl}$ III \\
\hline $\mathbf{B}_{24}$ & $=$ & $x_{5} \mathbf{a}_{1}+\left(x_{5}-y_{5}\right) \mathbf{a}_{2}-z_{5} \mathbf{a}_{3}$ & $=$ & $\left(x_{5}-\frac{1}{2} y_{5}\right) a \hat{\mathbf{x}}-\frac{\sqrt{3}}{2} y_{5} a \hat{\mathbf{y}}-z_{5} c \hat{\mathbf{z}}$ & $(6 c)$ & $\mathrm{Cl}$ III \\
\hline
\end{tabular}

References:

- N. Wooster, The Structure of Chromium Trichloride $\mathrm{CrCl}_{3}$, Zeitschrift für Kristallographie - Crystalline Materials 74, 363-374 (1930), doi:10.1524/zkri.1930.74.1.363. 


\section{Found in:}

- R. T. Downs and M. Hall-Wallace, The American Mineralogist Crystal Structure Database, Am. Mineral. 88, 247-250 (2003).

\section{Geometry files:}

- CIF: pp. 911

- POSCAR: pp. 911 


\section{S-II Structure: A_hP9_154_bc}

05
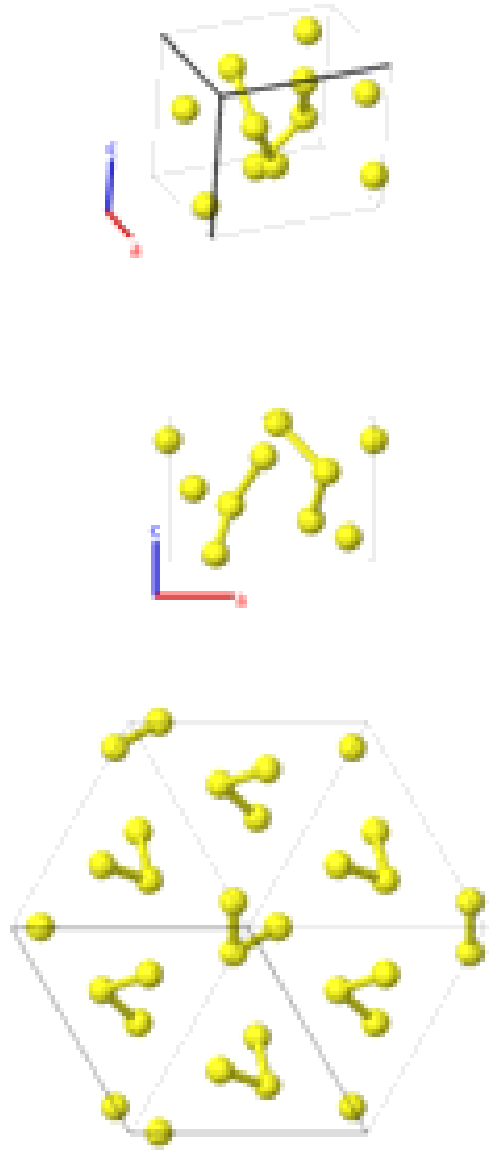

Prototype

AFLOW prototype label

Strukturbericht designation

Pearson symbol

Space group number

Space group symbol

AFLOW prototype command : aflow --proto=A_hP9_154_bc

- params $=a, c / a, x_{1}, x_{2}, y_{2}, z_{2}$

- The S-II phase is found when sulfur heated and pressurized above $3 \mathrm{GPa}$. This data was taken at $5.8 \mathrm{GPa}$ and $800 \mathrm{~K}$.

Trigonal Hexagonal primitive vectors:

$$
\begin{array}{ll}
\mathbf{a}_{1}= & \frac{1}{2} a \hat{\mathbf{x}}-\frac{\sqrt{3}}{2} a \hat{\mathbf{y}} \\
\mathbf{a}_{2}= & \frac{1}{2} a \hat{\mathbf{x}}+\frac{\sqrt{3}}{2} a \hat{\mathbf{y}} \\
\mathbf{a}_{3}= & c \hat{\mathbf{z}}
\end{array}
$$
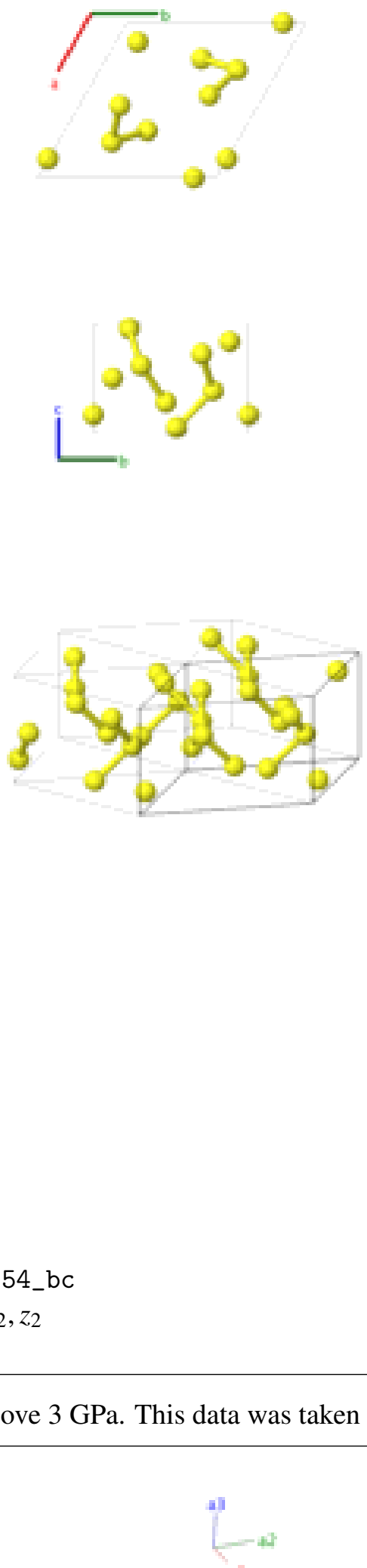

il

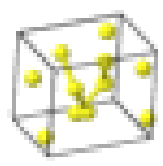




\section{Lattice Coordinates}

$$
\begin{aligned}
& \mathbf{B}_{1}= \\
& x_{1} \mathbf{a}_{1}+\frac{1}{6} \mathbf{a}_{3} \\
& \mathbf{B}_{2}= \\
& x_{1} \mathbf{a}_{2}+\frac{5}{6} \mathbf{a}_{3} \\
& \mathbf{B}_{3}= \\
& -x_{1} \mathbf{a}_{1}-x_{1} \mathbf{a}_{2}+\frac{1}{2} \mathbf{a}_{3} \\
& \mathbf{B}_{4}= \\
& x_{2} \mathbf{a}_{1}+y_{2} \mathbf{a}_{2}+z_{2} \mathbf{a}_{3} \\
& =\quad \frac{1}{2} x_{1} a \hat{\mathbf{x}}-\frac{\sqrt{3}}{2} x_{1} a \hat{\mathbf{y}}+\frac{1}{6} c \hat{\mathbf{z}} \\
& =\quad \frac{1}{2} x_{1} a \hat{\mathbf{x}}+\frac{\sqrt{3}}{2} x_{1} a \hat{\mathbf{y}}+\frac{5}{6} c \hat{\mathbf{z}} \\
& =\quad-x_{1} a \hat{\mathbf{x}}+\frac{1}{2} c \hat{\mathbf{z}} \\
& = \\
& \frac{1}{2}\left(x_{2}+y_{2}\right) a \hat{\mathbf{x}}+ \\
& \frac{\sqrt{3}}{2}\left(-x_{2}+y_{2}\right) a \hat{\mathbf{y}}+z_{2} c \hat{\mathbf{z}} \\
& \mathbf{B}_{5}=-y_{2} \mathbf{a}_{1}+\left(x_{2}-y_{2}\right) \mathbf{a}_{2}+\left(\frac{2}{3}+z_{2}\right) \mathbf{a}_{3}=\left(\frac{1}{2} x_{2}-y_{2}\right) a \hat{\mathbf{x}}+\frac{\sqrt{3}}{2} x_{2} a \hat{\mathbf{y}}+ \\
& \left(\frac{2}{3}+z_{2}\right) c \hat{\mathbf{z}} \\
& \mathbf{B}_{6}=\left(-x_{2}+y_{2}\right) \mathbf{a}_{1}-x_{2} \mathbf{a}_{2}+\left(\frac{1}{3}+z_{2}\right) \mathbf{a}_{3}=\left(-x_{2}+\frac{1}{2} y_{2}\right) a \hat{\mathbf{x}}-\frac{\sqrt{3}}{2} y_{2} a \hat{\mathbf{y}}+ \\
& \left(\frac{1}{3}+z_{2}\right) c \hat{\mathbf{z}} \\
& \mathbf{B}_{7}=y_{2} \mathbf{a}_{1}+x_{2} \mathbf{a}_{2}-z_{2} \mathbf{a}_{3} \\
& =\frac{1}{2}\left(x_{2}+y_{2}\right) a \hat{\mathbf{x}}+\frac{\sqrt{3}}{2}\left(x_{2}-y_{2}\right) a \hat{\mathbf{y}}- \\
& \mathbf{B}_{8}=\left(x_{2}-y_{2}\right) \mathbf{a}_{1}-y_{2} \mathbf{a}_{2}+\left(\frac{1}{3}-z_{2}\right) \mathbf{a}_{3}=\left(\frac{1}{2} x_{2}-y_{2}\right) a \hat{\mathbf{x}}-\frac{\sqrt{3}}{2} x_{2} a \hat{\mathbf{y}}+ \\
& \left(\frac{1}{3}-z_{2}\right) c \hat{\mathbf{z}} \\
& \mathbf{B}_{9}=-x_{2} \mathbf{a}_{1}+\left(-x_{2}+y_{2}\right) \mathbf{a}_{2}+\left(\frac{2}{3}-z_{2}\right) \mathbf{a}_{3}=\left(-x_{2}+\frac{1}{2} y_{2}\right) a \hat{\mathbf{x}}+\frac{\sqrt{3}}{2} y_{2} a \hat{\mathbf{y}}+ \\
& \left(\frac{2}{3}-z_{2}\right) c \hat{\mathbf{z}}
\end{aligned}
$$$$
\text { Cartesian Coordinates }
$$

\section{References:}

- O. Degtyareva, E. Gregoryanz, M. Somayazulu, P. Dera, H. Mao, and R. J. Hemley, Novel chain structures in group VI elements, Nat. Mater. 4, 152-155 (2005), doi:10.1038/nmat1294.

\section{Geometry files:}

- CIF: pp. 911

- POSCAR: pp. 911 


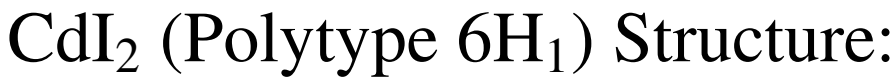 AB2_hP9_156_b2c_3a2bc}
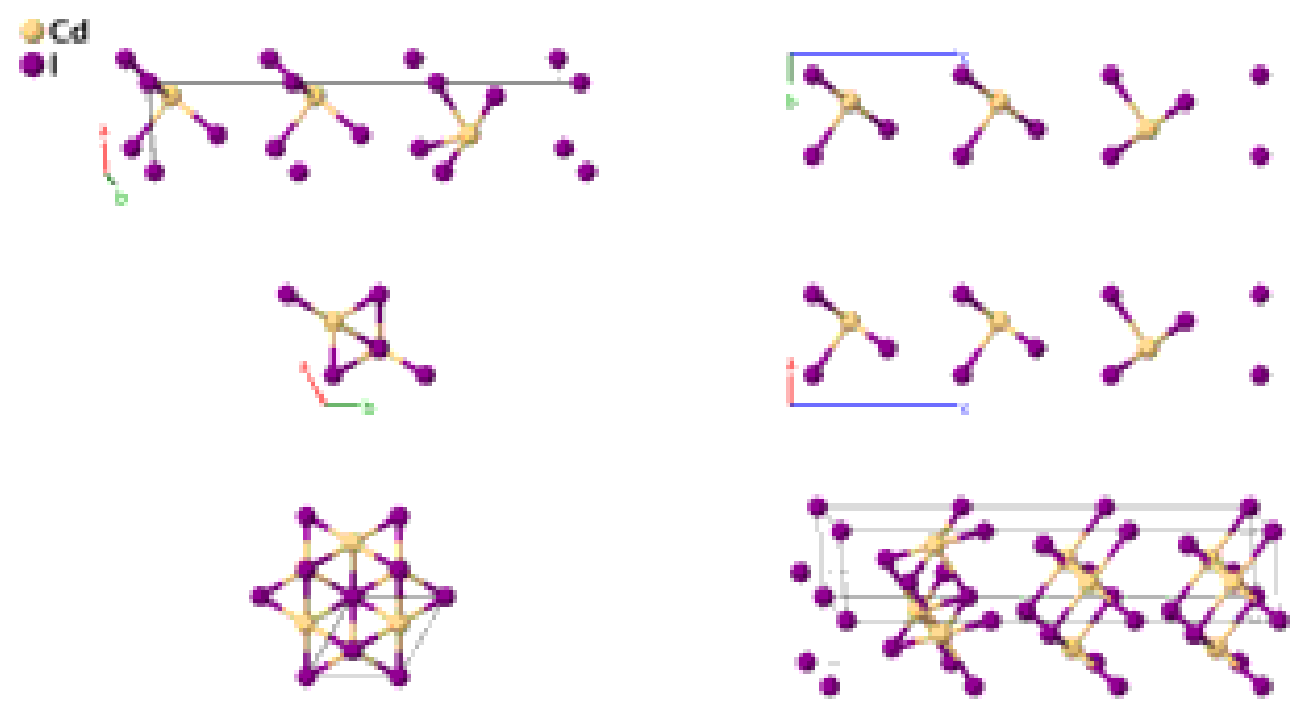

Prototype

AFLOW prototype label

: $\quad \mathrm{CdI}_{2}$

Strukturbericht designation

Pearson symbol

AB2_hP9_156_b2c_3a2bc

Space group number

None

Space group symbol

hP9

AFLOW prototype command

: 156

: $\quad P 3 m 1$

aflow - -proto=AB2_hP9_156_b2c_3a2bc

- -params $=a, c / a, z_{1}, z_{2}, z_{3}, z_{4}, z_{5}, z_{6}, z_{7}, z_{8}, z_{9}$

Trigonal Hexagonal primitive vectors:

$$
\begin{array}{lll}
\mathbf{a}_{1} & = & \frac{1}{2} a \hat{\mathbf{x}}-\frac{\sqrt{3}}{2} a \hat{\mathbf{y}} \\
\mathbf{a}_{2} & = & \frac{1}{2} a \hat{\mathbf{x}}+\frac{\sqrt{3}}{2} a \hat{\mathbf{y}} \\
\mathbf{a}_{3} & = & c \hat{\mathbf{z}}
\end{array}
$$

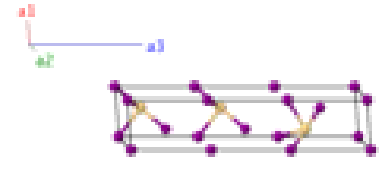

Basis vectors:

Lattice Coordinates

$\begin{array}{lcccc}\mathbf{B}_{1}= & z_{1} \mathbf{a}_{3} & = & z_{1} c \hat{\mathbf{z}} \\ \mathbf{B}_{2}= & z_{2} \mathbf{a}_{3} & = & z_{2} c \hat{\mathbf{z}} \\ \mathbf{B}_{3}= & z_{3} \mathbf{a}_{3} & = & z_{3} c \hat{\mathbf{z}} \\ \mathbf{B}_{4}= & \frac{1}{3} \mathbf{a}_{1}+\frac{2}{3} \mathbf{a}_{2}+z_{4} \mathbf{a}_{3} & = & \frac{1}{2} a \hat{\mathbf{x}}+\frac{1}{2 \sqrt{3}} a \hat{\mathbf{y}}+z_{4} c \hat{\mathbf{z}} \\ \mathbf{B}_{5}= & \frac{1}{3} \mathbf{a}_{1}+\frac{2}{3} \mathbf{a}_{2}+z_{5} \mathbf{a}_{3} & = & \frac{1}{2} a \hat{\mathbf{x}}+\frac{1}{2 \sqrt{3}} a \hat{\mathbf{y}}+z_{5} c \hat{\mathbf{z}} \\ \mathbf{B}_{6}= & \frac{1}{3} \mathbf{a}_{1}+\frac{2}{3} \mathbf{a}_{2}+z_{6} \mathbf{a}_{3} & = & \frac{1}{2} a \hat{\mathbf{x}}+\frac{1}{2 \sqrt{3}} a \hat{\mathbf{y}}+z_{6} c \hat{\mathbf{z}} \\ \mathbf{B}_{7}= & \frac{2}{3} \mathbf{a}_{1}+\frac{1}{3} \mathbf{a}_{2}+z_{7} \mathbf{a}_{3} & = & \frac{1}{2} a \hat{\mathbf{x}}-\frac{1}{2 \sqrt{3}} a \hat{\mathbf{y}}+z_{7} c \hat{\mathbf{z}} \\ \mathbf{B}_{8}= & \frac{2}{3} \mathbf{a}_{1}+\frac{1}{3} \mathbf{a}_{2}+z_{8} \mathbf{a}_{3} & = & \frac{1}{2} a \hat{\mathbf{x}}-\frac{1}{2 \sqrt{3}} a \hat{\mathbf{y}}+z_{8} c \hat{\mathbf{z}}\end{array}$

Wyckoff Position
Atom Type

I I

I II

I III

Cd I

I IV

I V

Cd II

Cd III 
$\mathbf{B}_{9} \quad=\quad \frac{2}{3} \mathbf{a}_{1}+\frac{1}{3} \mathbf{a}_{2}+z_{9} \mathbf{a}_{3} \quad=\quad \frac{1}{2} a \hat{\mathbf{x}}-\frac{1}{2 \sqrt{3}} a \hat{\mathbf{y}}+z_{9} c \hat{\mathbf{z}}$

$(1 c)$

I VI

\section{References:}

- R. S. Mitchell, Polytypism of Cadmium Iodide and its Relationship to Screw Dislocations: I. Cadmium Iodide Polytypes, Zeitschrift für Kristallographie - Crystalline Materials 108, 296-315 (1956), doi:10.1524/zkri.1956.108.3-4.296.

\section{Found in:}

- P. Villars and K. Cenzual, Pearson's Crystal Data - Crystal Structure Database for Inorganic Compounds, ASM International (2013).

\section{Geometry files:}

- CIF: pp. 911

- POSCAR: pp. 912 


\section{CuI Structure: AB_hP12_156_2ab3c_2ab3c}

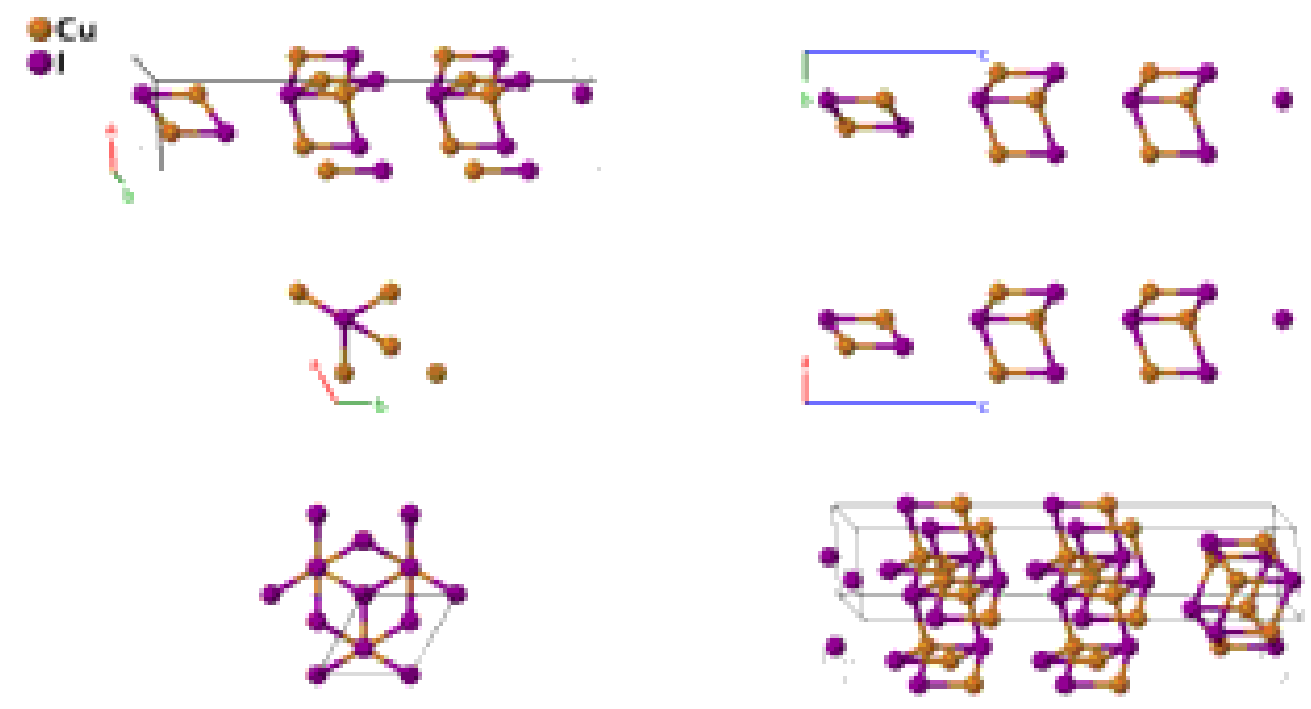

Prototype

$: \quad \mathrm{CuI}$

AFLOW prototype label

: $\quad$ AB_hP12_156_2ab3c_2ab3c

Strukturbericht designation : None

Pearson symbol $\quad: \quad \mathrm{hP} 12$

Space group number $\quad: 156$

Space group symbol $\quad: \quad P 3 m 1$

AFLOW prototype command : aflow --proto=AB_hP12_156_2ab3c_2ab3c

- params $=a, c / a, z_{1}, z_{2}, z_{3}, z_{4}, z_{5}, z_{6}, z_{7}, z_{8}, z_{9}, z_{10}, z_{11}, z_{12}$

Trigonal Hexagonal primitive vectors:

$$
\begin{array}{ll}
\mathbf{a}_{1}= & \frac{1}{2} a \hat{\mathbf{x}}-\frac{\sqrt{3}}{2} a \hat{\mathbf{y}} \\
\mathbf{a}_{2}= & \frac{1}{2} a \hat{\mathbf{x}}+\frac{\sqrt{3}}{2} a \hat{\mathbf{y}} \\
\mathbf{a}_{3}= & c \hat{\mathbf{z}}
\end{array}
$$

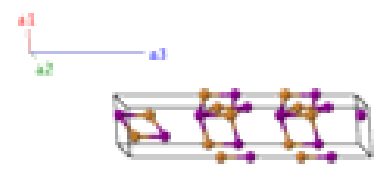

Basis vectors:

Lattice Coordinates

$\begin{array}{lcccc}\mathbf{B}_{1} & = & z_{1} \mathbf{a}_{3} & = & z_{1} c \hat{\mathbf{z}} \\ \mathbf{B}_{2} & z_{2} \mathbf{a}_{3} & = & z_{2} c \hat{\mathbf{z}} \\ \mathbf{B}_{3}= & z_{3} \mathbf{a}_{3} & = & z_{3} c \hat{\mathbf{z}} \\ \mathbf{B}_{4} & z_{4} \mathbf{a}_{3} & = & z_{4} c \hat{\mathbf{z}} \\ \mathbf{B}_{5} & = & \frac{1}{3} \mathbf{a}_{1}+\frac{2}{3} \mathbf{a}_{2}+z_{5} \mathbf{a}_{3} & = & \frac{1}{2} a \hat{\mathbf{x}}+\frac{1}{2 \sqrt{3}} a \hat{\mathbf{y}}+z_{5} c \hat{\mathbf{z}} \\ \mathbf{B}_{6}= & \frac{1}{3} \mathbf{a}_{1}+\frac{2}{3} \mathbf{a}_{2}+z_{6} \mathbf{a}_{3} & = & \frac{1}{2} a \hat{\mathbf{x}}+\frac{1}{2 \sqrt{3}} a \hat{\mathbf{y}}+z_{6} c \hat{\mathbf{z}} \\ \mathbf{B}_{7}= & \frac{2}{3} \mathbf{a}_{1}+\frac{1}{3} \mathbf{a}_{2}+z_{7} \mathbf{a}_{3} & = & \frac{1}{2} a \hat{\mathbf{x}}-\frac{1}{2 \sqrt{3}} a \hat{\mathbf{y}}+z_{7} c \hat{\mathbf{z}} \\ \mathbf{B}_{8} & = & \frac{2}{3} \mathbf{a}_{1}+\frac{1}{3} \mathbf{a}_{2}+z_{8} \mathbf{a}_{3} & = & \frac{1}{2} a \hat{\mathbf{x}}-\frac{1}{2 \sqrt{3}} a \hat{\mathbf{y}}+z_{8} c \hat{\mathbf{z}} \\ \mathbf{B}_{9} & = & \frac{2}{3} \mathbf{a}_{1}+\frac{1}{3} \mathbf{a}_{2}+z_{9} \mathbf{a}_{3} & = & \frac{1}{2} a \hat{\mathbf{x}}-\frac{1}{2 \sqrt{3}} a \hat{\mathbf{y}}+z_{9} c \hat{\mathbf{z}}\end{array}$

Wyckoff Position

Atom Type

$(1 a)$

$\mathrm{Cu} \mathrm{I}$

(1a)

(1a)

(1a)

(1b)

(1b)

(1c)
$\mathrm{Cu}$ II

I I

I II

$\mathrm{Cu}$ III

I III

$\mathrm{Cu}$ IV

$\mathrm{Cu}$ V

$\mathrm{Cu}$ VI 


$\begin{array}{llllll}\mathbf{B}_{10}= & \frac{2}{3} \mathbf{a}_{1}+\frac{1}{3} \mathbf{a}_{2}+z_{10} \mathbf{a}_{3} & = & \frac{1}{2} a \hat{\mathbf{x}}-\frac{1}{2 \sqrt{3}} a \hat{\mathbf{y}}+z_{10} c \hat{\mathbf{z}} & \text { (1c) } & \text { I IV } \\ \mathbf{B}_{11}= & \frac{2}{3} \mathbf{a}_{1}+\frac{1}{3} \mathbf{a}_{2}+z_{11} \mathbf{a}_{3}= & \frac{1}{2} a \hat{\mathbf{x}}-\frac{1}{2 \sqrt{3}} a \hat{\mathbf{y}}+z_{11} c \hat{\mathbf{z}} & \text { (1c) } & \text { I V } \\ \mathbf{B}_{12}= & \frac{2}{3} \mathbf{a}_{1}+\frac{1}{3} \mathbf{a}_{2}+z_{12} \mathbf{a}_{3}= & \frac{1}{2} a \hat{\mathbf{x}}-\frac{1}{2 \sqrt{3}} a \hat{\mathbf{y}}+z_{12} c \hat{\mathbf{z}} & \text { (1c) } & \text { I VI }\end{array}$

\section{References:}

- R. N. Kurdyumova and R. V. Baranova, An electron diffraction study of thin films of cuprous iodide, Sov. Phys. Crystallogr. 6, 318-321 (1961).

\section{Found in:}

- P. Villars and K. Cenzual, Pearson's Crystal Data - Crystal Structure Database for Inorganic Compounds, ASM International (2013).

\section{Geometry files:}

- CIF: pp. 912

- POSCAR: pp. 912 


\section{$\beta$-CuI Structure: AB_hP4_156_ac_ac}
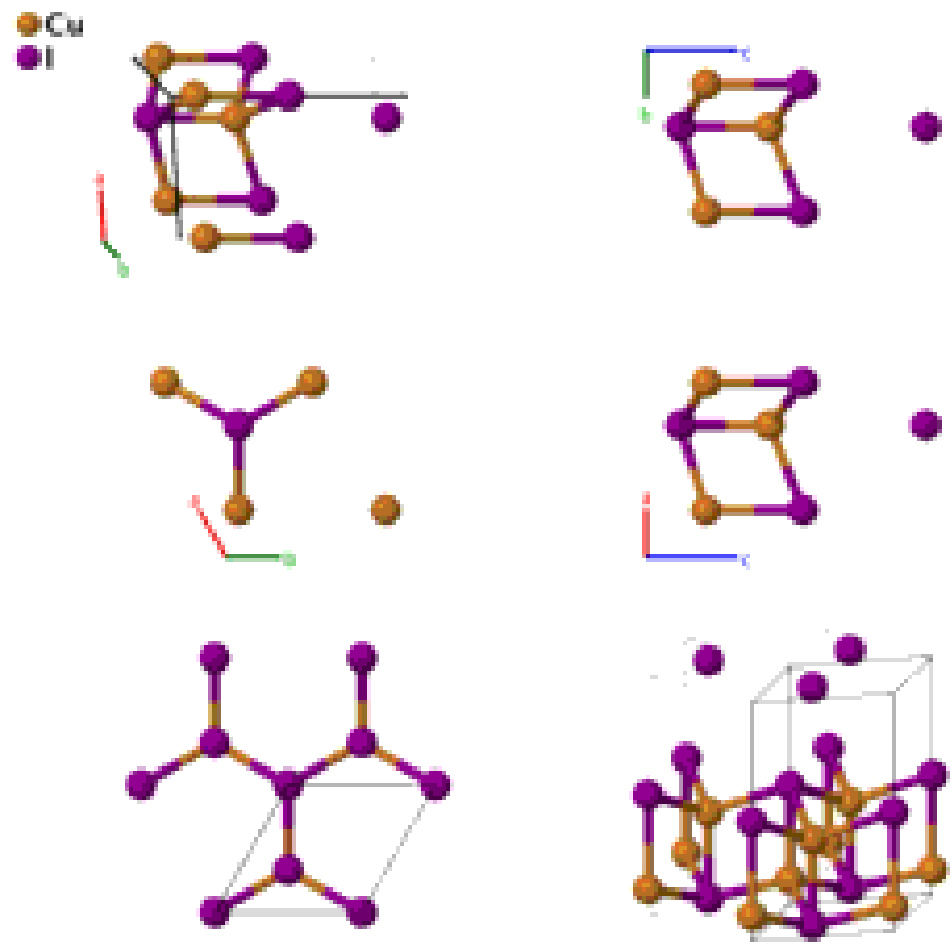

\section{Prototype}

AFLOW prototype label

Strukturbericht designation

Pearson symbol

$: \quad \beta$-CuI

Space group number

AB_hP4_156_ac_ac

Space group symbol

: None

AFLOW prototype command

: $\quad \mathrm{hP} 4$

: 156

: $\quad P 3 m 1$

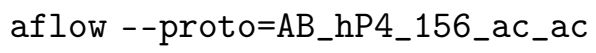

Trigonal Hexagonal primitive vectors:

$$
\begin{array}{ll}
\mathbf{a}_{1}= & \frac{1}{2} a \hat{\mathbf{x}}-\frac{\sqrt{3}}{2} a \hat{\mathbf{y}} \\
\mathbf{a}_{2}= & \frac{1}{2} a \hat{\mathbf{x}}+\frac{\sqrt{3}}{2} a \hat{\mathbf{y}} \\
\mathbf{a}_{3}= & c \hat{\mathbf{z}}
\end{array}
$$

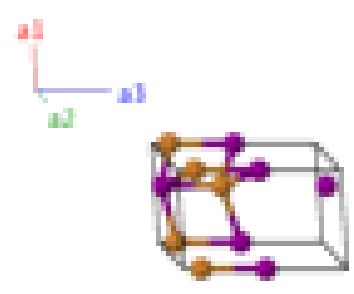

Basis vectors:

Lattice Coordinates

$\begin{array}{lcccc}\mathbf{B}_{1}= & z_{1} \mathbf{a}_{3} & = & z_{1} c \hat{\mathbf{z}} \\ \mathbf{B}_{2}= & z_{2} \mathbf{a}_{3} & = & z_{2} c \hat{\mathbf{z}} \\ \mathbf{B}_{3}= & \frac{2}{3} \mathbf{a}_{1}+\frac{1}{3} \mathbf{a}_{2}+z_{3} \mathbf{a}_{3} & = & \frac{1}{2} a \hat{\mathbf{x}}-\frac{1}{2 \sqrt{3}} a \hat{\mathbf{y}}+z_{3} c \hat{\mathbf{z}} \\ \mathbf{B}_{4}= & \frac{2}{3} \mathbf{a}_{1}+\frac{1}{3} \mathbf{a}_{2}+z_{4} \mathbf{a}_{3} & = & \frac{1}{2} a \hat{\mathbf{x}}-\frac{1}{2 \sqrt{3}} a \hat{\mathbf{y}}+z_{4} c \hat{\mathbf{z}}\end{array}$

Cartesian Coordinates 


\section{References:}

- T. Sakuma, Crystal structure of $\beta$-CuI, J. Phys. Soc. Jpn. 57, 565-569 (1988), doi:10.1143/JPSJ.57.565.

\section{Found in:}

- P. Villars and K. Cenzual, Pearson's Crystal Data - Crystal Structure Database for Inorganic Compounds, ASM International (2013).

\section{Geometry files:}

- CIF: pp. 912

- POSCAR: pp. 913 


\section{$\mathrm{Ag}_{5} \mathrm{~Pb}_{2} \mathrm{O}_{6}$ Structure: A5B6C2_hP13_157_2ac_2c_b}
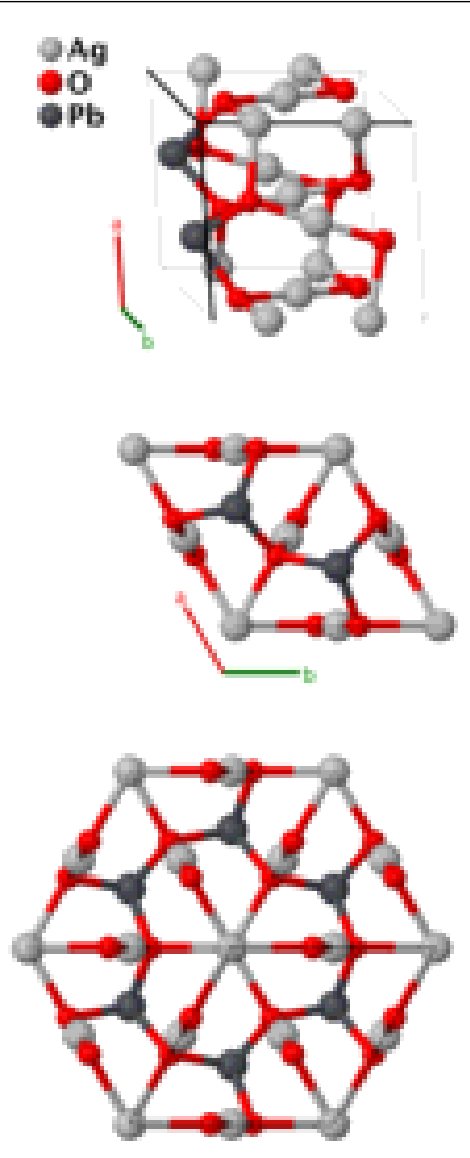
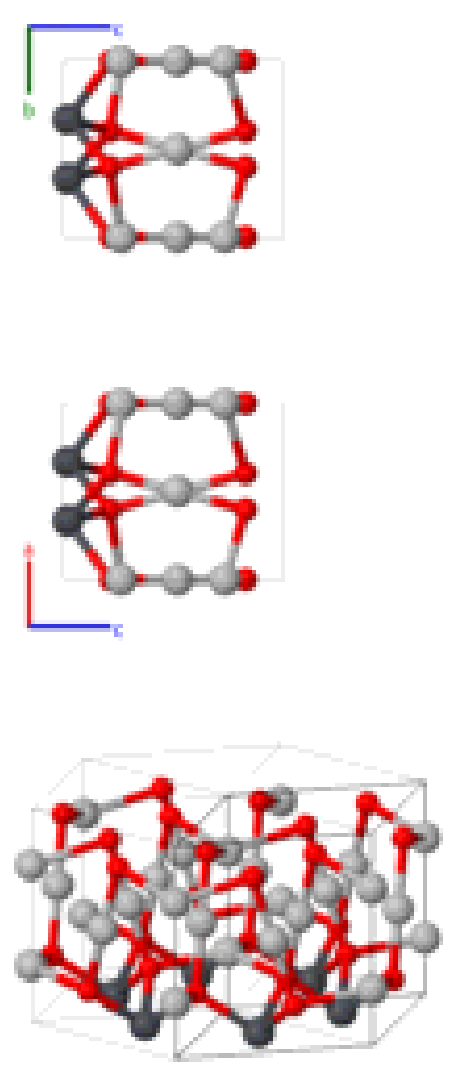

\section{Prototype}

\section{AFLOW prototype label}

Strukturbericht designation

Pearson symbol

Space group number

Space group symbol

AFLOW prototype command
: $\quad \mathrm{Ag}_{5} \mathrm{~Pb}_{2} \mathrm{O}_{6}$

: A5B6C2_hP13_157_2ac_2c_b

: None

: $\mathrm{hP} 13$

: $\quad 157$

: $\quad P 31 m$

- The original reference (Byström, 1950) lists this structure as $\mathrm{Ag}_{5} \mathrm{~Pb}_{2} \mathrm{O}_{6}$, while (Villars, 1985) lists it as $\mathrm{Ag} 2 \mathrm{PbO}_{3}$. (Byström, 1950) provides three Wyckoff positions for Ag (1a, 1a, and 3c), while (Villars, 195) only provides two (1a and $3 \mathrm{c}$ ), giving rise to the stoichiometry discrepancy. While both descriptions yield space group \#157, the authors use the structure and coordinates provided by the original reference (Byström, 1950).

\section{Trigonal Hexagonal primitive vectors:}

$$
\begin{array}{ll}
\mathbf{a}_{1}= & \frac{1}{2} a \hat{\mathbf{x}}-\frac{\sqrt{3}}{2} a \hat{\mathbf{y}} \\
\mathbf{a}_{2}= & \frac{1}{2} a \hat{\mathbf{x}}+\frac{\sqrt{3}}{2} a \hat{\mathbf{y}} \\
\mathbf{a}_{3}= & c \hat{\mathbf{z}}
\end{array}
$$

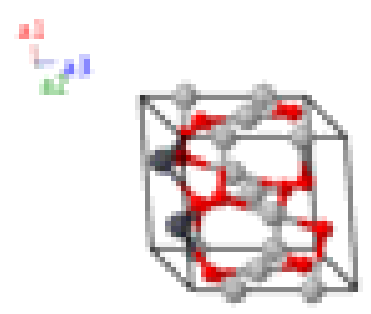




\section{Basis vectors:}

Lattice Coordinates

$\mathbf{B}_{1}=$

$\mathbf{B}_{2}=$

$\mathbf{B}_{3}=$

$\mathbf{B}_{4}=$

$\mathbf{B}_{5}=$

$\mathbf{B}_{6}=$

$\mathbf{B}_{7}=$

$\mathbf{B}_{8}=$

$\mathbf{B}_{9}=$

$\mathbf{B}_{10}=$

$\mathbf{B}_{11}=$

$\mathbf{B}_{12}=$

$\mathbf{B}_{13}=$

$$
z_{1} \mathbf{a}_{3}
$$$$
z_{2} \mathbf{a}_{3}
$$$$
\frac{1}{3} \mathbf{a}_{1}+\frac{2}{3} \mathbf{a}_{2}+z_{3} \mathbf{a}_{3}
$$$$
\frac{2}{3} \mathbf{a}_{1}+\frac{1}{3} \mathbf{a}_{2}+z_{3} \mathbf{a}_{3}
$$

$$
x_{4} \mathbf{a}_{1}+z_{4} \mathbf{a}_{3}
$$$$
x_{4} \mathbf{a}_{2}+z_{4} \mathbf{a}_{3}
$$$$
-x_{4} \mathbf{a}_{1}-x_{4} \mathbf{a}_{2}+z_{4} \mathbf{a}_{3}
$$

$$
x_{5} \mathbf{a}_{1}+z_{5} \mathbf{a}_{3}
$$

$$
x_{5} \mathbf{a}_{2}+z_{5} \mathbf{a}_{3}
$$

$$
-x_{5} \mathbf{a}_{1}-x_{5} \mathbf{a}_{2}+z_{5} \mathbf{a}_{3}
$$

$$
x_{6} \mathbf{a}_{1}+z_{6} \mathbf{a}_{3}
$$$$
x_{6} \mathbf{a}_{2}+z_{6} \mathbf{a}_{3}
$$$$
-x_{6} \mathbf{a}_{1}-x_{6} \mathbf{a}_{2}+z_{6} \mathbf{a}_{3}
$$

\section{Cartesian Coordinates}

$=$

$$
z_{1} c \hat{\mathbf{z}}
$$$$
z_{2} c \hat{\mathbf{z}}
$$

$=$

$=$

$=$

$=$

$=$
$=$

$$
\frac{1}{2} a \hat{\mathbf{x}}+\frac{1}{2 \sqrt{3}} a \hat{\mathbf{y}}+z_{3} c \hat{\mathbf{z}}
$$$$
\frac{1}{2} a \hat{\mathbf{x}}-\frac{1}{2 \sqrt{3}} a \hat{\mathbf{y}}+z_{3} c \hat{\mathbf{z}}
$$$$
\frac{1}{2} x_{4} a \hat{\mathbf{x}}-\frac{\sqrt{3}}{2} x_{4} a \hat{\mathbf{y}}+z_{4} c \hat{\mathbf{z}}
$$$$
\frac{1}{2} x_{4} a \hat{\mathbf{x}}+\frac{\sqrt{3}}{2} x_{4} a \hat{\mathbf{y}}+z_{4} c \hat{\mathbf{z}}
$$$$
-x_{4} a \hat{\mathbf{x}}+z_{4} c \hat{\mathbf{z}}
$$

$=$

$$
\frac{1}{2} x_{5} a \hat{\mathbf{x}}-\frac{\sqrt{3}}{2} x_{5} a \hat{\mathbf{y}}+z_{5} c \hat{\mathbf{z}}
$$$$
=\quad \frac{1}{2} x_{5} a \hat{\mathbf{x}}+\frac{\sqrt{3}}{2} x_{5} a \hat{\mathbf{y}}+z_{5} c \hat{\mathbf{z}}
$$$$
=\quad-x_{5} a \hat{\mathbf{x}}+z_{5} c \hat{\mathbf{z}}
$$$$
=\quad \frac{1}{2} x_{6} a \hat{\mathbf{x}}-\frac{\sqrt{3}}{2} x_{6} a \hat{\mathbf{y}}+z_{6} c \hat{\mathbf{z}}
$$$$
=\quad \frac{1}{2} x_{6} a \hat{\mathbf{x}}+\frac{\sqrt{3}}{2} x_{6} a \hat{\mathbf{y}}+z_{6} c \hat{\mathbf{z}}
$$$$
=
$$

Wyckoff Position

(1a)
Atom Type

Ag I

Ag II

$\mathrm{Pb}$

$\mathrm{Pb}$

Ag III

Ag III

Ag III

O I

O I

O I

O II

O II

O II

\section{References:}

- A. Byström and L. Evers, The Crystal Structures of $\mathrm{Ag}_{2} \mathrm{PbO}_{2}$ and $\mathrm{Ag}_{5} \mathrm{~Pb}_{2} \mathrm{O}_{6}$, Acta Chem. Scand. 4, 613-627 (1950), doi:10.3891/acta.chem.scand.04-0613.

\section{Found in:}

- P. Villars and L. D. Calvert, eds., Pearson's Handbook of Crystallographic Data for Intermetallic Phases, vol. 1 (American Society of Metals, Materials Park, Ohio, 1985).

\section{Geometry files:}

- CIF: pp. 913

- POSCAR: pp. 913 


\section{$\beta-\mathrm{RuCl}_{3}$ Structure: A3B_hP8_158_d_a}
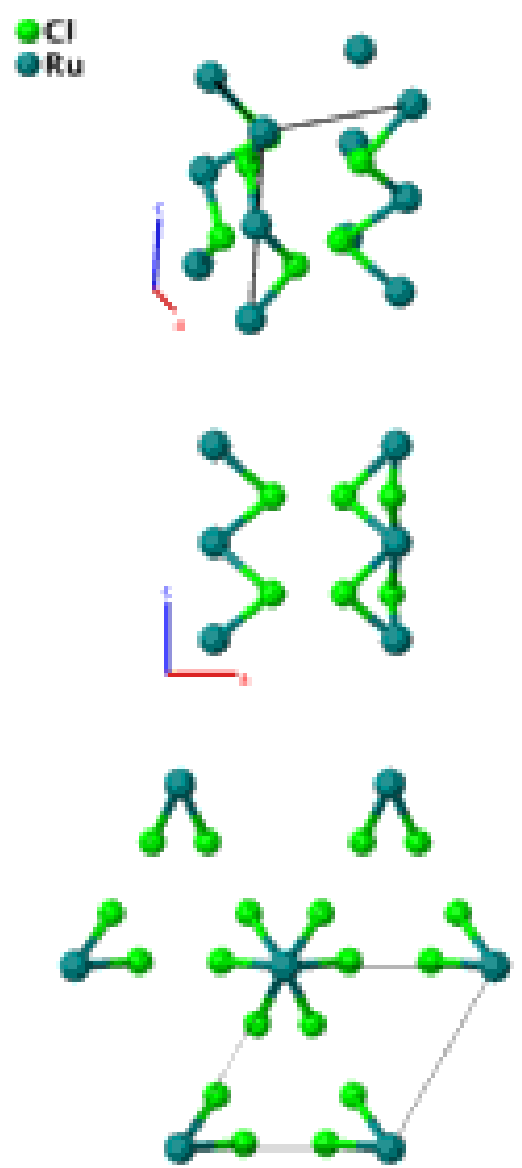
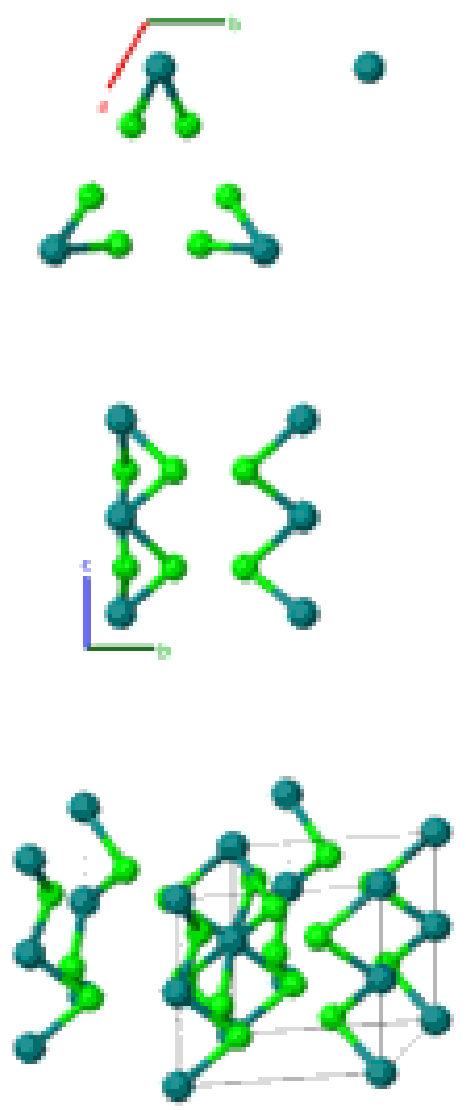

Prototype

AFLOW prototype label

Strukturbericht designation

Pearson symbol

Space group number

Space group symbol

AFLOW prototype command
$: \quad \beta-\mathrm{RuCl}_{3}$

: A3B_hP8_158_d_a

: None

: $\mathrm{hP8}$

: $\quad 158$

: $\quad P 3 c 1$

\footnotetext{
aflow --proto=A3B_hP8_158_d_a

- params $=a, c / a, z_{1}, x_{2}, y_{2}, z_{2}$
}

- Pearson comments that space groups \#185, \#188, \#193, could not be rejected, but this structure is consistent with space group \#158. We also provide the structure with space group \#185: $\beta-\mathrm{RuCl}_{3}$ (A3B_hP8_185_c_a) structure.

Trigonal Hexagonal primitive vectors:

$$
\begin{array}{ll}
\mathbf{a}_{1}= & \frac{1}{2} a \hat{\mathbf{x}}-\frac{\sqrt{3}}{2} a \hat{\mathbf{y}} \\
\mathbf{a}_{2}= & \frac{1}{2} a \hat{\mathbf{x}}+\frac{\sqrt{3}}{2} a \hat{\mathbf{y}} \\
\mathbf{a}_{3}= & c \hat{\mathbf{z}}
\end{array}
$$

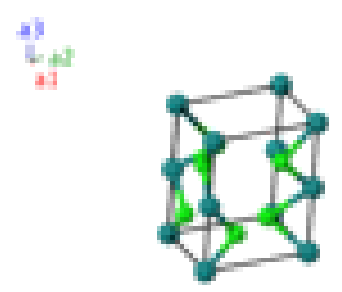

\section{Basis vectors:}




\section{Lattice Coordinates}
$\mathbf{B}_{1}=$
$z_{1} \mathbf{a}_{3}$
$\mathbf{B}_{2}=$
$\left(\frac{1}{2}+z_{1}\right) \mathbf{a}_{3}$
$\mathbf{B}_{3}=$
$x_{2} \mathbf{a}_{1}+y_{2} \mathbf{a}_{2}+z_{2} \mathbf{a}_{3}$

$\mathbf{B}_{4}=-y_{2} \mathbf{a}_{1}+\left(x_{2}-y_{2}\right) \mathbf{a}_{2}+z_{2} \mathbf{a}_{3}$

$\mathbf{B}_{5}=\left(-x_{2}+y_{2}\right) \mathbf{a}_{1}-x_{2} \mathbf{a}_{2}+z_{2} \mathbf{a}_{3}$

$\mathbf{B}_{6}=-y_{2} \mathbf{a}_{1}-x_{2} \mathbf{a}_{2}+\left(\frac{1}{2}+z_{2}\right) \mathbf{a}_{3}=$

$=$
$=$
$=$

Cartesian Coordinates

Wyckoff Position

Atom Type

$$
\begin{aligned}
& \begin{array}{r}
\frac{\sqrt{3}}{2}\left(-x_{2}+y_{2}\right) a \hat{\mathbf{y}}+\left(\frac{1}{2}+z_{2}\right) c \hat{\mathbf{z}}
\end{array} \\
& \mathbf{B}_{7}=\left(-x_{2}+y_{2}\right) \mathbf{a}_{1}+y_{2} \mathbf{a}_{2}+\left(\frac{1}{2}+z_{2}\right) \mathbf{a}_{3}=\left(-\frac{1}{2} x_{2}+y_{2}\right) a \hat{\mathbf{x}}+\frac{\sqrt{3}}{2} x_{2} a \hat{\mathbf{y}}+ \\
& \left(\frac{1}{2}+z_{2}\right) c \hat{\mathbf{z}} \\
& \mathbf{B}_{8}=x_{2} \mathbf{a}_{1}+\left(x_{2}-y_{2}\right) \mathbf{a}_{2}+\left(\frac{1}{2}+z_{2}\right) \mathbf{a}_{3}=\left(x_{2}-\frac{1}{2} y_{2}\right) a \hat{\mathbf{x}}-\frac{\sqrt{3}}{2} y_{2} a \hat{\mathbf{y}}+ \\
& \left(\frac{1}{2}+z_{2}\right) c \hat{\mathbf{z}}
\end{aligned}
$$

(2a)

$\mathrm{Ru}$

(2a)

$\mathrm{Ru}$

$(6 d)$

(6d)

$(6 d)$

$(6 d)$

$(6 d)$

$(6 d)$
$\mathrm{Cl}$

$\mathrm{Cl}$

$\mathrm{Cl}$

$\mathrm{Cl}$

$\mathrm{Cl}$

$\mathrm{Cl}$

\section{References:}

- J. M. Fletcher, W. E. Gardner, A. C. Fox, and G. Topping, X-Ray, infrared, and magnetic studies of $\alpha$ - and $\beta$-ruthenium trichloride, J. Chem. Soc. A pp. 1038-1045 (1967), doi:10.1039/J19670001038.

\section{Geometry files:}

- CIF: pp. 913

- POSCAR: pp. 913 
$\mathrm{Bi}_{2} \mathrm{O}_{3}$ (High-pressure) Structure:

A2B3_hP20_159_bc_2c
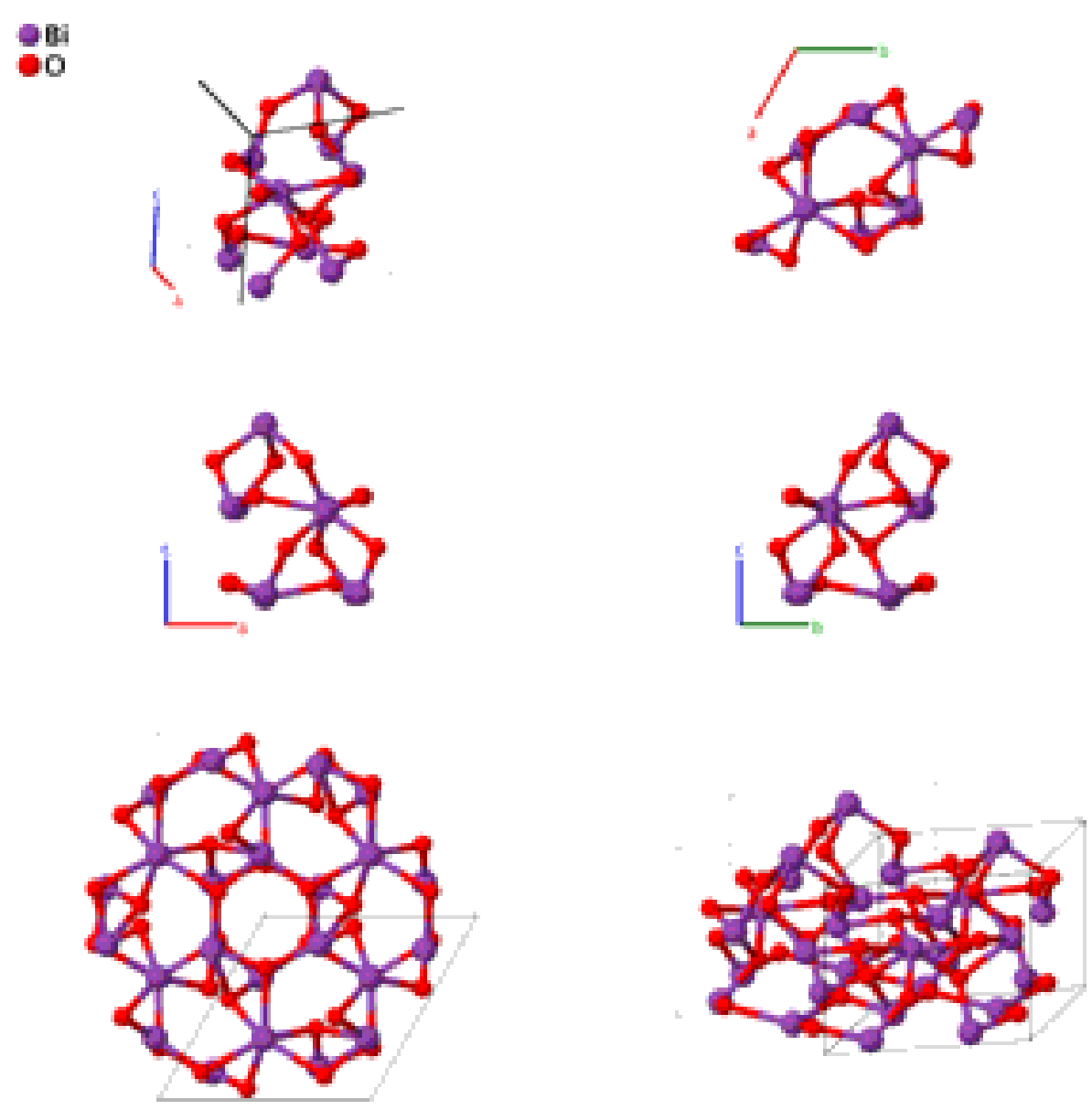

Prototype

$: \quad \mathrm{Bi}_{2} \mathrm{O}_{3}$

AFLOW prototype label

: A2B3_hP20_159_bc_2c

Strukturbericht designation : None

Pearson symbol $\quad: \quad h P 20$

Space group number : $\quad 159$

Space group symbol $\quad: \quad P 31 c$

AFLOW prototype command : aflow --proto=A2B3_hP20_159_bc_2c

- -params $=a, c / a, z_{1}, x_{2}, y_{2}, z_{2}, x_{3}, y_{3}, z_{3}, x_{4}, y_{4}, z_{4}$

Trigonal Hexagonal primitive vectors:

$$
\begin{array}{ll}
\mathbf{a}_{1}= & \frac{1}{2} a \hat{\mathbf{x}}-\frac{\sqrt{3}}{2} a \hat{\mathbf{y}} \\
\mathbf{a}_{2}= & \frac{1}{2} a \hat{\mathbf{x}}+\frac{\sqrt{3}}{2} a \hat{\mathbf{y}} \\
\mathbf{a}_{3}= & c \hat{\mathbf{z}}
\end{array}
$$

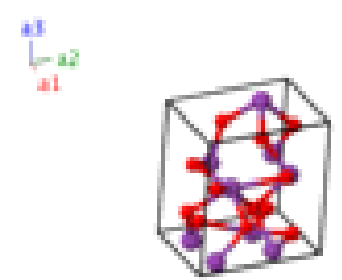

Basis vectors: 
Lattice Coordinates

$$
\begin{aligned}
& \mathbf{B}_{1}=\frac{1}{3} \mathbf{a}_{1}+\frac{2}{3} \mathbf{a}_{2}+z_{1} \mathbf{a}_{3} \\
& \mathbf{B}_{2}=\frac{2}{3} \mathbf{a}_{1}+\frac{1}{3} \mathbf{a}_{2}+\left(\frac{1}{2}+z_{1}\right) \mathbf{a}_{3} \\
& \mathbf{B}_{3}=x_{2} \mathbf{a}_{1}+y_{2} \mathbf{a}_{2}+z_{2} \mathbf{a}_{3} \\
& \mathbf{B}_{4}=-y_{2} \mathbf{a}_{1}+\left(x_{2}-y_{2}\right) \mathbf{a}_{2}+z_{2} \mathbf{a}_{3} \\
& \mathbf{B}_{5}=\left(-x_{2}+y_{2}\right) \mathbf{a}_{1}-x_{2} \mathbf{a}_{2}+z_{2} \mathbf{a}_{3} \\
& \mathbf{B}_{6}=y_{2} \mathbf{a}_{1}+x_{2} \mathbf{a}_{2}+\left(\frac{1}{2}+z_{2}\right) \mathbf{a}_{3} \\
& =\quad \frac{1}{2} a \hat{\mathbf{x}}+\frac{1}{2 \sqrt{3}} a \hat{\mathbf{y}}+z_{1} c \hat{\mathbf{z}} \\
& =\quad \frac{1}{2} a \hat{\mathbf{x}}-\frac{1}{2 \sqrt{3}} a \hat{\mathbf{y}}+\left(\frac{1}{2}+z_{1}\right) c \hat{\mathbf{z}} \\
& =\quad \frac{1}{2}\left(x_{2}+y_{2}\right) a \hat{\mathbf{x}}+ \\
& \frac{\sqrt{3}}{2}\left(-x_{2}+y_{2}\right) a \hat{\mathbf{y}}+z_{2} c \hat{\mathbf{z}} \\
& =\left(\frac{1}{2} x_{2}-y_{2}\right) a \hat{\mathbf{x}}+\frac{\sqrt{3}}{2} x_{2} a \hat{\mathbf{y}}+z_{2} c \hat{\mathbf{z}} \\
& =\left(-x_{2}+\frac{1}{2} y_{2}\right) a \hat{\mathbf{x}}-\frac{\sqrt{3}}{2} y_{2} a \hat{\mathbf{y}}+z_{2} c \hat{\mathbf{z}} \\
& =\frac{1}{2}\left(x_{2}+y_{2}\right) a \hat{\mathbf{x}}+\frac{\sqrt{3}}{2}\left(x_{2}-y_{2}\right) a \hat{\mathbf{y}}+ \\
& \left(\frac{1}{2}+z_{2}\right) c \hat{\mathbf{z}} \\
& \mathbf{B}_{7}=\left(x_{2}-y_{2}\right) \mathbf{a}_{1}-y_{2} \mathbf{a}_{2}+\left(\frac{1}{2}+z_{2}\right) \mathbf{a}_{3}=\left(\frac{1}{2} x_{2}-y_{2}\right) a \hat{\mathbf{x}}-\frac{\sqrt{3}}{2} x_{2} a \hat{\mathbf{y}}+ \\
& \left(\frac{1}{2}+z_{2}\right) c \hat{\mathbf{z}} \\
& \mathbf{B}_{8}=-x_{2} \mathbf{a}_{1}+\left(-x_{2}+y_{2}\right) \mathbf{a}_{2}+\left(\frac{1}{2}+z_{2}\right) \mathbf{a}_{3}=\left(-x_{2}+\frac{1}{2} y_{2}\right) a \hat{\mathbf{x}}+\frac{\sqrt{3}}{2} y_{2} a \hat{\mathbf{y}}+ \\
& \left(\frac{1}{2}+z_{2}\right) c \hat{\mathbf{z}} \\
& \mathbf{B}_{9} \quad=\quad x_{3} \mathbf{a}_{1}+y_{3} \mathbf{a}_{2}+z_{3} \mathbf{a}_{3} \\
& \mathbf{B}_{10}=-y_{3} \mathbf{a}_{1}+\left(x_{3}-y_{3}\right) \mathbf{a}_{2}+z_{3} \mathbf{a}_{3}=\left(\frac{1}{2} x_{3}-y_{3}\right) a \hat{\mathbf{x}}+\frac{\sqrt{3}}{2} x_{3} a \hat{\mathbf{y}}+z_{3} c \hat{\mathbf{z}} \\
& \mathbf{B}_{11}=\left(-x_{3}+y_{3}\right) \mathbf{a}_{1}-x_{3} \mathbf{a}_{2}+z_{3} \mathbf{a}_{3}=\left(-x_{3}+\frac{1}{2} y_{3}\right) a \hat{\mathbf{x}}-\frac{\sqrt{3}}{2} y_{3} a \hat{\mathbf{y}}+z_{3} c \hat{\mathbf{z}} \\
& \mathbf{B}_{12}=y_{3} \mathbf{a}_{1}+x_{3} \mathbf{a}_{2}+\left(\frac{1}{2}+z_{3}\right) \mathbf{a}_{3}=\frac{1}{2}\left(x_{3}+y_{3}\right) a \hat{\mathbf{x}}+\frac{\sqrt{3}}{2}\left(x_{3}-y_{3}\right) a \hat{\mathbf{y}}+ \\
& \left(\frac{1}{2}+z_{3}\right) c \hat{\mathbf{z}} \\
& \mathbf{B}_{13}=\left(x_{3}-y_{3}\right) \mathbf{a}_{1}-y_{3} \mathbf{a}_{2}+\left(\frac{1}{2}+z_{3}\right) \mathbf{a}_{3}=\left(\frac{1}{2} x_{3}-y_{3}\right) a \hat{\mathbf{x}}-\frac{\sqrt{3}}{2} x_{3} a \hat{\mathbf{y}}+ \\
& \left(\frac{1}{2}+z_{3}\right) c \hat{\mathbf{z}} \\
& \mathbf{B}_{14}=-x_{3} \mathbf{a}_{1}+\left(-x_{3}+y_{3}\right) \mathbf{a}_{2}+\left(\frac{1}{2}+z_{3}\right) \mathbf{a}_{3}=\left(-x_{3}+\frac{1}{2} y_{3}\right) a \hat{\mathbf{x}}+\frac{\sqrt{3}}{2} y_{3} a \hat{\mathbf{y}}+ \\
& \left(\frac{1}{2}+z_{3}\right) c \hat{\mathbf{z}} \\
& \mathbf{B}_{15}=x_{4} \mathbf{a}_{1}+y_{4} \mathbf{a}_{2}+z_{4} \mathbf{a}_{3} \quad=\quad \frac{1}{\sqrt{3}}\left(x_{4}+y_{4}\right) a \hat{\mathbf{x}}+ \\
& \frac{\sqrt{3}}{2}\left(-x_{4}+y_{4}\right) a \hat{\mathbf{y}}+z_{4} c \hat{\mathbf{z}} \\
& \mathbf{B}_{16}=-y_{4} \mathbf{a}_{1}+\left(x_{4}-y_{4}\right) \mathbf{a}_{2}+z_{4} \mathbf{a}_{3}=\left(\frac{1}{2} x_{4}-y_{4}\right) a \hat{\mathbf{x}}+\frac{\sqrt{3}}{2} x_{4} a \hat{\mathbf{y}}+z_{4} c \hat{\mathbf{z}} \\
& \mathbf{B}_{17}=\left(-x_{4}+y_{4}\right) \mathbf{a}_{1}-x_{4} \mathbf{a}_{2}+z_{4} \mathbf{a}_{3}=\left(-x_{4}+\frac{1}{2} y_{4}\right) a \hat{\mathbf{x}}-\frac{\sqrt{3}}{2} y_{4} a \hat{\mathbf{y}}+z_{4} c \hat{\mathbf{z}} \\
& \mathbf{B}_{18}=y_{4} \mathbf{a}_{1}+x_{4} \mathbf{a}_{2}+\left(\frac{1}{2}+z_{4}\right) \mathbf{a}_{3}=\frac{1}{2}\left(x_{4}+y_{4}\right) a \hat{\mathbf{x}}+\frac{\sqrt{3}}{2}\left(x_{4}-y_{4}\right) a \hat{\mathbf{y}}+ \\
& \left(\frac{1}{2}+z_{4}\right) c \hat{\mathbf{z}} \\
& \mathbf{B}_{19}=\left(x_{4}-y_{4}\right) \mathbf{a}_{1}-y_{4} \mathbf{a}_{2}+\left(\frac{1}{2}+z_{4}\right) \mathbf{a}_{3}=\left(\frac{1}{2} x_{4}-y_{4}\right) a \hat{\mathbf{x}}-\frac{\sqrt{3}}{2} x_{4} a \hat{\mathbf{y}}+ \\
& \left(\frac{1}{2}+z_{4}\right) c \hat{\mathbf{z}} \\
& \mathbf{B}_{20}=-x_{4} \mathbf{a}_{1}+\left(-x_{4}+y_{4}\right) \mathbf{a}_{2}+\left(\frac{1}{2}+z_{4}\right) \mathbf{a}_{3}=\left(-x_{4}+\frac{1}{2} y_{4}\right) a \hat{\mathbf{x}}+\frac{\sqrt{3}}{2} y_{4} a \hat{\mathbf{y}}+ \\
& \left(\frac{1}{2}+z_{4}\right) c \hat{\mathbf{z}}
\end{aligned}
$$

O II

O II

O II

O II

O II

\section{References:}

- T. Locherer, D. L. V. K. Prasad, R. Dinnebier, U. Wedig, M. Jansen, G. Garbarino, and T. Hansen, High-pressure structural evolution of $\mathrm{HP}_{-} \mathrm{Bi}_{2} \mathrm{O}_{3}$, Phys. Rev. B 83, 214102 (2011), doi:10.1103/PhysRevB.83.214102.

\section{Found in:}

- P. Villars and K. Cenzual, Pearson's Crystal Data - Crystal Structure Database for Inorganic Compounds, ASM International (2013). 
Geometry files:

- CIF: pp. 914

- POSCAR: pp. 914 


\section{Nierite $\left(\alpha-\mathrm{Si}_{3} \mathrm{~N}_{4}\right)$ Structure: A4B3_hP28_159_ab2c_2c}
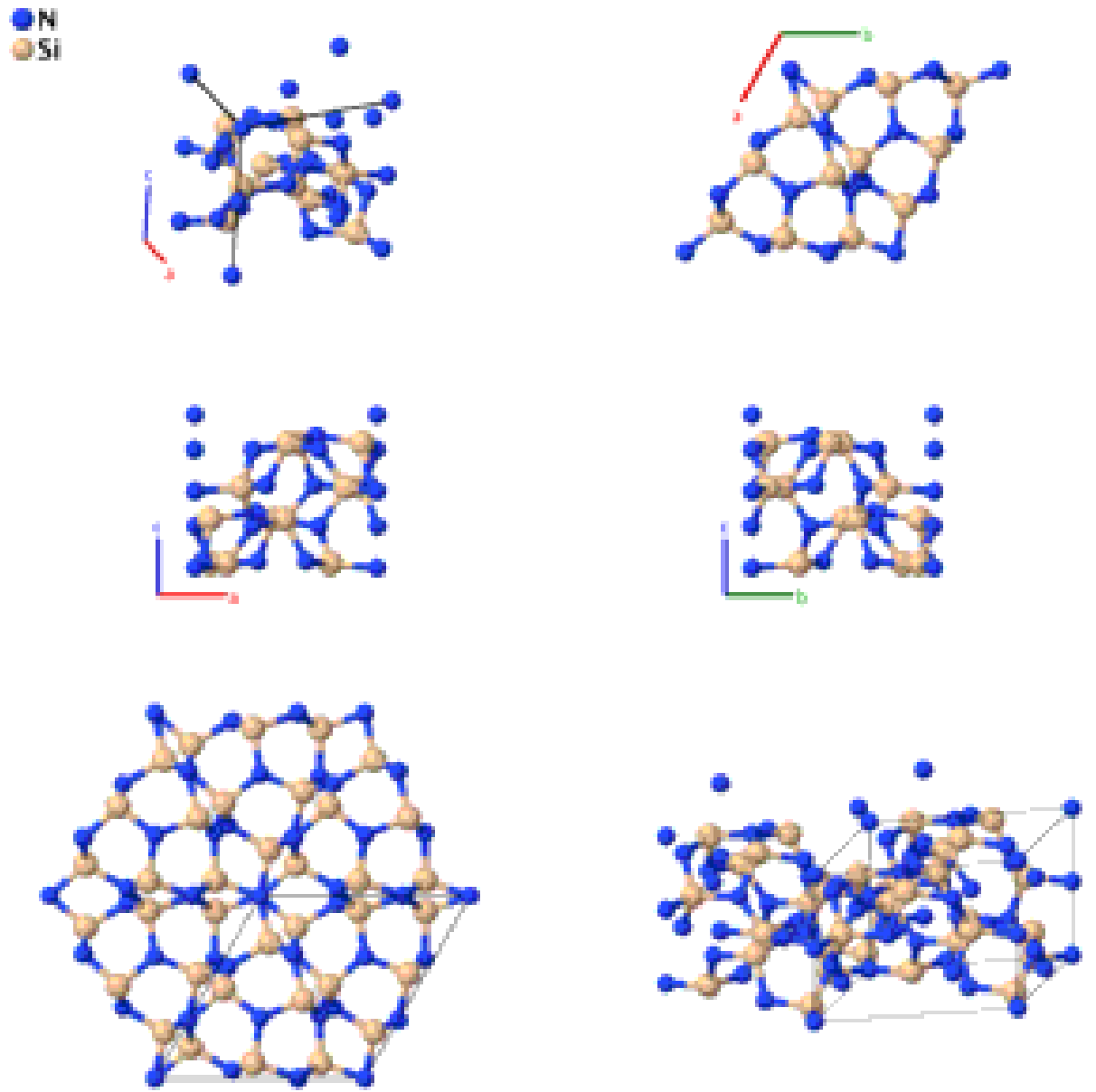

\section{Prototype}

AFLOW prototype label

$\quad \alpha-\mathrm{Si}_{3} \mathrm{~N}_{4}$

Strukturbericht designation

Pearson symbol

: A4B3_hP28_159_ab2c_2c

Space group number

None

Space group symbol

: $\mathrm{hP} 28$

AFLOW prototype command : aflow --proto=A4B3_hP28_159_ab2c_2c

- params $=a, c / a, z_{1}, z_{2}, x_{3}, y_{3}, z_{3}, x_{4}, y_{4}, z_{4}, x_{5}, y_{5}, z_{5}, x_{6}, y_{6}, z_{6}$

Trigonal Hexagonal primitive vectors:

$$
\begin{array}{ll}
\mathbf{a}_{1}= & \frac{1}{2} a \hat{\mathbf{x}}-\frac{\sqrt{3}}{2} a \hat{\mathbf{y}} \\
\mathbf{a}_{2}= & \frac{1}{2} a \hat{\mathbf{x}}+\frac{\sqrt{3}}{2} a \hat{\mathbf{y}} \\
\mathbf{a}_{3}= & c \hat{\mathbf{z}}
\end{array}
$$

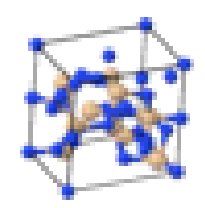

Basis vectors: 


\begin{tabular}{|c|c|c|c|c|c|c|}
\hline $\mathbf{B}_{1}$ & $=$ & $z_{1} \mathbf{a}_{3}$ & $=$ & $z_{1} c \hat{\mathbf{z}}$ & $(2 a)$ & $\mathrm{N} \mathrm{I}$ \\
\hline $\mathbf{B}_{2}$ & $=$ & $\left(\frac{1}{2}+z_{1}\right) \mathbf{a}_{3}$ & $=$ & $\left(\frac{1}{2}+z_{1}\right) c \hat{\mathbf{z}}$ & $(2 a)$ & N I \\
\hline $\mathbf{B}_{3}$ & $=$ & $\frac{1}{3} \mathbf{a}_{1}+\frac{2}{3} \mathbf{a}_{2}+z_{2} \mathbf{a}_{3}$ & $=$ & $\frac{1}{2} a \hat{\mathbf{x}}+\frac{1}{2 \sqrt{3}} a \hat{\mathbf{y}}+z_{2} c \hat{\mathbf{z}}$ & $(2 b)$ & $\mathrm{N}$ II \\
\hline $\mathbf{B}_{4}$ & $=$ & $\frac{2}{3} \mathbf{a}_{1}+\frac{1}{3} \mathbf{a}_{2}+\left(\frac{1}{2}+z_{2}\right) \mathbf{a}_{3}$ & $=$ & $\frac{1}{2} a \hat{\mathbf{x}}-\frac{1}{2 \sqrt{3}} a \hat{\mathbf{y}}+\left(\frac{1}{2}+z_{2}\right) c \hat{\mathbf{z}}$ & $(2 b)$ & $\mathrm{N}$ II \\
\hline $\mathbf{B}_{5}$ & $=$ & $x_{3} \mathbf{a}_{1}+y_{3} \mathbf{a}_{2}+z_{3} \mathbf{a}_{3}$ & $=$ & $\begin{array}{c}\frac{1}{2}\left(x_{3}+y_{3}\right) a \hat{\mathbf{x}}+ \\
\frac{\sqrt{3}}{2}\left(-x_{3}+y_{3}\right) a \hat{\mathbf{y}}+z_{3} c \hat{\mathbf{z}}\end{array}$ & $(6 c)$ & N III \\
\hline $\mathbf{B}_{6}$ & $=$ & $-y_{3} \mathbf{a}_{1}+\left(x_{3}-y_{3}\right) \mathbf{a}_{2}+z_{3} \mathbf{a}_{3}$ & $=$ & $\left(\frac{1}{2} x_{3}-y_{3}\right) a \hat{\mathbf{x}}+\frac{\sqrt{3}}{2} x_{3} a \hat{\mathbf{y}}+z_{3} c \hat{\mathbf{z}}$ & $(6 c)$ & N III \\
\hline $\mathbf{B}_{7}$ & $=$ & $\left(-x_{3}+y_{3}\right) \mathbf{a}_{1}-x_{3} \mathbf{a}_{2}+z_{3} \mathbf{a}_{3}$ & $=$ & $\left(-x_{3}+\frac{1}{2} y_{3}\right) a \hat{\mathbf{x}}-\frac{\sqrt{3}}{2} y_{3} a \hat{\mathbf{y}}+z_{3} c \hat{\mathbf{z}}$ & $(6 c)$ & $\mathrm{N}$ III \\
\hline $\mathbf{B}_{8}$ & $=$ & $y_{3} \mathbf{a}_{1}+x_{3} \mathbf{a}_{2}+\left(\frac{1}{2}+z_{3}\right) \mathbf{a}_{3}$ & $=$ & $\begin{array}{c}\frac{1}{2}\left(x_{3}+y_{3}\right) a \hat{\mathbf{x}}+\frac{\sqrt{3}}{2}\left(x_{3}-y_{3}\right) a \hat{\mathbf{y}}+ \\
\left(\frac{1}{2}+z_{3}\right) c \hat{\mathbf{z}}\end{array}$ & $(6 c)$ & N III \\
\hline $\mathbf{B}_{9}$ & $=$ & $\left(x_{3}-y_{3}\right) \mathbf{a}_{1}-y_{3} \mathbf{a}_{2}+\left(\frac{1}{2}+z_{3}\right) \mathbf{a}_{3}$ & $=$ & $\begin{array}{c}\left(\frac{1}{2} x_{3}-y_{3}\right) a \hat{\mathbf{x}}-\frac{\sqrt{3}}{2} x_{3} a \hat{\mathbf{y}}+ \\
\left(\frac{1}{2}+z_{3}\right) c \hat{\mathbf{z}}\end{array}$ & $(6 c)$ & N III \\
\hline $\mathbf{B}_{10}$ & $=$ & $-x_{3} \mathbf{a}_{1}+\left(-x_{3}+y_{3}\right) \mathbf{a}_{2}+\left(\frac{1}{2}+z_{3}\right) \mathbf{a}_{3}$ & $=$ & $\begin{array}{c}\left(-x_{3}+\frac{1}{2} y_{3}\right) a \hat{\mathbf{x}}+\frac{\sqrt{3}}{2} y_{3} a \hat{\mathbf{y}}+ \\
\left(\frac{1}{2}+z_{3}\right) c \hat{\mathbf{z}}\end{array}$ & $(6 c)$ & N III \\
\hline $\mathbf{B}_{11}$ & $=$ & $x_{4} \mathbf{a}_{1}+y_{4} \mathbf{a}_{2}+z_{4} \mathbf{a}_{3}$ & $=$ & $\begin{array}{c}\frac{1}{2}\left(x_{4}+y_{4}\right) a \hat{\mathbf{x}}+ \\
\frac{\sqrt{3}}{2}\left(-x_{4}+y_{4}\right) a \hat{\mathbf{y}}+z_{4} c \hat{\mathbf{z}}\end{array}$ & $(6 c)$ & N IV \\
\hline $\mathbf{B}_{12}$ & $=$ & $-y_{4} \mathbf{a}_{1}+\left(x_{4}-y_{4}\right) \mathbf{a}_{2}+z_{4} \mathbf{a}_{3}$ & $=$ & $\left(\frac{1}{2} x_{4}-y_{4}\right) a \hat{\mathbf{x}}+\frac{\sqrt{3}}{2} x_{4} a \hat{\mathbf{y}}+z_{4} c \hat{\mathbf{z}}$ & $(6 c)$ & N IV \\
\hline $\mathbf{B}_{13}$ & $=$ & $\left(-x_{4}+y_{4}\right) \mathbf{a}_{1}-x_{4} \mathbf{a}_{2}+z_{4} \mathbf{a}_{3}$ & $=$ & $\left(-x_{4}+\frac{1}{2} y_{4}\right) a \hat{\mathbf{x}}-\frac{\sqrt{3}}{2} y_{4} a \hat{\mathbf{y}}+z_{4} c \hat{\mathbf{z}}$ & $(6 c)$ & N IV \\
\hline $\mathbf{B}_{14}$ & $=$ & $y_{4} \mathbf{a}_{1}+x_{4} \mathbf{a}_{2}+\left(\frac{1}{2}+z_{4}\right) \mathbf{a}_{3}$ & $=$ & $\begin{array}{c}\frac{1}{2}\left(x_{4}+y_{4}\right) a \hat{\mathbf{x}}+\frac{\sqrt{3}}{2}\left(x_{4}-y_{4}\right) a \hat{\mathbf{y}}+ \\
\left(\frac{1}{2}+z_{4}\right) c \hat{\mathbf{z}}\end{array}$ & $(6 c)$ & N IV \\
\hline $\mathbf{B}_{15}$ & $=$ & $\left(x_{4}-y_{4}\right) \mathbf{a}_{1}-y_{4} \mathbf{a}_{2}+\left(\frac{1}{2}+z_{4}\right) \mathbf{a}_{3}$ & $=$ & $\begin{array}{c}\left(\frac{1}{2} x_{4}-y_{4}\right) a \hat{\mathbf{x}}-\frac{\sqrt{3}}{2} x_{4} a \hat{\mathbf{y}}+ \\
\left(\frac{1}{2}+z_{4}\right) c \hat{\mathbf{z}}\end{array}$ & $(6 c)$ & N IV \\
\hline $\mathbf{B}_{16}$ & $=$ & $-x_{4} \mathbf{a}_{1}+\left(-x_{4}+y_{4}\right) \mathbf{a}_{2}+\left(\frac{1}{2}+z_{4}\right) \mathbf{a}_{3}$ & $=$ & $\begin{array}{c}\left(-x_{4}+\frac{1}{2} y_{4}\right) a \hat{\mathbf{x}}+\frac{\sqrt{3}}{2} y_{4} a \hat{\mathbf{y}}+ \\
\left(\frac{1}{2}+z_{4}\right) c \hat{\mathbf{z}}\end{array}$ & $(6 c)$ & N IV \\
\hline $\mathbf{B}_{17}$ & $=$ & $x_{5} \mathbf{a}_{1}+y_{5} \mathbf{a}_{2}+z_{5} \mathbf{a}_{3}$ & $=$ & $\begin{array}{c}\frac{1}{2}\left(x_{5}+y_{5}\right) a \hat{\mathbf{x}}+ \\
\frac{\sqrt{3}}{2}\left(-x_{5}+y_{5}\right) a \hat{\mathbf{y}}+z_{5} c \hat{\mathbf{z}}\end{array}$ & $(6 c)$ & Si I \\
\hline $\mathbf{B}_{18}$ & $=$ & $-y_{5} \mathbf{a}_{1}+\left(x_{5}-y_{5}\right) \mathbf{a}_{2}+z_{5} \mathbf{a}_{3}$ & $=$ & $\left(\frac{1}{2} x_{5}-y_{5}\right) a \hat{\mathbf{x}}+\frac{\sqrt{3}}{2} x_{5} a \hat{\mathbf{y}}+z_{5} c \hat{\mathbf{z}}$ & $(6 c)$ & $\mathrm{Si} \mathrm{I}$ \\
\hline $\mathbf{B}_{19}$ & $=$ & $\left(-x_{5}+y_{5}\right) \mathbf{a}_{1}-x_{5} \mathbf{a}_{2}+z_{5} \mathbf{a}_{3}$ & $=$ & $\left(-x_{5}+\frac{1}{2} y_{5}\right) a \hat{\mathbf{x}}-\frac{\sqrt{3}}{2} y_{5} a \hat{\mathbf{y}}+z_{5} c \hat{\mathbf{z}}$ & $(6 c)$ & Si I \\
\hline $\mathbf{B}_{20}$ & $=$ & $y_{5} \mathbf{a}_{1}+x_{5} \mathbf{a}_{2}+\left(\frac{1}{2}+z_{5}\right) \mathbf{a}_{3}$ & $=$ & $\begin{array}{c}\frac{1}{2}\left(x_{5}+y_{5}\right) a \hat{\mathbf{x}}+\frac{\sqrt{3}}{2}\left(x_{5}-y_{5}\right) a \hat{\mathbf{y}}+ \\
\left(\frac{1}{2}+z_{5}\right) c \hat{\mathbf{z}}\end{array}$ & $(6 c)$ & Si I \\
\hline $\mathbf{B}_{21}$ & $=$ & $\left(x_{5}-y_{5}\right) \mathbf{a}_{1}-y_{5} \mathbf{a}_{2}+\left(\frac{1}{2}+z_{5}\right) \mathbf{a}_{3}$ & $=$ & $\begin{array}{c}\left(\frac{1}{2} x_{5}-y_{5}\right) a \hat{\mathbf{x}}-\frac{\sqrt{3}}{2} x_{5} a \hat{\mathbf{y}}+ \\
\left(\frac{1}{2}+z_{5}\right) c \hat{\mathbf{z}}\end{array}$ & $(6 c)$ & Si I \\
\hline $\mathbf{B}_{22}$ & $=$ & $-x_{5} \mathbf{a}_{1}+\left(-x_{5}+y_{5}\right) \mathbf{a}_{2}+\left(\frac{1}{2}+z_{5}\right) \mathbf{a}_{3}$ & $=$ & $\begin{array}{c}\left(-x_{5}+\frac{1}{2} y_{5}\right) a \hat{\mathbf{x}}+\frac{\sqrt{3}}{2} y_{5} a \hat{\mathbf{y}}+ \\
\left(\frac{1}{2}+z_{5}\right) c \hat{\mathbf{z}}\end{array}$ & $(6 c)$ & Si I \\
\hline $\mathbf{B}_{23}$ & $=$ & $x_{6} \mathbf{a}_{1}+y_{6} \mathbf{a}_{2}+z_{6} \mathbf{a}_{3}$ & $=$ & $\begin{array}{c}\frac{1}{2}\left(x_{6}+y_{6}\right) a \hat{\mathbf{x}}+ \\
\frac{\sqrt{3}}{2}\left(-x_{6}+y_{6}\right) a \hat{\mathbf{y}}+z_{6} c \hat{\mathbf{z}}\end{array}$ & $(6 c)$ & Si II \\
\hline $\mathbf{B}_{24}$ & $=$ & $-y_{6} \mathbf{a}_{1}+\left(x_{6}-y_{6}\right) \mathbf{a}_{2}+z_{6} \mathbf{a}_{3}$ & & $\left(\frac{1}{2} x_{6}-y_{6}\right) a \hat{\mathbf{x}}+\frac{\sqrt{3}}{2} x_{6} a \hat{\mathbf{y}}+z_{6} c \hat{\mathbf{z}}$ & $(6 c)$ & $\mathrm{Si}$ II \\
\hline $\mathbf{B}_{25}$ & $=$ & $\left(-x_{6}+y_{6}\right) \mathbf{a}_{1}-x_{6} \mathbf{a}_{2}+z_{6} \mathbf{a}_{3}$ & $=$ & $\left(-x_{6}+\frac{1}{2} y_{6}\right) a \hat{\mathbf{x}}-\frac{\sqrt{3}}{2} y_{6} a \hat{\mathbf{y}}+z_{6} c \hat{\mathbf{z}}$ & $(6 c)$ & $\mathrm{Si}$ II \\
\hline $\mathbf{B}_{26}$ & $=$ & $y_{6} \mathbf{a}_{1}+x_{6} \mathbf{a}_{2}+\left(\frac{1}{2}+z_{6}\right) \mathbf{a}_{3}$ & $=$ & $\begin{array}{c}\frac{1}{2}\left(x_{6}+y_{6}\right) a \hat{\mathbf{x}}+\frac{\sqrt{3}}{2}\left(x_{6}-y_{6}\right) a \hat{\mathbf{y}}+ \\
\left(\frac{1}{2}+z_{6}\right) c \hat{\mathbf{z}}\end{array}$ & $(6 c)$ & Si II \\
\hline
\end{tabular}




$$
\begin{aligned}
& \mathbf{B}_{27}=\left(x_{6}-y_{6}\right) \mathbf{a}_{1}-y_{6} \mathbf{a}_{2}+\left(\frac{1}{2}+z_{6}\right) \mathbf{a}_{3}=\left(\frac{1}{2} x_{6}-y_{6}\right) a \hat{\mathbf{x}}-\frac{\sqrt{3}}{2} x_{6} a \hat{\mathbf{y}}+ \\
& \left(\frac{1}{2}+z_{6}\right) c \hat{\mathbf{z}} \\
& \mathbf{B}_{28}=-x_{6} \mathbf{a}_{1}+\left(-x_{6}+y_{6}\right) \mathbf{a}_{2}+\left(\frac{1}{2}+z_{6}\right) \mathbf{a}_{3}=\left(-x_{6}+\frac{1}{2} y_{6}\right) a \hat{\mathbf{x}}+\frac{\sqrt{3}}{2} y_{6} a \hat{\mathbf{y}}+ \\
& \left(\frac{1}{2}+z_{6}\right) c \hat{\mathbf{z}}
\end{aligned}
$$

\section{References:}

- D. Hardie and K. H. Jack, Crystal structures of silicon nitride, Nature 180, 332-333 (1957), doi:doi:10.1038/180332a0.

\section{Found in:}

- P. Villars and K. Cenzual, Pearson's Crystal Data - Crystal Structure Database for Inorganic Compounds, ASM International (2013).

\section{Geometry files:}

- CIF: pp. 914

- POSCAR: pp. 914 


\section{$\mathrm{YbBaCo}_{4} \mathrm{O}_{7}$ Structure: AB4C7D_hP26_159_b_ac_a2c_b}
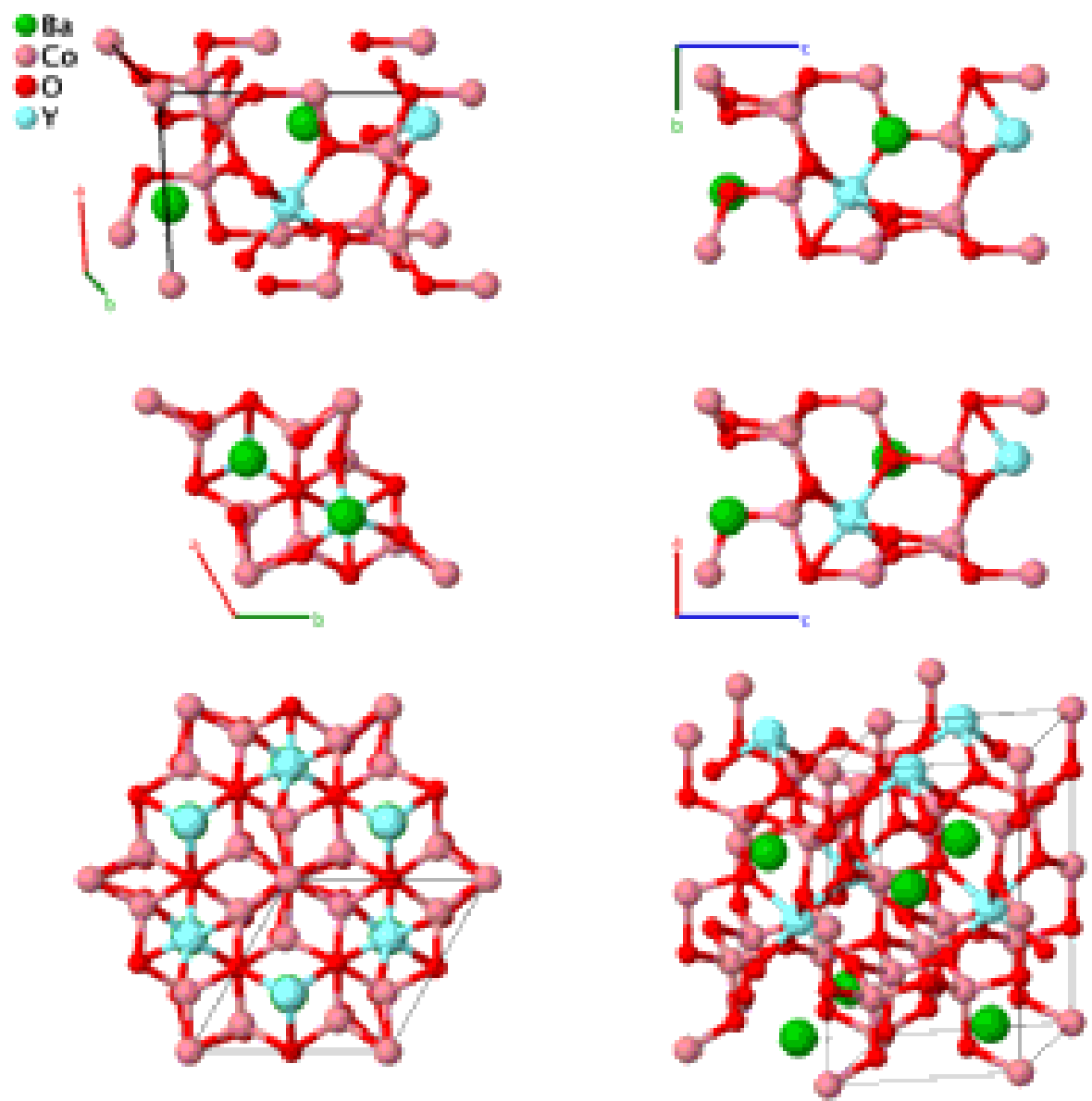

Prototype

AFLOW prototype label

: $\quad \mathrm{YbBaCo}_{4} \mathrm{O}_{7}$

Strukturbericht designation : None

Pearson symbol

: hP26

Space group number

159

Space group symbol

P31c

AFLOW prototype command

aflow --proto=AB4C7D_hP26_159_b_ac_a2c_b

- params $=a, c / a, z_{1}, z_{2}, z_{3}, z_{4}, x_{5}, y_{5}, z_{5}, x_{6}, y_{6}, z_{6}, x_{7}, y_{7}, z_{7}$

Trigonal Hexagonal primitive vectors:

$$
\begin{array}{ll}
\mathbf{a}_{1}= & \frac{1}{2} a \hat{\mathbf{x}}-\frac{\sqrt{3}}{2} a \hat{\mathbf{y}} \\
\mathbf{a}_{2}= & \frac{1}{2} a \hat{\mathbf{x}}+\frac{\sqrt{3}}{2} a \hat{\mathbf{y}} \\
\mathbf{a}_{3}= & c \hat{\mathbf{z}}
\end{array}
$$

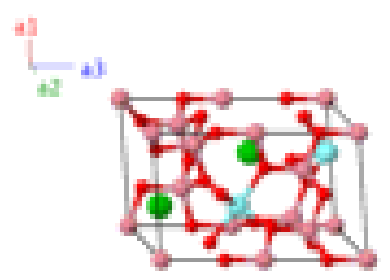

Basis vectors:

Lattice Coordinates

$\mathbf{B}_{1}=$
Cartesian Coordinates $=\quad z_{1} c \hat{\mathbf{z}}$
Wyckoff Position

(2a)
Atom Type

Co I 


\begin{tabular}{|c|c|c|c|c|c|}
\hline $\mathbf{B}_{2}$ & $=$ & $\left(\frac{1}{2}+z_{1}\right) \mathbf{a}_{3}$ & $\left(\frac{1}{2}+z_{1}\right) c \hat{\mathbf{z}}$ & $(2 a)$ & Co I \\
\hline $\mathbf{B}_{3}$ & $=$ & $z_{2} \mathbf{a}_{3}$ & $z_{2} c \hat{\mathbf{z}}$ & $(2 a)$ & O I \\
\hline $\mathbf{B}_{4}$ & $=$ & $\left(\frac{1}{2}+z_{2}\right) \mathbf{a}_{3}$ & $\left(\frac{1}{2}+z_{2}\right) c \hat{\mathbf{z}}$ & $(2 a)$ & O I \\
\hline $\mathbf{B}_{5}$ & $=$ & $\frac{1}{3} \mathbf{a}_{1}+\frac{2}{3} \mathbf{a}_{2}+z_{3} \mathbf{a}_{3}$ & $\frac{1}{2} a \hat{\mathbf{x}}+\frac{1}{2 \sqrt{3}} a \hat{\mathbf{y}}+z_{3} c \hat{\mathbf{z}}$ & $(2 b)$ & $\mathrm{Ba}$ \\
\hline $\mathbf{B}_{6}$ & $=$ & $\frac{2}{3} \mathbf{a}_{1}+\frac{1}{3} \mathbf{a}_{2}+\left(\frac{1}{2}+z_{3}\right) \mathbf{a}_{3}$ & $\frac{1}{2} a \hat{\mathbf{x}}-\frac{1}{2 \sqrt{3}} a \hat{\mathbf{y}}+\left(\frac{1}{2}+z_{3}\right) c \hat{\mathbf{z}}$ & $(2 b)$ & $\mathrm{Ba}$ \\
\hline $\mathbf{B}_{7}$ & $=$ & $\frac{1}{3} \mathbf{a}_{1}+\frac{2}{3} \mathbf{a}_{2}+z_{4} \mathbf{a}_{3}$ & $\frac{1}{2} a \hat{\mathbf{x}}+\frac{1}{2 \sqrt{3}} a \hat{\mathbf{y}}+z_{4} c \hat{\mathbf{z}}$ & $(2 b)$ & Y \\
\hline $\mathbf{B}_{8}$ & $=$ & $\frac{2}{3} \mathbf{a}_{1}+\frac{1}{3} \mathbf{a}_{2}+\left(\frac{1}{2}+z_{4}\right) \mathbf{a}_{3}$ & $=\quad \frac{1}{2} a \hat{\mathbf{x}}-\frac{1}{2 \sqrt{3}} a \hat{\mathbf{y}}+\left(\frac{1}{2}+z_{4}\right) c \hat{\mathbf{z}}$ & $(2 b)$ & $\mathrm{Y}$ \\
\hline $\mathbf{B}_{9}$ & $=$ & $x_{5} \mathbf{a}_{1}+y_{5} \mathbf{a}_{2}+z_{5} \mathbf{a}_{3}$ & 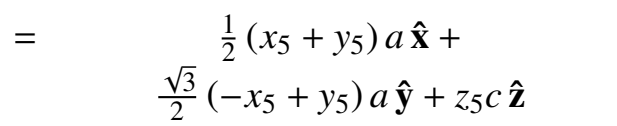 & $(6 c)$ & Co II \\
\hline $\mathbf{B}_{10}$ & $=$ & $-y_{5} \mathbf{a}_{1}+\left(x_{5}-y_{5}\right) \mathbf{a}_{2}+z_{5} \mathbf{a}_{3}$ & $=\left(\frac{1}{2} x_{5}-y_{5}\right) a \hat{\mathbf{x}}+\frac{\sqrt{3}}{2} x_{5} a \hat{\mathbf{y}}+z_{5} c \hat{\mathbf{z}}$ & $(6 c)$ & Co II \\
\hline $\mathbf{B}_{11}$ & $=$ & $\left(-x_{5}+y_{5}\right) \mathbf{a}_{1}-x_{5} \mathbf{a}_{2}+z_{5} \mathbf{a}_{3}$ & $=\left(-x_{5}+\frac{1}{2} y_{5}\right) a \hat{\mathbf{x}}-\frac{\sqrt{3}}{2} y_{5} a \hat{\mathbf{y}}+z_{5} c \hat{\mathbf{z}}$ & $(6 c)$ & Co II \\
\hline $\mathbf{B}_{12}$ & $=$ & $y_{5} \mathbf{a}_{1}+x_{5} \mathbf{a}_{2}+\left(\frac{1}{2}+z_{5}\right) \mathbf{a}_{3}$ & $\begin{array}{c}=\frac{1}{2}\left(x_{5}+y_{5}\right) a \hat{\mathbf{x}}+\frac{\sqrt{3}}{2}\left(x_{5}-y_{5}\right) a \hat{\mathbf{y}}+ \\
\left(\frac{1}{2}+z_{5}\right) c \hat{\mathbf{z}}\end{array}$ & $(6 c)$ & Co II \\
\hline $\mathbf{B}_{13}$ & $=$ & $\left(x_{5}-y_{5}\right) \mathbf{a}_{1}-y_{5} \mathbf{a}_{2}+\left(\frac{1}{2}+z_{5}\right) \mathbf{a}_{3}$ & $\begin{array}{c}=\left(\frac{1}{2} x_{5}-y_{5}\right) a \hat{\mathbf{x}}-\frac{\sqrt{3}}{2} x_{5} a \hat{\mathbf{y}}+ \\
\left(\frac{1}{2}+z_{5}\right) c \hat{\mathbf{z}}\end{array}$ & $(6 c)$ & Co II \\
\hline $\mathbf{B}_{14}$ & $=$ & $-x_{5} \mathbf{a}_{1}+\left(-x_{5}+y_{5}\right) \mathbf{a}_{2}+\left(\frac{1}{2}+z_{5}\right) \mathbf{a}_{3}$ & $\begin{array}{c}\left(-x_{5}+\frac{1}{2} y_{5}\right) a \hat{\mathbf{x}}+\frac{\sqrt{3}}{2} y_{5} a \hat{\mathbf{y}}+ \\
\left(\frac{1}{2}+z_{5}\right) c \hat{\mathbf{z}}\end{array}$ & $(6 c)$ & Co II \\
\hline $\mathbf{B}_{15}$ & $=$ & $x_{6} \mathbf{a}_{1}+y_{6} \mathbf{a}_{2}+z_{6} \mathbf{a}_{3}$ & $\begin{array}{c}=\quad \frac{1}{2}\left(x_{6}+y_{6}\right) a \hat{\mathbf{x}}+ \\
\frac{\sqrt{3}}{2}\left(-x_{6}+y_{6}\right) a \hat{\mathbf{y}}+z_{6} c \hat{\mathbf{z}}\end{array}$ & $(6 c)$ & O II \\
\hline $\mathbf{B}_{16}$ & $=$ & $-y_{6} \mathbf{a}_{1}+\left(x_{6}-y_{6}\right) \mathbf{a}_{2}+z_{6} \mathbf{a}_{3}$ & $=\left(\frac{1}{2} x_{6}-y_{6}\right) a \hat{\mathbf{x}}+\frac{\sqrt{3}}{2} x_{6} a \hat{\mathbf{y}}+z_{6} c \hat{\mathbf{z}}$ & $(6 c)$ & O II \\
\hline $\mathbf{B}_{17}$ & $=$ & $\left(-x_{6}+y_{6}\right) \mathbf{a}_{1}-x_{6} \mathbf{a}_{2}+z_{6} \mathbf{a}_{3}$ & $=\left(-x_{6}+\frac{1}{2} y_{6}\right) a \hat{\mathbf{x}}-\frac{\sqrt{3}}{2} y_{6} a \hat{\mathbf{y}}+z_{6} c \hat{\mathbf{z}}$ & $(6 c)$ & O II \\
\hline $\mathbf{B}_{18}$ & $=$ & $y_{6} \mathbf{a}_{1}+x_{6} \mathbf{a}_{2}+\left(\frac{1}{2}+z_{6}\right) \mathbf{a}_{3}$ & $\begin{array}{c}=\frac{1}{2}\left(x_{6}+y_{6}\right) a \hat{\mathbf{x}}+\frac{\sqrt{3}}{2}\left(x_{6}-y_{6}\right) a \hat{\mathbf{y}}+ \\
\left(\frac{1}{2}+z_{6}\right) c \hat{\mathbf{z}}\end{array}$ & $(6 c)$ & O II \\
\hline $\mathbf{B}_{19}$ & $=$ & $\left(x_{6}-y_{6}\right) \mathbf{a}_{1}-y_{6} \mathbf{a}_{2}+\left(\frac{1}{2}+z_{6}\right) \mathbf{a}_{3}$ & $\begin{array}{c}\left(\frac{1}{2} x_{6}-y_{6}\right) a \hat{\mathbf{x}}-\frac{\sqrt{3}}{2} x_{6} a \hat{\mathbf{y}}+ \\
\left(\frac{1}{2}+z_{6}\right) c \hat{\mathbf{z}}\end{array}$ & $(6 c)$ & O II \\
\hline $\mathbf{B}_{20}$ & $=$ & $-x_{6} \mathbf{a}_{1}+\left(-x_{6}+y_{6}\right) \mathbf{a}_{2}+\left(\frac{1}{2}+z_{6}\right) \mathbf{a}_{3}$ & $\begin{array}{c}\left(-x_{6}+\frac{1}{2} y_{6}\right) a \hat{\mathbf{x}}+\frac{\sqrt{3}}{2} y_{6} a \hat{\mathbf{y}}+ \\
\left(\frac{1}{2}+z_{6}\right) c \hat{\mathbf{z}}\end{array}$ & $(6 c)$ & O II \\
\hline $\mathbf{B}_{21}$ & $=$ & $x_{7} \mathbf{a}_{1}+y_{7} \mathbf{a}_{2}+z_{7} \mathbf{a}_{3}$ & 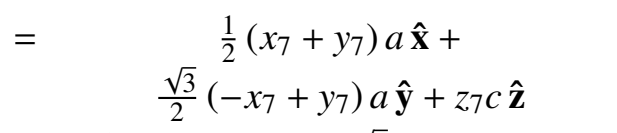 & $(6 c)$ & O III \\
\hline $\mathbf{B}_{22}$ & $=$ & $-y_{7} \mathbf{a}_{1}+\left(x_{7}-y_{7}\right) \mathbf{a}_{2}+z_{7} \mathbf{a}_{3}$ & $=\left(\frac{1}{2} x_{7}-y_{7}\right) a \hat{\mathbf{x}}+\frac{\sqrt{3}}{2} x_{7} a \hat{\mathbf{y}}+z_{7} c \hat{\mathbf{z}}$ & $(6 c)$ & O III \\
\hline $\mathbf{B}_{23}$ & $=$ & $\left(-x_{7}+y_{7}\right) \mathbf{a}_{1}-x_{7} \mathbf{a}_{2}+z_{7} \mathbf{a}_{3}$ & $=\left(-x_{7}+\frac{1}{2} y_{7}\right) a \hat{\mathbf{x}}-\frac{\sqrt{3}}{2} y_{7} a \hat{\mathbf{y}}+z_{7} c \hat{\mathbf{z}}$ & $(6 c)$ & O III \\
\hline $\mathbf{B}_{24}$ & $=$ & $y_{7} \mathbf{a}_{1}+x_{7} \mathbf{a}_{2}+\left(\frac{1}{2}+z_{7}\right) \mathbf{a}_{3}$ & $\begin{array}{c}=\frac{1}{2}\left(x_{7}+y_{7}\right) a \hat{\mathbf{x}}+\frac{\sqrt{3}}{2}\left(x_{7}-y_{7}\right) a \hat{\mathbf{y}}+ \\
\left(\frac{1}{2}+z_{7}\right) c \hat{\mathbf{z}}\end{array}$ & $(6 c)$ & O III \\
\hline $\mathbf{B}_{25}$ & $=$ & $\left(x_{7}-y_{7}\right) \mathbf{a}_{1}-y_{7} \mathbf{a}_{2}+\left(\frac{1}{2}+z_{7}\right) \mathbf{a}_{3}$ & $\begin{array}{c}\left(\frac{1}{2} x_{7}-y_{7}\right) a \hat{\mathbf{x}}-\frac{\sqrt{3}}{2} x_{7} a \hat{\mathbf{y}}+ \\
\left(\frac{1}{2}+z_{7}\right) c \hat{\mathbf{z}}\end{array}$ & $(6 c)$ & O III \\
\hline $\mathbf{B}_{26}$ & $=$ & $-x_{7} \mathbf{a}_{1}+\left(-x_{7}+y_{7}\right) \mathbf{a}_{2}+\left(\frac{1}{2}+z_{7}\right) \mathbf{a}_{3}$ & $\begin{array}{c}\left(-x_{7}+\frac{1}{2} y_{7}\right) a \hat{\mathbf{x}}+\frac{\sqrt{3}}{2} y_{7} a \hat{\mathbf{y}}+ \\
\left(\frac{1}{2}+z_{7}\right) c \hat{\mathbf{z}}\end{array}$ & $(6 c)$ & O III \\
\hline
\end{tabular}


properties of the Kagomé antiferromagnet $\mathrm{YbBaCo}_{4} \mathrm{O}_{7}$, J. Solid State Chem. 179, 1136-1145 (2006), doi:10.1016/j.jssc.2006.01.010.

\section{Found in:}

- P. Villars and K. Cenzual, Pearson's Crystal Data - Crystal Structure Database for Inorganic Compounds, ASM International (2013).

\section{Geometry files:}

- CIF: pp. 915

- POSCAR: pp. 915 


\section{H3S (130 GPa) Structure: A3B_hR4_160_b_a}

$\frac{14}{15}$
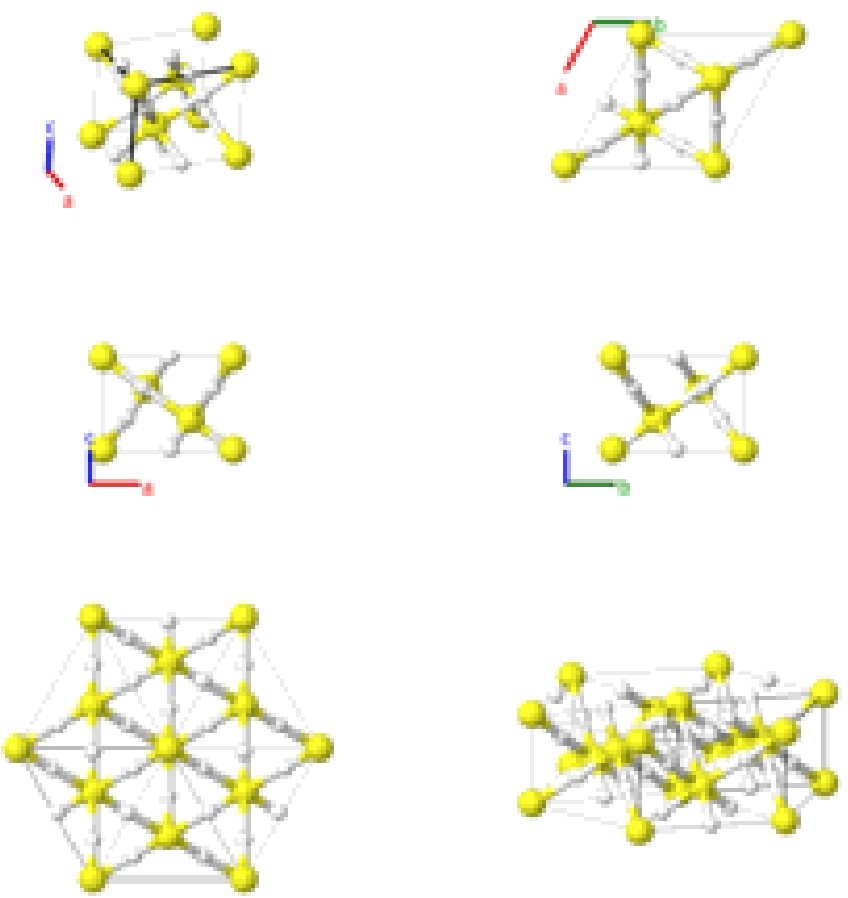

\section{Prototype}

AFLOW prototype label

Strukturbericht designation

Pearson symbol

Space group number

Space group symbol

AFLOW prototype command
: $\mathrm{H}_{3} \mathrm{~S}$

: A3B_hR4_160_b_a

: None

: $\quad \mathrm{hR} 4$

: 160

: $\quad R 3 m$

aflow - -proto=A3B_hR4_160_b_a [--hex]

- params $=a, c / a, x_{1}, x_{2}, z_{2}$

- This structure was found by first-principles electronic structure calculations and is predicted to be the stable structure of $\mathrm{H}_{3} \mathrm{~S}$ for pressures between 90 and $150 \mathrm{GPa}$. When $c / a \rightarrow \sqrt{8}, x_{2} \rightarrow 1 / 2$ and $z_{2} \rightarrow 0$ this structure continuously evolves into the cubic $200 \mathrm{GPa}_{3} \mathrm{~S}$ Structure. The data presented here was computed at $130 \mathrm{GPa}$.

\section{Rhombohedral primitive vectors:}

$$
\begin{array}{llc}
\mathbf{a}_{1}= & \frac{1}{2} a \hat{\mathbf{x}}-\frac{1}{2 \sqrt{3}} a \hat{\mathbf{y}}+\frac{1}{3} c \hat{\mathbf{z}} \\
\mathbf{a}_{2}= & \frac{1}{\sqrt{3}} a \hat{\mathbf{y}}+\frac{1}{3} c \hat{\mathbf{z}} \\
\mathbf{a}_{3}= & -\frac{1}{2} a \hat{\mathbf{x}}-\frac{1}{2 \sqrt{3}} a \hat{\mathbf{y}}+\frac{1}{3} c \hat{\mathbf{z}}
\end{array}
$$

\section{Basis vectors:}



$\mathbf{B}_{1}=x_{1} \mathbf{a}_{1}+x_{1} \mathbf{a}_{2}+x_{1} \mathbf{a}_{3}=$
$x_{1} c \hat{\mathbf{z}}$
(1a)
S
$\mathbf{B}_{2}=x_{2} \mathbf{a}_{1}+x_{2} \mathbf{a}_{2}+z_{2} \mathbf{a}_{3}=\frac{1}{2}\left(x_{2}-z_{2}\right) a \hat{\mathbf{x}}+\frac{1}{2 \sqrt{3}}\left(x_{2}-z_{2}\right) a \hat{\mathbf{y}}+\left(\frac{2}{3} x_{2}+\frac{1}{3} z_{2}\right) c \hat{\mathbf{z}}$
$(3 b)$
$\mathrm{H}$
$\mathbf{B}_{3}=z_{2} \mathbf{a}_{1}+x_{2} \mathbf{a}_{2}+x_{2} \mathbf{a}_{3}=$
$\frac{1}{2}\left(-x_{2}+z_{2}\right) a \hat{\mathbf{x}}+\frac{1}{2 \sqrt{3}}\left(x_{2}-z_{2}\right) a \hat{\mathbf{y}}+$
$(3 b)$
$\mathrm{H}$
$\mathbf{B}_{4}=x_{2} \mathbf{a}_{1}+z_{2} \mathbf{a}_{2}+x_{2} \mathbf{a}_{3}=\frac{1}{\sqrt{3}}\left(-x_{2}+z_{2}\right) a \hat{\mathbf{y}}+\left(\frac{2}{3} x_{2}+\frac{1}{3} z_{2}\right) c \hat{\mathbf{z}}$
$(3 b)$
$\mathrm{H}$

\section{References:}

- D. Duan, Y. Liu, F. Tian, D. Li, X. Huang, Z. Zhao, H. Yu, B. Liu, W. Tian, and T. Cui, Pressure-induced metallization of dense $\left(\mathrm{H}_{2} \mathrm{~S}_{2} \mathrm{H}_{2}\right.$ with high-T $\mathrm{T}_{c}$ superconductivity, Sci. Rep. 4, 6968 (2014), doi:10.1038/srep06968.

\section{Geometry files:}

- CIF: pp. 915

- POSCAR: pp. 916 


\section{$\mathrm{Al}_{8} \mathrm{Cr}_{5}\left(D 8_{10}\right)$ Structure: A8B5_hR26_160_a3bc_a3b}
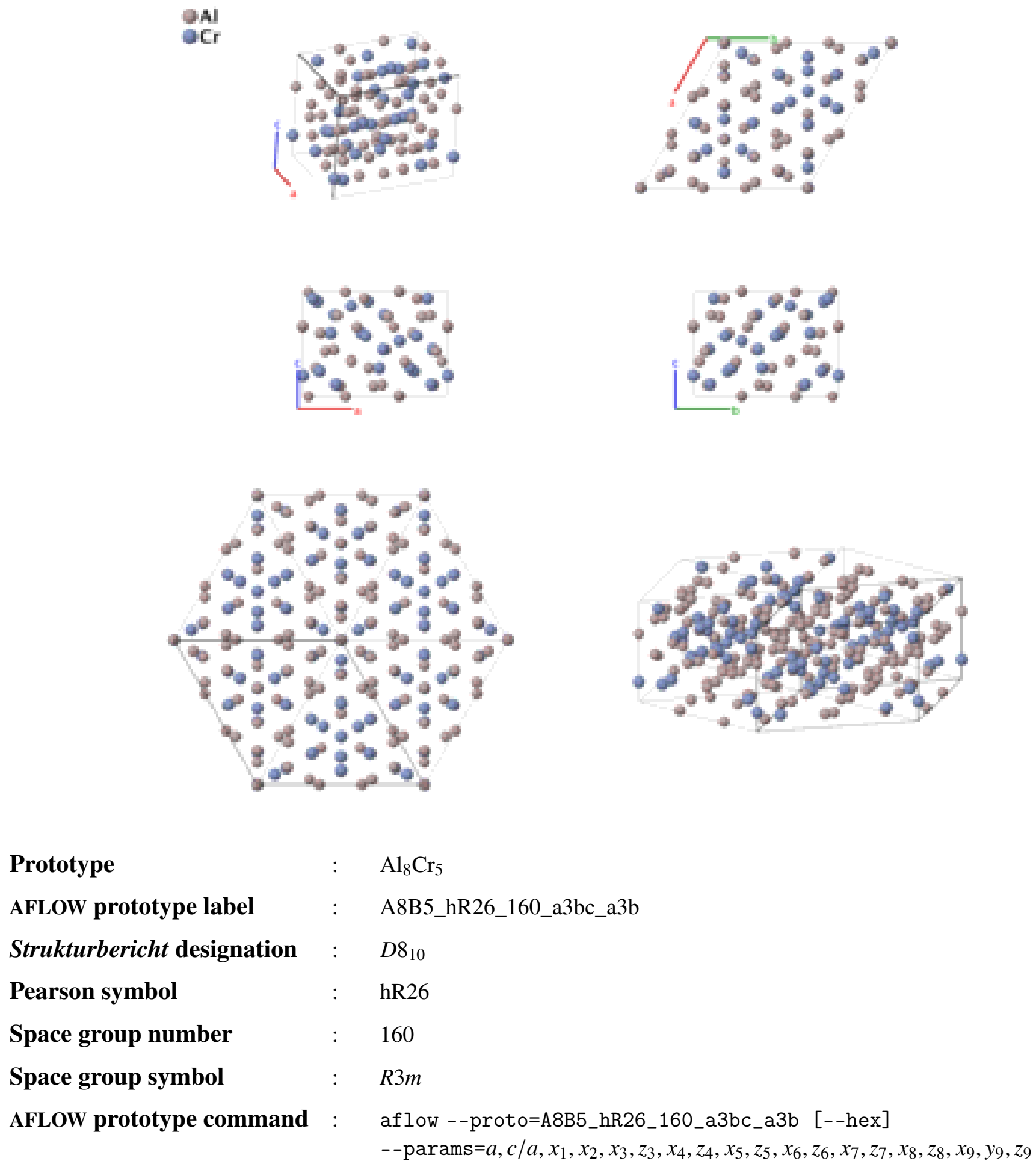

- (Bradley, 1937) notes that the positions here are very close to the positions of the atoms in the $\gamma$-brass $\left(\mathrm{Cu}_{5} \mathrm{Zn}_{8}, D 8_{2}\right)$ structure. 


\section{Rhombohedral primitive vectors:}

$$
\begin{array}{llc}
\mathbf{a}_{1}= & \frac{1}{2} a \hat{\mathbf{x}}-\frac{1}{2 \sqrt{3}} a \hat{\mathbf{y}}+\frac{1}{3} c \hat{\mathbf{z}} \\
\mathbf{a}_{2}= & \frac{1}{\sqrt{3}} a \hat{\mathbf{y}}+\frac{1}{3} c \hat{\mathbf{z}} \\
\mathbf{a}_{3}= & -\frac{1}{2} a \hat{\mathbf{x}}-\frac{1}{2 \sqrt{3}} a \hat{\mathbf{y}}+\frac{1}{3} c \hat{\mathbf{z}}
\end{array}
$$

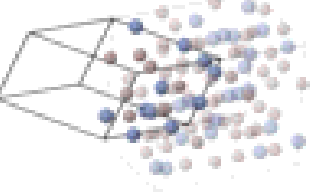

\section{Basis vectors:}

Lattice Coordinates

$\mathbf{B}_{1}=x_{1} \mathbf{a}_{1}+x_{1} \mathbf{a}_{2}+x_{1} \mathbf{a}_{3}=$
$\mathbf{B}_{2}=x_{2} \mathbf{a}_{1}+x_{2} \mathbf{a}_{2}+x_{2} \mathbf{a}_{3}=$
$\mathbf{B}_{3}=x_{3} \mathbf{a}_{1}+x_{3} \mathbf{a}_{2}+z_{3} \mathbf{a}_{3}=$
$\mathbf{B}_{4}=z_{3} \mathbf{a}_{1}+x_{3} \mathbf{a}_{2}+x_{3} \mathbf{a}_{3}=$

$x_{1} c \hat{\mathbf{z}}$

$x_{2} c \hat{\mathbf{z}}$

$=\frac{1}{2}\left(x_{3}-z_{3}\right) a \hat{\mathbf{x}}+\frac{1}{2 \sqrt{3}}\left(x_{3}-z_{3}\right) a \hat{\mathbf{y}}+\left(\frac{2}{3} x_{3}+\frac{1}{3} z_{3}\right) c \hat{\mathbf{z}}$

$=\frac{1}{2}\left(-x_{3}+z_{3}\right) a \hat{\mathbf{x}}+\frac{1}{2 \sqrt{3}}\left(x_{3}-z_{3}\right) a \hat{\mathbf{y}}+$

$$
\left(\frac{2}{3} x_{3}+\frac{1}{3} z_{3}\right) c \hat{\mathbf{z}}
$$

$\mathbf{B}_{5}=x_{3} \mathbf{a}_{1}+z_{3} \mathbf{a}_{2}+x_{3} \mathbf{a}_{3}=\frac{1}{\sqrt{3}}\left(-x_{3}+z_{3}\right) a \hat{\mathbf{y}}+\left(\frac{2}{3} x_{3}+\frac{1}{3} z_{3}\right) c \hat{\mathbf{z}}$

$\mathbf{B}_{6}=x_{4} \mathbf{a}_{1}+x_{4} \mathbf{a}_{2}+z_{4} \mathbf{a}_{3}=\frac{1}{2}\left(x_{4}-z_{4}\right) a \hat{\mathbf{x}}+\frac{1}{2 \sqrt{3}}\left(x_{4}-z_{4}\right) a \hat{\mathbf{y}}+\left(\frac{2}{3} x_{4}+\frac{1}{3} z_{4}\right) c \hat{\mathbf{z}}$

$\mathbf{B}_{7}=z_{4} \mathbf{a}_{1}+x_{4} \mathbf{a}_{2}+x_{4} \mathbf{a}_{3}=\frac{1}{2}\left(-x_{4}+z_{4}\right) a \hat{\mathbf{x}}+\frac{1}{2 \sqrt{3}}\left(x_{4}-z_{4}\right) a \hat{\mathbf{y}}+$

$$
\left(\frac{2}{3} x_{4}+\frac{1}{3} z_{4}\right) c \hat{\mathbf{z}}
$$

$\mathbf{B}_{8}=x_{4} \mathbf{a}_{1}+z_{4} \mathbf{a}_{2}+x_{4} \mathbf{a}_{3}=\frac{1}{\sqrt{3}}\left(-x_{4}+z_{4}\right) a \hat{\mathbf{y}}+\left(\frac{2}{3} x_{4}+\frac{1}{3} z_{4}\right) c \hat{\mathbf{z}}$

$\mathbf{B}_{9}=x_{5} \mathbf{a}_{1}+x_{5} \mathbf{a}_{2}+z_{5} \mathbf{a}_{3}=\frac{1}{2}\left(x_{5}-z_{5}\right) a \hat{\mathbf{x}}+\frac{1}{2 \sqrt{3}}\left(x_{5}-z_{5}\right) a \hat{\mathbf{y}}+\left(\frac{2}{3} x_{5}+\frac{1}{3} z_{5}\right) c \hat{\mathbf{z}}$

$\mathbf{B}_{10}=z_{5} \mathbf{a}_{1}+x_{5} \mathbf{a}_{2}+x_{5} \mathbf{a}_{3}=\frac{1}{2}\left(-x_{5}+z_{5}\right) a \hat{\mathbf{x}}+\frac{1}{2 \sqrt{3}}\left(x_{5}-z_{5}\right) a \hat{\mathbf{y}}+$

$$
\left(\frac{2}{3} x_{5}+\frac{1}{3} z_{5}\right) c \hat{\mathbf{z}}
$$

$\mathbf{B}_{11}=x_{5} \mathbf{a}_{1}+z_{5} \mathbf{a}_{2}+x_{5} \mathbf{a}_{3}=\frac{1}{\sqrt{3}}\left(-x_{5}+z_{5}\right) a \hat{\mathbf{y}}+\left(\frac{2}{3} x_{5}+\frac{1}{3} z_{5}\right) c \hat{\mathbf{z}}$

$\mathbf{B}_{12}=x_{6} \mathbf{a}_{1}+x_{6} \mathbf{a}_{2}+z_{6} \mathbf{a}_{3}=\frac{1}{2}\left(x_{6}-z_{6}\right) a \hat{\mathbf{x}}+\frac{1}{2 \sqrt{3}}\left(x_{6}-z_{6}\right) a \hat{\mathbf{y}}+\left(\frac{2}{3} x_{6}+\frac{1}{3} z_{6}\right) c \hat{\mathbf{z}}$

$\mathbf{B}_{13}=z_{6} \mathbf{a}_{1}+x_{6} \mathbf{a}_{2}+x_{6} \mathbf{a}_{3}=\quad \frac{1}{2}\left(-x_{6}+z_{6}\right) a \hat{\mathbf{x}}+\frac{1}{2 \sqrt{3}}\left(x_{6}-z_{6}\right) a \hat{\mathbf{y}}+$

$$
\left(\frac{2}{3} x_{6}+\frac{1}{3} z_{6}\right) c \hat{\mathbf{z}}
$$

$\mathbf{B}_{14}=x_{6} \mathbf{a}_{1}+z_{6} \mathbf{a}_{2}+x_{6} \mathbf{a}_{3}=\frac{1}{\sqrt{3}}\left(-x_{6}+z_{6}\right) a \hat{\mathbf{y}}+\left(\frac{2}{3} x_{6}+\frac{1}{3} z_{6}\right) c \hat{\mathbf{z}}$

$\mathbf{B}_{15}=x_{7} \mathbf{a}_{1}+x_{7} \mathbf{a}_{2}+z_{7} \mathbf{a}_{3}=\frac{1}{2}\left(x_{7}-z_{7}\right) a \hat{\mathbf{x}}+\frac{1}{2 \sqrt{3}}\left(x_{7}-z_{7}\right) a \hat{\mathbf{y}}+\left(\frac{2}{3} x_{7}+\frac{1}{3} z_{7}\right) c \hat{\mathbf{z}}$

$\mathbf{B}_{16}=z_{7} \mathbf{a}_{1}+x_{7} \mathbf{a}_{2}+x_{7} \mathbf{a}_{3}=\frac{1}{2}\left(-x_{7}+z_{7}\right) a \hat{\mathbf{x}}+\frac{1}{2 \sqrt{3}}\left(x_{7}-z_{7}\right) a \hat{\mathbf{y}}+$

$\mathbf{B}_{17}=x_{7} \mathbf{a}_{1}+z_{7} \mathbf{a}_{2}+x_{7} \mathbf{a}_{3}=\frac{1}{\sqrt{3}}\left(-x_{7}+z_{7}\right) a \hat{\mathbf{y}}+\left(\frac{2}{3} x_{7}+\frac{1}{3} z_{7}\right) c \hat{\mathbf{z}}$

$\mathbf{B}_{18}=x_{8} \mathbf{a}_{1}+x_{8} \mathbf{a}_{2}+z_{8} \mathbf{a}_{3}=\frac{1}{2}\left(x_{8}-z_{8}\right) a \hat{\mathbf{x}}+\frac{1}{2 \sqrt{3}}\left(x_{8}-z_{8}\right) a \hat{\mathbf{y}}+\left(\frac{2}{3} x_{8}+\frac{1}{3} z_{8}\right) c \hat{\mathbf{z}}$

$\mathbf{B}_{19}=z_{8} \mathbf{a}_{1}+x_{8} \mathbf{a}_{2}+x_{8} \mathbf{a}_{3}=\quad \frac{1}{2}\left(-x_{8}+z_{8}\right) a \hat{\mathbf{x}}+\frac{1}{2 \sqrt{3}}\left(x_{8}-z_{8}\right) a \hat{\mathbf{y}}+$

$\mathbf{B}_{20}=x_{8} \mathbf{a}_{1}+z_{8} \mathbf{a}_{2}+x_{8} \mathbf{a}_{3}=\frac{1}{\sqrt{3}}\left(-x_{8}+z_{8}\right) a \hat{\mathbf{y}}+\left(\frac{2}{3} x_{8}+\frac{1}{3} z_{8}\right) c \hat{\mathbf{z}}$

$\mathbf{B}_{21}=x_{9} \mathbf{a}_{1}+y_{9} \mathbf{a}_{2}+z_{9} \mathbf{a}_{3}=\frac{1}{2}\left(x_{9}-z_{9}\right) a \hat{\mathbf{x}}+\left(-\frac{1}{2 \sqrt{3}} x_{9}+\frac{1}{\sqrt{3}} y_{9}-\frac{1}{2 \sqrt{3}} z_{9}\right) a \hat{\mathbf{y}}+$ $\frac{1}{3}\left(x_{9}+y_{9}+z_{9}\right) c \hat{\mathbf{z}}$ (1a)

Al II

Al III

Al III

Al III

Al IV

Al IV

Al IV

Cr II

Cr II

Cr II

Cr III

Cr III

Cr III

Cr IV

Cr IV 


$$
\begin{aligned}
& \mathbf{B}_{22}=z_{9} \mathbf{a}_{1}+x_{9} \mathbf{a}_{2}+y_{9} \mathbf{a}_{3}=\frac{1}{2}\left(-y_{9}+z_{9}\right) a \hat{\mathbf{x}}+\left(\frac{1}{\sqrt{3}} x_{9}-\frac{1}{2 \sqrt{3}} y_{9}-\frac{1}{2 \sqrt{3}} z_{9}\right) a \hat{\mathbf{y}}+ \\
& \frac{1}{3}\left(x_{9}+y_{9}+z_{9}\right) c \hat{\mathbf{z}} \\
& \mathbf{B}_{23}=y_{9} \mathbf{a}_{1}+z_{9} \mathbf{a}_{2}+x_{9} \mathbf{a}_{3}=\frac{1}{2}\left(-x_{9}+y_{9}\right) a \hat{\mathbf{x}}+\left(-\frac{1}{2 \sqrt{3}} x_{9}-\frac{1}{2 \sqrt{3}} y_{9}+\frac{1}{\sqrt{3}} z_{9}\right) a \hat{\mathbf{y}}+ \\
& \frac{1}{3}\left(x_{9}+y_{9}+z_{9}\right) c \hat{\mathbf{z}} \\
& \mathbf{B}_{24}=z_{9} \mathbf{a}_{1}+y_{9} \mathbf{a}_{2}+x_{9} \mathbf{a}_{3}=\frac{1}{2}\left(-x_{9}+z_{9}\right) a \hat{\mathbf{x}}+\left(-\frac{1}{2 \sqrt{3}} x_{9}+\frac{1}{\sqrt{3}} y_{9}-\frac{1}{2 \sqrt{3}} z_{9}\right) a \hat{\mathbf{y}}+ \\
& \frac{1}{3}\left(x_{9}+y_{9}+z_{9}\right) c \hat{\mathbf{z}} \\
& \mathbf{B}_{25}=y_{9} \mathbf{a}_{1}+x_{9} \mathbf{a}_{2}+z_{9} \mathbf{a}_{3}=\frac{1}{2}\left(y_{9}-z_{9}\right) a \hat{\mathbf{x}}+\left(\frac{1}{\sqrt{3}} x_{9}-\frac{1}{2 \sqrt{3}} y_{9}-\frac{1}{2 \sqrt{3}} z_{9}\right) a \hat{\mathbf{y}}+ \\
& \frac{1}{3}\left(x_{9}+y_{9}+z_{9}\right) c \hat{\mathbf{z}} \\
& \begin{array}{c}
\mathbf{B}_{26}=\quad x_{9} \mathbf{a}_{1}+z_{9} \mathbf{a}_{2}+y_{9} \mathbf{a}_{3}=\frac{1}{2}\left(x_{9}-y_{9}\right) a \hat{\mathbf{x}}+\left(-\frac{1}{2 \sqrt{3}} x_{9}-\frac{1}{2 \sqrt{3}} y_{9}+\frac{1}{\sqrt{3}} z_{9}\right) a \hat{\mathbf{y}}+ \\
\frac{1}{3}\left(x_{9}+y_{9}+z_{9}\right) c \hat{\mathbf{z}}
\end{array}
\end{aligned}
$$

\section{References:}

- A. J. Bradley and S. S. Lu, The Crystal Structures of $\mathrm{Cr}_{2} A l$ and $\mathrm{Cr}_{5} A l_{8}$, Z. Kristallogr. 96, 20-37 (1937), doi:10.1524/zkri.1937.96.1.20.

\section{Found in:}

- S. Grazulis, Crystal Data (2014). Crystallography-online.com.

\section{Geometry files:}

- CIF: pp. 916

- POSCAR: pp. 916 


\section{Carbonyl Sulphide (COS, $\left.\mathrm{F0}_{2}\right)$ Structure:}

\section{ABC_hR3_160_a_a_a}
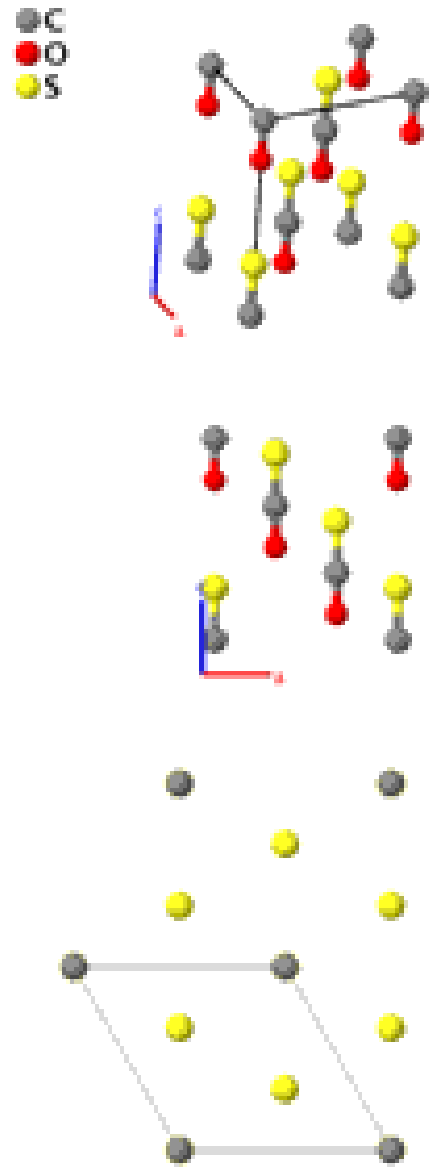

Prototype

AFLOW prototype label

Strukturbericht designation

Pearson symbol

Space group number

Space group symbol

AFLOW prototype command
: $\operatorname{COS}$

: ABC_hR3_160_a_a_a

: $\quad \mathrm{FO}_{2}$

: $\quad$ hR3

: 160

: $\quad R 3 m$
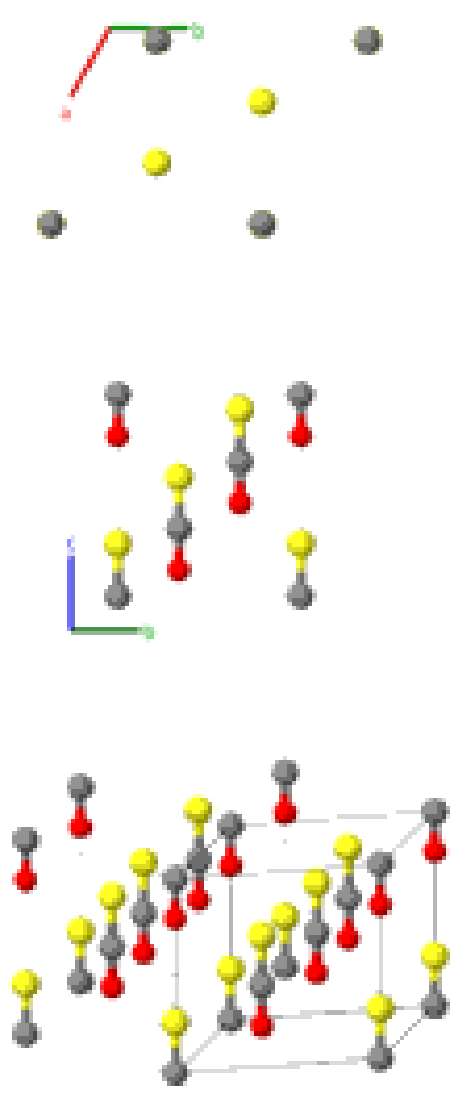

- (Overell, 1982) does not give the Wyckoff positions directly. They are inferred from the C-O and C-S bond lengths. The experimental data was obtained at $90 \mathrm{~K}$. 
Rhombohedral primitive vectors:

$$
\begin{array}{llc}
\mathbf{a}_{1}= & \frac{1}{2} a \hat{\mathbf{x}}-\frac{1}{2 \sqrt{3}} a \hat{\mathbf{y}}+\frac{1}{3} c \hat{\mathbf{z}} \\
\mathbf{a}_{2}= & \frac{1}{\sqrt{3}} a \hat{\mathbf{y}}+\frac{1}{3} c \hat{\mathbf{z}} \\
\mathbf{a}_{3}= & -\frac{1}{2} a \hat{\mathbf{x}}-\frac{1}{2 \sqrt{3}} a \hat{\mathbf{y}}+\frac{1}{3} c \hat{\mathbf{z}}
\end{array}
$$

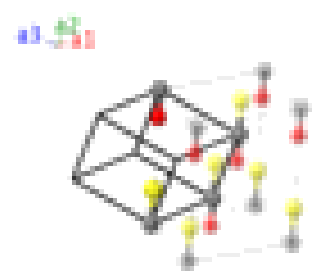

\section{Basis vectors:}

Lattice Coordinates

$\mathbf{B}_{1}=x_{1} \mathbf{a}_{1}+x_{1} \mathbf{a}_{2}+x_{1} \mathbf{a}_{3}=$

$\mathbf{B}_{2}=x_{2} \mathbf{a}_{1}+x_{2} \mathbf{a}_{2}+x_{2} \mathbf{a}_{3}=$

$\mathbf{B}_{3}=x_{3} \mathbf{a}_{1}+x_{3} \mathbf{a}_{2}+x_{3} \mathbf{a}_{3}=$

\section{Cartesian Coordinates}

$x_{1} c \hat{\mathbf{z}}$

$x_{2} c \hat{\mathbf{z}}$

$x_{3} c \hat{\mathbf{z}}$
Wyckoff Position

(1a)

(1a)

(1a)
Atom Type

$\mathrm{C}$

$\mathrm{O}$

S

\section{References:}

- J. S. W. Overell, G. S. Pawley, and B. M. Powell, Powder refinement of carbonyl sulphide, Acta Crystallogr. Sect. B Struct. Sci. 38, 1121-1123 (1982), doi:10.1107/S0567740882005111.

\section{Geometry files:}

- CIF: pp. 916

- POSCAR: pp. 917 


\section{Moissanite-15R (SiC, B7) Structure: AB_hR10_160_5a_5a}
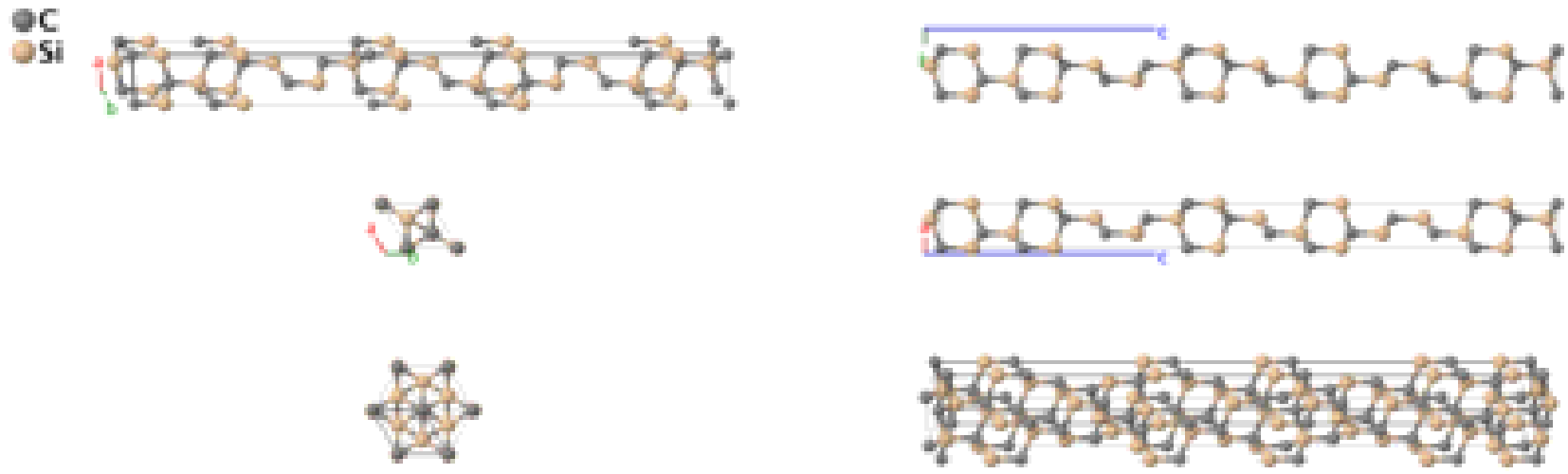

Prototype

: $\quad \mathrm{SiC}$

AFLOW prototype label

AB_hR10_160_5a_5a

Strukturbericht designation

$B 7$

Pearson symbol

hR10

Space group number

160

Space group symbol

$R 3 m$

AFLOW prototype command : aflow --proto=AB_hR10_160_5a_5a [--hex]

- - params $=a, c / a, x_{1}, x_{2}, x_{3}, x_{4}, x_{5}, x_{6}, x_{7}, x_{8}, x_{9}, x_{10}$

- (Ewald, 1931) and (Thibault, 1944) both call this structure "Type I $\alpha$-silicon carbide."

- The atomic positions are not well determined. We follow (Thibolt, 1944) and assume that the (0001) planes of carbon atoms are equally spaced, and that each carbon atom has a silicon atom at a distance of $c / 20$ along the $\hat{\mathbf{z}}$ axis.

Rhombohedral primitive vectors:

$$
\begin{array}{llc}
\mathbf{a}_{1}= & \frac{1}{2} a \hat{\mathbf{x}}-\frac{1}{2 \sqrt{3}} a \hat{\mathbf{y}}+\frac{1}{3} c \hat{\mathbf{z}} \\
\mathbf{a}_{2}= & \frac{1}{\sqrt{3}} a \hat{\mathbf{y}}+\frac{1}{3} c \hat{\mathbf{z}} \\
\mathbf{a}_{3}= & -\frac{1}{2} a \hat{\mathbf{x}}-\frac{1}{2 \sqrt{3}} a \hat{\mathbf{y}}+\frac{1}{3} c \hat{\mathbf{z}}
\end{array}
$$

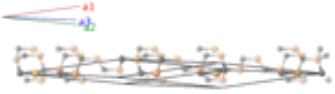

Basis vectors:

Lattice Coordinates

$\mathbf{B}_{1} \quad=\quad x_{1} \mathbf{a}_{1}+x_{1} \mathbf{a}_{2}+x_{1} \mathbf{a}_{3}$

$\mathbf{B}_{2}=x_{2} \mathbf{a}_{1}+x_{2} \mathbf{a}_{2}+x_{2} \mathbf{a}_{3}$

$\mathbf{B}_{3}=x_{3} \mathbf{a}_{1}+x_{3} \mathbf{a}_{2}+x_{3} \mathbf{a}_{3}$

$\mathbf{B}_{4}=x_{4} \mathbf{a}_{1}+x_{4} \mathbf{a}_{2}+x_{4} \mathbf{a}_{3}$

$\mathbf{B}_{5}=x_{5} \mathbf{a}_{1}+x_{5} \mathbf{a}_{2}+x_{5} \mathbf{a}_{3}$

$\mathbf{B}_{6}=x_{6} \mathbf{a}_{1}+x_{6} \mathbf{a}_{2}+x_{6} \mathbf{a}_{3}$

$\mathbf{B}_{7}=$

$x_{7} \mathbf{a}_{1}+x_{7} \mathbf{a}_{2}+x_{7} \mathbf{a}_{3}$
$=$

$=$

$=$

$=$

$=$

$=$

$=$
Cartesian Coordinates

$x_{1} c \hat{\mathbf{z}}$

$x_{2} c \hat{\mathbf{z}}$

$x_{3} c \hat{\mathbf{z}}$

$x_{4} c \hat{\mathbf{z}}$

$x_{5} c \hat{\mathbf{z}}$

$x_{6} c \hat{\mathbf{z}}$

$x_{7} c \hat{\mathbf{z}}$
Wyckoff Position

(1a)

(1a)

(1a)

(1a)

(1a)

(1a)

(1a)
Atom Type

C I

C II

C III

C IV

$\mathrm{C} \mathrm{V}$

Si I

Si II 


\begin{tabular}{|c|c|c|c|c|c|c|}
\hline $\mathbf{B}_{8}$ & $=$ & $x_{8} \mathbf{a}_{1}+x_{8} \mathbf{a}_{2}+x_{8} \mathbf{a}_{3}$ & $=$ & $x_{8} c \hat{\mathbf{z}}$ & $(1 a)$ & Si III \\
\hline $\mathbf{B}_{9}$ & $=$ & $x_{9} \mathbf{a}_{1}+x_{9} \mathbf{a}_{2}+x_{9} \mathbf{a}_{3}$ & $=$ & $x_{9} c \hat{\mathbf{z}}$ & $(1 a)$ & Si IV \\
\hline $\mathbf{B}_{10}$ & $=$ & $x_{10} \mathbf{a}_{1}+x_{10} \mathbf{a}_{2}+x_{10} \mathbf{a}_{3}$ & $=$ & $x_{10} c \hat{\mathbf{z}}$ & $(1 a)$ & Si V \\
\hline
\end{tabular}

\section{References:}

- N. W. Thibault, Morphological and Structural Crystallography and Optical Properties of Silicon Carbide (SiC) Part II: Structural Crystallography and Optical Properties, Am. Mineral. 29, 327-362 (1944).

- P. P. Ewald and K. Herrman, eds., Strukturbericht 1913-1928, vol. I (Akademische Verlagsgesellschaft M. B. H., 1931).

\section{Found in:}

- G. L. Harris, ed., Properties of Silicon Carbide (INSPEC, London, 1995).

\section{Geometry files:}

- CIF: pp. 917

- POSCAR: pp. 917 


\section{$\mathrm{La}_{2} \mathrm{O}_{3}\left(D 5_{2}\right)$ Structure: A2B3_hP5_164_d_ad}
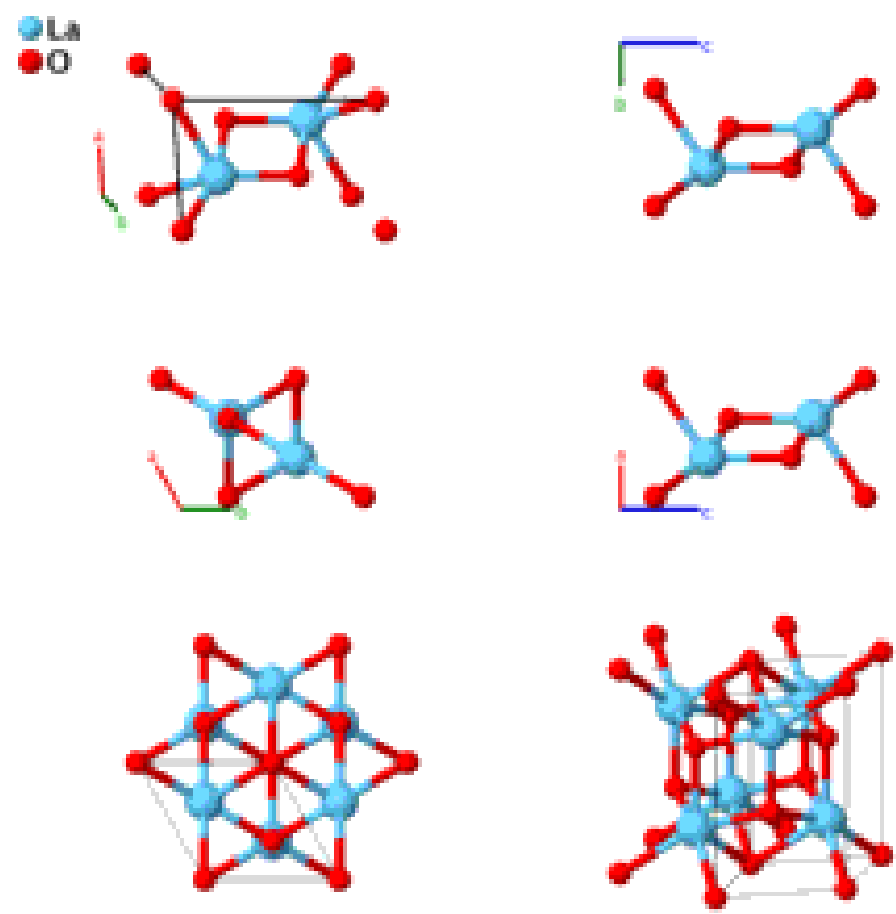

\section{Prototype}

AFLOW prototype label

Strukturbericht designation

Pearson symbol

Space group number

Space group symbol

AFLOW prototype command
: $\quad \mathrm{La}_{2} \mathrm{O}_{3}$

: A2B3_hP5_164_d_ad

$: \quad D 5_{2}$

: hP5

: 164

: $\quad P \overline{3} m 1$

aflow --proto=A2B3_hP5_164_d_ad

- params $=a, c / a, z_{2}, z_{3}$

\section{Other compounds with this structure:}

- $\mathrm{Ac}_{2} \mathrm{O}_{3}, \mathrm{Ce}_{2} \mathrm{O}_{3}, \mathrm{Nd}_{2} \mathrm{O}_{3}, \mathrm{Pr}_{2} \mathrm{O}_{3}, \mathrm{Th}_{2} \mathrm{~N}_{3}, \mathrm{U}_{2} \mathrm{~N}_{3}, \alpha-\mathrm{Bi}_{2} \mathrm{Mg}_{3}, \alpha-\mathrm{Sb}_{2} \mathrm{Mg}_{3}$

- This structure has proved rather controversial. (Zachariasen 1926, 1929) originally proposed that the crystal structure of $\mathrm{La}_{2} \mathrm{O}_{3}$ and other lanthanide series oxides belong to trigonal space group $P 321$ \#150. This was immediately criticized by (Pauling, 1928), who suggested the trigonal space group ( $P \overline{3} m 1$ \#164). This was confirmed by (Koehler, 1953).

- Much later, (Aldebert, 1979) stated that the structure was hexagonal, space group $P 6_{3} / m m c \# 194$. This would represent a doubling of the $P \overline{3} m 1$ unit cell in the $z$-direction. However, they give structural parameters, used by us and by (Villars, 1991, 2016), which have a density consistent with the $P \overline{3} m 1$ structure, and are in reasonable agreement with lattice parameters given in (Pearson, 1958) and (Koehler, 1953). We agree with (Villars, 1991, 2016) that these are close to the correct values for the structure.

- This structure is very similar to $\mathrm{Al}_{3} \mathrm{Ni}_{2}$ (D5 13 , A3B2_hP5_164_ad_d). We follow (Pearson, 1958) and assign the intermetallics as $D 5_{13}$, keeping $D 5_{2}$ for oxides and related compounds. 
Trigonal Hexagonal primitive vectors:

$$
\begin{array}{ll}
\mathbf{a}_{1}= & \frac{1}{2} a \hat{\mathbf{x}}-\frac{\sqrt{3}}{2} a \hat{\mathbf{y}} \\
\mathbf{a}_{2}= & \frac{1}{2} a \hat{\mathbf{x}}+\frac{\sqrt{3}}{2} a \hat{\mathbf{y}} \\
\mathbf{a}_{3}= & c \hat{\mathbf{z}}
\end{array}
$$

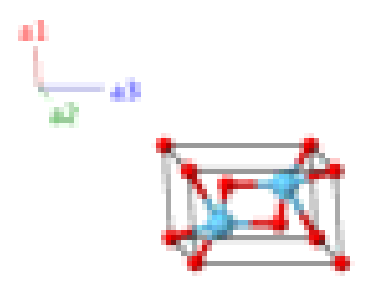

\section{Basis vectors:}

$\begin{array}{rllccc}\text { Lattice Coordinates } & & \text { Cartesian Coordinates } & \text { Wyckoff Position } & \text { Atom Type } \\ \mathbf{B}_{1}= & 0 \mathbf{a}_{1}+0 \mathbf{a}_{2}+0 \mathbf{a}_{3} & = & 0 \hat{\mathbf{x}}+0 \hat{\mathbf{y}}+0 \hat{\mathbf{z}} & (1 a) & \text { O I } \\ \mathbf{B}_{2}= & \frac{1}{3} \mathbf{a}_{1}+\frac{2}{3} \mathbf{a}_{2}+z_{2} \mathbf{a}_{3} & = & \frac{1}{2} a \hat{\mathbf{x}}+\frac{1}{2 \sqrt{3}} a \hat{\mathbf{y}}+z_{2} c \hat{\mathbf{z}} & (2 d) & \text { La } \\ \mathbf{B}_{3}= & \frac{2}{3} \mathbf{a}_{1}+\frac{1}{3} \mathbf{a}_{2}-z_{2} \mathbf{a}_{3} & = & \frac{1}{2} a \hat{\mathbf{x}}-\frac{1}{2 \sqrt{3}} a \hat{\mathbf{y}}-z_{2} c \hat{\mathbf{z}} & (2 d) & \text { La } \\ \mathbf{B}_{4}= & \frac{1}{3} \mathbf{a}_{1}+\frac{2}{3} \mathbf{a}_{2}+z_{3} \mathbf{a}_{3} & = & \frac{1}{2} a \hat{\mathbf{x}}+\frac{1}{2 \sqrt{3}} a \hat{\mathbf{y}}+z_{3} c \hat{\mathbf{z}} & (2 d) & \text { O II } \\ \mathbf{B}_{5}= & \frac{2}{3} \mathbf{a}_{1}+\frac{1}{3} \mathbf{a}_{2}-z_{3} \mathbf{a}_{3} & = & \frac{1}{2} a \hat{\mathbf{x}}-\frac{1}{2 \sqrt{3}} a \hat{\mathbf{y}}-z_{3} c \hat{\mathbf{z}} & \text { O II }\end{array}$

\section{References:}

- P. Aldebert and J. P. Traverse, Etude par diffraction neutronique des structures de haute temperature de $\mathrm{La}_{2} \mathrm{O}_{3}$ et $\mathrm{Nd}_{2} \mathrm{O}_{3}$, Mater. Res. Bull. 14, 303-323 (1979), doi:10.1016/0025-5408(79)90095-3.

- W. Zachariasen, Die Kristallstruktur der ÎS-Modifikation von den Sesquioxyden der seltenen Erdmetalle. $\left(\mathrm{La}_{2} \mathrm{O}_{3}, \mathrm{Ce}_{2} \mathrm{O}_{3}\right.$, $\mathrm{Pr}_{2} \mathrm{O}_{3}, \mathrm{Nd}_{2} \mathrm{O}_{3}$ ), Z. Phys. Chem. 123, 134 (1926), doi:10.1515/zpch-1926-12309.

- W. H. Zachariasen, Kürzere Originalmitteilungen und Notizen, Z. Kristallogr. 70, 187-188 (1929), doi:10.1524/zkri.1929.70.1.187.

- L. Pauling, The Crystal Structure of the A-Modification of the Rare Earth Sesquioxides, Z. Kristallogr. 69, 415 (1928), doi:10.1524/zkri.1929.69.1.415.

- W. C. Koehler and E. O. Wollan, Neutron-diffraction study of the structure of the A-form of the rare earth sesquioxides, Acta Cryst. 6, 741-742 (1953), doi:10.1107/S0365110X53002076.

- P. Villars, ed., PAULING FILE in: Inorganic Solid Phases (SpringerMaterials (online database), Heidelberg, 2016).

- W. B. Pearson, A Handbook of Lattice Spacings and Structures of Metals and Alloys, no. N.R.C. No. 4303 in International Series of Monographs on Metal Physics and Physical Metallurgy (Pergamon Press, Oxford, London, Edinburgh, New York, Paris, Frankfort, 1958), 1964 reprint with corrections edn.

\section{Geometry files:}

- CIF: pp. 917

- POSCAR: pp. 918 
$\delta_{H}^{I I}-\mathrm{NW}_{2}$ Structure:

AB2_hP9_164_bd_c2d
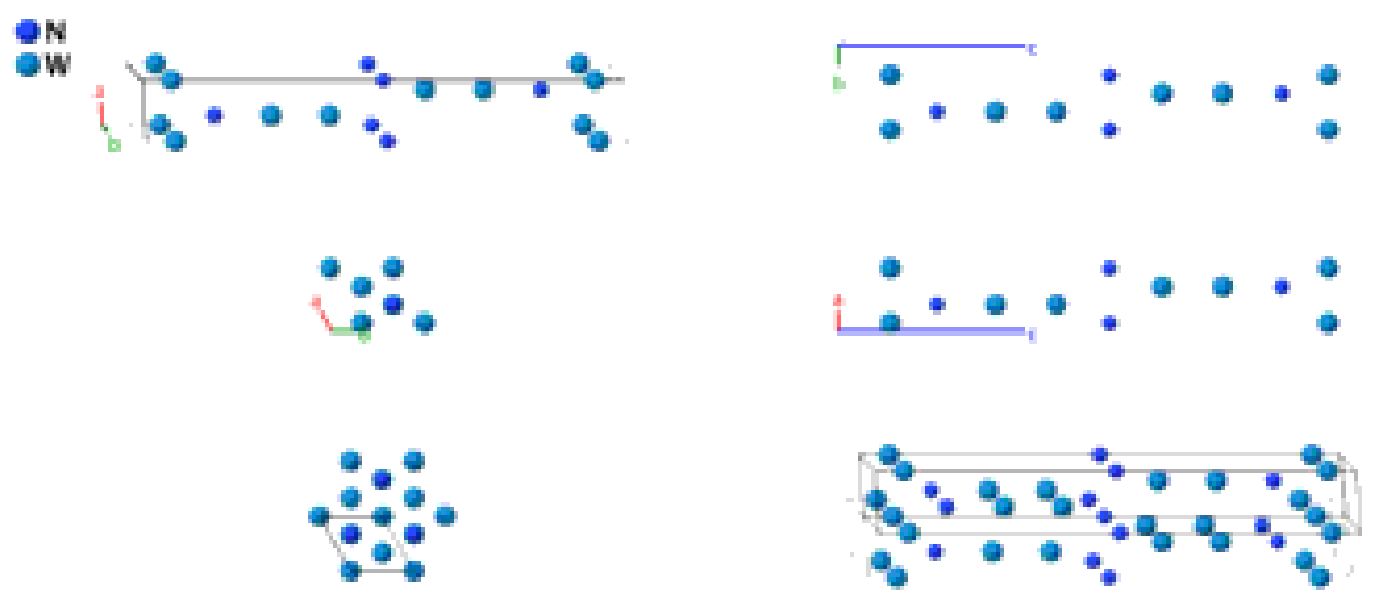

Prototype

AFLOW prototype label

$: \quad \delta_{H}^{I I}-\mathrm{NW}_{2}$

Strukturbericht designation

: AB2_hP9_164_bd_c2d

Pearson symbol

: None

Space group number

$: \mathrm{hP} 9$

Space group symbol

$: \quad 164$

AFLOW prototype command : aflow --proto=AB2_hP9_164_bd_c2d

: $\quad P \overline{3} m 1$

- params $=a, c / a, z_{2}, z_{3}, z_{4}, z_{5}$

- Khitrova and Pinkser put this structure in space group P $\overline{3}$ (\#147), but the Wyckoff positions used are identical with space group $\mathrm{P} \overline{3} \mathrm{~m} 1$ (\#164), so we assign this to the higher symmetry space group.

Trigonal Hexagonal primitive vectors:

$$
\begin{array}{lll}
\mathbf{a}_{1} & = & \frac{1}{2} a \hat{\mathbf{x}}-\frac{\sqrt{3}}{2} a \hat{\mathbf{y}} \\
\mathbf{a}_{2} & = & \frac{1}{2} a \hat{\mathbf{x}}+\frac{\sqrt{3}}{2} a \hat{\mathbf{y}} \\
\mathbf{a}_{3} & = & c \hat{\mathbf{z}}
\end{array}
$$

Basis vectors:

Lattice Coordinates

$\begin{array}{lcccc}\mathbf{B}_{1}= & \frac{1}{2} \mathbf{a}_{3} & = & \frac{1}{2} c \hat{\mathbf{z}} \\ \mathbf{B}_{2}= & z_{2} \mathbf{a}_{3} & = & z_{2} c \hat{\mathbf{z}} \\ \mathbf{B}_{3}= & -z_{2} \mathbf{a}_{3} & = & -z_{2} c \hat{\mathbf{z}} \\ \mathbf{B}_{4}= & \frac{1}{3} \mathbf{a}_{1}+\frac{2}{3} \mathbf{a}_{2}+z_{3} \mathbf{a}_{3} & = & \frac{1}{2} a \hat{\mathbf{x}}+\frac{1}{2 \sqrt{3}} a \hat{\mathbf{y}}+z_{3} c \hat{\mathbf{z}} \\ \mathbf{B}_{5}= & \frac{2}{3} \mathbf{a}_{1}+\frac{1}{3} \mathbf{a}_{2}-z_{3} \mathbf{a}_{3} & = & \frac{1}{2} a \hat{\mathbf{x}}-\frac{1}{2 \sqrt{3}} a \hat{\mathbf{y}}-z_{3} c \hat{\mathbf{z}} \\ \mathbf{B}_{6}= & \frac{1}{3} \mathbf{a}_{1}+\frac{2}{3} \mathbf{a}_{2}+z_{4} \mathbf{a}_{3} & = & \frac{1}{2} a \hat{\mathbf{x}}+\frac{1}{2 \sqrt{3}} a \hat{\mathbf{y}}+z_{4} c \hat{\mathbf{z}} \\ \mathbf{B}_{7}= & \frac{2}{3} \mathbf{a}_{1}+\frac{1}{3} \mathbf{a}_{2}-z_{4} \mathbf{a}_{3} & = & \frac{1}{2} a \hat{\mathbf{x}}-\frac{1}{2 \sqrt{3}} a \hat{\mathbf{y}}-z_{4} c \hat{\mathbf{z}}\end{array}$

Wyckoff Position
Atom Type

$\mathrm{N}$ I

W I

W I

N II

N II

W II

W II 


$\begin{array}{llllll}\mathbf{B}_{8}= & \frac{1}{3} \mathbf{a}_{1}+\frac{2}{3} \mathbf{a}_{2}+z_{5} \mathbf{a}_{3} & = & \frac{1}{2} a \hat{\mathbf{x}}+\frac{1}{2 \sqrt{3}} a \hat{\mathbf{y}}+z_{5} c \hat{\mathbf{z}} & (2 d) & \text { W III } \\ \mathbf{B}_{9}= & \frac{2}{3} \mathbf{a}_{1}+\frac{1}{3} \mathbf{a}_{2}-z_{5} \mathbf{a}_{3}= & \frac{1}{2} a \hat{\mathbf{x}}-\frac{1}{2 \sqrt{3}} a \hat{\mathbf{y}}-z_{5} c \hat{\mathbf{z}} & (2 d) & \text { W III }\end{array}$

\section{References:}

- V. I. Khitrova and Z. G. Pinkser, Chemical Crystallography of Tungsten Nitrides and of Some Other Interstitial Phases, Sov. Phys. Crystallogr. 6, 712-719 (1962).

\section{Geometry files:}

- CIF: pp. 918

- POSCAR: pp. 918 


\section{$\mathrm{CuNiSb} b_{2}$ Structure: ABC2_hP4_164_a_b_d}
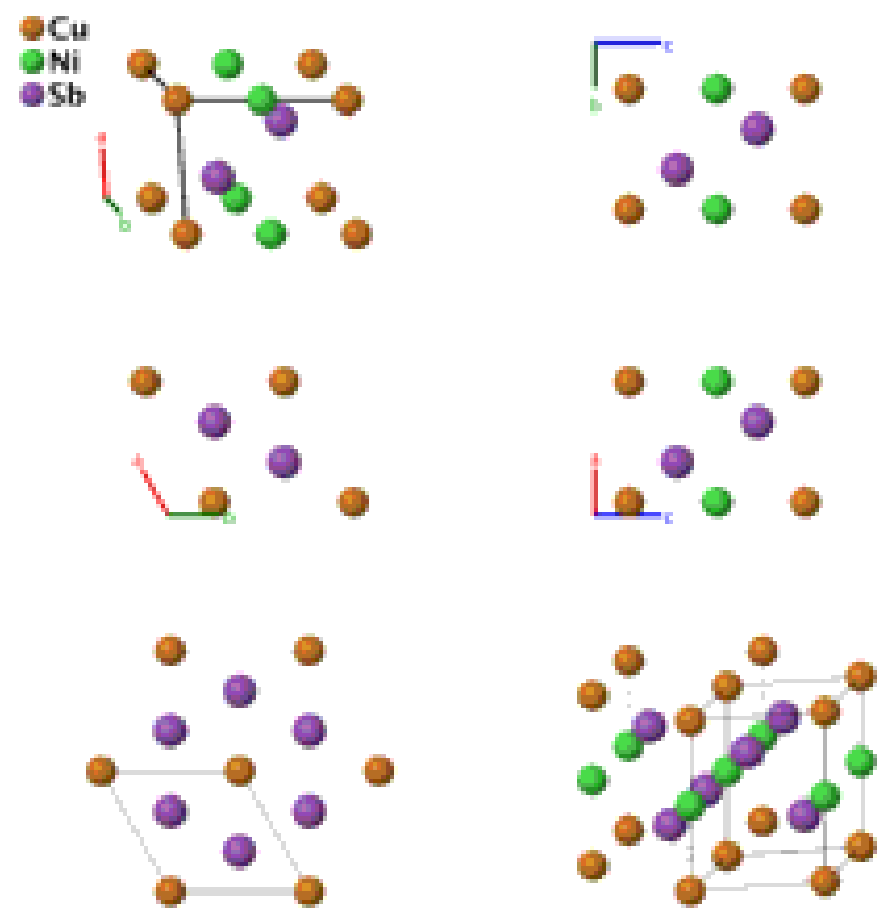

Prototype

AFLOW prototype label

: $\mathrm{CuNiSb}_{2}$

Strukturbericht designation

: ABC2_hP4_164_a_b_d

Pearson symbol

: None

Space group number

$: \mathrm{hP} 4$

Space group symbol

: 164

AFLOW prototype command : aflow --proto=ABC2_hP4_164_a_b_d

- params $=a, c / a, z_{3}$

Trigonal Hexagonal primitive vectors:

$$
\begin{array}{ll}
\mathbf{a}_{1}= & \frac{1}{2} a \hat{\mathbf{x}}-\frac{\sqrt{3}}{2} a \hat{\mathbf{y}} \\
\mathbf{a}_{2}= & \frac{1}{2} a \hat{\mathbf{x}}+\frac{\sqrt{3}}{2} a \hat{\mathbf{y}} \\
\mathbf{a}_{3}= & c \hat{\mathbf{z}}
\end{array}
$$
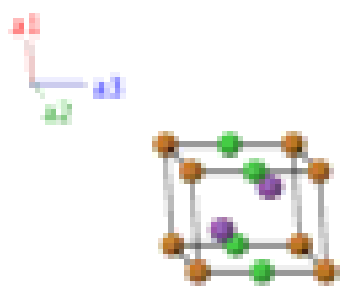

Basis vectors:

Lattice Coordinates

$\mathbf{B}_{1}=0 \mathbf{a}_{1}+0 \mathbf{a}_{2}+0 \mathbf{a}_{3}$

$\mathbf{B}_{2}=\frac{1}{2} \mathbf{a}_{3}$

$\mathbf{B}_{3}=\frac{1}{3} \mathbf{a}_{1}+\frac{2}{3} \mathbf{a}_{2}+z_{3} \mathbf{a}_{3}$

$\mathbf{B}_{4}=\frac{2}{3} \mathbf{a}_{1}+\frac{1}{3} \mathbf{a}_{2}-z_{3} \mathbf{a}_{3}$

$=$

$=$

$=$

$=$
Cartesian Coordinates

$$
\begin{gathered}
0 \hat{\mathbf{x}}+0 \hat{\mathbf{y}}+0 \hat{\mathbf{z}} \\
\frac{1}{2} c \hat{\mathbf{z}} \\
\frac{1}{2} a \hat{\mathbf{x}}+\frac{1}{2 \sqrt{3}} a \hat{\mathbf{y}}+z_{3} c \hat{\mathbf{z}} \\
\frac{1}{2} a \hat{\mathbf{x}}-\frac{1}{2 \sqrt{3}} a \hat{\mathbf{y}}-z_{3} c \hat{\mathbf{z}}
\end{gathered}
$$

Wyckoff Position

(1a)
Atom Type

$\mathrm{Cu}$

$\mathrm{Ni}$

$\mathrm{Sb}$

$\mathrm{Sb}$ 


\section{References:}

- R. L. Kift, Intermetallic Compounds by Reductive Annealing, Ph.D. thesis, University of Hull (2010).

\section{Geometry files:}

- CIF: pp. 918

- POSCAR: pp. 919 


\section{$\mathrm{Cu}_{3} \mathrm{P}\left(D 0_{21}\right)$ Structure: A3B_hP24_165_bdg_f}
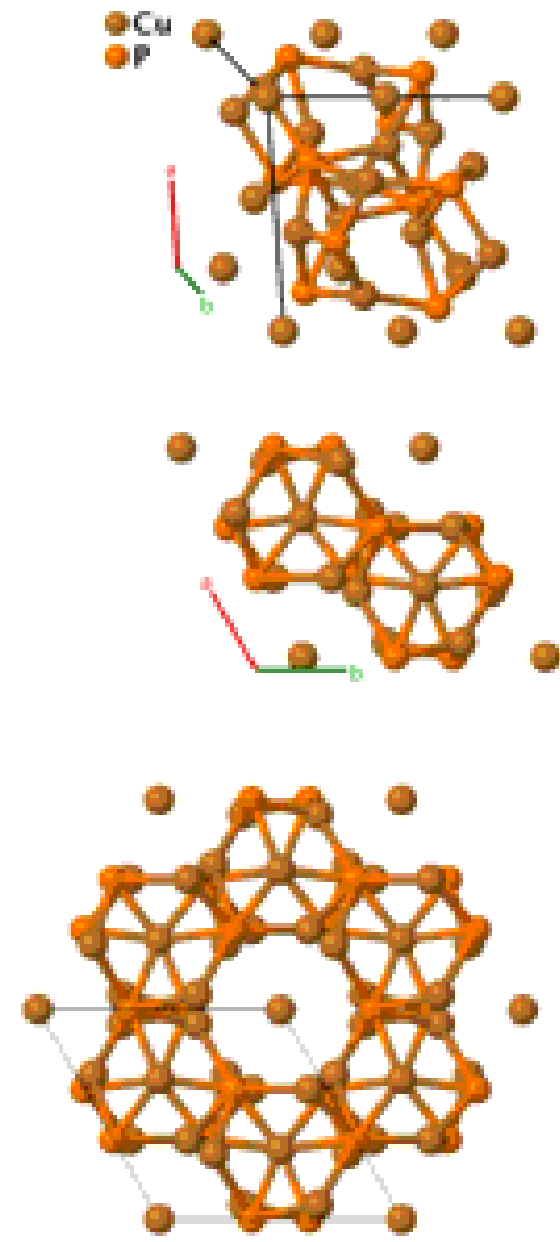

Prototype

AFLOW prototype label

Strukturbericht designation

Pearson symbol

Space group number

Space group symbol

AFLOW prototype command
: $\quad \mathrm{Cu}_{3} \mathrm{P}$

: A3B_hP24_165_bdg_f

: $\quad D 0_{21}$

: $\quad \mathrm{hP} 24$

: 165

: $\quad P \overline{3} c 1$

- params $=a, c / a, z_{2}, x_{3}, x_{4}, y_{4}, z_{4}$
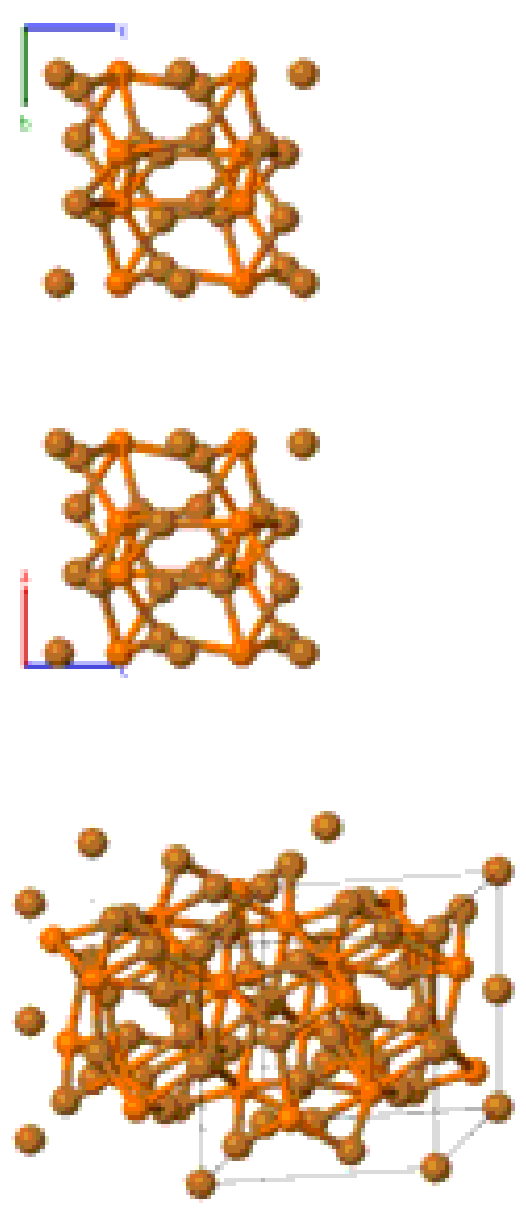

\section{Other compounds with this structure:}

- $\mathrm{Cu}_{3} \mathrm{As}, \mathrm{CeF}_{3}, \mathrm{LaF}_{3}$

- Range and Hafner (Range, 1993) and Olofsson (Olofson, 1972) argue that several compounds which were previously identified as having this structure actually take on the $\mathrm{Cu}_{3} \mathrm{P}$ structure, space group $P 6_{3} \mathrm{~cm}$, A3B_hP24_185_ab2c_c. These structures include $\mathrm{Cu}_{3} \mathrm{P}, \mathrm{Na}_{3} \mathrm{As}, \mathrm{AuMg}_{3}, \mathrm{Ir}_{3}$ and $\mathrm{Mg}_{3} \mathrm{Pt}$.

- The $D 1_{21}$ structure is crystallographically equivalent to the $\mathrm{H}_{3}$ Ho structure, A3B_hP24_165_adg_f. We have not found any evidence in the literature showing that either the hydride structure or the fluoride structures mentioned above should be in any other space group. 
- We were unable to obtain the original reference, so we use the crystallographic information presented in the American Mineralogist Crystal Structure Database (Downs, 2003). The original version of Pearson's Handbook (Pearson, 1958) states that the lattice constants for this structure should be slightly smaller.

- $\mathrm{Cu}_{3} \mathrm{P}$ (pp. 602) and $\mathrm{Na}_{3} \mathrm{As}$ (pp. 607) have similar AFLOW prototype labels (i.e., same symmetry and set of Wyckoff positions with different stoichiometry labels due to alphabetic ordering of atomic species). They are generated by the same symmetry operations with different sets of parameters (--params) specified in their corresponding CIF files.

\section{Trigonal Hexagonal primitive vectors:}

$$
\begin{array}{ll}
\mathbf{a}_{1}= & \frac{1}{2} a \hat{\mathbf{x}}-\frac{\sqrt{3}}{2} a \hat{\mathbf{y}} \\
\mathbf{a}_{2}= & \frac{1}{2} a \hat{\mathbf{x}}+\frac{\sqrt{3}}{2} a \hat{\mathbf{y}} \\
\mathbf{a}_{3}= & c \hat{\mathbf{z}}
\end{array}
$$

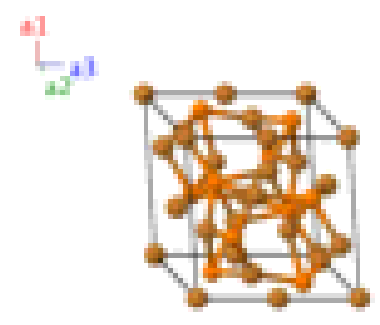

\section{Basis vectors:}

\section{Lattice Coordinates}

$\begin{array}{lcc}\mathbf{B}_{1}= & 0 \mathbf{a}_{1}+0 \mathbf{a}_{2}+0 \mathbf{a}_{3} \\ \mathbf{B}_{2}= & \frac{1}{2} \mathbf{a}_{3} \\ \mathbf{B}_{3}= & \frac{1}{3} \mathbf{a}_{1}+\frac{2}{3} \mathbf{a}_{2}+z_{2} \mathbf{a}_{3} \\ \mathbf{B}_{4}= & \frac{2}{3} \mathbf{a}_{1}+\frac{1}{3} \mathbf{a}_{2}+\left(\frac{1}{2}-z_{2}\right) \mathbf{a}_{3} \\ \mathbf{B}_{5}= & \frac{2}{3} \mathbf{a}_{1}+\frac{1}{3} \mathbf{a}_{2}-z_{2} \mathbf{a}_{3} \\ \mathbf{B}_{6}= & \frac{1}{3} \mathbf{a}_{1}+\frac{2}{3} \mathbf{a}_{2}+\left(\frac{1}{2}+z_{2}\right) \mathbf{a}_{3} \\ \mathbf{B}_{7}= & x_{3} \mathbf{a}_{1}+\frac{1}{4} \mathbf{a}_{3} \\ \mathbf{B}_{8}= & x_{3} \mathbf{a}_{2}+\frac{1}{4} \mathbf{a}_{3} \\ \mathbf{B}_{9}= & -x_{3} \mathbf{a}_{1}-x_{3} \mathbf{a}_{2}+\frac{1}{4} \mathbf{a}_{3} \\ \mathbf{B}_{10}= & -x_{3} \mathbf{a}_{1}+\frac{3}{4} \mathbf{a}_{3} \\ \mathbf{B}_{11}= & -x_{3} \mathbf{a}_{2}+\frac{3}{4} \mathbf{a}_{3} \\ \mathbf{B}_{12}= & x_{3} \mathbf{a}_{1}+x_{3} \mathbf{a}_{2}+\frac{3}{4} \mathbf{a}_{3} \\ \mathbf{B}_{13}= & x_{4} \mathbf{a}_{1}+y_{4} \mathbf{a}_{2}+z_{4} \mathbf{a}_{3}\end{array}$

$\mathbf{B}_{14}=-y_{4} \mathbf{a}_{1}+\left(x_{4}-y_{4}\right) \mathbf{a}_{2}+z_{4} \mathbf{a}_{3}$

$\mathbf{B}_{15}=\left(-x_{4}+y_{4}\right) \mathbf{a}_{1}-x_{4} \mathbf{a}_{2}+z_{4} \mathbf{a}_{3}$

$\mathbf{B}_{16}=y_{4} \mathbf{a}_{1}+x_{4} \mathbf{a}_{2}+\left(\frac{1}{2}-z_{4}\right) \mathbf{a}_{3}$
$=$

$=$

$=\quad \frac{1}{2} a \hat{\mathbf{x}}+\frac{1}{2 \sqrt{3}} a \hat{\mathbf{y}}+z_{2} c \hat{\mathbf{z}}$

$=\quad \frac{1}{2} a \hat{\mathbf{x}}-\frac{1}{2 \sqrt{3}} a \hat{\mathbf{y}}+\left(\frac{1}{2}-z_{2}\right) c \hat{\mathbf{z}}$

$=\quad \frac{1}{2} a \hat{\mathbf{x}}-\frac{1}{2 \sqrt{3}} a \hat{\mathbf{y}}-z_{2} c \hat{\mathbf{z}}$

$=\quad \frac{1}{2} a \hat{\mathbf{x}}+\frac{1}{2 \sqrt{3}} a \hat{\mathbf{y}}+\left(\frac{1}{2}+z_{2}\right) c \hat{\mathbf{z}}$

$=\quad \frac{1}{2} x_{3} a \hat{\mathbf{x}}-\frac{\sqrt{3}}{2} x_{3} a \hat{\mathbf{y}}+\frac{1}{4} c \hat{\mathbf{z}}$

$=\quad \frac{1}{2} x_{3} a \hat{\mathbf{x}}+\frac{\sqrt{3}}{2} x_{3} a \hat{\mathbf{y}}+\frac{1}{4} c \hat{\mathbf{z}}$

$=\quad-x_{3} a \hat{\mathbf{x}}+\frac{1}{4} c \hat{\mathbf{z}}$

$=\quad-\frac{1}{2} x_{3} a \hat{\mathbf{x}}+\frac{\sqrt{3}}{2} x_{3} a \hat{\mathbf{y}}+\frac{3}{4} c \hat{\mathbf{z}}$

$=\quad-\frac{1}{2} x_{3} a \hat{\mathbf{x}}-\frac{\sqrt{3}}{2} x_{3} a \hat{\mathbf{y}}+\frac{3}{4} c \hat{\mathbf{z}}$

$=\quad x_{3} a \hat{\mathbf{x}}+\frac{3}{4} c \hat{\mathbf{z}}$

$=$$$
\frac{1}{2}\left(x_{4}+y_{4}\right) a \hat{\mathbf{x}}+
$$

Cartesian Coordinates$$
\frac{\sqrt{3}}{2}\left(-x_{4}+y_{4}\right) a \hat{\mathbf{y}}+z_{4} c \hat{\mathbf{z}}
$$

$=\left(\frac{1}{2} x_{4}-y_{4}\right) a \hat{\mathbf{x}}+\frac{\sqrt{3}}{2} x_{4} a \hat{\mathbf{y}}+z_{4} c \hat{\mathbf{z}}$

$=\left(-x_{4}+\frac{1}{2} y_{4}\right) a \hat{\mathbf{x}}-\frac{\sqrt{3}}{2} y_{4} a \hat{\mathbf{y}}+z_{4} c \hat{\mathbf{z}}$

$=\frac{1}{2}\left(x_{4}+y_{4}\right) a \hat{\mathbf{x}}+\frac{\sqrt{3}}{2}\left(x_{4}-y_{4}\right) a \hat{\mathbf{y}}+$

$$
\left(\frac{1}{2}-z_{4}\right) c \hat{\mathbf{z}}
$$

$\mathbf{B}_{17}=\left(x_{4}-y_{4}\right) \mathbf{a}_{1}-y_{4} \mathbf{a}_{2}+\left(\frac{1}{2}-z_{4}\right) \mathbf{a}_{3}=\left(\frac{1}{2} x_{4}-y_{4}\right) a \hat{\mathbf{x}}-\frac{\sqrt{3}}{2} x_{4} a \hat{\mathbf{y}}+$

$$
\left(\frac{1}{2}-z_{4}\right) c \hat{\mathbf{z}}
$$

$\mathbf{B}_{18}=-x_{4} \mathbf{a}_{1}+\left(-x_{4}+y_{4}\right) \mathbf{a}_{2}+\left(\frac{1}{2}-z_{4}\right) \mathbf{a}_{3}=\left(-x_{4}+\frac{1}{2} y_{4}\right) a \hat{\mathbf{x}}+\frac{\sqrt{3}}{2} y_{4} a \hat{\mathbf{y}}+$

$$
\left(\frac{1}{2}-z_{4}\right) c \hat{\mathbf{z}}
$$

$\mathbf{B}_{19}=\quad-x_{4} \mathbf{a}_{1}-y_{4} \mathbf{a}_{2}-z_{4} \mathbf{a}_{3}$

$$
\begin{gathered}
-\frac{1}{2}\left(x_{4}+y_{4}\right) a \hat{\mathbf{x}}+ \\
\frac{\sqrt{3}}{2}\left(x_{4}-y_{4}\right) a \hat{\mathbf{y}}-z_{4} c \hat{\mathbf{z}}
\end{gathered}
$$

Wyckoff Position Atom Type

$\mathrm{Cu} \mathrm{I}$
$\mathrm{Cu}$ III

$\mathrm{Cu} \mathrm{I}$

$\mathrm{Cu}$ II

$\mathrm{Cu}$ II

$\mathrm{Cu}$ II

$\mathrm{Cu}$ II

$$
\mathrm{P}
$$

$$
\mathrm{P}
$$

$\mathrm{P}$

$\mathrm{P}$

$$
\mathrm{P}
$$

$$
\text { P }
$$

$\mathrm{Cu}$ III

$\mathrm{Cu}$ III

$\mathrm{Cu}$ III

$\mathrm{Cu}$ III

$\mathrm{Cu}$ III

$\mathrm{Cu}$ III 


\begin{tabular}{|c|c|c|c|c|c|c|}
\hline $\mathbf{B}_{20}$ & $=$ & $y_{4} \mathbf{a}_{1}+\left(-x_{4}+y_{4}\right) \mathbf{a}_{2}-z_{4} \mathbf{a}_{3}$ & $=$ & $\left(-\frac{1}{2} x_{4}+y_{4}\right) a \hat{\mathbf{x}}-\frac{\sqrt{3}}{2} x_{4} a \hat{\mathbf{y}}-z_{4} c \hat{\mathbf{z}}$ & $(12 g)$ & $\mathrm{Cu}$ III \\
\hline $\mathbf{B}_{21}$ & $=$ & $\left(x_{4}-y_{4}\right) \mathbf{a}_{1}+x_{4} \mathbf{a}_{2}-z_{4} \mathbf{a}_{3}$ & $=$ & $\left(x_{4}-\frac{1}{2} y_{4}\right) a \hat{\mathbf{x}}+\frac{\sqrt{3}}{2} y_{4} a \hat{\mathbf{y}}-z_{4} c \hat{\mathbf{z}}$ & $(12 g)$ & $\mathrm{Cu}$ III \\
\hline $\mathbf{B}_{22}$ & $=$ & $-y_{4} \mathbf{a}_{1}-x_{4} \mathbf{a}_{2}+\left(\frac{1}{2}+z_{4}\right) \mathbf{a}_{3}$ & $=$ & $\begin{array}{c}-\frac{1}{2}\left(x_{4}+y_{4}\right) a \hat{\mathbf{x}}+ \\
\frac{\sqrt{3}}{2}\left(-x_{4}+y_{4}\right) a \hat{\mathbf{y}}+\left(\frac{1}{2}+z_{4}\right) c \hat{\mathbf{z}}\end{array}$ & $(12 g)$ & $\mathrm{Cu}$ III \\
\hline $\mathbf{B}_{23}$ & $=$ & $\left(-x_{4}+y_{4}\right) \mathbf{a}_{1}+y_{4} \mathbf{a}_{2}+\left(\frac{1}{2}+z_{4}\right) \mathbf{a}_{3}$ & $=$ & $\begin{array}{c}\left(-\frac{1}{2} x_{4}+y_{4}\right) a \hat{\mathbf{x}}+\frac{\sqrt{3}}{2} x_{4} a \hat{\mathbf{y}}+ \\
\left(\frac{1}{2}+z_{4}\right) c \hat{\mathbf{z}}\end{array}$ & $(12 g)$ & $\mathrm{Cu}$ III \\
\hline $\mathbf{B}_{24}$ & $=$ & $x_{4} \mathbf{a}_{1}+\left(x_{4}-y_{4}\right) \mathbf{a}_{2}+\left(\frac{1}{2}+z_{4}\right) \mathbf{a}_{3}$ & $=$ & $\begin{array}{c}\left(x_{4}-\frac{1}{2} y_{4}\right) a \hat{\mathbf{x}}-\frac{\sqrt{3}}{2} y_{4} a \hat{\mathbf{y}}+ \\
\left(\frac{1}{2}+z_{4}\right) c \hat{\mathbf{z}}\end{array}$ & $(12 g)$ & $\mathrm{Cu}$ III \\
\hline
\end{tabular}

\section{References:}

- B. Steenberg, The Crystal Structure of $\mathrm{Cu}_{3} A s$ and $\mathrm{Cu}_{3} P$, Ark. Kem. Mineral. Geol. A12, 1-15 (1938).

\section{Found in:}

- W. B. Pearson, A Handbook of Lattice Spacings and Structures of Metals and Alloys, no. N.R.C. No. 4303 in International Series of Monographs on Metal Physics and Physical Metallurgy (Pergamon Press, Oxford, London, Edinburgh, New York, Paris, Frankfort, 1958), 1964 reprint with corrections edn.

- R. T. Downs and M. Hall-Wallace, The American Mineralogist Crystal Structure Database, Am. Mineral. 88, 247-250 (2003).

\section{Geometry files:}

- CIF: pp. 919

- POSCAR: pp. 919 


\section{$\mathrm{Al}_{4} \mathrm{C}_{3}\left(D 7_{1}\right)$ Structure: A4B3_hR7_166_2c_ac}
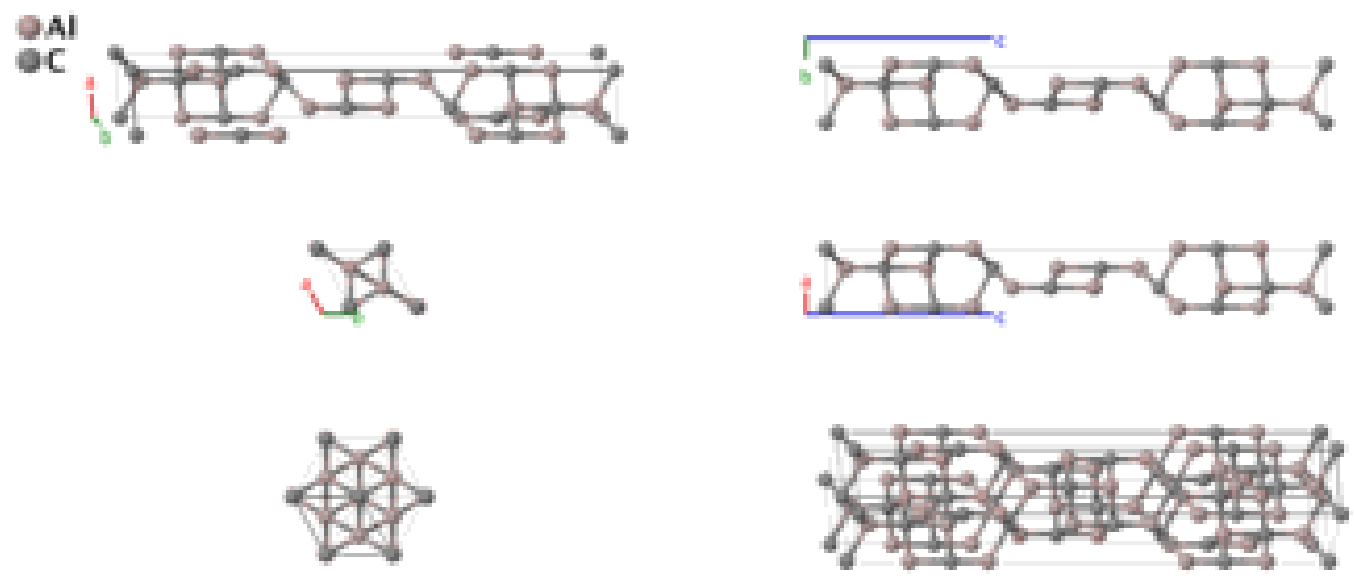

Prototype

AFLOW prototype label

Strukturbericht designation

Pearson symbol

Space group number

Space group symbol

AFLOW prototype command
: $\quad \mathrm{Al}_{4} \mathrm{C}_{3}$

: A4B3_hR7_166_2c_ac

: $\quad D 7_{1}$

: $\quad$ hR7

: $\quad 166$

: $R \overline{3} m$

aflow --proto=A4B3_hR7_166_2c_ac [--hex]

- - params $=a, c / a, x_{2}, x_{3}, x_{4}$

Rhombohedral primitive vectors:

$$
\begin{aligned}
& \mathbf{a}_{1}=\frac{1}{2} a \hat{\mathbf{x}}-\frac{1}{2 \sqrt{3}} a \hat{\mathbf{y}}+\frac{1}{3} c \hat{\mathbf{z}} \\
& \mathbf{a}_{2}=\frac{1}{\sqrt{3}} a \hat{\mathbf{y}}+\frac{1}{3} c \hat{\mathbf{z}} \\
& \mathbf{a}_{3}=-\frac{1}{2} a \hat{\mathbf{x}}-\frac{1}{2 \sqrt{3}} a \hat{\mathbf{y}}+\frac{1}{3} c \hat{\mathbf{z}}
\end{aligned}
$$

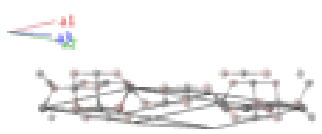

Basis vectors:

Lattice Coordinates

$\mathbf{B}_{1}=0 \mathbf{a}_{1}+0 \mathbf{a}_{2}+0 \mathbf{a}_{3}=$

$\mathbf{B}_{2}=x_{2} \mathbf{a}_{1}+x_{2} \mathbf{a}_{2}+x_{2} \mathbf{a}_{3}$

$\mathbf{B}_{3}=-x_{2} \mathbf{a}_{1}-x_{2} \mathbf{a}_{2}-x_{2} \mathbf{a}_{3}$

$\mathbf{B}_{4}=x_{3} \mathbf{a}_{1}+x_{3} \mathbf{a}_{2}+x_{3} \mathbf{a}_{3}$

$\mathbf{B}_{5}=-x_{3} \mathbf{a}_{1}-x_{3} \mathbf{a}_{2}-x_{3} \mathbf{a}_{3}$

$\mathbf{B}_{6}=x_{4} \mathbf{a}_{1}+x_{4} \mathbf{a}_{2}+x_{4} \mathbf{a}_{3}$

$\mathbf{B}_{7}=$
Cartesian Coordinates

$0 \hat{\mathbf{x}}+0 \hat{\mathbf{y}}+0 \hat{\mathbf{z}}$
$x_{2} c \hat{\mathbf{z}}$
$-x_{2} c \hat{\mathbf{z}}$
$x_{3} c \hat{\mathbf{z}}$
$-x_{3} c \hat{\mathbf{z}}$
$x_{4} c \hat{\mathbf{z}}$
$-x_{4} c \hat{\mathbf{z}}$

Wyckoff Position

(1a)
Atom Type

C I

Al I

Al I

Al II

Al II

C II

C II

\section{References:}

- T. M. Gesing and W. Jeitschko, The Crystal Structure and Chemical Properties of $U_{2} A_{3} C_{4}$ and Structure Refinement of $A l_{4} C_{3}$, Z. Naturforsch. B 50, 196-200 (1995), doi:10.1515/znb-1995-0206. 
Geometry files:

- CIF: pp. 919

- POSCAR: pp. 920 


\section{SmSI Structure: ABC_hR6_166_c_c_c}
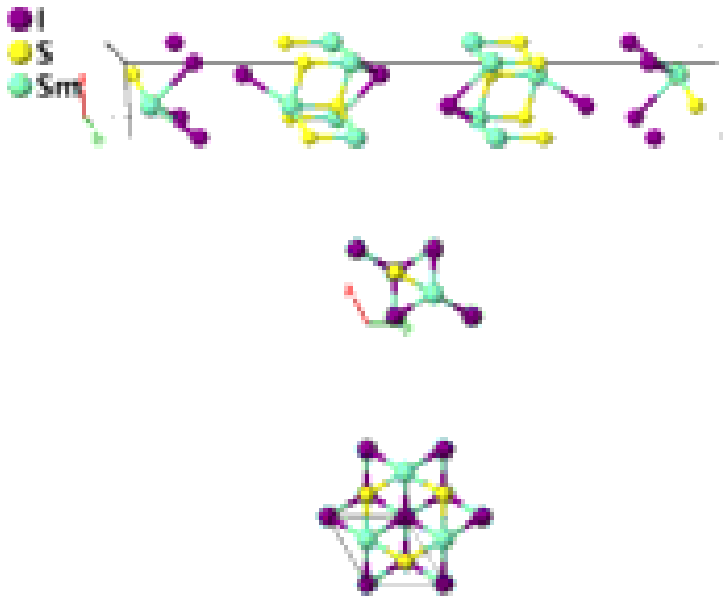
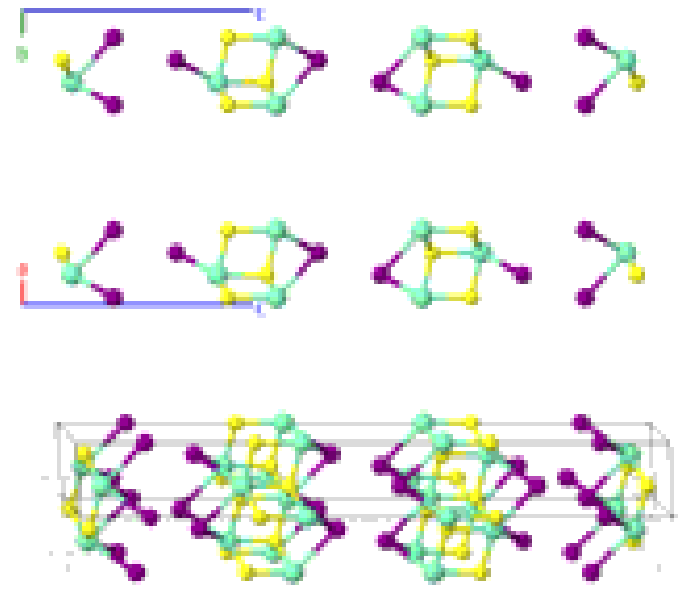

Prototype

AFLOW prototype label

Strukturbericht designation

Pearson symbol

Space group number

Space group symbol

AFLOW prototype command
: SmSI

: ABC_hR6_166_c_c_c

: None

: $\quad$ hR6

: 166

: $R \overline{3} m$

\section{Other compounds with this structure:}

- $\beta$-CeSI, PrSI, NdSI, and the superconducting alkali metal intercalcates $\beta-M \mathrm{~N} X(M=\mathrm{Zr}, \mathrm{Hf} ; X=\mathrm{Cl}, \mathrm{Br}, \mathrm{I})$.

- Although this has the same crystallographic structure as the C12 structure, the layering is substantially different.

\section{Rhombohedral primitive vectors:}

$$
\begin{array}{llc}
\mathbf{a}_{1}= & \frac{1}{2} a \hat{\mathbf{x}}-\frac{1}{2 \sqrt{3}} a \hat{\mathbf{y}}+\frac{1}{3} c \hat{\mathbf{z}} \\
\mathbf{a}_{2}= & \frac{1}{\sqrt{3}} a \hat{\mathbf{y}}+\frac{1}{3} c \hat{\mathbf{z}} \\
\mathbf{a}_{3}= & -\frac{1}{2} a \hat{\mathbf{x}}-\frac{1}{2 \sqrt{3}} a \hat{\mathbf{y}}+\frac{1}{3} c \hat{\mathbf{z}}
\end{array}
$$

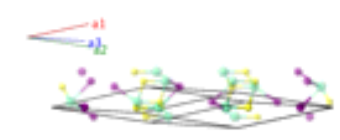

\section{Basis vectors:}

\section{Lattice Coordinates}

$\mathbf{B}_{1}=$

$\mathbf{B}_{2}=-x_{1} \mathbf{a}_{1}-x_{1} \mathbf{a}_{2}-x_{1} \mathbf{a}_{3}$

$\mathbf{B}_{3}=x_{2} \mathbf{a}_{1}+x_{2} \mathbf{a}_{2}+x_{2} \mathbf{a}_{3}$

$\mathbf{B}_{4}=-x_{2} \mathbf{a}_{1}-x_{2} \mathbf{a}_{2}-x_{2} \mathbf{a}_{3}$

$\mathbf{B}_{5}=x_{3} \mathbf{a}_{1}+x_{3} \mathbf{a}_{2}+x_{3} \mathbf{a}_{3}$

$\mathbf{B}_{6}=-x_{3} \mathbf{a}_{1}-x_{3} \mathbf{a}_{2}-x_{3} \mathbf{a}_{3}$
Cartesian Coordinates

$$
\begin{gathered}
x_{1} c \hat{\mathbf{z}} \\
-x_{1} c \hat{\mathbf{z}} \\
x_{2} c \hat{\mathbf{z}} \\
-x_{2} c \hat{\mathbf{z}} \\
x_{3} c \hat{\mathbf{z}} \\
-x_{3} c \hat{\mathbf{z}}
\end{gathered}
$$

Wyckoff Position

(2c)

$=$ $=\quad x_{3} c \hat{\mathbf{z}}$

(2c)
Atom Type$$
\text { I }
$$$$
\text { I }
$$$$
\text { S }
$$$$
\text { S }
$$

Sm

Sm 


\section{References:}

- H. P. Beck and C. Strobel, Zur Hochdruckpolymorphie der Seltenerdsulfidiodide LnSI, Z. Anorg. Allg. Chem. 535, 222-239 (1986), doi:10.1002/zaac.19865350427.

\section{Found in:}

- A. M. Fogg, J. S. O. Evens, and D. O'Hare, Crystal structure of $\beta$ - $M N X(M=Z r, H f ; X=C l, B r)$, Chem. Comm. 0, 2269-2270 (1998), doi:10.1039/A806415F.

\section{Geometry files:}

- CIF: pp. 920

- POSCAR: pp. 920 


\section{$\mathrm{PrNiO}_{3}$ Structure: AB3C_hR10_167_b_e_a}
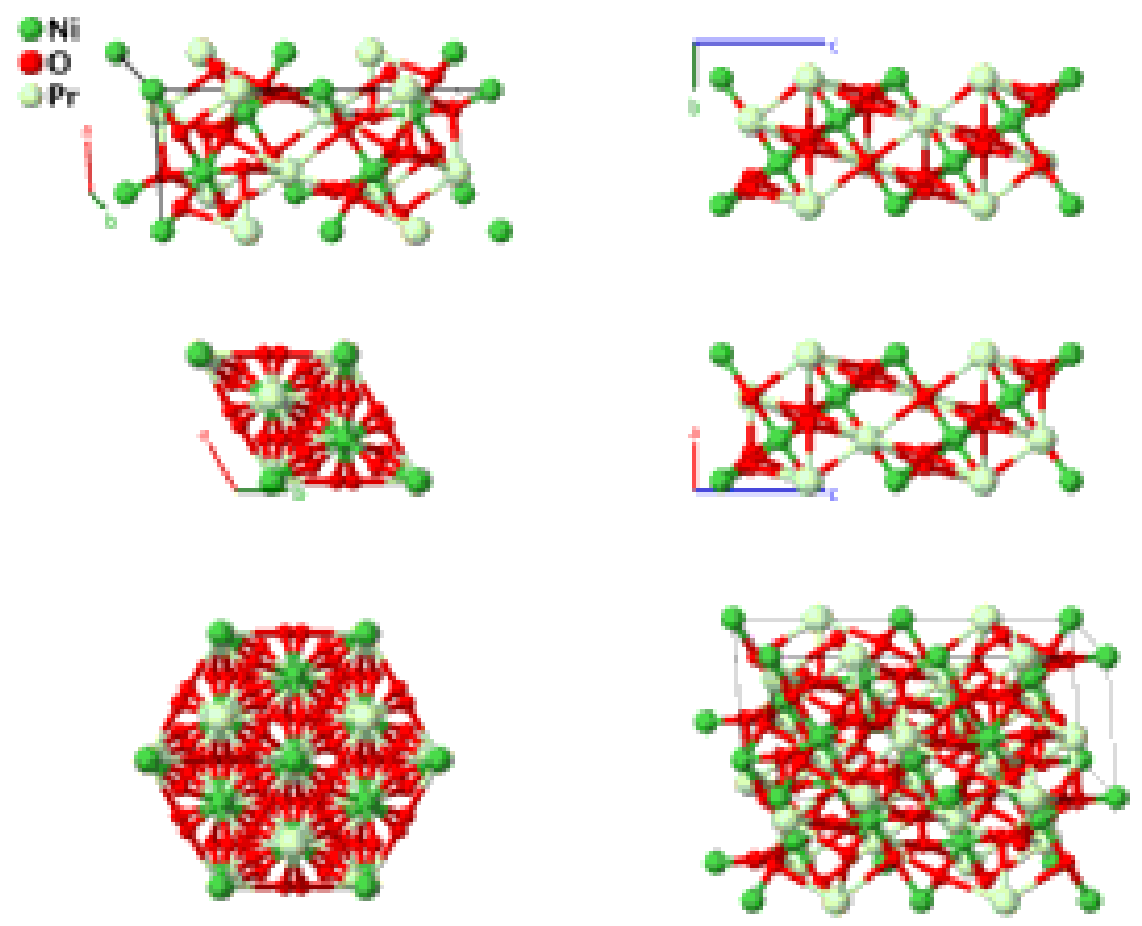

Prototype

AFLOW prototype label

Strukturbericht designation

Pearson symbol

Space group number

Space group symbol

AFLOW prototype command
: $\quad \mathrm{PrNiO}_{3}$

: AB3C_hR10_167_b_e_a

: None

: $\quad \mathrm{hR} 10$

: $\quad 167$

: $\quad R \overline{3} c$ aflow --proto=AB3C_hR10_167_b_e_a [--hex]

- -params $=a, c / a, x_{3}$

\section{Other compounds with this structure:}

- $\mathrm{LaNiO}_{3}$

- This structure has the same crystal structure and occupies the same Wyckoff positions as Calcite $\left(\mathrm{CaCO}_{3}\right.$ Strukturbericht $G 0_{1}, \mathrm{ABC} 3 \_$hR10_167_a_b_e), but $c / a$ and $x_{3}$ are different enough to warrant calling this a new structure. This is the high-temperature form of $\mathrm{PrNiO}_{3}$, and we present the data collected at $500^{\circ} \mathrm{C}$. Below $500^{\circ} \mathrm{C}$ this compound transforms to the orthorhombic perovskite structure (AB3C_oP20_62_c_cd_a).

\section{Rhombohedral primitive vectors:}

$$
\begin{array}{llc}
\mathbf{a}_{1}= & \frac{1}{2} a \hat{\mathbf{x}}-\frac{1}{2 \sqrt{3}} a \hat{\mathbf{y}}+\frac{1}{3} c \hat{\mathbf{z}} \\
\mathbf{a}_{2}= & \frac{1}{\sqrt{3}} a \hat{\mathbf{y}}+\frac{1}{3} c \hat{\mathbf{z}} \\
\mathbf{a}_{3}= & -\frac{1}{2} a \hat{\mathbf{x}}-\frac{1}{2 \sqrt{3}} a \hat{\mathbf{y}}+\frac{1}{3} c \hat{\mathbf{z}}
\end{array}
$$

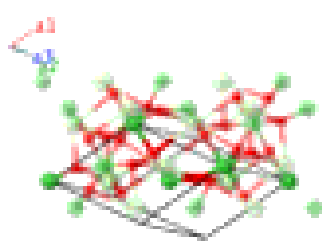

\section{Basis vectors:}


Lattice Coordinates
$\mathbf{B}_{1}=\frac{1}{4} \mathbf{a}_{1}+\frac{1}{4} \mathbf{a}_{2}+\frac{1}{4} \mathbf{a}_{3}$
$\mathbf{B}_{2}=\frac{3}{4} \mathbf{a}_{1}+\frac{3}{4} \mathbf{a}_{2}+\frac{3}{4} \mathbf{a}_{3}$
$=$
$\mathbf{B}_{3}=0 \mathbf{a}_{1}+0 \mathbf{a}_{2}+0 \mathbf{a}_{3}$
$\mathbf{B}_{4}=\frac{1}{2} \mathbf{a}_{1}+\frac{1}{2} \mathbf{a}_{2}+\frac{1}{2} \mathbf{a}_{3}$
$\mathbf{B}_{5}=x_{3} \mathbf{a}_{1}+\left(\frac{1}{2}-x_{3}\right) \mathbf{a}_{2}+\frac{1}{4} \mathbf{a}_{3}=$
$\mathbf{B}_{6}=\frac{1}{4} \mathbf{a}_{1}+x_{3} \mathbf{a}_{2}+\left(\frac{1}{2}-x_{3}\right) \mathbf{a}_{3}$
$\mathbf{B}_{7}=\left(\frac{1}{2}-x_{3}\right) \mathbf{a}_{1}+\frac{1}{4} \mathbf{a}_{2}+x_{3} \mathbf{a}_{3}=$
$\mathbf{B}_{8}=-x_{3} \mathbf{a}_{1}+\left(\frac{1}{2}+x_{3}\right) \mathbf{a}_{2}+\frac{3}{4} \mathbf{a}_{3}$
$\mathbf{B}_{9}=\frac{3}{4} \mathbf{a}_{1}-x_{3} \mathbf{a}_{2}+\left(\frac{1}{2}+x_{3}\right) \mathbf{a}_{3}$
$\mathbf{B}_{10}=\left(\frac{1}{2}+x_{3}\right) \mathbf{a}_{1}+\frac{3}{4} \mathbf{a}_{2}-x_{3} \mathbf{a}_{3}=$
$\frac{1}{4} c \hat{\mathbf{z}}$
$\frac{3}{4} c \hat{\mathbf{z}}$
$0 \hat{\mathbf{x}}+0 \hat{\mathbf{y}}+0 \hat{\mathbf{z}}$
$\frac{1}{2} c \hat{\mathbf{z}}$
$=\left(-\frac{1}{8}+\frac{1}{2} x_{3}\right) a \hat{\mathbf{x}}+\left(\frac{\sqrt{3}}{8}-\frac{\sqrt{3}}{2} x_{3}\right) a \hat{\mathbf{y}}+\frac{1}{4} c \hat{\mathbf{z}}$
$=\left(-\frac{1}{8}+\frac{1}{2} x_{3}\right) a \hat{\mathbf{x}}+\left(-\frac{\sqrt{3}}{8}+\frac{\sqrt{3}}{2} x_{3}\right) a \hat{\mathbf{y}}+\frac{1}{4} c \hat{\mathbf{z}}$
$\left(\frac{1}{4}-x_{3}\right) a \hat{\mathbf{x}}+\frac{1}{4} c \hat{\mathbf{z}}$
$=-a\left(\frac{1}{2} x_{3}+\frac{3}{8}\right) \hat{\mathbf{x}}+\left(\frac{1}{8 \sqrt{3}}+\frac{\sqrt{3}}{2} x_{3}\right) a \hat{\mathbf{y}}+\frac{5}{12} c \hat{\mathbf{z}}$
$=\left(\frac{1}{8}-\frac{1}{2} x_{3}\right) a \hat{\mathbf{x}}-a\left(\frac{\sqrt{3}}{2} x_{3}+\frac{5}{8 \sqrt{3}}\right) \hat{\mathbf{y}}+\frac{5}{12} c \hat{\mathbf{z}}$
$\left(\frac{1}{4}+x_{3}\right) a \hat{\mathbf{x}}+\frac{1}{2 \sqrt{3}} a \hat{\mathbf{y}}+\frac{5}{12} c \hat{\mathbf{z}}$

Cartesian Coordinates
Wyckoff Position Atom Type

(2a)

Pr

(2a)

Pr

$(2 b)$

$\mathrm{Ni}$

$(2 b)$

$\mathrm{Ni}$

(6e)

$\mathrm{O}$

(6e)

$\mathrm{O}$

$(6 e)$

$\mathrm{O}$

(6e)

$\mathrm{O}$

$(6 e)$

$\mathrm{O}$

(6e)

\section{References:}

- T. C. Huang, W. Parrish, H. Toraya, P. Lacorre, and J. B. Torrance, High-Temperature Crystal Structures of Orthorhombic and Rhombohedral $\mathrm{PrNiO}_{3}$, Mater. Res. Bull. 25, 1091-1098 (1990), doi:10.1016/0025-5408(90)90138-R.

\section{Geometry files:}

- CIF: pp. 920

- POSCAR: pp. 921 
$\mathrm{KBO}_{2}\left(F 5_{13}\right)$ Structure: ABC2_hR24_167_e_e_2e

$\begin{array}{r}20 \\ \hline 0\end{array}$
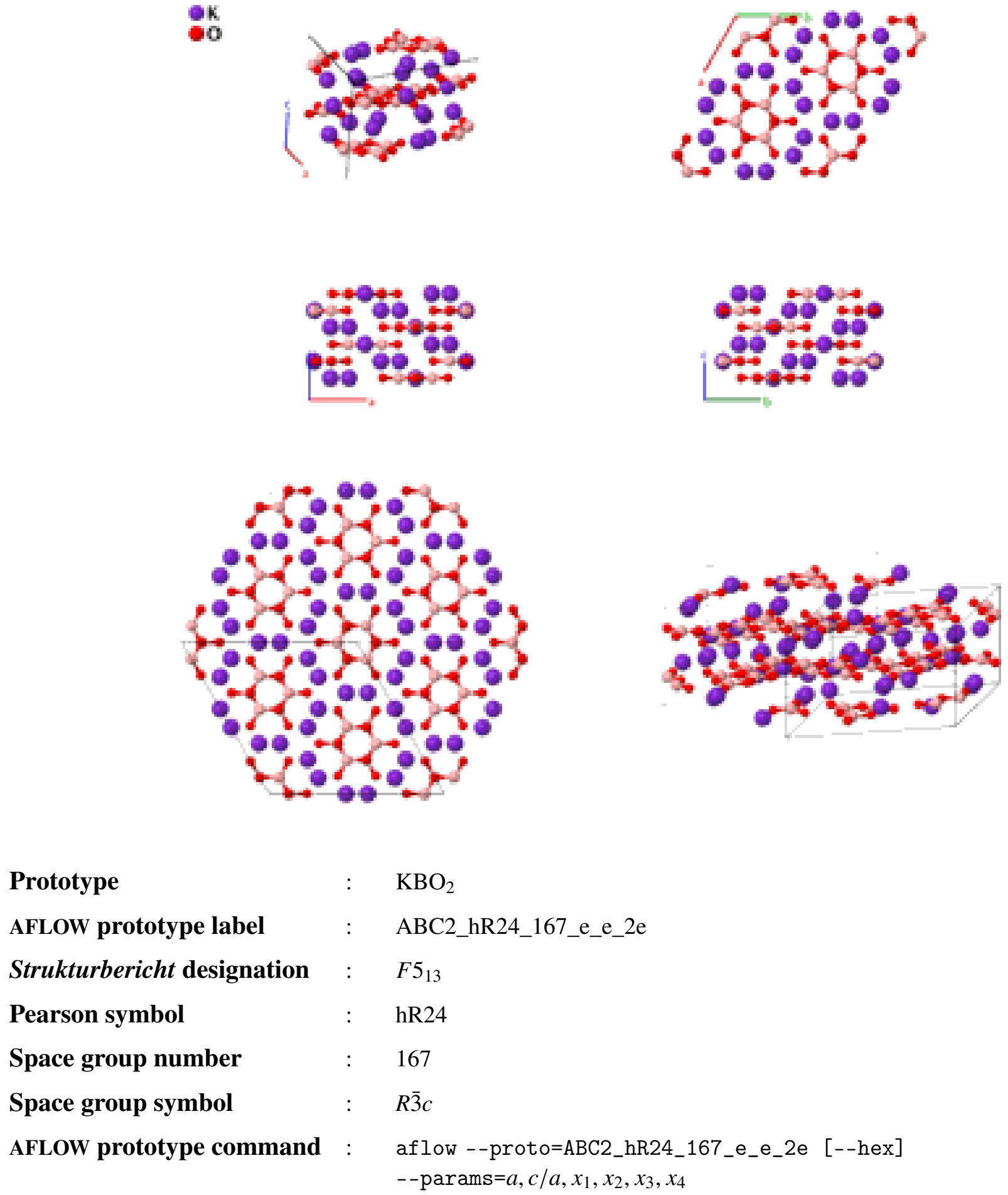

Other compounds with this structure:

- $\mathrm{NaBO}_{2}, \mathrm{NaBS}_{2}$ 


\section{Rhombohedral primitive vectors:}

$$
\begin{array}{llc}
\mathbf{a}_{1}= & \frac{1}{2} a \hat{\mathbf{x}}-\frac{1}{2 \sqrt{3}} a \hat{\mathbf{y}}+\frac{1}{3} c \hat{\mathbf{z}} \\
\mathbf{a}_{2}= & \frac{1}{\sqrt{3}} a \hat{\mathbf{y}}+\frac{1}{3} c \hat{\mathbf{z}} \\
\mathbf{a}_{3}= & -\frac{1}{2} a \hat{\mathbf{x}}-\frac{1}{2 \sqrt{3}} a \hat{\mathbf{y}}+\frac{1}{3} c \hat{\mathbf{z}}
\end{array}
$$

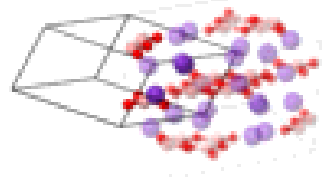

\section{Basis vectors:}

Lattice Coordinates

$\mathbf{B}_{1}=x_{1} \mathbf{a}_{1}+\left(\frac{1}{2}-x_{1}\right) \mathbf{a}_{2}+\frac{1}{4} \mathbf{a}_{3}$

$\mathbf{B}_{2}=\frac{1}{4} \mathbf{a}_{1}+x_{1} \mathbf{a}_{2}+\left(\frac{1}{2}-x_{1}\right) \mathbf{a}_{3}$

$\mathbf{B}_{3}=\left(\frac{1}{2}-x_{1}\right) \mathbf{a}_{1}+\frac{1}{4} \mathbf{a}_{2}+x_{1} \mathbf{a}_{3}=$

$\mathbf{B}_{4}=-x_{1} \mathbf{a}_{1}+\left(\frac{1}{2}+x_{1}\right) \mathbf{a}_{2}+\frac{3}{4} \mathbf{a}_{3}$

$\mathbf{B}_{5}=\frac{3}{4} \mathbf{a}_{1}-x_{1} \mathbf{a}_{2}+\left(\frac{1}{2}+x_{1}\right) \mathbf{a}_{3}$

$\mathbf{B}_{6}=\left(\frac{1}{2}+x_{1}\right) \mathbf{a}_{1}+\frac{3}{4} \mathbf{a}_{2}-x_{1} \mathbf{a}_{3}$

$\mathbf{B}_{7}=x_{2} \mathbf{a}_{1}+\left(\frac{1}{2}-x_{2}\right) \mathbf{a}_{2}+\frac{1}{4} \mathbf{a}_{3}$

$\mathbf{B}_{8}=\frac{1}{4} \mathbf{a}_{1}+x_{2} \mathbf{a}_{2}+\left(\frac{1}{2}-x_{2}\right) \mathbf{a}_{3}$

$\mathbf{B}_{9}=\left(\frac{1}{2}-x_{2}\right) \mathbf{a}_{1}+\frac{1}{4} \mathbf{a}_{2}+x_{2} \mathbf{a}_{3}$

$\mathbf{B}_{10}=-x_{2} \mathbf{a}_{1}+\left(\frac{1}{2}+x_{2}\right) \mathbf{a}_{2}+\frac{3}{4} \mathbf{a}_{3}$

$\mathbf{B}_{11}=\frac{3}{4} \mathbf{a}_{1}-x_{2} \mathbf{a}_{2}+\left(\frac{1}{2}+x_{2}\right) \mathbf{a}_{3}$

$\mathbf{B}_{12}=\left(\frac{1}{2}+x_{2}\right) \mathbf{a}_{1}+\frac{3}{4} \mathbf{a}_{2}-x_{2} \mathbf{a}_{3}$

$\mathbf{B}_{13}=x_{3} \mathbf{a}_{1}+\left(\frac{1}{2}-x_{3}\right) \mathbf{a}_{2}+\frac{1}{4} \mathbf{a}_{3}$

$\mathbf{B}_{14}=\frac{1}{4} \mathbf{a}_{1}+x_{3} \mathbf{a}_{2}+\left(\frac{1}{2}-x_{3}\right) \mathbf{a}_{3}$

$\mathbf{B}_{15}=\left(\frac{1}{2}-x_{3}\right) \mathbf{a}_{1}+\frac{1}{4} \mathbf{a}_{2}+x_{3} \mathbf{a}_{3}=$

$\mathbf{B}_{16}=-x_{3} \mathbf{a}_{1}+\left(\frac{1}{2}+x_{3}\right) \mathbf{a}_{2}+\frac{3}{4} \mathbf{a}_{3}$

$\mathbf{B}_{17}=\frac{3}{4} \mathbf{a}_{1}-x_{3} \mathbf{a}_{2}+\left(\frac{1}{2}+x_{3}\right) \mathbf{a}_{3}$

$\mathbf{B}_{18}=\left(\frac{1}{2}+x_{3}\right) \mathbf{a}_{1}+\frac{3}{4} \mathbf{a}_{2}-x_{3} \mathbf{a}_{3}=$

$\mathbf{B}_{19}=x_{4} \mathbf{a}_{1}+\left(\frac{1}{2}-x_{4}\right) \mathbf{a}_{2}+\frac{1}{4} \mathbf{a}_{3}$

$\mathbf{B}_{20}=\frac{1}{4} \mathbf{a}_{1}+x_{4} \mathbf{a}_{2}+\left(\frac{1}{2}-x_{4}\right) \mathbf{a}_{3}$

$\mathbf{B}_{21}=\left(\frac{1}{2}-x_{4}\right) \mathbf{a}_{1}+\frac{1}{4} \mathbf{a}_{2}+x_{4} \mathbf{a}_{3}=$

$\mathbf{B}_{22}=-x_{4} \mathbf{a}_{1}+\left(\frac{1}{2}+x_{4}\right) \mathbf{a}_{2}+\frac{3}{4} \mathbf{a}_{3}=$

$\mathbf{B}_{23}=\frac{3}{4} \mathbf{a}_{1}-x_{4} \mathbf{a}_{2}+\left(\frac{1}{2}+x_{4}\right) \mathbf{a}_{3}$

$\mathbf{B}_{24}=\left(\frac{1}{2}+x_{4}\right) \mathbf{a}_{1}+\frac{3}{4} \mathbf{a}_{2}-x_{4} \mathbf{a}_{3}=$
Cartesian Coordinates

$=\left(-\frac{1}{8}+\frac{1}{2} x_{1}\right) a \hat{\mathbf{x}}+\left(\frac{\sqrt{3}}{8}-\frac{\sqrt{3}}{2} x_{1}\right) a \hat{\mathbf{y}}+\frac{1}{4} c \hat{\mathbf{z}}$

$=\left(-\frac{1}{8}+\frac{1}{2} x_{1}\right) a \hat{\mathbf{x}}+\left(-\frac{\sqrt{3}}{8}+\frac{\sqrt{3}}{2} x_{1}\right) a \hat{\mathbf{y}}+\frac{1}{4} c \hat{\mathbf{z}}$

$\left(\frac{1}{4}-x_{1}\right) a \hat{\mathbf{x}}+\frac{1}{4} c \hat{\mathbf{z}}$

$-a\left(\frac{1}{2} x_{1}+\frac{3}{8}\right) \hat{\mathbf{x}}+\left(\frac{1}{8 \sqrt{3}}+\frac{\sqrt{3}}{2} x_{1}\right) a \hat{\mathbf{y}}+\frac{5}{12} c \hat{\mathbf{z}}$

$=\left(\frac{1}{8}-\frac{1}{2} x_{1}\right) a \hat{\mathbf{x}}-a\left(\frac{\sqrt{3}}{2} x_{1}+\frac{5}{8 \sqrt{3}}\right) \hat{\mathbf{y}}+\frac{5}{12} c \hat{\mathbf{z}}$

$\left(\frac{1}{4}+x_{1}\right) a \hat{\mathbf{x}}+\frac{1}{2 \sqrt{3}} a \hat{\mathbf{y}}+\frac{5}{12} c \hat{\mathbf{z}}$

$=\left(-\frac{1}{8}+\frac{1}{2} x_{2}\right) a \hat{\mathbf{x}}+\left(\frac{\sqrt{3}}{8}-\frac{\sqrt{3}}{2} x_{2}\right) a \hat{\mathbf{y}}+\frac{1}{4} c \hat{\mathbf{z}}$

$=\left(-\frac{1}{8}+\frac{1}{2} x_{2}\right) a \hat{\mathbf{x}}+\left(-\frac{\sqrt{3}}{8}+\frac{\sqrt{3}}{2} x_{2}\right) a \hat{\mathbf{y}}+\frac{1}{4} c \hat{\mathbf{z}}$

$\left(\frac{1}{4}-x_{2}\right) a \hat{\mathbf{x}}+\frac{1}{4} c \hat{\mathbf{z}}$

$-a\left(\frac{1}{2} x_{2}+\frac{3}{8}\right) \hat{\mathbf{x}}+\left(\frac{1}{8 \sqrt{3}}+\frac{\sqrt{3}}{2} x_{2}\right) a \hat{\mathbf{y}}+\frac{5}{12} c \hat{\mathbf{z}}$

$=\left(\frac{1}{8}-\frac{1}{2} x_{2}\right) a \hat{\mathbf{x}}-a\left(\frac{\sqrt{3}}{2} x_{2}+\frac{5}{8 \sqrt{3}}\right) \hat{\mathbf{y}}+\frac{5}{12} c \hat{\mathbf{z}}$

$=\quad\left(\frac{1}{4}+x_{2}\right) a \hat{\mathbf{x}}+\frac{1}{2 \sqrt{3}} a \hat{\mathbf{y}}+\frac{5}{12} c \hat{\mathbf{z}}$

$=\left(-\frac{1}{8}+\frac{1}{2} x_{3}\right) a \hat{\mathbf{x}}+\left(\frac{\sqrt{3}}{8}-\frac{\sqrt{3}}{2} x_{3}\right) a \hat{\mathbf{y}}+\frac{1}{4} c \hat{\mathbf{z}}$

$=\left(-\frac{1}{8}+\frac{1}{2} x_{3}\right) a \hat{\mathbf{x}}+\left(-\frac{\sqrt{3}}{8}+\frac{\sqrt{3}}{2} x_{3}\right) a \hat{\mathbf{y}}+\frac{1}{4} c \hat{\mathbf{z}}$

$\left(\frac{1}{4}-x_{3}\right) a \hat{\mathbf{x}}+\frac{1}{4} c \hat{\mathbf{z}}$

$=-a\left(\frac{1}{2} x_{3}+\frac{3}{8}\right) \hat{\mathbf{x}}+\left(\frac{1}{8 \sqrt{3}}+\frac{\sqrt{3}}{2} x_{3}\right) a \hat{\mathbf{y}}+\frac{5}{12} c \hat{\mathbf{z}}$

$=\left(\frac{1}{8}-\frac{1}{2} x_{3}\right) a \hat{\mathbf{x}}-a\left(\frac{\sqrt{3}}{2} x_{3}+\frac{5}{8 \sqrt{3}}\right) \hat{\mathbf{y}}+\frac{5}{12} c \hat{\mathbf{z}}$

$=\left(\frac{1}{4}+x_{3}\right) a \hat{\mathbf{x}}+\frac{1}{2 \sqrt{3}} a \hat{\mathbf{y}}+\frac{5}{12} c \hat{\mathbf{z}}$

$=\left(-\frac{1}{8}+\frac{1}{2} x_{4}\right) a \hat{\mathbf{x}}+\left(\frac{\sqrt{3}}{8}-\frac{\sqrt{3}}{2} x_{4}\right) a \hat{\mathbf{y}}+\frac{1}{4} c \hat{\mathbf{z}}$

$=\left(-\frac{1}{8}+\frac{1}{2} x_{4}\right) a \hat{\mathbf{x}}+\left(-\frac{\sqrt{3}}{8}+\frac{\sqrt{3}}{2} x_{4}\right) a \hat{\mathbf{y}}+\frac{1}{4} c \hat{\mathbf{z}}$

$\left(\frac{1}{4}-x_{4}\right) a \hat{\mathbf{x}}+\frac{1}{4} c \hat{\mathbf{Z}}$

$=-a\left(\frac{1}{2} x_{4}+\frac{3}{8}\right) \hat{\mathbf{x}}+\left(\frac{1}{8 \sqrt{3}}+\frac{\sqrt{3}}{2} x_{4}\right) a \hat{\mathbf{y}}+\frac{5}{12} c \hat{\mathbf{z}}$

$=\left(\frac{1}{8}-\frac{1}{2} x_{4}\right) a \hat{\mathbf{x}}-a\left(\frac{\sqrt{3}}{2} x_{4}+\frac{5}{8 \sqrt{3}}\right) \hat{\mathbf{y}}+\frac{5}{12} c \hat{\mathbf{z}}$

$\left(\frac{1}{4}+x_{4}\right) a \hat{\mathbf{x}}+\frac{1}{2 \sqrt{3}} a \hat{\mathbf{y}}+\frac{5}{12} c \hat{\mathbf{z}}$
Wyckoff Position Atom Type

(6e)

B

$(6 e)$

B

$(6 e)$

B

(6e)

B

$(6 e)$

B

(6e)

B

(6e)

K

(6e)

K

(6e)

K

(6e)

K

K

(6e)

K

(6e)

O I

O I

(6e)

O I

(6e)

O I

(6e)

O I

(6e)

O I

(6e)

O II

(6e)

O II

(6e)

O II

(6e)

O II

(6e)

O II

(6e)

\section{References:}

- W. Schneider and G. B. Carpenter, Bond lengths and thermal parameters of potassium metaborate, $\mathrm{K}_{3} \mathrm{~B}_{3} \mathrm{O}_{6}$, Acta Crystallogr. Sect. B Struct. Sci. 26, 1189-1191 (1970), doi:10.1107/S0567740870003849. 


\section{Found in:}

- P. Villars, K. Cenzual, J. Daams, R. Gladyshevskii, O. Shcherban, V. Dubenskyy, N. Melnichenko-Koblyuk, O. Pavlyuk, I. Savysyuk, S. Stoyko, and L. Sysa, Landolt-Börnstein - Group III Condensed Matter (Numerical Data and Functional Relationships in Science and Technology) (Springer, Berlin, Heidelberg, 2007), vol. 43A5, chap. KBO2 in Structure Types. Part 5: Space Groups (173) P63 - (166) R-3m.

\section{Geometry files:}

- CIF: pp. 921

- POSCAR: pp. 921 


\section{$\mathrm{K}_{2} \mathrm{Ta}_{4} \mathrm{O}_{9} \mathrm{~F}_{4}$ Structure: A2B13C4_hP57_168_d_c6d_2d}
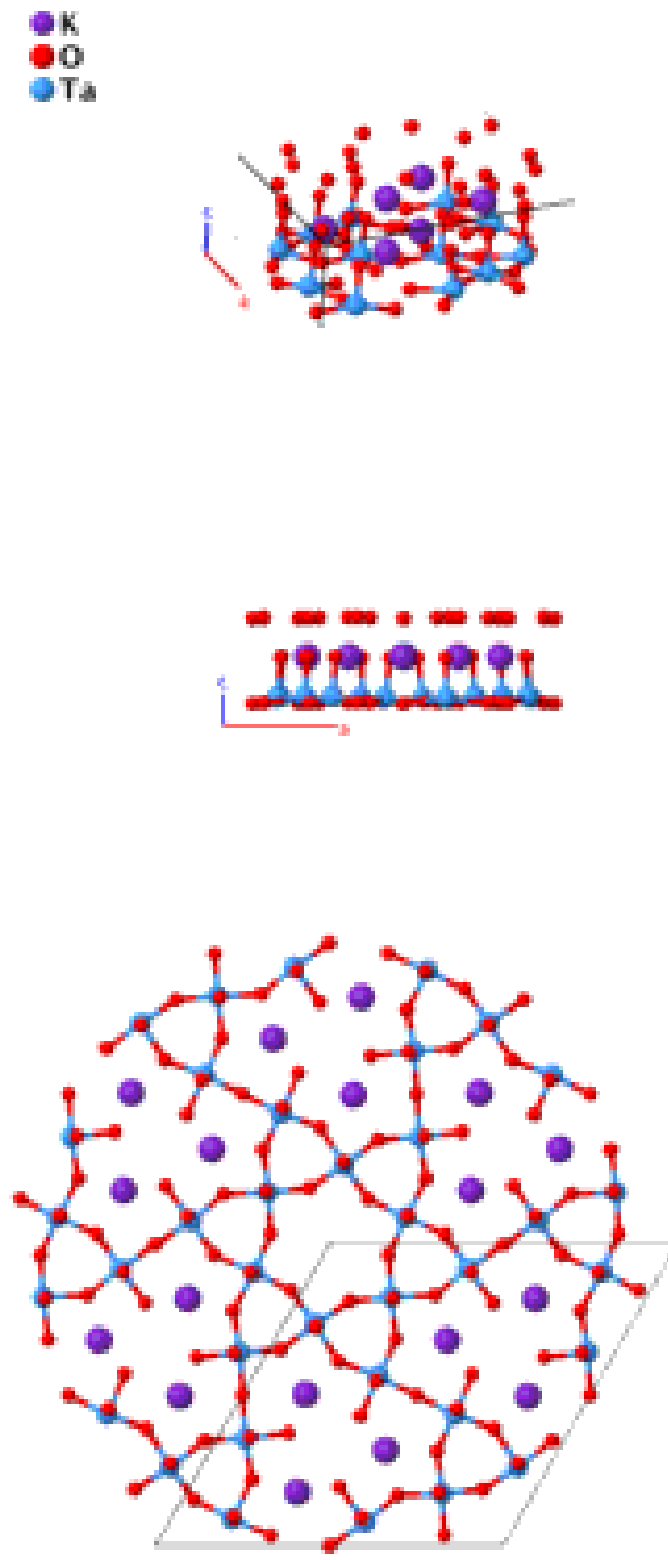
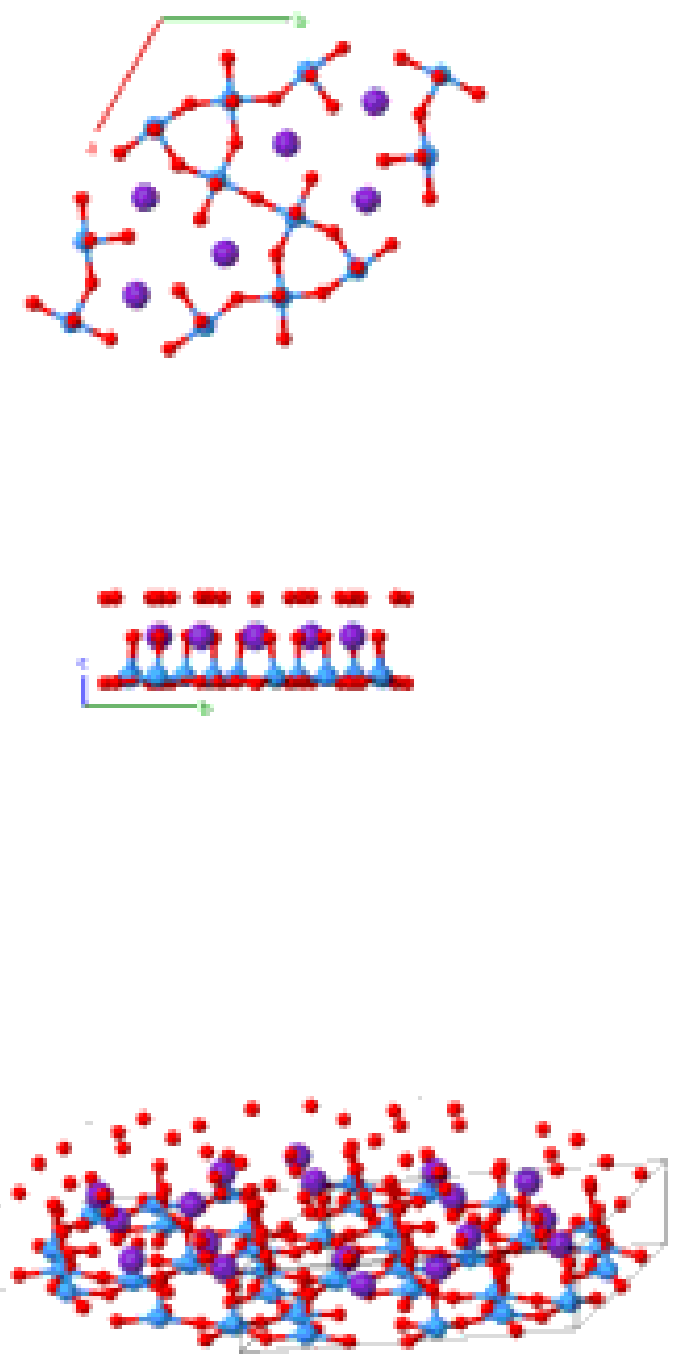

\section{Prototype}

AFLOW prototype label

Strukturbericht designation

Pearson symbol

Space group number

Space group symbol

AFLOW prototype command
: $\quad \mathrm{K}_{2} \mathrm{Ta}_{4} \mathrm{O}_{9} \mathrm{~F}_{4}$

: A2B13C4_hP57_168_d_c6d_2d

: None

: $\quad$ hP57

: 168

: $\quad P 6$

aflow --proto=A2B13C4_hP57_168_d_c6d_2d

- - params $=a, c / a, z_{1}, x_{2}, y_{2}, z_{2}, x_{3}, y_{3}, z_{3}, x_{4}, y_{4}, z_{4}, x_{5}, y_{5}, z_{5}, x_{6}, y_{6}, z_{6}, x_{7}, y_{7}, z_{7}$, $x_{8}, y_{8}, z_{8}, x_{9}, y_{9}, z_{9}, x_{10}, y_{10}, z_{10}$

- The $\mathrm{O}$ sites are partially occupied with the following concentration $0.692 \mathrm{O}+0.308 \mathrm{~F}$. 


\section{Hexagonal primitive vectors:}

$$
\begin{array}{ll}
\mathbf{a}_{1}= & \frac{1}{2} a \hat{\mathbf{x}}-\frac{\sqrt{3}}{2} a \hat{\mathbf{y}} \\
\mathbf{a}_{2}= & \frac{1}{2} a \hat{\mathbf{x}}+\frac{\sqrt{3}}{2} a \hat{\mathbf{y}} \\
\mathbf{a}_{3}= & c \hat{\mathbf{z}}
\end{array}
$$

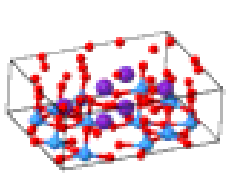

Basis vectors:

Lattice Coordinates

$$
\begin{aligned}
& \mathbf{B}_{1}= \\
& \frac{1}{2} \mathbf{a}_{1}+z_{1} \mathbf{a}_{3} \\
& \mathbf{B}_{2}= \\
& \frac{1}{2} \mathbf{a}_{2}+z_{1} \mathbf{a}_{3} \\
& \mathbf{B}_{3}= \\
& \frac{1}{2} \mathbf{a}_{1}+\frac{1}{2} \mathbf{a}_{2}+z_{1} \mathbf{a}_{3} \\
& \mathbf{B}_{4}= \\
& x_{2} \mathbf{a}_{1}+y_{2} \mathbf{a}_{2}+z_{2} \mathbf{a}_{3} \\
& \mathbf{B}_{5}=-y_{2} \mathbf{a}_{1}+\left(x_{2}-y_{2}\right) \mathbf{a}_{2}+z_{2} \mathbf{a}_{3} \\
& \mathbf{B}_{6}=\left(-x_{2}+y_{2}\right) \mathbf{a}_{1}-x_{2} \mathbf{a}_{2}+z_{2} \mathbf{a}_{3} \\
& \mathbf{B}_{7}= \\
& -x_{2} \mathbf{a}_{1}-y_{2} \mathbf{a}_{2}+z_{2} \mathbf{a}_{3} \\
& \mathbf{B}_{8}=y_{2} \mathbf{a}_{1}+\left(-x_{2}+y_{2}\right) \mathbf{a}_{2}+z_{2} \mathbf{a}_{3} \\
& \mathbf{B}_{9}=\left(x_{2}-y_{2}\right) \mathbf{a}_{1}+x_{2} \mathbf{a}_{2}+z_{2} \mathbf{a}_{3} \\
& \mathbf{B}_{10}= \\
& x_{3} \mathbf{a}_{1}+y_{3} \mathbf{a}_{2}+z_{3} \mathbf{a}_{3} \\
& \mathbf{B}_{11}=-y_{3} \mathbf{a}_{1}+\left(x_{3}-y_{3}\right) \mathbf{a}_{2}+z_{3} \mathbf{a}_{3} \\
& \mathbf{B}_{12}=\left(-x_{3}+y_{3}\right) \mathbf{a}_{1}-x_{3} \mathbf{a}_{2}+z_{3} \mathbf{a}_{3} \\
& \mathbf{B}_{13}= \\
& -x_{3} \mathbf{a}_{1}-y_{3} \mathbf{a}_{2}+z_{3} \mathbf{a}_{3} \\
& \mathbf{B}_{14}=y_{3} \mathbf{a}_{1}+\left(-x_{3}+y_{3}\right) \mathbf{a}_{2}+z_{3} \mathbf{a}_{3} \\
& \mathbf{B}_{15}=\left(x_{3}-y_{3}\right) \mathbf{a}_{1}+x_{3} \mathbf{a}_{2}+z_{3} \mathbf{a}_{3} \\
& \mathbf{B}_{16}= \\
& x_{4} \mathbf{a}_{1}+y_{4} \mathbf{a}_{2}+z_{4} \mathbf{a}_{3} \\
& \mathbf{B}_{17}=-y_{4} \mathbf{a}_{1}+\left(x_{4}-y_{4}\right) \mathbf{a}_{2}+z_{4} \mathbf{a}_{3} \\
& \mathbf{B}_{18}=\left(-x_{4}+y_{4}\right) \mathbf{a}_{1}-x_{4} \mathbf{a}_{2}+z_{4} \mathbf{a}_{3} \\
& \mathbf{B}_{19}=-x_{4} \mathbf{a}_{1}-y_{4} \mathbf{a}_{2}+z_{4} \mathbf{a}_{3} \\
& \mathbf{B}_{20}=y_{4} \mathbf{a}_{1}+\left(-x_{4}+y_{4}\right) \mathbf{a}_{2}+z_{4} \mathbf{a}_{3} \\
& \mathbf{B}_{21}=\left(x_{4}-y_{4}\right) \mathbf{a}_{1}+x_{4} \mathbf{a}_{2}+z_{4} \mathbf{a}_{3} \\
& \mathbf{B}_{22}= \\
& x_{5} \mathbf{a}_{1}+y_{5} \mathbf{a}_{2}+z_{5} \mathbf{a}_{3} \\
& \mathbf{B}_{23}=-y_{5} \mathbf{a}_{1}+\left(x_{5}-y_{5}\right) \mathbf{a}_{2}+z_{5} \mathbf{a}_{3} \\
& \mathbf{B}_{24}=\left(-x_{5}+y_{5}\right) \mathbf{a}_{1}-x_{5} \mathbf{a}_{2}+z_{5} \mathbf{a}_{3} \\
& \mathbf{B}_{25}= \\
& -x_{5} \mathbf{a}_{1}-y_{5} \mathbf{a}_{2}+z_{5} \mathbf{a}_{3} \\
& =\quad \frac{1}{4} a \hat{\mathbf{x}}+\frac{\sqrt{3}}{4} a \hat{\mathbf{y}}+z_{1} c \hat{\mathbf{z}} \\
& =\quad \frac{1}{2} a \hat{\mathbf{x}}+z_{1} c \hat{\mathbf{z}} \\
& =\frac{1}{2}\left(x_{2}+y_{2}\right) a \hat{\mathbf{x}}+\frac{\sqrt{3}}{2}\left(-x_{2}+y_{2}\right) a \hat{\mathbf{y}}+ \\
& z_{2} c \hat{\mathbf{z}} \\
& =\left(\frac{1}{2} x_{2}-y_{2}\right) a \hat{\mathbf{x}}+\frac{\sqrt{3}}{2} x_{2} a \hat{\mathbf{y}}+z_{2} c \hat{\mathbf{z}} \\
& =\left(-x_{2}+\frac{1}{2} y_{2}\right) a \hat{\mathbf{x}}-\frac{\sqrt{3}}{2} y_{2} a \hat{\mathbf{y}}+z_{2} c \hat{\mathbf{z}} \\
& =-\frac{1}{2}\left(x_{2}+y_{2}\right) a \hat{\mathbf{x}}+\frac{\sqrt{3}}{2}\left(x_{2}-y_{2}\right) a \hat{\mathbf{y}}+ \\
& =\left(-\frac{1}{2} x_{2}+y_{2}\right) a \hat{\mathbf{x}}-\frac{\sqrt{3}}{2} x_{2} a \hat{\mathbf{y}}+z_{2} c \hat{\mathbf{z}} \\
& =\left(x_{2}-\frac{1}{2} y_{2}\right) a \hat{\mathbf{x}}+\frac{\sqrt{3}}{2} y_{2} a \hat{\mathbf{y}}+z_{2} c \hat{\mathbf{z}} \\
& =\frac{1}{2}\left(x_{3}+y_{3}\right) a \hat{\mathbf{x}}+\frac{\sqrt{3}}{2}\left(-x_{3}+y_{3}\right) a \hat{\mathbf{y}}+ \\
& z_{3} c \hat{\mathbf{z}} \\
& =\left(\frac{1}{2} x_{3}-y_{3}\right) a \hat{\mathbf{x}}+\frac{\sqrt{3}}{2} x_{3} a \hat{\mathbf{y}}+z_{3} c \hat{\mathbf{z}} \\
& =\left(-x_{3}+\frac{1}{2} y_{3}\right) a \hat{\mathbf{x}}-\frac{\sqrt{3}}{2} y_{3} a \hat{\mathbf{y}}+z_{3} c \hat{\mathbf{z}} \\
& =-\frac{1}{2}\left(x_{3}+y_{3}\right) a \hat{\mathbf{x}}+\frac{\sqrt{3}}{2}\left(x_{3}-y_{3}\right) a \hat{\mathbf{y}}+ \\
& =\left(-\frac{1}{2} x_{3}+y_{3}\right) a \hat{\mathbf{x}}-\frac{\sqrt{3}}{2} x_{3} a \hat{\mathbf{y}}+z_{3} c \hat{\mathbf{z}} \\
& =\left(x_{3}-\frac{1}{2} y_{3}\right) a \hat{\mathbf{x}}+\frac{\sqrt{3}}{2} y_{3} a \hat{\mathbf{y}}+z_{3} c \hat{\mathbf{z}} \\
& =\frac{1}{2}\left(x_{4}+y_{4}\right) a \hat{\mathbf{x}}+\frac{\sqrt{3}}{2}\left(-x_{4}+y_{4}\right) a \hat{\mathbf{y}}+ \\
& =\left(\frac{1}{2} x_{4}-y_{4}\right) a \hat{\mathbf{x}}+\frac{\sqrt{3}}{2} x_{4} a \hat{\mathbf{y}}+z_{4} c \hat{\mathbf{z}} \\
& =\left(-x_{4}+\frac{1}{2} y_{4}\right) a \hat{\mathbf{x}}-\frac{\sqrt{3}}{2} y_{4} a \hat{\mathbf{y}}+z_{4} c \hat{\mathbf{z}} \\
& =\quad-\frac{1}{2}\left(x_{4}+y_{4}\right) a \hat{\mathbf{x}}+\frac{\sqrt{3}}{2}\left(x_{4}-y_{4}\right) a \hat{\mathbf{y}}+ \\
& { }_{24} c \hat{\mathbf{z}} \\
& =\left(-\frac{1}{2} x_{4}+y_{4}\right) a \hat{\mathbf{x}}-\frac{\sqrt{3}}{2} x_{4} a \hat{\mathbf{y}}+z_{4} c \hat{\mathbf{z}} \\
& =\left(x_{4}-\frac{1}{2} y_{4}\right) a \hat{\mathbf{x}}+\frac{\sqrt{3}}{2} y_{4} a \hat{\mathbf{y}}+z_{4} c \hat{\mathbf{z}} \\
& =\frac{1}{2}\left(x_{5}+y_{5}\right) a \hat{\mathbf{x}}+\frac{\sqrt{3}}{2}\left(-x_{5}+y_{5}\right) a \hat{\mathbf{y}}+ \\
& { }_{25} c \hat{\mathbf{z}} \\
& =\left(\frac{1}{2} x_{5}-y_{5}\right) a \hat{\mathbf{x}}+\frac{\sqrt{3}}{2} x_{5} a \hat{\mathbf{y}}+z_{5} c \hat{\mathbf{z}} \\
& =\left(-x_{5}+\frac{1}{2} y_{5}\right) a \hat{\mathbf{x}}-\frac{\sqrt{3}}{2} y_{5} a \hat{\mathbf{y}}+z_{5} c \hat{\mathbf{z}} \\
& =-\frac{1}{2}\left(x_{5}+y_{5}\right) a \hat{\mathbf{x}}+\frac{\sqrt{3}}{2}\left(x_{5}-y_{5}\right) a \hat{\mathbf{y}}+
\end{aligned}
$$

Cartesian Coordinates
Wyckoff Position Atom Type

O I

O I

$(6 d)$

$$
\text { K }
$$

K

K

K

K

K

O II

O II

O II

O II

O II

O II

O III

O III

O III

O III

O III

O III

O IV

(6d)

O IV

O IV

O IV 


$$
\begin{aligned}
& \mathbf{B}_{26}=y_{5} \mathbf{a}_{1}+\left(-x_{5}+y_{5}\right) \mathbf{a}_{2}+z_{5} \mathbf{a}_{3}=\left(-\frac{1}{2} x_{5}+y_{5}\right) a \hat{\mathbf{x}}-\frac{\sqrt{3}}{2} x_{5} a \hat{\mathbf{y}}+z_{5} c \hat{\mathbf{z}} \\
& \mathbf{B}_{27}=\left(x_{5}-y_{5}\right) \mathbf{a}_{1}+x_{5} \mathbf{a}_{2}+z_{5} \mathbf{a}_{3}=\left(x_{5}-\frac{1}{2} y_{5}\right) a \hat{\mathbf{x}}+\frac{\sqrt{3}}{2} y_{5} a \hat{\mathbf{y}}+z_{5} c \hat{\mathbf{z}} \\
& \mathbf{B}_{28}=x_{6} \mathbf{a}_{1}+y_{6} \mathbf{a}_{2}+z_{6} \mathbf{a}_{3} \quad=\frac{1}{2}\left(x_{6}+y_{6}\right) a \hat{\mathbf{x}}+\frac{\sqrt{3}}{2}\left(-x_{6}+y_{6}\right) a \hat{\mathbf{y}}+ \\
& \mathbf{B}_{29}=-y_{6} \mathbf{a}_{1}+\left(x_{6}-y_{6}\right) \mathbf{a}_{2}+z_{6} \mathbf{a}_{3}=\left(\frac{1}{2} x_{6}-y_{6}\right) a \hat{\mathbf{x}}+\frac{\sqrt{3}}{2} x_{6} a \hat{\mathbf{y}}+z_{6} c \hat{\mathbf{z}} \\
& \mathbf{B}_{30}=\left(-x_{6}+y_{6}\right) \mathbf{a}_{1}-x_{6} \mathbf{a}_{2}+z_{6} \mathbf{a}_{3}=\left(-x_{6}+\frac{1}{2} y_{6}\right) a \hat{\mathbf{x}}-\frac{\sqrt{3}}{2} y_{6} a \hat{\mathbf{y}}+z_{6} c \hat{\mathbf{z}} \\
& \mathbf{B}_{31}=-x_{6} \mathbf{a}_{1}-y_{6} \mathbf{a}_{2}+z_{6} \mathbf{a}_{3} \quad=\quad-\frac{1}{2}\left(x_{6}+y_{6}\right) a \hat{\mathbf{x}}+\frac{\sqrt{3}}{2}\left(x_{6}-y_{6}\right) a \hat{\mathbf{y}}+ \\
& \mathbf{B}_{32}=y_{6} \mathbf{a}_{1}+\left(-x_{6}+y_{6}\right) \mathbf{a}_{2}+z_{6} \mathbf{a}_{3}=\left(-\frac{1}{2} x_{6}+y_{6}\right) a \hat{\mathbf{x}}-\frac{\sqrt{3}}{2} x_{6} a \hat{\mathbf{y}}+z_{6} c \hat{\mathbf{z}} \\
& \mathbf{B}_{33}=\left(x_{6}-y_{6}\right) \mathbf{a}_{1}+x_{6} \mathbf{a}_{2}+z_{6} \mathbf{a}_{3}=\left(x_{6}-\frac{1}{2} y_{6}\right) a \hat{\mathbf{x}}+\frac{\sqrt{3}}{2} y_{6} a \hat{\mathbf{y}}+z_{6} c \hat{\mathbf{z}} \\
& \mathbf{B}_{34}=x_{7} \mathbf{a}_{1}+y_{7} \mathbf{a}_{2}+z_{7} \mathbf{a}_{3} \quad=\quad \frac{1}{2}\left(x_{7}+y_{7}\right) a \hat{\mathbf{x}}+\frac{\sqrt{3}}{2}\left(-x_{7}+y_{7}\right) a \hat{\mathbf{y}}+ \\
& z_{7} c \hat{\mathbf{z}} \\
& \mathbf{B}_{35}=-y_{7} \mathbf{a}_{1}+\left(x_{7}-y_{7}\right) \mathbf{a}_{2}+z_{7} \mathbf{a}_{3}=\left(\frac{1}{2} x_{7}-y_{7}\right) a \hat{\mathbf{x}}+\frac{\sqrt{3}}{2} x_{7} a \hat{\mathbf{y}}+z_{7} c \hat{\mathbf{z}} \\
& \mathbf{B}_{36}=\left(-x_{7}+y_{7}\right) \mathbf{a}_{1}-x_{7} \mathbf{a}_{2}+z_{7} \mathbf{a}_{3}=\left(-x_{7}+\frac{1}{2} y_{7}\right) a \hat{\mathbf{x}}-\frac{\sqrt{3}}{2} y_{7} a \hat{\mathbf{y}}+z_{7} c \hat{\mathbf{z}} \\
& \mathbf{B}_{37}=-x_{7} \mathbf{a}_{1}-y_{7} \mathbf{a}_{2}+z_{7} \mathbf{a}_{3}=-\frac{1}{2}\left(x_{7}+y_{7}\right) a \hat{\mathbf{x}}+\frac{\sqrt{3}}{2}\left(x_{7}-y_{7}\right) a \hat{\mathbf{y}}+ \\
& \mathbf{B}_{38}=y_{7} \mathbf{a}_{1}+\left(-x_{7}+y_{7}\right) \mathbf{a}_{2}+z_{7} \mathbf{a}_{3}=\left(-\frac{1}{2} x_{7}+y_{7}\right) a \hat{\mathbf{x}}-\frac{\sqrt{3}}{2} x_{7} a \hat{\mathbf{y}}+z_{7} c \hat{\mathbf{z}} \\
& \mathbf{B}_{39}=\left(x_{7}-y_{7}\right) \mathbf{a}_{1}+x_{7} \mathbf{a}_{2}+z_{7} \mathbf{a}_{3}=\left(x_{7}-\frac{1}{2} y_{7}\right) a \hat{\mathbf{x}}+\frac{\sqrt{3}}{2} y_{7} a \hat{\mathbf{y}}+z_{7} c \hat{\mathbf{z}} \\
& \mathbf{B}_{40}=x_{8} \mathbf{a}_{1}+y_{8} \mathbf{a}_{2}+z_{8} \mathbf{a}_{3}=\frac{1}{2}\left(x_{8}+y_{8}\right) a \hat{\mathbf{x}}+\frac{\sqrt{3}}{2}\left(-x_{8}+y_{8}\right) a \hat{\mathbf{y}}+ \\
& z_{8} c \hat{\mathbf{z}} \\
& \mathbf{B}_{41}=-y_{8} \mathbf{a}_{1}+\left(x_{8}-y_{8}\right) \mathbf{a}_{2}+z_{8} \mathbf{a}_{3}=\left(\frac{1}{2} x_{8}-y_{8}\right) a \hat{\mathbf{x}}+\frac{\sqrt{3}}{2} x_{8} a \hat{\mathbf{y}}+z_{8} c \hat{\mathbf{z}} \\
& \mathbf{B}_{42}=\left(-x_{8}+y_{8}\right) \mathbf{a}_{1}-x_{8} \mathbf{a}_{2}+z_{8} \mathbf{a}_{3}=\left(-x_{8}+\frac{1}{2} y_{8}\right) a \hat{\mathbf{x}}-\frac{\sqrt{3}}{2} y_{8} a \hat{\mathbf{y}}+z_{8} c \hat{\mathbf{z}} \\
& \mathbf{B}_{43}=-x_{8} \mathbf{a}_{1}-y_{8} \mathbf{a}_{2}+z_{8} \mathbf{a}_{3}=-\frac{1}{2}\left(x_{8}+y_{8}\right) a \hat{\mathbf{x}}+\frac{\sqrt{3}}{2}\left(x_{8}-y_{8}\right) a \hat{\mathbf{y}}+ \\
& \mathbf{B}_{44}=y_{8} \mathbf{a}_{1}+\left(-x_{8}+y_{8}\right) \mathbf{a}_{2}+z_{8} \mathbf{a}_{3}=\left(-\frac{1}{2} x_{8}+y_{8}\right) a \hat{\mathbf{x}}-\frac{\sqrt{3}}{2} x_{8} a \hat{\mathbf{y}}+z_{8} c \hat{\mathbf{z}} \\
& \mathbf{B}_{45}=\left(x_{8}-y_{8}\right) \mathbf{a}_{1}+x_{8} \mathbf{a}_{2}+z_{8} \mathbf{a}_{3}=\left(x_{8}-\frac{1}{2} y_{8}\right) a \hat{\mathbf{x}}+\frac{\sqrt{3}}{2} y_{8} a \hat{\mathbf{y}}+z_{8} c \hat{\mathbf{z}} \\
& \mathbf{B}_{46}=x_{9} \mathbf{a}_{1}+y_{9} \mathbf{a}_{2}+z_{9} \mathbf{a}_{3} \quad=\quad \frac{1}{2}\left(x_{9}+y_{9}\right) a \hat{\mathbf{x}}+\frac{\sqrt{3}}{2}\left(-x_{9}+y_{9}\right) a \hat{\mathbf{y}}+ \\
& \mathbf{B}_{47}=-y_{9} \mathbf{a}_{1}+\left(x_{9}-y_{9}\right) \mathbf{a}_{2}+z_{9} \mathbf{a}_{3}=\left(\frac{1}{2} x_{9}-y_{9}\right) a \hat{\mathbf{x}}+\frac{\sqrt{3}}{2} x_{9} a \hat{\mathbf{y}}+z_{9} c \hat{\mathbf{z}} \\
& \mathbf{B}_{48}=\left(-x_{9}+y_{9}\right) \mathbf{a}_{1}-x_{9} \mathbf{a}_{2}+z_{9} \mathbf{a}_{3}=\left(-x_{9}+\frac{1}{2} y_{9}\right) a \hat{\mathbf{x}}-\frac{\sqrt{3}}{2} y_{9} a \hat{\mathbf{y}}+z_{9} c \hat{\mathbf{z}} \\
& \mathbf{B}_{49}=-x_{9} \mathbf{a}_{1}-y_{9} \mathbf{a}_{2}+z_{9} \mathbf{a}_{3}=-\frac{1}{2}\left(x_{9}+y_{9}\right) a \hat{\mathbf{x}}+\frac{\sqrt{3}}{2}\left(x_{9}-y_{9}\right) a \hat{\mathbf{y}}+ \\
& \mathbf{B}_{50}=y_{9} \mathbf{a}_{1}+\left(-x_{9}+y_{9}\right) \mathbf{a}_{2}+z_{9} \mathbf{a}_{3}=\left(-\frac{1}{2} x_{9}+y_{9}\right) a \hat{\mathbf{x}}-\frac{\sqrt{3}}{2} x_{9} a \hat{\mathbf{y}}+z_{9} c \hat{\mathbf{z}} \\
& \mathbf{B}_{51}=\left(x_{9}-y_{9}\right) \mathbf{a}_{1}+x_{9} \mathbf{a}_{2}+z_{9} \mathbf{a}_{3}=\left(x_{9}-\frac{1}{2} y_{9}\right) a \hat{\mathbf{x}}+\frac{\sqrt{3}}{2} y_{9} a \hat{\mathbf{y}}+z_{9} c \hat{\mathbf{z}} \\
& \mathbf{B}_{52}=x_{10} \mathbf{a}_{1}+y_{10} \mathbf{a}_{2}+z_{10} \mathbf{a}_{3} \quad=\quad \frac{1}{2}\left(x_{10}+y_{10}\right) a \hat{\mathbf{x}}+ \\
& \frac{\sqrt{3}}{2}\left(-x_{10}+y_{10}\right) a \hat{\mathbf{y}}+z_{10} c \hat{\mathbf{z}} \\
& \mathbf{B}_{53}=-y_{10} \mathbf{a}_{1}+\left(x_{10}-y_{10}\right) \mathbf{a}_{2}+z_{10} \mathbf{a}_{3}=\left(\frac{1}{2} x_{10}-y_{10}\right) a \hat{\mathbf{x}}+\frac{\sqrt{3}}{2} x_{10} a \hat{\mathbf{y}}+z_{10} c \hat{\mathbf{z}} \\
& \mathbf{B}_{54}=\left(-x_{10}+y_{10}\right) \mathbf{a}_{1}-x_{10} \mathbf{a}_{2}+z_{10} \mathbf{a}_{3}=\left(-x_{10}+\frac{1}{2} y_{10}\right) a \hat{\mathbf{x}}-\frac{\sqrt{3}}{2} y_{10} a \hat{\mathbf{y}}+z_{10} c \hat{\mathbf{z}} \\
& \mathbf{B}_{55}=-x_{10} \mathbf{a}_{1}-y_{10} \mathbf{a}_{2}+z_{10} \mathbf{a}_{3} \quad=\quad-\frac{1}{2}\left(x_{10}+y_{10}\right) a \hat{\mathbf{x}}+ \\
& \frac{\sqrt{3}}{2}\left(x_{10}-y_{10}\right) a \hat{\mathbf{y}}+z_{10} c \hat{\mathbf{z}}
\end{aligned}
$$




\section{References:}

- A. Boukhari, J. P. Chaminade, M. Vlasse, and M. Pouchard, Structure cristalline de l'oxyfluorure de tantale et de potassium, $\mathrm{K}_{2} \mathrm{Ta}_{4} \mathrm{~F}_{4} \mathrm{O}_{9}$, Acta Crystallogr. Sect. B Struct. Sci. 35, 1983-1986 (1979), doi:10.1107/S056774087900830X.

\section{Found in:}

- P. Villars and K. Cenzual, Pearson's Crystal Data - Crystal Structure Database for Inorganic Compounds, ASM International (2013).

\section{Geometry files:}

- CIF: pp. 922

- POSCAR: pp. 922 


\section{$\mathrm{Al}\left[\mathrm{PO}_{4}\right]$ Structure: AB4C_hP72_168_2d_8d_2d}
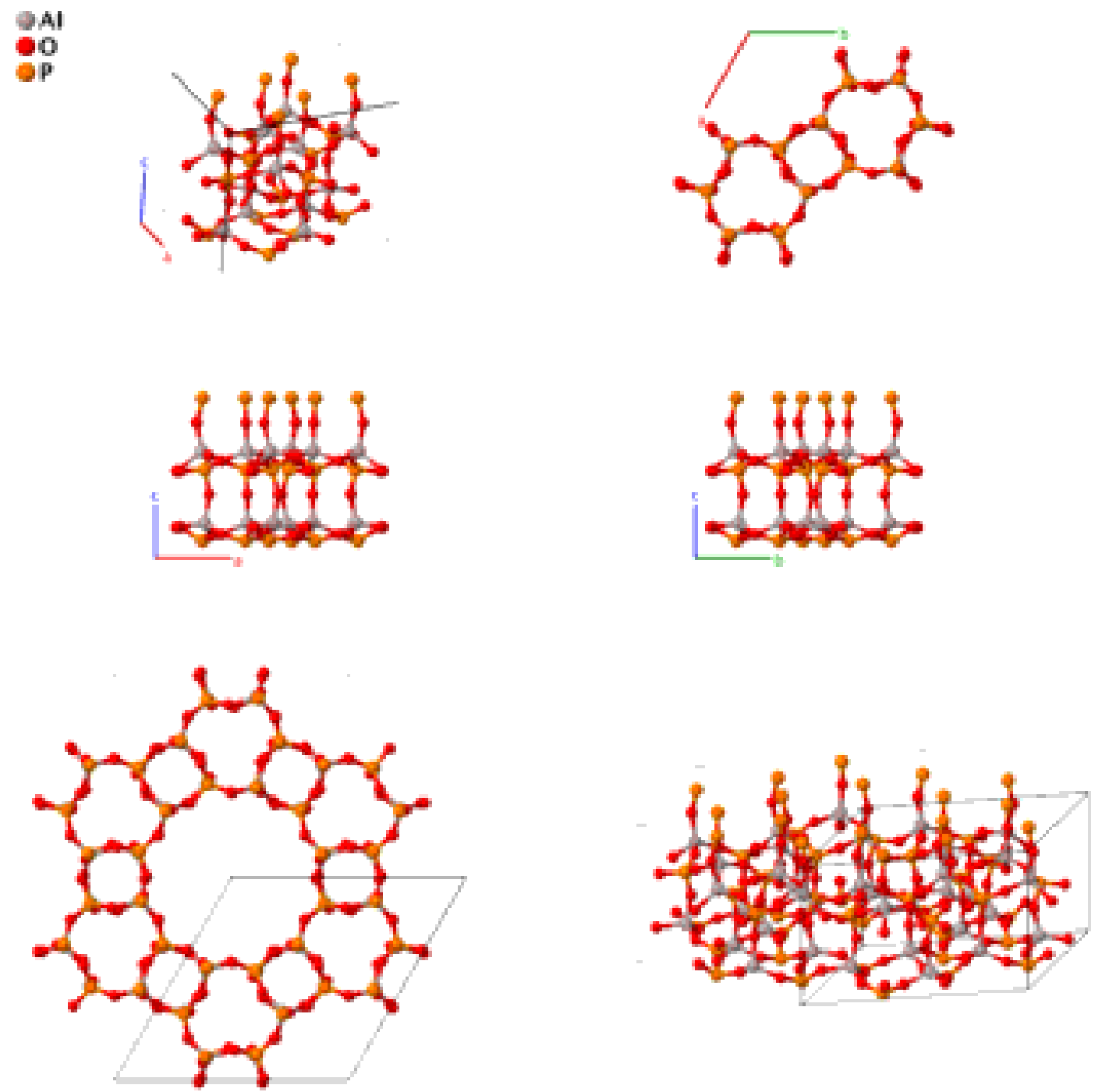

Prototype

AFLOW prototype label

$\mathrm{Al}\left[\mathrm{PO}_{4}\right]$

Strukturbericht designation : None

Pearson symbol : $\mathrm{hP72}$

Space group number $\quad: \quad 168$

Space group symbol $\quad: \quad P 6$

AFLOW prototype command : aflow --proto=AB4C_hP72_168_2d_8d_2d

- - params $=a, c / a, x_{1}, y_{1}, z_{1}, x_{2}, y_{2}, z_{2}, x_{3}, y_{3}, z_{3}, x_{4}, y_{4}, z_{4}, x_{5}, y_{5}, z_{5}, x_{6}, y_{6}, z_{6}, x_{7}$,

$y_{7}, z_{7}, x_{8}, y_{8}, z_{8}, x_{9}, y_{9}, z_{9}, x_{10}, y_{10}, z_{10}, x_{11}, y_{11}, z_{11}, x_{12}, y_{12}, z_{12}$

- The same compound appears also in a \#184 polytype. 


\section{Hexagonal primitive vectors:}

$$
\begin{array}{ll}
\mathbf{a}_{1}= & \frac{1}{2} a \hat{\mathbf{x}}-\frac{\sqrt{3}}{2} a \hat{\mathbf{y}} \\
\mathbf{a}_{2}= & \frac{1}{2} a \hat{\mathbf{x}}+\frac{\sqrt{3}}{2} a \hat{\mathbf{y}} \\
\mathbf{a}_{3}= & c \hat{\mathbf{z}}
\end{array}
$$

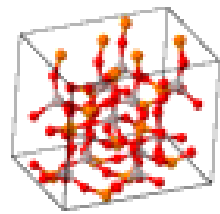

\section{Basis vectors:}

Lattice Coordinates

Cartesian Coordinates

$\mathbf{B}_{1}=$

$x_{1} \mathbf{a}_{1}+y_{1} \mathbf{a}_{2}+z_{1} \mathbf{a}_{3}$

$=\frac{1}{2}\left(x_{1}+y_{1}\right) a \hat{\mathbf{x}}+\frac{\sqrt{3}}{2}\left(-x_{1}+y_{1}\right) a \hat{\mathbf{y}}+$

$\mathbf{B}_{2}=-y_{1} \mathbf{a}_{1}+\left(x_{1}-y_{1}\right) \mathbf{a}_{2}+z_{1} \mathbf{a}_{3}$

$=\left(\frac{1}{2} x_{1}-y_{1}\right) a \hat{\mathbf{x}}+\frac{\sqrt{3}}{2} x_{1} a \hat{\mathbf{y}}+z_{1} c \hat{\mathbf{z}}$

$\mathbf{B}_{3}=\left(-x_{1}+y_{1}\right) \mathbf{a}_{1}-x_{1} \mathbf{a}_{2}+z_{1} \mathbf{a}_{3}$

$=\left(-x_{1}+\frac{1}{2} y_{1}\right) a \hat{\mathbf{x}}-\frac{\sqrt{3}}{2} y_{1} a \hat{\mathbf{y}}+z_{1} c \hat{\mathbf{z}}$

$\mathbf{B}_{4}=$

$-x_{1} \mathbf{a}_{1}-y_{1} \mathbf{a}_{2}+z_{1} \mathbf{a}_{3}$

$=-\frac{1}{2}\left(x_{1}+y_{1}\right) a \hat{\mathbf{x}}+\frac{\sqrt{3}}{2}\left(x_{1}-y_{1}\right) a \hat{\mathbf{y}}+$

$\mathbf{B}_{5}=y_{1} \mathbf{a}_{1}+\left(-x_{1}+y_{1}\right) \mathbf{a}_{2}+z_{1} \mathbf{a}_{3}$

$=$ $z_{1} c \hat{\mathbf{z}}$

$\mathbf{B}_{6}=\left(x_{1}-y_{1}\right) \mathbf{a}_{1}+x_{1} \mathbf{a}_{2}+z_{1} \mathbf{a}_{3}$

$=$

$x_{2} \mathbf{a}_{1}+y_{2} \mathbf{a}_{2}+z_{2} \mathbf{a}_{3}$

$\mathbf{B}_{7}=$

$$
x_{2} \mathbf{a}_{1}+y_{2} \mathbf{a}_{2}+z_{2} \mathbf{a}_{3}
$$

$\mathbf{B}_{8}=-y_{2} \mathbf{a}_{1}+\left(x_{2}-y_{2}\right) \mathbf{a}_{2}+z_{2} \mathbf{a}_{3}$

$\mathbf{B}_{9}=\left(-x_{2}+y_{2}\right) \mathbf{a}_{1}-x_{2} \mathbf{a}_{2}+z_{2} \mathbf{a}_{3}$

$\mathbf{B}_{10}=$

$$
-x_{2} \mathbf{a}_{1}-y_{2} \mathbf{a}_{2}+z_{2} \mathbf{a}_{3}
$$

$\mathbf{B}_{11}=y_{2} \mathbf{a}_{1}+\left(-x_{2}+y_{2}\right) \mathbf{a}_{2}+z_{2} \mathbf{a}_{3}$

$\mathbf{B}_{12}=\left(x_{2}-y_{2}\right) \mathbf{a}_{1}+x_{2} \mathbf{a}_{2}+z_{2} \mathbf{a}_{3}$

$\mathbf{B}_{13}=$

$$
x_{3} \mathbf{a}_{1}+y_{3} \mathbf{a}_{2}+z_{3} \mathbf{a}_{3}
$$

$\mathbf{B}_{14}=-y_{3} \mathbf{a}_{1}+\left(x_{3}-y_{3}\right) \mathbf{a}_{2}+z_{3} \mathbf{a}_{3}$

$\mathbf{B}_{15}=\left(-x_{3}+y_{3}\right) \mathbf{a}_{1}-x_{3} \mathbf{a}_{2}+z_{3} \mathbf{a}_{3}$

$\mathbf{B}_{16}=$

$-x_{3} \mathbf{a}_{1}-y_{3} \mathbf{a}_{2}+z_{3} \mathbf{a}_{3}$

$\mathbf{B}_{17}=y_{3} \mathbf{a}_{1}+\left(-x_{3}+y_{3}\right) \mathbf{a}_{2}+z_{3} \mathbf{a}_{3}$

$\mathbf{B}_{18}=\left(x_{3}-y_{3}\right) \mathbf{a}_{1}+x_{3} \mathbf{a}_{2}+z_{3} \mathbf{a}_{3}$

$\mathbf{B}_{19}=$

$$
x_{4} \mathbf{a}_{1}+y_{4} \mathbf{a}_{2}+z_{4} \mathbf{a}_{3}
$$

$\mathbf{B}_{20}=-y_{4} \mathbf{a}_{1}+\left(x_{4}-y_{4}\right) \mathbf{a}_{2}+z_{4} \mathbf{a}_{3}$

$\mathbf{B}_{21}=\left(-x_{4}+y_{4}\right) \mathbf{a}_{1}-x_{4} \mathbf{a}_{2}+z_{4} \mathbf{a}_{3}$

$\mathbf{B}_{22}=$

$$
-x_{4} \mathbf{a}_{1}-y_{4} \mathbf{a}_{2}+z_{4} \mathbf{a}_{3}
$$

$\mathbf{B}_{23}=y_{4} \mathbf{a}_{1}+\left(-x_{4}+y_{4}\right) \mathbf{a}_{2}+z_{4} \mathbf{a}_{3}$

$\mathbf{B}_{24}=\left(x_{4}-y_{4}\right) \mathbf{a}_{1}+x_{4} \mathbf{a}_{2}+z_{4} \mathbf{a}_{3}$

$$
=\frac{1}{2}\left(x_{2}+y_{2}\right) a \hat{\mathbf{x}}+\frac{\sqrt{3}}{2}\left(-x_{2}+y_{2}\right) a \hat{\mathbf{y}}+
$$$$
=\left(\frac{1}{2} x_{2}-y_{2}\right) a \hat{\mathbf{x}}+\frac{\sqrt{3}}{2} x_{2} a \hat{\mathbf{y}}+z_{2} c \hat{\mathbf{z}}
$$$$
=\left(-x_{2}+\frac{1}{2} y_{2}\right) a \hat{\mathbf{x}}-\frac{\sqrt{3}}{2} y_{2} a \hat{\mathbf{y}}+z_{2} c \hat{\mathbf{z}}
$$$$
=-\frac{1}{2}\left(x_{2}+y_{2}\right) a \hat{\mathbf{x}}+\frac{\sqrt{3}}{2}\left(x_{2}-y_{2}\right) a \hat{\mathbf{y}}+
$$$$
=\left(-\frac{1}{2} x_{2}+y_{2}\right) a \hat{\mathbf{x}}-\frac{\sqrt{3}}{2} x_{2} a \hat{\mathbf{y}}+z_{2} c \hat{\mathbf{z}}
$$$$
=\left(x_{2}-\frac{1}{2} y_{2}\right) a \hat{\mathbf{x}}+\frac{\sqrt{3}}{2} y_{2} a \hat{\mathbf{y}}+z_{2} c \hat{\mathbf{z}}
$$$$
=\frac{1}{2}\left(x_{3}+y_{3}\right) a \hat{\mathbf{x}}+\frac{\sqrt{3}}{2}\left(-x_{3}+y_{3}\right) a \hat{\mathbf{y}}+
$$

$$
z_{3} c \hat{\mathbf{z}}
$$$$
=\quad\left(\frac{1}{2} x_{3}-y_{3}\right) a \hat{\mathbf{x}}+\frac{\sqrt{3}}{2} x_{3} a \hat{\mathbf{y}}+z_{3} c \hat{\mathbf{z}}
$$$$
=\left(-x_{3}+\frac{1}{2} y_{3}\right) a \hat{\mathbf{x}}-\frac{\sqrt{3}}{2} y_{3} a \hat{\mathbf{y}}+z_{3} c \hat{\mathbf{z}}
$$$$
=-\frac{1}{2}\left(x_{3}+y_{3}\right) a \hat{\mathbf{x}}+\frac{\sqrt{3}}{2}\left(x_{3}-y_{3}\right) a \hat{\mathbf{y}}+
$$$$
=\left(-\frac{1}{2} x_{3}+y_{3}\right) a \hat{\mathbf{x}}-\frac{\sqrt{3}}{2} x_{3} a \hat{\mathbf{y}}+z_{3} c \hat{\mathbf{z}}
$$$$
=\left(x_{3}-\frac{1}{2} y_{3}\right) a \hat{\mathbf{x}}+\frac{\sqrt{3}}{2} y_{3} a \hat{\mathbf{y}}+z_{3} c \hat{\mathbf{z}}
$$$$
=\frac{1}{2}\left(x_{4}+y_{4}\right) a \hat{\mathbf{x}}+\frac{\sqrt{3}}{2}\left(-x_{4}+y_{4}\right) a \hat{\mathbf{y}}+
$$$$
=\left(\frac{1}{2} x_{4}-y_{4}\right) a \hat{\mathbf{x}}+\frac{\sqrt{3}}{2} x_{4} a \hat{\mathbf{y}}+z_{4} c \hat{\mathbf{z}}
$$$$
=\left(-x_{4}+\frac{1}{2} y_{4}\right) a \hat{\mathbf{x}}-\frac{\sqrt{3}}{2} y_{4} a \hat{\mathbf{y}}+z_{4} c \hat{\mathbf{z}}
$$$$
=-\frac{1}{2}\left(x_{4}+y_{4}\right) a \hat{\mathbf{x}}+\frac{\sqrt{3}}{2}\left(x_{4}-y_{4}\right) a \hat{\mathbf{y}}+
$$$$
=\left(-\frac{1}{2} x_{4}+y_{4}\right) a \hat{\mathbf{x}}-\frac{\sqrt{3}}{2} x_{4} a \hat{\mathbf{y}}+z_{4} c \hat{\mathbf{z}}
$$$$
=\left(x_{4}-\frac{1}{2} y_{4}\right) a \hat{\mathbf{x}}+\frac{\sqrt{3}}{2} y_{4} a \hat{\mathbf{y}}+z_{4} c \hat{\mathbf{z}}
$$

Wyckoff Position Atom Type

$(6 d)$

Al I
Al I

Al I

Al I

Al I

Al I

Al II

Al II

Al II

Al II

Al II

Al II

O I

O I

O I

O I

O I

O I

O II

O II

O II

O II

O II

O II 


\begin{tabular}{|c|c|c|c|c|c|}
\hline $\mathbf{B}_{25}$ & $=$ & $x_{5} \mathbf{a}_{1}+y_{5} \mathbf{a}_{2}+z_{5} \mathbf{a}_{3}$ & $=\frac{1}{2}\left(x_{5}+y_{5}\right) a \hat{\mathbf{x}}+\frac{\sqrt{3}}{2}\left(-x_{5}+y_{5}\right) a \hat{\mathbf{y}}+$ & $(6 d)$ & O III \\
\hline $\mathbf{B}_{26}$ & $=$ & $-y_{5} \mathbf{a}_{1}+\left(x_{5}-y_{5}\right) \mathbf{a}_{2}+z_{5} \mathbf{a}_{3}$ & $=\left(\frac{1}{2} x_{5}-y_{5}\right) a \hat{\mathbf{x}}+\frac{\sqrt{3}}{2} x_{5} a \hat{\mathbf{y}}+z_{5} c \hat{\mathbf{z}}$ & $(6 d)$ & O III \\
\hline $\mathbf{B}_{27}$ & $=$ & $\left(-x_{5}+y_{5}\right) \mathbf{a}_{1}-x_{5} \mathbf{a}_{2}+z_{5} \mathbf{a}_{3}$ & $=\left(-x_{5}+\frac{1}{2} y_{5}\right) a \hat{\mathbf{x}}-\frac{\sqrt{3}}{2} y_{5} a \hat{\mathbf{y}}+z_{5} c \hat{\mathbf{z}}$ & $(6 d)$ & O III \\
\hline $\mathbf{B}_{28}$ & $=$ & $-x_{5} \mathbf{a}_{1}-y_{5} \mathbf{a}_{2}+z_{5} \mathbf{a}_{3}$ & $=\frac{-\frac{1}{2}\left(x_{5}+y_{5}\right) a \hat{\mathbf{x}}+\frac{\sqrt{3}}{2}\left(x_{5}-y_{5}\right) a \hat{\mathbf{y}}+}{z_{5} c \hat{\mathbf{z}}}$ & $(6 d)$ & O III \\
\hline $\mathbf{B}_{29}$ & $=$ & $y_{5} \mathbf{a}_{1}+\left(-x_{5}+y_{5}\right) \mathbf{a}_{2}+z_{5} \mathbf{a}_{3}$ & $\left(-\frac{1}{2} x_{5}+y_{5}\right) a \hat{\mathbf{x}}-\frac{\sqrt{3}}{2} x_{5} a \hat{\mathbf{y}}+z_{5} c \hat{\mathbf{z}}$ & $(6 d)$ & O III \\
\hline $\mathbf{B}_{30}$ & $=$ & $\left(x_{5}-y_{5}\right) \mathbf{a}_{1}+x_{5} \mathbf{a}_{2}+z_{5} \mathbf{a}_{3}$ & $=\left(x_{5}-\frac{1}{2} y_{5}\right) a \hat{\mathbf{x}}+\frac{\sqrt{3}}{2} y_{5} a \hat{\mathbf{y}}+z_{5} c \hat{\mathbf{z}}$ & $(6 d)$ & O III \\
\hline $\mathbf{B}_{31}$ & $=$ & $x_{6} \mathbf{a}_{1}+y_{6} \mathbf{a}_{2}+z_{6} \mathbf{a}_{3}$ & 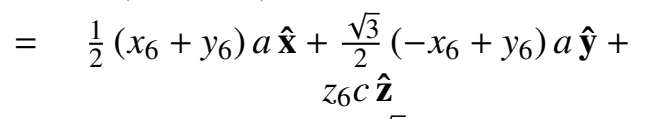 & $(6 d)$ & O IV \\
\hline $\mathbf{B}_{32}$ & $=$ & $-y_{6} \mathbf{a}_{1}+\left(x_{6}-y_{6}\right) \mathbf{a}_{2}+z_{6} \mathbf{a}_{3}$ & $=\left(\frac{1}{2} x_{6}-y_{6}\right) a \hat{\mathbf{x}}+\frac{\sqrt{3}}{2} x_{6} a \hat{\mathbf{y}}+z_{6} c \hat{\mathbf{z}}$ & $(6 d)$ & O IV \\
\hline $\mathbf{B}_{33}$ & $=$ & $\left(-x_{6}+y_{6}\right) \mathbf{a}_{1}-x_{6} \mathbf{a}_{2}+z_{6} \mathbf{a}_{3}$ & $=\left(-x_{6}+\frac{1}{2} y_{6}\right) a \hat{\mathbf{x}}-\frac{\sqrt{3}}{2} y_{6} a \hat{\mathbf{y}}+z_{6} c \hat{\mathbf{z}}$ & $(6 d)$ & O IV \\
\hline $\mathbf{B}_{34}$ & $=$ & $-x_{6} \mathbf{a}_{1}-y_{6} \mathbf{a}_{2}+z_{6} \mathbf{a}_{3}$ & $=\frac{-\frac{1}{2}\left(x_{6}+y_{6}\right) a \hat{\mathbf{x}}+\frac{\sqrt{3}}{2}\left(x_{6}-y_{6}\right) a \hat{\mathbf{y}}+}{z_{6} c \hat{\mathbf{z}}}$ & $(6 d)$ & O IV \\
\hline $\mathbf{B}_{35}$ & $=$ & $y_{6} \mathbf{a}_{1}+\left(-x_{6}+y_{6}\right) \mathbf{a}_{2}+z_{6} \mathbf{a}_{3}$ & $=\left(-\frac{1}{2} x_{6}+y_{6}\right) a \hat{\mathbf{x}}-\frac{\sqrt{3}}{2} x_{6} a \hat{\mathbf{y}}+z_{6} c \hat{\mathbf{z}}$ & $(6 d)$ & O IV \\
\hline $\mathbf{B}_{36}$ & $=$ & $\left(x_{6}-y_{6}\right) \mathbf{a}_{1}+x_{6} \mathbf{a}_{2}+z_{6} \mathbf{a}_{3}$ & $=\left(x_{6}-\frac{1}{2} y_{6}\right) a \hat{\mathbf{x}}+\frac{\sqrt{3}}{2} y_{6} a \hat{\mathbf{y}}+z_{6} c \hat{\mathbf{z}}$ & $(6 d)$ & O IV \\
\hline $\mathbf{B}_{37}$ & $=$ & $x_{7} \mathbf{a}_{1}+y_{7} \mathbf{a}_{2}+z_{7} \mathbf{a}_{3}$ & 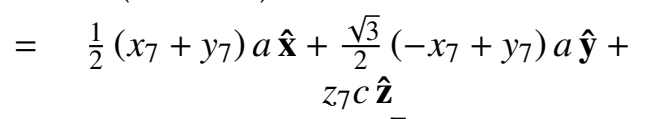 & $(6 d)$ & $\mathrm{OV}$ \\
\hline $\mathbf{B}_{38}$ & $=$ & $-y_{7} \mathbf{a}_{1}+\left(x_{7}-y_{7}\right) \mathbf{a}_{2}+z_{7} \mathbf{a}_{3}$ & $=\left(\frac{1}{2} x_{7}-y_{7}\right) a \hat{\mathbf{x}}+\frac{\sqrt{3}}{2} x_{7} a \hat{\mathbf{y}}+z_{7} c \hat{\mathbf{z}}$ & $(6 d)$ & $\mathrm{OV}$ \\
\hline $\mathbf{B}_{39}$ & $=$ & $\left(-x_{7}+y_{7}\right) \mathbf{a}_{1}-x_{7} \mathbf{a}_{2}+z_{7} \mathbf{a}_{3}$ & $=\left(-x_{7}+\frac{1}{2} y_{7}\right) a \hat{\mathbf{x}}-\frac{\sqrt{3}}{2} y_{7} a \hat{\mathbf{y}}+z_{7} c \hat{\mathbf{z}}$ & $(6 d)$ & $\mathrm{OV}$ \\
\hline $\mathbf{B}_{40}$ & $=$ & $-x_{7} \mathbf{a}_{1}-y_{7} \mathbf{a}_{2}+z_{7} \mathbf{a}_{3}$ & $=\frac{-\frac{1}{2}\left(x_{7}+y_{7}\right) a \hat{\mathbf{x}}+\frac{\sqrt{3}}{2}\left(x_{7}-y_{7}\right) a \hat{\mathbf{y}}+}{z_{7} c \hat{\mathbf{z}}}$ & $(6 d)$ & $\mathrm{OV}$ \\
\hline $\mathbf{B}_{41}$ & $=$ & $y_{7} \mathbf{a}_{1}+\left(-x_{7}+y_{7}\right) \mathbf{a}_{2}+z_{7} \mathbf{a}_{3}$ & $\left(-\frac{1}{2} x_{7}+y_{7}\right) a \hat{\mathbf{x}}-\frac{\sqrt{3}}{2} x_{7} a \hat{\mathbf{y}}+z_{7} c \hat{\mathbf{z}}$ & $(6 d)$ & $\mathrm{O} \mathrm{V}$ \\
\hline $\mathbf{B}_{42}$ & $=$ & $\left(x_{7}-y_{7}\right) \mathbf{a}_{1}+x_{7} \mathbf{a}_{2}+z_{7} \mathbf{a}_{3}$ & $=\left(x_{7}-\frac{1}{2} y_{7}\right) a \hat{\mathbf{x}}+\frac{\sqrt{3}}{2} y_{7} a \hat{\mathbf{y}}+z_{7} c \hat{\mathbf{z}}$ & $(6 d)$ & $\mathrm{OV}$ \\
\hline $\mathbf{B}_{43}$ & $=$ & $x_{8} \mathbf{a}_{1}+y_{8} \mathbf{a}_{2}+z_{8} \mathbf{a}_{3}$ & $\begin{array}{c}=\frac{1}{2}\left(x_{8}+y_{8}\right) a \hat{\mathbf{x}}+\frac{\sqrt{3}}{2}\left(-x_{8}+y_{8}\right) a \hat{\mathbf{y}}+ \\
z_{8} c \hat{\mathbf{z}}\end{array}$ & $(6 d)$ & O VI \\
\hline $\mathbf{B}_{44}$ & $=$ & $-y_{8} \mathbf{a}_{1}+\left(x_{8}-y_{8}\right) \mathbf{a}_{2}+z_{8} \mathbf{a}_{3}$ & $=\left(\frac{1}{2} x_{8}-y_{8}\right) a \hat{\mathbf{x}}+\frac{\sqrt{3}}{2} x_{8} a \hat{\mathbf{y}}+z_{8} c \hat{\mathbf{z}}$ & $(6 d)$ & O VI \\
\hline $\mathbf{B}_{45}$ & $=$ & $\left(-x_{8}+y_{8}\right) \mathbf{a}_{1}-x_{8} \mathbf{a}_{2}+z_{8} \mathbf{a}_{3}$ & $=\left(-x_{8}+\frac{1}{2} y_{8}\right) a \hat{\mathbf{x}}-\frac{\sqrt{3}}{2} y_{8} a \hat{\mathbf{y}}+z_{8} c \hat{\mathbf{z}}$ & $(6 d)$ & O VI \\
\hline $\mathbf{B}_{46}$ & $=$ & $-x_{8} \mathbf{a}_{1}-y_{8} \mathbf{a}_{2}+z_{8} \mathbf{a}_{3}$ & $=\frac{-\frac{1}{2}\left(x_{8}+y_{8}\right) a \hat{\mathbf{x}}+\frac{\sqrt{3}}{2}\left(x_{8}-y_{8}\right) a \hat{\mathbf{y}}+}{z_{8} c \hat{\mathbf{z}}}$ & $(6 d)$ & O VI \\
\hline $\mathbf{B}_{47}$ & $=$ & $y_{8} \mathbf{a}_{1}+\left(-x_{8}+y_{8}\right) \mathbf{a}_{2}+z_{8} \mathbf{a}_{3}$ & $\left(-\frac{1}{2} x_{8}+y_{8}\right) a \hat{\mathbf{x}}-\frac{\sqrt{3}}{2} x_{8} a \hat{\mathbf{y}}+z_{8} c \hat{\mathbf{z}}$ & $(6 d)$ & O VI \\
\hline $\mathbf{B}_{48}$ & $=$ & $\left(x_{8}-y_{8}\right) \mathbf{a}_{1}+x_{8} \mathbf{a}_{2}+z_{8} \mathbf{a}_{3}$ & $=\left(x_{8}-\frac{1}{2} y_{8}\right) a \hat{\mathbf{x}}+\frac{\sqrt{3}}{2} y_{8} a \hat{\mathbf{y}}+z_{8} c \hat{\mathbf{z}}$ & $(6 d)$ & $\mathrm{O} \mathrm{VI}$ \\
\hline $\mathbf{B}_{49}$ & $=$ & $x_{9} \mathbf{a}_{1}+y_{9} \mathbf{a}_{2}+z_{9} \mathbf{a}_{3}$ & $=\begin{array}{c}\frac{1}{2}\left(x_{9}+y_{9}\right) a \hat{\mathbf{x}}+\frac{\sqrt{3}}{2}\left(-x_{9}+y_{9}\right) a \hat{\mathbf{y}}+ \\
z_{9} c \hat{\mathbf{z}}\end{array}$ & $(6 d)$ & O VII \\
\hline $\mathbf{B}_{50}$ & $=$ & $-y_{9} \mathbf{a}_{1}+\left(x_{9}-y_{9}\right) \mathbf{a}_{2}+z_{9} \mathbf{a}_{3}$ & $=\left(\frac{1}{2} x_{9}-y_{9}\right) a \hat{\mathbf{x}}+\frac{\sqrt{3}}{2} x_{9} a \hat{\mathbf{y}}+z_{9} c \hat{\mathbf{z}}$ & $(6 d)$ & O VII \\
\hline $\mathbf{B}_{51}$ & $=$ & $\left(-x_{9}+y_{9}\right) \mathbf{a}_{1}-x_{9} \mathbf{a}_{2}+z_{9} \mathbf{a}_{3}$ & $=\left(-x_{9}+\frac{1}{2} y_{9}\right) a \hat{\mathbf{x}}-\frac{\sqrt{3}}{2} y_{9} a \hat{\mathbf{y}}+z_{9} c \hat{\mathbf{z}}$ & $(6 d)$ & O VII \\
\hline $\mathbf{B}_{52}$ & $=$ & $-x_{9} \mathbf{a}_{1}-y_{9} \mathbf{a}_{2}+z_{9} \mathbf{a}_{3}$ & $=\frac{-\frac{1}{2}\left(x_{9}+y_{9}\right) a \hat{\mathbf{x}}+\frac{\sqrt{3}}{2}\left(x_{9}-y_{9}\right) a \hat{\mathbf{y}}+}{z_{9} c \hat{\mathbf{z}}}$ & $(6 d)$ & O VII \\
\hline $\mathbf{B}_{53}$ & $=$ & $y_{9} \mathbf{a}_{1}+\left(-x_{9}+y_{9}\right) \mathbf{a}_{2}+z_{9} \mathbf{a}_{3}$ & $\left(-\frac{1}{2} x_{9}+y_{9}\right) a \hat{\mathbf{x}}-\frac{\sqrt{3}}{2} x_{9} a \hat{\mathbf{y}}+z_{9} c \hat{\mathbf{z}}$ & $(6 d)$ & O VII \\
\hline $\mathbf{B}_{54}$ & $=$ & $\left(x_{9}-y_{9}\right) \mathbf{a}_{1}+x_{9} \mathbf{a}_{2}+z_{9} \mathbf{a}_{3}$ & $\left(x_{9}-\frac{1}{2} y_{9}\right) a \hat{\mathbf{x}}+\frac{\sqrt{3}}{2} y_{9} a \hat{\mathbf{y}}+z_{9} c \hat{\mathbf{z}}$ & $(6 d)$ & O VII \\
\hline $\mathbf{B}_{55}$ & $=$ & $x_{10} \mathbf{a}_{1}+y_{10} \mathbf{a}_{2}+z_{10} \mathbf{a}_{3}$ & $\begin{array}{c}\frac{1}{2}\left(x_{10}+y_{10}\right) a \hat{\mathbf{x}}+ \\
\frac{\sqrt{3}}{2}\left(-x_{10}+y_{10}\right) a \hat{\mathbf{y}}+z_{10} c \hat{\mathbf{z}}\end{array}$ & $(6 d)$ & O VIII \\
\hline
\end{tabular}




$$
\begin{aligned}
& \mathbf{B}_{56}=-y_{10} \mathbf{a}_{1}+\left(x_{10}-y_{10}\right) \mathbf{a}_{2}+z_{10} \mathbf{a}_{3}=\left(\frac{1}{2} x_{10}-y_{10}\right) a \hat{\mathbf{x}}+\frac{\sqrt{3}}{2} x_{10} a \hat{\mathbf{y}}+z_{10} c \hat{\mathbf{z}} \\
& \mathbf{B}_{57}=\left(-x_{10}+y_{10}\right) \mathbf{a}_{1}-x_{10} \mathbf{a}_{2}+z_{10} \mathbf{a}_{3}=\left(-x_{10}+\frac{1}{2} y_{10}\right) a \hat{\mathbf{x}}-\frac{\sqrt{3}}{2} y_{10} a \hat{\mathbf{y}}+z_{10} c \hat{\mathbf{z}} \\
& \text { O VIII } \\
& \mathbf{B}_{58}=-x_{10} \mathbf{a}_{1}-y_{10} \mathbf{a}_{2}+z_{10} \mathbf{a}_{3} \quad=\quad-\frac{1}{2}\left(x_{10}+y_{10}\right) a \hat{\mathbf{x}}+ \\
& \frac{\sqrt{3}}{2}\left(x_{10}-y_{10}\right) a \hat{\mathbf{y}}+z_{10} c \hat{\mathbf{z}} \\
& \mathbf{B}_{59}=y_{10} \mathbf{a}_{1}+\left(-x_{10}+y_{10}\right) \mathbf{a}_{2}+z_{10} \mathbf{a}_{3}=\left(-\frac{1}{2} x_{10}+y_{10}\right) a \hat{\mathbf{x}}-\frac{\sqrt{3}}{2} x_{10} a \hat{\mathbf{y}}+z_{10} c \hat{\mathbf{z}} \\
& \text { O VIII } \\
& \mathbf{B}_{60}=\left(x_{10}-y_{10}\right) \mathbf{a}_{1}+x_{10} \mathbf{a}_{2}+z_{10} \mathbf{a}_{3}=\left(x_{10}-\frac{1}{2} y_{10}\right) a \hat{\mathbf{x}}+\frac{\sqrt{3}}{2} y_{10} a \hat{\mathbf{y}}+z_{10} c \hat{\mathbf{z}} \\
& \mathbf{B}_{61}=x_{11} \mathbf{a}_{1}+y_{11} \mathbf{a}_{2}+z_{11} \mathbf{a}_{3}= \\
& \frac{1}{2}\left(x_{11}+y_{11}\right) a \hat{\mathbf{x}}+ \\
& \frac{\sqrt{3}}{2}\left(-x_{11}+y_{11}\right) a \hat{\mathbf{y}}+z_{11} c \hat{\mathbf{z}} \\
& \mathbf{B}_{62}=-y_{11} \mathbf{a}_{1}+\left(x_{11}-y_{11}\right) \mathbf{a}_{2}+z_{11} \mathbf{a}_{3}=\left(\frac{1}{2} x_{11}-y_{11}\right) a \hat{\mathbf{x}}+\frac{\sqrt{3}}{2} x_{11} a \hat{\mathbf{y}}+z_{11} c \hat{\mathbf{z}} \\
& \text { O VIII } \\
& \text { P I } \\
& \mathbf{B}_{63}=\left(-x_{11}+y_{11}\right) \mathbf{a}_{1}-x_{11} \mathbf{a}_{2}+z_{11} \mathbf{a}_{3}=\left(-x_{11}+\frac{1}{2} y_{11}\right) a \hat{\mathbf{x}}-\frac{\sqrt{3}}{2} y_{11} a \hat{\mathbf{y}}+z_{11} c \hat{\mathbf{z}} \\
& \mathbf{B}_{64}=-x_{11} \mathbf{a}_{1}-y_{11} \mathbf{a}_{2}+z_{11} \mathbf{a}_{3} \quad=\quad-\frac{1}{2}\left(x_{11}+y_{11}\right) a \hat{\mathbf{x}}+ \\
& \frac{\sqrt{3}}{2}\left(x_{11}-y_{11}\right) a \hat{\mathbf{y}}+z_{11} c \hat{\mathbf{z}} \\
& \mathbf{B}_{65}=y_{11} \mathbf{a}_{1}+\left(-x_{11}+y_{11}\right) \mathbf{a}_{2}+z_{11} \mathbf{a}_{3}=\left(-\frac{1}{2} x_{11}+y_{11}\right) a \hat{\mathbf{x}}-\frac{\sqrt{3}}{2} x_{11} a \hat{\mathbf{y}}+z_{11} c \hat{\mathbf{z}} \quad(6 d) \\
& \text { P I } \\
& \mathbf{B}_{66}=\left(x_{11}-y_{11}\right) \mathbf{a}_{1}+x_{11} \mathbf{a}_{2}+z_{11} \mathbf{a}_{3}=\left(x_{11}-\frac{1}{2} y_{11}\right) a \hat{\mathbf{x}}+\frac{\sqrt{3}}{2} y_{11} a \hat{\mathbf{y}}+z_{11} c \hat{\mathbf{z}} \\
& \mathbf{B}_{67}=x_{12} \mathbf{a}_{1}+y_{12} \mathbf{a}_{2}+z_{12} \mathbf{a}_{3}= \\
& =\quad \frac{1}{2}\left(x_{12}+y_{12}\right) a \hat{\mathbf{x}}+ \\
& \frac{\sqrt{3}}{2}\left(-x_{12}+y_{12}\right) a \hat{\mathbf{y}}+z_{12} c \hat{\mathbf{z}} \\
& \mathbf{B}_{68}=-y_{12} \mathbf{a}_{1}+\left(x_{12}-y_{12}\right) \mathbf{a}_{2}+z_{12} \mathbf{a}_{3}=\left(\frac{1}{2} x_{12}-y_{12}\right) a \hat{\mathbf{x}}+\frac{\sqrt{3}}{2} x_{12} a \hat{\mathbf{y}}+z_{12} c \hat{\mathbf{z}} \\
& \mathbf{B}_{69}=\left(-x_{12}+y_{12}\right) \mathbf{a}_{1}-x_{12} \mathbf{a}_{2}+z_{12} \mathbf{a}_{3}=\left(-x_{12}+\frac{1}{2} y_{12}\right) a \hat{\mathbf{x}}-\frac{\sqrt{3}}{2} y_{12} a \hat{\mathbf{y}}+z_{12} c \hat{\mathbf{z}} \\
& \text { P II } \\
& \mathbf{B}_{70}=-x_{12} \mathbf{a}_{1}-y_{12} \mathbf{a}_{2}+z_{12} \mathbf{a}_{3} \quad=\quad-\frac{1}{2}\left(x_{12}+y_{12}\right) a \hat{\mathbf{x}}+ \\
& \mathbf{B}_{71}=y_{12} \mathbf{a}_{1}+\left(-x_{12}+y_{12}\right) \mathbf{a}_{2}+z_{12} \mathbf{a}_{3}=\left(-\frac{1}{2} x_{12}+y_{12}\right) a \hat{\mathbf{x}}-\frac{\sqrt{3}}{2} x_{12} a \hat{\mathbf{y}}+z_{12} c \hat{\mathbf{z}} \\
& \mathbf{B}_{72}=\left(x_{12}-y_{12}\right) \mathbf{a}_{1}+x_{12} \mathbf{a}_{2}+z_{12} \mathbf{a}_{3}=\left(x_{12}-\frac{1}{2} y_{12}\right) a \hat{\mathbf{x}}+\frac{\sqrt{3}}{2} y_{12} a \hat{\mathbf{y}}+z_{12} c \hat{\mathbf{z}}
\end{aligned}
$$

\section{References:}

- J. W. Richardson Jr., J. J. Pluth, and J. V. Smith, Aluminophosphate number 5: time-of-flight neutron powder diffraction study of calcined powder at 295 K, Acta Crystallogr. C 43, 1469-1472 (1987), doi:10.1107/S0108270187091418.

\section{Found in:}

- P. Villars and K. Cenzual, Pearson's Crystal Data - Crystal Structure Database for Inorganic Compounds, ASM International (2013).

\section{Geometry files:}

- CIF: pp. 922

- POSCAR: pp. 923 


\section{$\alpha-\mathrm{Al}_{2} \mathrm{~S}_{3}$ Structure: A2B3_hP30_169_2a_3a}
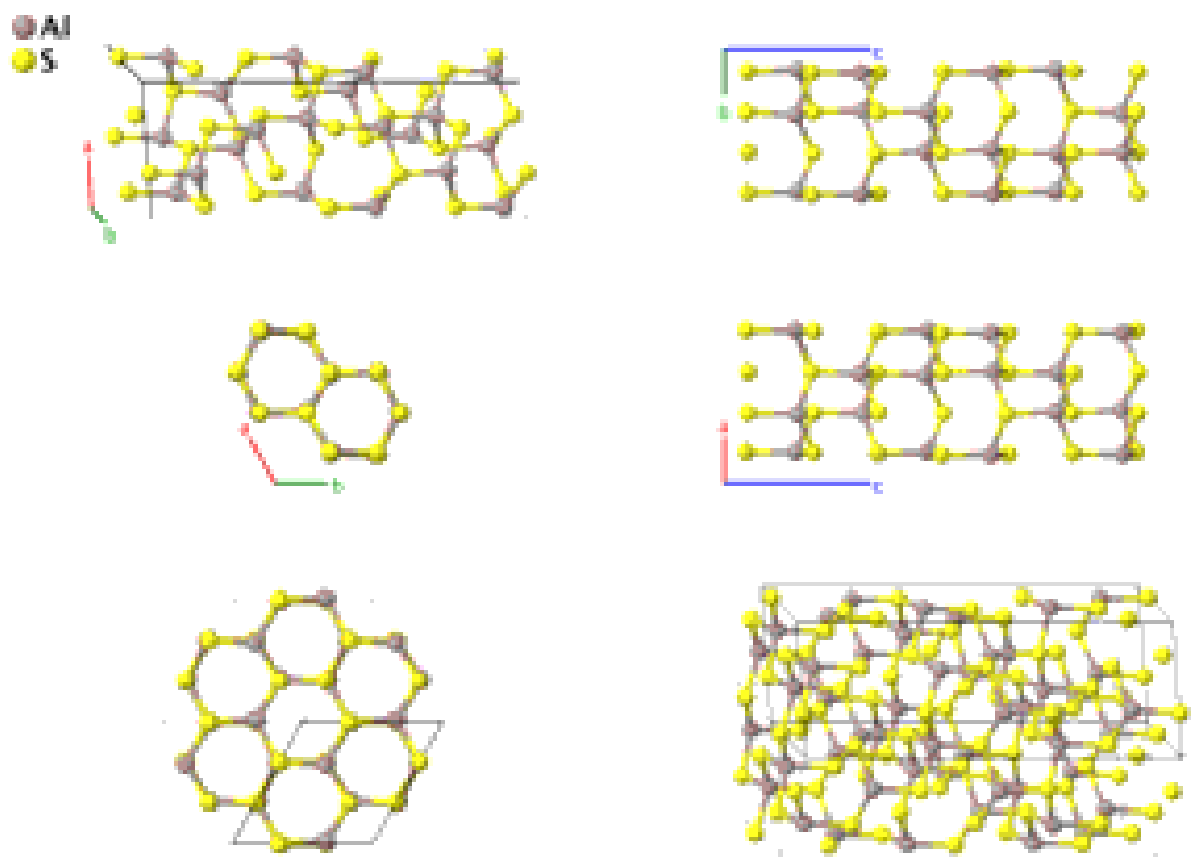

\section{Prototype}

AFLOW prototype label

Strukturbericht designation

Pearson symbol

Space group number

Space group symbol

AFLOW prototype command : aflow --proto=A2B3_hP30_169_2a_3a

- -params $=a, c / a, x_{1}, y_{1}, z_{1}, x_{2}, y_{2}, z_{2}, x_{3}, y_{3}, z_{3}, x_{4}, y_{4}, z_{4}, x_{5}, y_{5}, z_{5}$

\section{Hexagonal primitive vectors:}

$$
\begin{array}{ll}
\mathbf{a}_{1}= & \frac{1}{2} a \hat{\mathbf{x}}-\frac{\sqrt{3}}{2} a \hat{\mathbf{y}} \\
\mathbf{a}_{2}= & \frac{1}{2} a \hat{\mathbf{x}}+\frac{\sqrt{3}}{2} a \hat{\mathbf{y}} \\
\mathbf{a}_{3}= & c \hat{\mathbf{z}}
\end{array}
$$

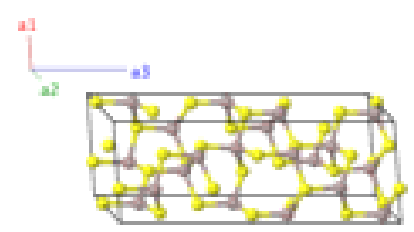

Basis vectors:

\section{Lattice Coordinates}

$\mathbf{B}_{1}=$

$x_{1} \mathbf{a}_{1}+y_{1} \mathbf{a}_{2}+z_{1} \mathbf{a}_{3}$

$$
=
$$

Cartesian Coordinates

$$
\begin{array}{ccc}
\mathbf{B}_{2}=-y_{1} \mathbf{a}_{1}+\left(x_{1}-y_{1}\right) \mathbf{a}_{2}+\left(\frac{1}{3}+z_{1}\right) \mathbf{a}_{3}= & \left(\frac{1}{2} x_{1}-y_{1}\right) a \hat{\mathbf{x}}+\frac{\sqrt{3}}{2} x_{1} a \hat{\mathbf{y}}+ \\
\mathbf{B}_{3}=\left(-x_{1}+y_{1}\right) \mathbf{a}_{1}-x_{1} \mathbf{a}_{2}+\left(\frac{2}{3}+z_{1}\right) \mathbf{a}_{3}= & \left(-x_{1}+\frac{1}{2} y_{1}\right) a \hat{\mathbf{x}}-\frac{\sqrt{3}}{2} y_{1} a \hat{\mathbf{y}}+ \\
\left(\frac{2}{3}+z_{1}\right) c \hat{\mathbf{z}}
\end{array}
$$

Wyckoff Position Atom Type

(6a)

Al I

(6a)

Al I

$(6 a)$

Al I 


\begin{tabular}{|c|c|c|c|c|c|c|}
\hline $\mathbf{B}_{4}$ & $=$ & $-x_{1} \mathbf{a}_{1}-y_{1} \mathbf{a}_{2}+\left(\frac{1}{2}+z_{1}\right) \mathbf{a}_{3}$ & $=$ & $\begin{array}{c}-\frac{1}{2}\left(x_{1}+y_{1}\right) a \hat{\mathbf{x}}+ \\
\frac{\sqrt{3}}{2}\left(x_{1}-y_{1}\right) a \hat{\mathbf{y}}+\left(\frac{1}{2}+z_{1}\right) c \hat{\mathbf{z}}\end{array}$ & $(6 a)$ & $\mathrm{Al} \mathrm{I}$ \\
\hline $\mathbf{B}_{5}$ & $=$ & $y_{1} \mathbf{a}_{1}+\left(-x_{1}+y_{1}\right) \mathbf{a}_{2}+\left(\frac{5}{6}+z_{1}\right) \mathbf{a}_{3}$ & $=$ & $\begin{array}{c}\left(-\frac{1}{2} x_{1}+y_{1}\right) a \hat{\mathbf{x}}-\frac{\sqrt{3}}{2} x_{1} a \hat{\mathbf{y}}+ \\
\left(\frac{5}{6}+z_{1}\right) c \hat{\mathbf{z}}\end{array}$ & $(6 a)$ & $\mathrm{Al} \mathrm{I}$ \\
\hline $\mathbf{B}_{6}$ & $=$ & $\left(x_{1}-y_{1}\right) \mathbf{a}_{1}+x_{1} \mathbf{a}_{2}+\left(\frac{1}{6}+z_{1}\right) \mathbf{a}_{3}$ & $=$ & $\begin{array}{c}\left(x_{1}-\frac{1}{2} y_{1}\right) a \hat{\mathbf{x}}+\frac{\sqrt{3}}{2} y_{1} a \hat{\mathbf{y}}+ \\
\left(\frac{1}{6}+z_{1}\right) c \hat{\mathbf{z}}\end{array}$ & $(6 a)$ & $\mathrm{Al} \mathrm{I}$ \\
\hline $\mathbf{B}_{7}$ & $=$ & $x_{2} \mathbf{a}_{1}+y_{2} \mathbf{a}_{2}+z_{2} \mathbf{a}_{3}$ & $=$ & $\begin{array}{c}\frac{1}{2}\left(x_{2}+y_{2}\right) a \hat{\mathbf{x}}+ \\
\frac{\sqrt{3}}{2}\left(-x_{2}+y_{2}\right) a \hat{\mathbf{y}}+z_{2} c \hat{\mathbf{z}}\end{array}$ & $(6 a)$ & $\mathrm{Al} \mathrm{II}$ \\
\hline $\mathbf{B}_{8}$ & $=$ & $-y_{2} \mathbf{a}_{1}+\left(x_{2}-y_{2}\right) \mathbf{a}_{2}+\left(\frac{1}{3}+z_{2}\right) \mathbf{a}_{3}$ & $=$ & $\begin{array}{c}\left(\frac{1}{2} x_{2}-y_{2}\right) a \hat{\mathbf{x}}+\frac{\sqrt{3}}{2} x_{2} a \hat{\mathbf{y}}+ \\
\left(\frac{1}{3}+z_{2}\right) c \hat{\mathbf{z}}\end{array}$ & $(6 a)$ & $\mathrm{Al}$ II \\
\hline $\mathbf{B}_{9}$ & $=$ & $\left(-x_{2}+y_{2}\right) \mathbf{a}_{1}-x_{2} \mathbf{a}_{2}+\left(\frac{2}{3}+z_{2}\right) \mathbf{a}_{3}$ & $=$ & $\begin{array}{c}\left(-x_{2}+\frac{1}{2} y_{2}\right) a \hat{\mathbf{x}}-\frac{\sqrt{3}}{2} y_{2} a \hat{\mathbf{y}}+ \\
\left(\frac{2}{3}+z_{2}\right) c \hat{\mathbf{z}}\end{array}$ & $(6 a)$ & $\mathrm{Al}$ II \\
\hline $\mathbf{B}_{10}$ & $=$ & $-x_{2} \mathbf{a}_{1}-y_{2} \mathbf{a}_{2}+\left(\frac{1}{2}+z_{2}\right) \mathbf{a}_{3}$ & $=$ & $\begin{array}{c}-\frac{1}{2}\left(x_{2}+y_{2}\right) a \hat{\mathbf{x}}+ \\
\frac{\sqrt{3}}{2}\left(x_{2}-y_{2}\right) a \hat{\mathbf{y}}+\left(\frac{1}{2}+z_{2}\right) c \hat{\mathbf{z}}\end{array}$ & $(6 a)$ & $\mathrm{Al}$ II \\
\hline $\mathbf{B}_{11}$ & $=$ & $y_{2} \mathbf{a}_{1}+\left(-x_{2}+y_{2}\right) \mathbf{a}_{2}+\left(\frac{5}{6}+z_{2}\right) \mathbf{a}_{3}$ & $=$ & $\begin{array}{c}\left(-\frac{1}{2} x_{2}+y_{2}\right) a \hat{\mathbf{x}}-\frac{\sqrt{3}}{2} x_{2} a \hat{\mathbf{y}}+ \\
\left(\frac{5}{6}+z_{2}\right) c \hat{\mathbf{z}}\end{array}$ & $(6 a)$ & $\mathrm{Al}$ II \\
\hline $\mathbf{B}_{12}$ & $=$ & $\left(x_{2}-y_{2}\right) \mathbf{a}_{1}+x_{2} \mathbf{a}_{2}+\left(\frac{1}{6}+z_{2}\right) \mathbf{a}_{3}$ & $=$ & $\begin{array}{c}\left(x_{2}-\frac{1}{2} y_{2}\right) a \hat{\mathbf{x}}+\frac{\sqrt{3}}{2} y_{2} a \hat{\mathbf{y}}+ \\
\left(\frac{1}{6}+z_{2}\right) c \hat{\mathbf{z}}\end{array}$ & $(6 a)$ & $\mathrm{Al}$ II \\
\hline $\mathbf{B}_{13}$ & $=$ & $x_{3} \mathbf{a}_{1}+y_{3} \mathbf{a}_{2}+z_{3} \mathbf{a}_{3}$ & $=$ & $\begin{array}{c}\frac{1}{2}\left(x_{3}+y_{3}\right) a \hat{\mathbf{x}}+ \\
\frac{\sqrt{3}}{2}\left(-x_{3}+y_{3}\right) a \hat{\mathbf{y}}+z_{3} c \hat{\mathbf{z}}\end{array}$ & $(6 a)$ & S I \\
\hline $\mathbf{B}_{14}$ & $=$ & $-y_{3} \mathbf{a}_{1}+\left(x_{3}-y_{3}\right) \mathbf{a}_{2}+\left(\frac{1}{3}+z_{3}\right) \mathbf{a}_{3}$ & $=$ & $\begin{array}{c}\left(\frac{1}{2} x_{3}-y_{3}\right) a \hat{\mathbf{x}}+\frac{\sqrt{3}}{2} x_{3} a \hat{\mathbf{y}}+ \\
\left(\frac{1}{3}+z_{3}\right) c \hat{\mathbf{z}}\end{array}$ & $(6 a)$ & S I \\
\hline $\mathbf{B}_{15}$ & $=$ & $\left(-x_{3}+y_{3}\right) \mathbf{a}_{1}-x_{3} \mathbf{a}_{2}+\left(\frac{2}{3}+z_{3}\right) \mathbf{a}_{3}$ & $=$ & $\begin{array}{c}\left(-x_{3}+\frac{1}{2} y_{3}\right) a \hat{\mathbf{x}}-\frac{\sqrt{3}}{2} y_{3} a \hat{\mathbf{y}}+ \\
\left(\frac{2}{3}+z_{3}\right) c \hat{\mathbf{z}}\end{array}$ & $(6 a)$ & S I \\
\hline $\mathbf{B}_{16}$ & $=$ & $-x_{3} \mathbf{a}_{1}-y_{3} \mathbf{a}_{2}+\left(\frac{1}{2}+z_{3}\right) \mathbf{a}_{3}$ & $=$ & $\begin{array}{c}-\frac{1}{2}\left(x_{3}+y_{3}\right) a \hat{\mathbf{x}}+ \\
\frac{\sqrt{3}}{2}\left(x_{3}-y_{3}\right) a \hat{\mathbf{y}}+\left(\frac{1}{2}+z_{3}\right) c \hat{\mathbf{z}}\end{array}$ & $(6 a)$ & S I \\
\hline $\mathbf{B}_{17}$ & $=$ & $y_{3} \mathbf{a}_{1}+\left(-x_{3}+y_{3}\right) \mathbf{a}_{2}+\left(\frac{5}{6}+z_{3}\right) \mathbf{a}_{3}$ & $=$ & $\begin{array}{c}\left(-\frac{1}{2} x_{3}+y_{3}\right) a \hat{\mathbf{x}}-\frac{\sqrt{3}}{2} x_{3} a \hat{\mathbf{y}}+ \\
\left(\frac{5}{6}+z_{3}\right) c \hat{\mathbf{z}}\end{array}$ & $(6 a)$ & S I \\
\hline $\mathbf{B}_{18}$ & $=$ & $\left(x_{3}-y_{3}\right) \mathbf{a}_{1}+x_{3} \mathbf{a}_{2}+\left(\frac{1}{6}+z_{3}\right) \mathbf{a}_{3}$ & $=$ & $\begin{array}{c}\left(x_{3}-\frac{1}{2} y_{3}\right) a \hat{\mathbf{x}}+\frac{\sqrt{3}}{2} y_{3} a \hat{\mathbf{y}}+ \\
\left(\frac{1}{6}+z_{3}\right) c \hat{\mathbf{z}}\end{array}$ & $(6 a)$ & S I \\
\hline $\mathbf{B}_{19}$ & $=$ & $x_{4} \mathbf{a}_{1}+y_{4} \mathbf{a}_{2}+z_{4} \mathbf{a}_{3}$ & $=$ & $\begin{array}{c}\frac{1}{2}\left(x_{4}+y_{4}\right) a \hat{\mathbf{x}}+ \\
\frac{\sqrt{3}}{2}\left(-x_{4}+y_{4}\right) a \hat{\mathbf{y}}+z_{4} c \hat{\mathbf{z}}\end{array}$ & $(6 a)$ & S II \\
\hline $\mathbf{B}_{20}$ & $=$ & $-y_{4} \mathbf{a}_{1}+\left(x_{4}-y_{4}\right) \mathbf{a}_{2}+\left(\frac{1}{3}+z_{4}\right) \mathbf{a}_{3}$ & $=$ & $\begin{array}{c}\left(\frac{1}{2} x_{4}-y_{4}\right) a \hat{\mathbf{x}}+\frac{\sqrt{3}}{2} x_{4} a \hat{\mathbf{y}}+ \\
\left(\frac{1}{3}+z_{4}\right) c \hat{\mathbf{z}}\end{array}$ & $(6 a)$ & S II \\
\hline $\mathbf{B}_{21}$ & $=$ & $\left(-x_{4}+y_{4}\right) \mathbf{a}_{1}-x_{4} \mathbf{a}_{2}+\left(\frac{2}{3}+z_{4}\right) \mathbf{a}_{3}$ & $=$ & $\begin{array}{c}\left(-x_{4}+\frac{1}{2} y_{4}\right) a \hat{\mathbf{x}}-\frac{\sqrt{3}}{2} y_{4} a \hat{\mathbf{y}}+ \\
\left(\frac{2}{3}+z_{4}\right) c \hat{\mathbf{z}}\end{array}$ & $(6 a)$ & S II \\
\hline $\mathbf{B}_{22}$ & $=$ & $-x_{4} \mathbf{a}_{1}-y_{4} \mathbf{a}_{2}+\left(\frac{1}{2}+z_{4}\right) \mathbf{a}_{3}$ & $=$ & $\begin{array}{c}-\frac{1}{2}\left(x_{4}+y_{4}\right) a \hat{\mathbf{x}}+ \\
\frac{\sqrt{3}}{2}\left(x_{4}-y_{4}\right) a \hat{\mathbf{y}}+\left(\frac{1}{2}+z_{4}\right) c \hat{\mathbf{z}}\end{array}$ & $(6 a)$ & S II \\
\hline $\mathbf{B}_{23}$ & $=$ & $y_{4} \mathbf{a}_{1}+\left(-x_{4}+y_{4}\right) \mathbf{a}_{2}+\left(\frac{5}{6}+z_{4}\right) \mathbf{a}_{3}$ & $=$ & $\begin{array}{c}\left(-\frac{1}{2} x_{4}+y_{4}\right) a \hat{\mathbf{x}}-\frac{\sqrt{3}}{2} x_{4} a \hat{\mathbf{y}}+ \\
\left(\frac{5}{6}+z_{4}\right) c \hat{\mathbf{z}}\end{array}$ & $(6 a)$ & S II \\
\hline
\end{tabular}




\begin{tabular}{|c|c|c|c|c|c|c|}
\hline $\mathbf{B}_{24}$ & $=$ & $\left(x_{4}-y_{4}\right) \mathbf{a}_{1}+x_{4} \mathbf{a}_{2}+\left(\frac{1}{6}+z_{4}\right) \mathbf{a}_{3}$ & $=$ & $\begin{array}{c}\left(x_{4}-\frac{1}{2} y_{4}\right) a \hat{\mathbf{x}}+\frac{\sqrt{3}}{2} y_{4} a \hat{\mathbf{y}}+ \\
\left(\frac{1}{6}+z_{4}\right) c \hat{\mathbf{z}}\end{array}$ & $(6 a)$ & S II \\
\hline $\mathbf{B}_{25}$ & $=$ & $x_{5} \mathbf{a}_{1}+y_{5} \mathbf{a}_{2}+z_{5} \mathbf{a}_{3}$ & $=$ & $\begin{array}{c}\frac{1}{2}\left(x_{5}+y_{5}\right) a \hat{\mathbf{x}}+ \\
\frac{\sqrt{3}}{2}\left(-x_{5}+y_{5}\right) a \hat{\mathbf{y}}+z_{5} c \hat{\mathbf{z}}\end{array}$ & $(6 a)$ & S III \\
\hline $\mathbf{B}_{26}$ & $=$ & $-y_{5} \mathbf{a}_{1}+\left(x_{5}-y_{5}\right) \mathbf{a}_{2}+\left(\frac{1}{3}+z_{5}\right) \mathbf{a}_{3}$ & $=$ & $\begin{array}{c}\left(\frac{1}{2} x_{5}-y_{5}\right) a \hat{\mathbf{x}}+\frac{\sqrt{3}}{2} x_{5} a \hat{\mathbf{y}}+ \\
\left(\frac{1}{3}+z_{5}\right) c \hat{\mathbf{z}}\end{array}$ & $(6 a)$ & S III \\
\hline $\mathbf{B}_{27}$ & $=$ & $\left(-x_{5}+y_{5}\right) \mathbf{a}_{1}-x_{5} \mathbf{a}_{2}+\left(\frac{2}{3}+z_{5}\right) \mathbf{a}_{3}$ & $=$ & $\begin{array}{c}\left(-x_{5}+\frac{1}{2} y_{5}\right) a \hat{\mathbf{x}}-\frac{\sqrt{3}}{2} y_{5} a \hat{\mathbf{y}}+ \\
\left(\frac{2}{3}+z_{5}\right) c \hat{\mathbf{z}}\end{array}$ & $(6 a)$ & S III \\
\hline $\mathbf{B}_{28}$ & $=$ & $-x_{5} \mathbf{a}_{1}-y_{5} \mathbf{a}_{2}+\left(\frac{1}{2}+z_{5}\right) \mathbf{a}_{3}$ & $=$ & $\begin{array}{c}-\frac{1}{2}\left(x_{5}+y_{5}\right) a \hat{\mathbf{x}}+ \\
\frac{\sqrt{3}}{2}\left(x_{5}-y_{5}\right) a \hat{\mathbf{y}}+\left(\frac{1}{2}+z_{5}\right) c \hat{\mathbf{z}}\end{array}$ & $(6 a)$ & S III \\
\hline $\mathbf{B}_{29}$ & $=$ & $y_{5} \mathbf{a}_{1}+\left(-x_{5}+y_{5}\right) \mathbf{a}_{2}+\left(\frac{5}{6}+z_{5}\right) \mathbf{a}_{3}$ & $=$ & $\begin{array}{c}\left(-\frac{1}{2} x_{5}+y_{5}\right) a \hat{\mathbf{x}}-\frac{\sqrt{3}}{2} x_{5} a \hat{\mathbf{y}}+ \\
\left(\frac{5}{6}+z_{5}\right) c \hat{\mathbf{z}}\end{array}$ & $(6 a)$ & S III \\
\hline $\mathbf{B}_{30}$ & $=$ & $\left(x_{5}-y_{5}\right) \mathbf{a}_{1}+x_{5} \mathbf{a}_{2}+\left(\frac{1}{6}+z_{5}\right) \mathbf{a}_{3}$ & $=$ & $\begin{array}{c}\left(x_{5}-\frac{1}{2} y_{5}\right) a \hat{\mathbf{x}}+\frac{\sqrt{3}}{2} y_{5} a \hat{\mathbf{y}}+ \\
\left(\frac{1}{6}+z_{5}\right) c \hat{\mathbf{z}}\end{array}$ & $(6 a)$ & S III \\
\hline
\end{tabular}

\section{References:}

- B. Eisenmann, Crystal structure of $\alpha$-dialuminium trisulfide, $A l_{2} S_{3}$, Z. Kristallogr. 198, 307-308 (1992).

\section{Found in:}

- P. Villars and K. Cenzual, Pearson's Crystal Data - Crystal Structure Database for Inorganic Compounds, ASM International (2013).

\section{Geometry files:}

- CIF: pp. 923

- POSCAR: pp. 924 


\section{$\mathrm{Al}_{2} \mathrm{~S}_{3}$ Structure: A2B3_hP30_170_2a_3a}
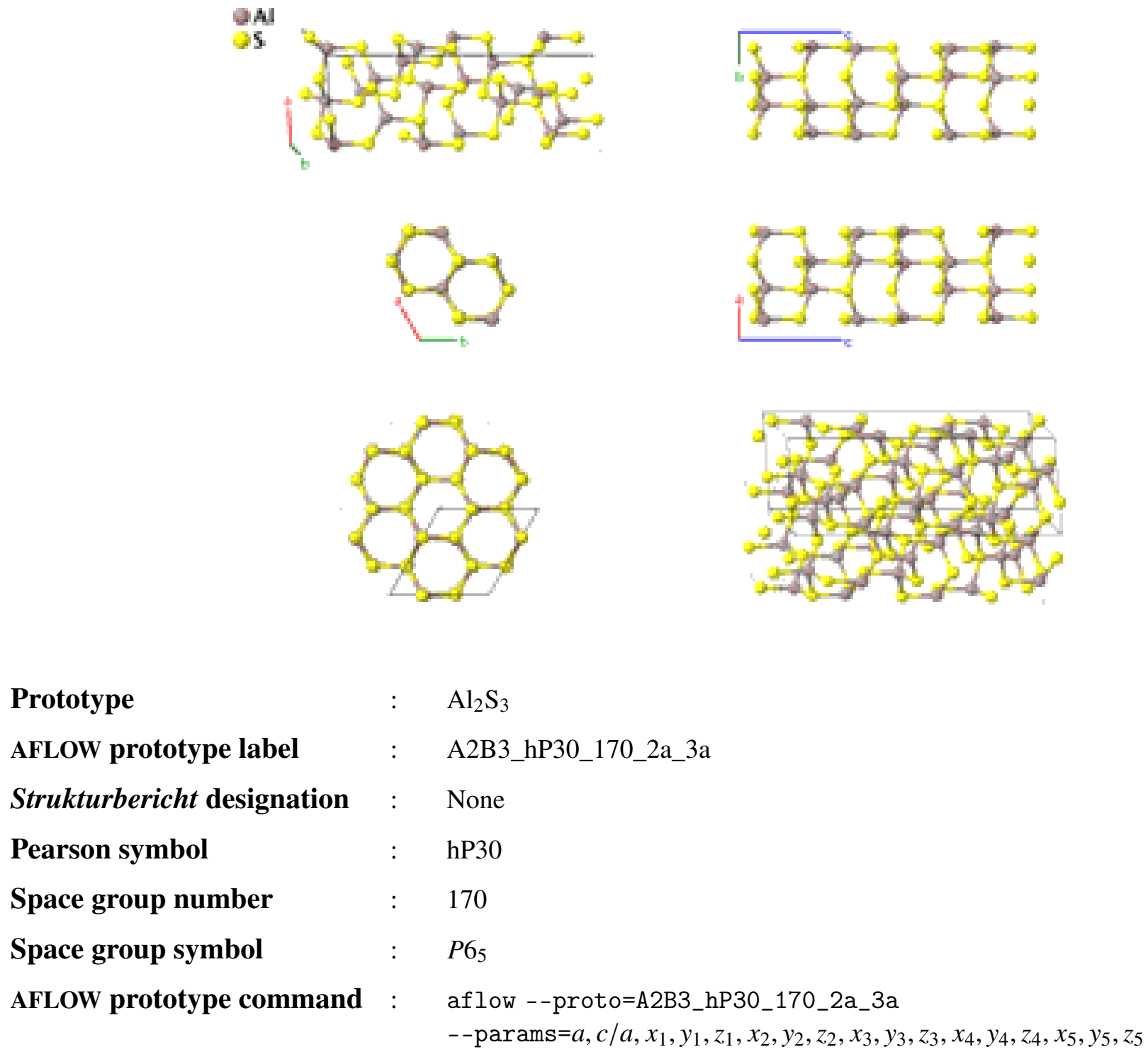

- This structure is the enantiomorph of the $\mathrm{Al}_{2} \mathrm{~S}_{3}$ (A2B3_hP30_169_2a_3a) structure, and was generated by reflecting the coordinates of the space group \#169 structure through the $z=0$ plane.

\section{Hexagonal primitive vectors:}

$$
\begin{array}{ll}
\mathbf{a}_{1}= & \frac{1}{2} a \hat{\mathbf{x}}-\frac{\sqrt{3}}{2} a \hat{\mathbf{y}} \\
\mathbf{a}_{2}= & \frac{1}{2} a \hat{\mathbf{x}}+\frac{\sqrt{3}}{2} a \hat{\mathbf{y}} \\
\mathbf{a}_{3}= & c \hat{\mathbf{z}}
\end{array}
$$

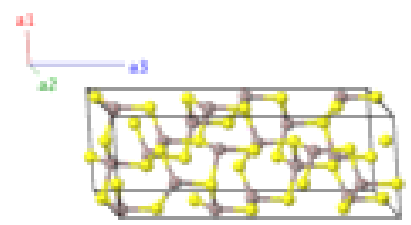

\section{Basis vectors:}

$\begin{array}{ccc}\mathbf{B}_{1}= & \begin{array}{c}\text { Lattice Coordinates } \\ x_{1} \mathbf{a}_{1}+y_{1} \mathbf{a}_{2}+z_{1} \mathbf{a}_{3}\end{array} & \text { Cartesian Coordinates } \\ \mathbf{B}_{2}=-y_{1} \mathbf{a}_{1}+\left(x_{1}-y_{1}\right) \mathbf{a}_{2}+\left(\frac{2}{3}+z_{1}\right) \mathbf{a}_{3} & = & \frac{1}{2}\left(x_{1}+y_{1}\right) a \hat{\mathbf{x}}+ \\ & & \left(\frac{1}{2} x_{1}-y_{1}\right) a \hat{\mathbf{x}}+\frac{\sqrt{3}}{2} x_{1} a \hat{\mathbf{y}}+ \\ & & \left(\frac{2}{3}+z_{1}\right) c \hat{\mathbf{z}}\end{array}$

Wyckoff Position

$(6 a)$

$(6 a)$ 


$$
\begin{aligned}
& \mathbf{B}_{3}=\left(-x_{1}+y_{1}\right) \mathbf{a}_{1}-x_{1} \mathbf{a}_{2}+\left(\frac{1}{3}+z_{1}\right) \mathbf{a}_{3}=\left(-x_{1}+\frac{1}{2} y_{1}\right) a \hat{\mathbf{x}}-\frac{\sqrt{3}}{2} y_{1} a \hat{\mathbf{y}}+ \\
& \left(\frac{1}{3}+z_{1}\right) c \hat{\mathbf{z}} \\
& \begin{aligned}
\mathbf{B}_{4}=-x_{1} \mathbf{a}_{1}-y_{1} \mathbf{a}_{2}+\left(\frac{1}{2}+z_{1}\right) \mathbf{a}_{3}= & -\frac{1}{2}\left(x_{1}+y_{1}\right) a \hat{\mathbf{x}}+ \\
& \frac{\sqrt{3}}{2}\left(x_{1}-y_{1}\right) a \hat{\mathbf{y}}+\left(\frac{1}{2}+z_{1}\right) c \hat{\mathbf{z}} \\
\mathbf{B}_{5}=\quad y_{1} \mathbf{a}_{1}+\left(-x_{1}+y_{1}\right) \mathbf{a}_{2}+\left(\frac{1}{6}+z_{1}\right) \mathbf{a}_{3}= & \left(-\frac{1}{2} x_{1}+y_{1}\right) a \hat{\mathbf{x}}-\frac{\sqrt{3}}{2} x_{1} a \hat{\mathbf{y}}+
\end{aligned} \\
& \mathbf{B}_{5}=y_{1} \mathbf{a}_{1}+\left(-x_{1}+y_{1}\right) \mathbf{a}_{2}+\left(\frac{1}{6}+z_{1}\right) \mathbf{a}_{3}=\left(-\frac{1}{2} x_{1}+y_{1}\right) a \hat{\mathbf{x}}-\frac{\sqrt{3}}{2} x_{1} a \hat{\mathbf{y}}+ \\
& \left(\frac{1}{6}+z_{1}\right) c \hat{\mathbf{z}} \\
& \mathbf{B}_{6}=\left(x_{1}-y_{1}\right) \mathbf{a}_{1}+x_{1} \mathbf{a}_{2}+\left(\frac{5}{6}+z_{1}\right) \mathbf{a}_{3}=\left(x_{1}-\frac{1}{2} y_{1}\right) a \hat{\mathbf{x}}+\frac{\sqrt{3}}{2} y_{1} a \hat{\mathbf{y}}+ \\
& \left(\frac{5}{6}+z_{1}\right) c \hat{\mathbf{z}} \\
& \mathbf{B}_{7}=\begin{array}{lc}
x_{2} \mathbf{a}_{1}+y_{2} \mathbf{a}_{2}+z_{2} \mathbf{a}_{3}= & \frac{1}{2}\left(x_{2}+y_{2}\right) a \hat{\mathbf{x}}+ \\
& \frac{\sqrt{3}}{2}\left(-x_{2}+y_{2}\right) a \hat{\mathbf{y}}+z_{2} c \hat{\mathbf{z}}
\end{array} \\
& \mathbf{B}_{8}=-y_{2} \mathbf{a}_{1}+\left(x_{2}-y_{2}\right) \mathbf{a}_{2}+\left(\frac{2}{3}+z_{2}\right) \mathbf{a}_{3}=\left(\frac{1}{2} x_{2}-y_{2}\right) a \hat{\mathbf{x}}+\frac{\sqrt{3}}{2} x_{2} a \hat{\mathbf{y}}+ \\
& \left(\frac{2}{3}+z_{2}\right) c \hat{\mathbf{z}} \\
& \mathbf{B}_{9}=\left(-x_{2}+y_{2}\right) \mathbf{a}_{1}-x_{2} \mathbf{a}_{2}+\left(\frac{1}{3}+z_{2}\right) \mathbf{a}_{3}=\left(-x_{2}+\frac{1}{2} y_{2}\right) a \hat{\mathbf{x}}-\frac{\sqrt{3}}{2} y_{2} a \hat{\mathbf{y}}+ \\
& \left(\frac{1}{3}+z_{2}\right) c \hat{\mathbf{z}} \\
& \mathbf{B}_{10}=-x_{2} \mathbf{a}_{1}-y_{2} \mathbf{a}_{2}+\left(\frac{1}{2}+z_{2}\right) \mathbf{a}_{3} \quad=\quad-\frac{1}{2}\left(x_{2}+y_{2}\right) a \hat{\mathbf{x}}+ \\
& \frac{\sqrt{3}}{2}\left(x_{2}-y_{2}\right) a \hat{\mathbf{y}}+\left(\frac{1}{2}+z_{2}\right) c \hat{\mathbf{z}} \\
& \mathbf{B}_{11}=y_{2} \mathbf{a}_{1}+\left(-x_{2}+y_{2}\right) \mathbf{a}_{2}+\left(\frac{1}{6}+z_{2}\right) \mathbf{a}_{3}=\left(-\frac{1}{2} x_{2}+y_{2}\right) a \hat{\mathbf{x}}-\frac{\sqrt{3}}{2} x_{2} a \hat{\mathbf{y}}+ \\
& \left(\frac{1}{6}+z_{2}\right) c \hat{\mathbf{z}} \\
& \mathbf{B}_{12}=\left(x_{2}-y_{2}\right) \mathbf{a}_{1}+x_{2} \mathbf{a}_{2}+\left(\frac{5}{6}+z_{2}\right) \mathbf{a}_{3}=\left(x_{2}-\frac{1}{2} y_{2}\right) a \hat{\mathbf{x}}+\frac{\sqrt{3}}{2} y_{2} a \hat{\mathbf{y}}+ \\
& \left(\frac{5}{6}+z_{2}\right) c \hat{\mathbf{z}} \\
& \mathbf{B}_{13}=x_{3} \mathbf{a}_{1}+y_{3} \mathbf{a}_{2}+z_{3} \mathbf{a}_{3} \quad=\quad \frac{1}{2}\left(x_{3}+y_{3}\right) a \hat{\mathbf{x}}+ \\
& \frac{\sqrt{3}}{2}\left(-x_{3}+y_{3}\right) a \hat{\mathbf{y}}+z_{3} c \hat{\mathbf{z}} \\
& \mathbf{B}_{14}=-y_{3} \mathbf{a}_{1}+\left(x_{3}-y_{3}\right) \mathbf{a}_{2}+\left(\frac{2}{3}+z_{3}\right) \mathbf{a}_{3}=\left(\frac{1}{2} x_{3}-y_{3}\right) a \hat{\mathbf{x}}+\frac{\sqrt{3}}{2} x_{3} a \hat{\mathbf{y}}+ \\
& \left(\frac{2}{3}+z_{3}\right) c \hat{\mathbf{z}} \\
& \mathbf{B}_{15}=\left(-x_{3}+y_{3}\right) \mathbf{a}_{1}-x_{3} \mathbf{a}_{2}+\left(\frac{1}{3}+z_{3}\right) \mathbf{a}_{3}=\left(-x_{3}+\frac{1}{2} y_{3}\right) a \hat{\mathbf{x}}-\frac{\sqrt{3}}{2} y_{3} a \hat{\mathbf{y}}+ \\
& \left(\frac{1}{3}+z_{3}\right) c \hat{\mathbf{z}} \\
& \mathbf{B}_{16}=-x_{3} \mathbf{a}_{1}-y_{3} \mathbf{a}_{2}+\left(\frac{1}{2}+z_{3}\right) \mathbf{a}_{3}=-\frac{1}{2}\left(x_{3}+y_{3}\right) a \hat{\mathbf{x}}+ \\
& \frac{\sqrt{3}}{2}\left(x_{3}-y_{3}\right) a \hat{\mathbf{y}}+\left(\frac{1}{2}+z_{3}\right) c \hat{\mathbf{z}} \\
& \mathbf{B}_{17}=y_{3} \mathbf{a}_{1}+\left(-x_{3}+y_{3}\right) \mathbf{a}_{2}+\left(\frac{1}{6}+z_{3}\right) \mathbf{a}_{3}=\left(-\frac{1}{2} x_{3}+y_{3}\right) a \hat{\mathbf{x}}-\frac{\sqrt{3}}{2} x_{3} a \hat{\mathbf{y}}+ \\
& \left(\frac{1}{6}+z_{3}\right) c \hat{\mathbf{z}} \\
& \mathbf{B}_{18}=\left(x_{3}-y_{3}\right) \mathbf{a}_{1}+x_{3} \mathbf{a}_{2}+\left(\frac{5}{6}+z_{3}\right) \mathbf{a}_{3}=\left(x_{3}-\frac{1}{2} y_{3}\right) a \hat{\mathbf{x}}+\frac{\sqrt{3}}{2} y_{3} a \hat{\mathbf{y}}+ \\
& \left(\frac{5}{6}+z_{3}\right) c \hat{\mathbf{z}} \\
& \mathbf{B}_{19}=x_{4} \mathbf{a}_{1}+y_{4} \mathbf{a}_{2}+z_{4} \mathbf{a}_{3} \quad=\quad \frac{1}{2}\left(x_{4}+y_{4}\right) a \hat{\mathbf{x}}+ \\
& \frac{\sqrt{3}}{2}\left(-x_{4}+y_{4}\right) a \hat{\mathbf{y}}+z_{4} c \hat{\mathbf{z}} \\
& \mathbf{B}_{20}=-y_{4} \mathbf{a}_{1}+\left(x_{4}-y_{4}\right) \mathbf{a}_{2}+\left(\frac{2}{3}+z_{4}\right) \mathbf{a}_{3}=\left(\frac{1}{2} x_{4}-y_{4}\right) a \hat{\mathbf{x}}+\frac{\sqrt{3}}{2} x_{4} a \hat{\mathbf{y}}+ \\
& \left(\frac{2}{3}+z_{4}\right) c \hat{\mathbf{z}} \\
& \mathbf{B}_{21}=\left(-x_{4}+y_{4}\right) \mathbf{a}_{1}-x_{4} \mathbf{a}_{2}+\left(\frac{1}{3}+z_{4}\right) \mathbf{a}_{3}=\left(-x_{4}+\frac{1}{2} y_{4}\right) a \hat{\mathbf{x}}-\frac{\sqrt{3}}{2} y_{4} a \hat{\mathbf{y}}+ \\
& \left(\frac{1}{3}+z_{4}\right) c \hat{\mathbf{z}} \\
& \mathbf{B}_{22}=-x_{4} \mathbf{a}_{1}-y_{4} \mathbf{a}_{2}+\left(\frac{1}{2}+z_{4}\right) \mathbf{a}_{3}=\begin{array}{c}
-\frac{1}{2}\left(x_{4}+y_{4}\right) a \hat{\mathbf{x}}+ \\
\frac{\sqrt{3}}{2}\left(x_{4}-y_{4}\right) a \hat{\mathbf{y}}+\left(\frac{1}{2}+z_{4}\right) c \hat{\mathbf{z}}
\end{array}
\end{aligned}
$$




\begin{tabular}{|c|c|c|c|c|c|c|}
\hline $\mathbf{B}_{23}$ & $=$ & $y_{4} \mathbf{a}_{1}+\left(-x_{4}+y_{4}\right) \mathbf{a}_{2}+\left(\frac{1}{6}+z_{4}\right) \mathbf{a}_{3}$ & $=$ & $\begin{array}{c}\left(-\frac{1}{2} x_{4}+y_{4}\right) a \hat{\mathbf{x}}-\frac{\sqrt{3}}{2} x_{4} a \hat{\mathbf{y}}+ \\
\left(\frac{1}{6}+z_{4}\right) c \hat{\mathbf{z}}\end{array}$ & $(6 a)$ & S II \\
\hline $\mathbf{B}_{24}$ & $=$ & $\left(x_{4}-y_{4}\right) \mathbf{a}_{1}+x_{4} \mathbf{a}_{2}+\left(\frac{5}{6}+z_{4}\right) \mathbf{a}_{3}$ & $=$ & $\begin{array}{c}\left(x_{4}-\frac{1}{2} y_{4}\right) a \hat{\mathbf{x}}+\frac{\sqrt{3}}{2} y_{4} a \hat{\mathbf{y}}+ \\
\left(\frac{5}{6}+z_{4}\right) c \hat{\mathbf{z}}\end{array}$ & $(6 a)$ & S II \\
\hline $\mathbf{B}_{25}$ & $=$ & $x_{5} \mathbf{a}_{1}+y_{5} \mathbf{a}_{2}+z_{5} \mathbf{a}_{3}$ & $=$ & $\begin{array}{c}\frac{1}{2}\left(x_{5}+y_{5}\right) a \hat{\mathbf{x}}+ \\
\frac{\sqrt{3}}{2}\left(-x_{5}+y_{5}\right) a \hat{\mathbf{y}}+z_{5} c \hat{\mathbf{z}}\end{array}$ & $(6 a)$ & S III \\
\hline $\mathbf{B}_{26}$ & $=$ & $-y_{5} \mathbf{a}_{1}+\left(x_{5}-y_{5}\right) \mathbf{a}_{2}+\left(\frac{2}{3}+z_{5}\right) \mathbf{a}_{3}$ & $=$ & $\begin{array}{c}\left(\frac{1}{2} x_{5}-y_{5}\right) a \hat{\mathbf{x}}+\frac{\sqrt{3}}{2} x_{5} a \hat{\mathbf{y}}+ \\
\left(\frac{2}{3}+z_{5}\right) c \hat{\mathbf{z}}\end{array}$ & $(6 a)$ & S III \\
\hline $\mathbf{B}_{27}$ & $=$ & $\left(-x_{5}+y_{5}\right) \mathbf{a}_{1}-x_{5} \mathbf{a}_{2}+\left(\frac{1}{3}+z_{5}\right) \mathbf{a}_{3}$ & $=$ & $\begin{array}{c}\left(-x_{5}+\frac{1}{2} y_{5}\right) a \hat{\mathbf{x}}-\frac{\sqrt{3}}{2} y_{5} a \hat{\mathbf{y}}+ \\
\left(\frac{1}{3}+z_{5}\right) c \hat{\mathbf{z}}\end{array}$ & $(6 a)$ & S III \\
\hline $\mathbf{B}_{28}$ & $=$ & $-x_{5} \mathbf{a}_{1}-y_{5} \mathbf{a}_{2}+\left(\frac{1}{2}+z_{5}\right) \mathbf{a}_{3}$ & $=$ & $\begin{array}{c}-\frac{1}{2}\left(x_{5}+y_{5}\right) a \hat{\mathbf{x}}+ \\
\frac{\sqrt{3}}{2}\left(x_{5}-y_{5}\right) a \hat{\mathbf{y}}+\left(\frac{1}{2}+z_{5}\right) c \hat{\mathbf{z}}\end{array}$ & $(6 a)$ & S III \\
\hline $\mathbf{B}_{29}$ & $=$ & $y_{5} \mathbf{a}_{1}+\left(-x_{5}+y_{5}\right) \mathbf{a}_{2}+\left(\frac{1}{6}+z_{5}\right) \mathbf{a}_{3}$ & $=$ & $\begin{array}{c}\left(-\frac{1}{2} x_{5}+y_{5}\right) a \hat{\mathbf{x}}-\frac{\sqrt{3}}{2} x_{5} a \hat{\mathbf{y}}+ \\
\left(\frac{1}{6}+z_{5}\right) c \hat{\mathbf{z}}\end{array}$ & $(6 a)$ & S III \\
\hline $\mathbf{B}_{30}$ & $=$ & $\left(x_{5}-y_{5}\right) \mathbf{a}_{1}+x_{5} \mathbf{a}_{2}+\left(\frac{5}{6}+z_{5}\right) \mathbf{a}_{3}$ & $=$ & $\begin{array}{c}\left(x_{5}-\frac{1}{2} y_{5}\right) a \hat{\mathbf{x}}+\frac{\sqrt{3}}{2} y_{5} a \hat{\mathbf{y}}+ \\
\left(\frac{5}{6}+z_{5}\right) c \hat{\mathbf{z}}\end{array}$ & $(6 a)$ & S III \\
\hline
\end{tabular}

\section{References:}

- B. Eisenmann, Crystal structure of $\alpha$-dialuminium trisulfide, $A l_{2} S_{3}$, Z. Kristallogr. 198, 307-308 (1992).

\section{Geometry files:}

- CIF: pp. 924

- POSCAR: pp. 924 


\section{$\mathrm{Sr}\left[\mathrm{S}_{2} \mathrm{O}_{6}\right]\left[\mathrm{H}_{2} \mathrm{O}\right]_{4}$ Structure: A10B2C_hP39_171_5c_c_a}
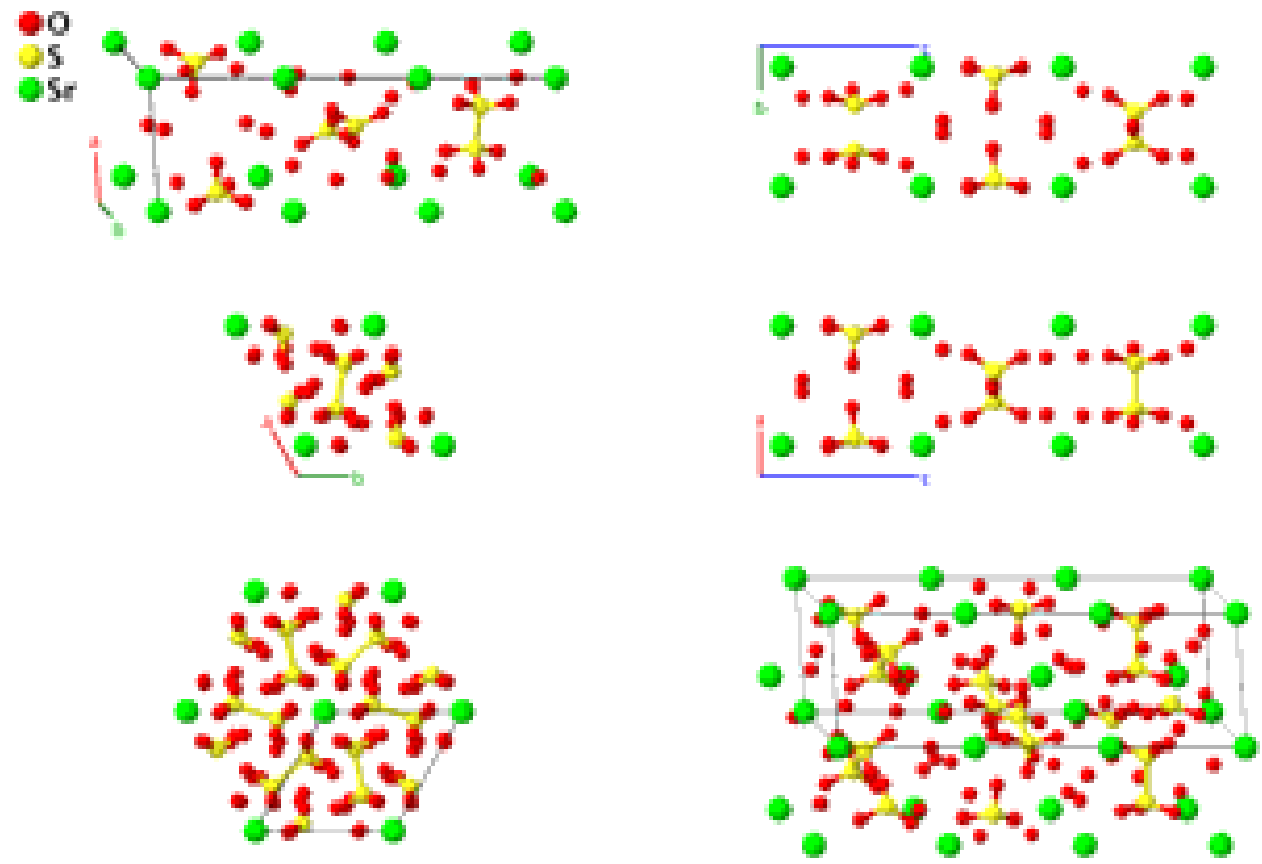

\section{Prototype}

AFLOW prototype label

$: \quad \mathrm{Sr}\left[\mathrm{S}_{2} \mathrm{O}_{6}\right]\left[\mathrm{H}_{2} \mathrm{O}\right]_{4}$

Strukturbericht designation

: A10B2C_hP39_171_5c_c_a

Pearson symbol

: None

Space group number

hP39

Space group symbol

AFLOW prototype command

$\mathrm{Pb}_{2}$

aflow - -proto=A10B2C_hP39_171_5c_c_a

- - params $=a, c / a, z_{1}, x_{2}, y_{2}, z_{2}, x_{3}, y_{3}, z_{3}, x_{4}, y_{4}, z_{4}, x_{5}, y_{5}, z_{5}, x_{6}, y_{6}, z_{6}, x_{7}, y_{7}, z_{7}$

- This structure is the enantiomorph of the $\mathrm{Sr}\left[\mathrm{S}_{2} \mathrm{O}_{6}\right]\left[\mathrm{H}_{2} \mathrm{O}\right]_{4}$ (A10B2C_hP39_172_5c_c_a) structure. Only the nonhydrogen atoms are included in the prototype.

\section{Hexagonal primitive vectors:}

$$
\begin{array}{ll}
\mathbf{a}_{1}= & \frac{1}{2} a \hat{\mathbf{x}}-\frac{\sqrt{3}}{2} a \hat{\mathbf{y}} \\
\mathbf{a}_{2}= & \frac{1}{2} a \hat{\mathbf{x}}+\frac{\sqrt{3}}{2} a \hat{\mathbf{y}} \\
\mathbf{a}_{3}= & c \hat{\mathbf{z}}
\end{array}
$$

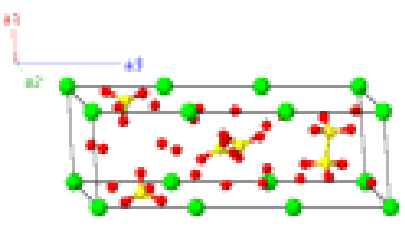

Basis vectors:

Lattice Coordinates
$\mathbf{B}_{1}=$
$z_{1} \mathbf{a}_{3}$
$\mathbf{B}_{2}=$
$\left(\frac{2}{3}+z_{1}\right) \mathbf{a}_{3}$
$\mathbf{B}_{3}=$
$\left(\frac{1}{3}+z_{1}\right) \mathbf{a}_{3}$

Cartesian Coordinates

$=$

$=$

$=$ $z_{1} c \hat{\mathbf{z}}$

$\left(\frac{2}{3}+z_{1}\right) c \hat{\mathbf{z}}$

$\left(\frac{1}{3}+z_{1}\right) c \hat{\mathbf{z}}$
Wyckoff Position Atom Type

(3a)

$\mathrm{Sr}$

(3a)

$\mathrm{Sr}$

(3a) 


\begin{tabular}{|c|c|c|c|c|c|c|}
\hline $\mathbf{B}_{4}$ & $=$ & $x_{2} \mathbf{a}_{1}+y_{2} \mathbf{a}_{2}+z_{2} \mathbf{a}_{3}$ & $=$ & $\begin{array}{c}\frac{1}{2}\left(x_{2}+y_{2}\right) a \hat{\mathbf{x}}+ \\
\frac{\sqrt{3}}{2}\left(-x_{2}+y_{2}\right) a \hat{\mathbf{y}}+z_{2} c \hat{\mathbf{z}}\end{array}$ & $(6 c)$ & O I \\
\hline $\mathbf{B}_{5}$ & $=$ & $-y_{2} \mathbf{a}_{1}+\left(x_{2}-y_{2}\right) \mathbf{a}_{2}+\left(\frac{2}{3}+z_{2}\right) \mathbf{a}_{3}$ & $=$ & $\begin{array}{c}\left(\frac{1}{2} x_{2}-y_{2}\right) a \hat{\mathbf{x}}+\frac{\sqrt{3}}{2} x_{2} a \hat{\mathbf{y}}+ \\
\left(\frac{2}{3}+z_{2}\right) c \hat{\mathbf{z}}\end{array}$ & $(6 c)$ & O I \\
\hline $\mathbf{B}_{6}$ & $=$ & $\left(-x_{2}+y_{2}\right) \mathbf{a}_{1}-x_{2} \mathbf{a}_{2}+\left(\frac{1}{3}+z_{2}\right) \mathbf{a}_{3}$ & $=$ & $\begin{array}{c}\left(-x_{2}+\frac{1}{2} y_{2}\right) a \hat{\mathbf{x}}-\frac{\sqrt{3}}{2} y_{2} a \hat{\mathbf{y}}+ \\
\left(\frac{1}{3}+z_{2}\right) c \hat{\mathbf{z}}\end{array}$ & $(6 c)$ & O I \\
\hline $\mathbf{B}_{7}$ & $=$ & $-x_{2} \mathbf{a}_{1}-y_{2} \mathbf{a}_{2}+z_{2} \mathbf{a}_{3}$ & $=$ & $\begin{array}{c}-\frac{1}{2}\left(x_{2}+y_{2}\right) a \hat{\mathbf{x}}+ \\
\frac{\sqrt{3}}{2}\left(x_{2}-y_{2}\right) a \hat{\mathbf{y}}+z_{2} c \hat{\mathbf{z}}\end{array}$ & $(6 c)$ & O I \\
\hline $\mathbf{B}_{8}$ & $=$ & $y_{2} \mathbf{a}_{1}+\left(-x_{2}+y_{2}\right) \mathbf{a}_{2}+\left(\frac{2}{3}+z_{2}\right) \mathbf{a}_{3}$ & $=$ & $\begin{array}{c}\left(-\frac{1}{2} x_{2}+y_{2}\right) a \hat{\mathbf{x}}-\frac{\sqrt{3}}{2} x_{2} a \hat{\mathbf{y}}+ \\
\left(\frac{2}{3}+z_{2}\right) c \hat{\mathbf{z}}\end{array}$ & $(6 c)$ & O I \\
\hline $\mathbf{B}_{9}$ & $=$ & $\left(x_{2}-y_{2}\right) \mathbf{a}_{1}+x_{2} \mathbf{a}_{2}+\left(\frac{1}{3}+z_{2}\right) \mathbf{a}_{3}$ & $=$ & $\begin{array}{c}\left(x_{2}-\frac{1}{2} y_{2}\right) a \hat{\mathbf{x}}+\frac{\sqrt{3}}{2} y_{2} a \hat{\mathbf{y}}+ \\
\left(\frac{1}{3}+z_{2}\right) c \hat{\mathbf{z}}\end{array}$ & $(6 c)$ & $\mathrm{O} \mathrm{I}$ \\
\hline $\mathbf{B}_{10}$ & $=$ & $x_{3} \mathbf{a}_{1}+y_{3} \mathbf{a}_{2}+z_{3} \mathbf{a}_{3}$ & $=$ & $\begin{array}{c}\frac{1}{2}\left(x_{3}+y_{3}\right) a \hat{\mathbf{x}}+ \\
\frac{\sqrt{3}}{2}\left(-x_{3}+y_{3}\right) a \hat{\mathbf{y}}+z_{3} c \hat{\mathbf{z}}\end{array}$ & $(6 c)$ & O II \\
\hline $\mathbf{B}_{11}$ & $=$ & $-y_{3} \mathbf{a}_{1}+\left(x_{3}-y_{3}\right) \mathbf{a}_{2}+\left(\frac{2}{3}+z_{3}\right) \mathbf{a}_{3}$ & $=$ & $\begin{array}{c}\left(\frac{1}{2} x_{3}-y_{3}\right) a \hat{\mathbf{x}}+\frac{\sqrt{3}}{2} x_{3} a \hat{\mathbf{y}}+ \\
\left(\frac{2}{3}+z_{3}\right) c \hat{\mathbf{z}}\end{array}$ & $(6 c)$ & O II \\
\hline $\mathbf{B}_{12}$ & $=$ & $\left(-x_{3}+y_{3}\right) \mathbf{a}_{1}-x_{3} \mathbf{a}_{2}+\left(\frac{1}{3}+z_{3}\right) \mathbf{a}_{3}$ & $=$ & $\begin{array}{c}\left(-x_{3}+\frac{1}{2} y_{3}\right) a \hat{\mathbf{x}}-\frac{\sqrt{3}}{2} y_{3} a \hat{\mathbf{y}}+ \\
\left(\frac{1}{3}+z_{3}\right) c \hat{\mathbf{z}}\end{array}$ & $(6 c)$ & O II \\
\hline $\mathbf{B}_{13}$ & $=$ & $-x_{3} \mathbf{a}_{1}-y_{3} \mathbf{a}_{2}+z_{3} \mathbf{a}_{3}$ & $=$ & $\begin{array}{c}-\frac{1}{2}\left(x_{3}+y_{3}\right) a \hat{\mathbf{x}}+ \\
\frac{\sqrt{3}}{2}\left(x_{3}-y_{3}\right) a \hat{\mathbf{y}}+z_{3} c \hat{\mathbf{z}}\end{array}$ & $(6 c)$ & O II \\
\hline $\mathbf{B}_{14}$ & $=$ & $y_{3} \mathbf{a}_{1}+\left(-x_{3}+y_{3}\right) \mathbf{a}_{2}+\left(\frac{2}{3}+z_{3}\right) \mathbf{a}_{3}$ & $=$ & $\begin{array}{c}\left(-\frac{1}{2} x_{3}+y_{3}\right) a \hat{\mathbf{x}}-\frac{\sqrt{3}}{2} x_{3} a \hat{\mathbf{y}}+ \\
\left(\frac{2}{3}+z_{3}\right) c \hat{\mathbf{z}}\end{array}$ & $(6 c)$ & O II \\
\hline $\mathbf{B}_{15}$ & $=$ & $\left(x_{3}-y_{3}\right) \mathbf{a}_{1}+x_{3} \mathbf{a}_{2}+\left(\frac{1}{3}+z_{3}\right) \mathbf{a}_{3}$ & $=$ & $\begin{array}{c}\left(x_{3}-\frac{1}{2} y_{3}\right) a \hat{\mathbf{x}}+\frac{\sqrt{3}}{2} y_{3} a \hat{\mathbf{y}}+ \\
\left(\frac{1}{3}+z_{3}\right) c \hat{\mathbf{z}}\end{array}$ & $(6 c)$ & O II \\
\hline $\mathbf{B}_{16}$ & $=$ & $x_{4} \mathbf{a}_{1}+y_{4} \mathbf{a}_{2}+z_{4} \mathbf{a}_{3}$ & $=$ & $\begin{array}{c}\frac{1}{2}\left(x_{4}+y_{4}\right) a \hat{\mathbf{x}}+ \\
\frac{\sqrt{3}}{2}\left(-x_{4}+y_{4}\right) a \hat{\mathbf{y}}+z_{4} c \hat{\mathbf{z}}\end{array}$ & $(6 c)$ & O III \\
\hline $\mathbf{B}_{17}$ & $=$ & $-y_{4} \mathbf{a}_{1}+\left(x_{4}-y_{4}\right) \mathbf{a}_{2}+\left(\frac{2}{3}+z_{4}\right) \mathbf{a}_{3}$ & $=$ & $\begin{array}{c}\left(\frac{1}{2} x_{4}-y_{4}\right) a \hat{\mathbf{x}}+\frac{\sqrt{3}}{2} x_{4} a \hat{\mathbf{y}}+ \\
\left(\frac{2}{3}+z_{4}\right) c \hat{\mathbf{z}}\end{array}$ & $(6 c)$ & O III \\
\hline $\mathbf{B}_{18}$ & $=$ & $\left(-x_{4}+y_{4}\right) \mathbf{a}_{1}-x_{4} \mathbf{a}_{2}+\left(\frac{1}{3}+z_{4}\right) \mathbf{a}_{3}$ & $=$ & $\begin{array}{c}\left(-x_{4}+\frac{1}{2} y_{4}\right) a \hat{\mathbf{x}}-\frac{\sqrt{3}}{2} y_{4} a \hat{\mathbf{y}}+ \\
\left(\frac{1}{3}+z_{4}\right) c \hat{\mathbf{z}}\end{array}$ & $(6 c)$ & O III \\
\hline $\mathbf{B}_{19}$ & $=$ & $-x_{4} \mathbf{a}_{1}-y_{4} \mathbf{a}_{2}+z_{4} \mathbf{a}_{3}$ & $=$ & $\begin{array}{c}-\frac{1}{2}\left(x_{4}+y_{4}\right) a \hat{\mathbf{x}}+ \\
\frac{\sqrt{3}}{2}\left(x_{4}-y_{4}\right) a \hat{\mathbf{y}}+z_{4} c \hat{\mathbf{z}}\end{array}$ & $(6 c)$ & O III \\
\hline $\mathbf{B}_{20}$ & $=$ & $y_{4} \mathbf{a}_{1}+\left(-x_{4}+y_{4}\right) \mathbf{a}_{2}+\left(\frac{2}{3}+z_{4}\right) \mathbf{a}_{3}$ & $=$ & $\begin{array}{c}\left(-\frac{1}{2} x_{4}+y_{4}\right) a \hat{\mathbf{x}}-\frac{\sqrt{3}}{2} x_{4} a \hat{\mathbf{y}}+ \\
\left(\frac{2}{3}+z_{4}\right) c \hat{\mathbf{z}}\end{array}$ & $(6 c)$ & O III \\
\hline $\mathbf{B}_{21}$ & $=$ & $\left(x_{4}-y_{4}\right) \mathbf{a}_{1}+x_{4} \mathbf{a}_{2}+\left(\frac{1}{3}+z_{4}\right) \mathbf{a}_{3}$ & $=$ & $\begin{array}{c}\left(x_{4}-\frac{1}{2} y_{4}\right) a \hat{\mathbf{x}}+\frac{\sqrt{3}}{2} y_{4} a \hat{\mathbf{y}}+ \\
\left(\frac{1}{3}+z_{4}\right) c \hat{\mathbf{z}}\end{array}$ & $(6 c)$ & O III \\
\hline $\mathbf{B}_{22}$ & $=$ & $x_{5} \mathbf{a}_{1}+y_{5} \mathbf{a}_{2}+z_{5} \mathbf{a}_{3}$ & $=$ & $\begin{array}{c}\frac{1}{2}\left(x_{5}+y_{5}\right) a \hat{\mathbf{x}}+ \\
\frac{\sqrt{3}}{2}\left(-x_{5}+y_{5}\right) a \hat{\mathbf{y}}+z_{5} c \hat{\mathbf{z}}\end{array}$ & $(6 c)$ & O IV \\
\hline $\mathbf{B}_{23}$ & $=$ & $-y_{5} \mathbf{a}_{1}+\left(x_{5}-y_{5}\right) \mathbf{a}_{2}+\left(\frac{2}{3}+z_{5}\right) \mathbf{a}_{3}$ & $=$ & $\begin{array}{c}\left(\frac{1}{2} x_{5}-y_{5}\right) a \hat{\mathbf{x}}+\frac{\sqrt{3}}{2} x_{5} a \hat{\mathbf{y}}+ \\
\left(\frac{2}{3}+z_{5}\right) c \hat{\mathbf{z}}\end{array}$ & $(6 c)$ & O IV \\
\hline
\end{tabular}




$$
\begin{aligned}
& \mathbf{B}_{24}=\left(-x_{5}+y_{5}\right) \mathbf{a}_{1}-x_{5} \mathbf{a}_{2}+\left(\frac{1}{3}+z_{5}\right) \mathbf{a}_{3}=\left(-x_{5}+\frac{1}{2} y_{5}\right) a \hat{\mathbf{x}}-\frac{\sqrt{3}}{2} y_{5} a \hat{\mathbf{y}}+ \\
& \left(\frac{1}{3}+z_{5}\right) c \hat{\mathbf{z}} \\
& \mathbf{B}_{25}=-x_{5} \mathbf{a}_{1}-y_{5} \mathbf{a}_{2}+z_{5} \mathbf{a}_{3} \quad=\quad-\frac{1}{2}\left(x_{5}+y_{5}\right) a \hat{\mathbf{x}}+\quad \text { (6c) } \\
& \frac{\sqrt{3}}{2}\left(x_{5}-y_{5}\right) a \hat{\mathbf{y}}+z_{5} c \hat{\mathbf{z}} \\
& \mathbf{B}_{26}=y_{5} \mathbf{a}_{1}+\left(-x_{5}+y_{5}\right) \mathbf{a}_{2}+\left(\frac{2}{3}+z_{5}\right) \mathbf{a}_{3}=\left(-\frac{1}{2} x_{5}+y_{5}\right) a \hat{\mathbf{x}}-\frac{\sqrt{3}}{2} x_{5} a \hat{\mathbf{y}}+ \\
& \left(\frac{2}{3}+z_{5}\right) c \hat{\mathbf{z}} \\
& \mathbf{B}_{27}=\left(x_{5}-y_{5}\right) \mathbf{a}_{1}+x_{5} \mathbf{a}_{2}+\left(\frac{1}{3}+z_{5}\right) \mathbf{a}_{3}=\left(x_{5}-\frac{1}{2} y_{5}\right) a \hat{\mathbf{x}}+\frac{\sqrt{3}}{2} y_{5} a \hat{\mathbf{y}}+ \\
& \left(\frac{1}{3}+z_{5}\right) c \hat{\mathbf{z}} \\
& \mathbf{B}_{28}=x_{6} \mathbf{a}_{1}+y_{6} \mathbf{a}_{2}+z_{6} \mathbf{a}_{3} \quad=\quad \frac{1}{2}\left(x_{6}+y_{6}\right) a \hat{\mathbf{x}}+ \\
& \frac{\sqrt{3}}{2}\left(-x_{6}+y_{6}\right) a \hat{\mathbf{y}}+z_{6} c \hat{\mathbf{z}} \\
& \mathbf{B}_{29}=-y_{6} \mathbf{a}_{1}+\left(x_{6}-y_{6}\right) \mathbf{a}_{2}+\left(\frac{2}{3}+z_{6}\right) \mathbf{a}_{3}=\left(\frac{1}{2} x_{6}-y_{6}\right) a \hat{\mathbf{x}}+\frac{\sqrt{3}}{2} x_{6} a \hat{\mathbf{y}}+ \\
& \left(\frac{2}{3}+z_{6}\right) c \hat{\mathbf{z}} \\
& \mathbf{B}_{30}=\left(-x_{6}+y_{6}\right) \mathbf{a}_{1}-x_{6} \mathbf{a}_{2}+\left(\frac{1}{3}+z_{6}\right) \mathbf{a}_{3}=\left(-x_{6}+\frac{1}{2} y_{6}\right) a \hat{\mathbf{x}}-\frac{\sqrt{3}}{2} y_{6} a \hat{\mathbf{y}}+ \\
& \left(\frac{1}{3}+z_{6}\right) c \hat{\mathbf{z}} \\
& \mathbf{B}_{31}=-x_{6} \mathbf{a}_{1}-y_{6} \mathbf{a}_{2}+z_{6} \mathbf{a}_{3} \quad=\quad-\frac{1}{2}\left(x_{6}+y_{6}\right) a \hat{\mathbf{x}}+\quad \text { (6c) } \\
& \frac{\sqrt{3}}{2}\left(x_{6}-y_{6}\right) a \hat{\mathbf{y}}+z_{6} c \hat{\mathbf{z}} \\
& \mathbf{B}_{32}=y_{6} \mathbf{a}_{1}+\left(-x_{6}+y_{6}\right) \mathbf{a}_{2}+\left(\frac{2}{3}+z_{6}\right) \mathbf{a}_{3}=\left(-\frac{1}{2} x_{6}+y_{6}\right) a \hat{\mathbf{x}}-\frac{\sqrt{3}}{2} x_{6} a \hat{\mathbf{y}}+ \\
& \left(\frac{2}{3}+z_{6}\right) c \hat{\mathbf{z}} \\
& \mathbf{B}_{33}=\left(x_{6}-y_{6}\right) \mathbf{a}_{1}+x_{6} \mathbf{a}_{2}+\left(\frac{1}{3}+z_{6}\right) \mathbf{a}_{3}=\left(x_{6}-\frac{1}{2} y_{6}\right) a \hat{\mathbf{x}}+\frac{\sqrt{3}}{2} y_{6} a \hat{\mathbf{y}}+ \\
& \left(\frac{1}{3}+z_{6}\right) c \hat{\mathbf{z}} \\
& \mathbf{B}_{34}=\quad x_{7} \mathbf{a}_{1}+y_{7} \mathbf{a}_{2}+z_{7} \mathbf{a}_{3} \quad=\quad \frac{1}{2}\left(x_{7}+y_{7}\right) a \hat{\mathbf{x}}+\quad \text { (6c) } \\
& \frac{\sqrt{3}}{2}\left(-x_{7}+y_{7}\right) a \hat{\mathbf{y}}+z_{7} c \hat{\mathbf{z}} \\
& \mathbf{B}_{35}=-y_{7} \mathbf{a}_{1}+\left(x_{7}-y_{7}\right) \mathbf{a}_{2}+\left(\frac{2}{3}+z_{7}\right) \mathbf{a}_{3}=\left(\frac{1}{2} x_{7}-y_{7}\right) a \hat{\mathbf{x}}+\frac{\sqrt{3}}{2} x_{7} a \hat{\mathbf{y}}+ \\
& \left(\frac{2}{3}+z_{7}\right) c \hat{\mathbf{z}} \\
& \mathbf{B}_{36}=\left(-x_{7}+y_{7}\right) \mathbf{a}_{1}-x_{7} \mathbf{a}_{2}+\left(\frac{1}{3}+z_{7}\right) \mathbf{a}_{3}=\left(-x_{7}+\frac{1}{2} y_{7}\right) a \hat{\mathbf{x}}-\frac{\sqrt{3}}{2} y_{7} a \hat{\mathbf{y}}+ \\
& \left(\frac{1}{3}+z_{7}\right) c \hat{\mathbf{z}} \\
& \mathbf{B}_{37}=\quad-x_{7} \mathbf{a}_{1}-y_{7} \mathbf{a}_{2}+z_{7} \mathbf{a}_{3} \quad=\quad-\frac{1}{2}\left(x_{7}+y_{7}\right) a \hat{\mathbf{x}}+ \\
& \frac{\sqrt{3}}{2}\left(x_{7}-y_{7}\right) a \hat{\mathbf{y}}+z_{7} c \hat{\mathbf{z}} \\
& \mathbf{B}_{38}=y_{7} \mathbf{a}_{1}+\left(-x_{7}+y_{7}\right) \mathbf{a}_{2}+\left(\frac{2}{3}+z_{7}\right) \mathbf{a}_{3}=\left(-\frac{1}{2} x_{7}+y_{7}\right) a \hat{\mathbf{x}}-\frac{\sqrt{3}}{2} x_{7} a \hat{\mathbf{y}}+ \\
& \left(\frac{2}{3}+z_{7}\right) c \hat{\mathbf{z}} \\
& \mathbf{B}_{39}=\left(x_{7}-y_{7}\right) \mathbf{a}_{1}+x_{7} \mathbf{a}_{2}+\left(\frac{1}{3}+z_{7}\right) \mathbf{a}_{3}=\left(x_{7}-\frac{1}{2} y_{7}\right) a \hat{\mathbf{x}}+\frac{\sqrt{3}}{2} y_{7} a \hat{\mathbf{y}}+ \\
& \left(\frac{1}{3}+z_{7}\right) c \hat{\mathbf{z}} \\
& \text { O V }
\end{aligned}
$$

\section{References:}

- R. N. Hargreaves and E. Stanley, The structure of strontium dithionate tetrahydrate, Zeitschrift für Kristallographie Crystalline Materials 135, 399-407 (1972), doi:10.1524/zkri.1972.135.5-6.399.

\section{Found in:}

- P. Villars and K. Cenzual, Pearson's Crystal Data - Crystal Structure Database for Inorganic Compounds, ASM International (2013).

\section{Geometry files:}

- CIF: pp. 924 
- POSCAR: pp. 925 


\section{$\mathrm{Sr}\left[\mathrm{S}_{2} \mathrm{O}_{6}\right]\left[\mathrm{H}_{2} \mathrm{O}\right]_{4}$ Structure: A10B2C_hP39_172_5c_c_a}
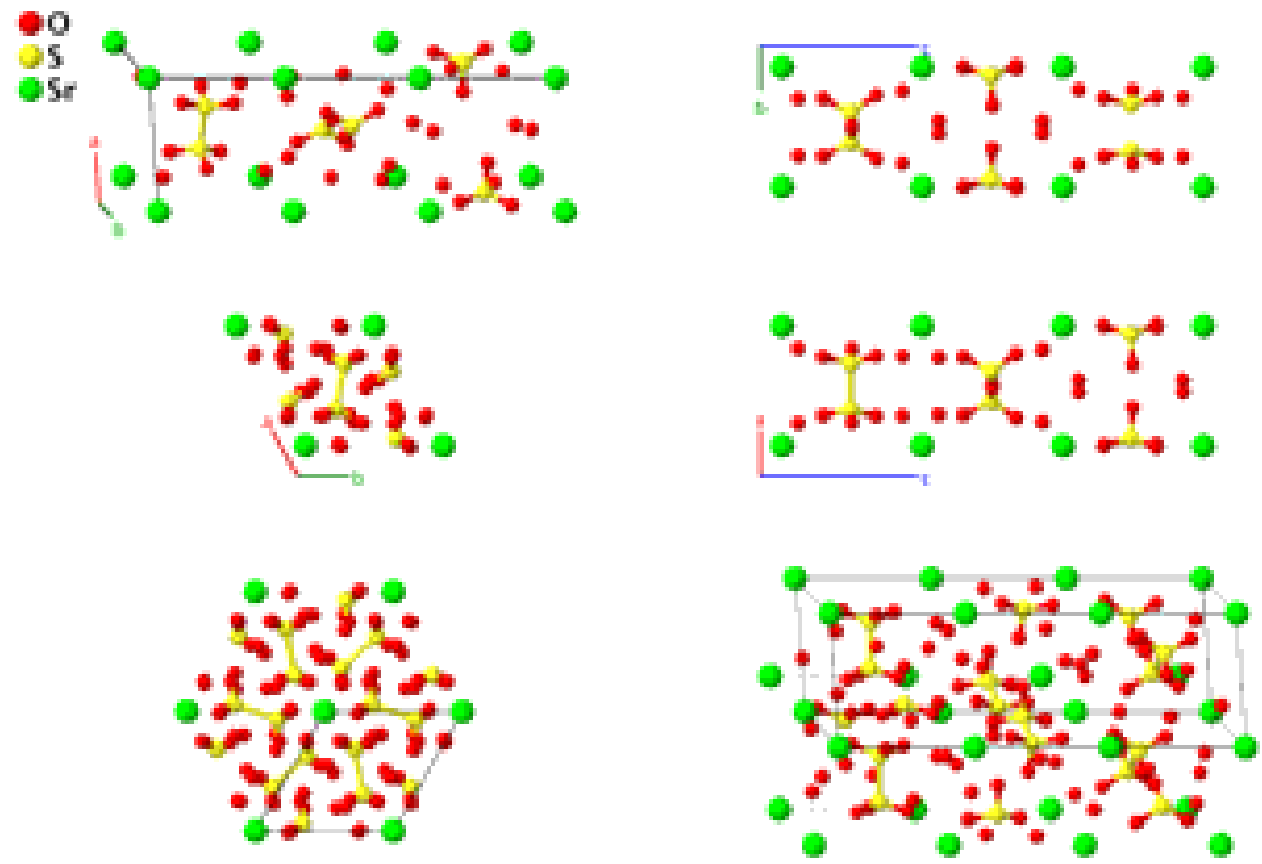

\section{Prototype}

AFLOW prototype label

Strukturbericht designation

Pearson symbol

Space group number

Space group symbol

AFLOW prototype command
: $\quad \mathrm{Sr}\left[\mathrm{S}_{2} \mathrm{O}_{6}\right]\left[\mathrm{H}_{2} \mathrm{O}\right]_{4}$

: A10B2C_hP39_172_5c_c_a

: None

: $\quad$ hP39

: 172

$: \quad P 6_{4}$

aflow - -proto $=$ A10B2C_hP39_172_5c_c_a

- -params $=a, c / a, z_{1}, x_{2}, y_{2}, z_{2}, x_{3}, y_{3}, z_{3}, x_{4}, y_{4}, z_{4}, x_{5}, y_{5}, z_{5}, x_{6}, y_{6}, z_{6}, x_{7}, y_{7}, z_{7}$

- This structure is the enantiomorph of the $\operatorname{Sr}\left[\mathrm{S}_{2} \mathrm{O}_{6}\right]\left[\mathrm{H}_{2} \mathrm{O}\right]_{4}$ (A10B2C_hP39_171_5c_c_a) structure, and was generated by reflecting the coordinates of the space group \#171 structure through the $z=0$ plane. Only the non-hydrogen atoms are included in the prototype.

\section{Hexagonal primitive vectors:}

$$
\begin{array}{ll}
\mathbf{a}_{1}= & \frac{1}{2} a \hat{\mathbf{x}}-\frac{\sqrt{3}}{2} a \hat{\mathbf{y}} \\
\mathbf{a}_{2}= & \frac{1}{2} a \hat{\mathbf{x}}+\frac{\sqrt{3}}{2} a \hat{\mathbf{y}} \\
\mathbf{a}_{3}= & c \hat{\mathbf{z}}
\end{array}
$$

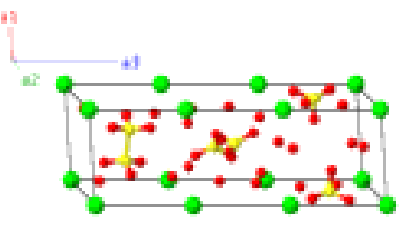

Basis vectors:

Lattice Coordinates

$\begin{array}{llc}\mathbf{B}_{1}= & z_{1} \mathbf{a}_{3} \\ \mathbf{B}_{2}= & \left(\frac{1}{3}+z_{1}\right) \mathbf{a}_{3} \\ \mathbf{B}_{3}= & \left(\frac{2}{3}+z_{1}\right) \mathbf{a}_{3}\end{array}$

$\mathbf{B}_{1}=$
Cartesian Coordinates

$=$

$=$

$=$ $z_{1} c \hat{\mathbf{z}}$

$\left(\frac{1}{3}+z_{1}\right) c \hat{\mathbf{z}}$

$\left(\frac{2}{3}+z_{1}\right) c \hat{\mathbf{z}}$
Wyckoff Position

Atom Type

(3a)

$\mathrm{Sr}$

(3a)

$\mathrm{Sr}$

(3a) 


\begin{tabular}{|c|c|c|c|c|c|c|}
\hline $\mathbf{B}_{4}$ & $=$ & $x_{2} \mathbf{a}_{1}+y_{2} \mathbf{a}_{2}+z_{2} \mathbf{a}_{3}$ & $=$ & $\begin{array}{c}\frac{1}{2}\left(x_{2}+y_{2}\right) a \hat{\mathbf{x}}+ \\
\frac{\sqrt{3}}{2}\left(-x_{2}+y_{2}\right) a \hat{\mathbf{y}}+z_{2} c \hat{\mathbf{z}}\end{array}$ & $(6 c)$ & O I \\
\hline $\mathbf{B}_{5}$ & $=$ & $-y_{2} \mathbf{a}_{1}+\left(x_{2}-y_{2}\right) \mathbf{a}_{2}+\left(\frac{1}{3}+z_{2}\right) \mathbf{a}_{3}$ & $=$ & $\begin{array}{c}\left(\frac{1}{2} x_{2}-y_{2}\right) a \hat{\mathbf{x}}+\frac{\sqrt{3}}{2} x_{2} a \hat{\mathbf{y}}+ \\
\left(\frac{1}{3}+z_{2}\right) c \hat{\mathbf{z}}\end{array}$ & $(6 c)$ & O I \\
\hline $\mathbf{B}_{6}$ & $=$ & $\left(-x_{2}+y_{2}\right) \mathbf{a}_{1}-x_{2} \mathbf{a}_{2}+\left(\frac{2}{3}+z_{2}\right) \mathbf{a}_{3}$ & $=$ & $\begin{array}{c}\left(-x_{2}+\frac{1}{2} y_{2}\right) a \hat{\mathbf{x}}-\frac{\sqrt{3}}{2} y_{2} a \hat{\mathbf{y}}+ \\
\left(\frac{2}{3}+z_{2}\right) c \hat{\mathbf{z}}\end{array}$ & $(6 c)$ & O I \\
\hline $\mathbf{B}_{7}$ & $=$ & $-x_{2} \mathbf{a}_{1}-y_{2} \mathbf{a}_{2}+z_{2} \mathbf{a}_{3}$ & $=$ & $\begin{array}{c}-\frac{1}{2}\left(x_{2}+y_{2}\right) a \hat{\mathbf{x}}+ \\
\frac{\sqrt{3}}{2}\left(x_{2}-y_{2}\right) a \hat{\mathbf{y}}+z_{2} c \hat{\mathbf{z}}\end{array}$ & $(6 c)$ & O I \\
\hline $\mathbf{B}_{8}$ & $=$ & $y_{2} \mathbf{a}_{1}+\left(-x_{2}+y_{2}\right) \mathbf{a}_{2}+\left(\frac{1}{3}+z_{2}\right) \mathbf{a}_{3}$ & $=$ & $\begin{array}{c}\left(-\frac{1}{2} x_{2}+y_{2}\right) a \hat{\mathbf{x}}-\frac{\sqrt{3}}{2} x_{2} a \hat{\mathbf{y}}+ \\
\left(\frac{1}{3}+z_{2}\right) c \hat{\mathbf{z}}\end{array}$ & $(6 c)$ & O I \\
\hline $\mathbf{B}_{9}$ & $=$ & $\left(x_{2}-y_{2}\right) \mathbf{a}_{1}+x_{2} \mathbf{a}_{2}+\left(\frac{2}{3}+z_{2}\right) \mathbf{a}_{3}$ & $=$ & $\begin{array}{c}\left(x_{2}-\frac{1}{2} y_{2}\right) a \hat{\mathbf{x}}+\frac{\sqrt{3}}{2} y_{2} a \hat{\mathbf{y}}+ \\
\left(\frac{2}{3}+z_{2}\right) c \hat{\mathbf{z}}\end{array}$ & $(6 c)$ & $\mathrm{O} \mathrm{I}$ \\
\hline $\mathbf{B}_{10}$ & $=$ & $x_{3} \mathbf{a}_{1}+y_{3} \mathbf{a}_{2}+z_{3} \mathbf{a}_{3}$ & $=$ & $\begin{array}{c}\frac{1}{2}\left(x_{3}+y_{3}\right) a \hat{\mathbf{x}}+ \\
\frac{\sqrt{3}}{2}\left(-x_{3}+y_{3}\right) a \hat{\mathbf{y}}+z_{3} c \hat{\mathbf{z}}\end{array}$ & $(6 c)$ & O II \\
\hline $\mathbf{B}_{11}$ & $=$ & $-y_{3} \mathbf{a}_{1}+\left(x_{3}-y_{3}\right) \mathbf{a}_{2}+\left(\frac{1}{3}+z_{3}\right) \mathbf{a}_{3}$ & $=$ & $\begin{array}{c}\left(\frac{1}{2} x_{3}-y_{3}\right) a \hat{\mathbf{x}}+\frac{\sqrt{3}}{2} x_{3} a \hat{\mathbf{y}}+ \\
\left(\frac{1}{3}+z_{3}\right) c \hat{\mathbf{z}}\end{array}$ & $(6 c)$ & O II \\
\hline $\mathbf{B}_{12}$ & $=$ & $\left(-x_{3}+y_{3}\right) \mathbf{a}_{1}-x_{3} \mathbf{a}_{2}+\left(\frac{2}{3}+z_{3}\right) \mathbf{a}_{3}$ & $=$ & $\begin{array}{c}\left(-x_{3}+\frac{1}{2} y_{3}\right) a \hat{\mathbf{x}}-\frac{\sqrt{3}}{2} y_{3} a \hat{\mathbf{y}}+ \\
\left(\frac{2}{3}+z_{3}\right) c \hat{\mathbf{z}}\end{array}$ & $(6 c)$ & O II \\
\hline $\mathbf{B}_{13}$ & $=$ & $-x_{3} \mathbf{a}_{1}-y_{3} \mathbf{a}_{2}+z_{3} \mathbf{a}_{3}$ & $=$ & $\begin{array}{c}-\frac{1}{2}\left(x_{3}+y_{3}\right) a \hat{\mathbf{x}}+ \\
\frac{\sqrt{3}}{2}\left(x_{3}-y_{3}\right) a \hat{\mathbf{y}}+z_{3} c \hat{\mathbf{z}}\end{array}$ & $(6 c)$ & O II \\
\hline $\mathbf{B}_{14}$ & $=$ & $y_{3} \mathbf{a}_{1}+\left(-x_{3}+y_{3}\right) \mathbf{a}_{2}+\left(\frac{1}{3}+z_{3}\right) \mathbf{a}_{3}$ & $=$ & $\begin{array}{c}\left(-\frac{1}{2} x_{3}+y_{3}\right) a \hat{\mathbf{x}}-\frac{\sqrt{3}}{2} x_{3} a \hat{\mathbf{y}}+ \\
\left(\frac{1}{3}+z_{3}\right) c \hat{\mathbf{z}}\end{array}$ & $(6 c)$ & O II \\
\hline $\mathbf{B}_{15}$ & $=$ & $\left(x_{3}-y_{3}\right) \mathbf{a}_{1}+x_{3} \mathbf{a}_{2}+\left(\frac{2}{3}+z_{3}\right) \mathbf{a}_{3}$ & $=$ & $\begin{array}{c}\left(x_{3}-\frac{1}{2} y_{3}\right) a \hat{\mathbf{x}}+\frac{\sqrt{3}}{2} y_{3} a \hat{\mathbf{y}}+ \\
\left(\frac{2}{3}+z_{3}\right) c \hat{\mathbf{z}}\end{array}$ & $(6 c)$ & $\mathrm{O}$ II \\
\hline $\mathbf{B}_{16}$ & $=$ & $x_{4} \mathbf{a}_{1}+y_{4} \mathbf{a}_{2}+z_{4} \mathbf{a}_{3}$ & $=$ & $\begin{array}{c}\frac{1}{2}\left(x_{4}+y_{4}\right) a \hat{\mathbf{x}}+ \\
\frac{\sqrt{3}}{2}\left(-x_{4}+y_{4}\right) a \hat{\mathbf{y}}+z_{4} c \hat{\mathbf{z}}\end{array}$ & $(6 c)$ & O III \\
\hline $\mathbf{B}_{17}$ & $=$ & $-y_{4} \mathbf{a}_{1}+\left(x_{4}-y_{4}\right) \mathbf{a}_{2}+\left(\frac{1}{3}+z_{4}\right) \mathbf{a}_{3}$ & $=$ & $\begin{array}{c}\left(\frac{1}{2} x_{4}-y_{4}\right) a \hat{\mathbf{x}}+\frac{\sqrt{3}}{2} x_{4} a \hat{\mathbf{y}}+ \\
\left(\frac{1}{3}+z_{4}\right) c \hat{\mathbf{z}}\end{array}$ & $(6 c)$ & O III \\
\hline $\mathbf{B}_{18}$ & $=$ & $\left(-x_{4}+y_{4}\right) \mathbf{a}_{1}-x_{4} \mathbf{a}_{2}+\left(\frac{2}{3}+z_{4}\right) \mathbf{a}_{3}$ & $=$ & $\begin{array}{c}\left(-x_{4}+\frac{1}{2} y_{4}\right) a \hat{\mathbf{x}}-\frac{\sqrt{3}}{2} y_{4} a \hat{\mathbf{y}}+ \\
\left(\frac{2}{3}+z_{4}\right) c \hat{\mathbf{z}}\end{array}$ & $(6 c)$ & O III \\
\hline $\mathbf{B}_{19}$ & $=$ & $-x_{4} \mathbf{a}_{1}-y_{4} \mathbf{a}_{2}+z_{4} \mathbf{a}_{3}$ & $=$ & $\begin{array}{c}-\frac{1}{2}\left(x_{4}+y_{4}\right) a \hat{\mathbf{x}}+ \\
\frac{\sqrt{3}}{2}\left(x_{4}-y_{4}\right) a \hat{\mathbf{y}}+z_{4} c \hat{\mathbf{z}}\end{array}$ & $(6 c)$ & O III \\
\hline $\mathbf{B}_{20}$ & $=$ & $y_{4} \mathbf{a}_{1}+\left(-x_{4}+y_{4}\right) \mathbf{a}_{2}+\left(\frac{1}{3}+z_{4}\right) \mathbf{a}_{3}$ & $=$ & $\begin{array}{c}\left(-\frac{1}{2} x_{4}+y_{4}\right) a \hat{\mathbf{x}}-\frac{\sqrt{3}}{2} x_{4} a \hat{\mathbf{y}}+ \\
\left(\frac{1}{3}+z_{4}\right) c \hat{\mathbf{z}}\end{array}$ & $(6 c)$ & O III \\
\hline $\mathbf{B}_{21}$ & $=$ & $\left(x_{4}-y_{4}\right) \mathbf{a}_{1}+x_{4} \mathbf{a}_{2}+\left(\frac{2}{3}+z_{4}\right) \mathbf{a}_{3}$ & $=$ & $\begin{array}{c}\left(x_{4}-\frac{1}{2} y_{4}\right) a \hat{\mathbf{x}}+\frac{\sqrt{3}}{2} y_{4} a \hat{\mathbf{y}}+ \\
\left(\frac{2}{3}+z_{4}\right) c \hat{\mathbf{z}}\end{array}$ & $(6 c)$ & O III \\
\hline $\mathbf{B}_{22}$ & $=$ & $x_{5} \mathbf{a}_{1}+y_{5} \mathbf{a}_{2}+z_{5} \mathbf{a}_{3}$ & $=$ & $\begin{array}{c}\frac{1}{2}\left(x_{5}+y_{5}\right) a \hat{\mathbf{x}}+ \\
\frac{\sqrt{3}}{2}\left(-x_{5}+y_{5}\right) a \hat{\mathbf{y}}+z_{5} c \hat{\mathbf{z}}\end{array}$ & $(6 c)$ & O IV \\
\hline $\mathbf{B}_{23}$ & $=$ & $-y_{5} \mathbf{a}_{1}+\left(x_{5}-y_{5}\right) \mathbf{a}_{2}+\left(\frac{1}{3}+z_{5}\right) \mathbf{a}_{3}$ & $=$ & $\begin{array}{c}\left(\frac{1}{2} x_{5}-y_{5}\right) a \hat{\mathbf{x}}+\frac{\sqrt{3}}{2} x_{5} a \hat{\mathbf{y}}+ \\
\left(\frac{1}{3}+z_{5}\right) c \hat{\mathbf{z}}\end{array}$ & $(6 c)$ & O IV \\
\hline
\end{tabular}




$$
\begin{aligned}
& \mathbf{B}_{24}=\left(-x_{5}+y_{5}\right) \mathbf{a}_{1}-x_{5} \mathbf{a}_{2}+\left(\frac{2}{3}+z_{5}\right) \mathbf{a}_{3}=\left(-x_{5}+\frac{1}{2} y_{5}\right) a \hat{\mathbf{x}}-\frac{\sqrt{3}}{2} y_{5} a \hat{\mathbf{y}}+ \\
& \left(\frac{2}{3}+z_{5}\right) c \hat{\mathbf{z}} \\
& \mathbf{B}_{25}=-x_{5} \mathbf{a}_{1}-y_{5} \mathbf{a}_{2}+z_{5} \mathbf{a}_{3} \quad=\quad-\frac{1}{2}\left(x_{5}+y_{5}\right) a \hat{\mathbf{x}}+\quad \text { (6c) } \\
& \frac{\sqrt{3}}{2}\left(x_{5}-y_{5}\right) a \hat{\mathbf{y}}+z_{5} c \hat{\mathbf{z}} \\
& \mathbf{B}_{26}=y_{5} \mathbf{a}_{1}+\left(-x_{5}+y_{5}\right) \mathbf{a}_{2}+\left(\frac{1}{3}+z_{5}\right) \mathbf{a}_{3}=\left(-\frac{1}{2} x_{5}+y_{5}\right) a \hat{\mathbf{x}}-\frac{\sqrt{3}}{2} x_{5} a \hat{\mathbf{y}}+ \\
& \left(\frac{1}{3}+z_{5}\right) c \hat{\mathbf{z}} \\
& \mathbf{B}_{27}=\left(x_{5}-y_{5}\right) \mathbf{a}_{1}+x_{5} \mathbf{a}_{2}+\left(\frac{2}{3}+z_{5}\right) \mathbf{a}_{3}=\left(x_{5}-\frac{1}{2} y_{5}\right) a \hat{\mathbf{x}}+\frac{\sqrt{3}}{2} y_{5} a \hat{\mathbf{y}}+ \\
& \left(\frac{2}{3}+z_{5}\right) c \hat{\mathbf{z}} \\
& \mathbf{B}_{28}=x_{6} \mathbf{a}_{1}+y_{6} \mathbf{a}_{2}+z_{6} \mathbf{a}_{3} \quad=\quad \frac{1}{2}\left(x_{6}+y_{6}\right) a \hat{\mathbf{x}}+ \\
& \frac{\sqrt{3}}{2}\left(-x_{6}+y_{6}\right) a \hat{\mathbf{y}}+z_{6} c \hat{\mathbf{z}} \\
& \mathbf{B}_{29}=-y_{6} \mathbf{a}_{1}+\left(x_{6}-y_{6}\right) \mathbf{a}_{2}+\left(\frac{1}{3}+z_{6}\right) \mathbf{a}_{3}=\begin{array}{c}
\left(\frac{1}{2} x_{6}-y_{6}\right) a \hat{\mathbf{x}}+\frac{\sqrt{3}}{2} x_{6} a \hat{\mathbf{y}}+ \\
\left(\frac{1}{3}+z_{6}\right) c \hat{\mathbf{z}}
\end{array} \\
& \mathbf{B}_{30}=\left(-x_{6}+y_{6}\right) \mathbf{a}_{1}-x_{6} \mathbf{a}_{2}+\left(\frac{2}{3}+z_{6}\right) \mathbf{a}_{3}=\left(-x_{6}+\frac{1}{2} y_{6}\right) a \hat{\mathbf{x}}-\frac{\sqrt{3}}{2} y_{6} a \hat{\mathbf{y}}+ \\
& \left(\frac{2}{3}+z_{6}\right) c \hat{\mathbf{z}} \\
& \mathbf{B}_{31}=-x_{6} \mathbf{a}_{1}-y_{6} \mathbf{a}_{2}+z_{6} \mathbf{a}_{3} \quad=\quad-\frac{1}{2}\left(x_{6}+y_{6}\right) a \hat{\mathbf{x}}+ \\
& \frac{\sqrt{3}}{2}\left(x_{6}-y_{6}\right) a \hat{\mathbf{y}}+z_{6} c \hat{\mathbf{z}} \\
& \mathbf{B}_{32}=y_{6} \mathbf{a}_{1}+\left(-x_{6}+y_{6}\right) \mathbf{a}_{2}+\left(\frac{1}{3}+z_{6}\right) \mathbf{a}_{3}=\left(-\frac{1}{2} x_{6}+y_{6}\right) a \hat{\mathbf{x}}-\frac{\sqrt{3}}{2} x_{6} a \hat{\mathbf{y}}+ \\
& \left(\frac{1}{3}+z_{6}\right) c \hat{\mathbf{z}} \\
& \mathbf{B}_{33}=\left(x_{6}-y_{6}\right) \mathbf{a}_{1}+x_{6} \mathbf{a}_{2}+\left(\frac{2}{3}+z_{6}\right) \mathbf{a}_{3}=\left(x_{6}-\frac{1}{2} y_{6}\right) a \hat{\mathbf{x}}+\frac{\sqrt{3}}{2} y_{6} a \hat{\mathbf{y}}+ \\
& \left(\frac{2}{3}+z_{6}\right) c \hat{\mathbf{z}} \\
& \mathbf{B}_{34}=\quad x_{7} \mathbf{a}_{1}+y_{7} \mathbf{a}_{2}+z_{7} \mathbf{a}_{3} \quad=\quad \frac{1}{2}\left(x_{7}+y_{7}\right) a \hat{\mathbf{x}}+\quad \text { (6c) } \\
& \frac{\sqrt{3}}{2}\left(-x_{7}+y_{7}\right) a \hat{\mathbf{y}}+z_{7} c \hat{\mathbf{z}} \\
& \mathbf{B}_{35}=-y_{7} \mathbf{a}_{1}+\left(x_{7}-y_{7}\right) \mathbf{a}_{2}+\left(\frac{1}{3}+z_{7}\right) \mathbf{a}_{3}=\left(\frac{1}{2} x_{7}-y_{7}\right) a \hat{\mathbf{x}}+\frac{\sqrt{3}}{2} x_{7} a \hat{\mathbf{y}}+ \\
& \left(\frac{1}{3}+z_{7}\right) c \hat{\mathbf{z}} \\
& \mathbf{B}_{36}=\left(-x_{7}+y_{7}\right) \mathbf{a}_{1}-x_{7} \mathbf{a}_{2}+\left(\frac{2}{3}+z_{7}\right) \mathbf{a}_{3}=\left(-x_{7}+\frac{1}{2} y_{7}\right) a \hat{\mathbf{x}}-\frac{\sqrt{3}}{2} y_{7} a \hat{\mathbf{y}}+ \\
& \left(\frac{2}{3}+z_{7}\right) c \hat{\mathbf{z}} \\
& \mathbf{B}_{37}=\quad-x_{7} \mathbf{a}_{1}-y_{7} \mathbf{a}_{2}+z_{7} \mathbf{a}_{3} \quad=\quad-\frac{1}{2}\left(x_{7}+y_{7}\right) a \hat{\mathbf{x}}+ \\
& \frac{\sqrt{3}}{2}\left(x_{7}-y_{7}\right) a \hat{\mathbf{y}}+z_{7} c \hat{\mathbf{z}} \\
& \mathbf{B}_{38}=y_{7} \mathbf{a}_{1}+\left(-x_{7}+y_{7}\right) \mathbf{a}_{2}+\left(\frac{1}{3}+z_{7}\right) \mathbf{a}_{3}=\left(-\frac{1}{2} x_{7}+y_{7}\right) a \hat{\mathbf{x}}-\frac{\sqrt{3}}{2} x_{7} a \hat{\mathbf{y}}+ \\
& \left(\frac{1}{3}+z_{7}\right) c \hat{\mathbf{z}} \\
& \mathbf{B}_{39}=\left(x_{7}-y_{7}\right) \mathbf{a}_{1}+x_{7} \mathbf{a}_{2}+\left(\frac{2}{3}+z_{7}\right) \mathbf{a}_{3}=\left(x_{7}-\frac{1}{2} y_{7}\right) a \hat{\mathbf{x}}+\frac{\sqrt{3}}{2} y_{7} a \hat{\mathbf{y}}+ \\
& \left(\frac{2}{3}+z_{7}\right) c \hat{\mathbf{z}} \\
& \text { O V }
\end{aligned}
$$

\section{References:}

- R. N. Hargreaves and E. Stanley, The structure of strontium dithionate tetrahydrate, Zeitschrift für Kristallographie Crystalline Materials 135, 399-407 (1972), doi:10.1524/zkri.1972.135.5-6.399.

\section{Geometry files:}

- CIF: pp. 925

- POSCAR: pp. 925 
$\mathrm{PI}_{3}$ Structure: A3B_hP8_173_c_b
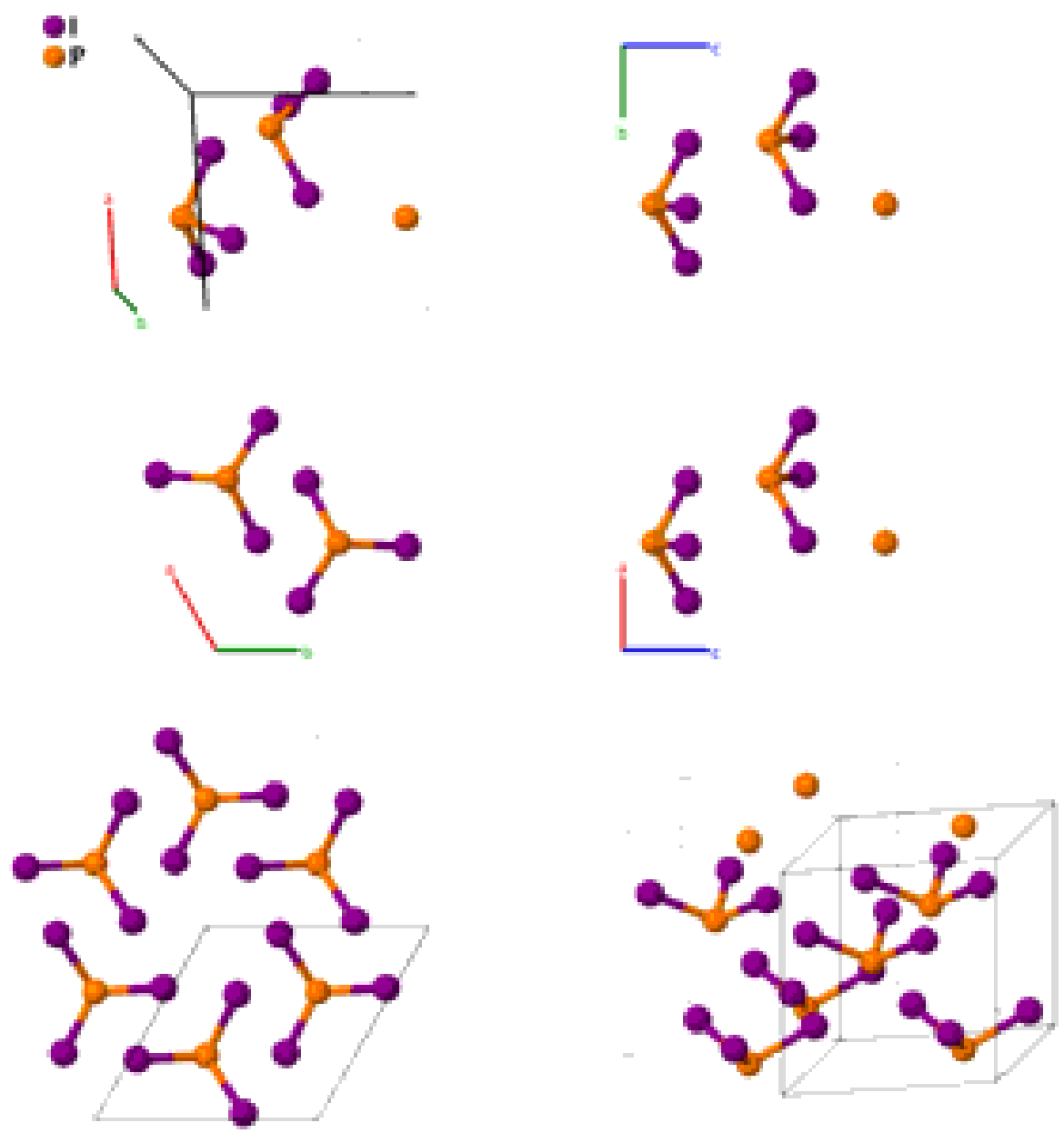

Prototype

AFLOW prototype label

: $\quad \mathrm{PI}_{3}$

Strukturbericht designation

Pearson symbol

A3B_hP8_173_c_b

Space group number

None

Space group symbol

: $\mathrm{hP8}$

AFLOW prototype command

173

: $\quad P_{3}$

aflow - -proto=A3B_hP8_173_c_b

- -params $=a, c / a, z_{1}, x_{2}, y_{2}, z_{2}$

Hexagonal primitive vectors:

$$
\begin{array}{ll}
\mathbf{a}_{1}= & \frac{1}{2} a \hat{\mathbf{x}}-\frac{\sqrt{3}}{2} a \hat{\mathbf{y}} \\
\mathbf{a}_{2}= & \frac{1}{2} a \hat{\mathbf{x}}+\frac{\sqrt{3}}{2} a \hat{\mathbf{y}} \\
\mathbf{a}_{3}= & c \hat{\mathbf{z}}
\end{array}
$$

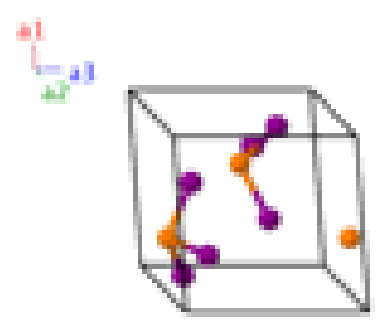




\section{Lattice Coordinates}

$$
\begin{aligned}
& \mathbf{B}_{1}=\quad \frac{1}{3} \mathbf{a}_{1}+\frac{2}{3} \mathbf{a}_{2}+z_{1} \mathbf{a}_{3} \\
& \mathbf{B}_{2}=\frac{2}{3} \mathbf{a}_{1}+\frac{1}{3} \mathbf{a}_{2}+\left(\frac{1}{2}+z_{1}\right) \mathbf{a}_{3} \\
& \mathbf{B}_{3}= \\
& x_{2} \mathbf{a}_{1}+y_{2} \mathbf{a}_{2}+z_{2} \mathbf{a}_{3} \\
& \mathbf{B}_{4}=-y_{2} \mathbf{a}_{1}+\left(x_{2}-y_{2}\right) \mathbf{a}_{2}+z_{2} \mathbf{a}_{3}= \\
& \mathbf{B}_{5}=\left(-x_{2}+y_{2}\right) \mathbf{a}_{1}-x_{2} \mathbf{a}_{2}+z_{2} \mathbf{a}_{3}= \\
& \mathbf{B}_{6}=-x_{2} \mathbf{a}_{1}-y_{2} \mathbf{a}_{2}+\left(\frac{1}{2}+z_{2}\right) \mathbf{a}_{3}= \\
& =\quad \frac{1}{2} a \hat{\mathbf{x}}+\frac{1}{2 \sqrt{3}} a \hat{\mathbf{y}}+z_{1} c \hat{\mathbf{z}} \\
& =\frac{1}{2} a \hat{\mathbf{x}}-\frac{1}{2 \sqrt{3}} a \hat{\mathbf{y}}+\left(\frac{1}{2}+z_{1}\right) c \hat{\mathbf{z}} \\
& = \\
& \frac{1}{2}\left(x_{2}+y_{2}\right) a \hat{\mathbf{x}}+ \\
& \frac{\sqrt{3}}{2}\left(-x_{2}+y_{2}\right) a \hat{\mathbf{y}}+z_{2} c \hat{\mathbf{z}} \\
& =\left(\frac{1}{2} x_{2}-y_{2}\right) a \hat{\mathbf{x}}+\frac{\sqrt{3}}{2} x_{2} a \hat{\mathbf{y}}+z_{2} c \hat{\mathbf{z}} \\
& =\left(-x_{2}+\frac{1}{2} y_{2}\right) a \hat{\mathbf{x}}-\frac{\sqrt{3}}{2} y_{2} a \hat{\mathbf{y}}+z_{2} c \hat{\mathbf{z}} \\
& =\quad-\frac{1}{2}\left(x_{2}+y_{2}\right) a \hat{\mathbf{x}}+ \\
& \frac{\sqrt{3}}{2}\left(x_{2}-y_{2}\right) a \hat{\mathbf{y}}+\left(\frac{1}{2}+z_{2}\right) c \hat{\mathbf{z}} \\
& \mathbf{B}_{7}=y_{2} \mathbf{a}_{1}+\left(-x_{2}+y_{2}\right) \mathbf{a}_{2}+\left(\frac{1}{2}+z_{2}\right) \mathbf{a}_{3}=\left(-\frac{1}{2} x_{2}+y_{2}\right) a \hat{\mathbf{x}}-\frac{\sqrt{3}}{2} x_{2} a \hat{\mathbf{y}}+ \\
& \left(\frac{1}{2}+z_{2}\right) c \hat{\mathbf{z}} \\
& \mathbf{B}_{8}=\left(x_{2}-y_{2}\right) \mathbf{a}_{1}+x_{2} \mathbf{a}_{2}+\left(\frac{1}{2}+z_{2}\right) \mathbf{a}_{3}=\left(x_{2}-\frac{1}{2} y_{2}\right) a \hat{\mathbf{x}}+\frac{\sqrt{3}}{2} y_{2} a \hat{\mathbf{y}}+ \\
& \left(\frac{1}{2}+z_{2}\right) c \hat{\mathbf{z}}
\end{aligned}
$$

\section{Atom Type}

$\mathrm{P}$

$\mathrm{P}$

I

I

I

I

I

I

\section{References:}

- E. T. Lance, J. M. Haschke, and D. R. Peacor, Crystal and molecular structure of phosphorus triiodide, Inorg. Chem. 15, 780-781 (1976), doi:10.1021/ic50158a007.

\section{Found in:}

- P. Villars and K. Cenzual, Pearson's Crystal Data - Crystal Structure Database for Inorganic Compounds, ASM International (2013).

\section{Geometry files:}

- CIF: pp. 925

- POSCAR: pp. 926 
$\beta-\mathrm{Si}_{3} \mathrm{~N}_{4}$ Structure: A4B3_hP14_173_bc_c

$9 \mathrm{~N}$
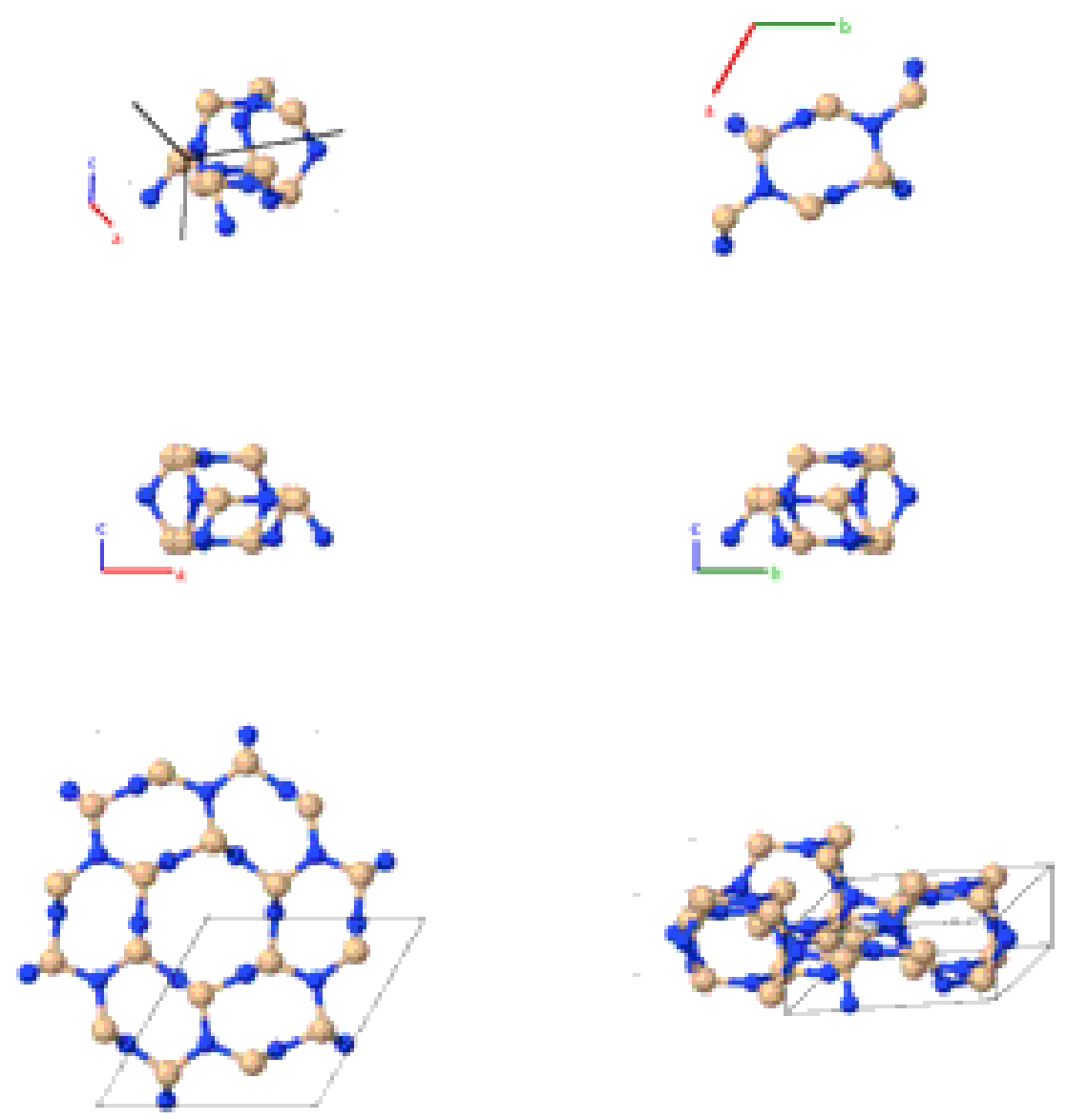

Prototype

$: \quad \beta-\mathrm{Si}_{3} \mathrm{~N}_{4}$

AFLOW prototype label

: A4B3_hP14_173_bc_c

Strukturbericht designation

Pearson symbol

None

Space group number

hP14

Space group symbol

173

AFLOW prototype command

$\mathrm{PG}_{3}$

aflow --proto=A4B3_hP14_173_bc_c

- -params $=a, c / a, z_{1}, x_{2}, y_{2}, z_{2}, x_{3}, y_{3}, z_{3}$

Hexagonal primitive vectors:

$$
\begin{array}{ll}
\mathbf{a}_{1}= & \frac{1}{2} a \hat{\mathbf{x}}-\frac{\sqrt{3}}{2} a \hat{\mathbf{y}} \\
\mathbf{a}_{2}= & \frac{1}{2} a \hat{\mathbf{x}}+\frac{\sqrt{3}}{2} a \hat{\mathbf{y}} \\
\mathbf{a}_{3}= & c \hat{\mathbf{z}}
\end{array}
$$




\begin{tabular}{|c|c|c|c|c|c|c|}
\hline $\mathbf{B}_{1}$ & $=$ & $\frac{1}{3} \mathbf{a}_{1}+\frac{2}{3} \mathbf{a}_{2}+z_{1} \mathbf{a}_{3}$ & $=$ & $\frac{1}{2} a \hat{\mathbf{x}}+\frac{1}{2 \sqrt{3}} a \hat{\mathbf{y}}+z_{1} c \hat{\mathbf{z}}$ & $(2 b)$ & $\mathrm{N} \mathrm{I}$ \\
\hline $\mathbf{B}_{2}$ & $=$ & $\frac{2}{3} \mathbf{a}_{1}+\frac{1}{3} \mathbf{a}_{2}+\left(\frac{1}{2}+z_{1}\right) \mathbf{a}_{3}$ & $=$ & $\frac{1}{2} a \hat{\mathbf{x}}-\frac{1}{2 \sqrt{3}} a \hat{\mathbf{y}}+\left(\frac{1}{2}+z_{1}\right) c \hat{\mathbf{z}}$ & $(2 b)$ & $\mathrm{N} \mathrm{I}$ \\
\hline $\mathbf{B}_{3}$ & $=$ & $x_{2} \mathbf{a}_{1}+y_{2} \mathbf{a}_{2}+z_{2} \mathbf{a}_{3}$ & $=$ & $\begin{array}{c}\frac{1}{2}\left(x_{2}+y_{2}\right) a \hat{\mathbf{x}}+ \\
\frac{\sqrt{3}}{2}\left(-x_{2}+y_{2}\right) a \hat{\mathbf{y}}+z_{2} c \hat{\mathbf{z}}\end{array}$ & $(6 c)$ & $\mathrm{N}$ II \\
\hline $\mathbf{B}_{4}$ & $=$ & $-y_{2} \mathbf{a}_{1}+\left(x_{2}-y_{2}\right) \mathbf{a}_{2}+z_{2} \mathbf{a}_{3}$ & $=$ & $\left(\frac{1}{2} x_{2}-y_{2}\right) a \hat{\mathbf{x}}+\frac{\sqrt{3}}{2} x_{2} a \hat{\mathbf{y}}+z_{2} c \hat{\mathbf{z}}$ & $(6 c)$ & $\mathrm{N}$ II \\
\hline $\mathbf{B}_{5}$ & $=$ & $\left(-x_{2}+y_{2}\right) \mathbf{a}_{1}-x_{2} \mathbf{a}_{2}+z_{2} \mathbf{a}_{3}$ & $=$ & $\left(-x_{2}+\frac{1}{2} y_{2}\right) a \hat{\mathbf{x}}-\frac{\sqrt{3}}{2} y_{2} a \hat{\mathbf{y}}+z_{2} c \hat{\mathbf{z}}$ & $(6 c)$ & N II \\
\hline $\mathbf{B}_{6}$ & $=$ & $-x_{2} \mathbf{a}_{1}-y_{2} \mathbf{a}_{2}+\left(\frac{1}{2}+z_{2}\right) \mathbf{a}_{3}$ & $=$ & $\begin{array}{c}-\frac{1}{2}\left(x_{2}+y_{2}\right) a \hat{\mathbf{x}}+ \\
\frac{\sqrt{3}}{2}\left(x_{2}-y_{2}\right) a \hat{\mathbf{y}}+\left(\frac{1}{2}+z_{2}\right) c \hat{\mathbf{z}}\end{array}$ & $(6 c)$ & N II \\
\hline $\mathbf{B}_{7}$ & $=$ & $y_{2} \mathbf{a}_{1}+\left(-x_{2}+y_{2}\right) \mathbf{a}_{2}+\left(\frac{1}{2}+z_{2}\right) \mathbf{a}_{3}$ & $=$ & $\begin{array}{c}\left(-\frac{1}{2} x_{2}+y_{2}\right) a \hat{\mathbf{x}}-\frac{\sqrt{3}}{2} x_{2} a \hat{\mathbf{y}}+ \\
\left(\frac{1}{2}+z_{2}\right) c \hat{\mathbf{z}}\end{array}$ & $(6 c)$ & $\mathrm{N}$ II \\
\hline $\mathbf{B}_{8}$ & $=$ & $\left(x_{2}-y_{2}\right) \mathbf{a}_{1}+x_{2} \mathbf{a}_{2}+\left(\frac{1}{2}+z_{2}\right) \mathbf{a}_{3}$ & $=$ & $\begin{array}{c}\left(x_{2}-\frac{1}{2} y_{2}\right) a \hat{\mathbf{x}}+\frac{\sqrt{3}}{2} y_{2} a \hat{\mathbf{y}}+ \\
\left(\frac{1}{2}+z_{2}\right) c \hat{\mathbf{z}}\end{array}$ & $(6 c)$ & $\mathrm{N}$ II \\
\hline $\mathbf{B}_{9}$ & $=$ & $x_{3} \mathbf{a}_{1}+y_{3} \mathbf{a}_{2}+z_{3} \mathbf{a}_{3}$ & $=$ & $\begin{array}{c}\frac{1}{2}\left(x_{3}+y_{3}\right) a \hat{\mathbf{x}}+ \\
\frac{\sqrt{3}}{2}\left(-x_{3}+y_{3}\right) a \hat{\mathbf{y}}+z_{3} c \hat{\mathbf{z}}\end{array}$ & $(6 c)$ & $\mathrm{Si}$ \\
\hline $\mathbf{B}_{10}$ & $=$ & $-y_{3} \mathbf{a}_{1}+\left(x_{3}-y_{3}\right) \mathbf{a}_{2}+z_{3} \mathbf{a}_{3}$ & $=$ & $\left(\frac{1}{2} x_{3}-y_{3}\right) a \hat{\mathbf{x}}+\frac{\sqrt{3}}{2} x_{3} a \hat{\mathbf{y}}+z_{3} c \hat{\mathbf{z}}$ & $(6 c)$ & $\mathrm{Si}$ \\
\hline $\mathbf{B}_{11}$ & $=$ & $\left(-x_{3}+y_{3}\right) \mathbf{a}_{1}-x_{3} \mathbf{a}_{2}+z_{3} \mathbf{a}_{3}$ & $=$ & $\left(-x_{3}+\frac{1}{2} y_{3}\right) a \hat{\mathbf{x}}-\frac{\sqrt{3}}{2} y_{3} a \hat{\mathbf{y}}+z_{3} c \hat{\mathbf{z}}$ & $(6 c)$ & $\mathrm{Si}$ \\
\hline $\mathbf{B}_{12}$ & $=$ & $-x_{3} \mathbf{a}_{1}-y_{3} \mathbf{a}_{2}+\left(\frac{1}{2}+z_{3}\right) \mathbf{a}_{3}$ & $=$ & $\begin{array}{c}-\frac{1}{2}\left(x_{3}+y_{3}\right) a \hat{\mathbf{x}}+ \\
\frac{\sqrt{3}}{2}\left(x_{3}-y_{3}\right) a \hat{\mathbf{y}}+\left(\frac{1}{2}+z_{3}\right) c \hat{\mathbf{z}}\end{array}$ & $(6 c)$ & $\mathrm{Si}$ \\
\hline $\mathbf{B}_{13}$ & $=$ & $y_{3} \mathbf{a}_{1}+\left(-x_{3}+y_{3}\right) \mathbf{a}_{2}+\left(\frac{1}{2}+z_{3}\right) \mathbf{a}_{3}$ & $=$ & $\begin{array}{c}\left(-\frac{1}{2} x_{3}+y_{3}\right) a \hat{\mathbf{x}}-\frac{\sqrt{3}}{2} x_{3} a \hat{\mathbf{y}}+ \\
\left(\frac{1}{2}+z_{3}\right) c \hat{\mathbf{z}}\end{array}$ & $(6 c)$ & $\mathrm{Si}$ \\
\hline $\mathbf{B}_{14}$ & $=$ & $\left(x_{3}-y_{3}\right) \mathbf{a}_{1}+x_{3} \mathbf{a}_{2}+\left(\frac{1}{2}+z_{3}\right) \mathbf{a}_{3}$ & $=$ & $\begin{array}{c}\left(x_{3}-\frac{1}{2} y_{3}\right) a \hat{\mathbf{x}}+\frac{\sqrt{3}}{2} y_{3} a \hat{\mathbf{y}}+ \\
\left(\frac{1}{2}+z_{3}\right) c \hat{\mathbf{z}}\end{array}$ & $(6 c)$ & $\mathrm{Si}$ \\
\hline
\end{tabular}

\section{References:}

- W. D. Forgeng and B. F. Decker, Nitrides of silicon, T. Am. I. Min. Met. Eng. 212, 343-348 (1958).

\section{Found in:}

- P. Villars and K. Cenzual, Pearson's Crystal Data - Crystal Structure Database for Inorganic Compounds, ASM International (2013).

\section{Geometry files:}

- CIF: pp. 926

- POSCAR: pp. 926 
$\mathrm{Fe}_{12} \mathrm{Zr}_{2} \mathrm{P}_{7}$ Structure: A12B7C2_hP21_174_2j2k_ajk_cf
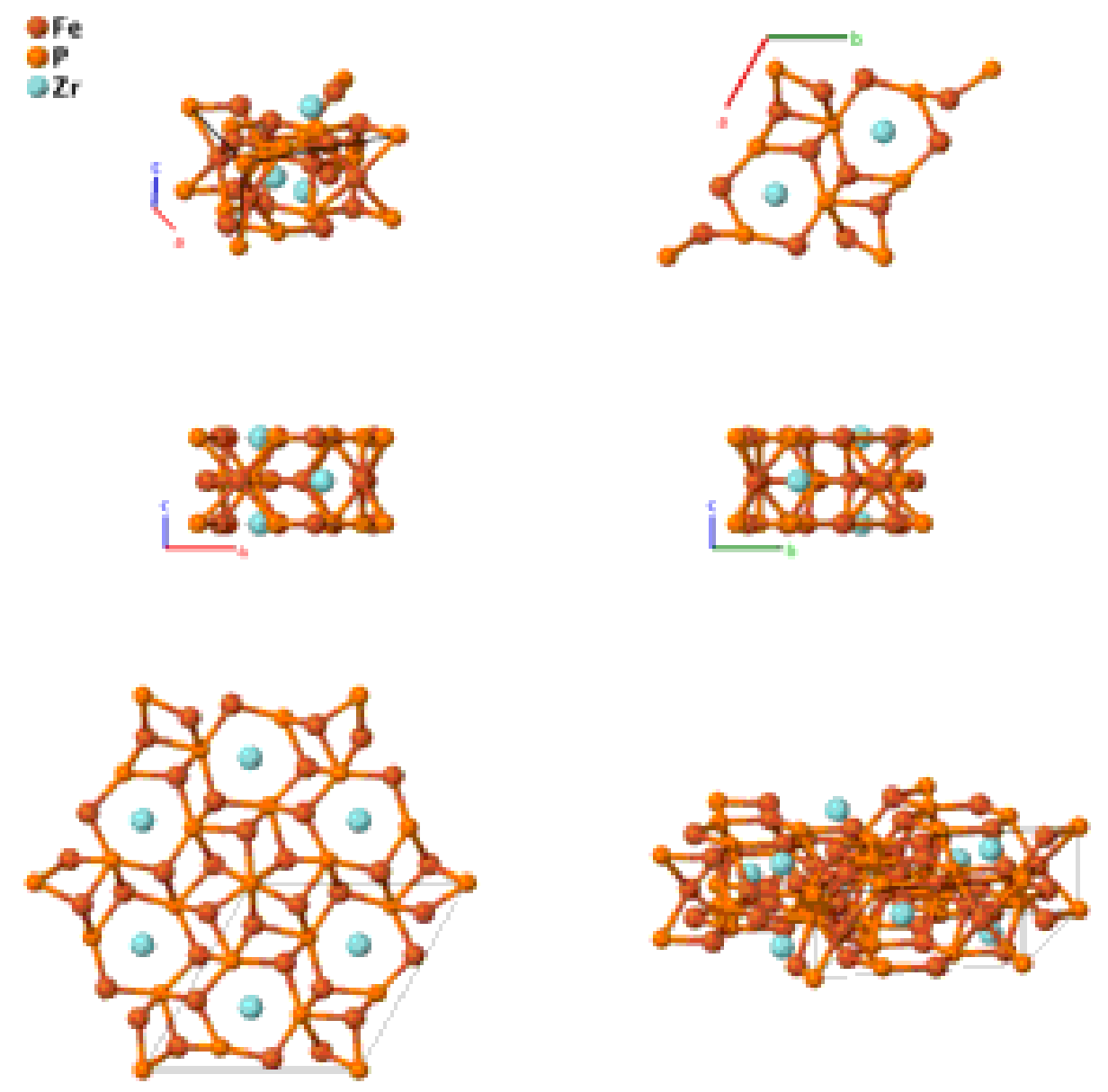

\section{Prototype}

AFLOW prototype label

Strukturbericht designation

Pearson symbol

: $\quad \mathrm{Fe}_{12} \mathrm{Zr}_{2} \mathrm{P}_{7}$

Space group number

Space group symbol

: A12B7C2_hP21_174_2j2k_ajk_cf

AFLOW prototype command

: None

: $\mathrm{hP} 21$

: 174

: $\quad P \overline{6}$

: $\quad$ aflow --proto=A12B7C2_hP21_174_2j2k_ajk_cf

- - params $=a, c / a, x_{4}, y_{4}, x_{5}, y_{5}, x_{6}, y_{6}, x_{7}, y_{7}, x_{8}, y_{8}, x_{9}, y_{9}$

Hexagonal primitive vectors:

$$
\begin{array}{ll}
\mathbf{a}_{1}= & \frac{1}{2} a \hat{\mathbf{x}}-\frac{\sqrt{3}}{2} a \hat{\mathbf{y}} \\
\mathbf{a}_{2}= & \frac{1}{2} a \hat{\mathbf{x}}+\frac{\sqrt{3}}{2} a \hat{\mathbf{y}} \\
\mathbf{a}_{3}= & c \hat{\mathbf{z}}
\end{array}
$$

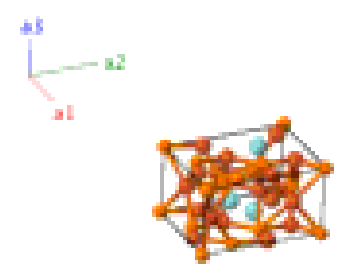

Basis vectors: 


\begin{tabular}{|c|c|c|c|c|c|c|}
\hline $\mathbf{B}_{1}$ & $=$ & $0 \mathbf{a}_{1}+0 \mathbf{a}_{2}+0 \mathbf{a}_{3}$ & $=$ & $0 \hat{\mathbf{x}}+0 \hat{\mathbf{y}}+0 \hat{\mathbf{z}}$ & $(1 a)$ & P I \\
\hline $\mathbf{B}_{2}$ & $=$ & $\frac{1}{3} \mathbf{a}_{1}+\frac{2}{3} \mathbf{a}_{2}$ & $=$ & $\frac{1}{2} a \hat{\mathbf{x}}+\frac{1}{2 \sqrt{3}} a \hat{\mathbf{y}}$ & $(1 c)$ & $\mathrm{Zr} \mathrm{I}$ \\
\hline $\mathbf{B}_{3}$ & $=$ & $\frac{2}{3} \mathbf{a}_{1}+\frac{1}{3} \mathbf{a}_{2}+\frac{1}{2} \mathbf{a}_{3}$ & $=$ & $\frac{1}{2} a \hat{\mathbf{x}}-\frac{1}{2 \sqrt{3}} a \hat{\mathbf{y}}+\frac{1}{2} c \hat{\mathbf{z}}$ & $(1 f)$ & $\mathrm{Zr}$ II \\
\hline $\mathbf{B}_{4}$ & $=$ & $x_{4} \mathbf{a}_{1}+y_{4} \mathbf{a}_{2}$ & $=$ & $\frac{1}{2}\left(x_{4}+y_{4}\right) a \hat{\mathbf{x}}+\frac{\sqrt{3}}{2}\left(-x_{4}+y_{4}\right) a \hat{\mathbf{y}}$ & $(3 j)$ & Fe I \\
\hline $\mathbf{B}_{5}$ & $=$ & $-y_{4} \mathbf{a}_{1}+\left(x_{4}-y_{4}\right) \mathbf{a}_{2}$ & $=$ & $\left(\frac{1}{2} x_{4}-y_{4}\right) a \hat{\mathbf{x}}+\frac{\sqrt{3}}{2} x_{4} a \hat{\mathbf{y}}$ & $(3 j)$ & $\mathrm{Fe} \mathrm{I}$ \\
\hline $\mathbf{B}_{6}$ & $=$ & $\left(-x_{4}+y_{4}\right) \mathbf{a}_{1}-x_{4} \mathbf{a}_{2}$ & $=$ & $\left(-x_{4}+\frac{1}{2} y_{4}\right) a \hat{\mathbf{x}}-\frac{\sqrt{3}}{2} y_{4} a \hat{\mathbf{y}}$ & $(3 j)$ & $\mathrm{Fe} \mathrm{I}$ \\
\hline $\mathbf{B}_{7}$ & $=$ & $x_{5} \mathbf{a}_{1}+y_{5} \mathbf{a}_{2}$ & $=$ & $\frac{1}{2}\left(x_{5}+y_{5}\right) a \hat{\mathbf{x}}+\frac{\sqrt{3}}{2}\left(-x_{5}+y_{5}\right) a \hat{\mathbf{y}}$ & $(3 j)$ & Fe II \\
\hline $\mathbf{B}_{8}$ & $=$ & $-y_{5} \mathbf{a}_{1}+\left(x_{5}-y_{5}\right) \mathbf{a}_{2}$ & $=$ & $\left(\frac{1}{2} x_{5}-y_{5}\right) a \hat{\mathbf{x}}+\frac{\sqrt{3}}{2} x_{5} a \hat{\mathbf{y}}$ & $(3 j)$ & $\mathrm{Fe} I I$ \\
\hline $\mathbf{B}_{9}$ & $=$ & $\left(-x_{5}+y_{5}\right) \mathbf{a}_{1}-x_{5} \mathbf{a}_{2}$ & $=$ & $\left(-x_{5}+\frac{1}{2} y_{5}\right) a \hat{\mathbf{x}}-\frac{\sqrt{3}}{2} y_{5} a \hat{\mathbf{y}}$ & $(3 j)$ & Fe II \\
\hline $\mathbf{B}_{10}$ & $=$ & $x_{6} \mathbf{a}_{1}+y_{6} \mathbf{a}_{2}$ & $=$ & $\frac{1}{2}\left(x_{6}+y_{6}\right) a \hat{\mathbf{x}}+\frac{\sqrt{3}}{2}\left(-x_{6}+y_{6}\right) a \hat{\mathbf{y}}$ & $(3 j)$ & P II \\
\hline $\mathbf{B}_{11}$ & $=$ & $-y_{6} \mathbf{a}_{1}+\left(x_{6}-y_{6}\right) \mathbf{a}_{2}$ & $=$ & $\left(\frac{1}{2} x_{6}-y_{6}\right) a \hat{\mathbf{x}}+\frac{\sqrt{3}}{2} x_{6} a \hat{\mathbf{y}}$ & $(3 j)$ & P II \\
\hline $\mathbf{B}_{12}$ & $=$ & $\left(-x_{6}+y_{6}\right) \mathbf{a}_{1}-x_{6} \mathbf{a}_{2}$ & $=$ & $\left(-x_{6}+\frac{1}{2} y_{6}\right) a \hat{\mathbf{x}}-\frac{\sqrt{3}}{2} y_{6} a \hat{\mathbf{y}}$ & $(3 j)$ & P II \\
\hline $\mathbf{B}_{13}$ & $=$ & $x_{7} \mathbf{a}_{1}+y_{7} \mathbf{a}_{2}+\frac{1}{2} \mathbf{a}_{3}$ & $=$ & $\frac{1}{2}\left(x_{7}+y_{7}\right) a \hat{\mathbf{x}}+\frac{\sqrt{3}}{2}\left(-x_{7}+y_{7}\right) a \hat{\mathbf{y}}+\frac{1}{2} c \hat{\mathbf{z}}$ & $(3 k)$ & Fe III \\
\hline $\mathbf{B}_{14}$ & $=$ & $-y_{7} \mathbf{a}_{1}+\left(x_{7}-y_{7}\right) \mathbf{a}_{2}+\frac{1}{2} \mathbf{a}_{3}$ & $=$ & $\left(\frac{1}{2} x_{7}-y_{7}\right) a \hat{\mathbf{x}}+\frac{\sqrt{3}}{2} x_{7} a \hat{\mathbf{y}}+\frac{1}{2} c \hat{\mathbf{z}}$ & $(3 k)$ & Fe III \\
\hline $\mathbf{B}_{15}$ & $=$ & $\left(-x_{7}+y_{7}\right) \mathbf{a}_{1}-x_{7} \mathbf{a}_{2}+\frac{1}{2} \mathbf{a}_{3}$ & $=$ & $\left(-x_{7}+\frac{1}{2} y_{7}\right) a \hat{\mathbf{x}}-\frac{\sqrt{3}}{2} y_{7} a \hat{\mathbf{y}}+\frac{1}{2} c \hat{\mathbf{z}}$ & $(3 k)$ & Fe III \\
\hline $\mathbf{B}_{16}$ & $=$ & $x_{8} \mathbf{a}_{1}+y_{8} \mathbf{a}_{2}+\frac{1}{2} \mathbf{a}_{3}$ & $=$ & $\frac{1}{2}\left(x_{8}+y_{8}\right) a \hat{\mathbf{x}}+\frac{\sqrt{3}}{2}\left(-x_{8}+y_{8}\right) a \hat{\mathbf{y}}+\frac{1}{2} c \hat{\mathbf{z}}$ & $(3 k)$ & Fe IV \\
\hline $\mathbf{B}_{17}$ & $=$ & $-y_{8} \mathbf{a}_{1}+\left(x_{8}-y_{8}\right) \mathbf{a}_{2}+\frac{1}{2} \mathbf{a}_{3}$ & $=$ & $\left(\frac{1}{2} x_{8}-y_{8}\right) a \hat{\mathbf{x}}+\frac{\sqrt{3}}{2} x_{8} a \hat{\mathbf{y}}+\frac{1}{2} c \hat{\mathbf{z}}$ & $(3 k)$ & Fe IV \\
\hline $\mathbf{B}_{18}$ & $=$ & $\left(-x_{8}+y_{8}\right) \mathbf{a}_{1}-x_{8} \mathbf{a}_{2}+\frac{1}{2} \mathbf{a}_{3}$ & $=$ & $\left(-x_{8}+\frac{1}{2} y_{8}\right) a \hat{\mathbf{x}}-\frac{\sqrt{3}}{2} y_{8} a \hat{\mathbf{y}}+\frac{1}{2} c \hat{\mathbf{z}}$ & $(3 k)$ & Fe IV \\
\hline $\mathbf{B}_{19}$ & $=$ & $x_{9} \mathbf{a}_{1}+y_{9} \mathbf{a}_{2}+\frac{1}{2} \mathbf{a}_{3}$ & $=$ & $\frac{1}{2}\left(x_{9}+y_{9}\right) a \hat{\mathbf{x}}+\frac{\sqrt{3}}{2}\left(-x_{9}+y_{9}\right) a \hat{\mathbf{y}}+\frac{1}{2} c \hat{\mathbf{z}}$ & $(3 k)$ & P III \\
\hline $\mathbf{B}_{20}$ & $=$ & $-y_{9} \mathbf{a}_{1}+\left(x_{9}-y_{9}\right) \mathbf{a}_{2}+\frac{1}{2} \mathbf{a}_{3}$ & $=$ & $\left(\frac{1}{2} x_{9}-y_{9}\right) a \hat{\mathbf{x}}+\frac{\sqrt{3}}{2} x_{9} a \hat{\mathbf{y}}+\frac{1}{2} c \hat{\mathbf{z}}$ & $(3 k)$ & P III \\
\hline $\mathbf{B}_{21}$ & $=$ & $\left(-x_{9}+y_{9}\right) \mathbf{a}_{1}-x_{9} \mathbf{a}_{2}+\frac{1}{2} \mathbf{a}_{3}$ & $=$ & $\left(-x_{9}+\frac{1}{2} y_{9}\right) a \hat{\mathbf{x}}-\frac{\sqrt{3}}{2} y_{9} a \hat{\mathbf{y}}+\frac{1}{2} c \hat{\mathbf{z}}$ & $(3 k)$ & P III \\
\hline
\end{tabular}

\section{References:}

- E. Ganglberger, Die Kristallstruktur von $\mathrm{Fe}_{12} \mathrm{Zr}_{2} \mathrm{P}_{7}$, Monatsh. Chem. 99, 557-565 (1968), doi:10.1007/BF00901204.

\section{Found in:}

- P. Villars and K. Cenzual, Pearson's Crystal Data - Crystal Structure Database for Inorganic Compounds, ASM International (2013).

\section{Geometry files:}

- CIF: pp. 926

- POSCAR: pp. 927 


\section{GdSI Structure: ABC_hP12_174_cj_fk_aj}
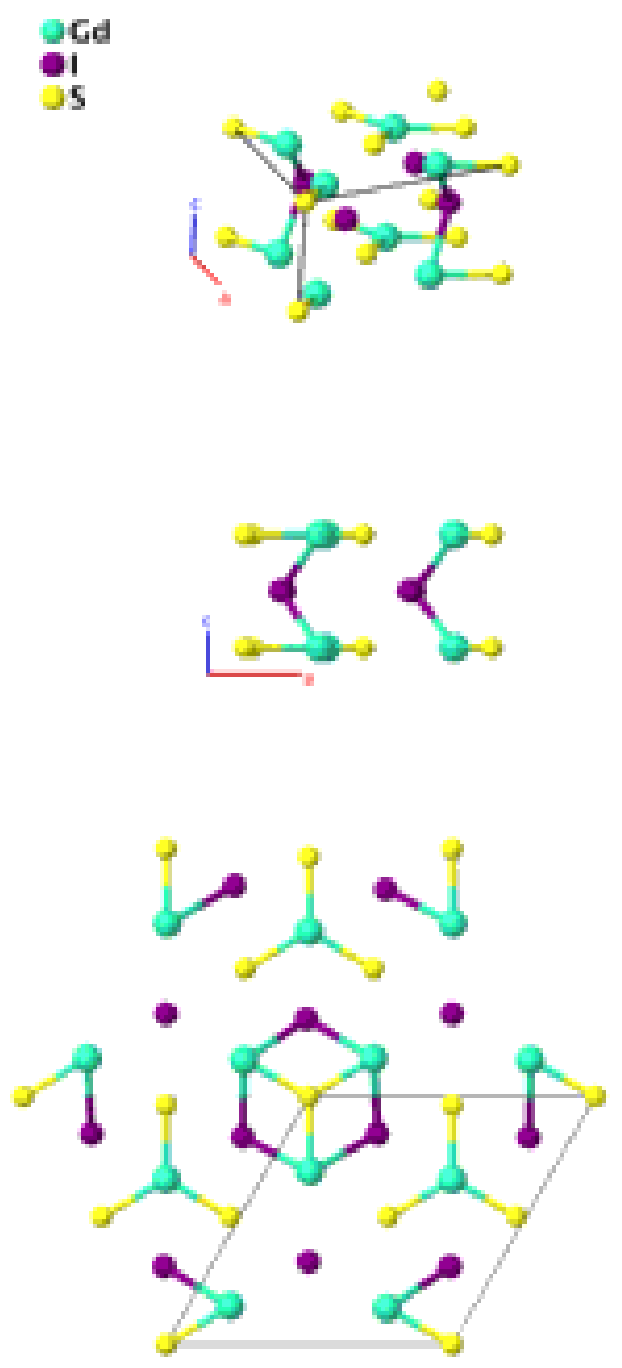
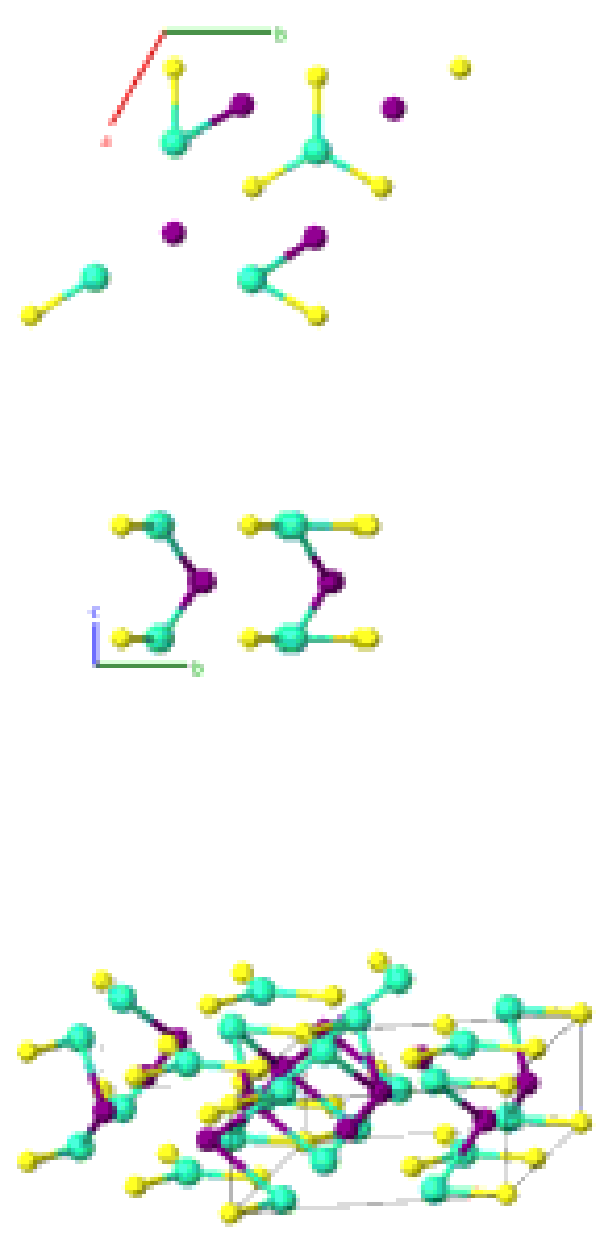

\section{Prototype}

: GdSI

AFLOW prototype label

: $\quad$ ABC_hP12_174_cj_fk_aj

Strukturbericht designation : None

Pearson symbol

: $\mathrm{hP} 12$

Space group number

: 174

Space group symbol

: $\quad P \overline{6}$

AFLOW prototype command : aflow --proto=ABC_hP12_174_cj_fk_aj

- params $=a, c / a, x_{4}, y_{4}, x_{5}, y_{5}, x_{6}, y_{6}$

Hexagonal primitive vectors:

$$
\begin{array}{ll}
\mathbf{a}_{1}= & \frac{1}{2} a \hat{\mathbf{x}}-\frac{\sqrt{3}}{2} a \hat{\mathbf{y}} \\
\mathbf{a}_{2}= & \frac{1}{2} a \hat{\mathbf{x}}+\frac{\sqrt{3}}{2} a \hat{\mathbf{y}} \\
\mathbf{a}_{3}= & c \hat{\mathbf{z}}
\end{array}
$$

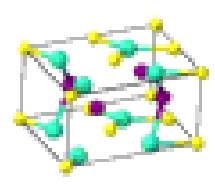




$$
\begin{array}{lcccc}
\mathbf{B}_{1}= & 0 \mathbf{a}_{1}+0 \mathbf{a}_{2}+0 \mathbf{a}_{3} & = & 0 \hat{\mathbf{x}}+0 \hat{\mathbf{y}}+0 \hat{\mathbf{z}} \\
\mathbf{B}_{2}= & \frac{1}{3} \mathbf{a}_{1}+\frac{2}{3} \mathbf{a}_{2} & = & \frac{1}{2} a \hat{\mathbf{x}}+\frac{1}{2 \sqrt{3}} a \hat{\mathbf{y}} \\
\mathbf{B}_{3}= & \frac{2}{3} \mathbf{a}_{1}+\frac{1}{3} \mathbf{a}_{2}+\frac{1}{2} \mathbf{a}_{3} & = & \frac{1}{2} a \hat{\mathbf{x}}-\frac{1}{2 \sqrt{3}} a \hat{\mathbf{y}}+\frac{1}{2} c \hat{\mathbf{z}} \\
\mathbf{B}_{4}= & x_{4} \mathbf{a}_{1}+y_{4} \mathbf{a}_{2} & = & \frac{1}{2}\left(x_{4}+y_{4}\right) a \hat{\mathbf{x}}+\frac{\sqrt{3}}{2}\left(-x_{4}+y_{4}\right) a \hat{\mathbf{y}} \\
\mathbf{B}_{5}= & -y_{4} \mathbf{a}_{1}+\left(x_{4}-y_{4}\right) \mathbf{a}_{2} & = & \left(\frac{1}{2} x_{4}-y_{4}\right) a \hat{\mathbf{x}}+\frac{\sqrt{3}}{2} x_{4} a \hat{\mathbf{y}} \\
\mathbf{B}_{6}= & \left(-x_{4}+y_{4}\right) \mathbf{a}_{1}-x_{4} \mathbf{a}_{2} & = & \left(-x_{4}+\frac{1}{2} y_{4}\right) a \hat{\mathbf{x}}-\frac{\sqrt{3}}{2} y_{4} a \hat{\mathbf{y}} \\
\mathbf{B}_{7}= & x_{5} \mathbf{a}_{1}+y_{5} \mathbf{a}_{2} & = & \frac{1}{2}\left(x_{5}+y_{5}\right) a \hat{\mathbf{x}}+\frac{\sqrt{3}}{2}\left(-x_{5}+y_{5}\right) a \hat{\mathbf{y}} \\
\mathbf{B}_{8}= & -y_{5} \mathbf{a}_{1}+\left(x_{5}-y_{5}\right) \mathbf{a}_{2} & = & \left(\frac{1}{2} x_{5}-y_{5}\right) a \hat{\mathbf{x}}+\frac{\sqrt{3}}{2} x_{5} a \hat{\mathbf{y}} \\
\mathbf{B}_{9}= & \left(-x_{5}+y_{5}\right) \mathbf{a}_{1}-x_{5} \mathbf{a}_{2} & = & \left(-x_{5}+\frac{1}{2} y_{5}\right) a \hat{\mathbf{x}}-\frac{\sqrt{3}}{2} y_{5} a \hat{\mathbf{y}} \\
\mathbf{B}_{10}= & x_{6} \mathbf{a}_{1}+y_{6} \mathbf{a}_{2}+\frac{1}{2} \mathbf{a}_{3} & = & \frac{1}{2}\left(x_{6}+y_{6}\right) a \hat{\mathbf{x}}+\frac{\sqrt{3}}{2}\left(-x_{6}+y_{6}\right) a \hat{\mathbf{y}}+\frac{1}{2} c \hat{\mathbf{z}} \\
\mathbf{B}_{11}= & -y_{6} \mathbf{a}_{1}+\left(x_{6}-y_{6}\right) \mathbf{a}_{2}+\frac{1}{2} \mathbf{a}_{3}= & & \left(\frac{1}{2} x_{6}-y_{6}\right) a \hat{\mathbf{x}}+\frac{\sqrt{3}}{2} x_{6} a \hat{\mathbf{y}}+\frac{1}{2} c \hat{\mathbf{z}} \\
\mathbf{B}_{12}= & \left(-x_{6}+y_{6}\right) \mathbf{a}_{1}-x_{6} \mathbf{a}_{2}+\frac{1}{2} \mathbf{a}_{3}= & \left(-x_{6}+\frac{1}{2} y_{6}\right) a \hat{\mathbf{x}}-\frac{\sqrt{3}}{2} y_{6} a \hat{\mathbf{y}}+\frac{1}{2} c \hat{\mathbf{z}}
\end{array}
$$

Gd I

I I

Gd II

Gd II

Gd II

S II

S II

S II

I II

I II

I II

\section{References:}

- C. Dagron and F. Thevet, Répartition des types cristallins dans la série des iodosulfures et fluorosulfures des éléments des terres rares et d'yttrium, C. R. Hebd. Séances Acad. Sci. C 268, 1867-1869 (1969).

\section{Found in:}

- P. Villars and K. Cenzual, Pearson's Crystal Data - Crystal Structure Database for Inorganic Compounds, ASM International (2013).

\section{Geometry files:}

- CIF: pp. 927

- POSCAR: pp. 927 


\section{$\mathrm{Nb}_{7} \mathrm{Ru}_{6} \mathrm{~B}_{8}$ Structure: A8B7C6_hP21_175_ck_aj_k}
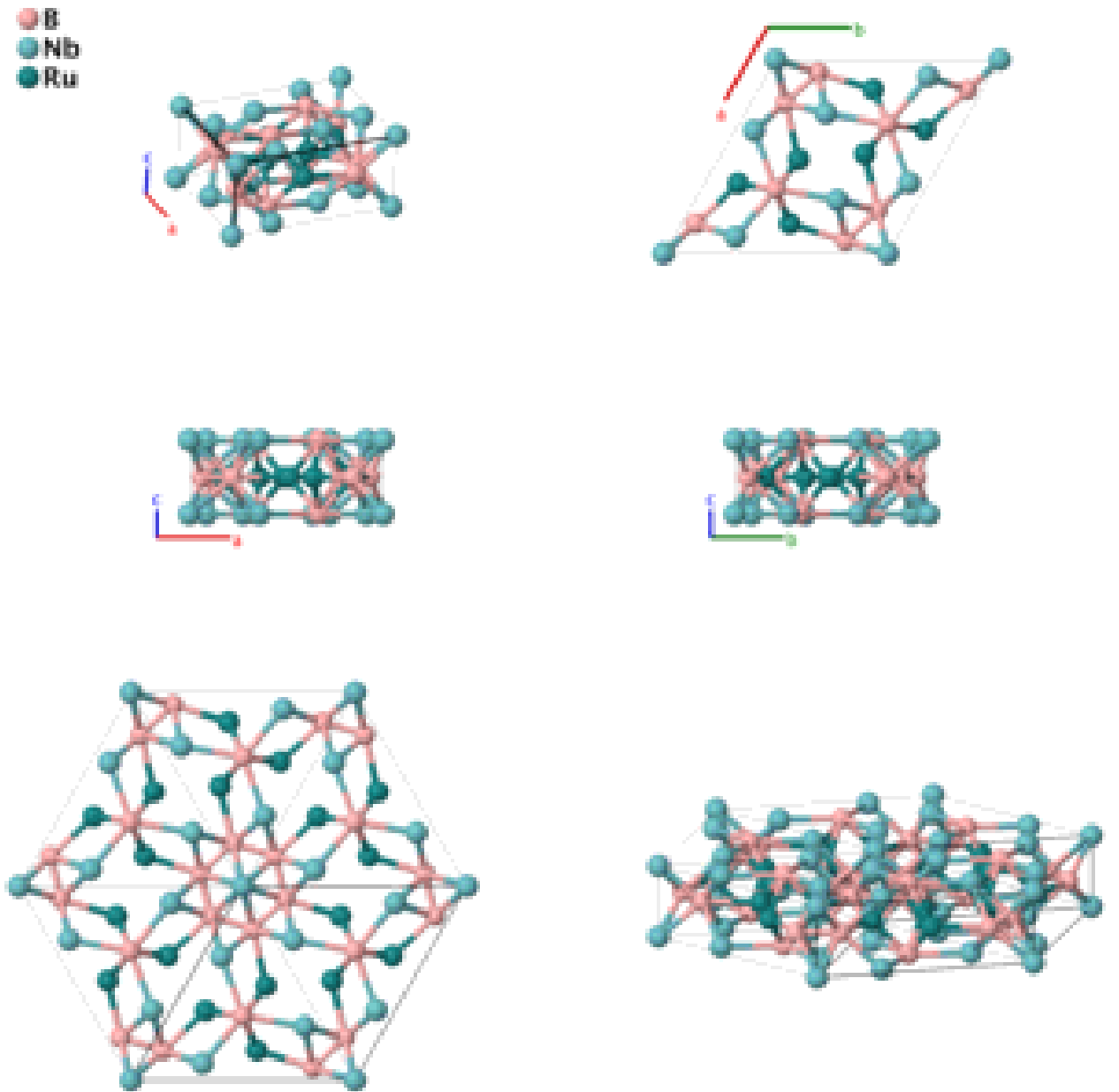

Prototype

AFLOW prototype label

: $\quad \mathrm{Nb}_{7} \mathrm{Ru}_{6} \mathrm{~B}_{8}$

Strukturbericht designation : None

Pearson symbol $\quad: \quad \mathrm{hP} 21$

Space group number $\quad: \quad 175$

Space group symbol $\quad: \quad P 6 / m$

AFLOW prototype command : aflow --proto=A8B7C6_hP21_175_ck_aj_k

- - params $=a, c / a, x_{3}, y_{3}, x_{4}, y_{4}, x_{5}, y_{5}$

Hexagonal primitive vectors:

$$
\begin{array}{ll}
\mathbf{a}_{1}= & \frac{1}{2} a \hat{\mathbf{x}}-\frac{\sqrt{3}}{2} a \hat{\mathbf{y}} \\
\mathbf{a}_{2}= & \frac{1}{2} a \hat{\mathbf{x}}+\frac{\sqrt{3}}{2} a \hat{\mathbf{y}} \\
\mathbf{a}_{3}= & c \hat{\mathbf{z}}
\end{array}
$$

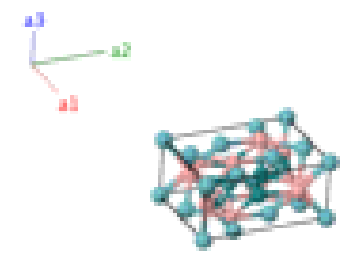

Basis vectors: 
Lattice Coordinates

$\begin{array}{lcc}\mathbf{B}_{1}= & 0 \mathbf{a}_{1}+0 \mathbf{a}_{2}+0 \mathbf{a}_{3} \\ \mathbf{B}_{2}= & \frac{1}{3} \mathbf{a}_{1}+\frac{2}{3} \mathbf{a}_{2} \\ \mathbf{B}_{3}= & \frac{2}{3} \mathbf{a}_{1}+\frac{1}{3} \mathbf{a}_{2} \\ \mathbf{B}_{4}= & x_{3} \mathbf{a}_{1}+y_{3} \mathbf{a}_{2} \\ \mathbf{B}_{5}= & -y_{3} \mathbf{a}_{1}+\left(x_{3}-y_{3}\right) \mathbf{a}_{2} \\ \mathbf{B}_{6}= & \left(-x_{3}+y_{3}\right) \mathbf{a}_{1}-x_{3} \mathbf{a}_{2} \\ \mathbf{B}_{7}= & -x_{3} \mathbf{a}_{1}-y_{3} \mathbf{a}_{2} \\ \mathbf{B}_{8}= & y_{3} \mathbf{a}_{1}+\left(-x_{3}+y_{3}\right) \mathbf{a}_{2} \\ \mathbf{B}_{9}= & \left(x_{3}-y_{3}\right) \mathbf{a}_{1}+x_{3} \mathbf{a}_{2} \\ \mathbf{B}_{10}= & x_{4} \mathbf{a}_{1}+y_{4} \mathbf{a}_{2}+\frac{1}{2} \mathbf{a}_{3} \\ \mathbf{B}_{11}= & -y_{4} \mathbf{a}_{1}+\left(x_{4}-y_{4}\right) \mathbf{a}_{2}+\frac{1}{2} \mathbf{a}_{3}= \\ \mathbf{B}_{12}= & \left(-x_{4}+y_{4}\right) \mathbf{a}_{1}-x_{4} \mathbf{a}_{2}+\frac{1}{2} \mathbf{a}_{3}= \\ \mathbf{B}_{13}= & -x_{4} \mathbf{a}_{1}-y_{4} \mathbf{a}_{2}+\frac{1}{2} \mathbf{a}_{3}= \\ \mathbf{B}_{14}= & y_{4} \mathbf{a}_{1}+\left(-x_{4}+y_{4}\right) \mathbf{a}_{2}+\frac{1}{2} \mathbf{a}_{3}= \\ \mathbf{B}_{15}= & \left(x_{4}-y_{4}\right) \mathbf{a}_{1}+x_{4} \mathbf{a}_{2}+\frac{1}{2} \mathbf{a}_{3}= \\ \mathbf{B}_{16}= & x_{5} \mathbf{a}_{1}+y_{5} \mathbf{a}_{2}+\frac{1}{2} \mathbf{a}_{3}= \\ \mathbf{B}_{17}= & -y_{5} \mathbf{a}_{1}+\left(x_{5}-y_{5}\right) \mathbf{a}_{2}+\frac{1}{2} \mathbf{a}_{3}= \\ \mathbf{B}_{18}= & \left(-x_{5}+y_{5}\right) \mathbf{a}_{1}-x_{5} \mathbf{a}_{2}+\frac{1}{2} \mathbf{a}_{3}= \\ \mathbf{B}_{19}= & -x_{5} \mathbf{a}_{1}-y_{5} \mathbf{a}_{2}+\frac{1}{2} \mathbf{a}_{3}= \\ \mathbf{B}_{20}= & y_{5} \mathbf{a}_{1}+\left(-x_{5}+y_{5}\right) \mathbf{a}_{2}+\frac{1}{2} \mathbf{a}_{3}= \\ \mathbf{B}_{21}= & \left(x_{5}-y_{5}\right) \mathbf{a}_{1}+x_{5} \mathbf{a}_{2}+\frac{1}{2} \mathbf{a}_{3}= \\ & = & =\end{array}$

Cartesian Coordinates

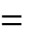

$=$

$=$

$=$

$=$

$=$

$=$

$=$

$=$

$=\frac{1}{2}\left(x_{4}+y_{4}\right) a \hat{\mathbf{x}}+\frac{\sqrt{3}}{2}\left(-x_{4}+y_{4}\right) a \hat{\mathbf{y}}+\frac{1}{2} c \hat{\mathbf{z}}$

$=\left(\frac{1}{2} x_{4}-y_{4}\right) a \hat{\mathbf{x}}+\frac{\sqrt{3}}{2} x_{4} a \hat{\mathbf{y}}+\frac{1}{2} c \hat{\mathbf{z}}$

$=\left(-x_{4}+\frac{1}{2} y_{4}\right) a \hat{\mathbf{x}}-\frac{\sqrt{3}}{2} y_{4} a \hat{\mathbf{y}}+\frac{1}{2} c \hat{\mathbf{z}}$

$=-\frac{1}{2}\left(x_{4}+y_{4}\right) a \hat{\mathbf{x}}+\frac{\sqrt{3}}{2}\left(x_{4}-y_{4}\right) a \hat{\mathbf{y}}+\frac{1}{2} c \hat{\mathbf{z}}$

$=\left(-\frac{1}{2} x_{4}+y_{4}\right) a \hat{\mathbf{x}}-\frac{\sqrt{3}}{2} x_{4} a \hat{\mathbf{y}}+\frac{1}{2} c \hat{\mathbf{z}}$

$=$

$=$

$\left(x_{4}-\frac{1}{2} y_{4}\right) a \hat{\mathbf{x}}+\frac{\sqrt{3}}{2} y_{4} a \hat{\mathbf{y}}+\frac{1}{2} c \hat{\mathbf{z}}$

$\frac{1}{2}\left(x_{5}+y_{5}\right) a \hat{\mathbf{x}}+\frac{\sqrt{3}}{2}\left(-x_{5}+y_{5}\right) a \hat{\mathbf{y}}+\frac{1}{2} c \hat{\mathbf{z}}$

$\left(\frac{1}{2} x_{5}-y_{5}\right) a \hat{\mathbf{x}}+\frac{\sqrt{3}}{2} x_{5} a \hat{\mathbf{y}}+\frac{1}{2} c \hat{\mathbf{z}}$

$\left(-x_{5}+\frac{1}{2} y_{5}\right) a \hat{\mathbf{x}}-\frac{\sqrt{3}}{2} y_{5} a \hat{\mathbf{y}}+\frac{1}{2} c \hat{\mathbf{z}}$

$=-\frac{1}{2}\left(x_{5}+y_{5}\right) a \hat{\mathbf{x}}+\frac{\sqrt{3}}{2}\left(x_{5}-y_{5}\right) a \hat{\mathbf{y}}+\frac{1}{2} c \hat{\mathbf{z}}$

$\left(-\frac{1}{2} x_{5}+y_{5}\right) a \hat{\mathbf{x}}-\frac{\sqrt{3}}{2} x_{5} a \hat{\mathbf{y}}+\frac{1}{2} c \hat{\mathbf{z}}$

$\left(x_{5}-\frac{1}{2} y_{5}\right) a \hat{\mathbf{x}}+\frac{\sqrt{3}}{2} y_{5} a \hat{\mathbf{y}}+\frac{1}{2} c \hat{\mathbf{z}}$
Wyckoff Position

(1a)
Atom Type

$\mathrm{Nb} \mathrm{I}$

B I

B I

$\mathrm{Nb}$ II

$\mathrm{Nb}$ II

$\mathrm{Nb}$ II

$\mathrm{Nb}$ II

$\mathrm{Nb}$ II

$\mathrm{Nb}$ II

B II

B II

B II

B II

B II

B II

$\mathrm{Ru}$

$\mathrm{Ru}$

$\mathrm{Ru}$

$\mathrm{Ru}$

$\mathrm{Ru}$

$\mathrm{Ru}$

\section{References:}

- Q. Zheng, M. Kohout, R. Gumeniuk, N. Abramchuk, H. Borrmann, Y. Prots, U. Burkhardt, W. Schnelle, L. Akselrud, H. Gu, A. Leithe-Jasper, and Y. Grin, $T M_{7} T M^{\prime}{ }_{6} B_{8}\left(T M=T a, N b ; T M^{\prime}=R u, R h\right.$, Ir): New Compounds with [B $B_{6}$ ] Ring Polyanions, Inorg. Chem. 51, 7472-7483 (2012), doi:10.1021/ic201978n.

\section{Found in:}

- P. Villars and K. Cenzual, Pearson's Crystal Data - Crystal Structure Database for Inorganic Compounds, ASM International (2013).

\section{Geometry files:}

- CIF: pp. 927

- POSCAR: pp. 928 
$\operatorname{Mg}[\mathrm{NH}]$ Structure: ABC_hP36_175_jk_jk_jk
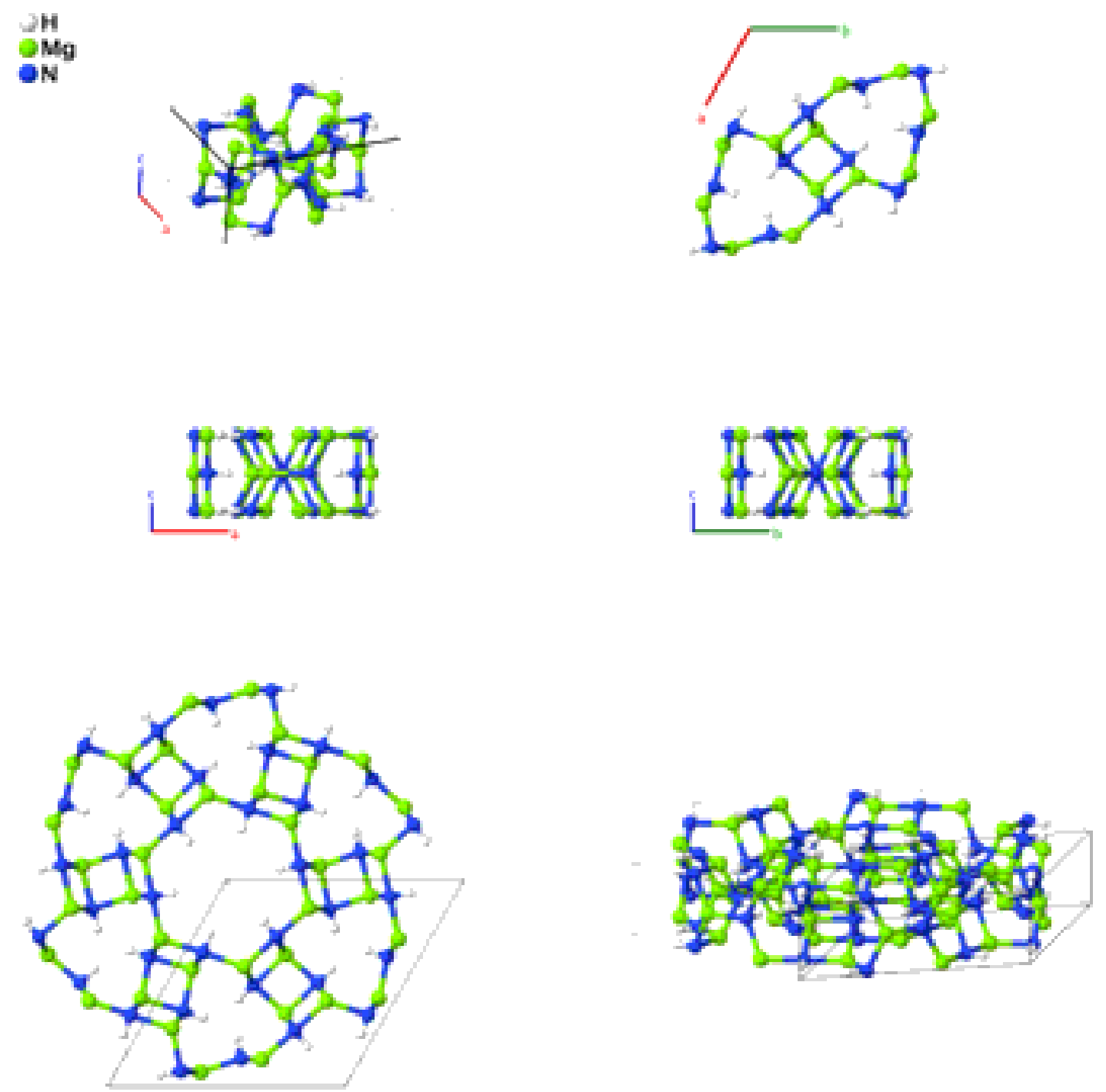

\section{Prototype}

AFLOW prototype label

Strukturbericht designation

Pearson symbol

: $\quad \mathrm{Mg}[\mathrm{NH}]$

Space group number

: $\quad$ ABC_hP36_175_jk_jk_jk

Space group symbol

: None

AFLOW prototype command : $\quad$ aflow --proto $=A B C \_h P 36 \_175-j k_{-} j k_{-} j k$

- -params $=a, c / a, x_{1}, y_{1}, x_{2}, y_{2}, x_{3}, y_{3}, x_{4}, y_{4}, x_{5}, y_{5}, x_{6}, y_{6}$

Hexagonal primitive vectors:

$$
\begin{aligned}
& \mathbf{a}_{1}=\frac{1}{2} a \hat{\mathbf{x}}-\frac{\sqrt{3}}{2} a \hat{\mathbf{y}} \\
& \mathbf{a}_{2}=\frac{1}{2} a \hat{\mathbf{x}}+\frac{\sqrt{3}}{2} a \hat{\mathbf{y}} \\
& \mathbf{a}_{3}=c \hat{\mathbf{z}}
\end{aligned}
$$

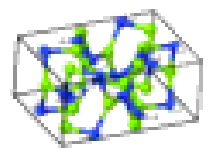


Lattice Coordinates

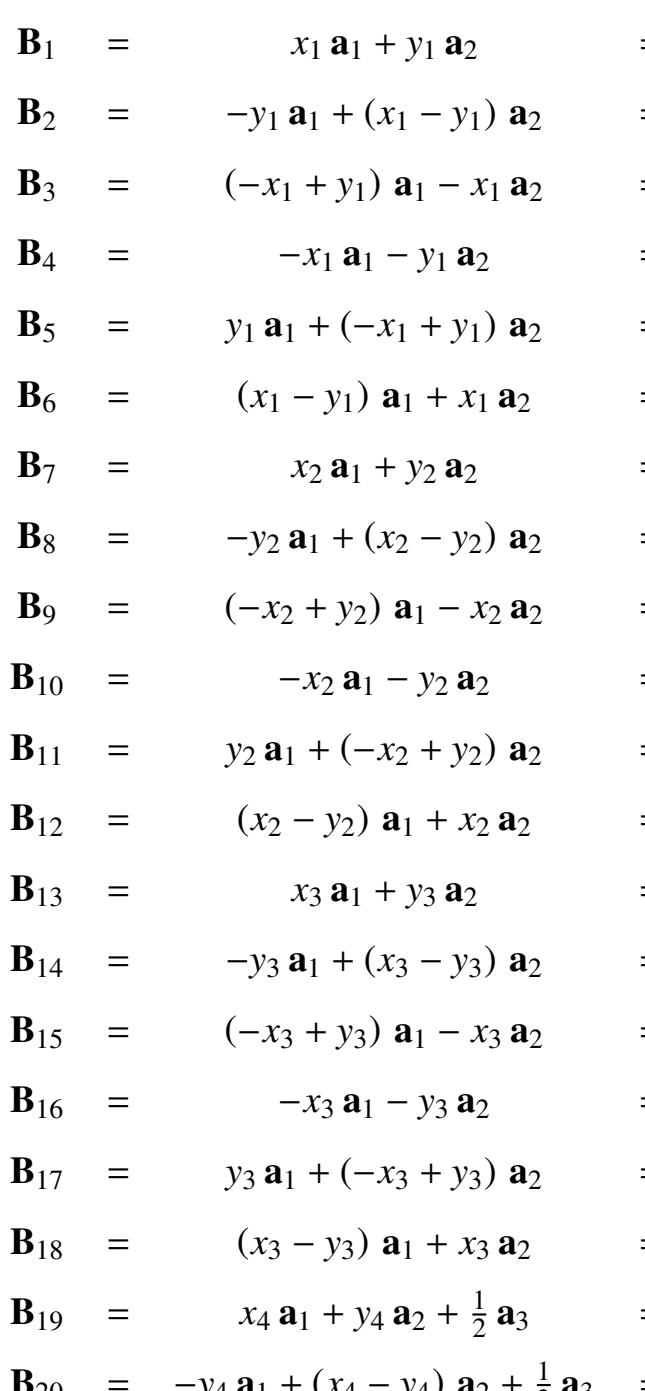

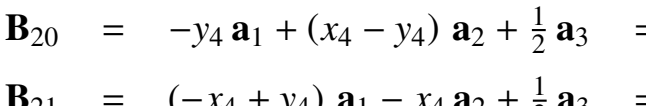

$\mathbf{B}_{21}=\left(-x_{4}+y_{4}\right) \mathbf{a}_{1}-x_{4} \mathbf{a}_{2}+\frac{1}{2} \mathbf{a}_{3}$
$\mathbf{B}_{22}=-\quad-x_{4} \mathbf{a}_{1}-y_{4} \mathbf{a}_{2}+\frac{1}{2} \mathbf{a}_{3}$

$\mathbf{B}_{23}=y_{4} \mathbf{a}_{1}+\left(-x_{4}+y_{4}\right) \mathbf{a}_{2}+\frac{1}{2} \mathbf{a}_{3}=$

$\mathbf{B}_{24}=\left(x_{4}-y_{4}\right) \mathbf{a}_{1}+x_{4} \mathbf{a}_{2}+\frac{1}{2} \mathbf{a}_{3}=$

$\mathbf{B}_{25}=x_{5} \mathbf{a}_{1}+y_{5} \mathbf{a}_{2}+\frac{1}{2} \mathbf{a}_{3}$

$\mathbf{B}_{26}=-y_{5} \mathbf{a}_{1}+\left(x_{5}-y_{5}\right) \mathbf{a}_{2}+\frac{1}{2} \mathbf{a}_{3}=$

$\mathbf{B}_{27}=\left(-x_{5}+y_{5}\right) \mathbf{a}_{1}-x_{5} \mathbf{a}_{2}+\frac{1}{2} \mathbf{a}_{3}=$

$\mathbf{B}_{28}=-x_{5} \mathbf{a}_{1}-y_{5} \mathbf{a}_{2}+\frac{1}{2} \mathbf{a}_{3}=$

$\mathbf{B}_{29}=y_{5} \mathbf{a}_{1}+\left(-x_{5}+y_{5}\right) \mathbf{a}_{2}+\frac{1}{2} \mathbf{a}_{3}=$

$\mathbf{B}_{30}=\left(x_{5}-y_{5}\right) \mathbf{a}_{1}+x_{5} \mathbf{a}_{2}+\frac{1}{2} \mathbf{a}_{3}=$

$\mathbf{B}_{31}=x_{6} \mathbf{a}_{1}+y_{6} \mathbf{a}_{2}+\frac{1}{2} \mathbf{a}_{3}=$

$\mathbf{B}_{32}=-y_{6} \mathbf{a}_{1}+\left(x_{6}-y_{6}\right) \mathbf{a}_{2}+\frac{1}{2} \mathbf{a}_{3}=$

$\mathbf{B}_{33}=\left(-x_{6}+y_{6}\right) \mathbf{a}_{1}-x_{6} \mathbf{a}_{2}+\frac{1}{2} \mathbf{a}_{3}=$

$\mathbf{B}_{34}=$ $=\quad \frac{1}{2}\left(x_{1}+y_{1}\right) a \hat{\mathbf{x}}+\frac{\sqrt{3}}{2}\left(-x_{1}+y_{1}\right) a \hat{\mathbf{y}}$

$=\quad\left(\frac{1}{2} x_{1}-y_{1}\right) a \hat{\mathbf{x}}+\frac{\sqrt{3}}{2} x_{1} a \hat{\mathbf{y}}$

$=\quad\left(-x_{1}+\frac{1}{2} y_{1}\right) a \hat{\mathbf{x}}-\frac{\sqrt{3}}{2} y_{1} a \hat{\mathbf{y}}$

$=\quad-\frac{1}{2}\left(x_{1}+y_{1}\right) a \hat{\mathbf{x}}+\frac{\sqrt{3}}{2}\left(x_{1}-y_{1}\right) a \hat{\mathbf{y}}$

$=\quad\left(-\frac{1}{2} x_{1}+y_{1}\right) a \hat{\mathbf{x}}-\frac{\sqrt{3}}{2} x_{1} a \hat{\mathbf{y}}$

$=\quad\left(x_{1}-\frac{1}{2} y_{1}\right) a \hat{\mathbf{x}}+\frac{\sqrt{3}}{2} y_{1} a \hat{\mathbf{y}}$

$=\quad \frac{1}{2}\left(x_{2}+y_{2}\right) a \hat{\mathbf{x}}+\frac{\sqrt{3}}{2}\left(-x_{2}+y_{2}\right) a \hat{\mathbf{y}}$

$=\quad\left(\frac{1}{2} x_{2}-y_{2}\right) a \hat{\mathbf{x}}+\frac{\sqrt{3}}{2} x_{2} a \hat{\mathbf{y}}$

$=\quad\left(-x_{2}+\frac{1}{2} y_{2}\right) a \hat{\mathbf{x}}-\frac{\sqrt{3}}{2} y_{2} a \hat{\mathbf{y}}$

$=\quad-\frac{1}{2}\left(x_{2}+y_{2}\right) a \hat{\mathbf{x}}+\frac{\sqrt{3}}{2}\left(x_{2}-y_{2}\right) a \hat{\mathbf{y}}$

$=\quad\left(-\frac{1}{2} x_{2}+y_{2}\right) a \hat{\mathbf{x}}-\frac{\sqrt{3}}{2} x_{2} a \hat{\mathbf{y}}$

$=\quad\left(x_{2}-\frac{1}{2} y_{2}\right) a \hat{\mathbf{x}}+\frac{\sqrt{3}}{2} y_{2} a \hat{\mathbf{y}}$

$=\quad \frac{1}{2}\left(x_{3}+y_{3}\right) a \hat{\mathbf{x}}+\frac{\sqrt{3}}{2}\left(-x_{3}+y_{3}\right) a \hat{\mathbf{y}}$

$=\quad\left(\frac{1}{2} x_{3}-y_{3}\right) a \hat{\mathbf{x}}+\frac{\sqrt{3}}{2} x_{3} a \hat{\mathbf{y}}$

$=\quad\left(-x_{3}+\frac{1}{2} y_{3}\right) a \hat{\mathbf{x}}-\frac{\sqrt{3}}{2} y_{3} a \hat{\mathbf{y}}$

$=\quad-\frac{1}{2}\left(x_{3}+y_{3}\right) a \hat{\mathbf{x}}+\frac{\sqrt{3}}{2}\left(x_{3}-y_{3}\right) a \hat{\mathbf{y}}$

$=\quad\left(-\frac{1}{2} x_{3}+y_{3}\right) a \hat{\mathbf{x}}-\frac{\sqrt{3}}{2} x_{3} a \hat{\mathbf{y}}$

$=\quad\left(x_{3}-\frac{1}{2} y_{3}\right) a \hat{\mathbf{x}}+\frac{\sqrt{3}}{2} y_{3} a \hat{\mathbf{y}}$

$=\frac{1}{2}\left(x_{4}+y_{4}\right) a \hat{\mathbf{x}}+\frac{\sqrt{3}}{2}\left(-x_{4}+y_{4}\right) a \hat{\mathbf{y}}+\frac{1}{2} c \hat{\mathbf{z}}$

$=\left(\frac{1}{2} x_{4}-y_{4}\right) a \hat{\mathbf{x}}+\frac{\sqrt{3}}{2} x_{4} a \hat{\mathbf{y}}+\frac{1}{2} c \hat{\mathbf{z}}$

$=\left(-x_{4}+\frac{1}{2} y_{4}\right) a \hat{\mathbf{x}}-\frac{\sqrt{3}}{2} y_{4} a \hat{\mathbf{y}}+\frac{1}{2} c \hat{\mathbf{z}}$

$=-\frac{1}{2}\left(x_{4}+y_{4}\right) a \hat{\mathbf{x}}+\frac{\sqrt{3}}{2}\left(x_{4}-y_{4}\right) a \hat{\mathbf{y}}+\frac{1}{2} c \hat{\mathbf{z}}$

$=\left(-\frac{1}{2} x_{4}+y_{4}\right) a \hat{\mathbf{x}}-\frac{\sqrt{3}}{2} x_{4} a \hat{\mathbf{y}}+\frac{1}{2} c \hat{\mathbf{z}}$

$=$

$\left(x_{4}-\frac{1}{2} y_{4}\right) a \hat{\mathbf{x}}+\frac{\sqrt{3}}{2} y_{4} a \hat{\mathbf{y}}+\frac{1}{2} c \hat{\mathbf{z}}$

$=\frac{1}{2}\left(x_{5}+y_{5}\right) a \hat{\mathbf{x}}+\frac{\sqrt{3}}{2}\left(-x_{5}+y_{5}\right) a \hat{\mathbf{y}}+\frac{1}{2} c \hat{\mathbf{z}}$

$=\left(\frac{1}{2} x_{5}-y_{5}\right) a \hat{\mathbf{x}}+\frac{\sqrt{3}}{2} x_{5} a \hat{\mathbf{y}}+\frac{1}{2} c \hat{\mathbf{z}}$

$=\quad\left(-x_{5}+\frac{1}{2} y_{5}\right) a \hat{\mathbf{x}}-\frac{\sqrt{3}}{2} y_{5} a \hat{\mathbf{y}}+\frac{1}{2} c \hat{\mathbf{z}}$

$=-\frac{1}{2}\left(x_{5}+y_{5}\right) a \hat{\mathbf{x}}+\frac{\sqrt{3}}{2}\left(x_{5}-y_{5}\right) a \hat{\mathbf{y}}+\frac{1}{2} c \hat{\mathbf{z}}$

$=\left(-\frac{1}{2} x_{5}+y_{5}\right) a \hat{\mathbf{x}}-\frac{\sqrt{3}}{2} x_{5} a \hat{\mathbf{y}}+\frac{1}{2} c \hat{\mathbf{z}}$

$=\left(x_{5}-\frac{1}{2} y_{5}\right) a \hat{\mathbf{x}}+\frac{\sqrt{3}}{2} y_{5} a \hat{\mathbf{y}}+\frac{1}{2} c \hat{\mathbf{z}}$

$=\frac{1}{2}\left(x_{6}+y_{6}\right) a \hat{\mathbf{x}}+\frac{\sqrt{3}}{2}\left(-x_{6}+y_{6}\right) a \hat{\mathbf{y}}+\frac{1}{2} c \hat{\mathbf{z}}$

$=\left(\frac{1}{2} x_{6}-y_{6}\right) a \hat{\mathbf{x}}+\frac{\sqrt{3}}{2} x_{6} a \hat{\mathbf{y}}+\frac{1}{2} c \hat{\mathbf{z}}$

$=\left(-x_{6}+\frac{1}{2} y_{6}\right) a \hat{\mathbf{x}}-\frac{\sqrt{3}}{2} y_{6} a \hat{\mathbf{y}}+\frac{1}{2} c \hat{\mathbf{z}}$

$=-\frac{1}{2}\left(x_{6}+y_{6}\right) a \hat{\mathbf{x}}+\frac{\sqrt{3}}{2}\left(x_{6}-y_{6}\right) a \hat{\mathbf{y}}+\frac{1}{2} c \hat{\mathbf{z}}$
Wyckoff Position Atom Type

(6j)

H I

(6j)

H I

H I

(6j)

H I

(6j)

H I

$(6 j)$

H I

(6j)

(6j)

(6j)

(6j)

(6j)
Mg I

Mg I

Mg I

Mg I

Mg I

Mg I

N I

N I

N I

N I

N I

N I

H II

H II

H II

H II

H II

H II

Mg II

Mg II

Mg II

Mg II

Mg II

Mg II

N II

N II

N II

N II 
$\mathbf{B}_{35}=y_{6} \mathbf{a}_{1}+\left(-x_{6}+y_{6}\right) \mathbf{a}_{2}+\frac{1}{2} \mathbf{a}_{3}=\left(-\frac{1}{2} x_{6}+y_{6}\right) a \hat{\mathbf{x}}-\frac{\sqrt{3}}{2} x_{6} a \hat{\mathbf{y}}+\frac{1}{2} c \hat{\mathbf{z}}$
$\mathbf{B}_{36}=\left(x_{6}-y_{6}\right) \mathbf{a}_{1}+x_{6} \mathbf{a}_{2}+\frac{1}{2} \mathbf{a}_{3}=\left(x_{6}-\frac{1}{2} y_{6}\right) a \hat{\mathbf{x}}+\frac{\sqrt{3}}{2} y_{6} a \hat{\mathbf{y}}+\frac{1}{2} c \hat{\mathbf{z}}$

$(6 k)$

$(6 k)$

\section{References:}

- F. Dolci, E. Napolitano, E. Weidner, S. Enzo, P. Moretto, M. Brunelli, T. Hansen, M. Fichtner, and W. Lohstroh, Magnesium imide: synthesis and structure determination of an unconventional alkaline earth imide from decomposition of magnesium amide, Inorg. Chem. 50, 1116-1122 (2010), doi:10.1021/ic1023778.

\section{Found in:}

- P. Villars and K. Cenzual, Pearson's Crystal Data - Crystal Structure Database for Inorganic Compounds, ASM International (2013).

\section{Geometry files:}

- CIF: pp. 928

- POSCAR: pp. 928 
$\mathrm{Er}_{3} \mathrm{Ru}_{2}$ Structure: A3B2_hP10_176_h_bd
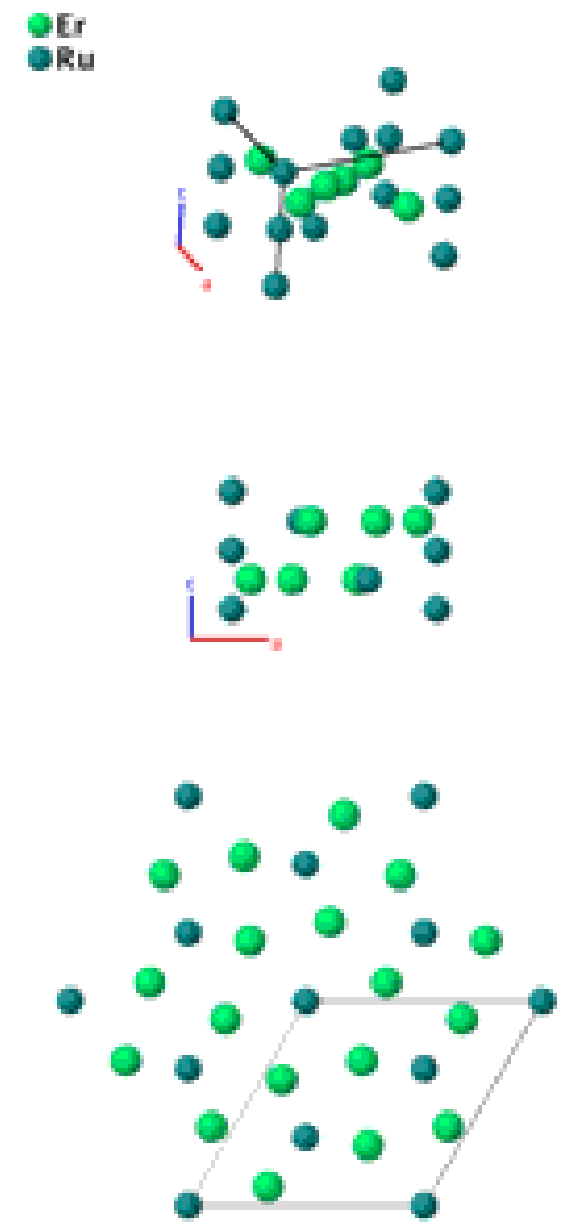
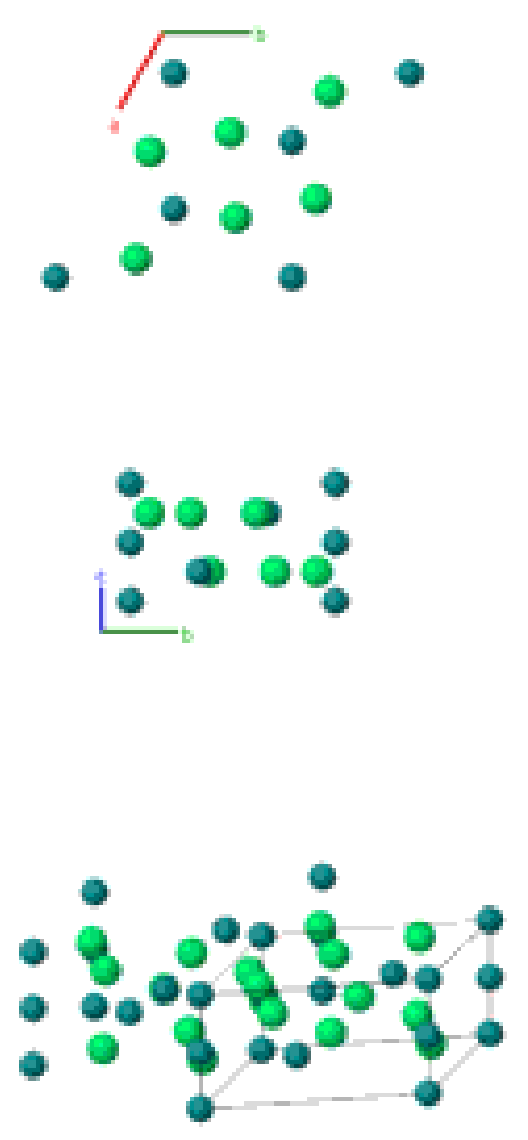

\section{Prototype}

AFLOW prototype label

Strukturbericht designation

Pearson symbol

Space group number

Space group symbol

AFLOW prototype command
: $\quad \mathrm{Er}_{3} \mathrm{Ru}_{2}$

: A3B2_hP10_176_h_bd

: None

: hP10

: 176

: $\quad P 6_{3} / m$

: aflow --proto=A3B2_hP10_176_h_bd

- - params $=a, c / a, x_{3}, y_{3}$

Hexagonal primitive vectors:

$$
\begin{array}{ll}
\mathbf{a}_{1}= & \frac{1}{2} a \hat{\mathbf{x}}-\frac{\sqrt{3}}{2} a \hat{\mathbf{y}} \\
\mathbf{a}_{2}= & \frac{1}{2} a \hat{\mathbf{x}}+\frac{\sqrt{3}}{2} a \hat{\mathbf{y}} \\
\mathbf{a}_{3}= & c \hat{\mathbf{z}}
\end{array}
$$

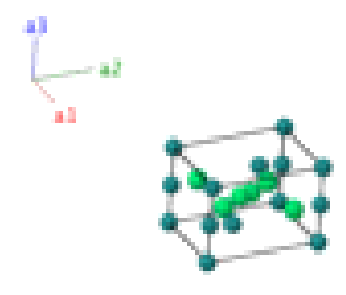

Basis vectors: 

$\mathbf{B}_{1}=$
$0 \mathbf{a}_{1}+0 \mathbf{a}_{2}+0 \mathbf{a}_{3}$
$=$
$0 \hat{\mathbf{x}}+0 \hat{\mathbf{y}}+0 \hat{\mathbf{z}}$
$\mathbf{B}_{2}=$ $\frac{1}{2} \mathbf{a}_{3}$ $\frac{1}{2} c \hat{\mathbf{z}}$
$\mathbf{B}_{3}=\frac{2}{3} \mathbf{a}_{1}+\frac{1}{3} \mathbf{a}_{2}+\frac{1}{4} \mathbf{a}_{3}$
$=$
$\mathbf{B}_{4}=\frac{1}{3} \mathbf{a}_{1}+\frac{2}{3} \mathbf{a}_{2}+\frac{3}{4} \mathbf{a}_{3}$
$=$
$\frac{1}{2} a \hat{\mathbf{x}}-\frac{1}{2 \sqrt{3}} a \hat{\mathbf{y}}+\frac{1}{4} c \hat{\mathbf{z}}$
$\mathbf{B}_{5}=x_{3} \mathbf{a}_{1}+y_{3} \mathbf{a}_{2}+\frac{1}{4} \mathbf{a}_{3}$
$=$
$\frac{1}{2} a \hat{\mathbf{x}}+\frac{1}{2 \sqrt{3}} a \hat{\mathbf{y}}+\frac{3}{4} c \hat{\mathbf{z}}$
$\mathbf{B}_{6}=-y_{3} \mathbf{a}_{1}+\left(x_{3}-y_{3}\right) \mathbf{a}_{2}+\frac{1}{4} \mathbf{a}_{3}=$
$=\frac{1}{2}\left(x_{3}+y_{3}\right) a \hat{\mathbf{x}}+\frac{\sqrt{3}}{2}\left(-x_{3}+y_{3}\right) a \hat{\mathbf{y}}+\frac{1}{4} c \hat{\mathbf{z}}$
$\mathbf{B}_{7}=\left(-x_{3}+y_{3}\right) \mathbf{a}_{1}-x_{3} \mathbf{a}_{2}+\frac{1}{4} \mathbf{a}_{3}=$

$$
\left(\frac{1}{2} x_{3}-y_{3}\right) a \hat{\mathbf{x}}+\frac{\sqrt{3}}{2} x_{3} a \hat{\mathbf{y}}+\frac{1}{4} c \hat{\mathbf{z}}
$$
$\mathbf{B}_{8}=-x_{3} \mathbf{a}_{1}-y_{3} \mathbf{a}_{2}+\frac{3}{4} \mathbf{a}_{3}=$

$$
\left(-x_{3}+\frac{1}{2} y_{3}\right) a \hat{\mathbf{x}}-\frac{\sqrt{3}}{2} y_{3} a \hat{\mathbf{y}}+\frac{1}{4} c \hat{\mathbf{z}}
$$
$\mathbf{B}_{9}=y_{3} \mathbf{a}_{1}+\left(-x_{3}+y_{3}\right) \mathbf{a}_{2}+\frac{3}{4} \mathbf{a}_{3}=$
$=-\frac{1}{2}\left(x_{3}+y_{3}\right) a \hat{\mathbf{x}}+\frac{\sqrt{3}}{2}\left(x_{3}-y_{3}\right) a \hat{\mathbf{y}}+\frac{3}{4} c \hat{\mathbf{z}}$
$\mathbf{B}_{10}=\left(x_{3}-y_{3}\right) \mathbf{a}_{1}+x_{3} \mathbf{a}_{2}+\frac{3}{4} \mathbf{a}_{3}=$
$\left(-\frac{1}{2} x_{3}+y_{3}\right) a \hat{\mathbf{x}}-\frac{\sqrt{3}}{2} x_{3} a \hat{\mathbf{y}}+\frac{3}{4} c \hat{\mathbf{z}}$

(2b)

$\mathrm{Ru} \mathrm{I}$

$\mathrm{Ru}$ I

$\mathrm{Ru}$ II

$(2 d)$

$\mathrm{Ru}$ II

Er

Er

Er

Er

Er

$(6 h)$

Er

\section{References:}

- A. Palenzona, The phase diagram of the Er-Ru system, J. Less-Common Met. 159, L21-L23 (1990), doi:10.1016/0022-5088(90)90169-K.

\section{Found in:}

- P. Villars and K. Cenzual, Pearson's Crystal Data - Crystal Structure Database for Inorganic Compounds, ASM International (2013).

\section{Geometry files:}

- CIF: pp. 929

- POSCAR: pp. 929 
$\mathrm{Fe}_{3} \mathrm{Te}_{3} \mathrm{Tl}$ Structure: A3B3C_hP14_176_h_h_d
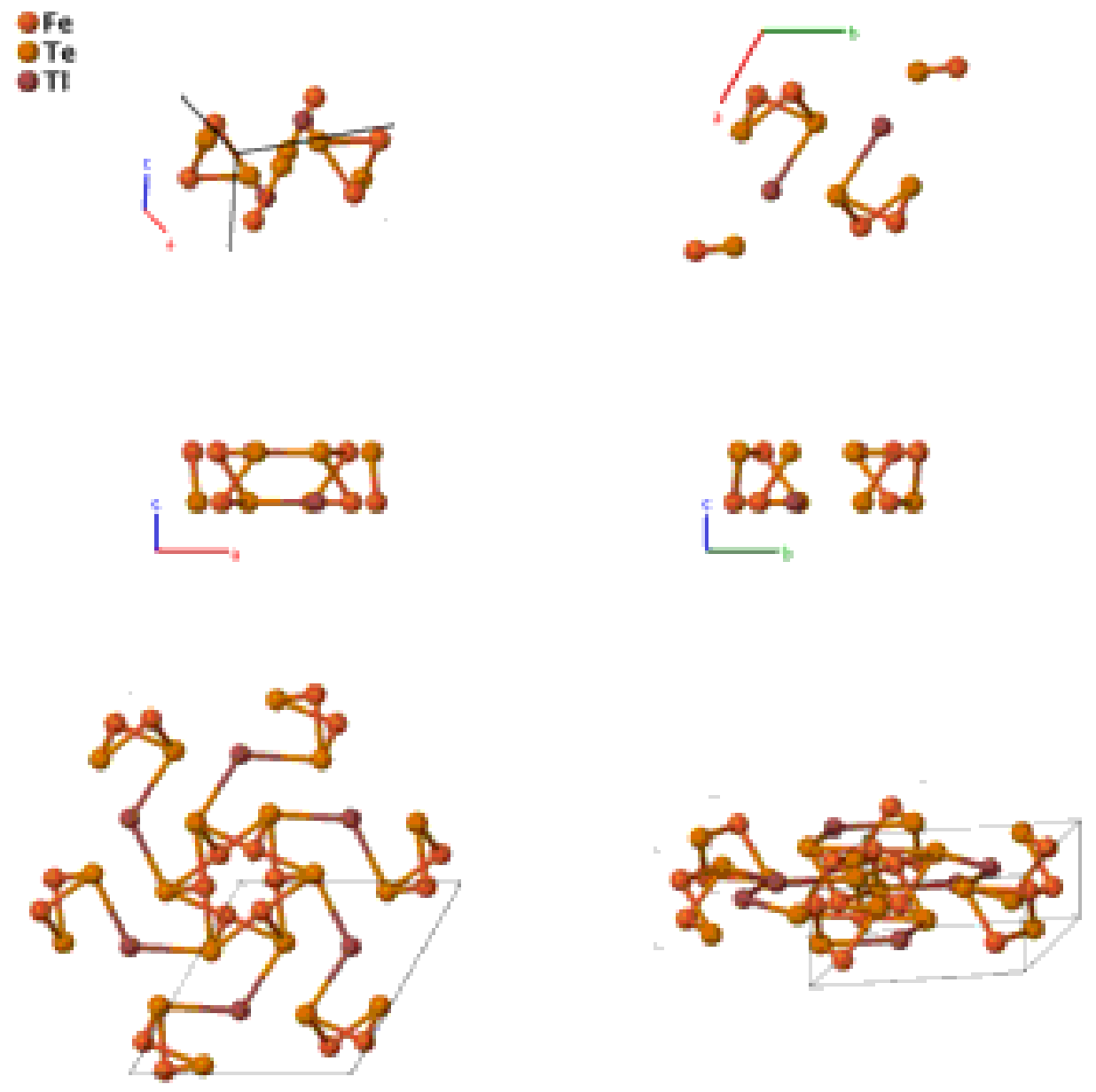

\section{Prototype}

AFLOW prototype label

Strukturbericht designation

Pearson symbol

Space group number

Space group symbol

AFLOW prototype command
$: \quad \mathrm{Fe}_{3} \mathrm{Te}_{3} \mathrm{Tl}$

: A3B3C_hP14_176_h_h_d

: None

: $\quad \mathrm{hP} 14$

: 176

: $\quad P 6_{3} / m$

: $\quad$ aflow --proto=A3B3C_hP14_176_h_h_d

- -params $=a, c / a, x_{2}, y_{2}, x_{3}, y_{3}$

Hexagonal primitive vectors:
$\mathbf{a}_{1}=\frac{1}{2} a \hat{\mathbf{x}}-\frac{\sqrt{3}}{2} a \hat{\mathbf{y}}$
$\mathbf{a}_{2}=\frac{1}{2} a \hat{\mathbf{x}}+\frac{\sqrt{3}}{2} a \hat{\mathbf{y}}$
$\mathbf{a}_{3}=c \hat{\mathbf{z}}$

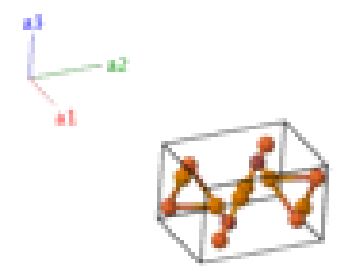

Basis vectors: 


\begin{tabular}{|c|c|c|c|c|c|}
\hline $\mathbf{B}_{1}$ & $=$ & $\frac{2}{3} \mathbf{a}_{1}+\frac{1}{3} \mathbf{a}_{2}+\frac{1}{4} \mathbf{a}_{3}$ & $=$ & $\frac{1}{2} a \hat{\mathbf{x}}-\frac{1}{2 \sqrt{3}} a \hat{\mathbf{y}}+\frac{1}{4} c \hat{\mathbf{z}}$ & $(2 d)$ \\
\hline $\mathbf{B}_{2}$ & $=$ & $\frac{1}{3} \mathbf{a}_{1}+\frac{2}{3} \mathbf{a}_{2}+\frac{3}{4} \mathbf{a}_{3}$ & $=$ & $\frac{1}{2} a \hat{\mathbf{x}}+\frac{1}{2 \sqrt{3}} a \hat{\mathbf{y}}+\frac{3}{4} c \hat{\mathbf{z}}$ & $(2 d)$ \\
\hline $\mathbf{B}_{3}$ & $=$ & $x_{2} \mathbf{a}_{1}+y_{2} \mathbf{a}_{2}+\frac{1}{4} \mathbf{a}_{3}$ & $=$ & $\frac{1}{2}\left(x_{2}+y_{2}\right) a \hat{\mathbf{x}}+\frac{\sqrt{3}}{2}\left(-x_{2}+y_{2}\right) a \hat{\mathbf{y}}+\frac{1}{4} c \hat{\mathbf{z}}$ & $(6 h)$ \\
\hline $\mathbf{B}_{4}$ & $=$ & $-y_{2} \mathbf{a}_{1}+\left(x_{2}-y_{2}\right) \mathbf{a}_{2}+\frac{1}{4} \mathbf{a}_{3}$ & $=$ & $\left(\frac{1}{2} x_{2}-y_{2}\right) a \hat{\mathbf{x}}+\frac{\sqrt{3}}{2} x_{2} a \hat{\mathbf{y}}+\frac{1}{4} c \hat{\mathbf{z}}$ & $(6 h)$ \\
\hline $\mathbf{B}_{5}$ & $=$ & $\left(-x_{2}+y_{2}\right) \mathbf{a}_{1}-x_{2} \mathbf{a}_{2}+\frac{1}{4} \mathbf{a}_{3}$ & $=$ & $\left(-x_{2}+\frac{1}{2} y_{2}\right) a \hat{\mathbf{x}}-\frac{\sqrt{3}}{2} y_{2} a \hat{\mathbf{y}}+\frac{1}{4} c \hat{\mathbf{z}}$ & $(6 h)$ \\
\hline $\mathbf{B}_{6}$ & $=$ & $-x_{2} \mathbf{a}_{1}-y_{2} \mathbf{a}_{2}+\frac{3}{4} \mathbf{a}_{3}$ & $=$ & $-\frac{1}{2}\left(x_{2}+y_{2}\right) a \hat{\mathbf{x}}+\frac{\sqrt{3}}{2}\left(x_{2}-y_{2}\right) a \hat{\mathbf{y}}+\frac{3}{4} c \hat{\mathbf{z}}$ & $(6 h)$ \\
\hline $\mathbf{B}_{7}$ & $=$ & $y_{2} \mathbf{a}_{1}+\left(-x_{2}+y_{2}\right) \mathbf{a}_{2}+\frac{3}{4} \mathbf{a}_{3}$ & $=$ & $\left(-\frac{1}{2} x_{2}+y_{2}\right) a \hat{\mathbf{x}}-\frac{\sqrt{3}}{2} x_{2} a \hat{\mathbf{y}}+\frac{3}{4} c \hat{\mathbf{z}}$ & $(6 h)$ \\
\hline $\mathbf{B}_{8}$ & $=$ & $\left(x_{2}-y_{2}\right) \mathbf{a}_{1}+x_{2} \mathbf{a}_{2}+\frac{3}{4} \mathbf{a}_{3}$ & $=$ & $\left(x_{2}-\frac{1}{2} y_{2}\right) a \hat{\mathbf{x}}+\frac{\sqrt{3}}{2} y_{2} a \hat{\mathbf{y}}+\frac{3}{4} c \hat{\mathbf{z}}$ & $(6 h)$ \\
\hline $\mathbf{B}_{9}$ & $=$ & $x_{3} \mathbf{a}_{1}+y_{3} \mathbf{a}_{2}+\frac{1}{4} \mathbf{a}_{3}$ & $=$ & $\frac{1}{2}\left(x_{3}+y_{3}\right) a \hat{\mathbf{x}}+\frac{\sqrt{3}}{2}\left(-x_{3}+y_{3}\right) a \hat{\mathbf{y}}+\frac{1}{4} c \hat{\mathbf{z}}$ & $(6 h)$ \\
\hline $\mathbf{B}_{10}$ & $=$ & $-y_{3} \mathbf{a}_{1}+\left(x_{3}-y_{3}\right) \mathbf{a}_{2}+\frac{1}{4} \mathbf{a}_{3}$ & $=$ & $\left(\frac{1}{2} x_{3}-y_{3}\right) a \hat{\mathbf{x}}+\frac{\sqrt{3}}{2} x_{3} a \hat{\mathbf{y}}+\frac{1}{4} c \hat{\mathbf{z}}$ & $(6 h)$ \\
\hline $\mathbf{B}_{11}$ & $=$ & $\left(-x_{3}+y_{3}\right) \mathbf{a}_{1}-x_{3} \mathbf{a}_{2}+\frac{1}{4} \mathbf{a}_{3}$ & $=$ & $\left(-x_{3}+\frac{1}{2} y_{3}\right) a \hat{\mathbf{x}}-\frac{\sqrt{3}}{2} y_{3} a \hat{\mathbf{y}}+\frac{1}{4} c \hat{\mathbf{z}}$ & $(6 h)$ \\
\hline $\mathbf{B}_{12}$ & $=$ & $-x_{3} \mathbf{a}_{1}-y_{3} \mathbf{a}_{2}+\frac{3}{4} \mathbf{a}_{3}$ & $=$ & $-\frac{1}{2}\left(x_{3}+y_{3}\right) a \hat{\mathbf{x}}+\frac{\sqrt{3}}{2}\left(x_{3}-y_{3}\right) a \hat{\mathbf{y}}+\frac{3}{4} c \hat{\mathbf{z}}$ & $(6 h)$ \\
\hline $\mathbf{B}_{13}$ & $=$ & $y_{3} \mathbf{a}_{1}+\left(-x_{3}+y_{3}\right) \mathbf{a}_{2}+\frac{3}{4} \mathbf{a}_{3}$ & $=$ & $\left(-\frac{1}{2} x_{3}+y_{3}\right) a \hat{\mathbf{x}}-\frac{\sqrt{3}}{2} x_{3} a \hat{\mathbf{y}}+\frac{3}{4} c \hat{\mathbf{z}}$ & $(6 h)$ \\
\hline $\mathbf{B}_{14}$ & $=$ & $\left(x_{3}-y_{3}\right) \mathbf{a}_{1}+x_{3} \mathbf{a}_{2}+\frac{3}{4} \mathbf{a}_{3}$ & $=$ & $\left(x_{3}-\frac{1}{2} y_{3}\right) a \hat{\mathbf{x}}+\frac{\sqrt{3}}{2} y_{3} a \hat{\mathbf{y}}+\frac{3}{4} c \hat{\mathbf{z}}$ & $(6 h)$ \\
\hline
\end{tabular}

\section{References:}

- K. Klepp and H. Boller, Crystal Structures of Thallium-Iron Chalcogenides, Acta Crystallogr. Sect. A 34, S160-S161 (1978).

\section{Found in:}

- P. Villars and K. Cenzual, Pearson's Crystal Data - Crystal Structure Database for Inorganic Compounds, ASM International (2013).

\section{Geometry files:}

- CIF: pp. 929

- POSCAR: pp. 929 


\section{$\mathrm{UCl}_{3}$ Structure: A3B_hP8_176_h_d}

\section{9 ㄷ}
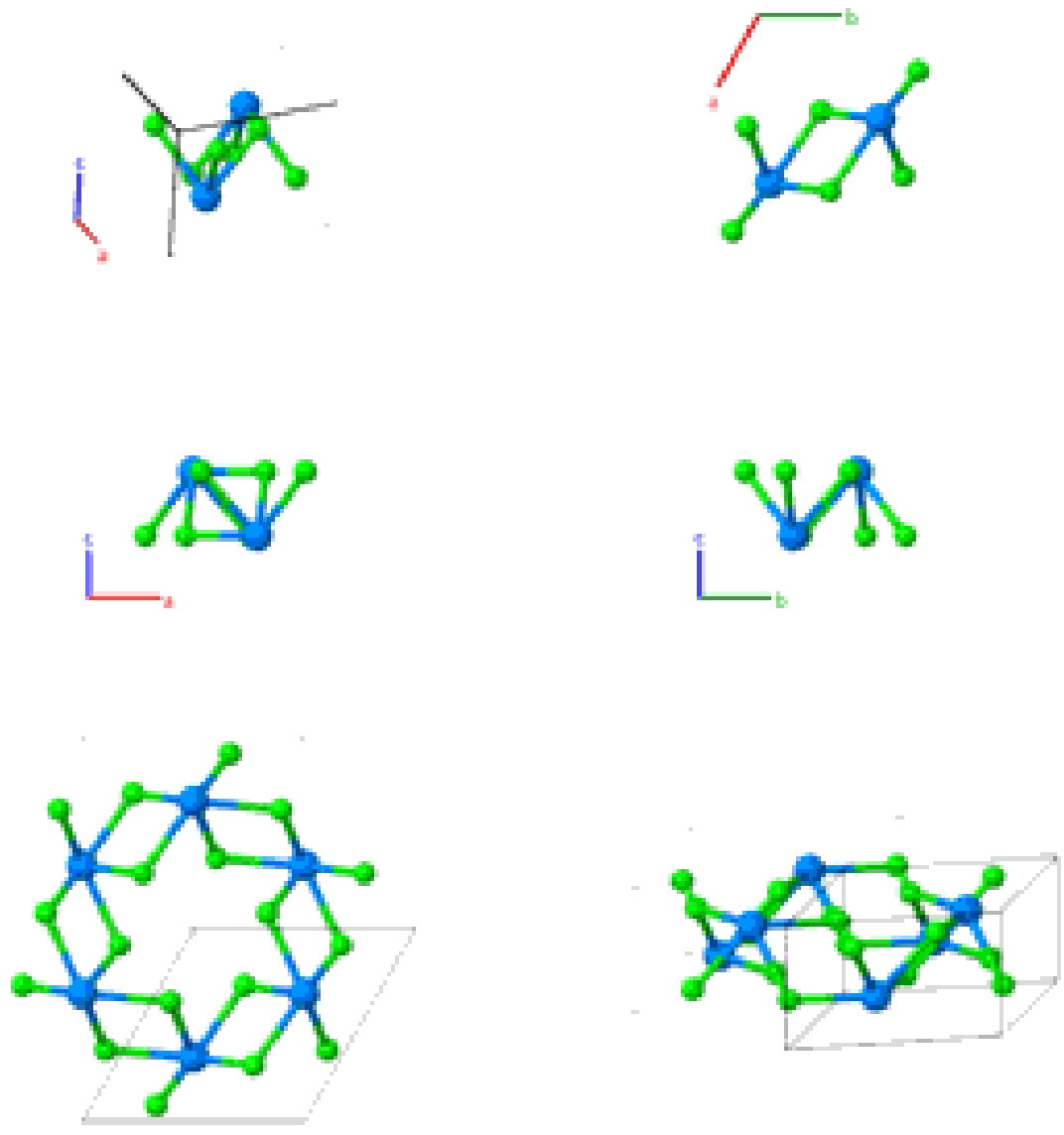

Prototype

: $\quad \mathrm{UCl}_{3}$

AFLOW prototype label

: A3B_hP8_176_h_d

Strukturbericht designation : None

Pearson symbol

: $\quad$ hP8

Space group number

: 176

Space group symbol

: $\quad P 6_{3} / m$

AFLOW prototype command : aflow --proto=A3B_hP8_176_h_d

- - params $=a, c / a, x_{2}, y_{2}$

Hexagonal primitive vectors:

$$
\begin{array}{ll}
\mathbf{a}_{1}= & \frac{1}{2} a \hat{\mathbf{x}}-\frac{\sqrt{3}}{2} a \hat{\mathbf{y}} \\
\mathbf{a}_{2}= & \frac{1}{2} a \hat{\mathbf{x}}+\frac{\sqrt{3}}{2} a \hat{\mathbf{y}} \\
\mathbf{a}_{3}= & c \hat{\mathbf{z}}
\end{array}
$$

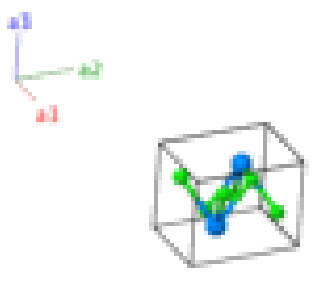

Basis vectors: 

$\mathbf{B}_{1}=\frac{2}{3} \mathbf{a}_{1}+\frac{1}{3} \mathbf{a}_{2}+\frac{1}{4} \mathbf{a}_{3}=$
$\frac{1}{2} a \hat{\mathbf{x}}-\frac{1}{2 \sqrt{3}} a \hat{\mathbf{y}}+\frac{1}{4} c \hat{\mathbf{z}}$
(2d)
$\mathbf{B}_{2}=\frac{1}{3} \mathbf{a}_{1}+\frac{2}{3} \mathbf{a}_{2}+\frac{3}{4} \mathbf{a}_{3}=$
$\frac{1}{2} a \hat{\mathbf{x}}+\frac{1}{2 \sqrt{3}} a \hat{\mathbf{y}}+\frac{3}{4} c \hat{\mathbf{z}}$
(2d)
$\mathbf{B}_{3}=x_{2} \mathbf{a}_{1}+y_{2} \mathbf{a}_{2}+\frac{1}{4} \mathbf{a}_{3}=$
$=\frac{1}{2}\left(x_{2}+y_{2}\right) a \hat{\mathbf{x}}+\frac{\sqrt{3}}{2}\left(-x_{2}+y_{2}\right) a \hat{\mathbf{y}}+\frac{1}{4} c \hat{\mathbf{z}}$
(6h)
$\mathrm{Cl}$
$\mathbf{B}_{4}=-y_{2} \mathbf{a}_{1}+\left(x_{2}-y_{2}\right) \mathbf{a}_{2}+\frac{1}{4} \mathbf{a}_{3}=$
$\left(\frac{1}{2} x_{2}-y_{2}\right) a \hat{\mathbf{x}}+\frac{\sqrt{3}}{2} x_{2} a \hat{\mathbf{y}}+\frac{1}{4} c \hat{\mathbf{z}}$
(6h)
$\mathrm{Cl}$
$\mathbf{B}_{5}=\left(-x_{2}+y_{2}\right) \mathbf{a}_{1}-x_{2} \mathbf{a}_{2}+\frac{1}{4} \mathbf{a}_{3}=$
$\left(-x_{2}+\frac{1}{2} y_{2}\right) a \hat{\mathbf{x}}-\frac{\sqrt{3}}{2} y_{2} a \hat{\mathbf{y}}+\frac{1}{4} c \hat{\mathbf{z}}$
(6h)
$\mathrm{Cl}$
$\mathbf{B}_{6}=-x_{2} \mathbf{a}_{1}-y_{2} \mathbf{a}_{2}+\frac{3}{4} \mathbf{a}_{3}=$
$\mathbf{B}_{7}=y_{2} \mathbf{a}_{1}+\left(-x_{2}+y_{2}\right) \mathbf{a}_{2}+\frac{3}{4} \mathbf{a}_{3}=$
$=-\frac{1}{2}\left(x_{2}+y_{2}\right) a \hat{\mathbf{x}}+\frac{\sqrt{3}}{2}\left(x_{2}-y_{2}\right) a \hat{\mathbf{y}}+\frac{3}{4} c \hat{\mathbf{z}}$
$\mathrm{Cl}$
$\mathbf{B}_{8}=\left(x_{2}-y_{2}\right) \mathbf{a}_{1}+x_{2} \mathbf{a}_{2}+\frac{3}{4} \mathbf{a}_{3}=$
$\left(-\frac{1}{2} x_{2}+y_{2}\right) a \hat{\mathbf{x}}-\frac{\sqrt{3}}{2} x_{2} a \hat{\mathbf{y}}+\frac{3}{4} c \hat{\mathbf{z}}$
$\mathrm{Cl}$
(6h)
$\mathrm{Cl}$

\section{References:}

- W. H. Zachariasen, Crystal chemical studies of the 5f-series of elements. I. New structure types, Acta Cryst. 1, 265-268 (1948), doi:10.1107/S0365110X48000703.

\section{Found in:}

- P. Villars and K. Cenzual, Pearson's Crystal Data - Crystal Structure Database for Inorganic Compounds, ASM International (2013).

\section{Geometry files:}

- CIF: pp. 930

- POSCAR: pp. 930 
$\mathrm{SiO}_{2}$ Structure: A2B_hP36_177_j2lm_n

95
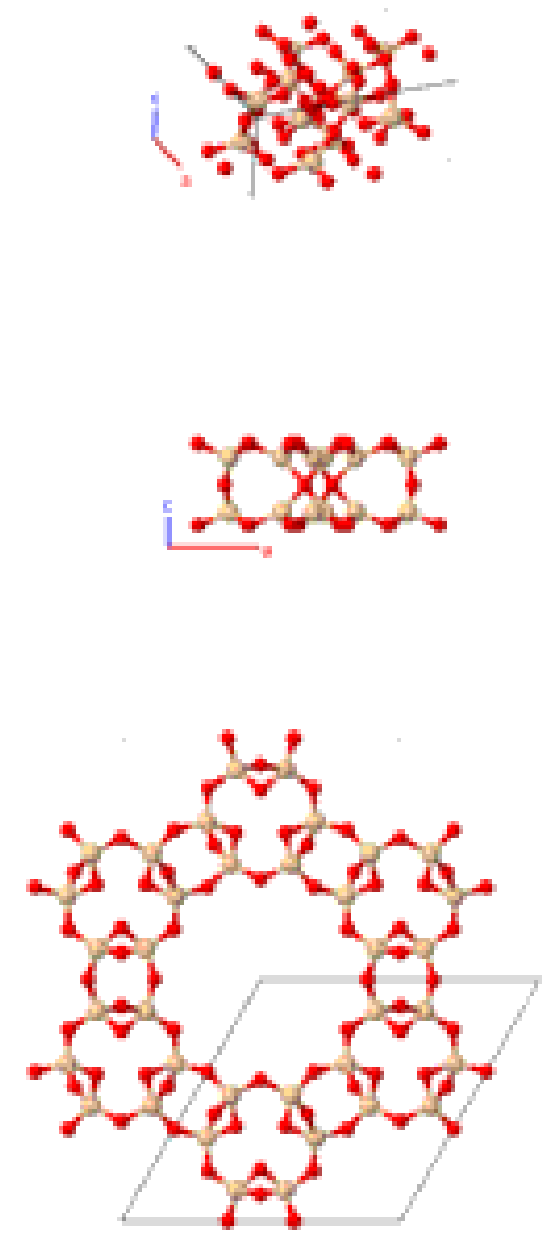
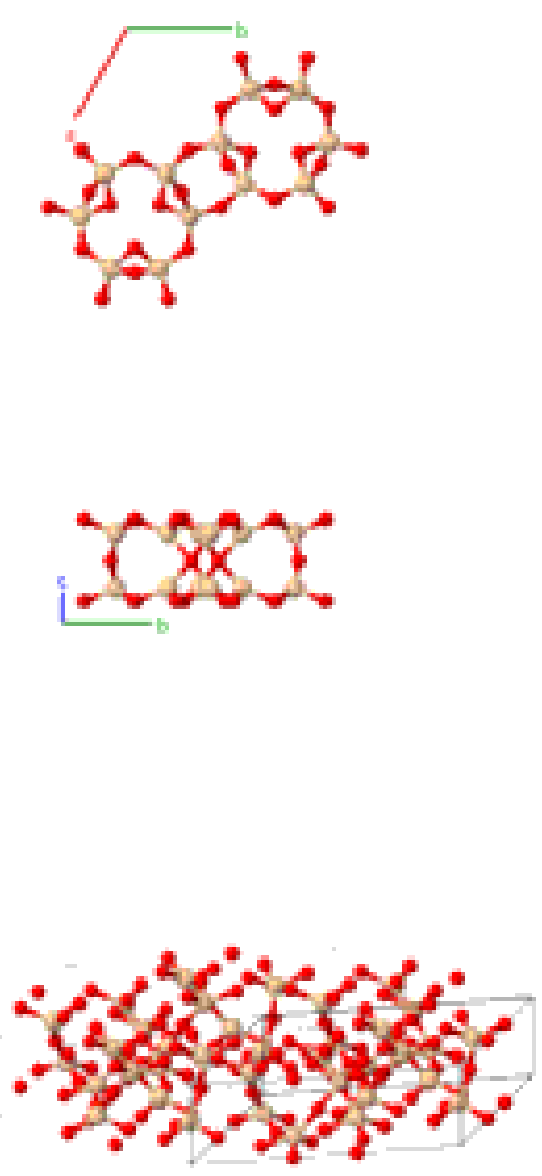

\section{Prototype}

AFLOW prototype label

Strukturbericht designation

Pearson symbol

Space group number

Space group symbol

AFLOW prototype command
$: \quad \mathrm{SiO}_{2}$

: A2B_hP36_177_j21m_n

: None

: $\mathrm{hP} 36$

: $\quad 177$

: $\quad P 622$

aflow --proto=A2B_hP36_177_j21m_n

- -params $=a, c / a, x_{1}, x_{2}, x_{3}, x_{4}, x_{5}, y_{5}, z_{5}$

Hexagonal primitive vectors:
$\mathbf{a}_{1}=\frac{1}{2} a \hat{\mathbf{x}}-\frac{\sqrt{3}}{2} a \hat{\mathbf{y}}$
$\mathbf{a}_{2}=\frac{1}{2} a \hat{\mathbf{x}}+\frac{\sqrt{3}}{2} a \hat{\mathbf{y}}$
$\mathbf{a}_{3}=$
$c \hat{\mathbf{z}}$ 
Lattice Coordinates
$\mathbf{B}_{1}=$
$\mathbf{B}_{2}=$
$\mathbf{B}_{3}=$
$\mathbf{B}_{4}=$
$\mathbf{B}_{5}=$
$\mathbf{B}_{6}=$
$\mathbf{B}_{7}=$
$\mathbf{B}_{8}=$
$\mathbf{B}_{9}=$
$\mathbf{B}_{10}=$
$\mathbf{B}_{11}=$
$\mathbf{B}_{12}=$
$\mathbf{B}_{13}=$
$\mathbf{B}_{14}=$
$\mathbf{B}_{15}=$
$\mathbf{B}_{16}=$
$\mathbf{B}_{17}=$
$\mathbf{B}_{18}=$
$\mathbf{B}_{19}=$
$\mathbf{B}_{20}=$
$\mathbf{B}_{21}=$
$\mathbf{B}_{22}=$
$\mathbf{B}_{23}=$
$\mathbf{B}_{24}=$
$\mathbf{B}_{25}=$
$x_{1} \mathbf{a}_{1}$
$x_{1} \mathbf{a}_{2}$
$-x_{1} \mathbf{a}_{1}-x_{1} \mathbf{a}_{2}$
$-x_{1} \mathbf{a}_{1}$
$-x_{1} \mathbf{a}_{2}$
$x_{1} \mathbf{a}_{1}+x_{1} \mathbf{a}_{2}$
$x_{2} \mathbf{a}_{1}-x_{2} \mathbf{a}_{2}$
$x_{2} \mathbf{a}_{1}+2 x_{2} \mathbf{a}_{2}$
$-2 x_{2} \mathbf{a}_{1}-x_{2} \mathbf{a}_{2}$
$-x_{2} \mathbf{a}_{1}+x_{2} \mathbf{a}_{2}$
$-x_{2} \mathbf{a}_{1}-2 x_{2} \mathbf{a}_{2}$
$2 x_{2} \mathbf{a}_{1}+x_{2} \mathbf{a}_{2}$
$x_{3} \mathbf{a}_{1}-x_{3} \mathbf{a}_{2}$
$x_{3} \mathbf{a}_{1}+2 x_{3} \mathbf{a}_{2}$
$-2 x_{3} \mathbf{a}_{1}-x_{3} \mathbf{a}_{2}$
$-x_{3} \mathbf{a}_{1}+x_{3} \mathbf{a}_{2}$
$-x_{3} \mathbf{a}_{1}-2 x_{3} \mathbf{a}_{2}$
$2 x_{3} \mathbf{a}_{1}+x_{3} \mathbf{a}_{2}$
$x_{4} \mathbf{a}_{1}-x_{4} \mathbf{a}_{2}+\frac{1}{2} \mathbf{a}_{3}$
$x_{4} \mathbf{a}_{1}+2 x_{4} \mathbf{a}_{2}+\frac{1}{2} \mathbf{a}_{3}$
$-2 x_{4} \mathbf{a}_{1}-x_{4} \mathbf{a}_{2}+\frac{1}{2} \mathbf{a}_{3}$
$-x_{4} \mathbf{a}_{1}+x_{4} \mathbf{a}_{2}+\frac{1}{2} \mathbf{a}_{3}$
$-x_{4} \mathbf{a}_{1}-2 x_{4} \mathbf{a}_{2}+\frac{1}{2} \mathbf{a}_{3}$
$2 x_{4} \mathbf{a}_{1}+x_{4} \mathbf{a}_{2}+\frac{1}{2} \mathbf{a}_{3}$
$x_{5} \mathbf{a}_{1}+y_{5} \mathbf{a}_{2}+z_{5} \mathbf{a}_{3}$
$\mathbf{B}_{26}=$
$\mathbf{B}_{27}=$
$\mathbf{B}_{28}=$
$-y_{5} \mathbf{a}_{1}+\left(x_{5}-y_{5}\right) \mathbf{a}_{2}+z_{5} \mathbf{a}_{3}$

$$
=
$$
$=$$$
=
$$$$
=
$$$$
=
$$$$
=
$$$$
=
$$$$
=
$$$$
=
$$$$
=
$$$$
=
$$$$
=
$$$$
=
$$$$
=
$$$$
=
$$$$
=
$$$$
=
$$$$
=
$$$$
=
$$$$
=
$$$$
=
$$$$
=
$$$$
=
$$$$
=
$$$$
=
$$$$
=
$$$$
=
$$$$
=
$$$$
=
$$$$
=\frac{1}{2}\left(x_{5}+y_{5}\right) a \hat{\mathbf{x}}+\frac{\sqrt{3}}{2}\left(-x_{5}+y_{5}\right) a \hat{\mathbf{y}}+
$$$$
\frac{1}{2} x_{1} a \hat{\mathbf{x}}-\frac{\sqrt{3}}{2} x_{1} a \hat{\mathbf{y}}
$$$$
\frac{1}{2} x_{1} a \hat{\mathbf{x}}+\frac{\sqrt{3}}{2} x_{1} a \hat{\mathbf{y}}
$$$$
-x_{1} a \hat{\mathbf{x}}
$$$$
-\frac{1}{2} x_{1} a \hat{\mathbf{x}}+\frac{\sqrt{3}}{2} x_{1} a \hat{\mathbf{y}}
$$$$
-\frac{1}{2} x_{1} a \hat{\mathbf{x}}-\frac{\sqrt{3}}{2} x_{1} a \hat{\mathbf{y}}
$$$$
x_{1} a \hat{\mathbf{x}}
$$$$
-\sqrt{3} x_{2} a \hat{\mathbf{y}}
$$$$
\frac{3}{2} x_{2} a \hat{\mathbf{x}}+\frac{\sqrt{3}}{2} x_{2} a \hat{\mathbf{y}}
$$$$
-\frac{3}{2} x_{2} a \hat{\mathbf{x}}+\frac{\sqrt{3}}{2} x_{2} a \hat{\mathbf{y}}
$$$$
\sqrt{3} x_{2} a \hat{\mathbf{y}}
$$$$
-\frac{3}{2} x_{2} a \hat{\mathbf{x}}-\frac{\sqrt{3}}{2} x_{2} a \hat{\mathbf{y}}
$$$$
\frac{3}{2} x_{2} a \hat{\mathbf{x}}-\frac{\sqrt{3}}{2} x_{2} a \hat{\mathbf{y}}
$$$$
-\sqrt{3} x_{3} a \hat{\mathbf{y}}
$$$$
\frac{3}{2} x_{3} a \hat{\mathbf{x}}+\frac{\sqrt{3}}{2} x_{3} a \hat{\mathbf{y}}
$$$$
-\frac{3}{2} x_{3} a \hat{\mathbf{x}}+\frac{\sqrt{3}}{2} x_{3} a \hat{\mathbf{y}}
$$$$
\sqrt{3} x_{3} a \hat{\mathbf{y}}
$$$$
-\frac{3}{2} x_{3} a \hat{\mathbf{x}}-\frac{\sqrt{3}}{2} x_{3} a \hat{\mathbf{y}}
$$$$
\frac{3}{2} x_{3} a \hat{\mathbf{x}}-\frac{\sqrt{3}}{2} x_{3} a \hat{\mathbf{y}}
$$$$
-\sqrt{3} x_{4} a \hat{\mathbf{y}}+\frac{1}{2} c \hat{\mathbf{z}}
$$$$
\frac{3}{2} x_{4} a \hat{\mathbf{x}}+\frac{\sqrt{3}}{2} x_{4} a \hat{\mathbf{y}}+\frac{1}{2} c \hat{\mathbf{z}}
$$$$
-\frac{3}{2} x_{4} a \hat{\mathbf{x}}+\frac{\sqrt{3}}{2} x_{4} a \hat{\mathbf{y}}+\frac{1}{2} c \hat{\mathbf{z}}
$$$$
\sqrt{3} x_{4} a \hat{\mathbf{y}}+\frac{1}{2} c \hat{\mathbf{z}}
$$$$
-\frac{3}{2} x_{4} a \hat{\mathbf{x}}-\frac{\sqrt{3}}{2} x_{4} a \hat{\mathbf{y}}+\frac{1}{2} c \hat{\mathbf{z}}
$$$$
\frac{3}{2} x_{4} a \hat{\mathbf{x}}-\frac{\sqrt{3}}{2} x_{4} a \hat{\mathbf{y}}+\frac{1}{2} c \hat{\mathbf{z}}
$$$$
z_{5} c \hat{\mathbf{z}}
$$$$
=\left(\frac{1}{2} x_{5}-y_{5}\right) a \hat{\mathbf{x}}+\frac{\sqrt{3}}{2} x_{5} a \hat{\mathbf{y}}+z_{5} c \hat{\mathbf{z}}
$$$$
=\left(-x_{5}+\frac{1}{2} y_{5}\right) a \hat{\mathbf{x}}-\frac{\sqrt{3}}{2} y_{5} a \hat{\mathbf{y}}+z_{5} c \hat{\mathbf{z}}
$$$$
=-\frac{1}{2}\left(x_{5}+y_{5}\right) a \hat{\mathbf{x}}+\frac{\sqrt{3}}{2}\left(x_{5}-y_{5}\right) a \hat{\mathbf{y}}+
$$$$
\mathbf{B}_{29}=y_{5} \mathbf{a}_{1}+\left(-x_{5}+y_{5}\right) \mathbf{a}_{2}+z_{5} \mathbf{a}_{3}=\left(-\frac{1}{2} x_{5}+y_{5}\right) a \hat{\mathbf{x}}-\frac{\sqrt{3}}{2} x_{5} a \hat{\mathbf{y}}+z_{5} c \hat{\mathbf{z}}
$$$$
\mathbf{B}_{30}=\left(x_{5}-y_{5}\right) \mathbf{a}_{1}+x_{5} \mathbf{a}_{2}+z_{5} \mathbf{a}_{3}=\left(x_{5}-\frac{1}{2} y_{5}\right) a \hat{\mathbf{x}}+\frac{\sqrt{3}}{2} y_{5} a \hat{\mathbf{y}}+z_{5} c \hat{\mathbf{z}}
$$$$
\mathbf{B}_{31}=y_{5} \mathbf{a}_{1}+x_{5} \mathbf{a}_{2}-z_{5} \mathbf{a}_{3}
$$$$
\mathbf{B}_{32}=\left(x_{5}-y_{5}\right) \mathbf{a}_{1}-y_{5} \mathbf{a}_{2}-z_{5} \mathbf{a}_{3}
$$$$
=\frac{1}{2}\left(x_{5}+y_{5}\right) a \hat{\mathbf{x}}+\frac{\sqrt{3}}{2}\left(x_{5}-y_{5}\right) a \hat{\mathbf{y}}-z_{5} c \hat{\mathbf{z}}
$$$$
=\left(\frac{1}{2} x_{5}-y_{5}\right) a \hat{\mathbf{x}}-\frac{\sqrt{3}}{2} x_{5} a \hat{\mathbf{y}}-z_{5} c \hat{\mathbf{z}}
$$$$
\mathbf{B}_{33}=-x_{5} \mathbf{a}_{1}+\left(-x_{5}+y_{5}\right) \mathbf{a}_{2}-z_{5} \mathbf{a}_{3}
$$
$\mathbf{B}_{34}=$

$$
-y_{5} \mathbf{a}_{1}-x_{5} \mathbf{a}_{2}-z_{5} \mathbf{a}_{3}
$$$$
=\left(-x_{5}+\frac{1}{2} y_{5}\right) a \hat{\mathbf{x}}+\frac{\sqrt{3}}{2} y_{5} a \hat{\mathbf{y}}-z_{5} c \hat{\mathbf{z}}
$$$$
=-\frac{1}{2}\left(x_{5}+y_{5}\right) a \hat{\mathbf{x}}+\frac{\sqrt{3}}{2}\left(-x_{5}+y_{5}\right) a \hat{\mathbf{y}}-
$$

$$
z_{5} c \hat{\mathbf{z}}
$$


$\mathbf{B}_{35}=\left(-x_{5}+y_{5}\right) \mathbf{a}_{1}+y_{5} \mathbf{a}_{2}-z_{5} \mathbf{a}_{3}=\left(-\frac{1}{2} x_{5}+y_{5}\right) a \hat{\mathbf{x}}+\frac{\sqrt{3}}{2} x_{5} a \hat{\mathbf{y}}-z_{5} c \hat{\mathbf{z}}$
$\mathbf{B}_{36}=x_{5} \mathbf{a}_{1}+\left(x_{5}-y_{5}\right) \mathbf{a}_{2}-z_{5} \mathbf{a}_{3}=\left(x_{5}-\frac{1}{2} y_{5}\right) a \hat{\mathbf{x}}-\frac{\sqrt{3}}{2} y_{5} a \hat{\mathbf{y}}-z_{5} c \hat{\mathbf{z}}$

$(12 n)$

$(12 n)$

\section{References:}

- M. D. Foster, O. Delgado Friedrichs, R. G. Bell, and F. A. Almeida Pazand J. Klinowski, Chemical Evaluation of Hypothetical Uninodal Zeolites, J. Am. Chem. Soc. 126, 9769-9775 (2004), doi:10.1021/ja037334j.

\section{Found in:}

- ICSD, Inorganic Crystal Structure Database. ID 170519.

\section{Geometry files:}

- CIF: pp. 930

- POSCAR: pp. 930 


\section{$\mathrm{AuF}_{3}$ Structure: AB3_hP24_178_b_ac}
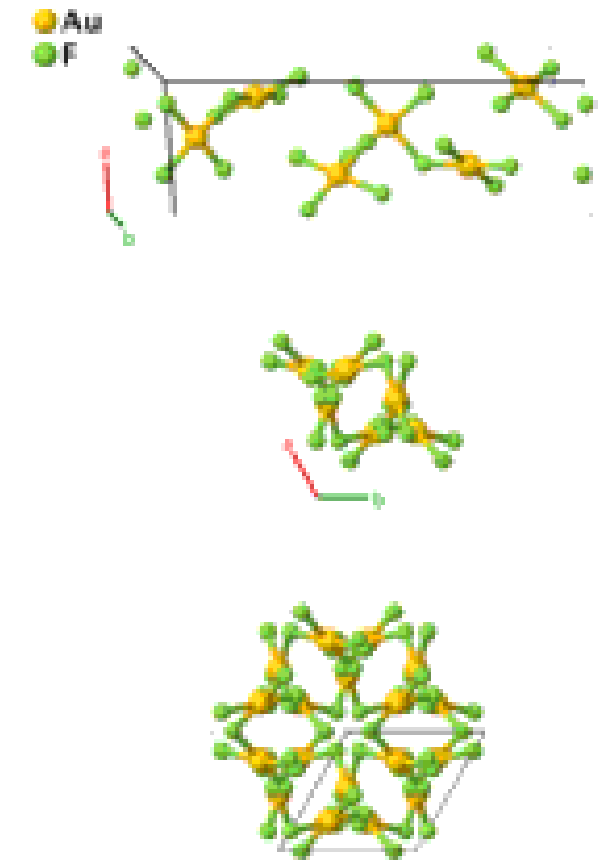

\section{Prototype}

AFLOW prototype label

Strukturbericht designation

Pearson symbol

Space group number

Space group symbol

AFLOW prototype command
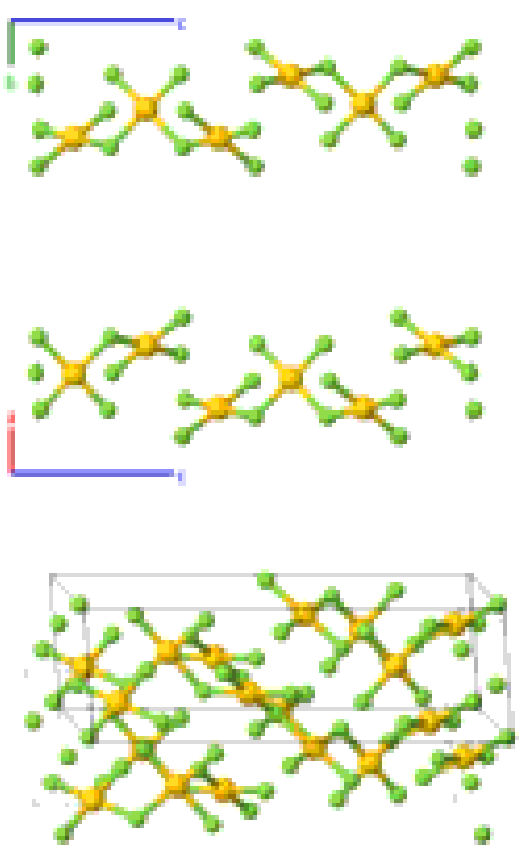

\section{Hexagonal primitive vectors:}

$$
\begin{array}{ll}
\mathbf{a}_{1}= & \frac{1}{2} a \hat{\mathbf{x}}-\frac{\sqrt{3}}{2} a \hat{\mathbf{y}} \\
\mathbf{a}_{2}= & \frac{1}{2} a \hat{\mathbf{x}}+\frac{\sqrt{3}}{2} a \hat{\mathbf{y}} \\
\mathbf{a}_{3}= & c \hat{\mathbf{z}}
\end{array}
$$

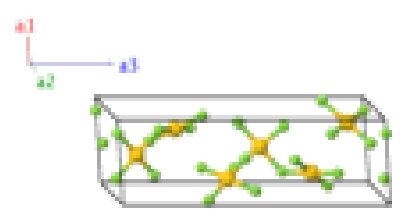

Basis vectors:

Lattice Coordinates
$\mathbf{B}_{1}=$
$x_{1} \mathbf{a}_{1}$
$\mathbf{B}_{2}=$
$\mathbf{B}_{3}=$
$\mathbf{B}_{4}=$
$\mathbf{B}_{5}=$
$\mathbf{B}_{6}=$

Cartesian Coordinates

$=\quad \frac{1}{2} x_{1} a \hat{\mathbf{x}}-\frac{\sqrt{3}}{2} x_{1} a \hat{\mathbf{y}}$

$=\quad \frac{1}{2} x_{1} a \hat{\mathbf{x}}+\frac{\sqrt{3}}{2} x_{1} a \hat{\mathbf{y}}+\frac{1}{3} c \hat{\mathbf{z}}$

$=\quad-x_{1} a \hat{\mathbf{x}}+\frac{2}{3} c \hat{\mathbf{z}}$

$=\quad-\frac{1}{2} x_{1} a \hat{\mathbf{x}}+\frac{\sqrt{3}}{2} x_{1} a \hat{\mathbf{y}}+\frac{1}{2} c \hat{\mathbf{z}}$

$=\quad-\frac{1}{2} x_{1} a \hat{\mathbf{x}}-\frac{\sqrt{3}}{2} x_{1} a \hat{\mathbf{y}}+\frac{5}{6} c \hat{\mathbf{z}}$

$=\quad x_{1} a \hat{\mathbf{x}}+\frac{1}{6} c \hat{\mathbf{z}}$
Wyckoff Position Atom Type

$(6 a)$

F I

(6a)

F I

(6a)

F I

(6a)

F I

(6a)

F I

(6a) 


\begin{tabular}{|c|c|c|c|c|c|c|}
\hline $\mathbf{B}_{7}$ & $=$ & $x_{2} \mathbf{a}_{1}+2 x_{2} \mathbf{a}_{2}+\frac{1}{4} \mathbf{a}_{3}$ & $=$ & $\frac{3}{2} x_{2} a \hat{\mathbf{x}}+\frac{\sqrt{3}}{2} x_{2} a \hat{\mathbf{y}}+\frac{1}{4} c \hat{\mathbf{z}}$ & $(6 b)$ & $\mathrm{Au}$ \\
\hline $\mathbf{B}_{8}$ & $=$ & $-2 x_{2} \mathbf{a}_{1}-x_{2} \mathbf{a}_{2}+\frac{7}{12} \mathbf{a}_{3}$ & $=$ & $-\frac{3}{2} x_{2} a \hat{\mathbf{x}}+\frac{\sqrt{3}}{2} x_{2} a \hat{\mathbf{y}}+\frac{7}{12} c \hat{\mathbf{z}}$ & $(6 b)$ & $\mathrm{Au}$ \\
\hline $\mathbf{B}_{9}$ & $=$ & $x_{2} \mathbf{a}_{1}-x_{2} \mathbf{a}_{2}+\frac{11}{12} \mathbf{a}_{3}$ & $=$ & $-\sqrt{3} x_{2} a \hat{\mathbf{y}}+\frac{11}{12} c \hat{\mathbf{z}}$ & $(6 b)$ & $\mathrm{Au}$ \\
\hline $\mathbf{B}_{10}$ & $=$ & $-x_{2} \mathbf{a}_{1}-2 x_{2} \mathbf{a}_{2}+\frac{3}{4} \mathbf{a}_{3}$ & $=$ & $-\frac{3}{2} x_{2} a \hat{\mathbf{x}}-\frac{\sqrt{3}}{2} x_{2} a \hat{\mathbf{y}}+\frac{3}{4} c \hat{\mathbf{z}}$ & $(6 b)$ & $\mathrm{Au}$ \\
\hline $\mathbf{B}_{11}$ & $=$ & $2 x_{2} \mathbf{a}_{1}+x_{2} \mathbf{a}_{2}+\frac{1}{12} \mathbf{a}_{3}$ & $=$ & $\frac{3}{2} x_{2} a \hat{\mathbf{x}}-\frac{\sqrt{3}}{2} x_{2} a \hat{\mathbf{y}}+\frac{1}{12} c \hat{\mathbf{z}}$ & $(6 b)$ & $\mathrm{Au}$ \\
\hline $\mathbf{B}_{12}$ & $=$ & $-x_{2} \mathbf{a}_{1}+x_{2} \mathbf{a}_{2}+\frac{5}{12} \mathbf{a}_{3}$ & $=$ & $\sqrt{3} x_{2} a \hat{\mathbf{y}}+\frac{5}{12} c \hat{\mathbf{z}}$ & $(6 b)$ & $\mathrm{Au}$ \\
\hline $\mathbf{B}_{13}$ & $=$ & $x_{3} \mathbf{a}_{1}+y_{3} \mathbf{a}_{2}+z_{3} \mathbf{a}_{3}$ & $=$ & $\begin{array}{c}\frac{1}{2}\left(x_{3}+y_{3}\right) a \hat{\mathbf{x}}+ \\
\frac{\sqrt{3}}{2}\left(-x_{3}+y_{3}\right) a \hat{\mathbf{y}}+z_{3} c \hat{\mathbf{z}}\end{array}$ & $(12 c)$ & F II \\
\hline $\mathbf{B}_{14}$ & $=$ & $-y_{3} \mathbf{a}_{1}+\left(x_{3}-y_{3}\right) \mathbf{a}_{2}+\left(\frac{1}{3}+z_{3}\right) \mathbf{a}_{3}$ & $=$ & $\begin{array}{c}\left(\frac{1}{2} x_{3}-y_{3}\right) a \hat{\mathbf{x}}+\frac{\sqrt{3}}{2} x_{3} a \hat{\mathbf{y}}+ \\
\left(\frac{1}{3}+z_{3}\right) c \hat{\mathbf{z}}\end{array}$ & $(12 c)$ & F II \\
\hline $\mathbf{B}_{15}$ & $=$ & $\left(-x_{3}+y_{3}\right) \mathbf{a}_{1}-x_{3} \mathbf{a}_{2}+\left(\frac{2}{3}+z_{3}\right) \mathbf{a}_{3}$ & $=$ & $\begin{array}{c}\left(-x_{3}+\frac{1}{2} y_{3}\right) a \hat{\mathbf{x}}-\frac{\sqrt{3}}{2} y_{3} a \hat{\mathbf{y}}+ \\
\left(\frac{2}{3}+z_{3}\right) c \hat{\mathbf{z}}\end{array}$ & $(12 c)$ & F II \\
\hline $\mathbf{B}_{16}$ & $=$ & $-x_{3} \mathbf{a}_{1}-y_{3} \mathbf{a}_{2}+\left(\frac{1}{2}+z_{3}\right) \mathbf{a}_{3}$ & $=$ & $\begin{array}{c}-\frac{1}{2}\left(x_{3}+y_{3}\right) a \hat{\mathbf{x}}+ \\
\frac{\sqrt{3}}{2}\left(x_{3}-y_{3}\right) a \hat{\mathbf{y}}+\left(\frac{1}{2}+z_{3}\right) c \hat{\mathbf{z}}\end{array}$ & $(12 c)$ & F II \\
\hline $\mathbf{B}_{17}$ & $=$ & $y_{3} \mathbf{a}_{1}+\left(-x_{3}+y_{3}\right) \mathbf{a}_{2}+\left(\frac{5}{6}+z_{3}\right) \mathbf{a}_{3}$ & $=$ & $\begin{array}{c}\left(-\frac{1}{2} x_{3}+y_{3}\right) a \hat{\mathbf{x}}-\frac{\sqrt{3}}{2} x_{3} a \hat{\mathbf{y}}+ \\
\left(\frac{5}{6}+z_{3}\right) c \hat{\mathbf{z}}\end{array}$ & $(12 c)$ & F II \\
\hline $\mathbf{B}_{18}$ & $=$ & $\left(x_{3}-y_{3}\right) \mathbf{a}_{1}+x_{3} \mathbf{a}_{2}+\left(\frac{1}{6}+z_{3}\right) \mathbf{a}_{3}$ & $=$ & $\begin{array}{c}\left(x_{3}-\frac{1}{2} y_{3}\right) a \hat{\mathbf{x}}+\frac{\sqrt{3}}{2} y_{3} a \hat{\mathbf{y}}+ \\
\left(\frac{1}{6}+z_{3}\right) c \hat{\mathbf{z}}\end{array}$ & $(12 c)$ & F II \\
\hline $\mathbf{B}_{19}$ & $=$ & $y_{3} \mathbf{a}_{1}+x_{3} \mathbf{a}_{2}+\left(\frac{1}{3}-z_{3}\right) \mathbf{a}_{3}$ & $=$ & $\begin{array}{c}\frac{1}{2}\left(x_{3}+y_{3}\right) a \hat{\mathbf{x}}+\frac{\sqrt{3}}{2}\left(x_{3}-y_{3}\right) a \hat{\mathbf{y}}+ \\
\left(\frac{1}{3}-z_{3}\right) c \hat{\mathbf{z}}\end{array}$ & $(12 c)$ & F II \\
\hline $\mathbf{B}_{20}$ & $=$ & $\left(x_{3}-y_{3}\right) \mathbf{a}_{1}-y_{3} \mathbf{a}_{2}-z_{3} \mathbf{a}_{3}$ & $=$ & $\left(\frac{1}{2} x_{3}-y_{3}\right) a \hat{\mathbf{x}}-\frac{\sqrt{3}}{2} x_{3} a \hat{\mathbf{y}}-z_{3} c \hat{\mathbf{z}}$ & $(12 c)$ & F II \\
\hline $\mathbf{B}_{21}$ & $=$ & $-x_{3} \mathbf{a}_{1}+\left(-x_{3}+y_{3}\right) \mathbf{a}_{2}+\left(\frac{2}{3}-z_{3}\right) \mathbf{a}_{3}$ & $=$ & $\begin{array}{c}\left(-x_{3}+\frac{1}{2} y_{3}\right) a \hat{\mathbf{x}}+\frac{\sqrt{3}}{2} y_{3} a \hat{\mathbf{y}}+ \\
\left(\frac{2}{3}-z_{3}\right) c \hat{\mathbf{z}}\end{array}$ & $(12 c)$ & F II \\
\hline $\mathbf{B}_{22}$ & $=$ & $-y_{3} \mathbf{a}_{1}-x_{3} \mathbf{a}_{2}+\left(\frac{5}{6}-z_{3}\right) \mathbf{a}_{3}$ & $=$ & $\begin{array}{c}-\frac{1}{2}\left(x_{3}+y_{3}\right) a \hat{\mathbf{x}}+ \\
\frac{\sqrt{3}}{2}\left(-x_{3}+y_{3}\right) a \hat{\mathbf{y}}+\left(\frac{5}{6}-z_{3}\right) c \hat{\mathbf{z}}\end{array}$ & $(12 c)$ & F II \\
\hline $\mathbf{B}_{23}$ & $=$ & $\left(-x_{3}+y_{3}\right) \mathbf{a}_{1}+y_{3} \mathbf{a}_{2}+\left(\frac{1}{2}-z_{3}\right) \mathbf{a}_{3}$ & $=$ & $\begin{array}{c}\left(-\frac{1}{2} x_{3}+y_{3}\right) a \hat{\mathbf{x}}+\frac{\sqrt{3}}{2} x_{3} a \hat{\mathbf{y}}+ \\
\left(\frac{1}{2}-z_{3}\right) c \hat{\mathbf{z}}\end{array}$ & $(12 c)$ & F II \\
\hline $\mathbf{B}_{24}$ & $=$ & $x_{3} \mathbf{a}_{1}+\left(x_{3}-y_{3}\right) \mathbf{a}_{2}+\left(\frac{1}{6}-z_{3}\right) \mathbf{a}_{3}$ & $=$ & $\begin{array}{c}\left(x_{3}-\frac{1}{2} y_{3}\right) a \hat{\mathbf{x}}-\frac{\sqrt{3}}{2} y_{3} a \hat{\mathbf{y}}+ \\
\left(\frac{1}{6}-z_{3}\right) c \hat{\mathbf{z}}\end{array}$ & $(12 c)$ & F II \\
\hline
\end{tabular}

\section{References:}

- L. B. Asprey, F. H. Kruse, K. H. Jack, and R. Maitland, Preparation and properties of crystalline gold trifluoride, Inorg. Chem. 3, 602-604 (1964), doi:10.1021/ic50014a037.

\section{Found in:}

- P. Villars and K. Cenzual, Pearson's Crystal Data - Crystal Structure Database for Inorganic Compounds, ASM International (2013).

\section{Geometry files:}

- CIF: pp. 930

- POSCAR: pp. 931 


\section{Sc-V (High-pressure) Structure: A_hP6_178_a}

$\Delta \mathrm{se}$
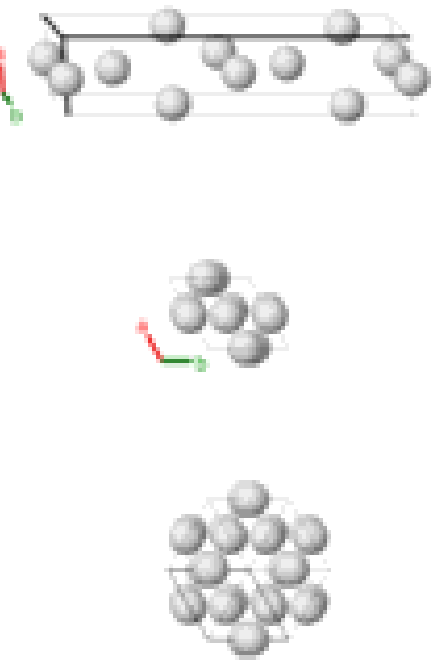
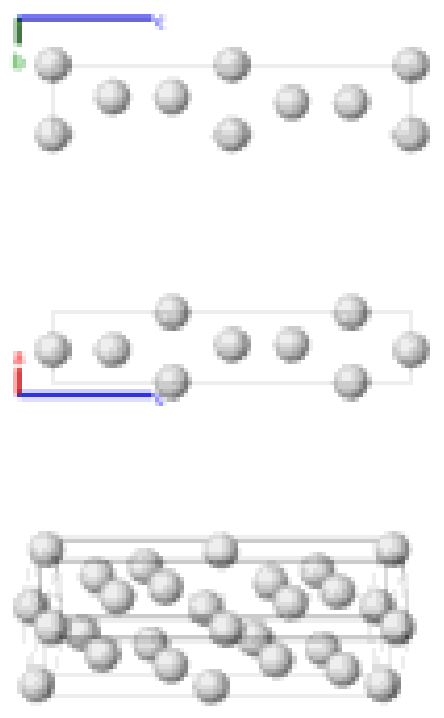

Prototype

AFLOW prototype label

Strukturbericht designation

Pearson symbol

Space group number

Space group symbol

AFLOW prototype command
: $\quad \mathrm{Sc}$

: A_hP6_178_a

: None

: $\quad$ hP6

: $\quad 178$

: $\quad P 6_{1} 22$

- This high pressure phase of scandium becomes stable at $240 \mathrm{GPa}$. We use the experimental data at $242 \mathrm{GPa}$ and $297 \mathrm{~K}$. This chiral structure could also have been presented in the enantiomorphic space group $P 6_{5} 22$ (\#179).

\section{Hexagonal primitive vectors:}

$$
\begin{array}{ll}
\mathbf{a}_{1}= & \frac{1}{2} a \hat{\mathbf{x}}-\frac{\sqrt{3}}{2} a \hat{\mathbf{y}} \\
\mathbf{a}_{2}= & \frac{1}{2} a \hat{\mathbf{x}}+\frac{\sqrt{3}}{2} a \hat{\mathbf{y}} \\
\mathbf{a}_{3}= & c \hat{\mathbf{z}}
\end{array}
$$
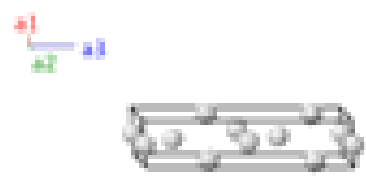

Basis vectors:

Lattice Coordinates

$\begin{array}{lcccc}\mathbf{B}_{1}= & x_{1} \mathbf{a}_{1} & = & \frac{1}{2} x_{1} a \hat{\mathbf{x}}-\frac{\sqrt{3}}{2} x_{1} a \hat{\mathbf{y}} \\ \mathbf{B}_{2}= & x_{1} \mathbf{a}_{2}+\frac{1}{3} \mathbf{a}_{3} & = & \frac{1}{2} x_{1} a \hat{\mathbf{x}}+\frac{\sqrt{3}}{2} x_{1} a \hat{\mathbf{y}}+\frac{1}{3} c \hat{\mathbf{z}} \\ \mathbf{B}_{3}= & -x_{1} \mathbf{a}_{1}-x_{1} \mathbf{a}_{2}+\frac{2}{3} \mathbf{a}_{3} & = & -x_{1} a \hat{\mathbf{x}}+\frac{2}{3} c \hat{\mathbf{z}} \\ \mathbf{B}_{4}= & -x_{1} \mathbf{a}_{1}+\frac{1}{2} \mathbf{a}_{3} & = & -\frac{1}{2} x_{1} a \hat{\mathbf{x}}+\frac{\sqrt{3}}{2} x_{1} a \hat{\mathbf{y}}+\frac{1}{2} c \hat{\mathbf{z}} \\ \mathbf{B}_{5}= & -x_{1} \mathbf{a}_{2}+\frac{5}{6} \mathbf{a}_{3} & = & -\frac{1}{2} x_{1} a \hat{\mathbf{x}}-\frac{\sqrt{3}}{2} x_{1} a \hat{\mathbf{y}}+\frac{5}{6} c \hat{\mathbf{z}} \\ \mathbf{B}_{6}= & x_{1} \mathbf{a}_{1}+x_{1} \mathbf{a}_{2}+\frac{1}{6} \mathbf{a}_{3} & & & x_{1} a \hat{\mathbf{x}}+\frac{1}{6} c \hat{\mathbf{z}}\end{array}$

$\mathbf{B}_{1}=$

$x_{1} \mathbf{a}_{1}+x_{1} \mathbf{a}_{2}+\frac{1}{6} \mathbf{a}_{3}$
Wyckoff Position

Atom Type

(6a)

$\mathrm{Sc}$

(6a)

Sc

(6a)

$\mathrm{Sc}$

(6a)

Sc

(6a)

$\mathrm{Sc}$

(6a)

Sc 


\section{References:}

- Y. Akahama, H. Fujihisa, and H. Kawamura, New Helical Chain Structure for Scandium at 240 GPa, Phys. Rev. Lett. 94, 195503 (2005), doi:10.1103/PhysRevLett.94.195503.

\section{Geometry files:}

- CIF: pp. 931

- POSCAR: pp. 931 


\section{$\mathrm{AuF}_{3}$ Structure: AB3_hP24_179_b_ac}
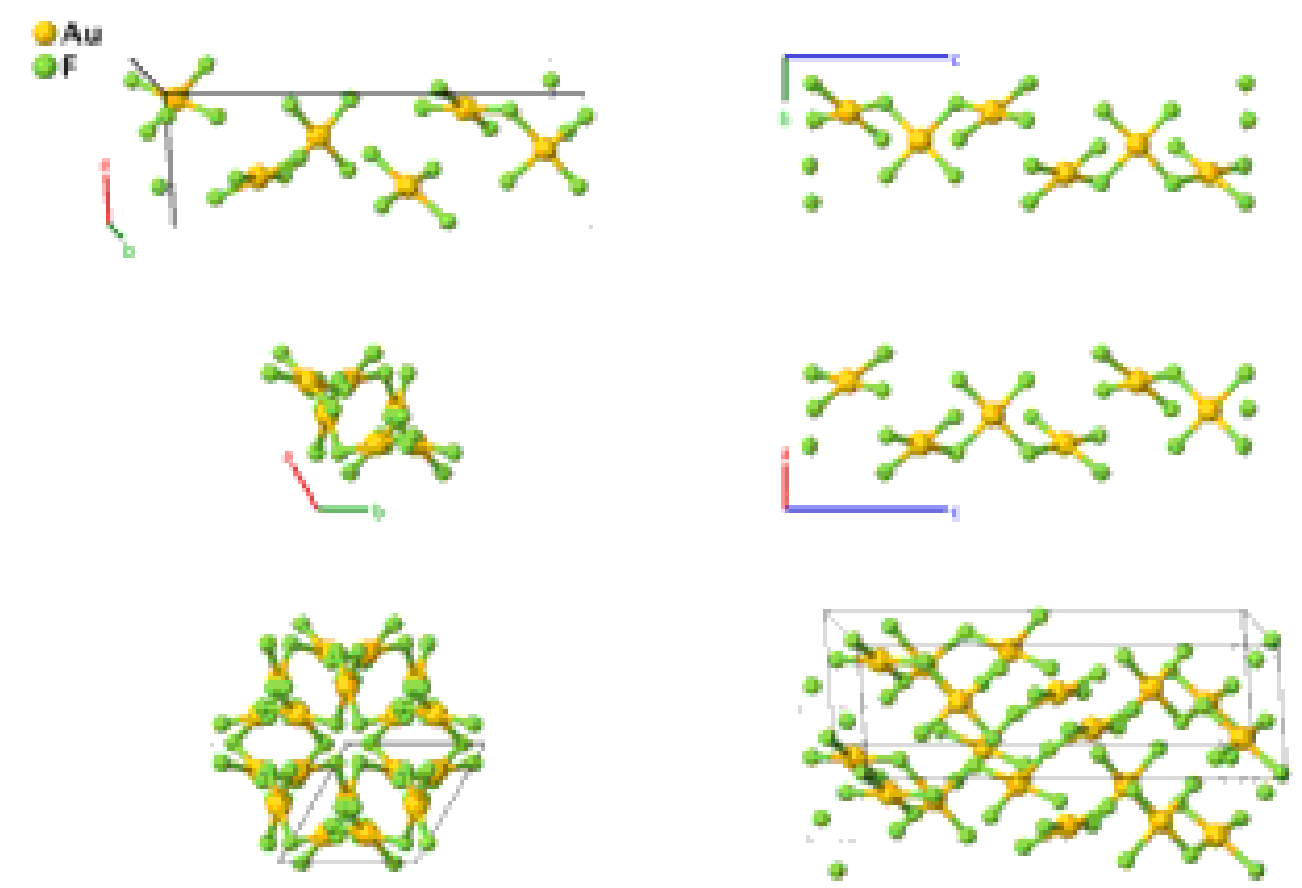

\section{Prototype}

AFLOW prototype label

Strukturbericht designation

Pearson symbol

Space group number

Space group symbol

AFLOW prototype command
: $\mathrm{AuF}_{3}$

: AB3_hP24_179_b_ac

: None

: $\mathrm{hP} 24$

: 179

: $\quad P 6_{5} 22$

\section{aflow - proto=AB3_hP24_179_b_ac \\ - -params $=a, c / a, x_{1}, x_{2}, x_{3}, y_{3}, z_{3}$}

- This structure is the enantiomorph of the $\mathrm{AuF}_{3}\left(\mathrm{AB} 3 \_\mathrm{hP} 24 \_178 \_\mathrm{b} \_\mathrm{ac}\right)$ structure, and was generated by reflecting the coordinates of the space group \#178 structure through the $z=0$ plane.

\section{Hexagonal primitive vectors:}

$$
\begin{array}{lll}
\mathbf{a}_{1} & = & \frac{1}{2} a \hat{\mathbf{x}}-\frac{\sqrt{3}}{2} a \hat{\mathbf{y}} \\
\mathbf{a}_{2} & = & \frac{1}{2} a \hat{\mathbf{x}}+\frac{\sqrt{3}}{2} a \hat{\mathbf{y}} \\
\mathbf{a}_{3} & = & c \hat{\mathbf{z}}
\end{array}
$$

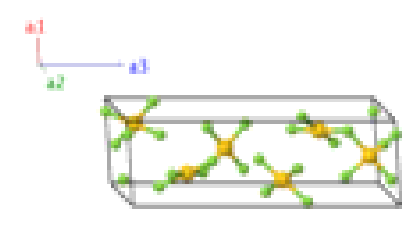

\section{Basis vectors:}

Lattice Coordinates

$\begin{array}{ccc}\mathbf{B}_{1}= & x_{1} \mathbf{a}_{1} \\ \mathbf{B}_{2}= & x_{1} \mathbf{a}_{2}+\frac{2}{3} \mathbf{a}_{3} \\ \mathbf{B}_{3}= & -x_{1} \mathbf{a}_{1}-x_{1} \mathbf{a}_{2}+\frac{1}{3} \mathbf{a}_{3}\end{array}$

Cartesian Coordinates

$=\quad \frac{1}{2} x_{1} a \hat{\mathbf{x}}-\frac{\sqrt{3}}{2} x_{1} a \hat{\mathbf{y}}$

$=\quad \frac{1}{2} x_{1} a \hat{\mathbf{x}}+\frac{\sqrt{3}}{2} x_{1} a \hat{\mathbf{y}}+\frac{2}{3} c \hat{\mathbf{z}}$

$=$
Wyckoff Position

Atom Type

(6a)

F I

$(6 a)$

F I

$(6 a)$ 


\begin{tabular}{|c|c|c|c|c|c|c|}
\hline $\mathbf{B}_{4}$ & $=$ & $-x_{1} \mathbf{a}_{1}+\frac{1}{2} \mathbf{a}_{3}$ & $=$ & $-\frac{1}{2} x_{1} a \hat{\mathbf{x}}+\frac{\sqrt{3}}{2} x_{1} a \hat{\mathbf{y}}+\frac{1}{2} c \hat{\mathbf{z}}$ & $(6 a)$ & F I \\
\hline $\mathbf{B}_{5}$ & $=$ & $-x_{1} \mathbf{a}_{2}+\frac{1}{6} \mathbf{a}_{3}$ & $=$ & $-\frac{1}{2} x_{1} a \hat{\mathbf{x}}-\frac{\sqrt{3}}{2} x_{1} a \hat{\mathbf{y}}+\frac{1}{6} c \hat{\mathbf{z}}$ & $(6 a)$ & F I \\
\hline $\mathbf{B}_{6}$ & $=$ & $x_{1} \mathbf{a}_{1}+x_{1} \mathbf{a}_{2}+\frac{5}{6} \mathbf{a}_{3}$ & $=$ & $x_{1} a \hat{\mathbf{x}}+\frac{5}{6} c \hat{\mathbf{z}}$ & $(6 a)$ & F I \\
\hline $\mathbf{B}_{7}$ & $=$ & $x_{2} \mathbf{a}_{1}+2 x_{2} \mathbf{a}_{2}+\frac{3}{4} \mathbf{a}_{3}$ & $=$ & $\frac{3}{2} x_{2} a \hat{\mathbf{x}}+\frac{\sqrt{3}}{2} x_{2} a \hat{\mathbf{y}}+\frac{3}{4} c \hat{\mathbf{z}}$ & $(6 b)$ & $\mathrm{Au}$ \\
\hline $\mathbf{B}_{8}$ & $=$ & $-2 x_{2} \mathbf{a}_{1}-x_{2} \mathbf{a}_{2}+\frac{5}{12} \mathbf{a}_{3}$ & $=$ & $-\frac{3}{2} x_{2} a \hat{\mathbf{x}}+\frac{\sqrt{3}}{2} x_{2} a \hat{\mathbf{y}}+\frac{5}{12} c \hat{\mathbf{z}}$ & $(6 b)$ & $\mathrm{Au}$ \\
\hline $\mathbf{B}_{9}$ & $=$ & $x_{2} \mathbf{a}_{1}-x_{2} \mathbf{a}_{2}+\frac{1}{12} \mathbf{a}_{3}$ & $=$ & $-\sqrt{3} x_{2} a \hat{\mathbf{y}}+\frac{1}{12} c \hat{\mathbf{z}}$ & $(6 b)$ & $\mathrm{Au}$ \\
\hline $\mathbf{B}_{10}$ & $=$ & $-x_{2} \mathbf{a}_{1}-2 x_{2} \mathbf{a}_{2}+\frac{1}{4} \mathbf{a}_{3}$ & $=$ & $-\frac{3}{2} x_{2} a \hat{\mathbf{x}}-\frac{\sqrt{3}}{2} x_{2} a \hat{\mathbf{y}}+\frac{1}{4} c \hat{\mathbf{z}}$ & $(6 b)$ & $\mathrm{Au}$ \\
\hline $\mathbf{B}_{11}$ & $=$ & $2 x_{2} \mathbf{a}_{1}+x_{2} \mathbf{a}_{2}+\frac{11}{12} \mathbf{a}_{3}$ & $=$ & $\frac{3}{2} x_{2} a \hat{\mathbf{x}}-\frac{\sqrt{3}}{2} x_{2} a \hat{\mathbf{y}}+\frac{11}{12} c \hat{\mathbf{z}}$ & $(6 b)$ & $\mathrm{Au}$ \\
\hline $\mathbf{B}_{12}$ & $=$ & $-x_{2} \mathbf{a}_{1}+x_{2} \mathbf{a}_{2}+\frac{7}{12} \mathbf{a}_{3}$ & $=$ & $\sqrt{3} x_{2} a \hat{\mathbf{y}}+\frac{7}{12} c \hat{\mathbf{z}}$ & $(6 b)$ & $\mathrm{Au}$ \\
\hline $\mathbf{B}_{13}$ & $=$ & $x_{3} \mathbf{a}_{1}+y_{3} \mathbf{a}_{2}+z_{3} \mathbf{a}_{3}$ & $=$ & $\begin{array}{c}\frac{1}{2}\left(x_{3}+y_{3}\right) a \hat{\mathbf{x}}+ \\
\frac{\sqrt{3}}{2}\left(-x_{3}+y_{3}\right) a \hat{\mathbf{y}}+z_{3} c \hat{\mathbf{z}}\end{array}$ & $(12 c)$ & F II \\
\hline $\mathbf{B}_{14}$ & $=$ & $-y_{3} \mathbf{a}_{1}+\left(x_{3}-y_{3}\right) \mathbf{a}_{2}+\left(\frac{2}{3}+z_{3}\right) \mathbf{a}_{3}$ & $=$ & $\begin{array}{c}\left(\frac{1}{2} x_{3}-y_{3}\right) a \hat{\mathbf{x}}+\frac{\sqrt{3}}{2} x_{3} a \hat{\mathbf{y}}+ \\
\left(\frac{2}{3}+z_{3}\right) c \hat{\mathbf{z}}\end{array}$ & $(12 c)$ & F II \\
\hline $\mathbf{B}_{15}$ & $=$ & $\left(-x_{3}+y_{3}\right) \mathbf{a}_{1}-x_{3} \mathbf{a}_{2}+\left(\frac{1}{3}+z_{3}\right) \mathbf{a}_{3}$ & $=$ & $\begin{array}{c}\left(-x_{3}+\frac{1}{2} y_{3}\right) a \hat{\mathbf{x}}-\frac{\sqrt{3}}{2} y_{3} a \hat{\mathbf{y}}+ \\
\left(\frac{1}{3}+z_{3}\right) c \hat{\mathbf{z}}\end{array}$ & $(12 c)$ & F II \\
\hline $\mathbf{B}_{16}$ & $=$ & $-x_{3} \mathbf{a}_{1}-y_{3} \mathbf{a}_{2}+\left(\frac{1}{2}+z_{3}\right) \mathbf{a}_{3}$ & $=$ & $\begin{array}{c}-\frac{1}{2}\left(x_{3}+y_{3}\right) a \hat{\mathbf{x}}+ \\
\frac{\sqrt{3}}{2}\left(x_{3}-y_{3}\right) a \hat{\mathbf{y}}+\left(\frac{1}{2}+z_{3}\right) c \hat{\mathbf{z}}\end{array}$ & $(12 c)$ & F II \\
\hline $\mathbf{B}_{17}$ & $=$ & $y_{3} \mathbf{a}_{1}+\left(-x_{3}+y_{3}\right) \mathbf{a}_{2}+\left(\frac{1}{6}+z_{3}\right) \mathbf{a}_{3}$ & $=$ & $\begin{array}{c}\left(-\frac{1}{2} x_{3}+y_{3}\right) a \hat{\mathbf{x}}-\frac{\sqrt{3}}{2} x_{3} a \hat{\mathbf{y}}+ \\
\left(\frac{1}{6}+z_{3}\right) c \hat{\mathbf{z}}\end{array}$ & $(12 c)$ & F II \\
\hline $\mathbf{B}_{18}$ & $=$ & $\left(x_{3}-y_{3}\right) \mathbf{a}_{1}+x_{3} \mathbf{a}_{2}+\left(\frac{5}{6}+z_{3}\right) \mathbf{a}_{3}$ & $=$ & $\begin{array}{c}\left(x_{3}-\frac{1}{2} y_{3}\right) a \hat{\mathbf{x}}+\frac{\sqrt{3}}{2} y_{3} a \hat{\mathbf{y}}+ \\
\left(\frac{5}{6}+z_{3}\right) c \hat{\mathbf{z}}\end{array}$ & $(12 c)$ & F II \\
\hline $\mathbf{B}_{19}$ & $=$ & $y_{3} \mathbf{a}_{1}+x_{3} \mathbf{a}_{2}+\left(\frac{2}{3}-z_{3}\right) \mathbf{a}_{3}$ & $=$ & $\begin{array}{c}\frac{1}{2}\left(x_{3}+y_{3}\right) a \hat{\mathbf{x}}+\frac{\sqrt{3}}{2}\left(x_{3}-y_{3}\right) a \hat{\mathbf{y}}+ \\
\left(\frac{2}{3}-z_{3}\right) c \hat{\mathbf{z}}\end{array}$ & $(12 c)$ & F II \\
\hline $\mathbf{B}_{20}$ & $=$ & $\left(x_{3}-y_{3}\right) \mathbf{a}_{1}-y_{3} \mathbf{a}_{2}-z_{3} \mathbf{a}_{3}$ & $=$ & $\left(\frac{1}{2} x_{3}-y_{3}\right) a \hat{\mathbf{x}}-\frac{\sqrt{3}}{2} x_{3} a \hat{\mathbf{y}}-z_{3} c \hat{\mathbf{z}}$ & $(12 c)$ & F II \\
\hline $\mathbf{B}_{21}$ & $=$ & $-x_{3} \mathbf{a}_{1}+\left(-x_{3}+y_{3}\right) \mathbf{a}_{2}+\left(\frac{1}{3}-z_{3}\right) \mathbf{a}_{3}$ & $=$ & $\begin{array}{c}\left(-x_{3}+\frac{1}{2} y_{3}\right) a \hat{\mathbf{x}}+\frac{\sqrt{3}}{2} y_{3} a \hat{\mathbf{y}}+ \\
\left(\frac{1}{3}-z_{3}\right) c \hat{\mathbf{z}}\end{array}$ & $(12 c)$ & F II \\
\hline $\mathbf{B}_{22}$ & $=$ & $-y_{3} \mathbf{a}_{1}-x_{3} \mathbf{a}_{2}+\left(\frac{1}{6}-z_{3}\right) \mathbf{a}_{3}$ & $=$ & $\begin{array}{c}-\frac{1}{2}\left(x_{3}+y_{3}\right) a \hat{\mathbf{x}}+ \\
\frac{\sqrt{3}}{2}\left(-x_{3}+y_{3}\right) a \hat{\mathbf{y}}+\left(\frac{1}{6}-z_{3}\right) c \hat{\mathbf{z}}\end{array}$ & $(12 c)$ & F II \\
\hline $\mathbf{B}_{23}$ & $=$ & $\left(-x_{3}+y_{3}\right) \mathbf{a}_{1}+y_{3} \mathbf{a}_{2}+\left(\frac{1}{2}-z_{3}\right) \mathbf{a}_{3}$ & $=$ & $\begin{array}{c}\left(-\frac{1}{2} x_{3}+y_{3}\right) a \hat{\mathbf{x}}+\frac{\sqrt{3}}{2} x_{3} a \hat{\mathbf{y}}+ \\
\left(\frac{1}{2}-z_{3}\right) c \hat{\mathbf{z}}\end{array}$ & $(12 c)$ & F II \\
\hline $\mathbf{B}_{24}$ & $=$ & $x_{3} \mathbf{a}_{1}+\left(x_{3}-y_{3}\right) \mathbf{a}_{2}+\left(\frac{5}{6}-z_{3}\right) \mathbf{a}_{3}$ & $=$ & $\begin{array}{c}\left(x_{3}-\frac{1}{2} y_{3}\right) a \hat{\mathbf{x}}-\frac{\sqrt{3}}{2} y_{3} a \hat{\mathbf{y}}+ \\
\left(\frac{5}{6}-z_{3}\right) c \hat{\mathbf{z}}\end{array}$ & $(12 c)$ & F II \\
\hline
\end{tabular}

\section{References:}

- L. B. Asprey, F. H. Kruse, K. H. Jack, and R. Maitland, Preparation and properties of crystalline gold trifluoride, Inorg. Chem. 3, 602-604 (1964), doi:10.1021/ic50014a037.

\section{Geometry files:}

- CIF: pp. 931

- POSCAR: pp. 932 

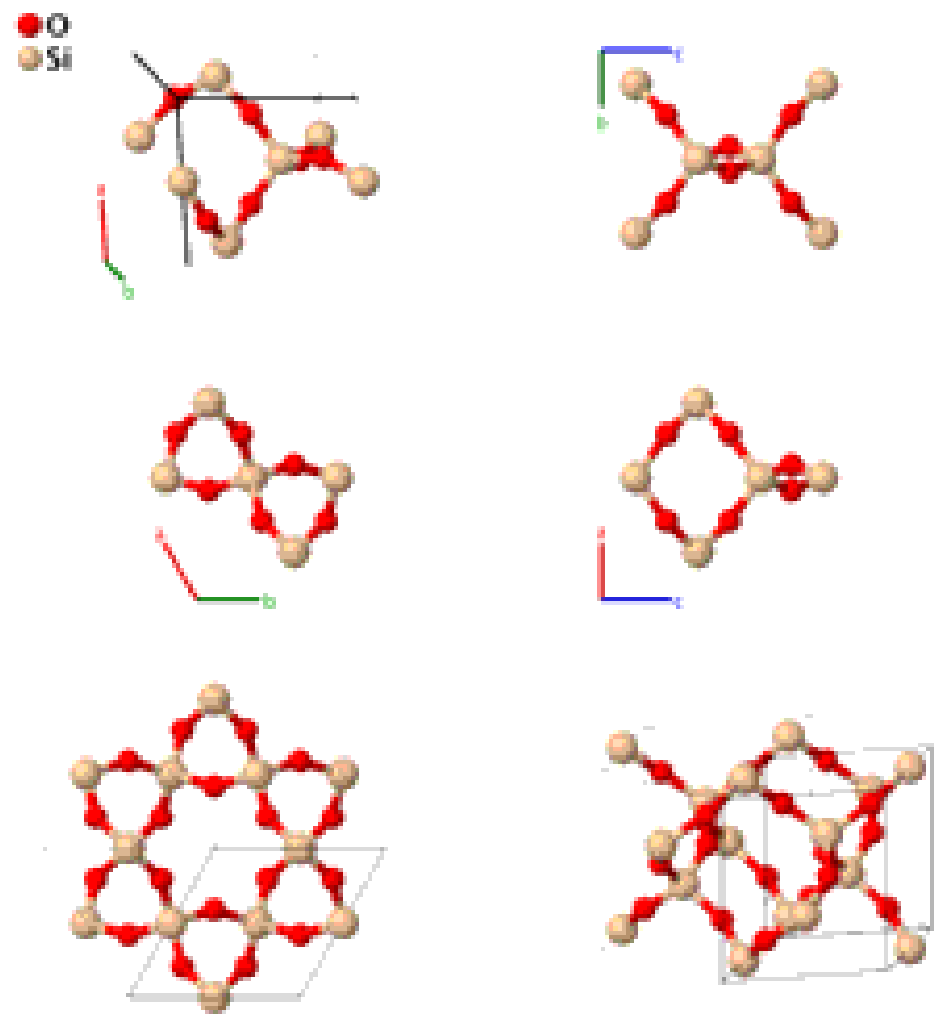

Prototype

AFLOW prototype label

Strukturbericht designation

Pearson symbol

Space group number

Space group symbol

AFLOW prototype command
: $\quad \beta-\mathrm{SiO}_{2}$

: A2B_hP9_181_j_c

: None

: $\mathrm{hP9}$

: $\quad 181$

$: \quad P 6_{4} 22$

aflow --proto=A2B_hP9_181_j_c

- params $=a, c / a, x_{2}$

- This structure is the enantiomorph of the $\beta$-Quartz (A2B_hP9_180_j_c) structure, and was generated by reflecting the coordinates of the space group \#180 structure through the $z=0$ plane.

\section{Hexagonal primitive vectors:}

$$
\begin{array}{ll}
\mathbf{a}_{1}= & \frac{1}{2} a \hat{\mathbf{x}}-\frac{\sqrt{3}}{2} a \hat{\mathbf{y}} \\
\mathbf{a}_{2}= & \frac{1}{2} a \hat{\mathbf{x}}+\frac{\sqrt{3}}{2} a \hat{\mathbf{y}} \\
\mathbf{a}_{3}= & c \hat{\mathbf{z}}
\end{array}
$$
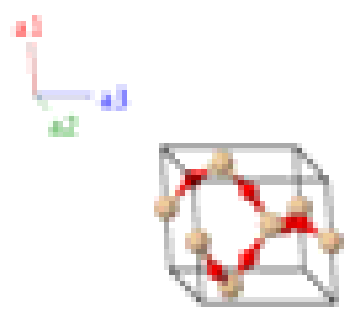

\section{Basis vectors:}


Lattice Coordinates

$\begin{array}{lcc}\mathbf{B}_{1}= & \frac{1}{2} \mathbf{a}_{1} \\ \mathbf{B}_{2}= & \frac{1}{2} \mathbf{a}_{2}+\frac{1}{3} \mathbf{a}_{3} \\ \mathbf{B}_{3}= & \frac{1}{2} \mathbf{a}_{1}+\frac{1}{2} \mathbf{a}_{2}+\frac{2}{3} \mathbf{a}_{3} \\ \mathbf{B}_{4}= & x_{2} \mathbf{a}_{1}+2 x_{2} \mathbf{a}_{2}+\frac{1}{2} \mathbf{a}_{3} \\ \mathbf{B}_{5}= & -2 x_{2} \mathbf{a}_{1}-x_{2} \mathbf{a}_{2}+\frac{5}{6} \mathbf{a}_{3} \\ \mathbf{B}_{6}= & x_{2} \mathbf{a}_{1}-x_{2} \mathbf{a}_{2}+\frac{1}{6} \mathbf{a}_{3} \\ \mathbf{B}_{7}= & -x_{2} \mathbf{a}_{1}-2 x_{2} \mathbf{a}_{2}+\frac{1}{2} \mathbf{a}_{3} \\ \mathbf{B}_{8}= & 2 x_{2} \mathbf{a}_{1}+x_{2} \mathbf{a}_{2}+\frac{5}{6} \mathbf{a}_{3} \\ \mathbf{B}_{9}= & -x_{2} \mathbf{a}_{1}+x_{2} \mathbf{a}_{2}+\frac{1}{6} \mathbf{a}_{3}\end{array}$

Cartesian Coordinates

$$
=
$$$$
\frac{1}{4} a \hat{\mathbf{x}}-\frac{\sqrt{3}}{4} a \hat{\mathbf{y}}
$$$$
=\quad \frac{1}{4} a \hat{\mathbf{x}}+\frac{\sqrt{3}}{4} a \hat{\mathbf{y}}+\frac{1}{3} c \hat{\mathbf{z}}
$$$$
=
$$$$
\frac{1}{2} a \hat{\mathbf{x}}+\frac{2}{3} c \hat{\mathbf{z}}
$$$$
=\quad \frac{3}{2} x_{2} a \hat{\mathbf{x}}+\frac{\sqrt{3}}{2} x_{2} a \hat{\mathbf{y}}+\frac{1}{2} c \hat{\mathbf{z}}
$$$$
=\quad-\frac{3}{2} x_{2} a \hat{\mathbf{x}}+\frac{\sqrt{3}}{2} x_{2} a \hat{\mathbf{y}}+\frac{5}{6} c \hat{\mathbf{z}}
$$

$=$

$=$

$$
-\sqrt{3} x_{2} a \hat{\mathbf{y}}+\frac{1}{6} c \hat{\mathbf{z}}
$$$$
-\frac{3}{2} x_{2} a \hat{\mathbf{x}}-\frac{\sqrt{3}}{2} x_{2} a \hat{\mathbf{y}}+\frac{1}{2} c \hat{\mathbf{z}}
$$$$
=\quad \frac{3}{2} x_{2} a \hat{\mathbf{x}}-\frac{\sqrt{3}}{2} x_{2} a \hat{\mathbf{y}}+\frac{5}{6} c \hat{\mathbf{z}}
$$$$
=
$$

$\sqrt{3} x_{2} a \hat{\mathbf{y}}+\frac{1}{6} c \hat{\mathbf{z}}$
Wyckoff Position

$(6 j)$
Atom Type

$\mathrm{O}$

$\mathrm{O}$

O

\section{References:}

- A. F. Wright and M. S. Lehmann, The Structure of Quartz at 25 and $590^{\circ}$ C Determined by Neutron Diffraction, J. Solid State Chem. 36, 371-380 (1981), doi:10.1016/0022-4596(81)90449-7.

\section{Geometry files:}

- CIF: pp. 932

- POSCAR: pp. 932 


\section{AuCN Structure: ABC_hP3_183_a_a_a}
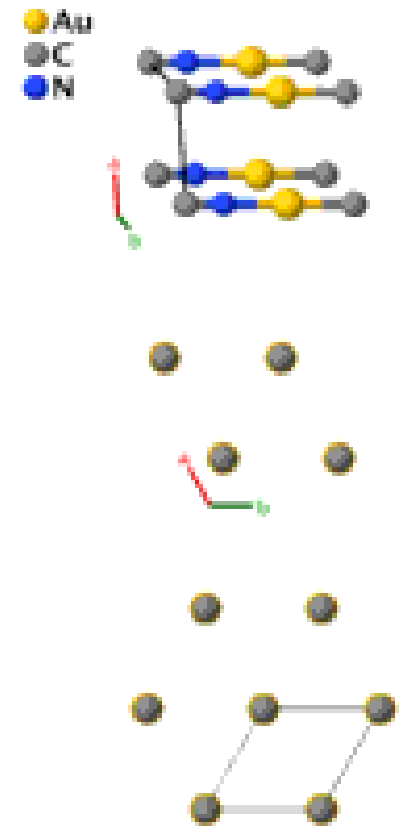
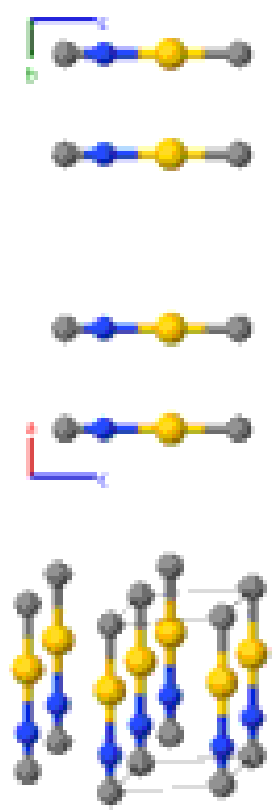

\section{Prototype}

$\mathrm{AuCN}$

AFLOW prototype label

: ABC_hP3_183_a_a_a

Strukturbericht designation

Pearson symbol

None

Space group number

hP3

Space group symbol

183

AFLOW prototype command

\section{P6mm}

aflow - -proto=ABC_hP3_183_a_a_a

- params $=a, c / a, z_{1}, z_{2}, z_{3}$

\section{Hexagonal primitive vectors:}

$$
\begin{array}{ll}
\mathbf{a}_{1}= & \frac{1}{2} a \hat{\mathbf{x}}-\frac{\sqrt{3}}{2} a \hat{\mathbf{y}} \\
\mathbf{a}_{2}= & \frac{1}{2} a \hat{\mathbf{x}}+\frac{\sqrt{3}}{2} a \hat{\mathbf{y}} \\
\mathbf{a}_{3}= & c \hat{\mathbf{z}}
\end{array}
$$

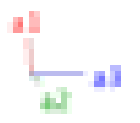

Basis vectors:

Lattice Coordinates

$\begin{array}{llll}\mathbf{B}_{1}= & z_{1} \mathbf{a}_{3} & = \\ \mathbf{B}_{2}= & z_{2} \mathbf{a}_{3} & = \\ \mathbf{B}_{3}= & z_{3} \mathbf{a}_{3} & =\end{array}$

\section{References:}


- S. J. Hibble, A. C. Hannon, and S. M. Cheyne, Structure of AuCN determined from total neutron diffraction, Inorg. Chem. 42, 4724-4730 (2003), doi:10.1021/ic0342043.

\section{Found in:}

- P. Villars and K. Cenzual, Pearson's Crystal Data - Crystal Structure Database for Inorganic Compounds, ASM International (2013).

\section{Geometry files:}

- CIF: pp. 932

- POSCAR: pp. 933 


\section{$\mathrm{CrFe}_{3} \mathrm{NiSn}_{5}$ Structure: AB_hP6_183_c_ab}
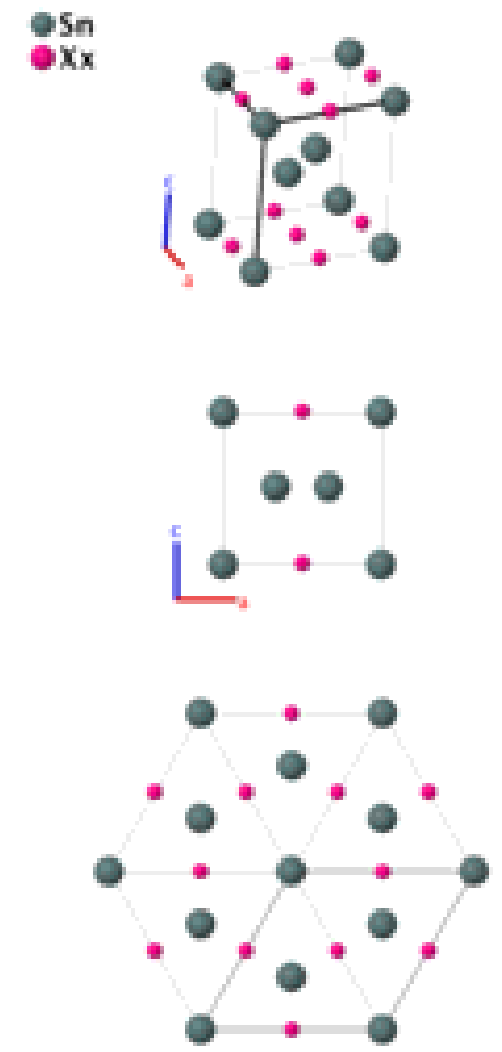

\section{Prototype}

AFLOW prototype label

Strukturbericht designation

Pearson symbol

Space group number

Space group symbol

AFLOW prototype command
: $\quad \mathrm{CrFe}_{3} \mathrm{NiSn}_{5}$

: AB_hP6_183_c_ab

: None

: $\mathrm{hP6}$

: $\quad 183$

: $\quad P 6 \mathrm{~mm}$

aflow --proto=AB_hP6_183_c_ab

- params $=a, c / a, z_{1}, z_{2}, z_{3}$
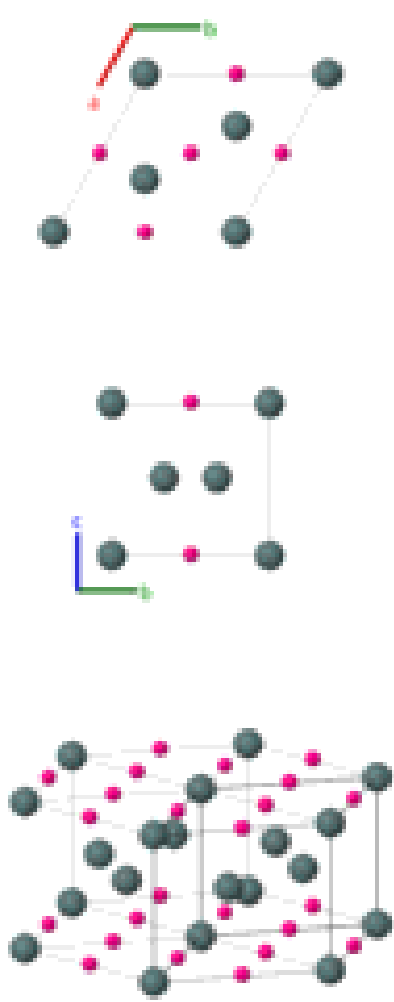

- Here, the $\mathrm{M}$ sites are mixed occupation $0.6 \mathrm{Fe}+0.2 \mathrm{Cr}+0.2 \mathrm{Ni}$ The Jmol image does not distinguish between the different $\mathrm{M}$ labels and is represented by the "Xx" atoms.

\section{Hexagonal primitive vectors:}

$$
\begin{array}{ll}
\mathbf{a}_{1}= & \frac{1}{2} a \hat{\mathbf{x}}-\frac{\sqrt{3}}{2} a \hat{\mathbf{y}} \\
\mathbf{a}_{2}= & \frac{1}{2} a \hat{\mathbf{x}}+\frac{\sqrt{3}}{2} a \hat{\mathbf{y}} \\
\mathbf{a}_{3}= & c \hat{\mathbf{z}}
\end{array}
$$

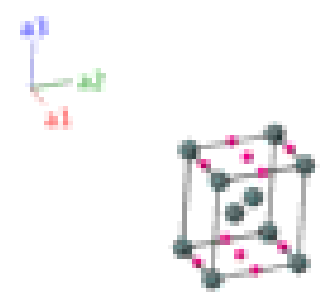

Basis vectors: 


$\begin{array}{lcccc}\mathbf{B}_{1}= & z_{1} \mathbf{a}_{3} & = & z_{1} c \hat{\mathbf{z}} \\ \mathbf{B}_{2}= & \frac{1}{3} \mathbf{a}_{1}+\frac{2}{3} \mathbf{a}_{2}+z_{2} \mathbf{a}_{3} & = & \frac{1}{2} a \hat{\mathbf{x}}+\frac{1}{2 \sqrt{3}} a \hat{\mathbf{y}}+z_{2} c \hat{\mathbf{z}} \\ \mathbf{B}_{3}= & \frac{2}{3} \mathbf{a}_{1}+\frac{1}{3} \mathbf{a}_{2}+z_{2} \mathbf{a}_{3} & = & \frac{1}{2} a \hat{\mathbf{x}}-\frac{1}{2 \sqrt{3}} a \hat{\mathbf{y}}+z_{2} c \hat{\mathbf{z}} \\ \mathbf{B}_{4}= & \frac{1}{2} \mathbf{a}_{1}+z_{3} \mathbf{a}_{3} & = & \frac{1}{4} a \hat{\mathbf{x}}-\frac{\sqrt{3}}{4} a \hat{\mathbf{y}}+z_{3} c \hat{\mathbf{z}} \\ \mathbf{B}_{5}= & \frac{1}{2} \mathbf{a}_{2}+z_{3} \mathbf{a}_{3} & = & \frac{1}{4} a \hat{\mathbf{x}}+\frac{\sqrt{3}}{4} a \hat{\mathbf{y}}+z_{3} c \hat{\mathbf{z}} \\ \mathbf{B}_{6}= & \frac{1}{2} \mathbf{a}_{1}+\frac{1}{2} \mathbf{a}_{2}+z_{3} \mathbf{a}_{3} & = & \frac{1}{2} a \hat{\mathbf{x}}+z_{3} c \hat{\mathbf{z}}\end{array}$

\section{References:}

- J. Huang, L. Zeng, and Z. Sun, $\mathrm{X}$-ray powder diffraction data and Rietveld refinement of $\mathrm{CrFe}_{3} \mathrm{NiSn}_{5}$, Powder Diffraction 19, 372-374 (2004), doi:10.1154/1.1763153.

\section{Found in:}

- P. Villars and K. Cenzual, Pearson's Crystal Data - Crystal Structure Database for Inorganic Compounds, ASM International (2013).

\section{Geometry files:}

- CIF: pp. 933

- POSCAR: pp. 933 


\section{$\mathrm{Al}\left[\mathrm{PO}_{4}\right]$ (Framework type AFI) Structure: AB4C_hP72_184_d_4d_d}
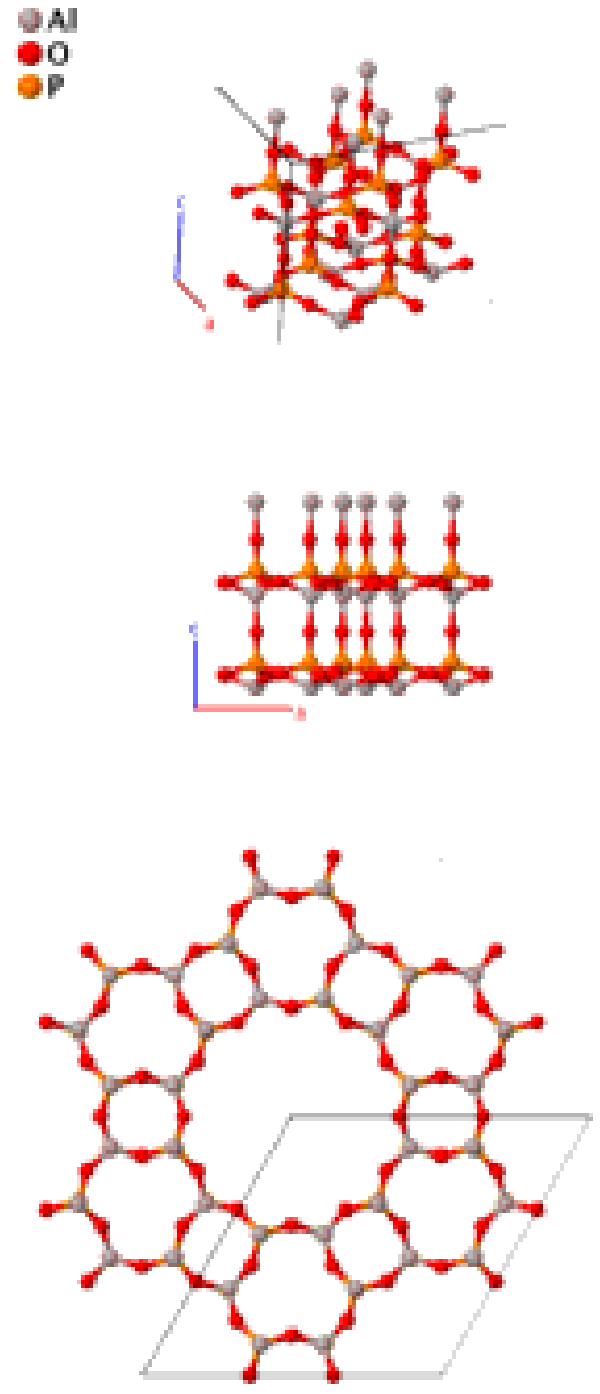

Prototype

AFLOW prototype label

Strukturbericht designation : None

Pearson symbol

Space group number

Space group symbol

AFLOW prototype command
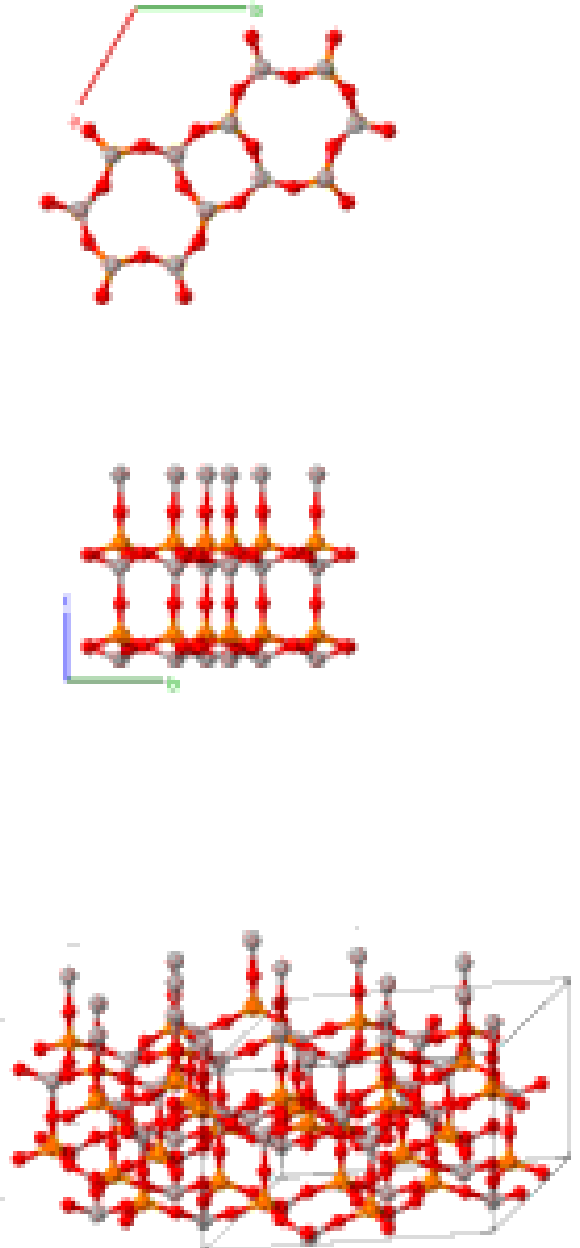

- This is the structure of $\mathrm{AlPO}_{4}-5$, which has the zeolite framework designation AFI. 


\section{Hexagonal primitive vectors:}

$$
\begin{array}{ll}
\mathbf{a}_{1}= & \frac{1}{2} a \hat{\mathbf{x}}-\frac{\sqrt{3}}{2} a \hat{\mathbf{y}} \\
\mathbf{a}_{2}= & \frac{1}{2} a \hat{\mathbf{x}}+\frac{\sqrt{3}}{2} a \hat{\mathbf{y}} \\
\mathbf{a}_{3}= & c \hat{\mathbf{z}}
\end{array}
$$

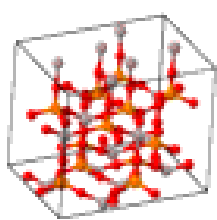

\section{Basis vectors:}

Lattice Coordinates

$\mathbf{B}_{1}=$

$x_{1} \mathbf{a}_{1}+y_{1} \mathbf{a}_{2}+z_{1} \mathbf{a}_{3}$

$\mathbf{B}_{2}=-y_{1} \mathbf{a}_{1}+\left(x_{1}-y_{1}\right) \mathbf{a}_{2}+z_{1} \mathbf{a}_{3}$

$\mathbf{B}_{3}=\left(-x_{1}+y_{1}\right) \mathbf{a}_{1}-x_{1} \mathbf{a}_{2}+z_{1} \mathbf{a}_{3}$

$\mathbf{B}_{4}=$

$-x_{1} \mathbf{a}_{1}-y_{1} \mathbf{a}_{2}+z_{1} \mathbf{a}_{3}$

$\mathbf{B}_{5}=y_{1} \mathbf{a}_{1}+\left(-x_{1}+y_{1}\right) \mathbf{a}_{2}+z_{1} \mathbf{a}_{3}$

$\mathbf{B}_{6}=\left(x_{1}-y_{1}\right) \mathbf{a}_{1}+x_{1} \mathbf{a}_{2}+z_{1} \mathbf{a}_{3}$

$\mathbf{B}_{7}=-y_{1} \mathbf{a}_{1}-x_{1} \mathbf{a}_{2}+\left(\frac{1}{2}+z_{1}\right) \mathbf{a}_{3}$

$\mathbf{B}_{8}=\left(-x_{1}+y_{1}\right) \mathbf{a}_{1}+y_{1} \mathbf{a}_{2}+\left(\frac{1}{2}+z_{1}\right) \mathbf{a}_{3}=$

$\mathbf{B}_{9}=x_{1} \mathbf{a}_{1}+\left(x_{1}-y_{1}\right) \mathbf{a}_{2}+\left(\frac{1}{2}+z_{1}\right) \mathbf{a}_{3}=$

$\mathbf{B}_{10}=\quad y_{1} \mathbf{a}_{1}+x_{1} \mathbf{a}_{2}+\left(\frac{1}{2}+z_{1}\right) \mathbf{a}_{3}$

$$
\begin{gathered}
\left(\frac{1}{2}+z_{1}\right) c \hat{\mathbf{z}} \\
=\quad \frac{1}{2}\left(x_{1}+y_{1}\right) a \hat{\mathbf{x}}+\frac{\sqrt{3}}{2}\left(x_{1}-y_{1}\right) a \hat{\mathbf{y}}+ \\
\left(\frac{1}{2}+z_{1}\right) c \hat{\mathbf{z}} \\
=\quad\left(\frac{1}{2} x_{1}-y_{1}\right) a \hat{\mathbf{x}}-\frac{\sqrt{3}}{2} x_{1} a \hat{\mathbf{y}}+ \\
\left(\frac{1}{2}+z_{1}\right) c \hat{\mathbf{z}}
\end{gathered}
$$

Cartesian Coordinates

$=$

$=\left(\frac{1}{2} x_{1}-y_{1}\right) a \hat{\mathbf{x}}+\frac{\sqrt{3}}{2} x_{1} a \hat{\mathbf{y}}+z_{1} c \hat{\mathbf{z}}$

$=\left(-x_{1}+\frac{1}{2} y_{1}\right) a \hat{\mathbf{x}}-\frac{\sqrt{3}}{2} y_{1} a \hat{\mathbf{y}}+z_{1} c \hat{\mathbf{z}}$

$=\quad-\frac{1}{2}\left(x_{1}+y_{1}\right) a \hat{\mathbf{x}}+$

$$
\frac{\sqrt{3}}{2}\left(x_{1}-y_{1}\right) a \hat{\mathbf{y}}+z_{1} c \hat{\mathbf{z}}
$$

$=\left(-\frac{1}{2} x_{1}+y_{1}\right) a \hat{\mathbf{x}}-\frac{\sqrt{3}}{2} x_{1} a \hat{\mathbf{y}}+z_{1} c \hat{\mathbf{z}}$

$=\left(x_{1}-\frac{1}{2} y_{1}\right) a \hat{\mathbf{x}}+\frac{\sqrt{3}}{2} y_{1} a \hat{\mathbf{y}}+z_{1} c \hat{\mathbf{z}}$

$=\quad-\frac{1}{2}\left(x_{1}+y_{1}\right) a \hat{\mathbf{x}}+$ $\frac{\sqrt{3}}{2}\left(-x_{1}+y_{1}\right) a \hat{\mathbf{y}}+\left(\frac{1}{2}+z_{1}\right) c \hat{\mathbf{z}}$

$=\quad\left(-\frac{1}{2} x_{1}+y_{1}\right) a \hat{\mathbf{x}}+\frac{\sqrt{3}}{2} x_{1} a \hat{\mathbf{y}}+$

$$
\left(\frac{1}{2}+z_{1}\right) c \hat{\mathbf{z}}
$$

$=\left(x_{1}-\frac{1}{2} y_{1}\right) a \hat{\mathbf{x}}-\frac{\sqrt{3}}{2} y_{1} a \hat{\mathbf{y}}+$

$\mathbf{B}_{11}=\left(x_{1}-y_{1}\right) \mathbf{a}_{1}-y_{1} \mathbf{a}_{2}+\left(\frac{1}{2}+z_{1}\right) \mathbf{a}_{3}=\left(\frac{1}{2} x_{1}-y_{1}\right) a \hat{\mathbf{x}}-\frac{\sqrt{3}}{2} x_{1} a \hat{\mathbf{y}}+$

$\mathbf{B}_{12}=-x_{1} \mathbf{a}_{1}+\left(-x_{1}+y_{1}\right) \mathbf{a}_{2}+\left(\frac{1}{2}+z_{1}\right) \mathbf{a}_{3}=\left(-x_{1}+\frac{1}{2} y_{1}\right) a \hat{\mathbf{x}}+\frac{\sqrt{3}}{2} y_{1} a \hat{\mathbf{y}}+$

$$
\begin{array}{lc} 
& \left(\frac{1}{2}+z_{1}\right) c \hat{\mathbf{z}} \\
= & \frac{1}{2}\left(x_{2}+y_{2}\right) a \hat{\mathbf{x}}+ \\
\frac{\sqrt{3}}{2}\left(-x_{2}+y_{2}\right) a \hat{\mathbf{y}}+z_{2} c \hat{\mathbf{z}} \\
= & \left(\frac{1}{2} x_{2}-y_{2}\right) a \hat{\mathbf{x}}+\frac{\sqrt{3}}{2} x_{2} a \hat{\mathbf{y}}+z_{2} c \hat{\mathbf{z}} \\
= & \left(-x_{2}+\frac{1}{2} y_{2}\right) a \hat{\mathbf{x}}-\frac{\sqrt{3}}{2} y_{2} a \hat{\mathbf{y}}+z_{2} c \hat{\mathbf{z}} \\
= & -\frac{1}{2}\left(x_{2}+y_{2}\right) a \hat{\mathbf{x}}+ \\
= & \frac{\sqrt{3}}{2}\left(x_{2}-y_{2}\right) a \hat{\mathbf{y}}+z_{2} c \hat{\mathbf{z}} \\
= & \left(x_{2}-\frac{1}{2} x_{2}+y_{2}\right) a \hat{\mathbf{x}}-\frac{\sqrt{3}}{2} x_{2} a \hat{\mathbf{y}}+\frac{\sqrt{3}}{2} y_{2} a \hat{\mathbf{y}}+z_{2} c \hat{\mathbf{z}} \\
= & -\frac{1}{2}\left(x_{2}+y_{2}\right) a \hat{\mathbf{x}}+ \\
& \frac{\sqrt{3}}{2}\left(-x_{2}+y_{2}\right) a \hat{\mathbf{y}}+\left(\frac{1}{2}+z_{2}\right) c \hat{\mathbf{z}}
\end{array}
$$

$\mathbf{B}_{13}=x_{2} \mathbf{a}_{1}+y_{2} \mathbf{a}_{2}+z_{2} \mathbf{a}_{3}$

$\mathbf{B}_{14}=-y_{2} \mathbf{a}_{1}+\left(x_{2}-y_{2}\right) \mathbf{a}_{2}+z_{2} \mathbf{a}_{3}$

$\mathbf{B}_{15}=\left(-x_{2}+y_{2}\right) \mathbf{a}_{1}-x_{2} \mathbf{a}_{2}+z_{2} \mathbf{a}_{3}$

$\mathbf{B}_{16}=$

$$
-x_{2} \mathbf{a}_{1}-y_{2} \mathbf{a}_{2}+z_{2} \mathbf{a}_{3}
$$

$\mathbf{B}_{17}=y_{2} \mathbf{a}_{1}+\left(-x_{2}+y_{2}\right) \mathbf{a}_{2}+z_{2} \mathbf{a}_{3}$

$\mathbf{B}_{18}=\left(x_{2}-y_{2}\right) \mathbf{a}_{1}+x_{2} \mathbf{a}_{2}+z_{2} \mathbf{a}_{3}$

$\mathbf{B}_{19}=$
Wyckoff Position Atom Type

$\mathrm{Al}$

Al

O I

O I

O I

O I

O I

O I 


\begin{tabular}{|c|c|c|c|c|c|c|}
\hline $\mathbf{B}_{20}$ & $=$ & $\left(-x_{2}+y_{2}\right) \mathbf{a}_{1}+y_{2} \mathbf{a}_{2}+\left(\frac{1}{2}+z_{2}\right) \mathbf{a}_{3}$ & $=$ & $\begin{array}{c}\left(-\frac{1}{2} x_{2}+y_{2}\right) a \hat{\mathbf{x}}+\frac{\sqrt{3}}{2} x_{2} a \hat{\mathbf{y}}+ \\
\left(\frac{1}{2}+z_{2}\right) c \hat{\mathbf{z}}\end{array}$ & $(12 d)$ & O I \\
\hline $\mathbf{B}_{21}$ & $=$ & $x_{2} \mathbf{a}_{1}+\left(x_{2}-y_{2}\right) \mathbf{a}_{2}+\left(\frac{1}{2}+z_{2}\right) \mathbf{a}_{3}$ & $=$ & $\begin{array}{c}\left(x_{2}-\frac{1}{2} y_{2}\right) a \hat{\mathbf{x}}-\frac{\sqrt{3}}{2} y_{2} a \hat{\mathbf{y}}+ \\
\left(\frac{1}{2}+z_{2}\right) c \hat{\mathbf{z}}\end{array}$ & $(12 d)$ & O I \\
\hline $\mathbf{B}_{22}$ & $=$ & $y_{2} \mathbf{a}_{1}+x_{2} \mathbf{a}_{2}+\left(\frac{1}{2}+z_{2}\right) \mathbf{a}_{3}$ & $=$ & $\begin{array}{c}\frac{1}{2}\left(x_{2}+y_{2}\right) a \hat{\mathbf{x}}+\frac{\sqrt{3}}{2}\left(x_{2}-y_{2}\right) a \hat{\mathbf{y}}+ \\
\left(\frac{1}{2}+z_{2}\right) c \hat{\mathbf{z}}\end{array}$ & $(12 d)$ & O I \\
\hline $\mathbf{B}_{23}$ & $=$ & $\left(x_{2}-y_{2}\right) \mathbf{a}_{1}-y_{2} \mathbf{a}_{2}+\left(\frac{1}{2}+z_{2}\right) \mathbf{a}_{3}$ & $=$ & $\begin{array}{c}\left(\frac{1}{2} x_{2}-y_{2}\right) a \hat{\mathbf{x}}-\frac{\sqrt{3}}{2} x_{2} a \hat{\mathbf{y}}+ \\
\left(\frac{1}{2}+z_{2}\right) c \hat{\mathbf{z}}\end{array}$ & $(12 d)$ & O I \\
\hline $\mathbf{B}_{24}$ & $=$ & $-x_{2} \mathbf{a}_{1}+\left(-x_{2}+y_{2}\right) \mathbf{a}_{2}+\left(\frac{1}{2}+z_{2}\right) \mathbf{a}_{3}$ & $=$ & $\begin{array}{c}\left(-x_{2}+\frac{1}{2} y_{2}\right) a \hat{\mathbf{x}}+\frac{\sqrt{3}}{2} y_{2} a \hat{\mathbf{y}}+ \\
\left(\frac{1}{2}+z_{2}\right) c \hat{\mathbf{z}}\end{array}$ & $(12 d)$ & $\mathrm{O} \mathrm{I}$ \\
\hline $\mathbf{B}_{25}$ & $=$ & $x_{3} \mathbf{a}_{1}+y_{3} \mathbf{a}_{2}+z_{3} \mathbf{a}_{3}$ & $=$ & $\begin{array}{c}\frac{1}{2}\left(x_{3}+y_{3}\right) a \hat{\mathbf{x}}+ \\
\frac{\sqrt{3}}{2}\left(-x_{3}+y_{3}\right) a \hat{\mathbf{y}}+z_{3} c \hat{\mathbf{z}}\end{array}$ & $(12 d)$ & O II \\
\hline $\mathbf{B}_{26}$ & $=$ & $-y_{3} \mathbf{a}_{1}+\left(x_{3}-y_{3}\right) \mathbf{a}_{2}+z_{3} \mathbf{a}_{3}$ & $=$ & $\left(\frac{1}{2} x_{3}-y_{3}\right) a \hat{\mathbf{x}}+\frac{\sqrt{3}}{2} x_{3} a \hat{\mathbf{y}}+z_{3} c \hat{\mathbf{z}}$ & $(12 d)$ & O II \\
\hline $\mathbf{B}_{27}$ & $=$ & $\left(-x_{3}+y_{3}\right) \mathbf{a}_{1}-x_{3} \mathbf{a}_{2}+z_{3} \mathbf{a}_{3}$ & $=$ & $\left(-x_{3}+\frac{1}{2} y_{3}\right) a \hat{\mathbf{x}}-\frac{\sqrt{3}}{2} y_{3} a \hat{\mathbf{y}}+z_{3} c \hat{\mathbf{z}}$ & $(12 d)$ & O II \\
\hline $\mathbf{B}_{28}$ & $=$ & $-x_{3} \mathbf{a}_{1}-y_{3} \mathbf{a}_{2}+z_{3} \mathbf{a}_{3}$ & $=$ & $\begin{array}{c}-\frac{1}{2}\left(x_{3}+y_{3}\right) a \hat{\mathbf{x}}+ \\
\frac{\sqrt{3}}{2}\left(x_{3}-y_{3}\right) a \hat{\mathbf{y}}+z_{3} c \hat{\mathbf{z}}\end{array}$ & $(12 d)$ & O II \\
\hline $\mathbf{B}_{29}$ & $=$ & $y_{3} \mathbf{a}_{1}+\left(-x_{3}+y_{3}\right) \mathbf{a}_{2}+z_{3} \mathbf{a}_{3}$ & $=$ & $\left(-\frac{1}{2} x_{3}+y_{3}\right) a \hat{\mathbf{x}}-\frac{\sqrt{3}}{2} x_{3} a \hat{\mathbf{y}}+z_{3} c \hat{\mathbf{z}}$ & $(12 d)$ & O II \\
\hline $\mathbf{B}_{30}$ & $=$ & $\left(x_{3}-y_{3}\right) \mathbf{a}_{1}+x_{3} \mathbf{a}_{2}+z_{3} \mathbf{a}_{3}$ & $=$ & $\left(x_{3}-\frac{1}{2} y_{3}\right) a \hat{\mathbf{x}}+\frac{\sqrt{3}}{2} y_{3} a \hat{\mathbf{y}}+z_{3} c \hat{\mathbf{z}}$ & $(12 d)$ & O II \\
\hline $\mathbf{B}_{31}$ & $=$ & $-y_{3} \mathbf{a}_{1}-x_{3} \mathbf{a}_{2}+\left(\frac{1}{2}+z_{3}\right) \mathbf{a}_{3}$ & $=$ & $\begin{array}{c}-\frac{1}{2}\left(x_{3}+y_{3}\right) a \hat{\mathbf{x}}+ \\
\frac{\sqrt{3}}{2}\left(-x_{3}+y_{3}\right) a \hat{\mathbf{y}}+\left(\frac{1}{2}+z_{3}\right) c \hat{\mathbf{z}}\end{array}$ & $(12 d)$ & O II \\
\hline $\mathbf{B}_{32}$ & $=$ & $\left(-x_{3}+y_{3}\right) \mathbf{a}_{1}+y_{3} \mathbf{a}_{2}+\left(\frac{1}{2}+z_{3}\right) \mathbf{a}_{3}$ & $=$ & $\begin{array}{c}\left(-\frac{1}{2} x_{3}+y_{3}\right) a \hat{\mathbf{x}}+\frac{\sqrt{3}}{2} x_{3} a \hat{\mathbf{y}}+ \\
\left(\frac{1}{2}+z_{3}\right) c \hat{\mathbf{z}}\end{array}$ & $(12 d)$ & O II \\
\hline $\mathbf{B}_{33}$ & $=$ & $x_{3} \mathbf{a}_{1}+\left(x_{3}-y_{3}\right) \mathbf{a}_{2}+\left(\frac{1}{2}+z_{3}\right) \mathbf{a}_{3}$ & $=$ & $\begin{array}{c}\left(x_{3}-\frac{1}{2} y_{3}\right) a \hat{\mathbf{x}}-\frac{\sqrt{3}}{2} y_{3} a \hat{\mathbf{y}}+ \\
\left(\frac{1}{2}+z_{3}\right) c \hat{\mathbf{z}}\end{array}$ & $(12 d)$ & O II \\
\hline $\mathbf{B}_{34}$ & $=$ & $y_{3} \mathbf{a}_{1}+x_{3} \mathbf{a}_{2}+\left(\frac{1}{2}+z_{3}\right) \mathbf{a}_{3}$ & $=$ & $\begin{array}{c}\frac{1}{2}\left(x_{3}+y_{3}\right) a \hat{\mathbf{x}}+\frac{\sqrt{3}}{2}\left(x_{3}-y_{3}\right) a \hat{\mathbf{y}}+ \\
\left(\frac{1}{2}+z_{3}\right) c \hat{\mathbf{z}}\end{array}$ & $(12 d)$ & O II \\
\hline $\mathbf{B}_{35}$ & $=$ & $\left(x_{3}-y_{3}\right) \mathbf{a}_{1}-y_{3} \mathbf{a}_{2}+\left(\frac{1}{2}+z_{3}\right) \mathbf{a}_{3}$ & $=$ & $\begin{array}{c}\left(\frac{1}{2} x_{3}-y_{3}\right) a \hat{\mathbf{x}}-\frac{\sqrt{3}}{2} x_{3} a \hat{\mathbf{y}}+ \\
\left(\frac{1}{2}+z_{3}\right) c \hat{\mathbf{z}}\end{array}$ & $(12 d)$ & O II \\
\hline $\mathbf{B}_{36}$ & $=$ & $-x_{3} \mathbf{a}_{1}+\left(-x_{3}+y_{3}\right) \mathbf{a}_{2}+\left(\frac{1}{2}+z_{3}\right) \mathbf{a}_{3}$ & $=$ & $\begin{array}{c}\left(-x_{3}+\frac{1}{2} y_{3}\right) a \hat{\mathbf{x}}+\frac{\sqrt{3}}{2} y_{3} a \hat{\mathbf{y}}+ \\
\left(\frac{1}{2}+z_{3}\right) c \hat{\mathbf{z}}\end{array}$ & $(12 d)$ & O II \\
\hline $\mathbf{B}_{37}$ & $=$ & $x_{4} \mathbf{a}_{1}+y_{4} \mathbf{a}_{2}+z_{4} \mathbf{a}_{3}$ & $=$ & $\begin{array}{c}\frac{1}{2}\left(x_{4}+y_{4}\right) a \hat{\mathbf{x}}+ \\
\frac{\sqrt{3}}{2}\left(-x_{4}+y_{4}\right) a \hat{\mathbf{y}}+z_{4} c \hat{\mathbf{z}}\end{array}$ & $(12 d)$ & O III \\
\hline $\mathbf{B}_{38}$ & $=$ & $-y_{4} \mathbf{a}_{1}+\left(x_{4}-y_{4}\right) \mathbf{a}_{2}+z_{4} \mathbf{a}_{3}$ & $=$ & $\left(\frac{1}{2} x_{4}-y_{4}\right) a \hat{\mathbf{x}}+\frac{\sqrt{3}}{2} x_{4} a \hat{\mathbf{y}}+z_{4} c \hat{\mathbf{z}}$ & $(12 d)$ & O III \\
\hline $\mathbf{B}_{39}$ & $=$ & $\left(-x_{4}+y_{4}\right) \mathbf{a}_{1}-x_{4} \mathbf{a}_{2}+z_{4} \mathbf{a}_{3}$ & $=$ & $\left(-x_{4}+\frac{1}{2} y_{4}\right) a \hat{\mathbf{x}}-\frac{\sqrt{3}}{2} y_{4} a \hat{\mathbf{y}}+z_{4} c \hat{\mathbf{z}}$ & $(12 d)$ & O III \\
\hline $\mathbf{B}_{40}$ & $=$ & $-x_{4} \mathbf{a}_{1}-y_{4} \mathbf{a}_{2}+z_{4} \mathbf{a}_{3}$ & $=$ & $\begin{array}{c}-\frac{1}{2}\left(x_{4}+y_{4}\right) a \hat{\mathbf{x}}+ \\
\frac{\sqrt{3}}{2}\left(x_{4}-y_{4}\right) a \hat{\mathbf{y}}+z_{4} c \hat{\mathbf{z}}\end{array}$ & $(12 d)$ & O III \\
\hline $\mathbf{B}_{41}$ & $=$ & $y_{4} \mathbf{a}_{1}+\left(-x_{4}+y_{4}\right) \mathbf{a}_{2}+z_{4} \mathbf{a}_{3}$ & $=$ & $\left(-\frac{1}{2} x_{4}+y_{4}\right) a \hat{\mathbf{x}}-\frac{\sqrt{3}}{2} x_{4} a \hat{\mathbf{y}}+z_{4} c \hat{\mathbf{z}}$ & $(12 d)$ & O III \\
\hline $\mathbf{B}_{42}$ & $=$ & $\left(x_{4}-y_{4}\right) \mathbf{a}_{1}+x_{4} \mathbf{a}_{2}+z_{4} \mathbf{a}_{3}$ & $=$ & $\left(x_{4}-\frac{1}{2} y_{4}\right) a \hat{\mathbf{x}}+\frac{\sqrt{3}}{2} y_{4} a \hat{\mathbf{y}}+z_{4} c \hat{\mathbf{z}}$ & $(12 d)$ & O III \\
\hline $\mathbf{B}_{43}$ & $=$ & $-y_{4} \mathbf{a}_{1}-x_{4} \mathbf{a}_{2}+\left(\frac{1}{2}+z_{4}\right) \mathbf{a}_{3}$ & $=$ & $\begin{array}{c}-\frac{1}{2}\left(x_{4}+y_{4}\right) a \hat{\mathbf{x}}+ \\
\frac{\sqrt{3}}{2}\left(-x_{4}+y_{4}\right) a \hat{\mathbf{y}}+\left(\frac{1}{2}+z_{4}\right) c \hat{\mathbf{z}}\end{array}$ & $(12 d)$ & O III \\
\hline
\end{tabular}




$$
\begin{aligned}
& \mathbf{B}_{44}=\left(-x_{4}+y_{4}\right) \mathbf{a}_{1}+y_{4} \mathbf{a}_{2}+\left(\frac{1}{2}+z_{4}\right) \mathbf{a}_{3}=\left(-\frac{1}{2} x_{4}+y_{4}\right) a \hat{\mathbf{x}}+\frac{\sqrt{3}}{2} x_{4} a \hat{\mathbf{y}}+ \\
& \left(\frac{1}{2}+z_{4}\right) c \hat{\mathbf{z}} \\
& \mathbf{B}_{45}=x_{4} \mathbf{a}_{1}+\left(x_{4}-y_{4}\right) \mathbf{a}_{2}+\left(\frac{1}{2}+z_{4}\right) \mathbf{a}_{3}=\left(x_{4}-\frac{1}{2} y_{4}\right) a \hat{\mathbf{x}}-\frac{\sqrt{3}}{2} y_{4} a \hat{\mathbf{y}}+ \\
& \left(\frac{1}{2}+z_{4}\right) c \hat{\mathbf{z}} \\
& \mathbf{B}_{46}=y_{4} \mathbf{a}_{1}+x_{4} \mathbf{a}_{2}+\left(\frac{1}{2}+z_{4}\right) \mathbf{a}_{3}=\frac{1}{2}\left(x_{4}+y_{4}\right) a \hat{\mathbf{x}}+\frac{\sqrt{3}}{2}\left(x_{4}-y_{4}\right) a \hat{\mathbf{y}}+
\end{aligned}
$$$$
\left(\frac{1}{2}+z_{4}\right) c \hat{\mathbf{z}}
$$

O III

$$
\mathbf{B}_{47}=\left(x_{4}-y_{4}\right) \mathbf{a}_{1}-y_{4} \mathbf{a}_{2}+\left(\frac{1}{2}+z_{4}\right) \mathbf{a}_{3}=\begin{array}{r}
\left(\frac{1}{2} x_{4}-y_{4}\right) a \hat{\mathbf{x}}-\frac{\sqrt{3}}{2} x_{4} a \hat{\mathbf{y}}+ \\
\left(\frac{1}{2}+z_{4}\right) c \hat{\mathbf{z}}
\end{array}
$$

O III

$\mathbf{B}_{48}=-x_{4} \mathbf{a}_{1}+\left(-x_{4}+y_{4}\right) \mathbf{a}_{2}+\left(\frac{1}{2}+z_{4}\right) \mathbf{a}_{3}=\left(-x_{4}+\frac{1}{2} y_{4}\right) a \hat{\mathbf{x}}+\frac{\sqrt{3}}{2} y_{4} a \hat{\mathbf{y}}+$

O III

$$
\begin{gathered}
\left(\frac{1}{2}+z_{4}\right) c \hat{\mathbf{z}} \\
\frac{1}{2}\left(x_{5}+y_{5}\right) a \hat{\mathbf{x}}+
\end{gathered}
$$

$\mathbf{B}_{49}=\quad x_{5} \mathbf{a}_{1}+y_{5} \mathbf{a}_{2}+z_{5} \mathbf{a}_{3}$

$$
=
$$$$
\frac{\sqrt{3}}{2}\left(-x_{5}+y_{5}\right) a \hat{\mathbf{y}}+z_{5} c \hat{\mathbf{z}}
$$$$
\mathbf{B}_{50}=-y_{5} \mathbf{a}_{1}+\left(x_{5}-y_{5}\right) \mathbf{a}_{2}+z_{5} \mathbf{a}_{3}=\left(\frac{1}{2} x_{5}-y_{5}\right) a \hat{\mathbf{x}}+\frac{\sqrt{3}}{2} x_{5} a \hat{\mathbf{y}}+z_{5} c \hat{\mathbf{z}}
$$

$\mathbf{B}_{51}=\left(-x_{5}+y_{5}\right) \mathbf{a}_{1}-x_{5} \mathbf{a}_{2}+z_{5} \mathbf{a}_{3}$

$=\left(-x_{5}+\frac{1}{2} y_{5}\right) a \hat{\mathbf{x}}-\frac{\sqrt{3}}{2} y_{5} a \hat{\mathbf{y}}+z_{5} c \hat{\mathbf{z}}$

O III

$\mathbf{B}_{52}=\quad-x_{5} \mathbf{a}_{1}-y_{5} \mathbf{a}_{2}+z_{5} \mathbf{a}_{3}$

$$
=
$$

$$
\begin{gathered}
-\frac{1}{2}\left(x_{5}+y_{5}\right) a \hat{\mathbf{x}}+ \\
\frac{\sqrt{3}}{2}\left(x_{5}-y_{5}\right) a \hat{\mathbf{y}}+z_{5} c \hat{\mathbf{z}}
\end{gathered}
$$

O IV

$\mathbf{B}_{53}=y_{5} \mathbf{a}_{1}+\left(-x_{5}+y_{5}\right) \mathbf{a}_{2}+z_{5} \mathbf{a}_{3}=\left(-\frac{1}{2} x_{5}+y_{5}\right) a \hat{\mathbf{x}}-\frac{\sqrt{3}}{2} x_{5} a \hat{\mathbf{y}}+z_{5} c \hat{\mathbf{z}}$

$\mathbf{B}_{54}=\left(x_{5}-y_{5}\right) \mathbf{a}_{1}+x_{5} \mathbf{a}_{2}+z_{5} \mathbf{a}_{3}$

$=\left(x_{5}-\frac{1}{2} y_{5}\right) a \hat{\mathbf{x}}+\frac{\sqrt{3}}{2} y_{5} a \hat{\mathbf{y}}+z_{5} c \hat{\mathbf{z}}$

$\mathbf{B}_{55}=-y_{5} \mathbf{a}_{1}-x_{5} \mathbf{a}_{2}+\left(\frac{1}{2}+z_{5}\right) \mathbf{a}_{3}=$

$$
-\frac{1}{2}\left(x_{5}+y_{5}\right) a \hat{\mathbf{x}}+
$$

O IV

O IV

O IV

$$
\frac{\sqrt{3}}{2}\left(-x_{5}+y_{5}\right) a \hat{\mathbf{y}}+\left(\frac{1}{2}+z_{5}\right) c \hat{\mathbf{z}}
$$

$\mathbf{B}_{56}=\left(-x_{5}+y_{5}\right) \mathbf{a}_{1}+y_{5} \mathbf{a}_{2}+\left(\frac{1}{2}+z_{5}\right) \mathbf{a}_{3}=\left(-\frac{1}{2} x_{5}+y_{5}\right) a \hat{\mathbf{x}}+\frac{\sqrt{3}}{2} x_{5} a \hat{\mathbf{y}}+$

$$
\left(\frac{1}{2}+z_{5}\right) c \hat{\mathbf{z}}
$$

$\mathbf{B}_{57}=x_{5} \mathbf{a}_{1}+\left(x_{5}-y_{5}\right) \mathbf{a}_{2}+\left(\frac{1}{2}+z_{5}\right) \mathbf{a}_{3}=\left(x_{5}-\frac{1}{2} y_{5}\right) a \hat{\mathbf{x}}-\frac{\sqrt{3}}{2} y_{5} a \hat{\mathbf{y}}+$

$\mathbf{B}_{58}=y_{5} \mathbf{a}_{1}+x_{5} \mathbf{a}_{2}+\left(\frac{1}{2}+z_{5}\right) \mathbf{a}_{3}=\frac{1}{2}\left(x_{5}+y_{5}\right) a \hat{\mathbf{x}}+\frac{\sqrt{3}}{2}\left(x_{5}-y_{5}\right) a \hat{\mathbf{y}}+$

O IV

O IV

O IV

O IV

O IV

$$
\begin{aligned}
& \left(\frac{1}{2}+z_{5}\right) c \hat{\mathbf{z}} \\
& a \hat{\mathbf{x}}+\frac{\sqrt{3}}{2}\left(x_{5}\right. \\
& \left(\frac{1}{2}+z_{5}\right) c \hat{\mathbf{z}}
\end{aligned}
$$

O IV

$\mathbf{B}_{59}=\left(x_{5}-y_{5}\right) \mathbf{a}_{1}-y_{5} \mathbf{a}_{2}+\left(\frac{1}{2}+z_{5}\right) \mathbf{a}_{3}=\begin{array}{r}\left(\frac{1}{2} x_{5}-y_{5}\right) a \hat{\mathbf{x}}-\frac{\sqrt{3}}{2} x_{5} a \hat{\mathbf{y}}+ \\ \left(\frac{1}{2}+z_{5}\right) c \hat{\mathbf{z}}\end{array}$

O IV

$\mathbf{B}_{60}=-x_{5} \mathbf{a}_{1}+\left(-x_{5}+y_{5}\right) \mathbf{a}_{2}+\left(\frac{1}{2}+z_{5}\right) \mathbf{a}_{3}=\left(-x_{5}+\frac{1}{2} y_{5}\right) a \hat{\mathbf{x}}+\frac{\sqrt{3}}{2} y_{5} a \hat{\mathbf{y}}+$

O IV

$$
\begin{aligned}
& \mathbf{B}_{61}=\quad x_{6} \mathbf{a}_{1}+y_{6} \mathbf{a}_{2}+z_{6} \mathbf{a}_{3} \\
& \mathbf{B}_{62}=-y_{6} \mathbf{a}_{1}+\left(x_{6}-y_{6}\right) \mathbf{a}_{2}+z_{6} \mathbf{a}_{3} \\
& \mathbf{B}_{63}=\left(-x_{6}+y_{6}\right) \mathbf{a}_{1}-x_{6} \mathbf{a}_{2}+z_{6} \mathbf{a}_{3} \\
& \mathbf{B}_{64}=\quad-x_{6} \mathbf{a}_{1}-y_{6} \mathbf{a}_{2}+z_{6} \mathbf{a}_{3} \\
& =\quad \frac{1}{2}\left(x_{6}+y_{6}\right) a \hat{\mathbf{x}}+ \\
& \frac{\sqrt{3}}{2}\left(-x_{6}+y_{6}\right) a \hat{\mathbf{y}}+z_{6} c \hat{\mathbf{z}} \\
& =\left(\frac{1}{2} x_{6}-y_{6}\right) a \hat{\mathbf{x}}+\frac{\sqrt{3}}{2} x_{6} a \hat{\mathbf{y}}+z_{6} c \hat{\mathbf{z}} \\
& =\left(-x_{6}+\frac{1}{2} y_{6}\right) a \hat{\mathbf{x}}-\frac{\sqrt{3}}{2} y_{6} a \hat{\mathbf{y}}+z_{6} c \hat{\mathbf{z}} \\
& =\quad-\frac{1}{2}\left(x_{6}+y_{6}\right) a \hat{\mathbf{x}}+ \\
& \frac{\sqrt{3}}{2}\left(x_{6}-y_{6}\right) a \hat{\mathbf{y}}+z_{6} c \hat{\mathbf{z}} \\
& \mathbf{B}_{65}=y_{6} \mathbf{a}_{1}+\left(-x_{6}+y_{6}\right) \mathbf{a}_{2}+z_{6} \mathbf{a}_{3}=\left(-\frac{1}{2} x_{6}+y_{6}\right) a \hat{\mathbf{x}}-\frac{\sqrt{3}}{2} x_{6} a \hat{\mathbf{y}}+z_{6} c \hat{\mathbf{z}} \\
& \mathbf{B}_{66}=\left(x_{6}-y_{6}\right) \mathbf{a}_{1}+x_{6} \mathbf{a}_{2}+z_{6} \mathbf{a}_{3}=\left(x_{6}-\frac{1}{2} y_{6}\right) a \hat{\mathbf{x}}+\frac{\sqrt{3}}{2} y_{6} a \hat{\mathbf{y}}+z_{6} c \hat{\mathbf{z}} \\
& \mathbf{B}_{67}=-y_{6} \mathbf{a}_{1}-x_{6} \mathbf{a}_{2}+\left(\frac{1}{2}+z_{6}\right) \mathbf{a}_{3}=-\frac{1}{2}\left(x_{6}+y_{6}\right) a \hat{\mathbf{x}}+ \\
& \frac{\sqrt{3}}{2}\left(-x_{6}+y_{6}\right) a \hat{\mathbf{y}}+\left(\frac{1}{2}+z_{6}\right) c \hat{\mathbf{z}}
\end{aligned}
$$$$
\left(\frac{1}{2}+z_{5}\right) c \hat{\mathbf{z}}
$$

$$
\begin{aligned}
& \mathrm{P} \\
& \mathrm{P} \\
& \mathrm{P} \\
& \mathrm{P} \\
& \mathrm{P} \\
& \mathrm{P} \\
& \mathrm{P}
\end{aligned}
$$




$$
\begin{aligned}
& \mathbf{B}_{68}=\left(-x_{6}+y_{6}\right) \mathbf{a}_{1}+y_{6} \mathbf{a}_{2}+\left(\frac{1}{2}+z_{6}\right) \mathbf{a}_{3}=\left(-\frac{1}{2} x_{6}+y_{6}\right) a \hat{\mathbf{x}}+\frac{\sqrt{3}}{2} x_{6} a \hat{\mathbf{y}}+ \\
& \left(\frac{1}{2}+z_{6}\right) c \hat{\mathbf{z}} \\
& \mathbf{B}_{69}=x_{6} \mathbf{a}_{1}+\left(x_{6}-y_{6}\right) \mathbf{a}_{2}+\left(\frac{1}{2}+z_{6}\right) \mathbf{a}_{3}=\left(x_{6}-\frac{1}{2} y_{6}\right) a \hat{\mathbf{x}}-\frac{\sqrt{3}}{2} y_{6} a \hat{\mathbf{y}}+ \\
& \left(\frac{1}{2}+z_{6}\right) c \hat{\mathbf{z}} \\
& \mathbf{B}_{70}=y_{6} \mathbf{a}_{1}+x_{6} \mathbf{a}_{2}+\left(\frac{1}{2}+z_{6}\right) \mathbf{a}_{3}=\frac{1}{2}\left(x_{6}+y_{6}\right) a \hat{\mathbf{x}}+\frac{\sqrt{3}}{2}\left(x_{6}-y_{6}\right) a \hat{\mathbf{y}}+ \\
& \left(\frac{1}{2}+z_{6}\right) c \hat{\mathbf{z}} \\
& \mathbf{B}_{71}=\left(x_{6}-y_{6}\right) \mathbf{a}_{1}-y_{6} \mathbf{a}_{2}+\left(\frac{1}{2}+z_{6}\right) \mathbf{a}_{3}=\left(\frac{1}{2} x_{6}-y_{6}\right) a \hat{\mathbf{x}}-\frac{\sqrt{3}}{2} x_{6} a \hat{\mathbf{y}}+ \\
& \left(\frac{1}{2}+z_{6}\right) c \hat{\mathbf{z}} \\
& \mathbf{B}_{72}=-x_{6} \mathbf{a}_{1}+\left(-x_{6}+y_{6}\right) \mathbf{a}_{2}+\left(\frac{1}{2}+z_{6}\right) \mathbf{a}_{3}=\left(-x_{6}+\frac{1}{2} y_{6}\right) a \hat{\mathbf{x}}+\frac{\sqrt{3}}{2} y_{6} a \hat{\mathbf{y}}+ \\
& \left(\frac{1}{2}+z_{6}\right) c \hat{\mathbf{z}}
\end{aligned}
$$

\section{References:}

- G. J. Klap, H. van Koningsveld, H. Graafsma, and A. M. M. Schreurs, Absolute configuration and domain structure of $\mathrm{AlPO}_{4}-5$ studied by single crystal X-ray diffraction, Microporous Mesoporous Mater. 38, 403-412 (2000), doi:10.1016/S1387-1811(00)00161-X.

\section{Found in:}

- P. Villars and K. Cenzual, Pearson's Crystal Data - Crystal Structure Database for Inorganic Compounds, ASM International (2013).

\section{Geometry files:}

- CIF: pp. 933

- POSCAR: pp. 934 
$\mathrm{KNiCl}_{3}$ (Room-temperature) Structure:

\section{A3BC_hP30_185_cd_c_ab}
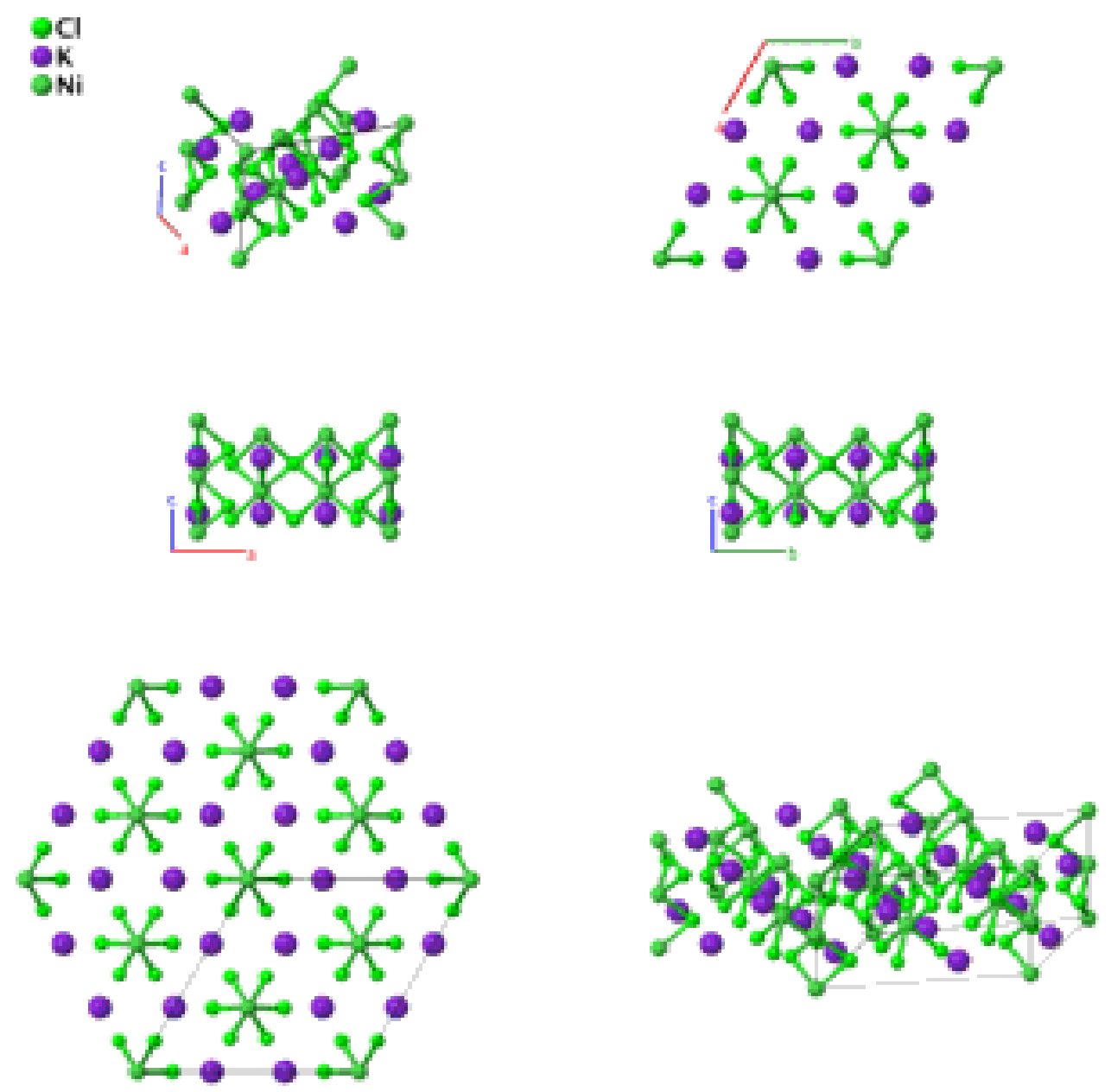

Prototype

: $\quad \mathrm{KNiCl}_{3}$

AFLOW prototype label

: A3BC_hP30_185_cd_c_ab

Strukturbericht designation : None

Pearson symbol : $\mathrm{hP} 30$

Space group number $\quad: \quad 185$

Space group symbol $\quad: \quad P 6_{3} \mathrm{~cm}$

AFLOW prototype command : aflow --proto=A3BC_hP30_185_cd_c_ab

- params $=a, c / a, z_{1}, z_{2}, x_{3}, z_{3}, x_{4}, z_{4}, x_{5}, y_{5}, z_{5}$

Hexagonal primitive vectors:

$$
\begin{array}{ll}
\mathbf{a}_{1}= & \frac{1}{2} a \hat{\mathbf{x}}-\frac{\sqrt{3}}{2} a \hat{\mathbf{y}} \\
\mathbf{a}_{2}= & \frac{1}{2} a \hat{\mathbf{x}}+\frac{\sqrt{3}}{2} a \hat{\mathbf{y}} \\
\mathbf{a}_{3}= & c \hat{\mathbf{z}}
\end{array}
$$

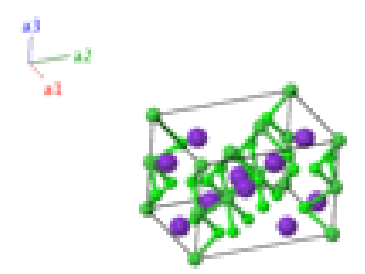


Lattice Coordinates

$\begin{array}{ccc}\mathbf{B}_{1}= & z_{1} \mathbf{a}_{3} \\ \mathbf{B}_{2}= & \left(\frac{1}{2}+z_{1}\right) \mathbf{a}_{3} \\ \mathbf{B}_{3}= & \frac{1}{3} \mathbf{a}_{1}+\frac{2}{3} \mathbf{a}_{2}+z_{2} \mathbf{a}_{3} \\ \mathbf{B}_{4}= & \frac{2}{3} \mathbf{a}_{1}+\frac{1}{3} \mathbf{a}_{2}+\left(\frac{1}{2}+z_{2}\right) \mathbf{a}_{3} \\ \mathbf{B}_{5}= & \frac{1}{3} \mathbf{a}_{1}+\frac{2}{3} \mathbf{a}_{2}+\left(\frac{1}{2}+z_{2}\right) \mathbf{a}_{3} \\ \mathbf{B}_{6}= & \frac{2}{3} \mathbf{a}_{1}+\frac{1}{3} \mathbf{a}_{2}+z_{2} \mathbf{a}_{3} \\ \mathbf{B}_{7}= & x_{3} \mathbf{a}_{1}+z_{3} \mathbf{a}_{3} \\ \mathbf{B}_{8}= & x_{3} \mathbf{a}_{2}+z_{3} \mathbf{a}_{3} \\ \mathbf{B}_{9}= & -x_{3} \mathbf{a}_{1}-x_{3} \mathbf{a}_{2}+z_{3} \mathbf{a}_{3} \\ \mathbf{B}_{10}= & -x_{3} \mathbf{a}_{1}+\left(\frac{1}{2}+z_{3}\right) \mathbf{a}_{3} \\ \mathbf{B}_{11}= & -x_{3} \mathbf{a}_{2}+\left(\frac{1}{2}+z_{3}\right) \mathbf{a}_{3} \\ \mathbf{B}_{12}= & x_{3} \mathbf{a}_{1}+x_{3} \mathbf{a}_{2}+\left(\frac{1}{2}+z_{3}\right) \mathbf{a}_{3} \\ \mathbf{B}_{13}= & x_{4} \mathbf{a}_{1}+z_{4} \mathbf{a}_{3} \\ \mathbf{B}_{14}= & x_{4} \mathbf{a}_{2}+z_{4} \mathbf{a}_{3} \\ \mathbf{B}_{15}= & -x_{4} \mathbf{a}_{1}-x_{4} \mathbf{a}_{2}+z_{4} \mathbf{a}_{3} \\ \mathbf{B}_{16}= & -x_{4} \mathbf{a}_{1}+\left(\frac{1}{2}+z_{4}\right) \mathbf{a}_{3} \\ \mathbf{B}_{17}= & -x_{4} \mathbf{a}_{2}+\left(\frac{1}{2}+z_{4}\right) \mathbf{a}_{3} \\ \mathbf{B}_{18}= & x_{4} \mathbf{a}_{1}+x_{4} \mathbf{a}_{2}+\left(\frac{1}{2}+z_{4}\right) \mathbf{a}_{3} \\ \mathbf{B}_{19}= & x_{5} \mathbf{a}_{1}+y_{5} \mathbf{a}_{2}+z_{5} \mathbf{a}_{3}\end{array}$

$$
=
$$$$
=
$$$$
=
$$$$
=
$$$$
=
$$$$
=
$$$$
=
$$$$
=
$$$$
=
$$$$
=
$$$$
=-\frac{1}{2} x_{3} a \hat{\mathbf{x}}+\frac{\sqrt{3}}{2} x_{3} a \hat{\mathbf{y}}+\left(\frac{1}{2}+z_{3}\right) c \hat{\mathbf{z}}
$$$$
=-\frac{1}{2} x_{3} a \hat{\mathbf{x}}-\frac{\sqrt{3}}{2} x_{3} a \hat{\mathbf{y}}+\left(\frac{1}{2}+z_{3}\right) c \hat{\mathbf{z}}
$$$$
=\quad x_{3} a \hat{\mathbf{x}}+\left(\frac{1}{2}+z_{3}\right) c \hat{\mathbf{z}}
$$$$
=\quad \frac{1}{2} x_{4} a \hat{\mathbf{x}}-\frac{\sqrt{3}}{2} x_{4} a \hat{\mathbf{y}}+z_{4} c \hat{\mathbf{z}}
$$$$
=\quad \frac{1}{2} x_{4} a \hat{\mathbf{x}}+\frac{\sqrt{3}}{2} x_{4} a \hat{\mathbf{y}}+z_{4} c \hat{\mathbf{z}}
$$$$
=\quad-x_{4} a \hat{\mathbf{x}}+z_{4} c \hat{\mathbf{z}}
$$$$
=-\frac{1}{2} x_{4} a \hat{\mathbf{x}}+\frac{\sqrt{3}}{2} x_{4} a \hat{\mathbf{y}}+\left(\frac{1}{2}+z_{4}\right) c \hat{\mathbf{z}}
$$$$
=-\frac{1}{2} x_{4} a \hat{\mathbf{x}}-\frac{\sqrt{3}}{2} x_{4} a \hat{\mathbf{y}}+\left(\frac{1}{2}+z_{4}\right) c \hat{\mathbf{z}}
$$$$
=\quad x_{4} a \hat{\mathbf{x}}+\left(\frac{1}{2}+z_{4}\right) c \hat{\mathbf{z}}
$$$$
=\quad \frac{1}{2}\left(x_{5}+y_{5}\right) a \hat{\mathbf{x}}+
$$$$
\frac{\sqrt{3}}{2}\left(-x_{5}+y_{5}\right) a \hat{\mathbf{y}}+z_{5} c \hat{\mathbf{z}}
$$$$
\mathbf{B}_{20}=-y_{5} \mathbf{a}_{1}+\left(x_{5}-y_{5}\right) \mathbf{a}_{2}+z_{5} \mathbf{a}_{3}=\left(\frac{1}{2} x_{5}-y_{5}\right) a \hat{\mathbf{x}}+\frac{\sqrt{3}}{2} x_{5} a \hat{\mathbf{y}}+z_{5} c \hat{\mathbf{z}}
$$$$
\mathbf{B}_{21}=\left(-x_{5}+y_{5}\right) \mathbf{a}_{1}-x_{5} \mathbf{a}_{2}+z_{5} \mathbf{a}_{3}=\left(-x_{5}+\frac{1}{2} y_{5}\right) a \hat{\mathbf{x}}-\frac{\sqrt{3}}{2} y_{5} a \hat{\mathbf{y}}+z_{5} c \hat{\mathbf{z}}
$$$$
\mathbf{B}_{22}=-x_{5} \mathbf{a}_{1}-y_{5} \mathbf{a}_{2}+\left(\frac{1}{2}+z_{5}\right) \mathbf{a}_{3} \quad=\quad-\frac{1}{2}\left(x_{5}+y_{5}\right) a \hat{\mathbf{x}}+
$$$$
\frac{\sqrt{3}}{2}\left(x_{5}-y_{5}\right) a \hat{\mathbf{y}}+\left(\frac{1}{2}+z_{5}\right) c \hat{\mathbf{z}}
$$$$
\mathbf{B}_{23}=y_{5} \mathbf{a}_{1}+\left(-x_{5}+y_{5}\right) \mathbf{a}_{2}+\left(\frac{1}{2}+z_{5}\right) \mathbf{a}_{3}=\left(-\frac{1}{2} x_{5}+y_{5}\right) a \hat{\mathbf{x}}-\frac{\sqrt{3}}{2} x_{5} a \hat{\mathbf{y}}+
$$$$
\left(\frac{1}{2}+z_{5}\right) c \hat{\mathbf{z}}
$$$$
\mathbf{B}_{24}=\left(x_{5}-y_{5}\right) \mathbf{a}_{1}+x_{5} \mathbf{a}_{2}+\left(\frac{1}{2}+z_{5}\right) \mathbf{a}_{3}=\left(x_{5}-\frac{1}{2} y_{5}\right) a \hat{\mathbf{x}}+\frac{\sqrt{3}}{2} y_{5} a \hat{\mathbf{y}}+
$$$$
\left(\frac{1}{2}+z_{5}\right) c \hat{\mathbf{z}}
$$$$
\mathbf{B}_{25}=-y_{5} \mathbf{a}_{1}-x_{5} \mathbf{a}_{2}+\left(\frac{1}{2}+z_{5}\right) \mathbf{a}_{3} \quad=\quad-\frac{1}{2}\left(x_{5}+y_{5}\right) a \hat{\mathbf{x}}+
$$$$
\frac{\sqrt{3}}{2}\left(-x_{5}+y_{5}\right) a \hat{\mathbf{y}}+\left(\frac{1}{2}+z_{5}\right) c \hat{\mathbf{z}}
$$$$
\mathbf{B}_{26}=\left(-x_{5}+y_{5}\right) \mathbf{a}_{1}+y_{5} \mathbf{a}_{2}+\left(\frac{1}{2}+z_{5}\right) \mathbf{a}_{3}=\left(-\frac{1}{2} x_{5}+y_{5}\right) a \hat{\mathbf{x}}+\frac{\sqrt{3}}{2} x_{5} a \hat{\mathbf{y}}+
$$$$
\left(\frac{1}{2}+z_{5}\right) c \hat{\mathbf{z}}
$$$$
\mathbf{B}_{27}=x_{5} \mathbf{a}_{1}+\left(x_{5}-y_{5}\right) \mathbf{a}_{2}+\left(\frac{1}{2}+z_{5}\right) \mathbf{a}_{3}=\left(x_{5}-\frac{1}{2} y_{5}\right) a \hat{\mathbf{x}}-\frac{\sqrt{3}}{2} y_{5} a \hat{\mathbf{y}}+
$$$$
\left(\frac{1}{2}+z_{5}\right) c \hat{\mathbf{z}}
$$

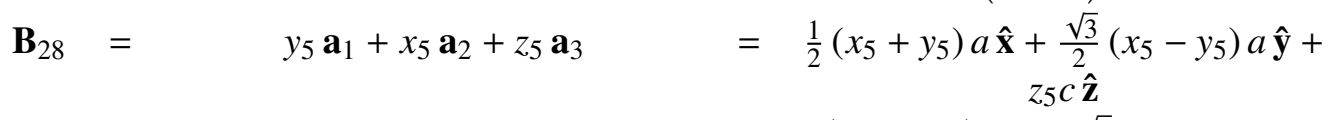$$
\mathbf{B}_{29}=\left(x_{5}-y_{5}\right) \mathbf{a}_{1}-y_{5} \mathbf{a}_{2}+z_{5} \mathbf{a}_{3}=\left(\frac{1}{2} x_{5}-y_{5}\right) a \hat{\mathbf{x}}-\frac{\sqrt{3}}{2} x_{5} a \hat{\mathbf{y}}+z_{5} c \hat{\mathbf{z}}
$$

Wyckoff Position Atom Type

Ni I

Ni I

Ni II

Cl II

Cl II

Cl II

Cl II

Cl II 
$\mathbf{B}_{30}=-x_{5} \mathbf{a}_{1}+\left(-x_{5}+y_{5}\right) \mathbf{a}_{2}+z_{5} \mathbf{a}_{3}=\left(-x_{5}+\frac{1}{2} y_{5}\right) a \hat{\mathbf{x}}+\frac{\sqrt{3}}{2} y_{5} a \hat{\mathbf{y}}+z_{5} c \hat{\mathbf{z}}$

$(12 d)$

Cl II

\section{References:}

- D. Visser, G. C. Verschoor, and D. J. W. IJdo, The structure of $\mathrm{KNiCl}_{3}$ at room temperature, Acta Crystallogr. Sect. B Struct. Sci. 36, 28-34 (1980), doi:10.1107/S0567740880002385.

\section{Found in:}

- P. Villars and K. Cenzual, Pearson's Crystal Data - Crystal Structure Database for Inorganic Compounds, ASM International (2013).

\section{Geometry files:}

- CIF: pp. 934

- POSCAR: pp. 934 


\section{$\mathrm{Cu}_{3} \mathrm{P}$ Structure: A3B_hP24_185_ab2c_c}
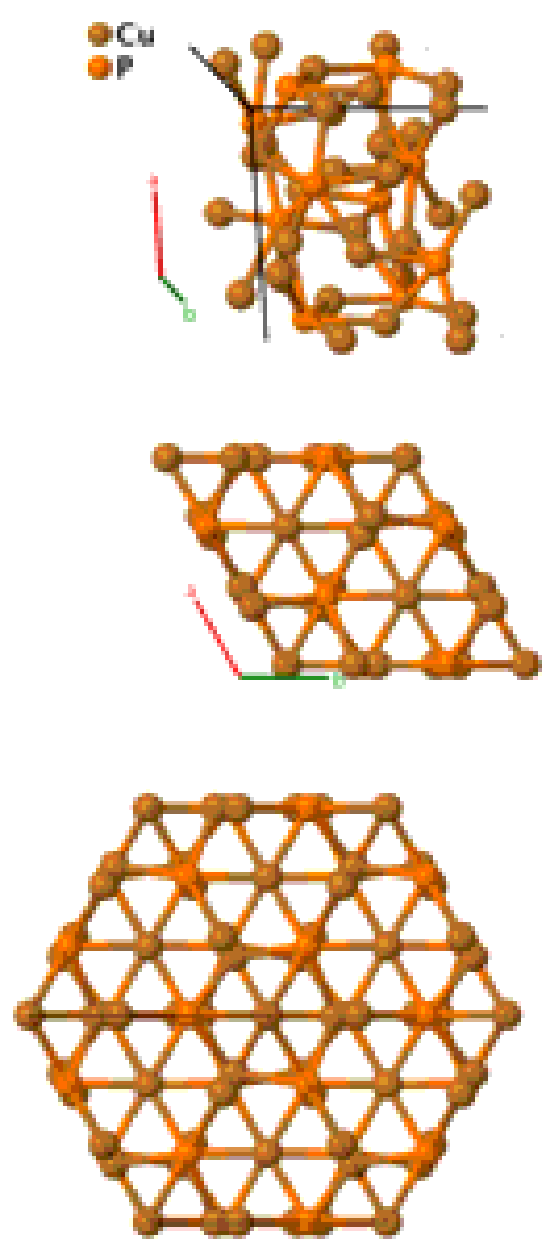

\section{Prototype}

AFLOW prototype label

Strukturbericht designation

Pearson symbol

Space group number

Space group symbol

AFLOW prototype command

$: \quad \mathrm{Cu}_{3} \mathrm{P}$

: None

: $\quad$ hP24

: 185

: $\quad P 6_{3} \mathrm{~cm}$
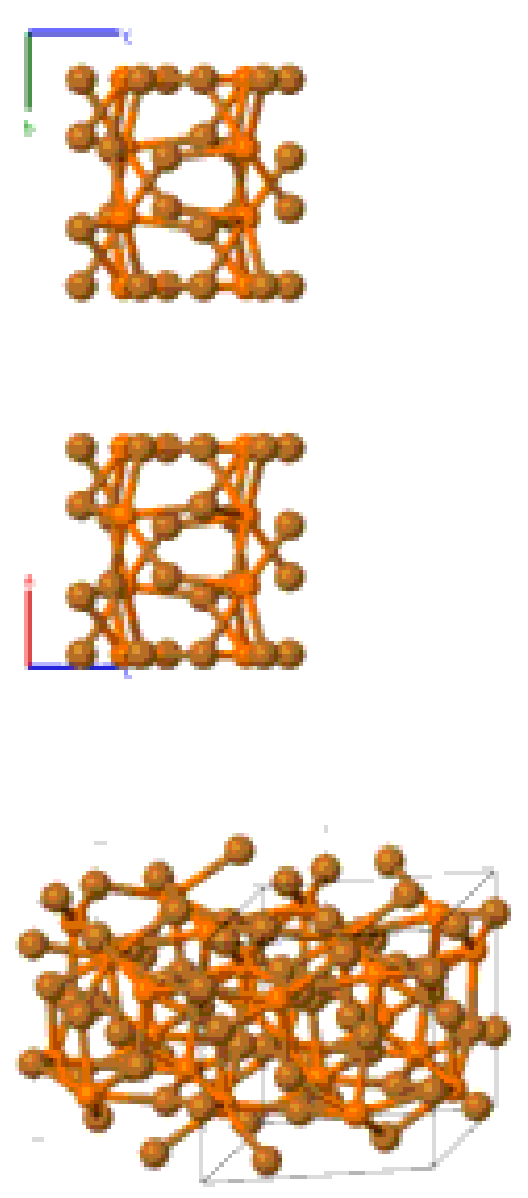

: A3B_hP24_185_ab2c_c

\footnotetext{
aflow --proto=A3B_hP24_185_ab2c_c
}

- params $=a, c / a, z_{1}, z_{2}, x_{3}, z_{3}, x_{4}, z_{4}, x_{5}, z_{5}$

- Olofsson (Olofsson, 1972) argues that this is the correct structure for $\mathrm{Cu}_{3} \mathrm{P}$ rather than the structure which was originally identified as Strukturbericht $D 0_{21}$, space group $P \overline{3} c 1$, A3B_hP24_165_bdg_f.

- Hafner and Range (Hafner, 1994) state that this is also the correct structure for $\mathrm{AsNa}_{3}$, rather than Strukturbericht $\mathrm{D0}_{18}$, space group $P 66_{3} / m m c$, AB3_hP8_194_c_bf.

- Finally, $\mathrm{AuMg}_{3}, \mathrm{IrMg}_{3}$ and $\mathrm{Mg}_{3} \mathrm{Pt}$, which have been described by both the $D 0_{18}$ and $D 0_{21}$ structures, are also claimed to actually be in this structure (Range, 1993). 


\section{Hexagonal primitive vectors:}

$$
\begin{array}{ll}
\mathbf{a}_{1}= & \frac{1}{2} a \hat{\mathbf{x}}-\frac{\sqrt{3}}{2} a \hat{\mathbf{y}} \\
\mathbf{a}_{2}= & \frac{1}{2} a \hat{\mathbf{x}}+\frac{\sqrt{3}}{2} a \hat{\mathbf{y}} \\
\mathbf{a}_{3}= & c \hat{\mathbf{z}}
\end{array}
$$

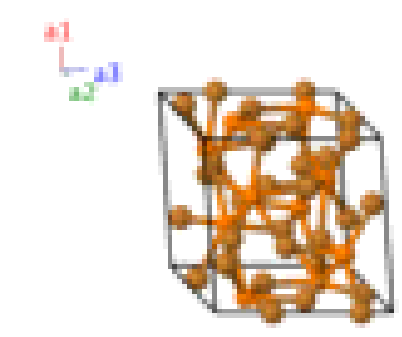

\section{Basis vectors:}

Lattice Coordinates

$\mathbf{B}_{1}=z_{1} \mathbf{a}_{3}=$

$\mathbf{B}_{2}=$

$\mathbf{B}_{3}=$

$\mathbf{B}_{4}=$

$\mathbf{B}_{5}$

$\mathbf{B}_{6}=$

$\mathbf{B}_{7}=$

$\mathbf{B}_{8}=$

$\mathbf{B}_{9}=-x_{3} \mathbf{a}_{1}-x_{3} \mathbf{a}_{2}+z_{3} \mathbf{a}_{3}$

$\mathbf{B}_{10}=$

$\mathbf{B}_{11}=$

$\mathbf{B}_{12}=$

$\mathbf{B}_{13}=$

$\mathbf{B}_{14}=$

$\mathbf{B}_{15}=$

$\mathbf{B}_{16}=$

$\mathbf{B}_{17}=$

$-x_{4} \mathbf{a}_{2}+\left(\frac{1}{2}+z_{4}\right) \mathbf{a}_{3}$

$\mathbf{B}_{18}=x_{4} \mathbf{a}_{1}+x_{4} \mathbf{a}_{2}+\left(\frac{1}{2}+z_{4}\right) \mathbf{a}_{3}$

$\mathbf{B}_{19}=$

$\mathbf{B}_{20}=$

$$
x_{5} \mathbf{a}_{1}+z_{5} \mathbf{a}_{3}
$$

$\mathbf{B}_{21}=-x_{5} \mathbf{a}_{1}-x_{5} \mathbf{a}_{2}+z_{5} \mathbf{a}_{3}$

$\mathbf{B}_{22}=$

$\mathbf{B}_{23}=$

$\mathbf{B}_{24}=$$$
=
$$$$
\text { (1) }
$$$$
\begin{aligned}
& = \\
& =
\end{aligned}
$$

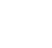$$
=
$$$$
=
$$$$
=
$$

$=$

$=$

$$
=
$$

$$
=
$$$$
=
$$$$
=
$$$$
=
$$$$
=
$$$$
=
$$$$
=
$$

$$
\begin{aligned}
& = \\
& =
\end{aligned}
$$$$
=
$$$$
=
$$$$
=
$$$$
=
$$$$
=
$$$$
=
$$$$
=
$$$$
=
$$$$
=
$$

Cartesian Coordinates

${ }_{1} c \hat{\mathbf{z}}$

$$
\left(\frac{1}{2}+z_{1}\right) c \hat{\mathbf{z}}
$$$$
\frac{1}{2} a \hat{\mathbf{x}}+\frac{1}{2 \sqrt{3}} a \hat{\mathbf{y}}+z_{2} c \hat{\mathbf{z}}
$$$$
\frac{1}{2} a \hat{\mathbf{x}}-\frac{1}{2 \sqrt{3}} a \hat{\mathbf{y}}+\left(\frac{1}{2}+z_{2}\right) c \hat{\mathbf{z}}
$$$$
\frac{1}{2} a \hat{\mathbf{x}}+\frac{1}{2 \sqrt{3}} a \hat{\mathbf{y}}+\left(\frac{1}{2}+z_{2}\right) c \hat{\mathbf{z}}
$$$$
\frac{1}{2} a \hat{\mathbf{x}}-\frac{1}{2 \sqrt{3}} a \hat{\mathbf{y}}+z_{2} c \hat{\mathbf{z}}
$$$$
\frac{1}{2} x_{3} a \hat{\mathbf{x}}-\frac{\sqrt{3}}{2} x_{3} a \hat{\mathbf{y}}+z_{3} c \hat{\mathbf{z}}
$$$$
\frac{1}{2} x_{3} a \hat{\mathbf{x}}+\frac{\sqrt{3}}{2} x_{3} a \hat{\mathbf{y}}+z_{3} c \hat{\mathbf{z}}
$$$$
-x_{3} a \hat{\mathbf{x}}+z_{3} c \hat{\mathbf{z}}
$$$$
-\frac{1}{2} x_{3} a \hat{\mathbf{x}}+\frac{\sqrt{3}}{2} x_{3} a \hat{\mathbf{y}}+\left(\frac{1}{2}+z_{3}\right) c \hat{\mathbf{z}}
$$$$
-\frac{1}{2} x_{3} a \hat{\mathbf{x}}-\frac{\sqrt{3}}{2} x_{3} a \hat{\mathbf{y}}+\left(\frac{1}{2}+z_{3}\right) c \hat{\mathbf{z}}
$$$$
x_{3} a \hat{\mathbf{x}}+\left(\frac{1}{2}+z_{3}\right) c \hat{\mathbf{z}}
$$$$
\frac{1}{2} x_{4} a \hat{\mathbf{x}}-\frac{\sqrt{3}}{2} x_{4} a \hat{\mathbf{y}}+z_{4} c \hat{\mathbf{z}}
$$$$
\frac{1}{2} x_{4} a \hat{\mathbf{x}}+\frac{\sqrt{3}}{2} x_{4} a \hat{\mathbf{y}}+z_{4} c \hat{\mathbf{z}}
$$$$
-x_{4} a \hat{\mathbf{x}}+z_{4} c \hat{\mathbf{z}}
$$$$
-\frac{1}{2} x_{4} a \hat{\mathbf{x}}+\frac{\sqrt{3}}{2} x_{4} a \hat{\mathbf{y}}+\left(\frac{1}{2}+z_{4}\right) c \hat{\mathbf{z}}
$$$$
-\frac{1}{2} x_{4} a \hat{\mathbf{x}}-\frac{\sqrt{3}}{2} x_{4} a \hat{\mathbf{y}}+\left(\frac{1}{2}+z_{4}\right) c \hat{\mathbf{z}}
$$$$
x_{4} a \hat{\mathbf{x}}+\left(\frac{1}{2}+z_{4}\right) c \hat{\mathbf{z}}
$$$$
\frac{1}{2} x_{5} a \hat{\mathbf{x}}-\frac{\sqrt{3}}{2} x_{5} a \hat{\mathbf{y}}+z_{5} c \hat{\mathbf{z}}
$$$$
\frac{1}{2} x_{5} a \hat{\mathbf{x}}+\frac{\sqrt{3}}{2} x_{5} a \hat{\mathbf{y}}+z_{5} c \hat{\mathbf{z}}
$$$$
-x_{5} a \hat{\mathbf{x}}+z_{5} c \hat{\mathbf{z}}
$$$$
-\frac{1}{2} x_{5} a \hat{\mathbf{x}}+\frac{\sqrt{3}}{2} x_{5} a \hat{\mathbf{y}}+\left(\frac{1}{2}+z_{5}\right) c \hat{\mathbf{z}}
$$$$
-\frac{1}{2} x_{5} a \hat{\mathbf{x}}-\frac{\sqrt{3}}{2} x_{5} a \hat{\mathbf{y}}+\left(\frac{1}{2}+z_{5}\right) c \hat{\mathbf{z}}
$$$$
x_{5} a \hat{\mathbf{x}}+\left(\frac{1}{2}+z_{5}\right) c \hat{\mathbf{z}}
$$

Wyckoff Position

Atom Type

(2a)
$\mathrm{Cu} \mathrm{I}$

$\mathrm{Cu} \mathrm{I}$

$\mathrm{Cu}$ II

$\mathrm{Cu}$ II

$\mathrm{Cu}$ II

$\mathrm{Cu}$ II

$\mathrm{Cu}$ III

$\mathrm{Cu}$ III

$\mathrm{Cu}$ III

$\mathrm{Cu}$ III

$\mathrm{Cu}$ III

$\mathrm{Cu}$ III

$\mathrm{Cu}$ IV

$\mathrm{Cu}$ IV

Cu IV

Cu IV

Cu IV

Cu IV

$\mathrm{P}$
$\mathrm{P}$
$\mathrm{P}$
$\mathrm{P}$
$\mathrm{P}$
$\mathrm{P}$

\section{References:}

- O. Olofsson, The Crystal Structure of $C u_{3} P$, Acta Chem. Scand. 26, 2777-2787 (1972), doi:10.3891/acta.chem.scand.26-2771. 
- P. Hafner and K.-J. Range, $\mathrm{Na}_{3} \mathrm{As}$ revisited: high-pressure synthesis of single crystals and structure refinement, J. Alloys Compd. 216, 7-10 (1994), doi:10.1016/0925-8388(94)91033-2.

- K.-J. Range and P. Hafner, Structure refinement of $\mathrm{AuMg}_{3}, \mathrm{IrMg}_{3}$ and $\mathrm{IrMg}_{2.8}$, J. Alloys Compd. 191, L5-L7 (1993), doi:10.1016/0925-8388(93)90053-P.

\section{Geometry files:}

- CIF: pp. 935

- POSCAR: pp. 935 


\section{$\beta-\mathrm{RuCl}_{3}$ Structure: A3B_hP8_185_c_a}
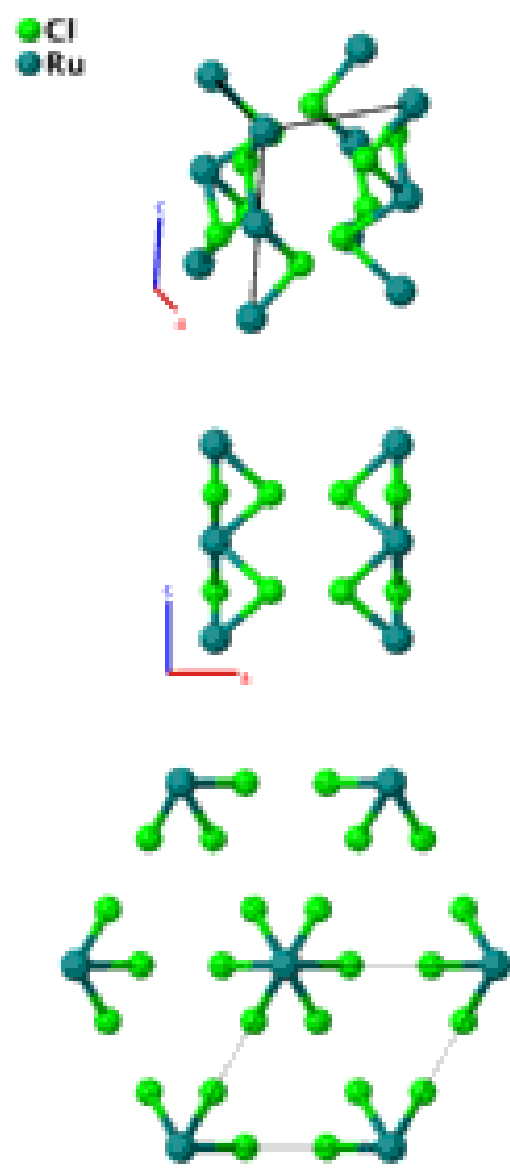
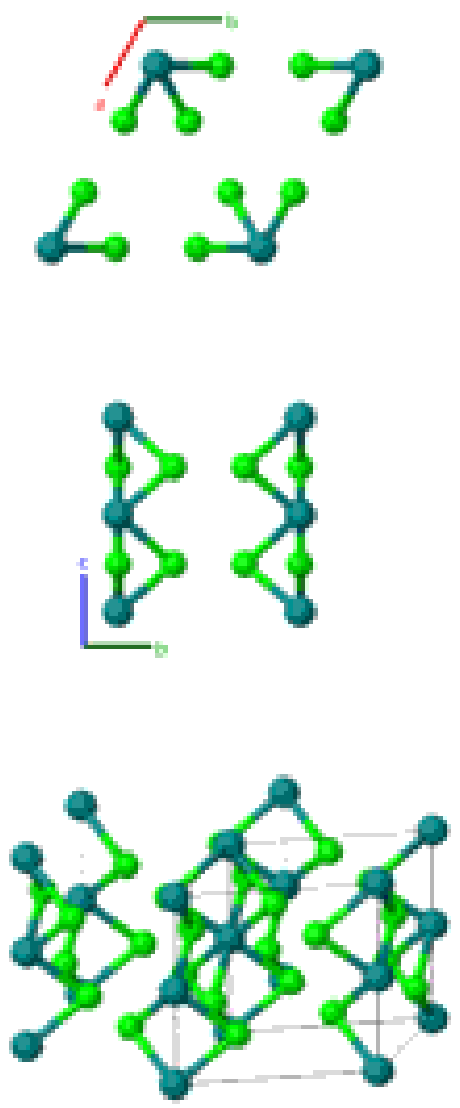

Prototype

AFLOW prototype label

Strukturbericht designation

Pearson symbol

Space group number

Space group symbol

AFLOW prototype command
$: \quad \beta-\mathrm{RuCl}_{3}$

: A3B_hP8_185_c_a

: None

: $\mathrm{hP8}$

: $\quad 185$

: $\quad \mathrm{Pb}_{3} \mathrm{~cm}$

aflow - - proto=A3B_hP8_185_C_a

- params $=a, c / a, z_{1}, x_{2}, z_{2}$

- Pearson comments that space groups \#158, \#188, \#193, could not be rejected, but this structure is consistent with space group \#185. We also provide the structure with space group \#158: $\beta-\mathrm{RuCl}_{3}$ (A3B_hP8_158_d_a) structure.

Hexagonal primitive vectors:

$$
\begin{array}{ll}
\mathbf{a}_{1}= & \frac{1}{2} a \hat{\mathbf{x}}-\frac{\sqrt{3}}{2} a \hat{\mathbf{y}} \\
\mathbf{a}_{2}= & \frac{1}{2} a \hat{\mathbf{x}}+\frac{\sqrt{3}}{2} a \hat{\mathbf{y}} \\
\mathbf{a}_{3}= & c \hat{\mathbf{z}}
\end{array}
$$

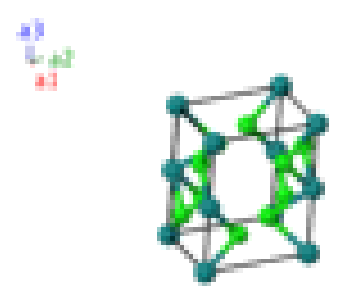

\section{Basis vectors:}




\begin{tabular}{|c|c|c|c|c|c|}
\hline $\mathbf{B}_{1}$ & $=$ & $z_{1} \mathbf{a}_{3}$ & $=$ & $z_{1} c \hat{\mathbf{z}}$ & $(2 a)$ \\
\hline $\mathbf{B}_{2}$ & $=$ & $\left(\frac{1}{2}+z_{1}\right) \mathbf{a}_{3}$ & $=$ & $\left(\frac{1}{2}+z_{1}\right) c \hat{\mathbf{z}}$ & $(2 a)$ \\
\hline $\mathbf{B}_{3}$ & $=$ & $x_{2} \mathbf{a}_{1}+z_{2} \mathbf{a}_{3}$ & $=$ & $\frac{1}{2} x_{2} a \hat{\mathbf{x}}-\frac{\sqrt{3}}{2} x_{2} a \hat{\mathbf{y}}+z_{2} c \hat{\mathbf{z}}$ & $(6 c)$ \\
\hline $\mathbf{B}_{4}$ & $=$ & $x_{2} \mathbf{a}_{2}+z_{2} \mathbf{a}_{3}$ & $=$ & $\frac{1}{2} x_{2} a \hat{\mathbf{x}}+\frac{\sqrt{3}}{2} x_{2} a \hat{\mathbf{y}}+z_{2} c \hat{\mathbf{z}}$ & $(6 c)$ \\
\hline $\mathbf{B}_{5}$ & $=$ & $-x_{2} \mathbf{a}_{1}-x_{2} \mathbf{a}_{2}+z_{2} \mathbf{a}_{3}$ & $=$ & $-x_{2} a \hat{\mathbf{x}}+z_{2} c \hat{\mathbf{z}}$ & $(6 c)$ \\
\hline $\mathbf{B}_{6}$ & $=$ & $-x_{2} \mathbf{a}_{1}+\left(\frac{1}{2}+z_{2}\right) \mathbf{a}_{3}$ & $=$ & $-\frac{1}{2} x_{2} a \hat{\mathbf{x}}+\frac{\sqrt{3}}{2} x_{2} a \hat{\mathbf{y}}+\left(\frac{1}{2}+z_{2}\right) c \hat{\mathbf{z}}$ & $(6 c)$ \\
\hline $\mathbf{B}_{7}$ & $=$ & $-x_{2} \mathbf{a}_{2}+\left(\frac{1}{2}+z_{2}\right) \mathbf{a}_{3}$ & $=$ & $-\frac{1}{2} x_{2} a \hat{\mathbf{x}}-\frac{\sqrt{3}}{2} x_{2} a \hat{\mathbf{y}}+\left(\frac{1}{2}+z_{2}\right) c \hat{\mathbf{z}}$ & $(6 c)$ \\
\hline $\mathbf{B}_{8}$ & $=$ & $x_{2} \mathbf{a}_{1}+x_{2} \mathbf{a}_{2}+\left(\frac{1}{2}+z_{2}\right) \mathbf{a}_{3}$ & $=$ & $x_{2} a \hat{\mathbf{x}}+\left(\frac{1}{2}+z_{2}\right) c \hat{\mathbf{z}}$ & $(6 c)$ \\
\hline
\end{tabular}

\section{References:}

- J. M. Fletcher, W. E. Gardner, A. C. Fox, and G. Topping, X-Ray, infrared, and magnetic studies of $\alpha$-and $\beta$-ruthenium trichloride, J. Chem. Soc. A pp. 1038-1045 (1967), doi:10.1039/J19670001038.

\section{Found in:}

- P. Villars and K. Cenzual, Pearson's Crystal Data - Crystal Structure Database for Inorganic Compounds, ASM International (2013).

\section{Geometry files:}

- CIF: pp. 935

- POSCAR: pp. 935 


\section{Na 3 As Structure: AB3_hP24_185_c_ab2c}
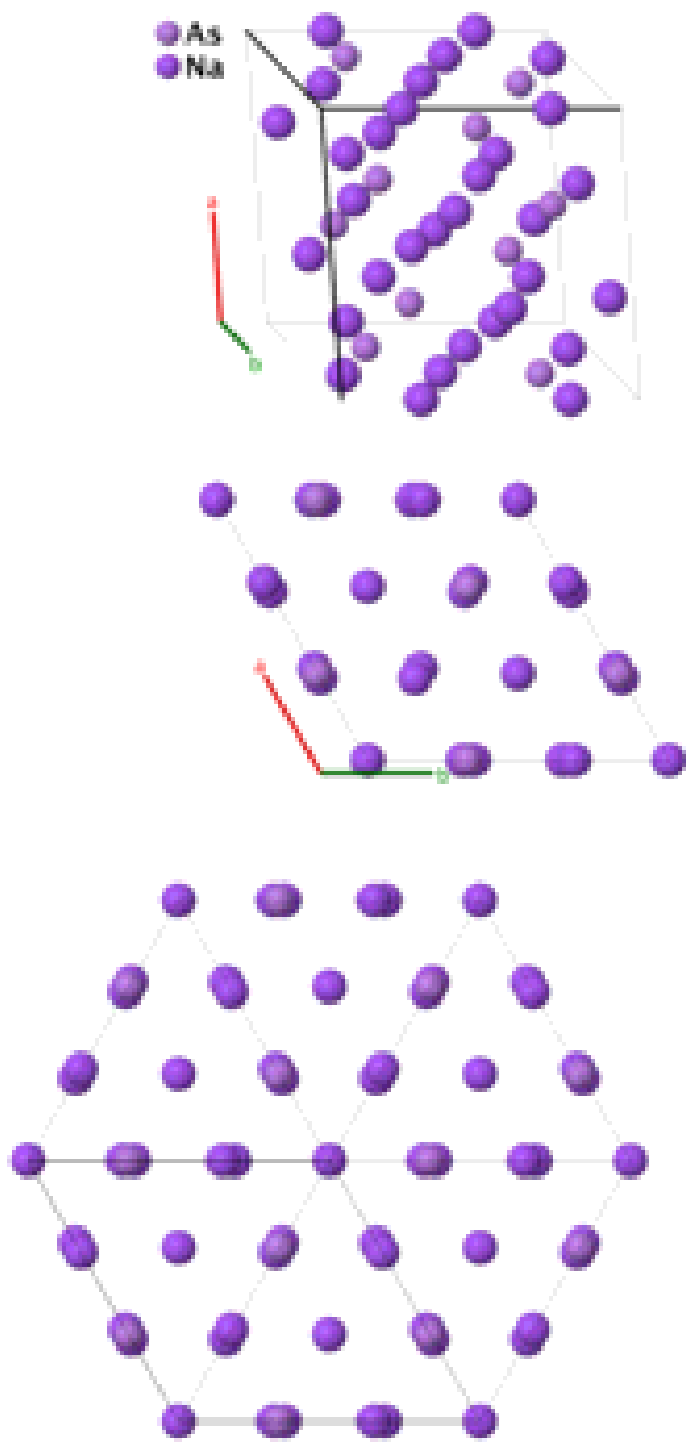
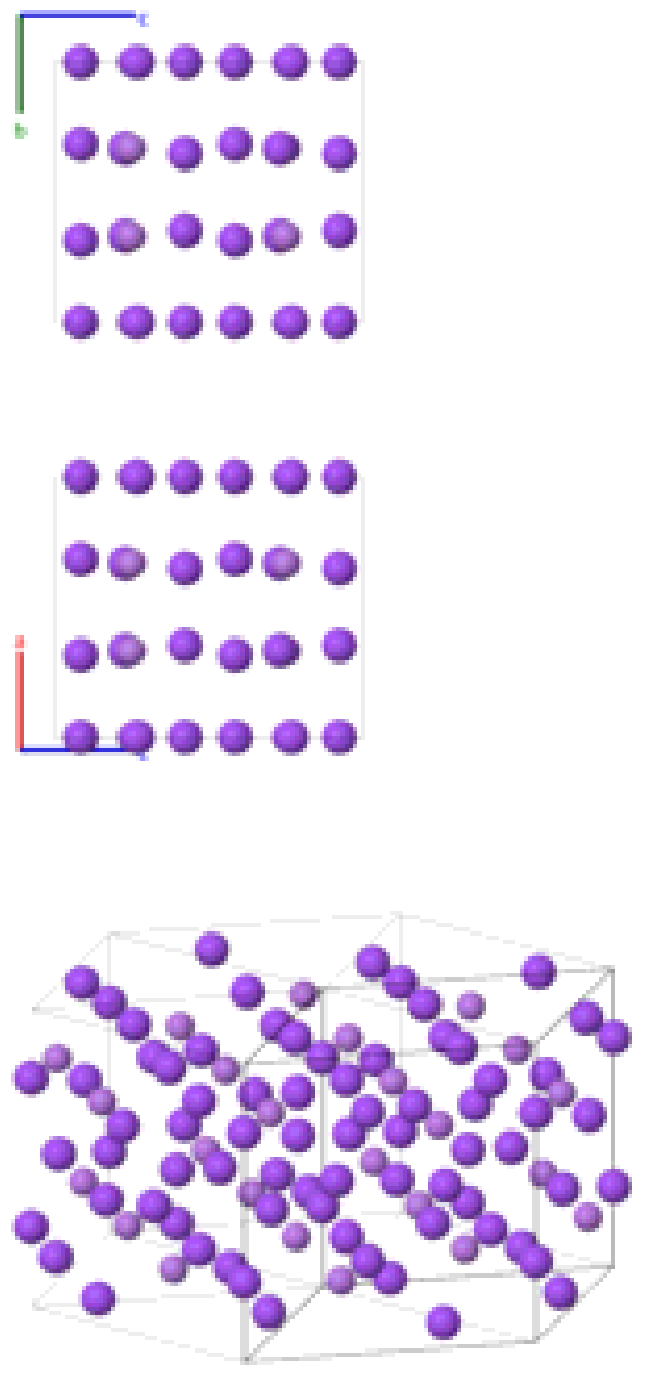

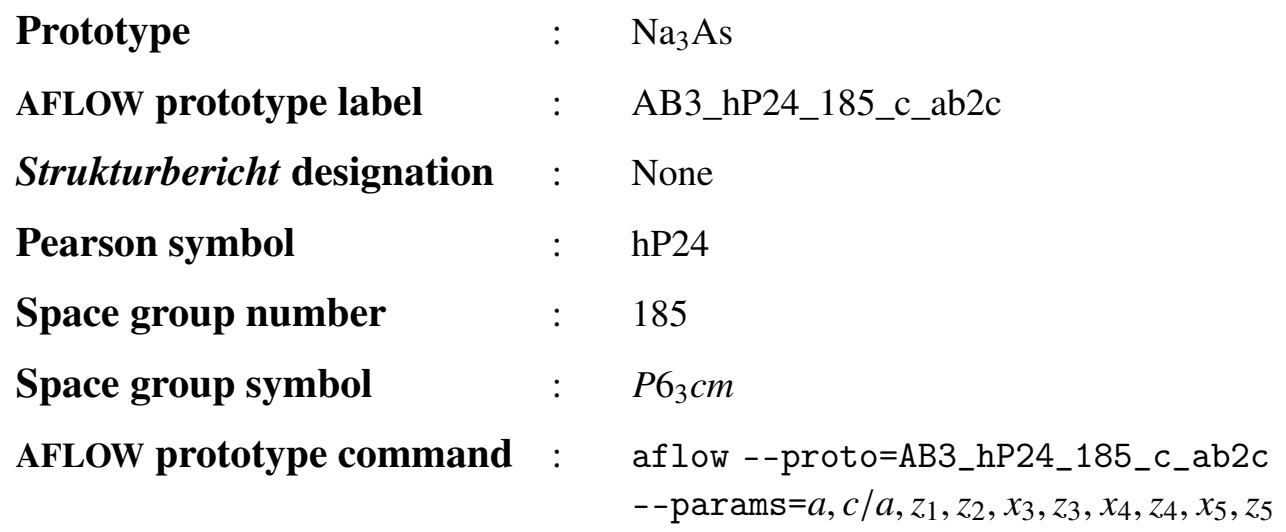

Prototype

$: \quad \mathrm{Na}_{3} \mathrm{As}$

AFLOW prototype label

: $\quad$ AB3_hP24_185_c_ab2c

Strukturbericht designation : None

Pearson symbol : $\quad$ hP24

Space group number $\quad: \quad 185$

Space group symbol $\quad: \quad \mathrm{P}_{3} \mathrm{~cm}$

AFLOW prototype command : aflow --proto=AB3_hP24_185_c_ab2c

- params $=a, c / a, z_{1}, z_{2}, x_{3}, z_{3}, x_{4}, z_{4}, x_{5}, z_{5}$

\section{Other compounds with the structure:}

- $\mathrm{Cu}_{3} \mathrm{P}$

- The authors state that this is a correction of the $D 0_{18} \mathrm{Na}_{3} \mathrm{As}$ structure. $\mathrm{Cu}_{3} \mathrm{P}$ (pp. 602) and $\mathrm{Na}_{3} \mathrm{As}$ (pp. 607) have similar AFLOW prototype labels (i.e., same symmetry and set of Wyckoff positions with different stoichiometry labels due to alphabetic ordering of atomic species). They are generated by the same symmetry operations with different sets of parameters (--params) specified in their corresponding CIF files. 


\section{Hexagonal primitive vectors:}

$$
\begin{array}{ll}
\mathbf{a}_{1}= & \frac{1}{2} a \hat{\mathbf{x}}-\frac{\sqrt{3}}{2} a \hat{\mathbf{y}} \\
\mathbf{a}_{2}= & \frac{1}{2} a \hat{\mathbf{x}}+\frac{\sqrt{3}}{2} a \hat{\mathbf{y}} \\
\mathbf{a}_{3}= & c \hat{\mathbf{z}}
\end{array}
$$

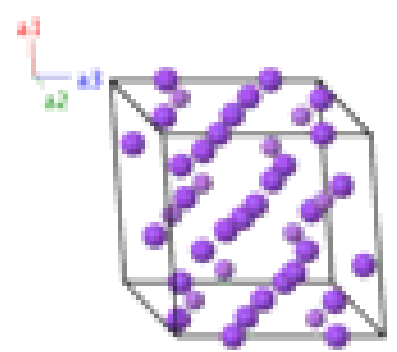

\section{Basis vectors:}

Lattice Coordinates

$\mathbf{B}_{1}=$

$\mathbf{B}_{2}=$

$\mathbf{B}_{3}=$

$\mathbf{B}_{4}=$

$\mathbf{B}_{5}$

$\mathbf{B}_{6}=$

$\mathbf{B}_{7}=$

$\mathbf{B}_{8}=$

$\mathbf{B}_{9}=-x_{3} \mathbf{a}_{1}-x_{3} \mathbf{a}_{2}+z_{3} \mathbf{a}_{3}$

$\mathbf{B}_{10}=-x_{3} \mathbf{a}_{1}+\left(\frac{1}{2}+z_{3}\right) \mathbf{a}_{3}$

$\mathbf{B}_{11}=$

$\mathbf{B}_{12}=$

$\mathbf{B}_{13}=$

$\mathbf{B}_{14}=$

$\mathbf{B}_{15}=$

$\mathbf{B}_{16}=$

$$
-x_{3} \mathbf{a}_{2}+\left(\frac{1}{2}+z_{3}\right) \mathbf{a}_{3}
$$

$x_{3} \mathbf{a}_{1}+x_{3} \mathbf{a}_{2}+\left(\frac{1}{2}+z_{3}\right) \mathbf{a}_{3}$

$x_{4} \mathbf{a}_{1}+z_{4} \mathbf{a}_{3}$

$x_{4} \mathbf{a}_{2}+z_{4} \mathbf{a}_{3}$

$\mathbf{B}_{17}=$

$\mathbf{B}_{18}=x_{4} \mathbf{a}_{1}+x_{4} \mathbf{a}_{2}+\left(\frac{1}{2}+z_{4}\right) \mathbf{a}_{3}$

$\mathbf{B}_{19}=$

$\mathbf{B}_{20}=$

$$
x_{5} \mathbf{a}_{1}+z_{5} \mathbf{a}_{3}
$$

$\mathbf{B}_{21}=$

$$
x_{5} \mathbf{a}_{2}+z_{5} \mathbf{a}_{3}
$$

$\mathbf{B}_{22}=$

$$
-x_{5} \mathbf{a}_{1}-x_{5} \mathbf{a}_{2}+z_{5} \mathbf{a}_{3}
$$$$
-x_{5} \mathbf{a}_{1}+\left(\frac{1}{2}+z_{5}\right) \mathbf{a}_{3}
$$

$\mathbf{B}_{23}=$

$$
-x_{5} \mathbf{a}_{2}+\left(\frac{1}{2}+z_{5}\right) \mathbf{a}_{3}
$$

$\mathbf{B}_{24}$
$=$

$=$

$$
=
$$

$$
=
$$$$
=
$$

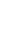$$
=
$$$$
=
$$$$
\begin{aligned}
& = \\
& =
\end{aligned}
$$$$
=
$$$$
=
$$$$
=
$$$$
=
$$$$
=
$$$$
=
$$$$
=
$$$$
=
$$$$
=
$$$$
=
$$$$
=
$$$$
=
$$$$
=
$$$$
=
$$$$
=
$$$$
=
$$$$
=
$$$$
=
$$$$
=
$$

Cartesian Coordinates

$z_{1} c \hat{\mathbf{z}}$

$$
\left(\frac{1}{2}+z_{1}\right) c \hat{\mathbf{z}}
$$$$
\frac{1}{2} a \hat{\mathbf{x}}+\frac{1}{2 \sqrt{3}} a \hat{\mathbf{y}}+z_{2} c \hat{\mathbf{z}}
$$$$
\frac{1}{2} a \hat{\mathbf{x}}-\frac{1}{2 \sqrt{3}} a \hat{\mathbf{y}}+\left(\frac{1}{2}+z_{2}\right) c \hat{\mathbf{z}}
$$$$
\frac{1}{2} a \hat{\mathbf{x}}+\frac{1}{2 \sqrt{3}} a \hat{\mathbf{y}}+\left(\frac{1}{2}+z_{2}\right) c \hat{\mathbf{z}}
$$$$
\frac{1}{2} a \hat{\mathbf{x}}-\frac{1}{2 \sqrt{3}} a \hat{\mathbf{y}}+z_{2} c \hat{\mathbf{z}}
$$$$
\frac{1}{2} x_{3} a \hat{\mathbf{x}}-\frac{\sqrt{3}}{2} x_{3} a \hat{\mathbf{y}}+z_{3} c \hat{\mathbf{z}}
$$$$
\frac{1}{2} x_{3} a \hat{\mathbf{x}}+\frac{\sqrt{3}}{2} x_{3} a \hat{\mathbf{y}}+z_{3} c \hat{\mathbf{z}}
$$$$
-x_{3} a \hat{\mathbf{x}}+z_{3} c \hat{\mathbf{z}}
$$$$
-\frac{1}{2} x_{3} a \hat{\mathbf{x}}+\frac{\sqrt{3}}{2} x_{3} a \hat{\mathbf{y}}+\left(\frac{1}{2}+z_{3}\right) c \hat{\mathbf{z}}
$$$$
-\frac{1}{2} x_{3} a \hat{\mathbf{x}}-\frac{\sqrt{3}}{2} x_{3} a \hat{\mathbf{y}}+\left(\frac{1}{2}+z_{3}\right) c \hat{\mathbf{z}}
$$$$
x_{3} a \hat{\mathbf{x}}+\left(\frac{1}{2}+z_{3}\right) c \hat{\mathbf{z}}
$$$$
\frac{1}{2} x_{4} a \hat{\mathbf{x}}-\frac{\sqrt{3}}{2} x_{4} a \hat{\mathbf{y}}+z_{4} c \hat{\mathbf{z}}
$$$$
\frac{1}{2} x_{4} a \hat{\mathbf{x}}+\frac{\sqrt{3}}{2} x_{4} a \hat{\mathbf{y}}+z_{4} c \hat{\mathbf{z}}
$$$$
-x_{4} a \hat{\mathbf{x}}+z_{4} c \hat{\mathbf{z}}
$$$$
-\frac{1}{2} x_{4} a \hat{\mathbf{x}}+\frac{\sqrt{3}}{2} x_{4} a \hat{\mathbf{y}}+\left(\frac{1}{2}+z_{4}\right) c \hat{\mathbf{z}}
$$$$
-\frac{1}{2} x_{4} a \hat{\mathbf{x}}-\frac{\sqrt{3}}{2} x_{4} a \hat{\mathbf{y}}+\left(\frac{1}{2}+z_{4}\right) c \hat{\mathbf{z}}
$$$$
x_{4} a \hat{\mathbf{x}}+\left(\frac{1}{2}+z_{4}\right) c \hat{\mathbf{z}}
$$$$
\frac{1}{2} x_{5} a \hat{\mathbf{x}}-\frac{\sqrt{3}}{2} x_{5} a \hat{\mathbf{y}}+z_{5} c \hat{\mathbf{z}}
$$$$
\frac{1}{2} x_{5} a \hat{\mathbf{x}}+\frac{\sqrt{3}}{2} x_{5} a \hat{\mathbf{y}}+z_{5} c \hat{\mathbf{z}}
$$$$
-x_{5} a \hat{\mathbf{x}}+z_{5} c \hat{\mathbf{z}}
$$$$
-\frac{1}{2} x_{5} a \hat{\mathbf{x}}+\frac{\sqrt{3}}{2} x_{5} a \hat{\mathbf{y}}+\left(\frac{1}{2}+z_{5}\right) c \hat{\mathbf{z}}
$$$$
-\frac{1}{2} x_{5} a \hat{\mathbf{x}}-\frac{\sqrt{3}}{2} x_{5} a \hat{\mathbf{y}}+\left(\frac{1}{2}+z_{5}\right) c \hat{\mathbf{z}}
$$$$
x_{5} a \hat{\mathbf{x}}+\left(\frac{1}{2}+z_{5}\right) c \hat{\mathbf{z}}
$$

Wyckoff Position

Atom Type

(2a)
$\mathrm{NaI}$

$\mathrm{Na} \mathrm{I}$

$\mathrm{Na}$ II

$\mathrm{Na}$ II

$\mathrm{Na}$ II

$\mathrm{Na}$ II

As

As

As

As

As

As

$\mathrm{Na}$ III

$\mathrm{Na}$ III

$\mathrm{Na}$ III

$\mathrm{Na}$ III

$\mathrm{Na}$ III

$\mathrm{Na}$ III

$\mathrm{Na}$ IV

$\mathrm{Na}$ IV

Na IV

Na IV

$\mathrm{Na}$ IV

Na IV

\section{References:}

- P. Hafner and K.-J. Range, $\mathrm{Na}_{3}$ As revisited: high-pressure synthesis of single crystals and structure refinement, J. Alloys Compd. 216, 7-10 (1994), doi:10.1016/0925-8388(94)91033-2. 
Geometry files:

- CIF: pp. 936

- POSCAR: pp. 936 
$\mathrm{Fe}_{3} \mathrm{Th}_{7}\left(D 10_{2}\right)$ Structure: A3B7_hP20_186_c_b2c
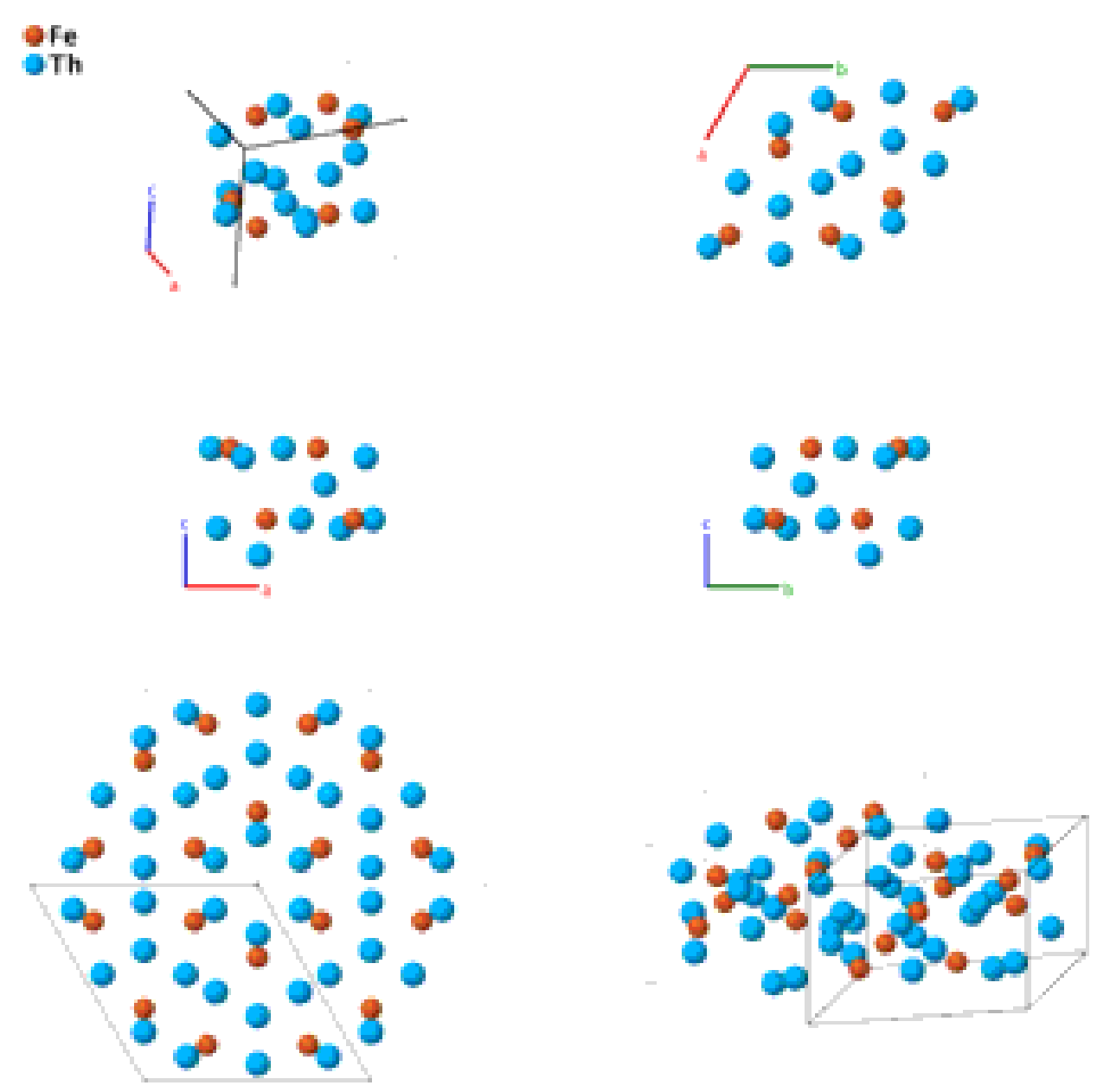

\section{Prototype}

AFLOW prototype label

Strukturbericht designation

Pearson symbol

Space group number

Space group symbol

AFLOW prototype command
$: \quad \mathrm{Fe}_{3} \mathrm{Th}_{7}$

: A3B7_hP20_186_c_b2c

: $\quad D 10_{2}$

: $\mathrm{hP} 20$

: 186

: $\quad P 6_{3} m c$

aflow - -proto=A3B7_hP20_186_c_b2c

- params $=a, c / a, z_{1}, x_{2}, z_{2}, x_{3}, z_{3}, x_{4}, z_{4}$

Other compounds with this structure:

- $\mathrm{Th}_{7} \mathrm{Co}_{3}, \mathrm{Th}_{7} \mathrm{Ni}_{3}$

Hexagonal primitive vectors:

$$
\begin{array}{ll}
\mathbf{a}_{1}= & \frac{1}{2} a \hat{\mathbf{x}}-\frac{\sqrt{3}}{2} a \hat{\mathbf{y}} \\
\mathbf{a}_{2}= & \frac{1}{2} a \hat{\mathbf{x}}+\frac{\sqrt{3}}{2} a \hat{\mathbf{y}} \\
\mathbf{a}_{3}= & c \hat{\mathbf{z}}
\end{array}
$$

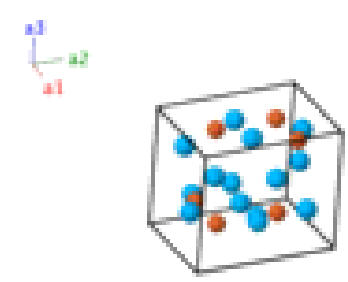




\section{Basis vectors:}

Lattice Coordinates

$\mathbf{B}_{1}=$

$\mathbf{B}_{2}=$

$\mathbf{B}_{3}=$

$\mathbf{B}_{4}=$

$\mathbf{B}_{5}=$

$\mathbf{B}_{6}=-x_{2} \mathbf{a}_{1}+x_{2} \mathbf{a}_{2}+\left(\frac{1}{2}+z_{2}\right) \mathbf{a}_{3}$

$\mathbf{B}_{7}=-x_{2} \mathbf{a}_{1}-2 x_{2} \mathbf{a}_{2}+\left(\frac{1}{2}+z_{2}\right) \mathbf{a}_{3}$

$\mathbf{B}_{8}=2 x_{2} \mathbf{a}_{1}+x_{2} \mathbf{a}_{2}+\left(\frac{1}{2}+z_{2}\right) \mathbf{a}_{3}$

$\mathbf{B}_{9}=x_{3} \mathbf{a}_{1}-x_{3} \mathbf{a}_{2}+z_{3} \mathbf{a}_{3}$

$\mathbf{B}_{10}=x_{3} \mathbf{a}_{1}+2 x_{3} \mathbf{a}_{2}+z_{3} \mathbf{a}_{3}$

$\mathbf{B}_{11}=-2 x_{3} \mathbf{a}_{1}-x_{3} \mathbf{a}_{2}+z_{3} \mathbf{a}_{3}$

$\mathbf{B}_{12}=-x_{3} \mathbf{a}_{1}+x_{3} \mathbf{a}_{2}+\left(\frac{1}{2}+z_{3}\right) \mathbf{a}_{3}$

$\mathbf{B}_{13}=-x_{3} \mathbf{a}_{1}-2 x_{3} \mathbf{a}_{2}+\left(\frac{1}{2}+z_{3}\right) \mathbf{a}_{3}$

$\mathbf{B}_{14}=2 x_{3} \mathbf{a}_{1}+x_{3} \mathbf{a}_{2}+\left(\frac{1}{2}+z_{3}\right) \mathbf{a}_{3}$

$\mathbf{B}_{15}=$

$\mathbf{B}_{16}=$

$\mathbf{B}_{17}=-2 x_{4} \mathbf{a}_{1}-x_{4} \mathbf{a}_{2}+z_{4} \mathbf{a}_{3}$

$\mathbf{B}_{18}=-x_{4} \mathbf{a}_{1}+x_{4} \mathbf{a}_{2}+\left(\frac{1}{2}+z_{4}\right) \mathbf{a}_{3}$

$\mathbf{B}_{19}=-x_{4} \mathbf{a}_{1}-2 x_{4} \mathbf{a}_{2}+\left(\frac{1}{2}+z_{4}\right) \mathbf{a}_{3}$

$\mathbf{B}_{20}=2 x_{4} \mathbf{a}_{1}+x_{4} \mathbf{a}_{2}+\left(\frac{1}{2}+z_{4}\right) \mathbf{a}_{3}$

\section{Cartesian Coordinates}

$$
=
$$

$$
=
$$$$
=
$$

$=$

$$
=
$$$$
=
$$$$
=
$$$$
=
$$

$$
=
$$

$$
=
$$$$
=
$$$$
=
$$$$
=
$$$$
=
$$$$
=
$$$$
=
$$$$
=
$$$$
\begin{gathered}
\frac{1}{2} a \hat{\mathbf{x}}+\frac{1}{2 \sqrt{3}} a \hat{\mathbf{y}}+z_{1} c \hat{\mathbf{z}} \\
\frac{1}{2} a \hat{\mathbf{x}}-\frac{1}{2 \sqrt{3}} a \hat{\mathbf{y}}+\left(\frac{1}{2}+z_{1}\right) c \hat{\mathbf{z}}
\end{gathered}
$$$$
-\sqrt{3} x_{2} a \hat{\mathbf{y}}+z_{2} c \hat{\mathbf{z}}
$$$$
\frac{3}{2} x_{2} a \hat{\mathbf{x}}+\frac{\sqrt{3}}{2} x_{2} a \hat{\mathbf{y}}+z_{2} c \hat{\mathbf{z}}
$$$$
-\frac{3}{2} x_{2} a \hat{\mathbf{x}}+\frac{\sqrt{3}}{2} x_{2} a \hat{\mathbf{y}}+z_{2} c \hat{\mathbf{z}}
$$$$
\sqrt{3} x_{2} a \hat{\mathbf{y}}+\left(\frac{1}{2}+z_{2}\right) c \hat{\mathbf{z}}
$$$$
-\frac{3}{2} x_{2} a \hat{\mathbf{x}}-\frac{\sqrt{3}}{2} x_{2} a \hat{\mathbf{y}}+\left(\frac{1}{2}+z_{2}\right) c \hat{\mathbf{z}}
$$$$
\frac{3}{2} x_{2} a \hat{\mathbf{x}}-\frac{\sqrt{3}}{2} x_{2} a \hat{\mathbf{y}}+\left(\frac{1}{2}+z_{2}\right) c \hat{\mathbf{z}}
$$$$
-\sqrt{3} x_{3} a \hat{\mathbf{y}}+z_{3} c \hat{\mathbf{z}}
$$$$
\frac{3}{2} x_{3} a \hat{\mathbf{x}}+\frac{\sqrt{3}}{2} x_{3} a \hat{\mathbf{y}}+z_{3} c \hat{\mathbf{z}}
$$$$
-\frac{3}{2} x_{3} a \hat{\mathbf{x}}+\frac{\sqrt{3}}{2} x_{3} a \hat{\mathbf{y}}+z_{3} c \hat{\mathbf{z}}
$$$$
\sqrt{3} x_{3} a \hat{\mathbf{y}}+\left(\frac{1}{2}+z_{3}\right) c \hat{\mathbf{z}}
$$$$
-\frac{3}{2} x_{3} a \hat{\mathbf{x}}-\frac{\sqrt{3}}{2} x_{3} a \hat{\mathbf{y}}+\left(\frac{1}{2}+z_{3}\right) c \hat{\mathbf{z}}
$$$$
\frac{3}{2} x_{3} a \hat{\mathbf{x}}-\frac{\sqrt{3}}{2} x_{3} a \hat{\mathbf{y}}+\left(\frac{1}{2}+z_{3}\right) c \hat{\mathbf{z}}
$$$$
-\sqrt{3} x_{4} a \hat{\mathbf{y}}+z_{4} c \hat{\mathbf{z}}
$$$$
\frac{3}{2} x_{4} a \hat{\mathbf{x}}+\frac{\sqrt{3}}{2} x_{4} a \hat{\mathbf{y}}+z_{4} c \hat{\mathbf{z}}
$$$$
-\frac{3}{2} x_{4} a \hat{\mathbf{x}}+\frac{\sqrt{3}}{2} x_{4} a \hat{\mathbf{y}}+z_{4} c \hat{\mathbf{z}}
$$$$
\sqrt{3} x_{4} a \hat{\mathbf{y}}+\left(\frac{1}{2}+z_{4}\right) c \hat{\mathbf{z}}
$$$$
-\frac{3}{2} x_{4} a \hat{\mathbf{x}}-\frac{\sqrt{3}}{2} x_{4} a \hat{\mathbf{y}}+\left(\frac{1}{2}+z_{4}\right) c \hat{\mathbf{z}}
$$$$
\frac{3}{2} x_{4} a \hat{\mathbf{x}}-\frac{\sqrt{3}}{2} x_{4} a \hat{\mathbf{y}}+\left(\frac{1}{2}+z_{4}\right) c \hat{\mathbf{z}}
$$

Wyckoff Position
Atom Type

Th I

Th I

$\mathrm{Fe}$

$\mathrm{Fe}$

$\mathrm{Fe}$

$\mathrm{Fe}$

$\mathrm{Fe}$

$\mathrm{Fe}$

Th II

Th II

Th II

Th II

Th II

Th II

Th III

Th III

Th III

Th III

Th III

Th III

\section{References:}

- J. V. Florio, N. C. Baenziger, and R. E. Rundle, Compounds of thorium with transition metals. II. Systems with iron, cobalt and nickel, Acta Cryst. 9, 367-372 (1956), doi:10.1107/S0365110X5600108X.

\section{Found in:}

- ICSD, Inorganic Crystal Structure Database. ID 401657.

\section{Geometry files:}

- CIF: pp. 936

- POSCAR: pp. 937 


\section{$\mathrm{Re}_{3} \mathrm{~N}$ Structure: AB3_hP4_187_e_fh}
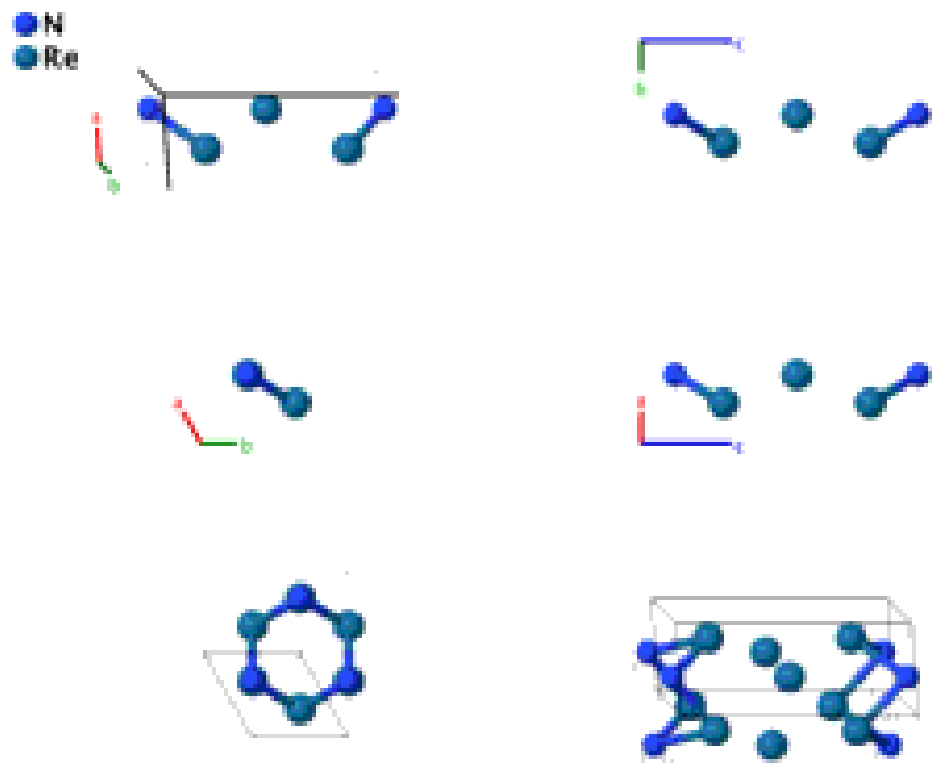

$\begin{array}{lll}\text { Prototype } & : & \mathrm{Re}_{3} \mathrm{~N} \\ \text { AFLOW prototype label } & : & \mathrm{AB} 3 \_\mathrm{hP} 4 \_187 \_\mathrm{e} \_f h \\ \text { Strukturbericht designation } & : & \mathrm{None} \\ \text { Pearson symbol } & : & \mathrm{hP} 4 \\ \text { Space group number } & : & 187 \\ \text { Space group symbol } & : & P \overline{6} m 2 \\ \text { AFLOW prototype command } & : & \text { aflow --proto }=\mathrm{AB} 3 \_\mathrm{hP} 4_{-} 187_{-} \mathrm{e}_{-} \mathrm{fh} \\ & & -- \text { params }=a, c / a, z_{3}\end{array}$

- The reference presents both experimental findings and the results of Density Functional Theory calculations. We obtain our data from the density functional theory calculations at equilibrium $(P=0)$, which are consistent with the lattice constants found experimentally.

Hexagonal primitive vectors:

$$
\begin{array}{ll}
\mathbf{a}_{1}= & \frac{1}{2} a \hat{\mathbf{x}}-\frac{\sqrt{3}}{2} a \hat{\mathbf{y}} \\
\mathbf{a}_{2}= & \frac{1}{2} a \hat{\mathbf{x}}+\frac{\sqrt{3}}{2} a \hat{\mathbf{y}} \\
\mathbf{a}_{3}= & c \hat{\mathbf{z}}
\end{array}
$$

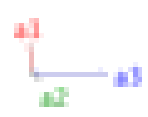

\section{Basis vectors:}

Lattice Coordinates

$$
\mathbf{B}_{1}=\quad \frac{2}{3} \mathbf{a}_{1}+\frac{1}{3} \mathbf{a}_{2}
$$

$\mathbf{B}_{2}=\frac{2}{3} \mathbf{a}_{1}+\frac{1}{3} \mathbf{a}_{2}+\frac{1}{2} \mathbf{a}_{3}$

$\mathbf{B}_{3}=\frac{1}{3} \mathbf{a}_{1}+\frac{2}{3} \mathbf{a}_{2}+z_{3} \mathbf{a}_{3}$

$\mathbf{B}_{4}=$
Cartesian Coordinates

$=$

$=$

$=$

$=$

$$
\begin{gathered}
\frac{1}{2} a \hat{\mathbf{x}}-\frac{1}{2 \sqrt{3}} a \hat{\mathbf{y}} \\
\frac{1}{2} a \hat{\mathbf{x}}-\frac{1}{2 \sqrt{3}} a \hat{\mathbf{y}}+\frac{1}{2} c \hat{\mathbf{z}} \\
\frac{1}{2} a \hat{\mathbf{x}}+\frac{1}{2 \sqrt{3}} a \hat{\mathbf{y}}+z_{3} c \hat{\mathbf{z}} \\
\frac{1}{2} a \hat{\mathbf{x}}+\frac{1}{2 \sqrt{3}} a \hat{\mathbf{y}}-z_{3} c \hat{\mathbf{z}}
\end{gathered}
$$




\section{References:}

- A. Friedrich, B. Winkler, L. Bayarjargal, W. Morgenroth, E. A. Juarez-Arellano, V. Milman, K. Refson, M. Kunz, and K. Chen, Novel Rhenium Nitrides, Phys. Rev. Lett. 105, 085504 (2010), doi:10.1103/PhysRevLett.105.085504.

\section{Geometry files:}

- CIF: pp. 937

- POSCAR: pp. 937 
$\mathrm{LiScI}_{3}$ Structure: A3BC_hP10_188_k_a_e
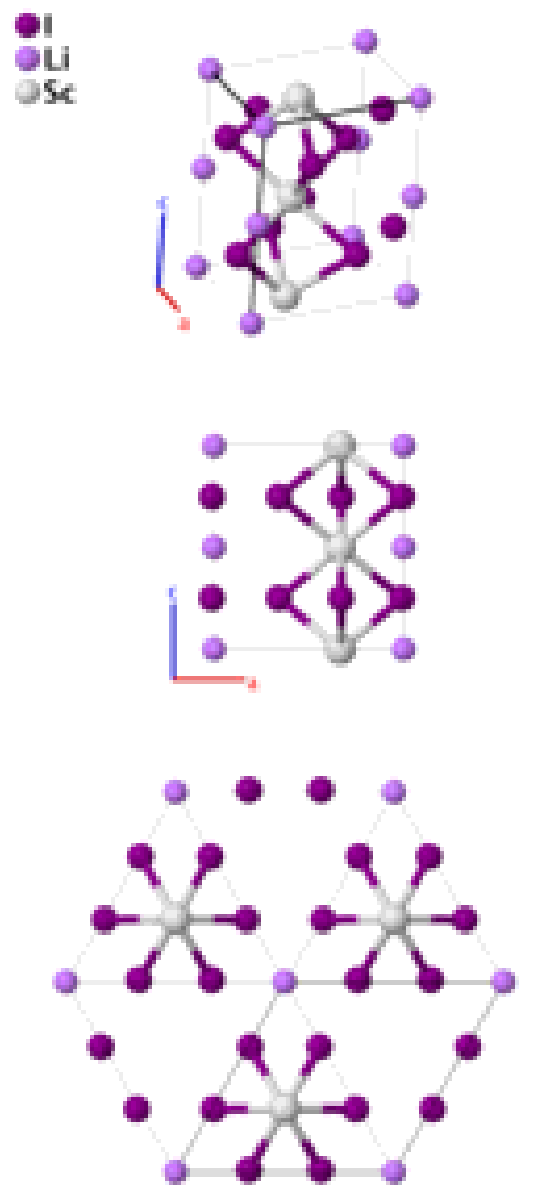
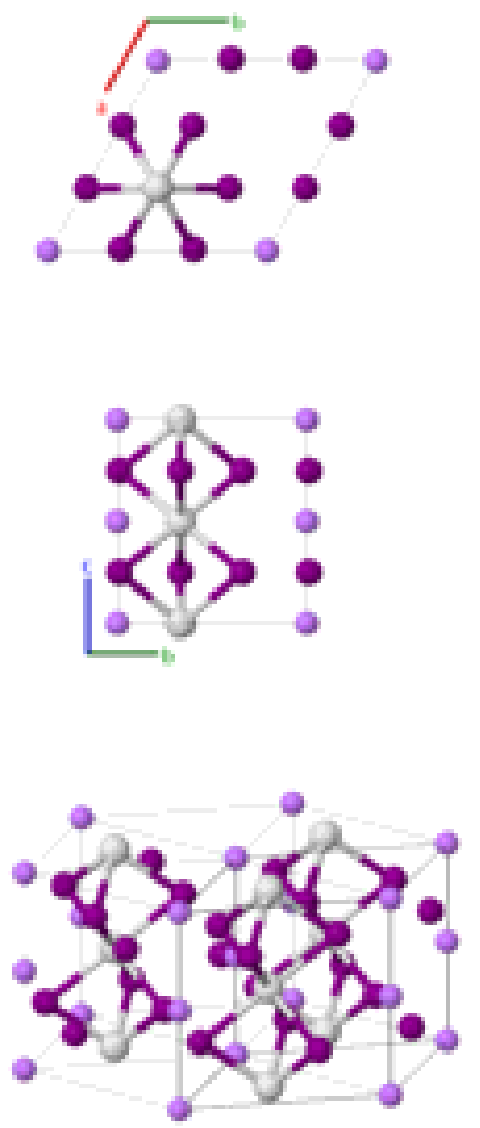

Prototype

AFLOW prototype label

Strukturbericht designation

Pearson symbol

Space group number

Space group symbol

AFLOW prototype command
: $\quad \mathrm{LiScI}_{3}$

: A3BC_hP10_188_k_a_e

: None

: $\mathrm{hP} 10$

: $\quad 188$

: $\quad P \overline{6} c 2$

Hexagonal primitive vectors:

$$
\begin{array}{lll}
\mathbf{a}_{1} & = & \frac{1}{2} a \hat{\mathbf{x}}-\frac{\sqrt{3}}{2} a \hat{\mathbf{y}} \\
\mathbf{a}_{2} & = & \frac{1}{2} a \hat{\mathbf{x}}+\frac{\sqrt{3}}{2} a \hat{\mathbf{y}} \\
\mathbf{a}_{3} & = & c \hat{\mathbf{z}}
\end{array}
$$

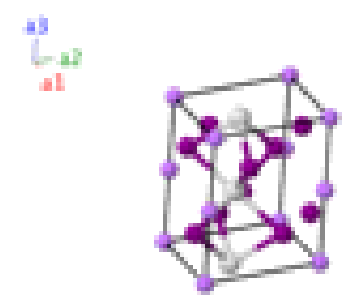

Basis vectors: 


$\begin{array}{llclll}\mathbf{B}_{1} & = & 0 \mathbf{a}_{1}+0 \mathbf{a}_{2}+0 \mathbf{a}_{3} & = & 0 \hat{\mathbf{x}}+0 \hat{\mathbf{y}}+0 \hat{\mathbf{z}} & \end{array}$

\section{References:}

- A. Lachgar, D. S. Dudis, P. K. Dorhout, and J. D. Corbett, Synthesis and properties of two novel line phases that contain linear scandium chains, lithium scandium iodide ( $\left.\mathrm{LiScI}_{3}\right)$ and sodium scandium iodide ( $\mathrm{Na}_{0.5} \mathrm{ScI}_{3}$ ), Inorg. Chem. 30, 3321-3326 (1991), doi:10.1021/ic00017a019.

\section{Found in:}

- P. Villars and K. Cenzual, Pearson's Crystal Data - Crystal Structure Database for Inorganic Compounds, ASM International (2013).

\section{Geometry files:}

- CIF: pp. 937

- POSCAR: pp. 938 


\section{$\mathrm{BaSi}_{4} \mathrm{O}_{9}$ Structure: AB9C4_hP28_188_e_kl_ak}
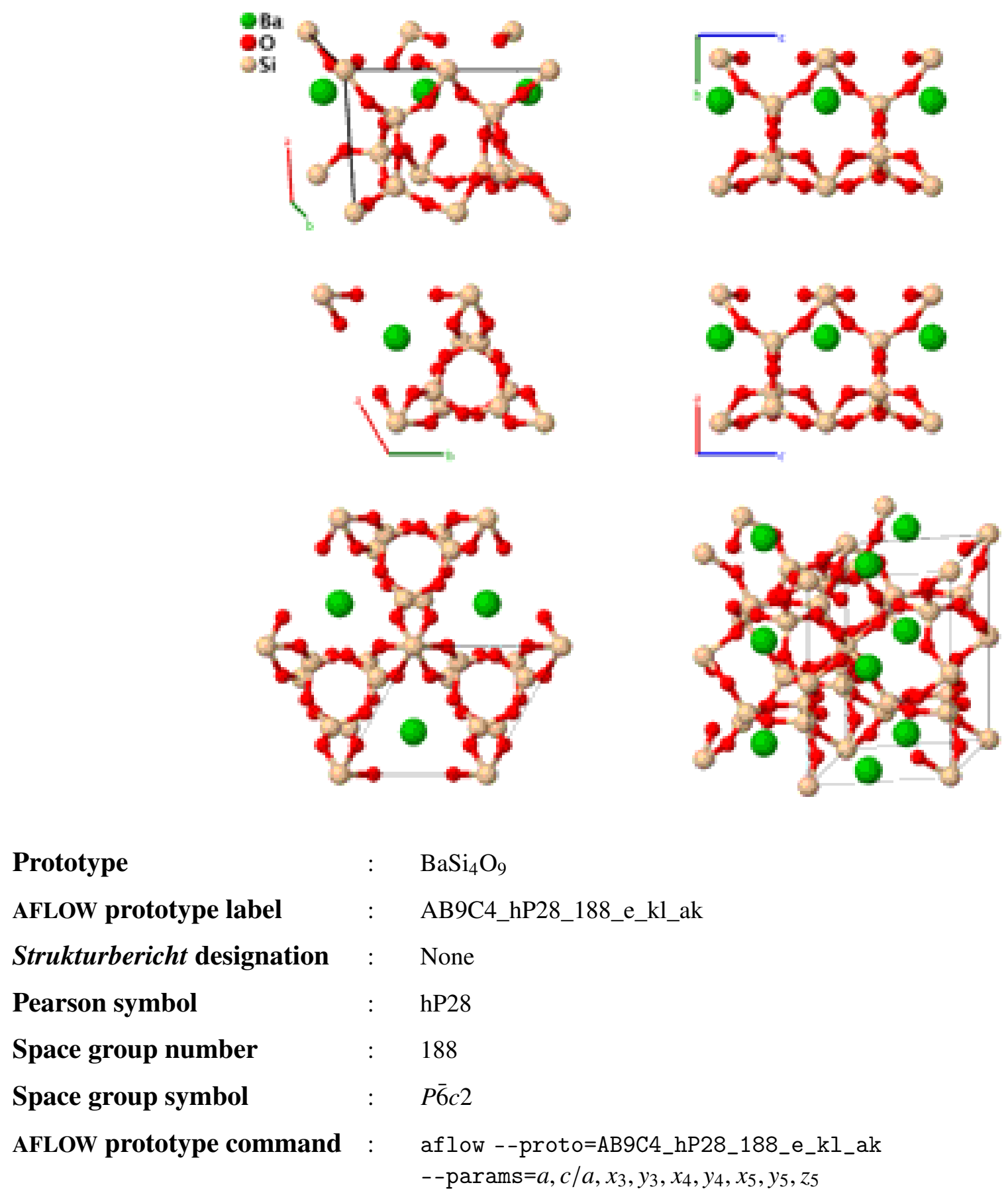

Hexagonal primitive vectors:

$$
\begin{array}{ll}
\mathbf{a}_{1}= & \frac{1}{2} a \hat{\mathbf{x}}-\frac{\sqrt{3}}{2} a \hat{\mathbf{y}} \\
\mathbf{a}_{2}= & \frac{1}{2} a \hat{\mathbf{x}}+\frac{\sqrt{3}}{2} a \hat{\mathbf{y}} \\
\mathbf{a}_{3}= & c \hat{\mathbf{z}}
\end{array}
$$

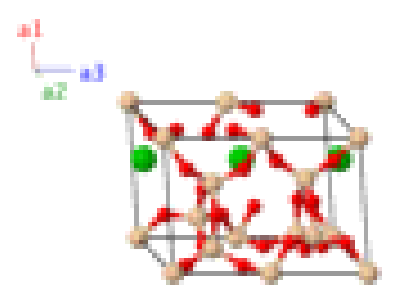

Basis vectors: 


\begin{tabular}{|c|c|c|c|c|c|c|}
\hline $\mathbf{B}_{1}$ & $=$ & $0 \mathbf{a}_{1}+0 \mathbf{a}_{2}+0 \mathbf{a}_{3}$ & $=$ & $0 \hat{\mathbf{x}}+0 \hat{\mathbf{y}}+0 \hat{\mathbf{z}}$ & $(2 a)$ & $\mathrm{Si} \mathrm{I}$ \\
\hline $\mathbf{B}_{2}$ & $=$ & $\frac{1}{2} \mathbf{a}_{3}$ & $=$ & $\frac{1}{2} c \hat{\mathbf{z}}$ & $(2 a)$ & $\mathrm{Si} \mathrm{I}$ \\
\hline $\mathbf{B}_{3}$ & $=$ & $\frac{2}{3} \mathbf{a}_{1}+\frac{1}{3} \mathbf{a}_{2}$ & $=$ & $\frac{1}{2} a \hat{\mathbf{x}}-\frac{1}{2 \sqrt{3}} a \hat{\mathbf{y}}$ & $(2 e)$ & $\mathrm{Ba}$ \\
\hline $\mathbf{B}_{4}$ & $=$ & $\frac{2}{3} \mathbf{a}_{1}+\frac{1}{3} \mathbf{a}_{2}+\frac{1}{2} \mathbf{a}_{3}$ & $=$ & $\frac{1}{2} a \hat{\mathbf{x}}-\frac{1}{2 \sqrt{3}} a \hat{\mathbf{y}}+\frac{1}{2} c \hat{\mathbf{z}}$ & $(2 e)$ & $\mathrm{Ba}$ \\
\hline $\mathbf{B}_{5}$ & $=$ & $x_{3} \mathbf{a}_{1}+y_{3} \mathbf{a}_{2}+\frac{1}{4} \mathbf{a}_{3}$ & $=$ & $\begin{array}{c}\frac{1}{2}\left(x_{3}+y_{3}\right) a \hat{\mathbf{x}}+ \\
\frac{\sqrt{3}}{2}\left(-x_{3}+y_{3}\right) a \hat{\mathbf{y}}+\frac{1}{4} c \hat{\mathbf{z}}\end{array}$ & $(6 k)$ & O I \\
\hline $\mathbf{B}_{6}$ & $=$ & $-y_{3} \mathbf{a}_{1}+\left(x_{3}-y_{3}\right) \mathbf{a}_{2}+\frac{1}{4} \mathbf{a}_{3}$ & $=$ & $\left(\frac{1}{2} x_{3}-y_{3}\right) a \hat{\mathbf{x}}+\frac{\sqrt{3}}{2} x_{3} a \hat{\mathbf{y}}+\frac{1}{4} c \hat{\mathbf{z}}$ & $(6 k)$ & O I \\
\hline $\mathbf{B}_{7}$ & $=$ & $\left(-x_{3}+y_{3}\right) \mathbf{a}_{1}-x_{3} \mathbf{a}_{2}+\frac{1}{4} \mathbf{a}_{3}$ & $=$ & $\left(-x_{3}+\frac{1}{2} y_{3}\right) a \hat{\mathbf{x}}-\frac{\sqrt{3}}{2} y_{3} a \hat{\mathbf{y}}+\frac{1}{4} c \hat{\mathbf{z}}$ & $(6 k)$ & O I \\
\hline $\mathbf{B}_{8}$ & $=$ & $-y_{3} \mathbf{a}_{1}-x_{3} \mathbf{a}_{2}+\frac{3}{4} \mathbf{a}_{3}$ & $=$ & $\begin{array}{c}-\frac{1}{2}\left(x_{3}+y_{3}\right) a \hat{\mathbf{x}}+ \\
\frac{\sqrt{3}}{2}\left(-x_{3}+y_{3}\right) a \hat{\mathbf{y}}+\frac{3}{4} c \hat{\mathbf{z}}\end{array}$ & $(6 k)$ & O I \\
\hline $\mathbf{B}_{9}$ & $=$ & $\left(-x_{3}+y_{3}\right) \mathbf{a}_{1}+y_{3} \mathbf{a}_{2}+\frac{3}{4} \mathbf{a}_{3}$ & $=$ & $\left(-\frac{1}{2} x_{3}+y_{3}\right) a \hat{\mathbf{x}}+\frac{\sqrt{3}}{2} x_{3} a \hat{\mathbf{y}}+\frac{3}{4} c \hat{\mathbf{z}}$ & $(6 k)$ & O I \\
\hline $\mathbf{B}_{10}$ & $=$ & $x_{3} \mathbf{a}_{1}+\left(x_{3}-y_{3}\right) \mathbf{a}_{2}+\frac{3}{4} \mathbf{a}_{3}$ & $=$ & $\left(x_{3}-\frac{1}{2} y_{3}\right) a \hat{\mathbf{x}}-\frac{\sqrt{3}}{2} y_{3} a \hat{\mathbf{y}}+\frac{3}{4} c \hat{\mathbf{z}}$ & $(6 k)$ & O I \\
\hline $\mathbf{B}_{11}$ & $=$ & $x_{4} \mathbf{a}_{1}+y_{4} \mathbf{a}_{2}+\frac{1}{4} \mathbf{a}_{3}$ & $=$ & $\begin{array}{c}\frac{1}{2}\left(x_{4}+y_{4}\right) a \hat{\mathbf{x}}+ \\
\frac{\sqrt{3}}{2}\left(-x_{4}+y_{4}\right) a \hat{\mathbf{y}}+\frac{1}{4} c \hat{\mathbf{z}}\end{array}$ & $(6 k)$ & Si II \\
\hline $\mathbf{B}_{12}$ & $=$ & $-y_{4} \mathbf{a}_{1}+\left(x_{4}-y_{4}\right) \mathbf{a}_{2}+\frac{1}{4} \mathbf{a}_{3}$ & $=$ & $\left(\frac{1}{2} x_{4}-y_{4}\right) a \hat{\mathbf{x}}+\frac{\sqrt{3}}{2} x_{4} a \hat{\mathbf{y}}+\frac{1}{4} c \hat{\mathbf{z}}$ & $(6 k)$ & Si II \\
\hline $\mathbf{B}_{13}$ & $=$ & $\left(-x_{4}+y_{4}\right) \mathbf{a}_{1}-x_{4} \mathbf{a}_{2}+\frac{1}{4} \mathbf{a}_{3}$ & $=$ & $\left(-x_{4}+\frac{1}{2} y_{4}\right) a \hat{\mathbf{x}}-\frac{\sqrt{3}}{2} y_{4} a \hat{\mathbf{y}}+\frac{1}{4} c \hat{\mathbf{z}}$ & $(6 k)$ & Si II \\
\hline $\mathbf{B}_{14}$ & $=$ & $-y_{4} \mathbf{a}_{1}-x_{4} \mathbf{a}_{2}+\frac{3}{4} \mathbf{a}_{3}$ & $=$ & $\begin{array}{c}-\frac{1}{2}\left(x_{4}+y_{4}\right) a \hat{\mathbf{x}}+ \\
\frac{\sqrt{3}}{2}\left(-x_{4}+y_{4}\right) a \hat{\mathbf{y}}+\frac{3}{4} c \hat{\mathbf{z}}\end{array}$ & $(6 k)$ & Si II \\
\hline $\mathbf{B}_{15}$ & $=$ & $\left(-x_{4}+y_{4}\right) \mathbf{a}_{1}+y_{4} \mathbf{a}_{2}+\frac{3}{4} \mathbf{a}_{3}$ & $=$ & $\left(-\frac{1}{2} x_{4}+y_{4}\right) a \hat{\mathbf{x}}+\frac{\sqrt{3}}{2} x_{4} a \hat{\mathbf{y}}+\frac{3}{4} c \hat{\mathbf{z}}$ & $(6 k)$ & Si II \\
\hline $\mathbf{B}_{16}$ & $=$ & $x_{4} \mathbf{a}_{1}+\left(x_{4}-y_{4}\right) \mathbf{a}_{2}+\frac{3}{4} \mathbf{a}_{3}$ & $=$ & $\left(x_{4}-\frac{1}{2} y_{4}\right) a \hat{\mathbf{x}}-\frac{\sqrt{3}}{2} y_{4} a \hat{\mathbf{y}}+\frac{3}{4} c \hat{\mathbf{z}}$ & $(6 k)$ & Si II \\
\hline $\mathbf{B}_{17}$ & $=$ & $x_{5} \mathbf{a}_{1}+y_{5} \mathbf{a}_{2}+z_{5} \mathbf{a}_{3}$ & $=$ & $\begin{array}{c}\frac{1}{2}\left(x_{5}+y_{5}\right) a \hat{\mathbf{x}}+ \\
\frac{\sqrt{3}}{2}\left(-x_{5}+y_{5}\right) a \hat{\mathbf{y}}+z_{5} c \hat{\mathbf{z}}\end{array}$ & $(12 l)$ & O II \\
\hline $\mathbf{B}_{18}$ & $=$ & $-y_{5} \mathbf{a}_{1}+\left(x_{5}-y_{5}\right) \mathbf{a}_{2}+z_{5} \mathbf{a}_{3}$ & $=$ & $\left(\frac{1}{2} x_{5}-y_{5}\right) a \hat{\mathbf{x}}+\frac{\sqrt{3}}{2} x_{5} a \hat{\mathbf{y}}+z_{5} c \hat{\mathbf{z}}$ & $(12 l)$ & O II \\
\hline $\mathbf{B}_{19}$ & $=$ & $\left(-x_{5}+y_{5}\right) \mathbf{a}_{1}-x_{5} \mathbf{a}_{2}+z_{5} \mathbf{a}_{3}$ & $=$ & $\left(-x_{5}+\frac{1}{2} y_{5}\right) a \hat{\mathbf{x}}-\frac{\sqrt{3}}{2} y_{5} a \hat{\mathbf{y}}+z_{5} c \hat{\mathbf{z}}$ & $(12 l)$ & O II \\
\hline $\mathbf{B}_{20}$ & $=$ & $x_{5} \mathbf{a}_{1}+y_{5} \mathbf{a}_{2}+\left(\frac{1}{2}-z_{5}\right) \mathbf{a}_{3}$ & $=$ & $\begin{array}{c}\frac{1}{2}\left(x_{5}+y_{5}\right) a \hat{\mathbf{x}}+ \\
\frac{\sqrt{3}}{2}\left(-x_{5}+y_{5}\right) a \hat{\mathbf{y}}+\left(\frac{1}{2}-z_{5}\right) c \hat{\mathbf{z}}\end{array}$ & $(12 l)$ & O II \\
\hline $\mathbf{B}_{21}$ & $=$ & $-y_{5} \mathbf{a}_{1}+\left(x_{5}-y_{5}\right) \mathbf{a}_{2}+\left(\frac{1}{2}-z_{5}\right) \mathbf{a}_{3}$ & $=$ & $\begin{array}{c}\left(\frac{1}{2} x_{5}-y_{5}\right) a \hat{\mathbf{x}}+\frac{\sqrt{3}}{2} x_{5} a \hat{\mathbf{y}}+ \\
\left(\frac{1}{2}-z_{5}\right) c \hat{\mathbf{z}}\end{array}$ & $(12 l)$ & O II \\
\hline $\mathbf{B}_{22}$ & $=$ & $\left(-x_{5}+y_{5}\right) \mathbf{a}_{1}-x_{5} \mathbf{a}_{2}+\left(\frac{1}{2}-z_{5}\right) \mathbf{a}_{3}$ & $=$ & $\begin{array}{c}\left(-x_{5}+\frac{1}{2} y_{5}\right) a \hat{\mathbf{x}}-\frac{\sqrt{3}}{2} y_{5} a \hat{\mathbf{y}}+ \\
\left(\frac{1}{2}-z_{5}\right) c \hat{\mathbf{z}}\end{array}$ & $(12 l)$ & O II \\
\hline $\mathbf{B}_{23}$ & $=$ & $-y_{5} \mathbf{a}_{1}-x_{5} \mathbf{a}_{2}+\left(\frac{1}{2}+z_{5}\right) \mathbf{a}_{3}$ & $=$ & $\begin{array}{c}-\frac{1}{2}\left(x_{5}+y_{5}\right) a \hat{\mathbf{x}}+ \\
\frac{\sqrt{3}}{2}\left(-x_{5}+y_{5}\right) a \hat{\mathbf{y}}+\left(\frac{1}{2}+z_{5}\right) c \hat{\mathbf{z}}\end{array}$ & $(12 l)$ & O II \\
\hline $\mathbf{B}_{24}$ & $=$ & $\left(-x_{5}+y_{5}\right) \mathbf{a}_{1}+y_{5} \mathbf{a}_{2}+\left(\frac{1}{2}+z_{5}\right) \mathbf{a}_{3}$ & $=$ & $\begin{array}{c}\left(-\frac{1}{2} x_{5}+y_{5}\right) a \hat{\mathbf{x}}+\frac{\sqrt{3}}{2} x_{5} a \hat{\mathbf{y}}+ \\
\left(\frac{1}{2}+z_{5}\right) c \hat{\mathbf{z}}\end{array}$ & $(12 l)$ & O II \\
\hline $\mathbf{B}_{25}$ & $=$ & $x_{5} \mathbf{a}_{1}+\left(x_{5}-y_{5}\right) \mathbf{a}_{2}+\left(\frac{1}{2}+z_{5}\right) \mathbf{a}_{3}$ & $=$ & $\begin{array}{c}\left(x_{5}-\frac{1}{2} y_{5}\right) a \hat{\mathbf{x}}-\frac{\sqrt{3}}{2} y_{5} a \hat{\mathbf{y}}+ \\
\left(\frac{1}{2}+z_{5}\right) c \hat{\mathbf{z}}\end{array}$ & $(12 l)$ & $\mathrm{O}$ II \\
\hline $\mathbf{B}_{26}$ & $=$ & $-y_{5} \mathbf{a}_{1}-x_{5} \mathbf{a}_{2}-z_{5} \mathbf{a}_{3}$ & $=$ & $\begin{array}{c}-\frac{1}{2}\left(x_{5}+y_{5}\right) a \hat{\mathbf{x}}+ \\
\frac{\sqrt{3}}{2}\left(-x_{5}+y_{5}\right) a \hat{\mathbf{y}}-z_{5} c \hat{\mathbf{z}}\end{array}$ & $(12 l)$ & O II \\
\hline $\mathbf{B}_{27}$ & $=$ & $\left(-x_{5}+y_{5}\right) \mathbf{a}_{1}+y_{5} \mathbf{a}_{2}-z_{5} \mathbf{a}_{3}$ & $=$ & $\left(-\frac{1}{2} x_{5}+y_{5}\right) a \hat{\mathbf{x}}+\frac{\sqrt{3}}{2} x_{5} a \hat{\mathbf{y}}-z_{5} c \hat{\mathbf{z}}$ & $(12 l)$ & O II \\
\hline
\end{tabular}


$\mathbf{B}_{28}=x_{5} \mathbf{a}_{1}+\left(x_{5}-y_{5}\right) \mathbf{a}_{2}-z_{5} \mathbf{a}_{3}=\left(x_{5}-\frac{1}{2} y_{5}\right) a \hat{\mathbf{x}}-\frac{\sqrt{3}}{2} y_{5} a \hat{\mathbf{y}}-z_{5} c \hat{\mathbf{z}}$

$(12 l)$

O II

\section{References:}

- L. W. Finger, R. M. Hazen, and B. A. Fursenko, Refinement of the crystal structure of $\mathrm{BaSi}_{4} \mathrm{O}_{9}$ in the benitoite form, J. Phys. Chem. Solids 56, 1389-1393 (1995), doi:10.1016/0022-3697(95)00075-5.

\section{Found in:}

- P. Villars and K. Cenzual, Pearson's Crystal Data - Crystal Structure Database for Inorganic Compounds, ASM International (2013).

\section{Geometry files:}

- CIF: pp. 938

- POSCAR: pp. 938 


\section{$\pi-\mathrm{FeMg}_{3} \mathrm{Al}_{8} \mathrm{Si}_{6}\left(E 9_{b}\right)$ Structure: A8BC3D6_hP18_189_bfh_a_g_i}
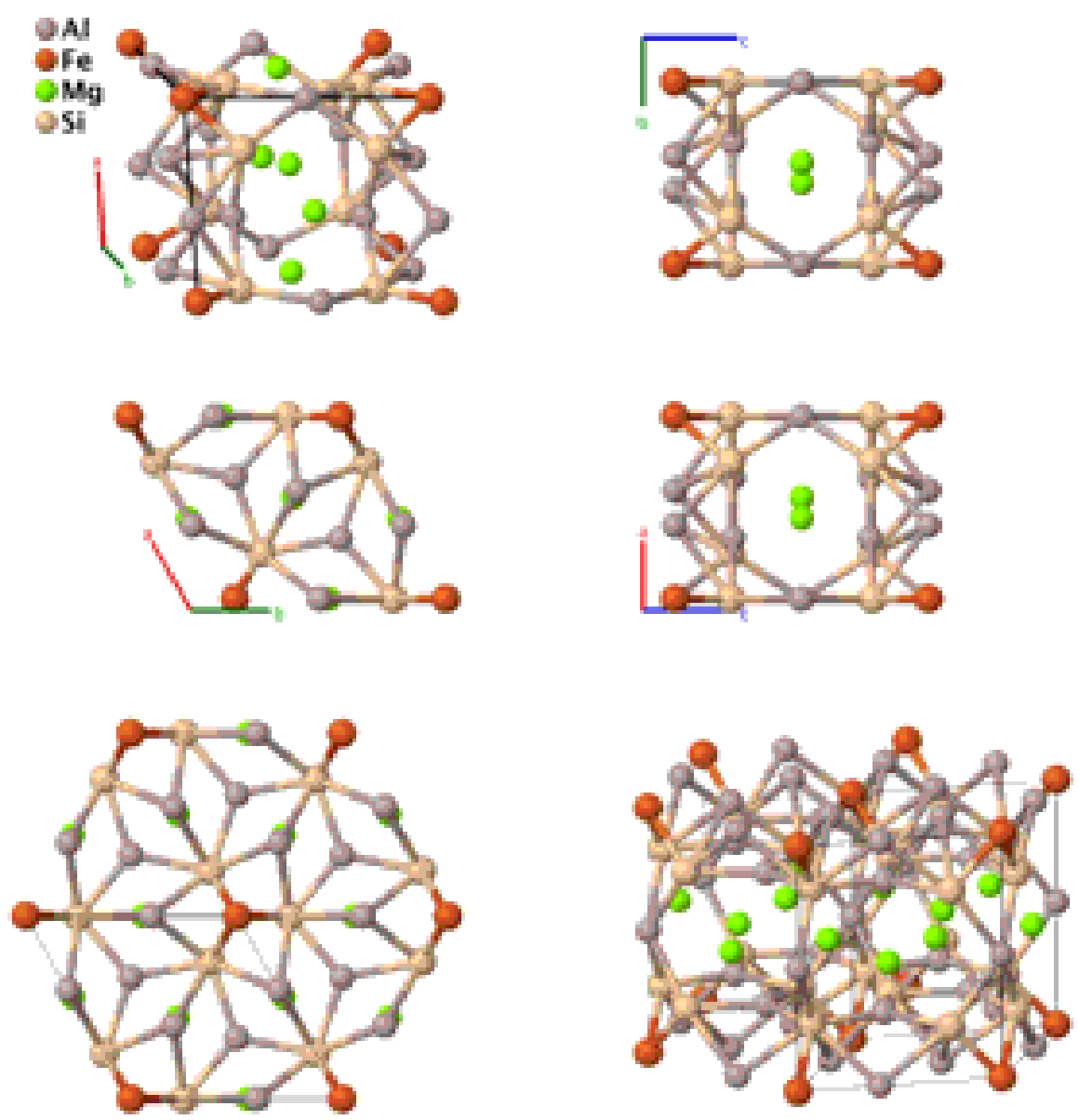

\begin{tabular}{|c|c|c|}
\hline Prototype & $\cdot$ & $\pi-\mathrm{FeMg}_{3} \mathrm{Al}_{8} \mathrm{Si}_{6}$ \\
\hline AFLOW prototype label & & A8BC3D6_hP18_189_bfh_a_g_i \\
\hline Strukturbericht designation & & $E 9_{b}$ \\
\hline Pearson symbol & & hP18 \\
\hline Space group number & $\cdot$ & 189 \\
\hline Space group symbol & & $P \overline{6} 2 m$ \\
\hline AFLOW prototype command & & $\begin{array}{l}\text { aflow --proto=A8BC3D6_hP18_189_bfh_a_g_i } \\
-- \text { params }=a, c / a, x_{3}, x_{4}, z_{5}, x_{6}, z_{6}\end{array}$ \\
\hline
\end{tabular}

- We have been unable to obtain a copy of (Perlitz, 1942), and use the data provided by (Brandes, 1992) and (Foss, 2003). Foss et al. argue that the actual compositition of this phase should be $\mathrm{FeMg}_{3} \mathrm{Al}_{9} \mathrm{Si}_{5}$. This requires a reordering of the atomic positions, as described in A9BC3D5_hP18_189_fi_a_g_bh. 


\section{Hexagonal primitive vectors:}

$$
\begin{array}{ll}
\mathbf{a}_{1}= & \frac{1}{2} a \hat{\mathbf{x}}-\frac{\sqrt{3}}{2} a \hat{\mathbf{y}} \\
\mathbf{a}_{2}= & \frac{1}{2} a \hat{\mathbf{x}}+\frac{\sqrt{3}}{2} a \hat{\mathbf{y}} \\
\mathbf{a}_{3}= & c \hat{\mathbf{z}}
\end{array}
$$

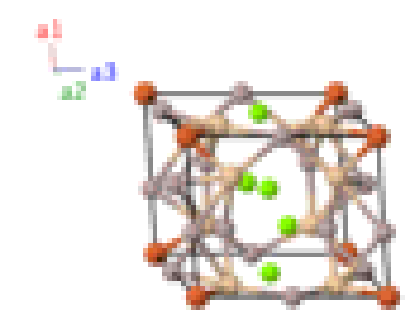

\section{Basis vectors:}

\section{Lattice Coordinates}

$\mathbf{B}_{1}=$

$\mathbf{B}_{2}=$

$\mathbf{B}_{3}=$

$\mathbf{B}_{4}=$

$\mathbf{B}_{5}=$

$\mathbf{B}_{6}=$

$\mathbf{B}_{7}=$

$\mathbf{B}_{8}=$

$\mathbf{B}_{9}=$

$\mathbf{B}_{10}=$

$\mathbf{B}_{11}=$

$\mathbf{B}_{12}=$

$\mathbf{B}_{13}=$

$\mathbf{B}_{14}=$

$\mathbf{B}_{15}=$

$\mathbf{B}_{16}=$

$\mathbf{B}_{17}=$

$\mathbf{B}_{18}=$

$$
0 \mathbf{a}_{1}+0 \mathbf{a}_{2}+0 \mathbf{a}_{3}
$$$$
\frac{1}{2} \mathbf{a}_{3}
$$

$x_{3} \mathbf{a}_{1}$

$x_{3} \mathbf{a}_{2}$

$-x_{3} \mathbf{a}_{1}-x_{3} \mathbf{a}_{2}$

$x_{4} \mathbf{a}_{1}+\frac{1}{2} \mathbf{a}_{3}$

$x_{4} \mathbf{a}_{2}+\frac{1}{2} \mathbf{a}_{3}$

$-x_{4} \mathbf{a}_{1}-x_{4} \mathbf{a}_{2}+\frac{1}{2} \mathbf{a}_{3}$

$$
\frac{1}{3} \mathbf{a}_{1}+\frac{2}{3} \mathbf{a}_{2}+z_{5} \mathbf{a}_{3}
$$

$$
\frac{1}{3} \mathbf{a}_{1}+\frac{2}{3} \mathbf{a}_{2}-z_{5} \mathbf{a}_{3}
$$

$$
\frac{2}{3} \mathbf{a}_{1}+\frac{1}{3} \mathbf{a}_{2}-z_{5} \mathbf{a}_{3}
$$$$
\frac{2}{3} \mathbf{a}_{1}+\frac{1}{3} \mathbf{a}_{2}+z_{5} \mathbf{a}_{3}
$$

$$
x_{6} \mathbf{a}_{1}+z_{6} \mathbf{a}_{3}
$$$$
x_{6} \mathbf{a}_{2}+z_{6} \mathbf{a}_{3}
$$$$
-x_{6} \mathbf{a}_{1}-x_{6} \mathbf{a}_{2}+z_{6} \mathbf{a}_{3}
$$

$$
x_{6} \mathbf{a}_{1}+-z_{6} \mathbf{a}_{3}
$$

$$
x_{6} \mathbf{a}_{2}-z_{6} \mathbf{a}_{3}
$$$$
-x_{6} \mathbf{a}_{1}-x_{6} \mathbf{a}_{2}-z_{6} \mathbf{a}_{3}
$$

\section{Cartesian Coordinates}

$=$

$=$

$=$

$=$

$=$

$=$

$=$

$=$

$=$
$=$

$=$

$=$

$=$

$=$

$=$

$=$

$=$

$=$

$=$

$=$

$=$

$$
0 \hat{\mathbf{x}}+0 \hat{\mathbf{y}}+0 \hat{\mathbf{z}}
$$$$
\frac{1}{2} c \hat{\mathbf{z}}
$$

$$
\frac{1}{2} x_{3} a \hat{\mathbf{x}}-\frac{\sqrt{3}}{2} x_{3} a \hat{\mathbf{y}}
$$$$
\frac{1}{2} x_{3} a \hat{\mathbf{x}}+\frac{\sqrt{3}}{2} x_{3} a \hat{\mathbf{y}}
$$$$
-x_{3} a \hat{\mathbf{x}}
$$$$
\frac{1}{2} x_{4} a \hat{\mathbf{x}}-\frac{\sqrt{3}}{2} x_{4} a \hat{\mathbf{y}}+\frac{1}{2} c \hat{\mathbf{z}}
$$$$
\frac{1}{2} x_{4} a \hat{\mathbf{x}}+\frac{\sqrt{3}}{2} x_{4} a \hat{\mathbf{y}}+\frac{1}{2} c \hat{\mathbf{z}}
$$$$
-x_{4} a \hat{\mathbf{x}}+\frac{1}{2} c \hat{\mathbf{z}}
$$

$$
\frac{1}{2} a \hat{\mathbf{x}}+\frac{1}{2 \sqrt{3}} a \hat{\mathbf{y}}+z_{5} c \hat{\mathbf{z}}
$$$$
\frac{1}{2} a \hat{\mathbf{x}}+\frac{1}{2 \sqrt{3}} a \hat{\mathbf{y}}-z_{5} c \hat{\mathbf{z}}
$$$$
\frac{1}{2} a \hat{\mathbf{x}}-\frac{1}{2 \sqrt{3}} a \hat{\mathbf{y}}-z_{5} c \hat{\mathbf{z}}
$$$$
\frac{1}{2} a \hat{\mathbf{x}}-\frac{1}{2 \sqrt{3}} a \hat{\mathbf{y}}+z_{5} c \hat{\mathbf{z}}
$$$$
\frac{1}{2} x_{6} a \hat{\mathbf{x}}-\frac{\sqrt{3}}{2} x_{6} a \hat{\mathbf{y}}+z_{6} c \hat{\mathbf{z}}
$$$$
\frac{1}{2} x_{6} a \hat{\mathbf{x}}+\frac{\sqrt{3}}{2} x_{6} a \hat{\mathbf{y}}+z_{6} c \hat{\mathbf{z}}
$$$$
-x_{6} a \hat{\mathbf{x}}+z_{6} c \hat{\mathbf{z}}
$$$$
\frac{1}{2} x_{6} a \hat{\mathbf{x}}-\frac{\sqrt{3}}{2} x_{6} a \hat{\mathbf{y}}-z_{6} c \hat{\mathbf{z}}
$$$$
\frac{1}{2} x_{6} a \hat{\mathbf{x}}+\frac{\sqrt{3}}{2} x_{6} a \hat{\mathbf{y}}-z_{6} c \hat{\mathbf{z}}
$$$$
-x_{6} a \hat{\mathbf{x}}+-z_{6} c \hat{\mathbf{z}}
$$

Wyckoff Position

(1a)
Atom Type

$\mathrm{Fe}$

Al I

Al II

Al II

Al II

$\mathrm{Mg}$

$\mathrm{Mg}$

$\mathrm{Mg}$

Al III

Al III

Al III

Al III

$\mathrm{Si}$

$\mathrm{Si}$

$\mathrm{Si}$

$\mathrm{Si}$

$\mathrm{Si}$

$\mathrm{Si}$

\section{References:}

- H. Perlitz and A. Westgren, The Crystal Structure of $\mathrm{Al}_{8} \mathrm{Si}_{6} \mathrm{Mg}_{3} \mathrm{Fe}$, Ark. Kem. Mineral. Geol. 15B, 1-8 (1942).

- E. A. Brandes and G. B. Brook, eds., Smithells Metals Reference Book (Butterworth Heinemann, Oxford, Auckland, Boston, Johannesburg, Melbourne, New Delhi, 1992), chap. 6, pp. 6-60, seventh edn.

\section{Found in:}

- S. Foss, A. Olsen, C. J. Simensen, and J. Tafto, Determination of the crystal structure of the $\pi$-AlFeMgSi phase using symmetry- and site-sensitive electron microscope techniques, Acta Crystallogr. Sect. B Struct. Sci. 59, 36-42 (2003), doi:10.1107/S0108768102022887.

\section{Geometry files:}

- CIF: pp. 938 
- POSCAR: pp. 939 


\section{$\pi$-FeMg${ }_{3} \mathrm{Al}_{9} \mathrm{Si}_{5}$ Structure: A9BC3D5_hP18_189_fi_a_g_bh}
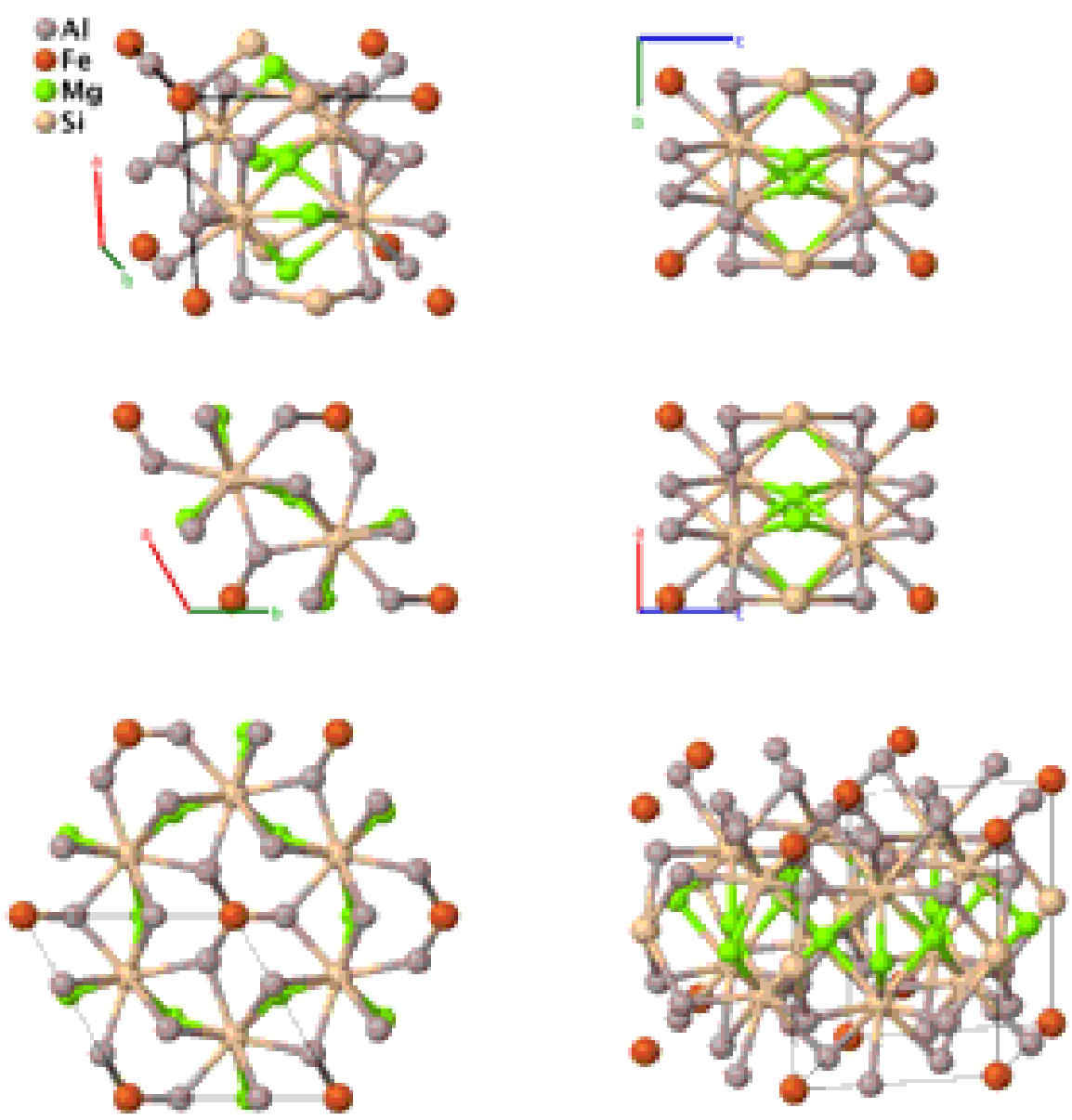

\section{Prototype}

AFLOW prototype label

Strukturbericht designation

Pearson symbol

Space group number

Space group symbol

AFLOW prototype command
: $\quad \pi-\mathrm{FeMg}_{3} \mathrm{Al}_{9} \mathrm{Si}_{5}$

: A9BC3D5_hP18_189_fi_a_g_bh

: None

: $\quad$ hP18

: $\quad 189$

: $\quad P \overline{6} 2 m$
: aflow --proto=A9BC3D5_hP18_189_fi_a_g_bh

- params $=a, c / a, x_{3}, x_{4}, z_{5}, x_{6}, z_{6}$

- This is a reanalysis of the $\pi$-FeMg $\mathrm{Al}_{8} \mathrm{Al}_{6}\left(E 9_{b}\right)$ structure. The space group and occupied Wyckoff positions are unchanged, but the ordering, stoichiometry, and atomic positions are different.

\section{Hexagonal primitive vectors:}

$$
\begin{array}{ll}
\mathbf{a}_{1}= & \frac{1}{2} a \hat{\mathbf{x}}-\frac{\sqrt{3}}{2} a \hat{\mathbf{y}} \\
\mathbf{a}_{2}= & \frac{1}{2} a \hat{\mathbf{x}}+\frac{\sqrt{3}}{2} a \hat{\mathbf{y}} \\
\mathbf{a}_{3}= & c \hat{\mathbf{z}}
\end{array}
$$

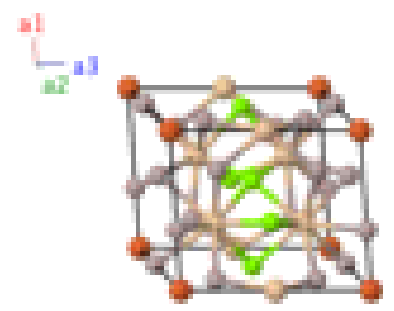




\section{Basis vectors:}

$$
\text { Lattice Coordinates }
$$

$\mathbf{B}_{1}=$

$\mathbf{B}_{2}=$

$\mathbf{B}_{3}=$

$\mathbf{B}_{4}=$

$\mathbf{B}_{5}=$

$\mathbf{B}_{6}=$

$\mathbf{B}_{7}=$

$\mathbf{B}_{8}=-x_{4} \mathbf{a}_{1}-x_{4} \mathbf{a}_{2}+\frac{1}{2} \mathbf{a}_{3}$

$\mathbf{B}_{9}=$

$\mathbf{B}_{10}=$

$\mathbf{B}_{11}=$

$\mathbf{B}_{12}=$

$\mathbf{B}_{13}=$

$\mathbf{B}_{14}=$

$\mathbf{B}_{15}=$

$\mathbf{B}_{16}=$

$\mathbf{B}_{17}=$

$\mathbf{B}_{18}=$

$$
0 \mathbf{a}_{1}+0 \mathbf{a}_{2}+0 \mathbf{a}_{3}
$$$$
\frac{1}{2} \mathbf{a}_{3}
$$$$
x_{3} \mathbf{a}_{1}
$$$$
x_{3} \mathbf{a}_{2}
$$$$
-x_{3} \mathbf{a}_{1}-x_{3} \mathbf{a}_{2}
$$$$
x_{4} \mathbf{a}_{1}+\frac{1}{2} \mathbf{a}_{3}
$$$$
x_{4} \mathbf{a}_{2}+\frac{1}{2} \mathbf{a}_{3}
$$

$$
\frac{1}{3} \mathbf{a}_{1}+\frac{2}{3} \mathbf{a}_{2}+z_{5} \mathbf{a}_{3}
$$

$$
\frac{1}{3} \mathbf{a}_{1}+\frac{2}{3} \mathbf{a}_{2}-z_{5} \mathbf{a}_{3}
$$$$
\frac{2}{3} \mathbf{a}_{1}+\frac{1}{3} \mathbf{a}_{2}-z_{5} \mathbf{a}_{3}
$$$$
\frac{2}{3} \mathbf{a}_{1}+\frac{1}{3} \mathbf{a}_{2}+z_{5} \mathbf{a}_{3}
$$$$
x_{6} \mathbf{a}_{1}+z_{6} \mathbf{a}_{3}
$$$$
x_{6} \mathbf{a}_{2}+z_{6} \mathbf{a}_{3}
$$$$
-x_{6} \mathbf{a}_{1}-x_{6} \mathbf{a}_{2}+z_{6} \mathbf{a}_{3}
$$

$$
x_{6} \mathbf{a}_{1}+-z_{6} \mathbf{a}_{3}
$$

$$
x_{6} \mathbf{a}_{2}-z_{6} \mathbf{a}_{3}
$$$$
-x_{6} \mathbf{a}_{1}-x_{6} \mathbf{a}_{2}-z_{6} \mathbf{a}_{3}
$$

$$
\text { Cartesian Coordinates }
$$

$=$

$=$

$=$

$=$

$$
0 \hat{\mathbf{x}}+0 \hat{\mathbf{y}}+0 \hat{\mathbf{z}}
$$$$
\frac{1}{2} c \hat{\mathbf{z}}
$$$$
\frac{1}{2} x_{3} a \hat{\mathbf{x}}-\frac{\sqrt{3}}{2} x_{3} a \hat{\mathbf{y}}
$$$$
\frac{1}{2} x_{3} a \hat{\mathbf{x}}+\frac{\sqrt{3}}{2} x_{3} a \hat{\mathbf{y}}
$$

$=$

$-x_{3} a \hat{\mathbf{x}}$

$=\quad \frac{1}{2} x_{4} a \hat{\mathbf{x}}-\frac{\sqrt{3}}{2} x_{4} a \hat{\mathbf{y}}+\frac{1}{2} c \hat{\mathbf{z}}$

$=\quad \frac{1}{2} x_{4} a \hat{\mathbf{x}}+\frac{\sqrt{3}}{2} x_{4} a \hat{\mathbf{y}}+\frac{1}{2} c \hat{\mathbf{z}}$

$=\quad-x_{4} a \hat{\mathbf{x}}+\frac{1}{2} c \hat{\mathbf{z}}$

$=\quad \frac{1}{2} a \hat{\mathbf{x}}+\frac{1}{2 \sqrt{3}} a \hat{\mathbf{y}}+z_{5} c \hat{\mathbf{z}}$

$=\quad \frac{1}{2} a \hat{\mathbf{x}}+\frac{1}{2 \sqrt{3}} a \hat{\mathbf{y}}-z_{5} c \hat{\mathbf{z}}$

$=\quad \frac{1}{2} a \hat{\mathbf{x}}-\frac{1}{2 \sqrt{3}} a \hat{\mathbf{y}}-z_{5} c \hat{\mathbf{z}}$

$=\quad \frac{1}{2} a \hat{\mathbf{x}}-\frac{1}{2 \sqrt{3}} a \hat{\mathbf{y}}+z_{5} c \hat{\mathbf{z}}$

$=\quad \frac{1}{2} x_{6} a \hat{\mathbf{x}}-\frac{\sqrt{3}}{2} x_{6} a \hat{\mathbf{y}}+z_{6} c \hat{\mathbf{z}}$

$=\quad \frac{1}{2} x_{6} a \hat{\mathbf{x}}+\frac{\sqrt{3}}{2} x_{6} a \hat{\mathbf{y}}+z_{6} c \hat{\mathbf{z}}$

$=$

$$
-x_{6} a \hat{\mathbf{x}}+z_{6} c \hat{\mathbf{z}}
$$

$=\quad \frac{1}{2} x_{6} a \hat{\mathbf{x}}-\frac{\sqrt{3}}{2} x_{6} a \hat{\mathbf{y}}-z_{6} c \hat{\mathbf{z}}$

$=\quad \frac{1}{2} x_{6} a \hat{\mathbf{x}}+\frac{\sqrt{3}}{2} x_{6} a \hat{\mathbf{y}}-z_{6} c \hat{\mathbf{z}}$

$-x_{6} a \hat{\mathbf{x}}+-z_{6} c \hat{\mathbf{z}}$
Wyckoff Position

(1a)
Atom Type

$\mathrm{Fe}$

Si I

Al I

Al I

Al I

$\mathrm{Mg}$

Mg

Mg

Si II

Si II

Si II

Si II

Al II

Al II

Al II

Al II

Al II

Al II

\section{References:}

- S. Foss, A. Olsen, C. J. Simensen, and J. Taftø, Determination of the crystal structure of the $\pi$-AlFeMgSi phase using symmetry- and site-sensitive electron microscope techniques, Acta Crystallogr. Sect. B Struct. Sci. 59, 36-42 (2003), doi:10.1107/S0108768102022887.

\section{Found in:}

- The Materials Project, $\mathrm{Mg}_{3} \mathrm{Al}_{9} \mathrm{FeSi}_{5}$, doi:10.17188/1286117. ID mp-7062.

- ICSD, Inorganic Crystal Structure Database. ID 96905.

\section{Geometry files:}

- CIF: pp. 939

- POSCAR: pp. 939 


$$
\text { QLi }
$$
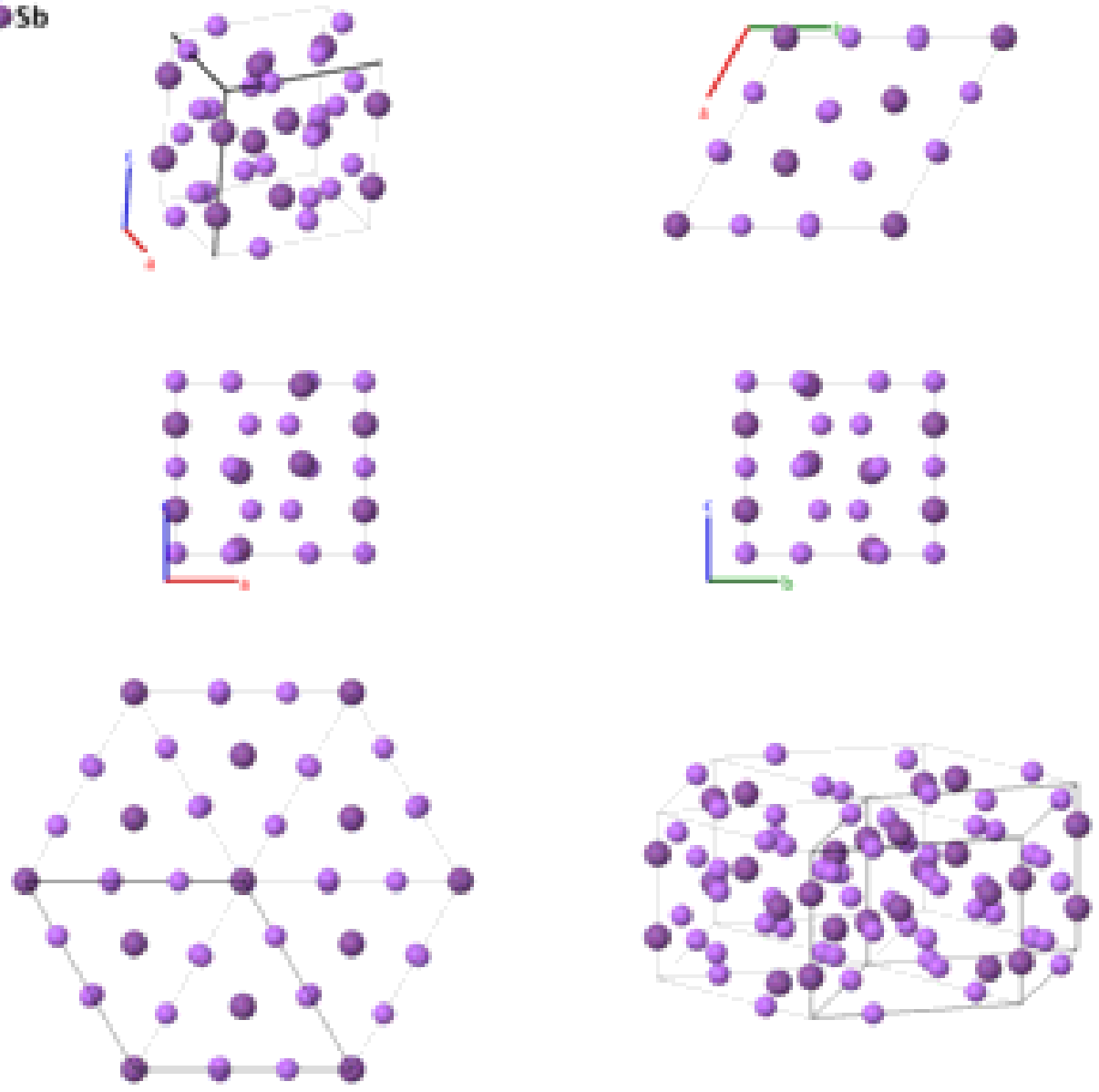

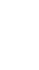

\section{Prototype}

AFLOW prototype label

Strukturbericht designation

Pearson symbol

Space group number

Space group symbol

AFLOW prototype command

: $\quad \mathrm{Li}_{2} \mathrm{Sb}$

: A2B_hP18_190_gh_bf

: None

: $\quad$ hP18

: 190

: $\quad P \overline{6} 2 c$ 
Lattice Coordinates
$\mathbf{B}_{1}=$
$\frac{1}{4} \mathbf{a}_{3}$
$\mathbf{B}_{2}=$
$\frac{3}{4} \mathbf{a}_{3}$
$\mathbf{B}_{3}=$
$\frac{1}{3} \mathbf{a}_{1}+\frac{2}{3} \mathbf{a}_{2}+z_{2} \mathbf{a}_{3}$
$\mathbf{B}_{4}=$
$\mathbf{B}_{5}=$
$\frac{1}{3} \mathbf{a}_{1}+\frac{2}{3} \mathbf{a}_{2}+\left(\frac{1}{2}-z_{2}\right) \mathbf{a}_{3}$
$\mathbf{B}_{6}=$
$\frac{2}{3} \mathbf{a}_{1}+\frac{1}{3} \mathbf{a}_{2}-z_{2} \mathbf{a}_{3}$
$\mathbf{B}_{7}=$
$\frac{2}{3} \mathbf{a}_{1}+\frac{1}{3} \mathbf{a}_{2}+\left(\frac{1}{2}+z_{2}\right) \mathbf{a}_{3}$
$\mathbf{B}_{8}=$
$x_{3} \mathbf{a}_{1}$
$\mathbf{B}_{9}=$
$x_{3} \mathbf{a}_{2}$
$\mathbf{B}_{10}=$
$-x_{3} \mathbf{a}_{1}-x_{3} \mathbf{a}_{2}$
$\mathbf{B}_{11}=$
$x_{3} \mathbf{a}_{1}+\frac{1}{2} \mathbf{a}_{3}$
$\mathbf{B}_{12}=$
$x_{3} \mathbf{a}_{2}+\frac{1}{2} \mathbf{a}_{3}$
$\mathbf{B}_{13}=$
$-x_{3} \mathbf{a}_{1}-x_{3} \mathbf{a}_{2}+\frac{1}{2} \mathbf{a}_{3}$
$x_{4} \mathbf{a}_{1}+y_{4} \mathbf{a}_{2}+\frac{1}{4} \mathbf{a}_{3}$
$\mathbf{B}_{14}=$
$\mathbf{B}_{15}=\left(-x_{4}+y_{4}\right) \mathbf{a}_{1}-x_{4} \mathbf{a}_{2}+\frac{1}{4} \mathbf{a}_{3}$
$\mathbf{B}_{16}=y_{4} \mathbf{a}_{1}+x_{4} \mathbf{a}_{2}+\frac{3}{4} \mathbf{a}_{3}=$
$\mathbf{B}_{17}=\left(x_{4}-y_{4}\right) \mathbf{a}_{1}-y_{4} \mathbf{a}_{2}+\frac{3}{4} \mathbf{a}_{3}=$
$\mathbf{B}_{18}=-x_{4} \mathbf{a}_{1}+\left(-x_{4}+y_{4}\right) \mathbf{a}_{2}+\frac{3}{4} \mathbf{a}_{3}=$

$$
=
$$$$
\frac{1}{4} c \hat{\mathbf{z}}
$$$$
=
$$
$=$
$=$
$=$
$=$
$=$
$=$
$=$
$=$
$=$
$=$
$=$
$=$
$=$
$=\frac{1}{2}\left(x_{4}+y_{4}\right) a \hat{\mathbf{x}}+\frac{\sqrt{3}}{2}\left(x_{4}-y_{4}\right) a \hat{\mathbf{y}}+\frac{3}{4} c \hat{\mathbf{z}}$
$=\left(\frac{1}{2} x_{4}-y_{4}\right) a \hat{\mathbf{x}}-\frac{\sqrt{3}}{2} x_{4} a \hat{\mathbf{y}}+\frac{3}{4} c \hat{\mathbf{z}}$

$$
\left(-x_{4}+\frac{1}{2} y_{4}\right) a \hat{\mathbf{x}}+\frac{\sqrt{3}}{2} y_{4} a \hat{\mathbf{y}}+\frac{3}{4} c \hat{\mathbf{z}}
$$

Cartesian Coordinates
Wyckoff Position

(2b)

(6h)
Atom Type

$\mathrm{Sb} \mathrm{I}$

$\mathrm{Sb} \mathrm{I}$

Sb II

Sb II

Sb II

$\mathrm{Sb}$ II

Li I

Li I

Li I

Li I

Li I

Li I

Li II

Li II

Li II

Li II

Li II

Li II

\section{References:}

- W. Müller, Darstellung und Struktur der Phase Li 2 Sb, Z. Naturforsch. B 32, 357-359 (1977).

\section{Geometry files:}

- CIF: pp. 939

- POSCAR: pp. 940 
$\alpha-\mathrm{Sm}_{3} \mathrm{Ge}_{5}$ (High-temperature) Structure:

A5B3_hP16_190_bdh_g
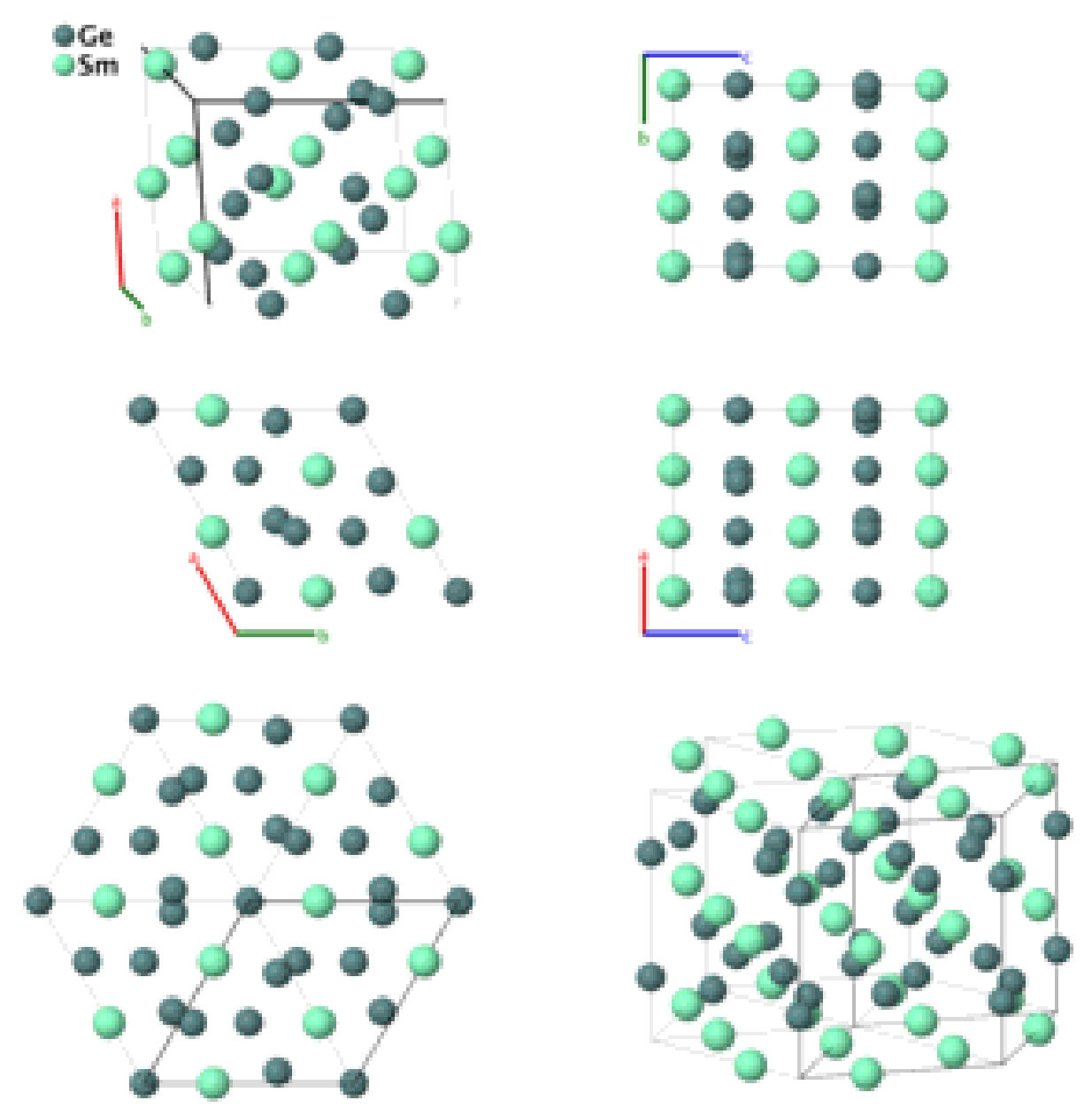

Prototype

AFLOW prototype label

: $\quad \alpha-\mathrm{Sm}_{3} \mathrm{Ge}_{5}$

Strukturbericht designation

Pearson symbol

: A5B3_hP16_190_bdh_g

Space group number

: None

Space group symbol

: $\quad \mathrm{hP} 16$

AFLOW prototype command

: 190

: $\quad P \overline{6} 2 c$

aflow --proto=A5B3_hP16_190_bdh_g

- params $=a, c / a, x_{3}, x_{4}, y_{4}$

Hexagonal primitive vectors:

$$
\begin{array}{ll}
\mathbf{a}_{1}= & \frac{1}{2} a \hat{\mathbf{x}}-\frac{\sqrt{3}}{2} a \hat{\mathbf{y}} \\
\mathbf{a}_{2}= & \frac{1}{2} a \hat{\mathbf{x}}+\frac{\sqrt{3}}{2} a \hat{\mathbf{y}} \\
\mathbf{a}_{3}= & c \hat{\mathbf{z}}
\end{array}
$$

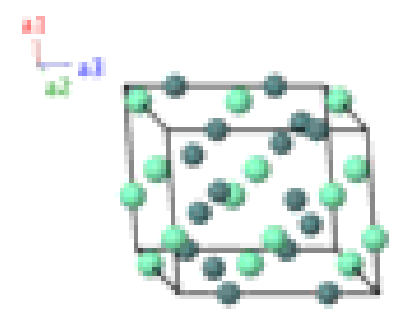

Basis vectors: 


$$
\begin{aligned}
& \mathbf{B}_{1}= \\
& \frac{1}{4} \mathbf{a}_{3} \\
& = \\
& \frac{1}{4} c \hat{\mathbf{z}} \\
& \mathbf{B}_{2}=\quad \frac{3}{4} \mathbf{a}_{3} \\
& = \\
& \frac{3}{4} c \hat{\mathbf{z}} \\
& \mathbf{B}_{11}=x_{4} \mathbf{a}_{1}+y_{4} \mathbf{a}_{2}+\frac{1}{4} \mathbf{a}_{3}= \\
& =\frac{1}{2}\left(x_{4}+y_{4}\right) a \hat{\mathbf{x}}+\frac{\sqrt{3}}{2}\left(-x_{4}+y_{4}\right) a \hat{\mathbf{y}}+\frac{1}{4} c \hat{\mathbf{z}} \\
& \left(-x_{4}+\frac{1}{2} y_{4}\right) a \hat{\mathbf{x}}+\frac{\sqrt{3}}{2} y_{4} a \hat{\mathbf{y}}+\frac{3}{4} c \hat{\mathbf{z}}
\end{aligned}
$$

Ge I

Ge I

Ge II

Ge II

$\mathrm{Sm}$

$\mathrm{Sm}$

$\mathrm{Sm}$

$\mathrm{Sm}$

$\mathrm{Sm}$

$\mathrm{Sm}$

Ge III

Ge III

Ge III

Ge III

Ge III

Ge III

\section{References:}

- P. H. Tobash, D. Lins, S. Bobev, N. Hur, J. D. Thompson, and J. L. Sarrao, Vacancy ordering in $S m G e_{2-x}$ and $G d G e_{2-x}(x$ = 0.33): Structure and properties of two $\mathrm{Sm}_{3} \mathrm{Ge}_{5}$ polymorphs and of $\mathrm{Gd}_{3} \mathrm{Ge}_{5}$, Inorg. Chem. 45, 7286-7294 (2006), doi:10.1021/ic060913f.

\section{Found in:}

- P. Villars and K. Cenzual, Pearson's Crystal Data - Crystal Structure Database for Inorganic Compounds, ASM International (2013).

\section{Geometry files:}

- CIF: pp. 940

- POSCAR: pp. 940 


\section{Troilite (FeS) Structure: AB_hP24_190_i_afh}
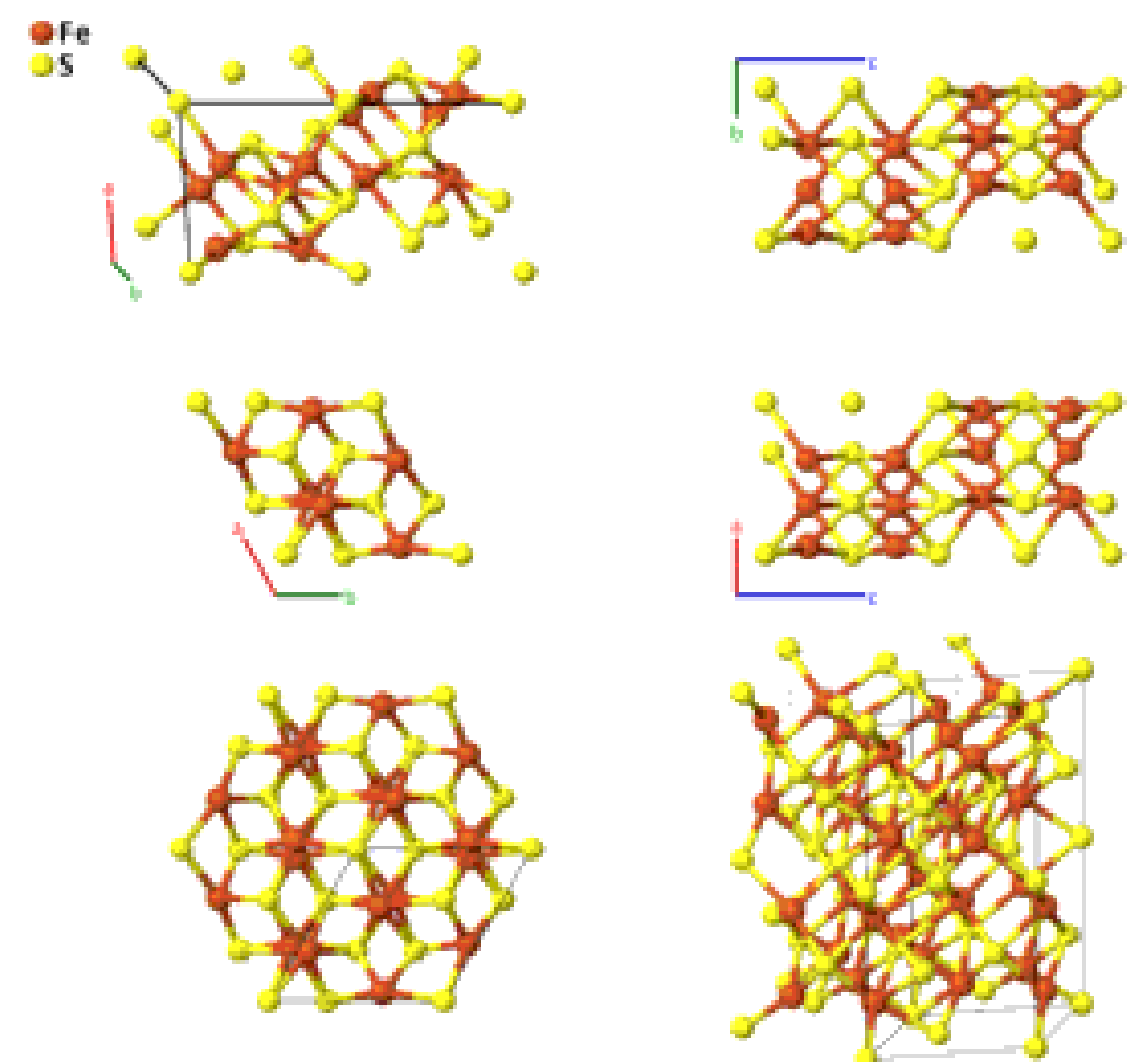

\section{Prototype}

AFLOW prototype label

Strukturbericht designation

Pearson symbol

Space group number

Space group symbol

AFLOW prototype command : aflow--proto=AB_hP24_190_i_afh

- params $=a, c / a, z_{2}, x_{3}, y_{3}, x_{4}, y_{4}, z_{4}$

Hexagonal primitive vectors:

$$
\begin{array}{ll}
\mathbf{a}_{1}= & \frac{1}{2} a \hat{\mathbf{x}}-\frac{\sqrt{3}}{2} a \hat{\mathbf{y}} \\
\mathbf{a}_{2}= & \frac{1}{2} a \hat{\mathbf{x}}+\frac{\sqrt{3}}{2} a \hat{\mathbf{y}} \\
\mathbf{a}_{3}= & c \hat{\mathbf{z}}
\end{array}
$$

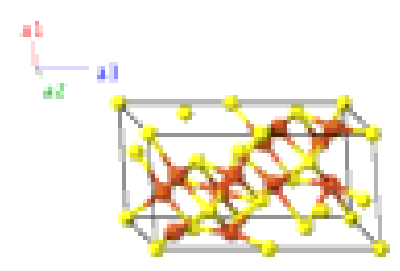

Basis vectors:

Lattice Coordinates

$\begin{array}{lcc}\mathbf{B}_{1}= & 0 \mathbf{a}_{1}+0 \mathbf{a}_{2}+0 \mathbf{a}_{3} \\ \mathbf{B}_{2}= & \frac{1}{2} \mathbf{a}_{3} \\ \mathbf{B}_{3}= & \frac{1}{3} \mathbf{a}_{1}+\frac{2}{3} \mathbf{a}_{2}+z_{2} \mathbf{a}_{3}\end{array}$

Cartesian Coordinates

$=$

$=$

$=$

$$
\begin{gathered}
0 \hat{\mathbf{x}}+0 \hat{\mathbf{y}}+0 \hat{\mathbf{z}} \\
\frac{1}{2} c \hat{\mathbf{z}} \\
\frac{1}{2} a \hat{\mathbf{x}}+\frac{1}{2 \sqrt{3}} a \hat{\mathbf{y}}+z_{2} c \hat{\mathbf{z}}
\end{gathered}
$$

Wyckoff Position

Atom Type

(2a)

(2a)

(4f) 


\begin{tabular}{|c|c|c|c|c|c|}
\hline $\mathbf{B}_{4}$ & $=$ & $\frac{1}{3} \mathbf{a}_{1}+\frac{2}{3} \mathbf{a}_{2}+\left(\frac{1}{2}-z_{2}\right) \mathbf{a}_{3}$ & $=\frac{1}{2} a \hat{\mathbf{x}}+\frac{1}{2 \sqrt{3}} a \hat{\mathbf{y}}+\left(\frac{1}{2}-z_{2}\right) c \hat{\mathbf{z}}$ & $(4 f)$ & S II \\
\hline $\mathbf{B}_{5}$ & $=$ & $\frac{2}{3} \mathbf{a}_{1}+\frac{1}{3} \mathbf{a}_{2}-z_{2} \mathbf{a}_{3}$ & $=\quad \frac{1}{2} a \hat{\mathbf{x}}-\frac{1}{2 \sqrt{3}} a \hat{\mathbf{y}}-z_{2} c \hat{\mathbf{z}}$ & $(4 f)$ & S II \\
\hline $\mathbf{B}_{6}$ & $=$ & $\frac{2}{3} \mathbf{a}_{1}+\frac{1}{3} \mathbf{a}_{2}+\left(\frac{1}{2}+z_{2}\right) \mathbf{a}_{3}$ & $\frac{1}{2} a \hat{\mathbf{x}}-\frac{1}{2 \sqrt{3}} a \hat{\mathbf{y}}+\left(\frac{1}{2}+z_{2}\right) c \hat{\mathbf{z}}$ & $(4 f)$ & S II \\
\hline $\mathbf{B}_{7}$ & $=$ & $x_{3} \mathbf{a}_{1}+y_{3} \mathbf{a}_{2}+\frac{1}{4} \mathbf{a}_{3}$ & $\begin{array}{c}=\quad \begin{array}{c}\frac{1}{2}\left(x_{3}+y_{3}\right) a \hat{\mathbf{x}}+ \\
\frac{\sqrt{3}}{2}\left(-x_{3}+y_{3}\right) a \hat{\mathbf{y}}+\frac{1}{4} c \hat{\mathbf{z}}\end{array}\end{array}$ & $(6 h)$ & S III \\
\hline $\mathbf{B}_{8}$ & $=$ & $-y_{3} \mathbf{a}_{1}+\left(x_{3}-y_{3}\right) \mathbf{a}_{2}+\frac{1}{4} \mathbf{a}_{3}$ & $=\left(\frac{1}{2} x_{3}-y_{3}\right) a \hat{\mathbf{x}}+\frac{\sqrt{3}}{2} x_{3} a \hat{\mathbf{y}}+\frac{1}{4} c \hat{\mathbf{z}}$ & $(6 h)$ & S III \\
\hline $\mathbf{B}_{9}$ & $=$ & $\left(-x_{3}+y_{3}\right) \mathbf{a}_{1}-x_{3} \mathbf{a}_{2}+\frac{1}{4} \mathbf{a}_{3}$ & $=\left(-x_{3}+\frac{1}{2} y_{3}\right) a \hat{\mathbf{x}}-\frac{\sqrt{3}}{2} y_{3} a \hat{\mathbf{y}}+\frac{1}{4} c \hat{\mathbf{z}}$ & $(6 h)$ & S III \\
\hline $\mathbf{B}_{10}$ & $=$ & $y_{3} \mathbf{a}_{1}+x_{3} \mathbf{a}_{2}+\frac{3}{4} \mathbf{a}_{3}$ & $=\frac{1}{2}\left(x_{3}+y_{3}\right) a \hat{\mathbf{x}}+\frac{\sqrt{3}}{2}\left(x_{3}-y_{3}\right) a \hat{\mathbf{y}}+$ & $(6 h)$ & S III \\
\hline $\mathbf{B}_{11}$ & $=$ & $\left(x_{3}-y_{3}\right) \mathbf{a}_{1}-y_{3} \mathbf{a}_{2}+\frac{3}{4} \mathbf{a}_{3}$ & $=\left(\frac{1}{2} x_{3}-y_{3}\right) a \hat{\mathbf{x}}-\frac{\sqrt{3}}{2} x_{3} a \hat{\mathbf{y}}+\frac{3}{4} c \hat{\mathbf{z}}$ & $(6 h)$ & S III \\
\hline $\mathbf{B}_{12}$ & $=$ & $-x_{3} \mathbf{a}_{1}+\left(-x_{3}+y_{3}\right) \mathbf{a}_{2}+\frac{3}{4} \mathbf{a}_{3}$ & $=\left(-x_{3}+\frac{1}{2} y_{3}\right) a \hat{\mathbf{x}}+\frac{\sqrt{3}}{2} y_{3} a \hat{\mathbf{y}}+\frac{3}{4} c \hat{\mathbf{z}}$ & $(6 h)$ & S III \\
\hline $\mathbf{B}_{13}$ & $=$ & $x_{4} \mathbf{a}_{1}+y_{4} \mathbf{a}_{2}+z_{4} \mathbf{a}_{3}$ & 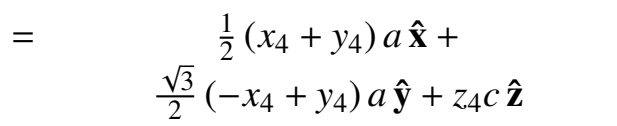 & $(12 i)$ & $\mathrm{Fe}$ \\
\hline $\mathbf{B}_{14}$ & $=$ & $-y_{4} \mathbf{a}_{1}+\left(x_{4}-y_{4}\right) \mathbf{a}_{2}+z_{4} \mathbf{a}_{3}$ & $=\left(\frac{1}{2} x_{4}-y_{4}\right) a \hat{\mathbf{x}}+\frac{\sqrt{3}}{2} x_{4} a \hat{\mathbf{y}}+z_{4} c \hat{\mathbf{z}}$ & $(12 i)$ & $\mathrm{Fe}$ \\
\hline $\mathbf{B}_{15}$ & $=$ & $\left(-x_{4}+y_{4}\right) \mathbf{a}_{1}-x_{4} \mathbf{a}_{2}+z_{4} \mathbf{a}_{3}$ & $=\left(-x_{4}+\frac{1}{2} y_{4}\right) a \hat{\mathbf{x}}-\frac{\sqrt{3}}{2} y_{4} a \hat{\mathbf{y}}+z_{4} c \hat{\mathbf{z}}$ & $(12 i)$ & $\mathrm{Fe}$ \\
\hline $\mathbf{B}_{16}$ & $=$ & $x_{4} \mathbf{a}_{1}+y_{4} \mathbf{a}_{2}+\left(\frac{1}{2}-z_{4}\right) \mathbf{a}_{3}$ & $\begin{array}{c}=\quad \frac{1}{2}\left(x_{4}+y_{4}\right) a \hat{\mathbf{x}}+ \\
\frac{\sqrt{3}}{2}\left(-x_{4}+y_{4}\right) a \hat{\mathbf{y}}+\left(\frac{1}{2}-z_{4}\right) c \hat{\mathbf{z}}\end{array}$ & $(12 i)$ & $\mathrm{Fe}$ \\
\hline $\mathbf{B}_{17}$ & $=$ & $-y_{4} \mathbf{a}_{1}+\left(x_{4}-y_{4}\right) \mathbf{a}_{2}+\left(\frac{1}{2}-z_{4}\right) \mathbf{a}_{3}$ & $\begin{array}{c}=\left(\frac{1}{2} x_{4}-y_{4}\right) a \hat{\mathbf{x}}+\frac{\sqrt{3}}{2} x_{4} a \hat{\mathbf{y}}+ \\
\left(\frac{1}{2}-z_{4}\right) c \hat{\mathbf{z}}\end{array}$ & $(12 i)$ & $\mathrm{Fe}$ \\
\hline $\mathbf{B}_{18}$ & $=$ & $\left(-x_{4}+y_{4}\right) \mathbf{a}_{1}-x_{4} \mathbf{a}_{2}+\left(\frac{1}{2}-z_{4}\right) \mathbf{a}_{3}$ & $\begin{array}{c}=\left(-x_{4}+\frac{1}{2} y_{4}\right) a \hat{\mathbf{x}}-\frac{\sqrt{3}}{2} y_{4} a \hat{\mathbf{y}}+ \\
\left(\frac{1}{2}-z_{4}\right) c \hat{\mathbf{z}}\end{array}$ & $(12 i)$ & $\mathrm{Fe}$ \\
\hline $\mathbf{B}_{19}$ & $=$ & $y_{4} \mathbf{a}_{1}+x_{4} \mathbf{a}_{2}-z_{4} \mathbf{a}_{3}$ & $=\frac{1}{2}\left(x_{4}+y_{4}\right) a \hat{\mathbf{x}}+\frac{\sqrt{3}}{2}\left(x_{4}-y_{4}\right) a \hat{\mathbf{y}}-$ & $(12 i)$ & $\mathrm{Fe}$ \\
\hline $\mathbf{B}_{20}$ & $=$ & $\left(x_{4}-y_{4}\right) \mathbf{a}_{1}-y_{4} \mathbf{a}_{2}-z_{4} \mathbf{a}_{3}$ & $=\left(\frac{1}{2} x_{4}-y_{4}\right) a \hat{\mathbf{x}}-\frac{\sqrt{3}}{2} x_{4} a \hat{\mathbf{y}}-z_{4} c \hat{\mathbf{z}}$ & $(12 i)$ & $\mathrm{Fe}$ \\
\hline $\mathbf{B}_{21}$ & $=$ & $-x_{4} \mathbf{a}_{1}+\left(-x_{4}+y_{4}\right) \mathbf{a}_{2}-z_{4} \mathbf{a}_{3}$ & $=\left(-x_{4}+\frac{1}{2} y_{4}\right) a \hat{\mathbf{x}}+\frac{\sqrt{3}}{2} y_{4} a \hat{\mathbf{y}}-z_{4} c \hat{\mathbf{z}}$ & $(12 i)$ & $\mathrm{Fe}$ \\
\hline $\mathbf{B}_{22}$ & $=$ & $y_{4} \mathbf{a}_{1}+x_{4} \mathbf{a}_{2}+\left(\frac{1}{2}+z_{4}\right) \mathbf{a}_{3}$ & $\begin{array}{c}=\frac{1}{2}\left(x_{4}+y_{4}\right) a \hat{\mathbf{x}}+\frac{\sqrt{3}}{2}\left(x_{4}-y_{4}\right) a \hat{\mathbf{y}}+ \\
\left(\frac{1}{2}+z_{4}\right) c \hat{\mathbf{z}}\end{array}$ & $(12 i)$ & $\mathrm{Fe}$ \\
\hline $\mathbf{B}_{23}$ & $=$ & $\left(x_{4}-y_{4}\right) \mathbf{a}_{1}-y_{4} \mathbf{a}_{2}+\left(\frac{1}{2}+z_{4}\right) \mathbf{a}_{3}$ & $\begin{array}{c}\left(\frac{1}{2} x_{4}-y_{4}\right) a \hat{\mathbf{x}}-\frac{\sqrt{3}}{2} x_{4} a \hat{\mathbf{y}}+ \\
\left(\frac{1}{2}+z_{4}\right) c \hat{\mathbf{z}}\end{array}$ & $(12 i)$ & $\mathrm{Fe}$ \\
\hline $\mathbf{B}_{24}$ & $=$ & $-x_{4} \mathbf{a}_{1}+\left(-x_{4}+y_{4}\right) \mathbf{a}_{2}+\left(\frac{1}{2}+z_{4}\right) \mathbf{a}_{3}$ & $\begin{array}{c}\left(-x_{4}+\frac{1}{2} y_{4}\right) a \hat{\mathbf{x}}+\frac{\sqrt{3}}{2} y_{4} a \hat{\mathbf{y}}+ \\
\left(\frac{1}{2}+z_{4}\right) c \hat{\mathbf{z}}\end{array}$ & $(12 i)$ & $\mathrm{Fe}$ \\
\hline
\end{tabular}

\section{References:}

- N. Morimoto, H. Nakazawa, K. Nishigucmi, and M. Tokonami, Pyrrhotites: Stoichiometric Compounds with Composition $\mathrm{Fe}_{n-1} S_{n}(n \geq 8)$, Science 168, 964-966 (1970), doi:10.1126/science.168.3934.964.

\section{Found in:}

- P. Villars and K. Cenzual, Pearson's Crystal Data - Crystal Structure Database for Inorganic Compounds, ASM International (2013).

\section{Geometry files:}

- CIF: pp. 940 
- POSCAR: pp. 941 


\section{Beryl $\left(\mathrm{Be}_{3} \mathrm{Al}_{2} \mathrm{Si}_{6} \mathrm{O}_{18}, G 3_{1}\right)$ Structure: A2B3C18D6_hP58_192_c_f_lm_1}
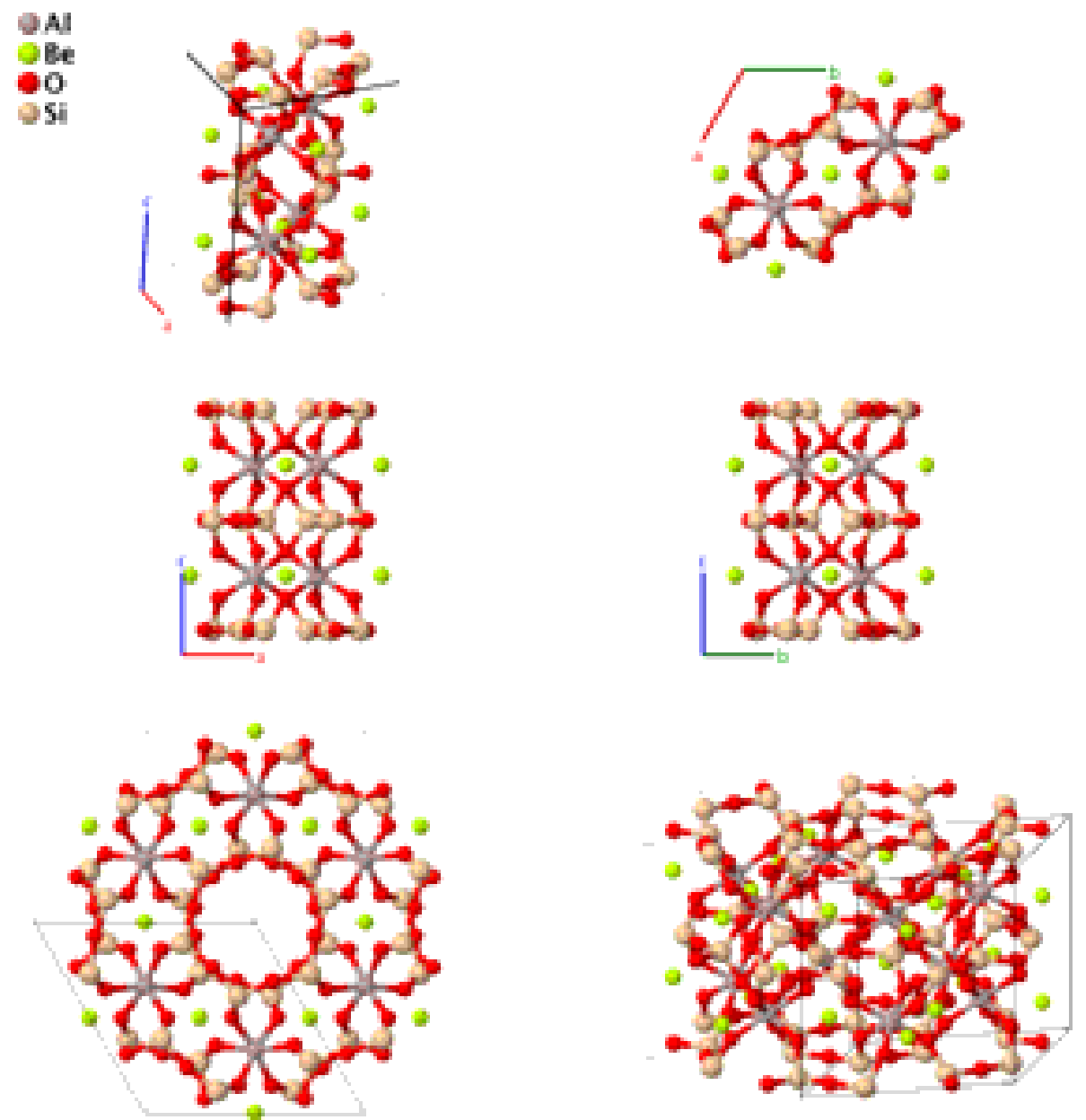

\section{Prototype}

AFLOW prototype label

Strukturbericht designation

Pearson symbol

Space group number

Space group symbol

AFLOW prototype command
$: \quad \mathrm{Be}_{3} \mathrm{Al}_{2} \mathrm{Si}_{6} \mathrm{O}_{18}$

: A2B3C18D6_hP58_192_c_f_lm_1

: $\quad G 3_{1}$

$: \quad \mathrm{hP58}$

: $\quad 192$

: $\quad P 6 / m c c$

aflow - -proto $=$ A2B3C18D6_hP58_192_C_f_lm_l

- params $=a, c / a, x_{3}, y_{3}, x_{4}, y_{4}, x_{5}, y_{5}, z_{5}$

- (Morosin, 1972) places oxygen atoms on the (2a) Wyckoff site (lattice coordinates $(0,0, \pm 1 / 4)$, with an occupation of (0.0991). We follow (Hazen, 1986) and ignore this small contribution to the structure. 


\section{Hexagonal primitive vectors:}

$$
\begin{array}{ll}
\mathbf{a}_{1}= & \frac{1}{2} a \hat{\mathbf{x}}-\frac{\sqrt{3}}{2} a \hat{\mathbf{y}} \\
\mathbf{a}_{2}= & \frac{1}{2} a \hat{\mathbf{x}}+\frac{\sqrt{3}}{2} a \hat{\mathbf{y}} \\
\mathbf{a}_{3}= & c \hat{\mathbf{z}}
\end{array}
$$

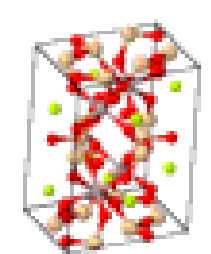

\begin{tabular}{|c|c|c|}
\hline $\mathbf{B}_{1}$ & $=$ & $\frac{1}{3} \mathbf{a}_{1}+\frac{2}{3} \mathbf{a}_{2}+\frac{1}{4} \mathbf{a}_{3}$ \\
\hline $\mathbf{B}_{2}$ & $=$ & $\frac{2}{3} \mathbf{a}_{1}+\frac{1}{3} \mathbf{a}_{2}+\frac{1}{4} \mathbf{a}_{3}$ \\
\hline $\mathbf{B}_{3}$ & $=$ & $\frac{2}{3} \mathbf{a}_{1}+\frac{1}{3} \mathbf{a}_{2}+\frac{3}{4} \mathbf{a}_{3}$ \\
\hline $\mathbf{B}_{4}$ & $=$ & $\frac{1}{3} \mathbf{a}_{1}+\frac{2}{3} \mathbf{a}_{2}+\frac{3}{4} \mathbf{a}_{3}$ \\
\hline $\mathbf{B}_{5}$ & $=$ & $\frac{1}{2} \mathbf{a}_{1}+\frac{1}{4} \mathbf{a}_{3}$ \\
\hline $\mathbf{B}_{6}$ & $=$ & $\frac{1}{2} \mathbf{a}_{2}+\frac{1}{4} \mathbf{a}_{3}$ \\
\hline $\mathbf{B}_{7}$ & $=$ & $\frac{1}{2} \mathbf{a}_{1}+\frac{1}{2} \mathbf{a}_{2}+\frac{1}{4} \mathbf{a}_{3}$ \\
\hline $\mathbf{B}_{8}$ & $=$ & $\frac{1}{2} \mathbf{a}_{1}+\frac{3}{4} \mathbf{a}_{3}$ \\
\hline $\mathbf{B}_{9}$ & $=$ & $\frac{1}{2} \mathbf{a}_{2}+\frac{3}{4} \mathbf{a}_{3}$ \\
\hline $\mathbf{B}_{10}$ & $=$ & $\frac{1}{2} \mathbf{a}_{1}+\frac{1}{2} \mathbf{a}_{2}+\frac{3}{4} \mathbf{a}_{3}$ \\
\hline $\mathbf{B}_{11}$ & $=$ & $x_{3} \mathbf{a}_{1}+y_{3} \mathbf{a}_{2}$ \\
\hline $\mathbf{B}_{12}$ & $=$ & $-y_{3} \mathbf{a}_{1}+\left(x_{3}-y_{3}\right) \mathbf{a}_{2}$ \\
\hline $\mathbf{B}_{13}$ & $=$ & $\left(-x_{3}+y_{3}\right) \mathbf{a}_{1}-x_{3} \mathbf{a}_{2}$ \\
\hline $\mathbf{B}_{14}$ & $=$ & $-x_{3} \mathbf{a}_{1}-y_{3} \mathbf{a}_{2}$ \\
\hline $\mathbf{B}_{15}$ & $=$ & $y_{3} \mathbf{a}_{1}+\left(-x_{3}+y_{3}\right) \mathbf{a}_{2}$ \\
\hline $\mathbf{B}_{16}$ & $=$ & $\left(x_{3}-y_{3}\right) \mathbf{a}_{1}+x_{3} \mathbf{a}_{2}$ \\
\hline $\mathbf{B}_{17}$ & $=$ & $y_{3} \mathbf{a}_{1}+x_{3} \mathbf{a}_{2}+\frac{1}{2} \mathbf{a}_{3}$ \\
\hline
\end{tabular}

\section{Basis vectors:}

Lattice Coordinates

$\mathbf{B}_{18}=\left(x_{3}-y_{3}\right) \mathbf{a}_{1}-y_{3} \mathbf{a}_{2}+\frac{1}{2} \mathbf{a}_{3}$

$\mathbf{B}_{19}=-x_{3} \mathbf{a}_{1}+\left(-x_{3}+y_{3}\right) \mathbf{a}_{2}+\frac{1}{2} \mathbf{a}_{3}$

$\mathbf{B}_{20}=$

$\mathbf{B}_{21}=\left(-x_{3}+y_{3}\right) \mathbf{a}_{1}+y_{3} \mathbf{a}_{2}+\frac{1}{2} \mathbf{a}_{3}$

$\mathbf{B}_{22}=$

$\mathbf{B}_{23}=$

$\mathbf{B}_{24}=$

$\mathbf{B}_{25}=$

$\mathbf{B}_{26}=$

$\mathbf{B}_{27}=$
$=$

$=$

$$
=
$$

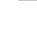$$
=
$$

$$
=
$$$$
=
$$$$
=
$$$$
=
$$$$
=\quad \frac{1}{4} a \hat{\mathbf{x}}-\frac{\sqrt{3}}{4} a \hat{\mathbf{y}}+\frac{3}{4} c \hat{\mathbf{z}}
$$$$
=\quad \frac{1}{4} a \hat{\mathbf{x}}+\frac{\sqrt{3}}{4} a \hat{\mathbf{y}}+\frac{3}{4} c \hat{\mathbf{z}}
$$$$
=\quad \frac{1}{2} a \hat{\mathbf{x}}+\frac{3}{4} c \hat{\mathbf{z}}
$$$$
=\frac{1}{2}\left(x_{3}+y_{3}\right) a \hat{\mathbf{x}}+\frac{\sqrt{3}}{2}\left(-x_{3}+y_{3}\right) a \hat{\mathbf{y}}
$$$$
=\quad\left(\frac{1}{2} x_{3}-y_{3}\right) a \hat{\mathbf{x}}+\frac{\sqrt{3}}{2} x_{3} a \hat{\mathbf{y}}
$$$$
=\quad\left(-x_{3}+\frac{1}{2} y_{3}\right) a \hat{\mathbf{x}}-\frac{\sqrt{3}}{2} y_{3} a \hat{\mathbf{y}}
$$$$
=-\frac{1}{2}\left(x_{3}+y_{3}\right) a \hat{\mathbf{x}}+\frac{\sqrt{3}}{2}\left(x_{3}-y_{3}\right) a \hat{\mathbf{y}}
$$$$
=\quad\left(-\frac{1}{2} x_{3}+y_{3}\right) a \hat{\mathbf{x}}-\frac{\sqrt{3}}{2} x_{3} a \hat{\mathbf{y}}
$$$$
=\quad\left(x_{3}-\frac{1}{2} y_{3}\right) a \hat{\mathbf{x}}+\frac{\sqrt{3}}{2} y_{3} a \hat{\mathbf{y}}
$$$$
=\frac{1}{2}\left(x_{3}+y_{3}\right) a \hat{\mathbf{x}}+\frac{\sqrt{3}}{2}\left(x_{3}-y_{3}\right) a \hat{\mathbf{y}}+
$$$$
=\left(\frac{1}{2} x_{3}-y_{3}\right) a \hat{\mathbf{x}}-\frac{\sqrt{3}}{2} x_{3} a \hat{\mathbf{y}}+\frac{1}{2} c \hat{\mathbf{z}}
$$$$
=\left(-x_{3}+\frac{1}{2} y_{3}\right) a \hat{\mathbf{x}}+\frac{\sqrt{3}}{2} y_{3} a \hat{\mathbf{y}}+\frac{1}{2} c \hat{\mathbf{z}}
$$$$
=
$$$$
-\frac{1}{2}\left(x_{3}+y_{3}\right) a \hat{\mathbf{x}}+
$$$$
\frac{\sqrt{3}}{2}\left(-x_{3}+y_{3}\right) a \hat{\mathbf{y}}+\frac{1}{2} c \hat{\mathbf{z}}
$$$$
=\left(-\frac{1}{2} x_{3}+y_{3}\right) a \hat{\mathbf{x}}+\frac{\sqrt{3}}{2} x_{3} a \hat{\mathbf{y}}+\frac{1}{2} c \hat{\mathbf{z}}
$$$$
=\left(x_{3}-\frac{1}{2} y_{3}\right) a \hat{\mathbf{x}}-\frac{\sqrt{3}}{2} y_{3} a \hat{\mathbf{y}}+\frac{1}{2} c \hat{\mathbf{z}}
$$$$
=\frac{1}{2}\left(x_{4}+y_{4}\right) a \hat{\mathbf{x}}+\frac{\sqrt{3}}{2}\left(-x_{4}+y_{4}\right) a \hat{\mathbf{y}}
$$$$
=\quad\left(\frac{1}{2} x_{4}-y_{4}\right) a \hat{\mathbf{x}}+\frac{\sqrt{3}}{2} x_{4} a \hat{\mathbf{y}}
$$$$
=\quad\left(-x_{4}+\frac{1}{2} y_{4}\right) a \hat{\mathbf{x}}-\frac{\sqrt{3}}{2} y_{4} a \hat{\mathbf{y}}
$$$$
=-\frac{1}{2}\left(x_{4}+y_{4}\right) a \hat{\mathbf{x}}+\frac{\sqrt{3}}{2}\left(x_{4}-y_{4}\right) a \hat{\mathbf{y}}
$$$$
=\quad\left(-\frac{1}{2} x_{4}+y_{4}\right) a \hat{\mathbf{x}}-\frac{\sqrt{3}}{2} x_{4} a \hat{\mathbf{y}}
$$

Wyckoff Position Atom Type
Al

Al

Al

Al

$\mathrm{Be}$

Be

Be

Be

Be

$\mathrm{Be}$

O I

O I

O I

O I

O I

O I

O I

O I

O I

O I

O I

O I

$\mathrm{Si}$

$\mathrm{Si}$

$\mathrm{Si}$

$\mathrm{Si}$

$\mathrm{Si}$ 


\begin{tabular}{|c|c|c|c|c|c|}
\hline $\mathbf{B}_{28}$ & $=$ & $\left(x_{4}-y_{4}\right) \mathbf{a}_{1}+x_{4} \mathbf{a}_{2}$ & $=\quad\left(x_{4}-\frac{1}{2} y_{4}\right) a \hat{\mathbf{x}}+\frac{\sqrt{3}}{2} y_{4} a \hat{\mathbf{y}}$ & $(12 l)$ & $\mathrm{Si}$ \\
\hline $\mathbf{B}_{29}$ & $=$ & $y_{4} \mathbf{a}_{1}+x_{4} \mathbf{a}_{2}+\frac{1}{2} \mathbf{a}_{3}$ & $=\frac{1}{2}\left(x_{4}+y_{4}\right) a \hat{\mathbf{x}}+\frac{\sqrt{3}}{2}\left(x_{4}-y_{4}\right) a \hat{\mathbf{y}}+$ & $(12 l)$ & $\mathrm{Si}$ \\
\hline $\mathbf{B}_{30}$ & $=$ & $\left(x_{4}-y_{4}\right) \mathbf{a}_{1}-y_{4} \mathbf{a}_{2}+\frac{1}{2} \mathbf{a}_{3}$ & $=\left(\frac{1}{2} x_{4}-y_{4}\right) a \hat{\mathbf{x}}-\frac{\sqrt{3}}{2} x_{4} a \hat{\mathbf{y}}+\frac{1}{2} c \hat{\mathbf{z}}$ & $(12 l)$ & $\mathrm{Si}$ \\
\hline $\mathbf{B}_{31}$ & $=$ & $-x_{4} \mathbf{a}_{1}+\left(-x_{4}+y_{4}\right) \mathbf{a}_{2}+\frac{1}{2} \mathbf{a}_{3}$ & $=\left(-x_{4}+\frac{1}{2} y_{4}\right) a \hat{\mathbf{x}}+\frac{\sqrt{3}}{2} y_{4} a \hat{\mathbf{y}}+\frac{1}{2} c \hat{\mathbf{z}}$ & $(12 l)$ & $\mathrm{Si}$ \\
\hline $\mathbf{B}_{32}$ & $=$ & $-y_{4} \mathbf{a}_{1}-x_{4} \mathbf{a}_{2}+\frac{1}{2} \mathbf{a}_{3}$ & $\begin{array}{c}-\frac{1}{2}\left(x_{4}+y_{4}\right) a \hat{\mathbf{x}}+ \\
\frac{\sqrt{3}}{2}\left(-x_{4}+y_{4}\right) a \hat{\mathbf{y}}+\frac{1}{2} c \hat{\mathbf{z}}\end{array}$ & $(12 l)$ & $\mathrm{Si}$ \\
\hline $\mathbf{B}_{33}$ & $=$ & $\left(-x_{4}+y_{4}\right) \mathbf{a}_{1}+y_{4} \mathbf{a}_{2}+\frac{1}{2} \mathbf{a}_{3}$ & $=\left(-\frac{1}{2} x_{4}+y_{4}\right) a \hat{\mathbf{x}}+\frac{\sqrt{3}}{2} x_{4} a \hat{\mathbf{y}}+\frac{1}{2} c \hat{\mathbf{z}}$ & $(12 l)$ & $\mathrm{Si}$ \\
\hline $\mathbf{B}_{34}$ & $=$ & $x_{4} \mathbf{a}_{1}+\left(x_{4}-y_{4}\right) \mathbf{a}_{2}+\frac{1}{2} \mathbf{a}_{3}$ & $=\left(x_{4}-\frac{1}{2} y_{4}\right) a \hat{\mathbf{x}}-\frac{\sqrt{3}}{2} y_{4} a \hat{\mathbf{y}}+\frac{1}{2} c \hat{\mathbf{z}}$ & $(12 l)$ & $\mathrm{Si}$ \\
\hline $\mathbf{B}_{35}$ & $=$ & $x_{5} \mathbf{a}_{1}+y_{5} \mathbf{a}_{2}+z_{5} \mathbf{a}_{3}$ & $\begin{array}{c}\frac{1}{2}\left(x_{5}+y_{5}\right) a \hat{\mathbf{x}}+ \\
\frac{\sqrt{3}}{2}\left(-x_{5}+y_{5}\right) a \hat{\mathbf{y}}+z_{5} c \hat{\mathbf{z}}\end{array}$ & $(24 m)$ & O II \\
\hline $\mathbf{B}_{36}$ & $=$ & $-y_{5} \mathbf{a}_{1}+\left(x_{5}-y_{5}\right) \mathbf{a}_{2}+z_{5} \mathbf{a}_{3}$ & $=\left(\frac{1}{2} x_{5}-y_{5}\right) a \hat{\mathbf{x}}+\frac{\sqrt{3}}{2} x_{5} a \hat{\mathbf{y}}+z_{5} c \hat{\mathbf{z}}$ & $(24 m)$ & O II \\
\hline $\mathbf{B}_{37}$ & $=$ & $\left(-x_{5}+y_{5}\right) \mathbf{a}_{1}-x_{5} \mathbf{a}_{2}+z_{5} \mathbf{a}_{3}$ & $=\left(-x_{5}+\frac{1}{2} y_{5}\right) a \hat{\mathbf{x}}-\frac{\sqrt{3}}{2} y_{5} a \hat{\mathbf{y}}+z_{5} c \hat{\mathbf{z}}$ & $(24 m)$ & O II \\
\hline $\mathbf{B}_{38}$ & $=$ & $-x_{5} \mathbf{a}_{1}-y_{5} \mathbf{a}_{2}+z_{5} \mathbf{a}_{3}$ & $\begin{array}{c}=-\frac{1}{2}\left(x_{5}+y_{5}\right) a \hat{\mathbf{x}}+ \\
\frac{\sqrt{3}}{2}\left(x_{5}-y_{5}\right) a \hat{\mathbf{y}}+z_{5} c \hat{\mathbf{z}}\end{array}$ & $(24 m)$ & O II \\
\hline $\mathbf{B}_{39}$ & $=$ & $y_{5} \mathbf{a}_{1}+\left(-x_{5}+y_{5}\right) \mathbf{a}_{2}+z_{5} \mathbf{a}_{3}$ & $=\left(-\frac{1}{2} x_{5}+y_{5}\right) a \hat{\mathbf{x}}-\frac{\sqrt{3}}{2} x_{5} a \hat{\mathbf{y}}+z_{5} c \hat{\mathbf{z}}$ & $(24 m)$ & O II \\
\hline $\mathbf{B}_{40}$ & $=$ & $\left(x_{5}-y_{5}\right) \mathbf{a}_{1}+x_{5} \mathbf{a}_{2}+z_{5} \mathbf{a}_{3}$ & $=\left(x_{5}-\frac{1}{2} y_{5}\right) a \hat{\mathbf{x}}+\frac{\sqrt{3}}{2} y_{5} a \hat{\mathbf{y}}+z_{5} c \hat{\mathbf{z}}$ & $(24 m)$ & O II \\
\hline $\mathbf{B}_{41}$ & $=$ & $y_{5} \mathbf{a}_{1}+x_{5} \mathbf{a}_{2}+\left(\frac{1}{2}-z_{5}\right) \mathbf{a}_{3}$ & $\begin{array}{c}=\frac{1}{2}\left(x_{5}+y_{5}\right) a \hat{\mathbf{x}}+\frac{\sqrt{3}}{2}\left(x_{5}-y_{5}\right) a \hat{\mathbf{y}}+ \\
\left(\frac{1}{2}-z_{5}\right) c \hat{\mathbf{z}}\end{array}$ & $(24 m)$ & O II \\
\hline $\mathbf{B}_{42}$ & $=$ & $\left(x_{5}-y_{5}\right) \mathbf{a}_{1}-y_{5} \mathbf{a}_{2}+\left(\frac{1}{2}-z_{5}\right) \mathbf{a}_{3}$ & $\begin{array}{c}\left(\frac{1}{2} x_{5}-y_{5}\right) a \hat{\mathbf{x}}-\frac{\sqrt{3}}{2} x_{5} a \hat{\mathbf{y}}+ \\
\left(\frac{1}{2}-z_{5}\right) c \hat{\mathbf{z}}\end{array}$ & $(24 m)$ & O II \\
\hline $\mathbf{B}_{43}$ & $=$ & $-x_{5} \mathbf{a}_{1}+\left(-x_{5}+y_{5}\right) \mathbf{a}_{2}+\left(\frac{1}{2}-z_{5}\right) \mathbf{a}_{3}$ & $\begin{array}{c}\left(-x_{5}+\frac{1}{2} y_{5}\right) a \hat{\mathbf{x}}+\frac{\sqrt{3}}{2} y_{5} a \hat{\mathbf{y}}+ \\
\left(\frac{1}{2}-z_{5}\right) c \hat{\mathbf{z}}\end{array}$ & $(24 m)$ & O II \\
\hline $\mathbf{B}_{44}$ & $=$ & $-y_{5} \mathbf{a}_{1}-x_{5} \mathbf{a}_{2}+\left(\frac{1}{2}-z_{5}\right) \mathbf{a}_{3}$ & $\begin{array}{c}-\frac{1}{2}\left(x_{5}+y_{5}\right) a \hat{\mathbf{x}}+ \\
\frac{\sqrt{3}}{2}\left(-x_{5}+y_{5}\right) a \hat{\mathbf{y}}+\left(\frac{1}{2}-z_{5}\right) c \hat{\mathbf{z}}\end{array}$ & $(24 m)$ & O II \\
\hline $\mathbf{B}_{45}$ & $=$ & $\left(-x_{5}+y_{5}\right) \mathbf{a}_{1}+y_{5} \mathbf{a}_{2}+\left(\frac{1}{2}-z_{5}\right) \mathbf{a}_{3}$ & $\begin{array}{c}\left(-\frac{1}{2} x_{5}+y_{5}\right) a \hat{\mathbf{x}}+\frac{\sqrt{3}}{2} x_{5} a \hat{\mathbf{y}}+ \\
\left(\frac{1}{2}-z_{5}\right) c \hat{\mathbf{z}}\end{array}$ & $(24 m)$ & O II \\
\hline $\mathbf{B}_{46}$ & $=$ & $x_{5} \mathbf{a}_{1}+\left(x_{5}-y_{5}\right) \mathbf{a}_{2}+\left(\frac{1}{2}-z_{5}\right) \mathbf{a}_{3}$ & $\begin{array}{c}\left(x_{5}-\frac{1}{2} y_{5}\right) a \hat{\mathbf{x}}-\frac{\sqrt{3}}{2} y_{5} a \hat{\mathbf{y}}+ \\
\left(\frac{1}{2}-z_{5}\right) c \hat{\mathbf{z}}\end{array}$ & $(24 m)$ & O II \\
\hline $\mathbf{B}_{47}$ & $=$ & $-x_{5} \mathbf{a}_{1}-y_{5} \mathbf{a}_{2}-z_{5} \mathbf{a}_{3}$ & $\begin{array}{c}-\frac{1}{2}\left(x_{5}+y_{5}\right) a \hat{\mathbf{x}}+ \\
\frac{\sqrt{3}}{2}\left(x_{5}-y_{5}\right) a \hat{\mathbf{y}}-z_{5} c \hat{\mathbf{z}}\end{array}$ & $(24 m)$ & O II \\
\hline $\mathbf{B}_{48}$ & $=$ & $y_{5} \mathbf{a}_{1}+\left(-x_{5}+y_{5}\right) \mathbf{a}_{2}-z_{5} \mathbf{a}_{3}$ & $=\left(-\frac{1}{2} x_{5}+y_{5}\right) a \hat{\mathbf{x}}-\frac{\sqrt{3}}{2} x_{5} a \hat{\mathbf{y}}-z_{5} c \hat{\mathbf{z}}$ & $(24 m)$ & O II \\
\hline $\mathbf{B}_{49}$ & $=$ & $\left(x_{5}-y_{5}\right) \mathbf{a}_{1}+x_{5} \mathbf{a}_{2}-z_{5} \mathbf{a}_{3}$ & $=\left(x_{5}-\frac{1}{2} y_{5}\right) a \hat{\mathbf{x}}+\frac{\sqrt{3}}{2} y_{5} a \hat{\mathbf{y}}-z_{5} c \hat{\mathbf{z}}$ & $(24 m)$ & O II \\
\hline $\mathbf{B}_{50}$ & $=$ & $x_{5} \mathbf{a}_{1}+y_{5} \mathbf{a}_{2}-z_{5} \mathbf{a}_{3}$ & $\begin{array}{c}\frac{1}{2}\left(x_{5}+y_{5}\right) a \hat{\mathbf{x}}+ \\
\frac{\sqrt{3}}{2}\left(-x_{5}+y_{5}\right) a \hat{\mathbf{y}}-z_{5} c \hat{\mathbf{z}}\end{array}$ & $(24 m)$ & O II \\
\hline $\mathbf{B}_{51}$ & $=$ & $-y_{5} \mathbf{a}_{1}+\left(x_{5}-y_{5}\right) \mathbf{a}_{2}-z_{5} \mathbf{a}_{3}$ & $=\left(\frac{1}{2} x_{5}-y_{5}\right) a \hat{\mathbf{x}}+\frac{\sqrt{3}}{2} x_{5} a \hat{\mathbf{y}}-z_{5} c \hat{\mathbf{z}}$ & $(24 m)$ & O II \\
\hline $\mathbf{B}_{52}$ & $=$ & $\left(-x_{5}+y_{5}\right) \mathbf{a}_{1}-x_{5} \mathbf{a}_{2}-z_{5} \mathbf{a}_{3}$ & $=\left(-x_{5}+\frac{1}{2} y_{5}\right) a \hat{\mathbf{x}}-\frac{\sqrt{3}}{2} y_{5} a \hat{\mathbf{y}}-z_{5} c \hat{\mathbf{z}}$ & $(24 m)$ & O II \\
\hline $\mathbf{B}_{53}$ & $=$ & $-y_{5} \mathbf{a}_{1}-x_{5} \mathbf{a}_{2}+\left(\frac{1}{2}+z_{5}\right) \mathbf{a}_{3}$ & $\begin{array}{c}-\frac{1}{2}\left(x_{5}+y_{5}\right) a \hat{\mathbf{x}}+ \\
\frac{\sqrt{3}}{2}\left(-x_{5}+y_{5}\right) a \hat{\mathbf{y}}+\left(\frac{1}{2}+z_{5}\right) c \hat{\mathbf{z}}\end{array}$ & $(24 m)$ & O II \\
\hline
\end{tabular}




$$
\begin{aligned}
& \mathbf{B}_{54}=\left(-x_{5}+y_{5}\right) \mathbf{a}_{1}+y_{5} \mathbf{a}_{2}+\left(\frac{1}{2}+z_{5}\right) \mathbf{a}_{3}=\left(-\frac{1}{2} x_{5}+y_{5}\right) a \hat{\mathbf{x}}+\frac{\sqrt{3}}{2} x_{5} a \hat{\mathbf{y}}+\quad \text { O II }
\end{aligned}
$$

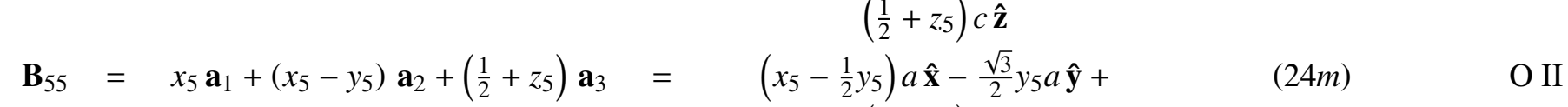

$$
\begin{aligned}
& \mathbf{B}_{56}=y_{5} \mathbf{a}_{1}+x_{5} \mathbf{a}_{2}+\left(\frac{1}{2}+z_{5}\right) \mathbf{a}_{3} \quad=\frac{1}{2}\left(x_{5}+y_{5}\right) a \hat{\mathbf{x}}+\frac{\sqrt{3}}{2}\left(x_{5}-y_{5}\right) a \hat{\mathbf{y}}+\quad \text { O II } \\
& \mathbf{B}_{57}=\left(x_{5}-y_{5}\right) \mathbf{a}_{1}-y_{5} \mathbf{a}_{2}+\left(\frac{1}{2}+z_{5}\right) \mathbf{a}_{3}=\left(\frac{1}{2} x_{5}-y_{5}\right) a \hat{\mathbf{x}}-\frac{\sqrt{3}}{2} x_{5} a \hat{\mathbf{y}}+\quad\left(\frac{1}{2}+z_{5}\right) \quad \text { (24m) } \\
& \mathbf{B}_{58}=-x_{5} \mathbf{a}_{1}+\left(-x_{5}+y_{5}\right) \mathbf{a}_{2}+\left(\frac{1}{2}+z_{5}\right) \mathbf{a}_{3}=\left(-x_{5}+\frac{1}{2} y_{5}\right) a \hat{\mathbf{x}}+\frac{\sqrt{3}}{2} y_{5} a \hat{\mathbf{y}}+\quad \text { O II } \\
& \left(\frac{1}{2}+z_{5}\right) c \hat{\mathbf{z}}
\end{aligned}
$$

\section{References:}

- R. M. Hazen, A. Y. Au, and L. W. Finger, High-pressure crystal chemistry of beryl $\left(\mathrm{Be}_{3} \mathrm{Al}_{2} \mathrm{Si}_{6} \mathrm{O}_{18}\right)$ and euclase ( $\left.\mathrm{BeAlSiO}_{4} \mathrm{OH}\right)$, Am. Mineral. 71, 977-984 (1986).

- B. Morosin, Structure and Thermal Expansion of Beryl, Acta Crystallogr. Sect. B Struct. Sci. 28, 1899-1903 (1972), doi:10.1107/S0567740872005199.

\section{Found in:}

- R. T. Downs and M. Hall-Wallace, The American Mineralogist Crystal Structure Database, Am. Mineral. 88, 247-250 (2003).

\section{Geometry files:}

- CIF: pp. 941

- POSCAR: pp. 941 


\section{$\mathrm{AlPO}_{4}$ Structure: AB2_hP72_192_m_j2kl}
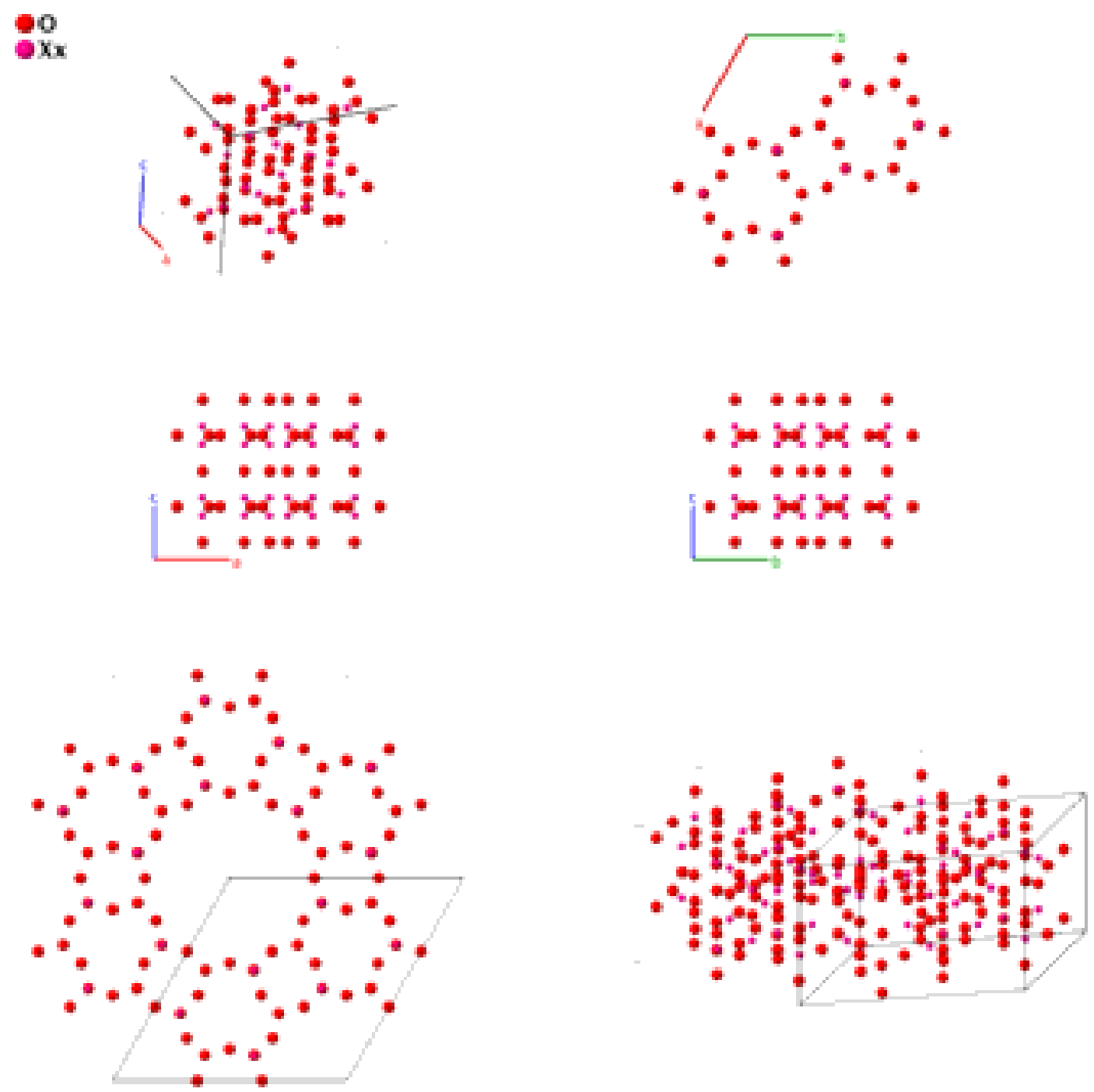

\section{Prototype}

AFLOW prototype label

Strukturbericht designation

Pearson symbol

Space group number

Space group symbol
$\mathrm{AlPO}_{4}$

: AB2_hP72_192_m_j2kl

: None

: $\mathrm{hP72}$

: 192

: $\quad P 6 / m c c$
AFLOW prototype command

- Here, the $\mathrm{M}$ sites are partially occupied with $0.5 \mathrm{Al}+0.5 \mathrm{P}$. The Jmol image does not distinguish between the different $\mathrm{M}$ labels and is represented by the "Xx" atoms. Polytypes of this compound also appear in a space groups \#168 and \#184. 


$$
\begin{array}{ll}
\mathbf{a}_{1}= & \frac{1}{2} a \hat{\mathbf{x}}-\frac{\sqrt{3}}{2} a \hat{\mathbf{y}} \\
\mathbf{a}_{2}= & \frac{1}{2} a \hat{\mathbf{x}}+\frac{\sqrt{3}}{2} a \hat{\mathbf{y}} \\
\mathbf{a}_{3}= & c \hat{\mathbf{z}}
\end{array}
$$

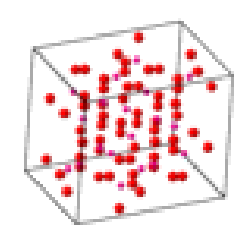

\begin{tabular}{|c|c|c|c|c|}
\hline $\mathbf{B}_{1}$ & $=$ & $x_{1} \mathbf{a}_{1}+\frac{1}{4} \mathbf{a}_{3}$ & $=$ & $\frac{1}{2} x_{1} a \hat{\mathbf{x}}-\frac{\sqrt{3}}{2} x_{1} a \hat{\mathbf{y}}+\frac{1}{4} c \hat{\mathbf{z}}$ \\
\hline $\mathbf{B}_{2}$ & $=$ & $x_{1} \mathbf{a}_{2}+\frac{1}{4} \mathbf{a}_{3}$ & $=$ & $\frac{1}{2} x_{1} a \hat{\mathbf{x}}+\frac{\sqrt{3}}{2} x_{1} a \hat{\mathbf{y}}+\frac{1}{4}$ \\
\hline $\mathbf{B}_{3}$ & $=$ & $-x_{1} \mathbf{a}_{1}-x_{1} \mathbf{a}_{2}+\frac{1}{4} \mathbf{a}_{3}$ & $=$ & $-x_{1} a \hat{\mathbf{x}}+\frac{1}{4} c \hat{\mathbf{z}}$ \\
\hline $\mathbf{B}_{4}$ & $=$ & $-x_{1} \mathbf{a}_{1}+\frac{1}{4} \mathbf{a}_{3}$ & $=$ & $-\frac{1}{2} x_{1} a \hat{\mathbf{x}}+\frac{\sqrt{3}}{2} x_{1} a \hat{\mathbf{y}}+$ \\
\hline $\mathbf{B}_{5}$ & $=$ & $-x_{1} \mathbf{a}_{2}+\frac{1}{4} \mathbf{a}_{3}$ & $=$ & $-\frac{1}{2} x_{1} a \hat{\mathbf{x}}-\frac{\sqrt{3}}{2} x_{1} a \hat{\mathbf{y}}+$ \\
\hline $\mathbf{B}_{6}$ & $=$ & $x_{1} \mathbf{a}_{1}+x_{1} \mathbf{a}_{2}+\frac{1}{4} \mathbf{a}_{3}$ & $=$ & $x_{1} a \hat{\mathbf{x}}+\frac{1}{4} c \hat{\mathbf{z}}$ \\
\hline $\mathbf{B}_{7}$ & $=$ & $-x_{1} \mathbf{a}_{1}+\frac{3}{4} \mathbf{a}_{3}$ & $=$ & $-\frac{1}{2} x_{1} a \hat{\mathbf{x}}+\frac{\sqrt{3}}{2} x_{1} a \hat{\mathbf{y}}+$ \\
\hline $\mathbf{B}_{8}$ & $=$ & $-x_{1} \mathbf{a}_{2}+\frac{3}{4} \mathbf{a}_{3}$ & $=$ & $-\frac{1}{2} x_{1} a \hat{\mathbf{x}}-\frac{\sqrt{3}}{2} x_{1} a \hat{\mathbf{y}}+$ \\
\hline $\mathbf{B}_{9}$ & $=$ & $x_{1} \mathbf{a}_{1}+x_{1} \mathbf{a}_{2}+\frac{3}{4} \mathbf{a}_{3}$ & $=$ & $x_{1} a \hat{\mathbf{x}}+\frac{3}{4} c \hat{\mathbf{z}}$ \\
\hline $\mathbf{B}_{10}$ & $=$ & $x_{1} \mathbf{a}_{1}+\frac{3}{4} \mathbf{a}_{3}$ & $=$ & $\frac{1}{2} x_{1} a \hat{\mathbf{x}}-\frac{\sqrt{3}}{2} x_{1} a \hat{\mathbf{y}}+\frac{3}{4}$ \\
\hline $\mathbf{B}_{11}$ & $=$ & $x_{1} \mathbf{a}_{2}+\frac{3}{4} \mathbf{a}_{3}$ & $=$ & $\frac{1}{2} x_{1} a \hat{\mathbf{x}}+\frac{\sqrt{3}}{2} x_{1} a \hat{\mathbf{y}}+\frac{3}{4}$ \\
\hline $\mathbf{B}_{12}$ & $=$ & $-x_{1} \mathbf{a}_{1}-x_{1} \mathbf{a}_{2}+\frac{3}{4} \mathbf{a}_{3}$ & $=$ & $-x_{1} a \hat{\mathbf{x}}+\frac{3}{4} c \hat{\mathbf{z}}$ \\
\hline $\mathbf{B}_{13}$ & $=$ & $x_{2} \mathbf{a}_{1}+2 x_{2} \mathbf{a}_{2}+\frac{1}{4} \mathbf{a}_{3}$ & $=$ & $\frac{3}{2} x_{2} a \hat{\mathbf{x}}+\frac{\sqrt{3}}{2} x_{2} a \hat{\mathbf{y}}+$ \\
\hline $\mathbf{B}_{14}$ & $=$ & $-2 x_{2} \mathbf{a}_{1}-x_{2} \mathbf{a}_{2}+\frac{1}{4} \mathbf{a}_{3}$ & $=$ & $-\frac{3}{2} x_{2} a \hat{\mathbf{x}}+\frac{\sqrt{3}}{2} x_{2} a \hat{\mathbf{y}}+$ \\
\hline $\mathbf{B}_{15}$ & $=$ & $x_{2} \mathbf{a}_{1}-x_{2} \mathbf{a}_{2}+\frac{1}{4} \mathbf{a}_{3}$ & $=$ & $-\sqrt{3} x_{2} a \hat{\mathbf{y}}+\frac{1}{4} c \hat{\mathbf{z}}$ \\
\hline $\mathbf{B}_{16}$ & $=$ & $-x_{2} \mathbf{a}_{1}-2 x_{2} \mathbf{a}_{2}+\frac{1}{4} \mathbf{a}_{3}$ & $=$ & $-\frac{3}{2} x_{2} a \hat{\mathbf{x}}-\frac{\sqrt{3}}{2} x_{2} a \hat{\mathbf{y}}+\frac{1}{4}$ \\
\hline $\mathbf{B}_{17}$ & $=$ & $2 x_{2} \mathbf{a}_{1}+x_{2} \mathbf{a}_{2}+\frac{1}{4} \mathbf{a}_{3}$ & $=$ & $\frac{3}{2} x_{2} a \hat{\mathbf{x}}-\frac{\sqrt{3}}{2} x_{2} a \hat{\mathbf{y}}+\frac{1}{4}$ \\
\hline $\mathbf{B}_{18}$ & $=$ & $-x_{2} \mathbf{a}_{1}+x_{2} \mathbf{a}_{2}+\frac{1}{4} \mathbf{a}_{3}$ & $=$ & $\sqrt{3} x_{2} a \hat{\mathbf{y}}+\frac{1}{4} c \hat{\mathbf{z}}$ \\
\hline $\mathbf{B}_{19}$ & $=$ & $-x_{2} \mathbf{a}_{1}-2 x_{2} \mathbf{a}_{2}+\frac{3}{4} \mathbf{a}_{3}$ & $=$ & $-\frac{3}{2} x_{2} a \hat{\mathbf{x}}-\frac{\sqrt{3}}{2} x_{2} a \hat{\mathbf{y}}+\frac{3}{4} c \hat{\mathbf{z}}$ \\
\hline $\mathbf{B}_{20}$ & $=$ & $2 x_{2} \mathbf{a}_{1}+x_{2} \mathbf{a}_{2}+\frac{3}{4} \mathbf{a}_{3}$ & $=$ & $\frac{3}{2} x_{2} a \hat{\mathbf{x}}-\frac{\sqrt{3}}{2} x_{2} a \hat{\mathbf{y}}+\frac{3}{4}$ \\
\hline $\mathbf{B}_{21}$ & $=$ & $-x_{2} \mathbf{a}_{1}+x_{2} \mathbf{a}_{2}+\frac{3}{4} \mathbf{a}_{3}$ & $=$ & $\sqrt{3} x_{2} a \hat{\mathbf{y}}+\frac{3}{4} c \hat{\mathbf{z}}$ \\
\hline $\mathbf{B}_{22}$ & $=$ & $x_{2} \mathbf{a}_{1}+2 x_{2} \mathbf{a}_{2}+\frac{3}{4} \mathbf{a}_{3}$ & $=$ & $\frac{3}{2} x_{2} a \hat{\mathbf{x}}+\frac{\sqrt{3}}{2} x_{2} a \hat{\mathbf{y}}+\frac{3}{4} c \hat{\mathbf{z}}$ \\
\hline $\mathbf{B}_{23}$ & $=$ & $-2 x_{2} \mathbf{a}_{1}-x_{2} \mathbf{a}_{2}+\frac{3}{4} \mathbf{a}_{3}$ & $=$ & $-\frac{3}{2} x_{2} a \hat{\mathbf{x}}+\frac{\sqrt{3}}{2} x_{2} a \hat{\mathbf{y}}+$ \\
\hline $\mathbf{B}_{24}$ & $=$ & $x_{2} \mathbf{a}_{1}-x_{2} \mathbf{a}_{2}+\frac{3}{4} \mathbf{a}_{3}$ & $=$ & $-\sqrt{3} x_{2} a \hat{\mathbf{y}}+\frac{3}{4} c \hat{\mathbf{z}}$ \\
\hline $\mathbf{B}_{25}$ & $=$ & $x_{3} \mathbf{a}_{1}+2 x_{3} \mathbf{a}_{2}+\frac{1}{4} \mathbf{a}_{3}$ & $=$ & $\frac{3}{2} x_{3} a \hat{\mathbf{x}}+\frac{\sqrt{3}}{2} x_{3} a \hat{\mathbf{y}}+\frac{1}{4} c \hat{\mathbf{z}}$ \\
\hline $\mathbf{B}_{26}$ & $=$ & $-2 x_{3} \mathbf{a}_{1}-x_{3} \mathbf{a}_{2}+\frac{1}{4} \mathbf{a}_{3}$ & $=$ & $-\frac{3}{2} x_{3} a \hat{\mathbf{x}}+\frac{\sqrt{3}}{2} x_{3} a \hat{\mathbf{y}}+\frac{1}{4} c \hat{\mathbf{z}}$ \\
\hline $\mathbf{B}_{27}$ & $=$ & $x_{3} \mathbf{a}_{1}-x_{3} \mathbf{a}_{2}+\frac{1}{4} \mathbf{a}_{3}$ & $=$ & $-\sqrt{3} x_{3} a \hat{\mathbf{y}}+\frac{1}{4} c \hat{\mathbf{z}}$ \\
\hline $\mathbf{B}_{28}$ & $=$ & $-x_{3} \mathbf{a}_{1}-2 x_{3} \mathbf{a}_{2}+\frac{1}{4} \mathbf{a}_{3}$ & $=$ & $-\frac{3}{2} x_{3} a \hat{\mathbf{x}}-\frac{\sqrt{3}}{2} x_{3} a \hat{\mathbf{y}}$ \\
\hline
\end{tabular}

\section{Basis vectors:}

Lattice Coordinates
Wyckoff Position Atom Type

O I

O I

O I

O I

O I

O I

O I

O I

O I

O II

O II

O II

O II

O II

O II

O II

O II

O II

O II

O II

O II

O III

O III

O III

O III 


\begin{tabular}{|c|c|c|c|c|c|c|}
\hline $\mathbf{B}_{29}$ & $=$ & $2 x_{3} \mathbf{a}_{1}+x_{3} \mathbf{a}_{2}+\frac{1}{4} \mathbf{a}_{3}$ & $=$ & $\frac{3}{2} x_{3} a \hat{\mathbf{x}}-\frac{\sqrt{3}}{2} x_{3} a \hat{\mathbf{y}}+\frac{1}{4} c \hat{\mathbf{z}}$ & $(12 k)$ & O III \\
\hline $\mathbf{B}_{30}$ & $=$ & $-x_{3} \mathbf{a}_{1}+x_{3} \mathbf{a}_{2}+\frac{1}{4} \mathbf{a}_{3}$ & $=$ & $\sqrt{3} x_{3} a \hat{\mathbf{y}}+\frac{1}{4} c \hat{\mathbf{z}}$ & $(12 k)$ & O III \\
\hline $\mathbf{B}_{31}$ & $=$ & $-x_{3} \mathbf{a}_{1}-2 x_{3} \mathbf{a}_{2}+\frac{3}{4} \mathbf{a}_{3}$ & $=$ & $-\frac{3}{2} x_{3} a \hat{\mathbf{x}}-\frac{\sqrt{3}}{2} x_{3} a \hat{\mathbf{y}}+\frac{3}{4} c \hat{\mathbf{z}}$ & $(12 k)$ & O III \\
\hline $\mathbf{B}_{32}$ & $=$ & $2 x_{3} \mathbf{a}_{1}+x_{3} \mathbf{a}_{2}+\frac{3}{4} \mathbf{a}_{3}$ & $=$ & $\frac{3}{2} x_{3} a \hat{\mathbf{x}}-\frac{\sqrt{3}}{2} x_{3} a \hat{\mathbf{y}}+\frac{3}{4} c \hat{\mathbf{z}}$ & $(12 k)$ & O III \\
\hline $\mathbf{B}_{33}$ & $=$ & $-x_{3} \mathbf{a}_{1}+x_{3} \mathbf{a}_{2}+\frac{3}{4} \mathbf{a}_{3}$ & $=$ & $\sqrt{3} x_{3} a \hat{\mathbf{y}}+\frac{3}{4} c \hat{\mathbf{z}}$ & $(12 k)$ & O III \\
\hline $\mathbf{B}_{34}$ & $=$ & $x_{3} \mathbf{a}_{1}+2 x_{3} \mathbf{a}_{2}+\frac{3}{4} \mathbf{a}_{3}$ & $=$ & $\frac{3}{2} x_{3} a \hat{\mathbf{x}}+\frac{\sqrt{3}}{2} x_{3} a \hat{\mathbf{y}}+\frac{3}{4} c \hat{\mathbf{z}}$ & $(12 k)$ & O III \\
\hline $\mathbf{B}_{35}$ & $=$ & $-2 x_{3} \mathbf{a}_{1}-x_{3} \mathbf{a}_{2}+\frac{3}{4} \mathbf{a}_{3}$ & $=$ & $-\frac{3}{2} x_{3} a \hat{\mathbf{x}}+\frac{\sqrt{3}}{2} x_{3} a \hat{\mathbf{y}}+\frac{3}{4} c \hat{\mathbf{z}}$ & $(12 k)$ & O III \\
\hline $\mathbf{B}_{36}$ & $=$ & $x_{3} \mathbf{a}_{1}-x_{3} \mathbf{a}_{2}+\frac{3}{4} \mathbf{a}_{3}$ & $=$ & $-\sqrt{3} x_{3} a \hat{\mathbf{y}}+\frac{3}{4} c \hat{\mathbf{z}}$ & $(12 k)$ & O III \\
\hline $\mathbf{B}_{37}$ & $=$ & $x_{4} \mathbf{a}_{1}+y_{4} \mathbf{a}_{2}$ & $=$ & $\frac{1}{2}\left(x_{4}+y_{4}\right) a \hat{\mathbf{x}}+\frac{\sqrt{3}}{2}\left(-x_{4}+y_{4}\right) a \hat{\mathbf{y}}$ & $(12 l)$ & O IV \\
\hline $\mathbf{B}_{38}$ & $=$ & $-y_{4} \mathbf{a}_{1}+\left(x_{4}-y_{4}\right) \mathbf{a}_{2}$ & $=$ & $\left(\frac{1}{2} x_{4}-y_{4}\right) a \hat{\mathbf{x}}+\frac{\sqrt{3}}{2} x_{4} a \hat{\mathbf{y}}$ & $(12 l)$ & O IV \\
\hline $\mathbf{B}_{39}$ & $=$ & $\left(-x_{4}+y_{4}\right) \mathbf{a}_{1}-x_{4} \mathbf{a}_{2}$ & $=$ & $\left(-x_{4}+\frac{1}{2} y_{4}\right) a \hat{\mathbf{x}}-\frac{\sqrt{3}}{2} y_{4} a \hat{\mathbf{y}}$ & $(12 l)$ & O IV \\
\hline $\mathbf{B}_{40}$ & $=$ & $-x_{4} \mathbf{a}_{1}-y_{4} \mathbf{a}_{2}$ & $=$ & $-\frac{1}{2}\left(x_{4}+y_{4}\right) a \hat{\mathbf{x}}+\frac{\sqrt{3}}{2}\left(x_{4}-y_{4}\right) a \hat{\mathbf{y}}$ & $(12 l)$ & O IV \\
\hline $\mathbf{B}_{41}$ & $=$ & $y_{4} \mathbf{a}_{1}+\left(-x_{4}+y_{4}\right) \mathbf{a}_{2}$ & $=$ & $\left(-\frac{1}{2} x_{4}+y_{4}\right) a \hat{\mathbf{x}}-\frac{\sqrt{3}}{2} x_{4} a \hat{\mathbf{y}}$ & $(12 l)$ & O IV \\
\hline $\mathbf{B}_{42}$ & $=$ & $\left(x_{4}-y_{4}\right) \mathbf{a}_{1}+x_{4} \mathbf{a}_{2}$ & $=$ & $\left(x_{4}-\frac{1}{2} y_{4}\right) a \hat{\mathbf{x}}+\frac{\sqrt{3}}{2} y_{4} a \hat{\mathbf{y}}$ & $(12 l)$ & O IV \\
\hline $\mathbf{B}_{43}$ & $=$ & $y_{4} \mathbf{a}_{1}+x_{4} \mathbf{a}_{2}+\frac{1}{2} \mathbf{a}_{3}$ & $=$ & $\begin{array}{c}\frac{1}{2}\left(x_{4}+y_{4}\right) a \hat{\mathbf{x}}+\frac{\sqrt{3}}{2}\left(x_{4}-y_{4}\right) a \hat{\mathbf{y}}+ \\
\frac{1}{2} c \hat{\mathbf{z}}\end{array}$ & $(12 l)$ & O IV \\
\hline $\mathbf{B}_{44}$ & $=$ & $\left(x_{4}-y_{4}\right) \mathbf{a}_{1}-y_{4} \mathbf{a}_{2}+\frac{1}{2} \mathbf{a}_{3}$ & $=$ & $\left(\frac{1}{2} x_{4}-y_{4}\right) a \hat{\mathbf{x}}-\frac{\sqrt{3}}{2} x_{4} a \hat{\mathbf{y}}+\frac{1}{2} c \hat{\mathbf{z}}$ & $(12 l)$ & O IV \\
\hline $\mathbf{B}_{45}$ & $=$ & $-x_{4} \mathbf{a}_{1}+\left(-x_{4}+y_{4}\right) \mathbf{a}_{2}+\frac{1}{2} \mathbf{a}_{3}$ & $=$ & $\left(-x_{4}+\frac{1}{2} y_{4}\right) a \hat{\mathbf{x}}+\frac{\sqrt{3}}{2} y_{4} a \hat{\mathbf{y}}+\frac{1}{2} c \hat{\mathbf{z}}$ & $(12 l)$ & O IV \\
\hline $\mathbf{B}_{46}$ & $=$ & $-y_{4} \mathbf{a}_{1}-x_{4} \mathbf{a}_{2}+\frac{1}{2} \mathbf{a}_{3}$ & $=$ & $\begin{array}{c}-\frac{1}{2}\left(x_{4}+y_{4}\right) a \hat{\mathbf{x}}+ \\
\frac{\sqrt{3}}{2}\left(-x_{4}+y_{4}\right) a \hat{\mathbf{y}}+\frac{1}{2} c \hat{\mathbf{z}}\end{array}$ & $(12 l)$ & O IV \\
\hline $\mathbf{B}_{47}$ & $=$ & $\left(-x_{4}+y_{4}\right) \mathbf{a}_{1}+y_{4} \mathbf{a}_{2}+\frac{1}{2} \mathbf{a}_{3}$ & $=$ & $\left(-\frac{1}{2} x_{4}+y_{4}\right) a \hat{\mathbf{x}}+\frac{\sqrt{3}}{2} x_{4} a \hat{\mathbf{y}}+\frac{1}{2} c \hat{\mathbf{z}}$ & $(12 l)$ & O IV \\
\hline $\mathbf{B}_{48}$ & $=$ & $x_{4} \mathbf{a}_{1}+\left(x_{4}-y_{4}\right) \mathbf{a}_{2}+\frac{1}{2} \mathbf{a}_{3}$ & $=$ & $\left(x_{4}-\frac{1}{2} y_{4}\right) a \hat{\mathbf{x}}-\frac{\sqrt{3}}{2} y_{4} a \hat{\mathbf{y}}+\frac{1}{2} c \hat{\mathbf{z}}$ & $(12 l)$ & O IV \\
\hline $\mathbf{B}_{49}$ & $=$ & $x_{5} \mathbf{a}_{1}+y_{5} \mathbf{a}_{2}+z_{5} \mathbf{a}_{3}$ & $=$ & $\begin{array}{c}\frac{1}{2}\left(x_{5}+y_{5}\right) a \hat{\mathbf{x}}+ \\
\frac{\sqrt{3}}{2}\left(-x_{5}+y_{5}\right) a \hat{\mathbf{y}}+z_{5} c \hat{\mathbf{z}}\end{array}$ & $(24 m)$ & $\mathrm{M}$ \\
\hline $\mathbf{B}_{50}$ & $=$ & $-y_{5} \mathbf{a}_{1}+\left(x_{5}-y_{5}\right) \mathbf{a}_{2}+z_{5} \mathbf{a}_{3}$ & $=$ & $\left(\frac{1}{2} x_{5}-y_{5}\right) a \hat{\mathbf{x}}+\frac{\sqrt{3}}{2} x_{5} a \hat{\mathbf{y}}+z_{5} c \hat{\mathbf{z}}$ & $(24 m)$ & M \\
\hline $\mathbf{B}_{51}$ & $=$ & $\left(-x_{5}+y_{5}\right) \mathbf{a}_{1}-x_{5} \mathbf{a}_{2}+z_{5} \mathbf{a}_{3}$ & $=$ & $\left(-x_{5}+\frac{1}{2} y_{5}\right) a \hat{\mathbf{x}}-\frac{\sqrt{3}}{2} y_{5} a \hat{\mathbf{y}}+z_{5} c \hat{\mathbf{z}}$ & $(24 m)$ & M \\
\hline $\mathbf{B}_{52}$ & $=$ & $-x_{5} \mathbf{a}_{1}-y_{5} \mathbf{a}_{2}+z_{5} \mathbf{a}_{3}$ & $=$ & $\begin{array}{c}-\frac{1}{2}\left(x_{5}+y_{5}\right) a \hat{\mathbf{x}}+ \\
\frac{\sqrt{3}}{2}\left(x_{5}-y_{5}\right) a \hat{\mathbf{y}}+z_{5} c \hat{\mathbf{z}}\end{array}$ & $(24 m)$ & M \\
\hline $\mathbf{B}_{53}$ & $=$ & $y_{5} \mathbf{a}_{1}+\left(-x_{5}+y_{5}\right) \mathbf{a}_{2}+z_{5} \mathbf{a}_{3}$ & $=$ & $\left(-\frac{1}{2} x_{5}+y_{5}\right) a \hat{\mathbf{x}}-\frac{\sqrt{3}}{2} x_{5} a \hat{\mathbf{y}}+z_{5} c \hat{\mathbf{z}}$ & $(24 m)$ & M \\
\hline $\mathbf{B}_{54}$ & $=$ & $\left(x_{5}-y_{5}\right) \mathbf{a}_{1}+x_{5} \mathbf{a}_{2}+z_{5} \mathbf{a}_{3}$ & $=$ & $\left(x_{5}-\frac{1}{2} y_{5}\right) a \hat{\mathbf{x}}+\frac{\sqrt{3}}{2} y_{5} a \hat{\mathbf{y}}+z_{5} c \hat{\mathbf{z}}$ & $(24 m)$ & M \\
\hline $\mathbf{B}_{55}$ & $=$ & $y_{5} \mathbf{a}_{1}+x_{5} \mathbf{a}_{2}+\left(\frac{1}{2}-z_{5}\right) \mathbf{a}_{3}$ & $=$ & $\begin{array}{c}\frac{1}{2}\left(x_{5}+y_{5}\right) a \hat{\mathbf{x}}+\frac{\sqrt{3}}{2}\left(x_{5}-y_{5}\right) a \hat{\mathbf{y}}+ \\
\left(\frac{1}{2}-z_{5}\right) c \hat{\mathbf{z}}\end{array}$ & $(24 m)$ & M \\
\hline $\mathbf{B}_{56}$ & $=$ & $\left(x_{5}-y_{5}\right) \mathbf{a}_{1}-y_{5} \mathbf{a}_{2}+\left(\frac{1}{2}-z_{5}\right) \mathbf{a}_{3}$ & $=$ & $\begin{array}{c}\left(\frac{1}{2} x_{5}-y_{5}\right) a \hat{\mathbf{x}}-\frac{\sqrt{3}}{2} x_{5} a \hat{\mathbf{y}}+ \\
\left(\frac{1}{2}-z_{5}\right) c \hat{\mathbf{z}}\end{array}$ & $(24 m)$ & M \\
\hline $\mathbf{B}_{57}$ & $=$ & $-x_{5} \mathbf{a}_{1}+\left(-x_{5}+y_{5}\right) \mathbf{a}_{2}+\left(\frac{1}{2}-z_{5}\right) \mathbf{a}_{3}$ & $=$ & $\begin{array}{c}\left(-x_{5}+\frac{1}{2} y_{5}\right) a \hat{\mathbf{x}}+\frac{\sqrt{3}}{2} y_{5} a \hat{\mathbf{y}}+ \\
\left(\frac{1}{2}-z_{5}\right) c \hat{\mathbf{z}}\end{array}$ & $(24 m)$ & M \\
\hline $\mathbf{B}_{58}$ & $=$ & $-y_{5} \mathbf{a}_{1}-x_{5} \mathbf{a}_{2}+\left(\frac{1}{2}-z_{5}\right) \mathbf{a}_{3}$ & $=$ & $\begin{array}{c}-\frac{1}{2}\left(x_{5}+y_{5}\right) a \hat{\mathbf{x}}+ \\
\frac{\sqrt{3}}{2}\left(-x_{5}+y_{5}\right) a \hat{\mathbf{y}}+\left(\frac{1}{2}-z_{5}\right) c \hat{\mathbf{z}}\end{array}$ & $(24 m)$ & $\mathrm{M}$ \\
\hline
\end{tabular}




\begin{tabular}{|c|c|c|c|c|c|}
\hline $\mathbf{B}_{59}$ & $=$ & $\left(-x_{5}+y_{5}\right) \mathbf{a}_{1}+y_{5} \mathbf{a}_{2}+\left(\frac{1}{2}-z_{5}\right) \mathbf{a}_{3}$ & $=$ & $\begin{array}{c}\left(-\frac{1}{2} x_{5}+y_{5}\right) a \hat{\mathbf{x}}+\frac{\sqrt{3}}{2} x_{5} a \hat{\mathbf{y}}+ \\
\left(\frac{1}{2}-z_{5}\right) c \hat{\mathbf{z}}\end{array}$ & $(24 m)$ \\
\hline $\mathbf{B}_{60}$ & $=$ & $x_{5} \mathbf{a}_{1}+\left(x_{5}-y_{5}\right) \mathbf{a}_{2}+\left(\frac{1}{2}-z_{5}\right) \mathbf{a}_{3}$ & $=$ & $\begin{array}{c}\left(x_{5}-\frac{1}{2} y_{5}\right) a \hat{\mathbf{x}}-\frac{\sqrt{3}}{2} y_{5} a \hat{\mathbf{y}}+ \\
\left(\frac{1}{2}-z_{5}\right) c \hat{\mathbf{z}}\end{array}$ & $(24 m)$ \\
\hline $\mathbf{B}_{61}$ & $=$ & $-x_{5} \mathbf{a}_{1}-y_{5} \mathbf{a}_{2}-z_{5} \mathbf{a}_{3}$ & $=$ & $\begin{array}{c}-\frac{1}{2}\left(x_{5}+y_{5}\right) a \hat{\mathbf{x}}+ \\
\frac{\sqrt{3}}{2}\left(x_{5}-y_{5}\right) a \hat{\mathbf{y}}-z_{5} c \hat{\mathbf{z}}\end{array}$ & $(24 m)$ \\
\hline $\mathbf{B}_{62}$ & $=$ & $y_{5} \mathbf{a}_{1}+\left(-x_{5}+y_{5}\right) \mathbf{a}_{2}-z_{5} \mathbf{a}_{3}$ & $=$ & $\left(-\frac{1}{2} x_{5}+y_{5}\right) a \hat{\mathbf{x}}-\frac{\sqrt{3}}{2} x_{5} a \hat{\mathbf{y}}-z_{5} c \hat{\mathbf{z}}$ & $(24 m)$ \\
\hline $\mathbf{B}_{63}$ & $=$ & $\left(x_{5}-y_{5}\right) \mathbf{a}_{1}+x_{5} \mathbf{a}_{2}-z_{5} \mathbf{a}_{3}$ & $=$ & $\left(x_{5}-\frac{1}{2} y_{5}\right) a \hat{\mathbf{x}}+\frac{\sqrt{3}}{2} y_{5} a \hat{\mathbf{y}}-z_{5} c \hat{\mathbf{z}}$ & $(24 m)$ \\
\hline $\mathbf{B}_{64}$ & $=$ & $x_{5} \mathbf{a}_{1}+y_{5} \mathbf{a}_{2}-z_{5} \mathbf{a}_{3}$ & $=$ & $\begin{array}{c}\frac{1}{2}\left(x_{5}+y_{5}\right) a \hat{\mathbf{x}}+ \\
\frac{\sqrt{3}}{2}\left(-x_{5}+y_{5}\right) a \hat{\mathbf{y}}-z_{5} c \hat{\mathbf{z}}\end{array}$ & $(24 m)$ \\
\hline $\mathbf{B}_{65}$ & $=$ & $-y_{5} \mathbf{a}_{1}+\left(x_{5}-y_{5}\right) \mathbf{a}_{2}-z_{5} \mathbf{a}_{3}$ & $=$ & $\left(\frac{1}{2} x_{5}-y_{5}\right) a \hat{\mathbf{x}}+\frac{\sqrt{3}}{2} x_{5} a \hat{\mathbf{y}}-z_{5} c \hat{\mathbf{z}}$ & $(24 m)$ \\
\hline $\mathbf{B}_{66}$ & $=$ & $\left(-x_{5}+y_{5}\right) \mathbf{a}_{1}-x_{5} \mathbf{a}_{2}-z_{5} \mathbf{a}_{3}$ & $=$ & $\left(-x_{5}+\frac{1}{2} y_{5}\right) a \hat{\mathbf{x}}-\frac{\sqrt{3}}{2} y_{5} a \hat{\mathbf{y}}-z_{5} c \hat{\mathbf{z}}$ & $(24 m)$ \\
\hline $\mathbf{B}_{67}$ & $=$ & $-y_{5} \mathbf{a}_{1}-x_{5} \mathbf{a}_{2}+\left(\frac{1}{2}+z_{5}\right) \mathbf{a}_{3}$ & $=$ & $\begin{array}{c}-\frac{1}{2}\left(x_{5}+y_{5}\right) a \hat{\mathbf{x}}+ \\
\frac{\sqrt{3}}{2}\left(-x_{5}+y_{5}\right) a \hat{\mathbf{y}}+\left(\frac{1}{2}+z_{5}\right) c \hat{\mathbf{z}}\end{array}$ & $(24 m)$ \\
\hline $\mathbf{B}_{68}$ & $=$ & $\left(-x_{5}+y_{5}\right) \mathbf{a}_{1}+y_{5} \mathbf{a}_{2}+\left(\frac{1}{2}+z_{5}\right) \mathbf{a}_{3}$ & $=$ & $\begin{array}{c}\left(-\frac{1}{2} x_{5}+y_{5}\right) a \hat{\mathbf{x}}+\frac{\sqrt{3}}{2} x_{5} a \hat{\mathbf{y}}+ \\
\left(\frac{1}{2}+z_{5}\right) c \hat{\mathbf{z}}\end{array}$ & $(24 m)$ \\
\hline $\mathbf{B}_{69}$ & $=$ & $x_{5} \mathbf{a}_{1}+\left(x_{5}-y_{5}\right) \mathbf{a}_{2}+\left(\frac{1}{2}+z_{5}\right) \mathbf{a}_{3}$ & $=$ & $\begin{array}{c}\left(x_{5}-\frac{1}{2} y_{5}\right) a \hat{\mathbf{x}}-\frac{\sqrt{3}}{2} y_{5} a \hat{\mathbf{y}}+ \\
\left(\frac{1}{2}+z_{5}\right) c \hat{\mathbf{z}}\end{array}$ & $(24 m)$ \\
\hline $\mathbf{B}_{70}$ & $=$ & $y_{5} \mathbf{a}_{1}+x_{5} \mathbf{a}_{2}+\left(\frac{1}{2}+z_{5}\right) \mathbf{a}_{3}$ & $=$ & $\begin{array}{c}\frac{1}{2}\left(x_{5}+y_{5}\right) a \hat{\mathbf{x}}+\frac{\sqrt{3}}{2}\left(x_{5}-y_{5}\right) a \hat{\mathbf{y}}+ \\
\left(\frac{1}{2}+z_{5}\right) c \hat{\mathbf{z}}\end{array}$ & $(24 m)$ \\
\hline $\mathbf{B}_{71}$ & $=$ & $\left(x_{5}-y_{5}\right) \mathbf{a}_{1}-y_{5} \mathbf{a}_{2}+\left(\frac{1}{2}+z_{5}\right) \mathbf{a}_{3}$ & $=$ & $\begin{array}{c}\left(\frac{1}{2} x_{5}-y_{5}\right) a \hat{\mathbf{x}}-\frac{\sqrt{3}}{2} x_{5} a \hat{\mathbf{y}}+ \\
\left(\frac{1}{2}+z_{5}\right) c \hat{\mathbf{z}}\end{array}$ & $(24 m)$ \\
\hline $\mathbf{B}_{72}$ & $=$ & $-x_{5} \mathbf{a}_{1}+\left(-x_{5}+y_{5}\right) \mathbf{a}_{2}+\left(\frac{1}{2}+z_{5}\right) \mathbf{a}_{3}$ & $=$ & $\begin{array}{c}\left(-x_{5}+\frac{1}{2} y_{5}\right) a \hat{\mathbf{x}}+\frac{\sqrt{3}}{2} y_{5} a \hat{\mathbf{y}}+ \\
\left(\frac{1}{2}+z_{5}\right) c \hat{\mathbf{z}}\end{array}$ & $(24 m)$ \\
\hline
\end{tabular}

\section{References:}

- J. W. Richardson Jr., J. J. Pluth, and J. V. Smith, Aluminophosphate number 5: time-of-flight neutron powder diffraction study of calcined powder at 295 K, Acta Crystallogr. C 43, 1469-1472 (1987), doi:10.1107/S0108270187091418.

\section{Found in:}

- P. Villars and K. Cenzual, Pearson's Crystal Data - Crystal Structure Database for Inorganic Compounds, ASM International (2013).

\section{Geometry files:}

- CIF: pp. 942

- POSCAR: pp. 942 


\section{Mavlyanovite $\left(\mathrm{Mn}_{5} \mathrm{Si}_{3}\right)$ Structure: A5B3_hP16_193_dg_g}
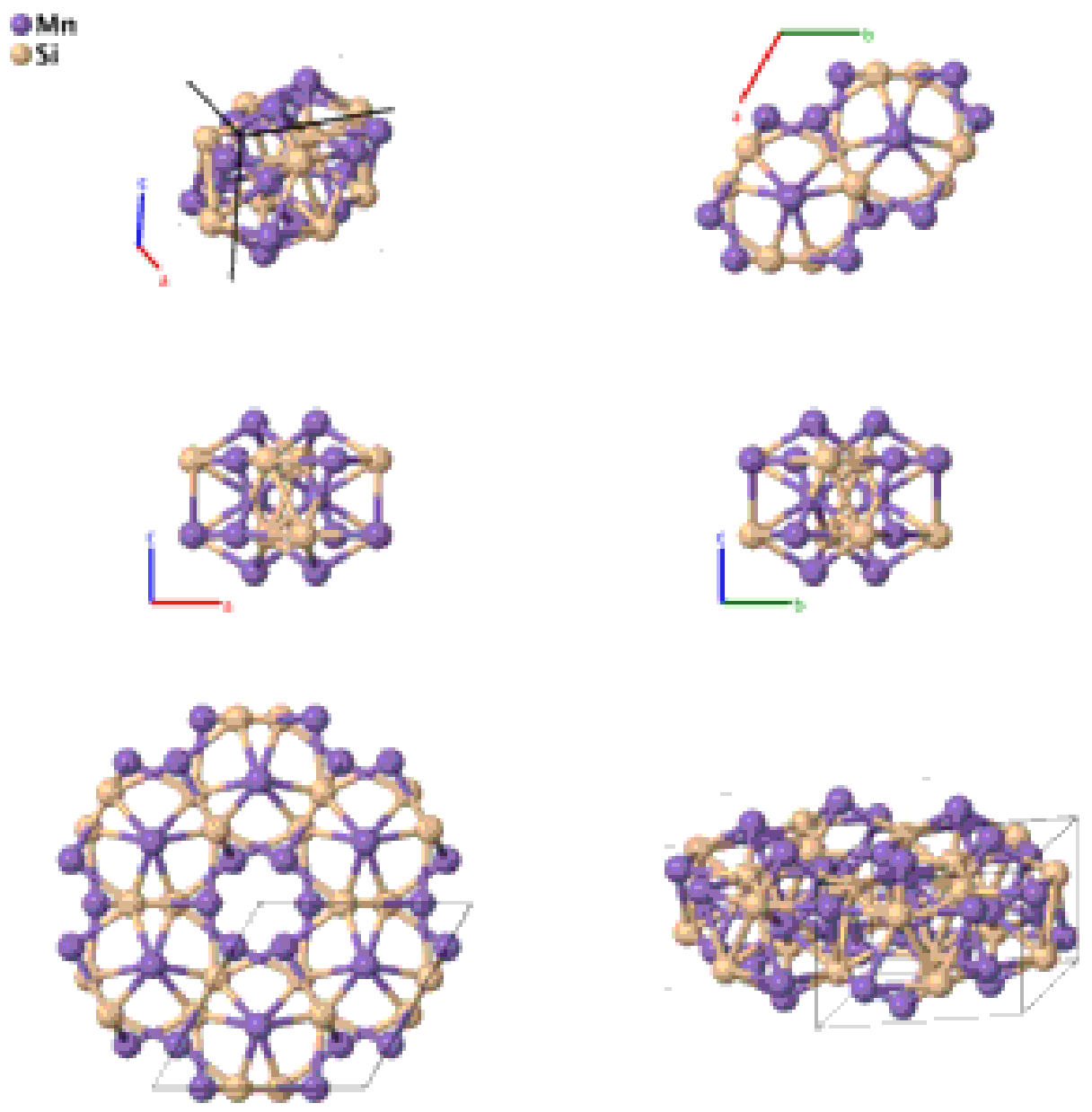

Prototype

$: \quad \mathrm{Mn}_{5} \mathrm{Si}_{3}$

AFLOW prototype label

: A5B3_hP16_193_dg_g

Strukturbericht designation

: None

Pearson symbol

: $\quad \mathrm{hP} 16$

Space group number

: 193

Space group symbol

: $\quad P 6_{3} / \mathrm{mcm}$

AFLOW prototype command : aflow --proto=A5B3_hP16_193_dg_g

- - params $=a, c / a, x_{2}, x_{3}$

Hexagonal primitive vectors:

$$
\begin{array}{ll}
\mathbf{a}_{1}= & \frac{1}{2} a \hat{\mathbf{x}}-\frac{\sqrt{3}}{2} a \hat{\mathbf{y}} \\
\mathbf{a}_{2}= & \frac{1}{2} a \hat{\mathbf{x}}+\frac{\sqrt{3}}{2} a \hat{\mathbf{y}} \\
\mathbf{a}_{3}= & c \hat{\mathbf{z}}
\end{array}
$$

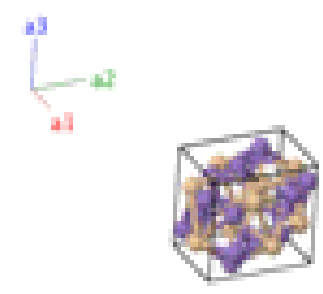

Basis vectors: 
Lattice Coordinates
$\mathbf{B}_{1}=$
$\frac{1}{3} \mathbf{a}_{1}+\frac{2}{3} \mathbf{a}_{2}$
$\mathbf{B}_{2}=$
$\frac{2}{3} \mathbf{a}_{1}+\frac{1}{3} \mathbf{a}_{2}+\frac{1}{2} \mathbf{a}_{3}$
$\mathbf{B}_{3}=$
$\frac{2}{3} \mathbf{a}_{1}+\frac{1}{3} \mathbf{a}_{2}$
$\mathbf{B}_{4}=\frac{1}{3} \mathbf{a}_{1}+\frac{2}{3} \mathbf{a}_{2}+\frac{1}{2} \mathbf{a}_{3}$
$\mathbf{B}_{5}=$
$x_{2} \mathbf{a}_{1}+\frac{1}{4} \mathbf{a}_{3}$
$\mathbf{B}_{6}=$
$x_{2} \mathbf{a}_{2}+\frac{1}{4} \mathbf{a}_{3}$
$\mathbf{B}_{7}=-x_{2} \mathbf{a}_{1}-x_{2} \mathbf{a}_{2}+\frac{1}{4} \mathbf{a}_{3}$
$\mathbf{B}_{8}=-x_{2} \mathbf{a}_{1}+\frac{3}{4} \mathbf{a}_{3}$
$\mathbf{B}_{9}=$
$-x_{2} \mathbf{a}_{2}+\frac{3}{4} \mathbf{a}_{3}$
$\mathbf{B}_{10}=$
$\mathbf{B}_{11}=$
$x_{2} \mathbf{a}_{1}+x_{2} \mathbf{a}_{2}+\frac{3}{4} \mathbf{a}_{3}$
$\mathbf{B}_{12}=$
$\mathbf{B}_{13}=$
$\mathbf{B}_{14}=$
$\mathbf{B}_{15}=$
$\mathbf{B}_{16}=$
$-x_{3} \mathbf{a}_{1}-x_{3} \mathbf{a}_{2}+\frac{1}{4} \mathbf{a}_{3}$
$x_{3} \mathbf{a}_{1}+\frac{1}{4} \mathbf{a}_{3}$
$x_{3} \mathbf{a}_{2}+\frac{1}{4} \mathbf{a}_{3}$
$-x_{3} \mathbf{a}_{1}+\frac{3}{4} \mathbf{a}_{3}$
$-x_{3} \mathbf{a}_{2}+\frac{3}{4} \mathbf{a}_{3}$
$x_{3} \mathbf{a}_{1}+x_{3} \mathbf{a}_{2}+\frac{3}{4} \mathbf{a}_{3}$

Cartesian Coordinates

$=$

$=$

$=$

$=$

$=$

$=$

$=$
$=$

$=$

$=$

$$
=
$$

$=$

$=$

$=$

$$
\frac{1}{2} a \hat{\mathbf{x}}+\frac{1}{2 \sqrt{3}} a \hat{\mathbf{y}}
$$$$
\frac{1}{2} a \hat{\mathbf{x}}-\frac{1}{2 \sqrt{3}} a \hat{\mathbf{y}}+\frac{1}{2} c \hat{\mathbf{z}}
$$$$
\frac{1}{2} a \hat{\mathbf{x}}-\frac{1}{2 \sqrt{3}} a \hat{\mathbf{y}}
$$$$
\frac{1}{2} a \hat{\mathbf{x}}+\frac{1}{2 \sqrt{3}} a \hat{\mathbf{y}}+\frac{1}{2} c \hat{\mathbf{z}}
$$$$
\frac{1}{2} x_{2} a \hat{\mathbf{x}}-\frac{\sqrt{3}}{2} x_{2} a \hat{\mathbf{y}}+\frac{1}{4} c \hat{\mathbf{z}}
$$$$
\frac{1}{2} x_{2} a \hat{\mathbf{x}}+\frac{\sqrt{3}}{2} x_{2} a \hat{\mathbf{y}}+\frac{1}{4} c \hat{\mathbf{z}}
$$$$
-x_{2} a \hat{\mathbf{x}}+\frac{1}{4} c \hat{\mathbf{z}}
$$$$
-\frac{1}{2} x_{2} a \hat{\mathbf{x}}+\frac{\sqrt{3}}{2} x_{2} a \hat{\mathbf{y}}+\frac{3}{4} c \hat{\mathbf{z}}
$$$$
-\frac{1}{2} x_{2} a \hat{\mathbf{x}}-\frac{\sqrt{3}}{2} x_{2} a \hat{\mathbf{y}}+\frac{3}{4} c \hat{\mathbf{z}}
$$$$
x_{2} a \hat{\mathbf{x}}+\frac{3}{4} c \hat{\mathbf{z}}
$$$$
\frac{1}{2} x_{3} a \hat{\mathbf{x}}-\frac{\sqrt{3}}{2} x_{3} a \hat{\mathbf{y}}+\frac{1}{4} c \hat{\mathbf{z}}
$$$$
\frac{1}{2} x_{3} a \hat{\mathbf{x}}+\frac{\sqrt{3}}{2} x_{3} a \hat{\mathbf{y}}+\frac{1}{4} c \hat{\mathbf{z}}
$$$$
-x_{3} a \hat{\mathbf{x}}+\frac{1}{4} c \hat{\mathbf{z}}
$$$$
=
$$$$
=
$$

$=$

$=$
Wyckoff Position
Atom Type

Mn I

Mn I

Mn I

Mn I

Mn II

Mn II

Mn II

Mn II

Mn II

Mn II

$\mathrm{Si}$

$\mathrm{Si}$

$\mathrm{Si}$

$\mathrm{Si}$

$\mathrm{Si}$

$\mathrm{Si}$

\section{References:}

- B. Aronsson, A note on the compositions and crystal structures of $\mathrm{MnB}_{2}, \mathrm{Mn}_{3} \mathrm{Si}, \mathrm{Mn}_{5} \mathrm{Si}_{3}$, and $\mathrm{FeSi}_{2}$, Acta Chem. Scand. 14, 1414-1418 (1960), doi:10.3891/acta.chem.scand.14-1414.

\section{Found in:}

- P. Villars and K. Cenzual, Pearson's Crystal Data - Crystal Structure Database for Inorganic Compounds, ASM International (2013).

\section{Geometry files:}

- CIF: pp. 943

- POSCAR: pp. 943 


\section{$\mathrm{Ni}_{3} \mathrm{Ti}\left(D 0_{24}\right)$ Structure: A3B_hP16_194_gh_ac}
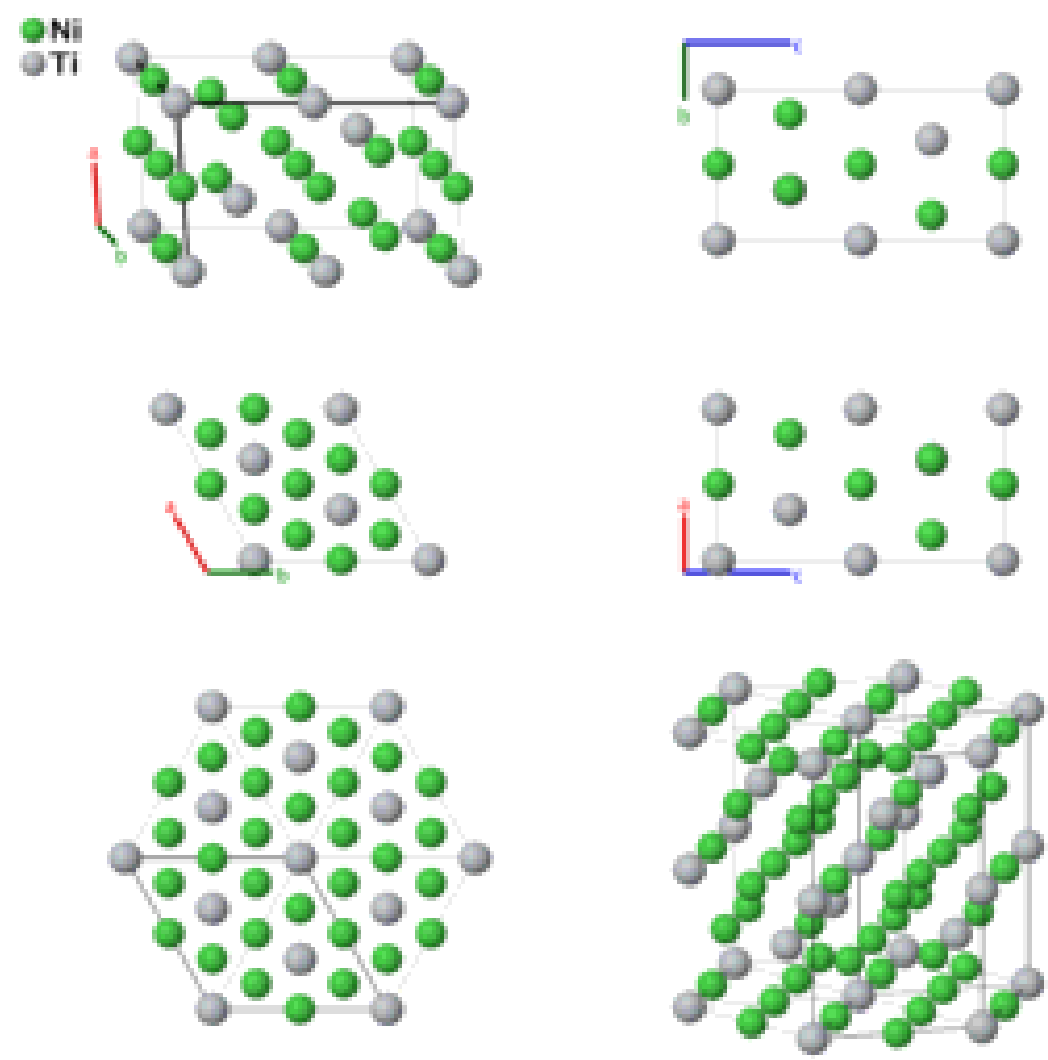

Prototype

AFLOW prototype label

Strukturbericht designation

Pearson symbol

Space group number

Space group symbol

AFLOW prototype command
: $\quad \mathrm{Ni}_{3} \mathrm{Ti}$

: A3B_hP16_194_gh_ac

: $\quad \mathrm{DO}_{24}$

: $\mathrm{hP} 16$

: $\quad 194$

: $\quad P 6_{3} / m m c$ aflow --proto=A3B_hP16_194_gh_ac

- params $=a, c / a, x_{4}$

\section{Other compounds with this structure:}

- $\mathrm{NpPd}_{3}, \mathrm{HfPd}_{3}, \mathrm{TiPd}_{3}, \mathrm{ZrPd}_{3}, \mathrm{HfPt}_{3}, \mathrm{ZrPt}_{3}$

- The internal coordinate $x_{4}$ was not determined by any reference we could find. We follow (Villars, 2016) and set $x_{4}=-1 / 6$, which places the $\mathrm{Nb}$ atoms in line with the Ti atoms in the $z=1 / 4$ and $z=3 / 4$ planes. This is not required by symmetry, and it is likely that the actual value of $x_{4}$ will be close, but not equal to $-1 / 6$.

\section{Hexagonal primitive vectors:}

$$
\begin{array}{ll}
\mathbf{a}_{1}= & \frac{1}{2} a \hat{\mathbf{x}}-\frac{\sqrt{3}}{2} a \hat{\mathbf{y}} \\
\mathbf{a}_{2}= & \frac{1}{2} a \hat{\mathbf{x}}+\frac{\sqrt{3}}{2} a \hat{\mathbf{y}} \\
\mathbf{a}_{3}= & c \hat{\mathbf{z}}
\end{array}
$$

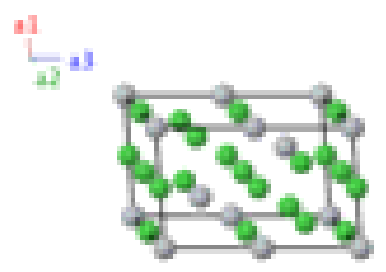




\section{Basis vectors:}

\section{Lattice Coordinates}

$\mathbf{B}_{1}=$

$\mathbf{B}_{2}=$

$\mathbf{B}_{3}=$

$\mathbf{B}_{4}=$

$\mathbf{B}_{5}=$

$\mathbf{B}_{6}=$

$\mathbf{B}_{7}=$

$\mathbf{B}_{8}=$

$\mathbf{B}_{9}=$

$\mathbf{B}_{10}=$

$\mathbf{B}_{11}=$

$\mathbf{B}_{12}=$

$\mathbf{B}_{13}=$

$\mathbf{B}_{14}=$

$\mathbf{B}_{15}=$

$\mathbf{B}_{16}=$

$$
0 \mathbf{a}_{1}+0 \mathbf{a}_{2}+0 \mathbf{a}_{3}
$$$$
\frac{1}{2} \mathbf{a}_{3}
$$$$
\frac{1}{3} \mathbf{a}_{1}+\frac{2}{3} \mathbf{a}_{2}+\frac{1}{4} \mathbf{a}_{3}
$$$$
\frac{2}{3} \mathbf{a}_{1}+\frac{1}{3} \mathbf{a}_{2}+\frac{3}{4} \mathbf{a}_{3}
$$$$
\frac{1}{2} \mathbf{a}_{1}
$$$$
\frac{1}{2} \mathbf{a}_{2}
$$

$$
\frac{1}{2} \mathbf{a}_{1}+\frac{1}{2} \mathbf{a}_{2}
$$$$
\frac{1}{2} \mathbf{a}_{1}+\frac{1}{2} \mathbf{a}_{3}
$$$$
\frac{1}{2} \mathbf{a}_{2}+\frac{1}{2} \mathbf{a}_{3}
$$

$$
\frac{1}{2} \mathbf{a}_{1}+\frac{1}{2} \mathbf{a}_{2}+\frac{1}{2} \mathbf{a}_{3}
$$

$$
x_{4} \mathbf{a}_{1}+2 x_{4} \mathbf{a}_{2}+\frac{1}{4} \mathbf{a}_{3}
$$$$
-2 x_{4} \mathbf{a}_{1}-x_{4} \mathbf{a}_{2}+\frac{1}{4} \mathbf{a}_{3}
$$$$
x_{4} \mathbf{a}_{1}-x_{4} \mathbf{a}_{2}+\frac{1}{4} \mathbf{a}_{3}
$$$$
-x_{4} \mathbf{a}_{1}-2 x_{4} \mathbf{a}_{2}+\frac{3}{4} \mathbf{a}_{3}
$$$$
2 x_{4} \mathbf{a}_{1}+x_{4} \mathbf{a}_{2}+\frac{3}{4} \mathbf{a}_{3}
$$$$
-x_{4} \mathbf{a}_{1}+x_{4} \mathbf{a}_{2}+\frac{3}{4} \mathbf{a}_{3}
$$

$$
\text { Cartesian Coordinates }
$$

$$
=
$$$$
0 \hat{\mathbf{x}}+0 \hat{\mathbf{y}}+0 \hat{\mathbf{z}}
$$$$
\frac{1}{2} c \hat{\mathbf{z}}
$$$$
\frac{1}{2} a \hat{\mathbf{x}}+\frac{1}{2 \sqrt{3}} a \hat{\mathbf{y}}+\frac{1}{4} c \hat{\mathbf{z}}
$$$$
\frac{1}{2} a \hat{\mathbf{x}}-\frac{1}{2 \sqrt{3}} a \hat{\mathbf{y}}+\frac{3}{4} c \hat{\mathbf{z}}
$$$$
\frac{1}{4} a \hat{\mathbf{x}}-\frac{\sqrt{3}}{4} a \hat{\mathbf{y}}
$$$$
\frac{1}{4} a \hat{\mathbf{x}}+\frac{\sqrt{3}}{4} a \hat{\mathbf{y}}
$$$$
\frac{1}{2} a \hat{\mathbf{x}}
$$$$
\frac{1}{4} a \hat{\mathbf{x}}-\frac{\sqrt{3}}{4} a \hat{\mathbf{y}}+\frac{1}{2} c \hat{\mathbf{z}}
$$$$
\frac{1}{4} a \hat{\mathbf{x}}+\frac{\sqrt{3}}{4} a \hat{\mathbf{y}}+\frac{1}{2} c \hat{\mathbf{z}}
$$$$
\frac{1}{2} a \hat{\mathbf{x}}+\frac{1}{2} c \hat{\mathbf{z}}
$$$$
\frac{3}{2} x_{4} a \hat{\mathbf{x}}+\frac{\sqrt{3}}{2} x_{4} a \hat{\mathbf{y}}+\frac{1}{4} c \hat{\mathbf{z}}
$$$$
-\frac{3}{2} x_{4} a \hat{\mathbf{x}}+\frac{\sqrt{3}}{2} x_{4} a \hat{\mathbf{y}}+\frac{1}{4} c \hat{\mathbf{z}}
$$$$
-\sqrt{3} x_{4} a \hat{\mathbf{y}}+\frac{1}{4} c \hat{\mathbf{z}}
$$$$
-\frac{3}{2} x_{4} a \hat{\mathbf{x}}-\frac{\sqrt{3}}{2} x_{4} a \hat{\mathbf{y}}+\frac{3}{4} c \hat{\mathbf{z}}
$$$$
\frac{3}{2} x_{4} a \hat{\mathbf{x}}-\frac{\sqrt{3}}{2} x_{4} a \hat{\mathbf{y}}+\frac{3}{4} c \hat{\mathbf{z}}
$$$$
\sqrt{3} x_{4} a \hat{\mathbf{y}}+\frac{3}{4} c \hat{\mathbf{z}}
$$

Wyckoff Position

(2a)

(6h)
Atom Type

Ti I

Ti I

Ti II

Ti II

$\mathrm{Ni}$ I

Ni I

Ni I

Ni I

Ni I

Ni I

Ni II

Ni II

Ni II

Ni II

Ni II

Ni II

\section{References:}

- F. Laves and H. J. Wallbaum, Die Kristallstruktur von $\mathrm{Ni}_{3} \mathrm{Ti}$ und $\mathrm{Si}_{2} \mathrm{Ti}$ (Zwei neue Typen.), Z. Kristallogr. 101, 78-93 (1939), doi:10.1524/zkri.1939.101.1.78.

- P. Villars, ed., PAULING FILE in: Inorganic Solid Phases (SpringerMaterials (online database), Heidelberg, 2016).

\section{Geometry files:}

- CIF: pp. 943

- POSCAR: pp. 943 


\section{$\mathrm{Co}_{2} \mathrm{Al}_{5}\left(D 8_{11}\right)$ Structure: A5B2_hP28_194_ahk_ch}
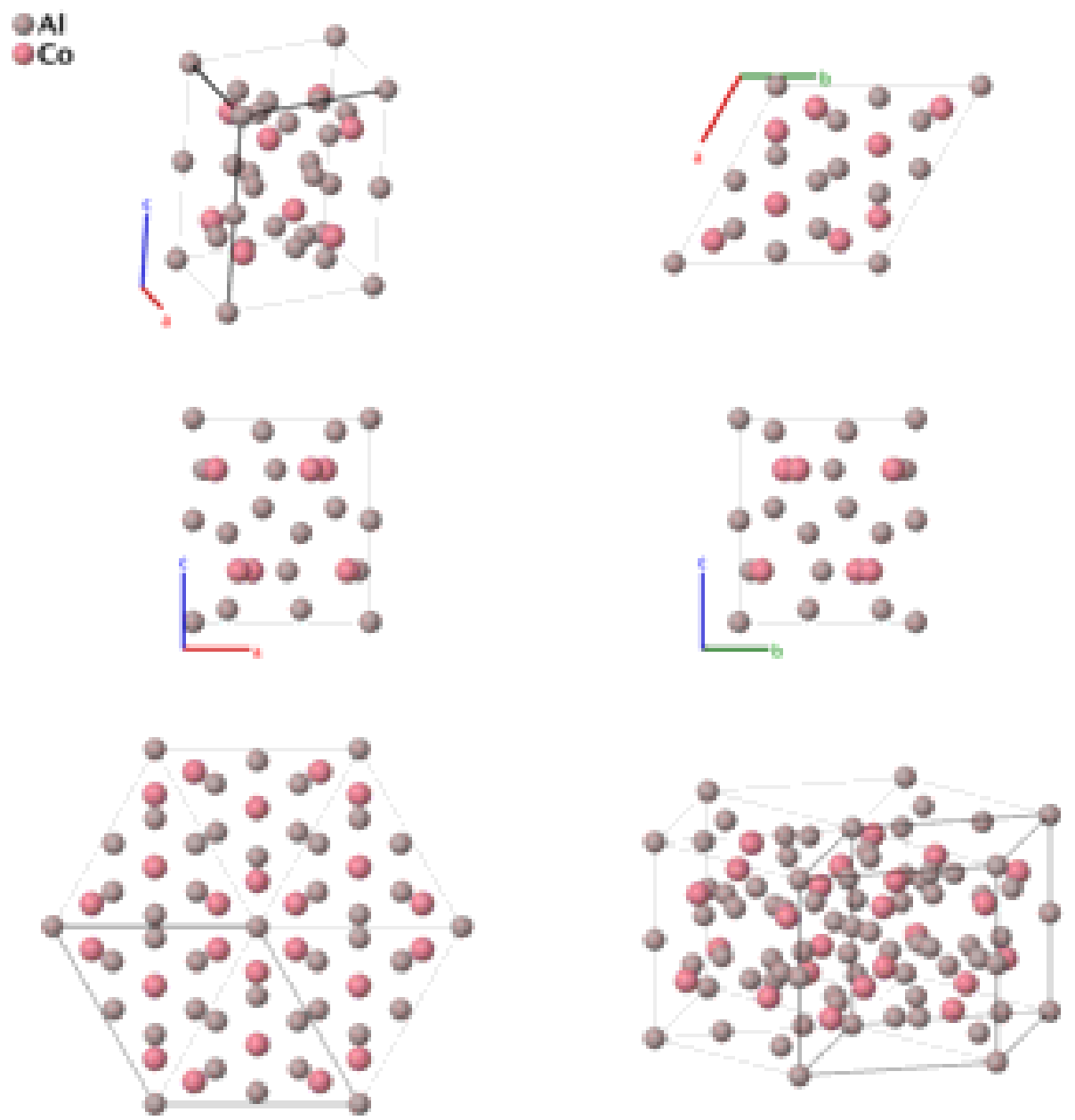

$\begin{array}{lll}\text { Prototype } & : & \mathrm{Co}_{2} \mathrm{Al}_{5} \\ \text { AFLOW prototype label } & : & \mathrm{A} 5 \mathrm{~B} 2 \_\mathrm{hP} 28 \_194 \_a h k \_c h \\ \text { Strukturbericht designation } & : & D 8_{11} \\ \text { Pearson symbol } & : & \mathrm{hP} 28 \\ \text { Space group number } & : & 194 \\ \text { Space group symbol } & : & P 6_{3} / m m c \\ \text { AFLOW prototype command } & : & \begin{array}{l}\text { aflow -proto }=\mathrm{A} 5 \mathrm{~B} 2 \_\mathrm{hP} 28_{-} 194 \_a h k_{-} \mathrm{ch} \\ \end{array} \\ & & -- \text { params }=a, c / a, x_{3}, x_{4}, x_{5}, z_{5}\end{array}$

\section{Other compounds with this structure:}

- $\mathrm{Rh}_{2} \mathrm{Mg}_{5}, \mathrm{Pd}_{2} \mathrm{Mg}_{5}$

- (Newkirk, 1961) puts the Co I atoms at the (2d) Wyckoff sites. We have shifted the origin by $1 / 2 c \hat{\mathbf{z}}$, which shifts the Co atoms to the (2c) sites. 


$$
\begin{array}{ll}
\mathbf{a}_{1}= & \frac{1}{2} a \hat{\mathbf{x}}-\frac{\sqrt{3}}{2} a \hat{\mathbf{y}} \\
\mathbf{a}_{2}= & \frac{1}{2} a \hat{\mathbf{x}}+\frac{\sqrt{3}}{2} a \hat{\mathbf{y}} \\
\mathbf{a}_{3}= & c \hat{\mathbf{z}}
\end{array}
$$

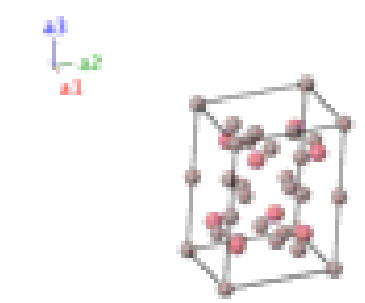

\section{Basis vectors:}

Lattice Coordinates
$\mathbf{B}_{1}=0 \mathbf{a}_{1}+0 \mathbf{a}_{2}+0 \mathbf{a}_{3}$
$\mathbf{B}_{2}=$
$\frac{1}{2} \mathbf{a}_{3}$
$\mathbf{B}_{3}=\quad \frac{1}{3} \mathbf{a}_{1}+\frac{2}{3} \mathbf{a}_{2}+\frac{1}{4} \mathbf{a}_{3}$
$\mathbf{B}_{4}=\quad \frac{2}{3} \mathbf{a}_{1}+\frac{1}{3} \mathbf{a}_{2}+\frac{3}{4} \mathbf{a}_{3}$
$\mathbf{B}_{5}=$
$x_{3} \mathbf{a}_{1}+2 x_{3} \mathbf{a}_{2}+\frac{1}{4} \mathbf{a}_{3}$
$\mathbf{B}_{6}=-2 x_{3} \mathbf{a}_{1}-x_{3} \mathbf{a}_{2}+\frac{1}{4} \mathbf{a}_{3}$
$\mathbf{B}_{7}=$$$
x_{3} \mathbf{a}_{1}-x_{3} \mathbf{a}_{2}+\frac{1}{4} \mathbf{a}_{3}
$$
$\mathbf{B}_{8}=-x_{3} \mathbf{a}_{1}-2 x_{3} \mathbf{a}_{2}+\frac{3}{4} \mathbf{a}_{3}$
$\mathbf{B}_{9}=$
$\mathbf{B}_{10}=$
$\mathbf{B}_{11}=\quad x_{4} \mathbf{a}_{1}+2 x_{4} \mathbf{a}_{2}+\frac{1}{4} \mathbf{a}_{3}$
$\mathbf{B}_{12}=-2 x_{4} \mathbf{a}_{1}-x_{4} \mathbf{a}_{2}+\frac{1}{4} \mathbf{a}_{3}$
$\mathbf{B}_{13}=x_{4} \mathbf{a}_{1}-x_{4} \mathbf{a}_{2}+\frac{1}{4} \mathbf{a}_{3}$
$\mathbf{B}_{14}=-x_{4} \mathbf{a}_{1}-2 x_{4} \mathbf{a}_{2}+\frac{3}{4} \mathbf{a}_{3}$
$\mathbf{B}_{15}=2 x_{4} \mathbf{a}_{1}+x_{4} \mathbf{a}_{2}+\frac{3}{4} \mathbf{a}_{3}$
$\mathbf{B}_{16}=-x_{4} \mathbf{a}_{1}+x_{4} \mathbf{a}_{2}+\frac{3}{4} \mathbf{a}_{3}$
$\mathbf{B}_{17}=x_{5} \mathbf{a}_{1}+2 x_{5} \mathbf{a}_{2}+z_{5} \mathbf{a}_{3}$
$\mathbf{B}_{18}=-2 x_{5} \mathbf{a}_{1}-x_{5} \mathbf{a}_{2}+z_{5} \mathbf{a}_{3}$
$\mathbf{B}_{19}=x_{5} \mathbf{a}_{1}-x_{5} \mathbf{a}_{2}+z_{5} \mathbf{a}_{3}$
$\mathbf{B}_{20}=-x_{5} \mathbf{a}_{1}-2 x_{5} \mathbf{a}_{2}+\left(\frac{1}{2}+z_{5}\right) \mathbf{a}_{3}$
$\mathbf{B}_{21}=2 x_{5} \mathbf{a}_{1}+x_{5} \mathbf{a}_{2}+\left(\frac{1}{2}+z_{5}\right) \mathbf{a}_{3}$
$\mathbf{B}_{22}=-x_{5} \mathbf{a}_{1}+x_{5} \mathbf{a}_{2}+\left(\frac{1}{2}+z_{5}\right) \mathbf{a}_{3}$
$\mathbf{B}_{23}=2 x_{5} \mathbf{a}_{1}+x_{5} \mathbf{a}_{2}-z_{5} \mathbf{a}_{3}$
$\mathbf{B}_{24}=-x_{5} \mathbf{a}_{1}-2 x_{5} \mathbf{a}_{2}-z_{5} \mathbf{a}_{3}$
$\mathbf{B}_{25}=-x_{5} \mathbf{a}_{1}+x_{5} \mathbf{a}_{2}-z_{5} \mathbf{a}_{3}$
$\mathbf{B}_{26}=-2 x_{5} \mathbf{a}_{1}-x_{5} \mathbf{a}_{2}+\left(\frac{1}{2}-z_{5}\right) \mathbf{a}_{3}$
$\mathbf{B}_{27}=x_{5} \mathbf{a}_{1}+2 x_{5} \mathbf{a}_{2}+\left(\frac{1}{2}-z_{5}\right) \mathbf{a}_{3}$
$\mathbf{B}_{28}=x_{5} \mathbf{a}_{1}-x_{5} \mathbf{a}_{2}+\left(\frac{1}{2}-z_{5}\right) \mathbf{a}_{3}$

$\begin{array}{cc}= & 0 \hat{\mathbf{x}}+0 \hat{\mathbf{y}}+0 \hat{\mathbf{z}} \\ = & \frac{1}{2} c \hat{\mathbf{z}}\end{array}$
$=\quad \frac{1}{2} a \hat{\mathbf{x}}+\frac{1}{2 \sqrt{3}} a \hat{\mathbf{y}}+\frac{1}{4} c \hat{\mathbf{z}}$
$=\quad \frac{1}{2} a \hat{\mathbf{x}}-\frac{1}{2 \sqrt{3}} a \hat{\mathbf{y}}+\frac{3}{4} c \hat{\mathbf{z}}$
$=\quad \frac{3}{2} x_{3} a \hat{\mathbf{x}}+\frac{\sqrt{3}}{2} x_{3} a \hat{\mathbf{y}}+\frac{1}{4} c \hat{\mathbf{z}}$
$=\quad-\frac{3}{2} x_{3} a \hat{\mathbf{x}}+\frac{\sqrt{3}}{2} x_{3} a \hat{\mathbf{y}}+\frac{1}{4} c \hat{\mathbf{z}}$
$=\quad-\sqrt{3} x_{3} a \hat{\mathbf{y}}+\frac{1}{4} c \hat{\mathbf{z}}$
$=\quad-\frac{3}{2} x_{3} a \hat{\mathbf{x}}-\frac{\sqrt{3}}{2} x_{3} a \hat{\mathbf{y}}+\frac{3}{4} c \hat{\mathbf{z}}$
$=\quad \frac{3}{2} x_{3} a \hat{\mathbf{x}}-\frac{\sqrt{3}}{2} x_{3} a \hat{\mathbf{y}}+\frac{3}{4} c \hat{\mathbf{z}}$

$$
\sqrt{3} x_{3} a \hat{\mathbf{y}}+\frac{3}{4} c \hat{\mathbf{z}}
$$
$=\quad \frac{3}{2} x_{4} a \hat{\mathbf{x}}+\frac{\sqrt{3}}{2} x_{4} a \hat{\mathbf{y}}+\frac{1}{4} c \hat{\mathbf{z}}$
$=\quad-\frac{3}{2} x_{4} a \hat{\mathbf{x}}+\frac{\sqrt{3}}{2} x_{4} a \hat{\mathbf{y}}+\frac{1}{4} c \hat{\mathbf{z}}$$$
=\quad-\sqrt{3} x_{4} a \hat{\mathbf{y}}+\frac{1}{4} c \hat{\mathbf{z}}
$$
$=\quad-\frac{3}{2} x_{4} a \hat{\mathbf{x}}-\frac{\sqrt{3}}{2} x_{4} a \hat{\mathbf{y}}+\frac{3}{4} c \hat{\mathbf{z}}$
$=\quad \frac{3}{2} x_{4} a \hat{\mathbf{x}}-\frac{\sqrt{3}}{2} x_{4} a \hat{\mathbf{y}}+\frac{3}{4} c \hat{\mathbf{z}}$
$=\sqrt{3} x_{4} a \hat{\mathbf{y}}+\frac{3}{4} c \hat{\mathbf{z}}$
$=\quad \frac{3}{2} x_{5} a \hat{\mathbf{x}}+\frac{\sqrt{3}}{2} x_{5} a \hat{\mathbf{y}}+z_{5} c \hat{\mathbf{z}}$
$=\quad-\frac{3}{2} x_{5} a \hat{\mathbf{x}}+\frac{\sqrt{3}}{2} x_{5} a \hat{\mathbf{y}}+z_{5} c \hat{\mathbf{z}}$

$$
-\sqrt{3} x_{5} a \hat{\mathbf{y}}+z_{5} c \hat{\mathbf{z}}
$$
$=\quad-\frac{3}{2} x_{5} a \hat{\mathbf{x}}-\frac{\sqrt{3}}{2} x_{5} a \hat{\mathbf{y}}+\left(\frac{1}{2}+z_{5}\right) c \hat{\mathbf{z}}$
$=\quad \frac{3}{2} x_{5} a \hat{\mathbf{x}}-\frac{\sqrt{3}}{2} x_{5} a \hat{\mathbf{y}}+\left(\frac{1}{2}+z_{5}\right) c \hat{\mathbf{z}}$
$=\sqrt{3} x_{5} a \hat{\mathbf{y}}+\left(\frac{1}{2}+z_{5}\right) c \hat{\mathbf{z}}$
$=\quad \frac{3}{2} x_{5} a \hat{\mathbf{x}}-\frac{\sqrt{3}}{2} x_{5} a \hat{\mathbf{y}}-z_{5} c \hat{\mathbf{z}}$
$=\quad-\frac{3}{2} x_{5} a \hat{\mathbf{x}}-\frac{\sqrt{3}}{2} x_{5} a \hat{\mathbf{y}}-z_{5} c \hat{\mathbf{z}}$
$=\sqrt{3} x_{5} a \hat{\mathbf{y}}-z_{5} c \hat{\mathbf{z}}$
$=\quad-\frac{3}{2} x_{5} a \hat{\mathbf{x}}+\frac{\sqrt{3}}{2} x_{5} a \hat{\mathbf{y}}+\left(\frac{1}{2}-z_{5}\right) c \hat{\mathbf{z}}$
$=\quad \frac{3}{2} x_{5} a \hat{\mathbf{x}}+\frac{\sqrt{3}}{2} x_{5} a \hat{\mathbf{y}}+\left(\frac{1}{2}-z_{5}\right) c \hat{\mathbf{z}}$
$-\sqrt{3} x_{5} a \hat{\mathbf{y}}+\left(\frac{1}{2}-z_{5}\right) c \hat{\mathbf{z}}$

Cartesian Coordinates
Wyckoff Position Atom Type

(2a)

Al I

Al I

Co I

Co I

Al II

(6h)

Al II

Al II

(6h)

Al II

(6h)

Al II
Al II

Co II

Co II

Co II

Co II

Co II

Co II

Al III

Al III

Al III

Al III

Al III

Al III

Al III

Al III

Al III

Al III

Al III

Al III 


\section{References:}

- J. B. Newkirk, P. J. Black, and A. Damjanovic, The refinement of the $\mathrm{Co}_{2} \mathrm{Al}_{5}$ structures, Acta Cryst. 14, 532-533 (1961), doi:10.1107/S0365110X61001637.

\section{Found in:}

- L. Westin, A Palladium-Magnesium Alloy Phase of $\mathrm{Co}_{2} \mathrm{Al}_{5}$ Type, Acta Chem. Scand. 22, 2574-2580 (1968), doi:10.3891/acta.chem.scand.22-2574.

\section{Geometry files:}

- CIF: pp. 944

- POSCAR: pp. 944 


\section{$\mathrm{Al}_{9} \mathrm{Mn}_{3} \mathrm{Si}\left(E 9_{c}\right)$ Structure: A9B3C_hP26_194_hk_h_a}
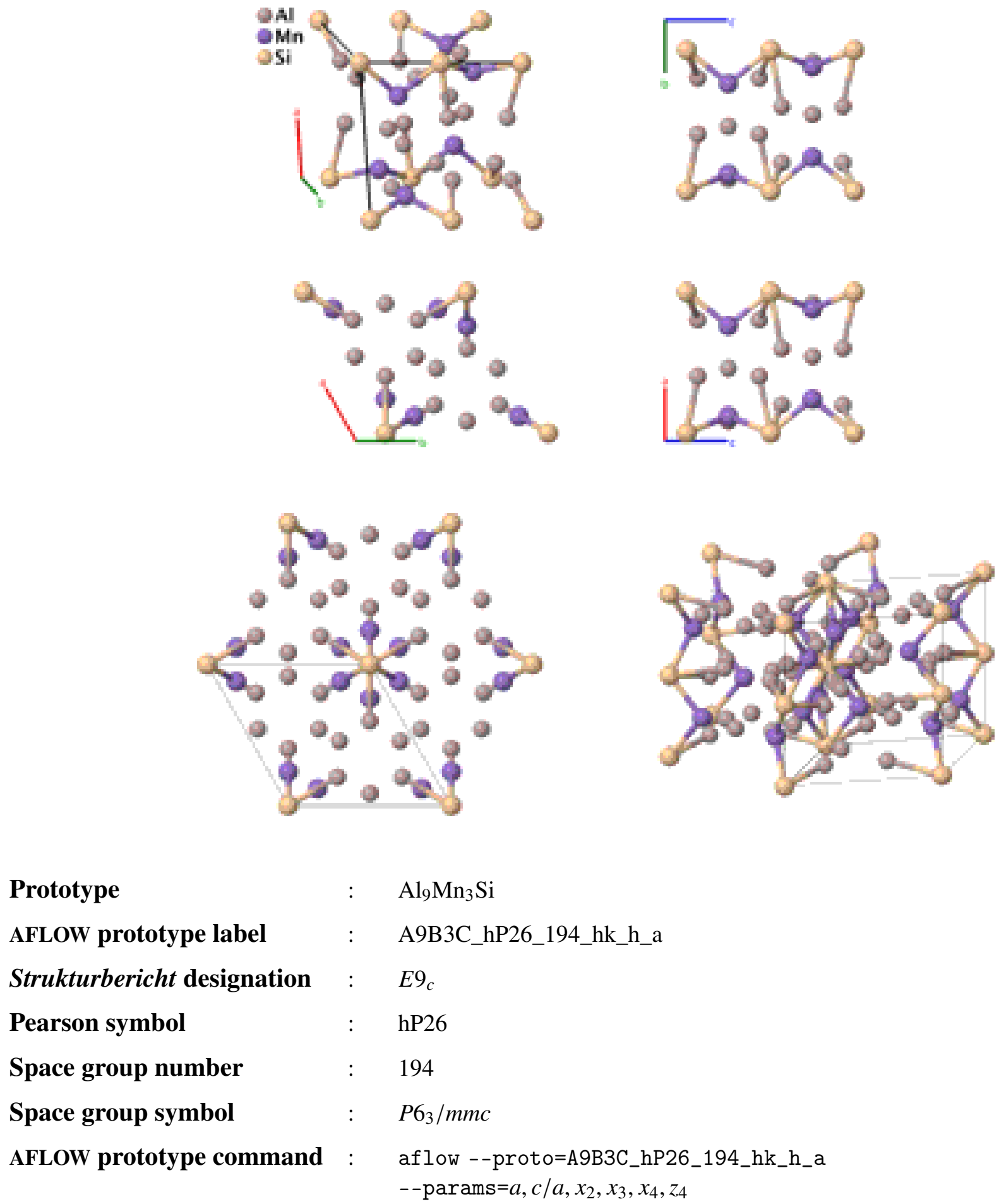

- (Pearson, 1958) quotes (Pratt, 1951) for this structure, but this paper does not contain useful structural information. Pearson also cites (Robinson, 1952) for this structure, but that reference actually discusses $\mathrm{Ni}_{4} \mathrm{Mn}_{11} \mathrm{Al}_{60}$. We use the lattice constants given by Pearson, who states that the structure is stabilized by vacancies. Pearson calls this structure $\beta$-Al-Mn-Si. The atomic postitions are taken from (Brandes, 1992), who give no reference. 


$$
\begin{array}{ll}
\mathbf{a}_{1}= & \frac{1}{2} a \hat{\mathbf{x}}-\frac{\sqrt{3}}{2} a \hat{\mathbf{y}} \\
\mathbf{a}_{2}= & \frac{1}{2} a \hat{\mathbf{x}}+\frac{\sqrt{3}}{2} a \hat{\mathbf{y}} \\
\mathbf{a}_{3}= & c \hat{\mathbf{z}}
\end{array}
$$

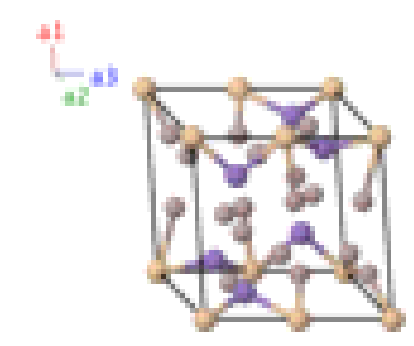

\section{Basis vectors:}

Lattice Coordinates

$\mathbf{B}_{1}=0 \mathbf{a}_{1}+0 \mathbf{a}_{2}+0 \mathbf{a}_{3}$

$\mathbf{B}_{2}=$

$\mathbf{B}_{3}=$

$\mathbf{B}_{4}=$

$\mathbf{B}_{5}=$

$\mathbf{B}_{6}=$

$\mathbf{B}_{7}=$

$\mathbf{B}_{8}=$

$\mathbf{B}_{9}=$

$\mathbf{B}_{10}=$

$\mathbf{B}_{11}=$

$\mathbf{B}_{12}=$

$\mathbf{B}_{13}=$

$\mathbf{B}_{14}=$

$\mathbf{B}_{15}=$

$\mathbf{B}_{16}=$

$\mathbf{B}_{17}=$

$\mathbf{B}_{18}=$

$\mathbf{B}_{19}=$

$\mathbf{B}_{20}=$

$\mathbf{B}_{21}=$

$\mathbf{B}_{21}=$
$\mathbf{B}_{22}=$

$\mathbf{B}_{23}=$

$\mathbf{B}_{24}=-2 x_{4} \mathbf{a}_{1}-x_{4} \mathbf{a}_{2}+\left(\frac{1}{2}-z_{4}\right) \mathbf{a}_{3}$

$\mathbf{B}_{25}=x_{4} \mathbf{a}_{1}+2 x_{4} \mathbf{a}_{2}+\left(\frac{1}{2}-z_{4}\right) \mathbf{a}_{3}$

$\mathbf{B}_{26}=x_{4} \mathbf{a}_{1}-x_{4} \mathbf{a}_{2}+\left(\frac{1}{2}-z_{4}\right) \mathbf{a}_{3}$
Cartesian Coordinates

$=$

$$
0 \hat{\mathbf{x}}+0 \hat{\mathbf{y}}+0 \hat{\mathbf{z}}
$$

$\frac{1}{2} c \hat{\mathbf{z}}$

$=$

$=$

$=$

$=$

$=$

$=$

$=$

$=$

$=$

$$
=
$$$$
=
$$$$
=\quad \frac{3}{2} x_{3} a \hat{\mathbf{x}}-\frac{\sqrt{3}}{2} x_{3} a \hat{\mathbf{y}}+\frac{3}{4} c \hat{\mathbf{z}}
$$$$
=\sqrt{3} x_{3} a \hat{\mathbf{y}}+\frac{3}{4} c \hat{\mathbf{z}}
$$$$
=\quad \frac{3}{2} x_{4} a \hat{\mathbf{x}}+\frac{\sqrt{3}}{2} x_{4} a \hat{\mathbf{y}}+z_{4} c \hat{\mathbf{z}}
$$$$
=\quad-\frac{3}{2} x_{4} a \hat{\mathbf{x}}+\frac{\sqrt{3}}{2} x_{4} a \hat{\mathbf{y}}+z_{4} c \hat{\mathbf{z}}
$$$$
=\quad-\sqrt{3} x_{4} a \hat{\mathbf{y}}+z_{4} c \hat{\mathbf{z}}
$$$$
=\quad-\frac{3}{2} x_{4} a \hat{\mathbf{x}}-\frac{\sqrt{3}}{2} x_{4} a \hat{\mathbf{y}}+\left(\frac{1}{2}+z_{4}\right) c \hat{\mathbf{z}}
$$$$
=\quad \frac{3}{2} x_{4} a \hat{\mathbf{x}}-\frac{\sqrt{3}}{2} x_{4} a \hat{\mathbf{y}}+\left(\frac{1}{2}+z_{4}\right) c \hat{\mathbf{z}}
$$$$
=\sqrt{3} x_{4} a \hat{\mathbf{y}}+\left(\frac{1}{2}+z_{4}\right) c \hat{\mathbf{z}}
$$$$
=\quad \frac{3}{2} x_{4} a \hat{\mathbf{x}}-\frac{\sqrt{3}}{2} x_{4} a \hat{\mathbf{y}}-z_{4} c \hat{\mathbf{z}}
$$$$
=\quad-\frac{3}{2} x_{4} a \hat{\mathbf{x}}-\frac{\sqrt{3}}{2} x_{4} a \hat{\mathbf{y}}-z_{4} c \hat{\mathbf{z}}
$$$$
=
$$$$
\sqrt{3} x_{4} a \hat{\mathbf{y}}-z_{4} c \hat{\mathbf{z}}
$$$$
=\quad-\frac{3}{2} x_{4} a \hat{\mathbf{x}}+\frac{\sqrt{3}}{2} x_{4} a \hat{\mathbf{y}}+\left(\frac{1}{2}-z_{4}\right) c \hat{\mathbf{z}}
$$$$
=\quad \frac{3}{2} x_{4} a \hat{\mathbf{x}}+\frac{\sqrt{3}}{2} x_{4} a \hat{\mathbf{y}}+\left(\frac{1}{2}-z_{4}\right) c \hat{\mathbf{z}}
$$$$
=\quad-\sqrt{3} x_{4} a \hat{\mathbf{y}}+\left(\frac{1}{2}-z_{4}\right) c \hat{\mathbf{z}}
$$

Wyckoff Position Atom Type

$\mathrm{Si}$
$\mathrm{Si}$

Al I

Al I

Al I

Al I

Al I

Al I

$\mathrm{Mn}$

Mn

Mn

Mn

$\mathrm{Mn}$

$\mathrm{Mn}$

Al II

Al II

Al II

Al II

Al II

Al II

Al II

Al II

Al II

Al II

Al II

Al II

\section{References:}


- K. Robinson, LXXIII. The unit cell and Brillouin Zones of $N i_{4} M n_{11} A l_{60}$ and belated compounds, Philos. Mag. 43, 775-782 (1952), doi:10.1080/14786440708520993.

- J. N. Pratt and G. V. Raynor, The intermetallic compounds in the alloys of aluminium and silicon with chromium, manganese, iron, cobalt and nickel, J. Inst. Met. 79, 211 (1951).

\section{Found in:}

- W. B. Pearson, A Handbook of Lattice Spacings and Structures of Metals and Alloys, no. N.R.C. No. 4303 in International Series of Monographs on Metal Physics and Physical Metallurgy (Pergamon Press, Oxford, London, Edinburgh, New York, Paris, Frankfort, 1958), 1964 reprint with corrections edn.

- E. A. Brandes and G. B. Brook, eds., Smithells Metals Reference Book (Butterworth Heinemann, Oxford, Auckland, Boston, Johannesburg, Melbourne, New Delhi, 1992), chap. 6, pp. 6-60, seventh edn.

\section{Geometry files:}

- CIF: pp. 944

- POSCAR: pp. 945 


\section{$\mathrm{PrRu}_{4} \mathrm{P}_{12}$ Structure: A12BC4_cP34_195_2j_ab_2e}
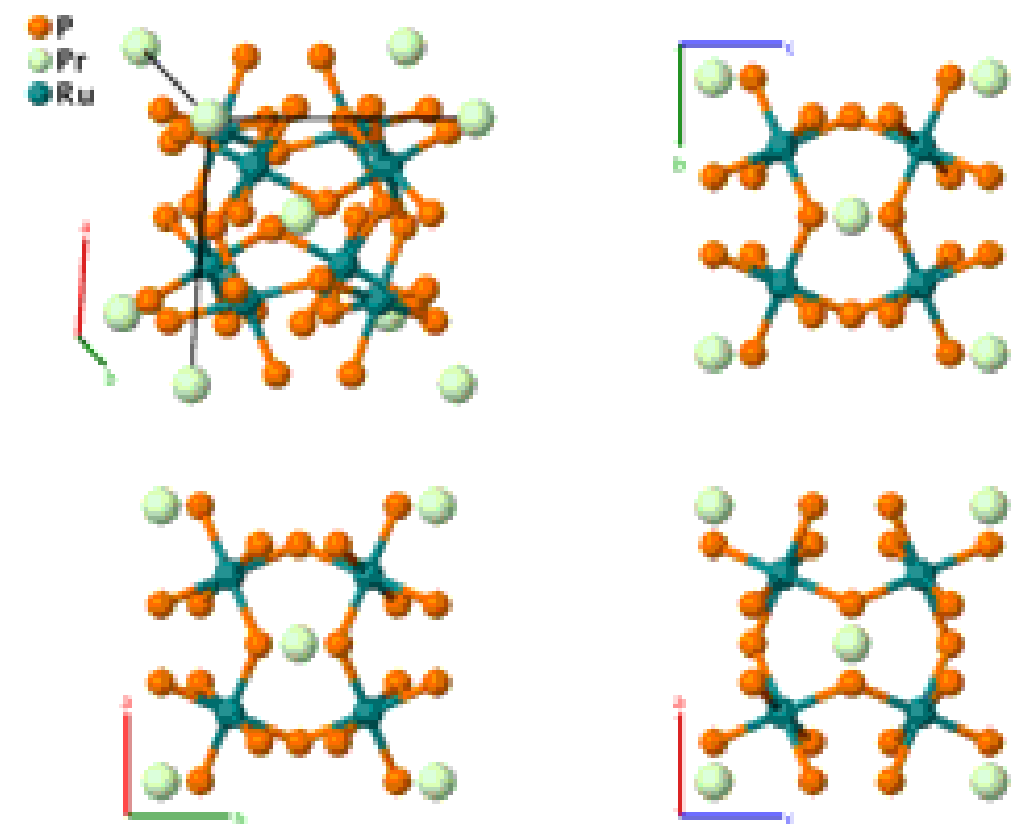

\section{Prototype}

AFLOW prototype label

: $\quad \mathrm{PrRu}_{4} \mathrm{P}_{12}$

Strukturbericht designation

Pearson symbol

: A12BC4_cP34_195_2j_ab_2e

Space group number

: None

Space group symbol

: $\quad \mathrm{cP} 34$

AFLOW prototype command

: 195

: $\quad P 23$

aflow --proto=A12BC4_cP34_195_2j_ab_2e

- -params $=a, x_{3}, x_{4}, x_{5}, y_{5}, z_{5}, x_{6}, y_{6}, z_{6}$

Simple Cubic primitive vectors:

$$
\begin{aligned}
& \mathbf{a}_{1}=a \hat{\mathbf{x}} \\
& \mathbf{a}_{2}=a \hat{\mathbf{y}} \\
& \mathbf{a}_{3}=a \hat{\mathbf{z}}
\end{aligned}
$$

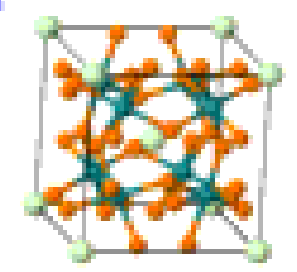

Basis vectors:

Lattice Coordinates

$\begin{array}{llllc}\mathbf{B}_{1}= & 0 \mathbf{a}_{1}+0 \mathbf{a}_{2}+0 \mathbf{a}_{3} & = & 0 \hat{\mathbf{x}}+0 \hat{\mathbf{y}}+0 \hat{\mathbf{z}} \\ \mathbf{B}_{2}= & \frac{1}{2} \mathbf{a}_{1}+\frac{1}{2} \mathbf{a}_{2}+\frac{1}{2} \mathbf{a}_{3} & = & \frac{1}{2} a \hat{\mathbf{x}}+\frac{1}{2} a \hat{\mathbf{y}}+\frac{1}{2} a \hat{\mathbf{z}} \\ \mathbf{B}_{3}= & x_{3} \mathbf{a}_{1}+x_{3} \mathbf{a}_{2}+x_{3} \mathbf{a}_{3} & = & x_{3} a \hat{\mathbf{x}}+x_{3} a \hat{\mathbf{y}}+x_{3} a \hat{\mathbf{z}} \\ \mathbf{B}_{4}= & -x_{3} \mathbf{a}_{1}-x_{3} \mathbf{a}_{2}+x_{3} \mathbf{a}_{3} & = & -x_{3} a \hat{\mathbf{x}}-x_{3} a \hat{\mathbf{y}}+x_{3} a \hat{\mathbf{z}}\end{array}$

Wyckoff Position
Atom Type

$\operatorname{Pr} I$

Pr II

$\mathrm{Ru} I$

$\mathrm{Ru}$ I 


\begin{tabular}{|c|c|c|c|c|c|c|}
\hline $\mathbf{B}_{5}$ & $=$ & $-x_{3} \mathbf{a}_{1}+x_{3} \mathbf{a}_{2}-x_{3} \mathbf{a}_{3}$ & $=$ & $-x_{3} a \hat{\mathbf{x}}+x_{3} a \hat{\mathbf{y}}-x_{3} a \hat{\mathbf{z}}$ & $(4 e)$ & $\mathrm{Ru} \mathrm{I}$ \\
\hline $\mathbf{B}_{6}$ & $=$ & $x_{3} \mathbf{a}_{1}-x_{3} \mathbf{a}_{2}-x_{3} \mathbf{a}_{3}$ & $=$ & $x_{3} a \hat{\mathbf{x}}-x_{3} a \hat{\mathbf{y}}-x_{3} a \hat{\mathbf{z}}$ & $(4 e)$ & $\mathrm{Ru} \mathrm{I}$ \\
\hline $\mathbf{B}_{7}$ & $=$ & $x_{4} \mathbf{a}_{1}+x_{4} \mathbf{a}_{2}+x_{4} \mathbf{a}_{3}$ & $=$ & $x_{4} a \hat{\mathbf{x}}+x_{4} a \hat{\mathbf{y}}+x_{4} a \hat{\mathbf{z}}$ & $(4 e)$ & $\mathrm{Ru}$ II \\
\hline $\mathbf{B}_{8}$ & $=$ & $-x_{4} \mathbf{a}_{1}-x_{4} \mathbf{a}_{2}+x_{4} \mathbf{a}_{3}$ & $=$ & $-x_{4} a \hat{\mathbf{x}}-x_{4} a \hat{\mathbf{y}}+x_{4} a \hat{\mathbf{z}}$ & $(4 e)$ & $\mathrm{Ru}$ II \\
\hline $\mathbf{B}_{9}$ & $=$ & $-x_{4} \mathbf{a}_{1}+x_{4} \mathbf{a}_{2}-x_{4} \mathbf{a}_{3}$ & $=$ & $-x_{4} a \hat{\mathbf{x}}+x_{4} a \hat{\mathbf{y}}-x_{4} a \hat{\mathbf{z}}$ & $(4 e)$ & Ru II \\
\hline $\mathbf{B}_{10}$ & $=$ & $x_{4} \mathbf{a}_{1}-x_{4} \mathbf{a}_{2}-x_{4} \mathbf{a}_{3}$ & $=$ & $x_{4} a \hat{\mathbf{x}}-x_{4} a \hat{\mathbf{y}}-x_{4} a \hat{\mathbf{z}}$ & $(4 e)$ & Ru II \\
\hline $\mathbf{B}_{11}$ & $=$ & $x_{5} \mathbf{a}_{1}+y_{5} \mathbf{a}_{2}+z_{5} \mathbf{a}_{3}$ & $=$ & $x_{5} a \hat{\mathbf{x}}+y_{5} a \hat{\mathbf{y}}+z_{5} a \hat{\mathbf{z}}$ & $(12 j)$ & P I \\
\hline $\mathbf{B}_{12}$ & $=$ & $-x_{5} \mathbf{a}_{1}-y_{5} \mathbf{a}_{2}+z_{5} \mathbf{a}_{3}$ & $=$ & $-x_{5} a \hat{\mathbf{x}}-y_{5} a \hat{\mathbf{y}}+z_{5} a \hat{\mathbf{z}}$ & $(12 j)$ & P I \\
\hline $\mathbf{B}_{13}$ & $=$ & $-x_{5} \mathbf{a}_{1}+y_{5} \mathbf{a}_{2}-z_{5} \mathbf{a}_{3}$ & $=$ & $-x_{5} a \hat{\mathbf{x}}+y_{5} a \hat{\mathbf{y}}-z_{5} a \hat{\mathbf{z}}$ & $(12 j)$ & P I \\
\hline $\mathbf{B}_{14}$ & $=$ & $x_{5} \mathbf{a}_{1}-y_{5} \mathbf{a}_{2}-z_{5} \mathbf{a}_{3}$ & $=$ & $x_{5} a \hat{\mathbf{x}}-y_{5} a \hat{\mathbf{y}}-z_{5} a \hat{\mathbf{z}}$ & $(12 j)$ & P I \\
\hline $\mathbf{B}_{15}$ & $=$ & $z_{5} \mathbf{a}_{1}+x_{5} \mathbf{a}_{2}+y_{5} \mathbf{a}_{3}$ & $=$ & $z_{5} a \hat{\mathbf{x}}+x_{5} a \hat{\mathbf{y}}+y_{5} a \hat{\mathbf{z}}$ & $(12 j)$ & P I \\
\hline $\mathbf{B}_{16}$ & $=$ & $z_{5} \mathbf{a}_{1}-x_{5} \mathbf{a}_{2}-y_{5} \mathbf{a}_{3}$ & $=$ & $z_{5} a \hat{\mathbf{x}}-x_{5} a \hat{\mathbf{y}}-y_{5} a \hat{\mathbf{z}}$ & $(12 j)$ & P I \\
\hline $\mathbf{B}_{17}$ & $=$ & $-z_{5} \mathbf{a}_{1}-x_{5} \mathbf{a}_{2}+y_{5} \mathbf{a}_{3}$ & $=$ & $-z_{5} a \hat{\mathbf{x}}-x_{5} a \hat{\mathbf{y}}+y_{5} a \hat{\mathbf{z}}$ & $(12 j)$ & P I \\
\hline $\mathbf{B}_{18}$ & $=$ & $-z_{5} \mathbf{a}_{1}+x_{5} \mathbf{a}_{2}-y_{5} \mathbf{a}_{3}$ & $=$ & $-z_{5} a \hat{\mathbf{x}}+x_{5} a \hat{\mathbf{y}}-y_{5} a \hat{\mathbf{z}}$ & $(12 j)$ & P I \\
\hline $\mathbf{B}_{19}$ & $=$ & $y_{5} \mathbf{a}_{1}+z_{5} \mathbf{a}_{2}+x_{5} \mathbf{a}_{3}$ & $=$ & $y_{5} a \hat{\mathbf{x}}+z_{5} a \hat{\mathbf{y}}+x_{5} a \hat{\mathbf{z}}$ & $(12 j)$ & P I \\
\hline $\mathbf{B}_{20}$ & $=$ & $-y_{5} \mathbf{a}_{1}+z_{5} \mathbf{a}_{2}-x_{5} \mathbf{a}_{3}$ & $=$ & $-y_{5} a \hat{\mathbf{x}}+z_{5} a \hat{\mathbf{y}}-x_{5} a \hat{\mathbf{z}}$ & $(12 j)$ & P I \\
\hline $\mathbf{B}_{21}$ & $=$ & $y_{5} \mathbf{a}_{1}-z_{5} \mathbf{a}_{2}-x_{5} \mathbf{a}_{3}$ & $=$ & $y_{5} a \hat{\mathbf{x}}-z_{5} a \hat{\mathbf{y}}-x_{5} a \hat{\mathbf{z}}$ & $(12 j)$ & P I \\
\hline $\mathbf{B}_{22}$ & $=$ & $-y_{5} \mathbf{a}_{1}-z_{5} \mathbf{a}_{2}+x_{5} \mathbf{a}_{3}$ & $=$ & $-y_{5} a \hat{\mathbf{x}}-z_{5} a \hat{\mathbf{y}}+x_{5} a \hat{\mathbf{z}}$ & $(12 j)$ & P I \\
\hline $\mathbf{B}_{23}$ & $=$ & $x_{6} \mathbf{a}_{1}+y_{6} \mathbf{a}_{2}+z_{6} \mathbf{a}_{3}$ & $=$ & $x_{6} a \hat{\mathbf{x}}+y_{6} a \hat{\mathbf{y}}+z_{6} a \hat{\mathbf{z}}$ & $(12 j)$ & P II \\
\hline $\mathbf{B}_{24}$ & $=$ & $-x_{6} \mathbf{a}_{1}-y_{6} \mathbf{a}_{2}+z_{6} \mathbf{a}_{3}$ & $=$ & $-x_{6} a \hat{\mathbf{x}}-y_{6} a \hat{\mathbf{y}}+z_{6} a \hat{\mathbf{z}}$ & $(12 j)$ & P II \\
\hline $\mathbf{B}_{25}$ & $=$ & $-x_{6} \mathbf{a}_{1}+y_{6} \mathbf{a}_{2}-z_{6} \mathbf{a}_{3}$ & $=$ & $-x_{6} a \hat{\mathbf{x}}+y_{6} a \hat{\mathbf{y}}-z_{6} a \hat{\mathbf{z}}$ & $(12 j)$ & P II \\
\hline $\mathbf{B}_{26}$ & $=$ & $x_{6} \mathbf{a}_{1}-y_{6} \mathbf{a}_{2}-z_{6} \mathbf{a}_{3}$ & $=$ & $x_{6} a \hat{\mathbf{x}}-y_{6} a \hat{\mathbf{y}}-z_{6} a \hat{\mathbf{z}}$ & $(12 j)$ & P II \\
\hline $\mathbf{B}_{27}$ & $=$ & $z_{6} \mathbf{a}_{1}+x_{6} \mathbf{a}_{2}+y_{6} \mathbf{a}_{3}$ & $=$ & $z_{6} a \hat{\mathbf{x}}+x_{6} a \hat{\mathbf{y}}+y_{6} a \hat{\mathbf{z}}$ & $(12 j)$ & P II \\
\hline $\mathbf{B}_{28}$ & $=$ & $z_{6} \mathbf{a}_{1}-x_{6} \mathbf{a}_{2}-y_{6} \mathbf{a}_{3}$ & $=$ & $z_{6} a \hat{\mathbf{x}}-x_{6} a \hat{\mathbf{y}}-y_{6} a \hat{\mathbf{z}}$ & $(12 j)$ & P II \\
\hline $\mathbf{B}_{29}$ & $=$ & $-z_{6} \mathbf{a}_{1}-x_{6} \mathbf{a}_{2}+y_{6} \mathbf{a}_{3}$ & $=$ & $-z_{6} a \hat{\mathbf{x}}-x_{6} a \hat{\mathbf{y}}+y_{6} a \hat{\mathbf{z}}$ & $(12 j)$ & P II \\
\hline $\mathbf{B}_{30}$ & $=$ & $-z_{6} \mathbf{a}_{1}+x_{6} \mathbf{a}_{2}-y_{6} \mathbf{a}_{3}$ & $=$ & $-z_{6} a \hat{\mathbf{x}}+x_{6} a \hat{\mathbf{y}}-y_{6} a \hat{\mathbf{z}}$ & $(12 j)$ & P II \\
\hline $\mathbf{B}_{31}$ & $=$ & $y_{6} \mathbf{a}_{1}+z_{6} \mathbf{a}_{2}+x_{6} \mathbf{a}_{3}$ & $=$ & $y_{6} a \hat{\mathbf{x}}+z_{6} a \hat{\mathbf{y}}+x_{6} a \hat{\mathbf{z}}$ & $(12 j)$ & P II \\
\hline $\mathbf{B}_{32}$ & $=$ & $-y_{6} \mathbf{a}_{1}+z_{6} \mathbf{a}_{2}-x_{6} \mathbf{a}_{3}$ & $=$ & $-y_{6} a \hat{\mathbf{x}}+z_{6} a \hat{\mathbf{y}}-x_{6} a \hat{\mathbf{z}}$ & $(12 j)$ & P II \\
\hline $\mathbf{B}_{33}$ & $=$ & $y_{6} \mathbf{a}_{1}-z_{6} \mathbf{a}_{2}-x_{6} \mathbf{a}_{3}$ & $=$ & $y_{6} a \hat{\mathbf{x}}-z_{6} a \hat{\mathbf{y}}-x_{6} a \hat{\mathbf{z}}$ & $(12 j)$ & P II \\
\hline $\mathbf{B}_{34}$ & $=$ & $-y_{6} \mathbf{a}_{1}-z_{6} \mathbf{a}_{2}+x_{6} \mathbf{a}_{3}$ & $=$ & $-y_{6} a \hat{\mathbf{x}}-z_{6} a \hat{\mathbf{y}}+x_{6} a \hat{\mathbf{z}}$ & $(12 j)$ & P II \\
\hline
\end{tabular}

\section{References:}

- C. H. Lee, H. Matsuhata, H. Yamaguchi, C. Sekine, K. Kihou, and I. Shirotani, A study of the crystal structure at low temperature in the metal-insulator transition compound $\operatorname{PrRu}_{4} P_{12}$, J. Magn. Magn. Mater. 272, 426-427 (2004), doi:10.1016/j.jmmm.2003.12.433.

- H. T. Stokes and D. M. Hatch, FINDSYM: Program for identifying the space group symmetry of a crystal, J. Appl. Crystallogr. 38, 237-238 (2005), doi:10.1107/S0021889804031528.

- D. Hicks, C. Oses, E. Gossett, G. Gomez, R. H. Taylor, C. Toher, M. J. Mehl, O. Levy, and S. Curtarolo, AFLOW-SYM: platform for the complete, automatic and self-consistent symmetry analysis of crystals, Acta Crystallogr. Sect. A 74, 
184-203 (2018), doi:10.1107/S2053273318003066.

- A. L. Spek, Single-crystal structure validation with the program PLATON, J. Appl. Crystallogr. 36, 7-13 (2003), doi:10.1107/S0021889802022112.

\section{Found in:}

- P. Villars and K. Cenzual, Pearson's Crystal Data - Crystal Structure Database for Inorganic Compounds, ASM International (2013).

\section{Geometry files:}

- CIF: pp. 945

- POSCAR: pp. 945 


\section{$\mathrm{Cu}_{2} \mathrm{Fe}[\mathrm{CN}]_{6}$ Structure: A12B2C_cF60_196_h_bc_a}
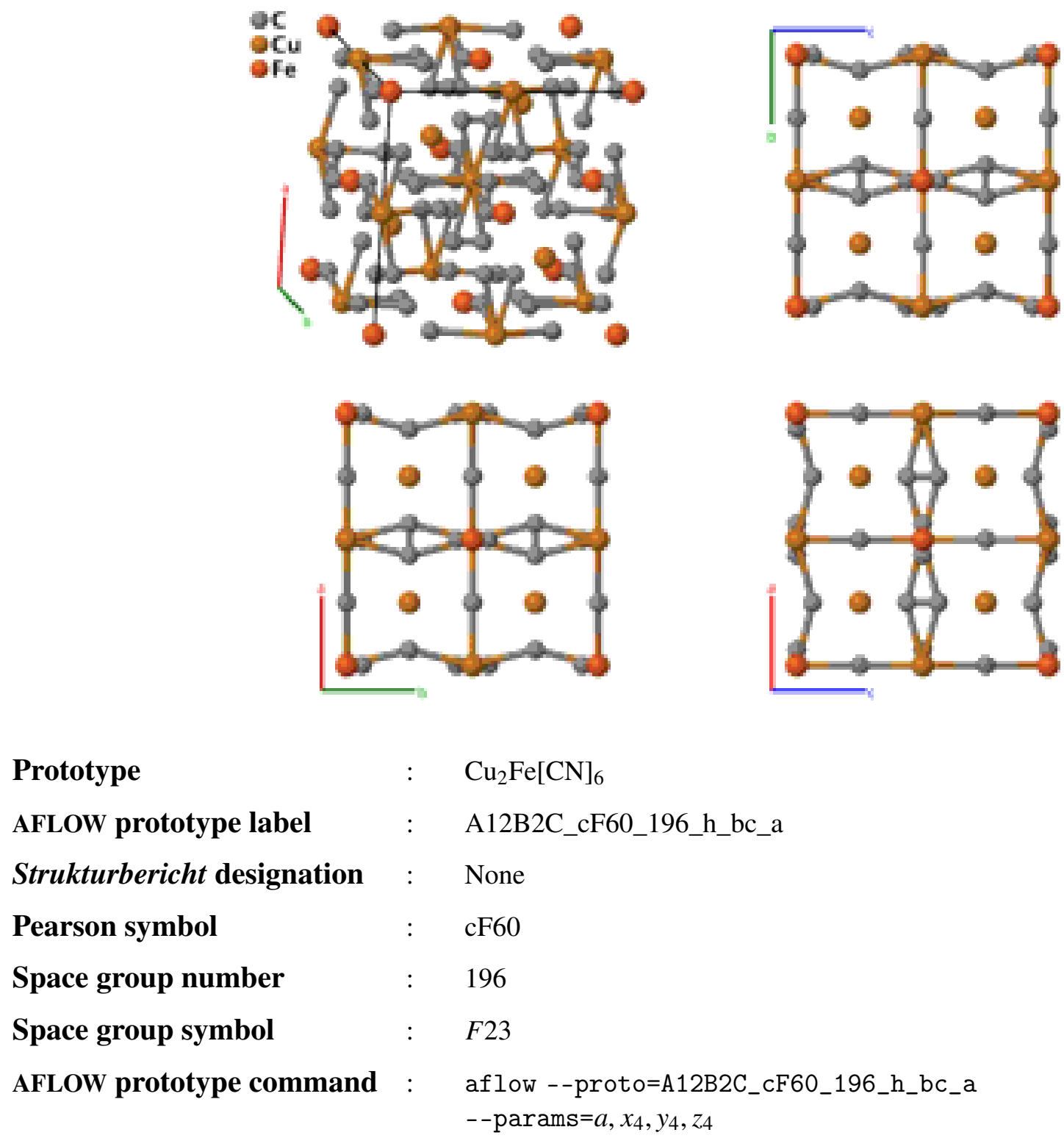

Prototype

AFLOW prototype label

$: \quad \mathrm{Cu}_{2} \mathrm{Fe}[\mathrm{CN}]_{6}$

Strukturbericht designation : None

Pearson symbol $\quad: \quad c F 60$

Space group number $\quad$ : 196

Space group symbol $\quad: \quad F 23$

AFLOW prototype command : aflow --proto=A12B2C_cF60_196_h_bc_a

- params $=a, x_{4}, y_{4}, z_{4}$

- The $\mathrm{C}$ sites are partially occupied with $0.5 \mathrm{C}+0.5 \mathrm{~N}$.

Face-centered Cubic primitive vectors:

$$
\begin{aligned}
& \mathbf{a}_{1}=\frac{1}{2} a \hat{\mathbf{y}}+\frac{1}{2} a \hat{\mathbf{z}} \\
& \mathbf{a}_{2}=\frac{1}{2} a \hat{\mathbf{x}}+\frac{1}{2} a \hat{\mathbf{z}} \\
& \mathbf{a}_{3}=\frac{1}{2} a \hat{\mathbf{x}}+\frac{1}{2} a \hat{\mathbf{y}}
\end{aligned}
$$

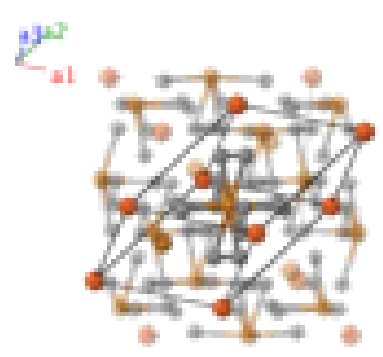

Basis vectors:

Lattice Coordinates

$\mathbf{B}_{1}=0 \mathbf{a}_{1}+0 \mathbf{a}_{2}+0 \mathbf{a}_{3}$
Cartesian Coordinates

Wyckoff Position

(4a)
Atom Type

$=\quad 0 \hat{\mathbf{x}}+0 \hat{\mathbf{y}}+0 \hat{\mathbf{z}}$

$\mathrm{Fe}$ 


\begin{tabular}{|c|c|c|c|c|c|c|}
\hline $\mathbf{B}_{2}$ & $=$ & $\frac{1}{2} \mathbf{a}_{1}+\frac{1}{2} \mathbf{a}_{2}+\frac{1}{2} \mathbf{a}_{3}$ & $=$ & $\frac{1}{2} a \hat{\mathbf{x}}+\frac{1}{2} a \hat{\mathbf{y}}+\frac{1}{2} a \hat{\mathbf{z}}$ & $(4 b)$ & $\mathrm{Cu} \mathrm{I}$ \\
\hline $\mathbf{B}_{3}$ & $=$ & $\frac{1}{4} \mathbf{a}_{1}+\frac{1}{4} \mathbf{a}_{2}+\frac{1}{4} \mathbf{a}_{3}$ & $=$ & $\frac{1}{4} a \hat{\mathbf{x}}+\frac{1}{4} a \hat{\mathbf{y}}+\frac{1}{4} a \hat{\mathbf{z}}$ & $(4 c)$ & $\mathrm{Cu}$ II \\
\hline $\mathbf{B}_{4}$ & $=$ & $\begin{array}{c}\left(-x_{4}+y_{4}+z_{4}\right) \mathbf{a}_{1}+\left(x_{4}-y_{4}+z_{4}\right) \mathbf{a}_{2}+ \\
\left(x_{4}+y_{4}-z_{4}\right) \mathbf{a}_{3}\end{array}$ & $=$ & $x_{4} a \hat{\mathbf{x}}+y_{4} a \hat{\mathbf{y}}+z_{4} a \hat{\mathbf{z}}$ & $(48 h)$ & $\mathrm{C}$ \\
\hline $\mathbf{B}_{5}$ & $=$ & $\begin{array}{c}\left(x_{4}-y_{4}+z_{4}\right) \mathbf{a}_{1}+\left(-x_{4}+y_{4}+z_{4}\right) \mathbf{a}_{2}+ \\
\left(-x_{4}-y_{4}-z_{4}\right) \mathbf{a}_{3}\end{array}$ & $=$ & $-x_{4} a \hat{\mathbf{x}}-y_{4} a \hat{\mathbf{y}}+z_{4} a \hat{\mathbf{z}}$ & $(48 h)$ & $\mathrm{C}$ \\
\hline $\mathbf{B}_{6}$ & $=$ & $\begin{array}{c}\left(x_{4}+y_{4}-z_{4}\right) \mathbf{a}_{1}+\left(-x_{4}-y_{4}-z_{4}\right) \mathbf{a}_{2}+ \\
\left(-x_{4}+y_{4}+z_{4}\right) \mathbf{a}_{3}\end{array}$ & $=$ & $-x_{4} a \hat{\mathbf{x}}+y_{4} a \hat{\mathbf{y}}-z_{4} a \hat{\mathbf{z}}$ & $(48 h)$ & $\mathrm{C}$ \\
\hline $\mathbf{B}_{7}$ & $=$ & $\begin{array}{c}\left(-x_{4}-y_{4}-z_{4}\right) \mathbf{a}_{1}+\left(x_{4}+y_{4}-z_{4}\right) \mathbf{a}_{2}+ \\
\left(x_{4}-y_{4}+z_{4}\right) \mathbf{a}_{3}\end{array}$ & $=$ & $x_{4} a \hat{\mathbf{x}}-y_{4} a \hat{\mathbf{y}}-z_{4} a \hat{\mathbf{z}}$ & $(48 h)$ & $\mathrm{C}$ \\
\hline $\mathbf{B}_{8}$ & $=$ & $\begin{array}{c}\left(x_{4}+y_{4}-z_{4}\right) \mathbf{a}_{1}+\left(-x_{4}+y_{4}+z_{4}\right) \mathbf{a}_{2}+ \\
\left(x_{4}-y_{4}+z_{4}\right) \mathbf{a}_{3}\end{array}$ & $=$ & $z_{4} a \hat{\mathbf{x}}+x_{4} a \hat{\mathbf{y}}+y_{4} a \hat{\mathbf{z}}$ & $(48 h)$ & $\mathrm{C}$ \\
\hline $\mathbf{B}_{9}$ & $=$ & $\begin{array}{c}\left(-x_{4}-y_{4}-z_{4}\right) \mathbf{a}_{1}+\left(x_{4}-y_{4}+z_{4}\right) \mathbf{a}_{2}+ \\
\left(-x_{4}+y_{4}+z_{4}\right) \mathbf{a}_{3}\end{array}$ & $=$ & $z_{4} a \hat{\mathbf{x}}-x_{4} a \hat{\mathbf{y}}-y_{4} a \hat{\mathbf{z}}$ & $(48 h)$ & $\mathrm{C}$ \\
\hline $\mathbf{B}_{10}$ & $=$ & $\begin{array}{c}\left(-x_{4}+y_{4}+z_{4}\right) \mathbf{a}_{1}+\left(x_{4}+y_{4}-z_{4}\right) \mathbf{a}_{2}+ \\
\left(-x_{4}-y_{4}-z_{4}\right) \mathbf{a}_{3}\end{array}$ & $=$ & $-z_{4} a \hat{\mathbf{x}}-x_{4} a \hat{\mathbf{y}}+y_{4} a \hat{\mathbf{z}}$ & $(48 h)$ & $\mathrm{C}$ \\
\hline $\mathbf{B}_{11}$ & $=$ & $\begin{array}{c}\left(x_{4}-y_{4}+z_{4}\right) \mathbf{a}_{1}+\left(-x_{4}-y_{4}-z_{4}\right) \mathbf{a}_{2}+ \\
\left(x_{4}+y_{4}-z_{4}\right) \mathbf{a}_{3}\end{array}$ & $=$ & $-z_{4} a \hat{\mathbf{x}}+x_{4} a \hat{\mathbf{y}}-y_{4} a \hat{\mathbf{z}}$ & $(48 h)$ & $\mathrm{C}$ \\
\hline $\mathbf{B}_{12}$ & $=$ & $\begin{array}{c}\left(x_{4}-y_{4}+z_{4}\right) \mathbf{a}_{1}+\left(x_{4}+y_{4}-z_{4}\right) \mathbf{a}_{2}+ \\
\left(-x_{4}+y_{4}+z_{4}\right) \mathbf{a}_{3}\end{array}$ & $=$ & $y_{4} a \hat{\mathbf{x}}+z_{4} a \hat{\mathbf{y}}+x_{4} a \hat{\mathbf{z}}$ & $(48 h)$ & $\mathrm{C}$ \\
\hline $\mathbf{B}_{13}$ & $=$ & $\begin{array}{c}\left(-x_{4}+y_{4}+z_{4}\right) \mathbf{a}_{1}+\left(-x_{4}-y_{4}-z_{4}\right) \mathbf{a}_{2}+ \\
\left(x_{4}-y_{4}+z_{4}\right) \mathbf{a}_{3}\end{array}$ & $=$ & $-y_{4} a \hat{\mathbf{x}}+z_{4} a \hat{\mathbf{y}}-x_{4} a \hat{\mathbf{z}}$ & $(48 h)$ & $\mathrm{C}$ \\
\hline $\mathbf{B}_{14}$ & $=$ & $\begin{array}{c}\left(-x_{4}-y_{4}-z_{4}\right) \mathbf{a}_{1}+\left(-x_{4}+y_{4}+z_{4}\right) \mathbf{a}_{2}+ \\
\left(x_{4}+y_{4}-z_{4}\right) \mathbf{a}_{3}\end{array}$ & $=$ & $y_{4} a \hat{\mathbf{x}}-z_{4} a \hat{\mathbf{y}}-x_{4} a \hat{\mathbf{z}}$ & $(48 h)$ & $\mathrm{C}$ \\
\hline $\mathbf{B}_{15}$ & $=$ & $\begin{array}{c}\left(x_{4}+y_{4}-z_{4}\right) \mathbf{a}_{1}+\left(x_{4}-y_{4}+z_{4}\right) \mathbf{a}_{2}+ \\
\left(-x_{4}-y_{4}-z_{4}\right) \mathbf{a}_{3}\end{array}$ & $=$ & $-y_{4} a \hat{\mathbf{x}}-z_{4} a \hat{\mathbf{y}}+x_{4} a \hat{\mathbf{z}}$ & $(48 h)$ & $\mathrm{C}$ \\
\hline
\end{tabular}

\section{References:}

- R. Rigamonti, Structure of Cupriferrocyanides I. Copper Ferrocyanide and Potassium Copper Ferrocyanide, Gazz. Chim. Ital. 67, 137-146 (1937).

\section{Found in:}

- P. Villars and K. Cenzual, Pearson's Crystal Data - Crystal Structure Database for Inorganic Compounds, ASM International (2013).

\section{Geometry files:}

- CIF: pp. 945

- POSCAR: pp. 946 
$\mathrm{MgB}_{12} \mathrm{H}_{12}\left[\mathrm{H}_{2} \mathrm{O}\right]_{12}$ Structure:

A12B36CD12_cF488_196_2h_6h_ac_fgh
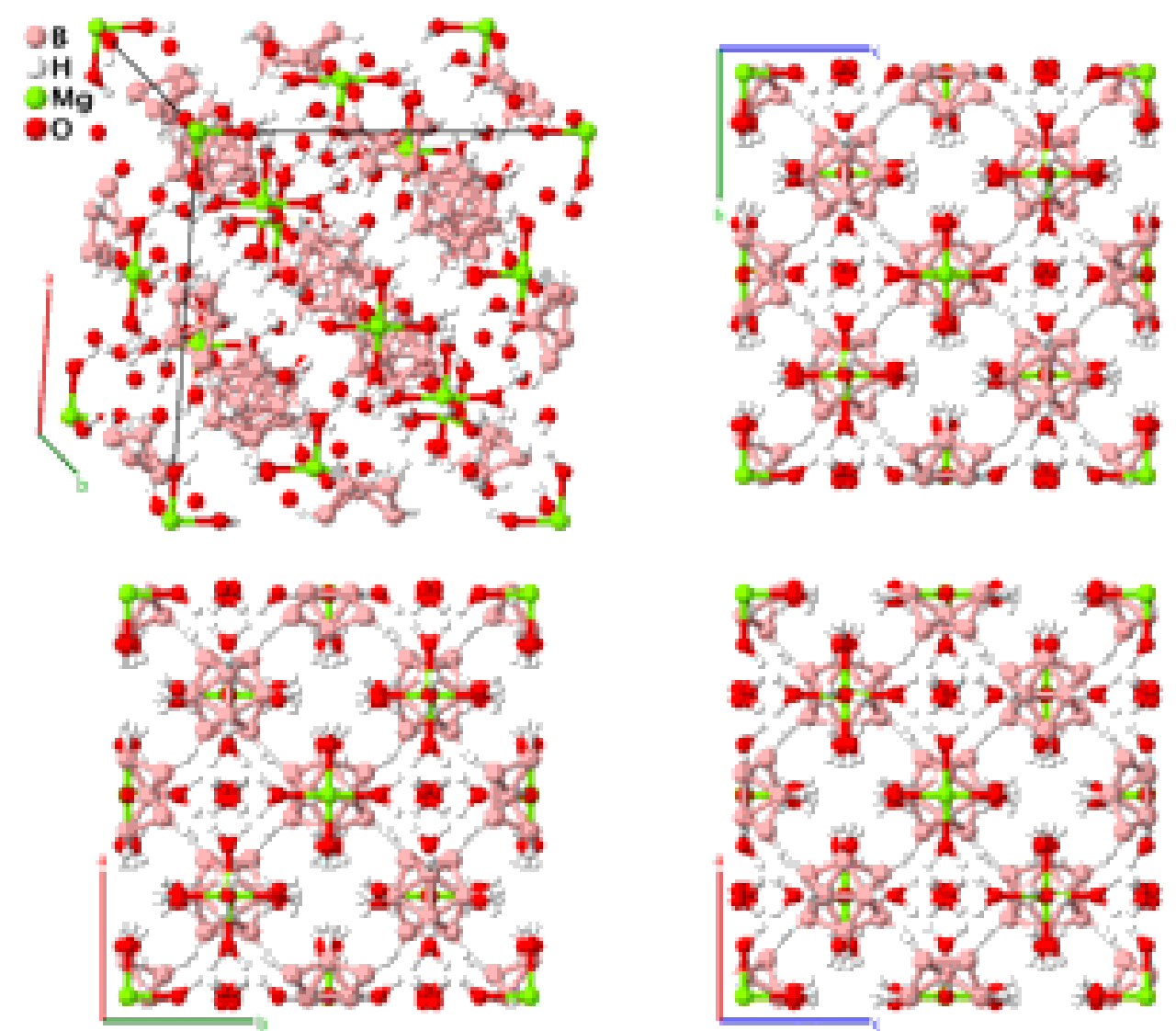

\section{Prototype}

AFLOW prototype label

$\mathrm{MgB}_{12} \mathrm{H}_{12}\left[\mathrm{H}_{2} \mathrm{O}\right]_{12}$

Strukturbericht designation : None

Pearson symbol

: $\quad \mathrm{cF} 488$

Space group number

: 196

Space group symbol

F23

AFLOW prototype command : aflow --proto=A12B36CD12_cF488_196_2h_6h_ac_fgh

- - params $=a, x_{3}, x_{4}, x_{5}, y_{5}, z_{5}, x_{6}, y_{6}, z_{6}, x_{7}, y_{7}, z_{7}, x_{8}, y_{8}, z_{8}, x_{9}, y_{9}, z_{9}, x_{10}, y_{10}, z_{10}, x_{11}$, $y_{11}, z_{11}, x_{12}, y_{12}, z_{12}, x_{13}, y_{13}, z_{13}$

Face-centered Cubic primitive vectors:

$$
\begin{aligned}
& \mathbf{a}_{1}=\frac{1}{2} a \hat{\mathbf{y}}+\frac{1}{2} a \hat{\mathbf{z}} \\
& \mathbf{a}_{2}=\frac{1}{2} a \hat{\mathbf{x}}+\frac{1}{2} a \hat{\mathbf{z}} \\
& \mathbf{a}_{3}=\frac{1}{2} a \hat{\mathbf{x}}+\frac{1}{2} a \hat{\mathbf{y}}
\end{aligned}
$$

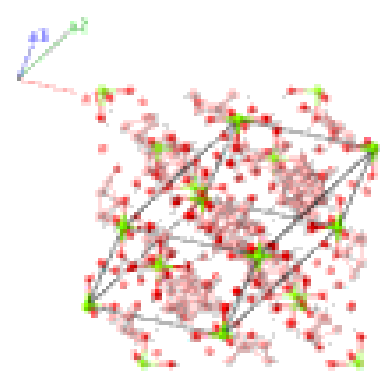

Basis vectors: 
Lattice Coordinates

$$
\begin{aligned}
& \mathbf{B}_{1}=\quad 0 \mathbf{a}_{1}+0 \mathbf{a}_{2}+0 \mathbf{a}_{3} \\
& \mathbf{B}_{2}=\quad \frac{1}{4} \mathbf{a}_{1}+\frac{1}{4} \mathbf{a}_{2}+\frac{1}{4} \mathbf{a}_{3} \\
& \mathbf{B}_{3}=-x_{3} \mathbf{a}_{1}+x_{3} \mathbf{a}_{2}+x_{3} \mathbf{a}_{3} \\
& \mathbf{B}_{4}=x_{3} \mathbf{a}_{1}-x_{3} \mathbf{a}_{2}-x_{3} \mathbf{a}_{3} \\
& \mathbf{B}_{5}=x_{3} \mathbf{a}_{1}-x_{3} \mathbf{a}_{2}+x_{3} \mathbf{a}_{3} \\
& \mathbf{B}_{6}=-x_{3} \mathbf{a}_{1}+x_{3} \mathbf{a}_{2}-x_{3} \mathbf{a}_{3} \\
& \mathbf{B}_{7}=x_{3} \mathbf{a}_{1}+x_{3} \mathbf{a}_{2}-x_{3} \mathbf{a}_{3} \\
& \mathbf{B}_{8}=-x_{3} \mathbf{a}_{1}-x_{3} \mathbf{a}_{2}+x_{3} \mathbf{a}_{3} \\
& \mathbf{B}_{9}=\left(\frac{1}{2}-x_{4}\right) \mathbf{a}_{1}+x_{4} \mathbf{a}_{2}+x_{4} \mathbf{a}_{3} \\
& \mathbf{B}_{10}=x_{4} \mathbf{a}_{1}+\left(\frac{1}{2}-x_{4}\right) \mathbf{a}_{2}+\left(\frac{1}{2}-x_{4}\right) \mathbf{a}_{3} \\
& \mathbf{B}_{11}=x_{4} \mathbf{a}_{1}+\left(\frac{1}{2}-x_{4}\right) \mathbf{a}_{2}+x_{4} \mathbf{a}_{3} \\
& \mathbf{B}_{12}=\left(\frac{1}{2}-x_{4}\right) \mathbf{a}_{1}+x_{4} \mathbf{a}_{2}+\left(\frac{1}{2}-x_{4}\right) \mathbf{a}_{3} \\
& \mathbf{B}_{13}=x_{4} \mathbf{a}_{1}+x_{4} \mathbf{a}_{2}+\left(\frac{1}{2}-x_{4}\right) \mathbf{a}_{3} \\
& \mathbf{B}_{14}=\left(\frac{1}{2}-x_{4}\right) \mathbf{a}_{1}+\left(\frac{1}{2}-x_{4}\right) \mathbf{a}_{2}+x_{4} \mathbf{a}_{3} \\
& \mathbf{B}_{15}=\left(-x_{5}+y_{5}+z_{5}\right) \mathbf{a}_{1}+\left(x_{5}-y_{5}+z_{5}\right) \mathbf{a}_{2}+= \\
& \left(x_{5}+y_{5}-z_{5}\right) \mathbf{a}_{3} \\
& \mathbf{B}_{16}=\left(x_{5}-y_{5}+z_{5}\right) \mathbf{a}_{1}+\left(-x_{5}+y_{5}+z_{5}\right) \mathbf{a}_{2}+=-x_{5} a \hat{\mathbf{x}}-y_{5} a \hat{\mathbf{y}}+z_{5} a \hat{\mathbf{z}} \\
& \left(-x_{5}-y_{5}-z_{5}\right) \mathbf{a}_{3} \\
& \mathbf{B}_{17}=\left(x_{5}+y_{5}-z_{5}\right) \mathbf{a}_{1}+\left(-x_{5}-y_{5}-z_{5}\right) \mathbf{a}_{2}+=-x_{5} a \hat{\mathbf{x}}+y_{5} a \hat{\mathbf{y}}-z_{5} a \hat{\mathbf{z}} \\
& \left(-x_{5}+y_{5}+z_{5}\right) \mathbf{a}_{3} \\
& \mathbf{B}_{18}=\left(-x_{5}-y_{5}-z_{5}\right) \mathbf{a}_{1}+\left(x_{5}+y_{5}-z_{5}\right) \mathbf{a}_{2}+=x_{5} a \hat{\mathbf{x}}-y_{5} a \hat{\mathbf{y}}-z_{5} a \hat{\mathbf{z}} \\
& \left(x_{5}-y_{5}+z_{5}\right) \mathbf{a}_{3} \\
& \mathbf{B}_{19}=\left(x_{5}+y_{5}-z_{5}\right) \mathbf{a}_{1}+\left(-x_{5}+y_{5}+z_{5}\right) \mathbf{a}_{2}+=z_{5} a \hat{\mathbf{x}}+x_{5} a \hat{\mathbf{y}}+y_{5} a \hat{\mathbf{z}} \\
& \left(x_{5}-y_{5}+z_{5}\right) \mathbf{a}_{3} \\
& \mathbf{B}_{20}=\left(-x_{5}-y_{5}-z_{5}\right) \mathbf{a}_{1}+\left(x_{5}-y_{5}+z_{5}\right) \mathbf{a}_{2}+\quad=\quad z_{5} a \hat{\mathbf{x}}-x_{5} a \hat{\mathbf{y}}-y_{5} a \hat{\mathbf{z}} \\
& \left(-x_{5}+y_{5}+z_{5}\right) \mathbf{a}_{3} \\
& \mathbf{B}_{21}=\left(-x_{5}+y_{5}+z_{5}\right) \mathbf{a}_{1}+\left(x_{5}+y_{5}-z_{5}\right) \mathbf{a}_{2}+=-z_{5} a \hat{\mathbf{x}}-x_{5} a \hat{\mathbf{y}}+y_{5} a \hat{\mathbf{z}} \\
& \left(-x_{5}-y_{5}-z_{5}\right) \mathbf{a}_{3} \\
& \mathbf{B}_{22}=\left(x_{5}-y_{5}+z_{5}\right) \mathbf{a}_{1}+\left(-x_{5}-y_{5}-z_{5}\right) \mathbf{a}_{2}+\quad=\quad-z_{5} a \hat{\mathbf{x}}+x_{5} a \hat{\mathbf{y}}-y_{5} a \hat{\mathbf{z}} \\
& \left(x_{5}+y_{5}-z_{5}\right) \mathbf{a}_{3} \\
& \mathbf{B}_{23}=\left(x_{5}-y_{5}+z_{5}\right) \mathbf{a}_{1}+\left(x_{5}+y_{5}-z_{5}\right) \mathbf{a}_{2}+=y_{5} a \hat{\mathbf{x}}+z_{5} a \hat{\mathbf{y}}+x_{5} a \hat{\mathbf{z}} \\
& \left(-x_{5}+y_{5}+z_{5}\right) \mathbf{a}_{3} \\
& \mathbf{B}_{24}=\left(-x_{5}+y_{5}+z_{5}\right) \mathbf{a}_{1}+\left(-x_{5}-y_{5}-z_{5}\right) \mathbf{a}_{2}+\quad=\quad-y_{5} a \hat{\mathbf{x}}+z_{5} a \hat{\mathbf{y}}-x_{5} a \hat{\mathbf{z}} \\
& \left(x_{5}-y_{5}+z_{5}\right) \mathbf{a}_{3} \\
& \mathbf{B}_{25}=\left(-x_{5}-y_{5}-z_{5}\right) \mathbf{a}_{1}+\left(-x_{5}+y_{5}+z_{5}\right) \mathbf{a}_{2}+=y_{5} a \hat{\mathbf{x}}-z_{5} a \hat{\mathbf{y}}-x_{5} a \hat{\mathbf{z}} \\
& \left(x_{5}+y_{5}-z_{5}\right) \mathbf{a}_{3} \\
& \mathbf{B}_{26}=\left(x_{5}+y_{5}-z_{5}\right) \mathbf{a}_{1}+\left(x_{5}-y_{5}+z_{5}\right) \mathbf{a}_{2}+\quad=\quad-y_{5} a \hat{\mathbf{x}}-z_{5} a \hat{\mathbf{y}}+x_{5} a \hat{\mathbf{z}} \\
& \left(-x_{5}-y_{5}-z_{5}\right) \mathbf{a}_{3} \\
& \mathbf{B}_{27}=\left(-x_{6}+y_{6}+z_{6}\right) \mathbf{a}_{1}+\left(x_{6}-y_{6}+z_{6}\right) \mathbf{a}_{2}+\quad=\quad x_{6} a \hat{\mathbf{x}}+y_{6} a \hat{\mathbf{y}}+z_{6} a \hat{\mathbf{z}} \\
& \left(x_{6}+y_{6}-z_{6}\right) \mathbf{a}_{3} \\
& \mathbf{B}_{28}=\left(x_{6}-y_{6}+z_{6}\right) \mathbf{a}_{1}+\left(-x_{6}+y_{6}+z_{6}\right) \mathbf{a}_{2}+\quad=\quad-x_{6} a \hat{\mathbf{x}}-y_{6} a \hat{\mathbf{y}}+z_{6} a \hat{\mathbf{z}} \\
& \left(-x_{6}-y_{6}-z_{6}\right) \mathbf{a}_{3}
\end{aligned}
$$

Wyckoff Position Atom Type
Mg I

Mg II

O I

O I

O I

O I

O I

O I

O II

O II

O II

O II

O II

O II

B I

B I

B I

B I

B I

B I

B I

B I

B I

B I

B I

B I

B II

B II 


$$
\begin{aligned}
& \mathbf{B}_{29}=\left(x_{6}+y_{6}-z_{6}\right) \mathbf{a}_{1}+\left(-x_{6}-y_{6}-z_{6}\right) \mathbf{a}_{2}+=-x_{6} a \hat{\mathbf{x}}+y_{6} a \hat{\mathbf{y}}-z_{6} a \hat{\mathbf{z}} \\
& \left(-x_{6}+y_{6}+z_{6}\right) \mathbf{a}_{3} \\
& \mathbf{B}_{30}=\left(-x_{6}-y_{6}-z_{6}\right) \mathbf{a}_{1}+\left(x_{6}+y_{6}-z_{6}\right) \mathbf{a}_{2}+=x_{6} a \hat{\mathbf{x}}-y_{6} a \hat{\mathbf{y}}-z_{6} a \hat{\mathbf{z}} \\
& \left(x_{6}-y_{6}+z_{6}\right) \mathbf{a}_{3} \\
& \mathbf{B}_{31}=\left(x_{6}+y_{6}-z_{6}\right) \mathbf{a}_{1}+\left(-x_{6}+y_{6}+z_{6}\right) \mathbf{a}_{2}+=z_{6} a \hat{\mathbf{x}}+x_{6} a \hat{\mathbf{y}}+y_{6} a \hat{\mathbf{z}} \\
& \left(x_{6}-y_{6}+z_{6}\right) \mathbf{a}_{3} \\
& \mathbf{B}_{32}=\left(-x_{6}-y_{6}-z_{6}\right) \mathbf{a}_{1}+\left(x_{6}-y_{6}+z_{6}\right) \mathbf{a}_{2}+=z_{6} a \hat{\mathbf{x}}-x_{6} a \hat{\mathbf{y}}-y_{6} a \hat{\mathbf{z}} \\
& \left(-x_{6}+y_{6}+z_{6}\right) \mathbf{a}_{3} \\
& \mathbf{B}_{33}=\left(-x_{6}+y_{6}+z_{6}\right) \mathbf{a}_{1}+\left(x_{6}+y_{6}-z_{6}\right) \mathbf{a}_{2}+=-z_{6} a \hat{\mathbf{x}}-x_{6} a \hat{\mathbf{y}}+y_{6} a \hat{\mathbf{z}} \\
& \left(-x_{6}-y_{6}-z_{6}\right) \mathbf{a}_{3} \\
& \mathbf{B}_{34}=\left(x_{6}-y_{6}+z_{6}\right) \mathbf{a}_{1}+\left(-x_{6}-y_{6}-z_{6}\right) \mathbf{a}_{2}+=-z_{6} a \hat{\mathbf{x}}+x_{6} a \hat{\mathbf{y}}-y_{6} a \hat{\mathbf{z}} \\
& \left(x_{6}+y_{6}-z_{6}\right) \mathbf{a}_{3} \\
& \mathbf{B}_{35}=\left(x_{6}-y_{6}+z_{6}\right) \mathbf{a}_{1}+\left(x_{6}+y_{6}-z_{6}\right) \mathbf{a}_{2}+\quad=\quad y_{6} a \hat{\mathbf{x}}+z_{6} a \hat{\mathbf{y}}+x_{6} a \hat{\mathbf{z}} \\
& \left(-x_{6}+y_{6}+z_{6}\right) \mathbf{a}_{3} \\
& \mathbf{B}_{36}=\left(-x_{6}+y_{6}+z_{6}\right) \mathbf{a}_{1}+\left(-x_{6}-y_{6}-z_{6}\right) \mathbf{a}_{2}+=-y_{6} a \hat{\mathbf{x}}+z_{6} a \hat{\mathbf{y}}-x_{6} a \hat{\mathbf{z}} \\
& \left(x_{6}-y_{6}+z_{6}\right) \mathbf{a}_{3} \\
& \mathbf{B}_{37}=\left(-x_{6}-y_{6}-z_{6}\right) \mathbf{a}_{1}+\left(-x_{6}+y_{6}+z_{6}\right) \mathbf{a}_{2}+=y_{6} a \hat{\mathbf{x}}-z_{6} a \hat{\mathbf{y}}-x_{6} a \hat{\mathbf{z}} \\
& \left(x_{6}+y_{6}-z_{6}\right) \mathbf{a}_{3} \\
& \mathbf{B}_{38}=\left(x_{6}+y_{6}-z_{6}\right) \mathbf{a}_{1}+\left(x_{6}-y_{6}+z_{6}\right) \mathbf{a}_{2}+\quad=\quad-y_{6} a \hat{\mathbf{x}}-z_{6} a \hat{\mathbf{y}}+x_{6} a \hat{\mathbf{z}} \\
& \left(-x_{6}-y_{6}-z_{6}\right) \mathbf{a}_{3} \\
& \mathbf{B}_{39}=\left(-x_{7}+y_{7}+z_{7}\right) \mathbf{a}_{1}+\left(x_{7}-y_{7}+z_{7}\right) \mathbf{a}_{2}+=\quad=\quad x_{7} a \hat{\mathbf{x}}+y_{7} a \hat{\mathbf{y}}+z_{7} a \hat{\mathbf{z}} \\
& \left(x_{7}+y_{7}-z_{7}\right) \mathbf{a}_{3} \\
& \mathbf{B}_{40}=\left(x_{7}-y_{7}+z_{7}\right) \mathbf{a}_{1}+\left(-x_{7}+y_{7}+z_{7}\right) \mathbf{a}_{2}+\quad=\quad-x_{7} a \hat{\mathbf{x}}-y_{7} a \hat{\mathbf{y}}+z_{7} a \hat{\mathbf{z}} \\
& \left(-x_{7}-y_{7}-z_{7}\right) \mathbf{a}_{3} \\
& \mathbf{B}_{41}=\left(x_{7}+y_{7}-z_{7}\right) \mathbf{a}_{1}+\left(-x_{7}-y_{7}-z_{7}\right) \mathbf{a}_{2}+=-x_{7} a \hat{\mathbf{x}}+y_{7} a \hat{\mathbf{y}}-z_{7} a \hat{\mathbf{z}} \\
& \left(-x_{7}+y_{7}+z_{7}\right) \mathbf{a}_{3} \\
& \mathbf{B}_{42}=\left(-x_{7}-y_{7}-z_{7}\right) \mathbf{a}_{1}+\left(x_{7}+y_{7}-z_{7}\right) \mathbf{a}_{2}+=x_{7} a \hat{\mathbf{x}}-y_{7} a \hat{\mathbf{y}}-z_{7} a \hat{\mathbf{z}} \\
& \left(x_{7}-y_{7}+z_{7}\right) \mathbf{a}_{3} \\
& \mathbf{B}_{43}=\left(x_{7}+y_{7}-z_{7}\right) \mathbf{a}_{1}+\left(-x_{7}+y_{7}+z_{7}\right) \mathbf{a}_{2}+=z_{7} a \hat{\mathbf{x}}+x_{7} a \hat{\mathbf{y}}+y_{7} a \hat{\mathbf{z}} \\
& \left(x_{7}-y_{7}+z_{7}\right) \mathbf{a}_{3} \\
& \mathbf{B}_{44}=\left(-x_{7}-y_{7}-z_{7}\right) \mathbf{a}_{1}+\left(x_{7}-y_{7}+z_{7}\right) \mathbf{a}_{2}+=z_{7} a \hat{\mathbf{x}}-x_{7} a \hat{\mathbf{y}}-y_{7} a \hat{\mathbf{z}} \\
& \left(-x_{7}+y_{7}+z_{7}\right) \mathbf{a}_{3} \\
& \mathbf{B}_{45}=\left(-x_{7}+y_{7}+z_{7}\right) \mathbf{a}_{1}+\left(x_{7}+y_{7}-z_{7}\right) \mathbf{a}_{2}+=-z_{7} a \hat{\mathbf{x}}-x_{7} a \hat{\mathbf{y}}+y_{7} a \hat{\mathbf{z}} \\
& \left(-x_{7}-y_{7}-z_{7}\right) \mathbf{a}_{3} \\
& \mathbf{B}_{46}=\left(x_{7}-y_{7}+z_{7}\right) \mathbf{a}_{1}+\left(-x_{7}-y_{7}-z_{7}\right) \mathbf{a}_{2}+=-z_{7} a \hat{\mathbf{x}}+x_{7} a \hat{\mathbf{y}}-y_{7} a \hat{\mathbf{z}} \\
& \left(x_{7}+y_{7}-z_{7}\right) \mathbf{a}_{3} \\
& \mathbf{B}_{47}=\left(x_{7}-y_{7}+z_{7}\right) \mathbf{a}_{1}+\left(x_{7}+y_{7}-z_{7}\right) \mathbf{a}_{2}+=y_{7} a \hat{\mathbf{x}}+z_{7} a \hat{\mathbf{y}}+x_{7} a \hat{\mathbf{z}} \\
& \left(-x_{7}+y_{7}+z_{7}\right) \mathbf{a}_{3} \\
& \mathbf{B}_{48}=\left(-x_{7}+y_{7}+z_{7}\right) \mathbf{a}_{1}+\left(-x_{7}-y_{7}-z_{7}\right) \mathbf{a}_{2}+=-y_{7} a \hat{\mathbf{x}}+z_{7} a \hat{\mathbf{y}}-x_{7} a \hat{\mathbf{z}} \\
& \left(x_{7}-y_{7}+z_{7}\right) \mathbf{a}_{3} \\
& \mathbf{B}_{49}=\left(-x_{7}-y_{7}-z_{7}\right) \mathbf{a}_{1}+\left(-x_{7}+y_{7}+z_{7}\right) \mathbf{a}_{2}+=y_{7} a \hat{\mathbf{x}}-z_{7} a \hat{\mathbf{y}}-x_{7} a \hat{\mathbf{z}} \\
& \left(x_{7}+y_{7}-z_{7}\right) \mathbf{a}_{3} \\
& \mathbf{B}_{50}=\left(x_{7}+y_{7}-z_{7}\right) \mathbf{a}_{1}+\left(x_{7}-y_{7}+z_{7}\right) \mathbf{a}_{2}+\quad=\quad-y_{7} a \hat{\mathbf{x}}-z_{7} a \hat{\mathbf{y}}+x_{7} a \hat{\mathbf{z}} \\
& \left(-x_{7}-y_{7}-z_{7}\right) \mathbf{a}_{3} \\
& \mathbf{B}_{51}=\left(-x_{8}+y_{8}+z_{8}\right) \mathbf{a}_{1}+\left(x_{8}-y_{8}+z_{8}\right) \mathbf{a}_{2}+\quad=\quad x_{8} a \hat{\mathbf{x}}+y_{8} a \hat{\mathbf{y}}+z_{8} a \hat{\mathbf{z}} \\
& \left(x_{8}+y_{8}-z_{8}\right) \mathbf{a}_{3} \\
& \mathbf{B}_{52}=\left(x_{8}-y_{8}+z_{8}\right) \mathbf{a}_{1}+\left(-x_{8}+y_{8}+z_{8}\right) \mathbf{a}_{2}+=-x_{8} a \hat{\mathbf{x}}-y_{8} a \hat{\mathbf{y}}+z_{8} a \hat{\mathbf{z}} \\
& \left(-x_{8}-y_{8}-z_{8}\right) \mathbf{a}_{3}
\end{aligned}
$$

B II

B II

B II

B II

B II

B II

B II

H I

H I

H I

H I

H I

H I

H I

H I

H I

H I

H I

H I

H II

H II 


\begin{tabular}{|c|c|c|c|c|c|c|}
\hline $\mathbf{B}_{53}$ & $=$ & $\begin{array}{c}\left(x_{8}+y_{8}-z_{8}\right) \mathbf{a}_{1}+\left(-x_{8}-y_{8}-z_{8}\right) \mathbf{a}_{2}+ \\
\left(-x_{8}+y_{8}+z_{8}\right) \mathbf{a}_{3}\end{array}$ & $=$ & $-x_{8} a \hat{\mathbf{x}}+y_{8} a \hat{\mathbf{y}}-z_{8} a \hat{\mathbf{z}}$ & $(48 h)$ & H II \\
\hline $\mathbf{B}_{54}$ & $=$ & $\begin{array}{c}\left(-x_{8}-y_{8}-z_{8}\right) \mathbf{a}_{1}+\left(x_{8}+y_{8}-z_{8}\right) \mathbf{a}_{2}+ \\
\left(x_{8}-y_{8}+z_{8}\right) \mathbf{a}_{3}\end{array}$ & $=$ & $x_{8} a \hat{\mathbf{x}}-y_{8} a \hat{\mathbf{y}}-z_{8} a \hat{\mathbf{z}}$ & $(48 h)$ & H II \\
\hline $\mathbf{B}_{55}$ & $=$ & $\begin{array}{c}\left(x_{8}+y_{8}-z_{8}\right) \mathbf{a}_{1}+\left(-x_{8}+y_{8}+z_{8}\right) \mathbf{a}_{2}+ \\
\left(x_{8}-y_{8}+z_{8}\right) \mathbf{a}_{3}\end{array}$ & $=$ & $z_{8} a \hat{\mathbf{x}}+x_{8} a \hat{\mathbf{y}}+y_{8} a \hat{\mathbf{z}}$ & $(48 h)$ & H II \\
\hline $\mathbf{B}_{56}$ & $=$ & $\begin{array}{c}\left(-x_{8}-y_{8}-z_{8}\right) \mathbf{a}_{1}+\left(x_{8}-y_{8}+z_{8}\right) \mathbf{a}_{2}+ \\
\left(-x_{8}+y_{8}+z_{8}\right) \mathbf{a}_{3}\end{array}$ & $=$ & $z_{8} a \hat{\mathbf{x}}-x_{8} a \hat{\mathbf{y}}-y_{8} a \hat{\mathbf{z}}$ & $(48 h)$ & H II \\
\hline $\mathbf{B}_{57}$ & $=$ & $\begin{array}{c}\left(-x_{8}+y_{8}+z_{8}\right) \mathbf{a}_{1}+\left(x_{8}+y_{8}-z_{8}\right) \mathbf{a}_{2}+ \\
\left(-x_{8}-y_{8}-z_{8}\right) \mathbf{a}_{3}\end{array}$ & $=$ & $-z_{8} a \hat{\mathbf{x}}-x_{8} a \hat{\mathbf{y}}+y_{8} a \hat{\mathbf{z}}$ & $(48 h)$ & H II \\
\hline $\mathbf{B}_{58}$ & $=$ & $\begin{array}{c}\left(x_{8}-y_{8}+z_{8}\right) \mathbf{a}_{1}+\left(-x_{8}-y_{8}-z_{8}\right) \mathbf{a}_{2}+ \\
\left(x_{8}+y_{8}-z_{8}\right) \mathbf{a}_{3}\end{array}$ & $=$ & $-z_{8} a \hat{\mathbf{x}}+x_{8} a \hat{\mathbf{y}}-y_{8} a \hat{\mathbf{z}}$ & $(48 h)$ & H II \\
\hline $\mathbf{B}_{59}$ & $=$ & $\begin{array}{c}\left(x_{8}-y_{8}+z_{8}\right) \mathbf{a}_{1}+\left(x_{8}+y_{8}-z_{8}\right) \mathbf{a}_{2}+ \\
\left(-x_{8}+y_{8}+z_{8}\right) \mathbf{a}_{3}\end{array}$ & $=$ & $y_{8} a \hat{\mathbf{x}}+z_{8} a \hat{\mathbf{y}}+x_{8} a \hat{\mathbf{z}}$ & $(48 h)$ & H II \\
\hline $\mathbf{B}_{60}$ & $=$ & $\begin{array}{c}\left(-x_{8}+y_{8}+z_{8}\right) \mathbf{a}_{1}+\left(-x_{8}-y_{8}-z_{8}\right) \mathbf{a}_{2}+ \\
\left(x_{8}-y_{8}+z_{8}\right) \mathbf{a}_{3}\end{array}$ & $=$ & $-y_{8} a \hat{\mathbf{x}}+z_{8} a \hat{\mathbf{y}}-x_{8} a \hat{\mathbf{z}}$ & $(48 h)$ & H II \\
\hline $\mathbf{B}_{61}$ & $=$ & $\begin{array}{c}\left(-x_{8}-y_{8}-z_{8}\right) \mathbf{a}_{1}+\left(-x_{8}+y_{8}+z_{8}\right) \mathbf{a}_{2}+ \\
\left(x_{8}+y_{8}-z_{8}\right) \mathbf{a}_{3}\end{array}$ & $=$ & $y_{8} a \hat{\mathbf{x}}-z_{8} a \hat{\mathbf{y}}-x_{8} a \hat{\mathbf{z}}$ & $(48 h)$ & H II \\
\hline $\mathbf{B}_{62}$ & $=$ & $\begin{array}{c}\left(x_{8}+y_{8}-z_{8}\right) \mathbf{a}_{1}+\left(x_{8}-y_{8}+z_{8}\right) \mathbf{a}_{2}+ \\
\left(-x_{8}-y_{8}-z_{8}\right) \mathbf{a}_{3}\end{array}$ & $=$ & $-y_{8} a \hat{\mathbf{x}}-z_{8} a \hat{\mathbf{y}}+x_{8} a \hat{\mathbf{z}}$ & $(48 h)$ & H II \\
\hline $\mathbf{B}_{63}$ & $=$ & $\begin{array}{c}\left(-x_{9}+y_{9}+z_{9}\right) \mathbf{a}_{1}+\left(x_{9}-y_{9}+z_{9}\right) \mathbf{a}_{2}+ \\
\left(x_{9}+y_{9}-z_{9}\right) \mathbf{a}_{3}\end{array}$ & $=$ & $x_{9} a \hat{\mathbf{x}}+y_{9} a \hat{\mathbf{y}}+z_{9} a \hat{\mathbf{z}}$ & $(48 h)$ & H III \\
\hline $\mathbf{B}_{64}$ & $=$ & $\begin{array}{c}\left(x_{9}-y_{9}+z_{9}\right) \mathbf{a}_{1}+\left(-x_{9}+y_{9}+z_{9}\right) \mathbf{a}_{2}+ \\
\left(-x_{9}-y_{9}-z_{9}\right) \mathbf{a}_{3}\end{array}$ & $=$ & $-x_{9} a \hat{\mathbf{x}}-y_{9} a \hat{\mathbf{y}}+z_{9} a \hat{\mathbf{z}}$ & $(48 h)$ & H III \\
\hline $\mathbf{B}_{65}$ & $=$ & $\begin{array}{c}\left(x_{9}+y_{9}-z_{9}\right) \mathbf{a}_{1}+\left(-x_{9}-y_{9}-z_{9}\right) \mathbf{a}_{2}+ \\
\left(-x_{9}+y_{9}+z_{9}\right) \mathbf{a}_{3}\end{array}$ & $=$ & $-x_{9} a \hat{\mathbf{x}}+y_{9} a \hat{\mathbf{y}}-z_{9} a \hat{\mathbf{z}}$ & $(48 h)$ & H III \\
\hline $\mathbf{B}_{66}$ & $=$ & $\begin{array}{c}\left(-x_{9}-y_{9}-z_{9}\right) \mathbf{a}_{1}+\left(x_{9}+y_{9}-z_{9}\right) \mathbf{a}_{2}+ \\
\left(x_{9}-y_{9}+z_{9}\right) \mathbf{a}_{3}\end{array}$ & $=$ & $x_{9} a \hat{\mathbf{x}}-y_{9} a \hat{\mathbf{y}}-z_{9} a \hat{\mathbf{z}}$ & $(48 h)$ & H III \\
\hline $\mathbf{B}_{67}$ & $=$ & $\begin{array}{c}\left(x_{9}+y_{9}-z_{9}\right) \mathbf{a}_{1}+\left(-x_{9}+y_{9}+z_{9}\right) \mathbf{a}_{2}+ \\
\left(x_{9}-y_{9}+z_{9}\right) \mathbf{a}_{3}\end{array}$ & $=$ & $z_{9} a \hat{\mathbf{x}}+x_{9} a \hat{\mathbf{y}}+y_{9} a \hat{\mathbf{z}}$ & $(48 h)$ & H III \\
\hline $\mathbf{B}_{68}$ & $=$ & $\begin{array}{c}\left(-x_{9}-y_{9}-z_{9}\right) \mathbf{a}_{1}+\left(x_{9}-y_{9}+z_{9}\right) \mathbf{a}_{2}+ \\
\left(-x_{9}+y_{9}+z_{9}\right) \mathbf{a}_{3}\end{array}$ & $=$ & $z_{9} a \hat{\mathbf{x}}-x_{9} a \hat{\mathbf{y}}-y_{9} a \hat{\mathbf{z}}$ & $(48 h)$ & H III \\
\hline $\mathbf{B}_{69}$ & $=$ & $\begin{array}{c}\left(-x_{9}+y_{9}+z_{9}\right) \mathbf{a}_{1}+\left(x_{9}+y_{9}-z_{9}\right) \mathbf{a}_{2}+ \\
\left(-x_{9}-y_{9}-z_{9}\right) \mathbf{a}_{3}\end{array}$ & $=$ & $-z_{9} a \hat{\mathbf{x}}-x_{9} a \hat{\mathbf{y}}+y_{9} a \hat{\mathbf{z}}$ & $(48 h)$ & H III \\
\hline $\mathbf{B}_{70}$ & $=$ & $\begin{array}{c}\left(x_{9}-y_{9}+z_{9}\right) \mathbf{a}_{1}+\left(-x_{9}-y_{9}-z_{9}\right) \mathbf{a}_{2}+ \\
\left(x_{9}+y_{9}-z_{9}\right) \mathbf{a}_{3}\end{array}$ & $=$ & $-z_{9} a \hat{\mathbf{x}}+x_{9} a \hat{\mathbf{y}}-y_{9} a \hat{\mathbf{z}}$ & $(48 h)$ & H III \\
\hline $\mathbf{B}_{71}$ & $=$ & $\begin{array}{c}\left(x_{9}-y_{9}+z_{9}\right) \mathbf{a}_{1}+\left(x_{9}+y_{9}-z_{9}\right) \mathbf{a}_{2}+ \\
\left(-x_{9}+y_{9}+z_{9}\right) \mathbf{a}_{3}\end{array}$ & $=$ & $y_{9} a \hat{\mathbf{x}}+z_{9} a \hat{\mathbf{y}}+x_{9} a \hat{\mathbf{z}}$ & $(48 h)$ & H III \\
\hline $\mathbf{B}_{72}$ & $=$ & $\begin{array}{c}\left(-x_{9}+y_{9}+z_{9}\right) \mathbf{a}_{1}+\left(-x_{9}-y_{9}-z_{9}\right) \mathbf{a}_{2}+ \\
\left(x_{9}-y_{9}+z_{9}\right) \mathbf{a}_{3}\end{array}$ & $=$ & $-y_{9} a \hat{\mathbf{x}}+z_{9} a \hat{\mathbf{y}}-x_{9} a \hat{\mathbf{z}}$ & $(48 h)$ & H III \\
\hline $\mathbf{B}_{73}$ & $=$ & $\begin{array}{c}\left(-x_{9}-y_{9}-z_{9}\right) \mathbf{a}_{1}+\left(-x_{9}+y_{9}+z_{9}\right) \mathbf{a}_{2}+ \\
\left(x_{9}+y_{9}-z_{9}\right) \mathbf{a}_{3}\end{array}$ & $=$ & $y_{9} a \hat{\mathbf{x}}-z_{9} a \hat{\mathbf{y}}-x_{9} a \hat{\mathbf{z}}$ & $(48 h)$ & H III \\
\hline $\mathbf{B}_{74}$ & $=$ & $\begin{array}{c}\left(x_{9}+y_{9}-z_{9}\right) \mathbf{a}_{1}+\left(x_{9}-y_{9}+z_{9}\right) \mathbf{a}_{2}+ \\
\left(-x_{9}-y_{9}-z_{9}\right) \mathbf{a}_{3}\end{array}$ & $=$ & $-y_{9} a \hat{\mathbf{x}}-z_{9} a \hat{\mathbf{y}}+x_{9} a \hat{\mathbf{z}}$ & $(48 h)$ & H III \\
\hline $\mathbf{B}_{75}$ & $=$ & $\begin{array}{c}\left(-x_{10}+y_{10}+z_{10}\right) \mathbf{a}_{1}+ \\
\left(x_{10}-y_{10}+z_{10}\right) \mathbf{a}_{2}+\left(x_{10}+y_{10}-z_{10}\right) \mathbf{a}_{3}\end{array}$ & $=$ & $x_{10} a \hat{\mathbf{x}}+y_{10} a \hat{\mathbf{y}}+z_{10} a \hat{\mathbf{z}}$ & $(48 h)$ & H IV \\
\hline $\mathbf{B}_{76}$ & $=$ & $\begin{array}{c}\left(x_{10}-y_{10}+z_{10}\right) \mathbf{a}_{1}+ \\
\left(-x_{10}+y_{10}+z_{10}\right) \mathbf{a}_{2}+ \\
\left(-x_{10}-y_{10}-z_{10}\right) \mathbf{a}_{3}\end{array}$ & $=$ & $-x_{10} a \hat{\mathbf{x}}-y_{10} a \hat{\mathbf{y}}+z_{10} a \hat{\mathbf{z}}$ & $(48 h)$ & H IV \\
\hline
\end{tabular}




$$
\begin{aligned}
& \begin{array}{r}
\mathbf{B}_{77=}\left(x_{10}+y_{10}-z_{10}\right) \mathbf{a}_{1}+ \\
\left(-x_{10}-y_{10}-z_{10}\right) \mathbf{a}_{2}+
\end{array} \\
& \left(-x_{10}+y_{10}+z_{10}\right) \mathbf{a}_{3} \\
& \mathbf{B}_{78}=\begin{array}{c}
\left(-x_{10}-y_{10}-z_{10}\right) \mathbf{a}_{1}+ \\
\left(x_{10}+y_{10}-z_{10}\right) \mathbf{a}_{2}+\left(x_{10}-y_{10}+z_{10}\right) \mathbf{a}_{3}
\end{array} \\
& \mathbf{B}_{79}=\begin{array}{c}
\left(x_{10}+y_{10}-z_{10}\right) \mathbf{a}_{1}+ \\
\left(-x_{10}+y_{10}+z_{10}\right) \mathbf{a}_{2}+\left(x_{10}-y_{10}+z_{10}\right) \mathbf{a}_{3}
\end{array} \\
& \begin{array}{c}
\mathbf{B}_{80}= \\
\left(x_{10}-y_{10}+z_{10}\right) \mathbf{a}_{2}+\left(-x_{10}+y_{10}+z_{10}\right) \mathbf{a}_{3}
\end{array} \\
& \mathbf{B}_{81}=\begin{array}{c}
\left(-x_{10}+y_{10}+z_{10}\right) \mathbf{a}_{1}+ \\
\left(x_{10}+y_{10}-z_{10}\right) \mathbf{a}_{2}+\left(-x_{10}-y_{10}-z_{10}\right) \mathbf{a}_{3}
\end{array} \\
& \begin{array}{c}
\mathbf{B}_{82}= \\
\left(-x_{10}-y_{10}-z_{10}\right) \mathbf{a}_{2}+\left(x_{10}+y_{10}-z_{10}\right) \mathbf{a}_{3}
\end{array} \\
& \mathbf{B}_{83}=\begin{array}{c}
\left(x_{10}-y_{10}+z_{10}\right) \mathbf{a}_{1}+ \\
\left(x_{10}+y_{10}-z_{10}\right) \mathbf{a}_{2}+\left(-x_{10}+y_{10}+z_{10}\right) \mathbf{a}_{3}
\end{array} \\
& \mathbf{B}_{84}=\underset{\left(-x_{10}-y_{10}-z_{10}\right) \mathbf{a}_{2}+\left(x_{10}-y_{10}+z_{10}\right) \mathbf{a}_{3}}{\left(-z_{10}\right) \mathbf{a}_{1}+}=-y_{10} a \hat{\mathbf{x}}+z_{10} a \hat{\mathbf{y}}-x_{10} a \hat{\mathbf{z}} \\
& \mathbf{B}_{85}=\left(-x_{10}-y_{10}-z_{10}\right) \mathbf{a}_{1}+\quad=\quad y_{10} a \hat{\mathbf{x}}-z_{10} a \hat{\mathbf{y}}-x_{10} a \hat{\mathbf{z}} \\
& \left(-x_{10}+y_{10}+z_{10}\right) \mathbf{a}_{2}+\left(x_{10}+y_{10}-z_{10}\right) \mathbf{a}_{3} \\
& \mathbf{B}_{86}=\begin{array}{c}
\left(x_{10}+y_{10}-z_{10}\right) \mathbf{a}_{1}+ \\
\left(x_{10}-y_{10}+z_{10}\right) \mathbf{a}_{2}+\left(-x_{10}-y_{10}-z_{10}\right) \mathbf{a}_{3}
\end{array} \\
& \mathbf{B}_{87}=\underset{\left(-x_{11}+y_{11}+z_{11}\right) \mathbf{a}_{1}+}{\left(x_{11}-y_{11}+z_{11}\right) \mathbf{a}_{2}+\left(x_{11}+y_{11}-z_{11}\right) \mathbf{a}_{3}}=x_{11} a \hat{\mathbf{x}}+y_{11} a \hat{\mathbf{y}}+z_{11} a \hat{\mathbf{z}}
\end{aligned}
$$

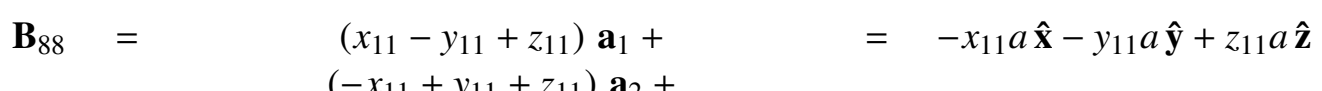

$$
\begin{aligned}
& \left(-x_{11}+y_{11}+z_{11}\right) \mathbf{a}_{2}+ \\
& \left(-x_{11}-y_{11}-z_{11}\right) \mathbf{a}_{3} \\
& \mathbf{B}_{89}=\left(x_{11}+y_{11}-z_{11}\right) \mathbf{a}_{1}+\quad=-x_{11} a \hat{\mathbf{x}}+y_{11} a \hat{\mathbf{y}}-z_{11} a \hat{\mathbf{z}} \\
& \left(-x_{11}-y_{11}-z_{11}\right) \mathbf{a}_{2}+ \\
& \left(-x_{11}+y_{11}+z_{11}\right) \mathbf{a}_{3} \\
& \begin{array}{c}
\mathbf{B}_{90}= \\
\left(-x_{11}-y_{11}-z_{11}\right) \mathbf{a}_{1}+ \\
\left(x_{11}+y_{11}-z_{11}\right) \mathbf{a}_{2}+\left(x_{11}-y_{11}+z_{11}\right) \mathbf{a}_{3}
\end{array} \\
& \begin{array}{l}
\mathbf{B}_{91}= \\
\mathbf{B}_{92}= \\
\left(-x_{11}+y_{11}+z_{11}\right) \mathbf{a}_{2}+\left(x_{11}-y_{11}+z_{11}\right) \mathbf{a}_{3}
\end{array} \quad \begin{array}{c}
\left(-x_{11}-y_{11}-z_{11}\right) \mathbf{a}_{1}+ \\
\left(x_{11}-y_{11}+z_{11}\right) \mathbf{a}_{2}+\left(-x_{11}+y_{11}+z_{11}\right) \mathbf{a}_{3}
\end{array}=z_{11} a \hat{\mathbf{x}}-x_{11} a \hat{\mathbf{y}}-y_{11} a \hat{\mathbf{z}} \\
& \mathbf{B}_{93}=\left(-x_{11}+y_{11}+z_{11}\right) \mathbf{a}_{1}+=-z_{11} a \hat{\mathbf{x}}-x_{11} a \hat{\mathbf{y}}+y_{11} a \hat{\mathbf{z}} \\
& \left(x_{11}+y_{11}-z_{11}\right) \mathbf{a}_{2}+\left(-x_{11}-y_{11}-z_{11}\right) \mathbf{a}_{3} \\
& \mathbf{B}_{94}=\left(x_{11}-y_{11}+z_{11}\right) \mathbf{a}_{1}+=-z_{11} a \hat{\mathbf{x}}+x_{11} a \hat{\mathbf{y}}-y_{11} a \hat{\mathbf{z}} \\
& \mathbf{B}_{95}=\left(x_{11}-y_{11}+z_{11}\right) \mathbf{a}_{1}+\quad=\quad y_{11} a \hat{\mathbf{x}}+z_{11} a \hat{\mathbf{y}}+x_{11} a \hat{\mathbf{z}} \\
& \left(x_{11}+y_{11}-z_{11}\right) \mathbf{a}_{2}+\left(-x_{11}+y_{11}+z_{11}\right) \mathbf{a}_{3} \\
& \mathbf{B}_{96}=\left(-x_{11}+y_{11}+z_{11}\right) \mathbf{a}_{1}+=-y_{11} a \hat{\mathbf{x}}+z_{11} a \hat{\mathbf{y}}-x_{11} a \hat{\mathbf{z}} \\
& \mathbf{B}_{97}=\left(-x_{11}-y_{11}-z_{11}\right) \mathbf{a}_{1}+\quad=\quad y_{11} a \hat{\mathbf{x}}-z_{11} a \hat{\mathbf{y}}-x_{11} a \hat{\mathbf{z}} \\
& \left(-x_{11}+y_{11}+z_{11}\right) \mathbf{a}_{2}+\left(x_{11}+y_{11}-z_{11}\right) \mathbf{a}_{3} \\
& \mathbf{B}_{98}=\begin{array}{c}
\left(x_{11}+y_{11}-z_{11}\right) \mathbf{a}_{1}+ \\
\left(x_{11}-y_{11}+z_{11}\right) \mathbf{a}_{2}+\left(-x_{11}-y_{11}-z_{11}\right) \mathbf{a}_{3}
\end{array}=-y_{11} a \hat{\mathbf{x}}-z_{11} a \hat{\mathbf{y}}+x_{11} a \hat{\mathbf{z}} \\
& \mathbf{B}_{99}=\left(-x_{12}+y_{12}+z_{12}\right) \mathbf{a}_{1}+=x_{12} a \hat{\mathbf{x}}+y_{12} a \hat{\mathbf{y}}+z_{12} a \hat{\mathbf{z}}
\end{aligned}
$$

H V

H V

H V

H V

H V

H V

H V

H V

H V

H V

H VI 


\begin{tabular}{|c|c|c|c|c|c|c|}
\hline $\mathbf{B}_{100}$ & $=$ & $\begin{array}{c}\left(x_{12}-y_{12}+z_{12}\right) \mathbf{a}_{1}+ \\
\left(-x_{12}+y_{12}+z_{12}\right) \mathbf{a}_{2}+ \\
\left(-x_{12}-y_{12}-z_{12}\right) \mathbf{a}_{3}\end{array}$ & $=$ & $-x_{12} a \hat{\mathbf{x}}-y_{12} a \hat{\mathbf{y}}+z_{12} a \hat{\mathbf{z}}$ & $(48 h)$ & H VI \\
\hline $\mathbf{B}_{101}$ & $=$ & $\begin{array}{c}\left(x_{12}+y_{12}-z_{12}\right) \mathbf{a}_{1}+ \\
\left(-x_{12}-y_{12}-z_{12}\right) \mathbf{a}_{2}+ \\
\left(-x_{12}+y_{12}+z_{12}\right) \mathbf{a}_{3}\end{array}$ & $=$ & $-x_{12} a \hat{\mathbf{x}}+y_{12} a \hat{\mathbf{y}}-z_{12} a \hat{\mathbf{z}}$ & $(48 h)$ & H VI \\
\hline $\mathbf{B}_{102}$ & $=$ & $\begin{array}{c}\left(-x_{12}-y_{12}-z_{12}\right) \mathbf{a}_{1}+ \\
\left(x_{12}+y_{12}-z_{12}\right) \mathbf{a}_{2}+\left(x_{12}-y_{12}+z_{12}\right) \mathbf{a}_{3}\end{array}$ & $=$ & $x_{12} a \hat{\mathbf{x}}-y_{12} a \hat{\mathbf{y}}-z_{12} a \hat{\mathbf{z}}$ & $(48 h)$ & H VI \\
\hline $\mathbf{B}_{103}$ & $=$ & $\begin{array}{c}\left(x_{12}+y_{12}-z_{12}\right) \mathbf{a}_{1}+ \\
\left(-x_{12}+y_{12}+z_{12}\right) \mathbf{a}_{2}+\left(x_{12}-y_{12}+z_{12}\right) \mathbf{a}_{3}\end{array}$ & $=$ & $z_{12} a \hat{\mathbf{x}}+x_{12} a \hat{\mathbf{y}}+y_{12} a \hat{\mathbf{z}}$ & $(48 h)$ & H VI \\
\hline $\mathbf{B}_{104}$ & $=$ & $\begin{array}{c}\left(-x_{12}-y_{12}-z_{12}\right) \mathbf{a}_{1}+ \\
\left(x_{12}-y_{12}+z_{12}\right) \mathbf{a}_{2}+\left(-x_{12}+y_{12}+z_{12}\right) \mathbf{a}_{3}\end{array}$ & $=$ & $z_{12} a \hat{\mathbf{x}}-x_{12} a \hat{\mathbf{y}}-y_{12} a \hat{\mathbf{z}}$ & $(48 h)$ & H VI \\
\hline $\mathbf{B}_{105}$ & $=$ & $\begin{array}{c}\left(-x_{12}+y_{12}+z_{12}\right) \mathbf{a}_{1}+ \\
\left(x_{12}+y_{12}-z_{12}\right) \mathbf{a}_{2}+\left(-x_{12}-y_{12}-z_{12}\right) \mathbf{a}_{3}\end{array}$ & $=$ & $-z_{12} a \hat{\mathbf{x}}-x_{12} a \hat{\mathbf{y}}+y_{12} a \hat{\mathbf{z}}$ & $(48 h)$ & H VI \\
\hline $\mathbf{B}_{106}$ & $=$ & $\begin{array}{c}\left(x_{12}-y_{12}+z_{12}\right) \mathbf{a}_{1}+ \\
\left(-x_{12}-y_{12}-z_{12}\right) \mathbf{a}_{2}+\left(x_{12}+y_{12}-z_{12}\right) \mathbf{a}_{3}\end{array}$ & $=$ & $-z_{12} a \hat{\mathbf{x}}+x_{12} a \hat{\mathbf{y}}-y_{12} a \hat{\mathbf{z}}$ & $(48 h)$ & H VI \\
\hline $\mathbf{B}_{107}$ & $=$ & $\begin{array}{c}\left(x_{12}-y_{12}+z_{12}\right) \mathbf{a}_{1}+ \\
\left(x_{12}+y_{12}-z_{12}\right) \mathbf{a}_{2}+\left(-x_{12}+y_{12}+z_{12}\right) \mathbf{a}_{3}\end{array}$ & $=$ & $y_{12} a \hat{\mathbf{x}}+z_{12} a \hat{\mathbf{y}}+x_{12} a \hat{\mathbf{z}}$ & $(48 h)$ & H VI \\
\hline $\mathbf{B}_{108}$ & $=$ & $\begin{array}{c}\left(-x_{12}+y_{12}+z_{12}\right) \mathbf{a}_{1}+ \\
\left(-x_{12}-y_{12}-z_{12}\right) \mathbf{a}_{2}+\left(x_{12}-y_{12}+z_{12}\right) \mathbf{a}_{3}\end{array}$ & $=$ & $-y_{12} a \hat{\mathbf{x}}+z_{12} a \hat{\mathbf{y}}-x_{12} a \hat{\mathbf{z}}$ & $(48 h)$ & H VI \\
\hline $\mathbf{B}_{109}$ & $=$ & $\begin{array}{c}\left(-x_{12}-y_{12}-z_{12}\right) \mathbf{a}_{1}+ \\
\left(-x_{12}+y_{12}+z_{12}\right) \mathbf{a}_{2}+\left(x_{12}+y_{12}-z_{12}\right) \mathbf{a}_{3}\end{array}$ & $=$ & $y_{12} a \hat{\mathbf{x}}-z_{12} a \hat{\mathbf{y}}-x_{12} a \hat{\mathbf{z}}$ & $(48 h)$ & H VI \\
\hline $\mathbf{B}_{110}$ & $=$ & $\begin{array}{c}\left(x_{12}+y_{12}-z_{12}\right) \mathbf{a}_{1}+ \\
\left(x_{12}-y_{12}+z_{12}\right) \mathbf{a}_{2}+\left(-x_{12}-y_{12}-z_{12}\right) \mathbf{a}_{3}\end{array}$ & $=$ & $-y_{12} a \hat{\mathbf{x}}-z_{12} a \hat{\mathbf{y}}+x_{12} a \hat{\mathbf{z}}$ & $(48 h)$ & H VI \\
\hline $\mathbf{B}_{111}$ & $=$ & $\begin{array}{c}\left(-x_{13}+y_{13}+z_{13}\right) \mathbf{a}_{1}+ \\
\left(x_{13}-y_{13}+z_{13}\right) \mathbf{a}_{2}+\left(x_{13}+y_{13}-z_{13}\right) \mathbf{a}_{3}\end{array}$ & $=$ & $x_{13} a \hat{\mathbf{x}}+y_{13} a \hat{\mathbf{y}}+z_{13} a \hat{\mathbf{z}}$ & $(48 h)$ & O III \\
\hline $\mathbf{B}_{112}$ & $=$ & $\begin{array}{c}\left(x_{13}-y_{13}+z_{13}\right) \mathbf{a}_{1}+ \\
\left(-x_{13}+y_{13}+z_{13}\right) \mathbf{a}_{2}+ \\
\left(-x_{13}-y_{13}-z_{13}\right) \mathbf{a}_{3}\end{array}$ & $=$ & $-x_{13} a \hat{\mathbf{x}}-y_{13} a \hat{\mathbf{y}}+z_{13} a \hat{\mathbf{z}}$ & $(48 h)$ & O III \\
\hline $\mathbf{B}_{113}$ & $=$ & $\begin{array}{c}\left(x_{13}+y_{13}-z_{13}\right) \mathbf{a}_{1}+ \\
\left(-x_{13}-y_{13}-z_{13}\right) \mathbf{a}_{2}+ \\
\left(-x_{13}+y_{13}+z_{13}\right) \mathbf{a}_{3}\end{array}$ & $=$ & $-x_{13} a \hat{\mathbf{x}}+y_{13} a \hat{\mathbf{y}}-z_{13} a \hat{\mathbf{z}}$ & $(48 h)$ & O III \\
\hline $\mathbf{B}_{114}$ & $=$ & $\begin{array}{c}\left(-x_{13}-y_{13}-z_{13}\right) \mathbf{a}_{1}+ \\
\left(x_{13}+y_{13}-z_{13}\right) \mathbf{a}_{2}+\left(x_{13}-y_{13}+z_{13}\right) \mathbf{a}_{3}\end{array}$ & $=$ & $x_{13} a \hat{\mathbf{x}}-y_{13} a \hat{\mathbf{y}}-z_{13} a \hat{\mathbf{z}}$ & $(48 h)$ & O III \\
\hline $\mathbf{B}_{115}$ & $=$ & $\begin{array}{c}\left(x_{13}+y_{13}-z_{13}\right) \mathbf{a}_{1}+ \\
\left(-x_{13}+y_{13}+z_{13}\right) \mathbf{a}_{2}+\left(x_{13}-y_{13}+z_{13}\right) \mathbf{a}_{3}\end{array}$ & $=$ & $z_{13} a \hat{\mathbf{x}}+x_{13} a \hat{\mathbf{y}}+y_{13} a \hat{\mathbf{z}}$ & $(48 h)$ & O III \\
\hline $\mathbf{B}_{116}$ & $=$ & $\begin{array}{c}\left(-x_{13}-y_{13}-z_{13}\right) \mathbf{a}_{1}+ \\
\left(x_{13}-y_{13}+z_{13}\right) \mathbf{a}_{2}+\left(-x_{13}+y_{13}+z_{13}\right) \mathbf{a}_{3}\end{array}$ & $=$ & $z_{13} a \hat{\mathbf{x}}-x_{13} a \hat{\mathbf{y}}-y_{13} a \hat{\mathbf{z}}$ & $(48 h)$ & O III \\
\hline $\mathbf{B}_{117}$ & $=$ & $\begin{array}{c}\left(-x_{13}+y_{13}+z_{13}\right) \mathbf{a}_{1}+ \\
\left(x_{13}+y_{13}-z_{13}\right) \mathbf{a}_{2}+\left(-x_{13}-y_{13}-z_{13}\right) \mathbf{a}_{3}\end{array}$ & $=$ & $-z_{13} a \hat{\mathbf{x}}-x_{13} a \hat{\mathbf{y}}+y_{13} a \hat{\mathbf{z}}$ & $(48 h)$ & O III \\
\hline $\mathbf{B}_{118}$ & $=$ & $\begin{array}{c}\left(x_{13}-y_{13}+z_{13}\right) \mathbf{a}_{1}+ \\
\left(-x_{13}-y_{13}-z_{13}\right) \mathbf{a}_{2}+\left(x_{13}+y_{13}-z_{13}\right) \mathbf{a}_{3}\end{array}$ & $=$ & $-z_{13} a \hat{\mathbf{x}}+x_{13} a \hat{\mathbf{y}}-y_{13} a \hat{\mathbf{z}}$ & $(48 h)$ & O III \\
\hline $\mathbf{B}_{119}$ & $=$ & $\begin{array}{c}\left(x_{13}-y_{13}+z_{13}\right) \mathbf{a}_{1}+ \\
\left(x_{13}+y_{13}-z_{13}\right) \mathbf{a}_{2}+\left(-x_{13}+y_{13}+z_{13}\right) \mathbf{a}_{3}\end{array}$ & $=$ & $y_{13} a \hat{\mathbf{x}}+z_{13} a \hat{\mathbf{y}}+x_{13} a \hat{\mathbf{z}}$ & $(48 h)$ & O III \\
\hline $\mathbf{B}_{120}$ & $=$ & $\begin{array}{c}\left(-x_{13}+y_{13}+z_{13}\right) \mathbf{a}_{1}+ \\
\left(-x_{13}-y_{13}-z_{13}\right) \mathbf{a}_{2}+\left(x_{13}-y_{13}+z_{13}\right) \mathbf{a}_{3}\end{array}$ & $=$ & $-y_{13} a \hat{\mathbf{x}}+z_{13} a \hat{\mathbf{y}}-x_{13} a \hat{\mathbf{z}}$ & $(48 h)$ & O III \\
\hline $\mathbf{B}_{121}$ & $=$ & $\begin{array}{c}\left(-x_{13}-y_{13}-z_{13}\right) \mathbf{a}_{1}+ \\
\left(-x_{13}+y_{13}+z_{13}\right) \mathbf{a}_{2}+\left(x_{13}+y_{13}-z_{13}\right) \mathbf{a}_{3}\end{array}$ & $=$ & $y_{13} a \hat{\mathbf{x}}-z_{13} a \hat{\mathbf{y}}-x_{13} a \hat{\mathbf{z}}$ & $(48 h)$ & O III \\
\hline $\mathbf{B}_{122}$ & $=$ & $\begin{array}{c}\left(x_{13}+y_{13}-z_{13}\right) \mathbf{a}_{1}+ \\
\left(x_{13}-y_{13}+z_{13}\right) \mathbf{a}_{2}+\left(-x_{13}-y_{13}-z_{13}\right) \mathbf{a}_{3}\end{array}$ & $=$ & $-y_{13} a \hat{\mathbf{x}}-z_{13} a \hat{\mathbf{y}}+x_{13} a \hat{\mathbf{z}}$ & $(48 h)$ & O III \\
\hline
\end{tabular}




\section{References:}

- I. Tiritiris and T. Schleid, Synthesis, Crystal Structure, and Thermal Decomposition of $\mathrm{Mg}\left(\mathrm{H}_{2} \mathrm{O}\right)_{6}\left[\mathrm{~B}_{12} \mathrm{H}_{12}\right] \times 6 \mathrm{H}_{2} \mathrm{O}$, ChemInform 35 (2004), doi:10.1002/chin.200425008.

\section{Found in:}

- P. Villars and K. Cenzual, Pearson's Crystal Data - Crystal Structure Database for Inorganic Compounds, ASM International (2013).

\section{Geometry files:}

- CIF: pp. 946

- POSCAR: pp. 947 
Sodium Chlorate $\left(\mathrm{NaClO}_{3}, \mathrm{G3}\right)$ Structure:

ABC3_cP20_198_a_a_b
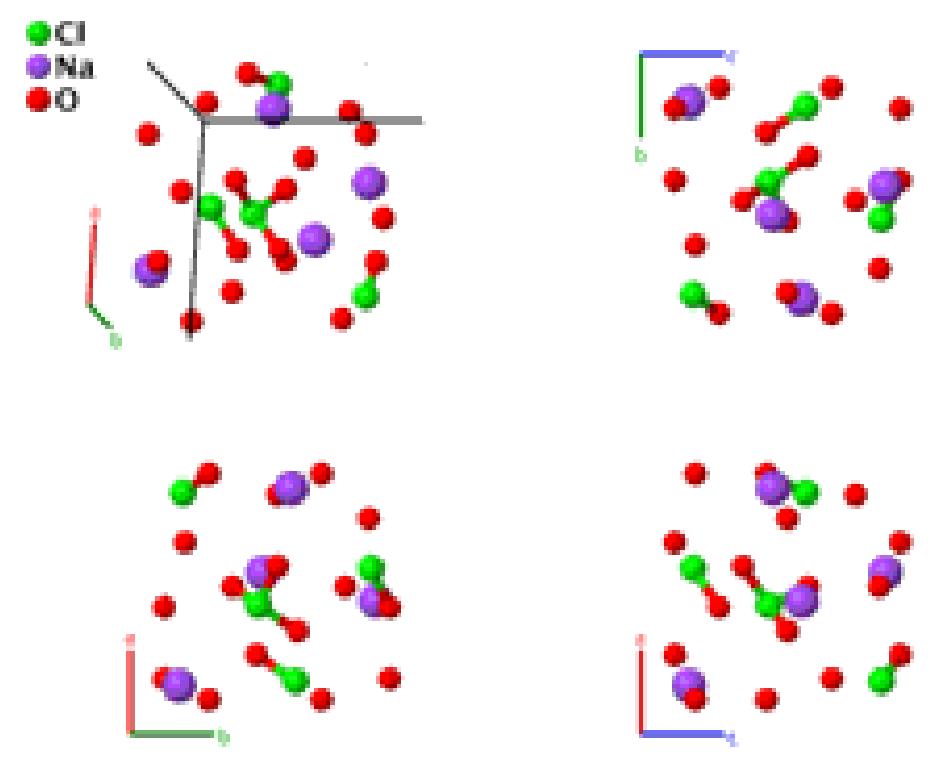

\section{Prototype}

AFLOW prototype label

$: \quad \mathrm{NaClO}_{3}$

Strukturbericht designation

: ABC3_cP20_198_a_a_b

Pearson symbol

: $\quad G 3$

Space group number

: $\quad \mathrm{cP} 20$

Space group symbol

: 198

AFLOW prototype command

$$
\text { : } \quad P 2{ }_{1} 3
$$

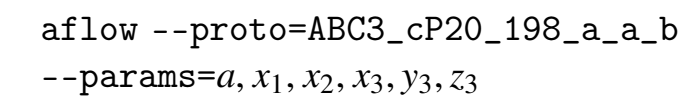

Other compounds with this structure:

- $\mathrm{NaBrO}_{3}$

Simple Cubic primitive vectors:

$$
\begin{aligned}
& \mathbf{a}_{1}=a \hat{\mathbf{x}} \\
& \mathbf{a}_{2}=a \hat{\mathbf{y}} \\
& \mathbf{a}_{3}=a \hat{\mathbf{z}}
\end{aligned}
$$
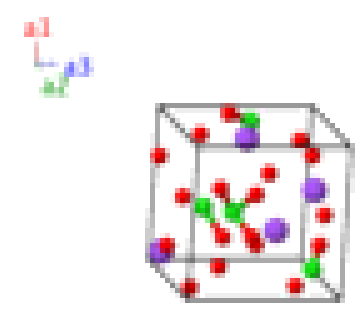

Basis vectors:

Lattice Coordinates

$\begin{array}{llccc}\mathbf{B}_{1}= & x_{1} \mathbf{a}_{1}+x_{1} \mathbf{a}_{2}+x_{1} \mathbf{a}_{3} & = & x_{1} a \hat{\mathbf{x}}+x_{1} a \hat{\mathbf{y}}+x_{1} a \hat{\mathbf{z}} \\ \mathbf{B}_{2}= & \left(\frac{1}{2}-x_{1}\right) \mathbf{a}_{1}-x_{1} \mathbf{a}_{2}+\left(\frac{1}{2}+x_{1}\right) \mathbf{a}_{3} & = & \left(\frac{1}{2}-x_{1}\right) a \hat{\mathbf{x}}-x_{1} a \hat{\mathbf{y}}+\left(\frac{1}{2}+x_{1}\right) a \hat{\mathbf{z}}\end{array}$
Wyckoff Position Atom Type

(4a)

$\mathrm{Cl}$

$(4 a)$ 


$\begin{aligned} \mathbf{B}_{3}=-x_{1} \mathbf{a}_{1}+\left(\frac{1}{2}+x_{1}\right) \mathbf{a}_{2}+\left(\frac{1}{2}-x_{1}\right) \mathbf{a}_{3} & =-x_{1} a \hat{\mathbf{x}}+\left(\frac{1}{2}+x_{1}\right) a \hat{\mathbf{y}}+\left(\frac{1}{2}-x_{1}\right) a \hat{\mathbf{z}} \\ \mathbf{B}_{4}=\left(\frac{1}{2}+x_{1}\right) \mathbf{a}_{1}+\left(\frac{1}{2}-x_{1}\right) \mathbf{a}_{2}-x_{1} \mathbf{a}_{3} & =\left(\frac{1}{2}+x_{1}\right) a \hat{\mathbf{x}}+\left(\frac{1}{2}-x_{1}\right) a \hat{\mathbf{y}}-x_{1} a \hat{\mathbf{z}} \\ \mathbf{B}_{5}= & =\end{aligned}$

\section{References:}

- G. N. Ramachandran and K. S. Chandrasekaran, The absolute configuration of sodium chlorate, Acta Cryst. 10, 671-675 (1957), doi:10.1107/S0365110X57002327.

\section{Found in:}

- A. A. Kaminskii, S. N. Bagayev, J. Hulliger, H. Eichler, J. Findeisen, and R. Macdonald, Acentric cubic $\mathrm{NaClO}_{3}-a$ new crystal for Raman lasers, Appl. Phys. B 67, 157-162 (1998), doi:10.1007/s003400050487.

\section{Geometry files:}

- CIF: pp. 947

- POSCAR: pp. 948 
$\mathrm{Mg}_{2} \mathrm{Zn}_{11}$ Structure: A2B11_cP39_200_f_aghij

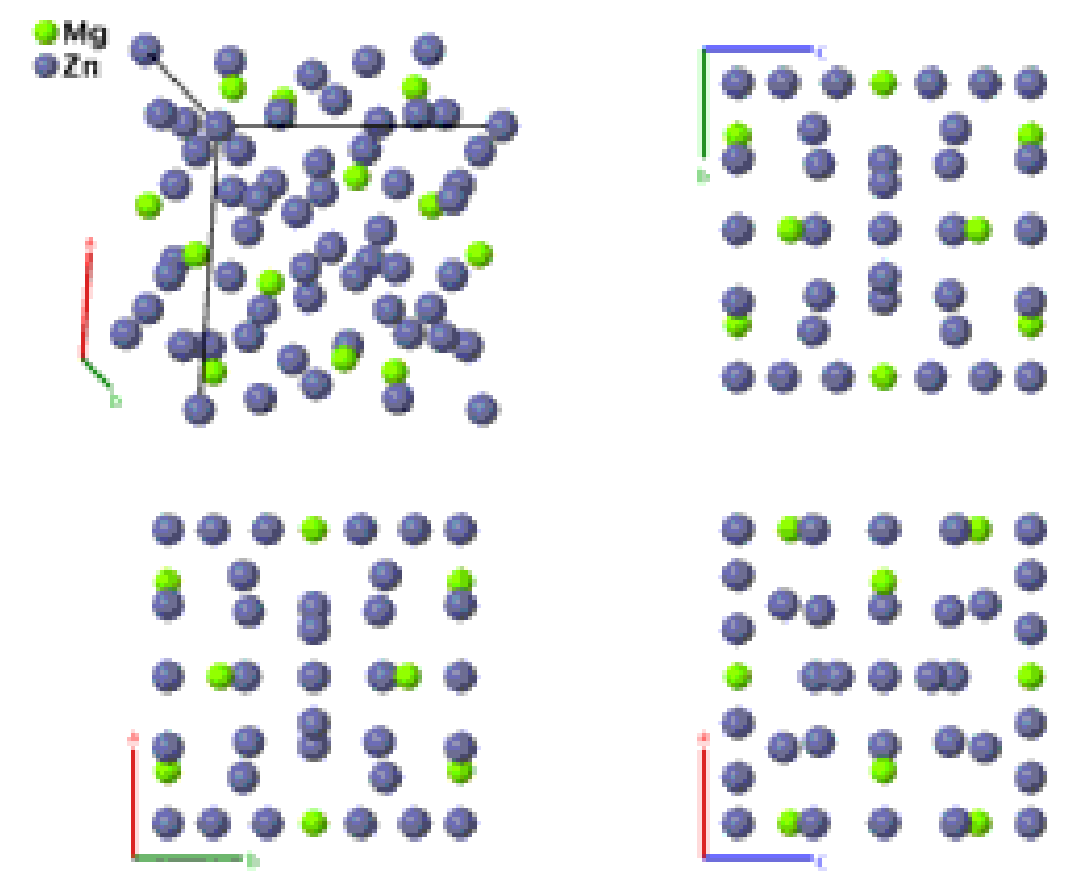

\section{Prototype}

AFLOW prototype label

Strukturbericht designation

Pearson symbol

Space group number

Space group symbol

AFLOW prototype command
: $\quad \mathrm{Mg}_{2} \mathrm{Zn}_{11}$

: A2B11_cP39_200_f_aghij

: None

: $\quad$ cP39

: 200

: $\quad P m \overline{3}$

aflow --proto=A2B11_cP39_200_f_aghij

- params $=a, x_{2}, x_{3}, x_{4}, x_{5}, y_{6}, z_{6}$

Simple Cubic primitive vectors:

$$
\begin{aligned}
& \mathbf{a}_{1}=a \hat{\mathbf{x}} \\
& \mathbf{a}_{2}=a \hat{\mathbf{y}} \\
& \mathbf{a}_{3}=a \hat{\mathbf{z}}
\end{aligned}
$$
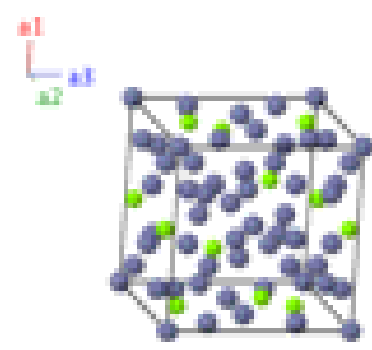

Basis vectors:

Lattice Coordinates
$\mathbf{B}_{1}=$
$\mathbf{B}_{2}=$
$\mathbf{B}_{3}=$
$\mathbf{B}_{4}=$

$$
0 \mathbf{a}_{1}+0 \mathbf{a}_{2}+0 \mathbf{a}_{3}
$$$$
x_{2} \mathbf{a}_{1}+\frac{1}{2} \mathbf{a}_{3}
$$$$
-x_{2} \mathbf{a}_{1}+\frac{1}{2} \mathbf{a}_{3}
$$$$
\frac{1}{2} \mathbf{a}_{1}+x_{2} \mathbf{a}_{2}
$$

Cartesian Coordinates

$=$

$=$

$=$

$=$

$$
\begin{array}{r}
0 \hat{\mathbf{x}}+0 \hat{\mathbf{y}}+0 \hat{\mathbf{z}} \\
x_{2} a \hat{\mathbf{x}}+\frac{1}{2} a \hat{\mathbf{z}} \\
-x_{2} a \hat{\mathbf{x}}+\frac{1}{2} a \hat{\mathbf{z}} \\
\frac{1}{2} a \hat{\mathbf{x}}+x_{2} a \hat{\mathbf{y}}
\end{array}
$$

Wyckoff Position

Atom Type

(1a)

Zn I

$(6 f)$

$\mathrm{Mg}$

$(6 f)$

$(6 f)$
$\mathrm{Mg}$

$\mathrm{Mg}$ 


\begin{tabular}{|c|c|c|c|c|c|c|}
\hline $\mathbf{B}_{5}$ & $=$ & $\frac{1}{2} \mathbf{a}_{1}-x_{2} \mathbf{a}_{2}$ & $=$ & $\frac{1}{2} a \hat{\mathbf{x}}-x_{2} a \hat{\mathbf{y}}$ & $(6 f)$ & $\mathrm{Mg}$ \\
\hline $\mathbf{B}_{6}$ & $=$ & $\frac{1}{2} \mathbf{a}_{2}+x_{2} \mathbf{a}_{3}$ & $=$ & $\frac{1}{2} a \hat{\mathbf{y}}+x_{2} a \hat{\mathbf{z}}$ & $(6 f)$ & $\mathrm{Mg}$ \\
\hline $\mathbf{B}_{7}$ & $=$ & $\frac{1}{2} \mathbf{a}_{2}-x_{2} \mathbf{a}_{3}$ & $=$ & $\frac{1}{2} a \hat{\mathbf{y}}-x_{2} a \hat{\mathbf{z}}$ & $(6 f)$ & $\mathrm{Mg}$ \\
\hline $\mathbf{B}_{8}$ & $=$ & $x_{3} \mathbf{a}_{1}+\frac{1}{2} \mathbf{a}_{2}$ & $=$ & $x_{3} a \hat{\mathbf{x}}+\frac{1}{2} a \hat{\mathbf{y}}$ & $(6 g)$ & Zn II \\
\hline $\mathbf{B}_{9}$ & $=$ & $-x_{3} \mathbf{a}_{1}+\frac{1}{2} \mathbf{a}_{2}$ & $=$ & $-x_{3} a \hat{\mathbf{x}}+\frac{1}{2} a \hat{\mathbf{y}}$ & $(6 g)$ & Zn II \\
\hline $\mathbf{B}_{10}$ & $=$ & $x_{3} \mathbf{a}_{2}+\frac{1}{2} \mathbf{a}_{3}$ & $=$ & $x_{3} a \hat{\mathbf{y}}+\frac{1}{2} a \hat{\mathbf{z}}$ & $(6 g)$ & Zn II \\
\hline $\mathbf{B}_{11}$ & $=$ & $-x_{3} \mathbf{a}_{2}+\frac{1}{2} \mathbf{a}_{3}$ & $=$ & $-x_{3} a \hat{\mathbf{y}}+\frac{1}{2} a \hat{\mathbf{z}}$ & $(6 g)$ & $\mathrm{Zn}$ II \\
\hline $\mathbf{B}_{12}$ & $=$ & $\frac{1}{2} \mathbf{a}_{1}+x_{3} \mathbf{a}_{3}$ & $=$ & $\frac{1}{2} a \hat{\mathbf{x}}+x_{3} a \hat{\mathbf{z}}$ & $(6 g)$ & Zn II \\
\hline $\mathbf{B}_{13}$ & $=$ & $\frac{1}{2} \mathbf{a}_{1}+-x_{3} \mathbf{a}_{3}$ & $=$ & $\frac{1}{2} a \hat{\mathbf{x}}+-x_{3} a \hat{\mathbf{z}}$ & $(6 g)$ & Zn II \\
\hline $\mathbf{B}_{14}$ & $=$ & $x_{4} \mathbf{a}_{1}+\frac{1}{2} \mathbf{a}_{2}+\frac{1}{2} \mathbf{a}_{3}$ & $=$ & $x_{4} a \hat{\mathbf{x}}+\frac{1}{2} a \hat{\mathbf{y}}+\frac{1}{2} a \hat{\mathbf{z}}$ & $(6 h)$ & Zn III \\
\hline $\mathbf{B}_{15}$ & $=$ & $-x_{4} \mathbf{a}_{1}+\frac{1}{2} \mathbf{a}_{2}+\frac{1}{2} \mathbf{a}_{3}$ & $=$ & $-x_{4} a \hat{\mathbf{x}}+\frac{1}{2} a \hat{\mathbf{y}}+\frac{1}{2} a \hat{\mathbf{z}}$ & $(6 h)$ & Zn III \\
\hline $\mathbf{B}_{16}$ & $=$ & $\frac{1}{2} \mathbf{a}_{1}+x_{4} \mathbf{a}_{2}+\frac{1}{2} \mathbf{a}_{3}$ & $=$ & $\frac{1}{2} a \hat{\mathbf{x}}+x_{4} a \hat{\mathbf{y}}+\frac{1}{2} a \hat{\mathbf{z}}$ & $(6 h)$ & Zn III \\
\hline $\mathbf{B}_{17}$ & $=$ & $\frac{1}{2} \mathbf{a}_{1}-x_{4} \mathbf{a}_{2}+\frac{1}{2} \mathbf{a}_{3}$ & $=$ & $\frac{1}{2} a \hat{\mathbf{x}}-x_{4} a \hat{\mathbf{y}}+\frac{1}{2} a \hat{\mathbf{z}}$ & $(6 h)$ & Zn III \\
\hline $\mathbf{B}_{18}$ & $=$ & $\frac{1}{2} \mathbf{a}_{1}+\frac{1}{2} \mathbf{a}_{2}+x_{4} \mathbf{a}_{3}$ & $=$ & $\frac{1}{2} a \hat{\mathbf{x}}+\frac{1}{2} a \hat{\mathbf{y}}+x_{4} a \hat{\mathbf{z}}$ & $(6 h)$ & Zn III \\
\hline $\mathbf{B}_{19}$ & $=$ & $\frac{1}{2} \mathbf{a}_{1}+\frac{1}{2} \mathbf{a}_{2}-x_{4} \mathbf{a}_{3}$ & $=$ & $\frac{1}{2} a \hat{\mathbf{x}}+\frac{1}{2} a \hat{\mathbf{y}}-x_{4} a \hat{\mathbf{z}}$ & $(6 h)$ & Zn III \\
\hline $\mathbf{B}_{20}$ & $=$ & $x_{5} \mathbf{a}_{1}+x_{5} \mathbf{a}_{2}+x_{5} \mathbf{a}_{3}$ & $=$ & $x_{5} a \hat{\mathbf{x}}+x_{5} a \hat{\mathbf{y}}+x_{5} a \hat{\mathbf{z}}$ & $(8 i)$ & Zn IV \\
\hline $\mathbf{B}_{21}$ & $=$ & $-x_{5} \mathbf{a}_{1}-x_{5} \mathbf{a}_{2}+x_{5} \mathbf{a}_{3}$ & $=$ & $-x_{5} a \hat{\mathbf{x}}-x_{5} a \hat{\mathbf{y}}+x_{5} a \hat{\mathbf{z}}$ & $(8 i)$ & $\mathrm{Zn} \mathrm{IV}$ \\
\hline $\mathbf{B}_{22}$ & $=$ & $-x_{5} \mathbf{a}_{1}+x_{5} \mathbf{a}_{2}-x_{5} \mathbf{a}_{3}$ & $=$ & $-x_{5} a \hat{\mathbf{x}}+x_{5} a \hat{\mathbf{y}}-x_{5} a \hat{\mathbf{z}}$ & $(8 i)$ & $\mathrm{Zn} \mathrm{IV}$ \\
\hline $\mathbf{B}_{23}$ & $=$ & $x_{5} \mathbf{a}_{1}-x_{5} \mathbf{a}_{2}-x_{5} \mathbf{a}_{3}$ & $=$ & $x_{5} a \hat{\mathbf{x}}-x_{5} a \hat{\mathbf{y}}-x_{5} a \hat{\mathbf{z}}$ & $(8 i)$ & $\mathrm{Zn} \mathrm{IV}$ \\
\hline $\mathbf{B}_{24}$ & $=$ & $-x_{5} \mathbf{a}_{1}-x_{5} \mathbf{a}_{2}-x_{5} \mathbf{a}_{3}$ & $=$ & $-x_{5} a \hat{\mathbf{x}}-x_{5} a \hat{\mathbf{y}}-x_{5} a \hat{\mathbf{z}}$ & $(8 i)$ & $\mathrm{Zn} \mathrm{IV}$ \\
\hline $\mathbf{B}_{25}$ & $=$ & $x_{5} \mathbf{a}_{1}+x_{5} \mathbf{a}_{2}-x_{5} \mathbf{a}_{3}$ & $=$ & $x_{5} a \hat{\mathbf{x}}+x_{5} a \hat{\mathbf{y}}-x_{5} a \hat{\mathbf{z}}$ & $(8 i)$ & Zn IV \\
\hline $\mathbf{B}_{26}$ & $=$ & $x_{5} \mathbf{a}_{1}-x_{5} \mathbf{a}_{2}+x_{5} \mathbf{a}_{3}$ & $=$ & $x_{5} a \hat{\mathbf{x}}-x_{5} a \hat{\mathbf{y}}+x_{5} a \hat{\mathbf{z}}$ & $(8 i)$ & $\mathrm{Zn} \mathrm{IV}$ \\
\hline $\mathbf{B}_{27}$ & $=$ & $-x_{5} \mathbf{a}_{1}+x_{5} \mathbf{a}_{2}+x_{5} \mathbf{a}_{3}$ & $=$ & $-x_{5} a \hat{\mathbf{x}}+x_{5} a \hat{\mathbf{y}}+x_{5} a \hat{\mathbf{z}}$ & $(8 i)$ & $\mathrm{Zn} \mathrm{IV}$ \\
\hline $\mathbf{B}_{28}$ & $=$ & $y_{6} \mathbf{a}_{2}+z_{6} \mathbf{a}_{3}$ & $=$ & $y_{6} a \hat{\mathbf{y}}+z_{6} a \hat{\mathbf{z}}$ & $(12 j)$ & $\mathrm{Zn} \mathrm{V}$ \\
\hline $\mathbf{B}_{29}$ & $=$ & $-y_{6} \mathbf{a}_{2}+z_{6} \mathbf{a}_{3}$ & $=$ & $-y_{6} a \hat{\mathbf{y}}+z_{6} a \hat{\mathbf{z}}$ & $(12 j)$ & $\mathrm{Zn} \mathrm{V}$ \\
\hline $\mathbf{B}_{30}$ & $=$ & $y_{6} \mathbf{a}_{2}-z_{6} \mathbf{a}_{3}$ & $=$ & $y_{6} a \hat{\mathbf{y}}-z_{6} a \hat{\mathbf{z}}$ & $(12 j)$ & $\mathrm{Zn} \mathrm{V}$ \\
\hline $\mathbf{B}_{31}$ & $=$ & $-y_{6} \mathbf{a}_{2}-z_{6} \mathbf{a}_{3}$ & $=$ & $-y_{6} a \hat{\mathbf{y}}-z_{6} a \hat{\mathbf{z}}$ & $(12 j)$ & $\mathrm{Zn} \mathrm{V}$ \\
\hline $\mathbf{B}_{32}$ & $=$ & $z_{6} \mathbf{a}_{1}+y_{6} \mathbf{a}_{3}$ & $=$ & $z_{6} a \hat{\mathbf{x}}+y_{6} a \hat{\mathbf{z}}$ & $(12 j)$ & $\mathrm{Zn} \mathrm{V}$ \\
\hline $\mathbf{B}_{33}$ & $=$ & $z_{6} \mathbf{a}_{1}+-y_{6} \mathbf{a}_{3}$ & $=$ & $z_{6} a \hat{\mathbf{x}}+-y_{6} a \hat{\mathbf{z}}$ & $(12 j)$ & $\mathrm{Zn} \mathrm{V}$ \\
\hline $\mathbf{B}_{34}$ & $=$ & $-z_{6} \mathbf{a}_{1}+y_{6} \mathbf{a}_{3}$ & $=$ & $-z_{6} a \hat{\mathbf{x}}+y_{6} a \hat{\mathbf{z}}$ & $(12 j)$ & $\mathrm{Zn} \mathrm{V}$ \\
\hline $\mathbf{B}_{35}$ & $=$ & $-z_{6} \mathbf{a}_{1}+-y_{6} \mathbf{a}_{3}$ & $=$ & $-z_{6} a \hat{\mathbf{x}}+-y_{6} a \hat{\mathbf{z}}$ & $(12 j)$ & $\mathrm{Zn} \mathrm{V}$ \\
\hline $\mathbf{B}_{36}$ & $=$ & $y_{6} \mathbf{a}_{1}+z_{6} \mathbf{a}_{2}$ & $=$ & $y_{6} a \hat{\mathbf{x}}+z_{6} a \hat{\mathbf{y}}$ & $(12 j)$ & $\mathrm{Zn} \mathrm{V}$ \\
\hline $\mathbf{B}_{37}$ & $=$ & $-y_{6} \mathbf{a}_{1}+z_{6} \mathbf{a}_{2}$ & $=$ & $-y_{6} a \hat{\mathbf{x}}+z_{6} a \hat{\mathbf{y}}$ & $(12 j)$ & $\mathrm{Zn} \mathrm{V}$ \\
\hline $\mathbf{B}_{38}$ & $=$ & $y_{6} \mathbf{a}_{1}-z_{6} \mathbf{a}_{2}$ & $=$ & $y_{6} a \hat{\mathbf{x}}-z_{6} a \hat{\mathbf{y}}$ & $(12 j)$ & $\mathrm{Zn} \mathrm{V}$ \\
\hline $\mathbf{B}_{39}$ & $=$ & $-y_{6} \mathbf{a}_{1}-z_{6} \mathbf{a}_{2}$ & $=$ & $-y_{6} a \hat{\mathbf{x}}-z_{6} a \hat{\mathbf{y}}$ & $(12 j)$ & $\mathrm{Zn} \mathrm{V}$ \\
\hline
\end{tabular}


- S. Samson, Die Kristallstruktur von $\mathrm{Mg}_{2} \mathrm{Zn}_{11}$ Isomorphie zwischen $\mathrm{Mg}_{2} \mathrm{Zn}_{11}$ und $\mathrm{Mg}_{2} \mathrm{Cu}_{6} \mathrm{Al}_{5}$, Acta Chem. Scand. 3, 835-843 (1949), doi:10.3891/acta.chem.scand.03-0835.

\section{Found in:}

- P. Villars and K. Cenzual, Pearson's Crystal Data - Crystal Structure Database for Inorganic Compounds, ASM International (2013).

\section{Geometry files:}

- CIF: pp. 948

- POSCAR: pp. 948 
$\mathrm{KSbO}_{3}$ (High-temperature) Structure:

AB3C_cP60_201_ce_fh_g

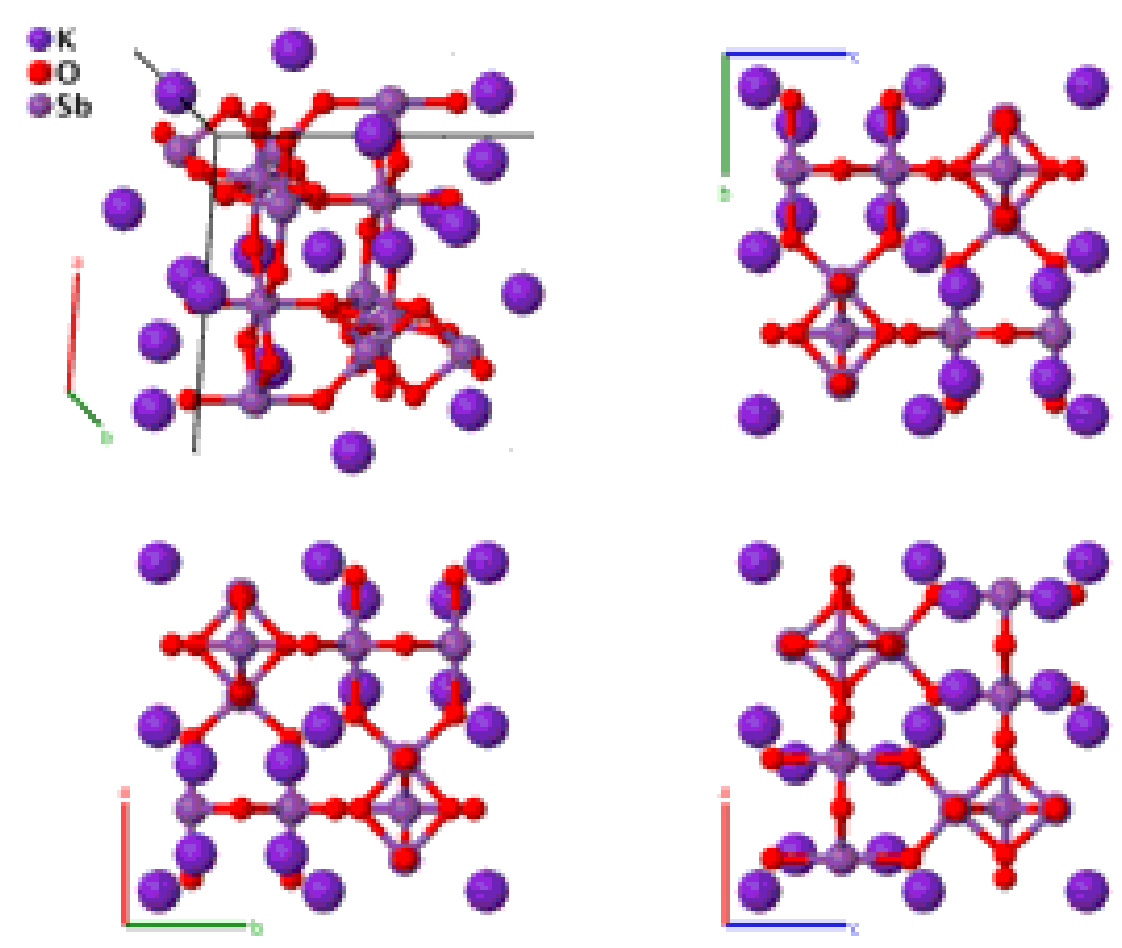

Prototype : $\mathrm{KSbO}_{3}$

AFLOW prototype label $\quad$ : AB3C_cP60_201_ce_fh_g

Strukturbericht designation : None

Pearson symbol $\quad: \quad \mathrm{cP} 60$

Space group number $\quad: \quad 201$

Space group symbol : $P n \overline{3}$

AFLOW prototype command : aflow --proto=AB3C_cP60_201_ce_fh_g

- params $=a, x_{2}, x_{3}, x_{4}, x_{5}, y_{5}, z_{5}$

Simple Cubic primitive vectors:

$$
\begin{aligned}
& \mathbf{a}_{1}=a \hat{\mathbf{x}} \\
& \mathbf{a}_{2}=a \hat{\mathbf{y}} \\
& \mathbf{a}_{3}=a \hat{\mathbf{z}}
\end{aligned}
$$

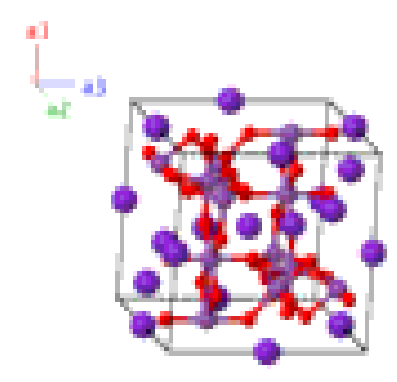

Basis vectors:

Lattice Coordinates

$\begin{array}{ccc}\mathbf{B}_{1}= & \frac{1}{2} \mathbf{a}_{1}+\frac{1}{2} \mathbf{a}_{2}+\frac{1}{2} \mathbf{a}_{3} \\ \mathbf{B}_{2}= & \frac{1}{2} \mathbf{a}_{3}\end{array}$

Cartesian Coordinates

$=\quad \frac{1}{2} a \hat{\mathbf{x}}+\frac{1}{2} a \hat{\mathbf{y}}+\frac{1}{2} a \hat{\mathbf{z}}$

$=\frac{1}{2} a \hat{\mathbf{z}}$
Wyckoff Position

Atom Type

$(4 c)$

K I

$(4 c)$ 


\begin{tabular}{|c|c|c|c|c|c|c|}
\hline $\mathbf{B}_{3}$ & $=$ & $\frac{1}{2} \mathbf{a}_{2}$ & $=$ & $\frac{1}{2} a \hat{\mathbf{y}}$ & $(4 c)$ & K I \\
\hline $\mathbf{B}_{4}$ & $=$ & $\frac{1}{2} \mathbf{a}_{1}$ & $=$ & $\frac{1}{2} a \hat{\mathbf{x}}$ & $(4 c)$ & K I \\
\hline $\mathbf{B}_{5}$ & $=$ & $x_{2} \mathbf{a}_{1}+x_{2} \mathbf{a}_{2}+x_{2} \mathbf{a}_{3}$ & $=$ & $x_{2} a \hat{\mathbf{x}}+x_{2} a \hat{\mathbf{y}}+x_{2} a \hat{\mathbf{z}}$ & $(8 e)$ & K II \\
\hline $\mathbf{B}_{6}$ & $=$ & $\left(\frac{1}{2}-x_{2}\right) \mathbf{a}_{1}+\left(\frac{1}{2}-x_{2}\right) \mathbf{a}_{2}+x_{2} \mathbf{a}_{3}$ & $=$ & $\left(\frac{1}{2}-x_{2}\right) a \hat{\mathbf{x}}+\left(\frac{1}{2}-x_{2}\right) a \hat{\mathbf{y}}+x_{2} a \hat{\mathbf{z}}$ & $(8 e)$ & K II \\
\hline $\mathbf{B}_{7}$ & $=$ & $\left(\frac{1}{2}-x_{2}\right) \mathbf{a}_{1}+x_{2} \mathbf{a}_{2}+\left(\frac{1}{2}-x_{2}\right) \mathbf{a}_{3}$ & $=$ & $\left(\frac{1}{2}-x_{2}\right) a \hat{\mathbf{x}}+x_{2} a \hat{\mathbf{y}}+\left(\frac{1}{2}-x_{2}\right) a \hat{\mathbf{z}}$ & $(8 e)$ & K II \\
\hline $\mathbf{B}_{8}$ & $=$ & $x_{2} \mathbf{a}_{1}+\left(\frac{1}{2}-x_{2}\right) \mathbf{a}_{2}+\left(\frac{1}{2}-x_{2}\right) \mathbf{a}_{3}$ & $=$ & $x_{2} a \hat{\mathbf{x}}+\left(\frac{1}{2}-x_{2}\right) a \hat{\mathbf{y}}+\left(\frac{1}{2}-x_{2}\right) a \hat{\mathbf{z}}$ & $(8 e)$ & K II \\
\hline $\mathbf{B}_{9}$ & $=$ & $-x_{2} \mathbf{a}_{1}-x_{2} \mathbf{a}_{2}-x_{2} \mathbf{a}_{3}$ & $=$ & $-x_{2} a \hat{\mathbf{x}}-x_{2} a \hat{\mathbf{y}}-x_{2} a \hat{\mathbf{z}}$ & $(8 e)$ & K II \\
\hline $\mathbf{B}_{10}$ & $=$ & $\left(\frac{1}{2}+x_{2}\right) \mathbf{a}_{1}+\left(\frac{1}{2}+x_{2}\right) \mathbf{a}_{2}-x_{2} \mathbf{a}_{3}$ & $=$ & $\left(\frac{1}{2}+x_{2}\right) a \hat{\mathbf{x}}+\left(\frac{1}{2}+x_{2}\right) a \hat{\mathbf{y}}-x_{2} a \hat{\mathbf{z}}$ & $(8 e)$ & K II \\
\hline $\mathbf{B}_{11}$ & $=$ & $\left(\frac{1}{2}+x_{2}\right) \mathbf{a}_{1}-x_{2} \mathbf{a}_{2}+\left(\frac{1}{2}+x_{2}\right) \mathbf{a}_{3}$ & $=$ & $\left(\frac{1}{2}+x_{2}\right) a \hat{\mathbf{x}}-x_{2} a \hat{\mathbf{y}}+\left(\frac{1}{2}+x_{2}\right) a \hat{\mathbf{z}}$ & $(8 e)$ & K II \\
\hline $\mathbf{B}_{12}$ & $=$ & $-x_{2} \mathbf{a}_{1}+\left(\frac{1}{2}+x_{2}\right) \mathbf{a}_{2}+\left(\frac{1}{2}+x_{2}\right) \mathbf{a}_{3}$ & $=$ & $-x_{2} a \hat{\mathbf{x}}+\left(\frac{1}{2}+x_{2}\right) a \hat{\mathbf{y}}+\left(\frac{1}{2}+x_{2}\right) a \hat{\mathbf{z}}$ & $(8 e)$ & K II \\
\hline $\mathbf{B}_{13}$ & $=$ & $x_{3} \mathbf{a}_{1}+\frac{1}{4} \mathbf{a}_{2}+\frac{1}{4} \mathbf{a}_{3}$ & $=$ & $x_{3} a \hat{\mathbf{x}}+\frac{1}{4} a \hat{\mathbf{y}}+\frac{1}{4} a \hat{\mathbf{z}}$ & $(12 f)$ & O I \\
\hline $\mathbf{B}_{14}$ & $=$ & $\left(\frac{1}{2}-x_{3}\right) \mathbf{a}_{1}+\frac{1}{4} \mathbf{a}_{2}+\frac{1}{4} \mathbf{a}_{3}$ & $=$ & $\left(\frac{1}{2}-x_{3}\right) a \hat{\mathbf{x}}+\frac{1}{4} a \hat{\mathbf{y}}+\frac{1}{4} a \hat{\mathbf{z}}$ & $(12 f)$ & O I \\
\hline $\mathbf{B}_{15}$ & $=$ & $\frac{1}{4} \mathbf{a}_{1}+x_{3} \mathbf{a}_{2}+\frac{1}{4} \mathbf{a}_{3}$ & $=$ & $\frac{1}{4} a \hat{\mathbf{x}}+x_{3} a \hat{\mathbf{y}}+\frac{1}{4} a \hat{\mathbf{z}}$ & $(12 f)$ & O I \\
\hline $\mathbf{B}_{16}$ & $=$ & $\frac{1}{4} \mathbf{a}_{1}+\left(\frac{1}{2}-x_{3}\right) \mathbf{a}_{2}+\frac{1}{4} \mathbf{a}_{3}$ & $=$ & $\frac{1}{4} a \hat{\mathbf{x}}+\left(\frac{1}{2}-x_{3}\right) a \hat{\mathbf{y}}+\frac{1}{4} a \hat{\mathbf{z}}$ & $(12 f)$ & O I \\
\hline $\mathbf{B}_{17}$ & $=$ & $\frac{1}{4} \mathbf{a}_{1}+\frac{1}{4} \mathbf{a}_{2}+x_{3} \mathbf{a}_{3}$ & $=$ & $\frac{1}{4} a \hat{\mathbf{x}}+\frac{1}{4} a \hat{\mathbf{y}}+x_{3} a \hat{\mathbf{z}}$ & $(12 f)$ & O I \\
\hline $\mathbf{B}_{18}$ & $=$ & $\frac{1}{4} \mathbf{a}_{1}+\frac{1}{4} \mathbf{a}_{2}+\left(\frac{1}{2}-x_{3}\right) \mathbf{a}_{3}$ & $=$ & $\frac{1}{4} a \hat{\mathbf{x}}+\frac{1}{4} a \hat{\mathbf{y}}+\left(\frac{1}{2}-x_{3}\right) a \hat{\mathbf{z}}$ & $(12 f)$ & O I \\
\hline $\mathbf{B}_{19}$ & $=$ & $-x_{3} \mathbf{a}_{1}+\frac{3}{4} \mathbf{a}_{2}+\frac{3}{4} \mathbf{a}_{3}$ & $=$ & $-x_{3} a \hat{\mathbf{x}}+\frac{3}{4} a \hat{\mathbf{y}}+\frac{3}{4} a \hat{\mathbf{z}}$ & $(12 f)$ & O I \\
\hline $\mathbf{B}_{20}$ & $=$ & $\left(\frac{1}{2}+x_{3}\right) \mathbf{a}_{1}+\frac{3}{4} \mathbf{a}_{2}+\frac{3}{4} \mathbf{a}_{3}$ & $=$ & $\left(\frac{1}{2}+x_{3}\right) a \hat{\mathbf{x}}+\frac{3}{4} a \hat{\mathbf{y}}+\frac{3}{4} a \hat{\mathbf{z}}$ & $(12 f)$ & O I \\
\hline $\mathbf{B}_{21}$ & $=$ & $\frac{3}{4} \mathbf{a}_{1}-x_{3} \mathbf{a}_{2}+\frac{3}{4} \mathbf{a}_{3}$ & $=$ & $\frac{3}{4} a \hat{\mathbf{x}}-x_{3} a \hat{\mathbf{y}}+\frac{3}{4} a \hat{\mathbf{z}}$ & $(12 f)$ & O I \\
\hline $\mathbf{B}_{22}$ & $=$ & $\frac{3}{4} \mathbf{a}_{1}+\left(\frac{1}{2}+x_{3}\right) \mathbf{a}_{2}+\frac{3}{4} \mathbf{a}_{3}$ & $=$ & $\frac{3}{4} a \hat{\mathbf{x}}+\left(\frac{1}{2}+x_{3}\right) a \hat{\mathbf{y}}+\frac{3}{4} a \hat{\mathbf{z}}$ & $(12 f)$ & O I \\
\hline $\mathbf{B}_{23}$ & $=$ & $\frac{3}{4} \mathbf{a}_{1}+\frac{3}{4} \mathbf{a}_{2}-x_{3} \mathbf{a}_{3}$ & $=$ & $\frac{3}{4} a \hat{\mathbf{x}}+\frac{3}{4} a \hat{\mathbf{y}}-x_{3} a \hat{\mathbf{z}}$ & $(12 f)$ & O I \\
\hline $\mathbf{B}_{24}$ & $=$ & $\frac{3}{4} \mathbf{a}_{1}+\frac{3}{4} \mathbf{a}_{2}+\left(\frac{1}{2}+x_{3}\right) \mathbf{a}_{3}$ & $=$ & $\frac{3}{4} a \hat{\mathbf{x}}+\frac{3}{4} a \hat{\mathbf{y}}+\left(\frac{1}{2}+x_{3}\right) a \hat{\mathbf{z}}$ & $(12 f)$ & O I \\
\hline $\mathbf{B}_{25}$ & $=$ & $x_{4} \mathbf{a}_{1}+\frac{3}{4} \mathbf{a}_{2}+\frac{1}{4} \mathbf{a}_{3}$ & $=$ & $x_{4} a \hat{\mathbf{x}}+\frac{3}{4} a \hat{\mathbf{y}}+\frac{1}{4} a \hat{\mathbf{z}}$ & $(12 g)$ & $\mathrm{Sb}$ \\
\hline $\mathbf{B}_{26}$ & $=$ & $\left(\frac{1}{2}-x_{4}\right) \mathbf{a}_{1}+\frac{3}{4} \mathbf{a}_{2}+\frac{1}{4} \mathbf{a}_{3}$ & $=$ & $\left(\frac{1}{2}-x_{4}\right) a \hat{\mathbf{x}}+\frac{3}{4} a \hat{\mathbf{y}}+\frac{1}{4} a \hat{\mathbf{z}}$ & $(12 g)$ & $\mathrm{Sb}$ \\
\hline $\mathbf{B}_{27}$ & $=$ & $\frac{1}{4} \mathbf{a}_{1}+x_{4} \mathbf{a}_{2}+\frac{3}{4} \mathbf{a}_{3}$ & $=$ & $\frac{1}{4} a \hat{\mathbf{x}}+x_{4} a \hat{\mathbf{y}}+\frac{3}{4} a \hat{\mathbf{z}}$ & $(12 g)$ & $\mathrm{Sb}$ \\
\hline $\mathbf{B}_{28}$ & $=$ & $\frac{1}{4} \mathbf{a}_{1}+\left(\frac{1}{2}-x_{4}\right) \mathbf{a}_{2}+\frac{3}{4} \mathbf{a}_{3}$ & $=$ & $\frac{1}{4} a \hat{\mathbf{x}}+\left(\frac{1}{2}-x_{4}\right) a \hat{\mathbf{y}}+\frac{3}{4} a \hat{\mathbf{z}}$ & $(12 g)$ & $\mathrm{Sb}$ \\
\hline $\mathbf{B}_{29}$ & $=$ & $\frac{3}{4} \mathbf{a}_{1}+\frac{1}{4} \mathbf{a}_{2}+x_{4} \mathbf{a}_{3}$ & $=$ & $\frac{3}{4} a \hat{\mathbf{x}}+\frac{1}{4} a \hat{\mathbf{y}}+x_{4} a \hat{\mathbf{z}}$ & $(12 g)$ & $\mathrm{Sb}$ \\
\hline $\mathbf{B}_{30}$ & $=$ & $\frac{3}{4} \mathbf{a}_{1}+\frac{1}{4} \mathbf{a}_{2}+\left(\frac{1}{2}-x_{4}\right) \mathbf{a}_{3}$ & $=$ & $\frac{3}{4} a \hat{\mathbf{x}}+\frac{1}{4} a \hat{\mathbf{y}}+\left(\frac{1}{2}-x_{4}\right) a \hat{\mathbf{z}}$ & $(12 g)$ & $\mathrm{Sb}$ \\
\hline $\mathbf{B}_{31}$ & $=$ & $-x_{4} \mathbf{a}_{1}+\frac{1}{4} \mathbf{a}_{2}+\frac{3}{4} \mathbf{a}_{3}$ & $=$ & $-x_{4} a \hat{\mathbf{x}}+\frac{1}{4} a \hat{\mathbf{y}}+\frac{3}{4} a \hat{\mathbf{z}}$ & $(12 g)$ & $\mathrm{Sb}$ \\
\hline $\mathbf{B}_{32}$ & $=$ & $\left(\frac{1}{2}+x_{4}\right) \mathbf{a}_{1}+\frac{1}{4} \mathbf{a}_{2}+\frac{3}{4} \mathbf{a}_{3}$ & $=$ & $\left(\frac{1}{2}+x_{4}\right) a \hat{\mathbf{x}}+\frac{1}{4} a \hat{\mathbf{y}}+\frac{3}{4} a \hat{\mathbf{z}}$ & $(12 g)$ & $\mathrm{Sb}$ \\
\hline $\mathbf{B}_{33}$ & $=$ & $\frac{3}{4} \mathbf{a}_{1}-x_{4} \mathbf{a}_{2}+\frac{1}{4} \mathbf{a}_{3}$ & $=$ & $\frac{3}{4} a \hat{\mathbf{x}}-x_{4} a \hat{\mathbf{y}}+\frac{1}{4} a \hat{\mathbf{z}}$ & $(12 g)$ & $\mathrm{Sb}$ \\
\hline $\mathbf{B}_{34}$ & $=$ & $\frac{3}{4} \mathbf{a}_{1}+\left(\frac{1}{2}+x_{4}\right) \mathbf{a}_{2}+\frac{1}{4} \mathbf{a}_{3}$ & $=$ & $\frac{3}{4} a \hat{\mathbf{x}}+\left(\frac{1}{2}+x_{4}\right) a \hat{\mathbf{y}}+\frac{1}{4} a \hat{\mathbf{z}}$ & $(12 g)$ & $\mathrm{Sb}$ \\
\hline $\mathbf{B}_{35}$ & $=$ & $\frac{1}{4} \mathbf{a}_{1}+\frac{3}{4} \mathbf{a}_{2}-x_{4} \mathbf{a}_{3}$ & $=$ & $\frac{1}{4} a \hat{\mathbf{x}}+\frac{3}{4} a \hat{\mathbf{y}}-x_{4} a \hat{\mathbf{z}}$ & $(12 g)$ & $\mathrm{Sb}$ \\
\hline $\mathbf{B}_{36}$ & $=$ & $\frac{1}{4} \mathbf{a}_{1}+\frac{3}{4} \mathbf{a}_{2}+\left(\frac{1}{2}+x_{4}\right) \mathbf{a}_{3}$ & $=$ & $\frac{1}{4} a \hat{\mathbf{x}}+\frac{3}{4} a \hat{\mathbf{y}}+\left(\frac{1}{2}+x_{4}\right) a \hat{\mathbf{z}}$ & $(12 g)$ & $\mathrm{Sb}$ \\
\hline $\mathbf{B}_{37}$ & $=$ & $x_{5} \mathbf{a}_{1}+y_{5} \mathbf{a}_{2}+z_{5} \mathbf{a}_{3}$ & $=$ & $x_{5} a \hat{\mathbf{x}}+y_{5} a \hat{\mathbf{y}}+z_{5} a \hat{\mathbf{z}}$ & $(24 h)$ & O II \\
\hline $\mathbf{B}_{38}$ & $=$ & $\left(\frac{1}{2}-x_{5}\right) \mathbf{a}_{1}+\left(\frac{1}{2}-y_{5}\right) \mathbf{a}_{2}+z_{5} \mathbf{a}_{3}$ & $=$ & $\left(\frac{1}{2}-x_{5}\right) a \hat{\mathbf{x}}+\left(\frac{1}{2}-y_{5}\right) a \hat{\mathbf{y}}+z_{5} a \hat{\mathbf{z}}$ & $(24 h)$ & O II \\
\hline
\end{tabular}




\begin{tabular}{|c|c|c|c|c|c|c|}
\hline $\mathbf{B}_{39}$ & $=$ & $\left(\frac{1}{2}-x_{5}\right) \mathbf{a}_{1}+y_{5} \mathbf{a}_{2}+\left(\frac{1}{2}-z_{5}\right) \mathbf{a}_{3}$ & $=$ & $\left(\frac{1}{2}-x_{5}\right) a \hat{\mathbf{x}}+y_{5} a \hat{\mathbf{y}}+\left(\frac{1}{2}-z_{5}\right) a \hat{\mathbf{z}}$ & $(24 h)$ & O II \\
\hline $\mathbf{B}_{40}$ & $=$ & $x_{5} \mathbf{a}_{1}+\left(\frac{1}{2}-y_{5}\right) \mathbf{a}_{2}+\left(\frac{1}{2}-z_{5}\right) \mathbf{a}_{3}$ & $=$ & $x_{5} a \hat{\mathbf{x}}+\left(\frac{1}{2}-y_{5}\right) a \hat{\mathbf{y}}+\left(\frac{1}{2}-z_{5}\right) a \hat{\mathbf{z}}$ & $(24 h)$ & O II \\
\hline $\mathbf{B}_{41}$ & $=$ & $z_{5} \mathbf{a}_{1}+x_{5} \mathbf{a}_{2}+y_{5} \mathbf{a}_{3}$ & $=$ & $z_{5} a \hat{\mathbf{x}}+x_{5} a \hat{\mathbf{y}}+y_{5} a \hat{\mathbf{z}}$ & $(24 h)$ & $\mathrm{O}$ II \\
\hline $\mathbf{B}_{42}$ & $=$ & $z_{5} \mathbf{a}_{1}+\left(\frac{1}{2}-x_{5}\right) \mathbf{a}_{2}+\left(\frac{1}{2}-y_{5}\right) \mathbf{a}_{3}$ & $=$ & $z_{5} a \hat{\mathbf{x}}+\left(\frac{1}{2}-x_{5}\right) a \hat{\mathbf{y}}+\left(\frac{1}{2}-y_{5}\right) a \hat{\mathbf{z}}$ & $(24 h)$ & O II \\
\hline $\mathbf{B}_{43}$ & $=$ & $\left(\frac{1}{2}-z_{5}\right) \mathbf{a}_{1}+\left(\frac{1}{2}-x_{5}\right) \mathbf{a}_{2}+y_{5} \mathbf{a}_{3}$ & $=$ & $\left(\frac{1}{2}-z_{5}\right) a \hat{\mathbf{x}}+\left(\frac{1}{2}-x_{5}\right) a \hat{\mathbf{y}}+y_{5} a \hat{\mathbf{z}}$ & $(24 h)$ & O II \\
\hline $\mathbf{B}_{44}$ & $=$ & $\left(\frac{1}{2}-z_{5}\right) \mathbf{a}_{1}+x_{5} \mathbf{a}_{2}+\left(\frac{1}{2}-y_{5}\right) \mathbf{a}_{3}$ & $=$ & $\left(\frac{1}{2}-z_{5}\right) a \hat{\mathbf{x}}+x_{5} a \hat{\mathbf{y}}+\left(\frac{1}{2}-y_{5}\right) a \hat{\mathbf{z}}$ & $(24 h)$ & O II \\
\hline $\mathbf{B}_{45}$ & $=$ & $y_{5} \mathbf{a}_{1}+z_{5} \mathbf{a}_{2}+x_{5} \mathbf{a}_{3}$ & $=$ & $y_{5} a \hat{\mathbf{x}}+z_{5} a \hat{\mathbf{y}}+x_{5} a \hat{\mathbf{z}}$ & $(24 h)$ & O II \\
\hline $\mathbf{B}_{46}$ & $=$ & $\left(\frac{1}{2}-y_{5}\right) \mathbf{a}_{1}+z_{5} \mathbf{a}_{2}+\left(\frac{1}{2}-x_{5}\right) \mathbf{a}_{3}$ & $=$ & $\left(\frac{1}{2}-y_{5}\right) a \hat{\mathbf{x}}+z_{5} a \hat{\mathbf{y}}+\left(\frac{1}{2}-x_{5}\right) a \hat{\mathbf{z}}$ & $(24 h)$ & O II \\
\hline $\mathbf{B}_{47}$ & $=$ & $y_{5} \mathbf{a}_{1}+\left(\frac{1}{2}-z_{5}\right) \mathbf{a}_{2}+\left(\frac{1}{2}-x_{5}\right) \mathbf{a}_{3}$ & $=$ & $y_{5} a \hat{\mathbf{x}}+\left(\frac{1}{2}-z_{5}\right) a \hat{\mathbf{y}}+\left(\frac{1}{2}-x_{5}\right) a \hat{\mathbf{z}}$ & $(24 h)$ & O II \\
\hline $\mathbf{B}_{48}$ & $=$ & $\left(\frac{1}{2}-y_{5}\right) \mathbf{a}_{1}+\left(\frac{1}{2}-z_{5}\right) \mathbf{a}_{2}+x_{5} \mathbf{a}_{3}$ & $=$ & $\left(\frac{1}{2}-y_{5}\right) a \hat{\mathbf{x}}+\left(\frac{1}{2}-z_{5}\right) a \hat{\mathbf{y}}+x_{5} a \hat{\mathbf{z}}$ & $(24 h)$ & O II \\
\hline $\mathbf{B}_{49}$ & $=$ & $-x_{5} \mathbf{a}_{1}-y_{5} \mathbf{a}_{2}-z_{5} \mathbf{a}_{3}$ & $=$ & $-x_{5} a \hat{\mathbf{x}}-y_{5} a \hat{\mathbf{y}}-z_{5} a \hat{\mathbf{z}}$ & $(24 h)$ & O II \\
\hline $\mathbf{B}_{50}$ & $=$ & $\left(\frac{1}{2}+x_{5}\right) \mathbf{a}_{1}+\left(\frac{1}{2}+y_{5}\right) \mathbf{a}_{2}-z_{5} \mathbf{a}_{3}$ & $=$ & $\left(\frac{1}{2}+x_{5}\right) a \hat{\mathbf{x}}+\left(\frac{1}{2}+y_{5}\right) a \hat{\mathbf{y}}-z_{5} a \hat{\mathbf{z}}$ & $(24 h)$ & O II \\
\hline $\mathbf{B}_{51}$ & $=$ & $\left(\frac{1}{2}+x_{5}\right) \mathbf{a}_{1}-y_{5} \mathbf{a}_{2}+\left(\frac{1}{2}+z_{5}\right) \mathbf{a}_{3}$ & $=$ & $\left(\frac{1}{2}+x_{5}\right) a \hat{\mathbf{x}}-y_{5} a \hat{\mathbf{y}}+\left(\frac{1}{2}+z_{5}\right) a \hat{\mathbf{z}}$ & $(24 h)$ & O II \\
\hline $\mathbf{B}_{52}$ & $=$ & $-x_{5} \mathbf{a}_{1}+\left(\frac{1}{2}+y_{5}\right) \mathbf{a}_{2}+\left(\frac{1}{2}+z_{5}\right) \mathbf{a}_{3}$ & $=$ & $-x_{5} a \hat{\mathbf{x}}+\left(\frac{1}{2}+y_{5}\right) a \hat{\mathbf{y}}+\left(\frac{1}{2}+z_{5}\right) a \hat{\mathbf{z}}$ & $(24 h)$ & O II \\
\hline $\mathbf{B}_{53}$ & $=$ & $-z_{5} \mathbf{a}_{1}-x_{5} \mathbf{a}_{2}-y_{5} \mathbf{a}_{3}$ & $=$ & $-z_{5} a \hat{\mathbf{x}}-x_{5} a \hat{\mathbf{y}}-y_{5} a \hat{\mathbf{z}}$ & $(24 h)$ & O II \\
\hline $\mathbf{B}_{54}$ & $=$ & $-z_{5} \mathbf{a}_{1}+\left(\frac{1}{2}+x_{5}\right) \mathbf{a}_{2}+\left(\frac{1}{2}+y_{5}\right) \mathbf{a}_{3}$ & $=$ & $-z_{5} a \hat{\mathbf{x}}+\left(\frac{1}{2}+x_{5}\right) a \hat{\mathbf{y}}+\left(\frac{1}{2}+y_{5}\right) a \hat{\mathbf{z}}$ & $(24 h)$ & O II \\
\hline $\mathbf{B}_{55}$ & $=$ & $\left(\frac{1}{2}+z_{5}\right) \mathbf{a}_{1}+\left(\frac{1}{2}+x_{5}\right) \mathbf{a}_{2}-y_{5} \mathbf{a}_{3}$ & $=$ & $\left(\frac{1}{2}+z_{5}\right) a \hat{\mathbf{x}}+\left(\frac{1}{2}+x_{5}\right) a \hat{\mathbf{y}}-y_{5} a \hat{\mathbf{z}}$ & $(24 h)$ & O II \\
\hline $\mathbf{B}_{56}$ & $=$ & $\left(\frac{1}{2}+z_{5}\right) \mathbf{a}_{1}-x_{5} \mathbf{a}_{2}+\left(\frac{1}{2}+y_{5}\right) \mathbf{a}_{3}$ & $=$ & $\left(\frac{1}{2}+z_{5}\right) a \hat{\mathbf{x}}-x_{5} a \hat{\mathbf{y}}+\left(\frac{1}{2}+y_{5}\right) a \hat{\mathbf{z}}$ & $(24 h)$ & O II \\
\hline $\mathbf{B}_{57}$ & $=$ & $-y_{5} \mathbf{a}_{1}-z_{5} \mathbf{a}_{2}-x_{5} \mathbf{a}_{3}$ & $=$ & $-y_{5} a \hat{\mathbf{x}}-z_{5} a \hat{\mathbf{y}}-x_{5} a \hat{\mathbf{z}}$ & $(24 h)$ & O II \\
\hline $\mathbf{B}_{58}$ & $=$ & $\left(\frac{1}{2}+y_{5}\right) \mathbf{a}_{1}-z_{5} \mathbf{a}_{2}+\left(\frac{1}{2}+x_{5}\right) \mathbf{a}_{3}$ & $=$ & $\left(\frac{1}{2}+y_{5}\right) a \hat{\mathbf{x}}-z_{5} a \hat{\mathbf{y}}+\left(\frac{1}{2}+x_{5}\right) a \hat{\mathbf{z}}$ & $(24 h)$ & O II \\
\hline $\mathbf{B}_{59}$ & $=$ & $-y_{5} \mathbf{a}_{1}+\left(\frac{1}{2}+z_{5}\right) \mathbf{a}_{2}+\left(\frac{1}{2}+x_{5}\right) \mathbf{a}_{3}$ & $=$ & $-y_{5} a \hat{\mathbf{x}}+\left(\frac{1}{2}+z_{5}\right) a \hat{\mathbf{y}}+\left(\frac{1}{2}+x_{5}\right) a \hat{\mathbf{z}}$ & $(24 h)$ & O II \\
\hline $\mathbf{B}_{60}$ & $=$ & $\left(\frac{1}{2}+y_{5}\right) \mathbf{a}_{1}+\left(\frac{1}{2}+z_{5}\right) \mathbf{a}_{2}-x_{5} \mathbf{a}_{3}$ & $=$ & $\left(\frac{1}{2}+y_{5}\right) a \hat{\mathbf{x}}+\left(\frac{1}{2}+z_{5}\right) a \hat{\mathbf{y}}-x_{5} a \hat{\mathbf{z}}$ & $(24 h)$ & O II \\
\hline
\end{tabular}

\section{References:}

- P. Spiegelberg, X-ray studies on potassium antimonates, Ark. Kem. Mineral. Geol. 14A, 1-12 (1940).

\section{Found in:}

- P. Villars and K. Cenzual, Pearson's Crystal Data - Crystal Structure Database for Inorganic Compounds, ASM International (2013).

\section{Geometry files:}

- CIF: pp. 949

- POSCAR: pp. 949 


\section{$\mathrm{KB}_{6} \mathrm{H}_{6}$ Structure: A6B6C_cF104_202_h_h_c}
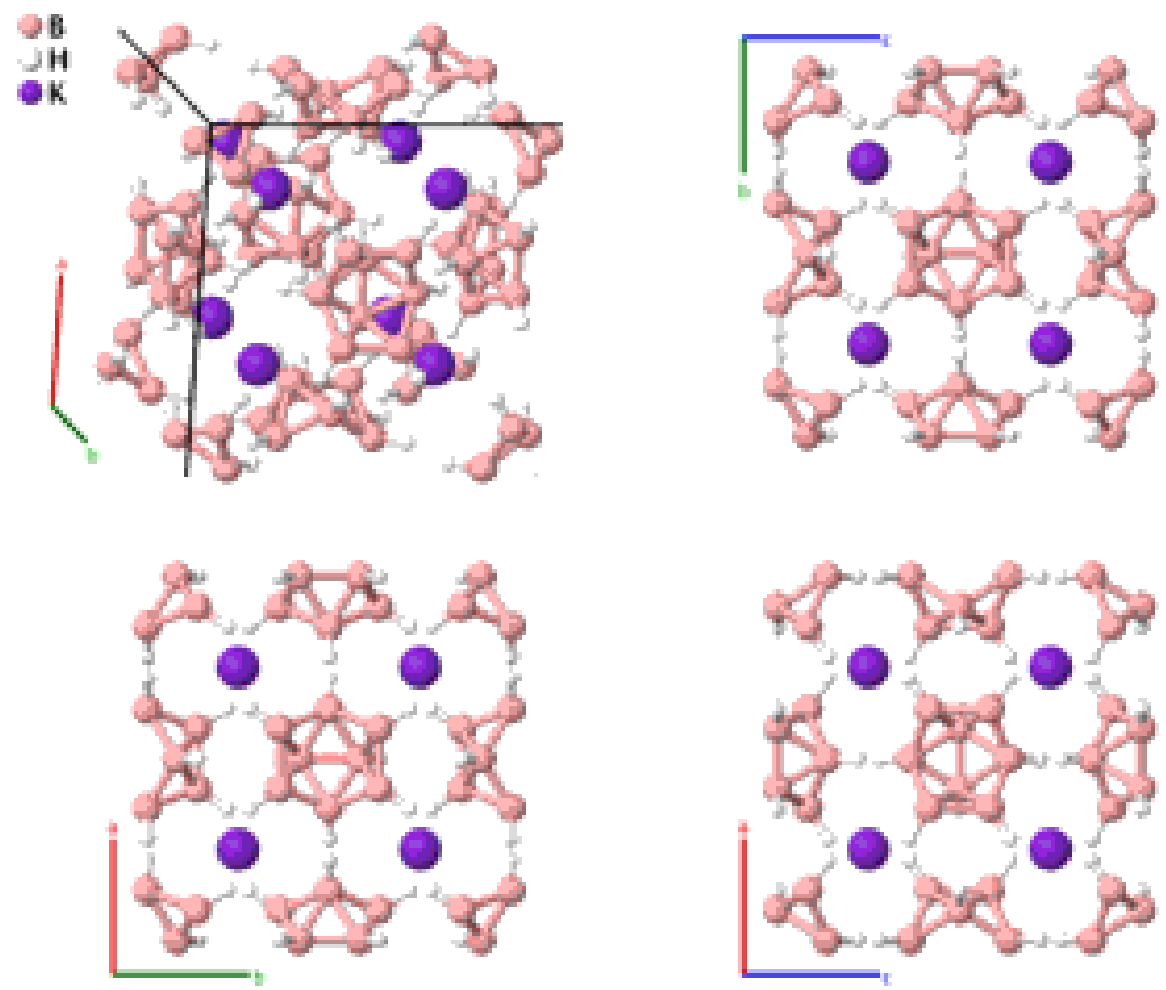

\section{Prototype}

AFLOW prototype label

: $\quad \mathrm{KB}_{6} \mathrm{H}_{6}$

Strukturbericht designation : None

Pearson symbol

: $\quad \mathrm{cF} 104$

Space group number $\quad: \quad 202$

Space group symbol $\quad: F m \overline{3}$

AFLOW prototype command : aflow --proto=A6B6C_cF104_202_h_h_c

- params $=a, y_{2}, z_{2}, y_{3}, z_{3}$

Face-centered Cubic primitive vectors:

$$
\begin{aligned}
& \mathbf{a}_{1}=\frac{1}{2} a \hat{\mathbf{y}}+\frac{1}{2} a \hat{\mathbf{z}} \\
& \mathbf{a}_{2}=\frac{1}{2} a \hat{\mathbf{x}}+\frac{1}{2} a \hat{\mathbf{z}} \\
& \mathbf{a}_{3}=\frac{1}{2} a \hat{\mathbf{x}}+\frac{1}{2} a \hat{\mathbf{y}}
\end{aligned}
$$

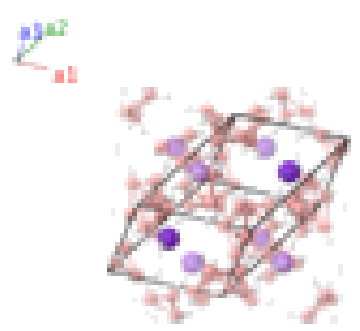

Basis vectors:

Lattice Coordinates

$\begin{array}{lll}\mathbf{B}_{1}= & \frac{1}{4} \mathbf{a}_{1}+\frac{1}{4} \mathbf{a}_{2}+\frac{1}{4} \mathbf{a}_{3} \\ \mathbf{B}_{2}= & \frac{3}{4} \mathbf{a}_{1}+\frac{3}{4} \mathbf{a}_{2}+\frac{3}{4} \mathbf{a}_{3}\end{array}$
Cartesian Coordinates

$=\frac{1}{4} a \hat{\mathbf{x}}+\frac{1}{4} a \hat{\mathbf{y}}+\frac{1}{4} a \hat{\mathbf{z}}$

$=\frac{3}{4} a \hat{\mathbf{x}}+\frac{3}{4} a \hat{\mathbf{y}}+\frac{3}{4} a \hat{\mathbf{z}}$
Wyckoff Position

Atom Type

K

$(8 c)$ 


\begin{tabular}{|c|c|c|c|c|c|}
\hline $\mathbf{B}_{3}$ & $=$ & $\left(y_{2}+z_{2}\right) \mathbf{a}_{1}+\left(-y_{2}+z_{2}\right) \mathbf{a}_{2}+\left(y_{2}-z_{2}\right) \mathbf{a}_{3}$ & $=$ & $y_{2} a \hat{\mathbf{y}}+z_{2} a \hat{\mathbf{z}}$ & $(48 h)$ \\
\hline $\mathbf{B}_{4}$ & $=$ & $\left(-y_{2}+z_{2}\right) \mathbf{a}_{1}+\left(y_{2}+z_{2}\right) \mathbf{a}_{2}+\left(-y_{2}-z_{2}\right) \mathbf{a}_{3}$ & $=$ & $-y_{2} a \hat{\mathbf{y}}+z_{2} a \hat{\mathbf{z}}$ & $(48 h)$ \\
\hline $\mathbf{B}_{5}$ & $=$ & $\left(y_{2}-z_{2}\right) \mathbf{a}_{1}+\left(-y_{2}-z_{2}\right) \mathbf{a}_{2}+\left(y_{2}+z_{2}\right) \mathbf{a}_{3}$ & $=$ & $y_{2} a \hat{\mathbf{y}}-z_{2} a \hat{\mathbf{z}}$ & $(48 h)$ \\
\hline $\mathbf{B}_{6}$ & $=$ & $\left(-y_{2}-z_{2}\right) \mathbf{a}_{1}+\left(y_{2}-z_{2}\right) \mathbf{a}_{2}+\left(-y_{2}+z_{2}\right) \mathbf{a}_{3}$ & $=$ & $-y_{2} a \hat{\mathbf{y}}-z_{2} a \hat{\mathbf{z}}$ & $(48 h)$ \\
\hline $\mathbf{B}_{7}$ & $=$ & $\left(y_{2}-z_{2}\right) \mathbf{a}_{1}+\left(y_{2}+z_{2}\right) \mathbf{a}_{2}+\left(-y_{2}+z_{2}\right) \mathbf{a}_{3}$ & $=$ & $z_{2} a \hat{\mathbf{x}}+y_{2} a \hat{\mathbf{z}}$ & $(48 h)$ \\
\hline $\mathbf{B}_{8}$ & $=$ & $\left(-y_{2}-z_{2}\right) \mathbf{a}_{1}+\left(-y_{2}+z_{2}\right) \mathbf{a}_{2}+\left(y_{2}+z_{2}\right) \mathbf{a}_{3}$ & $=$ & $z_{2} a \hat{\mathbf{x}}+-y_{2} a \hat{\mathbf{z}}$ & $(48 h)$ \\
\hline $\mathbf{B}_{9}$ & $=$ & $\left(y_{2}+z_{2}\right) \mathbf{a}_{1}+\left(y_{2}-z_{2}\right) \mathbf{a}_{2}+\left(-y_{2}-z_{2}\right) \mathbf{a}_{3}$ & $=$ & $-z_{2} a \hat{\mathbf{x}}+y_{2} a \hat{\mathbf{z}}$ & $(48 h)$ \\
\hline $\mathbf{B}_{10}$ & $=$ & $\left(-y_{2}+z_{2}\right) \mathbf{a}_{1}+\left(-y_{2}-z_{2}\right) \mathbf{a}_{2}+\left(y_{2}-z_{2}\right) \mathbf{a}_{3}$ & $=$ & $-z_{2} a \hat{\mathbf{x}}+-y_{2} a \hat{\mathbf{z}}$ & $(48 h)$ \\
\hline $\mathbf{B}_{11}$ & $=$ & $\left(-y_{2}+z_{2}\right) \mathbf{a}_{1}+\left(y_{2}-z_{2}\right) \mathbf{a}_{2}+\left(y_{2}+z_{2}\right) \mathbf{a}_{3}$ & $=$ & $y_{2} a \hat{\mathbf{x}}+z_{2} a \hat{\mathbf{y}}$ & $(48 h)$ \\
\hline $\mathbf{B}_{12}$ & $=$ & $\left(y_{2}+z_{2}\right) \mathbf{a}_{1}+\left(-y_{2}-z_{2}\right) \mathbf{a}_{2}+\left(-y_{2}+z_{2}\right) \mathbf{a}_{3}$ & $=$ & $-y_{2} a \hat{\mathbf{x}}+z_{2} a \hat{\mathbf{y}}$ & $(48 h)$ \\
\hline $\mathbf{B}_{13}$ & $=$ & $\left(-y_{2}-z_{2}\right) \mathbf{a}_{1}+\left(y_{2}+z_{2}\right) \mathbf{a}_{2}+\left(y_{2}-z_{2}\right) \mathbf{a}_{3}$ & $=$ & $y_{2} a \hat{\mathbf{x}}-z_{2} a \hat{\mathbf{y}}$ & $(48 h)$ \\
\hline $\mathbf{B}_{14}$ & $=$ & $\left(y_{2}-z_{2}\right) \mathbf{a}_{1}+\left(-y_{2}+z_{2}\right) \mathbf{a}_{2}+\left(-y_{2}-z_{2}\right) \mathbf{a}_{3}$ & $=$ & $-y_{2} a \hat{\mathbf{x}}-z_{2} a \hat{\mathbf{y}}$ & $(48 h)$ \\
\hline $\mathbf{B}_{15}$ & $=$ & $\left(y_{3}+z_{3}\right) \mathbf{a}_{1}+\left(-y_{3}+z_{3}\right) \mathbf{a}_{2}+\left(y_{3}-z_{3}\right) \mathbf{a}_{3}$ & $=$ & $y_{3} a \hat{\mathbf{y}}+z_{3} a \hat{\mathbf{z}}$ & $(48 h)$ \\
\hline $\mathbf{B}_{16}$ & $=$ & $\left(-y_{3}+z_{3}\right) \mathbf{a}_{1}+\left(y_{3}+z_{3}\right) \mathbf{a}_{2}+\left(-y_{3}-z_{3}\right) \mathbf{a}_{3}$ & $=$ & $-y_{3} a \hat{\mathbf{y}}+z_{3} a \hat{\mathbf{z}}$ & $(48 h)$ \\
\hline $\mathbf{B}_{17}$ & $=$ & $\left(y_{3}-z_{3}\right) \mathbf{a}_{1}+\left(-y_{3}-z_{3}\right) \mathbf{a}_{2}+\left(y_{3}+z_{3}\right) \mathbf{a}_{3}$ & $=$ & $y_{3} a \hat{\mathbf{y}}-z_{3} a \hat{\mathbf{z}}$ & $(48 h)$ \\
\hline $\mathbf{B}_{18}$ & $=$ & $\left(-y_{3}-z_{3}\right) \mathbf{a}_{1}+\left(y_{3}-z_{3}\right) \mathbf{a}_{2}+\left(-y_{3}+z_{3}\right) \mathbf{a}_{3}$ & $=$ & $-y_{3} a \hat{\mathbf{y}}-z_{3} a \hat{\mathbf{z}}$ & $(48 h)$ \\
\hline $\mathbf{B}_{19}$ & $=$ & $\left(y_{3}-z_{3}\right) \mathbf{a}_{1}+\left(y_{3}+z_{3}\right) \mathbf{a}_{2}+\left(-y_{3}+z_{3}\right) \mathbf{a}_{3}$ & $=$ & $z_{3} a \hat{\mathbf{x}}+y_{3} a \hat{\mathbf{z}}$ & $(48 h)$ \\
\hline $\mathbf{B}_{20}$ & $=$ & $\left(-y_{3}-z_{3}\right) \mathbf{a}_{1}+\left(-y_{3}+z_{3}\right) \mathbf{a}_{2}+\left(y_{3}+z_{3}\right) \mathbf{a}_{3}$ & $=$ & $z_{3} a \hat{\mathbf{x}}+-y_{3} a \hat{\mathbf{z}}$ & $(48 h)$ \\
\hline $\mathbf{B}_{21}$ & $=$ & $\left(y_{3}+z_{3}\right) \mathbf{a}_{1}+\left(y_{3}-z_{3}\right) \mathbf{a}_{2}+\left(-y_{3}-z_{3}\right) \mathbf{a}_{3}$ & $=$ & $-z_{3} a \hat{\mathbf{x}}+y_{3} a \hat{\mathbf{z}}$ & $(48 h)$ \\
\hline $\mathbf{B}_{22}$ & $=$ & $\left(-y_{3}+z_{3}\right) \mathbf{a}_{1}+\left(-y_{3}-z_{3}\right) \mathbf{a}_{2}+\left(y_{3}-z_{3}\right) \mathbf{a}_{3}$ & $=$ & $-z_{3} a \hat{\mathbf{x}}+-y_{3} a \hat{\mathbf{z}}$ & $(48 h)$ \\
\hline $\mathbf{B}_{23}$ & $=$ & $\left(-y_{3}+z_{3}\right) \mathbf{a}_{1}+\left(y_{3}-z_{3}\right) \mathbf{a}_{2}+\left(y_{3}+z_{3}\right) \mathbf{a}_{3}$ & $=$ & $y_{3} a \hat{\mathbf{x}}+z_{3} a \hat{\mathbf{y}}$ & $(48 h)$ \\
\hline $\mathbf{B}_{24}$ & $=$ & $\left(y_{3}+z_{3}\right) \mathbf{a}_{1}+\left(-y_{3}-z_{3}\right) \mathbf{a}_{2}+\left(-y_{3}+z_{3}\right) \mathbf{a}_{3}$ & $=$ & $-y_{3} a \hat{\mathbf{x}}+z_{3} a \hat{\mathbf{y}}$ & $(48 h)$ \\
\hline $\mathbf{B}_{25}$ & $=$ & $\left(-y_{3}-z_{3}\right) \mathbf{a}_{1}+\left(y_{3}+z_{3}\right) \mathbf{a}_{2}+\left(y_{3}-z_{3}\right) \mathbf{a}_{3}$ & $=$ & $y_{3} a \hat{\mathbf{x}}-z_{3} a \hat{\mathbf{y}}$ & $(48 h)$ \\
\hline $\mathbf{B}_{26}$ & $=$ & $\left(y_{3}-z_{3}\right) \mathbf{a}_{1}+\left(-y_{3}+z_{3}\right) \mathbf{a}_{2}+\left(-y_{3}-z_{3}\right) \mathbf{a}_{3}$ & $=$ & $-y_{3} a \hat{\mathbf{x}}-z_{3} a \hat{\mathbf{y}}$ & $(48 h)$ \\
\hline
\end{tabular}

\section{References:}

- J. A. Wunderlich and W. N. Lipscomb, Structure of $B_{12} H_{12}^{-2}$ Ion, J. Am. Ceram. Soc. 82, 4427-4428 (1960), doi:10.1021/ja01501a076.

\section{Found in:}

- P. Villars and K. Cenzual, Pearson's Crystal Data - Crystal Structure Database for Inorganic Compounds, ASM International (2013).

\section{Geometry files:}

- CIF: pp. 949

- POSCAR: pp. 950 


\section{FCC $\mathrm{C}_{60}$ Buckminsterfullerine Structure:}

\section{A_cF240_202_h2i}
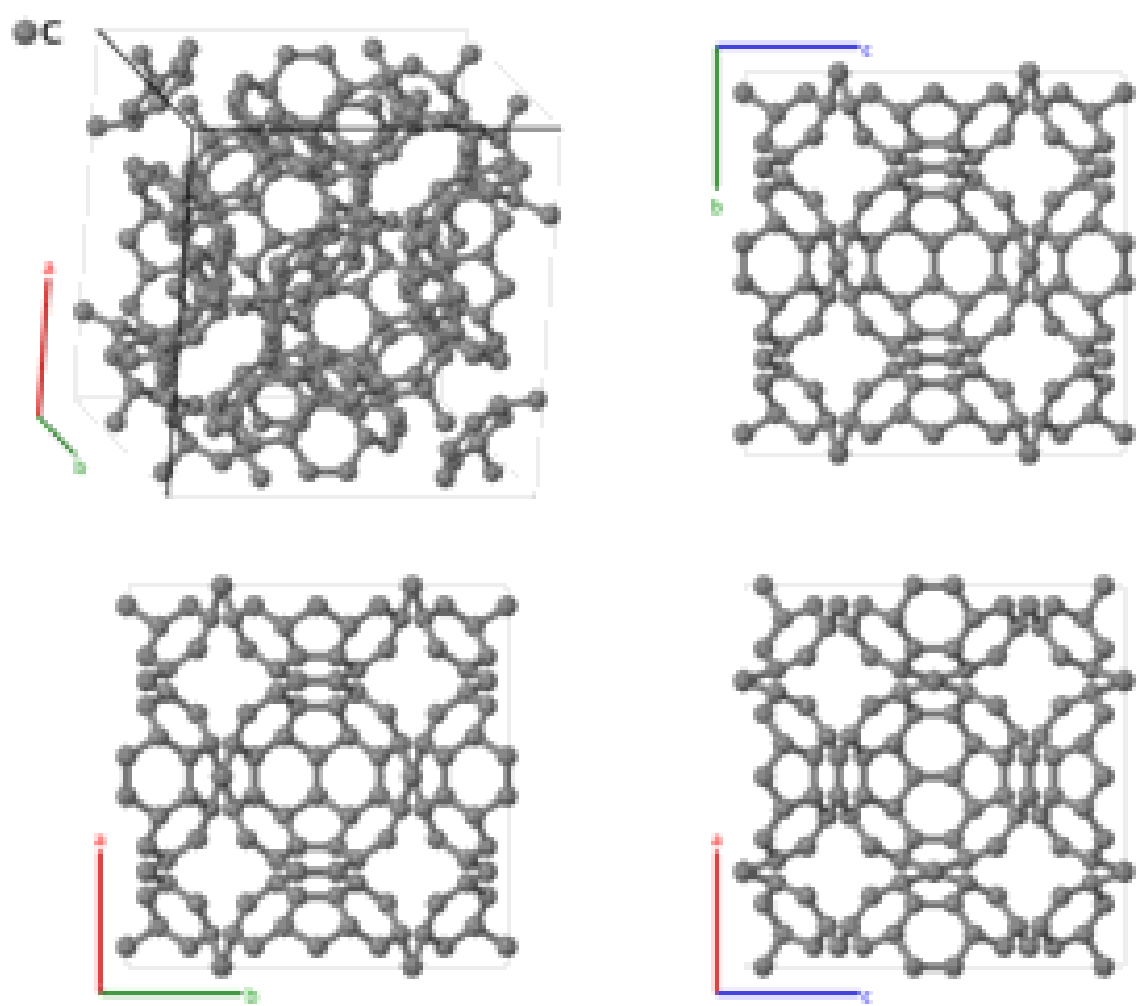

$\begin{array}{lll}\text { Prototype } & : & \text { C } \\ \text { AFLOW prototype label } & : & \text { A_cF240_202_h2i } \\ \text { Strukturbericht designation } & : & \text { None } \\ \text { Pearson symbol } & : & \mathrm{cF} 240 \\ \text { Space group number } & : & 202 \\ \text { Space group symbol } & : & F m \overline{3} \\ \text { AFLOW prototype command } & : & \text { aflow --proto }=A_{-} \text {cF } 240 \_202_{-} \mathrm{h} 2 \mathrm{i} \\ & & -- \text { params }=a, y_{1}, z_{1}, x_{2}, y_{2}, z_{2}, x_{3}, y_{3}, z_{3}\end{array}$

- This is an approximate representation of the structure of $\mathrm{C}_{60}$ buckminsterfullerene. As noted by the authors, "a careful analysis of the intensity data reveals that the molecules must pack in an uncorrelated array, in full agreement with the results from most previous diffraction and spectroscopic determinations." The $\mathrm{C}_{60}$ molecules are on the sites of an fcc lattice. Below $249 \mathrm{~K}$ there is a transition to a simple cubic phase of $\mathrm{C}_{60}$.

Face-centered Cubic primitive vectors:

$$
\begin{aligned}
& \mathbf{a}_{1}=\frac{1}{2} a \hat{\mathbf{y}}+\frac{1}{2} a \hat{\mathbf{z}} \\
& \mathbf{a}_{2}=\frac{1}{2} a \hat{\mathbf{x}}+\frac{1}{2} a \hat{\mathbf{z}} \\
& \mathbf{a}_{3}=\frac{1}{2} a \hat{\mathbf{x}}+\frac{1}{2} a \hat{\mathbf{y}}
\end{aligned}
$$

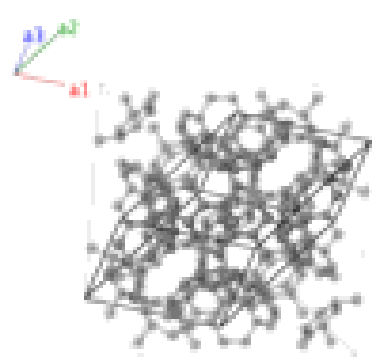




\section{Lattice Coordinates}

$$
\begin{aligned}
\mathbf{B}_{1}=\left(y_{1}+z_{1}\right) \mathbf{a}_{1}+\left(-y_{1}+z_{1}\right) \mathbf{a}_{2}+\left(y_{1}-z_{1}\right) \mathbf{a}_{3} \\
\mathbf{B}_{2}=\left(-y_{1}+z_{1}\right) \mathbf{a}_{1}+\left(y_{1}+z_{1}\right) \mathbf{a}_{2}+\left(-y_{1}-z_{1}\right) \mathbf{a}_{3} \\
\mathbf{B}_{3}=\left(y_{1}-z_{1}\right) \mathbf{a}_{1}+\left(-y_{1}-z_{1}\right) \mathbf{a}_{2}+\left(y_{1}+z_{1}\right) \mathbf{a}_{3} \\
\mathbf{B}_{4}=\left(-y_{1}-z_{1}\right) \mathbf{a}_{1}+\left(y_{1}-z_{1}\right) \mathbf{a}_{2}+\left(-y_{1}+z_{1}\right) \mathbf{a}_{3} \\
\mathbf{B}_{5}=\left(y_{1}-z_{1}\right) \mathbf{a}_{1}+\left(y_{1}+z_{1}\right) \mathbf{a}_{2}+\left(-y_{1}+z_{1}\right) \mathbf{a}_{3} \\
\mathbf{B}_{6}=\left(-y_{1}-z_{1}\right) \mathbf{a}_{1}+\left(-y_{1}+z_{1}\right) \mathbf{a}_{2}+\left(y_{1}+z_{1}\right) \mathbf{a}_{3} \\
\mathbf{B}_{7}=\left(y_{1}+z_{1}\right) \mathbf{a}_{1}+\left(y_{1}-z_{1}\right) \mathbf{a}_{2}+\left(-y_{1}-z_{1}\right) \mathbf{a}_{3} \\
\mathbf{B}_{8}=\left(-y_{1}+z_{1}\right) \mathbf{a}_{1}+\left(-y_{1}-z_{1}\right) \mathbf{a}_{2}+\left(y_{1}-z_{1}\right) \mathbf{a}_{3} \\
\mathbf{B}_{9}=\left(-y_{1}+z_{1}\right) \mathbf{a}_{1}+\left(y_{1}-z_{1}\right) \mathbf{a}_{2}+\left(y_{1}+z_{1}\right) \mathbf{a}_{3} \\
\mathbf{B}_{10}=\left(y_{1}+z_{1}\right) \mathbf{a}_{1}+\left(-y_{1}-z_{1}\right) \mathbf{a}_{2}+\left(-y_{1}+z_{1}\right) \mathbf{a}_{3} \\
\mathbf{B}_{11}=\left(-y_{1}-z_{1}\right) \mathbf{a}_{1}+\left(y_{1}+z_{1}\right) \mathbf{a}_{2}+\left(y_{1}-z_{1}\right) \mathbf{a}_{3} \\
\mathbf{B}_{12}=\left(y_{1}-z_{1}\right) \mathbf{a}_{1}+\left(-y_{1}+z_{1}\right) \mathbf{a}_{2}+\left(-y_{1}-z_{1}\right) \mathbf{a}_{3} \\
\mathbf{B}_{13}=\left(-x_{2}+y_{2}+z_{2}\right) \mathbf{a}_{1}+\left(x_{2}-y_{2}+z_{2}\right) \mathbf{a}_{2}+ \\
\left(x_{2}+y_{2}-z_{2}\right) \mathbf{a}_{3}
\end{aligned}
$$$$
\mathbf{B}_{14}=\left(x_{2}-y_{2}+z_{2}\right) \mathbf{a}_{1}+\left(-x_{2}+y_{2}+z_{2}\right) \mathbf{a}_{2}+
$$$$
\left(-x_{2}-y_{2}-z_{2}\right) \mathbf{a}_{3}
$$$$
\begin{gathered}
\mathbf{B}_{15}=\quad\left(x_{2}+y_{2}-z_{2}\right) \mathbf{a}_{1}+\left(-x_{2}-y_{2}-z_{2}\right) \mathbf{a}_{2}+ \\
\left(-x_{2}+y_{2}+z_{2}\right) \mathbf{a}_{3}
\end{gathered}
$$$$
\mathbf{B}_{16}=\left(-x_{2}-y_{2}-z_{2}\right) \mathbf{a}_{1}+\left(x_{2}+y_{2}-z_{2}\right) \mathbf{a}_{2}+
$$$$
\left(x_{2}-y_{2}+z_{2}\right) \mathbf{a}_{3}
$$$$
\mathbf{B}_{17}=\left(x_{2}+y_{2}-z_{2}\right) \mathbf{a}_{1}+\left(-x_{2}+y_{2}+z_{2}\right) \mathbf{a}_{2}+
$$$$
\left(x_{2}-y_{2}+z_{2}\right) \mathbf{a}_{3}
$$$$
\mathbf{B}_{18}=\left(-x_{2}-y_{2}-z_{2}\right) \mathbf{a}_{1}+\left(x_{2}-y_{2}+z_{2}\right) \mathbf{a}_{2}+
$$$$
\left(-x_{2}+y_{2}+z_{2}\right) \mathbf{a}_{3}
$$$$
\mathbf{B}_{19}=\left(-x_{2}+y_{2}+z_{2}\right) \mathbf{a}_{1}+\left(x_{2}+y_{2}-z_{2}\right) \mathbf{a}_{2}+
$$$$
\left(-x_{2}-y_{2}-z_{2}\right) \mathbf{a}_{3}
$$$$
\begin{gathered}
\mathbf{B}_{20}=\quad\left(x_{2}-y_{2}+z_{2}\right) \mathbf{a}_{1}+\left(-x_{2}-y_{2}-z_{2}\right) \mathbf{a}_{2}+ \\
\left(x_{2}+y_{2}-z_{2}\right) \mathbf{a}_{3}
\end{gathered}
$$$$
\mathbf{B}_{21}=\left(x_{2}-y_{2}+z_{2}\right) \mathbf{a}_{1}+\left(x_{2}+y_{2}-z_{2}\right) \mathbf{a}_{2}+
$$

\begin{tabular}{|c|c|c|c|}
\hline$=$ & $y_{1} a \hat{\mathbf{y}}+z_{1} a \hat{\mathbf{z}}$ & $(48 h)$ & $\mathrm{CI}$ \\
\hline$=$ & $-y_{1} a \hat{\mathbf{y}}+z_{1} a \hat{\mathbf{z}}$ & $(48 h)$ & C I \\
\hline$=$ & $y_{1} a \hat{\mathbf{y}}-z_{1} a \hat{\mathbf{z}}$ & $(48 h)$ & C I \\
\hline$=$ & $-y_{1} a \hat{\mathbf{y}}-z_{1} a \hat{\mathbf{z}}$ & $(48 h)$ & C I \\
\hline$=$ & $z_{1} a \hat{\mathbf{x}}+y_{1} a \hat{\mathbf{z}}$ & $(48 h)$ & $\mathrm{CI}$ \\
\hline$=$ & $z_{1} a \hat{\mathbf{x}}+-y_{1} a \hat{\mathbf{z}}$ & $(48 h)$ & $\mathrm{CI}$ \\
\hline$=$ & $-z_{1} a \hat{\mathbf{x}}+y_{1} a \hat{\mathbf{z}}$ & $(48 h)$ & $\mathrm{CI}$ \\
\hline$=$ & $-z_{1} a \hat{\mathbf{x}}+-y_{1} a \hat{\mathbf{z}}$ & $(48 h)$ & C I \\
\hline$=$ & $y_{1} a \hat{\mathbf{x}}+z_{1} a \hat{\mathbf{y}}$ & $(48 h)$ & C I \\
\hline$=$ & $-y_{1} a \hat{\mathbf{x}}+z_{1} a \hat{\mathbf{y}}$ & $(48 h)$ & $\mathrm{C} \mathrm{I}$ \\
\hline$=$ & $y_{1} a \hat{\mathbf{x}}-z_{1} a \hat{\mathbf{y}}$ & $(48 h)$ & $\mathrm{C} \mathrm{I}$ \\
\hline$=$ & $-y_{1} a \hat{\mathbf{x}}-z_{1} a \hat{\mathbf{y}}$ & $(48 h)$ & $\mathrm{C} \mathrm{I}$ \\
\hline$=$ & $x_{2} a \hat{\mathbf{x}}+y_{2} a \hat{\mathbf{y}}+z_{2} a \hat{\mathbf{z}}$ & $(96 i)$ & C II \\
\hline$=$ & $-x_{2} a \hat{\mathbf{x}}-y_{2} a \hat{\mathbf{y}}+z_{2} a \hat{\mathbf{z}}$ & $(96 i)$ & C II \\
\hline$=$ & $-x_{2} a \hat{\mathbf{x}}+y_{2} a \hat{\mathbf{y}}-z_{2} a \hat{\mathbf{z}}$ & $(96 i)$ & C II \\
\hline$=$ & $x_{2} a \hat{\mathbf{x}}-y_{2} a \hat{\mathbf{y}}-z_{2} a \hat{\mathbf{z}}$ & $(96 i)$ & C II \\
\hline$=$ & $z_{2} a \hat{\mathbf{x}}+x_{2} a \hat{\mathbf{y}}+y_{2} a \hat{\mathbf{z}}$ & $(96 i)$ & C II \\
\hline$=$ & $z_{2} a \hat{\mathbf{x}}-x_{2} a \hat{\mathbf{y}}-y_{2} a \hat{\mathbf{z}}$ & $(96 i)$ & C II \\
\hline$=$ & $-z_{2} a \hat{\mathbf{x}}-x_{2} a \hat{\mathbf{y}}+y_{2} a \hat{\mathbf{z}}$ & $(96 i)$ & C II \\
\hline$=$ & $-z_{2} a \hat{\mathbf{x}}+x_{2} a \hat{\mathbf{y}}-y_{2} a \hat{\mathbf{z}}$ & $(96 i)$ & C II \\
\hline$=$ & $y_{2} a \hat{\mathbf{x}}+z_{2} a \hat{\mathbf{y}}+x_{2} a \hat{\mathbf{z}}$ & $(96 i)$ & C II \\
\hline$=$ & $-y_{2} a \hat{\mathbf{x}}+z_{2} a \hat{\mathbf{y}}-x_{2} a \hat{\mathbf{z}}$ & $(96 i)$ & C II \\
\hline$=$ & $y_{2} a \hat{\mathbf{x}}-z_{2} a \hat{\mathbf{y}}-x_{2} a \hat{\mathbf{z}}$ & $(96 i)$ & C II \\
\hline$=$ & $-y_{2} a \hat{\mathbf{x}}-z_{2} a \hat{\mathbf{y}}+x_{2} a \hat{\mathbf{z}}$ & $(96 i)$ & C II \\
\hline$=$ & $-x_{2} a \hat{\mathbf{x}}-y_{2} a \hat{\mathbf{y}}-z_{2} a \hat{\mathbf{z}}$ & $(96 i)$ & C II \\
\hline$=$ & $x_{2} a \hat{\mathbf{x}}+y_{2} a \hat{\mathbf{y}}-z_{2} a \hat{\mathbf{z}}$ & $(96 i)$ & C II \\
\hline$=$ & $x_{2} a \hat{\mathbf{x}}-y_{2} a \hat{\mathbf{y}}+z_{2} a \hat{\mathbf{z}}$ & $(96 i)$ & C II \\
\hline
\end{tabular}$$
\left(-x_{2}+y_{2}+z_{2}\right) \mathbf{a}_{3}
$$$$
\mathbf{B}_{22}=\left(-x_{2}+y_{2}+z_{2}\right) \mathbf{a}_{1}+\left(-x_{2}-y_{2}-z_{2}\right) \mathbf{a}_{2}+
$$$$
\left(x_{2}-y_{2}+z_{2}\right) \mathbf{a}_{3}
$$$$
\mathbf{B}_{23}=\left(-x_{2}-y_{2}-z_{2}\right) \mathbf{a}_{1}+\left(-x_{2}+y_{2}+z_{2}\right) \mathbf{a}_{2}+
$$$$
\left(x_{2}+y_{2}-z_{2}\right) \mathbf{a}_{3}
$$$$
\mathbf{B}_{24}=\left(x_{2}+y_{2}-z_{2}\right) \mathbf{a}_{1}+\left(x_{2}-y_{2}+z_{2}\right) \mathbf{a}_{2}+
$$$$
\left(-x_{2}-y_{2}-z_{2}\right) \mathbf{a}_{3}
$$$$
\mathbf{B}_{25}=\left(x_{2}-y_{2}-z_{2}\right) \mathbf{a}_{1}+\left(-x_{2}+y_{2}-z_{2}\right) \mathbf{a}_{2}+
$$$$
\left(-x_{2}-y_{2}+z_{2}\right) \mathbf{a}_{3}
$$$$
\begin{gathered}
\mathbf{B}_{26}=\left(-x_{2}+y_{2}-z_{2}\right) \mathbf{a}_{1}+\left(x_{2}-y_{2}-z_{2}\right) \mathbf{a}_{2}+ \\
\left(x_{2}+y_{2}+z_{2}\right) \mathbf{a}_{3}
\end{gathered}
$$$$
\mathbf{B}_{27}=\left(-x_{2}-y_{2}+z_{2}\right) \mathbf{a}_{1}+\left(x_{2}+y_{2}+z_{2}\right) \mathbf{a}_{2}+
$$$$
\left(x_{2}-y_{2}-z_{2}\right) \mathbf{a}_{3}
$$

Cartesian Coordinates Wyckoff Position Atom Type 


\begin{tabular}{|c|c|c|c|c|c|c|}
\hline $\mathbf{B}_{28}$ & $=$ & $\begin{array}{c}\left(x_{2}+y_{2}+z_{2}\right) \mathbf{a}_{1}+\left(-x_{2}-y_{2}+z_{2}\right) \mathbf{a}_{2}+ \\
\left(-x_{2}+y_{2}-z_{2}\right) \mathbf{a}_{3}\end{array}$ & $=$ & $-x_{2} a \hat{\mathbf{x}}+y_{2} a \hat{\mathbf{y}}+z_{2} a \hat{\mathbf{z}}$ & $(96 i)$ & C II \\
\hline $\mathbf{B}_{29}$ & $=$ & $\begin{array}{c}\left(-x_{2}-y_{2}+z_{2}\right) \mathbf{a}_{1}+\left(x_{2}-y_{2}-z_{2}\right) \mathbf{a}_{2}+ \\
\left(-x_{2}+y_{2}-z_{2}\right) \mathbf{a}_{3}\end{array}$ & $=$ & $-z_{2} a \hat{\mathbf{x}}-x_{2} a \hat{\mathbf{y}}-y_{2} a \hat{\mathbf{z}}$ & $(96 i)$ & C II \\
\hline $\mathbf{B}_{30}$ & $=$ & $\begin{array}{c}\left(x_{2}+y_{2}+z_{2}\right) \mathbf{a}_{1}+\left(-x_{2}+y_{2}-z_{2}\right) \mathbf{a}_{2}+ \\
\left(x_{2}-y_{2}-z_{2}\right) \mathbf{a}_{3}\end{array}$ & $=$ & $-z_{2} a \hat{\mathbf{x}}+x_{2} a \hat{\mathbf{y}}+y_{2} a \hat{\mathbf{z}}$ & $(96 i)$ & C II \\
\hline $\mathbf{B}_{31}$ & $=$ & $\begin{array}{c}\left(x_{2}-y_{2}-z_{2}\right) \mathbf{a}_{1}+\left(-x_{2}-y_{2}+z_{2}\right) \mathbf{a}_{2}+ \\
\left(x_{2}+y_{2}+z_{2}\right) \mathbf{a}_{3}\end{array}$ & $=$ & $z_{2} a \hat{\mathbf{x}}+x_{2} a \hat{\mathbf{y}}-y_{2} a \hat{\mathbf{z}}$ & $(96 i)$ & C II \\
\hline $\mathbf{B}_{32}$ & $=$ & $\begin{array}{c}\left(-x_{2}+y_{2}-z_{2}\right) \mathbf{a}_{1}+\left(x_{2}+y_{2}+z_{2}\right) \mathbf{a}_{2}+ \\
\left(-x_{2}-y_{2}+z_{2}\right) \mathbf{a}_{3}\end{array}$ & $=$ & $z_{2} a \hat{\mathbf{x}}-x_{2} a \hat{\mathbf{y}}+y_{2} a \hat{\mathbf{z}}$ & $(96 i)$ & C II \\
\hline $\mathbf{B}_{33}$ & $=$ & $\begin{array}{c}\left(-x_{2}+y_{2}-z_{2}\right) \mathbf{a}_{1}+\left(-x_{2}-y_{2}+z_{2}\right) \mathbf{a}_{2}+ \\
\left(x_{2}-y_{2}-z_{2}\right) \mathbf{a}_{3}\end{array}$ & $=$ & $-y_{2} a \hat{\mathbf{x}}-z_{2} a \hat{\mathbf{y}}-x_{2} a \hat{\mathbf{z}}$ & $(96 i)$ & C II \\
\hline $\mathbf{B}_{34}$ & $=$ & $\begin{array}{c}\left(x_{2}-y_{2}-z_{2}\right) \mathbf{a}_{1}+\left(x_{2}+y_{2}+z_{2}\right) \mathbf{a}_{2}+ \\
\left(-x_{2}+y_{2}-z_{2}\right) \mathbf{a}_{3}\end{array}$ & $=$ & $y_{2} a \hat{\mathbf{x}}-z_{2} a \hat{\mathbf{y}}+x_{2} a \hat{\mathbf{z}}$ & $(96 i)$ & C II \\
\hline $\mathbf{B}_{35}$ & $=$ & $\begin{array}{c}\left(x_{2}+y_{2}+z_{2}\right) \mathbf{a}_{1}+\left(x_{2}-y_{2}-z_{2}\right) \mathbf{a}_{2}+ \\
\left(-x_{2}-y_{2}+z_{2}\right) \mathbf{a}_{3}\end{array}$ & $=$ & $-y_{2} a \hat{\mathbf{x}}+z_{2} a \hat{\mathbf{y}}+x_{2} a \hat{\mathbf{z}}$ & $(96 i)$ & C II \\
\hline $\mathbf{B}_{36}$ & $=$ & $\begin{array}{c}\left(-x_{2}-y_{2}+z_{2}\right) \mathbf{a}_{1}+\left(-x_{2}+y_{2}-z_{2}\right) \mathbf{a}_{2}+ \\
\left(x_{2}+y_{2}+z_{2}\right) \mathbf{a}_{3}\end{array}$ & $=$ & $y_{2} a \hat{\mathbf{x}}+z_{2} a \hat{\mathbf{y}}-x_{2} a \hat{\mathbf{z}}$ & $(96 i)$ & C II \\
\hline $\mathbf{B}_{37}$ & $=$ & $\begin{array}{c}\left(-x_{3}+y_{3}+z_{3}\right) \mathbf{a}_{1}+\left(x_{3}-y_{3}+z_{3}\right) \mathbf{a}_{2}+ \\
\left(x_{3}+y_{3}-z_{3}\right) \mathbf{a}_{3}\end{array}$ & $=$ & $x_{3} a \hat{\mathbf{x}}+y_{3} a \hat{\mathbf{y}}+z_{3} a \hat{\mathbf{z}}$ & $(96 i)$ & C III \\
\hline $\mathbf{B}_{38}$ & $=$ & $\begin{array}{c}\left(x_{3}-y_{3}+z_{3}\right) \mathbf{a}_{1}+\left(-x_{3}+y_{3}+z_{3}\right) \mathbf{a}_{2}+ \\
\left(-x_{3}-y_{3}-z_{3}\right) \mathbf{a}_{3}\end{array}$ & $=$ & $-x_{3} a \hat{\mathbf{x}}-y_{3} a \hat{\mathbf{y}}+z_{3} a \hat{\mathbf{z}}$ & $(96 i)$ & C III \\
\hline $\mathbf{B}_{39}$ & $=$ & $\begin{array}{c}\left(x_{3}+y_{3}-z_{3}\right) \mathbf{a}_{1}+\left(-x_{3}-y_{3}-z_{3}\right) \mathbf{a}_{2}+ \\
\left(-x_{3}+y_{3}+z_{3}\right) \mathbf{a}_{3}\end{array}$ & $=$ & $-x_{3} a \hat{\mathbf{x}}+y_{3} a \hat{\mathbf{y}}-z_{3} a \hat{\mathbf{z}}$ & $(96 i)$ & C III \\
\hline $\mathbf{B}_{40}$ & $=$ & $\begin{array}{c}\left(-x_{3}-y_{3}-z_{3}\right) \mathbf{a}_{1}+\left(x_{3}+y_{3}-z_{3}\right) \mathbf{a}_{2}+ \\
\left(x_{3}-y_{3}+z_{3}\right) \mathbf{a}_{3}\end{array}$ & $=$ & $x_{3} a \hat{\mathbf{x}}-y_{3} a \hat{\mathbf{y}}-z_{3} a \hat{\mathbf{z}}$ & $(96 i)$ & C III \\
\hline $\mathbf{B}_{41}$ & $=$ & $\begin{array}{c}\left(x_{3}+y_{3}-z_{3}\right) \mathbf{a}_{1}+\left(-x_{3}+y_{3}+z_{3}\right) \mathbf{a}_{2}+ \\
\left(x_{3}-y_{3}+z_{3}\right) \mathbf{a}_{3}\end{array}$ & $=$ & $z_{3} a \hat{\mathbf{x}}+x_{3} a \hat{\mathbf{y}}+y_{3} a \hat{\mathbf{z}}$ & $(96 i)$ & C III \\
\hline $\mathbf{B}_{42}$ & $=$ & $\begin{array}{c}\left(-x_{3}-y_{3}-z_{3}\right) \mathbf{a}_{1}+\left(x_{3}-y_{3}+z_{3}\right) \mathbf{a}_{2}+ \\
\left(-x_{3}+y_{3}+z_{3}\right) \mathbf{a}_{3}\end{array}$ & $=$ & $z_{3} a \hat{\mathbf{x}}-x_{3} a \hat{\mathbf{y}}-y_{3} a \hat{\mathbf{z}}$ & $(96 i)$ & C III \\
\hline $\mathbf{B}_{43}$ & $=$ & $\begin{array}{c}\left(-x_{3}+y_{3}+z_{3}\right) \mathbf{a}_{1}+\left(x_{3}+y_{3}-z_{3}\right) \mathbf{a}_{2}+ \\
\left(-x_{3}-y_{3}-z_{3}\right) \mathbf{a}_{3}\end{array}$ & $=$ & $-z_{3} a \hat{\mathbf{x}}-x_{3} a \hat{\mathbf{y}}+y_{3} a \hat{\mathbf{z}}$ & $(96 i)$ & C III \\
\hline $\mathbf{B}_{44}$ & $=$ & $\begin{array}{c}\left(x_{3}-y_{3}+z_{3}\right) \mathbf{a}_{1}+\left(-x_{3}-y_{3}-z_{3}\right) \mathbf{a}_{2}+ \\
\left(x_{3}+y_{3}-z_{3}\right) \mathbf{a}_{3}\end{array}$ & $=$ & $-z_{3} a \hat{\mathbf{x}}+x_{3} a \hat{\mathbf{y}}-y_{3} a \hat{\mathbf{z}}$ & $(96 i)$ & C III \\
\hline $\mathbf{B}_{45}$ & $=$ & $\begin{array}{c}\left(x_{3}-y_{3}+z_{3}\right) \mathbf{a}_{1}+\left(x_{3}+y_{3}-z_{3}\right) \mathbf{a}_{2}+ \\
\left(-x_{3}+y_{3}+z_{3}\right) \mathbf{a}_{3}\end{array}$ & $=$ & $y_{3} a \hat{\mathbf{x}}+z_{3} a \hat{\mathbf{y}}+x_{3} a \hat{\mathbf{z}}$ & $(96 i)$ & C III \\
\hline $\mathbf{B}_{46}$ & $=$ & $\begin{array}{c}\left(-x_{3}+y_{3}+z_{3}\right) \mathbf{a}_{1}+\left(-x_{3}-y_{3}-z_{3}\right) \mathbf{a}_{2}+ \\
\left(x_{3}-y_{3}+z_{3}\right) \mathbf{a}_{3}\end{array}$ & $=$ & $-y_{3} a \hat{\mathbf{x}}+z_{3} a \hat{\mathbf{y}}-x_{3} a \hat{\mathbf{z}}$ & $(96 i)$ & C III \\
\hline $\mathbf{B}_{47}$ & $=$ & $\begin{array}{c}\left(-x_{3}-y_{3}-z_{3}\right) \mathbf{a}_{1}+\left(-x_{3}+y_{3}+z_{3}\right) \mathbf{a}_{2}+ \\
\left(x_{3}+y_{3}-z_{3}\right) \mathbf{a}_{3}\end{array}$ & $=$ & $y_{3} a \hat{\mathbf{x}}-z_{3} a \hat{\mathbf{y}}-x_{3} a \hat{\mathbf{z}}$ & $(96 i)$ & C III \\
\hline $\mathbf{B}_{48}$ & $=$ & $\begin{array}{c}\left(x_{3}+y_{3}-z_{3}\right) \mathbf{a}_{1}+\left(x_{3}-y_{3}+z_{3}\right) \mathbf{a}_{2}+ \\
\left(-x_{3}-y_{3}-z_{3}\right) \mathbf{a}_{3}\end{array}$ & $=$ & $-y_{3} a \hat{\mathbf{x}}-z_{3} a \hat{\mathbf{y}}+x_{3} a \hat{\mathbf{z}}$ & $(96 i)$ & C III \\
\hline $\mathbf{B}_{49}$ & $=$ & $\begin{array}{c}\left(x_{3}-y_{3}-z_{3}\right) \mathbf{a}_{1}+\left(-x_{3}+y_{3}-z_{3}\right) \mathbf{a}_{2}+ \\
\left(-x_{3}-y_{3}+z_{3}\right) \mathbf{a}_{3}\end{array}$ & $=$ & $-x_{3} a \hat{\mathbf{x}}-y_{3} a \hat{\mathbf{y}}-z_{3} a \hat{\mathbf{z}}$ & $(96 i)$ & C III \\
\hline $\mathbf{B}_{50}$ & $=$ & $\begin{array}{c}\left(-x_{3}+y_{3}-z_{3}\right) \mathbf{a}_{1}+\left(x_{3}-y_{3}-z_{3}\right) \mathbf{a}_{2}+ \\
\left(x_{3}+y_{3}+z_{3}\right) \mathbf{a}_{3}\end{array}$ & $=$ & $x_{3} a \hat{\mathbf{x}}+y_{3} a \hat{\mathbf{y}}-z_{3} a \hat{\mathbf{z}}$ & $(96 i)$ & C III \\
\hline $\mathbf{B}_{51}$ & $=$ & $\begin{array}{c}\left(-x_{3}-y_{3}+z_{3}\right) \mathbf{a}_{1}+\left(x_{3}+y_{3}+z_{3}\right) \mathbf{a}_{2}+ \\
\left(x_{3}-y_{3}-z_{3}\right) \mathbf{a}_{3}\end{array}$ & $=$ & $x_{3} a \hat{\mathbf{x}}-y_{3} a \hat{\mathbf{y}}+z_{3} a \hat{\mathbf{z}}$ & $(96 i)$ & C III \\
\hline
\end{tabular}




\begin{tabular}{|c|c|c|c|c|c|c|}
\hline $\mathbf{B}_{52}$ & $=$ & $\begin{array}{c}\left(x_{3}+y_{3}+z_{3}\right) \mathbf{a}_{1}+\left(-x_{3}-y_{3}+z_{3}\right) \mathbf{a}_{2}+ \\
\left(-x_{3}+y_{3}-z_{3}\right) \mathbf{a}_{3}\end{array}$ & $=$ & $-x_{3} a \hat{\mathbf{x}}+y_{3} a \hat{\mathbf{y}}+z_{3} a \hat{\mathbf{z}}$ & $(96 i)$ & C III \\
\hline $\mathbf{B}_{53}$ & $=$ & $\begin{array}{c}\left(-x_{3}-y_{3}+z_{3}\right) \mathbf{a}_{1}+\left(x_{3}-y_{3}-z_{3}\right) \mathbf{a}_{2}+ \\
\left(-x_{3}+y_{3}-z_{3}\right) \mathbf{a}_{3}\end{array}$ & $=$ & $-z_{3} a \hat{\mathbf{x}}-x_{3} a \hat{\mathbf{y}}-y_{3} a \hat{\mathbf{z}}$ & $(96 i)$ & C III \\
\hline $\mathbf{B}_{54}$ & $=$ & $\begin{array}{c}\left(x_{3}+y_{3}+z_{3}\right) \mathbf{a}_{1}+\left(-x_{3}+y_{3}-z_{3}\right) \mathbf{a}_{2}+ \\
\left(x_{3}-y_{3}-z_{3}\right) \mathbf{a}_{3}\end{array}$ & $=$ & $-z_{3} a \hat{\mathbf{x}}+x_{3} a \hat{\mathbf{y}}+y_{3} a \hat{\mathbf{z}}$ & $(96 i)$ & C III \\
\hline $\mathbf{B}_{55}$ & $=$ & $\begin{array}{c}\left(x_{3}-y_{3}-z_{3}\right) \mathbf{a}_{1}+\left(-x_{3}-y_{3}+z_{3}\right) \mathbf{a}_{2}+ \\
\left(x_{3}+y_{3}+z_{3}\right) \mathbf{a}_{3}\end{array}$ & $=$ & $z_{3} a \hat{\mathbf{x}}+x_{3} a \hat{\mathbf{y}}-y_{3} a \hat{\mathbf{z}}$ & $(96 i)$ & C III \\
\hline $\mathbf{B}_{56}$ & $=$ & $\begin{array}{c}\left(-x_{3}+y_{3}-z_{3}\right) \mathbf{a}_{1}+\left(x_{3}+y_{3}+z_{3}\right) \mathbf{a}_{2}+ \\
\left(-x_{3}-y_{3}+z_{3}\right) \mathbf{a}_{3}\end{array}$ & $=$ & $z_{3} a \hat{\mathbf{x}}-x_{3} a \hat{\mathbf{y}}+y_{3} a \hat{\mathbf{z}}$ & $(96 i)$ & C III \\
\hline $\mathbf{B}_{57}$ & $=$ & $\begin{array}{c}\left(-x_{3}+y_{3}-z_{3}\right) \mathbf{a}_{1}+\left(-x_{3}-y_{3}+z_{3}\right) \mathbf{a}_{2}+ \\
\left(x_{3}-y_{3}-z_{3}\right) \mathbf{a}_{3}\end{array}$ & $=$ & $-y_{3} a \hat{\mathbf{x}}-z_{3} a \hat{\mathbf{y}}-x_{3} a \hat{\mathbf{z}}$ & $(96 i)$ & C III \\
\hline $\mathbf{B}_{58}$ & $=$ & $\begin{array}{c}\left(x_{3}-y_{3}-z_{3}\right) \mathbf{a}_{1}+\left(x_{3}+y_{3}+z_{3}\right) \mathbf{a}_{2}+ \\
\left(-x_{3}+y_{3}-z_{3}\right) \mathbf{a}_{3}\end{array}$ & $=$ & $y_{3} a \hat{\mathbf{x}}-z_{3} a \hat{\mathbf{y}}+x_{3} a \hat{\mathbf{z}}$ & $(96 i)$ & C III \\
\hline $\mathbf{B}_{59}$ & $=$ & $\begin{array}{c}\left(x_{3}+y_{3}+z_{3}\right) \mathbf{a}_{1}+\left(x_{3}-y_{3}-z_{3}\right) \mathbf{a}_{2}+ \\
\left(-x_{3}-y_{3}+z_{3}\right) \mathbf{a}_{3}\end{array}$ & $=$ & $-y_{3} a \hat{\mathbf{x}}+z_{3} a \hat{\mathbf{y}}+x_{3} a \hat{\mathbf{z}}$ & $(96 i)$ & C III \\
\hline $\mathbf{B}_{60}$ & $=$ & $\begin{array}{c}\left(-x_{3}-y_{3}+z_{3}\right) \mathbf{a}_{1}+\left(-x_{3}+y_{3}-z_{3}\right) \mathbf{a}_{2}+ \\
\left(x_{3}+y_{3}+z_{3}\right) \mathbf{a}_{3}\end{array}$ & $=$ & $y_{3} a \hat{\mathbf{x}}+z_{3} a \hat{\mathbf{y}}-x_{3} a \hat{\mathbf{z}}$ & $(96 i)$ & C III \\
\hline
\end{tabular}

\section{References:}

- D. L. Dorset and M. P. McCourt, Disorder and the molecular packing of $C_{60}$ buckminsterfullerene: a direct electron-crystallographic analysis, Acta Crystallogr. Sect. A 50, 344-351 (1994), doi:10.1107/S0108767393012607.

\section{Geometry files:}

- CIF: pp. 950

- POSCAR: pp. 951 


\section{Pyrochlore $\left(\mathrm{Na}_{3} \mathrm{Co}\left(\mathrm{CO}_{3}\right)_{2} \mathrm{Cl}\right)$ Structure: A2BCD3E6_cF208_203_e_c_d_f_g}
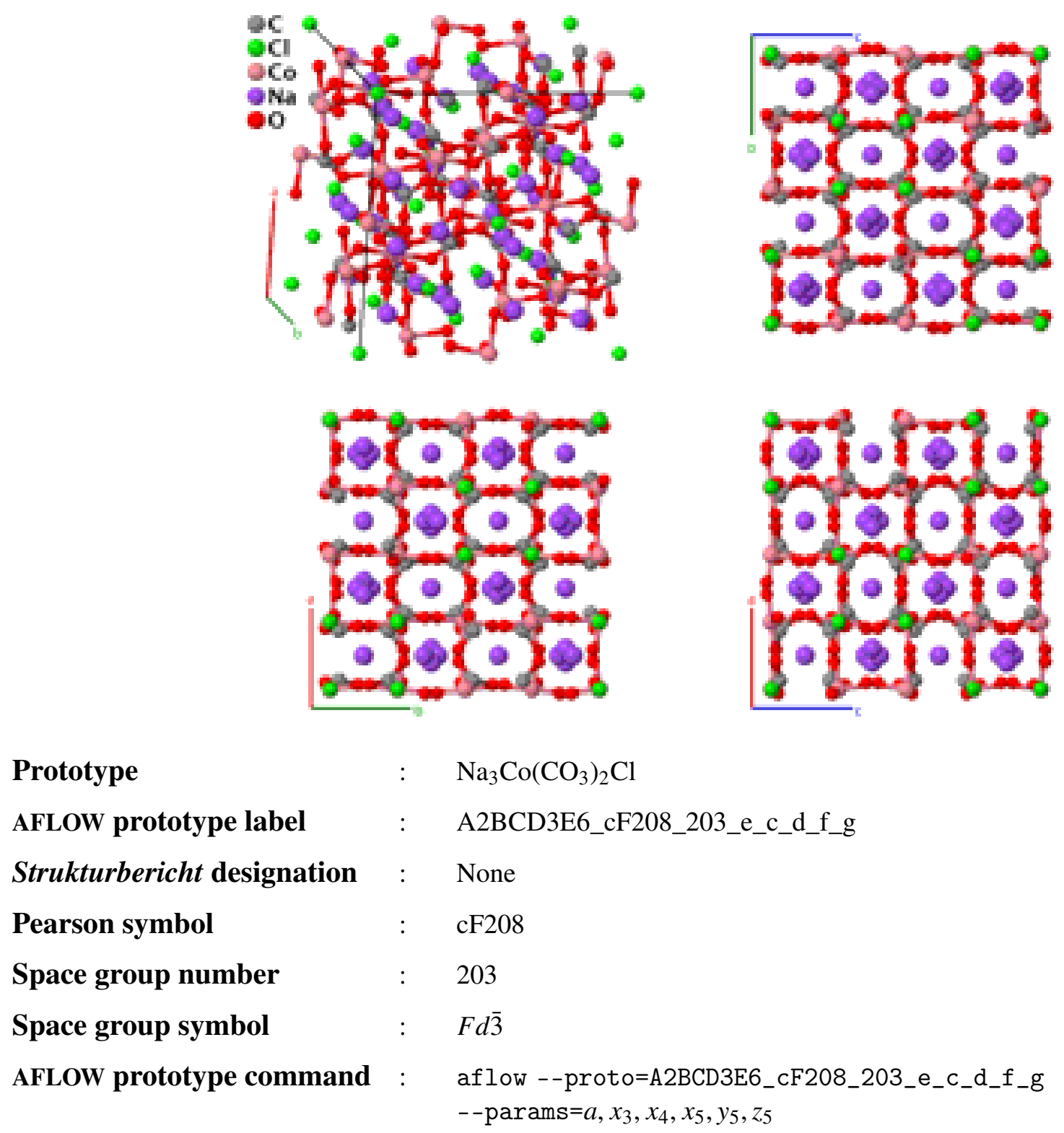

- This structure was suggested to us by Prof. Joel Helton, United States Naval Academy. This is a pyrochlore-like antiferromagnet, which we loosely define as a structure with magnetic ions on the corners of corner-sharing tetrahedra.

Face-centered Cubic primitive vectors:

$$
\begin{aligned}
& \mathbf{a}_{1}=\frac{1}{2} a \hat{\mathbf{y}}+\frac{1}{2} a \hat{\mathbf{z}} \\
& \mathbf{a}_{2}=\frac{1}{2} a \hat{\mathbf{x}}+\frac{1}{2} a \hat{\mathbf{z}} \\
& \mathbf{a}_{3}=\frac{1}{2} a \hat{\mathbf{x}}+\frac{1}{2} a \hat{\mathbf{y}}
\end{aligned}
$$

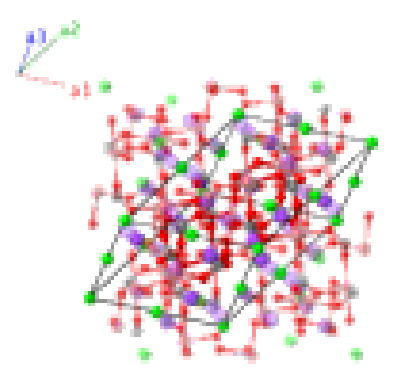


Lattice Coordinates

\begin{tabular}{|c|c|c|c|c|c|}
\hline $\mathbf{B}_{1}$ & $=$ & $0 \mathbf{a}_{1}+0 \mathbf{a}_{2}+0 \mathbf{a}_{3}$ & $=$ & $0 \hat{\mathbf{x}}+0 \hat{\mathbf{y}}+0 \hat{\mathbf{z}}$ & $(16 c)$ \\
\hline $\mathbf{B}_{2}$ & $=$ & $\frac{1}{2} \mathbf{a}_{3}$ & $=$ & $\frac{1}{4} a \hat{\mathbf{x}}+\frac{1}{4} a \hat{\mathbf{y}}$ & $(16 c)$ \\
\hline $\mathbf{B}_{3}$ & $=$ & $\frac{1}{2} \mathbf{a}_{2}$ & $=$ & $\frac{1}{4} a \hat{\mathbf{x}}+\frac{1}{4} a \hat{\mathbf{z}}$ & $(16 c)$ \\
\hline $\mathbf{B}_{4}$ & $=$ & $\frac{1}{2} \mathbf{a}_{1}$ & $=$ & $\frac{1}{4} a \hat{\mathbf{y}}+\frac{1}{4} a \hat{\mathbf{z}}$ & $(16 c)$ \\
\hline $\mathbf{B}_{5}$ & $=$ & $\frac{1}{2} \mathbf{a}_{1}+\frac{1}{2} \mathbf{a}_{2}+\frac{1}{2} \mathbf{a}_{3}$ & $=$ & $\frac{1}{2} a \hat{\mathbf{x}}+\frac{1}{2} a \hat{\mathbf{y}}+\frac{1}{2} a \hat{\mathbf{z}}$ & $(16 d)$ \\
\hline $\mathbf{B}_{6}$ & $=$ & $\frac{1}{2} \mathbf{a}_{1}+\frac{1}{2} \mathbf{a}_{2}$ & $=$ & $\frac{1}{4} a \hat{\mathbf{x}}+\frac{1}{4} a \hat{\mathbf{y}}+\frac{1}{2} a \hat{\mathbf{z}}$ & $(16 d)$ \\
\hline $\mathbf{B}_{7}$ & $=$ & $\frac{1}{2} \mathbf{a}_{1}+\frac{1}{2} \mathbf{a}_{3}$ & $=$ & $\frac{1}{4} a \hat{\mathbf{x}}+\frac{1}{2} a \hat{\mathbf{y}}+\frac{1}{4} a \hat{\mathbf{z}}$ & $(16 d)$ \\
\hline $\mathbf{B}_{8}$ & $=$ & $\frac{1}{2} \mathbf{a}_{2}+\frac{1}{2} \mathbf{a}_{3}$ & $=$ & $\frac{1}{2} a \hat{\mathbf{x}}+\frac{1}{4} a \hat{\mathbf{y}}+\frac{1}{4} a \hat{\mathbf{z}}$ & $(16 d)$ \\
\hline $\mathbf{B}_{9}$ & $=$ & $x_{3} \mathbf{a}_{1}+x_{3} \mathbf{a}_{2}+x_{3} \mathbf{a}_{3}$ & $=$ & $x_{3} a \hat{\mathbf{x}}+x_{3} a \hat{\mathbf{y}}+x_{3} a \hat{\mathbf{z}}$ & $(32 e)$ \\
\hline $\mathbf{B}_{10}$ & $=$ & $x_{3} \mathbf{a}_{1}+x_{3} \mathbf{a}_{2}+\left(\frac{1}{2}-3 x_{3}\right) \mathbf{a}_{3}$ & $=$ & $\left(\frac{1}{4}-x_{3}\right) a \hat{\mathbf{x}}+\left(\frac{1}{4}-x_{3}\right) a \hat{\mathbf{y}}+x_{3} a \hat{\mathbf{z}}$ & $(32 e)$ \\
\hline $\mathbf{B}_{11}$ & $=$ & $x_{3} \mathbf{a}_{1}+\left(\frac{1}{2}-3 x_{3}\right) \mathbf{a}_{2}+x_{3} \mathbf{a}_{3}$ & $=$ & $\left(\frac{1}{4}-x_{3}\right) a \hat{\mathbf{x}}+x_{3} a \hat{\mathbf{y}}+\left(\frac{1}{4}-x_{3}\right) a \hat{\mathbf{z}}$ & $(32 e)$ \\
\hline $\mathbf{B}_{12}$ & $=$ & $\left(\frac{1}{2}-3 x_{3}\right) \mathbf{a}_{1}+x_{3} \mathbf{a}_{2}+x_{3} \mathbf{a}_{3}$ & $=$ & $x_{3} a \hat{\mathbf{x}}+\left(\frac{1}{4}-x_{3}\right) a \hat{\mathbf{y}}+\left(\frac{1}{4}-x_{3}\right) a \hat{\mathbf{z}}$ & $(32 e)$ \\
\hline $\mathbf{B}_{13}$ & $=$ & $-x_{3} \mathbf{a}_{1}-x_{3} \mathbf{a}_{2}-x_{3} \mathbf{a}_{3}$ & $=$ & $-x_{3} a \hat{\mathbf{x}}-x_{3} a \hat{\mathbf{y}}-x_{3} a \hat{\mathbf{z}}$ & $(32 e)$ \\
\hline $\mathbf{B}_{14}$ & $=$ & $-x_{3} \mathbf{a}_{1}-x_{3} \mathbf{a}_{2}+\left(\frac{1}{2}+3 x_{3}\right) \mathbf{a}_{3}$ & $=$ & $\left(\frac{1}{4}+x_{3}\right) a \hat{\mathbf{x}}+\left(\frac{1}{4}+x_{3}\right) a \hat{\mathbf{y}}-x_{3} a \hat{\mathbf{z}}$ & $(32 e)$ \\
\hline $\mathbf{B}_{15}$ & $=$ & $-x_{3} \mathbf{a}_{1}+\left(\frac{1}{2}+3 x_{3}\right) \mathbf{a}_{2}-x_{3} \mathbf{a}_{3}$ & $=$ & $\left(\frac{1}{4}+x_{3}\right) a \hat{\mathbf{x}}-x_{3} a \hat{\mathbf{y}}+\left(\frac{1}{4}+x_{3}\right) a \hat{\mathbf{z}}$ & $(32 e)$ \\
\hline $\mathbf{B}_{16}$ & $=$ & $\left(\frac{1}{2}+3 x_{3}\right) \mathbf{a}_{1}-x_{3} \mathbf{a}_{2}-x_{3} \mathbf{a}_{3}$ & $=$ & $-x_{3} a \hat{\mathbf{x}}+\left(\frac{1}{4}+x_{3}\right) a \hat{\mathbf{y}}+\left(\frac{1}{4}+x_{3}\right) a \hat{\mathbf{z}}$ & $(32 e)$ \\
\hline $\mathbf{B}_{17}$ & $=$ & $\left(\frac{1}{4}-x_{4}\right) \mathbf{a}_{1}+x_{4} \mathbf{a}_{2}+x_{4} \mathbf{a}_{3}$ & $=$ & $x_{4} a \hat{\mathbf{x}}+\frac{1}{8} a \hat{\mathbf{y}}+\frac{1}{8} a \hat{\mathbf{z}}$ & $(48 f)$ \\
\hline $\mathbf{B}_{18}$ & $=$ & $x_{4} \mathbf{a}_{1}+\left(\frac{1}{4}-x_{4}\right) \mathbf{a}_{2}+\left(\frac{1}{4}-x_{4}\right) \mathbf{a}_{3}$ & $=$ & $\left(\frac{1}{4}-x_{4}\right) a \hat{\mathbf{x}}+\frac{1}{8} a \hat{\mathbf{y}}+\frac{1}{8} a \hat{\mathbf{z}}$ & $(48 f)$ \\
\hline $\mathbf{B}_{19}$ & $=$ & $x_{4} \mathbf{a}_{1}+\left(\frac{1}{4}-x_{4}\right) \mathbf{a}_{2}+x_{4} \mathbf{a}_{3}$ & $=$ & $\frac{1}{8} a \hat{\mathbf{x}}+x_{4} a \hat{\mathbf{y}}+\frac{1}{8} a \hat{\mathbf{z}}$ & $(48 f)$ \\
\hline $\mathbf{B}_{20}$ & $=$ & $\left(\frac{1}{4}-x_{4}\right) \mathbf{a}_{1}+x_{4} \mathbf{a}_{2}+\left(\frac{1}{4}-x_{4}\right) \mathbf{a}_{3}$ & $=$ & $\frac{1}{8} a \hat{\mathbf{x}}+\left(\frac{1}{4}-x_{4}\right) a \hat{\mathbf{y}}+\frac{1}{8} a \hat{\mathbf{z}}$ & $(48 f)$ \\
\hline $\mathbf{B}_{21}$ & $=$ & $x_{4} \mathbf{a}_{1}+x_{4} \mathbf{a}_{2}+\left(\frac{1}{4}-x_{4}\right) \mathbf{a}_{3}$ & $=$ & $\frac{1}{8} a \hat{\mathbf{x}}+\frac{1}{8} a \hat{\mathbf{y}}+x_{4} a \hat{\mathbf{z}}$ & $(48 f)$ \\
\hline $\mathbf{B}_{22}$ & $=$ & $\left(\frac{1}{4}-x_{4}\right) \mathbf{a}_{1}+\left(\frac{1}{4}-x_{4}\right) \mathbf{a}_{2}+x_{4} \mathbf{a}_{3}$ & $=$ & $\frac{1}{8} a \hat{\mathbf{x}}+\frac{1}{8} a \hat{\mathbf{y}}+\left(\frac{1}{4}-x_{4}\right) a \hat{\mathbf{z}}$ & $(48 f)$ \\
\hline $\mathbf{B}_{23}$ & $=$ & $\left(\frac{3}{4}+x_{4}\right) \mathbf{a}_{1}-x_{4} \mathbf{a}_{2}-x_{4} \mathbf{a}_{3}$ & $=$ & $-x_{4} a \hat{\mathbf{x}}+\frac{3}{8} a \hat{\mathbf{y}}+\frac{3}{8} a \hat{\mathbf{z}}$ & $(48 f)$ \\
\hline $\mathbf{B}_{24}$ & $=$ & $-x_{4} \mathbf{a}_{1}+\left(\frac{3}{4}+x_{4}\right) \mathbf{a}_{2}+\left(\frac{3}{4}+x_{4}\right) \mathbf{a}_{3}$ & $=$ & $\left(\frac{3}{4}+x_{4}\right) a \hat{\mathbf{x}}+\frac{3}{8} a \hat{\mathbf{y}}+\frac{3}{8} a \hat{\mathbf{z}}$ & $(48 f)$ \\
\hline $\mathbf{B}_{25}$ & $=$ & $-x_{4} \mathbf{a}_{1}+\left(\frac{3}{4}+x_{4}\right) \mathbf{a}_{2}-x_{4} \mathbf{a}_{3}$ & $=$ & $\frac{3}{8} a \hat{\mathbf{x}}-x_{4} a \hat{\mathbf{y}}+\frac{3}{8} a \hat{\mathbf{z}}$ & $(48 f)$ \\
\hline $\mathbf{B}_{26}$ & $=$ & $\left(\frac{3}{4}+x_{4}\right) \mathbf{a}_{1}-x_{4} \mathbf{a}_{2}+\left(\frac{3}{4}+x_{4}\right) \mathbf{a}_{3}$ & $=$ & $\frac{3}{8} a \hat{\mathbf{x}}+\left(\frac{3}{4}+x_{4}\right) a \hat{\mathbf{y}}+\frac{3}{8} a \hat{\mathbf{z}}$ & $(48 f)$ \\
\hline $\mathbf{B}_{27}$ & $=$ & $-x_{4} \mathbf{a}_{1}-x_{4} \mathbf{a}_{2}+\left(\frac{3}{4}+x_{4}\right) \mathbf{a}_{3}$ & $=$ & $\frac{3}{8} a \hat{\mathbf{x}}+\frac{3}{8} a \hat{\mathbf{y}}-x_{4} a \hat{\mathbf{z}}$ & $(48 f)$ \\
\hline $\mathbf{B}_{28}$ & $=$ & $\left(\frac{3}{4}+x_{4}\right) \mathbf{a}_{1}+\left(\frac{3}{4}+x_{4}\right) \mathbf{a}_{2}-x_{4} \mathbf{a}_{3}$ & $=$ & $\frac{3}{8} a \hat{\mathbf{x}}+\frac{3}{8} a \hat{\mathbf{y}}+\left(\frac{3}{4}+x_{4}\right) a \hat{\mathbf{z}}$ & $(48 f)$ \\
\hline $\mathbf{B}_{29}$ & $=$ & $\begin{array}{c}\left(-x_{5}+y_{5}+z_{5}\right) \mathbf{a}_{1}+ \\
\left(x_{5}-y_{5}+z_{5}\right) \mathbf{a}_{2}+ \\
\left(x_{5}+y_{5}-z_{5}\right) \mathbf{a}_{3}\end{array}$ & $=$ & $x_{5} a \hat{\mathbf{x}}+y_{5} a \hat{\mathbf{y}}+z_{5} a \hat{\mathbf{z}}$ & $(96 g)$ \\
\hline $\mathbf{B}_{30}$ & $=$ & $\begin{array}{c}\left(x_{5}-y_{5}+z_{5}\right) \mathbf{a}_{1}+ \\
\left(-x_{5}+y_{5}+z_{5}\right) \mathbf{a}_{2}+ \\
\left(\frac{1}{2}-x_{5}-y_{5}-z_{5}\right) \mathbf{a}_{3}\end{array}$ & & $\left(\frac{1}{4}-x_{5}\right) a \hat{\mathbf{x}}+\left(\frac{1}{4}-y_{5}\right) a \hat{\mathbf{y}}+z_{5} a \hat{\mathbf{z}}$ & $(96 g)$ \\
\hline $\mathbf{B}_{31}$ & $=$ & $\begin{array}{c}\left(x_{5}+y_{5}-z_{5}\right) \mathbf{a}_{1}+ \\
\left(\frac{1}{2}-x_{5}-y_{5}-z_{5}\right) \mathbf{a}_{2}+ \\
\left(-x_{5}+y_{5}+z_{5}\right) \mathbf{a}_{3}\end{array}$ & & $\left(\frac{1}{4}-x_{5}\right) a \hat{\mathbf{x}}+y_{5} a \hat{\mathbf{y}}+\left(\frac{1}{4}-z_{5}\right) a \hat{\mathbf{z}}$ & $(96 g)$ \\
\hline
\end{tabular}

Wyckoff Position Atom Type

$\mathrm{Cl}$

$\mathrm{Cl}$

$\mathrm{Cl}$

Co

Co

Co

Co

C

C

C

a

a

$\mathrm{Na}$

$\mathrm{Na}$

$\mathrm{Na}$

$\mathrm{Na}$

$\mathrm{Na}$

$\mathrm{Na}$

$\mathrm{Na}$

$\mathrm{Na}$

$\mathrm{Na}$

$\mathrm{Na}$

$\mathrm{O}$

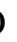

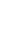




\begin{tabular}{|c|c|c|c|c|c|}
\hline $\mathbf{B}_{32}$ & $=$ & $\begin{array}{c}\left(\frac{1}{2}-x_{5}-y_{5}-z_{5}\right) \mathbf{a}_{1}+ \\
\left(x_{5}+y_{5}-z_{5}\right) \mathbf{a}_{2}+ \\
\left(x_{5}-y_{5}+z_{5}\right) \mathbf{a}_{3}\end{array}$ & $=$ & $x_{5} a \hat{\mathbf{x}}+\left(\frac{1}{4}-y_{5}\right) a \hat{\mathbf{y}}+\left(\frac{1}{4}-z_{5}\right) a \hat{\mathbf{z}}$ & $(96 g)$ \\
\hline $\mathbf{B}_{33}$ & $=$ & $\begin{array}{c}\left(x_{5}+y_{5}-z_{5}\right) \mathbf{a}_{1}+ \\
\left(-x_{5}+y_{5}+z_{5}\right) \mathbf{a}_{2}+ \\
\left(x_{5}-y_{5}+z_{5}\right) \mathbf{a}_{3}\end{array}$ & $=$ & $z_{5} a \hat{\mathbf{x}}+x_{5} a \hat{\mathbf{y}}+y_{5} a \hat{\mathbf{z}}$ & $(96 g)$ \\
\hline $\mathbf{B}_{34}$ & $=$ & $\begin{array}{c}\left(\frac{1}{2}-x_{5}-y_{5}-z_{5}\right) \mathbf{a}_{1}+ \\
\left(x_{5}-y_{5}+z_{5}\right) \mathbf{a}_{2}+ \\
\quad\left(-x_{5}+y_{5}+z_{5}\right) \mathbf{a}_{3}\end{array}$ & $=$ & $z_{5} a \hat{\mathbf{x}}+\left(\frac{1}{4}-x_{5}\right) a \hat{\mathbf{y}}+\left(\frac{1}{4}-y_{5}\right) a \hat{\mathbf{z}}$ & $(96 g)$ \\
\hline $\mathbf{B}_{35}$ & $=$ & $\begin{array}{c}\left(-x_{5}+y_{5}+z_{5}\right) \mathbf{a}_{1}+ \\
\left(x_{5}+y_{5}-z_{5}\right) \mathbf{a}_{2}+ \\
\left(\frac{1}{2}-x_{5}-y_{5}-z_{5}\right) \mathbf{a}_{3}\end{array}$ & $=$ & $\left(\frac{1}{4}-z_{5}\right) a \hat{\mathbf{x}}+\left(\frac{1}{4}-x_{5}\right) a \hat{\mathbf{y}}+y_{5} a \hat{\mathbf{z}}$ & $(96 g)$ \\
\hline $\mathbf{B}_{36}$ & $=$ & $\begin{array}{c}\left(x_{5}-y_{5}+z_{5}\right) \mathbf{a}_{1}+ \\
\left(\frac{1}{2}-x_{5}-y_{5}-z_{5}\right) \mathbf{a}_{2}+ \\
\left(x_{5}+y_{5}-z_{5}\right) \mathbf{a}_{3}\end{array}$ & $=$ & $\left(\frac{1}{4}-z_{5}\right) a \hat{\mathbf{x}}+x_{5} a \hat{\mathbf{y}}+\left(\frac{1}{4}-y_{5}\right) a \hat{\mathbf{z}}$ & $(96 g)$ \\
\hline $\mathbf{B}_{37}$ & $=$ & $\begin{array}{c}\left(x_{5}-y_{5}+z_{5}\right) \mathbf{a}_{1}+ \\
\left(x_{5}+y_{5}-z_{5}\right) \mathbf{a}_{2}+ \\
\left(-x_{5}+y_{5}+z_{5}\right) \mathbf{a}_{3}\end{array}$ & $=$ & $y_{5} a \hat{\mathbf{x}}+z_{5} a \hat{\mathbf{y}}+x_{5} a \hat{\mathbf{z}}$ & $(96 g)$ \\
\hline $\mathbf{B}_{38}$ & $=$ & $\begin{array}{c}\left(-x_{5}+y_{5}+z_{5}\right) \mathbf{a}_{1}+ \\
\left(\frac{1}{2}-x_{5}-y_{5}-z_{5}\right) \mathbf{a}_{2}+ \\
\left(x_{5}-y_{5}+z_{5}\right) \mathbf{a}_{3}\end{array}$ & $=$ & $\left(\frac{1}{4}-y_{5}\right) a \hat{\mathbf{x}}+z_{5} a \hat{\mathbf{y}}+\left(\frac{1}{4}-x_{5}\right) a \hat{\mathbf{z}}$ & $(96 g)$ \\
\hline $\mathbf{B}_{39}$ & $=$ & $\begin{array}{c}\left(\frac{1}{2}-x_{5}-y_{5}-z_{5}\right) \mathbf{a}_{1}+ \\
\left(-x_{5}+y_{5}+z_{5}\right) \mathbf{a}_{2}+ \\
\left(x_{5}+y_{5}-z_{5}\right) \mathbf{a}_{3}\end{array}$ & $=$ & $y_{5} a \hat{\mathbf{x}}+\left(\frac{1}{4}-z_{5}\right) a \hat{\mathbf{y}}+\left(\frac{1}{4}-x_{5}\right) a \hat{\mathbf{z}}$ & $(96 g)$ \\
\hline $\mathbf{B}_{40}$ & $=$ & $\begin{array}{c}\left(x_{5}+y_{5}-z_{5}\right) \mathbf{a}_{1}+ \\
\left(x_{5}-y_{5}+z_{5}\right) \mathbf{a}_{2}+ \\
\left(\frac{1}{2}-x_{5}-y_{5}-z_{5}\right) \mathbf{a}_{3}\end{array}$ & $=$ & $\left(\frac{1}{4}-y_{5}\right) a \hat{\mathbf{x}}+\left(\frac{1}{4}-z_{5}\right) a \hat{\mathbf{y}}+x_{5} a \hat{\mathbf{z}}$ & $(96 g)$ \\
\hline $\mathbf{B}_{41}$ & $=$ & $\begin{array}{c}\left(x_{5}-y_{5}-z_{5}\right) \mathbf{a}_{1}+ \\
\left(-x_{5}+y_{5}-z_{5}\right) \mathbf{a}_{2}+ \\
\left(-x_{5}-y_{5}+z_{5}\right) \mathbf{a}_{3}\end{array}$ & $=$ & $-x_{5} a \hat{\mathbf{x}}-y_{5} a \hat{\mathbf{y}}-z_{5} a \hat{\mathbf{z}}$ & $(96 g)$ \\
\hline $\mathbf{B}_{42}$ & $=$ & $\begin{array}{c}\left(-x_{5}+y_{5}-z_{5}\right) \mathbf{a}_{1}+ \\
\left(x_{5}-y_{5}-z_{5}\right) \mathbf{a}_{2}+ \\
\left(\frac{1}{2}+x_{5}+y_{5}+z_{5}\right) \mathbf{a}_{3}\end{array}$ & $=$ & $\left(\frac{1}{4}+x_{5}\right) a \hat{\mathbf{x}}+\left(\frac{1}{4}+y_{5}\right) a \hat{\mathbf{y}}-z_{5} a \hat{\mathbf{z}}$ & $(96 g)$ \\
\hline $\mathbf{B}_{43}$ & $=$ & $\begin{array}{c}\left(-x_{5}-y_{5}+z_{5}\right) \mathbf{a}_{1}+ \\
\left(\frac{1}{2}+x_{5}+y_{5}+z_{5}\right) \mathbf{a}_{2}+ \\
\left(x_{5}-y_{5}-z_{5}\right) \mathbf{a}_{3}\end{array}$ & $=$ & $\left(\frac{1}{4}+x_{5}\right) a \hat{\mathbf{x}}-y_{5} a \hat{\mathbf{y}}+\left(\frac{1}{4}+z_{5}\right) a \hat{\mathbf{z}}$ & $(96 g)$ \\
\hline $\mathbf{B}_{44}$ & $=$ & $\begin{array}{c}\left(\frac{1}{2}+x_{5}+y_{5}+z_{5}\right) \mathbf{a}_{1}+ \\
\left(-x_{5}-y_{5}+z_{5}\right) \mathbf{a}_{2}+ \\
\left(-x_{5}+y_{5}-z_{5}\right) \mathbf{a}_{3}\end{array}$ & $=$ & $-x_{5} a \hat{\mathbf{x}}+\left(\frac{1}{4}+y_{5}\right) a \hat{\mathbf{y}}+\left(\frac{1}{4}+z_{5}\right) a \hat{\mathbf{z}}$ & $(96 g)$ \\
\hline $\mathbf{B}_{45}$ & $=$ & $\begin{array}{c}\left(-x_{5}-y_{5}+z_{5}\right) \mathbf{a}_{1}+ \\
\left(x_{5}-y_{5}-z_{5}\right) \mathbf{a}_{2}+ \\
\left(-x_{5}+y_{5}-z_{5}\right) \mathbf{a}_{3}\end{array}$ & $=$ & $-z_{5} a \hat{\mathbf{x}}-x_{5} a \hat{\mathbf{y}}-y_{5} a \hat{\mathbf{z}}$ & $(96 g)$ \\
\hline $\mathbf{B}_{46}$ & $=$ & $\begin{array}{c}\left(\frac{1}{2}+x_{5}+y_{5}+z_{5}\right) \mathbf{a}_{1}+ \\
\left(-x_{5}+y_{5}-z_{5}\right) \mathbf{a}_{2}+ \\
\quad\left(x_{5}-y_{5}-z_{5}\right) \mathbf{a}_{3}\end{array}$ & $=$ & $-z_{5} a \hat{\mathbf{x}}+\left(\frac{1}{4}+x_{5}\right) a \hat{\mathbf{y}}+\left(\frac{1}{4}+y_{5}\right) a \hat{\mathbf{z}}$ & $(96 g)$ \\
\hline $\mathbf{B}_{47}$ & $=$ & $\begin{array}{c}\left(x_{5}-y_{5}-z_{5}\right) \mathbf{a}_{1}+ \\
\left(-x_{5}-y_{5}+z_{5}\right) \mathbf{a}_{2}+ \\
\left(\frac{1}{2}+x_{5}+y_{5}+z_{5}\right) \mathbf{a}_{3}\end{array}$ & $=$ & $\left(\frac{1}{4}+z_{5}\right) a \hat{\mathbf{x}}+\left(\frac{1}{4}+x_{5}\right) a \hat{\mathbf{y}}-y_{5} a \hat{\mathbf{z}}$ & $(96 g)$ \\
\hline
\end{tabular}




\begin{tabular}{|c|c|c|c|c|c|}
\hline $\mathbf{B}_{48}$ & $=$ & $\begin{array}{c}\left(-x_{5}+y_{5}-z_{5}\right) \mathbf{a}_{1}+ \\
\left(\frac{1}{2}+x_{5}+y_{5}+z_{5}\right) \mathbf{a}_{2}+ \\
\quad\left(-x_{5}-y_{5}+z_{5}\right) \mathbf{a}_{3}\end{array}$ & & $\left(\frac{1}{4}+z_{5}\right) a \hat{\mathbf{x}}-x_{5} a \hat{\mathbf{y}}+\left(\frac{1}{4}+y_{5}\right) a \hat{\mathbf{z}}$ & $(96 g)$ \\
\hline $\mathbf{B}_{49}$ & $=$ & $\begin{array}{c}\left(-x_{5}+y_{5}-z_{5}\right) \mathbf{a}_{1}+ \\
\left(-x_{5}-y_{5}+z_{5}\right) \mathbf{a}_{2}+ \\
\left(x_{5}-y_{5}-z_{5}\right) \mathbf{a}_{3}\end{array}$ & $=$ & $-y_{5} a \hat{\mathbf{x}}-z_{5} a \hat{\mathbf{y}}-x_{5} a \hat{\mathbf{z}}$ & $(96 g)$ \\
\hline $\mathbf{B}_{50}$ & $=$ & $\begin{array}{c}\left(x_{5}-y_{5}-z_{5}\right) \mathbf{a}_{1}+ \\
\left(\frac{1}{2}+x_{5}+y_{5}+z_{5}\right) \mathbf{a}_{2}+ \\
\left(-x_{5}+y_{5}-z_{5}\right) \mathbf{a}_{3}\end{array}$ & $=$ & $\left(\frac{1}{4}+y_{5}\right) a \hat{\mathbf{x}}-z_{5} a \hat{\mathbf{y}}+\left(\frac{1}{4}+x_{5}\right) a \hat{\mathbf{z}}$ & $(96 g)$ \\
\hline $\mathbf{B}_{51}$ & $=$ & $\begin{array}{c}\left(\frac{1}{2}+x_{5}+y_{5}+z_{5}\right) \mathbf{a}_{1}+ \\
\quad\left(x_{5}-y_{5}-z_{5}\right) \mathbf{a}_{2}+ \\
\quad\left(-x_{5}-y_{5}+z_{5}\right) \mathbf{a}_{3}\end{array}$ & $=$ & $-y_{5} a \hat{\mathbf{x}}+\left(\frac{1}{4}+z_{5}\right) a \hat{\mathbf{y}}+\left(\frac{1}{4}+x_{5}\right) a \hat{\mathbf{z}}$ & $(96 g)$ \\
\hline $\mathbf{B}_{52}$ & $=$ & $\begin{array}{l}\left(-x_{5}-y_{5}+z_{5}\right) \mathbf{a}_{1}+ \\
\left(-x_{5}+y_{5}-z_{5}\right) \mathbf{a}_{2}+ \\
\left(\frac{1}{2}+x_{5}+y_{5}+z_{5}\right) \mathbf{a}_{3}\end{array}$ & $=$ & $\left(\frac{1}{4}+y_{5}\right) a \hat{\mathbf{x}}+\left(\frac{1}{4}+z_{5}\right) a \hat{\mathbf{y}}-x_{5} a \hat{\mathbf{z}}$ & $(96 g)$ \\
\hline
\end{tabular}

\section{References:}

- Z. Fu, Y. Zheng, Y. Xiao, S. Bedanta, A. Senyshyn, G. G. Simeoni, Y. Su, U. Rücker, P. Kögerler, and T. Brückel, Coexistence of magnetic order and spin-glass-like phase in the pyrochlore antiferromagnet $\mathrm{Na}_{3} \mathrm{Co}\left(\mathrm{CO}_{3}\right)_{2} \mathrm{Cl}$, Phys. Rev. B 87, 214406 (2013), doi:10.1103/PhysRevB.87.214406.

\section{Geometry files:}

- CIF: pp. 951

- POSCAR: pp. 952 


\section{Tychite $\left(\mathrm{Na}_{6} \mathrm{Mg}_{2}\left(\mathrm{SO}_{4}\right)\left(\mathrm{CO}_{3}\right)_{4}\right)$ Structure:}

\section{A4B2C6D16E_cF232_203_e_d_f_eg_a}
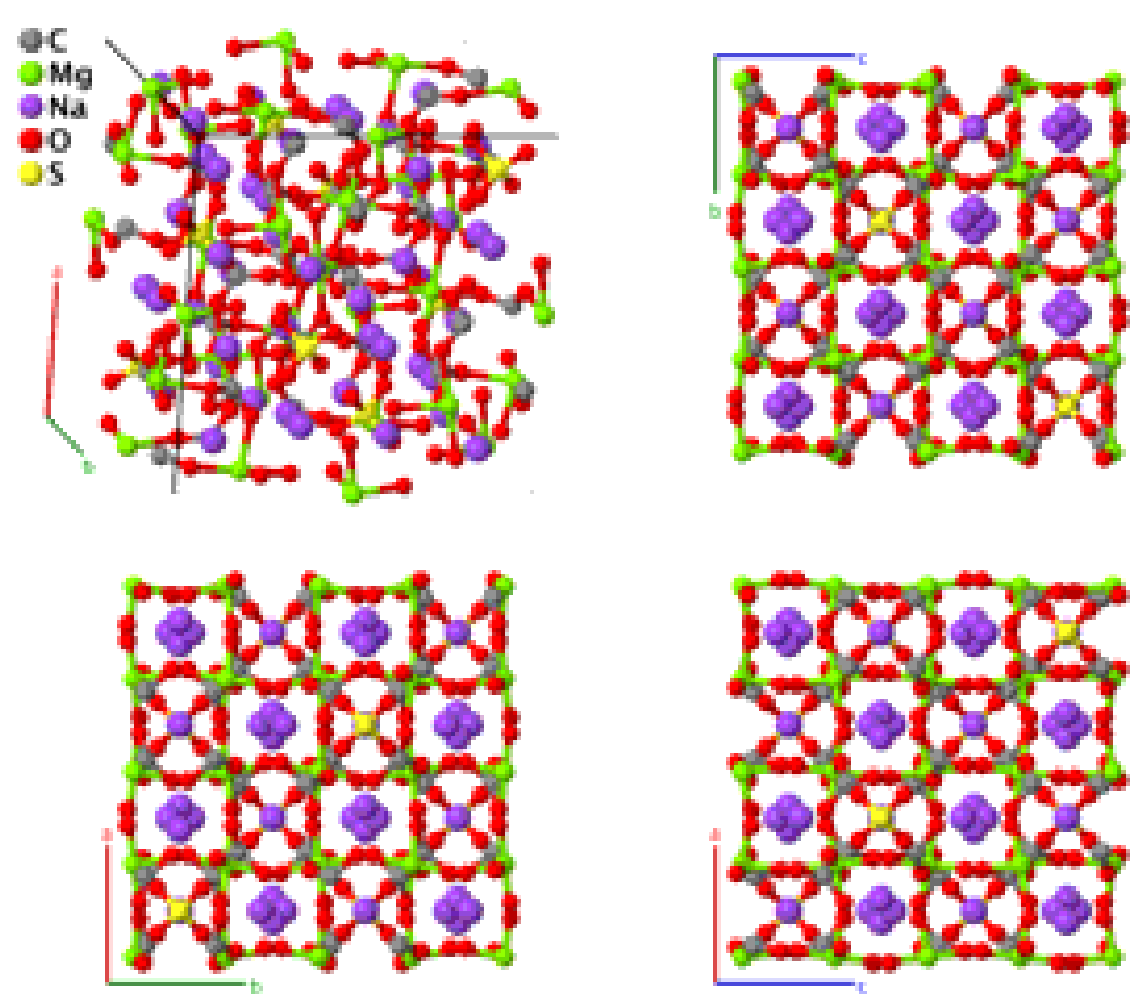

Prototype

AFLOW prototype label

$: \quad \mathrm{Na}_{6} \mathrm{Mg}_{2}\left(\mathrm{SO}_{4}\right)\left(\mathrm{CO}_{3}\right)_{4}$

Strukturbericht designation

A4B2C6D16E_cF232_203_e_d_f_eg_a

Pearson symbol

None

Space group number

$\mathrm{cF} 232$

Space group symbol

203

AFLOW prototype command

$F d \overline{3}$

aflow --proto=A4B2C6D16E_cF232_203_e_d_f_eg_a

- params $=a, x_{3}, x_{4}, x_{5}, x_{6}, y_{6}, z_{6}$

- The data was obtained from an X-ray diffraction study of a single crystal at room temperature (298 K).

Face-centered Cubic primitive vectors:

$$
\begin{aligned}
& \mathbf{a}_{1}=\frac{1}{2} a \hat{\mathbf{y}}+\frac{1}{2} a \hat{\mathbf{z}} \\
& \mathbf{a}_{2}=\frac{1}{2} a \hat{\mathbf{x}}+\frac{1}{2} a \hat{\mathbf{z}} \\
& \mathbf{a}_{3}=\frac{1}{2} a \hat{\mathbf{x}}+\frac{1}{2} a \hat{\mathbf{y}}
\end{aligned}
$$

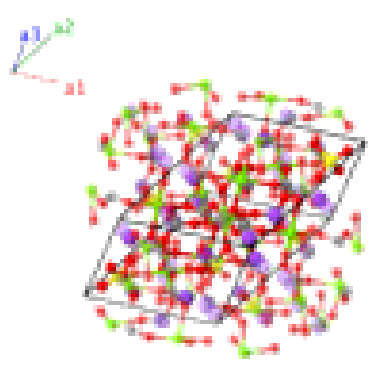

Basis vectors: 
Lattice Coordinates

\begin{tabular}{|c|c|c|c|c|c|}
\hline $\mathbf{B}_{1}$ & $=$ & $\frac{1}{8} \mathbf{a}_{1}+\frac{1}{8} \mathbf{a}_{2}+\frac{1}{8} \mathbf{a}_{3}$ & $=$ & $\frac{1}{8} a \hat{\mathbf{x}}+\frac{1}{8} a \hat{\mathbf{y}}+\frac{1}{8} a \hat{\mathbf{z}}$ & $(8 a)$ \\
\hline $\mathbf{B}_{2}$ & $=$ & $\frac{7}{8} \mathbf{a}_{1}+\frac{7}{8} \mathbf{a}_{2}+\frac{7}{8} \mathbf{a}_{3}$ & $=$ & $\frac{7}{8} a \hat{\mathbf{x}}+\frac{7}{8} a \hat{\mathbf{y}}+\frac{7}{8} a \hat{\mathbf{z}}$ & $(8 a)$ \\
\hline $\mathbf{B}_{3}$ & $=$ & $\frac{1}{2} \mathbf{a}_{1}+\frac{1}{2} \mathbf{a}_{2}+\frac{1}{2} \mathbf{a}_{3}$ & $=$ & $\frac{1}{2} a \hat{\mathbf{x}}+\frac{1}{2} a \hat{\mathbf{y}}+\frac{1}{2} a \hat{\mathbf{z}}$ & $(16 d)$ \\
\hline $\mathbf{B}_{4}$ & $=$ & $\frac{1}{2} \mathbf{a}_{1}+\frac{1}{2} \mathbf{a}_{2}$ & $=$ & $\frac{1}{4} a \hat{\mathbf{x}}+\frac{1}{4} a \hat{\mathbf{y}}+\frac{1}{2} a \hat{\mathbf{z}}$ & $(16 d)$ \\
\hline $\mathbf{B}_{5}$ & $=$ & $\frac{1}{2} \mathbf{a}_{1}+\frac{1}{2} \mathbf{a}_{3}$ & $=$ & $\frac{1}{4} a \hat{\mathbf{x}}+\frac{1}{2} a \hat{\mathbf{y}}+\frac{1}{4} a \hat{\mathbf{z}}$ & $(16 d)$ \\
\hline $\mathbf{B}_{6}$ & $=$ & $\frac{1}{2} \mathbf{a}_{2}+\frac{1}{2} \mathbf{a}_{3}$ & $=$ & $\frac{1}{2} a \hat{\mathbf{x}}+\frac{1}{4} a \hat{\mathbf{y}}+\frac{1}{4} a \hat{\mathbf{z}}$ & $(16 d)$ \\
\hline $\mathbf{B}_{7}$ & $=$ & $x_{3} \mathbf{a}_{1}+x_{3} \mathbf{a}_{2}+x_{3} \mathbf{a}_{3}$ & $=$ & $x_{3} a \hat{\mathbf{x}}+x_{3} a \hat{\mathbf{y}}+x_{3} a \hat{\mathbf{z}}$ & $(32 e)$ \\
\hline $\mathbf{B}_{8}$ & $=$ & $x_{3} \mathbf{a}_{1}+x_{3} \mathbf{a}_{2}+\left(\frac{1}{2}-3 x_{3}\right) \mathbf{a}_{3}$ & $=$ & $\left(\frac{1}{4}-x_{3}\right) a \hat{\mathbf{x}}+\left(\frac{1}{4}-x_{3}\right) a \hat{\mathbf{y}}+x_{3} a \hat{\mathbf{z}}$ & $(32 e)$ \\
\hline $\mathbf{B}_{9}$ & $=$ & $x_{3} \mathbf{a}_{1}+\left(\frac{1}{2}-3 x_{3}\right) \mathbf{a}_{2}+x_{3} \mathbf{a}_{3}$ & $=$ & $\left(\frac{1}{4}-x_{3}\right) a \hat{\mathbf{x}}+x_{3} a \hat{\mathbf{y}}+\left(\frac{1}{4}-x_{3}\right) a \hat{\mathbf{z}}$ & $(32 e)$ \\
\hline $\mathbf{B}_{10}$ & $=$ & $\left(\frac{1}{2}-3 x_{3}\right) \mathbf{a}_{1}+x_{3} \mathbf{a}_{2}+x_{3} \mathbf{a}_{3}$ & $=$ & $x_{3} a \hat{\mathbf{x}}+\left(\frac{1}{4}-x_{3}\right) a \hat{\mathbf{y}}+\left(\frac{1}{4}-x_{3}\right) a \hat{\mathbf{z}}$ & $(32 e)$ \\
\hline $\mathbf{B}_{11}$ & $=$ & $-x_{3} \mathbf{a}_{1}-x_{3} \mathbf{a}_{2}-x_{3} \mathbf{a}_{3}$ & $=$ & $-x_{3} a \hat{\mathbf{x}}-x_{3} a \hat{\mathbf{y}}-x_{3} a \hat{\mathbf{z}}$ & $(32 e)$ \\
\hline $\mathbf{B}_{12}$ & $=$ & $-x_{3} \mathbf{a}_{1}-x_{3} \mathbf{a}_{2}+\left(\frac{1}{2}+3 x_{3}\right) \mathbf{a}_{3}$ & $=$ & $\left(\frac{1}{4}+x_{3}\right) a \hat{\mathbf{x}}+\left(\frac{1}{4}+x_{3}\right) a \hat{\mathbf{y}}-x_{3} a \hat{\mathbf{z}}$ & $(32 e)$ \\
\hline $\mathbf{B}_{13}$ & $=$ & $-x_{3} \mathbf{a}_{1}+\left(\frac{1}{2}+3 x_{3}\right) \mathbf{a}_{2}-x_{3} \mathbf{a}_{3}$ & $=$ & $\left(\frac{1}{4}+x_{3}\right) a \hat{\mathbf{x}}-x_{3} a \hat{\mathbf{y}}+\left(\frac{1}{4}+x_{3}\right) a \hat{\mathbf{z}}$ & $(32 e)$ \\
\hline $\mathbf{B}_{14}$ & $=$ & $\left(\frac{1}{2}+3 x_{3}\right) \mathbf{a}_{1}-x_{3} \mathbf{a}_{2}-x_{3} \mathbf{a}_{3}$ & $=$ & $-x_{3} a \hat{\mathbf{x}}+\left(\frac{1}{4}+x_{3}\right) a \hat{\mathbf{y}}+\left(\frac{1}{4}+x_{3}\right) a \hat{\mathbf{z}}$ & $(32 e)$ \\
\hline $\mathbf{B}_{15}$ & $=$ & $x_{4} \mathbf{a}_{1}+x_{4} \mathbf{a}_{2}+x_{4} \mathbf{a}_{3}$ & $=$ & $x_{4} a \hat{\mathbf{x}}+x_{4} a \hat{\mathbf{y}}+x_{4} a \hat{\mathbf{z}}$ & $(32 e)$ \\
\hline $\mathbf{B}_{16}$ & $=$ & $x_{4} \mathbf{a}_{1}+x_{4} \mathbf{a}_{2}+\left(\frac{1}{2}-3 x_{4}\right) \mathbf{a}_{3}$ & $=$ & $\left(\frac{1}{4}-x_{4}\right) a \hat{\mathbf{x}}+\left(\frac{1}{4}-x_{4}\right) a \hat{\mathbf{y}}+x_{4} a \hat{\mathbf{z}}$ & $(32 e)$ \\
\hline $\mathbf{B}_{17}$ & $=$ & $x_{4} \mathbf{a}_{1}+\left(\frac{1}{2}-3 x_{4}\right) \mathbf{a}_{2}+x_{4} \mathbf{a}_{3}$ & $=$ & $\left(\frac{1}{4}-x_{4}\right) a \hat{\mathbf{x}}+x_{4} a \hat{\mathbf{y}}+\left(\frac{1}{4}-x_{4}\right) a \hat{\mathbf{z}}$ & $(32 e)$ \\
\hline $\mathbf{B}_{18}$ & $=$ & $\left(\frac{1}{2}-3 x_{4}\right) \mathbf{a}_{1}+x_{4} \mathbf{a}_{2}+x_{4} \mathbf{a}_{3}$ & $=$ & $x_{4} a \hat{\mathbf{x}}+\left(\frac{1}{4}-x_{4}\right) a \hat{\mathbf{y}}+\left(\frac{1}{4}-x_{4}\right) a \hat{\mathbf{z}}$ & $(32 e)$ \\
\hline $\mathbf{B}_{19}$ & $=$ & $-x_{4} \mathbf{a}_{1}-x_{4} \mathbf{a}_{2}-x_{4} \mathbf{a}_{3}$ & $=$ & $-x_{4} a \hat{\mathbf{x}}-x_{4} a \hat{\mathbf{y}}-x_{4} a \hat{\mathbf{z}}$ & $(32 e)$ \\
\hline $\mathbf{B}_{20}$ & $=$ & $-x_{4} \mathbf{a}_{1}-x_{4} \mathbf{a}_{2}+\left(\frac{1}{2}+3 x_{4}\right) \mathbf{a}_{3}$ & $=$ & $\left(\frac{1}{4}+x_{4}\right) a \hat{\mathbf{x}}+\left(\frac{1}{4}+x_{4}\right) a \hat{\mathbf{y}}-x_{4} a \hat{\mathbf{z}}$ & $(32 e)$ \\
\hline $\mathbf{B}_{21}$ & $=$ & $-x_{4} \mathbf{a}_{1}+\left(\frac{1}{2}+3 x_{4}\right) \mathbf{a}_{2}-x_{4} \mathbf{a}_{3}$ & $=$ & $\left(\frac{1}{4}+x_{4}\right) a \hat{\mathbf{x}}-x_{4} a \hat{\mathbf{y}}+\left(\frac{1}{4}+x_{4}\right) a \hat{\mathbf{z}}$ & $(32 e)$ \\
\hline $\mathbf{B}_{22}$ & $=$ & $\left(\frac{1}{2}+3 x_{4}\right) \mathbf{a}_{1}-x_{4} \mathbf{a}_{2}-x_{4} \mathbf{a}_{3}$ & $=$ & $-x_{4} a \hat{\mathbf{x}}+\left(\frac{1}{4}+x_{4}\right) a \hat{\mathbf{y}}+\left(\frac{1}{4}+x_{4}\right) a \hat{\mathbf{z}}$ & $(32 e)$ \\
\hline $\mathbf{B}_{23}$ & $=$ & $\left(\frac{1}{4}-x_{5}\right) \mathbf{a}_{1}+x_{5} \mathbf{a}_{2}+x_{5} \mathbf{a}_{3}$ & $=$ & $x_{5} a \hat{\mathbf{x}}+\frac{1}{8} a \hat{\mathbf{y}}+\frac{1}{8} a \hat{\mathbf{z}}$ & $(48 f)$ \\
\hline $\mathbf{B}_{24}$ & $=$ & $x_{5} \mathbf{a}_{1}+\left(\frac{1}{4}-x_{5}\right) \mathbf{a}_{2}+\left(\frac{1}{4}-x_{5}\right) \mathbf{a}_{3}$ & $=$ & $\left(\frac{1}{4}-x_{5}\right) a \hat{\mathbf{x}}+\frac{1}{8} a \hat{\mathbf{y}}+\frac{1}{8} a \hat{\mathbf{z}}$ & $(48 f)$ \\
\hline $\mathbf{B}_{25}$ & $=$ & $x_{5} \mathbf{a}_{1}+\left(\frac{1}{4}-x_{5}\right) \mathbf{a}_{2}+x_{5} \mathbf{a}_{3}$ & $=$ & $\frac{1}{8} a \hat{\mathbf{x}}+x_{5} a \hat{\mathbf{y}}+\frac{1}{8} a \hat{\mathbf{z}}$ & $(48 f)$ \\
\hline $\mathbf{B}_{26}$ & $=$ & $\left(\frac{1}{4}-x_{5}\right) \mathbf{a}_{1}+x_{5} \mathbf{a}_{2}+\left(\frac{1}{4}-x_{5}\right) \mathbf{a}_{3}$ & $=$ & $\frac{1}{8} a \hat{\mathbf{x}}+\left(\frac{1}{4}-x_{5}\right) a \hat{\mathbf{y}}+\frac{1}{8} a \hat{\mathbf{z}}$ & $(48 f)$ \\
\hline $\mathbf{B}_{27}$ & $=$ & $x_{5} \mathbf{a}_{1}+x_{5} \mathbf{a}_{2}+\left(\frac{1}{4}-x_{5}\right) \mathbf{a}_{3}$ & $=$ & $\frac{1}{8} a \hat{\mathbf{x}}+\frac{1}{8} a \hat{\mathbf{y}}+x_{5} a \hat{\mathbf{z}}$ & $(48 f)$ \\
\hline $\mathbf{B}_{28}$ & $=$ & $\left(\frac{1}{4}-x_{5}\right) \mathbf{a}_{1}+\left(\frac{1}{4}-x_{5}\right) \mathbf{a}_{2}+x_{5} \mathbf{a}_{3}$ & $=$ & $\frac{1}{8} a \hat{\mathbf{x}}+\frac{1}{8} a \hat{\mathbf{y}}+\left(\frac{1}{4}-x_{5}\right) a \hat{\mathbf{z}}$ & $(48 f)$ \\
\hline $\mathbf{B}_{29}$ & $=$ & $\left(\frac{3}{4}+x_{5}\right) \mathbf{a}_{1}-x_{5} \mathbf{a}_{2}-x_{5} \mathbf{a}_{3}$ & $=$ & $-x_{5} a \hat{\mathbf{x}}+\frac{3}{8} a \hat{\mathbf{y}}+\frac{3}{8} a \hat{\mathbf{z}}$ & $(48 f)$ \\
\hline $\mathbf{B}_{30}$ & $=$ & $-x_{5} \mathbf{a}_{1}+\left(\frac{3}{4}+x_{5}\right) \mathbf{a}_{2}+\left(\frac{3}{4}+x_{5}\right) \mathbf{a}_{3}$ & $=$ & $\left(\frac{3}{4}+x_{5}\right) a \hat{\mathbf{x}}+\frac{3}{8} a \hat{\mathbf{y}}+\frac{3}{8} a \hat{\mathbf{z}}$ & $(48 f)$ \\
\hline $\mathbf{B}_{31}$ & $=$ & $-x_{5} \mathbf{a}_{1}+\left(\frac{3}{4}+x_{5}\right) \mathbf{a}_{2}-x_{5} \mathbf{a}_{3}$ & $=$ & $\frac{3}{8} a \hat{\mathbf{x}}-x_{5} a \hat{\mathbf{y}}+\frac{3}{8} a \hat{\mathbf{z}}$ & $(48 f)$ \\
\hline $\mathbf{B}_{32}$ & $=$ & $\left(\frac{3}{4}+x_{5}\right) \mathbf{a}_{1}-x_{5} \mathbf{a}_{2}+\left(\frac{3}{4}+x_{5}\right) \mathbf{a}_{3}$ & $=$ & $\frac{3}{8} a \hat{\mathbf{x}}+\left(\frac{3}{4}+x_{5}\right) a \hat{\mathbf{y}}+\frac{3}{8} a \hat{\mathbf{z}}$ & $(48 f)$ \\
\hline $\mathbf{B}_{33}$ & $=$ & $-x_{5} \mathbf{a}_{1}-x_{5} \mathbf{a}_{2}+\left(\frac{3}{4}+x_{5}\right) \mathbf{a}_{3}$ & $=$ & $\frac{3}{8} a \hat{\mathbf{x}}+\frac{3}{8} a \hat{\mathbf{y}}-x_{5} a \hat{\mathbf{z}}$ & $(48 f)$ \\
\hline $\mathbf{B}_{34}$ & $=$ & $\left(\frac{3}{4}+x_{5}\right) \mathbf{a}_{1}+\left(\frac{3}{4}+x_{5}\right) \mathbf{a}_{2}-x_{5} \mathbf{a}_{3}$ & $=$ & $\frac{3}{8} a \hat{\mathbf{x}}+\frac{3}{8} a \hat{\mathbf{y}}+\left(\frac{3}{4}+x_{5}\right) a \hat{\mathbf{z}}$ & $(48 f)$ \\
\hline
\end{tabular}
S

$\mathrm{Mg}$

$\mathrm{Mg}$

$\mathrm{Mg}$

C

C

C

C

C

O I

O I

O I

O I

O I

O I

O I

O I

$\mathrm{Na}$

$\mathrm{Na}$

(a)

a

$\mathrm{Na}$

$\mathrm{Na}$

$\mathrm{Na}$

$\mathrm{Na}$

$\mathrm{Na}$

$\mathrm{Na}$

$\mathrm{Na}$

$\mathrm{Na}$ 


\begin{tabular}{|c|c|c|c|c|c|c|}
\hline $\mathbf{B}_{35}$ & $=$ & $\begin{array}{c}\left(-x_{6}+y_{6}+z_{6}\right) \mathbf{a}_{1}+ \\
\left(x_{6}-y_{6}+z_{6}\right) \mathbf{a}_{2}+ \\
\left(x_{6}+y_{6}-z_{6}\right) \mathbf{a}_{3}\end{array}$ & $=$ & $x_{6} a \hat{\mathbf{x}}+y_{6} a \hat{\mathbf{y}}+z_{6} a \hat{\mathbf{z}}$ & $(96 g)$ & O II \\
\hline $\mathbf{B}_{36}$ & $=$ & $\begin{array}{c}\left(x_{6}-y_{6}+z_{6}\right) \mathbf{a}_{1}+ \\
\left(-x_{6}+y_{6}+z_{6}\right) \mathbf{a}_{2}+ \\
\left(\frac{1}{2}-x_{6}-y_{6}-z_{6}\right) \mathbf{a}_{3}\end{array}$ & $=$ & $\left(\frac{1}{4}-x_{6}\right) a \hat{\mathbf{x}}+\left(\frac{1}{4}-y_{6}\right) a \hat{\mathbf{y}}+z_{6} a \hat{\mathbf{z}}$ & $(96 g)$ & O II \\
\hline $\mathbf{B}_{37}$ & $=$ & $\begin{array}{c}\left(x_{6}+y_{6}-z_{6}\right) \mathbf{a}_{1}+ \\
\left(\frac{1}{2}-x_{6}-y_{6}-z_{6}\right) \mathbf{a}_{2}+ \\
\quad\left(-x_{6}+y_{6}+z_{6}\right) \mathbf{a}_{3}\end{array}$ & $=$ & $\left(\frac{1}{4}-x_{6}\right) a \hat{\mathbf{x}}+y_{6} a \hat{\mathbf{y}}+\left(\frac{1}{4}-z_{6}\right) a \hat{\mathbf{z}}$ & $(96 g)$ & O II \\
\hline $\mathbf{B}_{38}$ & $=$ & $\begin{array}{c}\left(\frac{1}{2}-x_{6}-y_{6}-z_{6}\right) \mathbf{a}_{1}+ \\
\left(x_{6}+y_{6}-z_{6}\right) \mathbf{a}_{2}+ \\
\quad\left(x_{6}-y_{6}+z_{6}\right) \mathbf{a}_{3}\end{array}$ & $=$ & $x_{6} a \hat{\mathbf{x}}+\left(\frac{1}{4}-y_{6}\right) a \hat{\mathbf{y}}+\left(\frac{1}{4}-z_{6}\right) a \hat{\mathbf{z}}$ & $(96 g)$ & O II \\
\hline $\mathbf{B}_{39}$ & $=$ & $\begin{array}{c}\left(x_{6}+y_{6}-z_{6}\right) \mathbf{a}_{1}+ \\
\left(-x_{6}+y_{6}+z_{6}\right) \mathbf{a}_{2}+ \\
\left(x_{6}-y_{6}+z_{6}\right) \mathbf{a}_{3}\end{array}$ & $=$ & $z_{6} a \hat{\mathbf{x}}+x_{6} a \hat{\mathbf{y}}+y_{6} a \hat{\mathbf{z}}$ & $(96 g)$ & O II \\
\hline $\mathbf{B}_{40}$ & $=$ & $\begin{array}{l}\left(\frac{1}{2}-x_{6}-y_{6}-z_{6}\right) \mathbf{a}_{1}+ \\
\quad\left(x_{6}-y_{6}+z_{6}\right) \mathbf{a}_{2}+ \\
\quad\left(-x_{6}+y_{6}+z_{6}\right) \mathbf{a}_{3}\end{array}$ & $=$ & $z_{6} a \hat{\mathbf{x}}+\left(\frac{1}{4}-x_{6}\right) a \hat{\mathbf{y}}+\left(\frac{1}{4}-y_{6}\right) a \hat{\mathbf{z}}$ & $(96 g)$ & O II \\
\hline $\mathbf{B}_{41}$ & $=$ & $\begin{array}{c}\left(-x_{6}+y_{6}+z_{6}\right) \mathbf{a}_{1}+ \\
\left(x_{6}+y_{6}-z_{6}\right) \mathbf{a}_{2}+ \\
\left(\frac{1}{2}-x_{6}-y_{6}-z_{6}\right) \mathbf{a}_{3}\end{array}$ & $=$ & $\left(\frac{1}{4}-z_{6}\right) a \hat{\mathbf{x}}+\left(\frac{1}{4}-x_{6}\right) a \hat{\mathbf{y}}+y_{6} a \hat{\mathbf{z}}$ & $(96 g)$ & O II \\
\hline $\mathbf{B}_{42}$ & $=$ & $\begin{array}{c}\left(x_{6}-y_{6}+z_{6}\right) \mathbf{a}_{1}+ \\
\left(\frac{1}{2}-x_{6}-y_{6}-z_{6}\right) \mathbf{a}_{2}+ \\
\left(x_{6}+y_{6}-z_{6}\right) \mathbf{a}_{3}\end{array}$ & $=$ & $\left(\frac{1}{4}-z_{6}\right) a \hat{\mathbf{x}}+x_{6} a \hat{\mathbf{y}}+\left(\frac{1}{4}-y_{6}\right) a \hat{\mathbf{z}}$ & $(96 g)$ & O II \\
\hline $\mathbf{B}_{43}$ & $=$ & $\begin{array}{c}\left(x_{6}-y_{6}+z_{6}\right) \mathbf{a}_{1}+ \\
\left(x_{6}+y_{6}-z_{6}\right) \mathbf{a}_{2}+ \\
\left(-x_{6}+y_{6}+z_{6}\right) \mathbf{a}_{3}\end{array}$ & $=$ & $y_{6} a \hat{\mathbf{x}}+z_{6} a \hat{\mathbf{y}}+x_{6} a \hat{\mathbf{z}}$ & $(96 g)$ & O II \\
\hline $\mathbf{B}_{44}$ & $=$ & $\begin{array}{c}\left(-x_{6}+y_{6}+z_{6}\right) \mathbf{a}_{1}+ \\
\left(\frac{1}{2}-x_{6}-y_{6}-z_{6}\right) \mathbf{a}_{2}+ \\
\left(x_{6}-y_{6}+z_{6}\right) \mathbf{a}_{3}\end{array}$ & $=$ & $\left(\frac{1}{4}-y_{6}\right) a \hat{\mathbf{x}}+z_{6} a \hat{\mathbf{y}}+\left(\frac{1}{4}-x_{6}\right) a \hat{\mathbf{z}}$ & $(96 g)$ & O II \\
\hline $\mathbf{B}_{45}$ & $=$ & $\begin{array}{c}\left(\frac{1}{2}-x_{6}-y_{6}-z_{6}\right) \mathbf{a}_{1}+ \\
\left(-x_{6}+y_{6}+z_{6}\right) \mathbf{a}_{2}+ \\
\left(x_{6}+y_{6}-z_{6}\right) \mathbf{a}_{3}\end{array}$ & $=$ & $y_{6} a \hat{\mathbf{x}}+\left(\frac{1}{4}-z_{6}\right) a \hat{\mathbf{y}}+\left(\frac{1}{4}-x_{6}\right) a \hat{\mathbf{z}}$ & $(96 g)$ & O II \\
\hline $\mathbf{B}_{46}$ & $=$ & $\begin{array}{c}\left(x_{6}+y_{6}-z_{6}\right) \mathbf{a}_{1}+ \\
\left(x_{6}-y_{6}+z_{6}\right) \mathbf{a}_{2}+ \\
\left(\frac{1}{2}-x_{6}-y_{6}-z_{6}\right) \mathbf{a}_{3}\end{array}$ & $=$ & $\left(\frac{1}{4}-y_{6}\right) a \hat{\mathbf{x}}+\left(\frac{1}{4}-z_{6}\right) a \hat{\mathbf{y}}+x_{6} a \hat{\mathbf{z}}$ & $(96 g)$ & O II \\
\hline $\mathbf{B}_{47}$ & $=$ & $\begin{array}{c}\left(x_{6}-y_{6}-z_{6}\right) \mathbf{a}_{1}+ \\
\left(-x_{6}+y_{6}-z_{6}\right) \mathbf{a}_{2}+ \\
\left(-x_{6}-y_{6}+z_{6}\right) \mathbf{a}_{3}\end{array}$ & $=$ & $-x_{6} a \hat{\mathbf{x}}-y_{6} a \hat{\mathbf{y}}-z_{6} a \hat{\mathbf{z}}$ & $(96 g)$ & O II \\
\hline $\mathbf{B}_{48}$ & $=$ & $\begin{array}{c}\left(-x_{6}+y_{6}-z_{6}\right) \mathbf{a}_{1}+ \\
\left(x_{6}-y_{6}-z_{6}\right) \mathbf{a}_{2}+ \\
\left(\frac{1}{2}+x_{6}+y_{6}+z_{6}\right) \mathbf{a}_{3}\end{array}$ & $=$ & $\left(\frac{1}{4}+x_{6}\right) a \hat{\mathbf{x}}+\left(\frac{1}{4}+y_{6}\right) a \hat{\mathbf{y}}-z_{6} a \hat{\mathbf{z}}$ & $(96 g)$ & O II \\
\hline $\mathbf{B}_{49}$ & $=$ & $\begin{array}{c}\left(-x_{6}-y_{6}+z_{6}\right) \mathbf{a}_{1}+ \\
\left(\frac{1}{2}+x_{6}+y_{6}+z_{6}\right) \mathbf{a}_{2}+ \\
\quad\left(x_{6}-y_{6}-z_{6}\right) \mathbf{a}_{3}\end{array}$ & $=$ & $\left(\frac{1}{4}+x_{6}\right) a \hat{\mathbf{x}}-y_{6} a \hat{\mathbf{y}}+\left(\frac{1}{4}+z_{6}\right) a \hat{\mathbf{z}}$ & $(96 g)$ & O II \\
\hline $\mathbf{B}_{50}$ & $=$ & $\begin{array}{c}\left(\frac{1}{2}+x_{6}+y_{6}+z_{6}\right) \mathbf{a}_{1}+ \\
\left(-x_{6}-y_{6}+z_{6}\right) \mathbf{a}_{2}+ \\
\quad\left(-x_{6}+y_{6}-z_{6}\right) \mathbf{a}_{3}\end{array}$ & $=$ & $-x_{6} a \hat{\mathbf{x}}+\left(\frac{1}{4}+y_{6}\right) a \hat{\mathbf{y}}+\left(\frac{1}{4}+z_{6}\right) a \hat{\mathbf{z}}$ & $(96 g)$ & O II \\
\hline
\end{tabular}




\begin{tabular}{|c|c|c|c|c|c|c|}
\hline $\mathbf{B}_{51}$ & $=$ & $\begin{array}{c}\left(-x_{6}-y_{6}+z_{6}\right) \mathbf{a}_{1}+ \\
\left(x_{6}-y_{6}-z_{6}\right) \mathbf{a}_{2}+ \\
\left(-x_{6}+y_{6}-z_{6}\right) \mathbf{a}_{3}\end{array}$ & $=$ & $-z_{6} a \hat{\mathbf{x}}-x_{6} a \hat{\mathbf{y}}-y_{6} a \hat{\mathbf{z}}$ & $(96 g)$ & O II \\
\hline $\mathbf{B}_{52}$ & $=$ & $\begin{array}{c}\left(\frac{1}{2}+x_{6}+y_{6}+z_{6}\right) \mathbf{a}_{1}+ \\
\left(-x_{6}+y_{6}-z_{6}\right) \mathbf{a}_{2}+ \\
\quad\left(x_{6}-y_{6}-z_{6}\right) \mathbf{a}_{3}\end{array}$ & $=$ & $-z_{6} a \hat{\mathbf{x}}+\left(\frac{1}{4}+x_{6}\right) a \hat{\mathbf{y}}+\left(\frac{1}{4}+y_{6}\right) a \hat{\mathbf{z}}$ & $(96 g)$ & O II \\
\hline $\mathbf{B}_{53}$ & $=$ & $\begin{array}{c}\left(x_{6}-y_{6}-z_{6}\right) \mathbf{a}_{1}+ \\
\left(-x_{6}-y_{6}+z_{6}\right) \mathbf{a}_{2}+ \\
\left(\frac{1}{2}+x_{6}+y_{6}+z_{6}\right) \mathbf{a}_{3}\end{array}$ & $=$ & $\left(\frac{1}{4}+z_{6}\right) a \hat{\mathbf{x}}+\left(\frac{1}{4}+x_{6}\right) a \hat{\mathbf{y}}-y_{6} a \hat{\mathbf{z}}$ & $(96 g)$ & O II \\
\hline $\mathbf{B}_{54}$ & $=$ & $\begin{array}{c}\left(-x_{6}+y_{6}-z_{6}\right) \mathbf{a}_{1}+ \\
\left(\frac{1}{2}+x_{6}+y_{6}+z_{6}\right) \mathbf{a}_{2}+ \\
\left(-x_{6}-y_{6}+z_{6}\right) \mathbf{a}_{3}\end{array}$ & $=$ & $\left(\frac{1}{4}+z_{6}\right) a \hat{\mathbf{x}}-x_{6} a \hat{\mathbf{y}}+\left(\frac{1}{4}+y_{6}\right) a \hat{\mathbf{z}}$ & $(96 g)$ & O II \\
\hline $\mathbf{B}_{55}$ & $=$ & $\begin{array}{c}\left(-x_{6}+y_{6}-z_{6}\right) \mathbf{a}_{1}+ \\
\left(-x_{6}-y_{6}+z_{6}\right) \mathbf{a}_{2}+ \\
\left(x_{6}-y_{6}-z_{6}\right) \mathbf{a}_{3}\end{array}$ & $=$ & $-y_{6} a \hat{\mathbf{x}}-z_{6} a \hat{\mathbf{y}}-x_{6} a \hat{\mathbf{z}}$ & $(96 g)$ & O II \\
\hline $\mathbf{B}_{56}$ & $=$ & $\begin{array}{c}\left(x_{6}-y_{6}-z_{6}\right) \mathbf{a}_{1}+ \\
\left(\frac{1}{2}+x_{6}+y_{6}+z_{6}\right) \mathbf{a}_{2}+ \\
\left(-x_{6}+y_{6}-z_{6}\right) \mathbf{a}_{3}\end{array}$ & $=$ & $\left(\frac{1}{4}+y_{6}\right) a \hat{\mathbf{x}}-z_{6} a \hat{\mathbf{y}}+\left(\frac{1}{4}+x_{6}\right) a \hat{\mathbf{z}}$ & $(96 g)$ & O II \\
\hline $\mathbf{B}_{57}$ & $=$ & $\begin{array}{c}\left(\frac{1}{2}+x_{6}+y_{6}+z_{6}\right) \mathbf{a}_{1}+ \\
\left(x_{6}-y_{6}-z_{6}\right) \mathbf{a}_{2}+ \\
\left(-x_{6}-y_{6}+z_{6}\right) \mathbf{a}_{3}\end{array}$ & $=$ & $-y_{6} a \hat{\mathbf{x}}+\left(\frac{1}{4}+z_{6}\right) a \hat{\mathbf{y}}+\left(\frac{1}{4}+x_{6}\right) a \hat{\mathbf{z}}$ & $(96 g)$ & O II \\
\hline $\mathbf{B}_{58}$ & $=$ & $\begin{array}{l}\left(-x_{6}-y_{6}+z_{6}\right) \mathbf{a}_{1}+ \\
\left(-x_{6}+y_{6}-z_{6}\right) \mathbf{a}_{2}+ \\
\left(\frac{1}{2}+x_{6}+y_{6}+z_{6}\right) \mathbf{a}_{3}\end{array}$ & $=$ & $\left(\frac{1}{4}+y_{6}\right) a \hat{\mathbf{x}}+\left(\frac{1}{4}+z_{6}\right) a \hat{\mathbf{y}}-x_{6} a \hat{\mathbf{z}}$ & $(96 g)$ & O II \\
\hline
\end{tabular}

\section{References:}

- G. R. Schmidt, R. Jacqueline, H. Yang, and R. T. Downs, Tychite, $\mathrm{Na}_{6} \mathrm{Mg}_{2}\left(\mathrm{SO}_{4}\right)\left(\mathrm{CO}_{3}\right)_{4}$ : Structure analysis and Raman spectroscopic data, Acta Crystallogr. E 62, i207-i209 (2006), doi:10.1107/S160053680603491X.

\section{Found in:}

- R. T. Downs and M. Hall-Wallace, The American Mineralogist Crystal Structure Database, Am. Mineral. 88, 247-250 (2003).

\section{Geometry files:}

- CIF: pp. 953

- POSCAR: pp. 953 
$\mathrm{Rb}_{3} \mathrm{AsSe}_{16}$ Structure: AB3C16_cF160_203_b_ad_eg
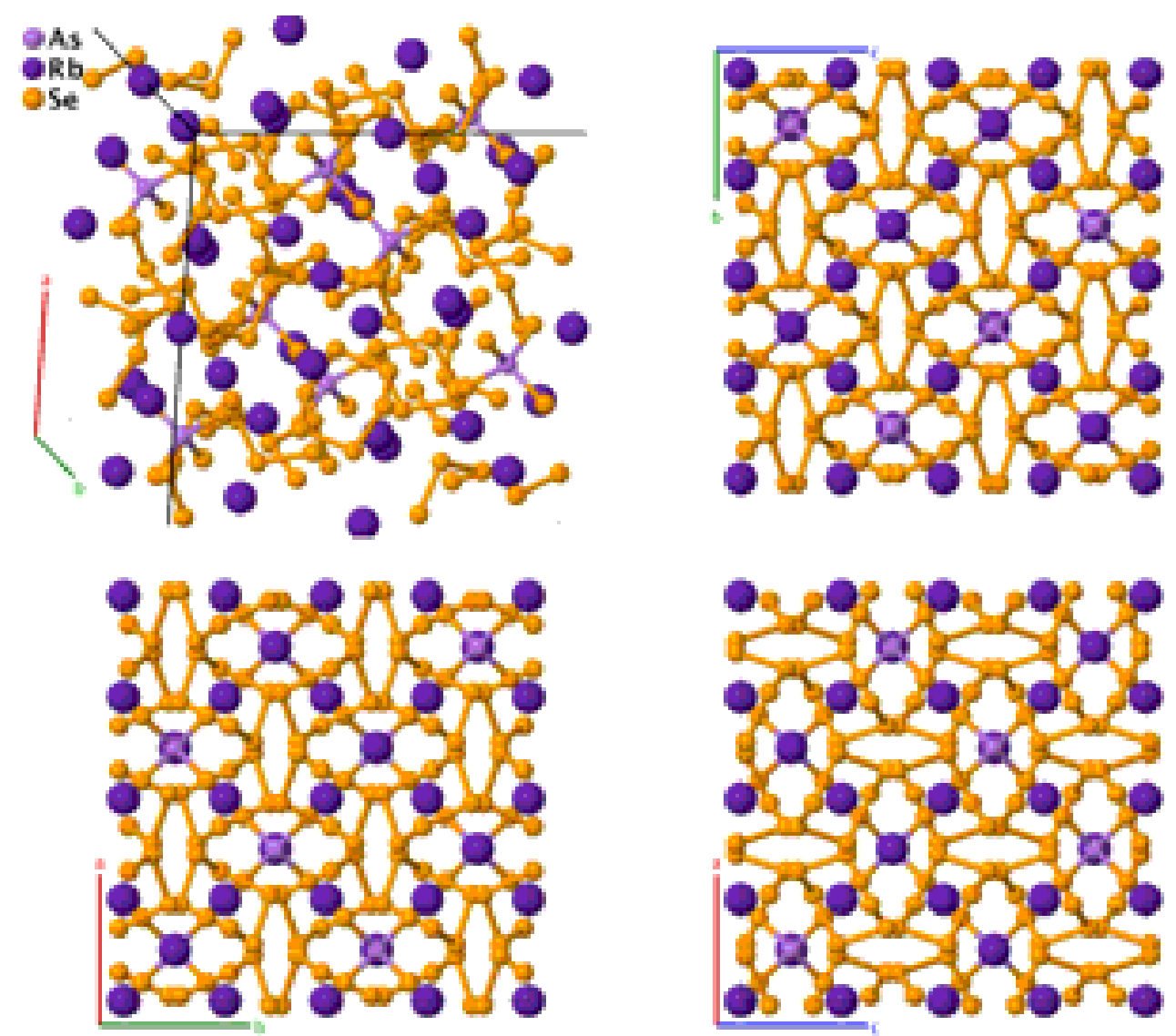

\section{Prototype}

AFLOW prototype label

Strukturbericht designation

Pearson symbol

: $\quad \mathrm{Rb}_{3} \mathrm{AsSe}_{16}$

Space group number

Space group symbol

AFLOW prototype command

: AB3C16_cF160_203_b_ad_eg

: None

: $\quad \mathrm{cF} 160$

: 203

: $F d \overline{3}$
aflow --proto=AB3C16_cF160_203_b_ad_eg
- params $=a, x_{4}, x_{5}, y_{5}, z_{5}$

Face-centered Cubic primitive vectors:

$$
\begin{aligned}
& \mathbf{a}_{1}=\frac{1}{2} a \hat{\mathbf{y}}+\frac{1}{2} a \hat{\mathbf{z}} \\
& \mathbf{a}_{2}=\frac{1}{2} a \hat{\mathbf{x}}+\frac{1}{2} a \hat{\mathbf{z}} \\
& \mathbf{a}_{3}=\frac{1}{2} a \hat{\mathbf{x}}+\frac{1}{2} a \hat{\mathbf{y}}
\end{aligned}
$$

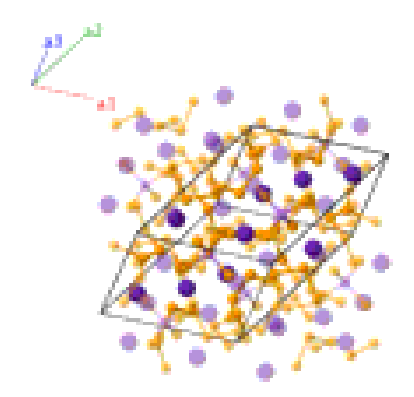

Basis vectors: 


\begin{tabular}{|c|c|c|c|c|c|c|}
\hline $\mathbf{B}_{1}$ & $=$ & $\frac{1}{8} \mathbf{a}_{1}+\frac{1}{8} \mathbf{a}_{2}+\frac{1}{8} \mathbf{a}_{3}$ & $=$ & $\frac{1}{8} a \hat{\mathbf{x}}+\frac{1}{8} a \hat{\mathbf{y}}+\frac{1}{8} a \hat{\mathbf{z}}$ & $(8 a)$ & $\mathrm{Rb} \mathrm{I}$ \\
\hline $\mathbf{B}_{2}$ & $=$ & $\frac{7}{8} \mathbf{a}_{1}+\frac{7}{8} \mathbf{a}_{2}+\frac{7}{8} \mathbf{a}_{3}$ & $=$ & $\frac{7}{8} a \hat{\mathbf{x}}+\frac{7}{8} a \hat{\mathbf{y}}+\frac{7}{8} a \hat{\mathbf{z}}$ & $(8 a)$ & $\mathrm{Rb} \mathrm{I}$ \\
\hline $\mathbf{B}_{3}$ & $=$ & $\frac{5}{8} \mathbf{a}_{1}+\frac{5}{8} \mathbf{a}_{2}+\frac{5}{8} \mathbf{a}_{3}$ & $=$ & $\frac{5}{8} a \hat{\mathbf{x}}+\frac{5}{8} a \hat{\mathbf{y}}+\frac{5}{8} a \hat{\mathbf{z}}$ & $(8 b)$ & As \\
\hline $\mathbf{B}_{4}$ & $=$ & $\frac{3}{8} \mathbf{a}_{1}+\frac{3}{8} \mathbf{a}_{2}+\frac{3}{8} \mathbf{a}_{3}$ & $=$ & $\frac{3}{8} a \hat{\mathbf{x}}+\frac{3}{8} a \hat{\mathbf{y}}+\frac{3}{8} a \hat{\mathbf{z}}$ & $(8 b)$ & As \\
\hline $\mathbf{B}_{5}$ & $=$ & $\frac{1}{2} \mathbf{a}_{1}+\frac{1}{2} \mathbf{a}_{2}+\frac{1}{2} \mathbf{a}_{3}$ & $=$ & $\frac{1}{2} a \hat{\mathbf{x}}+\frac{1}{2} a \hat{\mathbf{y}}+\frac{1}{2} a \hat{\mathbf{z}}$ & $(16 d)$ & $\mathrm{Rb} \mathrm{Il}$ \\
\hline $\mathbf{B}_{6}$ & $=$ & $\frac{1}{2} \mathbf{a}_{1}+\frac{1}{2} \mathbf{a}_{2}$ & $=$ & $\frac{1}{4} a \hat{\mathbf{x}}+\frac{1}{4} a \hat{\mathbf{y}}+\frac{1}{2} a \hat{\mathbf{z}}$ & $(16 d)$ & $\mathrm{Rb} \mathrm{II}$ \\
\hline $\mathbf{B}_{7}$ & $=$ & $\frac{1}{2} \mathbf{a}_{1}+\frac{1}{2} \mathbf{a}_{3}$ & $=$ & $\frac{1}{4} a \hat{\mathbf{x}}+\frac{1}{2} a \hat{\mathbf{y}}+\frac{1}{4} a \hat{\mathbf{z}}$ & $(16 d)$ & $\mathrm{Rb} \mathrm{II}$ \\
\hline $\mathbf{B}_{8}$ & $=$ & $\frac{1}{2} \mathbf{a}_{2}+\frac{1}{2} \mathbf{a}_{3}$ & $=$ & $\frac{1}{2} a \hat{\mathbf{x}}+\frac{1}{4} a \hat{\mathbf{y}}+\frac{1}{4} a \hat{\mathbf{z}}$ & $(16 d)$ & $\mathrm{Rb} \mathrm{II}$ \\
\hline $\mathbf{B}_{9}$ & $=$ & $x_{4} \mathbf{a}_{1}+x_{4} \mathbf{a}_{2}+x_{4} \mathbf{a}_{3}$ & $=$ & $x_{4} a \hat{\mathbf{x}}+x_{4} a \hat{\mathbf{y}}+x_{4} a \hat{\mathbf{z}}$ & $(32 e)$ & $\mathrm{Se} \mathrm{I}$ \\
\hline $\mathbf{B}_{10}$ & $=$ & $x_{4} \mathbf{a}_{1}+x_{4} \mathbf{a}_{2}+\left(\frac{1}{2}-3 x_{4}\right) \mathbf{a}_{3}$ & $=$ & $\left(\frac{1}{4}-x_{4}\right) a \hat{\mathbf{x}}+\left(\frac{1}{4}-x_{4}\right) a \hat{\mathbf{y}}+x_{4} a \hat{\mathbf{z}}$ & $(32 e)$ & Se I \\
\hline $\mathbf{B}_{11}$ & $=$ & $x_{4} \mathbf{a}_{1}+\left(\frac{1}{2}-3 x_{4}\right) \mathbf{a}_{2}+x_{4} \mathbf{a}_{3}$ & $=$ & $\left(\frac{1}{4}-x_{4}\right) a \hat{\mathbf{x}}+x_{4} a \hat{\mathbf{y}}+\left(\frac{1}{4}-x_{4}\right) a \hat{\mathbf{z}}$ & $(32 e)$ & Se I \\
\hline $\mathbf{B}_{12}$ & $=$ & $\left(\frac{1}{2}-3 x_{4}\right) \mathbf{a}_{1}+x_{4} \mathbf{a}_{2}+x_{4} \mathbf{a}_{3}$ & $=$ & $x_{4} a \hat{\mathbf{x}}+\left(\frac{1}{4}-x_{4}\right) a \hat{\mathbf{y}}+\left(\frac{1}{4}-x_{4}\right) a \hat{\mathbf{z}}$ & $(32 e)$ & Se I \\
\hline $\mathbf{B}_{13}$ & $=$ & $-x_{4} \mathbf{a}_{1}-x_{4} \mathbf{a}_{2}-x_{4} \mathbf{a}_{3}$ & $=$ & $-x_{4} a \hat{\mathbf{x}}-x_{4} a \hat{\mathbf{y}}-x_{4} a \hat{\mathbf{z}}$ & $(32 e)$ & $\mathrm{Se} \mathrm{I}$ \\
\hline $\mathbf{B}_{14}$ & $=$ & $-x_{4} \mathbf{a}_{1}-x_{4} \mathbf{a}_{2}+\left(\frac{1}{2}+3 x_{4}\right) \mathbf{a}_{3}$ & $=$ & $\left(\frac{1}{4}+x_{4}\right) a \hat{\mathbf{x}}+\left(\frac{1}{4}+x_{4}\right) a \hat{\mathbf{y}}-x_{4} a \hat{\mathbf{z}}$ & $(32 e)$ & Se I \\
\hline $\mathbf{B}_{15}$ & $=$ & $-x_{4} \mathbf{a}_{1}+\left(\frac{1}{2}+3 x_{4}\right) \mathbf{a}_{2}-x_{4} \mathbf{a}_{3}$ & $=$ & $\left(\frac{1}{4}+x_{4}\right) a \hat{\mathbf{x}}-x_{4} a \hat{\mathbf{y}}+\left(\frac{1}{4}+x_{4}\right) a \hat{\mathbf{z}}$ & $(32 e)$ & Se I \\
\hline $\mathbf{B}_{16}$ & $=$ & $\left(\frac{1}{2}+3 x_{4}\right) \mathbf{a}_{1}-x_{4} \mathbf{a}_{2}-x_{4} \mathbf{a}_{3}$ & $=$ & $-x_{4} a \hat{\mathbf{x}}+\left(\frac{1}{4}+x_{4}\right) a \hat{\mathbf{y}}+\left(\frac{1}{4}+x_{4}\right) a \hat{\mathbf{z}}$ & $(32 e)$ & Se I \\
\hline $\mathbf{B}_{17}$ & $=$ & $\begin{array}{c}\left(-x_{5}+y_{5}+z_{5}\right) \mathbf{a}_{1}+ \\
\left(x_{5}-y_{5}+z_{5}\right) \mathbf{a}_{2}+ \\
\left(x_{5}+y_{5}-z_{5}\right) \mathbf{a}_{3}\end{array}$ & $=$ & $x_{5} a \hat{\mathbf{x}}+y_{5} a \hat{\mathbf{y}}+z_{5} a \hat{\mathbf{z}}$ & $(96 g)$ & Se II \\
\hline $\mathbf{B}_{18}$ & $=$ & $\begin{array}{l}\left(x_{5}-y_{5}+z_{5}\right) \mathbf{a}_{1}+ \\
\left(-x_{5}+y_{5}+z_{5}\right) \mathbf{a}_{2}+ \\
\left(\frac{1}{2}-x_{5}-y_{5}-z_{5}\right) \mathbf{a}_{3}\end{array}$ & $=$ & $\left(\frac{1}{4}-x_{5}\right) a \hat{\mathbf{x}}+\left(\frac{1}{4}-y_{5}\right) a \hat{\mathbf{y}}+z_{5} a \hat{\mathbf{z}}$ & $(96 g)$ & Se II \\
\hline $\mathbf{B}_{19}$ & $=$ & $\begin{array}{c}\left(x_{5}+y_{5}-z_{5}\right) \mathbf{a}_{1}+ \\
\left(\frac{1}{2}-x_{5}-y_{5}-z_{5}\right) \mathbf{a}_{2}+ \\
\left(-x_{5}+y_{5}+z_{5}\right) \mathbf{a}_{3}\end{array}$ & $=$ & $\left(\frac{1}{4}-x_{5}\right) a \hat{\mathbf{x}}+y_{5} a \hat{\mathbf{y}}+\left(\frac{1}{4}-z_{5}\right) a \hat{\mathbf{z}}$ & $(96 g)$ & Se II \\
\hline $\mathbf{B}_{20}$ & $=$ & $\begin{array}{c}\left(\frac{1}{2}-x_{5}-y_{5}-z_{5}\right) \mathbf{a}_{1}+ \\
\left(x_{5}+y_{5}-z_{5}\right) \mathbf{a}_{2}+ \\
\left(x_{5}-y_{5}+z_{5}\right) \mathbf{a}_{3}\end{array}$ & $=$ & $x_{5} a \hat{\mathbf{x}}+\left(\frac{1}{4}-y_{5}\right) a \hat{\mathbf{y}}+\left(\frac{1}{4}-z_{5}\right) a \hat{\mathbf{z}}$ & $(96 g)$ & Se II \\
\hline $\mathbf{B}_{21}$ & $=$ & $\begin{array}{c}\left(x_{5}+y_{5}-z_{5}\right) \mathbf{a}_{1}+ \\
\left(-x_{5}+y_{5}+z_{5}\right) \mathbf{a}_{2}+ \\
\left(x_{5}-y_{5}+z_{5}\right) \mathbf{a}_{3}\end{array}$ & $=$ & $z_{5} a \hat{\mathbf{x}}+x_{5} a \hat{\mathbf{y}}+y_{5} a \hat{\mathbf{z}}$ & $(96 g)$ & Se II \\
\hline $\mathbf{B}_{22}$ & $=$ & $\begin{array}{c}\left(\frac{1}{2}-x_{5}-y_{5}-z_{5}\right) \mathbf{a}_{1}+ \\
\left(x_{5}-y_{5}+z_{5}\right) \mathbf{a}_{2}+ \\
\left(-x_{5}+y_{5}+z_{5}\right) \mathbf{a}_{3}\end{array}$ & $=$ & $z_{5} a \hat{\mathbf{x}}+\left(\frac{1}{4}-x_{5}\right) a \hat{\mathbf{y}}+\left(\frac{1}{4}-y_{5}\right) a \hat{\mathbf{z}}$ & $(96 g)$ & Se II \\
\hline $\mathbf{B}_{23}$ & $=$ & $\begin{array}{c}\left(-x_{5}+y_{5}+z_{5}\right) \mathbf{a}_{1}+ \\
\left(x_{5}+y_{5}-z_{5}\right) \mathbf{a}_{2}+ \\
\left(\frac{1}{2}-x_{5}-y_{5}-z_{5}\right) \mathbf{a}_{3}\end{array}$ & $=$ & $\left(\frac{1}{4}-z_{5}\right) a \hat{\mathbf{x}}+\left(\frac{1}{4}-x_{5}\right) a \hat{\mathbf{y}}+y_{5} a \hat{\mathbf{z}}$ & $(96 g)$ & Se II \\
\hline $\mathbf{B}_{24}$ & $=$ & $\begin{array}{c}\left(x_{5}-y_{5}+z_{5}\right) \mathbf{a}_{1}+ \\
\left(\frac{1}{2}-x_{5}-y_{5}-z_{5}\right) \mathbf{a}_{2}+ \\
\left(x_{5}+y_{5}-z_{5}\right) \mathbf{a}_{3}\end{array}$ & $=$ & $\left(\frac{1}{4}-z_{5}\right) a \hat{\mathbf{x}}+x_{5} a \hat{\mathbf{y}}+\left(\frac{1}{4}-y_{5}\right) a \hat{\mathbf{z}}$ & $(96 g)$ & Se II \\
\hline $\mathbf{B}_{25}$ & $=$ & $\begin{array}{l}\left(x_{5}-y_{5}+z_{5}\right) \mathbf{a}_{1}+ \\
\left(x_{5}+y_{5}-z_{5}\right) \mathbf{a}_{2}+ \\
\left(-x_{5}+y_{5}+z_{5}\right) \mathbf{a}_{3}\end{array}$ & $=$ & $y_{5} a \hat{\mathbf{x}}+z_{5} a \hat{\mathbf{y}}+x_{5} a \hat{\mathbf{z}}$ & $(96 g)$ & Se II \\
\hline
\end{tabular}




\begin{tabular}{|c|c|c|c|c|c|c|}
\hline $\mathbf{B}_{26}$ & $=$ & $\begin{array}{c}\left(-x_{5}+y_{5}+z_{5}\right) \mathbf{a}_{1}+ \\
\left(\frac{1}{2}-x_{5}-y_{5}-z_{5}\right) \mathbf{a}_{2}+ \\
\left(x_{5}-y_{5}+z_{5}\right) \mathbf{a}_{3}\end{array}$ & $=$ & $\left(\frac{1}{4}-y_{5}\right) a \hat{\mathbf{x}}+z_{5} a \hat{\mathbf{y}}+\left(\frac{1}{4}-x_{5}\right) a \hat{\mathbf{z}}$ & $(96 g)$ & Se II \\
\hline $\mathbf{B}_{27}$ & $=$ & $\begin{array}{c}\left(\frac{1}{2}-x_{5}-y_{5}-z_{5}\right) \mathbf{a}_{1}+ \\
\left(-x_{5}+y_{5}+z_{5}\right) \mathbf{a}_{2}+ \\
\left(x_{5}+y_{5}-z_{5}\right) \mathbf{a}_{3}\end{array}$ & $=$ & $y_{5} a \hat{\mathbf{x}}+\left(\frac{1}{4}-z_{5}\right) a \hat{\mathbf{y}}+\left(\frac{1}{4}-x_{5}\right) a \hat{\mathbf{z}}$ & $(96 g)$ & Se II \\
\hline $\mathbf{B}_{28}$ & $=$ & $\begin{array}{c}\left(x_{5}+y_{5}-z_{5}\right) \mathbf{a}_{1}+ \\
\left(x_{5}-y_{5}+z_{5}\right) \mathbf{a}_{2}+ \\
\left(\frac{1}{2}-x_{5}-y_{5}-z_{5}\right) \mathbf{a}_{3}\end{array}$ & $=$ & $\left(\frac{1}{4}-y_{5}\right) a \hat{\mathbf{x}}+\left(\frac{1}{4}-z_{5}\right) a \hat{\mathbf{y}}+x_{5} a \hat{\mathbf{z}}$ & $(96 g)$ & Se II \\
\hline $\mathbf{B}_{29}$ & $=$ & $\begin{array}{c}\left(x_{5}-y_{5}-z_{5}\right) \mathbf{a}_{1}+ \\
\left(-x_{5}+y_{5}-z_{5}\right) \mathbf{a}_{2}+ \\
\left(-x_{5}-y_{5}+z_{5}\right) \mathbf{a}_{3}\end{array}$ & $=$ & $-x_{5} a \hat{\mathbf{x}}-y_{5} a \hat{\mathbf{y}}-z_{5} a \hat{\mathbf{z}}$ & $(96 g)$ & Se II \\
\hline $\mathbf{B}_{30}$ & $=$ & $\begin{array}{c}\left(-x_{5}+y_{5}-z_{5}\right) \mathbf{a}_{1}+ \\
\left(x_{5}-y_{5}-z_{5}\right) \mathbf{a}_{2}+ \\
\left(\frac{1}{2}+x_{5}+y_{5}+z_{5}\right) \mathbf{a}_{3}\end{array}$ & $=$ & $\left(\frac{1}{4}+x_{5}\right) a \hat{\mathbf{x}}+\left(\frac{1}{4}+y_{5}\right) a \hat{\mathbf{y}}-z_{5} a \hat{\mathbf{z}}$ & $(96 g)$ & Se II \\
\hline $\mathbf{B}_{31}$ & $=$ & $\begin{array}{c}\left(-x_{5}-y_{5}+z_{5}\right) \mathbf{a}_{1}+ \\
\left(\frac{1}{2}+x_{5}+y_{5}+z_{5}\right) \mathbf{a}_{2}+ \\
\quad\left(x_{5}-y_{5}-z_{5}\right) \mathbf{a}_{3}\end{array}$ & $=$ & $\left(\frac{1}{4}+x_{5}\right) a \hat{\mathbf{x}}-y_{5} a \hat{\mathbf{y}}+\left(\frac{1}{4}+z_{5}\right) a \hat{\mathbf{z}}$ & $(96 g)$ & Se II \\
\hline $\mathbf{B}_{32}$ & $=$ & $\begin{array}{c}\left(\frac{1}{2}+x_{5}+y_{5}+z_{5}\right) \mathbf{a}_{1}+ \\
\left(-x_{5}-y_{5}+z_{5}\right) \mathbf{a}_{2}+ \\
\quad\left(-x_{5}+y_{5}-z_{5}\right) \mathbf{a}_{3}\end{array}$ & $=$ & $-x_{5} a \hat{\mathbf{x}}+\left(\frac{1}{4}+y_{5}\right) a \hat{\mathbf{y}}+\left(\frac{1}{4}+z_{5}\right) a \hat{\mathbf{z}}$ & $(96 g)$ & Se II \\
\hline $\mathbf{B}_{33}$ & $=$ & $\begin{array}{c}\left(-x_{5}-y_{5}+z_{5}\right) \mathbf{a}_{1}+ \\
\left(x_{5}-y_{5}-z_{5}\right) \mathbf{a}_{2}+ \\
\left(-x_{5}+y_{5}-z_{5}\right) \mathbf{a}_{3}\end{array}$ & $=$ & $-z_{5} a \hat{\mathbf{x}}-x_{5} a \hat{\mathbf{y}}-y_{5} a \hat{\mathbf{z}}$ & $(96 g)$ & Se II \\
\hline $\mathbf{B}_{34}$ & $=$ & $\begin{array}{c}\left(\frac{1}{2}+x_{5}+y_{5}+z_{5}\right) \mathbf{a}_{1}+ \\
\left(-x_{5}+y_{5}-z_{5}\right) \mathbf{a}_{2}+ \\
\quad\left(x_{5}-y_{5}-z_{5}\right) \mathbf{a}_{3}\end{array}$ & $=$ & $-z_{5} a \hat{\mathbf{x}}+\left(\frac{1}{4}+x_{5}\right) a \hat{\mathbf{y}}+\left(\frac{1}{4}+y_{5}\right) a \hat{\mathbf{z}}$ & $(96 g)$ & Se II \\
\hline $\mathbf{B}_{35}$ & $=$ & $\begin{array}{c}\left(x_{5}-y_{5}-z_{5}\right) \mathbf{a}_{1}+ \\
\left(-x_{5}-y_{5}+z_{5}\right) \mathbf{a}_{2}+ \\
\left(\frac{1}{2}+x_{5}+y_{5}+z_{5}\right) \mathbf{a}_{3}\end{array}$ & $=$ & $\left(\frac{1}{4}+z_{5}\right) a \hat{\mathbf{x}}+\left(\frac{1}{4}+x_{5}\right) a \hat{\mathbf{y}}-y_{5} a \hat{\mathbf{z}}$ & $(96 g)$ & Se II \\
\hline $\mathbf{B}_{36}$ & $=$ & $\begin{array}{c}\left(-x_{5}+y_{5}-z_{5}\right) \mathbf{a}_{1}+ \\
\left(\frac{1}{2}+x_{5}+y_{5}+z_{5}\right) \mathbf{a}_{2}+ \\
\quad\left(-x_{5}-y_{5}+z_{5}\right) \mathbf{a}_{3}\end{array}$ & $=$ & $\left(\frac{1}{4}+z_{5}\right) a \hat{\mathbf{x}}-x_{5} a \hat{\mathbf{y}}+\left(\frac{1}{4}+y_{5}\right) a \hat{\mathbf{z}}$ & $(96 g)$ & Se II \\
\hline $\mathbf{B}_{37}$ & $=$ & $\begin{array}{c}\left(-x_{5}+y_{5}-z_{5}\right) \mathbf{a}_{1}+ \\
\left(-x_{5}-y_{5}+z_{5}\right) \mathbf{a}_{2}+ \\
\left(x_{5}-y_{5}-z_{5}\right) \mathbf{a}_{3}\end{array}$ & $=$ & $-y_{5} a \hat{\mathbf{x}}-z_{5} a \hat{\mathbf{y}}-x_{5} a \hat{\mathbf{z}}$ & $(96 g)$ & Se II \\
\hline $\mathbf{B}_{38}$ & $=$ & $\begin{array}{c}\left(x_{5}-y_{5}-z_{5}\right) \mathbf{a}_{1}+ \\
\left(\frac{1}{2}+x_{5}+y_{5}+z_{5}\right) \mathbf{a}_{2}+ \\
\left(-x_{5}+y_{5}-z_{5}\right) \mathbf{a}_{3}\end{array}$ & $=$ & $\left(\frac{1}{4}+y_{5}\right) a \hat{\mathbf{x}}-z_{5} a \hat{\mathbf{y}}+\left(\frac{1}{4}+x_{5}\right) a \hat{\mathbf{z}}$ & $(96 g)$ & Se II \\
\hline $\mathbf{B}_{39}$ & $=$ & $\begin{array}{c}\left(\frac{1}{2}+x_{5}+y_{5}+z_{5}\right) \mathbf{a}_{1}+ \\
\quad\left(x_{5}-y_{5}-z_{5}\right) \mathbf{a}_{2}+ \\
\quad\left(-x_{5}-y_{5}+z_{5}\right) \mathbf{a}_{3}\end{array}$ & $=$ & $-y_{5} a \hat{\mathbf{x}}+\left(\frac{1}{4}+z_{5}\right) a \hat{\mathbf{y}}+\left(\frac{1}{4}+x_{5}\right) a \hat{\mathbf{z}}$ & $(96 g)$ & Se II \\
\hline $\mathbf{B}_{40}$ & $=$ & $\begin{array}{l}\left(-x_{5}-y_{5}+z_{5}\right) \mathbf{a}_{1}+ \\
\left(-x_{5}+y_{5}-z_{5}\right) \mathbf{a}_{2}+ \\
\left(\frac{1}{2}+x_{5}+y_{5}+z_{5}\right) \mathbf{a}_{3}\end{array}$ & $=$ & $\left(\frac{1}{4}+y_{5}\right) a \hat{\mathbf{x}}+\left(\frac{1}{4}+z_{5}\right) a \hat{\mathbf{y}}-x_{5} a \hat{\mathbf{z}}$ & $(96 g)$ & Se II \\
\hline
\end{tabular}

\section{References:}

- M. Wachhold and W. S. Sheldrick, Methanolothermale Synthese von $\mathrm{Rb}_{3} \mathrm{AsSe}_{4} \cdot 2 \mathrm{Se}_{6}$ und $\mathrm{Cs}_{3} \mathrm{AsSe}_{4} \cdot 2 \mathrm{Cs}_{2} \mathrm{As}_{2} \mathrm{Se}_{4}$. $6 \mathrm{Te}_{4} \mathrm{Se}_{2}$, zwei Selenidoarsenate mit sechsgliedrigen Chalkogenringen/Methanolothermal Synthesis of $\mathrm{Rb}_{3} \mathrm{AsSe}_{4} \cdot 2 \mathrm{Se}_{6}$ and 
$\mathrm{Cs}_{3} \mathrm{AsSe}_{4} \cdot 2 \mathrm{Cs}_{2} \mathrm{As}_{2} \mathrm{Se}_{4} \cdot 6 \mathrm{Te}_{4} \mathrm{Se}_{2}$. Two Selenidoarsenates with Six-Membered Chalcogen Rings, Z. Naturforsch. B 52, 169-175 (1997), doi:10.1515/znb-1997-0204.

\section{Found in:}

- P. Villars and K. Cenzual, Pearson's Crystal Data - Crystal Structure Database for Inorganic Compounds, ASM International (2013).

\section{Geometry files:}

- CIF: pp. 954

- POSCAR: pp. 954 


\section{$\mathrm{Ca}_{3} \mathrm{Al}_{2} \mathrm{O}_{6}$ Structure: A2B3C6_cP264_205_2d_ab2c2d_6d}
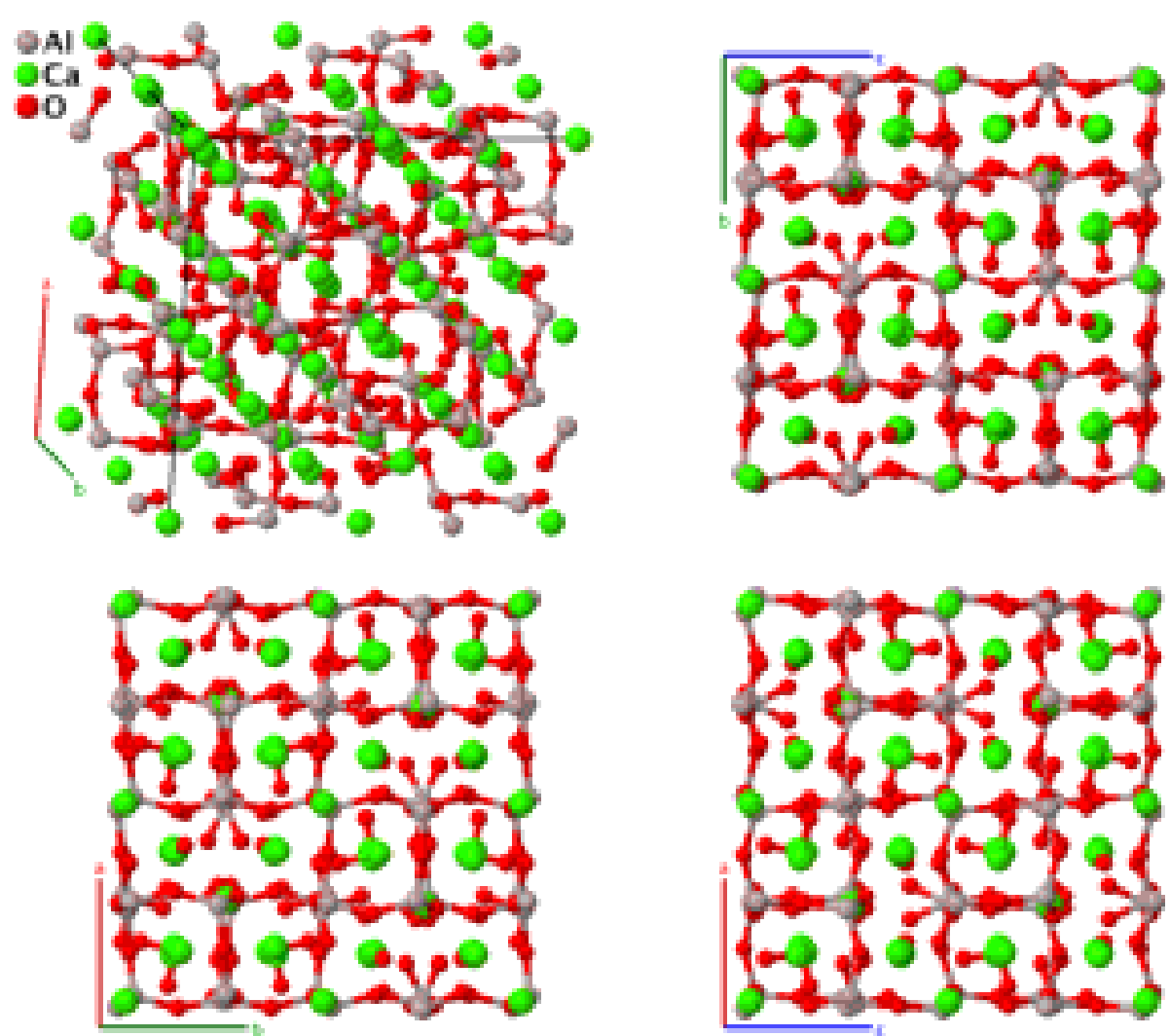

\section{Prototype}

AFLOW prototype label

: $\mathrm{Ca}_{3} \mathrm{Al}_{2} \mathrm{O}_{6}$

Strukturbericht designation

Pearson symbol

: A2B3C6_cP264_205_2d_ab2c2d_6d

\section{Space group number}

Space group symbol

AFLOW prototype command

: None

: $\quad \mathrm{cP} 264$

: 205

\section{: $\quad P a \overline{3}$}

aflow --proto=A2B3C6_cP264_205_2d_ab2c2d_6d

- params $=a, x_{3}, x_{4}, x_{5}, y_{5}, z_{5}, x_{6}, y_{6}, z_{6}, x_{7}, y_{7}, z_{7}, x_{8}, y_{8}, z_{8}, x_{9}, y_{9}, z_{9}, x_{10}, y_{10}, z_{10}, x_{11}$, $y_{11}, z_{11}, x_{12}, y_{12}, z_{12}, x_{13}, y_{13}, z_{13}, x_{14}, y_{14}, z_{14}$

- This is a redetermination of the $E 9_{1}\left(\mathrm{Ca}_{3} \mathrm{Al}_{2} \mathrm{O}_{6}\right)$ structure. The lattice constant of the new unit cell is twice the original, giving a volume eight times larger.

Simple Cubic primitive vectors:

$$
\begin{aligned}
& \mathbf{a}_{1}=a \hat{\mathbf{x}} \\
& \mathbf{a}_{2}=a \hat{\mathbf{y}} \\
& \mathbf{a}_{3}=a \hat{\mathbf{z}}
\end{aligned}
$$

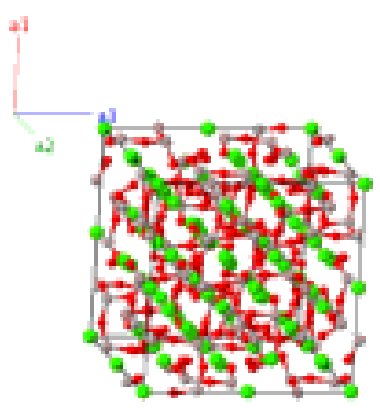


Lattice Coordinates

Cartesian Coordinates

$\begin{array}{lcc}\mathbf{B}_{1}= & 0 \mathbf{a}_{1}+0 \mathbf{a}_{2}+0 \mathbf{a}_{3} \\ \mathbf{B}_{2}= & \frac{1}{2} \mathbf{a}_{1}+\frac{1}{2} \mathbf{a}_{3} \\ \mathbf{B}_{3}= & \frac{1}{2} \mathbf{a}_{2}+\frac{1}{2} \mathbf{a}_{3} \\ \mathbf{B}_{4}= & \frac{1}{2} \mathbf{a}_{1}+\frac{1}{2} \mathbf{a}_{2} \\ \mathbf{B}_{5}= & \frac{1}{2} \mathbf{a}_{1}+\frac{1}{2} \mathbf{a}_{2}+\frac{1}{2} \mathbf{a}_{3} \\ \mathbf{B}_{6}= & \frac{1}{2} \mathbf{a}_{2} \\ \mathbf{B}_{7}= & \frac{1}{2} \mathbf{a}_{1} \\ \mathbf{B}_{8}= & \frac{1}{2} \mathbf{a}_{3}\end{array}$

$\mathbf{B}_{9}=$

$\mathbf{B}_{10}=$

$\mathbf{B}_{11}=-x_{3} \mathbf{a}_{1}+\left(\frac{1}{2}+x_{3}\right) \mathbf{a}_{2}+\left(\frac{1}{2}-x_{3}\right) \mathbf{a}_{3}$

$\mathbf{B}_{12}=\left(\frac{1}{2}+x_{3}\right) \mathbf{a}_{1}+\left(\frac{1}{2}-x_{3}\right) \mathbf{a}_{2}-x_{3} \mathbf{a}_{3}$

$\mathbf{B}_{13}=-x_{3} \mathbf{a}_{1}-x_{3} \mathbf{a}_{2}-x_{3} \mathbf{a}_{3}$

$\mathbf{B}_{14}=\left(\frac{1}{2}+x_{3}\right) \mathbf{a}_{1}+x_{3} \mathbf{a}_{2}+\left(\frac{1}{2}-x_{3}\right) \mathbf{a}_{3}$

$\mathbf{B}_{15}=x_{3} \mathbf{a}_{1}+\left(\frac{1}{2}-x_{3}\right) \mathbf{a}_{2}+\left(\frac{1}{2}+x_{3}\right) \mathbf{a}_{3}$

$\mathbf{B}_{16}=\left(\frac{1}{2}-x_{3}\right) \mathbf{a}_{1}+\left(\frac{1}{2}+x_{3}\right) \mathbf{a}_{2}+x_{3} \mathbf{a}_{3}$

$\mathbf{B}_{17}=$

$\mathbf{B}_{18}=\left(\frac{1}{2}-x_{4}\right) \mathbf{a}_{1}-x_{4} \mathbf{a}_{2}+\left(\frac{1}{2}+x_{4}\right) \mathbf{a}_{3}$

$\mathbf{B}_{19}=-x_{4} \mathbf{a}_{1}+\left(\frac{1}{2}+x_{4}\right) \mathbf{a}_{2}+\left(\frac{1}{2}-x_{4}\right) \mathbf{a}_{3}$

$\mathbf{B}_{20}=\left(\frac{1}{2}+x_{4}\right) \mathbf{a}_{1}+\left(\frac{1}{2}-x_{4}\right) \mathbf{a}_{2}-x_{4} \mathbf{a}_{3}$

$\mathbf{B}_{21}=$

$\mathbf{B}_{22}=\left(\frac{1}{2}+x_{4}\right) \mathbf{a}_{1}+x_{4} \mathbf{a}_{2}+\left(\frac{1}{2}-x_{4}\right) \mathbf{a}_{3}$

$\mathbf{B}_{23}=x_{4} \mathbf{a}_{1}+\left(\frac{1}{2}-x_{4}\right) \mathbf{a}_{2}+\left(\frac{1}{2}+x_{4}\right) \mathbf{a}_{3}$

$\mathbf{B}_{24}=\left(\frac{1}{2}-x_{4}\right) \mathbf{a}_{1}+\left(\frac{1}{2}+x_{4}\right) \mathbf{a}_{2}+x_{4} \mathbf{a}_{3}$

$\mathbf{B}_{25}=x_{5} \mathbf{a}_{1}+y_{5} \mathbf{a}_{2}+z_{5} \mathbf{a}_{3}$

$\mathbf{B}_{26}=\left(\frac{1}{2}-x_{5}\right) \mathbf{a}_{1}-y_{5} \mathbf{a}_{2}+\left(\frac{1}{2}+z_{5}\right) \mathbf{a}_{3}$

$\mathbf{B}_{27}=-x_{5} \mathbf{a}_{1}+\left(\frac{1}{2}+y_{5}\right) \mathbf{a}_{2}+\left(\frac{1}{2}-z_{5}\right) \mathbf{a}_{3}$

$\mathbf{B}_{28}=\left(\frac{1}{2}+x_{5}\right) \mathbf{a}_{1}+\left(\frac{1}{2}-y_{5}\right) \mathbf{a}_{2}-z_{5} \mathbf{a}_{3}$

$\mathbf{B}_{29}=z_{5} \mathbf{a}_{1}+x_{5} \mathbf{a}_{2}+y_{5} \mathbf{a}_{3}$

$\mathbf{B}_{30}=\left(\frac{1}{2}+z_{5}\right) \mathbf{a}_{1}+\left(\frac{1}{2}-x_{5}\right) \mathbf{a}_{2}-y_{5} \mathbf{a}_{3}$

$\mathbf{B}_{31}=\left(\frac{1}{2}-z_{5}\right) \mathbf{a}_{1}-x_{5} \mathbf{a}_{2}+\left(\frac{1}{2}+y_{5}\right) \mathbf{a}_{3}$

$\mathbf{B}_{32}=-z_{5} \mathbf{a}_{1}+\left(\frac{1}{2}+x_{5}\right) \mathbf{a}_{2}+\left(\frac{1}{2}-y_{5}\right) \mathbf{a}_{3}$

$\mathbf{B}_{33}=y_{5} \mathbf{a}_{1}+z_{5} \mathbf{a}_{2}+x_{5} \mathbf{a}_{3}$

$\mathbf{B}_{34}=-y_{5} \mathbf{a}_{1}+\left(\frac{1}{2}+z_{5}\right) \mathbf{a}_{2}+\left(\frac{1}{2}-x_{5}\right) \mathbf{a}_{3}$

$\mathbf{B}_{35}=\left(\frac{1}{2}+y_{5}\right) \mathbf{a}_{1}+\left(\frac{1}{2}-z_{5}\right) \mathbf{a}_{2}-x_{5} \mathbf{a}_{3}$

$$
=
$$

$$
=
$$

$$
=
$$$$
=
$$$$
=
$$$$
=
$$$$
=
$$$$
=
$$$$
=
$$$$
=\quad x_{3} a \hat{\mathbf{x}}+x_{3} a \hat{\mathbf{y}}+x_{3} a \hat{\mathbf{z}}
$$$$
=\left(\frac{1}{2}-x_{3}\right) a \hat{\mathbf{x}}-x_{3} a \hat{\mathbf{y}}+\left(\frac{1}{2}+x_{3}\right) a \hat{\mathbf{z}}
$$$$
=-x_{3} a \hat{\mathbf{x}}+\left(\frac{1}{2}+x_{3}\right) a \hat{\mathbf{y}}+\left(\frac{1}{2}-x_{3}\right) a \hat{\mathbf{z}}
$$$$
=\left(\frac{1}{2}+x_{3}\right) a \hat{\mathbf{x}}+\left(\frac{1}{2}-x_{3}\right) a \hat{\mathbf{y}}-x_{3} a \hat{\mathbf{z}}
$$$$
=\quad-x_{3} a \hat{\mathbf{x}}-x_{3} a \hat{\mathbf{y}}-x_{3} a \hat{\mathbf{z}}
$$$$
=\left(\frac{1}{2}+x_{3}\right) a \hat{\mathbf{x}}+x_{3} a \hat{\mathbf{y}}+\left(\frac{1}{2}-x_{3}\right) a \hat{\mathbf{z}}
$$$$
=x_{3} a \hat{\mathbf{x}}+\left(\frac{1}{2}-x_{3}\right) a \hat{\mathbf{y}}+\left(\frac{1}{2}+x_{3}\right) a \hat{\mathbf{z}}
$$$$
=\left(\frac{1}{2}-x_{3}\right) a \hat{\mathbf{x}}+\left(\frac{1}{2}+x_{3}\right) a \hat{\mathbf{y}}+x_{3} a \hat{\mathbf{z}}
$$$$
=\quad x_{4} a \hat{\mathbf{x}}+x_{4} a \hat{\mathbf{y}}+x_{4} a \hat{\mathbf{z}}
$$$$
=\left(\frac{1}{2}-x_{4}\right) a \hat{\mathbf{x}}-x_{4} a \hat{\mathbf{y}}+\left(\frac{1}{2}+x_{4}\right) a \hat{\mathbf{z}}
$$$$
=-x_{4} a \hat{\mathbf{x}}+\left(\frac{1}{2}+x_{4}\right) a \hat{\mathbf{y}}+\left(\frac{1}{2}-x_{4}\right) a \hat{\mathbf{z}}
$$$$
=\left(\frac{1}{2}+x_{4}\right) a \hat{\mathbf{x}}+\left(\frac{1}{2}-x_{4}\right) a \hat{\mathbf{y}}-x_{4} a \hat{\mathbf{z}}
$$$$
=\quad-x_{4} a \hat{\mathbf{x}}-x_{4} a \hat{\mathbf{y}}-x_{4} a \hat{\mathbf{z}}
$$$$
=\left(\frac{1}{2}+x_{4}\right) a \hat{\mathbf{x}}+x_{4} a \hat{\mathbf{y}}+\left(\frac{1}{2}-x_{4}\right) a \hat{\mathbf{z}}
$$$$
=x_{4} a \hat{\mathbf{x}}+\left(\frac{1}{2}-x_{4}\right) a \hat{\mathbf{y}}+\left(\frac{1}{2}+x_{4}\right) a \hat{\mathbf{z}}
$$$$
=\left(\frac{1}{2}-x_{4}\right) a \hat{\mathbf{x}}+\left(\frac{1}{2}+x_{4}\right) a \hat{\mathbf{y}}+x_{4} a \hat{\mathbf{z}}
$$$$
=\quad x_{5} a \hat{\mathbf{x}}+y_{5} a \hat{\mathbf{y}}+z_{5} a \hat{\mathbf{z}}
$$$$
=\left(\frac{1}{2}-x_{5}\right) a \hat{\mathbf{x}}-y_{5} a \hat{\mathbf{y}}+\left(\frac{1}{2}+z_{5}\right) a \hat{\mathbf{z}}
$$$$
=-x_{5} a \hat{\mathbf{x}}+\left(\frac{1}{2}+y_{5}\right) a \hat{\mathbf{y}}+\left(\frac{1}{2}-z_{5}\right) a \hat{\mathbf{z}}
$$$$
=\left(\frac{1}{2}+x_{5}\right) a \hat{\mathbf{x}}+\left(\frac{1}{2}-y_{5}\right) a \hat{\mathbf{y}}-z_{5} a \hat{\mathbf{z}}
$$$$
=\quad z_{5} a \hat{\mathbf{x}}+x_{5} a \hat{\mathbf{y}}+y_{5} a \hat{\mathbf{z}}
$$$$
=\left(\frac{1}{2}+z_{5}\right) a \hat{\mathbf{x}}+\left(\frac{1}{2}-x_{5}\right) a \hat{\mathbf{y}}-y_{5} a \hat{\mathbf{z}}
$$$$
=\left(\frac{1}{2}-z_{5}\right) a \hat{\mathbf{x}}-x_{5} a \hat{\mathbf{y}}+\left(\frac{1}{2}+y_{5}\right) a \hat{\mathbf{z}}
$$$$
=-z_{5} a \hat{\mathbf{x}}+\left(\frac{1}{2}+x_{5}\right) a \hat{\mathbf{y}}+\left(\frac{1}{2}-y_{5}\right) a \hat{\mathbf{z}}
$$$$
=\quad y_{5} a \hat{\mathbf{x}}+z_{5} a \hat{\mathbf{y}}+x_{5} a \hat{\mathbf{z}}
$$

Ca I

$\mathrm{Ca}$ I

$\mathrm{Ca} \mathrm{I}$

Ca I

Ca II

Ca II

Ca II

Ca II

Ca III

Ca III

Ca III

Ca III

Ca III

Ca III

Ca III

Ca III

Ca IV

Ca IV

Ca IV

Ca IV

Ca IV

Ca IV

Ca IV

Ca IV

Al I

Al I

Al I

Al I

Al I

Al I

Al I

Al I

Al I

Al I

Al I 


\begin{tabular}{|c|c|c|c|c|c|c|}
\hline $\mathbf{B}_{36}$ & $=$ & $\left(\frac{1}{2}-y_{5}\right) \mathbf{a}_{1}-z_{5} \mathbf{a}_{2}+\left(\frac{1}{2}+x_{5}\right) \mathbf{a}_{3}$ & $=$ & $\left(\frac{1}{2}-y_{5}\right) a \hat{\mathbf{x}}-z_{5} a \hat{\mathbf{y}}+\left(\frac{1}{2}+x_{5}\right) a \hat{\mathbf{z}}$ & $(24 d)$ & Al I \\
\hline $\mathbf{B}_{37}$ & $=$ & $-x_{5} \mathbf{a}_{1}-y_{5} \mathbf{a}_{2}-z_{5} \mathbf{a}_{3}$ & $=$ & $-x_{5} a \hat{\mathbf{x}}-y_{5} a \hat{\mathbf{y}}-z_{5} a \hat{\mathbf{z}}$ & $(24 d)$ & $\mathrm{Al} \mathrm{I}$ \\
\hline $\mathbf{B}_{38}$ & $=$ & $\left(\frac{1}{2}+x_{5}\right) \mathbf{a}_{1}+y_{5} \mathbf{a}_{2}+\left(\frac{1}{2}-z_{5}\right) \mathbf{a}_{3}$ & $=$ & $\left(\frac{1}{2}+x_{5}\right) a \hat{\mathbf{x}}+y_{5} a \hat{\mathbf{y}}+\left(\frac{1}{2}-z_{5}\right) a \hat{\mathbf{z}}$ & $(24 d)$ & Al I \\
\hline $\mathbf{B}_{39}$ & $=$ & $x_{5} \mathbf{a}_{1}+\left(\frac{1}{2}-y_{5}\right) \mathbf{a}_{2}+\left(\frac{1}{2}+z_{5}\right) \mathbf{a}_{3}$ & $=$ & $x_{5} a \hat{\mathbf{x}}+\left(\frac{1}{2}-y_{5}\right) a \hat{\mathbf{y}}+\left(\frac{1}{2}+z_{5}\right) a \hat{\mathbf{z}}$ & $(24 d)$ & Al I \\
\hline $\mathbf{B}_{40}$ & $=$ & $\left(\frac{1}{2}-x_{5}\right) \mathbf{a}_{1}+\left(\frac{1}{2}+y_{5}\right) \mathbf{a}_{2}+z_{5} \mathbf{a}_{3}$ & $=$ & $\left(\frac{1}{2}-x_{5}\right) a \hat{\mathbf{x}}+\left(\frac{1}{2}+y_{5}\right) a \hat{\mathbf{y}}+z_{5} a \hat{\mathbf{z}}$ & $(24 d)$ & $\mathrm{Al} \mathrm{I}$ \\
\hline $\mathbf{B}_{41}$ & $=$ & $-z_{5} \mathbf{a}_{1}-x_{5} \mathbf{a}_{2}-y_{5} \mathbf{a}_{3}$ & $=$ & $-z_{5} a \hat{\mathbf{x}}-x_{5} a \hat{\mathbf{y}}-y_{5} a \hat{\mathbf{z}}$ & $(24 d)$ & $\mathrm{Al} \mathrm{I}$ \\
\hline $\mathbf{B}_{42}$ & $=$ & $\left(\frac{1}{2}-z_{5}\right) \mathbf{a}_{1}+\left(\frac{1}{2}+x_{5}\right) \mathbf{a}_{2}+y_{5} \mathbf{a}_{3}$ & $=$ & $\left(\frac{1}{2}-z_{5}\right) a \hat{\mathbf{x}}+\left(\frac{1}{2}+x_{5}\right) a \hat{\mathbf{y}}+y_{5} a \hat{\mathbf{z}}$ & $(24 d)$ & Al I \\
\hline $\mathbf{B}_{43}$ & $=$ & $\left(\frac{1}{2}+z_{5}\right) \mathbf{a}_{1}+x_{5} \mathbf{a}_{2}+\left(\frac{1}{2}-y_{5}\right) \mathbf{a}_{3}$ & $=$ & $\left(\frac{1}{2}+z_{5}\right) a \hat{\mathbf{x}}+x_{5} a \hat{\mathbf{y}}+\left(\frac{1}{2}-y_{5}\right) a \hat{\mathbf{z}}$ & $(24 d)$ & $\mathrm{Al} \mathrm{I}$ \\
\hline $\mathbf{B}_{44}$ & $=$ & $z_{5} \mathbf{a}_{1}+\left(\frac{1}{2}-x_{5}\right) \mathbf{a}_{2}+\left(\frac{1}{2}+y_{5}\right) \mathbf{a}_{3}$ & $=$ & $z_{5} a \hat{\mathbf{x}}+\left(\frac{1}{2}-x_{5}\right) a \hat{\mathbf{y}}+\left(\frac{1}{2}+y_{5}\right) a \hat{\mathbf{z}}$ & $(24 d)$ & $\mathrm{Al} \mathrm{I}$ \\
\hline $\mathbf{B}_{45}$ & $=$ & $-y_{5} \mathbf{a}_{1}-z_{5} \mathbf{a}_{2}-x_{5} \mathbf{a}_{3}$ & $=$ & $-y_{5} a \hat{\mathbf{x}}-z_{5} a \hat{\mathbf{y}}-x_{5} a \hat{\mathbf{z}}$ & $(24 d)$ & $\mathrm{Al} \mathrm{I}$ \\
\hline $\mathbf{B}_{46}$ & $=$ & $y_{5} \mathbf{a}_{1}+\left(\frac{1}{2}-z_{5}\right) \mathbf{a}_{2}+\left(\frac{1}{2}+x_{5}\right) \mathbf{a}_{3}$ & $=$ & $y_{5} a \hat{\mathbf{x}}+\left(\frac{1}{2}-z_{5}\right) a \hat{\mathbf{y}}+\left(\frac{1}{2}+x_{5}\right) a \hat{\mathbf{z}}$ & $(24 d)$ & $\mathrm{Al} \mathrm{I}$ \\
\hline $\mathbf{B}_{47}$ & $=$ & $\left(\frac{1}{2}-y_{5}\right) \mathbf{a}_{1}+\left(\frac{1}{2}+z_{5}\right) \mathbf{a}_{2}+x_{5} \mathbf{a}_{3}$ & $=$ & $\left(\frac{1}{2}-y_{5}\right) a \hat{\mathbf{x}}+\left(\frac{1}{2}+z_{5}\right) a \hat{\mathbf{y}}+x_{5} a \hat{\mathbf{z}}$ & $(24 d)$ & Al I \\
\hline $\mathbf{B}_{48}$ & $=$ & $\left(\frac{1}{2}+y_{5}\right) \mathbf{a}_{1}+z_{5} \mathbf{a}_{2}+\left(\frac{1}{2}-x_{5}\right) \mathbf{a}_{3}$ & $=$ & $\left(\frac{1}{2}+y_{5}\right) a \hat{\mathbf{x}}+z_{5} a \hat{\mathbf{y}}+\left(\frac{1}{2}-x_{5}\right) a \hat{\mathbf{z}}$ & $(24 d)$ & Al I \\
\hline $\mathbf{B}_{49}$ & $=$ & $x_{6} \mathbf{a}_{1}+y_{6} \mathbf{a}_{2}+z_{6} \mathbf{a}_{3}$ & $=$ & $x_{6} a \hat{\mathbf{x}}+y_{6} a \hat{\mathbf{y}}+z_{6} a \hat{\mathbf{z}}$ & $(24 d)$ & $\mathrm{Al} \mathrm{II}$ \\
\hline $\mathbf{B}_{50}$ & $=$ & $\left(\frac{1}{2}-x_{6}\right) \mathbf{a}_{1}-y_{6} \mathbf{a}_{2}+\left(\frac{1}{2}+z_{6}\right) \mathbf{a}_{3}$ & $=$ & $\left(\frac{1}{2}-x_{6}\right) a \hat{\mathbf{x}}-y_{6} a \hat{\mathbf{y}}+\left(\frac{1}{2}+z_{6}\right) a \hat{\mathbf{z}}$ & $(24 d)$ & Al II \\
\hline $\mathbf{B}_{51}$ & $=$ & $-x_{6} \mathbf{a}_{1}+\left(\frac{1}{2}+y_{6}\right) \mathbf{a}_{2}+\left(\frac{1}{2}-z_{6}\right) \mathbf{a}_{3}$ & $=$ & $-x_{6} a \hat{\mathbf{x}}+\left(\frac{1}{2}+y_{6}\right) a \hat{\mathbf{y}}+\left(\frac{1}{2}-z_{6}\right) a \hat{\mathbf{z}}$ & $(24 d)$ & Al II \\
\hline $\mathbf{B}_{52}$ & $=$ & $\left(\frac{1}{2}+x_{6}\right) \mathbf{a}_{1}+\left(\frac{1}{2}-y_{6}\right) \mathbf{a}_{2}-z_{6} \mathbf{a}_{3}$ & $=$ & $\left(\frac{1}{2}+x_{6}\right) a \hat{\mathbf{x}}+\left(\frac{1}{2}-y_{6}\right) a \hat{\mathbf{y}}-z_{6} a \hat{\mathbf{z}}$ & $(24 d)$ & $\mathrm{Al}$ II \\
\hline $\mathbf{B}_{53}$ & $=$ & $z_{6} \mathbf{a}_{1}+x_{6} \mathbf{a}_{2}+y_{6} \mathbf{a}_{3}$ & $=$ & $z_{6} a \hat{\mathbf{x}}+x_{6} a \hat{\mathbf{y}}+y_{6} a \hat{\mathbf{z}}$ & $(24 d)$ & Al II \\
\hline $\mathbf{B}_{54}$ & $=$ & $\left(\frac{1}{2}+z_{6}\right) \mathbf{a}_{1}+\left(\frac{1}{2}-x_{6}\right) \mathbf{a}_{2}-y_{6} \mathbf{a}_{3}$ & $=$ & $\left(\frac{1}{2}+z_{6}\right) a \hat{\mathbf{x}}+\left(\frac{1}{2}-x_{6}\right) a \hat{\mathbf{y}}-y_{6} a \hat{\mathbf{z}}$ & $(24 d)$ & $\mathrm{Al}$ II \\
\hline $\mathbf{B}_{55}$ & $=$ & $\left(\frac{1}{2}-z_{6}\right) \mathbf{a}_{1}-x_{6} \mathbf{a}_{2}+\left(\frac{1}{2}+y_{6}\right) \mathbf{a}_{3}$ & $=$ & $\left(\frac{1}{2}-z_{6}\right) a \hat{\mathbf{x}}-x_{6} a \hat{\mathbf{y}}+\left(\frac{1}{2}+y_{6}\right) a \hat{\mathbf{z}}$ & $(24 d)$ & $\mathrm{Al} \mathrm{II}$ \\
\hline $\mathbf{B}_{56}$ & $=$ & $-z_{6} \mathbf{a}_{1}+\left(\frac{1}{2}+x_{6}\right) \mathbf{a}_{2}+\left(\frac{1}{2}-y_{6}\right) \mathbf{a}_{3}$ & $=$ & $-z_{6} a \hat{\mathbf{x}}+\left(\frac{1}{2}+x_{6}\right) a \hat{\mathbf{y}}+\left(\frac{1}{2}-y_{6}\right) a \hat{\mathbf{z}}$ & $(24 d)$ & $\mathrm{Al}$ II \\
\hline $\mathbf{B}_{57}$ & $=$ & $y_{6} \mathbf{a}_{1}+z_{6} \mathbf{a}_{2}+x_{6} \mathbf{a}_{3}$ & $=$ & $y_{6} a \hat{\mathbf{x}}+z_{6} a \hat{\mathbf{y}}+x_{6} a \hat{\mathbf{z}}$ & $(24 d)$ & $\mathrm{Al}$ II \\
\hline $\mathbf{B}_{58}$ & $=$ & $-y_{6} \mathbf{a}_{1}+\left(\frac{1}{2}+z_{6}\right) \mathbf{a}_{2}+\left(\frac{1}{2}-x_{6}\right) \mathbf{a}_{3}$ & $=$ & $-y_{6} a \hat{\mathbf{x}}+\left(\frac{1}{2}+z_{6}\right) a \hat{\mathbf{y}}+\left(\frac{1}{2}-x_{6}\right) a \hat{\mathbf{z}}$ & $(24 d)$ & Al II \\
\hline $\mathbf{B}_{59}$ & $=$ & $\left(\frac{1}{2}+y_{6}\right) \mathbf{a}_{1}+\left(\frac{1}{2}-z_{6}\right) \mathbf{a}_{2}-x_{6} \mathbf{a}_{3}$ & $=$ & $\left(\frac{1}{2}+y_{6}\right) a \hat{\mathbf{x}}+\left(\frac{1}{2}-z_{6}\right) a \hat{\mathbf{y}}-x_{6} a \hat{\mathbf{z}}$ & $(24 d)$ & Al II \\
\hline $\mathbf{B}_{60}$ & $=$ & $\left(\frac{1}{2}-y_{6}\right) \mathbf{a}_{1}-z_{6} \mathbf{a}_{2}+\left(\frac{1}{2}+x_{6}\right) \mathbf{a}_{3}$ & $=$ & $\left(\frac{1}{2}-y_{6}\right) a \hat{\mathbf{x}}-z_{6} a \hat{\mathbf{y}}+\left(\frac{1}{2}+x_{6}\right) a \hat{\mathbf{z}}$ & $(24 d)$ & $\mathrm{Al}$ II \\
\hline $\mathbf{B}_{61}$ & $=$ & $-x_{6} \mathbf{a}_{1}-y_{6} \mathbf{a}_{2}-z_{6} \mathbf{a}_{3}$ & $=$ & $-x_{6} a \hat{\mathbf{x}}-y_{6} a \hat{\mathbf{y}}-z_{6} a \hat{\mathbf{z}}$ & $(24 d)$ & Al II \\
\hline $\mathbf{B}_{62}$ & $=$ & $\left(\frac{1}{2}+x_{6}\right) \mathbf{a}_{1}+y_{6} \mathbf{a}_{2}+\left(\frac{1}{2}-z_{6}\right) \mathbf{a}_{3}$ & $=$ & $\left(\frac{1}{2}+x_{6}\right) a \hat{\mathbf{x}}+y_{6} a \hat{\mathbf{y}}+\left(\frac{1}{2}-z_{6}\right) a \hat{\mathbf{z}}$ & $(24 d)$ & Al II \\
\hline $\mathbf{B}_{63}$ & $=$ & $x_{6} \mathbf{a}_{1}+\left(\frac{1}{2}-y_{6}\right) \mathbf{a}_{2}+\left(\frac{1}{2}+z_{6}\right) \mathbf{a}_{3}$ & $=$ & $x_{6} a \hat{\mathbf{x}}+\left(\frac{1}{2}-y_{6}\right) a \hat{\mathbf{y}}+\left(\frac{1}{2}+z_{6}\right) a \hat{\mathbf{z}}$ & $(24 d)$ & Al II \\
\hline $\mathbf{B}_{64}$ & $=$ & $\left(\frac{1}{2}-x_{6}\right) \mathbf{a}_{1}+\left(\frac{1}{2}+y_{6}\right) \mathbf{a}_{2}+z_{6} \mathbf{a}_{3}$ & $=$ & $\left(\frac{1}{2}-x_{6}\right) a \hat{\mathbf{x}}+\left(\frac{1}{2}+y_{6}\right) a \hat{\mathbf{y}}+z_{6} a \hat{\mathbf{z}}$ & $(24 d)$ & Al II \\
\hline $\mathbf{B}_{65}$ & $=$ & $-z_{6} \mathbf{a}_{1}-x_{6} \mathbf{a}_{2}-y_{6} \mathbf{a}_{3}$ & $=$ & $-z_{6} a \hat{\mathbf{x}}-x_{6} a \hat{\mathbf{y}}-y_{6} a \hat{\mathbf{z}}$ & $(24 d)$ & Al II \\
\hline $\mathbf{B}_{66}$ & $=$ & $\left(\frac{1}{2}-z_{6}\right) \mathbf{a}_{1}+\left(\frac{1}{2}+x_{6}\right) \mathbf{a}_{2}+y_{6} \mathbf{a}_{3}$ & $=$ & $\left(\frac{1}{2}-z_{6}\right) a \hat{\mathbf{x}}+\left(\frac{1}{2}+x_{6}\right) a \hat{\mathbf{y}}+y_{6} a \hat{\mathbf{z}}$ & $(24 d)$ & Al II \\
\hline $\mathbf{B}_{67}$ & $=$ & $\left(\frac{1}{2}+z_{6}\right) \mathbf{a}_{1}+x_{6} \mathbf{a}_{2}+\left(\frac{1}{2}-y_{6}\right) \mathbf{a}_{3}$ & $=$ & $\left(\frac{1}{2}+z_{6}\right) a \hat{\mathbf{x}}+x_{6} a \hat{\mathbf{y}}+\left(\frac{1}{2}-y_{6}\right) a \hat{\mathbf{z}}$ & $(24 d)$ & Al II \\
\hline $\mathbf{B}_{68}$ & $=$ & $z_{6} \mathbf{a}_{1}+\left(\frac{1}{2}-x_{6}\right) \mathbf{a}_{2}+\left(\frac{1}{2}+y_{6}\right) \mathbf{a}_{3}$ & $=$ & $z_{6} a \hat{\mathbf{x}}+\left(\frac{1}{2}-x_{6}\right) a \hat{\mathbf{y}}+\left(\frac{1}{2}+y_{6}\right) a \hat{\mathbf{z}}$ & $(24 d)$ & 月11 \\
\hline $\mathbf{B}_{69}$ & $=$ & $-y_{6} \mathbf{a}_{1}-z_{6} \mathbf{a}_{2}-x_{6} \mathbf{a}_{3}$ & $=$ & $-y_{6} a \hat{\mathbf{x}}-z_{6} a \hat{\mathbf{y}}-x_{6} a \hat{\mathbf{z}}$ & $(24 d)$ & $\mathrm{Al} \mathrm{II}$ \\
\hline $\mathbf{B}_{70}$ & $=$ & $y_{6} \mathbf{a}_{1}+\left(\frac{1}{2}-z_{6}\right) \mathbf{a}_{2}+\left(\frac{1}{2}+x_{6}\right) \mathbf{a}_{3}$ & $=$ & $y_{6} a \hat{\mathbf{x}}+\left(\frac{1}{2}-z_{6}\right) a \hat{\mathbf{y}}+\left(\frac{1}{2}+x_{6}\right) a \hat{\mathbf{z}}$ & $(24 d)$ & $\mathrm{Al}$ \\
\hline $\mathbf{B}_{71}$ & $=$ & $\left(\frac{1}{2}-y_{6}\right) \mathbf{a}_{1}+\left(\frac{1}{2}+z_{6}\right) \mathbf{a}_{2}+x_{6} \mathbf{a}_{3}$ & $=$ & $\left(\frac{1}{2}-y_{6}\right) a \hat{\mathbf{x}}+\left(\frac{1}{2}+z_{6}\right) a \hat{\mathbf{y}}+x_{6} a \hat{\mathbf{z}}$ & $(24 d)$ & $\mathrm{Al}$ \\
\hline
\end{tabular}




\begin{tabular}{|c|c|c|c|c|c|c|}
\hline $\mathbf{B}_{72}$ & $=$ & $\left(\frac{1}{2}+y_{6}\right) \mathbf{a}_{1}+z_{6} \mathbf{a}_{2}+\left(\frac{1}{2}-x_{6}\right) \mathbf{a}_{3}$ & $=$ & $\left(\frac{1}{2}+y_{6}\right) a \hat{\mathbf{x}}+z_{6} a \hat{\mathbf{y}}+\left(\frac{1}{2}-x_{6}\right) a \hat{\mathbf{z}}$ & $(24 d)$ & Al II \\
\hline $\mathbf{B}_{73}$ & $=$ & $x_{7} \mathbf{a}_{1}+y_{7} \mathbf{a}_{2}+z_{7} \mathbf{a}_{3}$ & $=$ & $x_{7} a \hat{\mathbf{x}}+y_{7} a \hat{\mathbf{y}}+z_{7} a \hat{\mathbf{z}}$ & $(24 d)$ & $\mathrm{Ca} \mathrm{V}$ \\
\hline $\mathbf{B}_{74}$ & $=$ & $\left(\frac{1}{2}-x_{7}\right) \mathbf{a}_{1}-y_{7} \mathbf{a}_{2}+\left(\frac{1}{2}+z_{7}\right) \mathbf{a}_{3}$ & $=$ & $\left(\frac{1}{2}-x_{7}\right) a \hat{\mathbf{x}}-y_{7} a \hat{\mathbf{y}}+\left(\frac{1}{2}+z_{7}\right) a \hat{\mathbf{z}}$ & $(24 d)$ & $\mathrm{Ca} \mathrm{V}$ \\
\hline $\mathbf{B}_{75}$ & $=$ & $-x_{7} \mathbf{a}_{1}+\left(\frac{1}{2}+y_{7}\right) \mathbf{a}_{2}+\left(\frac{1}{2}-z_{7}\right) \mathbf{a}_{3}$ & $=$ & $-x_{7} a \hat{\mathbf{x}}+\left(\frac{1}{2}+y_{7}\right) a \hat{\mathbf{y}}+\left(\frac{1}{2}-z_{7}\right) a \hat{\mathbf{z}}$ & $(24 d)$ & $\mathrm{Ca} \mathrm{V}$ \\
\hline $\mathbf{B}_{76}$ & $=$ & $\left(\frac{1}{2}+x_{7}\right) \mathbf{a}_{1}+\left(\frac{1}{2}-y_{7}\right) \mathbf{a}_{2}-z_{7} \mathbf{a}_{3}$ & $=$ & $\left(\frac{1}{2}+x_{7}\right) a \hat{\mathbf{x}}+\left(\frac{1}{2}-y_{7}\right) a \hat{\mathbf{y}}-z_{7} a \hat{\mathbf{z}}$ & $(24 d)$ & $\mathrm{Ca} \mathrm{V}$ \\
\hline $\mathbf{B}_{77}$ & $=$ & $z_{7} \mathbf{a}_{1}+x_{7} \mathbf{a}_{2}+y_{7} \mathbf{a}_{3}$ & $=$ & $z_{7} a \hat{\mathbf{x}}+x_{7} a \hat{\mathbf{y}}+y_{7} a \hat{\mathbf{z}}$ & $(24 d)$ & $\mathrm{Ca} \mathrm{V}$ \\
\hline $\mathbf{B}_{78}$ & $=$ & $\left(\frac{1}{2}+z_{7}\right) \mathbf{a}_{1}+\left(\frac{1}{2}-x_{7}\right) \mathbf{a}_{2}-y_{7} \mathbf{a}_{3}$ & $=$ & $\left(\frac{1}{2}+z_{7}\right) a \hat{\mathbf{x}}+\left(\frac{1}{2}-x_{7}\right) a \hat{\mathbf{y}}-y_{7} a \hat{\mathbf{z}}$ & $(24 d)$ & $\mathrm{Ca} \mathrm{V}$ \\
\hline $\mathbf{B}_{79}$ & $=$ & $\left(\frac{1}{2}-z_{7}\right) \mathbf{a}_{1}-x_{7} \mathbf{a}_{2}+\left(\frac{1}{2}+y_{7}\right) \mathbf{a}_{3}$ & $=$ & $\left(\frac{1}{2}-z_{7}\right) a \hat{\mathbf{x}}-x_{7} a \hat{\mathbf{y}}+\left(\frac{1}{2}+y_{7}\right) a \hat{\mathbf{z}}$ & $(24 d)$ & $\mathrm{Ca} \mathrm{V}$ \\
\hline $\mathbf{B}_{80}$ & $=$ & $-z_{7} \mathbf{a}_{1}+\left(\frac{1}{2}+x_{7}\right) \mathbf{a}_{2}+\left(\frac{1}{2}-y_{7}\right) \mathbf{a}_{3}$ & $=$ & $-z_{7} a \hat{\mathbf{x}}+\left(\frac{1}{2}+x_{7}\right) a \hat{\mathbf{y}}+\left(\frac{1}{2}-y_{7}\right) a \hat{\mathbf{z}}$ & $(24 d)$ & $\mathrm{Ca} \mathrm{V}$ \\
\hline $\mathbf{B}_{81}$ & $=$ & $y_{7} \mathbf{a}_{1}+z_{7} \mathbf{a}_{2}+x_{7} \mathbf{a}_{3}$ & $=$ & $y_{7} a \hat{\mathbf{x}}+z_{7} a \hat{\mathbf{y}}+x_{7} a \hat{\mathbf{z}}$ & $(24 d)$ & $\mathrm{Ca} \mathrm{V}$ \\
\hline $\mathbf{B}_{82}$ & $=$ & $-y_{7} \mathbf{a}_{1}+\left(\frac{1}{2}+z_{7}\right) \mathbf{a}_{2}+\left(\frac{1}{2}-x_{7}\right) \mathbf{a}_{3}$ & $=$ & $-y_{7} a \hat{\mathbf{x}}+\left(\frac{1}{2}+z_{7}\right) a \hat{\mathbf{y}}+\left(\frac{1}{2}-x_{7}\right) a \hat{\mathbf{z}}$ & $(24 d)$ & $\mathrm{Ca} \mathrm{V}$ \\
\hline $\mathbf{B}_{83}$ & $=$ & $\left(\frac{1}{2}+y_{7}\right) \mathbf{a}_{1}+\left(\frac{1}{2}-z_{7}\right) \mathbf{a}_{2}-x_{7} \mathbf{a}_{3}$ & $=$ & $\left(\frac{1}{2}+y_{7}\right) a \hat{\mathbf{x}}+\left(\frac{1}{2}-z_{7}\right) a \hat{\mathbf{y}}-x_{7} a \hat{\mathbf{z}}$ & $(24 d)$ & $\mathrm{Ca} \mathrm{V}$ \\
\hline $\mathbf{B}_{84}$ & $=$ & $\left(\frac{1}{2}-y_{7}\right) \mathbf{a}_{1}-z_{7} \mathbf{a}_{2}+\left(\frac{1}{2}+x_{7}\right) \mathbf{a}_{3}$ & $=$ & $\left(\frac{1}{2}-y_{7}\right) a \hat{\mathbf{x}}-z_{7} a \hat{\mathbf{y}}+\left(\frac{1}{2}+x_{7}\right) a \hat{\mathbf{z}}$ & $(24 d)$ & $\mathrm{Ca} \mathrm{V}$ \\
\hline $\mathbf{B}_{85}$ & $=$ & $-x_{7} \mathbf{a}_{1}-y_{7} \mathbf{a}_{2}-z_{7} \mathbf{a}_{3}$ & $=$ & $-x_{7} a \hat{\mathbf{x}}-y_{7} a \hat{\mathbf{y}}-z_{7} a \hat{\mathbf{z}}$ & $(24 d)$ & $\mathrm{Ca} \mathrm{V}$ \\
\hline $\mathbf{B}_{86}$ & $=$ & $\left(\frac{1}{2}+x_{7}\right) \mathbf{a}_{1}+y_{7} \mathbf{a}_{2}+\left(\frac{1}{2}-z_{7}\right) \mathbf{a}_{3}$ & $=$ & $\left(\frac{1}{2}+x_{7}\right) a \hat{\mathbf{x}}+y_{7} a \hat{\mathbf{y}}+\left(\frac{1}{2}-z_{7}\right) a \hat{\mathbf{z}}$ & $(24 d)$ & $\mathrm{Ca} \mathrm{V}$ \\
\hline $\mathbf{B}_{87}$ & $=$ & $x_{7} \mathbf{a}_{1}+\left(\frac{1}{2}-y_{7}\right) \mathbf{a}_{2}+\left(\frac{1}{2}+z_{7}\right) \mathbf{a}_{3}$ & $=$ & $x_{7} a \hat{\mathbf{x}}+\left(\frac{1}{2}-y_{7}\right) a \hat{\mathbf{y}}+\left(\frac{1}{2}+z_{7}\right) a \hat{\mathbf{z}}$ & $(24 d)$ & $\mathrm{Ca} \mathrm{V}$ \\
\hline $\mathbf{B}_{88}$ & $=$ & $\left(\frac{1}{2}-x_{7}\right) \mathbf{a}_{1}+\left(\frac{1}{2}+y_{7}\right) \mathbf{a}_{2}+z_{7} \mathbf{a}_{3}$ & $=$ & $\left(\frac{1}{2}-x_{7}\right) a \hat{\mathbf{x}}+\left(\frac{1}{2}+y_{7}\right) a \hat{\mathbf{y}}+z_{7} a \hat{\mathbf{z}}$ & $(24 d)$ & $\mathrm{Ca} \mathrm{V}$ \\
\hline $\mathbf{B}_{89}$ & $=$ & $-z_{7} \mathbf{a}_{1}-x_{7} \mathbf{a}_{2}-y_{7} \mathbf{a}_{3}$ & $=$ & $-z_{7} a \hat{\mathbf{x}}-x_{7} a \hat{\mathbf{y}}-y_{7} a \hat{\mathbf{z}}$ & $(24 d)$ & $\mathrm{Ca} \mathrm{V}$ \\
\hline $\mathbf{B}_{90}$ & $=$ & $\left(\frac{1}{2}-z_{7}\right) \mathbf{a}_{1}+\left(\frac{1}{2}+x_{7}\right) \mathbf{a}_{2}+y_{7} \mathbf{a}_{3}$ & $=$ & $\left(\frac{1}{2}-z_{7}\right) a \hat{\mathbf{x}}+\left(\frac{1}{2}+x_{7}\right) a \hat{\mathbf{y}}+y_{7} a \hat{\mathbf{z}}$ & $(24 d)$ & $\mathrm{Ca} \mathrm{V}$ \\
\hline $\mathbf{B}_{91}$ & $=$ & $\left(\frac{1}{2}+z_{7}\right) \mathbf{a}_{1}+x_{7} \mathbf{a}_{2}+\left(\frac{1}{2}-y_{7}\right) \mathbf{a}_{3}$ & $=$ & $\left(\frac{1}{2}+z_{7}\right) a \hat{\mathbf{x}}+x_{7} a \hat{\mathbf{y}}+\left(\frac{1}{2}-y_{7}\right) a \hat{\mathbf{z}}$ & $(24 d)$ & $\mathrm{Ca} \mathrm{V}$ \\
\hline $\mathbf{B}_{92}$ & $=$ & $z_{7} \mathbf{a}_{1}+\left(\frac{1}{2}-x_{7}\right) \mathbf{a}_{2}+\left(\frac{1}{2}+y_{7}\right) \mathbf{a}_{3}$ & $=$ & $z_{7} a \hat{\mathbf{x}}+\left(\frac{1}{2}-x_{7}\right) a \hat{\mathbf{y}}+\left(\frac{1}{2}+y_{7}\right) a \hat{\mathbf{z}}$ & $(24 d)$ & $\mathrm{Ca} \mathrm{V}$ \\
\hline $\mathbf{B}_{93}$ & $=$ & $-y_{7} \mathbf{a}_{1}-z_{7} \mathbf{a}_{2}-x_{7} \mathbf{a}_{3}$ & $=$ & $-y_{7} a \hat{\mathbf{x}}-z_{7} a \hat{\mathbf{y}}-x_{7} a \hat{\mathbf{z}}$ & $(24 d)$ & $\mathrm{Ca} \mathrm{V}$ \\
\hline $\mathbf{B}_{94}$ & $=$ & $y_{7} \mathbf{a}_{1}+\left(\frac{1}{2}-z_{7}\right) \mathbf{a}_{2}+\left(\frac{1}{2}+x_{7}\right) \mathbf{a}_{3}$ & $=$ & $y_{7} a \hat{\mathbf{x}}+\left(\frac{1}{2}-z_{7}\right) a \hat{\mathbf{y}}+\left(\frac{1}{2}+x_{7}\right) a \hat{\mathbf{z}}$ & $(24 d)$ & $\mathrm{Ca} \mathrm{V}$ \\
\hline $\mathbf{B}_{95}$ & $=$ & $\left(\frac{1}{2}-y_{7}\right) \mathbf{a}_{1}+\left(\frac{1}{2}+z_{7}\right) \mathbf{a}_{2}+x_{7} \mathbf{a}_{3}$ & $=$ & $\left(\frac{1}{2}-y_{7}\right) a \hat{\mathbf{x}}+\left(\frac{1}{2}+z_{7}\right) a \hat{\mathbf{y}}+x_{7} a \hat{\mathbf{z}}$ & $(24 d)$ & $\mathrm{Ca} \mathrm{V}$ \\
\hline $\mathbf{B}_{96}$ & $=$ & $\left(\frac{1}{2}+y_{7}\right) \mathbf{a}_{1}+z_{7} \mathbf{a}_{2}+\left(\frac{1}{2}-x_{7}\right) \mathbf{a}_{3}$ & $=$ & $\left(\frac{1}{2}+y_{7}\right) a \hat{\mathbf{x}}+z_{7} a \hat{\mathbf{y}}+\left(\frac{1}{2}-x_{7}\right) a \hat{\mathbf{z}}$ & $(24 d)$ & $\mathrm{Ca} \mathrm{V}$ \\
\hline $\mathbf{B}_{97}$ & $=$ & $x_{8} \mathbf{a}_{1}+y_{8} \mathbf{a}_{2}+z_{8} \mathbf{a}_{3}$ & $=$ & $x_{8} a \hat{\mathbf{x}}+y_{8} a \hat{\mathbf{y}}+z_{8} a \hat{\mathbf{z}}$ & $(24 d)$ & $\mathrm{Ca} \mathrm{VI}$ \\
\hline $\mathbf{B}_{98}$ & $=$ & $\left(\frac{1}{2}-x_{8}\right) \mathbf{a}_{1}-y_{8} \mathbf{a}_{2}+\left(\frac{1}{2}+z_{8}\right) \mathbf{a}_{3}$ & $=$ & $\left(\frac{1}{2}-x_{8}\right) a \hat{\mathbf{x}}-y_{8} a \hat{\mathbf{y}}+\left(\frac{1}{2}+z_{8}\right) a \hat{\mathbf{z}}$ & $(24 d)$ & $\mathrm{Ca} \mathrm{VI}$ \\
\hline $\mathbf{B}_{99}$ & $=$ & $-x_{8} \mathbf{a}_{1}+\left(\frac{1}{2}+y_{8}\right) \mathbf{a}_{2}+\left(\frac{1}{2}-z_{8}\right) \mathbf{a}_{3}$ & $=$ & $-x_{8} a \hat{\mathbf{x}}+\left(\frac{1}{2}+y_{8}\right) a \hat{\mathbf{y}}+\left(\frac{1}{2}-z_{8}\right) a \hat{\mathbf{z}}$ & $(24 d)$ & $\mathrm{Ca} \mathrm{VI}$ \\
\hline $\mathbf{B}_{100}$ & $=$ & $\left(\frac{1}{2}+x_{8}\right) \mathbf{a}_{1}+\left(\frac{1}{2}-y_{8}\right) \mathbf{a}_{2}-z_{8} \mathbf{a}_{3}$ & $=$ & $\left(\frac{1}{2}+x_{8}\right) a \hat{\mathbf{x}}+\left(\frac{1}{2}-y_{8}\right) a \hat{\mathbf{y}}-z_{8} a \hat{\mathbf{z}}$ & $(24 d)$ & $\mathrm{Ca} \mathrm{VI}$ \\
\hline $\mathbf{B}_{101}$ & $=$ & $z_{8} \mathbf{a}_{1}+x_{8} \mathbf{a}_{2}+y_{8} \mathbf{a}_{3}$ & $=$ & $z_{8} a \hat{\mathbf{x}}+x_{8} a \hat{\mathbf{y}}+y_{8} a \hat{\mathbf{z}}$ & $(24 d)$ & $\mathrm{Ca} \mathrm{VI}$ \\
\hline $\mathbf{B}_{102}$ & $=$ & $\left(\frac{1}{2}+z_{8}\right) \mathbf{a}_{1}+\left(\frac{1}{2}-x_{8}\right) \mathbf{a}_{2}-y_{8} \mathbf{a}_{3}$ & $=$ & $\left(\frac{1}{2}+z_{8}\right) a \hat{\mathbf{x}}+\left(\frac{1}{2}-x_{8}\right) a \hat{\mathbf{y}}-y_{8} a \hat{\mathbf{z}}$ & $(24 d)$ & $\mathrm{Ca} \mathrm{VI}$ \\
\hline $\mathbf{B}_{103}$ & $=$ & $\left(\frac{1}{2}-z_{8}\right) \mathbf{a}_{1}-x_{8} \mathbf{a}_{2}+\left(\frac{1}{2}+y_{8}\right) \mathbf{a}_{3}$ & $=$ & $\left(\frac{1}{2}-z_{8}\right) a \hat{\mathbf{x}}-x_{8} a \hat{\mathbf{y}}+\left(\frac{1}{2}+y_{8}\right) a \hat{\mathbf{z}}$ & $(24 d)$ & $\mathrm{Ca} \mathrm{VI}$ \\
\hline $\mathbf{B}_{104}$ & $=$ & $-z_{8} \mathbf{a}_{1}+\left(\frac{1}{2}+x_{8}\right) \mathbf{a}_{2}+\left(\frac{1}{2}-y_{8}\right) \mathbf{a}_{3}$ & $=$ & $-z_{8} a \hat{\mathbf{x}}+\left(\frac{1}{2}+x_{8}\right) a \hat{\mathbf{y}}+\left(\frac{1}{2}-y_{8}\right) a \hat{\mathbf{z}}$ & $(24 d)$ & $\mathrm{Ca} \mathrm{VI}$ \\
\hline $\mathbf{B}_{105}$ & $=$ & $y_{8} \mathbf{a}_{1}+z_{8} \mathbf{a}_{2}+x_{8} \mathbf{a}_{3}$ & $=$ & $y_{8} a \hat{\mathbf{x}}+z_{8} a \hat{\mathbf{y}}+x_{8} a \hat{\mathbf{z}}$ & $(24 d)$ & $\mathrm{Ca} \mathrm{VI}$ \\
\hline $\mathbf{B}_{106}$ & $=$ & $-y_{8} \mathbf{a}_{1}+\left(\frac{1}{2}+z_{8}\right) \mathbf{a}_{2}+\left(\frac{1}{2}-x_{8}\right) \mathbf{a}_{3}$ & $=$ & $-y_{8} a \hat{\mathbf{x}}+\left(\frac{1}{2}+z_{8}\right) a \hat{\mathbf{y}}+\left(\frac{1}{2}-x_{8}\right) a \hat{\mathbf{z}}$ & $(24 d)$ & $\mathrm{Ca} \mathrm{VI}$ \\
\hline 107 & $=$ & $\left(\frac{1}{2}+y_{8}\right) \mathbf{a}_{1}+\left(\frac{1}{2}-z_{8}\right) \mathbf{a}_{2}-x_{8} \mathbf{a}_{3}$ & $=$ & $\left(\frac{1}{2}+y_{8}\right) a \hat{\mathbf{x}}+\left(\frac{1}{2}-z_{8}\right) a \hat{\mathbf{y}}-x_{8} a \hat{\mathbf{z}}$ & $(24 d)$ & $\mathrm{Ca} \mathrm{VI}$ \\
\hline
\end{tabular}




\begin{tabular}{|c|c|c|c|c|c|c|}
\hline $\mathbf{B}_{108}$ & $=$ & $\left(\frac{1}{2}-y_{8}\right) \mathbf{a}_{1}-z_{8} \mathbf{a}_{2}+\left(\frac{1}{2}+x_{8}\right) \mathbf{a}_{3}$ & $=$ & $\left(\frac{1}{2}-y_{8}\right) a \hat{\mathbf{x}}-z_{8} a \hat{\mathbf{y}}+\left(\frac{1}{2}+x_{8}\right) a \hat{\mathbf{z}}$ & $(24 d)$ & $\mathrm{Ca} \mathrm{VI}$ \\
\hline $\mathbf{B}_{109}$ & $=$ & $-x_{8} \mathbf{a}_{1}-y_{8} \mathbf{a}_{2}-z_{8} \mathbf{a}_{3}$ & $=$ & $-x_{8} a \hat{\mathbf{x}}-y_{8} a \hat{\mathbf{y}}-z_{8} a \hat{\mathbf{z}}$ & $(24 d)$ & $\mathrm{Ca} \mathrm{VI}$ \\
\hline $\mathbf{B}_{110}$ & $=$ & $\left(\frac{1}{2}+x_{8}\right) \mathbf{a}_{1}+y_{8} \mathbf{a}_{2}+\left(\frac{1}{2}-z_{8}\right) \mathbf{a}_{3}$ & $=$ & $\left(\frac{1}{2}+x_{8}\right) a \hat{\mathbf{x}}+y_{8} a \hat{\mathbf{y}}+\left(\frac{1}{2}-z_{8}\right) a \hat{\mathbf{z}}$ & $(24 d)$ & $\mathrm{Ca} \mathrm{VI}$ \\
\hline $\mathbf{B}_{111}$ & $=$ & $x_{8} \mathbf{a}_{1}+\left(\frac{1}{2}-y_{8}\right) \mathbf{a}_{2}+\left(\frac{1}{2}+z_{8}\right) \mathbf{a}_{3}$ & $=$ & $x_{8} a \hat{\mathbf{x}}+\left(\frac{1}{2}-y_{8}\right) a \hat{\mathbf{y}}+\left(\frac{1}{2}+z_{8}\right) a \hat{\mathbf{z}}$ & $(24 d)$ & $\mathrm{Ca} \mathrm{VI}$ \\
\hline $\mathbf{B}_{112}$ & $=$ & $\left(\frac{1}{2}-x_{8}\right) \mathbf{a}_{1}+\left(\frac{1}{2}+y_{8}\right) \mathbf{a}_{2}+z_{8} \mathbf{a}_{3}$ & $=$ & $\left(\frac{1}{2}-x_{8}\right) a \hat{\mathbf{x}}+\left(\frac{1}{2}+y_{8}\right) a \hat{\mathbf{y}}+z_{8} a \hat{\mathbf{z}}$ & $(24 d)$ & $\mathrm{Ca} \mathrm{VI}$ \\
\hline $\mathbf{B}_{113}$ & $=$ & $-z_{8} \mathbf{a}_{1}-x_{8} \mathbf{a}_{2}-y_{8} \mathbf{a}_{3}$ & $=$ & $-z_{8} a \hat{\mathbf{x}}-x_{8} a \hat{\mathbf{y}}-y_{8} a \hat{\mathbf{z}}$ & $(24 d)$ & $\mathrm{Ca} \mathrm{VI}$ \\
\hline $\mathbf{B}_{114}$ & $=$ & $\left(\frac{1}{2}-z_{8}\right) \mathbf{a}_{1}+\left(\frac{1}{2}+x_{8}\right) \mathbf{a}_{2}+y_{8} \mathbf{a}_{3}$ & $=$ & $\left(\frac{1}{2}-z_{8}\right) a \hat{\mathbf{x}}+\left(\frac{1}{2}+x_{8}\right) a \hat{\mathbf{y}}+y_{8} a \hat{\mathbf{z}}$ & $(24 d)$ & $\mathrm{Ca} \mathrm{VI}$ \\
\hline $\mathbf{B}_{115}$ & $=$ & $\left(\frac{1}{2}+z_{8}\right) \mathbf{a}_{1}+x_{8} \mathbf{a}_{2}+\left(\frac{1}{2}-y_{8}\right) \mathbf{a}_{3}$ & $=$ & $\left(\frac{1}{2}+z_{8}\right) a \hat{\mathbf{x}}+x_{8} a \hat{\mathbf{y}}+\left(\frac{1}{2}-y_{8}\right) a \hat{\mathbf{z}}$ & $(24 d)$ & $\mathrm{Ca}$ VI \\
\hline $\mathbf{B}_{116}$ & $=$ & $z_{8} \mathbf{a}_{1}+\left(\frac{1}{2}-x_{8}\right) \mathbf{a}_{2}+\left(\frac{1}{2}+y_{8}\right) \mathbf{a}_{3}$ & $=$ & $z_{8} a \hat{\mathbf{x}}+\left(\frac{1}{2}-x_{8}\right) a \hat{\mathbf{y}}+\left(\frac{1}{2}+y_{8}\right) a \hat{\mathbf{z}}$ & $(24 d)$ & $\mathrm{Ca} \mathrm{VI}$ \\
\hline $\mathbf{B}_{117}$ & $=$ & $-y_{8} \mathbf{a}_{1}-z_{8} \mathbf{a}_{2}-x_{8} \mathbf{a}_{3}$ & $=$ & $-y_{8} a \hat{\mathbf{x}}-z_{8} a \hat{\mathbf{y}}-x_{8} a \hat{\mathbf{z}}$ & $(24 d)$ & $\mathrm{Ca} \mathrm{VI}$ \\
\hline $\mathbf{B}_{118}$ & $=$ & $y_{8} \mathbf{a}_{1}+\left(\frac{1}{2}-z_{8}\right) \mathbf{a}_{2}+\left(\frac{1}{2}+x_{8}\right) \mathbf{a}_{3}$ & $=$ & $y_{8} a \hat{\mathbf{x}}+\left(\frac{1}{2}-z_{8}\right) a \hat{\mathbf{y}}+\left(\frac{1}{2}+x_{8}\right) a \hat{\mathbf{z}}$ & $(24 d)$ & $\mathrm{Ca} \mathrm{VI}$ \\
\hline $\mathbf{B}_{119}$ & $=$ & $\left(\frac{1}{2}-y_{8}\right) \mathbf{a}_{1}+\left(\frac{1}{2}+z_{8}\right) \mathbf{a}_{2}+x_{8} \mathbf{a}_{3}$ & $=$ & $\left(\frac{1}{2}-y_{8}\right) a \hat{\mathbf{x}}+\left(\frac{1}{2}+z_{8}\right) a \hat{\mathbf{y}}+x_{8} a \hat{\mathbf{z}}$ & $(24 d)$ & $\mathrm{Ca} \mathrm{VI}$ \\
\hline $\mathbf{B}_{120}$ & $=$ & $\left(\frac{1}{2}+y_{8}\right) \mathbf{a}_{1}+z_{8} \mathbf{a}_{2}+\left(\frac{1}{2}-x_{8}\right) \mathbf{a}_{3}$ & $=$ & $\left(\frac{1}{2}+y_{8}\right) a \hat{\mathbf{x}}+z_{8} a \hat{\mathbf{y}}+\left(\frac{1}{2}-x_{8}\right) a \hat{\mathbf{z}}$ & $(24 d)$ & $\mathrm{Ca} \mathrm{VI}$ \\
\hline $\mathbf{B}_{121}$ & $=$ & $x_{9} \mathbf{a}_{1}+y_{9} \mathbf{a}_{2}+z_{9} \mathbf{a}_{3}$ & $=$ & $x_{9} a \hat{\mathbf{x}}+y_{9} a \hat{\mathbf{y}}+z_{9} a \hat{\mathbf{z}}$ & $(24 d)$ & O I \\
\hline $\mathbf{B}_{122}$ & $=$ & $\left(\frac{1}{2}-x_{9}\right) \mathbf{a}_{1}-y_{9} \mathbf{a}_{2}+\left(\frac{1}{2}+z_{9}\right) \mathbf{a}_{3}$ & $=$ & $\left(\frac{1}{2}-x_{9}\right) a \hat{\mathbf{x}}-y_{9} a \hat{\mathbf{y}}+\left(\frac{1}{2}+z_{9}\right) a \hat{\mathbf{z}}$ & $(24 d)$ & O I \\
\hline $\mathbf{B}_{123}$ & $=$ & $-x_{9} \mathbf{a}_{1}+\left(\frac{1}{2}+y_{9}\right) \mathbf{a}_{2}+\left(\frac{1}{2}-z_{9}\right) \mathbf{a}_{3}$ & $=$ & $-x_{9} a \hat{\mathbf{x}}+\left(\frac{1}{2}+y_{9}\right) a \hat{\mathbf{y}}+\left(\frac{1}{2}-z_{9}\right) a \hat{\mathbf{z}}$ & $(24 d)$ & O I \\
\hline $\mathbf{B}_{124}$ & $=$ & $\left(\frac{1}{2}+x_{9}\right) \mathbf{a}_{1}+\left(\frac{1}{2}-y_{9}\right) \mathbf{a}_{2}-z_{9} \mathbf{a}_{3}$ & $=$ & $\left(\frac{1}{2}+x_{9}\right) a \hat{\mathbf{x}}+\left(\frac{1}{2}-y_{9}\right) a \hat{\mathbf{y}}-z_{9} a \hat{\mathbf{z}}$ & $(24 d)$ & O I \\
\hline $\mathbf{B}_{125}$ & $=$ & $z_{9} \mathbf{a}_{1}+x_{9} \mathbf{a}_{2}+y_{9} \mathbf{a}_{3}$ & $=$ & $z_{9} a \hat{\mathbf{x}}+x_{9} a \hat{\mathbf{y}}+y_{9} a \hat{\mathbf{z}}$ & $(24 d)$ & O I \\
\hline $\mathbf{B}_{126}$ & $=$ & $\left(\frac{1}{2}+z_{9}\right) \mathbf{a}_{1}+\left(\frac{1}{2}-x_{9}\right) \mathbf{a}_{2}-y_{9} \mathbf{a}_{3}$ & $=$ & $\left(\frac{1}{2}+z_{9}\right) a \hat{\mathbf{x}}+\left(\frac{1}{2}-x_{9}\right) a \hat{\mathbf{y}}-y_{9} a \hat{\mathbf{z}}$ & $(24 d)$ & O I \\
\hline $\mathbf{B}_{127}$ & $=$ & $\left(\frac{1}{2}-z_{9}\right) \mathbf{a}_{1}-x_{9} \mathbf{a}_{2}+\left(\frac{1}{2}+y_{9}\right) \mathbf{a}_{3}$ & $=$ & $\left(\frac{1}{2}-z_{9}\right) a \hat{\mathbf{x}}-x_{9} a \hat{\mathbf{y}}+\left(\frac{1}{2}+y_{9}\right) a \hat{\mathbf{z}}$ & $(24 d)$ & O I \\
\hline $\mathbf{B}_{128}$ & $=$ & $-z_{9} \mathbf{a}_{1}+\left(\frac{1}{2}+x_{9}\right) \mathbf{a}_{2}+\left(\frac{1}{2}-y_{9}\right) \mathbf{a}_{3}$ & $=$ & $-z_{9} a \hat{\mathbf{x}}+\left(\frac{1}{2}+x_{9}\right) a \hat{\mathbf{y}}+\left(\frac{1}{2}-y_{9}\right) a \hat{\mathbf{z}}$ & $(24 d)$ & O I \\
\hline $\mathbf{B}_{129}$ & $=$ & $y_{9} \mathbf{a}_{1}+z_{9} \mathbf{a}_{2}+x_{9} \mathbf{a}_{3}$ & $=$ & $y_{9} a \hat{\mathbf{x}}+z_{9} a \hat{\mathbf{y}}+x_{9} a \hat{\mathbf{z}}$ & $(24 d)$ & O I \\
\hline $\mathbf{B}_{130}$ & $=$ & $-y_{9} \mathbf{a}_{1}+\left(\frac{1}{2}+z_{9}\right) \mathbf{a}_{2}+\left(\frac{1}{2}-x_{9}\right) \mathbf{a}_{3}$ & $=$ & $-y_{9} a \hat{\mathbf{x}}+\left(\frac{1}{2}+z_{9}\right) a \hat{\mathbf{y}}+\left(\frac{1}{2}-x_{9}\right) a \hat{\mathbf{z}}$ & $(24 d)$ & O I \\
\hline $\mathbf{B}_{131}$ & $=$ & $\left(\frac{1}{2}+y_{9}\right) \mathbf{a}_{1}+\left(\frac{1}{2}-z 9\right) \mathbf{a}_{2}-x_{9} \mathbf{a}_{3}$ & $=$ & $\left(\frac{1}{2}+y_{9}\right) a \hat{\mathbf{x}}+\left(\frac{1}{2}-z_{9}\right) a \hat{\mathbf{y}}-x_{9} a \hat{\mathbf{z}}$ & $(24 d)$ & O I \\
\hline $\mathbf{B}_{132}$ & $=$ & $\left(\frac{1}{2}-y_{9}\right) \mathbf{a}_{1}-z_{9} \mathbf{a}_{2}+\left(\frac{1}{2}+x_{9}\right) \mathbf{a}_{3}$ & $=$ & $\left(\frac{1}{2}-y_{9}\right) a \hat{\mathbf{x}}-z_{9} a \hat{\mathbf{y}}+\left(\frac{1}{2}+x_{9}\right) a \hat{\mathbf{z}}$ & $(24 d)$ & O I \\
\hline $\mathbf{B}_{133}$ & $=$ & $-x_{9} \mathbf{a}_{1}-y_{9} \mathbf{a}_{2}-z_{9} \mathbf{a}_{3}$ & $=$ & $-x_{9} a \hat{\mathbf{x}}-y_{9} a \hat{\mathbf{y}}-z_{9} a \hat{\mathbf{z}}$ & $(24 d)$ & O I \\
\hline $\mathbf{B}_{134}$ & $=$ & $\left(\frac{1}{2}+x_{9}\right) \mathbf{a}_{1}+y_{9} \mathbf{a}_{2}+\left(\frac{1}{2}-z_{9}\right) \mathbf{a}_{3}$ & $=$ & $\left(\frac{1}{2}+x_{9}\right) a \hat{\mathbf{x}}+y_{9} a \hat{\mathbf{y}}+\left(\frac{1}{2}-z_{9}\right) a \hat{\mathbf{z}}$ & $(24 d)$ & O I \\
\hline $\mathbf{B}_{135}$ & $=$ & $x_{9} \mathbf{a}_{1}+\left(\frac{1}{2}-y_{9}\right) \mathbf{a}_{2}+\left(\frac{1}{2}+z_{9}\right) \mathbf{a}_{3}$ & $=$ & $x_{9} a \hat{\mathbf{x}}+\left(\frac{1}{2}-y_{9}\right) a \hat{\mathbf{y}}+\left(\frac{1}{2}+z_{9}\right) a \hat{\mathbf{z}}$ & $(24 d)$ & O I \\
\hline $\mathbf{B}_{136}$ & $=$ & $\left(\frac{1}{2}-x_{9}\right) \mathbf{a}_{1}+\left(\frac{1}{2}+y_{9}\right) \mathbf{a}_{2}+z_{9} \mathbf{a}_{3}$ & $=$ & $\left(\frac{1}{2}-x_{9}\right) a \hat{\mathbf{x}}+\left(\frac{1}{2}+y_{9}\right) a \hat{\mathbf{y}}+z_{9} a \hat{\mathbf{z}}$ & $(24 d)$ & O I \\
\hline $\mathbf{B}_{137}$ & $=$ & $-z_{9} \mathbf{a}_{1}-x_{9} \mathbf{a}_{2}-y_{9} \mathbf{a}_{3}$ & $=$ & $-z_{9} a \hat{\mathbf{x}}-x_{9} a \hat{\mathbf{y}}-y_{9} a \hat{\mathbf{z}}$ & $(24 d)$ & O I \\
\hline $\mathbf{B}_{138}$ & $=$ & $\left(\frac{1}{2}-z_{9}\right) \mathbf{a}_{1}+\left(\frac{1}{2}+x_{9}\right) \mathbf{a}_{2}+y_{9} \mathbf{a}_{3}$ & $=$ & $\left(\frac{1}{2}-z_{9}\right) a \hat{\mathbf{x}}+\left(\frac{1}{2}+x_{9}\right) a \hat{\mathbf{y}}+y_{9} a \hat{\mathbf{z}}$ & $(24 d)$ & O I \\
\hline $\mathbf{B}_{139}$ & $=$ & $\left(\frac{1}{2}+z_{9}\right) \mathbf{a}_{1}+x_{9} \mathbf{a}_{2}+\left(\frac{1}{2}-y_{9}\right) \mathbf{a}_{3}$ & $=$ & $\left(\frac{1}{2}+z_{9}\right) a \hat{\mathbf{x}}+x_{9} a \hat{\mathbf{y}}+\left(\frac{1}{2}-y_{9}\right) a \hat{\mathbf{z}}$ & $(24 d)$ & O I \\
\hline $\mathbf{B}_{140}$ & $=$ & $z_{9} \mathbf{a}_{1}+\left(\frac{1}{2}-x_{9}\right) \mathbf{a}_{2}+\left(\frac{1}{2}+y_{9}\right) \mathbf{a}_{3}$ & $=$ & $z_{9} a \hat{\mathbf{x}}+\left(\frac{1}{2}-x_{9}\right) a \hat{\mathbf{y}}+\left(\frac{1}{2}+y_{9}\right) a \hat{\mathbf{z}}$ & $(24 d)$ & O I \\
\hline $\mathbf{B}_{141}$ & $=$ & $-y_{9} \mathbf{a}_{1}-z_{9} \mathbf{a}_{2}-x_{9} \mathbf{a}_{3}$ & $=$ & $-y_{9} a \hat{\mathbf{x}}-z_{9} a \hat{\mathbf{y}}-x_{9} a \hat{\mathbf{z}}$ & $(24 d)$ & O I \\
\hline $\mathbf{B}_{142}$ & $=$ & $y_{9} \mathbf{a}_{1}+\left(\frac{1}{2}-z_{9}\right) \mathbf{a}_{2}+\left(\frac{1}{2}+x_{9}\right) \mathbf{a}_{3}$ & $=$ & $y_{9} a \hat{\mathbf{x}}+\left(\frac{1}{2}-z_{9}\right) a \hat{\mathbf{y}}+\left(\frac{1}{2}+x_{9}\right) a \hat{\mathbf{z}}$ & $(24 d)$ & O I \\
\hline $\mathbf{B}_{143}$ & $=$ & $\left(\frac{1}{2}-y_{9}\right) \mathbf{a}_{1}+\left(\frac{1}{2}+z_{9}\right) \mathbf{a}_{2}+x_{9} \mathbf{a}_{3}$ & $=$ & $\left(\frac{1}{2}-y_{9}\right) a \hat{\mathbf{x}}+\left(\frac{1}{2}+z_{9}\right) a \hat{\mathbf{y}}+x_{9} a \hat{\mathbf{z}}$ & $(24 d)$ & O I \\
\hline
\end{tabular}




\begin{tabular}{|c|c|c|c|c|c|c|}
\hline $\mathbf{B}_{144}$ & $=$ & $\left(\frac{1}{2}+y_{9}\right) \mathbf{a}_{1}+z_{9} \mathbf{a}_{2}+\left(\frac{1}{2}-x_{9}\right) \mathbf{a}_{3}$ & $=$ & $\left(\frac{1}{2}+y_{9}\right) a \hat{\mathbf{x}}+z_{9} a \hat{\mathbf{y}}+\left(\frac{1}{2}-x_{9}\right) a \hat{\mathbf{z}}$ & $(24 d)$ & O I \\
\hline $\mathbf{B}_{145}$ & $=$ & $x_{10} \mathbf{a}_{1}+y_{10} \mathbf{a}_{2}+z_{10} \mathbf{a}_{3}$ & $=$ & $x_{10} a \hat{\mathbf{x}}+y_{10} a \hat{\mathbf{y}}+z_{10} a \hat{\mathbf{z}}$ & $(24 d)$ & O II \\
\hline $\mathbf{B}_{146}$ & $=$ & $\left(\frac{1}{2}-x_{10}\right) \mathbf{a}_{1}-y_{10} \mathbf{a}_{2}+\left(\frac{1}{2}+z_{10}\right) \mathbf{a}_{3}$ & $=$ & $\left(\frac{1}{2}-x_{10}\right) a \hat{\mathbf{x}}-y_{10} a \hat{\mathbf{y}}+\left(\frac{1}{2}+z_{10}\right) a \hat{\mathbf{z}}$ & $(24 d)$ & O II \\
\hline $\mathbf{B}_{147}$ & $=$ & $\begin{array}{c}-x_{10} \mathbf{a}_{1}+\left(\frac{1}{2}+y_{10}\right) \mathbf{a}_{2}+ \\
\left(\frac{1}{2}-z_{10}\right) \mathbf{a}_{3}\end{array}$ & $=$ & $\begin{array}{c}-x_{10} a \hat{\mathbf{x}}+\left(\frac{1}{2}+y_{10}\right) a \hat{\mathbf{y}}+ \\
\left(\frac{1}{2}-z_{10}\right) a \hat{\mathbf{z}}\end{array}$ & $(24 d)$ & O II \\
\hline $\mathbf{B}_{148}$ & $=$ & $\left(\frac{1}{2}+x_{10}\right) \mathbf{a}_{1}+\left(\frac{1}{2}-y_{10}\right) \mathbf{a}_{2}-z_{10} \mathbf{a}_{3}$ & $=$ & $\left(\frac{1}{2}+x_{10}\right) a \hat{\mathbf{x}}+\left(\frac{1}{2}-y_{10}\right) a \hat{\mathbf{y}}-z_{10} a \hat{\mathbf{z}}$ & $(24 d)$ & O II \\
\hline $\mathbf{B}_{149}$ & $=$ & $z_{10} \mathbf{a}_{1}+x_{10} \mathbf{a}_{2}+y_{10} \mathbf{a}_{3}$ & $=$ & $z_{10} a \hat{\mathbf{x}}+x_{10} a \hat{\mathbf{y}}+y_{10} a \hat{\mathbf{z}}$ & $(24 d)$ & O II \\
\hline $\mathbf{B}_{150}$ & $=$ & $\left(\frac{1}{2}+z_{10}\right) \mathbf{a}_{1}+\left(\frac{1}{2}-x_{10}\right) \mathbf{a}_{2}-y_{10} \mathbf{a}_{3}$ & $=$ & $\left(\frac{1}{2}+z_{10}\right) a \hat{\mathbf{x}}+\left(\frac{1}{2}-x_{10}\right) a \hat{\mathbf{y}}-y_{10} a \hat{\mathbf{z}}$ & $(24 d)$ & O II \\
\hline $\mathbf{B}_{151}$ & $=$ & $\left(\frac{1}{2}-z_{10}\right) \mathbf{a}_{1}-x_{10} \mathbf{a}_{2}+\left(\frac{1}{2}+y_{10}\right) \mathbf{a}_{3}$ & $=$ & $\left(\frac{1}{2}-z_{10}\right) a \hat{\mathbf{x}}-x_{10} a \hat{\mathbf{y}}+\left(\frac{1}{2}+y_{10}\right) a \hat{\mathbf{z}}$ & $(24 d)$ & O II \\
\hline $\mathbf{B}_{152}$ & $=$ & $\begin{array}{c}-z_{10} \mathbf{a}_{1}+\left(\frac{1}{2}+x_{10}\right) \mathbf{a}_{2}+ \\
\left(\frac{1}{2}-y_{10}\right) \mathbf{a}_{3}\end{array}$ & $=$ & $\begin{array}{c}-z_{10} a \hat{\mathbf{x}}+\left(\frac{1}{2}+x_{10}\right) a \hat{\mathbf{y}}+ \\
\left(\frac{1}{2}-y_{10}\right) a \hat{\mathbf{z}}\end{array}$ & $(24 d)$ & O II \\
\hline $\mathbf{B}_{153}$ & $=$ & $y_{10} \mathbf{a}_{1}+z_{10} \mathbf{a}_{2}+x_{10} \mathbf{a}_{3}$ & $=$ & $y_{10} a \hat{\mathbf{x}}+z_{10} a \hat{\mathbf{y}}+x_{10} a \hat{\mathbf{z}}$ & $(24 d)$ & O II \\
\hline $\mathbf{B}_{154}$ & $=$ & $\begin{array}{c}-y_{10} \mathbf{a}_{1}+\left(\frac{1}{2}+z_{10}\right) \mathbf{a}_{2}+ \\
\left(\frac{1}{2}-x_{10}\right) \mathbf{a}_{3}\end{array}$ & $=$ & $\begin{array}{c}-y_{10} a \hat{\mathbf{x}}+\left(\frac{1}{2}+z_{10}\right) a \hat{\mathbf{y}}+ \\
\left(\frac{1}{2}-x_{10}\right) a \hat{\mathbf{z}}\end{array}$ & $(24 d)$ & O II \\
\hline $\mathbf{B}_{155}$ & $=$ & $\left(\frac{1}{2}+y_{10}\right) \mathbf{a}_{1}+\left(\frac{1}{2}-z_{10}\right) \mathbf{a}_{2}-x_{10} \mathbf{a}_{3}$ & $=$ & $\left(\frac{1}{2}+y_{10}\right) a \hat{\mathbf{x}}+\left(\frac{1}{2}-z_{10}\right) a \hat{\mathbf{y}}-x_{10} a \hat{\mathbf{z}}$ & $(24 d)$ & O II \\
\hline $\mathbf{B}_{156}$ & $=$ & $\left(\frac{1}{2}-y_{10}\right) \mathbf{a}_{1}-z_{10} \mathbf{a}_{2}+\left(\frac{1}{2}+x_{10}\right) \mathbf{a}_{3}$ & $=$ & $\left(\frac{1}{2}-y_{10}\right) a \hat{\mathbf{x}}-z_{10} a \hat{\mathbf{y}}+\left(\frac{1}{2}+x_{10}\right) a \hat{\mathbf{z}}$ & $(24 d)$ & O II \\
\hline $\mathbf{B}_{157}$ & $=$ & $-x_{10} \mathbf{a}_{1}-y_{10} \mathbf{a}_{2}-z_{10} \mathbf{a}_{3}$ & $=$ & $-x_{10} a \hat{\mathbf{x}}-y_{10} a \hat{\mathbf{y}}-z_{10} a \hat{\mathbf{z}}$ & $(24 d)$ & O II \\
\hline $\mathbf{B}_{158}$ & $=$ & $\left(\frac{1}{2}+x_{10}\right) \mathbf{a}_{1}+y_{10} \mathbf{a}_{2}+\left(\frac{1}{2}-z_{10}\right) \mathbf{a}_{3}$ & $=$ & $\left(\frac{1}{2}+x_{10}\right) a \hat{\mathbf{x}}+y_{10} a \hat{\mathbf{y}}+\left(\frac{1}{2}-z_{10}\right) a \hat{\mathbf{z}}$ & $(24 d)$ & O II \\
\hline $\mathbf{B}_{159}$ & $=$ & $x_{10} \mathbf{a}_{1}+\left(\frac{1}{2}-y_{10}\right) \mathbf{a}_{2}+\left(\frac{1}{2}+z_{10}\right) \mathbf{a}_{3}$ & $=$ & $x_{10} a \hat{\mathbf{x}}+\left(\frac{1}{2}-y_{10}\right) a \hat{\mathbf{y}}+\left(\frac{1}{2}+z_{10}\right) a \hat{\mathbf{z}}$ & $(24 d)$ & O II \\
\hline $\mathbf{B}_{160}$ & $=$ & $\left(\frac{1}{2}-x_{10}\right) \mathbf{a}_{1}+\left(\frac{1}{2}+y_{10}\right) \mathbf{a}_{2}+z_{10} \mathbf{a}_{3}$ & $=$ & $\left(\frac{1}{2}-x_{10}\right) a \hat{\mathbf{x}}+\left(\frac{1}{2}+y_{10}\right) a \hat{\mathbf{y}}+z_{10} a \hat{\mathbf{z}}$ & $(24 d)$ & O II \\
\hline $\mathbf{B}_{161}$ & $=$ & $-z_{10} \mathbf{a}_{1}-x_{10} \mathbf{a}_{2}-y_{10} \mathbf{a}_{3}$ & $=$ & $-z_{10} a \hat{\mathbf{x}}-x_{10} a \hat{\mathbf{y}}-y_{10} a \hat{\mathbf{z}}$ & $(24 d)$ & O II \\
\hline $\mathbf{B}_{162}$ & $=$ & $\left(\frac{1}{2}-z_{10}\right) \mathbf{a}_{1}+\left(\frac{1}{2}+x_{10}\right) \mathbf{a}_{2}+y_{10} \mathbf{a}_{3}$ & $=$ & $\left(\frac{1}{2}-z_{10}\right) a \hat{\mathbf{x}}+\left(\frac{1}{2}+x_{10}\right) a \hat{\mathbf{y}}+y_{10} a \hat{\mathbf{z}}$ & $(24 d)$ & O II \\
\hline $\mathbf{B}_{163}$ & $=$ & $\left(\frac{1}{2}+z_{10}\right) \mathbf{a}_{1}+x_{10} \mathbf{a}_{2}+\left(\frac{1}{2}-y_{10}\right) \mathbf{a}_{3}$ & $=$ & $\left(\frac{1}{2}+z_{10}\right) a \hat{\mathbf{x}}+x_{10} a \hat{\mathbf{y}}+\left(\frac{1}{2}-y_{10}\right) a \hat{\mathbf{z}}$ & $(24 d)$ & O II \\
\hline $\mathbf{B}_{164}$ & $=$ & $z_{10} \mathbf{a}_{1}+\left(\frac{1}{2}-x_{10}\right) \mathbf{a}_{2}+\left(\frac{1}{2}+y_{10}\right) \mathbf{a}_{3}$ & $=$ & $z_{10} a \hat{\mathbf{x}}+\left(\frac{1}{2}-x_{10}\right) a \hat{\mathbf{y}}+\left(\frac{1}{2}+y_{10}\right) a \hat{\mathbf{z}}$ & $(24 d)$ & O II \\
\hline $\mathbf{B}_{165}$ & $=$ & $-y_{10} \mathbf{a}_{1}-z_{10} \mathbf{a}_{2}-x_{10} \mathbf{a}_{3}$ & $=$ & $-y_{10} a \hat{\mathbf{x}}-z_{10} a \hat{\mathbf{y}}-x_{10} a \hat{\mathbf{z}}$ & $(24 d)$ & O II \\
\hline $\mathbf{B}_{166}$ & $=$ & $y_{10} \mathbf{a}_{1}+\left(\frac{1}{2}-z_{10}\right) \mathbf{a}_{2}+\left(\frac{1}{2}+x_{10}\right) \mathbf{a}_{3}$ & $=$ & $y_{10} a \hat{\mathbf{x}}+\left(\frac{1}{2}-z_{10}\right) a \hat{\mathbf{y}}+\left(\frac{1}{2}+x_{10}\right) a \hat{\mathbf{z}}$ & $(24 d)$ & O II \\
\hline $\mathbf{B}_{167}$ & $=$ & $\left(\frac{1}{2}-y_{10}\right) \mathbf{a}_{1}+\left(\frac{1}{2}+z_{10}\right) \mathbf{a}_{2}+x_{10} \mathbf{a}_{3}$ & $=$ & $\left(\frac{1}{2}-y_{10}\right) a \hat{\mathbf{x}}+\left(\frac{1}{2}+z_{10}\right) a \hat{\mathbf{y}}+x_{10} a \hat{\mathbf{z}}$ & $(24 d)$ & O II \\
\hline $\mathbf{B}_{168}$ & $=$ & $\left(\frac{1}{2}+y_{10}\right) \mathbf{a}_{1}+z_{10} \mathbf{a}_{2}+\left(\frac{1}{2}-x_{10}\right) \mathbf{a}_{3}$ & $=$ & $\left(\frac{1}{2}+y_{10}\right) a \hat{\mathbf{x}}+z_{10} a \hat{\mathbf{y}}+\left(\frac{1}{2}-x_{10}\right) a \hat{\mathbf{z}}$ & $(24 d)$ & O II \\
\hline $\mathbf{B}_{169}$ & $=$ & $x_{11} \mathbf{a}_{1}+y_{11} \mathbf{a}_{2}+z_{11} \mathbf{a}_{3}$ & $=$ & $x_{11} a \hat{\mathbf{x}}+y_{11} a \hat{\mathbf{y}}+z_{11} a \hat{\mathbf{z}}$ & $(24 d)$ & O III \\
\hline $\mathbf{B}_{170}$ & $=$ & $\left(\frac{1}{2}-x_{11}\right) \mathbf{a}_{1}-y_{11} \mathbf{a}_{2}+\left(\frac{1}{2}+z_{11}\right) \mathbf{a}_{3}$ & $=$ & $\left(\frac{1}{2}-x_{11}\right) a \hat{\mathbf{x}}-y_{11} a \hat{\mathbf{y}}+\left(\frac{1}{2}+z_{11}\right) a \hat{\mathbf{z}}$ & $(24 d)$ & O III \\
\hline $\mathbf{B}_{171}$ & $=$ & $\begin{array}{c}-x_{11} \mathbf{a}_{1}+\left(\frac{1}{2}+y_{11}\right) \mathbf{a}_{2}+ \\
\left(\frac{1}{2}-z_{11}\right) \mathbf{a}_{3}\end{array}$ & $=$ & $\begin{array}{c}-x_{11} a \hat{\mathbf{x}}+\left(\frac{1}{2}+y_{11}\right) a \hat{\mathbf{y}}+ \\
\left(\frac{1}{2}-z_{11}\right) a \hat{\mathbf{z}}\end{array}$ & $(24 d)$ & O III \\
\hline $\mathbf{B}_{172}$ & $=$ & $\left(\frac{1}{2}+x_{11}\right) \mathbf{a}_{1}+\left(\frac{1}{2}-y_{11}\right) \mathbf{a}_{2}-z_{11} \mathbf{a}_{3}$ & $=$ & $\left(\frac{1}{2}+x_{11}\right) a \hat{\mathbf{x}}+\left(\frac{1}{2}-y_{11}\right) a \hat{\mathbf{y}}-z_{11} a \hat{\mathbf{z}}$ & $(24 d)$ & O III \\
\hline $\mathbf{B}_{173}$ & $=$ & $z_{11} \mathbf{a}_{1}+x_{11} \mathbf{a}_{2}+y_{11} \mathbf{a}_{3}$ & $=$ & $z_{11} a \hat{\mathbf{x}}+x_{11} a \hat{\mathbf{y}}+y_{11} a \hat{\mathbf{z}}$ & $(24 d)$ & O III \\
\hline $\mathbf{B}_{174}$ & $=$ & $\left(\frac{1}{2}+z_{11}\right) \mathbf{a}_{1}+\left(\frac{1}{2}-x_{11}\right) \mathbf{a}_{2}-y_{11} \mathbf{a}_{3}$ & $=$ & $\left(\frac{1}{2}+z_{11}\right) a \hat{\mathbf{x}}+\left(\frac{1}{2}-x_{11}\right) a \hat{\mathbf{y}}-y_{11} a \hat{\mathbf{z}}$ & $(24 d)$ & O III \\
\hline $\mathbf{B}_{175}$ & $=$ & $\left(\frac{1}{2}-z_{11}\right) \mathbf{a}_{1}-x_{11} \mathbf{a}_{2}+\left(\frac{1}{2}+y_{11}\right) \mathbf{a}_{3}$ & $=$ & $\left(\frac{1}{2}-z_{11}\right) a \hat{\mathbf{x}}-x_{11} a \hat{\mathbf{y}}+\left(\frac{1}{2}+y_{11}\right) a \hat{\mathbf{z}}$ & $(24 d)$ & O III \\
\hline
\end{tabular}




\begin{tabular}{|c|c|c|c|c|}
\hline $\mathbf{B}_{176}$ & $\begin{array}{c}-z_{11} \mathbf{a}_{1}+\left(\frac{1}{2}+x_{11}\right) \mathbf{a}_{2}+ \\
\left(\frac{1}{2}-y_{11}\right) \mathbf{a}_{3}\end{array}$ & $\begin{array}{c}-z_{11} a \hat{\mathbf{x}}+\left(\frac{1}{2}+x_{11}\right) a \hat{\mathbf{y}}+ \\
\left(\frac{1}{2}-y_{11}\right) a \hat{\mathbf{z}}\end{array}$ & $(24 d)$ & O III \\
\hline $\mathbf{B}_{177}$ & $y_{11} \mathbf{a}_{1}+z_{11} \mathbf{a}_{2}+x_{11} \mathbf{a}_{3}$ & $y_{11} a \hat{\mathbf{x}}+z_{11} a \hat{\mathbf{y}}+x_{11} a \hat{\mathbf{z}}$ & $(24 d)$ & O III \\
\hline $\mathbf{B}_{178}$ & $\begin{array}{c}-y_{11} \mathbf{a}_{1}+\left(\frac{1}{2}+z_{11}\right) \mathbf{a}_{2}+ \\
\left(\frac{1}{2}-x_{11}\right) \mathbf{a}_{3}\end{array}$ & $\begin{array}{c}-y_{11} a \hat{\mathbf{x}}+\left(\frac{1}{2}+z_{11}\right) a \hat{\mathbf{y}}+ \\
\left(\frac{1}{2}-x_{11}\right) a \hat{\mathbf{z}}\end{array}$ & $(24 d)$ & O III \\
\hline $\mathbf{B}_{179}$ & $=\left(\frac{1}{2}+y_{11}\right) \mathbf{a}_{1}+\left(\frac{1}{2}-z_{11}\right) \mathbf{a}_{2}-x_{11} \mathbf{a}_{3}$ & $=\left(\frac{1}{2}+y_{11}\right) a \hat{\mathbf{x}}+\left(\frac{1}{2}-z_{11}\right) a \hat{\mathbf{y}}-x_{11} a \hat{\mathbf{z}}$ & $(24 d)$ & O III \\
\hline $\mathbf{B}_{180}$ & $=\left(\frac{1}{2}-y_{11}\right) \mathbf{a}_{1}-z_{11} \mathbf{a}_{2}+\left(\frac{1}{2}+x_{11}\right) \mathbf{a}_{3}$ & $=\left(\frac{1}{2}-y_{11}\right) a \hat{\mathbf{x}}-z_{11} a \hat{\mathbf{y}}+\left(\frac{1}{2}+x_{11}\right) a \hat{\mathbf{z}}$ & $(24 d)$ & O III \\
\hline $\mathbf{B}_{181}$ & $-x_{11} \mathbf{a}_{1}-y_{11} \mathbf{a}_{2}-z_{11} \mathbf{a}_{3}$ & $-x_{11} a \hat{\mathbf{x}}-y_{11} a \hat{\mathbf{y}}-z_{11} a \hat{\mathbf{z}}$ & $(24 d)$ & O III \\
\hline $\mathbf{B}_{182}$ & $=\left(\frac{1}{2}+x_{11}\right) \mathbf{a}_{1}+y_{11} \mathbf{a}_{2}+\left(\frac{1}{2}-z_{11}\right) \mathbf{a}_{3}$ & $=\left(\frac{1}{2}+x_{11}\right) a \hat{\mathbf{x}}+y_{11} a \hat{\mathbf{y}}+\left(\frac{1}{2}-z_{11}\right) a \hat{\mathbf{z}}$ & $(24 d)$ & O III \\
\hline $\mathbf{B}_{183}$ & $=x_{11} \mathbf{a}_{1}+\left(\frac{1}{2}-y_{11}\right) \mathbf{a}_{2}+\left(\frac{1}{2}+z_{11}\right) \mathbf{a}_{3}$ & $=\quad x_{11} a \hat{\mathbf{x}}+\left(\frac{1}{2}-y_{11}\right) a \hat{\mathbf{y}}+\left(\frac{1}{2}+z_{11}\right) a \hat{\mathbf{z}}$ & $(24 d)$ & O III \\
\hline $\mathbf{B}_{184}$ & $=\left(\frac{1}{2}-x_{11}\right) \mathbf{a}_{1}+\left(\frac{1}{2}+y_{11}\right) \mathbf{a}_{2}+z_{11} \mathbf{a}_{3}$ & $=\left(\frac{1}{2}-x_{11}\right) a \hat{\mathbf{x}}+\left(\frac{1}{2}+y_{11}\right) a \hat{\mathbf{y}}+z_{11} a \hat{\mathbf{z}}$ & $(24 d)$ & O III \\
\hline $\mathbf{B}_{185}$ & $-z_{11} \mathbf{a}_{1}-x_{11} \mathbf{a}_{2}-y_{11} \mathbf{a}_{3}$ & $-z_{11} a \hat{\mathbf{x}}-x_{11} a \hat{\mathbf{y}}-y_{11} a \hat{\mathbf{z}}$ & $(24 d)$ & O III \\
\hline $\mathbf{B}_{186}$ & $=\left(\frac{1}{2}-z_{11}\right) \mathbf{a}_{1}+\left(\frac{1}{2}+x_{11}\right) \mathbf{a}_{2}+y_{11} \mathbf{a}_{3}$ & $=\left(\frac{1}{2}-z_{11}\right) a \hat{\mathbf{x}}+\left(\frac{1}{2}+x_{11}\right) a \hat{\mathbf{y}}+y_{11} a \hat{\mathbf{z}}$ & $(24 d)$ & O III \\
\hline $\mathbf{B}_{187}$ & $=\left(\frac{1}{2}+z_{11}\right) \mathbf{a}_{1}+x_{11} \mathbf{a}_{2}+\left(\frac{1}{2}-y_{11}\right) \mathbf{a}_{3}$ & $=\left(\frac{1}{2}+z_{11}\right) a \hat{\mathbf{x}}+x_{11} a \hat{\mathbf{y}}+\left(\frac{1}{2}-y_{11}\right) a \hat{\mathbf{z}}$ & $(24 d)$ & O III \\
\hline $\mathbf{B}_{188}$ & $=z_{11} \mathbf{a}_{1}+\left(\frac{1}{2}-x_{11}\right) \mathbf{a}_{2}+\left(\frac{1}{2}+y_{11}\right) \mathbf{a}_{3}$ & $=z_{11} a \hat{\mathbf{x}}+\left(\frac{1}{2}-x_{11}\right) a \hat{\mathbf{y}}+\left(\frac{1}{2}+y_{11}\right) a \hat{\mathbf{z}}$ & $(24 d)$ & O III \\
\hline $\mathbf{B}_{189}$ & $-y_{11} \mathbf{a}_{1}-z_{11} \mathbf{a}_{2}-x_{11} \mathbf{a}_{3}$ & $-y_{11} a \hat{\mathbf{x}}-z_{11} a \hat{\mathbf{y}}-x_{11} a \hat{\mathbf{z}}$ & $(24 d)$ & O III \\
\hline $\mathbf{B}_{190}$ & $=y_{11} \mathbf{a}_{1}+\left(\frac{1}{2}-z_{11}\right) \mathbf{a}_{2}+\left(\frac{1}{2}+x_{11}\right) \mathbf{a}_{3}$ & $=y_{11} a \hat{\mathbf{x}}+\left(\frac{1}{2}-z_{11}\right) a \hat{\mathbf{y}}+\left(\frac{1}{2}+x_{11}\right) a \hat{\mathbf{z}}$ & $(24 d)$ & O III \\
\hline $\mathbf{B}_{191}$ & $=\left(\frac{1}{2}-y_{11}\right) \mathbf{a}_{1}+\left(\frac{1}{2}+z_{11}\right) \mathbf{a}_{2}+x_{11} \mathbf{a}_{3}$ & $=\left(\frac{1}{2}-y_{11}\right) a \hat{\mathbf{x}}+\left(\frac{1}{2}+z_{11}\right) a \hat{\mathbf{y}}+x_{11} a \hat{\mathbf{z}}$ & $(24 d)$ & O III \\
\hline $\mathbf{B}_{192}$ & $=\left(\frac{1}{2}+y_{11}\right) \mathbf{a}_{1}+z_{11} \mathbf{a}_{2}+\left(\frac{1}{2}-x_{11}\right) \mathbf{a}_{3}$ & $=\left(\frac{1}{2}+y_{11}\right) a \hat{\mathbf{x}}+z_{11} a \hat{\mathbf{y}}+\left(\frac{1}{2}-x_{11}\right) a \hat{\mathbf{z}}$ & $(24 d)$ & O III \\
\hline $\mathbf{B}_{193}$ & $x_{12} \mathbf{a}_{1}+y_{12} \mathbf{a}_{2}+z_{12} \mathbf{a}_{3}$ & $x_{12} a \hat{\mathbf{x}}+y_{12} a \hat{\mathbf{y}}+z_{12} a \hat{\mathbf{z}}$ & $(24 d)$ & O IV \\
\hline $\mathbf{B}_{194}$ & $=\left(\frac{1}{2}-x_{12}\right) \mathbf{a}_{1}-y_{12} \mathbf{a}_{2}+\left(\frac{1}{2}+z_{12}\right) \mathbf{a}_{3}$ & $=\left(\frac{1}{2}-x_{12}\right) a \hat{\mathbf{x}}-y_{12} a \hat{\mathbf{y}}+\left(\frac{1}{2}+z_{12}\right) a \hat{\mathbf{z}}$ & $(24 d)$ & O IV \\
\hline $\mathbf{B}_{195}$ & $\begin{array}{c}-x_{12} \mathbf{a}_{1}+\left(\frac{1}{2}+y_{12}\right) \mathbf{a}_{2}+ \\
\left(\frac{1}{2}-z_{12}\right) \mathbf{a}_{3}\end{array}$ & $\begin{array}{c}-x_{12} a \hat{\mathbf{x}}+\left(\frac{1}{2}+y_{12}\right) a \hat{\mathbf{y}}+ \\
\left(\frac{1}{2}-z_{12}\right) a \hat{\mathbf{z}}\end{array}$ & $(24 d)$ & O IV \\
\hline $\mathbf{B}_{196}$ & $=\left(\frac{1}{2}+x_{12}\right) \mathbf{a}_{1}+\left(\frac{1}{2}-y_{12}\right) \mathbf{a}_{2}-z_{12} \mathbf{a}_{3}$ & $=\left(\frac{1}{2}+x_{12}\right) a \hat{\mathbf{x}}+\left(\frac{1}{2}-y_{12}\right) a \hat{\mathbf{y}}-z_{12} a \hat{\mathbf{z}}$ & $(24 d)$ & O IV \\
\hline $\mathbf{B}_{197}$ & $z_{12} \mathbf{a}_{1}+x_{12} \mathbf{a}_{2}+y_{12} \mathbf{a}_{3}$ & $z_{12} a \hat{\mathbf{x}}+x_{12} a \hat{\mathbf{y}}+y_{12} a \hat{\mathbf{z}}$ & $(24 d)$ & O IV \\
\hline $\mathbf{B}_{198}$ & $=\left(\frac{1}{2}+z_{12}\right) \mathbf{a}_{1}+\left(\frac{1}{2}-x_{12}\right) \mathbf{a}_{2}-y_{12} \mathbf{a}_{3}$ & $=\left(\frac{1}{2}+z_{12}\right) a \hat{\mathbf{x}}+\left(\frac{1}{2}-x_{12}\right) a \hat{\mathbf{y}}-y_{12} a \hat{\mathbf{z}}$ & $(24 d)$ & O IV \\
\hline $\mathbf{B}_{199}$ & $=\left(\frac{1}{2}-z_{12}\right) \mathbf{a}_{1}-x_{12} \mathbf{a}_{2}+\left(\frac{1}{2}+y_{12}\right) \mathbf{a}_{3}$ & $=\left(\frac{1}{2}-z_{12}\right) a \hat{\mathbf{x}}-x_{12} a \hat{\mathbf{y}}+\left(\frac{1}{2}+y_{12}\right) a \hat{\mathbf{z}}$ & $(24 d)$ & O IV \\
\hline $\mathbf{B}_{200}$ & $\begin{array}{c}-z_{12} \mathbf{a}_{1}+\left(\frac{1}{2}+x_{12}\right) \mathbf{a}_{2}+ \\
\left(\frac{1}{2}-y_{12}\right) \mathbf{a}_{3}\end{array}$ & $\begin{array}{c}-z_{12} a \hat{\mathbf{x}}+\left(\frac{1}{2}+x_{12}\right) a \hat{\mathbf{y}}+ \\
\left(\frac{1}{2}-y_{12}\right) a \hat{\mathbf{z}}\end{array}$ & $(24 d)$ & O IV \\
\hline $\mathbf{B}_{201}$ & $y_{12} \mathbf{a}_{1}+z_{12} \mathbf{a}_{2}+x_{12} \mathbf{a}_{3}$ & $y_{12} a \hat{\mathbf{x}}+z_{12} a \hat{\mathbf{y}}+x_{12} a \hat{\mathbf{z}}$ & $(24 d)$ & $\mathrm{O}$ IV \\
\hline $\mathbf{B}_{202}$ & $\begin{array}{c}-y_{12} \mathbf{a}_{1}+\left(\frac{1}{2}+z_{12}\right) \mathbf{a}_{2}+ \\
\left(\frac{1}{2}-x_{12}\right) \mathbf{a}_{3}\end{array}$ & $\begin{array}{c}-y_{12} a \hat{\mathbf{x}}+\left(\frac{1}{2}+z_{12}\right) a \hat{\mathbf{y}}+ \\
\left(\frac{1}{2}-x_{12}\right) a \hat{\mathbf{z}}\end{array}$ & $(24 d)$ & O IV \\
\hline $\mathbf{B}_{203}$ & $=\left(\frac{1}{2}+y_{12}\right) \mathbf{a}_{1}+\left(\frac{1}{2}-z_{12}\right) \mathbf{a}_{2}-x_{12} \mathbf{a}_{3}$ & $=\left(\frac{1}{2}+y_{12}\right) a \hat{\mathbf{x}}+\left(\frac{1}{2}-z_{12}\right) a \hat{\mathbf{y}}-x_{12} a \hat{\mathbf{z}}$ & $(24 d)$ & O IV \\
\hline $\mathbf{B}_{204}$ & $=\left(\frac{1}{2}-y_{12}\right) \mathbf{a}_{1}-z_{12} \mathbf{a}_{2}+\left(\frac{1}{2}+x_{12}\right) \mathbf{a}_{3}$ & $=\left(\frac{1}{2}-y_{12}\right) a \hat{\mathbf{x}}-z_{12} a \hat{\mathbf{y}}+\left(\frac{1}{2}+x_{12}\right) a \hat{\mathbf{z}}$ & $(24 d)$ & O IV \\
\hline $\mathbf{B}_{205}$ & $-x_{12} \mathbf{a}_{1}-y_{12} \mathbf{a}_{2}-z_{12} \mathbf{a}_{3}$ & $-x_{12} a \hat{\mathbf{x}}-y_{12} a \hat{\mathbf{y}}-z_{12} a \hat{\mathbf{z}}$ & $(24 d)$ & O IV \\
\hline $\mathbf{B}_{206}$ & $=\left(\frac{1}{2}+x_{12}\right) \mathbf{a}_{1}+y_{12} \mathbf{a}_{2}+\left(\frac{1}{2}-z_{12}\right) \mathbf{a}_{3}$ & $=\left(\frac{1}{2}+x_{12}\right) a \hat{\mathbf{x}}+y_{12} a \hat{\mathbf{y}}+\left(\frac{1}{2}-z_{12}\right) a \hat{\mathbf{z}}$ & $(24 d)$ & O IV \\
\hline $\mathbf{B}_{207}$ & $=x_{12} \mathbf{a}_{1}+\left(\frac{1}{2}-y_{12}\right) \mathbf{a}_{2}+\left(\frac{1}{2}+z_{12}\right) \mathbf{a}_{3}$ & $=x_{12} a \hat{\mathbf{x}}+\left(\frac{1}{2}-y_{12}\right) a \hat{\mathbf{y}}+\left(\frac{1}{2}+z_{12}\right) a \hat{\mathbf{z}}$ & $(24 d)$ & O IV \\
\hline
\end{tabular}




\begin{tabular}{|c|c|c|c|c|c|c|}
\hline $\mathbf{B}_{208}$ & $=$ & $\left(\frac{1}{2}-x_{12}\right) \mathbf{a}_{1}+\left(\frac{1}{2}+y_{12}\right) \mathbf{a}_{2}+z_{12} \mathbf{a}_{3}$ & $=$ & $\left(\frac{1}{2}-x_{12}\right) a \hat{\mathbf{x}}+\left(\frac{1}{2}+y_{12}\right) a \hat{\mathbf{y}}+z_{12} a \hat{\mathbf{z}}$ & $(24 d)$ & O IV \\
\hline $\mathbf{B}_{209}$ & $=$ & $-z_{12} \mathbf{a}_{1}-x_{12} \mathbf{a}_{2}-y_{12} \mathbf{a}_{3}$ & $=$ & $-z_{12} a \hat{\mathbf{x}}-x_{12} a \hat{\mathbf{y}}-y_{12} a \hat{\mathbf{z}}$ & $(24 d)$ & O IV \\
\hline $\mathbf{B}_{210}$ & $=$ & $\left(\frac{1}{2}-z_{12}\right) \mathbf{a}_{1}+\left(\frac{1}{2}+x_{12}\right) \mathbf{a}_{2}+y_{12} \mathbf{a}_{3}$ & $=$ & $\left(\frac{1}{2}-z_{12}\right) a \hat{\mathbf{x}}+\left(\frac{1}{2}+x_{12}\right) a \hat{\mathbf{y}}+y_{12} a \hat{\mathbf{z}}$ & $(24 d)$ & O IV \\
\hline $\mathbf{B}_{211}$ & $=$ & $\left(\frac{1}{2}+z_{12}\right) \mathbf{a}_{1}+x_{12} \mathbf{a}_{2}+\left(\frac{1}{2}-y_{12}\right) \mathbf{a}_{3}$ & $=$ & $\left(\frac{1}{2}+z_{12}\right) a \hat{\mathbf{x}}+x_{12} a \hat{\mathbf{y}}+\left(\frac{1}{2}-y_{12}\right) a \hat{\mathbf{z}}$ & $(24 d)$ & O IV \\
\hline $\mathbf{B}_{212}$ & $=$ & $z_{12} \mathbf{a}_{1}+\left(\frac{1}{2}-x_{12}\right) \mathbf{a}_{2}+\left(\frac{1}{2}+y_{12}\right) \mathbf{a}_{3}$ & $=$ & $z_{12} a \hat{\mathbf{x}}+\left(\frac{1}{2}-x_{12}\right) a \hat{\mathbf{y}}+\left(\frac{1}{2}+y_{12}\right) a \hat{\mathbf{z}}$ & $(24 d)$ & O IV \\
\hline $\mathbf{B}_{213}$ & $=$ & $-y_{12} \mathbf{a}_{1}-z_{12} \mathbf{a}_{2}-x_{12} \mathbf{a}_{3}$ & $=$ & $-y_{12} a \hat{\mathbf{x}}-z_{12} a \hat{\mathbf{y}}-x_{12} a \hat{\mathbf{z}}$ & $(24 d)$ & O IV \\
\hline $\mathbf{B}_{214}$ & $=$ & $y_{12} \mathbf{a}_{1}+\left(\frac{1}{2}-z_{12}\right) \mathbf{a}_{2}+\left(\frac{1}{2}+x_{12}\right) \mathbf{a}_{3}$ & $=$ & $y_{12} a \hat{\mathbf{x}}+\left(\frac{1}{2}-z_{12}\right) a \hat{\mathbf{y}}+\left(\frac{1}{2}+x_{12}\right) a \hat{\mathbf{z}}$ & $(24 d)$ & O IV \\
\hline $\mathbf{B}_{215}$ & $=$ & $\left(\frac{1}{2}-y_{12}\right) \mathbf{a}_{1}+\left(\frac{1}{2}+z_{12}\right) \mathbf{a}_{2}+x_{12} \mathbf{a}_{3}$ & $=$ & $\left(\frac{1}{2}-y_{12}\right) a \hat{\mathbf{x}}+\left(\frac{1}{2}+z_{12}\right) a \hat{\mathbf{y}}+x_{12} a \hat{\mathbf{z}}$ & $(24 d)$ & O IV \\
\hline $\mathbf{B}_{216}$ & $=$ & $\left(\frac{1}{2}+y_{12}\right) \mathbf{a}_{1}+z_{12} \mathbf{a}_{2}+\left(\frac{1}{2}-x_{12}\right) \mathbf{a}_{3}$ & $=$ & $\left(\frac{1}{2}+y_{12}\right) a \hat{\mathbf{x}}+z_{12} a \hat{\mathbf{y}}+\left(\frac{1}{2}-x_{12}\right) a \hat{\mathbf{z}}$ & $(24 d)$ & O IV \\
\hline $\mathbf{B}_{217}$ & $=$ & $x_{13} \mathbf{a}_{1}+y_{13} \mathbf{a}_{2}+z_{13} \mathbf{a}_{3}$ & $=$ & $x_{13} a \hat{\mathbf{x}}+y_{13} a \hat{\mathbf{y}}+z_{13} a \hat{\mathbf{z}}$ & $(24 d)$ & $\mathrm{OV}$ \\
\hline $\mathbf{B}_{218}$ & $=$ & $\left(\frac{1}{2}-x_{13}\right) \mathbf{a}_{1}-y_{13} \mathbf{a}_{2}+\left(\frac{1}{2}+z_{13}\right) \mathbf{a}_{3}$ & $=$ & $\left(\frac{1}{2}-x_{13}\right) a \hat{\mathbf{x}}-y_{13} a \hat{\mathbf{y}}+\left(\frac{1}{2}+z_{13}\right) a \hat{\mathbf{z}}$ & $(24 d)$ & $\mathrm{OV}$ \\
\hline $\mathbf{B}_{219}$ & $=$ & $\begin{array}{c}-x_{13} \mathbf{a}_{1}+\left(\frac{1}{2}+y_{13}\right) \mathbf{a}_{2}+ \\
\left(\frac{1}{2}-z_{13}\right) \mathbf{a}_{3}\end{array}$ & $=$ & $\begin{array}{c}-x_{13} a \hat{\mathbf{x}}+\left(\frac{1}{2}+y_{13}\right) a \hat{\mathbf{y}}+ \\
\left(\frac{1}{2}-z_{13}\right) a \hat{\mathbf{z}}\end{array}$ & $(24 d)$ & $\mathrm{OV}$ \\
\hline $\mathbf{B}_{220}$ & $=$ & $\left(\frac{1}{2}+x_{13}\right) \mathbf{a}_{1}+\left(\frac{1}{2}-y_{13}\right) \mathbf{a}_{2}-z_{13} \mathbf{a}_{3}$ & $=$ & $\left(\frac{1}{2}+x_{13}\right) a \hat{\mathbf{x}}+\left(\frac{1}{2}-y_{13}\right) a \hat{\mathbf{y}}-z_{13} a \hat{\mathbf{z}}$ & $(24 d)$ & $\mathrm{OV}$ \\
\hline $\mathbf{B}_{221}$ & $=$ & $z_{13} \mathbf{a}_{1}+x_{13} \mathbf{a}_{2}+y_{13} \mathbf{a}_{3}$ & $=$ & $z_{13} a \hat{\mathbf{x}}+x_{13} a \hat{\mathbf{y}}+y_{13} a \hat{\mathbf{z}}$ & $(24 d)$ & $\mathrm{OV}$ \\
\hline $\mathbf{B}_{222}$ & $=$ & $\left(\frac{1}{2}+z_{13}\right) \mathbf{a}_{1}+\left(\frac{1}{2}-x_{13}\right) \mathbf{a}_{2}-y_{13} \mathbf{a}_{3}$ & $=$ & $\left(\frac{1}{2}+z_{13}\right) a \hat{\mathbf{x}}+\left(\frac{1}{2}-x_{13}\right) a \hat{\mathbf{y}}-y_{13} a \hat{\mathbf{z}}$ & $(24 d)$ & $\mathrm{OV}$ \\
\hline $\mathbf{B}_{223}$ & $=$ & $\left(\frac{1}{2}-z_{13}\right) \mathbf{a}_{1}-x_{13} \mathbf{a}_{2}+\left(\frac{1}{2}+y_{13}\right) \mathbf{a}_{3}$ & $=$ & $\left(\frac{1}{2}-z_{13}\right) a \hat{\mathbf{x}}-x_{13} a \hat{\mathbf{y}}+\left(\frac{1}{2}+y_{13}\right) a \hat{\mathbf{z}}$ & $(24 d)$ & $\mathrm{OV}$ \\
\hline $\mathbf{B}_{224}$ & $=$ & $\begin{array}{c}-z_{13} \mathbf{a}_{1}+\left(\frac{1}{2}+x_{13}\right) \mathbf{a}_{2}+ \\
\left(\frac{1}{2}-y_{13}\right) \mathbf{a}_{3}\end{array}$ & $=$ & $\begin{array}{c}-z_{13} a \hat{\mathbf{x}}+\left(\frac{1}{2}+x_{13}\right) a \hat{\mathbf{y}}+ \\
\left(\frac{1}{2}-y_{13}\right) a \hat{\mathbf{z}}\end{array}$ & $(24 d)$ & $\mathrm{OV}$ \\
\hline $\mathbf{B}_{225}$ & $=$ & $y_{13} \mathbf{a}_{1}+z_{13} \mathbf{a}_{2}+x_{13} \mathbf{a}_{3}$ & $=$ & $y_{13} a \hat{\mathbf{x}}+z_{13} a \hat{\mathbf{y}}+x_{13} a \hat{\mathbf{z}}$ & $(24 d)$ & $\mathrm{OV}$ \\
\hline $\mathbf{B}_{226}$ & $=$ & $\begin{array}{c}-y_{13} \mathbf{a}_{1}+\left(\frac{1}{2}+z_{13}\right) \mathbf{a}_{2}+ \\
\left(\frac{1}{2}-x_{13}\right) \mathbf{a}_{3}\end{array}$ & $=$ & $\begin{array}{c}-y_{13} a \hat{\mathbf{x}}+\left(\frac{1}{2}+z_{13}\right) a \hat{\mathbf{y}}+ \\
\left(\frac{1}{2}-x_{13}\right) a \hat{\mathbf{z}}\end{array}$ & $(24 d)$ & $\mathrm{OV}$ \\
\hline $\mathbf{B}_{227}$ & $=$ & $\left(\frac{1}{2}+y_{13}\right) \mathbf{a}_{1}+\left(\frac{1}{2}-z_{13}\right) \mathbf{a}_{2}-x_{13} \mathbf{a}_{3}$ & $=$ & $\left(\frac{1}{2}+y_{13}\right) a \hat{\mathbf{x}}+\left(\frac{1}{2}-z_{13}\right) a \hat{\mathbf{y}}-x_{13} a \hat{\mathbf{z}}$ & $(24 d)$ & $\mathrm{OV}$ \\
\hline $\mathbf{B}_{228}$ & $=$ & $\left(\frac{1}{2}-y_{13}\right) \mathbf{a}_{1}-z_{13} \mathbf{a}_{2}+\left(\frac{1}{2}+x_{13}\right) \mathbf{a}_{3}$ & $=$ & $\left(\frac{1}{2}-y_{13}\right) a \hat{\mathbf{x}}-z_{13} a \hat{\mathbf{y}}+\left(\frac{1}{2}+x_{13}\right) a \hat{\mathbf{z}}$ & $(24 d)$ & $\mathrm{OV}$ \\
\hline $\mathbf{B}_{229}$ & $=$ & $-x_{13} \mathbf{a}_{1}-y_{13} \mathbf{a}_{2}-z_{13} \mathbf{a}_{3}$ & $=$ & $-x_{13} a \hat{\mathbf{x}}-y_{13} a \hat{\mathbf{y}}-z_{13} a \hat{\mathbf{z}}$ & $(24 d)$ & $\mathrm{OV}$ \\
\hline $\mathbf{B}_{230}$ & $=$ & $\left(\frac{1}{2}+x_{13}\right) \mathbf{a}_{1}+y_{13} \mathbf{a}_{2}+\left(\frac{1}{2}-z_{13}\right) \mathbf{a}_{3}$ & $=$ & $\left(\frac{1}{2}+x_{13}\right) a \hat{\mathbf{x}}+y_{13} a \hat{\mathbf{y}}+\left(\frac{1}{2}-z_{13}\right) a \hat{\mathbf{z}}$ & $(24 d)$ & $\mathrm{OV}$ \\
\hline $\mathbf{B}_{231}$ & $=$ & $x_{13} \mathbf{a}_{1}+\left(\frac{1}{2}-y_{13}\right) \mathbf{a}_{2}+\left(\frac{1}{2}+z_{13}\right) \mathbf{a}_{3}$ & $=$ & $x_{13} a \hat{\mathbf{x}}+\left(\frac{1}{2}-y_{13}\right) a \hat{\mathbf{y}}+\left(\frac{1}{2}+z_{13}\right) a \hat{\mathbf{z}}$ & $(24 d)$ & $\mathrm{OV}$ \\
\hline $\mathbf{B}_{232}$ & $=$ & $\left(\frac{1}{2}-x_{13}\right) \mathbf{a}_{1}+\left(\frac{1}{2}+y_{13}\right) \mathbf{a}_{2}+z_{13} \mathbf{a}_{3}$ & $=$ & $\left(\frac{1}{2}-x_{13}\right) a \hat{\mathbf{x}}+\left(\frac{1}{2}+y_{13}\right) a \hat{\mathbf{y}}+z_{13} a \hat{\mathbf{z}}$ & $(24 d)$ & $\mathrm{OV}$ \\
\hline $\mathbf{B}_{233}$ & $=$ & $-z_{13} \mathbf{a}_{1}-x_{13} \mathbf{a}_{2}-y_{13} \mathbf{a}_{3}$ & $=$ & $-z_{13} a \hat{\mathbf{x}}-x_{13} a \hat{\mathbf{y}}-y_{13} a \hat{\mathbf{z}}$ & $(24 d)$ & $\mathrm{OV}$ \\
\hline $\mathbf{B}_{234}$ & $=$ & $\left(\frac{1}{2}-z_{13}\right) \mathbf{a}_{1}+\left(\frac{1}{2}+x_{13}\right) \mathbf{a}_{2}+y_{13} \mathbf{a}_{3}$ & $=$ & $\left(\frac{1}{2}-z_{13}\right) a \hat{\mathbf{x}}+\left(\frac{1}{2}+x_{13}\right) a \hat{\mathbf{y}}+y_{13} a \hat{\mathbf{z}}$ & $(24 d)$ & $\mathrm{OV}$ \\
\hline $\mathbf{B}_{235}$ & $=$ & $\left(\frac{1}{2}+z_{13}\right) \mathbf{a}_{1}+x_{13} \mathbf{a}_{2}+\left(\frac{1}{2}-y_{13}\right) \mathbf{a}_{3}$ & $=$ & $\left(\frac{1}{2}+z_{13}\right) a \hat{\mathbf{x}}+x_{13} a \hat{\mathbf{y}}+\left(\frac{1}{2}-y_{13}\right) a \hat{\mathbf{z}}$ & $(24 d)$ & $\mathrm{OV}$ \\
\hline $\mathbf{B}_{236}$ & $=$ & $z_{13} \mathbf{a}_{1}+\left(\frac{1}{2}-x_{13}\right) \mathbf{a}_{2}+\left(\frac{1}{2}+y_{13}\right) \mathbf{a}_{3}$ & $=$ & $z_{13} a \hat{\mathbf{x}}+\left(\frac{1}{2}-x_{13}\right) a \hat{\mathbf{y}}+\left(\frac{1}{2}+y_{13}\right) a \hat{\mathbf{z}}$ & $(24 d)$ & $\mathrm{OV}$ \\
\hline $\mathbf{B}_{237}$ & $=$ & $-y_{13} \mathbf{a}_{1}-z_{13} \mathbf{a}_{2}-x_{13} \mathbf{a}_{3}$ & $=$ & $-y_{13} a \hat{\mathbf{x}}-z_{13} a \hat{\mathbf{y}}-x_{13} a \hat{\mathbf{z}}$ & $(24 d)$ & $\mathrm{OV}$ \\
\hline $\mathbf{B}_{238}$ & $=$ & $y_{13} \mathbf{a}_{1}+\left(\frac{1}{2}-z_{13}\right) \mathbf{a}_{2}+\left(\frac{1}{2}+x_{13}\right) \mathbf{a}_{3}$ & $=$ & $y_{13} a \hat{\mathbf{x}}+\left(\frac{1}{2}-z_{13}\right) a \hat{\mathbf{y}}+\left(\frac{1}{2}+x_{13}\right) a \hat{\mathbf{z}}$ & $(24 d)$ & $\mathrm{OV}$ \\
\hline $\mathbf{B}_{239}$ & $=$ & $\left(\frac{1}{2}-y_{13}\right) \mathbf{a}_{1}+\left(\frac{1}{2}+z_{13}\right) \mathbf{a}_{2}+x_{13} \mathbf{a}_{3}$ & $=$ & $\left(\frac{1}{2}-y_{13}\right) a \hat{\mathbf{x}}+\left(\frac{1}{2}+z_{13}\right) a \hat{\mathbf{y}}+x_{13} a \hat{\mathbf{z}}$ & $(24 d)$ & $\mathrm{OV}$ \\
\hline $\mathbf{B}_{240}$ & $=$ & $\left(\frac{1}{2}+y_{13}\right) \mathbf{a}_{1}+z_{13} \mathbf{a}_{2}+\left(\frac{1}{2}-x_{13}\right) \mathbf{a}_{3}$ & $=$ & $\left(\frac{1}{2}+y_{13}\right) a \hat{\mathbf{x}}+z_{13} a \hat{\mathbf{y}}+\left(\frac{1}{2}-x_{13}\right) a \hat{\mathbf{z}}$ & $(24 d)$ & $\mathrm{OV}$ \\
\hline $\mathbf{B}_{241}$ & $=$ & $x_{14} \mathbf{a}_{1}+y_{14} \mathbf{a}_{2}+z_{14} \mathbf{a}_{3}$ & $=$ & $x_{14} a \hat{\mathbf{x}}+y_{14} a \hat{\mathbf{y}}+z_{14} a \hat{\mathbf{z}}$ & $(24 d)$ & $\mathrm{O}$ VI \\
\hline
\end{tabular}




\begin{tabular}{|c|c|c|c|c|}
\hline $\mathbf{B}_{242}$ & $=\left(\frac{1}{2}-x_{14}\right) \mathbf{a}_{1}-y_{14} \mathbf{a}_{2}+\left(\frac{1}{2}+z_{14}\right) \mathbf{a}_{3}$ & $=\left(\frac{1}{2}-x_{14}\right) a \hat{\mathbf{x}}-y_{14} a \hat{\mathbf{y}}+\left(\frac{1}{2}+z_{14}\right) a \hat{\mathbf{z}}$ & $(24 d)$ & $\mathrm{O} \mathrm{VI}$ \\
\hline $\mathbf{B}_{243}$ & $\begin{array}{c}-x_{14} \mathbf{a}_{1}+\left(\frac{1}{2}+y_{14}\right) \mathbf{a}_{2}+ \\
\left(\frac{1}{2}-z_{14}\right) \mathbf{a}_{3}\end{array}$ & $\begin{array}{c}-x_{14} a \hat{\mathbf{x}}+\left(\frac{1}{2}+y_{14}\right) a \hat{\mathbf{y}}+ \\
\left(\frac{1}{2}-z_{14}\right) a \hat{\mathbf{z}}\end{array}$ & $(24 d)$ & $\mathrm{O}$ VI \\
\hline $\mathbf{B}_{244}$ & $=\left(\frac{1}{2}+x_{14}\right) \mathbf{a}_{1}+\left(\frac{1}{2}-y_{14}\right) \mathbf{a}_{2}-z_{14} \mathbf{a}_{3}$ & $=\left(\frac{1}{2}+x_{14}\right) a \hat{\mathbf{x}}+\left(\frac{1}{2}-y_{14}\right) a \hat{\mathbf{y}}-z_{14} a \hat{\mathbf{z}}$ & $(24 d)$ & O VI \\
\hline $\mathbf{B}_{245}$ & $z_{14} \mathbf{a}_{1}+x_{14} \mathbf{a}_{2}+y_{14} \mathbf{a}_{3}$ & $z_{14} a \hat{\mathbf{x}}+x_{14} a \hat{\mathbf{y}}+y_{14} a \hat{\mathbf{z}}$ & $(24 d)$ & $\mathrm{O}$ VI \\
\hline $\mathbf{B}_{246}$ & $=\left(\frac{1}{2}+z_{14}\right) \mathbf{a}_{1}+\left(\frac{1}{2}-x_{14}\right) \mathbf{a}_{2}-y_{14} \mathbf{a}_{3}$ & $=\left(\frac{1}{2}+z_{14}\right) a \hat{\mathbf{x}}+\left(\frac{1}{2}-x_{14}\right) a \hat{\mathbf{y}}-y_{14} a \hat{\mathbf{z}}$ & $(24 d)$ & $\mathrm{O}$ VI \\
\hline $\mathbf{B}_{247}$ & $=\left(\frac{1}{2}-z_{14}\right) \mathbf{a}_{1}-x_{14} \mathbf{a}_{2}+\left(\frac{1}{2}+y_{14}\right) \mathbf{a}_{3}$ & $=\left(\frac{1}{2}-z_{14}\right) a \hat{\mathbf{x}}-x_{14} a \hat{\mathbf{y}}+\left(\frac{1}{2}+y_{14}\right) a \hat{\mathbf{z}}$ & $(24 d)$ & $\mathrm{O}$ VI \\
\hline $\mathbf{B}_{248}$ & $\begin{array}{c}-z_{14} \mathbf{a}_{1}+\left(\frac{1}{2}+x_{14}\right) \mathbf{a}_{2}+ \\
\left(\frac{1}{2}-y_{14}\right) \mathbf{a}_{3}\end{array}$ & $\begin{array}{c}-z_{14} a \hat{\mathbf{x}}+\left(\frac{1}{2}+x_{14}\right) a \hat{\mathbf{y}}+ \\
\left(\frac{1}{2}-y_{14}\right) a \hat{\mathbf{z}}\end{array}$ & $(24 d)$ & $\mathrm{O}$ VI \\
\hline $\mathbf{B}_{249}$ & $y_{14} \mathbf{a}_{1}+z_{14} \mathbf{a}_{2}+x_{14} \mathbf{a}_{3}$ & $y_{14} a \hat{\mathbf{x}}+z_{14} a \hat{\mathbf{y}}+x_{14} a \hat{\mathbf{z}}$ & $(24 d)$ & $\mathrm{O} \mathrm{VI}$ \\
\hline $\mathbf{B}_{250}$ & $\begin{array}{c}-y_{14} \mathbf{a}_{1}+\left(\frac{1}{2}+z_{14}\right) \mathbf{a}_{2}+ \\
\left(\frac{1}{2}-x_{14}\right) \mathbf{a}_{3}\end{array}$ & $\begin{array}{c}-y_{14} a \hat{\mathbf{x}}+\left(\frac{1}{2}+z_{14}\right) a \hat{\mathbf{y}}+ \\
\left(\frac{1}{2}-x_{14}\right) a \hat{\mathbf{z}}\end{array}$ & $(24 d)$ & $\mathrm{O}$ VI \\
\hline $\mathbf{B}_{251}$ & $=\left(\frac{1}{2}+y_{14}\right) \mathbf{a}_{1}+\left(\frac{1}{2}-z_{14}\right) \mathbf{a}_{2}-x_{14} \mathbf{a}_{3}$ & $=\left(\frac{1}{2}+y_{14}\right) a \hat{\mathbf{x}}+\left(\frac{1}{2}-z_{14}\right) a \hat{\mathbf{y}}-x_{14} a \hat{\mathbf{z}}$ & $(24 d)$ & O VI \\
\hline $\mathbf{B}_{252}$ & $=\left(\frac{1}{2}-y_{14}\right) \mathbf{a}_{1}-z_{14} \mathbf{a}_{2}+\left(\frac{1}{2}+x_{14}\right) \mathbf{a}_{3}$ & $=\left(\frac{1}{2}-y_{14}\right) a \hat{\mathbf{x}}-z_{14} a \hat{\mathbf{y}}+\left(\frac{1}{2}+x_{14}\right) a \hat{\mathbf{z}}$ & $(24 d)$ & $\mathrm{O}$ VI \\
\hline $\mathbf{B}_{253}$ & $-x_{14} \mathbf{a}_{1}-y_{14} \mathbf{a}_{2}-z_{14} \mathbf{a}_{3}$ & $-x_{14} a \hat{\mathbf{x}}-y_{14} a \hat{\mathbf{y}}-z_{14} a \hat{\mathbf{z}}$ & $(24 d)$ & $\mathrm{O}$ VI \\
\hline $\mathbf{B}_{254}$ & $=\left(\frac{1}{2}+x_{14}\right) \mathbf{a}_{1}+y_{14} \mathbf{a}_{2}+\left(\frac{1}{2}-z_{14}\right) \mathbf{a}_{3}$ & $=\left(\frac{1}{2}+x_{14}\right) a \hat{\mathbf{x}}+y_{14} a \hat{\mathbf{y}}+\left(\frac{1}{2}-z_{14}\right) a \hat{\mathbf{z}}$ & $(24 d)$ & $\mathrm{O}$ VI \\
\hline $\mathbf{B}_{255}$ & $=x_{14} \mathbf{a}_{1}+\left(\frac{1}{2}-y_{14}\right) \mathbf{a}_{2}+\left(\frac{1}{2}+z_{14}\right) \mathbf{a}_{3}$ & $=\quad x_{14} a \hat{\mathbf{x}}+\left(\frac{1}{2}-y_{14}\right) a \hat{\mathbf{y}}+\left(\frac{1}{2}+z_{14}\right) a \hat{\mathbf{z}}$ & $(24 d)$ & $\mathrm{O} \mathrm{VI}$ \\
\hline $\mathbf{B}_{256}$ & $=\left(\frac{1}{2}-x_{14}\right) \mathbf{a}_{1}+\left(\frac{1}{2}+y_{14}\right) \mathbf{a}_{2}+z_{14} \mathbf{a}_{3}$ & $=\left(\frac{1}{2}-x_{14}\right) a \hat{\mathbf{x}}+\left(\frac{1}{2}+y_{14}\right) a \hat{\mathbf{y}}+z_{14} a \hat{\mathbf{z}}$ & $(24 d)$ & $\mathrm{O} \mathrm{VI}$ \\
\hline $\mathbf{B}_{257}$ & $-z_{14} \mathbf{a}_{1}-x_{14} \mathbf{a}_{2}-y_{14} \mathbf{a}_{3}$ & $-z_{14} a \hat{\mathbf{x}}-x_{14} a \hat{\mathbf{y}}-y_{14} a \hat{\mathbf{z}}$ & $(24 d)$ & $\mathrm{O} \mathrm{VI}$ \\
\hline $\mathbf{B}_{258}$ & $=\left(\frac{1}{2}-z_{14}\right) \mathbf{a}_{1}+\left(\frac{1}{2}+x_{14}\right) \mathbf{a}_{2}+y_{14} \mathbf{a}_{3}$ & $=\left(\frac{1}{2}-z_{14}\right) a \hat{\mathbf{x}}+\left(\frac{1}{2}+x_{14}\right) a \hat{\mathbf{y}}+y_{14} a \hat{\mathbf{z}}$ & $(24 d)$ & $\mathrm{O}$ VI \\
\hline $\mathbf{B}_{259}$ & $=\left(\frac{1}{2}+z_{14}\right) \mathbf{a}_{1}+x_{14} \mathbf{a}_{2}+\left(\frac{1}{2}-y_{14}\right) \mathbf{a}_{3}$ & $=\left(\frac{1}{2}+z_{14}\right) a \hat{\mathbf{x}}+x_{14} a \hat{\mathbf{y}}+\left(\frac{1}{2}-y_{14}\right) a \hat{\mathbf{z}}$ & $(24 d)$ & $\mathrm{O}$ VI \\
\hline $\mathbf{B}_{260}$ & $=z_{14} \mathbf{a}_{1}+\left(\frac{1}{2}-x_{14}\right) \mathbf{a}_{2}+\left(\frac{1}{2}+y_{14}\right) \mathbf{a}_{3}$ & $=z_{14} a \hat{\mathbf{x}}+\left(\frac{1}{2}-x_{14}\right) a \hat{\mathbf{y}}+\left(\frac{1}{2}+y_{14}\right) a \hat{\mathbf{z}}$ & $(24 d)$ & $\mathrm{O} \mathrm{VI}$ \\
\hline $\mathbf{B}_{261}$ & $-y_{14} \mathbf{a}_{1}-z_{14} \mathbf{a}_{2}-x_{14} \mathbf{a}_{3}$ & $-y_{14} a \hat{\mathbf{x}}-z_{14} a \hat{\mathbf{y}}-x_{14} a \hat{\mathbf{z}}$ & $(24 d)$ & O VI \\
\hline $\mathbf{B}_{262}$ & $=y_{14} \mathbf{a}_{1}+\left(\frac{1}{2}-z_{14}\right) \mathbf{a}_{2}+\left(\frac{1}{2}+x_{14}\right) \mathbf{a}_{3}$ & $=y_{14} a \hat{\mathbf{x}}+\left(\frac{1}{2}-z_{14}\right) a \hat{\mathbf{y}}+\left(\frac{1}{2}+x_{14}\right) a \hat{\mathbf{z}}$ & $(24 d)$ & $\mathrm{O} \mathrm{VI}$ \\
\hline $\mathbf{B}_{263}$ & $=\left(\frac{1}{2}-y_{14}\right) \mathbf{a}_{1}+\left(\frac{1}{2}+z_{14}\right) \mathbf{a}_{2}+x_{14} \mathbf{a}_{3}$ & $=\left(\frac{1}{2}-y_{14}\right) a \hat{\mathbf{x}}+\left(\frac{1}{2}+z_{14}\right) a \hat{\mathbf{y}}+x_{14} a \hat{\mathbf{z}}$ & $(24 d)$ & $\mathrm{O}$ VI \\
\hline $\mathbf{B}_{264}$ & $=\left(\frac{1}{2}+y_{14}\right) \mathbf{a}_{1}+z_{14} \mathbf{a}_{2}+\left(\frac{1}{2}-x_{14}\right) \mathbf{a}_{3}$ & $=\left(\frac{1}{2}+y_{14}\right) a \hat{\mathbf{x}}+z_{14} a \hat{\mathbf{y}}+\left(\frac{1}{2}-x_{14}\right) a \hat{\mathbf{z}}$ & $(24 d)$ & $\mathrm{O}$ VI \\
\hline
\end{tabular}

\section{References:}

- P. Mondal and J. W. Jeffery, The crystal structure of tricalcium aluminate, $\mathrm{Ca}_{3} \mathrm{Al}_{2} \mathrm{O}_{6}$, Acta Crystallogr. Sect. B Struct. Sci. 31, 689-697 (1975), doi:10.1107/S0567740875003639.

\section{Geometry files:}

- CIF: pp. 955

- POSCAR: pp. 955 


\section{Simple Cubic $\mathrm{C}_{60}$ Buckminsterfullerine Structure:} A_cP240_205_10d
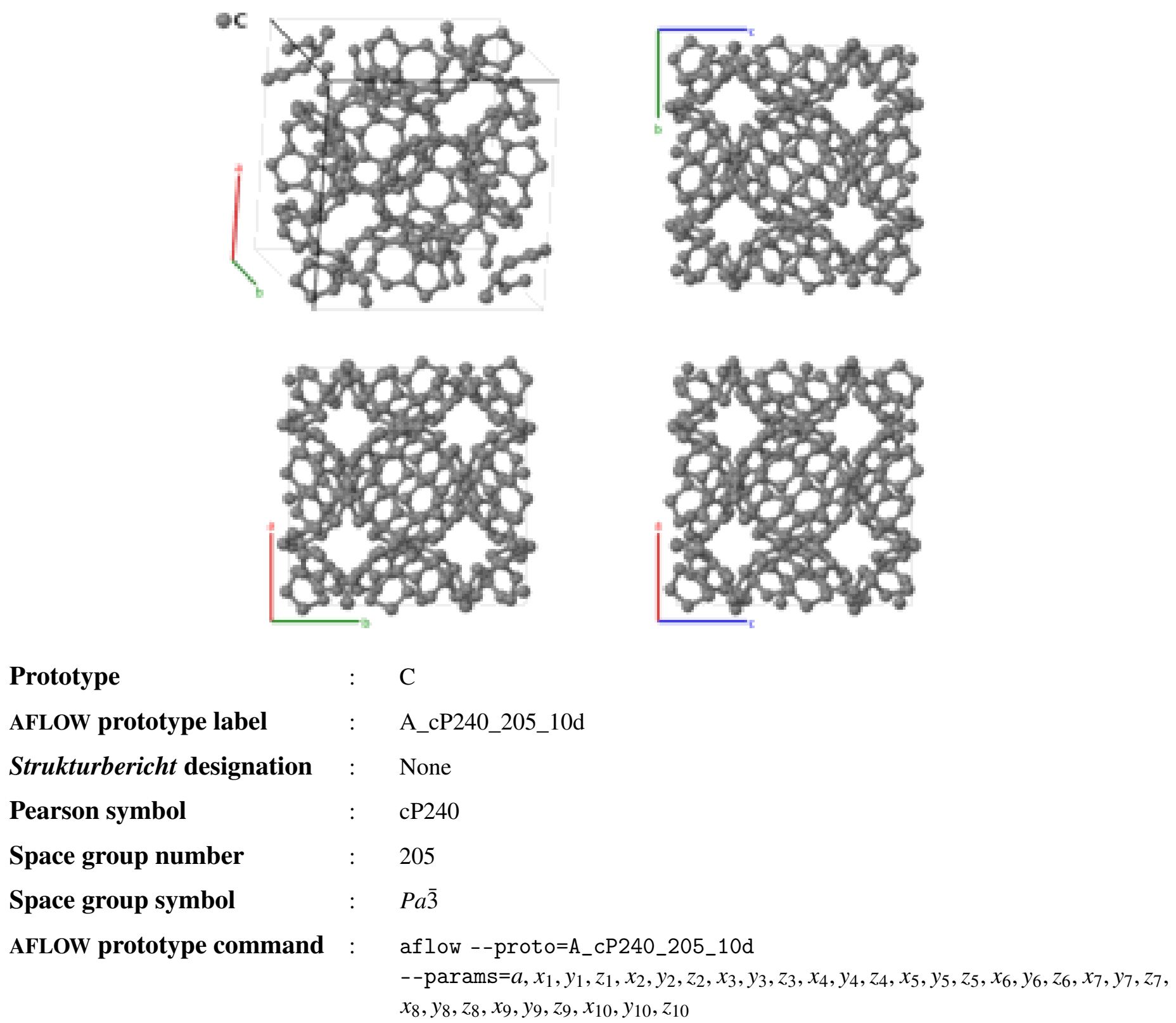

- This is the experimentally determined structure of $\mathrm{C}_{60}$ buckminsterfullerne (a.k.a "buckyballs") below $249 \mathrm{~K}$. Above that temperature the $\mathrm{C}_{60}$ molecules are orientationally disordered and set on a face-centered cubic lattice. For computational purposes that structure is approximated by the $\mathrm{FCC}_{60}$ buckminsterfullerine structure.

\section{Simple Cubic primitive vectors:}

$$
\begin{aligned}
& \mathbf{a}_{1}=a \hat{\mathbf{x}} \\
& \mathbf{a}_{2}=a \hat{\mathbf{y}} \\
& \mathbf{a}_{3}=a \hat{\mathbf{z}}
\end{aligned}
$$

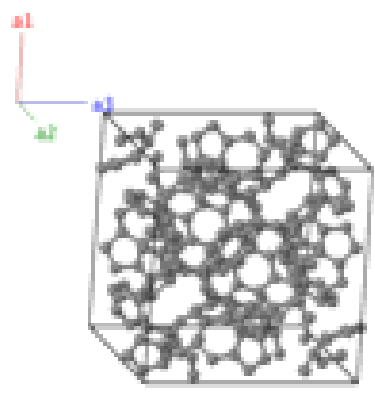




\section{Basis vectors:}

\section{Lattice Coordinates}

$\mathbf{B}_{1}=$

$x_{1} \mathbf{a}_{1}+y_{1} \mathbf{a}_{2}+z_{1} \mathbf{a}_{3}$

$\mathbf{B}_{2}$

$\mathbf{B}_{3}$

$\mathbf{B}_{4}$

$\mathbf{B}_{5}=$

$\mathbf{B}_{6}=\left(\frac{1}{2}+z_{1}\right) \mathbf{a}_{1}+\left(\frac{1}{2}-x_{1}\right) \mathbf{a}_{2}-y_{1} \mathbf{a}_{3}$

$\mathbf{B}_{7}=\left(\frac{1}{2}-z_{1}\right) \mathbf{a}_{1}-x_{1} \mathbf{a}_{2}+\left(\frac{1}{2}+y_{1}\right) \mathbf{a}_{3}$

$\mathbf{B}_{8}=-z_{1} \mathbf{a}_{1}+\left(\frac{1}{2}+x_{1}\right) \mathbf{a}_{2}+\left(\frac{1}{2}-y_{1}\right) \mathbf{a}_{3}$

$\mathbf{B}_{9}=y_{1} \mathbf{a}_{1}+z_{1} \mathbf{a}_{2}+x_{1} \mathbf{a}_{3}$

$\mathbf{B}_{10}=-y_{1} \mathbf{a}_{1}+\left(\frac{1}{2}+z_{1}\right) \mathbf{a}_{2}+\left(\frac{1}{2}-x_{1}\right) \mathbf{a}_{3}$

$\mathbf{B}_{11}=\left(\frac{1}{2}+y_{1}\right) \mathbf{a}_{1}+\left(\frac{1}{2}-z_{1}\right) \mathbf{a}_{2}-x_{1} \mathbf{a}_{3}$

$\mathbf{B}_{12}=\left(\frac{1}{2}-y_{1}\right) \mathbf{a}_{1}-z_{1} \mathbf{a}_{2}+\left(\frac{1}{2}+x_{1}\right) \mathbf{a}_{3}$

$\mathbf{B}_{13}=\quad-x_{1} \mathbf{a}_{1}-y_{1} \mathbf{a}_{2}-z_{1} \mathbf{a}_{3}$

$\mathbf{B}_{14}=\left(\frac{1}{2}+x_{1}\right) \mathbf{a}_{1}+y_{1} \mathbf{a}_{2}+\left(\frac{1}{2}-z_{1}\right) \mathbf{a}_{3}$

$\mathbf{B}_{15}=x_{1} \mathbf{a}_{1}+\left(\frac{1}{2}-y_{1}\right) \mathbf{a}_{2}+\left(\frac{1}{2}+z_{1}\right) \mathbf{a}_{3}$

$\mathbf{B}_{16}=\left(\frac{1}{2}-x_{1}\right) \mathbf{a}_{1}+\left(\frac{1}{2}+y_{1}\right) \mathbf{a}_{2}+z_{1} \mathbf{a}_{3}$

$\mathbf{B}_{17}=-z_{1} \mathbf{a}_{1}-x_{1} \mathbf{a}_{2}-y_{1} \mathbf{a}_{3}$

$\mathbf{B}_{18}=\left(\frac{1}{2}-z_{1}\right) \mathbf{a}_{1}+\left(\frac{1}{2}+x_{1}\right) \mathbf{a}_{2}+y_{1} \mathbf{a}_{3}$

$\mathbf{B}_{19}=\left(\frac{1}{2}+z_{1}\right) \mathbf{a}_{1}+x_{1} \mathbf{a}_{2}+\left(\frac{1}{2}-y_{1}\right) \mathbf{a}_{3}$

$\mathbf{B}_{20}=z_{1} \mathbf{a}_{1}+\left(\frac{1}{2}-x_{1}\right) \mathbf{a}_{2}+\left(\frac{1}{2}+y_{1}\right) \mathbf{a}_{3}$

$\mathbf{B}_{21}=-y_{1} \mathbf{a}_{1}-z_{1} \mathbf{a}_{2}-x_{1} \mathbf{a}_{3}$

$\mathbf{B}_{22}=y_{1} \mathbf{a}_{1}+\left(\frac{1}{2}-z_{1}\right) \mathbf{a}_{2}+\left(\frac{1}{2}+x_{1}\right) \mathbf{a}_{3}$

$\mathbf{B}_{23}=\left(\frac{1}{2}-y_{1}\right) \mathbf{a}_{1}+\left(\frac{1}{2}+z_{1}\right) \mathbf{a}_{2}+x_{1} \mathbf{a}_{3}$

$\mathbf{B}_{24}=\left(\frac{1}{2}+y_{1}\right) \mathbf{a}_{1}+z_{1} \mathbf{a}_{2}+\left(\frac{1}{2}-x_{1}\right) \mathbf{a}_{3}$

$\mathbf{B}_{25}=x_{2} \mathbf{a}_{1}+y_{2} \mathbf{a}_{2}+z_{2} \mathbf{a}_{3}$

$\mathbf{B}_{26}=\left(\frac{1}{2}-x_{2}\right) \mathbf{a}_{1}-y_{2} \mathbf{a}_{2}+\left(\frac{1}{2}+z_{2}\right) \mathbf{a}_{3}$

$\mathbf{B}_{27}=-x_{2} \mathbf{a}_{1}+\left(\frac{1}{2}+y_{2}\right) \mathbf{a}_{2}+\left(\frac{1}{2}-z_{2}\right) \mathbf{a}_{3}$

$\mathbf{B}_{28}=\left(\frac{1}{2}+x_{2}\right) \mathbf{a}_{1}+\left(\frac{1}{2}-y_{2}\right) \mathbf{a}_{2}-z_{2} \mathbf{a}_{3}$

$\mathbf{B}_{29}=z_{2} \mathbf{a}_{1}+x_{2} \mathbf{a}_{2}+y_{2} \mathbf{a}_{3}$

$\mathbf{B}_{30}=\left(\frac{1}{2}+z_{2}\right) \mathbf{a}_{1}+\left(\frac{1}{2}-x_{2}\right) \mathbf{a}_{2}-y_{2} \mathbf{a}_{3}$

$\mathbf{B}_{31}=\left(\frac{1}{2}-z_{2}\right) \mathbf{a}_{1}-x_{2} \mathbf{a}_{2}+\left(\frac{1}{2}+y_{2}\right) \mathbf{a}_{3}$

$\mathbf{B}_{32}=-z_{2} \mathbf{a}_{1}+\left(\frac{1}{2}+x_{2}\right) \mathbf{a}_{2}+\left(\frac{1}{2}-y_{2}\right) \mathbf{a}_{3}$

$\mathbf{B}_{33}=y_{2} \mathbf{a}_{1}+z_{2} \mathbf{a}_{2}+x_{2} \mathbf{a}_{3}$

$\mathbf{B}_{34}=-y_{2} \mathbf{a}_{1}+\left(\frac{1}{2}+z_{2}\right) \mathbf{a}_{2}+\left(\frac{1}{2}-x_{2}\right) \mathbf{a}_{3}$

\section{Cartesian Coordinates}

$=\quad x_{1} a \hat{\mathbf{x}}+y_{1} a \hat{\mathbf{y}}+z_{1} a \hat{\mathbf{z}}$

$=\left(\frac{1}{2}-x_{1}\right) a \hat{\mathbf{x}}-y_{1} a \hat{\mathbf{y}}+\left(\frac{1}{2}+z_{1}\right) a \hat{\mathbf{z}}$

$=-x_{1} a \hat{\mathbf{x}}+\left(\frac{1}{2}+y_{1}\right) a \hat{\mathbf{y}}+\left(\frac{1}{2}-z_{1}\right) a \hat{\mathbf{z}}$

$=\left(\frac{1}{2}+x_{1}\right) a \hat{\mathbf{x}}+\left(\frac{1}{2}-y_{1}\right) a \hat{\mathbf{y}}-z_{1} a \hat{\mathbf{z}}$

$=\quad z_{1} a \hat{\mathbf{x}}+x_{1} a \hat{\mathbf{y}}+y_{1} a \hat{\mathbf{z}}$

$=\left(\frac{1}{2}+z_{1}\right) a \hat{\mathbf{x}}+\left(\frac{1}{2}-x_{1}\right) a \hat{\mathbf{y}}-y_{1} a \hat{\mathbf{z}}$

$=\left(\frac{1}{2}-z_{1}\right) a \hat{\mathbf{x}}-x_{1} a \hat{\mathbf{y}}+\left(\frac{1}{2}+y_{1}\right) a \hat{\mathbf{z}}$

$=-z_{1} a \hat{\mathbf{x}}+\left(\frac{1}{2}+x_{1}\right) a \hat{\mathbf{y}}+\left(\frac{1}{2}-y_{1}\right) a \hat{\mathbf{z}}$

$=\quad y_{1} a \hat{\mathbf{x}}+z_{1} a \hat{\mathbf{y}}+x_{1} a \hat{\mathbf{z}}$

$=-y_{1} a \hat{\mathbf{x}}+\left(\frac{1}{2}+z_{1}\right) a \hat{\mathbf{y}}+\left(\frac{1}{2}-x_{1}\right) a \hat{\mathbf{z}}$

$=\left(\frac{1}{2}+y_{1}\right) a \hat{\mathbf{x}}+\left(\frac{1}{2}-z_{1}\right) a \hat{\mathbf{y}}-x_{1} a \hat{\mathbf{z}}$

$=\left(\frac{1}{2}-y_{1}\right) a \hat{\mathbf{x}}-z_{1} a \hat{\mathbf{y}}+\left(\frac{1}{2}+x_{1}\right) a \hat{\mathbf{z}}$

$=\quad-x_{1} a \hat{\mathbf{x}}-y_{1} a \hat{\mathbf{y}}-z_{1} a \hat{\mathbf{z}}$

$=\left(\frac{1}{2}+x_{1}\right) a \hat{\mathbf{x}}+y_{1} a \hat{\mathbf{y}}+\left(\frac{1}{2}-z_{1}\right) a \hat{\mathbf{z}}$

$=x_{1} a \hat{\mathbf{x}}+\left(\frac{1}{2}-y_{1}\right) a \hat{\mathbf{y}}+\left(\frac{1}{2}+z_{1}\right) a \hat{\mathbf{z}}$

$=\left(\frac{1}{2}-x_{1}\right) a \hat{\mathbf{x}}+\left(\frac{1}{2}+y_{1}\right) a \hat{\mathbf{y}}+z_{1} a \hat{\mathbf{z}}$

$=\quad-z_{1} a \hat{\mathbf{x}}-x_{1} a \hat{\mathbf{y}}-y_{1} a \hat{\mathbf{z}}$

$=\left(\frac{1}{2}-z_{1}\right) a \hat{\mathbf{x}}+\left(\frac{1}{2}+x_{1}\right) a \hat{\mathbf{y}}+y_{1} a \hat{\mathbf{z}}$

$=\left(\frac{1}{2}+z_{1}\right) a \hat{\mathbf{x}}+x_{1} a \hat{\mathbf{y}}+\left(\frac{1}{2}-y_{1}\right) a \hat{\mathbf{z}}$

$=z_{1} a \hat{\mathbf{x}}+\left(\frac{1}{2}-x_{1}\right) a \hat{\mathbf{y}}+\left(\frac{1}{2}+y_{1}\right) a \hat{\mathbf{z}}$

$=\quad-y_{1} a \hat{\mathbf{x}}-z_{1} a \hat{\mathbf{y}}-x_{1} a \hat{\mathbf{z}}$

$=y_{1} a \hat{\mathbf{x}}+\left(\frac{1}{2}-z_{1}\right) a \hat{\mathbf{y}}+\left(\frac{1}{2}+x_{1}\right) a \hat{\mathbf{z}}$

$=\left(\frac{1}{2}-y_{1}\right) a \hat{\mathbf{x}}+\left(\frac{1}{2}+z_{1}\right) a \hat{\mathbf{y}}+x_{1} a \hat{\mathbf{z}}$

$=\left(\frac{1}{2}+y_{1}\right) a \hat{\mathbf{x}}+z_{1} a \hat{\mathbf{y}}+\left(\frac{1}{2}-x_{1}\right) a \hat{\mathbf{z}}$

$=\quad x_{2} a \hat{\mathbf{x}}+y_{2} a \hat{\mathbf{y}}+z_{2} a \hat{\mathbf{z}}$

$=\left(\frac{1}{2}-x_{2}\right) a \hat{\mathbf{x}}-y_{2} a \hat{\mathbf{y}}+\left(\frac{1}{2}+z_{2}\right) a \hat{\mathbf{z}}$

$=-x_{2} a \hat{\mathbf{x}}+\left(\frac{1}{2}+y_{2}\right) a \hat{\mathbf{y}}+\left(\frac{1}{2}-z_{2}\right) a \hat{\mathbf{z}}$

$=\left(\frac{1}{2}+x_{2}\right) a \hat{\mathbf{x}}+\left(\frac{1}{2}-y_{2}\right) a \hat{\mathbf{y}}-z_{2} a \hat{\mathbf{z}}$

$=\quad z_{2} a \hat{\mathbf{x}}+x_{2} a \hat{\mathbf{y}}+y_{2} a \hat{\mathbf{z}}$

$=\left(\frac{1}{2}+z_{2}\right) a \hat{\mathbf{x}}+\left(\frac{1}{2}-x_{2}\right) a \hat{\mathbf{y}}-y_{2} a \hat{\mathbf{z}}$

$=\left(\frac{1}{2}-z_{2}\right) a \hat{\mathbf{x}}-x_{2} a \hat{\mathbf{y}}+\left(\frac{1}{2}+y_{2}\right) a \hat{\mathbf{z}}$

$=-z_{2} a \hat{\mathbf{x}}+\left(\frac{1}{2}+x_{2}\right) a \hat{\mathbf{y}}+\left(\frac{1}{2}-y_{2}\right) a \hat{\mathbf{z}}$

$=\quad y_{2} a \hat{\mathbf{x}}+z_{2} a \hat{\mathbf{y}}+x_{2} a \hat{\mathbf{z}}$

$=-y_{2} a \hat{\mathbf{x}}+\left(\frac{1}{2}+z_{2}\right) a \hat{\mathbf{y}}+\left(\frac{1}{2}-x_{2}\right) a \hat{\mathbf{z}}$
Wyckoff Position Atom Type

$$
(24 d)
$$

C I

(24d)

(24d)

(24d)

$(24 d)$

(24d)

(24d)

(24d)

(24d)

(24d)

(24d)

(24d)

(24d)

(24d)

(24d)

(24d)

(24d)

(24d)

(24d)

(24d)

(24d)

(24d)

(24d)

(24d)

(24d)

(24d)

(24d)

(24d)

(24d)

(24d)

(24d)

(24d)

(24d)

(24d)
C I

C I

C I

C I

C I

C I

C I

C I

C I

C I

C I

C I

C I

C I

C I

C I

C I

C I

C I

C I

C I

C I

C I

C II

C II

C II

C II

C II

C II

C II

C II

C II

C II 


\begin{tabular}{|c|c|c|c|c|c|c|}
\hline $\mathbf{B}_{35}$ & $=$ & $\left(\frac{1}{2}+y_{2}\right) \mathbf{a}_{1}+\left(\frac{1}{2}-z_{2}\right) \mathbf{a}_{2}-x_{2} \mathbf{a}_{3}$ & $=$ & $\left(\frac{1}{2}+y_{2}\right) a \hat{\mathbf{x}}+\left(\frac{1}{2}-z_{2}\right) a \hat{\mathbf{y}}-x_{2} a \hat{\mathbf{z}}$ & $(24 d)$ & C II \\
\hline $\mathbf{B}_{36}$ & $=$ & $\left(\frac{1}{2}-y_{2}\right) \mathbf{a}_{1}-z_{2} \mathbf{a}_{2}+\left(\frac{1}{2}+x_{2}\right) \mathbf{a}_{3}$ & $=$ & $\left(\frac{1}{2}-y_{2}\right) a \hat{\mathbf{x}}-z_{2} a \hat{\mathbf{y}}+\left(\frac{1}{2}+x_{2}\right) a \hat{\mathbf{z}}$ & $(24 d)$ & C II \\
\hline $\mathbf{B}_{37}$ & $=$ & $-x_{2} \mathbf{a}_{1}-y_{2} \mathbf{a}_{2}-z_{2} \mathbf{a}_{3}$ & $=$ & $-x_{2} a \hat{\mathbf{x}}-y_{2} a \hat{\mathbf{y}}-z_{2} a \hat{\mathbf{z}}$ & $(24 d)$ & C II \\
\hline $\mathbf{B}_{38}$ & $=$ & $\left(\frac{1}{2}+x_{2}\right) \mathbf{a}_{1}+y_{2} \mathbf{a}_{2}+\left(\frac{1}{2}-z_{2}\right) \mathbf{a}_{3}$ & $=$ & $\left(\frac{1}{2}+x_{2}\right) a \hat{\mathbf{x}}+y_{2} a \hat{\mathbf{y}}+\left(\frac{1}{2}-z_{2}\right) a \hat{\mathbf{z}}$ & $(24 d)$ & C II \\
\hline $\mathbf{B}_{39}$ & $=$ & $x_{2} \mathbf{a}_{1}+\left(\frac{1}{2}-y_{2}\right) \mathbf{a}_{2}+\left(\frac{1}{2}+z_{2}\right) \mathbf{a}_{3}$ & $=$ & $x_{2} a \hat{\mathbf{x}}+\left(\frac{1}{2}-y_{2}\right) a \hat{\mathbf{y}}+\left(\frac{1}{2}+z_{2}\right) a \hat{\mathbf{z}}$ & $(24 d)$ & C II \\
\hline $\mathbf{B}_{40}$ & $=$ & $\left(\frac{1}{2}-x_{2}\right) \mathbf{a}_{1}+\left(\frac{1}{2}+y_{2}\right) \mathbf{a}_{2}+z_{2} \mathbf{a}_{3}$ & $=$ & $\left(\frac{1}{2}-x_{2}\right) a \hat{\mathbf{x}}+\left(\frac{1}{2}+y_{2}\right) a \hat{\mathbf{y}}+z_{2} a \hat{\mathbf{z}}$ & $(24 d)$ & C II \\
\hline $\mathbf{B}_{41}$ & $=$ & $-z_{2} \mathbf{a}_{1}-x_{2} \mathbf{a}_{2}-y_{2} \mathbf{a}_{3}$ & $=$ & $-z_{2} a \hat{\mathbf{x}}-x_{2} a \hat{\mathbf{y}}-y_{2} a \hat{\mathbf{z}}$ & $(24 d)$ & C II \\
\hline $\mathbf{B}_{42}$ & $=$ & $\left(\frac{1}{2}-z_{2}\right) \mathbf{a}_{1}+\left(\frac{1}{2}+x_{2}\right) \mathbf{a}_{2}+y_{2} \mathbf{a}_{3}$ & $=$ & $\left(\frac{1}{2}-z_{2}\right) a \hat{\mathbf{x}}+\left(\frac{1}{2}+x_{2}\right) a \hat{\mathbf{y}}+y_{2} a \hat{\mathbf{z}}$ & $(24 d)$ & C II \\
\hline $\mathbf{B}_{43}$ & $=$ & $\left(\frac{1}{2}+z_{2}\right) \mathbf{a}_{1}+x_{2} \mathbf{a}_{2}+\left(\frac{1}{2}-y_{2}\right) \mathbf{a}_{3}$ & $=$ & $\left(\frac{1}{2}+z_{2}\right) a \hat{\mathbf{x}}+x_{2} a \hat{\mathbf{y}}+\left(\frac{1}{2}-y_{2}\right) a \hat{\mathbf{z}}$ & $(24 d)$ & C II \\
\hline $\mathbf{B}_{44}$ & $=$ & $z_{2} \mathbf{a}_{1}+\left(\frac{1}{2}-x_{2}\right) \mathbf{a}_{2}+\left(\frac{1}{2}+y_{2}\right) \mathbf{a}_{3}$ & $=$ & $z_{2} a \hat{\mathbf{x}}+\left(\frac{1}{2}-x_{2}\right) a \hat{\mathbf{y}}+\left(\frac{1}{2}+y_{2}\right) a \hat{\mathbf{z}}$ & $(24 d)$ & C II \\
\hline $\mathbf{B}_{45}$ & $=$ & $-y_{2} \mathbf{a}_{1}-z_{2} \mathbf{a}_{2}-x_{2} \mathbf{a}_{3}$ & $=$ & $-y_{2} a \hat{\mathbf{x}}-z_{2} a \hat{\mathbf{y}}-x_{2} a \hat{\mathbf{z}}$ & $(24 d)$ & C II \\
\hline $\mathbf{B}_{46}$ & $=$ & $y_{2} \mathbf{a}_{1}+\left(\frac{1}{2}-z_{2}\right) \mathbf{a}_{2}+\left(\frac{1}{2}+x_{2}\right) \mathbf{a}_{3}$ & $=$ & $y_{2} a \hat{\mathbf{x}}+\left(\frac{1}{2}-z_{2}\right) a \hat{\mathbf{y}}+\left(\frac{1}{2}+x_{2}\right) a \hat{\mathbf{z}}$ & $(24 d)$ & C II \\
\hline $\mathbf{B}_{47}$ & $=$ & $\left(\frac{1}{2}-y_{2}\right) \mathbf{a}_{1}+\left(\frac{1}{2}+z_{2}\right) \mathbf{a}_{2}+x_{2} \mathbf{a}_{3}$ & $=$ & $\left(\frac{1}{2}-y_{2}\right) a \hat{\mathbf{x}}+\left(\frac{1}{2}+z_{2}\right) a \hat{\mathbf{y}}+x_{2} a \hat{\mathbf{z}}$ & $(24 d)$ & C II \\
\hline $\mathbf{B}_{48}$ & $=$ & $\left(\frac{1}{2}+y_{2}\right) \mathbf{a}_{1}+z_{2} \mathbf{a}_{2}+\left(\frac{1}{2}-x_{2}\right) \mathbf{a}_{3}$ & $=$ & $\left(\frac{1}{2}+y_{2}\right) a \hat{\mathbf{x}}+z_{2} a \hat{\mathbf{y}}+\left(\frac{1}{2}-x_{2}\right) a \hat{\mathbf{z}}$ & $(24 d)$ & C II \\
\hline $\mathbf{B}_{49}$ & $=$ & $x_{3} \mathbf{a}_{1}+y_{3} \mathbf{a}_{2}+z_{3} \mathbf{a}_{3}$ & $=$ & $x_{3} a \hat{\mathbf{x}}+y_{3} a \hat{\mathbf{y}}+z_{3} a \hat{\mathbf{z}}$ & $(24 d)$ & C III \\
\hline $\mathbf{B}_{50}$ & $=$ & $\left(\frac{1}{2}-x_{3}\right) \mathbf{a}_{1}-y_{3} \mathbf{a}_{2}+\left(\frac{1}{2}+z_{3}\right) \mathbf{a}_{3}$ & $=$ & $\left(\frac{1}{2}-x_{3}\right) a \hat{\mathbf{x}}-y_{3} a \hat{\mathbf{y}}+\left(\frac{1}{2}+z_{3}\right) a \hat{\mathbf{z}}$ & $(24 d)$ & C III \\
\hline $\mathbf{B}_{51}$ & $=$ & $-x_{3} \mathbf{a}_{1}+\left(\frac{1}{2}+y_{3}\right) \mathbf{a}_{2}+\left(\frac{1}{2}-z_{3}\right) \mathbf{a}_{3}$ & $=$ & $-x_{3} a \hat{\mathbf{x}}+\left(\frac{1}{2}+y_{3}\right) a \hat{\mathbf{y}}+\left(\frac{1}{2}-z_{3}\right) a \hat{\mathbf{z}}$ & $(24 d)$ & III \\
\hline $\mathbf{B}_{52}$ & $=$ & $\left(\frac{1}{2}+x_{3}\right) \mathbf{a}_{1}+\left(\frac{1}{2}-y_{3}\right) \mathbf{a}_{2}-z_{3} \mathbf{a}_{3}$ & $=$ & $\left(\frac{1}{2}+x_{3}\right) a \hat{\mathbf{x}}+\left(\frac{1}{2}-y_{3}\right) a \hat{\mathbf{y}}-z_{3} a \hat{\mathbf{z}}$ & $(24 d)$ & C III \\
\hline $\mathbf{B}_{53}$ & $=$ & $z_{3} \mathbf{a}_{1}+x_{3} \mathbf{a}_{2}+y_{3} \mathbf{a}_{3}$ & $=$ & $z_{3} a \hat{\mathbf{x}}+x_{3} a \hat{\mathbf{y}}+y_{3} a \hat{\mathbf{z}}$ & $(24 d)$ & C III \\
\hline $\mathbf{B}_{54}$ & $=$ & $\left(\frac{1}{2}+z_{3}\right) \mathbf{a}_{1}+\left(\frac{1}{2}-x_{3}\right) \mathbf{a}_{2}-y_{3} \mathbf{a}_{3}$ & $=$ & $\left(\frac{1}{2}+z_{3}\right) a \hat{\mathbf{x}}+\left(\frac{1}{2}-x_{3}\right) a \hat{\mathbf{y}}-y_{3} a \hat{\mathbf{z}}$ & $(24 d)$ & C III \\
\hline $\mathbf{B}_{55}$ & $=$ & $\left(\frac{1}{2}-z_{3}\right) \mathbf{a}_{1}-x_{3} \mathbf{a}_{2}+\left(\frac{1}{2}+y_{3}\right) \mathbf{a}_{3}$ & $=$ & $\left(\frac{1}{2}-z_{3}\right) a \hat{\mathbf{x}}-x_{3} a \hat{\mathbf{y}}+\left(\frac{1}{2}+y_{3}\right) a \hat{\mathbf{z}}$ & $(24 d)$ & C III \\
\hline $\mathbf{B}_{56}$ & $=$ & $-z_{3} \mathbf{a}_{1}+\left(\frac{1}{2}+x_{3}\right) \mathbf{a}_{2}+\left(\frac{1}{2}-y_{3}\right) \mathbf{a}_{3}$ & $=$ & $-z_{3} a \hat{\mathbf{x}}+\left(\frac{1}{2}+x_{3}\right) a \hat{\mathbf{y}}+\left(\frac{1}{2}-y_{3}\right) a \hat{\mathbf{z}}$ & $(24 d)$ & C III \\
\hline $\mathbf{B}_{57}$ & $=$ & $y_{3} \mathbf{a}_{1}+z_{3} \mathbf{a}_{2}+x_{3} \mathbf{a}_{3}$ & $=$ & $y_{3} a \hat{\mathbf{x}}+z_{3} a \hat{\mathbf{y}}+x_{3} a \hat{\mathbf{z}}$ & $(24 d)$ & C III \\
\hline $\mathbf{B}_{58}$ & $=$ & $-y_{3} \mathbf{a}_{1}+\left(\frac{1}{2}+z_{3}\right) \mathbf{a}_{2}+\left(\frac{1}{2}-x_{3}\right) \mathbf{a}_{3}$ & $=$ & $-y_{3} a \hat{\mathbf{x}}+\left(\frac{1}{2}+z_{3}\right) a \hat{\mathbf{y}}+\left(\frac{1}{2}-x_{3}\right) a \hat{\mathbf{z}}$ & $(24 d)$ & C III \\
\hline $\mathbf{B}_{59}$ & $=$ & $\left(\frac{1}{2}+y_{3}\right) \mathbf{a}_{1}+\left(\frac{1}{2}-z_{3}\right) \mathbf{a}_{2}-x_{3} \mathbf{a}_{3}$ & $=$ & $\left(\frac{1}{2}+y_{3}\right) a \hat{\mathbf{x}}+\left(\frac{1}{2}-z_{3}\right) a \hat{\mathbf{y}}-x_{3} a \hat{\mathbf{z}}$ & $(24 d)$ & C III \\
\hline $\mathbf{B}_{60}$ & $=$ & $\left(\frac{1}{2}-y_{3}\right) \mathbf{a}_{1}-z_{3} \mathbf{a}_{2}+\left(\frac{1}{2}+x_{3}\right) \mathbf{a}_{3}$ & $=$ & $\left(\frac{1}{2}-y_{3}\right) a \hat{\mathbf{x}}-z_{3} a \hat{\mathbf{y}}+\left(\frac{1}{2}+x_{3}\right) a \hat{\mathbf{z}}$ & $(24 d)$ & C III \\
\hline $\mathbf{B}_{61}$ & $=$ & $-x_{3} \mathbf{a}_{1}-y_{3} \mathbf{a}_{2}-z_{3} \mathbf{a}_{3}$ & $=$ & $-x_{3} a \hat{\mathbf{x}}-y_{3} a \hat{\mathbf{y}}-z_{3} a \hat{\mathbf{z}}$ & $(24 d)$ & C III \\
\hline $\mathbf{B}_{62}$ & $=$ & $\left(\frac{1}{2}+x_{3}\right) \mathbf{a}_{1}+y_{3} \mathbf{a}_{2}+\left(\frac{1}{2}-z_{3}\right) \mathbf{a}_{3}$ & $=$ & $\left(\frac{1}{2}+x_{3}\right) a \hat{\mathbf{x}}+y_{3} a \hat{\mathbf{y}}+\left(\frac{1}{2}-z_{3}\right) a \hat{\mathbf{z}}$ & $(24 d)$ & C III \\
\hline $\mathbf{B}_{63}$ & $=$ & $x_{3} \mathbf{a}_{1}+\left(\frac{1}{2}-y_{3}\right) \mathbf{a}_{2}+\left(\frac{1}{2}+z_{3}\right) \mathbf{a}_{3}$ & $=$ & $x_{3} a \hat{\mathbf{x}}+\left(\frac{1}{2}-y_{3}\right) a \hat{\mathbf{y}}+\left(\frac{1}{2}+z_{3}\right) a \hat{\mathbf{z}}$ & $(24 d)$ & C III \\
\hline $\mathbf{B}_{64}$ & $=$ & $\left(\frac{1}{2}-x_{3}\right) \mathbf{a}_{1}+\left(\frac{1}{2}+y_{3}\right) \mathbf{a}_{2}+z_{3} \mathbf{a}_{3}$ & $=$ & $\left(\frac{1}{2}-x_{3}\right) a \hat{\mathbf{x}}+\left(\frac{1}{2}+y_{3}\right) a \hat{\mathbf{y}}+z_{3} a \hat{\mathbf{z}}$ & $(24 d)$ & C III \\
\hline $\mathbf{B}_{65}$ & $=$ & $-z_{3} \mathbf{a}_{1}-x_{3} \mathbf{a}_{2}-y_{3} \mathbf{a}_{3}$ & $=$ & $-z_{3} a \hat{\mathbf{x}}-x_{3} a \hat{\mathbf{y}}-y_{3} a \hat{\mathbf{z}}$ & $(24 d)$ & C III \\
\hline $\mathbf{B}_{66}$ & $=$ & $\left(\frac{1}{2}-z_{3}\right) \mathbf{a}_{1}+\left(\frac{1}{2}+x_{3}\right) \mathbf{a}_{2}+y_{3} \mathbf{a}_{3}$ & $=$ & $\left(\frac{1}{2}-z_{3}\right) a \hat{\mathbf{x}}+\left(\frac{1}{2}+x_{3}\right) a \hat{\mathbf{y}}+y_{3} a \hat{\mathbf{z}}$ & $(24 d)$ & C III \\
\hline $\mathbf{B}_{67}$ & $=$ & $\left(\frac{1}{2}+z_{3}\right) \mathbf{a}_{1}+x_{3} \mathbf{a}_{2}+\left(\frac{1}{2}-y_{3}\right) \mathbf{a}_{3}$ & $=$ & $\left(\frac{1}{2}+z_{3}\right) a \hat{\mathbf{x}}+x_{3} a \hat{\mathbf{y}}+\left(\frac{1}{2}-y_{3}\right) a \hat{\mathbf{z}}$ & $(24 d)$ & III \\
\hline $\mathbf{B}_{68}$ & $=$ & $z_{3} \mathbf{a}_{1}+\left(\frac{1}{2}-x_{3}\right) \mathbf{a}_{2}+\left(\frac{1}{2}+y_{3}\right) \mathbf{a}_{3}$ & $=$ & $z_{3} a \hat{\mathbf{x}}+\left(\frac{1}{2}-x_{3}\right) a \hat{\mathbf{y}}+\left(\frac{1}{2}+y_{3}\right) a \hat{\mathbf{z}}$ & $(24 d)$ & C III \\
\hline $\mathbf{B}_{69}$ & $=$ & $-y_{3} \mathbf{a}_{1}-z_{3} \mathbf{a}_{2}-x_{3} \mathbf{a}_{3}$ & $=$ & $-y_{3} a \hat{\mathbf{x}}-z_{3} a \hat{\mathbf{y}}-x_{3} a \hat{\mathbf{z}}$ & $(24 d)$ & C III \\
\hline $\mathbf{B}_{70}$ & $=$ & $y_{3} \mathbf{a}_{1}+\left(\frac{1}{2}-z_{3}\right) \mathbf{a}_{2}+\left(\frac{1}{2}+x_{3}\right) \mathbf{a}_{3}$ & $=$ & $y_{3} a \hat{\mathbf{x}}+\left(\frac{1}{2}-z_{3}\right) a \hat{\mathbf{y}}+\left(\frac{1}{2}+x_{3}\right) a \hat{\mathbf{z}}$ & $(24 d)$ & \\
\hline
\end{tabular}




\begin{tabular}{|c|c|c|c|c|c|c|}
\hline $\mathbf{B}_{71}$ & $=$ & $\left(\frac{1}{2}-y_{3}\right) \mathbf{a}_{1}+\left(\frac{1}{2}+z_{3}\right) \mathbf{a}_{2}+x_{3} \mathbf{a}_{3}$ & $=$ & $\left(\frac{1}{2}-y_{3}\right) a \hat{\mathbf{x}}+\left(\frac{1}{2}+z_{3}\right) a \hat{\mathbf{y}}+x_{3} a \hat{\mathbf{z}}$ & $(24 d)$ & C III \\
\hline $\mathbf{B}_{72}$ & $=$ & $\left(\frac{1}{2}+y_{3}\right) \mathbf{a}_{1}+z_{3} \mathbf{a}_{2}+\left(\frac{1}{2}-x_{3}\right) \mathbf{a}_{3}$ & $=$ & $\left(\frac{1}{2}+y_{3}\right) a \hat{\mathbf{x}}+z_{3} a \hat{\mathbf{y}}+\left(\frac{1}{2}-x_{3}\right) a \hat{\mathbf{z}}$ & $(24 d)$ & C III \\
\hline $\mathbf{B}_{73}$ & $=$ & $x_{4} \mathbf{a}_{1}+y_{4} \mathbf{a}_{2}+z_{4} \mathbf{a}_{3}$ & $=$ & $x_{4} a \hat{\mathbf{x}}+y_{4} a \hat{\mathbf{y}}+z_{4} a \hat{\mathbf{z}}$ & $(24 d)$ & C IV \\
\hline $\mathbf{B}_{74}$ & $=$ & $\left(\frac{1}{2}-x_{4}\right) \mathbf{a}_{1}-y_{4} \mathbf{a}_{2}+\left(\frac{1}{2}+z_{4}\right) \mathbf{a}_{3}$ & $=$ & $\left(\frac{1}{2}-x_{4}\right) a \hat{\mathbf{x}}-y_{4} a \hat{\mathbf{y}}+\left(\frac{1}{2}+z_{4}\right) a \hat{\mathbf{z}}$ & $(24 d)$ & C IV \\
\hline $\mathbf{B}_{75}$ & $=$ & $-x_{4} \mathbf{a}_{1}+\left(\frac{1}{2}+y_{4}\right) \mathbf{a}_{2}+\left(\frac{1}{2}-z_{4}\right) \mathbf{a}_{3}$ & $=$ & $-x_{4} a \hat{\mathbf{x}}+\left(\frac{1}{2}+y_{4}\right) a \hat{\mathbf{y}}+\left(\frac{1}{2}-z_{4}\right) a \hat{\mathbf{z}}$ & $(24 d)$ & C IV \\
\hline $\mathbf{B}_{76}$ & $=$ & $\left(\frac{1}{2}+x_{4}\right) \mathbf{a}_{1}+\left(\frac{1}{2}-y_{4}\right) \mathbf{a}_{2}-z_{4} \mathbf{a}_{3}$ & $=$ & $\left(\frac{1}{2}+x_{4}\right) a \hat{\mathbf{x}}+\left(\frac{1}{2}-y_{4}\right) a \hat{\mathbf{y}}-z_{4} a \hat{\mathbf{z}}$ & $(24 d)$ & C IV \\
\hline $\mathbf{B}_{77}$ & $=$ & $z_{4} \mathbf{a}_{1}+x_{4} \mathbf{a}_{2}+y_{4} \mathbf{a}_{3}$ & $=$ & $z_{4} a \hat{\mathbf{x}}+x_{4} a \hat{\mathbf{y}}+y_{4} a \hat{\mathbf{z}}$ & $(24 d)$ & C IV \\
\hline $\mathbf{B}_{78}$ & $=$ & $\left(\frac{1}{2}+z_{4}\right) \mathbf{a}_{1}+\left(\frac{1}{2}-x_{4}\right) \mathbf{a}_{2}-y_{4} \mathbf{a}_{3}$ & $=$ & $\left(\frac{1}{2}+z_{4}\right) a \hat{\mathbf{x}}+\left(\frac{1}{2}-x_{4}\right) a \hat{\mathbf{y}}-y_{4} a \hat{\mathbf{z}}$ & $(24 d)$ & C IV \\
\hline $\mathbf{B}_{79}$ & $=$ & $\left(\frac{1}{2}-z_{4}\right) \mathbf{a}_{1}-x_{4} \mathbf{a}_{2}+\left(\frac{1}{2}+y_{4}\right) \mathbf{a}_{3}$ & $=$ & $\left(\frac{1}{2}-z_{4}\right) a \hat{\mathbf{x}}-x_{4} a \hat{\mathbf{y}}+\left(\frac{1}{2}+y_{4}\right) a \hat{\mathbf{z}}$ & $(24 d)$ & C IV \\
\hline $\mathbf{B}_{80}$ & $=$ & $-z_{4} \mathbf{a}_{1}+\left(\frac{1}{2}+x_{4}\right) \mathbf{a}_{2}+\left(\frac{1}{2}-y_{4}\right) \mathbf{a}_{3}$ & $=$ & $-z_{4} a \hat{\mathbf{x}}+\left(\frac{1}{2}+x_{4}\right) a \hat{\mathbf{y}}+\left(\frac{1}{2}-y_{4}\right) a \hat{\mathbf{z}}$ & $(24 d)$ & C IV \\
\hline $\mathbf{B}_{81}$ & $=$ & $y_{4} \mathbf{a}_{1}+z_{4} \mathbf{a}_{2}+x_{4} \mathbf{a}_{3}$ & $=$ & $y_{4} a \hat{\mathbf{x}}+z_{4} a \hat{\mathbf{y}}+x_{4} a \hat{\mathbf{z}}$ & $(24 d)$ & C IV \\
\hline $\mathbf{B}_{82}$ & $=$ & $-y_{4} \mathbf{a}_{1}+\left(\frac{1}{2}+z_{4}\right) \mathbf{a}_{2}+\left(\frac{1}{2}-x_{4}\right) \mathbf{a}_{3}$ & $=$ & $-y_{4} a \hat{\mathbf{x}}+\left(\frac{1}{2}+z_{4}\right) a \hat{\mathbf{y}}+\left(\frac{1}{2}-x_{4}\right) a \hat{\mathbf{z}}$ & $(24 d)$ & C IV \\
\hline $\mathbf{B}_{83}$ & $=$ & $\left(\frac{1}{2}+y_{4}\right) \mathbf{a}_{1}+\left(\frac{1}{2}-z_{4}\right) \mathbf{a}_{2}-x_{4} \mathbf{a}_{3}$ & $=$ & $\left(\frac{1}{2}+y_{4}\right) a \hat{\mathbf{x}}+\left(\frac{1}{2}-z_{4}\right) a \hat{\mathbf{y}}-x_{4} a \hat{\mathbf{z}}$ & $(24 d)$ & C IV \\
\hline $\mathbf{B}_{84}$ & $=$ & $\left(\frac{1}{2}-y_{4}\right) \mathbf{a}_{1}-z_{4} \mathbf{a}_{2}+\left(\frac{1}{2}+x_{4}\right) \mathbf{a}_{3}$ & $=$ & $\left(\frac{1}{2}-y_{4}\right) a \hat{\mathbf{x}}-z_{4} a \hat{\mathbf{y}}+\left(\frac{1}{2}+x_{4}\right) a \hat{\mathbf{z}}$ & $(24 d)$ & C IV \\
\hline $\mathbf{B}_{85}$ & $=$ & $-x_{4} \mathbf{a}_{1}-y_{4} \mathbf{a}_{2}-z_{4} \mathbf{a}_{3}$ & $=$ & $-x_{4} a \hat{\mathbf{x}}-y_{4} a \hat{\mathbf{y}}-z_{4} a \hat{\mathbf{z}}$ & $(24 d)$ & C IV \\
\hline $\mathbf{B}_{86}$ & $=$ & $\left(\frac{1}{2}+x_{4}\right) \mathbf{a}_{1}+y_{4} \mathbf{a}_{2}+\left(\frac{1}{2}-z_{4}\right) \mathbf{a}_{3}$ & $=$ & $\left(\frac{1}{2}+x_{4}\right) a \hat{\mathbf{x}}+y_{4} a \hat{\mathbf{y}}+\left(\frac{1}{2}-z_{4}\right) a \hat{\mathbf{z}}$ & $(24 d)$ & C IV \\
\hline $\mathbf{B}_{87}$ & $=$ & $x_{4} \mathbf{a}_{1}+\left(\frac{1}{2}-y_{4}\right) \mathbf{a}_{2}+\left(\frac{1}{2}+z_{4}\right) \mathbf{a}_{3}$ & $=$ & $x_{4} a \hat{\mathbf{x}}+\left(\frac{1}{2}-y_{4}\right) a \hat{\mathbf{y}}+\left(\frac{1}{2}+z_{4}\right) a \hat{\mathbf{z}}$ & $(24 d)$ & C IV \\
\hline $\mathbf{B}_{88}$ & $=$ & $\left(\frac{1}{2}-x_{4}\right) \mathbf{a}_{1}+\left(\frac{1}{2}+y_{4}\right) \mathbf{a}_{2}+z_{4} \mathbf{a}_{3}$ & $=$ & $\left(\frac{1}{2}-x_{4}\right) a \hat{\mathbf{x}}+\left(\frac{1}{2}+y_{4}\right) a \hat{\mathbf{y}}+z_{4} a \hat{\mathbf{z}}$ & $(24 d)$ & C IV \\
\hline $\mathbf{B}_{89}$ & $=$ & $-z_{4} \mathbf{a}_{1}-x_{4} \mathbf{a}_{2}-y_{4} \mathbf{a}_{3}$ & $=$ & $-z_{4} a \hat{\mathbf{x}}-x_{4} a \hat{\mathbf{y}}-y_{4} a \hat{\mathbf{z}}$ & $(24 d)$ & C IV \\
\hline $\mathbf{B}_{90}$ & $=$ & $\left(\frac{1}{2}-z_{4}\right) \mathbf{a}_{1}+\left(\frac{1}{2}+x_{4}\right) \mathbf{a}_{2}+y_{4} \mathbf{a}_{3}$ & $=$ & $\left(\frac{1}{2}-z_{4}\right) a \hat{\mathbf{x}}+\left(\frac{1}{2}+x_{4}\right) a \hat{\mathbf{y}}+y_{4} a \hat{\mathbf{z}}$ & $(24 d)$ & C IV \\
\hline $\mathbf{B}_{91}$ & $=$ & $\left(\frac{1}{2}+z_{4}\right) \mathbf{a}_{1}+x_{4} \mathbf{a}_{2}+\left(\frac{1}{2}-y_{4}\right) \mathbf{a}_{3}$ & $=$ & $\left(\frac{1}{2}+z_{4}\right) a \hat{\mathbf{x}}+x_{4} a \hat{\mathbf{y}}+\left(\frac{1}{2}-y_{4}\right) a \hat{\mathbf{z}}$ & $(24 d)$ & C IV \\
\hline $\mathbf{B}_{92}$ & $=$ & $z_{4} \mathbf{a}_{1}+\left(\frac{1}{2}-x_{4}\right) \mathbf{a}_{2}+\left(\frac{1}{2}+y_{4}\right) \mathbf{a}_{3}$ & $=$ & $z_{4} a \hat{\mathbf{x}}+\left(\frac{1}{2}-x_{4}\right) a \hat{\mathbf{y}}+\left(\frac{1}{2}+y_{4}\right) a \hat{\mathbf{z}}$ & $(24 d)$ & C IV \\
\hline $\mathbf{B}_{93}$ & $=$ & $-y_{4} \mathbf{a}_{1}-z_{4} \mathbf{a}_{2}-x_{4} \mathbf{a}_{3}$ & $=$ & $-y_{4} a \hat{\mathbf{x}}-z_{4} a \hat{\mathbf{y}}-x_{4} a \hat{\mathbf{z}}$ & $(24 d)$ & IV \\
\hline $\mathbf{B}_{94}$ & $=$ & $y_{4} \mathbf{a}_{1}+\left(\frac{1}{2}-z_{4}\right) \mathbf{a}_{2}+\left(\frac{1}{2}+x_{4}\right) \mathbf{a}_{3}$ & $=$ & $y_{4} a \hat{\mathbf{x}}+\left(\frac{1}{2}-z_{4}\right) a \hat{\mathbf{y}}+\left(\frac{1}{2}+x_{4}\right) a \hat{\mathbf{z}}$ & $(24 d)$ & C IV \\
\hline $\mathbf{B}_{95}$ & $=$ & $\left(\frac{1}{2}-y_{4}\right) \mathbf{a}_{1}+\left(\frac{1}{2}+z_{4}\right) \mathbf{a}_{2}+x_{4} \mathbf{a}_{3}$ & $=$ & $\left(\frac{1}{2}-y_{4}\right) a \hat{\mathbf{x}}+\left(\frac{1}{2}+z_{4}\right) a \hat{\mathbf{y}}+x_{4} a \hat{\mathbf{z}}$ & $(24 d)$ & C IV \\
\hline $\mathbf{B}_{96}$ & $=$ & $\left(\frac{1}{2}+y_{4}\right) \mathbf{a}_{1}+z_{4} \mathbf{a}_{2}+\left(\frac{1}{2}-x_{4}\right) \mathbf{a}_{3}$ & $=$ & $\left(\frac{1}{2}+y_{4}\right) a \hat{\mathbf{x}}+z_{4} a \hat{\mathbf{y}}+\left(\frac{1}{2}-x_{4}\right) a \hat{\mathbf{z}}$ & $(24 d)$ & C IV \\
\hline $\mathbf{B}_{97}$ & - & $x_{5} \mathbf{a}_{1}+y_{5} \mathbf{a}_{2}+z_{5} \mathbf{a}_{3}$ & $=$ & $x_{5} a \hat{\mathbf{x}}+y_{5} a \hat{\mathbf{y}}+z_{5} a \hat{\mathbf{z}}$ & $(24 d)$ & $\mathrm{CV}$ \\
\hline $\mathbf{B}_{98}$ & $=$ & $\left(\frac{1}{2}-x_{5}\right) \mathbf{a}_{1}-y_{5} \mathbf{a}_{2}+\left(\frac{1}{2}+z_{5}\right) \mathbf{a}_{3}$ & $=$ & $\left(\frac{1}{2}-x_{5}\right) a \hat{\mathbf{x}}-y_{5} a \hat{\mathbf{y}}+\left(\frac{1}{2}+z_{5}\right) a \hat{\mathbf{z}}$ & $(24 d)$ & $C \mathrm{~V}$ \\
\hline $\mathbf{B}_{99}$ & $=$ & $-x_{5} \mathbf{a}_{1}+\left(\frac{1}{2}+y_{5}\right) \mathbf{a}_{2}+\left(\frac{1}{2}-z_{5}\right) \mathbf{a}_{3}$ & $=$ & $-x_{5} a \hat{\mathbf{x}}+\left(\frac{1}{2}+y_{5}\right) a \hat{\mathbf{y}}+\left(\frac{1}{2}-z_{5}\right) a \hat{\mathbf{z}}$ & $(24 d)$ & $C \mathrm{~V}$ \\
\hline $\mathbf{B}_{100}$ & $=$ & $\left(\frac{1}{2}+x_{5}\right) \mathbf{a}_{1}+\left(\frac{1}{2}-y_{5}\right) \mathbf{a}_{2}-z_{5} \mathbf{a}_{3}$ & $=$ & $\left(\frac{1}{2}+x_{5}\right) a \hat{\mathbf{x}}+\left(\frac{1}{2}-y_{5}\right) a \hat{\mathbf{y}}-z_{5} a \hat{\mathbf{z}}$ & $(24 d)$ & $\mathrm{C} \mathrm{V}$ \\
\hline $\mathbf{B}_{101}$ & $=$ & $z_{5} \mathbf{a}_{1}+x_{5} \mathbf{a}_{2}+y_{5} \mathbf{a}_{3}$ & $=$ & $z_{5} a \hat{\mathbf{x}}+x_{5} a \hat{\mathbf{y}}+y_{5} a \hat{\mathbf{z}}$ & $(24 d)$ & $\mathrm{C} \mathrm{V}$ \\
\hline $\mathbf{B}_{102}$ & - & $\left(\frac{1}{2}+z_{5}\right) \mathbf{a}_{1}+\left(\frac{1}{2}-x_{5}\right) \mathbf{a}_{2}-y_{5} \mathbf{a}_{3}$ & $=$ & $\left(\frac{1}{2}+z_{5}\right) a \hat{\mathbf{x}}+\left(\frac{1}{2}-x_{5}\right) a \hat{\mathbf{y}}-y_{5} a \hat{\mathbf{z}}$ & $(24 d)$ & $\mathrm{C} \mathrm{V}$ \\
\hline $\mathbf{B}_{103}$ & - & $\left(\frac{1}{2}-z_{5}\right) \mathbf{a}_{1}-x_{5} \mathbf{a}_{2}+\left(\frac{1}{2}+y_{5}\right) \mathbf{a}_{3}$ & $=$ & $\left(\frac{1}{2}-z_{5}\right) a \hat{\mathbf{x}}-x_{5} a \hat{\mathbf{y}}+\left(\frac{1}{2}+y_{5}\right) a \hat{\mathbf{z}}$ & $(24 d)$ & $v$ \\
\hline $\mathbf{B}_{104}$ & - & $-z_{5} \mathbf{a}_{1}+\left(\frac{1}{2}+x_{5}\right) \mathbf{a}_{2}+\left(\frac{1}{2}-y_{5}\right) \mathbf{a}_{3}$ & $=$ & $-z_{5} a \hat{\mathbf{x}}+\left(\frac{1}{2}+x_{5}\right) a \hat{\mathbf{y}}+\left(\frac{1}{2}-y_{5}\right) a \hat{\mathbf{z}}$ & $(24 d)$ & $r$ \\
\hline $\mathbf{B}_{105}$ & - & $y_{5} \mathbf{a}_{1}+z_{5} \mathbf{a}_{2}+x_{5} \mathbf{a}_{3}$ & $=$ & $y_{5} a \hat{\mathbf{x}}+z_{5} a \hat{\mathbf{y}}+x_{5} a \hat{\mathbf{z}}$ & $(24 d)$ & $\mathrm{C} \mathrm{V}$ \\
\hline $\mathbf{B}_{106}$ & $=$ & $-y_{5} \mathbf{a}_{1}+\left(\frac{1}{2}+z_{5}\right) \mathbf{a}_{2}+\left(\frac{1}{2}-x_{5}\right) \mathbf{a}_{3}$ & $=$ & $-y_{5} a \hat{\mathbf{x}}+\left(\frac{1}{2}+z_{5}\right) a \hat{\mathbf{y}}+\left(\frac{1}{2}-x_{5}\right) a \hat{\mathbf{z}}$ & $(24 d)$ & $\mathrm{CV}$ \\
\hline
\end{tabular}




\begin{tabular}{|c|c|c|c|c|c|c|}
\hline $\mathbf{B}_{107}$ & $=$ & $\left(\frac{1}{2}+y_{5}\right) \mathbf{a}_{1}+\left(\frac{1}{2}-z_{5}\right) \mathbf{a}_{2}-x_{5} \mathbf{a}_{3}$ & $=$ & $\left(\frac{1}{2}+y_{5}\right) a \hat{\mathbf{x}}+\left(\frac{1}{2}-z_{5}\right) a \hat{\mathbf{y}}-x_{5} a \hat{\mathbf{z}}$ & $(24 d)$ & $\mathrm{C} \mathrm{V}$ \\
\hline $\mathbf{B}_{108}$ & $=$ & $\left(\frac{1}{2}-y_{5}\right) \mathbf{a}_{1}-z_{5} \mathbf{a}_{2}+\left(\frac{1}{2}+x_{5}\right) \mathbf{a}_{3}$ & $=$ & $\left(\frac{1}{2}-y_{5}\right) a \hat{\mathbf{x}}-z_{5} a \hat{\mathbf{y}}+\left(\frac{1}{2}+x_{5}\right) a \hat{\mathbf{z}}$ & $(24 d)$ & $\mathrm{C} \mathrm{V}$ \\
\hline $\mathbf{B}_{109}$ & $=$ & $-x_{5} \mathbf{a}_{1}-y_{5} \mathbf{a}_{2}-z_{5} \mathbf{a}_{3}$ & $=$ & $-x_{5} a \hat{\mathbf{x}}-y_{5} a \hat{\mathbf{y}}-z_{5} a \hat{\mathbf{z}}$ & $(24 d)$ & $\mathrm{C} \mathrm{V}$ \\
\hline $\mathbf{B}_{110}$ & $=$ & $\left(\frac{1}{2}+x_{5}\right) \mathbf{a}_{1}+y_{5} \mathbf{a}_{2}+\left(\frac{1}{2}-z_{5}\right) \mathbf{a}_{3}$ & $=$ & $\left(\frac{1}{2}+x_{5}\right) a \hat{\mathbf{x}}+y_{5} a \hat{\mathbf{y}}+\left(\frac{1}{2}-z_{5}\right) a \hat{\mathbf{z}}$ & $(24 d)$ & $\mathrm{C} \mathrm{V}$ \\
\hline $\mathbf{B}_{111}$ & $=$ & $x_{5} \mathbf{a}_{1}+\left(\frac{1}{2}-y_{5}\right) \mathbf{a}_{2}+\left(\frac{1}{2}+z_{5}\right) \mathbf{a}_{3}$ & $=$ & $x_{5} a \hat{\mathbf{x}}+\left(\frac{1}{2}-y_{5}\right) a \hat{\mathbf{y}}+\left(\frac{1}{2}+z_{5}\right) a \hat{\mathbf{z}}$ & $(24 d)$ & $\mathrm{C} \mathrm{V}$ \\
\hline $\mathbf{B}_{112}$ & $=$ & $\left(\frac{1}{2}-x_{5}\right) \mathbf{a}_{1}+\left(\frac{1}{2}+y_{5}\right) \mathbf{a}_{2}+z_{5} \mathbf{a}_{3}$ & $=$ & $\left(\frac{1}{2}-x_{5}\right) a \hat{\mathbf{x}}+\left(\frac{1}{2}+y_{5}\right) a \hat{\mathbf{y}}+z_{5} a \hat{\mathbf{z}}$ & $(24 d)$ & $\mathrm{C} \mathrm{V}$ \\
\hline $\mathbf{B}_{113}$ & $=$ & $-z_{5} \mathbf{a}_{1}-x_{5} \mathbf{a}_{2}-y_{5} \mathbf{a}_{3}$ & $=$ & $-z_{5} a \hat{\mathbf{x}}-x_{5} a \hat{\mathbf{y}}-y_{5} a \hat{\mathbf{z}}$ & $(24 d)$ & $\mathrm{C} \mathrm{V}$ \\
\hline $\mathbf{B}_{114}$ & $=$ & $\left(\frac{1}{2}-z_{5}\right) \mathbf{a}_{1}+\left(\frac{1}{2}+x_{5}\right) \mathbf{a}_{2}+y_{5} \mathbf{a}_{3}$ & $=$ & $\left(\frac{1}{2}-z_{5}\right) a \hat{\mathbf{x}}+\left(\frac{1}{2}+x_{5}\right) a \hat{\mathbf{y}}+y_{5} a \hat{\mathbf{z}}$ & $(24 d)$ & $\mathrm{C} \mathrm{V}$ \\
\hline $\mathbf{B}_{115}$ & $=$ & $\left(\frac{1}{2}+z_{5}\right) \mathbf{a}_{1}+x_{5} \mathbf{a}_{2}+\left(\frac{1}{2}-y_{5}\right) \mathbf{a}_{3}$ & $=$ & $\left(\frac{1}{2}+z_{5}\right) a \hat{\mathbf{x}}+x_{5} a \hat{\mathbf{y}}+\left(\frac{1}{2}-y_{5}\right) a \hat{\mathbf{z}}$ & $(24 d)$ & $\mathrm{C} \mathrm{V}$ \\
\hline $\mathbf{B}_{116}$ & $=$ & $z_{5} \mathbf{a}_{1}+\left(\frac{1}{2}-x_{5}\right) \mathbf{a}_{2}+\left(\frac{1}{2}+y_{5}\right) \mathbf{a}_{3}$ & $=$ & $z_{5} a \hat{\mathbf{x}}+\left(\frac{1}{2}-x_{5}\right) a \hat{\mathbf{y}}+\left(\frac{1}{2}+y_{5}\right) a \hat{\mathbf{z}}$ & $(24 d)$ & $\mathrm{C} \mathrm{V}$ \\
\hline $\mathbf{B}_{117}$ & $=$ & $-y_{5} \mathbf{a}_{1}-z_{5} \mathbf{a}_{2}-x_{5} \mathbf{a}_{3}$ & $=$ & $-y_{5} a \hat{\mathbf{x}}-z_{5} a \hat{\mathbf{y}}-x_{5} a \hat{\mathbf{z}}$ & $(24 d)$ & $\mathrm{C} \mathrm{V}$ \\
\hline $\mathbf{B}_{118}$ & $=$ & $y_{5} \mathbf{a}_{1}+\left(\frac{1}{2}-z_{5}\right) \mathbf{a}_{2}+\left(\frac{1}{2}+x_{5}\right) \mathbf{a}_{3}$ & $=$ & $y_{5} a \hat{\mathbf{x}}+\left(\frac{1}{2}-z_{5}\right) a \hat{\mathbf{y}}+\left(\frac{1}{2}+x_{5}\right) a \hat{\mathbf{z}}$ & $(24 d)$ & $\mathrm{C} \mathrm{V}$ \\
\hline $\mathbf{B}_{119}$ & $=$ & $\left(\frac{1}{2}-y_{5}\right) \mathbf{a}_{1}+\left(\frac{1}{2}+z_{5}\right) \mathbf{a}_{2}+x_{5} \mathbf{a}_{3}$ & $=$ & $\left(\frac{1}{2}-y_{5}\right) a \hat{\mathbf{x}}+\left(\frac{1}{2}+z_{5}\right) a \hat{\mathbf{y}}+x_{5} a \hat{\mathbf{z}}$ & $(24 d)$ & $\mathrm{C} \mathrm{V}$ \\
\hline $\mathbf{B}_{120}$ & $=$ & $\left(\frac{1}{2}+y_{5}\right) \mathbf{a}_{1}+z_{5} \mathbf{a}_{2}+\left(\frac{1}{2}-x_{5}\right) \mathbf{a}_{3}$ & $=$ & $\left(\frac{1}{2}+y_{5}\right) a \hat{\mathbf{x}}+z_{5} a \hat{\mathbf{y}}+\left(\frac{1}{2}-x_{5}\right) a \hat{\mathbf{z}}$ & $(24 d)$ & $\mathrm{C} \mathrm{V}$ \\
\hline $\mathbf{B}_{121}$ & $=$ & $x_{6} \mathbf{a}_{1}+y_{6} \mathbf{a}_{2}+z_{6} \mathbf{a}_{3}$ & $=$ & $x_{6} a \hat{\mathbf{x}}+y_{6} a \hat{\mathbf{y}}+z_{6} a \hat{\mathbf{z}}$ & $(24 d)$ & C VI \\
\hline $\mathbf{B}_{122}$ & $=$ & $\left(\frac{1}{2}-x_{6}\right) \mathbf{a}_{1}-y_{6} \mathbf{a}_{2}+\left(\frac{1}{2}+z_{6}\right) \mathbf{a}_{3}$ & $=$ & $\left(\frac{1}{2}-x_{6}\right) a \hat{\mathbf{x}}-y_{6} a \hat{\mathbf{y}}+\left(\frac{1}{2}+z_{6}\right) a \hat{\mathbf{z}}$ & $(24 d)$ & C VI \\
\hline $\mathbf{B}_{123}$ & $=$ & $-x_{6} \mathbf{a}_{1}+\left(\frac{1}{2}+y_{6}\right) \mathbf{a}_{2}+\left(\frac{1}{2}-z_{6}\right) \mathbf{a}_{3}$ & $=$ & $-x_{6} a \hat{\mathbf{x}}+\left(\frac{1}{2}+y_{6}\right) a \hat{\mathbf{y}}+\left(\frac{1}{2}-z_{6}\right) a \hat{\mathbf{z}}$ & $(24 d)$ & C VI \\
\hline $\mathbf{B}_{124}$ & $=$ & $\left(\frac{1}{2}+x_{6}\right) \mathbf{a}_{1}+\left(\frac{1}{2}-y_{6}\right) \mathbf{a}_{2}-z_{6} \mathbf{a}_{3}$ & $=$ & $\left(\frac{1}{2}+x_{6}\right) a \hat{\mathbf{x}}+\left(\frac{1}{2}-y_{6}\right) a \hat{\mathbf{y}}-z_{6} a \hat{\mathbf{z}}$ & $(24 d)$ & C VI \\
\hline $\mathbf{B}_{125}$ & $=$ & $z_{6} \mathbf{a}_{1}+x_{6} \mathbf{a}_{2}+y_{6} \mathbf{a}_{3}$ & $=$ & $z_{6} a \hat{\mathbf{x}}+x_{6} a \hat{\mathbf{y}}+y_{6} a \hat{\mathbf{z}}$ & $(24 d)$ & C VI \\
\hline $\mathbf{B}_{126}$ & $=$ & $\left(\frac{1}{2}+z_{6}\right) \mathbf{a}_{1}+\left(\frac{1}{2}-x_{6}\right) \mathbf{a}_{2}-y_{6} \mathbf{a}_{3}$ & $=$ & $\left(\frac{1}{2}+z_{6}\right) a \hat{\mathbf{x}}+\left(\frac{1}{2}-x_{6}\right) a \hat{\mathbf{y}}-y_{6} a \hat{\mathbf{z}}$ & $(24 d)$ & C VI \\
\hline $\mathbf{B}_{127}$ & $=$ & $\left(\frac{1}{2}-z_{6}\right) \mathbf{a}_{1}-x_{6} \mathbf{a}_{2}+\left(\frac{1}{2}+y_{6}\right) \mathbf{a}_{3}$ & $=$ & $\left(\frac{1}{2}-z_{6}\right) a \hat{\mathbf{x}}-x_{6} a \hat{\mathbf{y}}+\left(\frac{1}{2}+y_{6}\right) a \hat{\mathbf{z}}$ & $(24 d)$ & C VI \\
\hline $\mathbf{B}_{128}$ & $=$ & $-z_{6} \mathbf{a}_{1}+\left(\frac{1}{2}+x_{6}\right) \mathbf{a}_{2}+\left(\frac{1}{2}-y_{6}\right) \mathbf{a}_{3}$ & $=$ & $-z_{6} a \hat{\mathbf{x}}+\left(\frac{1}{2}+x_{6}\right) a \hat{\mathbf{y}}+\left(\frac{1}{2}-y_{6}\right) a \hat{\mathbf{z}}$ & $(24 d)$ & C VI \\
\hline $\mathbf{B}_{129}$ & $=$ & $y_{6} \mathbf{a}_{1}+z_{6} \mathbf{a}_{2}+x_{6} \mathbf{a}_{3}$ & $=$ & $y_{6} a \hat{\mathbf{x}}+z_{6} a \hat{\mathbf{y}}+x_{6} a \hat{\mathbf{z}}$ & $(24 d)$ & C VI \\
\hline $\mathbf{B}_{130}$ & $=$ & $-y_{6} \mathbf{a}_{1}+\left(\frac{1}{2}+z_{6}\right) \mathbf{a}_{2}+\left(\frac{1}{2}-x_{6}\right) \mathbf{a}_{3}$ & $=$ & $-y_{6} a \hat{\mathbf{x}}+\left(\frac{1}{2}+z_{6}\right) a \hat{\mathbf{y}}+\left(\frac{1}{2}-x_{6}\right) a \hat{\mathbf{z}}$ & $(24 d)$ & C VI \\
\hline $\mathbf{B}_{131}$ & $=$ & $\left(\frac{1}{2}+y_{6}\right) \mathbf{a}_{1}+\left(\frac{1}{2}-z_{6}\right) \mathbf{a}_{2}-x_{6} \mathbf{a}_{3}$ & $=$ & $\left(\frac{1}{2}+y_{6}\right) a \hat{\mathbf{x}}+\left(\frac{1}{2}-z_{6}\right) a \hat{\mathbf{y}}-x_{6} a \hat{\mathbf{z}}$ & $(24 d)$ & C VI \\
\hline $\mathbf{B}_{132}$ & $=$ & $\left(\frac{1}{2}-y_{6}\right) \mathbf{a}_{1}-z_{6} \mathbf{a}_{2}+\left(\frac{1}{2}+x_{6}\right) \mathbf{a}_{3}$ & $=$ & $\left(\frac{1}{2}-y_{6}\right) a \hat{\mathbf{x}}-z_{6} a \hat{\mathbf{y}}+\left(\frac{1}{2}+x_{6}\right) a \hat{\mathbf{z}}$ & $(24 d)$ & C VI \\
\hline $\mathbf{B}_{133}$ & $=$ & $-x_{6} \mathbf{a}_{1}-y_{6} \mathbf{a}_{2}-z_{6} \mathbf{a}_{3}$ & $=$ & $-x_{6} a \hat{\mathbf{x}}-y_{6} a \hat{\mathbf{y}}-z_{6} a \hat{\mathbf{z}}$ & $(24 d)$ & C VI \\
\hline $\mathbf{B}_{134}$ & $=$ & $\left(\frac{1}{2}+x_{6}\right) \mathbf{a}_{1}+y_{6} \mathbf{a}_{2}+\left(\frac{1}{2}-z_{6}\right) \mathbf{a}_{3}$ & $=$ & $\left(\frac{1}{2}+x_{6}\right) a \hat{\mathbf{x}}+y_{6} a \hat{\mathbf{y}}+\left(\frac{1}{2}-z_{6}\right) a \hat{\mathbf{z}}$ & $(24 d)$ & C VI \\
\hline $\mathbf{B}_{135}$ & $=$ & $x_{6} \mathbf{a}_{1}+\left(\frac{1}{2}-y_{6}\right) \mathbf{a}_{2}+\left(\frac{1}{2}+z_{6}\right) \mathbf{a}_{3}$ & $=$ & $x_{6} a \hat{\mathbf{x}}+\left(\frac{1}{2}-y_{6}\right) a \hat{\mathbf{y}}+\left(\frac{1}{2}+z_{6}\right) a \hat{\mathbf{z}}$ & $(24 d)$ & C VI \\
\hline $\mathbf{B}_{136}$ & $=$ & $\left(\frac{1}{2}-x_{6}\right) \mathbf{a}_{1}+\left(\frac{1}{2}+y_{6}\right) \mathbf{a}_{2}+z_{6} \mathbf{a}_{3}$ & $=$ & $\left(\frac{1}{2}-x_{6}\right) a \hat{\mathbf{x}}+\left(\frac{1}{2}+y_{6}\right) a \hat{\mathbf{y}}+z_{6} a \hat{\mathbf{z}}$ & $(24 d)$ & C VI \\
\hline $\mathbf{B}_{137}$ & $=$ & $-z_{6} \mathbf{a}_{1}-x_{6} \mathbf{a}_{2}-y_{6} \mathbf{a}_{3}$ & $=$ & $-z_{6} a \hat{\mathbf{x}}-x_{6} a \hat{\mathbf{y}}-y_{6} a \hat{\mathbf{z}}$ & $(24 d)$ & C VI \\
\hline $\mathbf{B}_{138}$ & $=$ & $\left(\frac{1}{2}-z_{6}\right) \mathbf{a}_{1}+\left(\frac{1}{2}+x_{6}\right) \mathbf{a}_{2}+y_{6} \mathbf{a}_{3}$ & $=$ & $\left(\frac{1}{2}-z_{6}\right) a \hat{\mathbf{x}}+\left(\frac{1}{2}+x_{6}\right) a \hat{\mathbf{y}}+y_{6} a \hat{\mathbf{z}}$ & $(24 d)$ & C VI \\
\hline $\mathbf{B}_{139}$ & $=$ & $\left(\frac{1}{2}+z_{6}\right) \mathbf{a}_{1}+x_{6} \mathbf{a}_{2}+\left(\frac{1}{2}-y_{6}\right) \mathbf{a}_{3}$ & $=$ & $\left(\frac{1}{2}+z_{6}\right) a \hat{\mathbf{x}}+x_{6} a \hat{\mathbf{y}}+\left(\frac{1}{2}-y_{6}\right) a \hat{\mathbf{z}}$ & $(24 d)$ & C VI \\
\hline $\mathbf{B}_{140}$ & $=$ & $z_{6} \mathbf{a}_{1}+\left(\frac{1}{2}-x_{6}\right) \mathbf{a}_{2}+\left(\frac{1}{2}+y_{6}\right) \mathbf{a}_{3}$ & $=$ & $z_{6} a \hat{\mathbf{x}}+\left(\frac{1}{2}-x_{6}\right) a \hat{\mathbf{y}}+\left(\frac{1}{2}+y_{6}\right) a \hat{\mathbf{z}}$ & $(24 d)$ & C VI \\
\hline $\mathbf{B}_{141}$ & $=$ & $-y_{6} \mathbf{a}_{1}-z_{6} \mathbf{a}_{2}-x_{6} \mathbf{a}_{3}$ & $=$ & $-y_{6} a \hat{\mathbf{x}}-z_{6} a \hat{\mathbf{y}}-x_{6} a \hat{\mathbf{z}}$ & $(24 d)$ & C VI \\
\hline $\mathbf{B}_{142}$ & $=$ & $y_{6} \mathbf{a}_{1}+\left(\frac{1}{2}-z_{6}\right) \mathbf{a}_{2}+\left(\frac{1}{2}+x_{6}\right) \mathbf{a}_{3}$ & $=$ & $y_{6} a \hat{\mathbf{x}}+\left(\frac{1}{2}-z_{6}\right) a \hat{\mathbf{y}}+\left(\frac{1}{2}+x_{6}\right) a \hat{\mathbf{z}}$ & $(24 d)$ & C VI \\
\hline
\end{tabular}




\begin{tabular}{|c|c|c|c|c|c|c|}
\hline $\mathbf{B}_{143}$ & $=$ & $\left(\frac{1}{2}-y_{6}\right) \mathbf{a}_{1}+\left(\frac{1}{2}+z_{6}\right) \mathbf{a}_{2}+x_{6} \mathbf{a}_{3}$ & $=$ & $\left(\frac{1}{2}-y_{6}\right) a \hat{\mathbf{x}}+\left(\frac{1}{2}+z_{6}\right) a \hat{\mathbf{y}}+x_{6} a \hat{\mathbf{z}}$ & $(24 d)$ & C VI \\
\hline $\mathbf{B}_{144}$ & $=$ & $\left(\frac{1}{2}+y_{6}\right) \mathbf{a}_{1}+z_{6} \mathbf{a}_{2}+\left(\frac{1}{2}-x_{6}\right) \mathbf{a}_{3}$ & $=$ & $\left(\frac{1}{2}+y_{6}\right) a \hat{\mathbf{x}}+z_{6} a \hat{\mathbf{y}}+\left(\frac{1}{2}-x_{6}\right) a \hat{\mathbf{z}}$ & $(24 d)$ & C VI \\
\hline $\mathbf{B}_{145}$ & $=$ & $x_{7} \mathbf{a}_{1}+y_{7} \mathbf{a}_{2}+z_{7} \mathbf{a}_{3}$ & $=$ & $x_{7} a \hat{\mathbf{x}}+y_{7} a \hat{\mathbf{y}}+z_{7} a \hat{\mathbf{z}}$ & $(24 d)$ & C VII \\
\hline $\mathbf{B}_{146}$ & $=$ & $\left(\frac{1}{2}-x_{7}\right) \mathbf{a}_{1}-y_{7} \mathbf{a}_{2}+\left(\frac{1}{2}+z_{7}\right) \mathbf{a}_{3}$ & $=$ & $\left(\frac{1}{2}-x_{7}\right) a \hat{\mathbf{x}}-y_{7} a \hat{\mathbf{y}}+\left(\frac{1}{2}+z_{7}\right) a \hat{\mathbf{z}}$ & $(24 d)$ & C VII \\
\hline $\mathbf{B}_{147}$ & $=$ & $-x_{7} \mathbf{a}_{1}+\left(\frac{1}{2}+y_{7}\right) \mathbf{a}_{2}+\left(\frac{1}{2}-z_{7}\right) \mathbf{a}_{3}$ & $=$ & $-x_{7} a \hat{\mathbf{x}}+\left(\frac{1}{2}+y_{7}\right) a \hat{\mathbf{y}}+\left(\frac{1}{2}-z_{7}\right) a \hat{\mathbf{z}}$ & $(24 d)$ & C VII \\
\hline $\mathbf{B}_{148}$ & $=$ & $\left(\frac{1}{2}+x_{7}\right) \mathbf{a}_{1}+\left(\frac{1}{2}-y_{7}\right) \mathbf{a}_{2}-z_{7} \mathbf{a}_{3}$ & $=$ & $\left(\frac{1}{2}+x_{7}\right) a \hat{\mathbf{x}}+\left(\frac{1}{2}-y_{7}\right) a \hat{\mathbf{y}}-z_{7} a \hat{\mathbf{z}}$ & $(24 d)$ & C VII \\
\hline $\mathbf{B}_{149}$ & $=$ & $z_{7} \mathbf{a}_{1}+x_{7} \mathbf{a}_{2}+y_{7} \mathbf{a}_{3}$ & $=$ & $z_{7} a \hat{\mathbf{x}}+x_{7} a \hat{\mathbf{y}}+y_{7} a \hat{\mathbf{z}}$ & $(24 d)$ & C VII \\
\hline $\mathbf{B}_{150}$ & $=$ & $\left(\frac{1}{2}+z_{7}\right) \mathbf{a}_{1}+\left(\frac{1}{2}-x_{7}\right) \mathbf{a}_{2}-y_{7} \mathbf{a}_{3}$ & $=$ & $\left(\frac{1}{2}+z_{7}\right) a \hat{\mathbf{x}}+\left(\frac{1}{2}-x_{7}\right) a \hat{\mathbf{y}}-y_{7} a \hat{\mathbf{z}}$ & $(24 d)$ & C VII \\
\hline $\mathbf{B}_{151}$ & $=$ & $\left(\frac{1}{2}-z_{7}\right) \mathbf{a}_{1}-x_{7} \mathbf{a}_{2}+\left(\frac{1}{2}+y_{7}\right) \mathbf{a}_{3}$ & $=$ & $\left(\frac{1}{2}-z_{7}\right) a \hat{\mathbf{x}}-x_{7} a \hat{\mathbf{y}}+\left(\frac{1}{2}+y_{7}\right) a \hat{\mathbf{z}}$ & $(24 d)$ & C VII \\
\hline $\mathbf{B}_{152}$ & $=$ & $-z_{7} \mathbf{a}_{1}+\left(\frac{1}{2}+x_{7}\right) \mathbf{a}_{2}+\left(\frac{1}{2}-y_{7}\right) \mathbf{a}_{3}$ & $=$ & $-z_{7} a \hat{\mathbf{x}}+\left(\frac{1}{2}+x_{7}\right) a \hat{\mathbf{y}}+\left(\frac{1}{2}-y_{7}\right) a \hat{\mathbf{z}}$ & $(24 d)$ & C VII \\
\hline $\mathbf{B}_{153}$ & $=$ & $y_{7} \mathbf{a}_{1}+z_{7} \mathbf{a}_{2}+x_{7} \mathbf{a}_{3}$ & $=$ & $y_{7} a \hat{\mathbf{x}}+z_{7} a \hat{\mathbf{y}}+x_{7} a \hat{\mathbf{z}}$ & $(24 d)$ & C VII \\
\hline $\mathbf{B}_{154}$ & $=$ & $-y_{7} \mathbf{a}_{1}+\left(\frac{1}{2}+z_{7}\right) \mathbf{a}_{2}+\left(\frac{1}{2}-x_{7}\right) \mathbf{a}_{3}$ & $=$ & $-y_{7} a \hat{\mathbf{x}}+\left(\frac{1}{2}+z_{7}\right) a \hat{\mathbf{y}}+\left(\frac{1}{2}-x_{7}\right) a \hat{\mathbf{z}}$ & $(24 d)$ & C VII \\
\hline $\mathbf{B}_{155}$ & $=$ & $\left(\frac{1}{2}+y_{7}\right) \mathbf{a}_{1}+\left(\frac{1}{2}-z_{7}\right) \mathbf{a}_{2}-x_{7} \mathbf{a}_{3}$ & $=$ & $\left(\frac{1}{2}+y_{7}\right) a \hat{\mathbf{x}}+\left(\frac{1}{2}-z_{7}\right) a \hat{\mathbf{y}}-x_{7} a \hat{\mathbf{z}}$ & $(24 d)$ & C VII \\
\hline $\mathbf{B}_{156}$ & $=$ & $\left(\frac{1}{2}-y_{7}\right) \mathbf{a}_{1}-z_{7} \mathbf{a}_{2}+\left(\frac{1}{2}+x_{7}\right) \mathbf{a}_{3}$ & $=$ & $\left(\frac{1}{2}-y_{7}\right) a \hat{\mathbf{x}}-z_{7} a \hat{\mathbf{y}}+\left(\frac{1}{2}+x_{7}\right) a \hat{\mathbf{z}}$ & $(24 d)$ & C VII \\
\hline $\mathbf{B}_{157}$ & $=$ & $-x_{7} \mathbf{a}_{1}-y_{7} \mathbf{a}_{2}-z_{7} \mathbf{a}_{3}$ & $=$ & $-x_{7} a \hat{\mathbf{x}}-y_{7} a \hat{\mathbf{y}}-z_{7} a \hat{\mathbf{z}}$ & $(24 d)$ & C VII \\
\hline $\mathbf{B}_{158}$ & $=$ & $\left(\frac{1}{2}+x_{7}\right) \mathbf{a}_{1}+y_{7} \mathbf{a}_{2}+\left(\frac{1}{2}-z_{7}\right) \mathbf{a}_{3}$ & $=$ & $\left(\frac{1}{2}+x_{7}\right) a \hat{\mathbf{x}}+y_{7} a \hat{\mathbf{y}}+\left(\frac{1}{2}-z_{7}\right) a \hat{\mathbf{z}}$ & $(24 d)$ & C VII \\
\hline $\mathbf{B}_{159}$ & $=$ & $x_{7} \mathbf{a}_{1}+\left(\frac{1}{2}-y_{7}\right) \mathbf{a}_{2}+\left(\frac{1}{2}+z_{7}\right) \mathbf{a}_{3}$ & $=$ & $x_{7} a \hat{\mathbf{x}}+\left(\frac{1}{2}-y_{7}\right) a \hat{\mathbf{y}}+\left(\frac{1}{2}+z_{7}\right) a \hat{\mathbf{z}}$ & $(24 d)$ & C VII \\
\hline $\mathbf{B}_{160}$ & $=$ & $\left(\frac{1}{2}-x_{7}\right) \mathbf{a}_{1}+\left(\frac{1}{2}+y_{7}\right) \mathbf{a}_{2}+z_{7} \mathbf{a}_{3}$ & $=$ & $\left(\frac{1}{2}-x_{7}\right) a \hat{\mathbf{x}}+\left(\frac{1}{2}+y_{7}\right) a \hat{\mathbf{y}}+z_{7} a \hat{\mathbf{z}}$ & $(24 d)$ & C VII \\
\hline $\mathbf{B}_{161}$ & $=$ & $-z_{7} \mathbf{a}_{1}-x_{7} \mathbf{a}_{2}-y_{7} \mathbf{a}_{3}$ & $=$ & $-z_{7} a \hat{\mathbf{x}}-x_{7} a \hat{\mathbf{y}}-y_{7} a \hat{\mathbf{z}}$ & $(24 d)$ & C VII \\
\hline $\mathbf{B}_{162}$ & $=$ & $\left(\frac{1}{2}-z_{7}\right) \mathbf{a}_{1}+\left(\frac{1}{2}+x_{7}\right) \mathbf{a}_{2}+y_{7} \mathbf{a}_{3}$ & $=$ & $\left(\frac{1}{2}-z_{7}\right) a \hat{\mathbf{x}}+\left(\frac{1}{2}+x_{7}\right) a \hat{\mathbf{y}}+y_{7} a \hat{\mathbf{z}}$ & $(24 d)$ & C VII \\
\hline $\mathbf{B}_{163}$ & $=$ & $\left(\frac{1}{2}+z_{7}\right) \mathbf{a}_{1}+x_{7} \mathbf{a}_{2}+\left(\frac{1}{2}-y_{7}\right) \mathbf{a}_{3}$ & $=$ & $\left(\frac{1}{2}+z_{7}\right) a \hat{\mathbf{x}}+x_{7} a \hat{\mathbf{y}}+\left(\frac{1}{2}-y_{7}\right) a \hat{\mathbf{z}}$ & $(24 d)$ & C VII \\
\hline $\mathbf{B}_{164}$ & $=$ & $z_{7} \mathbf{a}_{1}+\left(\frac{1}{2}-x_{7}\right) \mathbf{a}_{2}+\left(\frac{1}{2}+y_{7}\right) \mathbf{a}_{3}$ & $=$ & $z_{7} a \hat{\mathbf{x}}+\left(\frac{1}{2}-x_{7}\right) a \hat{\mathbf{y}}+\left(\frac{1}{2}+y_{7}\right) a \hat{\mathbf{z}}$ & $(24 d)$ & C VII \\
\hline $\mathbf{B}_{165}$ & $=$ & $-y_{7} \mathbf{a}_{1}-z_{7} \mathbf{a}_{2}-x_{7} \mathbf{a}_{3}$ & $=$ & $-y_{7} a \hat{\mathbf{x}}-z_{7} a \hat{\mathbf{y}}-x_{7} a \hat{\mathbf{z}}$ & $(24 d)$ & C VII \\
\hline $\mathbf{B}_{166}$ & $=$ & $y_{7} \mathbf{a}_{1}+\left(\frac{1}{2}-z_{7}\right) \mathbf{a}_{2}+\left(\frac{1}{2}+x_{7}\right) \mathbf{a}_{3}$ & $=$ & $y_{7} a \hat{\mathbf{x}}+\left(\frac{1}{2}-z_{7}\right) a \hat{\mathbf{y}}+\left(\frac{1}{2}+x_{7}\right) a \hat{\mathbf{z}}$ & $(24 d)$ & C VII \\
\hline $\mathbf{B}_{167}$ & $=$ & $\left(\frac{1}{2}-y_{7}\right) \mathbf{a}_{1}+\left(\frac{1}{2}+z_{7}\right) \mathbf{a}_{2}+x_{7} \mathbf{a}_{3}$ & $=$ & $\left(\frac{1}{2}-y_{7}\right) a \hat{\mathbf{x}}+\left(\frac{1}{2}+z_{7}\right) a \hat{\mathbf{y}}+x_{7} a \hat{\mathbf{z}}$ & $(24 d)$ & C VII \\
\hline $\mathbf{B}_{168}$ & $=$ & $\left(\frac{1}{2}+y_{7}\right) \mathbf{a}_{1}+z_{7} \mathbf{a}_{2}+\left(\frac{1}{2}-x_{7}\right) \mathbf{a}_{3}$ & $=$ & $\left(\frac{1}{2}+y_{7}\right) a \hat{\mathbf{x}}+z_{7} a \hat{\mathbf{y}}+\left(\frac{1}{2}-x_{7}\right) a \hat{\mathbf{z}}$ & $(24 d)$ & C VII \\
\hline $\mathbf{B}_{169}$ & $=$ & $x_{8} \mathbf{a}_{1}+y_{8} \mathbf{a}_{2}+z_{8} \mathbf{a}_{3}$ & $=$ & $x_{8} a \hat{\mathbf{x}}+y_{8} a \hat{\mathbf{y}}+z_{8} a \hat{\mathbf{z}}$ & $(24 d)$ & VIII \\
\hline $\mathbf{B}_{170}$ & $=$ & $\left(\frac{1}{2}-x_{8}\right) \mathbf{a}_{1}-y_{8} \mathbf{a}_{2}+\left(\frac{1}{2}+z_{8}\right) \mathbf{a}_{3}$ & $=$ & $\left(\frac{1}{2}-x_{8}\right) a \hat{\mathbf{x}}-y_{8} a \hat{\mathbf{y}}+\left(\frac{1}{2}+z_{8}\right) a \hat{\mathbf{z}}$ & $(24 d)$ & VIII \\
\hline $\mathbf{B}_{171}$ & $=$ & $-x_{8} \mathbf{a}_{1}+\left(\frac{1}{2}+y_{8}\right) \mathbf{a}_{2}+\left(\frac{1}{2}-z_{8}\right) \mathbf{a}_{3}$ & $=$ & $-x_{8} a \hat{\mathbf{x}}+\left(\frac{1}{2}+y_{8}\right) a \hat{\mathbf{y}}+\left(\frac{1}{2}-z_{8}\right) a \hat{\mathbf{z}}$ & $(24 d)$ & C VIII \\
\hline $\mathbf{B}_{172}$ & $=$ & $\left(\frac{1}{2}+x_{8}\right) \mathbf{a}_{1}+\left(\frac{1}{2}-y_{8}\right) \mathbf{a}_{2}-z_{8} \mathbf{a}_{3}$ & $=$ & $\left(\frac{1}{2}+x_{8}\right) a \hat{\mathbf{x}}+\left(\frac{1}{2}-y_{8}\right) a \hat{\mathbf{y}}-z_{8} a \hat{\mathbf{z}}$ & $(24 d)$ & C VIII \\
\hline $\mathbf{B}_{173}$ & $=$ & $z_{8} \mathbf{a}_{1}+x_{8} \mathbf{a}_{2}+y_{8} \mathbf{a}_{3}$ & $=$ & $z_{8} a \hat{\mathbf{x}}+x_{8} a \hat{\mathbf{y}}+y_{8} a \hat{\mathbf{z}}$ & $(24 d)$ & C VIII \\
\hline $\mathbf{B}_{174}$ & $=$ & $\left(\frac{1}{2}+z_{8}\right) \mathbf{a}_{1}+\left(\frac{1}{2}-x_{8}\right) \mathbf{a}_{2}-y_{8} \mathbf{a}_{3}$ & $=$ & $\left(\frac{1}{2}+z_{8}\right) a \hat{\mathbf{x}}+\left(\frac{1}{2}-x_{8}\right) a \hat{\mathbf{y}}-y_{8} a \hat{\mathbf{z}}$ & $(24 d)$ & C VIII \\
\hline $\mathbf{B}_{175}$ & $=$ & $\left(\frac{1}{2}-z_{8}\right) \mathbf{a}_{1}-x_{8} \mathbf{a}_{2}+\left(\frac{1}{2}+y_{8}\right) \mathbf{a}_{3}$ & $=$ & $\left(\frac{1}{2}-z_{8}\right) a \hat{\mathbf{x}}-x_{8} a \hat{\mathbf{y}}+\left(\frac{1}{2}+y_{8}\right) a \hat{\mathbf{z}}$ & $(24 d)$ & C VIII \\
\hline $\mathbf{B}_{176}$ & $=$ & $-z_{8} \mathbf{a}_{1}+\left(\frac{1}{2}+x_{8}\right) \mathbf{a}_{2}+\left(\frac{1}{2}-y_{8}\right) \mathbf{a}_{3}$ & $=$ & $-z_{8} a \hat{\mathbf{x}}+\left(\frac{1}{2}+x_{8}\right) a \hat{\mathbf{y}}+\left(\frac{1}{2}-y_{8}\right) a \hat{\mathbf{z}}$ & $(24 d)$ & C VIII \\
\hline $\mathbf{B}_{177}$ & $=$ & $y_{8} \mathbf{a}_{1}+z_{8} \mathbf{a}_{2}+x_{8} \mathbf{a}_{3}$ & $=$ & $y_{8} a \hat{\mathbf{x}}+z_{8} a \hat{\mathbf{y}}+x_{8} a \hat{\mathbf{z}}$ & $(24 d)$ & C VIII \\
\hline $\mathbf{B}_{178}$ & $=$ & $-y_{8} \mathbf{a}_{1}+\left(\frac{1}{2}+z_{8}\right) \mathbf{a}_{2}+\left(\frac{1}{2}-x_{8}\right) \mathbf{a}_{3}$ & $=$ & $-y_{8} a \hat{\mathbf{x}}+\left(\frac{1}{2}+z_{8}\right) a \hat{\mathbf{y}}+\left(\frac{1}{2}-x_{8}\right) a \hat{\mathbf{z}}$ & $(24 d)$ & C VIII \\
\hline
\end{tabular}




\begin{tabular}{|c|c|c|c|c|c|c|}
\hline $\mathbf{B}_{179}$ & $=$ & $\left(\frac{1}{2}+y_{8}\right) \mathbf{a}_{1}+\left(\frac{1}{2}-z_{8}\right) \mathbf{a}_{2}-x_{8} \mathbf{a}_{3}$ & $=$ & $\left(\frac{1}{2}+y_{8}\right) a \hat{\mathbf{x}}+\left(\frac{1}{2}-z_{8}\right) a \hat{\mathbf{y}}-x_{8} a \hat{\mathbf{z}}$ & $(24 d)$ & C VIII \\
\hline $\mathbf{B}_{180}$ & $=$ & $\left(\frac{1}{2}-y_{8}\right) \mathbf{a}_{1}-z_{8} \mathbf{a}_{2}+\left(\frac{1}{2}+x_{8}\right) \mathbf{a}_{3}$ & $=$ & $\left(\frac{1}{2}-y_{8}\right) a \hat{\mathbf{x}}-z_{8} a \hat{\mathbf{y}}+\left(\frac{1}{2}+x_{8}\right) a \hat{\mathbf{z}}$ & $(24 d)$ & C VIII \\
\hline $\mathbf{B}_{181}$ & $=$ & $-x_{8} \mathbf{a}_{1}-y_{8} \mathbf{a}_{2}-z_{8} \mathbf{a}_{3}$ & $=$ & $-x_{8} a \hat{\mathbf{x}}-y_{8} a \hat{\mathbf{y}}-z_{8} a \hat{\mathbf{z}}$ & $(24 d)$ & $\mathrm{C} \mathrm{VI}$ \\
\hline $\mathbf{B}_{182}$ & $=$ & $\left(\frac{1}{2}+x_{8}\right) \mathbf{a}_{1}+y_{8} \mathbf{a}_{2}+\left(\frac{1}{2}-z_{8}\right) \mathbf{a}_{3}$ & $=$ & $\left(\frac{1}{2}+x_{8}\right) a \hat{\mathbf{x}}+y_{8} a \hat{\mathbf{y}}+\left(\frac{1}{2}-z_{8}\right) a \hat{\mathbf{z}}$ & $(24 d)$ & C VIII \\
\hline $\mathbf{B}_{183}$ & $=$ & $x_{8} \mathbf{a}_{1}+\left(\frac{1}{2}-y_{8}\right) \mathbf{a}_{2}+\left(\frac{1}{2}+z_{8}\right) \mathbf{a}_{3}$ & $=$ & $x_{8} a \hat{\mathbf{x}}+\left(\frac{1}{2}-y_{8}\right) a \hat{\mathbf{y}}+\left(\frac{1}{2}+z_{8}\right) a \hat{\mathbf{z}}$ & $(24 d)$ & $\mathrm{C} \mathrm{Vl}$ \\
\hline $\mathbf{B}_{184}$ & $=$ & $\left(\frac{1}{2}-x_{8}\right) \mathbf{a}_{1}+\left(\frac{1}{2}+y_{8}\right) \mathbf{a}_{2}+z_{8} \mathbf{a}_{3}$ & $=$ & $\left(\frac{1}{2}-x_{8}\right) a \hat{\mathbf{x}}+\left(\frac{1}{2}+y_{8}\right) a \hat{\mathbf{y}}+z_{8} a \hat{\mathbf{z}}$ & $(24 d)$ & $\mathrm{C} \mathrm{Vl}$ \\
\hline $\mathbf{B}_{185}$ & $=$ & $-z_{8} \mathbf{a}_{1}-x_{8} \mathbf{a}_{2}-y_{8} \mathbf{a}_{3}$ & $=$ & $-z_{8} a \hat{\mathbf{x}}-x_{8} a \hat{\mathbf{y}}-y_{8} a \hat{\mathbf{z}}$ & $(24 d)$ & C VI \\
\hline $\mathbf{B}_{186}$ & $=$ & $\left(\frac{1}{2}-z_{8}\right) \mathbf{a}_{1}+\left(\frac{1}{2}+x_{8}\right) \mathbf{a}_{2}+y_{8} \mathbf{a}_{3}$ & $=$ & $\left(\frac{1}{2}-z_{8}\right) a \hat{\mathbf{x}}+\left(\frac{1}{2}+x_{8}\right) a \hat{\mathbf{y}}+y_{8} a \hat{\mathbf{z}}$ & $(24 d)$ & $\mathrm{C} \mathrm{VI}$ \\
\hline $\mathbf{B}_{187}$ & $=$ & $\left(\frac{1}{2}+z_{8}\right) \mathbf{a}_{1}+x_{8} \mathbf{a}_{2}+\left(\frac{1}{2}-y_{8}\right) \mathbf{a}_{3}$ & $=$ & $\left(\frac{1}{2}+z_{8}\right) a \hat{\mathbf{x}}+x_{8} a \hat{\mathbf{y}}+\left(\frac{1}{2}-y_{8}\right) a \hat{\mathbf{z}}$ & $(24 d)$ & C VI \\
\hline $\mathbf{B}_{188}$ & $=$ & $z_{8} \mathbf{a}_{1}+\left(\frac{1}{2}-x_{8}\right) \mathbf{a}_{2}+\left(\frac{1}{2}+y_{8}\right) \mathbf{a}_{3}$ & $=$ & $z_{8} a \hat{\mathbf{x}}+\left(\frac{1}{2}-x_{8}\right) a \hat{\mathbf{y}}+\left(\frac{1}{2}+y_{8}\right) a \hat{\mathbf{z}}$ & $(24 d)$ & C VI \\
\hline $\mathbf{B}_{189}$ & $=$ & $-y_{8} \mathbf{a}_{1}-z_{8} \mathbf{a}_{2}-x_{8} \mathbf{a}_{3}$ & $=$ & $-y_{8} a \hat{\mathbf{x}}-z_{8} a \hat{\mathbf{y}}-x_{8} a \hat{\mathbf{z}}$ & $(24 d)$ & 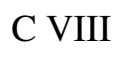 \\
\hline $\mathbf{B}_{190}$ & $=$ & $y_{8} \mathbf{a}_{1}+\left(\frac{1}{2}-z_{8}\right) \mathbf{a}_{2}+\left(\frac{1}{2}+x_{8}\right) \mathbf{a}_{3}$ & $=$ & $y_{8} a \hat{\mathbf{x}}+\left(\frac{1}{2}-z_{8}\right) a \hat{\mathbf{y}}+\left(\frac{1}{2}+x_{8}\right) a \hat{\mathbf{z}}$ & $(24 d)$ & $C \mathrm{~V}$ \\
\hline $\mathbf{B}_{191}$ & $=$ & $\left(\frac{1}{2}-y_{8}\right) \mathbf{a}_{1}+\left(\frac{1}{2}+z_{8}\right) \mathbf{a}_{2}+x_{8} \mathbf{a}_{3}$ & $=$ & $\left(\frac{1}{2}-y_{8}\right) a \hat{\mathbf{x}}+\left(\frac{1}{2}+z_{8}\right) a \hat{\mathbf{y}}+x_{8} a \hat{\mathbf{z}}$ & $(24 d)$ & $\mathrm{C} \mathrm{VI}$ \\
\hline $\mathbf{B}_{192}$ & $=$ & $\left(\frac{1}{2}+y_{8}\right) \mathbf{a}_{1}+z_{8} \mathbf{a}_{2}+\left(\frac{1}{2}-x_{8}\right) \mathbf{a}_{3}$ & $=$ & $\left(\frac{1}{2}+y_{8}\right) a \hat{\mathbf{x}}+z_{8} a \hat{\mathbf{y}}+\left(\frac{1}{2}-x_{8}\right) a \hat{\mathbf{z}}$ & $(24 d)$ & C VIII \\
\hline $\mathbf{B}_{193}$ & $=$ & $x_{9} \mathbf{a}_{1}+y_{9} \mathbf{a}_{2}+z_{9} \mathbf{a}_{3}$ & $=$ & $x_{9} a \hat{\mathbf{x}}+y_{9} a \hat{\mathbf{y}}+z_{9} a \hat{\mathbf{z}}$ & $(24 d)$ & C IX \\
\hline $\mathbf{B}_{194}$ & $=$ & $\left(\frac{1}{2}-x_{9}\right) \mathbf{a}_{1}-y_{9} \mathbf{a}_{2}+\left(\frac{1}{2}+z_{9}\right) \mathbf{a}_{3}$ & $=$ & $\left(\frac{1}{2}-x_{9}\right) a \hat{\mathbf{x}}-y_{9} a \hat{\mathbf{y}}+\left(\frac{1}{2}+z_{9}\right) a \hat{\mathbf{z}}$ & $(24 d)$ & C IX \\
\hline $\mathbf{B}_{195}$ & $=$ & $-x_{9} \mathbf{a}_{1}+\left(\frac{1}{2}+y_{9}\right) \mathbf{a}_{2}+\left(\frac{1}{2}-z_{9}\right) \mathbf{a}_{3}$ & $=$ & $-x_{9} a \hat{\mathbf{x}}+\left(\frac{1}{2}+y_{9}\right) a \hat{\mathbf{y}}+\left(\frac{1}{2}-z_{9}\right) a \hat{\mathbf{z}}$ & $(24 d)$ & C IX \\
\hline $\mathbf{B}_{196}$ & $=$ & $\left(\frac{1}{2}+x_{9}\right) \mathbf{a}_{1}+\left(\frac{1}{2}-y_{9}\right) \mathbf{a}_{2}-z_{9} \mathbf{a}_{3}$ & $=$ & $\left(\frac{1}{2}+x_{9}\right) a \hat{\mathbf{x}}+\left(\frac{1}{2}-y_{9}\right) a \hat{\mathbf{y}}-z_{9} a \hat{\mathbf{z}}$ & $(24 d)$ & C IX \\
\hline $\mathbf{B}_{197}$ & $=$ & $z_{9} \mathbf{a}_{1}+x_{9} \mathbf{a}_{2}+y_{9} \mathbf{a}_{3}$ & $=$ & $z_{9} a \hat{\mathbf{x}}+x_{9} a \hat{\mathbf{y}}+y_{9} a \hat{\mathbf{z}}$ & $(24 d)$ & C IX \\
\hline $\mathbf{B}_{198}$ & $=$ & $\left(\frac{1}{2}+z_{9}\right) \mathbf{a}_{1}+\left(\frac{1}{2}-x_{9}\right) \mathbf{a}_{2}-y_{9} \mathbf{a}_{3}$ & $=$ & $\left(\frac{1}{2}+z_{9}\right) a \hat{\mathbf{x}}+\left(\frac{1}{2}-x_{9}\right) a \hat{\mathbf{y}}-y_{9} a \hat{\mathbf{z}}$ & $(24 d)$ & C IX \\
\hline $\mathbf{B}_{199}$ & $=$ & $\left(\frac{1}{2}-z_{9}\right) \mathbf{a}_{1}-x_{9} \mathbf{a}_{2}+\left(\frac{1}{2}+y_{9}\right) \mathbf{a}_{3}$ & $=$ & $\left(\frac{1}{2}-z_{9}\right) a \hat{\mathbf{x}}-x_{9} a \hat{\mathbf{y}}+\left(\frac{1}{2}+y_{9}\right) a \hat{\mathbf{z}}$ & $(24 d)$ & C IX \\
\hline $\mathbf{B}_{200}$ & $=$ & $-z_{9} \mathbf{a}_{1}+\left(\frac{1}{2}+x_{9}\right) \mathbf{a}_{2}+\left(\frac{1}{2}-y_{9}\right) \mathbf{a}_{3}$ & $=$ & $-z_{9} a \hat{\mathbf{x}}+\left(\frac{1}{2}+x_{9}\right) a \hat{\mathbf{y}}+\left(\frac{1}{2}-y_{9}\right) a \hat{\mathbf{z}}$ & $(24 d)$ & C IX \\
\hline $\mathbf{B}_{201}$ & $=$ & $y_{9} \mathbf{a}_{1}+z_{9} \mathbf{a}_{2}+x_{9} \mathbf{a}_{3}$ & $=$ & $y_{9} a \hat{\mathbf{x}}+z_{9} a \hat{\mathbf{y}}+x_{9} a \hat{\mathbf{z}}$ & $(24 d)$ & C IX \\
\hline $\mathbf{B}_{202}$ & $=$ & $-y_{9} \mathbf{a}_{1}+\left(\frac{1}{2}+z 9\right) \mathbf{a}_{2}+\left(\frac{1}{2}-x_{9}\right) \mathbf{a}_{3}$ & $=$ & $-y_{9} a \hat{\mathbf{x}}+\left(\frac{1}{2}+z_{9}\right) a \hat{\mathbf{y}}+\left(\frac{1}{2}-x_{9}\right) a \hat{\mathbf{z}}$ & $(24 d)$ & C IX \\
\hline $\mathbf{B}_{203}$ & $=$ & $\left(\frac{1}{2}+y_{9}\right) \mathbf{a}_{1}+\left(\frac{1}{2}-z_{9}\right) \mathbf{a}_{2}-x_{9} \mathbf{a}_{3}$ & $=$ & $\left(\frac{1}{2}+y_{9}\right) a \hat{\mathbf{x}}+\left(\frac{1}{2}-z_{9}\right) a \hat{\mathbf{y}}-x_{9} a \hat{\mathbf{z}}$ & $(24 d)$ & C IX \\
\hline $\mathbf{B}_{204}$ & $=$ & $\left(\frac{1}{2}-y_{9}\right) \mathbf{a}_{1}-z_{9} \mathbf{a}_{2}+\left(\frac{1}{2}+x_{9}\right) \mathbf{a}_{3}$ & $=$ & $\left(\frac{1}{2}-y_{9}\right) a \hat{\mathbf{x}}-z_{9} a \hat{\mathbf{y}}+\left(\frac{1}{2}+x_{9}\right) a \hat{\mathbf{z}}$ & $(24 d)$ & C IX \\
\hline $\mathbf{B}_{205}$ & $=$ & $-x_{9} \mathbf{a}_{1}-y_{9} \mathbf{a}_{2}-z_{9} \mathbf{a}_{3}$ & $=$ & $-x_{9} a \hat{\mathbf{x}}-y_{9} a \hat{\mathbf{y}}-z_{9} a \hat{\mathbf{z}}$ & $(24 d)$ & C IX \\
\hline $\mathbf{B}_{206}$ & $=$ & $\left(\frac{1}{2}+x_{9}\right) \mathbf{a}_{1}+y_{9} \mathbf{a}_{2}+\left(\frac{1}{2}-z_{9}\right) \mathbf{a}_{3}$ & $=$ & $\left(\frac{1}{2}+x_{9}\right) a \hat{\mathbf{x}}+y_{9} a \hat{\mathbf{y}}+\left(\frac{1}{2}-z_{9}\right) a \hat{\mathbf{z}}$ & $(24 d)$ & C IX \\
\hline $\mathbf{B}_{207}$ & $=$ & $x_{9} \mathbf{a}_{1}+\left(\frac{1}{2}-y_{9}\right) \mathbf{a}_{2}+\left(\frac{1}{2}+z_{9}\right) \mathbf{a}_{3}$ & $=$ & $x_{9} a \hat{\mathbf{x}}+\left(\frac{1}{2}-y_{9}\right) a \hat{\mathbf{y}}+\left(\frac{1}{2}+z_{9}\right) a \hat{\mathbf{z}}$ & $(24 d)$ & C IX \\
\hline $\mathbf{B}_{208}$ & $=$ & $\left(\frac{1}{2}-x_{9}\right) \mathbf{a}_{1}+\left(\frac{1}{2}+y_{9}\right) \mathbf{a}_{2}+z_{9} \mathbf{a}_{3}$ & $=$ & $\left(\frac{1}{2}-x_{9}\right) a \hat{\mathbf{x}}+\left(\frac{1}{2}+y_{9}\right) a \hat{\mathbf{y}}+z_{9} a \hat{\mathbf{z}}$ & $(24 d)$ & C IX \\
\hline $\mathbf{B}_{209}$ & $=$ & $-z_{9} \mathbf{a}_{1}-x_{9} \mathbf{a}_{2}-y_{9} \mathbf{a}_{3}$ & $=$ & $-z_{9} a \hat{\mathbf{x}}-x_{9} a \hat{\mathbf{y}}-y_{9} a \hat{\mathbf{z}}$ & $(24 d)$ & C IX \\
\hline $\mathbf{B}_{210}$ & $=$ & $\left(\frac{1}{2}-z_{9}\right) \mathbf{a}_{1}+\left(\frac{1}{2}+x_{9}\right) \mathbf{a}_{2}+y_{9} \mathbf{a}_{3}$ & $=$ & $\left(\frac{1}{2}-z_{9}\right) a \hat{\mathbf{x}}+\left(\frac{1}{2}+x_{9}\right) a \hat{\mathbf{y}}+y_{9} a \hat{\mathbf{z}}$ & $(24 d)$ & C IX \\
\hline $\mathbf{B}_{211}$ & $=$ & $\left(\frac{1}{2}+z_{9}\right) \mathbf{a}_{1}+x_{9} \mathbf{a}_{2}+\left(\frac{1}{2}-y_{9}\right) \mathbf{a}_{3}$ & $=$ & $\left(\frac{1}{2}+z_{9}\right) a \hat{\mathbf{x}}+x_{9} a \hat{\mathbf{y}}+\left(\frac{1}{2}-y_{9}\right) a \hat{\mathbf{z}}$ & $(24 d)$ & C IX \\
\hline $\mathbf{B}_{212}$ & $=$ & $z_{9} \mathbf{a}_{1}+\left(\frac{1}{2}-x_{9}\right) \mathbf{a}_{2}+\left(\frac{1}{2}+y_{9}\right) \mathbf{a}_{3}$ & $=$ & $z_{9} a \hat{\mathbf{x}}+\left(\frac{1}{2}-x_{9}\right) a \hat{\mathbf{y}}+\left(\frac{1}{2}+y_{9}\right) a \hat{\mathbf{z}}$ & $(24 d)$ & C IX \\
\hline $\mathbf{B}_{213}$ & $=$ & $-y_{9} \mathbf{a}_{1}-z_{9} \mathbf{a}_{2}-x_{9} \mathbf{a}_{3}$ & $=$ & $-y_{9} a \hat{\mathbf{x}}-z_{9} a \hat{\mathbf{y}}-x_{9} a \hat{\mathbf{z}}$ & $(24 d)$ & C IX \\
\hline $\mathbf{B}_{214}$ & $=$ & $y_{9} \mathbf{a}_{1}+\left(\frac{1}{2}-z_{9}\right) \mathbf{a}_{2}+\left(\frac{1}{2}+x_{9}\right) \mathbf{a}_{3}$ & $=$ & $y_{9} a \hat{\mathbf{x}}+\left(\frac{1}{2}-z_{9}\right) a \hat{\mathbf{y}}+\left(\frac{1}{2}+x_{9}\right) a \hat{\mathbf{z}}$ & $(24 d)$ & C IX \\
\hline
\end{tabular}




\begin{tabular}{|c|c|c|c|c|}
\hline $\mathbf{B}_{215}$ & $=\left(\frac{1}{2}-y_{9}\right) \mathbf{a}_{1}+\left(\frac{1}{2}+z_{9}\right) \mathbf{a}_{2}+x_{9} \mathbf{a}_{3}$ & $=\left(\frac{1}{2}-y_{9}\right) a \hat{\mathbf{x}}+\left(\frac{1}{2}+z_{9}\right) a \hat{\mathbf{y}}+x_{9} a \hat{\mathbf{z}}$ & $(24 d)$ & C IX \\
\hline $\mathbf{B}_{216}$ & $=\left(\frac{1}{2}+y_{9}\right) \mathbf{a}_{1}+z_{9} \mathbf{a}_{2}+\left(\frac{1}{2}-x_{9}\right) \mathbf{a}_{3}$ & $=\left(\frac{1}{2}+y_{9}\right) a \hat{\mathbf{x}}+z_{9} a \hat{\mathbf{y}}+\left(\frac{1}{2}-x_{9}\right) a \hat{\mathbf{z}}$ & $(24 d)$ & C IX \\
\hline $\mathbf{B}_{217}$ & $x_{10} \mathbf{a}_{1}+y_{10} \mathbf{a}_{2}+z_{10} \mathbf{a}_{3}$ & $x_{10} a \hat{\mathbf{x}}+y_{10} a \hat{\mathbf{y}}+z_{10} a \hat{\mathbf{z}}$ & $(24 d)$ & $\mathrm{CX}$ \\
\hline $\mathbf{B}_{218}$ & $=\left(\frac{1}{2}-x_{10}\right) \mathbf{a}_{1}-y_{10} \mathbf{a}_{2}+\left(\frac{1}{2}+z_{10}\right) \mathbf{a}_{3}$ & $=\left(\frac{1}{2}-x_{10}\right) a \hat{\mathbf{x}}-y_{10} a \hat{\mathbf{y}}+\left(\frac{1}{2}+z_{10}\right) a \hat{\mathbf{z}}$ & $(24 d)$ & $\mathrm{CX}$ \\
\hline $\mathbf{B}_{219}$ & $\begin{array}{c}-x_{10} \mathbf{a}_{1}+\left(\frac{1}{2}+y_{10}\right) \mathbf{a}_{2}+ \\
\left(\frac{1}{2}-z_{10}\right) \mathbf{a}_{3}\end{array}$ & $\begin{array}{c}-x_{10} a \hat{\mathbf{x}}+\left(\frac{1}{2}+y_{10}\right) a \hat{\mathbf{y}}+ \\
\left(\frac{1}{2}-z_{10}\right) a \hat{\mathbf{z}}\end{array}$ & $(24 d)$ & $\mathrm{CX}$ \\
\hline $\mathbf{B}_{220}$ & $=\left(\frac{1}{2}+x_{10}\right) \mathbf{a}_{1}+\left(\frac{1}{2}-y_{10}\right) \mathbf{a}_{2}-z_{10} \mathbf{a}_{3}$ & $=\left(\frac{1}{2}+x_{10}\right) a \hat{\mathbf{x}}+\left(\frac{1}{2}-y_{10}\right) a \hat{\mathbf{y}}-z_{10} a \hat{\mathbf{z}}$ & $(24 d)$ & $\mathrm{CX}$ \\
\hline $\mathbf{B}_{221}$ & $z_{10} \mathbf{a}_{1}+x_{10} \mathbf{a}_{2}+y_{10} \mathbf{a}_{3}$ & $z_{10} a \hat{\mathbf{x}}+x_{10} a \hat{\mathbf{y}}+y_{10} a \hat{\mathbf{z}}$ & $(24 d)$ & $\mathrm{CX}$ \\
\hline $\mathbf{B}_{222}$ & $=\left(\frac{1}{2}+z_{10}\right) \mathbf{a}_{1}+\left(\frac{1}{2}-x_{10}\right) \mathbf{a}_{2}-y_{10} \mathbf{a}_{3}$ & $=\left(\frac{1}{2}+z_{10}\right) a \hat{\mathbf{x}}+\left(\frac{1}{2}-x_{10}\right) a \hat{\mathbf{y}}-y_{10} a \hat{\mathbf{z}}$ & $(24 d)$ & $\mathrm{CX}$ \\
\hline $\mathbf{B}_{223}$ & $=\left(\frac{1}{2}-z_{10}\right) \mathbf{a}_{1}-x_{10} \mathbf{a}_{2}+\left(\frac{1}{2}+y_{10}\right) \mathbf{a}_{3}$ & $=\left(\frac{1}{2}-z_{10}\right) a \hat{\mathbf{x}}-x_{10} a \hat{\mathbf{y}}+\left(\frac{1}{2}+y_{10}\right) a \hat{\mathbf{z}}$ & $(24 d)$ & $\mathrm{CX}$ \\
\hline $\mathbf{B}_{224}$ & $\begin{array}{c}-z_{10} \mathbf{a}_{1}+\left(\frac{1}{2}+x_{10}\right) \mathbf{a}_{2}+ \\
\left(\frac{1}{2}-y_{10}\right) \mathbf{a}_{3}\end{array}$ & $\begin{array}{c}-z_{10} a \hat{\mathbf{x}}+\left(\frac{1}{2}+x_{10}\right) a \hat{\mathbf{y}}+ \\
\left(\frac{1}{2}-y_{10}\right) a \hat{\mathbf{z}}\end{array}$ & $(24 d)$ & $\mathrm{CX}$ \\
\hline $\mathbf{B}_{225}$ & $y_{10} \mathbf{a}_{1}+z_{10} \mathbf{a}_{2}+x_{10} \mathbf{a}_{3}$ & $y_{10} a \hat{\mathbf{x}}+z_{10} a \hat{\mathbf{y}}+x_{10} a \hat{\mathbf{z}}$ & $(24 d)$ & $\mathrm{CX}$ \\
\hline $\mathbf{B}_{226}$ & $\begin{array}{c}-y_{10} \mathbf{a}_{1}+\left(\frac{1}{2}+z_{10}\right) \mathbf{a}_{2}+ \\
\left(\frac{1}{2}-x_{10}\right) \mathbf{a}_{3}\end{array}$ & $\begin{array}{c}-y_{10} a \hat{\mathbf{x}}+\left(\frac{1}{2}+z_{10}\right) a \hat{\mathbf{y}}+ \\
\left(\frac{1}{2}-x_{10}\right) a \hat{\mathbf{z}}\end{array}$ & $(24 d)$ & $\mathrm{CX}$ \\
\hline $\mathbf{B}_{227}$ & $=\left(\frac{1}{2}+y_{10}\right) \mathbf{a}_{1}+\left(\frac{1}{2}-z_{10}\right) \mathbf{a}_{2}-x_{10} \mathbf{a}_{3}$ & $=\left(\frac{1}{2}+y_{10}\right) a \hat{\mathbf{x}}+\left(\frac{1}{2}-z_{10}\right) a \hat{\mathbf{y}}-x_{10} a \hat{\mathbf{z}}$ & $(24 d)$ & $\mathrm{CX}$ \\
\hline $\mathbf{B}_{228}$ & $=\left(\frac{1}{2}-y_{10}\right) \mathbf{a}_{1}-z_{10} \mathbf{a}_{2}+\left(\frac{1}{2}+x_{10}\right) \mathbf{a}_{3}$ & $=\left(\frac{1}{2}-y_{10}\right) a \hat{\mathbf{x}}-z_{10} a \hat{\mathbf{y}}+\left(\frac{1}{2}+x_{10}\right) a \hat{\mathbf{z}}$ & $(24 d)$ & $\mathrm{CX}$ \\
\hline $\mathbf{B}_{229}$ & $-x_{10} \mathbf{a}_{1}-y_{10} \mathbf{a}_{2}-z_{10} \mathbf{a}_{3}$ & $-x_{10} a \hat{\mathbf{x}}-y_{10} a \hat{\mathbf{y}}-z_{10} a \hat{\mathbf{z}}$ & $(24 d)$ & $\mathrm{CX}$ \\
\hline $\mathbf{B}_{230}$ & $=\left(\frac{1}{2}+x_{10}\right) \mathbf{a}_{1}+y_{10} \mathbf{a}_{2}+\left(\frac{1}{2}-z_{10}\right) \mathbf{a}_{3}$ & $=\left(\frac{1}{2}+x_{10}\right) a \hat{\mathbf{x}}+y_{10} a \hat{\mathbf{y}}+\left(\frac{1}{2}-z_{10}\right) a \hat{\mathbf{z}}$ & $(24 d)$ & $\mathrm{CX}$ \\
\hline $\mathbf{B}_{231}$ & $=\quad x_{10} \mathbf{a}_{1}+\left(\frac{1}{2}-y_{10}\right) \mathbf{a}_{2}+\left(\frac{1}{2}+z_{10}\right) \mathbf{a}_{3}$ & $=x_{10} a \hat{\mathbf{x}}+\left(\frac{1}{2}-y_{10}\right) a \hat{\mathbf{y}}+\left(\frac{1}{2}+z_{10}\right) a \hat{\mathbf{z}}$ & $(24 d)$ & $\mathrm{CX}$ \\
\hline $\mathbf{B}_{232}$ & $=\left(\frac{1}{2}-x_{10}\right) \mathbf{a}_{1}+\left(\frac{1}{2}+y_{10}\right) \mathbf{a}_{2}+z_{10} \mathbf{a}_{3}$ & $=\left(\frac{1}{2}-x_{10}\right) a \hat{\mathbf{x}}+\left(\frac{1}{2}+y_{10}\right) a \hat{\mathbf{y}}+z_{10} a \hat{\mathbf{z}}$ & $(24 d)$ & $\mathrm{CX}$ \\
\hline $\mathbf{B}_{233}$ & $-z_{10} \mathbf{a}_{1}-x_{10} \mathbf{a}_{2}-y_{10} \mathbf{a}_{3}$ & $-z_{10} a \hat{\mathbf{x}}-x_{10} a \hat{\mathbf{y}}-y_{10} a \hat{\mathbf{z}}$ & $(24 d)$ & $\mathrm{CX}$ \\
\hline $\mathbf{B}_{234}$ & $=\left(\frac{1}{2}-z_{10}\right) \mathbf{a}_{1}+\left(\frac{1}{2}+x_{10}\right) \mathbf{a}_{2}+y_{10} \mathbf{a}_{3}$ & $=\left(\frac{1}{2}-z_{10}\right) a \hat{\mathbf{x}}+\left(\frac{1}{2}+x_{10}\right) a \hat{\mathbf{y}}+y_{10} a \hat{\mathbf{z}}$ & $(24 d)$ & $\mathrm{CX}$ \\
\hline $\mathbf{B}_{235}$ & $=\left(\frac{1}{2}+z_{10}\right) \mathbf{a}_{1}+x_{10} \mathbf{a}_{2}+\left(\frac{1}{2}-y_{10}\right) \mathbf{a}_{3}$ & $=\left(\frac{1}{2}+z_{10}\right) a \hat{\mathbf{x}}+x_{10} a \hat{\mathbf{y}}+\left(\frac{1}{2}-y_{10}\right) a \hat{\mathbf{z}}$ & $(24 d)$ & $\mathrm{CX}$ \\
\hline $\mathbf{B}_{236}$ & $=z_{10} \mathbf{a}_{1}+\left(\frac{1}{2}-x_{10}\right) \mathbf{a}_{2}+\left(\frac{1}{2}+y_{10}\right) \mathbf{a}_{3}$ & $=z_{10} a \hat{\mathbf{x}}+\left(\frac{1}{2}-x_{10}\right) a \hat{\mathbf{y}}+\left(\frac{1}{2}+y_{10}\right) a \hat{\mathbf{z}}$ & $(24 d)$ & $\mathrm{CX}$ \\
\hline $\mathbf{B}_{237}$ & $-y_{10} \mathbf{a}_{1}-z_{10} \mathbf{a}_{2}-x_{10} \mathbf{a}_{3}$ & $-y_{10} a \hat{\mathbf{x}}-z_{10} a \hat{\mathbf{y}}-x_{10} a \hat{\mathbf{z}}$ & $(24 d)$ & $\mathrm{CX}$ \\
\hline $\mathbf{B}_{238}$ & $=y_{10} \mathbf{a}_{1}+\left(\frac{1}{2}-z_{10}\right) \mathbf{a}_{2}+\left(\frac{1}{2}+x_{10}\right) \mathbf{a}_{3}$ & $=y_{10} a \hat{\mathbf{x}}+\left(\frac{1}{2}-z_{10}\right) a \hat{\mathbf{y}}+\left(\frac{1}{2}+x_{10}\right) a \hat{\mathbf{z}}$ & $(24 d)$ & $\mathrm{CX}$ \\
\hline $\mathbf{B}_{239}$ & $=\left(\frac{1}{2}-y_{10}\right) \mathbf{a}_{1}+\left(\frac{1}{2}+z_{10}\right) \mathbf{a}_{2}+x_{10} \mathbf{a}_{3}$ & $=\left(\frac{1}{2}-y_{10}\right) a \hat{\mathbf{x}}+\left(\frac{1}{2}+z_{10}\right) a \hat{\mathbf{y}}+x_{10} a \hat{\mathbf{z}}$ & $(24 d)$ & $\mathrm{CX}$ \\
\hline $\mathbf{B}_{240}$ & $=\left(\frac{1}{2}+y_{10}\right) \mathbf{a}_{1}+z_{10} \mathbf{a}_{2}+\left(\frac{1}{2}-x_{10}\right) \mathbf{a}_{3}$ & $=\left(\frac{1}{2}+y_{10}\right) a \hat{\mathbf{x}}+z_{10} a \hat{\mathbf{y}}+\left(\frac{1}{2}-x_{10}\right) a \hat{\mathbf{z}}$ & $(24 d)$ & $\mathrm{CX}$ \\
\hline
\end{tabular}

\section{References:}

- W. I. F. David, R. M. Ibberson, J. C. Matthewman, K. Prassides, T. J. S. Dennis, J. P. Hare, H. W. Kroto, R. Taylor, and D. R. M. Walton, Crystal structure and bonding of ordered $C_{60}$, Nature 353, 147-149 (1991), doi:10.1038/353147a0.

\section{Geometry files:}

- CIF: pp. 957

- POSCAR: pp. 957 


\section{$\mathrm{AlLi}_{3} \mathrm{~N}_{2}\left(E 9_{d}\right)$ Structure: AB3C2_cI96_206_c_e_ad}
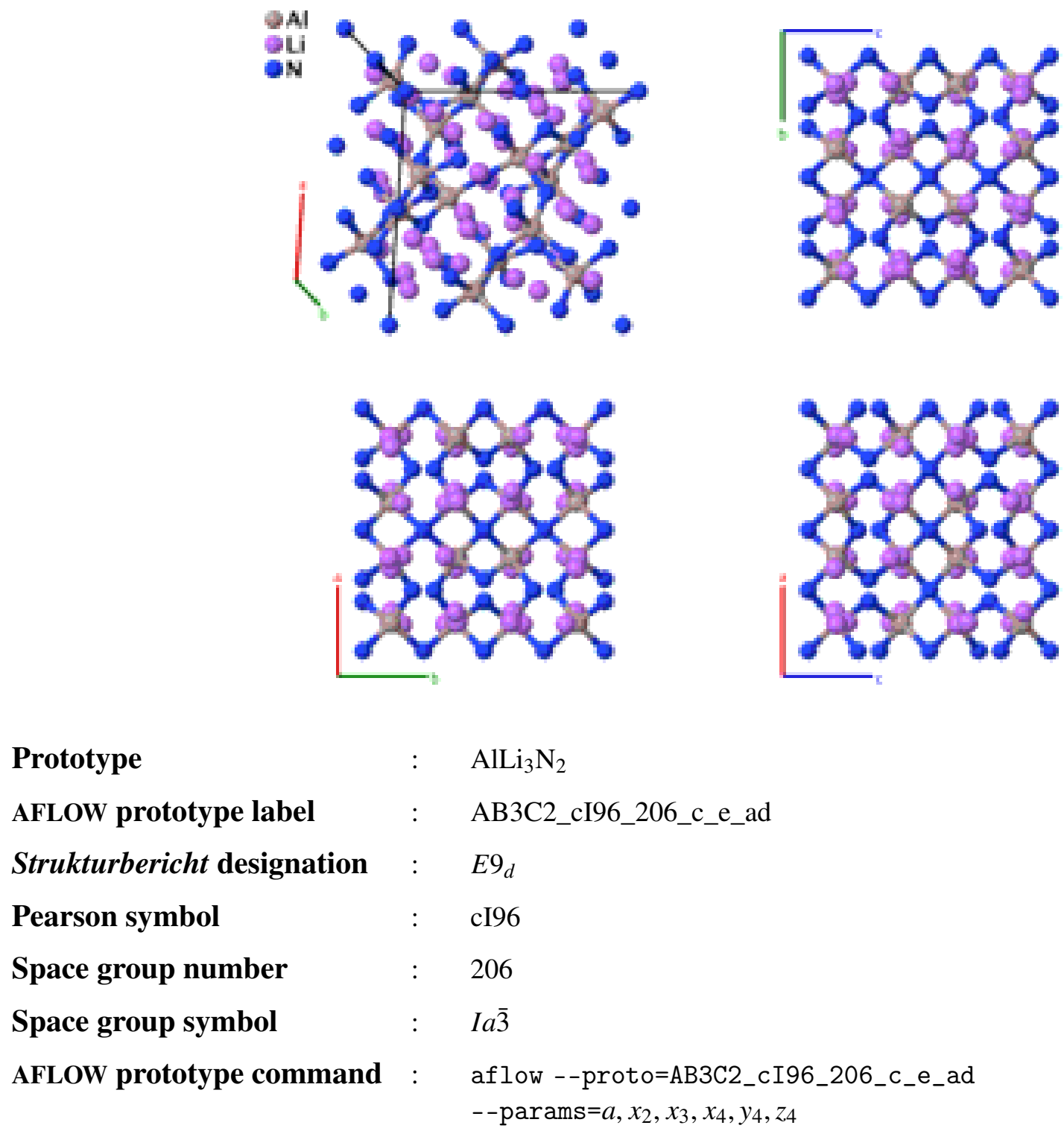

Other compounds with this structure:

- $\mathrm{GaLi}_{3} \mathrm{~N}_{2}, \mathrm{ScLi}_{3} \mathrm{~N}_{2}, \mathrm{TiLi}_{3} \mathrm{~N}_{2}, \mathrm{ZnLi}_{3} \mathrm{~N}_{2}, \mathrm{SiLi}_{3} \mathrm{~N}_{2}, \mathrm{GeLi}_{3} \mathrm{~N}_{2}$

Body-centered Cubic primitive vectors:

$$
\begin{aligned}
& \mathbf{a}_{1}=-\frac{1}{2} a \hat{\mathbf{x}}+\frac{1}{2} a \hat{\mathbf{y}}+\frac{1}{2} a \hat{\mathbf{z}} \\
& \mathbf{a}_{2}=\frac{1}{2} a \hat{\mathbf{x}}-\frac{1}{2} a \hat{\mathbf{y}}+\frac{1}{2} a \hat{\mathbf{z}} \\
& \mathbf{a}_{3}=\frac{1}{2} a \hat{\mathbf{x}}+\frac{1}{2} a \hat{\mathbf{y}}-\frac{1}{2} a \hat{\mathbf{z}}
\end{aligned}
$$

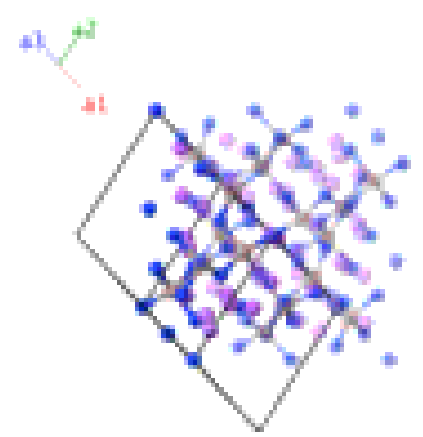

Basis vectors: 


\begin{tabular}{|c|c|c|c|c|c|c|}
\hline $\mathbf{B}_{1}$ & $=$ & $0 \mathbf{a}_{1}+0 \mathbf{a}_{2}+0 \mathbf{a}_{3}$ & $=$ & $0 \hat{\mathbf{x}}+0 \hat{\mathbf{y}}+0 \hat{\mathbf{z}}$ & $(8 a)$ & N I \\
\hline $\mathbf{B}_{2}$ & $=$ & $\frac{1}{2} \mathbf{a}_{1}+\frac{1}{2} \mathbf{a}_{3}$ & $=$ & $\frac{1}{2} a \hat{\mathbf{y}}$ & $(8 a)$ & $\mathrm{N} \mathrm{I}$ \\
\hline $\mathbf{B}_{3}$ & $=$ & $\frac{1}{2} \mathbf{a}_{2}+\frac{1}{2} \mathbf{a}_{3}$ & $=$ & $\frac{1}{2} a \hat{\mathbf{x}}$ & $(8 a)$ & $\mathrm{N} \mathrm{I}$ \\
\hline $\mathbf{B}_{4}$ & $=$ & $\frac{1}{2} \mathbf{a}_{1}+\frac{1}{2} \mathbf{a}_{2}$ & $=$ & $\frac{1}{2} a \hat{\mathbf{z}}$ & $(8 a)$ & $\mathrm{N} \mathrm{I}$ \\
\hline $\mathbf{B}_{5}$ & $=$ & $2 x_{2} \mathbf{a}_{1}+2 x_{2} \mathbf{a}_{2}+2 x_{2} \mathbf{a}_{3}$ & $=$ & $x_{2} a \hat{\mathbf{x}}+x_{2} a \hat{\mathbf{y}}+x_{2} a \hat{\mathbf{z}}$ & $(16 c)$ & $\mathrm{Al}$ \\
\hline $\mathbf{B}_{6}$ & $=$ & $\frac{1}{2} \mathbf{a}_{1}+\left(\frac{1}{2}-2 x_{2}\right) \mathbf{a}_{3}$ & $=$ & $-x_{2} a \hat{\mathbf{x}}+\left(\frac{1}{2}-x_{2}\right) a \hat{\mathbf{y}}+x_{2} a \hat{\mathbf{z}}$ & $(16 c)$ & $\mathrm{Al}$ \\
\hline $\mathbf{B}_{7}$ & $=$ & $\left(\frac{1}{2}-2 x_{2}\right) \mathbf{a}_{2}+\frac{1}{2} \mathbf{a}_{3}$ & $=$ & $\left(\frac{1}{2}-x_{2}\right) a \hat{\mathbf{x}}+x_{2} a \hat{\mathbf{y}}-x_{2} a \hat{\mathbf{z}}$ & $(16 c)$ & $\mathrm{Al}$ \\
\hline $\mathbf{B}_{8}$ & $=$ & $\left(\frac{1}{2}-2 x_{2}\right) \mathbf{a}_{1}+\frac{1}{2} \mathbf{a}_{2}$ & $=$ & $x_{2} a \hat{\mathbf{x}}-x_{2} a \hat{\mathbf{y}}+\left(\frac{1}{2}-x_{2}\right) a \hat{\mathbf{z}}$ & $(16 c)$ & $\mathrm{Al}$ \\
\hline $\mathbf{B}_{9}$ & $=$ & $-2 x_{2} \mathbf{a}_{1}-2 x_{2} \mathbf{a}_{2}-2 x_{2} \mathbf{a}_{3}$ & $=$ & $-x_{2} a \hat{\mathbf{x}}-x_{2} a \hat{\mathbf{y}}-x_{2} a \hat{\mathbf{z}}$ & $(16 c)$ & $\mathrm{Al}$ \\
\hline $\mathbf{B}_{10}$ & $=$ & $\frac{1}{2} \mathbf{a}_{1}+\left(\frac{1}{2}+2 x_{2}\right) \mathbf{a}_{3}$ & $=$ & $x_{2} a \hat{\mathbf{x}}+\left(\frac{1}{2}+x_{2}\right) a \hat{\mathbf{y}}-x_{2} a \hat{\mathbf{z}}$ & $(16 c)$ & $\mathrm{Al}$ \\
\hline $\mathbf{B}_{11}$ & $=$ & $\left(\frac{1}{2}+2 x_{2}\right) \mathbf{a}_{2}+\frac{1}{2} \mathbf{a}_{3}$ & $=$ & $\left(\frac{1}{2}+x_{2}\right) a \hat{\mathbf{x}}-x_{2} a \hat{\mathbf{y}}+x_{2} a \hat{\mathbf{z}}$ & $(16 c)$ & $\mathrm{Al}$ \\
\hline $\mathbf{B}_{12}$ & $=$ & $\left(\frac{1}{2}+2 x_{2}\right) \mathbf{a}_{1}+\frac{1}{2} \mathbf{a}_{2}$ & $=$ & $-x_{2} a \hat{\mathbf{x}}+x_{2} a \hat{\mathbf{y}}+\left(\frac{1}{2}+x_{2}\right) a \hat{\mathbf{z}}$ & $(16 c)$ & $\mathrm{Al}$ \\
\hline $\mathbf{B}_{13}$ & $=$ & $\frac{1}{4} \mathbf{a}_{1}+\left(\frac{1}{4}+x_{3}\right) \mathbf{a}_{2}+x_{3} \mathbf{a}_{3}$ & $=$ & $x_{3} a \hat{\mathbf{x}}+\frac{1}{4} a \hat{\mathbf{z}}$ & $(24 d)$ & N II \\
\hline $\mathbf{B}_{14}$ & $=$ & $\frac{3}{4} \mathbf{a}_{1}+\left(\frac{1}{4}-x_{3}\right) \mathbf{a}_{2}+\left(\frac{1}{2}-x_{3}\right) \mathbf{a}_{3}$ & $=$ & $-x_{3} a \hat{\mathbf{x}}+\frac{1}{2} a \hat{\mathbf{y}}+\frac{1}{4} a \hat{\mathbf{z}}$ & $(24 d)$ & $\mathrm{N}$ II \\
\hline $\mathbf{B}_{15}$ & $=$ & $x_{3} \mathbf{a}_{1}+\frac{1}{4} \mathbf{a}_{2}+\left(\frac{1}{4}+x_{3}\right) \mathbf{a}_{3}$ & $=$ & $\frac{1}{4} a \hat{\mathbf{x}}+x_{3} a \hat{\mathbf{y}}$ & $(24 d)$ & N II \\
\hline $\mathbf{B}_{16}$ & $=$ & $\left(\frac{1}{2}-x_{3}\right) \mathbf{a}_{1}+\frac{3}{4} \mathbf{a}_{2}+\left(\frac{1}{4}-x_{3}\right) \mathbf{a}_{3}$ & $=$ & $\frac{1}{4} a \hat{\mathbf{x}}-x_{3} a \hat{\mathbf{y}}+\frac{1}{2} a \hat{\mathbf{z}}$ & $(24 d)$ & N II \\
\hline $\mathbf{B}_{17}$ & $=$ & $\left(\frac{1}{4}+x_{3}\right) \mathbf{a}_{1}+x_{3} \mathbf{a}_{2}+\frac{1}{4} \mathbf{a}_{3}$ & $=$ & $\frac{1}{4} a \hat{\mathbf{y}}+x_{3} a \hat{\mathbf{z}}$ & $(24 d)$ & N II \\
\hline $\mathbf{B}_{18}$ & $=$ & $\left(\frac{1}{4}-x_{3}\right) \mathbf{a}_{1}+\left(\frac{1}{2}-x_{3}\right) \mathbf{a}_{2}+\frac{3}{4} \mathbf{a}_{3}$ & $=$ & $\frac{1}{2} a \hat{\mathbf{x}}+\frac{1}{4} a \hat{\mathbf{y}}-x_{3} a \hat{\mathbf{z}}$ & $(24 d)$ & N II \\
\hline $\mathbf{B}_{19}$ & $=$ & $\frac{3}{4} \mathbf{a}_{1}+\left(\frac{3}{4}-x_{3}\right) \mathbf{a}_{2}-x_{3} \mathbf{a}_{3}$ & $=$ & $-x_{3} a \hat{\mathbf{x}}+\frac{3}{4} a \hat{\mathbf{z}}$ & $(24 d)$ & $\mathrm{N}$ II \\
\hline $\mathbf{B}_{20}$ & $=$ & $\frac{1}{4} \mathbf{a}_{1}+\left(\frac{3}{4}+x_{3}\right) \mathbf{a}_{2}+\left(\frac{1}{2}+x_{3}\right) \mathbf{a}_{3}$ & $=$ & $\left(\frac{1}{2}+x_{3}\right) a \hat{\mathbf{x}}+\frac{1}{4} a \hat{\mathbf{z}}$ & $(24 d)$ & N II \\
\hline $\mathbf{B}_{21}$ & $=$ & $-x_{3} \mathbf{a}_{1}+\frac{3}{4} \mathbf{a}_{2}+\left(\frac{3}{4}-x_{3}\right) \mathbf{a}_{3}$ & $=$ & $\frac{3}{4} a \hat{\mathbf{x}}-x_{3} a \hat{\mathbf{y}}$ & $(24 d)$ & N II \\
\hline $\mathbf{B}_{22}$ & $=$ & $\left(\frac{1}{2}+x_{3}\right) \mathbf{a}_{1}+\frac{1}{4} \mathbf{a}_{2}+\left(\frac{3}{4}+x_{3}\right) \mathbf{a}_{3}$ & $=$ & $\frac{1}{4} a \hat{\mathbf{x}}+\left(\frac{1}{2}+x_{3}\right) a \hat{\mathbf{y}}$ & $(24 d)$ & N II \\
\hline $\mathbf{B}_{23}$ & $=$ & $\left(\frac{3}{4}-x_{3}\right) \mathbf{a}_{1}-x_{3} \mathbf{a}_{2}+\frac{3}{4} \mathbf{a}_{3}$ & $=$ & $\frac{3}{4} a \hat{\mathbf{y}}-x_{3} a \hat{\mathbf{z}}$ & $(24 d)$ & N II \\
\hline $\mathbf{B}_{24}$ & $=$ & $\left(\frac{3}{4}+x_{3}\right) \mathbf{a}_{1}+\left(\frac{1}{2}+x_{3}\right) \mathbf{a}_{2}+\frac{1}{4} \mathbf{a}_{3}$ & $=$ & $\frac{1}{4} a \hat{\mathbf{y}}+\left(\frac{1}{2}+x_{3}\right) a \hat{\mathbf{z}}$ & $(24 d)$ & N II \\
\hline $\mathbf{B}_{25}$ & $=$ & $\left(y_{4}+z_{4}\right) \mathbf{a}_{1}+\left(x_{4}+z_{4}\right) \mathbf{a}_{2}+\left(x_{4}+y_{4}\right) \mathbf{a}_{3}$ & $=$ & $x_{4} a \hat{\mathbf{x}}+y_{4} a \hat{\mathbf{y}}+z_{4} a \hat{\mathbf{z}}$ & $(48 e)$ & $\mathrm{Li}$ \\
\hline $\mathbf{B}_{26}$ & $=$ & $\begin{array}{c}\left(\frac{1}{2}-y_{4}+z_{4}\right) \mathbf{a}_{1}+\left(-x_{4}+z_{4}\right) \mathbf{a}_{2}+ \\
\left(\frac{1}{2}-x_{4}-y_{4}\right) \mathbf{a}_{3}\end{array}$ & $=$ & $-x_{4} a \hat{\mathbf{x}}+\left(\frac{1}{2}-y_{4}\right) a \hat{\mathbf{y}}+z_{4} a \hat{\mathbf{z}}$ & $(48 e)$ & $\mathrm{Li}$ \\
\hline $\mathbf{B}_{27}$ & $=$ & $\begin{array}{c}\left(y_{4}-z_{4}\right) \mathbf{a}_{1}+\left(\frac{1}{2}-x_{4}-z_{4}\right) \mathbf{a}_{2}+ \\
\left(\frac{1}{2}-x_{4}+y_{4}\right) \mathbf{a}_{3}\end{array}$ & $=$ & $\left(\frac{1}{2}-x_{4}\right) a \hat{\mathbf{x}}+y_{4} a \hat{\mathbf{y}}-z_{4} a \hat{\mathbf{z}}$ & $(48 e)$ & $\mathrm{Li}$ \\
\hline $\mathbf{B}_{28}$ & $=$ & $\begin{array}{c}\left(\frac{1}{2}-y_{4}-z_{4}\right) \mathbf{a}_{1}+\left(\frac{1}{2}+x_{4}-z_{4}\right) \mathbf{a}_{2}+ \\
\left(x_{4}-y_{4}\right) \mathbf{a}_{3}\end{array}$ & $=$ & $x_{4} a \hat{\mathbf{x}}-y_{4} a \hat{\mathbf{y}}+\left(\frac{1}{2}-z_{4}\right) a \hat{\mathbf{z}}$ & $(48 e)$ & $\mathrm{Li}$ \\
\hline $\mathbf{B}_{29}$ & $=$ & $\left(x_{4}+y_{4}\right) \mathbf{a}_{1}+\left(y_{4}+z_{4}\right) \mathbf{a}_{2}+\left(x_{4}+z_{4}\right) \mathbf{a}_{3}$ & $=$ & $z_{4} a \hat{\mathbf{x}}+x_{4} a \hat{\mathbf{y}}+y_{4} a \hat{\mathbf{z}}$ & $(48 e)$ & $\mathrm{Li}$ \\
\hline $\mathbf{B}_{30}$ & $=$ & $\begin{array}{c}\left(\frac{1}{2}-x_{4}-y_{4}\right) \mathbf{a}_{1}+\left(\frac{1}{2}-y_{4}+z_{4}\right) \mathbf{a}_{2}+ \\
\left(-x_{4}+z_{4}\right) \mathbf{a}_{3}\end{array}$ & $=$ & $z_{4} a \hat{\mathbf{x}}-x_{4} a \hat{\mathbf{y}}+\left(\frac{1}{2}-y_{4}\right) a \hat{\mathbf{z}}$ & $(48 e)$ & $\mathrm{Li}$ \\
\hline $\mathbf{B}_{31}$ & $=$ & $\begin{array}{c}\left(\frac{1}{2}-x_{4}+y_{4}\right) \mathbf{a}_{1}+\left(y_{4}-z_{4}\right) \mathbf{a}_{2}+ \\
\left(\frac{1}{2}-x_{4}-z_{4}\right) \mathbf{a}_{3}\end{array}$ & $=$ & $-z_{4} a \hat{\mathbf{x}}+\left(\frac{1}{2}-x_{4}\right) a \hat{\mathbf{y}}+y_{4} a \hat{\mathbf{z}}$ & $(48 e)$ & $\mathrm{Li}$ \\
\hline $\mathbf{B}_{32}$ & $=$ & $\begin{array}{c}\left(x_{4}-y_{4}\right) \mathbf{a}_{1}+\left(\frac{1}{2}-y_{4}-z_{4}\right) \mathbf{a}_{2}+ \\
\left(\frac{1}{2}+x_{4}-z_{4}\right) \mathbf{a}_{3}\end{array}$ & $=$ & $\left(\frac{1}{2}-z_{4}\right) a \hat{\mathbf{x}}+x_{4} a \hat{\mathbf{y}}-y_{4} a \hat{\mathbf{z}}$ & $(48 e)$ & $\mathrm{Li}$ \\
\hline
\end{tabular}




$$
\begin{aligned}
& \mathbf{B}_{33}=\left(x_{4}+z_{4}\right) \mathbf{a}_{1}+\left(x_{4}+y_{4}\right) \mathbf{a}_{2}+\left(y_{4}+z_{4}\right) \mathbf{a}_{3}=y_{4} a \hat{\mathbf{x}}+z_{4} a \hat{\mathbf{y}}+x_{4} a \hat{\mathbf{z}} \quad \text { (48e) } \\
& \mathbf{B}_{34}=\left(-x_{4}+z_{4}\right) \mathbf{a}_{1}+\left(\frac{1}{2}-x_{4}-y_{4}\right) \mathbf{a}_{2}+=\left(\frac{1}{2}-y_{4}\right) a \hat{\mathbf{x}}+z_{4} a \hat{\mathbf{y}}-x_{4} a \hat{\mathbf{z}} \\
& \left(\frac{1}{2}-y_{4}+z_{4}\right) \mathbf{a}_{3} \\
& \mathbf{B}_{35}=\left(\frac{1}{2}-x_{4}-z_{4}\right) \mathbf{a}_{1}+\left(\frac{1}{2}-x_{4}+y_{4}\right) \mathbf{a}_{2}+=y_{4} a \hat{\mathbf{x}}-z_{4} a \hat{\mathbf{y}}+\left(\frac{1}{2}-x_{4}\right) a \hat{\mathbf{z}} \\
& \left(y_{4}-z_{4}\right) \mathbf{a}_{3} \\
& \mathbf{B}_{36}=\left(\frac{1}{2}+x_{4}-z_{4}\right) \mathbf{a}_{1}+\left(x_{4}-y_{4}\right) \mathbf{a}_{2}+\quad=-y_{4} a \hat{\mathbf{x}}+\left(\frac{1}{2}-z_{4}\right) a \hat{\mathbf{y}}+x_{4} a \hat{\mathbf{z}} \\
& \left(\frac{1}{2}-y_{4}-z_{4}\right) \mathbf{a}_{3} \\
& \mathbf{B}_{37}=\left(-y_{4}-z_{4}\right) \mathbf{a}_{1}+\left(-x_{4}-z_{4}\right) \mathbf{a}_{2}+\quad=\quad-x_{4} a \hat{\mathbf{x}}-y_{4} a \hat{\mathbf{y}}-z_{4} a \hat{\mathbf{z}} \\
& \left(-x_{4}-y_{4}\right) \mathbf{a}_{3} \\
& \mathbf{B}_{38}=\left(\frac{1}{2}+y_{4}-z_{4}\right) \mathbf{a}_{1}+\left(x_{4}-z_{4}\right) \mathbf{a}_{2}+\quad=\quad x_{4} a \hat{\mathbf{x}}+\left(\frac{1}{2}+y_{4}\right) a \hat{\mathbf{y}}-z_{4} a \hat{\mathbf{z}} \\
& \left(\frac{1}{2}+x_{4}+y_{4}\right) \mathbf{a}_{3} \\
& \mathbf{B}_{39}=\left(-y_{4}+z_{4}\right) \mathbf{a}_{1}+\left(\frac{1}{2}+x_{4}+z_{4}\right) \mathbf{a}_{2}+=\left(\frac{1}{2}+x_{4}\right) a \hat{\mathbf{x}}-y_{4} a \hat{\mathbf{y}}+z_{4} a \hat{\mathbf{z}} \\
& \left(\frac{1}{2}+x_{4}-y_{4}\right) \mathbf{a}_{3} \\
& \mathbf{B}_{40}=\left(\frac{1}{2}+y_{4}+z_{4}\right) \mathbf{a}_{1}+\left(\frac{1}{2}-x_{4}+z_{4}\right) \mathbf{a}_{2}+=-x_{4} a \hat{\mathbf{x}}+y_{4} a \hat{\mathbf{y}}+\left(\frac{1}{2}+z_{4}\right) a \hat{\mathbf{z}} \\
& \left(-x_{4}+y_{4}\right) \mathbf{a}_{3} \\
& \mathbf{B}_{41}=\left(-x_{4}-y_{4}\right) \mathbf{a}_{1}+\left(-y_{4}-z_{4}\right) \mathbf{a}_{2}+\quad=\quad-z_{4} a \hat{\mathbf{x}}-x_{4} a \hat{\mathbf{y}}-y_{4} a \hat{\mathbf{z}} \\
& \left(-x_{4}-z_{4}\right) \mathbf{a}_{3} \\
& \mathbf{B}_{42}=\left(\frac{1}{2}+x_{4}+y_{4}\right) \mathbf{a}_{1}+\left(\frac{1}{2}+y_{4}-z_{4}\right) \mathbf{a}_{2}+=-z_{4} a \hat{\mathbf{x}}+x_{4} a \hat{\mathbf{y}}+\left(\frac{1}{2}+y_{4}\right) a \hat{\mathbf{z}} \\
& \mathbf{B}_{43}=\left(\frac{1}{2}+x_{4}-y_{4}\right) \mathbf{a}_{1}+\left(-y_{4}+z_{4}\right) \mathbf{a}_{2}+\quad=\quad z_{4} a \hat{\mathbf{x}}+\left(\frac{1}{2}+x_{4}\right) a \hat{\mathbf{y}}-y_{4} a \hat{\mathbf{z}} \\
& \left(\frac{1}{2}+x_{4}+z_{4}\right) \mathbf{a}_{3} \\
& \mathbf{B}_{44}=\left(-x_{4}+y_{4}\right) \mathbf{a}_{1}+\left(\frac{1}{2}+y_{4}+z_{4}\right) \mathbf{a}_{2}+=\left(\frac{1}{2}+z_{4}\right) a \hat{\mathbf{x}}-x_{4} a \hat{\mathbf{y}}+y_{4} a \hat{\mathbf{z}} \\
& \left(\frac{1}{2}-x_{4}+z_{4}\right) \mathbf{a}_{3} \\
& \mathbf{B}_{45}=\left(-x_{4}-z_{4}\right) \mathbf{a}_{1}+\left(-x_{4}-y_{4}\right) \mathbf{a}_{2}+\quad=\quad-y_{4} a \hat{\mathbf{x}}-z_{4} a \hat{\mathbf{y}}-x_{4} a \hat{\mathbf{z}} \\
& \left(-y_{4}-z_{4}\right) \mathbf{a}_{3} \\
& \mathbf{B}_{46}=\left(x_{4}-z_{4}\right) \mathbf{a}_{1}+\left(\frac{1}{2}+x_{4}+y_{4}\right) \mathbf{a}_{2}+\quad=\left(\frac{1}{2}+y_{4}\right) a \hat{\mathbf{x}}-z_{4} a \hat{\mathbf{y}}+x_{4} a \hat{\mathbf{z}} \\
& \left(\frac{1}{2}+y_{4}-z_{4}\right) \mathbf{a}_{3} \\
& \mathbf{B}_{47}=\left(\frac{1}{2}+x_{4}+z_{4}\right) \mathbf{a}_{1}+\left(\frac{1}{2}+x_{4}-y_{4}\right) \mathbf{a}_{2}+=-y_{4} a \hat{\mathbf{x}}+z_{4} a \hat{\mathbf{y}}+\left(\frac{1}{2}+x_{4}\right) a \hat{\mathbf{z}} \\
& \left(-y_{4}+z_{4}\right) \mathbf{a}_{3} \\
& \mathbf{B}_{48}=\left(\frac{1}{2}-x_{4}+z_{4}\right) \mathbf{a}_{1}+\left(-x_{4}+y_{4}\right) \mathbf{a}_{2}+\quad=\quad y_{4} a \hat{\mathbf{x}}+\left(\frac{1}{2}+z_{4}\right) a \hat{\mathbf{y}}-x_{4} a \hat{\mathbf{z}} \\
& \left(\frac{1}{2}+y_{4}+z_{4}\right) \mathbf{a}_{3}
\end{aligned}
$$

\section{References:}

- R. Juza and F. Hund, Die ternären Nitride $\mathrm{Li}_{3} \mathrm{AlN}_{2}$ und $\mathrm{Li}_{3} \mathrm{GaN}_{2}$. 17. Mitteilung über Metallamide und Metallnitride, $\mathrm{Z}$. Anorg. Allg. Chem. 257, 13-25 (1948), doi:10.1002/zaac.19482570102.

\section{Found in:}

- J. F. Herbst and L. G. Hector, Jr., Exploration of the formation of XLi $i_{3} N_{2}$ compounds $(X=S c-Z n)$ by means of density functional theory, Phys. Rev. B 85, 195137 (2012), doi:10.1103/PhysRevB.85.195137.

\section{Geometry files:}

- CIF: pp. 958

- POSCAR: pp. 959 


\section{$\mathrm{Pd}_{17} \mathrm{Se}_{15}$ Structure: A17B15_cP64_207_acfk_eij}
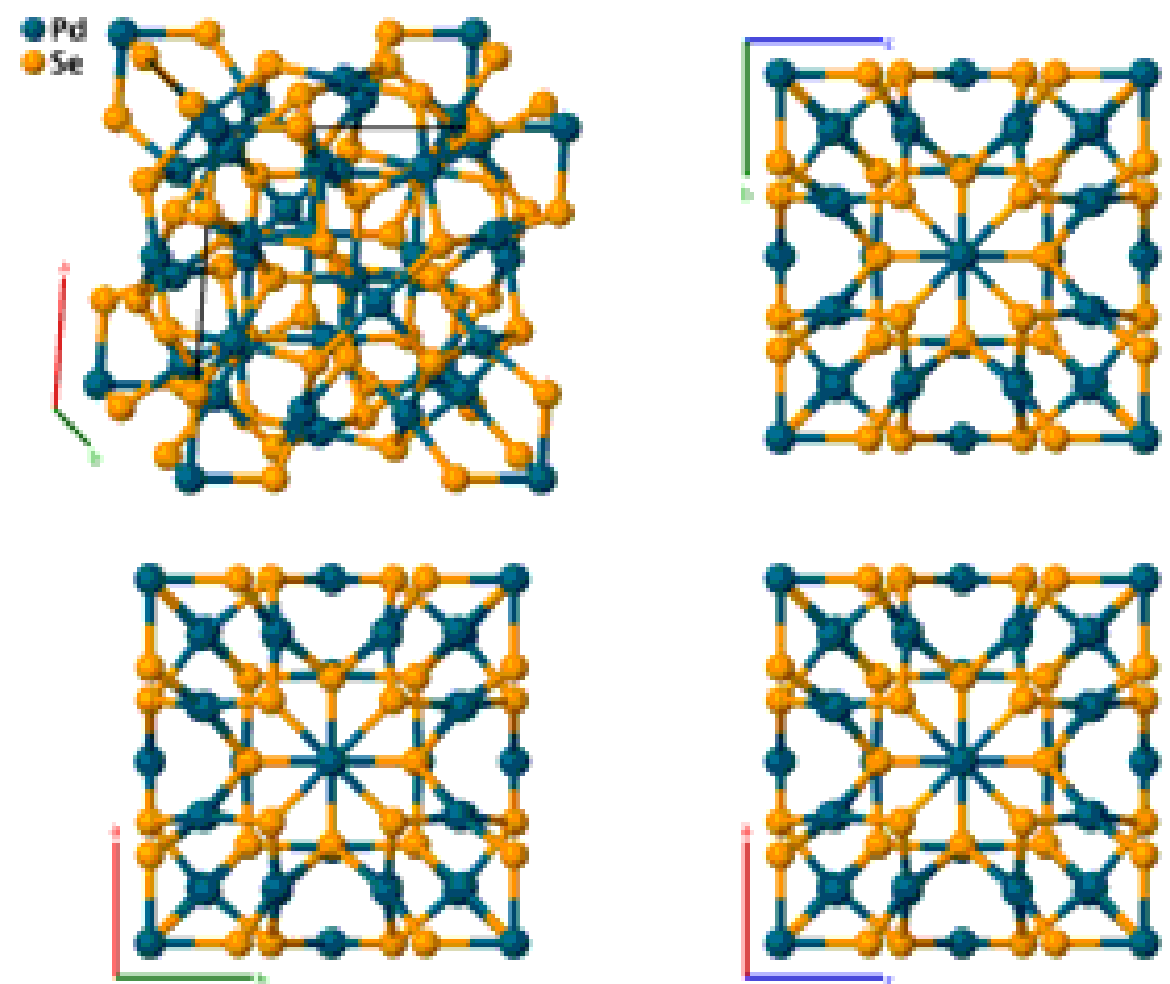

\section{Prototype}

AFLOW prototype label

: $\quad \mathrm{Pd}_{17} \mathrm{Se}_{15}$

Strukturbericht designation : None

Pearson symbol

: $\quad$ cP64

Space group number

: $\quad 207$

Space group symbol

$P 432$

AFLOW prototype command : aflow --proto=A17B15_cP64_207_acfk_eij

- - params $=a, x_{3}, x_{4}, y_{5}, y_{6}, x_{7}, y_{7}, z_{7}$

Simple Cubic primitive vectors:

$$
\begin{aligned}
& \mathbf{a}_{1}=a \hat{\mathbf{x}} \\
& \mathbf{a}_{2}=a \hat{\mathbf{y}} \\
& \mathbf{a}_{3}=a \hat{\mathbf{z}}
\end{aligned}
$$

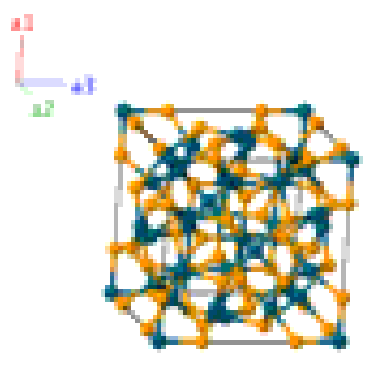

Basis vectors:

Lattice Coordinates
$\mathbf{B}_{1}=$
$0 \mathbf{a}_{1}+0 \mathbf{a}_{2}+0 \mathbf{a}_{3}$
$\mathbf{B}_{2}=$
$\frac{1}{2} \mathbf{a}_{2}+\frac{1}{2} \mathbf{a}_{3}$

Cartesian Coordinates

$=$

$=$

$$
\begin{gathered}
0 \hat{\mathbf{x}}+0 \hat{\mathbf{y}}+0 \hat{\mathbf{z}} \\
\frac{1}{2} a \hat{\mathbf{y}}+\frac{1}{2} a \hat{\mathbf{z}}
\end{gathered}
$$

Wyckoff Position

(1a)

$(3 c)$
Atom Type

Pd I

Pd II 


\begin{tabular}{|c|c|c|c|c|c|c|}
\hline $\mathbf{B}_{3}$ & $=$ & $\frac{1}{2} \mathbf{a}_{1}+\frac{1}{2} \mathbf{a}_{3}$ & $=$ & $\frac{1}{2} a \hat{\mathbf{x}}+\frac{1}{2} a \hat{\mathbf{z}}$ & $(3 c)$ & Pd II \\
\hline $\mathbf{B}_{4}$ & $=$ & $\frac{1}{2} \mathbf{a}_{1}+\frac{1}{2} \mathbf{a}_{2}$ & $=$ & $\frac{1}{2} a \hat{\mathbf{x}}+\frac{1}{2} a \hat{\mathbf{y}}$ & $(3 c)$ & Pd II \\
\hline $\mathbf{B}_{5}$ & $=$ & $x_{3} \mathbf{a}_{1}$ & $=$ & $x_{3} a \hat{\mathbf{x}}$ & $(6 e)$ & Se I \\
\hline $\mathbf{B}_{6}$ & $=$ & $-x_{3} \mathbf{a}_{1}$ & $=$ & $-x_{3} a \hat{\mathbf{x}}$ & $(6 e)$ & Se I \\
\hline $\mathbf{B}_{7}$ & $=$ & $x_{3} \mathbf{a}_{2}$ & $=$ & $x_{3} a \hat{\mathbf{y}}$ & $(6 e)$ & Se I \\
\hline $\mathbf{B}_{8}$ & $=$ & $-x_{3} \mathbf{a}_{2}$ & $=$ & $-x_{3} a \hat{\mathbf{y}}$ & $(6 e)$ & Se I \\
\hline $\mathbf{B}_{9}$ & $=$ & $x_{3} \mathbf{a}_{3}$ & $=$ & $x_{3} a \hat{\mathbf{z}}$ & $(6 e)$ & Se I \\
\hline $\mathbf{B}_{10}$ & $=$ & $-x_{3} \mathbf{a}_{3}$ & $=$ & $-x_{3} a \hat{\mathbf{z}}$ & $(6 e)$ & Se I \\
\hline $\mathbf{B}_{11}$ & $=$ & $x_{4} \mathbf{a}_{1}+\frac{1}{2} \mathbf{a}_{2}+\frac{1}{2} \mathbf{a}_{3}$ & $=$ & $x_{4} a \hat{\mathbf{x}}+\frac{1}{2} a \hat{\mathbf{y}}+\frac{1}{2} a \hat{\mathbf{z}}$ & $(6 f)$ & Pd III \\
\hline $\mathbf{B}_{12}$ & $=$ & $-x_{4} \mathbf{a}_{1}+\frac{1}{2} \mathbf{a}_{2}+\frac{1}{2} \mathbf{a}_{3}$ & $=$ & $-x_{4} a \hat{\mathbf{x}}+\frac{1}{2} a \hat{\mathbf{y}}+\frac{1}{2} a \hat{\mathbf{z}}$ & $(6 f)$ & Pd III \\
\hline $\mathbf{B}_{13}$ & $=$ & $\frac{1}{2} \mathbf{a}_{1}+x_{4} \mathbf{a}_{2}+\frac{1}{2} \mathbf{a}_{3}$ & $=$ & $\frac{1}{2} a \hat{\mathbf{x}}+x_{4} a \hat{\mathbf{y}}+\frac{1}{2} a \hat{\mathbf{z}}$ & $(6 f)$ & Pd III \\
\hline $\mathbf{B}_{14}$ & $=$ & $\frac{1}{2} \mathbf{a}_{1}-x_{4} \mathbf{a}_{2}+\frac{1}{2} \mathbf{a}_{3}$ & $=$ & $\frac{1}{2} a \hat{\mathbf{x}}-x_{4} a \hat{\mathbf{y}}+\frac{1}{2} a \hat{\mathbf{z}}$ & $(6 f)$ & Pd III \\
\hline $\mathbf{B}_{15}$ & $=$ & $\frac{1}{2} \mathbf{a}_{1}+\frac{1}{2} \mathbf{a}_{2}+x_{4} \mathbf{a}_{3}$ & $=$ & $\frac{1}{2} a \hat{\mathbf{x}}+\frac{1}{2} a \hat{\mathbf{y}}+x_{4} a \hat{\mathbf{z}}$ & $(6 f)$ & Pd III \\
\hline $\mathbf{B}_{16}$ & $=$ & $\frac{1}{2} \mathbf{a}_{1}+\frac{1}{2} \mathbf{a}_{2}-x_{4} \mathbf{a}_{3}$ & $=$ & $\frac{1}{2} a \hat{\mathbf{x}}+\frac{1}{2} a \hat{\mathbf{y}}-x_{4} a \hat{\mathbf{z}}$ & $(6 f)$ & Pd III \\
\hline $\mathbf{B}_{17}$ & $=$ & $y_{5} \mathbf{a}_{2}+y_{5} \mathbf{a}_{3}$ & $=$ & $y_{5} a \hat{\mathbf{y}}+y_{5} a \hat{\mathbf{z}}$ & $(12 i)$ & Se II \\
\hline $\mathbf{B}_{18}$ & $=$ & $-y_{5} \mathbf{a}_{2}+y_{5} \mathbf{a}_{3}$ & $=$ & $-y_{5} a \hat{\mathbf{y}}+y_{5} a \hat{\mathbf{z}}$ & $(12 i)$ & Se II \\
\hline $\mathbf{B}_{19}$ & $=$ & $y_{5} \mathbf{a}_{2}-y_{5} \mathbf{a}_{3}$ & $=$ & $y_{5} a \hat{\mathbf{y}}-y_{5} a \hat{\mathbf{z}}$ & $(12 i)$ & Se II \\
\hline $\mathbf{B}_{20}$ & $=$ & $-y_{5} \mathbf{a}_{2}-y_{5} \mathbf{a}_{3}$ & $=$ & $-y_{5} a \hat{\mathbf{y}}-y_{5} a \hat{\mathbf{z}}$ & $(12 i)$ & Se II \\
\hline $\mathbf{B}_{21}$ & $=$ & $y_{5} \mathbf{a}_{1}+y_{5} \mathbf{a}_{3}$ & $=$ & $y_{5} a \hat{\mathbf{x}}+y_{5} a \hat{\mathbf{z}}$ & $(12 i)$ & Se II \\
\hline $\mathbf{B}_{22}$ & $=$ & $y_{5} \mathbf{a}_{1}+-y_{5} \mathbf{a}_{3}$ & $=$ & $y_{5} a \hat{\mathbf{x}}+-y_{5} a \hat{\mathbf{z}}$ & $(12 i)$ & Se II \\
\hline $\mathbf{B}_{23}$ & $=$ & $-y_{5} \mathbf{a}_{1}+y_{5} \mathbf{a}_{3}$ & $=$ & $-y_{5} a \hat{\mathbf{x}}+y_{5} a \hat{\mathbf{z}}$ & $(12 i)$ & Se II \\
\hline $\mathbf{B}_{24}$ & $=$ & $-y_{5} \mathbf{a}_{1}+-y_{5} \mathbf{a}_{3}$ & $=$ & $-y_{5} a \hat{\mathbf{x}}+-y_{5} a \hat{\mathbf{z}}$ & $(12 i)$ & Se II \\
\hline $\mathbf{B}_{25}$ & $=$ & $y_{5} \mathbf{a}_{1}+y_{5} \mathbf{a}_{2}$ & $=$ & $y_{5} a \hat{\mathbf{x}}+y_{5} a \hat{\mathbf{y}}$ & $(12 i)$ & Se II \\
\hline $\mathbf{B}_{26}$ & $=$ & $-y_{5} \mathbf{a}_{1}+y_{5} \mathbf{a}_{2}$ & $=$ & $-y_{5} a \hat{\mathbf{x}}+y_{5} a \hat{\mathbf{y}}$ & $(12 i)$ & Se II \\
\hline $\mathbf{B}_{27}$ & $=$ & $y_{5} \mathbf{a}_{1}-y_{5} \mathbf{a}_{2}$ & $=$ & $y_{5} a \hat{\mathbf{x}}-y_{5} a \hat{\mathbf{y}}$ & $(12 i)$ & Se II \\
\hline $\mathbf{B}_{28}$ & $=$ & $-y_{5} \mathbf{a}_{1}-y_{5} \mathbf{a}_{2}$ & $=$ & $-y_{5} a \hat{\mathbf{x}}-y_{5} a \hat{\mathbf{y}}$ & $(12 i)$ & Se II \\
\hline $\mathbf{B}_{29}$ & $=$ & $\frac{1}{2} \mathbf{a}_{1}+y_{6} \mathbf{a}_{2}+y_{6} \mathbf{a}_{3}$ & $=$ & $\frac{1}{2} a \hat{\mathbf{x}}+y_{6} a \hat{\mathbf{y}}+y_{6} a \hat{\mathbf{z}}$ & $(12 j)$ & Se III \\
\hline $\mathbf{B}_{30}$ & $=$ & $\frac{1}{2} \mathbf{a}_{1}-y_{6} \mathbf{a}_{2}+y_{6} \mathbf{a}_{3}$ & $=$ & $\frac{1}{2} a \hat{\mathbf{x}}-y_{6} a \hat{\mathbf{y}}+y_{6} a \hat{\mathbf{z}}$ & $(12 j)$ & Se III \\
\hline $\mathbf{B}_{31}$ & $=$ & $\frac{1}{2} \mathbf{a}_{1}+y_{6} \mathbf{a}_{2}-y_{6} \mathbf{a}_{3}$ & $=$ & $\frac{1}{2} a \hat{\mathbf{x}}+y_{6} a \hat{\mathbf{y}}-y_{6} a \hat{\mathbf{z}}$ & $(12 j)$ & Se III \\
\hline $\mathbf{B}_{32}$ & $=$ & $\frac{1}{2} \mathbf{a}_{1}-y_{6} \mathbf{a}_{2}-y_{6} \mathbf{a}_{3}$ & $=$ & $\frac{1}{2} a \hat{\mathbf{x}}-y_{6} a \hat{\mathbf{y}}-y_{6} a \hat{\mathbf{z}}$ & $(12 j)$ & Se III \\
\hline $\mathbf{B}_{33}$ & $=$ & $y_{6} \mathbf{a}_{1}+\frac{1}{2} \mathbf{a}_{2}+y_{6} \mathbf{a}_{3}$ & $=$ & $y_{6} a \hat{\mathbf{x}}+\frac{1}{2} a \hat{\mathbf{y}}+y_{6} a \hat{\mathbf{z}}$ & $(12 j)$ & Se III \\
\hline $\mathbf{B}_{34}$ & $=$ & $y_{6} \mathbf{a}_{1}+\frac{1}{2} \mathbf{a}_{2}-y_{6} \mathbf{a}_{3}$ & $=$ & $y_{6} a \hat{\mathbf{x}}+\frac{1}{2} a \hat{\mathbf{y}}-y_{6} a \hat{\mathbf{z}}$ & $(12 j)$ & Se III \\
\hline $\mathbf{B}_{35}$ & $=$ & $-y_{6} \mathbf{a}_{1}+\frac{1}{2} \mathbf{a}_{2}+y_{6} \mathbf{a}_{3}$ & $=$ & $-y_{6} a \hat{\mathbf{x}}+\frac{1}{2} a \hat{\mathbf{y}}+y_{6} a \hat{\mathbf{z}}$ & $(12 j)$ & Se III \\
\hline $\mathbf{B}_{36}$ & $=$ & $-y_{6} \mathbf{a}_{1}+\frac{1}{2} \mathbf{a}_{2}-y_{6} \mathbf{a}_{3}$ & $=$ & $-y_{6} a \hat{\mathbf{x}}+\frac{1}{2} a \hat{\mathbf{y}}-y_{6} a \hat{\mathbf{z}}$ & $(12 j)$ & Se III \\
\hline $\mathbf{B}_{37}$ & $=$ & $y_{6} \mathbf{a}_{1}+y_{6} \mathbf{a}_{2}+\frac{1}{2} \mathbf{a}_{3}$ & $=$ & $y_{6} a \hat{\mathbf{x}}+y_{6} a \hat{\mathbf{y}}+\frac{1}{2} a \hat{\mathbf{z}}$ & $(12 j)$ & Se III \\
\hline $\mathbf{B}_{38}$ & $=$ & $-y_{6} \mathbf{a}_{1}+y_{6} \mathbf{a}_{2}+\frac{1}{2} \mathbf{a}_{3}$ & $=$ & $-y_{6} a \hat{\mathbf{x}}+y_{6} a \hat{\mathbf{y}}+\frac{1}{2} a \hat{\mathbf{z}}$ & $(12 j)$ & Se III \\
\hline
\end{tabular}




\begin{tabular}{|c|c|c|c|c|c|c|}
\hline $\mathbf{B}_{39}$ & $=$ & $y_{6} \mathbf{a}_{1}-y_{6} \mathbf{a}_{2}+\frac{1}{2} \mathbf{a}_{3}$ & $=$ & $y_{6} a \hat{\mathbf{x}}-y_{6} a \hat{\mathbf{y}}+\frac{1}{2} a \hat{\mathbf{z}}$ & $(12 j)$ & Se III \\
\hline $\mathbf{B}_{40}$ & $=$ & $-y_{6} \mathbf{a}_{1}-y_{6} \mathbf{a}_{2}+\frac{1}{2} \mathbf{a}_{3}$ & $=$ & $-y_{6} a \hat{\mathbf{x}}-y_{6} a \hat{\mathbf{y}}+\frac{1}{2} a \hat{\mathbf{z}}$ & $(12 j)$ & Se III \\
\hline $\mathbf{B}_{41}$ & $=$ & $x_{7} \mathbf{a}_{1}+y_{7} \mathbf{a}_{2}+z_{7} \mathbf{a}_{3}$ & $=$ & $x_{7} a \hat{\mathbf{x}}+y_{7} a \hat{\mathbf{y}}+z_{7} a \hat{\mathbf{z}}$ & $(24 k)$ & Pd IV \\
\hline $\mathbf{B}_{42}$ & $=$ & $-x_{7} \mathbf{a}_{1}-y_{7} \mathbf{a}_{2}+z_{7} \mathbf{a}_{3}$ & $=$ & $-x_{7} a \hat{\mathbf{x}}-y_{7} a \hat{\mathbf{y}}+z_{7} a \hat{\mathbf{z}}$ & $(24 k)$ & Pd IV \\
\hline $\mathbf{B}_{43}$ & $=$ & $-x_{7} \mathbf{a}_{1}+y_{7} \mathbf{a}_{2}-z_{7} \mathbf{a}_{3}$ & $=$ & $-x_{7} a \hat{\mathbf{x}}+y_{7} a \hat{\mathbf{y}}-z_{7} a \hat{\mathbf{z}}$ & $(24 k)$ & Pd IV \\
\hline $\mathbf{B}_{44}$ & $=$ & $x_{7} \mathbf{a}_{1}-y_{7} \mathbf{a}_{2}-z_{7} \mathbf{a}_{3}$ & $=$ & $x_{7} a \hat{\mathbf{x}}-y_{7} a \hat{\mathbf{y}}-z_{7} a \hat{\mathbf{z}}$ & $(24 k)$ & Pd IV \\
\hline $\mathbf{B}_{45}$ & $=$ & $z_{7} \mathbf{a}_{1}+x_{7} \mathbf{a}_{2}+y_{7} \mathbf{a}_{3}$ & $=$ & $z_{7} a \hat{\mathbf{x}}+x_{7} a \hat{\mathbf{y}}+y_{7} a \hat{\mathbf{z}}$ & $(24 k)$ & Pd IV \\
\hline $\mathbf{B}_{46}$ & $=$ & $z_{7} \mathbf{a}_{1}-x_{7} \mathbf{a}_{2}-y_{7} \mathbf{a}_{3}$ & $=$ & $z_{7} a \hat{\mathbf{x}}-x_{7} a \hat{\mathbf{y}}-y_{7} a \hat{\mathbf{z}}$ & $(24 k)$ & Pd IV \\
\hline $\mathbf{B}_{47}$ & $=$ & $-z_{7} \mathbf{a}_{1}-x_{7} \mathbf{a}_{2}+y_{7} \mathbf{a}_{3}$ & $=$ & $-z_{7} a \hat{\mathbf{x}}-x_{7} a \hat{\mathbf{y}}+y_{7} a \hat{\mathbf{z}}$ & $(24 k)$ & Pd IV \\
\hline $\mathbf{B}_{48}$ & $=$ & $-z_{7} \mathbf{a}_{1}+x_{7} \mathbf{a}_{2}-y_{7} \mathbf{a}_{3}$ & $=$ & $-z_{7} a \hat{\mathbf{x}}+x_{7} a \hat{\mathbf{y}}-y_{7} a \hat{\mathbf{z}}$ & $(24 k)$ & Pd IV \\
\hline $\mathbf{B}_{49}$ & $=$ & $y_{7} \mathbf{a}_{1}+z_{7} \mathbf{a}_{2}+x_{7} \mathbf{a}_{3}$ & $=$ & $y_{7} a \hat{\mathbf{x}}+z_{7} a \hat{\mathbf{y}}+x_{7} a \hat{\mathbf{z}}$ & $(24 k)$ & Pd IV \\
\hline $\mathbf{B}_{50}$ & $=$ & $-y_{7} \mathbf{a}_{1}+z_{7} \mathbf{a}_{2}-x_{7} \mathbf{a}_{3}$ & $=$ & $-y_{7} a \hat{\mathbf{x}}+z_{7} a \hat{\mathbf{y}}-x_{7} a \hat{\mathbf{z}}$ & $(24 k)$ & Pd IV \\
\hline $\mathbf{B}_{51}$ & $=$ & $y_{7} \mathbf{a}_{1}-z_{7} \mathbf{a}_{2}-x_{7} \mathbf{a}_{3}$ & $=$ & $y_{7} a \hat{\mathbf{x}}-z_{7} a \hat{\mathbf{y}}-x_{7} a \hat{\mathbf{z}}$ & $(24 k)$ & Pd IV \\
\hline $\mathbf{B}_{52}$ & $=$ & $-y_{7} \mathbf{a}_{1}-z_{7} \mathbf{a}_{2}+x_{7} \mathbf{a}_{3}$ & $=$ & $-y_{7} a \hat{\mathbf{x}}-z_{7} a \hat{\mathbf{y}}+x_{7} a \hat{\mathbf{z}}$ & $(24 k)$ & Pd IV \\
\hline $\mathbf{B}_{53}$ & $=$ & $y_{7} \mathbf{a}_{1}+x_{7} \mathbf{a}_{2}-z_{7} \mathbf{a}_{3}$ & $=$ & $y_{7} a \hat{\mathbf{x}}+x_{7} a \hat{\mathbf{y}}-z_{7} a \hat{\mathbf{z}}$ & $(24 k)$ & Pd IV \\
\hline $\mathbf{B}_{54}$ & $=$ & $-y_{7} \mathbf{a}_{1}-x_{7} \mathbf{a}_{2}-z_{7} \mathbf{a}_{3}$ & $=$ & $-y_{7} a \hat{\mathbf{x}}-x_{7} a \hat{\mathbf{y}}-z_{7} a \hat{\mathbf{z}}$ & $(24 k)$ & Pd IV \\
\hline $\mathbf{B}_{55}$ & $=$ & $y_{7} \mathbf{a}_{1}-x_{7} \mathbf{a}_{2}+z_{7} \mathbf{a}_{3}$ & $=$ & $y_{7} a \hat{\mathbf{x}}-x_{7} a \hat{\mathbf{y}}+z_{7} a \hat{\mathbf{z}}$ & $(24 k)$ & Pd IV \\
\hline $\mathbf{B}_{56}$ & $=$ & $-y_{7} \mathbf{a}_{1}+x_{7} \mathbf{a}_{2}+z_{7} \mathbf{a}_{3}$ & $=$ & $-y_{7} a \hat{\mathbf{x}}+x_{7} a \hat{\mathbf{y}}+z_{7} a \hat{\mathbf{z}}$ & $(24 k)$ & Pd IV \\
\hline $\mathbf{B}_{57}$ & $=$ & $x_{7} \mathbf{a}_{1}+z_{7} \mathbf{a}_{2}-y_{7} \mathbf{a}_{3}$ & $=$ & $x_{7} a \hat{\mathbf{x}}+z_{7} a \hat{\mathbf{y}}-y_{7} a \hat{\mathbf{z}}$ & $(24 k)$ & Pd IV \\
\hline $\mathbf{B}_{58}$ & $=$ & $-x_{7} \mathbf{a}_{1}+z_{7} \mathbf{a}_{2}+y_{7} \mathbf{a}_{3}$ & $=$ & $-x_{7} a \hat{\mathbf{x}}+z_{7} a \hat{\mathbf{y}}+y_{7} a \hat{\mathbf{z}}$ & $(24 k)$ & Pd IV \\
\hline $\mathbf{B}_{59}$ & $=$ & $-x_{7} \mathbf{a}_{1}-z_{7} \mathbf{a}_{2}-y_{7} \mathbf{a}_{3}$ & $=$ & $-x_{7} a \hat{\mathbf{x}}-z_{7} a \hat{\mathbf{y}}-y_{7} a \hat{\mathbf{z}}$ & $(24 k)$ & Pd IV \\
\hline $\mathbf{B}_{60}$ & $=$ & $x_{7} \mathbf{a}_{1}-z_{7} \mathbf{a}_{2}+y_{7} \mathbf{a}_{3}$ & $=$ & $x_{7} a \hat{\mathbf{x}}-z_{7} a \hat{\mathbf{y}}+y_{7} a \hat{\mathbf{z}}$ & $(24 k)$ & Pd IV \\
\hline $\mathbf{B}_{61}$ & $=$ & $z_{7} \mathbf{a}_{1}+y_{7} \mathbf{a}_{2}-x_{7} \mathbf{a}_{3}$ & $=$ & $z_{7} a \hat{\mathbf{x}}+y_{7} a \hat{\mathbf{y}}-x_{7} a \hat{\mathbf{z}}$ & $(24 k)$ & Pd IV \\
\hline $\mathbf{B}_{62}$ & $=$ & $z_{7} \mathbf{a}_{1}-y_{7} \mathbf{a}_{2}+x_{7} \mathbf{a}_{3}$ & $=$ & $z_{7} a \hat{\mathbf{x}}-y_{7} a \hat{\mathbf{y}}+x_{7} a \hat{\mathbf{z}}$ & $(24 k)$ & Pd IV \\
\hline $\mathbf{B}_{63}$ & $=$ & $-z_{7} \mathbf{a}_{1}+y_{7} \mathbf{a}_{2}+x_{7} \mathbf{a}_{3}$ & $=$ & $-z_{7} a \hat{\mathbf{x}}+y_{7} a \hat{\mathbf{y}}+x_{7} a \hat{\mathbf{z}}$ & $(24 k)$ & Pd IV \\
\hline $\mathbf{B}_{64}$ & $=$ & $-z_{7} \mathbf{a}_{1}-y_{7} \mathbf{a}_{2}-x_{7} \mathbf{a}_{3}$ & $=$ & $-z_{7} a \hat{\mathbf{x}}-y_{7} a \hat{\mathbf{y}}-x_{7} a \hat{\mathbf{z}}$ & $(24 k)$ & Pd IV \\
\hline
\end{tabular}

\section{References:}

- S. Geller, The crystal structure of $P d_{17} S e_{15}$, Acta Cryst. 15, 713-721 (1962), doi:10.1107/S0365110X62001929.

\section{Found in:}

- P. Villars and K. Cenzual, Pearson's Crystal Data - Crystal Structure Database for Inorganic Compounds, ASM International (2013).

\section{Geometry files:}

- CIF: pp. 959

- POSCAR: pp. 960 


\section{$\mathrm{PH}_{3}$ Structure: A3B_cP16_208_j_b}
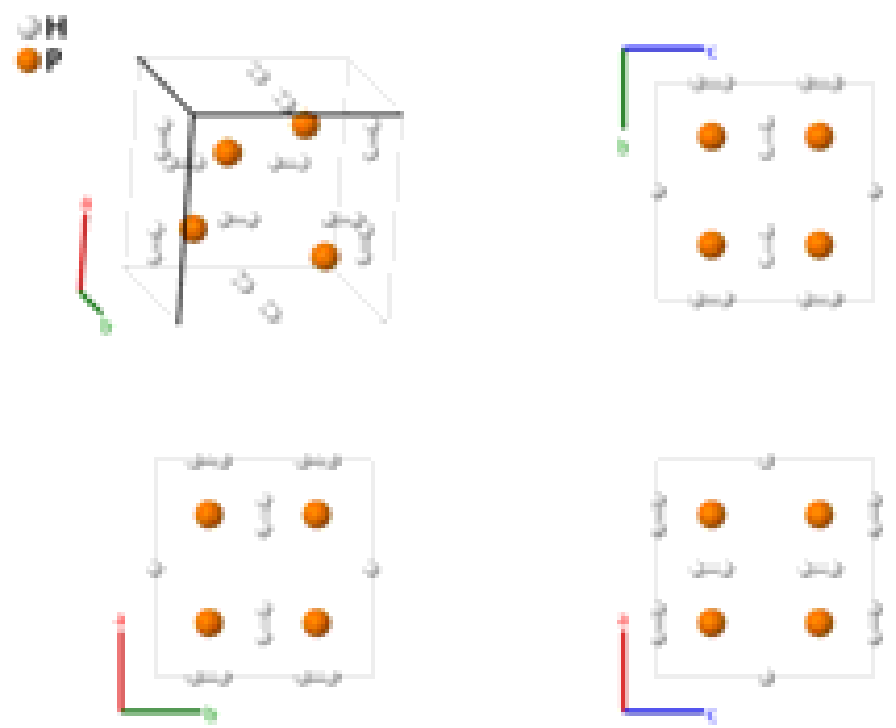

Prototype

AFLOW prototype label

Strukturbericht designation

Pearson symbol

Space group number

Space group symbol

AFLOW prototype command
: $\quad \mathrm{PH}_{3}$

: A3B_cP16_208_j_b

: None

: $\quad \mathrm{cP} 16$

: $\quad 208$

: $\quad P 4_{2} 32$ aflow - -proto=A3B_cP16_208_j_b

- -params $=a, x_{2}$

Simple Cubic primitive vectors:

$$
\begin{aligned}
& \mathbf{a}_{1}=a \hat{\mathbf{x}} \\
& \mathbf{a}_{2}=a \hat{\mathbf{y}} \\
& \mathbf{a}_{3}=a \hat{\mathbf{z}}
\end{aligned}
$$

Basis vectors:

$\begin{array}{rccccc} & \text { Lattice Coordinates } & & \text { Cartesian Coordinates } & \text { Wyckoff Position } & \text { Atom Type } \\ \mathbf{B}_{1}= & \frac{1}{4} \mathbf{a}_{1}+\frac{1}{4} \mathbf{a}_{2}+\frac{1}{4} \mathbf{a}_{3} & = & \frac{1}{4} a \hat{\mathbf{x}}+\frac{1}{4} a \hat{\mathbf{y}}+\frac{1}{4} a \hat{\mathbf{z}} & (4 b) & \mathrm{P} \\ \mathbf{B}_{2}= & \frac{3}{4} \mathbf{a}_{1}+\frac{3}{4} \mathbf{a}_{2}+\frac{1}{4} \mathbf{a}_{3} & = & \frac{3}{4} a \hat{\mathbf{x}}+\frac{3}{4} a \hat{\mathbf{y}}+\frac{1}{4} a \hat{\mathbf{z}} & (4 b) & \mathrm{P} \\ \mathbf{B}_{3}= & \frac{3}{4} \mathbf{a}_{1}+\frac{1}{4} \mathbf{a}_{2}+\frac{3}{4} \mathbf{a}_{3} & = & \frac{3}{4} a \hat{\mathbf{x}}+\frac{1}{4} a \hat{\mathbf{y}}+\frac{3}{4} a \hat{\mathbf{z}} & (4 b) & (4 b) \\ \mathbf{B}_{4}= & \frac{1}{4} \mathbf{a}_{1}+\frac{3}{4} \mathbf{a}_{2}+\frac{3}{4} \mathbf{a}_{3} & = & \frac{1}{4} a \hat{\mathbf{x}}+\frac{3}{4} a \hat{\mathbf{y}}+\frac{3}{4} a \hat{\mathbf{z}} & \mathrm{P} \\ \mathbf{B}_{5}= & x_{2} \mathbf{a}_{1}+\frac{1}{2} \mathbf{a}_{2} & = & x_{2} a \hat{\mathbf{x}}+\frac{1}{2} a \hat{\mathbf{y}} & \mathrm{P} \\ \mathbf{B}_{6}= & -x_{2} \mathbf{a}_{1}+\frac{1}{2} \mathbf{a}_{2} & = & -x_{2} a \hat{\mathbf{x}}+\frac{1}{2} a \hat{\mathbf{y}} & \mathrm{H}\end{array}$




\begin{tabular}{|c|c|c|c|c|c|}
\hline $\mathbf{B}_{7}$ & $=$ & $x_{2} \mathbf{a}_{2}+\frac{1}{2} \mathbf{a}_{3}$ & $=$ & $x_{2} a \hat{\mathbf{y}}+\frac{1}{2} a \hat{\mathbf{z}}$ & $(12 j)$ \\
\hline $\mathbf{B}_{8}$ & $=$ & $-x_{2} \mathbf{a}_{2}+\frac{1}{2} \mathbf{a}_{3}$ & $=$ & $-x_{2} a \hat{\mathbf{y}}+\frac{1}{2} a \hat{\mathbf{z}}$ & $(12 j)$ \\
\hline $\mathbf{B}_{9}$ & $=$ & $\frac{1}{2} \mathbf{a}_{1}+x_{2} \mathbf{a}_{3}$ & $=$ & $\frac{1}{2} a \hat{\mathbf{x}}+x_{2} a \hat{\mathbf{z}}$ & $(12 j)$ \\
\hline $\mathbf{B}_{10}$ & $=$ & $\frac{1}{2} \mathbf{a}_{1}+-x_{2} \mathbf{a}_{3}$ & $=$ & $\frac{1}{2} a \hat{\mathbf{x}}+-x_{2} a \hat{\mathbf{z}}$ & $(12 j)$ \\
\hline $\mathbf{B}_{11}$ & $=$ & $\left(\frac{1}{2}+x_{2}\right) \mathbf{a}_{2}+\frac{1}{2} \mathbf{a}_{3}$ & $=$ & $\left(\frac{1}{2}+x_{2}\right) a \hat{\mathbf{y}}+\frac{1}{2} a \hat{\mathbf{z}}$ & $(12 j)$ \\
\hline $\mathbf{B}_{12}$ & $=$ & $\left(\frac{1}{2}-x_{2}\right) \mathbf{a}_{2}+\frac{1}{2} \mathbf{a}_{3}$ & $=$ & $\left(\frac{1}{2}-x_{2}\right) a \hat{\mathbf{y}}+\frac{1}{2} a \hat{\mathbf{z}}$ & $(12 j)$ \\
\hline $\mathbf{B}_{13}$ & $=$ & $\left(\frac{1}{2}+x_{2}\right) \mathbf{a}_{1}+\frac{1}{2} \mathbf{a}_{2}$ & $=$ & $\left(\frac{1}{2}+x_{2}\right) a \hat{\mathbf{x}}+\frac{1}{2} a \hat{\mathbf{y}}$ & $(12 j)$ \\
\hline $\mathbf{B}_{14}$ & $=$ & $\left(\frac{1}{2}-x_{2}\right) \mathbf{a}_{1}+\frac{1}{2} \mathbf{a}_{2}$ & $=$ & $\left(\frac{1}{2}-x_{2}\right) a \hat{\mathbf{x}}+\frac{1}{2} a \hat{\mathbf{y}}$ & $(12 j)$ \\
\hline $\mathbf{B}_{15}$ & $=$ & $\frac{1}{2} \mathbf{a}_{1}+\left(\frac{1}{2}-x_{2}\right) \mathbf{a}_{3}$ & $=$ & $\frac{1}{2} a \hat{\mathbf{x}}+\left(\frac{1}{2}-x_{2}\right) a \hat{\mathbf{z}}$ & $(12 j)$ \\
\hline $\mathbf{B}_{16}$ & $=$ & $\frac{1}{2} \mathbf{a}_{1}+\left(\frac{1}{2}+x_{2}\right) \mathbf{a}_{3}$ & $=$ & $\frac{1}{2} a \hat{\mathbf{x}}+\left(\frac{1}{2}+x_{2}\right) a \hat{\mathbf{z}}$ & $(12 j)$ \\
\hline
\end{tabular}

\section{References:}

- G. Natta and E. Casazza, La struttura dell'idrogeno fosforato $\left(\mathrm{PH}_{3}\right)$ e dell'idrogeno arsenicale $\left(\mathrm{AsH}_{3}\right)$, Gazz. Chim. Ital. 60, 851-859 (1930).

\section{Found in:}

- R. T. Downs and M. Hall-Wallace, The American Mineralogist Crystal Structure Database, Am. Mineral. 88, 247-250 (2003).

\section{Geometry files:}

- CIF: pp. 960

- POSCAR: pp. 960 
$\mathrm{Cs}_{2} \mathrm{ZnFe}[\mathrm{CN}]_{6}$ Structure:

A6B2CD6E_cP64_208_m_ad_b_m_c
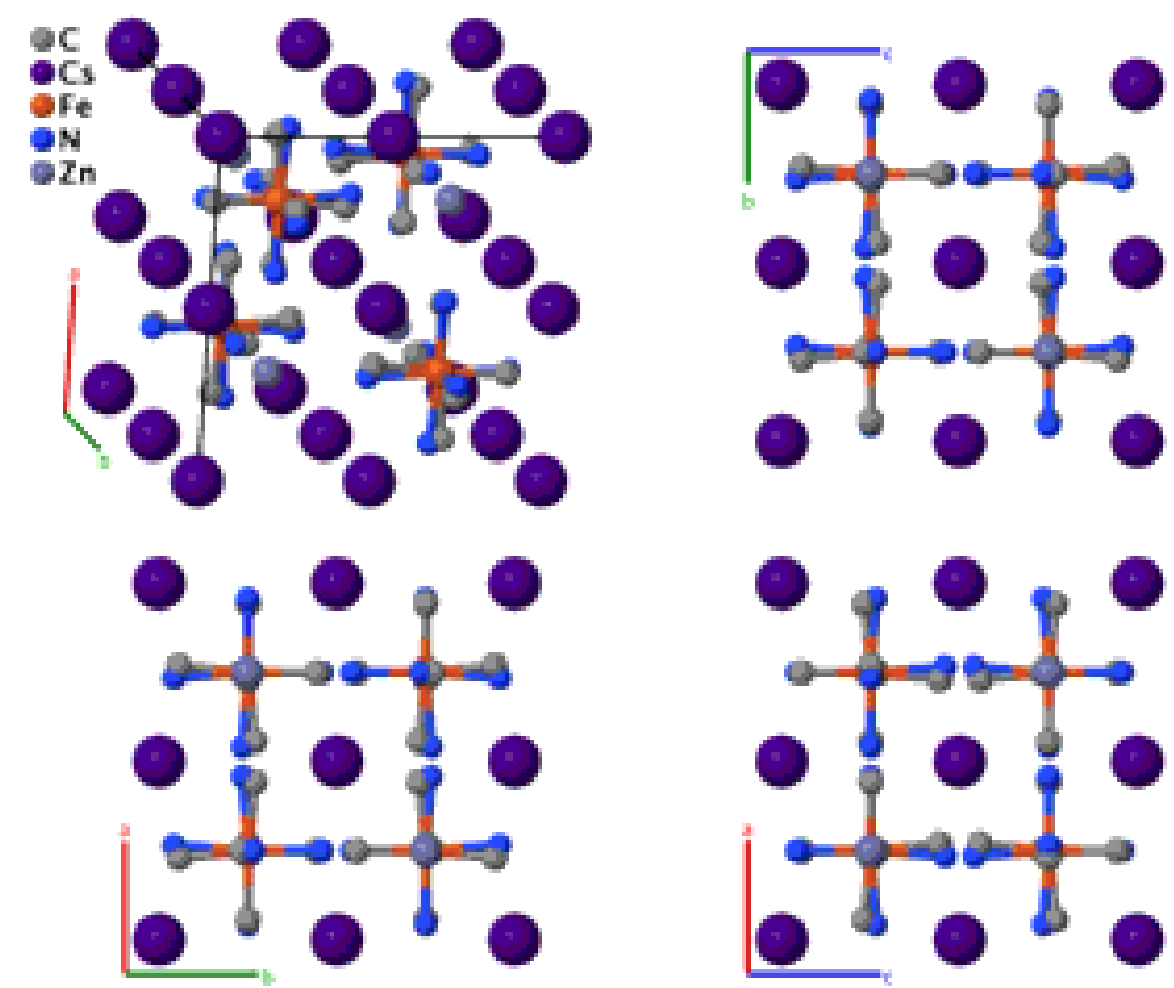

\section{Prototype}

: $\quad \mathrm{Cs}_{2} \mathrm{ZnFe}[\mathrm{CN}]_{6}$

AFLOW prototype label

: A6B2CD6E_cP64_208_m_ad_b_m_c

Strukturbericht designation : None

Pearson symbol

: $\quad$ cP64

Space group number

: 208

Space group symbol

: $\quad P 4_{2} 32$

AFLOW prototype command

aflow --proto=A6B2CD6E_cP64_208_m_ad_b_m_c

- - params $=a, x_{5}, y_{5}, z_{5}, x_{6}, y_{6}, z_{6}$

Simple Cubic primitive vectors:

$$
\begin{aligned}
& \mathbf{a}_{1}=a \hat{\mathbf{x}} \\
& \mathbf{a}_{2}=a \hat{\mathbf{y}} \\
& \mathbf{a}_{3}=a \hat{\mathbf{z}}
\end{aligned}
$$

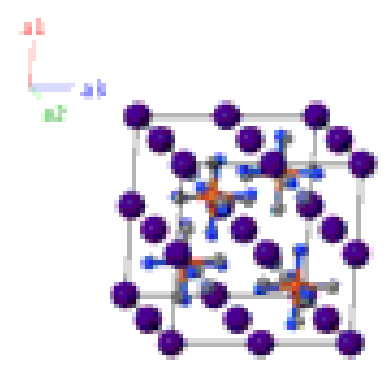

Basis vectors:

Lattice Coordinates

$\mathbf{B}_{1}=0 \mathbf{a}_{1}+0 \mathbf{a}_{2}+0 \mathbf{a}_{3}$
Cartesian Coordinates

$=$
$0 \hat{\mathbf{x}}+0 \hat{\mathbf{y}}+0 \hat{\mathbf{z}}$
Wyckoff Position

(2a)
Atom Type

Cs I 


\begin{tabular}{|c|c|c|c|c|c|c|}
\hline $\mathbf{B}_{2}$ & $=$ & $\frac{1}{2} \mathbf{a}_{1}+\frac{1}{2} \mathbf{a}_{2}+\frac{1}{2} \mathbf{a}_{3}$ & $=$ & $\frac{1}{2} a \hat{\mathbf{x}}+\frac{1}{2} a \hat{\mathbf{y}}+\frac{1}{2} a \hat{\mathbf{z}}$ & $(2 a)$ & Cs I \\
\hline $\mathbf{B}_{3}$ & $=$ & $\frac{1}{4} \mathbf{a}_{1}+\frac{1}{4} \mathbf{a}_{2}+\frac{1}{4} \mathbf{a}_{3}$ & $=$ & $\frac{1}{4} a \hat{\mathbf{X}}+\frac{1}{4} a \hat{\mathbf{y}}+\frac{1}{4} a \hat{\mathbf{z}}$ & $(4 b)$ & $\mathrm{Fe}$ \\
\hline $\mathbf{B}_{4}$ & $=$ & $\frac{3}{4} \mathbf{a}_{1}+\frac{3}{4} \mathbf{a}_{2}+\frac{1}{4} \mathbf{a}_{3}$ & $=$ & $\frac{3}{4} a \hat{\mathbf{x}}+\frac{3}{4} a \hat{\mathbf{y}}+\frac{1}{4} a \hat{\mathbf{z}}$ & $(4 b)$ & $\mathrm{Fe}$ \\
\hline $\mathbf{B}_{5}$ & $=$ & $\frac{3}{4} \mathbf{a}_{1}+\frac{1}{4} \mathbf{a}_{2}+\frac{3}{4} \mathbf{a}_{3}$ & $=$ & $\frac{3}{4} a \hat{\mathbf{x}}+\frac{1}{4} a \hat{\mathbf{y}}+\frac{3}{4} a \hat{\mathbf{z}}$ & $(4 b)$ & $\mathrm{Fe}$ \\
\hline $\mathbf{B}_{6}$ & $=$ & $\frac{1}{4} \mathbf{a}_{1}+\frac{3}{4} \mathbf{a}_{2}+\frac{3}{4} \mathbf{a}_{3}$ & $=$ & $\frac{1}{4} a \hat{\mathbf{x}}+\frac{3}{4} a \hat{\mathbf{y}}+\frac{3}{4} a \hat{\mathbf{z}}$ & $(4 b)$ & $\mathrm{Fe}$ \\
\hline $\mathbf{B}_{7}$ & $=$ & $\frac{3}{4} \mathbf{a}_{1}+\frac{3}{4} \mathbf{a}_{2}+\frac{3}{4} \mathbf{a}_{3}$ & $=$ & $\frac{3}{4} a \hat{\mathbf{x}}+\frac{3}{4} a \hat{\mathbf{y}}+\frac{3}{4} a \hat{\mathbf{z}}$ & $(4 c)$ & $\mathrm{Zn}$ \\
\hline $\mathbf{B}_{8}$ & $=$ & $\frac{1}{4} \mathbf{a}_{1}+\frac{1}{4} \mathbf{a}_{2}+\frac{3}{4} \mathbf{a}_{3}$ & $=$ & $\frac{1}{4} a \hat{\mathbf{x}}+\frac{1}{4} a \hat{\mathbf{y}}+\frac{3}{4} a \hat{\mathbf{z}}$ & $(4 c)$ & $\mathrm{Zn}$ \\
\hline $\mathbf{B}_{9}$ & $=$ & $\frac{1}{4} \mathbf{a}_{1}+\frac{3}{4} \mathbf{a}_{2}+\frac{1}{4} \mathbf{a}_{3}$ & $=$ & $\frac{1}{4} a \hat{\mathbf{x}}+\frac{3}{4} a \hat{\mathbf{y}}+\frac{1}{4} a \hat{\mathbf{z}}$ & $(4 c)$ & $\mathrm{Zn}$ \\
\hline $\mathbf{B}_{10}$ & $=$ & $\frac{3}{4} \mathbf{a}_{1}+\frac{1}{4} \mathbf{a}_{2}+\frac{1}{4} \mathbf{a}_{3}$ & $=$ & $\frac{3}{4} a \hat{\mathbf{x}}+\frac{1}{4} a \hat{\mathbf{y}}+\frac{1}{4} a \hat{\mathbf{z}}$ & $(4 c)$ & $\mathrm{Zn}$ \\
\hline $\mathbf{B}_{11}$ & $=$ & $\frac{1}{2} \mathbf{a}_{2}+\frac{1}{2} \mathbf{a}_{3}$ & $=$ & $\frac{1}{2} a \hat{\mathbf{y}}+\frac{1}{2} a \hat{\mathbf{z}}$ & $(6 d)$ & Cs II \\
\hline $\mathbf{B}_{12}$ & $=$ & $\frac{1}{2} \mathbf{a}_{1}+\frac{1}{2} \mathbf{a}_{3}$ & $=$ & $\frac{1}{2} a \hat{\mathbf{x}}+\frac{1}{2} a \hat{\mathbf{z}}$ & $(6 d)$ & Cs II \\
\hline $\mathbf{B}_{13}$ & $=$ & $\frac{1}{2} \mathbf{a}_{1}+\frac{1}{2} \mathbf{a}_{2}$ & $=$ & $\frac{1}{2} a \hat{\mathbf{x}}+\frac{1}{2} a \hat{\mathbf{y}}$ & $(6 d)$ & Cs II \\
\hline $\mathbf{B}_{14}$ & $=$ & $\frac{1}{2} \mathbf{a}_{2}$ & $=$ & $\frac{1}{2} a \hat{\mathbf{y}}$ & $(6 d)$ & Cs II \\
\hline $\mathbf{B}_{15}$ & $=$ & $\frac{1}{2} \mathbf{a}_{1}$ & $=$ & $\frac{1}{2} a \hat{\mathbf{x}}$ & $(6 d)$ & Cs II \\
\hline $\mathbf{B}_{16}$ & $=$ & $\frac{1}{2} \mathbf{a}_{3}$ & $=$ & $\frac{1}{2} a \hat{\mathbf{z}}$ & $(6 d)$ & Cs II \\
\hline $\mathbf{B}_{17}$ & $=$ & $x_{5} \mathbf{a}_{1}+y_{5} \mathbf{a}_{2}+z_{5} \mathbf{a}_{3}$ & $=$ & $x_{5} a \hat{\mathbf{x}}+y_{5} a \hat{\mathbf{y}}+z_{5} a \hat{\mathbf{z}}$ & $(24 m)$ & $\mathrm{C}$ \\
\hline $\mathbf{B}_{18}$ & $=$ & $-x_{5} \mathbf{a}_{1}-y_{5} \mathbf{a}_{2}+z_{5} \mathbf{a}_{3}$ & $=$ & $-x_{5} a \hat{\mathbf{x}}-y_{5} a \hat{\mathbf{y}}+z_{5} a \hat{\mathbf{z}}$ & $(24 m)$ & $\mathrm{C}$ \\
\hline $\mathbf{B}_{19}$ & $=$ & $-x_{5} \mathbf{a}_{1}+y_{5} \mathbf{a}_{2}-z_{5} \mathbf{a}_{3}$ & $=$ & $-x_{5} a \hat{\mathbf{x}}+y_{5} a \hat{\mathbf{y}}-z_{5} a \hat{\mathbf{z}}$ & $(24 m)$ & $\mathrm{C}$ \\
\hline $\mathbf{B}_{20}$ & $=$ & $x_{5} \mathbf{a}_{1}-y_{5} \mathbf{a}_{2}-z_{5} \mathbf{a}_{3}$ & $=$ & $x_{5} a \hat{\mathbf{x}}-y_{5} a \hat{\mathbf{y}}-z_{5} a \hat{\mathbf{z}}$ & $(24 m)$ & $\mathrm{C}$ \\
\hline $\mathbf{B}_{21}$ & $=$ & $z_{5} \mathbf{a}_{1}+x_{5} \mathbf{a}_{2}+y_{5} \mathbf{a}_{3}$ & $=$ & $z_{5} a \hat{\mathbf{x}}+x_{5} a \hat{\mathbf{y}}+y_{5} a \hat{\mathbf{z}}$ & $(24 m)$ & $\mathrm{C}$ \\
\hline $\mathbf{B}_{22}$ & $=$ & $z_{5} \mathbf{a}_{1}-x_{5} \mathbf{a}_{2}-y_{5} \mathbf{a}_{3}$ & $=$ & $z_{5} a \hat{\mathbf{x}}-x_{5} a \hat{\mathbf{y}}-y_{5} a \hat{\mathbf{z}}$ & $(24 m)$ & $\mathrm{C}$ \\
\hline $\mathbf{B}_{23}$ & $=$ & $-z_{5} \mathbf{a}_{1}-x_{5} \mathbf{a}_{2}+y_{5} \mathbf{a}_{3}$ & $=$ & $-z_{5} a \hat{\mathbf{x}}-x_{5} a \hat{\mathbf{y}}+y_{5} a \hat{\mathbf{z}}$ & $(24 m)$ & $\mathrm{C}$ \\
\hline $\mathbf{B}_{24}$ & $=$ & $-z_{5} \mathbf{a}_{1}+x_{5} \mathbf{a}_{2}-y_{5} \mathbf{a}_{3}$ & $=$ & $-z_{5} a \hat{\mathbf{x}}+x_{5} a \hat{\mathbf{y}}-y_{5} a \hat{\mathbf{z}}$ & $(24 m)$ & $\mathrm{C}$ \\
\hline $\mathbf{B}_{25}$ & $=$ & $y_{5} \mathbf{a}_{1}+z_{5} \mathbf{a}_{2}+x_{5} \mathbf{a}_{3}$ & $=$ & $y_{5} a \hat{\mathbf{x}}+z_{5} a \hat{\mathbf{y}}+x_{5} a \hat{\mathbf{z}}$ & $(24 m)$ & $\mathrm{C}$ \\
\hline $\mathbf{B}_{26}$ & $=$ & $-y_{5} \mathbf{a}_{1}+z_{5} \mathbf{a}_{2}-x_{5} \mathbf{a}_{3}$ & $=$ & $-y_{5} a \hat{\mathbf{x}}+z_{5} a \hat{\mathbf{y}}-x_{5} a \hat{\mathbf{z}}$ & $(24 m)$ & $\mathrm{C}$ \\
\hline $\mathbf{B}_{27}$ & $=$ & $y_{5} \mathbf{a}_{1}-z_{5} \mathbf{a}_{2}-x_{5} \mathbf{a}_{3}$ & $=$ & $y_{5} a \hat{\mathbf{x}}-z_{5} a \hat{\mathbf{y}}-x_{5} a \hat{\mathbf{z}}$ & $(24 m)$ & $\mathrm{C}$ \\
\hline $\mathbf{B}_{28}$ & $=$ & $-y_{5} \mathbf{a}_{1}-z_{5} \mathbf{a}_{2}+x_{5} \mathbf{a}_{3}$ & $=$ & $-y_{5} a \hat{\mathbf{x}}-z_{5} a \hat{\mathbf{y}}+x_{5} a \hat{\mathbf{z}}$ & $(24 m)$ & $\mathrm{C}$ \\
\hline $\mathbf{B}_{29}$ & $=$ & $\begin{array}{c}\left(\frac{1}{2}+y_{5}\right) \mathbf{a}_{1}+\left(\frac{1}{2}+x_{5}\right) \mathbf{a}_{2}+ \\
\left(\frac{1}{2}-z_{5}\right) \mathbf{a}_{3}\end{array}$ & $=$ & $\begin{array}{c}\left(\frac{1}{2}+y_{5}\right) a \hat{\mathbf{x}}+\left(\frac{1}{2}+x_{5}\right) a \hat{\mathbf{y}}+ \\
\left(\frac{1}{2}-z_{5}\right) a \hat{\mathbf{z}}\end{array}$ & $(24 m)$ & $\mathrm{C}$ \\
\hline $\mathbf{B}_{30}$ & $=$ & $\begin{array}{c}\left(\frac{1}{2}-y_{5}\right) \mathbf{a}_{1}+\left(\frac{1}{2}-x_{5}\right) \mathbf{a}_{2}+ \\
\left(\frac{1}{2}-z_{5}\right) \mathbf{a}_{3}\end{array}$ & $=$ & $\begin{array}{c}\left(\frac{1}{2}-y_{5}\right) a \hat{\mathbf{x}}+\left(\frac{1}{2}-x_{5}\right) a \hat{\mathbf{y}}+ \\
\left(\frac{1}{2}-z_{5}\right) a \hat{\mathbf{z}}\end{array}$ & $(24 m)$ & $\mathrm{C}$ \\
\hline $\mathbf{B}_{31}$ & $=$ & $\begin{array}{c}\left(\frac{1}{2}+y_{5}\right) \mathbf{a}_{1}+\left(\frac{1}{2}-x_{5}\right) \mathbf{a}_{2}+ \\
\left(\frac{1}{2}+z_{5}\right) \mathbf{a}_{3}\end{array}$ & $=$ & $\begin{array}{c}\left(\frac{1}{2}+y_{5}\right) a \hat{\mathbf{x}}+\left(\frac{1}{2}-x_{5}\right) a \hat{\mathbf{y}}+ \\
\left(\frac{1}{2}+z_{5}\right) a \hat{\mathbf{z}}\end{array}$ & $(24 m)$ & $\mathrm{C}$ \\
\hline $\mathbf{B}_{32}$ & $=$ & $\begin{array}{c}\left(\frac{1}{2}-y_{5}\right) \mathbf{a}_{1}+\left(\frac{1}{2}+x_{5}\right) \mathbf{a}_{2}+ \\
\left(\frac{1}{2}+z_{5}\right) \mathbf{a}_{3}\end{array}$ & $=$ & $\begin{array}{c}\left(\frac{1}{2}-y_{5}\right) a \hat{\mathbf{x}}+\left(\frac{1}{2}+x_{5}\right) a \hat{\mathbf{y}}+ \\
\left(\frac{1}{2}+z_{5}\right) a \hat{\mathbf{z}}\end{array}$ & $(24 m)$ & $\mathrm{C}$ \\
\hline $\mathbf{B}_{33}$ & $=$ & $\begin{array}{c}\left(\frac{1}{2}+x_{5}\right) \mathbf{a}_{1}+\left(\frac{1}{2}+z_{5}\right) \mathbf{a}_{2}+ \\
\left(\frac{1}{2}-y_{5}\right) \mathbf{a}_{3}\end{array}$ & $=$ & $\begin{array}{c}\left(\frac{1}{2}+x_{5}\right) a \hat{\mathbf{x}}+\left(\frac{1}{2}+z_{5}\right) a \hat{\mathbf{y}}+ \\
\left(\frac{1}{2}-y_{5}\right) a \hat{\mathbf{z}}\end{array}$ & $(24 m)$ & $\mathrm{C}$ \\
\hline
\end{tabular}




\begin{tabular}{|c|c|c|c|c|c|}
\hline $\mathbf{B}_{34}$ & $=$ & $\begin{array}{c}\left(\frac{1}{2}-x_{5}\right) \mathbf{a}_{1}+\left(\frac{1}{2}+z_{5}\right) \mathbf{a}_{2}+ \\
\left(\frac{1}{2}+y_{5}\right) \mathbf{a}_{3}\end{array}$ & $=$ & $\begin{array}{c}\left(\frac{1}{2}-x_{5}\right) a \hat{\mathbf{x}}+\left(\frac{1}{2}+z_{5}\right) a \hat{\mathbf{y}}+ \\
\left(\frac{1}{2}+y_{5}\right) a \hat{\mathbf{z}}\end{array}$ & $(24 m)$ \\
\hline $\mathbf{B}_{35}$ & $=$ & $\begin{array}{c}\left(\frac{1}{2}-x_{5}\right) \mathbf{a}_{1}+\left(\frac{1}{2}-z_{5}\right) \mathbf{a}_{2}+ \\
\left(\frac{1}{2}-y_{5}\right) \mathbf{a}_{3}\end{array}$ & $=$ & $\begin{array}{c}\left(\frac{1}{2}-x_{5}\right) a \hat{\mathbf{x}}+\left(\frac{1}{2}-z_{5}\right) a \hat{\mathbf{y}}+ \\
\left(\frac{1}{2}-y_{5}\right) a \hat{\mathbf{z}}\end{array}$ & $(24 m)$ \\
\hline $\mathbf{B}_{36}$ & $=$ & $\begin{array}{c}\left(\frac{1}{2}+x_{5}\right) \mathbf{a}_{1}+\left(\frac{1}{2}-z_{5}\right) \mathbf{a}_{2}+ \\
\left(\frac{1}{2}+y_{5}\right) \mathbf{a}_{3}\end{array}$ & $=$ & $\begin{array}{c}\left(\frac{1}{2}+x_{5}\right) a \hat{\mathbf{x}}+\left(\frac{1}{2}-z_{5}\right) a \hat{\mathbf{y}}+ \\
\left(\frac{1}{2}+y_{5}\right) a \hat{\mathbf{z}}\end{array}$ & $(24 m)$ \\
\hline $\mathbf{B}_{37}$ & $=$ & $\begin{array}{c}\left(\frac{1}{2}+z_{5}\right) \mathbf{a}_{1}+\left(\frac{1}{2}+y_{5}\right) \mathbf{a}_{2}+ \\
\left(\frac{1}{2}-x_{5}\right) \mathbf{a}_{3}\end{array}$ & $=$ & $\begin{array}{c}\left(\frac{1}{2}+z_{5}\right) a \hat{\mathbf{x}}+\left(\frac{1}{2}+y_{5}\right) a \hat{\mathbf{y}}+ \\
\left(\frac{1}{2}-x_{5}\right) a \hat{\mathbf{z}}\end{array}$ & $(24 m)$ \\
\hline $\mathbf{B}_{38}$ & $=$ & $\begin{array}{c}\left(\frac{1}{2}+z_{5}\right) \mathbf{a}_{1}+\left(\frac{1}{2}-y_{5}\right) \mathbf{a}_{2}+ \\
\left(\frac{1}{2}+x_{5}\right) \mathbf{a}_{3}\end{array}$ & $=$ & $\begin{array}{c}\left(\frac{1}{2}+z_{5}\right) a \hat{\mathbf{x}}+\left(\frac{1}{2}-y_{5}\right) a \hat{\mathbf{y}}+ \\
\left(\frac{1}{2}+x_{5}\right) a \hat{\mathbf{z}}\end{array}$ & $(24 m)$ \\
\hline $\mathbf{B}_{39}$ & $=$ & $\begin{array}{c}\left(\frac{1}{2}-z_{5}\right) \mathbf{a}_{1}+\left(\frac{1}{2}+y_{5}\right) \mathbf{a}_{2}+ \\
\left(\frac{1}{2}+x_{5}\right) \mathbf{a}_{3}\end{array}$ & $=$ & $\begin{array}{c}\left(\frac{1}{2}-z_{5}\right) a \hat{\mathbf{x}}+\left(\frac{1}{2}+y_{5}\right) a \hat{\mathbf{y}}+ \\
\left(\frac{1}{2}+x_{5}\right) a \hat{\mathbf{z}}\end{array}$ & $(24 m)$ \\
\hline $\mathbf{B}_{40}$ & $=$ & $\begin{array}{c}\left(\frac{1}{2}-z_{5}\right) \mathbf{a}_{1}+\left(\frac{1}{2}-y_{5}\right) \mathbf{a}_{2}+ \\
\left(\frac{1}{2}-x_{5}\right) \mathbf{a}_{3}\end{array}$ & $=$ & $\begin{array}{c}\left(\frac{1}{2}-z_{5}\right) a \hat{\mathbf{x}}+\left(\frac{1}{2}-y_{5}\right) a \hat{\mathbf{y}}+ \\
\left(\frac{1}{2}-x_{5}\right) a \hat{\mathbf{z}}\end{array}$ & $(24 m)$ \\
\hline $\mathbf{B}_{41}$ & $=$ & $x_{6} \mathbf{a}_{1}+y_{6} \mathbf{a}_{2}+z_{6} \mathbf{a}_{3}$ & $=$ & $x_{6} a \hat{\mathbf{x}}+y_{6} a \hat{\mathbf{y}}+z_{6} a \hat{\mathbf{z}}$ & $(24 m)$ \\
\hline $\mathbf{B}_{42}$ & $=$ & $-x_{6} \mathbf{a}_{1}-y_{6} \mathbf{a}_{2}+z_{6} \mathbf{a}_{3}$ & $=$ & $-x_{6} a \hat{\mathbf{x}}-y_{6} a \hat{\mathbf{y}}+z_{6} a \hat{\mathbf{z}}$ & $(24 m)$ \\
\hline $\mathbf{B}_{43}$ & $=$ & $-x_{6} \mathbf{a}_{1}+y_{6} \mathbf{a}_{2}-z_{6} \mathbf{a}_{3}$ & $=$ & $-x_{6} a \hat{\mathbf{x}}+y_{6} a \hat{\mathbf{y}}-z_{6} a \hat{\mathbf{z}}$ & $(24 m)$ \\
\hline $\mathbf{B}_{44}$ & $=$ & $x_{6} \mathbf{a}_{1}-y_{6} \mathbf{a}_{2}-z_{6} \mathbf{a}_{3}$ & $=$ & $x_{6} a \hat{\mathbf{x}}-y_{6} a \hat{\mathbf{y}}-z_{6} a \hat{\mathbf{z}}$ & $(24 m)$ \\
\hline $\mathbf{B}_{45}$ & $=$ & $z_{6} \mathbf{a}_{1}+x_{6} \mathbf{a}_{2}+y_{6} \mathbf{a}_{3}$ & $=$ & $z_{6} a \hat{\mathbf{x}}+x_{6} a \hat{\mathbf{y}}+y_{6} a \hat{\mathbf{z}}$ & $(24 m)$ \\
\hline $\mathbf{B}_{46}$ & $=$ & $z_{6} \mathbf{a}_{1}-x_{6} \mathbf{a}_{2}-y_{6} \mathbf{a}_{3}$ & $=$ & $z_{6} a \hat{\mathbf{x}}-x_{6} a \hat{\mathbf{y}}-y_{6} a \hat{\mathbf{z}}$ & $(24 m)$ \\
\hline $\mathbf{B}_{47}$ & $=$ & $-z_{6} \mathbf{a}_{1}-x_{6} \mathbf{a}_{2}+y_{6} \mathbf{a}_{3}$ & $=$ & $-z_{6} a \hat{\mathbf{x}}-x_{6} a \hat{\mathbf{y}}+y_{6} a \hat{\mathbf{z}}$ & $(24 m)$ \\
\hline $\mathbf{B}_{48}$ & $=$ & $-z_{6} \mathbf{a}_{1}+x_{6} \mathbf{a}_{2}-y_{6} \mathbf{a}_{3}$ & $=$ & $-z_{6} a \hat{\mathbf{x}}+x_{6} a \hat{\mathbf{y}}-y_{6} a \hat{\mathbf{z}}$ & $(24 m)$ \\
\hline $\mathbf{B}_{49}$ & $=$ & $y_{6} \mathbf{a}_{1}+z_{6} \mathbf{a}_{2}+x_{6} \mathbf{a}_{3}$ & $=$ & $y_{6} a \hat{\mathbf{x}}+z_{6} a \hat{\mathbf{y}}+x_{6} a \hat{\mathbf{z}}$ & $(24 m)$ \\
\hline $\mathbf{B}_{50}$ & $=$ & $-y_{6} \mathbf{a}_{1}+z_{6} \mathbf{a}_{2}-x_{6} \mathbf{a}_{3}$ & $=$ & $-y_{6} a \hat{\mathbf{x}}+z_{6} a \hat{\mathbf{y}}-x_{6} a \hat{\mathbf{z}}$ & $(24 m)$ \\
\hline $\mathbf{B}_{51}$ & $=$ & $y_{6} \mathbf{a}_{1}-z_{6} \mathbf{a}_{2}-x_{6} \mathbf{a}_{3}$ & $=$ & $y_{6} a \hat{\mathbf{x}}-z_{6} a \hat{\mathbf{y}}-x_{6} a \hat{\mathbf{z}}$ & $(24 m)$ \\
\hline $\mathbf{B}_{52}$ & $=$ & $-y_{6} \mathbf{a}_{1}-z_{6} \mathbf{a}_{2}+x_{6} \mathbf{a}_{3}$ & $=$ & $-y_{6} a \hat{\mathbf{x}}-z_{6} a \hat{\mathbf{y}}+x_{6} a \hat{\mathbf{z}}$ & $(24 m)$ \\
\hline $\mathbf{B}_{53}$ & $=$ & $\begin{array}{c}\left(\frac{1}{2}+y_{6}\right) \mathbf{a}_{1}+\left(\frac{1}{2}+x_{6}\right) \mathbf{a}_{2}+ \\
\left(\frac{1}{2}-z_{6}\right) \mathbf{a}_{3}\end{array}$ & $=$ & $\begin{array}{c}\left(\frac{1}{2}+y_{6}\right) a \hat{\mathbf{x}}+\left(\frac{1}{2}+x_{6}\right) a \hat{\mathbf{y}}+ \\
\left(\frac{1}{2}-z_{6}\right) a \hat{\mathbf{z}}\end{array}$ & $(24 m)$ \\
\hline $\mathbf{B}_{54}$ & $=$ & $\begin{array}{c}\left(\frac{1}{2}-y_{6}\right) \mathbf{a}_{1}+\left(\frac{1}{2}-x_{6}\right) \mathbf{a}_{2}+ \\
\left(\frac{1}{2}-z_{6}\right) \mathbf{a}_{3}\end{array}$ & $=$ & $\begin{array}{c}\left(\frac{1}{2}-y_{6}\right) a \hat{\mathbf{x}}+\left(\frac{1}{2}-x_{6}\right) a \hat{\mathbf{y}}+ \\
\left(\frac{1}{2}-z_{6}\right) a \hat{\mathbf{z}}\end{array}$ & $(24 m)$ \\
\hline $\mathbf{B}_{55}$ & $=$ & $\begin{array}{c}\left(\frac{1}{2}+y_{6}\right) \mathbf{a}_{1}+\left(\frac{1}{2}-x_{6}\right) \mathbf{a}_{2}+ \\
\left(\frac{1}{2}+z_{6}\right) \mathbf{a}_{3}\end{array}$ & $=$ & $\begin{array}{c}\left(\frac{1}{2}+y_{6}\right) a \hat{\mathbf{x}}+\left(\frac{1}{2}-x_{6}\right) a \hat{\mathbf{y}}+ \\
\left(\frac{1}{2}+z_{6}\right) a \hat{\mathbf{z}}\end{array}$ & $(24 m)$ \\
\hline $\mathbf{B}_{56}$ & $=$ & $\begin{array}{c}\left(\frac{1}{2}-y_{6}\right) \mathbf{a}_{1}+\left(\frac{1}{2}+x_{6}\right) \mathbf{a}_{2}+ \\
\left(\frac{1}{2}+z_{6}\right) \mathbf{a}_{3}\end{array}$ & $=$ & $\begin{array}{c}\left(\frac{1}{2}-y_{6}\right) a \hat{\mathbf{x}}+\left(\frac{1}{2}+x_{6}\right) a \hat{\mathbf{y}}+ \\
\left(\frac{1}{2}+z_{6}\right) a \hat{\mathbf{z}}\end{array}$ & $(24 m)$ \\
\hline $\mathbf{B}_{57}$ & $=$ & $\begin{array}{c}\left(\frac{1}{2}+x_{6}\right) \mathbf{a}_{1}+\left(\frac{1}{2}+z_{6}\right) \mathbf{a}_{2}+ \\
\left(\frac{1}{2}-y_{6}\right) \mathbf{a}_{3}\end{array}$ & $=$ & $\begin{array}{c}\left(\frac{1}{2}+x_{6}\right) a \hat{\mathbf{x}}+\left(\frac{1}{2}+z_{6}\right) a \hat{\mathbf{y}}+ \\
\left(\frac{1}{2}-y_{6}\right) a \hat{\mathbf{z}}\end{array}$ & $(24 m)$ \\
\hline $\mathbf{B}_{58}$ & $=$ & $\begin{array}{c}\left(\frac{1}{2}-x_{6}\right) \mathbf{a}_{1}+\left(\frac{1}{2}+z_{6}\right) \mathbf{a}_{2}+ \\
\left(\frac{1}{2}+y_{6}\right) \mathbf{a}_{3}\end{array}$ & $=$ & $\begin{array}{c}\left(\frac{1}{2}-x_{6}\right) a \hat{\mathbf{x}}+\left(\frac{1}{2}+z_{6}\right) a \hat{\mathbf{y}}+ \\
\left(\frac{1}{2}+y_{6}\right) a \hat{\mathbf{z}}\end{array}$ & $(24 m)$ \\
\hline
\end{tabular}




\begin{tabular}{|c|c|c|c|c|c|}
\hline $\mathbf{B}_{59}$ & $=$ & $\begin{array}{c}\left(\frac{1}{2}-x_{6}\right) \mathbf{a}_{1}+\left(\frac{1}{2}-z_{6}\right) \mathbf{a}_{2}+ \\
\left(\frac{1}{2}-y_{6}\right) \mathbf{a}_{3}\end{array}$ & $=$ & $\begin{array}{c}\left(\frac{1}{2}-x_{6}\right) a \hat{\mathbf{x}}+\left(\frac{1}{2}-z_{6}\right) a \hat{\mathbf{y}}+ \\
\left(\frac{1}{2}-y_{6}\right) a \hat{\mathbf{z}}\end{array}$ & $(24 m)$ \\
\hline $\mathbf{B}_{60}$ & $=$ & $\begin{array}{c}\left(\frac{1}{2}+x_{6}\right) \mathbf{a}_{1}+\left(\frac{1}{2}-z_{6}\right) \mathbf{a}_{2}+ \\
\left(\frac{1}{2}+y_{6}\right) \mathbf{a}_{3}\end{array}$ & $=$ & $\begin{array}{c}\left(\frac{1}{2}+x_{6}\right) a \hat{\mathbf{x}}+\left(\frac{1}{2}-z_{6}\right) a \hat{\mathbf{y}}+ \\
\left(\frac{1}{2}+y_{6}\right) a \hat{\mathbf{z}}\end{array}$ & $(24 m)$ \\
\hline $\mathbf{B}_{61}$ & $=$ & $\begin{array}{c}\left(\frac{1}{2}+z_{6}\right) \mathbf{a}_{1}+\left(\frac{1}{2}+y_{6}\right) \mathbf{a}_{2}+ \\
\left(\frac{1}{2}-x_{6}\right) \mathbf{a}_{3}\end{array}$ & $=$ & $\begin{array}{c}\left(\frac{1}{2}+z_{6}\right) a \hat{\mathbf{x}}+\left(\frac{1}{2}+y_{6}\right) a \hat{\mathbf{y}}+ \\
\left(\frac{1}{2}-x_{6}\right) a \hat{\mathbf{z}}\end{array}$ & $(24 m)$ \\
\hline $\mathbf{B}_{62}$ & $=$ & $\begin{array}{c}\left(\frac{1}{2}+z_{6}\right) \mathbf{a}_{1}+\left(\frac{1}{2}-y_{6}\right) \mathbf{a}_{2}+ \\
\left(\frac{1}{2}+x_{6}\right) \mathbf{a}_{3}\end{array}$ & $=$ & $\begin{array}{c}\left(\frac{1}{2}+z_{6}\right) a \hat{\mathbf{x}}+\left(\frac{1}{2}-y_{6}\right) a \hat{\mathbf{y}}+ \\
\left(\frac{1}{2}+x_{6}\right) a \hat{\mathbf{z}}\end{array}$ & $(24 m)$ \\
\hline $\mathbf{B}_{63}$ & $=$ & $\begin{array}{c}\left(\frac{1}{2}-z_{6}\right) \mathbf{a}_{1}+\left(\frac{1}{2}+y_{6}\right) \mathbf{a}_{2}+ \\
\left(\frac{1}{2}+x_{6}\right) \mathbf{a}_{3}\end{array}$ & $=$ & $\begin{array}{c}\left(\frac{1}{2}-z_{6}\right) a \hat{\mathbf{x}}+\left(\frac{1}{2}+y_{6}\right) a \hat{\mathbf{y}}+ \\
\left(\frac{1}{2}+x_{6}\right) a \hat{\mathbf{z}}\end{array}$ & $(24 m)$ \\
\hline $\mathbf{B}_{64}$ & $=$ & $\begin{array}{c}\left(\frac{1}{2}-z_{6}\right) \mathbf{a}_{1}+\left(\frac{1}{2}-y_{6}\right) \mathbf{a}_{2}+ \\
\left(\frac{1}{2}-x_{6}\right) \mathbf{a}_{3}\end{array}$ & $=$ & $\begin{array}{c}\left(\frac{1}{2}-z_{6}\right) a \hat{\mathbf{x}}+\left(\frac{1}{2}-y_{6}\right) a \hat{\mathbf{y}}+ \\
\left(\frac{1}{2}-x_{6}\right) a \hat{\mathbf{z}}\end{array}$ & $(24 m)$ \\
\hline
\end{tabular}

\section{References:}

- V. G. Kuznetsov, Z. V. Popova, and G. B. Seifer, X-ray diffraction study of ferrocyanides of copper, cobalt, nickel, trivalent iron and mixed copper ferrocyanide with potassium, Russ. J. Inorg. Chem. 15, 1084-1088 (1970).

\section{Found in:}

- P. Villars and K. Cenzual, Pearson's Crystal Data - Crystal Structure Database for Inorganic Compounds, ASM International (2013).

\section{Geometry files:}

- CIF: pp. 960

- POSCAR: pp. 961 


\section{F6 KP Structure: A24BC_cF104_209_j_a_b}
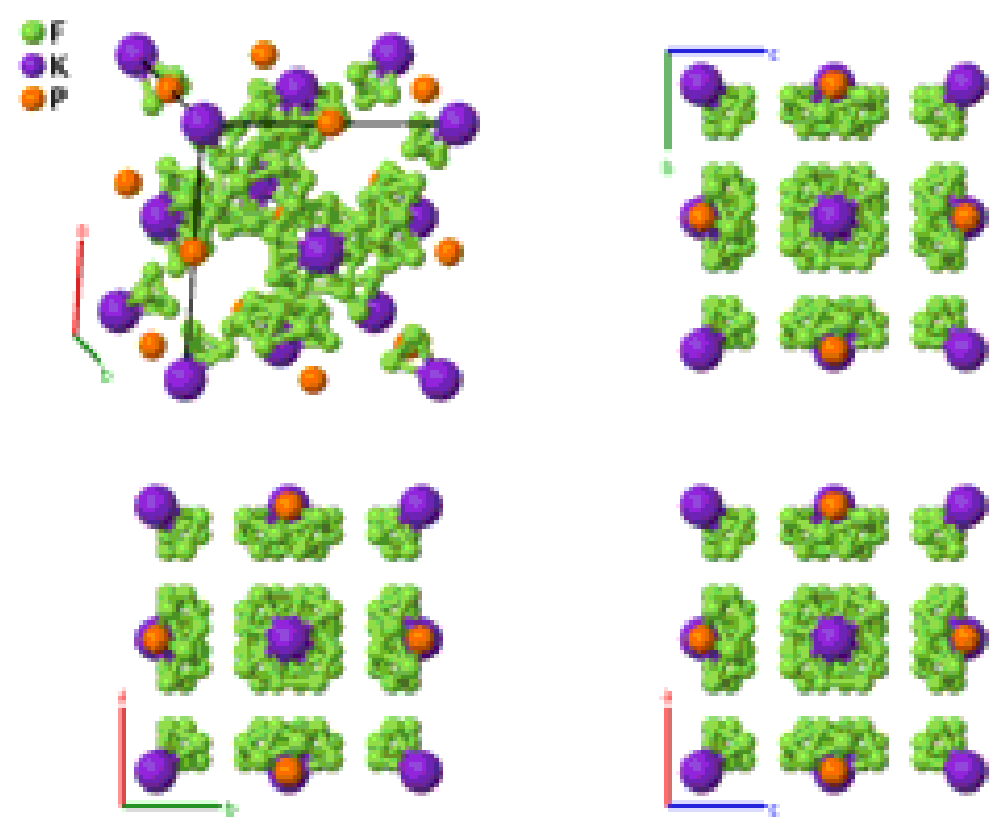

\section{Prototype}

AFLOW prototype label

Strukturbericht designation

Pearson symbol

Space group number

Space group symbol

AFLOW prototype command
$: \quad \mathrm{F}_{6} \mathrm{KP}$

: A24BC_cF104_209_j_a_b

: None

: $\quad$ cF104

: 209

: $\quad F 432$

: $\quad$ aflow --proto=A24BC_cF104_209_j_a_b

- params $=a, x_{3}, y_{3}, z_{3}$

- The (96j) Wyckoff positions are decorated by $\mathrm{F}$ atoms with a site occupation of 0.25 . Hence, the prototype material is $\mathrm{F}_{6} \mathrm{KP}$ as opposed to $\mathrm{F}_{24} \mathrm{KP}$.

Face-centered Cubic primitive vectors:

$$
\begin{aligned}
& \mathbf{a}_{1}=\frac{1}{2} a \hat{\mathbf{y}}+\frac{1}{2} a \hat{\mathbf{z}} \\
& \mathbf{a}_{2}=\frac{1}{2} a \hat{\mathbf{x}}+\frac{1}{2} a \hat{\mathbf{z}} \\
& \mathbf{a}_{3}=\frac{1}{2} a \hat{\mathbf{x}}+\frac{1}{2} a \hat{\mathbf{y}}
\end{aligned}
$$

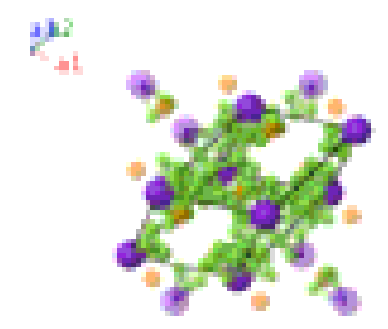

Basis vectors:

Lattice Coordinates
$\mathbf{B}_{1}=$
$0 \mathbf{a}_{1}+0 \mathbf{a}_{2}+0 \mathbf{a}_{3}$
$\mathbf{B}_{2}=$
$\frac{1}{2} \mathbf{a}_{1}+\frac{1}{2} \mathbf{a}_{2}+\frac{1}{2} \mathbf{a}_{3}$

Cartesian Coordinates

Wyckoff Position

Atom Type

$=\quad 0 \hat{\mathbf{x}}+0 \hat{\mathbf{y}}+0 \hat{\mathbf{z}}$

(4a)

K

$=\frac{1}{2} a \hat{\mathbf{x}}+\frac{1}{2} a \hat{\mathbf{y}}+\frac{1}{2} a \hat{\mathbf{z}}$

(4b) 


\begin{tabular}{|c|c|c|c|c|c|c|}
\hline $\mathbf{B}_{3}$ & $=$ & $\begin{array}{c}\left(-x_{3}+y_{3}+z_{3}\right) \mathbf{a}_{1}+\left(x_{3}-y_{3}+z_{3}\right) \mathbf{a}_{2}+ \\
\left(x_{3}+y_{3}-z_{3}\right) \mathbf{a}_{3}\end{array}$ & $=$ & $x_{3} a \hat{\mathbf{x}}+y_{3} a \hat{\mathbf{y}}+z_{3} a \hat{\mathbf{z}}$ & $(96 j)$ & $\mathrm{F}$ \\
\hline $\mathbf{B}_{4}$ & $=$ & $\begin{array}{c}\left(x_{3}-y_{3}+z_{3}\right) \mathbf{a}_{1}+\left(-x_{3}+y_{3}+z_{3}\right) \mathbf{a}_{2}+ \\
\left(-x_{3}-y_{3}-z_{3}\right) \mathbf{a}_{3}\end{array}$ & $=$ & $-x_{3} a \hat{\mathbf{x}}-y_{3} a \hat{\mathbf{y}}+z_{3} a \hat{\mathbf{z}}$ & $(96 j)$ & $\mathrm{F}$ \\
\hline $\mathbf{B}_{5}$ & $=$ & $\begin{array}{c}\left(x_{3}+y_{3}-z_{3}\right) \mathbf{a}_{1}+\left(-x_{3}-y_{3}-z_{3}\right) \mathbf{a}_{2}+ \\
\left(-x_{3}+y_{3}+z_{3}\right) \mathbf{a}_{3}\end{array}$ & $=$ & $-x_{3} a \hat{\mathbf{x}}+y_{3} a \hat{\mathbf{y}}-z_{3} a \hat{\mathbf{z}}$ & $(96 j)$ & $\mathrm{F}$ \\
\hline $\mathbf{B}_{6}$ & $=$ & $\begin{array}{c}\left(-x_{3}-y_{3}-z_{3}\right) \mathbf{a}_{1}+\left(x_{3}+y_{3}-z_{3}\right) \mathbf{a}_{2}+ \\
\left(x_{3}-y_{3}+z_{3}\right) \mathbf{a}_{3}\end{array}$ & $=$ & $x_{3} a \hat{\mathbf{x}}-y_{3} a \hat{\mathbf{y}}-z_{3} a \hat{\mathbf{z}}$ & $(96 j)$ & $\mathrm{F}$ \\
\hline $\mathbf{B}_{7}$ & $=$ & $\begin{array}{c}\left(x_{3}+y_{3}-z_{3}\right) \mathbf{a}_{1}+\left(-x_{3}+y_{3}+z_{3}\right) \mathbf{a}_{2}+ \\
\left(x_{3}-y_{3}+z_{3}\right) \mathbf{a}_{3}\end{array}$ & $=$ & $z_{3} a \hat{\mathbf{x}}+x_{3} a \hat{\mathbf{y}}+y_{3} a \hat{\mathbf{z}}$ & $(96 j)$ & $\mathrm{F}$ \\
\hline $\mathbf{B}_{8}$ & $=$ & $\begin{array}{c}\left(-x_{3}-y_{3}-z_{3}\right) \mathbf{a}_{1}+\left(x_{3}-y_{3}+z_{3}\right) \mathbf{a}_{2}+ \\
\left(-x_{3}+y_{3}+z_{3}\right) \mathbf{a}_{3}\end{array}$ & $=$ & $z_{3} a \hat{\mathbf{x}}-x_{3} a \hat{\mathbf{y}}-y_{3} a \hat{\mathbf{z}}$ & $(96 j)$ & $\mathrm{F}$ \\
\hline $\mathbf{B}_{9}$ & $=$ & $\begin{array}{c}\left(-x_{3}+y_{3}+z_{3}\right) \mathbf{a}_{1}+\left(x_{3}+y_{3}-z_{3}\right) \mathbf{a}_{2}+ \\
\left(-x_{3}-y_{3}-z_{3}\right) \mathbf{a}_{3}\end{array}$ & $=$ & $-z_{3} a \hat{\mathbf{x}}-x_{3} a \hat{\mathbf{y}}+y_{3} a \hat{\mathbf{z}}$ & $(96 j)$ & $\mathrm{F}$ \\
\hline $\mathbf{B}_{10}$ & $=$ & $\begin{array}{c}\left(x_{3}-y_{3}+z_{3}\right) \mathbf{a}_{1}+\left(-x_{3}-y_{3}-z_{3}\right) \mathbf{a}_{2}+ \\
\left(x_{3}+y_{3}-z_{3}\right) \mathbf{a}_{3}\end{array}$ & $=$ & $-z_{3} a \hat{\mathbf{x}}+x_{3} a \hat{\mathbf{y}}-y_{3} a \hat{\mathbf{z}}$ & $(96 j)$ & $\mathrm{F}$ \\
\hline $\mathbf{B}_{11}$ & $=$ & $\begin{array}{c}\left(x_{3}-y_{3}+z_{3}\right) \mathbf{a}_{1}+\left(x_{3}+y_{3}-z_{3}\right) \mathbf{a}_{2}+ \\
\left(-x_{3}+y_{3}+z_{3}\right) \mathbf{a}_{3}\end{array}$ & $=$ & $y_{3} a \hat{\mathbf{x}}+z_{3} a \hat{\mathbf{y}}+x_{3} a \hat{\mathbf{z}}$ & $(96 j)$ & $\mathrm{F}$ \\
\hline $\mathbf{B}_{12}$ & $=$ & $\begin{array}{c}\left(-x_{3}+y_{3}+z_{3}\right) \mathbf{a}_{1}+\left(-x_{3}-y_{3}-z_{3}\right) \mathbf{a}_{2}+ \\
\left(x_{3}-y_{3}+z_{3}\right) \mathbf{a}_{3}\end{array}$ & $=$ & $-y_{3} a \hat{\mathbf{x}}+z_{3} a \hat{\mathbf{y}}-x_{3} a \hat{\mathbf{z}}$ & $(96 j)$ & $\mathrm{F}$ \\
\hline $\mathbf{B}_{13}$ & $=$ & $\begin{array}{c}\left(-x_{3}-y_{3}-z_{3}\right) \mathbf{a}_{1}+\left(-x_{3}+y_{3}+z_{3}\right) \mathbf{a}_{2}+ \\
\left(x_{3}+y_{3}-z_{3}\right) \mathbf{a}_{3}\end{array}$ & $=$ & $y_{3} a \hat{\mathbf{x}}-z_{3} a \hat{\mathbf{y}}-x_{3} a \hat{\mathbf{z}}$ & $(96 j)$ & $\mathrm{F}$ \\
\hline $\mathbf{B}_{14}$ & $=$ & $\begin{array}{c}\left(x_{3}+y_{3}-z_{3}\right) \mathbf{a}_{1}+\left(x_{3}-y_{3}+z_{3}\right) \mathbf{a}_{2}+ \\
\left(-x_{3}-y_{3}-z_{3}\right) \mathbf{a}_{3}\end{array}$ & $=$ & $-y_{3} a \hat{\mathbf{x}}-z_{3} a \hat{\mathbf{y}}+x_{3} a \hat{\mathbf{z}}$ & $(96 j)$ & $\mathrm{F}$ \\
\hline $\mathbf{B}_{15}$ & $=$ & $\begin{array}{c}\left(x_{3}-y_{3}-z_{3}\right) \mathbf{a}_{1}+\left(-x_{3}+y_{3}-z_{3}\right) \mathbf{a}_{2}+ \\
\left(x_{3}+y_{3}+z_{3}\right) \mathbf{a}_{3}\end{array}$ & $=$ & $y_{3} a \hat{\mathbf{x}}+x_{3} a \hat{\mathbf{y}}-z_{3} a \hat{\mathbf{z}}$ & $(96 j)$ & $\mathrm{F}$ \\
\hline $\mathbf{B}_{16}$ & $=$ & $\begin{array}{c}\left(-x_{3}+y_{3}-z_{3}\right) \mathbf{a}_{1}+\left(x_{3}-y_{3}-z_{3}\right) \mathbf{a}_{2}+ \\
\left(-x_{3}-y_{3}+z_{3}\right) \mathbf{a}_{3}\end{array}$ & $=$ & $-y_{3} a \hat{\mathbf{x}}-x_{3} a \hat{\mathbf{y}}-z_{3} a \hat{\mathbf{z}}$ & $(96 j)$ & $\mathrm{F}$ \\
\hline $\mathbf{B}_{17}$ & $=$ & $\begin{array}{c}\left(-x_{3}-y_{3}+z_{3}\right) \mathbf{a}_{1}+\left(x_{3}+y_{3}+z_{3}\right) \mathbf{a}_{2}+ \\
\left(-x_{3}+y_{3}-z_{3}\right) \mathbf{a}_{3}\end{array}$ & $=$ & $y_{3} a \hat{\mathbf{x}}-x_{3} a \hat{\mathbf{y}}+z_{3} a \hat{\mathbf{z}}$ & $(96 j)$ & $\mathrm{F}$ \\
\hline $\mathbf{B}_{18}$ & $=$ & $\begin{array}{c}\left(x_{3}+y_{3}+z_{3}\right) \mathbf{a}_{1}+\left(-x_{3}-y_{3}+z_{3}\right) \mathbf{a}_{2}+ \\
\left(x_{3}-y_{3}-z_{3}\right) \mathbf{a}_{3}\end{array}$ & $=$ & $-y_{3} a \hat{\mathbf{x}}+x_{3} a \hat{\mathbf{y}}+z_{3} a \hat{\mathbf{z}}$ & $(96 j)$ & $\mathrm{F}$ \\
\hline $\mathbf{B}_{19}$ & $=$ & $\begin{array}{c}\left(-x_{3}-y_{3}+z_{3}\right) \mathbf{a}_{1}+\left(x_{3}-y_{3}-z_{3}\right) \mathbf{a}_{2}+ \\
\left(x_{3}+y_{3}+z_{3}\right) \mathbf{a}_{3}\end{array}$ & $=$ & $x_{3} a \hat{\mathbf{x}}+z_{3} a \hat{\mathbf{y}}-y_{3} a \hat{\mathbf{z}}$ & $(96 j)$ & $\mathrm{F}$ \\
\hline $\mathbf{B}_{20}$ & $=$ & $\begin{array}{c}\left(x_{3}+y_{3}+z_{3}\right) \mathbf{a}_{1}+\left(-x_{3}+y_{3}-z_{3}\right) \mathbf{a}_{2}+ \\
\left(-x_{3}-y_{3}+z_{3}\right) \mathbf{a}_{3}\end{array}$ & $=$ & $-x_{3} a \hat{\mathbf{x}}+z_{3} a \hat{\mathbf{y}}+y_{3} a \hat{\mathbf{z}}$ & $(96 j)$ & $\mathrm{F}$ \\
\hline $\mathbf{B}_{21}$ & $=$ & $\begin{array}{c}\left(x_{3}-y_{3}-z_{3}\right) \mathbf{a}_{1}+\left(-x_{3}-y_{3}+z_{3}\right) \mathbf{a}_{2}+ \\
\left(-x_{3}+y_{3}-z_{3}\right) \mathbf{a}_{3}\end{array}$ & $=$ & $-x_{3} a \hat{\mathbf{x}}-z_{3} a \hat{\mathbf{y}}-y_{3} a \hat{\mathbf{z}}$ & $(96 j)$ & $\mathrm{F}$ \\
\hline $\mathbf{B}_{22}$ & $=$ & $\begin{array}{c}\left(-x_{3}+y_{3}-z_{3}\right) \mathbf{a}_{1}+\left(x_{3}+y_{3}+z_{3}\right) \mathbf{a}_{2}+ \\
\left(x_{3}-y_{3}-z_{3}\right) \mathbf{a}_{3}\end{array}$ & $=$ & $x_{3} a \hat{\mathbf{x}}-z_{3} a \hat{\mathbf{y}}+y_{3} a \hat{\mathbf{z}}$ & $(96 j)$ & $\mathrm{F}$ \\
\hline $\mathbf{B}_{23}$ & $=$ & $\begin{array}{c}\left(-x_{3}+y_{3}-z_{3}\right) \mathbf{a}_{1}+\left(-x_{3}-y_{3}+z_{3}\right) \mathbf{a}_{2}+ \\
\left(x_{3}+y_{3}+z_{3}\right) \mathbf{a}_{3}\end{array}$ & $=$ & $z_{3} a \hat{\mathbf{x}}+y_{3} a \hat{\mathbf{y}}-x_{3} a \hat{\mathbf{z}}$ & $(96 j)$ & $\mathrm{F}$ \\
\hline $\mathbf{B}_{24}$ & $=$ & $\begin{array}{c}\left(x_{3}-y_{3}-z_{3}\right) \mathbf{a}_{1}+\left(x_{3}+y_{3}+z_{3}\right) \mathbf{a}_{2}+ \\
\left(-x_{3}-y_{3}+z_{3}\right) \mathbf{a}_{3}\end{array}$ & $=$ & $z_{3} a \hat{\mathbf{x}}-y_{3} a \hat{\mathbf{y}}+x_{3} a \hat{\mathbf{z}}$ & $(96 j)$ & $\mathrm{F}$ \\
\hline $\mathbf{B}_{25}$ & $=$ & $\begin{array}{c}\left(x_{3}+y_{3}+z_{3}\right) \mathbf{a}_{1}+\left(x_{3}-y_{3}-z_{3}\right) \mathbf{a}_{2}+ \\
\left(-x_{3}+y_{3}-z_{3}\right) \mathbf{a}_{3}\end{array}$ & $=$ & $-z_{3} a \hat{\mathbf{x}}+y_{3} a \hat{\mathbf{y}}+x_{3} a \hat{\mathbf{z}}$ & $(96 j)$ & $\mathrm{F}$ \\
\hline $\mathbf{B}_{26}$ & $=$ & $\begin{array}{c}\left(-x_{3}-y_{3}+z_{3}\right) \mathbf{a}_{1}+\left(-x_{3}+y_{3}-z_{3}\right) \mathbf{a}_{2}+ \\
\left(x_{3}-y_{3}-z_{3}\right) \mathbf{a}_{3}\end{array}$ & $=$ & $-z_{3} a \hat{\mathbf{x}}-y_{3} a \hat{\mathbf{y}}-x_{3} a \hat{\mathbf{z}}$ & $(96 j)$ & $\mathrm{F}$ \\
\hline
\end{tabular}




\section{References:}

- Y. P. Mascarenhas and S. H. Pulcinelli, A redetermination of the structure of $\alpha$-potassium fluorophosphate, Acta Crystallogr. Sect. A 37, C175 (1981), doi:10.1107/S0108767381094294.

\section{Found in:}

- P. Villars and K. Cenzual, Pearson's Crystal Data - Crystal Structure Database for Inorganic Compounds, ASM International (2013).

\section{Geometry files:}

- CIF: pp. 961

- POSCAR: pp. 962 


\section{$\mathrm{Te}[\mathrm{OH}]_{6}$ Structure: A12B6C_cF608_210_4h_2h_e}
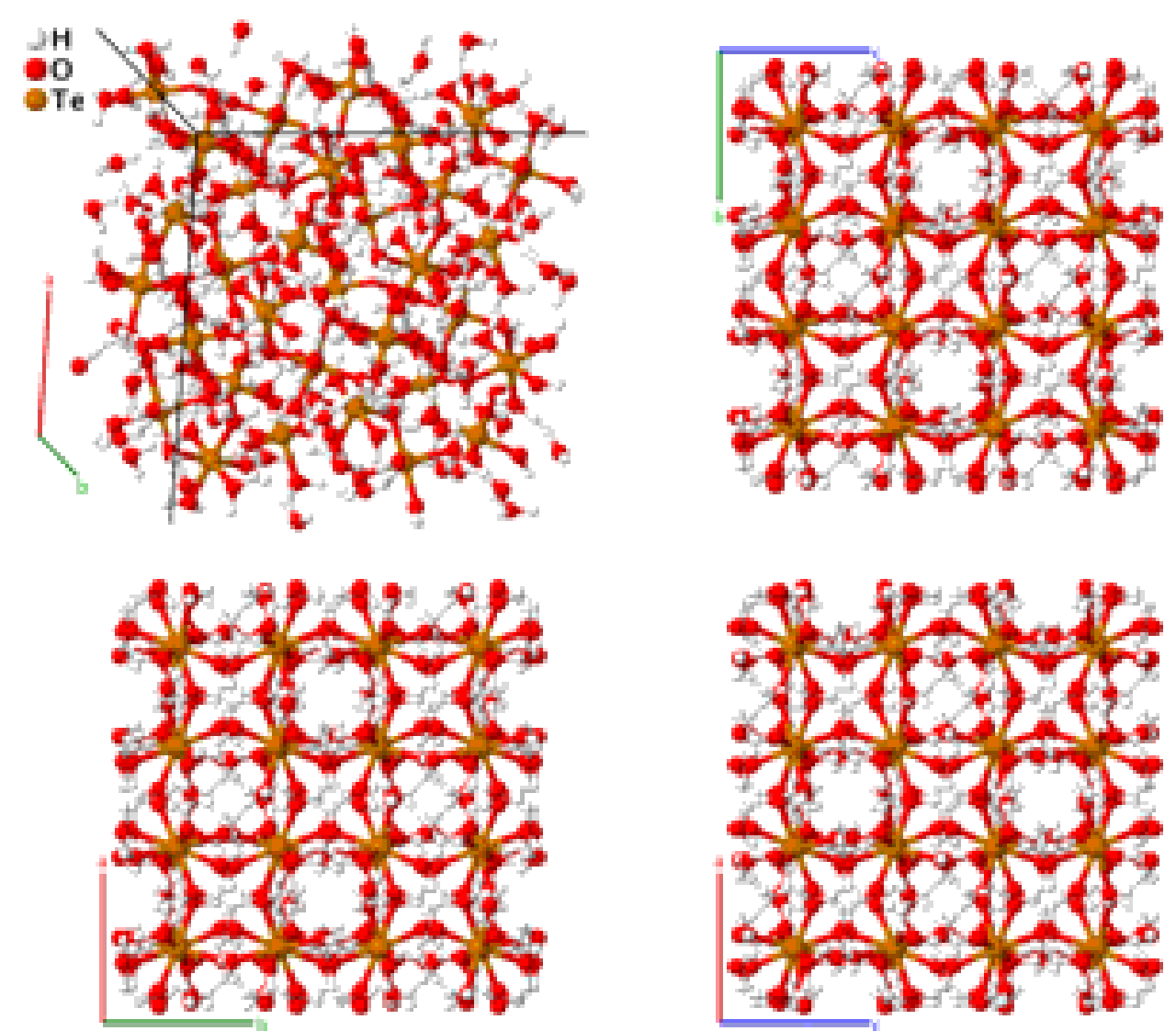

\section{Prototype}

AFLOW prototype label

Strukturbericht designation

Pearson symbol

Space group number

Space group symbol

AFLOW prototype command
: $\quad \mathrm{Te}[\mathrm{OH}]_{6}$

: A12B6C_cF608_210_4h_2h_e

: None

: $\quad$ cF608

: $\quad 210$

: $\quad F 4_{1} 32$

aflow --proto $=A 12 B 6 C \_c F 608 \_210 \_4 h \_2 h \_e$

- -params $=a, x_{1}, x_{2}, y_{2}, z_{2}, x_{3}, y_{3}, z_{3}, x_{4}, y_{4}, z_{4}, x_{5}, y_{5}, z_{5}, x_{6}, y_{6}, z_{6}, x_{7}, y_{7}, z_{7}$

- All H sites are half occupation. Polytypes appear in space groups \#14, \#228, and \#225.

Face-centered Cubic primitive vectors:

$$
\begin{aligned}
& \mathbf{a}_{1}=\frac{1}{2} a \hat{\mathbf{y}}+\frac{1}{2} a \hat{\mathbf{z}} \\
& \mathbf{a}_{2}=\frac{1}{2} a \hat{\mathbf{x}}+\frac{1}{2} a \hat{\mathbf{z}} \\
& \mathbf{a}_{3}=\frac{1}{2} a \hat{\mathbf{x}}+\frac{1}{2} a \hat{\mathbf{y}}
\end{aligned}
$$

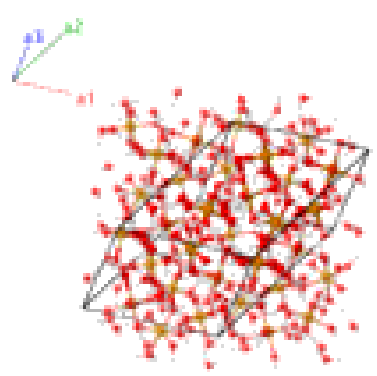




\section{Lattice Coordinates}

$$
\begin{aligned}
& \mathbf{B}_{1}=x_{1} \mathbf{a}_{1}+x_{1} \mathbf{a}_{2}+x_{1} \mathbf{a}_{3} \\
& = \\
& \mathbf{B}_{2}= \\
& x_{1} \mathbf{a}_{1}+x_{1} \mathbf{a}_{2}-3 x_{1} \mathbf{a}_{3} \\
& \mathbf{B}_{3}= \\
& x_{1} \mathbf{a}_{1}-3 x_{1} \mathbf{a}_{2}+x_{1} \mathbf{a}_{3} \\
& \mathbf{B}_{4}=-3 x_{1} \mathbf{a}_{1}+x_{1} \mathbf{a}_{2}+x_{1} \mathbf{a}_{3} \\
& \mathbf{B}_{5}=\left(\frac{1}{4}-x_{1}\right) \mathbf{a}_{1}+\left(\frac{1}{4}-x_{1}\right) \mathbf{a}_{2}+ \\
& \left(\frac{1}{4}+3 x_{1}\right) \mathbf{a}_{3} \\
& \mathbf{B}_{6}=\left(\frac{1}{4}-x_{1}\right) \mathbf{a}_{1}+\left(\frac{1}{4}-x_{1}\right) \mathbf{a}_{2}+=\left(\frac{1}{4}-x_{1}\right) a \hat{\mathbf{x}}+\left(\frac{1}{4}-x_{1}\right) a \hat{\mathbf{y}}+ \\
& \left(\frac{1}{4}-x_{1}\right) \mathbf{a}_{3} \\
& \mathbf{B}_{7}=\left(\frac{1}{4}-x_{1}\right) \mathbf{a}_{1}+\left(\frac{1}{4}+3 x_{1}\right) \mathbf{a}_{2}+ \\
& \left(\frac{1}{4}-x_{1}\right) \mathbf{a}_{3} \\
& \mathbf{B}_{8}=\left(\frac{1}{4}+3 x_{1}\right) \mathbf{a}_{1}+\left(\frac{1}{4}-x_{1}\right) \mathbf{a}_{2} \\
& \left(\frac{1}{4}-x_{1}\right) \mathbf{a}_{3} \\
& \mathbf{B}_{9}=\left(-x_{2}+y_{2}+z_{2}\right) \mathbf{a}_{1}+ \\
& \left(x_{2}-y_{2}+z_{2}\right) \mathbf{a}_{2}+ \\
& \left(x_{2}+y_{2}-z_{2}\right) \mathbf{a}_{3} \\
& \mathbf{B}_{10}=\left(x_{2}-y_{2}+z_{2}\right) \mathbf{a}_{1}+ \\
& \left(-x_{2}+y_{2}+z_{2}\right) \mathbf{a}_{2}+ \\
& \left(-x_{2}-y_{2}-z_{2}\right) \mathbf{a}_{3} \\
& \mathbf{B}_{11}=\quad\left(x_{2}+y_{2}-z_{2}\right) \mathbf{a}_{1}+ \\
& \left(-x_{2}-y_{2}-z_{2}\right) \mathbf{a}_{2}+ \\
& \left(-x_{2}+y_{2}+z_{2}\right) \mathbf{a}_{3} \\
& \mathbf{B}_{12}=\left(-x_{2}-y_{2}-z_{2}\right) \mathbf{a}_{1}+ \\
& \left(x_{2}+y_{2}-z_{2}\right) \mathbf{a}_{2}+ \\
& \left(x_{2}-y_{2}+z_{2}\right) \mathbf{a}_{3} \\
& \mathbf{B}_{13}=\left(x_{2}+y_{2}-z_{2}\right) \mathbf{a}_{1}+ \\
& \left(-x_{2}+y_{2}+z_{2}\right) \mathbf{a}_{2}+ \\
& \left(x_{2}-y_{2}+z_{2}\right) \mathbf{a}_{3} \\
& \mathbf{B}_{14}=\left(-x_{2}-y_{2}-z_{2}\right) \mathbf{a}_{1}+ \\
& \left(x_{2}-y_{2}+z_{2}\right) \mathbf{a}_{2}+ \\
& \left(-x_{2}+y_{2}+z_{2}\right) \mathbf{a}_{3} \\
& \mathbf{B}_{15}=\left(-x_{2}+y_{2}+z_{2}\right) \mathbf{a}_{1}+ \\
& \left(x_{2}+y_{2}-z_{2}\right) \mathbf{a}_{2}+ \\
& \left(-x_{2}-y_{2}-z_{2}\right) \mathbf{a}_{3} \\
& \mathbf{B}_{16}=\left(x_{2}-y_{2}+z_{2}\right) \mathbf{a}_{1}+ \\
& \left(-x_{2}-y_{2}-z_{2}\right) \mathbf{a}_{2}+ \\
& \left(x_{2}+y_{2}-z_{2}\right) \mathbf{a}_{3} \\
& \left(x_{2}-y_{2}+z_{2}\right) \mathbf{a}_{3} \\
& \begin{array}{r}
\mathbf{B}_{19}=\quad\left(-x_{2}-y_{2}-z_{2}\right) \mathbf{a}_{1}+ \\
\left(-x_{2}+y_{2}+z_{2}\right) \mathbf{a}_{2}+ \\
\left(x_{2}+y_{2}-z_{2}\right) \mathbf{a}_{3}
\end{array}
\end{aligned}
$$

Cartesian Coordinates

Wyckoff Position Atom Type

$(96 h)$

(96h)
H I

$\mathrm{Te}$

$\mathrm{Te}$

$\mathrm{Te}$

$\mathrm{Te}$

$\mathrm{Te}$

$\mathrm{Te}$

$\mathrm{Te}$

$\mathrm{Te}$

H I

H I

H I

H I

H I

H I

H I

H I

H I

H I 


\begin{tabular}{|c|c|c|c|c|c|c|}
\hline $\mathbf{B}_{20}$ & $=$ & $\begin{array}{l}\left(x_{2}+y_{2}-z_{2}\right) \mathbf{a}_{1}+ \\
\left(x_{2}-y_{2}+z_{2}\right) \mathbf{a}_{2}+ \\
\left(-x_{2}-y_{2}-z_{2}\right) \mathbf{a}_{3}\end{array}$ & $=$ & $-y_{2} a \hat{\mathbf{x}}-z_{2} a \hat{\mathbf{y}}+x_{2} a \hat{\mathbf{z}}$ & $(96 h)$ & H I \\
\hline $\mathbf{B}_{21}$ & $=$ & $\begin{array}{c}\left(\frac{1}{4}+x_{2}-y_{2}-z_{2}\right) \mathbf{a}_{1}+ \\
\left(\frac{1}{4}-x_{2}+y_{2}-z_{2}\right) \mathbf{a}_{2}+ \\
\left(\frac{1}{4}+x_{2}+y_{2}+z_{2}\right) \mathbf{a}_{3}\end{array}$ & $=$ & $\begin{array}{c}\left(\frac{1}{4}+y_{2}\right) a \hat{\mathbf{x}}+\left(\frac{1}{4}+x_{2}\right) a \hat{\mathbf{y}}+ \\
\left(\frac{1}{4}-z_{2}\right) a \hat{\mathbf{z}}\end{array}$ & $(96 h)$ & H I \\
\hline $\mathbf{B}_{22}$ & $=$ & $\begin{array}{c}\left(\frac{1}{4}-x_{2}+y_{2}-z_{2}\right) \mathbf{a}_{1}+ \\
\left(\frac{1}{4}+x_{2}-y_{2}-z_{2}\right) \mathbf{a}_{2}+ \\
\left(\frac{1}{4}-x_{2}-y_{2}+z_{2}\right) \mathbf{a}_{3}\end{array}$ & $=$ & $\begin{array}{c}\left(\frac{1}{4}-y_{2}\right) a \hat{\mathbf{x}}+\left(\frac{1}{4}-x_{2}\right) a \hat{\mathbf{y}}+ \\
\left(\frac{1}{4}-z_{2}\right) a \hat{\mathbf{z}}\end{array}$ & $(96 h)$ & H I \\
\hline $\mathbf{B}_{23}$ & $=$ & $\begin{array}{c}\left(\frac{1}{4}-x_{2}-y_{2}+z_{2}\right) \mathbf{a}_{1}+ \\
\left(\frac{1}{4}+x_{2}+y_{2}+z_{2}\right) \mathbf{a}_{2}+ \\
\left(\frac{1}{4}-x_{2}+y_{2}-z_{2}\right) \mathbf{a}_{3}\end{array}$ & $=$ & $\begin{array}{c}\left(\frac{1}{4}+y_{2}\right) a \hat{\mathbf{x}}+\left(\frac{1}{4}-x_{2}\right) a \hat{\mathbf{y}}+ \\
\left(\frac{1}{4}+z_{2}\right) a \hat{\mathbf{z}}\end{array}$ & $(96 h)$ & H I \\
\hline $\mathbf{B}_{24}$ & $=$ & $\begin{array}{c}\left(\frac{1}{4}+x_{2}+y_{2}+z_{2}\right) \mathbf{a}_{1}+ \\
\left(\frac{1}{4}-x_{2}-y_{2}+z_{2}\right) \mathbf{a}_{2}+ \\
\left(\frac{1}{4}+x_{2}-y_{2}-z_{2}\right) \mathbf{a}_{3}\end{array}$ & $=$ & $\begin{array}{c}\left(\frac{1}{4}-y_{2}\right) a \hat{\mathbf{x}}+\left(\frac{1}{4}+x_{2}\right) a \hat{\mathbf{y}}+ \\
\left(\frac{1}{4}+z_{2}\right) a \hat{\mathbf{z}}\end{array}$ & $(96 h)$ & H I \\
\hline $\mathbf{B}_{25}$ & $=$ & $\begin{array}{c}\left(\frac{1}{4}-x_{2}-y_{2}+z_{2}\right) \mathbf{a}_{1}+ \\
\left(\frac{1}{4}+x_{2}-y_{2}-z_{2}\right) \mathbf{a}_{2}+ \\
\left(\frac{1}{4}+x_{2}+y_{2}+z_{2}\right) \mathbf{a}_{3}\end{array}$ & $=$ & $\begin{array}{c}\left(\frac{1}{4}+x_{2}\right) a \hat{\mathbf{x}}+\left(\frac{1}{4}+z_{2}\right) a \hat{\mathbf{y}}+ \\
\left(\frac{1}{4}-y_{2}\right) a \hat{\mathbf{z}}\end{array}$ & $(96 h)$ & H I \\
\hline $\mathbf{B}_{26}$ & $=$ & $\begin{array}{c}\left(\frac{1}{4}+x_{2}+y_{2}+z_{2}\right) \mathbf{a}_{1}+ \\
\left(\frac{1}{4}-x_{2}+y_{2}-z_{2}\right) \mathbf{a}_{2}+ \\
\left(\frac{1}{4}-x_{2}-y_{2}+z_{2}\right) \mathbf{a}_{3}\end{array}$ & $=$ & $\begin{array}{c}\left(\frac{1}{4}-x_{2}\right) a \hat{\mathbf{x}}+\left(\frac{1}{4}+z_{2}\right) a \hat{\mathbf{y}}+ \\
\left(\frac{1}{4}+y_{2}\right) a \hat{\mathbf{z}}\end{array}$ & $(96 h)$ & H I \\
\hline $\mathbf{B}_{27}$ & $=$ & $\begin{array}{c}\left(\frac{1}{4}+x_{2}-y_{2}-z_{2}\right) \mathbf{a}_{1}+ \\
\left(\frac{1}{4}-x_{2}-y_{2}+z_{2}\right) \mathbf{a}_{2}+ \\
\left(\frac{1}{4}-x_{2}+y_{2}-z_{2}\right) \mathbf{a}_{3}\end{array}$ & $=$ & $\begin{array}{c}\left(\frac{1}{4}-x_{2}\right) a \hat{\mathbf{x}}+\left(\frac{1}{4}-z_{2}\right) a \hat{\mathbf{y}}+ \\
\left(\frac{1}{4}-y_{2}\right) a \hat{\mathbf{z}}\end{array}$ & $(96 h)$ & $\mathrm{H} \mathrm{I}$ \\
\hline $\mathbf{B}_{28}$ & $=$ & $\begin{array}{c}\left(\frac{1}{4}-x_{2}+y_{2}-z_{2}\right) \mathbf{a}_{1}+ \\
\left(\frac{1}{4}+x_{2}+y_{2}+z_{2}\right) \mathbf{a}_{2}+ \\
\left(\frac{1}{4}+x_{2}-y_{2}-z_{2}\right) \mathbf{a}_{3}\end{array}$ & $=$ & $\begin{array}{c}\left(\frac{1}{4}+x_{2}\right) a \hat{\mathbf{x}}+\left(\frac{1}{4}-z_{2}\right) a \hat{\mathbf{y}}+ \\
\left(\frac{1}{4}+y_{2}\right) a \hat{\mathbf{z}}\end{array}$ & $(96 h)$ & $\mathrm{H} \mathrm{I}$ \\
\hline $\mathbf{B}_{29}$ & $=$ & $\begin{array}{c}\left(\frac{1}{4}-x_{2}+y_{2}-z_{2}\right) \mathbf{a}_{1}+ \\
\left(\frac{1}{4}-x_{2}-y_{2}+z_{2}\right) \mathbf{a}_{2}+ \\
\left(\frac{1}{4}+x_{2}+y_{2}+z_{2}\right) \mathbf{a}_{3}\end{array}$ & $=$ & $\begin{array}{c}\left(\frac{1}{4}+z_{2}\right) a \hat{\mathbf{x}}+\left(\frac{1}{4}+y_{2}\right) a \hat{\mathbf{y}}+ \\
\left(\frac{1}{4}-x_{2}\right) a \hat{\mathbf{z}}\end{array}$ & $(96 h)$ & $\mathrm{H} \mathrm{I}$ \\
\hline $\mathbf{B}_{30}$ & $=$ & $\begin{array}{c}\left(\frac{1}{4}+x_{2}-y_{2}-z_{2}\right) \mathbf{a}_{1}+ \\
\left(\frac{1}{4}+x_{2}+y_{2}+z_{2}\right) \mathbf{a}_{2}+ \\
\left(\frac{1}{4}-x_{2}-y_{2}+z_{2}\right) \mathbf{a}_{3}\end{array}$ & $=$ & $\begin{array}{c}\left(\frac{1}{4}+z_{2}\right) a \hat{\mathbf{x}}+\left(\frac{1}{4}-y_{2}\right) a \hat{\mathbf{y}}+ \\
\left(\frac{1}{4}+x_{2}\right) a \hat{\mathbf{z}}\end{array}$ & $(96 h)$ & $\mathrm{H} \mathrm{I}$ \\
\hline $\mathbf{B}_{31}$ & $=$ & $\begin{array}{c}\left(\frac{1}{4}+x_{2}+y_{2}+z_{2}\right) \mathbf{a}_{1}+ \\
\left(\frac{1}{4}+x_{2}-y_{2}-z_{2}\right) \mathbf{a}_{2}+ \\
\left(\frac{1}{4}-x_{2}+y_{2}-z_{2}\right) \mathbf{a}_{3}\end{array}$ & $=$ & $\begin{array}{c}\left(\frac{1}{4}-z_{2}\right) a \hat{\mathbf{x}}+\left(\frac{1}{4}+y_{2}\right) a \hat{\mathbf{y}}+ \\
\left(\frac{1}{4}+x_{2}\right) a \hat{\mathbf{z}}\end{array}$ & $(96 h)$ & H I \\
\hline $\mathbf{B}_{32}$ & $=$ & $\begin{array}{c}\left(\frac{1}{4}-x_{2}-y_{2}+z_{2}\right) \mathbf{a}_{1}+ \\
\left(\frac{1}{4}-x_{2}+y_{2}-z_{2}\right) \mathbf{a}_{2}+ \\
\left(\frac{1}{4}+x_{2}-y_{2}-z_{2}\right) \mathbf{a}_{3}\end{array}$ & $=$ & $\begin{array}{c}\left(\frac{1}{4}-z_{2}\right) a \hat{\mathbf{x}}+\left(\frac{1}{4}-y_{2}\right) a \hat{\mathbf{y}}+ \\
\left(\frac{1}{4}-x_{2}\right) a \hat{\mathbf{z}}\end{array}$ & $(96 h)$ & $\mathrm{H} \mathrm{I}$ \\
\hline $\mathbf{B}_{33}$ & $=$ & $\begin{array}{c}\left(-x_{3}+y_{3}+z_{3}\right) \mathbf{a}_{1}+ \\
\left(x_{3}-y_{3}+z_{3}\right) \mathbf{a}_{2}+ \\
\left(x_{3}+y_{3}-z_{3}\right) \mathbf{a}_{3}\end{array}$ & $=$ & $x_{3} a \hat{\mathbf{x}}+y_{3} a \hat{\mathbf{y}}+z_{3} a \hat{\mathbf{z}}$ & $(96 h)$ & H II \\
\hline
\end{tabular}




\begin{tabular}{|c|c|c|c|c|c|c|}
\hline $\mathbf{B}_{34}$ & $=$ & $\begin{array}{c}\left(x_{3}-y_{3}+z_{3}\right) \mathbf{a}_{1}+ \\
\left(-x_{3}+y_{3}+z_{3}\right) \mathbf{a}_{2}+ \\
\left(-x_{3}-y_{3}-z_{3}\right) \mathbf{a}_{3}\end{array}$ & $=$ & $-x_{3} a \hat{\mathbf{x}}-y_{3} a \hat{\mathbf{y}}+z_{3} a \hat{\mathbf{z}}$ & $(96 h)$ & H II \\
\hline $\mathbf{B}_{35}$ & $=$ & $\begin{array}{c}\left(x_{3}+y_{3}-z_{3}\right) \mathbf{a}_{1}+ \\
\left(-x_{3}-y_{3}-z_{3}\right) \mathbf{a}_{2}+ \\
\left(-x_{3}+y_{3}+z_{3}\right) \mathbf{a}_{3}\end{array}$ & $=$ & $-x_{3} a \hat{\mathbf{x}}+y_{3} a \hat{\mathbf{y}}-z_{3} a \hat{\mathbf{z}}$ & $(96 h)$ & H II \\
\hline $\mathbf{B}_{36}$ & $=$ & $\begin{array}{c}\left(-x_{3}-y_{3}-z_{3}\right) \mathbf{a}_{1}+ \\
\left(x_{3}+y_{3}-z_{3}\right) \mathbf{a}_{2}+ \\
\left(x_{3}-y_{3}+z_{3}\right) \mathbf{a}_{3}\end{array}$ & $=$ & $x_{3} a \hat{\mathbf{x}}-y_{3} a \hat{\mathbf{y}}-z_{3} a \hat{\mathbf{z}}$ & $(96 h)$ & H II \\
\hline $\mathbf{B}_{37}$ & $=$ & $\begin{array}{c}\left(x_{3}+y_{3}-z_{3}\right) \mathbf{a}_{1}+ \\
\left(-x_{3}+y_{3}+z_{3}\right) \mathbf{a}_{2}+ \\
\left(x_{3}-y_{3}+z_{3}\right) \mathbf{a}_{3}\end{array}$ & $=$ & $z_{3} a \hat{\mathbf{x}}+x_{3} a \hat{\mathbf{y}}+y_{3} a \hat{\mathbf{z}}$ & $(96 h)$ & H II \\
\hline $\mathbf{B}_{38}$ & $=$ & $\begin{array}{c}\left(-x_{3}-y_{3}-z_{3}\right) \mathbf{a}_{1}+ \\
\left(x_{3}-y_{3}+z_{3}\right) \mathbf{a}_{2}+ \\
\left(-x_{3}+y_{3}+z_{3}\right) \mathbf{a}_{3}\end{array}$ & $=$ & $z_{3} a \hat{\mathbf{x}}-x_{3} a \hat{\mathbf{y}}-y_{3} a \hat{\mathbf{z}}$ & $(96 h)$ & H II \\
\hline $\mathbf{B}_{39}$ & $=$ & $\begin{array}{c}\left(-x_{3}+y_{3}+z_{3}\right) \mathbf{a}_{1}+ \\
\left(x_{3}+y_{3}-z_{3}\right) \mathbf{a}_{2}+ \\
\left(-x_{3}-y_{3}-z_{3}\right) \mathbf{a}_{3}\end{array}$ & $=$ & $-z_{3} a \hat{\mathbf{x}}-x_{3} a \hat{\mathbf{y}}+y_{3} a \hat{\mathbf{z}}$ & $(96 h)$ & H II \\
\hline $\mathbf{B}_{40}$ & $=$ & $\begin{array}{c}\left(x_{3}-y_{3}+z_{3}\right) \mathbf{a}_{1}+ \\
\left(-x_{3}-y_{3}-z_{3}\right) \mathbf{a}_{2}+ \\
\left(x_{3}+y_{3}-z_{3}\right) \mathbf{a}_{3}\end{array}$ & $=$ & $-z_{3} a \hat{\mathbf{x}}+x_{3} a \hat{\mathbf{y}}-y_{3} a \hat{\mathbf{z}}$ & $(96 h)$ & H II \\
\hline $\mathbf{B}_{41}$ & $=$ & $\begin{array}{l}\left(x_{3}-y_{3}+z_{3}\right) \mathbf{a}_{1}+ \\
\left(x_{3}+y_{3}-z_{3}\right) \mathbf{a}_{2}+ \\
\left(-x_{3}+y_{3}+z_{3}\right) \mathbf{a}_{3}\end{array}$ & $=$ & $y_{3} a \hat{\mathbf{x}}+z_{3} a \hat{\mathbf{y}}+x_{3} a \hat{\mathbf{z}}$ & $(96 h)$ & H II \\
\hline $\mathbf{B}_{42}$ & $=$ & $\begin{array}{l}\left(-x_{3}+y_{3}+z_{3}\right) \mathbf{a}_{1}+ \\
\left(-x_{3}-y_{3}-z_{3}\right) \mathbf{a}_{2}+ \\
\left(x_{3}-y_{3}+z_{3}\right) \mathbf{a}_{3}\end{array}$ & $=$ & $-y_{3} a \hat{\mathbf{x}}+z_{3} a \hat{\mathbf{y}}-x_{3} a \hat{\mathbf{z}}$ & $(96 h)$ & H II \\
\hline $\mathbf{B}_{43}$ & $=$ & $\begin{array}{l}\left(-x_{3}-y_{3}-z_{3}\right) \mathbf{a}_{1}+ \\
\left(-x_{3}+y_{3}+z_{3}\right) \mathbf{a}_{2}+ \\
\left(x_{3}+y_{3}-z_{3}\right) \mathbf{a}_{3}\end{array}$ & $=$ & $y_{3} a \hat{\mathbf{x}}-z_{3} a \hat{\mathbf{y}}-x_{3} a \hat{\mathbf{z}}$ & $(96 h)$ & H II \\
\hline $\mathbf{B}_{44}$ & $=$ & $\begin{array}{l}\left(x_{3}+y_{3}-z_{3}\right) \mathbf{a}_{1}+ \\
\left(x_{3}-y_{3}+z_{3}\right) \mathbf{a}_{2}+ \\
\left(-x_{3}-y_{3}-z_{3}\right) \mathbf{a}_{3}\end{array}$ & $=$ & $-y_{3} a \hat{\mathbf{x}}-z_{3} a \hat{\mathbf{y}}+x_{3} a \hat{\mathbf{z}}$ & $(96 h)$ & H II \\
\hline $\mathbf{B}_{45}$ & $=$ & $\begin{array}{c}\left(\frac{1}{4}+x_{3}-y_{3}-z_{3}\right) \mathbf{a}_{1}+ \\
\left(\frac{1}{4}-x_{3}+y_{3}-z_{3}\right) \mathbf{a}_{2}+ \\
\left(\frac{1}{4}+x_{3}+y_{3}+z_{3}\right) \mathbf{a}_{3}\end{array}$ & $=$ & $\begin{array}{c}\left(\frac{1}{4}+y_{3}\right) a \hat{\mathbf{x}}+\left(\frac{1}{4}+x_{3}\right) a \hat{\mathbf{y}}+ \\
\left(\frac{1}{4}-z_{3}\right) a \hat{\mathbf{z}}\end{array}$ & $(96 h)$ & H II \\
\hline $\mathbf{B}_{46}$ & $=$ & $\begin{array}{c}\left(\frac{1}{4}-x_{3}+y_{3}-z_{3}\right) \mathbf{a}_{1}+ \\
\left(\frac{1}{4}+x_{3}-y_{3}-z_{3}\right) \mathbf{a}_{2}+ \\
\left(\frac{1}{4}-x_{3}-y_{3}+z_{3}\right) \mathbf{a}_{3}\end{array}$ & $=$ & $\begin{array}{c}\left(\frac{1}{4}-y_{3}\right) a \hat{\mathbf{x}}+\left(\frac{1}{4}-x_{3}\right) a \hat{\mathbf{y}}+ \\
\left(\frac{1}{4}-z_{3}\right) a \hat{\mathbf{z}}\end{array}$ & $(96 h)$ & H II \\
\hline $\mathbf{B}_{47}$ & $=$ & $\begin{array}{c}\left(\frac{1}{4}-x_{3}-y_{3}+z_{3}\right) \mathbf{a}_{1}+ \\
\left(\frac{1}{4}+x_{3}+y_{3}+z_{3}\right) \mathbf{a}_{2}+ \\
\left(\frac{1}{4}-x_{3}+y_{3}-z_{3}\right) \mathbf{a}_{3}\end{array}$ & $=$ & $\begin{array}{c}\left(\frac{1}{4}+y_{3}\right) a \hat{\mathbf{x}}+\left(\frac{1}{4}-x_{3}\right) a \hat{\mathbf{y}}+ \\
\left(\frac{1}{4}+z_{3}\right) a \hat{\mathbf{z}}\end{array}$ & $(96 h)$ & H II \\
\hline $\mathbf{B}_{48}$ & $=$ & $\begin{array}{c}\left(\frac{1}{4}+x_{3}+y_{3}+z_{3}\right) \mathbf{a}_{1}+ \\
\left(\frac{1}{4}-x_{3}-y_{3}+z_{3}\right) \mathbf{a}_{2}+ \\
\left(\frac{1}{4}+x_{3}-y_{3}-z_{3}\right) \mathbf{a}_{3}\end{array}$ & $=$ & $\begin{array}{c}\left(\frac{1}{4}-y_{3}\right) a \hat{\mathbf{x}}+\left(\frac{1}{4}+x_{3}\right) a \hat{\mathbf{y}}+ \\
\left(\frac{1}{4}+z_{3}\right) a \hat{\mathbf{z}}\end{array}$ & $(96 h)$ & H II \\
\hline $\mathbf{B}_{49}$ & $=$ & $\begin{array}{c}\left(\frac{1}{4}-x_{3}-y_{3}+z_{3}\right) \mathbf{a}_{1}+ \\
\left(\frac{1}{4}+x_{3}-y_{3}-z_{3}\right) \mathbf{a}_{2}+ \\
\left(\frac{1}{4}+x_{3}+y_{3}+z_{3}\right) \mathbf{a}_{3}\end{array}$ & $=$ & $\begin{array}{c}\left(\frac{1}{4}+x_{3}\right) a \hat{\mathbf{x}}+\left(\frac{1}{4}+z_{3}\right) a \hat{\mathbf{y}}+ \\
\left(\frac{1}{4}-y_{3}\right) a \hat{\mathbf{z}}\end{array}$ & $(96 h)$ & H II \\
\hline
\end{tabular}




\begin{tabular}{|c|c|c|c|c|c|c|}
\hline $\mathbf{B}_{50}$ & $=$ & $\begin{array}{c}\left(\frac{1}{4}+x_{3}+y_{3}+z_{3}\right) \mathbf{a}_{1}+ \\
\left(\frac{1}{4}-x_{3}+y_{3}-z_{3}\right) \mathbf{a}_{2}+ \\
\left(\frac{1}{4}-x_{3}-y_{3}+z_{3}\right) \mathbf{a}_{3}\end{array}$ & $=$ & $\begin{array}{c}\left(\frac{1}{4}-x_{3}\right) a \hat{\mathbf{x}}+\left(\frac{1}{4}+z_{3}\right) a \hat{\mathbf{y}}+ \\
\left(\frac{1}{4}+y_{3}\right) a \hat{\mathbf{z}}\end{array}$ & $(96 h)$ & H II \\
\hline $\mathbf{B}_{51}$ & $=$ & $\begin{array}{c}\left(\frac{1}{4}+x_{3}-y_{3}-z_{3}\right) \mathbf{a}_{1}+ \\
\left(\frac{1}{4}-x_{3}-y_{3}+z_{3}\right) \mathbf{a}_{2}+ \\
\left(\frac{1}{4}-x_{3}+y_{3}-z_{3}\right) \mathbf{a}_{3}\end{array}$ & $=$ & $\begin{array}{c}\left(\frac{1}{4}-x_{3}\right) a \hat{\mathbf{x}}+\left(\frac{1}{4}-z_{3}\right) a \hat{\mathbf{y}}+ \\
\left(\frac{1}{4}-y_{3}\right) a \hat{\mathbf{z}}\end{array}$ & $(96 h)$ & H II \\
\hline $\mathbf{B}_{52}$ & $=$ & $\begin{array}{c}\left(\frac{1}{4}-x_{3}+y_{3}-z_{3}\right) \mathbf{a}_{1}+ \\
\left(\frac{1}{4}+x_{3}+y_{3}+z_{3}\right) \mathbf{a}_{2}+ \\
\left(\frac{1}{4}+x_{3}-y_{3}-z_{3}\right) \mathbf{a}_{3}\end{array}$ & $=$ & $\begin{array}{c}\left(\frac{1}{4}+x_{3}\right) a \hat{\mathbf{x}}+\left(\frac{1}{4}-z_{3}\right) a \hat{\mathbf{y}}+ \\
\left(\frac{1}{4}+y_{3}\right) a \hat{\mathbf{z}}\end{array}$ & $(96 h)$ & H II \\
\hline $\mathbf{B}_{53}$ & $=$ & $\begin{array}{c}\left(\frac{1}{4}-x_{3}+y_{3}-z_{3}\right) \mathbf{a}_{1}+ \\
\left(\frac{1}{4}-x_{3}-y_{3}+z_{3}\right) \mathbf{a}_{2}+ \\
\left(\frac{1}{4}+x_{3}+y_{3}+z_{3}\right) \mathbf{a}_{3}\end{array}$ & $=$ & $\begin{array}{c}\left(\frac{1}{4}+z_{3}\right) a \hat{\mathbf{x}}+\left(\frac{1}{4}+y_{3}\right) a \hat{\mathbf{y}}+ \\
\left(\frac{1}{4}-x_{3}\right) a \hat{\mathbf{z}}\end{array}$ & $(96 h)$ & H II \\
\hline $\mathbf{B}_{54}$ & $=$ & $\begin{array}{c}\left(\frac{1}{4}+x_{3}-y_{3}-z_{3}\right) \mathbf{a}_{1}+ \\
\left(\frac{1}{4}+x_{3}+y_{3}+z_{3}\right) \mathbf{a}_{2}+ \\
\left(\frac{1}{4}-x_{3}-y_{3}+z_{3}\right) \mathbf{a}_{3}\end{array}$ & $=$ & $\begin{array}{c}\left(\frac{1}{4}+z_{3}\right) a \hat{\mathbf{x}}+\left(\frac{1}{4}-y_{3}\right) a \hat{\mathbf{y}}+ \\
\left(\frac{1}{4}+x_{3}\right) a \hat{\mathbf{z}}\end{array}$ & $(96 h)$ & H II \\
\hline $\mathbf{B}_{55}$ & $=$ & $\begin{array}{c}\left(\frac{1}{4}+x_{3}+y_{3}+z_{3}\right) \mathbf{a}_{1}+ \\
\left(\frac{1}{4}+x_{3}-y_{3}-z_{3}\right) \mathbf{a}_{2}+ \\
\left(\frac{1}{4}-x_{3}+y_{3}-z_{3}\right) \mathbf{a}_{3}\end{array}$ & $=$ & $\begin{array}{c}\left(\frac{1}{4}-z_{3}\right) a \hat{\mathbf{x}}+\left(\frac{1}{4}+y_{3}\right) a \hat{\mathbf{y}}+ \\
\left(\frac{1}{4}+x_{3}\right) a \hat{\mathbf{z}}\end{array}$ & $(96 h)$ & H II \\
\hline $\mathbf{B}_{56}$ & $=$ & $\begin{array}{c}\left(\frac{1}{4}-x_{3}-y_{3}+z_{3}\right) \mathbf{a}_{1}+ \\
\left(\frac{1}{4}-x_{3}+y_{3}-z_{3}\right) \mathbf{a}_{2}+ \\
\left(\frac{1}{4}+x_{3}-y_{3}-z_{3}\right) \mathbf{a}_{3}\end{array}$ & $=$ & $\begin{array}{c}\left(\frac{1}{4}-z_{3}\right) a \hat{\mathbf{x}}+\left(\frac{1}{4}-y_{3}\right) a \hat{\mathbf{y}}+ \\
\left(\frac{1}{4}-x_{3}\right) a \hat{\mathbf{z}}\end{array}$ & $(96 h)$ & H II \\
\hline $\mathbf{B}_{57}$ & $=$ & $\begin{array}{c}\left(-x_{4}+y_{4}+z_{4}\right) \mathbf{a}_{1}+ \\
\left(x_{4}-y_{4}+z_{4}\right) \mathbf{a}_{2}+ \\
\left(x_{4}+y_{4}-z_{4}\right) \mathbf{a}_{3}\end{array}$ & $=$ & $x_{4} a \hat{\mathbf{x}}+y_{4} a \hat{\mathbf{y}}+z_{4} a \hat{\mathbf{z}}$ & $(96 h)$ & H III \\
\hline $\mathbf{B}_{58}$ & $=$ & $\begin{array}{c}\left(x_{4}-y_{4}+z_{4}\right) \mathbf{a}_{1}+ \\
\left(-x_{4}+y_{4}+z_{4}\right) \mathbf{a}_{2}+ \\
\left(-x_{4}-y_{4}-z_{4}\right) \mathbf{a}_{3}\end{array}$ & $=$ & $-x_{4} a \hat{\mathbf{x}}-y_{4} a \hat{\mathbf{y}}+z_{4} a \hat{\mathbf{z}}$ & $(96 h)$ & H III \\
\hline $\mathbf{B}_{59}$ & $=$ & $\begin{array}{c}\left(x_{4}+y_{4}-z_{4}\right) \mathbf{a}_{1}+ \\
\left(-x_{4}-y_{4}-z_{4}\right) \mathbf{a}_{2}+ \\
\left(-x_{4}+y_{4}+z_{4}\right) \mathbf{a}_{3}\end{array}$ & $=$ & $-x_{4} a \hat{\mathbf{x}}+y_{4} a \hat{\mathbf{y}}-z_{4} a \hat{\mathbf{z}}$ & $(96 h)$ & H III \\
\hline $\mathbf{B}_{60}$ & $=$ & $\begin{array}{c}\left(-x_{4}-y_{4}-z_{4}\right) \mathbf{a}_{1}+ \\
\left(x_{4}+y_{4}-z_{4}\right) \mathbf{a}_{2}+ \\
\left(x_{4}-y_{4}+z_{4}\right) \mathbf{a}_{3}\end{array}$ & $=$ & $x_{4} a \hat{\mathbf{x}}-y_{4} a \hat{\mathbf{y}}-z_{4} a \hat{\mathbf{z}}$ & $(96 h)$ & H III \\
\hline $\mathbf{B}_{61}$ & $=$ & $\begin{array}{c}\left(x_{4}+y_{4}-z_{4}\right) \mathbf{a}_{1}+ \\
\left(-x_{4}+y_{4}+z_{4}\right) \mathbf{a}_{2}+ \\
\left(x_{4}-y_{4}+z_{4}\right) \mathbf{a}_{3}\end{array}$ & $=$ & $z_{4} a \hat{\mathbf{x}}+x_{4} a \hat{\mathbf{y}}+y_{4} a \hat{\mathbf{z}}$ & $(96 h)$ & H III \\
\hline $\mathbf{B}_{62}$ & $=$ & $\begin{array}{c}\left(-x_{4}-y_{4}-z_{4}\right) \mathbf{a}_{1}+ \\
\left(x_{4}-y_{4}+z_{4}\right) \mathbf{a}_{2}+ \\
\left(-x_{4}+y_{4}+z_{4}\right) \mathbf{a}_{3}\end{array}$ & $=$ & $z_{4} a \hat{\mathbf{x}}-x_{4} a \hat{\mathbf{y}}-y_{4} a \hat{\mathbf{z}}$ & $(96 h)$ & H III \\
\hline $\mathbf{B}_{63}$ & $=$ & $\begin{array}{c}\left(-x_{4}+y_{4}+z_{4}\right) \mathbf{a}_{1}+ \\
\left(x_{4}+y_{4}-z_{4}\right) \mathbf{a}_{2}+ \\
\left(-x_{4}-y_{4}-z_{4}\right) \mathbf{a}_{3}\end{array}$ & $=$ & $-z_{4} a \hat{\mathbf{x}}-x_{4} a \hat{\mathbf{y}}+y_{4} a \hat{\mathbf{z}}$ & $(96 h)$ & H III \\
\hline $\mathbf{B}_{64}$ & $=$ & $\begin{array}{c}\left(x_{4}-y_{4}+z_{4}\right) \mathbf{a}_{1}+ \\
\left(-x_{4}-y_{4}-z_{4}\right) \mathbf{a}_{2}+ \\
\left(x_{4}+y_{4}-z_{4}\right) \mathbf{a}_{3}\end{array}$ & $=$ & $-z_{4} a \hat{\mathbf{x}}+x_{4} a \hat{\mathbf{y}}-y_{4} a \hat{\mathbf{z}}$ & $(96 h)$ & H III \\
\hline
\end{tabular}




\begin{tabular}{|c|c|c|c|c|c|c|}
\hline $\mathbf{B}_{65}$ & $=$ & $\begin{array}{c}\left(x_{4}-y_{4}+z_{4}\right) \mathbf{a}_{1}+ \\
\left(x_{4}+y_{4}-z_{4}\right) \mathbf{a}_{2}+ \\
\left(-x_{4}+y_{4}+z_{4}\right) \mathbf{a}_{3}\end{array}$ & $=$ & $y_{4} a \hat{\mathbf{x}}+z_{4} a \hat{\mathbf{y}}+x_{4} a \hat{\mathbf{z}}$ & $(96 h)$ & H III \\
\hline $\mathbf{B}_{66}$ & $=$ & $\begin{array}{c}\left(-x_{4}+y_{4}+z_{4}\right) \mathbf{a}_{1}+ \\
\left(-x_{4}-y_{4}-z_{4}\right) \mathbf{a}_{2}+ \\
\left(x_{4}-y_{4}+z_{4}\right) \mathbf{a}_{3}\end{array}$ & $=$ & $-y_{4} a \hat{\mathbf{x}}+z_{4} a \hat{\mathbf{y}}-x_{4} a \hat{\mathbf{z}}$ & $(96 h)$ & H III \\
\hline $\mathbf{B}_{67}$ & $=$ & $\begin{array}{c}\left(-x_{4}-y_{4}-z_{4}\right) \mathbf{a}_{1}+ \\
\left(-x_{4}+y_{4}+z_{4}\right) \mathbf{a}_{2}+ \\
\left(x_{4}+y_{4}-z_{4}\right) \mathbf{a}_{3}\end{array}$ & $=$ & $y_{4} a \hat{\mathbf{x}}-z_{4} a \hat{\mathbf{y}}-x_{4} a \hat{\mathbf{z}}$ & $(96 h)$ & H III \\
\hline $\mathbf{B}_{68}$ & $=$ & $\begin{array}{c}\left(x_{4}+y_{4}-z_{4}\right) \mathbf{a}_{1}+ \\
\left(x_{4}-y_{4}+z_{4}\right) \mathbf{a}_{2}+ \\
\left(-x_{4}-y_{4}-z_{4}\right) \mathbf{a}_{3}\end{array}$ & $=$ & $-y_{4} a \hat{\mathbf{x}}-z_{4} a \hat{\mathbf{y}}+x_{4} a \hat{\mathbf{z}}$ & $(96 h)$ & H III \\
\hline $\mathbf{B}_{69}$ & $=$ & $\begin{array}{c}\left(\frac{1}{4}+x_{4}-y_{4}-z_{4}\right) \mathbf{a}_{1}+ \\
\left(\frac{1}{4}-x_{4}+y_{4}-z_{4}\right) \mathbf{a}_{2}+ \\
\left(\frac{1}{4}+x_{4}+y_{4}+z_{4}\right) \mathbf{a}_{3}\end{array}$ & $=$ & $\begin{array}{c}\left(\frac{1}{4}+y_{4}\right) a \hat{\mathbf{x}}+\left(\frac{1}{4}+x_{4}\right) a \hat{\mathbf{y}}+ \\
\left(\frac{1}{4}-z_{4}\right) a \hat{\mathbf{z}}\end{array}$ & $(96 h)$ & H III \\
\hline $\mathbf{B}_{70}$ & $=$ & $\begin{array}{c}\left(\frac{1}{4}-x_{4}+y_{4}-z_{4}\right) \mathbf{a}_{1}+ \\
\left(\frac{1}{4}+x_{4}-y_{4}-z_{4}\right) \mathbf{a}_{2}+ \\
\left(\frac{1}{4}-x_{4}-y_{4}+z_{4}\right) \mathbf{a}_{3}\end{array}$ & $=$ & $\begin{array}{c}\left(\frac{1}{4}-y_{4}\right) a \hat{\mathbf{x}}+\left(\frac{1}{4}-x_{4}\right) a \hat{\mathbf{y}}+ \\
\left(\frac{1}{4}-z_{4}\right) a \hat{\mathbf{z}}\end{array}$ & $(96 h)$ & H III \\
\hline $\mathbf{B}_{71}$ & $=$ & $\begin{array}{c}\left(\frac{1}{4}-x_{4}-y_{4}+z_{4}\right) \mathbf{a}_{1}+ \\
\left(\frac{1}{4}+x_{4}+y_{4}+z_{4}\right) \mathbf{a}_{2}+ \\
\left(\frac{1}{4}-x_{4}+y_{4}-z_{4}\right) \mathbf{a}_{3}\end{array}$ & $=$ & $\begin{array}{c}\left(\frac{1}{4}+y_{4}\right) a \hat{\mathbf{x}}+\left(\frac{1}{4}-x_{4}\right) a \hat{\mathbf{y}}+ \\
\left(\frac{1}{4}+z_{4}\right) a \hat{\mathbf{z}}\end{array}$ & $(96 h)$ & H III \\
\hline $\mathbf{B}_{72}$ & $=$ & $\begin{array}{c}\left(\frac{1}{4}+x_{4}+y_{4}+z_{4}\right) \mathbf{a}_{1}+ \\
\left(\frac{1}{4}-x_{4}-y_{4}+z_{4}\right) \mathbf{a}_{2}+ \\
\left(\frac{1}{4}+x_{4}-y_{4}-z_{4}\right) \mathbf{a}_{3}\end{array}$ & $=$ & $\begin{array}{c}\left(\frac{1}{4}-y_{4}\right) a \hat{\mathbf{x}}+\left(\frac{1}{4}+x_{4}\right) a \hat{\mathbf{y}}+ \\
\left(\frac{1}{4}+z_{4}\right) a \hat{\mathbf{z}}\end{array}$ & $(96 h)$ & H III \\
\hline $\mathbf{B}_{73}$ & $=$ & $\begin{array}{c}\left(\frac{1}{4}-x_{4}-y_{4}+z_{4}\right) \mathbf{a}_{1}+ \\
\left(\frac{1}{4}+x_{4}-y_{4}-z_{4}\right) \mathbf{a}_{2}+ \\
\left(\frac{1}{4}+x_{4}+y_{4}+z_{4}\right) \mathbf{a}_{3}\end{array}$ & $=$ & $\begin{array}{c}\left(\frac{1}{4}+x_{4}\right) a \hat{\mathbf{x}}+\left(\frac{1}{4}+z_{4}\right) a \hat{\mathbf{y}}+ \\
\left(\frac{1}{4}-y_{4}\right) a \hat{\mathbf{z}}\end{array}$ & $(96 h)$ & H III \\
\hline $\mathbf{B}_{74}$ & $=$ & $\begin{array}{c}\left(\frac{1}{4}+x_{4}+y_{4}+z_{4}\right) \mathbf{a}_{1}+ \\
\left(\frac{1}{4}-x_{4}+y_{4}-z_{4}\right) \mathbf{a}_{2}+ \\
\left(\frac{1}{4}-x_{4}-y_{4}+z_{4}\right) \mathbf{a}_{3}\end{array}$ & $=$ & $\begin{array}{c}\left(\frac{1}{4}-x_{4}\right) a \hat{\mathbf{x}}+\left(\frac{1}{4}+z_{4}\right) a \hat{\mathbf{y}}+ \\
\left(\frac{1}{4}+y_{4}\right) a \hat{\mathbf{z}}\end{array}$ & $(96 h)$ & H III \\
\hline $\mathbf{B}_{75}$ & $=$ & $\begin{array}{c}\left(\frac{1}{4}+x_{4}-y_{4}-z_{4}\right) \mathbf{a}_{1}+ \\
\left(\frac{1}{4}-x_{4}-y_{4}+z_{4}\right) \mathbf{a}_{2}+ \\
\left(\frac{1}{4}-x_{4}+y_{4}-z_{4}\right) \mathbf{a}_{3}\end{array}$ & $=$ & $\begin{array}{c}\left(\frac{1}{4}-x_{4}\right) a \hat{\mathbf{x}}+\left(\frac{1}{4}-z_{4}\right) a \hat{\mathbf{y}}+ \\
\left(\frac{1}{4}-y_{4}\right) a \hat{\mathbf{z}}\end{array}$ & $(96 h)$ & H III \\
\hline $\mathbf{B}_{76}$ & $=$ & $\begin{array}{c}\left(\frac{1}{4}-x_{4}+y_{4}-z_{4}\right) \mathbf{a}_{1}+ \\
\left(\frac{1}{4}+x_{4}+y_{4}+z_{4}\right) \mathbf{a}_{2}+ \\
\left(\frac{1}{4}+x_{4}-y_{4}-z_{4}\right) \mathbf{a}_{3}\end{array}$ & $=$ & $\begin{array}{c}\left(\frac{1}{4}+x_{4}\right) a \hat{\mathbf{x}}+\left(\frac{1}{4}-z_{4}\right) a \hat{\mathbf{y}}+ \\
\left(\frac{1}{4}+y_{4}\right) a \hat{\mathbf{z}}\end{array}$ & $(96 h)$ & H III \\
\hline $\mathbf{B}_{77}$ & $=$ & $\begin{array}{c}\left(\frac{1}{4}-x_{4}+y_{4}-z_{4}\right) \mathbf{a}_{1}+ \\
\left(\frac{1}{4}-x_{4}-y_{4}+z_{4}\right) \mathbf{a}_{2}+ \\
\left(\frac{1}{4}+x_{4}+y_{4}+z_{4}\right) \mathbf{a}_{3}\end{array}$ & $=$ & $\begin{array}{c}\left(\frac{1}{4}+z_{4}\right) a \hat{\mathbf{x}}+\left(\frac{1}{4}+y_{4}\right) a \hat{\mathbf{y}}+ \\
\left(\frac{1}{4}-x_{4}\right) a \hat{\mathbf{z}}\end{array}$ & $(96 h)$ & H III \\
\hline $\mathbf{B}_{78}$ & $=$ & $\begin{array}{c}\left(\frac{1}{4}+x_{4}-y_{4}-z_{4}\right) \mathbf{a}_{1}+ \\
\left(\frac{1}{4}+x_{4}+y_{4}+z_{4}\right) \mathbf{a}_{2}+ \\
\left(\frac{1}{4}-x_{4}-y_{4}+z_{4}\right) \mathbf{a}_{3}\end{array}$ & $=$ & $\begin{array}{c}\left(\frac{1}{4}+z_{4}\right) a \hat{\mathbf{x}}+\left(\frac{1}{4}-y_{4}\right) a \hat{\mathbf{y}}+ \\
\left(\frac{1}{4}+x_{4}\right) a \hat{\mathbf{z}}\end{array}$ & $(96 h)$ & H III \\
\hline
\end{tabular}




\begin{tabular}{|c|c|c|c|c|c|c|}
\hline $\mathbf{B}_{79}$ & $=$ & $\begin{array}{c}\left(\frac{1}{4}+x_{4}+y_{4}+z_{4}\right) \mathbf{a}_{1}+ \\
\left(\frac{1}{4}+x_{4}-y_{4}-z_{4}\right) \mathbf{a}_{2}+ \\
\left(\frac{1}{4}-x_{4}+y_{4}-z_{4}\right) \mathbf{a}_{3}\end{array}$ & $=$ & $\begin{array}{c}\left(\frac{1}{4}-z_{4}\right) a \hat{\mathbf{x}}+\left(\frac{1}{4}+y_{4}\right) a \hat{\mathbf{y}}+ \\
\left(\frac{1}{4}+x_{4}\right) a \hat{\mathbf{z}}\end{array}$ & $(96 h)$ & H III \\
\hline $\mathbf{B}_{80}$ & $=$ & $\begin{array}{c}\left(\frac{1}{4}-x_{4}-y_{4}+z_{4}\right) \mathbf{a}_{1}+ \\
\left(\frac{1}{4}-x_{4}+y_{4}-z_{4}\right) \mathbf{a}_{2}+ \\
\left(\frac{1}{4}+x_{4}-y_{4}-z_{4}\right) \mathbf{a}_{3}\end{array}$ & $=$ & $\begin{array}{c}\left(\frac{1}{4}-z_{4}\right) a \hat{\mathbf{x}}+\left(\frac{1}{4}-y_{4}\right) a \hat{\mathbf{y}}+ \\
\left(\frac{1}{4}-x_{4}\right) a \hat{\mathbf{z}}\end{array}$ & $(96 h)$ & H III \\
\hline $\mathbf{B}_{81}$ & $=$ & $\begin{array}{c}\left(-x_{5}+y_{5}+z_{5}\right) \mathbf{a}_{1}+ \\
\left(x_{5}-y_{5}+z_{5}\right) \mathbf{a}_{2}+ \\
\left(x_{5}+y_{5}-z_{5}\right) \mathbf{a}_{3}\end{array}$ & $=$ & $x_{5} a \hat{\mathbf{x}}+y_{5} a \hat{\mathbf{y}}+z_{5} a \hat{\mathbf{z}}$ & $(96 h)$ & H IV \\
\hline $\mathbf{B}_{82}$ & $=$ & $\begin{array}{c}\left(x_{5}-y_{5}+z_{5}\right) \mathbf{a}_{1}+ \\
\left(-x_{5}+y_{5}+z_{5}\right) \mathbf{a}_{2}+ \\
\left(-x_{5}-y_{5}-z_{5}\right) \mathbf{a}_{3}\end{array}$ & $=$ & $-x_{5} a \hat{\mathbf{x}}-y_{5} a \hat{\mathbf{y}}+z_{5} a \hat{\mathbf{z}}$ & $(96 h)$ & H IV \\
\hline $\mathbf{B}_{83}$ & $=$ & $\begin{array}{c}\left(x_{5}+y_{5}-z_{5}\right) \mathbf{a}_{1}+ \\
\left(-x_{5}-y_{5}-z_{5}\right) \mathbf{a}_{2}+ \\
\left(-x_{5}+y_{5}+z_{5}\right) \mathbf{a}_{3}\end{array}$ & $=$ & $-x_{5} a \hat{\mathbf{x}}+y_{5} a \hat{\mathbf{y}}-z_{5} a \hat{\mathbf{z}}$ & $(96 h)$ & H IV \\
\hline $\mathbf{B}_{84}$ & $=$ & $\begin{array}{c}\left(-x_{5}-y_{5}-z_{5}\right) \mathbf{a}_{1}+ \\
\left(x_{5}+y_{5}-z_{5}\right) \mathbf{a}_{2}+ \\
\left(x_{5}-y_{5}+z_{5}\right) \mathbf{a}_{3}\end{array}$ & $=$ & $x_{5} a \hat{\mathbf{x}}-y_{5} a \hat{\mathbf{y}}-z_{5} a \hat{\mathbf{z}}$ & $(96 h)$ & H IV \\
\hline $\mathbf{B}_{85}$ & $=$ & $\begin{array}{c}\left(x_{5}+y_{5}-z_{5}\right) \mathbf{a}_{1}+ \\
\left(-x_{5}+y_{5}+z_{5}\right) \mathbf{a}_{2}+ \\
\left(x_{5}-y_{5}+z_{5}\right) \mathbf{a}_{3}\end{array}$ & $=$ & $z_{5} a \hat{\mathbf{x}}+x_{5} a \hat{\mathbf{y}}+y_{5} a \hat{\mathbf{z}}$ & $(96 h)$ & H IV \\
\hline $\mathbf{B}_{86}$ & $=$ & $\begin{array}{c}\left(-x_{5}-y_{5}-z_{5}\right) \mathbf{a}_{1}+ \\
\left(x_{5}-y_{5}+z_{5}\right) \mathbf{a}_{2}+ \\
\left(-x_{5}+y_{5}+z_{5}\right) \mathbf{a}_{3}\end{array}$ & $=$ & $z_{5} a \hat{\mathbf{x}}-x_{5} a \hat{\mathbf{y}}-y_{5} a \hat{\mathbf{z}}$ & $(96 h)$ & H IV \\
\hline $\mathbf{B}_{87}$ & $=$ & $\begin{array}{c}\left(-x_{5}+y_{5}+z_{5}\right) \mathbf{a}_{1}+ \\
\left(x_{5}+y_{5}-z_{5}\right) \mathbf{a}_{2}+ \\
\left(-x_{5}-y_{5}-z_{5}\right) \mathbf{a}_{3}\end{array}$ & $=$ & $-z_{5} a \hat{\mathbf{x}}-x_{5} a \hat{\mathbf{y}}+y_{5} a \hat{\mathbf{z}}$ & $(96 h)$ & H IV \\
\hline $\mathbf{B}_{88}$ & $=$ & $\begin{array}{c}\left(x_{5}-y_{5}+z_{5}\right) \mathbf{a}_{1}+ \\
\left(-x_{5}-y_{5}-z_{5}\right) \mathbf{a}_{2}+ \\
\left(x_{5}+y_{5}-z_{5}\right) \mathbf{a}_{3}\end{array}$ & $=$ & $-z_{5} a \hat{\mathbf{x}}+x_{5} a \hat{\mathbf{y}}-y_{5} a \hat{\mathbf{z}}$ & $(96 h)$ & H IV \\
\hline $\mathbf{B}_{89}$ & $=$ & $\begin{array}{l}\left(x_{5}-y_{5}+z_{5}\right) \mathbf{a}_{1}+ \\
\left(x_{5}+y_{5}-z_{5}\right) \mathbf{a}_{2}+ \\
\left(-x_{5}+y_{5}+z_{5}\right) \mathbf{a}_{3}\end{array}$ & $=$ & $y_{5} a \hat{\mathbf{x}}+z_{5} a \hat{\mathbf{y}}+x_{5} a \hat{\mathbf{z}}$ & $(96 h)$ & H IV \\
\hline $\mathbf{B}_{90}$ & $=$ & $\begin{array}{c}\left(-x_{5}+y_{5}+z_{5}\right) \mathbf{a}_{1}+ \\
\left(-x_{5}-y_{5}-z_{5}\right) \mathbf{a}_{2}+ \\
\left(x_{5}-y_{5}+z_{5}\right) \mathbf{a}_{3}\end{array}$ & $=$ & $-y_{5} a \hat{\mathbf{x}}+z_{5} a \hat{\mathbf{y}}-x_{5} a \hat{\mathbf{z}}$ & $(96 h)$ & H IV \\
\hline $\mathbf{B}_{91}$ & $=$ & $\begin{array}{c}\left(-x_{5}-y_{5}-z_{5}\right) \mathbf{a}_{1}+ \\
\left(-x_{5}+y_{5}+z_{5}\right) \mathbf{a}_{2}+ \\
\left(x_{5}+y_{5}-z_{5}\right) \mathbf{a}_{3}\end{array}$ & $=$ & $y_{5} a \hat{\mathbf{x}}-z_{5} a \hat{\mathbf{y}}-x_{5} a \hat{\mathbf{z}}$ & $(96 h)$ & H IV \\
\hline $\mathbf{B}_{92}$ & $=$ & $\begin{array}{l}\left(x_{5}+y_{5}-z_{5}\right) \mathbf{a}_{1}+ \\
\left(x_{5}-y_{5}+z_{5}\right) \mathbf{a}_{2}+ \\
\left(-x_{5}-y_{5}-z_{5}\right) \mathbf{a}_{3}\end{array}$ & $=$ & $-y_{5} a \hat{\mathbf{x}}-z_{5} a \hat{\mathbf{y}}+x_{5} a \hat{\mathbf{z}}$ & $(96 h)$ & H IV \\
\hline $\mathbf{B}_{93}$ & $=$ & $\begin{array}{c}\left(\frac{1}{4}+x_{5}-y_{5}-z_{5}\right) \mathbf{a}_{1}+ \\
\left(\frac{1}{4}-x_{5}+y_{5}-z_{5}\right) \mathbf{a}_{2}+ \\
\left(\frac{1}{4}+x_{5}+y_{5}+z_{5}\right) \mathbf{a}_{3}\end{array}$ & $=$ & $\begin{array}{c}\left(\frac{1}{4}+y_{5}\right) a \hat{\mathbf{x}}+\left(\frac{1}{4}+x_{5}\right) a \hat{\mathbf{y}}+ \\
\left(\frac{1}{4}-z_{5}\right) a \hat{\mathbf{z}}\end{array}$ & $(96 h)$ & H IV \\
\hline $\mathbf{B}_{94}$ & $=$ & $\begin{array}{c}\left(\frac{1}{4}-x_{5}+y_{5}-z_{5}\right) \mathbf{a}_{1}+ \\
\left(\frac{1}{4}+x_{5}-y_{5}-z_{5}\right) \mathbf{a}_{2}+ \\
\left(\frac{1}{4}-x_{5}-y_{5}+z_{5}\right) \mathbf{a}_{3}\end{array}$ & $=$ & $\begin{array}{c}\left(\frac{1}{4}-y_{5}\right) a \hat{\mathbf{x}}+\left(\frac{1}{4}-x_{5}\right) a \hat{\mathbf{y}}+ \\
\left(\frac{1}{4}-z_{5}\right) a \hat{\mathbf{z}}\end{array}$ & $(96 h)$ & H IV \\
\hline
\end{tabular}




\begin{tabular}{|c|c|c|c|c|c|c|}
\hline $\mathbf{B}_{95}$ & $=$ & $\begin{array}{c}\left(\frac{1}{4}-x_{5}-y_{5}+z_{5}\right) \mathbf{a}_{1}+ \\
\left(\frac{1}{4}+x_{5}+y_{5}+z_{5}\right) \mathbf{a}_{2}+ \\
\left(\frac{1}{4}-x_{5}+y_{5}-z_{5}\right) \mathbf{a}_{3}\end{array}$ & $=$ & $\begin{array}{c}\left(\frac{1}{4}+y_{5}\right) a \hat{\mathbf{x}}+\left(\frac{1}{4}-x_{5}\right) a \hat{\mathbf{y}}+ \\
\left(\frac{1}{4}+z_{5}\right) a \hat{\mathbf{z}}\end{array}$ & $(96 h)$ & H IV \\
\hline $\mathbf{B}_{96}$ & $=$ & $\begin{array}{c}\left(\frac{1}{4}+x_{5}+y_{5}+z_{5}\right) \mathbf{a}_{1}+ \\
\left(\frac{1}{4}-x_{5}-y_{5}+z_{5}\right) \mathbf{a}_{2}+ \\
\left(\frac{1}{4}+x_{5}-y_{5}-z_{5}\right) \mathbf{a}_{3}\end{array}$ & $=$ & $\begin{array}{c}\left(\frac{1}{4}-y_{5}\right) a \hat{\mathbf{x}}+\left(\frac{1}{4}+x_{5}\right) a \hat{\mathbf{y}}+ \\
\left(\frac{1}{4}+z_{5}\right) a \hat{\mathbf{z}}\end{array}$ & $(96 h)$ & H IV \\
\hline $\mathbf{B}_{97}$ & $=$ & $\begin{array}{c}\left(\frac{1}{4}-x_{5}-y_{5}+z_{5}\right) \mathbf{a}_{1}+ \\
\left(\frac{1}{4}+x_{5}-y_{5}-z_{5}\right) \mathbf{a}_{2}+ \\
\left(\frac{1}{4}+x_{5}+y_{5}+z_{5}\right) \mathbf{a}_{3}\end{array}$ & $=$ & $\begin{array}{c}\left(\frac{1}{4}+x_{5}\right) a \hat{\mathbf{x}}+\left(\frac{1}{4}+z_{5}\right) a \hat{\mathbf{y}}+ \\
\left(\frac{1}{4}-y_{5}\right) a \hat{\mathbf{z}}\end{array}$ & $(96 h)$ & H IV \\
\hline $\mathbf{B}_{98}$ & $=$ & $\begin{array}{c}\left(\frac{1}{4}+x_{5}+y_{5}+z_{5}\right) \mathbf{a}_{1}+ \\
\left(\frac{1}{4}-x_{5}+y_{5}-z_{5}\right) \mathbf{a}_{2}+ \\
\left(\frac{1}{4}-x_{5}-y_{5}+z_{5}\right) \mathbf{a}_{3}\end{array}$ & $=$ & $\begin{array}{c}\left(\frac{1}{4}-x_{5}\right) a \hat{\mathbf{x}}+\left(\frac{1}{4}+z_{5}\right) a \hat{\mathbf{y}}+ \\
\left(\frac{1}{4}+y_{5}\right) a \hat{\mathbf{z}}\end{array}$ & $(96 h)$ & H IV \\
\hline $\mathbf{B}_{99}$ & $=$ & $\begin{array}{c}\left(\frac{1}{4}+x_{5}-y_{5}-z_{5}\right) \mathbf{a}_{1}+ \\
\left(\frac{1}{4}-x_{5}-y_{5}+z_{5}\right) \mathbf{a}_{2}+ \\
\left(\frac{1}{4}-x_{5}+y_{5}-z_{5}\right) \mathbf{a}_{3}\end{array}$ & $=$ & $\begin{array}{c}\left(\frac{1}{4}-x_{5}\right) a \hat{\mathbf{x}}+\left(\frac{1}{4}-z_{5}\right) a \hat{\mathbf{y}}+ \\
\left(\frac{1}{4}-y_{5}\right) a \hat{\mathbf{z}}\end{array}$ & $(96 h)$ & H IV \\
\hline $\mathbf{B}_{100}$ & $=$ & $\begin{array}{c}\left(\frac{1}{4}-x_{5}+y_{5}-z_{5}\right) \mathbf{a}_{1}+ \\
\left(\frac{1}{4}+x_{5}+y_{5}+z_{5}\right) \mathbf{a}_{2}+ \\
\left(\frac{1}{4}+x_{5}-y_{5}-z_{5}\right) \mathbf{a}_{3}\end{array}$ & $=$ & $\begin{array}{c}\left(\frac{1}{4}+x_{5}\right) a \hat{\mathbf{x}}+\left(\frac{1}{4}-z_{5}\right) a \hat{\mathbf{y}}+ \\
\left(\frac{1}{4}+y_{5}\right) a \hat{\mathbf{z}}\end{array}$ & $(96 h)$ & H IV \\
\hline $\mathbf{B}_{101}$ & $=$ & $\begin{array}{c}\left(\frac{1}{4}-x_{5}+y_{5}-z_{5}\right) \mathbf{a}_{1}+ \\
\left(\frac{1}{4}-x_{5}-y_{5}+z_{5}\right) \mathbf{a}_{2}+ \\
\left(\frac{1}{4}+x_{5}+y_{5}+z_{5}\right) \mathbf{a}_{3}\end{array}$ & $=$ & $\begin{array}{c}\left(\frac{1}{4}+z_{5}\right) a \hat{\mathbf{x}}+\left(\frac{1}{4}+y_{5}\right) a \hat{\mathbf{y}}+ \\
\left(\frac{1}{4}-x_{5}\right) a \hat{\mathbf{z}}\end{array}$ & $(96 h)$ & H IV \\
\hline $\mathbf{B}_{102}$ & $=$ & $\begin{array}{c}\left(\frac{1}{4}+x_{5}-y_{5}-z_{5}\right) \mathbf{a}_{1}+ \\
\left(\frac{1}{4}+x_{5}+y_{5}+z_{5}\right) \mathbf{a}_{2}+ \\
\left(\frac{1}{4}-x_{5}-y_{5}+z_{5}\right) \mathbf{a}_{3}\end{array}$ & $=$ & $\begin{array}{c}\left(\frac{1}{4}+z_{5}\right) a \hat{\mathbf{x}}+\left(\frac{1}{4}-y_{5}\right) a \hat{\mathbf{y}}+ \\
\left(\frac{1}{4}+x_{5}\right) a \hat{\mathbf{z}}\end{array}$ & $(96 h)$ & H IV \\
\hline $\mathbf{B}_{103}$ & $=$ & $\begin{array}{c}\left(\frac{1}{4}+x_{5}+y_{5}+z_{5}\right) \mathbf{a}_{1}+ \\
\left(\frac{1}{4}+x_{5}-y_{5}-z_{5}\right) \mathbf{a}_{2}+ \\
\left(\frac{1}{4}-x_{5}+y_{5}-z_{5}\right) \mathbf{a}_{3}\end{array}$ & $=$ & $\begin{array}{c}\left(\frac{1}{4}-z_{5}\right) a \hat{\mathbf{x}}+\left(\frac{1}{4}+y_{5}\right) a \hat{\mathbf{y}}+ \\
\left(\frac{1}{4}+x_{5}\right) a \hat{\mathbf{z}}\end{array}$ & $(96 h)$ & H IV \\
\hline $\mathbf{B}_{104}$ & $=$ & $\begin{array}{c}\left(\frac{1}{4}-x_{5}-y_{5}+z_{5}\right) \mathbf{a}_{1}+ \\
\left(\frac{1}{4}-x_{5}+y_{5}-z_{5}\right) \mathbf{a}_{2}+ \\
\left(\frac{1}{4}+x_{5}-y_{5}-z_{5}\right) \mathbf{a}_{3}\end{array}$ & $=$ & $\begin{array}{c}\left(\frac{1}{4}-z_{5}\right) a \hat{\mathbf{x}}+\left(\frac{1}{4}-y_{5}\right) a \hat{\mathbf{y}}+ \\
\left(\frac{1}{4}-x_{5}\right) a \hat{\mathbf{z}}\end{array}$ & $(96 h)$ & H IV \\
\hline $\mathbf{B}_{105}$ & $=$ & $\begin{array}{c}\left(-x_{6}+y_{6}+z_{6}\right) \mathbf{a}_{1}+ \\
\left(x_{6}-y_{6}+z_{6}\right) \mathbf{a}_{2}+ \\
\left(x_{6}+y_{6}-z_{6}\right) \mathbf{a}_{3}\end{array}$ & $=$ & $x_{6} a \hat{\mathbf{x}}+y_{6} a \hat{\mathbf{y}}+z_{6} a \hat{\mathbf{z}}$ & $(96 h)$ & O I \\
\hline $\mathbf{B}_{106}$ & $=$ & $\begin{array}{c}\left(x_{6}-y_{6}+z_{6}\right) \mathbf{a}_{1}+ \\
\left(-x_{6}+y_{6}+z_{6}\right) \mathbf{a}_{2}+ \\
\left(-x_{6}-y_{6}-z_{6}\right) \mathbf{a}_{3}\end{array}$ & $=$ & $-x_{6} a \hat{\mathbf{x}}-y_{6} a \hat{\mathbf{y}}+z_{6} a \hat{\mathbf{z}}$ & $(96 h)$ & O I \\
\hline $\mathbf{B}_{107}$ & $=$ & $\begin{array}{c}\left(x_{6}+y_{6}-z_{6}\right) \mathbf{a}_{1}+ \\
\left(-x_{6}-y_{6}-z_{6}\right) \mathbf{a}_{2}+ \\
\left(-x_{6}+y_{6}+z_{6}\right) \mathbf{a}_{3}\end{array}$ & $=$ & $-x_{6} a \hat{\mathbf{x}}+y_{6} a \hat{\mathbf{y}}-z_{6} a \hat{\mathbf{z}}$ & $(96 h)$ & O I \\
\hline $\mathbf{B}_{108}$ & $=$ & $\begin{array}{c}\left(-x_{6}-y_{6}-z_{6}\right) \mathbf{a}_{1}+ \\
\left(x_{6}+y_{6}-z_{6}\right) \mathbf{a}_{2}+ \\
\left(x_{6}-y_{6}+z_{6}\right) \mathbf{a}_{3}\end{array}$ & $=$ & $x_{6} a \hat{\mathbf{x}}-y_{6} a \hat{\mathbf{y}}-z_{6} a \hat{\mathbf{z}}$ & $(96 h)$ & $\mathrm{O} \mathrm{I}$ \\
\hline
\end{tabular}




\begin{tabular}{|c|c|c|c|c|c|}
\hline $\mathbf{B}_{109}$ & $=$ & $\begin{array}{c}\left(x_{6}+y_{6}-z_{6}\right) \mathbf{a}_{1}+ \\
\left(-x_{6}+y_{6}+z_{6}\right) \mathbf{a}_{2}+ \\
\left(x_{6}-y_{6}+z_{6}\right) \mathbf{a}_{3}\end{array}$ & $=$ & $z_{6} a \hat{\mathbf{x}}+x_{6} a \hat{\mathbf{y}}+y_{6} a \hat{\mathbf{z}}$ & $(96 h)$ \\
\hline $\mathbf{B}_{110}$ & $=$ & $\begin{array}{c}\left(-x_{6}-y_{6}-z_{6}\right) \mathbf{a}_{1}+ \\
\left(x_{6}-y_{6}+z_{6}\right) \mathbf{a}_{2}+ \\
\left(-x_{6}+y_{6}+z_{6}\right) \mathbf{a}_{3}\end{array}$ & $=$ & $z_{6} a \hat{\mathbf{x}}-x_{6} a \hat{\mathbf{y}}-y_{6} a \hat{\mathbf{z}}$ & $(96 h)$ \\
\hline $\mathbf{B}_{111}$ & $=$ & $\begin{array}{c}\left(-x_{6}+y_{6}+z_{6}\right) \mathbf{a}_{1}+ \\
\left(x_{6}+y_{6}-z_{6}\right) \mathbf{a}_{2}+ \\
\left(-x_{6}-y_{6}-z_{6}\right) \mathbf{a}_{3}\end{array}$ & $=$ & $-z_{6} a \hat{\mathbf{x}}-x_{6} a \hat{\mathbf{y}}+y_{6} a \hat{\mathbf{z}}$ & $(96 h)$ \\
\hline $\mathbf{B}_{112}$ & $=$ & $\begin{array}{c}\left(x_{6}-y_{6}+z_{6}\right) \mathbf{a}_{1}+ \\
\left(-x_{6}-y_{6}-z_{6}\right) \mathbf{a}_{2}+ \\
\left(x_{6}+y_{6}-z_{6}\right) \mathbf{a}_{3}\end{array}$ & $=$ & $-z_{6} a \hat{\mathbf{x}}+x_{6} a \hat{\mathbf{y}}-y_{6} a \hat{\mathbf{z}}$ & $(96 h)$ \\
\hline $\mathbf{B}_{113}$ & $=$ & $\begin{array}{l}\left(x_{6}-y_{6}+z_{6}\right) \mathbf{a}_{1}+ \\
\left(x_{6}+y_{6}-z_{6}\right) \mathbf{a}_{2}+ \\
\left(-x_{6}+y_{6}+z_{6}\right) \mathbf{a}_{3}\end{array}$ & $=$ & $y_{6} a \hat{\mathbf{x}}+z_{6} a \hat{\mathbf{y}}+x_{6} a \hat{\mathbf{z}}$ & $(96 h)$ \\
\hline $\mathbf{B}_{114}$ & $=$ & $\begin{array}{c}\left(-x_{6}+y_{6}+z_{6}\right) \mathbf{a}_{1}+ \\
\left(-x_{6}-y_{6}-z_{6}\right) \mathbf{a}_{2}+ \\
\left(x_{6}-y_{6}+z_{6}\right) \mathbf{a}_{3}\end{array}$ & $=$ & $-y_{6} a \hat{\mathbf{x}}+z_{6} a \hat{\mathbf{y}}-x_{6} a \hat{\mathbf{z}}$ & $(96 h)$ \\
\hline $\mathbf{B}_{115}$ & $=$ & $\begin{array}{c}\left(-x_{6}-y_{6}-z_{6}\right) \mathbf{a}_{1}+ \\
\left(-x_{6}+y_{6}+z_{6}\right) \mathbf{a}_{2}+ \\
\left(x_{6}+y_{6}-z_{6}\right) \mathbf{a}_{3}\end{array}$ & $=$ & $y_{6} a \hat{\mathbf{x}}-z_{6} a \hat{\mathbf{y}}-x_{6} a \hat{\mathbf{z}}$ & $(96 h)$ \\
\hline $\mathbf{B}_{116}$ & $=$ & $\begin{array}{l}\left(x_{6}+y_{6}-z_{6}\right) \mathbf{a}_{1}+ \\
\left(x_{6}-y_{6}+z_{6}\right) \mathbf{a}_{2}+ \\
\left(-x_{6}-y_{6}-z_{6}\right) \mathbf{a}_{3}\end{array}$ & $=$ & $-y_{6} a \hat{\mathbf{x}}-z_{6} a \hat{\mathbf{y}}+x_{6} a \hat{\mathbf{z}}$ & $(96 h)$ \\
\hline $\mathbf{B}_{117}$ & $=$ & $\begin{array}{c}\left(\frac{1}{4}+x_{6}-y_{6}-z_{6}\right) \mathbf{a}_{1}+ \\
\left(\frac{1}{4}-x_{6}+y_{6}-z_{6}\right) \mathbf{a}_{2}+ \\
\left(\frac{1}{4}+x_{6}+y_{6}+z_{6}\right) \mathbf{a}_{3}\end{array}$ & $=$ & $\begin{array}{c}\left(\frac{1}{4}+y_{6}\right) a \hat{\mathbf{x}}+\left(\frac{1}{4}+x_{6}\right) a \hat{\mathbf{y}}+ \\
\left(\frac{1}{4}-z_{6}\right) a \hat{\mathbf{z}}\end{array}$ & $(96 h)$ \\
\hline $\mathbf{B}_{118}$ & $=$ & $\begin{array}{c}\left(\frac{1}{4}-x_{6}+y_{6}-z_{6}\right) \mathbf{a}_{1}+ \\
\left(\frac{1}{4}+x_{6}-y_{6}-z_{6}\right) \mathbf{a}_{2}+ \\
\left(\frac{1}{4}-x_{6}-y_{6}+z_{6}\right) \mathbf{a}_{3}\end{array}$ & $=$ & $\begin{array}{c}\left(\frac{1}{4}-y_{6}\right) a \hat{\mathbf{x}}+\left(\frac{1}{4}-x_{6}\right) a \hat{\mathbf{y}}+ \\
\left(\frac{1}{4}-z_{6}\right) a \hat{\mathbf{z}}\end{array}$ & $(96 h)$ \\
\hline $\mathbf{B}_{119}$ & $=$ & $\begin{array}{c}\left(\frac{1}{4}-x_{6}-y_{6}+z_{6}\right) \mathbf{a}_{1}+ \\
\left(\frac{1}{4}+x_{6}+y_{6}+z_{6}\right) \mathbf{a}_{2}+ \\
\left(\frac{1}{4}-x_{6}+y_{6}-z_{6}\right) \mathbf{a}_{3}\end{array}$ & $=$ & $\begin{array}{c}\left(\frac{1}{4}+y_{6}\right) a \hat{\mathbf{x}}+\left(\frac{1}{4}-x_{6}\right) a \hat{\mathbf{y}}+ \\
\left(\frac{1}{4}+z_{6}\right) a \hat{\mathbf{z}}\end{array}$ & $(96 h)$ \\
\hline $\mathbf{B}_{120}$ & $=$ & $\begin{array}{c}\left(\frac{1}{4}+x_{6}+y_{6}+z_{6}\right) \mathbf{a}_{1}+ \\
\left(\frac{1}{4}-x_{6}-y_{6}+z_{6}\right) \mathbf{a}_{2}+ \\
\left(\frac{1}{4}+x_{6}-y_{6}-z_{6}\right) \mathbf{a}_{3}\end{array}$ & $=$ & $\begin{array}{c}\left(\frac{1}{4}-y_{6}\right) a \hat{\mathbf{x}}+\left(\frac{1}{4}+x_{6}\right) a \hat{\mathbf{y}}+ \\
\left(\frac{1}{4}+z_{6}\right) a \hat{\mathbf{z}}\end{array}$ & $(96 h)$ \\
\hline $\mathbf{B}_{121}$ & $=$ & $\begin{array}{c}\left(\frac{1}{4}-x_{6}-y_{6}+z_{6}\right) \mathbf{a}_{1}+ \\
\left(\frac{1}{4}+x_{6}-y_{6}-z_{6}\right) \mathbf{a}_{2}+ \\
\left(\frac{1}{4}+x_{6}+y_{6}+z_{6}\right) \mathbf{a}_{3}\end{array}$ & $=$ & $\begin{array}{c}\left(\frac{1}{4}+x_{6}\right) a \hat{\mathbf{x}}+\left(\frac{1}{4}+z_{6}\right) a \hat{\mathbf{y}}+ \\
\left(\frac{1}{4}-y_{6}\right) a \hat{\mathbf{z}}\end{array}$ & $(96 h)$ \\
\hline $\mathbf{B}_{122}$ & $=$ & $\begin{array}{c}\left(\frac{1}{4}+x_{6}+y_{6}+z_{6}\right) \mathbf{a}_{1}+ \\
\left(\frac{1}{4}-x_{6}+y_{6}-z_{6}\right) \mathbf{a}_{2}+ \\
\left(\frac{1}{4}-x_{6}-y_{6}+z_{6}\right) \mathbf{a}_{3}\end{array}$ & $=$ & $\begin{array}{c}\left(\frac{1}{4}-x_{6}\right) a \hat{\mathbf{x}}+\left(\frac{1}{4}+z_{6}\right) a \hat{\mathbf{y}}+ \\
\left(\frac{1}{4}+y_{6}\right) a \hat{\mathbf{z}}\end{array}$ & $(96 h)$ \\
\hline $\mathbf{B}_{123}$ & $=$ & $\begin{array}{c}\left(\frac{1}{4}+x_{6}-y_{6}-z_{6}\right) \mathbf{a}_{1}+ \\
\left(\frac{1}{4}-x_{6}-y_{6}+z_{6}\right) \mathbf{a}_{2}+ \\
\left(\frac{1}{4}-x_{6}+y_{6}-z_{6}\right) \mathbf{a}_{3}\end{array}$ & $=$ & $\begin{array}{c}\left(\frac{1}{4}-x_{6}\right) a \hat{\mathbf{x}}+\left(\frac{1}{4}-z_{6}\right) a \hat{\mathbf{y}}+ \\
\left(\frac{1}{4}-y_{6}\right) a \hat{\mathbf{z}}\end{array}$ & $(96 h)$ \\
\hline
\end{tabular}




\begin{tabular}{|c|c|c|c|c|c|c|}
\hline $\mathbf{B}_{124}$ & $=$ & $\begin{array}{c}\left(\frac{1}{4}-x_{6}+y_{6}-z_{6}\right) \mathbf{a}_{1}+ \\
\left(\frac{1}{4}+x_{6}+y_{6}+z_{6}\right) \mathbf{a}_{2}+ \\
\left(\frac{1}{4}+x_{6}-y_{6}-z_{6}\right) \mathbf{a}_{3}\end{array}$ & $=$ & $\begin{array}{c}\left(\frac{1}{4}+x_{6}\right) a \hat{\mathbf{x}}+\left(\frac{1}{4}-z_{6}\right) a \hat{\mathbf{y}}+ \\
\left(\frac{1}{4}+y_{6}\right) a \hat{\mathbf{z}}\end{array}$ & $(96 h)$ & O I \\
\hline $\mathbf{B}_{125}$ & $=$ & $\begin{array}{c}\left(\frac{1}{4}-x_{6}+y_{6}-z_{6}\right) \mathbf{a}_{1}+ \\
\left(\frac{1}{4}-x_{6}-y_{6}+z_{6}\right) \mathbf{a}_{2}+ \\
\left(\frac{1}{4}+x_{6}+y_{6}+z_{6}\right) \mathbf{a}_{3}\end{array}$ & $=$ & $\begin{array}{c}\left(\frac{1}{4}+z_{6}\right) a \hat{\mathbf{x}}+\left(\frac{1}{4}+y_{6}\right) a \hat{\mathbf{y}}+ \\
\left(\frac{1}{4}-x_{6}\right) a \hat{\mathbf{z}}\end{array}$ & $(96 h)$ & O I \\
\hline $\mathbf{B}_{126}$ & $=$ & $\begin{array}{c}\left(\frac{1}{4}+x_{6}-y_{6}-z_{6}\right) \mathbf{a}_{1}+ \\
\left(\frac{1}{4}+x_{6}+y_{6}+z_{6}\right) \mathbf{a}_{2}+ \\
\left(\frac{1}{4}-x_{6}-y_{6}+z_{6}\right) \mathbf{a}_{3}\end{array}$ & $=$ & $\begin{array}{c}\left(\frac{1}{4}+z_{6}\right) a \hat{\mathbf{x}}+\left(\frac{1}{4}-y_{6}\right) a \hat{\mathbf{y}}+ \\
\left(\frac{1}{4}+x_{6}\right) a \hat{\mathbf{z}}\end{array}$ & $(96 h)$ & O I \\
\hline $\mathbf{B}_{127}$ & $=$ & $\begin{array}{c}\left(\frac{1}{4}+x_{6}+y_{6}+z_{6}\right) \mathbf{a}_{1}+ \\
\left(\frac{1}{4}+x_{6}-y_{6}-z_{6}\right) \mathbf{a}_{2}+ \\
\left(\frac{1}{4}-x_{6}+y_{6}-z_{6}\right) \mathbf{a}_{3}\end{array}$ & $=$ & $\begin{array}{c}\left(\frac{1}{4}-z_{6}\right) a \hat{\mathbf{x}}+\left(\frac{1}{4}+y_{6}\right) a \hat{\mathbf{y}}+ \\
\left(\frac{1}{4}+x_{6}\right) a \hat{\mathbf{z}}\end{array}$ & $(96 h)$ & O I \\
\hline $\mathbf{B}_{128}$ & $=$ & $\begin{array}{c}\left(\frac{1}{4}-x_{6}-y_{6}+z_{6}\right) \mathbf{a}_{1}+ \\
\left(\frac{1}{4}-x_{6}+y_{6}-z_{6}\right) \mathbf{a}_{2}+ \\
\left(\frac{1}{4}+x_{6}-y_{6}-z_{6}\right) \mathbf{a}_{3}\end{array}$ & $=$ & $\begin{array}{c}\left(\frac{1}{4}-z_{6}\right) a \hat{\mathbf{x}}+\left(\frac{1}{4}-y_{6}\right) a \hat{\mathbf{y}}+ \\
\left(\frac{1}{4}-x_{6}\right) a \hat{\mathbf{z}}\end{array}$ & $(96 h)$ & O I \\
\hline $\mathbf{B}_{129}$ & $=$ & $\begin{array}{c}\left(-x_{7}+y_{7}+z_{7}\right) \mathbf{a}_{1}+ \\
\left(x_{7}-y_{7}+z_{7}\right) \mathbf{a}_{2}+ \\
\left(x_{7}+y_{7}-z_{7}\right) \mathbf{a}_{3}\end{array}$ & $=$ & $x_{7} a \hat{\mathbf{x}}+y_{7} a \hat{\mathbf{y}}+z_{7} a \hat{\mathbf{z}}$ & $(96 h)$ & O II \\
\hline $\mathbf{B}_{130}$ & $=$ & $\begin{array}{c}\left(x_{7}-y_{7}+z_{7}\right) \mathbf{a}_{1}+ \\
\left(-x_{7}+y_{7}+z_{7}\right) \mathbf{a}_{2}+ \\
\left(-x_{7}-y_{7}-z_{7}\right) \mathbf{a}_{3}\end{array}$ & $=$ & $-x_{7} a \hat{\mathbf{x}}-y_{7} a \hat{\mathbf{y}}+z_{7} a \hat{\mathbf{z}}$ & $(96 h)$ & O II \\
\hline $\mathbf{B}_{131}$ & $=$ & $\begin{array}{c}\left(x_{7}+y_{7}-z_{7}\right) \mathbf{a}_{1}+ \\
\left(-x_{7}-y_{7}-z_{7}\right) \mathbf{a}_{2}+ \\
\left(-x_{7}+y_{7}+z_{7}\right) \mathbf{a}_{3}\end{array}$ & $=$ & $-x_{7} a \hat{\mathbf{x}}+y_{7} a \hat{\mathbf{y}}-z_{7} a \hat{\mathbf{z}}$ & $(96 h)$ & O II \\
\hline $\mathbf{B}_{132}$ & $=$ & $\begin{array}{c}\left(-x_{7}-y_{7}-z_{7}\right) \mathbf{a}_{1}+ \\
\left(x_{7}+y_{7}-z_{7}\right) \mathbf{a}_{2}+ \\
\left(x_{7}-y_{7}+z_{7}\right) \mathbf{a}_{3}\end{array}$ & $=$ & $x_{7} a \hat{\mathbf{x}}-y_{7} a \hat{\mathbf{y}}-z_{7} a \hat{\mathbf{z}}$ & $(96 h)$ & O II \\
\hline $\mathbf{B}_{133}$ & $=$ & $\begin{array}{c}\left(x_{7}+y_{7}-z_{7}\right) \mathbf{a}_{1}+ \\
\left(-x_{7}+y_{7}+z_{7}\right) \mathbf{a}_{2}+ \\
\left(x_{7}-y_{7}+z_{7}\right) \mathbf{a}_{3}\end{array}$ & $=$ & $z_{7} a \hat{\mathbf{x}}+x_{7} a \hat{\mathbf{y}}+y_{7} a \hat{\mathbf{z}}$ & $(96 h)$ & O II \\
\hline $\mathbf{B}_{134}$ & $=$ & $\begin{array}{c}\left(-x_{7}-y_{7}-z_{7}\right) \mathbf{a}_{1}+ \\
\left(x_{7}-y_{7}+z_{7}\right) \mathbf{a}_{2}+ \\
\left(-x_{7}+y_{7}+z_{7}\right) \mathbf{a}_{3}\end{array}$ & $=$ & $z_{7} a \hat{\mathbf{x}}-x_{7} a \hat{\mathbf{y}}-y_{7} a \hat{\mathbf{z}}$ & $(96 h)$ & O II \\
\hline $\mathbf{B}_{135}$ & $=$ & $\begin{array}{c}\left(-x_{7}+y_{7}+z_{7}\right) \mathbf{a}_{1}+ \\
\left(x_{7}+y_{7}-z_{7}\right) \mathbf{a}_{2}+ \\
\left(-x_{7}-y_{7}-z_{7}\right) \mathbf{a}_{3}\end{array}$ & $=$ & $-z_{7} a \hat{\mathbf{x}}-x_{7} a \hat{\mathbf{y}}+y_{7} a \hat{\mathbf{z}}$ & $(96 h)$ & O II \\
\hline $\mathbf{B}_{136}$ & $=$ & $\begin{array}{c}\left(x_{7}-y_{7}+z_{7}\right) \mathbf{a}_{1}+ \\
\left(-x_{7}-y_{7}-z_{7}\right) \mathbf{a}_{2}+ \\
\left(x_{7}+y_{7}-z_{7}\right) \mathbf{a}_{3}\end{array}$ & $=$ & $-z_{7} a \hat{\mathbf{x}}+x_{7} a \hat{\mathbf{y}}-y_{7} a \hat{\mathbf{z}}$ & $(96 h)$ & O II \\
\hline $\mathbf{B}_{137}$ & $=$ & $\begin{array}{c}\left(x_{7}-y_{7}+z_{7}\right) \mathbf{a}_{1}+ \\
\left(x_{7}+y_{7}-z_{7}\right) \mathbf{a}_{2}+ \\
\left(-x_{7}+y_{7}+z_{7}\right) \mathbf{a}_{3}\end{array}$ & $=$ & $y_{7} a \hat{\mathbf{x}}+z_{7} a \hat{\mathbf{y}}+x_{7} a \hat{\mathbf{z}}$ & $(96 h)$ & O II \\
\hline $\mathbf{B}_{138}$ & $=$ & $\begin{array}{c}\left(-x_{7}+y_{7}+z_{7}\right) \mathbf{a}_{1}+ \\
\left(-x_{7}-y_{7}-z_{7}\right) \mathbf{a}_{2}+ \\
\left(x_{7}-y_{7}+z_{7}\right) \mathbf{a}_{3}\end{array}$ & $=$ & $-y_{7} a \hat{\mathbf{x}}+z_{7} a \hat{\mathbf{y}}-x_{7} a \hat{\mathbf{z}}$ & $(96 h)$ & O II \\
\hline $\mathbf{B}_{139}$ & $=$ & $\begin{array}{c}\left(-x_{7}-y_{7}-z_{7}\right) \mathbf{a}_{1}+ \\
\left(-x_{7}+y_{7}+z_{7}\right) \mathbf{a}_{2}+ \\
\left(x_{7}+y_{7}-z_{7}\right) \mathbf{a}_{3}\end{array}$ & $=$ & $y_{7} a \hat{\mathbf{x}}-z_{7} a \hat{\mathbf{y}}-x_{7} a \hat{\mathbf{z}}$ & $(96 h)$ & O II \\
\hline
\end{tabular}




\begin{tabular}{|c|c|c|c|c|c|c|}
\hline $\mathbf{B}_{140}$ & $=$ & $\begin{array}{c}\left(x_{7}+y_{7}-z_{7}\right) \mathbf{a}_{1}+ \\
\left(x_{7}-y_{7}+z_{7}\right) \mathbf{a}_{2}+ \\
\left(-x_{7}-y_{7}-z_{7}\right) \mathbf{a}_{3}\end{array}$ & $=$ & $-y_{7} a \hat{\mathbf{x}}-z_{7} a \hat{\mathbf{y}}+x_{7} a \hat{\mathbf{z}}$ & $(96 h)$ & O II \\
\hline $\mathbf{B}_{141}$ & $=$ & $\begin{array}{c}\left(\frac{1}{4}+x_{7}-y_{7}-z_{7}\right) \mathbf{a}_{1}+ \\
\left(\frac{1}{4}-x_{7}+y_{7}-z_{7}\right) \mathbf{a}_{2}+ \\
\left(\frac{1}{4}+x_{7}+y_{7}+z_{7}\right) \mathbf{a}_{3}\end{array}$ & $=$ & $\begin{array}{c}\left(\frac{1}{4}+y_{7}\right) a \hat{\mathbf{x}}+\left(\frac{1}{4}+x_{7}\right) a \hat{\mathbf{y}}+ \\
\left(\frac{1}{4}-z_{7}\right) a \hat{\mathbf{z}}\end{array}$ & $(96 h)$ & O II \\
\hline $\mathbf{B}_{142}$ & $=$ & $\begin{array}{c}\left(\frac{1}{4}-x_{7}+y_{7}-z_{7}\right) \mathbf{a}_{1}+ \\
\left(\frac{1}{4}+x_{7}-y_{7}-z_{7}\right) \mathbf{a}_{2}+ \\
\left(\frac{1}{4}-x_{7}-y_{7}+z_{7}\right) \mathbf{a}_{3}\end{array}$ & $=$ & $\begin{array}{c}\left(\frac{1}{4}-y_{7}\right) a \hat{\mathbf{x}}+\left(\frac{1}{4}-x_{7}\right) a \hat{\mathbf{y}}+ \\
\left(\frac{1}{4}-z_{7}\right) a \hat{\mathbf{z}}\end{array}$ & $(96 h)$ & O II \\
\hline $\mathbf{B}_{143}$ & $=$ & $\begin{array}{c}\left(\frac{1}{4}-x_{7}-y_{7}+z_{7}\right) \mathbf{a}_{1}+ \\
\left(\frac{1}{4}+x_{7}+y_{7}+z_{7}\right) \mathbf{a}_{2}+ \\
\left(\frac{1}{4}-x_{7}+y_{7}-z_{7}\right) \mathbf{a}_{3}\end{array}$ & $=$ & $\begin{array}{c}\left(\frac{1}{4}+y_{7}\right) a \hat{\mathbf{x}}+\left(\frac{1}{4}-x_{7}\right) a \hat{\mathbf{y}}+ \\
\left(\frac{1}{4}+z_{7}\right) a \hat{\mathbf{z}}\end{array}$ & $(96 h)$ & O II \\
\hline $\mathbf{B}_{144}$ & $=$ & $\begin{array}{c}\left(\frac{1}{4}+x_{7}+y_{7}+z_{7}\right) \mathbf{a}_{1}+ \\
\left(\frac{1}{4}-x_{7}-y_{7}+z_{7}\right) \mathbf{a}_{2}+ \\
\left(\frac{1}{4}+x_{7}-y_{7}-z_{7}\right) \mathbf{a}_{3}\end{array}$ & $=$ & $\begin{array}{c}\left(\frac{1}{4}-y_{7}\right) a \hat{\mathbf{x}}+\left(\frac{1}{4}+x_{7}\right) a \hat{\mathbf{y}}+ \\
\left(\frac{1}{4}+z_{7}\right) a \hat{\mathbf{z}}\end{array}$ & $(96 h)$ & O II \\
\hline $\mathbf{B}_{145}$ & $=$ & $\begin{array}{c}\left(\frac{1}{4}-x_{7}-y_{7}+z_{7}\right) \mathbf{a}_{1}+ \\
\left(\frac{1}{4}+x_{7}-y_{7}-z_{7}\right) \mathbf{a}_{2}+ \\
\left(\frac{1}{4}+x_{7}+y_{7}+z_{7}\right) \mathbf{a}_{3}\end{array}$ & $=$ & $\begin{array}{c}\left(\frac{1}{4}+x_{7}\right) a \hat{\mathbf{x}}+\left(\frac{1}{4}+z_{7}\right) a \hat{\mathbf{y}}+ \\
\left(\frac{1}{4}-y_{7}\right) a \hat{\mathbf{z}}\end{array}$ & $(96 h)$ & O II \\
\hline $\mathbf{B}_{146}$ & $=$ & $\begin{array}{c}\left(\frac{1}{4}+x_{7}+y_{7}+z_{7}\right) \mathbf{a}_{1}+ \\
\left(\frac{1}{4}-x_{7}+y_{7}-z_{7}\right) \mathbf{a}_{2}+ \\
\left(\frac{1}{4}-x_{7}-y_{7}+z_{7}\right) \mathbf{a}_{3}\end{array}$ & $=$ & $\begin{array}{c}\left(\frac{1}{4}-x_{7}\right) a \hat{\mathbf{x}}+\left(\frac{1}{4}+z_{7}\right) a \hat{\mathbf{y}}+ \\
\left(\frac{1}{4}+y_{7}\right) a \hat{\mathbf{z}}\end{array}$ & $(96 h)$ & O II \\
\hline $\mathbf{B}_{147}$ & $=$ & $\begin{array}{c}\left(\frac{1}{4}+x_{7}-y_{7}-z_{7}\right) \mathbf{a}_{1}+ \\
\left(\frac{1}{4}-x_{7}-y_{7}+z_{7}\right) \mathbf{a}_{2}+ \\
\left(\frac{1}{4}-x_{7}+y_{7}-z_{7}\right) \mathbf{a}_{3}\end{array}$ & $=$ & $\begin{array}{c}\left(\frac{1}{4}-x_{7}\right) a \hat{\mathbf{x}}+\left(\frac{1}{4}-z_{7}\right) a \hat{\mathbf{y}}+ \\
\left(\frac{1}{4}-y_{7}\right) a \hat{\mathbf{z}}\end{array}$ & $(96 h)$ & O II \\
\hline $\mathbf{B}_{148}$ & $=$ & $\begin{array}{c}\left(\frac{1}{4}-x_{7}+y_{7}-z_{7}\right) \mathbf{a}_{1}+ \\
\left(\frac{1}{4}+x_{7}+y_{7}+z_{7}\right) \mathbf{a}_{2}+ \\
\left(\frac{1}{4}+x_{7}-y_{7}-z_{7}\right) \mathbf{a}_{3}\end{array}$ & $=$ & $\begin{array}{c}\left(\frac{1}{4}+x_{7}\right) a \hat{\mathbf{x}}+\left(\frac{1}{4}-z_{7}\right) a \hat{\mathbf{y}}+ \\
\left(\frac{1}{4}+y_{7}\right) a \hat{\mathbf{z}}\end{array}$ & $(96 h)$ & O II \\
\hline $\mathbf{B}_{149}$ & $=$ & $\begin{array}{c}\left(\frac{1}{4}-x_{7}+y_{7}-z_{7}\right) \mathbf{a}_{1}+ \\
\left(\frac{1}{4}-x_{7}-y_{7}+z_{7}\right) \mathbf{a}_{2}+ \\
\left(\frac{1}{4}+x_{7}+y_{7}+z_{7}\right) \mathbf{a}_{3}\end{array}$ & $=$ & $\begin{array}{c}\left(\frac{1}{4}+z_{7}\right) a \hat{\mathbf{x}}+\left(\frac{1}{4}+y_{7}\right) a \hat{\mathbf{y}}+ \\
\left(\frac{1}{4}-x_{7}\right) a \hat{\mathbf{z}}\end{array}$ & $(96 h)$ & O II \\
\hline $\mathbf{B}_{150}$ & $=$ & $\begin{array}{c}\left(\frac{1}{4}+x_{7}-y_{7}-z_{7}\right) \mathbf{a}_{1}+ \\
\left(\frac{1}{4}+x_{7}+y_{7}+z_{7}\right) \mathbf{a}_{2}+ \\
\left(\frac{1}{4}-x_{7}-y_{7}+z_{7}\right) \mathbf{a}_{3}\end{array}$ & $=$ & $\begin{array}{c}\left(\frac{1}{4}+z_{7}\right) a \hat{\mathbf{x}}+\left(\frac{1}{4}-y_{7}\right) a \hat{\mathbf{y}}+ \\
\left(\frac{1}{4}+x_{7}\right) a \hat{\mathbf{z}}\end{array}$ & $(96 h)$ & O II \\
\hline $\mathbf{B}_{151}$ & $=$ & $\begin{array}{c}\left(\frac{1}{4}+x_{7}+y_{7}+z_{7}\right) \mathbf{a}_{1}+ \\
\left(\frac{1}{4}+x_{7}-y_{7}-z_{7}\right) \mathbf{a}_{2}+ \\
\left(\frac{1}{4}-x_{7}+y_{7}-z_{7}\right) \mathbf{a}_{3}\end{array}$ & $=$ & $\begin{array}{c}\left(\frac{1}{4}-z_{7}\right) a \hat{\mathbf{x}}+\left(\frac{1}{4}+y_{7}\right) a \hat{\mathbf{y}}+ \\
\left(\frac{1}{4}+x_{7}\right) a \hat{\mathbf{z}}\end{array}$ & $(96 h)$ & O II \\
\hline $\mathbf{B}_{152}$ & $=$ & $\begin{array}{c}\left(\frac{1}{4}-x_{7}-y_{7}+z_{7}\right) \mathbf{a}_{1}+ \\
\left(\frac{1}{4}-x_{7}+y_{7}-z_{7}\right) \mathbf{a}_{2}+ \\
\left(\frac{1}{4}+x_{7}-y_{7}-z_{7}\right) \mathbf{a}_{3}\end{array}$ & $=$ & $\begin{array}{c}\left(\frac{1}{4}-z_{7}\right) a \hat{\mathbf{x}}+\left(\frac{1}{4}-y_{7}\right) a \hat{\mathbf{y}}+ \\
\left(\frac{1}{4}-x_{7}\right) a \hat{\mathbf{z}}\end{array}$ & $(96 h)$ & O II \\
\hline
\end{tabular}

\section{References:}

- D. F. Mullica, J. D. Korp, W. O. Milligan, G. W. Beall, and I. Bernal, Neutron structural refinement of cubic orthotelluric acid, Acta Crystallogr. Sect. B Struct. Sci. 36, 2565-2570 (1980), doi:10.1107/S0567740880009454. 


\section{Found in:}

- P. Villars and K. Cenzual, Pearson's Crystal Data - Crystal Structure Database for Inorganic Compounds, ASM International (2013).

\section{Geometry files:}

- CIF: pp. 962

- POSCAR: pp. 963 
$\mathrm{SiO}_{2}$ Structure: A2B_cI72_211_hi_i
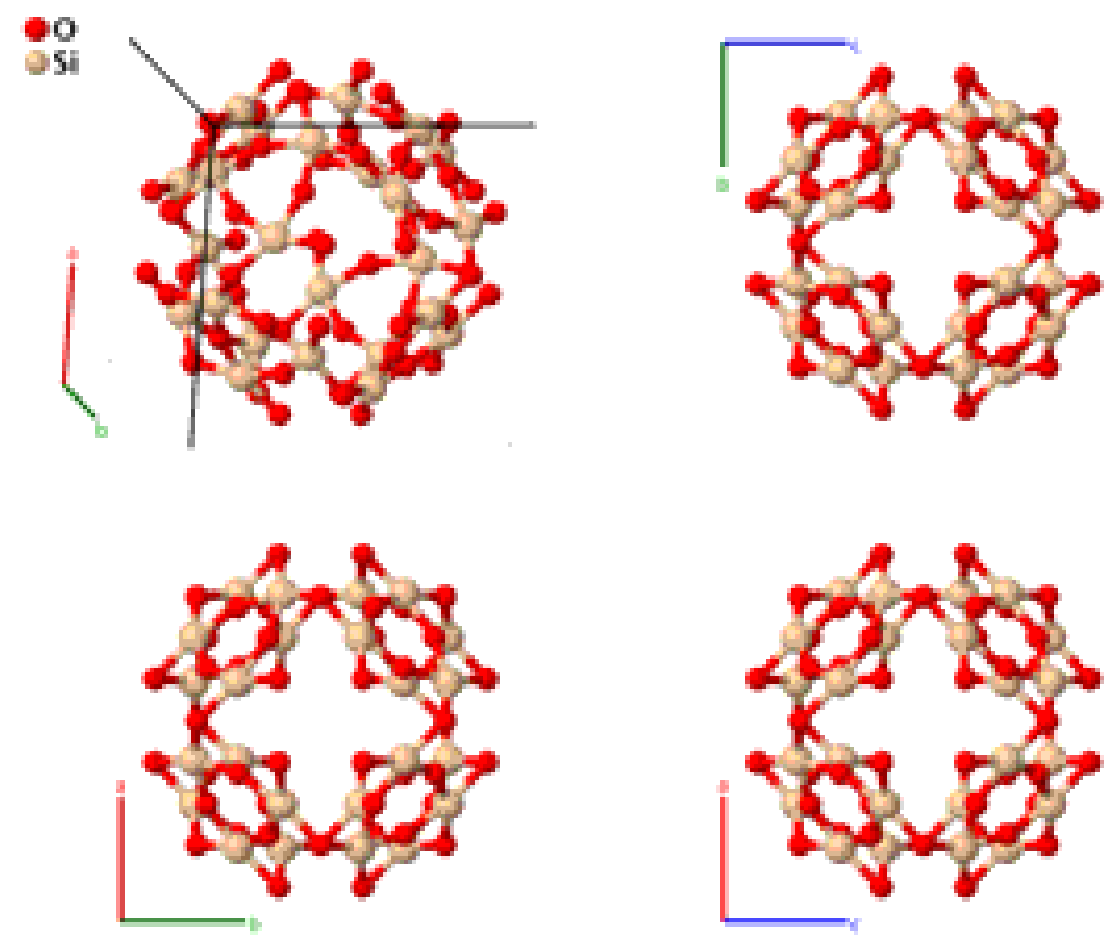

\section{Prototype}

AFLOW prototype label

$: \quad \mathrm{SiO}_{2}$

Strukturbericht designation

: A2B_cI72_211_hi_i

Pearson symbol

: None

Space group number

cI72

Space group symbol

11

AFLOW prototype command

I432

aflow - -proto=A2B_cI72_211_hi_i

- params $=a, y_{1}, y_{2}, y_{3}$

Body-centered Cubic primitive vectors:

$$
\begin{aligned}
& \mathbf{a}_{1}=-\frac{1}{2} a \hat{\mathbf{x}}+\frac{1}{2} a \hat{\mathbf{y}}+\frac{1}{2} a \hat{\mathbf{z}} \\
& \mathbf{a}_{2}=\frac{1}{2} a \hat{\mathbf{x}}-\frac{1}{2} a \hat{\mathbf{y}}+\frac{1}{2} a \hat{\mathbf{z}} \\
& \mathbf{a}_{3}=\frac{1}{2} a \hat{\mathbf{x}}+\frac{1}{2} a \hat{\mathbf{y}}-\frac{1}{2} a \hat{\mathbf{z}}
\end{aligned}
$$

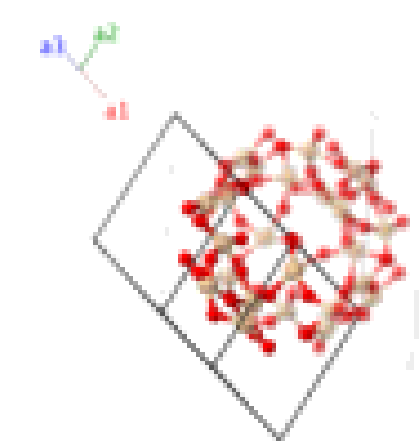

Basis vectors:

Lattice Coordinates
$\mathbf{B}_{1}=$
$\mathbf{B}_{2}=$
$\mathbf{B}_{3}=$

Cartesian Coordinates

$=$

$=\quad-y_{1} a \hat{\mathbf{y}}+y_{1} a \hat{\mathbf{z}}$

$=\quad y_{1} a \hat{\mathbf{y}}-y_{1} a \hat{\mathbf{z}}$
Wyckoff Position

(24h)

(24h)

(24h)
Atom Type

O I

O I

O I 


\begin{tabular}{|c|c|c|c|c|c|}
\hline $\mathbf{B}_{4}$ & $-2 y_{1} \mathbf{a}_{1}-y_{1} \mathbf{a}_{2}-y_{1} \mathbf{a}_{3}$ & $=$ & $-y_{1} a \hat{\mathbf{y}}-y_{1} a \hat{\mathbf{z}}$ & $(24 h)$ & $\mathrm{O}$ \\
\hline $\mathbf{B}_{5}$ & $y_{1} \mathbf{a}_{1}+2 y_{1} \mathbf{a}_{2}+y_{1} \mathbf{a}_{3}$ & $=$ & $y_{1} a \hat{\mathbf{x}}+y_{1} a \hat{\mathbf{z}}$ & $(24 h)$ & $\Omega$ \\
\hline $\mathbf{B}_{6}$ & $-y_{1} \mathbf{a}_{1}+y_{1} \mathbf{a}_{3}$ & $=$ & $y_{1} a \hat{\mathbf{x}}+-y_{1} a \hat{\mathbf{z}}$ & $(24 h)$ & O \\
\hline $\mathbf{B}_{7}$ & $y_{1} \mathbf{a}_{1}+-y_{1} \mathbf{a}_{3}$ & $=$ & $-y_{1} a \hat{\mathbf{x}}+y_{1} a \hat{\mathbf{z}}$ & $(24 h)$ & \\
\hline $\mathbf{B}_{8}$ & $-y_{1} \mathbf{a}_{1}-2 y_{1} \mathbf{a}_{2}-y_{1} \mathbf{a}_{3}$ & $=$ & $-y_{1} a \hat{\mathbf{x}}+-y_{1} a \hat{\mathbf{z}}$ & $(24 h)$ & \\
\hline $\mathbf{B}_{9}$ & $y_{1} \mathbf{a}_{1}+y_{1} \mathbf{a}_{2}+2 y_{1} \mathbf{a}_{3}$ & $=$ & $y_{1} a \hat{\mathbf{x}}+y_{1} a \hat{\mathbf{y}}$ & $(24 h)$ & \\
\hline $\mathbf{B}_{10}$ & $y_{1} \mathbf{a}_{1}-y_{1} \mathbf{a}_{2}$ & $=$ & $-y_{1} a \hat{\mathbf{x}}+y_{1} a \hat{\mathbf{y}}$ & $(24 h)$ & \\
\hline $\mathbf{B}_{11}$ & $-y_{1} \mathbf{a}_{1}+y_{1} \mathbf{a}_{2}$ & $=$ & $y_{1} a \hat{\mathbf{x}}-y_{1} a \hat{\mathbf{y}}$ & $(24 h)$ & \\
\hline $\mathbf{B}_{12}$ & $-y_{1} \mathbf{a}_{1}-y_{1} \mathbf{a}_{2}-2 y_{1} \mathbf{a}_{3}$ & $=$ & $-y_{1} a \hat{\mathbf{x}}-y_{1} a \hat{\mathbf{y}}$ & $(24 h)$ & \\
\hline $\mathbf{B}_{13}$ & $\frac{1}{2} \mathbf{a}_{1}+\left(\frac{3}{4}-y_{2}\right) \mathbf{a}_{2}+\left(\frac{1}{4}+y_{2}\right) \mathbf{a}_{3}$ & $=$ & $\frac{1}{4} a \hat{\mathbf{x}}+y_{2} a \hat{\mathbf{y}}+\left(\frac{1}{2}-y_{2}\right) a \hat{\mathbf{z}}$ & $(24 i)$ & \\
\hline $\mathbf{B}_{14}$ & $=\left(\frac{1}{2}-2 y_{2}\right) \mathbf{a}_{1}+\left(\frac{1}{4}-y_{2}\right) \mathbf{a}_{2}+\left(\frac{3}{4}-y_{2}\right) \mathbf{a}_{3}$ & $=$ & $\frac{1}{4} a \hat{\mathbf{x}}+\left(\frac{1}{2}-y_{2}\right) a \hat{\mathbf{y}}-y_{2} a \hat{\mathbf{z}}$ & $(24 i)$ & \\
\hline $\mathbf{B}_{15}$ & $=\left(\frac{1}{2}+2 y_{2}\right) \mathbf{a}_{1}+\left(\frac{1}{4}+y_{2}\right) \mathbf{a}_{2}+\left(\frac{3}{4}+y_{2}\right) \mathbf{a}_{3}$ & $=$ & $\frac{1}{4} a \hat{\mathbf{x}}+\left(\frac{1}{2}+y_{2}\right) a \hat{\mathbf{y}}+y_{2} a \hat{\mathbf{z}}$ & $(24 i)$ & \\
\hline $\mathbf{B}_{16}$ & $\frac{1}{2} \mathbf{a}_{1}+\left(\frac{3}{4}+y_{2}\right) \mathbf{a}_{2}+\left(\frac{1}{4}-y_{2}\right) \mathbf{a}_{3}$ & $=$ & $\frac{1}{4} a \hat{\mathbf{x}}-y_{2} a \hat{\mathbf{y}}+\left(\frac{1}{2}+y_{2}\right) a \hat{\mathbf{z}}$ & $(24 i)$ & \\
\hline $\mathbf{B}_{17}$ & $\left(\frac{1}{4}+y_{2}\right) \mathbf{a}_{1}+\frac{1}{2} \mathbf{a}_{2}+\left(\frac{3}{4}-y_{2}\right) \mathbf{a}_{3}$ & $=$ & $\left(\frac{1}{2}-y_{2}\right) a \hat{\mathbf{x}}+\frac{1}{4} a \hat{\mathbf{y}}+y_{2} a \hat{\mathbf{z}}$ & $(24 i)$ & \\
\hline $\mathbf{B}_{18}$ & $=\left(\frac{3}{4}-y_{2}\right) \mathbf{a}_{1}+\left(\frac{1}{2}-2 y_{2}\right) \mathbf{a}_{2}+\left(\frac{1}{4}-y_{2}\right) \mathbf{a}_{3}$ & $=$ & $-y_{2} a \hat{\mathbf{x}}+\frac{1}{4} a \hat{\mathbf{y}}+\left(\frac{1}{2}-y_{2}\right) a \hat{\mathbf{z}}$ & $(24 i)$ & \\
\hline $\mathbf{B}_{19}$ & $=\left(\frac{3}{4}+y_{2}\right) \mathbf{a}_{1}+\left(\frac{1}{2}+2 y_{2}\right) \mathbf{a}_{2}+\left(\frac{1}{4}+y_{2}\right) \mathbf{a}_{3}$ & $=$ & $y_{2} a \hat{\mathbf{x}}+\frac{1}{4} a \hat{\mathbf{y}}+\left(\frac{1}{2}+y_{2}\right) a \hat{\mathbf{z}}$ & $(24 i)$ & \\
\hline $\mathbf{B}_{20}$ & $=\left(\frac{1}{4}-y_{2}\right) \mathbf{a}_{1}+\frac{1}{2} \mathbf{a}_{2}+\left(\frac{3}{4}+y_{2}\right) \mathbf{a}_{3}$ & $=$ & $\left(\frac{1}{2}+y_{2}\right) a \hat{\mathbf{x}}+\frac{1}{4} a \hat{\mathbf{y}}-y_{2} a \hat{\mathbf{z}}$ & $(24 i)$ & \\
\hline $\mathbf{B}_{21}$ & $=\left(\frac{3}{4}-y_{2}\right) \mathbf{a}_{1}+\left(\frac{1}{4}+y_{2}\right) \mathbf{a}_{2}+\frac{1}{2} \mathbf{a}_{3}$ & $=$ & $y_{2} a \hat{\mathbf{x}}+\left(\frac{1}{2}-y_{2}\right) a \hat{\mathbf{y}}+\frac{1}{4} a \hat{\mathbf{z}}$ & $(24 i)$ & \\
\hline $\mathbf{B}_{22}$ & $=\left(\frac{1}{4}-y_{2}\right) \mathbf{a}_{1}+\left(\frac{3}{4}-y_{2}\right) \mathbf{a}_{2}+\left(\frac{1}{2}-2 y_{2}\right) \mathbf{a}_{3}$ & $=$ & $\left(\frac{1}{2}-y_{2}\right) a \hat{\mathbf{x}}-y_{2} a \hat{\mathbf{y}}+\frac{1}{4} a \hat{\mathbf{z}}$ & $(24 i)$ & \\
\hline $\mathbf{B}_{23}$ & $=\left(\frac{1}{4}+y_{2}\right) \mathbf{a}_{1}+\left(\frac{3}{4}+y_{2}\right) \mathbf{a}_{2}+\left(\frac{1}{2}+2 y_{2}\right) \mathbf{a}_{3}$ & $=$ & $\left(\frac{1}{2}+y_{2}\right) a \hat{\mathbf{x}}+y_{2} a \hat{\mathbf{y}}+\frac{1}{4} a \hat{\mathbf{z}}$ & $(24 i)$ & \\
\hline $\mathbf{B}_{24}$ & $=\left(\frac{3}{4}+y_{2}\right) \mathbf{a}_{1}+\left(\frac{1}{4}-y_{2}\right) \mathbf{a}_{2}+\frac{1}{2} \mathbf{a}_{3}$ & $=$ & $-y_{2} a \hat{\mathbf{x}}+\left(\frac{1}{2}+y_{2}\right) a \hat{\mathbf{y}}+\frac{1}{4} a \hat{\mathbf{z}}$ & $(24 i)$ & \\
\hline $\mathbf{B}_{25}$ & $\frac{1}{2} \mathbf{a}_{1}+\left(\frac{3}{4}-y_{3}\right) \mathbf{a}_{2}+\left(\frac{1}{4}+y_{3}\right) \mathbf{a}_{3}$ & $=$ & $\frac{1}{4} a \hat{\mathbf{x}}+y_{3} a \hat{\mathbf{y}}+\left(\frac{1}{2}-y_{3}\right) a \hat{\mathbf{z}}$ & $(24 i)$ & \\
\hline $\mathbf{B}_{26}$ & $=\left(\frac{1}{2}-2 y_{3}\right) \mathbf{a}_{1}+\left(\frac{1}{4}-y_{3}\right) \mathbf{a}_{2}+\left(\frac{3}{4}-y_{3}\right) \mathbf{a}_{3}$ & $=$ & $\frac{1}{4} a \hat{\mathbf{x}}+\left(\frac{1}{2}-y_{3}\right) a \hat{\mathbf{y}}-y_{3} a \hat{\mathbf{z}}$ & $(24 i)$ & \\
\hline $\mathbf{B}_{27}$ & $=\left(\frac{1}{2}+2 y_{3}\right) \mathbf{a}_{1}+\left(\frac{1}{4}+y_{3}\right) \mathbf{a}_{2}+\left(\frac{3}{4}+y_{3}\right) \mathbf{a}_{3}$ & $=$ & $\frac{1}{4} a \hat{\mathbf{x}}+\left(\frac{1}{2}+y_{3}\right) a \hat{\mathbf{y}}+y_{3} a \hat{\mathbf{z}}$ & $(24 i)$ & \\
\hline $\mathbf{B}_{28}$ & $\frac{1}{2} \mathbf{a}_{1}+\left(\frac{3}{4}+y_{3}\right) \mathbf{a}_{2}+\left(\frac{1}{4}-y_{3}\right) \mathbf{a}_{3}$ & $=$ & $\frac{1}{4} a \hat{\mathbf{x}}-y_{3} a \hat{\mathbf{y}}+\left(\frac{1}{2}+y_{3}\right) a \hat{\mathbf{z}}$ & $(24 i)$ & \\
\hline $\mathbf{B}_{29}$ & $\left(\frac{1}{4}+y_{3}\right) \mathbf{a}_{1}+\frac{1}{2} \mathbf{a}_{2}+\left(\frac{3}{4}-y_{3}\right) \mathbf{a}_{3}$ & $=$ & $\left(\frac{1}{2}-y_{3}\right) a \hat{\mathbf{x}}+\frac{1}{4} a \hat{\mathbf{y}}+y_{3} a \hat{\mathbf{z}}$ & $(24 i)$ & \\
\hline $\mathbf{B}_{30}$ & $=\left(\frac{3}{4}-y_{3}\right) \mathbf{a}_{1}+\left(\frac{1}{2}-2 y_{3}\right) \mathbf{a}_{2}+\left(\frac{1}{4}-y_{3}\right) \mathbf{a}_{3}$ & $=$ & $-y_{3} a \hat{\mathbf{x}}+\frac{1}{4} a \hat{\mathbf{y}}+\left(\frac{1}{2}-y_{3}\right) a \hat{\mathbf{z}}$ & $(24 i)$ & \\
\hline $\mathbf{B}_{31}$ & $=\left(\frac{3}{4}+y_{3}\right) \mathbf{a}_{1}+\left(\frac{1}{2}+2 y_{3}\right) \mathbf{a}_{2}+\left(\frac{1}{4}+y_{3}\right) \mathbf{a}_{3}$ & $=$ & $y_{3} a \hat{\mathbf{x}}+\frac{1}{4} a \hat{\mathbf{y}}+\left(\frac{1}{2}+y_{3}\right) a \hat{\mathbf{z}}$ & $(24 i)$ & \\
\hline $\mathbf{B}_{32}$ & $=\left(\frac{1}{4}-y_{3}\right) \mathbf{a}_{1}+\frac{1}{2} \mathbf{a}_{2}+\left(\frac{3}{4}+y_{3}\right) \mathbf{a}_{3}$ & $=$ & $\left(\frac{1}{2}+y_{3}\right) a \hat{\mathbf{x}}+\frac{1}{4} a \hat{\mathbf{y}}-y_{3} a \hat{\mathbf{z}}$ & $(24 i)$ & \\
\hline $\mathbf{B}_{33}$ & $\left(\frac{3}{4}-y_{3}\right) \mathbf{a}_{1}+\left(\frac{1}{4}+y_{3}\right) \mathbf{a}_{2}+\frac{1}{2} \mathbf{a}_{3}$ & $=$ & $y_{3} a \hat{\mathbf{x}}+\left(\frac{1}{2}-y_{3}\right) a \hat{\mathbf{y}}+\frac{1}{4} a \hat{\mathbf{z}}$ & $(24 i)$ & \\
\hline $\mathbf{B}_{34}$ & $=\left(\frac{1}{4}-y_{3}\right) \mathbf{a}_{1}+\left(\frac{3}{4}-y_{3}\right) \mathbf{a}_{2}+\left(\frac{1}{2}-2 y_{3}\right) \mathbf{a}_{3}$ & $=$ & $\left(\frac{1}{2}-y_{3}\right) a \hat{\mathbf{x}}-y_{3} a \hat{\mathbf{y}}+\frac{1}{4} a \hat{\mathbf{z}}$ & $(24 i)$ & \\
\hline $\mathbf{B}_{35}$ & $=\left(\frac{1}{4}+y_{3}\right) \mathbf{a}_{1}+\left(\frac{3}{4}+y_{3}\right) \mathbf{a}_{2}+\left(\frac{1}{2}+2 y_{3}\right) \mathbf{a}_{3}$ & $=$ & $\left(\frac{1}{2}+y_{3}\right) a \hat{\mathbf{x}}+y_{3} a \hat{\mathbf{y}}+\frac{1}{4} a \hat{\mathbf{z}}$ & $(24 i)$ & \\
\hline $\mathbf{B}_{36}$ & $\left(\frac{3}{4}+y_{3}\right) \mathbf{a}_{1}+\left(\frac{1}{4}-y_{3}\right) \mathbf{a}_{2}+\frac{1}{2} \mathbf{a}_{3}$ & $=$ & $-y_{3} a \hat{\mathbf{x}}+\left(\frac{1}{2}+y_{3}\right) a \hat{\mathbf{y}}+\frac{1}{4} a \hat{\mathbf{z}}$ & $(24 i)$ & \\
\hline
\end{tabular}

\section{References:}

- M. D. Foster, O. Delgado Friedrichs, R. G. Bell, and F. A. Almeida Pazand J. Klinowski, Chemical Evaluation of Hypothetical Uninodal Zeolites, J. Am. Chem. Soc. 126, 9769-9775 (2004), doi:10.1021/ja037334j. 


\section{Found in:}

- ICSD, Inorganic Crystal Structure Database. ID 170506.

\section{Geometry files:}

- CIF: pp. 964

- POSCAR: pp. 964 


\begin{tabular}{|c|c|c|c|c|c|}
\hline $\mathbf{B}_{4}$ & $=$ & $\frac{5}{8} \mathbf{a}_{1}+\frac{3}{8} \mathbf{a}_{2}+\frac{7}{8} \mathbf{a}_{3}$ & $=$ & $\frac{5}{8} a \hat{\mathbf{x}}+\frac{3}{8} a \hat{\mathbf{y}}+\frac{7}{8} a \hat{\mathbf{z}}$ & $(4 a)$ \\
\hline $\mathbf{B}_{5}$ & $=$ & $x_{2} \mathbf{a}_{1}+x_{2} \mathbf{a}_{2}+x_{2} \mathbf{a}_{3}$ & $=$ & $x_{2} a \hat{\mathbf{x}}+x_{2} a \hat{\mathbf{y}}+x_{2} a \hat{\mathbf{z}}$ & $(8 c)$ \\
\hline $\mathbf{B}_{6}$ & $=$ & $\left(\frac{1}{2}-x_{2}\right) \mathbf{a}_{1}-x_{2} \mathbf{a}_{2}+\left(\frac{1}{2}+x_{2}\right) \mathbf{a}_{3}$ & $=$ & $\left(\frac{1}{2}-x_{2}\right) a \hat{\mathbf{x}}-x_{2} a \hat{\mathbf{y}}+\left(\frac{1}{2}+x_{2}\right) a \hat{\mathbf{z}}$ & $(8 c)$ \\
\hline $\mathbf{B}_{7}$ & $=$ & $-x_{2} \mathbf{a}_{1}+\left(\frac{1}{2}+x_{2}\right) \mathbf{a}_{2}+\left(\frac{1}{2}-x_{2}\right) \mathbf{a}_{3}$ & $=$ & $-x_{2} a \hat{\mathbf{x}}+\left(\frac{1}{2}+x_{2}\right) a \hat{\mathbf{y}}+\left(\frac{1}{2}-x_{2}\right) a \hat{\mathbf{z}}$ & $(8 c)$ \\
\hline $\mathbf{B}_{8}$ & $=$ & $\left(\frac{1}{2}+x_{2}\right) \mathbf{a}_{1}+\left(\frac{1}{2}-x_{2}\right) \mathbf{a}_{2}-x_{2} \mathbf{a}_{3}$ & $=$ & $\left(\frac{1}{2}+x_{2}\right) a \hat{\mathbf{x}}+\left(\frac{1}{2}-x_{2}\right) a \hat{\mathbf{y}}-x_{2} a \hat{\mathbf{z}}$ & $(8 c)$ \\
\hline $\mathbf{B}_{9}$ & $=$ & $\begin{array}{c}\left(\frac{1}{4}+x_{2}\right) \mathbf{a}_{1}+\left(\frac{3}{4}+x_{2}\right) \mathbf{a}_{2}+ \\
\left(\frac{3}{4}-x_{2}\right) \mathbf{a}_{3}\end{array}$ & $=$ & $\begin{array}{c}\left(\frac{1}{4}+x_{2}\right) a \hat{\mathbf{x}}+\left(\frac{3}{4}+x_{2}\right) a \hat{\mathbf{y}}+ \\
\left(\frac{3}{4}-x_{2}\right) a \hat{\mathbf{z}}\end{array}$ & $(8 c)$ \\
\hline $\mathbf{B}_{10}$ & $=$ & $\begin{array}{c}\left(\frac{1}{4}-x_{2}\right) \mathbf{a}_{1}+\left(\frac{1}{4}-x_{2}\right) \mathbf{a}_{2}+ \\
\left(\frac{1}{4}-x_{2}\right) \mathbf{a}_{3}\end{array}$ & $=$ & $\begin{array}{c}\left(\frac{1}{4}-x_{2}\right) a \hat{\mathbf{x}}+\left(\frac{1}{4}-x_{2}\right) a \hat{\mathbf{y}}+ \\
\left(\frac{1}{4}-x_{2}\right) a \hat{\mathbf{z}}\end{array}$ & $(8 c)$ \\
\hline $\mathbf{B}_{11}$ & $=$ & $\begin{array}{c}\left(\frac{3}{4}+x_{2}\right) \mathbf{a}_{1}+\left(\frac{3}{4}-x_{2}\right) \mathbf{a}_{2}+ \\
\left(\frac{1}{4}+x_{2}\right) \mathbf{a}_{3}\end{array}$ & $=$ & $\begin{array}{c}\left(\frac{3}{4}+x_{2}\right) a \hat{\mathbf{x}}+\left(\frac{3}{4}-x_{2}\right) a \hat{\mathbf{y}}+ \\
\left(\frac{1}{4}+x_{2}\right) a \hat{\mathbf{z}}\end{array}$ & $(8 c)$ \\
\hline $\mathbf{B}_{12}$ & $=$ & $\begin{array}{c}\left(\frac{3}{4}-x_{2}\right) \mathbf{a}_{1}+\left(\frac{1}{4}+x_{2}\right) \mathbf{a}_{2}+ \\
\left(\frac{3}{4}+x_{2}\right) \mathbf{a}_{3}\end{array}$ & $=$ & $\begin{array}{c}\left(\frac{3}{4}-x_{2}\right) a \hat{\mathbf{x}}+\left(\frac{1}{4}+x_{2}\right) a \hat{\mathbf{y}}+ \\
\left(\frac{3}{4}+x_{2}\right) a \hat{\mathbf{z}}\end{array}$ & $(8 c)$ \\
\hline
\end{tabular}

\section{References:}

- K. Janzon, H. Schäfer, and A. Weiss, Kristallstruktur von Strontiumdisilicid SrSi 2 , Angew. Chem. Int. Ed. 77, 258-259 (1965), doi:10.1002/ange.19650770605.

\section{Found in:}

- P. Villars and L. D. Calvert, eds., Pearson's Handbook of Crystallographic Data for Intermetallic Phases, vol. 1 (American Society of Metals, Materials Park, Ohio, 1985).

\section{Geometry files:}

- CIF: pp. 965

- POSCAR: pp. 965 


\section{$\mathrm{Ca}_{3} \mathrm{PI}_{3}$ Structure: A3B3C_cI56_214_g_h_a}
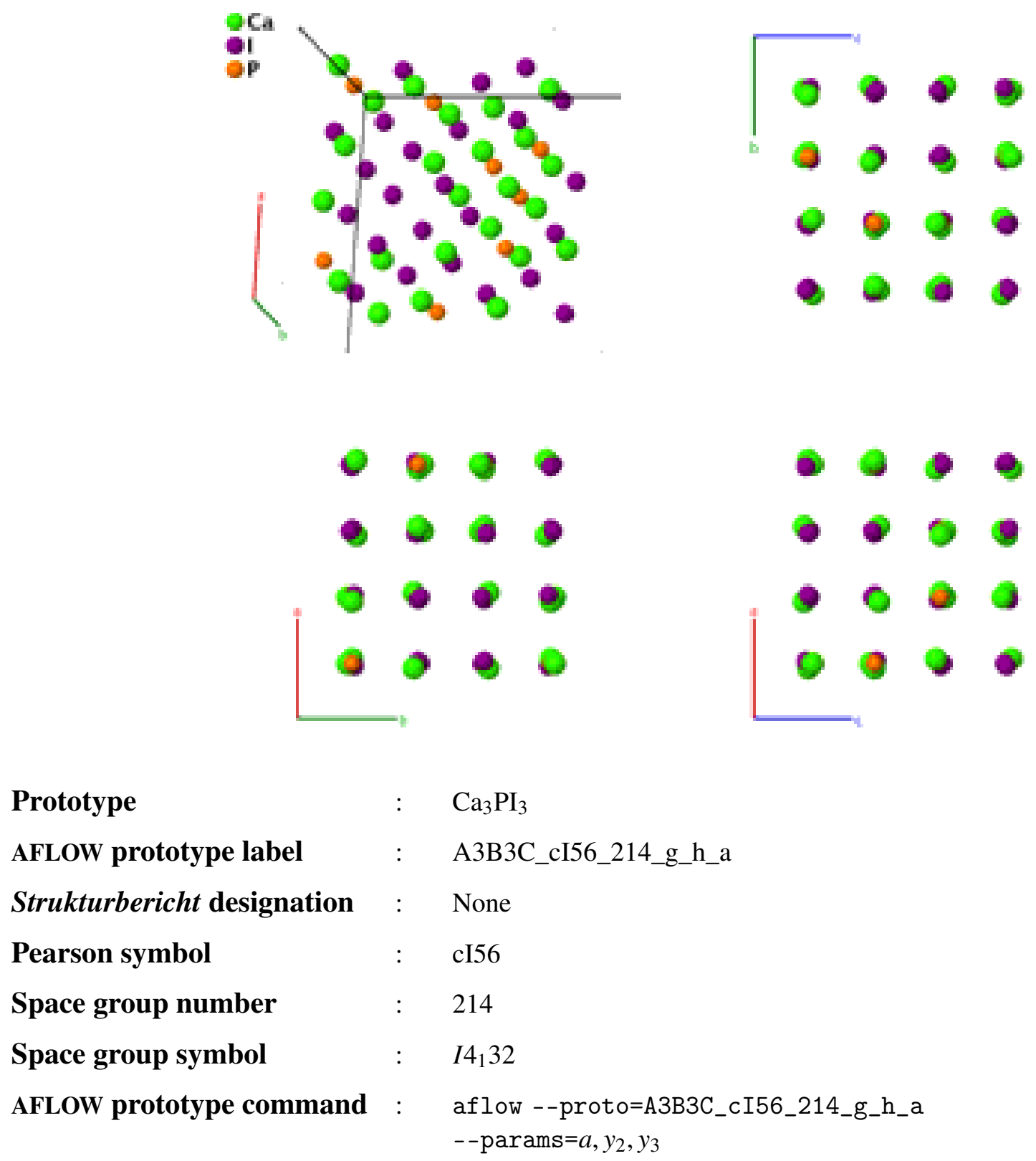

Body-centered Cubic primitive vectors:

$$
\begin{aligned}
& \mathbf{a}_{1}=-\frac{1}{2} a \hat{\mathbf{x}}+\frac{1}{2} a \hat{\mathbf{y}}+\frac{1}{2} a \hat{\mathbf{z}} \\
& \mathbf{a}_{2}=\frac{1}{2} a \hat{\mathbf{x}}-\frac{1}{2} a \hat{\mathbf{y}}+\frac{1}{2} a \hat{\mathbf{z}} \\
& \mathbf{a}_{3}=\frac{1}{2} a \hat{\mathbf{x}}+\frac{1}{2} a \hat{\mathbf{y}}-\frac{1}{2} a \hat{\mathbf{z}}
\end{aligned}
$$

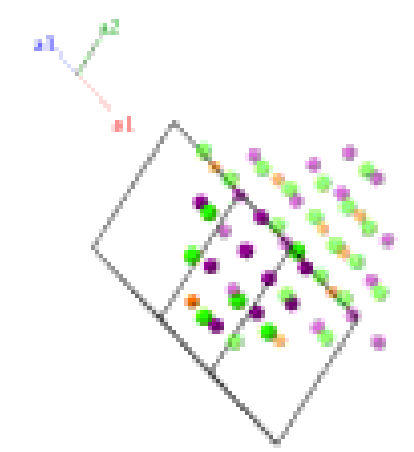

Basis vectors:

Lattice Coordinates

$\mathbf{B}_{1}=\quad \frac{1}{4} \mathbf{a}_{1}+\frac{1}{4} \mathbf{a}_{2}+\frac{1}{4} \mathbf{a}_{3}$
Cartesian Coordinates

$=\quad \frac{1}{8} a \hat{\mathbf{x}}+\frac{1}{8} a \hat{\mathbf{y}}+\frac{1}{8} a \hat{\mathbf{z}}$
Wyckoff Position Atom Type

$(8 a)$
$\mathrm{P}$ 


\begin{tabular}{|c|c|c|c|c|c|c|}
\hline $\mathbf{B}_{2}$ & $=$ & $\frac{1}{2} \mathbf{a}_{1}+\frac{1}{4} \mathbf{a}_{3}$ & $=$ & $-\frac{1}{8} a \hat{\mathbf{x}}+\frac{3}{8} a \hat{\mathbf{y}}+\frac{1}{8} a \hat{\mathbf{z}}$ & $(8 a)$ & $\mathrm{P}$ \\
\hline $\mathbf{B}_{3}$ & $=$ & $\frac{1}{4} \mathbf{a}_{2}+\frac{1}{2} \mathbf{a}_{3}$ & $=$ & $\frac{3}{8} a \hat{\mathbf{x}}+\frac{1}{8} a \hat{\mathbf{y}}-\frac{1}{8} a \hat{\mathbf{z}}$ & $(8 a)$ & $\mathrm{P}$ \\
\hline $\mathbf{B}_{4}$ & $=$ & $\frac{1}{4} \mathbf{a}_{1}+\frac{1}{2} \mathbf{a}_{2}$ & $=$ & $\frac{1}{8} a \hat{\mathbf{x}}-\frac{1}{8} a \hat{\mathbf{y}}+\frac{3}{8} a \hat{\mathbf{z}}$ & $(8 a)$ & $\mathrm{P}$ \\
\hline $\mathbf{B}_{5}$ & $=$ & $\begin{array}{c}\left(\frac{1}{4}+2 y_{2}\right) \mathbf{a}_{1}+\left(\frac{3}{8}+y_{2}\right) \mathbf{a}_{2}+ \\
\left(\frac{1}{8}+y_{2}\right) \mathbf{a}_{3}\end{array}$ & $=$ & $\frac{1}{8} a \hat{\mathbf{x}}+y_{2} a \hat{\mathbf{y}}+\left(\frac{1}{4}+y_{2}\right) a \hat{\mathbf{z}}$ & $(24 g)$ & $\mathrm{Ca}$ \\
\hline $\mathbf{B}_{6}$ & $=$ & $\frac{3}{4} \mathbf{a}_{1}+\left(\frac{1}{8}+y_{2}\right) \mathbf{a}_{2}+\left(\frac{3}{8}-y_{2}\right) \mathbf{a}_{3}$ & $=$ & $-\frac{1}{8} a \hat{\mathbf{x}}+\left(\frac{1}{2}-y_{2}\right) a \hat{\mathbf{y}}+\left(\frac{1}{4}+y_{2}\right) a \hat{\mathbf{z}}$ & $(24 g)$ & $\mathrm{Ca}$ \\
\hline $\mathbf{B}_{7}$ & $=$ & $\frac{3}{4} \mathbf{a}_{1}+\left(\frac{1}{8}-y_{2}\right) \mathbf{a}_{2}+\left(\frac{3}{8}+y_{2}\right) \mathbf{a}_{3}$ & $=$ & $\frac{7}{8} a \hat{\mathbf{x}}+\left(\frac{1}{2}+y_{2}\right) a \hat{\mathbf{y}}+\left(\frac{1}{4}-y_{2}\right) a \hat{\mathbf{z}}$ & $(24 g)$ & $\mathrm{Ca}$ \\
\hline $\mathbf{B}_{8}$ & $=$ & $\begin{array}{c}\left(\frac{1}{4}-2 y_{2}\right) \mathbf{a}_{1}+\left(\frac{3}{8}-y_{2}\right) \mathbf{a}_{2}+ \\
\left(\frac{1}{8}-y_{2}\right) \mathbf{a}_{3}\end{array}$ & $=$ & $\frac{1}{8} a \hat{\mathbf{x}}-y_{2} a \hat{\mathbf{y}}+\left(\frac{1}{4}-y_{2}\right) a \hat{\mathbf{z}}$ & $(24 g)$ & $\mathrm{Ca}$ \\
\hline $\mathbf{B}_{9}$ & $=$ & $\begin{array}{c}\left(\frac{1}{8}+y_{2}\right) \mathbf{a}_{1}+\left(\frac{1}{4}+2 y_{2}\right) \mathbf{a}_{2}+ \\
\left(\frac{3}{8}+y_{2}\right) \mathbf{a}_{3}\end{array}$ & $=$ & $\left(\frac{1}{4}+y_{2}\right) a \hat{\mathbf{x}}+\frac{1}{8} a \hat{\mathbf{y}}+y_{2} a \hat{\mathbf{z}}$ & $(24 g)$ & $\mathrm{Ca}$ \\
\hline $\mathbf{B}_{10}$ & $=$ & $\left(\frac{3}{8}-y_{2}\right) \mathbf{a}_{1}+\frac{3}{4} \mathbf{a}_{2}+\left(\frac{1}{8}+y_{2}\right) \mathbf{a}_{3}$ & $=$ & $\left(\frac{1}{4}+y_{2}\right) a \hat{\mathbf{x}}-\frac{1}{8} a \hat{\mathbf{y}}+\left(\frac{1}{2}-y_{2}\right) a \hat{\mathbf{z}}$ & $(24 g)$ & $\mathrm{Ca}$ \\
\hline $\mathbf{B}_{11}$ & $=$ & $\left(\frac{3}{8}+y_{2}\right) \mathbf{a}_{1}+\frac{3}{4} \mathbf{a}_{2}+\left(\frac{1}{8}-y_{2}\right) \mathbf{a}_{3}$ & $=$ & $\left(\frac{1}{4}-y_{2}\right) a \hat{\mathbf{x}}+\frac{7}{8} a \hat{\mathbf{y}}+\left(\frac{1}{2}+y_{2}\right) a \hat{\mathbf{z}}$ & $(24 g)$ & $\mathrm{Ca}$ \\
\hline $\mathbf{B}_{12}$ & $=$ & $\begin{array}{c}\left(\frac{1}{8}-y_{2}\right) \mathbf{a}_{1}+\left(\frac{1}{4}-2 y_{2}\right) \mathbf{a}_{2}+ \\
\left(\frac{3}{8}-y_{2}\right) \mathbf{a}_{3}\end{array}$ & $=$ & $\left(\frac{1}{4}-y_{2}\right) a \hat{\mathbf{x}}+\frac{1}{8} a \hat{\mathbf{y}}-y_{2} a \hat{\mathbf{z}}$ & $(24 g)$ & $\mathrm{Ca}$ \\
\hline $\mathbf{B}_{13}$ & $=$ & $\begin{array}{c}\left(\frac{3}{8}+y_{2}\right) \mathbf{a}_{1}+\left(\frac{1}{8}+y_{2}\right) \mathbf{a}_{2}+ \\
\left(\frac{1}{4}+2 y_{2}\right) \mathbf{a}_{3}\end{array}$ & $=$ & $y_{2} a \hat{\mathbf{x}}+\left(\frac{1}{4}+y_{2}\right) a \hat{\mathbf{y}}+\frac{1}{8} a \hat{\mathbf{z}}$ & $(24 g)$ & $\mathrm{Ca}$ \\
\hline $\mathbf{B}_{14}$ & $=$ & $\left(\frac{1}{8}+y_{2}\right) \mathbf{a}_{1}+\left(\frac{3}{8}-y_{2}\right) \mathbf{a}_{2}+\frac{3}{4} \mathbf{a}_{3}$ & $=$ & $\left(\frac{1}{2}-y_{2}\right) a \hat{\mathbf{x}}+\left(\frac{1}{4}+y_{2}\right) a \hat{\mathbf{y}}-\frac{1}{8} a \hat{\mathbf{z}}$ & $(24 g)$ & $\mathrm{Ca}$ \\
\hline $\mathbf{B}_{15}$ & $=$ & $\left(\frac{1}{8}-y_{2}\right) \mathbf{a}_{1}+\left(\frac{3}{8}+y_{2}\right) \mathbf{a}_{2}+\frac{3}{4} \mathbf{a}_{3}$ & $=$ & $\left(\frac{1}{2}+y_{2}\right) a \hat{\mathbf{x}}+\left(\frac{1}{4}-y_{2}\right) a \hat{\mathbf{y}}+\frac{7}{8} a \hat{\mathbf{z}}$ & $(24 g)$ & $\mathrm{Ca}$ \\
\hline $\mathbf{B}_{16}$ & $=$ & $\begin{array}{c}\left(\frac{3}{8}-y_{2}\right) \mathbf{a}_{1}+\left(\frac{1}{8}-y_{2}\right) \mathbf{a}_{2}+ \\
\left(\frac{1}{4}-2 y_{2}\right) \mathbf{a}_{3}\end{array}$ & $=$ & $-y_{2} a \hat{\mathbf{x}}+\left(\frac{1}{4}-y_{2}\right) a \hat{\mathbf{y}}+\frac{1}{8} a \hat{\mathbf{z}}$ & $(24 g)$ & $\mathrm{Ca}$ \\
\hline $\mathbf{B}_{17}$ & $=$ & $\frac{1}{4} \mathbf{a}_{1}+\left(\frac{3}{8}-y_{3}\right) \mathbf{a}_{2}+\left(\frac{1}{8}+y_{3}\right) \mathbf{a}_{3}$ & $=$ & $\frac{1}{8} a \hat{\mathbf{x}}+y_{3} a \hat{\mathbf{y}}+\left(\frac{1}{4}-y_{3}\right) a \hat{\mathbf{z}}$ & $(24 h)$ & I \\
\hline $\mathbf{B}_{18}$ & $=$ & $\begin{array}{c}\left(\frac{3}{4}-2 y_{3}\right) \mathbf{a}_{1}+\left(\frac{1}{8}-y_{3}\right) \mathbf{a}_{2}+ \\
\left(\frac{3}{8}-y_{3}\right) \mathbf{a}_{3}\end{array}$ & $=$ & $-\frac{1}{8} a \hat{\mathbf{x}}+\left(\frac{1}{2}-y_{3}\right) a \hat{\mathbf{y}}+\left(\frac{1}{4}-y_{3}\right) a \hat{\mathbf{z}}$ & $(24 h)$ & I \\
\hline $\mathbf{B}_{19}$ & $=$ & $\begin{array}{c}\left(\frac{3}{4}+2 y_{3}\right) \mathbf{a}_{1}+\left(\frac{1}{8}+y_{3}\right) \mathbf{a}_{2}+ \\
\left(\frac{3}{8}+y_{3}\right) \mathbf{a}_{3}\end{array}$ & $=$ & $\frac{7}{8} a \hat{\mathbf{x}}+\left(\frac{1}{2}+y_{3}\right) a \hat{\mathbf{y}}+\left(\frac{1}{4}+y_{3}\right) a \hat{\mathbf{z}}$ & $(24 h)$ & I \\
\hline $\mathbf{B}_{20}$ & $=$ & $\frac{1}{4} \mathbf{a}_{1}+\left(\frac{3}{8}+y_{3}\right) \mathbf{a}_{2}+\left(\frac{1}{8}-y_{3}\right) \mathbf{a}_{3}$ & $=$ & $\frac{1}{8} a \hat{\mathbf{x}}-y_{3} a \hat{\mathbf{y}}+\left(\frac{1}{4}+y_{3}\right) a \hat{\mathbf{z}}$ & $(24 h)$ & I \\
\hline $\mathbf{B}_{21}$ & $=$ & $\left(\frac{1}{8}+y_{3}\right) \mathbf{a}_{1}+\frac{1}{4} \mathbf{a}_{2}+\left(\frac{3}{8}-y_{3}\right) \mathbf{a}_{3}$ & $=$ & $\left(\frac{1}{4}-y_{3}\right) a \hat{\mathbf{x}}+\frac{1}{8} a \hat{\mathbf{y}}+y_{3} a \hat{\mathbf{z}}$ & $(24 h)$ & I \\
\hline $\mathbf{B}_{22}$ & $=$ & $\begin{array}{c}\left(\frac{3}{8}-y_{3}\right) \mathbf{a}_{1}+\left(\frac{3}{4}-2 y_{3}\right) \mathbf{a}_{2}+ \\
\left(\frac{1}{8}-y_{3}\right) \mathbf{a}_{3}\end{array}$ & $=$ & $\left(\frac{1}{4}-y_{3}\right) a \hat{\mathbf{x}}-\frac{1}{8} a \hat{\mathbf{y}}+\left(\frac{1}{2}-y_{3}\right) a \hat{\mathbf{z}}$ & $(24 h)$ & I \\
\hline $\mathbf{B}_{23}$ & $=$ & $\begin{array}{c}\left(\frac{3}{8}+y_{3}\right) \mathbf{a}_{1}+\left(\frac{3}{4}+2 y_{3}\right) \mathbf{a}_{2}+ \\
\left(\frac{1}{8}+y_{3}\right) \mathbf{a}_{3}\end{array}$ & $=$ & $\left(\frac{1}{4}+y_{3}\right) a \hat{\mathbf{x}}+\frac{7}{8} a \hat{\mathbf{y}}+\left(\frac{1}{2}+y_{3}\right) a \hat{\mathbf{z}}$ & $(24 h)$ & I \\
\hline $\mathbf{B}_{24}$ & $=$ & $\left(\frac{1}{8}-y_{3}\right) \mathbf{a}_{1}+\frac{1}{4} \mathbf{a}_{2}+\left(\frac{3}{8}+y_{3}\right) \mathbf{a}_{3}$ & $=$ & $\left(\frac{1}{4}+y_{3}\right) a \hat{\mathbf{x}}+\frac{1}{8} a \hat{\mathbf{y}}-y_{3} a \hat{\mathbf{z}}$ & $(24 h)$ & I \\
\hline $\mathbf{B}_{25}$ & $=$ & $\left(\frac{3}{8}-y_{3}\right) \mathbf{a}_{1}+\left(\frac{1}{8}+y_{3}\right) \mathbf{a}_{2}+\frac{1}{4} \mathbf{a}_{3}$ & $=$ & $y_{3} a \hat{\mathbf{x}}+\left(\frac{1}{4}-y_{3}\right) a \hat{\mathbf{y}}+\frac{1}{8} a \hat{\mathbf{z}}$ & $(24 h)$ & I \\
\hline $\mathbf{B}_{26}$ & $=$ & $\begin{array}{c}\left(\frac{1}{8}-y_{3}\right) \mathbf{a}_{1}+\left(\frac{3}{8}-y_{3}\right) \mathbf{a}_{2}+ \\
\left(\frac{3}{4}-2 y_{3}\right) \mathbf{a}_{3}\end{array}$ & $=$ & $\left(\frac{1}{2}-y_{3}\right) a \hat{\mathbf{x}}+\left(\frac{1}{4}-y_{3}\right) a \hat{\mathbf{y}}-\frac{1}{8} a \hat{\mathbf{z}}$ & $(24 h)$ & I \\
\hline $\mathbf{B}_{27}$ & $=$ & $\begin{array}{c}\left(\frac{1}{8}+y_{3}\right) \mathbf{a}_{1}+\left(\frac{3}{8}+y_{3}\right) \mathbf{a}_{2}+ \\
\left(\frac{3}{4}+2 y_{3}\right) \mathbf{a}_{3}\end{array}$ & $=$ & $\left(\frac{1}{2}+y_{3}\right) a \hat{\mathbf{x}}+\left(\frac{1}{4}+y_{3}\right) a \hat{\mathbf{y}}+\frac{7}{8} a \hat{\mathbf{z}}$ & $(24 h)$ & I \\
\hline $\mathbf{B}_{28}$ & $=$ & $\left(\frac{3}{8}+y_{3}\right) \mathbf{a}_{1}+\left(\frac{1}{8}-y_{3}\right) \mathbf{a}_{2}+\frac{1}{4} \mathbf{a}_{3}$ & $=$ & $-y_{3} a \hat{\mathbf{x}}+\left(\frac{1}{4}+y_{3}\right) a \hat{\mathbf{y}}+\frac{1}{8} a \hat{\mathbf{z}}$ & $(24 h)$ & I \\
\hline
\end{tabular}




\section{References:}

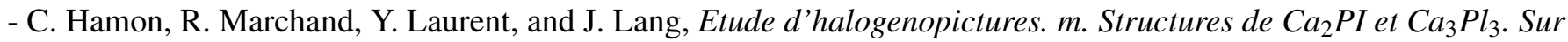
structures de type $\mathrm{NaCl}$, Bull. Soc. fr. Mineral. Crystallogr. 97, 6-12 (1974).

\section{Found in:}

- P. Villars and K. Cenzual, Pearson's Crystal Data - Crystal Structure Database for Inorganic Compounds, ASM International (2013).

\section{Geometry files:}

- CIF: pp. 965

- POSCAR: pp. 966 


\section{Petzite $\left(\mathrm{Ag}_{3} \mathrm{AuTe}_{2}\right)$ Structure: A3BC2_cI48_214_f_a_e}
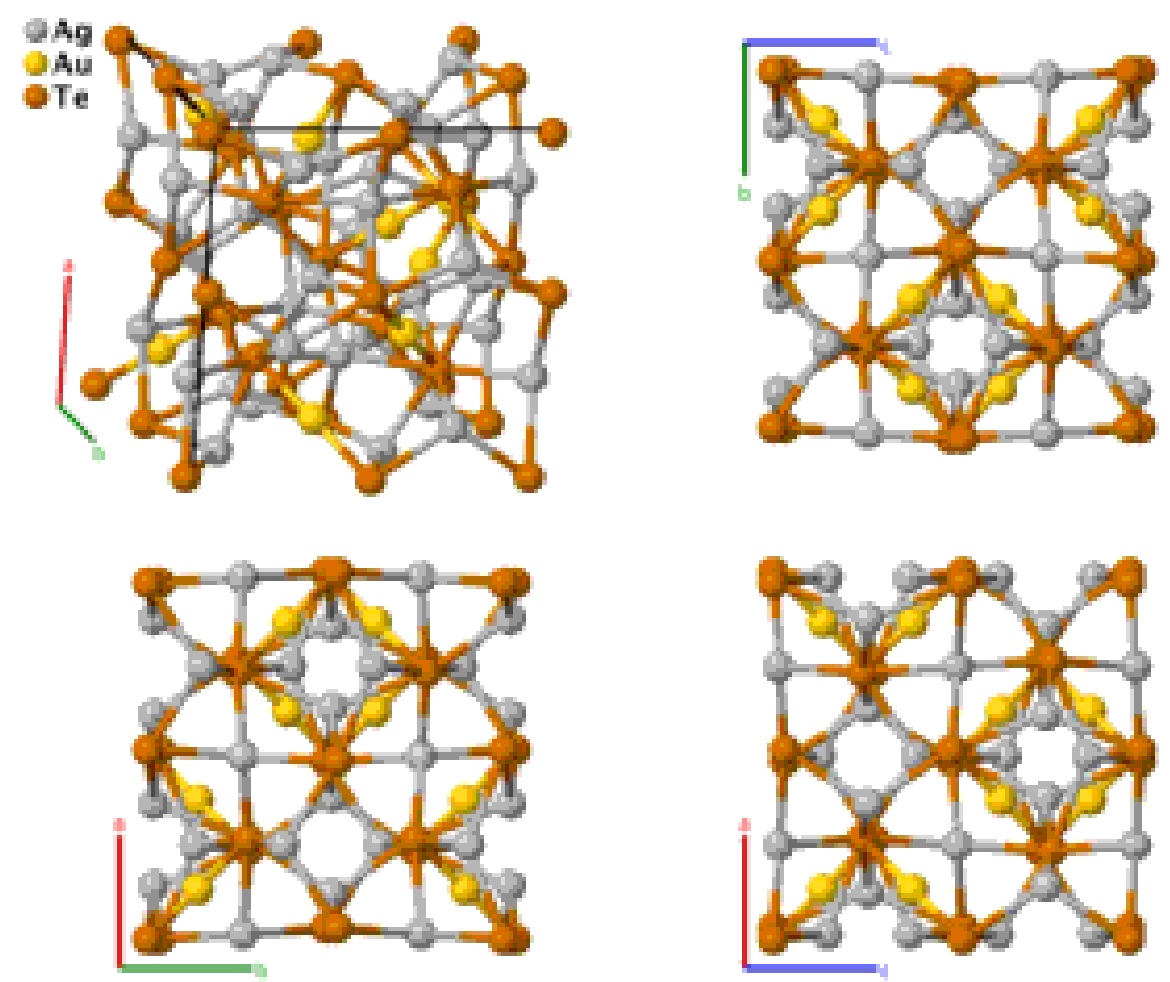

$\begin{array}{lll}\text { Prototype } & : & \mathrm{Ag}_{3} \mathrm{AuTe}_{2} \\ \text { AFLOW prototype label } & : & \mathrm{A} 3 \mathrm{BC} 2 \_c I 48 \_214 \_\mathrm{f} \_\mathrm{a} \_\mathrm{e} \\ \text { Strukturbericht designation } & : & \mathrm{None} \\ \text { Pearson symbol } & : & \mathrm{cI} 48 \\ \text { Space group number } & : & 214 \\ \text { Space group symbol } & : & I 4{ }_{1} 32 \\ \text { AFLOW prototype command } & : & \begin{array}{l}\text { aflow -proto=A3BC2_cI48_214_f_a_e } \\ \text {--params }=a, x_{2}, x_{3}\end{array}\end{array}$

- The Wyckoff positions given in the International Tables have been shifted by one or more body-centered cubic primitive lattice vectors to provide a more compact set of coordinates in both Cartesian and lattice space.

Body-centered Cubic primitive vectors:

$$
\begin{aligned}
& \mathbf{a}_{1}=-\frac{1}{2} a \hat{\mathbf{x}}+\frac{1}{2} a \hat{\mathbf{y}}+\frac{1}{2} a \hat{\mathbf{z}} \\
& \mathbf{a}_{2}=\frac{1}{2} a \hat{\mathbf{x}}-\frac{1}{2} a \hat{\mathbf{y}}+\frac{1}{2} a \hat{\mathbf{z}} \\
& \mathbf{a}_{3}=\frac{1}{2} a \hat{\mathbf{x}}+\frac{1}{2} a \hat{\mathbf{y}}-\frac{1}{2} a \hat{\mathbf{z}}
\end{aligned}
$$

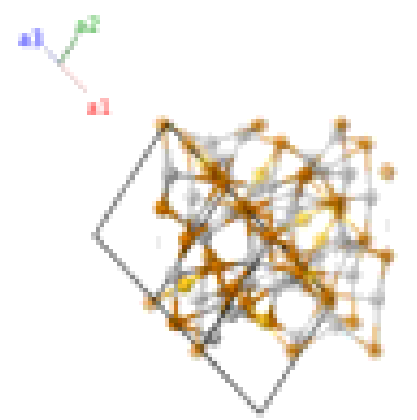

\section{Basis vectors:}


Lattice Coordinates

\begin{tabular}{|c|c|c|}
\hline $\mathbf{B}_{1}$ & $=$ & $\frac{1}{4} \mathbf{a}_{1}+\frac{1}{4} \mathbf{a}_{2}+\frac{1}{4} \mathbf{a}_{3}$ \\
\hline $\mathbf{B}_{2}$ & $=$ & $\frac{1}{2} \mathbf{a}_{1}+\frac{1}{4} \mathbf{a}_{3}$ \\
\hline $\mathbf{B}_{3}$ & $=$ & $\frac{1}{4} \mathbf{a}_{2}+\frac{1}{2} \mathbf{a}_{3}$ \\
\hline $\mathbf{B}_{4}$ & $=$ & $\frac{1}{4} \mathbf{a}_{1}+\frac{1}{2} \mathbf{a}_{2}$ \\
\hline $\mathbf{B}_{5}$ & $=$ & $2 x_{2} \mathbf{a}_{1}+2 x_{2} \mathbf{a}_{2}+2 x_{2} \mathbf{a}_{3}$ \\
\hline $\mathbf{B}_{6}$ & $=$ & $\frac{1}{2} \mathbf{a}_{1}+\left(\frac{1}{2}-2 x_{2}\right) \mathbf{a}_{3}$ \\
\hline $\mathbf{B}_{7}$ & $=$ & $\left(\frac{1}{2}-2 x_{2}\right) \mathbf{a}_{2}+\frac{1}{2} \mathbf{a}_{3}$ \\
\hline $\mathbf{B}_{8}$ & $=$ & $\left(\frac{1}{2}-2 x_{2}\right) \mathbf{a}_{1}+\frac{1}{2} \mathbf{a}_{2}$ \\
\hline $\mathbf{B}_{9}$ & $=$ & $\frac{1}{2} \mathbf{a}_{1}+2 x_{2} \mathbf{a}_{3}$ \\
\hline $\mathbf{B}_{10}$ & $=$ & $\begin{array}{c}\left(\frac{1}{2}-2 x_{2}\right) \mathbf{a}_{1}+\left(\frac{1}{2}-2 x_{2}\right) \mathbf{a}_{2}+ \\
\left(\frac{1}{2}-2 x_{2}\right) \mathbf{a}_{3}\end{array}$ \\
\hline $\mathbf{B}_{11}$ & $=$ & $2 x_{2} \mathbf{a}_{2}+\frac{1}{2} \mathbf{a}_{3}$ \\
\hline $\mathbf{B}_{12}$ & $=$ & $2 x_{2} \mathbf{a}_{1}+\frac{1}{2} \mathbf{a}_{2}$ \\
\hline $\mathbf{B}_{13}$ & $=$ & $\frac{1}{4} \mathbf{a}_{1}+\left(\frac{1}{4}+x_{3}\right) \mathbf{a}_{2}+x_{3} \mathbf{a}_{3}$ \\
\hline $\mathbf{B}_{14}$ & $=$ & $\frac{3}{4} \mathbf{a}_{1}+\left(\frac{1}{4}-x_{3}\right) \mathbf{a}_{2}+\left(\frac{1}{2}-x_{3}\right) \mathbf{a}_{3}$ \\
\hline $\mathbf{B}_{15}$ & $=$ & $x_{3} \mathbf{a}_{1}+\frac{1}{4} \mathbf{a}_{2}+\left(\frac{1}{4}+x_{3}\right) \mathbf{a}_{3}$ \\
\hline $\mathbf{B}_{16}$ & $=$ & $\left(\frac{1}{2}-x_{3}\right) \mathbf{a}_{1}+\frac{3}{4} \mathbf{a}_{2}+\left(\frac{1}{4}-x_{3}\right) \mathbf{a}_{3}$ \\
\hline $\mathbf{B}_{17}$ & $=$ & $\left(\frac{1}{4}+x_{3}\right) \mathbf{a}_{1}+x_{3} \mathbf{a}_{2}+\frac{1}{4} \mathbf{a}_{3}$ \\
\hline $\mathbf{B}_{18}$ & $=$ & $\left(\frac{1}{4}-x_{3}\right) \mathbf{a}_{1}+\left(\frac{1}{2}-x_{3}\right) \mathbf{a}_{2}+\frac{3}{4} \mathbf{a}_{3}$ \\
\hline $\mathbf{B}_{19}$ & $=$ & $\left(\frac{1}{4}+x_{3}\right) \mathbf{a}_{1}+\frac{3}{4} \mathbf{a}_{2}+x_{3} \mathbf{a}_{3}$ \\
\hline $\mathbf{B}_{20}$ & $=$ & $\left(\frac{1}{4}-x_{3}\right) \mathbf{a}_{1}+\frac{1}{4} \mathbf{a}_{2}+\left(\frac{1}{2}-x_{3}\right) \mathbf{a}_{3}$ \\
\hline $\mathbf{B}_{21}$ & $=$ & $\frac{3}{4} \mathbf{a}_{1}+x_{3} \mathbf{a}_{2}+\left(\frac{1}{4}+x_{3}\right) \mathbf{a}_{3}$ \\
\hline $\mathbf{B}_{22}$ & $=$ & $\frac{1}{4} \mathbf{a}_{1}+\left(\frac{1}{2}-x_{3}\right) \mathbf{a}_{2}+\left(\frac{1}{4}-x_{3}\right) \mathbf{a}_{3}$ \\
\hline $\mathbf{B}_{23}$ & $=$ & $\left(\frac{1}{2}-x_{3}\right) \mathbf{a}_{1}+\left(\frac{1}{4}-x_{3}\right) \mathbf{a}_{2}+\frac{1}{4} \mathbf{a}_{3}$ \\
\hline $\mathbf{B}_{24}$ & $=$ & $x_{3} \mathbf{a}_{1}+\left(\frac{1}{4}+x_{3}\right) \mathbf{a}_{2}+\frac{3}{4} \mathbf{a}_{3}$ \\
\hline
\end{tabular}

Cartesian Coordinates

$=\quad \frac{1}{8} a \hat{\mathbf{x}}+\frac{1}{8} a \hat{\mathbf{y}}+\frac{1}{8} a \hat{\mathbf{z}}$

$=\quad-\frac{1}{8} a \hat{\mathbf{x}}+\frac{3}{8} a \hat{\mathbf{y}}+\frac{1}{8} a \hat{\mathbf{z}}$

$=\quad \frac{3}{8} a \hat{\mathbf{x}}+\frac{1}{8} a \hat{\mathbf{y}}-\frac{1}{8} a \hat{\mathbf{z}}$

$=\quad \frac{1}{8} a \hat{\mathbf{x}}-\frac{1}{8} a \hat{\mathbf{y}}+\frac{3}{8} a \hat{\mathbf{z}}$

$=\quad x_{2} a \hat{\mathbf{x}}+x_{2} a \hat{\mathbf{y}}+x_{2} a \hat{\mathbf{z}}$

$=-x_{2} a \hat{\mathbf{x}}+\left(\frac{1}{2}-x_{2}\right) a \hat{\mathbf{y}}+x_{2} a \hat{\mathbf{z}}$

$=\left(\frac{1}{2}-x_{2}\right) a \hat{\mathbf{x}}+x_{2} a \hat{\mathbf{y}}-x_{2} a \hat{\mathbf{z}}$

$=\quad x_{2} a \hat{\mathbf{x}}-x_{2} a \hat{\mathbf{y}}+\left(\frac{1}{2}-x_{2}\right) a \hat{\mathbf{z}}$

$=\left(\frac{3}{4}+x_{2}\right) a \hat{\mathbf{x}}+\left(\frac{1}{4}+x_{2}\right) a \hat{\mathbf{y}}+$

$$
\left(\frac{1}{4}-x_{2}\right) a \hat{\mathbf{z}}
$$

$=\left(\frac{1}{4}-x_{2}\right) a \hat{\mathbf{x}}+\left(\frac{1}{4}-x_{2}\right) a \hat{\mathbf{y}}+$ $\left(\frac{1}{4}-x_{2}\right) a \hat{\mathbf{z}}$

$=\left(\frac{1}{4}+x_{2}\right) a \hat{\mathbf{x}}+\left(\frac{1}{4}-x_{2}\right) a \hat{\mathbf{y}}+$

$$
\left(\frac{3}{4}+x_{2}\right) a \hat{\mathbf{z}}
$$

$=\left(\frac{1}{4}-x_{2}\right) a \hat{\mathbf{x}}+\left(\frac{3}{4}+x_{2}\right) a \hat{\mathbf{y}}+$

$$
\left(\frac{1}{4}+x_{2}\right) a \hat{\mathbf{z}}
$$

$=$

$$
x_{3} a \hat{\mathbf{x}}+\frac{1}{4} a \hat{\mathbf{z}}
$$$$
-x_{3} a \hat{\mathbf{x}}+\frac{1}{2} a \hat{\mathbf{y}}+\frac{1}{4} a \hat{\mathbf{z}}
$$$$
\frac{1}{4} a \hat{\mathbf{x}}+x_{3} a \hat{\mathbf{y}}
$$$$
\frac{1}{4} a \hat{\mathbf{x}}-x_{3} a \hat{\mathbf{y}}+\frac{1}{2} a \hat{\mathbf{z}}
$$$$
\frac{1}{4} a \hat{\mathbf{y}}+x_{3} a \hat{\mathbf{z}}
$$$$
\frac{1}{2} a \hat{\mathbf{x}}+\frac{1}{4} a \hat{\mathbf{y}}-x_{3} a \hat{\mathbf{z}}
$$$$
\frac{1}{4} a \hat{\mathbf{x}}+\left(-\frac{1}{4}+x_{3}\right) a \hat{\mathbf{y}}+\frac{1}{2} a \hat{\mathbf{z}}
$$$$
\frac{1}{4} a \hat{\mathbf{x}}+\left(\frac{1}{4}-x_{3}\right) a \hat{\mathbf{y}}
$$$$
\left(\frac{3}{4}+x_{3}\right) a \hat{\mathbf{x}}+\frac{1}{2} a \hat{\mathbf{y}}+\frac{1}{4} a \hat{\mathbf{z}}
$$$$
\left(\frac{1}{4}-x_{3}\right) a \hat{\mathbf{x}}+\frac{1}{4} a \hat{\mathbf{z}}
$$$$
\frac{1}{4} a \hat{\mathbf{y}}+\left(\frac{1}{4}-x_{3}\right) a \hat{\mathbf{z}}
$$

$\frac{1}{2} a \hat{\mathbf{x}}+\frac{1}{4} a \hat{\mathbf{y}}+\left(\frac{3}{4}+x_{3}\right) a \hat{\mathbf{z}}$ (16e)

$\mathrm{Au}$

$\mathrm{Au}$

$\mathrm{Au}$

$\mathrm{Au}$

$\mathrm{Te}$

$\mathrm{Te}$

$\mathrm{Te}$

$\mathrm{Te}$

$\mathrm{Te}$

$\mathrm{Te}$

$\mathrm{Te}$

$\mathrm{Te}$

$\mathrm{Ag}$

$\mathrm{Ag}$

$\mathrm{Ag}$

$\mathrm{Ag}$

Ag

Ag

$\mathrm{Ag}$

$\mathrm{Ag}$

$\mathrm{Ag}$

$\mathrm{Ag}$

$\mathrm{Ag}$

$(24 f)$

$\mathrm{Ag}$

\section{References:}

- A. J. Frueh, Jr., Crystallography of petzite, $\mathrm{Ag}_{3} \mathrm{AuTe}_{2}$, Am. Mineral. 44, 693-701 (1959).

\section{Found in:}

- R. T. Downs and M. Hall-Wallace, The American Mineralogist Crystal Structure Database, Am. Mineral. 88, 247-250 (2003).

\section{Geometry files:}

- CIF: pp. 966

- POSCAR: pp. 966 


\section{$\gamma$-brass $\left(\mathrm{Cu}_{9} \mathrm{Al}_{4}, D 8_{3}\right)$ Structure: A4B9_cP52_215_ei_3efgi}
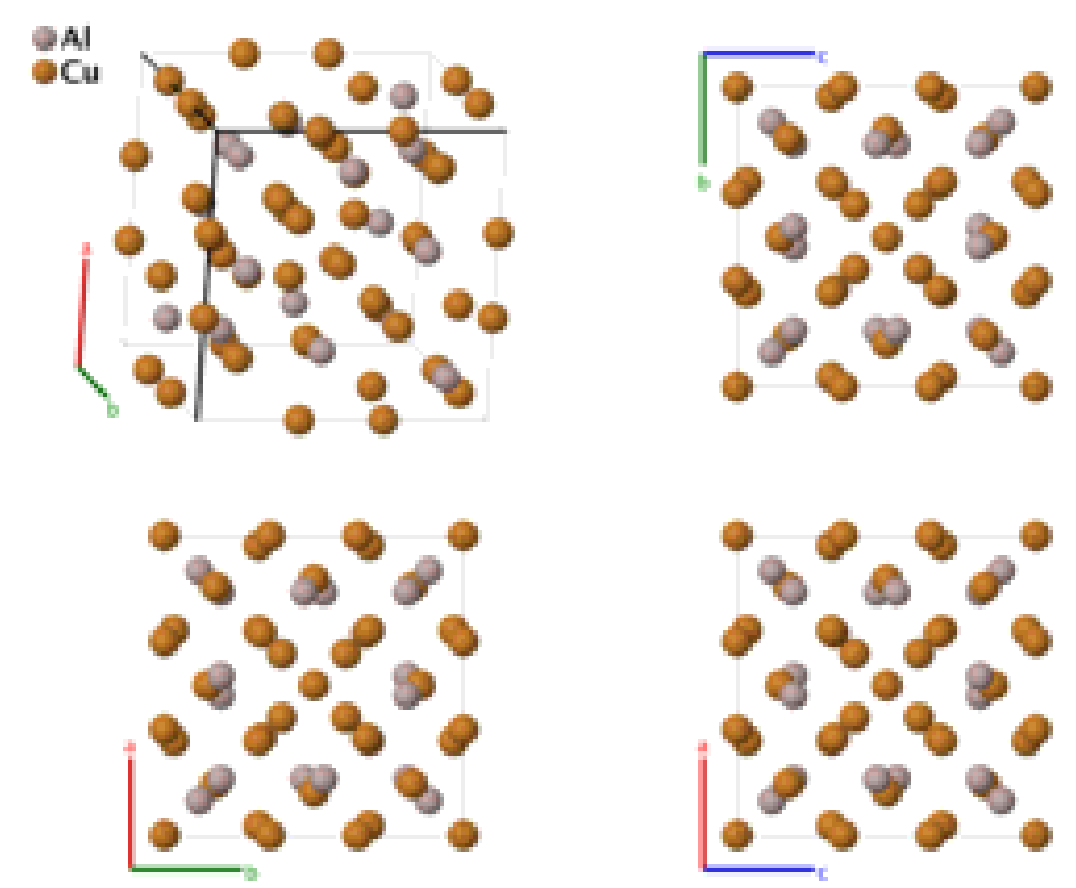

Prototype

AFLOW prototype label

Strukturbericht designation

Pearson symbol

Space group number

Space group symbol

AFLOW prototype command
$: \quad \gamma-\mathrm{Cu}_{9} \mathrm{Al}_{4}$

: A4B9_cP52_215_ei_3efgi

: $\quad D 8_{3}$

: $\quad$ cP52

: 215

: $\quad P \overline{4} 3 m$ aflow --proto=A4B9_cP52_215_ei_3efgi

- - params $=a, x_{1}, x_{2}, x_{3}, x_{4}, x_{5}, x_{6}, x_{7}, z_{7}, x_{8}, z_{8}$

\section{Other compounds with this structure:}

- $\mathrm{Cu}_{9} \mathrm{Ga}_{4}$. (Pearson, 1958), pp. 252, gives a list of compounds which can take on the $D 8_{1}, D 8_{2}$, or $D 8_{3}$ structure, depending on the exact composition.

- (Arnberg, 1978) give the Wyckoff positions of the $\mathrm{Cu} I V$ and $\mathrm{Cu} V$ atoms as $(6 \mathrm{~g})(x, 1 / 2,1 / 2)$, but give the coordinates in the form $(x, 0,0)$ corresponding to the (6f) site. (Stokhuyzen, 1974) used (6f) for both types of atoms in the isostructural system $\mathrm{Ga}_{9} \mathrm{Al}_{4}$. (Pearson, 1958) places the $\mathrm{Cu} \mathrm{IV}$ atoms on a (6f) site and $\mathrm{Cu} \mathrm{V}$ on $(6 \mathrm{~g})$, but does not give explicit coordinates.

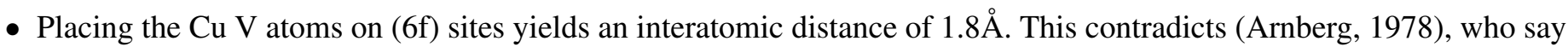
that the minimum interatomic distance is $2.48 \AA$ between the $\mathrm{Cu} \mathrm{IV}$ and $\mathrm{Cu} \mathrm{V}$ atoms. Placing the $\mathrm{Cu} \mathrm{V}$ atoms on $(6 \mathrm{~g})$ sites gives this distance, in agreement with (Pearson, 1958), so we make this choice for the crystal structure. This is a variety of $\gamma$-brass comparable to the $D 8_{2}$ structure. In fact, if we

- Replace the $\mathrm{Al}$ and $\mathrm{Cu}$ III atoms by $\mathrm{Zn}$, while setting $x_{4}=x_{1}+1 / 2$,

- Replace the $\mathrm{Al}$ II and $\mathrm{Cu}$ VI atoms by Zn, with $x_{8}=x_{7}+1 / 2$ and $z_{8}=z_{7}+1 / 2$, 
- Set $x_{3}=x_{2}+1 / 2$ and

- Set $x_{6}=x_{5}+1 / 2$,

then this structure is identical to $D 8_{2} \gamma$-brass.

\section{Simple Cubic primitive vectors:}

$$
\begin{aligned}
& \mathbf{a}_{1}=a \hat{\mathbf{x}} \\
& \mathbf{a}_{2}=a \hat{\mathbf{y}} \\
& \mathbf{a}_{3}=a \hat{\mathbf{z}}
\end{aligned}
$$

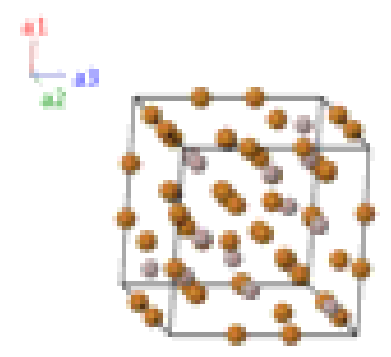

\section{Basis vectors:}

\section{Lattice Coordinates}

$\mathbf{B}_{1}=$

$\mathbf{B}_{2}=$

$\mathbf{B}_{3}=$

$\mathbf{B}_{4}=$

$\mathbf{B}_{5}=$

$\mathbf{B}_{6}=$

$\mathbf{B}_{7}=$

$\mathbf{B}_{8}=$

$\mathbf{B}_{9}=$

$\mathbf{B}_{10}=$

$\mathbf{B}_{11}=$

$\mathbf{B}_{12}=$

$\mathbf{B}_{13}=$

$\mathbf{B}_{14}=$

$\mathbf{B}_{15}=$

$\mathbf{B}_{16}=$

$\mathbf{B}_{17}=$

$\mathbf{B}_{18}=$

$\mathbf{B}_{19}=$

$\mathbf{B}_{20}=$

$\mathbf{B}_{21}=$

$\mathbf{B}_{22}=$

$\mathbf{B}_{23}=$

$\mathbf{B}_{24}=$

$$
x_{1} \mathbf{a}_{1}+x_{1} \mathbf{a}_{2}+x_{1} \mathbf{a}_{3}
$$$$
-x_{1} \mathbf{a}_{1}-x_{1} \mathbf{a}_{2}+x_{1} \mathbf{a}_{3}
$$$$
-x_{1} \mathbf{a}_{1}+x_{1} \mathbf{a}_{2}-x_{1} \mathbf{a}_{3}
$$$$
x_{1} \mathbf{a}_{1}-x_{1} \mathbf{a}_{2}-x_{1} \mathbf{a}_{3}
$$$$
x_{2} \mathbf{a}_{1}+x_{2} \mathbf{a}_{2}+x_{2} \mathbf{a}_{3}
$$$$
-x_{2} \mathbf{a}_{1}-x_{2} \mathbf{a}_{2}+x_{2} \mathbf{a}_{3}
$$$$
-x_{2} \mathbf{a}_{1}+x_{2} \mathbf{a}_{2}-x_{2} \mathbf{a}_{3}
$$$$
x_{2} \mathbf{a}_{1}-x_{2} \mathbf{a}_{2}-x_{2} \mathbf{a}_{3}
$$$$
x_{3} \mathbf{a}_{1}+x_{3} \mathbf{a}_{2}+x_{3} \mathbf{a}_{3}
$$$$
-x_{3} \mathbf{a}_{1}-x_{3} \mathbf{a}_{2}+x_{3} \mathbf{a}_{3}
$$$$
-x_{3} \mathbf{a}_{1}+x_{3} \mathbf{a}_{2}-x_{3} \mathbf{a}_{3}
$$$$
x_{3} \mathbf{a}_{1}-x_{3} \mathbf{a}_{2}-x_{3} \mathbf{a}_{3}
$$$$
x_{4} \mathbf{a}_{1}+x_{4} \mathbf{a}_{2}+x_{4} \mathbf{a}_{3}
$$$$
-x_{4} \mathbf{a}_{1}-x_{4} \mathbf{a}_{2}+x_{4} \mathbf{a}_{3}
$$$$
-x_{4} \mathbf{a}_{1}+x_{4} \mathbf{a}_{2}-x_{4} \mathbf{a}_{3}
$$$$
x_{4} \mathbf{a}_{1}-x_{4} \mathbf{a}_{2}-x_{4} \mathbf{a}_{3}
$$$$
x_{5} \mathbf{a}_{1}
$$$$
-x_{5} \mathbf{a}_{1}
$$$$
x_{5} \mathbf{a}_{2}
$$$$
-x_{5} \mathbf{a}_{2}
$$

$x_{5} \mathbf{a}_{3}$

$-x_{5} \mathbf{a}_{3}$

$x_{6} \mathbf{a}_{1}+\frac{1}{2} \mathbf{a}_{2}+\frac{1}{2} \mathbf{a}_{3}$

$-x_{6} \mathbf{a}_{1}+\frac{1}{2} \mathbf{a}_{2}+\frac{1}{2} \mathbf{a}_{3}$
$=$

$=$

$=$

$=$

$=$

$=$

$=$

$=$

$=$

$=$

$=$

$=$

$=$

$=$

$=$

$=$

$=$

$=$

$=$

$=$

$=$

$=$

$=$

$=$

$=$

$=$

$=$

$=$
Cartesian Coordinates

$x_{1} a \hat{\mathbf{x}}+x_{1} a \hat{\mathbf{y}}+x_{1} a \hat{\mathbf{z}}$

$-x_{1} a \hat{\mathbf{x}}-x_{1} a \hat{\mathbf{y}}+x_{1} a \hat{\mathbf{z}}$

$-x_{1} a \hat{\mathbf{x}}+x_{1} a \hat{\mathbf{y}}-x_{1} a \hat{\mathbf{z}}$

$x_{1} a \hat{\mathbf{x}}-x_{1} a \hat{\mathbf{y}}-x_{1} a \hat{\mathbf{z}}$

$x_{2} a \hat{\mathbf{x}}+x_{2} a \hat{\mathbf{y}}+x_{2} a \hat{\mathbf{z}}$

$-x_{2} a \hat{\mathbf{x}}-x_{2} a \hat{\mathbf{y}}+x_{2} a \hat{\mathbf{z}}$

$-x_{2} a \hat{\mathbf{x}}+x_{2} a \hat{\mathbf{y}}-x_{2} a \hat{\mathbf{z}}$

$x_{2} a \hat{\mathbf{x}}-x_{2} a \hat{\mathbf{y}}-x_{2} a \hat{\mathbf{z}}$

$x_{3} a \hat{\mathbf{x}}+x_{3} a \hat{\mathbf{y}}+x_{3} a \hat{\mathbf{z}}$

$-x_{3} a \hat{\mathbf{x}}-x_{3} a \hat{\mathbf{y}}+x_{3} a \hat{\mathbf{z}}$

$-x_{3} a \hat{\mathbf{x}}+x_{3} a \hat{\mathbf{y}}-x_{3} a \hat{\mathbf{z}}$

$x_{3} a \hat{\mathbf{x}}-x_{3} a \hat{\mathbf{y}}-x_{3} a \hat{\mathbf{z}}$

$x_{4} a \hat{\mathbf{x}}+x_{4} a \hat{\mathbf{y}}+x_{4} a \hat{\mathbf{z}}$

$-x_{4} a \hat{\mathbf{x}}-x_{4} a \hat{\mathbf{y}}+x_{4} a \hat{\mathbf{z}}$

$-x_{4} a \hat{\mathbf{x}}+x_{4} a \hat{\mathbf{y}}-x_{4} a \hat{\mathbf{z}}$

$x_{4} a \hat{\mathbf{x}}-x_{4} a \hat{\mathbf{y}}-x_{4} a \hat{\mathbf{z}}$

$x_{5} a \hat{\mathbf{x}}$

$-x_{5} a \hat{\mathbf{x}}$

$x_{5} a \hat{\mathbf{y}}$

$-x_{5} a \hat{\mathbf{y}}$

$x_{5} a \hat{\mathbf{z}}$

$-x_{5} a \hat{\mathbf{z}}$

$x_{6} a \hat{\mathbf{x}}+\frac{1}{2} a \hat{\mathbf{y}}+\frac{1}{2} a \hat{\mathbf{z}}$

$-x_{6} a \hat{\mathbf{x}}+\frac{1}{2} a \hat{\mathbf{y}}+\frac{1}{2} a \hat{\mathbf{z}}$
Wyckoff Position

$(4 e)$
Atom Type

Al I

Al I

Al I

Al I

$\mathrm{Cu} \mathrm{I}$

$\mathrm{Cu} \mathrm{I}$

$\mathrm{Cu} \mathrm{I}$

$\mathrm{Cu} \mathrm{I}$

$\mathrm{Cu}$ II

$\mathrm{Cu}$ II

$\mathrm{Cu}$ II

$\mathrm{Cu}$ II

$\mathrm{Cu}$ III

$\mathrm{Cu}$ III

$\mathrm{Cu}$ III

$\mathrm{Cu}$ III

$\mathrm{Cu}$ IV

$\mathrm{Cu}$ IV

$\mathrm{Cu}$ IV

Cu IV

$\mathrm{Cu}$ IV

$\mathrm{Cu}$ IV

$\mathrm{Cu}$ V

$\mathrm{Cu}$ V 


\begin{tabular}{|c|c|c|c|c|c|c|}
\hline $\mathbf{B}_{25}$ & $=$ & $\frac{1}{2} \mathbf{a}_{1}+x_{6} \mathbf{a}_{2}+\frac{1}{2} \mathbf{a}_{3}$ & $=$ & $\frac{1}{2} a \hat{\mathbf{x}}+x_{6} a \hat{\mathbf{y}}+\frac{1}{2} a \hat{\mathbf{z}}$ & $(6 g)$ & $\mathrm{Cu} \mathrm{V}$ \\
\hline $\mathbf{B}_{26}$ & $=$ & $\frac{1}{2} \mathbf{a}_{1}-x_{6} \mathbf{a}_{2}+\frac{1}{2} \mathbf{a}_{3}$ & $=$ & $\frac{1}{2} a \hat{\mathbf{x}}-x_{6} a \hat{\mathbf{y}}+\frac{1}{2} a \hat{\mathbf{z}}$ & $(6 g)$ & $\mathrm{Cu} \mathrm{V}$ \\
\hline $\mathbf{B}_{27}$ & $=$ & $\frac{1}{2} \mathbf{a}_{1}+\frac{1}{2} \mathbf{a}_{2}+x_{6} \mathbf{a}_{3}$ & $=$ & $\frac{1}{2} a \hat{\mathbf{x}}+\frac{1}{2} a \hat{\mathbf{y}}+x_{6} a \hat{\mathbf{z}}$ & $(6 g)$ & $\mathrm{Cu} \mathrm{V}$ \\
\hline $\mathbf{B}_{28}$ & $=$ & $\frac{1}{2} \mathbf{a}_{1}+\frac{1}{2} \mathbf{a}_{2}-x_{6} \mathbf{a}_{3}$ & $=$ & $\frac{1}{2} a \hat{\mathbf{x}}+\frac{1}{2} a \hat{\mathbf{y}}-x_{6} a \hat{\mathbf{z}}$ & $(6 g)$ & $\mathrm{Cu} \mathrm{V}$ \\
\hline $\mathbf{B}_{29}$ & $=$ & $x_{7} \mathbf{a}_{1}+x_{7} \mathbf{a}_{2}+z_{7} \mathbf{a}_{3}$ & $=$ & $x_{7} a \hat{\mathbf{x}}+x_{7} a \hat{\mathbf{y}}+z_{7} a \hat{\mathbf{z}}$ & $(12 i)$ & $\mathrm{Al} \mathrm{II}$ \\
\hline $\mathbf{B}_{30}$ & $=$ & $-x_{7} \mathbf{a}_{1}-x_{7} \mathbf{a}_{2}+z_{7} \mathbf{a}_{3}$ & $=$ & $-x_{7} a \hat{\mathbf{x}}-x_{7} a \hat{\mathbf{y}}+z_{7} a \hat{\mathbf{z}}$ & $(12 i)$ & $\mathrm{Al}$ II \\
\hline $\mathbf{B}_{31}$ & $=$ & $-x_{7} \mathbf{a}_{1}+x_{7} \mathbf{a}_{2}-z_{7} \mathbf{a}_{3}$ & $=$ & $-x_{7} a \hat{\mathbf{x}}+x_{7} a \hat{\mathbf{y}}-z_{7} a \hat{\mathbf{z}}$ & $(12 i)$ & $\mathrm{Al}$ II \\
\hline $\mathbf{B}_{32}$ & $=$ & $x_{7} \mathbf{a}_{1}-x_{7} \mathbf{a}_{2}-z_{7} \mathbf{a}_{3}$ & $=$ & $x_{7} a \hat{\mathbf{x}}-x_{7} a \hat{\mathbf{y}}-z_{7} a \hat{\mathbf{z}}$ & $(12 i)$ & $\mathrm{Al}$ II \\
\hline $\mathbf{B}_{33}$ & $=$ & $z_{7} \mathbf{a}_{1}+x_{7} \mathbf{a}_{2}+x_{7} \mathbf{a}_{3}$ & $=$ & $z_{7} a \hat{\mathbf{x}}+x_{7} a \hat{\mathbf{y}}+x_{7} a \hat{\mathbf{z}}$ & $(12 i)$ & $\mathrm{Al}$ II \\
\hline $\mathbf{B}_{34}$ & $=$ & $z_{7} \mathbf{a}_{1}-x_{7} \mathbf{a}_{2}-x_{7} \mathbf{a}_{3}$ & $=$ & $z_{7} a \hat{\mathbf{x}}-x_{7} a \hat{\mathbf{y}}-x_{7} a \hat{\mathbf{z}}$ & $(12 i)$ & Al II \\
\hline $\mathbf{B}_{35}$ & $=$ & $-z_{7} \mathbf{a}_{1}-x_{7} \mathbf{a}_{2}+x_{7} \mathbf{a}_{3}$ & $=$ & $-z_{7} a \hat{\mathbf{x}}-x_{7} a \hat{\mathbf{y}}+x_{7} a \hat{\mathbf{z}}$ & $(12 i)$ & $\mathrm{Al} \mathrm{II}$ \\
\hline $\mathbf{B}_{36}$ & $=$ & $-z_{7} \mathbf{a}_{1}+x_{7} \mathbf{a}_{2}-x_{7} \mathbf{a}_{3}$ & $=$ & $-z_{7} a \hat{\mathbf{x}}+x_{7} a \hat{\mathbf{y}}-x_{7} a \hat{\mathbf{z}}$ & $(12 i)$ & $\mathrm{Al}$ II \\
\hline $\mathbf{B}_{37}$ & $=$ & $x_{7} \mathbf{a}_{1}+z_{7} \mathbf{a}_{2}+x_{7} \mathbf{a}_{3}$ & $=$ & $x_{7} a \hat{\mathbf{x}}+z_{7} a \hat{\mathbf{y}}+x_{7} a \hat{\mathbf{z}}$ & $(12 i)$ & $\mathrm{Al}$ II \\
\hline $\mathbf{B}_{38}$ & $=$ & $-x_{7} \mathbf{a}_{1}+z_{7} \mathbf{a}_{2}-x_{7} \mathbf{a}_{3}$ & $=$ & $-x_{7} a \hat{\mathbf{x}}+z_{7} a \hat{\mathbf{y}}-x_{7} a \hat{\mathbf{z}}$ & $(12 i)$ & $\mathrm{Al}$ II \\
\hline $\mathbf{B}_{39}$ & $=$ & $x_{7} \mathbf{a}_{1}-z_{7} \mathbf{a}_{2}-x_{7} \mathbf{a}_{3}$ & $=$ & $x_{7} a \hat{\mathbf{x}}-z_{7} a \hat{\mathbf{y}}-x_{7} a \hat{\mathbf{z}}$ & $(12 i)$ & Al II \\
\hline $\mathbf{B}_{40}$ & $=$ & $-x_{7} \mathbf{a}_{1}-z_{7} \mathbf{a}_{2}+x_{7} \mathbf{a}_{3}$ & $=$ & $-x_{7} a \hat{\mathbf{x}}-z_{7} a \hat{\mathbf{y}}+x_{7} a \hat{\mathbf{z}}$ & $(12 i)$ & $\mathrm{Al}$ II \\
\hline $\mathbf{B}_{41}$ & $=$ & $x_{8} \mathbf{a}_{1}+x_{8} \mathbf{a}_{2}+z_{8} \mathbf{a}_{3}$ & $=$ & $x_{8} a \hat{\mathbf{x}}+x_{8} a \hat{\mathbf{y}}+z_{8} a \hat{\mathbf{z}}$ & $(12 i)$ & $\mathrm{Cu} \mathrm{Vl}$ \\
\hline $\mathbf{B}_{42}$ & $=$ & $-x_{8} \mathbf{a}_{1}-x_{8} \mathbf{a}_{2}+z_{8} \mathbf{a}_{3}$ & $=$ & $-x_{8} a \hat{\mathbf{x}}-x_{8} a \hat{\mathbf{y}}+z_{8} a \hat{\mathbf{z}}$ & $(12 i)$ & $\mathrm{Cu} \mathrm{Vl}$ \\
\hline $\mathbf{B}_{43}$ & $=$ & $-x_{8} \mathbf{a}_{1}+x_{8} \mathbf{a}_{2}-z_{8} \mathbf{a}_{3}$ & $=$ & $-x_{8} a \hat{\mathbf{x}}+x_{8} a \hat{\mathbf{y}}-z_{8} a \hat{\mathbf{z}}$ & $(12 i)$ & $\mathrm{Cu} \mathrm{Vl}$ \\
\hline $\mathbf{B}_{44}$ & $=$ & $x_{8} \mathbf{a}_{1}-x_{8} \mathbf{a}_{2}-z_{8} \mathbf{a}_{3}$ & $=$ & $x_{8} a \hat{\mathbf{x}}-x_{8} a \hat{\mathbf{y}}-z_{8} a \hat{\mathbf{z}}$ & $(12 i)$ & $\mathrm{Cu} \mathrm{Vl}$ \\
\hline $\mathbf{B}_{45}$ & $=$ & $z_{8} \mathbf{a}_{1}+x_{8} \mathbf{a}_{2}+x_{8} \mathbf{a}_{3}$ & $=$ & $z_{8} a \hat{\mathbf{x}}+x_{8} a \hat{\mathbf{y}}+x_{8} a \hat{\mathbf{z}}$ & $(12 i)$ & $\mathrm{Cu} \mathrm{Vl}$ \\
\hline $\mathbf{B}_{46}$ & $=$ & $z_{8} \mathbf{a}_{1}-x_{8} \mathbf{a}_{2}-x_{8} \mathbf{a}_{3}$ & $=$ & $z_{8} a \hat{\mathbf{x}}-x_{8} a \hat{\mathbf{y}}-x_{8} a \hat{\mathbf{z}}$ & $(12 i)$ & $\mathrm{Cu} \mathrm{Vl}$ \\
\hline $\mathbf{B}_{47}$ & $=$ & $-z_{8} \mathbf{a}_{1}-x_{8} \mathbf{a}_{2}+x_{8} \mathbf{a}_{3}$ & $=$ & $-z_{8} a \hat{\mathbf{x}}-x_{8} a \hat{\mathbf{y}}+x_{8} a \hat{\mathbf{z}}$ & $(12 i)$ & $\mathrm{Cu} \mathrm{Vl}$ \\
\hline $\mathbf{B}_{48}$ & $=$ & $-z_{8} \mathbf{a}_{1}+x_{8} \mathbf{a}_{2}-x_{8} \mathbf{a}_{3}$ & $=$ & $-z_{8} a \hat{\mathbf{x}}+x_{8} a \hat{\mathbf{y}}-x_{8} a \hat{\mathbf{z}}$ & $(12 i)$ & $\mathrm{Cu} \mathrm{Vl}$ \\
\hline $\mathbf{B}_{49}$ & $=$ & $x_{8} \mathbf{a}_{1}+z_{8} \mathbf{a}_{2}+x_{8} \mathbf{a}_{3}$ & $=$ & $x_{8} a \hat{\mathbf{x}}+z_{8} a \hat{\mathbf{y}}+x_{8} a \hat{\mathbf{z}}$ & $(12 i)$ & $\mathrm{Cu} \mathrm{Vl}$ \\
\hline $\mathbf{B}_{50}$ & $=$ & $-x_{8} \mathbf{a}_{1}+z_{8} \mathbf{a}_{2}-x_{8} \mathbf{a}_{3}$ & $=$ & $-x_{8} a \hat{\mathbf{x}}+z_{8} a \hat{\mathbf{y}}-x_{8} a \hat{\mathbf{z}}$ & $(12 i)$ & $\mathrm{Cu} \mathrm{Vl}$ \\
\hline $\mathbf{B}_{51}$ & $=$ & $x_{8} \mathbf{a}_{1}-z_{8} \mathbf{a}_{2}-x_{8} \mathbf{a}_{3}$ & $=$ & $x_{8} a \hat{\mathbf{x}}-z_{8} a \hat{\mathbf{y}}-x_{8} a \hat{\mathbf{z}}$ & $(12 i)$ & $\mathrm{Cu} \mathrm{Vl}$ \\
\hline $\mathbf{B}_{52}$ & $=$ & $-x_{8} \mathbf{a}_{1}-z_{8} \mathbf{a}_{2}+x_{8} \mathbf{a}_{3}$ & $=$ & $-x_{8} a \hat{\mathbf{x}}-z_{8} a \hat{\mathbf{y}}+x_{8} a \hat{\mathbf{z}}$ & $(12 i)$ & $\mathrm{Cu} \mathrm{Vl}$ \\
\hline
\end{tabular}

\section{References:}

- L. Arnberg and S. Westman, Crystal perfection in a noncentrosymmetric alloy. Refinement and test of twinning of the $\gamma \mathrm{Cu}_{9} \mathrm{Al}_{4}$ structure, Acta Crystallogr. Sect. A 34, 399-404 (1978), doi:10.1107/S0567739478000807.

- R. Stokhuyzen, J. K. Brandon, P. C. Chieh, and W. B. Pearson, Copper-Gallium, $\gamma_{1} C u_{9} G a_{4}$, Acta Crystallogr. Sect. B Struct. Sci. 30, 2910-2911 (1974), doi:10.1107/S0567740874008478.

- W. B. Pearson, A Handbook of Lattice Spacings and Structures of Metals and Alloys, no. N.R.C. No. 4303 in International Series of Monographs on Metal Physics and Physical Metallurgy (Pergamon Press, Oxford, London, Edinburgh, New York, Paris, Frankfort, 1958), 1964 reprint with corrections edn.

\section{Found in:}

- P. Villars and K. Cenuzal, eds., Structure Types (Springer, Berlin, Heidelberg, 2005), Landolt-Börnstein - Group III 
Condensed Matter (Numerical Data and Functional Relationships in Science and Technology), vol. 43A2, chap. $\mathrm{Cu}_{9} \mathrm{Al}_{4}$ in Part 2: Space Groups (218) P-43n - (195) P23.

\section{Geometry files:}

- CIF: pp. 966

- POSCAR: pp. 967 


\section{Quartenary Heusler (LiMgAuSn) Structure: ABCD_cF16_216_c_d_b_a}
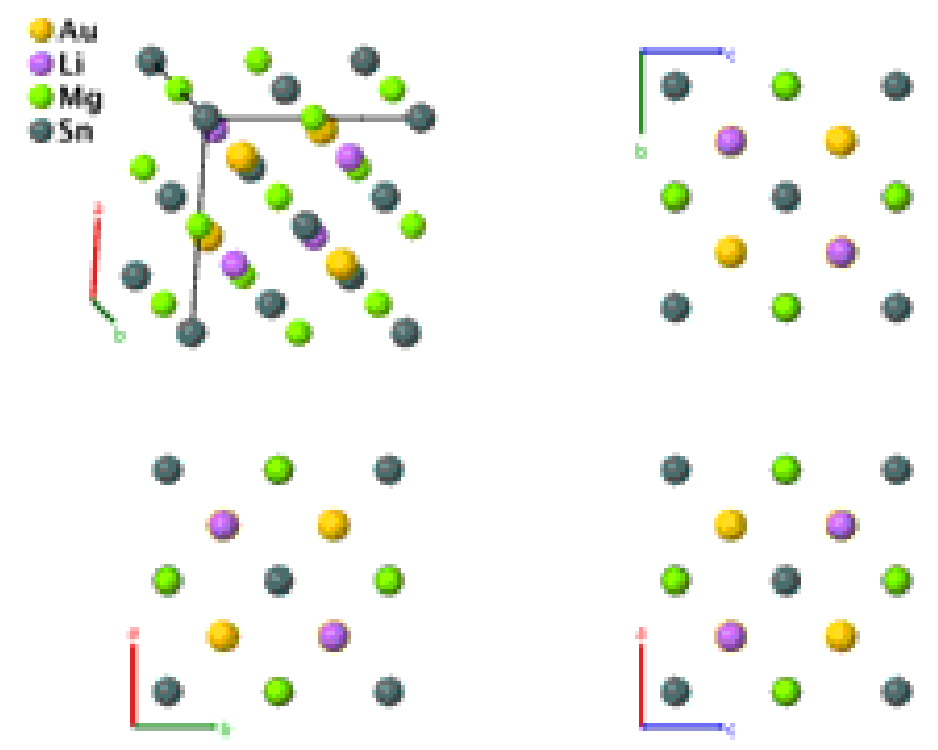

\section{Prototype}

AFLOW prototype label

Strukturbericht designation

Pearson symbol

Space group number

Space group symbol

AFLOW prototype command
: $\quad \operatorname{LiMgAuSn}$

: ABCD_cF16_216_c_d_b_a

$: \quad$ None

: $\quad$ cF16

: $\quad 216$

: $\quad F \overline{4} 3 m$ aflow --proto=ABCD_cF16_216_c_d_b_a

- - params $=a$

\section{Other compounds with this structure:}

- AuLiMgSn, CuMg $\mathrm{Ti}_{2} \mathrm{AuBiLi}_{2}, \mathrm{AgLi}_{2} \mathrm{Sn}, \mathrm{AuLi}_{2} \mathrm{Sn}, \mathrm{CuHfHg}{ }_{2}, \mathrm{MnPd}_{2} \mathrm{Sn}$, and many more. See (Eberz, 1980).

- This "quaternary-Heusler" structure can be considered as the parent of a wide variety of structures, depending on the occupancy of the (4a), (4b), (4c), and (4d) Wyckoff positions. Consider atoms of type A, B, C, D, distributed in this structure. By placing these atoms on the appropriate Wyckoff positions we find the following structures:

\begin{tabular}{|c|c|c|c|c|c|c|}
\hline Structure & Strukturbericht & AFLOW label & $(4 \mathrm{a})$ & (4b) & (4c) & (4d) \\
\hline \hline simple cubic & $A_{h}$ & A_cP1_221_a & A & A & - & - \\
\hline fcc & $A 1$ & A_cF4_225_a & A & - & - & - \\
\hline bcc & $A 2$ & A_cI2_229_a & A & A & - & - \\
\hline diamond & $A 4$ & A_cF8_227_a & A & - & A & - \\
\hline NaCl & $B 1$ & AB_cF8_225_a_b & A & B & - & - \\
\hline CsCl & $B 2$ & AB_cP2_221_a_b & A & B & - & - \\
\hline ZnS (zincblende) & $B 3$ & AB_cF8_216_c_a & B & - & A & - \\
\hline half-Heusler & $C 1_{b}$ & ABC_cF12_216_b_c_a & C & A & B & - \\
\hline Heusler & $L 2_{1}$ & AB2C_cF16_225_a_c_b & A & C & B & B \\
\hline
\end{tabular}


The ordering of this structure is somewhat arbitary. So long as Sn and Mg are on either the $(4 a) /(4 b)$ or $(4 c) /(4 d)$ sites, with $\mathrm{Au}$ and $\mathrm{Li}$ on the opposite sites, we will get the same structure.

\section{Face-centered Cubic primitive vectors:}

$$
\begin{aligned}
& \mathbf{a}_{1}=\frac{1}{2} a \hat{\mathbf{y}}+\frac{1}{2} a \hat{\mathbf{z}} \\
& \mathbf{a}_{2}=\frac{1}{2} a \hat{\mathbf{x}}+\frac{1}{2} a \hat{\mathbf{z}} \\
& \mathbf{a}_{3}=\frac{1}{2} a \hat{\mathbf{x}}+\frac{1}{2} a \hat{\mathbf{y}}
\end{aligned}
$$

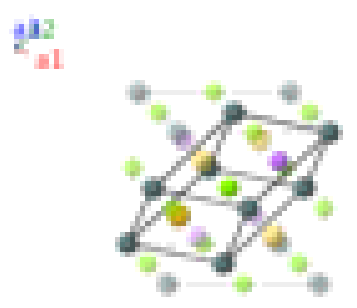

\section{Basis vectors:}

$\begin{array}{lllccc} & \text { Lattice Coordinates } & & \text { Cartesian Coordinates } & \text { Wyckoff Position } & \text { Atom Type } \\ \mathbf{B}_{1}= & 0 \mathbf{a}_{1}+0 \mathbf{a}_{2}+0 \mathbf{a}_{3} & = & 0 \hat{\mathbf{x}}+0 \hat{\mathbf{y}}+0 \hat{\mathbf{z}} & (4 a) & \mathrm{Sn} \\ \mathbf{B}_{2}= & \frac{1}{2} \mathbf{a}_{1}+\frac{1}{2} \mathbf{a}_{2}+\frac{1}{2} \mathbf{a}_{3} & = & \frac{1}{2} a \hat{\mathbf{x}}+\frac{1}{2} a \hat{\mathbf{y}}+\frac{1}{2} a \hat{\mathbf{z}} & (4 b) & \mathrm{Mg} \\ \mathbf{B}_{3}= & \frac{1}{4} \mathbf{a}_{1}+\frac{1}{4} \mathbf{a}_{2}+\frac{1}{4} \mathbf{a}_{3} & = & \frac{1}{4} a \hat{\mathbf{x}}+\frac{1}{4} a \hat{\mathbf{y}}+\frac{1}{4} a \hat{\mathbf{z}} & (4 c) & \mathrm{Au} \\ \mathbf{B}_{4}= & \frac{3}{4} \mathbf{a}_{1}+\frac{3}{4} \mathbf{a}_{2}+\frac{3}{4} \mathbf{a}_{3} & = & \frac{3}{4} a \hat{\mathbf{x}}+\frac{3}{4} a \hat{\mathbf{y}}+\frac{3}{4} a \hat{\mathbf{z}} & (4 d) & \mathrm{Li}\end{array}$

\section{References:}

- U. Eberz, W. Seelentag, and H.-U. Schuster, Zur Kenntnis farbiger ternärer und quaternärer Zintl-Phasen / Coloured Ternary and Quaternary Zintl-Phases, Z. Naturforsch. B 35, 1341-1343 (1980), doi:10.1515/znb-1980-1103.

\section{Found in:}

- P. V. C. Editor), ed., PAULING FILE in: Inorganic Solid Phases, SpringerMaterials (online database) (Springer Materials, Heigelberg, 2016), chap. LiMgPdSn Crystal Structure,

doi:https://materials.springer.com/isp/crystallographic/docs/sd_1703124.

\section{Geometry files:}

- CIF: pp. 967

- POSCAR: pp. 968 


\section{$\mathrm{Ag}_{3}\left[\mathrm{PO}_{4}\right]$ Structure: A3B4C_cP16_218_c_e_a}
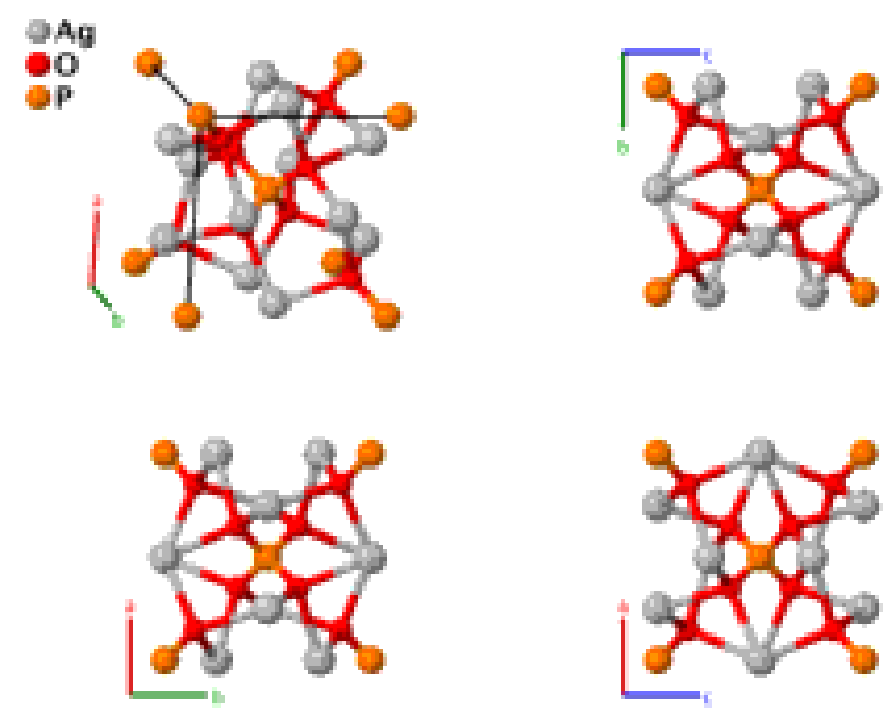

\section{Prototype}

\section{AFLOW prototype label}

Strukturbericht designation

Pearson symbol

Space group number

Space group symbol

AFLOW prototype command
$: \quad \mathrm{Ag}_{3}\left[\mathrm{PO}_{4}\right]$

: A3B4C_cP16_218_c_e_a

: None

: $\quad$ cP16

: $\quad 218$

: $\quad P \overline{4} 3 n$

aflow - -proto=A3B4C_cP16_218_c_e_a

- -params $=a, x_{3}$

Simple Cubic primitive vectors:

$$
\begin{aligned}
& \mathbf{a}_{1}=a \hat{\mathbf{x}} \\
& \mathbf{a}_{2}=a \hat{\mathbf{y}} \\
& \mathbf{a}_{3}=a \hat{\mathbf{z}}
\end{aligned}
$$

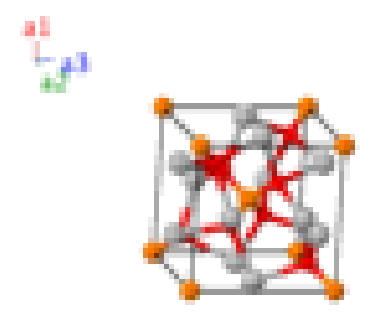

\section{Basis vectors:}

Lattice Coordinates

$\begin{array}{lcc}\mathbf{B}_{1}= & 0 \mathbf{a}_{1}+0 \mathbf{a}_{2}+0 \mathbf{a}_{3} \\ \mathbf{B}_{2}= & \frac{1}{2} \mathbf{a}_{1}+\frac{1}{2} \mathbf{a}_{2}+\frac{1}{2} \mathbf{a}_{3} \\ \mathbf{B}_{3}= & \frac{1}{4} \mathbf{a}_{1}+\frac{1}{2} \mathbf{a}_{2} \\ \mathbf{B}_{4}= & \frac{3}{4} \mathbf{a}_{1}+\frac{1}{2} \mathbf{a}_{2} \\ \mathbf{B}_{5}= & \frac{1}{4} \mathbf{a}_{2}+\frac{1}{2} \mathbf{a}_{3} \\ \mathbf{B}_{6}= & \frac{3}{4} \mathbf{a}_{2}+\frac{1}{2} \mathbf{a}_{3}\end{array}$

Cartesian Coordinates

$\begin{array}{lc}= & 0 \hat{\mathbf{x}}+0 \hat{\mathbf{y}}+0 \hat{\mathbf{z}} \\ = & \frac{1}{2} a \hat{\mathbf{x}}+\frac{1}{2} a \hat{\mathbf{y}}+\frac{1}{2} a \hat{\mathbf{z}} \\ = & \frac{1}{4} a \hat{\mathbf{x}}+\frac{1}{2} a \hat{\mathbf{y}} \\ = & \frac{3}{4} a \hat{\mathbf{x}}+\frac{1}{2} a \hat{\mathbf{y}} \\ = & \frac{1}{4} a \hat{\mathbf{y}}+\frac{1}{2} a \hat{\mathbf{z}} \\ = & \frac{3}{4} a \hat{\mathbf{y}}+\frac{1}{2} a \hat{\mathbf{z}}\end{array}$

Wyckoff Position

Atom Type

(2a)

P

(2a)

$\mathrm{P}$

(6c)

Ag

(6c)

Ag

(6c)

Ag

(6c) 


\begin{tabular}{|c|c|c|c|c|c|}
\hline $\mathbf{B}_{7}$ & $=$ & $\frac{1}{2} \mathbf{a}_{1}+\frac{1}{4} \mathbf{a}_{3}$ & $=$ & $\frac{1}{2} a \hat{\mathbf{x}}+\frac{1}{4} a \hat{\mathbf{z}}$ & $(6 c)$ \\
\hline $\mathbf{B}_{8}$ & $=$ & $\frac{1}{2} \mathbf{a}_{1}+\frac{3}{4} \mathbf{a}_{3}$ & $=$ & $\frac{1}{2} a \hat{\mathbf{x}}+\frac{3}{4} a \hat{\mathbf{z}}$ & $(6 c)$ \\
\hline $\mathbf{B}_{9}$ & $=$ & $x_{3} \mathbf{a}_{1}+x_{3} \mathbf{a}_{2}+x_{3} \mathbf{a}_{3}$ & $=$ & $x_{3} a \hat{\mathbf{x}}+x_{3} a \hat{\mathbf{y}}+x_{3} a \hat{\mathbf{z}}$ & $(8 e)$ \\
\hline $\mathbf{B}_{10}$ & $=$ & $-x_{3} \mathbf{a}_{1}-x_{3} \mathbf{a}_{2}+x_{3} \mathbf{a}_{3}$ & $=$ & $-x_{3} a \hat{\mathbf{x}}-x_{3} a \hat{\mathbf{y}}+x_{3} a \hat{\mathbf{z}}$ & $(8 e)$ \\
\hline $\mathbf{B}_{11}$ & $=$ & $-x_{3} \mathbf{a}_{1}+x_{3} \mathbf{a}_{2}-x_{3} \mathbf{a}_{3}$ & $=$ & $-x_{3} a \hat{\mathbf{x}}+x_{3} a \hat{\mathbf{y}}-x_{3} a \hat{\mathbf{z}}$ & $(8 e)$ \\
\hline $\mathbf{B}_{12}$ & $=$ & $x_{3} \mathbf{a}_{1}-x_{3} \mathbf{a}_{2}-x_{3} \mathbf{a}_{3}$ & $=$ & $x_{3} a \hat{\mathbf{x}}-x_{3} a \hat{\mathbf{y}}-x_{3} a \hat{\mathbf{z}}$ & $(8 e)$ \\
\hline $\mathbf{B}_{13}$ & $=$ & $\begin{array}{c}\left(\frac{1}{2}+x_{3}\right) \mathbf{a}_{1}+\left(\frac{1}{2}+x_{3}\right) \mathbf{a}_{2}+ \\
\left(\frac{1}{2}+x_{3}\right) \mathbf{a}_{3}\end{array}$ & $=$ & $\begin{array}{c}\left(\frac{1}{2}+x_{3}\right) a \hat{\mathbf{x}}+\left(\frac{1}{2}+x_{3}\right) a \hat{\mathbf{y}}+ \\
\left(\frac{1}{2}+x_{3}\right) a \hat{\mathbf{z}}\end{array}$ & $(8 e)$ \\
\hline $\mathbf{B}_{14}$ & $=$ & $\begin{array}{c}\left(\frac{1}{2}-x_{3}\right) \mathbf{a}_{1}+\left(\frac{1}{2}-x_{3}\right) \mathbf{a}_{2}+ \\
\left(\frac{1}{2}+x_{3}\right) \mathbf{a}_{3}\end{array}$ & $=$ & $\begin{array}{c}\left(\frac{1}{2}-x_{3}\right) a \hat{\mathbf{x}}+\left(\frac{1}{2}-x_{3}\right) a \hat{\mathbf{y}}+ \\
\left(\frac{1}{2}+x_{3}\right) a \hat{\mathbf{z}}\end{array}$ & $(8 e)$ \\
\hline $\mathbf{B}_{15}$ & $=$ & $\begin{array}{c}\left(\frac{1}{2}+x_{3}\right) \mathbf{a}_{1}+\left(\frac{1}{2}-x_{3}\right) \mathbf{a}_{2}+ \\
\left(\frac{1}{2}-x_{3}\right) \mathbf{a}_{3}\end{array}$ & $=$ & $\begin{array}{c}\left(\frac{1}{2}+x_{3}\right) a \hat{\mathbf{x}}+\left(\frac{1}{2}-x_{3}\right) a \hat{\mathbf{y}}+ \\
\left(\frac{1}{2}-x_{3}\right) a \hat{\mathbf{z}}\end{array}$ & $(8 e)$ \\
\hline $\mathbf{B}_{16}$ & $=$ & $\begin{array}{c}\left(\frac{1}{2}-x_{3}\right) \mathbf{a}_{1}+\left(\frac{1}{2}+x_{3}\right) \mathbf{a}_{2}+ \\
\left(\frac{1}{2}-x_{3}\right) \mathbf{a}_{3}\end{array}$ & $=$ & $\begin{array}{c}\left(\frac{1}{2}-x_{3}\right) a \hat{\mathbf{x}}+\left(\frac{1}{2}+x_{3}\right) a \hat{\mathbf{y}}+ \\
\left(\frac{1}{2}-x_{3}\right) a \hat{\mathbf{z}}\end{array}$ & $(8 e)$ \\
\hline
\end{tabular}

\section{References:}

- R. Masse, I. Tordjman, and A. Durif, Affinement de la structure cristalline du monophosphate d'argent $\mathrm{Ag}_{3} \mathrm{PO}_{4}$. Existence d'une forme haute temperature, Zeitschrift für Kristallographie - Crystalline Materials 144, 76-81 (1976), doi:10.1524/zkri.1976.144.1-6.76.

\section{Found in:}

- P. Villars and K. Cenzual, Pearson's Crystal Data - Crystal Structure Database for Inorganic Compounds, ASM International (2013).

\section{Geometry files:}

- CIF: pp. 968

- POSCAR: pp. 968 
Boracite $\left(\mathrm{Mg}_{3} \mathrm{~B}_{7} \mathrm{ClO}_{13}\right)$ Structure:

A7BC3D13_cF192_219_de_b_c_ah
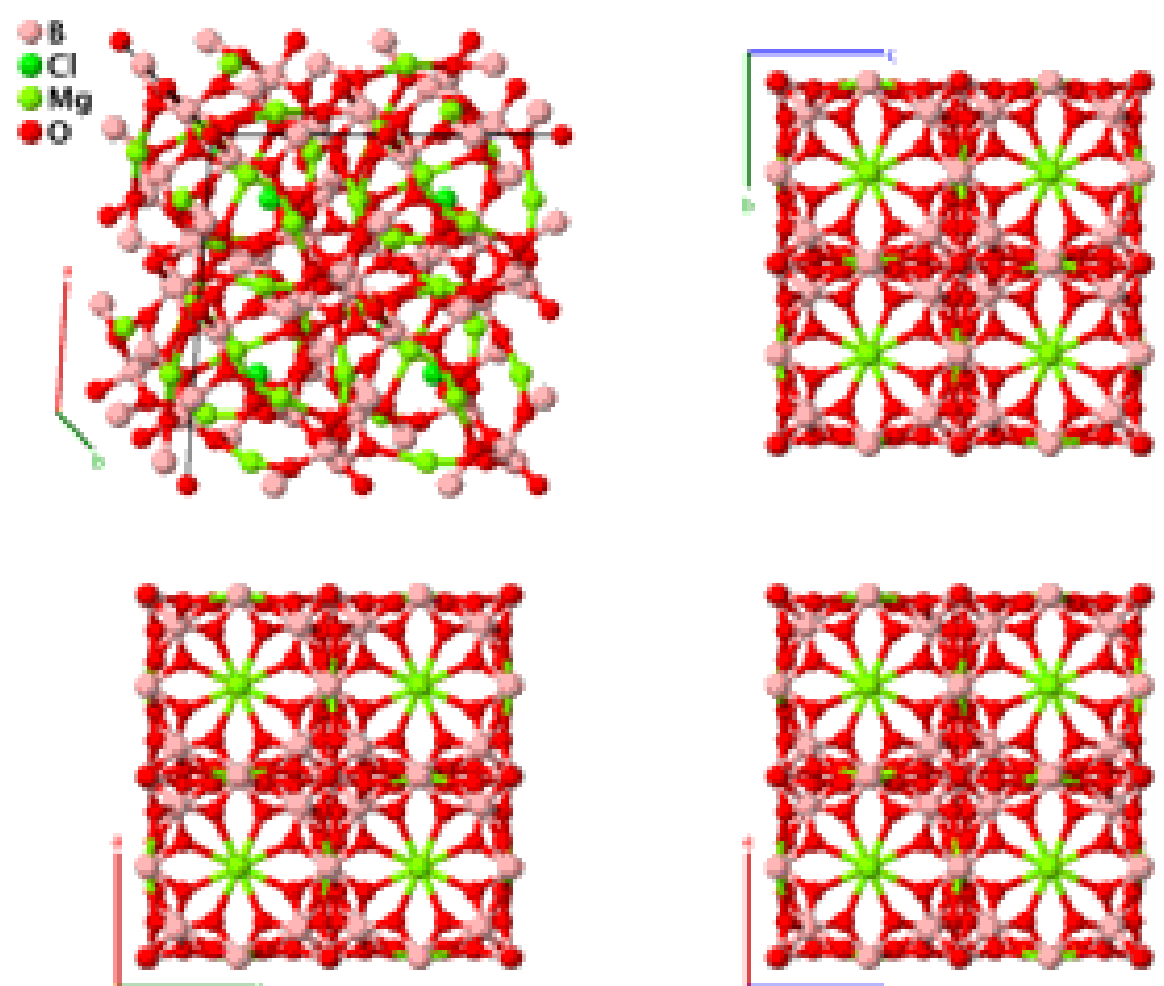

\section{Prototype}

AFLOW prototype label

Strukturbericht designation

Pearson symbol

Space group number

Space group symbol

AFLOW prototype command
$: \quad \mathrm{Mg}_{3} \mathrm{~B}_{7} \mathrm{ClO}_{13}$

: A7BC3D13_cF192_219_de_b_c_ah

: None

: $\quad$ cF192

: 219

: $\quad F \overline{4} 3 c$

- - params $=a, x_{5}, x_{6}, y_{6}, z_{6}$

\section{Other compounds with this structure:}

- $M_{3} \mathrm{~B}_{7} \mathrm{O}_{13} X ; M=\mathrm{Mg}, \mathrm{Cr}, \mathrm{Mn}, \mathrm{Fe}, \mathrm{Co} ; X=\mathrm{Cl}, \mathrm{Br}, \mathrm{I}$

- Experimental data was obtained at $400^{\circ} \mathrm{C}$.

Face-centered Cubic primitive vectors:

$$
\begin{aligned}
& \mathbf{a}_{1}=\frac{1}{2} a \hat{\mathbf{y}}+\frac{1}{2} a \hat{\mathbf{z}} \\
& \mathbf{a}_{2}=\frac{1}{2} a \hat{\mathbf{x}}+\frac{1}{2} a \hat{\mathbf{z}} \\
& \mathbf{a}_{3}=\frac{1}{2} a \hat{\mathbf{x}}+\frac{1}{2} a \hat{\mathbf{y}}
\end{aligned}
$$

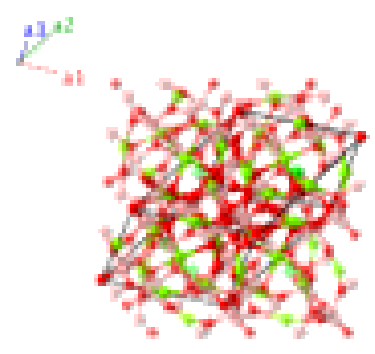


Lattice Coordinates

$\mathbf{B}_{1}=$
$\mathbf{B}_{2}=$
$\mathbf{B}_{3}=$
$\mathbf{B}_{4}=$
$\mathbf{B}_{5}=$
$\mathbf{B}_{6}=$
$\mathbf{B}_{7}=$
$\mathbf{B}_{8}=$
$\mathbf{B}_{9}=$

$\mathbf{B}_{10}=$

$\mathbf{B}_{11}=$

$\mathbf{B}_{12}=$

$\mathbf{B}_{13}=$

$\mathbf{B}_{14}=$

$\mathbf{B}_{15}=$

$\mathbf{B}_{16}=$

$\mathbf{B}_{17}=$

$\mathbf{B}_{18}=$

$\mathbf{B}_{19}=$

$\mathbf{B}_{20}=-3 x_{5} \mathbf{a}_{1}+x_{5} \mathbf{a}_{2}+x_{5} \mathbf{a}_{3}$

$\mathbf{B}_{21}=$

$$
\left(\frac{1}{2}+x_{5}\right) \mathbf{a}_{1}+\left(\frac{1}{2}+x_{5}\right) \mathbf{a}_{2}+
$$

$$
\left(\frac{1}{2}+x_{5}\right) \mathbf{a}_{3}
$$

$\mathbf{B}_{22}=\left(\frac{1}{2}+x_{5}\right) \mathbf{a}_{1}+\left(\frac{1}{2}+x_{5}\right) \mathbf{a}_{2}+$

$$
\left(\frac{1}{2}-3 x_{5}\right) \mathbf{a}_{3}
$$

$\mathbf{B}_{23}=\left(\frac{1}{2}-3 x_{5}\right) \mathbf{a}_{1}+\left(\frac{1}{2}+x_{5}\right) \mathbf{a}_{2}+=$

$$
\left(\frac{1}{2}+x_{5}\right) \mathbf{a}_{3}
$$

$\mathbf{B}_{24}=\left(\frac{1}{2}+x_{5}\right) \mathbf{a}_{1}+\left(\frac{1}{2}-3 x_{5}\right) \mathbf{a}_{2}+$

$$
\left(\frac{1}{2}+x_{5}\right) \mathbf{a}_{3}
$$

$$
\begin{aligned}
\mathbf{B}_{25}=\quad & \left(-x_{6}+y_{6}+z_{6}\right) \mathbf{a}_{1}+ \\
& \left(x_{6}-y_{6}+z_{6}\right) \mathbf{a}_{2}+ \\
& \left(x_{6}+y_{6}-z_{6}\right) \mathbf{a}_{3}
\end{aligned}
$$

$$
\begin{aligned}
\mathbf{B}_{26}=\quad & \left(x_{6}-y_{6}+z_{6}\right) \mathbf{a}_{1}+ \\
& \left(-x_{6}+y_{6}+z_{6}\right) \mathbf{a}_{2}+ \\
& \left(-x_{6}-y_{6}-z_{6}\right) \mathbf{a}_{3} \\
& \left(x_{6}+y_{6}-z_{6}\right) \mathbf{a}_{1}+ \\
& \left(-x_{6}-y_{6}-z_{6}\right) \mathbf{a}_{2}+ \\
& \left(-x_{6}+y_{6}+z_{6}\right) \mathbf{a}_{3}
\end{aligned}
$$

Cartesian Coordinates

$$
=
$$$$
=
$$$$
=
$$$$
=
$$$$
=
$$$$
=
$$$$
=
$$$$
=
$$$$
=
$$$$
=
$$$$
=
$$$$
=
$$$$
=
$$$$
=
$$$$
=
$$$$
=
$$$$
=
$$$$
=
$$$$
=
$$$$
=
$$$$
=
$$$$
=
$$$$
=
$$$$
=
$$$$
=
$$$$
=
$$$$
=
$$$$
=\left(\frac{1}{2}-x_{5}\right) a \hat{\mathbf{x}}+\left(\frac{1}{2}-x_{5}\right) a \hat{\mathbf{y}}+
$$$$
\left(\frac{1}{2}+x_{5}\right) a \hat{\mathbf{z}}
$$$$
0 \hat{\mathbf{x}}+0 \hat{\mathbf{y}}+0 \hat{\mathbf{z}}
$$$$
\frac{1}{2} a \hat{\mathbf{x}}+\frac{1}{2} a \hat{\mathbf{y}}+\frac{1}{2} a \hat{\mathbf{z}}
$$$$
\frac{1}{4} a \hat{\mathbf{x}}+\frac{1}{4} a \hat{\mathbf{y}}+\frac{1}{4} a \hat{\mathbf{z}}
$$$$
\frac{3}{4} a \hat{\mathbf{x}}+\frac{3}{4} a \hat{\mathbf{y}}+\frac{3}{4} a \hat{\mathbf{z}}
$$$$
\frac{1}{4} a \hat{\mathbf{y}}+\frac{1}{4} a \hat{\mathbf{z}}
$$$$
\frac{1}{2} a \hat{\mathbf{x}}+\frac{1}{4} a \hat{\mathbf{y}}+\frac{1}{4} a \hat{\mathbf{z}}
$$$$
\frac{1}{4} a \hat{\mathbf{x}}+\frac{1}{4} a \hat{\mathbf{z}}
$$$$
\frac{1}{4} a \hat{\mathbf{X}}+\frac{1}{2} a \hat{\mathbf{y}}+\frac{1}{4} a \hat{\mathbf{z}}
$$$$
\frac{1}{4} a \hat{\mathbf{x}}+\frac{1}{4} a \hat{\mathbf{y}}
$$$$
\frac{1}{4} a \hat{\mathbf{x}}+\frac{1}{4} a \hat{\mathbf{y}}+\frac{1}{2} a \hat{\mathbf{z}}
$$$$
\frac{1}{4} a \hat{\mathbf{x}}+\frac{1}{2} a \hat{\mathbf{y}}+\frac{1}{2} a \hat{\mathbf{z}}
$$$$
\frac{3}{4} a \hat{\mathbf{x}}+\frac{1}{2} a \hat{\mathbf{y}}+\frac{1}{2} a \hat{\mathbf{z}}
$$$$
\frac{1}{2} a \hat{\mathbf{x}}+\frac{1}{4} a \hat{\mathbf{y}}+\frac{1}{2} a \hat{\mathbf{z}}
$$$$
\frac{1}{2} a \hat{\mathbf{x}}+\frac{3}{4} a \hat{\mathbf{y}}+\frac{1}{2} a \hat{\mathbf{z}}
$$$$
\frac{1}{2} a \hat{\mathbf{x}}+\frac{1}{2} a \hat{\mathbf{y}}+\frac{1}{4} a \hat{\mathbf{z}}
$$$$
\frac{1}{2} a \hat{\mathbf{x}}+\frac{1}{2} a \hat{\mathbf{y}}+\frac{3}{4} a \hat{\mathbf{z}}
$$$$
x_{5} a \hat{\mathbf{x}}+x_{5} a \hat{\mathbf{y}}+x_{5} a \hat{\mathbf{z}}
$$$$
-x_{5} a \hat{\mathbf{x}}-x_{5} a \hat{\mathbf{y}}+x_{5} a \hat{\mathbf{z}}
$$$$
-x_{5} a \hat{\mathbf{x}}+x_{5} a \hat{\mathbf{y}}-x_{5} a \hat{\mathbf{z}}
$$$$
x_{5} a \hat{\mathbf{x}}-x_{5} a \hat{\mathbf{y}}-x_{5} a \hat{\mathbf{z}}
$$$$
\left(\frac{1}{2}+x_{5}\right) a \hat{\mathbf{x}}+\left(\frac{1}{2}+x_{5}\right) a \hat{\mathbf{y}}+
$$$$
\left(\frac{1}{2}+x_{5}\right) a \hat{\mathbf{z}}
$$$$
\left(\frac{1}{2}+x_{5}\right) a \hat{\mathbf{x}}+\left(\frac{1}{2}-x_{5}\right) a \hat{\mathbf{y}}+
$$$$
\left(\frac{1}{2}-x_{5}\right) a \hat{\mathbf{z}}
$$$$
\left(\frac{1}{2}-x_{5}\right) a \hat{\mathbf{x}}+\left(\frac{1}{2}+x_{5}\right) a \hat{\mathbf{y}}+
$$$$
\left(\frac{1}{2}-x_{5}\right) a \hat{\mathbf{z}}
$$$$
=\quad x_{6} a \hat{\mathbf{x}}+y_{6} a \hat{\mathbf{y}}+z_{6} a \hat{\mathbf{z}}
$$

O II

O II

(96h)

O II

O I

O I

$\mathrm{Cl}$

$\mathrm{Cl}$

$\mathrm{Mg}$

$\mathrm{Mg}$

$\mathrm{Mg}$

$\mathrm{Mg}$

$\mathrm{Mg}$

Mg

B I

B I

B I

B I

B I

B I

B II

B II

B II

B II

B II

B II

B II

B II 


\begin{tabular}{|c|c|c|c|c|c|c|}
\hline $\mathbf{B}_{28}$ & $=$ & $\begin{array}{c}\left(-x_{6}-y_{6}-z_{6}\right) \mathbf{a}_{1}+ \\
\left(x_{6}+y_{6}-z_{6}\right) \mathbf{a}_{2}+ \\
\left(x_{6}-y_{6}+z_{6}\right) \mathbf{a}_{3}\end{array}$ & $=$ & $x_{6} a \hat{\mathbf{x}}-y_{6} a \hat{\mathbf{y}}-z_{6} a \hat{\mathbf{z}}$ & $(96 h)$ & O II \\
\hline $\mathbf{B}_{29}$ & $=$ & $\begin{array}{c}\left(x_{6}+y_{6}-z_{6}\right) \mathbf{a}_{1}+ \\
\left(-x_{6}+y_{6}+z_{6}\right) \mathbf{a}_{2}+ \\
\left(x_{6}-y_{6}+z_{6}\right) \mathbf{a}_{3}\end{array}$ & $=$ & $z_{6} a \hat{\mathbf{x}}+x_{6} a \hat{\mathbf{y}}+y_{6} a \hat{\mathbf{z}}$ & $(96 h)$ & O II \\
\hline $\mathbf{B}_{30}$ & $=$ & $\begin{array}{c}\left(-x_{6}-y_{6}-z_{6}\right) \mathbf{a}_{1}+ \\
\left(x_{6}-y_{6}+z_{6}\right) \mathbf{a}_{2}+ \\
\left(-x_{6}+y_{6}+z_{6}\right) \mathbf{a}_{3}\end{array}$ & $=$ & $z_{6} a \hat{\mathbf{x}}-x_{6} a \hat{\mathbf{y}}-y_{6} a \hat{\mathbf{z}}$ & $(96 h)$ & O II \\
\hline $\mathbf{B}_{31}$ & $=$ & $\begin{array}{c}\left(-x_{6}+y_{6}+z_{6}\right) \mathbf{a}_{1}+ \\
\left(x_{6}+y_{6}-z_{6}\right) \mathbf{a}_{2}+ \\
\left(-x_{6}-y_{6}-z_{6}\right) \mathbf{a}_{3}\end{array}$ & $=$ & $-z_{6} a \hat{\mathbf{x}}-x_{6} a \hat{\mathbf{y}}+y_{6} a \hat{\mathbf{z}}$ & $(96 h)$ & O II \\
\hline $\mathbf{B}_{32}$ & $=$ & $\begin{array}{c}\left(x_{6}-y_{6}+z_{6}\right) \mathbf{a}_{1}+ \\
\left(-x_{6}-y_{6}-z_{6}\right) \mathbf{a}_{2}+ \\
\left(x_{6}+y_{6}-z_{6}\right) \mathbf{a}_{3}\end{array}$ & $=$ & $-z_{6} a \hat{\mathbf{x}}+x_{6} a \hat{\mathbf{y}}-y_{6} a \hat{\mathbf{z}}$ & $(96 h)$ & O II \\
\hline $\mathbf{B}_{33}$ & $=$ & $\begin{array}{c}\left(x_{6}-y_{6}+z_{6}\right) \mathbf{a}_{1}+ \\
\left(x_{6}+y_{6}-z_{6}\right) \mathbf{a}_{2}+ \\
\left(-x_{6}+y_{6}+z_{6}\right) \mathbf{a}_{3}\end{array}$ & $=$ & $y_{6} a \hat{\mathbf{x}}+z_{6} a \hat{\mathbf{y}}+x_{6} a \hat{\mathbf{z}}$ & $(96 h)$ & O II \\
\hline $\mathbf{B}_{34}$ & $=$ & $\begin{array}{c}\left(-x_{6}+y_{6}+z_{6}\right) \mathbf{a}_{1}+ \\
\left(-x_{6}-y_{6}-z_{6}\right) \mathbf{a}_{2}+ \\
\left(x_{6}-y_{6}+z_{6}\right) \mathbf{a}_{3}\end{array}$ & $=$ & $-y_{6} a \hat{\mathbf{x}}+z_{6} a \hat{\mathbf{y}}-x_{6} a \hat{\mathbf{z}}$ & $(96 h)$ & O II \\
\hline $\mathbf{B}_{35}$ & $=$ & $\begin{array}{c}\left(-x_{6}-y_{6}-z_{6}\right) \mathbf{a}_{1}+ \\
\left(-x_{6}+y_{6}+z_{6}\right) \mathbf{a}_{2}+ \\
\left(x_{6}+y_{6}-z_{6}\right) \mathbf{a}_{3}\end{array}$ & $=$ & $y_{6} a \hat{\mathbf{x}}-z_{6} a \hat{\mathbf{y}}-x_{6} a \hat{\mathbf{z}}$ & $(96 h)$ & O II \\
\hline $\mathbf{B}_{36}$ & $=$ & $\begin{array}{l}\left(x_{6}+y_{6}-z_{6}\right) \mathbf{a}_{1}+ \\
\left(x_{6}-y_{6}+z_{6}\right) \mathbf{a}_{2}+ \\
\left(-x_{6}-y_{6}-z_{6}\right) \mathbf{a}_{3}\end{array}$ & $=$ & $-y_{6} a \hat{\mathbf{x}}-z_{6} a \hat{\mathbf{y}}+x_{6} a \hat{\mathbf{z}}$ & $(96 h)$ & O II \\
\hline $\mathbf{B}_{37}$ & $=$ & $\begin{array}{c}\left(\frac{1}{2}+x_{6}-y_{6}+z_{6}\right) \mathbf{a}_{1}+ \\
\left(\frac{1}{2}-x_{6}+y_{6}+z_{6}\right) \mathbf{a}_{2}+ \\
\left(\frac{1}{2}+x_{6}+y_{6}-z_{6}\right) \mathbf{a}_{3}\end{array}$ & $=$ & $\begin{array}{c}\left(\frac{1}{2}+y_{6}\right) a \hat{\mathbf{x}}+\left(\frac{1}{2}+x_{6}\right) a \hat{\mathbf{y}}+ \\
\left(\frac{1}{2}+z_{6}\right) a \hat{\mathbf{z}}\end{array}$ & $(96 h)$ & O II \\
\hline $\mathbf{B}_{38}$ & $=$ & $\begin{array}{c}\left(\frac{1}{2}-x_{6}+y_{6}+z_{6}\right) \mathbf{a}_{1}+ \\
\left(\frac{1}{2}+x_{6}-y_{6}+z_{6}\right) \mathbf{a}_{2}+ \\
\left(\frac{1}{2}-x_{6}-y_{6}-z_{6}\right) \mathbf{a}_{3}\end{array}$ & $=$ & $\begin{array}{c}\left(\frac{1}{2}-y_{6}\right) a \hat{\mathbf{x}}+\left(\frac{1}{2}-x_{6}\right) a \hat{\mathbf{y}}+ \\
\left(\frac{1}{2}+z_{6}\right) a \hat{\mathbf{z}}\end{array}$ & $(96 h)$ & O II \\
\hline $\mathbf{B}_{39}$ & $=$ & $\begin{array}{c}\left(\frac{1}{2}-x_{6}-y_{6}-z_{6}\right) \mathbf{a}_{1}+ \\
\left(\frac{1}{2}+x_{6}+y_{6}-z_{6}\right) \mathbf{a}_{2}+ \\
\left(\frac{1}{2}-x_{6}+y_{6}+z_{6}\right) \mathbf{a}_{3}\end{array}$ & $=$ & $\begin{array}{c}\left(\frac{1}{2}+y_{6}\right) a \hat{\mathbf{x}}+\left(\frac{1}{2}-x_{6}\right) a \hat{\mathbf{y}}+ \\
\left(\frac{1}{2}-z_{6}\right) a \hat{\mathbf{z}}\end{array}$ & $(96 h)$ & O II \\
\hline $\mathbf{B}_{40}$ & $=$ & $\begin{array}{c}\left(\frac{1}{2}+x_{6}+y_{6}-z_{6}\right) \mathbf{a}_{1}+ \\
\left(\frac{1}{2}-x_{6}-y_{6}-z_{6}\right) \mathbf{a}_{2}+ \\
\left(\frac{1}{2}+x_{6}-y_{6}+z_{6}\right) \mathbf{a}_{3}\end{array}$ & $=$ & $\begin{array}{c}\left(\frac{1}{2}-y_{6}\right) a \hat{\mathbf{x}}+\left(\frac{1}{2}+x_{6}\right) a \hat{\mathbf{y}}+ \\
\left(\frac{1}{2}-z_{6}\right) a \hat{\mathbf{z}}\end{array}$ & $(96 h)$ & O II \\
\hline $\mathbf{B}_{41}$ & $=$ & $\begin{array}{c}\left(\frac{1}{2}-x_{6}+y_{6}+z_{6}\right) \mathbf{a}_{1}+ \\
\left(\frac{1}{2}+x_{6}+y_{6}-z_{6}\right) \mathbf{a}_{2}+ \\
\left(\frac{1}{2}+x_{6}-y_{6}+z_{6}\right) \mathbf{a}_{3}\end{array}$ & $=$ & $\begin{array}{c}\left(\frac{1}{2}+x_{6}\right) a \hat{\mathbf{x}}+\left(\frac{1}{2}+z_{6}\right) a \hat{\mathbf{y}}+ \\
\left(\frac{1}{2}+y_{6}\right) a \hat{\mathbf{z}}\end{array}$ & $(96 h)$ & O II \\
\hline $\mathbf{B}_{42}$ & $=$ & $\begin{array}{c}\left(\frac{1}{2}+x_{6}-y_{6}+z_{6}\right) \mathbf{a}_{1}+ \\
\left(\frac{1}{2}-x_{6}-y_{6}-z_{6}\right) \mathbf{a}_{2}+ \\
\left(\frac{1}{2}-x_{6}+y_{6}+z_{6}\right) \mathbf{a}_{3}\end{array}$ & $=$ & $\begin{array}{c}\left(\frac{1}{2}-x_{6}\right) a \hat{\mathbf{x}}+\left(\frac{1}{2}+z_{6}\right) a \hat{\mathbf{y}}+ \\
\left(\frac{1}{2}-y_{6}\right) a \hat{\mathbf{z}}\end{array}$ & $(96 h)$ & O II \\
\hline
\end{tabular}




\begin{tabular}{|c|c|c|c|c|c|c|}
\hline $\mathbf{B}_{43}$ & $=$ & $\begin{array}{c}\left(\frac{1}{2}+x_{6}+y_{6}-z_{6}\right) \mathbf{a}_{1}+ \\
\left(\frac{1}{2}-x_{6}+y_{6}+z_{6}\right) \mathbf{a}_{2}+ \\
\left(\frac{1}{2}-x_{6}-y_{6}-z_{6}\right) \mathbf{a}_{3}\end{array}$ & $=$ & $\begin{array}{c}\left(\frac{1}{2}-x_{6}\right) a \hat{\mathbf{x}}+\left(\frac{1}{2}-z_{6}\right) a \hat{\mathbf{y}}+ \\
\left(\frac{1}{2}+y_{6}\right) a \hat{\mathbf{z}}\end{array}$ & $(96 h)$ & O II \\
\hline $\mathbf{B}_{44}$ & $=$ & $\begin{array}{c}\left(\frac{1}{2}-x_{6}-y_{6}-z_{6}\right) \mathbf{a}_{1}+ \\
\left(\frac{1}{2}+x_{6}-y_{6}+z_{6}\right) \mathbf{a}_{2}+ \\
\left(\frac{1}{2}+x_{6}+y_{6}-z_{6}\right) \mathbf{a}_{3}\end{array}$ & $=$ & $\begin{array}{c}\left(\frac{1}{2}+x_{6}\right) a \hat{\mathbf{x}}+\left(\frac{1}{2}-z_{6}\right) a \hat{\mathbf{y}}+ \\
\left(\frac{1}{2}-y_{6}\right) a \hat{\mathbf{z}}\end{array}$ & $(96 h)$ & O II \\
\hline $\mathbf{B}_{45}$ & $=$ & $\begin{array}{c}\left(\frac{1}{2}+x_{6}+y_{6}-z_{6}\right) \mathbf{a}_{1}+ \\
\left(\frac{1}{2}+x_{6}-y_{6}+z_{6}\right) \mathbf{a}_{2}+ \\
\left(\frac{1}{2}-x_{6}+y_{6}+z_{6}\right) \mathbf{a}_{3}\end{array}$ & $=$ & $\begin{array}{c}\left(\frac{1}{2}+z_{6}\right) a \hat{\mathbf{x}}+\left(\frac{1}{2}+y_{6}\right) a \hat{\mathbf{y}}+ \\
\left(\frac{1}{2}+x_{6}\right) a \hat{\mathbf{z}}\end{array}$ & $(96 h)$ & O II \\
\hline $\mathbf{B}_{46}$ & $=$ & $\begin{array}{c}\left(\frac{1}{2}-x_{6}-y_{6}-z_{6}\right) \mathbf{a}_{1}+ \\
\left(\frac{1}{2}-x_{6}+y_{6}+z_{6}\right) \mathbf{a}_{2}+ \\
\left(\frac{1}{2}+x_{6}-y_{6}+z_{6}\right) \mathbf{a}_{3}\end{array}$ & $=$ & $\begin{array}{c}\left(\frac{1}{2}+z_{6}\right) a \hat{\mathbf{x}}+\left(\frac{1}{2}-y_{6}\right) a \hat{\mathbf{y}}+ \\
\left(\frac{1}{2}-x_{6}\right) a \hat{\mathbf{z}}\end{array}$ & $(96 h)$ & O II \\
\hline $\mathbf{B}_{47}$ & $=$ & $\begin{array}{c}\left(\frac{1}{2}-x_{6}+y_{6}+z_{6}\right) \mathbf{a}_{1}+ \\
\left(\frac{1}{2}-x_{6}-y_{6}-z_{6}\right) \mathbf{a}_{2}+ \\
\left(\frac{1}{2}+x_{6}+y_{6}-z_{6}\right) \mathbf{a}_{3}\end{array}$ & $=$ & $\begin{array}{c}\left(\frac{1}{2}-z_{6}\right) a \hat{\mathbf{x}}+\left(\frac{1}{2}+y_{6}\right) a \hat{\mathbf{y}}+ \\
\left(\frac{1}{2}-x_{6}\right) a \hat{\mathbf{z}}\end{array}$ & $(96 h)$ & O II \\
\hline $\mathbf{B}_{48}$ & $=$ & $\begin{array}{c}\left(\frac{1}{2}+x_{6}-y_{6}+z_{6}\right) \mathbf{a}_{1}+ \\
\left(\frac{1}{2}+x_{6}+y_{6}-z_{6}\right) \mathbf{a}_{2}+ \\
\left(\frac{1}{2}-x_{6}-y_{6}-z_{6}\right) \mathbf{a}_{3}\end{array}$ & $=$ & $\begin{array}{c}\left(\frac{1}{2}-z_{6}\right) a \hat{\mathbf{x}}+\left(\frac{1}{2}-y_{6}\right) a \hat{\mathbf{y}}+ \\
\left(\frac{1}{2}+x_{6}\right) a \hat{\mathbf{z}}\end{array}$ & $(96 h)$ & O II \\
\hline
\end{tabular}

\section{References:}

- S. Sueng, J. R. Clark, J. J. Papike, and J. A. Konnert, Crystal-Structure Refinement of Cubic Boracite, Am. Mineral. 58, 691-697 (1973).

\section{Geometry files:}

- CIF: pp. 969

- POSCAR: pp. 969 


\section{$\mathrm{Cu}_{15} \mathrm{Si}_{4}\left(D 8_{6}\right)$ Structure: A15B4_cI76_220_ae_c}
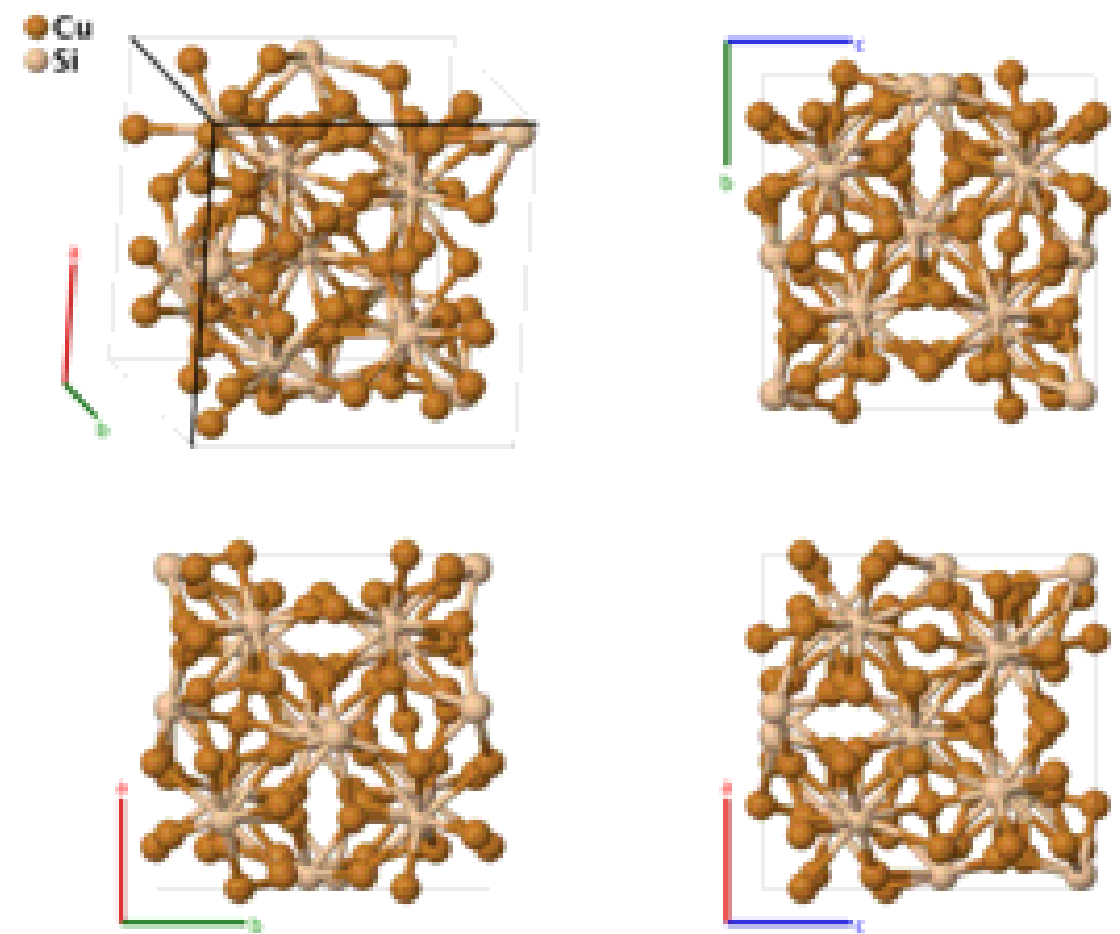

\begin{tabular}{|c|c|c|}
\hline Prototype & & $\mathrm{Cu}_{15} \mathrm{Si}_{4}$ \\
\hline AFLOW prototype label & & A15B4_cI76_220_ae_c \\
\hline Strukturbericht designation & & $D 8_{6}$ \\
\hline Pearson symbol & & cI76 \\
\hline Space group number & e & 220 \\
\hline Space group symbol & & $I \overline{4} 3 d$ \\
\hline AFLOW prototype command & $\cdot$ & $\begin{array}{l}\text { aflow --proto=A15B4_cI76_220_ae_c } \\
- \text {-params }=a, x_{2}, x_{3}, y_{3}, z_{3}\end{array}$ \\
\hline
\end{tabular}

Other compounds with this structure:

- $\mathrm{Cu}_{15} \mathrm{As}_{4}, \mathrm{Li}_{15} \mathrm{Si}_{4}, \mathrm{Na}_{15} \mathrm{~Pb}_{4}$

Body-centered Cubic primitive vectors:

$$
\begin{aligned}
& \mathbf{a}_{1}=-\frac{1}{2} a \hat{\mathbf{x}}+\frac{1}{2} a \hat{\mathbf{y}}+\frac{1}{2} a \hat{\mathbf{z}} \\
& \mathbf{a}_{2}=\frac{1}{2} a \hat{\mathbf{x}}-\frac{1}{2} a \hat{\mathbf{y}}+\frac{1}{2} a \hat{\mathbf{z}} \\
& \mathbf{a}_{3}=\frac{1}{2} a \hat{\mathbf{x}}+\frac{1}{2} a \hat{\mathbf{y}}-\frac{1}{2} a \hat{\mathbf{z}}
\end{aligned}
$$

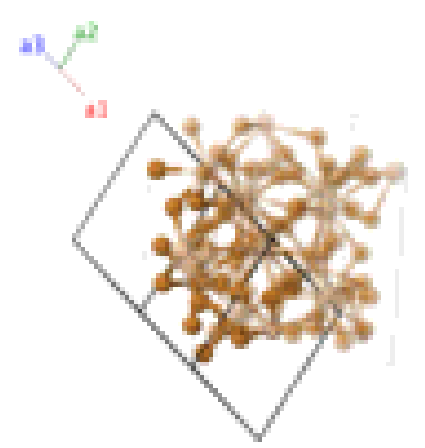

Basis vectors: 


\begin{tabular}{|c|c|c|c|c|c|c|}
\hline $\mathbf{B}_{1}$ & $=$ & $\frac{1}{4} \mathbf{a}_{1}+\frac{5}{8} \mathbf{a}_{2}+\frac{3}{8} \mathbf{a}_{3}$ & $=$ & $\frac{3}{8} a \hat{\mathbf{x}}+\frac{1}{4} a \hat{\mathbf{z}}$ & $(12 a)$ & $\mathrm{Cu} \mathrm{I}$ \\
\hline $\mathbf{B}_{2}$ & $=$ & $\frac{3}{4} \mathbf{a}_{1}+\frac{7}{8} \mathbf{a}_{2}+\frac{1}{8} \mathbf{a}_{3}$ & $=$ & $\frac{1}{8} a \hat{\mathbf{x}}+\frac{3}{4} a \hat{\mathbf{z}}$ & $(12 a)$ & $\mathrm{Cu} \mathrm{I}$ \\
\hline $\mathbf{B}_{3}$ & $=$ & $\frac{3}{8} \mathbf{a}_{1}+\frac{1}{4} \mathbf{a}_{2}+\frac{5}{8} \mathbf{a}_{3}$ & $=$ & $\frac{1}{4} a \hat{\mathbf{x}}+\frac{3}{8} a \hat{\mathbf{y}}$ & $(12 a)$ & $\mathrm{Cu} \mathrm{I}$ \\
\hline $\mathbf{B}_{4}$ & $=$ & $\frac{1}{8} \mathbf{a}_{1}+\frac{3}{4} \mathbf{a}_{2}+\frac{7}{8} \mathbf{a}_{3}$ & $=$ & $\frac{3}{4} a \hat{\mathbf{x}}+\frac{1}{8} a \hat{\mathbf{y}}$ & $(12 a)$ & $\mathrm{Cu} \mathrm{I}$ \\
\hline $\mathbf{B}_{5}$ & $=$ & $\frac{5}{8} \mathbf{a}_{1}+\frac{3}{8} \mathbf{a}_{2}+\frac{1}{4} \mathbf{a}_{3}$ & $=$ & $\frac{1}{4} a \hat{\mathbf{y}}+\frac{3}{8} a \hat{\mathbf{z}}$ & $(12 a)$ & $\mathrm{Cu} \mathrm{I}$ \\
\hline $\mathbf{B}_{6}$ & $=$ & $\frac{7}{8} \mathbf{a}_{1}+\frac{1}{8} \mathbf{a}_{2}+\frac{3}{4} \mathbf{a}_{3}$ & $=$ & $\frac{3}{4} a \hat{\mathbf{y}}+\frac{1}{8} a \hat{\mathbf{z}}$ & $(12 a)$ & $\mathrm{Cu} \mathrm{I}$ \\
\hline $\mathbf{B}_{7}$ & $=$ & $2 x_{2} \mathbf{a}_{1}+2 x_{2} \mathbf{a}_{2}+2 x_{2} \mathbf{a}_{3}$ & $=$ & $x_{2} a \hat{\mathbf{x}}+x_{2} a \hat{\mathbf{y}}+x_{2} a \hat{\mathbf{z}}$ & $(16 c)$ & $\mathrm{Si}$ \\
\hline $\mathbf{B}_{8}$ & $=$ & $\frac{1}{2} \mathbf{a}_{1}+\left(\frac{1}{2}-2 x_{2}\right) \mathbf{a}_{3}$ & $=$ & $-x_{2} a \hat{\mathbf{x}}+\left(\frac{1}{2}-x_{2}\right) a \hat{\mathbf{y}}+x_{2} a \hat{\mathbf{z}}$ & $(16 c)$ & $\mathrm{Si}$ \\
\hline $\mathbf{B}_{9}$ & $=$ & $\left(\frac{1}{2}-2 x_{2}\right) \mathbf{a}_{2}+\frac{1}{2} \mathbf{a}_{3}$ & $=$ & $\left(\frac{1}{2}-x_{2}\right) a \hat{\mathbf{x}}+x_{2} a \hat{\mathbf{y}}-x_{2} a \hat{\mathbf{z}}$ & $(16 c)$ & $\mathrm{Si}$ \\
\hline $\mathbf{B}_{10}$ & $=$ & $\left(\frac{1}{2}-2 x_{2}\right) \mathbf{a}_{1}+\frac{1}{2} \mathbf{a}_{2}$ & $=$ & $x_{2} a \hat{\mathbf{x}}-x_{2} a \hat{\mathbf{y}}+\left(\frac{1}{2}-x_{2}\right) a \hat{\mathbf{z}}$ & $(16 c)$ & $\mathrm{Si}$ \\
\hline $\mathbf{B}_{11}$ & $=$ & $\begin{array}{c}\left(\frac{1}{2}+2 x_{2}\right) \mathbf{a}_{1}+\left(\frac{1}{2}+2 x_{2}\right) \mathbf{a}_{2}+ \\
\left(\frac{1}{2}+2 x_{2}\right) \mathbf{a}_{3}\end{array}$ & $=$ & $\begin{array}{c}\left(\frac{1}{4}+x_{2}\right) a \hat{\mathbf{x}}+\left(\frac{1}{4}+x_{2}\right) a \hat{\mathbf{y}}+ \\
\left(\frac{1}{4}+x_{2}\right) a \hat{\mathbf{z}}\end{array}$ & $(16 c)$ & $\mathrm{Si}$ \\
\hline $\mathbf{B}_{12}$ & $=$ & $\frac{1}{2} \mathbf{a}_{1}+-2 x_{2} \mathbf{a}_{3}$ & $=$ & $\begin{array}{c}-a\left(x_{2}+\frac{1}{4}\right) \hat{\mathbf{x}}+\left(\frac{1}{4}-x_{2}\right) a \hat{\mathbf{y}}+ \\
\left(\frac{1}{4}+x_{2}\right) a \hat{\mathbf{z}}\end{array}$ & $(16 c)$ & $\mathrm{Si}$ \\
\hline $\mathbf{B}_{13}$ & $=$ & $-2 x_{2} \mathbf{a}_{1}+\frac{1}{2} \mathbf{a}_{2}$ & $=$ & $\begin{array}{c}\left(\frac{1}{4}+x_{2}\right) a \hat{\mathbf{x}}-a\left(x_{2}+\frac{1}{4}\right) \hat{\mathbf{y}}+ \\
\left(\frac{1}{4}-x_{2}\right) a \hat{\mathbf{z}}\end{array}$ & $(16 c)$ & $\mathrm{Si}$ \\
\hline $\mathbf{B}_{14}$ & $=$ & $-2 x_{2} \mathbf{a}_{2}+\frac{1}{2} \mathbf{a}_{3}$ & $=$ & $\begin{array}{c}\left(\frac{1}{4}-x_{2}\right) a \hat{\mathbf{x}}+\left(\frac{1}{4}+x_{2}\right) a \hat{\mathbf{y}}- \\
a\left(x_{2}+\frac{1}{4}\right) \hat{\mathbf{z}}\end{array}$ & $(16 c)$ & $\mathrm{Si}$ \\
\hline $\mathbf{B}_{15}$ & $=$ & $\begin{array}{c}\left(y_{3}+z_{3}\right) \mathbf{a}_{1}+\left(x_{3}+z_{3}\right) \mathbf{a}_{2}+ \\
\left(x_{3}+y_{3}\right) \mathbf{a}_{3}\end{array}$ & $=$ & $x_{3} a \hat{\mathbf{x}}+y_{3} a \hat{\mathbf{y}}+z_{3} a \hat{\mathbf{z}}$ & $(48 e)$ & $\mathrm{Cu}$ II \\
\hline $\mathbf{B}_{16}$ & $=$ & $\begin{array}{c}\left(\frac{1}{2}-y_{3}+z_{3}\right) \mathbf{a}_{1}+\left(-x_{3}+z_{3}\right) \mathbf{a}_{2}+ \\
\left(\frac{1}{2}-x_{3}-y_{3}\right) \mathbf{a}_{3}\end{array}$ & $=$ & $-x_{3} a \hat{\mathbf{x}}+\left(\frac{1}{2}-y_{3}\right) a \hat{\mathbf{y}}+z_{3} a \hat{\mathbf{z}}$ & $(48 e)$ & $\mathrm{Cu}$ II \\
\hline $\mathbf{B}_{17}$ & $=$ & $\begin{array}{c}\left(y_{3}-z_{3}\right) \mathbf{a}_{1}+\left(\frac{1}{2}-x_{3}-z_{3}\right) \mathbf{a}_{2}+ \\
\left(\frac{1}{2}-x_{3}+y_{3}\right) \mathbf{a}_{3}\end{array}$ & $=$ & $\left(\frac{1}{2}-x_{3}\right) a \hat{\mathbf{x}}+y_{3} a \hat{\mathbf{y}}-z_{3} a \hat{\mathbf{z}}$ & $(48 e)$ & $\mathrm{Cu}$ II \\
\hline $\mathbf{B}_{18}$ & $=$ & $\begin{array}{c}\left(\frac{1}{2}-y_{3}-z_{3}\right) \mathbf{a}_{1}+ \\
\left(\frac{1}{2}+x_{3}-z_{3}\right) \mathbf{a}_{2}+\left(x_{3}-y_{3}\right) \mathbf{a}_{3}\end{array}$ & $=$ & $x_{3} a \hat{\mathbf{x}}-y_{3} a \hat{\mathbf{y}}+\left(\frac{1}{2}-z_{3}\right) a \hat{\mathbf{z}}$ & $(48 e)$ & $\mathrm{Cu}$ II \\
\hline $\mathbf{B}_{19}$ & $=$ & $\begin{array}{c}\left(x_{3}+y_{3}\right) \mathbf{a}_{1}+\left(y_{3}+z_{3}\right) \mathbf{a}_{2}+ \\
\left(x_{3}+z_{3}\right) \mathbf{a}_{3}\end{array}$ & $=$ & $z_{3} a \hat{\mathbf{x}}+x_{3} a \hat{\mathbf{y}}+y_{3} a \hat{\mathbf{z}}$ & $(48 e)$ & $\mathrm{Cu}$ II \\
\hline $\mathbf{B}_{20}$ & $=$ & $\begin{array}{c}\left(\frac{1}{2}-x_{3}-y_{3}\right) \mathbf{a}_{1}+ \\
\left(\frac{1}{2}-y_{3}+z_{3}\right) \mathbf{a}_{2}+\left(-x_{3}+z_{3}\right) \mathbf{a}_{3}\end{array}$ & $=$ & $z_{3} a \hat{\mathbf{x}}-x_{3} a \hat{\mathbf{y}}+\left(\frac{1}{2}-y_{3}\right) a \hat{\mathbf{z}}$ & $(48 e)$ & $\mathrm{Cu}$ II \\
\hline $\mathbf{B}_{21}$ & $=$ & $\begin{array}{c}\left(\frac{1}{2}-x_{3}+y_{3}\right) \mathbf{a}_{1}+\left(y_{3}-z_{3}\right) \mathbf{a}_{2}+ \\
\left(\frac{1}{2}-x_{3}-z_{3}\right) \mathbf{a}_{3}\end{array}$ & $=$ & $-z_{3} a \hat{\mathbf{x}}+\left(\frac{1}{2}-x_{3}\right) a \hat{\mathbf{y}}+y_{3} a \hat{\mathbf{z}}$ & $(48 e)$ & $\mathrm{Cu}$ II \\
\hline $\mathbf{B}_{22}$ & $=$ & $\begin{array}{c}\left(x_{3}-y_{3}\right) \mathbf{a}_{1}+\left(\frac{1}{2}-y_{3}-z_{3}\right) \mathbf{a}_{2}+ \\
\left(\frac{1}{2}+x_{3}-z_{3}\right) \mathbf{a}_{3}\end{array}$ & $=$ & $\left(\frac{1}{2}-z_{3}\right) a \hat{\mathbf{x}}+x_{3} a \hat{\mathbf{y}}-y_{3} a \hat{\mathbf{z}}$ & $(48 e)$ & $\mathrm{Cu}$ II \\
\hline $\mathbf{B}_{23}$ & $=$ & $\begin{array}{c}\left(x_{3}+z_{3}\right) \mathbf{a}_{1}+\left(x_{3}+y_{3}\right) \mathbf{a}_{2}+ \\
\left(y_{3}+z_{3}\right) \mathbf{a}_{3}\end{array}$ & $=$ & $y_{3} a \hat{\mathbf{x}}+z_{3} a \hat{\mathbf{y}}+x_{3} a \hat{\mathbf{z}}$ & $(48 e)$ & $\mathrm{Cu}$ II \\
\hline $\mathbf{B}_{24}$ & $=$ & $\begin{array}{c}\left(-x_{3}+z_{3}\right) \mathbf{a}_{1}+\left(\frac{1}{2}-x_{3}-y_{3}\right) \mathbf{a}_{2}+ \\
\left(\frac{1}{2}-y_{3}+z_{3}\right) \mathbf{a}_{3}\end{array}$ & $=$ & $\left(\frac{1}{2}-y_{3}\right) a \hat{\mathbf{x}}+z_{3} a \hat{\mathbf{y}}-x_{3} a \hat{\mathbf{z}}$ & $(48 e)$ & $\mathrm{Cu}$ II \\
\hline $\mathbf{B}_{25}$ & $=$ & $\begin{array}{c}\left(\frac{1}{2}-x_{3}-z_{3}\right) \mathbf{a}_{1}+ \\
\left(\frac{1}{2}-x_{3}+y_{3}\right) \mathbf{a}_{2}+\left(y_{3}-z_{3}\right) \mathbf{a}_{3}\end{array}$ & $=$ & $y_{3} a \hat{\mathbf{x}}-z_{3} a \hat{\mathbf{y}}+\left(\frac{1}{2}-x_{3}\right) a \hat{\mathbf{z}}$ & $(48 e)$ & $\mathrm{Cu}$ II \\
\hline
\end{tabular}




$$
\begin{aligned}
& \mathbf{B}_{26}=\begin{array}{c}
\left(\frac{1}{2}+x_{3}-z_{3}\right) \mathbf{a}_{1}+\left(x_{3}-y_{3}\right) \mathbf{a}_{2}+ \\
\left(\frac{1}{2}-y_{3}-z_{3}\right) \mathbf{a}_{3}
\end{array} \\
& \mathbf{B}_{27}=\left(\frac{1}{2}+x_{3}+z_{3}\right) \mathbf{a}_{1}+\quad=\quad\left(\frac{1}{4}+y_{3}\right) a \hat{\mathbf{x}}+\left(\frac{1}{4}+x_{3}\right) a \hat{\mathbf{y}}+ \\
& \left(\frac{1}{2}+y_{3}+z_{3}\right) \mathbf{a}_{2}+\left(\frac{1}{2}+x_{3}+y_{3}\right) \mathbf{a}_{3} \quad\left(\frac{1}{4}+z_{3}\right) a \hat{\mathbf{z}} \\
& \mathbf{B}_{28}=\left(\frac{1}{2}-x_{3}+z_{3}\right) \mathbf{a}_{1}+\left(-y_{3}+z_{3}\right) \mathbf{a}_{2}+=-a\left(y_{3}+\frac{1}{4}\right) \hat{\mathbf{x}}+\left(\frac{1}{4}-x_{3}\right) a \hat{\mathbf{y}}+ \\
& \left(-x_{3}-y_{3}\right) \mathbf{a}_{3} \quad\left(\frac{1}{4}+z_{3}\right) a \hat{\mathbf{z}} \\
& \mathbf{B}_{29}=\left(-x_{3}-z_{3}\right) \mathbf{a}_{1}+\left(\frac{1}{2}+y_{3}-z_{3}\right) \mathbf{a}_{2}+=\left(\frac{1}{4}+y_{3}\right) a \hat{\mathbf{x}}-a\left(x_{3}+\frac{1}{4}\right) \hat{\mathbf{y}}+ \\
& \left(-x_{3}+y_{3}\right) \mathbf{a}_{3} \quad\left(\frac{1}{4}-z_{3}\right) a \hat{\mathbf{z}} \\
& \mathbf{B}_{30}=\left(x_{3}-z_{3}\right) \mathbf{a}_{1}+\left(-y_{3}-z_{3}\right) \mathbf{a}_{2}+=\left(\frac{1}{4}-y_{3}\right) a \hat{\mathbf{x}}+\left(\frac{1}{4}+x_{3}\right) a \hat{\mathbf{y}}- \\
& \left(\frac{1}{2}+x_{3}-y_{3}\right) \mathbf{a}_{3} \quad a\left(z_{3}+\frac{1}{4}\right) \hat{\mathbf{z}} \\
& \mathbf{B}_{31}=\left(\frac{1}{2}+y_{3}+z_{3}\right) \mathbf{a}_{1}+\quad=\left(\frac{1}{4}+x_{3}\right) a \hat{\mathbf{x}}+\left(\frac{1}{4}+z_{3}\right) a \hat{\mathbf{y}}+ \\
& \left(\frac{1}{2}+x_{3}+y_{3}\right) \mathbf{a}_{2}+\left(\frac{1}{2}+x_{3}+z_{3}\right) \mathbf{a}_{3} \quad\left(\frac{1}{4}+y_{3}\right) a \hat{\mathbf{z}} \\
& \mathbf{B}_{32}=\left(-y_{3}+z_{3}\right) \mathbf{a}_{1}+\left(-x_{3}-y_{3}\right) \mathbf{a}_{2}+=\left(\frac{1}{4}-x_{3}\right) a \hat{\mathbf{x}}+\left(\frac{1}{4}+z_{3}\right) a \hat{\mathbf{y}}- \\
& \left(\frac{1}{2}-x_{3}+z_{3}\right) \mathbf{a}_{3} \quad a\left(y_{3}+\frac{1}{4}\right) \hat{\mathbf{z}} \\
& \mathbf{B}_{33}=\left(\frac{1}{2}+y_{3}-z_{3}\right) \mathbf{a}_{1}+\left(-x_{3}+y_{3}\right) \mathbf{a}_{2}+=-a\left(x_{3}+\frac{1}{4}\right) \hat{\mathbf{x}}+\left(\frac{1}{4}-z_{3}\right) a \hat{\mathbf{y}}+ \\
& \left(-x_{3}-z_{3}\right) \mathbf{a}_{3} \quad\left(\frac{1}{4}+y_{3}\right) a \hat{\mathbf{z}} \\
& \mathbf{B}_{34}=\left(-y_{3}-z_{3}\right) \mathbf{a}_{1}+\left(\frac{1}{2}+x_{3}-y_{3}\right) \mathbf{a}_{2}+=\left(\frac{1}{4}+x_{3}\right) a \hat{\mathbf{x}}-a\left(z_{3}+\frac{1}{4}\right) \hat{\mathbf{y}}+ \\
& \left(x_{3}-z_{3}\right) \mathbf{a}_{3} \quad\left(\frac{1}{4}-y_{3}\right) a \hat{\mathbf{z}} \\
& \mathbf{B}_{35}=\left(\frac{1}{2}+x_{3}+y_{3}\right) \mathbf{a}_{1}+\quad=\quad\left(\frac{1}{4}+z_{3}\right) a \hat{\mathbf{x}}+\left(\frac{1}{4}+y_{3}\right) a \hat{\mathbf{y}}+ \\
& \left(\frac{1}{2}+x_{3}+z_{3}\right) \mathbf{a}_{2}+\left(\frac{1}{2}+y_{3}+z_{3}\right) \mathbf{a}_{3} \quad\left(\frac{1}{4}+x_{3}\right) a \hat{\mathbf{z}} \\
& \mathbf{B}_{36}=\left(-x_{3}-y_{3}\right) \mathbf{a}_{1}+\left(\frac{1}{2}-x_{3}+z_{3}\right) \mathbf{a}_{2}+=\left(\frac{1}{4}+z_{3}\right) a \hat{\mathbf{x}}-a\left(y_{3}+\frac{1}{4}\right) \hat{\mathbf{y}}+ \\
& \left(-y_{3}+z_{3}\right) \mathbf{a}_{3} \quad\left(\frac{1}{4}-x_{3}\right) a \hat{\mathbf{z}} \\
& \mathbf{B}_{37}=\left(-x_{3}+y_{3}\right) \mathbf{a}_{1}+\left(-x_{3}-z_{3}\right) \mathbf{a}_{2}+=\left(\frac{1}{4}-z_{3}\right) a \hat{\mathbf{x}}+\left(\frac{1}{4}+y_{3}\right) a \hat{\mathbf{y}}- \\
& \left(\frac{1}{2}+y_{3}-z_{3}\right) \mathbf{a}_{3} \quad a\left(x_{3}+\frac{1}{4}\right) \hat{\mathbf{z}} \\
& \mathbf{B}_{38}=\left(\frac{1}{2}+x_{3}-y_{3}\right) \mathbf{a}_{1}+\left(x_{3}-z_{3}\right) \mathbf{a}_{2}+=-a\left(z_{3}+\frac{1}{4}\right) \hat{\mathbf{x}}+\left(\frac{1}{4}-y_{3}\right) a \hat{\mathbf{y}}+ \\
& \left(-y_{3}-z_{3}\right) \mathbf{a}_{3} \quad\left(\frac{1}{4}+x_{3}\right) a \hat{\mathbf{z}}
\end{aligned}
$$

\section{References:}

- M. Mattern, R. Seyrich, L. Wilde, C. Baehtz, M. Knapp, and J. Acker, Phase formation of rapidly quenched Cu-Si alloys, J. Alloys Compd. 429, 211-215 (2007), doi:10.1016/j.jallcom.2006.04.046.

\section{Found in:}

- K. Sufryd, N. Ponweiser, P. Riani, K. W. Richter, and G. Cacciamani, Experimental investigation of the Cu-Si phase diagram at $x(C u)>0.72$, Intermetallics 19, 1479-1488 (2011), doi:10.1016/j.intermet.2011.05.017.

\section{Geometry files:}

- CIF: pp. 970

- POSCAR: pp. 970 


\section{$\mathrm{Th}_{3} \mathrm{P}_{4}\left(D 7_{3}\right)$ Structure: A4B3_cI28_220_c_a}
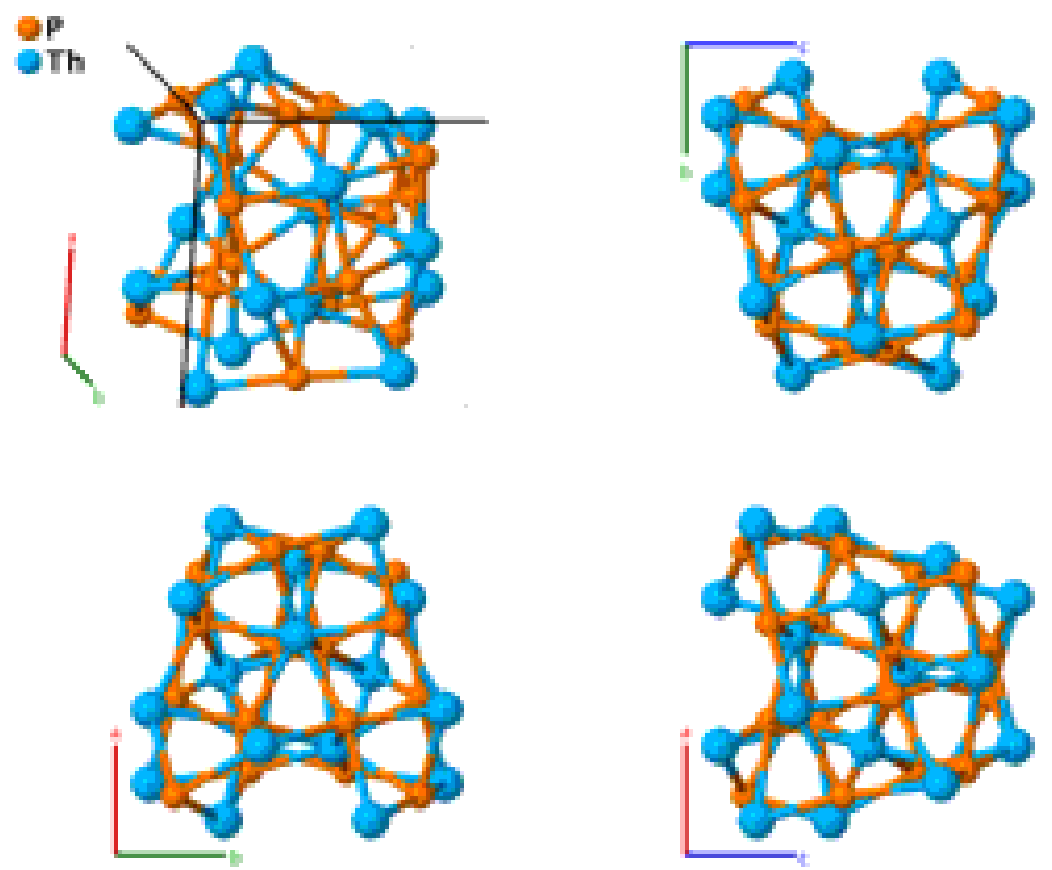

\section{Prototype}

AFLOW prototype label

Strukturbericht designation

Pearson symbol

Space group number

Space group symbol

AFLOW prototype command
: $\quad \mathrm{Th}_{3} \mathrm{P}_{4}$

: A4B3_cI28_220_c_a

: $\quad D 7_{3}$

: $\quad$ cI28

: 220

$: \quad I \overline{4} 3 d$

Other compounds with this structure:

- $\mathrm{Th}_{3} \mathrm{As}_{4}, \mathrm{U}_{3} \mathrm{AS}_{4}, \mathrm{U}_{3} \mathrm{Bi}_{4}, \mathrm{~N}_{3} \mathrm{P}_{4}, \mathrm{Th}_{3} \mathrm{P}_{4}, \mathrm{U}_{3} \mathrm{P}_{4}, \mathrm{Th}_{3} \mathrm{Sb}_{4}, \mathrm{U}_{3} \mathrm{Sb}_{4}, \mathrm{U}_{3} \mathrm{Te}_{4}$

Body-centered Cubic primitive vectors:

$$
\begin{aligned}
& \mathbf{a}_{1}=-\frac{1}{2} a \hat{\mathbf{x}}+\frac{1}{2} a \hat{\mathbf{y}}+\frac{1}{2} a \hat{\mathbf{z}} \\
& \mathbf{a}_{2}=\frac{1}{2} a \hat{\mathbf{x}}-\frac{1}{2} a \hat{\mathbf{y}}+\frac{1}{2} a \hat{\mathbf{z}} \\
& \mathbf{a}_{3}=\frac{1}{2} a \hat{\mathbf{x}}+\frac{1}{2} a \hat{\mathbf{y}}-\frac{1}{2} a \hat{\mathbf{z}}
\end{aligned}
$$

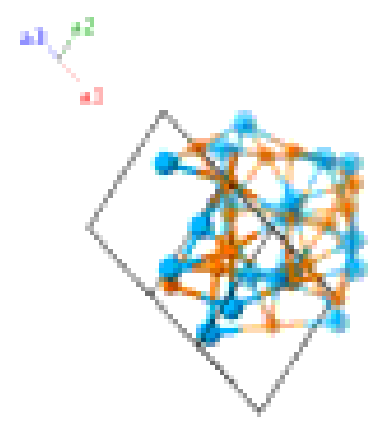

Basis vectors:

Lattice Coordinates

$\mathbf{B}_{1}=\quad \frac{1}{4} \mathbf{a}_{1}+\frac{5}{8} \mathbf{a}_{2}+\frac{3}{8} \mathbf{a}_{3}$
Cartesian Coordinates

$=$

$$
\frac{3}{8} a \hat{\mathbf{x}}+\frac{1}{4} a \hat{\mathbf{z}}
$$

Wyckoff Position

Atom Type

(12a)

Th 


\begin{tabular}{|c|c|c|c|c|c|}
\hline $\mathbf{B}_{2}$ & $=$ & $\frac{3}{4} \mathbf{a}_{1}+\frac{7}{8} \mathbf{a}_{2}+\frac{1}{8} \mathbf{a}_{3}$ & $=$ & $\frac{1}{8} a \hat{\mathbf{x}}+\frac{3}{4} a \hat{\mathbf{z}}$ & $(12 a)$ \\
\hline $\mathbf{B}_{3}$ & $=$ & $\frac{3}{8} \mathbf{a}_{1}+\frac{1}{4} \mathbf{a}_{2}+\frac{5}{8} \mathbf{a}_{3}$ & $=$ & $\frac{1}{4} a \hat{\mathbf{x}}+\frac{3}{8} a \hat{\mathbf{y}}$ & $(12 a)$ \\
\hline $\mathbf{B}_{4}$ & $=$ & $\frac{1}{8} \mathbf{a}_{1}+\frac{3}{4} \mathbf{a}_{2}+\frac{7}{8} \mathbf{a}_{3}$ & $=$ & $\frac{3}{4} a \hat{\mathbf{x}}+\frac{1}{8} a \hat{\mathbf{y}}$ & $(12 a)$ \\
\hline $\mathbf{B}_{5}$ & $=$ & $\frac{5}{8} \mathbf{a}_{1}+\frac{3}{8} \mathbf{a}_{2}+\frac{1}{4} \mathbf{a}_{3}$ & $=$ & $\frac{1}{4} a \hat{\mathbf{y}}+\frac{3}{8} a \hat{\mathbf{z}}$ & $(12 a)$ \\
\hline $\mathbf{B}_{6}$ & $=$ & $\frac{7}{8} \mathbf{a}_{1}+\frac{1}{8} \mathbf{a}_{2}+\frac{3}{4} \mathbf{a}_{3}$ & $=$ & $\frac{3}{4} a \hat{\mathbf{y}}+\frac{1}{8} a \hat{\mathbf{z}}$ & $(12 a)$ \\
\hline $\mathbf{B}_{7}$ & $=$ & $2 x_{2} \mathbf{a}_{1}+2 x_{2} \mathbf{a}_{2}+2 x_{2} \mathbf{a}_{3}$ & $=$ & $x_{2} a \hat{\mathbf{x}}+x_{2} a \hat{\mathbf{y}}+x_{2} a \hat{\mathbf{z}}$ & $(16 c)$ \\
\hline $\mathbf{B}_{8}$ & $=$ & $\frac{1}{2} \mathbf{a}_{1}+\left(\frac{1}{2}-2 x_{2}\right) \mathbf{a}_{3}$ & $=$ & $-x_{2} a \hat{\mathbf{x}}+\left(\frac{1}{2}-x_{2}\right) a \hat{\mathbf{y}}+x_{2} a \hat{\mathbf{z}}$ & $(16 c)$ \\
\hline $\mathbf{B}_{9}$ & $=$ & $\left(\frac{1}{2}-2 x_{2}\right) \mathbf{a}_{2}+\frac{1}{2} \mathbf{a}_{3}$ & $=$ & $\left(\frac{1}{2}-x_{2}\right) a \hat{\mathbf{x}}+x_{2} a \hat{\mathbf{y}}-x_{2} a \hat{\mathbf{z}}$ & $(16 c)$ \\
\hline $\mathbf{B}_{10}$ & $=$ & $\left(\frac{1}{2}-2 x_{2}\right) \mathbf{a}_{1}+\frac{1}{2} \mathbf{a}_{2}$ & $=$ & $x_{2} a \hat{\mathbf{x}}-x_{2} a \hat{\mathbf{y}}+\left(\frac{1}{2}-x_{2}\right) a \hat{\mathbf{z}}$ & $(16 c)$ \\
\hline $\mathbf{B}_{11}$ & $=$ & $\begin{array}{c}\left(\frac{1}{2}+2 x_{2}\right) \mathbf{a}_{1}+\left(\frac{1}{2}+2 x_{2}\right) \mathbf{a}_{2}+ \\
\left(\frac{1}{2}+2 x_{2}\right) \mathbf{a}_{3}\end{array}$ & $=$ & $\begin{array}{c}\left(\frac{1}{4}+x_{2}\right) a \hat{\mathbf{x}}+\left(\frac{1}{4}+x_{2}\right) a \hat{\mathbf{y}}+ \\
\left(\frac{1}{4}+x_{2}\right) a \hat{\mathbf{z}}\end{array}$ & $(16 c)$ \\
\hline $\mathbf{B}_{12}$ & $=$ & $\frac{1}{2} \mathbf{a}_{1}+-2 x_{2} \mathbf{a}_{3}$ & $=$ & $\begin{array}{c}-a\left(x_{2}+\frac{1}{4}\right) \hat{\mathbf{x}}+\left(\frac{1}{4}-x_{2}\right) a \hat{\mathbf{y}}+ \\
\left(\frac{1}{4}+x_{2}\right) a \hat{\mathbf{z}}\end{array}$ & $(16 c)$ \\
\hline $\mathbf{B}_{13}$ & $=$ & $-2 x_{2} \mathbf{a}_{1}+\frac{1}{2} \mathbf{a}_{2}$ & $=$ & $\begin{array}{c}\left(\frac{1}{4}+x_{2}\right) a \hat{\mathbf{x}}-a\left(x_{2}+\frac{1}{4}\right) \hat{\mathbf{y}}+ \\
\left(\frac{1}{4}-x_{2}\right) a \hat{\mathbf{z}}\end{array}$ & $(16 c)$ \\
\hline $\mathbf{B}_{14}$ & $=$ & $-2 x_{2} \mathbf{a}_{2}+\frac{1}{2} \mathbf{a}_{3}$ & $=$ & $\begin{array}{c}\left(\frac{1}{4}-x_{2}\right) a \hat{\mathbf{x}}+\left(\frac{1}{4}+x_{2}\right) a \hat{\mathbf{y}}- \\
a\left(x_{2}+\frac{1}{4}\right) \hat{\mathbf{z}}\end{array}$ & $(16 c)$ \\
\hline
\end{tabular}

\section{References:}

- K. Meisel, Kristallstrukturen von Thoriumphosphiden, Z. Anorg. Allg. Chem. 240, 300-312 (1939), doi:10.1002/zaac.19392400403.

\section{Geometry files:}

- CIF: pp. 970

- POSCAR: pp. 971 


\section{$\mathrm{Ca}_{3} \mathrm{Al}_{2} \mathrm{O}_{6}\left(E 9_{1}\right)$ Structure:}

\section{A2B3C6_cP33_221_cd_ag_fh}
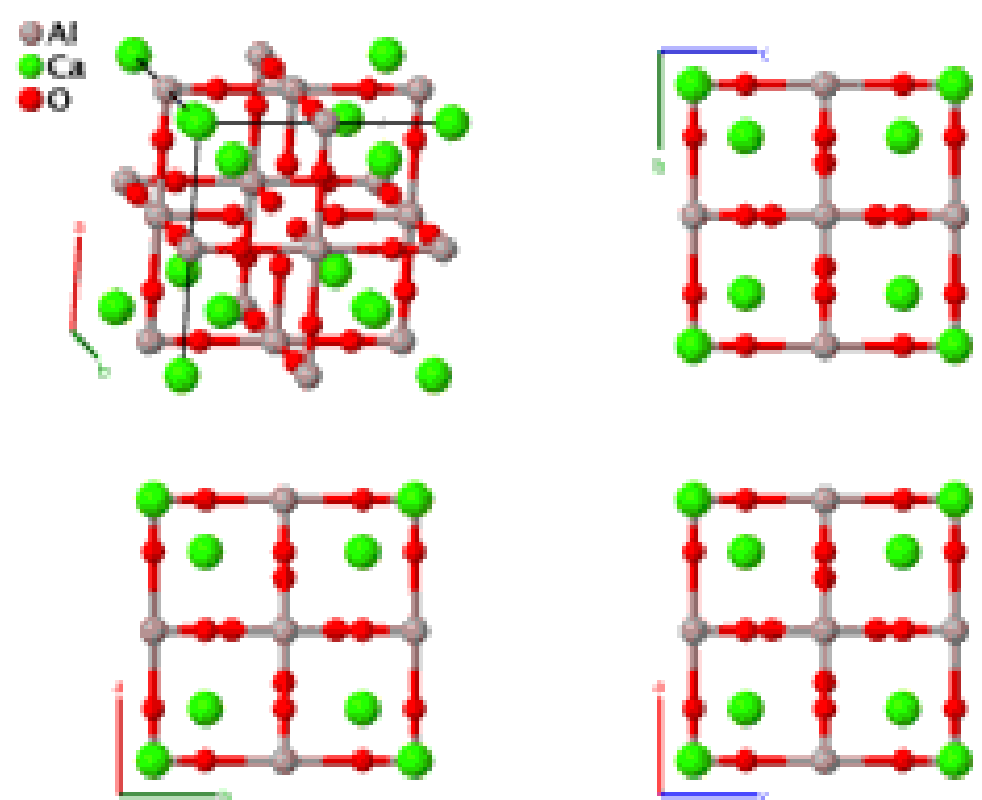

Prototype

AFLOW prototype label

Strukturbericht designation

Pearson symbol

Space group number

Space group symbol

AFLOW prototype command
: $\quad \mathrm{Ca}_{3} \mathrm{Al}_{2} \mathrm{O}_{6}$

: A2B3C6_cP33_221_cd_ag_fh

: $\quad E 9_{1}$

: $\quad \mathrm{cP} 33$

: $\quad 221$

: $\quad P m \overline{3} m$ aflow --proto=A2B3C6_cP33_221_cd_ag_fh

- - params $=a, x_{4}, x_{5}, x_{6}$

- (Steele, 1929) do not use the standard Wyckoff position notation to describe the atomic positions, so we use the parameters found in (Herman, 1937). An alternative description of the structure places the $\mathrm{O}$ I atoms on the $(6 \mathrm{e})( \pm x, 0,0) \ldots$ site rather than the $(6 \mathrm{f})$ site.

- (Mondal, 1975) reanalyzed this structure and concluded that the true structure was one where the lattice constant was doubled and contained 264 atoms. See the $\mathrm{Ca}_{3} \mathrm{Al}_{2} \mathrm{O}_{6}$ structure page.

\section{Simple Cubic primitive vectors:}

$$
\begin{aligned}
& \mathbf{a}_{1}=a \hat{\mathbf{x}} \\
& \mathbf{a}_{2}=a \hat{\mathbf{y}} \\
& \mathbf{a}_{3}=a \hat{\mathbf{z}}
\end{aligned}
$$

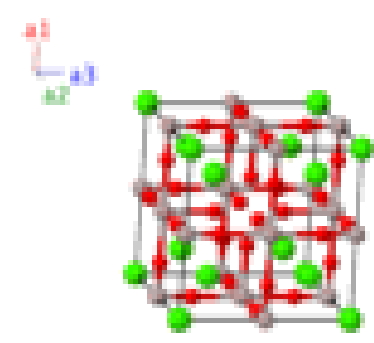

\section{Basis vectors:}


Lattice Coordinates
$\mathbf{B}_{1}=$
$0 \mathbf{a}_{1}+0 \mathbf{a}_{2}+0 \mathbf{a}_{3}$
$=$
$\mathbf{B}_{2}=$
$\frac{1}{2} \mathbf{a}_{2}+\frac{1}{2} \mathbf{a}_{3}$
$\mathbf{B}_{3}=$
$\mathbf{B}_{4}=$
$\mathbf{B}_{5}=$
$\mathbf{B}_{6}=$
$\mathbf{B}_{7}=$
$\frac{1}{2} \mathbf{a}_{1}+\frac{1}{2} \mathbf{a}_{3}$
$\frac{1}{2} \mathbf{a}_{1}+\frac{1}{2} \mathbf{a}_{2}$
$\frac{1}{2} \mathbf{a}_{1}$
$\frac{1}{2} \mathbf{a}_{2}$
$\mathbf{B}_{8}=$
$\frac{1}{2} \mathbf{a}_{3}$
$\mathbf{B}_{9}=$
$x_{4} \mathbf{a}_{1}+\frac{1}{2} \mathbf{a}_{2}+\frac{1}{2} \mathbf{a}_{3}$
$-x_{4} \mathbf{a}_{1}+\frac{1}{2} \mathbf{a}_{2}+\frac{1}{2} \mathbf{a}_{3}$
$\mathbf{B}_{10}=$
$\frac{1}{2} \mathbf{a}_{1}+x_{4} \mathbf{a}_{2}+\frac{1}{2} \mathbf{a}_{3}$
$\mathbf{B}_{11}=$
$\frac{1}{2} \mathbf{a}_{1}-x_{4} \mathbf{a}_{2}+\frac{1}{2} \mathbf{a}_{3}$
$\mathbf{B}_{12}=$
$\frac{1}{2} \mathbf{a}_{1}+\frac{1}{2} \mathbf{a}_{2}+x_{4} \mathbf{a}_{3}$
$\mathbf{B}_{13}=\frac{1}{2} \mathbf{a}_{1}+\frac{1}{2} \mathbf{a}_{2}-x_{4} \mathbf{a}_{3}$
$\mathbf{B}_{14}=x_{5} \mathbf{a}_{1}+x_{5} \mathbf{a}_{2}+x_{5} \mathbf{a}_{3}$
$\mathbf{B}_{15}=-x_{5} \mathbf{a}_{1}-x_{5} \mathbf{a}_{2}+x_{5} \mathbf{a}_{3}$
$\mathbf{B}_{16}=-x_{5} \mathbf{a}_{1}+x_{5} \mathbf{a}_{2}-x_{5} \mathbf{a}_{3}$
$\mathbf{B}_{17}=x_{5} \mathbf{a}_{1}-x_{5} \mathbf{a}_{2}-x_{5} \mathbf{a}_{3}$
$\mathbf{B}_{18}=x_{5} \mathbf{a}_{1}+x_{5} \mathbf{a}_{2}-x_{5} \mathbf{a}_{3}$
$\mathbf{B}_{19}=-x_{5} \mathbf{a}_{1}-x_{5} \mathbf{a}_{2}-x_{5} \mathbf{a}_{3}$
$\mathbf{B}_{20}=x_{5} \mathbf{a}_{1}-x_{5} \mathbf{a}_{2}+x_{5} \mathbf{a}_{3}$
$\mathbf{B}_{21}=$
$\mathbf{B}_{22}=$
$-x_{5} \mathbf{a}_{1}+x_{5} \mathbf{a}_{2}+x_{5} \mathbf{a}_{3}$
$x_{6} \mathbf{a}_{1}+\frac{1}{2} \mathbf{a}_{2}$
$\mathbf{B}_{23}=$
$\mathbf{B}_{24}=$
$\mathbf{B}_{25}=$
$\mathbf{B}_{26}=$
$\mathbf{B}_{27}=$
$\mathbf{B}_{28}=$
$\mathbf{B}_{29}=$
$\mathbf{B}_{30}=$
$\mathbf{B}_{31}=$
$\mathbf{B}_{32}=$
$\mathbf{B}_{33}=$

$$
\begin{gathered}
\frac{1}{2} a \hat{\mathbf{y}}+\frac{1}{2} a \hat{\mathbf{z}} \\
\frac{1}{2} a \hat{\mathbf{x}}+\frac{1}{2} a \hat{\mathbf{z}} \\
\frac{1}{2} a \hat{\mathbf{x}}+\frac{1}{2} a \hat{\mathbf{y}} \\
\frac{1}{2} a \hat{\mathbf{x}} \\
\frac{1}{2} a \hat{\mathbf{y}} \\
\frac{1}{2} a \hat{\mathbf{z}}
\end{gathered}
$$$$
0 \hat{\mathbf{x}}+0 \hat{\mathbf{y}}+0 \hat{\mathbf{z}}
$$$$
\frac{1}{2} a \hat{\mathbf{x}}+\frac{1}{2} a \hat{\mathbf{y}}
$$$$
x_{4} a \hat{\mathbf{x}}+\frac{1}{2} a \hat{\mathbf{y}}+\frac{1}{2} a \hat{\mathbf{z}}
$$$$
-x_{4} a \hat{\mathbf{x}}+\frac{1}{2} a \hat{\mathbf{y}}+\frac{1}{2} a \hat{\mathbf{z}}
$$$$
\frac{1}{2} a \hat{\mathbf{x}}+x_{4} a \hat{\mathbf{y}}+\frac{1}{2} a \hat{\mathbf{z}}
$$$$
\frac{1}{2} a \hat{\mathbf{x}}-x_{4} a \hat{\mathbf{y}}+\frac{1}{2} a \hat{\mathbf{z}}
$$$$
\frac{1}{2} a \hat{\mathbf{x}}+\frac{1}{2} a \hat{\mathbf{y}}+x_{4} a \hat{\mathbf{z}}
$$$$
\frac{1}{2} a \hat{\mathbf{x}}+\frac{1}{2} a \hat{\mathbf{y}}-x_{4} a \hat{\mathbf{z}}
$$$$
x_{5} a \hat{\mathbf{x}}+x_{5} a \hat{\mathbf{y}}+x_{5} a \hat{\mathbf{z}}
$$$$
-x_{5} a \hat{\mathbf{x}}-x_{5} a \hat{\mathbf{y}}+x_{5} a \hat{\mathbf{z}}
$$$$
-x_{5} a \hat{\mathbf{x}}+x_{5} a \hat{\mathbf{y}}-x_{5} a \hat{\mathbf{z}}
$$$$
x_{5} a \hat{\mathbf{x}}-x_{5} a \hat{\mathbf{y}}-x_{5} a \hat{\mathbf{z}}
$$$$
x_{5} a \hat{\mathbf{x}}+x_{5} a \hat{\mathbf{y}}-x_{5} a \hat{\mathbf{z}}
$$$$
-x_{5} a \hat{\mathbf{x}}-x_{5} a \hat{\mathbf{y}}-x_{5} a \hat{\mathbf{z}}
$$$$
x_{5} a \hat{\mathbf{x}}-x_{5} a \hat{\mathbf{y}}+x_{5} a \hat{\mathbf{z}}
$$$$
-x_{5} a \hat{\mathbf{x}}+x_{5} a \hat{\mathbf{y}}+x_{5} a \hat{\mathbf{z}}
$$

$$
x_{6} a \hat{\mathbf{x}}+\frac{1}{2} a \hat{\mathbf{y}}
$$$$
-x_{6} a \hat{\mathbf{x}}+\frac{1}{2} a \hat{\mathbf{y}}
$$$$
x_{6} a \hat{\mathbf{y}}+\frac{1}{2} a \hat{\mathbf{z}}
$$$$
-x_{6} a \hat{\mathbf{y}}+\frac{1}{2} a \hat{\mathbf{z}}
$$$$
\frac{1}{2} a \hat{\mathbf{x}}+x_{6} a \hat{\mathbf{z}}
$$$$
\frac{1}{2} a \hat{\mathbf{x}}+-x_{6} a \hat{\mathbf{z}}
$$$$
\frac{1}{2} a \hat{\mathbf{x}}+x_{6} a \hat{\mathbf{y}}
$$$$
\frac{1}{2} a \hat{\mathbf{x}}-x_{6} a \hat{\mathbf{y}}
$$$$
x_{6} a \hat{\mathbf{x}}+\frac{1}{2} a \hat{\mathbf{z}}
$$$$
-x_{6} a \hat{\mathbf{x}}+\frac{1}{2} a \hat{\mathbf{z}}
$$$$
\frac{1}{2} a \hat{\mathbf{y}}-x_{6} a \hat{\mathbf{z}}
$$$$
\frac{1}{2} a \hat{\mathbf{y}}+x_{6} a \hat{\mathbf{z}}
$$

Cartesian Coordinates
Wyckoff Position

(1a)
Atom Type

Ca I

Al I

Al I

Al I

Al II

Al II

Al II

O I

O I

O I

O I

O I

O I

Ca II

Ca II

Ca II

Ca II

Ca II

Ca II

Ca II

Ca II

O II

O II

O II

O II

O II

O II

O II

O II

O II

O II

O II

O II

\section{References:}

- F. A. Steele and W. P. Davey, The Crystal Structure of Tricalcium Aluminate, J. Am. Chem. Soc. 51, 689-697 (1929), 
doi:10.1021/ja01383a001.

- C. Hermann, O. Lohrmann, and H. Philipp, eds., Strukturbericht Band II, 1928-1932 (Akademsiche Verlagsgesellschaft M. B. H, Leipzig, 1937).

\section{Found in:}

- P. Mondal and J. W. Jeffery, The crystal structure of tricalcium aluminate, $\mathrm{Ca}_{3} \mathrm{Al}_{2} \mathrm{O}_{6}$, Acta Crystallogr. Sect. B Struct. Sci. 31, 689-697 (1975), doi:10.1107/S0567740875003639.

\section{Geometry files:}

- CIF: pp. 971

- POSCAR: pp. 972 


\section{$\mathrm{Ce}_{5} \mathrm{Mo}_{3} \mathrm{O}_{16}$ Structure: A5B3C16_cP96_222_ce_d_fi}
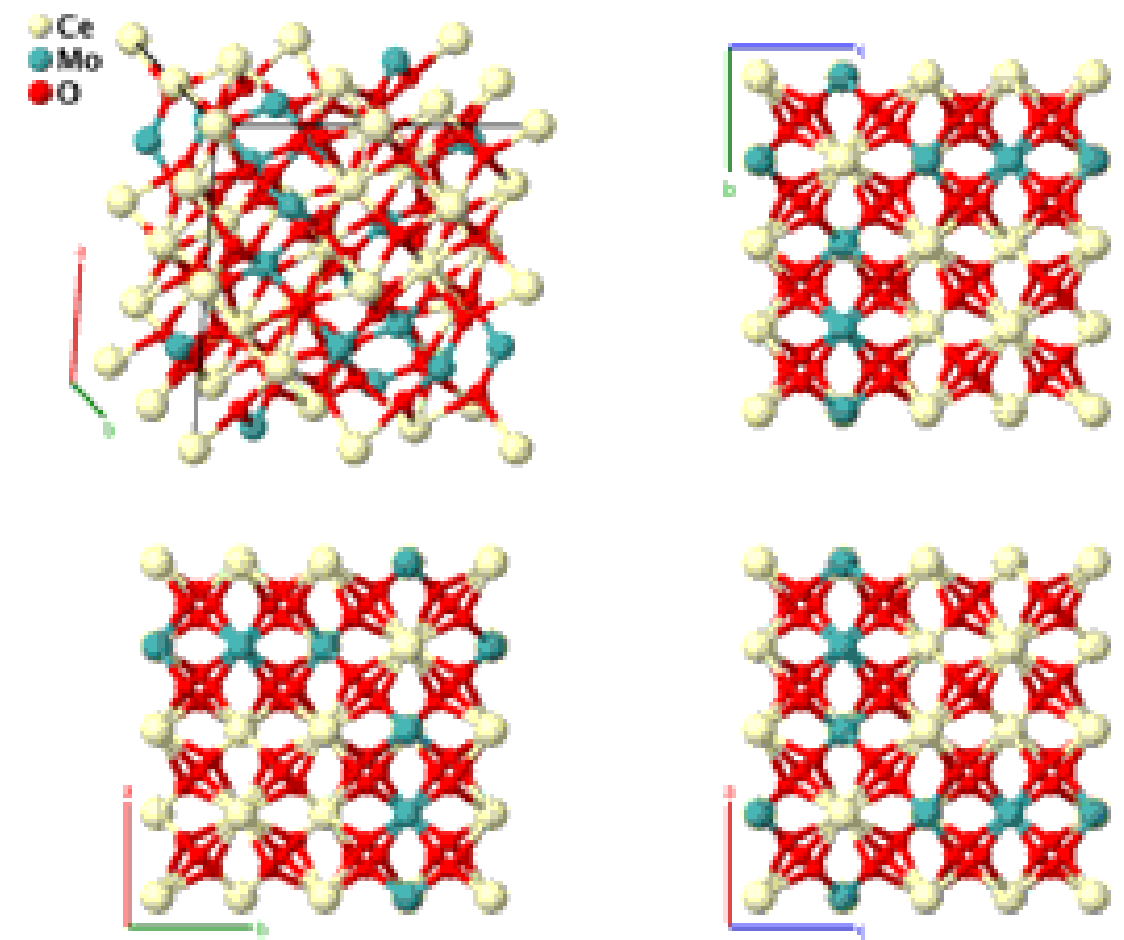

\section{Prototype}

AFLOW prototype label

$$
\text { : } \quad \mathrm{Ce}_{5} \mathrm{Mo}_{3} \mathrm{O}_{16}
$$

Strukturbericht designation

Pearson symbol

: A5B3C16_cP96_222_ce_d_fi

Space group number

: None

Space group symbol

: $\quad$ cP96

AFLOW prototype command

: 222

: $\quad P n \overline{3} n$

Aflow - -proto $=A 5 B 3 C 16_{-} c P 96 \_222_{-} c e_{-} d_{-} f i$

- -params $=a, x_{3}, x_{4}, x_{5}, y_{5}, z_{5}$

Simple Cubic primitive vectors:

$$
\begin{aligned}
& \mathbf{a}_{1}=a \hat{\mathbf{x}} \\
& \mathbf{a}_{2}=a \hat{\mathbf{y}} \\
& \mathbf{a}_{3}=a \hat{\mathbf{z}}
\end{aligned}
$$

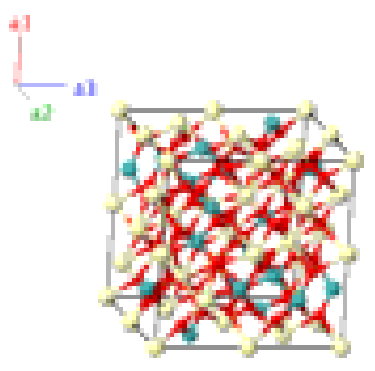

Basis vectors:

Lattice Coordinates
$\mathbf{B}_{1}=$
$0 \mathbf{a}_{1}+0 \mathbf{a}_{2}+0 \mathbf{a}_{3}$
$\mathbf{B}_{2}=$
$\frac{1}{2} \mathbf{a}_{1}+\frac{1}{2} \mathbf{a}_{2}$

Cartesian Coordinates

$=$

$=$

$$
\begin{gathered}
0 \hat{\mathbf{x}}+0 \hat{\mathbf{y}}+0 \hat{\mathbf{z}} \\
\frac{1}{2} a \hat{\mathbf{x}}+\frac{1}{2} a \hat{\mathbf{y}}
\end{gathered}
$$

Wyckoff Position

Atom Type

(8c)

Ce I

(8c)

Ce I 


\begin{tabular}{|c|c|c|c|c|c|c|}
\hline $\mathbf{B}_{3}$ & $=$ & $\frac{1}{2} \mathbf{a}_{1}+\frac{1}{2} \mathbf{a}_{3}$ & $=$ & $\frac{1}{2} a \hat{\mathbf{x}}+\frac{1}{2} a \hat{\mathbf{z}}$ & $(8 c)$ & $\mathrm{Ce} \mathrm{I}$ \\
\hline $\mathbf{B}_{4}$ & $=$ & $\frac{1}{2} \mathbf{a}_{2}+\frac{1}{2} \mathbf{a}_{3}$ & $=$ & $\frac{1}{2} a \hat{\mathbf{y}}+\frac{1}{2} a \hat{\mathbf{z}}$ & $(8 c)$ & $\mathrm{Ce} \mathrm{I}$ \\
\hline $\mathbf{B}_{5}$ & $=$ & $\frac{1}{2} \mathbf{a}_{3}$ & $=$ & $\frac{1}{2} a \hat{\mathbf{z}}$ & $(8 c)$ & $\mathrm{Ce} \mathrm{I}$ \\
\hline $\mathbf{B}_{6}$ & $=$ & $\frac{1}{2} \mathbf{a}_{1}+\frac{1}{2} \mathbf{a}_{2}+\frac{1}{2} \mathbf{a}_{3}$ & $=$ & $\frac{1}{2} a \hat{\mathbf{x}}+\frac{1}{2} a \hat{\mathbf{y}}+\frac{1}{2} a \hat{\mathbf{z}}$ & $(8 c)$ & $\mathrm{Ce} \mathrm{I}$ \\
\hline $\mathbf{B}_{7}$ & $=$ & $\frac{1}{2} \mathbf{a}_{2}$ & $=$ & $\frac{1}{2} a \hat{\mathbf{y}}$ & $(8 c)$ & $\mathrm{Ce} \mathrm{I}$ \\
\hline $\mathbf{B}_{8}$ & $=$ & $\frac{1}{2} \mathbf{a}_{1}$ & $=$ & $\frac{1}{2} a \hat{\mathbf{x}}$ & $(8 c)$ & $\mathrm{Ce} \mathrm{I}$ \\
\hline $\mathbf{B}_{9}$ & $=$ & $\frac{3}{4} \mathbf{a}_{2}+\frac{1}{4} \mathbf{a}_{3}$ & $=$ & $\frac{3}{4} a \hat{\mathbf{y}}+\frac{1}{4} a \hat{\mathbf{z}}$ & $(12 d)$ & Mo \\
\hline $\mathbf{B}_{10}$ & $=$ & $\frac{1}{2} \mathbf{a}_{1}+\frac{3}{4} \mathbf{a}_{2}+\frac{1}{4} \mathbf{a}_{3}$ & $=$ & $\frac{1}{2} a \hat{\mathbf{x}}+\frac{3}{4} a \hat{\mathbf{y}}+\frac{1}{4} a \hat{\mathbf{z}}$ & $(12 d)$ & Mo \\
\hline $\mathbf{B}_{11}$ & $=$ & $\frac{1}{4} \mathbf{a}_{1}+\frac{3}{4} \mathbf{a}_{3}$ & $=$ & $\frac{1}{4} a \hat{\mathbf{x}}+\frac{3}{4} a \hat{\mathbf{z}}$ & $(12 d)$ & Mo \\
\hline $\mathbf{B}_{12}$ & $=$ & $\frac{1}{4} \mathbf{a}_{1}+\frac{1}{2} \mathbf{a}_{2}+\frac{3}{4} \mathbf{a}_{3}$ & $=$ & $\frac{1}{4} a \hat{\mathbf{x}}+\frac{1}{2} a \hat{\mathbf{y}}+\frac{3}{4} a \hat{\mathbf{z}}$ & $(12 d)$ & Mo \\
\hline $\mathbf{B}_{13}$ & $=$ & $\frac{3}{4} \mathbf{a}_{1}+\frac{1}{4} \mathbf{a}_{2}$ & $=$ & $\frac{3}{4} a \hat{\mathbf{x}}+\frac{1}{4} a \hat{\mathbf{y}}$ & $(12 d)$ & Mo \\
\hline $\mathbf{B}_{14}$ & $=$ & $\frac{3}{4} \mathbf{a}_{1}+\frac{1}{4} \mathbf{a}_{2}+\frac{1}{2} \mathbf{a}_{3}$ & $=$ & $\frac{3}{4} a \hat{\mathbf{x}}+\frac{1}{4} a \hat{\mathbf{y}}+\frac{1}{2} a \hat{\mathbf{z}}$ & $(12 d)$ & Mo \\
\hline $\mathbf{B}_{15}$ & $=$ & $\frac{3}{4} \mathbf{a}_{1}+\frac{1}{4} \mathbf{a}_{3}$ & $=$ & $\frac{3}{4} a \hat{\mathbf{x}}+\frac{1}{4} a \hat{\mathbf{z}}$ & $(12 d)$ & Mo \\
\hline $\mathbf{B}_{16}$ & $=$ & $\frac{3}{4} \mathbf{a}_{1}+\frac{1}{2} \mathbf{a}_{2}+\frac{1}{4} \mathbf{a}_{3}$ & $=$ & $\frac{3}{4} a \hat{\mathbf{x}}+\frac{1}{2} a \hat{\mathbf{y}}+\frac{1}{4} a \hat{\mathbf{z}}$ & $(12 d)$ & Mo \\
\hline $\mathbf{B}_{17}$ & $=$ & $\frac{1}{4} \mathbf{a}_{2}+\frac{3}{4} \mathbf{a}_{3}$ & $=$ & $\frac{1}{4} a \hat{\mathbf{y}}+\frac{3}{4} a \hat{\mathbf{z}}$ & $(12 d)$ & Mo \\
\hline $\mathbf{B}_{18}$ & $=$ & $\frac{1}{2} \mathbf{a}_{1}+\frac{1}{4} \mathbf{a}_{2}+\frac{3}{4} \mathbf{a}_{3}$ & $=$ & $\frac{1}{2} a \hat{\mathbf{x}}+\frac{1}{4} a \hat{\mathbf{y}}+\frac{3}{4} a \hat{\mathbf{z}}$ & $(12 d)$ & Mo \\
\hline $\mathbf{B}_{19}$ & $=$ & $\frac{1}{4} \mathbf{a}_{1}+\frac{3}{4} \mathbf{a}_{2}+\frac{1}{2} \mathbf{a}_{3}$ & $=$ & $\frac{1}{4} a \hat{\mathbf{x}}+\frac{3}{4} a \hat{\mathbf{y}}+\frac{1}{2} a \hat{\mathbf{z}}$ & $(12 d)$ & Mo \\
\hline $\mathbf{B}_{20}$ & $=$ & $\frac{1}{4} \mathbf{a}_{1}+\frac{3}{4} \mathbf{a}_{2}$ & $=$ & $\frac{1}{4} a \hat{\mathbf{x}}+\frac{3}{4} a \hat{\mathbf{y}}$ & $(12 d)$ & Mo \\
\hline $\mathbf{B}_{21}$ & $=$ & $x_{3} \mathbf{a}_{1}+\frac{1}{4} \mathbf{a}_{2}+\frac{1}{4} \mathbf{a}_{3}$ & $=$ & $x_{3} a \hat{\mathbf{x}}+\frac{1}{4} a \hat{\mathbf{y}}+\frac{1}{4} a \hat{\mathbf{z}}$ & $(12 e)$ & Ce II \\
\hline $\mathbf{B}_{22}$ & $=$ & $\left(\frac{1}{2}-x_{3}\right) \mathbf{a}_{1}+\frac{1}{4} \mathbf{a}_{2}+\frac{1}{4} \mathbf{a}_{3}$ & $=$ & $\left(\frac{1}{2}-x_{3}\right) a \hat{\mathbf{x}}+\frac{1}{4} a \hat{\mathbf{y}}+\frac{1}{4} a \hat{\mathbf{z}}$ & $(12 e)$ & Ce II \\
\hline $\mathbf{B}_{23}$ & $=$ & $\frac{1}{4} \mathbf{a}_{1}+x_{3} \mathbf{a}_{2}+\frac{1}{4} \mathbf{a}_{3}$ & $=$ & $\frac{1}{4} a \hat{\mathbf{x}}+x_{3} a \hat{\mathbf{y}}+\frac{1}{4} a \hat{\mathbf{z}}$ & $(12 e)$ & Ce II \\
\hline $\mathbf{B}_{24}$ & $=$ & $\frac{1}{4} \mathbf{a}_{1}+\left(\frac{1}{2}-x_{3}\right) \mathbf{a}_{2}+\frac{1}{4} \mathbf{a}_{3}$ & $=$ & $\frac{1}{4} a \hat{\mathbf{x}}+\left(\frac{1}{2}-x_{3}\right) a \hat{\mathbf{y}}+\frac{1}{4} a \hat{\mathbf{z}}$ & $(12 e)$ & Ce II \\
\hline $\mathbf{B}_{25}$ & $=$ & $\frac{1}{4} \mathbf{a}_{1}+\frac{1}{4} \mathbf{a}_{2}+x_{3} \mathbf{a}_{3}$ & $=$ & $\frac{1}{4} a \hat{\mathbf{x}}+\frac{1}{4} a \hat{\mathbf{y}}+x_{3} a \hat{\mathbf{z}}$ & $(12 e)$ & Ce II \\
\hline $\mathbf{B}_{26}$ & $=$ & $\frac{1}{4} \mathbf{a}_{1}+\frac{1}{4} \mathbf{a}_{2}+\left(\frac{1}{2}-x_{3}\right) \mathbf{a}_{3}$ & $=$ & $\frac{1}{4} a \hat{\mathbf{x}}+\frac{1}{4} a \hat{\mathbf{y}}+\left(\frac{1}{2}-x_{3}\right) a \hat{\mathbf{z}}$ & $(12 e)$ & Ce II \\
\hline $\mathbf{B}_{27}$ & $=$ & $-x_{3} \mathbf{a}_{1}+\frac{3}{4} \mathbf{a}_{2}+\frac{3}{4} \mathbf{a}_{3}$ & $=$ & $-x_{3} a \hat{\mathbf{x}}+\frac{3}{4} a \hat{\mathbf{y}}+\frac{3}{4} a \hat{\mathbf{z}}$ & $(12 e)$ & Ce II \\
\hline $\mathbf{B}_{28}$ & $=$ & $\left(\frac{1}{2}+x_{3}\right) \mathbf{a}_{1}+\frac{3}{4} \mathbf{a}_{2}+\frac{3}{4} \mathbf{a}_{3}$ & $=$ & $\left(\frac{1}{2}+x_{3}\right) a \hat{\mathbf{x}}+\frac{3}{4} a \hat{\mathbf{y}}+\frac{3}{4} a \hat{\mathbf{z}}$ & $(12 e)$ & Ce II \\
\hline $\mathbf{B}_{29}$ & $=$ & $\frac{3}{4} \mathbf{a}_{1}-x_{3} \mathbf{a}_{2}+\frac{3}{4} \mathbf{a}_{3}$ & $=$ & $\frac{3}{4} a \hat{\mathbf{x}}-x_{3} a \hat{\mathbf{y}}+\frac{3}{4} a \hat{\mathbf{z}}$ & $(12 e)$ & Ce II \\
\hline $\mathbf{B}_{30}$ & $=$ & $\frac{3}{4} \mathbf{a}_{1}+\left(\frac{1}{2}+x_{3}\right) \mathbf{a}_{2}+\frac{3}{4} \mathbf{a}_{3}$ & $=$ & $\frac{3}{4} a \hat{\mathbf{x}}+\left(\frac{1}{2}+x_{3}\right) a \hat{\mathbf{y}}+\frac{3}{4} a \hat{\mathbf{z}}$ & $(12 e)$ & Ce II \\
\hline $\mathbf{B}_{31}$ & $=$ & $\frac{3}{4} \mathbf{a}_{1}+\frac{3}{4} \mathbf{a}_{2}-x_{3} \mathbf{a}_{3}$ & $=$ & $\frac{3}{4} a \hat{\mathbf{x}}+\frac{3}{4} a \hat{\mathbf{y}}-x_{3} a \hat{\mathbf{z}}$ & $(12 e)$ & Ce II \\
\hline $\mathbf{B}_{32}$ & $=$ & $\frac{3}{4} \mathbf{a}_{1}+\frac{3}{4} \mathbf{a}_{2}+\left(\frac{1}{2}+x_{3}\right) \mathbf{a}_{3}$ & $=$ & $\frac{3}{4} a \hat{\mathbf{x}}+\frac{3}{4} a \hat{\mathbf{y}}+\left(\frac{1}{2}+x_{3}\right) a \hat{\mathbf{z}}$ & $(12 e)$ & Ce II \\
\hline $\mathbf{B}_{33}$ & $=$ & $x_{4} \mathbf{a}_{1}+x_{4} \mathbf{a}_{2}+x_{4} \mathbf{a}_{3}$ & $=$ & $x_{4} a \hat{\mathbf{x}}+x_{4} a \hat{\mathbf{y}}+x_{4} a \hat{\mathbf{z}}$ & $(16 f)$ & O I \\
\hline $\mathbf{B}_{34}$ & $=$ & $\left(\frac{1}{2}-x_{4}\right) \mathbf{a}_{1}+\left(\frac{1}{2}-x_{4}\right) \mathbf{a}_{2}+x_{4} \mathbf{a}_{3}$ & $=$ & $\left(\frac{1}{2}-x_{4}\right) a \hat{\mathbf{x}}+\left(\frac{1}{2}-x_{4}\right) a \hat{\mathbf{y}}+x_{4} a \hat{\mathbf{z}}$ & $(16 f)$ & O I \\
\hline $\mathbf{B}_{35}$ & $=$ & $\left(\frac{1}{2}-x_{4}\right) \mathbf{a}_{1}+x_{4} \mathbf{a}_{2}+\left(\frac{1}{2}-x_{4}\right) \mathbf{a}_{3}$ & $=$ & $\left(\frac{1}{2}-x_{4}\right) a \hat{\mathbf{x}}+x_{4} a \hat{\mathbf{y}}+\left(\frac{1}{2}-x_{4}\right) a \hat{\mathbf{z}}$ & $(16 f)$ & O I \\
\hline $\mathbf{B}_{36}$ & $=$ & $x_{4} \mathbf{a}_{1}+\left(\frac{1}{2}-x_{4}\right) \mathbf{a}_{2}+\left(\frac{1}{2}-x_{4}\right) \mathbf{a}_{3}$ & $=$ & $x_{4} a \hat{\mathbf{x}}+\left(\frac{1}{2}-x_{4}\right) a \hat{\mathbf{y}}+\left(\frac{1}{2}-x_{4}\right) a \hat{\mathbf{z}}$ & $(16 f)$ & O I \\
\hline $\mathbf{B}_{37}$ & $=$ & $x_{4} \mathbf{a}_{1}+x_{4} \mathbf{a}_{2}+\left(\frac{1}{2}-x_{4}\right) \mathbf{a}_{3}$ & $=$ & $x_{4} a \hat{\mathbf{x}}+x_{4} a \hat{\mathbf{y}}+\left(\frac{1}{2}-x_{4}\right) a \hat{\mathbf{z}}$ & $(16 f)$ & O I \\
\hline $\mathbf{B}_{38}$ & $=$ & $\begin{array}{c}\left(\frac{1}{2}-x_{4}\right) \mathbf{a}_{1}+\left(\frac{1}{2}-x_{4}\right) \mathbf{a}_{2}+ \\
\left(\frac{1}{2}-x_{4}\right) \mathbf{a}_{3}\end{array}$ & $=$ & $\begin{array}{c}\left(\frac{1}{2}-x_{4}\right) a \hat{\mathbf{x}}+\left(\frac{1}{2}-x_{4}\right) a \hat{\mathbf{y}}+ \\
\left(\frac{1}{2}-x_{4}\right) a \hat{\mathbf{z}}\end{array}$ & $(16 f)$ & O I \\
\hline
\end{tabular}




\begin{tabular}{|c|c|c|c|c|c|c|}
\hline $\mathbf{B}_{39}$ & $=$ & $x_{4} \mathbf{a}_{1}+\left(\frac{1}{2}-x_{4}\right) \mathbf{a}_{2}+x_{4} \mathbf{a}_{3}$ & $=$ & $x_{4} a \hat{\mathbf{x}}+\left(\frac{1}{2}-x_{4}\right) a \hat{\mathbf{y}}+x_{4} a \hat{\mathbf{z}}$ & $(16 f)$ & O I \\
\hline $\mathbf{B}_{40}$ & $=$ & $\left(\frac{1}{2}-x_{4}\right) \mathbf{a}_{1}+x_{4} \mathbf{a}_{2}+x_{4} \mathbf{a}_{3}$ & $=$ & $\left(\frac{1}{2}-x_{4}\right) a \hat{\mathbf{x}}+x_{4} a \hat{\mathbf{y}}+x_{4} a \hat{\mathbf{z}}$ & $(16 f)$ & $\mathrm{O} \mathrm{I}$ \\
\hline $\mathbf{B}_{41}$ & $=$ & $-x_{4} \mathbf{a}_{1}-x_{4} \mathbf{a}_{2}-x_{4} \mathbf{a}_{3}$ & $=$ & $-x_{4} a \hat{\mathbf{x}}-x_{4} a \hat{\mathbf{y}}-x_{4} a \hat{\mathbf{z}}$ & $(16 f)$ & $\mathrm{O} \mathrm{I}$ \\
\hline $\mathbf{B}_{42}$ & $=$ & $\left(\frac{1}{2}+x_{4}\right) \mathbf{a}_{1}+\left(\frac{1}{2}+x_{4}\right) \mathbf{a}_{2}-x_{4} \mathbf{a}_{3}$ & $=$ & $\left(\frac{1}{2}+x_{4}\right) a \hat{\mathbf{x}}+\left(\frac{1}{2}+x_{4}\right) a \hat{\mathbf{y}}-x_{4} a \hat{\mathbf{z}}$ & $(16 f)$ & O I \\
\hline $\mathbf{B}_{43}$ & $=$ & $\left(\frac{1}{2}+x_{4}\right) \mathbf{a}_{1}-x_{4} \mathbf{a}_{2}+\left(\frac{1}{2}+x_{4}\right) \mathbf{a}_{3}$ & $=$ & $\left(\frac{1}{2}+x_{4}\right) a \hat{\mathbf{x}}-x_{4} a \hat{\mathbf{y}}+\left(\frac{1}{2}+x_{4}\right) a \hat{\mathbf{z}}$ & $(16 f)$ & $\mathrm{O} \mathrm{I}$ \\
\hline $\mathbf{B}_{44}$ & $=$ & $-x_{4} \mathbf{a}_{1}+\left(\frac{1}{2}+x_{4}\right) \mathbf{a}_{2}+\left(\frac{1}{2}+x_{4}\right) \mathbf{a}_{3}$ & $=$ & $-x_{4} a \hat{\mathbf{x}}+\left(\frac{1}{2}+x_{4}\right) a \hat{\mathbf{y}}+\left(\frac{1}{2}+x_{4}\right) a \hat{\mathbf{z}}$ & $(16 f)$ & O I \\
\hline $\mathbf{B}_{45}$ & $=$ & $-x_{4} \mathbf{a}_{1}-x_{4} \mathbf{a}_{2}+\left(\frac{1}{2}+x_{4}\right) \mathbf{a}_{3}$ & $=$ & $-x_{4} a \hat{\mathbf{x}}-x_{4} a \hat{\mathbf{y}}+\left(\frac{1}{2}+x_{4}\right) a \hat{\mathbf{z}}$ & $(16 f)$ & $\mathrm{O} \mathrm{I}$ \\
\hline $\mathbf{B}_{46}$ & $=$ & $\begin{array}{c}\left(\frac{1}{2}+x_{4}\right) \mathbf{a}_{1}+\left(\frac{1}{2}+x_{4}\right) \mathbf{a}_{2}+ \\
\left(\frac{1}{2}+x_{4}\right) \mathbf{a}_{3}\end{array}$ & $=$ & $\begin{array}{c}\left(\frac{1}{2}+x_{4}\right) a \hat{\mathbf{x}}+\left(\frac{1}{2}+x_{4}\right) a \hat{\mathbf{y}}+ \\
\left(\frac{1}{2}+x_{4}\right) a \hat{\mathbf{z}}\end{array}$ & $(16 f)$ & O I \\
\hline $\mathbf{B}_{47}$ & $=$ & $-x_{4} \mathbf{a}_{1}+\left(\frac{1}{2}+x_{4}\right) \mathbf{a}_{2}-x_{4} \mathbf{a}_{3}$ & $=$ & $-x_{4} a \hat{\mathbf{x}}+\left(\frac{1}{2}+x_{4}\right) a \hat{\mathbf{y}}-x_{4} a \hat{\mathbf{z}}$ & $(16 f)$ & $\mathrm{O} \mathrm{I}$ \\
\hline $\mathbf{B}_{48}$ & $=$ & $\left(\frac{1}{2}+x_{4}\right) \mathbf{a}_{1}-x_{4} \mathbf{a}_{2}-x_{4} \mathbf{a}_{3}$ & $=$ & $\left(\frac{1}{2}+x_{4}\right) a \hat{\mathbf{x}}-x_{4} a \hat{\mathbf{y}}-x_{4} a \hat{\mathbf{z}}$ & $(16 f)$ & $\mathrm{O} \mathrm{I}$ \\
\hline $\mathbf{B}_{49}$ & $=$ & $x_{5} \mathbf{a}_{1}+y_{5} \mathbf{a}_{2}+z_{5} \mathbf{a}_{3}$ & $=$ & $x_{5} a \hat{\mathbf{x}}+y_{5} a \hat{\mathbf{y}}+z_{5} a \hat{\mathbf{z}}$ & $(48 i)$ & O II \\
\hline $\mathbf{B}_{50}$ & $=$ & $\left(\frac{1}{2}-x_{5}\right) \mathbf{a}_{1}+\left(\frac{1}{2}-y_{5}\right) \mathbf{a}_{2}+z_{5} \mathbf{a}_{3}$ & $=$ & $\left(\frac{1}{2}-x_{5}\right) a \hat{\mathbf{x}}+\left(\frac{1}{2}-y_{5}\right) a \hat{\mathbf{y}}+z_{5} a \hat{\mathbf{z}}$ & $(48 i)$ & O II \\
\hline $\mathbf{B}_{51}$ & $=$ & $\left(\frac{1}{2}-x_{5}\right) \mathbf{a}_{1}+y_{5} \mathbf{a}_{2}+\left(\frac{1}{2}-z_{5}\right) \mathbf{a}_{3}$ & $=$ & $\left(\frac{1}{2}-x_{5}\right) a \hat{\mathbf{x}}+y_{5} a \hat{\mathbf{y}}+\left(\frac{1}{2}-z_{5}\right) a \hat{\mathbf{z}}$ & $(48 i)$ & O II \\
\hline $\mathbf{B}_{52}$ & $=$ & $x_{5} \mathbf{a}_{1}+\left(\frac{1}{2}-y_{5}\right) \mathbf{a}_{2}+\left(\frac{1}{2}-z_{5}\right) \mathbf{a}_{3}$ & $=$ & $x_{5} a \hat{\mathbf{x}}+\left(\frac{1}{2}-y_{5}\right) a \hat{\mathbf{y}}+\left(\frac{1}{2}-z_{5}\right) a \hat{\mathbf{z}}$ & $(48 i)$ & O II \\
\hline $\mathbf{B}_{53}$ & $=$ & $z_{5} \mathbf{a}_{1}+x_{5} \mathbf{a}_{2}+y_{5} \mathbf{a}_{3}$ & $=$ & $z_{5} a \hat{\mathbf{x}}+x_{5} a \hat{\mathbf{y}}+y_{5} a \hat{\mathbf{z}}$ & $(48 i)$ & O II \\
\hline $\mathbf{B}_{54}$ & $=$ & $z_{5} \mathbf{a}_{1}+\left(\frac{1}{2}-x_{5}\right) \mathbf{a}_{2}+\left(\frac{1}{2}-y_{5}\right) \mathbf{a}_{3}$ & $=$ & $z_{5} a \hat{\mathbf{x}}+\left(\frac{1}{2}-x_{5}\right) a \hat{\mathbf{y}}+\left(\frac{1}{2}-y_{5}\right) a \hat{\mathbf{z}}$ & $(48 i)$ & O II \\
\hline $\mathbf{B}_{55}$ & $=$ & $\left(\frac{1}{2}-z_{5}\right) \mathbf{a}_{1}+\left(\frac{1}{2}-x_{5}\right) \mathbf{a}_{2}+y_{5} \mathbf{a}_{3}$ & $=$ & $\left(\frac{1}{2}-z_{5}\right) a \hat{\mathbf{x}}+\left(\frac{1}{2}-x_{5}\right) a \hat{\mathbf{y}}+y_{5} a \hat{\mathbf{z}}$ & $(48 i)$ & O II \\
\hline $\mathbf{B}_{56}$ & $=$ & $\left(\frac{1}{2}-z_{5}\right) \mathbf{a}_{1}+x_{5} \mathbf{a}_{2}+\left(\frac{1}{2}-y_{5}\right) \mathbf{a}_{3}$ & $=$ & $\left(\frac{1}{2}-z_{5}\right) a \hat{\mathbf{x}}+x_{5} a \hat{\mathbf{y}}+\left(\frac{1}{2}-y_{5}\right) a \hat{\mathbf{z}}$ & $(48 i)$ & O II \\
\hline $\mathbf{B}_{57}$ & $=$ & $y_{5} \mathbf{a}_{1}+z_{5} \mathbf{a}_{2}+x_{5} \mathbf{a}_{3}$ & $=$ & $y_{5} a \hat{\mathbf{x}}+z_{5} a \hat{\mathbf{y}}+x_{5} a \hat{\mathbf{z}}$ & $(48 i)$ & O II \\
\hline $\mathbf{B}_{58}$ & $=$ & $\left(\frac{1}{2}-y_{5}\right) \mathbf{a}_{1}+z_{5} \mathbf{a}_{2}+\left(\frac{1}{2}-x_{5}\right) \mathbf{a}_{3}$ & $=$ & $\left(\frac{1}{2}-y_{5}\right) a \hat{\mathbf{x}}+z_{5} a \hat{\mathbf{y}}+\left(\frac{1}{2}-x_{5}\right) a \hat{\mathbf{z}}$ & $(48 i)$ & O II \\
\hline $\mathbf{B}_{59}$ & $=$ & $y_{5} \mathbf{a}_{1}+\left(\frac{1}{2}-z_{5}\right) \mathbf{a}_{2}+\left(\frac{1}{2}-x_{5}\right) \mathbf{a}_{3}$ & $=$ & $y_{5} a \hat{\mathbf{x}}+\left(\frac{1}{2}-z_{5}\right) a \hat{\mathbf{y}}+\left(\frac{1}{2}-x_{5}\right) a \hat{\mathbf{z}}$ & $(48 i)$ & O II \\
\hline $\mathbf{B}_{60}$ & $=$ & $\left(\frac{1}{2}-y_{5}\right) \mathbf{a}_{1}+\left(\frac{1}{2}-z_{5}\right) \mathbf{a}_{2}+x_{5} \mathbf{a}_{3}$ & $=$ & $\left(\frac{1}{2}-y_{5}\right) a \hat{\mathbf{x}}+\left(\frac{1}{2}-z_{5}\right) a \hat{\mathbf{y}}+x_{5} a \hat{\mathbf{z}}$ & $(48 i)$ & O II \\
\hline $\mathbf{B}_{61}$ & $=$ & $y_{5} \mathbf{a}_{1}+x_{5} \mathbf{a}_{2}+\left(\frac{1}{2}-z_{5}\right) \mathbf{a}_{3}$ & $=$ & $y_{5} a \hat{\mathbf{x}}+x_{5} a \hat{\mathbf{y}}+\left(\frac{1}{2}-z_{5}\right) a \hat{\mathbf{z}}$ & $(48 i)$ & O II \\
\hline $\mathbf{B}_{62}$ & $=$ & $\begin{array}{c}\left(\frac{1}{2}-y_{5}\right) \mathbf{a}_{1}+\left(\frac{1}{2}-x_{5}\right) \mathbf{a}_{2}+ \\
\left(\frac{1}{2}-z_{5}\right) \mathbf{a}_{3}\end{array}$ & $=$ & $\begin{array}{c}\left(\frac{1}{2}-y_{5}\right) a \hat{\mathbf{x}}+\left(\frac{1}{2}-x_{5}\right) a \hat{\mathbf{y}}+ \\
\left(\frac{1}{2}-z_{5}\right) a \hat{\mathbf{z}}\end{array}$ & $(48 i)$ & O II \\
\hline $\mathbf{B}_{63}$ & $=$ & $y_{5} \mathbf{a}_{1}+\left(\frac{1}{2}-x_{5}\right) \mathbf{a}_{2}+z_{5} \mathbf{a}_{3}$ & $=$ & $y_{5} a \hat{\mathbf{x}}+\left(\frac{1}{2}-x_{5}\right) a \hat{\mathbf{y}}+z_{5} a \hat{\mathbf{z}}$ & $(48 i)$ & O II \\
\hline $\mathbf{B}_{64}$ & $=$ & $\left(\frac{1}{2}-y_{5}\right) \mathbf{a}_{1}+x_{5} \mathbf{a}_{2}+z_{5} \mathbf{a}_{3}$ & $=$ & $\left(\frac{1}{2}-y_{5}\right) a \hat{\mathbf{x}}+x_{5} a \hat{\mathbf{y}}+z_{5} a \hat{\mathbf{z}}$ & $(48 i)$ & O II \\
\hline $\mathbf{B}_{65}$ & $=$ & $x_{5} \mathbf{a}_{1}+z_{5} \mathbf{a}_{2}+\left(\frac{1}{2}-y_{5}\right) \mathbf{a}_{3}$ & $=$ & $x_{5} a \hat{\mathbf{x}}+z_{5} a \hat{\mathbf{y}}+\left(\frac{1}{2}-y_{5}\right) a \hat{\mathbf{z}}$ & $(48 i)$ & O II \\
\hline $\mathbf{B}_{66}$ & $=$ & $\left(\frac{1}{2}-x_{5}\right) \mathbf{a}_{1}+z_{5} \mathbf{a}_{2}+y_{5} \mathbf{a}_{3}$ & $=$ & $\left(\frac{1}{2}-x_{5}\right) a \hat{\mathbf{x}}+z_{5} a \hat{\mathbf{y}}+y_{5} a \hat{\mathbf{z}}$ & $(48 i)$ & O II \\
\hline $\mathbf{B}_{67}$ & $=$ & $\begin{array}{c}\left(\frac{1}{2}-x_{5}\right) \mathbf{a}_{1}+\left(\frac{1}{2}-z_{5}\right) \mathbf{a}_{2}+ \\
\left(\frac{1}{2}-y_{5}\right) \mathbf{a}_{3}\end{array}$ & $=$ & $\begin{array}{c}\left(\frac{1}{2}-x_{5}\right) a \hat{\mathbf{x}}+\left(\frac{1}{2}-z_{5}\right) a \hat{\mathbf{y}}+ \\
\left(\frac{1}{2}-y_{5}\right) a \hat{\mathbf{z}}\end{array}$ & $(48 i)$ & O II \\
\hline $\mathbf{B}_{68}$ & $=$ & $x_{5} \mathbf{a}_{1}+\left(\frac{1}{2}-z_{5}\right) \mathbf{a}_{2}+y_{5} \mathbf{a}_{3}$ & $=$ & $x_{5} a \hat{\mathbf{x}}+\left(\frac{1}{2}-z_{5}\right) a \hat{\mathbf{y}}+y_{5} a \hat{\mathbf{z}}$ & $(48 i)$ & O II \\
\hline $\mathbf{B}_{69}$ & $=$ & $z_{5} \mathbf{a}_{1}+y_{5} \mathbf{a}_{2}+\left(\frac{1}{2}-x_{5}\right) \mathbf{a}_{3}$ & $=$ & $z_{5} a \hat{\mathbf{x}}+y_{5} a \hat{\mathbf{y}}+\left(\frac{1}{2}-x_{5}\right) a \hat{\mathbf{z}}$ & $(48 i)$ & O II \\
\hline $\mathbf{B}_{70}$ & $=$ & $z_{5} \mathbf{a}_{1}+\left(\frac{1}{2}-y_{5}\right) \mathbf{a}_{2}+x_{5} \mathbf{a}_{3}$ & $=$ & $z_{5} a \hat{\mathbf{x}}+\left(\frac{1}{2}-y_{5}\right) a \hat{\mathbf{y}}+x_{5} a \hat{\mathbf{z}}$ & $(48 i)$ & O II \\
\hline $\mathbf{B}_{71}$ & $=$ & $\left(\frac{1}{2}-z_{5}\right) \mathbf{a}_{1}+y_{5} \mathbf{a}_{2}+x_{5} \mathbf{a}_{3}$ & $=$ & $\left(\frac{1}{2}-z_{5}\right) a \hat{\mathbf{x}}+y_{5} a \hat{\mathbf{y}}+x_{5} a \hat{\mathbf{z}}$ & $(48 i)$ & O II \\
\hline
\end{tabular}




\begin{tabular}{|c|c|c|c|c|c|}
\hline $\mathbf{B}_{72}$ & $=$ & $\begin{array}{c}\left(\frac{1}{2}-z_{5}\right) \mathbf{a}_{1}+\left(\frac{1}{2}-y_{5}\right) \mathbf{a}_{2}+ \\
\left(\frac{1}{2}-x_{5}\right) \mathbf{a}_{3}\end{array}$ & $=$ & $\begin{array}{c}\left(\frac{1}{2}-z_{5}\right) a \hat{\mathbf{x}}+\left(\frac{1}{2}-y_{5}\right) a \hat{\mathbf{y}}+ \\
\left(\frac{1}{2}-x_{5}\right) a \hat{\mathbf{z}}\end{array}$ & $(48 i)$ \\
\hline $\mathbf{B}_{73}$ & $=$ & $-x_{5} \mathbf{a}_{1}-y_{5} \mathbf{a}_{2}-z_{5} \mathbf{a}_{3}$ & $=$ & $-x_{5} a \hat{\mathbf{x}}-y_{5} a \hat{\mathbf{y}}-z_{5} a \hat{\mathbf{z}}$ & $(48 i)$ \\
\hline $\mathbf{B}_{74}$ & $=$ & $\left(\frac{1}{2}+x_{5}\right) \mathbf{a}_{1}+\left(\frac{1}{2}+y_{5}\right) \mathbf{a}_{2}-z_{5} \mathbf{a}_{3}$ & $=$ & $\left(\frac{1}{2}+x_{5}\right) a \hat{\mathbf{x}}+\left(\frac{1}{2}+y_{5}\right) a \hat{\mathbf{y}}-z_{5} a \hat{\mathbf{z}}$ & $(48 i)$ \\
\hline $\mathbf{B}_{75}$ & $=$ & $\left(\frac{1}{2}+x_{5}\right) \mathbf{a}_{1}-y_{5} \mathbf{a}_{2}+\left(\frac{1}{2}+z_{5}\right) \mathbf{a}_{3}$ & $=$ & $\left(\frac{1}{2}+x_{5}\right) a \hat{\mathbf{x}}-y_{5} a \hat{\mathbf{y}}+\left(\frac{1}{2}+z_{5}\right) a \hat{\mathbf{z}}$ & $(48 i)$ \\
\hline $\mathbf{B}_{76}$ & $=$ & $-x_{5} \mathbf{a}_{1}+\left(\frac{1}{2}+y_{5}\right) \mathbf{a}_{2}+\left(\frac{1}{2}+z_{5}\right) \mathbf{a}_{3}$ & $=$ & $-x_{5} a \hat{\mathbf{x}}+\left(\frac{1}{2}+y_{5}\right) a \hat{\mathbf{y}}+\left(\frac{1}{2}+z_{5}\right) a \hat{\mathbf{z}}$ & $(48 i)$ \\
\hline $\mathbf{B}_{77}$ & $=$ & $-z_{5} \mathbf{a}_{1}-x_{5} \mathbf{a}_{2}-y_{5} \mathbf{a}_{3}$ & $=$ & $-z_{5} a \hat{\mathbf{x}}-x_{5} a \hat{\mathbf{y}}-y_{5} a \hat{\mathbf{z}}$ & $(48 i)$ \\
\hline $\mathbf{B}_{78}$ & $=$ & $-z_{5} \mathbf{a}_{1}+\left(\frac{1}{2}+x_{5}\right) \mathbf{a}_{2}+\left(\frac{1}{2}+y_{5}\right) \mathbf{a}_{3}$ & $=$ & $-z_{5} a \hat{\mathbf{x}}+\left(\frac{1}{2}+x_{5}\right) a \hat{\mathbf{y}}+\left(\frac{1}{2}+y_{5}\right) a \hat{\mathbf{z}}$ & $(48 i)$ \\
\hline $\mathbf{B}_{79}$ & $=$ & $\left(\frac{1}{2}+z_{5}\right) \mathbf{a}_{1}+\left(\frac{1}{2}+x_{5}\right) \mathbf{a}_{2}-y_{5} \mathbf{a}_{3}$ & $=$ & $\left(\frac{1}{2}+z_{5}\right) a \hat{\mathbf{x}}+\left(\frac{1}{2}+x_{5}\right) a \hat{\mathbf{y}}-y_{5} a \hat{\mathbf{z}}$ & $(48 i)$ \\
\hline $\mathbf{B}_{80}$ & $=$ & $\left(\frac{1}{2}+z_{5}\right) \mathbf{a}_{1}-x_{5} \mathbf{a}_{2}+\left(\frac{1}{2}+y_{5}\right) \mathbf{a}_{3}$ & $=$ & $\left(\frac{1}{2}+z_{5}\right) a \hat{\mathbf{x}}-x_{5} a \hat{\mathbf{y}}+\left(\frac{1}{2}+y_{5}\right) a \hat{\mathbf{z}}$ & $(48 i)$ \\
\hline $\mathbf{B}_{81}$ & $=$ & $-y_{5} \mathbf{a}_{1}-z_{5} \mathbf{a}_{2}-x_{5} \mathbf{a}_{3}$ & $=$ & $-y_{5} a \hat{\mathbf{x}}-z_{5} a \hat{\mathbf{y}}-x_{5} a \hat{\mathbf{z}}$ & $(48 i)$ \\
\hline $\mathbf{B}_{82}$ & $=$ & $\left(\frac{1}{2}+y_{5}\right) \mathbf{a}_{1}-z_{5} \mathbf{a}_{2}+\left(\frac{1}{2}+x_{5}\right) \mathbf{a}_{3}$ & $=$ & $\left(\frac{1}{2}+y_{5}\right) a \hat{\mathbf{x}}-z_{5} a \hat{\mathbf{y}}+\left(\frac{1}{2}+x_{5}\right) a \hat{\mathbf{z}}$ & $(48 i)$ \\
\hline $\mathbf{B}_{83}$ & $=$ & $-y_{5} \mathbf{a}_{1}+\left(\frac{1}{2}+z_{5}\right) \mathbf{a}_{2}+\left(\frac{1}{2}+x_{5}\right) \mathbf{a}_{3}$ & $=$ & $-y_{5} a \hat{\mathbf{x}}+\left(\frac{1}{2}+z_{5}\right) a \hat{\mathbf{y}}+\left(\frac{1}{2}+x_{5}\right) a \hat{\mathbf{z}}$ & $(48 i)$ \\
\hline $\mathbf{B}_{84}$ & $=$ & $\left(\frac{1}{2}+y_{5}\right) \mathbf{a}_{1}+\left(\frac{1}{2}+z_{5}\right) \mathbf{a}_{2}-x_{5} \mathbf{a}_{3}$ & $=$ & $\left(\frac{1}{2}+y_{5}\right) a \hat{\mathbf{x}}+\left(\frac{1}{2}+z_{5}\right) a \hat{\mathbf{y}}-x_{5} a \hat{\mathbf{z}}$ & $(48 i)$ \\
\hline $\mathbf{B}_{85}$ & $=$ & $-y_{5} \mathbf{a}_{1}-x_{5} \mathbf{a}_{2}+\left(\frac{1}{2}+z_{5}\right) \mathbf{a}_{3}$ & $=$ & $-y_{5} a \hat{\mathbf{x}}-x_{5} a \hat{\mathbf{y}}+\left(\frac{1}{2}+z_{5}\right) a \hat{\mathbf{z}}$ & $(48 i)$ \\
\hline $\mathbf{B}_{86}$ & $=$ & $\begin{array}{c}\left(\frac{1}{2}+y_{5}\right) \mathbf{a}_{1}+\left(\frac{1}{2}+x_{5}\right) \mathbf{a}_{2}+ \\
\left(\frac{1}{2}+z_{5}\right) \mathbf{a}_{3}\end{array}$ & $=$ & $\begin{array}{c}\left(\frac{1}{2}+y_{5}\right) a \hat{\mathbf{x}}+\left(\frac{1}{2}+x_{5}\right) a \hat{\mathbf{y}}+ \\
\left(\frac{1}{2}+z_{5}\right) a \hat{\mathbf{z}}\end{array}$ & $(48 i)$ \\
\hline $\mathbf{B}_{87}$ & $=$ & $-y_{5} \mathbf{a}_{1}+\left(\frac{1}{2}+x_{5}\right) \mathbf{a}_{2}-z_{5} \mathbf{a}_{3}$ & $=$ & $-y_{5} a \hat{\mathbf{x}}+\left(\frac{1}{2}+x_{5}\right) a \hat{\mathbf{y}}-z_{5} a \hat{\mathbf{z}}$ & $(48 i)$ \\
\hline $\mathbf{B}_{88}$ & $=$ & $\left(\frac{1}{2}+y_{5}\right) \mathbf{a}_{1}-x_{5} \mathbf{a}_{2}-z_{5} \mathbf{a}_{3}$ & $=$ & $\left(\frac{1}{2}+y_{5}\right) a \hat{\mathbf{x}}-x_{5} a \hat{\mathbf{y}}-z_{5} a \hat{\mathbf{z}}$ & $(48 i)$ \\
\hline $\mathbf{B}_{89}$ & $=$ & $-x_{5} \mathbf{a}_{1}-z_{5} \mathbf{a}_{2}+\left(\frac{1}{2}+y_{5}\right) \mathbf{a}_{3}$ & $=$ & $-x_{5} a \hat{\mathbf{x}}-z_{5} a \hat{\mathbf{y}}+\left(\frac{1}{2}+y_{5}\right) a \hat{\mathbf{z}}$ & $(48 i)$ \\
\hline $\mathbf{B}_{90}$ & $=$ & $\left(\frac{1}{2}+x_{5}\right) \mathbf{a}_{1}-z_{5} \mathbf{a}_{2}-y_{5} \mathbf{a}_{3}$ & $=$ & $\left(\frac{1}{2}+x_{5}\right) a \hat{\mathbf{x}}-z_{5} a \hat{\mathbf{y}}-y_{5} a \hat{\mathbf{z}}$ & $(48 i)$ \\
\hline $\mathbf{B}_{91}$ & $=$ & $\begin{array}{c}\left(\frac{1}{2}+x_{5}\right) \mathbf{a}_{1}+\left(\frac{1}{2}+z_{5}\right) \mathbf{a}_{2}+ \\
\left(\frac{1}{2}+y_{5}\right) \mathbf{a}_{3}\end{array}$ & $=$ & $\begin{array}{c}\left(\frac{1}{2}+x_{5}\right) a \hat{\mathbf{x}}+\left(\frac{1}{2}+z_{5}\right) a \hat{\mathbf{y}}+ \\
\left(\frac{1}{2}+y_{5}\right) a \hat{\mathbf{z}}\end{array}$ & $(48 i)$ \\
\hline $\mathbf{B}_{92}$ & $=$ & $-x_{5} \mathbf{a}_{1}+\left(\frac{1}{2}+z_{5}\right) \mathbf{a}_{2}-y_{5} \mathbf{a}_{3}$ & $=$ & $-x_{5} a \hat{\mathbf{x}}+\left(\frac{1}{2}+z_{5}\right) a \hat{\mathbf{y}}-y_{5} a \hat{\mathbf{z}}$ & $(48 i)$ \\
\hline $\mathbf{B}_{93}$ & $=$ & $-z_{5} \mathbf{a}_{1}-y_{5} \mathbf{a}_{2}+\left(\frac{1}{2}+x_{5}\right) \mathbf{a}_{3}$ & $=$ & $-z_{5} a \hat{\mathbf{x}}-y_{5} a \hat{\mathbf{y}}+\left(\frac{1}{2}+x_{5}\right) a \hat{\mathbf{z}}$ & $(48 i)$ \\
\hline $\mathbf{B}_{94}$ & $=$ & $-z_{5} \mathbf{a}_{1}+\left(\frac{1}{2}+y_{5}\right) \mathbf{a}_{2}-x_{5} \mathbf{a}_{3}$ & $=$ & $-z_{5} a \hat{\mathbf{x}}+\left(\frac{1}{2}+y_{5}\right) a \hat{\mathbf{y}}-x_{5} a \hat{\mathbf{z}}$ & $(48 i)$ \\
\hline $\mathbf{B}_{95}$ & $=$ & $\left(\frac{1}{2}+z_{5}\right) \mathbf{a}_{1}-y_{5} \mathbf{a}_{2}-x_{5} \mathbf{a}_{3}$ & $=$ & $\left(\frac{1}{2}+z_{5}\right) a \hat{\mathbf{x}}-y_{5} a \hat{\mathbf{y}}-x_{5} a \hat{\mathbf{z}}$ & $(48 i)$ \\
\hline $\mathbf{B}_{96}$ & $=$ & $\begin{array}{c}\left(\frac{1}{2}+z_{5}\right) \mathbf{a}_{1}+\left(\frac{1}{2}+y_{5}\right) \mathbf{a}_{2}+ \\
\left(\frac{1}{2}+x_{5}\right) \mathbf{a}_{3}\end{array}$ & $=$ & $\begin{array}{c}\left(\frac{1}{2}+z_{5}\right) a \hat{\mathbf{x}}+\left(\frac{1}{2}+y_{5}\right) a \hat{\mathbf{y}}+ \\
\left(\frac{1}{2}+x_{5}\right) a \hat{\mathbf{z}}\end{array}$ & $(48 i)$ \\
\hline
\end{tabular}

\section{References:}

- P. H. Hubert, Contribution à l'étude des molybdites des terres rares: 1. Bimolybdites $\mathrm{Ln}_{2} \mathrm{Mo}_{2} \mathrm{O}_{7}$, Bull. Soc. Chim. Fr pp. 2385-2386 (1974).

\section{Found in:}

- P. Villars and K. Cenzual, Pearson's Crystal Data - Crystal Structure Database for Inorganic Compounds, ASM International (2013).

\section{Geometry files:}

- CIF: pp. 972 
- POSCAR: pp. 972 


\section{$\operatorname{Th}_{6} \mathrm{Mn}_{23}\left(D 8_{a}\right)$ Structure: A23B6_cF116_225_bd2f_e}
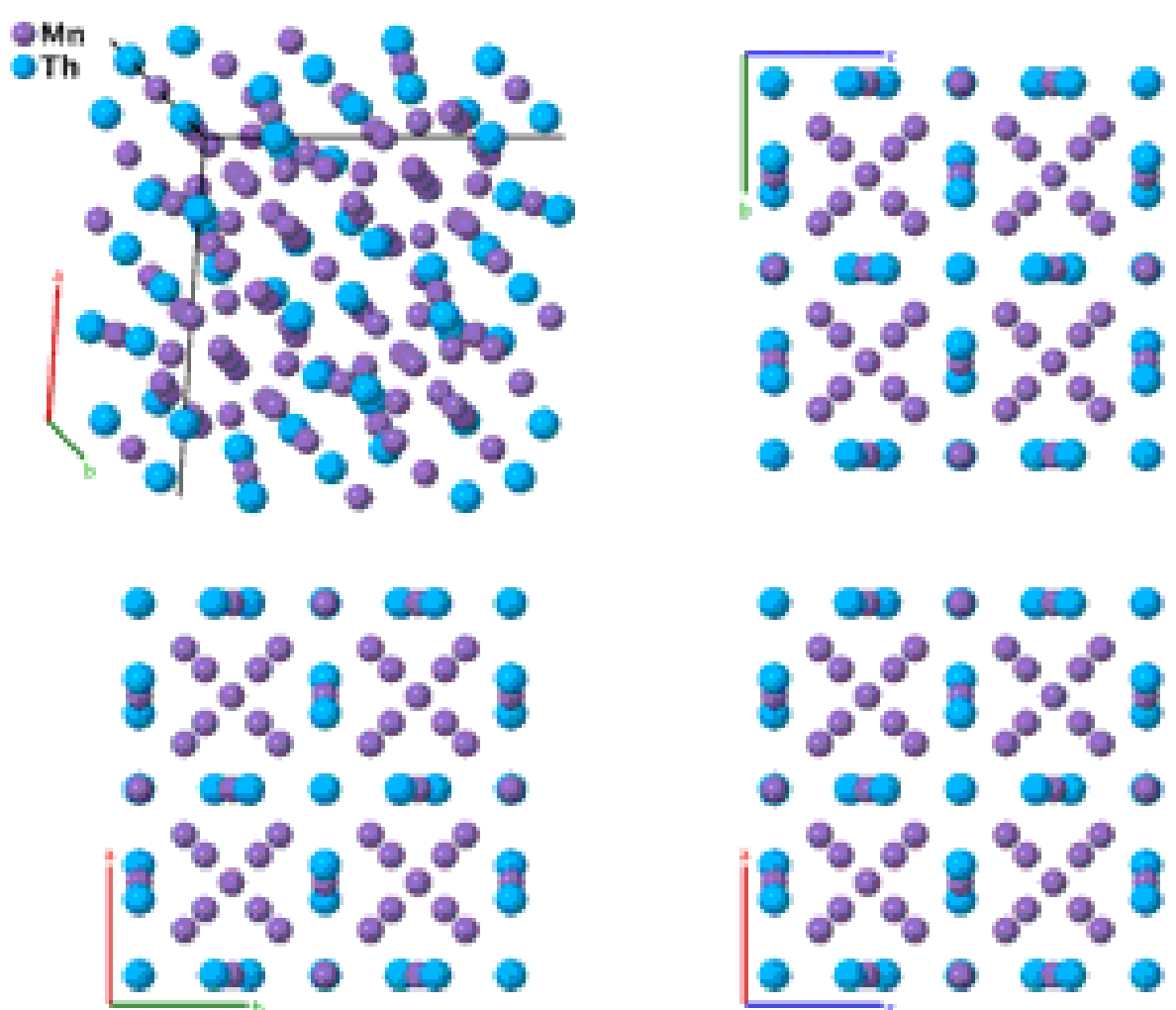

\section{Prototype}

$: \quad \mathrm{Th}_{6} \mathrm{Mn}_{23}$

AFLOW prototype label

: A23B6_cF116_225_bd2f_e

Strukturbericht designation : $D 8_{a}$

Pearson symbol

: $\quad \mathrm{cF} 116$

Space group number $\quad: \quad 225$

Space group symbol

: $F m \overline{3} m$

AFLOW prototype command : aflow --proto=A23B6_cF116_225_bd2f_e

- - params $=a, x_{3}, x_{4}, x_{5}$

\section{Other compounds with this structure:}

- $\mathrm{Ba}_{6} \mathrm{Mg}_{23}, \mathrm{Cu}_{16} \mathrm{Mg}_{4} \mathrm{Si}_{7}, \mathrm{Er6Fe} 23, \mathrm{Fe}_{10} \mathrm{Ge}_{13} \mathrm{Ti} 6, \mathrm{Fe} 3 \mathrm{Zn}, \mathrm{Ho6Fe} 23, \mathrm{Mn}_{6} \mathrm{Ni}_{16} \mathrm{Si}_{7}, \mathrm{Sm6Fe} 23, \mathrm{Sr}_{6} \mathrm{Li}_{23}, \mathrm{~Tb} 6 \mathrm{Fe} 23$, $\mathrm{Th}_{6-x} \mathrm{Y}_{x} \mathrm{Mn}_{23}, \mathrm{Th}_{6} \mathrm{Mn}_{23}, \mathrm{Y}_{6} \mathrm{Mn}_{23}$

Face-centered Cubic primitive vectors:

$$
\begin{aligned}
& \mathbf{a}_{1}=\frac{1}{2} a \hat{\mathbf{y}}+\frac{1}{2} a \hat{\mathbf{z}} \\
& \mathbf{a}_{2}=\frac{1}{2} a \hat{\mathbf{x}}+\frac{1}{2} a \hat{\mathbf{z}} \\
& \mathbf{a}_{3}=\frac{1}{2} a \hat{\mathbf{x}}+\frac{1}{2} a \hat{\mathbf{y}}
\end{aligned}
$$

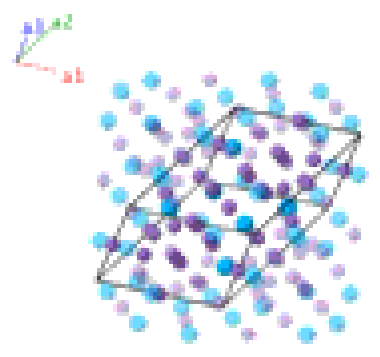


Lattice Coordinates

$\begin{array}{lcc}\mathbf{B}_{1}= & \frac{1}{2} \mathbf{a}_{1}+\frac{1}{2} \mathbf{a}_{2}+\frac{1}{2} \mathbf{a}_{3} \\ \mathbf{B}_{2}= & \frac{1}{2} \mathbf{a}_{1} \\ \mathbf{B}_{3}= & \frac{1}{2} \mathbf{a}_{2}+\frac{1}{2} \mathbf{a}_{3} \\ \mathbf{B}_{4}= & \frac{1}{2} \mathbf{a}_{2} \\ \mathbf{B}_{5}= & \frac{1}{2} \mathbf{a}_{1}+\frac{1}{2} \mathbf{a}_{3} \\ \mathbf{B}_{6}= & \frac{1}{2} \mathbf{a}_{3} \\ \mathbf{B}_{7}= & \frac{1}{2} \mathbf{a}_{1}+\frac{1}{2} \mathbf{a}_{2}\end{array}$

$\mathbf{B}_{8}=-x_{3} \mathbf{a}_{1}+x_{3} \mathbf{a}_{2}+x_{3} \mathbf{a}_{3}$

$\mathbf{B}_{9}=x_{3} \mathbf{a}_{1}-x_{3} \mathbf{a}_{2}-x_{3} \mathbf{a}_{3}$

$\mathbf{B}_{10}=$

$\mathbf{B}_{11}=$

$\mathbf{B}_{12}=$

$\mathbf{B}_{13}=$

$\mathbf{B}_{14}=$

$\mathbf{B}_{15}=x_{4} \mathbf{a}_{1}+x_{4} \mathbf{a}_{2}-3 x_{4} \mathbf{a}_{3}$

$\mathbf{B}_{16}=x_{4} \mathbf{a}_{1}-3 x_{4} \mathbf{a}_{2}+x_{4} \mathbf{a}_{3}$

$\mathbf{B}_{17}=-3 x_{4} \mathbf{a}_{1}+x_{4} \mathbf{a}_{2}+x_{4} \mathbf{a}_{3}$

$\mathbf{B}_{18}=-x_{4} \mathbf{a}_{1}-x_{4} \mathbf{a}_{2}+3 x_{4} \mathbf{a}_{3}$

$\mathbf{B}_{19}=-x_{4} \mathbf{a}_{1}-x_{4} \mathbf{a}_{2}-x_{4} \mathbf{a}_{3}$

$\mathbf{B}_{20}=-x_{4} \mathbf{a}_{1}+3 x_{4} \mathbf{a}_{2}-x_{4} \mathbf{a}_{3}$

$\mathbf{B}_{21}=3 x_{4} \mathbf{a}_{1}-x_{4} \mathbf{a}_{2}-x_{4} \mathbf{a}_{3}$

$\mathbf{B}_{22}=$

$\mathbf{B}_{23}=$

$\mathbf{B}_{24}=$

$\mathbf{B}_{25}=$

$\mathbf{B}_{26}=-x_{5} \mathbf{a}_{1}-x_{5} \mathbf{a}_{2}+3 x_{5} \mathbf{a}_{3}$

$\mathbf{B}_{27}=-x_{5} \mathbf{a}_{1}-x_{5} \mathbf{a}_{2}-x_{5} \mathbf{a}_{3}$

$\mathbf{B}_{28}=-x_{5} \mathbf{a}_{1}+3 x_{5} \mathbf{a}_{2}-x_{5} \mathbf{a}_{3}$

$\mathbf{B}_{29}=$

$$
=
$$$$
=
$$$$
=
$$$$
=
$$$$
=
$$$$
=
$$$$
=
$$$$
=
$$$$
=
$$$$
=
$$$$
=
$$$$
=
$$$$
=
$$$$
=
$$$$
=
$$$$
=
$$$$
=
$$$$
=
$$$$
=
$$$$
=
$$$$
=
$$$$
=
$$$$
=
$$$$
=
$$$$
=
$$$$
=
$$$$
=
$$$$
=
$$$$
=
$$$$
=
$$$$
=
$$$$
=
$$$$
=
$$$$
=
$$

Cartesian Coordinates

$$
\frac{1}{2} a \hat{\mathbf{x}}+\frac{1}{2} a \hat{\mathbf{y}}+\frac{1}{2} a \hat{\mathbf{z}}
$$$$
\frac{1}{4} a \hat{\mathbf{y}}+\frac{1}{4} a \hat{\mathbf{z}}
$$$$
\frac{1}{2} a \hat{\mathbf{x}}+\frac{1}{4} a \hat{\mathbf{y}}+\frac{1}{4} a \hat{\mathbf{z}}
$$$$
\frac{1}{4} a \hat{\mathbf{x}}+\frac{1}{4} a \hat{\mathbf{z}}
$$$$
\frac{1}{4} a \hat{\mathbf{x}}+\frac{1}{2} a \hat{\mathbf{y}}+\frac{1}{4} a \hat{\mathbf{z}}
$$$$
\frac{1}{4} a \hat{\mathbf{x}}+\frac{1}{4} a \hat{\mathbf{y}}
$$$$
\frac{1}{4} a \hat{\mathbf{x}}+\frac{1}{4} a \hat{\mathbf{y}}+\frac{1}{2} a \hat{\mathbf{z}}
$$$$
x_{3} a \hat{\mathbf{x}}
$$$$
-x_{3} a \hat{\mathbf{x}}
$$$$
x_{3} a \hat{\mathbf{y}}
$$$$
-x_{3} a \hat{\mathbf{y}}
$$$$
x_{3} a \hat{\mathbf{z}}
$$$$
-x_{3} a \hat{\mathbf{z}}
$$$$
x_{4} a \hat{\mathbf{x}}+x_{4} a \hat{\mathbf{y}}+x_{4} a \hat{\mathbf{z}}
$$$$
-x_{4} a \hat{\mathbf{x}}-x_{4} a \hat{\mathbf{y}}+x_{4} a \hat{\mathbf{z}}
$$$$
-x_{4} a \hat{\mathbf{x}}+x_{4} a \hat{\mathbf{y}}-x_{4} a \hat{\mathbf{z}}
$$$$
x_{4} a \hat{\mathbf{x}}-x_{4} a \hat{\mathbf{y}}-x_{4} a \hat{\mathbf{z}}
$$$$
x_{4} a \hat{\mathbf{x}}+x_{4} a \hat{\mathbf{y}}-x_{4} a \hat{\mathbf{z}}
$$$$
-x_{4} a \hat{\mathbf{x}}-x_{4} a \hat{\mathbf{y}}-x_{4} a \hat{\mathbf{z}}
$$$$
x_{4} a \hat{\mathbf{x}}-x_{4} a \hat{\mathbf{y}}+x_{4} a \hat{\mathbf{z}}
$$$$
-x_{4} a \hat{\mathbf{x}}+x_{4} a \hat{\mathbf{y}}+x_{4} a \hat{\mathbf{z}}
$$$$
x_{5} a \hat{\mathbf{x}}+x_{5} a \hat{\mathbf{y}}+x_{5} a \hat{\mathbf{z}}
$$$$
-x_{5} a \hat{\mathbf{x}}-x_{5} a \hat{\mathbf{y}}+x_{5} a \hat{\mathbf{z}}
$$$$
-x_{5} a \hat{\mathbf{x}}+x_{5} a \hat{\mathbf{y}}-x_{5} a \hat{\mathbf{z}}
$$$$
x_{5} a \hat{\mathbf{x}}-x_{5} a \hat{\mathbf{y}}-x_{5} a \hat{\mathbf{z}}
$$$$
x_{5} a \hat{\mathbf{x}}+x_{5} a \hat{\mathbf{y}}-x_{5} a \hat{\mathbf{z}}
$$$$
-x_{5} a \hat{\mathbf{x}}-x_{5} a \hat{\mathbf{y}}-x_{5} a \hat{\mathbf{z}}
$$$$
x_{5} a \hat{\mathbf{x}}-x_{5} a \hat{\mathbf{y}}+x_{5} a \hat{\mathbf{z}}
$$$$
-x_{5} a \hat{\mathbf{x}}+x_{5} a \hat{\mathbf{y}}+x_{5} a \hat{\mathbf{z}}
$$

Atom Type

Mn I

Mn II

Mn II

Mn II

Mn II

Mn II

Mn II

Th

Th

Th

Th

Th

Th

Mn III

Mn III

Mn III

Mn III

Mn III

Mn III

Mn III

Mn III

Mn IV

Mn IV

Mn IV

Mn IV

Mn IV

Mn IV

Mn IV

Mn IV

\section{References:}

- J. V. Florio, R. E. Rundle, and A. I. Snow, Compounds of thorium with transition metals. I. The thorium-manganese system, Acta Cryst. 5, 445-457 (1952), doi:10.1107/S0365110X52001337.

\section{Found in:}

- W. B. Pearson, A Handbook of Lattice Spacings and Structures of Metals and Alloys, no. N.R.C. No. 4303 in International Series of Monographs on Metal Physics and Physical Metallurgy (Pergamon Press, Oxford, London, Edinburgh, New York, Paris, Frankfort, 1958), 1964 reprint with corrections edn. 
Geometry files:

- CIF: pp. 973

- POSCAR: pp. 974 


\section{$\mathrm{K}_{2} \mathrm{PtCl}_{6}\left(J 1_{1}\right)$ Structure: A6B2C_cF36_225_e_c_a}
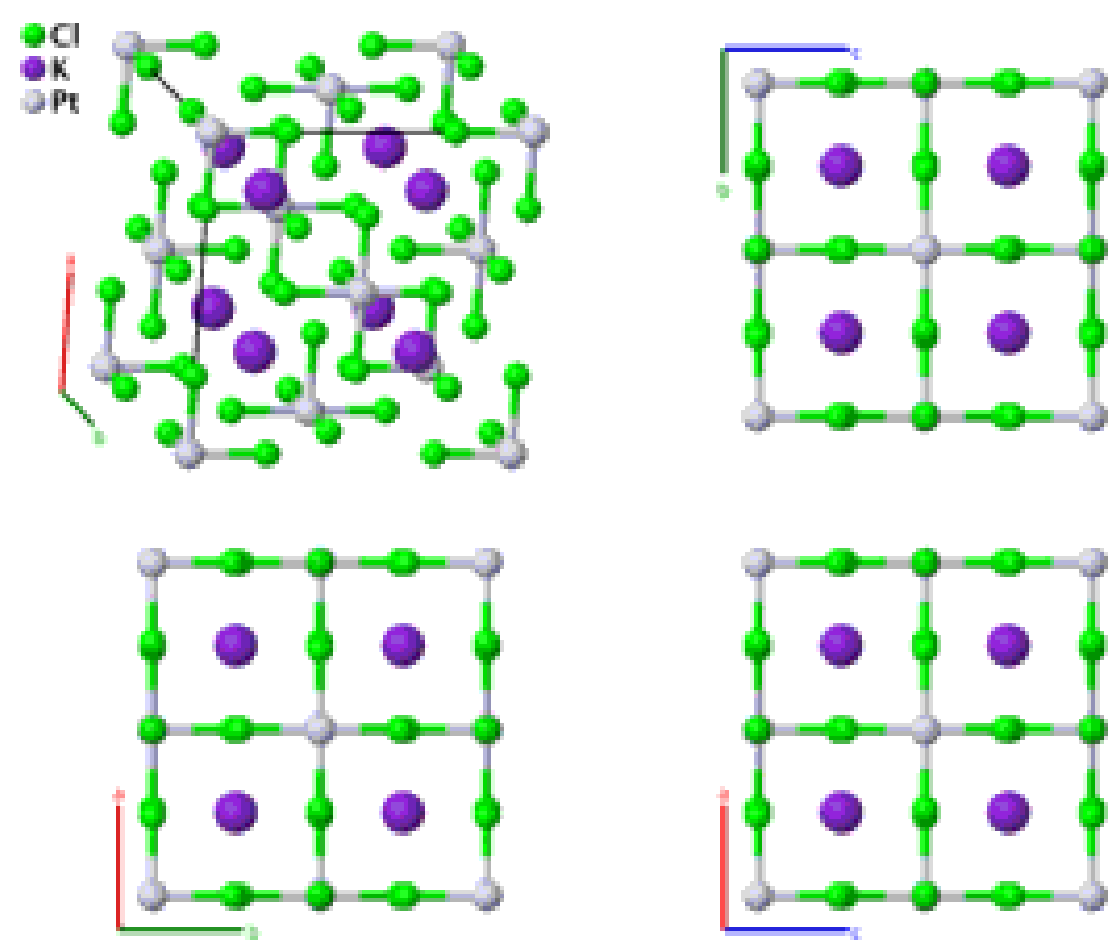

$\begin{array}{lll}\text { Prototype } & : & \mathrm{K}_{2} \mathrm{PtCl}_{6} \\ \text { AFLOW prototype label } & : & \mathrm{A} 6 \mathrm{~B} 2 \mathrm{C} \_\mathrm{cF} 36 \_225 \_\mathrm{e} \_\mathrm{c} \_\mathrm{a} \\ \text { Strukturbericht designation } & : & J 1_{1} \\ \text { Pearson symbol } & : & \mathrm{cF} 36 \\ \text { Space group number } & : & 225 \\ \text { Space group symbol } & : & F m \overline{3} m \\ \text { AFLOW prototype command } & : & \text { aflow --proto=A6B2C_cF36_225_e_c_a } \\ & & -- \text { params }=a, x_{3}\end{array}$

\section{Other compounds with this structure:}

- $\mathrm{Gd}_{2} \mathrm{MnGa}_{6}, \mathrm{~K}_{2} \mathrm{TeBr}_{6}, \mathrm{Sr}_{2} \mathrm{RuH}_{6}$

- In (Douglas, 2006), Table 6.6 provides an extensive list of compounds with this structure. Most have the formula $A_{2} M X_{6}$, where $A$ is an alkali metal, $M$ is a metal, and $X$ is a halide. An ammonium ion $\left(\mathrm{NH}_{4}^{+}\right)$can also substitute for the alkali.

Face-centered Cubic primitive vectors:

$$
\begin{aligned}
& \mathbf{a}_{1}=\frac{1}{2} a \hat{\mathbf{y}}+\frac{1}{2} a \hat{\mathbf{z}} \\
& \mathbf{a}_{2}=\frac{1}{2} a \hat{\mathbf{x}}+\frac{1}{2} a \hat{\mathbf{z}} \\
& \mathbf{a}_{3}=\frac{1}{2} a \hat{\mathbf{x}}+\frac{1}{2} a \hat{\mathbf{y}}
\end{aligned}
$$

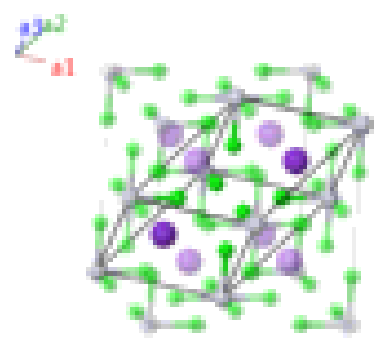


Lattice Coordinates

$\begin{array}{llc}\mathbf{B}_{1} & = & 0 \mathbf{a}_{1}+0 \mathbf{a}_{2}+0 \mathbf{a}_{3} \\ \mathbf{B}_{2} & = & \frac{1}{4} \mathbf{a}_{1}+\frac{1}{4} \mathbf{a}_{2}+\frac{1}{4} \mathbf{a}_{3} \\ \mathbf{B}_{3} & = & \frac{3}{4} \mathbf{a}_{1}+\frac{3}{4} \mathbf{a}_{2}+\frac{3}{4} \mathbf{a}_{3} \\ \mathbf{B}_{4}= & -x_{3} \mathbf{a}_{1}+x_{3} \mathbf{a}_{2}+x_{3} \mathbf{a}_{3} \\ \mathbf{B}_{5}= & x_{3} \mathbf{a}_{1}-x_{3} \mathbf{a}_{2}-x_{3} \mathbf{a}_{3} \\ \mathbf{B}_{6}= & x_{3} \mathbf{a}_{1}-x_{3} \mathbf{a}_{2}+x_{3} \mathbf{a}_{3} \\ \mathbf{B}_{7}= & -x_{3} \mathbf{a}_{1}+x_{3} \mathbf{a}_{2}-x_{3} \mathbf{a}_{3} \\ \mathbf{B}_{8}= & x_{3} \mathbf{a}_{1}+x_{3} \mathbf{a}_{2}-x_{3} \mathbf{a}_{3} \\ \mathbf{B}_{9}= & -x_{3} \mathbf{a}_{1}-x_{3} \mathbf{a}_{2}+x_{3} \mathbf{a}_{3}\end{array}$

Cartesian Coordinates

$=$

$=\quad \frac{1}{4} a \hat{\mathbf{x}}+\frac{1}{4} a \hat{\mathbf{y}}+\frac{1}{4} a \hat{\mathbf{z}}$

$=\quad \frac{3}{4} a \hat{\mathbf{x}}+\frac{3}{4} a \hat{\mathbf{y}}+\frac{3}{4} a \hat{\mathbf{z}}$

$x_{3} a \hat{\mathbf{x}}$

$-x_{3} a \hat{\mathbf{x}}$

$x_{3} a \hat{\mathbf{y}}$

$-x_{3} a \hat{\mathbf{y}}$

$x_{3} a \hat{\mathbf{z}}$

$-x_{3} a \hat{\mathbf{z}}$
Wyckoff Position

(4a)

$(24 e)$
Atom Type

$\mathrm{Pt}$

K

K

$\mathrm{Cl}$

$\mathrm{Cl}$

$\mathrm{Cl}$

$\mathrm{Cl}$

$\mathrm{Cl}$

$\mathrm{Cl}$

\section{References:}

- G. Engel, Die Kristallstrukturen einiger Hexachlorokomplexsalze, Z. Kristallogr. 90, 341-373 (1935), doi:10.1524/zkri.1935.90.1.341.

- B. Douglas and S.-M. Ho, Structure and Chemistry of Crystalline Solids (Springer, 2006).

\section{Found in:}

- R. T. Downs and M. Hall-Wallace, The American Mineralogist Crystal Structure Database, Am. Mineral. 88, 247-250 (2003).

\section{Geometry files:}

- CIF: pp. 974

- POSCAR: pp. 976 
$\mathrm{NaZn}_{13}\left(D 2_{3}\right)$ Structure: AB13_cF112_226_a_bi

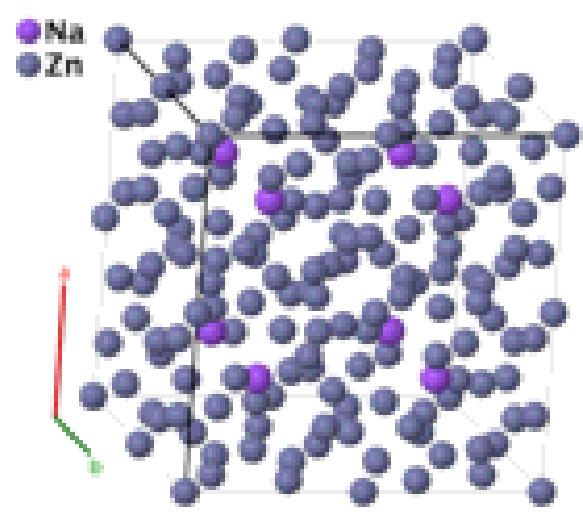

- 000000
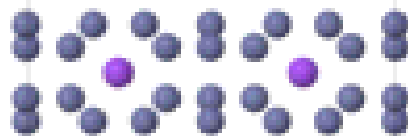

- 000000

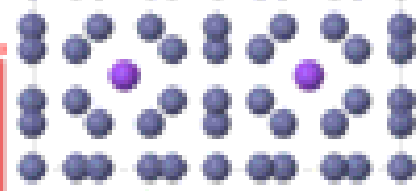

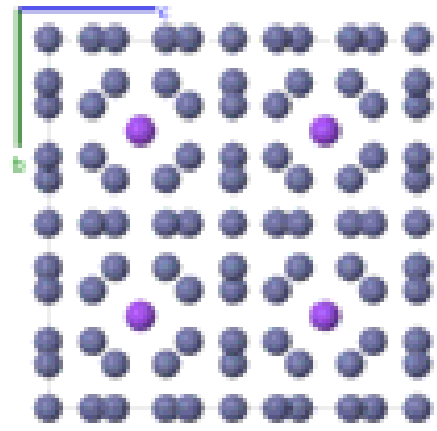

- 000000
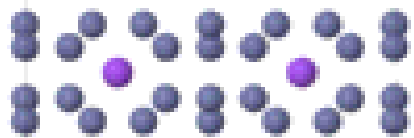

- 000000

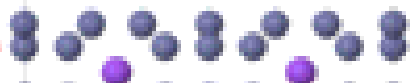

89.996 .09

00000000

\section{Prototype}

AFLOW prototype label

Strukturbericht designation

Pearson symbol

Space group number

Space group symbol

AFLOW prototype command : aflow--proto=AB13_cF112_226_a_bi

- params $=a, y_{3}, z_{3}$

Other compounds with this structure:

- $\mathrm{AmBe}_{13}, \mathrm{BaZn}_{13}, \mathrm{CaBe}_{13}, \mathrm{CaZn}_{13}, \mathrm{CdZn}_{13}, \mathrm{CeBe}_{13}, \mathrm{CsCd}_{13}, \mathrm{KCd}_{13}, \mathrm{KZn}_{13}, \mathrm{MgBe}_{13}, \mathrm{NbBe}_{13}, \mathrm{RbCd}_{13}, \mathrm{SrZn}_{13}$, $\mathrm{ThBe}_{13}, \mathrm{UBe}_{13}, \mathrm{VBe}_{13}, \mathrm{ZrBe}_{13}, \mathrm{CeNi}_{8.5} \mathrm{Si}_{4.5}, \mathrm{LaFe}_{13-x-y} \mathrm{Co}_{y} \mathrm{Al}_{x}, \mathrm{LaFe}_{13-x-y} \mathrm{Co}_{y} \mathrm{Si}_{x}, \mathrm{NdFe}_{13-x-y} \mathrm{Co}_{y} \mathrm{Si}_{x}$

Face-centered Cubic primitive vectors:

$$
\begin{aligned}
& \mathbf{a}_{1}=\frac{1}{2} a \hat{\mathbf{y}}+\frac{1}{2} a \hat{\mathbf{z}} \\
& \mathbf{a}_{2}=\frac{1}{2} a \hat{\mathbf{x}}+\frac{1}{2} a \hat{\mathbf{z}} \\
& \mathbf{a}_{3}=\frac{1}{2} a \hat{\mathbf{x}}+\frac{1}{2} a \hat{\mathbf{y}}
\end{aligned}
$$

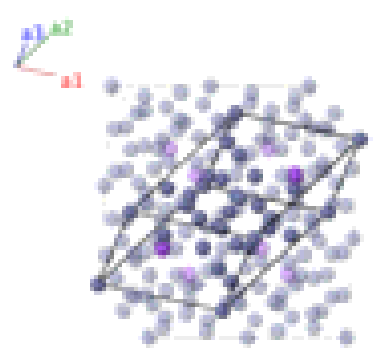

Basis vectors: 
Lattice Coordinates

$$
\begin{aligned}
& \mathbf{B}_{1}=\quad \frac{1}{4} \mathbf{a}_{1}+\frac{1}{4} \mathbf{a}_{2}+\frac{1}{4} \mathbf{a}_{3} \\
& \mathbf{B}_{2}=\quad \frac{3}{4} \mathbf{a}_{1}+\frac{3}{4} \mathbf{a}_{2}+\frac{3}{4} \mathbf{a}_{3} \\
& \mathbf{B}_{3}=0 \mathbf{a}_{1}+0 \mathbf{a}_{2}+0 \mathbf{a}_{3} \\
& \mathbf{B}_{4}=\quad \frac{1}{2} \mathbf{a}_{1}+\frac{1}{2} \mathbf{a}_{2}+\frac{1}{2} \mathbf{a}_{3} \\
& \mathbf{B}_{5}=\left(y_{3}+z_{3}\right) \mathbf{a}_{1}+\left(-y_{3}+z_{3}\right) \mathbf{a}_{2}+ \\
& \left(y_{3}-z_{3}\right) \mathbf{a}_{3} \\
& \mathbf{B}_{6}=\left(-y_{3}+z_{3}\right) \mathbf{a}_{1}+\left(y_{3}+z_{3}\right) \mathbf{a}_{2}+= \\
& \left(-y_{3}-z_{3}\right) \mathbf{a}_{3} \\
& \mathbf{B}_{7}=\left(y_{3}-z_{3}\right) \mathbf{a}_{1}+\left(-y_{3}-z_{3}\right) \mathbf{a}_{2}+= \\
& \left(y_{3}+z_{3}\right) \mathbf{a}_{3} \\
& \mathbf{B}_{8}=\left(-y_{3}-z_{3}\right) \mathbf{a}_{1}+\left(y_{3}-z_{3}\right) \mathbf{a}_{2}+= \\
& \left(-y_{3}+z_{3}\right) \mathbf{a}_{3} \\
& \mathbf{B}_{9}=\left(y_{3}-z_{3}\right) \mathbf{a}_{1}+\left(y_{3}+z_{3}\right) \mathbf{a}_{2}+= \\
& \left(-y_{3}+z_{3}\right) \mathbf{a}_{3} \\
& \mathbf{B}_{10}=\left(-y_{3}-z_{3}\right) \mathbf{a}_{1}+\left(-y_{3}+z_{3}\right) \mathbf{a}_{2}+= \\
& \left(y_{3}+z_{3}\right) \mathbf{a}_{3} \\
& \mathbf{B}_{11}=\left(y_{3}+z_{3}\right) \mathbf{a}_{1}+\left(y_{3}-z_{3}\right) \mathbf{a}_{2}+= \\
& \left(-y_{3}-z_{3}\right) \mathbf{a}_{3} \\
& \mathbf{B}_{12}=\left(-y_{3}+z_{3}\right) \mathbf{a}_{1}+\left(-y_{3}-z_{3}\right) \mathbf{a}_{2}+= \\
& \left(y_{3}-z_{3}\right) \mathbf{a}_{3} \\
& \mathbf{B}_{13}=\left(-y_{3}+z_{3}\right) \mathbf{a}_{1}+\left(y_{3}-z_{3}\right) \mathbf{a}_{2}+= \\
& \left(y_{3}+z_{3}\right) \mathbf{a}_{3} \\
& \mathbf{B}_{14}=\left(y_{3}+z_{3}\right) \mathbf{a}_{1}+\left(-y_{3}-z_{3}\right) \mathbf{a}_{2}+= \\
& \left(-y_{3}+z_{3}\right) \mathbf{a}_{3} \\
& \mathbf{B}_{15}=\left(-y_{3}-z_{3}\right) \mathbf{a}_{1}+\left(y_{3}+z_{3}\right) \mathbf{a}_{2}+= \\
& \left(y_{3}-z_{3}\right) \mathbf{a}_{3} \\
& \mathbf{B}_{16}=\left(y_{3}-z_{3}\right) \mathbf{a}_{1}+\left(-y_{3}+z_{3}\right) \mathbf{a}_{2}+= \\
& \left(-y_{3}-z_{3}\right) \mathbf{a}_{3} \\
& \mathbf{B}_{17}=\left(\frac{1}{2}-y_{3}-z_{3}\right) \mathbf{a}_{1}+\left(\frac{1}{2}+y_{3}-z_{3}\right) \mathbf{a}_{2}+=\left(\frac{1}{2}+y_{3}\right) a \hat{\mathbf{x}}+\frac{1}{2} a \hat{\mathbf{y}}+\left(\frac{1}{2}-z_{3}\right) a \hat{\mathbf{z}} \\
& \left(\frac{1}{2}+y_{3}+z_{3}\right) \mathbf{a}_{3} \\
& \frac{1}{4} a \hat{\mathbf{x}}+\frac{1}{4} a \hat{\mathbf{y}}+\frac{1}{4} a \hat{\mathbf{z}} \\
& \frac{3}{4} a \hat{\mathbf{x}}+\frac{3}{4} a \hat{\mathbf{y}}+\frac{3}{4} a \hat{\mathbf{z}} \\
& 0 \hat{\mathbf{x}}+0 \hat{\mathbf{y}}+0 \hat{\mathbf{z}} \\
& \frac{1}{2} a \hat{\mathbf{x}}+\frac{1}{2} a \hat{\mathbf{y}}+\frac{1}{2} a \hat{\mathbf{z}} \\
& y_{3} a \hat{\mathbf{y}}+z_{3} a \hat{\mathbf{z}} \\
& -y_{3} a \hat{\mathbf{y}}+z_{3} a \hat{\mathbf{z}} \\
& y_{3} a \hat{\mathbf{y}}-z_{3} a \hat{\mathbf{z}} \\
& -y_{3} a \hat{\mathbf{y}}-z_{3} a \hat{\mathbf{z}} \\
& z_{3} a \hat{\mathbf{x}}+y_{3} a \hat{\mathbf{z}} \\
& z_{3} a \hat{\mathbf{x}}+-y_{3} a \hat{\mathbf{z}} \\
& -z_{3} a \hat{\mathbf{x}}+y_{3} a \hat{\mathbf{z}} \\
& -z_{3} a \hat{\mathbf{x}}+-y_{3} a \hat{\mathbf{z}} \\
& y_{3} a \hat{\mathbf{x}}+z_{3} a \hat{\mathbf{y}} \\
& -y_{3} a \hat{\mathbf{x}}+z_{3} a \hat{\mathbf{y}} \\
& y_{3} a \hat{\mathbf{x}}-z_{3} a \hat{\mathbf{y}} \\
& -y_{3} a \hat{\mathbf{x}}-z_{3} a \hat{\mathbf{y}}
\end{aligned}
$$

Cartesian Coordinates
$\mathrm{Na}$

$\mathrm{Na}$

Zn I

Zn I

Zn II

Zn II

Zn II

Zn II

Zn II

Zn II

Zn II

Zn II

Zn II

Zn II

Zn II

Zn II

Zn II

Zn II

Zn II

Zn II

Zn II

Zn II

Zn II 


\begin{tabular}{|c|c|c|c|}
\hline $\mathbf{B}_{24}$ & $\begin{array}{c}=\left(\frac{1}{2}+y_{3}-z_{3}\right) \mathbf{a}_{1}+\left(\frac{1}{2}+y_{3}+z_{3}\right) \mathbf{a}_{2}+ \\
\left(\frac{1}{2}-y_{3}-z_{3}\right) \mathbf{a}_{3}\end{array}$ & $=\frac{1}{2} a \hat{\mathbf{x}}+\left(\frac{1}{2}-z_{3}\right) a \hat{\mathbf{y}}+\left(\frac{1}{2}+y_{3}\right) a \hat{\mathbf{z}}$ & $(96 i)$ \\
\hline $\mathbf{B}_{25}$ & $\begin{array}{c}=\left(\frac{1}{2}+y_{3}-z_{3}\right) \mathbf{a}_{1}+\left(\frac{1}{2}-y_{3}+z_{3}\right) \mathbf{a}_{2}+ \\
\left(\frac{1}{2}+y_{3}+z_{3}\right) \mathbf{a}_{3}\end{array}$ & $=\left(\frac{1}{2}+z_{3}\right) a \hat{\mathbf{x}}+\left(\frac{1}{2}+y_{3}\right) a \hat{\mathbf{y}}+\frac{1}{2} a \hat{\mathbf{z}}$ & $(96 i)$ \\
\hline $\mathbf{B}_{26}$ & $\begin{array}{c}=\left(\frac{1}{2}-y_{3}-z_{3}\right) \mathbf{a}_{1}+\left(\frac{1}{2}+y_{3}+z_{3}\right) \mathbf{a}_{2}+ \\
\left(\frac{1}{2}-y_{3}+z_{3}\right) \mathbf{a}_{3}\end{array}$ & $=\left(\frac{1}{2}+z_{3}\right) a \hat{\mathbf{x}}+\left(\frac{1}{2}-y_{3}\right) a \hat{\mathbf{y}}+\frac{1}{2} a \hat{\mathbf{z}}$ & $(96 i)$ \\
\hline $\mathbf{B}_{27}$ & $\begin{array}{c}=\left(\frac{1}{2}+y_{3}+z_{3}\right) \mathbf{a}_{1}+\left(\frac{1}{2}-y_{3}-z_{3}\right) \mathbf{a}_{2}+ \\
\left(\frac{1}{2}+y_{3}-z_{3}\right) \mathbf{a}_{3}\end{array}$ & $=\left(\frac{1}{2}-z_{3}\right) a \hat{\mathbf{x}}+\left(\frac{1}{2}+y_{3}\right) a \hat{\mathbf{y}}+\frac{1}{2} a \hat{\mathbf{z}}$ & $(96 i)$ \\
\hline $\mathbf{B}_{28}$ & $\begin{array}{c}=\left(\frac{1}{2}-y_{3}+z_{3}\right) \mathbf{a}_{1}+\left(\frac{1}{2}+y_{3}-z_{3}\right) \mathbf{a}_{2}+ \\
\left(\frac{1}{2}-y_{3}-z_{3}\right) \mathbf{a}_{3}\end{array}$ & $=\left(\frac{1}{2}-z_{3}\right) a \hat{\mathbf{x}}+\left(\frac{1}{2}-y_{3}\right) a \hat{\mathbf{y}}+\frac{1}{2} a \hat{\mathbf{z}}$ & $(96 i)$ \\
\hline
\end{tabular}

\section{References:}

- D. P. Shoemaker, R. E. Marsh, F. J. Ewing, and L. Pauling, Interatomic distances and atomic valences in NaZn $n_{13}$, Acta Cryst. 5, 637-644 (1952), doi:10.1107/S0365110X52001763.

\section{Geometry files:}

- CIF: pp. 976

- POSCAR: pp. 977 


\section{Pyrochlore Iridate $\left(\mathrm{Eu}_{2} \mathrm{Ir}_{2} \mathrm{O}_{7}\right)$ Structure:}

\section{A2B2C7_cF88_227_c_d_af}
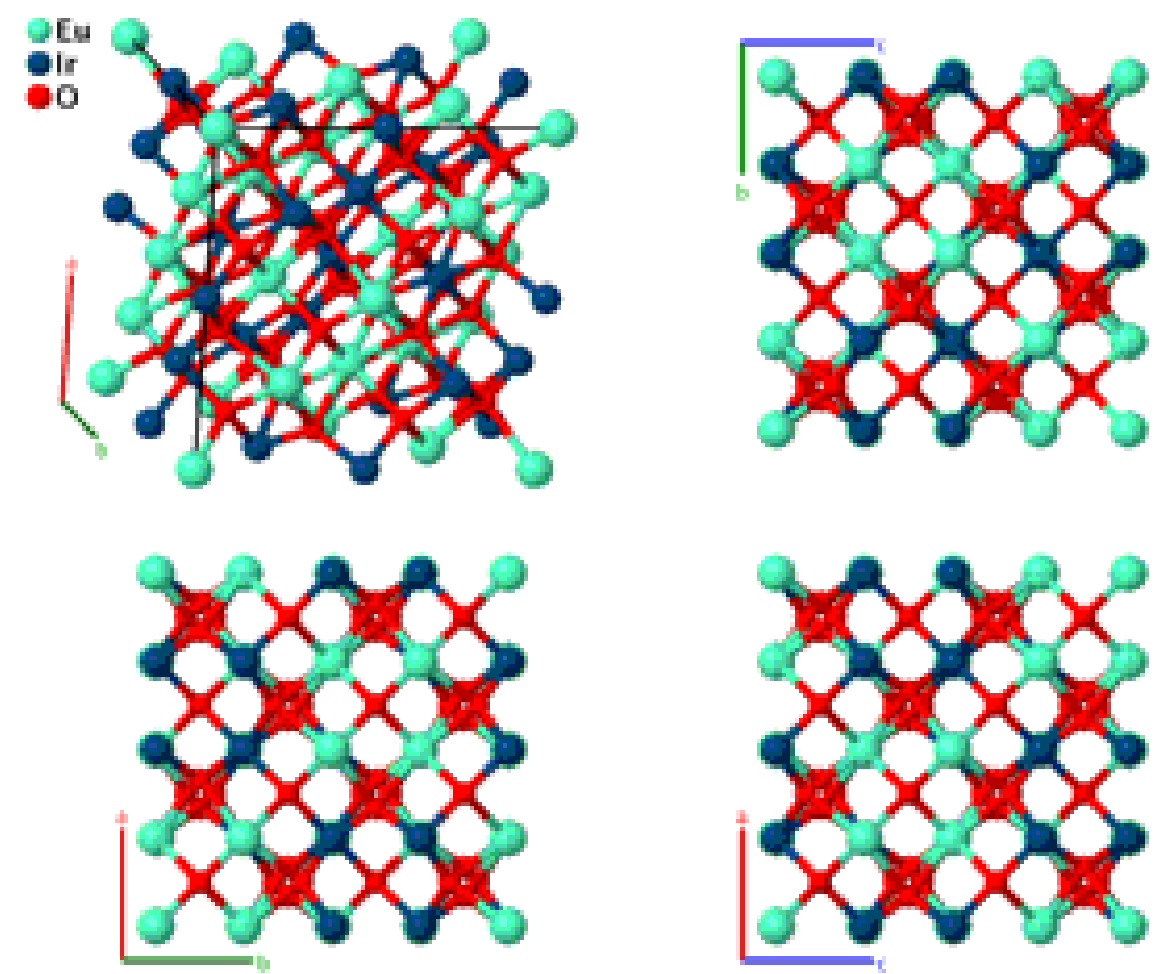

Prototype

AFLOW prototype label

$\mathrm{Eu}_{2} \mathrm{Ir}_{2} \mathrm{O}_{7}$

Strukturbericht designation : None

Pearson symbol

cF88

Space group number

227

Space group symbol

$F d \overline{3} m$

AFLOW prototype command

aflow --proto=A2B2C7_cF88_227_c_d_af

- params $=a, x_{4}$

Face-centered Cubic primitive vectors:

$$
\begin{aligned}
& \mathbf{a}_{1}=\frac{1}{2} a \hat{\mathbf{y}}+\frac{1}{2} a \hat{\mathbf{z}} \\
& \mathbf{a}_{2}=\frac{1}{2} a \hat{\mathbf{x}}+\frac{1}{2} a \hat{\mathbf{z}} \\
& \mathbf{a}_{3}=\frac{1}{2} a \hat{\mathbf{x}}+\frac{1}{2} a \hat{\mathbf{y}}
\end{aligned}
$$

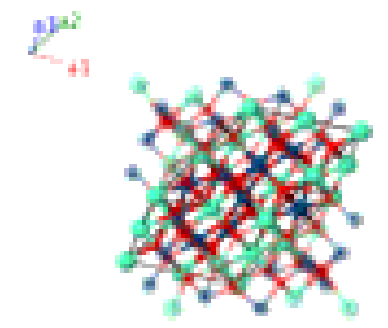

Basis vectors:

Lattice Coordinates

$\mathbf{B}_{1}=\quad \frac{1}{8} \mathbf{a}_{1}+\frac{1}{8} \mathbf{a}_{2}+\frac{1}{8} \mathbf{a}_{3}$
Cartesian Coordinates

$=\quad \frac{1}{8} a \hat{\mathbf{x}}+\frac{1}{8} a \hat{\mathbf{y}}+\frac{1}{8} a \hat{\mathbf{z}}$
Wyckoff Position

$(8 a)$
Atom Type

O I 


\begin{tabular}{|c|c|c|c|c|c|c|}
\hline $\mathbf{B}_{2}$ & $=$ & $\frac{7}{8} \mathbf{a}_{1}+\frac{7}{8} \mathbf{a}_{2}+\frac{7}{8} \mathbf{a}_{3}$ & $=$ & $\frac{7}{8} a \hat{\mathbf{x}}+\frac{7}{8} a \hat{\mathbf{y}}+\frac{7}{8} a \hat{\mathbf{z}}$ & $(8 a)$ & O I \\
\hline $\mathbf{B}_{3}$ & $=$ & $0 \mathbf{a}_{1}+0 \mathbf{a}_{2}+0 \mathbf{a}_{3}$ & $=$ & $0 \hat{\mathbf{x}}+0 \hat{\mathbf{y}}+0 \hat{\mathbf{z}}$ & $(16 c)$ & $\mathrm{Eu}$ \\
\hline $\mathbf{B}_{4}$ & $=$ & $\frac{1}{2} \mathbf{a}_{3}$ & $=$ & $\frac{1}{4} a \hat{\mathbf{x}}+\frac{1}{4} a \hat{\mathbf{y}}$ & $(16 c)$ & $\mathrm{Eu}$ \\
\hline $\mathbf{B}_{5}$ & $=$ & $\frac{1}{2} \mathbf{a}_{2}$ & $=$ & $\frac{1}{4} a \hat{\mathbf{x}}+\frac{1}{4} a \hat{\mathbf{z}}$ & $(16 c)$ & $\mathrm{Eu}$ \\
\hline $\mathbf{B}_{6}$ & $=$ & $\frac{1}{2} \mathbf{a}_{1}$ & $=$ & $\frac{1}{4} a \hat{\mathbf{y}}+\frac{1}{4} a \hat{\mathbf{z}}$ & $(16 c)$ & $\mathrm{Eu}$ \\
\hline $\mathbf{B}_{7}$ & $=$ & $\frac{1}{2} \mathbf{a}_{1}+\frac{1}{2} \mathbf{a}_{2}+\frac{1}{2} \mathbf{a}_{3}$ & $=$ & $\frac{1}{2} a \hat{\mathbf{x}}+\frac{1}{2} a \hat{\mathbf{y}}+\frac{1}{2} a \hat{\mathbf{z}}$ & $(16 d)$ & Ir \\
\hline $\mathbf{B}_{8}$ & $=$ & $\frac{1}{2} \mathbf{a}_{1}+\frac{1}{2} \mathbf{a}_{2}$ & $=$ & $\frac{1}{4} a \hat{\mathbf{x}}+\frac{1}{4} a \hat{\mathbf{y}}+\frac{1}{2} a \hat{\mathbf{z}}$ & $(16 d)$ & Ir \\
\hline $\mathbf{B}_{9}$ & $=$ & $\frac{1}{2} \mathbf{a}_{1}+\frac{1}{2} \mathbf{a}_{3}$ & $=$ & $\frac{1}{4} a \hat{\mathbf{x}}+\frac{1}{2} a \hat{\mathbf{y}}+\frac{1}{4} a \hat{\mathbf{z}}$ & $(16 d)$ & Ir \\
\hline $\mathbf{B}_{10}$ & $=$ & $\frac{1}{2} \mathbf{a}_{2}+\frac{1}{2} \mathbf{a}_{3}$ & $=$ & $\frac{1}{2} a \hat{\mathbf{x}}+\frac{1}{4} a \hat{\mathbf{y}}+\frac{1}{4} a \hat{\mathbf{z}}$ & $(16 d)$ & Ir \\
\hline $\mathbf{B}_{11}$ & $=$ & $\left(\frac{1}{4}-x_{4}\right) \mathbf{a}_{1}+x_{4} \mathbf{a}_{2}+x_{4} \mathbf{a}_{3}$ & $=$ & $x_{4} a \hat{\mathbf{x}}+\frac{1}{8} a \hat{\mathbf{y}}+\frac{1}{8} a \hat{\mathbf{z}}$ & $(48 f)$ & O II \\
\hline $\mathbf{B}_{12}$ & $=$ & $x_{4} \mathbf{a}_{1}+\left(\frac{1}{4}-x_{4}\right) \mathbf{a}_{2}+\left(\frac{1}{4}-x_{4}\right) \mathbf{a}_{3}$ & $=$ & $\left(\frac{1}{4}-x_{4}\right) a \hat{\mathbf{x}}+\frac{1}{8} a \hat{\mathbf{y}}+\frac{1}{8} a \hat{\mathbf{z}}$ & $(48 f)$ & O II \\
\hline $\mathbf{B}_{13}$ & $=$ & $x_{4} \mathbf{a}_{1}+\left(\frac{1}{4}-x_{4}\right) \mathbf{a}_{2}+x_{4} \mathbf{a}_{3}$ & $=$ & $\frac{1}{8} a \hat{\mathbf{x}}+x_{4} a \hat{\mathbf{y}}+\frac{1}{8} a \hat{\mathbf{z}}$ & $(48 f)$ & O II \\
\hline $\mathbf{B}_{14}$ & $=$ & $\left(\frac{1}{4}-x_{4}\right) \mathbf{a}_{1}+x_{4} \mathbf{a}_{2}+\left(\frac{1}{4}-x_{4}\right) \mathbf{a}_{3}$ & $=$ & $\frac{1}{8} a \hat{\mathbf{x}}+\left(\frac{1}{4}-x_{4}\right) a \hat{\mathbf{y}}+\frac{1}{8} a \hat{\mathbf{z}}$ & $(48 f)$ & O II \\
\hline $\mathbf{B}_{15}$ & $=$ & $x_{4} \mathbf{a}_{1}+x_{4} \mathbf{a}_{2}+\left(\frac{1}{4}-x_{4}\right) \mathbf{a}_{3}$ & $=$ & $\frac{1}{8} a \hat{\mathbf{x}}+\frac{1}{8} a \hat{\mathbf{y}}+x_{4} a \hat{\mathbf{z}}$ & $(48 f)$ & O II \\
\hline $\mathbf{B}_{16}$ & $=$ & $\left(\frac{1}{4}-x_{4}\right) \mathbf{a}_{1}+\left(\frac{1}{4}-x_{4}\right) \mathbf{a}_{2}+x_{4} \mathbf{a}_{3}$ & $=$ & $\frac{1}{8} a \hat{\mathbf{x}}+\frac{1}{8} a \hat{\mathbf{y}}+\left(\frac{1}{4}-x_{4}\right) a \hat{\mathbf{z}}$ & $(48 f)$ & O II \\
\hline $\mathbf{B}_{17}$ & $=$ & $\left(\frac{3}{4}+x_{4}\right) \mathbf{a}_{1}-x_{4} \mathbf{a}_{2}+\left(\frac{3}{4}+x_{4}\right) \mathbf{a}_{3}$ & $=$ & $\frac{3}{8} a \hat{\mathbf{x}}+\left(\frac{3}{4}+x_{4}\right) a \hat{\mathbf{y}}+\frac{3}{8} a \hat{\mathbf{z}}$ & $(48 f)$ & O II \\
\hline $\mathbf{B}_{18}$ & $=$ & $-x_{4} \mathbf{a}_{1}+\left(\frac{3}{4}+x_{4}\right) \mathbf{a}_{2}-x_{4} \mathbf{a}_{3}$ & $=$ & $\frac{3}{8} a \hat{\mathbf{x}}-x_{4} a \hat{\mathbf{y}}+\frac{3}{8} a \hat{\mathbf{z}}$ & $(48 f)$ & O II \\
\hline $\mathbf{B}_{19}$ & $=$ & $-x_{4} \mathbf{a}_{1}+\left(\frac{3}{4}+x_{4}\right) \mathbf{a}_{2}+\left(\frac{3}{4}+x_{4}\right) \mathbf{a}_{3}$ & $=$ & $\left(\frac{3}{4}+x_{4}\right) a \hat{\mathbf{x}}+\frac{3}{8} a \hat{\mathbf{y}}+\frac{3}{8} a \hat{\mathbf{z}}$ & $(48 f)$ & O II \\
\hline $\mathbf{B}_{20}$ & $=$ & $\left(\frac{3}{4}+x_{4}\right) \mathbf{a}_{1}-x_{4} \mathbf{a}_{2}-x_{4} \mathbf{a}_{3}$ & $=$ & $-x_{4} a \hat{\mathbf{x}}+\frac{3}{8} a \hat{\mathbf{y}}+\frac{3}{8} a \hat{\mathbf{z}}$ & $(48 f)$ & O II \\
\hline $\mathbf{B}_{21}$ & $=$ & $-x_{4} \mathbf{a}_{1}-x_{4} \mathbf{a}_{2}+\left(\frac{3}{4}+x_{4}\right) \mathbf{a}_{3}$ & $=$ & $\frac{3}{8} a \hat{\mathbf{x}}+\frac{3}{8} a \hat{\mathbf{y}}-x_{4} a \hat{\mathbf{z}}$ & $(48 f)$ & O II \\
\hline $\mathbf{B}_{22}$ & $=$ & $\left(\frac{3}{4}+x_{4}\right) \mathbf{a}_{1}+\left(\frac{3}{4}+x_{4}\right) \mathbf{a}_{2}-x_{4} \mathbf{a}_{3}$ & $=$ & $\frac{3}{8} a \hat{\mathbf{x}}+\frac{3}{8} a \hat{\mathbf{y}}+\left(\frac{3}{4}+x_{4}\right) a \hat{\mathbf{z}}$ & $(48 f)$ & O II \\
\hline
\end{tabular}

\section{References:}

- H. Sagayama, D. Uematsu, T. Arima, K. Sugimoto, J. J. Ishikawa, E. O’Farrell, and S. Nakatsuji, Determination of long-range all-in-all-out ordering of $\mathrm{Ir}^{4+}$ moments in a pyrochlore iridate $\mathrm{Eu}_{2} \mathrm{Ir}_{2} \mathrm{O}_{7}$ by resonant $x$-ray diffraction, Phys. Rev. B 87, 100403 (2013), doi:10.1103/PhysRevB.87.100403.

\section{Found in:}

- S. H. Chun, B. Yuan, D. Casa, J. Kim, C.-Y. Kim, Z. Tian, Y. Qiu, S. Nakatsuji, and Y.-J. Kim, Magnetic Excitations across the Metal-Insulator Transition in the Pyrochlore Iridate $\mathrm{Eu}_{2} \mathrm{Ir}_{2} \mathrm{O}_{7}$, Phys. Rev. Lett. 120, 177203 (2018), doi:10.1103/PhysRevLett.120.177203.

\section{Geometry files:}

- CIF: pp. 977

- POSCAR: pp. 978 


\section{Spinel $\left(\mathrm{Co}_{3} \mathrm{O}_{4}, D 7_{2}\right)$ Structure:}

\section{A3B4_cF56_227_ad_e}
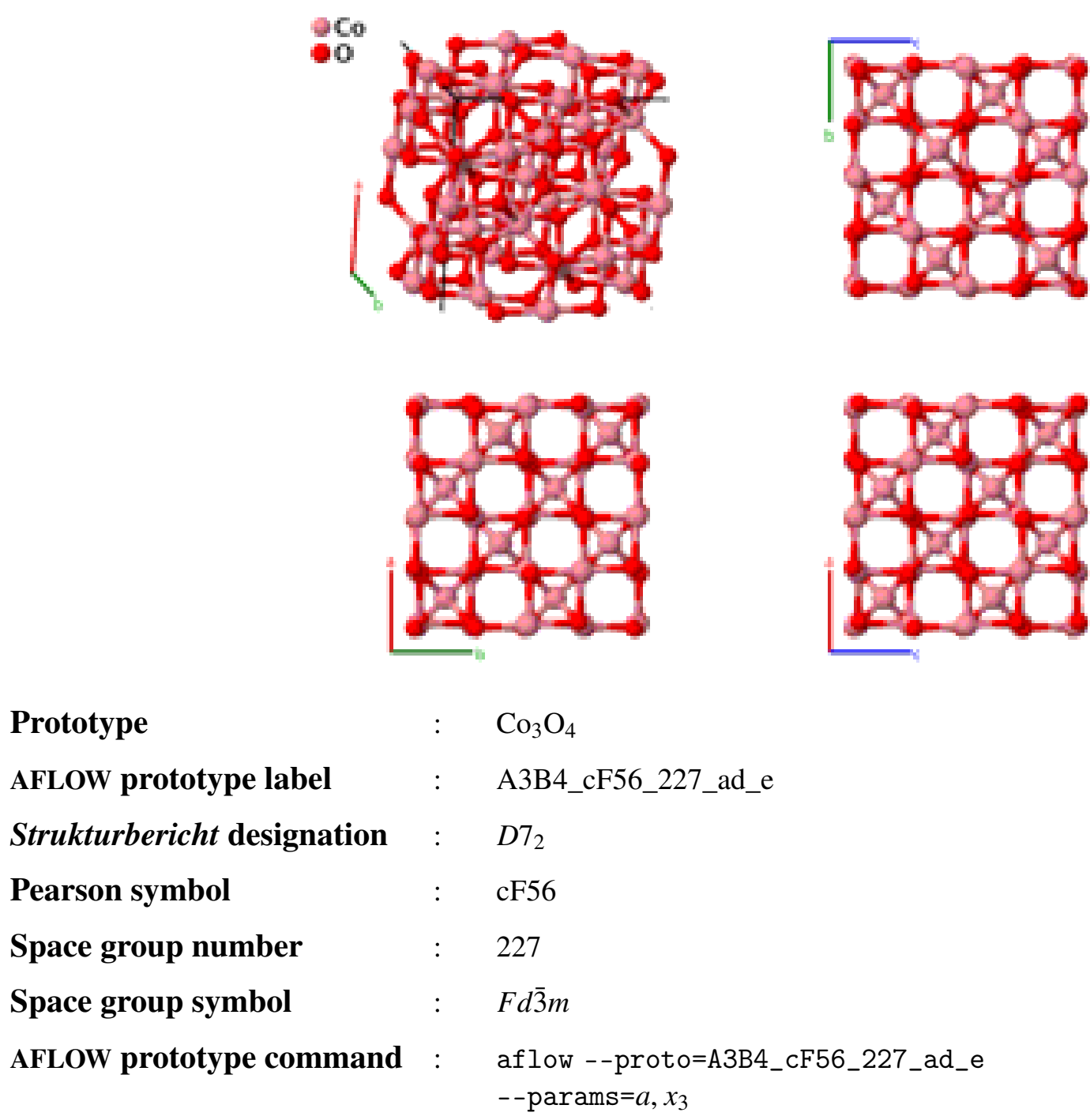

\section{Other compounds with this structure:}

- $\mathrm{NiCo}_{2} \mathrm{O}_{4}, \mathrm{Co}_{3} \mathrm{~S}_{4}, \mathrm{NiCo}_{2} \mathrm{~S}_{4}, \mathrm{FeNi}_{2} \mathrm{~S}_{4}$

- The $D 7_{2}$ and $H 1_{1}$ Spinel structures are for all intents and purposes identical. We could use $D 7_{3}$ for the binary spinels and $H 1_{1}$ for the ternaries, but historically this has not been the case. We dual-list this structure only to keep the historical record intact. (Hahn, 1955) has an extensive list of ternary spinels and inverse spinels.

Face-centered Cubic primitive vectors:

$$
\begin{aligned}
& \mathbf{a}_{1}=\frac{1}{2} a \hat{\mathbf{y}}+\frac{1}{2} a \hat{\mathbf{z}} \\
& \mathbf{a}_{2}=\frac{1}{2} a \hat{\mathbf{x}}+\frac{1}{2} a \hat{\mathbf{z}} \\
& \mathbf{a}_{3}=\frac{1}{2} a \hat{\mathbf{x}}+\frac{1}{2} a \hat{\mathbf{y}}
\end{aligned}
$$

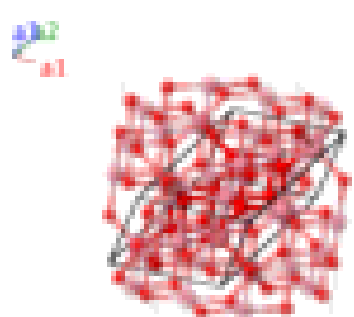




\section{Lattice Coordinates}

$\begin{array}{lcc}\mathbf{B}_{1}= & \frac{1}{8} \mathbf{a}_{1}+\frac{1}{8} \mathbf{a}_{2}+\frac{1}{8} \mathbf{a}_{3} \\ \mathbf{B}_{2}= & \frac{7}{8} \mathbf{a}_{1}+\frac{7}{8} \mathbf{a}_{2}+\frac{7}{8} \mathbf{a}_{3} \\ \mathbf{B}_{3}= & \frac{1}{2} \mathbf{a}_{1}+\frac{1}{2} \mathbf{a}_{2}+\frac{1}{2} \mathbf{a}_{3} \\ \mathbf{B}_{4}= & \frac{1}{2} \mathbf{a}_{1}+\frac{1}{2} \mathbf{a}_{2} \\ \mathbf{B}_{5}= & \frac{1}{2} \mathbf{a}_{1}+\frac{1}{2} \mathbf{a}_{3} \\ \mathbf{B}_{6}= & \frac{1}{2} \mathbf{a}_{2}+\frac{1}{2} \mathbf{a}_{3} \\ \mathbf{B}_{7}= & x_{3} \mathbf{a}_{1}+x_{3} \mathbf{a}_{2}+x_{3} \mathbf{a}_{3} \\ \mathbf{B}_{8}= & x_{3} \mathbf{a}_{1}+x_{3} \mathbf{a}_{2}+\left(\frac{1}{2}-3 x_{3}\right) \mathbf{a}_{3} \\ \mathbf{B}_{9}= & x_{3} \mathbf{a}_{1}+\left(\frac{1}{2}-3 x_{3}\right) \mathbf{a}_{2}+x_{3} \mathbf{a}_{3} \\ \mathbf{B}_{10}= & \left(\frac{1}{2}-3 x_{3}\right) \mathbf{a}_{1}+x_{3} \mathbf{a}_{2}+x_{3} \mathbf{a}_{3} \\ \mathbf{B}_{11}= & -x_{3} \mathbf{a}_{1}-x_{3} \mathbf{a}_{2}+\left(\frac{1}{2}+3 x_{3}\right) \mathbf{a}_{3} \\ \mathbf{B}_{12}= & -x_{3} \mathbf{a}_{1}-x_{3} \mathbf{a}_{2}-x_{3} \mathbf{a}_{3} \\ \mathbf{B}_{13}= & -x_{3} \mathbf{a}_{1}+\left(\frac{1}{2}+3 x_{3}\right) \mathbf{a}_{2}-x_{3} \mathbf{a}_{3}= \\ \mathbf{B}_{14}= & \left(\frac{1}{2}+3 x_{3}\right) \mathbf{a}_{1}-x_{3} \mathbf{a}_{2}-x_{3} \mathbf{a}_{3}=\end{array}$

Cartesian Coordinates

$=$

$=$

$=$

$=$

$=$

$=$

$=$

$=$

$=$

$=$

$=$

$=$

$=$

$$
=
$$

$=$

$$
\frac{1}{8} a \hat{\mathbf{x}}+\frac{1}{8} a \hat{\mathbf{y}}+\frac{1}{8} a \hat{\mathbf{z}}
$$$$
\frac{7}{8} a \hat{\mathbf{x}}+\frac{7}{8} a \hat{\mathbf{y}}+\frac{7}{8} a \hat{\mathbf{z}}
$$$$
\frac{1}{2} a \hat{\mathbf{x}}+\frac{1}{2} a \hat{\mathbf{y}}+\frac{1}{2} a \hat{\mathbf{z}}
$$$$
\frac{1}{4} a \hat{\mathbf{x}}+\frac{1}{4} a \hat{\mathbf{y}}+\frac{1}{2} a \hat{\mathbf{z}}
$$$$
\frac{1}{4} a \hat{\mathbf{x}}+\frac{1}{2} a \hat{\mathbf{y}}+\frac{1}{4} a \hat{\mathbf{z}}
$$$$
\frac{1}{2} a \hat{\mathbf{x}}+\frac{1}{4} a \hat{\mathbf{y}}+\frac{1}{4} a \hat{\mathbf{z}}
$$$$
x_{3} a \hat{\mathbf{x}}+x_{3} a \hat{\mathbf{y}}+x_{3} a \hat{\mathbf{z}}
$$$$
\left(\frac{1}{4}-x_{3}\right) a \hat{\mathbf{x}}+\left(\frac{1}{4}-x_{3}\right) a \hat{\mathbf{y}}+x_{3} a \hat{\mathbf{z}}
$$$$
\left(\frac{1}{4}-x_{3}\right) a \hat{\mathbf{x}}+x_{3} a \hat{\mathbf{y}}+\left(\frac{1}{4}-x_{3}\right) a \hat{\mathbf{z}}
$$$$
x_{3} a \hat{\mathbf{x}}+\left(\frac{1}{4}-x_{3}\right) a \hat{\mathbf{y}}+\left(\frac{1}{4}-x_{3}\right) a \hat{\mathbf{z}}
$$$$
\left(\frac{1}{4}+x_{3}\right) a \hat{\mathbf{x}}+\left(\frac{1}{4}+x_{3}\right) a \hat{\mathbf{y}}-x_{3} a \hat{\mathbf{z}}
$$$$
-x_{3} a \hat{\mathbf{x}}-x_{3} a \hat{\mathbf{y}}-x_{3} a \hat{\mathbf{z}}
$$$$
\left(\frac{1}{4}+x_{3}\right) a \hat{\mathbf{x}}-x_{3} a \hat{\mathbf{y}}+\left(\frac{1}{4}+x_{3}\right) a \hat{\mathbf{z}}
$$$$
-x_{3} a \hat{\mathbf{x}}+\left(\frac{1}{4}+x_{3}\right) a \hat{\mathbf{y}}+\left(\frac{1}{4}+x_{3}\right) a \hat{\mathbf{z}}
$$

Wyckoff Position

(16d)
Atom Type

Co I

Co I

Co II

Co II

Co II

Co II

$\mathrm{O}$

$\mathrm{O}$

$\mathrm{O}$

$\mathrm{O}$

$\mathrm{O}$

$\mathrm{O}$

$\mathrm{O}$

$\mathrm{O}$

\section{References:}

- O. Knop, K. I. G. Reid, Sutarno, and Y. Nakagawa, Chalkogenides of the transition elements. VI. X-Ray, neutron, and magnetic investigation of the spinels $\mathrm{Co}_{3} \mathrm{O}_{4}, \mathrm{NiCo}_{2} \mathrm{O}_{4}, \mathrm{Co}_{3} \mathrm{~S}_{4}$, and $\mathrm{NiCo}_{2} \mathrm{~S}_{4}$, Can. J. Chem. 46, 3463-3476 (1968), doi:10.1139/v68-576.

- H. Hahn, G. Frank, W. Klingler, A. D. Störger, and G. Störger, Chalkogenide. VI. Über Ternäre Chalkogenide des Aluminiums, Galliums und Indiums mit Zink, Cadmium und Quecksilber, Z. Anorg. Allg. Chem. 279, 241-270 (1955), doi:10.1002/zaac.19552790502.

\section{Geometry files:}

- CIF: pp. 978

- POSCAR: pp. 980 


\section{$\mathrm{CuCrCl}_{5}\left[\mathrm{NH}_{3}\right]_{6}$ Structure: A5BCD6_cF416_228_eg_c_b_h}

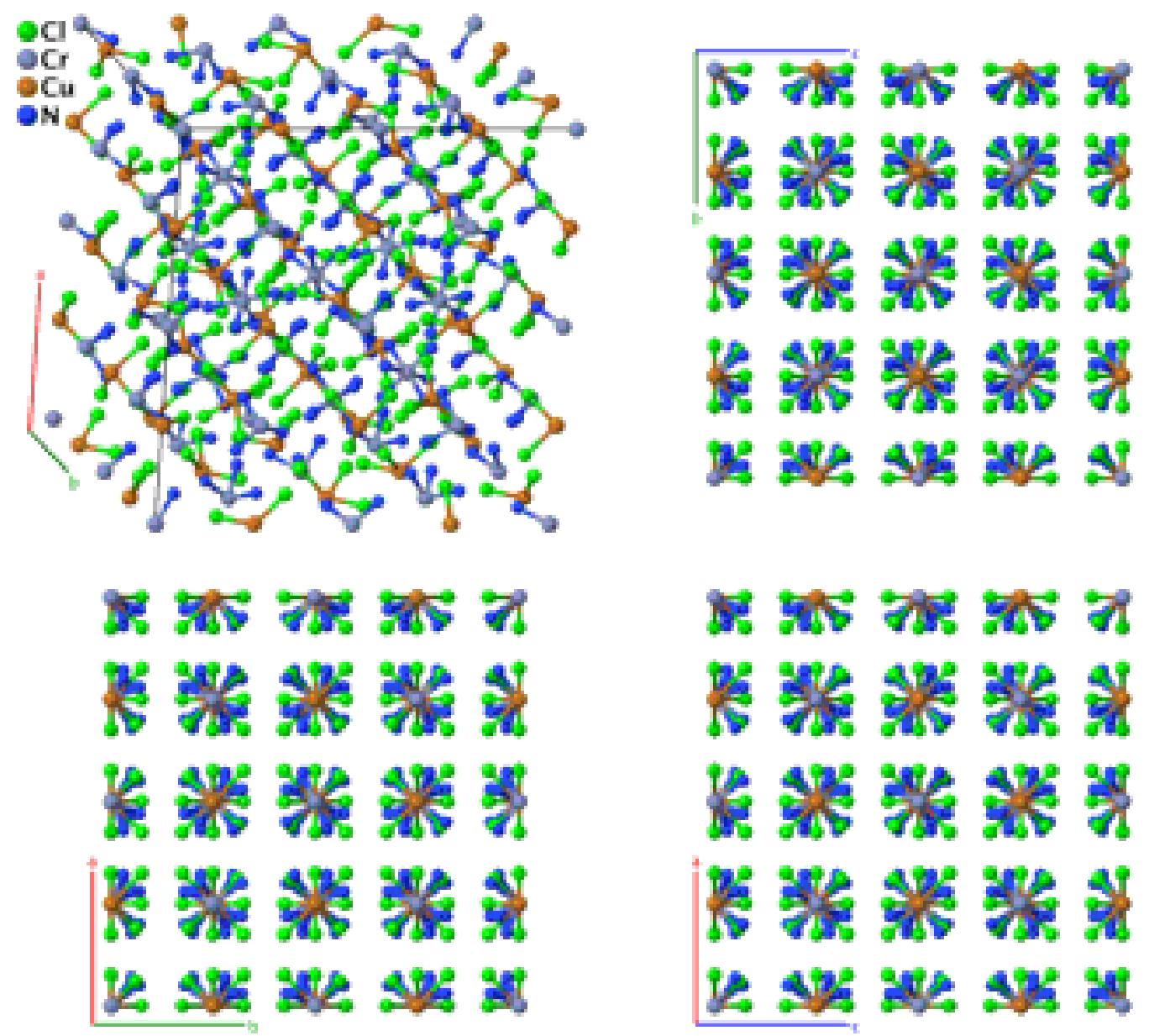

Prototype $\quad: \quad \mathrm{CuCrCl}_{5}\left[\mathrm{NH}_{3}\right]_{6}$

AFLOW prototype label $\quad$ : A5BCD6_cF416_228_eg_c_b_h

Strukturbericht designation : None

Pearson symbol $\quad: \quad c F 416$

Space group number $\quad: \quad 228$

Space group symbol $\quad: \quad F d \overline{3} c$

AFLOW prototype command : aflow --proto=A5BCD6_cF416_228_eg_c_b_h

- - params $=a, x_{3}, y_{4}, x_{5}, y_{5}, z_{5}$

- The $\mathrm{N}$ atoms correspond to $\mathrm{NH}_{3}$ units centered on the (192h) Wyckoff positions. 


$$
\begin{aligned}
& \mathbf{a}_{1}=\frac{1}{2} a \hat{\mathbf{y}}+\frac{1}{2} a \hat{\mathbf{z}} \\
& \mathbf{a}_{2}=\frac{1}{2} a \hat{\mathbf{x}}+\frac{1}{2} a \hat{\mathbf{z}} \\
& \mathbf{a}_{3}=\frac{1}{2} a \hat{\mathbf{x}}+\frac{1}{2} a \hat{\mathbf{y}}
\end{aligned}
$$

\begin{tabular}{|c|c|c|c|c|}
\hline $\mathbf{B}_{1}$ & $=$ & $\frac{1}{4} \mathbf{a}_{1}+\frac{1}{4} \mathbf{a}_{2}+\frac{1}{4} \mathbf{a}_{3}$ & $=$ & $\frac{1}{4} a \hat{\mathbf{x}}+\frac{1}{4} a \hat{\mathbf{y}}+\frac{1}{4} a \hat{\mathbf{z}}$ \\
\hline $\mathbf{B}_{2}$ & $=$ & $\frac{1}{4} \mathbf{a}_{1}+\frac{1}{4} \mathbf{a}_{2}+\frac{3}{4} \mathbf{a}_{3}$ & $=$ & $\frac{1}{2} a \hat{\mathbf{x}}+\frac{1}{2} a \hat{\mathbf{y}}+\frac{1}{4} a \hat{\mathbf{z}}$ \\
\hline $\mathbf{B}_{3}$ & $=$ & $\frac{1}{4} \mathbf{a}_{1}+\frac{3}{4} \mathbf{a}_{2}+\frac{1}{4} \mathbf{a}_{3}$ & $=$ & $\frac{1}{2} a \hat{\mathbf{x}}+\frac{1}{4} a \hat{\mathbf{y}}+\frac{1}{2} a \hat{\mathbf{z}}$ \\
\hline $\mathbf{B}_{4}$ & $=$ & $\frac{3}{4} \mathbf{a}_{1}+\frac{1}{4} \mathbf{a}_{2}+\frac{1}{4} \mathbf{a}_{3}$ & $=$ & $\frac{1}{4} a \hat{\mathbf{x}}+\frac{1}{2} a \hat{\mathbf{y}}+\frac{1}{2} a \hat{\mathbf{z}}$ \\
\hline $\mathbf{B}_{5}$ & $=$ & $\frac{3}{4} \mathbf{a}_{1}+\frac{3}{4} \mathbf{a}_{2}+\frac{3}{4} \mathbf{a}_{3}$ & $=$ & $\frac{3}{4} a \hat{\mathbf{x}}+\frac{3}{4} a \hat{\mathbf{y}}+\frac{3}{4} a \hat{\mathbf{z}}$ \\
\hline $\mathbf{B}_{6}$ & $=$ & $\frac{3}{4} \mathbf{a}_{1}+\frac{3}{4} \mathbf{a}_{2}+\frac{1}{4} \mathbf{a}_{3}$ & $=$ & $\frac{1}{2} a \hat{\mathbf{x}}+\frac{1}{2} a \hat{\mathbf{y}}+\frac{3}{4} a \hat{\mathbf{z}}$ \\
\hline $\mathbf{B}_{7}$ & $=$ & $\frac{3}{4} \mathbf{a}_{1}+\frac{1}{4} \mathbf{a}_{2}+\frac{3}{4} \mathbf{a}_{3}$ & $=$ & $\frac{1}{2} a \hat{\mathbf{x}}+\frac{3}{4} a \hat{\mathbf{y}}+\frac{1}{2} a \hat{\mathbf{z}}$ \\
\hline $\mathbf{B}_{8}$ & $=$ & $\frac{1}{4} \mathbf{a}_{1}+\frac{3}{4} \mathbf{a}_{2}+\frac{3}{4} \mathbf{a}_{3}$ & $=$ & $\frac{3}{4} a \hat{\mathbf{x}}+\frac{1}{2} a \hat{\mathbf{y}}+\frac{1}{2} a \hat{\mathbf{z}}$ \\
\hline $\mathbf{B}_{9}$ & $=$ & $0 \mathbf{a}_{1}+0 \mathbf{a}_{2}+0 \mathbf{a}_{3}$ & $=$ & $0 \hat{\mathbf{x}}+0 \hat{\mathbf{y}}+0 \hat{\mathbf{z}}$ \\
\hline $\mathbf{B}_{10}$ & $=$ & $\frac{1}{2} \mathbf{a}_{3}$ & $=$ & $\frac{1}{4} a \hat{\mathbf{x}}+\frac{1}{4} a \hat{\mathbf{y}}$ \\
\hline $\mathbf{B}_{11}$ & $=$ & $\frac{1}{2} \mathbf{a}_{2}$ & $=$ & $\frac{1}{4} a \hat{\mathbf{x}}+\frac{1}{4} a \hat{\mathbf{z}}$ \\
\hline $\mathbf{B}_{12}$ & $=$ & $\frac{1}{2} \mathbf{a}_{1}$ & $=$ & $\frac{1}{4} a \hat{\mathbf{y}}+\frac{1}{4} a \hat{\mathbf{z}}$ \\
\hline $\mathbf{B}_{13}$ & $=$ & $\frac{1}{2} \mathbf{a}_{1}+\frac{1}{2} \mathbf{a}_{2}$ & $=$ & $\frac{1}{4} a \hat{\mathbf{x}}+\frac{1}{4} a \hat{\mathbf{y}}+\frac{1}{2} a \hat{\mathbf{z}}$ \\
\hline $\mathbf{B}_{14}$ & $=$ & $\frac{1}{2} \mathbf{a}_{1}+\frac{1}{2} \mathbf{a}_{2}+\frac{1}{2} \mathbf{a}_{3}$ & $=$ & $\frac{1}{2} a \hat{\mathbf{x}}+\frac{1}{2} a \hat{\mathbf{y}}+\frac{1}{2} a \hat{\mathbf{z}}$ \\
\hline $\mathbf{B}_{15}$ & $=$ & $\frac{1}{2} \mathbf{a}_{1}+\frac{1}{2} \mathbf{a}_{3}$ & $=$ & $\frac{1}{4} a \hat{\mathbf{x}}+\frac{1}{2} a \hat{\mathbf{y}}+\frac{1}{4} a \hat{\mathbf{z}}$ \\
\hline $\mathbf{B}_{16}$ & $=$ & $\frac{1}{2} \mathbf{a}_{2}+\frac{1}{2} \mathbf{a}_{3}$ & $=$ & $\frac{1}{2} a \hat{\mathbf{x}}+\frac{1}{4} a \hat{\mathbf{y}}+\frac{1}{4} a \hat{\mathbf{z}}$ \\
\hline $\mathbf{B}_{17}$ & $=$ & $x_{3} \mathbf{a}_{1}+x_{3} \mathbf{a}_{2}+x_{3} \mathbf{a}_{3}$ & $=$ & $x_{3} a \hat{\mathbf{x}}+x_{3} a \hat{\mathbf{y}}+x_{3} a \hat{\mathbf{z}}$ \\
\hline $\mathbf{B}_{18}$ & $=$ & $x_{3} \mathbf{a}_{1}+x_{3} \mathbf{a}_{2}+\left(\frac{1}{2}-3 x_{3}\right) \mathbf{a}_{3}$ & $=$ & $\left(\frac{1}{4}-x_{3}\right) a \hat{\mathbf{x}}+\left(\frac{1}{4}-x_{3}\right) a \hat{\mathbf{y}}+x_{3} a \hat{\mathbf{z}}$ \\
\hline $\mathbf{B}_{19}$ & $=$ & $x_{3} \mathbf{a}_{1}+\left(\frac{1}{2}-3 x_{3}\right) \mathbf{a}_{2}+x_{3} \mathbf{a}_{3}$ & $=$ & $\left(\frac{1}{4}-x_{3}\right) a \hat{\mathbf{x}}+x_{3} a \hat{\mathbf{y}}+\left(\frac{1}{4}-x_{3}\right) a \hat{\mathbf{z}}$ \\
\hline $\mathbf{B}_{20}$ & $=$ & $\left(\frac{1}{2}-3 x_{3}\right) \mathbf{a}_{1}+x_{3} \mathbf{a}_{2}+x_{3} \mathbf{a}_{3}$ & $=$ & $x_{3} a \hat{\mathbf{x}}+\left(\frac{1}{4}-x_{3}\right) a \hat{\mathbf{y}}+\left(\frac{1}{4}-x_{3}\right) a \hat{\mathbf{z}}$ \\
\hline $\mathbf{B}_{21}$ & $=$ & $\left(\frac{1}{2}-x_{3}\right) \mathbf{a}_{1}+\left(\frac{1}{2}-x_{3}\right) \mathbf{a}_{2}+3 x_{3} \mathbf{a}_{3}$ & $=$ & $\begin{array}{c}\left(\frac{1}{4}+x_{3}\right) a \hat{\mathbf{x}}+\left(\frac{1}{4}+x_{3}\right) a \hat{\mathbf{y}}+ \\
\left(\frac{1}{2}-x_{3}\right) a \hat{\mathbf{z}}\end{array}$ \\
\hline $\mathbf{B}_{22}$ & $=$ & $\begin{array}{c}\left(\frac{1}{2}-x_{3}\right) \mathbf{a}_{1}+\left(\frac{1}{2}-x_{3}\right) \mathbf{a}_{2}+ \\
\left(\frac{1}{2}-x_{3}\right) \mathbf{a}_{3}\end{array}$ & $=$ & $\begin{array}{c}\left(\frac{1}{2}-x_{3}\right) a \hat{\mathbf{x}}+\left(\frac{1}{2}-x_{3}\right) a \hat{\mathbf{y}}+ \\
\left(\frac{1}{2}-x_{3}\right) a \hat{\mathbf{z}}\end{array}$ \\
\hline $\mathbf{B}_{23}$ & 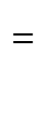 & $\left(\frac{1}{2}-x_{3}\right) \mathbf{a}_{1}+3 x_{3} \mathbf{a}_{2}+\left(\frac{1}{2}-x_{3}\right) \mathbf{a}_{3}$ & $=$ & $\begin{array}{c}\left(\frac{1}{4}+x_{3}\right) a \hat{\mathbf{x}}+\left(\frac{1}{2}-x_{3}\right) a \hat{\mathbf{y}}+ \\
\left(\frac{1}{4}+x_{3}\right) a \hat{\mathbf{z}}\end{array}$ \\
\hline
\end{tabular}

\section{Basis vectors:}

Lattice Coordinates
Wyckoff Position Atom Type

$\mathrm{Cu}$

$\mathrm{Cu}$

$\mathrm{Cu}$

$\mathrm{Cu}$

$\mathrm{Cu}$

$\mathrm{Cu}$

$\mathrm{Cu}$

$\mathrm{Cu}$

$\mathrm{Cr}$

$\mathrm{Cr}$

$\mathrm{Cr}$

$\mathrm{Cr}$

Cr

$\mathrm{Cr}$

Cr

$\mathrm{Cr}$

Cl I

$\mathrm{Cl}$ I

Cl I

Cl I

Cl I

Cl I

(64e)

Cl I 


\begin{tabular}{|c|c|c|c|c|c|c|}
\hline $\mathbf{B}_{24}$ & $=$ & $3 x_{3} \mathbf{a}_{1}+\left(\frac{1}{2}-x_{3}\right) \mathbf{a}_{2}+\left(\frac{1}{2}-x_{3}\right) \mathbf{a}_{3}$ & $=$ & $\begin{array}{c}\left(\frac{1}{2}-x_{3}\right) a \hat{\mathbf{x}}+\left(\frac{1}{4}+x_{3}\right) a \hat{\mathbf{y}}+ \\
\left(\frac{1}{4}+x_{3}\right) a \hat{\mathbf{z}}\end{array}$ & $(64 e)$ & $\mathrm{Cl} \mathrm{I}$ \\
\hline $\mathbf{B}_{25}$ & $=$ & $-x_{3} \mathbf{a}_{1}-x_{3} \mathbf{a}_{2}-x_{3} \mathbf{a}_{3}$ & $=$ & $-x_{3} a \hat{\mathbf{x}}-x_{3} a \hat{\mathbf{y}}-x_{3} a \hat{\mathbf{z}}$ & $(64 e)$ & $\mathrm{Cl} \mathrm{I}$ \\
\hline $\mathbf{B}_{26}$ & $=$ & $-x_{3} \mathbf{a}_{1}-x_{3} \mathbf{a}_{2}+\left(\frac{1}{2}+3 x_{3}\right) \mathbf{a}_{3}$ & $=$ & $\left(\frac{1}{4}+x_{3}\right) a \hat{\mathbf{x}}+\left(\frac{1}{4}+x_{3}\right) a \hat{\mathbf{y}}-x_{3} a \hat{\mathbf{z}}$ & $(64 e)$ & $\mathrm{Cl} \mathrm{I}$ \\
\hline $\mathbf{B}_{27}$ & $=$ & $-x_{3} \mathbf{a}_{1}+\left(\frac{1}{2}+3 x_{3}\right) \mathbf{a}_{2}-x_{3} \mathbf{a}_{3}$ & $=$ & $\left(\frac{1}{4}+x_{3}\right) a \hat{\mathbf{x}}-x_{3} a \hat{\mathbf{y}}+\left(\frac{1}{4}+x_{3}\right) a \hat{\mathbf{z}}$ & $(64 e)$ & $\mathrm{Cl} \mathrm{I}$ \\
\hline $\mathbf{B}_{28}$ & $=$ & $\left(\frac{1}{2}+3 x_{3}\right) \mathbf{a}_{1}-x_{3} \mathbf{a}_{2}-x_{3} \mathbf{a}_{3}$ & $=$ & $-x_{3} a \hat{\mathbf{x}}+\left(\frac{1}{4}+x_{3}\right) a \hat{\mathbf{y}}+\left(\frac{1}{4}+x_{3}\right) a \hat{\mathbf{z}}$ & $(64 e)$ & $\mathrm{Cl} \mathrm{I}$ \\
\hline $\mathbf{B}_{29}$ & $=$ & $\left(\frac{1}{2}+x_{3}\right) \mathbf{a}_{1}+\left(\frac{1}{2}+x_{3}\right) \mathbf{a}_{2}-3 x_{3} \mathbf{a}_{3}$ & $=$ & $\begin{array}{c}\left(\frac{1}{4}-x_{3}\right) a \hat{\mathbf{x}}+\left(\frac{1}{4}-x_{3}\right) a \hat{\mathbf{y}}+ \\
\left(\frac{1}{2}+x_{3}\right) a \hat{\mathbf{z}}\end{array}$ & $(64 e)$ & $\mathrm{Cl} \mathrm{I}$ \\
\hline $\mathbf{B}_{30}$ & $=$ & $\begin{array}{c}\left(\frac{1}{2}+x_{3}\right) \mathbf{a}_{1}+\left(\frac{1}{2}+x_{3}\right) \mathbf{a}_{2}+ \\
\left(\frac{1}{2}+x_{3}\right) \mathbf{a}_{3}\end{array}$ & $=$ & $\begin{array}{c}\left(\frac{1}{2}+x_{3}\right) a \hat{\mathbf{x}}+\left(\frac{1}{2}+x_{3}\right) a \hat{\mathbf{y}}+ \\
\left(\frac{1}{2}+x_{3}\right) a \hat{\mathbf{z}}\end{array}$ & $(64 e)$ & $\mathrm{Cl} \mathrm{I}$ \\
\hline $\mathbf{B}_{31}$ & $=$ & $\left(\frac{1}{2}+x_{3}\right) \mathbf{a}_{1}-3 x_{3} \mathbf{a}_{2}+\left(\frac{1}{2}+x_{3}\right) \mathbf{a}_{3}$ & $=$ & $\begin{array}{c}\left(\frac{1}{4}-x_{3}\right) a \hat{\mathbf{x}}+\left(\frac{1}{2}+x_{3}\right) a \hat{\mathbf{y}}+ \\
\left(\frac{1}{4}-x_{3}\right) a \hat{\mathbf{z}}\end{array}$ & $(64 e)$ & $\mathrm{Cl} \mathrm{I}$ \\
\hline $\mathbf{B}_{32}$ & $=$ & $-3 x_{3} \mathbf{a}_{1}+\left(\frac{1}{2}+x_{3}\right) \mathbf{a}_{2}+\left(\frac{1}{2}+x_{3}\right) \mathbf{a}_{3}$ & $=$ & $\begin{array}{c}\left(\frac{1}{2}+x_{3}\right) a \hat{\mathbf{x}}+\left(\frac{1}{4}-x_{3}\right) a \hat{\mathbf{y}}+ \\
\left(\frac{1}{4}-x_{3}\right) a \hat{\mathbf{z}}\end{array}$ & $(64 e)$ & $\mathrm{Cl} \mathrm{I}$ \\
\hline $\mathbf{B}_{33}$ & $=$ & $\left(\frac{1}{2}-2 y_{4}\right) \mathbf{a}_{2}+2 y_{4} \mathbf{a}_{3}$ & $=$ & $\frac{1}{4} a \hat{\mathbf{x}}+y_{4} a \hat{\mathbf{y}}+\left(\frac{1}{4}-y_{4}\right) a \hat{\mathbf{z}}$ & $(96 g)$ & $\mathrm{Cl}$ II \\
\hline $\mathbf{B}_{34}$ & $=$ & $\left(\frac{1}{4}-2 y_{4}\right) \mathbf{a}_{1}+\frac{3}{4} \mathbf{a}_{2}+\frac{1}{4} \mathbf{a}_{3}$ & $=$ & $\frac{1}{2} a \hat{\mathbf{x}}+\left(\frac{1}{4}-y_{4}\right) a \hat{\mathbf{y}}+\left(\frac{1}{2}-y_{4}\right) a \hat{\mathbf{z}}$ & $(96 g)$ & $\mathrm{Cl} \mathrm{II}$ \\
\hline $\mathbf{B}_{35}$ & $=$ & $\left(\frac{1}{4}+2 y_{4}\right) \mathbf{a}_{1}+\frac{1}{4} \mathbf{a}_{2}+\frac{3}{4} \mathbf{a}_{3}$ & $=$ & $\frac{1}{2} a \hat{\mathbf{x}}+\left(\frac{1}{2}+y_{4}\right) a \hat{\mathbf{y}}+\left(\frac{1}{4}+y_{4}\right) a \hat{\mathbf{z}}$ & $(96 g)$ & $\mathrm{Cl}$ II \\
\hline $\mathbf{B}_{36}$ & $=$ & $\frac{1}{4} \mathbf{a}_{1}+\left(\frac{1}{4}+2 y_{4}\right) \mathbf{a}_{2}+\left(\frac{1}{4}-2 y_{4}\right) \mathbf{a}_{3}$ & $=$ & $\frac{1}{4} a \hat{\mathbf{x}}+\left(\frac{1}{4}-y_{4}\right) a \hat{\mathbf{y}}+\left(\frac{1}{4}+y_{4}\right) a \hat{\mathbf{z}}$ & $(96 g)$ & $\mathrm{Cl}$ II \\
\hline $\mathbf{B}_{37}$ & $=$ & $\left(\frac{1}{4}+2 y_{4}\right) \mathbf{a}_{1}+\frac{3}{4} \mathbf{a}_{2}+\left(\frac{1}{4}-2 y_{4}\right) \mathbf{a}_{3}$ & $=$ & $\left(\frac{1}{2}-y_{4}\right) a \hat{\mathbf{x}}+\frac{1}{4} a \hat{\mathbf{y}}+\left(\frac{1}{2}+y_{4}\right) a \hat{\mathbf{z}}$ & $(96 g)$ & $\mathrm{Cl}$ II \\
\hline $\mathbf{B}_{38}$ & $=$ & $\frac{1}{4} \mathbf{a}_{1}+\left(\frac{1}{4}-2 y_{4}\right) \mathbf{a}_{2}+\frac{3}{4} \mathbf{a}_{3}$ & $=$ & $\left(\frac{1}{2}-y_{4}\right) a \hat{\mathbf{x}}+\frac{1}{2} a \hat{\mathbf{y}}+\left(\frac{1}{4}-y_{4}\right) a \hat{\mathbf{z}}$ & $(96 g)$ & $\mathrm{Cl} \mathrm{II}$ \\
\hline $\mathbf{B}_{39}$ & $=$ & $\frac{3}{4} \mathbf{a}_{1}+\left(\frac{1}{4}+2 y_{4}\right) \mathbf{a}_{2}+\frac{1}{4} \mathbf{a}_{3}$ & $=$ & $\left(\frac{1}{4}+y_{4}\right) a \hat{\mathbf{x}}+\frac{1}{2} a \hat{\mathbf{y}}+\left(\frac{1}{2}+y_{4}\right) a \hat{\mathbf{z}}$ & $(96 g)$ & $\mathrm{Cl} \mathrm{II}$ \\
\hline $\mathbf{B}_{40}$ & $=$ & $\left(\frac{1}{4}-2 y_{4}\right) \mathbf{a}_{1}+\frac{1}{4} \mathbf{a}_{2}+\left(\frac{1}{4}+2 y_{4}\right) \mathbf{a}_{3}$ & $=$ & $\left(\frac{1}{4}+y_{4}\right) a \hat{\mathbf{x}}+\frac{1}{4} a \hat{\mathbf{y}}+\left(\frac{1}{4}-y_{4}\right) a \hat{\mathbf{z}}$ & $(96 g)$ & $\mathrm{Cl}$ II \\
\hline $\mathbf{B}_{41}$ & $=$ & $\left(\frac{1}{4}-2 y_{4}\right) \mathbf{a}_{1}+\left(\frac{1}{4}+2 y_{4}\right) \mathbf{a}_{2}+\frac{3}{4} \mathbf{a}_{3}$ & $=$ & $\left(\frac{1}{2}+y_{4}\right) a \hat{\mathbf{x}}+\left(\frac{1}{2}-y_{4}\right) a \hat{\mathbf{y}}+\frac{1}{4} a \hat{\mathbf{z}}$ & $(96 g)$ & $\mathrm{Cl}$ II \\
\hline $\mathbf{B}_{42}$ & $=$ & $\frac{3}{4} \mathbf{a}_{1}+\frac{1}{4} \mathbf{a}_{2}+\left(\frac{1}{4}-2 y_{4}\right) \mathbf{a}_{3}$ & $=$ & $\left(\frac{1}{4}-y_{4}\right) a \hat{\mathbf{x}}+\left(\frac{1}{2}-y_{4}\right) a \hat{\mathbf{y}}+\frac{1}{2} a \hat{\mathbf{z}}$ & $(96 g)$ & $\mathrm{Cl} \mathrm{II}$ \\
\hline $\mathbf{B}_{43}$ & $=$ & $\frac{1}{4} \mathbf{a}_{1}+\frac{3}{4} \mathbf{a}_{2}+\left(\frac{1}{4}+2 y_{4}\right) \mathbf{a}_{3}$ & $=$ & $\left(\frac{1}{2}+y_{4}\right) a \hat{\mathbf{x}}+\left(\frac{1}{4}+y_{4}\right) a \hat{\mathbf{y}}+\frac{1}{2} a \hat{\mathbf{z}}$ & $(96 g)$ & $\mathrm{Cl}$ II \\
\hline $\mathbf{B}_{44}$ & $=$ & $\left(\frac{1}{4}+2 y_{4}\right) \mathbf{a}_{1}+\left(\frac{1}{4}-2 y_{4}\right) \mathbf{a}_{2}+\frac{1}{4} \mathbf{a}_{3}$ & $=$ & $\left(\frac{1}{4}-y_{4}\right) a \hat{\mathbf{x}}+\left(\frac{1}{4}+y_{4}\right) a \hat{\mathbf{y}}+\frac{1}{4} a \hat{\mathbf{z}}$ & $(96 g)$ & $\mathrm{Cl}$ II \\
\hline $\mathbf{B}_{45}$ & $=$ & $\frac{1}{4} \mathbf{a}_{1}+\left(\frac{3}{4}+2 y_{4}\right) \mathbf{a}_{2}+\left(\frac{3}{4}-2 y_{4}\right) \mathbf{a}_{3}$ & $=$ & $\frac{3}{4} a \hat{\mathbf{x}}+\left(\frac{1}{2}-y_{4}\right) a \hat{\mathbf{y}}+\left(\frac{1}{2}+y_{4}\right) a \hat{\mathbf{z}}$ & $(96 g)$ & $\mathrm{Cl} \mathrm{II}$ \\
\hline $\mathbf{B}_{46}$ & $=$ & $\left(\frac{3}{4}+2 y_{4}\right) \mathbf{a}_{1}+\frac{1}{4} \mathbf{a}_{2}+\frac{3}{4} \mathbf{a}_{3}$ & $=$ & $\frac{1}{2} a \hat{\mathbf{x}}+\left(\frac{3}{4}+y_{4}\right) a \hat{\mathbf{y}}+\left(\frac{1}{2}+y_{4}\right) a \hat{\mathbf{z}}$ & $(96 g)$ & $\mathrm{Cl} \mathrm{II}$ \\
\hline $\mathbf{B}_{47}$ & $=$ & $\left(\frac{3}{4}-2 y_{4}\right) \mathbf{a}_{1}+\frac{3}{4} \mathbf{a}_{2}+\frac{1}{4} \mathbf{a}_{3}$ & $=$ & $\frac{1}{2} a \hat{\mathbf{x}}+\left(\frac{1}{2}-y_{4}\right) a \hat{\mathbf{y}}+\left(\frac{3}{4}-y_{4}\right) a \hat{\mathbf{z}}$ & $(96 g)$ & $\mathrm{Cl}$ II \\
\hline $\mathbf{B}_{48}$ & $=$ & $\frac{3}{4} \mathbf{a}_{1}+\left(\frac{3}{4}-2 y_{4}\right) \mathbf{a}_{2}+\left(\frac{3}{4}+2 y_{4}\right) \mathbf{a}_{3}$ & $=$ & $\frac{3}{4} a \hat{\mathbf{x}}+\left(\frac{3}{4}+y_{4}\right) a \hat{\mathbf{y}}+\left(\frac{3}{4}-y_{4}\right) a \hat{\mathbf{z}}$ & $(96 g)$ & $\mathrm{Cl}$ II \\
\hline $\mathbf{B}_{49}$ & $=$ & $\left(\frac{3}{4}-2 y_{4}\right) \mathbf{a}_{1}+\frac{1}{4} \mathbf{a}_{2}+\left(\frac{3}{4}+2 y_{4}\right) \mathbf{a}_{3}$ & $=$ & $\left(\frac{1}{2}+y_{4}\right) a \hat{\mathbf{x}}+\frac{3}{4} a \hat{\mathbf{y}}+\left(\frac{1}{2}-y_{4}\right) a \hat{\mathbf{z}}$ & $(96 g)$ & $\mathrm{Cl}$ II \\
\hline $\mathbf{B}_{50}$ & $=$ & $\frac{3}{4} \mathbf{a}_{1}+\left(\frac{3}{4}+2 y_{4}\right) \mathbf{a}_{2}+\frac{1}{4} \mathbf{a}_{3}$ & $=$ & $\left(\frac{1}{2}+y_{4}\right) a \hat{\mathbf{x}}+\frac{1}{2} a \hat{\mathbf{y}}+\left(\frac{3}{4}+y_{4}\right) a \hat{\mathbf{z}}$ & $(96 g)$ & $\mathrm{Cl} \mathrm{II}$ \\
\hline $\mathbf{B}_{51}$ & $=$ & $\frac{1}{4} \mathbf{a}_{1}+\left(\frac{3}{4}-2 y_{4}\right) \mathbf{a}_{2}+\frac{3}{4} \mathbf{a}_{3}$ & $=$ & $\left(\frac{3}{4}-y_{4}\right) a \hat{\mathbf{x}}+\frac{1}{2} a \hat{\mathbf{y}}+\left(\frac{1}{2}-y_{4}\right) a \hat{\mathbf{z}}$ & $(96 g)$ & $\mathrm{Cl}$ II \\
\hline $\mathbf{B}_{52}$ & $=$ & $\left(\frac{3}{4}+2 y_{4}\right) \mathbf{a}_{1}+\frac{3}{4} \mathbf{a}_{2}+\left(\frac{3}{4}-2 y_{4}\right) \mathbf{a}_{3}$ & $=$ & $\left(\frac{3}{4}-y_{4}\right) a \hat{\mathbf{x}}+\frac{3}{4} a \hat{\mathbf{y}}+\left(\frac{3}{4}+y_{4}\right) a \hat{\mathbf{z}}$ & $(96 g)$ & $\mathrm{Cl}$ II \\
\hline $\mathbf{B}_{53}$ & $=$ & $\left(\frac{3}{4}+2 y_{4}\right) \mathbf{a}_{1}+\left(\frac{3}{4}-2 y_{4}\right) \mathbf{a}_{2}+\frac{1}{4} \mathbf{a}_{3}$ & $=$ & $\left(\frac{1}{2}-y_{4}\right) a \hat{\mathbf{x}}+\left(\frac{1}{2}+y_{4}\right) a \hat{\mathbf{y}}+\frac{3}{4} a \hat{\mathbf{z}}$ & $(96 g)$ & $\mathrm{Cl}$ II \\
\hline $\mathbf{B}_{54}$ & $=$ & $\frac{1}{4} \mathbf{a}_{1}+\frac{3}{4} \mathbf{a}_{2}+\left(\frac{3}{4}+2 y_{4}\right) \mathbf{a}_{3}$ & $=$ & $\left(\frac{3}{4}+y_{4}\right) a \hat{\mathbf{x}}+\left(\frac{1}{2}+y_{4}\right) a \hat{\mathbf{y}}+\frac{1}{2} a \hat{\mathbf{z}}$ & $(96 g)$ & $\mathrm{Cl}$ II \\
\hline $\mathbf{B}_{55}$ & $=$ & $\frac{3}{4} \mathbf{a}_{1}+\frac{1}{4} \mathbf{a}_{2}+\left(\frac{3}{4}-2 y_{4}\right) \mathbf{a}_{3}$ & $=$ & $\left(\frac{1}{2}-y_{4}\right) a \hat{\mathbf{x}}+\left(\frac{3}{4}-y_{4}\right) a \hat{\mathbf{y}}+\frac{1}{2} a \hat{\mathbf{z}}$ & $(96 g)$ & Cli \\
\hline
\end{tabular}




\begin{tabular}{|c|c|c|c|c|c|c|}
\hline $\mathbf{B}_{56}$ & $=$ & $\left.-2 y_{4}\right) \mathbf{a}_{1}+\left(\frac{3}{4}+2 y_{4}\right) \mathbf{a}_{2}+\frac{3}{4} \mathbf{a}_{3}$ & $=$ & $\left(\frac{3}{4}+y_{4}\right) a \hat{\mathbf{x}}+\left(\frac{3}{4}-y_{4}\right) a \hat{\mathbf{y}}+\frac{3}{4} a \hat{\mathbf{z}}$ & $(96 g)$ & $\mathrm{Cl} \mathrm{II}$ \\
\hline $\mathbf{B}_{57}$ & $=$ & $\begin{array}{c}\left(-x_{5}+y_{5}+z_{5}\right) \mathbf{a}_{1}+ \\
\left(x_{5}-y_{5}+z_{5}\right) \mathbf{a}_{2}+ \\
\left(x_{5}+y_{5}-z_{5}\right) \mathbf{a}_{3}\end{array}$ & $=$ & $x_{5} a \hat{\mathbf{x}}+y_{5} a \hat{\mathbf{y}}+z_{5} a \hat{\mathbf{z}}$ & $(192 h)$ & $\mathrm{N}$ \\
\hline $\mathbf{B}_{58}$ & $=$ & $\begin{array}{c}\left(x_{5}-y_{5}+z_{5}\right) \mathbf{a}_{1}+ \\
\left(-x_{5}+y_{5}+z_{5}\right) \mathbf{a}_{2}+ \\
\left(\frac{1}{2}-x_{5}-y_{5}-z_{5}\right) \mathbf{a}_{3}\end{array}$ & $=$ & $\left(\frac{1}{4}-x_{5}\right) a \hat{\mathbf{x}}+\left(\frac{1}{4}-y_{5}\right) a \hat{\mathbf{y}}+z_{5} a \hat{\mathbf{z}}$ & $(192 h)$ & $\mathrm{N}$ \\
\hline $\mathbf{B}_{59}$ & $=$ & $\begin{array}{c}\left(x_{5}+y_{5}-z_{5}\right) \mathbf{a}_{1}+ \\
\left(\frac{1}{2}-x_{5}-y_{5}-z_{5}\right) \mathbf{a}_{2}+ \\
\left(-x_{5}+y_{5}+z_{5}\right) \mathbf{a}_{3}\end{array}$ & $=$ & $\left(\frac{1}{4}-x_{5}\right) a \hat{\mathbf{x}}+y_{5} a \hat{\mathbf{y}}+\left(\frac{1}{4}-z_{5}\right) a \hat{\mathbf{z}}$ & $(192 h)$ & $\mathrm{N}$ \\
\hline $\mathbf{B}_{60}$ & $=$ & $\begin{array}{c}\left(\frac{1}{2}-x_{5}-y_{5}-z_{5}\right) \mathbf{a}_{1}+ \\
\left(x_{5}+y_{5}-z_{5}\right) \mathbf{a}_{2}+ \\
\left(x_{5}-y_{5}+z_{5}\right) \mathbf{a}_{3}\end{array}$ & $=$ & $x_{5} a \hat{\mathbf{x}}+\left(\frac{1}{4}-y_{5}\right) a \hat{\mathbf{y}}+\left(\frac{1}{4}-z_{5}\right) a \hat{\mathbf{z}}$ & $(192 h)$ & $\mathrm{N}$ \\
\hline $\mathbf{B}_{61}$ & $=$ & $\begin{array}{c}\left(x_{5}+y_{5}-z_{5}\right) \mathbf{a}_{1}+ \\
\left(-x_{5}+y_{5}+z_{5}\right) \mathbf{a}_{2}+ \\
\left(x_{5}-y_{5}+z_{5}\right) \mathbf{a}_{3}\end{array}$ & $=$ & $z_{5} a \hat{\mathbf{x}}+x_{5} a \hat{\mathbf{y}}+y_{5} a \hat{\mathbf{z}}$ & $(192 h)$ & $\mathrm{N}$ \\
\hline $\mathbf{B}_{62}$ & $=$ & $\begin{array}{c}\left(\frac{1}{2}-x_{5}-y_{5}-z_{5}\right) \mathbf{a}_{1}+ \\
\left(x_{5}-y_{5}+z_{5}\right) \mathbf{a}_{2}+ \\
\left(-x_{5}+y_{5}+z_{5}\right) \mathbf{a}_{3}\end{array}$ & $=$ & $z_{5} a \hat{\mathbf{x}}+\left(\frac{1}{4}-x_{5}\right) a \hat{\mathbf{y}}+\left(\frac{1}{4}-y_{5}\right) a \hat{\mathbf{z}}$ & $(192 h)$ & $\mathrm{N}$ \\
\hline $\mathbf{B}_{63}$ & $=$ & $\begin{array}{c}\left(-x_{5}+y_{5}+z_{5}\right) \mathbf{a}_{1}+ \\
\left(x_{5}+y_{5}-z_{5}\right) \mathbf{a}_{2}+ \\
\left(\frac{1}{2}-x_{5}-y_{5}-z_{5}\right) \mathbf{a}_{3}\end{array}$ & $=$ & $\left(\frac{1}{4}-z_{5}\right) a \hat{\mathbf{x}}+\left(\frac{1}{4}-x_{5}\right) a \hat{\mathbf{y}}+y_{5} a \hat{\mathbf{z}}$ & $(192 h)$ & $\mathrm{N}$ \\
\hline $\mathbf{B}_{64}$ & $=$ & $\begin{array}{c}\left(x_{5}-y_{5}+z_{5}\right) \mathbf{a}_{1}+ \\
\left(\frac{1}{2}-x_{5}-y_{5}-z_{5}\right) \mathbf{a}_{2}+ \\
\left(x_{5}+y_{5}-z_{5}\right) \mathbf{a}_{3}\end{array}$ & $=$ & $\left(\frac{1}{4}-z_{5}\right) a \hat{\mathbf{x}}+x_{5} a \hat{\mathbf{y}}+\left(\frac{1}{4}-y_{5}\right) a \hat{\mathbf{z}}$ & $(192 h)$ & $\mathrm{N}$ \\
\hline $\mathbf{B}_{65}$ & $=$ & $\begin{array}{l}\left(x_{5}-y_{5}+z_{5}\right) \mathbf{a}_{1}+ \\
\left(x_{5}+y_{5}-z_{5}\right) \mathbf{a}_{2}+ \\
\left(-x_{5}+y_{5}+z_{5}\right) \mathbf{a}_{3}\end{array}$ & $=$ & $y_{5} a \hat{\mathbf{x}}+z_{5} a \hat{\mathbf{y}}+x_{5} a \hat{\mathbf{z}}$ & $(192 h)$ & $\mathrm{N}$ \\
\hline $\mathbf{B}_{66}$ & $=$ & $\begin{array}{c}\left(-x_{5}+y_{5}+z_{5}\right) \mathbf{a}_{1}+ \\
\left(\frac{1}{2}-x_{5}-y_{5}-z_{5}\right) \mathbf{a}_{2}+ \\
\quad\left(x_{5}-y_{5}+z_{5}\right) \mathbf{a}_{3}\end{array}$ & $=$ & $\left(\frac{1}{4}-y_{5}\right) a \hat{\mathbf{x}}+z_{5} a \hat{\mathbf{y}}+\left(\frac{1}{4}-x_{5}\right) a \hat{\mathbf{z}}$ & $(192 h)$ & $\mathrm{N}$ \\
\hline $\mathbf{B}_{67}$ & $=$ & $\begin{array}{c}\left(\frac{1}{2}-x_{5}-y_{5}-z_{5}\right) \mathbf{a}_{1}+ \\
\left(-x_{5}+y_{5}+z_{5}\right) \mathbf{a}_{2}+ \\
\left(x_{5}+y_{5}-z_{5}\right) \mathbf{a}_{3}\end{array}$ & $=$ & $y_{5} a \hat{\mathbf{x}}+\left(\frac{1}{4}-z_{5}\right) a \hat{\mathbf{y}}+\left(\frac{1}{4}-x_{5}\right) a \hat{\mathbf{z}}$ & $(192 h)$ & $\mathrm{N}$ \\
\hline $\mathbf{B}_{68}$ & $=$ & $\begin{array}{c}\left(x_{5}+y_{5}-z_{5}\right) \mathbf{a}_{1}+ \\
\left(x_{5}-y_{5}+z_{5}\right) \mathbf{a}_{2}+ \\
\left(\frac{1}{2}-x_{5}-y_{5}-z_{5}\right) \mathbf{a}_{3}\end{array}$ & $=$ & $\left(\frac{1}{4}-y_{5}\right) a \hat{\mathbf{x}}+\left(\frac{1}{4}-z_{5}\right) a \hat{\mathbf{y}}+x_{5} a \hat{\mathbf{z}}$ & $(192 h)$ & $\mathrm{N}$ \\
\hline $\mathbf{B}_{69}$ & $=$ & $\begin{array}{c}\left(\frac{1}{2}+x_{5}-y_{5}-z_{5}\right) \mathbf{a}_{1}+ \\
\left(\frac{1}{2}-x_{5}+y_{5}-z_{5}\right) \mathbf{a}_{2}+ \\
\left(x_{5}+y_{5}+z_{5}\right) \mathbf{a}_{3}\end{array}$ & $=$ & $\begin{array}{c}\left(\frac{1}{4}+y_{5}\right) a \hat{\mathbf{x}}+\left(\frac{1}{4}+x_{5}\right) a \hat{\mathbf{y}}+ \\
\left(\frac{1}{2}-z_{5}\right) a \hat{\mathbf{z}}\end{array}$ & $(192 h)$ & $\mathrm{N}$ \\
\hline $\mathbf{B}_{70}$ & $=$ & $\begin{array}{c}\left(\frac{1}{2}-x_{5}+y_{5}-z_{5}\right) \mathbf{a}_{1}+ \\
\left(\frac{1}{2}+x_{5}-y_{5}-z_{5}\right) \mathbf{a}_{2}+ \\
\left(\frac{1}{2}-x_{5}-y_{5}+z_{5}\right) \mathbf{a}_{3}\end{array}$ & $=$ & $\begin{array}{c}\left(\frac{1}{2}-y_{5}\right) a \hat{\mathbf{x}}+\left(\frac{1}{2}-x_{5}\right) a \hat{\mathbf{y}}+ \\
\left(\frac{1}{2}-z_{5}\right) a \hat{\mathbf{z}}\end{array}$ & $(192 h)$ & $\mathrm{N}$ \\
\hline $\mathbf{B}_{71}$ & $=$ & $\begin{array}{c}\left(\frac{1}{2}-x_{5}-y_{5}+z_{5}\right) \mathbf{a}_{1}+ \\
\left(x_{5}+y_{5}+z_{5}\right) \mathbf{a}_{2}+ \\
\left(\frac{1}{2}-x_{5}+y_{5}-z_{5}\right) \mathbf{a}_{3}\end{array}$ & $=$ & $\begin{array}{c}\left(\frac{1}{4}+y_{5}\right) a \hat{\mathbf{x}}+\left(\frac{1}{2}-x_{5}\right) a \hat{\mathbf{y}}+ \\
\left(\frac{1}{4}+z_{5}\right) a \hat{\mathbf{z}}\end{array}$ & $(192 h)$ & $\mathrm{N}$ \\
\hline $\mathbf{B}_{72}$ & $=$ & $\begin{array}{c}\left(x_{5}+y_{5}+z_{5}\right) \mathbf{a}_{1}+ \\
\left(\frac{1}{2}-x_{5}-y_{5}+z_{5}\right) \mathbf{a}_{2}+ \\
\left(\frac{1}{2}+x_{5}-y_{5}-z_{5}\right) \mathbf{a}_{3}\end{array}$ & $=$ & $\begin{array}{c}\left(\frac{1}{2}-y_{5}\right) a \hat{\mathbf{x}}+\left(\frac{1}{4}+x_{5}\right) a \hat{\mathbf{y}}+ \\
\left(\frac{1}{4}+z_{5}\right) a \hat{\mathbf{z}}\end{array}$ & $(192 h)$ & $\mathrm{N}$ \\
\hline
\end{tabular}




$$
\begin{array}{r}
\mathbf{B}_{73}=\quad\left(\frac{1}{2}-x_{5}-y_{5}+z_{5}\right) \mathbf{a}_{1}+ \\
\left(\frac{1}{2}+x_{5}-y_{5}-z_{5}\right) \mathbf{a}_{2}+ \\
\left(x_{5}+y_{5}+z_{5}\right) \mathbf{a}_{3}
\end{array}
$$$$
\mathbf{B}_{74}=
$$$$
\left(x_{5}+y_{5}+z_{5}\right) \mathbf{a}_{1}+
$$$$
\left(\frac{1}{2}-x_{5}+y_{5}-z_{5}\right) \mathbf{a}_{2}+
$$$$
\left(\frac{1}{2}-x_{5}-y_{5}+z_{5}\right) \mathbf{a}_{3}
$$$$
\mathbf{B}_{75}=\left(\frac{1}{2}+x_{5}-y_{5}-z_{5}\right) \mathbf{a}_{1}+
$$$$
\left(\frac{1}{2}-x_{5}-y_{5}+z_{5}\right) \mathbf{a}_{2}+
$$$$
\left(\frac{1}{2}-x_{5}+y_{5}-z_{5}\right) \mathbf{a}_{3}
$$$$
\mathbf{B}_{76}=\left(\frac{1}{2}-x_{5}+y_{5}-z_{5}\right) \mathbf{a}_{1}+
$$$$
\left(x_{5}+y_{5}+z_{5}\right) \mathbf{a}_{2}+
$$$$
\left(\frac{1}{2}+x_{5}-y_{5}-z_{5}\right) \mathbf{a}_{3}
$$

$\mathbf{B}_{77}=\left(\frac{1}{2}-x_{5}+y_{5}-z_{5}\right) \mathbf{a}_{1}+$ $\left(\frac{1}{2}-x_{5}-y_{5}+z_{5}\right) \mathbf{a}_{2}+$

$$
\left(x_{5}+y_{5}+z_{5}\right) \mathbf{a}_{3}
$$

$\mathbf{B}_{78}=\left(\frac{1}{2}+x_{5}-y_{5}-z_{5}\right) \mathbf{a}_{1}+$ $\left(x_{5}+y_{5}+z_{5}\right) \mathbf{a}_{2}+$ $\left(\frac{1}{2}-x_{5}-y_{5}+z_{5}\right) \mathbf{a}_{3}$

$\mathbf{B}_{79}=$

$$
\begin{gathered}
\left(x_{5}+y_{5}+z_{5}\right) \mathbf{a}_{1}+ \\
\left(\frac{1}{2}+x_{5}-y_{5}-z_{5}\right) \mathbf{a}_{2}+ \\
\left(\frac{1}{2}-x_{5}+y_{5}-z_{5}\right) \mathbf{a}_{3}
\end{gathered}
$$

$\mathbf{B}_{80}=\left(\frac{1}{2}-x_{5}-y_{5}+z_{5}\right) \mathbf{a}_{1}+$ $\left(\frac{1}{2}-x_{5}+y_{5}-z_{5}\right) \mathbf{a}_{2}+$ $\left(\frac{1}{2}+x_{5}-y_{5}-z_{5}\right) \mathbf{a}_{3}$

$\mathbf{B}_{81}=\left(x_{5}-y_{5}-z_{5}\right) \mathbf{a}_{1}+$ $\left(-x_{5}+y_{5}-z_{5}\right) \mathbf{a}_{2}+$ $\left(-x_{5}-y_{5}+z_{5}\right) \mathbf{a}_{3}$

$\mathbf{B}_{82}=\left(-x_{5}+y_{5}-z_{5}\right) \mathbf{a}_{1}+$ $\left(x_{5}-y_{5}-z_{5}\right) \mathbf{a}_{2}+$ $\left(\frac{1}{2}+x_{5}+y_{5}+z_{5}\right) \mathbf{a}_{3}$

$\mathbf{B}_{83}=\left(-x_{5}-y_{5}+z_{5}\right) \mathbf{a}_{1}+$ $\left(\frac{1}{2}+x_{5}+y_{5}+z_{5}\right) \mathbf{a}_{2}+$ $\left(x_{5}-y_{5}-z_{5}\right) \mathbf{a}_{3}$

$\mathbf{B}_{84}=\left(\frac{1}{2}+x_{5}+y_{5}+z_{5}\right) \mathbf{a}_{1}+$ $\left(-x_{5}-y_{5}+z_{5}\right) \mathbf{a}_{2}+$ $\left(-x_{5}+y_{5}-z_{5}\right) \mathbf{a}_{3}$

$$
\begin{aligned}
\mathbf{B}_{85}=\quad & \left(-x_{5}-y_{5}+z_{5}\right) \mathbf{a}_{1}+ \\
& \left(x_{5}-y_{5}-z_{5}\right) \mathbf{a}_{2}+ \\
& \left(-x_{5}+y_{5}-z_{5}\right) \mathbf{a}_{3}
\end{aligned}
$$

$\mathbf{B}_{86}=\left(\frac{1}{2}+x_{5}+y_{5}+z_{5}\right) \mathbf{a}_{1}+$

$$
\left(-x_{5}+y_{5}-z_{5}\right) \mathbf{a}_{2}+
$$

$$
\left(x_{5}-y_{5}-z_{5}\right) \mathbf{a}_{3}
$$

$$
\begin{aligned}
\mathbf{B}_{87}=\quad & \left(x_{5}-y_{5}-z_{5}\right) \mathbf{a}_{1}+ \\
& \left(-x_{5}-y_{5}+z_{5}\right) \mathbf{a}_{2}+ \\
& \left(\frac{1}{2}+x_{5}+y_{5}+z_{5}\right) \mathbf{a}_{3}
\end{aligned}
$$

$$
\begin{aligned}
& \mathbf{B}_{88}=\left(-x_{5}+y_{5}-z_{5}\right) \mathbf{a}_{1}+ \\
& \left(\frac{1}{2}+x_{5}+y_{5}+z_{5}\right) \mathbf{a}_{2}+ \\
& \left(-x_{5}-y_{5}+z_{5}\right) \mathbf{a}_{3}
\end{aligned}
$$

$$
\begin{gathered}
=\quad\left(\frac{1}{4}+x_{5}\right) a \hat{\mathbf{x}}+\left(\frac{1}{4}+z_{5}\right) a \hat{\mathbf{y}}+ \\
\left(\frac{1}{2}-y_{5}\right) a \hat{\mathbf{z}}
\end{gathered}
$$

$=\left(\frac{1}{2}-x_{5}\right) a \hat{\mathbf{x}}+\left(\frac{1}{4}+z_{5}\right) a \hat{\mathbf{y}}+$ $\left(\frac{1}{4}+y_{5}\right) a \hat{\mathbf{z}}$

$=\left(\frac{1}{2}-x_{5}\right) a \hat{\mathbf{x}}+\left(\frac{1}{2}-z_{5}\right) a \hat{\mathbf{y}}+$

$\mathrm{N}$

$$
\left(\frac{1}{2}-y_{5}\right) a \hat{\mathbf{z}}
$$

$=\left(\frac{1}{4}+x_{5}\right) a \hat{\mathbf{x}}+\left(\frac{1}{2}-z_{5}\right) a \hat{\mathbf{y}}+$

$\mathrm{N}$

$$
\left(\frac{1}{4}+y_{5}\right) a \hat{\mathbf{z}}
$$

$=\left(\frac{1}{4}+z_{5}\right) a \hat{\mathbf{x}}+\left(\frac{1}{4}+y_{5}\right) a \hat{\mathbf{y}}+$

$\mathrm{N}$

$$
\left(\frac{1}{2}-x_{5}\right) a \hat{\mathbf{z}}
$$

$=\left(\frac{1}{4}+z_{5}\right) a \hat{\mathbf{x}}+\left(\frac{1}{2}-y_{5}\right) a \hat{\mathbf{y}}+$

(192h)

$\mathrm{N}$

$$
\left(\frac{1}{4}+x_{5}\right) a \hat{\mathbf{z}}
$$

$=\left(\frac{1}{2}-z_{5}\right) a \hat{\mathbf{x}}+\left(\frac{1}{4}+y_{5}\right) a \hat{\mathbf{y}}+$

(192h)

$\mathrm{N}$

$$
\left(\frac{1}{4}+x_{5}\right) a \hat{\mathbf{z}}
$$

$=\quad\left(\frac{1}{2}-z_{5}\right) a \hat{\mathbf{x}}+\left(\frac{1}{2}-y_{5}\right) a \hat{\mathbf{y}}+$

$\mathrm{N}$

$$
\left(\frac{1}{2}-x_{5}\right) a \hat{\mathbf{z}}
$$

$=\quad-x_{5} a \hat{\mathbf{x}}-y_{5} a \hat{\mathbf{y}}-z_{5} a \hat{\mathbf{z}}$

$\mathrm{N}$

$=\left(\frac{1}{4}+x_{5}\right) a \hat{\mathbf{x}}+\left(\frac{1}{4}+y_{5}\right) a \hat{\mathbf{y}}-z_{5} a \hat{\mathbf{z}}$

$\mathrm{N}$

$=\left(\frac{1}{4}+x_{5}\right) a \hat{\mathbf{x}}-y_{5} a \hat{\mathbf{y}}+\left(\frac{1}{4}+z_{5}\right) a \hat{\mathbf{z}}$

$\mathrm{N}$

$=-x_{5} a \hat{\mathbf{x}}+\left(\frac{1}{4}+y_{5}\right) a \hat{\mathbf{y}}+\left(\frac{1}{4}+z_{5}\right) a \hat{\mathbf{z}}$

$\mathrm{N}$

$=\quad-z_{5} a \hat{\mathbf{x}}-x_{5} a \hat{\mathbf{y}}-y_{5} a \hat{\mathbf{z}}$

$\mathrm{N}$

$=-z_{5} a \hat{\mathbf{x}}+\left(\frac{1}{4}+x_{5}\right) a \hat{\mathbf{y}}+\left(\frac{1}{4}+y_{5}\right) a \hat{\mathbf{z}}$

$\mathrm{N}$

$=\left(\frac{1}{4}+z_{5}\right) a \hat{\mathbf{x}}+\left(\frac{1}{4}+x_{5}\right) a \hat{\mathbf{y}}-y_{5} a \hat{\mathbf{z}}$

$\mathrm{N}$

$=\left(\frac{1}{4}+z_{5}\right) a \hat{\mathbf{x}}-x_{5} a \hat{\mathbf{y}}+\left(\frac{1}{4}+y_{5}\right) a \hat{\mathbf{z}}$

(192h)
$\mathrm{N}$

$\mathrm{N}$

$\mathrm{N}$

N

N

$\mathrm{N}$

N

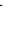

$\mathrm{N}$

N

N

$\mathrm{N}$

N

$\mathrm{N}$

$N$

$\mathrm{N}$ 


\begin{tabular}{|c|c|c|c|c|c|c|}
\hline $\mathbf{B}_{89}$ & $=$ & $\begin{array}{c}\left(-x_{5}+y_{5}-z_{5}\right) \mathbf{a}_{1}+ \\
\left(-x_{5}-y_{5}+z_{5}\right) \mathbf{a}_{2}+ \\
\left(x_{5}-y_{5}-z_{5}\right) \mathbf{a}_{3}\end{array}$ & $=$ & $-y_{5} a \hat{\mathbf{x}}-z_{5} a \hat{\mathbf{y}}-x_{5} a \hat{\mathbf{z}}$ & $(192 h)$ & $\mathrm{N}$ \\
\hline $\mathbf{B}_{90}$ & $=$ & $\begin{array}{c}\left(x_{5}-y_{5}-z_{5}\right) \mathbf{a}_{1}+ \\
\left(\frac{1}{2}+x_{5}+y_{5}+z_{5}\right) \mathbf{a}_{2}+ \\
\left(-x_{5}+y_{5}-z_{5}\right) \mathbf{a}_{3}\end{array}$ & $=$ & $\left(\frac{1}{4}+y_{5}\right) a \hat{\mathbf{x}}-z_{5} a \hat{\mathbf{y}}+\left(\frac{1}{4}+x_{5}\right) a \hat{\mathbf{z}}$ & $(192 h)$ & $\mathrm{N}$ \\
\hline $\mathbf{B}_{91}$ & $=$ & $\begin{array}{c}\left(\frac{1}{2}+x_{5}+y_{5}+z_{5}\right) \mathbf{a}_{1}+ \\
\left(x_{5}-y_{5}-z_{5}\right) \mathbf{a}_{2}+ \\
\left(-x_{5}-y_{5}+z_{5}\right) \mathbf{a}_{3}\end{array}$ & $=$ & $-y_{5} a \hat{\mathbf{x}}+\left(\frac{1}{4}+z_{5}\right) a \hat{\mathbf{y}}+\left(\frac{1}{4}+x_{5}\right) a \hat{\mathbf{z}}$ & $(192 h)$ & $\mathrm{N}$ \\
\hline $\mathbf{B}_{92}$ & $=$ & $\begin{array}{l}\left(-x_{5}-y_{5}+z_{5}\right) \mathbf{a}_{1}+ \\
\left(-x_{5}+y_{5}-z_{5}\right) \mathbf{a}_{2}+ \\
\left(\frac{1}{2}+x_{5}+y_{5}+z_{5}\right) \mathbf{a}_{3}\end{array}$ & $=$ & $\left(\frac{1}{4}+y_{5}\right) a \hat{\mathbf{x}}+\left(\frac{1}{4}+z_{5}\right) a \hat{\mathbf{y}}-x_{5} a \hat{\mathbf{z}}$ & $(192 h)$ & $\mathrm{N}$ \\
\hline $\mathbf{B}_{93}$ & $=$ & $\begin{array}{c}\left(\frac{1}{2}-x_{5}+y_{5}+z_{5}\right) \mathbf{a}_{1}+ \\
\left(\frac{1}{2}+x_{5}-y_{5}+z_{5}\right) \mathbf{a}_{2}+ \\
\left(-x_{5}-y_{5}-z_{5}\right) \mathbf{a}_{3}\end{array}$ & $=$ & $\begin{array}{c}\left(\frac{1}{4}-y_{5}\right) a \hat{\mathbf{x}}+\left(\frac{1}{4}-x_{5}\right) a \hat{\mathbf{y}}+ \\
\left(\frac{1}{2}+z_{5}\right) a \hat{\mathbf{z}}\end{array}$ & $(192 h)$ & $\mathrm{N}$ \\
\hline $\mathbf{B}_{94}$ & $=$ & $\begin{array}{c}\left(\frac{1}{2}+x_{5}-y_{5}+z_{5}\right) \mathbf{a}_{1}+ \\
\left(\frac{1}{2}-x_{5}+y_{5}+z_{5}\right) \mathbf{a}_{2}+ \\
\left(\frac{1}{2}+x_{5}+y_{5}-z_{5}\right) \mathbf{a}_{3}\end{array}$ & $=$ & $\begin{array}{c}\left(\frac{1}{2}+y_{5}\right) a \hat{\mathbf{x}}+\left(\frac{1}{2}+x_{5}\right) a \hat{\mathbf{y}}+ \\
\left(\frac{1}{2}+z_{5}\right) a \hat{\mathbf{z}}\end{array}$ & $(192 h)$ & $\mathrm{N}$ \\
\hline $\mathbf{B}_{95}$ & $=$ & $\begin{array}{c}\left(\frac{1}{2}+x_{5}+y_{5}-z_{5}\right) \mathbf{a}_{1}+ \\
\left(-x_{5}-y_{5}-z_{5}\right) \mathbf{a}_{2}+ \\
\left(\frac{1}{2}+x_{5}-y_{5}+z_{5}\right) \mathbf{a}_{3}\end{array}$ & $=$ & $\begin{array}{c}\left(\frac{1}{4}-y_{5}\right) a \hat{\mathbf{x}}+\left(\frac{1}{2}+x_{5}\right) a \hat{\mathbf{y}}+ \\
\left(\frac{1}{4}-z_{5}\right) a \hat{\mathbf{z}}\end{array}$ & $(192 h)$ & $\mathrm{N}$ \\
\hline $\mathbf{B}_{96}$ & $=$ & $\begin{array}{c}\left(-x_{5}-y_{5}-z_{5}\right) \mathbf{a}_{1}+ \\
\left(\frac{1}{2}+x_{5}+y_{5}-z_{5}\right) \mathbf{a}_{2}+ \\
\left(\frac{1}{2}-x_{5}+y_{5}+z_{5}\right) \mathbf{a}_{3}\end{array}$ & $=$ & $\begin{array}{c}\left(\frac{1}{2}+y_{5}\right) a \hat{\mathbf{x}}+\left(\frac{1}{4}-x_{5}\right) a \hat{\mathbf{y}}+ \\
\left(\frac{1}{4}-z_{5}\right) a \hat{\mathbf{z}}\end{array}$ & $(192 h)$ & $\mathrm{N}$ \\
\hline $\mathbf{B}_{97}$ & $=$ & $\begin{array}{c}\left(\frac{1}{2}+x_{5}+y_{5}-z_{5}\right) \mathbf{a}_{1}+ \\
\left(\frac{1}{2}-x_{5}+y_{5}+z_{5}\right) \mathbf{a}_{2}+ \\
\left(-x_{5}-y_{5}-z_{5}\right) \mathbf{a}_{3}\end{array}$ & $=$ & $\begin{array}{c}\left(\frac{1}{4}-x_{5}\right) a \hat{\mathbf{x}}+\left(\frac{1}{4}-z_{5}\right) a \hat{\mathbf{y}}+ \\
\left(\frac{1}{2}+y_{5}\right) a \hat{\mathbf{z}}\end{array}$ & $(192 h)$ & $\mathrm{N}$ \\
\hline $\mathbf{B}_{98}$ & $=$ & $\begin{array}{c}\left(-x_{5}-y_{5}-z_{5}\right) \mathbf{a}_{1}+ \\
\left(\frac{1}{2}+x_{5}-y_{5}+z_{5}\right) \mathbf{a}_{2}+ \\
\left(\frac{1}{2}+x_{5}+y_{5}-z_{5}\right) \mathbf{a}_{3}\end{array}$ & $=$ & $\begin{array}{c}\left(\frac{1}{2}+x_{5}\right) a \hat{\mathbf{x}}+\left(\frac{1}{4}-z_{5}\right) a \hat{\mathbf{y}}+ \\
\left(\frac{1}{4}-y_{5}\right) a \hat{\mathbf{z}}\end{array}$ & $(192 h)$ & $\mathrm{N}$ \\
\hline $\mathbf{B}_{99}$ & $=$ & $\begin{array}{c}\left(\frac{1}{2}-x_{5}+y_{5}+z_{5}\right) \mathbf{a}_{1}+ \\
\left(\frac{1}{2}+x_{5}+y_{5}-z_{5}\right) \mathbf{a}_{2}+ \\
\left(\frac{1}{2}+x_{5}-y_{5}+z_{5}\right) \mathbf{a}_{3}\end{array}$ & $=$ & $\begin{array}{c}\left(\frac{1}{2}+x_{5}\right) a \hat{\mathbf{x}}+\left(\frac{1}{2}+z_{5}\right) a \hat{\mathbf{y}}+ \\
\left(\frac{1}{2}+y_{5}\right) a \hat{\mathbf{z}}\end{array}$ & $(192 h)$ & $\mathrm{N}$ \\
\hline $\mathbf{B}_{100}$ & $=$ & $\begin{array}{c}\left(\frac{1}{2}+x_{5}-y_{5}+z_{5}\right) \mathbf{a}_{1}+ \\
\left(-x_{5}-y_{5}-z_{5}\right) \mathbf{a}_{2}+ \\
\left(\frac{1}{2}-x_{5}+y_{5}+z_{5}\right) \mathbf{a}_{3}\end{array}$ & $=$ & $\begin{array}{c}\left(\frac{1}{4}-x_{5}\right) a \hat{\mathbf{x}}+\left(\frac{1}{2}+z_{5}\right) a \hat{\mathbf{y}}+ \\
\left(\frac{1}{4}-y_{5}\right) a \hat{\mathbf{z}}\end{array}$ & $(192 h)$ & $\mathrm{N}$ \\
\hline $\mathbf{B}_{101}$ & $=$ & $\begin{array}{c}\left(\frac{1}{2}+x_{5}-y_{5}+z_{5}\right) \mathbf{a}_{1}+ \\
\left(\frac{1}{2}+x_{5}+y_{5}-z_{5}\right) \mathbf{a}_{2}+ \\
\left(-x_{5}-y_{5}-z_{5}\right) \mathbf{a}_{3}\end{array}$ & $=$ & $\begin{array}{c}\left(\frac{1}{4}-z_{5}\right) a \hat{\mathbf{x}}+\left(\frac{1}{4}-y_{5}\right) a \hat{\mathbf{y}}+ \\
\left(\frac{1}{2}+x_{5}\right) a \hat{\mathbf{z}}\end{array}$ & $(192 h)$ & $\mathrm{N}$ \\
\hline $\mathbf{B}_{102}$ & $=$ & $\begin{array}{c}\left(\frac{1}{2}-x_{5}+y_{5}+z_{5}\right) \mathbf{a}_{1}+ \\
\left(-x_{5}-y_{5}-z_{5}\right) \mathbf{a}_{2}+ \\
\left(\frac{1}{2}+x_{5}+y_{5}-z_{5}\right) \mathbf{a}_{3}\end{array}$ & $=$ & $\begin{array}{c}\left(\frac{1}{4}-z_{5}\right) a \hat{\mathbf{x}}+\left(\frac{1}{2}+y_{5}\right) a \hat{\mathbf{y}}+ \\
\left(\frac{1}{4}-x_{5}\right) a \hat{\mathbf{z}}\end{array}$ & $(192 h)$ & $\mathrm{N}$ \\
\hline $\mathbf{B}_{103}$ & $=$ & $\begin{array}{c}\left(-x_{5}-y_{5}-z_{5}\right) \mathbf{a}_{1}+ \\
\left(\frac{1}{2}-x_{5}+y_{5}+z_{5}\right) \mathbf{a}_{2}+ \\
\left(\frac{1}{2}+x_{5}-y_{5}+z_{5}\right) \mathbf{a}_{3}\end{array}$ & $=$ & $\begin{array}{c}\left(\frac{1}{2}+z_{5}\right) a \hat{\mathbf{x}}+\left(\frac{1}{4}-y_{5}\right) a \hat{\mathbf{y}}+ \\
\left(\frac{1}{4}-x_{5}\right) a \hat{\mathbf{z}}\end{array}$ & $(192 h)$ & $\mathrm{N}$ \\
\hline
\end{tabular}




$$
\begin{aligned}
\mathbf{B}_{104}= & \left(\frac{1}{2}+x_{5}+y_{5}-z_{5}\right) \mathbf{a}_{1}+ \\
\left(\frac{1}{2}+x_{5}-y_{5}+z_{5}\right) \mathbf{a}_{2}+ & =
\end{aligned} \quad \begin{gathered}
\left(\frac{1}{2}+z_{5}\right) a \hat{\mathbf{x}}+\left(\frac{1}{2}+y_{5}\right) a \hat{\mathbf{y}}+ \\
\left(\frac{1}{2}-x_{5}+y_{5}+z_{5}\right) \mathbf{a}_{3}
\end{gathered}
$$

\section{References:}

- M. Masayasu, S. Yoshihiko, and W. Tokunosuké, The Crystal Structure of [Cr( $\left.\mathrm{NH}_{3}\right)_{6}$ ] [CuCl 5$]$, Bull. Chem. Soc. Jpn. 34, 295-296 (1961), doi:10.1246/bcsj.34.295.

\section{Found in:}

- P. Villars and K. Cenzual, Pearson's Crystal Data - Crystal Structure Database for Inorganic Compounds, ASM International (2013).

\section{Geometry files:}

- CIF: pp. 980

- POSCAR: pp. 981 


\section{$\mathrm{TeO}_{6} \mathrm{H}_{6}$ Structure: A6B_cF224_228_h_c}
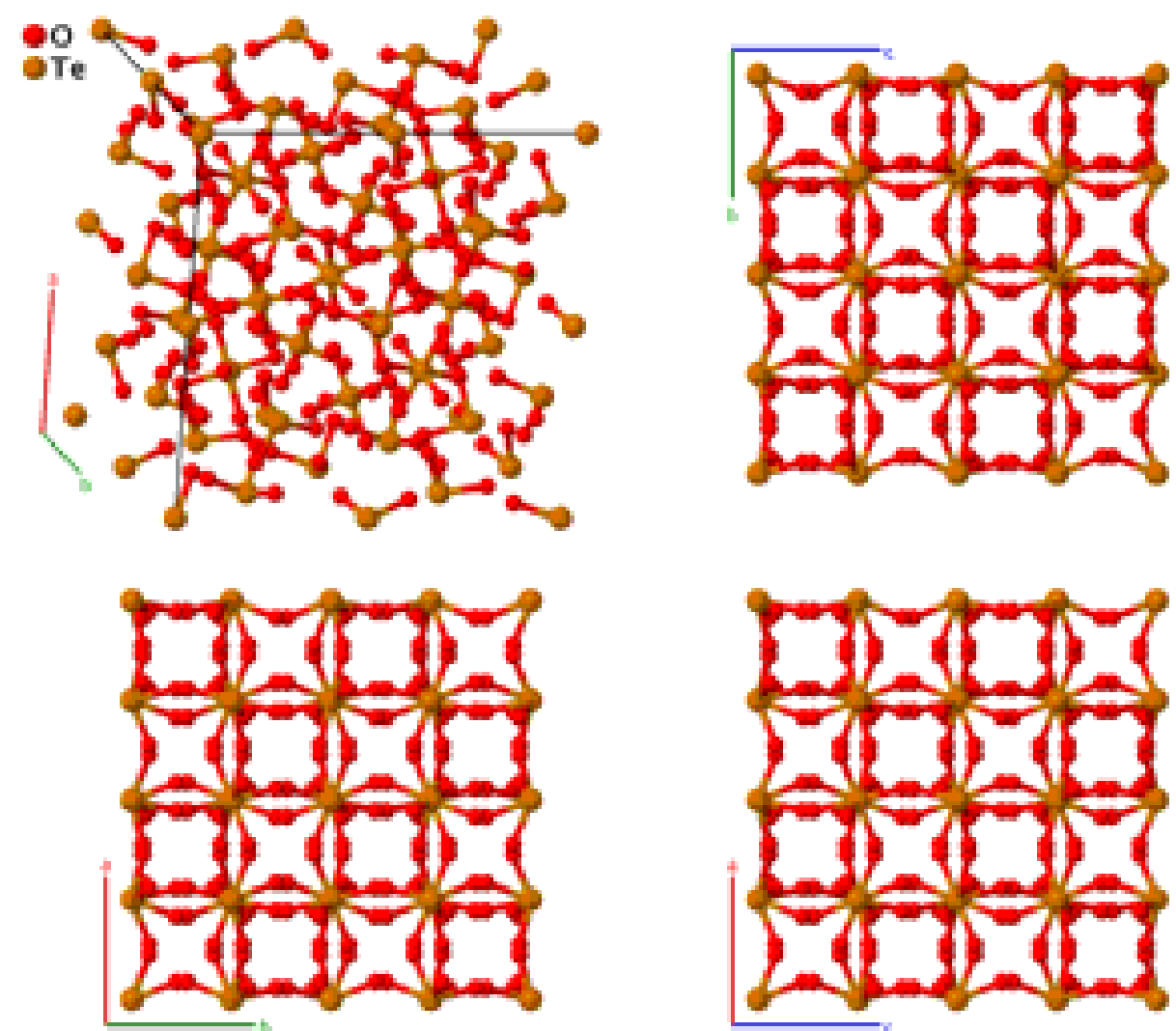

Prototype

: $\quad \mathrm{TeO}_{6} \mathrm{H}_{6}$

AFLOW prototype label

: A6B_cF224_228_h_c

Strukturbericht designation : None

Pearson symbol $\quad: \quad c F 224$

Space group number $\quad: \quad 228$

Space group symbol $\quad: \quad F d \overline{3} c$

AFLOW prototype command : aflow--proto=A6B_cF224_228_h_c

- params $=a, x_{2}, y_{2}, z_{2}$

- Polytypes appear in space groups \#14, \#210 and \#225. Only the non-hydrogen atoms are listed.

Face-centered Cubic primitive vectors:

$$
\begin{aligned}
& \mathbf{a}_{1}=\frac{1}{2} a \hat{\mathbf{y}}+\frac{1}{2} a \hat{\mathbf{z}} \\
& \mathbf{a}_{2}=\frac{1}{2} a \hat{\mathbf{x}}+\frac{1}{2} a \hat{\mathbf{z}} \\
& \mathbf{a}_{3}=\frac{1}{2} a \hat{\mathbf{x}}+\frac{1}{2} a \hat{\mathbf{y}}
\end{aligned}
$$

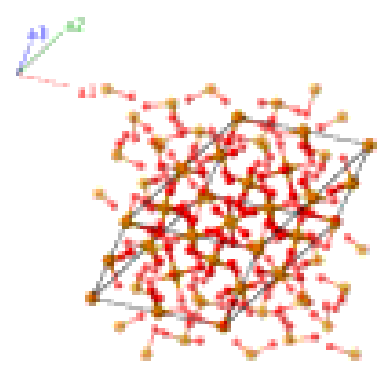

Basis vectors: 
Lattice Coordinates
$\mathbf{B}_{1}=$
$\mathbf{B}_{2}=$
$\mathbf{B}_{3}=$
$\mathbf{B}_{4}=$
$\mathbf{B}_{5}=$
$\mathbf{B}_{6}=$
$\mathbf{B}_{7}=$
$\mathbf{B}_{8}=$
$\mathbf{B}_{9}=$

$0 \mathbf{a}_{1}+0 \mathbf{a}_{2}+0 \mathbf{a}_{3}$

$$
\left(-x_{2}+y_{2}+z_{2}\right) \mathbf{a}_{1}+
$$$$
\left(x_{2}-y_{2}+z_{2}\right) \mathbf{a}_{2}+
$$$$
\left(x_{2}+y_{2}-z_{2}\right) \mathbf{a}_{3}
$$

$\mathbf{B}_{10}=$

$$
\begin{gathered}
\left(x_{2}-y_{2}+z_{2}\right) \mathbf{a}_{1}+ \\
\left(-x_{2}+y_{2}+z_{2}\right) \mathbf{a}_{2}+ \\
\left(\frac{1}{2}-x_{2}-y_{2}-z_{2}\right) \mathbf{a}_{3}
\end{gathered}
$$

$\mathbf{B}_{11}=$

$$
\begin{gathered}
\left(x_{2}+y_{2}-z_{2}\right) \mathbf{a}_{1}+ \\
\left(\frac{1}{2}-x_{2}-y_{2}-z_{2}\right) \mathbf{a}_{2}+ \\
\left(-x_{2}+y_{2}+z_{2}\right) \mathbf{a}_{3}
\end{gathered}
$$

$\mathbf{B}_{12}=\left(\frac{1}{2}-x_{2}-y_{2}-z_{2}\right) \mathbf{a}_{1}+$

$\left(x_{2}+y_{2}-z_{2}\right) \mathbf{a}_{2}+$

$$
\left(x_{2}-y_{2}+z_{2}\right) \mathbf{a}_{3}
$$

$\mathbf{B}_{13}=$

$$
\begin{gathered}
\left(x_{2}+y_{2}-z_{2}\right) \mathbf{a}_{1}+ \\
\left(-x_{2}+y_{2}+z_{2}\right) \mathbf{a}_{2}+ \\
\left(x_{2}-y_{2}+z_{2}\right) \mathbf{a}_{3}
\end{gathered}
$$

$\mathbf{B}_{14}=$

$$
\left(\frac{1}{2}-x_{2}-y_{2}-z_{2}\right) \mathbf{a}_{1}+
$$$$
\left(x_{2}-y_{2}+z_{2}\right) \mathbf{a}_{2}+
$$$$
\left(-x_{2}+y_{2}+z_{2}\right) \mathbf{a}_{3}
$$

$\mathbf{B}_{15}=$

$$
\left(-x_{2}+y_{2}+z_{2}\right) \mathbf{a}_{1}+
$$$$
\left(x_{2}+y_{2}-z_{2}\right) \mathbf{a}_{2}+
$$$$
\left(\frac{1}{2}-x_{2}-y_{2}-z_{2}\right) \mathbf{a}_{3}
$$

$\mathbf{B}_{16}=$

$$
\begin{gathered}
\left(x_{2}-y_{2}+z_{2}\right) \mathbf{a}_{1}+ \\
\left(\frac{1}{2}-x_{2}-y_{2}-z_{2}\right) \mathbf{a}_{2}+ \\
\left(x_{2}+y_{2}-z_{2}\right) \mathbf{a}_{3}
\end{gathered}
$$

$\mathbf{B}_{17}=$

$$
\left(x_{2}-y_{2}+z_{2}\right) \mathbf{a}_{1}+
$$$$
\left(x_{2}+y_{2}-z_{2}\right) \mathbf{a}_{2}+
$$$$
\left(-x_{2}+y_{2}+z_{2}\right) \mathbf{a}_{3}
$$

$\mathbf{B}_{18}=$

$$
\begin{gathered}
\left(-x_{2}+y_{2}+z_{2}\right) \mathbf{a}_{1}+ \\
\left(\frac{1}{2}-x_{2}-y_{2}-z_{2}\right) \mathbf{a}_{2}+ \\
\left(x_{2}-y_{2}+z_{2}\right) \mathbf{a}_{3}
\end{gathered}
$$

$\mathbf{B}_{19}=$

$$
\left(\frac{1}{2}-x_{2}-y_{2}-z_{2}\right) \mathbf{a}_{1}+
$$$$
\left(-x_{2}+y_{2}+z_{2}\right) \mathbf{a}_{2}+
$$$$
\left(x_{2}+y_{2}-z_{2}\right) \mathbf{a}_{3}
$$

$$
\begin{array}{r}
\mathbf{B}_{20}=\quad\left(x_{2}+y_{2}-z_{2}\right) \mathbf{a}_{1}+ \\
\left(x_{2}-y_{2}+z_{2}\right) \mathbf{a}_{2}+ \\
\left(\frac{1}{2}-x_{2}-y_{2}-z_{2}\right) \mathbf{a}_{3}
\end{array}
$$

Wyckoff Position Atom Type

$$
=\quad 0 \hat{\mathbf{x}}+0 \hat{\mathbf{y}}+0 \hat{\mathbf{z}}
$$

$$
=\left(\frac{1}{4}-x_{2}\right) a \hat{\mathbf{x}}+\left(\frac{1}{4}-y_{2}\right) a \hat{\mathbf{y}}+z_{2} a \hat{\mathbf{z}}
$$

$=\left(\frac{1}{4}-x_{2}\right) a \hat{\mathbf{x}}+y_{2} a \hat{\mathbf{y}}+\left(\frac{1}{4}-z_{2}\right) a \hat{\mathbf{z}}$

$=\quad x_{2} a \hat{\mathbf{x}}+\left(\frac{1}{4}-y_{2}\right) a \hat{\mathbf{y}}+\left(\frac{1}{4}-z_{2}\right) a \hat{\mathbf{z}}$

$$
=\quad z_{2} a \hat{\mathbf{x}}+\left(\frac{1}{4}-x_{2}\right) a \hat{\mathbf{y}}+\left(\frac{1}{4}-y_{2}\right) a \hat{\mathbf{z}}
$$

$$
=\left(\frac{1}{4}-z_{2}\right) a \hat{\mathbf{x}}+\left(\frac{1}{4}-x_{2}\right) a \hat{\mathbf{y}}+y_{2} a \hat{\mathbf{z}}
$$

$$
=\left(\frac{1}{4}-z_{2}\right) a \hat{\mathbf{x}}+x_{2} a \hat{\mathbf{y}}+\left(\frac{1}{4}-y_{2}\right) a \hat{\mathbf{z}} \quad(192 h) \quad \mathrm{O}
$$

$=\left(\frac{1}{4}-y_{2}\right) a \hat{\mathbf{x}}+z_{2} a \hat{\mathbf{y}}+\left(\frac{1}{4}-x_{2}\right) a \hat{\mathbf{z}} \quad(192 h)$

$=y_{2} a \hat{\mathbf{x}}+\left(\frac{1}{4}-z_{2}\right) a \hat{\mathbf{y}}+\left(\frac{1}{4}-x_{2}\right) a \hat{\mathbf{z}}$

$=\left(\frac{1}{4}-y_{2}\right) a \hat{\mathbf{x}}+\left(\frac{1}{4}-z_{2}\right) a \hat{\mathbf{y}}+x_{2} a \hat{\mathbf{z}}$

(192h)
$\mathrm{O}$

$\mathrm{O}$

$\mathrm{O}$

$\mathrm{O}$

$\mathrm{O}$

$\mathrm{Te}$

$\mathrm{Te}$

$\mathrm{Te}$

$\mathrm{Te}$

$\mathrm{Te}$

$\mathrm{Te}$

$\mathrm{Te}$

$\mathrm{Te}$

$\mathrm{O}$

$\mathrm{O}$

$\mathrm{O}$

$\mathrm{O}$

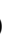

O

$\mathrm{O}$ 
$\mathbf{B}_{21}=\quad$
$\left(\frac{1}{2}+x_{2}-y_{2}-z_{2}\right) \mathbf{a}_{1}+$
$\left(\frac{1}{2}-x_{2}+y_{2}-z_{2}\right) \mathbf{a}_{2}+$
$\left(x_{2}+y_{2}+z_{2}\right) \mathbf{a}_{3}$

$\mathbf{B}_{22}=\left(\frac{1}{2}-x_{2}+y_{2}-z_{2}\right) \mathbf{a}_{1}+$

$\left(\frac{1}{2}+x_{2}-y_{2}-z_{2}\right) \mathbf{a}_{2}+$

$\left(\frac{1}{2}-x_{2}-y_{2}+z_{2}\right) \mathbf{a}_{3}$

$\mathbf{B}_{23}=\left(\frac{1}{2}-x_{2}-y_{2}+z_{2}\right) \mathbf{a}_{1}+$

$\left(x_{2}+y_{2}+z_{2}\right) \mathbf{a}_{2}+$

$\left(\frac{1}{2}-x_{2}+y_{2}-z_{2}\right) \mathbf{a}_{3}$

$\mathbf{B}_{24}=\left(x_{2}+y_{2}+z_{2}\right) \mathbf{a}_{1}+$

$\left(\frac{1}{2}-x_{2}-y_{2}+z_{2}\right) \mathbf{a}_{2}+$

$\left(\frac{1}{2}+x_{2}-y_{2}-z_{2}\right) \mathbf{a}_{3}$

$\mathbf{B}_{25}=\left(\frac{1}{2}-x_{2}-y_{2}+z_{2}\right) \mathbf{a}_{1}+$

$\left(\frac{1}{2}+x_{2}-y_{2}-z_{2}\right) \mathbf{a}_{2}+$

$\left(x_{2}+y_{2}+z_{2}\right) \mathbf{a}_{3}$

$\mathbf{B}_{26}=$

$\left(x_{2}+y_{2}+z_{2}\right) \mathbf{a}_{1}+$
$\left(\frac{1}{2}-x_{2}+y_{2}-z_{2}\right) \mathbf{a}_{2}+$

$\left(\frac{1}{2}-x_{2}-y_{2}+z_{2}\right) \mathbf{a}_{3}$

$\mathbf{B}_{27}=\left(\frac{1}{2}+x_{2}-y_{2}-z_{2}\right) \mathbf{a}_{1}+$

$\left(\frac{1}{2}-x_{2}-y_{2}+z_{2}\right) \mathbf{a}_{2}+$

$\left(\frac{1}{2}-x_{2}+y_{2}-z_{2}\right) \mathbf{a}_{3}$

$\mathbf{B}_{28}=\left(\frac{1}{2}-x_{2}+y_{2}-z_{2}\right) \mathbf{a}_{1}+$

$\left(x_{2}+y_{2}+z_{2}\right) \mathbf{a}_{2}+$

$\left(\frac{1}{2}+x_{2}-y_{2}-z_{2}\right) \mathbf{a}_{3}$

$\mathbf{B}_{29}=\left(\frac{1}{2}-x_{2}+y_{2}-z_{2}\right) \mathbf{a}_{1}+$ $\left(\frac{1}{2}-x_{2}-y_{2}+z_{2}\right) \mathbf{a}_{2}+$ $\left(x_{2}+y_{2}+z_{2}\right) \mathbf{a}_{3}$

$\mathbf{B}_{30}=\left(\frac{1}{2}+x_{2}-y_{2}-z_{2}\right) \mathbf{a}_{1}+$ $\left(x_{2}+y_{2}+z_{2}\right) \mathbf{a}_{2}+$ $\left(\frac{1}{2}-x_{2}-y_{2}+z_{2}\right) \mathbf{a}_{3}$

$\mathbf{B}_{31}=\left(x_{2}+y_{2}+z_{2}\right) \mathbf{a}_{1}+$ $\left(\frac{1}{2}+x_{2}-y_{2}-z_{2}\right) \mathbf{a}_{2}+$ $\left(\frac{1}{2}-x_{2}+y_{2}-z_{2}\right) \mathbf{a}_{3}$

$\mathbf{B}_{32}=\left(\frac{1}{2}-x_{2}-y_{2}+z_{2}\right) \mathbf{a}_{1}+$ $\left(\frac{1}{2}-x_{2}+y_{2}-z_{2}\right) \mathbf{a}_{2}+$ $\left(\frac{1}{2}+x_{2}-y_{2}-z_{2}\right) \mathbf{a}_{3}$

$\mathbf{B}_{33}=\left(x_{2}-y_{2}-z_{2}\right) \mathbf{a}_{1}+$ $\left(-x_{2}+y_{2}-z_{2}\right) \mathbf{a}_{2}+$ $\left(-x_{2}-y_{2}+z_{2}\right) \mathbf{a}_{3}$

$\mathbf{B}_{34}=\left(-x_{2}+y_{2}-z_{2}\right) \mathbf{a}_{1}+$ $\left(x_{2}-y_{2}-z_{2}\right) \mathbf{a}_{2}+$ $\left(\frac{1}{2}+x_{2}+y_{2}+z_{2}\right) \mathbf{a}_{3}$

$\mathbf{B}_{35}=\left(-x_{2}-y_{2}+z_{2}\right) \mathbf{a}_{1}+$ $\left(\frac{1}{2}+x_{2}+y_{2}+z_{2}\right) \mathbf{a}_{2}+$ $\left(x_{2}-y_{2}-z_{2}\right) \mathbf{a}_{3}$ $=\quad\left(\frac{1}{4}+y_{2}\right) a \hat{\mathbf{x}}+\left(\frac{1}{4}+x_{2}\right) a \hat{\mathbf{y}}+$

(192h)

$\mathrm{O}$

$=\quad\left(\frac{1}{2}-y_{2}\right) a \hat{\mathbf{x}}+\left(\frac{1}{2}-x_{2}\right) a \hat{\mathbf{y}}+$

(192h)

$\mathrm{O}$ $\left(\frac{1}{2}-z_{2}\right) a \hat{\mathbf{z}}$

$=\left(\frac{1}{4}+y_{2}\right) a \hat{\mathbf{x}}+\left(\frac{1}{2}-x_{2}\right) a \hat{\mathbf{y}}+$

(192h)

$\mathrm{O}$ $\left(\frac{1}{4}+z_{2}\right) a \hat{\mathbf{z}}$

$=\left(\frac{1}{2}-y_{2}\right) a \hat{\mathbf{x}}+\left(\frac{1}{4}+x_{2}\right) a \hat{\mathbf{y}}+$

(192h)

$\mathrm{O}$ $\left(\frac{1}{4}+z_{2}\right) a \hat{\mathbf{z}}$

$=\left(\frac{1}{4}+x_{2}\right) a \hat{\mathbf{x}}+\left(\frac{1}{4}+z_{2}\right) a \hat{\mathbf{y}}+$

$\mathrm{O}$

$$
\left(\frac{1}{2}-y_{2}\right) a \hat{\mathbf{z}}
$$

$=\left(\frac{1}{2}-x_{2}\right) a \hat{\mathbf{x}}+\left(\frac{1}{4}+z_{2}\right) a \hat{\mathbf{y}}+$

(192h)

$\mathrm{O}$

$$
\left(\frac{1}{4}+y_{2}\right) a \hat{\mathbf{z}}
$$

$=\left(\frac{1}{2}-x_{2}\right) a \hat{\mathbf{x}}+\left(\frac{1}{2}-z_{2}\right) a \hat{\mathbf{y}}+$

(192h)

O $\left(\frac{1}{2}-y_{2}\right) a \hat{\mathbf{z}}$

$=\left(\frac{1}{4}+x_{2}\right) a \hat{\mathbf{x}}+\left(\frac{1}{2}-z_{2}\right) a \hat{\mathbf{y}}+$

(192h)

O

$$
\left(\frac{1}{4}+y_{2}\right) a \hat{\mathbf{z}}
$$

$=\quad\left(\frac{1}{4}+z_{2}\right) a \hat{\mathbf{x}}+\left(\frac{1}{4}+y_{2}\right) a \hat{\mathbf{y}}+$

(192h)

O

$$
\left(\frac{1}{2}-x_{2}\right) a \hat{\mathbf{z}}
$$

$=\quad\left(\frac{1}{4}+z_{2}\right) a \hat{\mathbf{x}}+\left(\frac{1}{2}-y_{2}\right) a \hat{\mathbf{y}}+$

(192h)

O

$$
\left(\frac{1}{4}+x_{2}\right) a \hat{\mathbf{z}}
$$

$=\quad\left(\frac{1}{2}-z_{2}\right) a \hat{\mathbf{x}}+\left(\frac{1}{4}+y_{2}\right) a \hat{\mathbf{y}}+$

$$
\left(\frac{1}{4}+x_{2}\right) a \hat{\mathbf{z}}
$$

O

$=\left(\frac{1}{2}-z_{2}\right) a \hat{\mathbf{x}}+\left(\frac{1}{2}-y_{2}\right) a \hat{\mathbf{y}}+$ $\left(\frac{1}{2}-x_{2}\right) a \hat{\mathbf{z}}$

(192h)

O

$=\quad-x_{2} a \hat{\mathbf{x}}-y_{2} a \hat{\mathbf{y}}-z_{2} a \hat{\mathbf{z}}$

(192h)

$\mathrm{O}$

$=\left(\frac{1}{4}+x_{2}\right) a \hat{\mathbf{x}}+\left(\frac{1}{4}+y_{2}\right) a \hat{\mathbf{y}}-z_{2} a \hat{\mathbf{z}}$

(192h)

$\mathrm{O}$

$=\left(\frac{1}{4}+x_{2}\right) a \hat{\mathbf{x}}-y_{2} a \hat{\mathbf{y}}+\left(\frac{1}{4}+z_{2}\right) a \hat{\mathbf{z}}$

(192h)

O 


$$
\begin{array}{cc}
\mathbf{B}_{36}=\quad & \left(\frac{1}{2}+x_{2}+y_{2}+z_{2}\right) \mathbf{a}_{1}+ \\
& \left(-x_{2}-y_{2}+z_{2}\right) \mathbf{a}_{2}+ \\
& \left(-x_{2}+y_{2}-z_{2}\right) \mathbf{a}_{3} \\
& \left(-x_{2}-y_{2}+z_{2}\right) \mathbf{a}_{1}+ \\
& \left(x_{2}-y_{2}-z_{2}\right) \mathbf{a}_{2}+ \\
& \left(-x_{2}+y_{2}-z_{2}\right) \mathbf{a}_{3}
\end{array}
$$

$\mathbf{B}_{38}=\left(\frac{1}{2}+x_{2}+y_{2}+z_{2}\right) \mathbf{a}_{1}+$

$$
\left(-x_{2}+y_{2}-z_{2}\right) \mathbf{a}_{2}+
$$$$
\left(x_{2}-y_{2}-z_{2}\right) \mathbf{a}_{3}
$$

$\mathbf{B}_{39}=\quad\left(x_{2}-y_{2}-z_{2}\right) \mathbf{a}_{1}+$ $\left(-x_{2}-y_{2}+z_{2}\right) \mathbf{a}_{2}+$ $\left(\frac{1}{2}+x_{2}+y_{2}+z_{2}\right) \mathbf{a}_{3}$

$\mathbf{B}_{40}=\left(-x_{2}+y_{2}-z_{2}\right) \mathbf{a}_{1}+$ $\left(\frac{1}{2}+x_{2}+y_{2}+z_{2}\right) \mathbf{a}_{2}+$ $\left(-x_{2}-y_{2}+z_{2}\right) \mathbf{a}_{3}$

$\mathbf{B}_{41}=\left(-x_{2}+y_{2}-z_{2}\right) \mathbf{a}_{1}+$ $\left(-x_{2}-y_{2}+z_{2}\right) \mathbf{a}_{2}+$ $\left(x_{2}-y_{2}-z_{2}\right) \mathbf{a}_{3}$

$\mathbf{B}_{42}=$ $\left(x_{2}-y_{2}-z_{2}\right) \mathbf{a}_{1}+$ $\left(\frac{1}{2}+x_{2}+y_{2}+z_{2}\right) \mathbf{a}_{2}+$ $\left(-x_{2}+y_{2}-z_{2}\right) \mathbf{a}_{3}$

$\mathbf{B}_{43}=\left(\frac{1}{2}+x_{2}+y_{2}+z_{2}\right) \mathbf{a}_{1}+$ $\left(x_{2}-y_{2}-z_{2}\right) \mathbf{a}_{2}+$ $\left(-x_{2}-y_{2}+z_{2}\right) \mathbf{a}_{3}$

$\mathbf{B}_{44}=\left(-x_{2}-y_{2}+z_{2}\right) \mathbf{a}_{1}+$ $\left(-x_{2}+y_{2}-z_{2}\right) \mathbf{a}_{2}+$ $\left(\frac{1}{2}+x_{2}+y_{2}+z_{2}\right) \mathbf{a}_{3}$

$\mathbf{B}_{45}=\left(\frac{1}{2}-x_{2}+y_{2}+z_{2}\right) \mathbf{a}_{1}+$ $\left(\frac{1}{2}+x_{2}-y_{2}+z_{2}\right) \mathbf{a}_{2}+$ $\left(-x_{2}-y_{2}-z_{2}\right) \mathbf{a}_{3}$

$\mathbf{B}_{46}=\left(\frac{1}{2}+x_{2}-y_{2}+z_{2}\right) \mathbf{a}_{1}+$ $\left(\frac{1}{2}-x_{2}+y_{2}+z_{2}\right) \mathbf{a}_{2}+$ $\left(\frac{1}{2}+x_{2}+y_{2}-z_{2}\right) \mathbf{a}_{3}$

$\mathbf{B}_{47}=\left(\frac{1}{2}+x_{2}+y_{2}-z_{2}\right) \mathbf{a}_{1}+$ $\left(-x_{2}-y_{2}-z_{2}\right) \mathbf{a}_{2}+$ $\left(\frac{1}{2}+x_{2}-y_{2}+z_{2}\right) \mathbf{a}_{3}$

$\mathbf{B}_{48}=\left(-x_{2}-y_{2}-z_{2}\right) \mathbf{a}_{1}+$ $\left(\frac{1}{2}+x_{2}+y_{2}-z_{2}\right) \mathbf{a}_{2}+$ $\left(\frac{1}{2}-x_{2}+y_{2}+z_{2}\right) \mathbf{a}_{3}$

$\mathbf{B}_{49}=\left(\frac{1}{2}+x_{2}+y_{2}-z_{2}\right) \mathbf{a}_{1}+$ $\left(\frac{1}{2}-x_{2}+y_{2}+z_{2}\right) \mathbf{a}_{2}+$ $\left(-x_{2}-y_{2}-z_{2}\right) \mathbf{a}_{3}$

$\mathbf{B}_{50}=\left(-x_{2}-y_{2}-z_{2}\right) \mathbf{a}_{1}+$ $\left(\frac{1}{2}+x_{2}-y_{2}+z_{2}\right) \mathbf{a}_{2}+$ $\left(\frac{1}{2}+x_{2}+y_{2}-z_{2}\right) \mathbf{a}_{3}$

$\mathbf{B}_{51}=\left(\frac{1}{2}-x_{2}+y_{2}+z_{2}\right) \mathbf{a}_{1}+$ $\left(\frac{1}{2}+x_{2}+y_{2}-z_{2}\right) \mathbf{a}_{2}+$ $\left(\frac{1}{2}+x_{2}-y_{2}+z_{2}\right) \mathbf{a}_{3}$

$$
=-x_{2} a \hat{\mathbf{x}}+\left(\frac{1}{4}+y_{2}\right) a \hat{\mathbf{y}}+\left(\frac{1}{4}+z_{2}\right) a \hat{\mathbf{z}}
$$

$=\quad-z_{2} a \hat{\mathbf{x}}-x_{2} a \hat{\mathbf{y}}-y_{2} a \hat{\mathbf{z}}$

$\mathrm{O}$

$=-z_{2} a \hat{\mathbf{x}}+\left(\frac{1}{4}+x_{2}\right) a \hat{\mathbf{y}}+\left(\frac{1}{4}+y_{2}\right) a \hat{\mathbf{z}}$

$\mathrm{O}$

$=\left(\frac{1}{4}+z_{2}\right) a \hat{\mathbf{x}}+\left(\frac{1}{4}+x_{2}\right) a \hat{\mathbf{y}}-y_{2} a \hat{\mathbf{z}}$

$\mathrm{O}$

$=\left(\frac{1}{4}+z_{2}\right) a \hat{\mathbf{x}}-x_{2} a \hat{\mathbf{y}}+\left(\frac{1}{4}+y_{2}\right) a \hat{\mathbf{z}}$

(192h)

$\mathrm{O}$

$=\quad-y_{2} a \hat{\mathbf{x}}-z_{2} a \hat{\mathbf{y}}-x_{2} a \hat{\mathbf{z}}$

$\mathrm{O}$

$=\left(\frac{1}{4}+y_{2}\right) a \hat{\mathbf{x}}-z_{2} a \hat{\mathbf{y}}+\left(\frac{1}{4}+x_{2}\right) a \hat{\mathbf{z}}$

$\mathrm{O}$

$=-y_{2} a \hat{\mathbf{x}}+\left(\frac{1}{4}+z_{2}\right) a \hat{\mathbf{y}}+\left(\frac{1}{4}+x_{2}\right) a \hat{\mathbf{z}}$

(192h)

$\mathrm{O}$

$=\left(\frac{1}{4}+y_{2}\right) a \hat{\mathbf{x}}+\left(\frac{1}{4}+z_{2}\right) a \hat{\mathbf{y}}-x_{2} a \hat{\mathbf{z}}$

(192h)

$\mathrm{O}$

$=\left(\frac{1}{4}-y_{2}\right) a \hat{\mathbf{x}}+\left(\frac{1}{4}-x_{2}\right) a \hat{\mathbf{y}}+$

(192h)

$\mathrm{O}$

$$
\left(\frac{1}{2}+z_{2}\right) a \hat{\mathbf{z}}
$$

$=\left(\frac{1}{2}+y_{2}\right) a \hat{\mathbf{x}}+\left(\frac{1}{2}+x_{2}\right) a \hat{\mathbf{y}}+$

$\mathrm{O}$

$$
\left(\frac{1}{2}+z_{2}\right) a \hat{\mathbf{z}}
$$

$=\left(\frac{1}{4}-y_{2}\right) a \hat{\mathbf{x}}+\left(\frac{1}{2}+x_{2}\right) a \hat{\mathbf{y}}+$

$\mathrm{O}$

$$
\left(\frac{1}{4}-z_{2}\right) a \hat{\mathbf{z}}
$$

$=\left(\frac{1}{2}+y_{2}\right) a \hat{\mathbf{x}}+\left(\frac{1}{4}-x_{2}\right) a \hat{\mathbf{y}}+$

$\mathrm{O}$

$$
\left(\frac{1}{4}-z_{2}\right) a \hat{\mathbf{z}}
$$

$=\left(\frac{1}{4}-x_{2}\right) a \hat{\mathbf{x}}+\left(\frac{1}{4}-z_{2}\right) a \hat{\mathbf{y}}+$

$\mathrm{O}$

$$
\left(\frac{1}{2}+y_{2}\right) a \hat{\mathbf{z}}
$$

$=\left(\frac{1}{2}+x_{2}\right) a \hat{\mathbf{x}}+\left(\frac{1}{4}-z_{2}\right) a \hat{\mathbf{y}}+$

$\mathrm{O}$

$$
\left(\frac{1}{4}-y_{2}\right) a \hat{\mathbf{z}}
$$

$=\left(\frac{1}{2}+x_{2}\right) a \hat{\mathbf{x}}+\left(\frac{1}{2}+z_{2}\right) a \hat{\mathbf{y}}+$

(192h)

$\mathrm{O}$ 


\begin{tabular}{|c|c|c|c|c|c|}
\hline $\mathbf{B}_{52}$ & $=$ & $\begin{array}{c}\left(\frac{1}{2}+x_{2}-y_{2}+z_{2}\right) \mathbf{a}_{1}+ \\
\left(-x_{2}-y_{2}-z_{2}\right) \mathbf{a}_{2}+ \\
\left(\frac{1}{2}-x_{2}+y_{2}+z_{2}\right) \mathbf{a}_{3}\end{array}$ & $=$ & $\begin{array}{c}\left(\frac{1}{4}-x_{2}\right) a \hat{\mathbf{x}}+\left(\frac{1}{2}+z_{2}\right) a \hat{\mathbf{y}}+ \\
\left(\frac{1}{4}-y_{2}\right) a \hat{\mathbf{z}}\end{array}$ & $(192 h)$ \\
\hline $\mathbf{B}_{53}$ & $=$ & $\begin{array}{l}\left(\frac{1}{2}+x_{2}-y_{2}+z_{2}\right) \mathbf{a}_{1}+ \\
\left(\frac{1}{2}+x_{2}+y_{2}-z_{2}\right) \mathbf{a}_{2}+ \\
\quad\left(-x_{2}-y_{2}-z_{2}\right) \mathbf{a}_{3}\end{array}$ & $=$ & $\begin{array}{c}\left(\frac{1}{4}-z_{2}\right) a \hat{\mathbf{x}}+\left(\frac{1}{4}-y_{2}\right) a \hat{\mathbf{y}}+ \\
\left(\frac{1}{2}+x_{2}\right) a \hat{\mathbf{z}}\end{array}$ & $(192 h)$ \\
\hline $\mathbf{B}_{54}$ & $=$ & $\begin{array}{c}\left(\frac{1}{2}-x_{2}+y_{2}+z_{2}\right) \mathbf{a}_{1}+ \\
\left(-x_{2}-y_{2}-z_{2}\right) \mathbf{a}_{2}+ \\
\left(\frac{1}{2}+x_{2}+y_{2}-z_{2}\right) \mathbf{a}_{3}\end{array}$ & $=$ & $\begin{array}{c}\left(\frac{1}{4}-z_{2}\right) a \hat{\mathbf{x}}+\left(\frac{1}{2}+y_{2}\right) a \hat{\mathbf{y}}+ \\
\left(\frac{1}{4}-x_{2}\right) a \hat{\mathbf{z}}\end{array}$ & $(192 h)$ \\
\hline $\mathbf{B}_{55}$ & $=$ & $\begin{array}{c}\left(-x_{2}-y_{2}-z_{2}\right) \mathbf{a}_{1}+ \\
\left(\frac{1}{2}-x_{2}+y_{2}+z_{2}\right) \mathbf{a}_{2}+ \\
\left(\frac{1}{2}+x_{2}-y_{2}+z_{2}\right) \mathbf{a}_{3}\end{array}$ & $=$ & $\begin{array}{c}\left(\frac{1}{2}+z_{2}\right) a \hat{\mathbf{x}}+\left(\frac{1}{4}-y_{2}\right) a \hat{\mathbf{y}}+ \\
\left(\frac{1}{4}-x_{2}\right) a \hat{\mathbf{z}}\end{array}$ & $(192 h)$ \\
\hline $\mathbf{B}_{56}$ & $=$ & $\begin{array}{c}\left(\frac{1}{2}+x_{2}+y_{2}-z_{2}\right) \mathbf{a}_{1}+ \\
\left(\frac{1}{2}+x_{2}-y_{2}+z_{2}\right) \mathbf{a}_{2}+ \\
\left(\frac{1}{2}-x_{2}+y_{2}+z_{2}\right) \mathbf{a}_{3}\end{array}$ & $=$ & $\begin{array}{c}\left(\frac{1}{2}+z_{2}\right) a \hat{\mathbf{x}}+\left(\frac{1}{2}+y_{2}\right) a \hat{\mathbf{y}}+ \\
\left(\frac{1}{2}+x_{2}\right) a \hat{\mathbf{z}}\end{array}$ & $(192 h)$ \\
\hline
\end{tabular}

\section{References:}

- L. M. Kirkpatrick and L. Pauling, XXVIII. Über die Kristallstruktur der kubischen Tellursäure, Zeitschrift für Kristallographie - Crystalline Materials 63, 502-506 (1926), doi:10.1524/zkri.1926.63.1.502.

\section{Found in:}

- P. Villars and K. Cenzual, Pearson's Crystal Data - Crystal Structure Database for Inorganic Compounds, ASM International (2013).

\section{Geometry files:}

- CIF: pp. 982

- POSCAR: pp. 983 


\section{$\gamma$-brass $\left(\mathrm{Fe}_{3} \mathrm{Zn}_{10}, D 8_{1}\right)$ Structure: A3B10_cI52_229_e_fh}
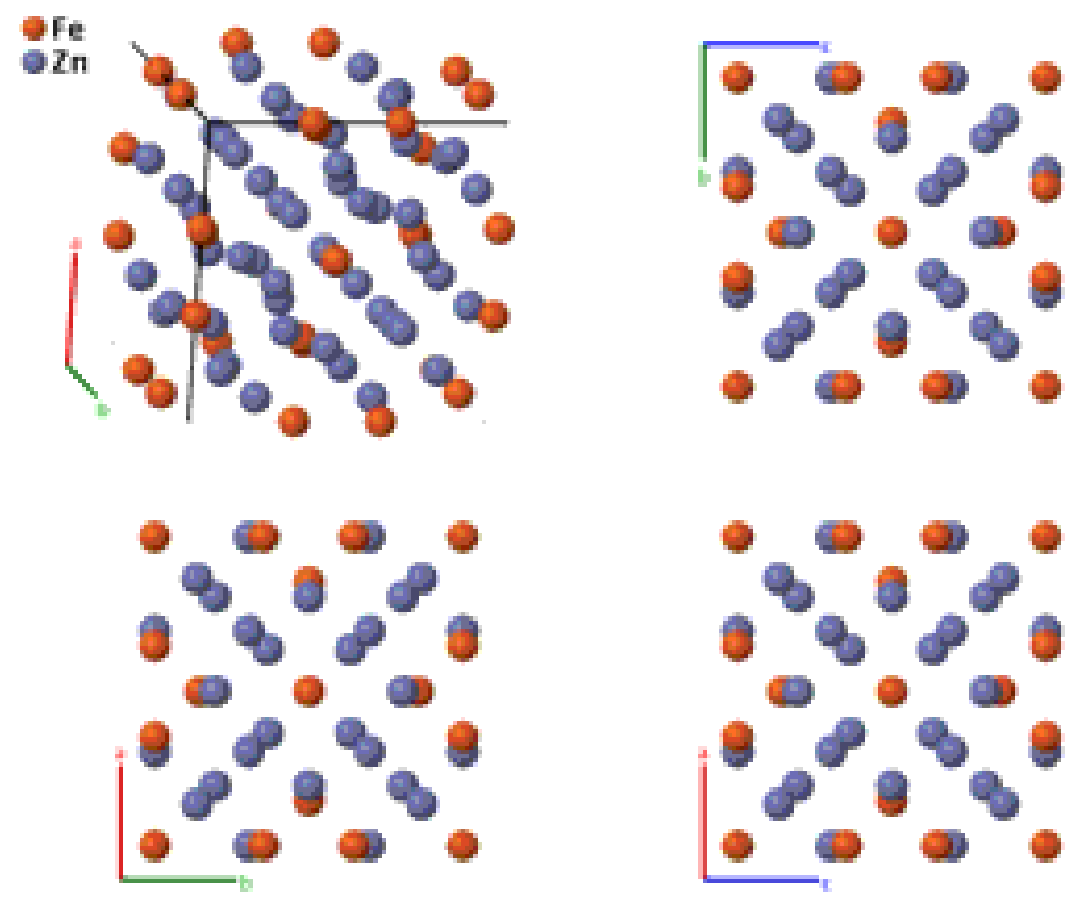

Prototype

AFLOW prototype label

Strukturbericht designation

Pearson symbol

Space group number

Space group symbol

AFLOW prototype command
: $\quad \gamma-\mathrm{Fe}_{3} \mathrm{Zn}_{10}$

: A3B10_cI52_229_e_fh

: $\quad D 8_{1}$

: $\quad$ cI52

: 229

: $\quad \operatorname{Im} \overline{3} m$
: aflow --proto=A3B10_cI52_229_e_fh

- params $=a, x_{1}, x_{2}, y_{3}$

\section{Other compounds with this structure:}

- (Pearson, 1958), p. 252, gives a list of compounds which can take on the $D 8_{1}, D 8_{2}$, or $D 8_{3}$ structure, depending on the exact composition.

- Adding another atom at the origin changes this to the $L 2_{2}$ structure. This structure is defined in (Pearson, 1958) quoting (Schramm, 1938). More recent investigations such as (Johannsson, 1968), (Brandon, 1974) and (Yu, 2005) find that $\gamma$ - $\mathrm{Fe}_{3} \mathrm{Zn}_{10}$ forms in the $D 8_{2}$ structure, with $\mathrm{Fe}$ atoms on one (8c) site, $\mathrm{Zn}$ atoms on the other (8e) site and the (24g) sites, and a 50-50 alloy of Fe and Zn on the other (8e) site. We use Brandon's data, mapping (12g) $\rightarrow(12 \mathrm{e}),(24 \mathrm{~g}) \rightarrow$ (24h), and averaging the two (8e) sites to produce the (12e) coordinate here. 


$$
\begin{aligned}
& \mathbf{a}_{1}=-\frac{1}{2} a \hat{\mathbf{x}}+\frac{1}{2} a \hat{\mathbf{y}}+\frac{1}{2} a \hat{\mathbf{z}} \\
& \mathbf{a}_{2}=\frac{1}{2} a \hat{\mathbf{x}}-\frac{1}{2} a \hat{\mathbf{y}}+\frac{1}{2} a \hat{\mathbf{z}} \\
& \mathbf{a}_{3}=\frac{1}{2} a \hat{\mathbf{x}}+\frac{1}{2} a \hat{\mathbf{y}}-\frac{1}{2} a \hat{\mathbf{z}}
\end{aligned}
$$

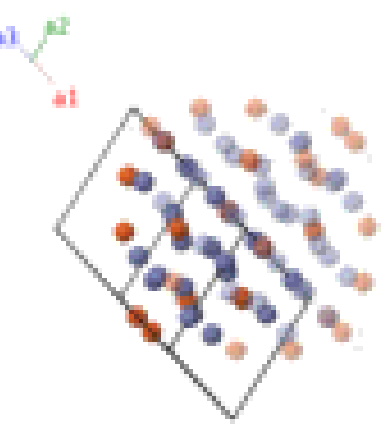

\section{Basis vectors:}

Lattice Coordinates

$\mathbf{B}_{1}=$

$\mathbf{B}_{2}=$

$\mathbf{B}_{3}=$

$\mathbf{B}_{4}=$

$\mathbf{B}_{5}=$

$\mathbf{B}_{6}=$

$\mathbf{B}_{7}=$

$\mathbf{B}_{8}=$

$\mathbf{B}_{9}=$

$\mathbf{B}_{10}=$

$\mathbf{B}_{11}=$

$\mathbf{B}_{12}=$

$\mathbf{B}_{13}=$

$\mathbf{B}_{14}=$

$\mathbf{B}_{15}=$

$\mathbf{B}_{16}=$

$\mathbf{B}_{17}=$

$\mathbf{B}_{18}=$

$\mathbf{B}_{19}=$

$\mathbf{B}_{20}=$

$\mathbf{B}_{21}=$

$\mathbf{B}_{22}=$

$\mathbf{B}_{23}=$

$\mathbf{B}_{24}=$

$\mathbf{B}_{25}=$

$\mathbf{B}_{26}=$

$$
x_{1} \mathbf{a}_{2}+x_{1} \mathbf{a}_{3}
$$$$
-x_{1} \mathbf{a}_{2}-x_{1} \mathbf{a}_{3}
$$$$
x_{1} \mathbf{a}_{1}+x_{1} \mathbf{a}_{3}
$$$$
-x_{1} \mathbf{a}_{1}+-x_{1} \mathbf{a}_{3}
$$$$
x_{1} \mathbf{a}_{1}+x_{1} \mathbf{a}_{2}
$$$$
-x_{1} \mathbf{a}_{1}-x_{1} \mathbf{a}_{2}
$$

$$
2 x_{2} \mathbf{a}_{1}+2 x_{2} \mathbf{a}_{2}+2 x_{2} \mathbf{a}_{3}
$$

$$
-2 x_{2} \mathbf{a}_{3}
$$$$
-2 x_{2} \mathbf{a}_{2}
$$$$
-2 x_{2} \mathbf{a}_{1}
$$$$
2 x_{2} \mathbf{a}_{3}
$$$$
-2 x_{2} \mathbf{a}_{1}-2 x_{2} \mathbf{a}_{2}-2 x_{2} \mathbf{a}_{3}
$$$$
2 x_{2} \mathbf{a}_{2}
$$$$
2 x_{2} \mathbf{a}_{1}
$$

$$
2 y_{3} \mathbf{a}_{1}+y_{3} \mathbf{a}_{2}+y_{3} \mathbf{a}_{3}
$$$$
y_{3} \mathbf{a}_{2}-y_{3} \mathbf{a}_{3}
$$$$
-y_{3} \mathbf{a}_{2}+y_{3} \mathbf{a}_{3}
$$

$$
-2 y_{3} \mathbf{a}_{1}-y_{3} \mathbf{a}_{2}-y_{3} \mathbf{a}_{3}
$$

$$
y_{3} \mathbf{a}_{1}+2 y_{3} \mathbf{a}_{2}+y_{3} \mathbf{a}_{3}
$$$$
-y_{3} \mathbf{a}_{1}+y_{3} \mathbf{a}_{3}
$$

$y_{3} \mathbf{a}_{1}+-y_{3} \mathbf{a}_{3}$

$$
-y_{3} \mathbf{a}_{1}-2 y_{3} \mathbf{a}_{2}-y_{3} \mathbf{a}_{3}
$$$$
y_{3} \mathbf{a}_{1}+y_{3} \mathbf{a}_{2}+2 y_{3} \mathbf{a}_{3}
$$$$
y_{3} \mathbf{a}_{1}-y_{3} \mathbf{a}_{2}
$$$$
-y_{3} \mathbf{a}_{1}+y_{3} \mathbf{a}_{2}
$$

$-y_{3} \mathbf{a}_{1}-y_{3} \mathbf{a}_{2}-2 y_{3} \mathbf{a}_{3}$
Cartesian Coordinates

$=$

$=$

$=$

$=$

$=$

$=$
$=$

$=$
$=$

$=$

$=$

$=$

$=$

$=$

$=$

$=$

$=$

$=$

$=$

$=$

$=$

$=$

$=$

$=$

$=$

$=$

$=$

$=$

$=$

$=$

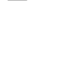

$=$

$=$

$$
\begin{gathered}
x_{1} a \hat{\mathbf{x}} \\
-x_{1} a \hat{\mathbf{x}} \\
x_{1} a \hat{\mathbf{y}} \\
-x_{1} a \hat{\mathbf{y}} \\
x_{1} a \hat{\mathbf{z}} \\
-x_{1} a \hat{\mathbf{z}} \\
x_{2} a \hat{\mathbf{x}}+x_{2} a \hat{\mathbf{y}}+x_{2} a \hat{\mathbf{z}} \\
-x_{2} a \hat{\mathbf{x}}-x_{2} a \hat{\mathbf{y}}+x_{2} a \hat{\mathbf{z}} \\
-x_{2} a \hat{\mathbf{x}}+x_{2} a \hat{\mathbf{y}}-x_{2} a \hat{\mathbf{z}} \\
x_{2} a \hat{\mathbf{x}}-x_{2} a \hat{\mathbf{y}}-x_{2} a \hat{\mathbf{z}} \\
x_{2} a \hat{\mathbf{x}}+x_{2} a \hat{\mathbf{y}}-x_{2} a \hat{\mathbf{z}} \\
-x_{2} a \hat{\mathbf{x}}-x_{2} a \hat{\mathbf{y}}-x_{2} a \hat{\mathbf{z}} \\
x_{2} a \hat{\mathbf{x}}-x_{2} a \hat{\mathbf{y}}+x_{2} a \hat{\mathbf{z}} \\
-x_{2} a \hat{\mathbf{x}}+x_{2} a \hat{\mathbf{y}}+x_{2} a \hat{\mathbf{z}} \\
y_{3} a \hat{\mathbf{y}}+y_{3} a \hat{\mathbf{z}} \\
-y_{3} a \hat{\mathbf{y}}+y_{3} a \hat{\mathbf{z}} \\
y_{3} a \hat{\mathbf{x}}+y_{3} a \hat{\mathbf{y}}+y_{3} a \hat{\mathbf{y}} \\
-y_{3} a \hat{\mathbf{y}}-y_{3} a \hat{\mathbf{z}}+y_{3} a \hat{\mathbf{z}} \\
y_{3} a \hat{\mathbf{y}}-y_{3} a \hat{\mathbf{z}} \\
y_{3} a \hat{\mathbf{x}}+y_{3} a \hat{\mathbf{z}} \\
-y_{3} a \hat{\mathbf{z}} \\
-y_{3} \\
-y_{3} \\
-y_{3}
\end{gathered}
$$

Wyckoff Position
Atom Type

$\mathrm{Fe}$

$\mathrm{Fe}$

$\mathrm{Fe}$

$\mathrm{Fe}$

$\mathrm{Fe}$

$\mathrm{Fe}$

Zn I

Zn I

Zn I

Zn I

Zn I

Zn I

Zn I

Zn I

Zn II

Zn II

Zn II

Zn II

Zn II

Zn II

Zn II

Zn II

Zn II

Zn II

Zn II

Zn II 


\section{References:}

- J. Schramm, X-Ray Investigation of Phases and Phase Limits of the Zn Alloy Systems with Fe, Co and Ni, Z. Metallkd. 30, 122-130 (1938).

- A. Johansson, H. Ljung, and S. Westman, X-Ray and Neutron Diffraction Studies on Gamma-Ni,Zn and Gamma-Fe,Zn, Acta Chem. Scand. 22, 2743-2753 (1968), doi:10.3891/acta.chem.scand.22-2743.

- J. K. Brandon, R. Y. Brizard, P. C. Chieh, R. K. McMillan, and W. B. Pearson, New refinements of the $\gamma$-brass type structures $\mathrm{Cu}_{5} \mathrm{Zn}_{8}, \mathrm{Cu}_{5} \mathrm{Cd} d_{8}$ and $\mathrm{Fe}_{3} \mathrm{Zn}_{10}$, Acta Crystallogr. Sect. B Struct. Sci. 30, 1412-1417 (1974), doi:10.1107/S0567740874004997.

- J. Yu, J. Liu, J. Zhang, and J. Wu, Electron Diffraction Study on Fe-Zn $\Gamma$ intermetallic Phase of a Galvannealed IF Steel Sheet, Mater. Trans. 46, 1079-1082 (2005).

\section{Found in:}

- W. B. Pearson, A Handbook of Lattice Spacings and Structures of Metals and Alloys, no. N.R.C. No. 4303 in International Series of Monographs on Metal Physics and Physical Metallurgy (Pergamon Press, Oxford, London, Edinburgh, New York, Paris, Frankfort, 1958), 1964 reprint with corrections edn.

\section{Geometry files:}

- CIF: pp. 983

- POSCAR: pp. 984 


\section{$\beta$-Hg ${ }_{4} \mathrm{Pt}$ Structure: A4B_cI10_229_c_a}
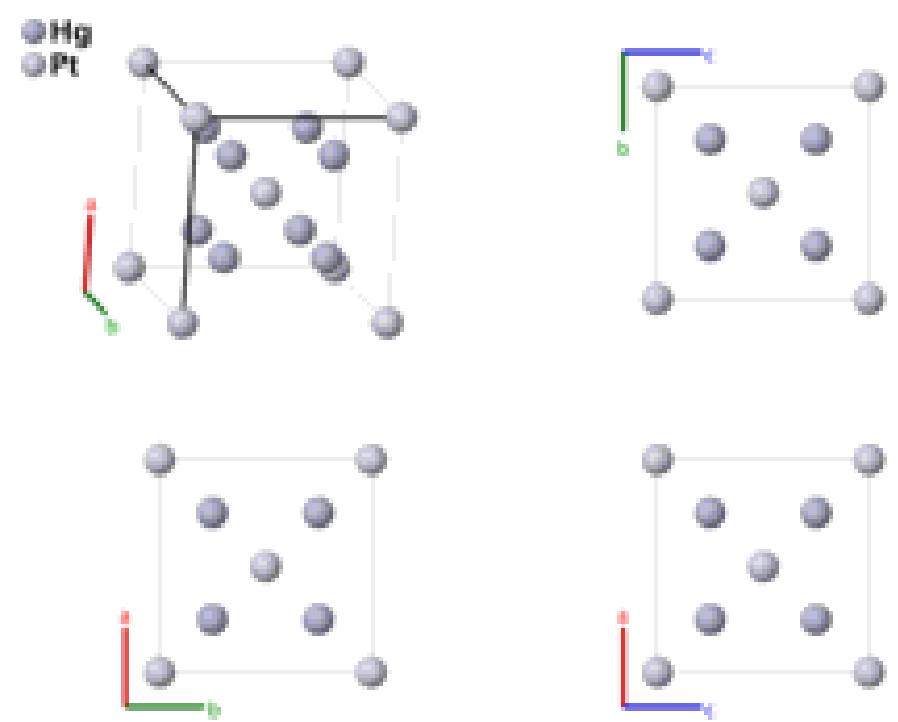

\section{Prototype}

AFLOW prototype label

$: \quad \beta-\mathrm{Hg}_{4} \mathrm{Pt}$

Strukturbericht designation

: A4B_cI10_229_c_a

Pearson symbol

: None

Space group number

: $\quad \mathrm{cI} 10$

Space group symbol

: $\quad 229$

AFLOW prototype command : $\begin{aligned} & \text { aflow --proto=A4B_cI10_229_c_a } \\ & -- \text { params }=a\end{aligned}$

: $\operatorname{Im} \overline{3} m$

- params $=a$

\section{Other compounds with this structure:}

- $\mathrm{Hg}_{4} \mathrm{Pd}, \mathrm{Hg}_{4} \mathrm{U}$

Body-centered Cubic primitive vectors:

$$
\begin{aligned}
& \mathbf{a}_{1}=-\frac{1}{2} a \hat{\mathbf{x}}+\frac{1}{2} a \hat{\mathbf{y}}+\frac{1}{2} a \hat{\mathbf{z}} \\
& \mathbf{a}_{2}=\frac{1}{2} a \hat{\mathbf{x}}-\frac{1}{2} a \hat{\mathbf{y}}+\frac{1}{2} a \hat{\mathbf{z}} \\
& \mathbf{a}_{3}=\frac{1}{2} a \hat{\mathbf{x}}+\frac{1}{2} a \hat{\mathbf{y}}-\frac{1}{2} a \hat{\mathbf{z}}
\end{aligned}
$$

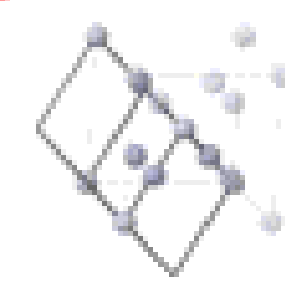

Basis vectors:

Lattice Coordinates

$\mathbf{B}_{1}=0 \mathbf{a}_{1}+0 \mathbf{a}_{2}+0 \mathbf{a}_{3}$

$\mathbf{B}_{2}=\frac{1}{2} \mathbf{a}_{1}+\frac{1}{2} \mathbf{a}_{2}+\frac{1}{2} \mathbf{a}_{3}$

$\mathbf{B}_{3}=$
Cartesian Coordinates

$=$ $0 \hat{\mathbf{x}}+0 \hat{\mathbf{y}}+0 \hat{\mathbf{z}}$

$\frac{1}{4} a \hat{\mathbf{x}}+\frac{1}{4} a \hat{\mathbf{y}}+\frac{1}{4} a \hat{\mathbf{z}}$

$=\quad \frac{1}{4} a \hat{\mathbf{x}}+\frac{1}{4} a \hat{\mathbf{y}}-\frac{1}{4} a \hat{\mathbf{z}}$
Wyckoff Position
Atom Type

$\mathrm{Pt}$

$\mathrm{Hg}$

$\mathrm{Hg}$ 


$\begin{array}{llllll}\mathbf{B}_{4}= & \frac{1}{2} \mathbf{a}_{2} & = & \frac{1}{4} a \hat{\mathbf{x}}-\frac{1}{4} a \hat{\mathbf{y}}+\frac{1}{4} a \hat{\mathbf{z}} & (8 c) & \mathrm{Hg} \\ \mathbf{B}_{5}= & \frac{1}{2} \mathbf{a}_{1} & = & -\frac{1}{4} a \hat{\mathbf{x}}+\frac{1}{4} a \hat{\mathbf{y}}+\frac{1}{4} a \hat{\mathbf{z}} & (8 c) & \mathrm{Hg}\end{array}$

\section{References:}

- E. Bauer, H. Nowotny, and A. Stempfl, Röntgenographische Untersuchungen im System: Platin-Quecksilber, Monatsh. Chem. 84, 211-212 (1953), doi:10.1007/BF00899140.

\section{Geometry files:}

- CIF: pp. 984

- POSCAR: pp. 985 


\section{$\mathrm{Ir}_{3} \mathrm{Ge}_{7}\left(D 8_{f}\right)$ Structure: A7B3_cI40_229_df_e}
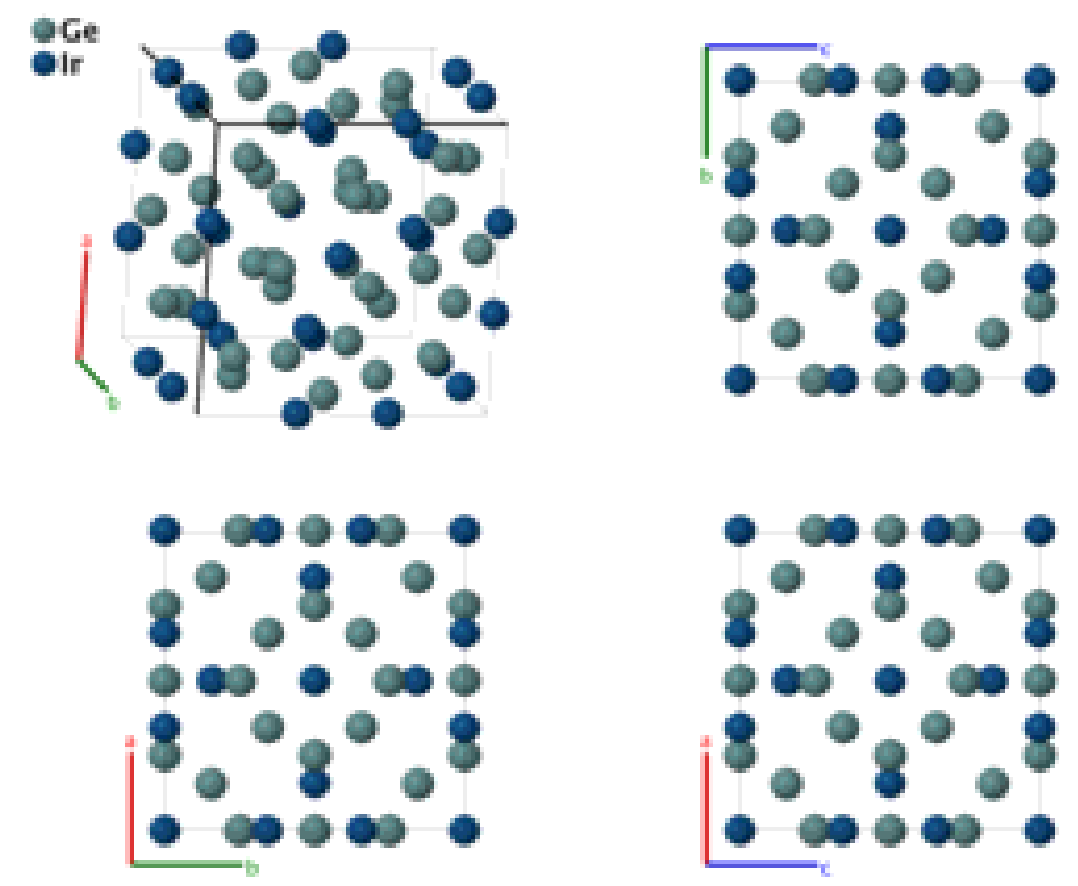

\section{Prototype}

AFLOW prototype label

$: \quad \mathrm{Ir}_{3} \mathrm{Ge}_{7}$

Strukturbericht designation

Pearson symbol

: A7B3_cI40_229_df_e

Space group number

Space group symbol

AFLOW prototype command

: $\quad D 8_{f}$

: $\quad$ cI40

: 229

: $\quad \operatorname{Im} \overline{3} m$

aflow --proto=A7B3_cI40_229_df_e

- - params $=a, x_{2}, x_{3}$

\section{Other compounds with this structure}

- $\mathrm{Ga}_{7} \mathrm{Ni}_{3}, \mathrm{In}_{7} \mathrm{Pd}_{3}, \mathrm{Sb}_{7} \mathrm{Mo}_{3}, \mathrm{As}_{7} \mathrm{Re}_{3}, \mathrm{Sn}_{7} \mathrm{Ru}_{3}$

Body-centered Cubic primitive vectors:

$$
\begin{aligned}
& \mathbf{a}_{1}=-\frac{1}{2} a \hat{\mathbf{x}}+\frac{1}{2} a \hat{\mathbf{y}}+\frac{1}{2} a \hat{\mathbf{z}} \\
& \mathbf{a}_{2}=\frac{1}{2} a \hat{\mathbf{x}}-\frac{1}{2} a \hat{\mathbf{y}}+\frac{1}{2} a \hat{\mathbf{z}} \\
& \mathbf{a}_{3}=\frac{1}{2} a \hat{\mathbf{x}}+\frac{1}{2} a \hat{\mathbf{y}}-\frac{1}{2} a \hat{\mathbf{z}}
\end{aligned}
$$

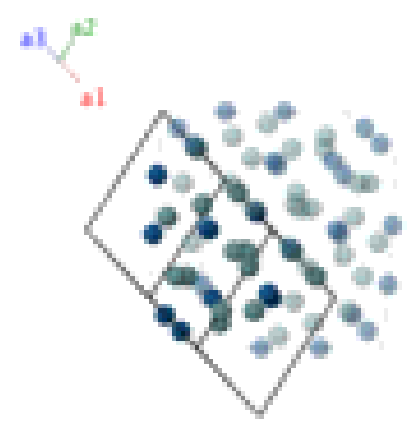

Basis vectors:

Lattice Coordinates

$\mathbf{B}_{1}=\frac{1}{2} \mathbf{a}_{1}+\frac{3}{4} \mathbf{a}_{2}+\frac{1}{4} \mathbf{a}_{3}$
Cartesian Coordinates

$\frac{1}{4} a \hat{\mathbf{x}}+\frac{1}{2} a \hat{\mathbf{z}}$
Wyckoff Position

$(12 d)$
Atom Type

Ge I 


\begin{tabular}{|c|c|c|c|c|c|c|}
\hline $\mathbf{B}_{2}$ & $=$ & $\frac{1}{2} \mathbf{a}_{1}+\frac{1}{4} \mathbf{a}_{2}+\frac{3}{4} \mathbf{a}_{3}$ & $=$ & $\frac{1}{4} a \hat{\mathbf{x}}+\frac{1}{2} a \hat{\mathbf{y}}$ & $(12 d)$ & Ge I \\
\hline $\mathbf{B}_{3}$ & $=$ & $\frac{1}{4} \mathbf{a}_{1}+\frac{1}{2} \mathbf{a}_{2}+\frac{3}{4} \mathbf{a}_{3}$ & $=$ & $\frac{1}{2} a \hat{\mathbf{x}}+\frac{1}{4} a \hat{\mathbf{y}}$ & $(12 d)$ & Ge I \\
\hline $\mathbf{B}_{4}$ & $=$ & $\frac{3}{4} \mathbf{a}_{1}+\frac{1}{2} \mathbf{a}_{2}+\frac{1}{4} \mathbf{a}_{3}$ & $=$ & $\frac{1}{4} a \hat{\mathbf{y}}+\frac{1}{2} a \hat{\mathbf{z}}$ & $(12 d)$ & Ge I \\
\hline $\mathbf{B}_{5}$ & $=$ & $\frac{3}{4} \mathbf{a}_{1}+\frac{1}{4} \mathbf{a}_{2}+\frac{1}{2} \mathbf{a}_{3}$ & $=$ & $\frac{1}{2} a \hat{\mathbf{y}}+\frac{1}{4} a \hat{\mathbf{z}}$ & $(12 d)$ & Ge I \\
\hline $\mathbf{B}_{6}$ & $=$ & $\frac{1}{4} \mathbf{a}_{1}+\frac{3}{4} \mathbf{a}_{2}+\frac{1}{2} \mathbf{a}_{3}$ & $=$ & $\frac{1}{2} a \hat{\mathbf{x}}+\frac{1}{4} a \hat{\mathbf{z}}$ & $(12 d)$ & Ge I \\
\hline $\mathbf{B}_{7}$ & $=$ & $x_{2} \mathbf{a}_{2}+x_{2} \mathbf{a}_{3}$ & $=$ & $x_{2} a \hat{\mathbf{x}}$ & $(12 e)$ & $\mathrm{Ir}$ \\
\hline $\mathbf{B}_{8}$ & $=$ & $-x_{2} \mathbf{a}_{2}-x_{2} \mathbf{a}_{3}$ & $=$ & $-x_{2} a \hat{\mathbf{x}}$ & $(12 e)$ & $\mathrm{Ir}$ \\
\hline $\mathbf{B}_{9}$ & $=$ & $x_{2} \mathbf{a}_{1}+x_{2} \mathbf{a}_{3}$ & $=$ & $x_{2} a \hat{\mathbf{y}}$ & $(12 e)$ & Ir \\
\hline $\mathbf{B}_{10}$ & $=$ & $-x_{2} \mathbf{a}_{1}+-x_{2} \mathbf{a}_{3}$ & $=$ & $-x_{2} a \hat{\mathbf{y}}$ & $(12 e)$ & Ir \\
\hline $\mathbf{B}_{11}$ & $=$ & $x_{2} \mathbf{a}_{1}+x_{2} \mathbf{a}_{2}$ & $=$ & $x_{2} a \hat{\mathbf{z}}$ & $(12 e)$ & Ir \\
\hline $\mathbf{B}_{12}$ & $=$ & $-x_{2} \mathbf{a}_{1}-x_{2} \mathbf{a}_{2}$ & $=$ & $-x_{2} a \hat{\mathbf{z}}$ & $(12 e)$ & $\mathrm{Ir}$ \\
\hline $\mathbf{B}_{13}$ & $=$ & $2 x_{3} \mathbf{a}_{1}+2 x_{3} \mathbf{a}_{2}+2 x_{3} \mathbf{a}_{3}$ & $=$ & $x_{3} a \hat{\mathbf{x}}+x_{3} a \hat{\mathbf{y}}+x_{3} a \hat{\mathbf{z}}$ & $(16 f)$ & Ge II \\
\hline $\mathbf{B}_{14}$ & $=$ & $-2 x_{3} \mathbf{a}_{3}$ & $=$ & $-x_{3} a \hat{\mathbf{x}}-x_{3} a \hat{\mathbf{y}}+x_{3} a \hat{\mathbf{z}}$ & $(16 f)$ & Ge II \\
\hline $\mathbf{B}_{15}$ & $=$ & $-2 x_{3} \mathbf{a}_{2}$ & $=$ & $-x_{3} a \hat{\mathbf{x}}+x_{3} a \hat{\mathbf{y}}-x_{3} a \hat{\mathbf{z}}$ & $(16 f)$ & Ge II \\
\hline $\mathbf{B}_{16}$ & $=$ & $-2 x_{3} \mathbf{a}_{1}$ & $=$ & $x_{3} a \hat{\mathbf{x}}-x_{3} a \hat{\mathbf{y}}-x_{3} a \hat{\mathbf{z}}$ & $(16 f)$ & Ge II \\
\hline $\mathbf{B}_{17}$ & $=$ & $2 x_{3} \mathbf{a}_{3}$ & $=$ & $x_{3} a \hat{\mathbf{x}}+x_{3} a \hat{\mathbf{y}}-x_{3} a \hat{\mathbf{z}}$ & $(16 f)$ & Ge II \\
\hline $\mathbf{B}_{18}$ & $=$ & $-2 x_{3} \mathbf{a}_{1}-2 x_{3} \mathbf{a}_{2}-2 x_{3} \mathbf{a}_{3}$ & $=$ & $-x_{3} a \hat{\mathbf{x}}-x_{3} a \hat{\mathbf{y}}-x_{3} a \hat{\mathbf{z}}$ & $(16 f)$ & Ge II \\
\hline $\mathbf{B}_{19}$ & $=$ & $2 x_{3} \mathbf{a}_{2}$ & $=$ & $x_{3} a \hat{\mathbf{x}}-x_{3} a \hat{\mathbf{y}}+x_{3} a \hat{\mathbf{z}}$ & $(16 f)$ & Ge II \\
\hline $\mathbf{B}_{20}$ & $=$ & $2 x_{3} \mathbf{a}_{1}$ & $=$ & $-x_{3} a \hat{\mathbf{x}}+x_{3} a \hat{\mathbf{y}}+x_{3} a \hat{\mathbf{z}}$ & $(16 f)$ & Ge II \\
\hline
\end{tabular}

\section{References:}

- U. Häussermann, M. Elding-Pontén, C. Svensson, and S. Lidin, Compounds with the $\mathrm{Ir}_{3} \mathrm{Ge}_{7}$ Structure Type: Interpenetrating Frameworks with Flexible Bonding Properties, Chem. Euro. J. 4, 1007-1015 (1998), doi:10.1002/(SICI)1521-3765(19980615)4:6<1007::AID-CHEM1007>3.0.CO;2-7.

\section{Found in:}

- F. Selim, J. P. Bevington, and G. S. Collins, Diffusion of ${ }^{111} \mathrm{Cd}$ probes in $\mathrm{Ga} 7 \mathrm{Pt}_{3}$ studied via nuclear quadrupole relaxation, Hyperfine Interact. 178, 87-90 (2007), doi:10.1007/s10751-008-9663-3.

\section{Geometry files:}

- CIF: pp. 985

- POSCAR: pp. 986 


\section{Garnet $\left(\mathrm{Co}_{3} \mathrm{Al}_{2} \mathrm{Si}_{3} \mathrm{O}_{12}, S 1_{4}\right)$ Structure: A2B3C12D3_cI160_230_a_c_h_d}
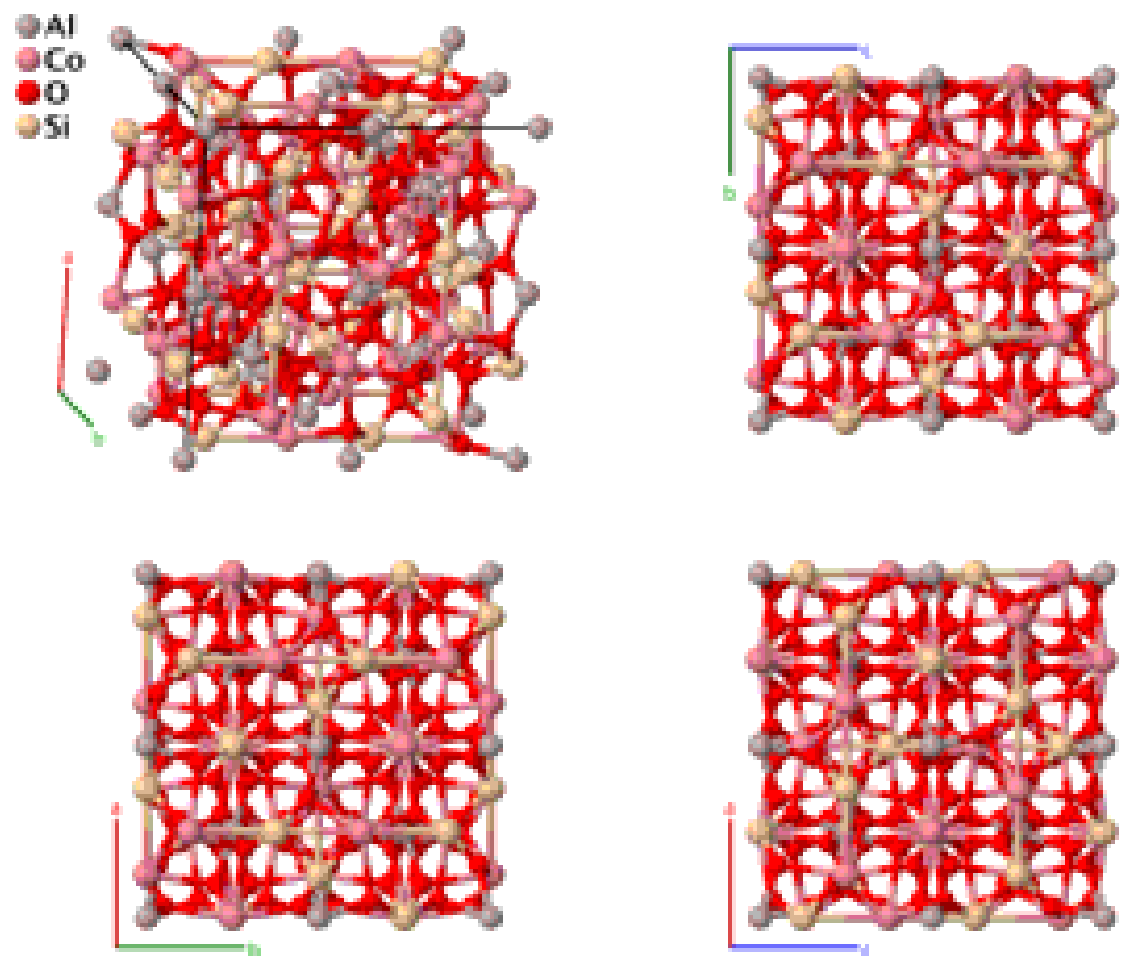

Prototype

AFLOW prototype label

$: \quad \mathrm{Co}_{3} \mathrm{Al}_{2} \mathrm{Si}_{3} \mathrm{O}_{12}$

Strukturbericht designation

: A2B3C12D3_cI160_230_a_c_h_d

Pearson symbol

$: \quad S 1_{4}$

Space group number

cI160

Space group symbol

230

AFLOW prototype command

$I a \overline{3} d$

aflow --proto=A2B3C12D3_cI160_230_a_c_h_d

- params $=a, x_{4}, y_{4}, z_{4}$

\section{Other compounds with this structure:}

- $\mathrm{Al}_{2}\left(\mathrm{Mg}, \mathrm{Ni}_{3} \mathrm{Si}_{3} \mathrm{O}_{12}, \mathrm{Al}_{2} \mathrm{Ca}_{3} \mathrm{Si}_{3} \mathrm{O}_{12}, \mathrm{Al}_{2} \mathrm{Co}_{3} \mathrm{Si}_{3} \mathrm{O}_{12}, \mathrm{Al}_{2} \mathrm{Mg}_{3} \mathrm{Si}_{3} \mathrm{O}_{12}, \mathrm{Al}_{2} \mathrm{Mn}_{3} \mathrm{Si}_{3} \mathrm{O}_{12}, \mathrm{Cr}_{2} \mathrm{Ca}_{3} \mathrm{Si}_{3} \mathrm{O}_{12}, \mathrm{Fe}_{2} \mathrm{Ca}_{3} \mathrm{Si}_{3} \mathrm{O}_{12}\right.$, $\mathrm{Fe}_{2} \mathrm{Mn}_{3} \mathrm{Ge}_{3} \mathrm{O}_{12}, \mathrm{Mn}_{5} \mathrm{Si}_{3} \mathrm{O}_{12}, \mathrm{Sc}_{2} \mathrm{Ca}_{3} \mathrm{Si}_{3} \mathrm{O}_{12}$

- (Ross, 1996) does not explicitly give the positions of the $\mathrm{Al}$ and $\mathrm{Si}$ atoms, which we take from (Downs, 2003). 


$$
\begin{aligned}
& \mathbf{a}_{1}=-\frac{1}{2} a \hat{\mathbf{x}}+\frac{1}{2} a \hat{\mathbf{y}}+\frac{1}{2} a \hat{\mathbf{z}} \\
& \mathbf{a}_{2}=\frac{1}{2} a \hat{\mathbf{x}}-\frac{1}{2} a \hat{\mathbf{y}}+\frac{1}{2} a \hat{\mathbf{z}} \\
& \mathbf{a}_{3}=\frac{1}{2} a \hat{\mathbf{x}}+\frac{1}{2} a \hat{\mathbf{y}}-\frac{1}{2} a \hat{\mathbf{z}}
\end{aligned}
$$

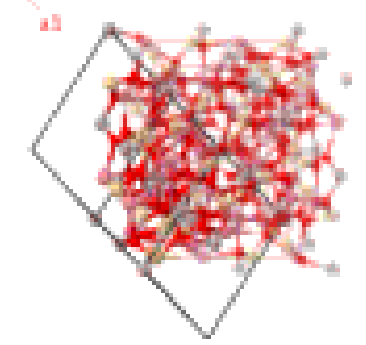

\begin{tabular}{|c|c|c|}
\hline $\mathbf{B}_{1}$ & $=$ & $0 \mathbf{a}_{1}+0 \mathbf{a}_{2}+0 \mathbf{a}_{3}$ \\
\hline $\mathbf{B}_{2}$ & $=$ & $\frac{1}{2} \mathbf{a}_{1}+\frac{1}{2} \mathbf{a}_{3}$ \\
\hline $\mathbf{B}_{3}$ & $=$ & $\frac{1}{2} \mathbf{a}_{2}+\frac{1}{2} \mathbf{a}_{3}$ \\
\hline $\mathbf{B}_{4}$ & $=$ & $\frac{1}{2} \mathbf{a}_{1}+\frac{1}{2} \mathbf{a}_{2}$ \\
\hline $\mathbf{B}_{5}$ & $=$ & $\frac{1}{2} \mathbf{a}_{1}$ \\
\hline $\mathbf{B}_{6}$ & $=$ & $\frac{1}{2} \mathbf{a}_{1}+\frac{1}{2} \mathbf{a}_{2}+\frac{1}{2}$ \\
\hline $\mathbf{B}_{7}$ & $=$ & $\frac{1}{2} \mathbf{a}_{3}$ \\
\hline $\mathbf{B}_{8}$ & $=$ & $\frac{1}{2} \mathbf{a}_{2}$ \\
\hline $\mathbf{B}_{9}$ & $=$ & $\frac{1}{4} \mathbf{a}_{1}+\frac{3}{8} \mathbf{a}_{2}+$ \\
\hline $\mathbf{B}_{10}$ & $=$ & $\mathbf{a}_{2}+$ \\
\hline $\mathbf{B}_{11}$ & $=$ & $\frac{1}{8} \mathbf{a}_{1}+\frac{1}{4} \mathbf{a}_{2}+\frac{3}{8}$ \\
\hline $\mathbf{B}_{12}$ & $=$ & $\frac{3}{8} \mathbf{a}_{1}+\frac{3}{4} \mathbf{a}_{2}+\frac{1}{8}$ \\
\hline $\mathbf{B}_{13}$ & $=$ & $\frac{3}{8} \mathbf{a}_{1}+\frac{1}{8} \mathbf{a}_{2}+\frac{1}{4}$ \\
\hline $\mathbf{B}_{14}$ & $=$ & $\frac{1}{8} \mathbf{a}_{1}+\frac{3}{8} \mathbf{a}_{2}+\frac{3}{4}$ \\
\hline $\mathbf{B}_{15}$ & $=$ & $\mathbf{a}_{2}+$ \\
\hline $\mathbf{B}_{16}$ & $=$ & $\frac{1}{4} \mathbf{a}_{1}+\frac{7}{8} \mathbf{a}_{2}+$ \\
\hline $\mathbf{B}_{17}$ & $=$ & $\frac{7}{8} \mathbf{a}_{1}+\frac{3}{4} \mathbf{a}_{2}+\frac{5}{8}$ \\
\hline $\mathbf{B}_{18}$ & $=$ & $+\frac{1}{4} \mathbf{a}_{2}+$ \\
\hline $\mathbf{B}_{19}$ & $=$ & $+\frac{7}{8} \mathbf{a}_{2}+\frac{3}{4}$ \\
\hline $\mathbf{B}_{20}$ & $=$ & $\frac{7}{8} \mathbf{a}_{1}+\frac{5}{8} \mathbf{a}_{2}+\frac{1}{4}$ \\
\hline $\mathbf{B}_{21}$ & $=$ & $\frac{1}{4} \mathbf{a}_{1}+\frac{5}{8} \mathbf{a}_{2}+$ \\
\hline $\mathbf{B}_{22}$ & $=$ & $\frac{3}{4} \mathbf{a}_{1}+\frac{7}{8} \mathbf{a}_{2}+\frac{1}{8}$ \\
\hline $\mathbf{B}_{23}$ & $=$ & $+\frac{1}{4} \mathbf{a}_{2}+\frac{5}{8}$ \\
\hline $\mathbf{B}_{24}$ & $=$ & $\frac{1}{8} \mathbf{a}_{1}+\frac{3}{4} \mathbf{a}_{2}+\frac{7}{8}$ \\
\hline $\mathbf{B}_{25}$ & $=$ & $\frac{5}{8} \mathbf{a}_{1}+\frac{3}{8} \mathbf{a}_{2}+\frac{1}{4}$ \\
\hline $\mathbf{B}_{26}$ & $=$ & \\
\hline $\mathbf{B}_{27}$ & $=$ & \\
\hline
\end{tabular}

Basis vectors:

Lattice Coordinates
Cartesian Coordinates

$$
=
$$$$
=
$$$$
=
$$$$
=
$$$$
=
$$$$
=
$$$$
=
$$$$
=
$$$$
=
$$$$
=
$$$$
=
$$$$
=
$$$$
=
$$$$
=
$$$$
=
$$$$
=
$$$$
=
$$$$
=
$$$$
=
$$$$
=
$$$$
=
$$$$
=
$$$$
=
$$$$
=
$$$$
=
$$$$
=
$$$$
=
$$$$
=
$$$$
=
$$$$
=
$$$$
=
$$$$
=
$$$$
=
$$$$
=
$$$$
=\quad \frac{1}{4} a \hat{\mathbf{x}}+\frac{1}{8} a \hat{\mathbf{y}}+\frac{1}{2} a \hat{\mathbf{z}}
$$

Wyckoff Position Atom Type

(16a)

$\mathrm{Al}$

(16a)

$\mathrm{Al}$

(16a)

Al

(16a)

Al

(16a)

$\mathrm{Al}$

(16a)

(16a)

(16a)
Al

$\mathrm{Al}$

$\mathrm{Al}$

Co

Co

Co

Co

Co

Co

Co

Co

Co

Co

Co

Co

$\mathrm{Si}$

$\mathrm{Si}$

Si

$\mathrm{Si}$

$\mathrm{Si}$

$\mathrm{Si}$

$\mathrm{Si}$ 


\begin{tabular}{|c|c|c|c|c|c|c|}
\hline $\mathbf{B}_{28}$ & $=$ & $\frac{7}{8} \mathbf{a}_{1}+\frac{1}{4} \mathbf{a}_{2}+\frac{1}{8} \mathbf{a}_{3}$ & $=$ & $\frac{3}{4} a \hat{\mathbf{x}}+\frac{3}{8} a \hat{\mathbf{y}}+\frac{1}{2} a \hat{\mathbf{z}}$ & $(24 d)$ & $\mathrm{Si}$ \\
\hline $\mathbf{B}_{29}$ & $=$ & $\frac{3}{4} \mathbf{a}_{1}+\frac{3}{8} \mathbf{a}_{2}+\frac{5}{8} \mathbf{a}_{3}$ & $=$ & $\frac{1}{8} a \hat{\mathbf{x}}+\frac{1}{2} a \hat{\mathbf{y}}+\frac{1}{4} a \hat{\mathbf{z}}$ & $(24 d)$ & $\mathrm{Si}$ \\
\hline $\mathbf{B}_{30}$ & $=$ & $\frac{1}{4} \mathbf{a}_{1}+\frac{1}{8} \mathbf{a}_{2}+\frac{7}{8} \mathbf{a}_{3}$ & $=$ & $\frac{3}{8} a \hat{\mathbf{x}}+\frac{1}{2} a \hat{\mathbf{y}}-\frac{1}{4} a \hat{\mathbf{z}}$ & $(24 d)$ & $\mathrm{Si}$ \\
\hline $\mathbf{B}_{31}$ & $=$ & $\frac{1}{8} \mathbf{a}_{1}+\frac{7}{8} \mathbf{a}_{2}+\frac{1}{4} \mathbf{a}_{3}$ & $=$ & $\frac{1}{2} a \hat{\mathbf{x}}-\frac{1}{4} a \hat{\mathbf{y}}+\frac{3}{8} a \hat{\mathbf{z}}$ & $(24 d)$ & $\mathrm{Si}$ \\
\hline $\mathbf{B}_{32}$ & $=$ & $\frac{3}{8} \mathbf{a}_{1}+\frac{5}{8} \mathbf{a}_{2}+\frac{3}{4} \mathbf{a}_{3}$ & $=$ & $\frac{1}{2} a \hat{\mathbf{x}}+\frac{1}{4} a \hat{\mathbf{y}}+\frac{1}{8} a \hat{\mathbf{z}}$ & $(24 d)$ & $\mathrm{Si}$ \\
\hline $\mathbf{B}_{33}$ & $=$ & $\begin{array}{c}\left(y_{4}+z_{4}\right) \mathbf{a}_{1}+\left(x_{4}+z_{4}\right) \mathbf{a}_{2}+ \\
\left(x_{4}+y_{4}\right) \mathbf{a}_{3}\end{array}$ & $=$ & $x_{4} a \hat{\mathbf{x}}+y_{4} a \hat{\mathbf{y}}+z_{4} a \hat{\mathbf{z}}$ & $(96 h)$ & $\mathrm{O}$ \\
\hline $\mathbf{B}_{34}$ & $=$ & $\begin{array}{c}\left(\frac{1}{2}-y_{4}+z_{4}\right) \mathbf{a}_{1}+\left(-x_{4}+z_{4}\right) \mathbf{a}_{2}+ \\
\left(\frac{1}{2}-x_{4}-y_{4}\right) \mathbf{a}_{3}\end{array}$ & $=$ & $-x_{4} a \hat{\mathbf{x}}+\left(\frac{1}{2}-y_{4}\right) a \hat{\mathbf{y}}+z_{4} a \hat{\mathbf{z}}$ & $(96 h)$ & $\mathrm{O}$ \\
\hline $\mathbf{B}_{35}$ & $=$ & $\begin{array}{c}\left(y_{4}-z_{4}\right) \mathbf{a}_{1}+\left(\frac{1}{2}-x_{4}-z_{4}\right) \mathbf{a}_{2}+ \\
\left(\frac{1}{2}-x_{4}+y_{4}\right) \mathbf{a}_{3}\end{array}$ & $=$ & $\left(\frac{1}{2}-x_{4}\right) a \hat{\mathbf{x}}+y_{4} a \hat{\mathbf{y}}-z_{4} a \hat{\mathbf{z}}$ & $(96 h)$ & $\mathrm{O}$ \\
\hline $\mathbf{B}_{36}$ & $=$ & $\begin{array}{c}\left(\frac{1}{2}-y_{4}-z_{4}\right) \mathbf{a}_{1}+ \\
\left(\frac{1}{2}+x_{4}-z_{4}\right) \mathbf{a}_{2}+\left(x_{4}-y_{4}\right) \mathbf{a}_{3}\end{array}$ & $=$ & $x_{4} a \hat{\mathbf{x}}-y_{4} a \hat{\mathbf{y}}+\left(\frac{1}{2}-z_{4}\right) a \hat{\mathbf{z}}$ & $(96 h)$ & $\mathrm{O}$ \\
\hline $\mathbf{B}_{37}$ & $=$ & $\begin{array}{c}\left(x_{4}+y_{4}\right) \mathbf{a}_{1}+\left(y_{4}+z_{4}\right) \mathbf{a}_{2}+ \\
\left(x_{4}+z_{4}\right) \mathbf{a}_{3}\end{array}$ & $=$ & $z_{4} a \hat{\mathbf{x}}+x_{4} a \hat{\mathbf{y}}+y_{4} a \hat{\mathbf{z}}$ & $(96 h)$ & $\mathrm{O}$ \\
\hline $\mathbf{B}_{38}$ & $=$ & $\begin{array}{c}\left(\frac{1}{2}-x_{4}-y_{4}\right) \mathbf{a}_{1}+ \\
\left(\frac{1}{2}-y_{4}+z_{4}\right) \mathbf{a}_{2}+\left(-x_{4}+z_{4}\right) \mathbf{a}_{3}\end{array}$ & $=$ & $z_{4} a \hat{\mathbf{x}}-x_{4} a \hat{\mathbf{y}}+\left(\frac{1}{2}-y_{4}\right) a \hat{\mathbf{z}}$ & $(96 h)$ & $\mathrm{O}$ \\
\hline $\mathbf{B}_{39}$ & $=$ & $\begin{array}{c}\left(\frac{1}{2}-x_{4}+y_{4}\right) \mathbf{a}_{1}+\left(y_{4}-z_{4}\right) \mathbf{a}_{2}+ \\
\left(\frac{1}{2}-x_{4}-z_{4}\right) \mathbf{a}_{3}\end{array}$ & $=$ & $-z_{4} a \hat{\mathbf{x}}+\left(\frac{1}{2}-x_{4}\right) a \hat{\mathbf{y}}+y_{4} a \hat{\mathbf{z}}$ & $(96 h)$ & $\mathrm{O}$ \\
\hline $\mathbf{B}_{40}$ & $=$ & $\begin{array}{c}\left(x_{4}-y_{4}\right) \mathbf{a}_{1}+\left(\frac{1}{2}-y_{4}-z_{4}\right) \mathbf{a}_{2}+ \\
\left(\frac{1}{2}+x_{4}-z_{4}\right) \mathbf{a}_{3}\end{array}$ & $=$ & $\left(\frac{1}{2}-z_{4}\right) a \hat{\mathbf{x}}+x_{4} a \hat{\mathbf{y}}-y_{4} a \hat{\mathbf{z}}$ & $(96 h)$ & $\mathrm{O}$ \\
\hline $\mathbf{B}_{41}$ & $=$ & $\begin{array}{c}\left(x_{4}+z_{4}\right) \mathbf{a}_{1}+\left(x_{4}+y_{4}\right) \mathbf{a}_{2}+ \\
\left(y_{4}+z_{4}\right) \mathbf{a}_{3}\end{array}$ & $=$ & $y_{4} a \hat{\mathbf{x}}+z_{4} a \hat{\mathbf{y}}+x_{4} a \hat{\mathbf{z}}$ & $(96 h)$ & $\mathrm{O}$ \\
\hline $\mathbf{B}_{42}$ & $=$ & $\begin{array}{c}\left(-x_{4}+z_{4}\right) \mathbf{a}_{1}+\left(\frac{1}{2}-x_{4}-y_{4}\right) \mathbf{a}_{2}+ \\
\left(\frac{1}{2}-y_{4}+z_{4}\right) \mathbf{a}_{3}\end{array}$ & $=$ & $\left(\frac{1}{2}-y_{4}\right) a \hat{\mathbf{x}}+z_{4} a \hat{\mathbf{y}}-x_{4} a \hat{\mathbf{z}}$ & $(96 h)$ & $\mathrm{O}$ \\
\hline $\mathbf{B}_{43}$ & $=$ & $\begin{array}{c}\left(\frac{1}{2}-x_{4}-z_{4}\right) \mathbf{a}_{1}+ \\
\left(\frac{1}{2}-x_{4}+y_{4}\right) \mathbf{a}_{2}+\left(y_{4}-z_{4}\right) \mathbf{a}_{3}\end{array}$ & $=$ & $y_{4} a \hat{\mathbf{x}}-z_{4} a \hat{\mathbf{y}}+\left(\frac{1}{2}-x_{4}\right) a \hat{\mathbf{z}}$ & $(96 h)$ & $\mathrm{O}$ \\
\hline $\mathbf{B}_{44}$ & $=$ & $\begin{array}{c}\left(\frac{1}{2}+x_{4}-z_{4}\right) \mathbf{a}_{1}+\left(x_{4}-y_{4}\right) \mathbf{a}_{2}+ \\
\left(\frac{1}{2}-y_{4}-z_{4}\right) \mathbf{a}_{3}\end{array}$ & $=$ & $-y_{4} a \hat{\mathbf{x}}+\left(\frac{1}{2}-z_{4}\right) a \hat{\mathbf{y}}+x_{4} a \hat{\mathbf{z}}$ & $(96 h)$ & $\mathrm{O}$ \\
\hline $\mathbf{B}_{45}$ & $=$ & $\begin{array}{c}\left(\frac{1}{2}+x_{4}-z_{4}\right) \mathbf{a}_{1}+\left(y_{4}-z_{4}\right) \mathbf{a}_{2}+ \\
\left(x_{4}+y_{4}\right) \mathbf{a}_{3}\end{array}$ & $=$ & $\begin{array}{c}\left(\frac{3}{4}+y_{4}\right) a \hat{\mathbf{x}}+\left(\frac{1}{4}+x_{4}\right) a \hat{\mathbf{y}}+ \\
\left(\frac{1}{4}-z_{4}\right) a \hat{\mathbf{z}}\end{array}$ & $(96 h)$ & $\mathrm{O}$ \\
\hline $\mathbf{B}_{46}$ & $=$ & $\begin{array}{c}\left(\frac{1}{2}-x_{4}-z_{4}\right) \mathbf{a}_{1}+ \\
\left(\frac{1}{2}-y_{4}-z_{4}\right) \mathbf{a}_{2}+\left(\frac{1}{2}-x_{4}-y_{4}\right) \mathbf{a}_{3}\end{array}$ & $=$ & $\begin{array}{c}\left(\frac{1}{4}-y_{4}\right) a \hat{\mathbf{x}}+\left(\frac{1}{4}-x_{4}\right) a \hat{\mathbf{y}}+ \\
\left(\frac{1}{4}-z_{4}\right) a \hat{\mathbf{z}}\end{array}$ & $(96 h)$ & $\mathrm{O}$ \\
\hline $\mathbf{B}_{47}$ & $=$ & $\begin{array}{c}\left(-x_{4}+z_{4}\right) \mathbf{a}_{1}+\left(y_{4}+z_{4}\right) \mathbf{a}_{2}+ \\
\left(\frac{1}{2}-x_{4}+y_{4}\right) \mathbf{a}_{3}\end{array}$ & $=$ & $\begin{array}{c}\left(\frac{1}{4}+y_{4}\right) a \hat{\mathbf{x}}+\left(\frac{1}{4}-x_{4}\right) a \hat{\mathbf{y}}+ \\
\left(\frac{3}{4}+z_{4}\right) a \hat{\mathbf{z}}\end{array}$ & $(96 h)$ & $\mathrm{O}$ \\
\hline $\mathbf{B}_{48}$ & $=$ & $\begin{array}{c}\left(x_{4}+z_{4}\right) \mathbf{a}_{1}+\left(\frac{1}{2}-y_{4}+z_{4}\right) \mathbf{a}_{2}+ \\
\left(x_{4}-y_{4}\right) \mathbf{a}_{3}\end{array}$ & $=$ & $\begin{array}{c}\left(\frac{1}{4}-y_{4}\right) a \hat{\mathbf{x}}+\left(\frac{3}{4}+x_{4}\right) a \hat{\mathbf{y}}+ \\
\left(\frac{1}{4}+z_{4}\right) a \hat{\mathbf{z}}\end{array}$ & $(96 h)$ & $\mathrm{O}$ \\
\hline $\mathbf{B}_{49}$ & $=$ & $\begin{array}{c}\left(\frac{1}{2}-y_{4}+z_{4}\right) \mathbf{a}_{1}+\left(x_{4}-y_{4}\right) \mathbf{a}_{2}+ \\
\left(x_{4}+z_{4}\right) \mathbf{a}_{3}\end{array}$ & $=$ & $\begin{array}{c}\left(\frac{3}{4}+x_{4}\right) a \hat{\mathbf{x}}+\left(\frac{1}{4}+z_{4}\right) a \hat{\mathbf{y}}+ \\
\left(\frac{1}{4}-y_{4}\right) a \hat{\mathbf{z}}\end{array}$ & $(96 h)$ & $\mathrm{O}$ \\
\hline $\mathbf{B}_{50}$ & $=$ & $\begin{array}{c}\left(y_{4}+z_{4}\right) \mathbf{a}_{1}+\left(\frac{1}{2}-x_{4}+y_{4}\right) \mathbf{a}_{2}+ \\
\left(-x_{4}+z_{4}\right) \mathbf{a}_{3}\end{array}$ & $=$ & $\begin{array}{c}\left(\frac{1}{4}-x_{4}\right) a \hat{\mathbf{x}}+\left(\frac{3}{4}+z_{4}\right) a \hat{\mathbf{y}}+ \\
\left(\frac{1}{4}+y_{4}\right) a \hat{\mathbf{z}}\end{array}$ & $(96 h)$ & $\mathrm{O}$ \\
\hline
\end{tabular}


$\mathbf{B}_{51}=\begin{gathered}\left(\frac{1}{2}-y_{4}-z_{4}\right) \mathbf{a}_{1}+ \\ \left(\frac{1}{2}-x_{4}-y_{4}\right) \mathbf{a}_{2}+\left(\frac{1}{2}-x_{4}-z_{4}\right) \mathbf{a}_{3}\end{gathered} \quad=\quad \begin{gathered}\left(\frac{1}{4}-x_{4}\right) a \hat{\mathbf{x}}+\left(\frac{1}{4}-z_{4}\right) a \hat{\mathbf{y}}+ \\ \left(\frac{1}{4}-y_{4}\right) a \hat{\mathbf{z}}\end{gathered}$

$(96 h)$

O

$\mathbf{B}_{52}=\left(y_{4}-z_{4}\right) \mathbf{a}_{1}+\left(x_{4}+y_{4}\right) \mathbf{a}_{2}+=\left(\frac{1}{4}+x_{4}\right) a \hat{\mathbf{x}}+\left(\frac{1}{4}-z_{4}\right) a \hat{\mathbf{y}}+$

$(96 h)$

$\mathrm{O}$

$\left(\frac{1}{2}+x_{4}-z_{4}\right) \mathbf{a}_{3}$

$\left(\frac{3}{4}+y_{4}\right) a \hat{\mathbf{z}}$

$\mathbf{B}_{53}=\left(\frac{1}{2}-x_{4}+y_{4}\right) \mathbf{a}_{1}+\left(-x_{4}+z_{4}\right) \mathbf{a}_{2}+=\left(\frac{3}{4}+z_{4}\right) a \hat{\mathbf{x}}+\left(\frac{1}{4}+y_{4}\right) a \hat{\mathbf{y}}+$

(96h)

$\mathrm{O}$

$\mathbf{B}_{54}=\left(x_{4}-y_{4}\right) \mathbf{a}_{1}+\left(x_{4}+z_{4}\right) \mathbf{a}_{2}+\quad=\quad\left(\frac{1}{4}+z_{4}\right) a \hat{\mathbf{x}}+\left(\frac{1}{4}-y_{4}\right) a \hat{\mathbf{y}}+$

$\mathrm{O}$

$\left(\frac{1}{2}-y_{4}+z_{4}\right) \mathbf{a}_{3}$

$\left(\frac{3}{4}+x_{4}\right) a \hat{\mathbf{z}}$

$\mathbf{B}_{55}=\left(x_{4}+y_{4}\right) \mathbf{a}_{1}+\left(\frac{1}{2}+x_{4}-z_{4}\right) \mathbf{a}_{2}+=\left(\frac{1}{4}-z_{4}\right) a \hat{\mathbf{x}}+\left(\frac{3}{4}+y_{4}\right) a \hat{\mathbf{y}}+$

$\mathrm{O}$

$\left(y_{4}-z_{4}\right) \mathbf{a}_{3}$ $\left(\frac{1}{4}+x_{4}\right) a \hat{\mathbf{z}}$

$\mathbf{B}_{56}=\begin{gathered}\left(\frac{1}{2}-x_{4}-y_{4}\right) \mathbf{a}_{1}+ \\ \left(\frac{1}{2}-x_{4}-z_{4}\right) \mathbf{a}_{2}+\left(\frac{1}{2}-y_{4}-z_{4}\right) \mathbf{a}_{3}\end{gathered}$

$=\left(\frac{1}{4}-z_{4}\right) a \hat{\mathbf{x}}+\left(\frac{1}{4}-y_{4}\right) a \hat{\mathbf{y}}+$

(96h)

$\mathbf{B}_{57}=\left(-y_{4}-z_{4}\right) \mathbf{a}_{1}+\left(-x_{4}-z_{4}\right) \mathbf{a}_{2}+=$

$\left(-x_{4}-y_{4}\right) \mathbf{a}_{3}$

$\mathbf{B}_{58}=\left(\frac{1}{2}+y_{4}-z_{4}\right) \mathbf{a}_{1}+\left(x_{4}-z_{4}\right) \mathbf{a}_{2}+=x_{4} a \hat{\mathbf{x}}+\left(\frac{1}{2}+y_{4}\right) a \hat{\mathbf{y}}-z_{4} a \hat{\mathbf{z}}$

$\left(\frac{1}{2}+x_{4}+y_{4}\right) \mathbf{a}_{3}$

$\mathbf{B}_{59}=\left(-y_{4}+z_{4}\right) \mathbf{a}_{1}+\left(\frac{1}{2}+x_{4}+z_{4}\right) \mathbf{a}_{2}+=\left(\frac{1}{2}+x_{4}\right) a \hat{\mathbf{x}}-y_{4} a \hat{\mathbf{y}}+z_{4} a \hat{\mathbf{z}}$

$\mathrm{O}$

$\left(\frac{1}{2}+x_{4}-y_{4}\right) \mathbf{a}_{3}$

$\mathbf{B}_{60}=\begin{gathered}\left(\frac{1}{2}+y_{4}+z_{4}\right) \mathbf{a}_{1}+ \\ \left(\frac{1}{2}-x_{4}+z_{4}\right) \mathbf{a}_{2}+\left(-x_{4}+y_{4}\right) \mathbf{a}_{3}\end{gathered}$

$\mathbf{B}_{61}=\left(-x_{4}-y_{4}\right) \mathbf{a}_{1}+\left(-y_{4}-z_{4}\right) \mathbf{a}_{2}+=$

$\left(-x_{4}-z_{4}\right) \mathbf{a}_{3}$

$\mathbf{B}_{62}=$

$\left(\frac{1}{2}+x_{4}+y_{4}\right) \mathbf{a}_{1}+$

$=\quad-x_{4} a \hat{\mathbf{x}}+y_{4} a \hat{\mathbf{y}}+\left(\frac{1}{2}+z_{4}\right) a \hat{\mathbf{z}}$

(96h)

$\mathrm{O}$

(96h)

O

$-z_{4} a \hat{\mathbf{x}}-x_{4} a \hat{\mathbf{y}}-y_{4} a \hat{\mathbf{z}}$

O

$\mathrm{O}$

$\mathrm{O}$

$\left(\frac{1}{2}+y_{4}-z_{4}\right) \mathbf{a}_{2}+\left(x_{4}-z_{4}\right) \mathbf{a}_{3}$

$\mathbf{B}_{63}=\left(\frac{1}{2}+x_{4}-y_{4}\right) \mathbf{a}_{1}+\left(-y_{4}+z_{4}\right) \mathbf{a}_{2}+=z_{4} a \hat{\mathbf{x}}+\left(\frac{1}{2}+x_{4}\right) a \hat{\mathbf{y}}-y_{4} a \hat{\mathbf{z}}$

$\mathrm{O}$

$\left(\frac{1}{2}+x_{4}+z_{4}\right) \mathbf{a}_{3}$

$\mathbf{B}_{64}=\left(-x_{4}+y_{4}\right) \mathbf{a}_{1}+\left(\frac{1}{2}+y_{4}+z_{4}\right) \mathbf{a}_{2}+=\left(\frac{1}{2}+z_{4}\right) a \hat{\mathbf{x}}-x_{4} a \hat{\mathbf{y}}+y_{4} a \hat{\mathbf{z}}$ $\left(\frac{1}{2}-x_{4}+z_{4}\right) \mathbf{a}_{3}$

$\mathbf{B}_{65}=\left(-x_{4}-z_{4}\right) \mathbf{a}_{1}+\left(-x_{4}-y_{4}\right) \mathbf{a}_{2}+=\quad-y_{4} a \hat{\mathbf{x}}-z_{4} a \hat{\mathbf{y}}-x_{4} a \hat{\mathbf{z}}$

$\mathrm{O}$

$\mathbf{B}_{66}=\left(x_{4}-z_{4}\right) \mathbf{a}_{1}+\left(\frac{1}{2}+x_{4}+y_{4}\right) \mathbf{a}_{2}+=\left(\frac{1}{2}+y_{4}\right) a \hat{\mathbf{x}}-z_{4} a \hat{\mathbf{y}}+x_{4} a \hat{\mathbf{z}}$

$\mathrm{O}$

$$
\left(\frac{1}{2}+y_{4}-z_{4}\right) \mathbf{a}_{3}
$$

$\mathbf{B}_{67}=\left(\frac{1}{2}+x_{4}+z_{4}\right) \mathbf{a}_{1}+\quad=\quad-y_{4} a \hat{\mathbf{x}}+z_{4} a \hat{\mathbf{y}}+\left(\frac{1}{2}+x_{4}\right) a \hat{\mathbf{z}}$

(96h)

O

$$
\left(\frac{1}{2}+x_{4}-y_{4}\right) \mathbf{a}_{2}+\left(-y_{4}+z_{4}\right) \mathbf{a}_{3}
$$

$\mathbf{B}_{68}=\left(\frac{1}{2}-x_{4}+z_{4}\right) \mathbf{a}_{1}+\left(-x_{4}+y_{4}\right) \mathbf{a}_{2}+=y_{4} a \hat{\mathbf{x}}+\left(\frac{1}{2}+z_{4}\right) a \hat{\mathbf{y}}-x_{4} a \hat{\mathbf{z}}$

$$
\left(\frac{1}{2}+y_{4}+z_{4}\right) \mathbf{a}_{3}
$$

$\mathbf{B}_{69}=\left(\frac{1}{2}-x_{4}+z_{4}\right) \mathbf{a}_{1}+\left(-y_{4}+z_{4}\right) \mathbf{a}_{2}+=-a\left(y_{4}+\frac{1}{4}\right) \hat{\mathbf{x}}+\left(\frac{1}{4}-x_{4}\right) a \hat{\mathbf{y}}+$ $\left(-x_{4}-y_{4}\right) \mathbf{a}_{3}$

(96h)

$\mathrm{O}$

$\mathbf{B}_{70}=\left(\frac{1}{2}+x_{4}+z_{4}\right) \mathbf{a}_{1}+\quad=\quad\left(\frac{1}{4}+y_{4}\right) a \hat{\mathbf{x}}+\left(\frac{1}{4}+x_{4}\right) a \hat{\mathbf{y}}+$

(96h)

$\mathrm{O}$ $\left(\frac{1}{2}+y_{4}+z_{4}\right) \mathbf{a}_{2}+\left(\frac{1}{2}+x_{4}+y_{4}\right) \mathbf{a}_{3}$

$$
\left(\frac{1}{4}+z_{4}\right) a \hat{\mathbf{z}}
$$

$\mathbf{B}_{71}=\left(x_{4}-z_{4}\right) \mathbf{a}_{1}+\left(-y_{4}-z_{4}\right) \mathbf{a}_{2}+\quad=\quad\left(\frac{1}{4}-y_{4}\right) a \hat{\mathbf{x}}+\left(\frac{1}{4}+x_{4}\right) a \hat{\mathbf{y}}-$ $\left(\frac{1}{2}+x_{4}-y_{4}\right) \mathbf{a}_{3}$

$a\left(z_{4}+\frac{1}{4}\right) \hat{\mathbf{z}}$

(96h)

O 


$$
\begin{aligned}
& \mathbf{B}_{72}=\left(-x_{4}-z_{4}\right) \mathbf{a}_{1}+\left(\frac{1}{2}+y_{4}-z_{4}\right) \mathbf{a}_{2}+=\left(\frac{1}{4}+y_{4}\right) a \hat{\mathbf{x}}-a\left(x_{4}+\frac{1}{4}\right) \hat{\mathbf{y}}+ \\
& \left(-x_{4}+y_{4}\right) \mathbf{a}_{3} \quad\left(\frac{1}{4}-z_{4}\right) a \hat{\mathbf{z}} \\
& \mathbf{B}_{73}=\begin{array}{cc}
\left(\frac{1}{2}+y_{4}-z_{4}\right) \mathbf{a}_{1}+\left(-x_{4}+y_{4}\right) \mathbf{a}_{2}+ & = \\
\left(-x_{4}-z_{4}\right) \mathbf{a}_{3} & -a\left(x_{4}+\frac{1}{4}\right) \hat{\mathbf{x}}+\left(\frac{1}{4}-z_{4}\right) a \hat{\mathbf{y}}+ \\
\left(\frac{1}{4}+y_{4}\right) a \hat{\mathbf{z}}
\end{array} \\
& \mathbf{B}_{74}=\left(-y_{4}-z_{4}\right) \mathbf{a}_{1}+\left(\frac{1}{2}+x_{4}-y_{4}\right) \mathbf{a}_{2}+=\left(\frac{1}{4}+x_{4}\right) a \hat{\mathbf{x}}-a\left(z_{4}+\frac{1}{4}\right) \hat{\mathbf{y}}+ \\
& \left(x_{4}-z_{4}\right) \mathbf{a}_{3} \quad\left(\frac{1}{4}-y_{4}\right) a \hat{\mathbf{z}} \\
& \mathbf{B}_{75}=\left(\frac{1}{2}+y_{4}+z_{4}\right) \mathbf{a}_{1}+\quad=\quad\left(\frac{1}{4}+x_{4}\right) a \hat{\mathbf{x}}+\left(\frac{1}{4}+z_{4}\right) a \hat{\mathbf{y}}+ \\
& \left(\frac{1}{2}+x_{4}+y_{4}\right) \mathbf{a}_{2}+\left(\frac{1}{2}+x_{4}+z_{4}\right) \mathbf{a}_{3} \quad\left(\frac{1}{4}+y_{4}\right) a \hat{\mathbf{z}} \\
& \mathbf{B}_{76}=\left(-y_{4}+z_{4}\right) \mathbf{a}_{1}+\left(-x_{4}-y_{4}\right) \mathbf{a}_{2}+=\left(\frac{1}{4}-x_{4}\right) a \hat{\mathbf{x}}+\left(\frac{1}{4}+z_{4}\right) a \hat{\mathbf{y}}- \\
& \left(\frac{1}{2}-x_{4}+z_{4}\right) \mathbf{a}_{3} \quad a\left(y_{4}+\frac{1}{4}\right) \hat{\mathbf{z}} \\
& \mathbf{B}_{77}=\left(\frac{1}{2}+x_{4}-y_{4}\right) \mathbf{a}_{1}+\left(x_{4}-z_{4}\right) \mathbf{a}_{2}+=-a\left(z_{4}+\frac{1}{4}\right) \hat{\mathbf{x}}+\left(\frac{1}{4}-y_{4}\right) a \hat{\mathbf{y}}+ \\
& \left(-y_{4}-z_{4}\right) \mathbf{a}_{3} \quad\left(\frac{1}{4}+x_{4}\right) a \hat{\mathbf{z}} \\
& \mathbf{B}_{78}=\left(-x_{4}+y_{4}\right) \mathbf{a}_{1}+\left(-x_{4}-z_{4}\right) \mathbf{a}_{2}+=\left(\frac{1}{4}-z_{4}\right) a \hat{\mathbf{x}}+\left(\frac{1}{4}+y_{4}\right) a \hat{\mathbf{y}}- \\
& \left(\frac{1}{2}+y_{4}-z_{4}\right) \mathbf{a}_{3} \quad a\left(x_{4}+\frac{1}{4}\right) \hat{\mathbf{z}} \\
& \mathbf{B}_{79}=\left(-x_{4}-y_{4}\right) \mathbf{a}_{1}+\left(\frac{1}{2}-x_{4}+z_{4}\right) \mathbf{a}_{2}+=\left(\frac{1}{4}+z_{4}\right) a \hat{\mathbf{x}}-a\left(y_{4}+\frac{1}{4}\right) \hat{\mathbf{y}}+ \\
& \left(-y_{4}+z_{4}\right) \mathbf{a}_{3} \quad\left(\frac{1}{4}-x_{4}\right) a \hat{\mathbf{z}} \\
& \mathbf{B}_{80}=\begin{array}{c}
\left(\frac{1}{2}+x_{4}+y_{4}\right) \mathbf{a}_{1}+ \\
\left(\frac{1}{2}+x_{4}+z_{4}\right) \mathbf{a}_{2}+\left(\frac{1}{2}+y_{4}+z_{4}\right) \mathbf{a}_{3}
\end{array} \quad=\quad \begin{array}{c}
\left(\frac{1}{4}+z_{4}\right) a \hat{\mathbf{x}}+\left(\frac{1}{4}+y_{4}\right) a \hat{\mathbf{y}}+ \\
\left(\frac{1}{4}+x_{4}\right) a \hat{\mathbf{z}}
\end{array}
\end{aligned}
$$

\section{References:}

- C. R. Ross II, H. Keppler, D. Canil, and H. S. C. O'Neill, Structure and crystal-field spectra of $\mathrm{Co}_{3} \mathrm{Al}_{2}\left(\mathrm{SiO}_{4}\right)_{3}$ and $\left(\mathrm{Mg}, \mathrm{Ni}_{3} \mathrm{Al}_{2}\left(\mathrm{SiO}_{4}\right)_{3}\right.$ garnet, Am. Mineral. 81, 61-66 (1996).

\section{Found in:}

- R. T. Downs and M. Hall-Wallace, The American Mineralogist Crystal Structure Database, Am. Mineral. 88, 247-250 (2003).

\section{Geometry files:}

- CIF: pp. 986

- POSCAR: pp. 987 


\section{CIF and POSCAR Files}

$\mathrm{H}_{2} \mathrm{~S}$ (90 GPa): A2B_aP6_2_aei_i - CIF

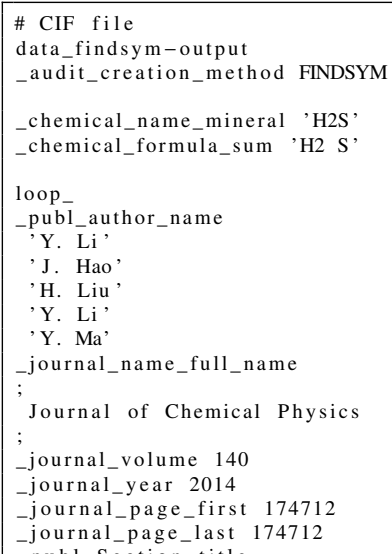

$\mathrm{H}_{2} \mathrm{~S}(90 \mathrm{GPa})$ : A2B_aP6_2_aei_i - POSCAR

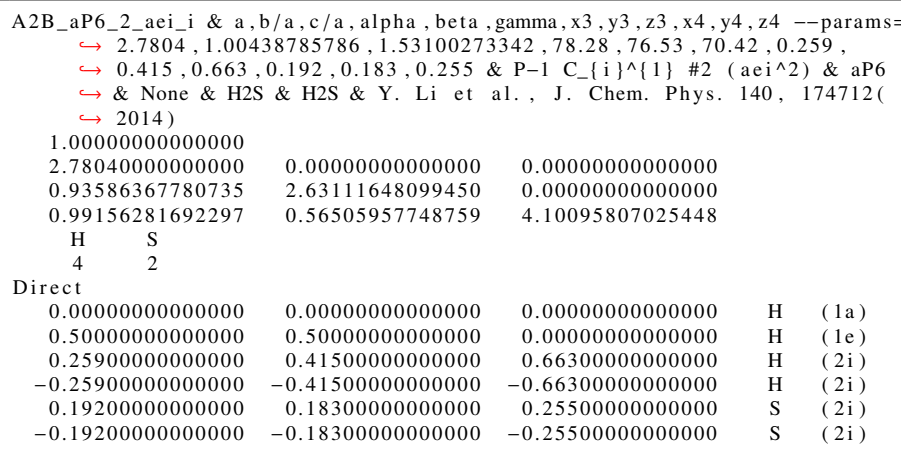

$\mathrm{Mo}_{8} \mathrm{P}_{5}$ (High-temperature): A8B5_mP13_6_a7b_3a2b - CIF

\# CIF file

data_findsym-output

-audit_creation_method FINDSYM

chemical name mineral 'Mo8P5,

chemical formula sum 'Mo8 P5,

loop

publ_author_name

'T. Johnsson'

journal_name_full_name
Acta Chemica Scandinavica

journal_volume 26

journal_year 1972

journal_page_first 365

journal_page_last 382

publ_Section_title

The crystal structure of $\operatorname{Mo}_{-}\{8\} \$ P \$ \_\{5\} \$$ from twin-crystal data

Found in Pearson's Crystal Data - Crystal Structure Database for $\hookrightarrow$ Inorganic Compounds, 2013

aflow title 'Mo\$ $\{8\}$ P $\$\{5\} \$$ (High-temperature) Structure'

aflow proto 'A8B5 mP13 6 a a b 3 a 2 b

aflow_params $, a, b / a, c / a, \backslash$ beta, $x_{-}\{1\}, z_{-}\{1\}, x_{-}\{2\}, z_{-}\{2\}, x_{-}\{3\}, z_{-}\{3\}, x_{-}\{4$ $\hookrightarrow\}, z_{-}\{4\}, x_{-}\{5\}, z_{-}\{5\}, x_{-}\{6\}, z_{-}\{6\}, x_{-}\{7\}, z_{-}\{7\}, x_{-}\{8\}, z_{-}\{8\}, x_{-}\{9\}$

$\longrightarrow z_{-}\{9\}, x_{-}\{10\}, z_{-}\{10\}, x_{-}\{11\}, z_{-}\{11\}, x_{-}\{12\}, z_{-}\{12\}, x_{-}\{13\}, z_{-}\{13\}$ $\leftrightarrow 0.5119,0.7172,0.4546,0.4285,0.044,0.5911,0.37,0.0053,0.3887$

$\hookrightarrow 0.2195,0.6277,0.0001,0.1818,0.4663,0.7456,0.5256,0.1826,0.7901$,

$\hookrightarrow 0.7824,0.2724,0.0,0.0,0.8188,0.8026,0.0123,0.2051$,

aflow_Strukturbericht 'None

aflow_Pearson ' $\mathrm{mP13}$ '

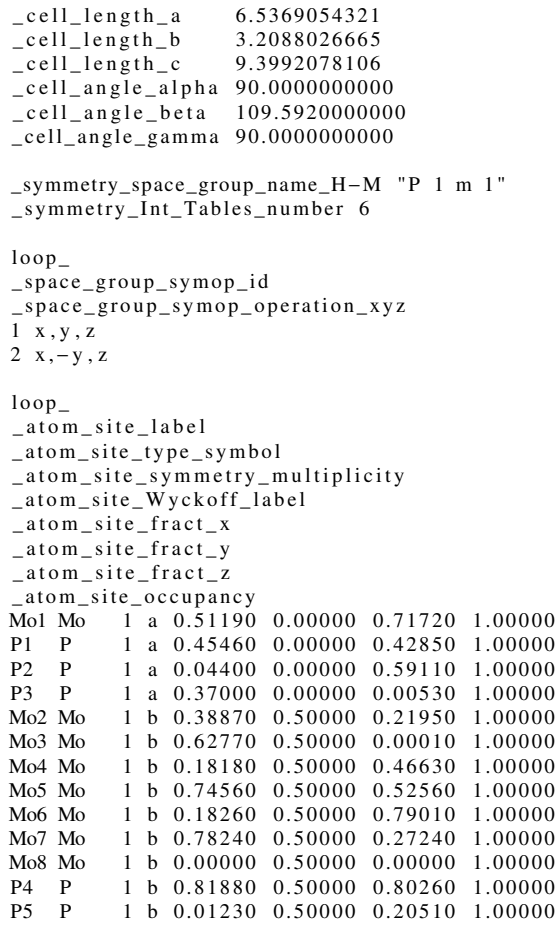

$\mathrm{Mo}_{8} \mathrm{P}_{5}$ (High-temperature): A8B5_mP13_6_a7b_3a2b - POSCAR

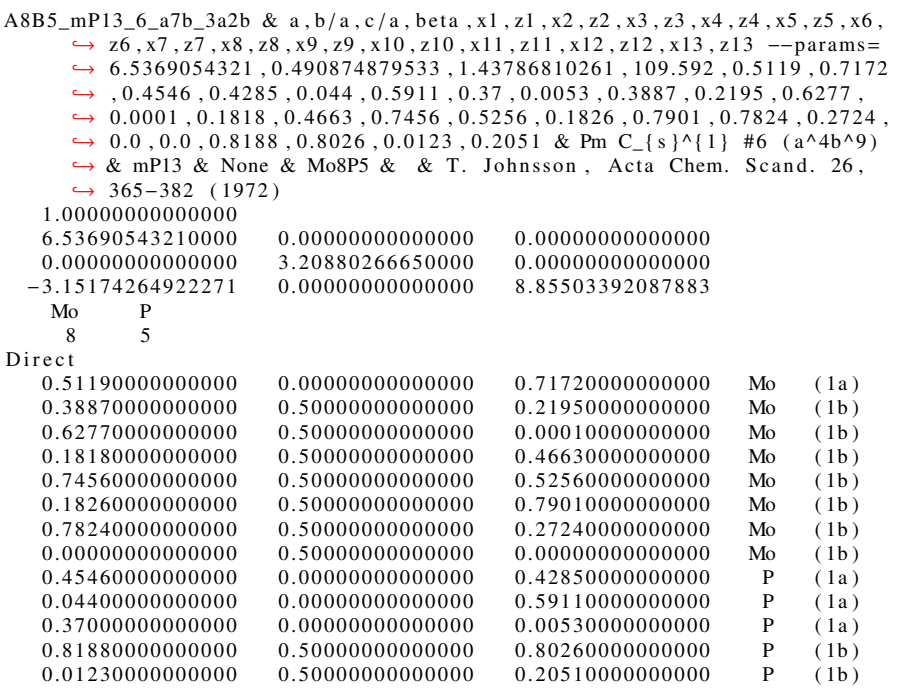

FeNi: AB_mP4_6_2b_2a - CIF

\section{\# CIF file}

data findsym-output

audit_creation_method FINDSYM

chemical_name_mineral ' $\mathrm{FeNi}$,

_chemical_formula_sum ' $\mathrm{Fe} \mathrm{Ni}$, 
loop

_publ_author_name

T. Tagai,

'H. Takeda',

journal_name_full_name

Zeitschrift $f\{\backslash " u\} r$ Kristallographie - Crystalline Materials

_journal_volume 210

journal year 1995

_journal_page_first 14

journal_page_first 14

- publ_Section_title

Superstructure of tetrataenite from the Saint Severin meteorite

\# Found in Pearson's Crystal Data - Crystal Structure Database fo $\hookrightarrow$ Inorganic Compounds, 2013

_aflow_title 'FeNi Structure'

_aflow_proto 'AB_mP4_6_2b_2a'

aflow_params $, a, b / a, c / a, \backslash$ beta $, x_{-}\{1\}, z_{-}\{1\}, x_{-}\{2\}, z_{-}\{2\}, x_{-}\{3\}, z_{-}\{3\}, x_{-}\{4$ $\hookrightarrow\}, z_{-}\{4\}$,

aflow_params_values ' $3.580975428,1.00027925162,1.00167550965,90.04,0.0$ $\hookrightarrow 0.0,0.518,0.507,0.026,0.501,0.529,0.027$,

(aflow Strukturbericht 'None'

-aflow_Pearson 'mP4'

_cell_length_a $\quad 3.5809754280$

_cell_length_b 3.5819754212

_cell_length_c $\quad 3.5869753869$

_cell_angle_alpha 90.0000000000

_cell_angle_beta 90.0400000000

_cell_angle_gamma 90.0000000000

_symmetry_space_group_name_H-M "P $1 \mathrm{~m} 1$ "

_symmetry_Int_Tables_number 6

loop

_space_group_symop_id

_space_group_symop_operation_xyz

$1 \mathrm{x}, \mathrm{y}, \mathrm{z}$

$2 \mathrm{x},-\mathrm{y}, \mathrm{z}$

loop_

atom site label

-atom_site_type_symbol

_atom_site_symmetry_multiplicity

_atom_site_Wyckoff_labe

_atom_site_fract_x

-atom_site_fract_y

_atom_site_fract_z

$\begin{array}{llllll}\mathrm{Ni} 1 \mathrm{Ni} & 1 \text { a } & 0.00000 & 0.00000 & 0.00000 & 1.00000\end{array}$

$\begin{array}{lllllllll}\mathrm{Ni} 2 & \mathrm{Ni} & 1 & \text { a } & 0.51800 & 0.00000 & 0.50700 & 1.0000\end{array}$

$\begin{array}{llllllll}\mathrm{Fe} 1 \mathrm{Fe} & 1 & \mathrm{~b} & 0.02600 & 0.50000 & 0.50100 & 1.00000\end{array}$

$\begin{array}{llllllll}\mathrm{Fe} 2 & \mathrm{Fe} & 1 & \text { b } & 0.52900 & 0.50000 & 0.02700 & 1.00000\end{array}$

FeNi: AB_mP4_6_2b_2a - POSCAR

AB_mP4_6_2b_2a \& a , b/a,c/a, beta , x1, z1 , x2, z2, x3, z3, x4, z4 --params= $\hookrightarrow 3.580975428,1.00027925162,1.00167550965,90.04,0.0,0.0,0.518$, $\hookrightarrow 0.507,0.026,0.501,0.529,0.027 \& \mathrm{Pm} \mathrm{C}_{-}\{\mathrm{s}\}^{\wedge}\{1\} \# 6\left(\mathrm{a}^{\wedge} 2 \mathrm{~b}^{\wedge} 2\right) \& \mathrm{mP4}$ $\hookrightarrow$ \& None \& FeNi \& \& T. Tagai and H. Takeda and T. Fukuda,

$\hookrightarrow$ Zeitschrift f" $\{$ u $\}$ r Kristallographie - Crystalline Materials 210 $\hookrightarrow, 14-18$ (1995)

1.00000000000000

$3.58097542800000 \quad 0.00000000000000$

3.58197542120000

$-0.00250418102416 \quad 0.00000000000000$

0.00000000000000

0.00000000000000

3.58697451277589

$\mathrm{Fe} \quad \mathrm{Ni}$

Direct

0.02600000000000

0.52900000000000

0.00000000000000

0.51800000000000

0.50000000000000 0.50000000000000 0.0000000000000 0.00000000000000

0.50100000000000 0.02700000000000 0.00000000000000 0.50700000000000

$\mathrm{H}_{2} \mathrm{~S}$ IV: A2B_mP12_7_4a_2a - CIF

\section{\# CIF file}

data_findsym-output

_audit_creation_method FINDSYM

_chemical_name_mineral 'H2S IV

_chemical_formula_sum 'H2 S'

loop

_publ_author_name

'Y. $\mathrm{Li}$ ',

'J. Hao',

'H. Liu,

'Y. Li,

'Y. Ma'

_journal_name_full_name

Journal of Chemical Physics

journal_volume 140

-journal year 2014

_journal_page_first 174712

_journal_page_last 174712
- publ Section_title

The metallization and superconductivity of dense hydrogen sulfide

aflow_title 'H\$_ $\{2\} \$$ S IV Structure

aflow_proto 'A2B_mP12_7_4a_2a

aflow_params $, a, b / a, c / a, \backslash$ beta $, x_{-}\{1\}, y_{-}\{1\}, z_{-}\{1\}, x_{-}\{2\}, y_{-}\{2\}, z_{-}\{2\}, x_{-}\{3$ $\hookrightarrow\}, y_{-}\{3\}$,

_aflow_params_values ' $5.0942,0.627360527659,1.04603274312,90.38,0.498,-$ $\hookrightarrow 0.062,0.472,-0.023,0.574,0.143,0.777,-0.052,0.799,0.271,0.151$,

$\hookrightarrow 0.261,-0.001,0.808,0.36,0.494,0.649,0.658$

aflow_Strukturbericht 'None'

aflow_Pearson 'mP12'

symmetry_space_group_name_H-M "P 1 c 1 "

symmetry_Int_Tables_number 7

cell_length_a $\quad 5.09420$

cell_length_b 3.19590

cell_length_c 5.32870

cell_angle_alpha 90.00000

cell_angle_beta 90.38000

cell_angle_gamma 90.00000

loop

space_group_symop_id

space_group_symop_operation_xyz

$\mathrm{x}, \mathrm{y}, \mathrm{z}$

$2 \mathrm{x},-\mathrm{y}, \mathrm{z}+1 / 2$

loop

atom_site_label

atom_site_type_symbol

atom_site_symmetry_multiplicity

atom_site_Wyckoff_label

atom_site_fract_x

atom_site_fract_y

atom_site_fract_z

atom_site_occupancy

$\begin{array}{lllll}\mathrm{H} 1 \mathrm{H} & 2 \text { a } 0.49800 & -0.06200 & 0.47200 & 1.00000\end{array}$

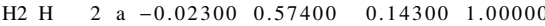

$\begin{array}{llllllll}\mathrm{H} 3 & \mathrm{H} & 2 & \mathrm{a} & 0.77700 & -0.05200 & 0.79900 & 1.00000\end{array}$

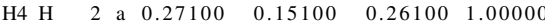

$\begin{array}{llllllll}\mathrm{S} 1 \mathrm{~S} & 2 & \mathrm{a} & -0.00100 & 0.80800 & 0.36000 & 1.00000\end{array}$

$\begin{array}{lllllllll} & \mathrm{S} 1 \mathrm{~S} & 2 & \mathrm{a} & 0.2710 .00100 & 0.15100 & 0.26100 & 1.00000\end{array}$

$\mathrm{H}_{2} \mathrm{~S}$ IV: A2B_mP12_7_4a_2a - POSCAR

A2B_mP12_7_4a_2a \& a, b/a,c/a, beta $, \mathrm{x} 1, \mathrm{y} 1, \mathrm{z} 1, \mathrm{x} 2, \mathrm{y} 2, \mathrm{z} 2, \mathrm{x} 3, \mathrm{y} 3, \mathrm{z} 3, \mathrm{x} 4, \mathrm{y} 4, \mathrm{z} 4, \mathrm{x} 5$ $\hookrightarrow, \mathrm{y} 5, \mathrm{z} 5, \mathrm{x} 6, \mathrm{y} 6, \mathrm{z} 6--$ params $=5.0942,0.627360527659,1.04603274312$,

$\leftrightarrow 90.38,0.498,-0.062,0.472,-0.023,0.574,0.143,0.777,-0.052,0.799$

$\hookrightarrow 0.271,0.151,0.261,-0.001,0.808,0.36,0.494,0.649,0.658 \&$ Pc $C_{-}\{\mathrm{s}$

$\hookrightarrow\}^{\wedge}\{2\} \# 7 \quad\left(a^{\wedge} 6\right) \& \mathrm{mP12} \&$ None \& H2S \& H2S IV \& Y. Li et al., J.

$\hookrightarrow$ Chem. Phys. 140, 174712(2014)

1.00000000000000

$5.09420000000000-0.00000000000000$

$\begin{array}{llll}0.03534101765261 & 0.00000000000000 & 5.32858280431779\end{array}$

$\begin{array}{ll}\mathrm{H} & \mathrm{S} \\ 8 & 4\end{array}$

$0.49800000000000-0.06200000000000$

$0.49800000000000 \quad 0.06200000000000$

$-0.02300000000000 \quad 0.57400000000000$

$-0.02300000000000-0.57400000000000$

$0.77700000000000-0.05200000000000$

$0.77700000000000 \quad 0.05200000000000$

$0.27100000000000 \quad 0.15100000000000$

$0.27100000000000-0.15100000000000$

$0.2710000000000-0.15100000000000$

$-0.0010000000000-0.80800000000000$

$-0.001000000000--0.8080000000000$

$\begin{array}{ll}0.49400000000000 & -0.64900000000000\end{array}$

0.47200000000000 0.97200000000000 0.14300000000000 0.64300000000000 0.79900000000000 1.29900000000000 0.26100000000000 0.76100000000000 0.3600000000000 0.86000000000000 0.86000000000000 1.15800000000000

As 2 Ba: A2B_mP18_7_6a_3a - CIF

\section{\# CIF file}

data_findsym-outpu

audit_creation_method FINDSYM

chemical_name_mineral 'As2Ba'

chemical_formula_sum 'As2 Ba'

loop

publ_author_name

'F. Emmerling'

'D. Petri,

'C. R $\{\backslash " \mathrm{o}\} \mathrm{hr}$

journal_name_full_name

Zeitschrift fur Anorganische und Allgemeine Chemie

journal_volume 630

journal year 2004

journal_page_first 2490

journal_page_last 2501

publ_Section_title

Neue Arsenide mit $A s \$^{\wedge}\{-\} \$-$ Ketten und -Ringen: $B a A s \$_{-}\{2\} \$$ und $\$ A^{\wedge}\{I\}$ $\hookrightarrow \$ B a \$_{-}\{2\} \$ \mathrm{As}_{-}\{5\} \$\left(\$ \mathrm{~A}^{\wedge}\{\mathrm{I}\} \$=\mathrm{K}, \mathrm{Rb}\right)$ 
\# Found in Pearson's Crystal Data - Crystal Structure Database for $\hookrightarrow$ Inorganic Compounds, 2013

_aflow_title 'As\$_ $\{2\} \$ B$ B Structure

_aflow_proto 'A2B_mP18_7_6a_3a

_aflow_params $, a, b / a, c / a, \backslash$ beta $, x_{-}\{1\}, y_{-}\{1\}, z_{-}\{1\}, x_{-}\{2\}, y_{-}\{2\}, z_{-}\{2\}, x_{-}\{3$ $\hookrightarrow\}, \mathrm{y}_{-}\{3\}, \mathrm{z}_{-}\{3\}, \mathrm{x}_{-}\{4\}, \mathrm{y}_{-}\{4\}, \mathrm{z}_{-}\{4\}, \mathrm{x}_{-}\{5\}, \mathrm{y}_{-}\{5\}, \mathrm{z}_{-}\{5\}, \mathrm{x}_{-}\{6\}, \mathrm{y}_{-}\{6\}$, $\hookrightarrow \mathrm{z}_{-}\{6\}, \mathrm{x}_{-}\{7\}, \mathrm{y}_{-}\{7\}, \mathrm{z}_{-}\{7\}, \mathrm{x}_{-}\{8\}, \mathrm{y}_{-}\{8\}, \mathrm{z}_{-}\{8\}, \mathrm{x}_{-}\{9\}, \mathrm{y}_{-}\{9\}, \mathrm{z}_{-}\{9\}$,

_aflow_params_values ' $6.5499839723,1.91328244277,1.22717557252,127.75$,

$\hookrightarrow 0.2883,0.1551,0.3432,0.1472,0.1112,0.5582,0.0,0.0746,0.0,0.5693$

$\hookrightarrow, 0.07852,0.0933,0.1184,0.41567,0.2938,0.8473,0.27155,0.6656$,

aflow_Strukturbericht 'None'

_aflow_Pearson 'mP18,

_cell_length_a $\quad 6.5499839723$

_cell_length_b 12.5319693346

_cell_length_c $\quad 8.0379803312$

_cell_angle_alpha 90.0000000000

_cell_angle_beta 127.7500000000

_cell_angle_gamma 90.0000000000

_symmetry_space_group_name_H-M "P 1 c 1 "

_symmetry_Int_Tables_number 7

loop

_space_group_symop_id

_space_group_symop_operation_xyz

$1 \mathrm{x}, \mathrm{y}, \mathrm{z}$

$2 \mathrm{x},-\mathrm{y}, \mathrm{z}+1 / 2$

loop_

atom_site_label

-atom_site_type_symbol

_atom_site_symmetry_multiplicity

_atom_site_Wyckoff_label

-atom_site_fract_x

-atom_site_fract_y
atom_site_fract_z

- Atom_site_fract_z

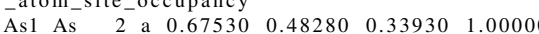

As2 As 2 a $0.47110 \quad 0.64980 \quad 0.30670 \quad 1.00000$

As3 As 2 a $0.41740 \quad 0.34330 \quad 0.08930 \quad 1.00000$

As4 As 2 a $0.28830 \quad 0.15510 \quad 0.34320 \quad 1.00000$

As5 As 2 a $0.14720 \quad 0.11120 \quad 0.55820 \quad 1.00000$

As6 As 2 a $0.00000 \quad 0.07460 \quad 0.00000 \quad 1.00000$

$\begin{array}{lllllllll}\mathrm{Ba} & \mathrm{Ba} & 2 & \mathrm{a} & 0.56930 & 0.07852 & 0.09330 & 1.00000\end{array}$

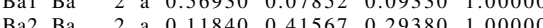

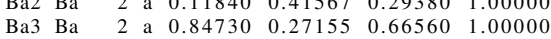

As 2 Ba: A2B_mP18_7_6a_3a - POSCAR

A2B_mP18_7_6a_3a \& a , b/a,c/a , beta , x1,y1, z1 , x2, y2, z2, x3, y3 , z3 , x4,y4, z4, x5 $\hookrightarrow, \mathrm{y} 5, \mathrm{z} 5, \mathrm{x} 6, \mathrm{y6}, \mathrm{z6}, \mathrm{x} 7, \mathrm{y} 7, \mathrm{z7}, \mathrm{x} 8, \mathrm{y} 8, \mathrm{z8}, \mathrm{x} 9, \mathrm{y9}, \mathrm{z} 9-\mathrm{params}=$

$\hookrightarrow 6.5499839723,1.91328244277,1.22717557252,127.75,0.6753,0.4828$

$\hookrightarrow 0.3393,0.4711,0.6498,0.3067,0.4174,0.3433,0.0893,0.2883,0.1551$

$\hookrightarrow 0.3432,0.1472,0.1112,0.5582,0.0,0.0746,0.0,0.5693,0.07852$,

$\hookrightarrow 0.0933,0.1184,0.41567,0.2938,0.8473,0.27155,0.6656 \& \mathrm{Pc}_{\mathrm{C}} \mathrm{C}_{-}\{\mathrm{s}\}^{\wedge}\{$

$\hookrightarrow 2\} \# 7 \quad\left(a^{\wedge} 9\right)$ \& mP18 \& None \& As2Ba \& \& F. Emmerling and D.

$\hookrightarrow$ Petri and C. R $\{\backslash " \mathrm{o}\} \mathrm{hr}$, Z. Anorg. Allg. Chem. 630, 2490-2501 $\hookrightarrow 2004)$

1.00000000000000

$\begin{array}{lll}6.54998397230000 & 0.00000000000000 & 0.00000000000000\end{array}$

$\begin{array}{lrl}0.00000000000000 & 12.53196933460000 & 0.00000000000000\end{array}$

$\begin{array}{rrr}-4.92099045533766 & 0.00000000000000 & 6.35554724183793\end{array}$

$\begin{array}{rr}\text { As } & \text { Ba } \\ 12 & 6\end{array}$

Direct

$0.67530000000000 \quad 0.48280000000000$ $0.67530000000000-0.48280000000000$ $0.47110000000000 \quad 0.64980000000000$ $0.47110000000000-0.64980000000000$ $0.4174000000000-0.3433000000000$ $0.4174000000000-0.34330000000000$ $0.41740000000000-0.34330000000000$ 0.28830000000000 .1551000000000 $0.28330000000000-0.1551000000000$ $0.14720000000000 \quad 0.11120000000000$ $0.14720000000000 \quad-0.11120000000000$ $0.00000000000000 \quad 0.07460000000000$ $0.00000000000000-0.07460000000000$ $0.56930000000000 \quad 0.07852000000000$ $\begin{array}{ll}0.56930000000000 & -0.07852000000000\end{array}$ $0.11840000000000 \quad 0.41567000000000$ $0.11840000000000-0.41567000000000$ $0.84730000000000 \quad 0.27155000000000$ $0.84730000000000 \quad-0.27155000000000$

$\epsilon-\mathrm{WO}_{3}$ (Low-temperature): A3B_mP16_7_6a_2a - CIF

\section{\# CIF file}

data_findsym-output

-audit_creation_method FINDSYM

_chemical_name_mineral 'epsilon-WO3,

chemical formula sum ' $\mathrm{O} 3 \mathrm{~W}$ '

loop

_publ_author_name

'P. M. Woodward

'A. W. Sleight

'T. Vogt,

_journal_name_full_name

Journal of Solid State Chemistry

0.58930000000000 As $\quad(2 \mathrm{a})$

0.34320000000000 As (2a)

0.55820000000000 As (2a)

1.05820000000000 As (2a)

$\begin{array}{lll}.00000000000000 & \text { As } \quad(2 a)\end{array}$

$\begin{array}{lll}0.09330000000000 & \mathrm{Ba} & (2 \mathrm{a}) \\ & & \end{array}$

$0.59330000000000 \quad \mathrm{Ba} \quad(2 \mathrm{a})$

$0.29380000000000 \quad \mathrm{Ba} \quad(2 \mathrm{a})$

$0.79380000000000 \quad \mathrm{Ba} \quad(2 \mathrm{a})$

$0.66560000000000 \quad \mathrm{Ba} \quad(2 \mathrm{a})$

$1.16560000000000 \quad \mathrm{Ba} \quad(2 \mathrm{a})$ _journal_volume 131

_ournal_year 1997

_journal_page_first 9

journal_page_last 17

_publ_Section_title

Ferroelectric tungsten trioxide

\# Found in Pearson's Crystal Data - Crystal Structure Database for $\hookrightarrow$ Inorganic Compounds, 2013

aflow_title $\$ \backslash$ epsilon $\$-W O \$ \_\{3\} \$$ (Low-temperature) Structure,

-aflow_proto, A3B_mP16_7_6a_2a

-aflow_params $, a, b / a, c / a, \backslash$ beta, $x_{-}\{1\}, y_{-}\{1\}, z_{-}\{1\}, x_{-}\{2\}, y_{-}\{2\}, z_{-}\{2\}, x_{-}\{3$ $\hookrightarrow\}, y_{-}\{3\}, z_{-}\{3\}, x_{-}\{4\}, y_{-}\{4\}, z_{-}\{4\}, x_{-}\{5\}, y_{-}\{5\}, z_{-}\{5\}, x_{-}\{6\}, y_{-}\{6\}$ $\hookrightarrow \mathrm{z}_{-}\{6\}, \mathrm{x}_{-}\{\overline{7}\}, \mathrm{y}_{-}\{\overline{7}\}, \mathrm{z}_{-}\{\overline{7}\}, \mathrm{x}_{-}\{\overline{8}\}, \mathrm{y}_{-}\{\overline{8}\}, \mathrm{z}_{-}\{\overline{8}\}$

.

$\hookrightarrow 0.5044,0.292,0.01,0.5764,0.215,0.586,0.0,0.209,0.0,0.0864,0.29$,

$\hookrightarrow 0.58,0.2874,0.0717,0.287,0.7924,0.4201,0.301,0.2874,0.014$,

$\hookrightarrow 0.0012,0.7994,0.528,0.078$,

_aflow_Strukturbericht 'None

_aflow_Pearson 'mP16'

_cell_length_a $\quad \begin{aligned} & 5.2778002048 \\ & \text { _cell_length_b }\end{aligned}$

_cell_length_b $\quad 5.1559002001$

cell angle alpha 90.0000000000

-cell_angle_beta 91.7620000000

cell_angle_gamma 90.0000000000

symmetry_space_group_name_H-M "P 1 c 1 "

_symmetry_Int_Tables_number 7

loop

_space_group_symop_id

_space_group_symop_operation_xy

$1 \mathrm{x}, \mathrm{y}, \mathrm{z}$

$2 \mathrm{x},-\mathrm{y}, \mathrm{z}+1 / 2$

loop

atom_site_label

atom_site_type_symbol

_atom_site_symmetry_multiplicity

_atom_site_Wyckoff_label

atom_site_fract_x

atom_site_fract_y

atom_site_fract_z

atom site occupancy

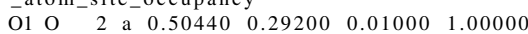

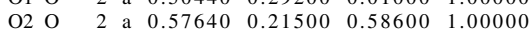

$\mathrm{O} 3 \mathrm{O} \quad 2$ a $20.00000 \quad 0.20900 \quad 0.00000 \quad 1.00000$

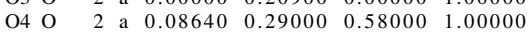

$\begin{array}{llllllll}\mathrm{O} 5 & \mathrm{O} & 2 & \text { a } & 0.28740 & 0.07170 & 0.28700 & 1.00000\end{array}$

$\begin{array}{lllllll}\mathrm{O} 6 \mathrm{O} & 2 & \text { a } & 0.79240 & 0.42010 & 0.30100 & 1.00000\end{array}$

W1 W 2 a $0.28740 \quad 0.01400 \quad 0.00120 \quad 1.00000$

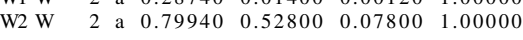

$\epsilon-\mathrm{WO}_{3}$ (Low-temperature): A3B_mP16_7_6a_2a - POSCAR

A3B_mP16_7_6a_2a \& a , b/a,c/a, beta , x1,y1, z1 , x2, y2, z2, x3,y3,z3, x4,y4,z4, x5 $\hookrightarrow, \mathrm{y} 5, \mathrm{z5}, \mathrm{x} 6, \mathrm{y} 6, \mathrm{z6}, \mathrm{x} 7, \mathrm{y} 7, \mathrm{z7}, \mathrm{x} 8, \mathrm{y} 8, \mathrm{z} 8-$-params $=5.2778002048$,

$\hookrightarrow 0.97690325515,1.45210125431,91.762,0.5044,0.292,0.01,0.5764$

$\hookrightarrow 0.215,0.586,0.0,0.209,0.0,0.0864,0.29,0.58,0.2874,0.0717,0.287$,

$\hookrightarrow 0.7924,0.4201,0.301,0.2874,0.014,0.0012,0.7994,0.528,0.078 \& \mathrm{Pc}$

$\hookrightarrow \mathrm{C}_{-}\{\mathrm{s}\}^{\wedge}\{2\} \# 7\left(\mathrm{a}^{\wedge} 8\right) \& \mathrm{mP} 16$ \& None \& WO3 \& epsilon \& P. M.

$\hookrightarrow$ Woodward and A. W. Sleight and T. Vogt, J. Solid State Chem.

$\hookrightarrow 131,9-17$ (1997)

1.00000000000000

$\begin{array}{lll}5.27780020480000 & 0.00000000000000 & 0.00000000000000\end{array}$

$0.00000000000000 \quad 5.15590020010000-0.00000000000000$

$\begin{array}{rrr}-0.23564849020651 & 0.00000000000000 & 7.66027659797942\end{array}$

O W

Direct

0.08930000000000 As $\quad(2 \mathrm{a})$

.50000000000000 As (2a)

$\begin{array}{ll}0.50440000000000 & -0.29200000000000\end{array}$

$\begin{array}{lll}0.57640000000000 & 0.21500000000000\end{array}$

$0.57640000000000-0.21500000000000$

$0.00000000000000 \quad 0.20900000000000$

$0.00000000000000 \quad-0.20900000000000$

$0.08640000000000 \quad 0.29000000000000$

$0.08640000000000-0.29000000000000$

$0.28740000000000 \quad 0.07170000000000$

$0.28740000000000-0.07170000000000$

$\begin{array}{lll}0.79240000000000 & 0.42010000000000\end{array}$

$0.79240000000000-0.42010000000000$

$0.28740000000000-0.01400000000000$

$0.28740000000000-0.0140000000000$

$0.2874000000000-0.01400000000000$

$\begin{array}{rr}0.79940000000000 & 0.52800000000000 \\ 0.79940000000000 & -0.52800000000000\end{array}$

0.01000000000000

0.51000000000000

0.58600000000000

1.08600000000000

0.00000000000000

0.50000000000000

0.58000000000000

.28700000000000

0.78700000000000

.30100000000000

80100000000000

00120000000000

.50120000000000

0.07800000000000

0.57800000000000

O (2a)

O (2a)

$\mathrm{O} \quad(2 \mathrm{a})$

O (2a)

$\begin{array}{ll}\mathrm{O} & (2 \mathrm{a}) \\ \mathrm{O} & (2 \mathrm{a})\end{array}$

O (2a)

O (2a)

O (2a)

(2a)

O (2a)

W (2a)

W (2a)

W (2a)

W (2a)

$\mathrm{Rh}_{2} \mathrm{Ga9}$ : A9B2_mP22_7_9a_2a - CIF

\# CIF file

data findsym-output

audit_creation_method FINDSYM

chemical name mineral 'Rh2Ga9'

chemical formula sum ' Ga9 Rh2'

loop

publ_author_nam

M. Bostr $\{\backslash$ "o $\} \mathrm{m}$

H. Rosner, 
'Y. Prots

urkhardt

Y. Grin

journal_name_full_name

Zeitschrift fur Anorganische und Allgemeine Chemie

_journal_volume 631

journal_year 2005

_journal_page_first 534

_journal_page_last 54

publ_Section_title

The Co\$_\{2\}\$Al\$_\{9\}\$ structure type revisited

Found in Pearson's Crystal Data - Crystal Structure Database for $\hookrightarrow$ Inorganic Compounds, 2013

aflow_title 'Rh\$_ $\{2\} \$ G a \$_{-}\{9\} \$$ Structure,

aflow_proto 'A9B2_mP22_7_9a_2a,

aflow_params $, a, b / a, c / a, \backslash$ beta, $x_{-}\{1\}, y_{-}\{1\}, z_{-}\{1\}, x_{-}\{2\}, y_{-}\{2\}, z_{-}\{2\}, x_{-}\{3$ $\hookrightarrow\}, y_{-}\{3\}, z_{-}\{3\}, x_{-}\{4\}, y_{-}\{4\}, z_{-}\{4\}, x_{-}\{5\}, y_{-}\{5\}, z_{-}\{5\}, x_{-}\{6\}, y_{-}\{6\}$, $\hookrightarrow \mathrm{z}_{-}\{6\}, \mathrm{x}_{-}\{7\}, \mathrm{y}_{-}\{7\}, \mathrm{z}_{-}\{7\}, \mathrm{x}_{-}\{8\}, \mathrm{y}_{-}\{8\}, \mathrm{z}_{-}\{8\}, \mathrm{x}_{-}\{9\}, \mathrm{y}_{-}\{9\}, \mathrm{z}_{-}\{9\}, \mathrm{x}_{-}$ $\hookrightarrow 10\}, \mathrm{y}_{-}\{10\}, \mathrm{z}_{-}\{10\}, \mathrm{x}_{-}\{11\}, \mathrm{y}_{-}\{11\}, \mathrm{z}_{-}\{11\}$

aflow_params_values '6.4165085102,0.999298672153,1.36910105356,93.39, $\hookrightarrow 0.4944,0.2369,0.0135,0.2965,0.2623,0.5568,0.4955,0.4453,0.2912$, $\hookrightarrow 0.2817,0.0557,0.2805,0.8067,0.5491,0.0506,0.0,0.0309,0.0,0.6774$ $\hookrightarrow, 0.1539,0.7442,0.096,0.6343,0.3258,0.8638,0.2389,0.2526,0.6359$

$\hookrightarrow 0.1217,0.4513,0.156,0.3761,0.11377$

aflow Strukturbericht 'None'

aflow_Pearson 'mP22'

cell_length_a $\quad 6.4165085102$

cell_length_b 6.4120084341

$\begin{array}{ll}\text { cell_length_c } & 8.7848485615\end{array}$

cell_angle_alpha 90.0000000000

cell_angle_beta 93.3900000000

_cell_angle_gamma 90.0000000000

_symmetry_space_group_name_H-M "P 1 c c 1 "

_symmetry_Int_Tables_number

loop

_space_group_symop_id

_space_group_symop_operation_xyz

$1 \mathrm{x}, \mathrm{y}, \mathrm{z}$

$2 \mathrm{x},-\mathrm{y}, \mathrm{z}+1 / 2$

loop

atom site label

_atom_site_type_symbol

_atom_site_symmetry_multiplicity

_atom_site_Wyckoff_label

atom_site_fract_x

atom_site_fract_y

atom_site_fract_z

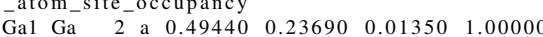

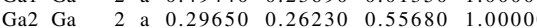

$\begin{array}{llllllll}\mathrm{Ga} 3 \mathrm{Ga} & 2 & \text { a } & 0.49550 & 0.44530 & 0.29120 & 1.00000\end{array}$

Ga4 Ga 2 a $0.28170 \quad 0.05570 \quad 0.28050 \quad 1.00000$

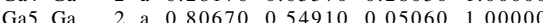

$\begin{array}{lllllll} & \text { Gas Ga } 2 \text { a } & 0.80670 & 0.54910 & 0.05060 & 1.00000\end{array}$

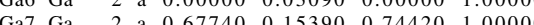

$\begin{array}{lllllll}\mathrm{Ga} 7 \mathrm{Ga} & 2 & \mathrm{a} & 0.67740 & 0.15390 & 0.74420 & 1.00000\end{array}$

Ga8 Ga 2 a $0.09600 \quad 0.63430-0.32580 \quad 1.00000$

$\mathrm{Ga} \mathrm{Ga}, 2 \mathrm{a} 0.863800 .23890-0.252601 .00000$

$\begin{array}{llllllll}\mathrm{Rh} & \mathrm{Rh} & 2 & \mathrm{a} & 0.63590 & 0.12170 & 0.45130 & 1.00000 \\ \mathrm{Rh} 2 & \mathrm{Rh} & 2 & \mathrm{a} & 0.15600 & 0.37610 & 0.11377 & 1.00000\end{array}$

$\mathrm{Rh}_{2} \mathrm{Ga9}$ : A9B2_mP22_7_9a_2a - POSCAR

A9B2_mP22_7_9a_2a \& a,b/a,c/a, beta, x1,y1,z1, x2, y2, z2, x3,y3,z3, x4,y4,z4 $\hookrightarrow \mathrm{x} 5, \mathrm{y} 5, \mathrm{z} 5, \mathrm{x} 6, \mathrm{y} 6, \mathrm{z} 6, \mathrm{x} 7, \mathrm{y} 7, \mathrm{z} 7, \mathrm{x} 8, \mathrm{y} 8, \mathrm{z} 8, \mathrm{x} 9, \mathrm{y} 9, \mathrm{z} 9, \mathrm{x} 10, \mathrm{y} 10, \mathrm{z} 10, \mathrm{x} 11$

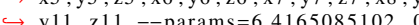

$\hookrightarrow 93.39,0.4944,0.2369,0.0135,0.2965,0.2623,0.5568,0.4955,0.4453$

$\hookrightarrow 0.2912,0.2817,0.0557,0.2805,0.8067,0.5491,0.0506,0.0,0.0309,0.0$

$\hookrightarrow, 0.6774,0.1539,0.7442,0.096,0.6343,0.3258,0.8638,0.2389,0.2526$

$\hookrightarrow 0.6359,0.1217,0.4513,0.156,0.3761,0.11377 \& \mathrm{Pc}_{\mathrm{C}} \mathrm{C}_{-}\{\mathrm{s}\}^{\wedge}\{2\} \# 7 \mathrm{a}^{\wedge}$

$\hookrightarrow$ 11) \& mP22 \& None \& Rh2Ga9 \& \& M. Bostr $\{\backslash " \mathrm{o}\} \mathrm{m}$ et al., Z.

$\hookrightarrow$ Anorg. Allg. Chem. 631, 534-541 (2005)

1.00000000000000

6.41650851020000

$0.00000000000000 \quad 0.00000000000000$

$\begin{array}{rr}0.00000000000000 & 6.41200843410000 \\ -0.51946695612781 & 0.00000000000000\end{array}$

0.00000000000000

00000000000000

$\begin{array}{rr}\mathrm{Ga} & \mathrm{Rh} \\ 18 & 4\end{array}$

Direct

$0.49440000000000 \quad 0.23690000000000$ $0.49440000000000-0.23690000000000$ $0.29650000000000 \quad 0.26230000000000$ $\begin{array}{ll}0.29650000000000 & -0.26230000000000\end{array}$ $\begin{array}{ll}0.49550000000000 & 0.44530000000000\end{array}$ $\begin{array}{rr}0.49550000000000 & -0.44530000000000\end{array}$ $\begin{array}{rr}0.49550000000000 & -0.44530000000000 \\ 0.28170000000000 & 0.05570000000000\end{array}$ $\begin{array}{rr}0.28170000000000 & 0.05570000000000 \\ 0.28170000000000 & -0.05570000000000\end{array}$ $0.28170000000000-0.05570000000000$ $0.80670000000000 \quad 0.54910000000000$ $0.80670000000000-0.54910000000000$ $0.00000000000000 \quad 0.03090000000000$ $0.00000000000000-0.03090000000000$ $0.67740000000000 \quad 0.15390000000000$ $0.67740000000000-0.15390000000000$ $0.09600000000000 \quad 0.63430000000000$ $0.09600000000000-0.63430000000000$ $\begin{array}{rr}0.86380000000000 & 0.23890000000000\end{array}$
$0.86380000000000-0.23890000000000$ $\begin{array}{lll}0.63590000000000 & 0.12170000000000\end{array}$ $0.63590000000000-0.12170000000000$ $0.15600000000000 \quad 0.37610000000000$ $0.15600000000000-0.37610000000000$ 0.45130000000000 0.95130000000000 0.11377000000000 0.61377000000000

$\alpha-\mathrm{P}_{3} \mathrm{~N}_{5}$ : A5B3_mC32_9_5a_3a-CIF

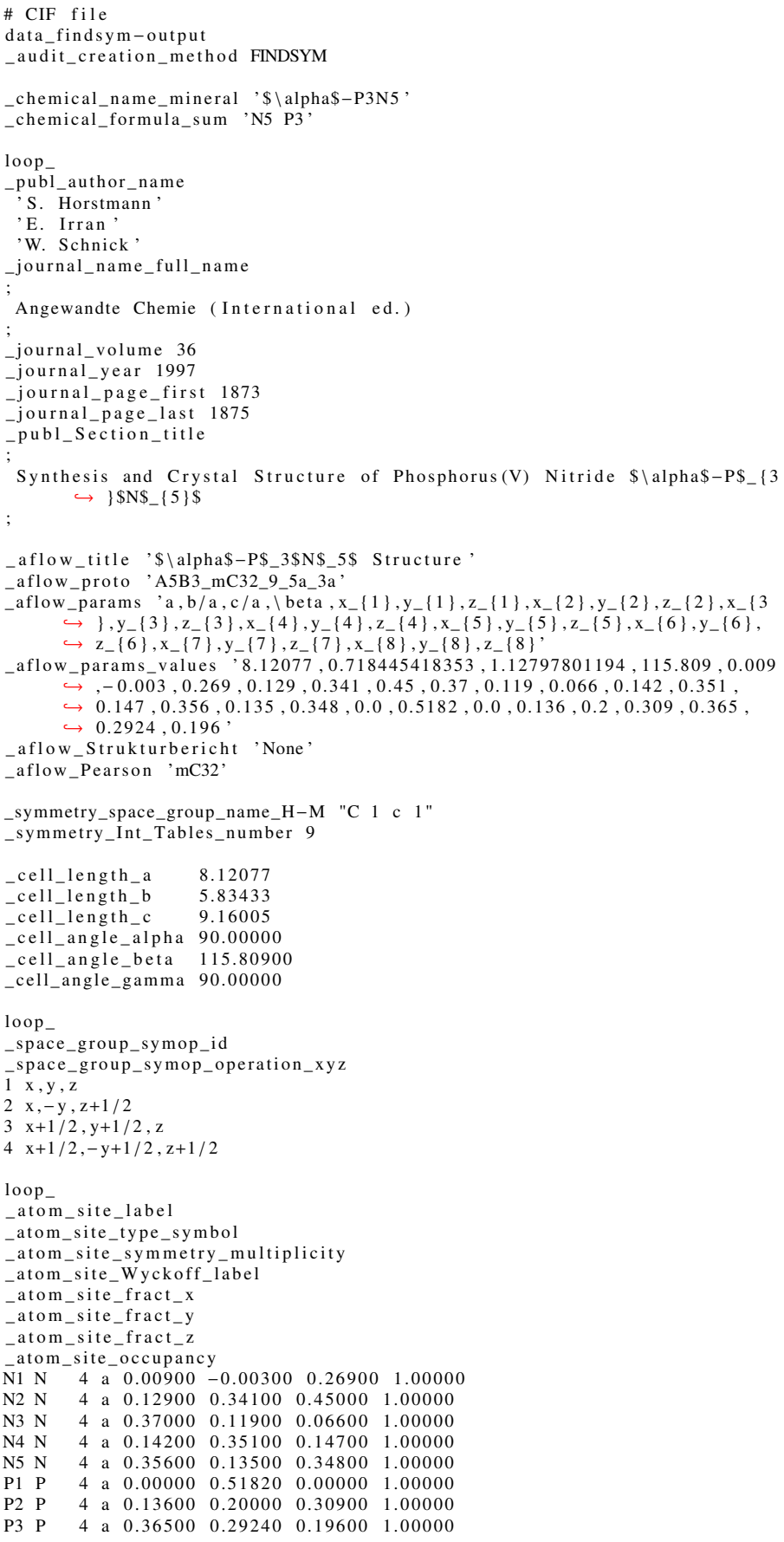

$\alpha-\mathrm{P}_{3} \mathrm{~N}_{5}$ : A5B3_mC32_9_5a_3a - POSCAR

A5B3_mC32_9_5a_3a \& a , b/a,c/a, beta , x1, y1, z1, x2, y2, z2 , x3, y3, z3, x4, y4, z4 $\hookrightarrow \mathrm{x} 5, \mathrm{y} 5, \mathrm{z} 5, \mathrm{x} 6, \mathrm{y} 6, \mathrm{z} 6, \mathrm{x} 7, \mathrm{y} 7, \mathrm{z} 7, \mathrm{x} 8, \mathrm{y} 8, \mathrm{z} 8--\mathrm{params}=8.12077$

$\hookrightarrow 0.718445418353,1.12797801194,115,809,0.009,-0.003,0.269,0.129$ $\hookrightarrow 0.341,0.45,0.37,0.119,0.066,0.142,0.351,0.147,0.356,0.135,0.348$ $\hookrightarrow, 0.0,0.5182,0.0,0.136,0.2,0.309,0.365,0.2924,0.196 \& \mathrm{Cc}_{-} \mathrm{C}_{-}\{\mathrm{s}\}^{\wedge}\{$ $\hookrightarrow 4\} \# 9\left(a^{\wedge} 8\right)$ \& mC32 \& None \& P3N5 \& \$ \alpha\$-P3N5 \& S. Horstmann $\hookrightarrow$ and E. Irran and W. Schnick, Angew. Chem. Int. Ed. 36, $\hookrightarrow 1873-1875(1997)$

1.00000000000000

$\begin{array}{lll}4.06038500000000 & -2.91716500000000 & 0.00000000000000\end{array}$

$\begin{array}{lll}4.06038500000000 & 2.91716500000000 & 0.00000000000000\end{array}$

$\begin{array}{rrr}-3.98803401284269 & 0.00000000000000 & 8.24633862480252\end{array}$

$\mathrm{N} \quad \mathrm{P}$

Direct

0.01200000000000 0.00600000000000 $-0.21200000000000$ 0.47000000000000 0.25100000000000
0.48900000000000
0.00600000000000 0.01200000000000 0.47000000000000 0.48900000000000 0.25100000000000 $-0.21200000000000$
0.26900000000000 0.76900000000000 0.45000000000000 0.95000000000000 0.06600000000000 0.56600000000000
$\mathrm{N} \quad(4 \mathrm{a})$ $\mathrm{N}$ (4a) $\mathrm{N} \quad(4 \mathrm{a})$ $\mathrm{N} \quad(4 \mathrm{a})$ $\mathrm{N} \quad(4 \mathrm{a})$ 


\begin{tabular}{|rrrrrr}
-0.20900000000000 & 0.49300000000000 & 0.14700000000000 & $\mathrm{~N}$ & $(4 \mathrm{a})$ \\
0.49300000000000 & -0.20900000000000 & 0.64700000000000 & $\mathrm{~N}$ & $(4 \mathrm{a})$ \\
0.22100000000000 & 0.49100000000000 & 0.34800000000000 & $\mathrm{~N}$ & $(4 \mathrm{a})$ \\
0.49100000000000 & 0.22100000000000 & 0.84800000000000 & $\mathrm{~N}$ & $(4 \mathrm{a})$ \\
-0.51820000000000 & 0.51820000000000 & 0.00000000000000 & $\mathrm{P}$ & $(4 \mathrm{a})$ \\
0.51820000000000 & -0.51820000000000 & 0.50000000000000 & $\mathrm{P}$ & $(4 \mathrm{a})$ \\
-0.06400000000000 & 0.33600000000000 & 0.30900000000000 & $\mathrm{P}$ & $(4 \mathrm{a})$ \\
0.33600000000000 & -0.06400000000000 & 0.80900000000000 & $\mathrm{P}$ & $(4 \mathrm{a})$ \\
0.07260000000000 & 0.65740000000000 & 0.19600000000000 & $\mathrm{P}$ & $(4 \mathrm{a})$ \\
0.65740000000000 & 0.07260000000000 & 0.69600000000000 & $\mathrm{P}$ & $(4 \mathrm{a})$ \\
\hline
\end{tabular}

$\mathrm{H}_{3} \mathrm{Cl}$ (20 GPa): AB3_mC16_9_a_3a - CIF

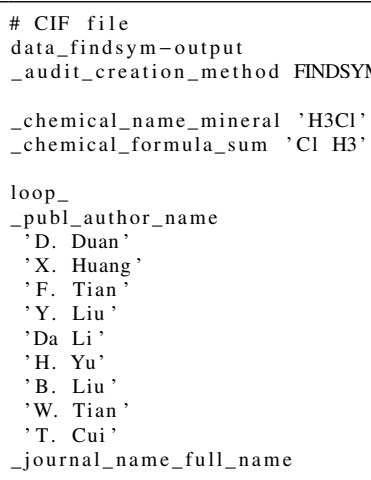

$\mathrm{H}_{3} \mathrm{Cl}$ (20 GPa): AB3_mC16_9_a_3a - POSCAR

AB3_mC16_9_a_3a \& a , b/a,c/a , beta , x1,y1,z1, x2, y2 , z2, x3,y3,z3, x4, y4, z4 $\hookrightarrow$ params $=3.5367,2.67777872028,0.986823875364,93.018,0.50691$ $\hookrightarrow 0.35551,0.49997,0.28659,0.26502,0.27913,0.57076,0.06256,0.41996$ $\hookrightarrow, 0.42173,0.07037,0.56036 \& \mathrm{Cc}_{-}\{\mathrm{s}\}^{\wedge}\{4\} \# 9\left(\mathrm{a}^{\wedge} 4\right) \& \mathrm{mCl} 16$ \& None $\hookrightarrow \& \mathrm{H} 3 \mathrm{Cl} \& \mathrm{H} 3 \mathrm{Cl} \& \mathrm{D}$. Duan et al., J. Phys. Chem. A 119 , $\hookrightarrow 11059-11065(2015)$

$\begin{array}{lll}1.76835000000000 & -4.73525000000000 & 0.00000000000000\end{array}$

$\begin{array}{rrr}1.76835000000000 & 4.73525000000000 & 0.00000000000000\end{array}$

$\begin{array}{rrr}-0.18375265646087 & 0.00000000000000 & 3.48525938363898\end{array}$

$$
\begin{array}{rr}
\mathrm{Cl} & \mathrm{H} \\
2 & 6
\end{array}
$$

Direct

0.15140000000000 0.86242000000000 0.02157000000000

0.86242000000000 0.15140000000000 0.55161000000000 0.99997000000000 0.27913000000000
0.49997000000000
0.55161000000000 0.5082000000000 0.63332000000000 0.35136000000000 0.49210000000000 0.63332000000000 0.50820000000000 0.49210000000000

$\delta$-PdCl 2 : A2B_mP6_10_mn_bg - CIF

\section{\# CIF file}

data_findsym-outpu

audit_creation_method FINDSYM

chemical_name_mineral ' $\$ \backslash$ delta $\$-P d C l 2$

chemical_formula_sum 'C12 Pd'

loop

publ_author_name

'J. Evers,

'W. Beck'

'M. G\"\{o\}bel,

'S. Jakob'

P. Mayer'

'G. Oehlinge

T. M. Klap $\backslash$ " $\{$ o $\}$ tke,

journal_name_full_name

Angewandte Chemie (International ed.)

journal_volume 49

journal_year 2010

journal_page_first 5677

journal_page_last 5682

_publ_Section_title

The Structures of $\$ \backslash$ delta $\$-P d C l \$ \_\{2\} \$$ and $\$ \backslash$ gamma $\$-P d C l \$ \_\{2\} \$:$ Phases $\hookrightarrow$ with Negative Thermal Expansion in One Direction

aflow_title $\$ \backslash$ delta $\$-P d C l \$ \_\{2\} \$$ Structure

aflow_proto , A2B_mP6_10_mn_bg

aflow_params a,b/a,c/a, $\backslash$ beta, $x_{-}\{3\}, z_{-}\{3\}, x_{-}\{4\}, z_{-}\{4\}$,

aflow_params_values ' $4.012,0.819541375872,2.93668993021,97.03,0.843$, $\stackrel{-P}{\longrightarrow} 0.126,0.558,0.644$

aflow_Strukturbericht 'None'

_aflow_Pearson 'mP6'

symmetry_space_group_name_H-M "P $12 / \mathrm{m} \quad 1$ "

_symmetry_Int_Tables_number 10

_cell_length_a $\quad 4.01200$

_cell_length_b $\quad 3.28800$

Ccell_length_c 11.78200

-cell-angle alpha 90.00000

_cell_angle_alpha 90.00000

cell_angle_bela 97.03000

loop

space_group_symop_id

space_group_symop_operation_xy

$1 \mathrm{x}, \mathrm{y}, \mathrm{z}$

$2-x, y,-z$
$3-x,-y,-z$

$4 \mathrm{x},-\mathrm{y}, \mathrm{z}$

loop

atom_site_label

_atom_site_type_symbol

atom_site_symmetry_multiplicity

-atom_site_Wyckoff_label

atom_site_fract_x

atom_site_fract_y

atom_site_fract_z

atom_site_occupancy

$\begin{array}{lllllll}\text { Pd1 Pd } & 1 \text { b } & 0.00000 & 0.50000 & 0.00000 & 1.00000\end{array}$

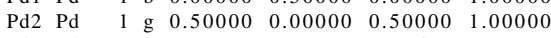

$\begin{array}{llllllll}\mathrm{Cl1} & \mathrm{Cl} & 2 & \mathrm{~m} & 0.84300 & 0.00000 & 0.12600 & 1.00000\end{array}$

$\begin{array}{lllllllll}\mathrm{C} 12 & \mathrm{Cl} & 2 & \mathrm{n} & 0.55800 & 0.50000 & 0.64400 & 1.00000\end{array}$

$\delta$-PdCl 2 : A2B_mP6_10_mn_bg - POSCAR

A2B_mP6_10_mn_bg \& a , b/a, c/a, beta , x3, z3, x4, z4 --params $=4.012$

$\hookrightarrow 0.819541375872,2.93668993021,97.03,0.843,0.126,0.558,0.644 \&$ P2 $\hookrightarrow / \mathrm{m} \mathrm{C}_{-}\{2 \mathrm{~h}\}^{\wedge}\{1\} \# 10$ (bgmn) \& mP6 \& None \& PdCl2 \& \$ \delta \$-PdCl2 $\hookrightarrow \&$ J. Evers et al., Angew. Chem. Int. Ed. 49, 5677-5682 (2010)

1.00000000000000

$\begin{array}{lll}4.01200000000000 & 0.00000000000000 & 0.00000000000000\end{array}$

$\begin{array}{lll}0.00000000000000 & 3.28800000000000 & 0.00000000000000\end{array}$

$\begin{array}{rrr}-1.44198746457411 & 0.00000000000000 & 11.69342533871110\end{array}$

$\begin{array}{rr}\mathrm{Cl} & \mathrm{Pd} \\ 4 & 2\end{array}$

Direct

$\begin{array}{lll}0.84300000000000 & 0.00000000000000 & 0.12600000000000\end{array}$ $\begin{array}{rrr}-0.84300000000000 & 0.00000000000000 & -0.12600000000000\end{array}$ $\begin{array}{rrr}-0.84300000000000 & 0.00000000000000 & -0.12600000000000 \\ -0.55800000000000 & 0.50000000000000 & 0.64400000000000\end{array}$ $\begin{array}{rrr}-0.5580000000000 & 0.50000000000000 & -0.64400000000000\end{array}$ $\begin{array}{lll}0.00000000000000 & 0.50000000000000 & 0.00000000000000 \\ 0.50000000000000 & 0.00000000000000 & 0.50000000000000\end{array}$

$\mathrm{H}_{3} \mathrm{Cl}(400 \mathrm{GPa}): \mathrm{AB} 3 \_\mathrm{mP16} \_10 \_\mathrm{mn} \_3 \mathrm{~m} 3 \mathrm{n}$ - CIF

\section{\# CIF fil}

data_findsym-output

audit_creation_method FINDSYM 
chemical_name_mineral ' $\mathrm{H} 3 \mathrm{Cl}$,

_chemical_formula_sum ' $\mathrm{Cl} \mathrm{H} 3$

loop

publ_author_name

'Q. Zeng'

' S. $\mathrm{Yu}$ '

A. R. Oganov

'G. Frapper'

_journal_name_full_name

Physical Chemistry Chemical Physics

journal_volume 19

journal year 2017

_journal_page_first 8236

_journal_page_last 82

publ_Section_title

Emergence of novel hydrogen chlorides under high pressure

aflow_title 'H\$_ $\$ 3\} \$ C l$ (400 GPa) Structure

aflow_proto 'AB3_mP16_10_mn_3m3n'

aflow_params 'a,b/a,c/a, $\backslash$ beta, $x_{-}\{1\}, z_{-}\{1\}, x_{-}\{2\}, z_{-}\{2\}, x_{-}\{3\}, z_{-}\{3\}, x_{-}\{4$ $\hookrightarrow\}, z_{-}\{4\}, x_{-}\{5\}, z_{-}\{5\}, x_{-}\{6\}, z_{-}\{6\}, x_{-}\{7\}, z_{-}\{7\}, x_{-}\{8\}, z_{-}\{8\}$

aflow_params_values '3.678,0.71098423056, $1.23817292007,90.0,0.263,0.339$

$\hookrightarrow, 0.08,0.059,0.795,0.341,0.438,-0.069,0.763,0.161,0.705,0.841$,

$\hookrightarrow \quad 0.08,0.059,0.795,0.341,0$

aflow Strukturbericht 'None'

aflow Pearson 'mP16'

_symmetry_space_group_name_H-M "P $1 \quad 2 / \mathrm{m}^{\prime} 1 "$

_symmetry_Int_Tables_number 10

_cell_length_a $\quad 3.67800$

_cell_length_b 2.61500

_cell_length_c $\quad 4.55400$

_cell_angle_alpha 90.00000

_cell_angle_beta 90.00000

_cell_angle_gamma 90.00000

loop

_space_group_symop_id

_space_group_symop_operation_xyz

$1 \mathrm{x}, \mathrm{y}, \mathrm{z}$

$2-\mathrm{x}, \mathrm{y},-\mathrm{z}$

$3-\mathrm{x},-\mathrm{y},-\mathrm{z}$

$4 \mathrm{x},-\mathrm{y}, \mathrm{z}$

loop

_atom_site_labe

atom_site_type_symbol

atom_site_symmetry_multiplicity

atom_site_Wyckoff_label

atom_site_fract_x

atom_site_fract_y

atom_site_fract_z

$\begin{array}{llllll}\mathrm{C}_{11} \mathrm{Cl} & 2 \mathrm{~m} & 0.26300 & 0.00000 & 0.33900 & 1.00000\end{array}$

$\begin{array}{llllllll}\mathrm{H} 1 & \mathrm{H} & 2 \mathrm{~m} & 0.08000 & 0.00000 & 0.05900 & 1.00000\end{array}$

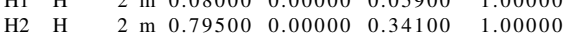

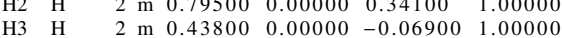

$\begin{array}{llllllll}\mathrm{H} 3 & \mathrm{H} & 2 & \mathrm{~m} & 0.43800 & 0.00000 & -0.06900 & 1.00000 \\ \mathrm{Cl} 2 & \mathrm{Cl} & 2 & \mathrm{n} & 0.76300 & 0.50000 & 0.16100 & 1.00000\end{array}$

$\begin{array}{llllllll}\mathrm{Cl} 2 & \mathrm{Cl} & 2 & \mathrm{n} & 0.76300 & 0.50000 & 0.16100 & 1.00000 \\ \mathrm{H} 4 & \mathrm{H} & 2 & \mathrm{n} & 0.70500 & 0.50000 & 0.84100 & 1.00000\end{array}$

$\begin{array}{llllllll}\mathrm{H} 4 & \mathrm{H} & 2 & \mathrm{n} & 0.70500 & 0.50000 & 0.84100 & 1.00000 \\ \mathrm{H} 5 & \mathrm{H} & 2 & \mathrm{n} & 0.58000 & 0.50000 & 0.44100 & 1.00000\end{array}$

$\begin{array}{llllllll}\mathrm{H} 6 & \mathrm{H} & 2 & \mathrm{n} & 0.06200 & 0.50000 & 0.43100 & 1.00000\end{array}$

$\mathrm{H}_{3} \mathrm{Cl}(400 \mathrm{GPa})$ : AB3_mP16_10_mn_3m3n - POSCAR

AB3_mP16_10_mn_3m3n \& a , b/a,c/a , beta , x1, z1 , x2, z2, x3, z3 , x4, z4, x5, z5, x6, z6 $\hookrightarrow, \mathrm{x} 7, \mathrm{z7}, \mathrm{x} 8, \mathrm{z} 8$--params $=3.678,0.71098423056,1.23817292007,90.0$

$\hookrightarrow 0.705,0.841,0.58,0.441,0.062,0.431 \& \mathrm{P} 2 / \mathrm{m} \mathrm{C}\{2 \mathrm{~h}\}^{\wedge}\{1\} \# 10\left(\mathrm{~m}^{\wedge} 4 \mathrm{n}^{\wedge}\right.$

$\hookrightarrow 4) \& \mathrm{mP16} \&$ None \& $\mathrm{H} 3 \mathrm{Cl} \& \mathrm{H} 3 \mathrm{Cl} \&$ Q. Zeng et al., Phys. Chem. $\hookrightarrow$ Chem. Phys. 19, 8236-8242 (2017)

1.00000000000000

$\begin{array}{lll}3.67800000000000 & 0.00000000000000 & 0.00000000000000\end{array}$

$\begin{array}{lll}0.00000000000000 & 2.61500000000000 & 0.00000000000000\end{array}$

$\begin{array}{lll}0.00000000000000 & 0.00000000000000 & 4.55400000000000\end{array}$

$\begin{array}{rrr}\mathrm{Cl} & \mathrm{H} \\ 4 & 12\end{array}$

Direct

0.26300000000000

$-0.26300000000000$

0.76300000000000

$-0.76300000000000$

0.08000000000000

$-0.08000000000000$

0.79500000000000

$-0.79500000000000$

0.43800000000000

$-0.43800000000000$

0.70500000000000

$-0.70500000000000$

0.58000000000000

$-0.58000000000000$

0.06200000000000

$-0.06200000000000$

$0.00000000000000 \quad 0.33900000000000$

0.00000000000000

0.50000000000000

0.50000000000000

0.00000000000000

0.00000000000000

0.00000000000000

0.00000000000000

0.00000000000000

0.50000000000000

0.50000000000000

0.50000000000000

0.50000000000000

0.50000000000000 0.16100000000000 $-0.16100000000000$ $-0.05900000000000$ $-0.05900000000000$ 0.3410000000000 0.34100000000000 $-0.3410000000000$ 0.06900000000000 .06900000000000 0.84100000000000 $-0.84100000000000$ 0.44100000000000 $-0.44100000000000$ .43100000000000

$\begin{aligned} \mathrm{Cl} & (2 \mathrm{~m}) \\ \mathrm{Cl} & (2 \mathrm{~m}) \\ \mathrm{Cl} & (2 \mathrm{n}) \\ \mathrm{Cl} & (2 \mathrm{n}) \\ \mathrm{H} & (2 \mathrm{~m}) \\ \mathrm{H} & (2 \mathrm{~m}) \\ \mathrm{H} & (2 \mathrm{~m}) \\ \mathrm{H} & (2 \mathrm{~m}) \\ \mathrm{H} & (2 \mathrm{~m}) \\ \mathrm{H} & (2 \mathrm{~m}) \\ \mathrm{H} & (2 \mathrm{n}) \\ \mathrm{H} & (2 \mathrm{n}) \\ \mathrm{H} & (2 \mathrm{n}) \\ \mathrm{H} & (2 \mathrm{n}) \\ \mathrm{H} & (2 \mathrm{n}) \\ \mathrm{H} & (2 \mathrm{n})\end{aligned}$

Muthmannite $(\mathrm{AuAgTe} 2)$ : ABC2 $2 \mathrm{mP} 8 \_10 \_$ac_eh_mn - CIF

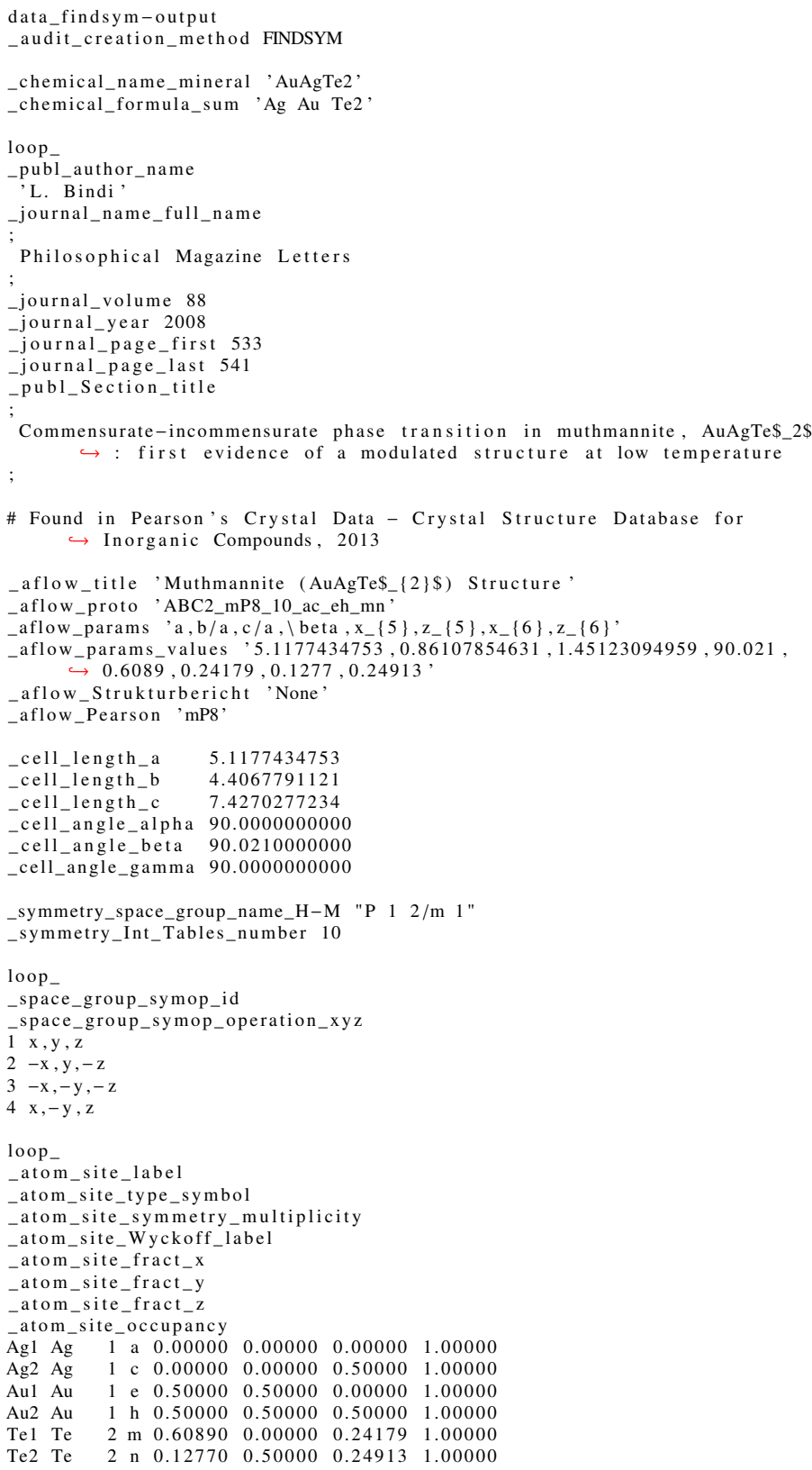

Muthmannite (AuAgTe 2$)$ : ABC2_mP8_10_ac_eh_mn - POSCAR

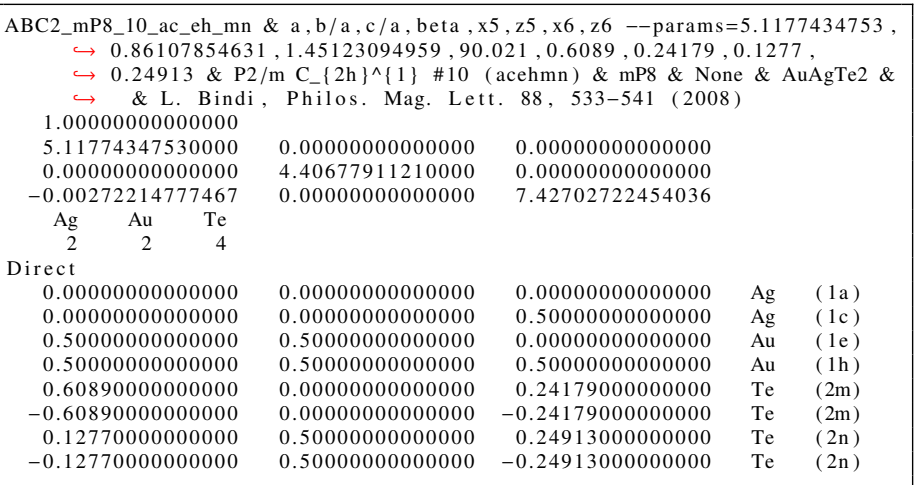

LiSn: AB_mP6_10_en_am - CIF

\# CIF file

data findsym-output

audit creation method FINDSYM

chemical_name_mineral 'LiSn'

chemical_formula_sum , $\mathrm{Li} \mathrm{Sn}$,

loop

publ_author_nam

W. M $\{\backslash$ u $\} 1$ ller,

H. Sch $\{\backslash$ a a fer

_journal_name_full_name

\# CIF file 
Zeitschrift $f\{\backslash " u\} r$ Naturforschung B

_journal_volume 28

_journal_year 1973

_journal_page_first 246

journal_page_last 248

publ_Section_title

Die Kristallstruktur der Phase LiSn

\# Found in Pearson's Crystal Data - Crystal Structure Database for $\hookrightarrow$ Inorganic Compounds, 2013

aflow title 'LiSn Structure,

aflow_proto 'AB_mP6_10_en_am,

aflow_params , a,b/a,c/a, $\backslash$ beta, $x_{-}\{3\}, z_{-}\{3\}, x_{-}\{4\}, z_{-}\{4\}$

aflow_params_values $5.1700416367,0.61508704062,1.49709864605,104.5$ $\hookrightarrow 0.234,0.66,0.263,0.336$

aflow_Strukturbericht 'None'

_aflow_Pearson 'mP6'

\section{$\begin{array}{ll}\text { _cell_length_a } & 5.1700416367 \\ \text { _cell_length_b } & 3.1800256102\end{array}$}

_cell_length_c $\quad 7.7400623343$

_cell_angle_alpha 90.0000000000

cell angle gamma 90.0000000000

symmetry_space_group_name_H-M "P $1 \quad 2 / \mathrm{m}^{1}$

symmetry_Int_Tables_number 10

loop

space_group_symop_id

_space_group_symop_operation_xyz

$1 \mathrm{x}, \mathrm{y}, \mathrm{z}$

$2-\mathrm{x}, \mathrm{y},-\mathrm{z}$

$3-x,-y,-z$
$4 x,-y, z$

loop

_atom_site_label

_atom_site_type_symbol

_atom_site_symmetry_multiplicity

_atom_site_Wyckoff_label

_atom_site_fract_x

_atom_site_fract_y

_atom_site_fract_z

atom_site_occupancy

\begin{tabular}{llllllll}
\hline Sn 1 & Sn & l a & 0.00000 & 0.00000 & 0.00000 & 1.00000
\end{tabular}

$\begin{array}{lllllllll}\mathrm{Li} 1 & \mathrm{Li} & 1 & \mathrm{e} & 0.50000 & 0.50000 & 0.00000 & 1.00000\end{array}$

$\begin{array}{llllllll}\mathrm{Sn} 2 & \mathrm{Sn} & 2 \mathrm{~m} & 0.23400 & 0.00000 & 0.66000 & 1.00000\end{array}$

$\begin{array}{llllllll}\mathrm{Li} 2 & \mathrm{Li} & 2 & \mathrm{n} & 0.26300 & 0.50000 & 0.33600 & 1.00000\end{array}$

LiSn: AB_mP6_10_en_am - POSCAR

$\mathrm{AB}$ mP6 10 en $\_\mathrm{am} \& \mathrm{a}, \mathrm{b} / \mathrm{a}, \mathrm{c} / \mathrm{a}, \mathrm{beta}, \mathrm{x} 3, \mathrm{z} 3, \mathrm{x} 4, \mathrm{z} 4--\mathrm{params}=5.1700416367$

$\hookrightarrow 0.61508704062,1.49709864605,104.5,0.234,0.66,0.263,0.336 \& \mathrm{P} 2 / \mathrm{m}$

$\hookrightarrow \mathrm{C}_{-}\{2 \mathrm{~h}\}^{\wedge}\{1\} \# 10(\mathrm{aemn}) \& \mathrm{mP6} \&$ None \& LiSn \& \& W. M $\{1 \mathrm{u}\} 11 \mathrm{e}$

$\hookrightarrow$ and H. Sch $\{\backslash "$ a \} fer, Z. Naturforsch. B 28, 246-248 (1973)

1.00000000000000

$\begin{array}{lll}5.17004163670000 & 0.00000000000000 & 0.00000000000000\end{array}$

$\begin{array}{rrr}5.17004163670000 & 0.00000000000000 & 0.00000000000000\end{array}$

$\begin{array}{rrr}-1.93795683864366 & 0.00000000000000 & 7.49352308533201\end{array}$

$\begin{array}{rr}\mathrm{Li} & \mathrm{Sn} \\ 3 & 3\end{array}$

Direct

$0.50000000000000 \quad 0.50000000000000 \quad 0.00000000000000$

0.26300000000000

$-0.26300000000000$

0.00000000000000

0.23400000000000

0.50000000000000

$0.50000000000000-0.33600000000000$

$0.00000000000000-0.33600000000000$

0.6600000000000

$-0.23400000000000$

$\begin{array}{ll}\mathrm{Li} & (1 \mathrm{e}) \\ \mathrm{Li} & (2 \mathrm{n}) \\ \mathrm{Li} & (2 \mathrm{n}) \\ \mathrm{Sn} & (1 \mathrm{a}) \\ \mathrm{Sn} & (2 \mathrm{~m}) \\ \mathrm{Sn} & (2 \mathrm{~m})\end{array}$

S-carbon: A_mP8_10_2m2n - CIF

\# CIF file

data_findsym-output

audit creation method FINDSYM

chemical_name_mineral 'S-carbon'

chemical_formula_sum 'C'

loop

-publ_author_name

'H. Niu

'X.-Q. Chen'

'S. Wang'

'D. $\mathrm{Li}$ '

'W. L. Mao'

'Y. Li,

journal_name_full_name

Physical Review Letter

_journal_volume 108

journal year 2012

-journal_page_first 135501

-journal_page_first 135501

-journal_page_last
-publ_Section_title

Families of Superhard Crystalline Carbon Allotropes Constructed via $\hookrightarrow$ Cold Compression of Graphite and Nanotubes

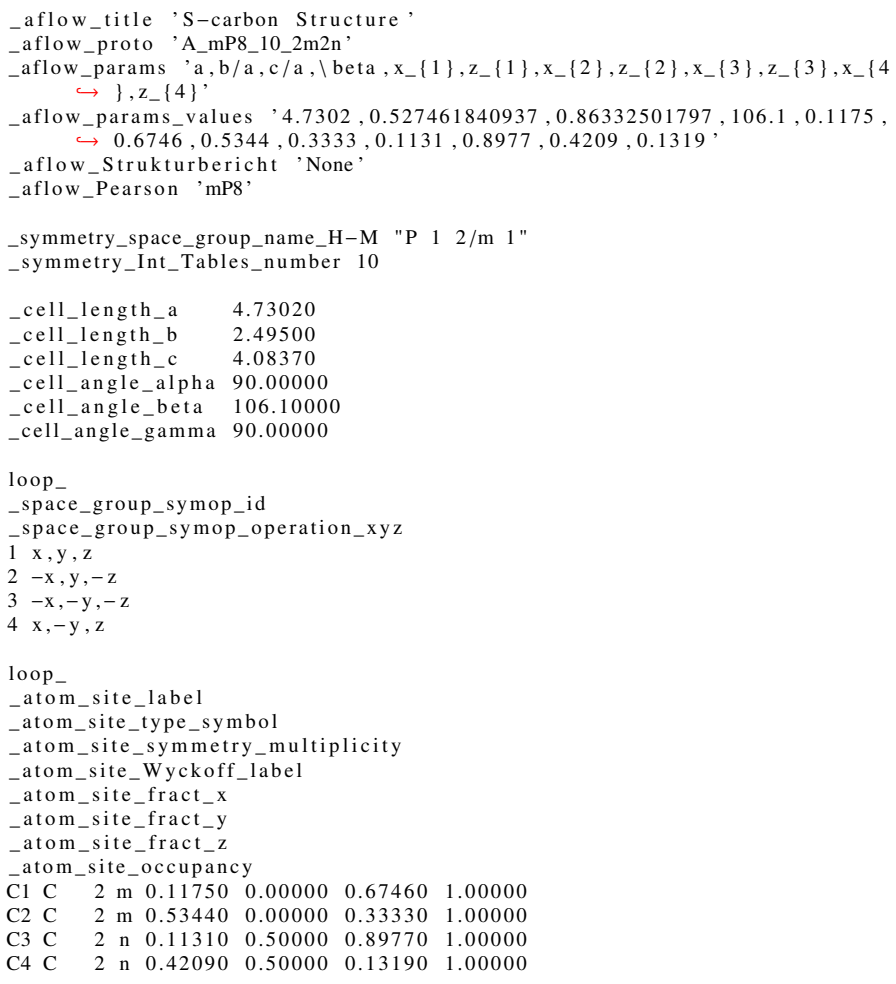

S-carbon: A_mP8_10_2m2n - POSCAR

\begin{tabular}{|c|c|c|c|c|}
\hline \multicolumn{5}{|c|}{$\begin{aligned} \text { A_mP8_10_2m } 2 \mathrm{n} \& \mathrm{a}, \mathrm{b} / \mathrm{a}, \mathrm{c} / \mathrm{a}, \mathrm{beta}, \mathrm{x} 1, \mathrm{z} 1, \mathrm{x} 2, \mathrm{z} 2, \mathrm{x} 3, \mathrm{z} 3, \mathrm{x} 4, \mathrm{z} 4--\mathrm{params}=4.7302, & \\
& \hookrightarrow 0.527461840937,0.86332501797,106.1,0.1175,0.6746,0.5344,0.3333, \\
& \hookrightarrow 0.1131,0.8977,0.4209,0.1319 \& \text { P } 2 / \mathrm{m} \mathrm{C} \mathrm{C}_{-}\{2 \mathrm{~h}\}^{\wedge}\{1\} \# 10\left(\mathrm{~m}^{\wedge} 2 \mathrm{n}^{\wedge} 2\right) \& \\
& \hookrightarrow \mathrm{mP8} \& \text { None \& C \& S-carbon \& H. Niu et al., Phys. Rev. Lett. } 108 \\
& \hookrightarrow, 135501(2012)\end{aligned}$} \\
\hline \multicolumn{5}{|l|}{1.00000000000000} \\
\hline 4.73020000000000 & 0.00000000000000 & 0.00000000000000 & & \\
\hline 0.00000000000000 & 2.49500000000000 & 0.00000000000000 & & \\
\hline-1.13246984969092 & 0.00000000000000 & 3.92353383183337 & & \\
\hline \multicolumn{5}{|l|}{ 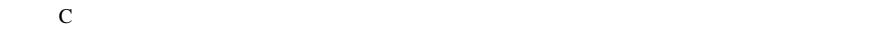 } \\
\hline \multirow{2}{*}{\multicolumn{5}{|c|}{ rect }} \\
\hline & & & & \\
\hline 0.11750000000000 & 0.00000000000000 & 0.67460000000000 & $\mathrm{C}$ & $(2 \mathrm{~m})$ \\
\hline-0.11750000000000 & 0.00000000000000 & -0.67460000000000 & $\mathrm{C}$ & $(2 \mathrm{~m})$ \\
\hline 0.53440000000000 & 0.00000000000000 & 0.33330000000000 & $\mathrm{C}$ & $(2 \mathrm{~m})$ \\
\hline-0.53440000000000 & 0.00000000000000 & -0.33330000000000 & $\mathrm{C}$ & $(2 \mathrm{~m})$ \\
\hline 0.11310000000000 & 0.50000000000000 & 0.89770000000000 & $\mathrm{C}$ & $(2 n)$ \\
\hline-0.11310000000000 & 0.50000000000000 & -0.89770000000000 & $\mathrm{C}$ & $(2 n)$ \\
\hline 0.42090000000000 & 0.50000000000000 & 0.13190000000000 & $\mathrm{C}$ & $(2 n)$ \\
\hline-0.42090000000000 & 0.50000000000000 & -0.13190000000000 & $\mathrm{C}$ & $(2 n)$ \\
\hline
\end{tabular}

Thortveitite ([Sc,Y $\left.]_{2} \mathrm{Si}_{2} \mathrm{O}_{7}, S 22_{1}\right):$ A7B2C2_mC22_12_aij_h_i - CIF

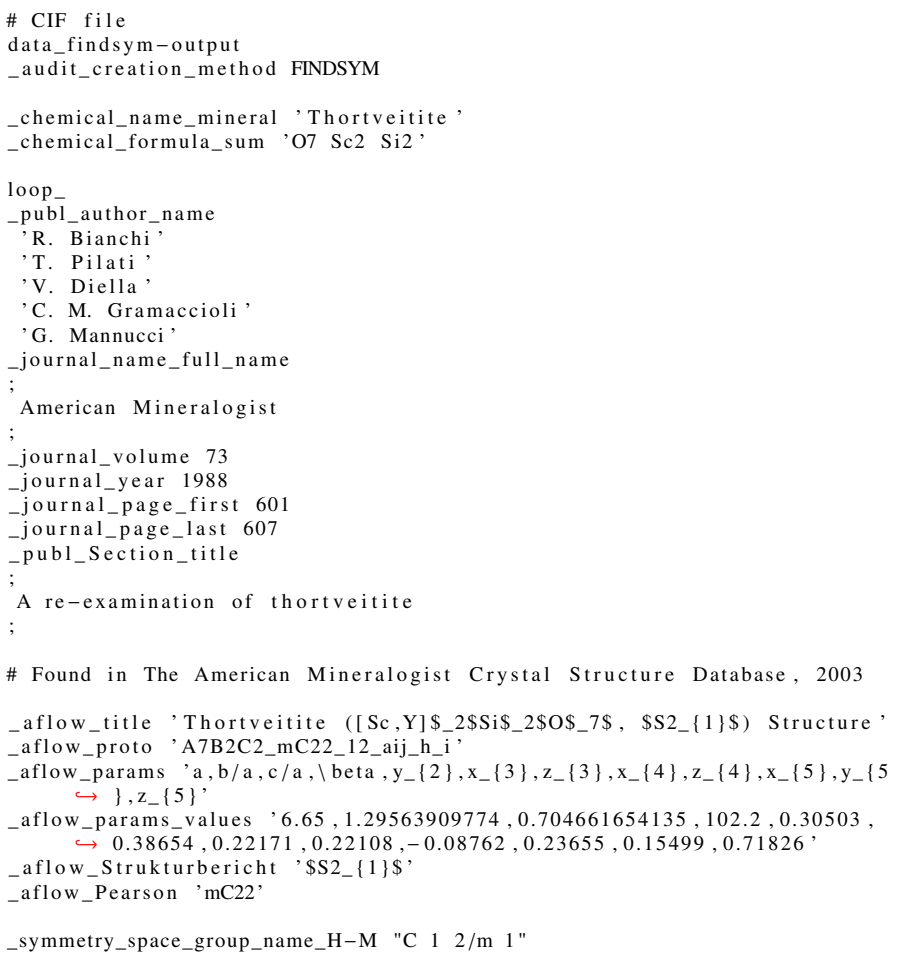




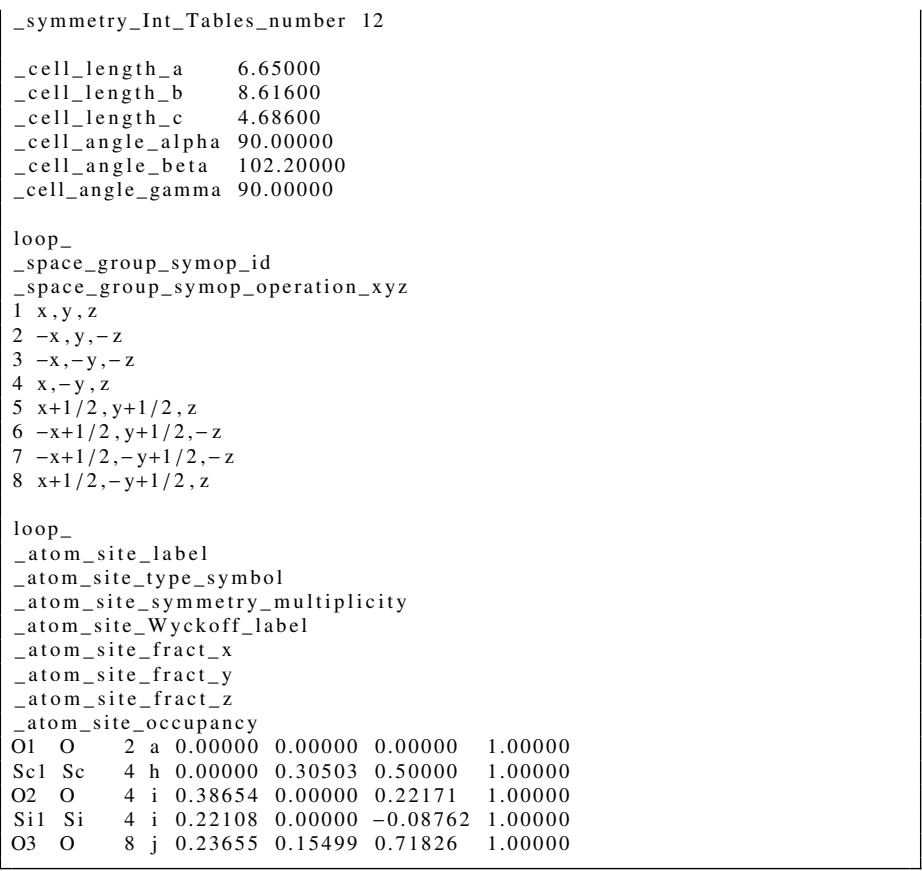

Thortveitite ([Sc,Y $\left.]_{2} \mathrm{Si}_{2} \mathrm{O}_{7}, S 2_{1}\right)$ : A7B2C2_mC22_12_aij_h_i - POSCAR

\begin{tabular}{|c|c|c|c|c|}
\hline \multicolumn{5}{|c|}{ 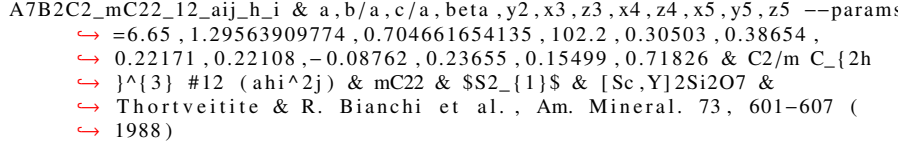 } \\
\hline \multicolumn{5}{|l|}{1.00000000000000} \\
\hline \multirow{2}{*}{$\begin{array}{l}3.32500000000000 \\
3.32500000000000\end{array}$} & -4.30800000000000 & 0.00000000000000 & & \\
\hline & 4.30800000000000 & 0.00000000000000 & & \\
\hline-0.99026799618995 & 0.00000000000000 & 4.58017088062465 & & \\
\hline $\mathrm{Sc}$ & & & & \\
\hline 7 & & & & \\
\hline \multicolumn{5}{|l|}{ Direct } \\
\hline 0.00000000000000 & 0.00000000000000 & 0.00000000000000 & $\mathrm{O}$ & (2a) \\
\hline 0.38654000000000 & 0.38654000000000 & 0.22171000000000 & $\mathrm{O}$ & (4i) \\
\hline-0.38654000000000 & -0.38654000000000 & -0.22171000000000 & $\mathrm{O}$ & (4i) \\
\hline 0.08156000000000 & 0.39154000000000 & 0.71826000000000 & $\mathrm{O}$ & $(8 \mathrm{j})$ \\
\hline-0.39154000000000 & -0.08156000000000 & -0.71826000000000 & $\mathrm{O}$ & $(8 \mathrm{j})$ \\
\hline-0.08156000000000 & -0.39154000000000 & -0.71826000000000 & $\mathrm{O}$ & $(8 \mathrm{j})$ \\
\hline 0.39154000000000 & 0.08156000000000 & 0.71826000000000 & $\mathrm{O}$ & $(8 \mathrm{j})$ \\
\hline-0.30503000000000 & 0.30503000000000 & 0.50000000000000 & $\mathrm{Sc}$ & (4h) \\
\hline 0.30503000000000 & -0.30503000000000 & 0.50000000000000 & $\mathrm{Sc}$ & (4h) \\
\hline 0.22108000000000 & 0.22108000000000 & -0.08762000000000 & $\mathrm{Si}$ & (4i) \\
\hline-0.22108000000000 & -0.22108000000000 & 0.08762000000000 & $\mathrm{Si}$ & (4i) \\
\hline
\end{tabular}

M-carbon: A_mC16_12_4i - CIF

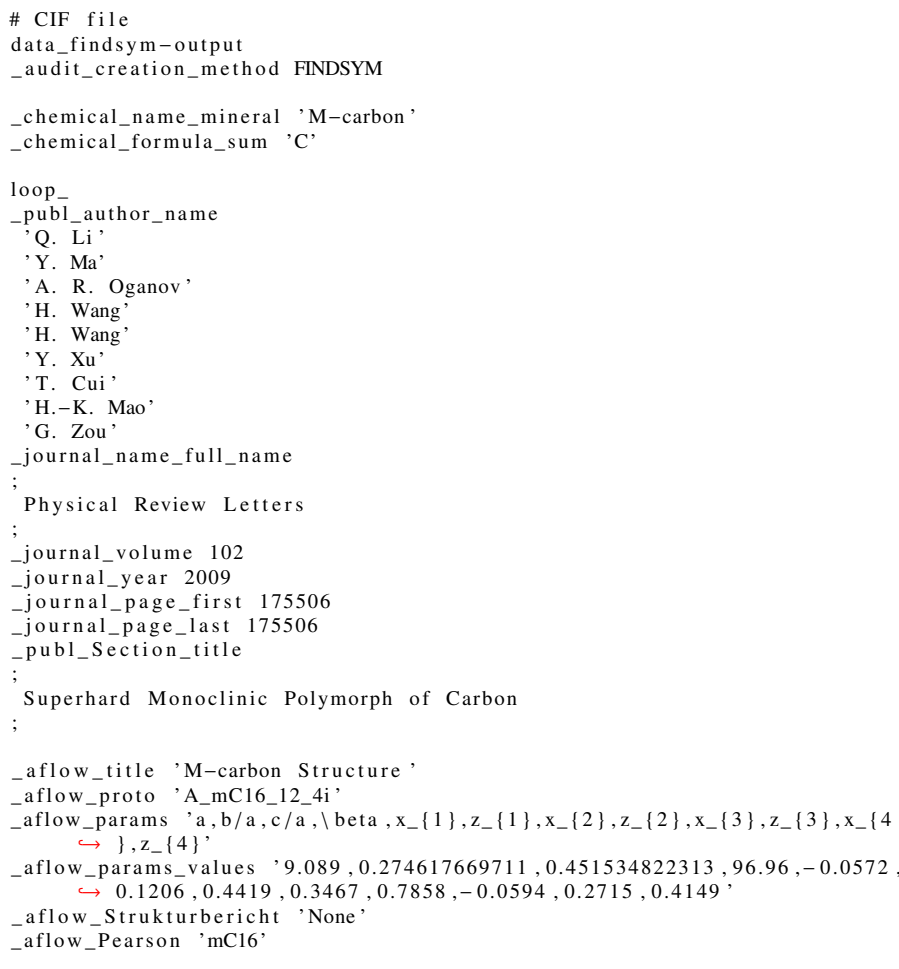

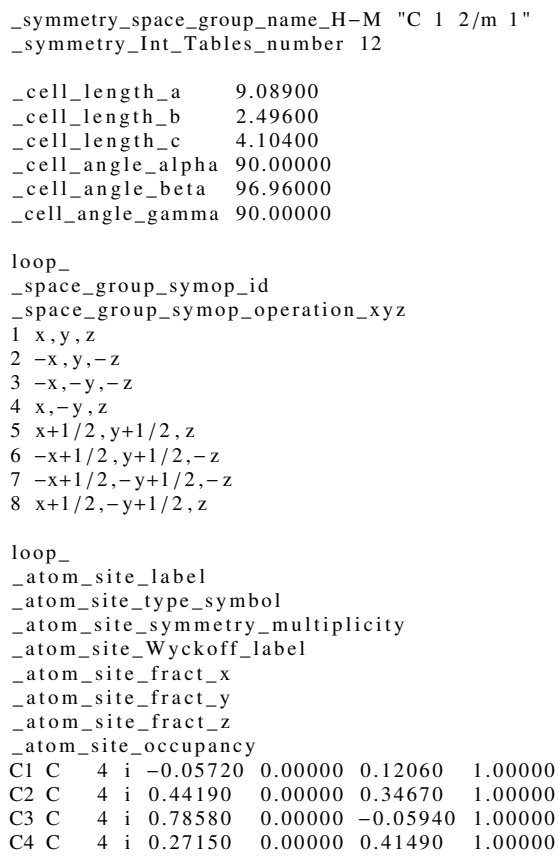

M-carbon: A_mC16_12_4i - POSCAR

\begin{tabular}{|c|c|c|c|c|}
\hline $\begin{aligned} \text { A_mC16_12_4i \& a,b/ } & \text { (12 } 0.274617669 \\
& \hookrightarrow 0.3467,0.78 \\
& \hookrightarrow \& \mathrm{mC} 16 \& \mathrm{I} \\
& \hookrightarrow 102.175506\end{aligned}$ & $\begin{array}{l}, c / a, b e t a, x 1, z 1, x 2, \\
11,0.451534822313, \\
8,-0.0594,0.2715,0 \\
\text { one \& C \& M-carbon } \\
(2009)\end{array}$ & $\begin{array}{l}\mathrm{z} 2, \mathrm{x} 3, \mathrm{z} 3, \mathrm{x} 4, \mathrm{z} 4--\mathrm{pa} \\
6.96,-0.0572,0.1206 \\
4149 \& \text { C } 2 / \mathrm{m} \mathrm{C}_{-}\{2 \mathrm{~h}\}^{\prime} \\
\text { Q. Li et al., Phy }\end{array}$ & $\begin{array}{l}3\} \# \\
\operatorname{Re}\end{array}$ & $\begin{array}{l}9 \\
2\left(i^{\wedge} 4\right) \\
\text { Lett. }\end{array}$ \\
\hline 1.00000000000000 & & & & \\
\hline 4.54450000000000 & -1.24800000000000 & 0.00000000000000 & & \\
\hline 4.54450000000000 & 1.24800000000000 & 0.00000000000000 & & \\
\hline-0.49730788744489 & 0.00000000000000 & 4.07375758545904 & & \\
\hline $\mathrm{C}$ & & & & \\
\hline 8 & & & & \\
\hline Direct & & & & \\
\hline-0.05720000000000 & -0.05720000000000 & 0.12060000000000 & $\mathrm{C}$ & (4i) \\
\hline 0.05720000000000 & 0.05720000000000 & -0.12060000000000 & $\mathrm{C}$ & (4i) \\
\hline 0.44190000000000 & 0.44190000000000 & 0.34670000000000 & $\mathrm{C}$ & (4i) \\
\hline-0.44190000000000 & -0.44190000000000 & -0.34670000000000 & $\mathrm{C}$ & (4i) \\
\hline 0.78580000000000 & 0.78580000000000 & -0.05940000000000 & $\mathrm{C}$ & (4i) \\
\hline-0.78580000000000 & -0.78580000000000 & 0.05940000000000 & $\mathrm{C}$ & (4i) \\
\hline 0.27150000000000 & 0.27150000000000 & 0.41490000000000 & $\mathrm{C}$ & (4i) \\
\hline-0.27150000000000 & -0.27150000000000 & -0.41490000000000 & $\mathrm{C}$ & (4i) \\
\hline
\end{tabular}

$\mathrm{H}_{2} \mathrm{~S}$ (15 GPa): A2B_mP12_13_2g_ef - CIF

\# CIF file

data findsym-output

_audit_creation_method FINDSYM

chemical name mineral 'H2S'

chemical formula sum 'H2 S'

loop

publ_author_nam

'Y. Li,

'J. Hao'

'H. Liu,

Y. $\mathrm{Li}$

'Y. Ma'

journal_name_full_name

Journal of Chemical Physics

journal_volume 140

journal_year 2014

journal page first 174712

-journal_page_first 174712

publ Section title

The metallization and superconductivity of dense hydrogen sulfide

aflow_title 'H\$_\{2\}\$S (15 GPa) Structure'

aflow_proto 'A2B_mP12_13_2g_ef '

aflow_params 'a,b/a,c/a, $\backslash$ beta $, y_{-}\{1\}, y_{-}\{2\}, x_{-}\{3\}, y_{-}\{3\}, z_{-}\{3\}, x_{-}\{4\}, y_{-}\{4$ $\hookrightarrow\}, z_{-}\{4\}$

aflow_params_values ' $5.6255,0.61198115723,1.23780997245,127.44,0.1808,-$ $\hookrightarrow 0.004,0.155,0.346,0.225,0.345,0.273,0.573$

aflow_Strukturbericht 'None'

-aflow_Pearson 'mP12'

symmetry space group name $\mathrm{H}-\mathrm{M}$ "P $12 / \mathrm{c} \quad 1$ "

symmetry_Int_Tables_number 13

cell_length_a $\quad 5.62550$

cell_length_b 3.44270

cell_length_c $\quad 6.96330$

cell_angle_alpha 90.00000

_cell_angle_beta 127.44000 


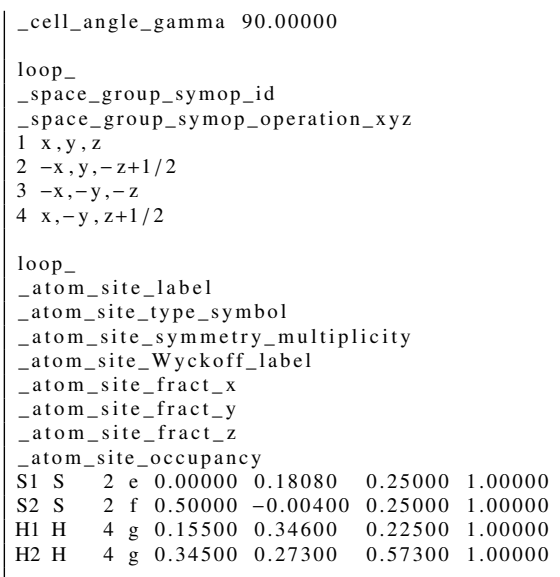

$\mathrm{H}_{2} \mathrm{~S}$ (15 GPa): A2B_mP12_13_2g_ef - POSCAR

A2B_mP12_13_2g_ef \& a b/a,c/a, beta , y1, y2, x3,y3, z3, x4,y4,z4 --params= $\hookrightarrow 5.6255,0.61198115723,1.23780997245,127.44,0.1808,-0.004,0.155$ $\hookrightarrow 0.346,0.225,0.345,0.273,0.573 \& \mathrm{P} 2 / \mathrm{c} \mathrm{C}_{-}\{2 \mathrm{~h}\} \wedge\{4\} \# 13\left(\mathrm{efg}^{\wedge} 2\right) \&$

$\hookrightarrow \mathrm{mP} 12$ \& None \& H2S \& H2S \& Y. Li et al., J. Chem. Phys. 140 ,

$\hookrightarrow 174712(2014)$

1.00000000000000

$\begin{array}{lll}5.62550000000000 & 0.00000000000000 & 0.00000000000000\end{array}$

$0.00000000000000 \quad 3.44270000000000 \quad 0.00000000000000$

$\begin{array}{rrr}-4.23320104193685 & 0.00000000000000 & 5.52879334290447\end{array}$

$$
\begin{array}{ll}
\mathrm{H} & \mathrm{S} \\
8 & 4
\end{array}
$$

Direct

0.15500000000000 $-0.15500000000000$

$-0.15500000000000$

0.1550000000000

0.34500000000000

$-0.34500000000000$

$-0.34500000000000$

0.34500000000000

0.00000000000000

0.00000000000000

0.50000000000000

0.50000000000000 6000000000 0.34600000000000 0.34600000000000 0.27300000000000 0.27300000000000 $-0.27300000000000$ $-0.27300000000000$ 0.1808000000000 $-0.18080000000000$ $-0.00400000000000$ 0.00400000000000

0.22500000000000 00000000 $-0.22500000000000$ 0.57300000000000 0.57300000000000 $-0.07300000000000$ $-0.57300000000000$ 1.07300000000000 0.25000000000000 0.75000000000000 0.25000000000000 0.75000000000000

$\begin{array}{ll}\mathrm{H} & (4 \mathrm{~g}) \\ \mathrm{H} & (4 \mathrm{~g}) \\ \mathrm{H} & (4 \mathrm{~g}) \\ \mathrm{H} & (4 \mathrm{~g}) \\ \mathrm{H} & (4 \mathrm{~g}) \\ \mathrm{H} & (4 \mathrm{~g}) \\ \mathrm{H} & (4 \mathrm{~g}) \\ \mathrm{H} & (4 \mathrm{~g}) \\ \mathrm{S} & (2 \mathrm{e}) \\ \mathrm{S} & (2 \mathrm{e}) \\ \mathrm{S} & (2 \mathrm{f}) \\ \mathrm{S} & (2 \mathrm{f})\end{array}$

$\gamma-\mathrm{PdCl}_{2}$ : A2B_mP6_14_e_a - CIF

\# CIF file

data_findsym-output

audit_creation_method FINDSYM

chemical_name_mineral ' $\$ \backslash$ gamma $\$-P d C l 2$,

chemical_formula_sum ' $\mathrm{Cl} 2 \mathrm{Pd}$

loop

publ_author_name

J. Evers

W. Beck'

M. G\" \{o\}bel

S. Jakob

G. Oehlinger

'M. Rotter,

T. M. Klap $\backslash "\{$ o $\}$ tke ,

journal_name_full_name

Angewandte Chemie (International ed.)

journal_volume 49

journal_year 2010

journal_page_first 5677

journal page last 5682

publ_Section_title

The Structures of $\$ \backslash$ delta $\$-P d C l \$\{2\} \$$ and $\$ \backslash$ gamma $\$-P d C l \$\{2\} \$$ : Phases $\hookrightarrow$ with Negative Thermal Expansion in One Direction

aflow_title, $\$ \backslash$ gamma\$-PdCl\$_ $\{2\} \$$ Structure

aflow_proto 'A2B_mP6_14_e_a'

aflow_params 'a,b/a,c/a, $\backslash$ beta, $x_{-}\{2\}, y_{-}\{2\}, z_{-}\{2\}$

aflow_params_values ' $5.5496,0.695689779444,1.15512829753,107.151,0.255$, $\hookrightarrow 0.2573,0.3141$

aflow_Strukturbericht 'None'

aflow_Pearson 'mP6'

symmetry_space_group_name_H-M "P 1 21/c 1"

symmetry Int Tables_number 14

cell_length_a $\quad 5.54960$

cell_length_b 3.86080

cell_length_c $\quad 6.41050$

cell_angle_alpha 90.00000

_cell_angle_alpha 90.00000

cell_angle gamma 90.00000

loop

space_group_symop_id

_space_group_symop_operation_xyz

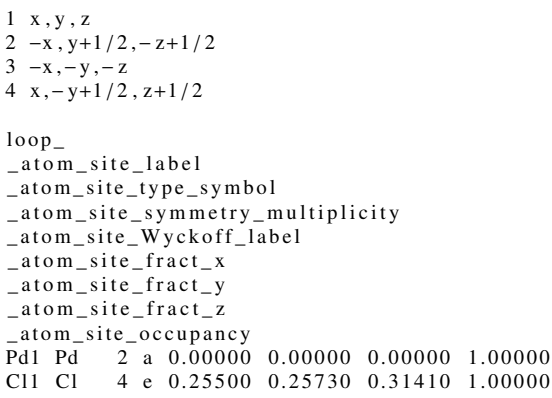

$\gamma$-PdCl 2 : A2B_mP6_14_e_a - POSCAR

\begin{tabular}{|c|c|c|c|c|}
\hline \multicolumn{5}{|c|}{ A2B_mP6_14_e_a \& a,b/a,c/a, beta } \\
\hline$\hookrightarrow 1.155128297$ & $3,107.151,0.255$ & $573,0.3141 \& P 2 \_\{$ & & $h\}^{\wedge}\{5\}$ \\
\hline$\hookrightarrow \# 14(\mathrm{ae}) \&$ & $\mathrm{mP6} \&$ None \& $\mathrm{PdCl}$ & $\& \$ \backslash$ gamma $\$-\mathrm{PdCl} 2$ \& & & ers et \\
\hline$\hookrightarrow$ al. & Chem. Int. Ed. 49 & $5677-5682(2010)$ & & \\
\hline 1.00000000000000 & & & & \\
\hline 5.54960000000000 & 0.00000000000000 & 0.00000000000000 & & \\
\hline 0.00000000000000 & 3.86080000000000 & 0.00000000000000 & & \\
\hline-1.89039860884891 & 0.00000000000000 & 6.12543087053165 & & \\
\hline $\mathrm{Pd}$ & & & & \\
\hline 4 & & & & \\
\hline irect & & & & \\
\hline 0.25500000000000 & 0.25730000000000 & 0.31410000000000 & $\mathrm{Cl}$ & $(4 \mathrm{e})$ \\
\hline-0.25500000000000 & 0.75730000000000 & 0.18590000000000 & $\mathrm{Cl}$ & $(4 \mathrm{e})$ \\
\hline-0.25500000000000 & -0.25730000000000 & -0.31410000000000 & $\mathrm{Cl}$ & $(4 \mathrm{e})$ \\
\hline 0.25500000000000 & 0.24270000000000 & 0.81410000000000 & $\mathrm{Cl}$ & $(4 \mathrm{e})$ \\
\hline 0.00000000000000 & 0.00000000000000 & 0.00000000000000 & $\mathrm{Pd}$ & (2a) \\
\hline 0.00000000000000 & 0.50000000000000 & 0.50000000000000 & $\mathrm{Pd}$ & $(2 a)$ \\
\hline
\end{tabular}

$\alpha$-Toluene: A7B8_mP120_14_14e_16e - CIF

\# CIF file

data_findsym-output

audit_creation_method FINDSYM

chemical_name_mineral 'alpha-Toluene,

chemical_formula_sum 'C7 $\mathrm{H}_{8}$ '

loop

publ_author_name

'S. K Nayak',

'R. Sathishkumar,

T. N. \{Guru Row\},

journal_name_full_name

CrystEngComm

journal_volume 12

journal_year 2010

journal_page_first 3112

journal_page_last 3118

-publ_Section_title

Directing role of functional groups in selective generation of $\mathrm{C}-\mathrm{H}-\$$ $\hookrightarrow$ pi\$ interactions: In situ cryo-crystallographic studies on

$\hookrightarrow$ benzyl derivatives

\# Found in Cambridge Structural Database, \{CSD Entry: TOLUEN03\},

-aflow_title $\$ \backslash$ alpha\$-Toluene Structure

aflow_proto 'A7B8_mP120_14_14e_16e'

aflow_params $, a, b / a, c / a, \backslash$ beta, $x_{-}\{1\}, y_{-}\{1\}, z_{-}\{1\}, x_{-}\{2\}, y_{-}\{2\}, z_{-}\{2\}, x_{-}\{3$ $\hookrightarrow\}, \mathrm{y}_{-}\{3\}, \mathrm{z}_{-}\{3\}, \mathrm{x}_{-}\{4\}, \mathrm{y}_{-}\{4\}, \mathrm{z}_{-}\{4\}, \mathrm{x}_{-}\{5\}, \mathrm{y}_{-}\{5\}, \mathrm{z}_{-}\{5\}, \mathrm{x}_{-}\{6\}, \mathrm{y}_{-}\{6\}$ $\hookrightarrow \mathrm{z}_{-}\{6\}, \mathrm{x}_{-}\{7\}, \mathrm{y}_{-}\{7\}, \mathrm{z}_{-}\{7\}, \mathrm{x}_{-}\{8\}, \mathrm{y}_{-}\{8\}, \mathrm{z}_{-}\{8\}, \mathrm{x}_{-}\{9\}, \mathrm{y}_{-}\{9\}, \mathrm{z}_{-}\{9\}, \mathrm{x}_{-}\{$ $\hookrightarrow 10\}, \mathrm{y}_{-}\{10\}, \mathrm{z}_{-}\{10\}, \mathrm{x}_{-}\{11\}, \mathrm{y}_{-}\{11\}, \mathrm{z}_{-}\{11\}, \mathrm{x}_{-}\{12\}, \mathrm{y}_{-}\{12\}, \mathrm{z}_{-}\{12\}, \mathrm{x}_{-}\{$ $\hookrightarrow 13\}, \mathrm{y}_{-}\{13\}, \mathrm{z}_{-}\{13\}, \mathrm{x}_{-}\{14\}, \mathrm{y}_{-}\{14\}, \mathrm{z}_{-}\{14\}, \mathrm{x}_{-}\{15\}, \mathrm{y}_{-}\{15\}, \mathrm{z}_{-}\{15\}, \mathrm{x}_{-}\{$ $\hookrightarrow 16\}, \mathrm{y}_{-}\{16\}, \mathrm{z}_{-}\{16\}, \mathrm{x}_{-}\{17\}, \mathrm{y}_{-}\{17\}, \mathrm{z}_{-}\{17\}, \mathrm{x}_{-}\{18\}, \mathrm{y}_{-}\{18\}, \mathrm{z}_{-}\{18\}, \mathrm{x}_{-}\{$ $\hookrightarrow 19\}, y_{-}\{19\}, z_{-}\{19\}, x_{-}\{20\}, y_{-}\{20\}, z_{-}\{20\}, x_{-}\{21\}, y_{-}\{21\}, z_{-}\{21\}, x_{-}\{$ $\hookrightarrow 22\}, \mathrm{y}_{-}\{22\}, \mathrm{z}_{-}\{22\}, \mathrm{x}_{-}\{23\}, \mathrm{y}_{-}\{23\}, \mathrm{z}_{-}\{23\}, \mathrm{x}_{-}\{24\}, \mathrm{y}_{-}\{24\}, \mathrm{z}_{-}\{24\}, \mathrm{x}_{-} \mid$

$\hookrightarrow 25\}, y_{-}\{25\}, z_{-}\{25\}, x_{-}\{26\}, y_{-}\{26\}, z_{-}\{26\}, x_{-}\{27\}, y_{-}\{27\}, z_{-}\{27\}, x_{-}\{$ $\hookrightarrow 28\}, y_{-}\{28\}, z_{-}\{28\}, x_{-}\{29\}, y_{0}\{29\}, z_{-}\{29\}, x_{-}\{30\}, y_{-}\{30\}, z_{-}\{30\}$

aflow_params_values $, 7.5889,0.766725085322,3.55545599494,106.136$.

$\hookrightarrow 0.25252,0.5751,0.3402,0.76924,0.888,0.47034,0.13489,0.3866$

$\hookrightarrow 0.33222,0.8218,0.8146,0.42747,0.12506,0.2343,0.29191,0.78423$,

$\hookrightarrow 0.9464,0.38285,0.23276,0.2673,0.25885,0.69189,0.1533,0.38027$,

$\hookrightarrow 0.34844,0.4547,0.26591,0.63874,0.2281,0.42248,0.35819,0.6069$,

$\hookrightarrow 0.3061,0.67752,0.0969,0.46714,0.26777,0.7386,0.3843,0.8119$

$\leftrightarrow 0.7461,0.51889,0.0602,0.3617,0.3547,0.8843,0.6723,0.4287,0.0438$

$\hookrightarrow, 0.1068,0.2871,0.822,0.8945,0.354,0.2273,0.1621,0.2315,0.6653$

$\hookrightarrow 0.243,0.3497,0.4219,0.4796,0.2431,0.5754,0.3699,0.4209,0.4385$

$\hookrightarrow 0.7352,0.3104,0.6409,0.1506,0.496,0.9409,0.7672,0.5381,0.7891$

$\leftrightarrow 0.5835,0.5099,0.7334,0.7952,0.5403,0.2081,0.8842,0.3711,0.3975$

$\hookrightarrow 0.7664,0.4019,0.2077,0.6717,0.4087$

aflow_Strukturbericht 'None

aflow_Pearson 'mP120'

symmetry_space group name_H-M "P $121 / c \quad 1$ "

symmetry_Int_Tables_number 14

cell_length_a $\quad 7.58890$

cell_length_b $\quad 5.81860$

cell_length_c 26.98200

cell angle alpha 90.00000

cell angle beta 106.13600

cell_angle_gamma 90.00000 


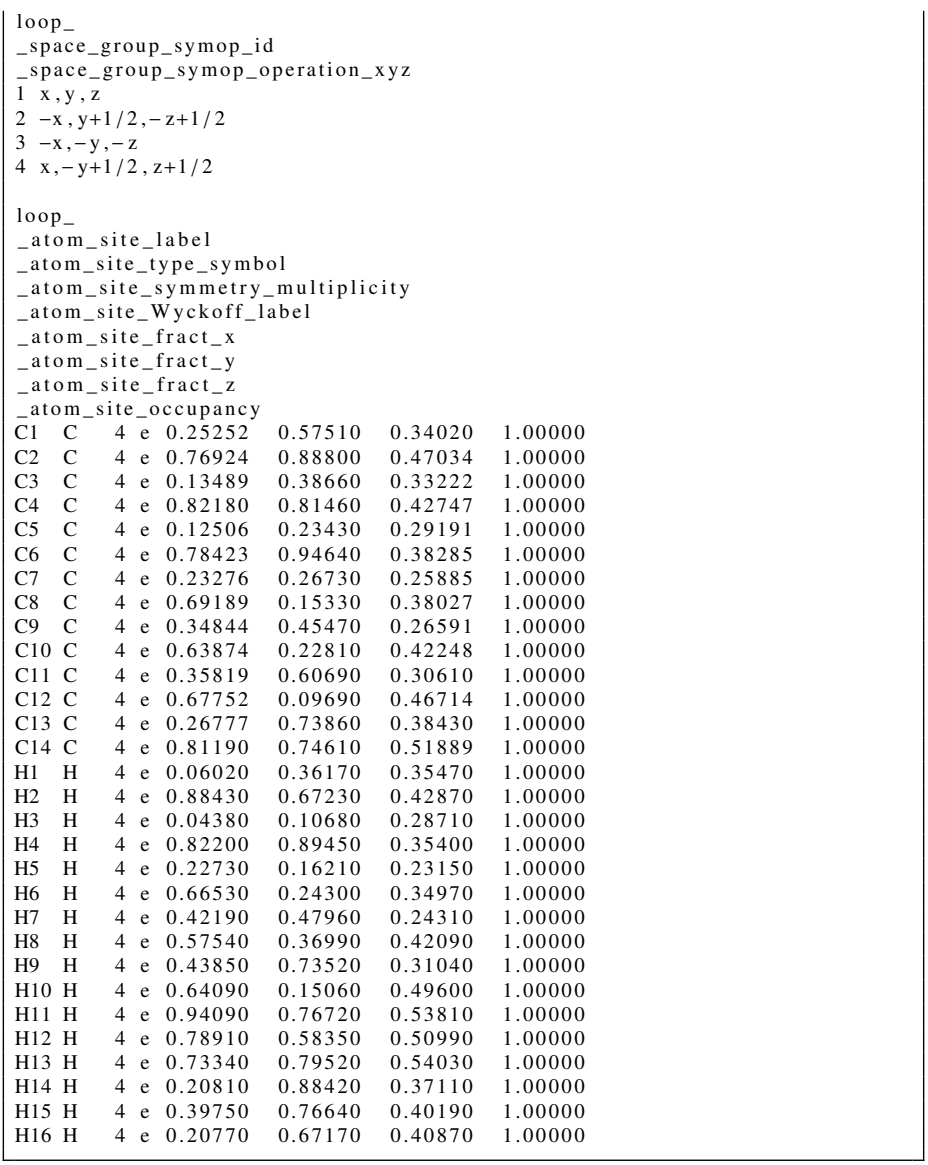

$\alpha$-Toluene: A7B8_mP120_14_14e_16e - POSCAR

A7B8_mP120_14_14e_16e \& a,b/a,c/a, beta , x1, y1, z1, x2, y2, z2, x3,y3,z3,x4,y4 $\hookrightarrow \mathrm{z} 4, \mathrm{x} 5, \mathrm{y} 5, \mathrm{z} 5, \mathrm{x} 6, \mathrm{y} 6, \mathrm{z} 6, \mathrm{x} 7, \mathrm{y} 7, \mathrm{z} 7, \mathrm{x} 8, \mathrm{y} 8, \mathrm{z} 8, \mathrm{x} 9, \mathrm{y} 9, \mathrm{z} 9, \mathrm{x} 10, \mathrm{y} 10, \mathrm{z} 10, \mathrm{x} 11$ $\hookrightarrow, \mathrm{y} 11, \mathrm{z} 11, \mathrm{x} 12, \mathrm{y} 12, \mathrm{z} 12, \mathrm{x} 13, \mathrm{y} 13, \mathrm{z} 13, \mathrm{x} 14, \mathrm{y} 14, \mathrm{z} 14, \mathrm{x} 15, \mathrm{y} 15, \mathrm{z} 15, \mathrm{x} 16$

$\hookrightarrow \mathrm{y} 16, \mathrm{z} 16, \mathrm{x} 17, \mathrm{y} 17, \mathrm{z} 17, \mathrm{x} 18, \mathrm{y} 18, \mathrm{z} 18, \mathrm{x} 19, \mathrm{y} 19, \mathrm{z} 19, \mathrm{x} 20, \mathrm{y} 20, \mathrm{z} 20, \mathrm{x} 21, \mathrm{y} 2$

$\hookrightarrow, \mathrm{z} 21, \mathrm{x} 22, \mathrm{y} 22, \mathrm{z} 22, \mathrm{x} 23, \mathrm{y} 23, \mathrm{z} 23, \mathrm{x} 24, \mathrm{y} 24, \mathrm{z} 24, \mathrm{x} 25, \mathrm{y} 25, \mathrm{z} 25, \mathrm{x} 26, \mathrm{y} 26$

$\hookrightarrow \mathrm{z} 26, \mathrm{x} 27, \mathrm{y} 27, \mathrm{z} 27, \mathrm{x} 28, \mathrm{y} 28, \mathrm{z} 28, \mathrm{x} 29, \mathrm{y} 29, \mathrm{z} 29, \mathrm{x} 30, \mathrm{y} 30, \mathrm{z} 30--\mathrm{param}$

$\rightarrow 0.3402,0.76924,0.888,0.47034,0.13489,0.3866,0.33222,0.8218$

$\hookrightarrow 0.8146,0.42747,0.12506,0.2343,0.29191,0.78423,0.9464,0.38285$,

$\hookrightarrow 0.23276,0.2673,0.25885,0.69189,0.1533,0.38027,0.34844,0.4547$

$\hookrightarrow 0.26591,0.63874,0.2281,0.42248,0.35819,0.6069,0.3061,0.67752$,

$\hookrightarrow 0.0969,0.46714,0.26777,0.7386,0.3843,0.8119,0.7461,0.51889$,

$\hookrightarrow 0.0602,0.3617,0.3547,0.8843,0.6723,0.4287,0.0438,0.1068,0.2871$

$\hookrightarrow 0.4219,0.4796,0.2431,0.5754,0.3699,0.4209,0.4385,0.7352,0.3104$

$\hookrightarrow 0.6409,0.1506,0.496,0.9409,0.7672,0.5381,0.7891,0.5835,0.5099$

$\hookrightarrow 0.7334,0.7952,0.5403,0.2081,0.8842,0.3711,0.3975,0.7664,0.4019$

$\hookrightarrow 0.2077,0.6717,0.4087 \& \mathrm{P}_{-}\{1\} / \mathrm{c} \mathrm{C}_{-}\{2 \mathrm{~h}\}^{\wedge}\{5\} \# 14\left(\mathrm{e}^{\wedge} 30\right) \& \mathrm{mP} 120$

$\hookrightarrow$ None \& C7H8 \& alpha-Toluene \& S. K Nayak and R. Sathishkuma

$\hookrightarrow$ and T. N. \{Guru Row\}, CrystEngComm 12, 3112-3118 (2010)

1.0000000000000

7.58890000000000

$\begin{array}{llll}0.0000000000000 & 5.81860000000000 & 0.00000000000000\end{array}$

$\begin{array}{llr}-7.49879086554484 & 0.00000000000000 & 25.91903662474400\end{array}$

$$
\begin{array}{rr}
\mathrm{C} & \mathrm{H} \\
56 & 64
\end{array}
$$

$$
\text { irect }
$$

0.25252000000000

$-0.25252000000000$

$-0.25252000000000$

0.25252000000000

0.76924000000000

0.76924000000000

0.76924000000000

0.13489000000000

$-0.13489000000000$

$-0.13489000000000$

0.13489000000000

0.82180000000000

$-0.82180000000000$

0.82180000000000

0.82180000000000

0.12506000000000

$-0.12506000000000$

0.12506000000000

0.12506000000000

0.78423000000000

$-0.78423000000000$

$-0.78423000000000$

0.23276000000000

$-0.23276000000000$

$-0.23276000000000$

0.23276000000000

0.57510000000000 1.07510000000000 $-0.5751000000000$ $-0.07510000000000$ 1.38800000000000 $-0.88800000000000$ $-0.38800000000000$ 0.38660000000000 0.88660000000000 $-0.38660000000000$ 0.11340000000000 0.81460000000000 $-0.31460000000000$ 0.23430000000000 0.7343000000000 $-0.23430000000000$ 0.26570000000000 0.94640000000000 (46000000000 $-0.9464000000000$ 0.44640000000000 0.26730000000000 0.76730000000000 $-0.26730000000000$
0.34020000000000 0.15980000000000 $-0.34020000000000$ 0.84020000000000 0.4703400000000 0.02966000000000 $-0.47034000000000$ 0.97034000000000 0.33222000000000 0.33222000000000 0.832200000000 0.8322200000000 0.92747000000000 0.29191000000000 0.20809000000000 0.79191000000000 0.11715000000000 0.38285000000000 0.25885000000000 0.24115000000000 $-0.25885000000000$
0.69189000000000 $-0.69189000000000$ $-0.69189000000000$ 0.69189000000000 0.34844000000000 $-0.34844000000000$ $-0.34844000000000$ 0.34844000000000 0.63874000000000 $-0.63874000000000$ 0.63874000000000 0.35819000000000 $-0.35819000000000$ $-0.35819000000000$ 0.35819000000000 0.67752000000000 $-0.67752000000000$ $-0.67752000000000$ 0.67752000000000 0.26777000000000 $-0.26777000000000$ $-0.26777000000000$ 0.81190000000000 $-0.81190000000000$ 0.81190000000000 0.06020000000000

$-0.06020000000000$

0.06020000000000

0.88430000000000 $-0.88430000000000$ $-0.88430000000000$ 0.88430000000000 0.04380000000000 $-0.04380000000000$

$-0.04380000000000$ 0.04380000000000 0.82200000000000 $-0.82200000000000$ 0.82200000000000 0.22730000000000 $-0.22730000000000$ $-0.2273000000000$ 0.22730000000000 0.66530000000000 $-0.66530000000000$ $-0.66530000000000$ 0.66530000000000 0.42190000000000 $-0.42190000000000$ $-0.42190000000000$ 0.42190000000000 0.57540000000000 $-0.57540000000000$ $-0.57540000000000$ 0.43850000000000 $-0.43850000000000$ $-0.43850000000000$ 0.43850000000000 0.43850000000000 0.64090000000000 $-0.64090000000000$ 0.64090000000000 0.94090000000000 $-0.94090000000000$ -0.94090000000000
0.94090000000000 0.94090000000000
0.78910000000000 0.78910000000000 $-0.78910000000000$ $-0.78910000000000$ 0.73340000000000 $-0.73340000000000$ $-0.73340000000000$ 0.73340000000000 0.20810000000000 0.20810000000000
-0.20810000000000 $-0.20810000000000$ 0.20810000000000 0.39750000000000 $-0.39750000000000$ $-0.39750000000000$ 0.39750000000000 0.20770000000000 $-0.20770000000000$ $-0.20770000000000$ 0.20770000000000

0.15330000000000 0.65330000000000 $-0.15330000000000$ 0.34670000000000 0.95470000000000 0.45470000000000 0.04530000000000 0.22810000000000 0.72810000000000 0.22810000000000 0.27190000000000 0.60690000000000 $-0.60690000000000$ 0.10690000000000 0.09690000000000 .59690000000000 0.09690000000000 0.40310000000000 0.73860000000000 1.23860000000000 $-0.73860000000000$ $-0.23860000000000$ .74610000000000 1.24610000000000 $-0.24610000000000$ 0.36170000000000 0.86170000000000 $-0.36170000000000$ 0.13830000000000 0.67230000000000 (2) (2) 0.17230000000000 0.10680000000000 0.60680000000000 $-0.10680000000000$ 0.39320000000000 0.89450000000000 . $-0.89450000000000$ 0.16210000000000 0.66210000000000 $-0.16210000000000$ 0.33790000000000 0.24300000000000 0.2430000000 0.24300000000000 0.2430000000000 0.25700000000000 0.47960000000000 0.97960000000000 $-0.47960000000000$ 0.02040000000000 0.36990000000000 0.86990000000000 $-0.36990000000000$ 0.13010000000000 0.73520000000000 1.23520000000000 $-0.23520000000000$ .15060000000000 0.65060000000000 0.34940000000000 0.76720000000000 1.26720000000000 $-0.76720000000000$ $-0.26720000000000$ 0.58350000000000 1.08350000000000 $-0.58350000000000$ $-0.08350000000000$ 0.79520000000000 1.29520000000000 $-0.79520000000000$ $-0.29520000000000$ 0.88420000000000

1.384200000000 $-0.88420000000000$ 0.38420000000000 0.76640000000000 1.26640000000000 $-0.76640000000000$ $-0.26640000000000$ 0.67170000000000 1.17170000000000 $-0.67170000000000$

$\mathrm{H}_{3} \mathrm{Cl}(50 \mathrm{GPa})$ : AB3_mC16_15_e_cf - CIF

\# CIF file

data_findsym-output
-audit_creation_method FINDSYM

chemical_name_mineral, ' $\mathrm{H} 3 \mathrm{Cl}$ '

loop

Di__author_name 


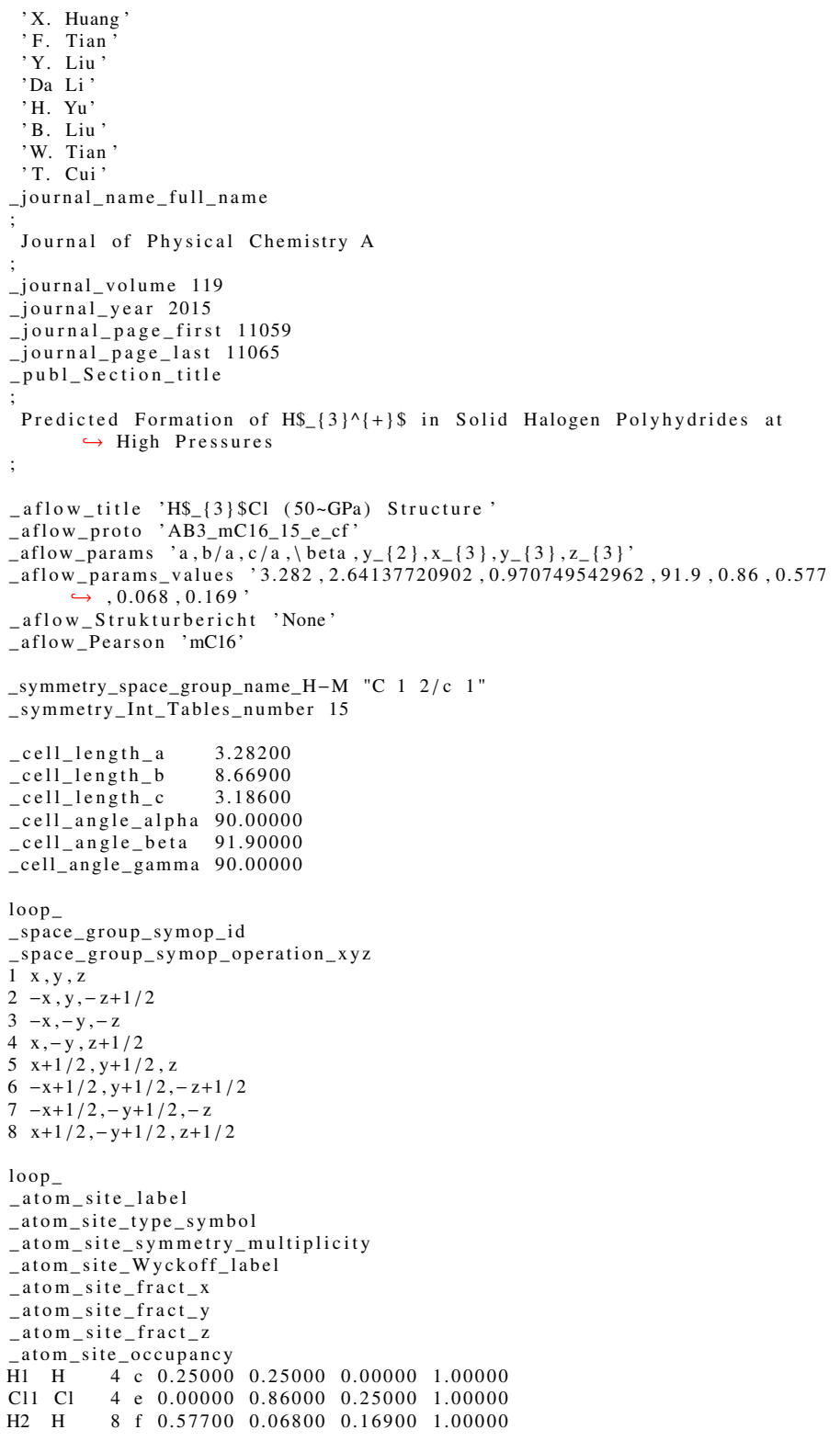

$\mathrm{H}_{3} \mathrm{Cl}$ (50 GPa): AB3_mC16_15_e_cf - POSCAR

\begin{tabular}{|c|c|c|c|c|}
\hline \multirow{4}{*}{\multicolumn{5}{|c|}{ 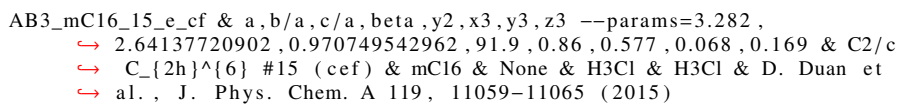 }} \\
\hline & & & & \\
\hline & & & & \\
\hline & & & & \\
\hline \multicolumn{5}{|c|}{1.00000000000000} \\
\hline 1.64100000000000 & -4.33450000000000 & 0.00000000000000 & & \\
\hline \multirow{2}{*}{$\begin{array}{r}1.64100000000000 \\
-0.10563239834584\end{array}$} & 4.33450000000000 & 0.0000000 & & \\
\hline & & & & \\
\hline \multicolumn{5}{|l|}{$\begin{array}{ll}\mathrm{Cl} & \mathrm{H}\end{array}$} \\
\hline 2 & & & & \\
\hline \multicolumn{5}{|l|}{ irect } \\
\hline-0.86000000000000 & & 0.25 & $\mathrm{Cl}$ & $(4 \mathrm{e})$ \\
\hline 0.86000000000000 & -0.8 & 0.75 & $\mathrm{Cl}$ & $(4 \mathrm{e})$ \\
\hline 0.000000000 & & & $\mathrm{H}$ & $(4 \mathrm{c})$ \\
\hline 0.500 & & 0.5 & $\mathrm{H}$ & $(4 \mathrm{c})$ \\
\hline 0.509000000 & & 0.16 & $\mathrm{H}$ & (8f) \\
\hline-0.64500000000000 & 000000 & 0.33100000 & $\mathrm{H}$ & \\
\hline-0.50900000000000 & -0.64500000000000 & -0.16900000000000 & $\mathrm{H}$ & $(8 \mathrm{f})$ \\
\hline 0.64500000000000 & 0.50900000000000 & 0.66900000000000 & $\mathrm{H}$ & $(8 \mathrm{f})$ \\
\hline
\end{tabular}

H-III (300 GPa): A_mC24_15_2e2f - CIF

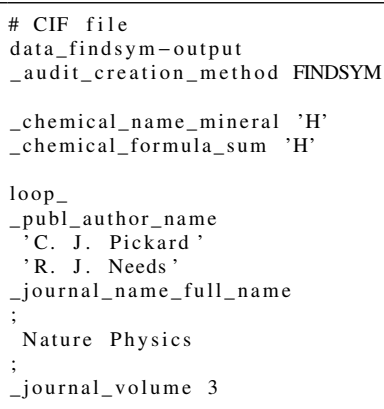

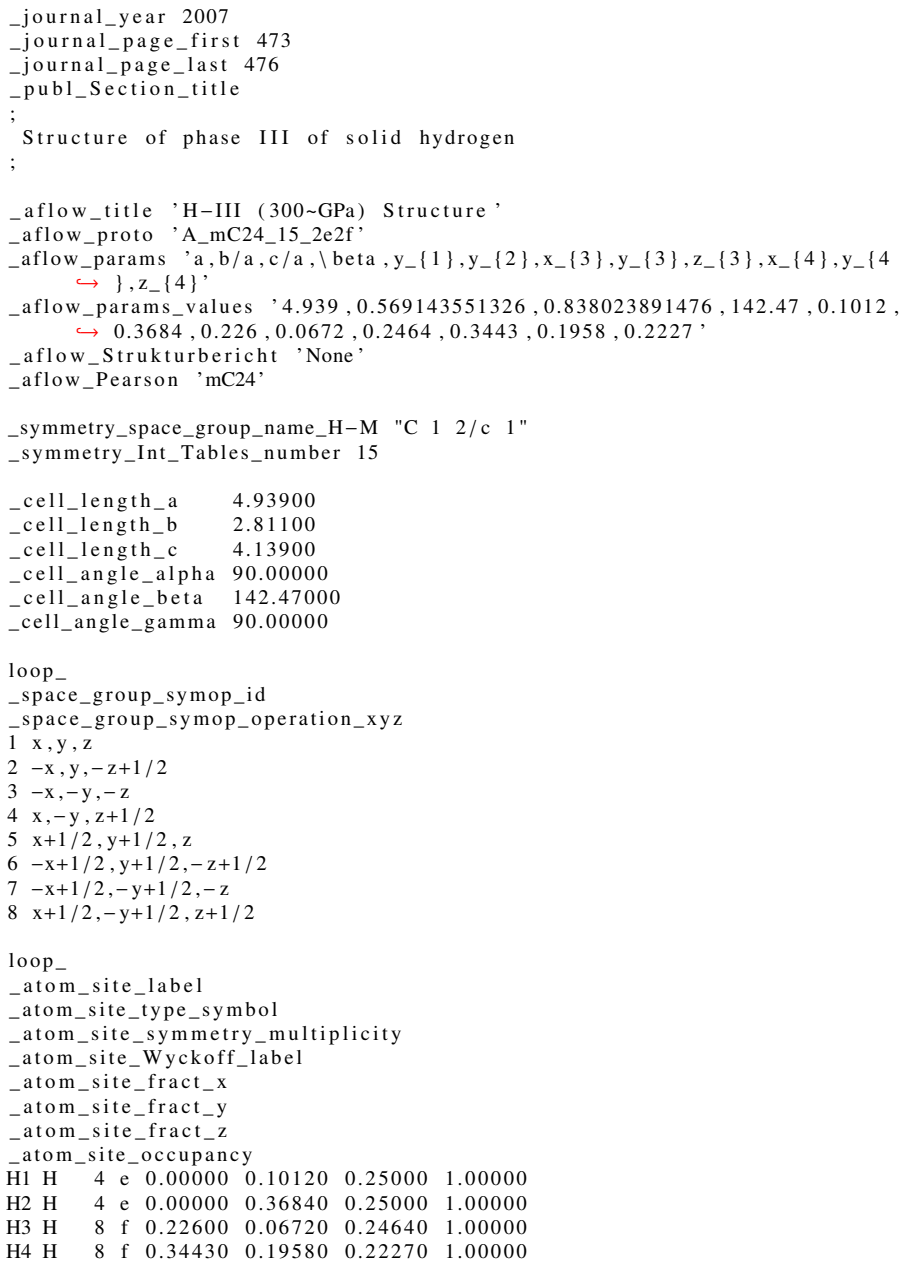

H-III (300 GPa): A_mC24_15_2e2f - POSCAR

\begin{tabular}{|c|c|c|c|c|}
\hline \\
\hline $\begin{array}{l}\hookrightarrow, 0.2464,0.3443,0.1958,0.2227 \& \mathrm{C} 2 / \mathrm{c} \mathrm{C}_{-}\{2 \mathrm{~h}\}^{\wedge}\{6\} \# 15\left(\mathrm{e}^{\wedge} 2 \mathrm{f}^{\wedge} 2\right) \& \\
\hookrightarrow \text { mC24 \& None \& H \& H \& C. J. Pickard and R. J. Needs, Nat. Phys. } \\
\hookrightarrow 3,473-476(2007)\end{array}$ & \multicolumn{4}{|c|}{ 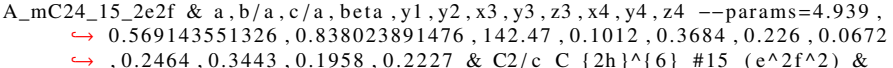 } \\
\hline \multicolumn{5}{|c|}{$\begin{array}{l}\hookrightarrow .3,473-476(2007) \\
1.00000000000000\end{array}$} \\
\hline 2.46950000000000 & -1.40550000000000 & 0.00000000000000 & & \\
\hline 2.46950000000000 & 1.40550000000000 & 0.00000000000000 & & \\
\hline-3.28236973265251 & 0.00000000000000 & 2.52138254498731 & & \\
\hline \multicolumn{5}{|l|}{$\mathrm{H}$} \\
\hline 12 & & & & \\
\hline \multicolumn{5}{|l|}{ Direct } \\
\hline-0.10120000000000 & 0.10120000000000 & 0.25000000000000 & $\mathrm{H}$ & (4e) \\
\hline 0.10120000000000 & -0.10120000000000 & 0.75000000000000 & $\mathrm{H}$ & $(4 \mathrm{e})$ \\
\hline-0.36840000000000 & 0.36840000000000 & 0.25000000000000 & $\mathrm{H}$ & (4e) \\
\hline 0.36840000000000 & -0.36840000000000 & 0.75000000000000 & $\mathrm{H}$ & (4e) \\
\hline 0.15880000000000 & 0.29320000000000 & 0.24640000000000 & $\mathrm{H}$ & $(8 \mathrm{f})$ \\
\hline-0.29320000000000 & -0.15880000000000 & 0.25360000000000 & $\mathrm{H}$ & (8f) \\
\hline-0.15880000000000 & -0.29320000000000 & -0.24640000000000 & $\mathrm{H}$ & (8f) \\
\hline 0.29320000000000 & 0.15880000000000 & 0.74640000000000 & $\mathrm{H}$ & (8f) \\
\hline 0.14850000000000 & 0.54010000000000 & 0.22270000000000 & $\mathrm{H}$ & (8f) \\
\hline-0.54010000000000 & -0.14850000000000 & 0.27730000000000 & $\mathrm{H}$ & $(8 \mathrm{f})$ \\
\hline-0.14850000000000 & -0.54010000000000 & -0.22270000000000 & $\mathrm{H}$ & ( $8 \mathrm{f})$ \\
\hline 0.54010000000000 & 0.14850000000000 & 0.72270000000000 & $\mathrm{H}$ & (8f) \\
\hline
\end{tabular}

$\alpha$-Naumannite $\left(\mathrm{Ag}_{2} \mathrm{Se}\right)$ : A2B_oP12_17_abe_e - CIF

\# CIF file
data_findsym-output
_audit_creation_method FINDSYM
_chemical_name_mineral 'alpha-A
_chemical_formula_sum 'Ag2 Se,
loop_-
_publ_author_name
'Z. G. Pinsker,
'C. \{Ching-liang\},
'R. M. Imamov'
'E. L. Lapidus,
_journal_name_full_name
;
Soviet Physics Crystallography
;
_journal_volume 10
-journal_year 1965
-journal_page_first 225
-journal_page_last 231
-publ_Section_title
;




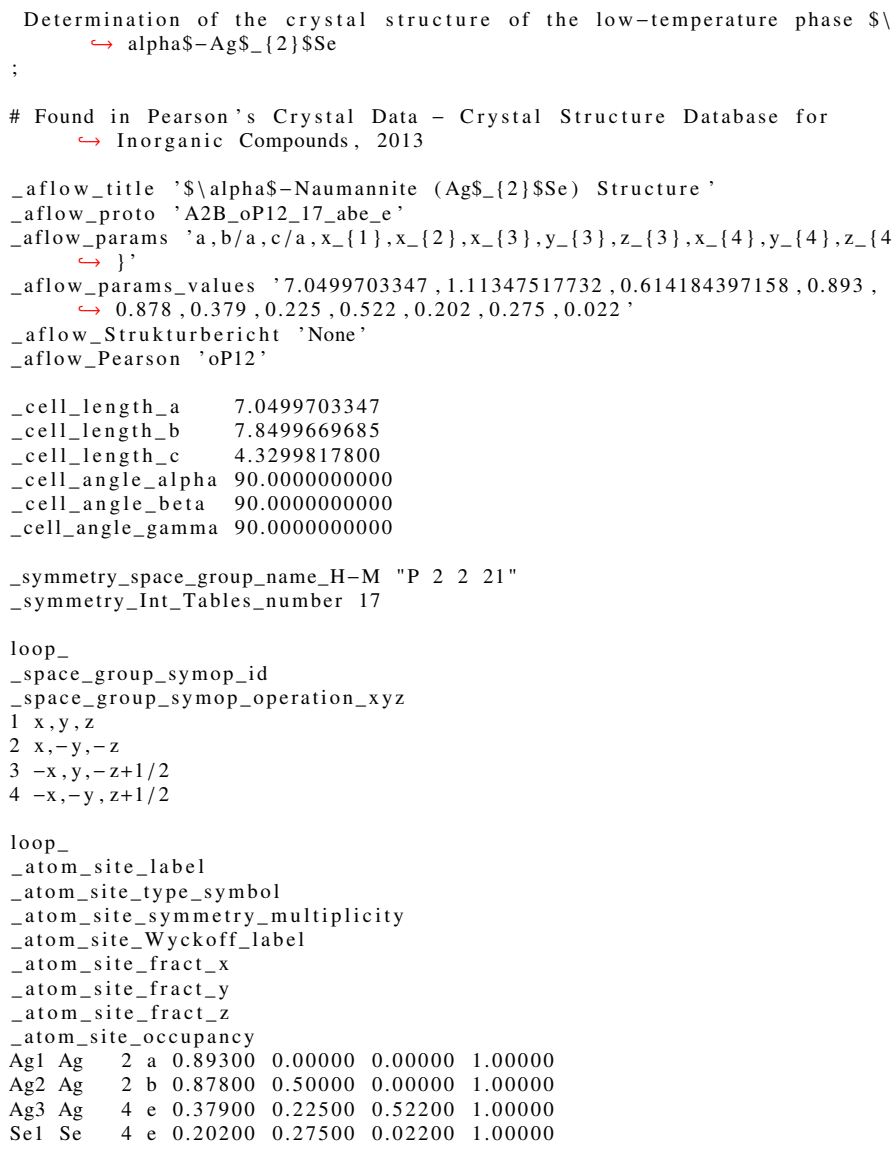

Found in Pearson's Crystal Data - Crystal Structure Database fo $\hookrightarrow$ Inorganic Compounds, 2013

aflow_title $\$ \backslash$ alpha $\$-N a u m a n n i t e ~\left(A g \$ \_\{2\} \$ S e\right)$ Structure

aflow_proto 'A2B_oP12_17_abe_e'

aflow_params 'a, b/a,c/a, $x_{-}\{1\}, x_{-}\{2\}, x_{-}\{3\}, y_{-}\{3\}, z_{-}\{3\}, x_{-}\{4\}, y_{-}\{4\}, z_{-}\{4$ $\hookrightarrow\}$

aflow_params_values ' $7.0499703347,1.11347517732,0.614184397158,0.893$ $\hookrightarrow 0.878,0.379,0.225,0.522,0.202,0.275,0.022$,

aflow_Strukturbericht 'None'

aflow_Pearson oP12,

cell_length_a $\quad 7.0499703347$

cell_length_b 7.8499669685

cell_length_c $\quad 4.3299817800$

cell_angle_alpha 90.0000000000

cell_angle_beta 90.0000000000

cell_angle_gamma 90.0000000000

symmetry_space_group_name_H-M "P 2221 "

symmetry_Int_Tables_number 17

loop_

_space_group_symop_id

_space_group_symop_operation_xyz

$1 \mathrm{x}, \mathrm{y}, \mathrm{z}$

$2 \mathrm{x},-\mathrm{y},-\mathrm{z}$

$3-\mathrm{x}, \mathrm{y},-\mathrm{z}+1 / 2$

$4-\mathrm{x},-\mathrm{y}, \mathrm{z}+1 / 2$

loop

atom_site_label

_atom_site_type_symbol

_atom_site_symmetry_multiplicity

atom_site_Wyckoff_label

atom_site_fract_x

atom_site_fract_y

atom_site_fract_z

atom_site_occupancy

\begin{tabular}{llllllll}
\hline $\mathrm{Ag} 1$ & $\mathrm{Ag}$ & 2 & $\mathrm{a}$ & 0.89300 & 0.00000 & 0.00000 & 1.00000
\end{tabular}

$\begin{array}{llllllll}\mathrm{Ag} 2 & \mathrm{Ag} & 2 & \mathrm{~b} & 0.87800 & 0.50000 & 0.00000 & 1.00000\end{array}$

$\begin{array}{lllllllll}\mathrm{Ag} 3 & \mathrm{Ag} & 4 & \mathrm{e} & 0.37900 & 0.22500 & 0.52200 & 1.00000\end{array}$

$\begin{array}{lllllllll}\mathrm{Ag} 3 & \mathrm{Ag} & 4 & \mathrm{e} & 0.37900 & 0.22500 & 0.52200 & 1.00000 \\ \mathrm{Se} 1 & \mathrm{Se} & 4 & \mathrm{e} & 0.20200 & 0.27500 & 0.02200 & 1.00000\end{array}$

$\alpha$-Naumannite $\left(\mathrm{Ag}_{2} \mathrm{Se}\right)$ : A2B_oP12_17_abe_e - POSCAR

A2B_oP12_17_abe_e \& a , b/a, c /a , x1, x2, x3,y3, z3, x4,y4, z4 --params =

$\hookrightarrow 7.0499703347,1.11347517732,0.614184397158,0.893,0.878,0.379$,

$\hookrightarrow 0.225,0.522,0.202,0.275,0.022 \& \mathrm{P} 222 \_\{1\} \mathrm{D}_{-}\{2\}^{\wedge}\{2\} \# 17 \quad\left(\mathrm{abe}^{\wedge} 2\right)$

$\hookrightarrow \&$ oP12 \& None \& Ag2Se \& alpha \& Z. G. Pinsker et al., Sov.

$\hookrightarrow$ Phys. Crystallogr. 10, 225-231 (1965)

$7.04997033470000 \quad 0.00000000000000 \quad 0.00000000000000$

$\begin{array}{lll}0.00000000000000 & 7.84996696850000 & 0.00000000000000\end{array}$

$\begin{array}{lll}0.00000000000000 & 0.00000000000000 & 4.32998178000000\end{array}$

$\mathrm{Ag} \quad \mathrm{Se}$

Direct

0.89300000000000

$-0.89300000000000$

0.87800000000000

$-0.87800000000000$

0.37900000000000

$-0.37900000000000$

$-0.37900000000000$

0.37900000000000

0.20200000000000

$-0.20200000000000$

$-0.20200000000000$

0.20200000000000

$0.00000000000000 \quad 0.00000000000000$

0.00000000000000

0.5000000000000

0.50000000000000

0.22500000000000

$-0.22500000000000$

0.22500000000000

0.22500000000000

$-0.27500000000000$

0.2750000000000

$-0.27500000000000$

0.50000000000000

0.00000000000000

0.50000000000000

0.52200000000000

1.02200000000000

$-0.02200000000000$

$-0.52200000000000$

0.02200000000000

0.52200000000000

0.47800000000000

0.47800000000000
-0.02200000000000

$\begin{array}{ll}\mathrm{Ag} & (2 \mathrm{a}) \\ \mathrm{Ag} & (2 \mathrm{a}) \\ \mathrm{Ag} & (2 \mathrm{~b}) \\ \mathrm{Ag} & (2 \mathrm{~b}) \\ \mathrm{Ag} & (4 \mathrm{e}) \\ \mathrm{Ag} & (4 \mathrm{e}) \\ \mathrm{Ag} & (4 \mathrm{e}) \\ \mathrm{Ag} & (4 \mathrm{e}) \\ \mathrm{Se} & (4 \mathrm{e}) \\ \mathrm{Se} & (4 \mathrm{e}) \\ \mathrm{Se} & (4 \mathrm{e}) \\ \mathrm{Se} & (4 \mathrm{e})\end{array}$

H3 Cl (100 GPa): AB3_oP16_19_a_3a - CIF

\# CIF file

data findsym-output

-audit_creation_method FINDSYM

chemical name mineral ' $\mathrm{H} 3 \mathrm{Cl}$ '

chemical formula sum ${ }^{\circ} \mathrm{Cl} \mathrm{H}$,

loop

_publ_author_name

'D. Duan'

'X. Huang,

'F. Tian,

'Y. Liu'

' $\mathrm{Da} \mathrm{Li}$ ',

H. Yu,

'B. Liu',

'W. Tian,

T. Cui

journal_name_full_name

Journal of Physical Chemistry A

journal volume 119

journal year 2015

journal_page_first 11059

_journal_page_last 11065

-publ_Section_title
Predicted Formation of $\mathrm{HS}\{3\}^{\wedge}\{+\} \$$ in Solid Halogen Polyhydrides at $\hookrightarrow$ High Pressures

aflow_title 'H\$_ $\{3\} \$ C \mathrm{Cl}(100 \sim \mathrm{GPa})$ Structure'

-aflow_proto 'AB3_oP16_19_a_3a'

aflow_params 'a, b/a,c/a, $x_{-}\{1\}, y_{-}\{1\}, z_{-}\{1\}, x_{-}\{2\}, y_{-}\{2\}, z_{-}\{2\}, x_{-}\{3\}, y_{-}\{3$ $\hookrightarrow\}, z_{-}\{3\}, x_{-}\{4\}, y_{-}\{4\}, z_{-}\{4\}$

aflow_params_values ' $5.668,0.758997882851,0.528757939308,0.584,0.123$, $\hookrightarrow 0.027,0.31,0.159,0.417,0.257,0.073,0.603,0.983,0.124,0.227$,

-aflow_Strukturbericht 'None'

aflow Pearson, $\mathrm{OP} 16$

symmetry_space_group_name_H-M "P 212121 "

symmetry_Int Tables_number 19

Cell_length_a $\quad 5.66800$

-cell_length_b $\quad 4.30200$

_cell_length_c 2.99700

_cell_angle_alpha 90.00000

cell_angle_beta 90.00000

_cell_angle_gamma 90.00000

loop

space_group_symop_id

space_group_symop_operation_xyz

$1 \mathrm{x}, \mathrm{y}, \mathrm{z}$

$2 \mathrm{x}+1 / 2,-\mathrm{y}+1 / 2,-\mathrm{z}$

$3-x, y+1 / 2,-z+1 / 2$

$4-\mathrm{x}+1 / 2,-\mathrm{y}, \mathrm{z}+1 / 2$

loop

atom_site_label

atom_site type symbol

atom_site_symmetry_multiplicity

-atom_site_Wyckoff_label

atom_site_fract_x

atom_site_fract_y

-atom_site_fract_z

_atom_site_occupancy

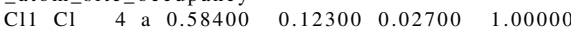

$\begin{array}{llllllll}\mathrm{H} 1 & \mathrm{H} & 4 & \mathrm{a} & 0.31000 & 0.15900 & 0.41700 & 1.00000\end{array}$

$\begin{array}{llllllll}\mathrm{H} 2 & \mathrm{H} & 4 & \mathrm{a} & 0.25700 & 0.07300 & 0.60300 & 1.00000\end{array}$

$\begin{array}{llllllll}\mathrm{H} 3 & \mathrm{H} & 4 & \mathrm{a} & 0.98300 & 0.12400 & 0.22700 & 1.00000\end{array}$

$\mathrm{H}_{3} \mathrm{Cl}(100 \mathrm{GPa})$ : AB3_oP16_19_a_3a - POSCAR

\begin{tabular}{|c|c|c|c|c|}
\hline \multicolumn{5}{|c|}{$\begin{aligned} & \text { AB3_oP16_19_a_3a \& a }, \mathrm{b} / \mathrm{a}, \mathrm{c} / \mathrm{a}, \mathrm{x} 1, \mathrm{y} 1, \mathrm{z} 1, \mathrm{x} 2, \mathrm{y} 2, \mathrm{z} 2, \mathrm{x} 3, \mathrm{y} 3, \mathrm{z} 3, \mathrm{x} 4, \mathrm{y} 4, \mathrm{z} 4-- \\
& \hookrightarrow \text { params }=5.668,0.758997882851,0.528757939308,0.584,0.123,0.027, \\
& \hookrightarrow 0.31,0.159,0.417,0.257,0.073,0.603,0.983,0.124,0.227 \& \mathrm{P} 2 \_\{1\} 2_{-}- \\
& \hookrightarrow\{1\} 2 \_\{1\} D_{-}\{2\} \wedge\{4\} \# 19\left(\mathrm{a}^{\wedge} 4\right) \& \text { oP } 16 \& \text { None \& H3Cl \& H3Cl \& D. } \\
& \hookrightarrow \text { Duan et al., J. Phys. Chem. A } 119,11059-11065(2015)\end{aligned}$} \\
\hline 5.66800000000000 & 0.00000000000000 & 0.00000000000000 & & \\
\hline \multirow{2}{*}{$\begin{array}{l}0.00000000000000 \\
0.00000000000000\end{array}$} & 4.30200000000000 & 0.00000000000000 & & \\
\hline & 0.00000000000000 & 2.99700000000000 & & \\
\hline $\begin{array}{r}\mathrm{Cl} \\
4\end{array}$ & & & & \\
\hline \multicolumn{5}{|l|}{ Direct } \\
\hline 0.58400000000000 & 0.12300000000000 & 0.02700000000000 & $\mathrm{Cl}$ & (4a) \\
\hline-0.08400000000000 & -0.12300000000000 & 0.52700000000000 & $\mathrm{Cl}$ & (4a) \\
\hline-0.58400000000000 & 0.62300000000000 & 0.47300000000000 & $\mathrm{Cl}$ & (4a) \\
\hline 1.08400000000000 & 0.37700000000000 & -0.02700000000000 & $\mathrm{Cl}$ & (4a) \\
\hline 0.31000000000000 & 0.15900000000000 & 0.41700000000000 & $\mathrm{H}$ & $(4 a)$ \\
\hline 0.19000000000000 & -0.15900000000000 & 0.91700000000000 & $\mathrm{H}$ & $(4 a)$ \\
\hline-0.31000000000000 & 0.65900000000000 & 0.08300000000000 & $\mathrm{H}$ & (4a) \\
\hline 0.81000000000000 & 0.34100000000000 & -0.41700000000000 & $\mathrm{H}$ & (4a) \\
\hline 0.25700000000000 & 0.07300000000000 & 0.60300000000000 & $\mathrm{H}$ & $(4 a)$ \\
\hline 0.24300000000000 & -0.07300000000000 & 1.10300000000000 & $\mathrm{H}$ & (4a) \\
\hline-0.25700000000000 & 0.57300000000000 & -0.10300000000000 & $\mathrm{H}$ & $(4 a)$ \\
\hline 0.75700000000000 & 0.42700000000000 & -0.60300000000000 & $\mathrm{H}$ & (4a) \\
\hline 0.98300000000000 & 0.12400000000000 & 0.22700000000000 & $\mathrm{H}$ & (4a) \\
\hline-0.48300000000000 & -0.12400000000000 & 0.72700000000000 & $\mathrm{H}$ & $(4 a)$ \\
\hline-0.98300000000000 & 0.62400000000000 & 0.27300000000000 & $\mathrm{H}$ & $(4 a)$ \\
\hline 1.48300000000000 & 0.37600000000000 & -0.22700000000000 & $\mathrm{H}$ & $(4 a)$ \\
\hline
\end{tabular}

Ta2 2 H: AB2_oC6_21_a_k - CIF

\section{\# CIF file}

data_findsym-output

audit_creation_method FINDSYM

chemical_name_mineral ' $\mathrm{Ta} 2 \mathrm{H}$ ',

chemical_formula_sum 'H Ta2,

loop

publ_author_name

H. Asano'

'Y. Ishikawa'

M. Hirabayashi,

journal_name_full_name

Journal of Applied Crystallography

journal volume 11

journal year 1978

journal_page_first 681

journal_page_last 683

publ_Section_title

Single-crystal $\mathrm{X}$-ray diffraction study on the hydrogen ordering in Ta\$ $\hookrightarrow\{2\} \$ H$ 


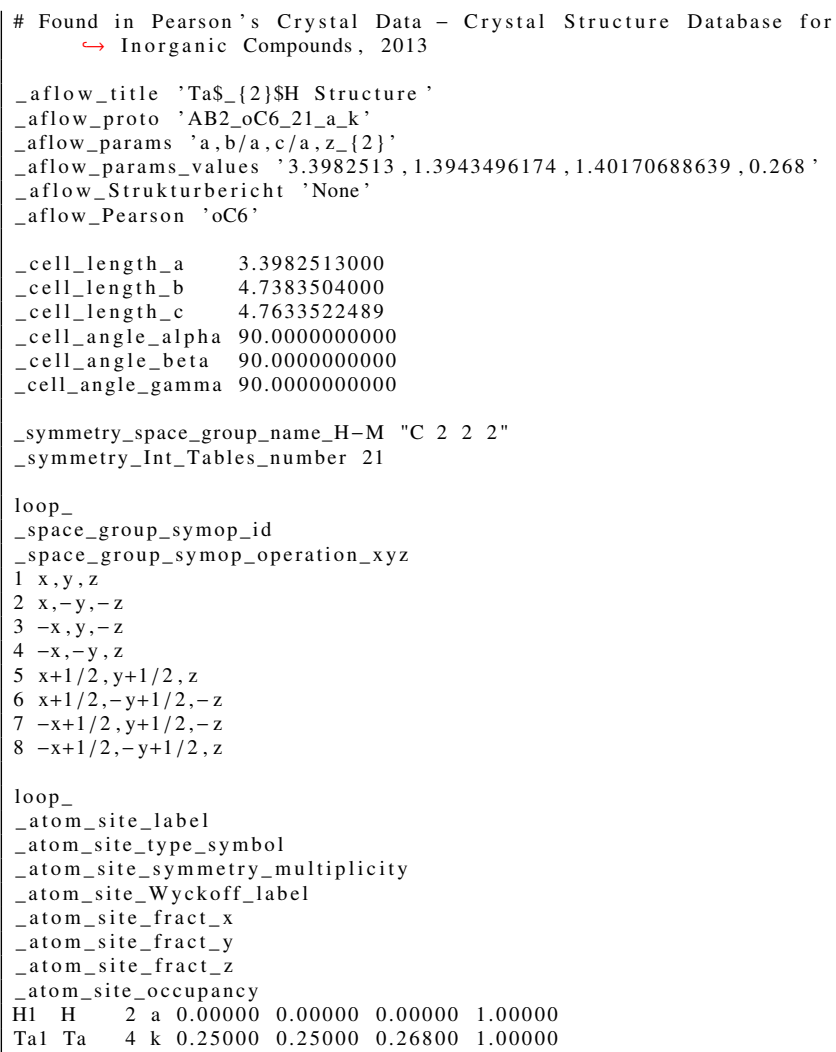

$\mathrm{Ta}_{2} \mathrm{H}$ : AB2_oC6_21_a_k - POSCAR

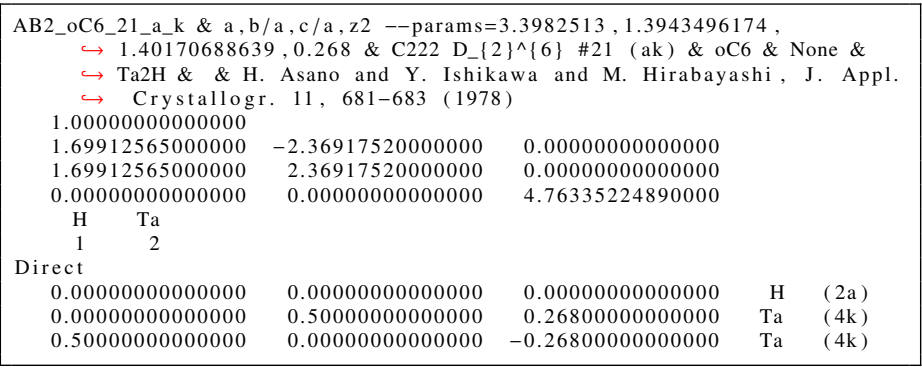

$\mathrm{CeRu}_{2} \mathrm{~B}_{2}$ : A2BC2_oF40_22_fi_ad_gh - CIF

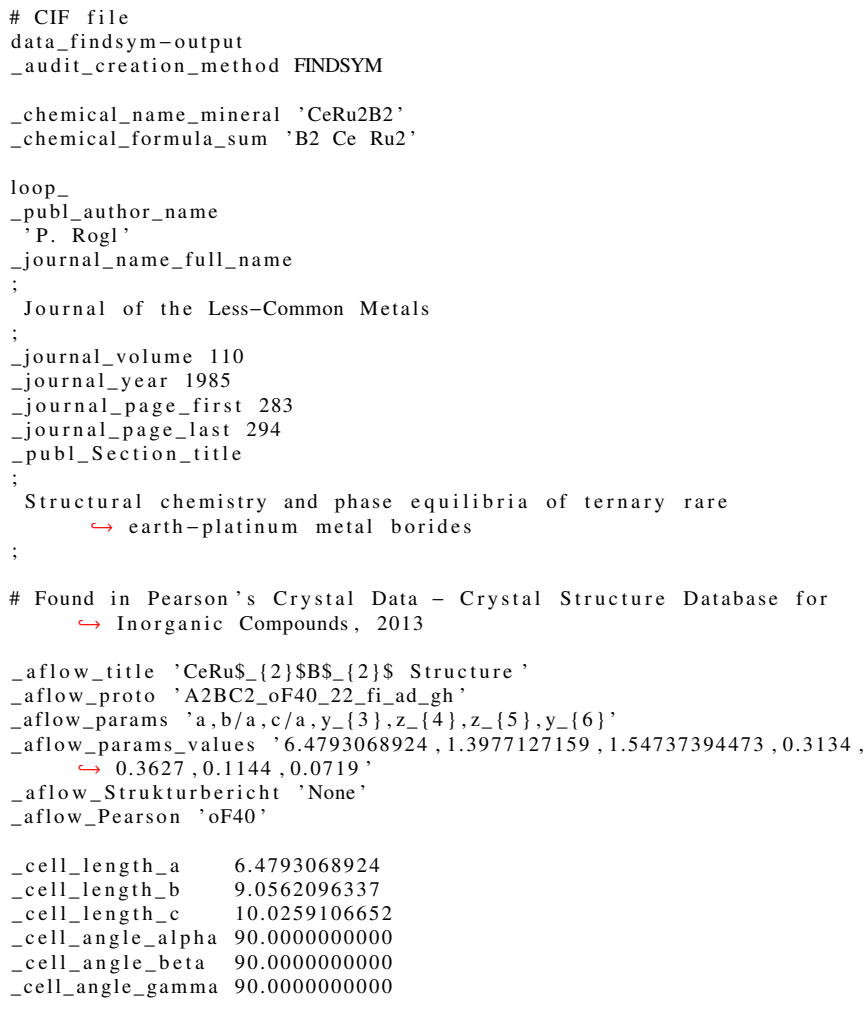

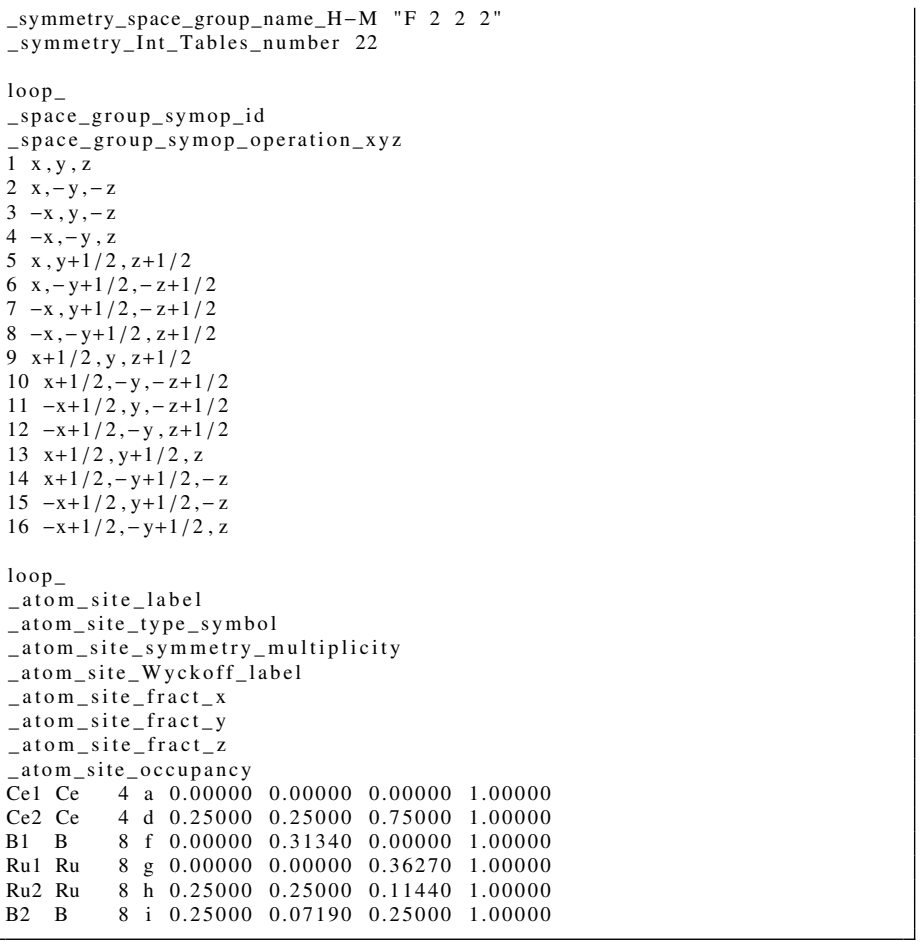

$\mathrm{CeRu}_{2} \mathrm{~B}_{2}$ : A2BC2_oF40_22_fi_ad_gh - POSCAR

\begin{tabular}{|c|c|c|c|c|}
\hline \\
\hline 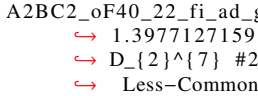 & $\begin{array}{l}\mathrm{gh} \& \mathrm{a}, \mathrm{b} / \mathrm{a}, \mathrm{c} / \mathrm{a}, \mathrm{y} 3, \mathrm{z} \\
1.54737394473,0.31 \\
2 \quad(\text { adfghi }) \& \text { oF } 40 \\
\text { Met. 110, 283-294 }\end{array}$ & $\begin{array}{l}4, \mathrm{z} 5, \mathrm{y} 6-- \text { params }=6 . \\
34,0.3627,0.1144,0 \\
\text { None \& CeRu2B2 \& } \\
(1985)\end{array}$ & $\begin{array}{l}719 \\
8 \mathrm{P} .\end{array}$ & $\begin{array}{l}8924, \\
\text { Fogl, J. }\end{array}$ \\
\hline \multicolumn{5}{|l|}{1.00000000000000} \\
\hline 0.00000000000000 & 4.52810481685000 & 5.01295533260000 & & \\
\hline \multirow{2}{*}{$\begin{array}{l}3.23965344620000 \\
3.23965344620000\end{array}$} & 0.00000000000000 & 5.01295533260000 & & \\
\hline & 4.52810481685000 & 0.00000000000000 & & \\
\hline $\mathrm{Ru}$ & & & & \\
\hline 4 & & & & \\
\hline \multicolumn{5}{|l|}{ Direct } \\
\hline 0.31340000000000 & -0.31340000000000 & 0.31340000000000 & B & $(8 \mathrm{f})$ \\
\hline-0.31340000000000 & 0.31340000000000 & -0.31340000000000 & B & $(8 \mathrm{f})$ \\
\hline 0.07190000000000 & 0.42810000000000 & 0.07190000000000 & B & ( $8 \mathrm{i})$ \\
\hline 0.42810000000000 & 0.07190000000000 & 0.42810000000000 & B & ( $8 \mathrm{i})$ \\
\hline \multirow{2}{*}{$\begin{array}{l}0.00000000000000 \\
0.75000000000000\end{array}$} & 0.00000000000000 & 0.00000000000000 & $\mathrm{Ce}$ & (4a) \\
\hline & 0.75000000000000 & 0.75000000000000 & $\mathrm{Ce}$ & $(4 d)$ \\
\hline 0.36270000000000 & 0.36270000000000 & -0.36270000000000 & $\mathrm{Ru}$ & $(8 \mathrm{~g})$ \\
\hline \multirow{2}{*}{-0.36270000000000} & -0.36270000000000 & 0.36270000000000 & $\mathrm{Ru}$ & $(8 \mathrm{~g})$ \\
\hline & 0.11440000000000 & 0.38560000000000 & $\mathrm{Ru}$ & $(8 \mathrm{~h})$ \\
\hline 0.38560000000000 & 0.38560000000000 & 0.11440000000000 & $\mathrm{Ru}$ & $(8 \mathrm{~h})$ \\
\hline
\end{tabular}

FeS (Low-temperature): AB_oF8_22_a_c - CIF

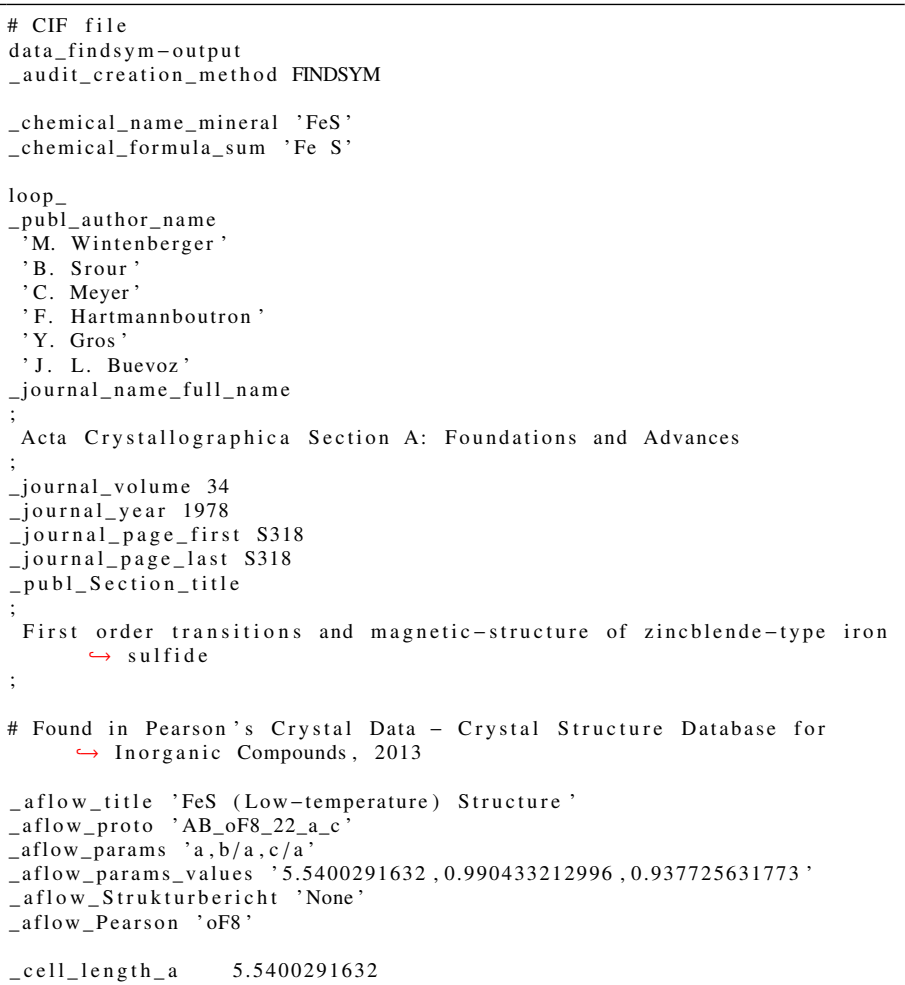


_cell_length_b $\quad 5.4870288842$

$\begin{array}{ll}\text { _cell_length_c } & 5.1950273471 \\ \text { cell_angle_alpha } & 90.0000000000\end{array}$

_cell_angle_alpha 90.0000000000
cell_angle_beta $\quad 90.0000000000$

_cell_angle_gamma 90.0000000000

_symmetry_space_group_name_H-M "F 222

symmetry_Int_Tables_number 22

loop_

_space_group_symop_id

_space_group_symop_operation_xyz

$1 \mathrm{x}, \mathrm{y}, \mathrm{z}$

$2 \mathrm{x},-\mathrm{y},-\mathrm{z}$

$3-\mathrm{x}, \mathrm{y},-\mathrm{z}$

$4-\mathrm{x},-\mathrm{y}, \mathrm{z}$

$5 \mathrm{x}, \mathrm{y}+1 / 2, \mathrm{z}+1 / 2$

$6 \mathrm{x},-\mathrm{y}+1 / 2,-\mathrm{z}+1 / 2$

$7-\mathrm{x}, \mathrm{y}+1 / 2,-\mathrm{z}+1 / 2$

$8-\mathrm{x},-\mathrm{y}+1 / 2, \mathrm{z}+1 / 2$

$9 \mathrm{x}+1 / 2, \mathrm{y}, \mathrm{z}+1 / 2$

$10 \mathrm{x}+1 / 2,-\mathrm{y},-\mathrm{z}+1 / 2$

$11-\mathrm{x}+1 / 2, \mathrm{y},-\mathrm{z}+1 / 2$

$12-x+1 / 2,-y, z+1 / 2$

$13 \mathrm{x}+1 / 2, \mathrm{y}+1 / 2, \mathrm{z}$

$14 x+1 / 2,-y+1 / 2,-z$

$6-x+1 / 2$

loop

ato site label

atom_site_type_symbol

atom_site_symmetry_multiplicity

atom_site_Wyckoff_label

atom_site_fract_x

-atom_site_fract_y

atom_site_fract_z

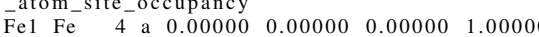

$\begin{array}{llllllll}\mathrm{S} 1 \mathrm{~S} & 4 & \mathrm{c} & 0.25000 & 0.25000 & 0.25000 & 1.00000\end{array}$

FeS (Low-temperature): AB_oF8_22_a_c - POSCAR

AB_oF8_22_a_c \& a,b/a,c / a --params $=5.5400291632,0.990433212996$

$\hookrightarrow 0.937725631773 \&$ \& $222 D_{-}\{2\}^{\wedge}\{7\}$ \#22 (ac) \& oF8 \& None \& FeS \&

$\hookrightarrow \&$ M. Wintenberger et al., Acta Crystallogr. Sect. A 34

$\hookrightarrow \mathrm{S} 318-\mathrm{S} 318(1978)$

1.00000000000000

$0.00000000000000 \quad 2.74351444210000 \quad 2.59751367355000$

$\begin{array}{lll}2.77001458160000 & 0.00000000000000 & 2.59751367355000\end{array}$

$\begin{array}{lll}2.77001458160000 & 2.74351444210000 & 0.00000000000000\end{array}$

$\mathrm{Fe} \quad \mathrm{S}$

Direct

$\begin{array}{llllll}0.00000000000000 & 0.00000000000000 & 0.00000000000000 & \mathrm{Fe} & \text { (4a) }\end{array}$ $\begin{array}{rrrrr}0.25000000000000 & 0.25000000000000 & 0.25000000000000 & \mathrm{~S} & \text { (4c) }\end{array}$

$\mathrm{H}_{3} \mathrm{~S}$ (5 GPa): A3B_ol32_23_ij2k_k - CIF

\# CIF file

data_findsym-output

audit creation method FINDSYM

chemical_name_mineral 'H3S'

chemical formula sum ' $\mathrm{H} 3 \mathrm{~S}$ '

loop

publ_author_name

T. A. Strobe

P. Ganesh

M. Somayazulu

'P. R. C. Kent'

'R. J. Hemley'

_journal_name_full_name

Physical Review Letter

_journal_volume 107

journal year 2011

journal page first 255503

journal_page_first 255503

publ_Section_title

Novel Cooperative Interactions and Structural Ordering in $\mathrm{H} \$ \$_{-}\{2\} \$ \$-\mathrm{H} \$ \$_{-}\{$

$$
\hookrightarrow 2\} \$
$$

aflow_title'H\$_\{3\}\$S (5 GPa) Structure',

aflow_proto A3B_oI32_23_ij2k_k

aflow_params $, a, b / a, c / a, z_{-}\{1\}, z_{-}\{2\}, x_{-}\{3\}, y_{-}\{3\}, z_{-}\{3\}, x_{-}\{4\}, y_{-}\{4\}, z_{-}\{4$ $\hookrightarrow\}, x_{-}\{5\}, y_{-}\{5\}, z_{-}\{5\}$

aflow_params_values $, 5.82463,1.24369101557,1.32254065924,0.04851$

$\hookrightarrow 0.45153,0.75475,0.49255,0.20405,0.4421,0.23223,0.28616,0.76005$ $\hookrightarrow 0.8216,0.36488$

aflow_Strukturbericht 'None'

_aflow_Pearson 'oI32,

ymmetry space group name $\mathrm{H}-\mathrm{M}$ "II $22^{2}$

symmetry_Int_Tables_number 23

cell_length_a $\quad 5.82463$

cell_length_b 7.24404

$\begin{array}{ll}\text { cell_length_c } & 7.70331\end{array}$

cell_angle_alpha 90.00000

_cell_angle_beta 90.00000 _cell_angle_gamma 90.00000

loop

space_group_symop_i

_space_group_symop_operation_xyz

$1 \mathrm{x}, \mathrm{y}, \mathrm{z}$

$2 \mathrm{x},-\mathrm{y},-\mathrm{z}$

$3-\mathrm{x}, \mathrm{y},-\mathrm{z}$

$4-\mathrm{x},-\mathrm{y}, \mathrm{z}$

$5 \mathrm{x}+1 / 2, \mathrm{y}+1 / 2, \mathrm{z}+1 / 2$

$6 \mathrm{x}+1 / 2,-\mathrm{y}+1 / 2,-\mathrm{z}+1 / 2$

$7-x+1 / 2, y+1 / 2,-z+1 / 2$

loop

atom_site_label

atom_site_type_symbol

atom_site_symmetry_multiplicity

-atom_site_Wyckoff_label

${ }_{-}$atom_site_fract_x

atom_site_fract_Z

_atom_site_occupancy

$\begin{array}{llllllll}\mathrm{H} 1 \mathrm{H} & 4 & \mathrm{i} & 0.00000 & 0.00000 & 0.04851 & 1.00000\end{array}$

$\begin{array}{lllllll}\mathrm{H} 2 \mathrm{H} & 4 \mathrm{j} & 0.00000 & 0.50000 & 0.45153 & 1.00000\end{array}$

\begin{tabular}{lllllllll}
$\mathrm{H} 3$ & $\mathrm{H}$ & 8 & $\mathrm{k}$ & 0.75475 & 0.49255 & 0.20405 & 1.00000 \\
\hline
\end{tabular}

$\begin{array}{lllllllll}\mathrm{S} 1 \mathrm{~S} & 8 & \mathrm{k} & 0.76005 & 0.23223 & 0.28616 & 1.00000\end{array}$

$\mathrm{H}_{3} \mathrm{~S}$ (5 GPa): A3B_oI32_23_ij2k_k - POSCAR

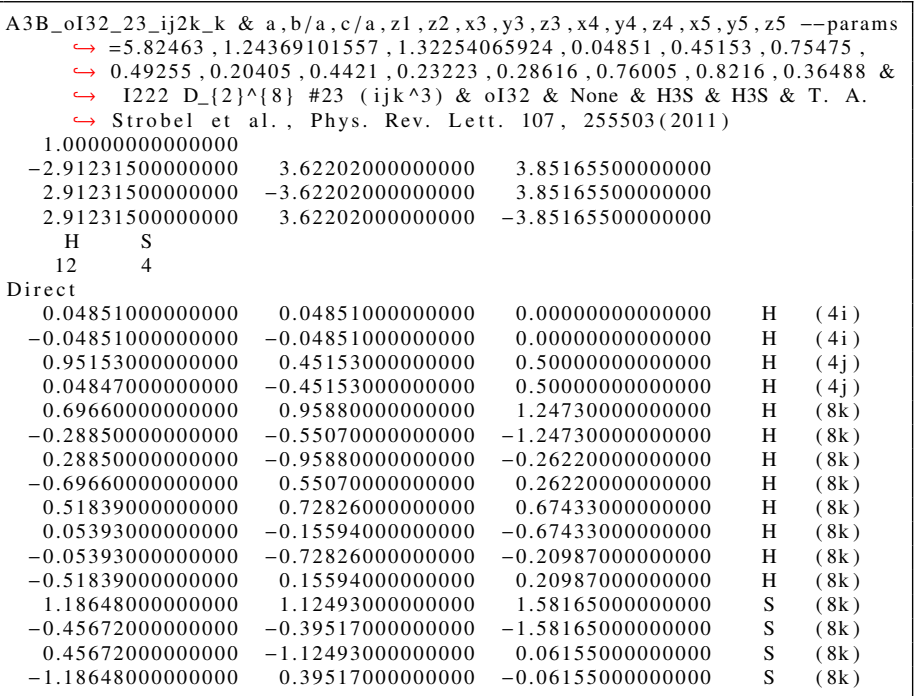

Stannoidite $\left(\mathrm{Cu}_{8}(\mathrm{Fe}, \mathrm{Zn})_{3} \mathrm{Sn}_{2} \mathrm{~S}_{12}\right)$ : A8B2C12D2E_OI50_23_bcfk_i_3k_j_a - CIF

\# CIF file

data findsym-output

audit_creation_method FINDSYM

chemical name mineral 'Stannoidite'

$\begin{array}{lllll}\text { chemical formula sum ' } \mathrm{Cu} 8 & \mathrm{Fe} 2 & \mathrm{~S} 12 & \mathrm{Sn} 2 & \mathrm{Zn}\end{array}$

loop

publ_author_nam

Y. Kudoh,

Y. Tak $\backslash \backslash,\{$ e $\}$ uchi

journal_name_full_name

Zeitschrift f $\{\backslash "$ u $\}$ r Kristallographie - Crystalline Materials

journal_volume 144

journal year 1976

journal_page_first 145

journal_page_first 145

publ_Section_title

The superstructure of stannoidite

_aflow_title 'Stannoidite $\left(\mathrm{Cu} \$_{-}\{8\} \$(\mathrm{Fe}, \mathrm{Zn}) \$_{-}\{3\} \$ \mathrm{Sn} \$_{-}\{2\} \$ S \$ \$_{-}\{12\} \$\right)$ $\hookrightarrow$ Structure

aflow_proto A8B2C12D2E_oI50_23_bcfk_i_3k_j_a

aflow_params $, a, b / a, c / a, x_{-}\{4\}, z_{-}\{5\}, z_{-}\{6\}, x_{-}\{7\}, y_{-}\{7\}, z_{-}\{7\}, x_{-}\{8\}, y_{-}\{8$ $\hookrightarrow\}, z_{-}\{8\}, x_{-}\{9\}, y_{-}\{9\}, z_{-}\{9\}, x_{-}\{10\}, y_{-}\{10\}, z_{-}\{10\}$

aflow_params_values $, 10.767,0.502554100492,1.4969815176,0.2511,0.3298$, $\hookrightarrow 0.1693,0.2465,0.0107,0.1695,0.1308,0.2443,0.0826,0.3792,0.7558$, $\hookrightarrow 0.0801,0.1294,0.7488,0.2546$,

aflow_Strukturbericht 'None'

aflow_Pearson, 0 I50

symmetry space group name_H-M "I 222 2"

symmetry Int Tables_number 23

cell_length_a $\quad 10.76700$

5.41100

16.11800
cell_length_c

cell_angle_alpha 90.00000

_cell_angle_beta 90.00000 


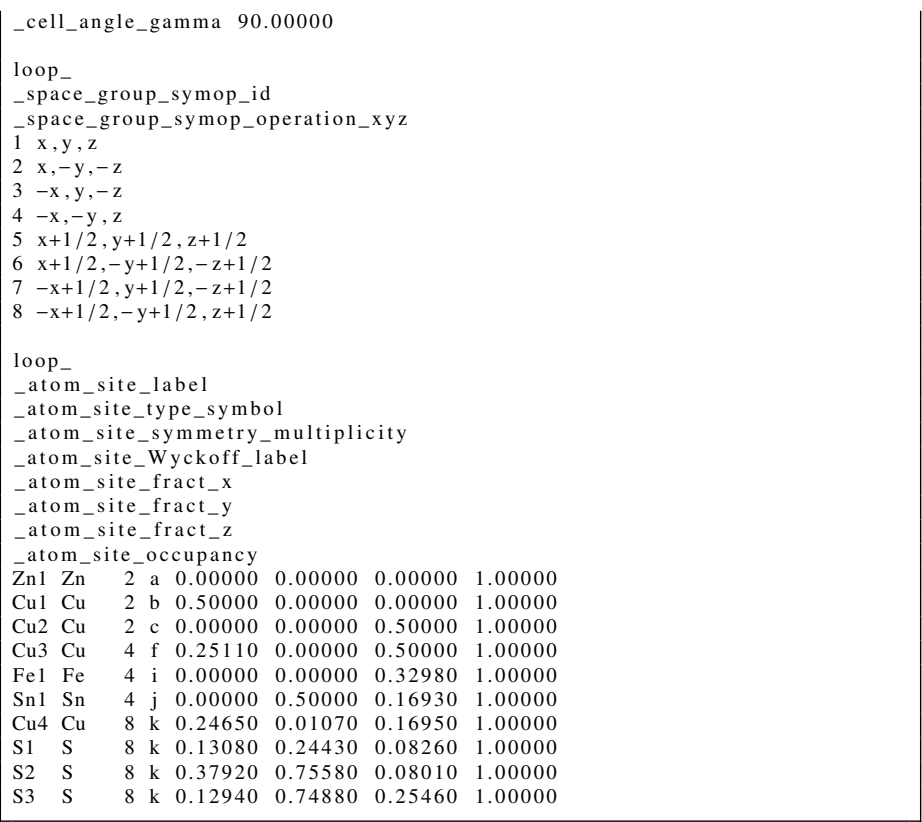

Stannoidite $\left(\mathrm{Cu}_{8}(\mathrm{Fe}, \mathrm{Zn})_{3} \mathrm{Sn}_{2} \mathrm{~S}_{12}\right)$ : A8B2C12D2E_oI50_23_bcfk_i_3k_j_a - POSCAR

A8B2C12D2E_oI50_23_bcfk_i_3k_j_a \& a , b/a,c/a, x4,z5, z6, x7,y7, z7, x8,y8,z8, $\hookrightarrow \mathrm{x} 9, \mathrm{y} 9, \mathrm{z} 9, \mathrm{x} 10, \mathrm{y} 10, \mathrm{z} 10-$ params $=10.767,0.502554100492$

$\hookrightarrow 1.4969815176,0.2511,0.3298,0.1693,0.2465,0.0107,0.1695,0.1308$

$\hookrightarrow 0.2443,0.0826,0.3792,0.7558,0.0801,0.1294,0.7488,0.2546 \&$ I 222

$\hookrightarrow \mathrm{D}\{2\}^{\wedge}\{8\} \# 23(\mathrm{abcfijk} \wedge 4) \& \mathrm{OI} 50$ \& None \& $\mathrm{Cu} 8(\mathrm{Fe}, \mathrm{Zn}) 3 \mathrm{Sn} 2 \mathrm{~S} 12$ \&

$\hookrightarrow$ Stannoidite \& Y. Kudoh and Y. Tak $\backslash\{$ e $\}$ uchi, Zeitschrift f" $\{u\} r$

$\hookrightarrow$ Kristallographie - Crystalline Materials 144, 145-160 (1976) 1.00000000000000

$-5.38350000000000$

$-5.38350000000000$

5.38350000000000

5.38350000000000

2.70550000000000

$2.70550000000000 \quad 8.05900000000000$

$\begin{array}{rrr}8 & \mathrm{Fe} & \mathrm{S} \\ 8 & 2 & 12\end{array}$

Direct

$\begin{array}{lll}0.00000000000000 & 0.50000000000000 & 0.50000000000000\end{array}$

0.50000000000000

0.50000000000000

0.50000000000000

0.18020000000000

0.1588000000000

$-0.1588000000000$

$-0.15880000000000$

0.32980000000000

$-0.32980000000000$

0.32690000000000

$-0.16170000000000$

0.16170000000000

$-0.32690000000000$

0.83590000000000

$-0.67570000000000$

0.67570000000000

$-0.83590000000000$

1.00340000000000

$-0.49420000000000$

0.49420000000000

$-1.00340000000000$

0.66930000000000

0.33070000000000

0.00000000000000

$\mathrm{Sn} \quad \mathrm{Zn}$ .50000000000000 0.75110000000000 0.24890000000000 0.41600000000000 $-0.07700000000000$ 0.41600000000000 0.07700000000000 0.32980000000000 0.329000000 . 0.2134000000 0.04820000000000 0.21340000000000 0.04820000000000 0.45930000000000 0.29910000000000 $-0.45930000000000$ 0.29910000000000 0.38400000000000 0.12520000000000 $-0.38400000000000$ $-0.12520000000000$ 0.1693000000000 0.1693000000000 0.00000000000000 0.2511000000000 $-0.25110000000000$ $-0.25720000000000$ $-0.2572000000000$ $-0.23580000000000$ 0.2358000000000 . 23580000000000 0.00000000000 0.00000000000000 0.37510000000000 $-0.37510000000000$ 0.11350000000000 $-0.1135000000000$ 1.13500000000000 $-1.13500000000000$ 0.37660000000000 $-0.3766000000000$ 0.8782000000000 $-0.87820000000000$ 0.61940000000000 $-0.6194000000000$ 0.5000000000000 0.50000000000000 0.00000000000000

$\begin{array}{ll}\mathrm{Cu} & (2 \mathrm{~b}) \\ \mathrm{Cu} & (2 \mathrm{c}) \\ \mathrm{Cu} & (4 \mathrm{f}) \\ \mathrm{Cu} & (4 \mathrm{f}) \\ \mathrm{Cu} & (8 \mathrm{k}) \\ \mathrm{Cu} & (8 \mathrm{k}) \\ \mathrm{Cu} & (8 \mathrm{k}) \\ \mathrm{Cu} & (8 \mathrm{k}) \\ \mathrm{Fe} & (4 \mathrm{i}) \\ \mathrm{Fe} & (4 \mathrm{i}) \\ \mathrm{S} & (8 \mathrm{k}) \\ \mathrm{S} & (8 \mathrm{k}) \\ \mathrm{S} & (8 \mathrm{k}) \\ \mathrm{S} & (8 \mathrm{k}) \\ \mathrm{S} & (8 \mathrm{k}) \\ \mathrm{S} & (8 \mathrm{k}) \\ \mathrm{S} & (8 \mathrm{k}) \\ \mathrm{S} & (8 \mathrm{k}) \\ \mathrm{S} & (8 \mathrm{k}) \\ \mathrm{S} & (8 \mathrm{k}) \\ \mathrm{S} & (8 \mathrm{k}) \\ \mathrm{S} & (8 \mathrm{k}) \\ \mathrm{Sn} & (4 \mathrm{j}) \\ \mathrm{Sn} & (4 \mathrm{j}) \\ \mathrm{Zn} & (2 \mathrm{a})\end{array}$

$\mathrm{NaFeS}_{2}: \mathrm{ABC} 2$ _ol16_23_ab_i_k - CIF

\# CIF file

data_findsym-output

audit_creation_method FINDSYM

chemical name mineral 'NaFeS2'

chemical formula_sum ' $\mathrm{Fe} \mathrm{NaS2}$,

loop

publ_author_name

'H. Boller
'H. Blaha'

journal_name_full_name

Monatshefte $\mathrm{f}\{\backslash \mathrm{lu}\} \mathrm{r}$ Chemie - Chemical Monthly

_journal_volume 114

_journal_year 198

_journal_page_first 145

journal_page_last 154

publ_Section_title

Zur Kenntnis des Natriumthioferrates (III)

\# Found in Pearson's Crystal Data - Crystal Structure Database fo $\hookrightarrow$ Inorganic Compounds, 2013

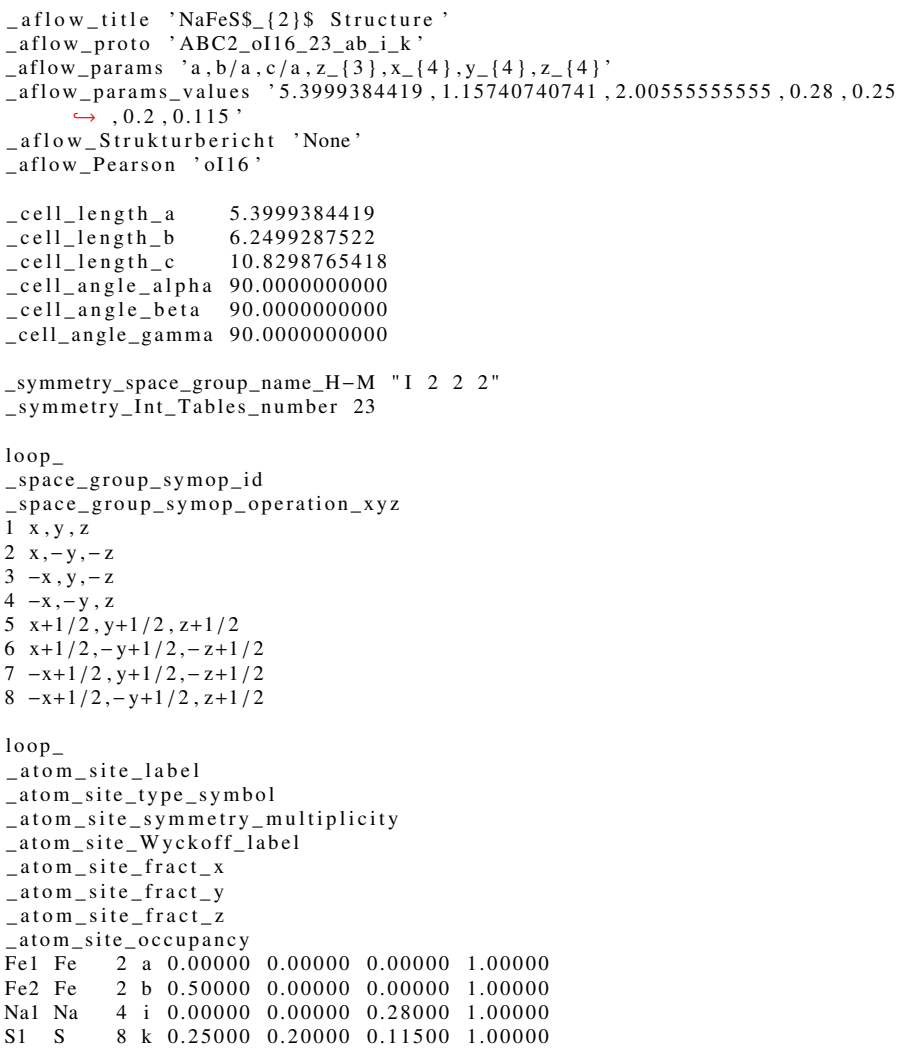

$\mathrm{NaFeS}_{2}$ : ABC2_oI16_23_ab_i_k - POSCAR

ABC2_oI16_23_ab_i_k \& a,b/a,c /a ,z3 , x4,y4, z4 --params=5.3999384419, $\hookrightarrow 1.15740740741,2.00555555555,0.28,0.25,0.2,0.115 \&$ I 222 D_ $\{2\}^{\wedge}\{8$ $\hookrightarrow$ \} \#23 (abik) \& oI16 \& None \& NaFeS2 \& \& H. Boller and H. Blaha Monatsh. Chem. 114, 145-154 (1983)

1.00000000000000

$\begin{array}{rrr}-2.69996922095000 & 3.12496437610000 & 5.41493827090000\end{array}$

$2.69996922095000 \quad-3.12496437610000 \quad 5.41493827090000$

$2.69996922095000-3.12496437610000-5.41493827090000$

$\mathrm{Fe} \quad \mathrm{Na} \quad \mathrm{S}$

Direct

$0.00000000000000-0.00000000000000-0.00000000000000$

0.00000000000000

0.28000000000000

$-0.28000000000000-0.28000000000000$

$0.31500000000000-0.36500000000000$

$-0.08500000000000-0.13500000000000$

$0.08500000000000-0.36500000000000$ 0.00000000000000 0.00000000000000 0.45000000000000 $-0.45000000000000$ $-0.05000000000000$ 0.05000000000000

BPS 4 : ABC4_oI12_23_a_b_k - CIF

\# CIF file

data_findsym-outpu

-audit_creation_method FINDSYM

chemical_name_mineral 'BPS4'

_chemical_formula_sum 'B P S4,

loop

publ_author_name

'A. Weiss'

'H. Sch $\{\backslash$ "a\} fer'

journal_name_full_name

Zeitschrift $f\{\backslash$ u $\}$ r Naturforschung B

journal_volume 18

journal year 1963

journal_page_first 81

journal_page_last 82

-publ_Section_title

Zur Kenntnis von Bortetrathiophosphat BPS\$_ $\{4\} \$$

\# Found in Pearson's Crystal Data - Crystal Structure Database for $\hookrightarrow$ Inorganic Compounds, 2013

aflow_title 'BPS\$_ $\{4\} \$$ Structure

aflow_proto 'ABC4_oI12_23_a_b_k'

aflow params, a, b/a,c/a, x $\{3\}, y\{3\}, z\{3\}$

aflow_params_values $, 5.2501580231,1.06666666665,1.7219047619,0.21,0.2$ $\hookrightarrow 0.115$,

aflow Strukturbericht 'None,

aflow_Pearson,oI12,

cell_length_a $\quad 5.250158023$

_cell_length_b 5.6001685579 
_cell_length_c $\quad 9.0402721007$

cell_angle_alpha 90.0000000000

cell_angle_beta 90.0000000000

_cell_angle_gamma 90.0000000000

_symmetry_space_group_name_H-M "I 2

_symmetry_Int_Tables_number 23

loop

_space_group_symop_id

_space_group_symop_operation_xyz

$1 \mathrm{x}, \mathrm{y}, \mathrm{z}$

$2 \mathrm{x},-\mathrm{y},-\mathrm{z}$

$3-x, y,-z$

$\begin{array}{ll}4 & -x,-y, z \\ 5 & x+1 / 2, y+1 / 2, z+1 / 2\end{array}$

$6 \mathrm{x}+1 / 2,-\mathrm{y}+1 / 2,-\mathrm{z}+1 / 2$

$7-x+1 / 2, y+1 / 2,-z+1 / 2$

$8-x+1 / 2,-y+1 / 2, z+1 / 2$

loop

_atom_site_labe

atom_site_type_symbol

atom_site_symmetry_multiplicity

atom_site_Wyckoff_label

atom_site_fract_x

atom_site_fract_y

atom_site_fract_z

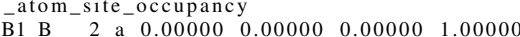

$\begin{array}{lllllllllll} & \text { P1 P } \quad 2 \text { b } & 0.50000 & 0.00000 & 0.00000 & 1.00000\end{array}$

S1 S 8 k $0.21000 \quad 0.200000 .11500 \quad 1.00000$

$\mathrm{BPS}_{4}$ : ABC4_oI12_23_a_b_k - POSCAR

ABC4_oI12_23_a_b_k \& a , b/a,c/a, x3, y3, z3 --params $=5.2501580231$,

$\hookrightarrow 1.06666666665,1.7219047619,0.21,0.2,0.115 \&$ I $222 D_{-}\{2\}^{\wedge}\{8\} \# 23$

$\hookrightarrow$ (abk) \& OI12 \& None \& BPS4 \& \& A. Weiss and H. Sch $\{1 "$ a fer, Z

$\hookrightarrow$ Naturforsch. B 18, 81-82 (1963)

1.00000000000000

$\begin{array}{rrr}-2.62507901155000 & 2.80008427895000 & 4.52013605035000\end{array}$

$\begin{array}{lll}2.62507901155000 & -2.80008427895000 & 4.52013605035000\end{array}$

$2.62507901155000-2.80008427895000-4.52013605035000$

B $\quad P \quad S$

Direct

0.00000000000000

0.00000000000000

0.31500000000000

$-0.08500000000000$

0.08500000000000

$-0.31500000000000$

$0.00000000000000 \quad 0.00000000000000$

$0.50000000000000 \quad 0.50000000000000$

$0.32500000000000 \quad 0.41000000000000$

$-0.09500000000000-0.41000000000000$

$-0.32500000000000-0.01000000000000$

0.01000000000000

B (2a)

$(8 \mathrm{k})$

Weberite $\left(\mathrm{Na}_{2} \mathrm{MgAlF}_{7}\right)$ : AB7CD2_oI44_24_a_b3d_c_ac - CIF

\section{\# CIF file}

data_findsym-output

audit_creation_method FINDSYM

chemical_name_mineral 'Na2MgAlF7,

chemical formula sum 'Al $\mathrm{F} 7 \mathrm{Mg} \mathrm{Na} 2$

loop_

_publ_author_name

'O. Knop'

'T. S. Cameron'

'K. Jochem'

_journal_name_full_name

Journal of Solid State Chemistry

journal_volume 43

journal_year 198

journal_page_first 213

journal_page_last 221

publ_Section_title

What is the true space group of weberite?

\# Found in Pearson's Crystal Data - Crystal Structure Database fo

$\hookrightarrow$ Inorganic Compounds, 2013

aflow_title 'Weberite (Na\$_ $\left.\{2\} \$ M g A l F \$ \_\{7\} \$\right)$ Structure

_aflow_proto 'AB7CD2_oI44_24_a_b3d_c_ac

-aflow_params ,a , b/a, c/a, $x_{-}\{1\}, x_{-}\{2\}, y_{-}\{3\}, z_{-}\{4\}, z_{-}\{5\}, x_{-}\{6\}, y_{-}\{6\}, z_{-}\{6$ $\hookrightarrow\}, x_{-}\{7\}, y_{-}\{7\}, z_{-}\{7\}, x_{-}\{8\}, y_{-}\{8\}, z_{-}\{8\}$

aflow_params_values $7.0501914381,1.035035461,1.41546099291,0.7511$

$\hookrightarrow 0.2496,0.1361,-0.0003,0.5002,0.7501,0.2213,0.3356,0.5662,0.0681$

$\hookrightarrow, 0.1362,-0.0648,0.0709,0.1385$,

aflow_Strukturbericht 'None'

aflow_Pearson 'oI44,

_cell_length_a $\quad 7.0501914381$

_cell_length_b $\quad 7.2971981453$

cell_length_c 9.9792709732

cell_angle_alpha 90.0000000000

cell angle gamma 90.0000000000

symmetry_space_group_name_H-M "I 212121 "

symmetry_Int_Tables_number 24

loop

_space_group_symop_id

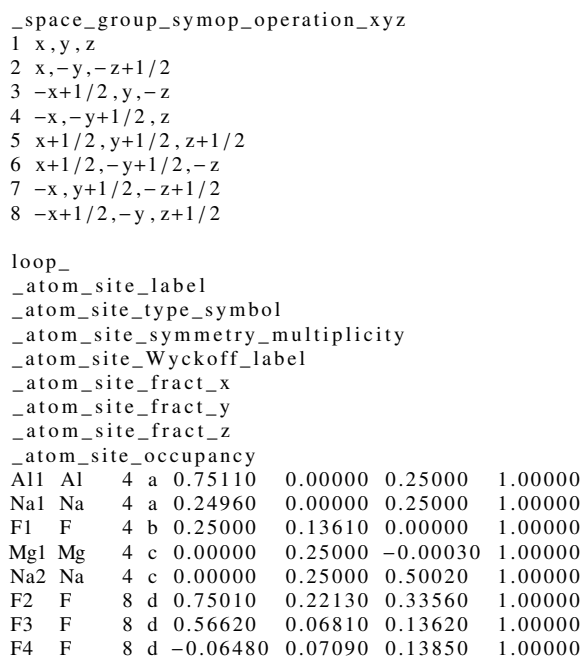

Weberite $\left(\mathrm{Na}_{2} \mathrm{MgAlF}_{7}\right)$ : AB7CD2_oI44_24_a_b3d_c_ac - POSCAR

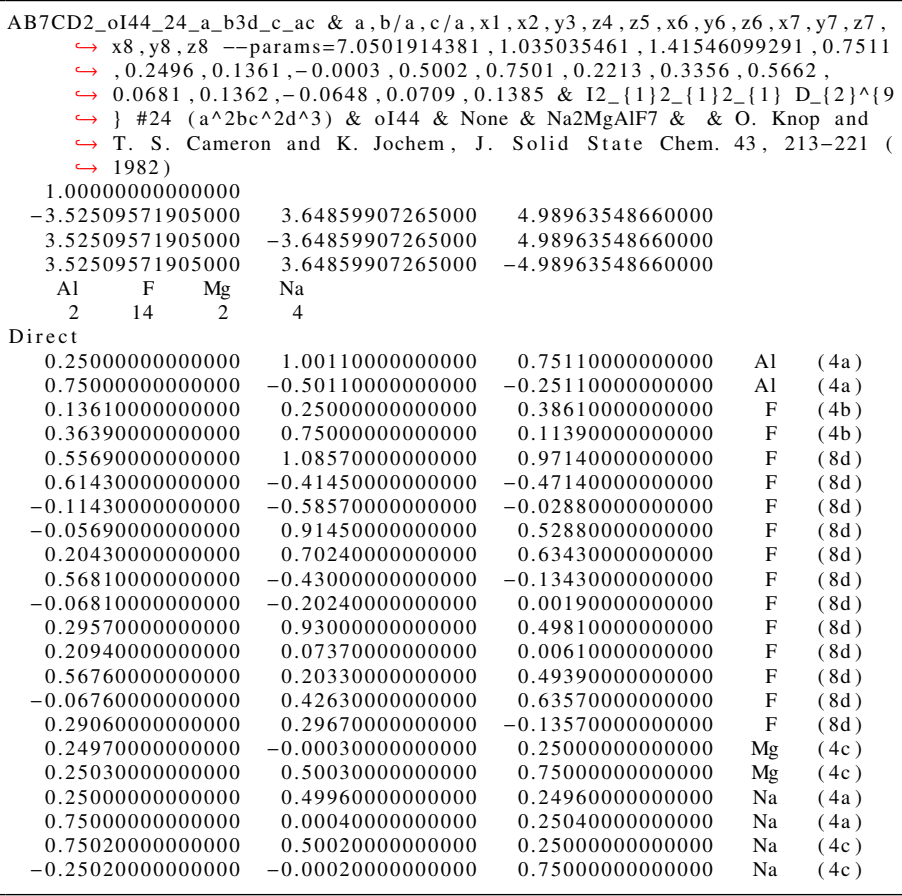

$\mathrm{H}_{2} \mathrm{~S}$ (70 GPa): A2B_oP12_26 abc $\_$ab - CIF

\# CIF file

data_findsym-output

audit_creation_method FINDSYM

chemical_name_mineral 'H2S'

chemical_formula_sum ' $\mathrm{H} 2 \mathrm{~S}$

loop

publ_author_name

'Y. Li'

'J. Hao',

'H. Liu'

Y. Li

journal_name_full_name

Journal of Chemical Physics

journal_volume 140

journal_year 2014

journal_page_first 174712

journal_page_last 174712

-publ_Section_title

The metallization and superconductivity of dense hydrogen sulfide

aflow title 'H\$_2\}\$S (70 GPa) Structure,

aflow_proto 'A2B_oP12_26_abc_ab,

aflow_params $, \mathrm{a}, \mathrm{b} / \mathrm{a}, \mathrm{c} / \mathrm{a}, \mathrm{y}_{-}\{1\}, \mathrm{z}_{-}\{1\}, \mathrm{y}_{-}\{2\}, \mathrm{z}_{-}\{2\}, \mathrm{y}_{-}\{3\}, \mathrm{z}_{-}\{3\}, \mathrm{y}_{-}\{4\}, \mathrm{z}_{-}\{4$ $\hookrightarrow\}, x_{-}\{5\}, y_{-}\{5\}, z_{-}\{5\}$

aflow params values $4.6806,0.627034995513,1.05710806307,0.455,0.858$ $\longrightarrow 0.179,0.623,0.048,0.545,0.375,0.355,0.751,0.119,0.213$,

aflow_Strukturbericht 'None'

aflow_Pearson 'oP12, 


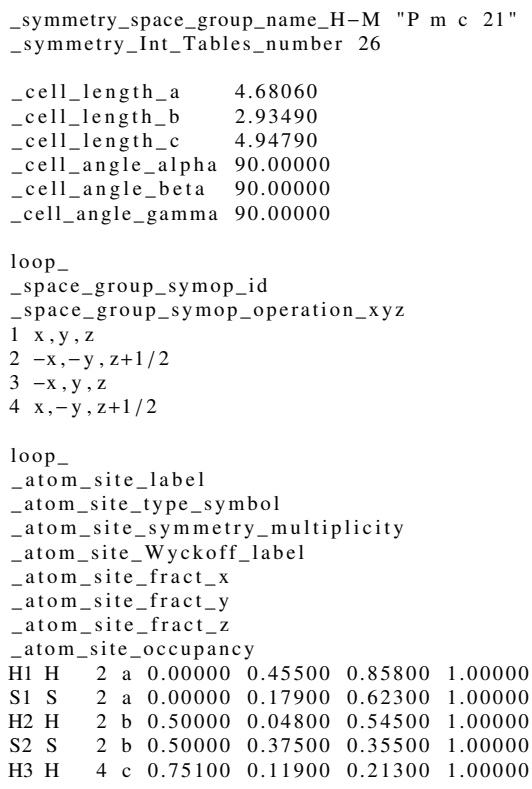

$\mathrm{H}_{2} \mathrm{~S}(70 \mathrm{GPa})$ : A2B_oP12_26_abc_ab - POSCAR

\begin{tabular}{|c|c|c|c|c|}
\hline \multirow{2}{*}{\multicolumn{5}{|c|}{$\begin{array}{c}\text { A2B_oP12_26_abc_ab \& a , b/a,c/a, y1 }, \mathrm{z} 1, \mathrm{y} 2, \mathrm{z} 2, \mathrm{y} 3, \mathrm{z} 3, \mathrm{y} 4, \mathrm{z} 4, \mathrm{x} 5, \mathrm{y} 5, \mathrm{z} 5--\mathrm{params} \\
\hookrightarrow=4.6806,0.627034995513,1.05710806307,0.455,0.858,0.179,0.623\end{array}$}} \\
\hline & & & & \\
\hline \multicolumn{5}{|c|}{$\begin{array}{l}\hookrightarrow=4.6806,0.627034995513,1.05710806307,0.455,0.858,0.179,0.623, \\
\hookrightarrow 0.048,0.545,0.375,0.355,0.751,0.119,0.213 \& P_{0} 2_{-}\{1\} C_{-}\{2 \mathrm{v}\}^{\wedge}\{2\}\end{array}$} \\
\hline \multirow{2}{*}{\multicolumn{5}{|c|}{$\begin{array}{l}\left.a^{\wedge} 2 b^{\wedge} 2 c\right) \& \\
\text { Phys. } 140,\end{array}$}} \\
\hline & & & & \\
\hline \multicolumn{5}{|l|}{1.00000000000000} \\
\hline 4.68060000000000 & 0.00000000000000 & 0.00000000000000 & & \\
\hline 0.00000000000000 & 2.93490000000000 & 0.00000000000000 & & \\
\hline 0.00000000000000 & 0.00000000000000 & 4.94790000000000 & & \\
\hline $\mathrm{H}$ & & & & \\
\hline 8 & & & & \\
\hline \multicolumn{5}{|l|}{ Direct } \\
\hline 0.00000000000000 & 0.45500000000000 & 0.85800000000000 & $\mathrm{H}$ & (2a) \\
\hline 0.00000000000000 & -0.45500 & 00000000 & $H$ & (2a) \\
\hline 0.50000000 & 0.04 & 0.5 & $\mathrm{H}$ & (2 \\
\hline 0.500000000 & 000000 & 00000000 & $\mathrm{H}$ & $(2 b)$ \\
\hline 0.75100000000000 & 0.11900000000000 & 0.21300000000000 & $\mathrm{H}$ & $(4 c)$ \\
\hline-0.75100000000000 & -0.11900000000000 & 0.71300000000000 & $\mathrm{H}$ & $(4 c)$ \\
\hline 0.75100000000000 & -0.11900000000000 & 0.71300000000000 & $\mathrm{H}$ & $(4 c)$ \\
\hline-0.75100000000000 & 0.11900000000000 & 0.21300000000000 & $\mathrm{H}$ & $(4 c)$ \\
\hline 0.00000000000000 & 0.17900000000000 & 0.62300000000000 & $\mathrm{~s}$ & (2a) \\
\hline 0.00000000000000 & -0.17900000000000 & 1.12300000000000 & $\mathrm{~s}$ & (2a) \\
\hline 0.50000000000000 & 0.37500000000000 & 0.35500000000000 & $\mathrm{~s}$ & $(2 b)$ \\
\hline 0.50000000000000 & -0.37500000000000 & 0.85500000000000 & $\mathrm{~s}$ & (2b) \\
\hline
\end{tabular}

$\beta-\mathrm{SeO}_{2}:$ A2B_oP12_26_abc_ab - CIF

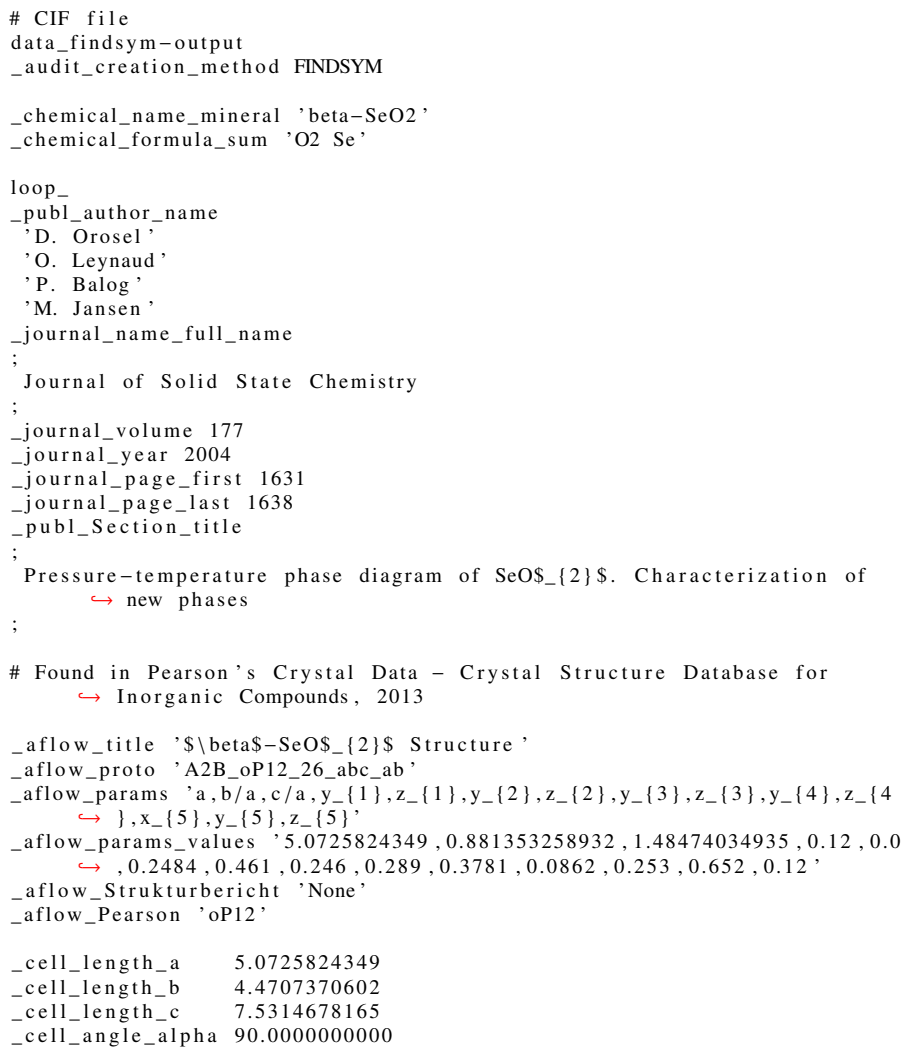

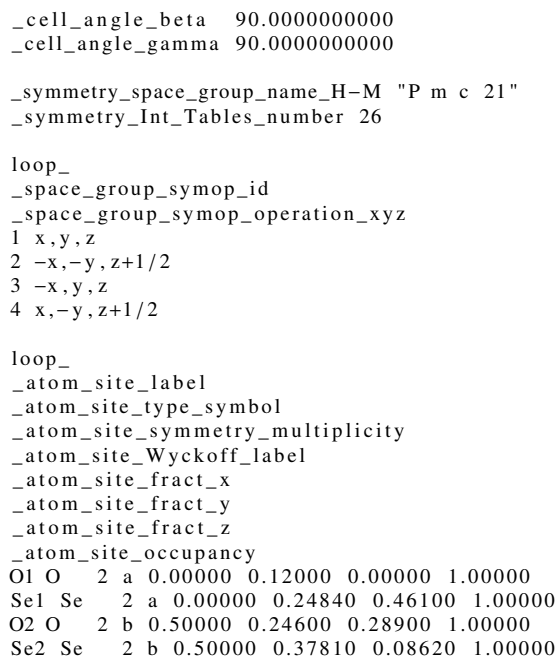

$\beta-\mathrm{SeO}_{2}$ : A2B_oP12_26_abc_ab - POSCAR

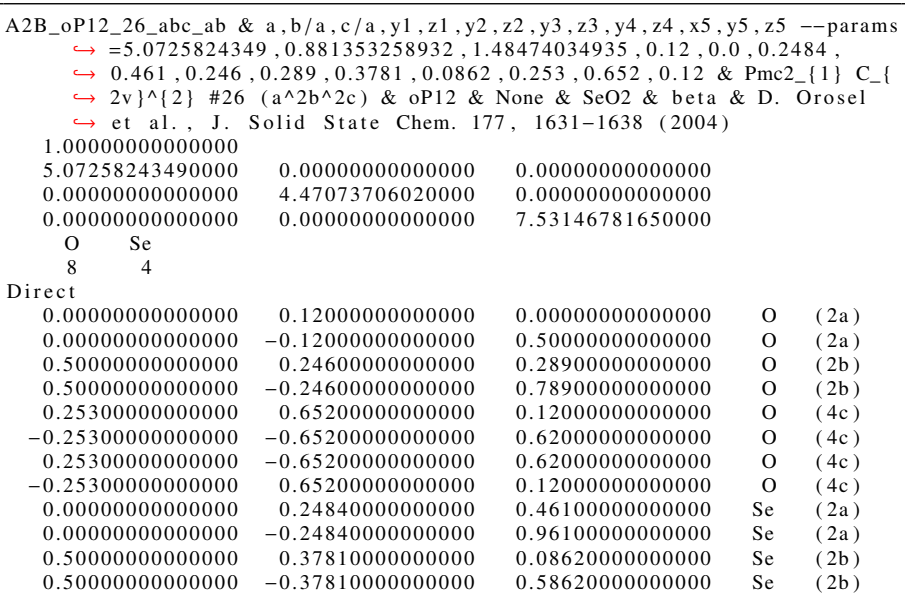

TIP 5 : A5B_oP24_26_3a3b2c_ab - CIF

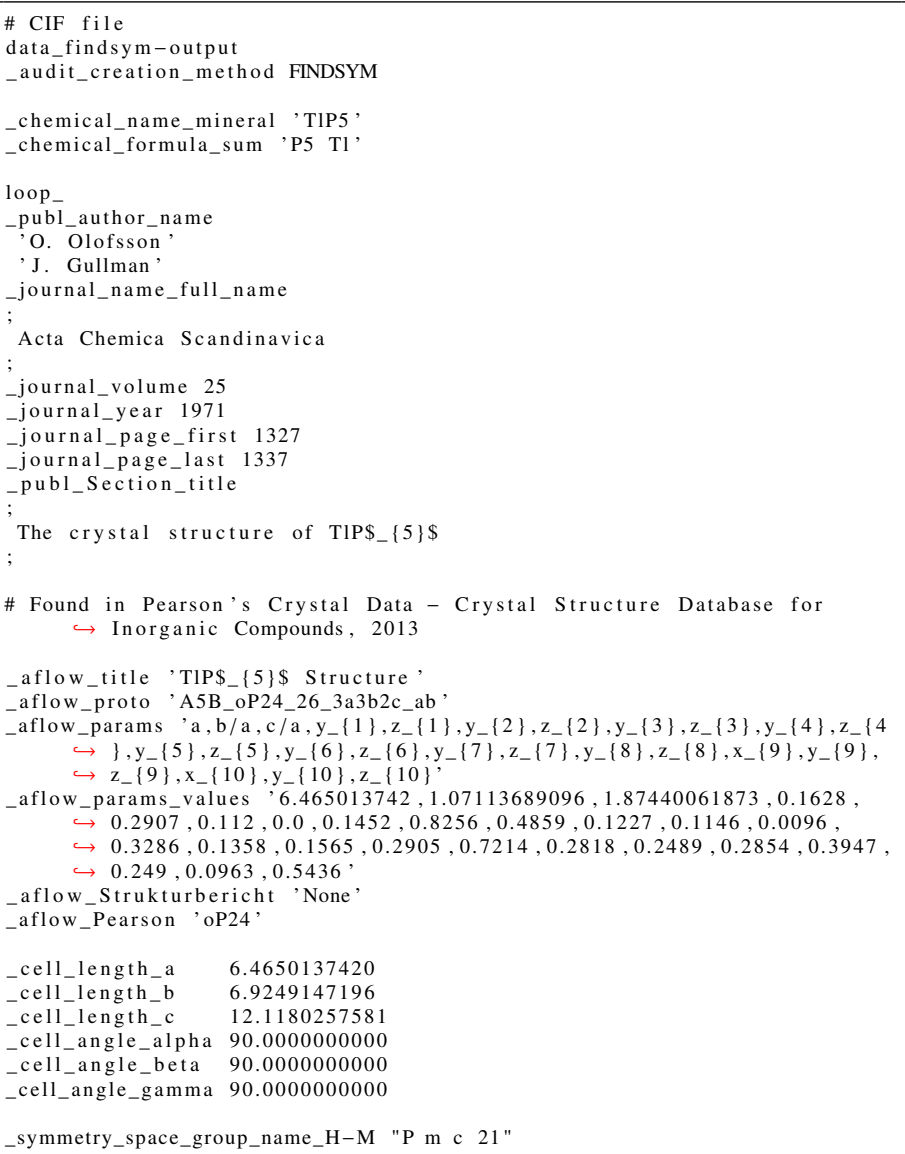




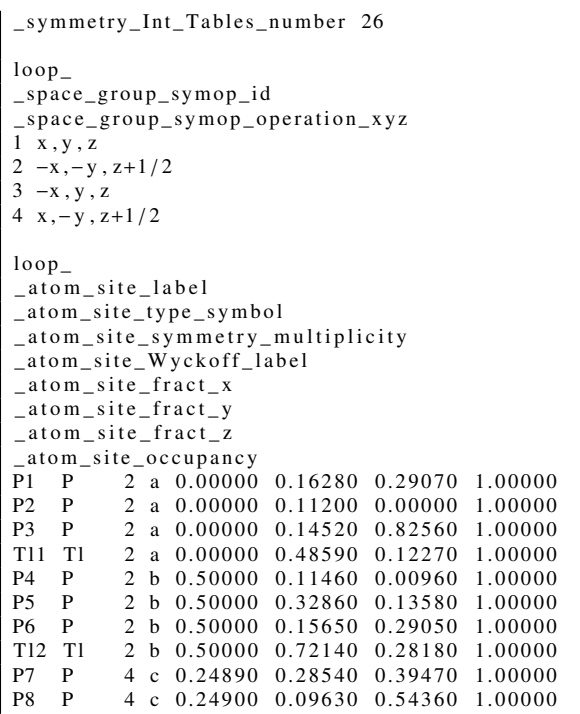

$\mathrm{TIP}_{5}$ : A5B_oP24_26_3a3b2c_ab - POSCAR

\begin{tabular}{|c|c|c|c|c|}
\hline \multirow{2}{*}{\multicolumn{5}{|c|}{$\hookrightarrow, \mathrm{z} 7, \mathrm{y} 8, \mathrm{z} 8, \mathrm{x} 9, \mathrm{y} 9, \mathrm{z} 9, \mathrm{x} 10, \mathrm{y} 10, \mathrm{z} 10-$-params $=6.465013742}}$, \\
\hline & & & & \\
\hline \multicolumn{5}{|c|}{$\hookrightarrow 1.07113689096,1.87440061873,0.1628,0.2907,0.112,0.0,0.1452}$, \\
\hline$\hookrightarrow 0.8256,0.485$ & \multicolumn{4}{|c|}{$9,0.1227,0.1146,0.0096,0.3286,0.1358,0.1565,0.2905$, } \\
\hline$\hookrightarrow 0.7214,0.2818$ & \\
\hline$\hookrightarrow 1\} \mathrm{C}_{-}\{2 \mathrm{v}\}^{\wedge}\{2$ & & & & \\
\hline$\hookrightarrow$ Olofsson and & \multicolumn{4}{|c|}{ 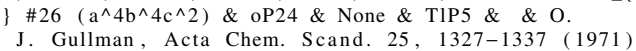 } \\
\hline 1.00000000000000 & \\
\hline 6.46501374200000 & \multicolumn{2}{|c|}{$0.00000000000000 \quad 0.00000000000000$} & & \\
\hline 0.00000000000000 & \multicolumn{4}{|c|}{$6.92491471960000 \quad 0.00000000000000$} \\
\hline 0.00000000000000 & \multirow[t]{3}{*}{0.00000000000000} & \multirow{2}{*}{\multicolumn{3}{|c|}{12.11802575810000}} \\
\hline & & & & \\
\hline 20 & & & & \\
\hline \multicolumn{5}{|l|}{ Direct } \\
\hline 0.00000000000000 & 0.16280000000000 & 0.29070000000000 & $\mathrm{P}$ & (2a) \\
\hline 0.00000000000000 & -0.16280000000000 & 0.79070000000000 & $\mathrm{P}$ & (2a) \\
\hline 0.00000000000000 & 0.11200000000000 & 0.00000000000000 & $\mathrm{P}$ & (2a) \\
\hline 0.00000000000000 & -0.11200000000000 & 0.50000000000000 & $\mathrm{P}$ & (2a) \\
\hline 0.00000000000000 & 0.14520000000000 & 0.82560000000000 & $\mathrm{P}$ & (2a) \\
\hline 0.00000000000000 & -0.14520000000000 & 1.32560000000000 & $\mathrm{P}$ & (2a) \\
\hline 0.50000000000000 & 0.11460000000000 & 0.00960000000000 & $\mathrm{P}$ & $(2 b)$ \\
\hline 0.50000000000000 & -0.11460000000000 & 0.50960000000000 & $\mathrm{P}$ & $(2 b)$ \\
\hline 0.50000000000000 & 0.32860000000000 & 0.13580000000000 & $\mathrm{P}$ & $(2 b)$ \\
\hline 0.50000000000000 & -0.32860000000000 & 0.63580000000000 & $\mathrm{P}$ & $(2 b)$ \\
\hline 0.50000000000000 & 0.15650000000000 & 0.29050000000000 & & $(2 b)$ \\
\hline 0.50000000000000 & -0.15650000000000 & 0.79050000000000 & $\mathrm{P}$ & $(2 b)$ \\
\hline 0.24890000000000 & 0.28540000000000 & 0.39470000000000 & $\mathrm{P}$ & $(4 c)$ \\
\hline-0.24890000000000 & -0.28540000000000 & 0.89470000000000 & $\mathrm{P}$ & $(4 c)$ \\
\hline 0.24890000000000 & -0.28540000000000 & 0.89470000000000 & $\mathrm{P}$ & $(4 c)$ \\
\hline-0.24890000000000 & 0.28540000000000 & 0.39470000000000 & $\mathrm{P}$ & $(4 c)$ \\
\hline 0.24900000000000 & 0.09630000000000 & 0.54360000000000 & $\mathrm{P}$ & $(4 c)$ \\
\hline-0.24900000000000 & -0.09630000000000 & 1.04360000000000 & $\mathrm{P}$ & $(4 c)$ \\
\hline 0.24900000000000 & -0.09630000000000 & 1.04360000000000 & $\mathrm{P}$ & $(4 c)$ \\
\hline-0.24900000000000 & 0.09630000000000 & 0.54360000000000 & $\mathrm{P}$ & $(4 c)$ \\
\hline 0.00000000000000 & 0.48590000000000 & 0.12270000000000 & $\mathrm{Tl}$ & (2a) \\
\hline 0.00000000000000 & -0.48590000000000 & 0.62270000000000 & 11 & $(2 a)$ \\
\hline 0.50000000000000 & 0.72140000000000 & 0.28180000000000 & $\mathrm{Tl}$ & (2b) \\
\hline 0.50000000000000 & -0.72140000000000 & 0.78180000000000 & $\mathrm{Tl}$ & $(2 b)$ \\
\hline
\end{tabular}

$\mathrm{Ca}_{4} \mathrm{Al}_{6} \mathrm{O}_{16} \mathrm{~S}$ : A6B4C16D_oP108_27_abcd4e_4e_16e_e - CIF aflow params, a, b/a,c/a, z $\left.\{1\}, z_{-}\{2\}, z\{3\}, z_{-}\{4\}, x_{-}\{5\}, y_{-}\{5\}, z_{-15}\right\}, x_{-}\{6$ $\hookrightarrow\}, y_{-}\{6\}, z_{-}\{6\}, x_{-}\{7\}, y_{-}\{7\}, z_{-}\{7\}, x_{-}\{8\}, y_{-}\{8\}, z_{-}\{8\}, x_{-}\{9\}, y_{-}\{9\}$ $\hookrightarrow \mathrm{z}_{-}\{9\}, \mathrm{x}_{-}\{10\}, \mathrm{y}_{-}\{10\}, \mathrm{z}_{-}\{10\}, \mathrm{x}_{-}\{11\}, \mathrm{y}_{-}\{11\}, \mathrm{z}_{-}\{11\}, \mathrm{x}_{-}\{12\}, \mathrm{y}_{-}\{12\}$ $\hookrightarrow \mathrm{z}_{-}\{12\}, \mathrm{x}_{-}\{13\}, \mathrm{y}_{-}\{13\}, \mathrm{z}_{-}\{13\}, \mathrm{x}_{-}\{14\}, \mathrm{y}_{-}\{14\}, \mathrm{z}_{-}\{14\}, \mathrm{x}_{-}\{15\}, \mathrm{y}_{-}\{15\}$ $\rightarrow \mathrm{z}_{-}\{15\}, \mathrm{x}_{-}\{16\}, \mathrm{y}_{-}\{16\}, \mathrm{z}_{-}\{16\}, \mathrm{x}_{-}\{17\}, \mathrm{y}_{-}\{17\}, \mathrm{z}_{-}\{17\}, \mathrm{x}_{-}\{18\}, \mathrm{y}_{-}\{18\}$ $\rightarrow \mathrm{z}_{-}\{18\}, \mathrm{x}_{-}\{19\}, \mathrm{y}_{-}\{19\}, \mathrm{z}_{-}\{19\}, \mathrm{x}_{-}\{20\}, \mathrm{y}_{-}\{20\}, \mathrm{z}_{-}\{20\}, \mathrm{x}_{-}\{21\}, \mathrm{y}_{-}\{21\}$, $\hookrightarrow \mathrm{z}_{-}\{21\}, \mathrm{x}_{-}\{22\}, \mathrm{y}_{-}\{22\}, \mathrm{z}_{-}\{22\}, \mathrm{x}_{-}\{23\}, \mathrm{y}_{-}\{23\}, \mathrm{z}_{-}\{23\}, \mathrm{x}_{-}\{24\}, \mathrm{y}_{-}\{24\}$, $\rightarrow \mathrm{z}_{-}\{24\}, \mathrm{x}_{-}\{25\}, \mathrm{y}_{-}\{25\}, \mathrm{z}_{-}\{25\}, \mathrm{x}_{-}\{26\}, \mathrm{y}_{-}\{26\}, \mathrm{z}_{-}\{26\}, \mathrm{x}_{-}\{27\}, \mathrm{y}_{-}\{27\}$, $\hookrightarrow \mathrm{z}_{-}\{27\}, \mathrm{x}_{-}\{28\}, \mathrm{y}_{-}\{28\}, \mathrm{z}_{-}\{28\}, \mathrm{x}_{-}\{29\}, \mathrm{y}_{-}\{29\}, \mathrm{z}_{-}\{29\}$

aflow params values $, 13.0409854622,0.999877309088,0.703110981603,0.0$, $\hookrightarrow 0.005,0.03,0.05,0.373,0.379,0.296,0.122,0.125,0.249,0.369,0.129$ $\hookrightarrow, 0.28,0.122,0.376,0.26,0.058,0.751,0.449,0.237,0.574,0.086$,

$\hookrightarrow 0.257,0.012,0.554,0.483,0.247,0.028,0.729,0.183,0.412,0.162$

$\hookrightarrow 0.684,0.26,0.23,0.164,0.679,0.651,0.299,0.6217,0.4,0.254,0.238$, $\hookrightarrow 0.433,0.091,0.44,0.445,0.395,0.455,0.245,0.09,0.304,0.404,0.057$ $\hookrightarrow, 0.126,0.093,0.054,0.102,0.243,0.402,0.336,0.099,0.466,0.124$

$\rightarrow 0.392,0.467,0.154,0.103,0.253,0.191,0.047,0.385,0.425,0.052$

$\hookrightarrow 0.111,0.41,0.74634,0.2513,0.28218$, aflow Strukturbericht 'None, aflow_Pearson, oP108,

$\begin{array}{ll} & 13.0393854518 \\ \text { cell_length_c } & 9.1692600894\end{array}$

cell_angle_alpha 90.0000000000

cell angle beta 90.0000000000

cell_angle_gamma 90.0000000000

symmetry_space_group_name_H-M "P c c 2"

symmetry_Int_Tables_number 27

loop

space_group_symop_id

space_group_symop_operation_xyz

$\mathrm{x}, \mathrm{y}, \mathrm{z}$

$2-\mathrm{x},-\mathrm{y}, \mathrm{z}$

$3-\mathrm{x}, \mathrm{y}, \mathrm{z}+1 / 2$

$4 \mathrm{x},-\mathrm{y}, \mathrm{z}+1 / 2$

loop

atom_site_label

atom_site_type_symbol

atom_site_symmetry_multiplicity

_atom_site_Wyckoff_label

atom_site_fract_x

atom_site_fract_y

atom_site_fract_z

tom_site_occupancy

$\begin{array}{lllllllll}\text { All } & \mathrm{Al} & 2 & \text { a } & 0.00000 & 0.00000 & 0.00000 & 1.00000\end{array}$

$\begin{array}{llllllll}\mathrm{Al} 2 & \mathrm{Al} & 2 & \mathrm{~b} & 0.00000 & 0.50000 & 0.00500 & 1.00000\end{array}$

$\begin{array}{lllllllll}\mathrm{Al} 3 & \mathrm{Al} & 2 & \mathrm{c} & 0.50000 & 0.00000 & 0.03000 & 1.00000\end{array}$

$\begin{array}{lllllllll}\mathrm{Al} 4 & \mathrm{Al} & 2 & \mathrm{~d} & 0.50000 & 0.50000 & 0.05000 & 1.00000\end{array}$

$\begin{array}{llllllll}\mathrm{A} 15 & \mathrm{Al} & 4 & \mathrm{e} & 0.37300 & 0.37900 & 0.29600 & 1.00000\end{array}$

$\begin{array}{llllllll}\mathrm{A} 16 & \mathrm{Al} & 4 & \mathrm{e} & 0.12200 & 0.12500 & 0.24900 & 1.00000\end{array}$

$\begin{array}{llllllll}\mathrm{Al} 7 \mathrm{Al} & 4 & \mathrm{e} & 0.36900 & 0.12900 & 0.28000 & 1.00000\end{array}$

$\begin{array}{llllllll}\mathrm{A} 18 & \mathrm{Al} & 4 & \mathrm{e} & 0.12200 & 0.37600 & 0.26000 & 1.00000\end{array}$

$\begin{array}{llllllll}\mathrm{Ca} 1 \mathrm{Ca} & 4 & \mathrm{e} & 0.05800 & 0.75100 & 0.44900 & 1.00000\end{array}$

$\mathrm{Ca} 2 \mathrm{Ca} \quad 4$ e $0.23700 \quad 0.57400 \quad 0.08600 \quad 1.00000$

$\begin{array}{llllllll}\mathrm{Ca} 3 & \mathrm{Ca} & 4 & \mathrm{e} & 0.25700 & 0.01200 & 0.55400 & 1.00000\end{array}$

$\begin{array}{lllllll}\mathrm{Ca} 4 \mathrm{Ca} & 4 & \text { e } & 0.48300 & 0.24700 & 0.02800 & 1.00000\end{array}$

$\begin{array}{lllllllll}\mathrm{O} 1 & \mathrm{O} & 4 & \mathrm{e} & 0.72900 & 0.18300 & 0.41200 & 1.00000\end{array}$

$\begin{array}{lllllllll}\mathrm{O} 2 & \mathrm{O} & 4 & \mathrm{e} & 0.16200 & 0.68400 & 0.26000 & 1.00000\end{array}$

$\begin{array}{lllllllll}\mathrm{O} 3 & \mathrm{O} & 4 & \mathrm{e} & 0.23000 & 0.16400 & 0.67900 & 1.00000\end{array}$

$\begin{array}{lllllllllll}\mathrm{O} 4 & \mathrm{O} & 4 & \mathrm{e} & 0.23000 & 0.16400 & 0.67900 & 1.00000\end{array}$

$\begin{array}{llllllll}04 & \mathrm{O} & 4 & \mathrm{e} & 0.65100 & 0.29500 & 0.62170 & 1.00000 \\ 05 & \mathrm{O} & 4 & \mathrm{e} & 0.40000 & 0.25400 & 0.23800 & 1.00000\end{array}$

$\begin{array}{llllllllll}05 & \mathrm{O} & 4 & \mathrm{e} & 0.45000 & 0.25400 & 0.23800 & 1.00000\end{array}$

$\begin{array}{lllllllll}06 & 0 & 4 & e & 0.43300 & 0.09100 & 0.44000 & 1.00000\end{array}$

08 O 4 - 4 e $0.245000 .090000 .30400-1.00000$

$\begin{array}{llllllllll}08 & \mathrm{O} & 4 & \mathrm{e} & 0.24500 & 0.09000 & 0.30400 & 1.00000\end{array}$

$\begin{array}{llllllllll}09 & \mathrm{O} & 4 & \mathrm{e} & 0.40400 & 0.05700 & 0.12600 & 1.00000\end{array}$

$\begin{array}{llllllllll}\mathrm{O} 10 & \mathrm{O} & 4 & \mathrm{e} & 0.09300 & 0.05400 & 0.10200 & 1.00000\end{array}$

$\begin{array}{llllllllll}\mathrm{O} 11 & \mathrm{O} & 4 & \mathrm{e} & 0.24300 & 0.40200 & 0.33600 & 1.00000\end{array}$

$\begin{array}{lllllllll}\mathrm{O} 12 & \mathrm{O} & 4 & \mathrm{e} & 0.09900 & 0.46600 & 0.12400 & 1.00000\end{array}$

$\begin{array}{lllllllll}\mathrm{O} 13 & \mathrm{O} & 4 & \mathrm{e} & 0.39200 & 0.46700 & 0.15400 & 1.00000\end{array}$ 4 e $0.10300 \quad 0.25300 \quad 0.19100 \quad 1.00000$ 4 e $0.04700 \quad 0.38500 \quad 0.42500 \quad 1.00000$

$\begin{array}{llllllll}016 & \mathrm{O} & 4 & \mathrm{e} & 0.05200 & 0.11100 & 0.41000 & 1.00000\end{array}$

$\begin{array}{llllllll}\mathrm{S} 1 & \mathrm{~S} & 4 & \mathrm{e} & 0.74634 & 0.25130 & 0.28218 & 1.00000\end{array}$

$\mathrm{Ca}_{4} \mathrm{Al}_{6} \mathrm{O}_{16} \mathrm{~S}:$ A6B4C16D_oP108_27_abcd4e_4e_16e_e - POSCAR

A6B4C16D_oP108_27_abcd4e_4e_16e_e \& a , b/a,c/a,z1,z2,z3, z4,x5,y5,z5, x6,y6 $\hookrightarrow, \mathrm{z} 6, \mathrm{x} 7, \mathrm{y} 7, \mathrm{z} 7, \mathrm{x} 8, \mathrm{y} 8, \mathrm{z} 8, \mathrm{x} 9, \mathrm{y} 9, \mathrm{z} 9, \mathrm{x} 10, \mathrm{y} 10, \mathrm{z} 10, \mathrm{x} 11, \mathrm{y} 11, \mathrm{z} 11, \mathrm{x} 12, \mathrm{y} 12$, $\hookrightarrow \mathrm{z} 12, \mathrm{x} 13, \mathrm{y} 13, \mathrm{z} 13, \mathrm{x} 14, \mathrm{y} 14, \mathrm{z} 14, \mathrm{x} 15, \mathrm{y} 15, \mathrm{z} 15, \mathrm{x} 16, \mathrm{y} 16, \mathrm{z} 16, \mathrm{x} 17, \mathrm{y} 17, \mathrm{z} 17$ $\hookrightarrow, \mathrm{x} 18, \mathrm{y} 18, \mathrm{z} 18, \mathrm{x} 19, \mathrm{y} 19, \mathrm{z} 19, \mathrm{x} 20, \mathrm{y} 20, \mathrm{z} 20, \mathrm{x} 21, \mathrm{y} 21, \mathrm{z} 21, \mathrm{x} 22, \mathrm{y} 22, \mathrm{z} 22$, $\hookrightarrow \mathrm{x} 23, \mathrm{y} 23, \mathrm{z} 23, \mathrm{x} 24, \mathrm{y} 24, \mathrm{z} 24, \mathrm{x} 25, \mathrm{y} 25, \mathrm{z} 25, \mathrm{x} 26, \mathrm{y} 26, \mathrm{z} 26, \mathrm{x} 27, \mathrm{y} 27, \mathrm{z} 27, \mathrm{x} 28$

$\hookrightarrow, \mathrm{y} 28, \mathrm{z} 28, \mathrm{x} 29, \mathrm{y} 29, \mathrm{z} 29--$ params $=13.0409854622,0.999877309088$,

$\hookrightarrow 0.125,0.249,0.369,0.129,0.28,0.122,0.376,0.26,0.058,0.751,0.449$

$\hookrightarrow, 0.237,0.574,0.086,0.257,0.012,0.554,0.483,0.247,0.028,0.729$,

$\hookrightarrow 0.183,0.412,0.162,0.684,0.26,0.23,0.164,0.679,0.651,0.299$

$\hookrightarrow 0.6217,0.4,0.254,0.238,0.433,0.091,0.44,0.445,0.395,0.455,0.245$

$\hookrightarrow, 0.09,0.304,0.404,0.057,0.126,0.093,0.054,0.102,0.243,0.402$

$\hookrightarrow 0.336,0.099,0.466,0.124,0.392,0.467,0.154,0.103,0.253,0.191$

$\hookrightarrow 0.047,0.385,0.425,0.052,0.111,0.41,0.74634,0.2513,0.28218 \&$

$\hookrightarrow \operatorname{Pcc} 2 \mathrm{C}_{-}\{2 \mathrm{v}\}^{\wedge}\{3\} \# 27\left(\mathrm{abcde}^{\wedge} 25\right)$ \& oP108 \& None \& Ca4A16O16S \& \&

$\hookrightarrow$ N. J. Calos et al., J. Solid State Chem. 119, 1-7 (1995)

1.00000000000000

$\begin{array}{lll}13.04098546220000 & 0.00000000000000 & 0.00000000000000\end{array}$

$\begin{array}{lll}0.00000000000000 & 13.03938545180000 & 0.00000000000000\end{array}$

$\begin{array}{lll}0.00000000000000 & 0.00000000000000 & 9.16926008940000\end{array}$

$\begin{array}{llrr}\mathrm{Al} & \mathrm{Ca} & \mathrm{O} & \mathrm{S} \\ 24 & 16 & 64 & 4\end{array}$

Dire

0.00000000000000

0.00000000000000

0.00000000000000

0.00000000000000

Al (2a)

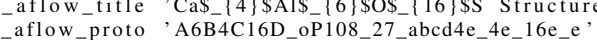

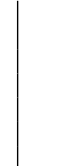


0.00000000000000 0.00000000000000 0.50000000000000 0.50000000000000 0.50000000000000 0.50000000000000 0.37300000000000 $-0.37300000000000$ 0.37300000000000 $-0.37300000000000$ 0.12200000000000 0.12200000000000 0.36900000000000 0.36900000000000 0.36900000000000 0.36900000000000 0.12200000000000 0.12200000000000 0.12200000000000 0.12200000000000 0.05800000000000 $-0.05800000000000$ 0.05800000000000 $-0.05800000000000$ 0.23700000000000 $-0.23700000000000$ 0.23700000000000 $-0.23700000000000$ 0.25700000000000 $-0.25700000000000$ 0.25700000000000 0.25700000000000 0.48300000000000 $-0.48300000000000$ 0.48300000000000 $-0.48300000000000$ 0.72900000000000 $-0.72900000000000$ 0.72900000000000 $-0.72900000000000$ 0.16200000000000 $-0.16200000000000$ 0.16200000000000 $-0.16200000000000$ 0.23000000000000 $-0.23000000000000$ 0.23000000000000 $-0.23000000000000$ 0.65100000000000 $-0.65100000000000$ 0.65100000000000 $-0.65100000000000$ 0.40000000000000 $-0.40000000000000$ 0.40000000000000 $-0.40000000000000$ 0.43300000000000 $-0.43300000000000$ 0.43300000000000 $-0.43300000000000$ 0.44500000000000 $-0.44500000000000$ 0.44500000000000 $-0.44500000000000$ 0.24500000000000 $-0.24500000000000$ 0.24500000000000 $-0.24500000000000$ 0.40400000000000 $-0.40400000000000$ 0.40400000000000 $-0.40400000000000$ 0.09300000000000 $-0.09300000000000$ 0.09300000000000 $-0.09300000000000$ 0.24300000000000 $-0.24300000000000$ 0.24300000000000 $-0.24300000000000$ 0.09900000000000 0.09900000000000 0.09900000000000 $-0.09900000000000$ 0.39200000000000 0.39200000000000 0.39200000000000 $-0.39200000000000$ 0.10300000000000 $-0.10300000000000$ 0.10300000000000 $-0.10300000000000$ 0.04700000000000 $-0.04700000000000$ 0.04700000000000 $-0.04700000000000$ 0.05200000000000 $-0.05200000000000$ 0.05200000000000 $-0.05200000000000$ 0.74634000000000 0.74634000000000
0.74634000000000
0.50000000000000 0.00000000000000 0.00000000000000 0.50000000000000 0.50000000000000 0.37900000000000 $-0.3790000000000$ $-0.37900000000000$ 0.37900000000000 0.12500000000000 $-0.12500000000000$ 0.1250000000000 0.12900000000000 $-0.12900000000000$ $-0.12900000000000$ 0.12900000000000 0.37600000000000 $-0.37600000000000$ $-0.37600000000000$ 0.37600000000000 0.7510000000000 51000000000 0.7510000000000 $-.751000000000$ 0.57400000000000 $-0.57400000000000$ $-0.57400000000000$ 0.57400000000000 0.01200000000000 $-0.01200000000000$ $-0.01200000000000$ 0.0120000000000 0.0120000000 $-0.24700000000000$ $-0.24700000000000$ 0.2470000000000 0.1830000000000 $-0.1830000000000$ $-0.18300000000000$ 0.1830000000000 .68400000000000 $-0.68400000000000$ $-0.68400000000000$ 0.68400000000000 0.16400000000000 $-0.16400000000000$ $-0.16400000000000$ 0.16400000000000 0.29900000000000 $-0.29900000000000$ $-0.29900000000000$ 0.29900000000000 0.25400000000000 $-0.25400000000000$ $-0.25400000000000$ 0.25400000000000 0.09100000000000 $-0.09100000000000$ $-0.09100000000000$ 0.3950000000000 $-0.39500000000000$ $-0.39500000000000$ 0.3950000000000 0.0900000000000 $-0.09000000000000$ $-0.09000000000000$ 0.09000000000000 0.05700000000000 0.05700000000000 $-0.0570000000000$ 0.05700000000 作 $-0.05400000000000$ $-0.0540000000000$ 0.40200000000000 $-0.40200000000000$ $-0.40200000000000$ 0.40200000000000 0.46600000000000 $-0.466000$ $-0.46600000000000$ 0.46600000000000 0.46700000000000 $-0.46700000000000$ $-0.46700000000000$ 0.4670000000000 0.25300000000000 $-0.25300000000000$ $-0.2530000000000$ 0.25300000000000 0.38500000000000 $-0.38500000000000$ 0.38500000000000 0.11100000000000 $-0.11100000000000$ 0.11100000000000 0.11100000000000 0.2513000000000 $-0.2513000000000$ $-0.25130000000000$

0.0050000000000 0.5050000000000 0.53000000000 0.53000000000000 0.0500000000000 0.55000000000000 0.29600000000000 0.29600000000000 0.79600000000000 0.79600000000000 0.24900000000000 0.24900000000000 0.74900000000000 0.7490000000000 0.28000000000000 0.28000000000000 0.78000000000000 0.780000000000 0.780000000000 0.26000000000000 0.26000000000000 0.76000000000000 0.7600000000000 0.44900000000000 0.44900000000000 0.94900000000000 0.9490000000000 0.08600000000000 0.0860000000000 0.58600000000000 0.58600000000000 0.55400000000000 0.5540000000000 . 1.05400000000000 0.02800000000000 0.0280000000000 0.02800000000000 0.52800000000000 0.5280000000000 0.41200000000000 0.4120000000000 0.91200000000000 0.91200000000000 0.26000000000000 0.26000000000000 0.76000000000000 0.76000000000000 0.67900000000000 0.67900000000000 1.17900000000000 1.17900000000000 0.62170000000000 0.62170000000000 .621700000000 1.12170000000000 1.12170000000000 0.2380000000000 0.23800000000000 0.73800000000000 0.73800000000000 0.4400000000000 0.44000000000000 0.94000000000000 0.94000000000000 0.45500000000000 0.4550000000000 0.9550000000000 0.95500000000000 0.30400000000000 0.30400000000000 0.80400000000000 0.80400000000000 0.80400000000000 0.12600000000000 0.12600000000000 0.62600000000000 0.6260000000000 0.10200000000000 0.10200000000000 0.60200000000000 0.60200000000000 0.33600000000000 0.33600000000000 0.8360000000000 


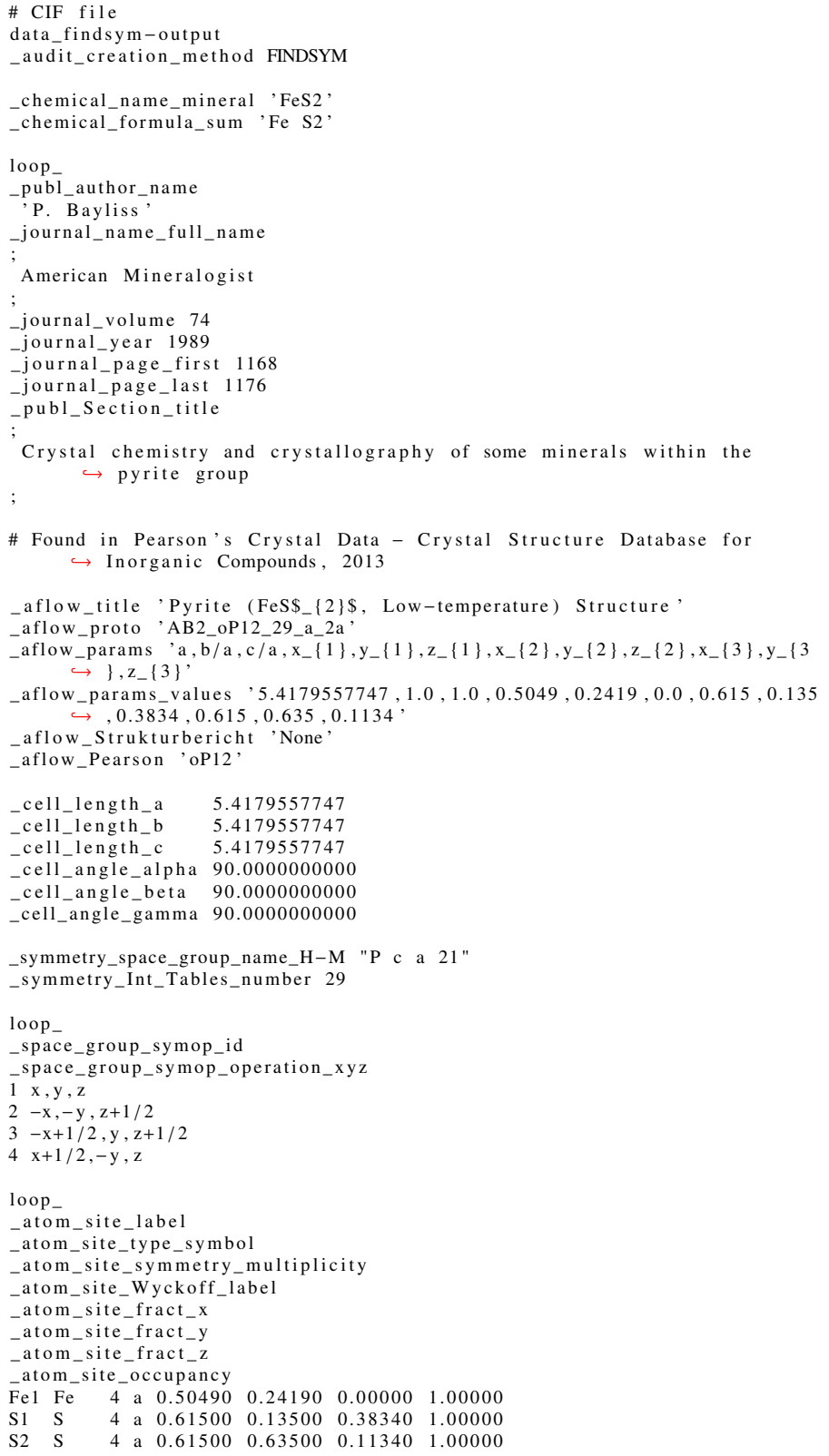

Pyrite ( $\mathrm{FeS}_{2}$, Low-temperature): AB2_oP12_29_a_2a - POSCAR

AB2_oP12_29_a_2a \& a,b/a,c/a, x1,y1,z1, x2,y2,z2, x3,y3,z3 --params= $\hookrightarrow 5.4179557747,1.0,1.0,0.5049,0.2419,0.0,0.615,0.135,0.3834,0.615$ $\hookrightarrow, 0.635,0.1134 \& \mathrm{Pca}_{-}\{1\} \mathrm{C}_{-}\{2 \mathrm{v}\}^{\wedge}\{5\} \# 29\left(\mathrm{a}^{\wedge} 3\right) \& \mathrm{oP} 12 \&$ None \& $\hookrightarrow \mathrm{FeS} 2$ \& \& P. Bayliss, Am. Mineral. 74, 1168-1176 (1989)

$5.41795577470000-0.00000000000000-0.00000000000000$ $\begin{array}{lll}0.00000000000000 & 5.41795577470000 & 0.00000000000000\end{array}$

$\begin{array}{lll}0.00000000000000 & 0.00000000000000 & 5.41795577470000\end{array}$ $\mathrm{Fe} \quad \mathrm{S}$

Direct

0.50490000000000 $-0.50490000000000$ 1.00490000000000 $-0.00490000000000$ 0.61500000000000 $-0.61500000000000$ 1.11500000000000 $-0.11500000000000$ 0.61500000000000 $-0.61500000000000$ 1.11500000000000 $-0.11500000000000$

0.24190000000000 0.24190000000000 0.2419000000000 0.24190000000000 0.13500000000000 $-0.1350000000000$ $-0.1350000000000$ 0.1350000000000 0.63500000000000 $-0.63500000000000$ $-0.63500000000000$ 0.63500000000000
0.00000000000000 0.50000000000000 .00000000000000 0.50000000000000 0.3834000000000 0.88340000000000 0.38340000000000 0.88340000000000 0.11340000000000 0.61340000000000 0.1134000000000 0.61340000000000
M. E. Fleet,

'P. C. Burns

_journal_name_full_name

Canadian Mineralogist

journal_volume 28

journal year 1990

journal_page_first 719

journal_page_last 723

publ Section

Structure and twinning of cobaltite

Found in Pearson's Crystal Data - Crystal Structure Database for $\hookrightarrow$ Inorganic Compounds, 2013

-aflow_title Cobaltite (CoAsS) Structure,

aflow_proto 'ABC_oP12_29_a_a_a

aflow_params $, a, b / a, c / a, x_{-}\{1\}, y_{-}\{1\}, z_{-}\{1\}, x_{-}\{2\}, y_{-}\{2\}, z_{-}\{2\}, x_{-}\{3\}, y_{-}\{3$ $\hookrightarrow\}, z_{-}\{3\}$ '

aflow_params_values ' $5.2594682584,0.963498098863,0.965209125484,0.61885$ $\hookrightarrow, 0.63065,0.11668,0.50496,0.24091,0.0,0.61734,0.13129,0.37996$ '

aflow_Strukturbericht 'None'

aflow_Pearson 'oP12

_cell_length_a $\quad 5.2594682584$

5.0674876680

-

_cell_angle_alpha 90.0000000000

cecli_angle_bela 90.000000000

symmetry_space_group_name_H-M "P c a 21 "

symmetry_Int_Tables_number 29

loop

space_group_symop_id

space_group_symop_operation_xyz

$\mathrm{x}, \mathrm{y}, \mathrm{z}$

$-\mathrm{x},-\mathrm{y}, \mathrm{z}+1 / 2$

$-\mathrm{x}+1 / 2, \mathrm{y}, \mathrm{z}+1 / 2$

$4 \mathrm{x}+1 / 2,-\mathrm{y}, \mathrm{z}$

loop

atom site label

atom_site_type_symbol

atom_site_symmetry_multiplicity

atom site Wyckoff_label

atom_site_fract_x

atom_site_fract_y

atom_site_fract_z

-atom_site_occupancy

As1 As $\quad \begin{array}{llllll}\text { a } & 0.61885 & 0.63065 & 0.11668 & 1.00000\end{array}$

$\begin{array}{llllllll}\text { Co1 Co } & 4 & \text { a } & 0.50496 & 0.24091 & 0.00000 & 1.00000\end{array}$

$\begin{array}{lllllllll}\mathrm{S} 1 & \mathrm{~S} & 4 & \mathrm{a} & 0.61734 & 0.13129 & 0.37996 & 1.00000\end{array}$

Cobaltite (CoAsS): ABC_oP12_29_a_a_a - POSCAR

ABC_oP12_29_a_a_a \& a , b/a,c/a, x1, y1, z1, x2, y2, z2, x3, y3, z3 --params =

$\hookrightarrow 5.2594682584,0.963498098863,0.965209125484,0.61885,0.63065$

$\hookrightarrow 0.11668,0.50496,0.24091,0.0,0.61734,0.13129,0.37996 \&$ Pca2_ $\{1\}$

$\hookrightarrow \mathrm{C}_{-}\{2 \mathrm{v}\}^{\wedge}\{5\} \# 29\left(\mathrm{a}^{\wedge} 3\right) \&$ oP12 \& None \& CoAsS \& \& M. E. Fleet and

$\hookrightarrow$ P. C. Burns, Can. Mineral. 28, 719-723 (1990)

1.00000000000000

$\begin{array}{lll}5.25946825840000 & 0.00000000000000 & 0.00000000000000\end{array}$

$\begin{array}{lll}0.00000000000000 & 5.06748766800000 & 0.00000000000000\end{array}$

$0.00000000000000 \quad 0.00000000000000 \quad 5.07648675820000$

$\begin{array}{rrr}\text { As } & \text { Co } & \text { S } \\ 4 & 4 & 4\end{array}$

Dire

$\begin{array}{lll}0.61885000000000 & 0.63065000000000 & 0.11668000000000\end{array}$

$-0.61885000000000-0.63065000000000 \quad 0.61668000000000$

$1.11885000000000-0.63065000000000 \quad 0.11668000000000$

$0.11885000000000 \quad 0.63065000000000$

$0.50496000000000 \quad 0.24091000000000$

$-0.50496000000000-0.24091000000000$

$1.00496000000000-0.24091000000000$

$-0.00496000000000 \quad 0.24091000000000$

$0.61734000000000 \quad 0.13129000000000$

$-0.61734000000000-0.13129000000000$

$1.11734000000000-0.13129000000000$ 0.61668000000000 0.00000000000000 0.50000000000000 0.00000000000000 0.50000000000000 0.37996000000000 0.37996000000000 0.87996000000000
As $\quad(4 a)$

(4a)

(4a)

Co $\quad(4 \mathrm{a})$

Co $(4 \mathrm{a})$

Co $(4 \mathrm{a})$

Co (4a)

$\mathrm{S} \quad(4 \mathrm{a})$

(4a)
$\mathrm{Bi}_{5} \mathrm{Nb}_{3} \mathrm{O}_{15}: \mathrm{A} 5 \mathrm{~B} 3 \mathrm{C} 15 \_$oP46 $30 \mathrm{a} 2 \mathrm{c}$ bc $\_$a7 $-\mathrm{CIF}$

\# CIF file

data_findsym-outpu

audit_creation_method FINDSYM

chemical_name mineral 'Bi5Nb3O15'

chemical_formula_sum 'Bi5 Nb3 O15

loop

publ_author_name

S. Tahara'

A. Shimada,

N. Kumada

Y. Sugahara

journal name_full name

Journal of Solid State Chemistry

_journal_volume 180

loop

pubi_author_name 
_journal_year 2007

_journal_page_first 251

-journal_page_last 252
_publ_Section_title

Characterization of $\mathrm{Bi}_{-}\{5\} \$ \mathrm{Nb} \$_{-}\{3\} \$ O{ }_{-}\{15\} \$$ by refinement of neutron $\hookrightarrow$ diffraction pattern, acid treatment and reaction of the

$\hookrightarrow$ acid-treated product with $\$$ n $\$-$ alkylamines

\# Found in Pearson's Crystal Data - Crystal Structure Database for

$\hookrightarrow$ Inorganic Compounds, 2013

aflow title, Bi\$_ $\{5\} \$ N b \$\{3\} \$ O{ }_{2}\{15\} \$$ Structure

aflow_proto 'A5B3C15_oP46_30_a2c_bc_a7c

aflow_params $, a, b / a, c / a, z_{-}\{1\}, z_{-}\{2\}, z_{-}\{3\}, x_{-}\{4\}, y_{-}\{4\}, z_{-}\{4\}, x_{-}\{5\}, y_{-}\{5$ $\hookrightarrow\}, \mathrm{z}_{-}\{5\}, \mathrm{x}_{-}\{6\}, \mathrm{y}_{-}\{6\}, \mathrm{z}_{-}\{6\}, \mathrm{x}_{-}\{7\}, \mathrm{y}_{-}\{7\}, \mathrm{z}_{-}\{7\}, \mathrm{x}_{-}\{8\}, \mathrm{y}_{-}\{8\}, \mathrm{z}_{-}\{8\}$, $\left.\hookrightarrow \mathrm{x}_{-}\{9\}, \mathrm{y}_{-}\{9\}, \mathrm{z}_{-}\{9\}, \mathrm{x}_{-}\{10\}, \mathrm{y}_{-}\{10\}, \mathrm{z}_{-}\{10\}, \mathrm{x}_{-}\{11\}, \mathrm{y}_{-}\{11\}, \mathrm{z}_{-}\{11\}, \mathrm{x}_{-}\right\}$ $\hookrightarrow 12\}, y_{-}\{12\}, z_{-}\{12\}, x_{-}\{13\}, y_{-}\{13\}, z_{-}\{13\}$

年, $\hookrightarrow, 0.523,0.2442,0.513,0.019,0.3675,0.026,0.016,0.1074,0.003,0.02$ $\hookrightarrow 0.1949,0.05,0.074,0.097,0.687,0.22,0.0844,0.254,0.226,0.413$ $\hookrightarrow 0.52,0.105,0.505,0.718,0.31,0.305,0.763,0.271,0.695,0.752,0.289$ flow_Strukturbericht 'None'

aflow_Pearson 'oP46,

cell_length_a $\quad 5.4630061801$

cell_length_b 5.4730061914

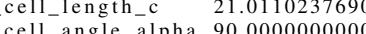

_cell_angle_alpha 90.0000000000

cell_angle gamma 90.0000000000

symmetry_space_group_name_H-M "P n c 2"

_symmetry_Int_Tables_number 30

loop

_space_group_symop_id

_space_group_symop_operation_xyz

$1 \mathrm{x}, \mathrm{y}, \mathrm{z}$

$2-\mathrm{x},-\mathrm{y}$,

$3-\mathrm{x}, \mathrm{y}+1 / 2, \mathrm{z}+1 / 2$

$4 \mathrm{x},-\mathrm{y}+1 / 2, \mathrm{z}+1 / 2$

loop

_atom_site_label

atom_site_type_symbol

atom_site_symmetry_multiplicity

atom_site_Wyckoff_label

atom_site_fract_x

atom_site_fract_y
atom_site_fract_z

(atom_site_fract $Z$

$\begin{array}{lllllll}\mathrm{Bi}_{\mathrm{B}} \mathrm{Bi} & 2 & \mathrm{a} & 0.00000 & 0.00000 & 0.50000 & 1.00000\end{array}$

$\begin{array}{lllllllll}\mathrm{O} 1 & \mathrm{O} & 2 & \mathrm{a} & 0.00000 & 0.00000 & 0.10700 & 1.00000\end{array}$

$\begin{array}{llllllll}\mathrm{Nb} 1 \mathrm{Nb} & 2 & \mathrm{~b} & 0.50000 & 0.00000 & 0.52300 & 1.00000\end{array}$

$\begin{array}{llllllll}\mathrm{Bi} 2 & \mathrm{Bi} & 4 & \mathrm{c} & 0.24420 & 0.51300 & 0.01900 & 1.00000\end{array}$

$\begin{array}{llllllll}\mathrm{Bi} 3 & \mathrm{Bi} & 4 & \mathrm{c} & 0.36750 & 0.02600 & 0.01600 & 1.00000\end{array}$

$\begin{array}{llllllll}\mathrm{Nb} 2 & \mathrm{Nb} & 4 & \mathrm{c} & 0.10740 & 0.00300 & 0.02000 & 1.00000\end{array}$

$\begin{array}{lllllllll}\mathrm{O} 2 & \mathrm{O} & 4 & \mathrm{c} & 0.19490 & 0.05000 & 0.07400 & 1.00000\end{array}$

$\begin{array}{lllllllll}\mathrm{O} 3 & \mathrm{O} & 4 & \mathrm{c} & 0.09700 & 0.68700 & 0.22000 & 1.00000\end{array}$

$\begin{array}{lllllllll}\mathrm{O} 4 & \mathrm{O} & 4 & \mathrm{c} & 0.08440 & 0.25400 & 0.22600 & 1.00000\end{array}$

$\begin{array}{llllllllll}05 & \mathrm{O} & 4 & \mathrm{c} & 0.41300 & 0.52000 & 0.10500 & 1.00000\end{array}$

$\begin{array}{lllllllll}06 & 0 & 4 & c & 0.41300 & 0.52000 & 0.10500 & 1.00000\end{array}$

$\begin{array}{lllllllll}06 & \mathrm{O} & & 4 & \mathrm{c} & 0.50500 & 0.71800 & 0.31000 & 1.00000\end{array}$

$\begin{array}{lllllllll}\mathrm{O} 7 & \mathrm{O} & & 4 & \mathrm{c} & 0.30500 & 0.76300 & 0.27100 & 1.00000 \\ \mathrm{O} 8 & \mathrm{O} & & 4 & \mathrm{c} & 0.69500 & 0.75200 & 0.28900 & 1.00000\end{array}$

$\mathrm{Bi}_{5} \mathrm{Nb}_{3} \mathrm{O}_{15}$ : A5B3C15_oP46_30_a2c_bc_a7c - POSCAR

A5B3C15 0 P $46,30, a 2 c, b c a 7 c \& a, b / a, c / a, z 1, z 2, z 3, x 4, y 4, z 4, x 5, y 5, z 5, x 6, y 6$ $\hookrightarrow \mathrm{z} 6, \mathrm{x} 7, \mathrm{y} 7, \mathrm{z} 7, \mathrm{x} 8, \mathrm{y} 8, \mathrm{z} 8, \mathrm{x} 9, \mathrm{y} 9, \mathrm{z} 9, \mathrm{x} 10, \mathrm{y} 10, \mathrm{z} 10, \mathrm{x} 11, \mathrm{y} 11, \mathrm{z} 11, \mathrm{x} 12, \mathrm{y} 12$, $\hookrightarrow \mathrm{z} 12, \mathrm{x} 13, \mathrm{y} 13, \mathrm{z} 13-$-params $=5.4630061801,1.00183049606$

$\hookrightarrow 3.84605528098,0.5,0.107,0.523,0.2442,0.513,0.019,0.3675,0.026$

$\hookrightarrow 0.016,0.1074,0.003,0.02,0.1949,0.05,0.074,0.097,0.687,0.22$

$\hookrightarrow 0.0844,0.254,0.226,0.413,0.52,0.105,0.505,0.718,0.31,0.305$

$\hookrightarrow 0.763,0.271,0.695,0.752,0.289 \&$ Pnc2 $C_{-}\{2 \mathrm{v}\} \wedge\{6\} \# 30\left(\mathrm{a}^{\wedge} 2 \mathrm{bc}^{\wedge} 10\right)$

$\hookrightarrow$ \& oP46 \& None \& Bi5Nb3O15 \& \& S. Tahara et al., J. Solid State $\hookrightarrow$ Chem. 180, 2517-2524 (2007)

1.00000000000000

$\begin{array}{lll}5.46300618010000 & 0.00000000000000 & 0.00000000000000\end{array}$

$\begin{array}{llll}0.00000000000000 & 5.47300619140000 & 0.00000000000000\end{array}$

$\begin{array}{lll}0.00000000000000 & 0.00000000000000 & 21.01102376900000\end{array}$

$$
\begin{array}{rrr}
\mathrm{Bi} & \mathrm{Nb} & \mathrm{O} \\
10 & 6 & 30
\end{array}
$$

Direct

0.00000000000000 0.00000000000000 0.0000000000000 (1) 0.24420000000000 0.24420000000000 $-0.24420000000000$ 0.36750000000000 $-0.36750000000000$ 0.36750000000000 $-0.36750000000000$ 0.50000000000000 0.50000000000000 0.10740000000000 $-0.10740000000000$ 0.10740000000000 $-0.10740000000000$ 0.00000000000000 0.00000000000000 0.19490000000000

0.00000000000000 0.50000000000000 0.51300000000000 $-0.51300000000000$ 0.01300000000000 1.01300000000000 0.02600000000000 $-0.02600000000000$ 0.47400000000000 0.52600000000000 0.00000000000000 0.50000000000000 0.00300000000000 $-0.00300000000000$ 0.49700000000000 0.50300000000000 0.00000000000000 0.50000000000000 0.05000000000000
0.50000000000000 1.00000000000000 0.01900000000000 0.01900000000000 0.51900000000000 0.51900000000000 0.01600000000000 0.01600000000000 0.51600000000000 0.51600000000000 0.51600000000000 0.52300000000000 1.02300000000000 0.02000000000000 0.0200000000000 0.52000000000000 0.52000000000000 0.10700000000000 0.6070000000000 0.07400000000000
$-0.19490000000000$ 0.19490000000000 $-0.19490000000000$ 0.09700000000000 $-0.0970000000000$ 0.09700000000000 $-0.09700000000000$ 0.08440000000000 $-0.08440000000000$ 0.08440000000000 $-0.08440000000000$ 0.41300000000000 $-0.41300000000000$ 0.4130000000000 0.41300000000000 $-0.41300000000000$ 0.50500000000000 0.50500000000000 0.50500000000000 $-0.50500000000000$ 0.30500000000000

$-0.30500000000000$

0.30500000000000

$-0.3050000000000$

0.69500000000000 $-0.69500000000000$ 0.69500000000000 $-0.69500000000000$
0.05000000000000 .45000000000000 0.55000000000000 0.68700000000000 0.68700000000000 $-0.18700000000000$ 1.18700000000000 0.25400000000000 $-0.25400000000000$ 0.24600000000000 0.75400000000000 0.52000000000000 $-0.52000000000000$ $-0.0200000000000$ 1.02000000000000 0.71800000000000 0.71800000000000 . . 1.21800000000000 0.76300000000000 0.76300000000000 .26300000000000 1.26300000000000 $-0.75200000000000$ .25200000000000 0.57400000000000 0.57400000000000 0.22000000000000 0.22000000000000 0.72000000000000 0.72000000000000 0.22600000000000 0.22600000000000 0.72600000000000 0.72600000000000 0.10500000000000 0.10500000000000 0.60500000000000 0.60500000000000 0.31000000000000 0.31000000000000 0.31000000000000 0.8100000000000 0.27100000000000 0.27100000000000 0.77100000000000 0.77100000000000 0.28900000000000 0.28900000000000 0.78900000000000 0.78900000000000 0.81000000000000

$\mathrm{O} \quad(4 \mathrm{c})$

$\mathrm{O} \quad(4 \mathrm{c})$

$\mathrm{O} \quad(4 \mathrm{c})$

$\mathrm{O} \quad(4 \mathrm{c})$

$\mathrm{O} \quad(4 \mathrm{c})$

$\mathrm{O} \quad(4 \mathrm{c})$

$\begin{array}{ll}\mathrm{O} & (4 \mathrm{c}) \\ \mathrm{O} & (4 \mathrm{c})\end{array}$

CuBrSe 3 : ABC3_oP20_30_2a_c_3c - CIF

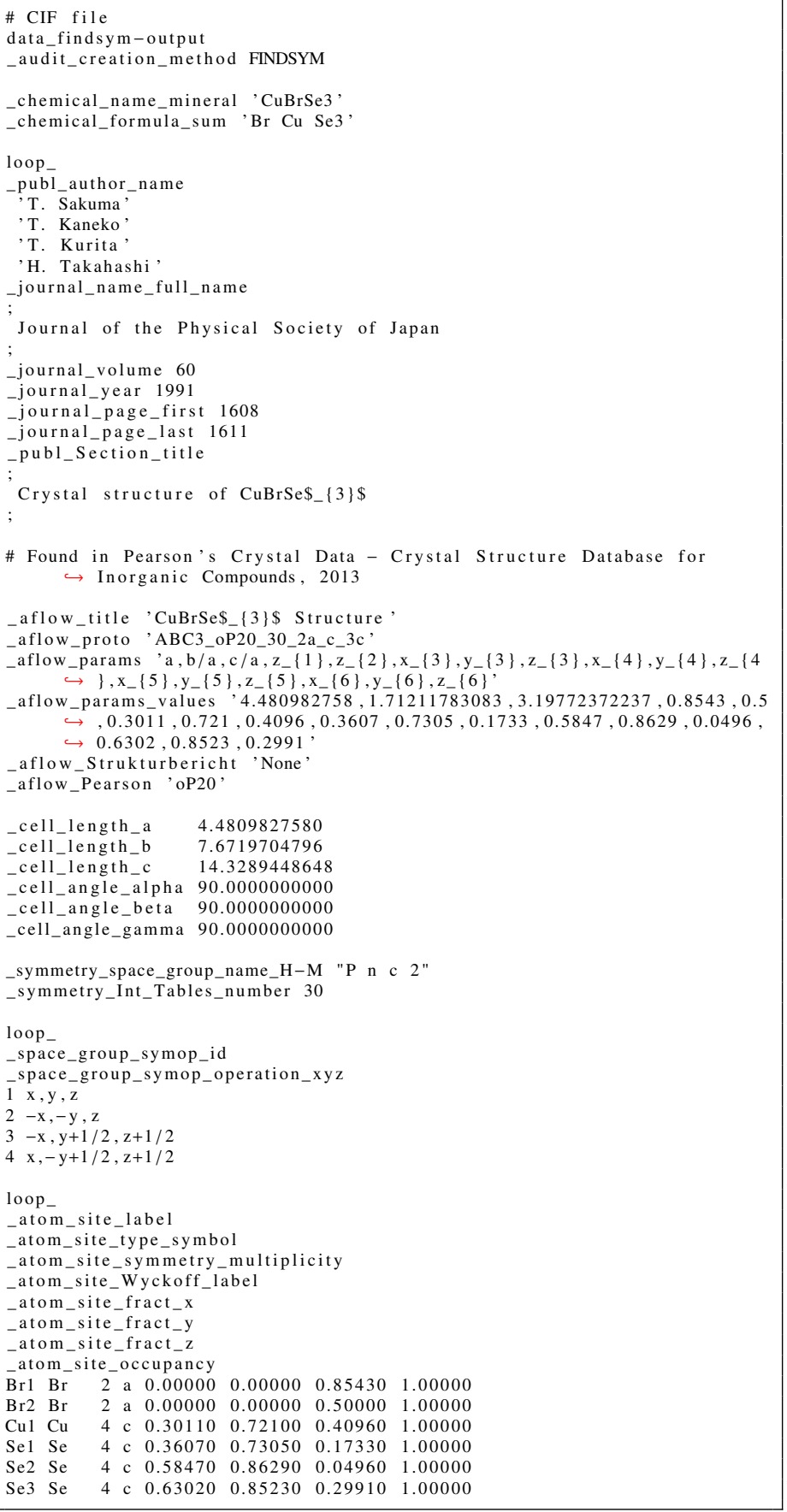

$\mathrm{CuBrSe}_{3}: \mathrm{ABC} 3$ oP20_30_2a_c $\_3 c-$ POSCAR 
$A B C 3$ $\hookrightarrow \mathrm{z} 6-$ params $=4.480982758,1.71211783083,3,19772372237,0.8543,0.5$ $\hookrightarrow 0.3011,0.721,0.4096,0.3607,0.7305,0.1733,0.5847,0.8629,0.0496$, $\hookrightarrow 0.6302,0.8523,0.2991 \&$ Pnc2 $\mathrm{C}_{-}\{2 \mathrm{v}\}^{\wedge}\{6\} \# 30\left(\mathrm{a}^{\wedge} 2 \mathrm{c}^{\wedge} 4\right) \& \mathrm{OP} 20 \&$

$\hookrightarrow$ None \& $\mathrm{CuBrSe}_{3} \&$ \& T. Sakuma et al., J. Phys. Soc. Jpn.60, $\hookrightarrow 1608-1611(1991)$

1.00000000000000

$\begin{array}{lll}4.48098275800000 & 0.00000000000000 & 0.00000000000000\end{array}$

$\begin{array}{llll}0.00000000000000 & 7.67197047960000 & 0.00000000000000\end{array}$

$\begin{array}{lllll}0.00000000000000 & 0.00000000000000 & 14.32894486480000\end{array}$

$$
\begin{array}{rrr}
\mathrm{Br} & \mathrm{Cu} & \mathrm{Se} \\
4 & 4 & 12
\end{array}
$$

Direct

0.00000000000000 0.00000000000000 0.0000000000000 0.00000000000 0.0000000000 0.30110000000000 $-0.30110000000000$ 0.30110000000000 $-0.30110000000000$ 0.36070000000000 $-0.36070000000000$ 0.36070000000000 $-0.36070000000000$ 0.58470000000000 $-0.58470000000000$ 0.58470000000000 $-0.58470000000000$ 0.63020000000000 $-0.63020000000000$ 0.63020000000000 0.63020000000000
-0.63020000000000

0.00000000000000 0.50000000000000 0.00000000000000 0.50000000000000 0.7210000000000 $-0.72100000000000$ $-0.22100000000000$ 1.22100000000000 0.7305000000000 0.730500000000 0.73050000000000 .2305000000000 0.86290000000000 $-0.86290000000000$ $-0.36290000000000$ 1.36290000000000 0.85230000000000 $-0.85230000000000$ $-0.35230000000000$ 1.35230000000000

$\begin{array}{lll}0.85430000000000 & \mathrm{Br} & (2 \mathrm{a}) \\ 1.35430000000000 & \mathrm{Br} & (2 \mathrm{a}) \\ 0.50000000000000 & \mathrm{Br} & (2 \mathrm{a}) \\ 1.00000000000000 & \mathrm{Br} & (2 \mathrm{a}) \\ 0.40960000000000 & \mathrm{Cu} & (4 \mathrm{c}) \\ 0.40960000000000 & \mathrm{Cu} & (4 \mathrm{c}) \\ 0.90960000000000 & \mathrm{Cu} & (4 \mathrm{c}) \\ 0.90960000000000 & \mathrm{Cu} & (4 \mathrm{c}) \\ 0.17330000000000 & \mathrm{Se} & (4 \mathrm{c}) \\ 0.17330000000000 & \mathrm{Se} & (4 \mathrm{c}) \\ 0.67330000000000 & \mathrm{Se} & (4 \mathrm{c}) \\ 0.67330000000000 & \mathrm{Se} & (4 \mathrm{c}) \\ 0.04960000000000 & \mathrm{Se} & (4 \mathrm{c}) \\ 0.04960000000000 & \mathrm{Se} & (4 \mathrm{c}) \\ 0.54960000000000 & \mathrm{Se} & (4 \mathrm{c}) \\ 0.54960000000000 & \mathrm{Se} & (4 \mathrm{c}) \\ 0.29910000000000 & \mathrm{Se} & (4 \mathrm{c}) \\ 0.29910000000000 & \mathrm{Se} & (4 \mathrm{c}) \\ 0.79910000000000 & \mathrm{Se} & (4 \mathrm{c}) \\ 0.79910000000000 & \mathrm{Se} & (4 \mathrm{c})\end{array}$

$\mathrm{Re}_{2} \mathrm{O}_{5}\left[\mathrm{SO}_{4}\right]_{2}: \mathrm{A} 13 \mathrm{~B} 2 \mathrm{C} 2 \_\mathrm{oP} 34 \_32 \_\mathrm{a} 6 \mathrm{c} \_\mathrm{c} \_\mathrm{c}-\mathrm{CIF}$

\# CIF file

data_findsym-output

audit_creation_method FINDSYM

chemical_name_mineral 'Re2O5[SO4]2'

chemical_formula_sum, $\mathrm{O} 13 \mathrm{Re} 2 \mathrm{~S} 2$,

loop

_pubi__author_name

U. Betke

'M. S. Wickleder'

_journal_name_full_name

Inorganic Chemistry

_journal_volume 50

_journal_year 2011

_journal_page_first 858

-journal_page_first 858
journal_page_last 872

_publ_Section_title

Sulfates of the Refractory Metals: Crystal Structure and Thermal

$\hookrightarrow$ Behavior of $\mathrm{Nb}_{-}\{2\} \$ O \$_{-}\{2\} \$\left(\operatorname{SO}_{-}\{4\} \$\right) \$_{-}\{3\} \$, \operatorname{MoO}_{-}\{2\} \$\left(\operatorname{SO} \$_{-}\{4\}\right.$

$\hookrightarrow \$), \operatorname{WO}\left(\operatorname{SO} \$_{-}\{4\} \$\right) \$ \_\{2\} \$$, and Two Modifications of $\operatorname{Re} \$_{-}\{2\} \$ O \$_{-}\{5$ $\hookrightarrow\} \$\left(\operatorname{SO}_{-}\{4\} \$\right) \$_{-}\{2\} \$$

\# Found in Pearson's Crystal Data - Crystal Structure Database for $\hookrightarrow$ Inorganic Compounds, 2013

aflow_title $\operatorname{Re} \$_{-}\{2\} \$ O{ }_{-}\{5\} \$\left[\operatorname{SO} \$_{-}\{4\} \$\right] \$ \_\{2\} \$$ Structure'

aflow_proto 'A13B2C2_oP34_32_a6c_c_c

aflow_params $, a, b / a, c / a, z_{-}\{1\}, x_{-}\{2\}, y_{-}\{2\}, z_{-}\{2\}, x_{-}\{3\}, y_{-}\{3\}, z_{-}\{3\}, x_{-}\{4$ $\hookrightarrow\}, y_{-}\{4\}, z_{-}\{4\}, x_{-}\{5\}, y_{-}\{5\}, z_{-}\{5\}, x_{-}\{6\}, y_{-}\{6\}, z_{-}\{6\}, x_{-}\{7\}, y_{-}\{7\}$, $\hookrightarrow \begin{aligned} & \hookrightarrow y_{-}\{4\}, z_{-}\{4\}, x_{-}\{5\}, y_{-}\{5\}, z_{-}\{5\}, x_{-}\{6\}, y_{-}\{6\}, \\ & \hookrightarrow z_{-}\{7\}, x_{-}\{8\}, y_{-}\{8\}, z_{-}\{8\}, x_{-}\{9\}, y_{-}\{9\}, z_{-}\{9\}\end{aligned}$

aflow_params_values $8.4261784988,1.1450866366,0.701103726564,0.0,0.808$ $\hookrightarrow, 0.55,0.8946,0.614,0.7595,-0.0255,0.8197,0.8044,0.642,0.5367$,

$\hookrightarrow 0.6481,0.3894,0.7689,-0.0703,0.2917,0.8392,0.688,0.2793,0.67666$ $\hookrightarrow \quad 0.60773,0.0844,0.8643,0.8235,0.4147^{\circ}$.

aflow_Strukturbericht 'None'

aflow_Pearson 'oP34,

cell_length_a $\quad 8.4261784988$

cell_length_b 9.6487043966

cell_length_c $\quad 5.9076251462$

cell_angle_alpha 90.0000000000

cell_angle_beta 90.0000000000

cell_angle_gamma 90.0000000000

symmetry_space_group_name_H-M "P b a 2 "

_symmetry_Int_Tables_number 32

loop

space_group_symop_id

_space_group_symop_operation_xyz

$1 \mathrm{x}, \mathrm{y}, \mathrm{z}$

$2-\mathrm{x},-\mathrm{y}$,

$3-x+1 / 2, y+1 / 2, z$

$4 \mathrm{x}+1 / 2,-\mathrm{y}+1 / 2, z$

loop

atom_site_label

atom_site_type_symbol

atom_site_symmetry_multiplicity

atom_site_Wyckoff_label

atom_site_fract_x

at om_site_fract_y

atom_site_occupancy

$\begin{array}{lllllllll}\mathrm{O} 1 & \mathrm{O} & 2 & \mathrm{a} & 0.00000 & 0.00000 & 0.00000 & 1.00000\end{array}$

$\begin{array}{llllllll}\mathrm{O} 2 & \mathrm{O} & 4 & \mathrm{c} & 0.80800 & 0.55000 & 0.89460 & 1.00000\end{array}$

$\begin{array}{lllllllll}\mathrm{O} 3 & \mathrm{O} & & 4 & \mathrm{c} & 0.61400 & 0.75950 & -0.02550 & 1.00000\end{array}$

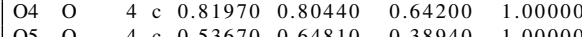

$\begin{array}{lllllllll}\mathrm{O} 5 & \mathrm{O} & 4 & \mathrm{c} & 0.53670 & 0.64810 & 0.38940 & 1.00000\end{array}$

$\begin{array}{lllllllll}\mathrm{O} 6 & \mathrm{O} & 4 & \mathrm{c} & 0.76890 & -0.07030 & 0.29170 & 1.00000\end{array}$

$\begin{array}{llllllll}\mathrm{O} 7 & \mathrm{O} & 4 & \mathrm{c} & 0.83920 & 0.68800 & 0.27930 & 1.00000\end{array}$

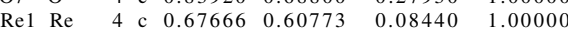

$\begin{array}{llllllll}\mathrm{S} 1 & \mathrm{~S} & 4 & \mathrm{c} & 0.86430 & 0.82350 & 0.41470 & 1.00000\end{array}$

$\mathrm{Re}_{2} \mathrm{O}_{5}\left[\mathrm{SO}_{4}\right]_{2}:$ A13B2C2_oP34_32_a6c_c_c - POSCAR

A13B2C2_oP34_32_a6c_c_c \& a , b/a, c/a , z1 , x2 , y2 , z2 , x3 , y3 , z3 , x4 , y4, z4 , x5 , y5

$\hookrightarrow \mathrm{z} 5, \mathrm{x} 6, \mathrm{y} 6, \mathrm{z} 6, \mathrm{x} 7, \mathrm{y} 7, \mathrm{z} 7, \mathrm{x} 8, \mathrm{y} 8, \mathrm{z} 8, \mathrm{x} 9, \mathrm{y} 9, \mathrm{z} 9--$ params $=8.4261784988$,

$\hookrightarrow 1.1450866366,0.701103726564,0.0,0.808,0.55,0.8946,0.614,0.7595$

$\hookrightarrow,-0.0255,0.8197,0.8044,0.642,0.5367,0.6481,0.3894,0.7689,-$

$\hookrightarrow 0.0703,0.2917,0.8392,0.688,0.2793,0.67666,0.60773,0.0844,0.8643$

$\hookrightarrow, 0.8235,0.4147 \&$ Pba2 $\mathrm{C}_{-}\{2 \mathrm{v}\}^{\wedge}\{8\} \# 32\left(\mathrm{ac}^{\wedge} 8\right) \&$ oP 34 \& None \&

$\hookrightarrow \operatorname{Re} 2 \mathrm{O} 5[\mathrm{SO} 4] 2$ \& \& U. Betke and M. S. Wickleder, Inorg. Chem. 50,

$\hookrightarrow \quad 858-872(2011)$

1.00000000000000

$0.00000000000000-0.00000000000000$

$0.00000000000000-9.64870439660000-0.00000000000000$

$\begin{array}{lll}0.00000000000000 & 0.00000000000000 & 5.90762514620000\end{array}$

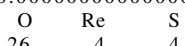

Direct

0.00000000000000

0.50000000000000

0.80800000000000

$-0.80800000000000$

1.30800000000000

$-0.30800000000000$

0.61400000000000

$-0.61400000000000$

1.11400000000000

$-0.11400000000000$

0.81970000000000

$-0.81970000000000$

1.31970000000000

$-0.31970000000000$

$-0.53670000000000$

1.03670000000000

$-0.03670000000000$

0.76890000000000
-0.76890000000000

-0.76890000000000
1.26890000000000

$-0.2689000000000$

0.83920000000000

$-0.83920000000000$

1.33920000000000

$-0.33920000000000$

0.67666000000000

-0.67666000000000
1.17666000000000

1.17666000000000
-0.17666000000000

0.86430000000000

$-0.8643000000000$

1.36430000000000

$-0.36430000000000$

0.00000000000000 0.50000000000000 0.55000000000000 $-0.55000000000000$ 0.05000000000000 1.05000000000000 0.75950000000000 $-0.75950000000000$ $-0.25950000000000$ 1.25950000000000 0.80440000000000 $-0.80440000000000$ $-0.30440000000000$ 1.30440000000000 0.64810000000000 . 0.14810000000000 1.14810000000000 0.07030000000000 0.07030000000000 0.57030000000000 0.42970000000000 0.68800000000000 $-0.68800000000000$ $-0.18800000000000$ 1.18800000000000 0.60773000000000 $-0.60773000000000$ $-0.1077300000000$ 1.10773000000000 0.8235000000000 $-0.82350000000000$ $-0.82350000000000$ 0.32350000000000
1.32350000000000

0.00000000000000 0.00000000000000 0.89460000000000 0.89460000000000 0.89460000000000 0.89460000000000 $-0.02550000000000$ $-0.02550000000000$ $-0.02550000000000$ $-0.02550000000000$ 0.64200000000000 0.64200000000000 0.64200000000000 0.642000000000 0.6420000000 0.389400000000 0.38940000000000 0.38940000000000 0.38940000000000 0.29170000000000 0.29170000000000 0.29170000000000 0.29170000000000 0.27930000000000 0.27930000000000 0.27930000000000 0.27930000000000 0.08440000000000 0.08440000000000 0.0844000000000 0.08440000000000 0.41470000000000 0.41470000000000 0.41470000000000

O (2a

$\mathrm{O} \quad(4 \mathrm{c})$

$\mathrm{O} \quad(4 \mathrm{c})$

$\mathrm{O} \quad(4 \mathrm{c})$

$\mathrm{O} \quad(4 \mathrm{c})$

$\mathrm{O} \quad(4 \mathrm{c})$

$\mathrm{O} \quad(4 \mathrm{c})$

$\mathrm{O} \quad(4 \mathrm{c})$

$\mathrm{O} \quad(4 \mathrm{c})$

$\mathrm{O} \quad(4 \mathrm{c})$

$\mathrm{O} \quad(4 \mathrm{c})$

O $(4 \mathrm{c})$

O (4c)

(4c)

O (4c)

O $(4 \mathrm{c}$

$\mathrm{O} \quad(4 \mathrm{c})$

$\mathrm{O} \quad(4 \mathrm{c})$

$\mathrm{O} \quad(4 \mathrm{c})$

$\mathrm{O} \quad(4 \mathrm{c})$

$\mathrm{O} \quad(4 \mathrm{c})$

$\mathrm{O} \quad(4 \mathrm{c})$

$\mathrm{O}$ (4c)

O (4c)

$\mathrm{O} \quad(4 \mathrm{c})$

$\mathrm{O} \quad(4 \mathrm{c})$

$\operatorname{Re}(4 c)$

$\mathrm{Re} \quad(4 \mathrm{c})$

$\operatorname{Re}(4 c)$

S (4c)

$S$ (4c)

$\kappa$-alumina $\left(\mathrm{Al}_{2} \mathrm{O}_{3}\right)$ : A2B3_oP40_33_4a_6a - CIF

\section{\# CIF file}

data findsym-output

audit_creation_method FINDSYM

chemical_name_mineral ' $\$ \backslash$ kappa $\$-a l u m i n a '$

chemical_formula_sum, A12 O3,

loop

publ_author_name

B. Ollivier

R. Retoux',

P. Lacorre,

G. $F \backslash \backslash,\{$ e $\}$ rey

journal name full name

Journal of Materials Chemistry

journal_volume 7

journal year 1997

journal_page_first 1049

journal_page_last 105

publ_Section_title

Crystal structure of $\$ \backslash$ kappa-alumina: an $X$-ray powder diffraction, TEM $\rightarrow$ and NMR study 
$\hookrightarrow 0.6381,0.5145,0.6728,0.1212,0.8608,0.3301,0.8662,0.336,0.4992$, $\hookrightarrow 0.9$ _aflow_Strukturbericht 'None

_aflow_Pearson 'oP40,

_symmetry_space_group_name_H-M "P n a 21 "

_symmetry_Int_Tables_number 33

\section{_cell_length_a $\quad 4.84370$}

-cell_length_b $\quad 8.33000$

cell_angle_alpha 90.00000

cell_angle_beta 90.00000

-cell_angle_gamma 90.00000

loop

_space_group_symop_id

_space_group_symop_operation_xyz

$1 \mathrm{x}, \mathrm{y}, \mathrm{z}$

$2-\mathrm{x},-\mathrm{y}, \mathrm{z}+1 / 2$

$3-x+1 / 2, y+1 / 2, z+1 / 2$

$4 \mathrm{x}+1 / 2,-\mathrm{y}+1 / 2, \mathrm{z}$

loop_

_atom_site_label

_atom_site_type_symbol

_atom_site_symmetry_multiplicity

_atom_site_Wyckoff_label

-atom_site_fract_x

- at om_site_fract_y

atom_site_occupancy

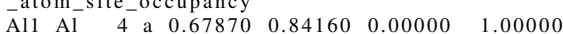

$\begin{array}{llllllll}\mathrm{A} 12 & \mathrm{Al} & 4 & \mathrm{a} & 0.18460 & 0.34320 & 0.78680 & 1.00000\end{array}$

$\begin{array}{lllllll}\mathrm{Al} 3 \mathrm{Al} & 4 & \mathrm{a} & 0.81150 & 0.64890 & 0.69720 & 1.00000\end{array}$

$\begin{array}{llllllll}\mathrm{Al} 4 & \mathrm{Al} & 4 & \mathrm{a} & 0.66770 & 0.46960 & 0.99930 & 1.00000\end{array}$

$\begin{array}{lllllllll}\mathrm{O} 1 & \mathrm{O} & 4 & \mathrm{a} & 0.32900 & 0.83130 & 0.89270 & 1.00000\end{array}$

$\begin{array}{llllllll}\mathrm{O} 2 & \mathrm{O} & 4 & \text { a } & 0.02480 & 0.49080 & 0.62920 & 1.00000\end{array}$

$\begin{array}{lllllllll}\mathrm{O} 3 & \mathrm{O} & 4 & \text { a } & 0.47170 & 0.66470 & 0.63810 & 1.00000\end{array}$

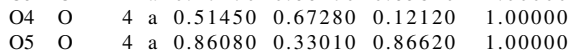

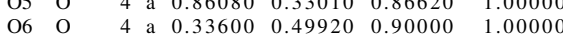

$\kappa$-alumina $\left(\mathrm{Al}_{2} \mathrm{O}_{3}\right)$ : A2B3_oP40_33_4a_6a - POSCAR

A2B3_oP40_33_4a_6a \& a,b/a,c/a, x1,y1,z1, x2 ,y2, z2, x3,y3,z3, x4, y4, z4, x5 , y5 $\hookrightarrow, \mathrm{z} 5, \mathrm{x} 6, \mathrm{y} 6, \mathrm{z} 6, \mathrm{x} 7, \mathrm{y} 7, \mathrm{z} 7, \mathrm{x} 8, \mathrm{y} 8, \mathrm{z} 8, \mathrm{x} 9, \mathrm{y} 9, \mathrm{z} 9, \mathrm{x} 10, \mathrm{y} 10, \mathrm{z} 10-\mathrm{params}=$

$\hookrightarrow 4.8437,1.71975968784,1.84873134174,0.6787,0.8416,0.0,0.1846$,

$\hookrightarrow 0.3432,0.7868,0.8115,0.6489,0.6972,0.6677,0.4696,0.9993,0.329$,

$\hookrightarrow 0.8313,0.8927,0.0248,0.4908,0.6292,0.4717,0.6647,0.6381,0.5145$

$\hookrightarrow 0.6728,0.1212,0.8608,0.3301,0.8662,0.336,0.4992,0.9 \& \mathrm{Pna}_{2}\{1\}$

$\hookrightarrow \mathrm{C}_{-}\{2 \mathrm{v}\}^{\wedge}\{9\} \# 33\left(\mathrm{a}^{\wedge} 10\right) \&$ oP40 \& None \& Al2O3 \& \$ kappa $\$-$ alumina

$\hookrightarrow$ \& B. Ollivier et al., J. Mater. Chem. 7, 1049-1056 (1997)

1.00000000000000

0.00000000000000

0.00000000000000

0.00000000000000

8.33000000000000

0.00000000000000

$\begin{array}{ll}\mathrm{Al} & \mathrm{O} \\ 16 & 24\end{array}$

Direct

$16 \mathrm{ct}$
0.67870000000000

$-0.67870000000000$

1.17870000000000

$-0.17870000000000$

0.18460000000000

$-0.18460000000000$

0.68460000000000

0.31540000000000 0.81150000000000

$-0.81150000000000$

1.31150000000000

$-0.31150000000000$

0.66770000000000

$-0.66770000000000$

$-.66770000000000$

1.16770000000000

0.32900000000000

$-0.32900000000000$

0.82900000000000

0.17100000000000

0.02480000000000

0.02480000000000

0.52480000000000

0.47520000000000

0.47170000000000

$-0.47170000000000$

0.97170000000000

0.02830000000000

0.51450000000000

0.51450000000000
-0.51450000000000

1.01450000000000

0.86080000000000

$-0.86080000000000$

1.36080000000000

$-0.36080000000000$

0.33600000000000

$-0.33600000000000$

0.83600000000000

0.16400000000000

0.84160000000000

$-0.84160000000000$

0.34160000000000

1.34160000000000

0.34320000000000

0.34320000000000

.15680000000000

0.84320000000000

64890000000000

$-0.64890000000000$

$-0.14890000000000$

1.14890000000000

0.46960000000000

.46960000000000

0.4630000000

0.96960000000000

0.83130000000000

$-0.83130000000000$

$-0.33130000000000$

1.33130000000000

0.49080000000000

$-0.49080000000000$

0.00920000000000

-

.66470000000000

0.66470000000000

1.16470000000000

0.67280000000000

$-0.67280000000000$

$-0.17280000000000$

1.17280000000000

. $-0.33010000000000$ 0.16990000000000 0.83010000000000 0.49920000000000 $-0.49920000000000$

0.0008000000000

0.99920000000000

.00000000000000

0.00000000000000

0.50000000000000

0.00000000000000

0.50000000000000

0.78680000000000

1.28680000000000

.78680000000000

.28680000000000 0.69720000000000 0.69720000000000

1.19720000000000

1.49930000000000

0.99930000000000

0.89270000000000

1.39270000000000

0.89270000000000

0.62920000000000

1.12920000000000

.62920000000000

.12920000000000

.63810000000000

1.13810000000000

0.63810000000000

1.13810000000000

0.1212000000000

0.62120000000000

0.86620000000000

- 1.366200000000

1.36620000000000

.86620000000000

1.36620000000000

0.90000000000000

1.40000000000000
Al (4a)

Al (4a)

$(4 \mathrm{a})$
(4a)

(4a)

(4a)

(4a)

(4a)

(4a)

(4a)

(4a)

(4a)

(4a)

Al (4a)

(4a)

O (4a)

$(4 a)$
$0 \quad(4 a)$

$\mathrm{O} \quad$ (4a)

O (4a)

$\mathrm{O} \quad(4 \mathrm{a})$

O $\quad(4 a)$

O (4a)

O $\quad(4 a)$

$\mathrm{O} \quad(4 \mathrm{a})$

$\begin{array}{ll}0 & (4 \mathrm{a})\end{array}$

O (4a)

O (4a)

O (4a)

O (4a)

O (4a)

$\mathrm{O} \quad(4 \mathrm{a})$

$\begin{array}{ll}\mathrm{O} & (4 \mathrm{a}) \\ \mathrm{O} & (4 \mathrm{a})\end{array}$

O (4a)

O $\quad(4 \mathrm{a})$

O (4a)

O (4a)
$\mathrm{TiAl}_{2} \mathrm{Br}_{8}$ : A2B8C_oP22_34_c_4c_a - CIF

\# CIF file

data_findsym-output

audit_creation_method FINDSYM

chemical_name_mineral 'TiAl2Br8,

chemical_formula_sum, Al2 Br8 Ti

loop

publ_author_name

'S. I. Troyanov'

V. M. Ionov'

journal_name_full_name

Russian Journal of Inorganic Chemistry

journal_volume 35

journal year 1990

journal_page_first 882

journal_page_last 887

Synthesis and Crystal Structure of TiBr\$_ $\{4\} \$, \operatorname{TiBr} \$_{-}\{3\} \$$ and $\operatorname{Ti}(\operatorname{AlBr} \$$ $\hookrightarrow\{4\} \$) \$ \_\{2\} \$$

\# Found in Pearson's Crystal Data - Crystal Structure Database for

$\rightarrow$ Inorganic Compounds, 2013

aflow_title $T$ TiAl\$_ $\{2\} \$ B r \$_{-}\{8\} \$$ Structure

aflow_proto 'A2B8C_oP22_34_c 4c

aflow_params 'a, b/a, c/a, z $\{1\}, x_{-}\{2\}, y_{-}\{2\}, z_{-}\{2\}, x_{-}\{3\}, y_{-}\{3\}, z_{-}\{3\}, x_{-}\{4$ $\stackrel{-p a r a m s}{\hookrightarrow}\}, y_{-}\{4\}, z_{-}\{4\}, x_{-}\{5\}, y_{-}\{5\}, z_{-}\{5\}, x_{-}\{6\}, y_{-}\{6\}, z_{-}\{6\}$,

aflow params values $x_{-} 2740008991,2.04032515143,1,38284985656,0.5,0.605$ $\hookrightarrow, 0.8135,0.499,0.7349,0.6796,0.009,0.743,-0.0925,0.3064,0.2388$

$\hookrightarrow 0.5935,0.2196,0.7131,0.6459,0.513$;

aflow Strukturbericht , None,

_aflow_Pearson, oP22,

_cell_length_a $\quad 6.2740008991$

cell_length_b $\quad 12.8010018345$

_cell_length_c $\quad 8.6760012434$

cell_angle_alpha 90.0000000000

cell_angle_beta $\quad 90.0000000000$

cell_angle_gamma 90.0000000000

symmetry_space_group_name_H-M "P n n 2"

_symmetry_Int_Tables_number 34

loop

space_group_symop_id

space_group_symop_operation_xyz

$1 \mathrm{x}, \mathrm{y}, \mathrm{z}$

$-\mathrm{x},-\mathrm{y}, \mathrm{z}$

$-\mathrm{x}+1 / 2, \mathrm{y}+1 / 2, \mathrm{z}+1 / 2$

$4 \mathrm{x}+1 / 2,-\mathrm{y}+1 / 2, \mathrm{z}+1 / 2$

loop_

atom_site_label

atom_site_type_symbol

atom_site_symmetry_multiplicity

atom_site_Wyckoff_label

atom_site_fract_x

atom_site_fract_y

atom_site_fract_z

atom_site_occupancy

$\begin{array}{llllllll}\mathrm{Ti} 1 & \mathrm{Ti} & 2 & \mathrm{a} & 0.00000 & 0.00000 & 0.50000 & 1.00000\end{array}$

$\begin{array}{lllllllll}\mathrm{All} & \mathrm{Al} & 4 & \mathrm{c} & 0.60500 & 0.81350 & 0.49900 & 1.00000\end{array}$

$\begin{array}{llllllll}\mathrm{Br} 1 \mathrm{Br} & 4 & \mathrm{c} & 0.73490 & 0.67960 & 0.00900 & 1.00000\end{array}$

$\begin{array}{lllllllll}\mathrm{Br} 2 & \mathrm{Br} & 4 & \mathrm{c} & 0.74300 & -0.09250 & 0.30640 & 1.00000\end{array}$

$\begin{array}{llllllll}\mathrm{Br} 3 & \mathrm{Br} & 4 & \mathrm{c} & 0.23880 & 0.59350 & 0.21960 & 1.00000\end{array}$

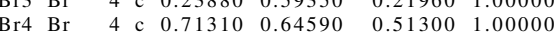

$\mathrm{TiAl}_{2} \mathrm{Br}_{8}:$ A2B8C_oP22_34_c 4c a - POSCAR

A2B8C_oP22_34_c_4c_a \& a , b/a,c/a, z1 , x2 , y2 , z2 , x3 $, \mathrm{y} 3, \mathrm{z} 3, \mathrm{x} 4, \mathrm{y} 4, \mathrm{z} 4, \mathrm{x} 5, \mathrm{y} 5, \mathrm{z} 5$

$\hookrightarrow \mathrm{x} 6, \mathrm{y} 6, \mathrm{z} 6--$ params $=6.2740008991,2.04032515143,1.38284985656,0.5$,

$\hookrightarrow 0.605,0.8135,0.499,0.7349,0.6796,0.009,0.743,-0.0925,0.3064$

$\hookrightarrow 0.2388,0.5935,0.2196,0.7131,0.6459,0.513 \&$ Pnn2 $C_{-}\{2 v\}^{\wedge}\{10\} \# 34$

$\hookrightarrow \quad\left(\mathrm{ac}^{\wedge} 5\right) \& \mathrm{oP} 22$ \& None \& TiAl2Br8 \& \& S. I. Troyanov and V. B.

$\hookrightarrow$ Rybakov and V. M. Ionov, Russ. J. Inorg. Chem. 35, 882-887 (

$\hookrightarrow 1990$ )

1.00000000000000

$\begin{array}{lll}6.27400089910000 & 0.00000000000000 & 0.00000000000000\end{array}$

$\begin{array}{lll}0.00000000000000 & 12.80100183450000 & 0.00000000000000\end{array}$

$\begin{array}{lll}0.00000000000000 & 0.00000000000000 & 8.67600124340000\end{array}$

$\begin{array}{rrr}\mathrm{Al} & \mathrm{Br} & \mathrm{Ti} \\ 4 & 16 & \end{array}$

Direct 500000000000

0.6050000000000

1.1050000000000

1.1050000000000

0.73490000000000

$-0.73490000000000$

1.2349000000000

$-0.23490000000000$

0.74300000000000

$-0.74300000000000$ 
1.21310000000000 $-0.21310000000000$ 0.00000000000000 0.50000000000000

$\mathrm{FeSb}_{2}$ : AB2_oP6_34_a_c - CIF

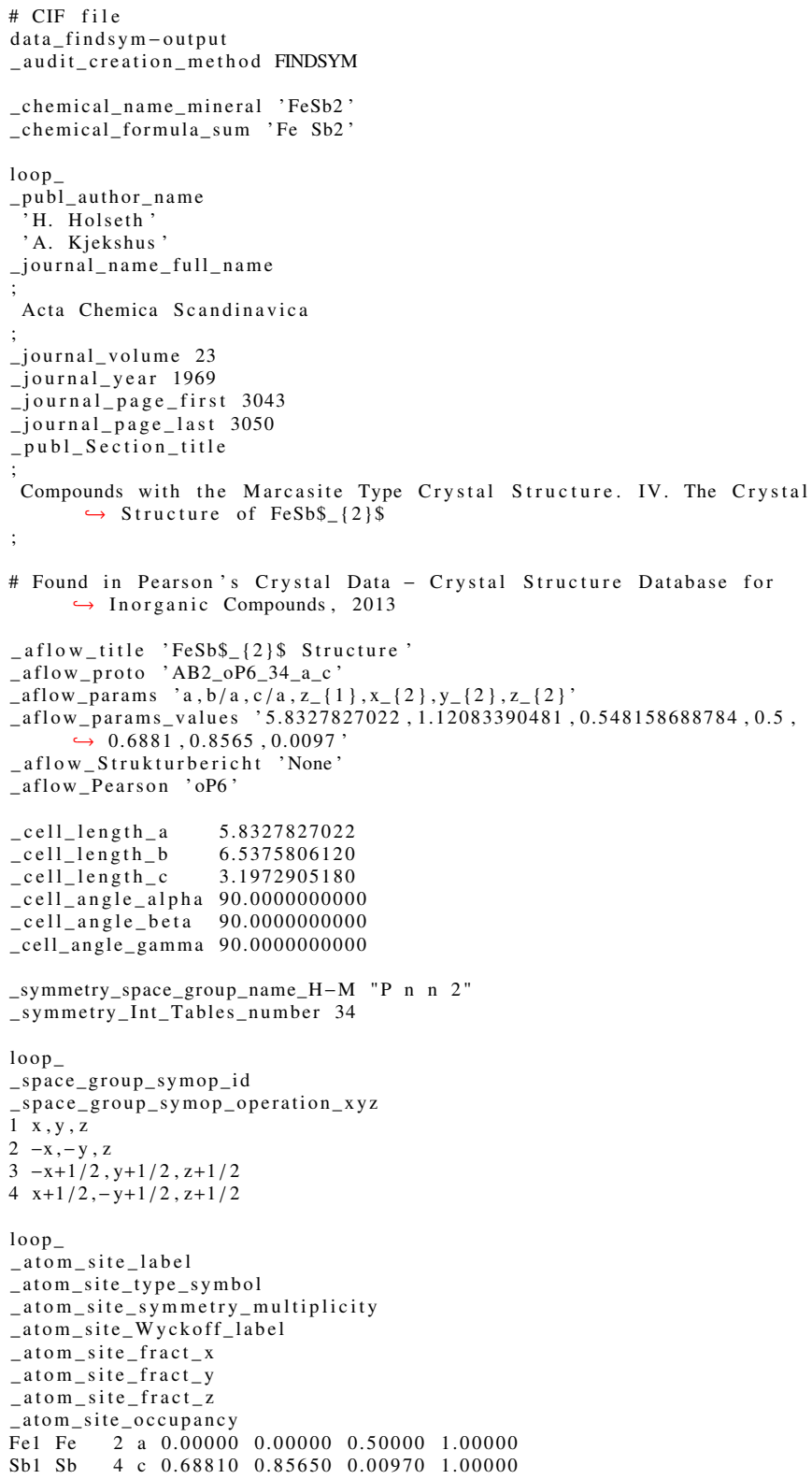

$\mathrm{FeSb}_{2}$ : AB2_oP6_34_a_c - POSCAR

\begin{tabular}{|c|c|c|c|c|}
\hline \multicolumn{5}{|c|}{ 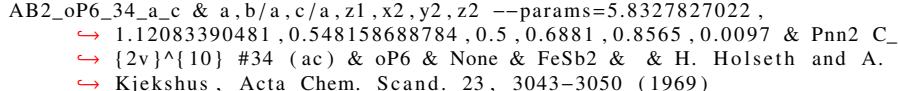 } \\
\hline \\
\hline 5.83278270220000 & 0.00000000000000 & 0.00000000000000 & & \\
\hline 0.00000000000000 & 6.53758061200000 & 0.00000000000000 & & \\
\hline 0.00000000000000 & 0.00000000000000 & 3.19729051800000 & & \\
\hline $\mathrm{Fe}$ & & & & \\
\hline 4 & & & & \\
\hline \multicolumn{5}{|l|}{ Direct } \\
\hline 0.00000000000000 & 0.00000000000000 & 0.50000000000000 & $\mathrm{Fe}$ & (2a) \\
\hline 0.50000000000000 & 0.50000000000000 & 1.00000000000000 & $\mathrm{Fe}$ & $(2 a)$ \\
\hline 0.68810000000000 & 0.85650000000000 & 0.00970000000000 & $\mathrm{Sb}$ & $(4 c)$ \\
\hline-0.68810000000000 & -0.85650000000000 & 0.00970000000000 & $\mathrm{Sb}$ & $(4 c)$ \\
\hline 1.18810000000000 & $0-0.35650000000000$ & 0.50970000000000 & $\mathrm{Sb}$ & $(4 c)$ \\
\hline-0.18810000000000 & 1.35650000000000 & 0.50970000000000 & $\mathrm{Sb}$ & $(4 c)$ \\
\hline
\end{tabular}

$\mathrm{V}_{2} \mathrm{MoO}_{8}$ : AB8C2_oC22_35_a_ab3e_e - CIF

\section{\# CIF file}

data findsym-output

audit_creation method FINDSYM

chemical_name_mineral 'V2MoO8'

chemical formula sum 'Mo O8 V2'

loop

publ_author_name
'P. Mah\{ll'e\}-Pailleret'

journal_year 1970

_publ_Section_title

Contribution $\{\backslash$ 'a $\} 1 \backslash,\{\backslash$ 'e $\}$ tude chimique et structurale des compos $\{\backslash$ $\hookrightarrow$ e $\}$ s AB\$_\{2\}\$O rencontr $\left\{1 \backslash^{\prime} e\right\}$ s dans les syst $\left\{\backslash^{\prime} e\right\}$ mes Mo-VO, UVO $\hookrightarrow$ et $\mathrm{U}-\mathrm{Mo}-\mathrm{O}$

\# Found in Pearson's Crystal Data - Crystal Structure Database for $\hookrightarrow$ Inorganic Compounds, 2013

aflow_title $V \$ \_\{2\} \$ M o O \$_{-}\{8\} \$$ Structure

aflow_proto , AB8C2_oC22_35_a_ab3e_e

aflow_params $, a, b / a, c / a, z_{-}\{1\}, z_{-}\{2\}, z_{-}\{3\}, y_{-}\{4\}, z_{-}\{4\}, y_{-}\{5\}, z_{-}\{5\}, y_{-}\{6$ $\longrightarrow$ params a , b/a, c/a, z

aflow params values $, 4.534758023,1.1408839779,2.72580110498,0.0,0.5838$ $\hookrightarrow,-0.042,0.189,0.5461,0.0961,0.122,0.2982,0.1399,0.1866,0.0038$, aflow_Strukturbericht 'None, -aflow_Pearson 'oC22,

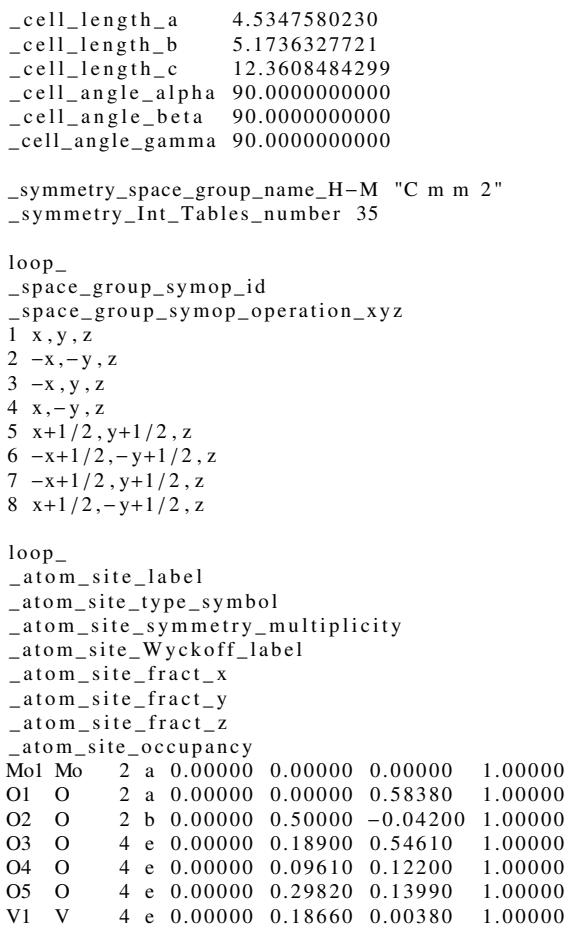

$\mathrm{V}_{2} \mathrm{MoO}_{8}$ : AB8C2_oC22_35_a_ab3e_e - POSCAR

\begin{tabular}{|c|c|c|c|c|}
\hline \multicolumn{5}{|c|}{ AB8C2_oC22_35_a_ab3e_e \& a,b/a,c/a,z1,z2,z3,y4,z4,y5,z5,y6,z6,y7,z7 -- } \\
\hline \multirow{4}{*}{\multicolumn{5}{|c|}{$\begin{array}{l}\hookrightarrow \text { params }=4.534758023,1.1408839779,2.72580110498,0.0,0.5838,-0.042 \\
\hookrightarrow, 0.189,0.5461,0.0961,0.122,0.2982,0.1399,0.1866,0.0038 \& \text { Cmm2 } \\
\hookrightarrow C_{-}\{2 \mathrm{v}\}^{\wedge}\{11\} \# 35\left(\mathrm{a}^{\wedge} 2 \mathrm{be}^{\wedge} 4\right) \& \text { oC22 \& None \& V2MoO8 \& \& P. Mah }\{\backslash, \\
\hookrightarrow \text { e }\}- \text { Pailleret },(1970)\end{array}$}} \\
\hline & & & & \\
\hline & & & & \\
\hline & & & & \\
\hline \multicolumn{5}{|l|}{1.00000000000000} \\
\hline 2.26737901150000 & -2.58681638605000 & 0.00000000000000 & & \\
\hline 2.26737901150000 & 2.58681638605000 & 0.00000000000000 & & \\
\hline 0.00000000000000 & 0.00000000000000 & 12.36084842990000 & & \\
\hline Mo & & & & \\
\hline 1 & & & & \\
\hline \multicolumn{5}{|l|}{ Direct } \\
\hline 0.00000000000000 & 0.0 & 0.0 & Mo & (2a) \\
\hline 0.00000000000000 & 0.00000000000000 & 0000000 & $\mathrm{O}$ & (2a) \\
\hline 0.50000000000000 & 0.50000000000000 & -0.04200000000000 & $\mathrm{O}$ & (2b) \\
\hline-0.18900000000000 & 0.18900000000000 & 00000000 & $\mathrm{O}$ & $(4 \mathrm{e})$ \\
\hline 0.18900000000000 & -0.18900000000000 & 0.54610000000000 & $\mathrm{O}$ & $(4 \mathrm{e})$ \\
\hline-0.09610000000000 & 0.09610000000000 & 0.12200000000000 & $\mathrm{O}$ & $(4 \mathrm{e})$ \\
\hline 0.09610000000000 & -0.09610000000000 & 0.12200000000000 & $\mathrm{O}$ & $(4 \mathrm{e})$ \\
\hline-0.29820000000000 & 0.29820000000000 & 0.13990000000000 & $\mathrm{O}$ & $(4 \mathrm{e})$ \\
\hline 0.29820000000000 & -0.29820000000000 & 0.13990000000000 & $\mathrm{O}$ & $(4 \mathrm{e})$ \\
\hline-0.18660000000000 & 0.18660000000000 & 0.00380000000000 & $\mathrm{~V}$ & $(4 \mathrm{e})$ \\
\hline 0.18660000000000 & -0.18660000000000 & 0.00380000000000 & $\mathrm{~V}$ & $(4 \mathrm{e})$ \\
\hline
\end{tabular}

HCl: AB_oC8_36_a_a - CIF

\# CIF file
data_findsym-output
_audit_creation_method
_chemical_name_mineral,
_chemical_formula_sum
loop_
_publ_author_name
'E. S $\backslash \backslash$, $\{$ a\}ndor,
'R. F. C. Farrow,
;ournal_name_full_name
; Nature
; journal_volume 213
_journal_year 1967




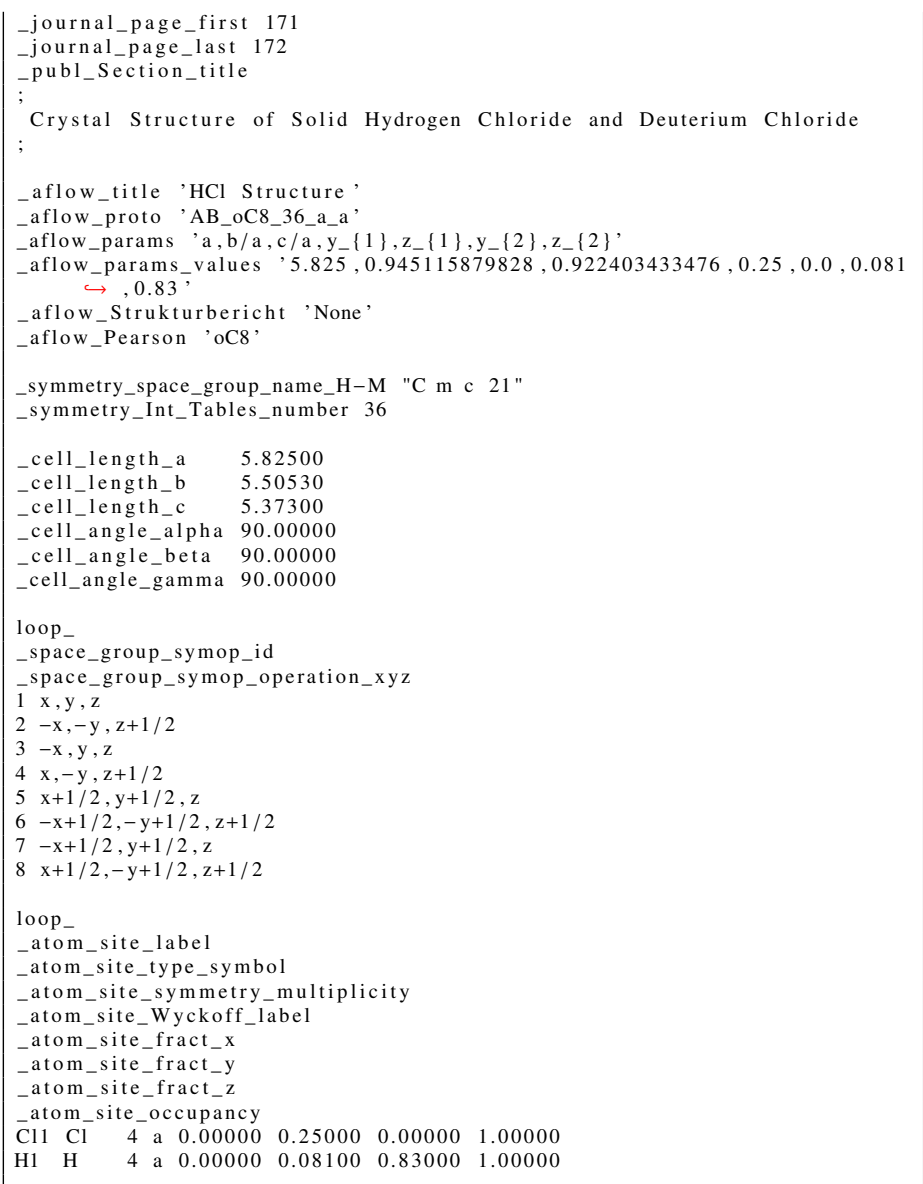

HCl: AB_oC8_36_a_a - POSCAR

\begin{tabular}{|c|c|c|c|c|}
\hline \multirow{2}{*}{\multicolumn{5}{|c|}{ 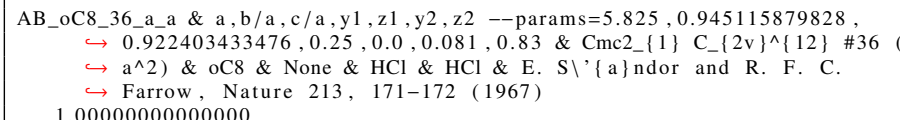 }} \\
\hline & & & & \\
\hline \multirow{2}{*}{2.91250000000000} & -2.75265000000000 & 0.00000000000000 & & \\
\hline & 2.75265000000000 & 0.00000000000000 & & \\
\hline 0.00000000000000 & 0.00000000000000 & 5.37300000000000 & & \\
\hline $\mathrm{Cl}$ & & & & \\
\hline 2 & & & & \\
\hline \multicolumn{5}{|l|}{ Direct } \\
\hline-0.25000000000000 & 0.25000000000000 & 0.00000000000000 & $\mathrm{Cl}$ & (4a) \\
\hline 0.25000000000000 & -0.25000000000000 & 0.50000000000000 & $\mathrm{Cl}$ & (4a) \\
\hline-0.08100000000000 & 0.08100000000000 & 0.83000000000000 & $\mathrm{H}$ & (4a) \\
\hline 0.08100000000000 & -0.08100000000000 & 1.33000000000000 & $\mathrm{H}$ & (4a) \\
\hline
\end{tabular}

$\mathrm{Li}_{2} \mathrm{Si}_{2} \mathrm{O}_{5}$ : A2B5C2_oC36_37_d_c2d_d - CIF

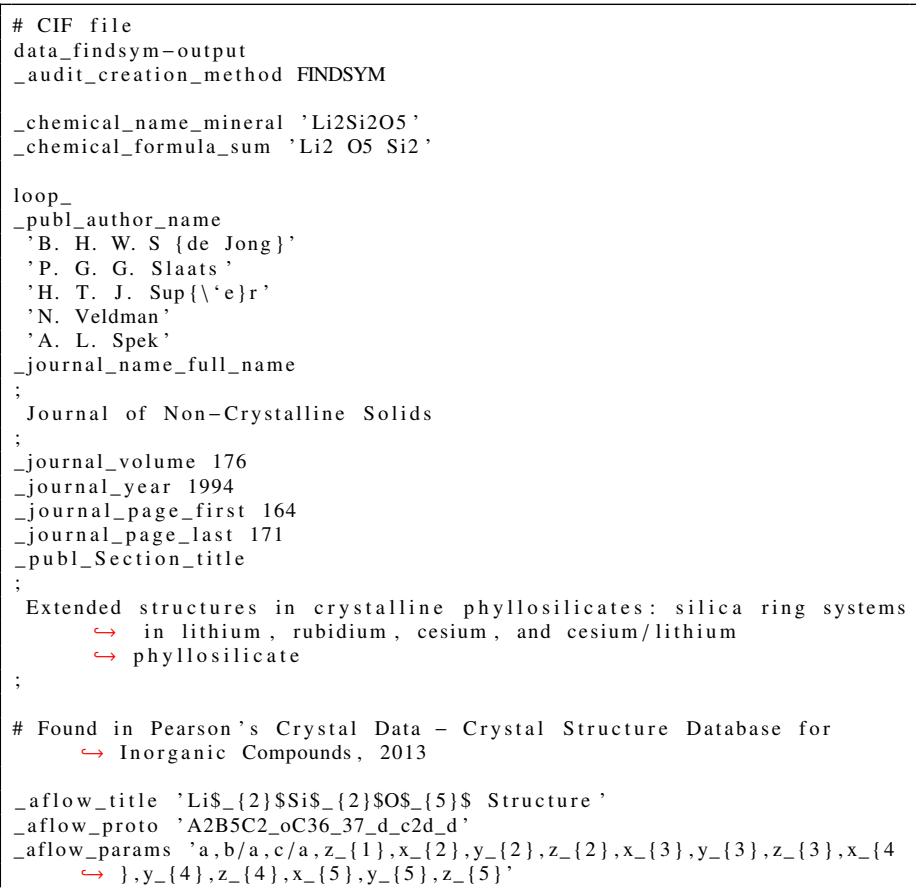

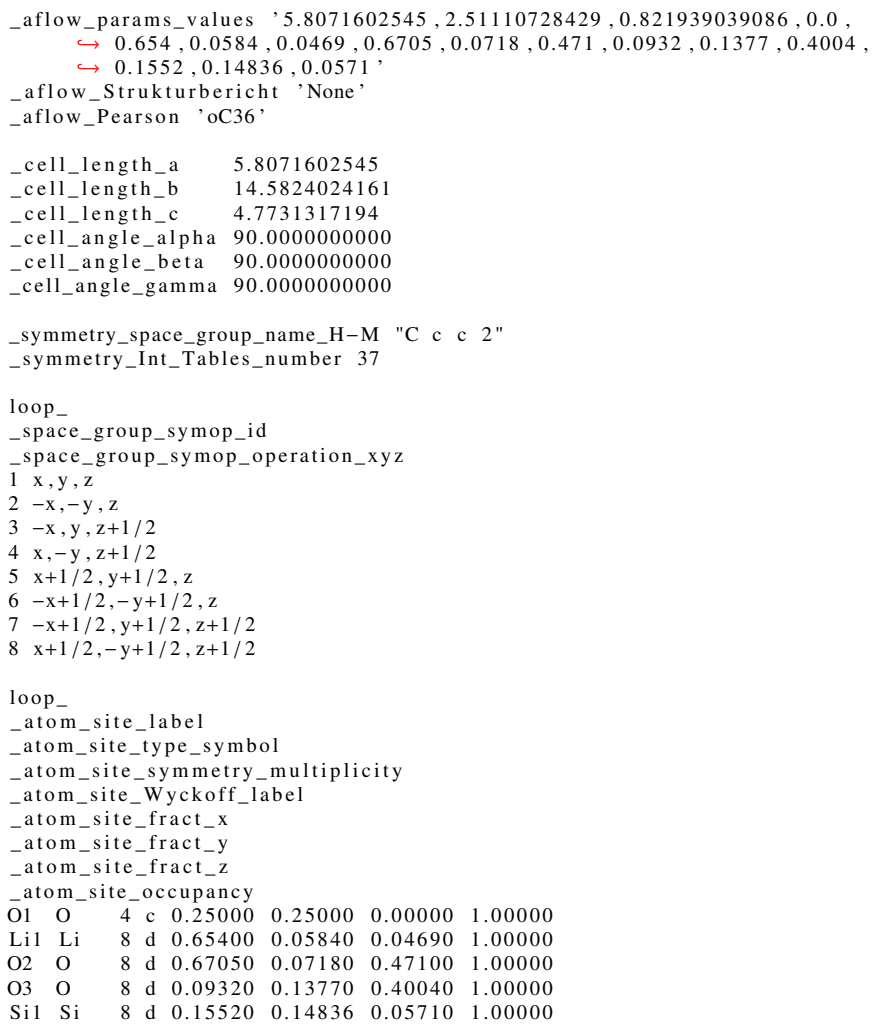

$\mathrm{Li}_{2} \mathrm{Si}_{2} \mathrm{O}_{5}$ : A2B5C2_oC36_37_d_c2d_d - POSCAR

\begin{tabular}{|c|c|c|c|c|}
\hline \multirow{2}{*}{\multicolumn{5}{|c|}{ A2B5C2_oC36_37_d_c2d_d \& a a b/a,c/a, z1, x2,y2, z2, x3,y3,z3, x4,y4, z4, x5, y5 }} \\
\hline & & & & \\
\hline \multicolumn{5}{|c|}{$\hookrightarrow, 0.0584,0.0469,0.6705,0.0718,0.471,0.0932,0.1377,0.4004,0.1552}$, \\
\hline \multirow{3}{*}{\multicolumn{5}{|c|}{$\begin{array}{l}\hookrightarrow 0.14836,0.0571 \& \text { C Cc2 } 2 \mathrm{C}_{-}\{2 \mathrm{v}\}^{\wedge}\{13\} \# 37\left(\mathrm{~cd}^{\wedge} 4\right) \& \text { oC } 36 \& \text { None \& } \\
\hookrightarrow \text { Li2Si2O5 \& \& B. H. W. S }\{\text { de Jong\} et al., J. Non Cryst. Solids }\end{array}$}} \\
\hline & & & & \\
\hline & & & & \\
\hline \multicolumn{5}{|c|}{1.00000000000000} \\
\hline 2.90358012725000 & -7.29120120805000 & \multicolumn{3}{|l|}{0.00000000000000} \\
\hline \multirow{2}{*}{$\begin{array}{l}2.90358012725000 \\
0.00000000000000\end{array}$} & 7.29120120805000 & \multicolumn{3}{|l|}{0.00000000000000} \\
\hline & 0000 & 4.77313171940000 & & \\
\hline $\mathrm{Li}$ & & & & \\
\hline \multirow{2}{*}{\multicolumn{5}{|c|}{ Direct }} \\
\hline & & & & \\
\hline 0.59560000000000 & 0.71 & 0.0469 & $\mathrm{Li}$ & $(8 \mathrm{~d})$ \\
\hline-0.59560000000000 & -0.71 & 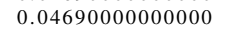 & $\mathrm{Li}$ & $(8 \mathrm{~d})$ \\
\hline & 0000 & 0.54690000000000 & $\mathrm{Li}$ & $(8 \mathrm{~d})$ \\
\hline & 0000000 & 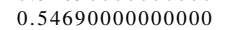 & $\mathrm{Li}$ & $(8 \mathrm{~d})$ \\
\hline 0.00000000000000 & & & $\mathrm{O}$ & \\
\hline 0.50000000000000 & & 0.5 & $\mathrm{O}$ & $(4 \mathrm{c})$ \\
\hline 0.59870000000000 & & & $\mathrm{O}$ & \\
\hline-0.59870000000000 & -0.74230000000000 & 0.471 & $\mathrm{O}$ & (8d) \\
\hline 0.74230000000000 & 0000000 & 0.97 & $\mathrm{O}$ & \\
\hline-0.74230000000000 & -0.59870 & 0.97100000000000 & & \\
\hline-0.04450000000000 & 0.23090000000000 & 0.40040000000000 & $\mathrm{O}$ & $(8 \mathrm{~d})$ \\
\hline 0.04450000000000 & -0.23090000000000 & 0.40040000000000 & $\mathrm{O}$ & \\
\hline 0.23090000000000 & -0.04450000000000 & 0.90040000000000 & $\mathrm{O}$ & $(8 \mathrm{~d})$ \\
\hline & 0.04450000000000 & 0.90040000000000 & $\mathrm{O}$ & \\
\hline 0.00684000000000 & 0.30356000000000 & 0.05710000000000 & $\mathrm{Si}$ & $(8 \mathrm{~d})$ \\
\hline-0.00684000000000 & -0.30356000000000 & 0.05710000000000 & $\mathrm{Si}$ & (8d) \\
\hline $\begin{array}{r}-0.00084000000000 \\
0.30356000000000\end{array}$ & 0.006840 & 0.55710000000000 & $\mathrm{Si}$ & (8d) \\
\hline-0.30356000000000 & -0.00684000000000 & 0.55710000000000 & $\mathrm{Si}$ & $(8 \mathrm{~d})$ \\
\hline
\end{tabular}

$\mathrm{Ta}_{3} \mathrm{~S}_{2}$ : A2B3_oC40_39_2d_2c2d - CIF

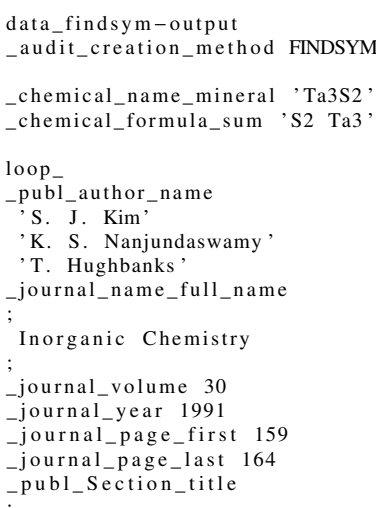


Single-crystal structure of tantalum sulfide (Ta\$_ $\left.\{3\} \$ S \$ \$_{-}\{2\} \$\right)$

$\rightarrow$ Structure and bonding in the $\operatorname{Ta} \$\{\{6\} \$ S n(\$ n \$=1,3,4,5$ ?)

$\hookrightarrow$ pentagonal-antiprismatic chain compounds

\# Found in Pearson's Crystal Data - Crystal Structure Database fo $\hookrightarrow$ Inorganic Compounds, 2013

aflow title 'Ta\$ $\{3\} \$ S \$\{2\} \$$ Structure

aflow_proto, A2B3_oC40 392 2d 2c2d

aflow_params $, a, b / a, c / a, x_{-}\{1\}, z_{-}\{1\}, x_{-}\{2\}, z_{-}\{2\}, x_{-}\{3\}, y_{-}\{3\}, z_{-}\{3\}, x_{-}\{4$ $\hookrightarrow\}, y_{-}\{4\}, z_{-}\{4\}, x_{-}\{5\}, y_{-}\{5\}, z_{-}\{5\}, x_{-}\{6\}, y_{-}\{6\}, z_{-}\{6\}$

aflow_params_values $7.47831,2.30292940517,0.749515599113,0.3449,0.5$

$\hookrightarrow 0.0058,0.7654,0.173,0.5398,0.3889,0.5843,0.6154,0.3917,0.28104$

$\hookrightarrow 0.0058,0.7654,0.173,0.5398,0.3889,0.584$

aflow Strukturbericht 'None'

aflow_Pearson 'oC40,

_symmetry_space_group_name_H-M "A b m 2"

-symmetry_Int_Tables_number 39

_cell_length_a 7.4783

_cell_length_b $\quad 17.22202$

_cell_length_c $\quad 5.60511$

_cell_angle_alpha 90.00000

_cell_angle_beta 90.00000

cell_angle_gamma 90.00000

loop

_space_group_symop_id

_space_group_symop_operation_xyz

$1 \mathrm{x}, \mathrm{y}, \mathrm{z}$

$2-x,-y, z$

$3 \mathrm{x},-\mathrm{y}, \mathrm{z}+1 / 2$
$4-\mathrm{x}, \mathrm{y}, \mathrm{z}+1 / 2$

$5 \mathrm{x}, \mathrm{y}+1 / 2, \mathrm{z}+1 / 2$

$6-\mathrm{x},-\mathrm{y}+1 / 2, \mathrm{z}+1 / 2$

$7 x,-y+1 / 2, z$

loop

_atom_site_label

_atom_site_type_symbol

_atom_site_symmetry_multiplicity

_atom_site_Wyckoff_label

_atom_site_fract_x

atom_site_fract_y

atom_site_fract_z

Tate site occupancy

$\begin{array}{llllllll}\mathrm{T}_{1} 1 \mathrm{Ta} & 4 & \mathrm{c} & 0.34490 & 0.25000 & 0.50000 & 1.00000\end{array}$

$\begin{array}{lllllllll}\mathrm{Ta} 2 & \mathrm{Ta} & 4 & \mathrm{c} & 0.00580 & 0.25000 & 0.76540 & 1.00000\end{array}$

$\begin{array}{llllllllll}\mathrm{S} 1 & \mathrm{~S} & 8 & \mathrm{~d} & 0.17300 & 0.53980 & 0.38890 & 1.00000\end{array}$

$\begin{array}{llllllll}\mathrm{S} 2 & \mathrm{~S} & 8 & \mathrm{~d} & 0.58430 & 0.61540 & 0.39170 & 1.00000\end{array}$

$\begin{array}{lllllllll}\mathrm{Ta} 3 & \mathrm{Ta} & 8 & \mathrm{~d} & 0.28104 & 0.16619 & 0.02060 & 1.00000\end{array}$

$\begin{array}{llllllll}\mathrm{Ta} 4 & \mathrm{Ta} & 8 & \mathrm{~d} & 0.10455 & 0.60730 & 0.01440 & 1.00000\end{array}$

$\mathrm{Ta}_{3} \mathrm{~S}_{2}$ : A2B3_oC40_39_2d_2c2d - POSCAR

A2B3_oC40_39_2d_2c2d \& a , b/a,c/a, x1, z1 $, \mathrm{x} 2, \mathrm{z2}, \mathrm{x} 3, \mathrm{y} 3, \mathrm{z} 3, \mathrm{x} 4, \mathrm{y} 4, \mathrm{z} 4, \mathrm{x} 5, \mathrm{y} 5, \mathrm{z} 5$

$\hookrightarrow \mathrm{x} 6, \mathrm{y} 6, \mathrm{z} 6-$ params $=7.47831,2.30292940517,0.749515599113,0.3449$,

$\hookrightarrow 0.5,0.0058,0.7654,0.173,0.5398,0.3889,0.5843,0.6154,0.3917$

$\hookrightarrow 0.28104,0.16619,0.0206,0.10455,0.6073,0.0144 \&$ Abm2 $C_{-}\{2 v\}^{\wedge}\{15\}$

$\hookrightarrow \# 39\left(\mathrm{c}^{\wedge} 2 \mathrm{~d}^{\wedge} 4\right) \& \mathrm{oC} 40$ \& None \& Ta3S2 \& \& S. J. Kim and K. S.

$\hookrightarrow$ Nanjundaswamy and T. Hughbanks, Inorg. Chem. 30, 159-164 (1991)

1.00000000000000

$\begin{array}{lll}7.47831000000000 & 0.00000000000000 & 0.00000000000000\end{array}$

$\begin{array}{llr}0.00000000000000 & 8.61101000000000 & -2.80255500000000\end{array}$

$\begin{array}{rrr}0.00000000000000 & 8.61101000000000 & 2.80255500000000\end{array}$

$\mathrm{S} \quad \mathrm{Ta}$

Direct

0.17300000000000

0.17300000000000
-0.17300000000000

0.17300000000000
0.17300000000000

$-0.17300000000000$

0.58430000000000

$-0.58430000000000$

0.58430000000000

$-0.58430000000000$

0.34490000000000

$-0.34490000000000$

0.00580000000000

$-0.00580000000000$

0.28104000000000

$-0.28104000000000$

0.28104000000000

$-0.28104000000000$

0.10455000000000

$-0.10455000000000$

0.10455000000000

0.10455000000000
-0.10455000000000

0.15090000000000

0.92870000000000

$-0.15090000000000$

0.34910000000000

1.42870000000000
1.00710000000000

1.00710000000000
-0.22370000000000 -0.22370000000000
0.27630000000000 0.27630000000000
1.50710000000000 1.50710000000000
0.75000000000000 0.75000000000000
1.25000000000000 1.01540000000000 1.51540000000000 0.18679000000000 $-0.14559000000000$ 0.35441000000000 0.68679000000000 0.62170000000000 $-0.5929000000000$ $-0.0929000000000$ -0.09290000000000
1.12170000000000 0.6455900 $-0.62170000000000$ $-0.12170000000000$

VPCl9: A9BC_oC44_39_3c3d_a_c - CIF

\# CIF file

data findsym-output

audit_creation_method FINDSYM

chemical name mineral 'VPCl9'

chemical formula_sum ' $\mathrm{Cl9} \mathrm{P} \mathrm{V}$ '

loop

publ_author_name

M. L. Ziegler

'B. Nuber'

K. Weidenhammer,

G. Hoch

journal_name_full_name

Zeitschrift $f \backslash \backslash " u\} r$ Naturforschung $B$

journal_volume 32

journal_year 1977

journal_page_first 18

journal_page_last 21

publ_section title

Die Molek $\{\backslash$ u $\} l$-und Kristallstruktur von

$\hookrightarrow$ Tetrachlorophosphoniumpentachlorovanadat (IV), [ $\left.\mathrm{PCl} \$ \_\{4\} \$\right][$

$\hookrightarrow$ Tetrachlorophosphoniumpentachlorovanadat (IV),

$\hookrightarrow$ Tetrachlorophosphoniumpentachlorovanadate (IV) , [ PCl\$_\{4\}\$]

$\hookrightarrow \mathrm{VCl}_{-}\{5\} \$ 1$

\# Found in Pearson's Crystal Data - Crystal Structure Database for

$\hookrightarrow$ Inorganic Compounds, 2013

aflow_title, $\mathrm{VPCl}_{-}\{9\} \$$ Structure

aflow_proto 'A9BC_oC44_39_3c3d_a_c

aflow_params 'a,b/a,c/a, $z_{-}\{1\}, x_{-}\{2\}, z_{-}\{2\}, x_{-}\{3\}, z_{-}\{3\}, x_{-}\{4\}, z_{-}\{4\}, x_{-}\{5$ $\hookrightarrow\}, z_{-}\{5\}, x_{-}\{6\}, y_{-}\{6\}, z_{-}\{6\}, x_{-}\{7\}, y_{-}\{7\}, z_{-}\{7\}, x_{-}\{8\}, y_{-}\{8\}, z_{-}\{8\}$

aflow_params_values $, 6.2123847492,1.90215711527,2.62509658728,0.5$

$\hookrightarrow 0.2673,0.7493,0.6176,0.8879,0.6246,0.6059,0.6191,0.7476,0.2266$,

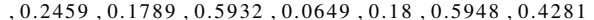

aflow Strukturbericht 'None'

-aflow_Pearson 'oC44'

cell_length_a $\quad 6.2123847492$

cell_length_b 11.8169318535

cell_length_c 16.3081100040

cell_angle_alpha 90.0000000000

_cell_angle_beta 90.0000000000

_cell_angle_gamma 90.0000000000

symmetry_space_group_name_H-M "A b m 2"

_symmetry_Int_Tables_number 39

loop

space_group_symop_id

space_group_symop_operation_xyz

$\mathrm{x}, \mathrm{y}, \mathrm{z}$

$2-x,-y, z$
$3-x,-y, z+1 / 2$

$\mathrm{x},-\mathrm{y}, \mathrm{z}+1 / 2$

$-\mathrm{x}, \mathrm{y}, \mathrm{z}+1 / 2$
$\mathrm{x}, \mathrm{y}+1 / 2, \mathrm{z}+1 / 2$

$\mathrm{x}, \mathrm{y}+1 / 2, \mathrm{z}+1 / 2$
$-\mathrm{x},-\mathrm{y}+1 / 2, \mathrm{z}+1 / 2$

$-\mathrm{x},-\mathrm{y}+1 / 2, \mathrm{z}+1$
$\mathrm{x},-\mathrm{y}+1 / 2, \mathrm{z}$

$8-x, y+1 / 2, z$

loop_

atom_site_label

atom_site_type_symbol

atom_site_symmetry_multiplicity

atom_site_Wyckoff_label

atom_site_fract_x

atom_site_fract_y

atom_site_fract_z

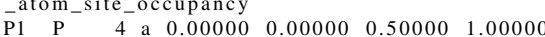

$\begin{array}{llllllll}\mathrm{Cl} 1 \mathrm{Cl} & 4 \mathrm{c} & 0.026730 & 0.25000 & 0.74930 & 1.00000\end{array}$

$\begin{array}{llllllll}\mathrm{Cl} & \mathrm{Cl} & 4 & \mathrm{c} & 0.26730 & 0.25000 & 0.74930 & 1.00000\end{array}$

$\begin{array}{lllllllll}\mathrm{Cl} & \mathrm{Cl} & 4 & \mathrm{c} & 0.61760 & 0.25000 & 0.88790 & 1.00000\end{array}$

$\begin{array}{lllllllll}\mathrm{Cl} & \mathrm{Cl} & 4 \mathrm{c} & 0.61760 & 0.25000 & 0.88790 & 1.00000\end{array}$

$\begin{array}{lllllllll}\mathrm{V} 1 & \mathrm{~V} & 4 & \mathrm{C} & \mathrm{c} & 0.61910 & 0.25000 & 0.74760 & 1.00000\end{array}$

$\begin{array}{llllllllll}\mathrm{Cl} 14 & \mathrm{Cl} & 8 & \mathrm{~d} & 0.22660 & 0.08570 & 0.24590 & 1.00000\end{array}$

$\begin{array}{llllllll}\mathrm{Cl} 5 & \mathrm{Cl} & 8 & \mathrm{~d} & 0.17890 & 0.59320 & 0.06490 & 1.00000 \\ \mathrm{Cl} 6 & \mathrm{Cl} & 8 & \mathrm{~d} & 0.18000 & 0.59480 & 0.42810 & 1.00000\end{array}$

VPCl 9 : A9BC_oC44_39_3c3d_a_c - POSCAR

A9BC_oC44 39 3c3d_a_c \& a , b/a,c/a, z1, x2, z2, x3, z3, x4, z4 x5 , z5, x6, y6, z6 , x7

$\hookrightarrow, \mathrm{y} 7, \mathrm{z} 7, \mathrm{x} 8, \mathrm{y} 8, \mathrm{z} 8-$-params $=6.2123847492,1.90215711527$,

$\longrightarrow 2.62509658728,0.5,0.2673,0.7493,0.6176,0.8879,0.6246,0.6059$

$\hookrightarrow 0.6191,0.7476,0.2266,0.0857,0.2459,0.1789,0.5932,0.0649,0.18$,

$\rightarrow 0.5948,0.4281 \& \mathrm{Abm} 2 \mathrm{C}_{-}\{2 \mathrm{v}\}^{\wedge}\{15\} \# 39\left(\mathrm{ac}^{\wedge} 4 \mathrm{~d}^{\wedge} 3\right) \& \mathrm{oC} 44 \&$ None \&

$\rightarrow$ VPCl9 \& \& M. L. Ziegler et al., Z. Naturforsch. B 32, 18-21 $\hookrightarrow$ 1977)

1.00000000000000

$\begin{array}{lll}6.21238474920000 & 0.00000000000000 & 0.00000000000000\end{array}$

$\begin{array}{lll}0.00000000000000 & 5.90846592675000 & -8.15405500200000\end{array}$

$\begin{array}{rrr}0.00000000000000 & 5.90846592675000 & 8.15405500200000\end{array}$

$\mathrm{Cl} \quad \mathrm{P}$

Direct

0.26730000000000

$-0.26730000000000$

0.61760000000000

$-0.61760000000000$

0.62460000000000

$-0.62460000000000$

0.22660000000000

$-0.22660000000000$

0.22660000000000

$-0.22660000000000$

0.17890000000000

$-0.17890000000000$

0.17890000000000

$-0.17890000000000$

0.18000000000000

$-0.18000000000000$

0.18000000000000

$-49930000000000$ 0.63790000000000 $-0.637900000000$ . 0.35590000000000 0.14410000000000 $-0.16020000000000$ 0.33160000000000 0.16840000000000 0.33980000000000 0.52830000000000 $-0.65810000000000$ 1.02830000000000 0.16670000000000 $-0.52290000000000$

0.99930000000000 1.49930000000000 1.13790000000000 1.63790000000000 0.85590000000000 1.35590000000000 0.33160000000000 0.16020000000000 0.16020000000000 0.66020000000000 0.83160000000000 0.65810000000000 $-0.52830000000000$ $-0.02830000000000$ $-0.16670000000000$ 0.33330000000000
$\mathrm{Cl} \quad(4 \mathrm{c})$

$\mathrm{Cl}(4 \mathrm{c})$

$\mathrm{Cl} \quad(4 \mathrm{c})$

$\mathrm{Cl}(4 \mathrm{c})$

$\mathrm{Cl} \quad(4 \mathrm{c})$

$\mathrm{Cl}$ (8d)

$\mathrm{Cl}$ (8d)

$\mathrm{Cl}$ (8d)

$\begin{array}{ll}\mathrm{Cl} & (8 \mathrm{~d}) \\ \mathrm{Cl} & (8 \mathrm{~d})\end{array}$

$\mathrm{Cl}(8 \mathrm{~d})$

$\begin{array}{ll}\mathrm{Cl} & (8 \mathrm{~d}) \\ \mathrm{Cl} & (8 \mathrm{~d})\end{array}$

Cl (8d)

$\begin{array}{ll}\mathrm{Cl} & (8 \mathrm{~d}) \\ \mathrm{Cl} & (8 \mathrm{~d})\end{array}$

$\mathrm{Cl} \quad(8 \mathrm{~d})$

$\mathrm{Cl}$
$\mathrm{Cl}$ 


$\begin{array}{rrrrr}-0.18000000000000 & 0.66670000000000 & 1.52290000000000 & \mathrm{Cl} & (8 \mathrm{~d}) \\ 0.00000000000000 & -0.50000000000000 & 0.50000000000000 & \mathrm{P} & (4 \mathrm{a}) \\ 0.00000000000000 & 0.00000000000000 & 1.00000000000000 & \mathrm{P} & (4 \mathrm{a}) \\ 0.61910000000000 & -0.49760000000000 & 0.99760000000000 & \mathrm{~V} & (4 \mathrm{c}) \\ -0.61910000000000 & 0.00240000000000 & 1.49760000000000 & \mathrm{~V} & (4 \mathrm{c})\end{array}$

$\mathrm{K}_{2} \mathrm{CdPb}:$ AB2C_oC16_40_a_2b_b - CIF

\section{\# CIF file}

data_findsym-output

_audit_creation_method FINDSYM

chemical_name mineral ' $\mathrm{K} 2 \mathrm{CdPb}$

_chemical_formula_sum ' $\mathrm{Cd} \mathrm{K} 2 \mathrm{~Pb}$ '

loop

_publ_author_name

R. Matthes'

'H.-U. Schuster'

_journal_name_full_name

Zeitschrift $f\{\backslash " u\}$ r Naturforschung B

_journal_volume 34

journal year 1979

_journal_page_first 541

_journal_page_last 543

-publ_Section_title

Synthese und Struktur der Phasen $K \$ \_\{2\} \$ C$ dSn und $K \$ \_\{2\} \$ C d P b /$ Synthesis

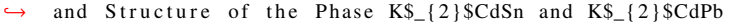

\# Found in Pearson's Crystal Data - Crystal Structure Database for $\hookrightarrow$ Inorganic Compounds, 2013

_aflow_title $K \$ \_\{2\} \$ C \mathrm{CdPb}$ Structure,

-aflow_proto 'AB2C_oC16_40_a_2b_b '

aflow_params 'a,b/a,c/a, z $\{1\}, y_{-}\{2\}, z_{-}\{2\}, y_{-}\{3\}, z_{-}\{3\}, y_{-}\{4\}, z_{-}\{4\}$,

aflow_params values $6.4580033647,1.33199132859,1.69634561784,0.0$, $\hookrightarrow 0.3467,0.1773,0.8661,0.3301,0.7281,0.0093$,

_aflow_Strukturbericht 'None'

_aflow_Pearson 'oC16,

_cell_length_a $\quad 6.4580033647$

_cell_length_b 8.6020044818

_cell_length_c $\quad 10.9550057077$

_cell_angle_alpha 90.0000000000

_cell_angle_beta 90.0000000000

cell_angle_gamma 90.0000000000

_symmetry_space_group_name_H-M "A m a 2"

_symmetry_Int_Tables_number 40

loop

_space_group_symop_id

_space_group_symop_operation_xyz

$1 \mathrm{x}, \mathrm{y}, \mathrm{z}$

$2-\mathrm{x},-\mathrm{y}, \mathrm{z}$

$3 \mathrm{x}+1 / 2,-\mathrm{y}, \mathrm{z}$
$4-\mathrm{x}+1 / 2, \mathrm{y}, \mathrm{z}$

$5 \mathrm{x}, \mathrm{y}+1 / 2, \mathrm{z}+1 / 2$

$6-x,-y+1 / 2, z+1 / 2$

$7 \mathrm{x}+1 / 2,-\mathrm{y}+1 / 2, \mathrm{z}+1 / 2$

$8-\mathrm{x}+1 / 2, \mathrm{y}+1 / 2, \mathrm{z}+1 / 2$

loop

_atom_site_type_symbol

atom_site_symmetry_multiplicity

_atom_site_Wyckoff_label

atom_site_fract_x

atom_site_fract_y

atom_site_fract_z

atom site occupancy

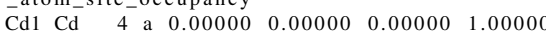

$\begin{array}{llllllll}\mathrm{K} 1 & \mathrm{~K} & 4 & \mathrm{~b} & 0.25000 & 0.34670 & 0.17730 & 1.00000\end{array}$

$\begin{array}{lllllllll}\mathrm{K} 2 & \mathrm{~K} & 4 & \mathrm{~b} & 0.25000 & 0.86610 & 0.33010 & 1.00000\end{array}$

$\begin{array}{llllllll}\mathrm{Pb} 1 & \mathrm{~Pb} & 4 & \mathrm{~b} & 0.25000 & 0.72810 & 0.00930 & 1.00000\end{array}$

$\mathrm{K}_{2} \mathrm{CdPb}$ : AB2C_oC16_40_a_2b_b - POSCAR

AB2C_oC16_40_a_2b_b \& a , b/a,c/a, z1, y2, z2,y3,z3,y4, z4 --params =

$\hookrightarrow 6.4580033647,1.33199132859,1.69634561784,0.0,0.3467,0.1773$

$\hookrightarrow 0.8661,0.3301,0.7281,0.0093 \&$ \& Ama2 $C_{-}\{2 \mathrm{v}\}^{\wedge}\{16\} \# 40\left(\mathrm{ab}^{\wedge} 3\right) \&$

$\hookrightarrow$ oC16 \& None \& $\mathrm{K} 2 \mathrm{CdPb} \&$ \& R. Matthes and H.-U. Schuster, Z

$\hookrightarrow$ Naturforsch. B 34,541-543(1979)

1.00000000000000

$\begin{array}{lll}6.45800336470000 & 0.00000000000000 & 0.00000000000000\end{array}$

$\begin{array}{rrr}0.00000000000000 & 4.30100224090000 & -5.47750285385000\end{array}$

$0.00000000000000 \quad 4.30100224090000 \quad 5.47750285385000$

$\mathrm{Cd} \quad \mathrm{K} \quad \mathrm{Pb}$

Direct

$\begin{array}{lll}0.00000000000000 & 0.00000000000000 & 0.00000000000000\end{array}$ $\begin{array}{lll}0.50000000000000 & 0.00000000000000 & 0.00000000000000\end{array}$ 0.25000000000000 0.75000000000000 0.25000000000000 0.75000000000000 0.25000000000000
0.75000000000000 0.1694000000000 $-0.5240000000000$ 0.53600000000000 $-1.19620000000000$ 0.71880000000000
-0.73740000000000 0.524000 0.52400000000000
-0.16940000000000 -0.16940000000000
1.19620000000000 1.19620000000000
-0.53600000000000 -0.53600000000000
0.73740000000000 0.73740000000000
-0.71880000000000

$\begin{aligned} \mathrm{Cd} & (4 \mathrm{a}) \\ \mathrm{Cd} & (4 \mathrm{a}) \\ \mathrm{K} & (4 \mathrm{~b}) \\ \mathrm{K} & (4 \mathrm{~b}) \\ \mathrm{K} & (4 \mathrm{~b}) \\ \mathrm{K} & (4 \mathrm{~b}) \\ \mathrm{Pb} & (4 \mathrm{~b}) \\ \mathrm{Pb} & (4 \mathrm{~b})\end{aligned}$

CeTe 3 : AB3_oC16_40_b_3b - CIF

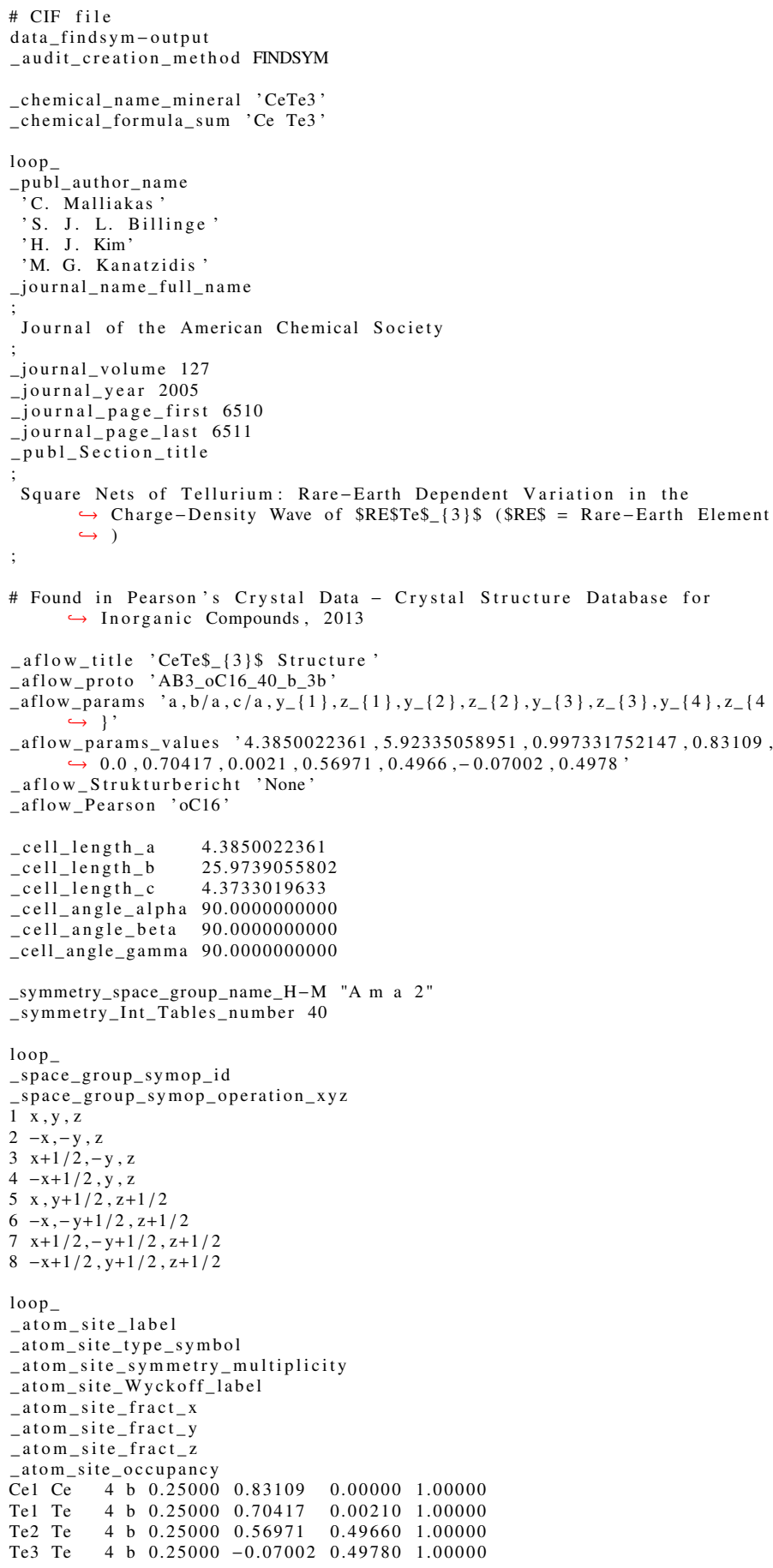

\# Found in Pearson's Crystal Data - Crystal Structure Database for $\hookrightarrow$ Inorganic Compounds, 2013

aflow_title 'CeTe\$_\{3\}\$ Structure'

aflow_proto , AB3_oC 16 40 b 3b

aflow_params 'a, b/a,c/a, y- $\{1\}, \mathrm{z}_{-}\{1\}, \mathrm{y}_{-}\{2\}, \mathrm{z}_{-}\{2\}, \mathrm{y}_{-}\{3\}, \mathrm{z}_{-}\{3\}, \mathrm{y}_{-}\{4\}, \mathrm{z}_{-}\{4$ $\hookrightarrow\}$

aflow_params_values ' $4.3850022361,5.92335058951,0.997331752147,0.83109$, $\hookrightarrow 0.0,0.70417,0.0021,0.56971,0.4966,-0.07002,0.4978$,

aflow_Strukturbericht 'None'

aflow_Pearson 'oC16,

_cell_length_a 4.3850022361

cell_length_b 25.9739055802

_cell_length_c 4.3733019633

cell_angle_alpha 90.0000000000

cell_angle_beta 90.0000000000

_cell_angle_gamma 90.0000000000

_symmetry_space_group_name_H-M "A m a 2"

_symmetry_Int_Tables_number 40

loop

space_group_symop_id

space_group_symop_operation_xyz

$1 \mathrm{x}, \mathrm{y}, \mathrm{z}$

$2-\mathrm{x},-\mathrm{y}, \mathrm{z}$

$3 \mathrm{x}+1 / 2,-\mathrm{y}, \mathrm{z}$

$4-\mathrm{x}+1 / 2, \mathrm{y}, \mathrm{z}$

$5 \mathrm{x}, \mathrm{y}+1 / 2, \mathrm{z}+1 / 2$

$6-\mathrm{x},-\mathrm{y}+1 / 2, \mathrm{z}+1 / 2$

$7 \mathrm{x}+1 / 2,-\mathrm{y}+1 / 2, \mathrm{z}+1 / 2$

$8-x+1 / 2, y+1 / 2, z+1 / 2$

loop

atom_site_label

atom_site_type_symbol

atom_site_symmetry_multiplicity

_atom_site_Wyckoff_label

atom_site_fract_x

atom_site_fract_y

atom_site_fract_z

atom_site_occupancy

$\begin{array}{lllllllll} & \mathrm{Ce} 1 \mathrm{Ce} & 4 & \mathrm{~b} & 0.25000 & 0.83109 & 0.00000 & 1.00000\end{array}$

$\begin{array}{llllllll}\mathrm{Te} 1 & \mathrm{Te} & 4 & \mathrm{~b} & 0.25000 & 0.70417 & 0.00210 & 1.00000\end{array}$

$\begin{array}{llllllll}\mathrm{Te} 2 \mathrm{Te} & 4 & \mathrm{~b} & 0.25000 & 0.56971 & 0.49660 & 1.00000\end{array}$

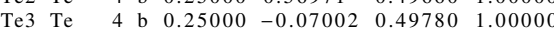

CeTe 3 : AB3_oC16_40_b_3b - POSCAR

AB3_oC16_40_b_3b \& a , b/a, c/a , y1, z1 ,y2, z2, y3 , z3,y4, z4 --params=

$\hookrightarrow 4.3850022361,5.92335058951,0.997331752147,0.83109,0.0,0.70417$ $\hookrightarrow 0.0021,0.56971,0.4966,-0.07002,0.4978 \&$ Ama2 $C_{-}\{2 \mathrm{v}\}^{\wedge}\{16\} \# 40$ (b $\hookrightarrow \wedge 4)$ \& oC16 \& None \& CeTe3 \& \& C. Malliakas et al., J. Am. $\hookrightarrow$ Chem. Soc. $127,6510-6511$ (2005)

1.00000000000000

$\begin{array}{lll}4.38500223610000 & 0.00000000000000 & 0.00000000000000\end{array}$

$\begin{array}{lll}0.00000000000000 & 12.98695279010000 & -2.18665098165000\end{array}$

$\begin{array}{lll}0.00000000000000 & 12.98695279010000 & 2.18665098165000\end{array}$

$\mathrm{Ce} \quad \mathrm{Te}$

Direct

$\begin{array}{lll}0.25000000000000 & 0.83109000000000 & 0.83109000000000\end{array}$ $\begin{array}{lrr}0.75000000000000 & -0.83109000000000 & -0.83109000000000\end{array}$ $0.7500000000000-0.831090000000-0.83109000000000$ $\begin{array}{lll}0.25000000000000 & 0.70207000000000 & 0.70627000000000\end{array}$ $-0.70207000000000$ $\begin{array}{rrr}0.25000000000000 & 0.07311000000000 & 1.06631000000000\end{array}$ $\begin{array}{rrr}0.75000000000000 & -1.06631000000000 & -0.07311000000000 \\ 0.25000000000000 & -0.56782000000000 & 0.42778000000000\end{array}$ $\begin{array}{lll}0.25000000000000 & -0.56782000000000 & 0.42778000000000 \\ 0.75000000000000 & -0.42778000000000 & 0.56782000000000\end{array}$

$\mathrm{W}_{3} \mathrm{O}_{10}$ : A10B3_oF52_42_2abce_ab - CIF

\section{\# CIF file}

data_findsym-output

_audit_creation_method FINDSYM 
chemical_name_mineral 'W3O10'

_chemical_formula_sum 'O10 W3'

loop

publ_author_name

'B. Gerand'

G. Nowogrocki

'M. Figlarz'

journal_name_full_name

Journal of Solid State Chemistry

journal_volume 38

journal year 1981

journal_page_first 312

journal_page_last 320

publ_Section_title

A new tungsten trioxide hydrate, $\operatorname{WO}_{-}\{3\} \$\{\backslash$ textperiodcentered $\} 1 / 3 \mathrm{H} \$ \_\{2\}$ $\hookrightarrow$ \$O: Preparation, characterization, and crystallographic study

\# Found in Pearson's Crystal Data - Crystal Structure Database for $\hookrightarrow$ Inorganic Compounds, 2013

aflow_title'W\$_\{3\}\$O\$_ $\{10\} \$$ Structure'

aflow proto, A10B3 oF52 42 2abce ab

aflow_params 'a,b/a,c/a, z $\{1\}, \mathrm{z}_{-}\{2\}, \mathrm{z}_{-}\{3\}, \mathrm{z}_{-}\{4\}, \mathrm{z}_{-}\{5\}, \mathrm{y}_{-}\{6\}, \mathrm{z}_{-}\{6\}, \mathrm{x}_{-}\{7$ $\hookrightarrow\}, y_{-}\{7\}, z_{-}\{7\}$,

aflow_params_values $, 7.4494846573,1.70036689767,1.04688136975,0.76,0.27$ $\hookrightarrow, 0.0,0.31,0.06,0.79,0.6,0.17,0.11,0.07$,

aflow Strukturbericht 'None'

aflow_Pearson 'oF52,

cell_length_a 7.4494846573

cell_length_b 12.6668571160

cell_length_c $\quad 7.7987267020$

cell_angle_alpha 90.000000000

cell_angle_beta 90.0000000000

cell_angle_gamma 90.0000000000

_symmetry_space_group_name_H-M "F $\mathrm{m} \mathrm{m} \mathrm{2"}$

symmetry Int Tables_number 42

loop_

space_group_symop_id

space_group_symop_operation_xyz

$1 \mathrm{x}, \mathrm{y}, \mathrm{z}$

$2-x,-y, z$

$3-x, y, z$

- $\mathrm{x},-\mathrm{y}, \mathrm{z}$

$5 \mathrm{x}, \mathrm{y}+1 / 2, \mathrm{z}+1 / 2$

$6-x,-y+1 / 2, z+1 / 2$

$7-\mathrm{x}, \mathrm{y}+1 / 2, \mathrm{z}+1 / 2$

$8 \mathrm{x},-\mathrm{y}+1 / 2, \mathrm{z}+1 / 2$

$9 \mathrm{x}+1 / 2, \mathrm{y}, \mathrm{z}+1 / 2$

$10-x+1 / 2,-y, z+1 / 2$

$11-x+1 / 2, y, z+1 / 2$

$12 \mathrm{x}+1 / 2,-\mathrm{y}, \mathrm{z}+1 / 2$

$13 \mathrm{x}+1 / 2, \mathrm{y}+1 / 2, \mathrm{z}$

$14-\mathrm{x}+1 / 2,-\mathrm{y}+1 / 2, \mathrm{z}$

$15-x+1 / 2, y+1 / 2, z$

$16 \mathrm{x}+1 / 2,-\mathrm{y}+1 / 2, \mathrm{z}$

loop

atom site label

_atom_site_type_symbol

_atom_site_symmetry_multiplicity

_atom_site_Wyckoff_labe

atom_site_fract_x

atom_site_fract_y

atom_site_fract_z

_atom_site_occupancy

$\mathrm{O} 1 \quad \mathrm{O} \quad 4 \quad \mathrm{a} \quad 0.00000 \quad 0.00000 \quad 0.76000 \quad 1.00000$

$\mathrm{O} 2 \mathrm{O} \quad 4 \quad \mathrm{a} \quad 0.00000 \quad 0.00000 \quad 0.27000 \quad 1.00000$

W1 $\quad \mathrm{W} \quad 4 \quad \mathrm{a} \quad 0.00000 \quad 0.00000 \quad 0.00000 \quad 1.00000$

$\begin{array}{llllllllll}\mathrm{O} 3 & \mathrm{O} & 8 & \mathrm{~b} & 0.25000 & 0.25000 & 0.31000 & 1.00000\end{array}$

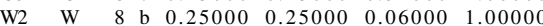

$\begin{array}{lllllllll}\mathrm{O} 4 & \mathrm{O} & 8 & \mathrm{c} & 0.00000 & 0.79000 & 0.60000 & 1.00000\end{array}$

$\begin{array}{lllllllll}\mathrm{O} 5 & \mathrm{O} & 16 & \mathrm{e} & 0.17000 & 0.11000 & 0.07000 & 1.00000\end{array}$

$\mathrm{W}_{3} \mathrm{O}_{10}$ : A10B3_oF52_42_2abce_ab - POSCAR

A10B3_oF52_42_2abce_ab \& a , b/a,c/a, z1 , z2, z3, z4, z5, y6, z6, x7, y7, z7 --

$\hookrightarrow$ params $=7.4494846573,1.70036689767,1.04688136975,0.76,0.27,0.0$,

$\hookrightarrow 0.31,0.06,0.79,0.6,0.17,0.11,0.07 \& \mathrm{Fmm} 2 \mathrm{C}\{2 \mathrm{v}\}^{\wedge}\{18\} \# 42\left(\mathrm{a}^{\wedge} 3 \mathrm{~b}^{\wedge}\right.$

$\hookrightarrow 2 \mathrm{ce}) \&$ oF52 \& None \& W3O10 \& \& B. Gerand and G. Nowogrocki and

$\hookrightarrow$ 2ce $) \&$ oF52 \& None \& W3O10 \& \& B. Gerand and G. Now
$\hookrightarrow$ M. Figlarz, J. Solid State Chem. 38, 312-320 (1981)

1.00000000000000

$0.00000000000000 \quad 6.33342855800000 \quad 3.89936335100000$

$\begin{array}{lll}. .72474232865000 & 0.00000000000000 & 3.89936335100000\end{array}$

$\begin{array}{lll}3.72474232865000 & 6.33342855800000 & 0.00000000000000\end{array}$

$\mathrm{O}$ W

Direct

0.76000000000000 0.27000000000000 0.31000000000000 0.81000000000000 1.39000000000000 $-0.19000000000000$ 0.01000000000000 0.13000000000000

$-0.21000000000000$

$0.76000000000000 \quad-0.7600000000000$ $0.27000000000000-0.27000000000000$ $0.31000000000000 \quad 0.19000000000000$ $0.81000000000000-0.31000000000000$ $-0.19000000000000 \quad 0.19000000000000$ $1.39000000000000-1.39000000000000$ $0.13000000000000 \quad 0.21000000000000$ $0.01000000000000-0.35000000000000$ $0.0100000000000-0.3500000000000$
.35000000000000 0.00000000000000

06000000000000

0.56000000000000

$-0.2100000000000$ 0.00000000000000 0.06000000000000

$-0.13000000000000$ 0.00000000000000 0.44000000000000 $-0.06000000000000$

BN (High-pressure, high-temperature): AB_oF8_42_a_a - CIF

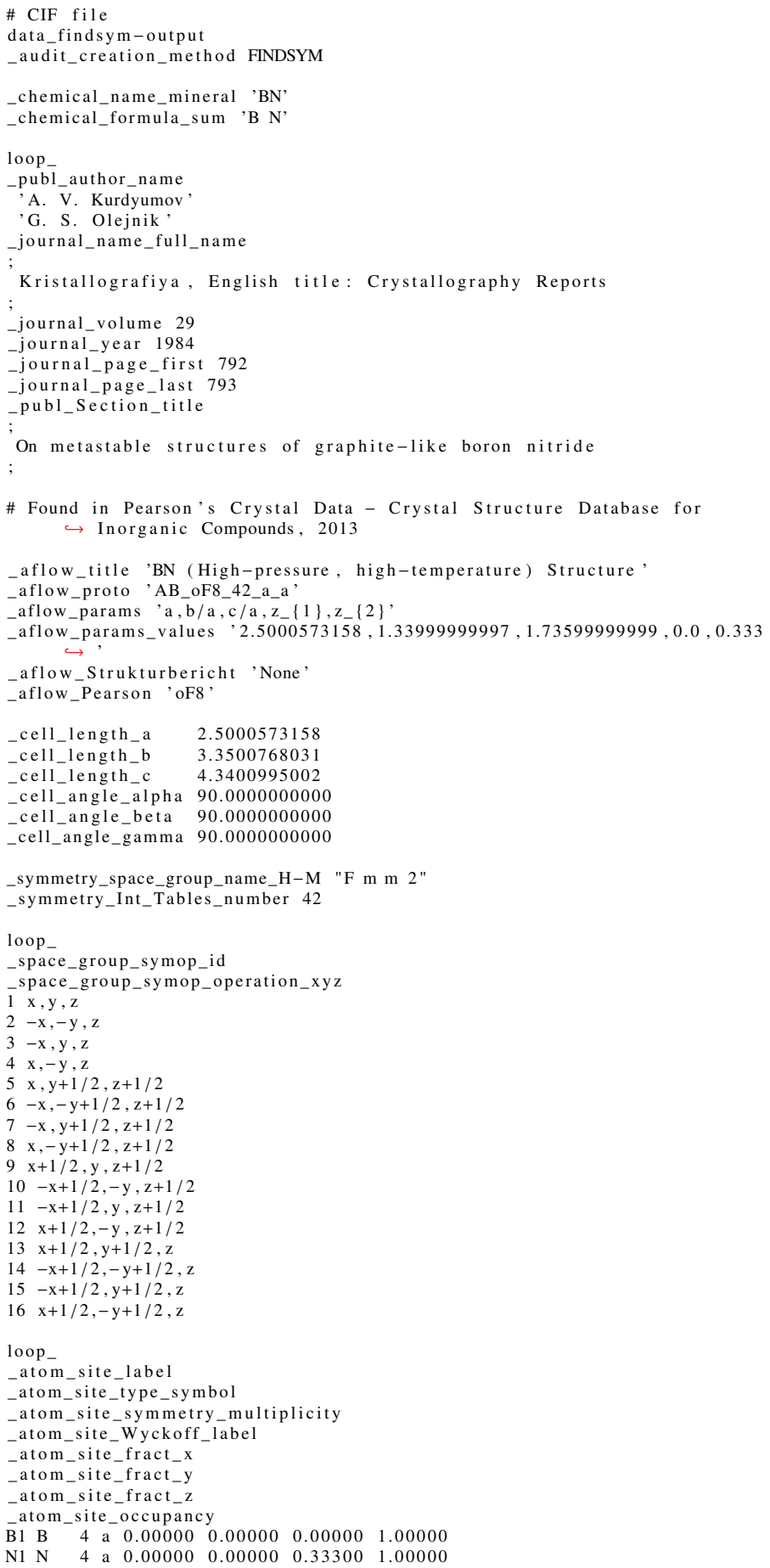

BN (High-pressure, high-temperature): AB_oF8_42_a_a - POSCAR

AB_oF8_42_a_a \& a,b/a,c/a,z1, z2 --params $=2.5000573158,1.33999999997$ $\rightarrow 1.73599999999,0.0,0.333 \& \mathrm{Fmm} 2 \mathrm{C}_{-}\{2 \mathrm{v}\}^{\wedge}\{18\} \# 42 \quad\left(\mathrm{a}^{\wedge} 2\right) \& \mathrm{oF} 8$ \& $\hookrightarrow$ None \& BN \& \& A. V. Kurdyumov and G. S. Olejnik

$\rightarrow$ Kristallografiya $29,792-793$ (1984)

1.00000000000000

$\begin{array}{lll}0.00000000000000 & 1.67503840155000 & 2.17004975010000\end{array}$

$1.25002865790000-0.00000000000000-2.17004975010000$

.25002865790000

1.675038

B $\quad \mathrm{N}$

Direc

$0.00000000000000 \quad 0.00000000000000-0.00000000000000$ $\begin{array}{llr}0.33300000000000 & 0.33300000000000 & -0.33300000000000\end{array}$

$\mathrm{MnGa}_{2} \mathrm{Sb}_{2}:$ A2BC2_oI20_45_c_b_c - CIF

\# CIF file

data_findsym-output 


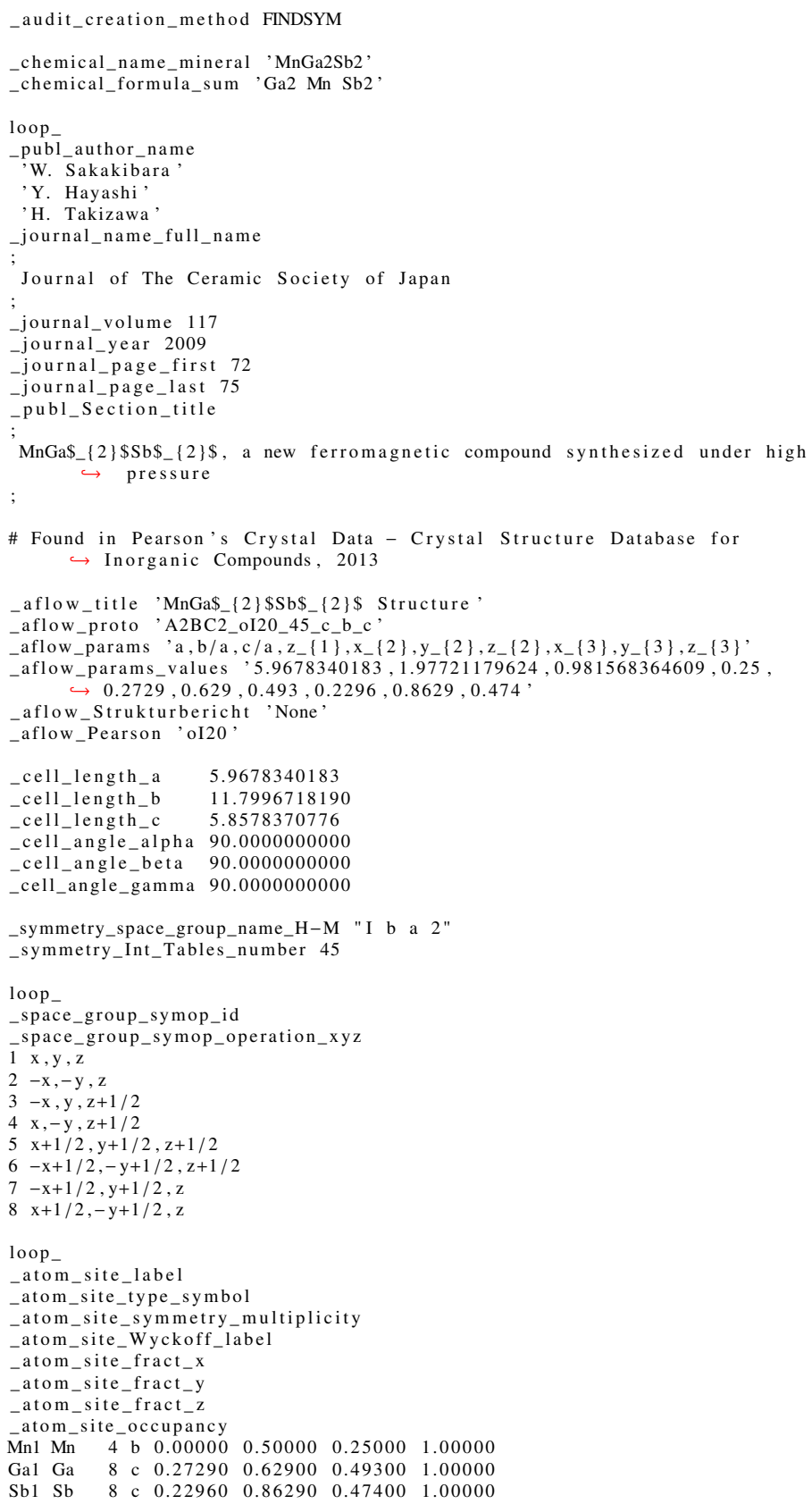

$\mathrm{MnGa}_{2} \mathrm{Sb}_{2}$ : A2BC2_oI20_45_c_b_c - POSCAR

A2BC2_oI20_45_c_b_c \& a , b /a,c/a, z1 , x2 , y2 , z2, x3, y3, z3 --params = $\hookrightarrow 5.9678340183,1.97721179624,0.981568364609,0.25,0.2729,0.629$ $\hookrightarrow 5.9678340183,1.97721179624,0.981568364609,0.25,0.2729,0.629$,

$\hookrightarrow 0.493,0.2296,0.8629,0.474 \&$ Iba2 $\mathrm{C}_{-}\{2 \mathrm{v}\}^{\wedge}\{21\} \# 45\left(\mathrm{bc}^{\wedge} 2\right) \&$ ol
$\hookrightarrow$ \& None \& MnGa2Sb2 \& \& W. Sakakibara and Y. Hayashi and H. 1.00000000000000

$\begin{array}{rrr}1.00000000015000 & 5.89983590950000 & 2.92891853880000\end{array}$

$\begin{array}{lrr}2.98391700915000 & -5.89983590950000 & 2.92891853880000\end{array}$

$\begin{array}{lll}2.98391700915000 & 5.89983590950000 & -2.92891853880000\end{array}$

$\begin{array}{rrr}\mathrm{Ga} & \mathrm{Mn} & \mathrm{Sb} \\ 4 & 2 & 4\end{array}$

Direct

1.12200000000000

$-0.13600000000000$

0.36400000000000

1.62200000000000

1.6220000000000

0.2500000000000

1.33690000000000

1.33690000000000

0.38890000000000

1.83690000000000

$\begin{array}{lrrr}0.76590000000000 & 0.90190000000000 & \mathrm{Ga} & (8 \mathrm{c}) \\ 0.22010000000000 & -0.90190000000000 & \mathrm{Ga} & (8 \mathrm{c}) \\ 1.26590000000000 & -0.35610000000000 & \mathrm{Ga} & (8 \mathrm{c}) \\ 0.72010000000000 & 0.35610000000000 & \mathrm{Ga} & (8 \mathrm{c}) \\ 0.25000000000000 & 0.50000000000000 & \mathrm{Mn} & (4 \mathrm{~b}) \\ 0.75000000000000 & 0.50000000000000 & \mathrm{Mn} & (4 \mathrm{~b}) \\ 0.70360000000000 & 1.09250000000000 & \mathrm{Sb} & (8 \mathrm{c}) \\ 0.24440000000000 & -1.09250000000000 & \mathrm{Sb} & (8 \mathrm{c}) \\ 1.20360000000000 & -0.63330000000000 & \mathrm{Sb} & (8 \mathrm{c}) \\ 0.74440000000000 & 0.63330000000000 & \mathrm{Sb} & (8 \mathrm{c})\end{array}$

TiFeSi: ABC_ol36_46_ac_bc_3b - CIF

\section{\# CIF file}

data_findsym-output

audit_creation_method FINDSYM

chemical name mineral ' TiFeSi,

_chemical_formula_sum ' Fe Si Ti,

loop_

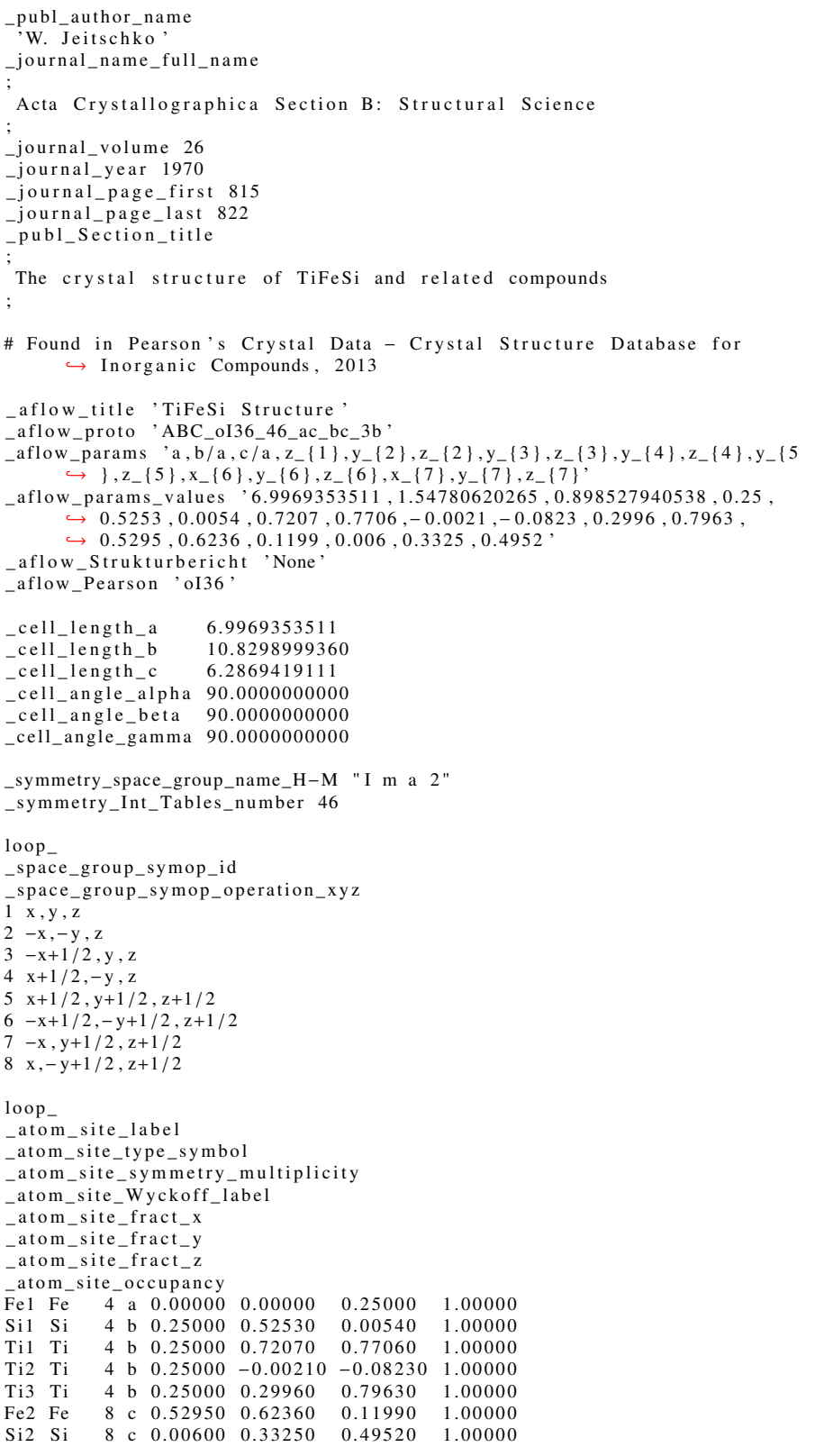

TiFeSi: ABC_ol36_46_ac_bc_3b - POSCAR

\begin{tabular}{|c|c|c|c|c|}
\hline \multirow{2}{*}{\multicolumn{5}{|c|}{ ABC_oI36_46_ac_bc_3b \& a,b/a, c/a, z1 , y2 , z2 $, y 3, \mathrm{z} 3, \mathrm{y} 4, \mathrm{z} 4, \mathrm{y} 5, \mathrm{z} 5, \mathrm{x} 6, \mathrm{y} 6, \mathrm{z} 6, \mathrm{x} 7}}$, \\
\hline & & & & \\
\hline \multicolumn{5}{|c|}{ ABC_oI36_46_ac_bc_3b \& a , b/a , c/a , z1 $, \mathrm{y} 2, \mathrm{z2}, \mathrm{y3}, \mathrm{z3}, \mathrm{y} 4, \mathrm{z} 4, \mathrm{y} 5, \mathrm{z5}, \mathrm{x} 6, \mathrm{y} 6, \mathrm{z6}, \mathrm{x} 7$} \\
\hline \multirow{2}{*}{\multicolumn{5}{|c|}{$\begin{array}{l}\hookrightarrow 0.5253,0.0054,0.7207,0.7706,-0.0021,-0.0823,0.2996,0.7963, \\
\hookrightarrow \\
\hookrightarrow 0.5295,0.6236,0.1199,0.006,0.3325,0.4952 \& \operatorname{Ima} 2 \mathrm{C}\{2 \mathrm{v}\}^{\wedge}\{22\} \# 46\end{array}$}} \\
\hline & & & & $\hookrightarrow 0.5295,0.6236,0.1199,0.006,0.3325,0.4952 \& \operatorname{Ima} 2 C_{-}\{2 v\}^{\wedge}\{22\} \# 46$ \\
\hline \multicolumn{5}{|c|}{$\begin{array}{l}\hookrightarrow \quad\left(a b^{\wedge} 4 c^{\wedge} 2\right) \& \text { oI } 36 \& \text { No } \\
\hookrightarrow \text { Crystallogr. Sect. B S S }\end{array}$} \\
\hline \multicolumn{5}{|l|}{$\begin{array}{l}\hookrightarrow \text { Crystallogr. } \\
1.00000000000000\end{array}$} \\
\hline-3.49846767555000 & 5.41494996800000 & 3.14347095555000 & & \\
\hline 3.49846767555000 & -5.41494996800000 & 3.14347095555000 & & \\
\hline 3.49846767555000 & 5.41494996800000 & -3.14347095555000 & & \\
\hline $\mathrm{Fe}$ & & & & \\
\hline 6 & & & & \\
\hline \multicolumn{5}{|l|}{ Direct } \\
\hline 0.25000000000000 & 0.25000000000000 & 0.0 & $\mathrm{Fe}$ & (4a) \\
\hline 0.25000000000000 & 00000 & 0.50000000000000 & $\mathrm{Fe}$ & (4a) \\
\hline 0.74350000000000 & 0.64940000000000 & 1.15310000000000 & $\mathrm{Fe}$ & $(8 c)$ \\
\hline-0.50370000000000 & -0.40960000000000 & -1.15310000000000 & $\mathrm{Fe}$ & $(8 c)$ \\
\hline-0.50370000000000 & 1.14940000000000 & 0.40590000000000 & $\mathrm{Fe}$ & $(8 \mathrm{c})$ \\
\hline 0.74350000000000 & 0.09040000000000 & 0.59410000000000 & $\mathrm{Fe}$ & $(8 \mathrm{c})$ \\
\hline \multirow{2}{*}{$\begin{array}{r}0.53070000000000 \\
-0.51990000000000\end{array}$} & & 0.77530000000000 & $\mathrm{Si}$ & (4b) \\
\hline & 0.75540000000000 & 0.22470000000000 & $\mathrm{Si}$ & (4b) \\
\hline 0.82770000000000 & 0.50120 & 0.3385000 & $\mathrm{Si}$ & $(8 c)$ \\
\hline 0.16270000000000 & 0.48920000000000 & -0.33850000000000 & $\mathrm{Si}$ & $(8 c)$ \\
\hline \multirow{2}{*}{$\begin{array}{l}0.16270000000000 \\
0.82770000000000\end{array}$} & 1.001200 & 0.17350000000000 & $\mathrm{Si}$ & $(8 c)$ \\
\hline & 0.98920000000000 & 0.82650000000000 & $\mathrm{Si}$ & $(8 \mathrm{c})$ \\
\hline $\begin{array}{l}0.82770000000000 \\
1.49130000000000\end{array}$ & 1.02060000000000 & 0.97070000000000 & $\mathrm{Ti}$ & (4b) \\
\hline 0.04990000000000 & 000000 & 0.02930000000000 & $\mathrm{Ti}$ & (4b) \\
\hline-0.08440000000000 & 0.16770000000000 & 0.24790000000000 & $\mathrm{Ti}$ & (4b) \\
\hline \multirow{2}{*}{$\begin{array}{r}-0.08020000000000 \\
1.09590000000000\end{array}$} & 0.66770000000000 & 0.75210000000000 & $\mathrm{Ti}$ & $(4 b)$ \\
\hline & 1.04630000000000 & 0.54960000000000 & $\mathrm{Ti}$ & $(4 b)$ \\
\hline 0.49670000000000 & 1.54630000000000 & 0.45040000000000 & $\mathrm{Ti}$ & (4b) \\
\hline
\end{tabular}

$\alpha-\operatorname{RbPr}\left[\mathrm{MoO}_{4}\right]_{2}: \mathrm{A} 2 \mathrm{~B} 8 \mathrm{CD} \_$oP24_48_k_2m_d_b - CIF

\# CIF file
data_findsym-output 


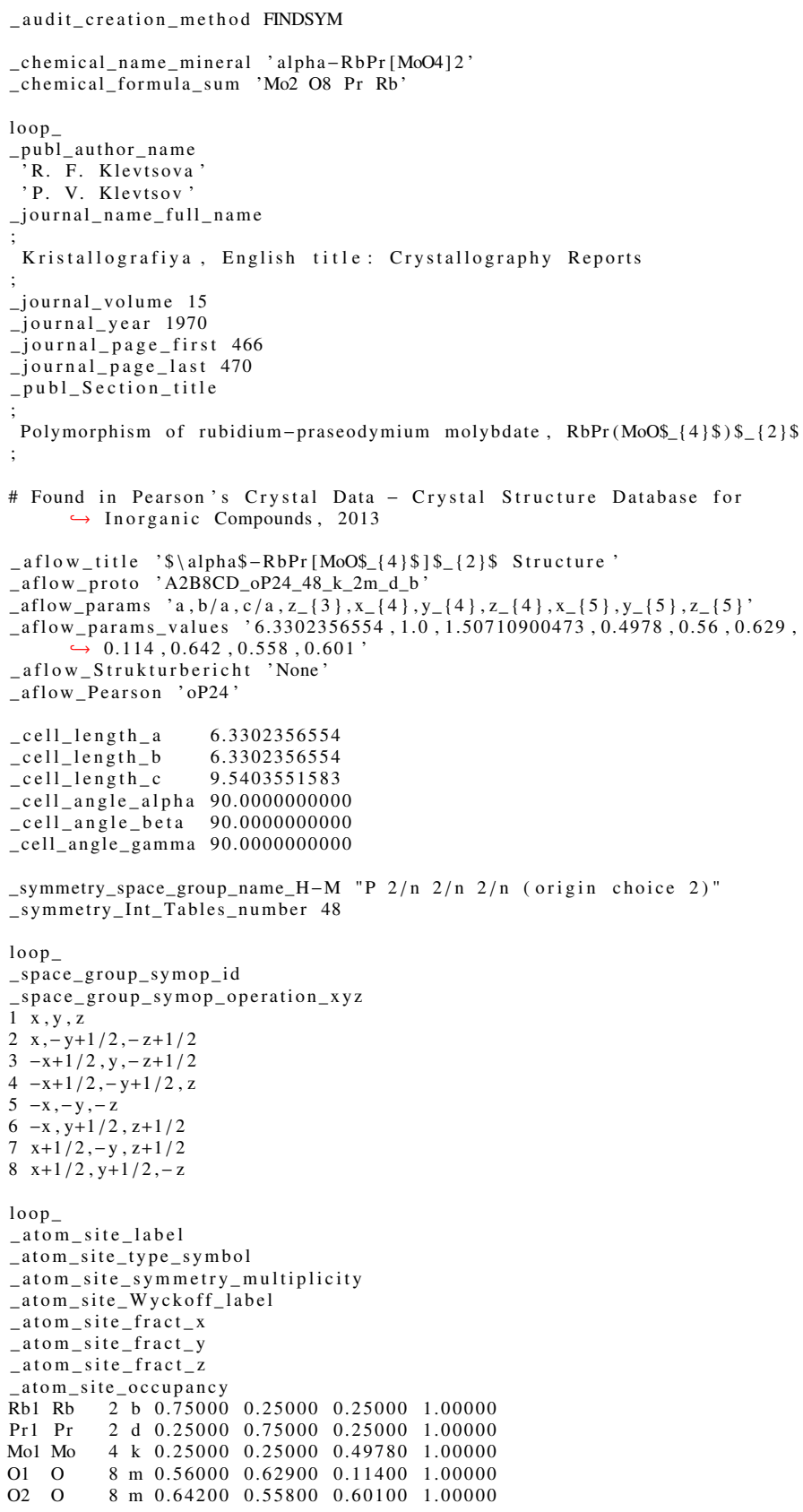

$\alpha-\mathrm{RbPr}\left[\mathrm{MoO}_{4}\right]_{2}$ : A2B8CD_oP24_48_k_2m_d_b - POSCAR

A2B8CD_oP24_48_k_2m_d_b \& a , b/a , c/a, z3 , x4,y4, z4 , x5 , y5 , z5 --params

$\hookrightarrow 6.3302356554,1.0,1.50710900473,0.4978,0.56,0.629$

$\hookrightarrow 0.558,0.601 \&$ \&nnn $\mathrm{D}\{2 \mathrm{~h}\}^{\wedge}\{2\} \# 48\left(\mathrm{bdkm}^{\wedge} 2\right) \&$ oP2 4 \& None \& RbP

$\hookrightarrow[\mathrm{MoO} 4] 2 \&$ alpha \& R. F. Klevtsova and P. V. Klevtsov,

$\hookrightarrow$ Kristallografiya $15,466-470(1970)$

1.00000000000000

6.33023565540000

0.00000000000000

6.33023565540000

0.00000000000000

$\begin{array}{rrrr}\mathrm{Mo} & \mathrm{O} & \mathrm{Pr} & \mathrm{Rb} \\ 4 & 16 & 2 & 2\end{array}$

Direct

0.25000000000000 0.25000000000000 0.750000000000 0.75000000000000 0.75000000000000 0.56000000000000 0.06000000000000 0.06000000000000 0.56000000000000 $-0.56000000000000$ 1.06000000000000 1.06000000000000 $-0.56000000000000$ 0.64200000000000 $-0.14200000000000$ $-0.14200000000000$ 0.64200000000000 $-0.64200000000000$ 1.14200000000000 1.14200000000000 $-0.64200000000000$ 0.25000000000000

$0.25000000000000 \quad 0.49780000000000$ $0.25000000000000 \quad 0.00220000000000$ $\begin{array}{ll}0.75000000000000 & -0.49780000000000\end{array}$ $0.75000000000000 \quad 0.99780000000000$ $0.62900000000000 \quad 0.11400000000000$ $-0.12900000000000 \quad 0.11400000000000$ $0.62900000000000-0.38600000000000$ $-0.12900000000000 \quad 0.38600000000000$ $-0.62900000000000-0.11400000000000$ $1.12900000000000-0.11400000000000$ $\begin{array}{rr}1.62900000000000 & 0.61400000000000\end{array}$ $\begin{array}{ll}1.62900000000000 & 0.61400000000000 \\ 1.12900000000000 & 0.61400000000000\end{array}$ $-0.05800000000000-0.60100000000000$ $0.55800000000000-0.10100000000000$ $-0.05800000000000-0.10100000000000$ $-0.55800000000000-0.60100000000000$ $1.05800000000000-0.60100000000000$ $-0.55800000000000-1.10100000000000$ $1.05800000000000-1.10100000000000$ $\begin{array}{ll}0.75000000000000 & 0.25000000000000\end{array}$
.75000000000000 0.25000000000000

$\beta-\mathrm{Ta}_{2} \mathrm{O}_{5}$ : A5B2_oP14_49_dehq_ab - CIF

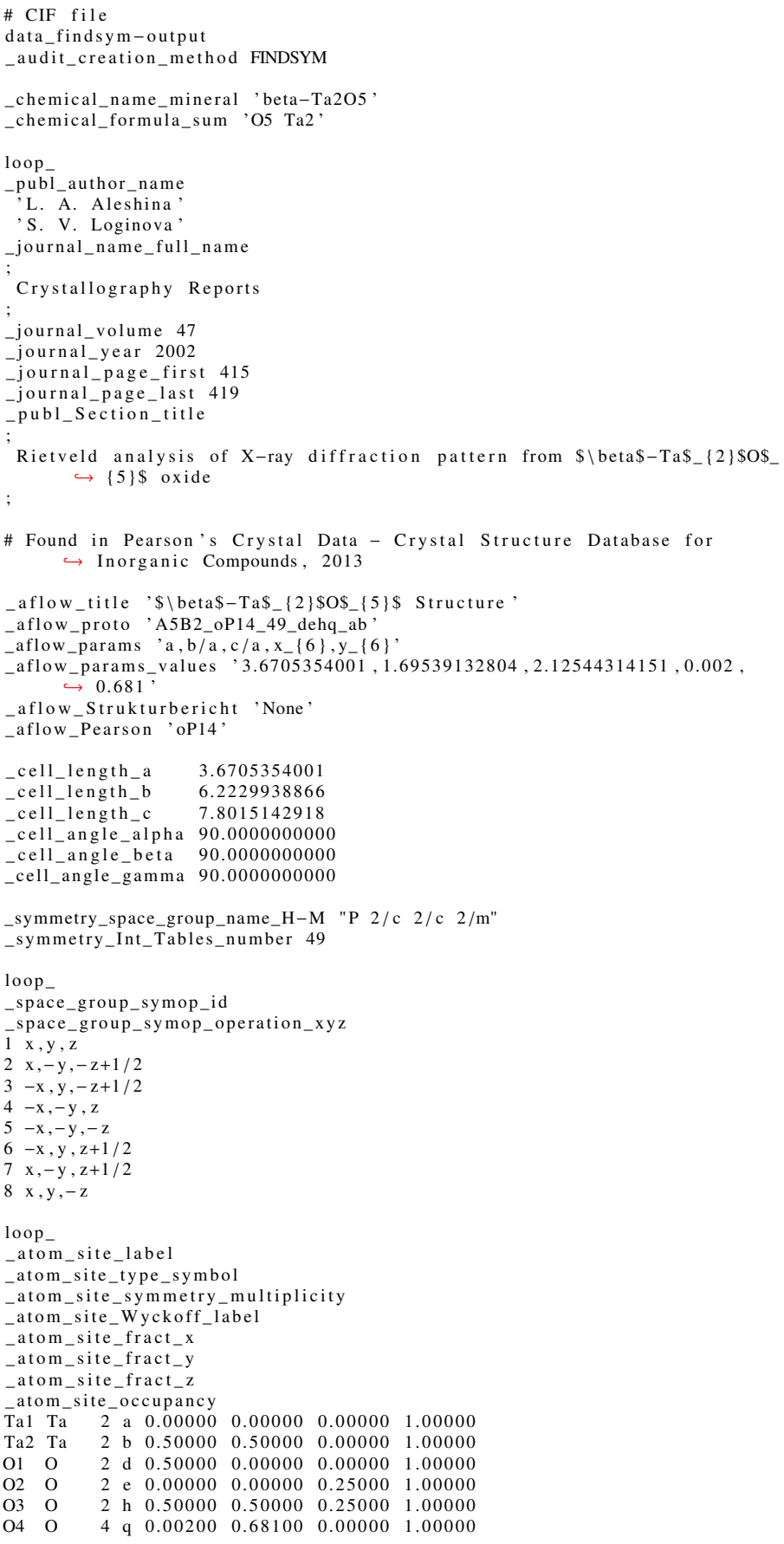

$\beta-\mathrm{Ta}_{2} \mathrm{O}_{5}$ : A5B2_oP14_49_dehq_ab - POSCAR

A5B2_oP14_49 dehq ab \& a, b/a,c/a, x6, y6 --params $=3.6705354001$

$\hookrightarrow 1.69539132804,2.12544314151,0.002,0.681 \&$ Pccm D $\{2 \mathrm{~h}\}^{\wedge}\{3\} \# 49$

$\rightarrow$ abdehq) \& oP14 \& None \& Ta2O5 \& beta \& L. A. Aleshina and S. V. Loginova, Crystallogr. Rep. 47, 415-419 (2002) $1.12900000000000 \quad 0.61400000000000$ $0.55800000000000 \quad 0.60100000000000$
1.00000000000000

$\begin{array}{lll}3.67053540010000 & 0.00000000000000 & 0.00000000000000\end{array}$

$\begin{array}{lll}0.00000000000000 & 6.22299388660000 & 0.00000000000000\end{array}$

$\begin{array}{lll}0.00000000000000 & 0.00000000000000 & 7.80151429180000\end{array}$

$$
\begin{array}{rr}
\mathrm{O} & \mathrm{Ta} \\
10 & 4
\end{array}
$$

Direct

0.50000000000000

0.50000000000000

0.0000000000000

0.5000000000000

0.50000000000000

0.002000000000

0.00200000000000

$-0.00200000000000$

$-0.00200000000000$

0.00200000000000

0.00000000000000

0.00000000000000
0.00000000000000 0.00000000000000 0.00000000000000 0.00000000000000 0.50000000000000 0.50000000000000 0.68100000000000 $-0.68100000000000$ 0.68100000000000 0.68100000000000 0.00000000000000 0.00000000000000
0.00000000000000 0.50000000000000 0.25000000000000 0.75000000000000 0.25000000000000 0.75000000000000 0.00000000000000 0.00000000000000 0.50000000000000 0.50000000000000 0.50000000000000 0.00000000000000 
$\mathrm{CsPr}\left[\mathrm{MoO}_{4}\right]_{2}:$ AB2C8D_oP24_49_g_q_2qr_e - CIF

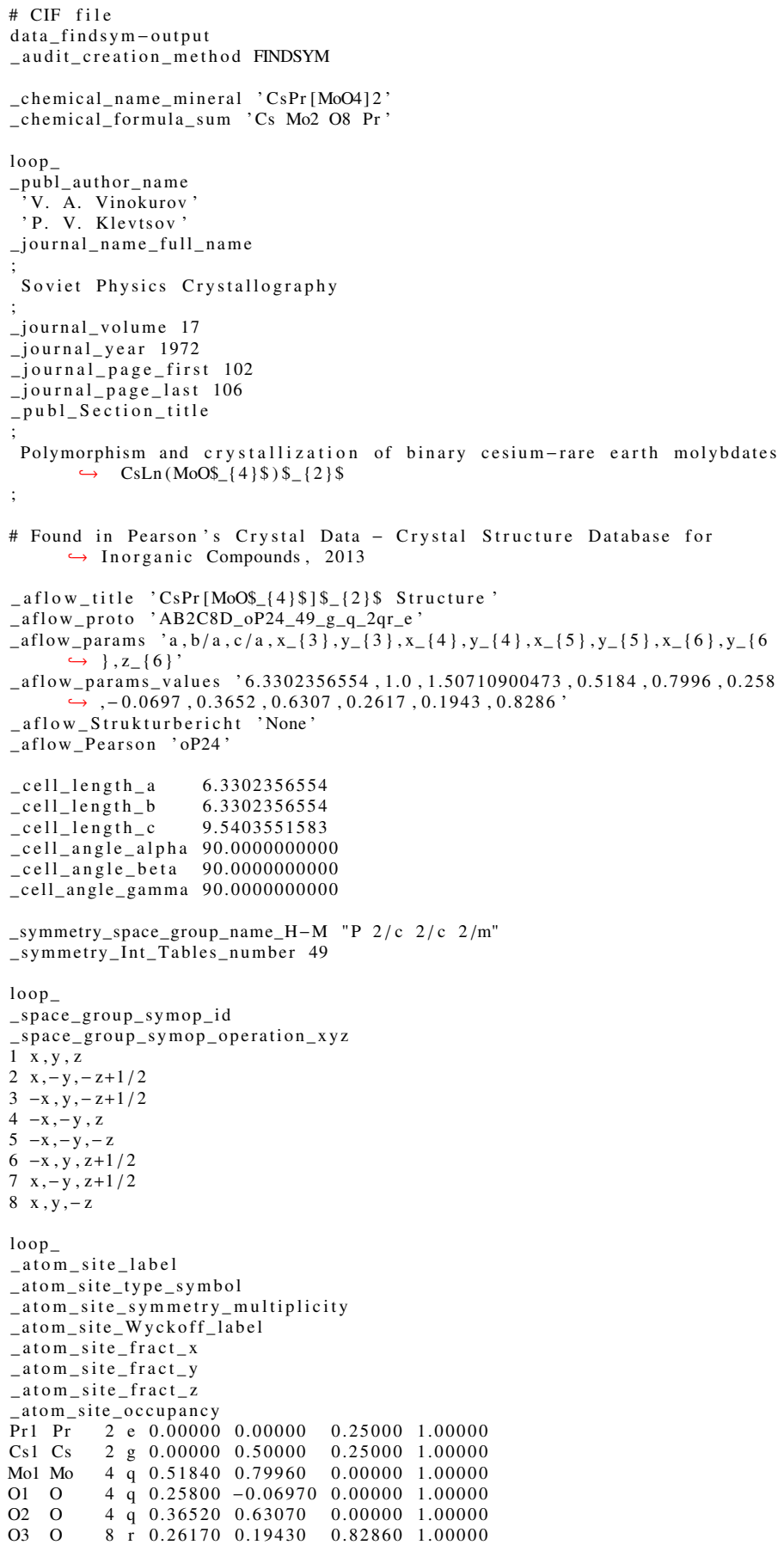

$\mathrm{CsPr}\left[\mathrm{MoO}_{4}\right]_{2}$ : AB2C8D_oP24_49_g_q_2qr_e - POSCAR

AB2C8D_oP24_49_g_q_2qr_e \& a,b/a,c/a,x3,y3, x4,y4,x5,y5,x6,y6,z6 --param $\hookrightarrow=6.3302356554,1.0,1.50710900473,0.5184,0.7996,0.258,-0.0697$

$\hookrightarrow 0.3652,0.6307,0.2617,0.1943,0.8286 \&$ Pccm D $\{2 \mathrm{~h}\}^{\wedge}\{3\} \# 49\left(\mathrm{egq}^{\wedge}\right.$

$\hookrightarrow 3 \mathrm{r})$ \& oP24 \& None \& $\mathrm{CsPr}[\mathrm{MoO} 4] 2$ \& \& V. A. Vinokurov and P. V. $\hookrightarrow$ Klevtsov, Sov. Phys. Crystallogr. 17, 102-106 (1972)

1.00000000000000

$6.33023565540000 \quad 0.00000000000000 \quad 0.00000000000000$

$\begin{array}{lll}0.00000000000000 & 6.33023565540000 & 0.00000000000000\end{array}$

$\begin{array}{lll}0.00000000000000 & 0.00000000000000 & 9.54035515830000\end{array}$

$\begin{array}{cccc}\text { Cs } & \text { Mo } & \mathrm{O} & \mathrm{Pr} \\ 2 & 4 & 16 & 2\end{array}$

Direct

$0.00000000000000 \quad 0.50000000000000$ $0.00000000000000 \quad 0.50000000000000$ $\begin{array}{lll}0.51840000000000 & 0.79960000000000\end{array}$ $\begin{array}{rr}-0.51840000000000 & -0.79960000000000\end{array}$ $\begin{array}{rr}-0.51840000000000 & 0.79960000000000\end{array}$ $\begin{array}{rr}0.51840000000000 & -0.79960000000000\end{array}$ $0.25800000000000-0.06970000000000$ $-0.25800000000000 \quad 0.06970000000000$ $-0.25800000000000-0.06970000000000$ $0.25800000000000 \quad 0.0697000000000$ $0.36520000000000 \quad 0.63070000000000$
0.25000000000000 0.75000000000000 0.00000000000000 0.0000000000000 0.00000000000000 0.50000000000000 . 0.00000000000000 0.50000000000000 0.50000000000000 0.00000000000000 0.50000000000000

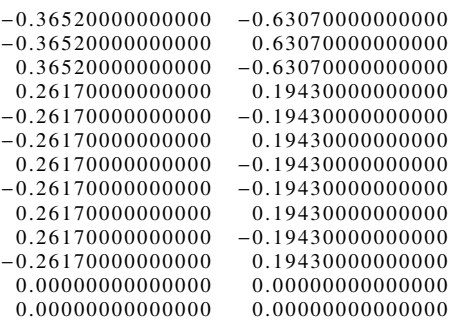

.63070000000000 0.63070000000000 0.63070000000000 0.19430000000000 $-0.19430000000000$ 0.19430000000000 $-0.19430000000000$ $-0.19430000000000$ 0.19430000000000 $-0.19430000000000$ 0.19430000000000 0.00000000000000 0.00000000000000

0.00000000000000 0.50000000000000 0.50000000000000 0.82860000000000 0.82860000000000 $-0.32860000000000$ $-0.32860000000000$ $-0.32860000000000$ $-0.82860000000000$ 0.82860000000000 1.32860000000000 .32860000000000 0.25000000000000 0.75000000000000

$\mathrm{La}_{2} \mathrm{NiO}_{4}$ : A2BC4_oP28_50_ij_ac_ijm - CIF

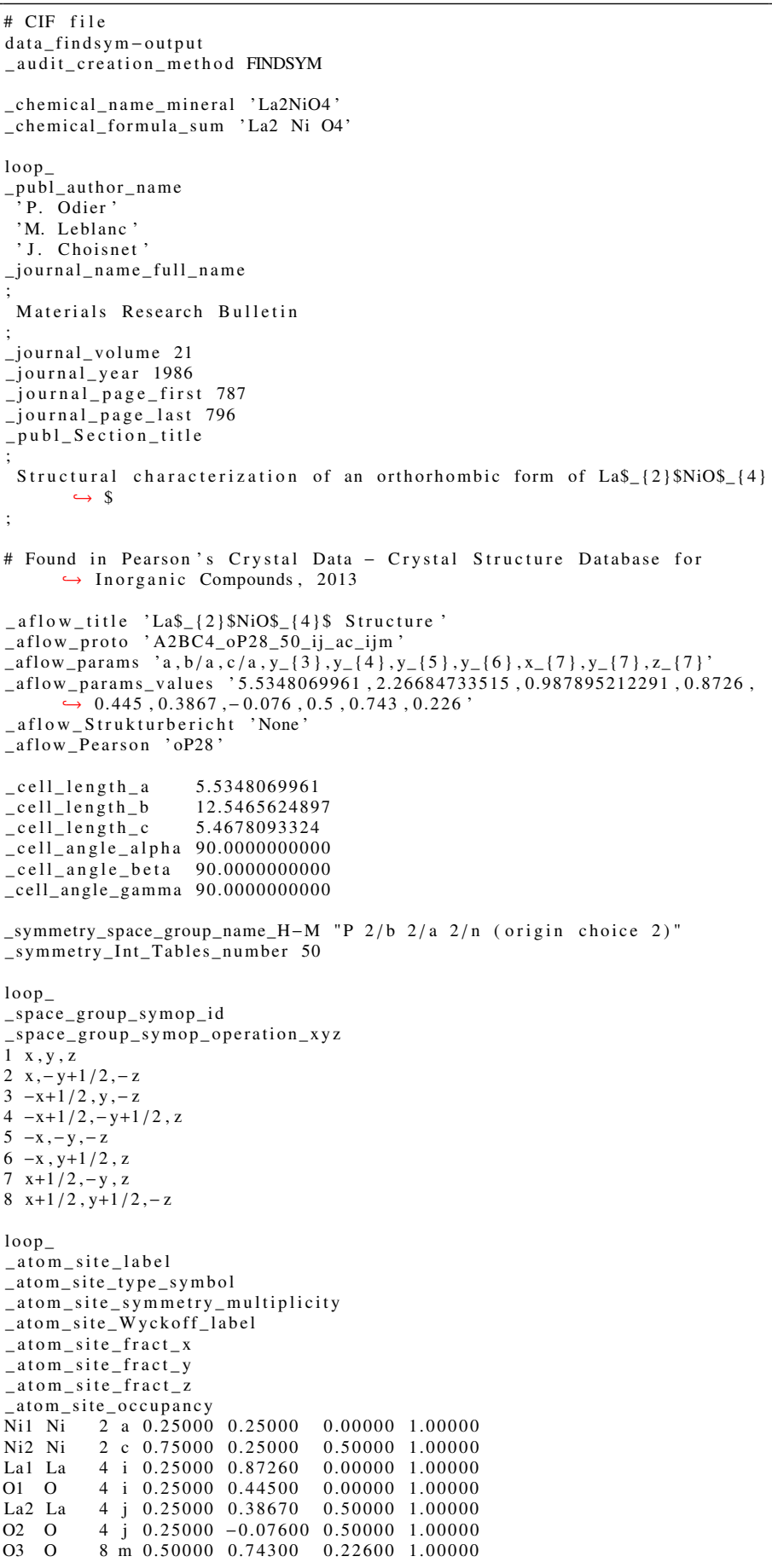

$\mathrm{La}_{2} \mathrm{NiO}_{4}$ : A2BC4_oP28_50_ij_ac_ijm - POSCAR

A2BC4_oP28_50_ij_ac_ijm \& a , b/a, c/a , y3 , y4 ,y5 , y6 , x7,y7, z7 --params= $\hookrightarrow 5.5348069961,2.26684733515,0.987895212291,0.8726,0.445,0.3867,-$ $\hookrightarrow 0.076,0.5,0.743,0.226 \&$ Pban D_ $\{2 \mathrm{~h}\}^{\wedge}\{4\} \# 50\left(\mathrm{aci}^{\wedge} 2 \mathrm{j}^{\wedge} 2 \mathrm{~m}\right) \&$ oP 28 $\hookrightarrow$ \& None \& La2NiO4 \& \& P. Odier and M. Leblanc and J. Choisnet, $\hookrightarrow$ Mater. Res. Bull.21, 787-796 (1986)

1.00000000000000

$5.53480699610000 \quad 0.00000000000000 \quad 0.00000000000000$ $\begin{array}{lrl}0.00000000000000 & 12.54656248970000 & 0.00000000000000\end{array}$ $\begin{array}{rrr}0.00000000000000 & 0.00000000000000 & 5.46780933240000\end{array}$ $\begin{array}{rrr}\mathrm{La} & \mathrm{Ni} & \mathrm{O} \\ 8 & 4 & 16\end{array}$ 
Direct

0.25000000000000 0.25000000000000 0.75000000000000 0.75000000000000 0.25000000000000 0.25000000000000 0.75000000000000 0.75000000000000 0.25000000000000 0.75000000000000 0.75000000000000 0.25000000000000 0.25000000000000 0.25000000000000 0.25000000000000 0.75000000000000 0.25000000000000 0.25000000000000 0.25000000000000 0.75000000000000 0.75000000000000 0.50000000000000 0.00000000000000 0.00000000000000 $-0.50000000000000$ 1.00000000000000 1.00000000000000 $-0.50000000000000$
0.87260000000000 $-0.37260000000000$ 1.37260000000000 0.38670000000000 0.11330000000000 $-0.38670000000000$ 0.88670000000000 0.25000000000000 0.75000000000000 0.25000000000000 0.75000000000000 0.4450000000000 0.05500000000000 0.44500000000000 0.94500000000000 0.07600000000000 $-0.57600000000000$ 0.57600000000000 0.42400000000000 0.74300000000000 $-0.24300000000000$ 0.74300000000000 $-0.24300000000000$ $-0.74300000000000$ 1.24300000000000 $-0.74300000000000$ 1.24300000000000 $-0.87260000000000$ 0.07600000000000
0.00000000000000 0.0000000000000 0.00000000000000 0.50000000000000 0.50000000000000 0.50000000000000 0.50000000000000 0.00000000000000 0.00000000000000 0.50000000000000 0.50000000000000 0.00000000000000 0.0000000000000 0.00000000000000 0.00000000000000 0.50000000000000 0.5000000000000 0.50000000000000 0.50000000000000 0.50000000000000 0.22600000000000 0.22600000000000 0.22600000000000 $-0.22600000000000$ $-0.22600000000000$ $-0.22600000000000$ 22600000000000 0.00000000000000

\begin{tabular}{ll}
$\mathrm{La}$ & $(4 \mathrm{i})$ \\
$\mathrm{La}$ & $(4 \mathrm{i})$ \\
$\mathrm{La}$ & $(4 \mathrm{i})$ \\
$\mathrm{La}$ & $(4 \mathrm{i})$ \\
$\mathrm{La}$ & $(4 \mathrm{j})$ \\
$\mathrm{La}$ & $(4 \mathrm{j})$ \\
$\mathrm{La}$ & $(4 \mathrm{j})$ \\
$\mathrm{La}$ & $(4 \mathrm{j})$ \\
$\mathrm{Ni}$ & $(2 \mathrm{a})$ \\
$\mathrm{Ni}$ & $(2 \mathrm{a})$ \\
$\mathrm{Ni}$ & $(2 \mathrm{c})$ \\
$\mathrm{Ni}$ & $(2 \mathrm{c})$ \\
$\mathrm{O}$ & $(4 \mathrm{i})$ \\
$\mathrm{O}$ & $(4 \mathrm{i})$ \\
$\mathrm{O}$ & $(4 \mathrm{i})$ \\
$\mathrm{O}$ & $(4 \mathrm{i})$ \\
$\mathrm{O}$ & $(4 \mathrm{j})$ \\
$\mathrm{O}$ & $(4 \mathrm{j})$ \\
$\mathrm{O}$ & $(4 \mathrm{j})$ \\
$\mathrm{O}$ & $(4 \mathrm{j})$ \\
$\mathrm{O}$ & $(8 \mathrm{~m})$ \\
$\mathrm{O}$ & $(8 \mathrm{~m})$ \\
$\mathrm{O}$ & $(8 \mathrm{~m})$ \\
$\mathrm{O}$ & $(8 \mathrm{~m})$ \\
$\mathrm{O}$ & $(8 \mathrm{~m})$ \\
$\mathrm{O}$ & $(8 \mathrm{~m})$ \\
$\mathrm{O}$ & $(8 \mathrm{~m})$ \\
$\mathrm{O}$ & $(8 \mathrm{~m})$ \\
\hline &
\end{tabular}

$\alpha-\mathrm{Tl}_{2} \mathrm{TeO}_{3}:$ A3BC2_oP48_50_3m_m_2m - CIF

\section{\# CIF file}

data findsym-output

audit_creation method FINDSYM

chemical name mineral 'alpha- $\mathrm{Tl} 2 \mathrm{TeO} 3$ '

chemical_formula_sum ${ }^{O} \mathrm{O} 3 \mathrm{Te}$ T12

loop

publ_author_name

F. Rieger

A.-V. Mudring

journal_name_full_name

Inorganic Chemistry

journal_volume 46

journal year 2007

journal_page_first 446

journal_page_last 452

publ_Section_title

Phase transition in TI\$_\{2\}\$TeO\$ $\{3\} \$$ : Influence and origin of the $\hookrightarrow$ thallium lone pair distortion

\# Found in Pearson's Crystal Data - Crystal Structure Database for $\hookrightarrow$ Inorganic Compounds, 2013

aflow_title $\$ \backslash$ alpha\$-TI\$_ $\{2\} \$ T$ TeO $\$_{-}\{3\} \$$ Structure

aflow_proto 'A3BC2_oP48_50_3m_m_2m,

aflow_params $, a, b / a, c / a, x_{-}\{1\}, y_{-}\{1\}, z_{-}\{1\}, x_{-}\{2\}, y_{-}\{2\}, z_{-}\{2\}, x_{-}\{3\}, y_{-}\{3$ $\hookrightarrow\}, z_{-}\{3\}, x_{-}\{4\}, y_{-}\{4\}, z_{-}\{4\}, x_{-}\{5\}, y_{-}\{5\}, z_{-}\{5\}, x_{-}\{6\}, y_{-}\{6\}, z_{-}\{6\}$,

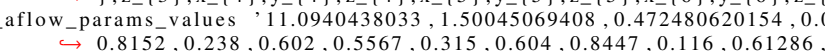

$\hookrightarrow 0.66266,0.2344,0.11964,0.66839,0.2479,0.63183,0.00412,0.2439$

aflow_Strukturbericht 'None'

aflow Pearson, oP48,

\section{cell_length_a $\quad 11.0940438033$ \\ cell_length_b $\quad 16.646065724$}

cell_length_c 5.2417206962

_cell_angle_alpha 90.0000000000

cell_angle_gamma 90.0000000000

symmetry_space_group_name_H-M "P 2/b 2/a 2/n (origin choice 2)"

symmetry_Int_Tables_number 50

loop

space_group_symop_id

space group symop operation $x y z$

$1 \mathrm{x}, \mathrm{y}, \mathrm{z}$

$2 \mathrm{x},-\mathrm{y}+1 / 2,-\mathrm{z}$

$\begin{array}{ll}2 & \mathrm{x},-\mathrm{y}+1 / 2,-\mathrm{z} \\ - & -\mathrm{x}+1 / 2, \mathrm{y},-\mathrm{z}\end{array}$

$4-x+1 / 2,-y+1 / 2, z$

$5-\mathrm{x},-\mathrm{y},-\mathrm{z}$

$6-\mathrm{x}, \mathrm{y}+1 / 2, \mathrm{z}$

$8 x+1 / 2, y+1 / 2,-z$

loop

_atom_site_label

atom_site_type_symbo

atom_site_symmetry_multiplicity

atom_site_Wyckoff_labe

atom_site_fract_x

atom_site_fract_y

atom_site_fract_z

$\begin{array}{lllllll}\mathrm{O} 1 & \mathrm{O} & 8 \mathrm{~m} & 0.05150 & 0.81520 & 0.23800 & 1.00000\end{array}$

$\begin{array}{lllllllll}\mathrm{O} 2 & \mathrm{O} & 8 \mathrm{~m} & 0.60200 & 0.55670 & 0.31500 & 1.00000\end{array}$

$\begin{array}{lllllllll}\mathrm{O} 3 & \mathrm{O} & 8 \mathrm{~m} & 0.60400 & 0.84470 & 0.11600 & 1.00000\end{array}$

$\begin{array}{lllllllll}\mathrm{O} 3 & \mathrm{O} & & 8 \mathrm{~m} & 0.60400 & 0.84470 & 0.11600 & 1.00000 \\ \mathrm{Te} 1 & \mathrm{Te} & 8 & \mathrm{~m} & 0.61286 & 0.66266 & 0.23440 & 1.00000\end{array}$
$\begin{array}{lllllllll}\mathrm{T} 11 & \mathrm{Tl} & 8 \mathrm{~m} & 0.11964 & 0.66839 & 0.24790 & 1.00000\end{array}$

$\begin{array}{lllllllll}\mathrm{T} 12 & \mathrm{Tl} & 8 \mathrm{~m} & 0.63183 & 0.00412 & 0.24390 & 1.00000\end{array}$

$\alpha-\mathrm{Tl}_{2} \mathrm{TeO}_{3}$ : A3BC2_oP48_50_3m_m_2m - POSCAR

\begin{tabular}{|c|c|c|c|c|}
\hline & & & & \\
\hline & & & & \\
\hline$\hookrightarrow 0.47248062$ & $54,0.0515,0.8152$ & $238,0.602,0.5567$ & & \\
\hline$\hookrightarrow 0.8447,0.11$ & $0.61286,0.66266$ & $2344,0.11964,0.6$ & & \\
\hline$\hookrightarrow 0.63183,0.0$ & $412,0.2439 \& \mathrm{Pban}$ & $\{2 \mathrm{~h}\}^{\wedge}\{4\} \# 50 \quad\left(\mathrm{~m}^{\wedge}\right.$ & & \\
\hline$\hookrightarrow$ None \& $\mathrm{Tl} 2 \mathrm{Te}$ & $\mathrm{O} 3$ \& alpha \& F. Ri & ger and A.-V. Mudri & & org. \\
\hline & $6-452(2007)$ & & & \\
\hline 1.00000000000000 & & & & \\
\hline 11.09404380330000 & 0.00000000000000 & 0.00000000000000 & & \\
\hline 0.00000000000000 & 16.64606572480000 & 0.00000000000000 & & \\
\hline 0.00000000000000 & 0.00000000000000 & 5.24172069620000 & & \\
\hline $\mathrm{Tl}$ & & & & \\
\hline 24 & & & & \\
\hline rect & & & & \\
\hline 0.05150000000000 & 0.81520000000000 & 0.23800000000000 & $\mathrm{O}$ & $(8 \mathrm{~m})$ \\
\hline 0.44850000000000 & -0.31520000000000 & 0.23800000000000 & $\mathrm{O}$ & $(8 \mathrm{~m})$ \\
\hline 0.44850000000000 & 0.81520000000000 & -0.23800000000000 & $\mathrm{O}$ & $(8 \mathrm{~m})$ \\
\hline 0.05150000000000 & -0.31520000000000 & -0.23800000000000 & $\mathrm{O}$ & $(8 \mathrm{~m})$ \\
\hline-0.05150000000000 & -0.81520000000000 & -0.23800000000000 & $\mathrm{O}$ & $(8 \mathrm{~m})$ \\
\hline 0.55150000000000 & 1.31520000000000 & -0.23800000000000 & $\mathrm{O}$ & $(8 \mathrm{~m})$ \\
\hline 0.55150000000000 & -0.81520000000000 & 0.23800000000000 & $\mathrm{O}$ & $(8 \mathrm{~m})$ \\
\hline-0.05150000000000 & 1.31520000000000 & 0.23800000000000 & $\mathrm{O}$ & $(8 \mathrm{~m})$ \\
\hline 0.60200000000000 & 0.55670000000000 & 0.31500000000000 & $\mathrm{O}$ & $(8 \mathrm{~m})$ \\
\hline-0.10200000000000 & -0.05670000000000 & 0.31500000000000 & $\mathrm{O}$ & $(8 \mathrm{~m})$ \\
\hline-0.10200000000000 & 0.55670000000000 & -0.31500000000000 & $\mathrm{O}$ & $(8 \mathrm{~m})$ \\
\hline 0.60200000000000 & -0.05670000000000 & -0.31500000000000 & $\mathrm{O}$ & $(8 \mathrm{~m})$ \\
\hline-0.60200000000000 & -0.55670000000000 & -0.31500000000000 & $\mathrm{O}$ & $(8 \mathrm{~m})$ \\
\hline 1.10200000000000 & 1.05670000000000 & -0.31500000000000 & $\mathrm{O}$ & $(8 \mathrm{~m})$ \\
\hline 1.10200000000000 & -0.55670000000000 & 0.31500000000000 & $\mathrm{O}$ & $(8 \mathrm{~m})$ \\
\hline-0.60200000000000 & 1.05670000000000 & 0.31500000000000 & $\mathrm{O}$ & $(8 \mathrm{~m})$ \\
\hline 0.60400000000000 & 0.84470000000000 & 0.11600000000000 & $\mathrm{O}$ & $(8 \mathrm{~m})$ \\
\hline-0.10400000000000 & -0.34470000000000 & 0.11600000000000 & $\mathrm{O}$ & $(8 \mathrm{~m})$ \\
\hline-0.10400000000000 & 0.84470000000000 & -0.11600000000000 & $\mathrm{O}$ & $(8 \mathrm{~m})$ \\
\hline 0.60400000000000 & -0.34470000000000 & -0.11600000000000 & $\mathrm{O}$ & $(8 \mathrm{~m})$ \\
\hline-0.60400000000000 & -0.84470000000000 & -0.11600000000000 & $\mathrm{O}$ & $(8 \mathrm{~m})$ \\
\hline 1.10400000000000 & 1.34470000000000 & -0.11600000000000 & $\mathrm{O}$ & $(8 \mathrm{~m})$ \\
\hline 1.10400000000000 & -0.84470000000000 & 0.11600000000000 & $\mathrm{O}$ & $(8 \mathrm{~m})$ \\
\hline-0.60400000000000 & 1.34470000000000 & 0.11600000000000 & $\mathrm{O}$ & $(8 \mathrm{~m})$ \\
\hline 0.61286000000000 & 0.66266000000000 & 0.23440000000000 & $\mathrm{Te}$ & $(8 \mathrm{~m})$ \\
\hline-0.11286000000000 & -0.16266000000000 & 0.23440000000000 & $\mathrm{Te}$ & $(8 \mathrm{~m})$ \\
\hline-0.11286000000000 & 0.66266000000000 & -0.23440000000000 & $\mathrm{Te}$ & $(8 \mathrm{~m})$ \\
\hline 0.61286000000000 & -0.16266000000000 & -0.23440000000000 & $\mathrm{Te}$ & $(8 \mathrm{~m})$ \\
\hline-0.61286000000000 & -0.66266000000000 & -0.23440000000000 & $\mathrm{Te}$ & $(8 \mathrm{~m})$ \\
\hline 1.11286000000000 & 1.16266000000000 & -0.23440000000000 & $\mathrm{Te}$ & $(8 \mathrm{~m})$ \\
\hline 1.11286000000000 & -0.66266000000000 & 0.23440000000000 & $\mathrm{Te}$ & $(8 \mathrm{~m})$ \\
\hline-0.61286000000000 & 1.16266000000000 & 0.23440000000000 & $\mathrm{Te}$ & $(8 \mathrm{~m})$ \\
\hline 0.11964000000000 & 0.66839000000000 & 0.24790000000000 & $\mathrm{Tl}$ & $(8 \mathrm{~m})$ \\
\hline 0.38036000000000 & -0.16839000000000 & 0.24790000000000 & $\mathrm{Tl}$ & $(8 \mathrm{~m})$ \\
\hline 0.38036000000000 & 0.66839000000000 & -0.24790000000000 & $\mathrm{Tl}$ & $(8 \mathrm{~m})$ \\
\hline 0.11964000000000 & -0.16839000000000 & -0.24790000000000 & $\mathrm{Tl}$ & $(8 \mathrm{~m})$ \\
\hline-0.11964000000000 & -0.66839000000000 & -0.24790000000000 & $\mathrm{Tl}$ & $(8 \mathrm{~m})$ \\
\hline 0.61964000000000 & 1.16839000000000 & -0.24790000000000 & $\mathrm{Tl}$ & $(8 \mathrm{~m})$ \\
\hline 0.61964000000000 & -0.66839000000000 & 0.24790000000000 & $\mathrm{Tl}$ & $(8 \mathrm{~m})$ \\
\hline-0.11964000000000 & 1.16839000000000 & 0.24790000000000 & $\mathrm{Tl}$ & $(8 \mathrm{~m})$ \\
\hline 0.63183000000000 & 0.00412000000000 & 0.24390000000000 & $\mathrm{Tl}$ & $(8 \mathrm{~m})$ \\
\hline-0.13183000000000 & 0.49588000000000 & 0.24390000000000 & $\mathrm{Tl}$ & $(8 \mathrm{~m})$ \\
\hline-0.13183000000000 & 0.00412000000000 & -0.24390000000000 & $\mathrm{Tl}$ & $(8 \mathrm{~m})$ \\
\hline 0.63183000000000 & 0.49588000000000 & -0.24390000000000 & $\mathrm{Tl}$ & $(8 \mathrm{~m})$ \\
\hline-0.63183000000000 & -0.00412000000000 & -0.24390000000000 & $\mathrm{Tl}$ & $(8 \mathrm{~m})$ \\
\hline 1.13183000000000 & 0.50412000000000 & -0.24390000000000 & $\mathrm{Tl}$ & $(8 \mathrm{~m})$ \\
\hline 1.13183000000000 & -0.00412000000000 & 0.24390000000000 & $\mathrm{Tl}$ & $(8 \mathrm{~m})$ \\
\hline-0.63183000000000 & 0.50412000000000 & 0.24390000000000 & $\mathrm{Tl}$ & $(8 \mathrm{~m})$ \\
\hline
\end{tabular}

$\mathrm{GaCl}_{2}$ (High-temperature): A2B_oP24_52_2e_cd - CIF

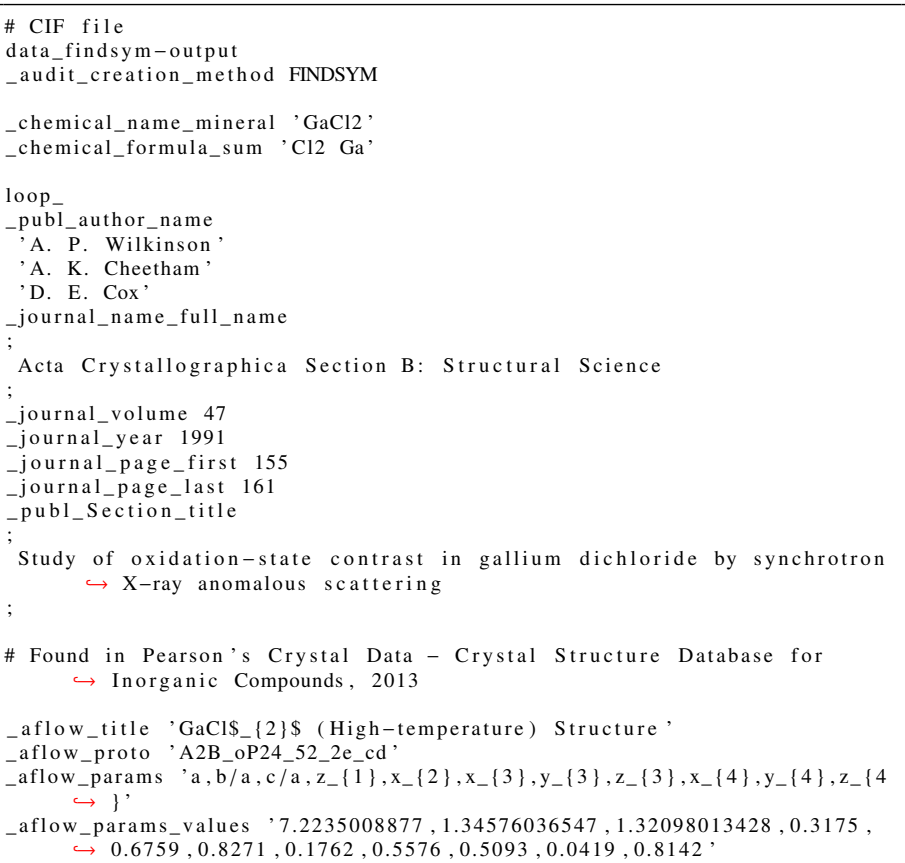




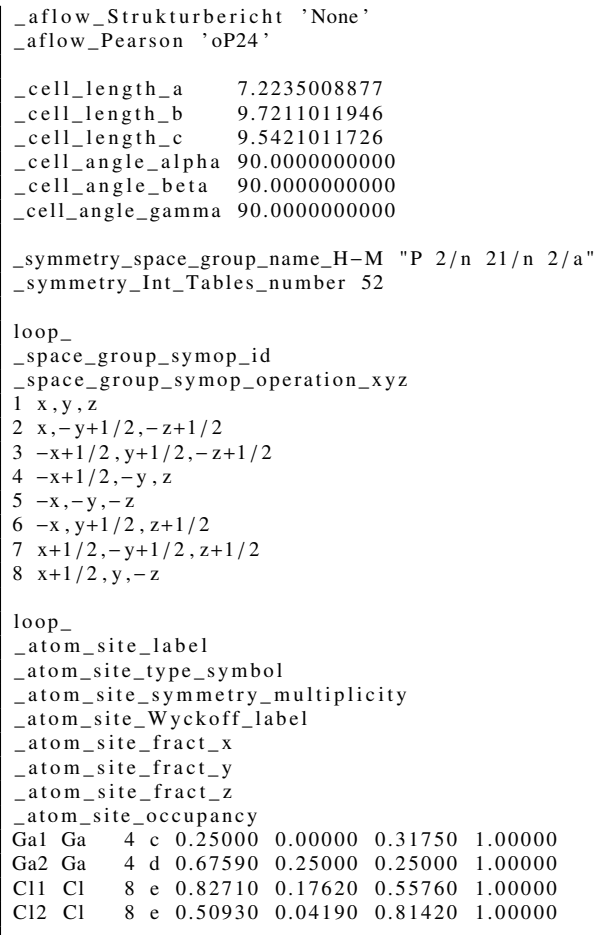

$\mathrm{GaCl}_{2}$ (High-temperature): A2B_oP24_52_2e_cd - POSCAR

A2B_oP24_52_2e_cd \& a , b/a , c/a , z1 , x2, x3, y3 , z3, x4, y4, z4 --params $=$

$9,0.8271$

$\hookrightarrow 0.1762,0.5576,0.5093,0.0419,0.8142 \&$ \&nna D_ $\{2 \mathrm{~h}\}^{\wedge}\{6\} \# 52\left(\mathrm{cde}^{\wedge}\right.$

$\hookrightarrow)$ \& oP24 \& None \& GaCl2 \& \& A. P. Wilkinson and A. K. Cheetha

$\hookrightarrow$ and D. E. Cox, Acta Crystallogr. Sect. B Struct. Sci. 47, $\hookrightarrow 155-161$ (1991)

1.00000000000000

$\begin{array}{lll}7.22350088770000 & 0.00000000000000 & 0.00000000000000\end{array}$

$\begin{array}{lll}0.00000000000000 & 9.72110119460000 & 0.00000000000000\end{array}$

$\begin{array}{llll}0.00000000000000 & 0.00000000000000 & 9.54210117260000\end{array}$

$$
\begin{array}{rr}
\mathrm{Cl} & \mathrm{Ga} \\
16 & 8
\end{array}
$$

Direct

0.82710000000000 $-0.32710000000000$ $-0.32710000000000$ 0.32710000000000 $-0.82710000000000$ 1.32710000000000 1.32710000000000 $-0.82710000000000$ 0.50930000000000 $-0.00930000000000$ $-0.00930000000000$ 0.50930000000000 $-0.50930000000000$ 1.00930000000000 1.00930000000000 $-0.50930000000000$ 0.25000000000000 0.25000000000000 0.75000000000000 0.75000000000000 0.75000000000000 0.1759000000000 $-0.17590000000000$ -0.67590000000000
1.17590000000000

$\begin{array}{rrrl}0.17620000000000 & 0.55760000000000 & \mathrm{Cl} & (8 \mathrm{e}) \\ -0.17620000000000 & 0.55760000000000 & \mathrm{Cl} & (8 \mathrm{e}) \\ 0.67620000000000 & -0.05760000000000 & \mathrm{Cl} & (8 \mathrm{e}) \\ 0.32380000000000 & -0.05760000000000 & \mathrm{Cl} & (8 \mathrm{e}) \\ -0.17620000000000 & -0.55760000000000 & \mathrm{Cl} & (8 \mathrm{e}) \\ 0.17620000000000 & -0.55760000000000 & \mathrm{Cl} & (8 \mathrm{e}) \\ 0.32380000000000 & 1.05760000000000 & \mathrm{Cl} & (8 \mathrm{e}) \\ 0.67620000000000 & 1.05760000000000 & \mathrm{Cl} & (8 \mathrm{e}) \\ 0.04190000000000 & 0.81420000000000 & \mathrm{Cl} & (8 \mathrm{e}) \\ -0.04190000000000 & 0.81420000000000 & \mathrm{Cl} & (8 \mathrm{e}) \\ 0.54190000000000 & -0.31420000000000 & \mathrm{Cl} & (8 \mathrm{e}) \\ 0.45810000000000 & -0.31420000000000 & \mathrm{Cl} & (8 \mathrm{e}) \\ -0.04190000000000 & -0.81420000000000 & \mathrm{Cl} & (8 \mathrm{e}) \\ 0.04190000000000 & -0.81420000000000 & \mathrm{Cl} & (8 \mathrm{e}) \\ 0.45810000000000 & 1.31420000000000 & \mathrm{Cl} & (8 \mathrm{e}) \\ 0.54190000000000 & 1.31420000000000 & \mathrm{Cl} & (8 \mathrm{e}) \\ 0.00000000000000 & 0.31750000000000 & \mathrm{Ga} & (4 \mathrm{c}) \\ 0.50000000000000 & 0.18250000000000 & \mathrm{Ga} & (4 \mathrm{c}) \\ 0.00000000000000 & -0.31750000000000 & \mathrm{Ga} & (4 \mathrm{c}) \\ 0.50000000000000 & 0.81750000000000 & \mathrm{Ga} & (4 \mathrm{c}) \\ 0.25000000000000 & 0.25000000000000 & \mathrm{Ga} & (4 \mathrm{~d}) \\ 0.75000000000000 & 0.25000000000000 & \mathrm{Ga} & (4 \mathrm{~d}) \\ 0.75000000000000 & 0.75000000000000 & \mathrm{Ga} & (4 \mathrm{~d}) \\ 0.25000000000000 & 0.75000000000000 & \mathrm{Ga} & (4 \mathrm{~d})\end{array}$

$\mathrm{Sr}_{2} \mathrm{Bi}_{3}$ : A3B2_oP20_52_de_cd - CIF

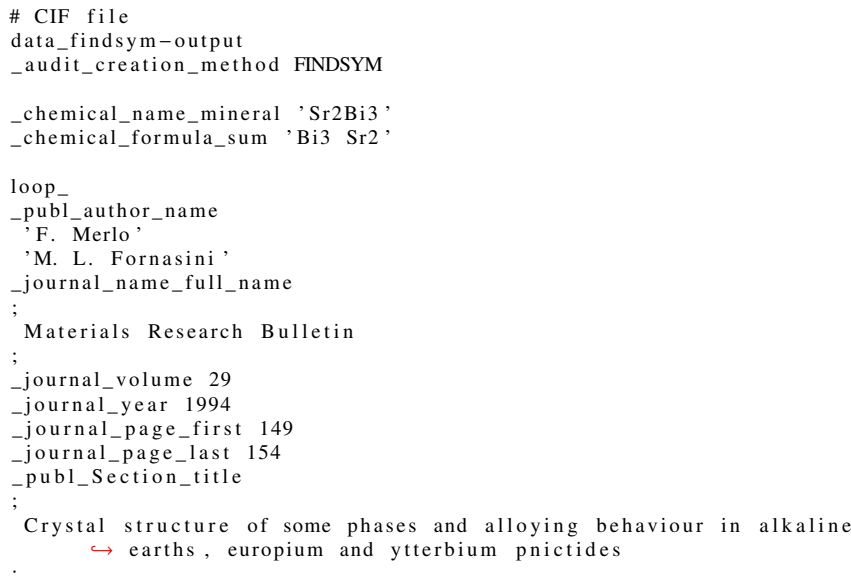
;
F Found in Pearson's Crystal Data - Crystal Structure Database for $\hookrightarrow$ Inorganic Compounds, 2013

aflow_title 'Sr\$_ $\{2\} \$ B i \$ \$_{-}\{3\} \$$ Structure

aflow_proto A3B2_oP20_52_de_cd

aflow_params 'a,b/a,c/a, $z_{-}\{1\}, x_{-}\{2\}, x_{-}\{3\}, x_{-}\{4\}, y_{-}\{4\}, z_{-}\{4\}$

aflow_params_values ' $15.5832022616,0.434585119311,0.426069989123,0.443$, $\hookrightarrow 0.4294,0.0001,0.6539,0.064,0.0788$

_aflow_Strukturbericht 'None'

aflow_Pearson 'oP20

cell_length_a $\quad 15.5832022616$

_cell_length_b 6.7722278141

cell_length_c 6.6395348181

_cell_angle_alpha

_cell_angle_beta 90.0000000000

_symmetry_space_group_name_H-M "P 2/n 21/n 2/a"

_symmetry_Int_Tables_number 52

loop

space_group_symop_id

space_group_symop_operation_xyz

$1 \mathrm{x}, \mathrm{y}, \mathrm{z}$

$2 \mathrm{x},-\mathrm{y}+1 / 2,-\mathrm{z}+1 / 2$

$3-\mathrm{x}+1 / 2, \mathrm{y}+1 / 2,-\mathrm{z}+1 / 2$

$4-\mathrm{x}+1 / 2,-\mathrm{y}, \mathrm{z}$

$5-\mathrm{x},-\mathrm{y},-\mathrm{z}$

$6-\mathrm{x}, \mathrm{y}+1 / 2, \mathrm{z}+1 / 2$

$7 \mathrm{x}+1 / 2,-\mathrm{y}+1 / 2, \mathrm{z}+1 / 2$

$8 \mathrm{x}+1 / 2, \mathrm{y},-\mathrm{z}$

loop

-atom_site_label

atom_site_type_symbol

atom_site_symmetry_multiplicity

atom_site_Wyckoff_label

atom_site_fract_x

atom_site_fract_y

atom_site_fract_z

atom_site_occupancy

$\begin{array}{llllllll}\mathrm{Sr} 1 & \mathrm{Sr} & 4 & \mathrm{c} & 0.25000 & 0.00000 & 0.44300 & 1.00000\end{array}$

$\begin{array}{lllllllll}\mathrm{Bi} 1 \mathrm{Bi} & 4 & \mathrm{~d} & 0.42940 & 0.25000 & 0.25000 & 1.00000\end{array}$

$\begin{array}{lllllllll}\mathrm{Sr} 2 & \mathrm{Sr} & 4 & \mathrm{~d} & 0.00010 & 0.25000 & 0.25000 & 1.00000\end{array}$

$\begin{array}{lllllllll}\mathrm{Bi} 2 & \mathrm{Bi} & 8 & \mathrm{e} & 0.65390 & 0.06400 & 0.07880 & 1.00000\end{array}$

$\mathrm{Sr}_{2} \mathrm{Bi}_{3}$ : A3B2_oP20_52_de_cd - POSCAR

\begin{tabular}{|c|c|c|c|c|}
\hline \multirow{2}{*}{\multicolumn{5}{|c|}{$\begin{aligned} \text { A3B2_oP20_52_de_cd \& a }, \mathrm{b} / \mathrm{a}, \mathrm{c} / \mathrm{a}, \mathrm{z} 1, \mathrm{x} 2, \mathrm{x} 3, \mathrm{x} 4, \mathrm{y} 4, \mathrm{z} 4-\mathrm{params}=15.5832022616, & \\
& \hookrightarrow 0.434585119311,0.426069989123,0.443,0.4294,0.0001,0.6539,0.064, \\
& \hookrightarrow 0.0788 \& \text { Pnna D_ }\{2 \mathrm{~h}\} \wedge\{6\} \# 52(\mathrm{~cd} \wedge 2 \mathrm{e}) \& \text { oP20 \& None \& Sr2Bi3\& } \\
& \hookrightarrow \& \text { F. Merlo and M. L. Fornasini, Mater. Res. Bull. 29, 149-154 } \\
& \hookrightarrow 1994)\end{aligned}$}} \\
\hline & & & & \\
\hline \multicolumn{5}{|c|}{1.00000000000000} \\
\hline 15.58320226160000 & 0.00000000000000 & 0.00000000000000 & & \\
\hline 0.00000000000000 & 6.77222781410000 & 0.00000000000000 & & \\
\hline 0.00000000000000 & 0.00000000000000 & 6.63953481810000 & & \\
\hline $\mathrm{Bi}$ & & & & \\
\hline 12 & & & & \\
\hline \multicolumn{5}{|l|}{ Direct } \\
\hline 0.42940000000000 & 0.25000000000000 & 0.25000000000000 & $\mathrm{Bi}$ & $(4 d)$ \\
\hline 0.07060000000000 & 0.75000000000000 & 0.25000000000000 & $\mathrm{Bi}$ & $(4 \mathrm{~d})$ \\
\hline-0.42940000000000 & 0.75000000000000 & 0.75000000000000 & $\mathrm{Bi}$ & $(4 \mathrm{~d})$ \\
\hline 0.92940000000000 & 0.25000000000000 & 0.75000000000000 & $\mathrm{Bi}$ & $(4 \mathrm{~d})$ \\
\hline 0.65390000000000 & 0.06400000000000 & 0.07880000000000 & $\mathrm{Bi}$ & $(8 \mathrm{e})$ \\
\hline-0.15390000000000 & -0.06400000000000 & 0.07880000000000 & $\mathrm{Bi}$ & $(8 \mathrm{e})$ \\
\hline-0.15390000000000 & 0.56400000000000 & 0.42120000000000 & $\mathrm{Bi}$ & $(8 \mathrm{e})$ \\
\hline 0.65390000000000 & 0.43600000000000 & 0.42120000000000 & $\mathrm{Bi}$ & $(8 \mathrm{e})$ \\
\hline-0.65390000000000 & -0.06400000000000 & -0.07880000000000 & $\mathrm{Bi}$ & $(8 \mathrm{e})$ \\
\hline 1.15390000000000 & 0.06400000000000 & -0.07880000000000 & $\mathrm{Bi}$ & $(8 \mathrm{e})$ \\
\hline 1.15390000000000 & 0.43600000000000 & 0.57880000000000 & $\mathrm{Bi}$ & $(8 \mathrm{e})$ \\
\hline-0.65390000000000 & 0.56400000000000 & 0.57880000000000 & $\mathrm{Bi}$ & $(8 \mathrm{e})$ \\
\hline 0.25000000000000 & 0.00000000000000 & 0.44300000000000 & $\mathrm{Sr}$ & $(4 c)$ \\
\hline 0.25000000000000 & 0.50000000000000 & 0.05700000000000 & $\mathrm{Sr}$ & $(4 c)$ \\
\hline 0.75000000000000 & 0.00000000000000 & -0.44300000000000 & $\mathrm{Sr}$ & $(4 c)$ \\
\hline 0.75000000000000 & 0.50000000000000 & 0.94300000000000 & $\mathrm{Sr}$ & $(4 \mathrm{c})$ \\
\hline 0.00010000000000 & 0.25000000000000 & 0.25000000000000 & $\mathrm{Sr}$ & (4d) \\
\hline 0.49990000000000 & 0.75000000000000 & 0.25000000000000 & $\mathrm{Sr}$ & (4d) \\
\hline-0.00010000000000 & 0.75000000000000 & 0.75000000000000 & $\mathrm{Sr}$ & $(4 d)$ \\
\hline 0.50010000000000 & 0.25000000000000 & 0.75000000000000 & $\mathrm{Sr}$ & (4d) \\
\hline
\end{tabular}

TaNiTe2: ABC2_oP16_53_h_e_gh - CIF

\# CIF file
data_findsym-output
_audit_creation_method FINDSYM
_chemical_name_mineral 'TaNiTe2,
_chemical_formula_sum,'Ni Ta Te2,
loop_
-publ_author_name
'W. Tremel,
_journal_name_full_name
; Angewandte Chemie (International ed.)
;
_journal_volume 30
-journal_year 1991
-journal_page_first 840
-journal_page_last 843
-publ_Section_title
;


Isolated and Condensed Ta $\$_{-}\{2\} \$ N i \$_{-}\{2\} \$$ Clusters in the Layered

$\rightarrow$ Tellurides Ta\$_ $\{2\} \$ N i \$_{-}\{2\} \$ T$ Te $\$_{-}\{4\} \$$ and $\mathrm{Ta}_{-}\{2\} \$ \mathrm{Ni} \$_{-}\{3\} \$ \mathrm{Te} \$_{-}\{5$ $\rightarrow$ \}\$

\# Found in Pearson's Crystal Data - Crystal Structure Database fo $\hookrightarrow$ Inorganic Compounds, 2013

-aflow_title 'TaNiTe\$_\{2\}\$ Structure

-aflow_proto 'ABC2 oP16 53 h_e gh '

-aflow_params 'a,b/a,c/a, $x_{-}\{1\}, y_{-}\{2\}, y_{-}\{3\}, z_{-}\{3\}, y_{-}\{4\}, z_{-}\{4\}$,

_aflow_params_values , $11.6904618594,0.282132455361,0.78890717994,0.79514$ $\hookrightarrow, 0.3193,0.1198,0.3549,0.224,0.7493$

aflow_Strukturbericht 'None'

-aflow_Pearson oP16,

_cell_length_a $\quad 11.6904618594$

_cell_length_b 3.2982587087

_cell_length_c $\quad 9.2226892977$

_cell_angle_alpha 90.0000000000

_cell_angle_beta 90.0000000000

_cell_angle_gamma 90.0000000000

_symmetry_space_group_name_H-M "P 2/m 2/n 21/a"

_symmetry_Int_Tables_number 53

loop

_space_group_symop_id

_space_group_symop_operation_xyz

$1 \mathrm{x}, \mathrm{y}, \mathrm{z}$

$2 \mathrm{x},-\mathrm{y},-\mathrm{z}$

$3-x+1 / 2, y,-z+1 / 2$

$4-x+1 / 2,-y, z+1 / 2$

$5-\mathrm{x},-\mathrm{y},-\mathrm{z}$

$6-\mathrm{x}, \mathrm{y}, \mathrm{z}$

$7 \mathrm{x}+1 / 2,-\mathrm{y}, \mathrm{z}+1 / 2$

loop_

_atom_site_label

_atom_site_type_symbol

_atom_site_symmetry_multiplicity

_atom_site_Wyckoff_label

-atom_site_fract_x

_atom_site_fract_y

_atom_site_fract_z

atom_site_occupancy

Tal Ta 4 e $0.79514-0.00000 \quad 0.00000 \quad 1.00000$

$\begin{array}{llllllllll} & \mathrm{Te} & \mathrm{Te} & 4 & \mathrm{~g} & 0.25000 & 0.31930 & 0.25000 & 1.00000\end{array}$

$\begin{array}{llllllll}\mathrm{Ni} & \mathrm{Ni} & 4 & \mathrm{~h} & 0.000000 & 0.11980 & 0.35490 & 1.00000 \\ \mathrm{Te} 2 & \mathrm{Te} & 4 & \mathrm{~h} & 0.00000 & 0.22400 & 0.74930 & 1.00000\end{array}$

TaNiTe 2 : ABC2_oP16_53_h_e_gh - POSCAR

ABC2_oP16_53_h_e_gh \& a,b/a,c/a, x1,y2,y3,z3,y4,z4 --params $=11.6904618594$ $\hookrightarrow, 0.282132455361,0.78890717994,0.79514,0.3193,0.1198,0.3549$ $\hookrightarrow, 0.224,0.7493 \&$ Pmna D $\{2 \mathrm{~h}\}^{\wedge}\{7\} \# 53(\mathrm{egh} \wedge 2) \&$ oP16 \& None \&

$\hookrightarrow$ TaNiTe2 \& \& W. Tremel, Angew. Chem. Int. Ed. 30, 840-843 (1991 $\hookrightarrow$ )

1.00000000000000

11.69046185940000

0.00000000000000

0.00000000000000

0.00000000000000 3.29825870870000 0.00000000000000

0.00000000000000 9.22268929770000

$\begin{array}{rrr}\mathrm{Ni} & \mathrm{Ta} & \mathrm{Te} \\ 4 & 4 & 8\end{array}$

Direct

$0.0000000000000 \quad 0.11980000000000$ $0.50000000000000-0.11980000000000$ $0.50000000000000 \quad 0.11980000000000$ $0.00000000000000-0.11980000000000$ $0.79514000000000 \quad 0.00000000000000$ $-0.29514000000000 \quad 0.00000000000000$ $-0.2951400000000-0.0000000000000$ $1.29514000000000-0.00000000000000$ $1.29514000000000-0.00000000000000$ $0.25000000000000-0.31930000000000$ $0.25000000000000-0.31930000000000$ $0.75000000000000-0.31930000000000$ $0.75000000000000 \quad 0.31930000000000$ $0.00000000000000 \quad 0.22400000000000$ $0.50000000000000-0.22400000000000$

$\begin{array}{rr}0.50000000000000 & 0.22400000000000 \\ 0.00000000000000 & -0.22400000000000\end{array}$

0.35490000000000 0.85490000000000 0.14510000000000 $-0.35490000000000$ 0.00000000000000 .50000000000000 0.000000000000 .5000000000000 0.50000000000000 0.25000000000000 0.75000000000000 0.75000000000000 0.25000000000000 0.74930000000000 1.24930000000000 $-0.24930000000000$ $-0.74930000000000$

$\begin{array}{ll}\mathrm{Ni} & (4 \mathrm{~h}) \\ \mathrm{Ni} & (4 \mathrm{~h}) \\ \mathrm{Ni} & (4 \mathrm{~h}) \\ \mathrm{Ni} & (4 \mathrm{~h}) \\ \mathrm{Ta} & (4 \mathrm{e}) \\ \mathrm{Ta} & (4 \mathrm{e}) \\ \mathrm{Ta} & (4 \mathrm{e}) \\ \mathrm{Ta} & (4 \mathrm{e}) \\ \mathrm{Te} & (4 \mathrm{~g}) \\ \mathrm{Te} & (4 \mathrm{~g}) \\ \mathrm{Te} & (4 \mathrm{~g}) \\ \mathrm{Te} & (4 \mathrm{~g}) \\ \mathrm{Te} & (4 \mathrm{~h}) \\ \mathrm{Te} & (4 \mathrm{~h}) \\ \mathrm{Te} & (4 \mathrm{~h}) \\ \mathrm{Te} & (4 \mathrm{~h})\end{array}$

CuBrSe 3 : ABC3_oP20_53_e_g_hi - CIF

\section{\# CIF file}

data_findsym-output

_audit_creation_method FINDSYM

_chemical_name_mineral 'CuBrSe3,

_chemical_formula_sum ' $\mathrm{Br} \mathrm{Cu} \mathrm{Se} 3$

loop

_publ_author_name

'H. M. Haendler'

'P. M. Carkner'

_journal_name_full_name

Journal of Solid State Chemistry

_journal_volume 29

journal year 1979

_journal_page_first 35

-journal_page_last 39
_publ_Section_title
The crystal structure of copper bromide triselenide, CuBrSe\$_\{3\}\$

\# Found in Pearson's Crystal Data - Crystal Structure Database for $\hookrightarrow$ Inorganic Compounds, 2013

aflow_title 'CuBrSe\$_\{3\}\$ Structure'

aflow_proto 'ABC3_oP20_53_e_g_hi '

aflow_params 'a, b/a,c/a, $x_{-}\{1\}, y_{-}\{2\}, y_{-}\{3\}, z_{-}\{3\}, x_{-}\{4\}, y_{-}\{4\}, z_{-}\{4\}$

aflow_params_values ' $14.3630679002,0.312469539787,0.535821207264,0.6826$ $\hookrightarrow, 0.2856,0.3458,0.2708,0.6247,0.6057,0.3575$

aflow_Strukturbericht 'None'

aflow_Pearson 'oP20,

cell_length_a 14.3630679002

cell_length_b 4.4880212167

cell_length_c 7.6960363823

_cell_angle_alpha 90.0000000000

cell_angle_beta 90.0000000000

cell_angle_gamma 90.0000000000

symmetry_space_group_name_H-M "P $2 / \mathrm{m} 2 / \mathrm{n} 21 / \mathrm{a} "$

symmetry_Int_Tables_number 53

loop

space_group_symop_id

space_group_symop_operation_xyz

$\mathrm{x}, \mathrm{y}, \mathrm{z}$

$3-x+1 / 2, y,-z+1 / 2$

$4-\mathrm{x}+1 / 2,-\mathrm{y}, \mathrm{z}+1 / 2$

$5-\mathrm{x},-\mathrm{y},-\mathrm{z}$

$6-\mathrm{x}, \mathrm{y}, \mathrm{z}$

$\mathrm{x}+1 / 2,-\mathrm{y}, \mathrm{z}+1 / 2$

$8 \mathrm{x}+1 / 2, \mathrm{y},-\mathrm{z}+1 / 2$

loop

atom_site_label

atom_site_type_symbol

atom_site_symmetry_multiplicity

atom_site_Wyckoff_label

atom_site_fract_x

atom_site_fract_y

atom_site_fract_z

$\begin{array}{llllllll}\mathrm{Br} 1 & \mathrm{Br} & 4 & \mathrm{e} & 0.68260 & 0.00000 & 0.00000 & 1.00000\end{array}$

$\begin{array}{llllllll}\mathrm{Cu} 1 \mathrm{Cu} & 4 & \mathrm{~g} & 0.25000 & 0.28560 & 0.25000 & 1.00000\end{array}$

$\begin{array}{llllllll}\mathrm{Cu} & \mathrm{Cu} & 4 & \mathrm{~g} & 0.25000 & 0.28560 & 0.25000 & 1.00000 \\ \mathrm{Se} 1 \mathrm{Se} & 4 & \mathrm{~h} & 0.00000 & 0.34580 & 0.27080 & 1.00000\end{array}$

$\begin{array}{lllllllll}\mathrm{Se} 2 & \mathrm{Se} & 8 & \mathrm{i} & 0.62470 & 0.60570 & 0.35750 & 1.00000\end{array}$

CuBrSe 3 : ABC3_oP20_53_e_g_hi - POSCAR

ABC3_oP20_53_e_g_hi \& a, b/a,c/a, x1,y2,y3, z3, x4,y4,z4 --params $=$

$\hookrightarrow 14.3630679002,0.312469539787,0.535821207264,0.6826,0.2856$

$\hookrightarrow 0.3458,0.2708,0.6247,0.6057,0.3575 \&$ Pmna D_ $\{2 \mathrm{~h}\}^{\wedge}\{7\} \# 53$ (eghi)

$\hookrightarrow$ \& oP20 \& None \& CuBrSe3 \& \& H. M. Haendler and P. M. Carkner

$\hookrightarrow$ J. Solid State Chem. 29, 35-39 (1979)

1.00000000000000

$14.36306790020000 \quad 0.00000000000000 \quad 0.00000000000000$

$\begin{array}{rrr}1.00000000000000 & 4.48802121670000 & 0.00000000000000\end{array}$

$\begin{array}{lll}0.00000000000000 & 4.48802121670000 & 0.00000000000000 \\ 0.00000000000000 & 0.00000000000000 & 7.69603638230000\end{array}$

$\mathrm{Br} \quad \mathrm{Cu} \quad \mathrm{Se}$

Direct

0.68260000000000

$-0.18260000000000$

$-0.68260000000000$

1.18260000000000

0.25000000000000

0.25000000000000

0.75000000000000

0.75000000000000

0.7500000000000

0.00000000000000

0.50000000000000

0.00000000000000

0.62470000000000

-0.12470000000000
-0.12470000000000

$-0.12470000000000$

0.62470000000000
-0.62470000000000

1.12470000000000

1.12470000000000
-0.62470000000000

0.00000000000000

0.00000000000000

0.00000000000000

0.00000000000000

28560000000000

0.28560000000000

.28560000000000

0.28560000000000

0.34580000000000

$-0.34580000000000$

0.34580000000000

0.34580000000000

0.60570000000000

$-0.60570000000000$

0.60570000000000

$-0.60570000000000$

$-0.60570000000000$

0.60570000000000

$-0.60570000000000$

0.60570000000000

0.00000000000000 0.50000000000000 0.00000000000000 0.50000000000000 .25000000000000 0.75000000000000 0.75000000000000 0.25000000000000 0.27080000000000 0.77080000000000 0.22920000000000 $-0.27080000000000$ 0.35750000000000 0.85750000000000 0.14250000000000 $-0.35750000000000$ $-0.35750000000000$ 0.14250000000000 0.85750000000000 0.35750000000000

$\begin{array}{ll}\mathrm{Br} & (4 \mathrm{e}) \\ \mathrm{Br} & (4 \mathrm{e}) \\ \mathrm{Br} & (4 \mathrm{e}) \\ \mathrm{Br} & (4 \mathrm{e}) \\ \mathrm{Cu} & (4 \mathrm{~g}) \\ \mathrm{Cu} & (4 \mathrm{~g}) \\ \mathrm{Cu} & (4 \mathrm{~g}) \\ \mathrm{Cu} & (4 \mathrm{~g}) \\ \mathrm{Se} & (4 \mathrm{~h}) \\ \mathrm{Se} & (4 \mathrm{~h}) \\ \mathrm{Se} & (4 \mathrm{~h}) \\ \mathrm{Se} & (4 \mathrm{~h}) \\ \mathrm{Se} & (8 \mathrm{i}) \\ \mathrm{Se} & (8 \mathrm{i}) \\ \mathrm{Se} & (8 \mathrm{i}) \\ \mathrm{Se} & (8 \mathrm{i}) \\ \mathrm{Se} & (8 \mathrm{i}) \\ \mathrm{Se} & (8 \mathrm{i}) \\ \mathrm{Se} & (8 \mathrm{i}) \\ \mathrm{Se} & (8 \mathrm{i})\end{array}$

$\mathrm{BiGaO}_{3}$ : ABC3_oP20_54_e_d_cf - CIF

\section{\# CIF file}

data_findsym-outpu

audit_creation_method FINDSYM

chemical_name_mineral 'BiGaO3'

chemical formula_sum ' $\mathrm{Bi} \mathrm{Ga} \mathrm{O}^{\prime}$

loop

'A. A. Belik'

E. \{Takayama-Muromachi\}

'N. Hirao'

journal_name_full_name

Physical Review B 
journal_volume 80

journal_year 2009

_journal_page_first 214103

journal_page_last 214103

publ_Section_title

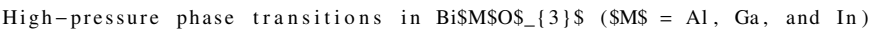
$\longrightarrow$ Tit In situ

Found in Pearson's Crystal Data - Crystal Structure Database for $\hookrightarrow$ Inorganic Compounds, 2013

aflow title' $\mathrm{BiGaO} \$ 3\}$ S Structure'

aflow_proto 'ABC3_oP20_54_e_d_cf,

aflow_params ,a,b/a,c/a, y- $\{1\}, z_{-}\{2\}, z_{-}\{3\}, x_{-}\{4\}, y_{-}\{4\}, z_{-}\{4\}$,

aflow_params values, $5.3467489374,0.956627452436,1.81710213776,0.8667$ $\hookrightarrow 0.6417,0.8902,0.4055,0.2686,0.5503$

aflow_Strukturbericht 'None'

_aflow_Pearson 'oP20,

\section{$\begin{array}{ll}\text { _cell_length_a } & 5.3467489374 \\ \text { _cell_length_b } & 5.1148468148\end{array}$}

_cell_length_c $\quad 9.7155889242$

_cell_angle_alpha 90.0000000000

_cell_angle_beta 90.0000000000

cell angle gamma 90.0000000000

symmetry_space group_name_H-M "P $21 / \mathrm{c} 2 / \mathrm{c} 2 / \mathrm{a} "$

-symmetry_Int_Tables_number 54

loop

_space_group_symop_id

_space_group_symop_operation_xyz

$1 \mathrm{x}, \mathrm{y}, \mathrm{z}$

$2 \mathrm{x}+1 / 2,-\mathrm{y},-\mathrm{z}+1 / 2$

$3-\mathrm{x}, \mathrm{y},-\mathrm{z}+1 / 2$

$4-x+1 / 2,-y, z$

$5-\mathrm{x},-\mathrm{y},-\mathrm{z}$

$6-x+1 / 2, y, z+1 / 2$

$\begin{array}{ll}7 & x,-y, z+1 / 2 \\ 8 & x+1 / 2, y,-z\end{array}$

loop_

atom site label

atom_site_type_symbol

atom_site_symmetry_multiplicity

atom_site_Wyckoff_label

atom_site_fract_x

-atom_site_fract_y
atom_site_fract_z

- atom_site_fract_z

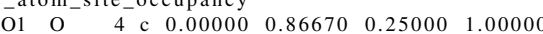

$\begin{array}{llllllll}\mathrm{Ga} 1 \mathrm{Ga} & 4 & \mathrm{~d} & 0.25000 & 0.00000 & 0.64170 & 1.00000\end{array}$

$\begin{array}{llllllll}\mathrm{Bi} 1 & \mathrm{Bi} & 4 & \mathrm{e} & 0.25000 & 0.50000 & 0.89020 & 1.00000\end{array}$

$\begin{array}{lllllllll}\mathrm{O} 2 & \mathrm{O} & 8 & \mathrm{f} & 0.40550 & 0.26860 & 0.55030 & 1.00000\end{array}$

$\mathrm{BiGaO}_{3}$ : ABC3_oP20_54_e_d_cf - POSCAR

ABC3_oP20_54_e_d_cf \& a , b/a , c/a, y1 , z2 , z3 , x4,y4, z4 --params $=5.3467489374$ $\hookrightarrow 0.956627452436,1.81710213776,0.8667,0.6417,0.8902,0.4055,0.2686$

$\hookrightarrow, 0.5503 \&$ Pcca D_ $\{2 \mathrm{~h}\}^{\wedge}\{8\} \# 54$ (cdef) \& oP20 \& None \& BiGaO3 \&

$\hookrightarrow$ \& H. Yusa et al., Phys. Rev. B 80, 214103(2009)

1.00000000000000

$\begin{array}{lll}5.34674893740000 & 0.00000000000000 & 0.00000000000000\end{array}$

$\begin{array}{lll}0.00000000000000 & 5.11484681480000 & 0.00000000000000\end{array}$

$0.00000000000000 \quad 0.00000000000000 \quad 9.71558892420000$

$\begin{array}{rrr}\mathrm{Bi} & \mathrm{Ga} & \mathrm{O} \\ 4 & 4 & 12\end{array}$

Direct

0.25000000000000

0.25000000000000

0.75000000000000

0.75000000000000

0.25000000000000

0.25000000000000

0.75000000000000

0.75000000000000

0.25000000000000

0.00000000000000

0.50000000000000

0.00000000000000

0.50000000000000

0.40550000000000

0.09450000000000

$-0.40550000000000$

0.90550000000000

$-0.40550000000000$

0.90550000000000

0.40550000000000

0.40550000000000

0.50000000000000

.5000000000000

0.00000000000000

0.00000000000000

0.00000000000000

0.00000000000000

0.86670000000000

$-0.86670000000000$

$-0.86670000000000$

.26860000000000

0.26860000000000

0.26860000000000

$-0.26860000000000$

$-0.26860000000000$

0.26860000000000

0.89020000000000 $-0.39020000000000$ 0.89020000000000 .39020000000000 0.64170000000000 $-0.14170000000000$ $-0.64170000000000$ 1.1417000000000 0.25000000000000 0.25000000000000 0.75000000000000 0.75000000000000 0.55030000000000 0.55030000000000 $-0.05030000000000$ $-0.05030000000000$ $-0.55030000000000$

$-0.55030000000000$

1.05030000000000 1.05030000000000

$\mathrm{Bi} \quad(4 \mathrm{e})$

$\mathrm{Bi} \quad(4 \mathrm{e})$

$\mathrm{Bi} \quad(4 \mathrm{e})$

$(4 \mathrm{e})$
$(4 \mathrm{~d})$

$(4 d)$
$(4 d)$

$\begin{array}{ll}\mathrm{Ga} & (4 \mathrm{~d}) \\ \mathrm{Ga} & (4 \mathrm{~d})\end{array}$

$\begin{array}{ll}\mathrm{Ga} & (4 \mathrm{~d}) \\ \mathrm{Ga} & (4 \mathrm{~d})\end{array}$

$\mathrm{O} \quad(4 \mathrm{c})$

$\mathrm{O} \quad(4 \mathrm{c})$

$\mathrm{O} \quad(4 \mathrm{c})$

$(4 c)$

$(8 \mathrm{f})$

$\mathrm{O} \quad(8 \mathrm{f})$

$\mathrm{O} \quad(8 \mathrm{f})$

$\mathrm{O} \quad(8 \mathrm{f})$

O ( 8f

( $8 \mathrm{f})$

$\mathrm{GeAs}_{2}$ : A2B_oP24_55_2g2h_gh - CIF

\# CIF file

data findsym-output

audit_creation_method FINDSYM

chemical name mineral 'GeAs2,

chemical formula_sum , As2 Ge,

loop

_publ_author_name

'T. Wadsten,"

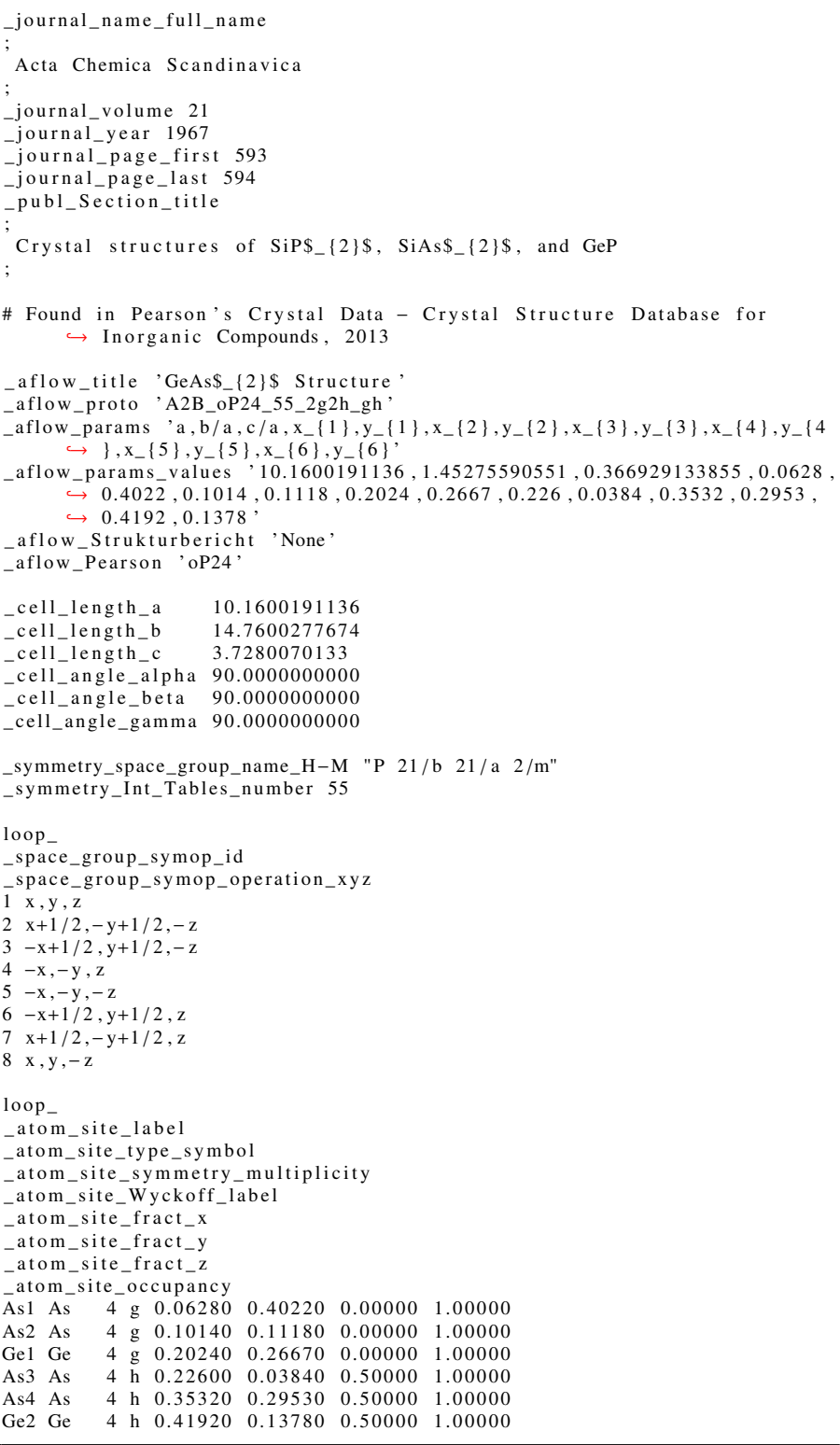

$\mathrm{GeAs}_{2}$ : A2B_oP24_55_2g2h_gh - POSCAR

A2B_oP24_55_2g2h_gh \& a , b/a,c/a, x1,y1, x2, y2, x3,y3, x4,y4, x5,y5, x6, y6 $\longrightarrow$ params $=10.1600191136,1.45275590551,0.366929133855,0.0628,0.4022$ $\hookrightarrow, 0.1014,0.1118,0.2024,0.2667,0.226,0.0384,0.3532,0.2953,0.4192$ $\hookrightarrow 0.1378$ \& Pbam D_ $\{2 \mathrm{~h}\}^{\wedge}\{9\} \# 55\left(\mathrm{~g}^{\wedge} 3 \mathrm{~h}^{\wedge} 3\right)$ \& $\mathrm{oP} 24$ \& None \& GeAs2 \& $\hookrightarrow \&$ T. Wadsten, Acta Chem. Scand. 21, 593-594 (1967) 1.00000000000000

10.16001911360000

14.76002776740000

As Ge

Direct

$0.06280000000000 \quad 0.40220000000000$

$-0.06280000000000-0.40220000000000$

$0.43720000000000 \quad 0.90220000000000$

$\begin{array}{ll}0.56280000000000 & 0.09780000000000\end{array}$

$0.10140000000000 \quad 0.11180000000000$

$-0.10140000000000-0.11180000000000$

$0.39860000000000 \quad 0.61180000000000$

$0.60140000000000 \quad 0.38820000000000$

$0.22600000000000-0.03840000000000$

$-0.22600000000000-0.03840000000000$

$0.27400000000000 \quad 0.53840000000000$

$0.72600000000000 \quad 0.46160000000000$

$0.35320000000000 \quad 0.29530000000000$

$\begin{array}{ll}-0.35320000000000 & -0.29530000000000\end{array}$

$0.14680000000000 \quad 0.79530000000000$

$0.85320000000000 \quad 0.20470000000000$

$0.20240000000000 \quad 0.26670000000000$

$-0.20240000000000-0.26670000000000$

$\begin{array}{rr}0.29760000000000 & 0.76670000000000\end{array}$

$0.70240000000000 \quad 0.23330000000000$

$\begin{array}{lll}0.41920000000000 & 0.13780000000000\end{array}$

$-0.41920000000000-0.13780000000000$

$0.08080000000000 \quad 0.63780000000000$

$0.91920000000000 \quad 0.36220000000000$

0.00000000000000

0.00000000000000

3.72800701330000

0.00000000000000

0.00000000000000

0.00000000000000

0.00000000000000

0.00000000000000

0.00000000000000

0.00000000000000

0.00000000000000

0.00000000000000

0.50000000000000

0.50000000000000

0.50000000000000

0.50000000000000

0.50000000000000

0.50000000000000

0.50000000000000

0.50000000000000

0.00000000000000

0.00000000000000

0.00000000000000

0.00000000000000

0.50000000000000

0.50000000000000

0.50000000000000

0.50000000000000

As $\quad(4 \mathrm{~g})$

As $\quad(4 \mathrm{~g})$

As $(4 \mathrm{~g})$

As $(4 \mathrm{~g})$

As $(4 \mathrm{~g})$

$\mathrm{Rh}_{5} \mathrm{Ge}_{3}$ : A3B5 _oP16_55_ch_agh - CIF 


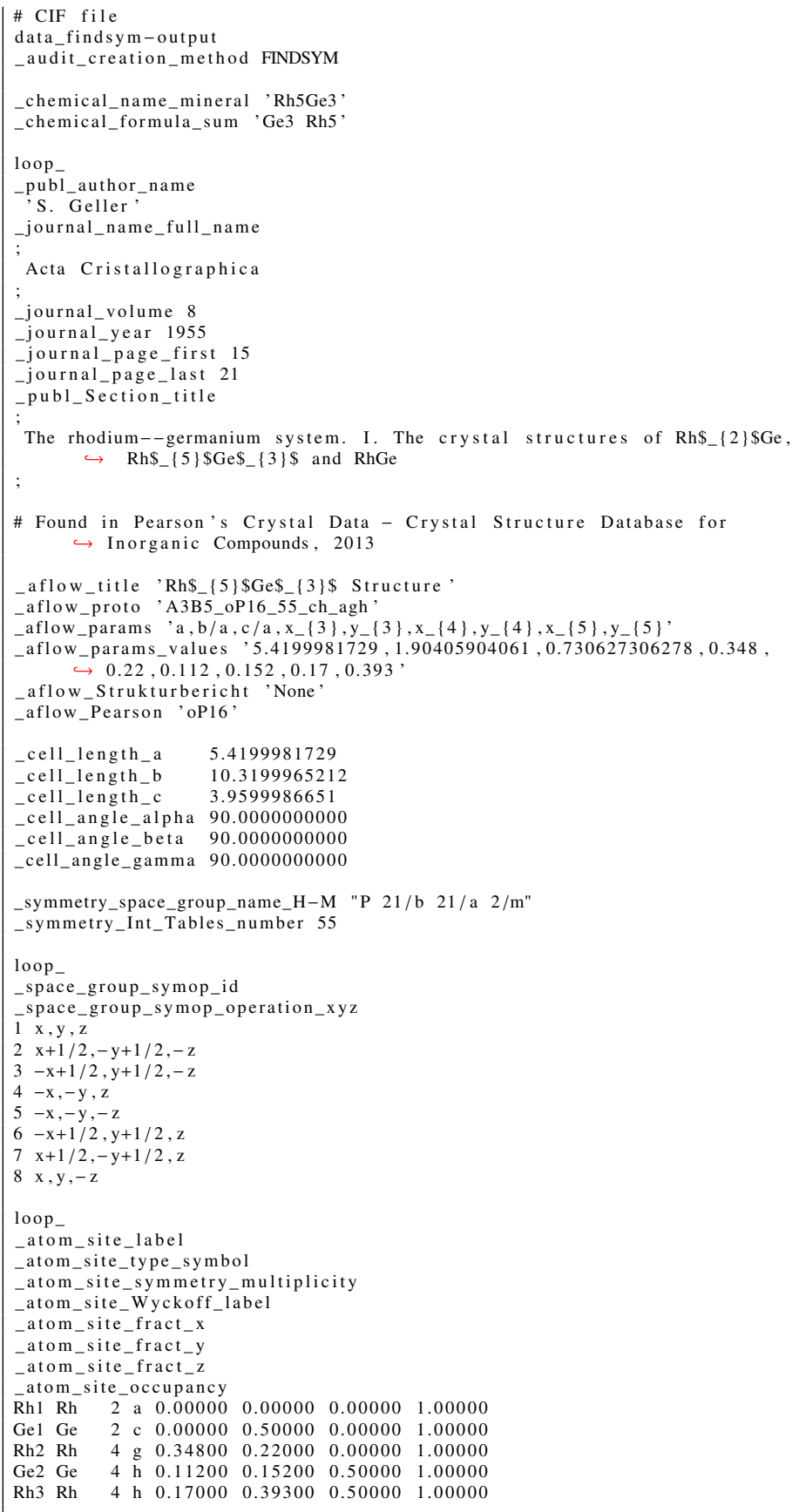

$\mathrm{Rh}_{5} \mathrm{Ge}_{3}$ : A3B5_oP16_55_ch_agh - POSCAR

A3B5_oP16_55_ch_agh \& a , b/a,c/a, x3,y3, x4,y4, x5,y5 --params $=5.4199981729$ $\hookrightarrow 1.90405904061,0.730627306278,0.348,0.22,0.112,0.152,0.17,0.393$ $\hookrightarrow \&$ Pbam D_ $\{2 \mathrm{~h}\}^{\wedge}\{9\} \# 55(\operatorname{acgh} \wedge 2) \&$ oP16 \& None \& Rh5Ge3 \& \& S $\hookrightarrow$ Geller, Acta Cryst. 8, 15-21 (1955)

1.00000000000000

$\begin{array}{lll}5.41999817290000 & 0.00000000000000 & 0.00000000000000\end{array}$

$\begin{array}{lrl}0.00000000000000 & 10.31999652120000 & 0.00000000000000\end{array}$

$\begin{array}{rrr}0.00000000000000 & 0.00000000000000 & 3.95999866510000\end{array}$

$$
\begin{array}{rr}
\mathrm{Ge} & \mathrm{Rh} \\
6 & 10
\end{array}
$$

Direct

0.00000000000000 0.50000000000000 0.11200000000000 $-0.11200000000000$ 0.3880000000000 0.6120000000000 0.00000000000000 0.500000000000 0.34800000000000 $-0.34800000000000$ 0.15200000000000 0.84800000000000 0.17000000000000 $-0.17000000000000$ 0.33000000000000 0.50000000000000 0.00000000000000 0.1520000000000 $-0.15200000000000$ 0.65200000000000 0.3480000000000 0.00000000000 0.5000000000000 0.2200000000000 $-0.22000000000000$ 0.72000000000000 0.2800000000000 0.3930000000000 $-0.39300000000000$ 0.89300000000000 0.10700000000000

0.00000000000000 0.00000000000000 0.50000000000000 0.5000000000000 0.50000000000000 0.50000000000000 0.50000000000 0.000000000000 0.00000000000000 0.00000000000000 0.00000000000000 0.00000000000000 0.00000000000000 0.50000000000000 0.5000000000000 0.5000000000000 0.50000000000000

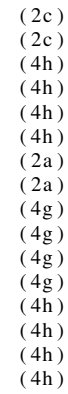

$(2 \mathrm{c})$

(4h)

(4h

(4h)

(2a)

$(4 \mathrm{~g})$

$(4 \mathrm{~g})$

$(4 \mathrm{~g})$

$(4 \mathrm{~h})$

(4h)

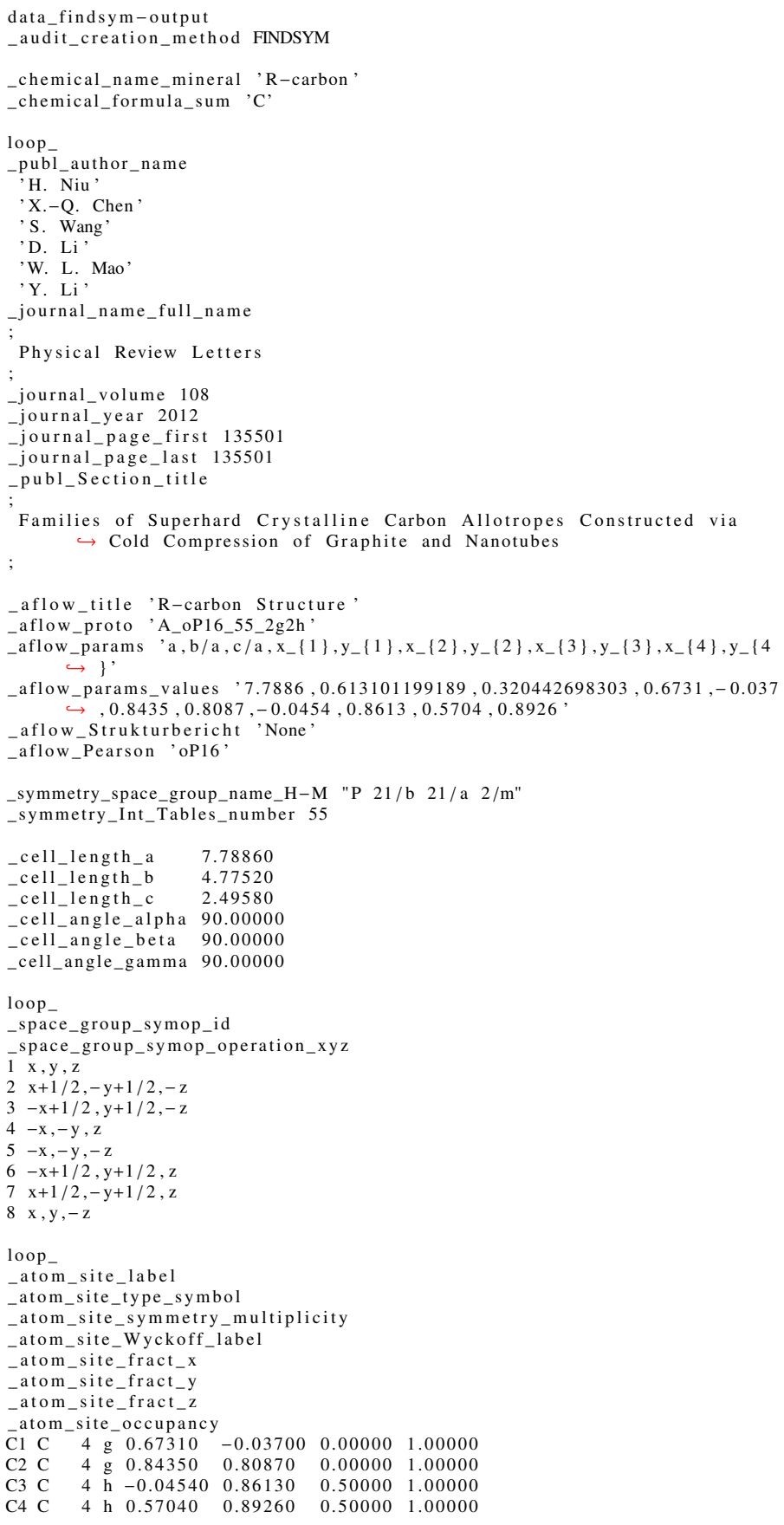

R-carbon: A_oP16_55_2g2h - POSCAR

\begin{tabular}{|c|c|c|c|c|}
\hline \multicolumn{5}{|c|}{ 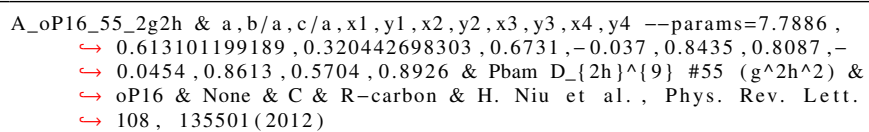 } \\
\hline \multicolumn{5}{|c|}{1.00000000000000} \\
\hline 7.78860000000000 & 0.00000000000000 & 0.00000000000000 & & \\
\hline 0.00000000000000 & 4.77520000000000 & 0.00000000000000 & & \\
\hline 0.00000000000000 & 0.00000000000000 & 2.49580000000000 & & \\
\hline \multicolumn{5}{|l|}{ 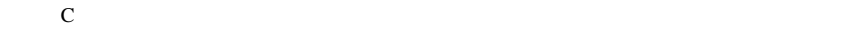 } \\
\hline \multicolumn{5}{|l|}{ Direct } \\
\hline 0.67310000000000 & -0.03700000000000 & 0.00000000000000 & $\mathrm{C}$ & $(4 \mathrm{~g})$ \\
\hline-0.67310000000000 & 0.03700000000000 & 0.00000000000000 & $\mathrm{C}$ & $(4 \mathrm{~g})$ \\
\hline-0.17310000000000 & 0.46300000000000 & 0.00000000000000 & $\mathrm{C}$ & $(4 \mathrm{~g})$ \\
\hline 1.17310000000000 & 0.53700000000000 & 0.00000000000000 & $\mathrm{C}$ & $(4 \mathrm{~g})$ \\
\hline 0.84350000000000 & 0.80870000000000 & 0.00000000000000 & $\mathrm{C}$ & $(4 \mathrm{~g})$ \\
\hline-0.84350000000000 & -0.80870000000000 & 0.00000000000000 & $\mathrm{C}$ & $(4 \mathrm{~g})$ \\
\hline-0.34350000000000 & 1.30870000000000 & 0.00000000000000 & $\mathrm{C}$ & $(4 \mathrm{~g})$ \\
\hline 1.34350000000000 & -0.30870000000000 & 0.00000000000000 & $\mathrm{C}$ & $(4 \mathrm{~g})$ \\
\hline-0.04540000000000 & 0.86130000000000 & 0.50000000000000 & $\mathrm{C}$ & (4h) \\
\hline 0.04540000000000 & -0.86130000000000 & 0.50000000000000 & $\mathrm{C}$ & (4h) \\
\hline 0.54540000000000 & 1.36130000000000 & 0.50000000000000 & $\mathrm{C}$ & (4h) \\
\hline 0.45460000000000 & -0.36130000000000 & 0.50000000000000 & $\mathrm{C}$ & (4h) \\
\hline 0.57040000000000 & 0.89260000000000 & 0.50000000000000 & $\mathrm{C}$ & (4h) \\
\hline-0.57040000000000 & -0.89260000000000 & 0.50000000000000 & $\mathrm{C}$ & (4h) \\
\hline-0.07040000000000 & 1.39260000000000 & 0.50000000000000 & $\mathrm{C}$ & (4h) \\
\hline 1.07040000000000 & -0.39260000000000 & 0.50000000000000 & $\mathrm{C}$ & (4h) \\
\hline
\end{tabular}

$\alpha-\mathrm{PdCl}_{2}(C 50):$ A2B_oP6_58_g_a - CIF

R-carbon: A_oP16_55_2g2h - CIF 


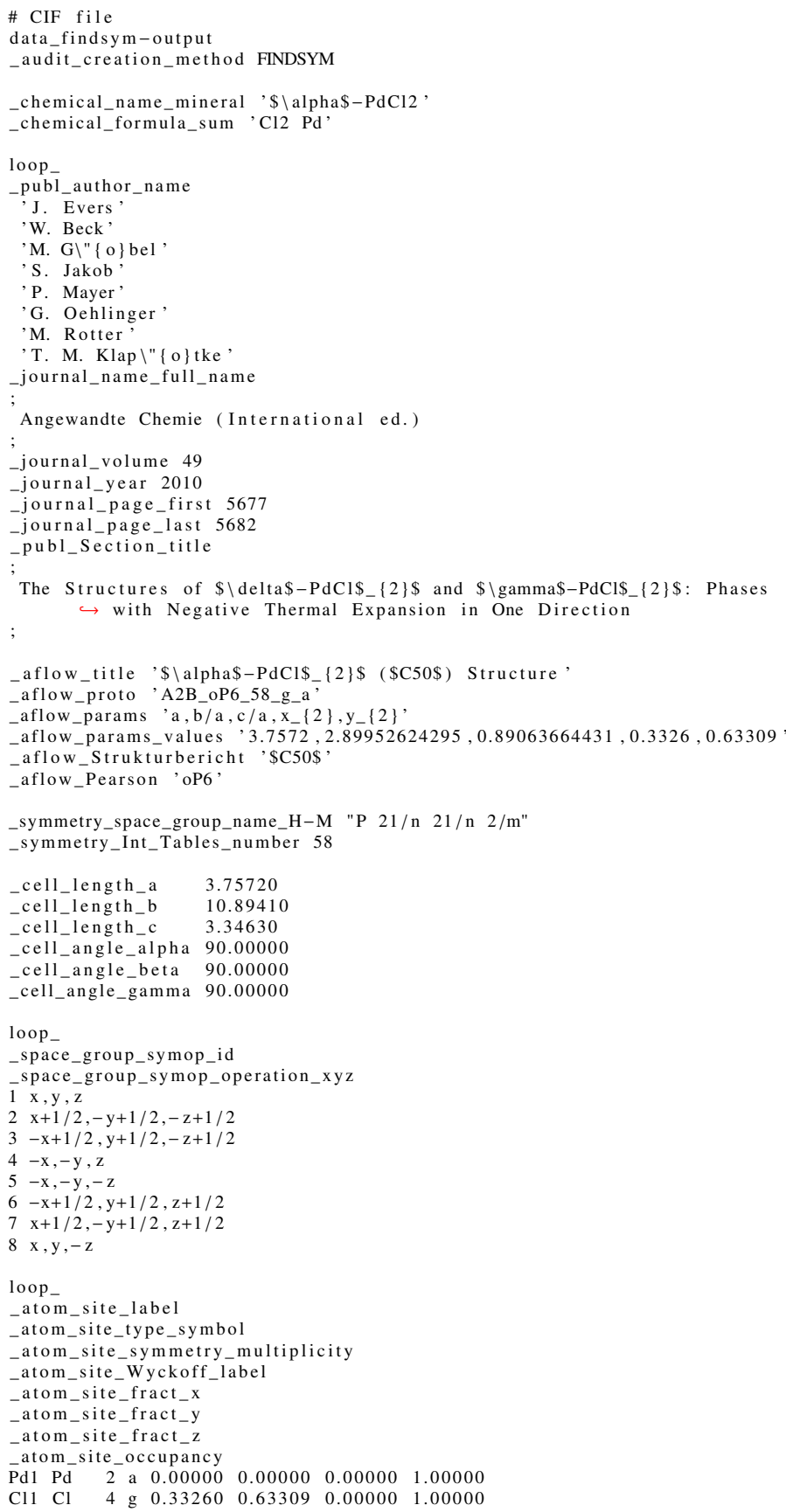

$\alpha-\mathrm{PdCl}_{2}(C 50)$ : A2B_oP6_58_g_a - POSCAR

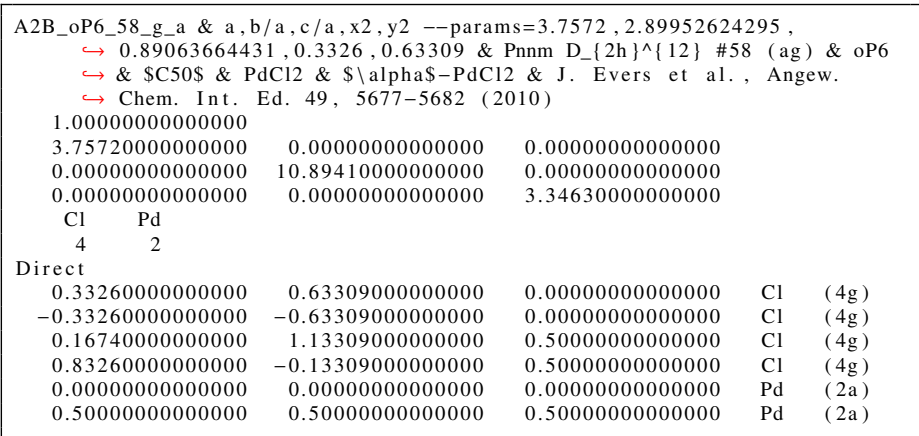

FeOCl: ABC_oP6_59_a_b_a - CIF

\# CIF file

data_findsym-output

-audit_creation_method FINDSYM

chemical name mineral ' $\mathrm{FeOCl}$ '

chemical formula_sum ${ }^{\prime} \mathrm{Cl} \mathrm{Fe} \mathrm{O}^{\prime}$

loop

-publ_author_name

S. M. Kauzlarich,
'J. L. Stanton

J. Faber,

'B. A. Averill,

_journal_name_full_name

Journal of the American Chemical Society

journal_volume 108

journal year 1986

journal_page_first 7946

journal page last 7951

publ_Section_title

Neutron profile refinement of the structure of $\mathrm{FeOCl}$ and $\mathrm{FeOCl}(\mathrm{TTF}) \$_{-}\{1$ $\hookrightarrow / 8.5\} \$$

aflow title ' $\mathrm{FeOCl}$ Structure'

aflow proto, $\mathrm{ABC}$-P6 59 a b a '

aflow_params a, a/a,c/a, z_ $\{1\}, z_{-}\{2\}, z_{-}\{3\}$

aflow_params_values ' $3.301,1.14298697364,2.39612238716,0.32961,-0.04795$ $\hookrightarrow, 0.89243$

-aflow_Strukturbericht 'None'

_aflow_Pearson 'oP6'

symmetry_space_group_name_H-M "P $\mathrm{m} \mathrm{m} \mathrm{n:2"}$

symmetry_Int_Tables_number 59

cell_length_a $\quad 3.30100$

cell_length_b $\quad 3.77300$

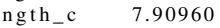

cell_angle_alpha 90.00000

cell-angle_beta 90.00000

_cell_angle_gamma 90.00000

loop

_space_group_symop_id

_space_group_symop_operation_xyz

$1 \mathrm{x}, \mathrm{y}, \mathrm{z}$

$2 \mathrm{x}+1 / 2,-\mathrm{y},-\mathrm{z}$

$3-x, y+1 / 2,-z$

$4-x+1 / 2,-y+1 / 2, z$

$5-x,-y,-z$

$7 x,-y+1 / 2, z$

$8 \mathrm{x}+1 / 2, \mathrm{y}+1 / 2,-\mathrm{z}$

loop

atom_site_label

atom_site_type_symbol

atom_site_symmetry_multiplicity

atom_site_Wyckoff_label

atom_site_fract_x

atom_site_fract_y

atom_site_fract_z

-atom_site_occupancy

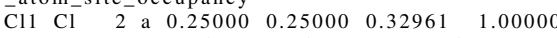

$\begin{array}{lllllllll}\mathrm{O} 1 & \mathrm{O} & 2 & \mathrm{a} & 0.25000 & 0.25000 & -0.04795 & 1.00000\end{array}$

$\begin{array}{llllllll}\mathrm{Fe} 1 & \mathrm{Fe} & 2 & \mathrm{~b} & 0.25000 & 0.75000 & 0.89243 & 1.00000\end{array}$

FeOCl: ABC_oP6_59_a_b_a - POSCAR

ABC_oP6_59_a_b_a \& a , b/a , c/a , z1 , z2 , z3 --params $=3.301,1.14298697364$ $\hookrightarrow 2.39612238716,0.32961,-0.04795,0.89243 \&$ \&mmn $D_{-}\{2 \mathrm{~h}\}^{\wedge}\{13\} \# 59$ $\left.\hookrightarrow \mathrm{a}^{\wedge} 2 \mathrm{~b}\right) \& \mathrm{oP} 6$ \& None \& $\mathrm{FeOCl} \& \mathrm{FeOCl} \& \mathrm{~S}$. M. Kauzlarich et al. , $\hookrightarrow$ J. Am. Chem. Soc. 108, 7946-7951 (1986)

1.00000000000000

$\begin{array}{lll}3.30100000000000 & 0.00000000000000 & 0.00000000000000\end{array}$

$\begin{array}{lll}0.00000000000000 & 3.77300000000000 & 0.00000000000000\end{array}$

$0.00000000000000 \quad 0.00000000000000 \quad 7.90960000000000$

$\mathrm{Cl} \quad \mathrm{Fe} \quad \mathrm{O}$

Direct

$\begin{array}{lll}0.25000000000000 & 0.25000000000000 & 0.32961000000000\end{array}$

$\begin{array}{lll}0.75000000000000 & 0.75000000000000 & -0.32961000000000\end{array}$

$\begin{array}{lll}0.25000000000000 & 0.75000000000000 & 0.89243000000000\end{array}$

$\begin{array}{lll}0.75000000000000 & 0.25000000000000 & -0.89243000000000\end{array}$

$\begin{array}{llll}0.25000000000000 & 0.25000000000000 & -0.04795000000000\end{array}$

$\begin{array}{rrr}0.75000000000000 & 0.75000000000000 & 0.04795000000000\end{array}$

$\mathrm{Cl} \quad(2 \mathrm{a})$

$\mathrm{Cl} \quad(2 \mathrm{a})$

$\mathrm{Fe} \quad(2 \mathrm{~b})$

$\mathrm{Fe} \quad(2 \mathrm{~b})$

O $\quad(2 \mathrm{a})$

$\mathrm{Rh}_{2} \mathrm{~S}_{3}:$ A2B3_oP20_60_d_cd - CIF

\section{\# CIF file}

data_findsym-output

_audit_creation_method FINDSYM

chemical_name_mineral 'Rh2S3,

_chemical_formula_sum 'Rh2 S3

loop

publ_author_name

'E. Parth $\{\backslash$ ' e $\}$ F.

'Hulliger

-journal year 1966

-publ_Section_title

The crystal structure of $\operatorname{Rh} \$ \_\{2\} \$ S \$ \_\{3\} \$$

\# Found in Pearson's Crystal Data - Crystal Structure Database for $\hookrightarrow$ Inorganic Compounds, 2013

aflow_title 'Rh\$_\{2\}\$S\$_\{3\}\$ Structure'

aflow_proto 'A2B3_oP20_60_d_cd

-aflow_params a, a/a,c/a, y_ $\{1\}, x_{-}\{2\}, y_{-}\{2\}, z_{-}\{2\}, x_{-}\{3\}, y_{-}\{3\}, z_{-}\{3\}$, 


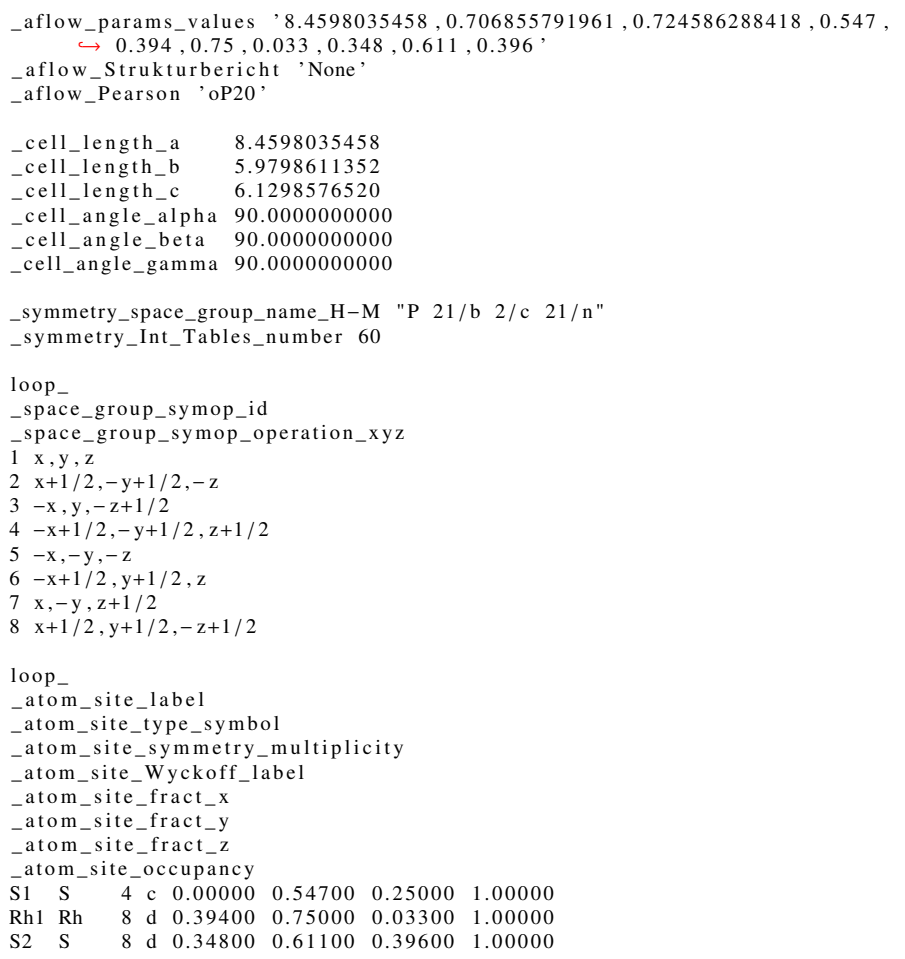

$\mathrm{Rh}_{2} \mathrm{~S}_{3}$ : A2B3_oP20_60_d_cd - POSCAR

\begin{tabular}{|c|c|c|c|c|}
\hline \multirow{3}{*}{\multicolumn{5}{|c|}{ 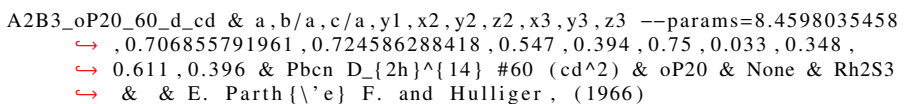 }} \\
\hline & & & & \\
\hline & & & & \\
\hline \multicolumn{5}{|c|}{1.00000000000000} \\
\hline 8.45980354580000 & 0.00000000000000 & 0.00000000000000 & & \\
\hline 0.00000000000000 & 5.97986113520000 & 0.00000000000000 & & \\
\hline 0.00000000000000 & 0.00000000000000 & 6.12985765200000 & & \\
\hline $\mathrm{Rh}$ & & & & \\
\hline 12 & & & & \\
\hline \multicolumn{5}{|l|}{ Direct } \\
\hline 0.39400000000000 & 0.75000000000000 & 0.03300000000000 & $\mathrm{Rh}$ & $(8 d)$ \\
\hline \multirow{2}{*}{$\begin{array}{r}0.10600000000000 \\
-0.39400000000000\end{array}$} & -0.25000000000000 & 0.53300000000000 & $\mathrm{Rh}$ & $(8 \mathrm{~d})$ \\
\hline & 0.75000000000000 & 00000000 & $\mathrm{R}$ & $(8 \mathrm{~d})$ \\
\hline $\begin{array}{r}-0.39400000000000 \\
0.89400000000000\end{array}$ & -0.25000000000000 & -0.03300000000000 & $\mathrm{R}$ & $(8 \mathrm{~d})$ \\
\hline-0.39400000000000 & -0.75000000000000 & -0.03300000000000 & $\mathrm{R}$ & (ed \\
\hline 0.89400000000000 & 1.25000000000000 & 0.467000000000 & & 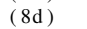 \\
\hline 0.39400000000000 & -0.75000000000000 & 0.533000000000 & $\mathrm{Rh}$ & $(8 d)$ \\
\hline \multirow{2}{*}{0.10600000000000} & 1.25000000000000 & 0.03300000000000 & $\mathrm{Rh}$ & $(8 \mathrm{~d})$ \\
\hline & 0.54700000000000 & 0.250000000000 & $\mathrm{~S}$ & (4c) \\
\hline \multirow{2}{*}{$\begin{array}{l}0.50000000000000 \\
0.00000000000000\end{array}$} & -0.04700000000000 & 0.75000000000000 & $\mathrm{~s}$ & $(4 c)$ \\
\hline & -0.54700000000000 & 0.750000000000 & $\mathrm{~s}$ & $(4 \mathrm{c})$ \\
\hline 0.50000000000000 & 1.0470 & 0.25000 & $\mathrm{~s}$ & $(4 \mathrm{c})$ \\
\hline 0.34800000000000 & 0.61100000000000 & 0.396000 & $\mathrm{~s}$ & $(8 d)$ \\
\hline \multirow{2}{*}{0.15200000000000} & -0.11100000000000 & 0.896000000000 & $\mathrm{~s}$ & \\
\hline & 0.61100000000000 & 0.10400000000000 & $\mathrm{~s}$ & $(8 d)$ \\
\hline $\begin{array}{r}-0.34800000000000 \\
0.84800000000000\end{array}$ & -0.11100000000000 & -0.39600000000000 & $\mathrm{~s}$ & \\
\hline-0.34800000000000 & -0.61100000000000 & -0.39600000000000 & $\mathrm{~s}$ & $(8 d)$ \\
\hline 0.84800000000000 & 1.11100000000000 & 0.10400000000000 & $\mathrm{~s}$ & $(8$ \\
\hline 0.34800000000000 & -0.61100000000000 & 0.89600000000000 & $\mathrm{~s}$ & $(8 d)$ \\
\hline 0.15200000000000 & 1.11100000000000 & 0.39600000000000 & $\mathrm{~s}$ & $(8 d)$ \\
\hline
\end{tabular}

$\mathrm{WO}_{3}$ : A3B_oP32_60_3d_d - CIF

\footnotetext{
\# CIF file

data findsym-output

audit_creation_method FINDSYM

chemical_name_mineral 'WO3'

chemical formula_sum 'O3 W

loop

publ_author_name

T. Vogt'

P. M. Woodwar

'B. A. Hunter'

_journal_name_full_name

Journal of Solid State Chemistry

_journal_volume 144

journal year 1999

journal_page_first 209

journal_page_last 215

publ_Section_title

The high-temperature phases of WOS $\{3\} \$$

\# Found in Pearson's Crystal Data - Crystal Structure Database fo

$\hookrightarrow$ Inorganic Compounds, 2013

_aflow_title 'WO\$_\{3\}\$ Structure
}

aflow_proto 'A3B_oP32_60_3d_d,

aflow_params $, a, b / a, c / a, x_{-}\{1\}, y_{-}\{1\}, z_{-}\{1\}, x_{-}\{2\}, y_{-}\{2\}, z_{-}\{2\}, x_{-}\{3\}, y_{-}\{3$ $\hookrightarrow\}, z_{-}\{3\}, x_{-}\{4\}, y_{-}\{4\}, z_{-}\{4\}$,

aflow_params_values $7.3397836195,1.05524748967,1.03197678378,0.5016$, $\hookrightarrow 0.7205,0.0322,0.2167,0.7591,0.2582,0.2197,0.5016,0.013,0.248$, $\hookrightarrow 0.783,0.0291$

aflow_Strukturbericht 'None'

aflow_Pearson 'oP32

cell_length_a 7.3397836195

cell_length_b $\quad 7.7452882392$

cell_length_c $\quad 7.5744862933$

cell angle alpha 90.0000000000

_cell_angle_alpha 90.00000000

symmetry_space_group_name_H-M "P $21 / \mathrm{b} \quad 2 / \mathrm{c} \quad 21 / \mathrm{n}$ "

symmetry_Int_Tables_number 60

loop

space_group_symop_id

space_group_symop_operation_xyz

$\mathrm{x}, \mathrm{y}, \mathrm{z}$

$2 x+1 / 2,-y+1 / 2,-z$

$3-\mathrm{x}, \mathrm{y},-\mathrm{z}+1 / 2$

$4-x+1 / 2,-y+1 / 2, z+1 / 2$

$5-\mathrm{x},-\mathrm{y},-\mathrm{z}$

$-x+1 / 2, y+1 / 2$

$8 \mathrm{x}+1 / 2, \mathrm{y}+1 / 2,-\mathrm{z}+1 / 2$

loop

atom_site_label

atom_site type symbol

atom_site_symmetry_multiplicity

atom_site_Wyckoff_label

atom_site_fract_x

atom_site_fract_y

atom_site_fract_z

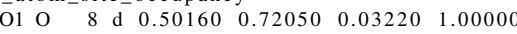

$\begin{array}{llllllllll}\mathrm{O} 2 & \mathrm{O} & 8 & \mathrm{~d} & 0.21670 & 0.75910 & 0.25820 & 1.00000\end{array}$

$\begin{array}{lllllllll}\mathrm{O} 3 & \mathrm{O} & 8 & \mathrm{~d} & 0.21970 & 0.50160 & 0.01300 & 1.00000\end{array}$

$\begin{array}{llllllll}\mathrm{W} 1 \mathrm{~W} & 8 & \mathrm{~d} & 0.24800 & 0.78300 & 0.02910 & 1.00000\end{array}$

$\mathrm{WO}_{3}$ : A3B_oP32_60_3d_d - POSCAR

A3B_oP32_60_3d_d \& a , b/a,c/a, x1,y1, z1 , x2 , y2 , z2, x3, y3 , z3 , x4 , y4, z4 --

$\rightarrow$ params $=7.3397836195,1.05524748967,1.03197678378,0.5016,0.7205$

$\hookrightarrow 0.0322,0.2167,0.7591,0.2582,0.2197,0.5016,0.013,0.248,0.783$

$\hookrightarrow 0.0291 \&$ Pben D_ $\{2 \mathrm{~h}\}^{\wedge}\{14\}$ \#60 $\left(\mathrm{d}^{\wedge} 4\right)$ \& oP32 \& None \& WO3 \& \& $\mathrm{T}$

$\hookrightarrow$ Vogt and P. M. Woodward and B. A. Hunter, J. Solid State Chem.

$\hookrightarrow \quad 144, \quad 209-215 \quad(1999)$

1.00000000000000

$\begin{array}{lll}7.33978361950000 & 0.00000000000000 & 0.00000000000000\end{array}$

$\begin{array}{lll}0.00000000000000 & 7.74528823920000 & 0.00000000000000\end{array}$

$\begin{array}{lll}0.00000000000000 & 0.00000000000000 & 7.57448629330000\end{array}$

$\mathrm{O} \quad \mathrm{W}$

Direct

0.50160000000000

$-0.00160000000000$

$-0.50160000000000$

1.00160000000000
-0.50160000000000

$-0.50160000000000$

0.50160000000000

$-0.00160000000000$

0.21670000000000

0.28330000000000

$-0.21670000000000$

0.71670000000000

$-0.21670000000000$

0.71670000000000
0.71670000000000

0.21670000000000

0.28330000000000

0.21970000000000

0.28030000000000

$-0.21970000000000$

0.71970000000000

$-0.21970000000000$

0.71970000000000

0.21970000000000

0.28030000000000

0.24800000000000

0.25200000000000

0.74800000000000

0.74800000000000
-0.24800000000000

0.74800000000000

0.24800000000000

0.25200000000000

0.72050000000000 0.22050000000000 0.72050000000000 0.22050000000000 .72050000000000 1.22050000000000 $-0.72050000000000$ 1.22050000000000 0.75910000000000 $-0.25910000000000$ 0.75910000000000 $-0.25910000000000$ $-0.75910000000000$ 1.25910000000000 . 75910000000000 1.25910000000000 0.50160000000000 0.00160000000000 0.50160000000000 0.00160000000000 $-0.50160000000000$ 1.00160000000000 0.50160000000000 1.00160000000000 0.78300000000000 $-0.28300000000000$ 0.78300000000000 $-0.28300000000000$ $-0.78300000000000$ 1.28300000000000 $-0.7830000000000$ 1.28300000000000

0.03220000000000 0.53220000000000 0.46780000000000 $-0.03220000000000$ $-0.03220000000000$ 0.46780000000000 0.53220000000000 0.03220000000000 0.25820000000000 0.75820000000000 0.24180000000000 $-0.25820000000000$ $-0.25820000000000$ 0.24180000000000 0.758200000000 0.75820000000000 0.0130000000000 0.51300000000000 0.48700000000000 $-0.01300000000000$ $-0.01300000000000$ 0.48700000000000 0.51300000000000 0.01300000000000 0.02910000000000 0.52910000000000 0.47090000000000 $-0.02910000000000$ $-0.02910000000000$ 0.47090000000000 0.52910000000000

( $8 d)$

$\mathrm{O} \quad(8 \mathrm{~d})$

$\begin{array}{ll}\mathrm{O} & (8 \mathrm{~d}) \\ \mathrm{O} & (8 \mathrm{~d})\end{array}$

$\mathrm{O} \quad(8 \mathrm{~d})$

O (8d)

$\mathrm{O} \quad(8 \mathrm{~d})$

$\mathrm{O} \quad(8 \mathrm{~d})$

$\mathrm{O} \quad(8 \mathrm{~d})$

$\mathrm{O} \quad(8 \mathrm{~d})$

$\begin{array}{ll}\mathrm{O} & (8 \mathrm{~d})\end{array}$

$\mathrm{O} \quad(8 \mathrm{~d})$

$\mathrm{O} \quad(8 \mathrm{~d})$

$\mathrm{O} \quad(8 \mathrm{~d})$

$\mathrm{O} \quad(8 \mathrm{~d})$

O (8d)

O (8d)

$\mathrm{O} \quad(8 \mathrm{~d})$

$\mathrm{O} \quad(8 \mathrm{~d})$

$\mathrm{O} \quad(8 \mathrm{~d})$

$\mathrm{O} \quad(8 \mathrm{~d})$

$\mathrm{O} \quad(8 \mathrm{~d})$

$\mathrm{O} \quad(8 \mathrm{~d})$

O (8d)

\# CIF file

data findsym-output

audit_creation_method FINDSYM

chemical name mineral 'beta-Toluene'

chemical_formula_sum 'C7 H8,

loop

_publ_author_nam

D. Andre' 


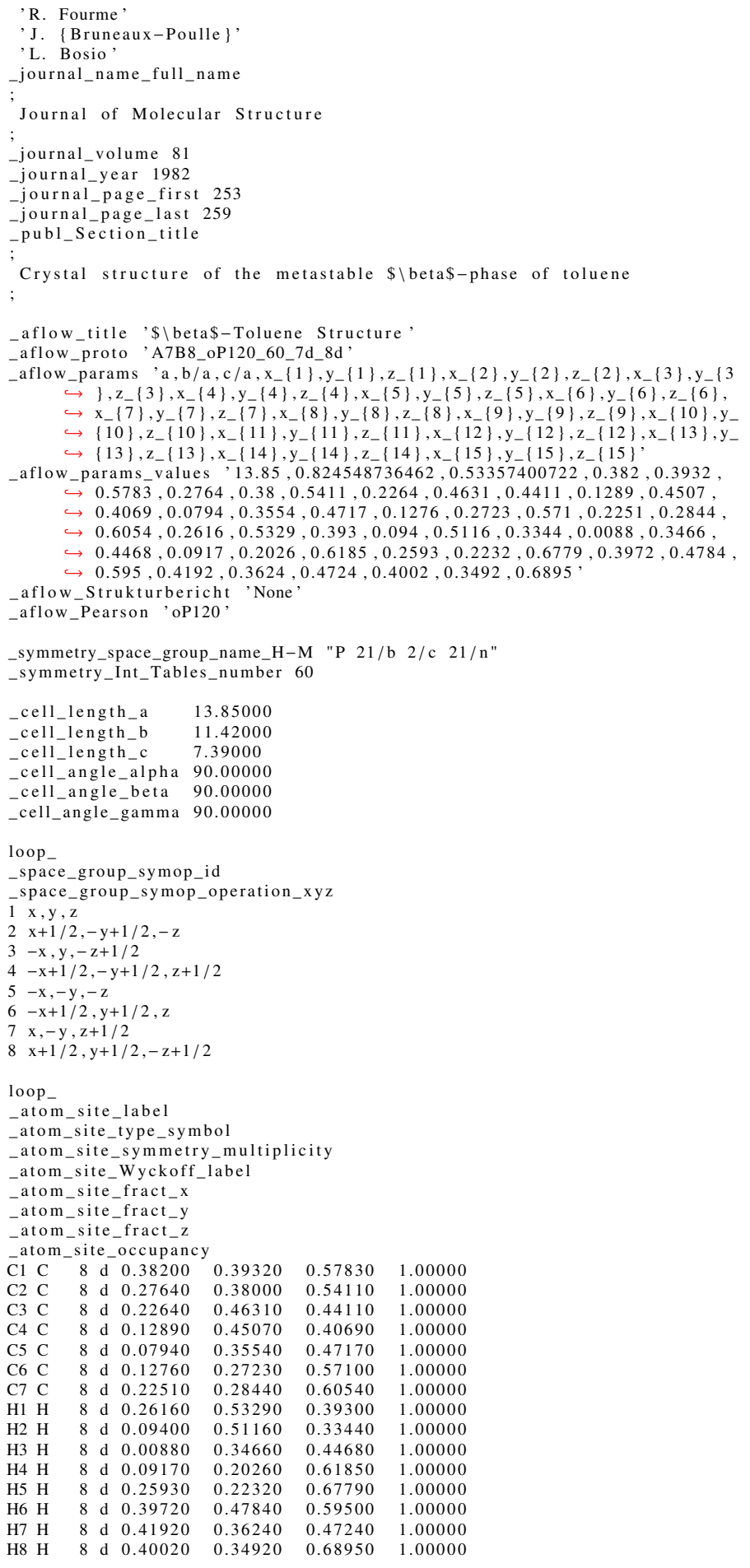

$\beta$-Toluene: A7B8_oP120_60_7d_8d - POSCAR

A7B8_oP120_60_7d_8d \& a , b/a,c/a , x1,y1, z1 , x2 , y2 , z2 , x3 $, y 3, \mathrm{z3}, \mathrm{x} 4, \mathrm{y} 4, \mathrm{z} 4, \mathrm{x} 5$, $\hookrightarrow \mathrm{y} 5, \mathrm{z} 5, \mathrm{x} 6, \mathrm{y} 6, \mathrm{z} 6, \mathrm{x} 7, \mathrm{y} 7, \mathrm{z7}, \mathrm{x} 8, \mathrm{y} 8, \mathrm{z} 8, \mathrm{x} 9, \mathrm{y} 9, \mathrm{z} 9, \mathrm{x} 10, \mathrm{y} 10, \mathrm{z} 10, \mathrm{x} 11, \mathrm{y} 11$,

$\hookrightarrow \mathrm{z} 11, \mathrm{x} 12, \mathrm{y} 12, \mathrm{z} 12, \mathrm{x} 13, \mathrm{y} 13, \mathrm{z} 13, \mathrm{x} 14, \mathrm{y} 14, \mathrm{z} 14, \mathrm{x} 15, \mathrm{y} 15, \mathrm{z} 15-\mathrm{p}$ arams $=$

$\hookrightarrow 0.38,0.5411,0.2264,0.4631,0.4411,0.1289,0.4507,0.4069,0.0794$,

$\hookrightarrow 0.3554,0.4717,0.1276,0.2723,0.571,0.2251,0.2844,0.6054,0.2616$

$\hookrightarrow 0.5329,0.393,0.094,0.5116,0.3344,0.0088,0.3466,0.4468,0.0917$,

$\hookrightarrow 0.2026,0.6185,0.2593,0.2232,0.6779,0.3972,0.4784,0.595,0.4192$,

$\hookrightarrow 0.3624,0.4724,0.4002,0.3492,0.6895 \&$ Pben D_ $\{2 \mathrm{~h}\} \wedge\{14\} \# 60\left(\mathrm{~d}^{\wedge} 15\right.$

$\hookrightarrow$ ) \& oP120 \& None \& C7H8 \& beta-Toluene \& D. Andre et al., J.

$\hookrightarrow$ Mol. Struct. 81, 253-259(1982)

1.00000000000000

$\begin{array}{lll}3.85000000000000 & 0.00000000000000 & 0.00000000000000\end{array}$

$\begin{array}{lrl}0.00000000000000 & 11.42000000000000 & 0.00000000000000\end{array}$

$\begin{array}{rrr}0.00000000000000 & 0.00000000000000 & 7.39000000000000\end{array}$

$\begin{array}{cc}\mathrm{C} & \mathrm{H} \\ 56 & 64\end{array}$

Direct

0.38200000000000 0.11800000000000 $-0.38200000000000$ 0.88200000000000

0.38200000000000

0.39320000000000 $0.39320000000000-0.07830000000000$ $0.10680000000000-0.57830000000000$ $-0.39320000000000-0.57830000000000$ $\begin{array}{rr}-0.39320000000000 & -0.57830000000000 \\ 0.89320000000000 & -0.07830000000000\end{array}$
0.38200000000000 .11800000000000 0.27640000000000 0.22360000000000 $-0.2764000000000$ 0.77640000000000 $-0.27640000000000$ 0.77640000000000 0.27640000000000 0.22360000000000 0.22640000000000 0.27360000000000 $-0.22640000000000$

0.72640000000000

0.72640000000000

0.22640000000000 0.27360000000000 0.12890000000000 0.37110000000000 $-0.12890000000000$ 0.62890000000000 $-0.12890000000000$ 0.62890000000000 0.12890000000000 0.37110000000000 0.07940000000000 0.42060000000000 $-0.07940000000000$ 0.57940000000000 $-0.07940000000000$ 0.57940000000000 0.07940000000000 0.42060000000000 0.12760000000000 0.37240000000000 $-0.12760000000000$ 0.62760000000000 $-0.1276000000000$ 0.62760000000000 0.12760000000000 0.37240000000000 0.22510000000000 0.27490000000000 $-0.22510000000000$ 0.72510000000000 $-0.2251000000000$ 0.7251000000000 0.72510000000000 0.27490000000000 0.26160000000000 0.23840000000000 $-0.26160000000000$ 0.76160000000000 $-0.26160000000000$ 0.76160000000000 0.26160000000000 0.2384000000000 0.09400000000000 0.40600000000000 $-0.09400000000000$ 0.59400000000000 $-0.09400000000000$ 0.594000000000 0.0940000000000 0.09400000000000 0.4060000000 0.088000000 0.49120000000000 $-0.00880000000000$ 0.50880000000000 $-0.0088000000000$ 0.50880000000000 0.00880000000000 0.49120000000000 0.09170000000000 0.40830000000000 $-0.09170000000000$ 0.59170000000000 $-0.09170000000000$ 0.59170000000000 0.09170000000000 0.40830000000000 0.40830000000000 0.25930000000000 0.240700000000 $-0.2593000000000$ 0.75930000000000 $-0.25930000000000$ 0.75930000000000 0.25930000000000 0.24070000000000 0.39720000000000 0.10280000000000 $-0.39720000000000$ 0.89720000000000 $-0.39720000000000$ 0.89720000000000 0.39720000000000 0.1028000000000 0.10280000000000 0.41920000000000 0.0808000000000 0.91920000000000 $-0.41920000000000$ 0.91920000000000 0.41920000000000
0.39320000000000 0.89320000000000 0.38000000000000 0.12000000000000 0.38000000000000 0.12000000000000 0.38000000000000 0.88000000000000 .38000000000000 0.46310000000000 0.03690000000000 0.46310000000000 0.03690000000000 $-0.46310000000000$ 0.96310000000000 0.46310000000000 0.96310000000000 0.45070000000000 0.04930000000000 0.45070000000000 0.04930000000000 $-0.45070000000000$ 0.95070000000000 $-0.45070000000000$ 0.95070000000000 0.35540000000000 0.14460000000000 0.35540000000000 0.14460000000000 $-0.35540000000000$ 0.85540000000000 0.85540000000 0.855400000000 0.85540000000000 0.27230000000000 0.22770000000000 0.27230000000000 0.22770000000000 $-0.27230000000000$ 0.77230000000000 $-0.27230000000000$ 0.77230000000000 0.28440000000000 0.21560000000000 0.28440000000000 0.21560000000000 $-0.28440000000000$ 0.78440000000000 0.784400000000 0.78440000000000 0.78440000000 0.532900000000 $-0.03290000000000$ 0.53290000000000 $-0.03290000000000$ $-0.53290000000000$ 1.03290000000000 0.53290000000000 1.03290000000000 0.51160000000000 $-0.01160000000000$ 0.51160000000000 $-0.01160000000000$ 0.51160000000000 $-0.5116000000000$ 1.01160000000000 0.34660000000000 0.15340000000000 0.34660000000000 0.15340000000000 $-0.34660000000000$ 0.84660000000000 $-0.34660000000000$ 0.84660000000000 0.20260000000000 0.29740000000000 0.20260000000000 0.29740000000000 $-0.20260000000000$ 0.70260000000000 $-0.20260000000000$ 0.20260000000000 0.7026000000 0.223200000000 0.27680000000 0.22320000000000 0.27680000000000 $-0.22320000000000$ 0.72320000000000 $-0.22320000000000$ 0.72320000000000 0.47840000000000 0.02160000000000 0.47840000000000 0.02160000000000 $-0.47840000000000$ 0.97840000000000 0.47840000000000 0.97840000000000 0.36240000000000 0.13760000000000 0.36240000000000 0.13760000000000 0.36240000000000 0.86240000000000 $-0.36240000000000$

1.07830000000000 0.57830000000000 0.54110000000000 1.04110000000000 00000000 0.5411000000000 0.54110000000000 $-0.54110000000000$ .04110000000000 1.04110000000000 0.54110000000000 0.94110000000000 0.05890000000000 $-0.44110000000000$ $-0.44110000000000$ 0.05890000000000 0.94110000000000 0.44110000000000 0.40690000000000 0.90690000000000 0.09310000000000 $-0.40690000000000$ 0.4060000000000 . 0.09310000000000 0.90690000000000 0.40690000000000 0.47170000000000 0.02830000000000 $-0.47170000000000$ $-0.47170000000000$ 0.02830000000000 0.97170000000000 0.47170000000000 0.57100000000000 1.07100000000000 $-0.07100000000000$ $-0.57100000000000$ $-0.5710000000000$ $-0.57100000000000$ 0.07100000000000 1.07100000000000 0.57100000000000 1.60540000000000 $-0.10540000000000$ $-0.60540000000000$ $-0.60540000000000$ $-0.10540000000000$ 1.10540000000000 0.60540000000000 
0.08080000000000 0.40020000000000 0.09980000000000 0.40020000000000 0.90020000000000 $-0.40020000000000$ 0.90020000000000 0.40020000000000 0.09980000000000

0.86240000000000 0.34920000000000 0.15080000000000 0.34920000000000 0.1508000000000 0.34920000000000 0.84920000000000 $-0.34920000000000$ 0.84920000000000

Benzene: $\mathrm{AB} \_$oP $48 \quad 61 \_3 \mathrm{c} \_3 \mathrm{c}-\mathrm{CIF}$

\section{\# CIF file}

data_findsym-output

audit_creation_method FINDSYM

chemical_name_mineral 'Benzene'

chemical_formula_sum ' $\mathrm{C}$ H'

loop

publ_author_name

'S. K Nayak'

R. Sathishkumar,

journal_name_full_name

CrystEngComm

journal_volume 12

journal_year 2010

journal_page_first 3112

journal_page_last 3118

publ_Section_title

Directing role of functional groups in selective generation of $\mathrm{C}-\mathrm{H}-\$$ $\rightarrow$ pi\$ interactions: In situ cryo-crystallographic studies on

$\hookrightarrow$ benzyl derivatives

aflow_title 'Benzene Structure

aflow proto 'AB oP48 61 3c 3c

aflow_params $, a, b / a, c / a, x_{-}\{1\}, y_{-}\{1\}, z_{-}\{1\}, x_{-}\{2\}, y_{-}\{2\}, z_{-}\{2\}, x_{-}\{3\}, y_{-}\{3$ $\hookrightarrow\}, \mathrm{z}_{-}\{3\}, \mathrm{x}_{-}\{4\}, \mathrm{y}_{-}\{4\}, \mathrm{z}_{-}\{4\}, \mathrm{x}_{-}\{5\}, \mathrm{y}_{-}\{5\}, \mathrm{z}_{-}\{5\}, \mathrm{x}_{-}\{6\}, \mathrm{y}_{-}\{6\}, \mathrm{z}_{-}\{6\}$

$0.1297,0.5762$

$\hookrightarrow 0.40803,0.1235,0.6328,0.54518,0.0057,0.4432,0.36289,0.2172$

$\hookrightarrow 0.6275,0.346,0.2068,0.7225,0.5756,0.0095,0.4051,0.2704$,

aflow_Strukturbericht 'None

_aflow_Pearson 'oP48,

symmetry_space_group_name_H-M "P 21/b 21/c 21/a"

symmetry_Int_Tables_number 61

cell_length_a $\quad 6.91400$

cell_length_c 9.56300

cell angle alpha 90.00000

_cell_angle_alpha 90.00000

cell_angle gamma 90.00000

loop

space_group_symop_id

_space_group_symop_operation_xyz

$1 \mathrm{x}, \mathrm{y}, \mathrm{z}$

$2 x+1 / 2,-y+1 / 2,-z$

$3-\mathrm{x}, \mathrm{y}+1 / 2,-\mathrm{z}+1 / 2$

$4-x+1 / 2,-y, z+1 / 2$

$5-\mathrm{x},-\mathrm{y},-\mathrm{z}$

$6-x+1 / 2, y+1 / 2, z$

$8 x+1 / 2, y,-$

loop

atom site label

atom_site type symbol

atom_site_symmetry_multiplicity

_site_Wyckoff_label

atom_site_fract_x

-atom_site_fract_y

Com site occupancy

$\begin{array}{lllllll}\mathrm{C} 1 \mathrm{C} & 8 & \mathrm{c} & 0.12970 & 0.57620 & 0.40803 & 1.00000\end{array}$

$\begin{array}{llllllll}\mathrm{C} 2 & \mathrm{C} & 8 & \mathrm{c} & 0.12350 & 0.63280 & 0.54518 & 1.00000\end{array}$

$\begin{array}{llllllll}\mathrm{C} 3 & \mathrm{C} & 8 & \mathrm{c} & 0.00570 & 0.44320 & 0.36289 & 1.00000\end{array}$

$\begin{array}{llllllll}\mathrm{H} 1 \mathrm{H} & 8 & \mathrm{c} & 0.21720 & 0.62750 & 0.34600 & 1.00000\end{array}$

$\begin{array}{llllllll}\mathrm{H} 3 & \mathrm{H} & 8 & \mathrm{c} & 0.00950 & 0.40510 & 0.27040 & 1.00000\end{array}$

Benzene: AB_oP48_61_3c_3c - POSCAR

AB_oP48_61_3c_3c \& a , b/a, c/a , x1,y1, z1, x2 , y2 , z2 , x3 , y3 , z3 , x4 ,y4 , z4 , x5 , y5 , $\hookrightarrow \mathrm{z} 5, \mathrm{x} 6, \mathrm{y} 6, \mathrm{z} 6-$ params $=6.914,1.08128435059,1.38313566676,0.1297$

$\hookrightarrow 0.5762,0.40803,0.1235,0.6328,0.54518,0.0057,0.4432,0.36289$

$\hookrightarrow 0.2172,0.6275,0.346,0.2068,0.7225,0.5756,0.0095,0.4051,0.2704 \&$

$\hookrightarrow$ Pbca D $\{2 \mathrm{~h}\}^{\wedge}\{15\} \# 61 \quad\left(\mathrm{c}^{\wedge} 6\right)$ \& $\mathrm{oP} 48$ \& None \& Benzene \& Benzene \&

$\hookrightarrow$ S. K Nayak and R. Sathishkumar and T. N. \{Guru Row\},

$\hookrightarrow$ CrystEngComm 12,3112-3118 (2010)

1.00000000000000

$6.91400000000000-0.00000000000000-0.00000000000000$

$0.00000000000000 \quad 7.47600000000000 \quad 0.00000000000000$

$\begin{array}{rrr}0.000000000000000 & 0.00000000000000 & 9.56300000000000\end{array}$

$$
\begin{array}{rr}
\mathrm{C} & \mathrm{H} \\
24 & 24
\end{array}
$$

Direct

$0.12970000000000 \quad 0.57620000000000$

$0.37030000000000-0.57620000000000$
.40803000000000 0.90803000000000

$-0.12970000000000$

0.6297000000000

-0.12970000000000
0.62970000000000

0.1297000000000

0.37030000000000

0.12350000000000

0.37650000000000

$-0.12350000000000$

0.62350000000000

0.62350000000000

0.12350000000000

0.37650000000000

0.0057000000000

0.494300000000

$-0.00570000000000$

0.50570000000000

$-0.00570000000000$

0.50570000000000

0.00570000000000

0.49430000000000

0.21720000000000

0.28280000000000

$-0.21720000000000$

0.71720000000000

$-0.21720000000000$

0.71720000000000

0.21720000000000

0.28280000000000

0.20680000000000

0.29320000000000

$-0.20680000000000$

0.70680000000000

$-0.20680000000000$

0.70680000000000

0.20680000000000

0.29320000000000

0.00950000000000

0.49050000000000

$-0.00950000000000$

0.50950000000000

$-0.00950000000000$

0.50950000000000

0.00950000000000

0.49050000000000

1.07620000000000 .07620000000000 . .57620000000000 0.57620000000000 $-0.07620000000000$ 1.07620000000000 0.63280000000000 0.63280000000000 1.13280000000000 $-0.13280000000000$ $-0.63280000000000$ 0.63280000000000 . 13280000000000 13280000000000 . 0.94320000000000 .05680000000000 0.44320000000000 0.4320000000 .44320000000000

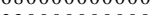
320000000000 2750000000000 0.12750000000000 0.62750000000000 0.62750000000000 .12750000000000 12750000000000 .72250000000000 1.22250000000000 1.22250000000000 . 0.72250000000000 . 0.22250000000000 1.22250000000000 0.40510000000000 $-0.40510000000000$ 0.90510000000000 0.09490000000000 $-0.40510000000000$ 0.40510000000000 0.09490000000000 0.90510000000000

0.09197000000000 0.40803000000000 $-0.40803000000000$ 0.09197000000000 0.90803000000000 0.40803000000000 0.5451800000000 .54518000000000 1.0451800000000 $-0.04518000000000$ $-0.54518000000000$ $-0.54518000000000$ $-0.04518000000000$ 1.04518000000000 0.54518000000000 0.36289000000000 0.8628900 0.137110 $-0.36289000000$ 0.36289000000000 0.1371100000000 0.86289000000000 0.36289000000000 0.34600000000000 0.84600000000000 0.15400000000000 $-0.34600000000000$ $-0.34600000000000$ 0.15400000000000 0.84600000000000 0.34600000000000 0.57560000000000 1.0756000000000 $-0.57560000000000$ $-0.57560000000000$ 0.07560000 .075600000000 0.57560000000000 0.27040000000000 0.77040000000000 0.22960000000000 $-0.27040000000000$ $-0.27040000000000$ 0.22960000000000 0.77040000000000 0.27040000000000

Tongbaite $\left(\mathrm{Cr}_{3} \mathrm{C}_{2}, D 5_{10}\right)$ : A2B3_oP20_62_2c_3c - CIF

\# CIF file

data_findsym-output

audit creation method FINDSYM

chemical_name_mineral 'Tongbaite

chemical_formula_sum ' $\mathrm{C} 2 \mathrm{Cr} 3$,

loop

publ author name

S. Rundqvist

G. Runnsj $\{\backslash " \circ\}$

journal_name_full_name

Acta Chemica Scandinavica

journal_volume 23

journal_year 1969

journal_page_first 1191

journal page last 1199

publ_Section_title

Crystal Structure Refinement of $\mathrm{Cr} \$\{3\} \$ C \$\{2\} \$$ 
atom site label

_atom_site_type_symbol

_atom_site_symmetry_multiplicity

_atom_site_Wyckoff_label

atom_site_fract_x

atom_site_fract_y

atom_site_fract_z

- atom_site_occupancy

$\begin{array}{llllllll}\mathrm{C} 1 & \mathrm{C} & 4 & \mathrm{c} & 0.10080 & 0.25000 & 0.20550 & 1.00000\end{array}$

$\begin{array}{lllllllll}\mathrm{C} 2 & \mathrm{C} & 4 & \mathrm{c} & 0.24320 & 0.25000 & -0.04640 & 1.00000\end{array}$

$\begin{array}{lllllllll}\mathrm{Cr} 1 & \mathrm{Cr} & 4 & \mathrm{c} & 0.01570 & 0.25000 & 0.40150 & 1.00000\end{array}$

$\begin{array}{lllllllll}\mathrm{Cr} 2 & \mathrm{Cr} & 4 & \mathrm{c} & 0.18080 & 0.25000 & 0.77370 & 1.00000\end{array}$

$\begin{array}{llllllll}\mathrm{Cr} 3 & \mathrm{Cr} & 4 & \mathrm{c} & 0.86910 & 0.25000 & -0.06880 & 1.00000\end{array}$

Tongbaite $\left(\mathrm{Cr}_{3} \mathrm{C}_{2}, D 5_{10}\right)$ : A2B3_oP20_62_2c_3c - POSCAR

A2B3_oP20_62_2c_3c \& a,b/a, c/a, x1, z1 , x2, z2, x3, z3, x4, z4, x5, z5 --params= $\hookrightarrow 5.5329,0.511305102207,2.07339731425,0.1008,0.2055,0.2432,-$

$\hookrightarrow 0.0464,0.0157,0.4015,0.1808,0.7737,0.8691,-0.0688 \&$ Pnma D $\{2 h$

$\hookrightarrow\}^{\wedge}\{16\} \# 62\left(\mathrm{c}^{\wedge} 5\right) \&$ oP20 \& \$D5 $\{10\} \$ \&$ Cr3C2 \& Tongbaite \& S.

$\hookrightarrow$ Rundqvist and G. Runnsj \{\"o\}, Acta Chem. Scand. 23, 1191-1199

$$
\hookrightarrow 1969 \text { ) }
$$

1.00000000000000

5.53290000000000

0.00000000000000

$0.00000000000000 \quad 0.00000000000000$

0.0000000000

$2.82900000000000 \quad 0.00000000000000$

$\mathrm{C} \quad \mathrm{Cr}$

8
Direct$$
\mathrm{Cr}
$$

0.10080000000000

0.39920000000000

$-0.10080000000000$

0.60080000000000

0.24320000000000

0.25680000000000

$-0.24320000000000$

0.74320000000000

0.01570000000000

0.48430000000000

0.48430000000000

0.5157000000000

0.18080000000000

0.31920000000000

$-0.18080000000000$

0.68080000000000

0.86910000000000

$-0.36910000000000$

$-0.86910000000000$

1.36910000000000

$0.25000000000000 \quad 0.20550000000000$

0.75000000000000

0.75000000000000

0.25000000000000

0.25000000000000

0.75000000000000

0.75000000000000

0.2500000000000

0.7500000000000

0.75000000000000

0.2500000000000

0.2500000000000

0.2500000000000

0.7500000000000

0.75000000000000

0.25000000000000
0.25000000000000

0.25000000000000
0.75000000000000

0.75000000000000

0.75000000000000
0.25000000000000

0.70550000000000

0.20550000000000

0.29450000000000

$-0.04640000000000$

0.45360000000000

0.04640000000000

0.54640000000000

0.40150000000000

.90150000000000

0.40150000000000

0.09850000000000

0.77370000000000

.

$-0.77370000000000$

$-0.77370000000000$

0.06880000000000

0.56880000000000

C $(4 c)$

$(4 c)$

C (4c)

C (4c)

(4c)

$\begin{array}{ll}\mathrm{C} & (4 \mathrm{c}) \\ \mathrm{C} & (4 \mathrm{c})\end{array}$

$\begin{array}{ll}\mathrm{C} & (4 \mathrm{c}) \\ \mathrm{C} & (4 \mathrm{c})\end{array}$

$\mathrm{Cr}(4 \mathrm{c})$

$\mathrm{Cr} \quad(4 \mathrm{c})$

$\mathrm{Cr} \quad(4 \mathrm{c})$

$\mathrm{Cr} \quad(4 \mathrm{c})$

$\mathrm{Cr} \quad(4 \mathrm{c})$

$\begin{array}{ll}\mathrm{Cr} & (4 \mathrm{c}) \\ \mathrm{Cr} & (4 \mathrm{c})\end{array}$

$\mathrm{Cr} \quad(4 \mathrm{c})$

$\mathrm{Cr} \quad(4 \mathrm{c})$

$\mathrm{Cr} \quad(4 \mathrm{c})$

$\begin{array}{ll}\mathrm{Cr} & (4 \mathrm{c}) \\ \mathrm{Cr} & (4 \mathrm{c})\end{array}$

Forsterite $\left(\mathrm{Mg}_{2} \mathrm{SiO}_{4}, S 1_{2}\right)$ : A2B4C_oP28_62_ac_2cd_c - CIF

\# CIF file

data_findsym-output

_audit_creation_method FINDSYM

chemical_name_mineral 'Forsterite

chemical_formula_sum ' $\mathrm{Mg} 2 \mathrm{O} 4 \mathrm{Si}$

loop

publ_author_name

'R. M. Hazen'

journal_name_full_name

American Mineralogist

journal_volume 61

-journal year 1976

_journal_page_first 128

_journal_page_last 1293

-publ_Section_title

Effects of temperature and pressure on the crystal structure of $\hookrightarrow$ forsterite

aflow_title, Forsterite $\left(\operatorname{Mg} \$ \_\{2\} \$ S i O \$ \_\{4\} \$, \$ S 1_{-}\{2\} \$\right)$ Structure,

aflow_proto 'A2B4C_oP28_62_ac_2cd_c

aflow_params 'a,b/a,c/a, $x_{-}\{2\}, z_{-}\{2\}, x_{-}\{3\}, z_{-}\{3\}, x_{-}\{4\}, z_{-}\{4\}, x_{-}\{5\}, z_{-}\{5$ $\hookrightarrow\}, x_{-}\{6\}, y_{-}\{6\}, z_{-}\{6\}$,

aflow params values , $10.193,0.586382811734,0.466202295693,0.2774,-$

$\hookrightarrow 0.0085,0.0913,0.7657,0.4474,0.2215,0.094,0.4262,0.1628,0.0331$,

$\hookrightarrow 0.2777$,

aflow Strukturbericht, $\$ S 1 \_\{2\} \$$

_aflow_Pearson, oP28,

_symmetry_space_group_name_H-M "P 21/n 21/m 21/a"

_symmetry_Int_Tables_number 62

_cell_length_a $\quad 10.19300$

_cell_length_b $\quad 5.97700$

cell_length_c 4.75200

cell_angle_alpha 90.00000

cell_angle_beta 90.00000

cell angle gamma 90.00000

loop

space_group_symop_id

space_group_symop_operation_xyz

$1 \mathrm{x}, \mathrm{y}, \mathrm{z}$

$2 \mathrm{x}+1 / 2,-\mathrm{y}+1 / 2,-\mathrm{z}+1 / 2$

$3-\mathrm{x}, \mathrm{y}+1 / 2,-\mathrm{z}$

$4-x+1 / 2,-y, z+1 / 2$

$5-x,-y,-z$
$6-x+1 / 2, y+1 / 2, z+1 / 2$

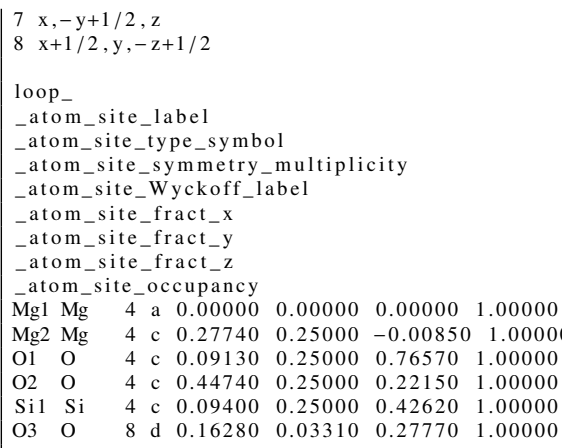

Forsterite $\left(\mathrm{Mg}_{2} \mathrm{SiO}_{4}, S 1_{2}\right)$ : A2B4C_oP28_62_ac_2cd_c - POSCAR

\begin{tabular}{|c|c|c|c|c|}
\hline \multirow{7}{*}{\multicolumn{5}{|c|}{ 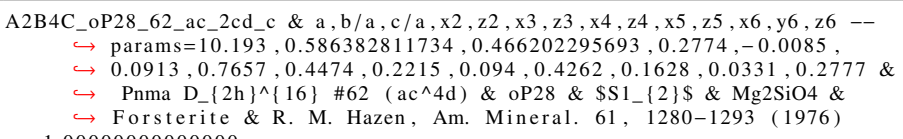 }} \\
\hline & & & & \\
\hline & & & & \\
\hline & & & & \\
\hline & & & & \\
\hline & & & & \\
\hline & & & & \\
\hline 10.19300000000000 & 0.00000000000000 & 0.00000000000000 & & \\
\hline 0.00000000000000 & 5.97700000000000 & 0.00000000000000 & & \\
\hline 0.00000000000000 & 0.00000000000000 & 4.75200000000000 & & \\
\hline $\mathrm{Mg}$ & & & & \\
\hline 16 & & & & \\
\hline \multicolumn{5}{|l|}{ Direct } \\
\hline 0.00000000000000 & 0.00000000000000 & 0.00000000000000 & $\mathrm{Mg}$ & (4a) \\
\hline 0.50000000000000 & 0.00000000000000 & 0.50000000000000 & $\mathrm{Mg}$ & (4a) \\
\hline 0.00000000000000 & 0.50000000000000 & 0.00000000000000 & $\mathrm{Mg}$ & (4a) \\
\hline 0.50000000000000 & 0.50000000000000 & 0.50000000000000 & $\mathrm{Mg}$ & $(4 a)$ \\
\hline 0.27740000000000 & 0.25000000000000 & -0.00850000000000 & $\mathrm{Mg}$ & $(4 c)$ \\
\hline 0.22260000000000 & 0.75000000000000 & 0.49150000000000 & $\mathrm{Mg}$ & $(4 c)$ \\
\hline-0.27740000000000 & 0.75000000000000 & 0.00850000000000 & $\mathrm{Mg}$ & $(4 \mathrm{c})$ \\
\hline 0.77740000000000 & 0.25000000000000 & 0.50850000000000 & $\mathrm{Mg}$ & $(4 c)$ \\
\hline 0.09130000000000 & 0.25000000000000 & 0.76570000000000 & $\mathrm{O}$ & $(4 c)$ \\
\hline \multirow{2}{*}{$\begin{array}{r}0.40870000000000 \\
-0.09130000000000\end{array}$} & 0.75000000000000 & 1.26570000000000 & $\mathrm{O}$ & $(4 c)$ \\
\hline & 0.75000000000000 & -0.76570000000000 & $\mathrm{O}$ & $(4 c)$ \\
\hline $\begin{array}{r}-0.09130000000000 \\
0.59130000000000\end{array}$ & 0.25000000000000 & -0.26570000000000 & $\mathrm{O}$ & $(4 \mathrm{c})$ \\
\hline 0.44740000000000 & 0.25000000000000 & 0.22150000000000 & $\mathrm{O}$ & $(4 \mathrm{c})$ \\
\hline 0.05260000000000 & 0.75000000000000 & 0.72150000000000 & $\mathrm{O}$ & $(4 \mathrm{c})$ \\
\hline-0.44740000000000 & 0.75000000000000 & -0.22150000000000 & $\mathrm{O}$ & $(4 c)$ \\
\hline 0.94740000000000 & 0.25000000000000 & 0.27850000000000 & $\mathrm{O}$ & $(4 c)$ \\
\hline 0.16280000000000 & 0.03310000000000 & 0.27770000000000 & $\mathrm{O}$ & \\
\hline 0.33720000000000 & -0.03310000000000 & 0.77770000000000 & $\mathrm{O}$ & $(8 \mathrm{~d})$ \\
\hline-0.16280000000000 & 0.53310000000000 & -0.27770000000000 & $\mathrm{O}$ & $(8 \mathrm{~d})$ \\
\hline \multirow{2}{*}{$\begin{array}{r}0.66280000000000 \\
-0.16280000000000\end{array}$} & 0.46690000000000 & 0.22230000000000 & $\mathrm{O}$ & $(8 \mathrm{~d})$ \\
\hline & -0.03310000000000 & -0.27770000000000 & $\mathrm{O}$ & $(8 \mathrm{~d})$ \\
\hline 0.66280000000000 & 0.03310000000000 & 0.22230000000000 & $\mathrm{O}$ & $(8 \mathrm{~d})$ \\
\hline 0.16280000000000 & 0.46690000000000 & 0.27770000000000 & $\mathrm{O}$ & (8d) \\
\hline 0.33720000000000 & 0.53310000000000 & 0.77770000000000 & $\mathrm{O}$ & $(8 \mathrm{~d})$ \\
\hline 0.09400000000000 & 0.25000000000000 & 0.42620000000000 & $\mathrm{Si}$ & $(4 c)$ \\
\hline 0.40600000000000 & 0.75000000000000 & 0.92620000000000 & $\mathrm{Si}$ & $(4 c)$ \\
\hline-0.09400000000000 & 0.75000000000000 & -0.42620000000000 & $\mathrm{Si}$ & $(4 c)$ \\
\hline 0.59400000000000 & 0.25000000000000 & 0.07380000000000 & $\mathrm{Si}$ & $(4 \mathrm{c})$ \\
\hline
\end{tabular}

$\mathrm{SrH}_{2}(C 29):$ A2B_oP12_62_2c_c - CIF

\section{\# CIF file}

data findsym-output

audit_creation_method FINDSYM

chemical_name_mineral ' $\mathrm{SrH} 2$,

chemical_formula_sum ' $\mathrm{H} 2 \mathrm{Sr}$,

loop

publ_author_name

'R. C. Ropp'

journal year 2013

publ_Section_title

Encyclopedia of the Alkaline Earth Compounds

aflow title 'SrH\$ $\{2\} \$(\$ C 29 \$)$ Structure,

aflow_proto 'A2B_oP12_62_2c_c

aflow params 'a, b/a,c/a, $x_{-}\{1\}, z_{-}\{1\}, x_{-}\{2\}, z_{-}\{2\}, x_{-}\{3\}, z_{-}\{3\}$

aflow params values $3.875,1.64232258065,1.89496774194,0.004,0.758,0.24$ P $0.07,0.24,0.39$

aflow_Strukturbericht $\$ \mathrm{C} 29 \$$

-aflow_Pearson 'oP12,

symmetry_space_group_name_H-M "P 21/n 21/m 21/a"

_symmetry_Int_Tables_number 62

cell_length_a $\quad 3.87500$

-cell_length_b 6.36400

_cell_length_c $\quad 7.34300$

$\begin{array}{ll}\text { cell_angle_alpha } & 90.00000 \\ \text { cell_angle_beta } & 90.00000\end{array}$

loop

space_group_symop_id

space group_symop_operation_xyz

$\mathrm{x}, \mathrm{y}, \mathrm{z}$

$\mathrm{x}+1 / 2,-\mathrm{y}+1 / 2,-\mathrm{z}+1 / 2$

$3-\mathrm{x}, \mathrm{y}+1 / 2,-\mathrm{z}$
$4-\mathrm{x}+1 / 2,-\mathrm{y}, \mathrm{z}+1 / 2$ 
$5-\mathrm{x},-\mathrm{y},-\mathrm{z}$

$-x+1 / 2, y+1 / 2, z+1 / 2$

$7 \mathrm{x},-\mathrm{y}+1 / 2, \mathrm{z}$

$8 \mathrm{x}+1 / 2, \mathrm{y},-\mathrm{z}+1 / 2$

loop_

atom_site_label

atom_site_type_symbol

atom_site_symmetry_multiplicity

atom_site_Wyckoff_label

atom_site_fract_x

atom_site_fract_y

atom_site_fract_z

$\begin{array}{lllllll}\overline{\mathrm{H}} 1 \mathrm{H} & 4 & \mathrm{c} & 0.00400 & 0.25000 & 0.75800 & 1.00000\end{array}$

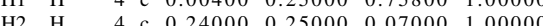

$\begin{array}{lllllllll}\mathrm{S} r 1 & \mathrm{Sr} & 4 & \mathrm{c} & 0.24000 & 0.25000 & 0.39000 & 1.00000\end{array}$

$\mathrm{SrH}_{2}($ C29): A2B_oP12_62_2c_c - POSCAR

A2B_oP12_62_2c_c \& a , b/a,c/a, x1, z1, x2, z2, x3, z3 --params $=3.875$

$\hookrightarrow 1.64232258065,1.89496774194,0.004,0.758,0.24,0.07,0.24,0.39 \&$

$\hookrightarrow$ Pnma D_ $\{2 \mathrm{~h}\}^{\wedge}\{16\} \# 62\left(\mathrm{c}^{\wedge} 3\right) \&$ oP12 \& \$C29\$ \& SrH2 \& SrH2 \& R. C.

$\hookrightarrow$ Pnma D_ $\{2 \mathrm{~h}\}^{\wedge}\{$
$\hookrightarrow \operatorname{Ropp}, \quad(2013)$

1.00000000000000

$\begin{array}{lll}3.87500000000000 & 0.00000000000000 & 0.00000000000000 \\ 0.00000000000000 & 6.36400000000000 & 0.00000000000000\end{array}$ $\begin{array}{lll}0.00000000000000 & 6.36400000000000 & 0.00000000000000 \\ 0.00000000000000 & 0.00000000000000 & 7.34300000000000\end{array}$

$\begin{array}{ccc}0.0000000000 \\ \mathrm{H} & \mathrm{Sr}\end{array}$

8

Direct

0.49600000000000

$-0.00400000000000$

0.50400000000000

0.24000000000000

0.26000000000000

$-0.24000000000000$

0.74000000000000

0.24000000000000

0.2400000000000

0.26000000000000

0.74000000000000

$0.25000000000000 \quad 0.75800000000000$ 0.75000000000000 0.75000000000000 0.25000000000000 0.25000000000000 0.75000000000000 0.75000000000000 0.25000000000000 0.2500000000000 0.7500000000000 0.75000000000000 0.25000000000000

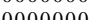
0.75800000000000 $-0.25800000000000$ 0.07000000000000 0.57000000000000 $-0.07000000000000$ 0.43000000000000 0.39000000000000 0.39000000000 $-0.39000000000000$ 0.11000000000000

$\begin{array}{cc}\mathrm{H} & (4 \mathrm{c}) \\ \mathrm{H} & (4 \mathrm{c}) \\ \mathrm{H} & (4 \mathrm{c}) \\ \mathrm{H} & (4 \mathrm{c}) \\ \mathrm{H} & (4 \mathrm{c}) \\ \mathrm{H} & (4 \mathrm{c}) \\ \mathrm{H} & (4 \mathrm{c}) \\ \mathrm{H} & (4 \mathrm{c}) \\ \mathrm{Sr} & (4 \mathrm{c}) \\ \mathrm{Sr} & (4 \mathrm{c}) \\ \mathrm{Sr} & (4 \mathrm{c}) \\ \mathrm{Sr} & (4 \mathrm{c})\end{array}$

$\epsilon-\mathrm{NiAl}_{3}\left(D 0_{20}\right):$ A3B_oP16_62_cd_c - CIF

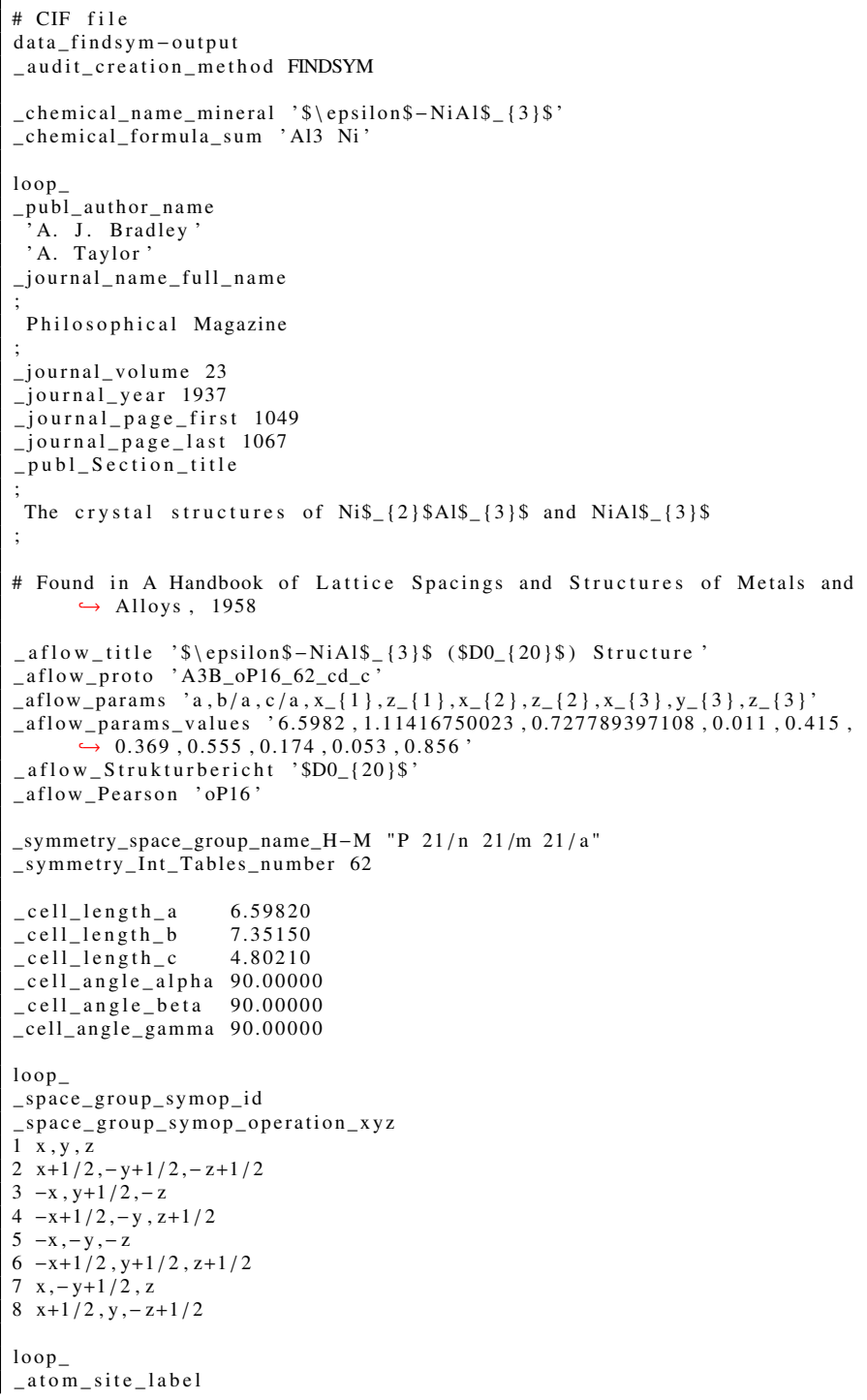

\# Found in A Handbook of Lattice Spacings and Structures of Metals and $\hookrightarrow$ Alloys , 1958

_aflow_title, $\$ \backslash$ epsilon\$-NiAl\$_\{3\}\$(\$D0_\{20\}\$) Structure,

-aflow_proto 'A3B_oP16_62_cd_c'

-aflow_params 'a,b/a,c/a, $x_{-}\{1\}, z_{-}\{1\}, x_{-}\{2\}, z_{-}\{2\}, x_{-}\{3\}, y_{-}\{3\}, z_{-}\{3\}$,

aflow_params_values ' $6.5982,1.11416750023,0.727789397108,0.011,0.415$ $\hookrightarrow 0.369,0.555,0.174,0.053,0.856$

_aflow_Strukturbericht '\$D0_\{20\}\$,

_aflow_Pearson, oP16,

symmetry_space group_name_H-M "P $21 / \mathrm{n} 21 / \mathrm{m} \mathrm{21/a"}$

_symmetry_Int_Tables_number 62

_cell_length_a $\quad 6.59820$

_cell_length_b 7.35150

_cell_length_c $\quad 4.80210$

_cell_angle_alpha 90.00000

_cell_angle_beta 90.00000

_cell_angle_gamma 90.00000

loop_

_space_group_symop_id

_space_group_symop_operation_xyz

$1 \mathrm{x}, \mathrm{y}, \mathrm{z}$

$2 \mathrm{x}+1 / 2,-\mathrm{y}+1 / 2,-\mathrm{z}+1 / 2$

$3-x, y+1 / 2,-z$

$4-x+1 / 2,-y, z+1 / 2$

$5-\mathrm{x},-\mathrm{y},-\mathrm{z}$

$6-\mathrm{x}+1 / 2, \mathrm{y}+1 / 2, \mathrm{z}+1 / 2$

$8 x+1 / 2, y,-z+1 / 2$

loop

_atom_site_label

\begin{tabular}{|c|c|c|c|c|}
\hline \multicolumn{5}{|c|}{ 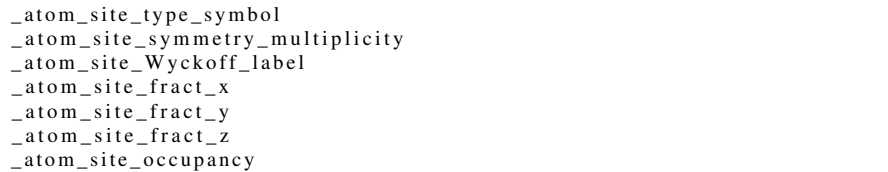 } \\
\hline \multicolumn{5}{|c|}{ 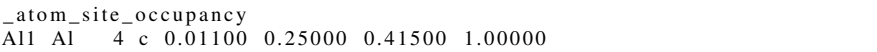 } \\
\hline 4 c 0.36900 & \\
\hline 8 d 0.17400 & $\begin{array}{llll}0.05300 & 0.85600 & 1.0\end{array}$ & & & \\
\hline \multicolumn{5}{|c|}{$\epsilon-\mathrm{NiAl}_{3}\left(D 0_{20}\right):$ A3B_oP16_62_cd_c - POSCAR } \\
\hline \multicolumn{5}{|c|}{ 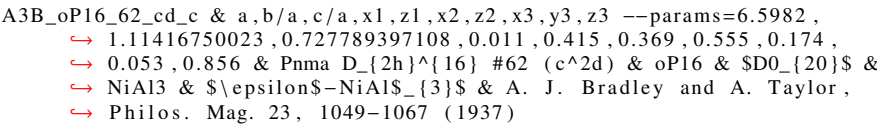 } \\
\hline 6.59820000000000 & 0.00000000000000 & 0.00000000000000 & & \\
\hline 0.00000000000000 & \multirow{2}{*}{$\begin{array}{l}7.35150000000000 \\
0.00000000000000\end{array}$} & & & \\
\hline 0.00000000000000 & & \multirow[t]{2}{*}{4.80210000000000} & & \\
\hline $\mathrm{Al}$ & & & & \\
\hline 12 & & & & \\
\hline \multicolumn{5}{|l|}{ Direct } \\
\hline 0.01100000000000 & 0.25000000000000 & 0.41500000000000 & $\mathrm{Al}$ & $(4 c)$ \\
\hline 0.48900000000000 & 0.75000000000000 & 0.91500000000000 & $\mathrm{Al}$ & $(4 c)$ \\
\hline-0.01100000000000 & 0.75000000000000 & -0.41500000000000 & $\mathrm{Al}$ & $(4 c)$ \\
\hline 0.51100000000000 & 0.25000000000000 & 0.08500000000000 & $\mathrm{Al}$ & $(4 c)$ \\
\hline 0.17400000000000 & 0.05300000000000 & 0.85600000000000 & $\mathrm{Al}$ & (8d) \\
\hline 0.32600000000000 & -0.05300000000000 & 1.35600000000000 & $\mathrm{Al}$ & $(8 \mathrm{~d})$ \\
\hline-0.17400000000000 & 0.55300000000000 & -0.85600000000000 & $\mathrm{Al}$ & $(8 \mathrm{~d})$ \\
\hline 0.67400000000000 & 0.44700000000000 & -0.35600000000000 & $\mathrm{Al}$ & $(8 \mathrm{~d})$ \\
\hline-0.17400000000000 & -0.05300000000000 & -0.85600000000000 & $\mathrm{Al}$ & (8d) \\
\hline 0.67400000000000 & 0.05300000000000 & -0.35600000000000 & $\mathrm{Al}$ & $(8 \mathrm{~d})$ \\
\hline 0.17400000000000 & 0.44700000000000 & 0.85600000000000 & $\mathrm{Al}$ & (8d) \\
\hline 0.32600000000000 & 0.55300000000000 & 1.35600000000000 & $\mathrm{Al}$ & (8d) \\
\hline 0.36900000000000 & 0.25000000000000 & 0.55500000000000 & $\mathrm{Ni}$ & $(4 \mathrm{c})$ \\
\hline 0.13100000000000 & 0.75000000000000 & 1.05500000000000 & $\mathrm{Ni}$ & $(4 c)$ \\
\hline-0.36900000000000 & 0.75000000000000 & -0.55500000000000 & $\mathrm{Ni}$ & \\
\hline 0.86900000000000 & 0.25000000000000 & -0.05500000000000 & $\mathrm{Ni}$ & $(4 c)$ \\
\hline
\end{tabular}

Cubanite $\left(\mathrm{CuFe}_{2} \mathrm{~S}_{3}, E 9_{e}\right)$ : AB2C3_oP24_62_c_d_cd - CIF

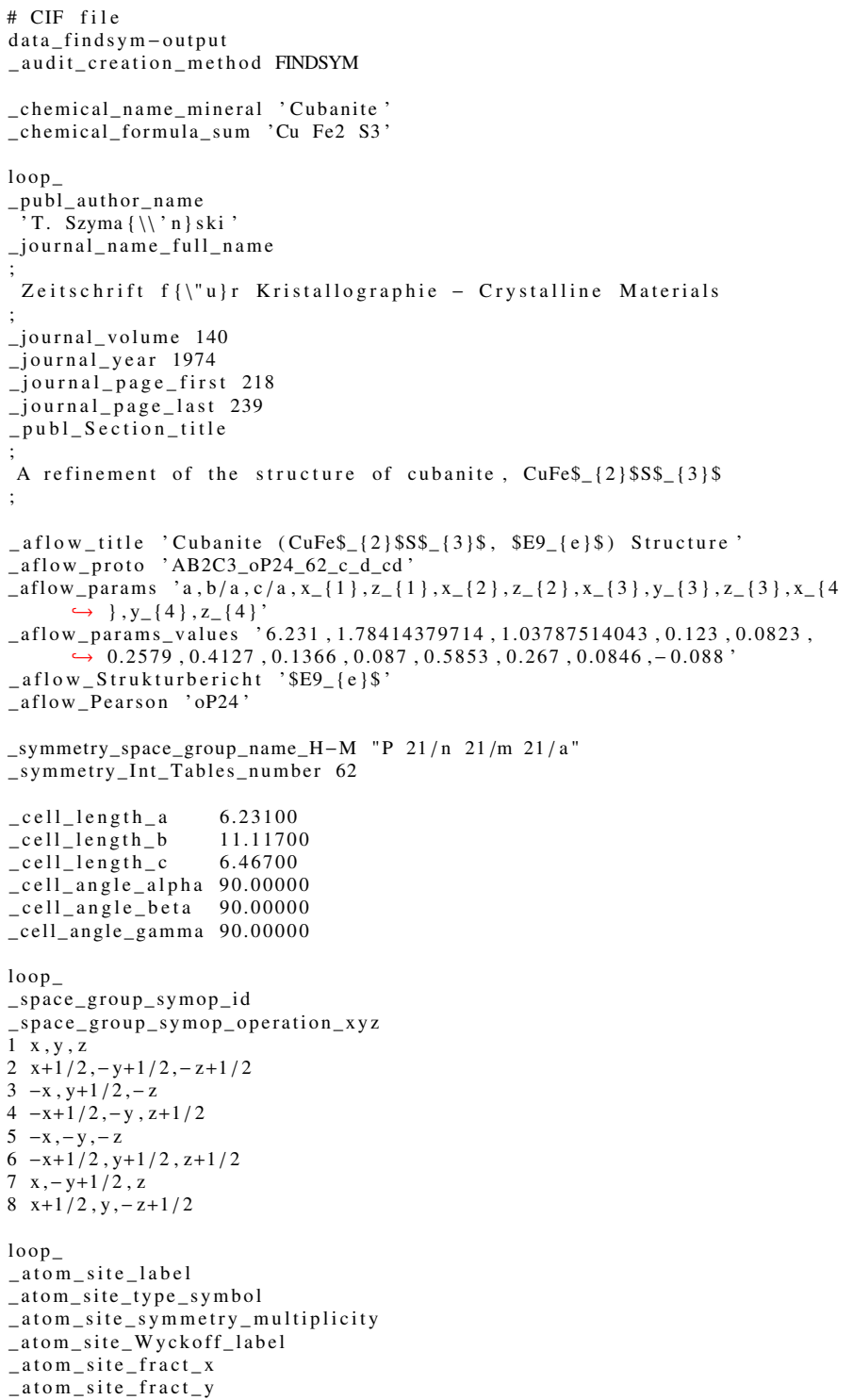


AB2C3_oP24_62_c_d_cd \& a , b/a,c/a, x1, z1, x2, z2, x3,y3,z3, x4,y4,z4 --params $\hookrightarrow 6.231,1.78414379714,1.03787514043,0.123,0.0823,0.2579,0.4127$

$\hookrightarrow 0.1366,0.087,0.5853,0.267,0.0846,-0.088 \&$ Pnma $D_{-}\{2 \mathrm{~h}\}^{\wedge}\{16\} \# 62$

$\hookrightarrow\left(\mathrm{c}^{\wedge} 2 \mathrm{~d}^{\wedge} 2\right) \&$ oP24 \& \$E9_ $\{\mathrm{e}\} \$ \&$ CuFe2S3 \& Cubanite \& T. Szyma $\left\{\backslash^{\prime} n\right\}$

$\hookrightarrow$ ski, Zeitschrift f" $\{\mathrm{u}\} \mathrm{r}$ Kristallographie - Crystalline

$\hookrightarrow$ Materials 140, 218-239(1974)

\subsection{0}

$6.23100000000000-0.00000000000000$

$0.00000000000000 \quad 11.11700000000000$

0.00000000000000 .00000000000000

$\mathrm{Cu} \quad \mathrm{Fe} \quad \mathrm{S}$

Direct

0.12300000000000

0.12300000000000

0.37700000000000

0.12300000000000

0.62300000000000

0.13660000000000

0.36340000000000

$-0.13660000000000$

0.63660000000000

$-0.13660000000000$

0.63660000000000

0.13660000000000

0.36340000000000

0.25790000000000

0.24210000000000

$-0.25790000000000$

0.75790000000000

0.26700000000000

0.23300000000000

0.2330000000000

0.7670000000000

0.767000000000

-0.26700000000000
0.76700000000000

0.26700000000000

0.23300000000000

0.25000000000000 0.08700000000000 0.08700000000 0.08700000000000 0.58700000000000 0.41300000000000 $-0.08700000000000$ 0.08700000000000 0.41300000000000 0.58700000000000 0.2500000000000 0.75000000000000 0.75000000000000 0.25000000000000 0.0846000000000 $-0.08460000000000$ 0.58460000000 0.415400000000 $-0.0846000000000$ 0.0846000000000 0.41540000000000
0.00000000000000 0.00000000000000 6.46700000000000

0.08230000000000 0.58230000000000 0.08230000000000 0.41770000000000 0.58530000000000 .08530000000000 $-0.58530000000000$ $-0.08530000000000$ $-0.5853000000000$ $-0.58530000000000$ 0.58530000000000 .58530000000000 08530000000000 0.41270000000000 0.91270000000000 $-0.41270000000000$ 0.08730000000000 $-0.08800000000000$ 0.41200000000000 0.08800000000000 0.58800000000000 0.08800000000000 0.58800000000000 $-0.08800000000000$ $-0.41200000000000$
$\mathrm{Cu} \quad(4 \mathrm{c})$

$\mathrm{Cu} \quad(4 \mathrm{c})$

$\mathrm{Cu} \quad(4 \mathrm{c})$

( $8 \mathrm{~d})$

$(8 d)$

$(8 \mathrm{~d})$

$\begin{array}{ll}\mathrm{Fe} & (8 \mathrm{~d}) \\ \mathrm{Fe} & (8 \mathrm{~d})\end{array}$

(8d)

(8d)

Molybdite $\left(\mathrm{MoO}_{3}, \mathrm{D0} 0_{8}\right)$ : AB3_oP16_62_c_3c - CIF

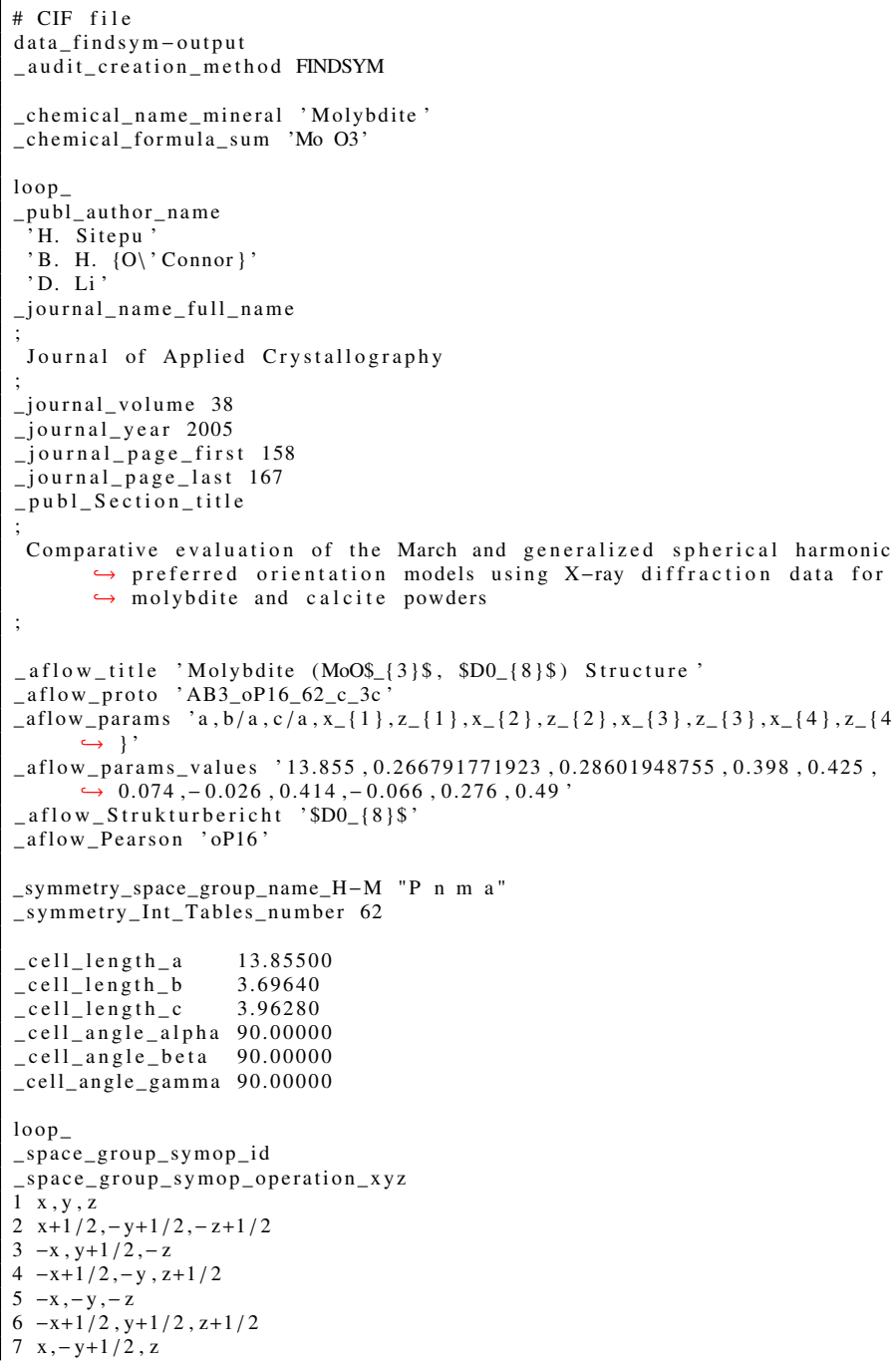

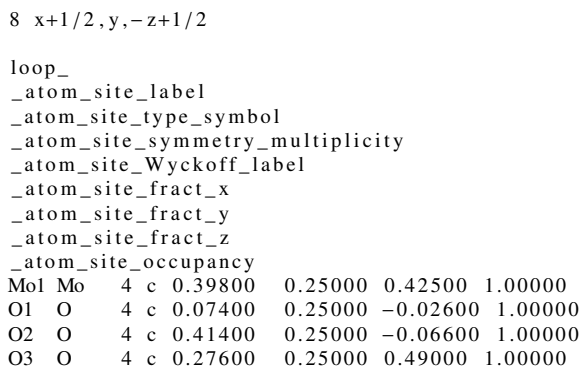

Molybdite $\left(\mathrm{MoO}_{3}, D 0_{8}\right)$ : AB3_oP16_62_c_3c - POSCAR

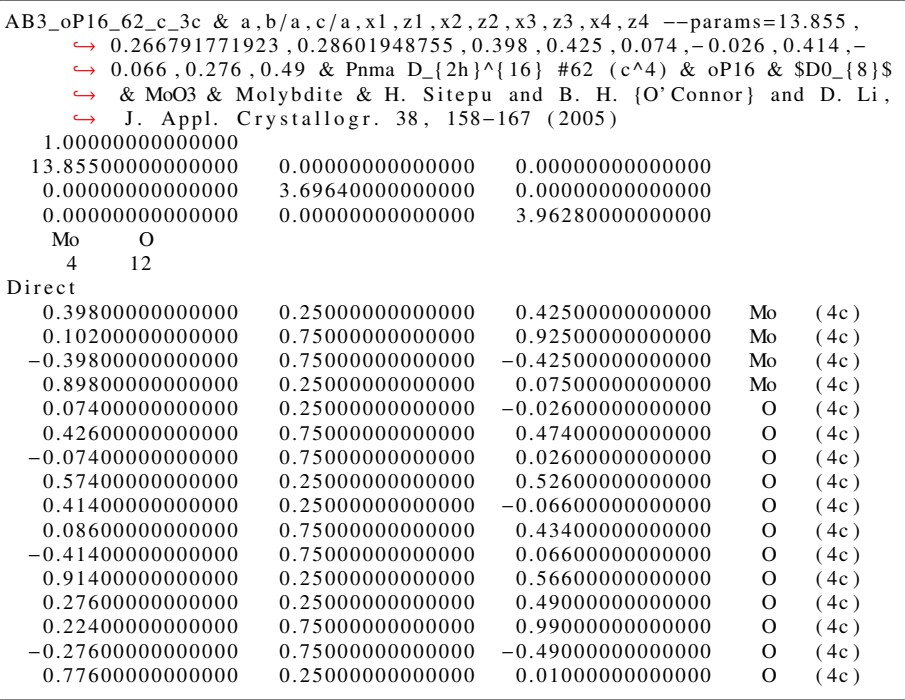

Barite $\left(\mathrm{BaSO}_{4}, \mathrm{H0}_{2}\right)$ : AB4C_oP24_62_c_2cd_c - CIF

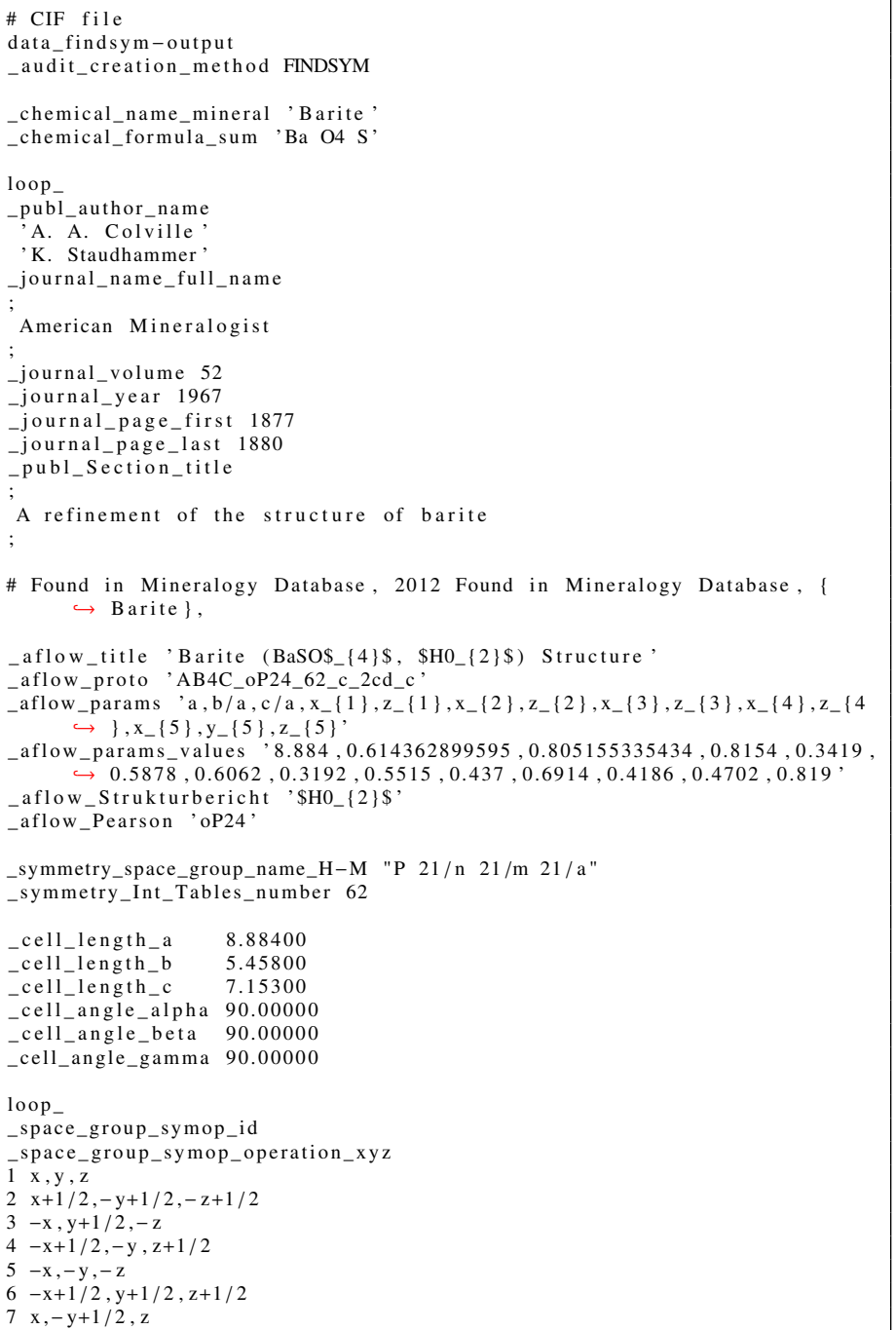




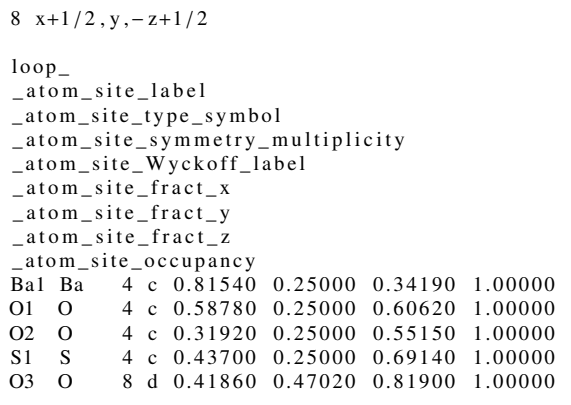

Barite $\left(\mathrm{BaSO}_{4}, \mathrm{H0}_{2}\right)$ : AB4C_oP24_62_c_2cd_c - POSCAR

AB4C_oP24_62_c_2cd_c \& a , b/a, c/a , x1, z1, x2, z2, x3, z3, x4, z4, x5, y5, z5 $\hookrightarrow$ params $=8.884,0.614362899595,0.805155335434,0.8154,0.3419,0.5878$ $40.6062,0.3192,0.5515,0.437,0.6914,0.4186,0.4702,0.819 \&$ Pnma

$\hookrightarrow D_{-}\{2 \mathrm{~h}\}^{\wedge}\{16\} \# 62\left(\mathrm{c}^{\wedge} 4 \mathrm{~d}\right) \&$ oP24 \& \$H0_ $\{2\} \$ \&$ BaSO4 \& Barite \& A.

$\hookrightarrow$ A. Colville and K. Staudhammer, Am. Mineral. 52, 1877-1880( $\hookrightarrow 1967$ )

1.00000000000000

8.88400000000000 0.00000000000000 0.00000000000000

0.00000000000000 0.00000000000000 $\begin{array}{rrr}\mathrm{Ba} & \mathrm{O} & \mathrm{S} \\ 4 & 16 & 4\end{array}$

Direct

0.81540000000000 $-0.31540000000000$ $-0.81540000000000$ 1.31540000000000 0.58780000000000 $-0.08780000000000$ $-0.58780000000000$ $-0.58780000000000$ 1.08780000000000 0.1808000000000 0.18080000000 0.31920000000000 0.81920000000000 0.41860000000000 0.08140000000000 $-0.41860000000000$ 0.91860000000000 $-0.41860000000000$ 0.91860000000000 0.41860000000000 0.08140000000000 0.43700000000000 0.06300000000000 $-0.43700000000000$ 0.93700000000000 0.00000000000000 0.00000000000000 7.15300000000000

$0.25000000000000 \quad 0.34190000000000$ 0.75000000000000 0.75000000000000 0.25000000000000 0.25000000000000 0.75000000000000 0.7500000000000 0.2500000000000 0.25000000000000 0.2500000000000 0.7500000000000 0.75000000000000 0.25000000000000 0.47020000000000 $-0.47020000000000$ 0.97020000000000 0.02980000000000 $-0.47020000000000$ 0.47020000000000 0.02980000000000 0.97020000000000 0.25000000000000 0.75000000000000 0.75000000000000 0.25000000000000 0.84190000000000 0.34190000000000 0.15810000000000 0.60620000000000 1.10620000000000 $-0.60620000000000$ $-0.10620000000000$ 0.55150000000000 1.05150000000000 $-0.55150000000000$ $-0.05150000000000$ 0.8190000000000 1.31900000000000 $-0.81900000000000$ $-0.31900000000000$ $-0.81900000000000$ $-0.31900000000000$ 0.81900000000000 1.31900000000000 0.69140000000000 1.19140000000000 $-0.69140000000000$ $-0.19140000000000$

$\begin{aligned} \mathrm{Ba} & (4 \mathrm{c}) \\ \mathrm{Ba} & (4 \mathrm{c}) \\ \mathrm{Ba} & (4 \mathrm{c}) \\ \mathrm{Ba} & (4 \mathrm{c}) \\ \mathrm{O} & (4 \mathrm{c}) \\ \mathrm{O} & (4 \mathrm{c}) \\ \mathrm{O} & (4 \mathrm{c}) \\ \mathrm{O} & (4 \mathrm{c}) \\ \mathrm{O} & (4 \mathrm{c}) \\ \mathrm{O} & (4 \mathrm{c}) \\ \mathrm{O} & (4 \mathrm{c}) \\ \mathrm{O} & (4 \mathrm{c}) \\ \mathrm{O} & (8 \mathrm{~d}) \\ \mathrm{O} & (8 \mathrm{~d}) \\ \mathrm{O} & (8 \mathrm{~d}) \\ \mathrm{O} & (8 \mathrm{~d}) \\ \mathrm{O} & (8 \mathrm{~d}) \\ \mathrm{O} & (8 \mathrm{~d}) \\ \mathrm{O} & (8 \mathrm{~d}) \\ \mathrm{O} & (8 \mathrm{~d}) \\ \mathrm{S} & (4 \mathrm{c}) \\ \mathrm{S} & (4 \mathrm{c}) \\ \mathrm{S} & (4 \mathrm{c}) \\ \mathrm{S} & (4 \mathrm{c})\end{aligned}$

Westerveldite (FeAs, B14): AB_oP8_62_c_c - CIF

\section{\# CIF file}

data_findsym-output

audit creation method FINDSYM

chemical name mineral 'Westerveldite'

chemical_formula_sum 'As $\mathrm{Fe}$

loop

publ_author_name

K. Selte

A. Kjekshus'

'A. F. Andresen,

_journal_name_full_name

Acta Chemica Scandinavica

_journal_volume 26

journal year 1972

_journal_page_first 3101

journal_page_last 3113

publ_Section_title

Magnetic Structure and Properties of FeAs

\# Found in Interplay between magnetism, structure, and strong

$\hookrightarrow$ electron-phonon coupling in binary FeAs under pressure, 2011

aflow_title, Westerveldite (FeAs, \$B14\$) Structure'

aflow_proto AB_oP8_62_c_c

aflow_params $, a, b / a, c / a, x_{-}\{1\}, z_{-}\{1\}, x_{-}\{2\}, z_{-}\{2\}$

aflow_params_values ' $5.454,0.609644297763,1.10542720939,0.2005,0.5741$, $\hookrightarrow 0.005 \overline{8}, 0.1993$,

aflow_Strukturbericht '\$B14\$,

aflow_Pearson, oP8

symmetry_space_group_name_H-M "P 21/n 21/m 21/a"

symmetry_Int_Tables_number 62

cell_length_a $\quad 5.45400$

cell_length_b 3.32500

cell_length_c 6.02900

cell_angle_alpha 90.00000

cell_angle_beta 90.00000

_cell_angle_gamma 90.00000 loop

space_group_symop_id

_space_group_symop_operation_xyz

$1 \mathrm{x}, \mathrm{y}, \mathrm{z}$

$2 \mathrm{x}+1 / 2,-\mathrm{y}+1 / 2,-\mathrm{z}+1 / 2$

$3-\mathrm{x}, \mathrm{y}+1 / 2,-\mathrm{z}$

$4-\mathrm{x}+1 / 2,-\mathrm{y}, \mathrm{z}+1 / 2$

$-\mathrm{x},-\mathrm{y},-\mathrm{z}$

$-x+1 / 2, y+1 / 2, z+1 / 2$

$7 \mathrm{x},-\mathrm{y}+1 / 2, \mathrm{z}$

$8 \mathrm{x}+1 / 2, \mathrm{y},-\mathrm{z}+1 / 2$

loop

atom_site_label

atom_site_type_symbol

_atom_site_symmetry_multiplicity

site Wyckoff label

atom_site_fract_x

-atom_site_fract_y

atom_site_fract_z

atom_site_occupancy

As1 As 4 c $0.20050 \quad 0.25000 \quad 0.57410 \quad 1.00000$

$\begin{array}{llllllll}\mathrm{Fe} 1 & \mathrm{Fe} & 4 & \mathrm{c} & 0.00580 & 0.25000 & 0.19930 & 1.00000\end{array}$

Westerveldite (FeAs, B14): AB_oP8_62_c_c - POSCAR

AB_oP8_62_c_c \& a , b/a,c/a, x1, z1 , x2, z2 --params $=5.454,0.609644297763$,

$\hookrightarrow 1.10542720939,0.2005,0.5741,0.0058,0.1993 \&$ Pnma D_ $\{2 \mathrm{~h}\}^{\wedge}\{16\}$ \#

$\hookrightarrow 62\left(\mathrm{c}^{\wedge} 2\right) \&$ oP8 \& $\$ B 14 \$ \&$ FeAs \& Westerveldite \& K. Selte and A.

$\hookrightarrow$ Kjekshus and A. F. Andresen, Acta Chem. Scand. 26, 3101-3113 ( $\hookrightarrow$ 1972)

1.00000000000000

$\begin{array}{lll}5.45400000000000 & 0.00000000000000 & 0.00000000000000\end{array}$

$\begin{array}{lll}0.00000000000000 & 3.32500000000000 & 0.00000000000000\end{array}$

$\begin{array}{lll}0.00000000000000 & 0.00000000000000 & 6.02900000000000\end{array}$

As $\mathrm{Fe}$

Direct

$\begin{array}{lllll}0.20050000000000 & 0.25000000000000 & 0.57410000000000 & \text { As } \quad(4 \mathrm{c})\end{array}$

$\begin{array}{lllll}0.29950000000000 & 0.75000000000000 & 1.07410000000000 & \text { As }(4 \mathrm{c})\end{array}$

$-0.20050000000000 \quad 0.75000000000000-0.57410000000000$ As $\quad(4 \mathrm{c})$

$\begin{array}{lllll}0.70050000000000 & 0.25000000000000 & -0.07410000000000 & \text { As } & (4 \mathrm{c})\end{array}$

$\begin{array}{lllll}0.00580000000000 & 0.25000000000000 & 0.19930000000000 & \mathrm{Fe} & (4 \mathrm{c})\end{array}$

$\begin{array}{llllll}0.49420000000000 & 0.75000000000000 & 0.69930000000000 & \mathrm{Fe} & (4 \mathrm{c})\end{array}$

$\begin{array}{lllll}-0.00580000000000 & 0.75000000000000 & -0.19930000000000 & \mathrm{Fe} & (4 \mathrm{c})\end{array}$

$\begin{array}{rrrrr}0.50580000000000 & 0.25000000000000 & 0.30070000000000 & \mathrm{Fe} & (4 \mathrm{c})\end{array}$

Rasvumite $\left(\mathrm{KFe}_{2} \mathrm{~S}_{3}\right)$ : A2BC3_oC24_63_e_c_cg - CIF

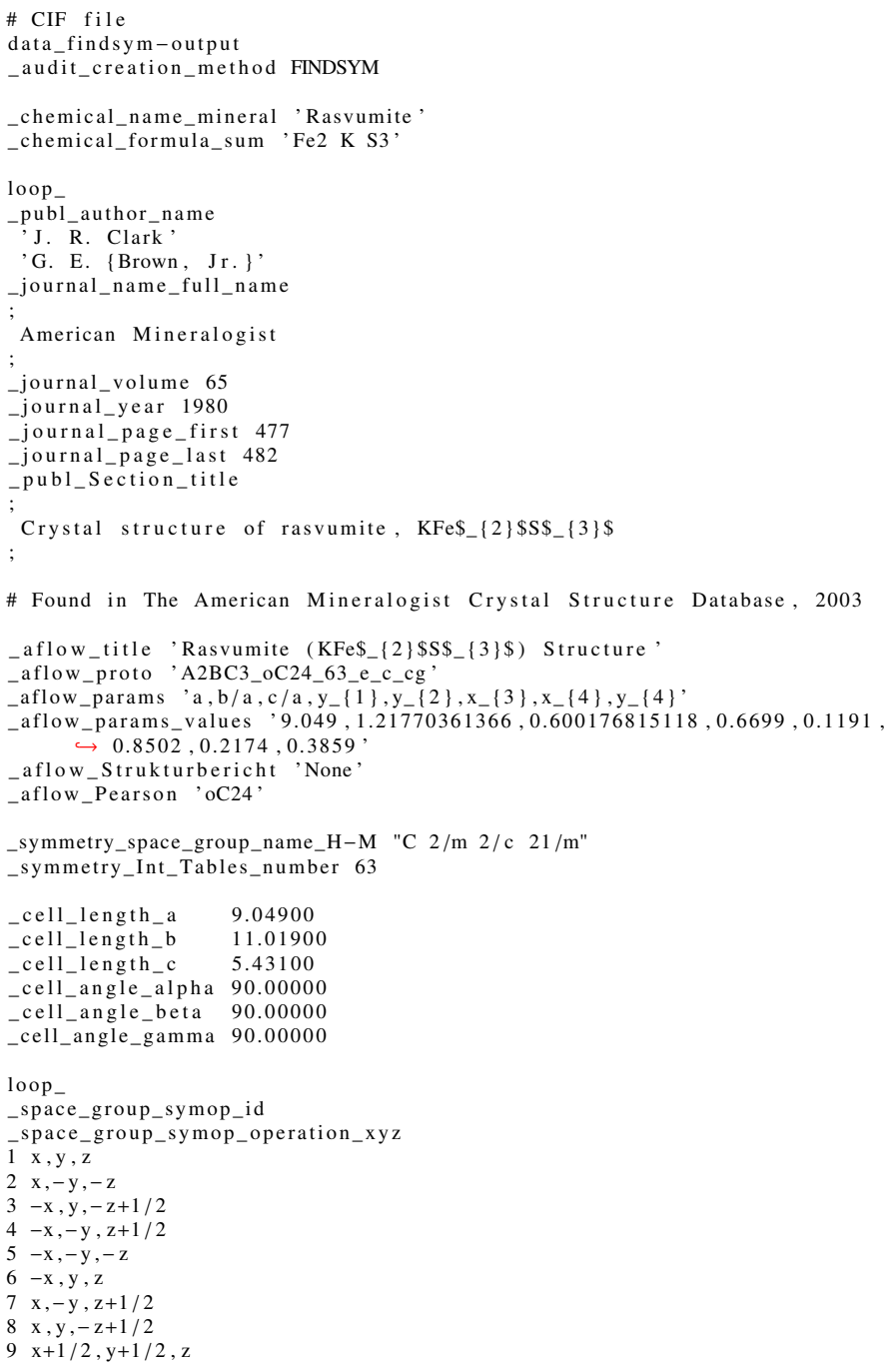


$10 \mathrm{x}+1 / 2,-\mathrm{y}+1 / 2,-\mathrm{z}$

$11-\mathrm{x}+1 / 2, \mathrm{y}+1 / 2,-\mathrm{z}+1 / 2$

$12-x+1 / 2,-y+1 / 2, z+1 / 2$

$13-x+1 / 2,-y+1 / 2,-z$

$14-\mathrm{x}+1 / 2, \mathrm{y}+1 / 2, \mathrm{z}$
$15 \mathrm{x}+1 / 2,-\mathrm{y}+1 / 2, \mathrm{z}+1 / 2$
$16 \mathrm{x}+1 / 2, \mathrm{t}+1 / 2,-z+1 / 2$

$16 \mathrm{x}+1 / 2, \mathrm{y}+1 / 2,-\mathrm{z}+1 / 2$

loop

_atom_site_label

-atom_site_type_symbol

atom_site_symmetry_multiplicity

atom_site_Wyckoff_label

atom_site_fract_x

atom_site_fract_y

atom_site_fract_z

atom_site_occupancy

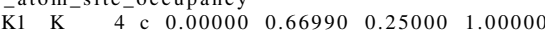

$\begin{array}{llllllll}\mathrm{S} 1 & \mathrm{~S} & 4 & \mathrm{c} & 0.00000 & 0.11910 & 0.25000 & 1.00000\end{array}$

$\begin{array}{lllllllll}\mathrm{Fe} 1 & \mathrm{Fe} & 8 & \text { e } & 0.85020 & 0.00000 & 0.00000 & 1.00000\end{array}$

$\begin{array}{lllllllll}\mathrm{S} 2 & \mathrm{~S} & 8 & \mathrm{~g} & 0.21740 & 0.38590 & 0.25000 & 1.00000\end{array}$

Rasvumite $\left(\mathrm{KFe}_{2} \mathrm{~S}_{3}\right)$ : A2BC3_oC24_63_e_c_cg - POSCAR

A2BC3_oC24_63_e_c_cg \& a , b/a , c/a , y1 , y2 , x3 , x4, y4 --params $=9.049$

$1.21770361366,0.600176815118,0.6699,0.1191,0.8502,0.2174,0.3859$

$\hookrightarrow$ \& Cmcm D_ $\{2 \mathrm{~h}\}^{\wedge}\{17\} \# 63\left(\mathrm{c}^{\wedge} 2 \mathrm{eg}\right) \& \mathrm{oC} 24 \&$ None \& $\mathrm{KFe} 2 \mathrm{~S} 3$ \&

$\hookrightarrow$ Rasvumite \& J. R. Clark and G. E. \{Brown, Jr.\}, Am. Mineral. 65 $477-482(1980)$

1.00000000000000

$\begin{array}{rrr}4.52450000000000 & -5.50950000000000 & 0.00000000000000 \\ 4.52450000000000 & 5.50950000000000 & 0.00000000000000\end{array}$

$\begin{array}{rrr}4.52450000000000 & 5.50950000000000 & 0.00000000000000 \\ 0.00000000000000 & 0.00000000000000 & 5.43100000000000\end{array}$

$\mathrm{Fe} \mathrm{K} \quad \mathrm{S}$

Direct

0.85020000000000

0.85020000000000
-0.85020000000000

0.8502000000000

0.85020000000000

$-0.6699000000000$

0.66900000000

0.1191000000000

0.11910000000000

$-0.16850000000000$

0.16850000000000

$-0.60330000000000$

0.60330000000000

0.85020000000000

$-0.85020000000000$

$-0.85020000000000$

0.85020000000000 0.66990000000000 $-0.66990000000000$ 0.11910000000000 $-0.11910000000000$ 0.6033000000000 $-0.60330000000000$

0.16850000000000

$-0.16850000000000$

0.00000000000000 0.50000000000000

0.00000000000000

0.50000000000000

0.25000000000000

0.75000000000000

0.75000000000000

0.75000000000000

0.25000000000000

.7500000000000

0.75000000000000

$\begin{array}{ll}\mathrm{Fe} & (8 \mathrm{e}) \\ \mathrm{Fe} & (8 \mathrm{e}) \\ \mathrm{Fe} & (8 \mathrm{e}) \\ \mathrm{Fe} & (8 \mathrm{e}) \\ \mathrm{K} & (4 \mathrm{c}) \\ \mathrm{K} & (4 \mathrm{c}) \\ \mathrm{S} & (4 \mathrm{c}) \\ \mathrm{S} & (4 \mathrm{c}) \\ \mathrm{S} & (8 \mathrm{~g}) \\ \mathrm{S} & (8 \mathrm{~g}) \\ \mathrm{S} & (8 \mathrm{~g}) \\ \mathrm{S} & (8 \mathrm{~g})\end{array}$

$-0.850200000000$

La43 $\mathrm{Ni}_{17} \mathrm{Mg}_{5}$ : A43B5C17_oC260_63_c8fg6h_cfg_ce3f2h - CIF

\section{\# CIF file}

data_findsym-output

_audit_creation_method FINDSYM

chemical_name_mineral 'La43Ni17Mg5

_chemical_formula_sum 'La43 Mg5 Ni17

loop_

publ_author_name

P. Solokha

'S. \{De Negri\}'

V. Pavlyuk

journal_name_full_name

Inorganic Chemistry

journal_volume 48

al year 2009

_journal_page_first 11586

journal_page_last 11593

publ_Section_title

Anti-Mackay Polyicosahedral Clusters in $\{\mathrm{La}-\mathrm{Ni}-\mathrm{Mg}\}$ Ternary Compounds: $\rightarrow$ Synthesis and Crystal Structure of the La\$_ $\{43\} \$ N_{i} \$\{17\} \$ M g \$$ $\hookrightarrow 5$ \}\$ New Intermetallic Phase

aflow_title 'La\$_ $\{43\} \$ N i \$_{-}\{17\} \$ M g \$_{-}\{5\} \$$ Structure

aflow proto A43B5C17_oC260_63_c8fg6h_cfg_ce 3f $2 \mathrm{~h}$

aflow_params $, a, b / a, c / a, y_{-}\{1\}, y_{-}\{2\}, y_{-}\{3\}, x_{-}\{4\}, y_{-}\{5\}, z_{-}\{5\}, y_{-}\{6\}, z_{-}\{6$ $\hookrightarrow\}, y_{-}\{7\}, z_{-}\{7\}, y_{-}\{8\}, z_{-}\{8\}, y_{-}\{9\}, z_{-}\{9\}, y_{-}\{10\}, z_{-}\{10\}, y_{-}\{11\}, z_{-}\{$

$\hookrightarrow 11\}, y_{-}\{12\}, z_{-}\{12\}, y_{-}\{13\}, z_{-}\{13\}, y_{-}\{14\}, z_{-}\{14\}, y_{-}\{15\}, z_{-}\{15\}, y_{-}$

$\hookrightarrow 16\}, \mathrm{z}_{-}\{16\}, \mathrm{x}_{-}\{17\}, \mathrm{y}_{-}\{17\}, \mathrm{x}_{-}\{18\}, \mathrm{y}_{-}\{18\}, \mathrm{x}_{-}\{19\}, \mathrm{y}_{-}\{19\}, \mathrm{z}_{-}\{19\}, \mathrm{x}_{-}$

$\hookrightarrow 20\}, y_{-}\{20\}, z_{-}\{20\}, x_{-}\{21\}, y_{-}\{21\}, z_{-}\{21\}, x_{-}\{22\}, y_{-}\{22\}, z_{-}\{22\}, x_{-}$
$\hookrightarrow 23\}, y_{-}\{23\}, z_{-}\{23\}, x_{-}\{24\}, y_{-}\{24\}, z_{-}\{24\}, x_{-}\{25\}, y_{-}\{25\}, z_{-}\{25\}, x_{-}$

low params values $, 10.11895,1.73974572461,4.16742843872,0.38998$,

$\hookrightarrow 0.0432,0.5987,0.1275,0.13992,0.17412,0.7278,0.20648,0.19723$

$\hookrightarrow 0.62632,0.1388,0.02884,0.39065,0.0988,0.06788,0.55569,0.59876$,

$\hookrightarrow 0.1328,0.28079,0.54761,0.0678,0.6912,0.07797,0.09224,0.45337$

$\hookrightarrow 0.1675,0.43146,0.0091,0.18607,0.20422,0.3338,0.3774,0.18828$

$\hookrightarrow 0.32898,0.174,0.19344,0.2014,0.10123,0.30731,0.03499,0.20655$

$\hookrightarrow 0.30495,0.49596,0.12681,0.19578,0.33018,0.026,0.31697,0.03483$

$\hookrightarrow 0.04522,0.2812,0.17213,0.16828,0.2807,0.35936,0.09067$ '

aflow_Strukturbericht 'None'

aflow_Pearson 'oC260

symmetry_space_group_name_H-M "C $2 / \mathrm{m} 2 / \mathrm{c} 21 / \mathrm{m}$ "

symmetry_Int_Tables_number 63

cell_length_a $\quad 10.11895$

cell_length_b 17.60440

$\begin{array}{ll}\text { cell_length_c } & 42.17000\end{array}$

-cell angle alpha 90.00000

cell angle beta 90.00000

cell_angle_gamma 90.00000

loop

space_group_symop_id

space_group_symop_operation_xyz

$\mathrm{x}, \mathrm{y}, \mathrm{z}$

$\mathrm{x},-\mathrm{y},-\mathrm{z}$

$3-\mathrm{x}, \mathrm{y},-\mathrm{z}+1 / 2$

$5-x,-y,-z$

$6-\mathrm{x}, \mathrm{y}, \mathrm{z}$

$7 \mathrm{x},-\mathrm{y}, \mathrm{z}+1 / 2$

$8 \mathrm{x}, \mathrm{y},-\mathrm{z}+1 / 2$

$9 \mathrm{x}+1 / 2, \mathrm{y}+1 / 2, \mathrm{z}$

$10 \mathrm{x}+1 / 2,-\mathrm{y}+1 / 2,-\mathrm{z}$

(1/2,y+1/2,-z+1/2

$12-x+1 / 2,-y+1 / 2, z+1 / 2$

$13-x+1 / 2,-y+1 / 2,-z$

$14-x+1 / 2, y+1 / 2, z$

$15 \mathrm{x}+1 / 2,-\mathrm{y}+1 / 2, \mathrm{z}+1 / 2$

$6 \mathrm{x}+1 / 2, \mathrm{y}+1 / 2,-\mathrm{z}+1 / 2$

loop

atom_site_type_symbol

atom_site_symmetry_multiplicity

atom_site_Wyckoff_label

atom_site_fract_x

atom_site_fract_y

atom_site_fract_z

tom site occupancy

$\begin{array}{lllllllll}\mathrm{L} a 1 & \mathrm{La} & 4 & \mathrm{c} & 0.00000 & 0.38998 & 0.25000 & 1.00000\end{array}$

$\begin{array}{lllllllll}\mathrm{Mg} 1 & \mathrm{Mg} & 4 & \mathrm{c} & 0.00000 & 0.04320 & 0.25000 & 1.00000\end{array}$

$\begin{array}{lllllllll}\mathrm{Ni} 1 & \mathrm{Ni} & 4 & \mathrm{c} & 0.00000 & 0.59870 & 0.25000 & 1.00000\end{array}$

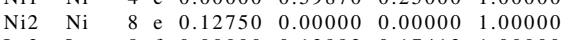

$\begin{array}{lllllllll}\mathrm{La} 2 & \mathrm{La} & 8 & \mathrm{f} & 0.00000 & 0.13992 & 0.17412 & 1.00000\end{array}$

$\begin{array}{lllllllll}\text { La3 } & \mathrm{La} & 8 & \mathrm{f} & 0.00000 & 0.72780 & 0.20648 & 1.00000\end{array}$

$\begin{array}{llllllll}\mathrm{La} 4 & \mathrm{La} & 8 & \mathrm{f} & 0.00000 & 0.19723 & 0.62632 & 1.00000\end{array}$

$\begin{array}{lllllllll}\mathrm{La} 5 & \mathrm{La} & 8 & \mathrm{f} & 0.00000 & 0.13880 & 0.02884 & 1.00000\end{array}$

$\begin{array}{lllllllll}\text { La6 } & \mathrm{La} & 8 & \mathrm{f} & 0.00000 & 0.39065 & 0.09880 & 1.00000\end{array}$

$\begin{array}{llllllllll}\mathrm{La} 7 & \mathrm{La} & 8 & \mathrm{f} & 0.00000 & 0.06788 & 0.55569 & 1.00000\end{array}$

$\begin{array}{llllllllll}\mathrm{La} 8 & \mathrm{La} & 8 & \mathrm{f} & 0.00000 & 0.59876 & 0.13280 & 1.00000\end{array}$

$\begin{array}{lllllllll}\mathrm{La} 9 & \mathrm{La} & 8 & \mathrm{f} & 0.00000 & 0.28079 & 0.54761 & 1.00000\end{array}$

$\begin{array}{lllllllll}\mathrm{Mg} 2 & \mathrm{Mg} & 8 & \mathrm{f} & 0.00000 & 0.06780 & 0.69120 & 1.00000\end{array}$

$\begin{array}{llllllllll}\mathrm{Ni} 3 & \mathrm{Ni} & 8 & \mathrm{f} & 0.00000 & 0.07797 & 0.09224 & 1.00000\end{array}$

$\begin{array}{llllllllll}\mathrm{Ni} 4 & \mathrm{Ni} & 8 & \mathrm{f} & 0.00000 & 0.45337 & 0.16750 & 1.00000\end{array}$

$\begin{array}{llllllll}\mathrm{Ni} & \mathrm{Ni} & 8 & \mathrm{f} & 0.00000 & 0.45337 & 0.16750 & 1.00000 \\ \mathrm{Ni} 5 & \mathrm{Ni} & 8 & \mathrm{f} & 0.00000 & 0.43146 & 0.00910 & 1.00000\end{array}$

$\begin{array}{llllllll}\mathrm{Ni5} & \mathrm{Ni} & 8 & \mathrm{f} & 0.00000 & 0.43146 & 0.00910 & 1.00000\end{array}$

$\begin{array}{llllllll}\text { La10 } & \mathrm{La} & 8 & \mathrm{~g} & 0.18607 & 0.20422 & 0.25000 & 1.00000\end{array}$

$\begin{array}{llllllll}\mathrm{Mg} 3 & \mathrm{Mg} & 8 & \mathrm{~g} & 0.33380 & 0.37740 & 0.25000 & 1.00000\end{array}$

$\begin{array}{lllllllll}\text { La11 La } & 16 & \mathrm{~h} & 0.18828 & 0.32898 & 0.17400 & 1.00000\end{array}$

$\begin{array}{lllllllll}\text { La12 } & \mathrm{La} & 16 & \mathrm{~h} & 0.19344 & 0.20140 & 0.10123 & 1.00000\end{array}$

$\begin{array}{lllllllll}\text { La13 } & \text { La } & 16 & \text { h } & 0.30731 & 0.03499 & 0.20655 & 1.00000\end{array}$

$\begin{array}{lllllllll}\text { La } 14 & \mathrm{La} & 16 & \mathrm{~h} & 0.30495 & 0.49596 & 0.12681 & 1.00000\end{array}$

$\begin{array}{lllllllll}\text { La } 15 & \mathrm{La} & 16 & \mathrm{~h} & 0.19578 & 0.33018 & 0.02600 & 1.00000\end{array}$

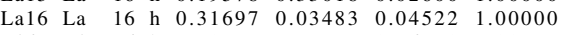

$\begin{array}{lllllllll}\mathrm{N} i 6 & \mathrm{Ni} & 16 & \mathrm{~h} & 0.28120 & 0.17213 & 0.16828 & 1.00000\end{array}$

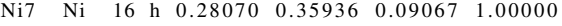

La $43 \mathrm{Ni}_{17} \mathrm{Mg}_{5}$ : A43B5C17_oC260_63_c8fg6h_cfg_ce3f2h - POSCAR

A43B5C17_oC260_63_c8fg6h_cfg_ce3f2h \& a,b/a,c/a,y1,y2,y3, x4,y5, z5, y6, z6 , $\hookrightarrow \mathrm{y} 7, \mathrm{z} 7, \mathrm{y} 8, \mathrm{z} 8, \mathrm{y} 9, \mathrm{z} 9, \mathrm{y} 10, \mathrm{z} 10, \mathrm{y} 11, \mathrm{z} 11, \mathrm{y} 12, \mathrm{z} 12, \mathrm{y} 13, \mathrm{z} 13, \mathrm{y} 14, \mathrm{z} 14, \mathrm{y} 15$

$\hookrightarrow \mathrm{z} 15, \mathrm{y} 16, \mathrm{z} 16, \mathrm{x} 17, \mathrm{y} 17, \mathrm{x} 18, \mathrm{y} 18, \mathrm{x} 19, \mathrm{y} 19, \mathrm{z} 19, \mathrm{x} 20, \mathrm{y} 20, \mathrm{z} 20, \mathrm{x} 21, \mathrm{y} 21, \mathrm{z} 21$

$\hookrightarrow, \mathrm{x} 22, \mathrm{y} 22, \mathrm{z} 22, \mathrm{x} 23, \mathrm{y} 23, \mathrm{z} 23, \mathrm{x} 24, \mathrm{y} 24, \mathrm{z} 24, \mathrm{x} 25, \mathrm{y} 25, \mathrm{z} 25, \mathrm{x} 26, \mathrm{y} 26, \mathrm{z} 26-$

$\hookrightarrow$ params $=10.11895,1.73974572461,4.16742843872,0.38998,0.0432$,

$\hookrightarrow 0.5987,0.1275,0.13992,0.17412,0.7278,0.20648,0.19723,0.62632$,

$\rightarrow 0.1388,0.02884,0.39065,0.0988,0.06788,0.55569,0.59876,0.1328$,

$\hookrightarrow 0.28079,0.54761,0.0678,0.6912,0.07797,0.09224,0.45337,0.1675$,

$\hookrightarrow 0.43146,0.0091,0.18607,0.20422,0.3338,0.3774,0.18828,0.32898$,

$\hookrightarrow 0.174,0.19344,0.2014,0.10123,0.30731,0.03499,0.20655,0.30495$,

$\hookrightarrow 0.49596,0.12681,0.19578,0.33018,0.026,0.31697,0.03483,0.04522$,

$\hookrightarrow 0.2812,0.17213,0.16828,0.2807,0.35936,0.09067^{\wedge} \& \mathrm{Cmcm} \mathrm{D}\{2 \mathrm{~h}\}^{\wedge}\{17$

$\hookrightarrow\} \# 63\left(\mathrm{c}^{\wedge} 3 \mathrm{ef}^{\wedge} 12 \mathrm{~g}^{\wedge} 2 \mathrm{~h}^{\wedge} 8\right) \& \mathrm{oC} 260$ \& None \& La43Ni17Mg5 \&

$\hookrightarrow$ La43Ni17Mg5 \& P Solokha et al. Inorg. Chem. 48, 11586-11593 $\hookrightarrow 2009$ )

1.00000000000000

5.05947500000000

5.05947500000000

0.00000000000000

$\begin{array}{lll}\mathrm{La} & \mathrm{Mg} & \mathrm{Ni} \\ 86 & 10 & 34\end{array}$

Direc

$-0.38998000000000$

0.38998000000000

$-0.13992000000000$

0.13992000000000

$-0.13992000000000$

0.13992000000000

$-0.72780000000000$

0.72780000000000

$-0.72780000000000$

0.72780000000000

$-0.19723000000000$

0.19723000000000

$-0.19723000000000$

0.19723000000000

$-0.13880000000000$

0.13880000000000

$-0.13880000000000$

0.13880000000000

$-0.39065000000000$

0.39065000000000

$-0.39065000000000$

8.80220000000000 8.80220000000000 0.00000000000000

0.00000000000000 2.17000000000000

0.38998000000000 $-0.38998000000000$ 0.13992000000000 $-0.13992000000000$ 0.13992000000000 $-0.13992000000000$ 0.72780000000000 0.72780000000000 0.72780000000000 0.72780000000000 0.19723000000000 0.19723000000000 0.19723000000000 $-0.19723000000000$ 0.13880000000000 0.13880000000000 0.13880000000000 0.39065000000000 0.39065000000000 0.39065000000000
0.25000000000000 0.75000000000000 0.17412000000000 0.67412000000000 0.32588000000000 $-0.17412000000000$ 0.20648000000000 0.70648000000000 0.29352000000000 $-0.20648000000000$ 0.62632000000000 0.62632000000000 1.12632000000000 $-0.12632000000000$ 0.62632000000000 .02884000000000 .52884000000000 0.47116000000000 $-0.02884000000000$ 0.09880000000000 0.59880000000000 0.40120000000000
$\mathrm{La} \quad(4 \mathrm{c})$

$\mathrm{La} \quad(4 \mathrm{c})$

$\mathrm{La} \quad(8 \mathrm{f})$

$\mathrm{La} \quad(8 \mathrm{f})$

$\mathrm{La} \quad(8 \mathrm{f})$

$\mathrm{La}(8 \mathrm{f})$

$\mathrm{La}$ (8f)

$\mathrm{La}(8 \mathrm{f})$

$\mathrm{La}(8 \mathrm{f})$

$\begin{array}{ll}\mathrm{La} & (8 \mathrm{f}) \\ \mathrm{La} & (8 \mathrm{f})\end{array}$

$\begin{array}{ll}\mathrm{La} & (8 \mathrm{f}) \\ \mathrm{La} & (8 \mathrm{f})\end{array}$

$\mathrm{La}(8 \mathrm{f})$

$\mathrm{La} \quad(8 \mathrm{f})$

$\mathrm{La} \quad(8 \mathrm{f})$

$\mathrm{La} \quad(8 \mathrm{f})$

$\begin{array}{ll}\mathrm{La} & (8 \mathrm{f}) \\ \mathrm{La} & (8 \mathrm{f})\end{array}$

$\mathrm{La} \quad(8 \mathrm{f})$

La (8f) 
0.39065000000000 0.06788000000000 0.06788000000000 $-0.06788000000000$ 0.06788000000000 $-0.59876000000000$ 0.59876000000000 0.59876000000000 0.59876000000000 $-0.28079000000000$ 0.28079000000000 0.28079000000000 $-0.0181500000000$ 0.01815000000000 0.01815000000000 0.39029000000000 0.14070000000000 0.14070000000000 $-0.51726000000000$ 0.51726000000000 0.14070000000000 $-0.14070000000000$ 0.51726000000000 $-0.51726000000000$ $-0.00796000000000$ 0.00796000000000 0.39484000000000 0.00796000000000 $-0.00796000000000$ 0.39484000000000 $-0.39484000000000$ 0.27232000000000 0.27232000000000 0.34230000000000 0.34230000000000 $-0.27232000000000$ 0.27232000000000 0.34230000000000 $-0.34230000000000$ $-0.19101000000000$ 0.19101000000000 $-0.80091000000000$ 0.80091000000000 0.19101000000000

$-0.19101000000000$ 0.80091000000000 $-0.8009100000000$ 0.13440000000000 $-0.52596000000000$ 0.52596000000000 0.13440000000000 0.13440000000000 0.52596000000000 $-0.52596000000000$ 0.28214000000000 $-0.28214000000000$ $-0.35180000000000$ 0.35180000000000 $-0.28214000000000$ 0.28214000000000 0.35180000000000 $-0.35180000000000$ $-0.35180000000000$ 0.04320000000 $-0.06780000000000$ 0.06780000000000 $-0.06780000000000$ 0.06780000000000 $-0.04360000000000$ 0.04360000000000 $-0.71120000000000$ 0.71120000000000 $-0.59870000000000$ 0.59870000000000 0.12750000000000 $-0.12750000000000$ 0.12750000000000 0.1275000000000 0.07797000000000 0.07797000000000 0.07797000000000 $-0.45337000000000$ 0.45337000000000 $-0.45337000000000$ 0.45337000000000 $-0.43146000000000$ 0.43146000000000 $-0.43146000000000$ 0.43146000000000 0.10907000000000 $-0.10907000000000$ $-0.45333000000000$ 0.45333000000000 $-0.10907000000000$ 0.1090700000000 0.45333000000000 0.453330000000 $-0.07866000000000$ 0.07866000000000 0.07866000000000
-0.64006000000000 0.64006000000000
$-0.39065000000000$ 0.0678800000000 $-0.06788000000000$ 0.06788000000000 0.06788000000 0.5987600000000 $-0.59876000000000$ 0.59876000000000 $-0.5987600000000$ 0.28079000000000 $-0.28079000000000$ 0.28079000000000 $-0.28079000000000$ 0.39029000000000 $-0.39029000000000$ 0.01815000000000 $-0.01815000000000$ 0.51726000000000 $-0.51726000000000$ 0.14070000000000 $-0.14070000000000$ $-0.5172600000000$ 0.51726000000000 0.14070000000000 0.14070000000000 0.39484000000000 $-0.39484000000000$ 0.00796000000000 $-0.00796000000000$ $-0.39484000000000$ 0.39484000000000 0.00796000000000 0.00796000000000 0.34230000000000 $-0.34230000000000$ $-0.27232000000000$ 0.27232000000000 $-0.34230000000000$ 0.3423000000000 0.2723200000000 $-0.27232000000000$ 0.80091000000000 $-0.80091000000000$ 0.19101000000000 $-0.19101000000000$ $-0.80091000000000$ 0.80091000000000 $-0.19101000000000$ 0.19101000000000 0.52596000000000 $-0.52596000000000$ 0.13440000000000 $-0.13440000000000$ $-0.52596000000000$ 0.52596000000000 $-0.13440000000000$ 0.13440000000000 0.35180000000000 $-0.3518000000000$ $-0.28214000000000$ 0.28214000000000 $-0.35180000000000$ 0.35180000000000 0.28214000000000 $-0.28214000000000$ 0.04320000000000 $-0.04320000000000$ 0.06780000000000 $-0.06780000000000$ 0.0678000000000 $-0.06780000000000$ 0.7112000000000 $-0.7112000000000$ 0.04360000000000 $-0.04360000000000$ 0.59870000000000 $-0.59870000000000$ 0.12750000000000 $-0.12750000000000$ 0.07797000000000 $-0.07797000000000$ 0.07797000000000 0.07797000000000 0.45337000000000 $-0.45337000000000$ 0.45337000000000 $-0.45337000000000$ 0.43146000000000 $-0.43146000000000$ 0.43146000000000 0.45333000000000 $-0.45333000000000$ $-0.10907000000000$ 0.10907000000000 $-0.4533300000000$ 0.45333000000000 0.10907000000000 0.10907000000000 0.64006000000000 $-0.64006000000000$ 0.0786600000000 $-0.07866000000000$

$-0.09880000000000$ 0.55569000000000 1.05569000000000 $-0.0556900000000$ .55569000000000 0.1328000000000 0.6328000000000 0.36720000000000 0.1328000000000 0.54761000000000 1.04761000000000 $-0.04761000000000$ $-0.54761000000000$ 0.25000000000000 0.75000000000000 0.25000000000000 0.75000000000000 0.750000000000 0.67400000000000 0.67400000000000 0.32600000000000 $-0.17400000000000$ $-0.17400000000000$ 0.32600000000000 0.67400000000000 0.17400000000000 0.10123000000000 0.60123000000000 0.39877000000000 $-0.10123000000000$ $-0.10123000000000$ 0.39877000000000 0.60123000000000 0.10123000000000 0.20655000000000 0.70655000000000 . 0.2934500000000 $-0.20655000000000$ $-0.20655000000000$ 0.29345000000000 0.70655000000000 0.20655000000000 0.12681000000000 0.62681000000000 0.37319000000000 $-0.12681000000000$ $-0.12681000000000$ 0.3731900000000 0.62681000000000 0.12681000000000 0.52600000000000 0.47400000000000 0.474000000000 $-0.0260000000000$ $-0.0260000000000$ 0.4740000000000 0.5260000000000 0.02600000000000 0.04522000000000 0.54522000000000 0.45478000000000 $-0.04522000000000$ $-0.04522000000000$ 0.45478000000000 0.54522000000000 0.04522000000000 0.25000000000000 0.75000000000000 0.6912000000000 19120000000000 $-0.19120000000000$ $-0.6912000000000$ 0.2500000000000 0.75000000000000 0.25000000000000 0.75000000000000 0.25000000000000 0.75000000000000 0.00000000000000 0.50000000000000 0.00000000000000 0.5000000000000 0.09224000000000 0.59224000000000 0.5922400000000 0.0924000000000 0.16750000000000 
0.03146000000000

$-0.60390000000000-0.60390000000000$

$-0.60390000000$

0.60390000000000

$-0.45686000000000$

$-0.03146000000000$

0.03146000000000

0.45686000000000

$-0.45686000000000$

0.25000000000000 0.75000000000000 0.25000000000000 0.75000000000000 0.25000000000000 0.75000000000000

$\begin{array}{ll}\mathrm{Al} & (8 \mathrm{~g}) \\ \mathrm{Al} & (8 \mathrm{~g}) \\ \mathrm{Al} & (8 \mathrm{~g}) \\ \mathrm{Al} & (8 \mathrm{~g}) \\ \mathrm{Mn} & (4 \mathrm{c}) \\ \mathrm{Mn} & (4 \mathrm{c})\end{array}$

Post-perovskite $\left(\mathrm{MgSiO}_{3}\right)$ : AB3C_oC20_63_a_cf_c - CIF

\section{\# CIF file}

data_findsym-output

_audit_creation_method FINDSYM

chemical_name_mineral 'Post-perovskite $\mathrm{MgSiO}_{-}\{3\} \$$,

chemical_formula_sum 'Mg O3 $\mathrm{Si}$,

loop

publ_author_name

'M. Murakami',

K. Hirose

'K. Kawamura'

N. Sata,

Y. Ohishi,

journal_name_full_name

Science

journal_volume 304

journal_year 2004

_journal_page_first 855

journal_page_last 858

publ_Section_title

Post-Perovskite Phase Transition in $\operatorname{MgSiO}_{-}\{3\} \$$

aflow title Post-perovskite (MgSiO\$_\{3\}\$) Structure,

_aflow_proto 'AB3C_oC20_63_a_cf_c'

aflow params , a, b/a,c/a,y $\{2\}, y\{3\}, y,\{4\}, z\{4\}$

aflow_params a, b/a, c/a, $y_{-}\{2\}, y_{-}\{3\}, y_{-}\{4\}, z_{-}\{4\}$
aflow_params_values , $2.456,3.27442996743,2.48086319218,0.077,0.747$, $\hookrightarrow 0.631,-0.064$

aflow Strukturbericht 'None'

aflow Pearson, oC20,

symmetry_space_group_name_H-M "C m c m"

_symmetry_Int_Tables_number 63

cell_length_a $\quad 2.45600$

cell_length_b 8.04200

cell_length_c 6.09300

cell_angle_alpha 90.00000

cell_angle_beta 90.00000

cell angle gamma 90.00000

loop

space_group_symop_id

_space_group_symop_operation_xyz

$1 \mathrm{x}, \mathrm{y}, \mathrm{z}$

$2 x,-y,-z$

$3-x, y,-z+1 / 2$

$4-\mathrm{x},-\mathrm{y}, \mathrm{z}+1 / 2$

$5-\mathrm{x},-\mathrm{y},-$

$6-\mathrm{x}, \mathrm{y}, \mathrm{z}$

$7 \mathrm{x},-\mathrm{y}, \mathrm{z}+1 / 2$

$8 \mathrm{x}, \mathrm{y},-\mathrm{z}+1 / 2$

$\mathrm{x}+1 / 2, \mathrm{y}+1 / 2, \mathrm{z}$

$0 x+1 / 2,-y+1 / 2,-z$

$1-\mathrm{x}+1 / 2, \mathrm{y}+1 / 2,-\mathrm{z}+1 / 2$

$12-x+1 / 2,-y+1 / 2, z+1 / 2$

$13-\mathrm{x}+1 / 2,-\mathrm{y}+1 / 2,-\mathrm{z}$

$14-x+1 / 2, y+1 / 2, z$

$15 \mathrm{x}+1 / 2,-\mathrm{y}+1 / 2, \mathrm{z}+1 / 2$

$16 \mathrm{x}+1 / 2, \mathrm{y}+1 / 2,-\mathrm{z}+1 / 2$

loop

atom site label

_atom_site_type_symbol

_atom_site_symmetry_multiplicity

_atom_site_Wyckoff_labe

-atom_site_fract_x

atom_site_fract_y

atom_site_fract_z

atom_site_occupancy

\begin{tabular}{lllllll}
\hline $\mathrm{Mg} 1 \mathrm{Mg}$ & 4 & $\mathrm{a}$ & 0.00000 & 0.00000 & 0.00000 & 1.00000
\end{tabular}

$\begin{array}{lllllllll}\mathrm{O} 1 & \mathrm{O} & 4 & \mathrm{c} & 0.00000 & 0.07700 & 0.25000 & 1.00000\end{array}$

$\begin{array}{lllllllll}\mathrm{Si} 1 & \mathrm{Si} & 4 & \mathrm{c} & 0.00000 & 0.74700 & 0.25000 & 1.00000\end{array}$

$\begin{array}{llllllllll}02 & 0 & 8 & \text { f } & 0.00000 & 0.63100 & -0.06400 & 1.00000\end{array}$

Post-perovskite $\left(\mathrm{MgSiO}_{3}\right)$ : AB3C_oC20_63_a_cf_c - POSCAR

AB3C_oC20_63_a_cf_c \& a , b/a,c/a, y2 ,y3,y4, z4 --params $=2.456,3.27442996743$ $\hookrightarrow, 2.48086319218,0.077,0.747,0.631,-0.064 \&$ Cmcm D_ $\{2 \mathrm{~h}\}^{\wedge}\{17\} \# 63$

$\hookrightarrow\left(\mathrm{ac}^{\wedge} 2 \mathrm{f}\right)$ \& $\mathrm{oC} 20$ \& None \& $\mathrm{MgSiO} 3$ \& Post-perovskite MgSiO\$_\{3\}\$ \&

$\hookrightarrow$ M. Murakami et al., Science 304, 855-858 (2004) 1.00000000000000

$1.22800000000000-4.02100000000000-0.00000000000000$

$1.22800000000000-4.02100000000000-0.0000000000000$

1.22800000000000

$\mathrm{Mg} \quad \mathrm{O} \quad \mathrm{Si}$

Direct

$\begin{array}{lll}0.00000000000000 & 0.00000000000000 & 0.00000000000000\end{array}$

$0.00000000000000 \quad 0.00000000000000 \quad 0.5000000000000$

$-0.07700000000000 \quad 0.07700000000000$

$0.07700000000000-0.07700000000000$

0.75000000000000

$\mathrm{Mg} \quad(4 \mathrm{a})$

$\mathrm{Mg} \quad(4 \mathrm{a})$

$\mathrm{O} \quad(4 \mathrm{c})$

$\mathrm{O} \quad(4 \mathrm{c})$
$\mathrm{MgSO}_{4}: \mathrm{AB} 4 \mathrm{C} \_$oC24_63_a_fg_c $-\mathrm{CIF}$

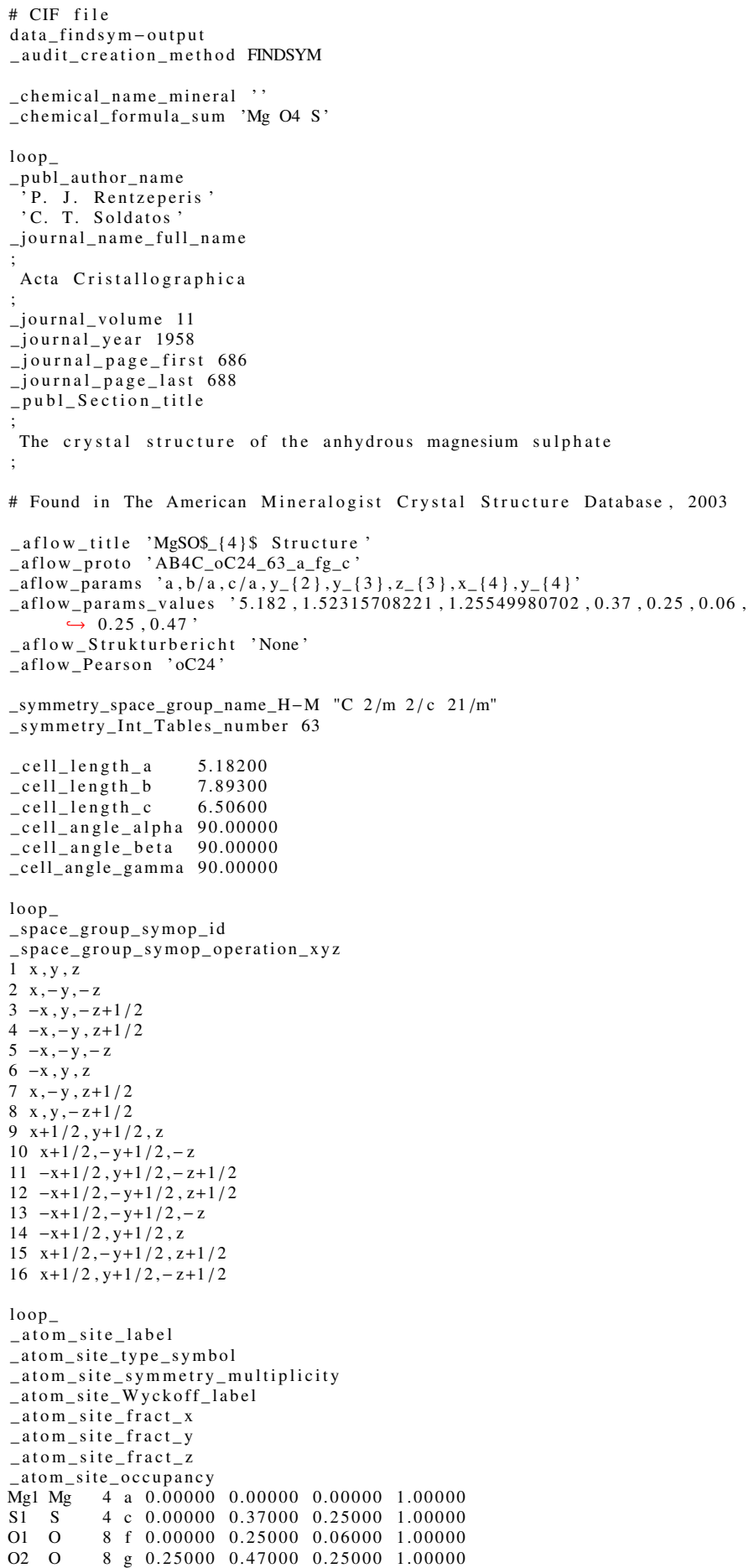

$\mathrm{MgSO}_{4}$ : AB4C_oC24_63_a_fg_c - POSCAR

\begin{tabular}{|c|c|c|c|c|c|c|}
\hline \multirow{3}{*}{\multicolumn{7}{|c|}{$\begin{aligned} & \text { AB4C_oC24_63_a_fg_c \& a }, \mathrm{b} / \mathrm{a}, \mathrm{c} / \mathrm{a}, \mathrm{y} 2, \mathrm{y} 3, \mathrm{z} 3, \mathrm{x} 4, \mathrm{y} 4--\mathrm{params}=5.182, \\
& \\
& \hookrightarrow 1.52315708221,1.25549980702,0.37,0.25,0.06,0.25,0.47 \& \text { Cmcm D_ }\{ \\
&\hookrightarrow 2 \mathrm{~h}\}^{\wedge}\{17\} \# 63(\mathrm{acfg}) \& \text { oC24 \& None \& MgO4S \& \& P. J. } \\
& \hookrightarrow \text { Rentzeperis and C. T. Soldatos, Acta Cryst. 11, 686-688 }\end{aligned}$}} \\
\hline & & & & & & \\
\hline & & & & & & \\
\hline \\
\hline \multicolumn{3}{|c|}{2.59100000000000} & -3.94650000000000 & 0.00000000000000 & & \\
\hline \multicolumn{3}{|c|}{2.59100000000000} & & & & \\
\hline \multicolumn{3}{|c|}{0.00000000000000} & 0.00 & 6.50 & & \\
\hline $\mathrm{Mg}$ & $\mathrm{O}$ & $\mathrm{S}$ & & & & \\
\hline 2 & 8 & 2 & & & & \\
\hline \multicolumn{7}{|l|}{ irect } \\
\hline \multicolumn{3}{|c|}{0.00000000000000} & 0.00 & & $\mathrm{Mg}$ & (4a) \\
\hline \multirow{2}{*}{\multicolumn{3}{|c|}{$\begin{array}{r}0.000000000000000 \\
-0.25000000000000\end{array}$}} & & & $\mathrm{Mg}$ & \\
\hline & & & 0.250 & 0 & $\mathrm{O}$ & $(8 \mathrm{f})$ \\
\hline \multicolumn{3}{|c|}{0.25000000000000} & -0.2 & & $\mathrm{O}$ & \\
\hline \multicolumn{3}{|c|}{-0.25000000000000} & 0.25000000000000 & 0.44000000000000 & $\mathrm{O}$ & $(8 \mathrm{f})$ \\
\hline
\end{tabular}




$\begin{array}{rrrrr}0.25000000000000 & -0.25000000000000 & -0.06000000000000 & \mathrm{O} & (8 \mathrm{f}) \\ -0.22000000000000 & 0.72000000000000 & 0.25000000000000 & \mathrm{O} & (8 \mathrm{~g}) \\ 0.22000000000000 & -0.72000000000000 & 0.75000000000000 & \mathrm{O} & (8 \mathrm{~g}) \\ -0.72000000000000 & 0.22000000000000 & 0.25000000000000 & \mathrm{O} & (8 \mathrm{~g}) \\ 0.72000000000000 & -0.22000000000000 & 0.75000000000000 & \mathrm{O} & (8 \mathrm{~g}) \\ -0.37000000000000 & 0.37000000000000 & 0.25000000000000 & \mathrm{~S} & (4 \mathrm{c}) \\ 0.37000000000000 & -0.37000000000000 & 0.75000000000000 & \mathrm{~S} & (4 \mathrm{c})\end{array}$

Anhydrite $\left(\mathrm{CaSO}_{4}, \mathrm{H}_{1}\right)$ : AB4C_oC24_63_c_fg_c - CIF

\section{\# CIF file}

data_findsym-output

_audit_creation_method FINDSYM

_chemical_name_mineral 'Anhydrite'

_chemical_formula_sum ' $\mathrm{CaO} \mathrm{O} 4 \mathrm{~S}$

loop

_publ_author_name

'F. C. Hawthorne'

R. B. Ferguson

_journal_name_full_name

Canadian Mineralogist

_journal_volume 13

journal year 1975

_journal_page_first 289

_journal_page_last 292

_publ_Section_title

Anhydrous sulphates. II. Refinement of the crystal structure of $\hookrightarrow$ anhydrit

aflow_title Anhydrite (CaSO\$_\{4\}\$, \$H0_\{1\}\$) Structure

aflow_proto 'AB4C_oC24_63_c_fg_c'

aflow_params a, b/a,c/a,y_\{1\},y_ $\{2\}, y_{-}\{3\}, z_{0}\{3\}, x_{-}\{4\}, y_{-}\{4\}$,

aflow_params_values $6.995,0.892780557541,0.999714081487,0.6524,0.15556$ $\hookrightarrow \quad 0.7025,-0.0819,0.1699,0.0162$

aflow Strukturbericht '\$HO $\{1\} \$$

aflow Pearson, oC24,

_symmetry_space_group_name_H-M "C $2 / \mathrm{m} 2 / \mathrm{c} 21 / \mathrm{m}$ "

_symmetry_Int_Tables_number 63

_cell_length_a $\quad 6.99500$

_cell_length_b 6.24500

_cell_length_c 6.99300

_cell_angle_alpha 90.00000

_cell_angle_beta 90.00000

_cell_angle_gamma 90.00000

loop

_space_group_symop_id

_space_group_symop_operation_xyz

$1 \mathrm{x}, \mathrm{y}, \mathrm{z}$

$2 \mathrm{x},-\mathrm{y},-\mathrm{z}$

$3-x, y,-z+1 / 2$

$4-\mathrm{x},-\mathrm{y}, \mathrm{z}+1 / 2$

$5-\mathrm{x},-\mathrm{y},-\mathrm{z}$

$7 \mathrm{x},-\mathrm{y}, \mathrm{z}+1 / 2$

$8 \mathrm{x}, \mathrm{y},-\mathrm{z}+1 / 2$

$9 \mathrm{x}+1 / 2, \mathrm{y}+1 / 2, \mathrm{z}$

$10 \mathrm{x}+1 / 2,-\mathrm{y}+1 / 2,-\mathrm{z}$

$11-x+1 / 2, y+1 / 2,-z+1 / 2$

$12-x+1 / 2,-y+1 / 2, z+1 / 2$

$13-x+1 / 2,-y+1 / 2,-z$

$14-\mathrm{x}+1 / 2, \mathrm{y}+1 / 2, \mathrm{z}$

$15 \mathrm{x}+1 / 2,-\mathrm{y}+1 / 2, \mathrm{z}+1 / 2$

$16 \mathrm{x}+1 / 2, \mathrm{y}+1 / 2,-\mathrm{z}+1 / 2$

loop

atom site label

-atom_site_type_symbol

_atom_site_symmetry_multiplicity

_atom_site_Wyckoff_labe

_atom_site_fract_x

atom_site_fract_y

atom_site_fract_z

atom_site_occupancy

$\begin{array}{lllllll}\mathrm{Ca} 1 \mathrm{Ca} & 4 & \mathrm{c} & 0.00000 & 0.65240 & 0.25000 & 1.00000\end{array}$

$\begin{array}{lllllllll}\mathrm{S} 1 & \mathrm{~S} & 4 & \mathrm{c} & 0.00000 & 0.15556 & 0.25000 & 1.00000\end{array}$

$\begin{array}{lllllllll}\mathrm{O} 1 & \mathrm{O} & 8 & \mathrm{f} & 0.00000 & 0.70250 & -0.08190 & 1.00000\end{array}$

$\begin{array}{llllllllll}\mathrm{O} 2 & \mathrm{O} & 8 & \mathrm{~g} & 0.16990 & 0.01620 & 0.25000 & 1.00000\end{array}$

Anhydrite $\left(\mathrm{CaSO}_{4}, \mathrm{HO}_{1}\right)$ : AB4C_oC24_63_c_fg_c - POSCAR

AB4C_oC24_63_c_fg_c \& a , b/a , c/a , y1,y2,y3, z3 , x4,y4 --params $=6.995$, $\hookrightarrow 0.892780557541,0.999714081487,0.6524,0.15556,0.7025,-0.0819$,

$\hookrightarrow 0.1699,0.0162 \&$ Cmcm D_ $\{2 \mathrm{~h}\}^{\wedge}\{17\} \# 63\left(\mathrm{c}^{\wedge} 2 \mathrm{fg}\right) \&$ oC24\& \$HO_ $\{1\} \$$

$\hookrightarrow$ \& CaSO4 \& Anhydrite \& F. C. Hawthorne and R. B. Ferguson, Can.

$\hookrightarrow$ Mineral. 13, 289-292 (1975)

1.00000000000000

3.49750000000000

$-3.12250000000000$

3.49750000000000

3.12250000000000

0.00000000000000

0.00000000000000

$\begin{array}{ccc}\mathrm{Ca} & \mathrm{O} & \mathrm{S} \\ 2 & 8 & 2\end{array}$

Direct

$-0.65240000000000$

0.65240000000000

0.70250000000000

0.65240000000000 $-0.65240000000000$ 0.70250000000000 $0.70250000000000 \quad-0.70250000000000$

0.25000000000000 0.75000000000000 $-0.08190000000000$ 0.41810000000000
$\mathrm{Ca} \quad(4 \mathrm{c})$

$\mathrm{Ca} \quad(4 \mathrm{c})$

$\begin{array}{ll}\mathrm{O} & (8 \mathrm{f}) \\ \mathrm{O} & (8 \mathrm{f})\end{array}$
$-0.70250000000000$ 0.70250000000000 0.15370000000000 $-0.15370000000000$ $-0.18610000000000$

0.18610000000000

$-0.15556000000000$

0.15556000000000

0.70250000000000 0.70250000000000 0.18610000000000 $-0.18610000000000$ 0.15370000000000 0.1537000000000 0.15556000000000 $-0.15556000000000$ 0.25000000000000 0.75000000000000 0.25000000000000 0.75000000000000 0.25000000000000 0.75000000000000

$\mathrm{H}_{2} \mathrm{~S}$ (170 GPa): A2B_oC24_64_2f_f - CIF

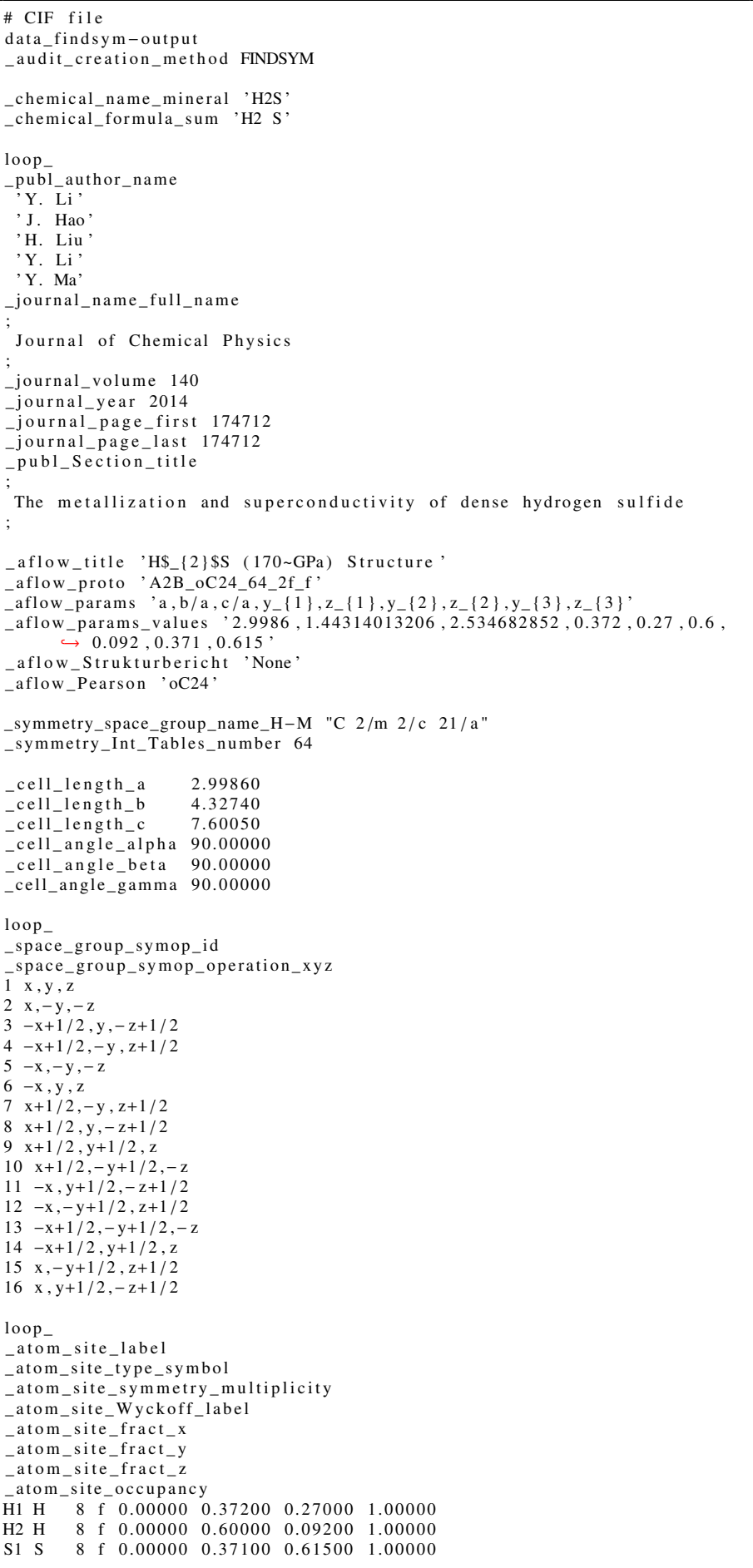

$\mathrm{H}_{2} \mathrm{~S}$ (170 GPa): A2B_oC24_64_2f_f - POSCAR

A2B_oC24_64_2f_f \& a , b/a, c/a, y1, z1 , y2 , z2 , y3 , z3 --params $=2.9986$ $\leftrightarrow 1.44314013206,2.534682852,0.372,0.27,0.6,0.092,0.371,0.615 \&$ $\hookrightarrow$ Cmca $D_{-}\{2 \mathrm{~h}\}^{\wedge}\{18\} \# 64 \quad\left(\mathrm{f}^{\wedge} 3\right) \&$ oC24 \& None \& H2S \& H2S \& Y. Li et $\hookrightarrow$ al., J. Chem. Phys. $140,174712(2014)$

1.00000000000000

$\begin{array}{lll}1.49930000000000 & -2.16370000000000 & 0.00000000000000\end{array}$

$1.49930000000000-2.16370000000000-0.00000000000000$

$0.0000000000000 \quad 0.0000000000000-7.60050000000000$

$\mathrm{H} \quad \mathrm{S}$

Direc

$-0.37200000000000$

0.87200000000000

0.12800000000000

0.37200000000000 0.12800000000000 0.87200000000000

0.27000000000000 0.77000000000000 0.23000000000000 
0.37200000000000 $-0.60000000000000$ 1.10000000000000
-0.10000000000000 0.60000000000000 $-0.37100000000000$ 0.87100000000000 0.12900000000000 0.37100000000000

$-0.37200000000000$ 0.60000000000000 $-0.10000000000000$ 1.10000000000000 $-0.6000000000000$ 0.37100000000000 0.1290000000000 0.87100000000000 $-0.37100000000000$

$-0.27000000000000$ 0.09200000000000 0.59200000000000 0.4080000000000 $-0.0920000000000$ 0.61500000000000 1.11500000000000 $-0.11500000000000$ $-0.61500000000000$

$\mathrm{SrAl}_{2} \mathrm{Se}_{4}: \mathrm{A} 2 \mathrm{~B} 4 \mathrm{C}$ oC28 $66 \_\mathrm{l} \mathrm{kl} \_$- $\mathrm{CIF}$

\section{\# CIF file}

data_findsym-output

audit_creation_method FINDSYM

chemical_name mineral 'SrAl2Se4'

chemical_formula_sum ' $\mathrm{Al} 2 \mathrm{Se} 4 \mathrm{Sr}$

loop

publ_author_name

'W. Klee'

'H. Sch $\{\backslash "$ a $\}$ fer

journal name full name

Zeitschrift $f\{\backslash " u\} r$ Naturforschung B

journal_volume 33

journal year 1978

journal_page_first 829

journal_page_last 833

publ_Section_title

CaAl\$_ $\{2\} \$$ Se $\$ \_\{4\} \$$ und SrAl\$_ $\{2\} \$ S e \$ \&\{4\} \$-$ Strukturvarianten des $\rightarrow$ TISe-Typs / CaAl\$_ $\{2\} \$ S e \$_{-}\{4\} \$$ and $\mathrm{SrAl}_{-}\{2\} \$ S e \$_{-}\{4\} \$-V$ ariants $\hookrightarrow$ of the TISe-Structure

\# Found in Pearson's Crystal Data - Crystal Structure Database for $\hookrightarrow$ Inorganic Compounds, 2013

aflow_title 'SrAl\$_ $\{2\} \$ S e \$_{-}\{4\} \$$ Structure,

aflow_proto, A2B4C_oC28_66_1_kl_a'

aflow_params $, a, b / a, c / a, z_{-}\{2\}, x_{-}\{3\}, y_{-}\{3\}, x_{-}\{4\}, y_{-}\{4\}$

aflow_params_values $6.2700590867,1.72567783094,1.73046251993,0.833$, $\hookrightarrow 0.005,0.268,0.737,0.42$

aflow_Strukturbericht 'None'

_aflow_Pearson 'oC28,

cell_length_a $\quad 6.2700590867$

cell_length_b $\quad 10.8201019646$

cell_length_c $\quad 10.8501022473$

cell_angle_alpha 90.0000000000

cell angle beta 90.0000000000

cell angle gamma 90.000000000

symmetry_space group_name_H-M "C $2 / \mathrm{c} 2 / \mathrm{c} 2 / \mathrm{m}$

symmetry_Int_Tables_number 66

loop

_space_group_symop_id

_space_group_symop_operation_xyz

$1 \mathrm{x}, \mathrm{y}, \mathrm{z}$

$2 \mathrm{x},-\mathrm{y},-\mathrm{z}+1 / 2$

$3-\mathrm{x}, \mathrm{y},-\mathrm{z}+1 / 2$

$4-\mathrm{x},-\mathrm{y}, \mathrm{z}$

$5-x,-y,-z$
$6-x, y, z+1 / 2$

$7 \mathrm{x},-\mathrm{y}, \mathrm{z}+1 / 2$

$8 \mathrm{x}, \mathrm{y},-\mathrm{z}$

$9 \mathrm{x}+1 / 2, \mathrm{y}+1 / 2, \mathrm{z}$

$10 \mathrm{x}+1 / 2,-\mathrm{y}+1 / 2,-\mathrm{z}+1 / 2$

$11-x+1 / 2, y+1 / 2,-z+1 / 2$

$12-x+1 / 2,-y+1 / 2, z$

$13-x+1 / 2,-y+1 / 2,-z$

$14-x+1 / 2, y+1 / 2, z+1 / 2$

$15 x+1 / 2,-y+1 / 2, z+1 / 2$

$16 \mathrm{x}+1 / 2, \mathrm{y}+1 / 2,-\mathrm{z}$

loop

atom_site_label

atom_site_type_symbol

atom_site_symmetry_multiplicity

atom_site_Wyckoff_label

atom_site_fract_x

atom_site_fract_y

atom_site_fract_z

$\begin{array}{llllll}\text { atom_site_occupancy } & & & & & \\ \text { Srl Sr } & 4 & 0.00000 & 0.00000 & 0.25000 & 1.00000\end{array}$

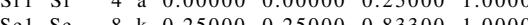

$\begin{array}{lllllllllll} & \text { Sel } & \text { Se } & 8 & k & 0.25000 & 0.25000 & 0.83300 & 1.00000\end{array}$

$\begin{array}{lllllllll}\mathrm{Al1} & \mathrm{Al} & 8 & 1 & 0.00500 & 0.26800 & 0.00000 & 1.00000 \\ \mathrm{Se} 2 & \mathrm{Se} & 8 & 1 & 0.73700 & 0.42000 & 0.00000 & 1.00000\end{array}$

$\mathrm{SrAl}_{2} \mathrm{Se}_{4}$ : A2B4C_oC28_66_l_kl_a - POSCAR

A2B4C_oC28_66_1_k1_a \& a , b/a,c/a ,z2, x3 , y3, x4,y4 --params $=6.2700590867$

$\hookrightarrow 1.72567783094,1.73046251993,0.833,0.005,0.268,0.737,0.42 \& \mathrm{Cccm}$

$\hookrightarrow$ D_ $\{2 \mathrm{~h}\}^{\wedge}\{20\} \# 66\left(\mathrm{akl} \mathbf{A}^{\wedge} 2\right) \&$ oC28 \& None \& SrAl2Se4 \& \& W. Klee

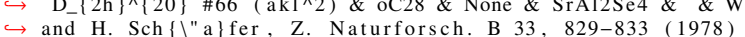

1.00000000000000

$\begin{array}{lll}3.13502954335000 & -5.41005098230000 & 0.00000000000000\end{array}$

$\begin{array}{rrr}3.13502954335000 & 5.41005098230000 & 0.00000000000000\end{array}$

$\begin{array}{rrr}0.00000000000000 & 0.00000000000000 & 10.85010224730000\end{array}$

$\begin{array}{rrr}\mathrm{Al} & \mathrm{Se} & \mathrm{Sr} \\ 4 & 8 & 2\end{array}$ \begin{tabular}{|rr} 
Direct \\
-0.26300000000000 & 0.27300000000000 \\
0.26300000000000 & -0.27300000000000 \\
-0.27300000000000 & 0.26300000000000 \\
0.27300000000000 & -0.26300000000000 \\
0.00000000000000 & 0.50000000000000 \\
0.50000000000000 & 0.00000000000000 \\
0.00000000000000 & 0.50000000000000 \\
0.50000000000000 & 0.00000000000000 \\
0.31700000000000 & 1.15700000000000 \\
-0.31700000000000 & -1.15700000000000 \\
-1.15700000000000 & -0.31700000000000 \\
1.15700000000000 & 0.31700000000000 \\
0.00000000000000 & 0.0000000000000 \\
0.00000000000000 & 0.0000000000000
\end{tabular}

0.00000000000000 0.00000000000000 0.50000000000000 0.50000000000000 0.83300000000000 $-0.33300000000000$ $-0.83300000000000$ 1.33300000000000 0.00000000000000 0.00000000000000 0.50000000000000 0.50000000000000 0.25000000000000 0.75000000000000

Al (81)

Al $\quad(81)$

Al (81)

Al $\quad(81)$

$\mathrm{Se} \quad(8 \mathrm{k})$

$\mathrm{H}_{3} \mathrm{~S}$ (60 GPa): A3B_oC64_66_gi2lm_2l - CIF

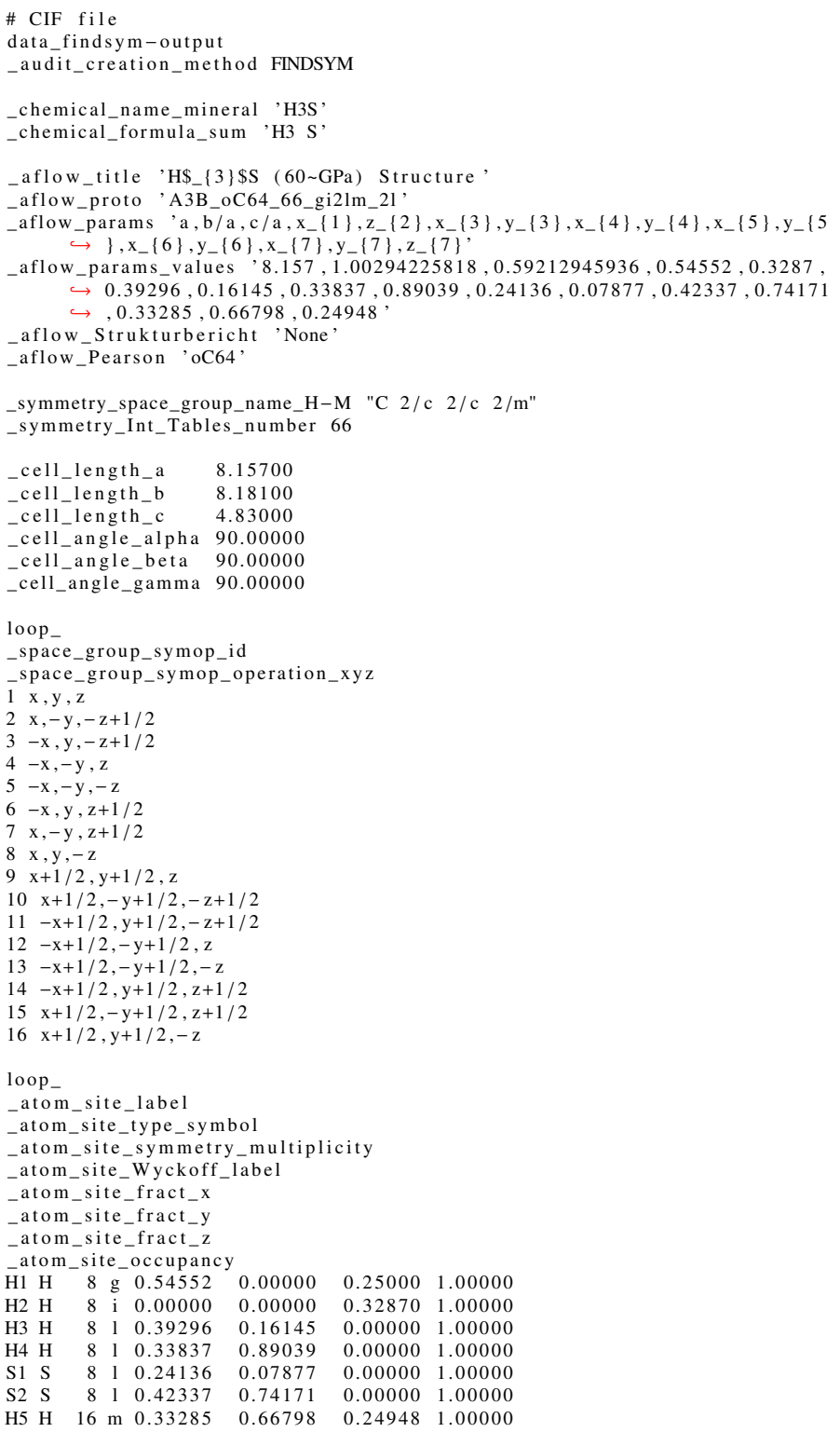

$\mathrm{H}_{3} \mathrm{~S}$ (60 GPa): A3B_oC64_66_gi2lm_2l - POSCAR

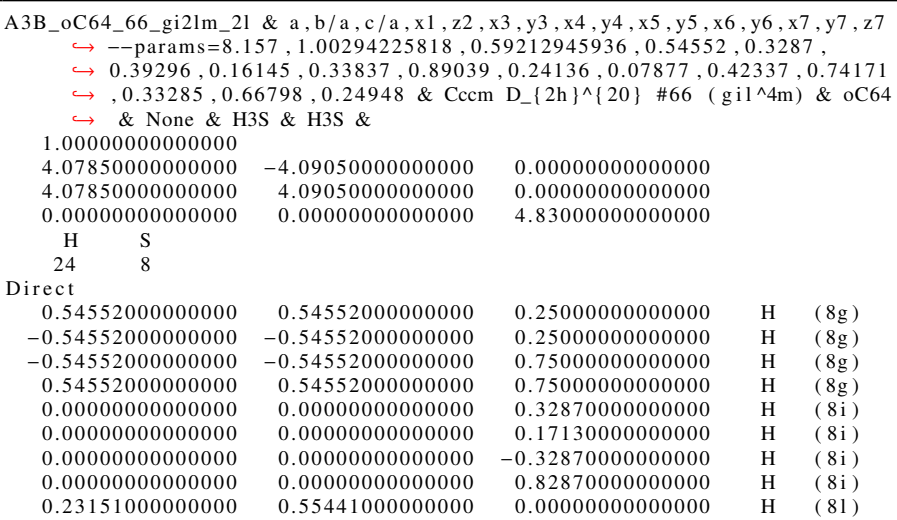




$\begin{array}{rrrrr}-0.23151000000000 & -0.55441000000000 & 0.00000000000000 & \mathrm{H} & (81) \\ -0.55441000000000 & -0.23151000000000 & 0.50000000000000 & \mathrm{H} & (81) \\ 0.55441000000000 & 0.23151000000000 & 0.50000000000000 & \mathrm{H} & (81) \\ -0.55202000000000 & 1.22876000000000 & 0.00000000000000 & \mathrm{H} & (81) \\ 0.55202000000000 & -1.22876000000000 & 0.00000000000000 & \mathrm{H} & (81) \\ -1.22876000000000 & 0.55202000000000 & 0.50000000000000 & \mathrm{H} & (81) \\ 1.22876000000000 & -0.55202000000000 & 0.50000000000000 & \mathrm{H} & (81) \\ -0.33513000000000 & 1.00083000000000 & 0.24948000000000 & \mathrm{H} & (16 \mathrm{~m}) \\ 0.33513000000000 & -1.00083000000000 & 0.24948000000000 & \mathrm{H} & (16 \mathrm{~m}) \\ -1.00083000000000 & 0.33513000000000 & 0.25052000000000 & \mathrm{H} & (16 \mathrm{~m}) \\ 1.00083000000000 & -0.33513000000000 & 0.25052000000000 & \mathrm{H} & (16 \mathrm{~m}) \\ 0.33513000000000 & -1.00083000000000 & -0.24948000000000 & \mathrm{H} & (16 \mathrm{~m}) \\ -0.33513000000000 & 1.00083000000000 & -0.24948000000000 & \mathrm{H} & (16 \mathrm{~m}) \\ 1.00083000000000 & -0.33513000000000 & 0.74948000000000 & \mathrm{H} & (16 \mathrm{~m}) \\ -1.00083000000000 & 0.33513000000000 & 0.74948000000000 & \mathrm{H} & (16 \mathrm{~m}) \\ 0.16259000000000 & 0.32013000000000 & 0.00000000000000 & \mathrm{~S} & (81) \\ -0.16259000000000 & -0.32013000000000 & 0.00000000000000 & \mathrm{~S} & (81) \\ -0.32013000000000 & -0.16259000000000 & 0.50000000000000 & \mathrm{~S} & (81) \\ 0.32013000000000 & 0.16259000000000 & 0.50000000000000 & \mathrm{~S} & (81) \\ -0.31834000000000 & 1.16508000000000 & 0.00000000000000 & \mathrm{~S} & (81) \\ 0.31834000000000 & -1.16508000000000 & 0.00000000000000 & \mathrm{~S} & (81) \\ -1.16508000000000 & 0.31834000000000 & 0.50000000000000 & \mathrm{~S} & (81) \\ 1.16508000000000 & -0.31834000000000 & 0.50000000000000 & \mathrm{~S} & (81)\end{array}$

$\beta$-ThI 3 : A3B_oC64_66_kl2m_bdl - CIF

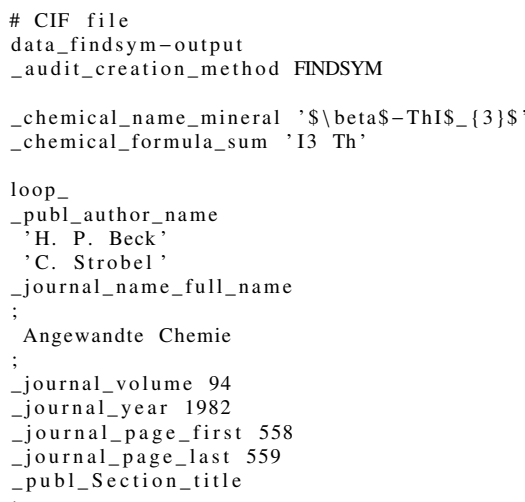

\# Found in The American Mineralogist Crystal Structure Database, 2003

aflow title, $\$ \backslash$ beta $\$-T h I \$\{3\}$ S Structure

aflow_proto 'A3B_oC64_66_kl2m_bdl'

aflow params $, a, b / a, c / a, z_{-}\{3\}, x_{-}\{4\}, y_{-}\{4\}, x_{-}\{5\}, y_{-}\{5\}, x_{-}\{6\}, y_{-}\{6\}, z_{-}\{6$ $\hookrightarrow\}, x_{-}\{7\}, y_{-}\{7\}, z_{-}\{7\}$,

aflow params values $, 8.735,2.32364052662,1.67842014883,0.1826,-0.0318$ $\rightarrow 0.1994,0.327,0.1716,0.2894,0.451,0.1302,0.1133,0.3773,0.3708$, aflow Strukturbericht 'None'

aflow_Pearson 'oC64,

symmetry_space_group_name_H-M "C $2 / \mathrm{c} \quad 2 / \mathrm{c} \quad 2 / \mathrm{m}$

symmetry_Int_Tables_number 66

cell_length_a $\quad 8.73500$

cell_length_b $\quad 20.29700$

cell_angle alpha 90.00000

cell_angle beta 90.00000

cell_angle_gamma 90.00000

loop

space_group_symop_id

_space_group_symop_operation_xyz

$1 \mathrm{x}, \mathrm{y}, \mathrm{z}$

$2 \mathrm{x},-\mathrm{y},-\mathrm{z}+1 / 2$

$3-\mathrm{x}, \mathrm{y},-\mathrm{z}+1 / 2$

$4-\mathrm{x},-\mathrm{y}, \mathrm{z}$

$5-x,-y,-z$
$6-x, y, z+1 / 2$

$\mathrm{x},-\mathrm{y}, \mathrm{z}+1 / 2$

$\mathrm{x}, \mathrm{y},-\mathrm{z}$

$\mathrm{x}+1 / 2, \mathrm{y}+1 / 2, \mathrm{z}$

$10 \mathrm{x}+1 / 2,-\mathrm{y}+1 / 2,-\mathrm{z}+1 / 2$

$11-\mathrm{x}+1 / 2, \mathrm{y}+1 / 2,-\mathrm{z}+1 / 2$

$12-x+1 / 2,-y+1 / 2, z$

$14-x+1 / 2,-y+1 / 2,-z$

14 $x+1 / 2, y+1 / 2, z+1 / 2$

$x+1 / 2,-y+1 / 2, z+1 / 2$
$x+1 / 2, y+1 / 2,-z$

loop

atom site labe

atom_site_type_symbol

atom_site_symmetry_multiplicity

atom_site_Wyckoff_label

atom_site_fract_x

atom_site_fract_y

atom_site_fract_z

$\begin{array}{lllllll}\text { Th1 Th } & 4 & \text { b } & 0.00000 & 0.50000 & 0.25000 & 1.00000\end{array}$

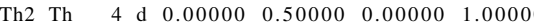

$\begin{array}{llllllllll} & \text { I1 } & \mathrm{I} & 8 & \mathrm{k} & 0.25000 & 0.25000 & 0.18260 & 1.00000\end{array}$

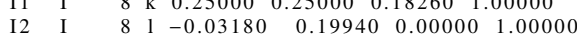

$\begin{array}{lllllllll}\text { Th3 } & \text { Th } & 8 & 1 & 0.32700 & 0.17160 & 0.00000 & 1.00000\end{array}$

$\begin{array}{lllllll}\text { I } & 16 \mathrm{~m} & 0.28940 & 0.45100 & 0.13020 & 1.00000\end{array}$

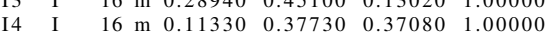

$\beta$-ThI $I_{3}$ : A3B_oC64_66_kl2m_bdl - POSCAR

A3B_oC64 66 kl2m bdl \& a,b/a,c/a,z3, x4,y4, x5, y5, x6, y6, z6 $, \mathrm{x} 7, \mathrm{y} 7, \mathrm{z} 7$

$\leftrightarrow$ params $=8.735,2.32364052662,1.67842014883,0.1826,-0.0318,0.1994$

列

$\longrightarrow\{2 \mathrm{~h}\}^{\wedge}\{20\} \# 66\left(\mathrm{bdkl}{ }^{\wedge} 2 \mathrm{~m}^{\wedge} 2\right) \&$ oC64 \& None \& ThI3 \& $\$ \backslash$ beta\$

$\hookrightarrow 3\} \$ \&$ H. P. Beck and C. Strobel, Angew. Chem. 94, 558-559 (1982

1.00000000000000

$4.36750000000000-10.14850000000000 \quad 0.00000000000000$

$\begin{array}{rrr}4.36750000000000 & 10.14850000000000 & 0.00000000000000\end{array}$

$\begin{array}{rrr}0.00000000000000 & 0.00000000000000 & 14.66100000000000\end{array}$

$\begin{array}{rr}\text { I } & \text { Th } \\ 24 & 8\end{array}$

Direct

0.00000000000000

0.50000000000000

0.00000000000000

0.50000000000000

$-0.23120000000000$

0.23120000000000

$-0.16760000000000$

0.16760000000000

$-0.16160000000000$

0.1616000000000

$-0.74040000000000$

0.74040000000000

0.16160000000000

0.74040000000000

$-0.74040000000000$

$-0.26400000000000$

0.26400000000000

$-0.49060000000000$

0.49060000000000

0.26400000000000

0.49060000000000
-0.49060000000000

$-0.49060000000000$

-0.50000000000000
0.5000000000000

0.5000000000000

0.50000000000000

0.50000000000000
0.15540000000000

0.15540000000000

$-0.15540000000000$

0.49860000000000

0.50000000000000 0.00000000000000 0.50000000000000 0.00000000000000 0.16760000000000 0.16760000000000 0.23120000000000 0.23120000000000 0.74040000000000 .74040000000000 0.16160000000000 $-0.16160000000000$ 0.74040000000000 0.74040000000000 $-0.16160000000000$ 0.16160000000000 0.49060000000000 $-0.49060000000000$ 0.26400000000000 0.26400000000000 $-0.49060000000000$ $-0.49060000000000$ 0.400000000 0.264000000000 0.500000000000 0.50000000000000 0.50000000000000 0.50000000000000 0.50000000000000 0.49860000000000 $-0.49860000000000$ $-0.15540000000000$ 0.15540000000000

0.18260000000000 0.31740000000000 $-0.18260000000000$ 0.68260000000000 0.00000000000000 0.00000000000000 0.50000000000000 0.50000000000000 0.13020000000000 0.13020000000000 0.36980000000000 0.36980000000000 0.36980000000000 $-0.13020000000000$ $-0.1302000000000$ 0.63020000000000 0.63020000000000 0.37080000000000 0.37080000000000 0.12920000000000 0.12920000000000 $-0.37080000000000$ $-0.37080000000000$ 0.8708000000000 0.87080000000000 0.25000000000000 0.75000000000000 0.00000000000000 0.50000000000000 0.00000000000000 0.00000000000000 0.50000000000000 0.50000000000000
I $\quad(8 \mathrm{k})$

I $\quad(8 \mathrm{k})$

I $\quad(8 \mathrm{k})$

$\begin{array}{ll}\text { I } & (8 \mathrm{k}) \\ \mathrm{I} & (81)\end{array}$

(81)

(81)

(81)

I $(16 \mathrm{~m})$

I $(16 \mathrm{~m})$

I (16m)

I $(16 \mathrm{~m})$

I $(16 \mathrm{~m})$

I (16m)

I $(16 \mathrm{~m})$

I $(16 \mathrm{~m})$

I $(16 \mathrm{~m})$

I (16m)

I $(16 \mathrm{~m})$

I $(16 \mathrm{~m})$

I $(16 \mathrm{~m})$

I $(16 \mathrm{~m})$

Th (4b)

Th (4b)

Th $(4 d)$

Th $(4 d)$

Th (81)

Th (81)

Th $(81)$
Al 2 CuIr: A2BC_oC16_67_ag_b_g - CIF

\# CIF file

data_findsym-outpu

audit_creation_method FINDSYM

chemical_name mineral 'Al2CuIr'

chemical_formula_sum ' $\mathrm{Al} 2 \mathrm{Cu} \mathrm{Ir}$

loop

publ_author_name

L. Meshi,

'V. Ezersky,

'D. Kapush',

'B. Grushko'

journal_name_full_name

Journal of Alloys and Compounds

journal_volume 496

journal_year 2010

journal_page_first 208

journal_page_last 211

-publ_Section_title

Crystal structure of the Al\$_ $\{2\} \$$ CuIr phase

Found in Pearson's Crystal Data - Crystal Structure Database for

$\hookrightarrow$ Inorganic Compounds, 2013

aflow title'Al\$_\{2\}\$CuIr Structure

-aflow_proto 'A2BC_oC16_67_ag_b_g

aflow_params ,a,b/a,c/a, z $\{3\}, z_{-}\{4\}$

aflow_params_values $, 5.0644067238,1.60320657112,1.02379259962,0.3305$. $\hookrightarrow 0.8198$

aflow_Strukturbericht 'None'

aflow_Pearson 'oC16'

cell_length_a 5.0644067238

cell_length_b 8.1192901384

_cell_length_c $\quad 5.1849021253$

_cell_angle_alpha 90.0000000000

cell_angle_beta 90.0000000000

cell_angle_gamma 90.0000000000

symmetry_space group_name $\mathrm{H}-\mathrm{M}$ "C $2 / \mathrm{m} 2 / \mathrm{m} 2 / \mathrm{a} "$

symmetry_Int Tables_number 67

loop_

space_group_symop_id

_space_group_symop_operation_xyz

$\overline{1} \mathrm{x}, \mathrm{y}, \mathrm{z}$ 


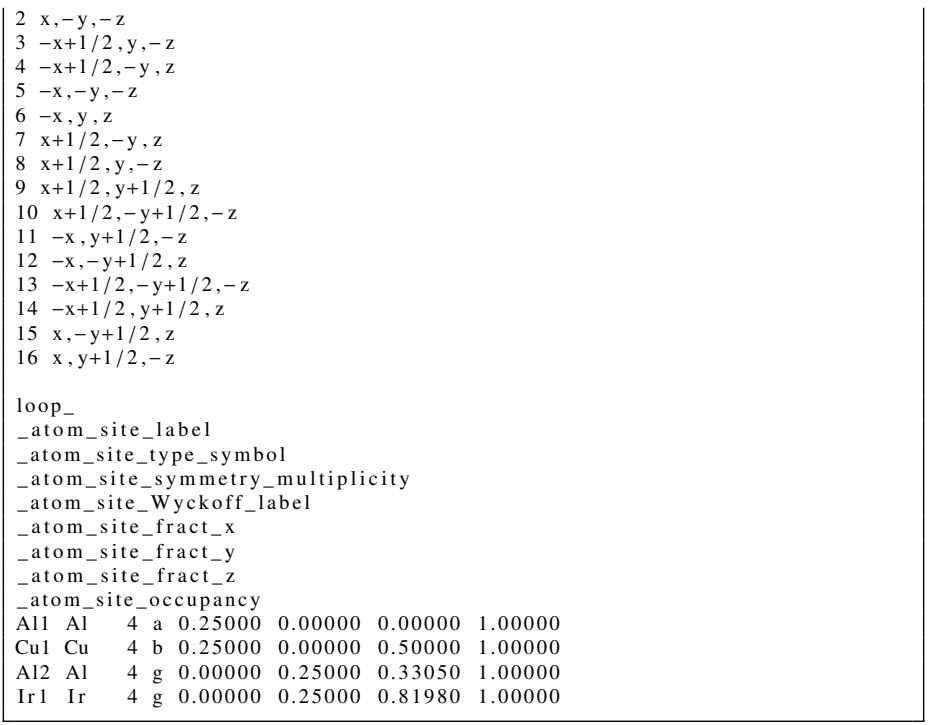

Al 2 CuIr: A2BC_oC16_67_ag_b_g - POSCAR

A2BC_oC16_67_ag_b_g \& a , b /a, c /a , z3 , z4 --params $=5.0644067238$, $\hookrightarrow 1.60320657112,1.02379259962,0.3305,0.8198 \&$ Cmma D_ $\{2 \mathrm{~h}\}^{\wedge}\{21\}$ \# $\hookrightarrow 67(\mathrm{abg} \wedge 2) \& \mathrm{oC} 16$ \& None \& Al2CuIr \& \& L. Meshi et al., J. $\hookrightarrow$ Alloys Compd. 496, 208-211 (2010) 1.00000000000000

$\begin{array}{rrr}2.53220336190000 & -4.05964506920000 & 0.00000000000000 \\ 2.53220336190000 & 4.05964506920000 & 0.00000000000000 \\ 0.00000000000000 & 0.00000000000000 & 5.18490212530000\end{array}$

$\mathrm{Al} \quad \mathrm{Cu} \quad \mathrm{Ir}$

Direct

0.25000000000000

0.75000000000000

0.75000000000000

0.25000000000000

0.25000000000000

0.75000000000000

0.75000000000000

0.25000000000000

0.25000000000000

0.75000000000000

0.25000000000000

0.75000000000000

0.25000000000000

0.75000000000000

$\begin{array}{rr}0.25000000000000 & 0.81980000000000 \\ 0.75000000000000 & -0.81980000000000\end{array}$

0.00000000000000

0.00000000000000

0.33050000000000

0.33050000000000

0.50000000000000000000

Al (4a)

$\begin{array}{ll}\mathrm{Al} & (4 \mathrm{a}) \\ \mathrm{Al} & (4 \mathrm{~g})\end{array}$

$\mathrm{Al} \quad(4 \mathrm{~g})$

$\mathrm{Cu}(4 \mathrm{~b})$

$\mathrm{Cu} \quad(4 \mathrm{~b})$

$\begin{array}{ll}\text { Ir } & (4 \mathrm{~g}) \\ \mathrm{Ir} & (4 \mathrm{~g})\end{array}$

HoCuP 2 : ABC2_oC16_67_b_g_ag - CIF

\section{\# CIF file}

data_findsym-output

_audit_creation_method FINDSYM

chemical_name_mineral 'HoCuP2'

chemical_formula_sum ' $\mathrm{Cu}$ Ho P2'

$\operatorname{loop}_{-}$

_publ_author_name

'Y. Mozharivsky'

D. Kaczorowski,

'H. F. Franzen'

journal_name_full_name

Zeitschrift fur Anorganische und Allgemeine Chemie

_journal_volume 627

journal_year 200

_journal_page_first 2163

_ournal_page_last 2172

publ_Section_title

Symmetry-Breaking Transitions in HoCuAs\$_ $\{2-\mathrm{x}\} \$ P \$_{-}\{\mathrm{x}\} \$$ and $\operatorname{ErCuAs} \$ \_\{2-\mathrm{x}$ $\rightarrow\} \$ P \$\{x\} \$(\$ x=0-2 \$)$ : Crystal Structure, Application of Landau

$\hookrightarrow$ Theory, Magnetic and Electrical Properties

\# Found in Pearson's Crystal Data - Crystal Structure Database for

$\hookrightarrow$ Inorganic Compounds, 2013

aflow_title 'HoCuP\$_\{2\}\$ Structure

aflow_proto 'ABC2_oC16_67_b_g_ag'

_aflow_params ,a,b/a,c/a, z- $\{3\}, z_{-}\{4\}$,

aflow_params_values ' $5.2729860526,1.00606865161,1.82912952778,0.765$, $\hookrightarrow 0.3403$

aflow_Strukturbericht 'None'

_aflow_Pearson 'oC16'

\section{_cell_length_a $\quad 5.2729860526$}

_cell_length_b $\quad 5.3049859679$

_cell_length_c 9.6449744884

_cell_angle_alpha 90.0000000000

symmetry_space_group_name_H-M "C $2 / \mathrm{m} 2 / \mathrm{m} 2 / \mathrm{a}$ "

_symmetry_Int_Tables_number 67

loop

_space_group_symop_id

_space_group_symop_operation_xyz

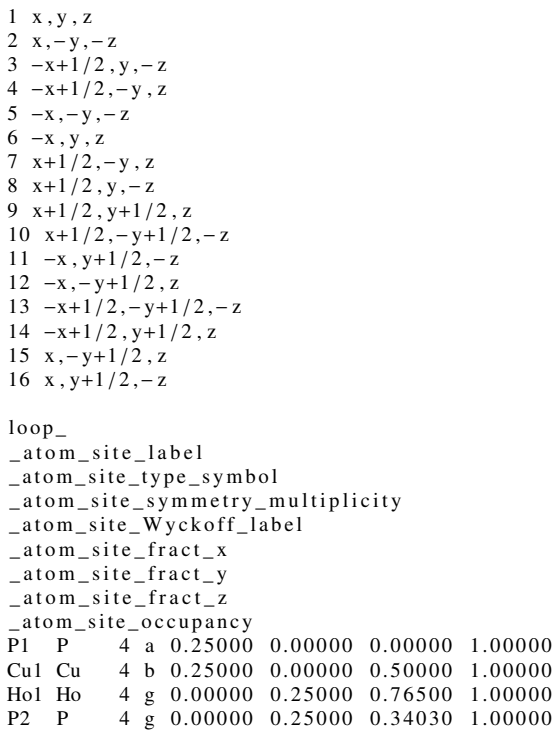

$\mathrm{HoCuP}_{2}$ : ABC2_oC16_67_b_g_ag - POSCAR

\begin{tabular}{|c|c|c|c|c|}
\hline \\
\hline \multicolumn{5}{|c|}{$\begin{aligned} \text { ABC2_oC16_67_b_g_ag \& a }, \mathrm{b} / \mathrm{a}, \mathrm{c} / \mathrm{a}, \mathrm{z3}, \mathrm{z} 4--\mathrm{params}=5.2729860526, & \\
& \hookrightarrow 1.00606865161,1.82912952778,0.765,0.3403 \& \text { Cmma D_ }\{2 \mathrm{~h}\}^{\wedge}\{21\} \# 67 \\
& \hookrightarrow(\mathrm{abg} \wedge 2) \& \text { oC16 \& None \& HoCuP2 \& \& Y. Mozharivsky and D. } \\
& \hookrightarrow \text { Kaczorowski and H. F. Franzen, Z. Anorg. Allg. Chem. 627, } \\
& \hookrightarrow 2163-2172(2001)\end{aligned}$} \\
\hline \multicolumn{5}{|c|}{1.00000000000000} \\
\hline 2.63649302630000 & -2.65249298395000 & 0.00000000000000 & & \\
\hline \multirow{2}{*}{$\begin{array}{l}2.63649302630000 \\
0.00000000000000\end{array}$} & 2.65249298395000 & 0.00000000000000 & & \\
\hline & 0.00000000000000 & 9.64497448840000 & & \\
\hline Ho & & & & \\
\hline 2 & & & & \\
\hline \multicolumn{5}{|l|}{ Direct } \\
\hline 0.25000000000000 & 0.25000000000000 & 0.50000000000000 & $\mathrm{Cu}$ & (4b) \\
\hline 0.75000000000000 & 0.75000000000000 & 0.50000000000000 & $\mathrm{Cu}$ & (4b) \\
\hline 0.75000000000000 & 0.25000000000000 & 0.76500000000000 & Но & $(4 \mathrm{~g})$ \\
\hline 0.25000000000000 & 0.75000000000000 & -0.76500000000000 & Но & $(4 \mathrm{~g})$ \\
\hline 0.25000000000000 & 0.25000000000000 & 0.00000000000000 & $\mathrm{P}$ & (4a) \\
\hline 0.75000000000000 & 0.75000000000000 & 0.00000000000000 & $\mathrm{P}$ & (4a) \\
\hline 0.75000000000000 & 0.25000000000000 & 0.34030000000000 & $\mathrm{P}$ & $(4 \mathrm{~g})$ \\
\hline 0.25000000000000 & 0.75000000000000 & -0.34030000000000 & $\mathrm{P}$ & $(4 \mathrm{~g})$ \\
\hline
\end{tabular}

$\alpha$-FeSe: AB_oC8_67_a_g - CIF

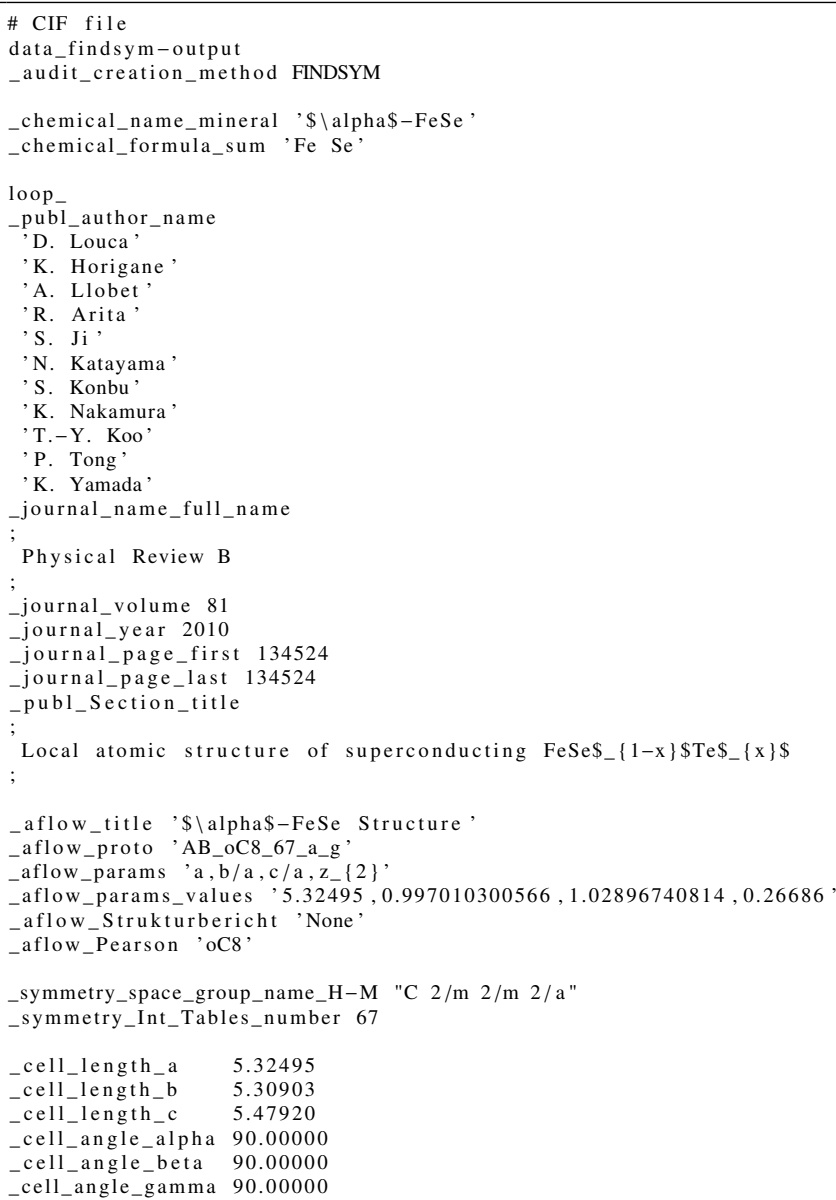


oop

_space_group_symop_id

_space_group_symop_operation_xyz

$1 \mathrm{x}, \mathrm{y}, \mathrm{z}$

$2 \mathrm{x},-\mathrm{y},-\mathrm{z}$

$3-x+1 / 2, y,-z$

$4-x+1 / 2,-y, z$

$5-\mathrm{x},-\mathrm{y},-\mathrm{z}$

$6-\mathrm{x}, \mathrm{y}, \mathrm{z}$

$7 \mathrm{x}+1 / 2,-\mathrm{y}, \mathrm{z}$

$\begin{array}{ll}8 & x+1 / 2, y,-z \\ 9 & x+1 / 2, y+1 / 2\end{array}$

$10 \mathrm{x}+1 / 2,-\mathrm{y}+1 / 2,-\mathrm{z}$

$11-x, y+1 / 2,-z$

$11-\mathrm{x}, \mathrm{y}+1 / 2,-\mathrm{z}$

$13-x+1 / 2,-y+1 / 2,-z$

$14-x+1 / 2, y+1 / 2, z$

$15 \mathrm{x},-\mathrm{y}+1 / 2, \mathrm{z}$

$16 \mathrm{x}, \mathrm{y}+1 / 2,-\mathrm{z}$

loop_

_atom_site_label

atom_site_type_symbol

atom_site_symmetry_multiplicity

_atom_site_Wyckoff_label

_atom_site_fract_x

_atom_site_fract_y

_atom_site_fract_z

Fe1 Fe 4 accupancy

$\begin{array}{lllllllll}\mathrm{Se} 1 \mathrm{Se} & 4 & \mathrm{~g} & 0.00000 & 0.25000 & 0.26686 & 1.00000\end{array}$

$\alpha$-FeSe: AB_oC8_67_a_g - POSCAR

AB_oC8_67_a_g \& a,b/a,c/a, z2 --params $=5.32495,0.997010300566$

$\hookrightarrow 1.02896740814,0.26686 \&$ Cmma D_ $\{2 \mathrm{~h}\}^{\wedge}\{21\} \# 67$ (ag) \& oC8 \& None

$\hookrightarrow \&$ FeSe \& $\$ \backslash$ alpha\$-FeSe \& D. Louca et al., Phys. Rev. B 81

$\hookrightarrow 134524(2010)$

0000000

$2.66247500000000 \quad-2.65451500000000 \quad 0.00000000000000$

$\begin{array}{rrr}2.66247500000000 & 2.65451500000000 & 0.00000000000000\end{array}$

$\begin{array}{lll}0.00000000000000 & 0.00000000000000 & 5.47920000000000\end{array}$

$\mathrm{Fe} \quad \mathrm{Se}$

Direct

0.25000000000000

0.75000000000000

0.75000000000000

0.25000000000000

0.75000000000000

0.00000000000000

0.26686000000000

$0.75000000000000-0.26686000000000$

$\begin{array}{ll}\mathrm{Fe} & (4 \mathrm{a}) \\ \mathrm{Fe} & (4 \mathrm{a})\end{array}$

Se $\quad(4 \mathrm{~g})$

( $4 \mathrm{~g})$

$\alpha$-PbO: AB_oC8_67_a_g - CIF

\# CIF file

data_findsym-output

_audit_creation_method FINDSYM

_chemical_name_mineral 'alpha-PbO'

chemical_formula_sum 'O $\mathrm{Pb}$ '

loop

publ_author_name

P. Boher,

'P. Garnier'

J. R. Gavarri

'A. W. Hewat'

journal_name_full_name

Journal of Solid State Chemistry

_journal_volume 57

journal_year 198

_journal_page_first 343

_journal_page_last 350

publ_Section_title

Monoxyde quadratique $\mathrm{PbO} \backslash \backslash$ alpha $\$$ (I): Description de la transition $\rightarrow$ structurale ferro $\left\{\backslash{ }^{\prime}\right.$ e $\}$ lastique

\# Found in Pearson's Crystal Data - Crystal Structure Database for $\hookrightarrow$ Inorganic Compounds, 2013

aflow title '\$ $\$$ alpha $\$-P b O$ Structure

_aflow_proto 'AB_oC8_67_a_g'

aflow params 'a,b/a,c/a,z $\{2\}$ '

-aflow_params_values 5.6124272786,0.999376380873,0.8895303257,0.7642,

-aflow_Strukturbericht 'None'

_aflow_Pearson oC8,

_cell_length_a $\quad 5.6124272786$

_cell_length_b 5.6089272616

_cell_length_c $\quad 4.9924242651$

_cell_angle_alpha 90.0000000000

cell angle beta 90.0000000000

cell angle gamma 90.0000000000

symmetry space group name $\mathrm{H}-\mathrm{M}$ "C $2 / \mathrm{m} 2 / \mathrm{m} \mathrm{2} 2 / \mathrm{a}$ "

symmetry_Int Tables_number 67

loop_

_space_group_symop_id

-space_group_symop_operation_xyz

$1 \mathrm{x}, \mathrm{y}, \mathrm{z}$

$2 \mathrm{x},-\mathrm{y},-\mathrm{z}$

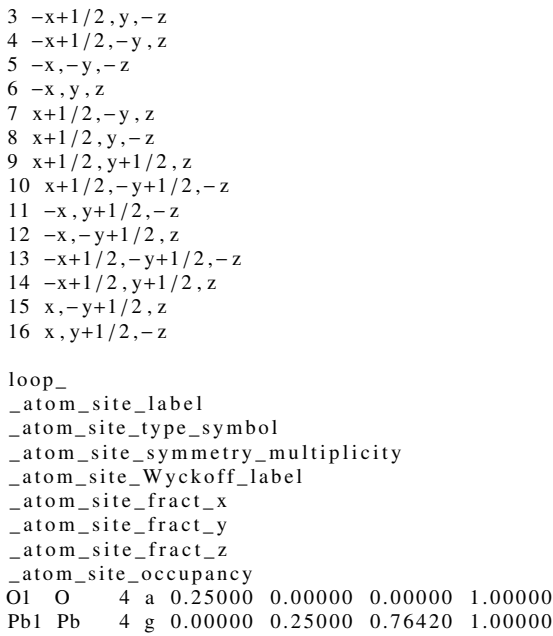

$\alpha$-PbO: AB_oC8_67_a_g - POSCAR

AB_oC8_67_a_g \& a,b/a,c/a, z2 --params $=5.6124272786,0.999376380873$, $\hookrightarrow 0.8895303257,0.7642$ \& Cmma D_ $\{2 \mathrm{~h}\}^{\wedge}\{21\}$ \#67 (ag) \& oC8 \& None \& $\hookrightarrow$ PbO \& alpha \& P. Boher et al., J. Solid State Chem. 57, 343-350 $\hookrightarrow \quad(1985)$

1.00000000000000

$\begin{array}{lll}2.80621363930000 & -2.80446363080000 & 0.00000000000000\end{array}$

$\begin{array}{lll}2.80621363930000 & 2.80446363080000 & 0.00000000000000\end{array}$

$0.00000000000000 \quad 0.00000000000000 \quad 4.99242426510000$

$\begin{array}{rr}\mathrm{O} & \mathrm{Pb} \\ 2 & 2\end{array}$

Direct

0.25000000000000

0.75000000000000

0.75000000000000

0.25000000000000

$0.25000000000000-0.00000000000000$ $0.75000000000000 \quad 0.00000000000000$ $0.25000000000000 \quad 0.76420000000000$ $0.75000000000000-0.76420000000000$

$\mathrm{PdSn}_{4}$ : AB4_oC20_68_a_i - CIF

\# CIF file\# This file was generated by FINDSYM

\# Harold T. Stokes, Branton J. Campbell, Dorian M. Hatch

\# Brigham Young University, Provo, Utah, USA

data_findsym-output

_audit_creation_method FINDSYM

chemical_name_mineral 'PdSn4

_chemical_formula_sum 'Pd Sn4

loop

publ_author_nam

, J. Nyl \{II'e\}n'

F. J. $\{\operatorname{Garc}\{\backslash \backslash, i\}$ a $\operatorname{Garc}\{\backslash \backslash, i\} a\}$ '

'B. D. Mosel'

R. P $\{\backslash$ "o\}ttgen

U. $\mathrm{H}\{\backslash$ " a ussermann

journal_name_full_name

Solid State Sciences

journal_volume 6

journal_year 2004

journal_page_first 147

journal_page_last 155

publ_Section_title

Structural relationships, phase stability and bonding of compound $\hookrightarrow \operatorname{PdSn} \$\{\mathrm{n}\} \$(\$ \mathrm{n} \$=2,3,4)$

\# Found in Pearson's Crystal Data - Crystal Structure Database for $\hookrightarrow$ Inorganic Compounds, 2013

aflow title 'PdSn\$_\{4\}\$ Structure

aflow proto 'AB4_oC20 68 a i

aflow params 'a,b/a,c/a, $x_{-}\{2\}, y\{2\}, z_{-}\{2\}$

aflow_params_values '6.44222, $1.77662048176,0.991771470083,0.3313,0.1233$

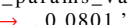

aflow_Strukturbericht 'None'

_aflow_Pearson, oC20

symmetry_space_group_name_H-M "C 2/c 2/c 2/a (origin choice 2)" symmetry_Int_Tables_number 68

cell_length_a 6.44222

cell_length_b $\quad 11.44538$

cell_length_c 6.38921

cell_angle alpha 90.00000

cell angle beta 90.00000

cell_angle_gamma

loop

space_group_symop_i

space_group_symop_operation_xy

$\mathrm{x}, \mathrm{y}, \mathrm{z}$

$2 \mathrm{x},-\mathrm{y}+1 / 2,-\mathrm{z}+1 / 2$

$3-x, y,-z+1 / 2$ 
$4-\mathrm{x},-\mathrm{y}+1 / 2, \mathrm{z}$

$5-x+1 / 2,-y+1 / 2,-$

$6-\mathrm{x}+1 / 2, \mathrm{y}, \mathrm{z}+1 / 2$

$7 \mathrm{x}+1 / 2,-\mathrm{y}+1 / 2, \mathrm{z}+1 / 2$

$8 \mathrm{x}+1 / 2, \mathrm{y},-\mathrm{z}$

$9 \mathrm{x}+1 / 2, \mathrm{y}+1 / 2, \mathrm{z}$

$10 \mathrm{x}+1 / 2,-\mathrm{y},-\mathrm{z}+1 / 2$

$11-\mathrm{x}+1 / 2, \mathrm{y}+1 / 2,-\mathrm{z}+1 / 2$

$12-\mathrm{x}+1 / 2,-\mathrm{y}, \mathrm{z}$

$14-\mathrm{x}, \mathrm{y}+1 / 2, \mathrm{z}+1 / 2$

$15 x,-y, z+1 / 2$

$16 \mathrm{x}, \mathrm{y}+1 / 2,-\mathrm{z}$

loop

atom site label

_atom_site_type_symbol

_atom_site_symmetry_multiplicity

_atom_site_Wyckoff_label

_atom_site_fract_x

-atom_site_fract_y
-atom_site_fract_z

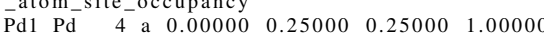

$\begin{array}{llllllll}\text { Sn } 1 & \text { Sn } & 16 & \text { i } & 0.33130 & 0.12330 & 0.08010 & 1.00000\end{array}$

PdSn 4 : AB4_oC20_68_a_i - POSCAR

AB4_oC20_68_a_i \& a , b/a , c /a, x2,y2, z2 --params $=6.44222,1.77662048176$ $\hookrightarrow 0.991771470083,0.3313,0.1233,0.0801 \&$ Ccca D_ $\{2 \mathrm{~h}\}^{\wedge}\{22\} \# 68$ (ai) $\hookrightarrow$ \& oC20 \& None \& PdSn4 \& \& J. Nyl\{ $\{$ 'e $\}$ n et al., Solid State $\hookrightarrow \mathrm{Sci}$. 6, 147-155 (2004)

1.00000000000000

$3.22111000000000 \quad-5.72269000000000 \quad 0.00000000000000$

$\begin{array}{lll}3.22111000000000 & 5.72269000000000 & 0.00000000000000\end{array}$

$\begin{array}{lll}0.00000000000000 & 0.00000000000000 & 6.38921000000000\end{array}$

$$
\begin{array}{rr}
\mathrm{Pd} & \mathrm{Sn} \\
2 & 8
\end{array}
$$

Direct

0.25000000000000

0.20800000000000

0.29200000000000

$-0.45460000000000$

0.95460000000000

$-0.20800000000000$

0.70800000000000

0.45460000000000

0.04540000000000

0.25000000000000 0.75000000000000 0.45460000000000 0.04540000000000 $-0.20800000000000$ 0.70800000000000 $-0.45460000000000$ 0.95460000000000 0.2080000000000 0.29200000000000

0.25000000000000 0.75000000000000 0.08010000000000 0.08010000000000 0.41990000000000 .41990000000000 $-0.08010000000000$ $-0.08010000000000$ 0.58010000000000 0.58010000000000

$\begin{array}{ll}\mathrm{Pd} & (4 \mathrm{a}) \\ \mathrm{Pd} & (4 \mathrm{a}) \\ \mathrm{Sn} & (16 \mathrm{i}) \\ \mathrm{Sn} & (16 \mathrm{i}) \\ \mathrm{Sn} & (16 \mathrm{i}) \\ \mathrm{Sn} & (16 \mathrm{i}) \\ \mathrm{Sn} & (16 \mathrm{i}) \\ \mathrm{Sn} & (16 \mathrm{i}) \\ \mathrm{Sn} & (16 \mathrm{i}) \\ \mathrm{Sn} & (16 \mathrm{i})\end{array}$

$\mathrm{Mn}_{2} \mathrm{~B}\left(D 1_{f}\right)$ : AB2_oF48_70_f_fg - CIF

\# CIF file

data_findsym-output

audit_creation_method FINDSYM

chemical_name_mineral 'Mn2B'

chemical_formula_sum 'B Mn2'

loop

publ_author_name

'L.-E. Tergenius

_journal_name_full_name

Journal of the Less-Common Metals

journal_volume 82

journal year 1981

journal_page_first 335

_ournal_page_last 340

-publ_Section_title

Refinement of the crystal structure of orthorhombic Mn\$_\{2\}\$B (formerly

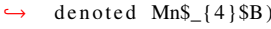

aflow_title 'Mn\$_ $\{2\} \$ B\left(\$ D 1 \_\{f\} \$\right)$ Structure

aflow_proto 'AB2_oF48_70_f_fg'

aflow_params, a,b/a,c/a, y $\{1\}, y,\{2\}, z \_\{3\}$,

aflow_params_values ' $4.2082,3.45504015969,1.7326647973,0.2495,0.54337$, $\mathrm{w}_{-} \mathrm{params} \mathrm{s}_{-} \mathrm{va}$
$\hookrightarrow 0.29445$

aflow_Strukturbericht '\$D1_\{f\}\$

aflow Pearson, oF48,

symmetry_space_group_name_H-M "F 2/d 2/d 2/d (origin choice 2)"

_symmetry_Int_Tables_number 70

cell_length_a $\quad 4.20820$

cell_length_b $\quad 14.53950$

cell_length_c $\quad 7.29140$

cell_angle_alpha 90.00000

cell_angle_beta 90.00000

cell_angle_gamma 90.00000

loop_

_space_group_symop_id

_space_group_symop_operation_xyz

$1 \mathrm{x}, \mathrm{y}, \mathrm{z}$

$2 \mathrm{x},-\mathrm{y}+3 / 4,-\mathrm{z}+3 / 4$

$3-\mathrm{x}+3 / 4, \mathrm{y},-\mathrm{z}+3 / 4$

$4-x+3 / 4,-y+3 / 4, z$

$5-\mathrm{x},-\mathrm{y},-\mathrm{z}$

$6-\mathrm{x}, \mathrm{y}+1 / 4, \mathrm{z}+1 / 4$

$\begin{array}{ll}7 & x+1 / 4,-y, z+1 / 4 \\ 8 & x+1 / 4, y+1 / 4,-z\end{array}$
$9 \mathrm{x}, \mathrm{y}+1 / 2, \mathrm{z}+1 / 2$

$0 \mathrm{x},-\mathrm{y}+1 / 4,-\mathrm{z}+1 / 4$

$11-x+3 / 4, y+1 / 2,-z+1 / 4$

$12-x+3 / 4,-y+1 / 4, z+1 / 2$

$13-\mathrm{x},-\mathrm{y}+1 / 2,-\mathrm{z}+1 / 2$

$14-x, y+3 / 4, z+3 / 4$

$15 \mathrm{x}+1 / 4,-\mathrm{y}+1 / 2, \mathrm{z}+3 / 4$

$16 \mathrm{x}+1 / 4, \mathrm{y}+3 / 4,-\mathrm{z}+1 / 2$

$17 \mathrm{x}+1 / 2, \mathrm{y}, \mathrm{z}+1 / 2$

$18 \mathrm{x}+1 / 2,-\mathrm{y}+3 / 4,-\mathrm{z}+1 / 4$

$19-\mathrm{x}+1 / 4, \mathrm{y},-\mathrm{z}+1 / 4$

$20-x+1 / 4,-y+3 / 4, z+1 / 2$

$21-x+1 / 2,-y,-z+1 / 2$

$22-x+1 / 2, y+1 / 4, z+3 / 4$

$23 \mathrm{x}+3 / 4,-\mathrm{y}, \mathrm{z}+3 / 4$

$25 x+3 / 4, y+1 / 4,-z+1$

$25 \mathrm{x}+1 / 2, \mathrm{y}+1 / 2, \mathrm{z}$

$26 x+1 / 2,-y+1 / 4,-z+3 / 4$

$27-\mathrm{x}+1 / 4, \mathrm{y}+1 / 2,-\mathrm{z}+3 / 4$

$28-x+1 / 4,-y+1 / 4, z$

$29-\mathrm{x}+1 / 2,-\mathrm{y}+1 / 2,-\mathrm{z}$

$30-x+1 / 2, y+3 / 4, z+1 / 4$

$31 \mathrm{x}+3 / 4,-\mathrm{y}+1 / 2, \mathrm{z}+1 / 4$

$32 \mathrm{x}+3 / 4, \mathrm{y}+3 / 4,-\mathrm{z}$

loop

atom_site_label

atom_site_type_symbol

atom_site_symmetry_multiplicity

atom_site_Wyckoff_label

atom_site_fract_x

atom_site_fract_y

atom_site_fract_z

atom_site_occupancy

$\begin{array}{lll}0.24950 & 0.12500 & 1.00000\end{array}$

$\begin{array}{llllllll}\mathrm{Mn} 1 \mathrm{Mn} & 16 & \mathrm{f} & 0.12500 & 0.54337 & 0.12500 & 1.00000\end{array}$

$\begin{array}{lllllll}\mathrm{Mn} 2 \mathrm{Mn} & 16 & \mathrm{~g} & 0.12500 & 0.12500 & 0.29445 & 1.00000\end{array}$

$\mathrm{Mn}_{2} \mathrm{~B}\left(D 1_{f}\right): \mathrm{AB} 2 \_\mathrm{oF} 48 \_70 \_f \_f g-$ POSCAR

AB2_oF48_70_f_fg \& a,b/a,c/a,y1,y2,z3 --params $=4.2082,3.45504015969$, $\hookrightarrow 1.7326647973,0.2495,0.54337,0.29445 \&$ Fddd $D,\{2 \mathrm{~h}\} \wedge\{24\} \# 70(\mathrm{f} \wedge$ $\hookrightarrow 2 \mathrm{~g}) \&$ oF48 \& \$D1_\{f\}\$ \& Mn2B \& Mn2B \& L.-E. Tergenius, J.

$\leftrightarrow$ Less-Common Met. 82, 335-340 (1981)

1.00000000000000

$\begin{array}{lll}0.00000000000000 & 7.26975000000000 & 3.64570000000000\end{array}$

$\begin{array}{lll}2.10410000000000 & 0.00000000000000 & 3.64570000000000\end{array}$

$\begin{array}{lll}2.10410000000000 & 7.26975000000000 & 0.00000000000000\end{array}$

$\begin{array}{cc}\text { B } & \text { Mn } \\ 4 & 8\end{array}$

Direct

0.24950000000000

0.00050000000000

$-0.24950000000000$

0.99950000000000

0.54337000000000

$-0.2933700000000$

$-0.2933700000000$

$-0.54337000000000$

0.29445000000000

$-0.04445000000000$

$-0.2944500000000$

1.04445000000000

$0.00050000000000 \quad 0.24950000000000$ 0.24950000000000 0.99950000000000 $-0.24950000000000$ 0.29337000000000 0.5433700000000

1.29337000000000 $-0.54337000000000$ 0.537000000 $-0.04445000000000$ $-0.29445000000000$ 1.04445000000000 $-0.24950000000000$ 0.99950000000000

0.54337000000000 $-0.29337000000000$ $-0.54337000000000$ 1.29337000000000 1.2933700000000 0.04445000000000 0.29445000000000 1.04445000000000 $-0.29445000000000$

$\mathrm{Ta}_{3} \mathrm{~B}_{4}\left(D 7_{b}\right)$ : A4B3_oI14_71_gh_cg - CIF

\section{\# CIF file}

data findsym-output

audit_creation_method FINDSYM

chemical_name_mineral 'Ta3B4,

chemical_formula_sum 'B4 Ta3

loop

publ_author_nam

R. Kiessling'

journal name full_name

Acta Chemica Scandinavica

journal volume 3

journal year 1949

journal_page_first 603

journal page last 615

publ_Section_title

The Borides of Tantalum

\# Found in Transition-metal borides with the tantalum boride ( $\mathrm{Ta} \$_{-}\{3\}$ $\left.\left.\rightarrow \$ B \$ \$_{-} 4\right\} \$\right)$ crystal structure: their electronic and bonding

$\hookrightarrow$ properties, 1991

aflow_title $T a \$_{-}\{3\} \$ B \$_{-}\{4\} \$\left(\$ D 7 \_\{b\} \$\right)$ Structure,

-aflow_proto 'A4B3_oI14_71_gh_cg'

aflow params , a, b/a,c/a,y $\{2\}, y\{3\}, y-\{4\}$

aflow_params_values $3.29,4.25531914894,0.951367781155,0.375,0.18,0.444$

aflow Strukturbericht, \$D7_\{b\}\$

aflow Pearson, oI14

symmetry_space_group_name_H-M "I $2 / \mathrm{m} 2 / \mathrm{m} 2 / \mathrm{m}^{\prime \prime}$

symmetry_Int_Tables_number 7

_cell_length_a $\quad 3.29000$ 
_cell_length_b $\quad 14.00000$ _cell_length_c $\quad 3.13000$ _cell_angle_alpha 90.00000 _cell_angle_beta 90.00000 _cell_angle_gamma 90.00000

loop

_space_group_symop_id

_space_group_symop_operation_xyz

$1 \mathrm{x}, \mathrm{y}, \mathrm{z}$

$2 \mathrm{x},-\mathrm{y},-\mathrm{z}$

$3-\mathrm{x}, \mathrm{y},-\mathrm{z}$

$4-\mathrm{x},-\mathrm{y}, \mathrm{z}$

$5-\mathrm{x},-\mathrm{y},-$

$6-\mathrm{x}, \mathrm{y}, \mathrm{z}$

$7 \mathrm{x},-\mathrm{y}, \mathrm{z}$
$8 \mathrm{x}, \mathrm{y},-\mathrm{z}$

$9 \mathrm{x}+1 / 2, \mathrm{y}+1 / 2, \mathrm{z}+1 / 2$

$10 \mathrm{x}+1 / 2,-\mathrm{y}+1 / 2,-\mathrm{z}+1 / 2$

$11-x+1 / 2, y+1 / 2,-z+1 / 2$

$12-\mathrm{x}+1 / 2,-\mathrm{y}+1 / 2, \mathrm{z}+1 / 2$
$13-\mathrm{x}+1 / 2,-\mathrm{y}+1 / 2,-\mathrm{z}+1 / 2$

$14-\mathrm{x}+1 / 2, \mathrm{y}+1 / 2, \mathrm{z}+1 / 2$

$15 \mathrm{x}+1 / 2,-\mathrm{y}+1 / 2, \mathrm{z}+1 / 2$

$16 \mathrm{x}+1 / 2, \mathrm{y}+1 / 2,-\mathrm{z}+1 / 2$

loop_

_atom_site_label

_atom_site_type_symbol

atom_site_symmetry_multiplicity

_atom_site_Wyckoff_label

atom_site_fract_x

atom_site_fract_y

atom_site_fract_z

atom site occupancy

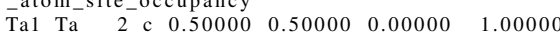

$\begin{array}{lllllllll}\text { B1 } & \text { B } & 4 & \text { g } & 0.00000 & 0.37500 & 0.00000 & 1.00000\end{array}$

$\begin{array}{llllllll}\mathrm{Ta} 2 & \mathrm{Ta} & 4 & \mathrm{~g} & 0.00000 & 0.18000 & 0.00000 & 1.00000\end{array}$

$\begin{array}{lllllllll}\mathrm{B} 2 & \mathrm{~B} & 4 & \mathrm{~h} & 0.00000 & 0.44400 & 0.50000 & 1.00000\end{array}$

$\mathrm{Ta}_{3} \mathrm{~B}_{4}\left(D 7_{b}\right)$ : A4B3_oI14_71_gh_cg - POSCAR

A4B3_oI14_71_gh_cg \& a,b/a,c/a,y2,y3,y4 --params $=3.29,4.25531914894$ $\hookrightarrow 0.951367781155,0.375,0.18,0.444 \&$ Immm D_ $\{2 \mathrm{~h}\}^{\wedge}\{25\} \# 71 \quad(\mathrm{cg} \wedge 2 \mathrm{~h})$ $\hookrightarrow$ \& oI14 \& \$D7_\{b\}\$\& Ta3B4 \& Ta3B4 \& R. Kiessling, Acta Chem. $\hookrightarrow$ Scand. 3, 603-615 (1949)

1.00000000000000

$\begin{array}{rrr}1.64500000000000 & 7.00000000000000 & 1.56500000000000\end{array}$

$\begin{array}{lll}1.64500000000000 & -7.00000000000000 & 1.56500000000000\end{array}$

$\begin{array}{rrr}1.64500000000000 & 7.00000000000000 & -1.56500000000000\end{array}$

B $\quad$ Ta

Direct

0.37500000000000

$-0.37500000000000$

0.94400000000000

0.05600000000000

0.50000000000000

0.18000000000000

$-0.18000000000000$

$\begin{array}{lrrr}0.00000000000000 & 0.37500000000000 & \mathrm{~B} & (4 \mathrm{~g}) \\ 0.00000000000000 & -0.37500000000000 & \mathrm{~B} & (4 \mathrm{~g}) \\ 0.50000000000000 & 0.44400000000000 & \mathrm{~B} & (4 \mathrm{~h}) \\ 0.50000000000000 & -0.44400000000000 & \mathrm{~B} & (4 \mathrm{~h}) \\ 0.50000000000000 & 0.00000000000000 & \mathrm{Ta} & (2 \mathrm{c}) \\ 0.00000000000000 & 0.18000000000000 & \mathrm{Ta} & (4 \mathrm{~g}) \\ 0.00000000000000 & -0.18000000000000 & \mathrm{Ta} & (4 \mathrm{~g})\end{array}$

NbPS: ABC_oI12_71_h_j_g - CIF

\section{\# CIF file}

data_findsym-output

-audit_creation_method FINDSYM

chemical name mineral,

_chemical_formula_sum 'Nb P S,

loop

_publ_author_name

'P. C. Donohue,
'P. E. Bierstedt

_journal_name_full_name

Inorganic Chemistry

journal_volume 8

journal year 1969

journal_page_first 2690

publ_Section_title

Synthesis, crystal structure, and superconducting properties of niobium

$\hookrightarrow$ phosphorus sulfide, niobium phosphorus selenide and tantalum

$\hookrightarrow$ phosphorus sulfide

aflow_title 'NbPS Structure'

aflow_proto 'ABC_oI12_71_h_j_g,

aflow_params 'a,b/a,c/a, y_ $\{1\}, y_{-}\{2\}, z_{-}\{3\}$,

aflow_params_values ' $3.438,3.4554973822,1.37434554974,0.212,0.1232$ $\hookrightarrow 0.235$,

aflow_Strukturbericht 'None'

aflow_Pearson 'oI12,

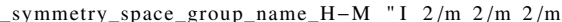

_symmetry_Int_Tables_number 71

_cell_length_a $\quad 3.43800$

cell-length b 11.88000

$\begin{array}{ll}\text { cell_length_c } & 4.72500\end{array}$

cell_angle_alpha 90.00000

_cell_angle_beta 90.00000 _cell_angle_gamma 90.00000

loop

space_group_symop_i

_space_group_symop_operation_xyz

$1 \mathrm{x}, \mathrm{y}, \mathrm{z}$

$2 \mathrm{x},-\mathrm{y},-\mathrm{z}$

$3-x, y,-z$

$4-x,-y, z$
$5-x,-y,-z$

$6-\mathrm{x}, \mathrm{y}, \mathrm{z}$

$7 \mathrm{x},-\mathrm{y}, \mathrm{z}$

$8 \mathrm{x}, \mathrm{y},-\mathrm{z}$

$\mathrm{x}+1 / 2, \mathrm{y}+1 / 2, \mathrm{z}+1 / 2$

$\mathrm{x}+1 / 2, \mathrm{y}+1 / 2, \mathrm{z}+1 / 2$

$10 \mathrm{x}+1 / 2,-\mathrm{y}+1 / 2,-\mathrm{z}+1 / 2$

$11-x+1 / 2, y+1 / 2,-z+1 / 2$

$12-\mathrm{x}+1 / 2,-\mathrm{y}+1 / 2, \mathrm{z}+1 / 2$

$13-x+1 / 2,-y+1 / 2,-z+1 / 2$

$14-\mathrm{x}+1 / 2, \mathrm{y}+1 / 2, \mathrm{z}+1 / 2$

$15 \mathrm{x}+1 / 2,-\mathrm{y}+1 / 2, \mathrm{z}+1 / 2$

$16 \mathrm{x}+1 / 2, \mathrm{y}+1 / 2,-\mathrm{z}+1 / 2$

loop

atom_site_label

atom_site_type_symbol

atom_site_symmetry_multiplicity

_atom_site_Wyckoff_label

-atom_site_fract_x

atom_site_fract_y

atom_site_fract_z

atom site occupancy

$\begin{array}{llllllll}\text { S1 } & \mathrm{S} & 4 & \mathrm{~g} & 0.00000 & 0.21200 & 0.00000 & 1.00000\end{array}$

$\begin{array}{llllllll}\mathrm{Nb} 1 \mathrm{Nb} & 4 & \mathrm{~h} & 0.00000 & 0.12320 & 0.50000 & 1.00000\end{array}$

$\begin{array}{lllllllll}\mathrm{P} 1 & \mathrm{P} & 4 & \mathrm{j} & 0.50000 & 0.00000 & 0.23500 & 1.00000\end{array}$

NbPS: ABC_oI12_71_h_j_g - POSCAR

ABC_oI12 71 h j g \& a , b/a,c/a,y1,y2,z3 --params $=3.438,3.4554973822$ $\hookrightarrow 1.37434554974,0.212,0.1232,0.235 \&$ Immm D_ $\{2 \mathrm{~h}\}^{\wedge}\{25\} \# 71(\mathrm{ghj}) \&$ $\hookrightarrow \quad$ oI12 \& None \& NbPS \& \& P. C. Donohue and P. E. Bierstedt,

$\hookrightarrow$ Inorg. Chem. 8, 2690-2694 (1969)

1.00000000000000

$-1.71900000000000 \quad 5.94000000000000 \quad 2.36250000000000$

$\begin{array}{rrr}1.71900000000000 & -5.94000000000000 & 2.36250000000000\end{array}$ $\begin{array}{rrr}1.71900000000000 & 5.94000000000000 & -2.36250000000000\end{array}$

$\mathrm{Nb} \quad \mathrm{P} \quad \mathrm{S}$

Direct

0.62320000000000

0.37680000000000

0.23500000000000

$-0.23500000000000$

0.21200000000000

0000000000 $-0.12320000000000$ 0.50000000000000

0.21200000000000

0.21200000000000

$\mathrm{KAg}\left[\mathrm{CO}_{3}\right]$ : $\mathrm{ABCD} 3$ _oI48_73_d_e_e_ef - CIF

\# CIF file

data_findsym-output

audit_creation_method FINDSYM

chemical_name_mineral ' $\mathrm{KAg}[\mathrm{CO} 3]$ '

chemical_formula_sum ' $\mathrm{Ag} \mathrm{C} \mathrm{K} \mathrm{O3}$,

loop

publ_author_nam

'Y.-Q. Zheng'

L. -X. Zhou

J.-L. Lin'

'S.-W. Zhang

journal_name_full_name

Zeitschrift $f \backslash \backslash "$ u $\}$ r Kristallographie - New Crystal Structures

journal_volume 215

journal year 2000

journal_page_first 467

journal page last 468

publ Section title

Refinement of the crystal structure of potassium \{ $\{$ it catena\}-$\hookrightarrow$ carbonato--argentate $(\mathrm{I}), \mathrm{K}\left[\mathrm{Ag}\left(\mathrm{CO}_{-}\{3\} \$\right)\right]$

\# Found in Pearson's Crystal Data - Crystal Structure Database for $\hookrightarrow$ Inorganic Compounds, 2013

aflow_title 'KAg[CO\$_\{3\}\$] Structure'

aflow_proto 'ABCD3_oI48_73_d_e_e_ef

aflow_params 'a, b/a,c/a, y_ $\{1\}, z_{-}\{2\}, z_{-}\{3\}, z_{-}\{4\}, x_{-}\{5\}, y_{-}\{5\}, z_{-}\{5\}$

flow_params_values ' $5.7750411261,1.02926406926,3.53056277057,0.63427$, $\hookrightarrow 0.3734,0.18032,0.311,0.1349,0.6124,0.0967$

aflow_Strukturbericht 'None'

aflow_Pearson ,oI48,

cell_length_a $\quad 5.775041126$

cell_length_b 5.9440423296

cell_length_c 20.3891451983

cell angle alpha 90.0000000000

cell angle beta 90.0000000000

cell_angle_gamma 90.0000000000

symmetry_space_group_name_H-M "I 21/b 21/c 21/a"

symmetry_Int_Tables_number 73 
loop

_space_group_symop_id

_space_group_symop_operation_xyz

$1 \mathrm{x}, \mathrm{y}, \mathrm{z}$

$2 \mathrm{x},-\mathrm{y},-\mathrm{z}+1 / 2$

$3-x+1 / 2, y,-z$

$4-x,-y+1 / 2, z$

$5-\mathrm{x},-\mathrm{y},-\mathrm{z}$

$6-\mathrm{x}, \mathrm{y}, \mathrm{z}+1 / 2$

$7 \mathrm{x}+1 / 2,-\mathrm{y}, \mathrm{z}$

$9 \mathrm{x}+1 / 2, \mathrm{y}+1 / 2, \mathrm{z}+1 / 2$

$10 x+1 / 2,-y+1 / 2,-z$

$11-x+y+1 / 2,-z+1 / 2$

$12-x+1 / 2,-y, z+1 / 2$

$13-\mathrm{x}+1 / 2,-\mathrm{y}+1 / 2,-\mathrm{z}+1 / 2$

$14-x+1 / 2, y+1 / 2, z$

$15 x+y+1 / 2, z+1 / 2$

$16 \mathrm{x}+1 / 2, \mathrm{y},-\mathrm{z}+1 / 2$

loop

_atom_site_label

atom_site_type_symbol

atom_site_symmetry_multiplicity

atom_site_Wyckoff_label

atom_site_fract_x

atom_site_fract_y

atom_site_fract_z

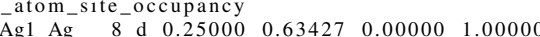

$\begin{array}{llllllllll}\mathrm{Agl} & \mathrm{Ag} & 8 & \mathrm{~d} & 0.25000 & 0.63427 & 0.00000 & 1.00000 \\ \mathrm{C} 1 & \mathrm{C} & 8 & \mathrm{e} & 0.00000 & 0.25000 & 0.37340 & 1.00000\end{array}$

$\begin{array}{lllllllll}\mathrm{Cl} & \mathrm{C} & 8 & \mathrm{e} & 0.00000 & 0.25000 & 0.37340 & 1.00000\end{array}$

$\begin{array}{lllllllll}\mathrm{K} 1 & \mathrm{~K} & -8 & \mathrm{e} & 0.00000 & 0.25000 & 0.18032 & 1.00000\end{array}$

$\begin{array}{llrllllll}\mathrm{O} 2 & \mathrm{O} & 16 & \mathrm{f} & 0.13490 & 0.61240 & 0.09670 & 1.00000\end{array}$

$\mathrm{KAg}\left[\mathrm{CO}_{3}\right]$ : ABCD3_ol48_73_d_e_e_ef - POSCAR

ABCD3_oI48_73_d_e_e_ef \& a , b/a, c / a , y1 , z2 , z3, z4, x5, y5, z5 --params= $\hookrightarrow 5.7750411261,1.02926406926,3.53056277057,0.63427,0.3734,0.18032$ $\hookrightarrow$ oI48 \& None \& KAg[CO3] \& \& Y.-Q. Zheng et al. Zeitschrift f"

$\hookrightarrow$ oI 48 \& None \& KAg $[$ CO3] \& \& Y.-Q. Zheng et al. Zeitschrift f
$\hookrightarrow$ u $\mathrm{r}$ Kristallographie - New Crystal Structures 215, 467-468 (

1.00000000000000

$\begin{array}{lll}-2.88752056305000 & 2.97202116480000 & 10.19457259915000\end{array}$

$\begin{array}{lll}2.88752056305000 & -2.97202116480000 & 10.19457259915000\end{array}$

$2.88752056305000 \quad 2.97202116480000-10.19457259915000$

$\begin{array}{rrrr}\mathrm{Ag} & \mathrm{C} & \mathrm{K} & \mathrm{O} \\ 4 & 4 & 4 & 12\end{array}$

Direct

0.63427000000000

$-0.13427000000000$

$-0.63427000000000$

1.13427000000000

0.62340000000000

0.6234000000000

0.3766000000000

1.1234000000000

1.12340000000000

0.43032000000000

0.06968000000000

0.56968000000000

0.93032000000000

0.56100000000000

$-0.06100000000000$

0.43900000000000

1.06100000000000

0.70910000000000

$-0.01570000000000$

0.51570000000000

$-0.20910000000000$

$-0.70910000000000$

1.01570000000000

$-0.5157000000000$

-0.51570000000000
1.20910000000000

$0.25000000000000 \quad 0.88427000000000$ 0.75000000000000 0.75000000000000 0.25000000000000 0.37340000000000 0.1266000000000 $-0.3734000000000$ 0.8734000000000 0.87340000000000 0.1803200000000 0.3196800000000 0.18032000000000 0.68032000000000 0.31100000000000 0.18900000000000 $-0.3110000000000$ 0.81100000000000 0.23160000000000 0.03820000000000 0.26840000000000 0.53820000000000 $-0.23160000000000$ 0.03820000000000 0.7316000000000 0.46180000000000 .38427000000000 1.38427000000000 .25000000000000 0.7500000000000 0.75000000000000 0.75000000000000000 0.2500000000000 0.25000000000000 0.75000000000000 .75000000000000 .25000000000000 0.25000000000000 0.7500000000000 0.75000000000000 0.25000000000000 0.7473000000000 $-0.24730000000000$ 0.97750000000000 $-0.47750000000000$ $-0.74730000000000$ 1.24730000000000 0.02250000000000 0.47750000000000

$\begin{aligned} \mathrm{Ag} & (8 \mathrm{~d}) \\ \mathrm{Ag} & (8 \mathrm{~d}) \\ \mathrm{Ag} & (8 \mathrm{~d}) \\ \mathrm{Ag} & (8 \mathrm{~d}) \\ \mathrm{C} & (8 \mathrm{e}) \\ \mathrm{C} & (8 \mathrm{e}) \\ \mathrm{C} & (8 \mathrm{e}) \\ \mathrm{C} & (8 \mathrm{e}) \\ \mathrm{K} & (8 \mathrm{e}) \\ \mathrm{K} & (8 \mathrm{e}) \\ \mathrm{K} & (8 \mathrm{e}) \\ \mathrm{K} & (8 \mathrm{e}) \\ \mathrm{O} & (8 \mathrm{e}) \\ \mathrm{O} & (8 \mathrm{e}) \\ \mathrm{O} & (8 \mathrm{e}) \\ \mathrm{O} & (8 \mathrm{e}) \\ \mathrm{O} & (16 \mathrm{f}) \\ \mathrm{O} & (16 \mathrm{f}) \\ \mathrm{O} & (16 \mathrm{f}) \\ \mathrm{O} & (16 \mathrm{f}) \\ \mathrm{O} & (16 \mathrm{f}) \\ \mathrm{O} & (16 \mathrm{f}) \\ \mathrm{O} & (16 \mathrm{f}) \\ \mathrm{O} & (16 \mathrm{f})\end{aligned}$

$\mathrm{KHg}_{2}$ : A2B_OI12_74_h_e - CIF

\# CIF file

data findsym-output

audit_creation_method FINDSYM

chemical name mineral ' $\mathrm{KHg} 2$

chemical formula sum ' $\mathrm{Hg} 2 \mathrm{~K}$ '

loop

publ_author_nam

E. J. Duwell

'N. C. Baenziger

_journal_name_full_name

Acta Cristallographica

_journal_volume 8

journal year 1955

_journal_page_first 705

journal page last 710

publ_Section_title

The crystal structures of $\mathrm{KHg}$ and $\mathrm{KHg} \$ \_\{2\} \$$

\# Found in Pearson's Crystal Data - Crystal Structure Database for

$\hookrightarrow$ Inorganic Compounds, 2013

_aflow_title 'KHg\$_\{2\}\$ Structure, aflow proto 'A2B oI12 74 h

aflow_params, a, b/a,c/a, z $\{1\}, y_{-}\{2\}, z_{-}\{2\}$

aflow_params_values ' $5.158858099,1.56976744188,1.70096899227,-0.047$ $\hookrightarrow 0.56,0.663$

_aflow_Strukturbericht 'None'

-aflow_Pearson 'oI12,

cell_length_a $\quad 5.1588580990$

cell_length_b $\quad 8.098207481$

cell_length_c 8.7750576619

cell_angle_alpha 90.0000000000

cell angle beta 90.0000000000

symmetry_space_group_name_H-M "I $21 / \mathrm{m} \mathrm{21/m} \mathrm{21/a"}$

symmetry_Int_Tables_number 74

loop

space_group_symop_i

_space_group_symop_operation_xyz

$1 \mathrm{x}, \mathrm{y}, \mathrm{z}$

$2 \mathrm{x},-\mathrm{y},-\mathrm{z}$

$3-x, y+1 / 2,-z$

$4-\mathrm{x},-\mathrm{y}+1 / 2, \mathrm{z}$

$5-\mathrm{x},-\mathrm{y},-\mathrm{z}$

-x, $y$,

$8 \mathrm{x}, \mathrm{y}+1 / 2,-\mathrm{z}$

$\mathrm{x}+1 / 2, \mathrm{y}+1 / 2, \mathrm{z}+1 / 2$

$10 \mathrm{x}+1 / 2,-\mathrm{y}+1 / 2,-\mathrm{z}+1 / 2$

$11-\mathrm{x}+1 / 2, \mathrm{y},-\mathrm{z}+1 / 2$

$/ 2,-y, z+1 / 2$

$13-x+1 / 2,-y+1 / 2,-z+1 / 2$

$14-\mathrm{x}+1 / 2, \mathrm{y}+1 / 2, \mathrm{z}+1 / 2$

$15 \mathrm{x}+1 / 2,-\mathrm{y}, \mathrm{z}+1 / 2$

$16 \mathrm{x}+1 / 2, \mathrm{y},-\mathrm{z}+1 / 2$

loop

atom_site_label

atom_site_type_symbol

atom_site_symmetry_multiplicity

atom_site_Wyckoff_label

atom_site_fract_x

atom_site_fract_y

_atom_site_fract_z

$\begin{array}{llllllll}\mathrm{K} 1 & \mathrm{~K} & 4 & \mathrm{e} & 0.00000 & 0.25000 & -0.04700 & 1.00000\end{array}$

$\begin{array}{llllllll}\mathrm{Hg} 1 \mathrm{Hg} & 8 & \mathrm{~h} & 0.00000 & 0.56000 & 0.66300 & 1.00000\end{array}$

$\mathrm{KHg}_{2}$ : A2B_OI12_74_h_e - POSCAR

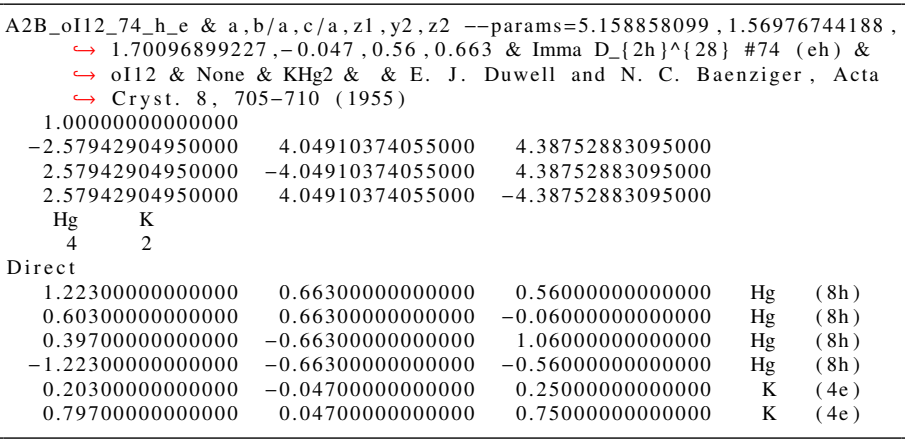

$\mathrm{Al}_{4} \mathrm{U}\left(D 1_{b}\right)$ : A4B_ol20_74_beh_e - CIF

\# CIF file

data_findsym-output

audit_creation_method FINDSYM

chemical_name_mineral ,

chemical_formula_sum 'Al4 U',

loop

publ_author_name

H. U. Borgsted

'H. Wedemeyer'

journal year 1989

publ Section title

Gmelin Handbook of Inorganic Chemistry

aflow_title, Al\$_ $\{4\} \$$ U (\$D1_\{b\}\$) Structure

aflow_proto 'A4B_oI20_74_beh_e'

aflow_params a, b/a,c/a, z_ $\{2\}, z_{-}\{3\}, y_{-}\{4\}, z_{-}\{4\}$,

_aflow_params_values ' $4.39,1.42369020501,3.12528473804,-0.111,0.111,-$ $\hookrightarrow 0.033,0.314$

aflow_Strukturbericht '\$D1_\{b\}\$,

aflow Pearson 'oI20

symmetry_space group_name_H-M "I $21 / \mathrm{m} \mathrm{21/m} \mathrm{21/a"}$

symmetry_Int Tables_number 74

cell_length_a $\quad 4.39000$

cell_length_b $\quad 6.25000$

cell_length_c 13.72000

cell angle alpha 90.00000

cell_angle_beta 90.00000

_cell_angle_gamma 90.00000 
loop

_space_group_symop_id

_space_group_symop_operation_xyz

$1 \mathrm{x}, \mathrm{y}, \mathrm{z}$

$2 \mathrm{x},-\mathrm{y},-\mathrm{z}$

$3-x, y+1 / 2,-z$

$4-x,-y+1 / 2, z$

$5-\mathrm{x},-\mathrm{y},-\mathrm{z}$

$6-\mathrm{x}, \mathrm{y}, \mathrm{z}$

$7 x,-y+1 / 2, z$

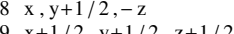

$10 \mathrm{x}+1 / 2,-\mathrm{y}+1 / 2,-\mathrm{z}+1 / 2$

$\begin{array}{ll}10 & \mathrm{x}+1 / 2,-\mathrm{y}+1 / 2,-\mathrm{z}+1 \\ 11 & -\mathrm{x}+1 / 2, \mathrm{y},-\mathrm{z}+1 / 2\end{array}$

$12-x+1 / 2,-y, z+1 / 2$

$13-\mathrm{x}+1 / 2,-\mathrm{y}+1 / 2,-\mathrm{z}+1 / 2$

$14-\mathrm{x}+1 / 2, \mathrm{y}+1 / 2, \mathrm{z}+1 / 2$

$15 \mathrm{x}+1 / 2,-\mathrm{y}, \mathrm{z}+1 / 2$

loop

atom_site_label

atom_site_type_symbol

atom_site_symmetry_multiplicity

atom_site_Wyckoff_label

atom_site_fract_x

atom_site_fract_y

atom_site_fract_z

$\begin{array}{llllll}\text {-atom_site_occupancy } & & & & \end{array}$

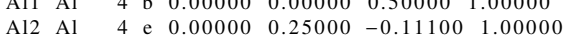

$\begin{array}{llllllll}\mathrm{Al} 2 & \mathrm{Al} & 4 & \mathrm{e} & 0.00000 & 0.25000 & -0.11100 & 1.00000 \\ \mathrm{U} 1 & \mathrm{U} & 4 & \mathrm{e} & 0.00000 & 0.25000 & 0.11100 & 1.00000\end{array}$

$\begin{array}{llllllll}\mathrm{Al} 3 & \mathrm{Al} & 8 & \mathrm{~h} & 0.00000 & -0.03300 & 0.31400 & 1.00000\end{array}$

$\mathrm{Al}_{4} \mathrm{U}\left(D 1_{b}\right)$ : A4B_oI20_74_beh_e - POSCAR

A4B_oI20_74_beh_e \& a , b/a , c/a, z2 , z3 , y4, z4 --params $=4.39,1.42369020501$

$\hookrightarrow 3.12528473804,-0.111,0.111,-0.033,0.314 \&$ Imma D_ $\{2 \mathrm{~h}\}^{\wedge}\{28\} \# 74$
$\hookrightarrow\left(b^{\wedge} 2 \mathrm{~h}\right) \&$ oI20 \& $\$ D 1_{-}\{$b $\} \$ \&$ Al4U \& \& H. U. Borgstedt and H.

$\hookrightarrow($ be^ $2 \mathrm{~h}) \&$ oI $20 \&$ \&

1.00000000000000

$\begin{array}{rrr}-2.19500000000000 & 3.12500000000000 & 6.86000000000000 \\ 2.19500000000000 & -3.12500000000000 & 6.86000000000000\end{array}$

$\begin{array}{rrr}2.19500000000000 & -3.12500000000000 & 6.86000000000000 \\ 2.19500000000000 & 3.12500000000000 & -6.86000000000000\end{array}$

Al U

Direct

0.13900000000000

0.86100000000000

0.28100000000000

0.84700000000000

0.15300000000000

0.36100000000000

0.63900000000000

50000000000000 0.50000000000000 $-0.11100000000000$ 0.11100000000000 0.31400000000000 0.31400000000000 $-0.31400000000000$ $-0.3140000000000$ $-0.11100000000000$ $-0.11100000000000$

0.00000000000000 0.5000000000000 0.2500000000000 0.75000000000000 0.03300000000000 0.53300000000000 0.46700000000000 0.03300000000000 0.25000000000000 0.75000000000000

$\begin{array}{cl}\mathrm{Al} & (4 \mathrm{~b}) \\ \mathrm{Al} & (4 \mathrm{~b}) \\ \mathrm{Al} & (4 \mathrm{e}) \\ \mathrm{Al} & (4 \mathrm{e}) \\ \mathrm{Al} & (8 \mathrm{~h}) \\ \mathrm{Al} & (8 \mathrm{~h}) \\ \mathrm{Al} & (8 \mathrm{~h}) \\ \mathrm{Al} & (8 \mathrm{~h}) \\ \mathrm{U} & (4 \mathrm{e}) \\ \mathrm{U} & (4 \mathrm{e})\end{array}$

$\mathrm{BaCr}_{2} \mathrm{Ru}_{4} \mathrm{O}_{12}$ : AB2C12D4_tP76_75_2a2b_2d_12d_4d - CIF

\# CIF file

data findsym-output

audit_creation_method FINDSYM

chemical name mineral ' $\mathrm{BaCr} 2 \mathrm{Ru} 4 \mathrm{O} 12$ '

chemical formula_sum 'Ba Cr2 O12 Ru4

loop

publ_author_name

M. C. $\operatorname{Cad}\{\backslash \backslash, \mathrm{e}\} \mathrm{e}$,

'A. Prodan

_journal_name_full_name

Materials Research Bulletin

_journal_volume 14

journal year 1979

_journal_page_first 613

journal_page_last 618

publ_Section_title

Tripling of the short axis in the hollandite structure

\# Found in Pearson's Crystal Data - Crystal Structure Database for $\hookrightarrow$ Inorganic Compounds, 2013

aflow_title 'BaCr\$_ $\{2\} \$ R u \$_{-}\{4\} \$ O \$_{-}\{12\} \$$ Structure

aflow_proto 'AB2C12D4_tP76_75_2a2b_2d_12d_4d

aflow_params 'a,c/a, z $\{1\}, \mathrm{z}_{-}\{2\}, \mathrm{z}_{-}\{3\}, \mathrm{z}_{-}\{4\}, \mathrm{x}_{-}\{5\}, \mathrm{y}_{-}\{5\}, \mathrm{z}_{-}\{5\}, \mathrm{x}_{-}\{6\}, \mathrm{y}_{-}\{$ $\hookrightarrow 6\}, z_{-}\{6\}, x_{-}\{7\}, y_{-}\{7\}, z_{-}\{7\}, x_{-}\{8\}, y_{-}\{8\}, z_{-}\{8\}, x_{-}\{9\}, y_{-}\{9\}, z_{-}\{9\}$, $\hookrightarrow \mathrm{x}_{-}\{10\}, \mathrm{y}_{-}\{10\}, \mathrm{z}_{-}\{10\}, \mathrm{x}_{-}\{11\}, \mathrm{y}_{-}\{11\}, \mathrm{z}_{-}\{11\}, \mathrm{x}_{-}\{12\}, \mathrm{y}_{-}\{12\}, \mathrm{z}_{-}\{12\}$,

$\hookrightarrow \mathrm{x}_{-}\{13\}, \mathrm{y}_{-}\{13\}, \mathrm{z}_{-}\{13\}, \mathrm{x}_{-}\{14\}, \mathrm{y}_{-}\{14\}, \mathrm{z}_{-}\{14\}, \mathrm{x}_{-}\{15\}, \mathrm{y}_{-}\{15\}, \mathrm{z}_{-}\{15\}$,

$\hookrightarrow \mathrm{x}_{-}\{16\}, \mathrm{y}_{-}\{16\}, \mathrm{z}_{-}\{16\}, \mathrm{x}_{-}\{17\}, \mathrm{y}_{-}\{17\}, \mathrm{z}_{-}\{17\}, \mathrm{x}_{-}\{18\}, \mathrm{y}_{-}\{18\}, \mathrm{z}_{-}\{18\}$

$\hookrightarrow \mathrm{x}_{-}\{19\}, \mathrm{y}_{-}\{19\}, \mathrm{z}_{-}\{19\}, \mathrm{x}_{-}$

aflow_params_values $9.8880614494,0.922431229769,0.3333,0.0,0.5003$

$\hookrightarrow 0.1666,0.167,0.348,0.1666,0.333,0.152,0.0,0.208,0.152,0.8333$,

$\hookrightarrow 0.458,0.152,0.8333,0.292,0.348,0.6666,0.042,0.348,0.6666,0.208$

$\hookrightarrow 0.152,0.5,0.458,0.152,0.5,0.292,0.348,0.3333,0.042,0.348,0.3333$

$\hookrightarrow \quad 0.208,0.152,0.1666,0.458,0.152,0.1666,0.292,0.348,0.0,0.042$

$\hookrightarrow 0.348,0.0,0.167,0.348,0.8333,0.333,0.152,0.6666,0.167,0.348,0.5$ $\hookrightarrow, 0.333,0.152,0.3333$
0.50000000000000

0.00000000000000

$-0.28100000000000$

$\hookrightarrow x_{-}\{22\}, y_{-}\{22\}, z_{-}\{22\}$

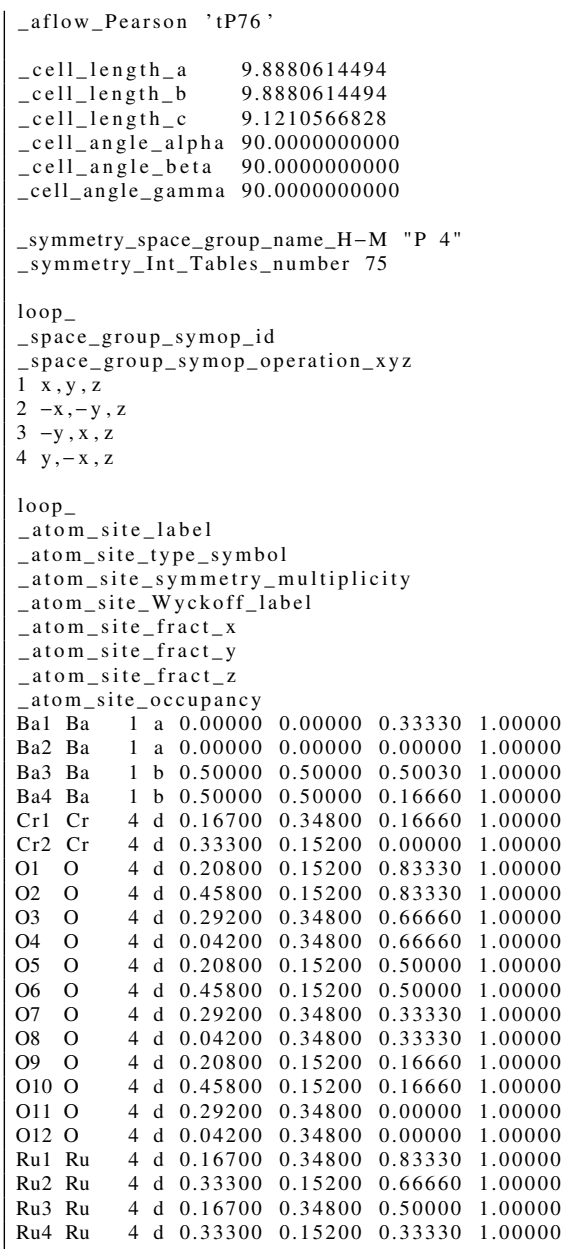

$\begin{array}{lllllll}\mathrm{Ba} 1 \mathrm{Ba} & 1 & 0.00000 & 0.00000 & 0.33330 & 1.00000\end{array}$

Ba $2 \mathrm{Ba} 1$ a $0.00000 \quad 0.00000 \quad 0.00000-1.00000$

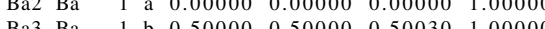

$\begin{array}{lllllll}\mathrm{Ba} 3 & \mathrm{Ba} & 1 & 0.50000 & 0.50000 & 0.50030 & 1.00000\end{array}$

$\begin{array}{llllllll}\mathrm{Ba} 4 & \mathrm{Ba} & 1 & \mathrm{~b} & 0.50000 & 0.50000 & 0.50030 & 1.00000\end{array}$

$\begin{array}{lllllllll}\mathrm{Cr} 1 & \mathrm{Cr}_{\mathrm{r}} 4 & 4 & \mathrm{~d} & 0.16700 & 0.34800 & 0.16660 & 1.00000\end{array}$

$\begin{array}{llllllll}\mathrm{Cr} 2 & \mathrm{Cr} & 4 & \mathrm{~d} & 0.36300 & 0.34800 & 0.16660 & 1.00000 \\ 01 & \mathrm{O} & 4 & \mathrm{~d} & 0.20800 & 0.15200 & 0.00000 & 1.00000\end{array}$

$\begin{array}{lllllllll}\mathrm{O} 1 & \mathrm{O} & 4 & \mathrm{~d} & 0.20800 & 0.15200 & 0.83330 & 1.00000 \\ \mathrm{O} 2 & \mathrm{O} & & 4 & \mathrm{~d} & 0.45800 & 0.15200 & 0.83330 & 1.00000\end{array}$

$\begin{array}{lllllllll}\mathrm{O} 2 & \mathrm{O} & 4 & \mathrm{~d} & 0.45800 & 0.15200 & 0.83330 & 1.00000 \\ \mathrm{O} 3 & \mathrm{O} & & 4 & \mathrm{~d} & 0.29200 & 0.34800 & 0.66660 & 1.00000\end{array}$

$\begin{array}{llllllllll}\mathrm{O} 3 & \mathrm{O} & & 4 & \mathrm{~d} & 0.29200 & 0.34800 & 0.66660 & 1.00000\end{array}$

$\begin{array}{llllllllll}\mathrm{O} 4 & \mathrm{O} & & 4 & \mathrm{~d} & 0.04200 & 0.34800 & 0.66660 & 1.00000\end{array}$

$\begin{array}{lllllllll}\mathrm{O} 5 & \mathrm{O} & & 4 & \mathrm{~d} & 0.20800 & 0.15200 & 0.50000 & 1.00000\end{array}$

$\begin{array}{lllllllll}\mathrm{O} 6 & \mathrm{O} & 4 & \mathrm{~d} & 0.45800 & 0.15200 & 0.50000 & 1.00000\end{array}$

$\begin{array}{lllllllll}07 & \mathrm{O} & 4 & \mathrm{~d} & 0.29200 & 0.34800 & 0.33330 & 1.00000\end{array}$

$\begin{array}{lllllllll}\mathrm{O} 8 & \mathrm{O} & & 4 & \mathrm{~d} & 0.04200 & 0.34800 & 0.33330 & 1.00000\end{array}$

$\begin{array}{lllllllll}\mathrm{O} 9 & \mathrm{O} & 4 & \mathrm{~d} & 0.20800 & 0.15200 & 0.16660 & 1.00000\end{array}$

$\begin{array}{lllllllll}\mathrm{O} 10 & \mathrm{O} & 4 & \mathrm{~d} & 0.45800 & 0.15200 & 0.16660 & 1.00000\end{array}$

$\begin{array}{lllllllll}\mathrm{O} 11 \mathrm{O} & 4 & \mathrm{~d} & 0.29200 & 0.34800 & 0.00000 & 1.00000\end{array}$

$\begin{array}{llllllll}\mathrm{O} 11 & \mathrm{O} & 4 & \mathrm{~d} & 0.29200 & 0.34800 & 0.00000 & 1.00000 \\ \mathrm{O} 12 & \mathrm{O} & 4 & \mathrm{~d} & 0.04200 & 0.34800 & 0.00000 & 1.00000\end{array}$

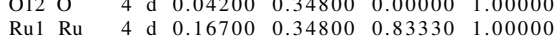

$\begin{array}{lllllllll}\mathrm{Ru} 1 & \mathrm{Ru} & 4 & \mathrm{~d} & 0.16700 & 0.34800 & 0.83330 & 1.00000 \\ \mathrm{R} 42 & \mathrm{Ru} & 4 & \mathrm{~d} & 0.33300 & 0.15200 & 0.66660 & 1.00000\end{array}$

$\begin{array}{lllllll}\text { Ru2 Ru } & 4 & \text { d } & 0.33300 & 0.15200 & 0.66660 & 1.00000\end{array}$

$\begin{array}{llllllll}\mathrm{Ru} 3 & \mathrm{Ru} & 4 & \mathrm{~d} & 0.16700 & 0.34800 & 0.50000 & 1.00000 \\ \mathrm{Ru} 4 & \mathrm{Ru} & 4 & \mathrm{~d} & 0.33300 & 0.15200 & 0.33330 & 1.00000\end{array}$

$\mathrm{BaCr}_{2} \mathrm{Ru}_{4} \mathrm{O}_{12}$ : AB2C12D4_tP76_75_2a2b_2d_12d_4d - POSCAR

AB2C12D4 tP76 75 2a2b 2d 12d 4d \& a c c/a, z1, z2, z3, z4, x 5, y5 , z5, x6, y6, z6, x7 $\hookrightarrow, \mathrm{y} 7, \mathrm{z} 7, \mathrm{x} 8, \mathrm{y} 8, \mathrm{z} 8, \mathrm{x} 9, \mathrm{y} 9, \mathrm{z} 9, \mathrm{x} 10, \mathrm{y} 10, \mathrm{z} 10, \mathrm{x} 11, \mathrm{y} 11, \mathrm{z} 11, \mathrm{x} 12, \mathrm{y} 12, \mathrm{z} 12$ $\hookrightarrow \mathrm{x} 13, \mathrm{y} 13, \mathrm{z} 13, \mathrm{x} 14, \mathrm{y} 14, \mathrm{z} 14, \mathrm{x} 15, \mathrm{y} 15, \mathrm{z} 15, \mathrm{x} 16, \mathrm{y} 16, \mathrm{z} 16, \mathrm{x} 17, \mathrm{y} 17, \mathrm{z} 17, \mathrm{x} 18$ $\hookrightarrow, \mathrm{y} 18, \mathrm{z} 18, \mathrm{x} 19, \mathrm{y} 19, \mathrm{z} 19, \mathrm{x} 20, \mathrm{y} 20, \mathrm{z} 20, \mathrm{x} 21, \mathrm{y} 21, \mathrm{z} 21, \mathrm{x} 22, \mathrm{y} 22, \mathrm{z} 22-{ }_{-}$ $\longrightarrow$ para $\hookrightarrow 0.167,0.348,0.1666,0.333,0.152,0.0,0.208,0.152,0.8333,0.458$ $\hookrightarrow 0.152,0.8333,0.292,0.348,0.6666,0.042,0.348,0.6666,0.208,0.152$, $\hookrightarrow 0.5,0.458,0.152,0.5,0.292,0.348,0.3333,0.042,0.348,0.3333,0.208$ $\hookrightarrow, 0.152,0.1666,0.458,0.152,0.1666,0.292,0.348,0.0,0.042,0.348$, $\hookrightarrow 0.0,0.167,0.348,0.8333,0.333,0.152,0.6666,0.167,0.348,0.5,0.333$ $\hookrightarrow, 0.152,0.3333 \&$ \& $4 \mathrm{C}_{-}\{4\}^{\wedge}\{1\} \# 75\left(\mathrm{a}^{\wedge} 2 \mathrm{~b}^{\wedge} 2 \mathrm{~d}^{\wedge} 18\right) \& \mathrm{tP} 76$ \& None \& $\hookrightarrow$ BaCr2Ru4O12 \& \& M. C. Cad $\{\backslash$ 'e $\}$ e and A. Prodan, Mater. Res. $\hookrightarrow$ Bull. 14, 613-618 (1979)

1.00000000000000

9.88806144940000
0.00000000000000 $\quad 0.00000000000000$

9.88806144940000

$0.0000000000000 \quad 0.00000000000000$ $\mathrm{Ru}$

Direct

$0.00000000000000 \quad 0.00000000000000$ $0.00000000000000 \quad 0.00000000000000$ $0.50000000000000 \quad 0.50000000000000$ $\begin{array}{ll}0.50000000000000 & 0.50000000000000\end{array}$ $0.16700000000000 \quad 0.34800000000000$ $-0.16700000000000-0.34800000000000$ $-0.34800000000000 \quad 0.16700000000000$ $0.34800000000000-0.16700000000000$ $0.33300000000000 \quad 0.15200000000000$ $-0.33300000000000 \quad-0.15200000000000$ $\begin{array}{ll}-0.15200000000000 & 0.33300000000000\end{array}$ $0.15200000000000-0.33300000000000$ $0.20800000000000-0.15200000000000$ $\begin{array}{rr}0.20800000000000 & 0.15200000000000 \\ -0.20800000000000 & -0.15200000000000\end{array}$ $-0.20800000000000 \quad-0.15200000000000$ $-0.15200000000000 \quad 0.20800000000000$ $0.15200000000000-0.20800000000000$ $0.45800000000000 \quad 0.15200000000000$ $-0.45800000000000-0.15200000000000$ $-0.15200000000000 \quad 0.45800000000000$ $0.15200000000000-0.45800000000000$ $0.29200000000000 \quad 0.34800000000000$ $-0.29200000000000 \quad-0.34800000000000$ $-0.34800000000000 \quad 0.29200000000000$ $\begin{array}{ll}0.34800000000000 & -0.29200000000000\end{array}$ $0.04200000000000 \quad 0.34800000000000$ $-0.04200000000000 \quad-0.34800000000000$ $-0.34800000000000 \quad 0.04200000000000$ $0.34800000000000-0.04200000000000$ $\begin{array}{lll}0.20800000000000 & 0.15200000000000\end{array}$

$-0.2080000000000-0.15200000000000$

$\begin{array}{rr}-0.20800000000000 & -0.15200000000000 \\ -0.15200000000000 & 0.2080000000000\end{array}$

0.33330000000000 0.00000000000000 0.50030000000000 0.16660000000000 0.16660000000000 0.16660000000000 0.16660000000000 0.16660000000000 0.16660000000000 0.00000000000000 0.00000000000000 0.00000000000000 0.00000000000000 0.83330000000000 0.83330000000000 0.83330000000000 0.83330000000000 0.83330000000000 0.83330000000000 0.83330000000000 0.83330000000000 0.66660000000000 0.66660000000000 0.66660000000000 0.66660000000000 0.66660000000000 0.66660000000000 0.66660000000000 0.66660000000000 0.50000000000000 0.5000000000000 0.50000000000000
Ba (1a) $\mathrm{Ba}$ (1a) $\mathrm{Ba} \quad(1 \mathrm{~b})$ $\mathrm{Ba} \quad(1 \mathrm{~b})$ $\mathrm{Cr} \quad(4 \mathrm{~d})$ $\mathrm{Cr} \quad(4 \mathrm{~d})$ $\mathrm{Cr} \quad(4 \mathrm{~d})$ $\mathrm{Cr} \quad(4 \mathrm{~d})$ $\mathrm{Cr} \quad(4 \mathrm{~d})$ (4d) $\mathrm{Cr} \quad(4 \mathrm{~d})$ $\begin{array}{ll}\mathrm{Cr} & (4 \mathrm{~d}) \\ \mathrm{O} & (4 \mathrm{~d})\end{array}$ $\begin{array}{ll}\mathrm{O} & (4 \mathrm{~d}) \\ \mathrm{O} & (4 \mathrm{~d})\end{array}$ O (4d) (4d) (4d) O (4d) O (4d) $\mathrm{O} \quad(4 \mathrm{~d})$ $\mathrm{O} \quad(4 \mathrm{~d})$ O (4d) $\mathrm{O} \quad(4 \mathrm{~d})$ $\mathrm{O} \quad(4 \mathrm{~d})$ $\mathrm{O} \quad(4 \mathrm{~d})$ O (4d) O (4d) O (4d) $\mathrm{O} \quad(4 \mathrm{~d})$ O (4d)

$\begin{array}{ll}\mathrm{O} & (4 \mathrm{~d}) \\ \mathrm{O} & (4 \mathrm{~d})\end{array}$ 
0.15200000000000 0.45800000000000 $-0.45800000000000$ $-0.15200000000000$ 0.15200000000000 0.29200000000000 $-0.29200000000000$ $-0.34800000000000$ 0.34800000000000 0.04200000000000 $-0.04200000000000$ $-0.34800000000000$ 0.34800000000000 0.20800000000000 $-0.20800000000000$ $-0.2080000000000$ 0.4580000000000 0.4580000000 $-0.45800000000000$ 0.1520000000000 0.15200000000000 0.29200000000000 $-0.29200000000000$ 0.34800000000000 0.34800000000000 0.04200000000000 $-0.04200000000000$ 0.34800000000000 0.34800000000000 $-0.16700000000000$ $-0.3480000000000$ 0.3480000000 0.348000000 0.333000000000 $-0.33300000000000$ $-.15200000000000$ 0.15200000000000 0.16700000000000 $-0.16700000000000$ $-0.34800000000000$ 0.34800000000000 0.33300000000000 $-0.33300000000000$ $-0.15200000000000$ 0.15200000000000

$-0.20800000000000$ 0.15200000000000 0.1520000000000 0.45800000000000 0.4580000000000 0.34800000000000 0.34800000000000 0.29200000000000 $-0.29200000000000$ 0.34800000000000 $-0.34800000000000$ 0.04200000000000 $-0.0420000000000$ 0.1520000000000 0.15200000000000 0.20800000000000 . $-0.2080000000000$ 0.1520000000000 $-0.15200000000000$ 0.45800000000000 $-0.4580000000000$ 0.34800000000000 0.34800000000000 0.2920000000000 $-0.29200000000000$ 0.34800000000000 $-0.34800000000000$ 0.04200000000000 $-0.04200000000000$ 0.34800000000000 $-0.34800000000000$ 0.16700000000000 0.1670000000000 0.15200000000000 0.15200000000000 0.1520000000 0.3330000000000 $-0.33300000000000$ 0.34800000000000 $-0.3480000000000$ 0.16700000000000 $-0.16700000000000$ 0.15200000000000 $-0.15200000000000$ 0.33300000000000 $-0.33300000000000$

0.50000000000000 0.50000000000000 .50000000000000 0.50000000000000 0.50000000000000 0.33330000000000 0.33330000000000 0.3333000000000 0.33330000000000 0.33330000000000 0.33330000000000 0.33330000000000 0.33330000000000 0.33330000000000 0.16660000000000 0.16660000000000 0.16660000000000 .166600000000000 0.16660000000000 0.16660000000000 0.166600000000 0.16660000000000 0.16660000000000 0.0000000000000 0.0000000000000 0.00000000000000 0.00000000000000 0.00000000000000 0.00000000000000 0.00000000000000 0.00000000000000 0.83330000000000 0.83330000000000 0.83330000000000 0.83330000000000 0.66660000000000 0.66660000000000 0.66660000000000 0.66660000000000 0.66660000000000 0.50000000000000 0.50000000000000 0.50000000000000 0.50000000000000 0.33330000000000 0.33330000000000 0.33330000000000 0.33330000000000

$\mathrm{LaRhC}_{2}$ : A2BC_tP16_76_2a_a_a - CIF

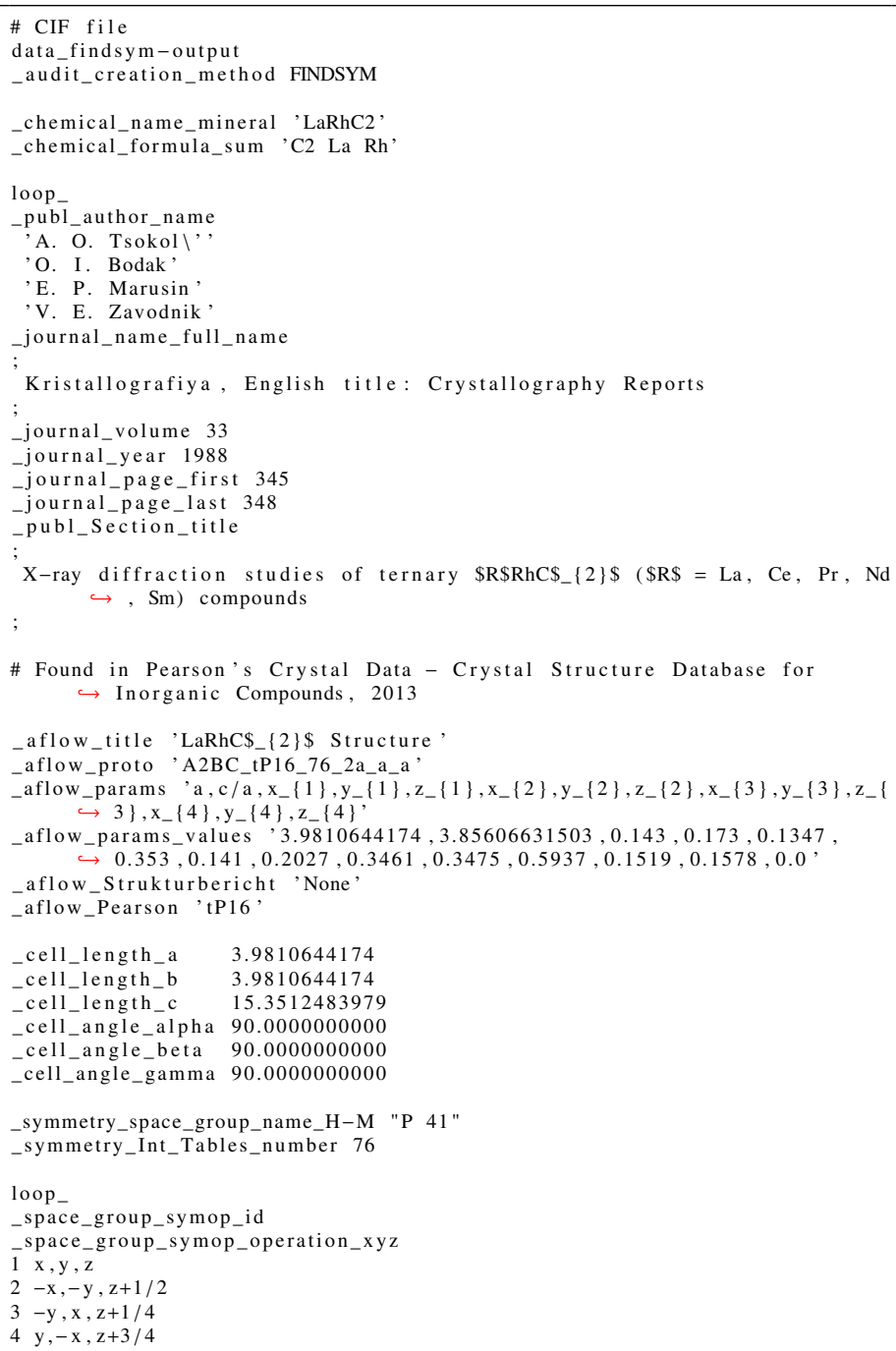

loop

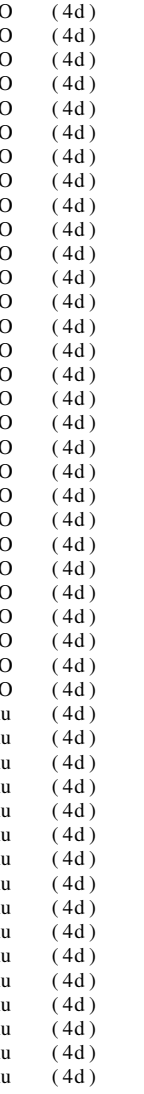

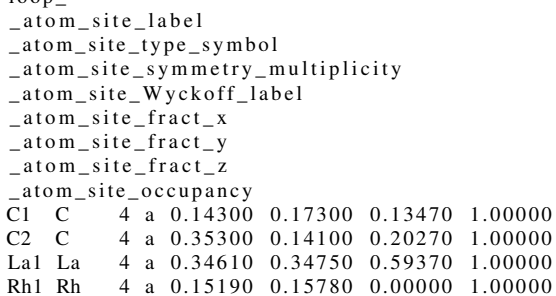

LaRhC 2 : A2BC_tP16_76_2a_a_a - POSCAR

\begin{tabular}{|c|c|c|c|c|}
\hline \multicolumn{5}{|c|}{ A2BC_tP16_76_2a_a_a \& a,c/a, x1,y1,z1,x2,y2, z2, x3,y3,z3, x4,y4,z4--params } \\
\hline & & & & \\
\hline$\hookrightarrow 0.2027,0.346$ & $1,0.3475,0.5937$ & $519,0.1578,0.0 \&$ & & $-\{4\}^{\wedge}\{$ \\
\hline$\hookrightarrow\} \# 76\left(a^{\wedge} 4\right)$ & \& tP16 \& None \& & $\mathrm{hC} 2 \& \&$ A. O. $\mathrm{T}$ & & al., \\
\hline$\hookrightarrow \mathrm{Kristallogr}$ & fiy a $33,345-348$ & & & \\
\hline 1.00000000000000 & & & & \\
\hline 3.98106441740000 & 0.00000000000000 & 0.00000000000000 & & \\
\hline 0.00000000000000 & 3.98106441740000 & 0.00000000000000 & & \\
\hline 0.00000000000000 & 0.00000000000000 & 15.35124839790000 & & \\
\hline $\mathrm{La} \quad \mathrm{Rh}$ & & & & \\
\hline 4 & & & & \\
\hline $\mathrm{ct}$ & & & & \\
\hline 0.14300000000000 & 0.17300000000000 & 0.13470000000000 & $\mathrm{C}$ & (4a) \\
\hline-0.14300000000000 & -0.17300000000000 & 0.63470000000000 & $\mathrm{C}$ & (4a) \\
\hline-0.17300000000000 & 0.14300000000000 & 0.38470000000000 & $\mathrm{C}$ & (4a) \\
\hline 0.17300000000000 & -0.14300000000000 & 0.88470000000000 & $\mathrm{C}$ & (4a) \\
\hline 0.35300000000000 & 0.14100000000000 & 0.20270000000000 & $\mathrm{C}$ & (4a) \\
\hline-0.35300000000000 & -0.14100000000000 & 0.70270000000000 & $\mathrm{C}$ & (4a) \\
\hline-0.14100000000000 & 0.35300000000000 & 0.45270000000000 & $\mathrm{C}$ & (4a) \\
\hline 0.14100000000000 & -0.35300000000000 & 0.95270000000000 & $\mathrm{C}$ & (4a) \\
\hline 0.34610000000000 & 0.34750000000000 & 0.59370000000000 & $\mathrm{La}$ & (4a) \\
\hline-0.34610000000000 & -0.34750000000000 & 1.09370000000000 & $\mathrm{La}$ & (4a) \\
\hline-0.34750000000000 & 0.34610000000000 & 0.84370000000000 & $\mathrm{La}$ & (4a) \\
\hline 0.34750000000000 & -0.34610000000000 & 1.34370000000000 & $\mathrm{La}$ & (4a) \\
\hline 0.15190000000000 & 0.15780000000000 & 0.00000000000000 & $\mathrm{Rh}$ & (4a) \\
\hline-0.15190000000000 & -0.15780000000000 & 0.50000000000000 & $\mathrm{Rh}$ & (4a) \\
\hline-0.15780000000000 & 0.15190000000000 & 0.25000000000000 & $\mathrm{Rh}$ & (4a) \\
\hline 0.15780000000000 & -0.15190000000000 & 0.75000000000000 & $\mathrm{Rh}$ & (4a) \\
\hline
\end{tabular}

$\mathrm{Cs}_{3} \mathrm{P}_{7}$ : A3B7_tP40_76_3a_7a - CIF

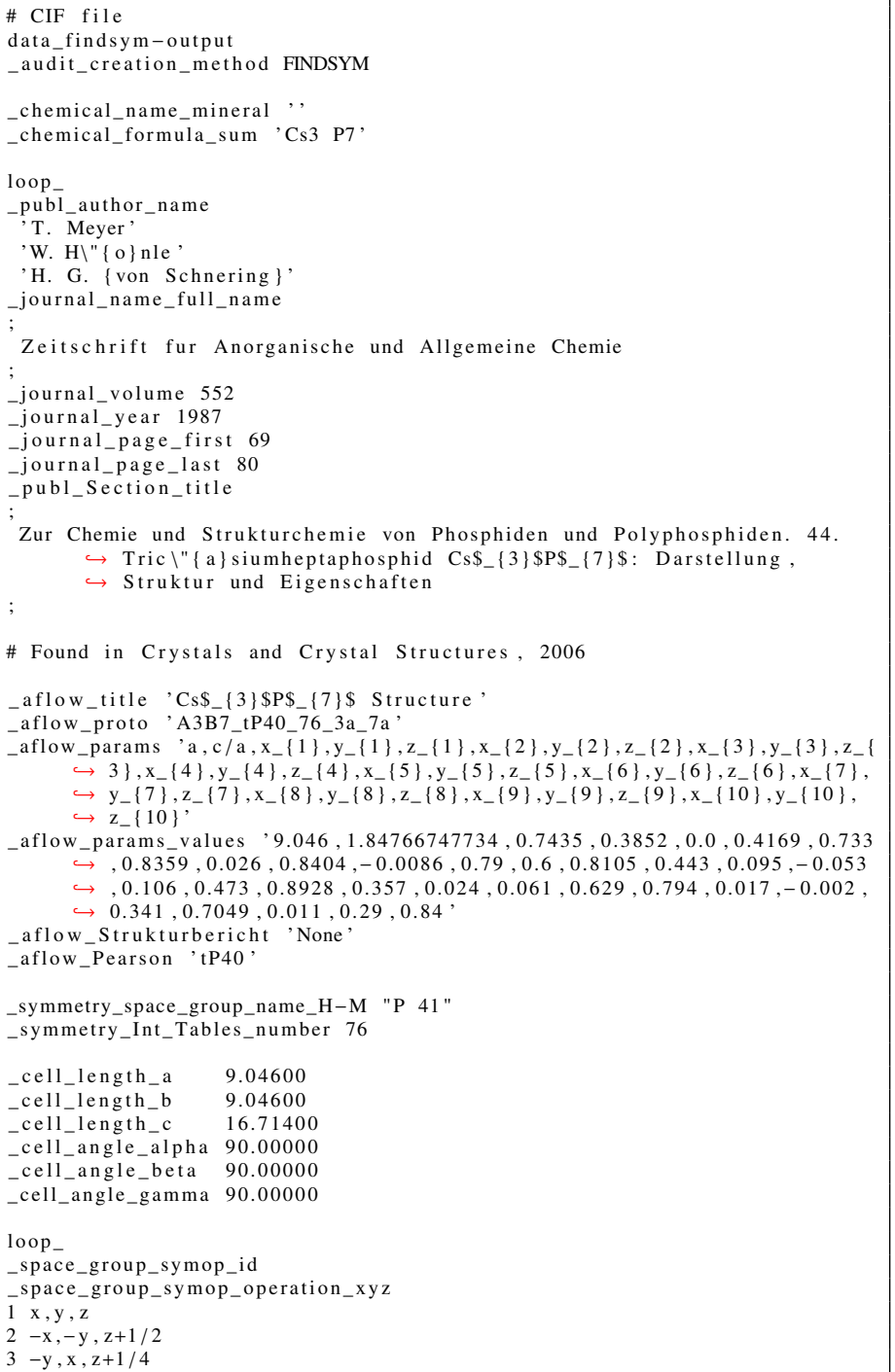




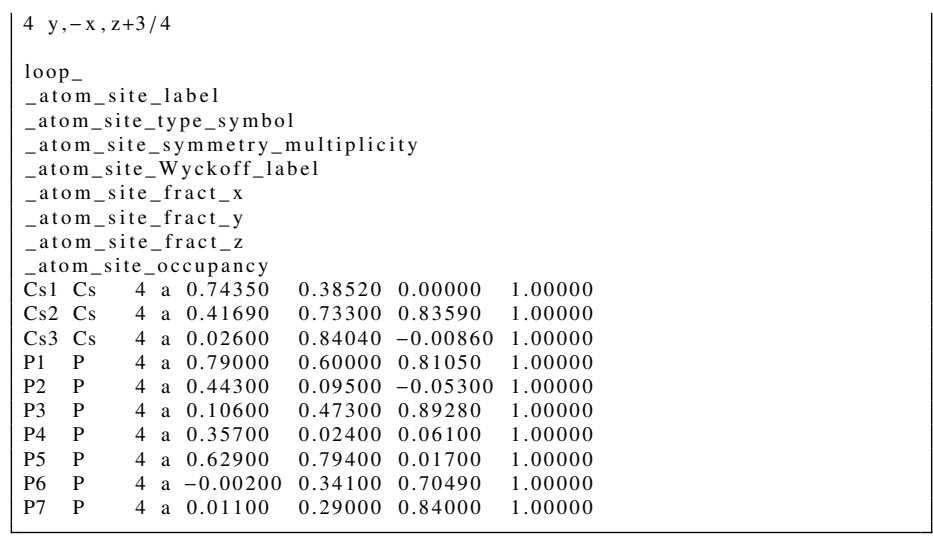

$\mathrm{Cs}_{3} \mathrm{P}_{7}$ : A3B7_tP40_76_3a_7a - POSCAR

A3B7_tP40_76_3a_7a \& a,c/a, x1,y1, z1 , x2, y2 , z2, x3,y3,z3, x4,y4,z4, x5, y5, z5 $\hookrightarrow \mathrm{x} 6, \mathrm{y} 6, \mathrm{z} 6, \mathrm{x} 7, \mathrm{y} 7, \mathrm{z7}, \mathrm{x} 8, \mathrm{y} 8, \mathrm{z8}, \mathrm{x} 9, \mathrm{y} 9, \mathrm{z} 9, \mathrm{x} 10, \mathrm{y} 10, \mathrm{z} 10--$ params $=9.046$

$\hookrightarrow 1.84766747734,0.7435,0.3852,0.0,0.4169,0.733,0.8359,0.026$,

$\hookrightarrow 0.8404,-0.0086,0.79,0.6,0.8105,0.443,0.095,-0.053,0.106,0.473$,

$\hookrightarrow 0.8928,0.357,0.024,0.061,0.629,0.794,0.017,-0.002,0.341,0.7049$

$\hookrightarrow 0.011,0.29,0.84 \&$ P $4\left\{\{1\} C_{-}\{4\}^{\wedge}\{2\} \# 76\left(a^{\wedge} 10\right) \&\right.$ tP40\& None \&
$\hookrightarrow$ Cs3P7 \& \& T. Meyer and W. H $\backslash^{\prime}\{0\}$ nle and H. G. \{von Schnering $\}$

$\hookrightarrow$ Z. Anorg. Allg. Chem. 552, 69-80 (1987)

1.00000000000000

$\begin{array}{lll}9.04600000000000 & 0.00000000000000 & 0.00000000000000\end{array}$

$0.00000000000000 \quad 9.04600000000000 \quad 0.00000000000000$

$\begin{array}{llll}0.00000000000000 & 0.00000000000000 & 16.71400000000000\end{array}$

$$
\begin{array}{lr}
\text { Cs } & \text { P } \\
12 & 28
\end{array}
$$

Direct

0.74350000000000 $-0.74350000000000$

$-0.74350000000000$

0.38520000000000

0.41690000000000

$-0.41690000000000$

$-0.73300000000000$

0.73300000000000

0.02600000000000

$-0.02600000000000$

$-0.84040000000000$

0.84040000000000

0.79000000000000

$-0.79000000000000$

$-0.60000000000000$

0.60000000000000

0.4430000000000

$-0.443000000000$

$-0.09500000000000$

0.09500000000000

0.10600000000000

$-0.10600000000000$

$-0.47300000000000$

0.47300000000000

0.35700000000000

$-0.35700000000000$

0.02400000000000

0.02400000000000

0.62900000000000

$-0.62900000000000$

$-0.79400000000000$

0.79400000000000

$-0.00200000000000$

0.0020000000000

0.00200000000000

0.34100000000000

0.01100000000000

$-0.01100000000000$

$-0.29000000000000$

0.29000000000000

0.38520000000000 0.3852000000000 0.74350000000000 0.74350000000000 0.73300000000000 $-0.73300000000000$ .4169000000000 $-0.41690000000000$ 0.84040000000000 $-0.84040000000000$ 0.02600000000000 $-0.02600000000000$ 0.60000000000000 $-0.60000000000000$ 0.79000000000000 $-0.79000000000000$ 0.09500000000000 $-0.0950000000000$ 0.4430000000000 0.44300000000000 0.47300000000000 $-0.47300000000000$ 0.10600000000000 0.10600000000000 0.02400000000000 $-0.02400000000000$ 0.35700000000000 $-0.35700000000000$ 0.79400000000000 $-0.79400000000000$ 0.62900000000000 $-0.62900000000000$ 0.34100000000000 $-34100000000000$ $-0.0020000000000$ 0.00200000000000 0.29000000000000 $-0.29000000000000$ 0.01100000000000

0.00000000000000 0.50000000000000 0.25000000000000 0.75000000000000 0.83590000000000 1.33590000000000 1.08590000000000 1.58590000000000 $-0.00860000000000$ 0.49140000000000 0.24140000000000 0.74140000000000 0.81050000000000 1.31050000000000 1.06050000000000 1.56050000000000 $-0.05300000000000$ $-0.05300000000000$ 0.44700000000000 0.1970000000000 0.6970000000000 0.89280000000000 1.39280000000000 1.14280000000000 1.64280000000000 0.06100000000000 0.56100000000000 0.31100000000000 0.8110000000000 0.01700000000000 0.5170000000000 0.26700000000000 0.76700000000000 0.70490000000000 0.70490000000000
1.20490000000000 .20490000000000 1.4549000000000 1.454900000 1.34000000000000 1.34000000000000 1.09000000000000
1.59000000000000

$\begin{array}{ll}C s & (4 a) \\ C s & (4 a) \\ C s & (4 a) \\ C s & (4 a) \\ C s & (4 a) \\ C s & (4 a) \\ C s & (4 a) \\ C s & (4 a) \\ C s & (4 a) \\ C s & (4 a) \\ C s & (4 a) \\ C s & (4 a) \\ P & (4 a) \\ P & (4 a) \\ P & (4 a) \\ P & (4 a) \\ P & (4 a) \\ P & (4 a) \\ P & (4 a) \\ P & (4 a) \\ P & (4 a) \\ P & (4 a) \\ P & (4 a) \\ P & (4 a) \\ P & (4 a) \\ P & (4 a) \\ P & (4 a) \\ P & (4 a) \\ P & (4 a) \\ P & (4 a) \\ P & (4 a) \\ P & (4 a) \\ P & (4 a) \\ P & (4 a) \\ P & (4 a) \\ P & (4 a) \\ P & (4 a) \\ P & (4 a) \\ P & (4 a) \\ P & (4 a) \\ & \end{array}$

Pinnoite $\left(\mathrm{MgB}_{2} \mathrm{O}(\mathrm{OH})_{6}\right)$ : A2B6CD7_tP64_77_2d_6d_d_ab6d - CIF

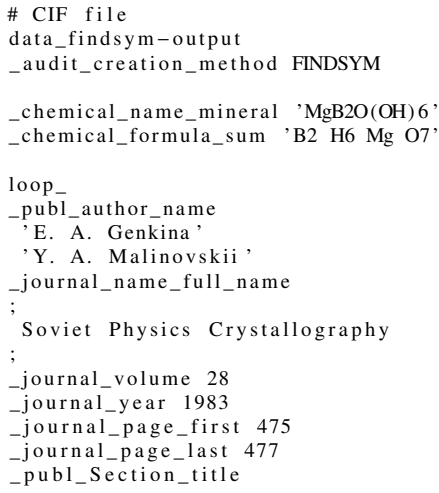

Found in Pearson's Crystal Data - Crystal Structure Database for $\hookrightarrow$ Inorganic Compounds, 2013

aflow_title 'Pinnoite $\left(\mathrm{MgB} \$ \_\{2\} \$ O(O H) \$ \_\{6\} \$\right)$ Structure

aflow_proto 'A2B6CD7_tP64_77_2d_6d_d_ab6d

aflow_params a , c/a, $z_{-}\{1\}, \mathrm{z}_{-}\{2\}, \mathrm{x}_{-}\{3\}, \mathrm{y}_{-}\{3\}, \mathrm{z}_{-}\{3\}, \mathrm{x}_{-}\{4\}, \mathrm{y}_{-}\{4\}, \mathrm{z}_{-}\{4\}, \mathrm{x}_{-}\{$ $\hookrightarrow 5\}, \mathrm{y}_{-}\{5\}, \mathrm{z}_{-}\{5\}, \mathrm{x}_{-}\{6\}, \mathrm{y}_{-}\{6\}, \mathrm{z}_{-}\{6\}, \mathrm{x}_{-}\{7\}, \mathrm{y}_{-}\{7\}, \mathrm{z}_{-}\{7\}, \mathrm{x}_{-}\{8\}, \mathrm{y}_{-}\{8\}$, $\hookrightarrow \mathrm{z}_{-}\{8\}, \mathrm{x}_{-}\{9\}, \mathrm{y}_{-}\{9\}, \mathrm{z}_{-}\{9\}, \mathrm{x}_{-}\{10\}, \mathrm{y}_{-}\{10\}, \mathrm{z}_{-}\{10\}, \mathrm{x}_{-}\{11\}, \mathrm{y}_{-}\{11\}, \mathrm{z}_{-}\{$ $\hookrightarrow 11\}, x_{-}\{12\}, y_{-}\{12\}, z_{-}\{12\}, x_{-}\{13\}, y_{-}\{13\}, z_{-}\{13\}, x_{-}\{14\}, y_{-}\{14\}, z_{-}\{$ $\hookrightarrow 14\}, x_{-}\{15\}, y_{-}\{15\}, z_{-}\{15\}, x_{-}\{16\}, y_{-}\{16\}, z_{-}\{16\}, x_{-}\{17\}, y_{-}\{17\}, z_{-}$ $\hookrightarrow 17\}$

aflow params values $, 7.6159522234,1.07480314961,0.0,0.035,0.1167,0.1203$ $\hookrightarrow, 0.418,0.392,0.3817,0.132,0.362,0.099,0.309,0.136,0.393,0.161$

$\hookrightarrow 0.192,0.355,0.442,0.312,0.133,0.03,0.028,0.14,0.14,0.47,0.35$,

$0.32,0.2491,0.7483,0.273,0.2602,0.0188,0.013,0.2316,0.4834$

$\hookrightarrow 0.184,0.1644,0.2577,0.529,0.3406,0.2365,0.012,0.0182,0.2119$

$\hookrightarrow 0.275,0.4808,0.3008,0.268$,

aflow_Strukturbericht 'None,

-aflow_Pearson, tP64

cell_length_a $\quad 7.615952223$

cell_length_b 7.6159522234

-cell_length_c 8.1856494370

cell_angle_alpha 90.0000000000

_cell_angle_beta 90.0000000000

symmetry_space_group_name_H-M "P 42"

symmetry_Int_Tables_number 77

loop

space_group_symop_id

space_group_symop_operation_xyz

$\mathrm{x}, \mathrm{y}, \mathrm{z}$

$2-\mathrm{x},-\mathrm{y}, \mathrm{z}$

$3-\mathrm{y}, \mathrm{x}, \mathrm{z}+1 / 2$

$4 \mathrm{y},-\mathrm{x}, \mathrm{z}+1 / 2$

loop

atom_site_label

atom_site_type_symbol

atom_site_symmetry_multiplicity

atom_site_Wyckoff_label

atom_site_fract_x

atom_site_fract_y

atom_site_fract_z

m_site_occupancy

$\begin{array}{lllllllll}01 & \mathrm{O} & 2 & \mathrm{a} & 0.00000 & 0.00000 & 0.00000 & 1.00000\end{array}$

$\begin{array}{lllllllll}\mathrm{O} 2 & \mathrm{O} & 2 & \mathrm{~b} & 0.50000 & 0.50000 & 0.03500 & 1.00000\end{array}$

$\begin{array}{llllll}4 & d & 0.11670 & 0.12030 & 0.41800 & 1.00000\end{array}$

$\begin{array}{lllllllll}\mathrm{B} 2 & \mathrm{~B} & 4 & \mathrm{~d} & 0.39200 & 0.38170 & 0.13200 & 1.00000\end{array}$

$\begin{array}{lllllllll}\mathrm{H} 1 & \mathrm{H} & 4 & \mathrm{~d} & 0.36200 & 0.09900 & 0.30900 & 1.00000\end{array}$

$\begin{array}{lllllllll}\mathrm{H} 2 & \mathrm{H} & 4 & \mathrm{~d} & 0.13600 & 0.39300 & 0.16100 & 1.00000\end{array}$

$\begin{array}{lllllllll}\mathrm{H} 3 & \mathrm{H} & 4 & \mathrm{~d} & 0.19200 & 0.35500 & 0.44200 & 1.00000\end{array}$

$\begin{array}{lllllllll}\mathrm{H} 4 & \mathrm{H} & 4 & \mathrm{~d} & 0.31200 & 0.13300 & 0.03000 & 1.00000\end{array}$

$\begin{array}{lllllllll}\mathrm{H} 5 & \mathrm{H} & 4 & \mathrm{~d} & 0.02800 & 0.14000 & 0.14000 & 1.00000\end{array}$

$\begin{array}{lllllllll}\mathrm{H} 6 & \mathrm{H} & 4 & \mathrm{~d} & 0.47000 & 0.35000 & 0.32000 & 1.00000\end{array}$

$\begin{array}{llllllll}\mathrm{Mg} 1 \mathrm{Mg} & 4 & \mathrm{~d} & 0.24910 & 0.74830 & 0.27300 & 1.00000\end{array}$

$\begin{array}{lllllllll}\mathrm{O} 3 & \mathrm{O} & 4 & \mathrm{~d} & 0.26020 & 0.01880 & 0.01300 & 1.00000\end{array}$

$\begin{array}{lllllllll}\mathrm{O} 4 & \mathrm{O} & 4 & \mathrm{~d} & 0.23160 & 0.48340 & 0.18400 & 1.00000\end{array}$

$\begin{array}{lllllllll}\mathrm{O} 5 & \mathrm{O} & 4 & \mathrm{~d} & 0.16440 & 0.25770 & 0.52900 & 1.00000\end{array}$

$\begin{array}{llllllllll}\mathrm{O} 6 & \mathrm{O} & 4 & \mathrm{~d} & 0.34060 & 0.23650 & 0.01200 & 1.00000\end{array}$

$\begin{array}{llllllll}07 & 0 & 4 & d & 0.01820 & 0.21190 & 0.27500 & 1.00000\end{array}$

Pinnoite $\left(\mathrm{MgB}_{2} \mathrm{O}(\mathrm{OH})_{6}\right)$ : A2B6CD7_tP64_77_2d_6d_d_ab6d - POSCAR

\begin{tabular}{|c|c|c|c|c|}
\hline \multicolumn{5}{|c|}{ A2B6CD7_tP64_77_2d_6d_d_ab6d \& a,c/a, z1, z2, x3, y3, z3, x4, y4, z4, x5, y5, z5, x6 } \\
\hline \multicolumn{5}{|c|}{$\hookrightarrow, \mathrm{y} 6, \mathrm{z} 6, \mathrm{x} 7, \mathrm{y} 7, \mathrm{z} 7, \mathrm{x} 8, \mathrm{y} 8, \mathrm{z} 8, \mathrm{x} 9, \mathrm{y} 9, \mathrm{z} 9, \mathrm{x} 10, \mathrm{y} 10, \mathrm{z} 10, \mathrm{x} 11, \mathrm{y} 11, \mathrm{z} 11, \mathrm{x} 12$} \\
\hline \multirow{2}{*}{\multicolumn{5}{|c|}{$\begin{array}{l}\mathrm{y} 12, \mathrm{z} 12, \mathrm{x} 13, \mathrm{y} 13, \mathrm{z} 13, \mathrm{x} 14, \mathrm{y} 14, \mathrm{z} 14, \mathrm{x} 15, \mathrm{y} 15, \mathrm{z} 15, \mathrm{x} 16, \mathrm{y} 16, \mathrm{z} 16, \mathrm{x} 17, \mathrm{y} 17 \\
\mathrm{z} 17-\text {-params }=7.6159522234,1.07480314961,0.0,0.035,0.1167\end{array}$}} \\
\hline & & & & \\
\hline \\
\hline \multicolumn{3}{|c|}{$0.161,0.192,0.355,0.442,0.312,0.133,0.03,0.028,0.14,0.14,0.47}$, & \multicolumn{2}{|c|}{$\begin{array}{l}0.1203,0.418,0.392,0.3817,0.132,0.362,0.099,0.309,0.136,0.393 \\
0.161,0.192,0.355,0.442,0.312,0.133,0.03,0.028,0.14,0.14,0.47\end{array}$} \\
\hline$\hookrightarrow 0.35,0.32,0$ & \multicolumn{4}{|c|}{$0.35,0.32,0.2491,0.7483,0.273,0.2602,0.0188,0.013,0.2316,0.4834$} \\
\hline \multicolumn{5}{|c|}{$0.184,0.1644,0.2577,0.529,0.3406,0.2365,0.012,0.0182,0.2119$} \\
\hline \multicolumn{5}{|c|}{$0.275,0.4808,0.3008,0.268 \& \mathrm{P}_{-}\{2\} \mathrm{C}_{-}\{4\}^{\wedge}\{3\} \# 77(\mathrm{abd} \wedge 15) \&$} \\
\hline \multicolumn{5}{|c|}{ tP64 \& None \& $\mathrm{MgB} 2 \mathrm{O}(\mathrm{OH}) 6$ \& \& E. A. Genkina and Y. A. } \\
\hline \multicolumn{5}{|c|}{ Malinovskii, Sov. Phys. Crystallogr. 28, 475-477 (1983) } \\
\hline \multicolumn{5}{|c|}{1.00000000000000} \\
\hline 7.61595222340000 & 0.00 & \multicolumn{3}{|l|}{0.00000000000000} \\
\hline \multirow{2}{*}{$\begin{array}{l}0.00000000000000 \\
0.00000000000000\end{array}$} & 22340000 & \multirow{2}{*}{\multicolumn{3}{|c|}{$\begin{array}{l}0.00000000000000 \\
8.18564943700000\end{array}$}} \\
\hline & 0.000 & & & \\
\hline $\mathrm{Mg}$ & $\mathrm{O}$ & & & \\
\hline 24 & & & & \\
\hline \multicolumn{5}{|l|}{ Direct } \\
\hline 0.11670000 & & & B & \\
\hline 000000000 & 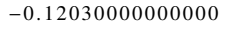 & 0000000 & B & $(4 a)$ \\
\hline-0.12030000000000 & & & & \\
\hline 0.12030000000000 & -0.11 & 0.9 & B & $(4$ \\
\hline 0.39200000000000 & & & B & \\
\hline-0.39200000000000 & 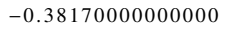 & & B & $(4$ \\
\hline-0.38170000000000 & & & B & \\
\hline 0.38170000000000 & -0.39 & & B & $(4$ \\
\hline 0.36200000000000 & & & $\mathrm{H}$ & \\
\hline-0.36200000000000 & -0 & & $\mathrm{H}$ & \\
\hline-0.09900000000000 & 0000 & 0000000 & $\mathrm{H}$ & $(4 d)$ \\
\hline 0.09900000000000 & -0.36 & & $\mathrm{H}$ & \\
\hline 0.13600000000000 & 0.3930 & 0.16100000000000 & $\mathrm{H}$ & $(4 d)$ \\
\hline-0.13600000000000 & -0.3930 & & $\mathrm{H}$ & \\
\hline-0.39300000000000 & 0.136 & 0.66100000000000 & $\mathrm{H}$ & $(4$ \\
\hline & & & $\mathrm{H}$ & \\
\hline 0.19200000000000 & 0.35500000000000 & 0.44200000000000 & $\mathrm{H}$ & $(4 d)$ \\
\hline-0.19200000000000 & -0.35500000000000 & 0.44200000000000 & $\mathrm{H}$ & $(4 d)$ \\
\hline
\end{tabular}


0.35500000000000 0.3120000000000 $-0.31200000000000$ $-0.13300000000000$ 0.13300000000000 0.02800000000000 $-0.02800000000000$ $-0.14000000000000$ 0.14000000000000 0.47000000000000 $-0.47000000000000$ $-0.35000000000000$ 0.35000000000000 0.24910000000000 $-0.24910000000000$ 0.74830000000000 0.74830000000000 (2) 0.00000000000 0.50000000000000 0.50000000000000 0.26020000000000 $-0.26020000000000$ 0.01880000000000 0.01880000000000 0.23160000000000 $-0.23160000000000$ 0.48340000000000 0.16440000000000 $-0.16440000000000$ 0.25770000000000 0.25770000000000 0.34060000000000 $-0.34060000000000$ $-0.23650000000000$ 0.23650000000000 0.01820000000000 $-0.01820000000000$ $-0.21190000000000$ 0.21190000000000 0.48080000000000 $-0.48080000000000$ $-0.30080000000000$ 0.30080000000000
$-0.19200000000000$ 0.13300000000000 0.13300000000000 0.31200000000000 0.31200000000000 0.14000000000000 $-0.14000000000000$ 0.02800000000000 $-0.02800000000000$ 0.35000000000000 $-0.35000000000000$ $-0.47000000000000$ 0.74830000000000 $-0.74830000000000$ 0.24910000000000 0.24910000000000 0.000000000 0.500000000000 0.50000000000000 0.50000000000000 0.01880000000000 $-0.01880000000000$ 0.2602000000000 $-0.26020000000000$ 0.48340000000000 $-0.48340000000000$ 0.23160000000000 $-0.23160000000000$ 0.25770000000000 $-0.25770000000000$ 0.16440000000000 $-0.16440000000000$ 0.23650000000000 0.23650000000 0.34060000000000 $-0.34060000000000$ 0.21190000000000 $-0.2119000000000$ 0.01820000000000 $-0.01820000000000$ 0.30080000000000 $-0.3008000000000$ 0.48080000000000 $-0.48080000000000$
0.94200000000000 0.0300000000000 0.0300000000000 0.5300000000000 0.5300000000000 0.1400000000000 0.14000000000000 0.64000000000000 0.64000000000000 0.3200000000000 0.32000000000000 0.82000000000000 0.82000000000000 0.27300000000000 0.27300000000000 0.7730000000000 0.77300000000000 0.00000000000000 0.500000000 0.5000000000000 0.03500000000000 0.53500000000000 0.01300000000000 0.0130000000000 0.51300000000000 0.51300000000000 0.18400000000000 0.18400000000000 0.68400000000000 0.68400000000000 0.52900000000000 0.52900000000000 1. 0290000000000 1. 02900000000000 .029000000000 0.01200000000000 0.51200000000000 0.51200000000000 0.27500000000000 0.27500000000000 0.77500000000000 0.77500000000000 0.26800000000000 0.26800000000000 0.76800000000000

\begin{tabular}{|c|c|}
\hline $\mathrm{H}$ & $(4 d)$ \\
\hline $\mathrm{H}$ & (4d) \\
\hline $\mathrm{H}$ & (4d) \\
\hline H & (4d) \\
\hline $\mathrm{H}$ & $(4 d)$ \\
\hline H & $(4 d)$ \\
\hline $\mathrm{H}$ & $(4 d)$ \\
\hline $\mathrm{H}$ & (4d) \\
\hline $\mathrm{H}$ & (4d) \\
\hline $\mathrm{H}$ & (4d) \\
\hline $\mathrm{H}$ & (4d) \\
\hline $\mathrm{H}$ & (4d) \\
\hline $\mathrm{H}$ & (4d) \\
\hline $\mathrm{Mg}$ & (4d) \\
\hline $\mathrm{Mg}$ & (4d) \\
\hline $\mathrm{Mg}$ & $(4 d)$ \\
\hline $\mathrm{Mg}$ & $(4 d)$ \\
\hline $\mathrm{O}$ & (2a) \\
\hline $\mathrm{O}$ & (2a) \\
\hline O & (2b) \\
\hline O & (2b) \\
\hline O & $(4 d)$ \\
\hline O & $(4 d)$ \\
\hline O & $(4 d)$ \\
\hline $\mathrm{O}$ & (4d) \\
\hline $\mathrm{O}$ & $(4 d)$ \\
\hline $\mathrm{O}$ & $(4 \mathrm{~d})$ \\
\hline $\mathrm{O}$ & $(4 d)$ \\
\hline $\mathrm{O}$ & $(4 d)$ \\
\hline $\mathrm{O}$ & $(4 d)$ \\
\hline $\mathrm{O}$ & $(4 d)$ \\
\hline $\mathrm{O}$ & (4d) \\
\hline $\mathrm{O}$ & $(4 d)$ \\
\hline $\mathrm{O}$ & $(4 d)$ \\
\hline $\mathrm{O}$ & $(4 d)$ \\
\hline $\mathrm{O}$ & (4d) \\
\hline $\mathrm{O}$ & $(4 d)$ \\
\hline $\mathrm{O}$ & $(4 d)$ \\
\hline $\mathrm{O}$ & $(4 d)$ \\
\hline $\mathrm{O}$ & $(4 d)$ \\
\hline $\mathrm{O}$ & (4d) \\
\hline $\mathrm{O}$ & $(4 d)$ \\
\hline $\mathrm{O}$ & (4d) \\
\hline $\mathrm{O}$ & $(4 d)$ \\
\hline $\mathrm{O}$ & $(4 d)$ \\
\hline
\end{tabular}

H2S III: A2B_tP48_77_8d_4d - CIF

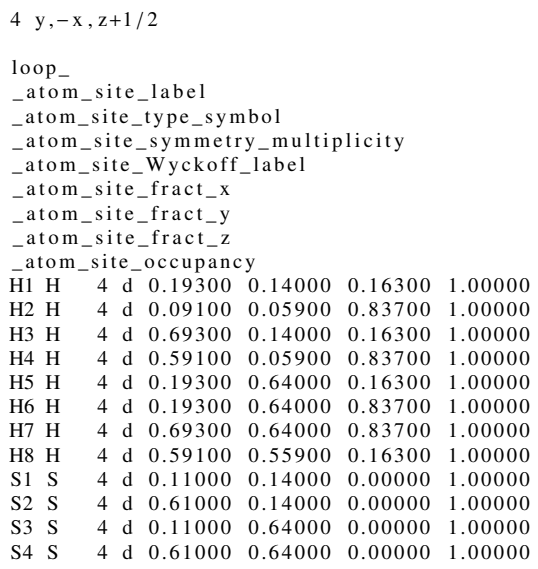

H 2 S III: A2B_tP48_77_8d_4d - POSCAR

\begin{tabular}{|c|c|c|c|c|}
\hline \multirow{2}{*}{\multicolumn{5}{|c|}{ A2B_tP48_77_8d_4d \& a,c/a, x1,y1,z1, x2, }} \\
\hline & & & & \\
\hline \multirow{2}{*}{\multicolumn{5}{|c|}{$\begin{array}{l}\hookrightarrow \mathrm{x} 6, \mathrm{y} 6, \mathrm{z} 6, \mathrm{x} 7, \mathrm{y} 7, \mathrm{z} 7, \mathrm{x} 8, \mathrm{y} 8, \mathrm{z} 8, \mathrm{x} 9, \mathrm{y} 9, \mathrm{z} 9, \mathrm{x} 10, \mathrm{y} 10, \mathrm{z} 10, \mathrm{x} 11, \mathrm{y} 11, \mathrm{z} 11, \mathrm{x} 12 \\
\hookrightarrow \mathrm{y} 12, \mathrm{z} 12-\text { params }=13.47,0.304380103935,0.193,0.14,0.163,0.091\end{array}$}} \\
\hline & & & & \\
\hline \multicolumn{5}{|c|}{$0.059,0.837,0.693,0.14,0.163,0.591,0.059,0.837,0.193,0.64,0.163$} \\
\hline \multicolumn{5}{|c|}{$0.193,0.64,0.837,0.693,0.64,0.837,0.591,0.559,0.163,0.11,0.14$} \\
\hline \multicolumn{5}{|c|}{$0.0,0.61,0.14,0.0,0.11,0.64,0.0,0.61,0.64,0.0 \& \mathrm{P} 4\{2\} \mathrm{C}\{4\} \wedge\{3$} \\
\hline \multirow{2}{*}{\multicolumn{5}{|c|}{ 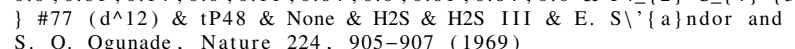 }} \\
\hline & & & & \\
\hline \multicolumn{5}{|l|}{1.00000000000000} \\
\hline 13.47000000000000 & 0.00000000000000 & 0.00000000000000 & & \\
\hline 0.00000000000000 & 13.47000000000000 & 0.00000000000000 & & \\
\hline 0.00000000000000 & 0.00000000000000 & 4.10000000000000 & & \\
\hline $\mathrm{S}$ & & & & \\
\hline 32 & & & & \\
\hline \multicolumn{5}{|l|}{ Direct } \\
\hline 0.19300000000000 & 0.14000000000000 & 0.16300000000000 & $\mathrm{H}$ & $(4 \mathrm{~d})$ \\
\hline-0.19300000000000 & -0.14000000000000 & 0.16300000000000 & $\mathrm{H}$ & (4d) \\
\hline-0.14000000000000 & 0.19300000000000 & 0.66300000000000 & $\mathrm{H}$ & (4d) \\
\hline 0.14000000000000 & -0.19300000000000 & 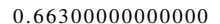 & $\mathrm{H}$ & $(4 \mathrm{~d})$ \\
\hline 0.09100000000000 & 0.05900000000000 & 0.83700000000000 & $\mathrm{H}$ & (4d) \\
\hline-0.09100000000000 & -0.059000 & 0000 & $\mathrm{H}$ & (4d) \\
\hline-0.05900000000000 & 0.09100000000000 & 1.33700000000000 & $\mathrm{H}$ & (4d) \\
\hline 0.05900000000000 & -0.09100000000000 & 000000000 & $\mathrm{H}$ & $(4 \mathrm{~d})$ \\
\hline 0.69300000000000 & 0.14000000000000 & 0.16300000000000 & $\mathrm{H}$ & (4d) \\
\hline-0.69300000000000 & 0000000000 & 0.163 & $\mathrm{H}$ & (4d) \\
\hline-0.14000000000000 & 0.69300000000000 & 0.66300000000000 & $\mathrm{H}$ & $(4 d)$ \\
\hline 0.14000000000000 & -0.69300000000000 & 0.66300000000000 & $\mathrm{H}$ & (4d) \\
\hline 0.59100000000000 & 0.05900000000000 & 0.83700000000000 & $\mathrm{H}$ & $(4 \mathrm{~d})$ \\
\hline-0.59100000000000 & -0.05900000000000 & 0.83700000000000 & $\mathrm{H}$ & $(4 \mathrm{~d})$ \\
\hline-0.05900000000000 & 0.59100000000000 & 1.33700000000000 & $\mathrm{H}$ & $(4 \mathrm{~d})$ \\
\hline 0.05900000000000 & -0.59100000000000 & 1.33700000000000 & $\mathrm{H}$ & (4d) \\
\hline 0.19300000000000 & 0000000000 & 0.16300000000000 & $\mathrm{H}$ & $(4 \mathrm{~d})$ \\
\hline-0.19300000000000 & -0.64000000000000 & 00000000 & $\mathrm{H}$ & $(4 d)$ \\
\hline-0.64000000000000 & 0.19300000000000 & 0.663 & $\mathrm{H}$ & $(4 \mathrm{~d})$ \\
\hline 0.64000000000000 & 00000000 & 0.663 & $\mathrm{H}$ & $(4 \mathrm{~d})$ \\
\hline 0.19300000000000 & 0000000000 & 0.8370 & $\mathrm{H}$ & $(4 \mathrm{~d})$ \\
\hline-0.19300000000000 & -0.640 & 0.837 & $\mathrm{H}$ & \\
\hline-0.64000000000000 & 0000000000 & 1.33700000000000 & $\mathrm{H}$ & $(4 \mathrm{~d})$ \\
\hline 0.64000000000000 & -0.19300000000000 & 1.337 & $\mathrm{H}$ & $(4 \mathrm{~d})$ \\
\hline 0.69300000000000 & 0.64000000000000 & 0.83700000000 & $\mathrm{H}$ & $(4 \mathrm{~d})$ \\
\hline-0.69300000000000 & -0.64000000000000 & 0.83700000000000 & $\mathrm{H}$ & $(4 \mathrm{~d})$ \\
\hline-0.64000000000000 & 0.69300000000000 & 1337 & $\mathrm{H}$ & \\
\hline 0.64000000000000 & -0.69300000000000 & 1.33700000000000 & $\mathrm{H}$ & $(4 \mathrm{~d})$ \\
\hline 0.59100000000000 & & 016 & $\mathrm{H}$ & \\
\hline-0.59100000000000 & -0.55900000000000 & 0.16300000000000 & $\mathrm{H}$ & $(4 \mathrm{~d})$ \\
\hline-0.55900000000000 & & & $\mathrm{H}$ & \\
\hline 0.55900000000000 & 00000000 & 0.6 & $\mathrm{H}$ & (4d) \\
\hline 0.11000000000000 & 0.140000000000 & 0.000 & $\mathrm{~S}$ & (4d) \\
\hline-0.11000000000000 & -0.14000000000000 & 0.00000000000000 & $\mathrm{~s}$ & (4d) \\
\hline-0.14000000000000 & 0.11000000000000 & 0.50000000000000 & $\mathrm{~s}$ & $(4 d)$ \\
\hline 0.14000000000000 & -0.11000000000000 & 0.50000000000000 & 些 & (4d) \\
\hline 0.61000000000000 & 0.14000000000000 & 0.00000000000000 & $\mathrm{~S}$ & (4d) \\
\hline-0.61000000000000 & -0.14000000000000 & 0.00000000000000 & $\mathrm{~s}$ & \\
\hline-0.14000000000000 & 0.61000000000000 & 0.50000000000000 & $\mathrm{~s}$ & (4d) \\
\hline 0.14000000000000 & -0.61000000000000 & 0.50000000000000 & $\mathrm{~S}$ & $(4 \mathrm{~d})$ \\
\hline 0.11000000000000 & 0.64000000000000 & 0.00000000000000 & $\mathrm{~s}$ & $(4 \mathrm{~d})$ \\
\hline-0.11000000000000 & -0.64000000000000 & 0.00000000000000 & $\mathrm{~S}$ & $(4 \mathrm{~d})$ \\
\hline-0.64000000000000 & 0.11000000000000 & 0.50000000000000 & $\mathrm{~S}$ & (4d) \\
\hline 0.64000000000000 & -0.11000000000000 & 0.50000000000000 & $\mathrm{~s}$ & $(4 d)$ \\
\hline 0.61000000000000 & 0.64000000000000 & 0.00000000000000 & $\mathrm{~s}$ & (4d) \\
\hline-0.61000000000000 & -0.64000000000000 & 0.00000000000000 & $\mathrm{~S}$ & $(4 c)$ \\
\hline-0.64000000000000 & 0.61000000000000 & 0.50000000000000 & $\mathrm{~S}$ & $(4 \mathrm{~d})$ \\
\hline 0.64000000000000 & -0.61000000000000 & 0.50000000000000 & $\mathrm{~S}$ & (4d) \\
\hline
\end{tabular}

$\mathrm{Sr}_{2} \mathrm{As}_{2} \mathrm{O}_{7}$ : A2B7C2_tP88_78_4a_14a_4a - CIF

\# CIF file
data_findsym-output
_audit_creation_method FINDSYM
_chemical_name_mineral 'Sr2As2O7,
_chemical_formula_sum 'As2 O7 Sr2,
loop__
-publ_author_name
,A. Mbarek,
'F. Edhokkar,
_journal_name_full_name
;


Acta Crystallographica Section E. Crystallographic Communications

_journal_volume 69

_journal_year 2013

journal_page_first i84

journal_page_last i 84

The P4\$_ $\{3\} \$$ enantiomorph of $\mathrm{Sr}_{-}\{2\} \$ \mathrm{As}_{-}\{2\} \$ \mathrm{O}_{-}\{7\}$

Found in Pearson's Crystal Data - Crystal Structure Database for $\hookrightarrow$ Inorganic Compounds, 2013

aflow_title'Sr\$_\{2\}\$As\$_\{2\}\$O\$_ 7$\}$ \$ Structure

aflow proto 'A2B7C2_tP88_78_4a_14a_4a

aflow_params $, a, c / a, x_{-}\{1\}, y_{-}\{1\}, z_{-}\{1\}, x_{-}\{2\}, y_{-}\{2\}, z_{-}\{2\}, x_{-}\{3\}, y_{-}\{3\}, z_{-}\{$ $\hookrightarrow 3\}, x_{-}\{4\}, y_{-}\{4\}, z_{-}\{4\}, x_{-}\{5\}, y_{-}\{5\}, z_{-}\{5\}, x_{-}\{6\}, y_{-}\{6\}, z_{-}\{6\}, x_{-}\{7\}$ $\hookrightarrow \mathrm{y}_{-}\{7\}, \mathrm{z}_{-}\{7\}, \mathrm{x}_{-}\{8\}, \mathrm{y}_{-}\{8\}, \mathrm{z}_{-}\{8\}, \mathrm{x}_{-}\{9\}, \mathrm{y}_{-}\{9\}, \mathrm{z}_{-}\{9\}, \mathrm{x}_{-}\{10\}, \mathrm{y}_{-}\{10\}$,
$\hookrightarrow \mathrm{z}_{-}\{10\}, \mathrm{x}_{-}\{11\}, \mathrm{y}_{-}\{11\}, \mathrm{z}_{-}\{11\}, \mathrm{x}_{-}\{12\}, \mathrm{y}_{-}\{12\}, \mathrm{z}_{-}\{12\}, \mathrm{x}_{-}\{13\}, \mathrm{y}_{-}\{13\}$,

$\hookrightarrow \mathrm{z}_{-}\{10\}, \mathrm{x}_{-}\{11\}, \mathrm{y}_{-}\{11\}, \mathrm{z}_{-}\{11\}, \mathrm{x}_{-}\{12\}, \mathrm{y}_{-}\{12\}, \mathrm{z}_{-}\{12\}, \mathrm{x}_{-}\{13\}, \mathrm{y}_{-}\{13\}$,

$\hookrightarrow \mathrm{z}_{-}\{16\}, \mathrm{x}_{-}\{17\}, \mathrm{y}_{-}\{17\}, \mathrm{z}_{-}\{17\}, \mathrm{x}_{-}\{18\}, \mathrm{y}_{-}\{18\}, \mathrm{z}_{-}\{18\}, \mathrm{x}_{-}\{19\}, \mathrm{y}_{-}\{19\}$

$\hookrightarrow \mathrm{z}_{-}\{19\}, \mathrm{x}_{-}\{20\}, \mathrm{y}_{-}\{20\}, \mathrm{z}_{-}\{20\}, \mathrm{x}_{-}\{21\}, \mathrm{y}_{-}\{21\}, \mathrm{z}_{-}\{21\}, \mathrm{x}_{-}\{22\}, \mathrm{y}_{-}\{22\}$, $\hookrightarrow \mathrm{z}_{-}\{22\}$

aflow_params_values $7.1088964505,3.60337042297,0.14561,0.23414,0.69696$ $\leftrightarrow, 0.22057,0.48276,0.79885,0.18673,0.10525,0.29124,0.24906$,

$\hookrightarrow 0.35443,0.19001,0.4586,0.3299,0.02876,0.0936,0.3899,0.1424$

$\hookrightarrow 0.4187,0.2147,0.16739,0.1085,0.2261,0.23449,0.2999,0.2626$,

$\hookrightarrow 0.32782,0.0777,0.3681,0.7514,0.2725,0.3751,0.65898,0.0556$,

$\hookrightarrow 0.0026,0.0009,0.31632,0.3813,0.3334,0.82115,0.2817,0.0576$

$\hookrightarrow 0.71776,0.168,0.0503,-0.08181,0.26434,0.22687,0.42638,0.02701$

$\hookrightarrow 0.34822,0.57318,0.15408,0.39584,-0.07135,0.37469,0.25769$, $\hookrightarrow 0.06464$

aflow_Strukturbericht 'None'

_aflow_Pearson 'tP88,

cell_length_a 7.1088964505

cell_length_b $\quad 7.1088964505$

cell_length_c $\quad 25.6159872097$

_el_angle_alpha 90.0000000000

cell_angle_gamma 90.0000000000

symmetry_space_group_name_H-M "P 43"

symmetry_Int_Tables_number 78

loop

space group symop id

_space_group_symop_operation_xyz

$1 \mathrm{x}, \mathrm{y}, \mathrm{z}$

$2-\mathrm{x},-\mathrm{y}, \mathrm{z}+1 / 2$

$3-\mathrm{y}, \mathrm{x}, \mathrm{z}+3 / 4$
$4 \mathrm{y},-\mathrm{x}, \mathrm{z}+1 / 4$

loop

atom_site_label

atom_site_type_symbol

atom_site_symmetry_multiplicity

atom_site_Wyckoff_label

atom_site_fract_x

atom_site_fract_y

_tom_site_fract_z

$\begin{array}{lllllll}\text { As1 As } & 4 \text { a } & 0.14561 & 0.23414 & 0.69696 & 1.00000\end{array}$

$\begin{array}{llllllll}\text { As1 As } & 4 & \text { a } & 0.14561 & 0.23414 & 0.69696 & 1.00000 \\ \text { As2 As } & 4 & \text { a } & 0.22057 & 0.48276 & 0.79885 & 1.00000\end{array}$

$\begin{array}{lllllll}\text { As3 As } \quad 4 \text { a } & 0.18673 & 0.10525 & 0.29124 & 1.00000\end{array}$

As4 As $\quad 4$ a $0.24906 \quad 0.354430 .19001 \quad 1.00000$

$\begin{array}{lllll}4 \text { a } & 0.45860 & 0.32990 & 0.02876 & 1.00000\end{array}$

$\begin{array}{llllllll}\mathrm{O} 2 & \mathrm{O} & 4 & \mathrm{a} & 0.09360 & 0.38990 & 0.14240 & 1.00000\end{array}$

$\begin{array}{lllllllll}\mathrm{O} 3 & \mathrm{O} & & 4 & \text { a } & 0.41870 & 0.21470 & 0.16739 & 1.00000 \\ \mathrm{O} 4 & \mathrm{O} & & 4 & \mathrm{a} & 0.10850 & 0.22610 & 0.23449 & 1.00000\end{array}$

$\begin{array}{llllllll}\mathrm{O} 4 & \mathrm{O} & 4 & \mathrm{a} & 0.10850 & 0.22610 & 0.23449 & 1.00000\end{array}$

$\begin{array}{lllllllll}\mathrm{O} 5 & \mathrm{O} & 4 & \mathrm{a} & 0.29990 & 0.26260 & 0.32782 & 1.00000\end{array}$

$\begin{array}{llllllll}\mathrm{O} 6 & \mathrm{O} & 4 & \mathrm{a} & 0.07770 & 0.36810 & 0.75140 & 1.00000\end{array}$

$\begin{array}{lllllllll}07 & \mathrm{O} & 4 & \text { a } & 0.27250 & 0.37510 & 0.65898 & 1.00000\end{array}$

$\begin{array}{lllllllll}\mathrm{O} 8 & \mathrm{O} & 4 & \mathrm{a} & 0.05560 & 0.34270 & 0.02051 & 1.00000\end{array}$

$\begin{array}{lllllllll}\mathrm{O} 9 & \mathrm{O} & 4 & \mathrm{a} & 0.32510 & 0.30370 & 0.52047 & 1.00000\end{array}$

$\begin{array}{lllllllll}010 & 0 & 4 & \text { a } & 0.48110 & 0.05460 & 0.59334 & 1.00000\end{array}$

$\begin{array}{lllllllll}\mathrm{O} 11 & \mathrm{O} & 4 & \mathrm{a} & 0.00260 & 0.00090 & 0.31632 & 1.00000\end{array}$

$\begin{array}{lllllllll}\mathrm{O} 12 & \mathrm{O} & 4 & 4 & \text { a } & 0.38130 & 0.33340 & 0.82115 & 1.00000\end{array}$

$\begin{array}{llllllll}012 & \mathrm{O} & 4 & \mathrm{a} & 0.38130 & 0.33340 & 0.82115 & 1.00000 \\ \mathrm{O} 13 & \mathrm{O} & 4 & \text { a } & 0.28170 & 0.05760 & 0.71776 & 1.00000\end{array}$

$\begin{array}{lllllllll}\mathrm{O} 13 & \mathrm{O} & 4 & 4 & \mathrm{a} & 0.28170 & 0.05760 & 0.71776 & 1.00000 \\ 014 & \mathrm{O} & 4 & 4 & 0.16800 & 0.05030 & -0.08181 & 1.00000\end{array}$

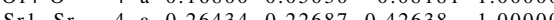

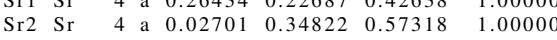

$\begin{array}{lllllllll}\mathrm{Sr} 3 & \mathrm{Sr} & 4 & \text { a } & 0.15408 & 0.39584 & -0.07135 & 1.00000 \\ \mathrm{Sr} 4 & \mathrm{Sr} & 4 & \mathrm{a} & 0.37469 & 0.25769 & 0.06464 & 1.00000\end{array}$

$\mathrm{Sr}_{2} \mathrm{As}_{2} \mathrm{O}_{7}$ : A2B7C2_tP88_78_4a_14a_4a - POSCAR

A2B7C2_tP88_78_4a_14a_4a \& a , c/a, x1, y1, z1 , x2 , y2 , z2 , x3 , y3 , z3 , x4, y4, z4 , x5 , $\hookrightarrow \mathrm{y} 5, \mathrm{z} 5, \mathrm{x} 6, \mathrm{y} 6, \mathrm{z} 6, \mathrm{x} 7, \mathrm{y} 7, \mathrm{z} 7, \mathrm{x} 8, \mathrm{y} 8, \mathrm{z} 8, \mathrm{x} 9, \mathrm{y} 9, \mathrm{z} 9, \mathrm{x} 10, \mathrm{y} 10, \mathrm{z} 10, \mathrm{x} 11, \mathrm{y} 11$,
$\hookrightarrow \mathrm{z} 11, \mathrm{x} 12, \mathrm{y} 12, \mathrm{z} 12, \mathrm{x} 13, \mathrm{y} 13, \mathrm{z} 13, \mathrm{x} 14, \mathrm{y} 14, \mathrm{z} 14, \mathrm{x} 15, \mathrm{y} 15, \mathrm{z} 15, \mathrm{x} 16, \mathrm{y} 16, \mathrm{z} 16$

$\hookrightarrow \mathrm{z} 11, \mathrm{x} 12, \mathrm{y} 12, \mathrm{z} 12, \mathrm{x} 13, \mathrm{y} 13, \mathrm{z} 13, \mathrm{x} 14, \mathrm{y} 14, \mathrm{z} 14, \mathrm{x} 15, \mathrm{y} 15, \mathrm{z} 15, \mathrm{x} 16, \mathrm{y} 16, \mathrm{z} 16$

$\hookrightarrow, \mathrm{x} 17, \mathrm{y} 17, \mathrm{z} 17, \mathrm{x} 18, \mathrm{y} 18, \mathrm{z} 18, \mathrm{x} 19, \mathrm{y} 19, \mathrm{z} 19, \mathrm{x} 20, \mathrm{y} 20, \mathrm{z} 20, \mathrm{x} 21, \mathrm{y} 21, \mathrm{z} 21$,

$\hookrightarrow \mathrm{x} 22, \mathrm{y} 22, \mathrm{z} 22-\mathrm{p}$ -

$\hookrightarrow 0.24906,0.35443,0.19001,0.4586,0.3299,0.02876,0.0936,0.3899$,

$\hookrightarrow 0.1424,0.4187,0.2147,0.16739,0.1085,0.2261,0.23449,0.2999$

$\hookrightarrow 0.2626,0.32782,0.0777,0.3681,0.7514,0.2725,0.3751,0.65898$

$\hookrightarrow 0.0556,0.3427,0.02051,0.3251,0.3037,0.52047,0.4811,0.0546$

$\hookrightarrow 0.59334,0.0026,0.0009,0.31632,0.3813,0.3334,0.82115,0.2817$,

$\hookrightarrow 0.02701,0.34822,0.57318,0.15408,0.39584,-0.07135,0.37469$.

$\hookrightarrow 0.25769,0.06464 \&$ P $4 \_\{3\} C_{-}\{4\}^{\wedge}\{4\} \# 78\left(a^{\wedge} 22\right) \&$ tP88 \& None \&

$\hookrightarrow$ Sr2As2O7\& \& A. Mbarek and F. Edhokkar, Acta Crystallogr. E 69

1.00000000000000

.10889645050000

0.00000000000000

$\begin{array}{rrr}\text { As } & \mathrm{O} & \mathrm{Sr} \\ 16 & 56 & 16\end{array}$

Direct

0.14561000000000 $-0.14561000000000$ $-0.23414000000000$ 0.23414000000000

$-0.22057000000000$

$-0.48276000000000$

0.48276000000000

0.18673000000000

$-0.18673000000000$

$-0.10525000000000$

0.10525000000000

0.24906000000000

$-0.24906000000000$

$-0.35443000000000$

0.35443000000000

$-0.45860000000000$

$-0.32990000000000$

0.09360000000000

$-0.09360000000000$

$-0.38990000000000$

0.38990000000000

0.4187000000000

$-0.41870000000000$

0.21470000000000

0.10850000000000

$-0.10850000000000$

-0.22610000000000
0.22610000000000

0.29990000000000

$-0.29990000000000$

$-0.26260000000000$

0.26260000000000

0.07770000000000

$-0.07770000000000$

$-0.36810000000000$

0.36810000000000

0.27250000000000

$-0.27250000000000$

$-0.37510000000000$

0.37510000000000

0.05560000000000

$-0.05560000000000$

-0.34270000000000
0.34270000000000

0.32510000000000

$-0.3251000000000$

$-0.30370000000000$

0.30370000000000

0.48110000000000

$-0.05460000000000$

0.05460000000000

0.00260000000000

0.00260000000000

$-0.00260000000000$

0.00090000000000

0.38130000000000

$-0.38130000000000$

$-0.33340000000000$

0.33340000000000

0.2817000000000

$-0.28170000000000$

$-0.05760000000000$

0.05760000000000

$-0.16800000000000$

$-0.05030000000000$

0.05030000000000

0.26434000000000

$-0.2643400000000$

$-0.2643400000000$

0.22687000000000

0.02701000000000

$-0.02701000000000$

$-0.34822000000000$

0.34822000000000

0.15408000000000

$-0.15408000000000$

$-0.39584000000000$

0.39584000000000

0.37469000000000

$-0.37469000000000$

$-0.25769000000000$

0.25769000000000

0000000000000

.10889645050000

0.00000000000000

0.00000000000000

25.61598720970000

0.23414000000000 .23414000000000

.14561000000000

0.48276000000000

.48276000000000

0.005700000000

.22057000000000

.10525000000000

.18673000000000

0.18673000000000

0.35443000000000

0.35443000000000

.24906000000000

.24906000000000

0.32990000000000

0.32990000000000

.

.45860000000000

0.38990000000000

0.09360000000000

0.09360000000000

.

21470000000000

41870000000000

.41870000000000

0.22610000000000

$-0.22610000000000$

0.10850000000000

0.10850000000000

0.26260000000000

0.26260000000000

90000000000

.

0.36810000000000

0.36810000000000

0.07770000000000

0.37510000000000

0.27250000000000

0.27250000000000

0.34270000000000

0.34270000000000

0.05560000000000

$-0.05560000000000$

0.30370000000000

0.30370000000000

0.32510000000000

.3510000000000 0.05460000000000 .05460000000000 0.48110000000000

0.48110000000000

.

0.00260000000000

0.00260000000000

0.33340000000000

0.33340000000000

0.38130000000000

$-0.38130000000000$

0.05760000000000

$-0.05760000000000$

0.28170000000000

28170000000000

.05030000000000

0.05030000000000

0.16800000000000

0.16800000000000

.22687000000000

0.2687000000000

0.26434000000000

$-0.26434000000000$

0.2643400000000

0.34822000000000

0.02701000000000

0.02701000000000

0.39584000000000

$-0.39584000000000$

0.15408000000000

0.15408000000000

. 25769000000000

.25769000000000

$-.37469000000000$

0.69696000000000 . 44696000000000 .94696000000000 0.79885000000000 1.29885000000000

1.54885000000000

1.04885000000000

.

79124000000000

4124000000000

54124000000000

19001000000000

69001000000000

0.94001000000000

.

. 44001000000000

.

作876000000000

.

.

. 14240000000000

64240000000000

89240000000000

.39240000000000

.16739000000000

.66739000000000

.91739000000000

41739000000000

23449000000000

.73449000000000

.

0.48449000000000

0.48449000000000

.

. 82782000000000

. 


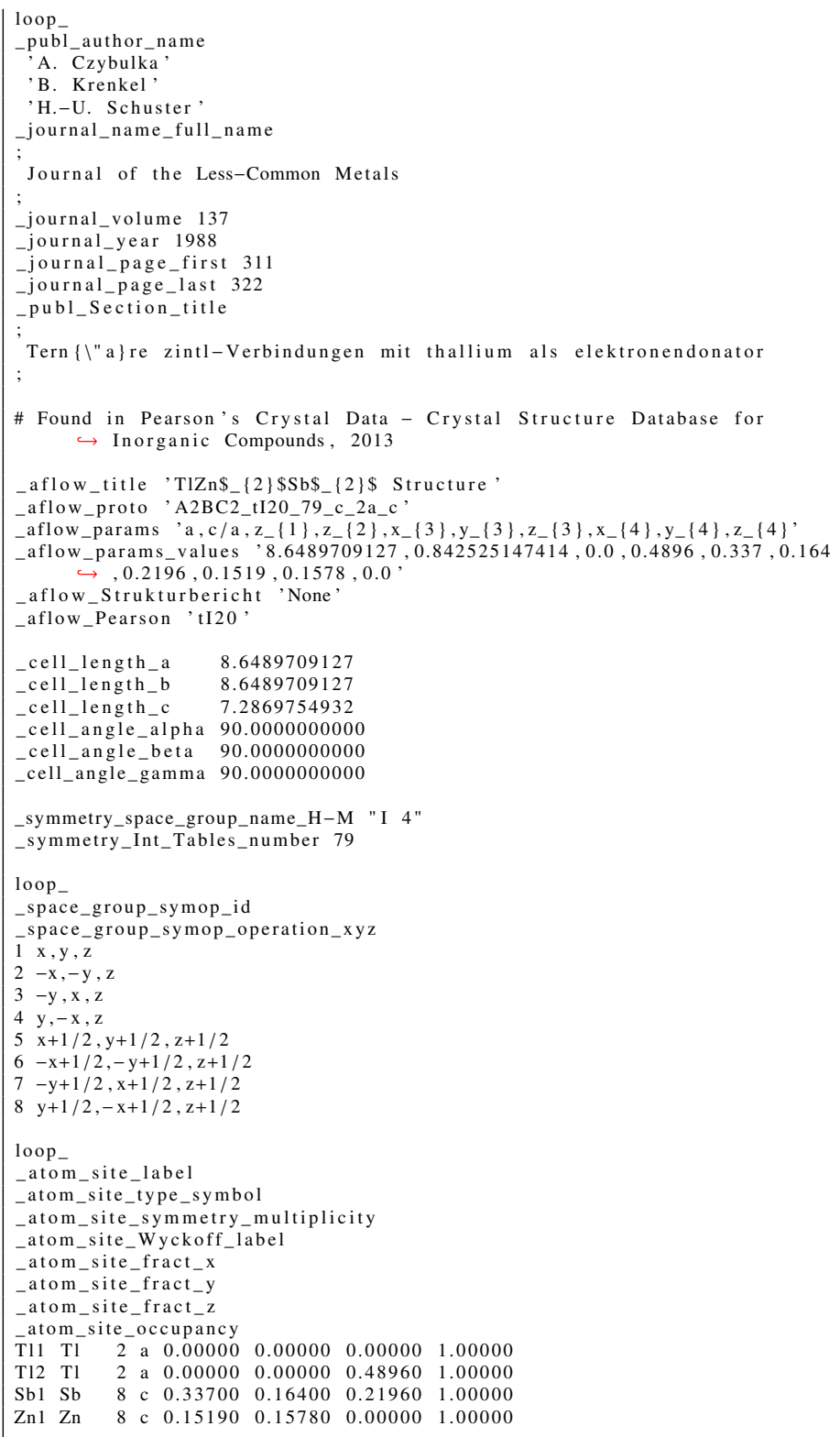

$\mathrm{TIZn}_{2} \mathrm{Sb}_{2}$ : A2BC2_tI20_79_c_2a_c - POSCAR

\begin{tabular}{|c|c|c|c|c|}
\hline \\
\hline & & & & \\
\hline \multirow{4}{*}{\multicolumn{5}{|c|}{ 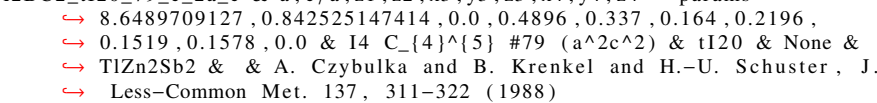 }} \\
\hline & & & & \\
\hline & & & & \\
\hline & & & & \\
\hline-4.32448545635000 & 4.32448545635000 & 3.64348774660000 & & \\
\hline 4.32448545635000 & -4.3244 & 3.64348774660000 & & \\
\hline 4.32448545635000 & 4.32448545635000 & 60000 & & \\
\hline $\mathrm{Zn}$ & & & & \\
\hline 4 & & & & \\
\hline \multicolumn{5}{|l|}{ Direct } \\
\hline 0.38360000000000 & 0. & 0.5 & $\mathrm{Sb}$ & $(8$ \\
\hline 0.05560000000000 & -0.1 & -0.5 & $\mathrm{Sb}$ & , \\
\hline 0.55660000000000 & 0.0556 & 00000 & $\mathrm{Sb}$ & $(8 \mathrm{c})$ \\
\hline-0.11740000000000 & & -0.17 & $\mathrm{Sb}$ & \\
\hline 0.00000000000000 & 0.00000000000000 & 0.00000000000000 & $\mathrm{Tl}$ & (2a) \\
\hline \multirow{2}{*}{$\begin{array}{l}0.48960000000000 \\
0.15780000000000\end{array}$} & & & $\mathrm{Tl}$ & \\
\hline & 0.15190000000000 & 0.30970000000000 & $\mathrm{Zn}$ & $(8 \mathrm{c})$ \\
\hline-0.15780000000000 & -0.15190000000000 & -0.30970000000000 & $\mathrm{Zn}$ & \\
\hline 0.15190000000000 & -0.1578 & -0.005 & $\mathrm{Zn}$ & $(8 \mathrm{c})$ \\
\hline-0.15190000000000 & 0.15780000000000 & 0.00590000000000 & $\mathrm{Zn}$ & $(8 \mathrm{c})$ \\
\hline
\end{tabular}

$\beta-\mathrm{NbO}_{2}:$ AB2_tI $48 \_80 \_2 \mathrm{~b} \_4 \mathrm{~b}-\mathrm{CIF}$

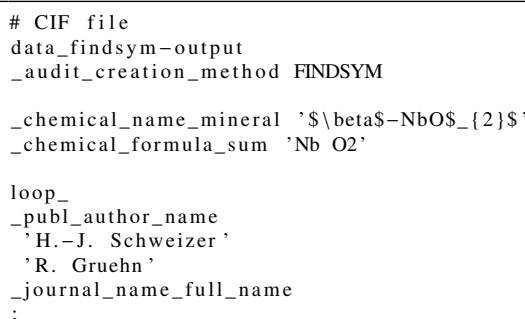

Zeitschrift $f\{\backslash$ u $\}$ r Naturforschung B

journal_volume 37

journal_year 1982

journal_page_first 136

journal_page_last 1368

publ_Section_title

Zur Darstellung und Kristallstruktur von $\$ \backslash$ beta $\$-N b O \$\{\{2\} \$$ / Synthesis $\hookrightarrow$ and Crystal Structure of $\$ \backslash$ beta $\$-\mathrm{NbO} \$\{2\} \$$

\# Found in Pearson's Handbook of Crystallographic Data, 1991

aflow title, $\$ \backslash$ beta $\$-N b O \$\{2\} \$$ Structure,

aflow proto 'AB2 tI 48 80 2 b $4 \mathrm{~b}$,

aflow_params 'a, c/a, $x_{-}\{1\}, y_{-}\{1\}, z_{-}\{1\}, x_{-}\{2\}, y_{-}\{2\}, z_{-}\{2\}, x_{-}\{3\}, y_{-}\{3\}, z_{-}$ $\hookrightarrow 3\}, x_{-}\{4\}, y_{-}\{4\}, z_{-}\{4\}, x_{-}\{5\}, y_{-}\{5\}, z_{-}\{5\}, x_{-}\{6\}, y_{-}\{6\}, z_{-}\{6\}$ $\stackrel{0}{\hookrightarrow} 0.4909,0.4804,0.3974,0.1497,0.0077,0.1102,0.3642,-0.0098,0.6086$ $\hookrightarrow, 0.3609,0.5064,0.65,0.1038,0.2484$,

-aflow_Strukturbericht 'None'

aflow_Pearson 'tI48

symmetry_space_group_name_H-M "I 41 "

symmetry_Int_Tables_number 80

cell_length_a $\quad 9.69300$

cell length_c 5.98500

cell angle

_cell_angle_alpha 90.00000

loop

space_group_symop_id

space_group_symop_operation_xy

$1 \mathrm{x}, \mathrm{y}, \mathrm{z}$

$-\mathrm{x},-\mathrm{y}, \mathrm{z}$

$3-\mathrm{y}, \mathrm{x}+1 / 2, \mathrm{z}+1 / 4$

y, $-\mathrm{x}+1 / 2, \mathrm{z}+1 / 4$

$5 \mathrm{x}+1 / 2, \mathrm{y}+1 / 2, \mathrm{z}+1 / 2$

$-x+1 / 2,-y+1 / 2, z+1 / 2$

$7-\mathrm{y}+1 / 2, \mathrm{x}, \mathrm{z}+3 / 4$

$8 \mathrm{y}+1 / 2,-\mathrm{x}, \mathrm{z}+3 / 4$

loop

atom_site label

atom_site type symbol

atom_site_symmetry_multiplicity

atom_site_Wyckoff_label

atom_site_fract_x

atom_site_fract_y

atom_site_fract_z

\begin{tabular}{llllllll}
\hline Nb1 Nb & 8 & b & 0.26210 & 0.50760 & 0.02990 & 1.00000
\end{tabular}

$\begin{array}{llllllll}\mathrm{Nb} 2 & \mathrm{Nb} & 8 & \mathrm{~b} & 0.24550 & 0.49090 & 0.48040 & 1.00000\end{array}$

$\begin{array}{lllllllll}\mathrm{O} 1 & \mathrm{O} & 8 & \mathrm{~b} & 0.39740 & 0.14970 & 0.00770 & 1.00000\end{array}$

$\begin{array}{lllllllll}\mathrm{O} 2 & \mathrm{O} & 8 & \mathrm{~b} & 0.11020 & 0.36420 & -0.00980 & 1.00000\end{array}$

$\begin{array}{lllllllll}\mathrm{O} 3 & \mathrm{O} & 8 & \mathrm{~b} & 0.60860 & 0.36090 & 0.50640 & 1.00000\end{array}$

$\begin{array}{lllllllll}\mathrm{O} 4 & \mathrm{O} & 8 & \mathrm{~b} & 0.65000 & 0.10380 & 0.24840 & 1.00000\end{array}$

$\beta-\mathrm{NbO}_{2}$ : AB2_tI $48 \_80 \_2 \mathrm{~b} \_4 \mathrm{~b}$ - POSCAR

\begin{tabular}{|c|c|c|c|c|}
\hline $\mathrm{I} 48 \_80 \_2 \mathrm{~b} \_4 \mathrm{~b} \quad \&$ & $, \mathrm{c} / \mathrm{a}, \mathrm{x} 1, \mathrm{y1}, \mathrm{z} 1, \mathrm{xz}$ & $2 L, \mathrm{AJ}$ & & \\
\hline$\hookrightarrow \mathrm{x} 6, \mathrm{y} 6, \mathrm{z} 6--\mathrm{p}$ & arams $=9.693,0.617$ & 896007,0 & & 299 \\
\hline$\hookrightarrow 0.2455,0.490$ & $9,0.4804,0.3974,0.1$ & $497,0.0077,0.1102$ & $.3642,-$ & -0.0098 \\
\hline$\hookrightarrow, 0.6086,0.36$ & $09,0.5064,0.65,0.10$ & $38,0.2484 \& \mathrm{I} 4-\{1\}$ & $C_{-}\{4\}^{\wedge}$ & $\{6\} \# 80$ \\
\hline$\hookrightarrow \quad\left(\mathrm{b}^{\wedge} 6\right) \& \mathrm{tI} 4$ & 8 \& None \& $\mathrm{NbO} 2 \&$ & $\$$ \beta $\$-\mathrm{NbO} \$\{\{2\} \$ \&$ & H. $-\mathrm{J}$. & \\
\hline $\begin{array}{l}\hookrightarrow \text { Schweizer an } \\
1.00000000000000\end{array}$ & d R. Gruehn, Z. Na & turforsch. B 37, & $1-1368$ & ( 1982$)$ \\
\hline 1.00000000000000 & & & & \\
\hline-4.84650000000000 & 4.84650000000000 & 2.99250000000000 & & \\
\hline 4.84650000000000 & -4.84650000000000 & 2.99250000000000 & & \\
\hline 4.84650000000000 & 4.84650000000000 & -2.99250000000000 & & \\
\hline $\mathrm{Nb}$ & & & & \\
\hline 16 & & & & \\
\hline irect & & & & \\
\hline 0.53750000000000 & 0.29200000000000 & 0.76970000000000 & $\mathrm{Nb}$ & $(8 b)$ \\
\hline-0.47770000000000 & -0.23220000000000 & -0.76970000000000 & $\mathrm{Nb}$ & $(8 \mathrm{~b})$ \\
\hline 1.04200000000000 & -0.22770000000000 & 0.25450000000000 & $\mathrm{Nb}$ & $(8 b)$ \\
\hline 0.51780000000000 & 0.78750000000000 & 0.74550000000000 & $\mathrm{Nb}$ & $(8 b)$ \\
\hline 0.97130000000000 & 0.72590000000000 & 0.73640000000000 & $\mathrm{Nb}$ & $(8 b)$ \\
\hline-0.01050000000000 & 0.23490000000000 & -0.73640000000000 & $\mathrm{Nb}$ & $(8 b)$ \\
\hline 1.47590000000000 & 0.23950000000000 & 0.25460000000000 & $\mathrm{Nb}$ & $(8 b)$ \\
\hline 0.98490000000000 & 1.22130000000000 & 0.74540000000000 & $\mathrm{Nb}$ & $(8 b)$ \\
\hline 0.15740000000000 & 0.40510000000000 & 0.54710000000000 & $\mathrm{O}$ & $(8 b)$ \\
\hline-0.14200000000000 & -0.38970000000000 & -0.54710000000000 & $\mathrm{O}$ & $(8 b)$ \\
\hline 1.15510000000000 & 0.10800000000000 & 0.74770000000000 & $\mathrm{O}$ & $(8 b)$ \\
\hline 0.36030000000000 & 0.40740000000000 & 0.25230000000000 & $\mathrm{O}$ & $(8 b)$ \\
\hline 0.35440000000000 & 0.10040000000000 & 0.47440000000000 & $\mathrm{O}$ & $(8 b)$ \\
\hline-0.37400000000000 & -0.12000000000000 & -0.47440000000000 & $\mathrm{O}$ & $(8 b)$ \\
\hline 0.85040000000000 & -0.12400000000000 & 0.24600000000000 & $\mathrm{O}$ & $(8 b)$ \\
\hline 0.63000000000000 & 0.60440000000000 & 0.75400000000000 & $\mathrm{O}$ & $(8 b)$ \\
\hline 0.86730000000000 & 1.11500000000000 & 0.96950000000000 & $\mathrm{O}$ & $(8 b)$ \\
\hline 0.14550000000000 & -0.10220000000000 & -0.96950000000000 & $\mathrm{O}$ & ( $8 b)$ \\
\hline 1.86500000000000 & 0.39550000000000 & 0.74770000000000 & $\mathrm{O}$ & $(8 b)$ \\
\hline 0.64780000000000 & 1.11730000000000 & 0.25230000000000 & $\mathrm{O}$ & $(8 b)$ \\
\hline 0.35220000000000 & 0.89840000000000 & 0.75380000000000 & $\mathrm{O}$ & $(8 b)$ \\
\hline 0.14460000000000 & -0.40160000000000 & -0.75380000000000 & $\mathrm{O}$ & $(8 b)$ \\
\hline 1.64840000000000 & 0.39460000000000 & 1.04620000000000 & $\mathrm{O}$ & $(8 \mathrm{~b})$ \\
\hline 0.34840000000000 & 0.60220000000000 & -0.04620000000000 & $\mathrm{O}$ & $(8 b)$ \\
\hline
\end{tabular}

$\mathrm{GeSe}_{2}$ (High-pressure): AB2_tP12_81_adg_2h - CIF 


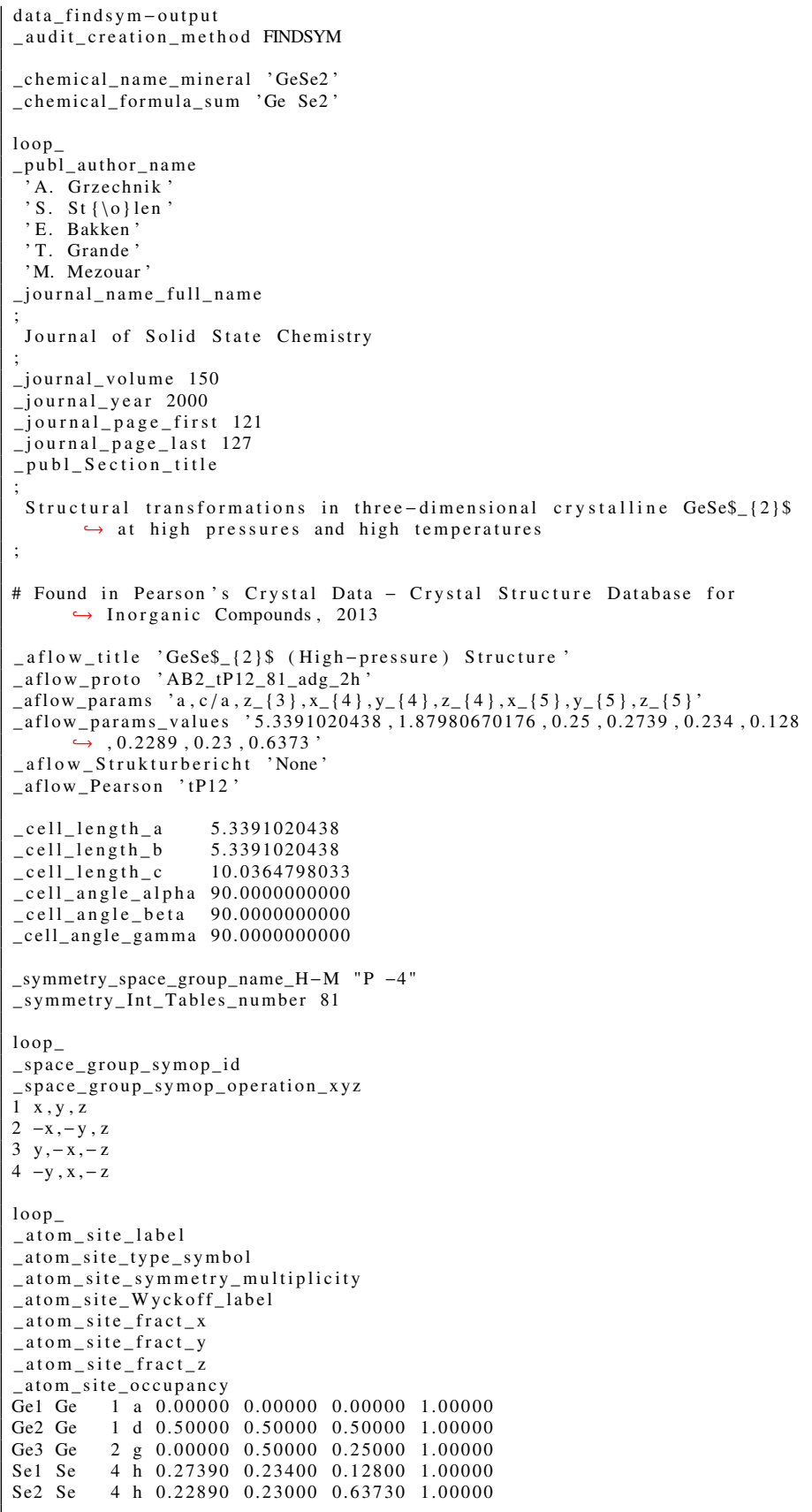

$\mathrm{GeSe}_{2}$ (High-pressure): AB2_tP12_81_adg_2h - POSCAR

AB2_tP12_81_adg_2h \& a , c/a, z3, x4,y4, z4 , x5,y5, z5 --params $=5.3391020438$, $\hookrightarrow 1.87980670176,0.25,0.2739,0.234,0.128,0.2289,0.23,0.6373 \& \mathrm{P}-4$ $\hookrightarrow \mathrm{S}_{\{}\{4\}^{\wedge}\{1\} \# 81\left(\operatorname{adgh}^{\wedge} 2\right) \& \mathrm{tP} 12$ \& None \& GeSe2 \& \& A. Grzechnik $\hookrightarrow$ et al. J. Solid State Chem. 150, 121-127(2000) 1.00000000000000

$5.33910204380000 \quad 0.00000000000000 \quad 0.00000000000000$

$\begin{array}{lll}0.00000000000000 & 5.33910204380000 & 0.00000000000000\end{array}$

$\begin{array}{lll}0.00000000000000 & 0.00000000000000 & 10.03647980330000\end{array}$

$$
\begin{array}{rr}
\mathrm{Ge} & \mathrm{Se} \\
4 & 8
\end{array}
$$

Direct

$\begin{array}{rrrrr}0.00000000000000 & 0.00000000000000 & 0.00000000000000 & \mathrm{Ge} & (1 \mathrm{a}) \\ 0.50000000000000 & 0.50000000000000 & 0.50000000000000 & \mathrm{Ge} & (1 \mathrm{~d}) \\ 0.00000000000000 & 0.50000000000000 & 0.25000000000000 & \mathrm{Ge} & (2 \mathrm{~g}) \\ 0.50000000000000 & 0.00000000000000 & -0.25000000000000 & \mathrm{Ge} & (2 \mathrm{~g}) \\ 0.27390000000000 & 0.23400000000000 & 0.12800000000000 & \mathrm{Se} & (4 \mathrm{~h}) \\ -0.27390000000000 & -0.23400000000000 & 0.12800000000000 & \mathrm{Se} & (4 \mathrm{~h}) \\ 0.23400000000000 & -0.27390000000000 & -0.12800000000000 & \mathrm{Se} & (4 \mathrm{~h}) \\ -0.23400000000000 & 0.27390000000000 & -0.12800000000000 & \mathrm{Se} & (4 \mathrm{~h}) \\ 0.22890000000000 & 0.23000000000000 & 0.63730000000000 & \mathrm{Se} & (4 \mathrm{~h}) \\ -0.22890000000000 & -0.23000000000000 & 0.63730000000000 & \mathrm{Se} & (4 \mathrm{~h}) \\ 0.23000000000000 & -0.22890000000000 & -0.63730000000000 & \mathrm{Se} & (4 \mathrm{~h}) \\ -0.23000000000000 & 0.22890000000000 & -0.63730000000000 & \mathrm{Se} & (4 \mathrm{~h})\end{array}$

$\mathrm{Ni}_{3} \mathrm{P}\left(D 0_{e}\right):$ A3B_tI32_82_3g_g - CIF

\section{\# CIF file}

data_findsym-output

_audit_creation_method FINDSYM

_chemical_name_mineral,

_chemical_formula_sum 'Ni3 P' oop

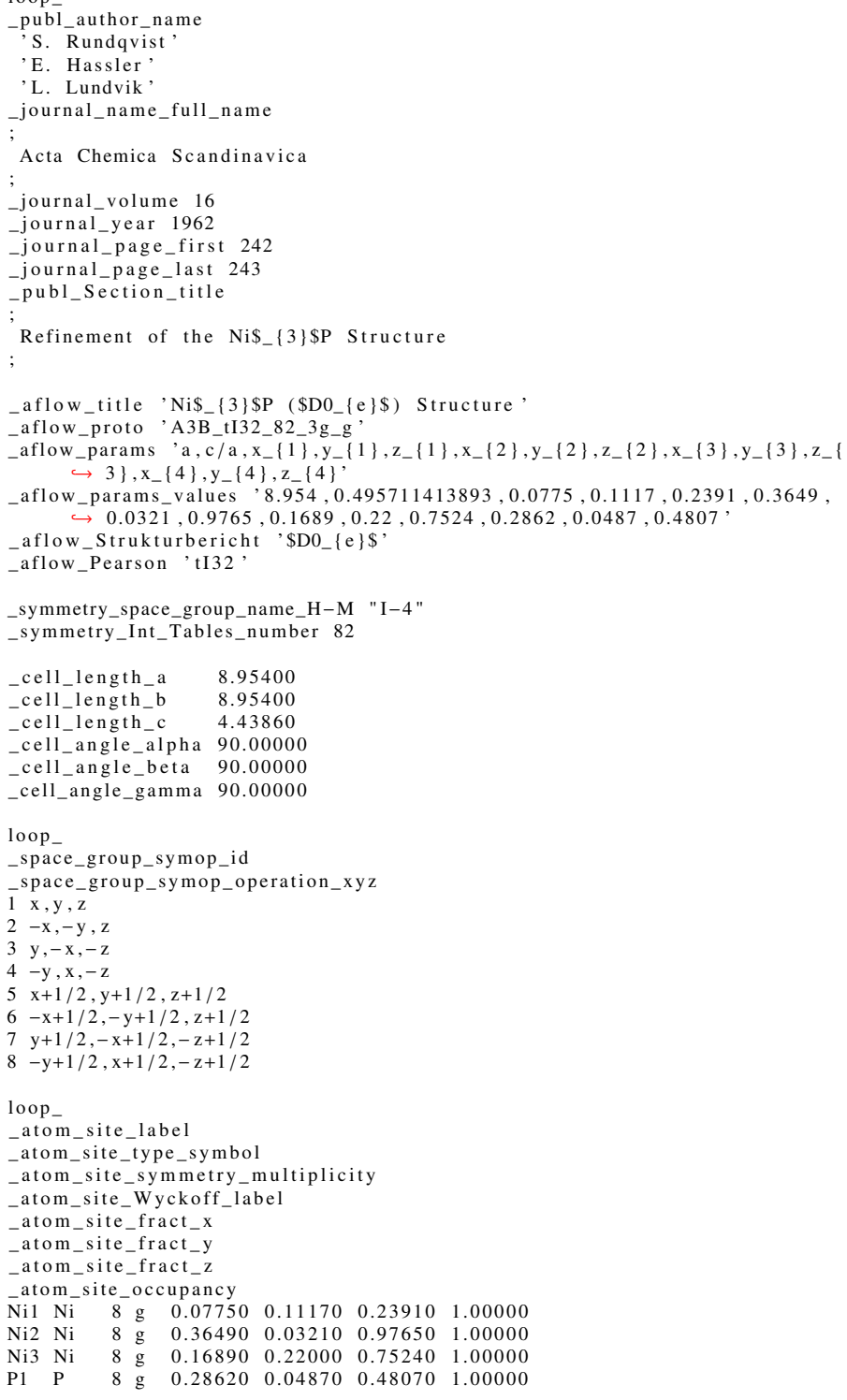

$\begin{array}{llllllll}\mathrm{Ni} 3 & \mathrm{Ni} & 8 & \mathrm{~g} & 0.16890 & 0.22000 & 0.75240 & 1.00000 \\ \mathrm{P} 1 & \mathrm{P} & 8 & \mathrm{~g} & 0.28620 & 0.04870 & 0.48070 & 1.00000\end{array}$

$\mathrm{Ni}_{3} \mathrm{P}\left(D 0_{e}\right):$ A3B_tI32_82_3g_g - POSCAR

\begin{tabular}{|c|c|c|c|c|}
\hline $\begin{aligned} & \text { A3B_tI32_82 } 83 g_{-} g \text { g } ~ \\
& \hookrightarrow 8.954,0.495 \\
& \hookrightarrow 0.1689,0.22 \\
&\hookrightarrow 4) \& \text { tI32 \& } \\
& \hookrightarrow \text { and L. Lund }\end{aligned}$ & $\begin{array}{l}\mathrm{c} / \mathrm{a}, \mathrm{x} 1, \mathrm{y} 1, \mathrm{z} 1, \mathrm{x} 2, \mathrm{y} 2 \\
11413893,0.0775,0.1 \\
0.7524,0.2862,0.048 \\
\$ \mathrm{D} 0 \_\{\mathrm{e}\} \$ \text { \& Ni3P \& } \\
\text { ik, Acta Chem. Sca }\end{array}$ & $\begin{array}{l}17,0.2391,0.3649, \\
7,0.4807 \text { \& }-4 \text { S } \\
\text { \& S. Rundqvist and } \\
\text { d. } 16,242-243 \quad(19\end{array}$ & $\begin{array}{l}0321 \\
\}^{\wedge}\left\{\begin{array}{l}2 \\
\text { E. } H\end{array}\right. \\
\text { ?) }\end{array}$ & $\begin{array}{l}0.9765, \\
\# 82(\mathrm{~g} \wedge \\
\text { ssler }\end{array}$ \\
\hline & 47700000000000 & & & \\
\hline-4.47700000000000 & 4.47700000000000 & 2.21930000000000 & & \\
\hline $\begin{array}{l}4.47700000000000 \\
4.47700000000000\end{array}$ & $\begin{array}{r}-4.47700000000000 \\
4.47700000000000\end{array}$ & $\begin{array}{r}2.21930000000000 \\
-2.21930000000000\end{array}$ & & \\
\hline $\mathrm{Ni}$ & & & & \\
\hline 12 & & & & \\
\hline Direct & & & & \\
\hline 0.35080000000000 & 0.31660000000000 & 0.18920000000000 & $\mathrm{Ni}$ & $(8 \mathrm{~g})$ \\
\hline 0.12740000000000 & 0.16160000000000 & -0.18920000000000 & $\mathrm{Ni}$ & $(8 g)$ \\
\hline-0.31660000000000 & -0.12740000000000 & 0.03420000000000 & $\mathrm{Ni}$ & $(8 \mathrm{~g})$ \\
\hline-0.16160000000000 & -0.35080000000000 & -0.03420000000000 & $\mathrm{Ni}$ & $(8 \mathrm{~g})$ \\
\hline 1.00860000000000 & 1.34140000000000 & 0.39700000000000 & $\mathrm{Ni}$ & $(8 \mathrm{~g})$ \\
\hline 0.94440000000000 & 0.61160000000000 & -0.39700000000000 & $\mathrm{Ni}$ & $(8 \mathrm{~g})$ \\
\hline-1.34140000000000 & -0.94440000000000 & -0.33280000000000 & $\mathrm{Ni}$ & $(8 \mathrm{~g})$ \\
\hline-0.61160000000000 & -1.00860000000000 & 0.33280000000000 & $\mathrm{Ni}$ & $(8 g)$ \\
\hline 0.97240000000000 & 0.92130000000000 & 0.38890000000000 & $\mathrm{Ni}$ & $(8 \mathrm{~g})$ \\
\hline 0.53240000000000 & 0.58350000000000 & -0.38890000000000 & $\mathrm{Ni}$ & $(8 \mathrm{~g})$ \\
\hline-0.92130000000000 & -0.53240000000000 & 0.05110000000000 & $\mathrm{Ni}$ & $(8 \mathrm{~g})$ \\
\hline-0.58350000000000 & -0.97240000000000 & -0.05110000000000 & $\mathrm{Ni}$ & $(8 \mathrm{~g})$ \\
\hline 0.52940000000000 & 0.76690000000000 & 0.33490000000000 & $\mathrm{P}$ & $(8 \mathrm{~g})$ \\
\hline 0.43200000000000 & 0.19450000000000 & -0.33490000000000 & $P$ & $(8 \mathrm{~g})$ \\
\hline-0.76690000000000 & -0.43200000000000 & -0.23750000000000 & $P$ & $(8 \mathrm{~g})$ \\
\hline-0.19450000000000 & -0.52940000000000 & 0.23750000000000 & $P$ & $(8 \mathrm{~g})$ \\
\hline
\end{tabular}

$\mathrm{Ti}_{2} \mathrm{Ge}_{3}$ : A3B2_tP10_83_adk_j - CIF

\section{\# CIF file}

data_findsym-output

audit_creation_method FINDSYM

chemical name mineral 'Ti2Ge3,

_chemical_formula_sum, Ge3 Ti2,

loop_ 


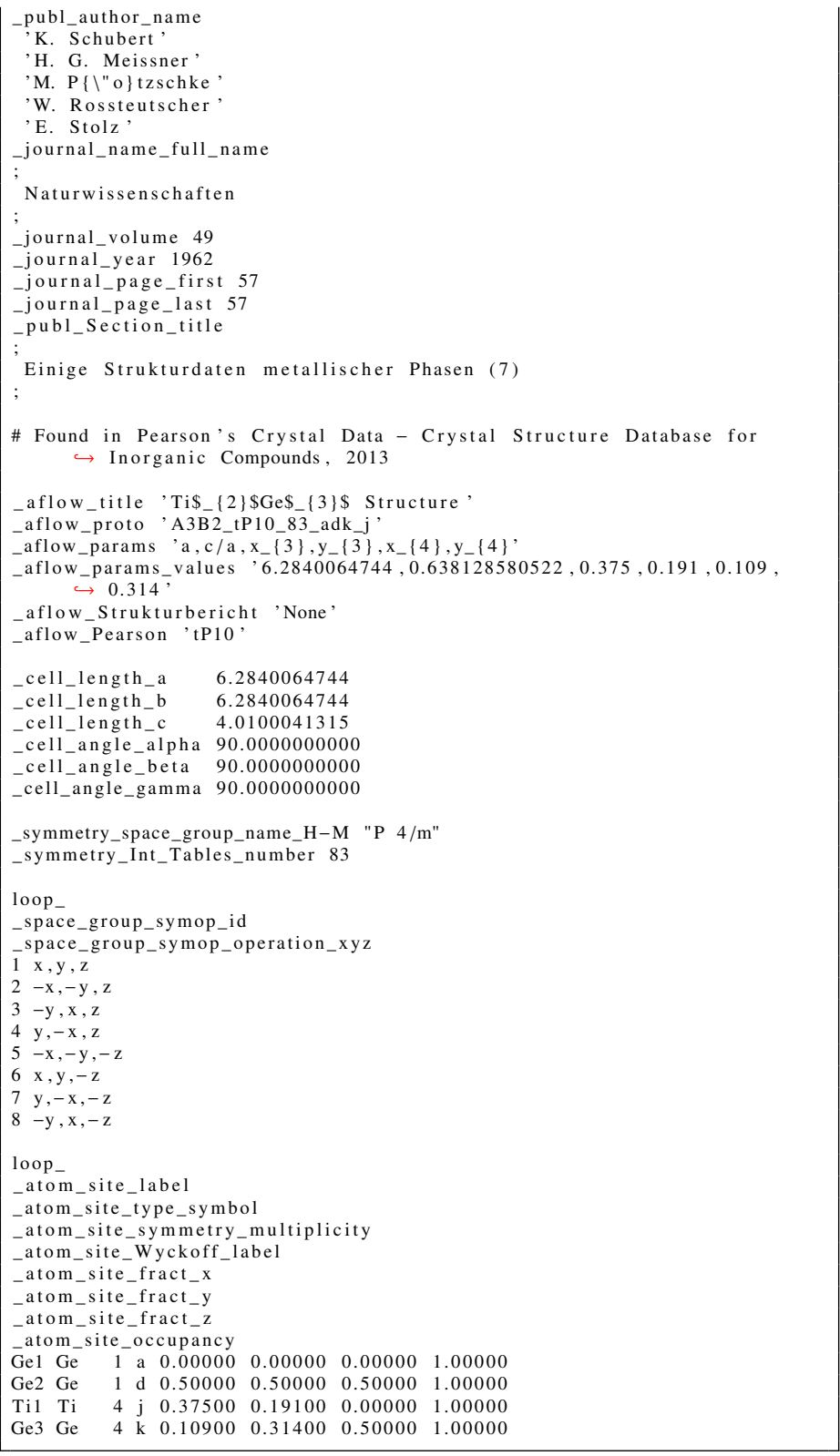

$\mathrm{Ti}_{2} \mathrm{Ge}_{3}$ : A3B2_tP10_83_adk_j - POSCAR

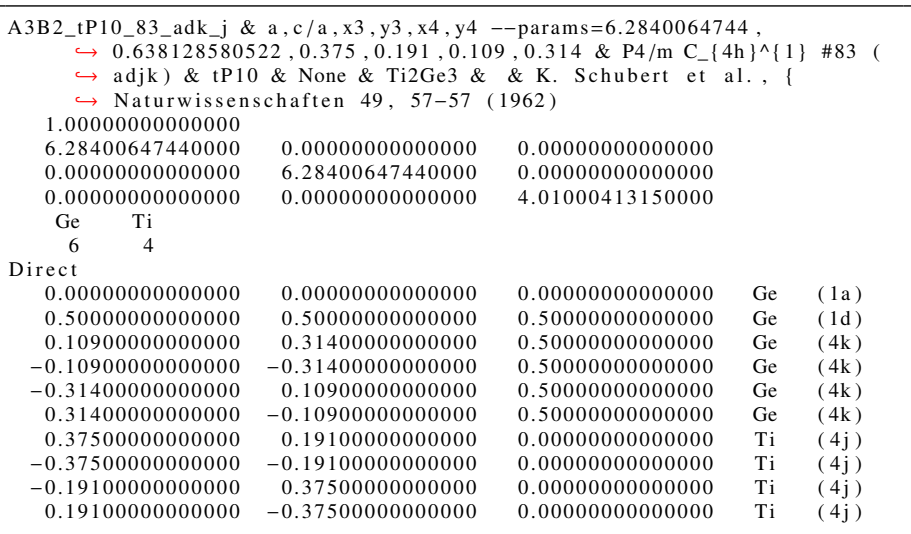

$\mathrm{SrBr}_{2}$ : A2B_tP30_85_ab2g_cg - CIF

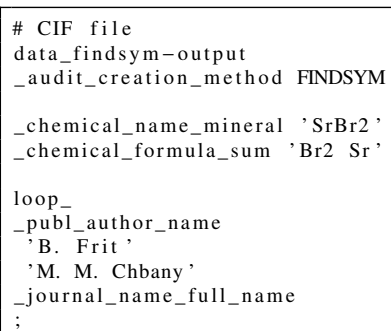

Journal of Inorganic and Nuclear Chemistry

_journal_volume 31

journal_year 1969

_journal_page_first 268

journal_page_last 2693

publ_Section_title

Les halogeno-carbonates de strontium

\# Found in Pearson's Crystal Data - Crystal Structure Database for $\hookrightarrow$ Inorganic Compounds, 2013

aflow_title 'SrBr\$_ $\{2\} \$$ Structure

aflow proto 'A2B tP30_85_ab2g_cg

aflow_params $\left., a, c / a, z_{-}\{3\}, x_{-}\{4\}, y_{-}\{4\}, z_{-}\{4\}, x_{-}\{5\}, y_{-}\{5\}, z_{-}\{5\}, x_{-}\{6\}, y_{-}\right\}$ $\hookrightarrow 6\}, z_{-}\{6\}$ '

6. $11.6179902668,0.614391461525,0.6517,0.5428,0.6612$ $\hookrightarrow 0.5963,0.6531,0.541,0.1258,0.5856,0.1045,0.2524$,

-aflow_Strukturbericht 'None'

_aflow_Pearson 'tP30'

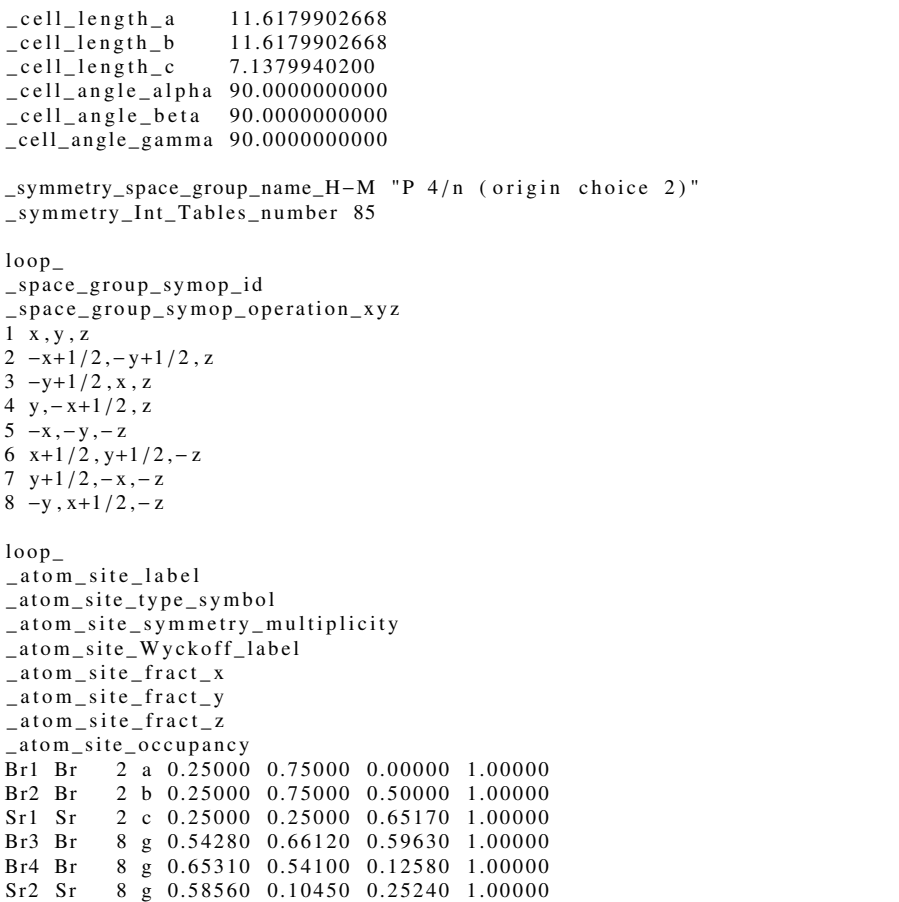

$\begin{array}{lllllllll}\mathrm{Sr} 2 & \mathrm{Sr} & 8 & \mathrm{~g} & 0.58560 & 0.10450 & 0.25240 & 1.00000\end{array}$

$\mathrm{SrBr}_{2}$ : A2B_tP30_85_ab2g_cg - POSCAR

A2B_tP30_85_ab2g_cg \& a , c / a , z3 $, \mathrm{x} 4, \mathrm{y} 4, \mathrm{z} 4, \mathrm{x} 5, \mathrm{y} 5, \mathrm{z} 5, \mathrm{x} 6, \mathrm{y} 6, \mathrm{z} 6--$ params $=$ $\hookrightarrow 11.6179902668,0.614391461525,0.6517,0.5428,0.6612,0.5963,0.6531$ $\hookrightarrow, 0.541,0.1258,0.5856,0.1045,0.2524 \& \mathrm{P} 4 / \mathrm{n} \mathrm{C}_{-}\{4 \mathrm{~h}\}^{\wedge}\{3\} \# 85(\mathrm{abc}\}^{\wedge}$ $\hookrightarrow$ 3) \& $\mathrm{tP} 30$ \& None \& SrBr2 \& \& B. Frit and M. M. Chbany, J. $\hookrightarrow$ Inorg. Nucl. Chem. 31, 2685-2693 (1969)

1.00000000000000

$\begin{array}{lll}11.61799026680000 & 0.00000000000000 & 0.00000000000000\end{array}$

$0.00000000000000-11.61799026680000-0.00000000000000$

0.0000000000000

0.00000000000000 7.13799402000000

$\mathrm{Br} \quad \mathrm{Sr}$

Direct

0.25000000000000 0.75000000000000 0.25000000000000 0.75000000000000 0.54280000000000 $-0.04280000000000$ $-0.16120000000000$ 0.66120000000000 $-0.54280000000000$

1.04280000000000

1.16120000000000 $-0.66120000000000$ 0.65310000000000 $-0.15310000000000$ $-0.0410000000000$ 0.04100000000000 0.54100000000000 $-0.65310000000000$ 1.15310000000000 1.04100000000000 $-0.54100000000000$ 0.25000000000000 0.75000000000000 0.58560000000000 $-0.08560000000000$ 0.39550000000000 0.10450000000000 $-0.58560000000000$ 1.08560000000000

0.60450000000000 $-0.10450000000000$

75000000000000 0.25000000000000 0.75000000000000 0.25000000000000 0.66120000000000 0.16120000000000 0.54280000000000 $-0.04280000000000$ $-0.66120000000000$ 1.16120000000000 $-0.54280000000000$ 1.04280000000000 0.54100000000000 $-0.0410000000000$ 0.65310000000000 $-0.65310000000000$ $-0.15310000000000$ 1.04100000000000 1.04100000000000 0.65310000000000 1.15310000000000 0.25000000000000 0.75000000000000 0.10450000000000 0.39550000000000 0.58560000000000 $-0.08560000000000$ $-0.10450000000000$ 0.60450000000000 $-0.58560000000000$ 1.08560000000000
0.00000000000000 0.00000000000000 0.50000000000000 0.5000000000000 0.59630000000000 0.59630000000000 0.59630000000000 0.59630000000000 $-0.59630000000000$ $-0.59630000000000$ $-0.59630000000000$ 0.1258000000000 0.12580000000000 0.125800000000 0.12580000000000 $-0.12580000000000$ 0.125800000000 $-0.12580000000000$ 0.12580000000000 0.65170000000000 $-0.65170000000000$ 0.25240000000000 0.25240000000000 0.25240000000000 0.25240000000000 $-0.25240000000000$ $-0.25240000000000$ $-0.25240000000000$ $-0.25240000000000$ $-0.59630000000000$

$\begin{array}{ll}\mathrm{Br} & (2 \mathrm{a}) \\ \mathrm{Br} & (2 \mathrm{a}) \\ \mathrm{Br} & (2 \mathrm{~b}) \\ \mathrm{Br} & (2 \mathrm{~b}) \\ \mathrm{Br} & (8 \mathrm{~g}) \\ \mathrm{Br} & (8 \mathrm{~g}) \\ \mathrm{Br} & (8 \mathrm{~g}) \\ \mathrm{Br} & (8 \mathrm{~g}) \\ \mathrm{Br} & (8 \mathrm{~g}) \\ \mathrm{Br} & (8 \mathrm{~g}) \\ \mathrm{Br} & (8 \mathrm{~g}) \\ \mathrm{Br} & (8 \mathrm{~g}) \\ \mathrm{Br} & (8 \mathrm{~g}) \\ \mathrm{Br} & (8 \mathrm{~g}) \\ \mathrm{Br} & (8 \mathrm{~g}) \\ \mathrm{Br} & (8 \mathrm{~g}) \\ \mathrm{Br} & (8 \mathrm{~g}) \\ \mathrm{Br} & (8 \mathrm{~g}) \\ \mathrm{Br} & (8 \mathrm{~g}) \\ \mathrm{Br} & (8 \mathrm{~g}) \\ \mathrm{Sr} & (2 \mathrm{c}) \\ \mathrm{Sr} & (2 \mathrm{c}) \\ \mathrm{Sr} & (8 \mathrm{~g}) \\ \mathrm{Sr} & (8 \mathrm{~g}) \\ \mathrm{Sr} & (8 \mathrm{~g}) \\ \mathrm{Sr} & (8 \mathrm{~g}) \\ \mathrm{Sr} & (8 \mathrm{~g}) \\ \mathrm{Sr} & (8 \mathrm{~g}) \\ \mathrm{Sr} & (8 \mathrm{~g}) \\ \mathrm{Sr} & (8 \mathrm{~g}) \\ & \end{array}$


Direct

Ti 3 P: AB3_tP32_86_g_3g - CIF

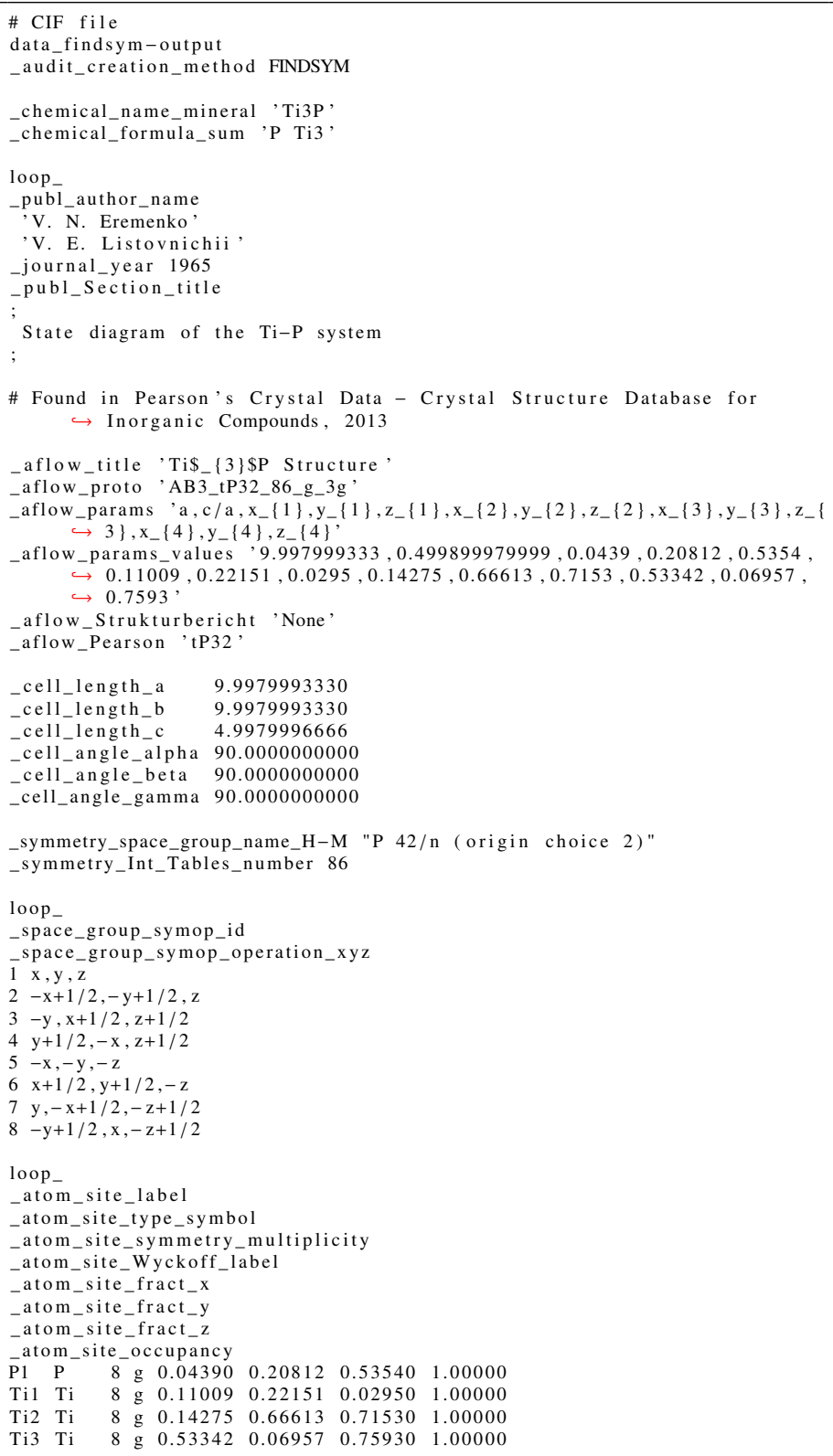

Ti 3 P: AB3_tP32_86_g_3g - POSCAR

AB3_tP32_86_g_3g \& a,c/a, x1, y1, z1 , x2 , y2 , z2, x3,y3, z3, x4,y4, z4 --params $\hookrightarrow 9.997999333,0.499899979999,0.0439,0.20812,0.5354,0.11009$

$\hookrightarrow 0.22151,0.0295,0.14275,0.66613,0.7153,0.53342,0.06957,0.7593 \&$

$\hookrightarrow \mathrm{P}_{-}\{2\} / \mathrm{n} \mathrm{C}_{-}\{4 \mathrm{~h}\}^{\wedge}\{4\} \# 86\left(\mathrm{~g}^{\wedge} 4\right) \& \mathrm{tP} 32 \&$ None \& Ti3P \& \& V. N.

$\hookrightarrow$ Eremenko and V. E. Listovnichii, (1965)

1.00000000000000

$9.99799933300000 \quad 0.00000000000000 \quad 0.00000000000000$

$\begin{array}{lll}0.00000000000000 & 9.99799933300000 & 0.00000000000000\end{array}$

$\begin{array}{llll}0.00000000000000 & 0.00000000000000 & 4.99799966660000\end{array}$

$\begin{array}{ll}\mathrm{P} & \mathrm{Ti} \\ 8 & 24\end{array}$

0.04390000000000 0.45610000000000 0.45610000000000 0.20812000000000 0.70812000000000 $-0.04390000000000$ 0.54390000000000 0.20812000000000 0.29188000000000 0.11009000000000 0.38991000000000 $-0.22151000000000$ 0.72151000000000 $-0.11009000000000$ 0.61009000000000 0.22151000000000 0.27849000000000 0.14275000000000 0.35725000000000 $-0.66613000000000$ 1.1661300000000 1.16613000000000 0.6427500000000 0.66613000000000
0.20812000000000 0.29188000000000 0.5439000000000 0.04390000000000 $-0.20812000000000$ 0.70812000000000 0.45610000000000 0.45610000000000 0.04390000000000 0.22151000000000 0.27849000000000 0.61009000000000 $-0.11009000000000$ $-0.22151000000000$ 0.72151000000000 0.38991000000000 0.11009000000000 0.66613000000000 $-0.16613000000000$ $-0.1427500000000$ $-0.66613000000000$ 1.16613000000000
0.35725000000000 0.64275000000000 $-0.66613000000000$
0.53540000000000 0.53540000000000 1.03540000000000 1.03540000000000 $-0.53540000000000$ $-0.53540000000000$ $-0.03540000000000$ $-0.03540000000000$ 0.02950000000000 0.02950000000000 0.52950000000000 0.52950000000000 $-0.02950000000000$ $-0.02950000000000$ 0.47050000000000 0.47050000000000 0.71530000000000 0.71530000000000 1.21530000000000 $-0.71530000000000$ $-0.71530000000000$ $-0.21530000000000$ 1.2153000000000
$-0.16613000000000$ 0.53342000000000 $-0.03342000000000$ $-0.06957000000000$ 0.56957000000000 $-0.53342000000000$ 1.03342000000000 0.06957000000000 0.43043000000000

.14275000000000 0.06957000000000 0.43043000000000 .03342000000000 -.53342000000000 0.06957000000000 0.56957000000000 -0.03342000000000
0.53342000000000 $-0.21530000000000$ 0.75930000000000 0.75930000000000 1.25930000000000 1.25930000000000 $-0.75930000000000$ $-0.75930000000000$ $-0.25930000000000$ $-0.25930000000000$

$\mathrm{ThCl}_{4}: \mathrm{A} 4 \mathrm{~B}$ tI20 $88 \mathrm{f}$ a - CIF

\# CIF file

data_findsym-outpu

audit_creation_method FINDSYM

chemical_name_mineral 'ThCl4,

chemical_formula_sum 'Cl4 Th'

loop

J. T. Mason'

M. C. Jha

journal_name_full_name

Journal of the Less-Common Metal

journal_volume 34

journal_year 1974

journal_page_last 151

publ_Section_title

Crystal structures of $\mathrm{ThCl} \$ \_\{4\} \$$ polymorphs

Found in Pearson's Crystal Data - Crystal Structure Database for $\hookrightarrow$ Inorganic Compounds, 2013

aflow title 'ThCl\$ $\{4\} \$$ Structure'

aflow proto, A4B tI20 88 f a

flow params, a, c/a, x $\{2\}, y_{-}\{2\}, z_{-}\{2\}$

aflow_params_values , $6.407994829,2.01685393259,0.147,0.017,0.298$,

aflow_Strukturbericht 'None,

aflow_Pearson tI 20 ,

cell_length_a $\quad 6.4079948290$

cell_length_b 6.4079948290

cell_length_c $\quad 12.9239895709$

cell_angle_alpha 90.0000000000

cell_angle_beta 90.0000000000

cell angle gamma 90.0000000000

symmetry space group name_H-M "I 41/a (origin choice 2)"

symmetry_Int_Tables_number 88

loop

space_group_symop_id

space_group_symop_operation_xy

$1 \mathrm{x}, \mathrm{y}, \mathrm{z}$

$-x,-y+1 / 2, z$

$3-\mathrm{y}+3 / 4, \mathrm{x}+1 / 4, \mathrm{z}+1 / 4$

$y+1 / 4,-x+1 / 4, z+1 / 4$

$5-\mathrm{x},-\mathrm{y},-\mathrm{z}$

$6 \mathrm{x}, \mathrm{y}+1 / 2,-\mathrm{z}$

$7 \mathrm{y}+1 / 4,-\mathrm{x}+3 / 4,-\mathrm{z}+3 / 4$

$9 \mathrm{x}+1 / 2, \mathrm{y}+1 / 2, \mathrm{z}+1 / 2$

$10-x+1 / 2,-y, z+1 / 2$

$11-\mathrm{y}+1 / 4, \mathrm{x}+3 / 4, \mathrm{z}+3 / 4$

$12 \mathrm{y}+3 / 4,-\mathrm{x}+3 / 4, \mathrm{z}+3 / 4$

$13-x+1 / 2,-y+1 / 2,-z+1 / 2$

$14 \mathrm{x}+1 / 2, \mathrm{y},-\mathrm{z}+1 / 2$

$14 x+1 / 2, y,-z+1 / 2$

$16-\mathrm{y}+1 / 4, \mathrm{x}+1 / 4,-\mathrm{z}+1 / 4$

loop

atom_site_label

atom_site_type_symbol

atom_site_symmetry_multiplicity

atom_site_Wyckoff_label

atom_site_fract_x

-atom_site_fract_y

-atom_site_fract_z

atom_site_occupancy

$\begin{array}{llllllll}\text { Th1 Th } & 4 & \text { a } & 0.00000 & 0.25000 & 0.12500 & 1.00000\end{array}$

A4B_tI20_88_f_a \& a , c/a, x2,y2,z2 --params $=6.407994829,2.01685393259$ $\hookrightarrow 0.147,0.017,0.298 \&$ I $4 \_\{1\} /$ a $C_{-}\{4 \mathrm{~h}\}^{\wedge}\{6\} \# 88$ (af) \& tI $20 \&$ None $\hookrightarrow \& \mathrm{ThCl} 4$ \& \& J. T. Mason and M. C. Jha and P. Chiotti, J. $\hookrightarrow$ Less-Common Met. 34, 143-151 (1974)

1.00000000000000

$3.20399741450000-3.20399741450000-6.46199478545000$

$\begin{array}{lll}3.20399741450000 & 3.20399741450000 & -6.46199478545000\end{array}$

$\mathrm{Cl}$ Th

Direc

$\begin{array}{lll}0.31500000000000 & 0.44500000000000 & 0.16400000000000\end{array}$ $\begin{array}{lll}0.78100000000000 & 0.15100000000000 & 0.33600000000000 \\ 0.94500000000000 & 0.28100000000000 & 0.13000000000000\end{array}$
$\mathrm{Ti} \quad(8 \mathrm{~g})$ -publ_author_nam

journal_page_first 143

$8-\mathrm{y}+3 / 4, \mathrm{x}+3 / 4,-\mathrm{z}+3 / 4$

$\mathrm{ThCl}_{4}$ : A4B_tI20_88_f_a - POSCAR

$\begin{array}{lll}-3.20399741450000 & 3.20399741450000 & 6.46199478545000\end{array}$ 
0.65100000000000 $-0.31500000000000$ 0.21900000000000 0.05500000000000 0.34900000000000 0.37500000000000 0.62500000000000

0.81500000000000 $-0.44500000000000$ $-0.15100000000000$ $-0.2810000000000$ 0.1850000000000 0.1250000000000 0.87500000000000

$\alpha-\mathrm{NbO}_{2}: \mathrm{AB} 2 \_\mathrm{tI} 96 \_88 \_2 \mathrm{f} \_4 \mathrm{f}-\mathrm{CIF}$

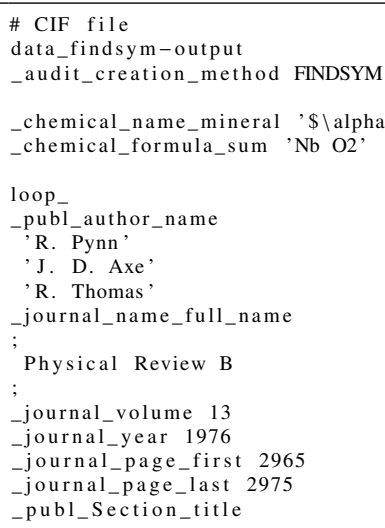

Found in A Powder Neutron Diffraction Study of Semiconducting and $\hookrightarrow$ Metallic Niobium Dioxide, 1994

aflow_title $\$ \backslash$ alpha\$-NbO\$_ $\{2\} \$$ Structure,

aflow_proto, AB2_tI96_88_2f_4f,

aflow_params 'a, c/a, $x_{-}\{1\}, y_{-}\{1\}, z_{-}\{1\}, x_{-}\{2\}, y_{-}\{2\}, z_{-}\{2\}, x_{-}\{3\}, y_{-}\{3\}, z_{-}\{$ $\hookrightarrow 3\}, x_{-}\{4\}, y_{-}\{4\}, z_{-}\{4\}, x_{-}\{5\}, y_{-}\{5\}, z_{-}\{5\}, x_{-}\{6\}, y_{-}\{6\}, z_{-}\{6\}$

aflow params_values, $13.66,0.436603221083,0.1155,0.1249,0.4746,0.1356$,

$\hookrightarrow 0.125,0.0267,-0.0134,0.1262,-0.0046,-0.0251,0.1252,0.5,0.2739$,

$\hookrightarrow 0.1245,-0.0002,0.2631,0.1241,0.5043$,

_aflow_Strukturbericht 'None

_aflow_Pearson 'tI96,

symmetry_space_group_name_H-M "I 41/a:2"

_symmetry_Int_Tables_number 88

$$
\begin{array}{ll}
\text { cell_length_a } & 13.66000 \\
\text { cell_length_b } & 13.66000 \\
\text { cell_length_c } & 5.96400 \\
\text { cell_angle_alpha } & 90.00000 \\
\text { cell_angle_beta } & 90.00000 \\
\text { cell_angle } &
\end{array}
$$

loop

_space_group_symop_id

-space_group_symop_operation_xyz

$1 \mathrm{x}, \mathrm{y}, \mathrm{z}$

$2-\mathrm{x},-\mathrm{y}+1 / 2, \mathrm{z}$

$3-y+3 / 4, x+1 / 4, z+1 / 4$

$4 \mathrm{y}+1 / 4,-x+1 / 4, z+1 / 4$

$5-\mathrm{x},-\mathrm{y},-\mathrm{z}$

$\begin{array}{ll}6 & \mathrm{x}, \mathrm{y}+1 / 2,-\mathrm{z} \\ 7 & \mathrm{y}+1 / 4,-\mathrm{x}+3 / 4,-\mathrm{z}+3 / 4 \\ 8 & -\mathrm{y}+3 / 4, \mathrm{x}+3 / 4,-2+3 / 4\end{array}$

$8-\mathrm{y}+3 / 4, \mathrm{x}+3 / 4,-\mathrm{z}+3 / 4$

$9 \mathrm{x}+1 / 2, \mathrm{y}+1 / 2, \mathrm{z}+1 / 2$

$10-\mathrm{x}+1 / 2,-\mathrm{y}, \mathrm{z}+1 / 2$

$11-\mathrm{y}+1 / 4, \mathrm{x}+3 / 4, \mathrm{z}+3 / 4$

$12 \mathrm{y}+3 / 4,-\mathrm{x}+3 / 4, \mathrm{z}+3 / 4$

$13-x+1 / 2,-y+1 / 2,-z+1 / 2$

$14 \mathrm{x}+1 / 2, \mathrm{y},-\mathrm{z}+1 / 2$

$15-y+3 / 4,-x+1 / 4,-z+1 / 4$

$16-\mathrm{y}+1 / 4, \mathrm{x}+1 / 4,-\mathrm{z}+1 / 4$

loop

_atom_site_labe

atom_site_type_symbol

atom_site_symmetry_multiplicity

atom_site_Wyckoff_label

atom_site_fract_x

atom_site_fract_y

atom_site_fract_z

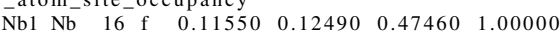

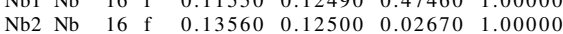

$\begin{array}{llllllll}\mathrm{Nb} 2 & \mathrm{Nb} & 16 & \mathrm{f} & 0.13560 & 0.12500 & 0.02670 & 1.00000 \\ \mathrm{O} 1 & \mathrm{O} & 16 & \mathrm{f} & -0.01340 & 0.12620 & -0.00460 & 1.00000\end{array}$

$\begin{array}{llllllll}\mathrm{O} 1 & \mathrm{O} & 16 & \mathrm{f} & -0.01340 & 0.12620 & -0.00460 & 1.00000 \\ \mathrm{O} 2 & \mathrm{O} & 16 & \mathrm{f} & -0.02510 & 0.12520 & 0.50000 & 1.00000\end{array}$

$\begin{array}{llllrrrr}\mathrm{O} 2 & \mathrm{O} & 16 & \mathrm{f} & -0.02510 & 0.12520 & 0.50000 & 1.00000 \\ \mathrm{O} 3 & \mathrm{O} & 16 & \mathrm{f} & 0.27390 & 0.12450 & -0.00020 & 1.00000\end{array}$

\begin{tabular}{llllllll}
$\mathrm{O} 3$ & $\mathrm{O}$ & 16 & $\mathrm{f}$ & 0.27390 & 0.12450 & -0.00020 & 1.00000 \\
\hline
\end{tabular}

$\alpha-\mathrm{NbO}_{2}:$ AB2_tI96_88_2f_4f - POSCAR

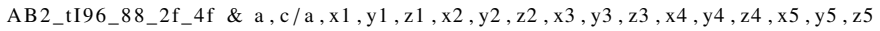

$\hookrightarrow \mathrm{x} 6, \mathrm{y} 6, \mathrm{z} 6-$ params $=13.66,0.436603221083,0.1155,0.1249,0.4746$

$\hookrightarrow 0.1356,0.125,0.0267,-0.0134,0.1262,-0.0046,-0.0251,0.1252,0.5$

$\hookrightarrow 0.2739,0.1245,-0.0002,0.2631,0.1241,0.5043 \& I_{-}\{1\} /$ a $C_{-}\{4 h\}^{\wedge}\{6$

$\hookrightarrow\} \# 88\left(f^{\wedge} 6\right)$ \& tI96 \& None \& NbO2 \& \$ \alpha\$-NbO\$ \{2\}\$\& R. Pynn

$\rightarrow$ and J. D. Axe and R. Thomas, Phys. Rev. B 13, 2965-2975 (1976) 1.00000000000000

$-6.83000000000000$

$6.8300000000000-6.83000000000000$

$-6.83000000000000$

.98200000000000

2.98200000000000
$6.83000000000000 \quad 6.83000000000000 \quad-2.98200000000000$

$\mathrm{Nb} \quad \mathrm{O}$

16
Direct

$\begin{array}{lll}0.59950000000000 & 0.59010000000000 & 0.24040000000000\end{array}$

0.84970000000000

1.09010000000000

0.85910000000000

$-0.59950000000000$

0.15030000000000

$-0.09010000000000$

0.14090000000000

0.15170000000000

0.4017000000000

0.66230000000000
0.39110000000000

$-0.15170000000000$

0.59830000000000

0.33770000000000

0.60890000000000

0.12160000000000

0.36920000000000

0.48200000000000

0.50880000000000

$-0.12160000000000$

0.63080000000000

0.49120000000000

0.62520000000000

0.87480000000000

0.8748000000000

0.9749000000000

1.02510000000000

0.12520000000000

0.02510000000000

$-0.02510000000000$

0.12430000000000

0.37530000000000
0.77370000000000

0.77370000000000
0.22590000000000

0.22590000000000

$-0.12430000000000$

0.22630000000000

0.77410000000000

0.62840000000000

0.88020000000000

1.2674000000000

1.26740000000000

0.7412000000000

0.11980000000000

$-0.26740000000000$

0.25880000000000

35910000000000

0.34970000000000

1.09950000000000

$-0.59010000000000$

$-0.35910000000000$

$-0.34970000000000$

.09950000000000

0.16230000000000

$-0.10890000000000$

0.09830000000000

0.65170000000000

0.16230000000000

0.10890000000000

0.09830000000000

0.34830000000000

.01800000000000

0.00880000000000

0.13080000000000

0.6216000000000

.01800000000000

.00880000000000 .13080000000000 0.37840000000000 0.47490000000000 .52510000000000 .37480000000000

12520000000000

.

$-0.52510000000000$

.

.12520000000000

0.27370000000000

0.27410000000000

0.12470000000000

0.62430000000000

$-0.27370000000000$

0.27410000000000

0.12470000000000 0.37570000000000 0.76740000000000 .24120000000000 0.38020000000000 1.12840000000000

$-0.76740000000000$

$-0.7674000000$

$-0.24120000000000$

$-0.12840000000000$

. 0.00940000000000 5094000000000 0.24040000000000 0.74040000000000 0.00940000000000 0.49060000000000 0.26060000000000 0.23940000000000 0.01060000000000 0.48940000000000 (26060000000 .760600 0.76060000000000 0.51060000000000 0.11280000000000 0.38720000000000 0.13960000000000 0.13960000000000 0.63960000000000 0.1280000000000 0.61280000000000 0.13960000000000 0.36040000000000 0.39990000000000 $-0.15030000000000$ 0.65030000000000 0.65030000000 0.60010000000000 0.15030000000000 0.34970000000000 0.39840000000000 0.10160000000000 0.14940000000000 0.35060000000000 $-0.39840000000000$ 0.89840000000000 $-0.14940000000000$ 0.64940000000000 0.38720000000000 0.11280000000000 0.13900000000000 0.36100000000000 $-0.38720000000000$ 0.88720000000000 0.88720000000000 0.63900000000000

$\mathrm{Nb}$ (16f) $\mathrm{Nb}$ (16f) $\mathrm{Nb}$ (16f) $\mathrm{Nb}$ (16f) $\mathrm{Nb}$ (16f) $\mathrm{Nb}$ (16f) $\mathrm{Nb}$ (16f) $\mathrm{Nb}$ (16f) $\mathrm{Nb} \quad(16 \mathrm{f})$ $\mathrm{Nb}$ (16f) $\mathrm{Nb}$ (16f) (16f) (16f) b (161) (16f) (16f) b (16f) O (16f) O (16f) O (16f) O (16f) O (16f) O (16f) O (16f) O (16f) O (16f)

O (16f)

O (16f)

O (16f)

(16f)

(16f)

(16f)

O (16f)

O (16f)

$\mathrm{O}$ (16f)

$\mathrm{O}$ (16f)

O (16f)

O (16f)

O (16f)

O (16f)

(16f)

O (16f)

(16f)

O (16f)

$\mathrm{O}$ (16f)

O (16f)

O (16f)

O (16f)

$\mathrm{O}$ (16f)

O (16f)

\# CIF file

data findsym-output

audit creation method FINDSYM

chemical_name_mineral ' $\mathrm{C} 17 \mathrm{FeO} 4 \mathrm{Pt}$ '

chemical_formula_sum ' $\mathrm{C} 17 \mathrm{Fe}$ O4 Pt 


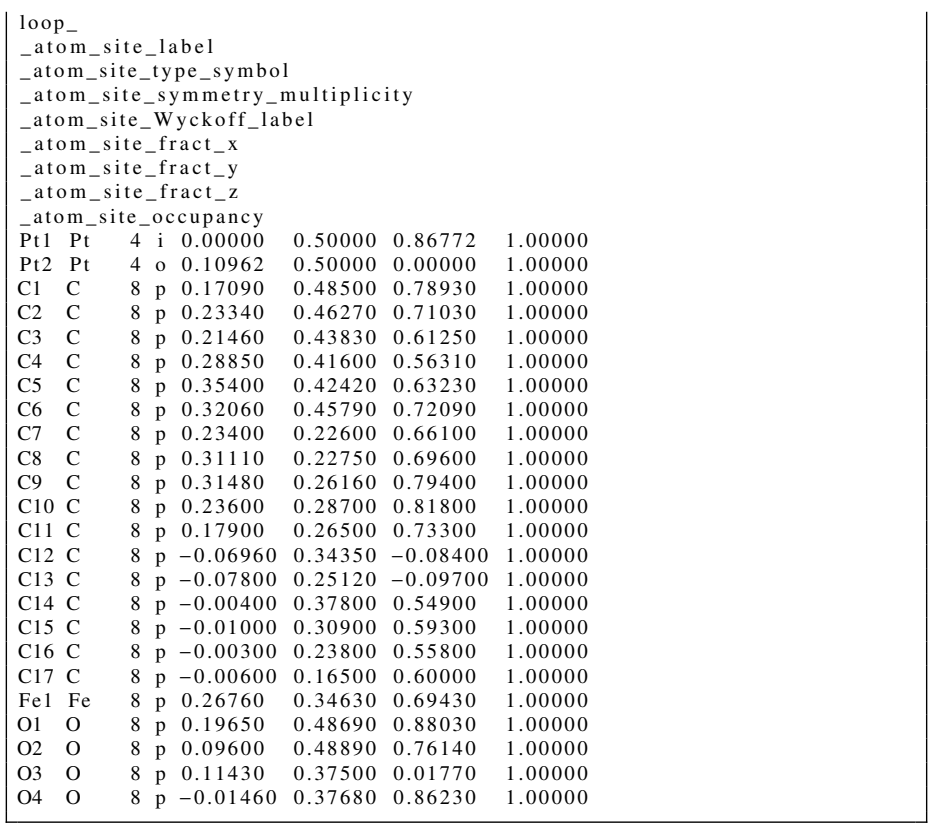

$\mathrm{C}_{17} \mathrm{FeO}_{4} \mathrm{Pt}$ : A17BC4D_tP184_89_17p_p_4p_io - POSCAR

A17BC4D_tP184_89_17p_p_4p_io \& a, c/a, z1, x2, x3,y3,z3, x4, y4, z4, x5, y5 , z5, x6 $\longrightarrow, \mathrm{y} 6, \mathrm{z6}, \mathrm{x} 7, \mathrm{y} 7, \mathrm{z} 7, \mathrm{x} 8, \mathrm{y} 8, \mathrm{z8}, \mathrm{x} 9, \mathrm{y} 9, \mathrm{z9}, \mathrm{x} 10, \mathrm{y} 10, \mathrm{z} 10, \mathrm{x} 11, \mathrm{y} 11, \mathrm{z} 11, \mathrm{x} 12$

$\hookrightarrow \mathrm{y} 117, \mathrm{x} 18, \mathrm{y} 18, \mathrm{z} 18, \mathrm{x} 19, \mathrm{y} 19, \mathrm{z} 19, \mathrm{x} 20, \mathrm{y} 20, \mathrm{z} 20, \mathrm{x} 21, \mathrm{y} 21, \mathrm{z} 21, \mathrm{x} 22, \mathrm{y} 22$

$\hookrightarrow, \mathrm{z} 17, \mathrm{x} 18, \mathrm{y} 18, \mathrm{z} 18, \mathrm{x} 19, \mathrm{y} 19, \mathrm{z} 19, \mathrm{x} 20, \mathrm{y} 20, \mathrm{z} 20, \mathrm{x} 21, \mathrm{y} 21, \mathrm{z} 21, \mathrm{x} 22, \mathrm{y} 22$

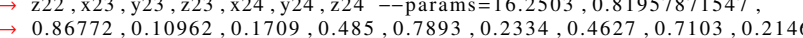

$, 0.4383,0.6125,0.2885,0.416,0.5631,0.354,0.4242,0.6323,0.3206$

$\hookrightarrow 0.4579,0.7209,0.234,0.226,0.661,0.3111,0.2275,0.696,0.3148$

$\longrightarrow 0.2616,0.794,0.236,0.287,0.818,0.179,0.265,0.733,-0.0696,0.3435$

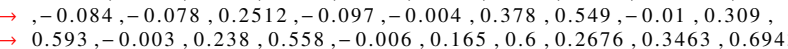

$\rightarrow 0.1965,0.4869,0.8803,0.096,0.4889,0.7614,0.1143,0.375,0.0177$,

$\hookrightarrow 0.0146,0.3768,0.8623 \&$ \& P422 D_ $\{4\}^{\wedge}\{1\} \# 89$ (iop^22) \& tP184 \&

$\longrightarrow$ None \& $\mathrm{C} 17 \mathrm{FeO} 4 \mathrm{Pt} \&$ \&

1.00000000000000

0.00000000000000

$0.00000000000000 \quad 0.00000000000000$

0.00000000000000

0.00000000000000

$\begin{array}{rrrr}\mathrm{C} & \mathrm{Fe} & \mathrm{O} & \mathrm{Pt} \\ 136 & 8 & 32 & 8\end{array}$

Direct

0.17090000000000

$-0.17090000000000$

$-0.48500000000000$

0.48500000000000

0.17090000000000
0.17090000000000

0.17090000000000

0.48500000000000

23340000000000

$-0.23340000000000$

$-0.46270000000000$

0.46270000000000

$-0.23340000000000$

0.23340000000000

0.46270000000000

0.46270000000000

0.21460000000000

$-0.21460000000000$

$-0.43830000000000$

0.43830000000000

$-0.21460000000000$

0.21460000000000

0.43830000000000

.

0.28850000000000

$-0.28850000000000$

0.41600000000000

$-0.28850000000000$

0.28850000000000

0.28850000000000

0.41600000000000

0.31600000000000
0.35400000000000

0.35400000000000

0.42420000000000

0.42420000000000

$-0.35400000000000$

0.35400000000000

0.42420000000000

0.32060000000000

$-0.32060000000000$

$-0.45790000000000$

0.45790000000000

0.32060000000000

0.4579000000000

$-0.45790000000000$
23400000000000 $-0.23400000000000$

$-0.22600000000000$

0.2260000000000

$-0.23400000000000$

0.23400000000000

0.3111000000000

$-0.31110000000000$

$-0.22750000000000$

0.22750000000000

$-0.31110000000000$

0.31110000000000

0.22750000000000

$-0.22750000000000$

0.31480000000000

$-0.31480000000000$

$-0.26160000000000$

0.26160000000000

$-0.31480000000000$

0.26160000000000

$-0.26160000000000$

0.23600000000000

$-0.23600000000000$

0.28700000000000

$-0.23600000000000$

0.23600000000000

$-0.28700000000000$

0.17900000000000

$-0.17900000000000$

0.26500000000000

0.26500000000000

$-0.17900000000000$

0.1790000000000

0.2650000000000

$-0.2650000000000$

$-0.06960000000000$

0.06960000000000

0.34350000000000

0.06960000000000

$-0.06960000000000$

0.34350000000000

$-0.3435000000000$

0.07800000000000

$-0.25120000000000$

0.25120000000000

0.07800000000000

$-0.07800000000000$

0.2512000000000

$-0.25120000000000$

$-0.00400000000000$

0.00400000000000

$-0.37800000000000$

0.00400000000000

$-0.00400000000000$

0.37800000000000

$-0.37800000000000$

$-0.01000000000000$

0.01000000000000

$-0.30900000000000$

0.30900000000000

0.01000000000000

-0.01000000000000
0.30900000000000

$-0.30900000000000$

$-0.00300000000000$

0.00300000000000

0.2380000000000

0.00300000000000

$-0.00300000000000$

0.23800000000000

$-0.23800000000000$

$-0.00600000000000$

0.00600000000000

$-0.16500000000000$

0.16500000000000

0.00600000000000

-0.00600000000000
-0.16500000000000

0.16500000000000
-0.16500000000000

0.26760000000000

$-0.26760000000000$

$-0.34630000000000$

0.3463000000000

$-0.26760000000000$

0.26760000000000

0.34630000000000

$-0.34630000000000$

0.19650000000000

0.1965000000000

$-0.19650000000000$

0.48690000000000

$-0.19650000000000$

0.19650000000000

0.4869000000000

-0.48690000000000
0.09600000000000
22600000000000 .23400000000000 .23400000000000
0.23400000000000 .2260000000000 .22600000000000 0.23400000000000 23400000000000 .22750000000000 .22750000000000 0.31110000000000 .31110000000000 .22750000000000 .31110000000000 0.31110000000000 .26160000000000 26160000000000 31480000000000 .31480000000000 0.26160000000000 .26160000000000 0.31480000000000 .31480000000000 .28700000000000 .28700000000000 .23600000000000 0.28700000000000 $-0.28700000000000$ 0.23600000000000 0.23600000000000 0.26500000000000 .26500000000000 17900000000000 0.17900000000000 0.26500000000000 $-0.26500000000000$ 0.17900000000000 0.17900000000000 0.34350000000000 0.34350000000000 0.06960000000000 0.06960000000000 0.34350000000000 $-0.34350000000000$ $-0.06960000000000$ 0.06960000000000 0.25120000000000 $-0.25120000000000$ 0.07800000000000 0.07800000000000 0.25120000000000 $-0.25120000000000$ $-0.07800000000000$ 0.07800000000000 0.37800000000000 $-0.37800000000000$ 0.00400000000000 0.00400000000000 $-0.37800000000000$ $-0.00400000000000$ 0.00400000000000 0.30900000000000 0.3090000000000 0.01000000000000 0.01000000000000 0.30900000000000 $-0.30900000000000$ 0.01000000000000 0.01000000000000 0.23800000000000 0.23800000000000 0.00300000000000 0.238000000000 $-0.23800000000000$ $-0.00300000000000$ 0.00300000000000 0.16500000000000 0.16500000000000 0.00600000000000 0.00600000000000 0.16500000000000 0.16500000000000 $-0.00600000000000$ 0.00600000000000 0.34630000000000 $-0.34630000000000$ 0.26760000000000 0.3463000000000 $-0.34630000000000$ 0.26760000000000 0.26760000000000 $-0.48690000000000$ 0.19650000000000 0.19650000000000 0.48690000000000 0.48690000000000 0.19650000000000 0.19650000000000
0.48890000000000

0.66100000000000 0.6610000000000 0.66100000000000 $-0.6610000000000$ $-0.66100000000000$ $-0.66100000000000$ 0.66100000000000 0.69600000000000 0.69600000000000 0.69600000000000 $-0.69600000000000$ $-0.69600000000000$ $-0.69600000000000$ 0.79400000000000 0.79400000000000 $-0.79400000000000$ 0.79400000000000 $-0.79400000000000$ 0.79400000000000 $-.79400000000000$ 0.81800000000000 0.81800000000000 0.81800000000000 $-0.8180000000000$ $-0.81800000000000$ $-0.81800000000000$ $-0.81800000000000$ 


$\begin{array}{rrrrr}-0.09600000000000 & -0.48890000000000 & 0.76140000000000 & \mathrm{O} & (8 \mathrm{p}) \\ -0.48890000000000 & 0.09600000000000 & 0.76140000000000 & \mathrm{O} & (8 \mathrm{p}) \\ 0.48890000000000 & -0.09600000000000 & 0.76140000000000 & \mathrm{O} & (8 \mathrm{p}) \\ -0.09600000000000 & 0.48890000000000 & -0.76140000000000 & \mathrm{O} & (8 \mathrm{p}) \\ 0.09600000000000 & -0.48890000000000 & -0.76140000000000 & \mathrm{O} & (8 \mathrm{p}) \\ 0.48890000000000 & 0.09600000000000 & -0.76140000000000 & \mathrm{O} & (8 \mathrm{p}) \\ -0.48890000000000 & -0.09600000000000 & -0.76140000000000 & \mathrm{O} & (8 \mathrm{p}) \\ 0.11430000000000 & 0.37500000000000 & 0.01770000000000 & \mathrm{O} & (8 \mathrm{p}) \\ -0.11430000000000 & -0.37500000000000 & 0.01770000000000 & \mathrm{O} & (8 \mathrm{p}) \\ -0.37500000000000 & 0.11430000000000 & 0.01770000000000 & \mathrm{O} & (8 \mathrm{p}) \\ 0.37500000000000 & -0.11430000000000 & 0.01770000000000 & \mathrm{O} & (8 \mathrm{p}) \\ -0.11430000000000 & 0.37500000000000 & -0.01770000000000 & \mathrm{O} & (8 \mathrm{p}) \\ 0.11430000000000 & -0.37500000000000 & -0.01770000000000 & \mathrm{O} & (8 \mathrm{p}) \\ 0.37500000000000 & 0.11430000000000 & -0.01770000000000 & \mathrm{O} & (8 \mathrm{p}) \\ -0.37500000000000 & -0.11430000000000 & -0.01770000000000 & \mathrm{O} & (8 \mathrm{p}) \\ -0.01460000000000 & 0.37680000000000 & 0.86230000000000 & \mathrm{O} & (8 \mathrm{p}) \\ 0.01460000000000 & -0.37680000000000 & 0.86230000000000 & \mathrm{O} & (8 \mathrm{p}) \\ -0.37680000000000 & -0.01460000000000 & 0.86230000000000 & \mathrm{O} & (8 \mathrm{p}) \\ 0.37680000000000 & 0.01460000000000 & 0.86230000000000 & \mathrm{O} & (8 \mathrm{p}) \\ 0.01460000000000 & 0.37680000000000 & -0.86230000000000 & \mathrm{O} & (8 \mathrm{p}) \\ -0.01460000000000 & -0.37680000000000 & -0.86230000000000 & \mathrm{O} & (8 \mathrm{p}) \\ 0.37680000000000 & -0.01460000000000 & -0.86230000000000 & \mathrm{O} & (8 \mathrm{p}) \\ -0.37680000000000 & 0.01460000000000 & -0.86230000000000 & \mathrm{O} & (8 \mathrm{p}) \\ 0.00000000000000 & 0.50000000000000 & 0.86772000000000 & \mathrm{Pt} & (4 \mathrm{i}) \\ 0.50000000000000 & 0.00000000000000 & 0.86772000000000 & \mathrm{Pt} & (4 \mathrm{i}) \\ 0.00000000000000 & 0.50000000000000 & -0.86772000000000 & \mathrm{Pt} & (4 \mathrm{i}) \\ 0.50000000000000 & 0.00000000000000 & -0.86772000000000 & \mathrm{Pt} & (4 \mathrm{i}) \\ 0.10962000000000 & 0.50000000000000 & 0.00000000000000 & \mathrm{Pt} & (4 \mathrm{o}) \\ -0.10962000000000 & 0.50000000000000 & 0.00000000000000 & \mathrm{Pt} & (4 \mathrm{o}) \\ 0.50000000000000 & 0.10962000000000 & 0.00000000000000 & \mathrm{Pt} & (4 \mathrm{o}) \\ 0.50000000000000 & -0.10962000000000 & 0.00000000000000 & \mathrm{Pt} & (4 \mathrm{o})\end{array}$

$\mathrm{Na}_{4} \mathrm{Ti}_{2} \mathrm{Si}_{8} \mathrm{O}_{22}\left[\mathrm{H}_{2} \mathrm{O}\right]_{4}$ : A4B2C13D_tP40_90_g_d_cef2g_c - CIF

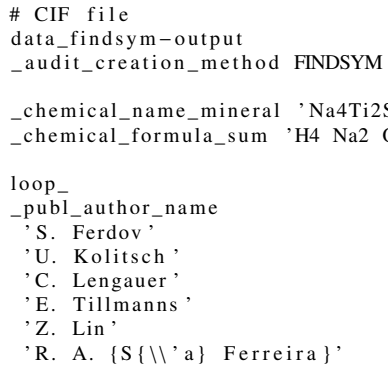

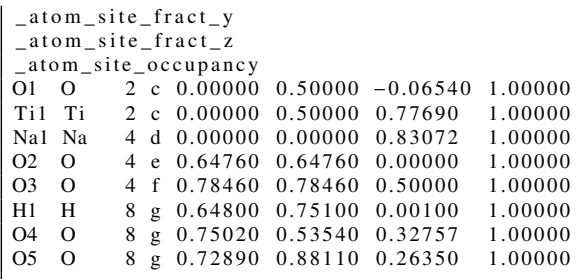

$\mathrm{Na}_{4} \mathrm{Ti}_{2} \mathrm{Si}_{8} \mathrm{O}_{22}\left[\mathrm{H}_{2} \mathrm{O}\right]_{4}$ : A4B2C13D_tP40_90_g_d_cef2g_c - POSCAR

\begin{tabular}{|c|c|c|c|c|}
\hline \multicolumn{5}{|c|}{ A4B2C13D_tP40_90_g_d_cef2g_c \& a , c/a , z1 ,z2, z3, x4, x5, x6, y6, z6 , x7,y7, z7, x8 } \\
\hline \multirow{5}{*}{\multicolumn{5}{|c|}{$\begin{array}{l}\hookrightarrow, \mathrm{y} 8, \mathrm{z} 8-\text { - params }=7.3739946979,1.45226471387,-0.0654,0.7769, \\
\hookrightarrow 0.83072,0.6476,0.7846,0.648,0.751,0.001,0.7502,0.5354,0.32757 \\
\hookrightarrow 0.7289,0.8811,0.2635 \& \mathrm{P} 42 \_\{1\} 2 \mathrm{D}_{-}\{4\}^{\wedge}\{2\} \# 90\left(\mathrm{c}^{\wedge} 2 \mathrm{defg} \wedge 3\right) \& \\
\hookrightarrow \text { tP40\& None \& Na4Ti2Si8O22[H2O } \& \& \& \text { S. Ferdov et al., Acta }\end{array}$}} \\
\hline & & & & \\
\hline & & & & \\
\hline & & & & \\
\hline \multirow{2}{*}{\multicolumn{5}{|c|}{100000000000000}} \\
\hline & & & & \\
\hline 7.37399469790000 & 0.00000000000000 & 0.00000000000000 & & \\
\hline \multirow{2}{*}{$\begin{array}{l}0.00000000000000 \\
0.00000000000000\end{array}$} & 7.37399469790000 & 0.00000000000000 & & \\
\hline & 0.00000000000000 & 10.70899230000000 & & \\
\hline $\mathrm{Na}$ & $\mathrm{Ti}$ & & & \\
\hline 26 & 2 & & & \\
\hline \multicolumn{5}{|l|}{ Direct } \\
\hline 0.64800000000000 & 0.75100000000000 & 0.00100000000000 & $\mathrm{H}$ & $(8 \mathrm{~g})$ \\
\hline-0.64800000000000 & -0.75100000000000 & 0.00100000000000 & $\mathrm{H}$ & $(8 \mathrm{~g})$ \\
\hline-0.25100000000000 & 1.14800000000000 & 0.00100000000000 & $\mathrm{H}$ & $(8 \mathrm{~g})$ \\
\hline 1.25100000000000 & -0.14800000000000 & 0.00100000000000 & $\mathrm{H}$ & $(8 \mathrm{~g})$ \\
\hline-0.14800000000000 & 1.25100000000000 & -0.00100000000000 & $\mathrm{H}$ & $(8 \mathrm{~g})$ \\
\hline 1.14800000000000 & -0.25100000000000 & -0.00100000000000 & $\mathrm{H}$ & $(8 \mathrm{~g})$ \\
\hline 0.75100000000000 & 0.64800000000000 & -0.00100000000000 & $\mathrm{H}$ & $(8 \mathrm{~g})$ \\
\hline-0.75100000000000 & -0.64800000000000 & -0.00100000000000 & $\mathrm{H}$ & $(8 \mathrm{~g})$ \\
\hline 0.00000000000000 & 0.00000000000000 & 0.83072000000000 & $\mathrm{Na}$ & (4d) \\
\hline 0.50000000000000 & 0.50000000000000 & 0.83072000000000 & $\mathrm{Na}$ & (4d) \\
\hline 0.50000000000000 & 0.50000000000000 & -0.83072000000000 & $\mathrm{Na}$ & (4d) \\
\hline 0.00000000000000 & 0.00000000000000 & -0.83072000000000 & $\mathrm{Na}$ & (4d) \\
\hline 0.00000000000000 & 0.50000000000000 & -0.06540000000000 & $\mathrm{O}$ & (2c) \\
\hline 0.50000000000000 & 0.00000000000000 & 0.06540000000000 & $\mathrm{O}$ & $(2 c)$ \\
\hline 0.64760000000000 & 0.64760000000000 & 0.00000000000000 & $\mathrm{O}$ & $(4 \mathrm{e})$ \\
\hline-0.64760000000000 & -0.64760000000000 & 0.00000000000000 & $\mathrm{O}$ & $(4 \mathrm{e})$ \\
\hline-0.14760000000000 & 1.14760000000000 & 0.00000000000000 & $\mathrm{O}$ & $(4 \mathrm{e})$ \\
\hline 1.14760000000000 & -0.14760000000000 & 0.00000000000000 & $\mathrm{O}$ & $(4 \mathrm{e})$ \\
\hline 0.78460000000000 & 0.78460000000000 & 0.50000000000000 & $\mathrm{O}$ & $(4 f)$ \\
\hline-0.78460000000000 & -0.78460000000000 & 0.50000000000000 & $\mathrm{O}$ & $(4 f)$ \\
\hline-0.28460000000000 & 1.28460000000000 & 0.50000000000000 & $\mathrm{O}$ & $(4 \mathrm{f})$ \\
\hline 1.28460000000000 & -0.28460000000000 & 0.50000000000000 & $\mathrm{O}$ & (4f) \\
\hline 0.75020000000000 & 0.53540000000000 & 0.32757000000000 & $\mathrm{O}$ & $(8 \mathrm{~g})$ \\
\hline-0.75020000000000 & -0.53540000000000 & 0.32757000000000 & $\mathrm{O}$ & $(8 \mathrm{~g})$ \\
\hline-0.03540000000000 & 1.25020000000000 & 0.32757000000000 & $\mathrm{O}$ & $(8 \mathrm{~g})$ \\
\hline 1.03540000000000 & -0.25020000000000 & 0.32757000000000 & $\mathrm{O}$ & $(8 \mathrm{~g})$ \\
\hline-0.25020000000000 & 1.03540000000000 & -0.32757000000000 & $\mathrm{O}$ & $(8 \mathrm{~g})$ \\
\hline 1.25020000000000 & -0.03540000000000 & -0.32757000000000 & $\mathrm{O}$ & $(8 \mathrm{~g})$ \\
\hline 0.53540000000000 & 0.75020000000000 & -0.32757000000000 & $\mathrm{O}$ & $(8 \mathrm{~g})$ \\
\hline-0.53540000000000 & -0.75020000000000 & -0.32757000000000 & $\mathrm{O}$ & $(8 \mathrm{~g})$ \\
\hline 0.72890000000000 & 0.88110000000000 & 0.26350000000000 & $\mathrm{O}$ & $(8 \mathrm{~g})$ \\
\hline-0.72890000000000 & -0.88110000000000 & 0.26350000000000 & $\mathrm{O}$ & \\
\hline-0.38110000000000 & 1.22890000000000 & 0.26350000000000 & $\mathrm{O}$ & $(8 \mathrm{~g})$ \\
\hline 1.38110000000000 & -0.22890000000000 & 0.26350000000000 & $\mathrm{O}$ & $(8 \mathrm{~g})$ \\
\hline-0.22890000000000 & 1.38110000000000 & -0.26350000000000 & $\mathrm{O}$ & $(8 \mathrm{~g})$ \\
\hline 1.22890000000000 & -0.38110000000000 & -0.26350000000000 & $\mathrm{O}$ & $(8 \mathrm{~g})$ \\
\hline 0.88110000000000 & 0.72890000000000 & -0.26350000000000 & $\mathrm{O}$ & $(8 \mathrm{~g})$ \\
\hline-0.88110000000000 & -0.72890000000000 & -0.26350000000000 & $\mathrm{O}$ & $(8 \mathrm{~g})$ \\
\hline 0.00000000000000 & 0.50000000000000 & 0.77690000000000 & $\mathrm{Ti}$ & $(2 \mathrm{c})$ \\
\hline 0.50000000000000 & 0.00000000000000 & -0.77690000000000 & $\mathrm{Ti}$ & $(2 \mathrm{c})$ \\
\hline
\end{tabular}

$\mathrm{BaCu}_{4}[\mathrm{VO}]\left[\mathrm{PO}_{4}\right]_{4}$ : AB4C17D4E_tP54_90_a_g_c4g_g_c - CIF

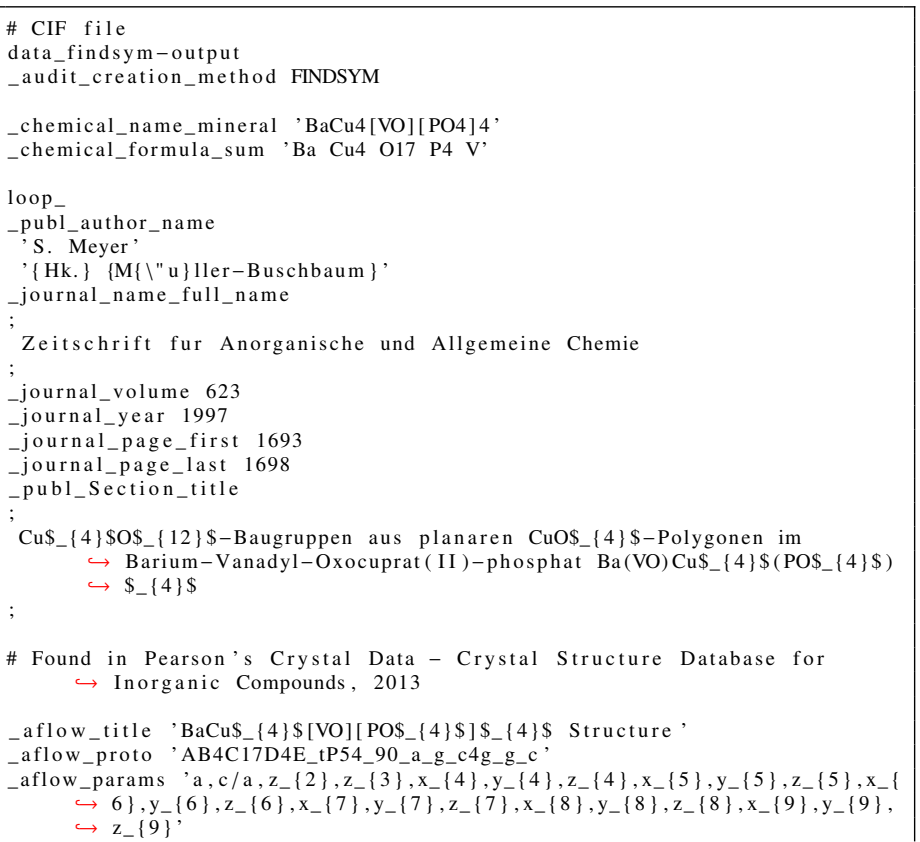




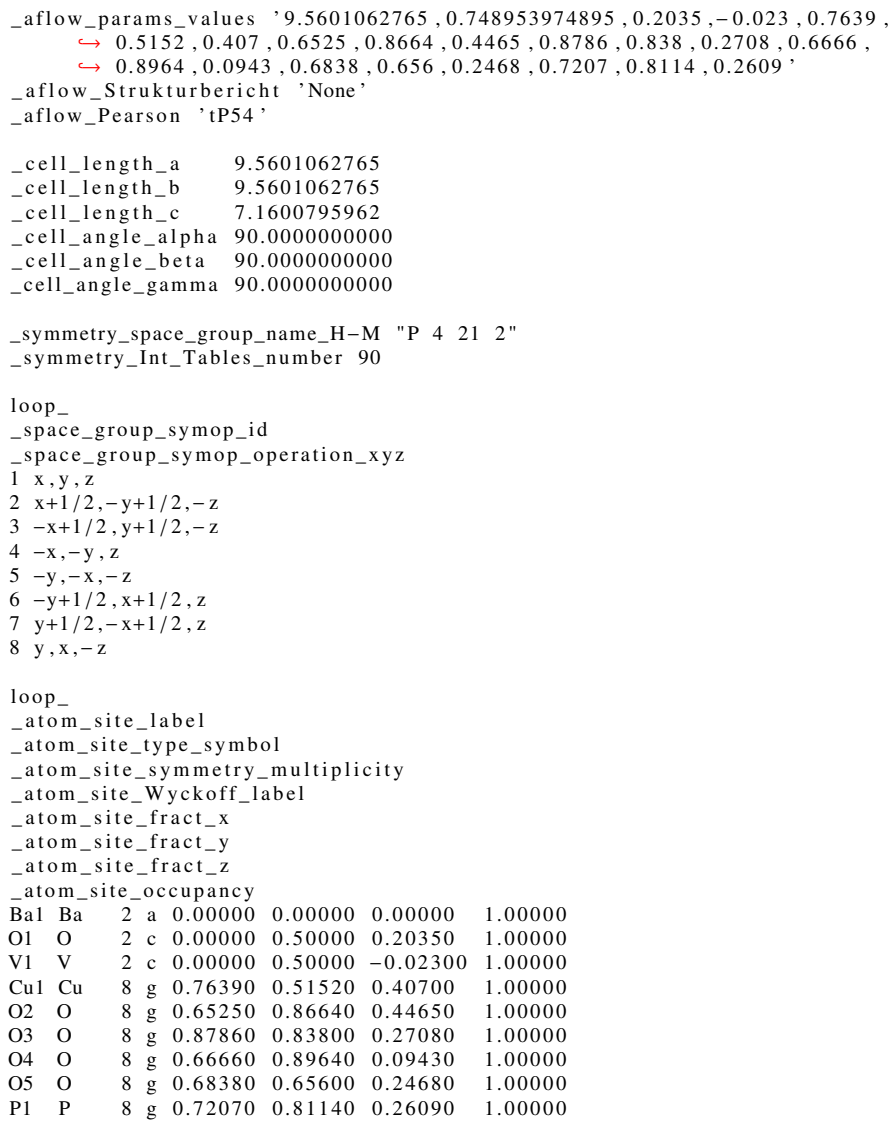

$\mathrm{BaCu}_{4}[\mathrm{VO}]\left[\mathrm{PO}_{4}\right]_{4}:$ AB4C17D4E_tP54_90_a_g_c4g_g_c - POSCAR

$\mathrm{AB} 4 \mathrm{C} 17 \mathrm{D} 4 \mathrm{E}$ tP54 $90, \mathrm{a} g \mathrm{c} 4 \mathrm{~g} \mathrm{~g} \mathrm{c} \& \mathrm{a}, \mathrm{c} / \mathrm{a}, \mathrm{z2}, \mathrm{z} 3, \mathrm{x} 4, \mathrm{y} 4, \mathrm{z} 4, \mathrm{x} 5, \mathrm{y} 5, \mathrm{z} 5, \mathrm{x} 6, \mathrm{y} 6, \mathrm{z6}$ $\hookrightarrow \mathrm{x} 7, \mathrm{y} 7, \mathrm{z} 7, \mathrm{x} 8, \mathrm{y} 8, \mathrm{z} 8, \mathrm{x} 9, \mathrm{y} 9, \mathrm{z} 9--\mathrm{params}=9.5601062765,0.748953974895$ $\hookrightarrow, 0.2035,-0.023,0.7639,0.5152,0.407,0.6525,0.8664,0.4465,0.8786$

$\hookrightarrow 0.838,0.2708,0.6666,0.8964,0.0943,0.6838,0.656,0.2468,0.7207$

$\hookrightarrow 0.8114,0.2609 \& \mathrm{P} 42\{11\} 2 \mathrm{D}\{4\}^{\wedge}\{2\} \# 90\left(\mathrm{ac}^{\wedge} 2 \mathrm{~g}^{\wedge} 6\right) \& \mathrm{tP} 54$ \& None

$\hookrightarrow$ \& BaCu4[VO][PO4]4 \& \& S. Meyer and $\{$ Hk. $\}\{\mathrm{M}\{\backslash " \mathrm{u}\}$ ller-Buschbaum

$\hookrightarrow \& \mathrm{BaCu} 4[\mathrm{VO}][\mathrm{PO} 4] 4$ \& \& S. Meyer and \{Hk.\}$\{\mathrm{M}\{\backslash "$
$\hookrightarrow$ \}, Z. Anorg. Allg. Chem. 623, 1693-1698 (1997) 1.00000000000000 9.56010627650000 0.00000000000000 0.00000000000000 $0.00000000000000 \quad 0.00000000000000$ $9.56010627650000 \quad 0.00000000000000$

$$
\begin{array}{rrrrr}
\mathrm{Ba} & \mathrm{Cu} & \mathrm{O} & \mathrm{P} & \mathrm{V} \\
2 & 8 & 34 & 8 & 2
\end{array}
$$

0.00000000000000 0.50000000000000 0.76390000000000 $-0.76390000000000$ $-0.01520000000000$ 1.01520000000000 $-0.26390000000000$ 1.26390000000000 1.515000000000 0.51520000000000 0.00000000000 0.652500000000 0.65250000000000 $-0.65250000000000$ $-0.36640000000000$ 1.36640000000000 $-0.15250000000000$ 1.15250000000000 0.86640000000000 $-0.86640000000000$ 0.87860000000000 $-0.87860000000000$ $-0.33800000000000$ 1.33800000000000 $-0.37860000000000$ $-0.37860000000000$ .83800000000000 $-0.83800000000000$ 0.66660000000000 $-0.66660000000000$ $-0.39640000000000$ 1.39640000000000 $-0.16660000000000$ 1.16660000000000 0.89640000000000 $-0.89640000000000$ 0.68380000000000 $-0.68380000000000$ $-0.15600000000000$ 1.15600000000000 $-0.18380000000000$ 1.18380000000000

0.00000000000000 0.50000000000000 0.51520000000000 $-0.5152000000000$ 1.26390000000000 $-0.26390000000000$ 1.01520000000000 $-0.01520000000000$ 0.76390000000000 $-0.76390000000000$ 0.50000000000000 0.00000000000000 0.86640000 0.8664000000000 $-0.86640000000000$ 1.15250000000000 $-0.15250000000000$ 1.36640000000000 $-0.36640000000000$ 0.65250000000000 $-0.65250000000000$ 0.83800000000000 $-0.83800000000000$ 1.37860000000000 $-0.37860000000000$ 1.33800000000000 $-0.33800000000000$ 0.87860000000000 0.87860000000000 0.8964000000000 0.896400000000 1.16660000000000 $-0.16660000000000$ 1.39640000000000 $-0.39640000000000$ 0.66660000000000 $-0.66660000000000$ 0.65600000000000 $-0.65600000000000$ 1.18380000000000 $-0.18380000000000$ 1.15600000000000 $-0.15600000000000$ 7.16007959620000

0.65600000000000 $-0.65600000000000$ 0.72070000000000 $-0.72070000000000$ $-0.31140000000000$ 1.31140000000000 $-0.22070000000000$ 1.22070000000000 0.81140000000000 $-0.81140000000000$ 0.00000000000000 0.50000000000000

0.68380000000000 0.68380000000000 0.81140000000000 $-0.81140000000000$ 1.2207000000000 $-0.22070000000000$ 1.31140000000000 $-0.31140000000000$ 0.72070000000000 $-0.72070000000000$ 0.50000000000000 0.00000000000000

$-0.24680000000000$ 0.24680000000000 0.26090000000000 0.26090000000000 0.26090000000000 0.26090000000 $-0.26090000000000$ $-0.26090000000000$ $-0.26090000000000$ $-0.26090000000000$ $-0.02300000000000$ 0.02300000000000

ThBC: ABC_tP24_91_d_d_d - CIF

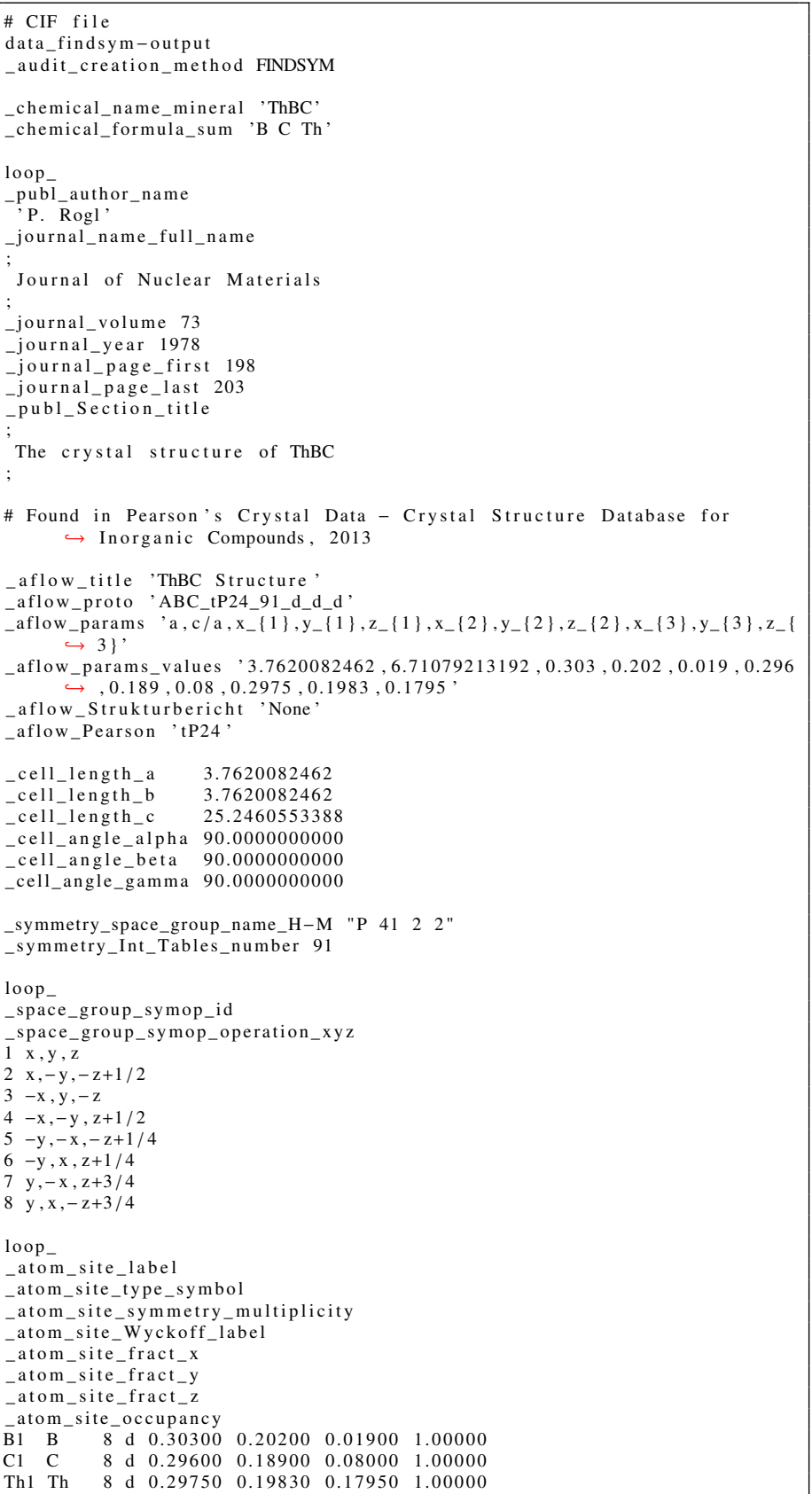

ThBC: ABC_tP24_91_d_d_d - POSCAR

ABC_tP24_91_d_d_d \& a , c /a, x1,y1, z1, x2,y2, z2, x3,y3, z3 --params= $\hookrightarrow 0.2975,0.1983,0.1795 \& P_{-}\{1\} 22 D_{-}\{4\}^{\wedge}\{3\} \# 91\left(\mathrm{~d}^{\wedge} 3\right) \& \operatorname{tP} 24 \&$ $\hookrightarrow$ None \& ThBC \& \& P. Rogl, J. Nucl. Mat. 73, 198-203 (1978) 1.00000000000000 \begin{tabular}{lll|llr}
0.09430000000000 & $\mathrm{O}$ & $(8 \mathrm{~g})$ & 1.00000000000000 & & \\
0.09430000000000 & $\mathrm{O}$ & $(8 \mathrm{~g})$ & 0.76200824620000 & 0.00000000000000 & 0.00000000000000 \\
0.09430000000000 & $\mathrm{O}$ & $(8 \mathrm{~g})$ & 0.000000000000 & 3.76200824620000 & 0.00000000000000
\end{tabular} \begin{tabular}{lll|llr}
0.09430000000000 & $\mathrm{O}$ & $(8 \mathrm{~g})$ & 1.00000000000000 & & \\
0.09430000000000 & $\mathrm{O}$ & $(8 \mathrm{~g})$ & 3.76200824620000 & 0.00000000000000 & 0.00000000000000 \\
0.09430000000000 & $\mathrm{O}$ & $(8 \mathrm{~g})$ & 0.00000000000000 & 3.76200824620000 & 0.00000000000000
\end{tabular}

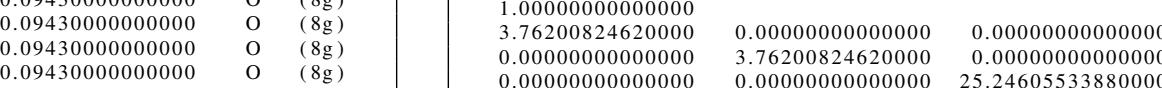
$0.09430000000000 \quad \mathrm{O} \quad(8 \mathrm{~g})$ $-0.09430000000000 \quad \mathrm{O} \quad(8 \mathrm{~g})$ $-0.09430000000000 \quad \mathrm{O} \quad(8 \mathrm{~g})$ $-0.09430000000000 \quad \mathrm{O} \quad(8 \mathrm{~g})$ $-0.09430000000000 \quad \mathrm{O} \quad(8 \mathrm{~g})$ $0.24680000000000 \quad \mathrm{O} \quad(8 \mathrm{~g})$ $0.24680000000000 \quad \mathrm{O} \quad(8 \mathrm{~g})$ $0.24680000000000 \quad \mathrm{O} \quad(8 \mathrm{~g})$ $-0.24680000000000-0.08$ $-0.24680000000000$ $\begin{array}{rrr}\text { B } & \text { C } & \text { Th } \\ 8 & 8 & 8\end{array}$

Direct

0.30300000000000 $-0.30300000000000-0.20200000000000$ $-0.20200000000000 \quad 0.30300000000000$ $0.20200000000000-0.30300000000000$

$-0.30300000000000$

0.30300000000000 0.20200000000000 $-0.20200000000000$ 0.30300000000000
0.01900000000000 0.51900000000000 0.26900000000000 0.76900000000000 $-0.01900000000000$ 0.48100000000000 0.73100000000000
B $\quad(8 d)$

B $\quad(8 \mathrm{~d})$ B $\quad(8 \mathrm{~d})$ B $\quad(8 \mathrm{~d})$ B $\quad(8 \mathrm{~d})$ B $\quad(8 \mathrm{~d})$ 
$-0.20200000000000$ 0.29600000000000 $-0.29600000000000$ $-0.18900000000000$ 0.18900000000000 $-0.29600000000000$ 0.29600000000000 0.18900000000000 $-0.18900000000000$ 0.29750000000000 $-0.29750000000000$ $-0.19830000000000$ 0.19830000000000 $-0.29750000000000$ 0.29750000000000 0.19830000000000 $-0.19830000000000$
$-0.30300000000000$ 0.18900000000000 $-0.18900000000000$ 0.29600000000000 $-0.18900000000000$ 0.29600000000000 0.29600000000000 0.19830000000000 $-0.19830000000000$ 0.29750000000000 $-0.29750000000000$ 0.19830000000000 0.29750000000000 $-0.29750000000000$ 0.29600000000000 0.18900000000000
0.23100000000000 0.0800000000000 0.58000000000000 0.33000000000000 0.83000000000000 0.08000000000000 0.4200000000000 0.67000000000000 0.17000000000000 0.17950000000000 0.67950000000000 0.42950000000000 0.92950000000000 $-0.17950000000000$ 0.32050000000000 0.57050000000000 0.07050000000000

$\begin{array}{ll}\text { B } & (8 d) \\ \text { C } & (8 d) \\ \text { C } & (8 d) \\ \text { C } & (8 d) \\ \text { C } & (8 d) \\ \text { C } & (8 d) \\ \text { C } & (8 d) \\ \text { C } & (8 d) \\ \text { C } & (8 d) \\ \text { Th } & (8 d) \\ \text { Th } & (8 d) \\ \text { Th } & (8 d) \\ \text { Th } & (8 d) \\ \text { Th } & (8 d) \\ \text { Th } & (8 d) \\ \text { Th } & (8 d) \\ \text { Th } & (8 d)\end{array}$

$\mathrm{AsPh}_{4} \mathrm{CeS}_{8} \mathrm{P}_{4} \mathrm{Me}_{8}$ : AB32CD4E8_tP184_93_i_16p_af_2p_4p - CIF

\# CIF file

data findsym-output

audit_creation method FINDSYM

chemical name mineral 'AsPh4CeS8P4Me8'

chemical_formula_sum 'As C32 Ce P4 S8,

aflow_title' $\mathrm{AsPh} \$_{-}\{4\} \$ \mathrm{CeS} \$_{-}\{8\} \$ \mathrm{P} \$_{-}\{4\} \$ \mathrm{Me} \$_{-}\{8\} \$$ Structure

aflow_proto 'AB32CD4E8_tP184_93_i_16p_af_2p_4p

aflow_params 'a, c/a, $z_{-}\{3\}, x_{-}\{4\}, y_{-}\{4\}, z_{-}\{4\}, x_{-}\{5\}, y_{-}\{5\}, z_{-}\{5\}, x_{-}\{6\}, y_{-}\{$ $\hookrightarrow 6\}, z_{-}\{6\}, x_{-}\{7\}, y_{-}\{7\}, z_{-}\{7\}, x_{-}\{8\}, y_{-}\{8\}, z_{-}\{8\}, x_{-}\{9\}, y_{-}\{9\}, z_{-}\{9\}$,

$\hookrightarrow \mathrm{x}_{-}\{10\}, \mathrm{y}_{-}\{10\}, \mathrm{z}_{-}\{10\}, \mathrm{x}_{-}\{11\}, \mathrm{y}_{-}\{11\}, \mathrm{z}_{-}\{11\}, \mathrm{x}_{-}\{12\}, \mathrm{y}_{-}\{12\}, \mathrm{z}_{-}\{12\}$,

$\hookrightarrow \mathrm{x}_{-}\{13\}, \mathrm{y}_{-}\{13\}, \mathrm{z}_{-}\{13\}, \mathrm{x}_{-}\{14\}, \mathrm{y}_{-}\{14\}, \mathrm{z}_{-}\{14\}, \mathrm{x}_{-}\{15\}, \mathrm{y}_{-}\{15\}, \mathrm{z}_{-}\{15\}$,

$\hookrightarrow \mathrm{x}_{-}\{16\}, \mathrm{y}_{-}\{16\}, \mathrm{z}_{-}\{16\}, \mathrm{x}_{-}\{17\}, \mathrm{y}_{-}\{17\}, \mathrm{z}_{-}\{17\}, \mathrm{x}_{-}\{18\}, \mathrm{y}_{-}\{18\}, \mathrm{z}_{-}\{18\}$,

$\hookrightarrow \mathrm{x}_{-}\{22\}, \mathrm{y}_{-}\{22\}, \mathrm{z}_{-}\{22\}, \mathrm{x}_{-}\{23\}, \mathrm{y}_{-}\{23\}, \mathrm{z}_{-}\{23\}, \mathrm{x}_{-}\{24\}, \mathrm{y}_{-}\{24\}, \mathrm{z}_{-}\{24\}$

$\hookrightarrow \mathrm{x}_{-}\{25\}, \mathrm{y}_{-}\{25\}, \mathrm{z}_{-}\{25\}$;

aflow_params_values ' $11.461,2.92112381119,0.62279,0.369,0.14,0.5403$,

$\hookrightarrow 0.251,0.129,0.6173,0.138,0.647,0.7882,0.215,0.58,0.8645,0.138$

$\hookrightarrow 0.517,0.591,0.132,0.58,0.5541,0.244,0.605,0.536,0.345,0.554$

$\hookrightarrow 0.5495,0.343,0.485,0.5842,0.237,0.463,0.6031,0.008,0.366,0.6554$

$\hookrightarrow \quad 0.077,0.371,0.6892,0.082,0.273,0.7151,0.023,0.17,0.7031,-0.04$

$\hookrightarrow 0.165,0.6678,-0.05,0.265,0.6408,0.2272,0.1019,0.5636,0.2601$,

$\hookrightarrow 0.6758,0.8049,0.2809,0.418,0.7912$

aflow_Strukturbericht 'None'

_aflow_Pearson 'tP184,

\section{cell_length_a $\quad 11.4610000000$ \\ $\begin{array}{ll}\text { cell_length_b } & 11.4610000000\end{array}$

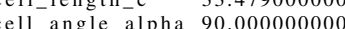 \\ cell_angle_beta 90.0000000000}

cell_angle_gamma 90.0000000000

symmetry_space_group_name_H-M "P $42 \quad 2 \quad 2 "$

symmetry_Int_Tables_number 93

loop

space group symop id

space_group_symop_operation_xyz

$1 \mathrm{x}, \mathrm{y}, \mathrm{z}$

$2 \mathrm{x},-\mathrm{y},-$

$3-\mathrm{x}, \mathrm{y},-\mathrm{z}$
$4-\mathrm{x},-\mathrm{y}, \mathrm{z}$
$5-\mathrm{y},-\mathrm{x},-2+4$

$5-\mathrm{y},-\mathrm{x},-\mathrm{z}+1 / 2$

$6-\mathrm{y}, \mathrm{x}, \mathrm{z}+1 / 2$

$7 \mathrm{y},-\mathrm{x}, \mathrm{z}+1 / 2$

loop

atom_site label

atom_site_type_symbol

atom_site_symmetry_multiplicity

atom_site_Wyckoff_label

atom_site_fract_x

atom_site_fract_y

atom_site_occupanc

$\begin{array}{lllllll}\mathrm{Ce} 1 \mathrm{Ce} & 2 & \mathrm{a} & 0.00000 & 0.00000 & 0.00000 & 1.00000\end{array}$

$\begin{array}{lllllll}\mathrm{Ce} 2 \mathrm{Ce} & 2 & \mathrm{f} & 0.50000 & 0.50000 & 0.25000 & 1.00000\end{array}$

As1 As 4 i $0.00000 \quad 0.50000 \quad 0.62279 \quad 1.00000$

$\begin{array}{llllllll}\mathrm{C} 1 & \mathrm{C} & 8 & \mathrm{p} & 0.36900 & 0.14000 & 0.54030 & 1.00000 \\ \mathrm{C} 2 & \mathrm{C} & 8 & \mathrm{p} & 0.25100 & 0.12900 & 0.61730 & 1.00000\end{array}$

$\begin{array}{llllllll}\mathrm{C} 2 & \mathrm{C} & 8 & \mathrm{p} & 0.25100 & 0.12900 & 0.61730 & 1.00000 \\ \mathrm{C} 3 & \mathrm{C} & 8 & \mathrm{p} & 0.13800 & 0.64700 & 0.78820 & 1.00000\end{array}$

$\begin{array}{llllllll}\mathrm{C} 4 & \mathrm{C} & 8 & \mathrm{p} & 0.21500 & 0.58000 & 0.86450 & 1.00000\end{array}$

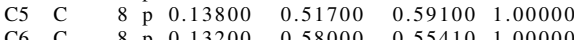

$\begin{array}{lllllllll}\mathrm{C} 6 & \mathrm{C} & 8 & \mathrm{p} & 0.13200 & 0.58000 & 0.55410 & 1.00000 \\ \mathrm{C} 7 & \mathrm{C} & 8 & \mathrm{p} & 0.24400 & 0.60500 & 0.53600 & 1.00000\end{array}$

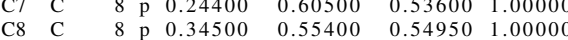

$\begin{array}{llllllll}\mathrm{C} 8 & \mathrm{C} & 8 & \mathrm{p} & 0.34500 & 0.55400 & 0.54950 & 1.00000 \\ \mathrm{C} 9 & \mathrm{C} & 8 & \mathrm{p} & 0.34300 & 0.48500 & 0.58420 & 1.00000\end{array}$

$\begin{array}{llllllll}\mathrm{C} 9 & \mathrm{C} & 8 & \mathrm{p} & 0.34300 & 0.48500 & 0.58420 & 1.00000 \\ \mathrm{C} 10 & \mathrm{C} & 8 & \mathrm{p} & 0.23700 & 0.46300 & 0.60310 & 1.00000\end{array}$

$\begin{array}{llllllll}\mathrm{C} 10 & \mathrm{C} & 8 & \mathrm{p} & 0.23700 & 0.46300 & 0.60310 & 1.00000 \\ \mathrm{C} 11 & \mathrm{C} & 8 & \mathrm{p} & 0.00800 & 0.36600 & 0.65540 & 1.00000 \\ \mathrm{C} 12 & \mathrm{C} & 8 & \mathrm{p} & 0.07700 & 0.37100 & 0.68920 & 1.00000\end{array}$

$\begin{array}{llllllll}\mathrm{C} 12 & \mathrm{C} & 8 & \mathrm{p} & 0.07700 & 0.37100 & 0.68920 & 1.00000\end{array}$

$\begin{array}{llllllll}\mathrm{C} 13 & \mathrm{C} & 8 & \mathrm{p} & 0.08200 & 0.27300 & 0.71510 & 1.00000\end{array}$

$\begin{array}{lllllllll}\mathrm{C} 14 & \mathrm{C} & 8 & \mathrm{p} & 0.02300 & 0.17000 & 0.70310 & 1.00000\end{array}$

$\begin{array}{llllllll}\mathrm{C} 15 & \mathrm{C} & 8 & \mathrm{p} & -0.04000 & 0.16500 & 0.66780 & 1.00000 \\ \mathrm{C} 16 & \mathrm{C} & 8 & \mathrm{p} & -0.05000 & 0.26500 & 0.64080 & 1.00000 \\ \mathrm{P} 1 & \mathrm{P} & 8 & \mathrm{p} & 0.22720 & 0.10190 & 0.56360 & 1.00000\end{array}$

$\begin{array}{llllllll}\mathrm{C} 16 & \mathrm{C} & 8 & \mathrm{p} & -0.05000 & 0.26500 & 0.64080 & 1.00000\end{array}$

$\begin{array}{llllllll}\mathrm{P} 1 & \mathrm{P} & 8 & \mathrm{p} & 0.22720 & 0.10190 & 0.56360 & 1.00000 \\ \mathrm{P} 2 & \mathrm{P} & 8 & \mathrm{p} & 0.26010 & 0.58020 & 0.81200 & 1.00000 \\ \mathrm{~S} 1 & \mathrm{~S} & 8 & \mathrm{P} & 0.10210 & 0.20500 & 0.54360 & 1.00000\end{array}$

$\begin{array}{llllll}8 & \mathrm{p} & 0.10210 & 0.20500 & 0.54360 & 1.00000\end{array}$

$\begin{array}{llllll}8 & \mathrm{p} & 0.19360 & -0.06760 & 0.55580 & 1.0000\end{array}$

$\begin{array}{llllllll}\mathrm{S} 3 & \mathrm{~S} & 8 & \mathrm{p} & 0.40400 & 0.67580 & 0.80490 & 1.00000\end{array}$

$\begin{array}{llllllll}\mathrm{S} 4 & \mathrm{~S} & 8 & \mathrm{p} & 0.28090 & 0.41800 & 0.79120 & 1.00000\end{array}$

$\mathrm{AsPh}_{4} \mathrm{CeS}_{8} \mathrm{P}_{4} \mathrm{Me}_{8}$ : AB32CD4E8_tP184_93_i_16p_af_2p_4p - POSCAR

AB32CD4E8_tP184_93_i_16p_af_2p_4p \& a , c/a , z3, x4, y4, z4, x5, y5, z5, x6, y6, z6 , $\hookrightarrow \mathrm{x} 7, \mathrm{y} 7, \mathrm{z} 7, \mathrm{x} 8, \mathrm{y} 8, \mathrm{z} 8, \mathrm{x} 9, \mathrm{y} 9, \mathrm{z} 9, \mathrm{x} 10, \mathrm{y} 10, \mathrm{z} 10, \mathrm{x} 11, \mathrm{y} 11, \mathrm{z} 11, \mathrm{x} 12, \mathrm{y} 12, \mathrm{z} 12$, $\hookrightarrow, \mathrm{y} 18, \mathrm{z} 18, \mathrm{x} 19, \mathrm{y} 19, \mathrm{z} 19, \mathrm{x} 20, \mathrm{y} 20, \mathrm{z} 20, \mathrm{x} 21, \mathrm{y} 21, \mathrm{z} 21, \mathrm{x} 22, \mathrm{y} 22, \mathrm{z} 22, \mathrm{x} 23$, $\hookrightarrow \mathrm{y} 23, \mathrm{z} 23, \mathrm{x} 24, \mathrm{y} 24, \mathrm{z} 24, \mathrm{x} 25, \mathrm{y} 25, \mathrm{z} 25-\mathrm{params}=11.461,2.92112381119$ $\hookrightarrow 0.62279,0.369,0.14,0.5403,0.251,0.129,0.6173,0.138,0.647,0.7882$ $\hookrightarrow, 0.215,0.58,0.8645,0.138,0.517,0.591,0.132,0.58,0.5541,0.244$, $\longrightarrow 0.605,0.536,0.345,0.554,0.5495,0.343,0.485,0.5842,0.237,0.463$, $0.023,0.17,0.7031,-0.04,0.165,0.6678,-0.05,0.265,0.6408,0.2272$ $0.1019,0.5636,0.2601,0.5802,0.812,0.1021,0.205,0.5436,0.1936$ $\rightarrow 0.0676,0.5558,0.404,0.6758,0.8049,0.2809,0.418,0.7912 \&$ P4_\{2\}

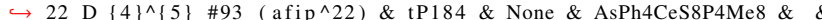
1.00000000000000

$11.46100000000000-0.00000000000000-0.00000000000000$ $\begin{array}{lrl}0.00000000000000 & 11.46100000000000 & 0.00000000000000\end{array}$ $\begin{array}{rrr}0.00000000000000 & 0.00000000000000 & 33.47900000000000\end{array}$

$$
\begin{array}{rrrrr}
\text { As } & \text { C } & \text { Ce } & \text { P } & \text { S } \\
4 & 128 & 4 & 16 & 32
\end{array}
$$

irect

0.00000000000000

0.50000000000000

0.00000000000000

0.50000000000000

0.36900000000000

$-0.36900000000000$

0.14000000000000

$-0.36900000000000$

0.36900000000000

0.14000000000000

$-0.14000000000000$

0.25100000000000

$-0.25100000000000$

$-0.12900000000000$

0.12900000000000
-0.25100000000000

$-0.25100000000000$

0.25100000000000
0.12900000000000

$-0.1290000000000$

0.13800000000000

$-0.13800000000000$

0.64700000000000

$-0.13800000000000$

0.13800000000000

0.64700000000000

$-0.64700000000000$

0.21500000000000

$-0.21500000000000$

$-0.5800000000000$

0.58000000000000

-0.21500000000000
0.21500000000000

0.21500000000000
0.58000000000000

0.58000000000000
-0.58000000000000

$-0.58000000000000$

$-0.13800000000000$

$-0.51700000000000$

0.51700000000000

$-0.13800000000000$

0.13800000000000

0.51700000000000

$-0.51700000000000$

0.13200000000000

$-0.13200000000000$

$-0.58000000000000$

0.58000000000000

$-0.1320000000000$

0.13200000000000

0.5800000000000

$-0.58000000000000$

0.2440000000000

$-0.24400000000000$

$-0.60500000000000$

0.60500000000000
-0.24400000000000

0.24400000000000

0.60500000000000

$-0.60500000000000$

$-0.6050000000000$

0.34500000000000

$-0.55400000000000$

0.55400000000000

$-0.34500000000000$

0.34500000000000

0.55400000000000

$-0.5540000000000$

0.34300000000000

$-0.34300000000000$

$-0.48500000000000$

$-0.34300000000000$

0.34300000000000

0.48500000000000

$-0.48500000000000$

0.2370000000000

0.23700000000000

$-0.23700000000000$

0.46300000000000

$-0.23700000000000$

0.23700000000000

0.46300000000000

$-0.46300000000000$

0.50000000000000 0.00000000000000 0.50000000000000 0.00000000000000 0.14000000000000 0.14000000000000 0.36900000000000 $-0.36900000000000$ 0.14000000000000 $-0.14000000000000$ 0.3690000000000 0.3600000000000 0.12900000000000 .12900000000000 0.25100000000000 0.25100000000000 0.12900000000000 $-0.12900000000000$ 0.25100000000000 0.25100000000000 0.64700000000000 0.64700000000000 0.13800000000000 $-0.13800000000000$ 0.64700000000000 $-0.64700000000000$ 0.1380000000000 0.1380000000000 0.5800000000000 0.58000000000000 .21500000000000 0.21500000000000 0.58000000000000 $-.58000000000000$ 0.21500000000000 $-0.21500000000000$ 0.51700000000000 $-0.51700000000000$ 000000000 0.13800000000000 $-0.51700000000000$ 0.13800000000000 $-0.13800000000000$ 0.58000000000000 0.58000000000000 0.13200000000000 .13200000000000 0.58000000000000 .58000000000000 0.13200000000000 $-0.13200000000000$ 0.60500000000000 $-0.60500000000000$ .24400000000000 .24400000000000 $-0.60500000000000$ 0.24400000000000 $-0.2440000000000$ 0.5540000000000 $-0.55400000000000$ 0.34500000000000 0.34500000000000 0.55400000000000 0.55400000000000 0.34500000000000 0.34500000000000 0.48500000000000 $-0.48500000000000$ 
0.00800000000000 0.00800000000000 $-0.36600000000000$ 0.36600000000000 $-0.00800000000000$ 0.00800000000000 0.36600000000000 $-0.36600000000000$ 0.07700000000000 $-0.07700000000000$ $-0.37100000000000$ 0.37100000000000 $-0.07700000000000$ 0.07700000000000 0.37100000000000 $-0.37100000000000$ 0.08200000000000 $-0.08200000000000$ 0.27300000000000 0.27300000000000 $-0.08200000000000$ 0.08200000000000 0.27300000000000 0.27300000000000 0.02300000000000 $-0.02300000000000$ $-0.17000000000000$ 0.17000000000000 $-0.02300000000000$ 0.02300000000000 0.17000000000000 $-0.17000000000000$ $-0.04000000000000$ 0.04000000000000 0.16500000000000 0.16500000000000 0.04000000000000 $-0.04000000000000$ 0.16500000000000 $-0.16500000000000$ $-0.05000000000000$ 0.05000000000000 $-0.26500000000000$ 0.05000000000000 $-0.05000000000000$ 0.26500000000000 $-0.26500000000000$ 0.00000000000000 0.00000000000000 0.00000000000000 0.50000000000000 0.50000000000000 0.22720000000000 $-0.22720000000000$ 0.10190000000000
-0.22720000000000 $-0.22720000000000$ 0.22720000000000 0.10190000000000 $-0.10190000000000$ $-0.26010000000000$ $-0.58020000000000$ 0.58020000000000 0.58020000000000 0.260100000000 0.580200000000 0.58020000000 0.58020000000000 0.10210000000000 $-0.10210000000000$ 0.20500000000000 0.20500000000000 $-0.10210000000000$ 0.10210000000000 0.20500000000000 $-0.20500000000000$ 0.19360000000000 $-0.19360000000000$ 0.06760000000000 $-0.06760000000000$ $-0.1936000000000$ 0.19360000000000 $-0.06760000000000$ 0.06760000000000 0.40400000000000 $-0.40400000000000$ $-0.67580000000000$ 0.67580000000000 0.40400000000000 0.40400000000000 0.67580000000000 $-0.67580000000000$ 0.28090000000000 $-0.28090000000000$ $-0.41800000000000$ 0.41800000000000 0.28090000000000

0.28090000000000

.41800000000000 $-0.41800000000000$

0.36600000000000 0.36600000000000 0.00800000000000 $-0.00800000000000$ 0.36600000000000 $-0.36600000000000$ 0.00800000000000 $-0.00800000000000$ 0.37100000000000 $-0.37100000000000$ 0.07700000000000 $-0.07700000000000$ 0.3710000000000 $-0.3710000000000$ 0.0770000000000 0.07700000000000 0.27300000000000 0.27300000000000 0.08200000000000 $-0.08200000000000$ 0.27300000000000 $-0.2730000000000$ 0.08200000000000 $-0.0820000000000$ 0.17000000000000 $-0.17000000000000$ 0.02300000000000 $-0.02300000000000$ 0.17000000000000 $-0.17000000000000$ 0.02300000000000 0.02300000000000 0.16500000000000 $-0.16500000000000$ $-0.04000000000000$ 0.04000000000000 0.16500000000000 $-0.16500000000000$ $-0.0400000000000$ 0.04000000000000 0.26500000000000 $-0.2650000000000$ $-0.0500000000000$ 0.05000000000000 0.26500000000000 $-0.05000000000000$ 0.05000000000000 0.0000000000000 0.00000000000000 0.5000000000 (2) 0.1019000000000 $-0.10190000000000$ 0.2272000000000 $-0.22720000000000$ 0.1019000000000 $-0.10190000000000$ 0.22720000000000 $-0.22720000000000$ $-0.58020000000000$ 0.2601000000000 $-0.26010000000000$ 0.58020000000000 0.58020000000 0.26010000000000 $-0.26010000000000$ 0.20500000000000 $-0.20500000000000$ 0.10210000000000 0.10210000000000 0.20500000000000 0.20500000000000 0.10210000000000 $-0.10210000000000$ 0.06760000000000 0.0676000000000 $-0.19360000000000$ $-0.06760000000000$ 0.06760000000000 0.19360000000000 0.67580000000000 $-0.67580000000000$ 0.40400000000000 $-0.40400000000000$ 0.67580000000000 $-0.67580000000000$ 0.4040000000000 $-0.40400000000000$ 0.41800000000000 $-0.41800000000000$ 0.28090000000000 $-0.28090000000000$ 0.41800000000000 0.28090000000000 $-0.28090000000000$

0.65540000000000 .65540000000000 15540000000000 1.15540000000000 1.15540000000 $-0.65540000000000$ $-0.15540000000000$ 0.155400000000 0.15540000000000 .68920000000000 .68920000000000 1.18920000000000 $-0.68920000000000$ $-0.68920000000000$ $-0.18920000000000$ $-0.1892000000000$ 0.71510000000000 0.7151000000000 .7151000000 1.215100000000 1.21510000000000 $-0.71510000000000$ $-0.7151000000000$ $-0.21510000000000$ 0.21510000000000 0.70310000000000 0.70310000000000 1.20310000000000 1.20310000000000 $-0.70310000000000$ $-0.20310000000000$ 0.20310000000000 0.66780000000000 0.66780000000000 1.16780000000000 1.16780000 1.16780000000000 $-0.6678000000000$ 0.16780000000000 0.64080000000000 0.64080000000000 1.14080000000000 1.14080000000000 $-0.64080000000000$ $-0.64080000000000$ $-0.14080000000000$ $-0.14080000000000$ 0.00000000000000 0.50000000000000 0.7500000000000 0.56360000000 0.56360000000000 1.06360000000000 1.06360000000000 $-0.56360000000000$ $-0.5636000000000$ $-0.06360000000000$ 0.06360000000000 0.81200000000000 0.81200000000000 1.31200000000000 1.31200000000000 $-0.81200000000000$ $-0.81200000000000$ $-0.31200000000000$ $-0.31200000000000$ 0.54360000000000 .54360000000000 .04360000000000 .0436000000000 $-0.54360000000000$ . $-0.04360000000000$ $-0.04360000000000$ 0.55580000000000 0.55580000000000 1.05580000000000 1.05580000000000 $-0.55580000000000$ $-0.55580000$ $-0.05580000000000$ 0.0558000000000 0.80490000000000 . 1.30490000000000 1.30490000000000 $-0.8049000000000$ $-0.80490000000000$ $-0.30490000000000$ 0.79120000000000 


\begin{tabular}{|c|c|c|c|c|}
\hline 0.00240000000000 & 0.70860000000000 & -0.62810000000000 & $\mathrm{~F}$ & $(8 g)$ \\
\hline 0.00240000000000 & -0.70860000000000 & -0.62810000000000 & $\mathrm{~F}$ & $(8 \mathrm{~g})$ \\
\hline-0.02870000000000 & 0.65750000000000 & 0.62420000000000 & $\mathrm{~F}$ & $(8 \mathrm{~g})$ \\
\hline 0.02870000000000 & -0.65750000000000 & 0.62420000000000 & $\mathrm{~F}$ & $(8 \mathrm{~g})$ \\
\hline-0.15750000000000 & 0.47130000000000 & 1.12420000000000 & $\mathrm{~F}$ & $(8 \mathrm{~g})$ \\
\hline 1.15750000000000 & 0.52870000000000 & 1.12420000000000 & $\mathrm{~F}$ & $(8 \mathrm{~g})$ \\
\hline 0.52870000000000 & 1.15750000000000 & -0.12420000000000 & $\mathrm{~F}$ & $(8 \mathrm{~g})$ \\
\hline 0.47130000000000 & -0.15750000000000 & -0.12420000000000 & $\mathrm{~F}$ & $(8 \mathrm{~g})$ \\
\hline 0.65750000000000 & -0.02870000000000 & -0.62420000000000 & $\mathrm{~F}$ & $(8 \mathrm{~g})$ \\
\hline-0.65750000000000 & 0.02870000000000 & -0.62420000000000 & $\mathrm{~F}$ & $(8 \mathrm{~g})$ \\
\hline 0.54000000000000 & 0.75000000000000 & 0.54480000000000 & $\mathrm{~F}$ & $(8 \mathrm{~g})$ \\
\hline-0.54000000000000 & -0.75000000000000 & 0.54480000000000 & $\mathrm{~F}$ & $(8 \mathrm{~g})$ \\
\hline-0.25000000000000 & 1.04000000000000 & 1.04480000000000 & $\mathrm{~F}$ & $(8 \mathrm{~g})$ \\
\hline 1.25000000000000 & -0.04000000000000 & 1.04480000000000 & $\mathrm{~F}$ & $(8 \mathrm{~g})$ \\
\hline-0.04000000000000 & 1.25000000000000 & -0.04480000000000 & $\mathrm{~F}$ & $(8 \mathrm{~g})$ \\
\hline 1.04000000000000 & -0.25000000000000 & -0.04480000000000 & $\mathrm{~F}$ & $(8 \mathrm{~g})$ \\
\hline 0.75000000000000 & 0.54000000000000 & -0.54480000000000 & $\mathrm{~F}$ & $(8 \mathrm{~g})$ \\
\hline-0.75000000000000 & -0.54000000000000 & -0.54480000000000 & $\mathrm{~F}$ & $(8 \mathrm{~g})$ \\
\hline 0.00000000000000 & 0.00000000000000 & 0.00000000000000 & $\mathrm{Fe}$ & (2a) \\
\hline 0.50000000000000 & 0.50000000000000 & 0.50000000000000 & $\mathrm{Fe}$ & (2a) \\
\hline 0.00000000000000 & 0.50000000000000 & -0.00160000000000 & $\mathrm{Fe}$ & $(4 d)$ \\
\hline 0.00000000000000 & 0.50000000000000 & 0.49840000000000 & $\mathrm{Fe}$ & $(4 d)$ \\
\hline 0.50000000000000 & 0.00000000000000 & 0.50160000000000 & $\mathrm{Fe}$ & $(4 \mathrm{~d})$ \\
\hline 0.50000000000000 & 0.00000000000000 & 0.00160000000000 & $\mathrm{Fe}$ & $(4 \mathrm{~d})$ \\
\hline 0.00000000000000 & 0.00000000000000 & 0.50000000000000 & $\mathrm{Na}$ & (2b) \\
\hline 0.50000000000000 & 0.50000000000000 & 0.00000000000000 & $\mathrm{Na}$ & (2b) \\
\hline 0.73120000000000 & 0.73480000000000 & 0.74030000000000 & $\mathrm{Na}$ & $(8 \mathrm{~g})$ \\
\hline-0.73120000000000 & -0.73480000000000 & 0.74030000000000 & $\mathrm{Na}$ & $(8 \mathrm{~g})$ \\
\hline-0.23480000000000 & 1.23120000000000 & 1.24030000000000 & $\mathrm{Na}$ & $(8 \mathrm{~g})$ \\
\hline 1.23480000000000 & -0.23120000000000 & 1.24030000000000 & $\mathrm{Na}$ & $(8 \mathrm{~g})$ \\
\hline-0.23120000000000 & 1.23480000000000 & -0.24030000000000 & $\mathrm{Na}$ & $(8 \mathrm{~g})$ \\
\hline 1.23120000000000 & -0.23480000000000 & -0.24030000000000 & $\mathrm{Na}$ & $(8 \mathrm{~g})$ \\
\hline 0.73480000000000 & 0.73120000000000 & -0.74030000000000 & $\mathrm{Na}$ & $(8 \mathrm{~g})$ \\
\hline-0.73480000000000 & -0.73120000000000 & -0.74030000000000 & $\mathrm{Na}$ & $(8 \mathrm{~g})$ \\
\hline
\end{tabular}

$\mathrm{Li}_{2} \mathrm{MoF}_{6}$ : A6B2C_tP18_94_eg_c_a - CIF $\begin{array}{llllllll}\text { F2 } & \text { F } & 8 & \text { g } & 0.68400 & 0.70700 & 0.65790 & 1.00000\end{array}$

$\mathrm{Li}_{2} \mathrm{MoF}_{6}$ : A6B2C_tP18_94_eg_c_a - POSCAR

\begin{tabular}{|c|c|c|c|c|}
\hline \multirow{3}{*}{\multicolumn{5}{|c|}{ 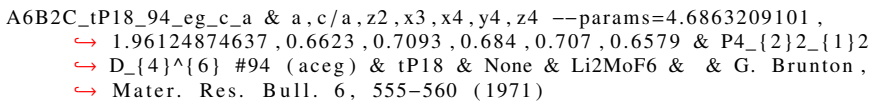 }} \\
\hline & & & & \\
\hline & & & & \\
\hline 4.68632091010000 & 0.00000000000000 & 0.00000000000000 & & \\
\hline \multirow{2}{*}{$\begin{array}{l}0.00000000000000 \\
0.00000000000000\end{array}$} & 4.68632091010000 & 0.00000000000000 & & \\
\hline & 0.00000000000000 & & & \\
\hline Mo & & & & \\
\hline 12 & & & & \\
\hline \multicolumn{5}{|l|}{ Direct } \\
\hline \multirow{2}{*}{0.70930000000000} & 0.70930000000000 & 0.00000000000000 & $\mathrm{~F}$ & $(4 \mathrm{e})$ \\
\hline & -0.70930000000000 & 0.00000000000000 & $\mathrm{~F}$ & (4e) \\
\hline-0.20930000000000 & 1.20930000000000 & 0.50000000000000 & $\mathrm{~F}$ & (4e) \\
\hline 1.20930000000000 & -0.20930000000000 & 0.50000000000000 & $\mathrm{~F}$ & $(4 \mathrm{e})$ \\
\hline 0.68400000000000 & 0.70700000000000 & 0.65790000000000 & $\mathrm{~F}$ & $(8 \mathrm{~g})$ \\
\hline-0.68400000000000 & -0.70700000000000 & 0.65790000000000 & $\mathrm{~F}$ & $(8 \mathrm{~g})$ \\
\hline-0.20700000000000 & 1.18400000000000 & 1.15790000000000 & $\mathrm{~F}$ & \\
\hline \multirow{2}{*}{$\begin{array}{r}1.20700000000000 \\
-0.1840000000000\end{array}$} & -0.18400000000000 & 1.15790000000000 & $\mathrm{~F}$ & $(8 \mathrm{~g})$ \\
\hline & 1.20700000000000 & -0.15790000000000 & $\mathrm{~F}$ & $(8 \mathrm{~g})$ \\
\hline 1.18400000000000 & -0.20700000000000 & -0.15790000000000 & 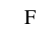 & \\
\hline 0.70700000000000 & 0.68400000000000 & -0.65790000000000 & $\mathrm{~F}$ & $(8 \mathrm{~g})$ \\
\hline \multirow{2}{*}{$\begin{array}{r}-0.70700000000000 \\
0.00000000000000\end{array}$} & -0.68400000000000 & -0.65790000000000 & & \\
\hline & 0.00000000000000 & 0.66230000000000 & $\mathrm{Li}$ & $(4 c)$ \\
\hline 0.50000000000000 & 0.50000000000000 & 1.16230000000000 & $\mathrm{Li}$ & \\
\hline 0.50000000000000 & 0.50000000000000 & -0.16230000000000 & $\mathrm{Li}$ & $(4 \mathrm{c})$ \\
\hline 0.00000000000000 & 0.00000000000000 & -0.66230000000000 & $\mathrm{Li}$ & \\
\hline \multirow{2}{*}{$\begin{array}{l}0.00000000000000 \\
0.50000000000000\end{array}$} & 0.00000000000000 & 0.00000000000000 & Mo & (2a) \\
\hline & 0.50000000000000 & 0.50000000000000 & Mo & (2a) \\
\hline
\end{tabular}

ThBC: ABC_tP24_95_d_d_d - CIF

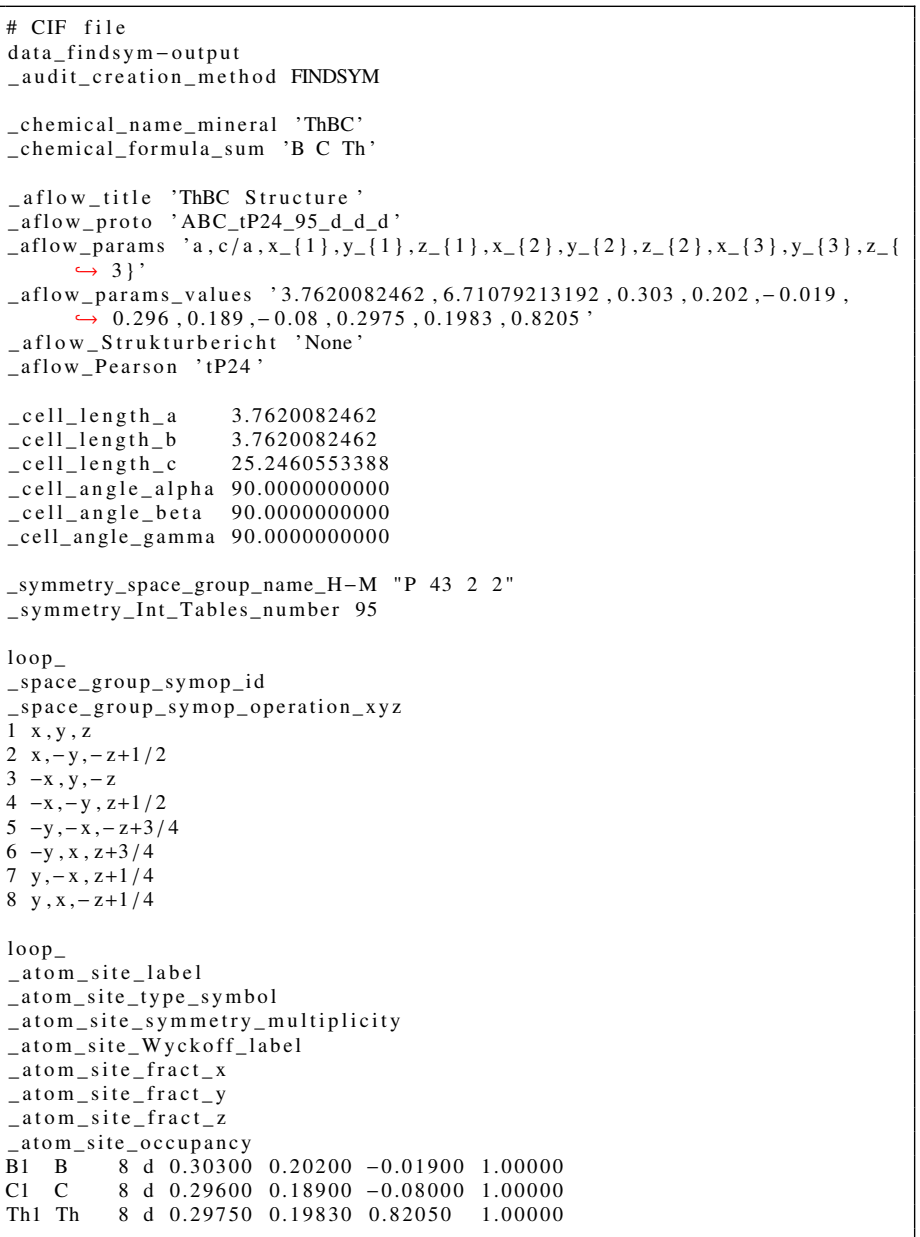

ThBC: ABC_tP24_95_d_d_d - POSCAR

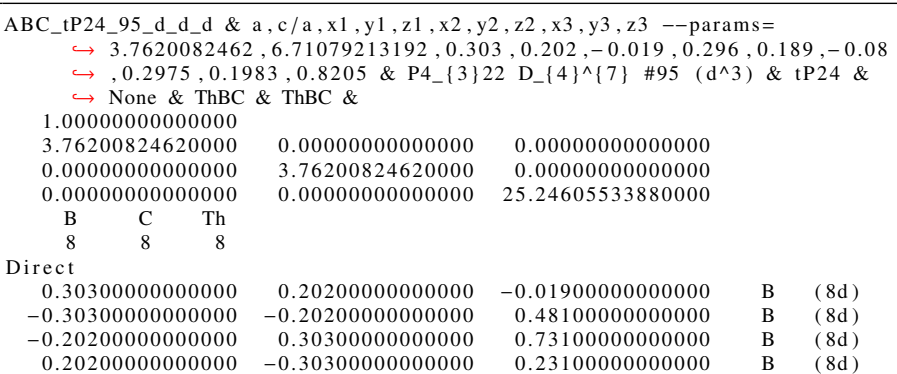




$\begin{array}{rrrrr}-0.30300000000000 & 0.20200000000000 & 0.01900000000000 & \mathrm{~B} & (8 \mathrm{~d}) \\ 0.30300000000000 & -0.20200000000000 & 0.51900000000000 & \mathrm{~B} & (8 \mathrm{~d}) \\ 0.20200000000000 & 0.30300000000000 & 0.26900000000000 & \mathrm{~B} & (8 \mathrm{~d}) \\ -0.20200000000000 & -0.30300000000000 & 0.76900000000000 & \mathrm{~B} & (8 \mathrm{~d}) \\ 0.29600000000000 & 0.18900000000000 & -0.08000000000000 & \mathrm{C} & (8 \mathrm{~d}) \\ -0.29600000000000 & -0.18900000000000 & 0.42000000000000 & \mathrm{C} & (8 \mathrm{~d}) \\ -0.18900000000000 & 0.29600000000000 & 0.67000000000000 & \mathrm{C} & (8 \mathrm{~d}) \\ 0.18900000000000 & -0.29600000000000 & 0.17000000000000 & \mathrm{C} & (8 \mathrm{~d}) \\ -0.29600000000000 & 0.18900000000000 & 0.08000000000000 & \mathrm{C} & (8 \mathrm{~d}) \\ 0.29600000000000 & -0.18900000000000 & 0.58000000000000 & \mathrm{C} & (8 \mathrm{~d}) \\ 0.18900000000000 & 0.29600000000000 & 0.33000000000000 & \mathrm{C} & (8 \mathrm{~d}) \\ -0.18900000000000 & -0.29600000000000 & 0.83000000000000 & \mathrm{C} & (8 \mathrm{~d}) \\ 0.29750000000000 & 0.19830000000000 & 0.82050000000000 & \text { Th } & (8 \mathrm{~d}) \\ -0.29750000000000 & -0.19830000000000 & 1.32050000000000 & \text { Th } & (8 \mathrm{~d}) \\ -0.19830000000000 & 0.29750000000000 & 1.57050000000000 & \text { Th } & (8 \mathrm{~d}) \\ 0.19830000000000 & -0.29750000000000 & 1.07050000000000 & \text { Th } & (8 \mathrm{~d}) \\ -0.29750000000000 & 0.19830000000000 & -0.82050000000000 & \text { Th } & (8 \mathrm{~d}) \\ 0.29750000000000 & -0.19830000000000 & -0.32050000000000 & \text { Th } & (8 \mathrm{~d}) \\ 0.19830000000000 & 0.29750000000000 & -0.57050000000000 & \text { Th } & (8 \mathrm{~d}) \\ -0.19830000000000 & -0.29750000000000 & -0.07050000000000 & \text { Th } & (8 \mathrm{~d})\end{array}$

NaGdCu $2 \mathrm{~F}_{8}$ : A2B8CD_tI24_97_d_k_a_b - CIF

\section{CIF file}

data_findsym-output

_audit_creation_method FINDSYM

chemical_name_mineral 'NaGdCu2F8,

_chemical_formula_sum ' $\mathrm{Cu} 2$ F8 Gd Na'

loop

publ_author_name

C. $\{$ De Nada $\backslash "$ i $\}$

A. Demourgues

'L. Lozano'

'P. Gravereau'

journal_name_full_name

Journal of Materials Chemistry

journal_volume 8

year 1998

_journal_page_first 248

_journal_page_last 2491

publ_Section_title

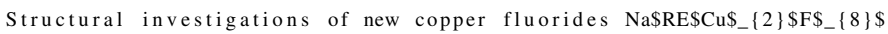
$\rightarrow \quad\left(\$ \operatorname{RE}^{\wedge}\{3+\} \$=\operatorname{Sm} \$^{\wedge}\{3+\} \$, \operatorname{Eu}^{\wedge}\{3+\} \$, \quad \operatorname{Gd}^{\wedge}\{3+\} \$, \mathrm{Y}^{\wedge}\{3+\} \$, \operatorname{Er} \$ \wedge\{\right.$ $\hookrightarrow 3+\} \$, \quad Y b \$ \wedge\{3+\} \$$

\# Found in Pearson's Crystal Data - Crystal Structure Database for $\hookrightarrow$ Inorganic Compounds, 2013

aflow title 'NaGdCu\$ $\{2\} \$ F \$\{8\} \$$ Structure'

aflow_proto 'A2B8CD_tI24_97_d_k_a_b'

aflow_params $, a, c / a, x_{-}\{4\}, y_{-}\{4\}, z_{-}\{4\}$,

aflow_params_values ' $5.4068544677,1.92010356944,0.1697,0.3128,0.1237$, aflow_Strukturbericht 'None

aflow_Pearson 'tI24,

cell_length_a $\quad 5.4068544677$

cell_length_b 5.4068544677

cell_length_c $\quad 10.3817205629$

cell_angle_alpha 90.0000000000

cell_angle_beta 90.0000000000

cell_angle_gamma 90.0000000000

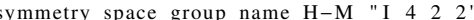

symmetry_Int_Tables_number 97

loop_

space_group_symop_id

_space_group_symop_operation_xyz

$1 \mathrm{x}, \mathrm{y}, \mathrm{z}$

$2 \mathrm{x},-\mathrm{y},-\mathrm{z}$

$3-x, y,-z$

$4-x,-y, z$

$5-\mathrm{y},-\mathrm{x},-\mathrm{z}$

$6-\mathrm{y}, \mathrm{x}, \mathrm{z}$
$7 \mathrm{y},-\mathrm{x}, \mathrm{z}$

$\mathrm{y}, \mathrm{x},-\mathrm{z}$

$\mathrm{x}+1 / 2, \mathrm{y}+1 / 2, \mathrm{z}+1 / 2$

$0 \mathrm{x}+1 / 2,-\mathrm{y}+1 / 2,-\mathrm{z}+1 / 2$

$11-\mathrm{x}+1 / 2, \mathrm{y}+1 / 2,-\mathrm{z}+1 / 2$

$12-x+1 / 2,-y+1 / 2, z+1 / 2$

$13-\mathrm{y}+1 / 2,-\mathrm{x}+1 / 2,-\mathrm{z}+1 / 2$

$14-\mathrm{y}+1 / 2, \mathrm{x}+1 / 2, \mathrm{z}+1 / 2$

$16 \mathrm{y}+1 / 2, \mathrm{x}+1 / 2,-\mathrm{z}+1 / 2$

loop

atom site label

_atom_site_type_symbol

atom_site_symmetry_multiplicity

atom_site_Wyckoff_label

atom_site_fract_x

atom_site_fract_y

atom_site_fract

atom_site_occupancy

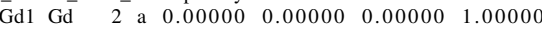

$\begin{array}{llllllll}\mathrm{Na} 1 & \mathrm{Na} & 2 & \mathrm{~b} & 0.00000 & 0.00000 & 0.50000 & 1.00000\end{array}$

$\begin{array}{llllllll}\mathrm{Cu} 1 \mathrm{Cu} & 4 & \mathrm{~d} & 0.00000 & 0.50000 & 0.25000 & 1.00000\end{array}$

$\begin{array}{llrllllll}\text { F1 } & \mathrm{F} & 16 & \mathrm{k} & 0.16970 & 0.31280 & 0.12370 & 1.00000\end{array}$
NaGdCu $2 F_{8}$ : A2B8CD_tI24_97_d_k_a_b - POSCAR

\begin{tabular}{|c|c|c|c|c|}
\hline \multirow{2}{*}{\multicolumn{5}{|c|}{ 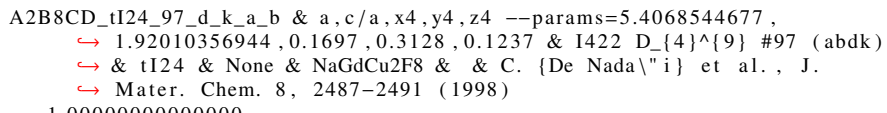 }} \\
\hline 1.00000000000000 & & & & \\
\hline-2.70342723385000 & 2.70342723385000 & 5.19086028145000 & & \\
\hline 2.70342723385000 & -2.70342723385000 & 5.19086028145000 & & \\
\hline 2.70342723385000 & 2.70342723385000 & -5.19086028145000 & & \\
\hline $\mathrm{Gd}$ & $\mathrm{Na}$ & & & \\
\hline 1 & 1 & & & \\
\hline \multicolumn{5}{|l|}{ Direct } \\
\hline 0.75000000000000 & 0.25000000000000 & 0.50000000000000 & $\mathrm{Cu}$ & $(4 d)$ \\
\hline 0.25000000000000 & 0.75000000000000 & 0.50000000000000 & $\mathrm{Cu}$ & (4d) \\
\hline 0.43650000000000 & 000000 & 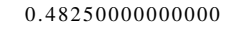 & $\mathrm{F}$ & $(16 \mathrm{k})$ \\
\hline-0.18910000000000 & -0.04600000000000 & -0.48250000000000 & $\mathrm{~F}$ & $(16 \mathrm{k})$ \\
\hline 0.29340000000000 & -0.18910000000000 & -0.14310000000000 & $\mathrm{~F}$ & $(16 \mathrm{k})$ \\
\hline-0.04600000000000 & 000000 & 0.143 & $\mathrm{~F}$ & $(16 \mathrm{k})$ \\
\hline 0.18910000000000 & 000000 & 0.143 & $\mathrm{~F}$ & $(16 \mathrm{k})$ \\
\hline-0.43650000000000 & 00000000 & -0.14310000000000 & $\mathrm{~F}$ & $(16 \mathrm{k})$ \\
\hline 0.04600000000000 & 000000 & 0.48250000000000 & $\mathrm{~F}$ & $(16 \mathrm{k})$ \\
\hline-0.29340000000000 & -0.43650000000000 & -0.48250000000000 & $\mathrm{~F}$ & $(16 \mathrm{k})$ \\
\hline 0.00000000000000 & 0.00000000000000 & 0.00000000000000 & $\mathrm{Gd}$ & $(2 a)$ \\
\hline 0.50000000000000 & 0.50000000000000 & 0.00000000000000 & $\mathrm{Na}$ & (2b) \\
\hline
\end{tabular}

$\mathrm{Ta}_{2} \mathrm{Se}_{8} \mathrm{I}$ : AB8C2_tI44_97_e_2k_cd - CIF

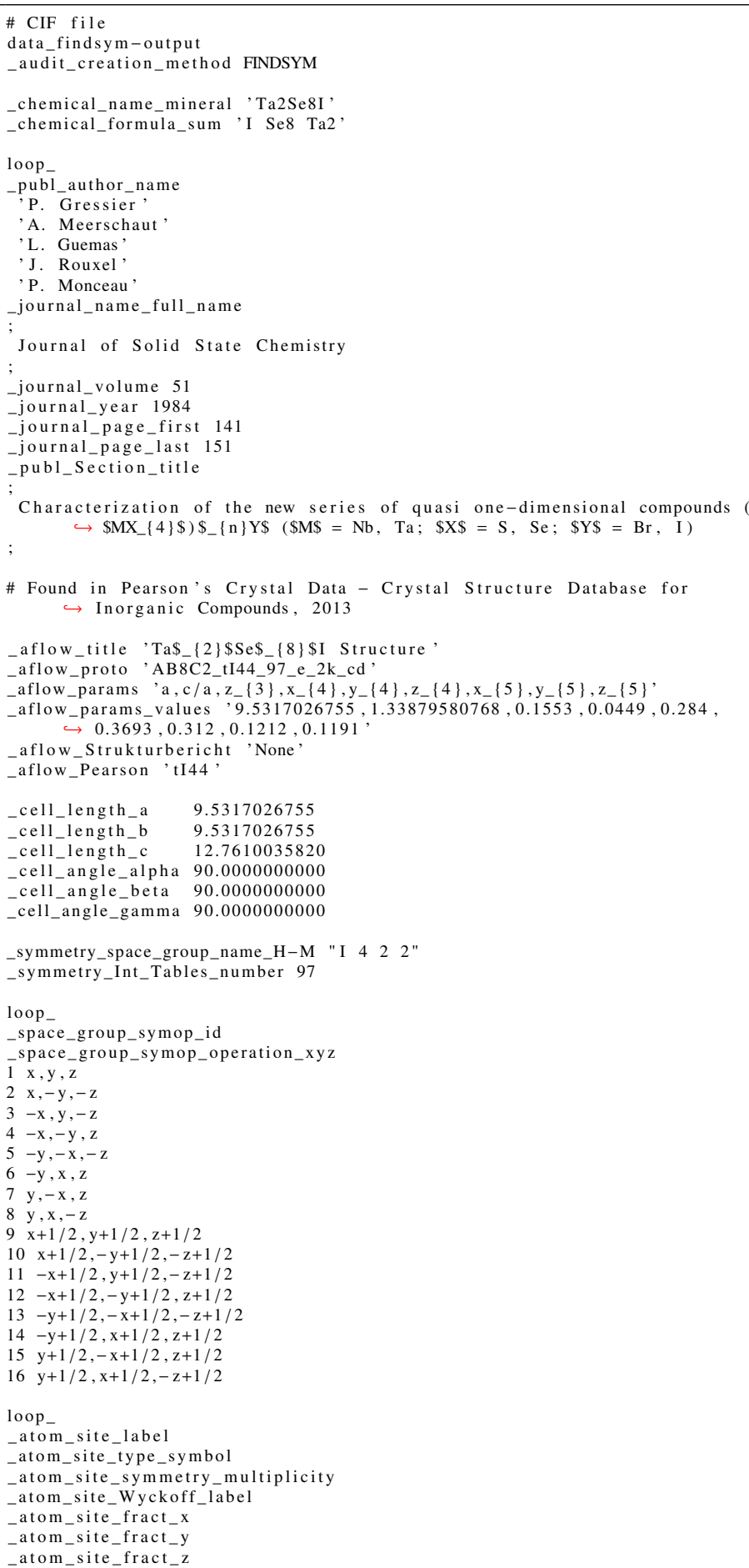


-atom_site_occupancy

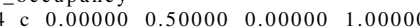

Ta2 Ta 4 d $0.00000 \quad 0.50000 \quad 0.25000 \quad 1.00000$

$\begin{array}{llllllllll}\text { II } & \mathrm{I} & 4 & \mathrm{e} & 0.00000 & 0.00000 & 0.15530 & 1.00000\end{array}$

$\begin{array}{llllllllll}\mathrm{Se} 1 & \mathrm{Se} & 16 & \mathrm{k} & 0.04490 & 0.28400 & 0.36930 & 1.00000\end{array}$

$\begin{array}{lllllllll}\mathrm{Se} 2 & \mathrm{Se} & 16 & \mathrm{k} & 0.31200 & 0.12120 & 0.11910 & 1.00000\end{array}$

$\mathrm{Ta}_{2} \mathrm{Se}_{8} \mathrm{I}$ : AB8C2_tI44_97_e_2k_cd - POSCAR

AB8C2_tI44_97_e_2k_cd \& a , c /a , z3 , x4,y4, z4, x5,y5, z5 --params $=9.5317026755$

$\hookrightarrow, 1.33879580768,0.1553,0.0449,0.284,0.3693,0.312,0.1212,0.1191$

$\hookrightarrow$ I422 D_ $\{4\}^{\wedge}\{9\} \# 97\left(\operatorname{cdek}^{\wedge} 2\right) \&$ tI44 \& None \& Ta2Se8I \& \& P.

$\hookrightarrow$ Gressier et al., J. Solid State Chem. 51, 141-151 (1984)

1.00000000000000

$\begin{array}{rrr}-4.76585133775000 & 4.76585133775000 & 6.38050179100000\end{array}$

$\begin{array}{llll}4.76585133775000 & -4.76585133775000 & 6.38050179100000\end{array}$

$\begin{array}{rrr}4.76585133775000 & 4.76585133775000 & -6.38050179100000\end{array}$

I $\mathrm{Se} \quad \mathrm{Ta}$

irect

0.15530000000000

$-0.1553000000000$

0.65330000000000

0.0853000000000

0.08530000000000

0.4142000000000

0.32440000000000

$-0.08530000000000$

$-0.65330000000000$

$-0.32440000000000$

$-0.41420000000000$

0.24030000000000

$-0.00210000000000$

0.43110000000000

$-0.19290000000000$

0.00210000000000

$-0.24030000000000$

0.19290000000000

$-0.43110000000000$

0.50000000000000

0.00000000000000

0.75000000000000

0.25000000000000

0.15530000000000

0.0000000000000

0.32890000000000

$-0.32890000000000$

41420000000000

0.32440000000000

0.08530000000000

0.65330000000000

$-0.41420000000000$

$-0.32440000000000$ $-0.239100000$ 0.2391000000000

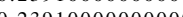
0.239100000000 $-0.23910000000000$ 0.32890000000000 $-0.3289000000000$ 0.4332000000000 $-0.43320000000000$ 0.19080000000000 $-0.1908000000000$ $-0.1908000000000$ 0.1908000000000 0.43320000000000 $-0.43320000000000$ 0.50000000000000

0.50000000000000

0.5000000000000

0.50000000000000

\begin{tabular}{cc}
$\mathrm{I}$ & $(4 \mathrm{e})$ \\
$\mathrm{I}$ & $(4 \mathrm{e})$ \\
$\mathrm{Se}$ & $(16 \mathrm{k})$ \\
$\mathrm{Se}$ & $(16 \mathrm{k})$ \\
$\mathrm{Se}$ & $(16 \mathrm{k})$ \\
$\mathrm{Se}$ & $(16 \mathrm{k})$ \\
$\mathrm{Se}$ & $(16 \mathrm{k})$ \\
$\mathrm{Se}$ & $(16 \mathrm{k})$ \\
$\mathrm{Se}$ & $(16 \mathrm{k})$ \\
$\mathrm{Se}$ & $(16 \mathrm{k})$ \\
$\mathrm{Se}$ & $(16 \mathrm{k})$ \\
$\mathrm{Se}$ & $(16 \mathrm{k})$ \\
$\mathrm{Se}$ & $(16 \mathrm{k})$ \\
$\mathrm{Se}$ & $(16 \mathrm{k})$ \\
$\mathrm{Se}$ & $(16 \mathrm{k})$ \\
$\mathrm{Se}$ & $(16 \mathrm{k})$ \\
$\mathrm{Se}$ & $(16 \mathrm{k})$ \\
$\mathrm{Se}$ & $(16 \mathrm{k})$ \\
$\mathrm{Ta}$ & $(4 \mathrm{c})$ \\
$\mathrm{Ta}$ & $(4 \mathrm{c})$ \\
$\mathrm{Ta}$ & $(4 \mathrm{~d})$ \\
$\mathrm{Ta}$ & $(4 \mathrm{~d})$ \\
\hline
\end{tabular}

$\mathrm{CdAs}_{2}$ : A2B_tI12_98_f_a - CIF

\# CIF file

data findsym-output

audit_creation_method FINDSYM

chemical name mineral 'CdAs2'

chemical formula_sum , As2 Cd,

loop

publ_author_name

'V. N. Yakimovich

V. A. Rubtsov

'V. M. Trukhan

journal_name_full_name

Inorganic Materials

journal_volume 32

journal year 1996

journal_page_first 579

journal_page_first 579

publ_Section_title

Phase Relationships in the CdP\$_ $\{4\} \$-Z n P \$ \_\{2\} \$-C d A s \$ \_\{2\} \$-Z n A s \$ \_\{2\} \$$ $\hookrightarrow$ System

Found in Pearson's Crystal Data - Crystal Structure Database fo $\hookrightarrow$ Inorganic Compounds, 2013

aflow_title CdAs\$_\{2\}\$ Structure

aflow_proto 'A2B_tI12_98_f_a'

aflow_params 'a, c/a, x $\{2\}$,

aflow_params values $77.953376649,0.587954231111,0.44$,

aflow_Strukturbericht 'None'

aflow_Pearson, tI12,

cell_length_a $\quad 7.9533766490$

cell length_b 7.9533766490

cell_length_c 4.6762214524

cell angle alpha 90.0000000000

cell_angle_beta 90.0000000000

cell_angle_gamma 90.0000000000

symmetry_space_group_name_H-M "I $4 \begin{array}{llll}4 & 2 & 2\end{array}$

symmetry_Int_Tables_number 98

loop_

space_group_symop_id

space_group_symop_operation_xyz

$1 \mathrm{x}, \mathrm{y}, \mathrm{z}$

$2 \mathrm{x},-\mathrm{y}+1 / 2,-\mathrm{z}+1 / 4$

$3-x, y+1 / 2,-z+1 / 4$

$4-\mathrm{x},-\mathrm{y}, \mathrm{z}$

$-\mathrm{y},-\mathrm{x},-\mathrm{z}$

$-y, x+1 / 2, z+1 / 4$

$\mathrm{y},-\mathrm{x}+1 / 2, \mathrm{z}+1 / 4$

$\mathrm{y}, \mathrm{x},-\mathrm{z}$

$\mathrm{x}+1 / 2, \mathrm{y}+1 / 2, \mathrm{z}+1 / 2$

$0 \mathrm{x}+1 / 2,-\mathrm{y},-\mathrm{z}+3 / 4$

$11-\mathrm{x}+1 / 2, \mathrm{y},-\mathrm{z}+3 / 4$ $12-\mathrm{x}+1 / 2,-\mathrm{y}+1 / 2, \mathrm{z}+1 / 2$

$13-\mathrm{y}+1 / 2,-\mathrm{x}+1 / 2,-\mathrm{z}+1 / 2$

$14-\mathrm{y}+1 / 2, \mathrm{x}, \mathrm{z}+3 / 4$

$15 \mathrm{y}+1 / 2,-\mathrm{x}, \mathrm{z}+3 / 4$

$16 \mathrm{y}+1 / 2, \mathrm{x}+1 / 2,-\mathrm{z}+1 / 2$

loop

atom_site_label

atom_site_type_symbol

atom_site_symmetry_multiplicity

atom_site_Wyckoff_label

atom_site_fract_x

atom_site_fract_y

atom_site_fract_z

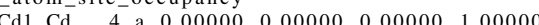

As1 As 8 f $0.44000 \quad 0.25000 \quad 0.12500 \quad 1.00000$

$\mathrm{CdAs}_{2}$ : A2B_tI12_98_f_a - POSCAR

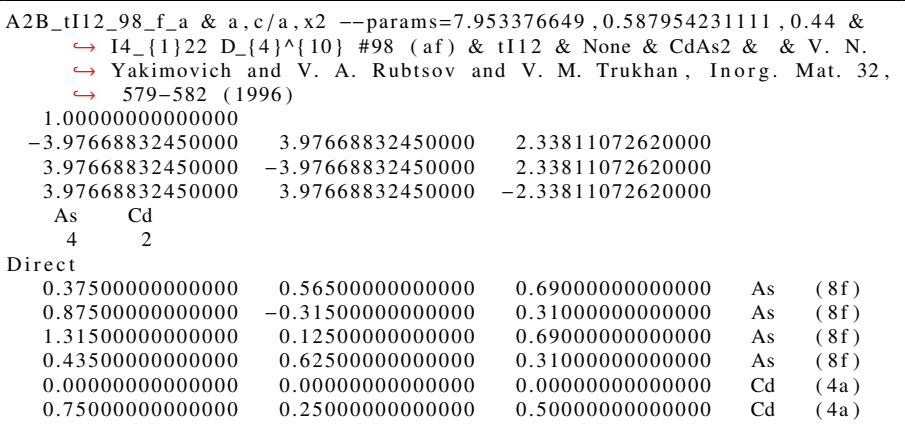

Fresnoite $\left(\mathrm{Ba}_{2} \mathrm{TiSi}_{2} \mathrm{O}_{8}\right)$ : A2B8C2D_tP26_100_c_abcd_c_a - CIF

\# CIF file

data_findsym-output

audit_creation_method FINDSYM

chemical name mineral 'Fresnoite'

chemical_formula_sum 'Ba2 O8 $\mathrm{Si} 2 \mathrm{Ti}$

loop

publ_author_name

S. A. Markgra

A. Halliya

A. S. Bhalla'

R. E. Newnham'

C. T. Prewitt'

journal_name_full_name

Ferroelectrics

journal_volume 62

journal year 1985

journal_page_first 17

journal page last 26

$X$-ray structure refinement and pyroelectric investigation of fresnoite $\hookrightarrow \quad \mathrm{Ba}_{-}\{2\} \$ \mathrm{TiSi}_{-}\{2\} \$ \mathrm{~S}_{-}\{8\} \$$

aflow title, Fresnoite (Ba\$ $\{2\} \$ T$ TiSi\$_\{2\}\$O\$_\{8\}\$) Structure,

aflow proto, A2B8C2D tP26 100

aflow_params a, c/a, z- $\{1\}, z_{-}\{2\}, z_{-}\{3\}, x_{-}\{4\}, z_{-}\{4\}, x_{-}\{5\}, z_{-}\{5\}, x_{-}\{6\}, z_{-}\{$ $\rightarrow 6\}, x_{-}\{7\}, y_{-}\{7\}, z_{-}\{7\}$

aflow_params_values $8.527,0.611047261639,0.7904,0.4646,0.3707,0.32701$, $\hookrightarrow 0.0,0.1259,0.7949,0.1282,0.4871,0.2924,0.5772,0.3571$

aflow_Strukturbericht 'None'

aflow_Pearson 'tP26'

symmetry_space group_name_H-M "P 4 b m"

symmetry_Int Tables_number 100

cell_length_a $\quad 8.52700$

cell_length_b 8.52700

_cell_length_c 5.21040

_cell_angle_alpha 90.00000

cell_angle_gamma 90.00000

loop

_space_group_symop_id

_space_group_symop_operation_xyz

$\mathrm{x}, \mathrm{y}, \mathrm{z}$

$2-\mathrm{x},-\mathrm{y}, \mathrm{z}$

$3-\mathrm{y}, \mathrm{x}, \mathrm{z}$

$4 \mathrm{y},-\mathrm{x}, \mathrm{z}$

$5-x+1 / 2, y+1 / 2, z$

$6 \mathrm{x}+1 / 2,-\mathrm{y}+1 / 2, \mathrm{z}$

$7 \mathrm{y}+1 / 2, x+1 / 2, z$

$8-y+1 / 2,-x+1 / 2$

loop

atom site label

atom_site_type_symbol

atom_site_symmetry_multiplicity

atom_site_Wyckoff_labe

_atom_site_fract_x 
-atom_site_fract_y

-atom_site_fract_z

$\begin{array}{llllllll}\mathrm{O} 1 & \mathrm{O} & 2 & \text { a } & 0.00000 & 0.00000 & 0.79040 & 1.00000\end{array}$

$\begin{array}{lllllllll}\text { Ti1 } & \text { Ti } & 2 & \text { a } & 0.00000 & 0.00000 & 0.46460 & 1.00000\end{array}$

$\begin{array}{llllllllll}\mathrm{O} 2 & \mathrm{O} & 2 & \mathrm{~b} & 0.50000 & 0.00000 & 0.37070 & 1.00000\end{array}$

$\mathrm{Ba} 1 \mathrm{Ba} \quad 4$ c $0.32701 \quad 0.82701 \quad 0.00000 \quad 1.00000$

$\begin{array}{lllllllll}\mathrm{O} 3 & \mathrm{O} & 4 & \mathrm{c} & 0.12590 & 0.62590 & 0.79490 & 1.00000\end{array}$

$\begin{array}{lllllllll}\mathrm{Si} 1 & \mathrm{Si} & 4 & \mathrm{c} & 0.12820 & 0.62820 & 0.48710 & 1.00000\end{array}$

$\begin{array}{lllllllll}\mathrm{O} 4 & \mathrm{O} & 8 & \mathrm{~d} & 0.29240 & 0.57720 & 0.35710 & 1.00000\end{array}$

Fresnoite $\left(\mathrm{Ba}_{2} \mathrm{TiSi}_{2} \mathrm{O}_{8}\right)$ : A2B8C2D_tP26_100_c_abcd_c_a - POSCAR

A2B8C2D_tP26_100_c_abcd_c_a \& a,c/a, z1 , z2, z3, x4, z4, x5, z5 , x6, z6, x7, y7, z7 --params $=8.527,0.611047261639,0.7904,0.4646,0.3707,0.32701,0.0$

$\hookrightarrow 0.1259,0.7949,0.1282,0.4871,0.2924,0.5772,0.3571 \&$ P $4 b m C_{-}\{4 \mathrm{v}$

$\hookrightarrow\}^{\wedge}\{2\} \# 100\left(a^{\wedge} 2 b^{\wedge} 3 d\right) \&$ tP26 \& None \& Ba2TiSi2O8 \& Fresnoite \&

$\hookrightarrow$ S. A. Markgraf et al., Ferroelectrics 62, 17-26 (1985)

1.00000000000000

8.52700000000000

0.00000000000000

0.00000000000000

0.00000000000000

0.00000000000000

. 52700000000000

0.00000000000000

$\begin{array}{rrrr}\mathrm{Ba} & \mathrm{O} & \mathrm{Si} & \mathrm{Ti} \\ 4 & 16 & 4 & \end{array}$

Direct

0.32701000000000 $-0.32701000000000$ 0.17299000000000 0.82701000000000 0.00000000000000 0.50000000000000 0.50000000000000 0.00000000000000 0.12590000000000 $-0.12590000000000$ 0.37410000000000 0.62590000000000 0.29240000000000 $-0.29240000000000$ $-0.2924000000000$ 0.57720000000000 0.772000000000 0.20760000000000 0.20760000000000 0.07720000000000 1.07720000000000 0.12820000000000 0.12820000000000 0.37180000000000 0.62820000000000 0.00000000000000 0.00000000000000 5.2104000

0.82701000000000 0.17299000000000 0.32701000000000 $-0.3270100000000$ 0.0000000000000 0.50000000000000 0.00000000000000 0.5000000000000 0.62590000000000 0.37410000000000 0.12590000000000 $-0.12590000000000$ 0.57720000000000 $-0.57720000000000$ 0.29240000000000 $-0.29240000000000$ $-0.07720000000000$ 1.07720000000000 0.20760000 0.2076000000000 0.79240000000000 0.62820000000000 0.3718000000000 0.1282000000000 $-0.12820000000000$ 0.00000000000000 0.50000000000000 0.50000000000000

0.00000000000000 0.00000000000000 0.0000000000000 0.00000000000000 0.79040000000000 0.79040000000000 0.37070000000000 0.37070000000000 0.79490000000000 0.79490000000000 0.79490000000000 0.79490000000000 0.35710000000000 0.35710000000000 0.3571000000000 0.35710000000000 0.35710000000000 0.35710000000000 0.35710000000000 0.35710000000000 0.35710000000000 0.48710000000000 0.48710000000000 0.4871000000000 0.48710000000000 0.46460000000000 0.46460000000000

$\mathrm{Ce}_{3} \mathrm{Si}_{6} \mathrm{~N}_{11}$ : A3B11C6_tP40_100_ac_bc2d_cd - CIF

\section{\# CIF file}

data_findsym-output

audit_creation_method FINDSYM

chemical_name_mineral 'Ce3Si6N11'

chemical_formula_sum 'Ce3 N11 Si6'

loop_

publ_author_name

M. Woike'

'W. Jeitschko'

journal_name_full_name

Inorganic Chemistry

journal_volume 34

ournal_year 199

journal_page_first 510

journal_page_last 5108

publ_Section_title

Preparation and Crystal Structure of the Nitridosilicates $\$ \operatorname{Ln}_{-}\{3\} \$$ Si $\$$ _ $\rightarrow 6\} \$ N \$\{11\} \$(\$ \operatorname{Ln} \$=\mathrm{La}, \mathrm{Ce}, \operatorname{Pr}, \mathrm{Nd}, \mathrm{Sm})$ and $\$ \operatorname{Ln} \$ \mathrm{Si} \$$ S $_{-}\{3\} \$ N \$ \$_{-}\{5\}$

$\hookrightarrow \$(\$ \operatorname{Ln} \$=\mathrm{Ce}, \operatorname{Pr}, \mathrm{Nd})$

\# Found in Pearson's Crystal Data - Crystal Structure Database for

$\hookrightarrow$ Inorganic Compounds, 2013

aflow_title ' $\mathrm{Ce} \$_{-}\{3\} \$ \mathrm{Si}_{-}\{6\} \$ N \$ \$_{-}\{11\} \$$ Structure

aflow_proto , A3B11C6_tP40_100 ac bc2d_cd

aflow_params $, a, c / a, z_{-}\{1\}, z_{-}\{2\}, x_{-}\{3\}, z_{-}\{3\}, x_{-}\{4\}, z_{-}\{4\}, x_{-}\{5\}, z_{-}\{5\}, x_{-}\{$ $\hookrightarrow 6\}, y_{-}\{6\}, z_{-}\{6\}, x_{-}\{7\}, y_{-}\{7\}, z_{-}\{7\}, x_{-}\{8\}, y_{-}\{8\}, z_{-}\{8\}$

aflow_params_values $, 10.1311200179,0.47843253381,0.0,0.0672,0.18111$

$\hookrightarrow 0.0154,0.6536,0.6912,0.6174,0.0432,0.5814,0.678,0.6402,0.7288$,

$\hookrightarrow 0.574,0.1742,0.7098,0.5782,0.5334$

aflow_Strukturbericht 'None

aflow_Pearson 'tP40'

cell_length_a $\quad 10.1311200179$

cell_length_b 10.1311200179

cell angle

(cello

cell_angle_gamma 90.0000000000

symmetry_space group name_H-M "P 4 b m"

symmetry_Int_Tables_number 100

loop

_space_group_symop_id

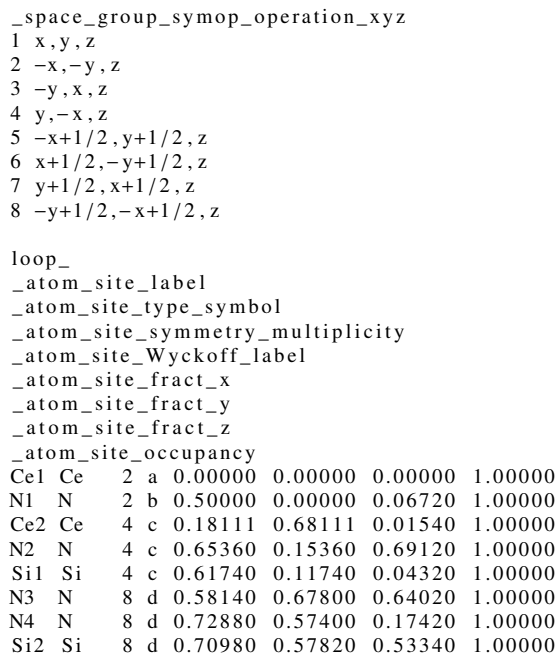

$\mathrm{Ce}_{3} \mathrm{Si}_{6} \mathrm{~N}_{11}$ : A3B11C6_tP40_100_ac_bc2d_cd - POSCAR

A3B11C6_tP40_100_ac_bc2d_cd \& a,c/a, z1 , z2 , x3 $, \mathrm{z} 3, \mathrm{x} 4, \mathrm{z} 4, \mathrm{x} 5, \mathrm{z} 5, \mathrm{x} 6, \mathrm{y} 6, \mathrm{z} 6, \mathrm{x} 7$, $\rightarrow \mathrm{y} 7, \mathrm{z} 7, \mathrm{x} 8, \mathrm{y} 8, \mathrm{z} 8--$ params $=10.1311200179,0.47843253381,0.0,0.0672$, $\hookrightarrow 0.18111,0.0154,0.6536,0.6912,0.6174,0.0432,0.5814,0.678,0.6402$. $\hookrightarrow 0.7288,0.574,0.1742,0.7098,0.5782,0.5334 \& \mathrm{P}_{\mathrm{b}} \mathrm{bm} \mathrm{C}_{-}\{4 \mathrm{v}\}^{\wedge}\{2\} \# 100$ $\hookrightarrow \quad\left(a b c^{\wedge} 3 \mathrm{~d}^{\wedge} 3\right) \& \mathrm{tP} 40$ \& None \& Ce3Si6N11 \& \& M. Woike and W.

$\hookrightarrow$ Jeitschko , Inorg. Chem. 34, 5105-5108 (1995)

1.00000000000000

$\begin{array}{lll}10.13112001790000 & 0.00000000000000 & 0.00000000000000\end{array}$

$\begin{array}{rrr}0.00000000000000 & 10.13112001790000 & 0.00000000000000\end{array}$

$\begin{array}{lll}0.00000000000000 & 0.00000000000000 & 4.84705742050000\end{array}$

$\begin{array}{lll}\mathrm{Ce} & \mathrm{N} & \mathrm{Si} \\ 6 & 22 & 12\end{array}$

Direct

0.00000000000000

0.50000000000000

0.18111000000000

$-0.1811100000000$

0.31889000000000

0.68111000000000

0.50000000000000

0.00000000000000

$-0.6536000000000$

$-0.15360000000000$

1.15360000000000

0.58140000000000

$-0.58140000000000$

0.67800000000000

1.08140000000000

$-0.08140000000000$

$-0.17800000000000$

1.17800000000000

0.72880000000000

$-0.72880000000000$

$-0.57400000000000$

0.57400000000000

1.22880000000000

$-0.22880000000000$

1.07400000000000

0.61740000000000

$-0.61740000000000$

$-0.617400000000$

$-0.11740000000000$

0.70980000000000

$-0.70980000000000$

$-0.57820000000000$

0.57820000000000

1.2098000000000

$-0.20980000000000$

$-0.07820000000000$

0.00000000000000 0.50000000000000 0.68111000000000 0.31889000000000 0.18111000000000 $-0.18111000000000$ 0.00000000000000 0.50000000000000 1.15360000000000 $-0.15360000000000$ 0.65360000000000 $-0.65360000000000$ 0.67800000000000 0.67800000000000 0.58140000000000 0.58140000000000 . 1.17800000000000 1.17800000000 .08140000000000 1.08140000000000 0.57400000000000 400000000000 0.72880000000000 0.07400000000000 07400000000000 0.22880000000000 1.22880000000000 1.11740000000000 . 11740000000000 0.61740000000000 0.61740000000000 0.57820000000000 $-0.57820000000000$ 0.70980000000000 0.70980000000000 $-0.07820000000000$ 1.07820000000000 $-0.20980000000000$ 1.20980000000000

0.00000000000000 0.00000000000000 0.01540000000000 0.01540000000000 0.01540000000000 0.01540000000000 0.06720000000000 0.69120000000000 0.69120000000000 0.69120000000000 0.69120000000000 0.64020000000000 0.64020000000000 0.64020000 0.64020000000000 0.64020000000000 0.64200000000 0.6420000000 0.64020000000000 0.64020000000000 0.17420000000000 0.17420000000000 .17420000000000 


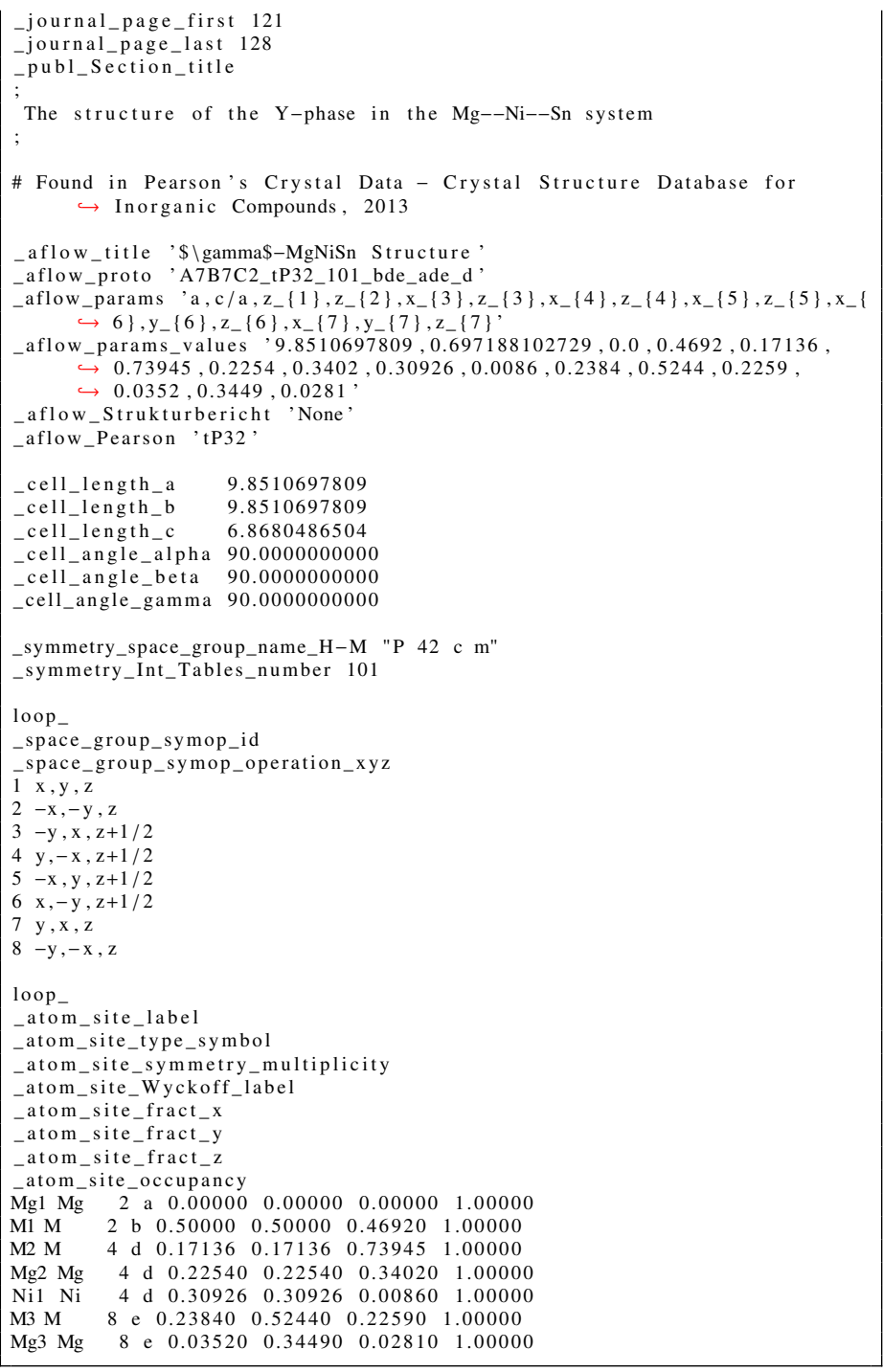

$\gamma$-MgNiSn: A7B7C2_tP32_101_bde_ade_d - POSCAR

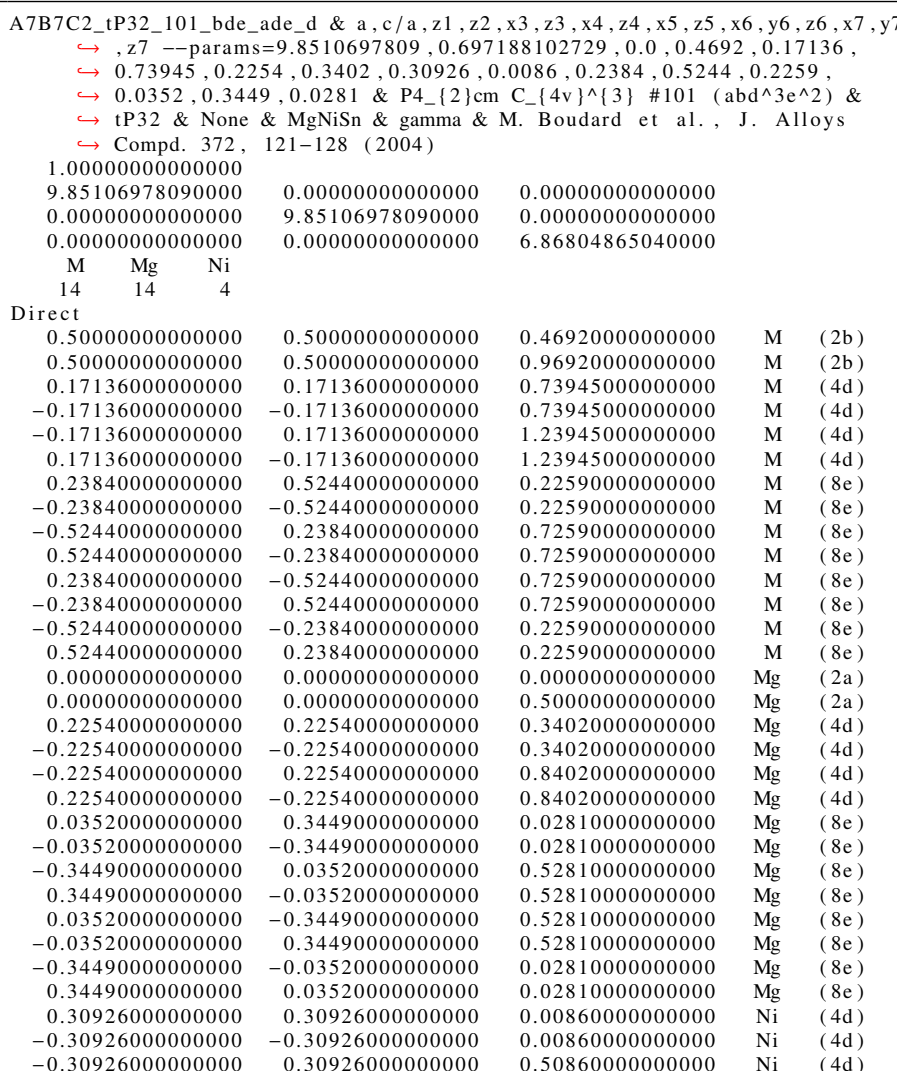

$\begin{array}{llllll}0.30926000000000 & -0.30926000000000 & 0.50860000000000 & \mathrm{Ni} & (4 \mathrm{~d})\end{array}$

$\mathrm{Gd}_{3} \mathrm{Al}_{2}:$ A2B3_tP20_102_2c_b2c - CIF

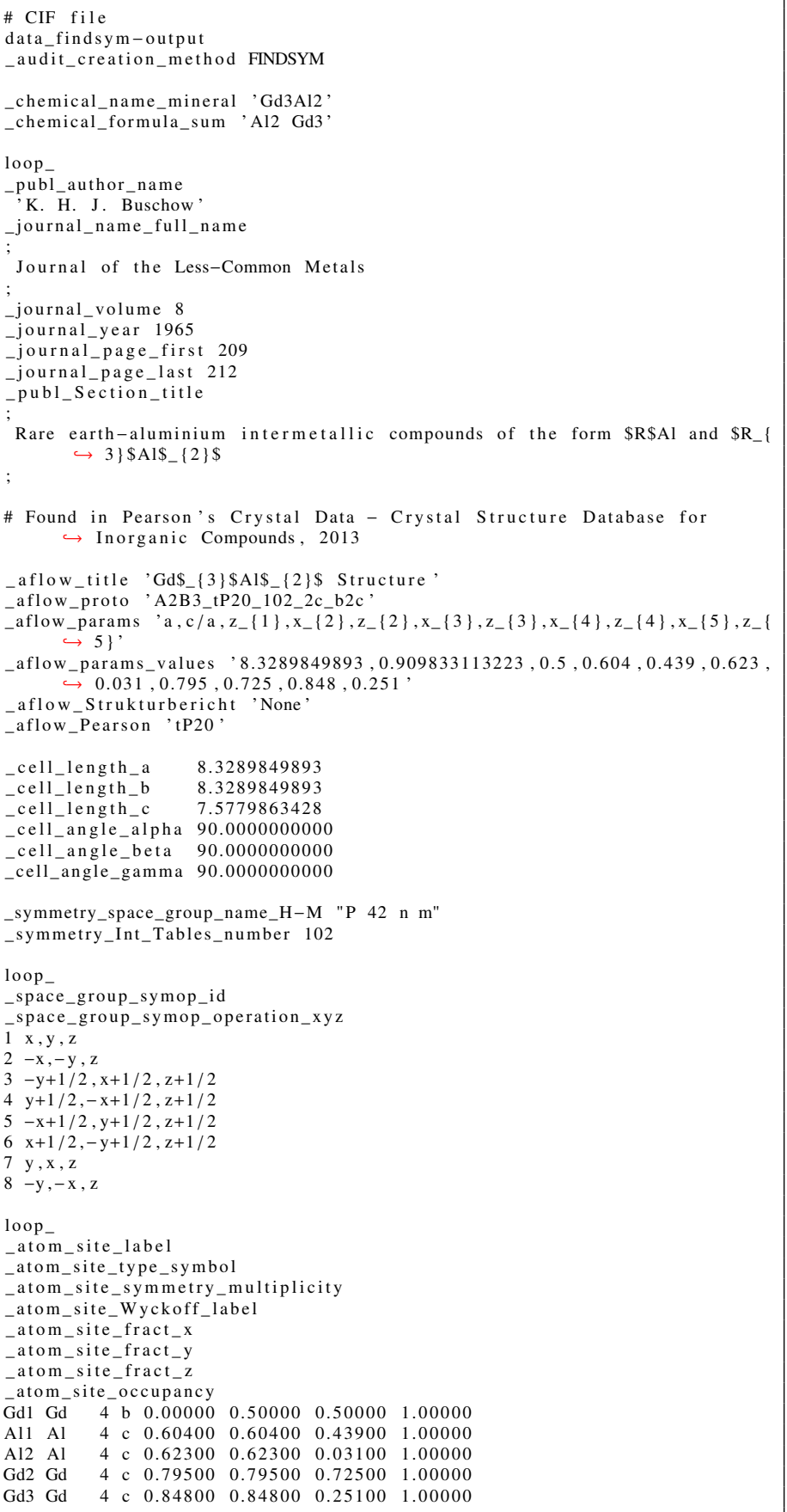

$\mathrm{Gd}_{3} \mathrm{Al}_{2}$ : A2B3_tP20_102_2c_b2c - POSCAR

\begin{tabular}{|c|c|c|c|c|}
\hline \multicolumn{5}{|c|}{ 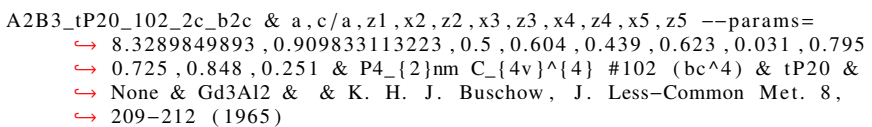 } \\
\hline \multicolumn{5}{|l|}{1.00000000000000} \\
\hline 8.32898498930000 & 0.00000000000000 & 0.00000000000000 & & \\
\hline \multirow{2}{*}{$\begin{array}{l}0.00000000000000 \\
0.00000000000000\end{array}$} & 8.32898498930000 & 0.00000000000000 & & \\
\hline & 0.00000000000000 & 7.57798634280000 & & \\
\hline $\mathrm{Gd}$ & & & & \\
\hline 12 & & & & \\
\hline \multicolumn{5}{|l|}{ Direct } \\
\hline 0.60400000000000 & 0.60400000000000 & 0.43900000000000 & $\mathrm{Al}$ & $(4 c)$ \\
\hline-0.60400000000000 & -0.60400000000000 & 0.43900000000000 & $\mathrm{Al}$ & $(4 c)$ \\
\hline-0.10400000000000 & 1.10400000000000 & 0.93900000000000 & $\mathrm{Al}$ & $(4 c)$ \\
\hline 1.10400000000000 & -0.10400000000000 & 0.93900000000000 & $\mathrm{Al}$ & $(4 c)$ \\
\hline 0.62300000000000 & 000000000 & 0.03100000000000 & $\mathrm{Al}$ & $(4 c)$ \\
\hline-0.62300000000000 & -0.62300000000000 & 0.031000000 & $\mathrm{Al}$ & $(4 c)$ \\
\hline-0.12300000000000 & 1.12300000000000 & 0.53100000000000 & $\mathrm{Al}$ & $(4 c)$ \\
\hline 1.12300000000000 & -0.12300000000000 & 0.53100000000000 & $\mathrm{Al}$ & \\
\hline 0.00000000000000 & 0.50000000000000 & 0.50000000000000 & $\mathrm{Gd}$ & \\
\hline 0.00000000000000 & 0.50000000000000 & 1.00000000000000 & $\mathrm{Gd}$ & $(4 b)$ \\
\hline 0.50000000000000 & 0.00000000000000 & 1.00000000000000 & $\mathrm{Gd}$ & \\
\hline 0.50000000000000 & 0.00000000000000 & 0.50000000000000 & $\mathrm{Gd}$ & $(4 b)$ \\
\hline 0.79500000000000 & 0.79500000000000 & 0.72500000000000 & $\mathrm{Gd}$ & \\
\hline & & & $\mathrm{Gd}$ & \\
\hline
\end{tabular}




\begin{tabular}{|rrrrr|}
-0.29500000000000 & 1.29500000000000 & 1.22500000000000 & $\mathrm{Gd}$ & $(4 \mathrm{c})$ \\
1.29500000000000 & -0.29500000000000 & 1.22500000000000 & $\mathrm{Gd}$ & $(4 \mathrm{c})$ \\
0.84800000000000 & 0.84800000000000 & 0.25100000000000 & $\mathrm{Gd}$ & $(4 \mathrm{c})$ \\
-0.84800000000000 & -0.84800000000000 & 0.25100000000000 & $\mathrm{Gd}$ & $(4 \mathrm{c})$ \\
-0.34800000000000 & 1.34800000000000 & 0.75100000000000 & $\mathrm{Gd}$ & $(4 \mathrm{c})$ \\
1.34800000000000 & -0.34800000000000 & 0.75100000000000 & $\mathrm{Gd}$ & $(4 \mathrm{c})$ \\
\hline
\end{tabular}

NbTe 4 : AB4_tP10_103_a_d - CIF

\# CIF file

data_findsym-output

_audit_creation_method FINDSYM

_chemical_name_mineral 'NbTe4,

_chemical_formula_sum ' $\mathrm{Nb} \mathrm{Te} 4$,

loop

_publ_author_name

H. B $\{1$ " o $\}$ hm

_journal_name_full_name

Zeitschrift $f\{\backslash " u\} r$ Kristallographie - Crystalline Materials

journal volume 180

journal year 1987

_journal_page_first 113

_journal_page_last 122

-publ_Section_title

The high temperature modification of niobium tetratelluride NbTe\$_\{4\}\$

\# Found in Pearson's Crystal Data - Crystal Structure Database for

$\hookrightarrow$ Inorganic Compounds, 2013

_aflow_title 'NbTe\$_\{4\}\$ Structure

-aflow_proto, AB4_tP10_103_a_d

-aflow_params a, a $/ a, z_{-}\{1\}, x_{-}\{2\}, y_{-}\{2\}, z_{-}\{2\}$

-aflow_params_values $6.5509768136,1.04518394138,0.0,0.144,0.3276,0.242$, aflow Strukturbericht 'None'

-aflow_Pearson 'tP10,

_cell_length_a $\quad 6.5509768136$

_cell_length_b 6.5509768136

_cell_length_c $\quad 6.8469757659$

_cell_angle_alpha 90.0000000000

_cell_angle_beta 90.0000000000

_cell_angle_gamma 90.0000000000

_symmetry_space_group_name_H-M "P 4 c c"

_symmetry_Int_Tables_number 103

loop

_space_group_symop_id

_space_group_symop_operation_xyz

$1 \mathrm{x}, \mathrm{y}, \mathrm{z}$

$2-x,-y, z$

$3-\mathrm{y}, \mathrm{x}, \mathrm{z}$
$4-\mathrm{y},-\mathrm{x}, \mathrm{z}$

$5-\mathrm{x}, \mathrm{y}, \mathrm{z}+1 / 2$

$6 \mathrm{x},-\mathrm{y}, \mathrm{z}+1 / 2$

$7 \mathrm{y}, \mathrm{x}, \mathrm{z}+1 / 2$
$8-\mathrm{y},-\mathrm{x}, \mathrm{z}+1 / 2$

loop

_atom_site_label

_atom_site_type_symbol

_atom_site_symmetry_multiplicity

_atom_site_Wyckoff_label

_atom_site_fract_x

-atom_site_fract_y

-atom_site_fract_z

atom_site_occupancy

$\begin{array}{lllllll}\overline{\mathrm{N} b} 1 \mathrm{Nb} & 2 & \mathrm{a} & 0.00000 & 0.00000 & 0.00000 & 1.00000\end{array}$

$\begin{array}{llllllll}\mathrm{Te} 1 & \mathrm{Te} & 8 & \mathrm{~d} & 0.14400 & 0.32760 & 0.24200 & 1.00000\end{array}$

$\mathrm{NbTe}_{4}$ : AB4_tP10_103_a_d - POSCAR

AB4 tP10 103 a d \& a , c $/ \mathrm{a}, \mathrm{z} 1, \mathrm{x} 2, \mathrm{y} 2, \mathrm{z} 2--$ params $=6.5509768136,1.04518394138$ $\hookrightarrow, 0.0,0.144,0.3276,0.242 \&$ P P cc C $\{4 \mathrm{v}\}^{\wedge}\{5\} \# 103(\mathrm{ad}) \& \mathrm{tP} 10 \&$ $\hookrightarrow$ None \& NbTe4 \& \& H. B $\{\backslash " \mathrm{o}\} \mathrm{hm}$, Zeitschrift f" $\{\mathrm{u}\} \mathrm{r}$

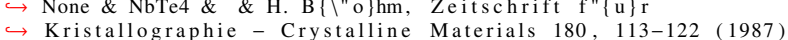
$\hookrightarrow$ Kristallograph

1.00000000000000

$\begin{array}{lll}6.55097681360000 & 0.00000000000000 & 0.00000000000000\end{array}$

$\begin{array}{lll}0.00000000000000 & 6.55097681360000 & 0.00000000000000\end{array}$

$\begin{array}{lll}0.00000000000000 & 0.00000000000000 & 6.84697576590000\end{array}$

$\begin{array}{rr}\mathrm{Nb} & \mathrm{Te} \\ 2 & 8\end{array}$

Direct

$\begin{array}{lll}0.00000000000000 & 0.00000000000000 & 0.00000000000000\end{array}$

$0.00000000000000 \quad 0.00000000000000$

$0.14400000000000-0.32760000000000$

$-0.14400000000000-0.32760000000000$

$-0.32760000000000-0.14400000000000$

$0.32760000000000-0.14400000000000$

$0.1440000000000-0.3276000000000$

$0.1440000000000-0.32760000000000$

$-0.14400000000000-0.32760000000000$

$\begin{array}{rr}-0.32760000000000 & -0.14400000000000 \\ 0.32760000000000 & 0.14400000000000\end{array}$

0.50000000000000

0.24200000000000

0.24200000000000

0.24200000000000

0.24200000000000

0.74200000000000

0.74200000000000

0.74200000000000

0.74200000000000

$\begin{array}{ll}\mathrm{Nb} & (2 \mathrm{a}) \\ \mathrm{Nb} & (2 \mathrm{a}) \\ \mathrm{Te} & (8 \mathrm{~d}) \\ \mathrm{Te} & (8 \mathrm{~d}) \\ \mathrm{Te} & (8 \mathrm{~d}) \\ \mathrm{Te} & (8 \mathrm{~d}) \\ \mathrm{Te} & (8 \mathrm{~d}) \\ \mathrm{Te} & (8 \mathrm{~d}) \\ \mathrm{Te} & (8 \mathrm{~d}) \\ \mathrm{Te} & (8 \mathrm{~d})\end{array}$

Ba $\mathrm{In}_{4} \mathrm{Bi}_{5}$ : A5B5C4_tP28_104_ac_ac_c - CIF

\# CIF file

data_findsym-output
audit_creation_method_FINDSYM

chemical_name_mineral 'Ba5In4Bi5

chemical_formula_sum 'Ba5 Bi5 In4'

loop

publ_author_name

'S. Ponou'

T. F. F $\{\backslash$ "a\}ssler,

G. Tob $\{\backslash \backslash$, i $\}$ as

'E. Canadell'

'A. Cho

'S. C. Sevov'

journal_name_full_name

Chemistry - A European Journal

journal_volume 10

journal_year

journal_page_first 3615

journal_page_last 3621

-publ_Section_title

Synthesis, Characterization, and Electronic Structure of Ba\$_\{5\}\$In\$_ $\{4$ $\hookrightarrow\} \$ B i \$\{\{5\}$ : An Acentric and One-Electron Deficient Phase

Found in Pearson's Crystal Data - Crystal Structure Database for $\hookrightarrow$ Inorganic Compounds, 2013

aflow_title 'Ba\$_ $\{5\} \$ I n \$_{-}\{4\} \$ B i \$_{-}\{5\} \$$ Structure,

aflow_proto 'A5B5C4_tP28_104_ac_ac_c,

aflow_params $, a, c / a, z_{-}\{1\}, z_{-}\{2\}, x_{-}\{3\}, y_{-}\{3\}, z_{-}\{3\}, x_{-}\{4\}, y_{-}\{4\}, z_{-}\{4\}, x_{-}\{$ $\hookrightarrow 5\}, y_{-}\{5\}, z_{-}\{5\}$,

aflow params

$\hookrightarrow 0.6057,0.3261,0.60942,0.80921,0.00978,0.8116,-0.072,0.1681$,

aflow_Strukturbericht 'None'

_aflow_Pearson 'tP28,

_cell_length_a $\quad 10.6225961282$

cell_length_b $\quad 10.6225961282$

cell_length_c $\quad 9.0112023088$

cell_angle_alpha 90.0000000000

_cell_angle_beta 90.0000000000

cell angle gamma 90.0000000000

_symmetry_space_group_name_H-M "P $4 \mathrm{n} \mathrm{c}$ "

symmetry_Int_Tables_number 104

loop

space_group_symop_id

-space_group_symop_operation_xy

$1 \mathrm{x}, \mathrm{y}, \mathrm{z}$

$2-\mathrm{x},-\mathrm{y}, \mathrm{z}$

$3-\mathrm{y}, \mathrm{x}, \mathrm{z}$

$4 \mathrm{y},-\mathrm{x}, \mathrm{z}$

$5-\mathrm{x}+1 / 2, \mathrm{y}+1 / 2, \mathrm{z}+1 / 2$

$6 \mathrm{x}+1 / 2,-\mathrm{y}+1 / 2, \mathrm{z}+1 / 2$

$7 \mathrm{y}+1 / 2, \mathrm{x}+1 / 2, \mathrm{z}+1 / 2$

$8-\mathrm{y}+1 / 2,-\mathrm{x}+1 / 2, \mathrm{z}+1 / 2$

loop

atom site label

_atom_site_type_symbol

atom_site_symmetry_multiplicity

atom_site Wyckoff_label

atom_site_fract_x

atom_site_fract_y

atom_site_fract_z

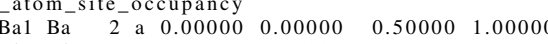

$\begin{array}{llllllll}\mathrm{Bi} 1 \mathrm{Bi} & 2 & \mathrm{a} & 0.00000 & 0.00000 & 0.88210 & 1.00000\end{array}$

$\begin{array}{lllllllll}\mathrm{Ba} 2 & \mathrm{Ba} & 8 & \mathrm{c} & 0.81160 & 0.60570 & 0.32610 & 1.00000\end{array}$

$\begin{array}{lllllllll}\mathrm{Bi} 2 & \mathrm{Bi} & 8 & \mathrm{c} & 0.60942 & 0.80921 & 0.00978 & 1.00000\end{array}$

$\begin{array}{llllllll}\text { In } 1 \text { In } & 8 & \text { c } & 0.81160 & -0.07200 & 0.16810 & 1.00000\end{array}$

Ba $\mathrm{In}_{4} \mathrm{Bi}_{5}:$ A5B5C4_tP28_104_ac_ac_c - POSCAR

A5B5C4_tP28_104_ac_ac_c \& a , c / a , z1 $, \mathrm{z} 2, \mathrm{x} 3, \mathrm{y3}, \mathrm{z3}, \mathrm{x} 4, \mathrm{y} 4, \mathrm{z} 4, \mathrm{x} 5, \mathrm{y} 5, \mathrm{z} 5--$ $\hookrightarrow$ params $=10.6225961282,0.84830508475,0.5,0.8821,0.8116,0.6057$, $\hookrightarrow 0.3261,0.60942,0.80921,0.00978,0.8116,-0.072,0.1681 \&$ P4nc C -\{ $\hookrightarrow 4 \mathrm{v}\}^{\wedge}\{6\} \# 104\left(\mathrm{a}^{\wedge} 2 \mathrm{c}^{\wedge} 3\right) \& \mathrm{tP} 28$ \& None \& Ba5In4Bi5 \& \& S. Ponou $\hookrightarrow$ et al., Chem. Euro. J. 10, 3615-3621 (2004)

1.00000000000000

$\begin{array}{rr}10.62259612820000 & 0.00000000000000\end{array}$

0.000000000000000 .62259612820000

$\mathrm{Ba} \quad \mathrm{Bi} \quad$ In

irect

0.00000000000000

0.50000000000000

0.81160000000000

$-0.81160000000000$

$-0.60570000000000$

0.60570000000000

1.31160000000000

$-0.31160000000000$

$-0.10570000000000$

1.10570000000000 0.00000000000000

0.50000000000000

0.60942000000000

$-0.60942000000000$

$-0.80921000000000$

0.00000000000000

9.01120230880000
0.00000000000000 0.60570000000000 $-0.60570000000000$ 0.81160000000000 $-0.81160000000000$ 1.10570000000000 $-0.31160000000000$ 1.31160000000000 0.50000000000000 0.80921000000000 $-0.8092100000000$ 0.60942000000000 0.50000000000000 $-0.10570000000000$
0.50000000000000 1.00000000000000 0.32610000000000 0.32610000000000 0.32610000000000 0.32610000000000 0.32610000000000 0.82610000000000 0.82610000000000 0.82610000000000 0.82610000000000 0.88210000000000 1.38210000000000 0.00978000000000 0.00978000000000 0.00978000000000
$\mathrm{Ba} \quad(2 \mathrm{a})$ $\mathrm{Ba} \quad(2 \mathrm{a})$ $\mathrm{Ba} \quad(8 \mathrm{c})$ $\mathrm{Ba} \quad(8 \mathrm{c})$ $\mathrm{Ba} \quad(8 \mathrm{c})$ $\mathrm{Ba} \quad(8 \mathrm{c})$ $\mathrm{Ba} \quad(8 \mathrm{c})$ $\mathrm{Ba} \quad(8 \mathrm{c})$ $\mathrm{Ba} \quad(8 \mathrm{c})$ $\mathrm{Ba} \quad(8 \mathrm{c})$ $\mathrm{Bi}$ (2a) $\mathrm{Bi} \quad(2 \mathrm{a})$ $\mathrm{Bi} \quad(8 \mathrm{c})$ $\begin{array}{ll}\mathrm{Bi} & (8 \mathrm{c}) \\ \mathrm{Bi} & (8 \mathrm{c})\end{array}$ $\mathrm{Bi} \quad(8 \mathrm{c})$ 
$0.80921000000000 \quad-0.60942000000000$ 1.1094200000000 $-0.1094200000000$ $-0.1094200000000$ 1.30921000000000 0.81160000000000 0.81160000000000 0.07200000000000 $-0.07200000000000$ 1.31160000000000 $-0.31160000000000$ 0.57200000000000 0.42800000 1.30921000000000 $-0.10942000000000$ 1.1094200000000 $-0.07200000000000$ 0.07200000000000 0.8116000000000 $-0.81160000000000$ 0.57200000000000 0.42800000000000 $-0.31160000000000$ 1.31160000000000

$\mathrm{Tl}_{4} \mathrm{HgI}_{6}$ : AB6C4_tP22_104_a_2ac_c - CIF
0.00978000000000 0.50978000000000 .50978000000000 0.5097800000000 0.581000000000 0.16810000000000 0.1681000000000 0.16810000000000 0.16810000000000 0.66810000000000 0.66810000000000 0.66810000000000 0.66810000000000 0.50978000000000

$\begin{array}{ll}\mathrm{Bi} & (8 \mathrm{c}) \\ \mathrm{Bi} & (8 \mathrm{c}) \\ \mathrm{Bi} & (8 \mathrm{c}) \\ \mathrm{Bi} & (8 \mathrm{c}) \\ \mathrm{Bi} & (8 \mathrm{c}) \\ \mathrm{In} & (8 \mathrm{c}) \\ \mathrm{In} & (8 \mathrm{c}) \\ \mathrm{In} & (8 \mathrm{c}) \\ \mathrm{In} & (8 \mathrm{c}) \\ \mathrm{In} & (8 \mathrm{c}) \\ \mathrm{In} & (8 \mathrm{c}) \\ \mathrm{In} & (8 \mathrm{c}) \\ \mathrm{In} & (8 \mathrm{c})\end{array}$

(8c) data_findsym-output

audit creation method FINDSYM

chemical_name_mineral 'Tl4HgI6,

chemical formula sum ' $\mathrm{Hg}$ I6 Tl4

oop

publ_author_name

G. M. $\{$ Kuz $\backslash$ 'micheva $\}$ '

V. L. Panyutin

V. B. Rybakov'

'V. I. Chizhikov'

'G. S. Shevyrdyaeva,

_journal_name_full_name

\section{Inorganic Materials}

journal_volume 40

journal_year 2004

journal_page_first 314

-journal_page_first 314

publ_Section_title

Growth and X-ray diffraction study of $\mathrm{TI} \$_{-}\{4\} \$ \mathrm{HgI}_{-}\{6\} \$$ crystals

\# Found in Pearson's Crystal Data - Crystal Structure Database fo $\hookrightarrow$ Inorganic Compounds, 2013

aflow_title TI\$_ $\{4\} \$ \mathrm{HgI}_{-}\{6\} \$$ Structure

aflow_proto 'AB6C4_tP22_104_a_2ac_c

aflow_params 'a, c/a, z- $\{1\}, \mathrm{z}_{-}\{2\}, \mathrm{z}_{-}\{3\}, \mathrm{x}_{-}\{4\}, \mathrm{y}_{-}\{4\}, \mathrm{z}_{-}\{4\}, \mathrm{x}_{-}\{5\}, \mathrm{y}_{-}\{5\}, \mathrm{z}_{-}\{$ $\hookrightarrow 5\}$

aflow_params_values ' $9.3940153509,0.981690440703,0.786,0.5,0.0649$, $\hookrightarrow 0.8297,0.6458,0.286,0.6491,0.8588,0.036^{\prime}$

aflow_Strukturbericht 'None'

aflow_Pearson 'tP22,

cell_length_a $\quad 9.3940153509$

cell_length_b 9.3940153509

cell_length_c $\quad 9.2220150698$

cell_angle_alpha 90.0000000000

cell_angle_beta 90.0000000000

cell_angle_gamma 90.0000000000

symmetry_space_group_name_H-M "P $4 \mathrm{n} \mathrm{c}$

symmetry_Int_Tables_number 104

loop

_space_group_symop_id

_space_group_symop_operation_xyz

$1 \mathrm{x}, \mathrm{y}, \mathrm{z}$

$2-x,-y$,

$-\mathrm{y}, \mathrm{x}, \mathrm{z}$

$\mathrm{y},-\mathrm{x}$

$-\mathrm{x}+1 / 2, \mathrm{y}+1 / 2, \mathrm{z}+1 / 2$

$\mathrm{x}+1 / 2,-\mathrm{y}+1 / 2, \mathrm{z}+1 / 2$

$y+1 / 2, x+1 / 2, z+1 / 2$
$-y+1 / 2,-x+1 / 2, z+1 / 2$

loop

atom_site_labe

atom_site_type_symbol

atom_site_symmetry_multiplicity

atom_site_Wyckoff_label

atom_site_fract_x

atom_site_fract_y

atom_site_fract_z

Catom_site_occupancy

$\begin{array}{llllllllll} & \mathrm{Hgl} \mathrm{Hg} & 2 & \mathrm{a} & 0.00000 & 0.00000 & 0.78600 & 1.00000\end{array}$

$\begin{array}{llllllllll}\text { I1 } & \text { I } & 2 & \text { a } & 0.00000 & 0.00000 & 0.50000 & 1.00000\end{array}$

$\begin{array}{llllllllll}12 & 1 & 2 & \mathrm{a} & 0.00000 & 0.00000 & 0.06490 & 1.00000\end{array}$

$\begin{array}{lllllllll}\mathrm{I} 3 & \mathrm{I} & 8 & \mathrm{c} & 0.82970 & 0.64580 & 0.28600 & 1.00000 \\ \mathrm{~T} 11 & \mathrm{Tl} & 8 & \mathrm{c} & 0.64910 & 0.85880 & 0.03600 & 1.00000\end{array}$

$\mathrm{Tl}_{4} \mathrm{HgI}_{6}:$ AB6C4_tP22_104_a_2ac_c - POSCAR

$\mathrm{AB} 6 \mathrm{C} 4 \_\mathrm{tP} 22 \_104 \_\mathrm{a} \_2 \mathrm{ac} \_\mathrm{c} \& \mathrm{a}, \mathrm{c} / \mathrm{a}, \mathrm{z} 1, \mathrm{z} 2, \mathrm{z} 3, \mathrm{x} 4, \mathrm{y} 4, \mathrm{z} 4, \mathrm{x} 5, \mathrm{y} 5, \mathrm{z} 5--\mathrm{params}=$

$\hookrightarrow 9.3940153509,0.981690440703,0.786,0.5,0.0649,0.8297,0.6458$

$\hookrightarrow 9.3940153509,0.981690440703,0.786,0.5,0.0649,0.8297,0.6458$,
$\hookrightarrow 0.286,0.6491,0.8588,0.036 \&$ P $4 n c C_{-}\{4 \mathrm{v}\}^{\wedge}\{6\} \# 104\left(\mathrm{a}^{\wedge} 3 \mathrm{c}^{\wedge} 2\right) \&$

$\hookrightarrow$ tP22 \& None \& T14HgI6 \& \& D. V. Badikov et al., Inorg. Mat. 40

$\hookrightarrow$ tP22 \& None \& T14

1.00000000000000

$9.39401535090000 \quad 0.00000000000000 \quad 0.00000000000000$

$\begin{array}{lll}0.00000000000000 & 9.39401535090000 & 0.00000000000000\end{array}$

\begin{tabular}{rrrrrrl}
\multicolumn{2}{l}{$\begin{array}{l}0.00000000000000 \\
\mathrm{Hg}\end{array} \mathrm{I}$} & 0.00000000000000 & 9.22201506980000 & & \\
$2 \mathrm{Tl}$ & & & & & \\
Direct & 8 & 8 & & & & \\
0.00000000000000 & 0.00000000000000 & 0.78600000000000 & $\mathrm{Hg}$ & $(2 \mathrm{a})$ \\
0.50000000000000 & 0.50000000000000 & 1.28600000000000 & $\mathrm{Hg}$ & $(2 \mathrm{a})$ \\
0.00000000000000 & 0.00000000000000 & 0.50000000000000 & $\mathrm{I}$ & $(2 \mathrm{a})$ \\
0.50000000000000 & 0.50000000000000 & 1.00000000000000 & $\mathrm{I}$ & $(2 \mathrm{a})$ \\
0.00000000000000 & 0.00000000000000 & 0.06490000000000 & $\mathrm{I}$ & $(2 \mathrm{a})$ \\
0.50000000000000 & 0.50000000000000 & 0.56490000000000 & $\mathrm{I}$ & $(2 \mathrm{a})$ \\
0.82970000000000 & 0.64580000000000 & 0.28600000000000 & $\mathrm{I}$ & $(8 \mathrm{c})$ \\
-0.82970000000000 & -0.64580000000000 & 0.28600000000000 & $\mathrm{I}$ & $(8 \mathrm{c})$ \\
-0.64580000000000 & 0.82970000000000 & 0.28600000000000 & $\mathrm{I}$ & $(8 \mathrm{c})$ \\
0.64580000000000 & -0.82970000000000 & 0.28600000000000 & $\mathrm{I}$ & $(8 \mathrm{c})$ \\
1.32970000000000 & -0.14580000000000 & 0.78600000000000 & $\mathrm{I}$ & $(8 \mathrm{c})$ \\
-0.32970000000000 & 1.14580000000000 & 0.78600000000000 & $\mathrm{I}$ & $(8 \mathrm{c})$ \\
-0.14580000000000 & -0.32970000000000 & 0.78600000000000 & $\mathrm{I}$ & $(8 \mathrm{c})$ \\
1.14580000000000 & 1.32970000000000 & 0.78600000000000 & $\mathrm{I}$ & $(8 \mathrm{c})$ \\
0.64910000000000 & 0.85880000000000 & 0.03600000000000 & $\mathrm{Tl}$ & $(8 \mathrm{c})$ \\
-0.64910000000000 & -0.85880000000000 & 0.03600000000000 & $\mathrm{Tl}$ & $(8 \mathrm{c})$ \\
-0.85880000000000 & 0.64910000000000 & 0.03600000000000 & $\mathrm{Tl}$ & $(8 \mathrm{c})$ \\
0.85880000000000 & -0.64910000000000 & 0.03600000000000 & $\mathrm{Tl}$ & $(8 \mathrm{c})$ \\
1.14910000000000 & -0.35880000000000 & 0.53600000000000 & $\mathrm{Tl}$ & $(8 \mathrm{c})$ \\
-0.14910000000000 & 1.35880000000000 & 0.53600000000000 & $\mathrm{Tl}$ & $(8 \mathrm{c})$ \\
-0.35880000000000 & -0.14910000000000 & 0.53600000000000 & $\mathrm{Tl}$ & $(8 \mathrm{c})$ \\
1.35880000000000 & 1.14910000000000 & 0.53600000000000 & $\mathrm{Tl}$ & $(8 \mathrm{c})$ \\
\hline
\end{tabular}

$\mathrm{BaGe}_{2} \mathrm{As}_{2}$ : A2BC2_tP20_105_f_ac_2e - CIF

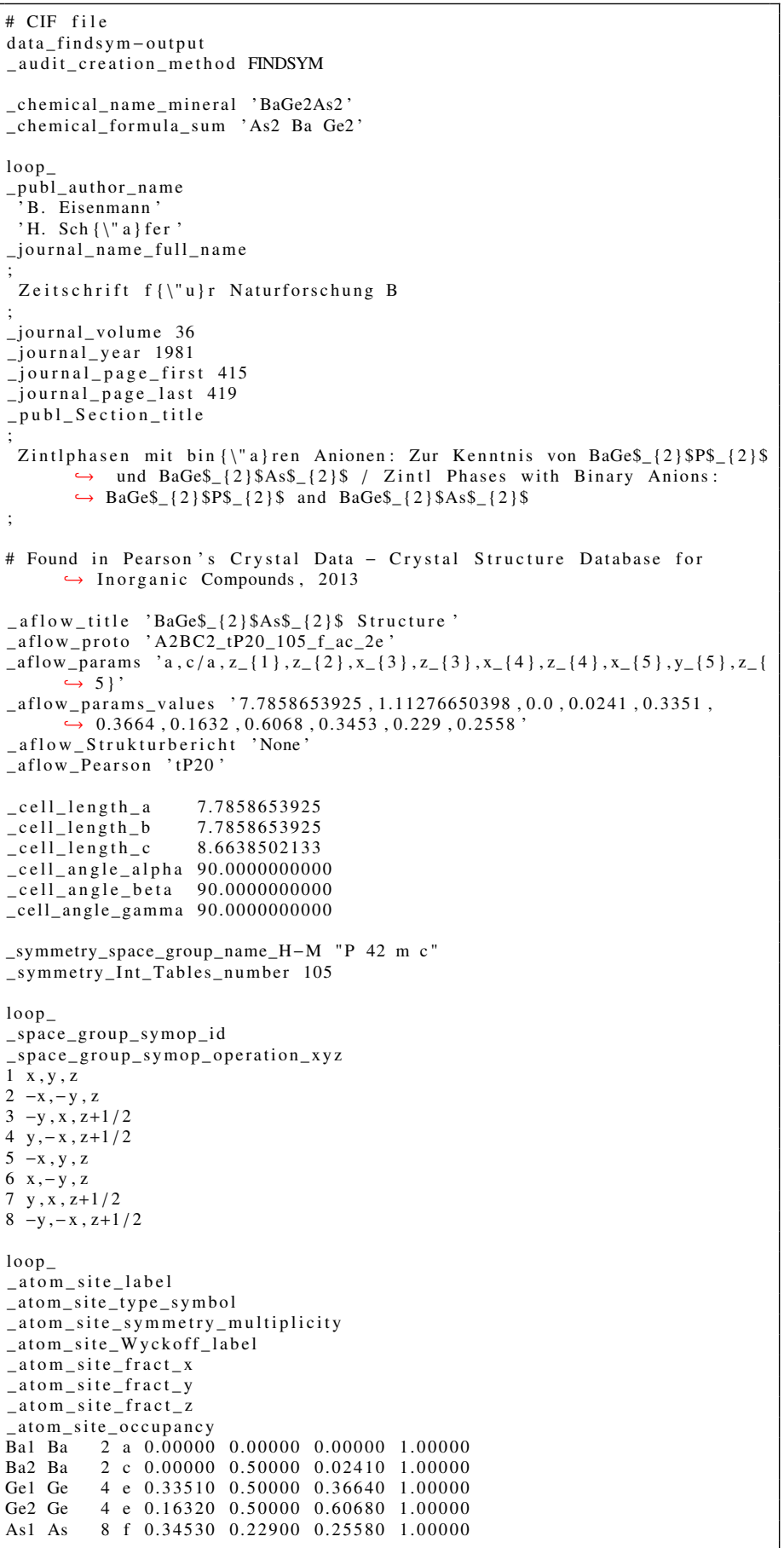

$\mathrm{BaGe}_{2} \mathrm{As}_{2}$ : A2BC2_tP20_105_f_ac_2e - POSCAR 


\begin{tabular}{|c|c|c|c|c|}
\hline \multicolumn{5}{|c|}{ 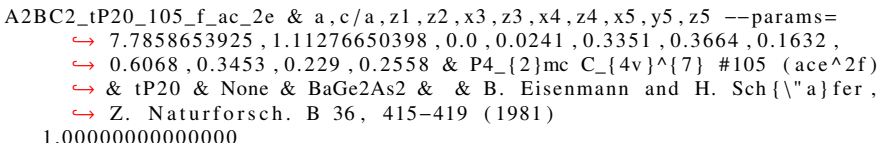 } \\
\hline 7.78586539250000 & 0.00000000000000 & 0.00000000000000 & & \\
\hline \multirow{2}{*}{$\begin{array}{l}0.00000000000000 \\
0.00000000000000\end{array}$} & 7.78586539250000 & 0.00000000000000 & & \\
\hline & 0.00000000000000 & 8.66385021330000 & & \\
\hline $\mathrm{Ge}$ & & & & \\
\hline 8 & & & & \\
\hline \multicolumn{5}{|l|}{ Direct } \\
\hline 0.34530000000000 & 0.22900000000000 & 0.25580000000000 & As & $(8 f)$ \\
\hline-0.34530000000000 & -0.22900000000000 & 0.25580000000000 & As & ( $8 f)$ \\
\hline-0.22900000000000 & 0.34530000000000 & 0.75580000000000 & As & (8f) \\
\hline 0.22900000000000 & -0.34530000000000 & 0.75580000000000 & As & ( $8 f)$ \\
\hline 0.34530000000000 & -0.22900000000000 & 0.25580000000000 & As & ( $8 f)$ \\
\hline-0.34530000000000 & 0.22900000000000 & 0.25580000000000 & As & (8f) \\
\hline-0.22900000000000 & -0.34530000000000 & 0.75580000000000 & As & (8f) \\
\hline 0.22900000000000 & 0.34530000000000 & 0.75580000000000 & As & (8f) \\
\hline 0.00000000000000 & 0.00000000000000 & 0.00000000000000 & $\mathrm{Ba}$ & (2a) \\
\hline 0.00000000000000 & 0.00000000000000 & 0.50000000000000 & $\mathrm{Ba}$ & (2a) \\
\hline 0.00000000000000 & 0.50000000000000 & 0.02410000000000 & $\mathrm{Ba}$ & (2c) \\
\hline 0.50000000000000 & 0.00000000000000 & 0.52410000000000 & $\mathrm{Ba}$ & $(2 \mathrm{c})$ \\
\hline 0.33510000000000 & 0.50000000000000 & 0.36640000000000 & $\mathrm{Ge}$ & $(4 \mathrm{e})$ \\
\hline-0.33510000000000 & 0.50000000000000 & 0.36640000000000 & $\mathrm{Ge}$ & (4e) \\
\hline 0.50000000000000 & 0.33510000000000 & 0.86640000000000 & $\mathrm{Ge}$ & (4e) \\
\hline 0.50000000000000 & -0.33510000000000 & 0.86640000000000 & $\mathrm{Ge}$ & (4e) \\
\hline 0.16320000000000 & 0.50000000000000 & 0.60680000000000 & $\mathrm{Ge}$ & $(4 \mathrm{e})$ \\
\hline-0.16320000000000 & 0.50000000000000 & 0.60680000000000 & $\mathrm{Ge}$ & $(4 \mathrm{e})$ \\
\hline 0.50000000000000 & 0.16320000000000 & 1.10680000000000 & $\mathrm{Ge}$ & $(4 \mathrm{e})$ \\
\hline 0.50000000000000 & -0.16320000000000 & 1.10680000000000 & $\mathrm{Ge}$ & (4e) \\
\hline
\end{tabular}

$\mathrm{NaZn}[\mathrm{OH}]_{3}:$ A3BC3D_tP64_106_3c_c_3c_c - CIF

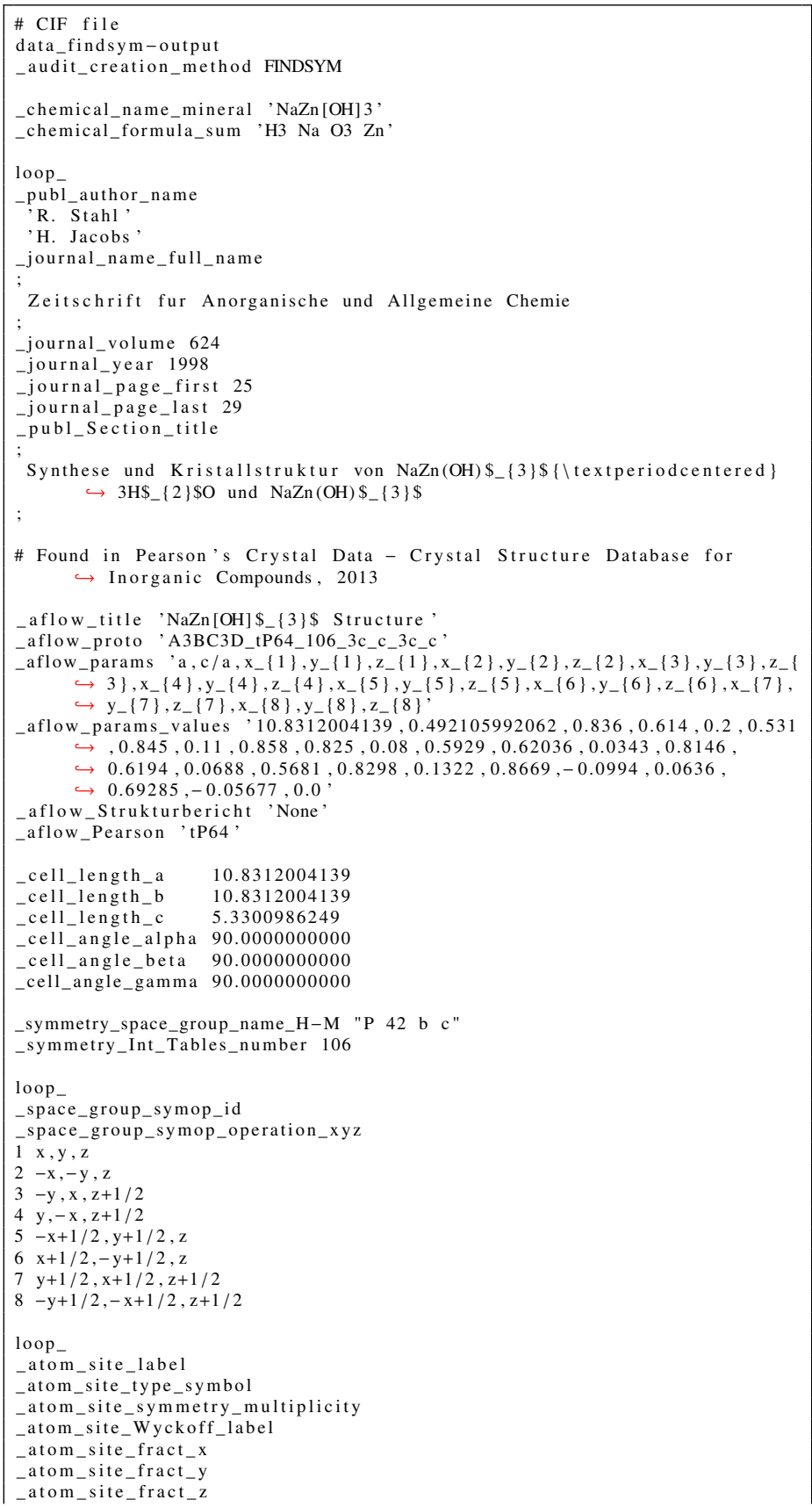

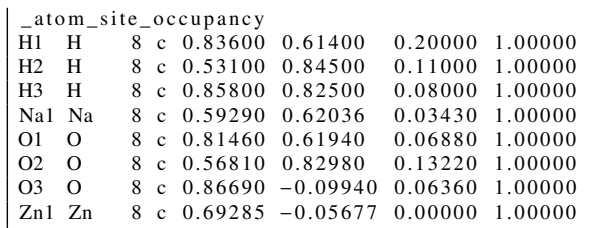

$\mathrm{NaZn}[\mathrm{OH}]_{3}$ : A3BC3D_tP64_106_3c_c_3c_c - POSCAR

\begin{tabular}{|c|c|c|c|c|}
\hline & & & & \\
\hline \multicolumn{5}{|c|}{ 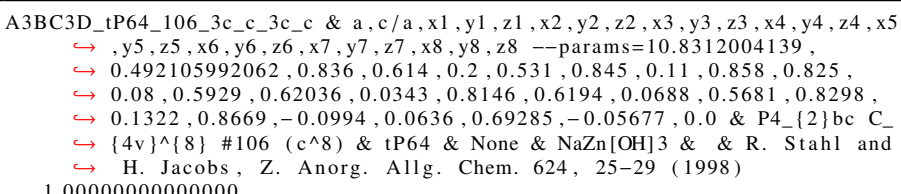 } \\
\hline \multicolumn{5}{|l|}{1.00000000000000} \\
\hline 10.83120041390000 & 0.00000000000000 & 0.00000000000000 & & \\
\hline \multirow{2}{*}{$\begin{array}{l}0.00000000000000 \\
0.00000000000000\end{array}$} & 10.83120041390000 & 0.00000000000000 & & \\
\hline & 0.00000000000000 & 5.33009862490000 & & \\
\hline $\mathrm{Na}$ & $\mathrm{Zn}$ & & & \\
\hline 24 & 8 & & & \\
\hline \multicolumn{5}{|l|}{ Direct } \\
\hline 0.83600000000000 & 0.61400000000000 & 0.20000000000000 & $\mathrm{H}$ & $(8 \mathrm{c})$ \\
\hline-0.83600000000000 & -0.61400000000000 & 0.20000000000000 & $\mathrm{H}$ & $(8 \mathrm{c})$ \\
\hline-0.61400000000000 & 0.83600000000000 & 0.70000000000000 & $\mathrm{H}$ & $(8 \mathrm{c})$ \\
\hline 0.61400000000000 & -0.83600000000000 & 0.70000000000000 & $\mathrm{H}$ & (8c) \\
\hline 1.33600000000000 & -0.11400000000000 & 0.20000000000000 & $\mathrm{H}$ & $(8 \mathrm{c})$ \\
\hline-0.33600000000000 & 1.11400000000000 & 0.20000000000000 & $\mathrm{H}$ & (8c) \\
\hline-0.11400000000000 & -0.33600000000000 & 0.70000000000000 & $\mathrm{H}$ & (8c) \\
\hline 1.11400000000000 & 1.33600000000000 & 0.70000000000000 & $\mathrm{H}$ & \\
\hline 0.53100000000000 & 0.84500000000000 & 0.11000000000000 & $\mathrm{H}$ & (8c) \\
\hline-0.53100000000000 & -0.84500000000000 & 0.11000000000000 & $\mathrm{H}$ & \\
\hline-0.84500000000000 & 0.53100000000000 & 0.61000000000000 & $\mathrm{H}$ & $(8 \mathrm{c})$ \\
\hline 0.84500000000000 & -0.53100000000000 & 0.61000000000000 & $\mathrm{H}$ & $(8 c)$ \\
\hline 1.03100000000000 & -0.34500000000000 & 0.11000000000000 & $\mathrm{H}$ & $(8 \mathrm{c})$ \\
\hline-0.03100000000000 & 1.34500000000000 & 0.11000000000000 & $\mathrm{H}$ & (8c) \\
\hline-0.34500000000000 & -0.03100000000000 & 0.61000000000000 & $\mathrm{H}$ & (8c) \\
\hline 1.34500000000000 & 1.03100000000000 & 0.61000000000000 & $\mathrm{H}$ & (8c) \\
\hline 0.85800000000000 & 0.82500000000000 & 0.08000000000000 & $\mathrm{H}$ & (8c) \\
\hline-0.85800000000000 & -0.82500000000000 & 0.08000000000000 & $\mathrm{H}$ & $(8 \mathrm{c})$ \\
\hline-0.82500000000000 & 0.85800000000000 & 0.58000000000000 & $\mathrm{H}$ & $(8 \mathrm{c})$ \\
\hline 0.82500000000000 & -0.85800000000000 & 0.58000000000000 & $\mathrm{H}$ & (8c) \\
\hline 1.35800000000000 & -0.32500000000000 & 0.08000000000000 & $\mathrm{H}$ & $(8 c)$ \\
\hline-0.35800000000000 & 1.32500000000000 & 0.08000000000000 & $\mathrm{H}$ & $(8 \mathrm{c})$ \\
\hline-0.32500000000000 & -0.35800000000000 & 0.58000000000000 & $\mathrm{H}$ & $(8 \mathrm{c})$ \\
\hline 1.32500000000000 & 1.35800000000000 & 0.58000000000000 & $\mathrm{H}$ & (8c) \\
\hline 0.59290000000000 & 0.62036000000000 & 0.03430000000000 & $\mathrm{Na}$ & $(8 \mathrm{c})$ \\
\hline-0.59290000000000 & -0.62036000000000 & 0.03430000000000 & $\mathrm{Na}$ & $(8 \mathrm{c})$ \\
\hline-0.62036000000000 & 0.59290000000000 & 0.53430000000000 & $\mathrm{Na}$ & $(8 \mathrm{c})$ \\
\hline 0.62036000000000 & -0.59290000000000 & 0.53430000000000 & $\mathrm{Na}$ & (8c) \\
\hline 1.09290000000000 & -0.12036000000000 & 0.03430000000000 & $\mathrm{Na}$ & $(8 \mathrm{c})$ \\
\hline-0.09290000000000 & 1.12036000000000 & 0.03430000000000 & $\mathrm{Na}$ & (8c) \\
\hline-0.12036000000000 & -0.09290000000000 & 0.53430000000000 & $\mathrm{Na}$ & $(8 \mathrm{c})$ \\
\hline 1.12036000000000 & 1.09290000000000 & 0.53430000000000 & $\mathrm{Na}$ & $(8 c)$ \\
\hline 0.81460000000000 & 0.61940000000000 & 0.06880000000000 & $\mathrm{O}$ & $(8 \mathrm{c})$ \\
\hline-0.81460000000000 & -0.61940000000000 & 0.06880000000000 & $\mathrm{O}$ & $(8 c)$ \\
\hline-0.61940000000000 & 0.81460000000000 & 0.56880000000000 & $\mathrm{O}$ & $(8 c)$ \\
\hline 0.61940000000000 & -0.81460000000000 & 0.56880000000000 & $\mathrm{O}$ & $(8 \mathrm{c})$ \\
\hline 1.31460000000000 & -0.11940000000000 & 0.06880000000000 & $\mathrm{O}$ & (8c) \\
\hline-0.31460000000000 & 1.11940000000000 & 0000000 & $\mathrm{O}$ & $(8 \mathrm{c})$ \\
\hline-0.11940000000000 & -0.31460000000000 & 0.56880000000000 & $\mathrm{O}$ & (8c) \\
\hline 1.11940000000000 & 1.31460000000000 & 0.56880000000000 & $\mathrm{O}$ & $(8 c)$ \\
\hline 0.56810000000000 & 0.82980000000000 & 0.13220000000000 & $\mathrm{O}$ & $(8 \mathrm{c})$ \\
\hline-0.56810000000000 & -0.82980000000000 & 0.13220000000000 & o & (8c) \\
\hline-0.82980000000000 & 0.56810000000000 & 0.63220000000000 & $\mathrm{O}$ & $(8 c)$ \\
\hline 0.82980000000000 & -0.56810000000000 & 0.63220000000000 & o & $(8 \mathrm{c})$ \\
\hline 1.06810000000000 & -0.32980000000000 & 0.13220000000000 & $\mathrm{O}$ & $8 c$ \\
\hline-0.06810000000000 & 1.32980000000000 & 0.13220000000000 & $\mathrm{O}$ & $(8 \mathrm{c})$ \\
\hline-0.32980000000000 & -0.06810000000000 & 0.63220000000000 & $\mathrm{O}$ & \\
\hline 1.32980000000000 & 1.06810000000000 & 0.63220000000000 & $\mathrm{O}$ & $(8 \mathrm{c})$ \\
\hline 0.86690000000000 & -0.09940000000000 & 0.06360000000000 & $\mathrm{O}$ & $(8 \mathrm{c})$ \\
\hline-0.86690000000000 & 0.09940000000000 & 0.06360000000000 & $\mathrm{O}$ & $(8 \mathrm{c})$ \\
\hline 0.09940000000000 & 0.86690000000000 & 0.56360000000000 & $\mathrm{O}$ & $(8 \mathrm{c})$ \\
\hline-0.09940000000000 & -0.86690000000000 & 0.56360000000000 & $\mathrm{O}$ & $(8 \mathrm{c})$ \\
\hline 1.36690000000000 & 0.59940000000000 & 0.06360000000000 & $\mathrm{O}$ & $(8 \mathrm{c})$ \\
\hline-0.36690000000000 & 0.40060000000000 & 0.06360000000000 & $\mathrm{O}$ & $(8 \mathrm{c})$ \\
\hline 0.59940000000000 & -0.36690000000000 & 0.56360000000000 & $\mathrm{O}$ & $(8 \mathrm{c})$ \\
\hline 0.40060000000000 & 1.36690000000000 & 0.56360000000000 & $\mathrm{O}$ & $(8 \mathrm{c})$ \\
\hline 0.69285000000000 & -0.05677000000000 & 0.00000000000000 & $\mathrm{Zn}$ & $(8 \mathrm{c})$ \\
\hline-0.69285000000000 & 0.05677000000000 & 0.00000000000000 & $\mathrm{Zn}$ & \\
\hline 0.05677000000000 & 0.69285000000000 & 0.50000000000000 & $\mathrm{Zn}$ & $(8 \mathrm{c})$ \\
\hline-0.05677000000000 & -0.69285000000000 & 0.50000000000000 & $\mathrm{Zn}$ & $(8 \mathrm{c})$ \\
\hline 1.19285000000000 & 0.55677000000000 & 0.00000000000000 & $\mathrm{Zn}$ & (8c) \\
\hline-0.19285000000000 & 0.44323000000000 & 0.00000000000000 & $\mathrm{Zn}$ & (8c) \\
\hline 0.55677000000000 & -0.19285000000000 & 0.50000000000000 & $\mathrm{Zn}$ & (8c) \\
\hline 0.44323000000000 & 1.19285000000000 & 0.50000000000000 & $\mathrm{Zn}$ & (8c) \\
\hline
\end{tabular}

$\mathrm{Co}_{5} \mathrm{Ge}_{7}$ : A5B7_tI24_107_ac_abd - CIF 
'H. G. Meissner'

M. P $\{\backslash$ "o $\}$ tzschke,

'W. Rossteutscher,

'E. Stolz'

_journal_name_full_name

Naturwissenschaften

_journal_volume 47

journal year 1960

_journal_page_first 512

-journal_page_last 512

publ_Section_title

Einige strukturelle Ergebnisse an metallischen Phasen (6)

\# Found in Pearson's Crystal Data - Crystal Structure Database for $\hookrightarrow$ Inorganic Compounds, 2013

_aflow_title, $C_{-} \$_{-}\{5\} \$ G e \$ \_\{7\} \$$ Structure,

_aflow_proto, A5B7_tI24_107_ac_abd

_aflow_params 'a,c/a, $z_{-}\{1\}, z_{-}\{2\}, z_{-}\{3\}, x_{-}\{4\}, z_{-}\{4\}, x_{-}\{5\}, z_{-}\{5\}$, aflow_params_values $7.6400197048,0.760471204184,0.0,0.056,0.04,0.22$, $\hookrightarrow 0.0,0.243,0.29$,

_aflow_Strukturbericht 'None'

aflow_Pearson 'tI24

\section{_cell_length_a $\quad 7.6400197048$}

_cell_length_b $\quad 7.6400197048$

_cell_length_c $\quad 5.8100149849$

cell_angle_alpha 90.0000000000

cell_angle beta 90.0000000000

cell_angle_gamma 90.0000000000

_symmetry_space_group_name_H-M "I $4 \mathrm{~m} \mathrm{~m}$

_symmetry_Int_Tables_number 107

loop

_space_group_symop_id

_space_group_symop_operation_xyz

$1 \mathrm{x}, \mathrm{y}, \mathrm{z}$

$2-\mathrm{x},-\mathrm{y}, \mathrm{z}$

$3-\mathrm{y}, \mathrm{x}, \mathrm{z}$

$4 \mathrm{y},-\mathrm{x}, \mathrm{z}$

$5-\mathrm{x}, \mathrm{y}, \mathrm{z}$

$6 \mathrm{x},-\mathrm{y}, \mathrm{z}$

$7 \mathrm{y}, \mathrm{x}, \mathrm{z}$

$8-\mathrm{y},-\mathrm{x}, \mathrm{z}$
$9 \mathrm{x}+1 / 2, \mathrm{y}+1 / 2, \mathrm{z}+1 / 2$
10

$9 \mathrm{x}+1 / 2, \mathrm{y}+1 / 2, \mathrm{z}+1 / 2$
$10-\mathrm{x}+1 / 2,-\mathrm{y}+1 / 2, \mathrm{z}+1 / 2$

$11-\mathrm{y}+1 / 2, \mathrm{x}+1 / 2, \mathrm{z}+1 / 2$

$12 \mathrm{y}+1 / 2,-\mathrm{x}+1 / 2, \mathrm{z}+1 / 2$

$13-\mathrm{x}+1 / 2, \mathrm{y}+1 / 2, \mathrm{z}+1 / 2$

$14 \mathrm{x}+1 / 2,-\mathrm{y}+1 / 2, \mathrm{z}+1 / 2$

$15 \mathrm{y}+1 / 2, \mathrm{x}+1 / 2, \mathrm{z}+1 / 2$

$16-\mathrm{y}+1 / 2,-\mathrm{x}+1 / 2, \mathrm{z}+1 / 2$

loop_

atom_site_label

_atom_site_type_symbol

_atom_site_symmetry_multiplicity

_atom_site_Wyckoff_label

_atom_site_fract_x

-atom_site_fract_y

atom_site_fract_z

- atom site occupancy

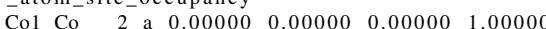

$\begin{array}{lllllll}\mathrm{Ge} 1 \mathrm{Ge} & 2 & \mathrm{a} & 0.00000 & 0.00000 & 0.05600 & 1.00000\end{array}$

$\begin{array}{lllllll}\mathrm{Ge} 2 \mathrm{Ge} & 4 & \mathrm{~b} & 0.00000 & 0.50000 & 0.04000 & 1.00000\end{array}$

$\begin{array}{llllllll}\mathrm{Co} 2 & \mathrm{Co} & 8 & \mathrm{c} & 0.22000 & 0.22000 & 0.00000 & 1.00000\end{array}$

$\begin{array}{lllllll}\mathrm{Ge} 3 \mathrm{Ge} & 8 & \mathrm{~d} & 0.24300 & 0.00000 & 0.29000 & 1.00000\end{array}$

$\mathrm{Co}_{5} \mathrm{Ge}_{7}$ : A5B7_tI24_107_ac_abd - POSCAR

A5B7_tI24_107_ac abd \& a , c/a , z1 , z2, z3, x4, z4, x5, z5 --params $=7.6400197048$

$\hookrightarrow 0.760471204184,0.0,0.056,0.04,0.22,0.0,0.243,0.29 \& \mathrm{I}_{4 m m ~ \mathrm{C}_{-}}\{4 \mathrm{v}$

$\hookrightarrow\}^{\wedge}\{9\} \# 107 \quad\left(a^{\wedge} 2 \mathrm{bcd}\right) \&$ tI 24 \& None \& Co5Ge7 \& \& K. Schubert et

$\hookrightarrow$ al., \{Naturwissenschaften $47,512(1960)$

1.00000000000000

$\begin{array}{rrr}-3.82000985240000 & 3.82000985240000 & 2.90500749245000\end{array}$

$\begin{array}{lrr}3.82000985240000 & -3.82000985240000 & 2.90500749245000\end{array}$

$\begin{array}{lll}3.82000985240000 & 3.82000985240000 & -2.90500749245000\end{array}$

$\begin{array}{rr}\text { Co } & \mathrm{Ge} \\ 5 & 7\end{array}$

Direct

$\begin{array}{lll}0.00000000000000 & 0.00000000000000 & 0.00000000000000\end{array}$

$\begin{array}{lll}0.22000000000000 & 0.22000000000000 & 0.44000000000000\end{array}$

$-0.22000000000000-0.22000000000000-0.44000000000000$

$0.22000000000000-0.22000000000000 \quad 0.00000000000000$

$\begin{array}{rrr}-0.22000000000000 & 0.22000000000000 & 0.00000000000000\end{array}$

$\begin{array}{lll}0.05600000000000 & 0.05600000000000 & 0.00000000000000\end{array}$

$\begin{array}{lll}0.05600000000000 & 0.05600000000000 & 0.00000000000000 \\ 0.54000000000000 & 0.04000000000000 & 0.50000000000000\end{array}$

$\begin{array}{lll}0.54000000000000 & 0.04000000000000 & 0.50000000000000 \\ 0.04000000000000 & 0.54000000000000 & 0.50000000000000\end{array}$

$\begin{array}{lll}0.04000000000000 & 0.54000000000000 & 0.50000000000000 \\ 0.29000000000000 & 0.53300000000000 & 0.24300000000000\end{array}$

$\begin{array}{llll}0.29000000000000 & 0.04700000000000 & -0.24300000000000\end{array}$

$\begin{array}{lllll}0.53300000000000 & 0.29000000000000 & 0.24300000000000\end{array}$

$\begin{array}{lll}0.04700000000000 & 0.29000000000000 & -0.24300000000000\end{array}$

Co $(2 \mathrm{a})$

Co $(8 \mathrm{c})$

Co $(8 \mathrm{c})$

Co $\quad(8 \mathrm{c})$

Co $\quad(8 \mathrm{c})$

$\mathrm{Ge} \quad(4 \mathrm{~b})$

$\mathrm{Ge} \quad(4 \mathrm{~b})$

$\mathrm{Ge} \quad(8 \mathrm{~d})$

$\mathrm{Ge} \quad(8 \mathrm{~d})$

$\mathrm{Ge} \quad(8 \mathrm{~d})$

(8d)

GeP (High-pressure, superconducting): AB_tI4_107_a_a - CIF

\section{\# CIF file}

data_findsym-output

-audit_creation_method FINDSYM

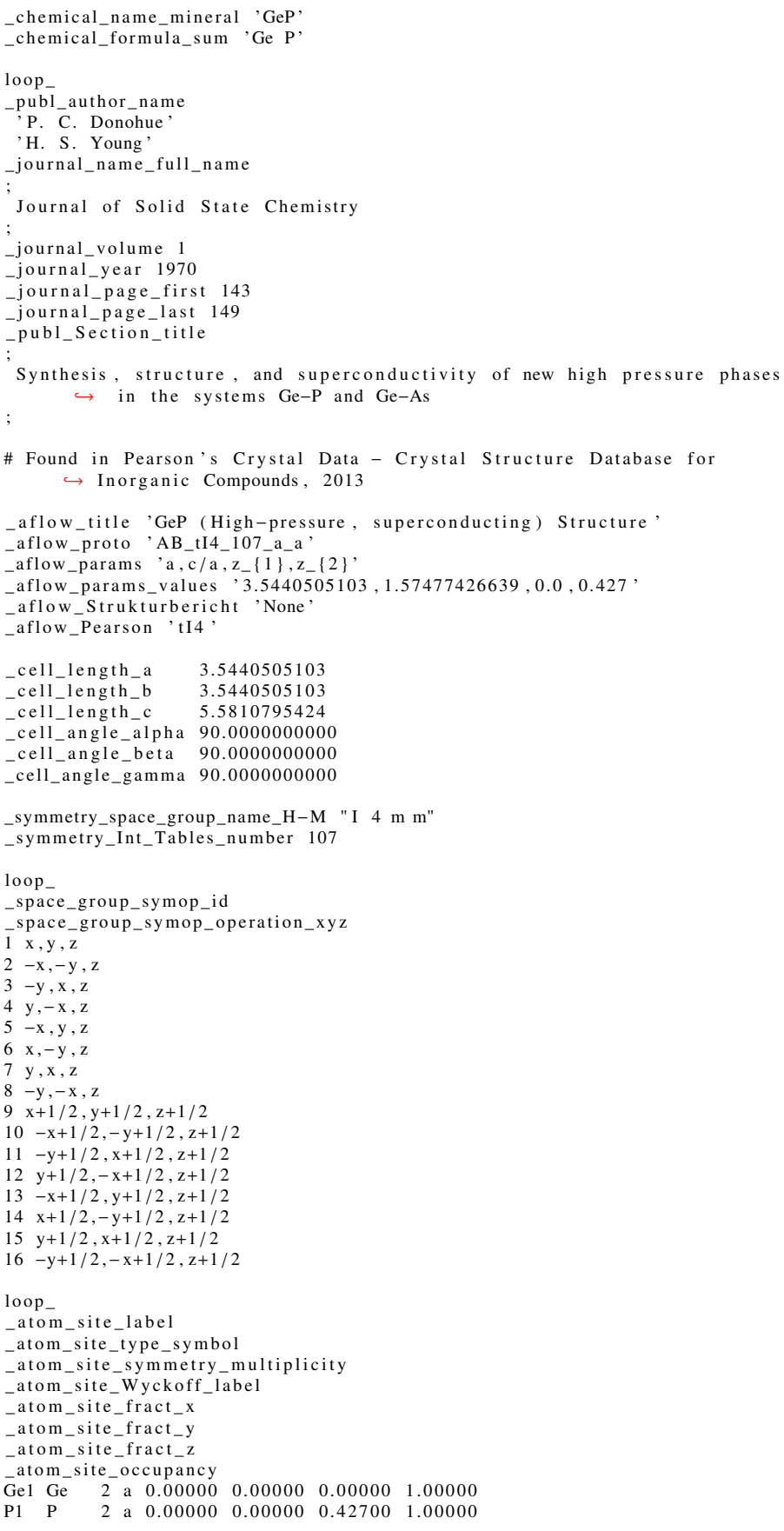

GeP (High-pressure, superconducting): AB_tI4_107_a_a - POSCAR

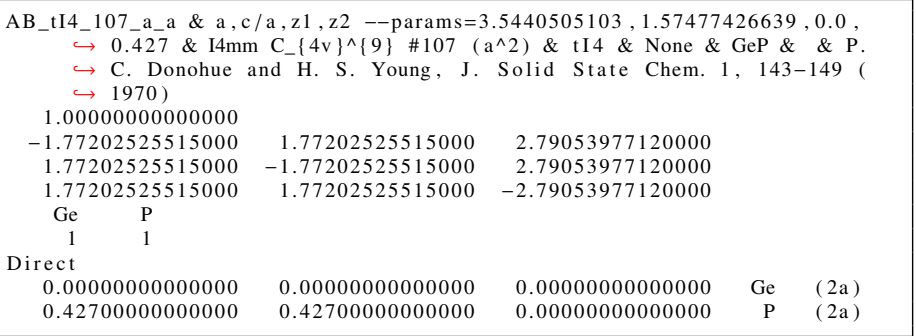

$\mathrm{Sr}_{5} \mathrm{Si}_{3}$ : A3B5_tI32_108_ac_a2c - CIF

\# CIF file

data_findsym-output

audit_creation_method FINDSYM

chemical_name_mineral 'Sr5Si3,

chemical_formula_sum 'Si3 $\mathrm{Sr} 5$,

loop

publ_author_name

G. Nagorsen

G. Rockt $\{\backslash " \mathrm{a}\}$ schel

H. Sch $\{\backslash$ "a $\}$ fer

A. Weiss

_journal_name_full_name 


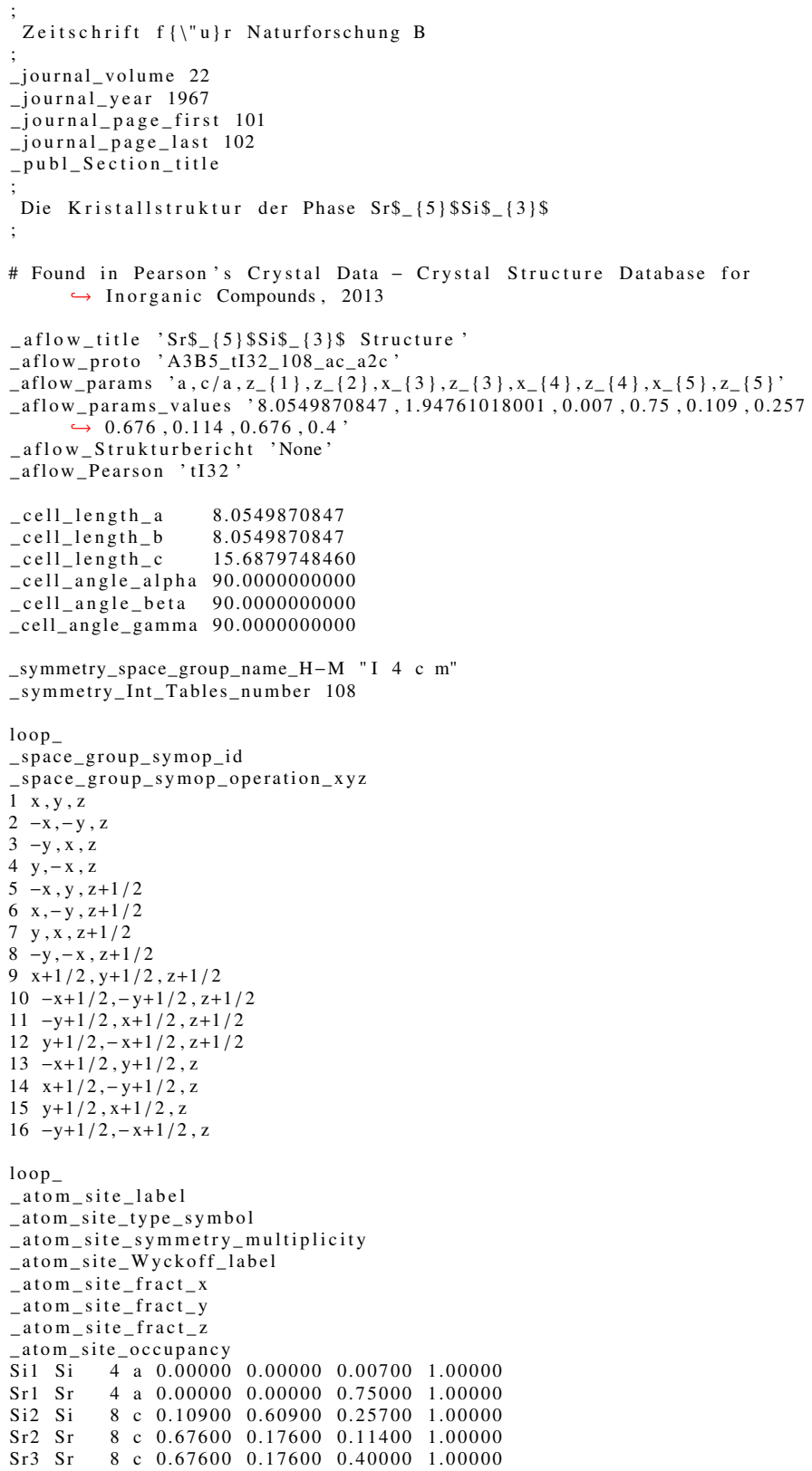

$\mathrm{Sr}_{5} \mathrm{Si}_{3}$ : A3B5_tI32_108_ac_a2c - POSCAR

\begin{tabular}{|c|c|c|c|c|}
\hline \multicolumn{5}{|c|}{ A3B5_tI32_108_ac_a2c \& a,c/a, z1, z2, x3, z3, x4,z4, x5, z5 --params $=$} \\
\hline$\hookrightarrow 8.0549870847$ & 1.747010100 & $109,0.25$ & . & \\
\hline$\hookrightarrow 0.676,0.4 \&$ & $\mathrm{I} 44 \mathrm{~cm} \mathrm{C}_{-}\{4 \mathrm{v}\}^{\wedge}\{10\}$ & $108\left(a^{\wedge} 2 c^{\wedge} 3\right) \& t I 32$ & & \\
\hline $\begin{array}{l}\hookrightarrow \operatorname{Sr} 5 \operatorname{Si3} \& \text { \& } \\
\hookrightarrow 1967)\end{array}$ & G. Nagorsen et al. & Z. Naturforsch. B & & $1-102($ \\
\hline 1.00000000000000 & & & & \\
\hline-4.02749354235000 & 4.02749354235000 & 7.84398742300000 & & \\
\hline 4.02749354235000 & -4.02749354235000 & 7.84398742300000 & & \\
\hline 4.02749354235000 & 4.02749354235000 & -7.84398742300000 & & \\
\hline $\mathrm{Si} \quad \mathrm{Sr}$ & & & & \\
\hline 10 & & & & \\
\hline Direct & & & & \\
\hline 0.00700000000000 & 0.00700000000000 & 0.00000000000000 & $\mathrm{Si}$ & (4a) \\
\hline 0.50700000000000 & 0.50700000000000 & 0.00000000000000 & $\mathrm{Si}$ & (4a) \\
\hline 0.86600000000000 & 0.36600000000000 & 0.71800000000000 & $\mathrm{Si}$ & $(8 c)$ \\
\hline 0.64800000000000 & 0.14800000000000 & 0.28200000000000 & $\mathrm{Si}$ & $(8 c)$ \\
\hline 0.36600000000000 & 0.64800000000000 & 0.50000000000000 & $\mathrm{Si}$ & $(8 c)$ \\
\hline 0.14800000000000 & 0.86600000000000 & 0.50000000000000 & $\mathrm{Si}$ & $(8 c)$ \\
\hline 0.75000000000000 & 0.75000000000000 & 0.00000000000000 & $\mathrm{Sr}$ & (4a) \\
\hline 1.25000000000000 & 1.25000000000000 & 0.00000000000000 & $\mathrm{Sr}$ & (4a) \\
\hline 1.29000000000000 & 0.79000000000000 & 1.85200000000000 & $\mathrm{Sr}$ & $(8 c)$ \\
\hline-0.06200000000000 & -0.56200000000000 & -0.85200000000000 & $\mathrm{Sr}$ & $(8 c)$ \\
\hline 0.79000000000000 & -0.06200000000000 & 0.50000000000000 & $\mathrm{Sr}$ & $(8 c)$ \\
\hline-0.56200000000000 & 1.29000000000000 & 0.50000000000000 & $\mathrm{Sr}$ & $(8 c)$ \\
\hline 1.57600000000000 & 1.07600000000000 & 1.85200000000000 & $\mathrm{Sr}$ & $(8 c)$ \\
\hline 0.22400000000000 & -0.27600000000000 & -0.85200000000000 & $\mathrm{Sr}$ & $(8 c)$ \\
\hline 1.07600000000000 & 0.22400000000000 & 0.50000000000000 & $\mathrm{Sr}$ & $(8 c)$ \\
\hline-0.27600000000000 & 1.57600000000000 & 0.50000000000000 & $\mathrm{Sr}$ & $(8 c)$ \\
\hline
\end{tabular}

LaPtSi: ABC_tI12_109_a_a_a - CIF

\# CIF file

data_findsym-output

-audit_creation_method FINDSYM

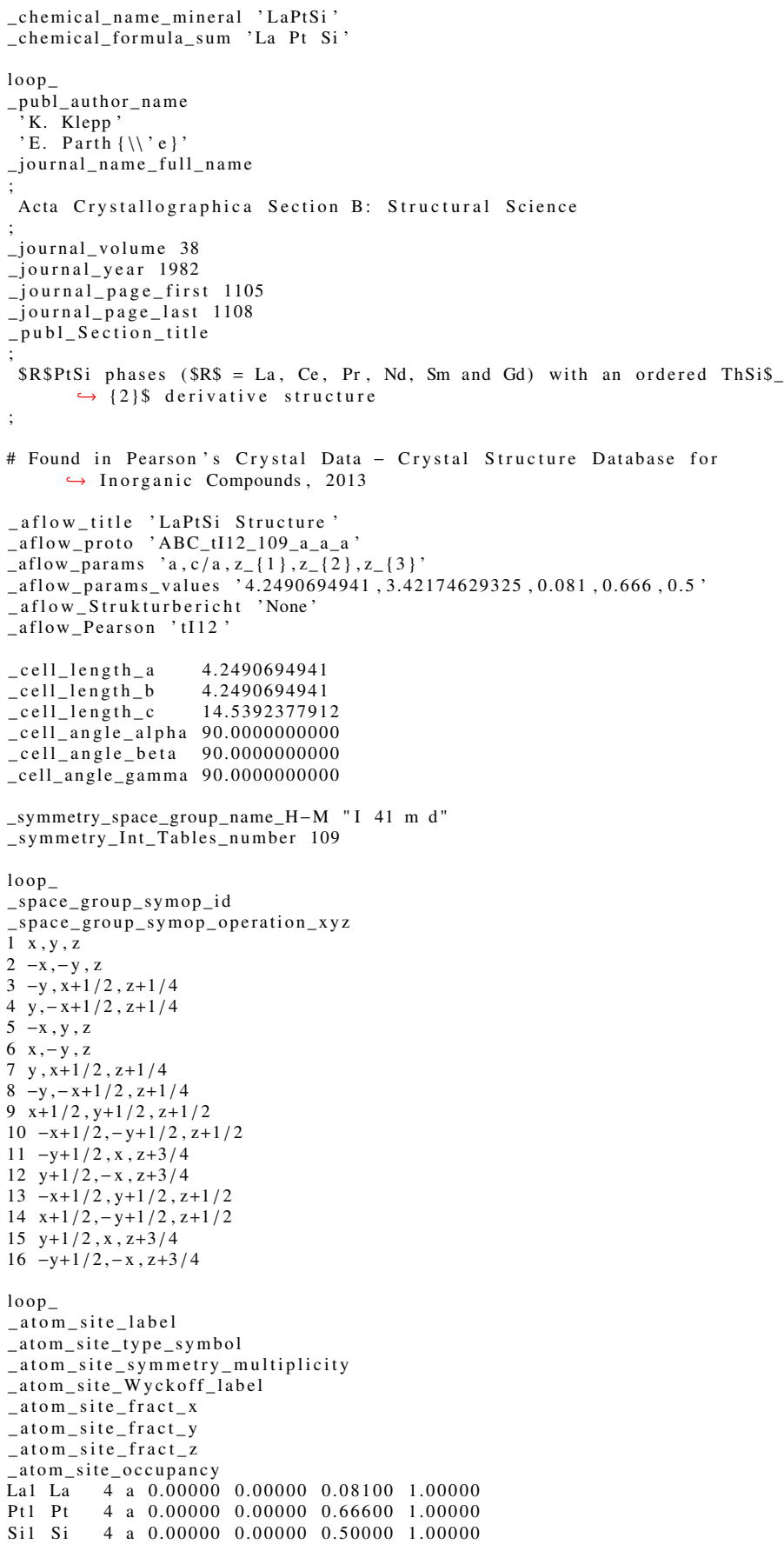

LaPtSi: ABC_tI12_109_a_a_a - POSCAR

\begin{tabular}{|c|c|c|c|c|}
\hline \multicolumn{5}{|c|}{ 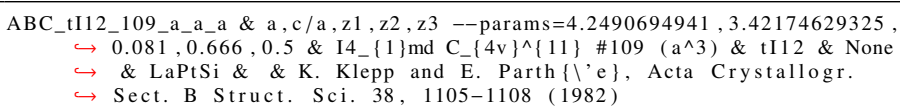 } \\
\hline \multicolumn{5}{|c|}{1.00000000000000} \\
\hline-2.12453474705000 & 2.12453474705000 & 7.26961889560000 & & \\
\hline 2.12453474705000 & -2.12453474705000 & 7.26961889560000 & & \\
\hline 2.12453474705000 & 2.12453474705000 & -7.26961889560000 & & \\
\hline $\mathrm{La}$ & & & & \\
\hline 2 & & & & \\
\hline \multicolumn{5}{|l|}{ Direct } \\
\hline 0.08100000000000 & 0.08100000000000 & 0.00000000000000 & $\mathrm{La}$ & (4a) \\
\hline 0.83100000000000 & 0.33100000000000 & 0.50000000000000 & $\mathrm{La}$ & (4a) \\
\hline 0.66600000000000 & 0.66600000000000 & 0.00000000000000 & $\mathrm{Pt}$ & (4a) \\
\hline 1.41600000000000 & 0.91600000000000 & 0.50000000000000 & $\mathrm{Pt}$ & (4a) \\
\hline 0.50000000000000 & 0.50000000000000 & 0.00000000000000 & $\mathrm{Si}$ & $(4 a)$ \\
\hline 1.25000000000000 & 0.75000000000000 & 0.50000000000000 & $\mathrm{Si}$ & $(4 a)$ \\
\hline
\end{tabular}

NbAs: AB_tI8_109_a_a - CIF

\# CIF file

data findsym-output

audit_creation_method FINDSYM

chemical name mineral 'NbAs'

_chemical_formula_sum 'As $\mathrm{Nb}$,

loop

_publ_author_name 


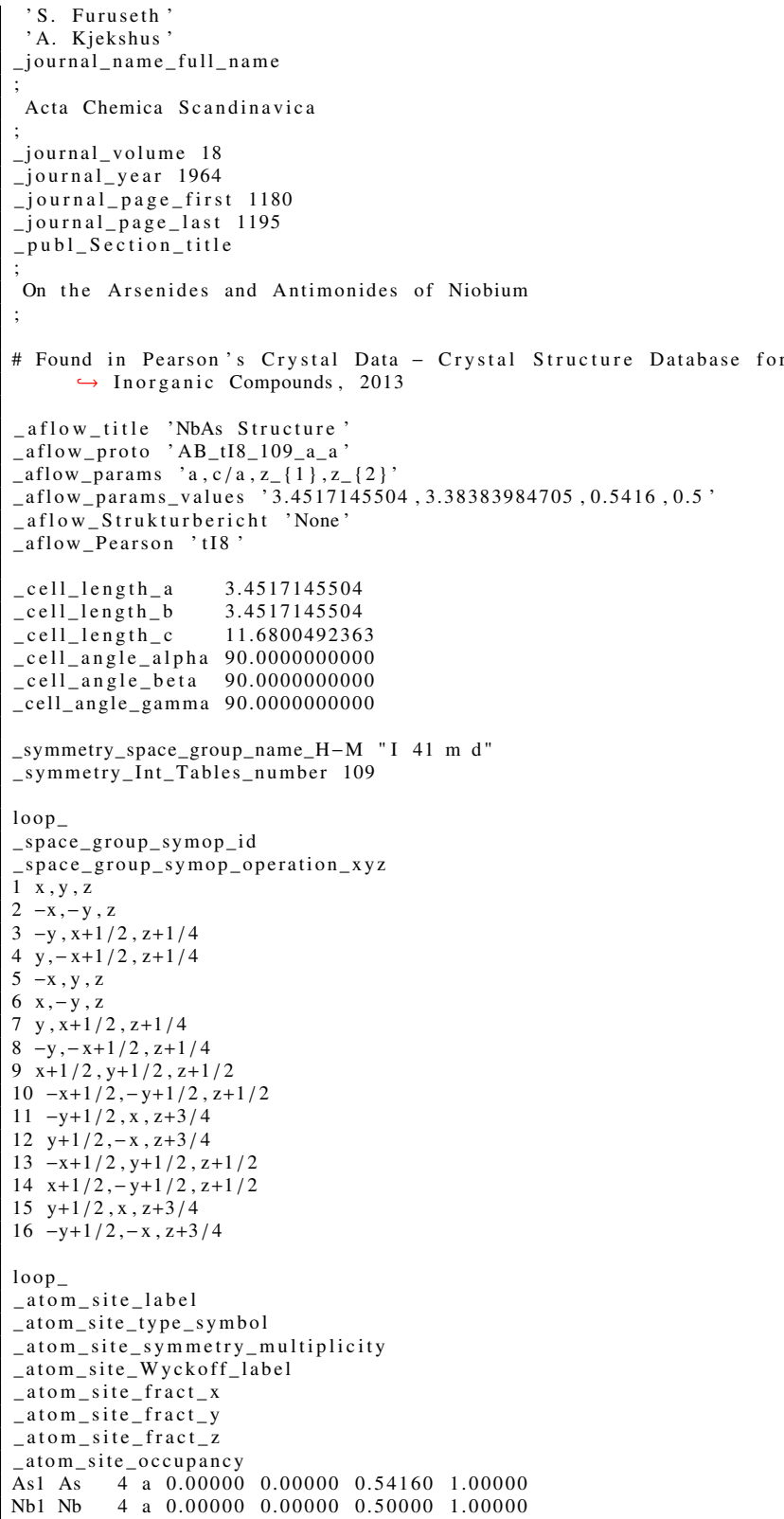

AB_tI8_109_a_a \& a, c/a, z1, z2 --params $=3.4517145504,3.38383984705,0.5416$, $\hookrightarrow 0.5$ \& I4_\{1\} md C_ $\{4 \mathrm{v}\}^{\wedge}\{11\} \# 109 \quad\left(\mathrm{a}^{\wedge} 2\right) \& \mathrm{tI} 8$ \& None \& NbAs \& \&

$\hookrightarrow$ S. Furuseth and A. Kjekshus, Acta Chem. Scand. 18, 1180-1195 ( $\hookrightarrow$ S. Fu

1.00000000000000

$\begin{array}{lll}1.72585727520000 & 1.72585727520000 & 5.84002461815000\end{array}$

$\begin{array}{lll}1.72585727520000 & -1.72585727520000 & 5.84002461815000\end{array}$

$\begin{array}{lrr}1.72585727520000 & 1.72585727520000 & -5.84002461815000\end{array}$

As $\mathrm{Nb}$

Direct

$\begin{array}{lll}0.54160000000000 & 0.54160000000000 & 0.00000000000000\end{array}$

$\begin{array}{lll}1.29160000000000 & 0.79160000000000 & 0.50000000000000\end{array}$

$\begin{array}{llll}0.50000000000000 & 0.50000000000000 & 0.00000000000000\end{array}$

1.25000000000000

0.75000000000000

0.5000000000000

$\mathrm{Be}\left[\mathrm{BH}_{4}\right]_{2}$ : A2BC8_tI176_110_2b_b_8b - CIF

\# CIF file\# This file was generated by FINDSYM

\# Harold T. Stokes, Branton J. Campbell, Dorian M. Hatch

\# Brigham Young University, Provo, Utah, USA

data_findsym-output

-audit_creation_method FINDSYM

_chemical_name_mineral 'Be [BH4]2

chemical_formula_sum 'B2 Be H8,

loop

_publ_author_name

D. S. Marynick'

W. N. Lipscomb

journal_name_full_name

Inorganic Chemistry

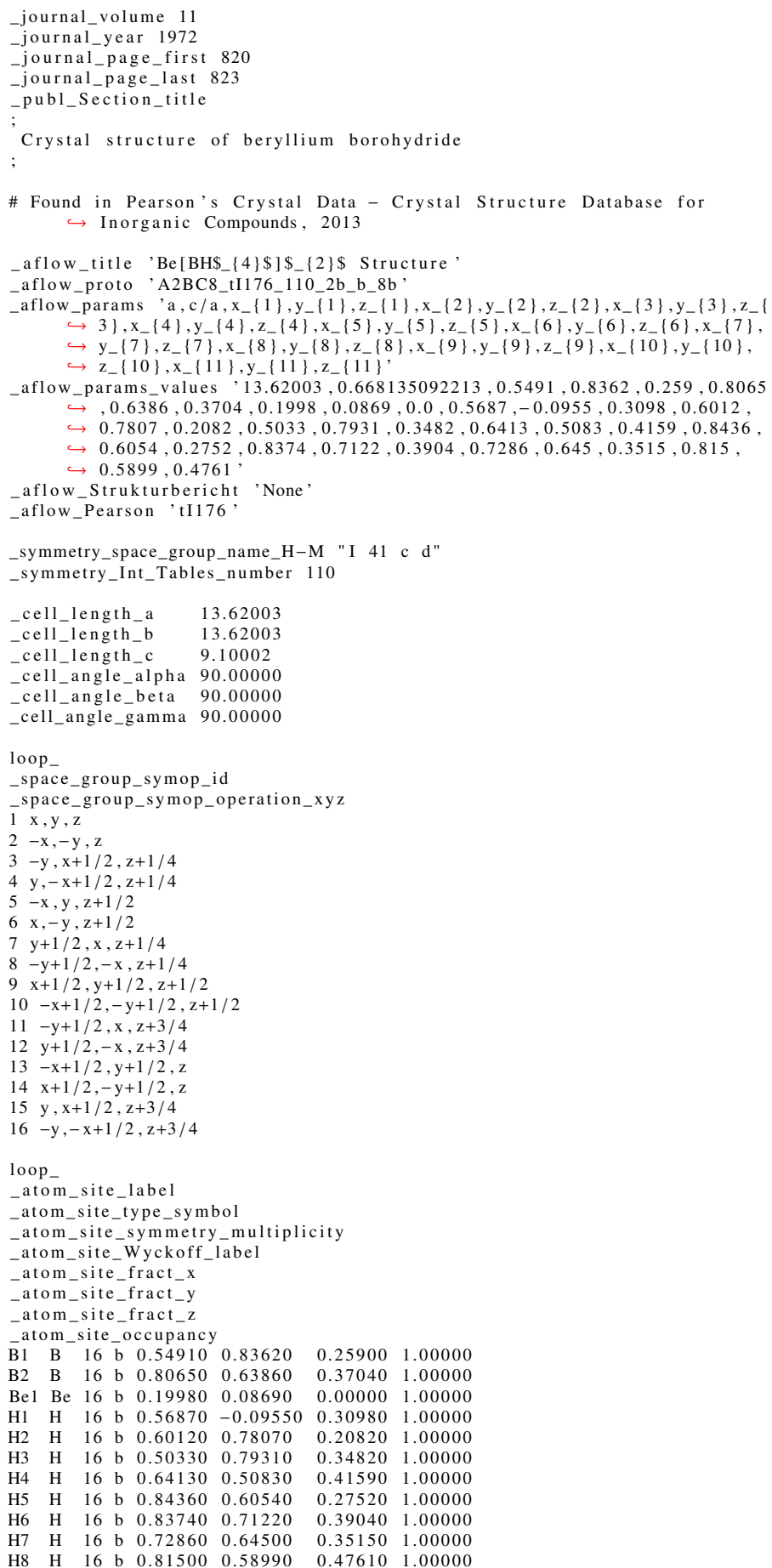

$\mathrm{Be}\left[\mathrm{BH}_{4}\right]_{2}$ : A2BC8_tI176_110_2b_b_8b - POSCAR

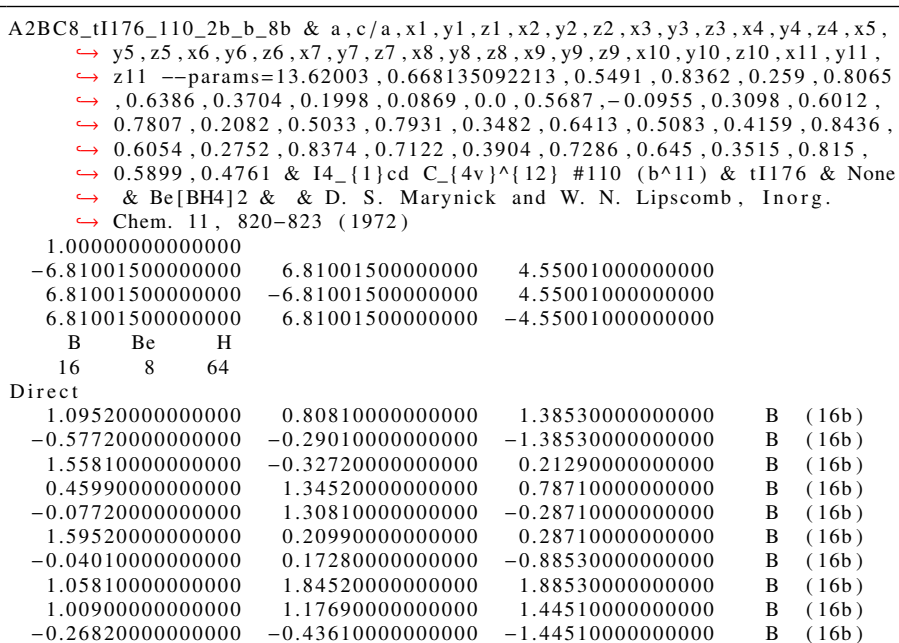


1.92690000000000 0.31390000000000 0.23180000000000 1.50900000000000 0.18610000000000 1.42690000000000 0.08690000000000 $-0.08690000000000$ 0.94980000000000 0.55020000000000 0.41310000000000 0.58690000000000 0.05020000000000 0.44980000000000 0.44980000000000 0.21430000000000 1.62850000000000 1.62850000000000 0.49110000000000 0.90530000000000 0.71430000000000 $-0.00890000000000$ 1.12850000000000 0.98890000000000 $-0.57250000000000$ 1.55940000000000 0.35700000000000 $-0.07250000000000$ 1.48890000000000 $-0.14300000000000$ 1.05940000000000 1.14130000000000 $-0.44490000000000$ 1.60150000000000 0.59490000000000 0.05510000000000 1.64130000000000 0.09490000000000 1.10150000000000 0.92420000000000 $-0.09240000000000$ 1.80720000000000 0.52460000000000 0.40760000000000 1.42420000000000 0.02460000000000 1.30720000000000 0.88060000000000 $-0.330200000000$ 1.86880000000000 1.86880000000000 0.18160000000000 0.16980000000000 1.38060000000000 $-0.31840000000000$ 1.36880000000000 1.10260000000000 $-0.32180000000000$ 1.97780000000000 0.30300000000000 0.17820000000000 1.60260000000000 $-0.19700000000000$ 1.47780000000000 0.99650000000000 0.29350000000000 1.8301000000000 1.83010000000000 0.37290000000 0.2065000000000 1.49650000000000 $-0.12710000000000$ 1.33010000000000 1.06600000000000 0.11380000000000 2.04110000000000 0.41110000000000 0.38620000000000 1.56600000000000 $-0.08890000000000$ 1.54110000000000

$-0.01820000000000$ 1.2590000000000 1.6769000000000 0.06390000000000 0.48180000000000 1.75900000000000 0.19980000000000 $-0.1998000000000$ 0.16310000000000 0.33690000000000 0.69980000000000 0.30020000000000 0.66310000000000 0.6636900000000 0.8785000000000 0.2589000000000 0.65530000000000 0.46430000 0.4643000000000 1.378500000000 0.24110000000000 1.1553000000000 0.9643000000000 0.8094000000000 $-0.39300000000000$ .32250000000000 1.23890000000000 1.30940000000000 0.10700000000000 1.73890000000000 0.85150000000000 $-0.15510000000000$ $-0.19490000000000$ 1.39130000000000 1.35150000000000 . 34.35000000000 0.30510000000000 1.8913000000000 1.05720000000000 1.05720000000000 0.15760000000000 1.17420000000000 1.55720000000000 0.27460000000000 0.65760000000000 1.67420000000000 1.1188000000000 $-0.568400000000$ $-0.0802000000000$ 1.1306000000000 1.61880000000 0.4198000000000 0.41980000000000 1.22780000 1.35260000000000 1.72780000000000 0.05300000000000 0.42820000000000 1.08010000000000 $-0.37710000000000$ $-0.04350000000000$ 1.24650000000000 1.58010000000000 0.12290000000000 1.74650000000000 0.13620000000000 1.31600000000000 1.79110000000000 0.16110000000000 1.8160000000000

.66790000000000 0.33210000000000 .16790000000000 $-0.16790000000000$ $-0.94510000000000$ 94510000000000 28670000000000 $-0.28670000000000$ 0.61290000000000 0.3871000000000 0.11290000000000 $-0.11290000000000$ 0.21330000000000 0.78670000000000 0.4732000000000 0.47320000000000 1.16420000000000 0.16420000000000 0.66420000000000 0.6642000000000 0.66420000000000 0.02680000000000 0.97320000000000 1.38190000000000 1.38190000000000 0.3205000000000 0.67950000000000 $-0.17950000000000$ 0.17950000000000 $-0.88190000000000$ 1.88190000000000 1.29640000000000 $-1.2964000000000$ $-1.29640000000$ 0.78980000000000 0.789800000000 0.28980000000000 0.28980000000000 $-0.79640000000000$ 1.79640000000000 1.14960000000000 $-1.14960000000000$ 0.6330000000000 0.3670000000000 0.13300000000000 $-0.13300000000000$ $-0.64960000000000$ 1.64960000000000 1.44900000000000 $-1.44900000000000$ 0.73820000000000 0.73820000000 0.23820000000000 0.238200000000 $-0.23820000000000$ 0.94900000000000 1.94900000000000 1.54960000000000 $-1.54960000000000$ 0.62520000000000 0.3748000000000 0.12520000000000 $-0.12520000000000$ $-1.04960000000000$ 2.04960000000000 1.37360000000000 $-1.37360000000000$ $-1.3736000000000$ 0.5836000000 0.08360000000000 . $-0.0836000000000$ 0.87360000000000 1.87360000000000 1.40490000000000 1.40490000000000 0.7251000000000 0.27490000000000 0.22510000000000 0.22510000000000 $-0.90490000000000$

B (16b)

B (16b)

B $(16 b)$

B (16b)

B $\quad(16 \mathrm{~b})$

B (16b)

Be $\quad(16 b)$

Be $(16 b)$

Be (16b)

Be (16b)

$\mathrm{Be} \quad(16 \mathrm{~b})$

$\mathrm{Be}(16 \mathrm{~b})$

$\begin{array}{cl}\mathrm{Be} & (16 \mathrm{~b}) \\ \mathrm{H} & (16 \mathrm{~b})\end{array}$

$\mathrm{H}(16 \mathrm{~b})$

H (16b)

H (16b)

$\mathrm{H} \quad(16 \mathrm{~b})$

$\mathrm{H}$ (16b)

$\mathrm{H}$ (16b)

$\mathrm{H}$ (16b)

$\mathrm{H}$ (16b)

(16b)

$(16 \mathrm{~b})$

(16b)

(16b)

(16b)

$\mathrm{H} \quad(16 \mathrm{~b})$

$\mathrm{H}$ (16b)

H (16b)

H (16b)

$\mathrm{H}$ (16b)

$\mathrm{H}$ (16b)

$\begin{array}{ll}\mathrm{H} & (16 \mathrm{~b}) \\ \mathrm{H} & (16 \mathrm{~b})\end{array}$

H (16b

H (16b)

$\mathrm{H}$ (16b)

(16b)

$\mathrm{H} \quad(16 \mathrm{~b})$

$\mathrm{H}$ (16b)

$\mathrm{H}$ (16b)

$\mathrm{H} \quad(16 \mathrm{~b})$

$\mathrm{H}$ (16b)

$\mathrm{H} \quad(16 \mathrm{~b})$

$\mathrm{H}$ (16b)

$\mathrm{H}$ (16b)

$\begin{array}{ll}\mathrm{H} & (16 \mathrm{~b}) \\ \mathrm{H} & (16 \mathrm{~b})\end{array}$

$\mathrm{H} \quad(16 \mathrm{~b})$

$\begin{array}{ll}\mathrm{H} & (16 \mathrm{~b}) \\ \mathrm{H} & (16 \mathrm{~b})\end{array}$

H (16b)

H (16b)

$\mathrm{H}$ (16b) 


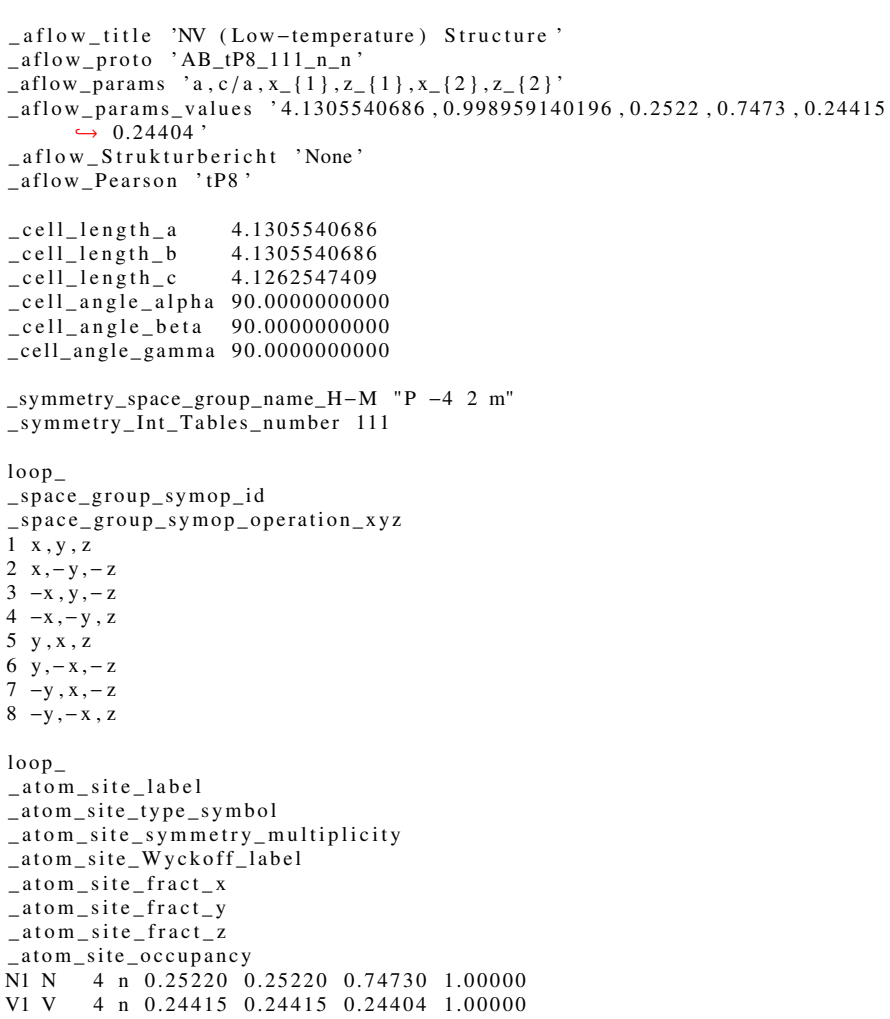

NV (Low-temperature): AB_tP8_111_n_n - POSCAR

AB_tP8_111_n_n \& a , c/a, x1, z1 , x2, z2 --params $=4.1305540686,0.998959140196$, $\hookrightarrow 0.2522,0.7473,0.24415,0.24404 \& \mathrm{P}-42 \mathrm{~m} \mathrm{D} \mathrm{D}_{-}\{2 \mathrm{~d}\}^{\wedge}\{1\} \# 111 \quad\left(\mathrm{n}^{\wedge} 2\right) \&$

$\hookrightarrow$ tP8 \& None \& VN \& \& F. Kubel et al., Phys. Rev. B 38, 12908( $\hookrightarrow 1988$ )

1.00000000000000

$\begin{array}{lll}4.13055406860000 & 0.00000000000000 & 0.00000000000000\end{array}$

$0.00000000000000 \quad 4.13055406860000 \quad 0.00000000000000$

$0.00000000000000 \quad 0.00000000000000 \quad 4.12625474090000$

N V

Direct

$\begin{array}{rrr}0.25220000000000 & 0.25220000000000 & 0.74730000000000\end{array}$

$\begin{array}{lll}-0.25220000000000 & -0.25220000000000 & 0.74730000000000\end{array}$

$\begin{array}{llll}0.25220000000000 & -0.25220000000000 & -0.74730000000000\end{array}$

$\begin{array}{rrr}-0.25220000000000 & 0.25220000000000 & -0.74730000000000\end{array}$

$\begin{array}{rrr}0.24415000000000 & 0.24415000000000 & 0.24404000000000\end{array}$

$\begin{array}{rrr}0.24415000000000 & -0.24415000000000 & 0.24404000000000\end{array}$

$\begin{array}{rrr}0.24415000000000 & -0.24415000000000 & -0.24404000000000\end{array}$

$\begin{array}{rrr}-0.24415000000000 & 0.24415000000000 & -0.24404000000000\end{array}$

$\alpha$-CuAlCl $4:$ AB4C_tP12_112_b_n_e - CIF

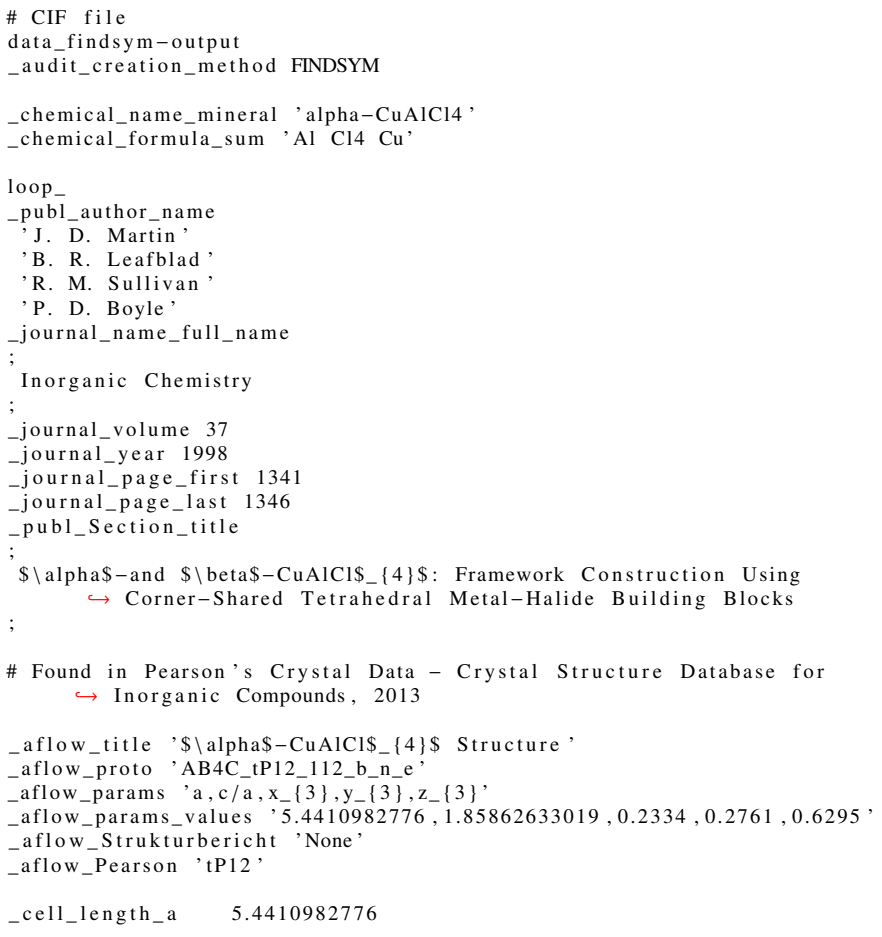

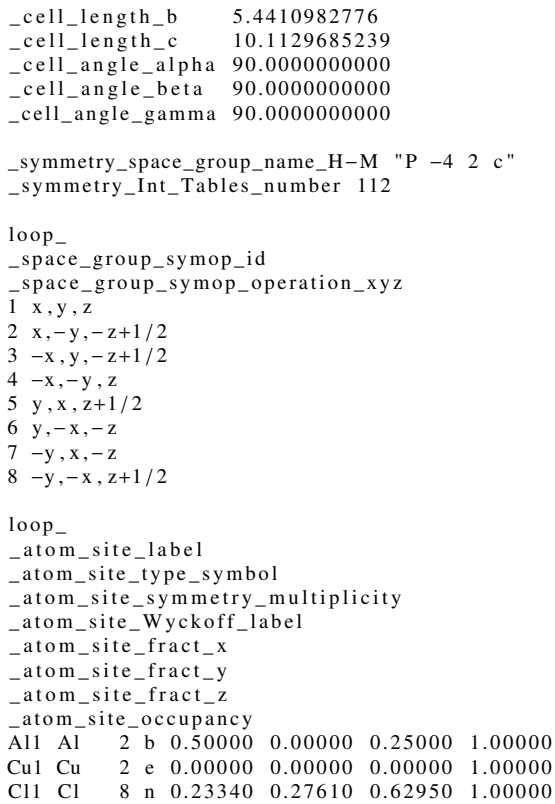

$\alpha$-CuAlCl 4 : AB4C_tP12_112_b_n_e - POSCAR

\begin{tabular}{|c|c|c|c|c|}
\hline \multirow{2}{*}{\multicolumn{5}{|c|}{ 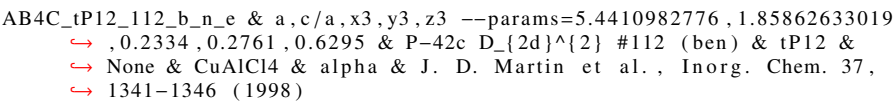 }} \\
\hline & & & & \\
\hline \multicolumn{5}{|l|}{1.00000000000000} \\
\hline 5.44109827760000 & 0.00000000000000 & 0.00000000000000 & & \\
\hline 0.00000000000000 & 5.44109827760000 & 0.00000000000000 & & \\
\hline 0.00000000000000 & 0.00000000000000 & 10.11296852390000 & & \\
\hline $\mathrm{Cu}$ & & & & \\
\hline \multirow{2}{*}{\multicolumn{5}{|c|}{ Direct }} \\
\hline & & & & \\
\hline 0.50000000000000 & 0.00000000000000 & 0.25000000000000 & $\mathrm{Al}$ & (2b) \\
\hline 0.00000000000000 & 0.50000000000000 & 0.75000000000000 & $\mathrm{Al}$ & $(2 b)$ \\
\hline 0.23340000000000 & 0.27610000000000 & 0.62950000000000 & $\mathrm{Cl}$ & $(8 n)$ \\
\hline-0.23340000000000 & -0.27610000000000 & 0.62950000000000 & $\mathrm{Cl}$ & $(8 \mathrm{n})$ \\
\hline 0.27610000000000 & -0.23340000000000 & -0.62950000000000 & $\mathrm{Cl}$ & $(8 n)$ \\
\hline-0.27610000000000 & 0.23340000000000 & -0.62950000000000 & $\mathrm{Cl}$ & $(8 n)$ \\
\hline-0.23340000000000 & 0.27610000000000 & -0.12950000000000 & $\mathrm{Cl}$ & $(8 n)$ \\
\hline 0.23340000000000 & -0.27610000000000 & -0.12950000000000 & $\mathrm{Cl}$ & $(8 n)$ \\
\hline-0.27610000000000 & -0.23340000000000 & 1.12950000000000 & $\mathrm{Cl}$ & $(8 n)$ \\
\hline 0.27610000000000 & 0.23340000000000 & 1.12950000000000 & $\mathrm{Cl}$ & $(8 n)$ \\
\hline 0.00000000000000 & 0.00000000000000 & 0.00000000000000 & $\mathrm{Cu}$ & (2e) \\
\hline 0.00000000000000 & 0.00000000000000 & 0.50000000000000 & $\mathrm{Cu}$ & $(2 \mathrm{e})$ \\
\hline
\end{tabular}

Akermanite $\left(\mathrm{Ca}_{2} \mathrm{MgSi}_{2} \mathrm{O}_{7}, S 5_{3}\right)$ : A2BC7D2_tP24_113_e_a_cef_e - CIF

\# CIF fil

data findsym-output

audit_creation_method FINDSYM

chemical name mineral 'Akermanite'

che $\mathrm{Mg}$ O7 $\mathrm{Si} 2$

loop

publ_author_name

H. Yang '

R. M. Hazen

R. T. Downs'

'L. W. Finger

journal_name_full_name

Physics and Chemistry of Minerals

journal_volume 24

journal_page_first 510

journal_page_first 510
journal_page_last 519

publ_Section title

Structural change associated with the incommensurate-normal phase $\hookrightarrow$ transition in akermanite, $\mathrm{Ca}_{-}\{2\} \$ M g S i \$_{-}\{2\} \$ O \$_{-}\{7\} \$$, at high $\hookrightarrow$ pressure

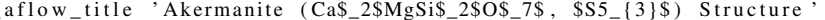
aflow_proto 'A2BC7D2_tP24_113_e_a_cef_e'

aflow_params $\left., a, c / a, z_{-}\{2\}, x_{-}\{3\}, z_{-}\{3\}, x_{-}\{4\}, z_{-}\{4\}, x_{-}\{5\}, z_{-}\{5\}, x_{-}\{6\}, y_{-}\right\}$ $\hookrightarrow 6\}, z_{-}\{6\}$ '

aflow params values $, 7.8338,0.639306594501,0.8201,0.8324,0.4935,0.6407$, $\hookrightarrow 0.7471,0.6396,0.0642,0.0798,0.1862,0.7856$

, aflow Strukturbericht $\$ \$ 55$

- aflow_Parson,

symmetry_space_group_name_H-M "P $-4 \quad 21 \mathrm{~m} "$

symmetry_Int_Tables_number 113

cell_length_a $\quad 7.83380$

cell_length_b $\quad 7.83380$

_cell_length_c $\quad 5.00820$ 
_cell_angle_alpha 90.00000

loop

_space_group_symop_id

_space_group_symop_operation_xyz

$1 \mathrm{x}, \mathrm{y}, \mathrm{z}$

$2 \mathrm{x}+1 / 2,-\mathrm{y}+1 / 2,-\mathrm{z}$

$3-x+1 / 2, y+1 / 2,-z$

$4-\mathrm{x},-\mathrm{y}, \mathrm{z}$

$6 \mathrm{y},-\mathrm{x},-\mathrm{z}$

$8-y+1 / 2,-x+1 / 2, z$

loop

atom site label

_atom_site_type_symbol

_atom_site_symmetry_multiplicity

atom_site_Wyckoff_label

atom_site_fract_x

atom_site_fract_y

atom_site_fract_z

atom_site_occupancy

$\begin{array}{lllllll}\mathrm{Mg} 1 \mathrm{Mg} & 2 & \mathrm{a} & 0.00000 & 0.00000 & 0.00000 & 1.00000\end{array}$

$\begin{array}{llllllllll}\mathrm{O} 1 & \mathrm{O} & 2 & \mathrm{c} & 0.00000 & 0.50000 & 0.82010 & 1.00000\end{array}$

$\begin{array}{lllllllll}\mathrm{Ca} 1 \mathrm{Ca} & 4 & \mathrm{e} & 0.83240 & 0.33240 & 0.49350 & 1.00000\end{array}$

$\begin{array}{lllllllll}\mathrm{O} 2 & \mathrm{O} & 4 & \text { e } & 0.64070 & 0.14070 & 0.74710 & 1.00000\end{array}$

$\begin{array}{llllllllll}\mathrm{S} i 1 & \mathrm{Si} & 4 & \mathrm{e} & 0.63960 & 0.13960 & 0.06420 & 1.00000\end{array}$

$\begin{array}{llllllllll}\mathrm{O} 3 & \mathrm{O} & 8 & \mathrm{f} & 0.07980 & 0.18620 & 0.78560 & 1.00000\end{array}$

Akermanite $\left(\mathrm{Ca}_{2} \mathrm{MgSi}_{2} \mathrm{O}_{7}, S 5_{3}\right)$ : A2BC7D2_tP24_113_e_a_cef_e - POSCAR

\begin{tabular}{|c|c|c|c|c|}
\hline \multirow{2}{*}{\multicolumn{5}{|c|}{ A2BC7D2_tP24_113_e_a_cef_e \& a,c/a, z2, x3, z3, x4, z4, x5, z5, x6,y6, z6 -- }} \\
\hline & & & & \\
\hline$\hookrightarrow \quad 0.6396,0.06$ & $42,0.0798,0.1862,0$. & $7856 \&$ \& $-42\{11\} \mathrm{m} \mathrm{D}$ & $2 \mathrm{~d}\} 1$ & 3\} $\# 113$ \\
\hline$\hookrightarrow \quad\left(\operatorname{ace}^{\wedge} 3 \mathrm{f}\right) \&$ & tP24\&\$S5_\{3\}\$\& & $\mathrm{a} 2 \mathrm{MgSi} 2 \mathrm{O} 7$ \& Akerma & ite $\&$ & H. Yang \\
\hline & Chem. Miner. 24, & $510-519$ & & \\
\hline 1.00000000000000 & & & & \\
\hline 7.83380000000000 & 0.00000000000000 & 0.00000000000000 & & \\
\hline 0.00000000000000 & 7.83380000000000 & 0.00000000000000 & & \\
\hline 0.00000000000000 & 0.00000000000000 & 5.00820000000000 & & \\
\hline $\mathrm{Mg}$ & $\mathrm{Si}$ & & & \\
\hline 14 & 4 & & & \\
\hline Direct & & & & \\
\hline 0.83240000000000 & 1.33240000000000 & 0.49350000000000 & $\mathrm{Ca}$ & $(4 \mathrm{e})$ \\
\hline-0.83240000000000 & -0.33240000000000 & 0.49350000000000 & $\mathrm{Ca}$ & $(4 \mathrm{e})$ \\
\hline 1.33240000000000 & -0.83240000000000 & -0.49350000000000 & $\mathrm{Ca}$ & (4e) \\
\hline-0.33240000000000 & 0.83240000000000 & -0.49350000000000 & $\mathrm{Ca}$ & $(4 \mathrm{e})$ \\
\hline 0.00000000000000 & 0.00000000000000 & 0.00000000000000 & $\mathrm{Mg}$ & (2a) \\
\hline 0.50000000000000 & 0.50000000000000 & 0.00000000000000 & $\mathrm{Mg}$ & (2a) \\
\hline 0.00000000000000 & 0.50000000000000 & 0.82010000000000 & $\mathrm{O}$ & $(2 \mathrm{c})$ \\
\hline 0.50000000000000 & 0.00000000000000 & -0.82010000000000 & $\mathrm{O}$ & $(2 \mathrm{c})$ \\
\hline 0.64070000000000 & 1.14070000000000 & 0.74710000000000 & $\mathrm{O}$ & (4e) \\
\hline-0.64070000000000 & -0.14070000000000 & 0.74710000000000 & $\mathrm{O}$ & (4e) \\
\hline 1.14070000000000 & -0.64070000000000 & -0.74710000000000 & $\mathrm{O}$ & $(4 \mathrm{e})$ \\
\hline-0.14070000000000 & 0.64070000000000 & -0.74710000000000 & $\mathrm{O}$ & $(4 \mathrm{e})$ \\
\hline 0.07980000000000 & 0.18620000000000 & 0.78560000000000 & $\mathrm{O}$ & (8f) \\
\hline-0.07980000000000 & -0.18620000000000 & 0.78560000000000 & $\mathrm{O}$ & (8f) \\
\hline 0.18620000000000 & -0.07980000000000 & -0.78560000000000 & $\mathrm{O}$ & (8f) \\
\hline-0.18620000000000 & 0.07980000000000 & -0.78560000000000 & $\mathrm{O}$ & (8f) \\
\hline 0.42020000000000 & 0.68620000000000 & -0.78560000000000 & $\mathrm{O}$ & (8f) \\
\hline 0.57980000000000 & 0.31380000000000 & -0.78560000000000 & $\mathrm{O}$ & (8f) \\
\hline 0.31380000000000 & 0.42020000000000 & 0.78560000000000 & $\mathrm{O}$ & (8f) \\
\hline 0.68620000000000 & 0.57980000000000 & 0.78560000000000 & $\mathrm{O}$ & (8f) \\
\hline 0.63960000000000 & 1.13960000000000 & 0.06420000000000 & $\mathrm{Si}$ & (4e) \\
\hline-0.63960000000000 & -0.13960000000000 & 0.06420000000000 & $\mathrm{Si}$ & (4e) \\
\hline 1.13960000000000 & -0.63960000000000 & -0.06420000000000 & $\mathrm{Si}$ & (4e) \\
\hline-0.13960000000000 & 0.63960000000000 & -0.06420000000000 & $\mathrm{Si}$ & $(4 \mathrm{e})$ \\
\hline
\end{tabular}

$\mathrm{SeO}_{3}$ : A3B_tP32_114_3e_e - CIF

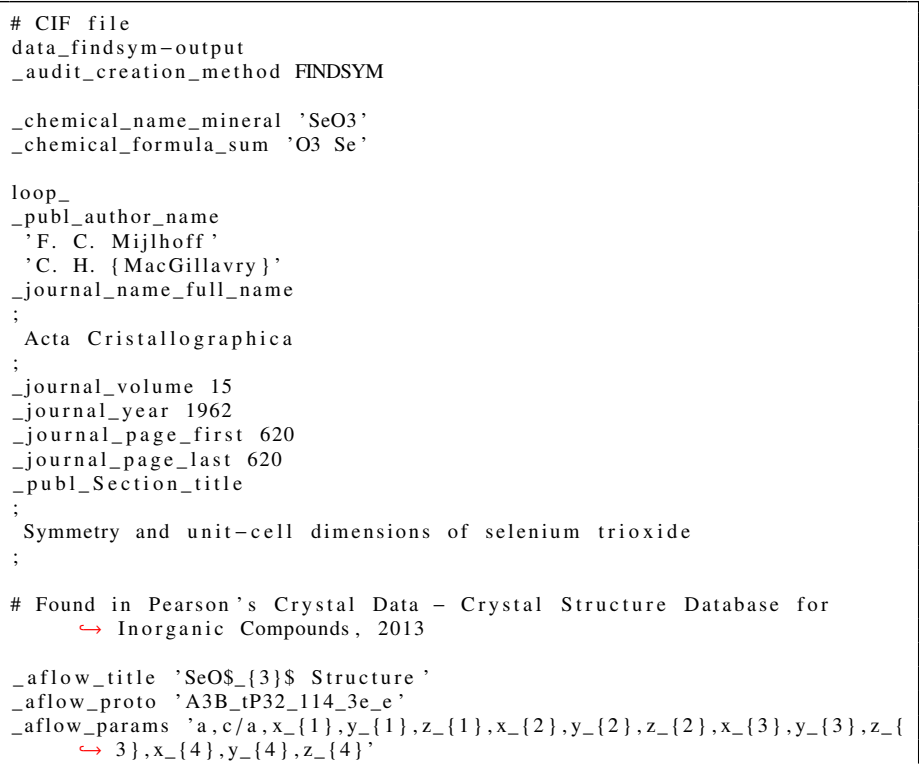

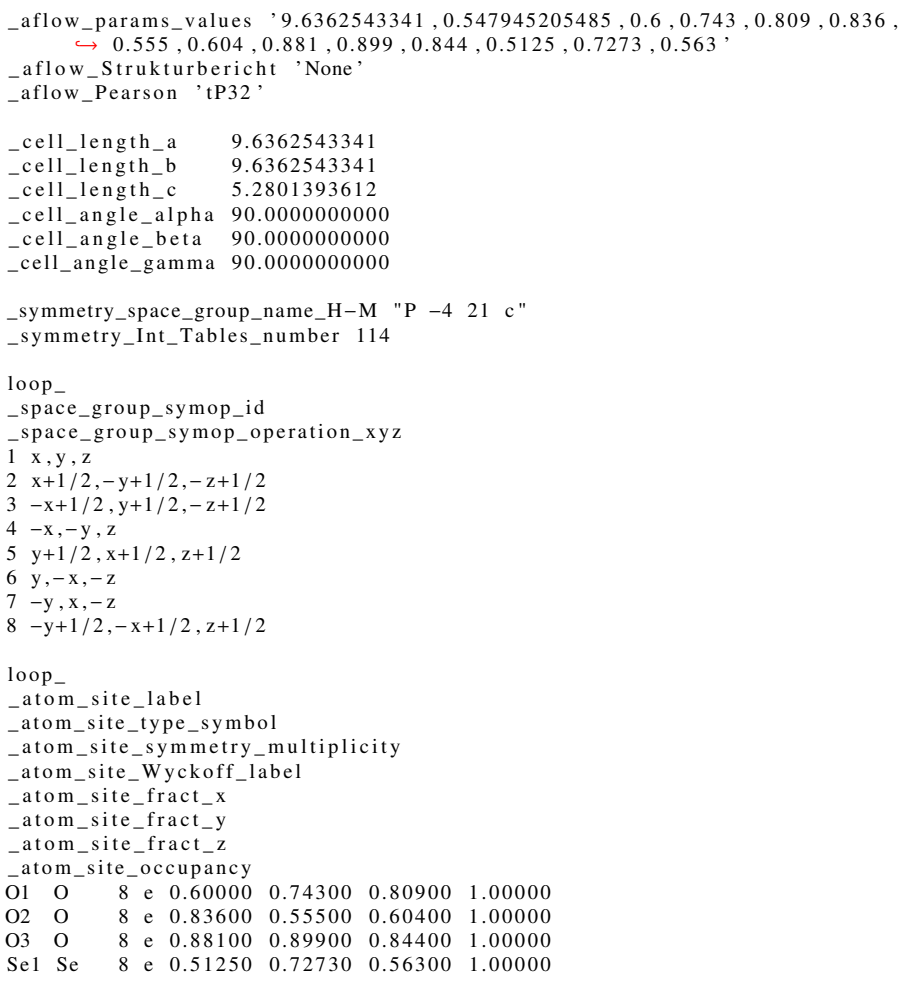

$\mathrm{SeO}_{3}$ : A3B_tP32_114_3e_e - POSCAR

\begin{tabular}{|c|c|c|c|c|}
\hline \multicolumn{5}{|c|}{ 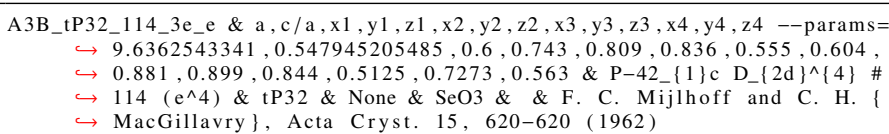 } \\
\hline 1.00000000000000 & & & & \\
\hline 9.63625433410000 & 0.00000000000000 & 0.00000000000000 & & \\
\hline \multirow{2}{*}{$\begin{array}{l}0.00000000000000 \\
0.00000000000000\end{array}$} & 9.63625433410000 & 0.00000000000000 & & \\
\hline & 0.00000000000000 & 5.28013936120000 & & \\
\hline $\begin{array}{rr}\mathrm{O} & \mathrm{Se} \\
24 & 8\end{array}$ & & & & \\
\hline \multicolumn{5}{|l|}{ Direct } \\
\hline 0.60000000000000 & 0.74300000000000 & 0.80900000000000 & $\mathrm{O}$ & $(8 \mathrm{e})$ \\
\hline-0.60000000000000 & -0.74300000000000 & 0.809000000 & $\mathrm{O}$ & $(8 \mathrm{e})$ \\
\hline 0.74300000000000 & -0.60000000000000 & -0.80900000000000 & $\mathrm{O}$ & $(8 \mathrm{e})$ \\
\hline-0.74300000000000 & 0.60000000000000 & -0.80900000000000 & $\mathrm{O}$ & \\
\hline-0.10000000000000 & 1.24300000000000 & -0.30900000000000 & $\mathrm{O}$ & $(8 \mathrm{e})$ \\
\hline 1.10000000000000 & -0.24 & 000000000 & o & $(8 \mathrm{e})$ \\
\hline-0.24300000000000 & -0.10000000000000 & 1.30900000000000 & o & $(8 \mathrm{e})$ \\
\hline 1.24300000000000 & 1.10000000000000 & 1.30900000000000 & $\mathrm{O}$ & $(8 \mathrm{e})$ \\
\hline 0.83600000000000 & 0.55500000000000 & 0.60400000000000 & $\mathrm{O}$ & $(8 \mathrm{e})$ \\
\hline-0.83600000000000 & -0.55500000000000 & 0.60400000000000 & $\mathrm{O}$ & $(8 \mathrm{e})$ \\
\hline 0.55500000000000 & -0.83600000000000 & -0.60400000000000 & $\mathrm{O}$ & $(8 \mathrm{e})$ \\
\hline-0.55500000000000 & 0.83600000000000 & -0.60400000000000 & $\mathrm{O}$ & $(8 \mathrm{e})$ \\
\hline-0.33600000000000 & 0000000000 & -0.10400000000000 & $\mathrm{O}$ & $(8 \mathrm{e})$ \\
\hline 1.33600000000000 & -0.05500000000000 & -0.10400000000000 & $\mathrm{O}$ & $(8 \mathrm{e})$ \\
\hline-0.05500000000000 & -0.33600000000000 & 1.10400000000000 & $\mathrm{O}$ & $(8 \mathrm{e})$ \\
\hline 1.05500000000000 & 1.33600000000000 & 1.10400000000000 & $\mathrm{O}$ & $(8 \mathrm{e})$ \\
\hline 0.88100000000000 & 0.89900000000000 & 0.84400000000000 & $\mathrm{O}$ & $(8 \mathrm{e})$ \\
\hline-0.88100000000000 & -0.89900000000000 & 0.84400000000000 & $\mathrm{O}$ & $(8 \mathrm{e})$ \\
\hline 0.89900000000000 & -0.88100000000000 & -0.84400000000000 & $\mathrm{O}$ & $(8 \mathrm{e})$ \\
\hline-0.89900000000000 & 0.88100000000000 & -0.84400000000000 & $\mathrm{O}$ & $(8 \mathrm{e})$ \\
\hline-0.38100000000000 & 1.39900000000000 & -0.34400000000000 & $\mathrm{O}$ & $(8 \mathrm{e})$ \\
\hline 1.38100000000000 & -0.39900000000000 & -0.34400000000000 & $\mathrm{O}$ & $(8 \mathrm{e})$ \\
\hline-0.39900000000000 & -0.38100000000000 & 1.34400000000000 & $\mathrm{O}$ & $(8 \mathrm{e})$ \\
\hline 1.39900000000000 & 1.38100000000000 & 1.34400000000000 & $\mathrm{O}$ & $(8 \mathrm{e})$ \\
\hline 0.51250000000000 & 0.72730000000000 & 0.56300000000000 & $\mathrm{Se}$ & $(8 \mathrm{e})$ \\
\hline-0.51250000000000 & -0.72730000000000 & 0.56300000000000 & $\mathrm{Se}$ & $(8 \mathrm{e})$ \\
\hline 0.72730000000000 & -0.51250000000000 & -0.56300000000000 & $\mathrm{Se}$ & $(8 \mathrm{e})$ \\
\hline-0.72730000000000 & 0.51250000000000 & -0.56300000000000 & $\mathrm{Se}$ & $(8 \mathrm{e})$ \\
\hline-0.01250000000000 & 1.22730000000000 & -0.06300000000000 & e & $(8 \mathrm{e})$ \\
\hline 1.01250000000000 & -0.22730000000000 & -0.06300000000000 & $\mathrm{Se}$ & $(8 \mathrm{e})$ \\
\hline-0.22730000000000 & -0.01250000000000 & 1.06300000000000 & $\mathrm{Se}$ & $(8 \mathrm{e})$ \\
\hline 1.22730000000000 & 1.01250000000000 & 1.06300000000000 & $\mathrm{Se}$ & $(8 \mathrm{e})$ \\
\hline
\end{tabular}

$\mathrm{Pd}_{4} \mathrm{Se}$ : A4B_tP10_114_e_a - CIF

\# CIF file

data findsym-output

audit_creation_method FINDSYM

chemical_name_mineral 'Pd4Se'

chemical_formula_sum ' $\mathrm{Pd} 4 \mathrm{Se}$

loop

publ_author_name

, F. Gr $\{\backslash 0\} n v$,

journal_name_full_name

Acta Chemica Scandinavica 


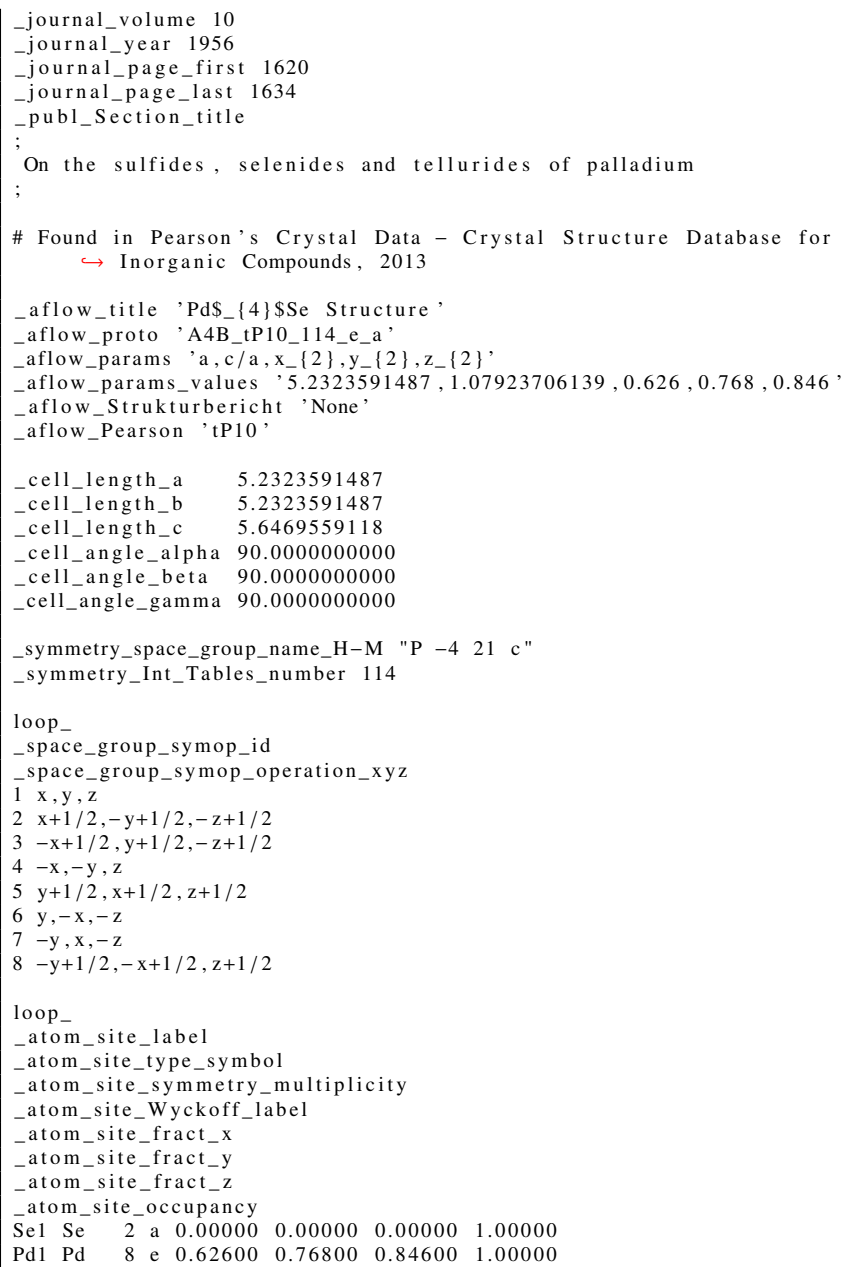

$\mathrm{Pd}_{4} \mathrm{Se}$ : A4B_tP10_114_e_a - POSCAR

\begin{tabular}{|c|c|c|c|c|}
\hline \multirow{4}{*}{\multicolumn{5}{|c|}{ 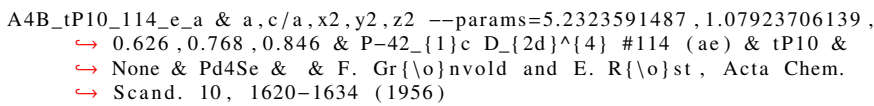 }} \\
\hline & & & & \\
\hline & & & & \\
\hline & & & & \\
\hline \multicolumn{5}{|c|}{1.0000000000000} \\
\hline 5.23235914870000 & 0.00000000000000 & \multicolumn{3}{|l|}{0.00000000000000} \\
\hline 0.00000000000000 & \multirow{2}{*}{$\begin{array}{l}5.23235914870000 \\
0.00000000000000\end{array}$} & \multicolumn{2}{|l|}{0.00000000000000} & \\
\hline 0.00000000000000 & & 5.64695591180000 & & \\
\hline $\mathrm{Pd}$ & & & & \\
\hline 8 & & & & \\
\hline \multicolumn{5}{|l|}{ Direct } \\
\hline 0.62600000000000 & 0.76800000000000 & 0.84600000000000 & $\mathrm{Pd}$ & $(8 \mathrm{e})$ \\
\hline-0.62600000000000 & -0.76800000000000 & 0.84600000000000 & & \\
\hline 0.76800000000000 & -0.62600000000000 & -0.84600000000000 & $P$ & \\
\hline-0.76800000000000 & 0.62600000000000 & -0.84600000000000 & & \\
\hline-0.12600000000000 & 1.26800000000000 & -0.34600000000000 & $\mathrm{Pd}$ & $(8 \mathrm{e})$ \\
\hline 1.12600000000000 & -0.26800000000000 & -0.34600000000000 & $\mathrm{Pd}$ & \\
\hline-0.26800000000000 & -0.12600000000000 & 1.34600000000000 & $\mathrm{Pd}$ & $(8 \mathrm{e})$ \\
\hline 1.26800000000000 & 1.12600000000000 & 1.34600000000000 & $\mathrm{Pd}$ & \\
\hline 0.00000000000000 & 0.00000000000000 & 0.00000000000000 & $\mathrm{Se}$ & (2a) \\
\hline 0.50000000000000 & 0.50000000000000 & 0.50000000000000 & $\mathrm{Se}$ & (2a) \\
\hline
\end{tabular}

$\mathrm{Rh}_{3} \mathrm{P}_{2}$ : A2B3_tP5_115_g_ag - CIF

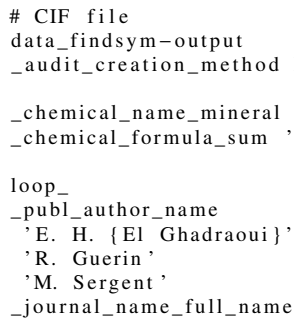

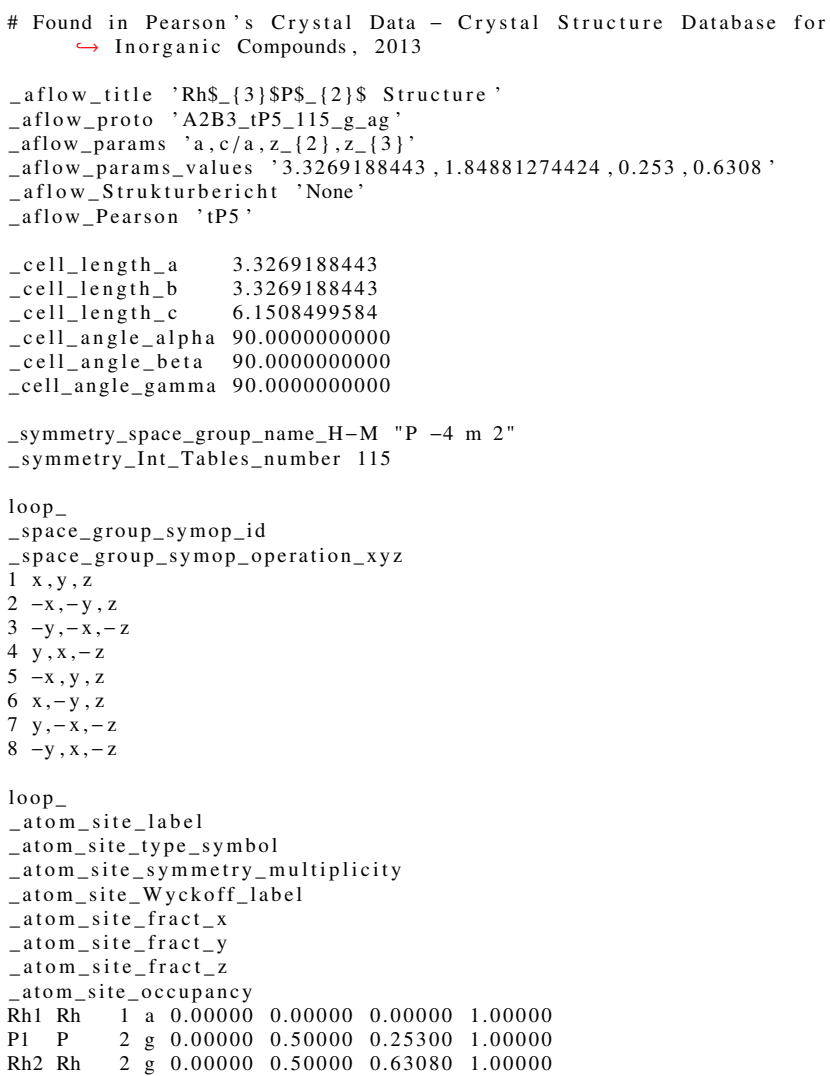

$\mathrm{Rh}_{3} \mathrm{P}_{2}$ : A2B3_tP5_115_g_ag - POSCAR

\begin{tabular}{|c|c|c|c|c|}
\hline $\begin{aligned} \text { A2B3_tP5_115_g_ag \& } & \\
& \hookrightarrow 0.253,0.630 \\
& \hookrightarrow \text { Rh3P2 \& \& } \\
& \hookrightarrow \text { Acta Crysta }\end{aligned}$ & $\begin{array}{l}, \mathrm{c} / \mathrm{a}, \mathrm{z} 2, \mathrm{z} 3-- \text { param } \\
\& \text { P-4m2 D_ }\{2 \mathrm{~d}\}^{\wedge}\{5 \\
\text { H. }\{\text { El Ghadraoui } \\
\text { ogr. C } 39,1493-1\end{array}$ & $\begin{array}{l}=3.3269188443,1.84 \\
\# 115\left(\mathrm{ag}^{\wedge} 2\right) \& \text { tP } 5 \\
\text { and R. Guerin and } \\
94(1983)\end{array}$ & & $\begin{array}{l}\& \\
\text { gent }\end{array}$ \\
\hline 1.00000000000000 & & & & \\
\hline 3.32691884430000 & 0.00000000000000 & 0.00000000000000 & & \\
\hline 0.00000000000000 & 3.32691884430000 & 0.00000000000000 & & \\
\hline 0.00000000000000 & 0.00000000000000 & 6.15084995840000 & & \\
\hline $\mathrm{Rh}$ & & & & \\
\hline 2 & & & & \\
\hline Direct & & & & \\
\hline 0.00000000000000 & 0.50000000000000 & 0.25300000000000 & $\mathrm{P}$ & $(2 \mathrm{~g})$ \\
\hline 0.50000000000000 & 0.00000000000000 & -0.25300000000000 & $\mathrm{P}$ & $(2 \mathrm{~g})$ \\
\hline 0.00000000000000 & 0.00000000000000 & 0.00000000000000 & $\mathrm{Rh}$ & (1a) \\
\hline 0.00000000000000 & 0.50000000000000 & 0.63080000000000 & $\mathrm{Rh}$ & $(2 g)$ \\
\hline 0.50000000000000 & 0.00000000000000 & -0.63080000000000 & $\mathrm{Rh}$ & $(2 \mathrm{~g})$ \\
\hline
\end{tabular}

HgI 2 : AB2_tP12_115_j_egi - CIF

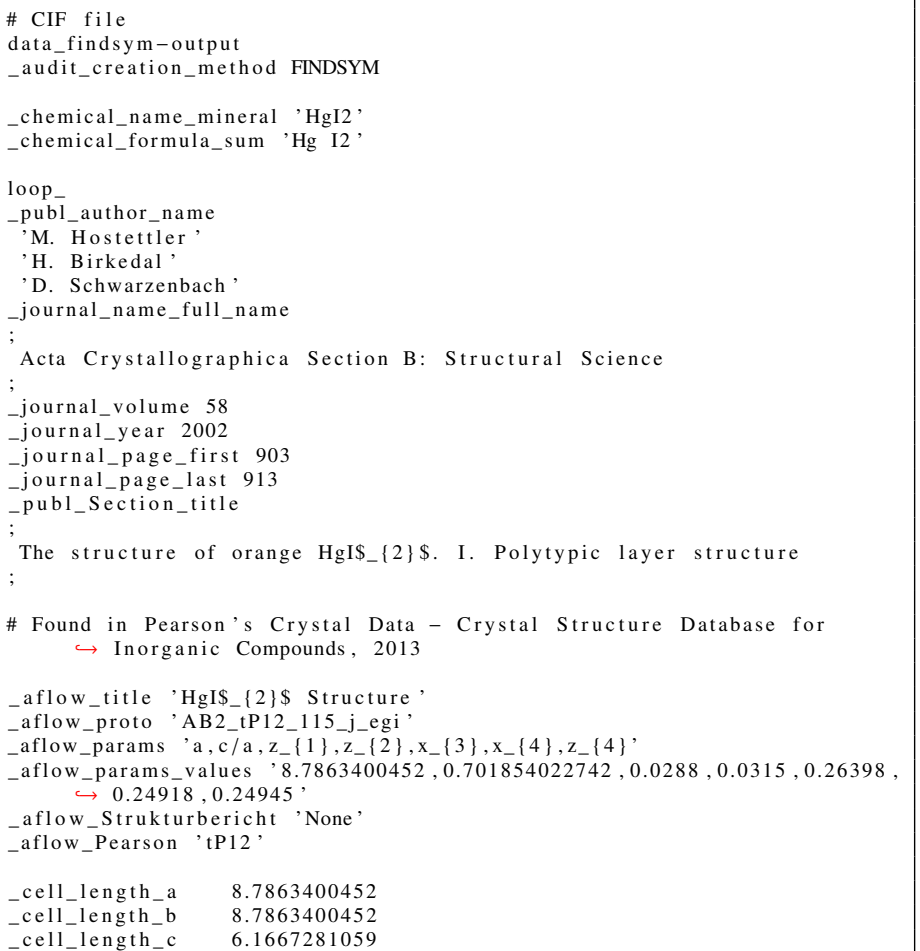


cell_angle_alpha 90.0000000000

cell_angle_beta 90.0000000000

_cell_angle_gamma 90.0000000000

_symmetry_space_group_name_H-M "P -4 m 2"

_symmetry_Int_Tables_number 115

loop

_space_group_symop_id

_space_group_symop_operation_xyz

$1 \mathrm{x}, \mathrm{y}, \mathrm{z}$

$2-\mathrm{x},-\mathrm{y}, \mathrm{z}$

$4, y, x,-z$

$4-y, x,-z$
$5-x, y, z$

$6 x,-y, z$

$7 \mathrm{y},-\mathrm{x},-\mathrm{z}$

$8-\mathrm{y}, \mathrm{x},-\mathrm{z}$

loop

_atom_site_labe

atom_site_type_symbol

atom_site_symmetry_multiplicity

atom_site_Wyckoff_label

atom_site_fract_x

atom_site_fract_y

atom_site_fract_z

$\begin{array}{lllllll}\mathrm{I}^{2} & \mathrm{I} & 2 \mathrm{e} & 0.00000 & 0.00000 & 0.02880 & 1.00000\end{array}$

$\begin{array}{llllllllll}\text { I2 } & \text { I } & 2 & \text { g } & 0.00000 & 0.50000 & 0.03150 & 1.00000\end{array}$

$\begin{array}{llllllll}13 & \text { I } & 4 & \mathrm{i} & 0.26398 & 0.26398 & 0.50000 & 1.00000\end{array}$

$\begin{array}{llllllll}\mathrm{Hg} 1 \mathrm{Hg} & 4 & \mathrm{j} & 0.24918 & 0.00000 & 0.24945 & 1.00000\end{array}$

$\mathrm{HgI}_{2}$ : AB2_tP12_115_j_egi - POSCAR

$\mathrm{AB} 2$ tP12_115_j_egi \& a, c/a, z1, z2, x3, x4, z4 --params $=8.7863400452$

$\hookrightarrow 0.701854022742,0.0288,0.0315,0.26398,0.24918,0.24945 \& \mathrm{P}-4 \mathrm{~m} 2 \mathrm{D}$

$\hookrightarrow\{2 \mathrm{~d}\}^{\wedge}\{5\} \# 115(\mathrm{egij}) \& \operatorname{tP} 12$ \& None \& HgI2 \& \& M. Hostettler

$\hookrightarrow$ and H. Birkedal and D. Schwarzenbach, Acta Crystallogr. Sect. B

$\hookrightarrow$ Struct. Sci. 58, 903-913(2002)

1.00000000000000

$\begin{array}{lll}8.78634004520000 & 0.00000000000000 & 0.00000000000000\end{array}$

$\begin{array}{lll}0.00000000000000 & 8.78634004520000 & 0.00000000000000 \\ 0.00000000000000 & 0.00000000000000 & 6.16672810590000\end{array}$

$\begin{array}{rr}\mathrm{Hg} & \mathrm{I} \\ 4 & 8\end{array}$

Direct

$0.24918000000000 \quad 0.00000000000000 \quad 0.24945000000000$

$-0.24918000000000$

0.00000000000000

0.00000000000000

0.00000000000000

0.00000000000000

0.00000000000000

0.50000000000000

0.5639800000000

$-0.263980000000$

0.26398000000000

$-0.26398000000000$

(2)

$-0.24918000000000$ 0.00000000000000

0.00000000000000

0.50000000000000

0.00000000000000

0.26398000000000

$-0.26398000000000$

$-0.26398000000000$

0.26398000000000

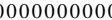
$-0.02880000000000$ 0.03150000000000 $-0.0315000000000$ 0.5000000000000

0.5000000000000

0.500000000000000

0.50000000000000

$\begin{aligned} \mathrm{Hg} & (4 \mathrm{j}) \\ \mathrm{Hg} & (4 \mathrm{j}) \\ \mathrm{Hg} & (4 \mathrm{j}) \\ \mathrm{Hg} & (4 \mathrm{j}) \\ \mathrm{I} & (2 \mathrm{e}) \\ \mathrm{I} & (2 \mathrm{e}) \\ \mathrm{I} & (2 \mathrm{~g}) \\ \mathrm{I} & (2 \mathrm{~g}) \\ \mathrm{I} & (4 \mathrm{i}) \\ \mathrm{I} & (4 \mathrm{i}) \\ \mathrm{I} & (4 \mathrm{i}) \\ \mathrm{I} & (4 \mathrm{i})\end{aligned}$

$\mathrm{Ru}_{2} \mathrm{Sn}_{3}$ : A2B3_tP20_116_bci_fj - CIF

\section{\# CIF file}

data_findsym-output

audit_creation_method FINDSYM

chemical name mineral 'Ru2Sn3'

chemical formula_sum , Ru2 Sn3,

loop

_publ_author_name

'O. Schwomma'

'H. Nowotny',

journal_name_full_name

Monatshefte f $\{\backslash " u\} r$ Chemie - Chemical Monthly

_journal_volume 95

-journal year

journal page first 1538

journal_page_last 1543

publ_Section_title

Untersuchungen im System: Ru--Sn

\# Found in Pearson's Crystal Data - Crystal Structure Database for $\hookrightarrow$ Inorganic Compounds, 2013

aflow_title 'Ru\$_ $\{2\} \$ S n \$_{-}\{3\} \$$ Structure,

aflow_proto 'A2B3_tP20_116_bci_fj

aflow_params 'a, c/a, $x_{-}\{3\}, z_{-}\{4\}, x_{-}\{5\}, y_{-}\{5\}, z_{-}\{5\}$ '

aflow_params_values ' $6.1720115185,1.606448477,0.177,0.625,0.655,0.216$, $\hookrightarrow 0.582$

aflow Strukturbericht 'None'

aflow_Pearson 'tP20,

cell_length_a 6.1720115185

cell_length_b 6.1720115185

cell_length_c 9.9150185039

cell_angle_alpha 90.0000000000

_cell_angle_beta 90.0000000000

cell_angle_gamma 90.0000000000

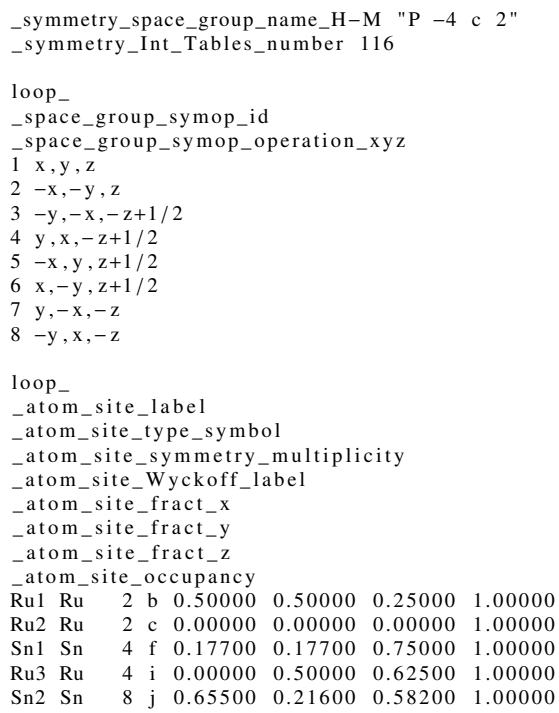

$\begin{array}{lll}6.17201151850000 & 0.00000000000000 & 0.00000000000000\end{array}$

$0.00000000000000-6.17201151850000-0.00000000000000$

$\begin{array}{lll}0.00000000000000 & 6.17201151850000 & 0.00000000000000 \\ 0.00000000000000 & 0.00000000000000 & 9.91501850390000\end{array}$

$\mathrm{Ru} \quad \mathrm{Sn}$

Direct

0.50000000000000

0.50000000000000

0.00000000000000

0.0000000000000

0.00000000000000

0.50000000000000

0.00000000000000

0.50000000000000

0.17700000000000

$-0.17700000000000$

0.17700000000000

$-0.17700000000000$

0.65500000000000

$-0.65500000000000$

0.21600000000000

$-0.21600000000000$

0.65500000000000

$-0.65500000000000$

0.21600000000000
-0.21600000000000

0.50000000000000

0.50000000000000

0.00000000000000

0.00000000000000

0.50000000000000

0.00000000000000

0.50000000000000

0.00000000000000

$-0.17700000000000$

$-0.17700000000000$

0.17700000000000

0.21600000000000

$-0.2160000000000$

$-0.65500000000000$

0.65500000000000

$-0.21600000000000$

0.21600000000000

0.65500000000000

0.25000000000000

0.75000000000000

0.00000000000000

0.50000000000000

0.62500000000000

0.62500000000000

.12500000000000

0.12500000000000

0.75000000000000

0.75000000000000

0.25000000000000

0.5820000000000

0.58200000000000

$-0.58200000000000$

$-0.58200000000000$

1.08200000000000

1.08200000000000

$-0.08200000000000$

$-0.08200000000000$

Ru (2b)

$\mathrm{Ru} \quad(2 \mathrm{~b}$

$\mathrm{Ru}$ (2c)

$\mathrm{Ru} \quad(2 \mathrm{c})$

$\beta-\mathrm{Bi}_{2} \mathrm{O}_{3}$ (High-temperature): $\mathrm{A} 2 \mathrm{~B} 3 \_\mathrm{tP} 20 \_117 \_\mathrm{i} \_$adgh - CIF

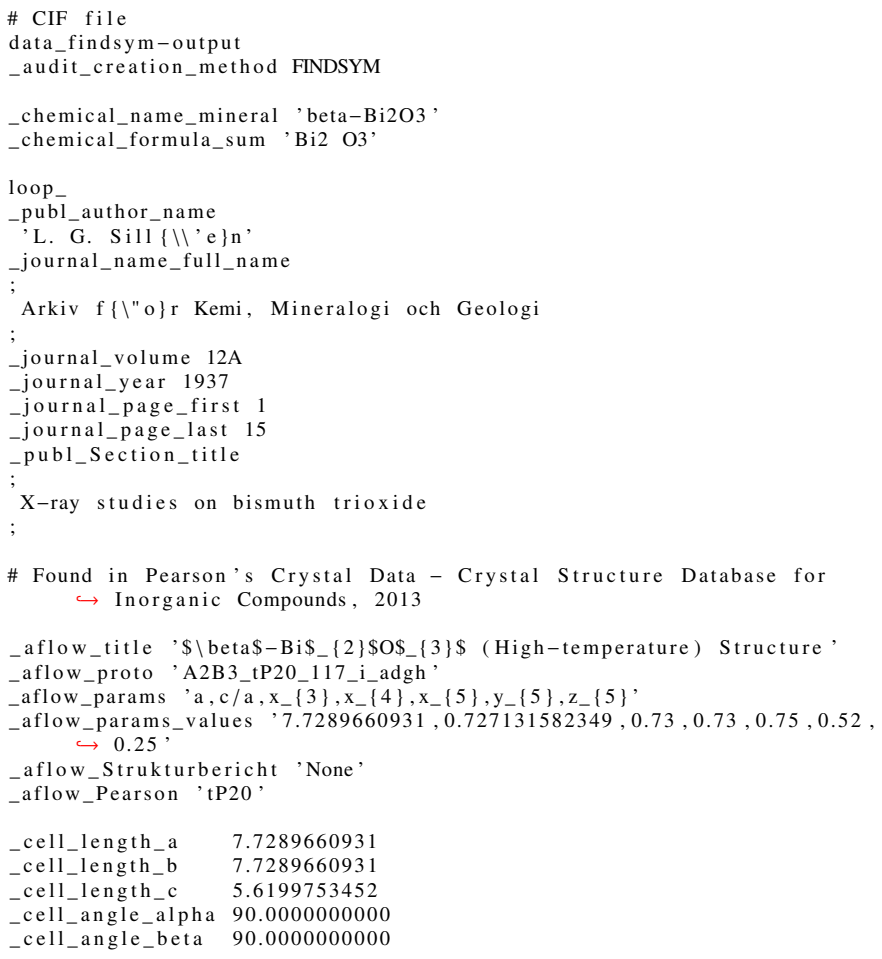




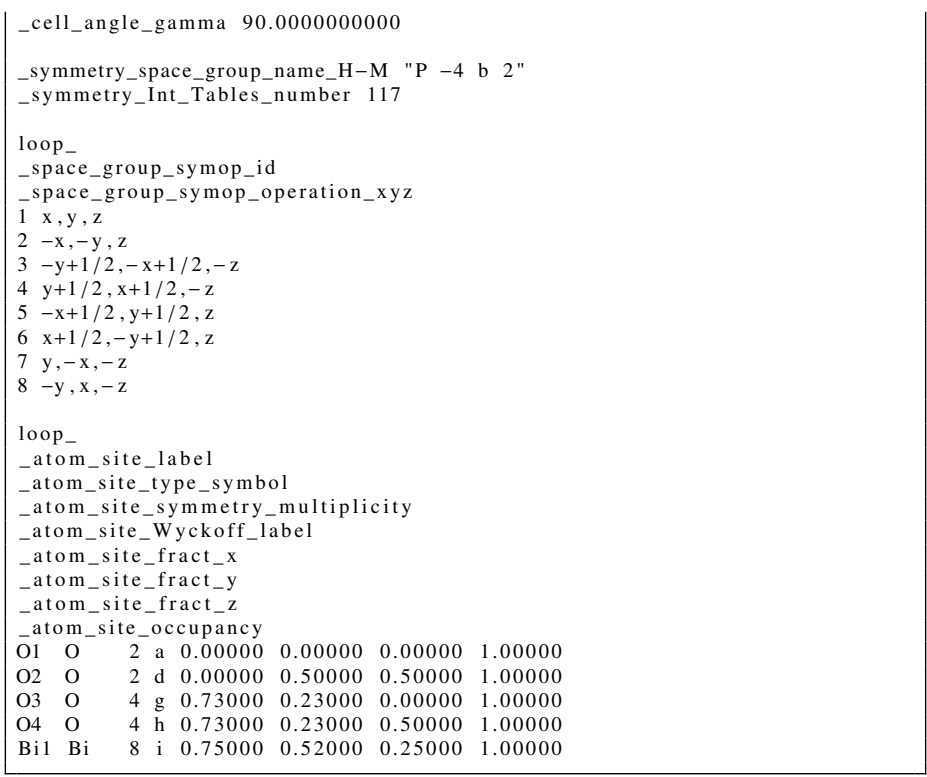

$\beta-\mathrm{Bi}_{2} \mathrm{O}_{3}$ (High-temperature): A2B3_tP20_117_i_adgh - POSCAR

\begin{tabular}{|c|c|c|c|c|}
\hline \multicolumn{5}{|c|}{ 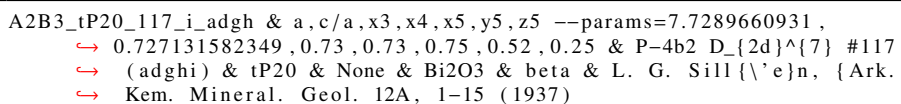 } \\
\hline \multicolumn{5}{|c|}{1.00000000000000} \\
\hline 7.72896609310000 & 0.00000000000000 & 0.00000000000000 & & \\
\hline \multirow{2}{*}{$\begin{array}{l}0.00000000000000 \\
0.00000000000000\end{array}$} & 7.72896609310000 & 0.00000000000000 & & \\
\hline & 0.00000000000000 & 5.61997534520000 & & \\
\hline $\mathrm{Bi}$ & & & & \\
\hline 12 & & & & \\
\hline \multicolumn{5}{|l|}{ Direct } \\
\hline 0.75000000000000 & 0.52000000000000 & 0.25000000000000 & & ( $8 \mathrm{i})$ \\
\hline-0.75000000000000 & -0.52000000000000 & 0.25000000000000 & $\mathrm{Bi}$ & $(8 \mathrm{i})$ \\
\hline 0.52000000000000 & -0.75000000000000 & -0.25000000000000 & $\mathrm{Bi}$ & $(8 \mathrm{i})$ \\
\hline-0.52000000000000 & 0.75000000000000 & -0.25000000000000 & $\mathrm{Bi}$ & $(8 \mathrm{i})$ \\
\hline 1.25000000000000 & -0.02000000000000 & 0.25000000000000 & $\mathrm{Bi}$ & ( $8 \mathrm{i})$ \\
\hline-0.25000000000000 & 1.02000000000000 & 0.25000000000000 & $\mathrm{Bi}$ & ( $8 \mathrm{i})$ \\
\hline 1.02000000000000 & 1.25000000000000 & -0.25000000000000 & $\mathrm{Bi}$ & $(8 \mathrm{i})$ \\
\hline-0.02000000000000 & -0.25000000000000 & -0.25000000000000 & $\mathrm{Bi}$ & $(8 \mathrm{i})$ \\
\hline 0.00000000000000 & 0.00000000000000 & 0.00000000000000 & $\mathrm{O}$ & (2a) \\
\hline 0.50000000000000 & 0.50000000000000 & 0.00000000000000 & $\mathrm{O}$ & (2a) \\
\hline 0.00000000000000 & 0.50000000000000 & 0.50000000000000 & $\mathrm{O}$ & (2d) \\
\hline 0.50000000000000 & 0.00000000000000 & 0.50000000000000 & $\mathrm{O}$ & (2d) \\
\hline 0.73000000000000 & 1.23000000000000 & 0.00000000000000 & $\mathrm{O}$ & $(4 \mathrm{~g})$ \\
\hline-0.73000000000000 & -0.23000000000000 & 0.00000000000000 & $\mathrm{O}$ & $(4 \mathrm{~g})$ \\
\hline 1.23000000000000 & -0.73000000000000 & 0.00000000000000 & $\mathrm{O}$ & $(4 \mathrm{~g})$ \\
\hline-0.23000000000000 & 0.73000000000000 & 0.00000000000000 & $\mathrm{O}$ & $(4 \mathrm{~g})$ \\
\hline 0.73000000000000 & 1.23000000000000 & 0.50000000000000 & $\mathrm{O}$ & $(4 \mathrm{~h})$ \\
\hline-0.73000000000000 & -0.23000000000000 & 0.50000000000000 & $\mathrm{O}$ & $(4 \mathrm{~h})$ \\
\hline 1.23000000000000 & -0.73000000000000 & 0.50000000000000 & $\mathrm{O}$ & (4h) \\
\hline-0.23000000000000 & 0.73000000000000 & 0.50000000000000 & $\mathrm{O}$ & $(4 \mathrm{~h})$ \\
\hline
\end{tabular}

RuIn 3 : A3B_tP16_118_ei_f - CIF

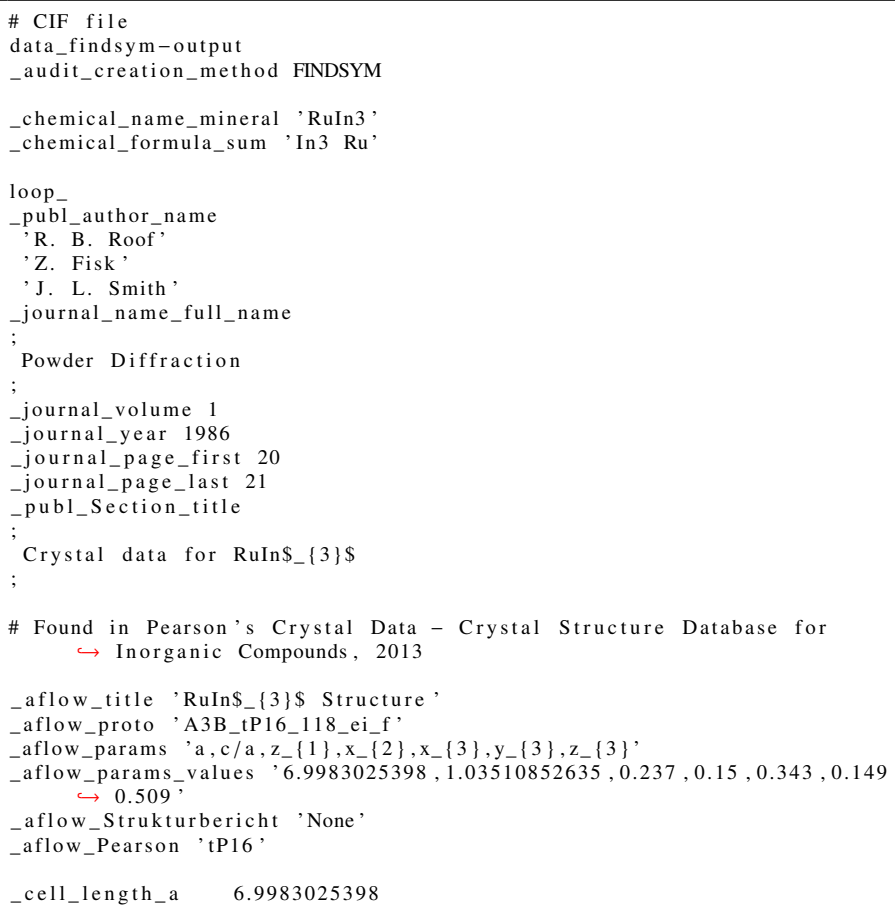

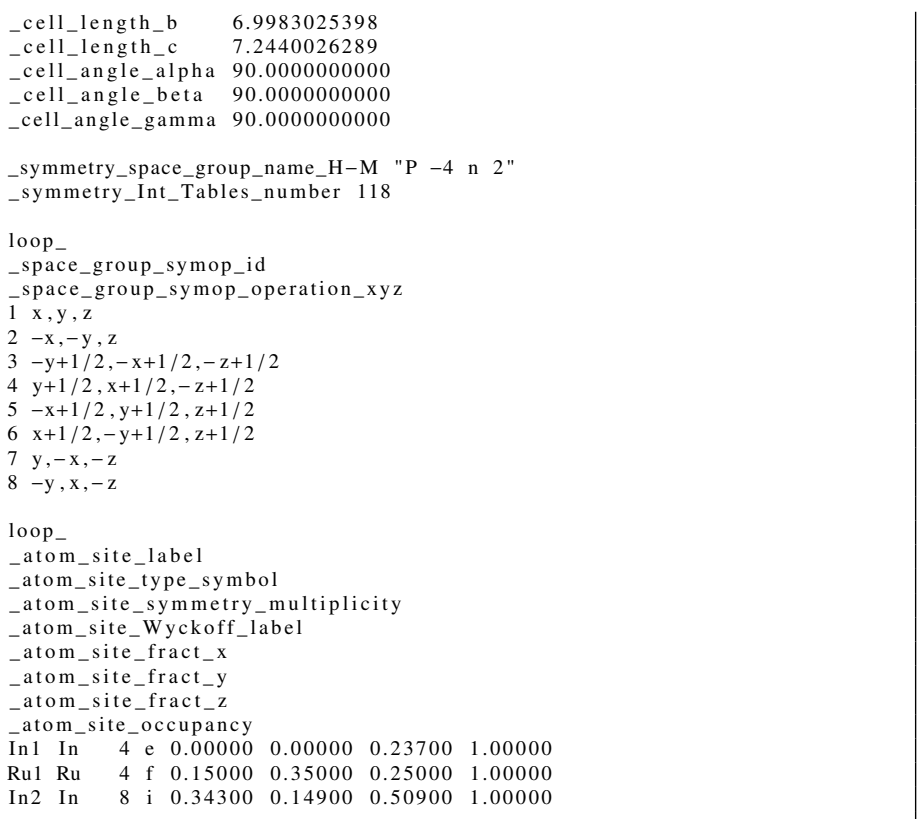

RuIn 3 : A3B_tP16_118_ei_f - POSCAR

\begin{tabular}{|c|c|c|c|c|}
\hline \multicolumn{5}{|c|}{ 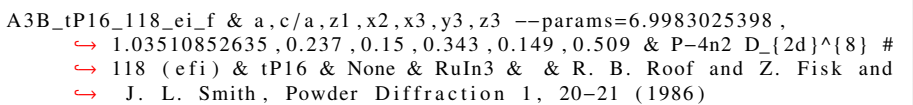 } \\
\hline \multicolumn{5}{|c|}{1.00000000000000} \\
\hline 6.99830253980000 & 0.00000000000000 & 0.00000000000000 & & \\
\hline 0.00000000000000 & 6.99830253980000 & 0.00000000000000 & & \\
\hline 0.00000000000000 & 0.00000000000000 & & & \\
\hline In & & & & \\
\hline 12 & & & & \\
\hline \multicolumn{5}{|l|}{ Direct } \\
\hline 0.000000000 & 0 & 0.23700 & In & $(4 \mathrm{e})$ \\
\hline 0.00000000000000 & 0.00000000000000 & -0.23700000000000 & In & $(4 \mathrm{e})$ \\
\hline 0.50000000000000 & & 0.73 & & \\
\hline 0.50000000000000 & 0.5 & 0.26 & $\mathrm{I}_{1}$ & $(4 \mathrm{e})$ \\
\hline 0.34300000000000 & & 0.50 & & ( $8 \mathrm{i})$ \\
\hline-0.34300000000000 & -0.1 & 0.50 & In & i) \\
\hline 0.14900000000000 & -0.3 & -0 & & \\
\hline-0.14900000000000 & 0000 & -0.50 & In & ) \\
\hline 0.84300000000000 & 0.351 & 1.00 & & \\
\hline 0.15700000000000 & 0 & 1.009 & & (0. \\
\hline 0.64900000000000 & 0.84300000000000 & -0.00900000000000 & In & ( $8 \mathrm{i})$ \\
\hline 0.35100000000000 & 0.15700000000000 & -0.00900000000 & & \\
\hline 0.15000000000000 & 0.35000000000000 & 0.25000000000000 & $\mathrm{R}$ & (4f) \\
\hline-0.15000000000000 & 0.65000000000000 & & & \\
\hline 0.35000000000000 & -0.15000000000000 & 0.75000000000 & $\mathrm{u}$ & (4f) \\
\hline 0.65000000000000 & 0.15000000000000 & 0.75000000000000 & $\mathrm{Ru}$ & (4f) \\
\hline
\end{tabular}

Ir 3 Ga5: A5B3_tP32_118_g2i_aceh - CIF

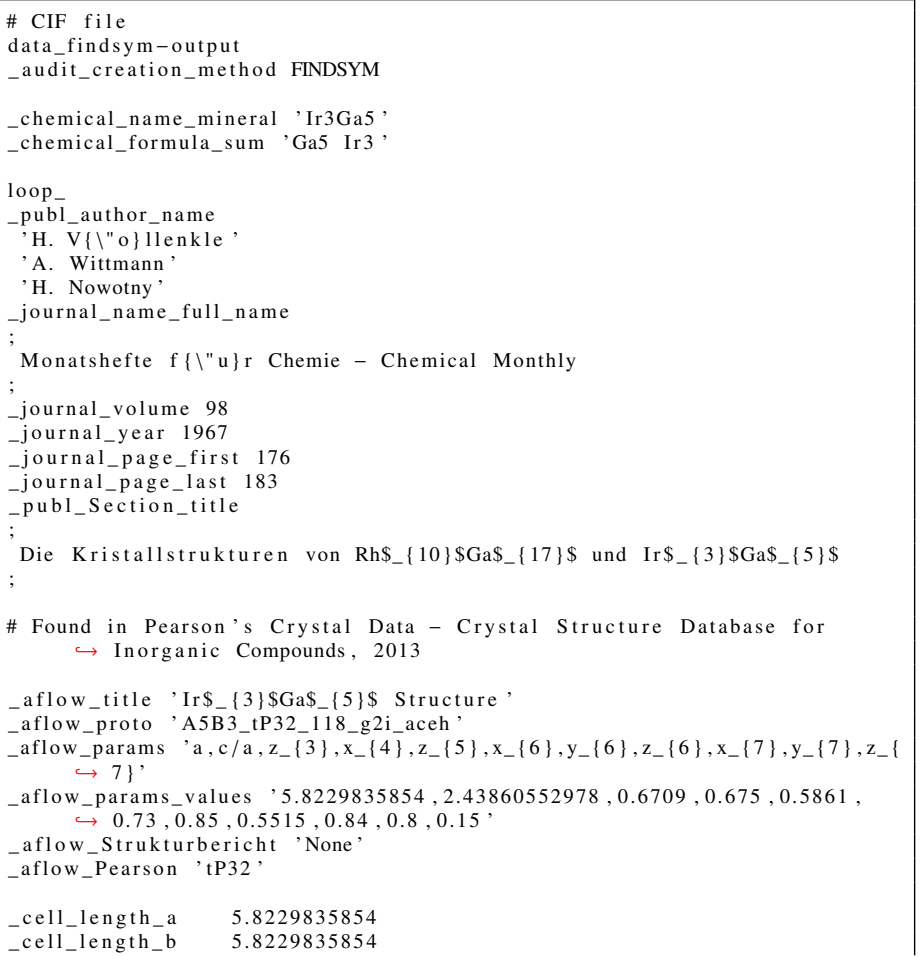


_cell_length_c 14.1999599712

_cell_angle_alpha 90.0000000000

_cell_angle_beta_ 90.000000000

_cell_angle_gamma 90.0000000000

_symmetry_space_group_name_H-M "P $\begin{array}{cccc}-4 & \mathrm{n} & 2 \text { " }\end{array}$

_symmetry_Int_Tables_number 118

loop

_space_group_symop_id

_space_group_symop_operation_xyz

$1 \mathrm{x}, \mathrm{y}, \mathrm{z}$

$2-x,-y$,

$3-\mathrm{y}+1 / 2,-\mathrm{x}+1 / 2,-\mathrm{z}+1 / 2$

$4 \mathrm{y}+1 / 2, \mathrm{x}+1 / 2,-\mathrm{z}+1 / 2$

$5-\mathrm{x}+1 / 2, \mathrm{y}+1 / 2, \mathrm{z}+1 / 2$

$6 \mathrm{x}+1 / 2,-\mathrm{y}+1 / 2, \mathrm{z}+1 / 2$

$7 \mathrm{y},-\mathrm{x},-\mathrm{z}$

$8-\mathrm{y}, \mathrm{x},-\mathrm{z}$

loop

_atom_site_labe

atom_site_type_symbol

atom_site_symmetry_multiplicity

atom_site_Wyckoff_label

atom_site_fract_x

atom_site_fract_y

atom_site_fract_z

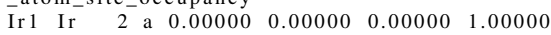

$\begin{array}{lllllllll}\mathrm{Ir} 2 & \mathrm{Ir} & 2 & \mathrm{c} & 0.00000 & 0.50000 & 0.25000 & 1.00000\end{array}$

$\begin{array}{llllllll}\mathrm{Ir} 2 & \mathrm{Ir} & 2 & \mathrm{c} & 0.00000 & 0.50000 & 0.25009 & 1.00000 \\ \mathrm{Ir} 3 & \mathrm{Ir} & 4 & \mathrm{e} & 0.00000 & 0.00000 & 0.67090 & 1.00000\end{array}$

$\begin{array}{llllllll}\mathrm{Ga} 1 \mathrm{Ga} & 4 & \mathrm{~g} & 0.67500 & 0.17500 & 0.25000 & 1.00000\end{array}$

$\begin{array}{llllllll}\mathrm{Ir} 4 & \mathrm{Ir} & 4 & \mathrm{~h} & 0.00000 & 0.50000 & 0.58610 & 1.00000\end{array}$

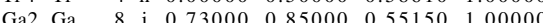

$\begin{array}{llllllll}\mathrm{Ga} 3 & \mathrm{Ga} & 8 & \mathrm{i} & 0.84000 & 0.80000 & 0.15000 & 1.00000\end{array}$

Ir 3 Ga5: A5B3_tP32_118_g2i_aceh - POSCAR

A5B3_tP32_118_g2i_aceh \& a, c/a, z3, x4, z5, x6, y6, z6, x7,y7, z7 --params =

$\hookrightarrow 5.8229835854,2.43860552978,0.6709,0.675,0.5861,0.73,0.85,0.5515$

$\hookrightarrow \quad 0.84,0.8,0.15 \& \mathrm{P}-4 \mathrm{n} 2 \mathrm{D}\{2 \mathrm{~d}\}^{\wedge}\{8\} \# 118(\mathrm{aceghi} \wedge 2) \& \mathrm{tP} 32$ \& None

$\hookrightarrow$ \& Ir3Ga5 \& \& H. V\{\"o\}llenkle and A. Wittmann and H. Nowotny, Monatsh. Chem. 98, 176-183 (1967)

1.00000000000000

5.82298358540000

0.00000000000000

$0.00000000000000 \quad 0.00000000000000$

$5.82298358540000 \quad 0.00000000000000$

$\begin{array}{lll}0.00000000000000 & 0.00000000000000 & 14.19995997120000\end{array}$

$\begin{array}{ll}\mathrm{Ga} & \mathrm{Ir} \\ 20 & 12\end{array}$

Direct

0.67500000000000

$-0.67500000000000$

1.17500000000000

$-0.17500000000000$

0.73000000000000

$-0.73000000000000$

0.85000000000000

$-0.85000000000000$

1.23000000000000

$-0.23000000000000$

1.35000000000000

$-0.35000000000000$

0.84000000000000

$-0.84000000000000$

0.80000000000000

$-0.80000000000000$

1.34000000000000

$-0.34000000000000$

1.30000000000000

$-0.30000000000000$

0.00000000000000

0.50000000000000

0.00000000000000

0.50000000000

0.500000000

0.000000000000

0.00000000000000

0.50000000000000

0.50000000000000

0.00000000000000

0.50000000000000

0.50000000000000

0.00000000000000

1.17500000000000 0.67500000000000 .85000000000000 0.8500000000000 0.73000000000000 0.35000000000000 1.35000000000000 .3500000000000000000000 1.23000000000000 0.80000000000000 $-0.80000000000000$ $-0.84000000000000$ 0.84000000000000 $-0.30000000000000$ 1.30000000000000 1.34000000000000 $-0.34000000000000$ 0.00000000000000 0.50000000000000 0.50000000000000 0.50000000000000 0.00000000000000 0.00000000000000 0.00000000000000 0.50000000000000 0.50000000000000 0.50000000000000 0.00000000000000 0.00000000000000

0.25000000000000 0.25000000000000 0.75000000000000 0.75000000000000 0.5515000000000 0.5515000000000 0.55150000000000 $-0.5515000000000$ $-0.55150000000000$ 1.051500000000 1.05150000000000 $-0.05150000000000$ $-0.05150000000000$ 0.1500000000000 0.15000000000000 $-0.15000000000000$ $-0.15000000000000$ 0.65000000000000 0.65000000000000 0.35000000000000 0.35000000000000 0.0000000000000 0.00000000000000 0.2500000000000 0.7500000000000 0.750000000 0.6709000000000 $-67090000000000$ 1.17090000000000 $-0.17090000000000$ 0.58610000000000 $-0.58610000000000$ 1.08610000000000 $-0.08610000000000$

$\begin{array}{ll}\mathrm{Ga} & (4 \mathrm{~g}) \\ \mathrm{Ga} & (4 \mathrm{~g}) \\ \mathrm{Ga} & (4 \mathrm{~g}) \\ \mathrm{Ga} & (4 \mathrm{~g}) \\ \mathrm{Ga} & (8 \mathrm{i}) \\ \mathrm{Ga} & (8 \mathrm{i}) \\ \mathrm{Ga} & (8 \mathrm{i}) \\ \mathrm{Ga} & (8 \mathrm{i}) \\ \mathrm{Ga} & (8 \mathrm{i}) \\ \mathrm{Ga} & (8 \mathrm{i}) \\ \mathrm{Ga} & (8 \mathrm{i}) \\ \mathrm{Ga} & (8 \mathrm{i}) \\ \mathrm{Ga} & (8 \mathrm{i}) \\ \mathrm{Ga} & (8 \mathrm{i}) \\ \mathrm{Ga} & (8 \mathrm{i}) \\ \mathrm{Ga} & (8 \mathrm{i}) \\ \mathrm{Ga} & (8 \mathrm{i}) \\ \mathrm{Ga} & (8 \mathrm{i}) \\ \mathrm{Ga} & (8 \mathrm{i}) \\ \mathrm{Ga} & (8 \mathrm{i}) \\ \mathrm{Ir} & (2 \mathrm{a}) \\ \mathrm{Ir} & (2 \mathrm{a}) \\ \mathrm{Ir} & (2 \mathrm{c}) \\ \mathrm{Ir} & (2 \mathrm{c}) \\ \mathrm{Ir} & (4 \mathrm{e}) \\ \mathrm{Ir} & (4 \mathrm{e}) \\ \mathrm{Ir} & (4 \mathrm{e}) \\ \mathrm{Ir} & (4 \mathrm{e}) \\ \mathrm{Ir} & (4 \mathrm{~h}) \\ \mathrm{Ir} & (4 \mathrm{~h}) \\ \mathrm{Ir} & (4 \mathrm{~h}) \\ \mathrm{Ir} & (4 \mathrm{~h}) \\ \mathrm{I} & \end{array}$

RbGa3: A3B tI24_119 b2i_af - CIF

\section{\# CIF file}

data_findsym-output

audit_creation_method FINDSYM

chemical_name_mineral 'RbGa3'

chemical_formula_sum ' $\mathrm{Ga} 3 \mathrm{Rb}$ '

loop

publ_author_name

R. G. Ling

C. Belin

journal_name_full_name

Zeitschrift fur Anorganische und Allgemeine Chemie

journal_volume 480

-journal-year 1981

_journal_page_first 18

_journal_page_last 185
publ_Section_title

Preparation and Crystal Structur

$\hookrightarrow$ Intermetallic Compound RbGa\$_\{3\}\$

\# Found in Pearson's Handbook of Crystallographic Data for Intermetallic $\hookrightarrow$ Phases, 1991

aflow title 'RbGa\$_\{3\}\$ Structure'

aflow_proto, A3B_tI24_119_b2i_af'

aflow params $, a, c / a, z_{-}\{3\}, x_{-}\{4\}, z_{2}\{4\}, x_{-}\{5\}, z_{-}\{5\}$,

aflow_params values $, 6.315,2.37529691211,0.372,0.2068,0.2229,0.3067$ $\rightarrow 0.3917$

, None'

aflow_Pearson 'tI24,

symmetry_space_group_name_H-M "I $-4 \mathrm{~m} \mathrm{2}$ "

symmetry_Int_Tables_number 119

_cell_length_a $\quad 6.31500$

-cell_length_b 6.31500

cell_length_c 15.00000

_cell_angle_alpha 90.00000

cell_angle_beta 90.00000

cell_angle_gamma 90.00000

loop

space_group_symop_id

space_group_symop_operation_xyz

$\mathrm{x}, \mathrm{y}, \mathrm{z}$

$2-x,-y, z$

$3-\mathrm{y},-\mathrm{x},-$
$4 \mathrm{y}, \mathrm{x},-\mathrm{z}$

, $y, x,-z$

(6) $x, y, z$

$6 \mathrm{x},-\mathrm{y}, \mathrm{z}$

$7 \mathrm{y},-\mathrm{x},-\mathrm{z}$

$8-y, x,-z$

$\mathrm{y}+1 / 2, \mathrm{z}+1 / 2$

$-x+1 / 2,-y+1 / 2, z+1 / 2$

$-\mathrm{y}+1 / 2,-\mathrm{x}+1 / 2,-\mathrm{z}+1 / 2$

$\mathrm{y}+1 / 2, \mathrm{x}+1 / 2,-\mathrm{z}+1 / 2$

$14 \mathrm{x}+1 / 2,-\mathrm{y}+1 / 2, \mathrm{z}+1 / 2$

$15 \mathrm{y}+1 / 2,-\mathrm{x}+1 / 2,-\mathrm{z}+1 / 2$

$16-y+1 / 2, x+1 / 2$,

loop

atom_site label

atom_site type symbol

atom_site_symmetry_multiplicity

atom_site_Wyckoff_labe

atom_site_fract_x

atom_site_fract_y

-atom_site_fract_z

-atom_site_occupancy

$\begin{array}{llllllll}\mathrm{Rb} 1 \mathrm{Rb} & 2 & \mathrm{a} & 0.00000 & 0.00000 & 0.00000 & 1.00000\end{array}$

$\begin{array}{llllllll}\mathrm{Ga} & \mathrm{Ga} & 2 & \mathrm{~b} & 0.00000 & 0.00000 & 0.50000 & 1.00000\end{array}$

$\begin{array}{llllllll}\mathrm{Rb} 2 \mathrm{Rb} & 4 & \mathrm{f} & 0.00000 & 0.50000 & 0.37200 & 1.00000\end{array}$

$\begin{array}{lllllllll}\mathrm{Ga} 2 & \mathrm{Ga} & 8 & \mathrm{i} & 0.20680 & 0.00000 & 0.22290 & 1.00000\end{array}$

Ga3 Ga $\quad \begin{array}{lllllllll} & \text { i } & 0.30670 & 0.00000 & 0.39170 & 1.00000\end{array}$

$\mathrm{RbGa}_{3}$ : A3B_tI24_119_b2i_af - POSCAR

A3B_tI24_119_b2i_af \& a,c/a, z3, x4, z4, x5 , z5 --params $=6.315,2.37529691211$ $\hookrightarrow 0.372,0.2068,0.2229,0.3067,0.3917 \& \mathrm{I}-4 \mathrm{~m} 2 \mathrm{D}_{-}\{2 \mathrm{~d}\}^{\wedge}\{9\} \# 119$ (abfi $\left.\hookrightarrow \wedge^{\wedge}\right)$ \& tI24 \& None \& RbGa3 \& RbGa3 \& R. G. Ling and C. Belin, Z. $\hookrightarrow$ Anorg. Allg. Chem. 480, 181-185 (1981)

1.00000000000000

$-3.15750000000000$

$3.15750000000000 \quad 7.50000000000000$

$-3.15750000000000 \quad 7.50000000000000$

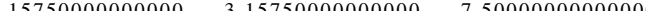

$\mathrm{Ga} \quad \mathrm{Rb}$

Direct

0.22290000000000

0.22290000000000

$-0.42970000000000$

$-0.01610000000000$

0.39170000000000

0.39170000000000

$-0.69840000000000$

$-0.08500000000000$

0.00000000000000

.87200000000000

$-7.50000000000000$

0.50000000000000 0.42970000000000 0.01610000000000 $-0.22290000000000$ $-0.22290000000000$ 0.69840000000000 0.08500000000000 $-0.39170000000000$ $-0.39170000000000$ 0.00000000000000 0.37200000000000 0.12800000000000

0.00000000000000 0.20680000000000 $-0.20680000000000$ $-0.20680000000000$ 0.20680000000000 0.30670000000000 $-0.30670000000000$ $-0.30670000000000$ 0.30670000000000 0.00000000000000 0.50000000000000 0.50000000000000

$\begin{array}{ll}\mathrm{Ga} & (2 \mathrm{~b}) \\ \mathrm{Ga} & (8 \mathrm{i}) \\ \mathrm{Ga} & (8 \mathrm{i}) \\ \mathrm{Ga} & (8 \mathrm{i}) \\ \mathrm{Ga} & (8 \mathrm{i}) \\ \mathrm{Ga} & (8 \mathrm{i}) \\ \mathrm{Ga} & (8 \mathrm{i}) \\ \mathrm{Ga} & (8 \mathrm{i}) \\ \mathrm{Ga} & (8 \mathrm{i}) \\ \mathrm{Rb} & (2 \mathrm{a}) \\ \mathrm{Rb} & (4 \mathrm{f}) \\ \mathrm{Rb} & (4 \mathrm{f})\end{array}$

GaSb: AB_tI4_119_c_a - CIF

\# CIF file

data_findsym-output

audit_creation_method FINDSYM

chemical_name_mineral 'GaSb'

chemical formula sum 'c

loop_

publ_author_name

T. R. R. McDonald,

R. Sard,

E. Gregory,

journal_name_full_name

Journal of Applied Physics 
_journal_volume 36

_journal_year 1965

_journal_page_first 1498

_journal_page_last 1499

-publ_Section_title

Retention of GaSb (II) at low temperatures and one atmosphere pressure

\# Found in Pearson's Crystal Data - Crystal Structure Database for

$\hookrightarrow$ Inorganic Compounds, 2013

_aflow_title 'GaSb Structure'

-aflow_proto 'AB_tI4_119_c_a',

aflow params 'a,c/a,

_aflow_params values '5.4790101504,0.558496075934,

-aflow_Strukturbericht 'None'

_aflow_Pearson, tI4,

_cell_length_a $\quad 5.4790101504$

_cell_length_b 5.4790101504

_cell_length_c $\quad 3.0600056690$

_cell_angle_alpha 90.0000000000

_cell_angle_beta 90.0000000000

cell_angle_gamma 90.0000000000

_symmetry_space_group_name_H-M "I $-4 \mathrm{~m} \mathrm{2}$ "

_symmetry_Int_Tables_number 119

loop

_space_group_symop_id

_space_group_symop_operation_xyz

$1 \mathrm{x}, \mathrm{y}, \mathrm{z}$

$2-\mathrm{x},-\mathrm{y}, \mathrm{z}$

$3-\mathrm{y},-\mathrm{x},-\mathrm{z}$
$4 \mathrm{y}, \mathrm{x},-\mathrm{z}$

$4 \mathrm{y}, \mathrm{x},-\mathrm{z}$

$5-\mathrm{x}, \mathrm{y}, \mathrm{z}$

$6 \mathrm{x},-\mathrm{y}, \mathrm{z}$

$7 \mathrm{y},-\mathrm{x},-\mathrm{z}$

$8-\mathrm{y}, \mathrm{x},-$

$9 \mathrm{x}+1 / 2, \mathrm{y}+1 / 2, \mathrm{z}+1 / 2$

$10-\mathrm{x}+1 / 2,-\mathrm{y}+1 / 2, \mathrm{z}+1 / 2$

$11-\mathrm{y}+1 / 2,-\mathrm{x}+1 / 2,-\mathrm{z}+1 / 2$

$12 \mathrm{y}+1 / 2, \mathrm{x}+1 / 2,-\mathrm{z}+1 / 2$

$13-x+1 / 2, y+1 / 2, z+1 / 2$

$14 \mathrm{x}+1 / 2,-\mathrm{y}+1 / 2, \mathrm{z}+1 / 2$

$14 \mathrm{x}+1 / 2,-\mathrm{y}+1 / 2, \mathrm{z}+1 / 2$

$16-\mathrm{y}+1 / 2, \mathrm{x}+1 / 2,-\mathrm{z}+1 / 2$

loop_

atom_site_label

_atom_site_type_symbol

_atom_site_symmetry_multiplicity

_atom_site_Wyckoff_label

-atom_site_fract_x

-atom_site_fract_y

-atom_site_fract_z

atom_site occupancy

\begin{tabular}{lllllll}
\hline Sb1 Sb & 2 & a & 0.00000 & 0.00000 & 0.00000 & 1.00000
\end{tabular}

Ga1 Ga 2 c $0.00000 \quad 0.50000 \quad 0.25000 \quad 1.00000$

GaSb: AB_tI4_119_c_a - POSCAR

AB_tI4_119_c_a \& a , c/a --params $=5.4790101504,0.558496075934 \&$ I-4m2 D_f

$\hookrightarrow 2 \mathrm{~d}\}^{\wedge}\{9\} \# 119$ (ac) \& tI4 \& None \& GaSb \& \& T. R. R. McDonald

$\hookrightarrow$ and R. Sard and E. Gregory, J. Appl. Phys. 36, 1498-1499 (1965)

1.00000000000000

$\begin{array}{rrr}-2.73950507520000 & 2.73950507520000 & 1.53000283450000\end{array}$

$\begin{array}{rrr}2.73950507520000 & -2.73950507520000 & 1.53000283450000\end{array}$

$\begin{array}{lll}2.73950507520000 & 2.73950507520000 & -1.53000283450000\end{array}$

$\mathrm{Ga} \quad \mathrm{Sb}$

Direct

$0.75000000000000-0.25000000000000=0.50000000000000$ 0.00000000

$0.50000000000000 \quad \mathrm{Ga} \quad(2 \mathrm{c})$ 0.00000000000000

$\mathrm{KAu}_{4} \mathrm{Sn}_{2}:$ A4BC2_tI28_120_i_d_e - CIF

\section{\# CIF file}

data_findsym-output

audit_creation_method FINDSYM

_chemical_name_mineral 'KAu4Sn2'

_chemical_formula_sum, Au4 K Sn2

loop

publ_author_name

'H.-D. Sinnen'

'H.-U. Schuster,

journal_name_full_name

Zeitschrift $f\{\backslash " u\}$ r Naturforschung B

journal volume 33

-journal_page_first 1077

_journal_page_last 1079

-publ_Section_title

Darstellung und Struktur des KAu\$_ $\{4\} \$ S n \$_{-}\{2\} \$$ / Preparation and

$\hookrightarrow$ Crystal Structure of KAu\$_\{4\}\$Sn\$_ $\{2\} \$$
\# Found in Pearson's Crystal Data - Crystal Structure Database for $\hookrightarrow$ Inorganic Compounds, 2013

aflow_title $K A u \$_{-}\{4\} \$ S n \$_{-}\{2\} \$$ Structure

aflow_proto 'A4BC2_tI28_120_i_d_e

aflow_params $, a, c / a, x_{-}\{2\}, x_{-}\{3\}, y_{-}\{3\}, z_{-}\{3\}$

aflow_params_values $, 8.8470588481,0.924381146154,0.856,0.6452,0.6575$, $\hookrightarrow 0.0851$,

-aflow_Strukturbericht 'None'

-aflow_Pearson 'tI28'

_cell_length_a $\quad 8.8470588481$

-cell_length_b $\quad 8.8470588481$

_cell_length_c 8.1780543981

_cell_angle_alpha 90.0000000000

theta

_symmetry_space_group_name_H-M "I -4 c 2 "

_symmetry_Int_Tables_number 120

loop_

space_group_symop_id

_space_group_symop_operation_xyz

$1 \mathrm{x}, \mathrm{y}, \mathrm{z}$

$-\mathrm{x},-\mathrm{y}, \mathrm{z}$
$3-\mathrm{y},-\mathrm{x},-\mathrm{z}+1 / 2$
$4 \mathrm{y}, \mathrm{x}, \mathrm{x}, \mathrm{z}+1 / 2$

$4 \mathrm{y}, \mathrm{x},-\mathrm{z}+1 / 2$

$5-\mathrm{x}, \mathrm{y}, \mathrm{z}+1 / 2$

$6 \mathrm{x},-\mathrm{y}, \mathrm{z}+1 / 2$

$7 \mathrm{y},-\mathrm{x},-\mathrm{z}$

$\begin{array}{ll}8 & -y, x,-z \\ 9 & x+1 / 2, y+1 / 2, z+1 / 2\end{array}$

$10-x+1 / 2,-y+1 / 2, z+1 / 2$

$11-y+1 / 2,-x+1 / 2,-z$

$12 \mathrm{y}+1 / 2, \mathrm{x}+1 / 2,-\mathrm{z}$

$13-x+1 / 2, y+1 / 2$,

$14 x+1 / 2,-y+1 / 2, z$

$15 \mathrm{y}+1 / 2,-\mathrm{x}+1 / 2,-\mathrm{z}+1 / 2$

$16-\mathrm{y}+1 / 2, \mathrm{x}+1 / 2,-\mathrm{z}+1 / 2$

loop

atom_site_label

atom_site_type_symbol

atom_site_symmetry_multiplicity

atom_site_Wyckoff_label

atom_site_fract_x

atom_site_fract_y

atom_site_fract_z

atom_site_occupancy

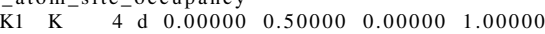

$\begin{array}{lllllllll}\mathrm{Sn} 1 & \text { Sn } & 8 & \text { e } & 0.85600 & 0.85600 & 0.25000 & 1.00000\end{array}$

Au1 Au 16 i $\begin{array}{llllll}0.64520 & 0.65750 & 0.08510 & 1.00000\end{array}$

$\mathrm{KAu}_{4} \mathrm{Sn}_{2}$ : A4BC2_tI28_120_i_d_e - POSCAR

A4BC2 tI28 120_i_d e \& a, c/a , x2, x3,y3, z3 --params $=8.8470588481$

$\hookrightarrow 0.924381146154,0.856,0.6452,0.6575,0.0851 \&$ I $-4 c 2$ D_ $\{2 \mathrm{~d}\}^{\wedge}\{10\}$

$\hookrightarrow 120$ (dei) \& $\mathrm{TI} 28$ \& None \& KAu4Sn2 \& \& H.-D Sinnen and H.-U.

$\hookrightarrow$ Schuster, Z. Naturforsch. B 33, 1077-1079 (1978)

1.00000000000000

$-4.42352942405000 \quad 4.42352942405000 \quad 4.08902719905000$

$\begin{array}{rrr}4.42352942405000 & -4.42352942405000 & 4.08902719905000\end{array}$

$\begin{array}{rrr}4.42352942405000 & 4.42352942405000 & -4.08902719905000\end{array}$

$\begin{array}{rrr}\mathrm{Au} & \mathrm{K} & \mathrm{Sn} \\ 8 & 2 & 4\end{array}$

Direct

$\begin{array}{lll}0.74260000000000 & 0.73030000000000 & 1.30270000000000\end{array}$

$-0.57240000000000-0.56010000000000-1.30270000000000$

$\begin{array}{lll}-0.73030000000000 & 0.57240000000000 & 0.01230000000000\end{array}$

$\begin{array}{lll}0.56010000000000 & -0.74260000000000 & -0.01230000000000\end{array}$

$-0.07240000000000-1.23030000000000-0.01230000000000$

$\begin{array}{lll}-0.07240000000000 & 1.23030000000000 & -0.01230000000000\end{array}$

$\begin{array}{lll}1.24260000000000 & -0.06010000000000 & 0.01230000000000\end{array}$

$\begin{array}{lll}1.06010000000000-1.07240000000000 & 1.30270000000000\end{array}$

$\begin{array}{rrr}-0.23030000000000 & -0.24260000000000 & -1.30270000000000\end{array}$

$\begin{array}{lll}0.50000000000000 & 0.00000000000000 & 0.50000000000000 \\ 0.00000000000000 & 0.50000000000000 & 0.50000000000000\end{array}$

$\begin{array}{rrr}0.00000000000000 & 0.50000000000000 & 0.50000000000000 \\ 1.10600000000000 & 1.10600000000000 & 1.71200000000000\end{array}$

$\begin{array}{rrr}1.10600000000000 & 1.10600000000000 & 1.71200000000000 \\ -0.60600000000000 & -0.60600000000000 & -1.71200000000000\end{array}$

$\begin{array}{rrr}-0.60600000000000 & -0.60600000000000 & -1.71200000000000 \\ -0.10600000000000 & 1.60600000000000 & 0.00000000000000\end{array}$

$\begin{array}{rrr}-0.10600000000000 & 1.60600000000000 & 0.00000000000000 \\ 1.60600000000000 & -0.10600000000000 & 0.00000000000000\end{array}$

$\mathrm{CaRbFe}_{4} \mathrm{As}_{4}$ (Superconducting): A4BC4D_tP10_123_gh_a_i_d - CIF

\# CIF file

data_findsym-output

_audit_creation_method FINDSYM

chemical_name_mineral 'CsRbFe4As4'

_chemical_formula_sum 'As4 Cs Fe4 Rb

loop

publ_author_name

'A. Iyo,

'K. Kawashima'

'T. Kinjo',

'T. Nishio'

'H. Fujihisa'

'H. Fujihisa,

'Y. Kotoh,

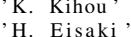

'Y. Yoshida,

_journal_name_full_name 
Journal of the American Chemical Society

_journal_volume 138

journal_year 2016

_journal_page_first 3410

_journal_page_last 3415

_publ_Section_title

New-Structure-Type Fe-Based Superconductors: CaAFe\$_\{4\}\$As\$_\{4\}\$ (A = K $\hookrightarrow, \mathrm{Rb}, \mathrm{Cs})$ and $\mathrm{SrAFe} \$ \_\{4\} \$ \mathrm{As}_{-}\{4\} \$(\mathrm{~A}=\mathrm{Rb}, \mathrm{Cs})$

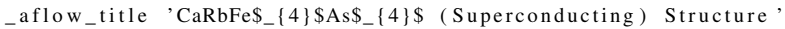

_aflow_proto A4BC4D_tP10_123_gh_a_i_d

_aflow_params 'a, c/a, z_ $\{3\}, z_{-}\{4\}, z_{-}\{5\}$ '

- aflow params values ' $3.8757,3.38106664603,0.3336,0.1193,0.2246$ '

- aflow_Strukturbericht 'None'

aflow_Pearson 'tP10,

_symmetry_space_group_name_H-M "P $4 / \mathrm{m} 2 / \mathrm{m} 2 / \mathrm{m}$

_symmetry_Int_Tables_number 123

$\begin{array}{ll}\text {-cell_length_a } & 3.87570 \\ \text {-cell_length_b } & 3.87570\end{array}$

-cell_length_b $\quad 3.87570$

cell_angle_alpha 90.00000

_cell_angle_beta 90.00000

cell angle gamma 90.00000

loop

space_group_symop_id

_space_group_symop_operation_xyz

$1 \mathrm{x}, \mathrm{y}, \mathrm{z}$

$2 \mathrm{x},-\mathrm{y},-\mathrm{z}$

$3-\mathrm{x}, \mathrm{y},-\mathrm{z}$

$\begin{array}{ll}4 & -\mathrm{x},-\mathrm{y}, \mathrm{z} \\ 5 & -\mathrm{y},-\mathrm{x},-\mathrm{z}\end{array}$

$6-\mathrm{y}, \mathrm{x}, \mathrm{z}$

$7 \mathrm{y},-\mathrm{x}, \mathrm{z}$

$8 \mathrm{y}, \mathrm{x},-\mathrm{z}$

$9-\mathrm{x},-\mathrm{y},-\mathrm{z}$

$10-\mathrm{x}, \mathrm{y}, \mathrm{z}$

$11 \mathrm{x},-\mathrm{y}, \mathrm{z}$

$13 \mathrm{y}, \mathrm{x}, \mathrm{z}$

$14 \mathrm{y},-\mathrm{x},-\mathrm{z}$

$15-\mathrm{y}, \mathrm{x},-\mathrm{z}$

$16-\mathrm{y},-\mathrm{x}, \mathrm{z}$

loop

atom site label

_atom_site_type_symbol

_atom_site_symmetry_multiplicity

_atom_site_Wyckoff_label

_atom_site_fract_x

atom_site_fract_y

atom_site_fract_z

_atom_site_occupancy

Cs1 Cs $\begin{array}{llllll}1 & 0.00000 & 0.00000 & 0.00000 & 1.00000\end{array}$

$\begin{array}{lllllll}\mathrm{Rb} 1 \mathrm{Rb} & 1 & \mathrm{~d} & 0.50000 & 0.50000 & 0.50000 & 1.00000\end{array}$

As1 As $\quad 2$ g $0.00000 \quad 0.00000 \quad 0.33360 \quad 1.00000$

$\begin{array}{llllllll}\text { As2 As } & 2 & \text { h } & 0.50000 & 0.50000 & 0.11930 & 1.00000\end{array}$

$\begin{array}{lllllllll}\mathrm{Fe} 1 & \mathrm{Fe} & 4 & \mathrm{i} & 0.00000 & 0.50000 & 0.22460 & 1.00000\end{array}$

$\mathrm{CaRbFe}_{4} \mathrm{As}_{4}$ (Superconducting): A4BC4D_tP10_123_gh_a_i_d - POSCAR

A4BC4D_tP10_123_gh_a_i_d \& a , c/a, z3 , z4, z5 --params $=3.8757,3.38106664603$, $\hookrightarrow 0.3336,0.1193,0.2246 \&$ P $4 / \mathrm{mmm} \mathrm{D}_{-}\{4 \mathrm{~h}\}^{\wedge}\{1\} \# 123$ (adghi) \& tP10\&

$\hookrightarrow$ None \& CsRbFe4As4 \& CsRbFe4As4 \& A. Iyo et al., J. Am. Chem.

$\hookrightarrow$ Soc. $138,3410-3415(2016)$

1.00000000000000

$\begin{array}{lll}3.87570000000000 & 0.00000000000000 & 0.00000000000000\end{array}$

$\begin{array}{lll}0.00000000000000 & 3.87570000000000 & 0.00000000000000\end{array}$

$\begin{array}{rrr}0.00000000000000 & 0.00000000000000 & 13.10400000000000\end{array}$

As $\quad \mathrm{Cs} \quad \mathrm{Fe} \quad \mathrm{Rb}$

Direct

0.00000000000000

0.00000000000000

0.50000000000000

0.50000000000000

0.00000000000000

0.00000000000000

0.50000000000000

0.00000000000000

0.50000000000000

0.50000000000000

0.00000000000000

0.00000000000000

0.5000000000000

0.50000000000000

0.00000000000000

.

0.50000000000000

0.00000000000000

0.50000000000000

0.33360000000000

$-0.33360000000000$

0.11930000000000

$-0.11930000000000$

0.00000000000000

.22460000000000

0.22460000000000

$-0.22460000000000$

0.50000000000000

$\begin{array}{ll}\text { As } & (2 \mathrm{~g}) \\ \mathrm{As} & (2 \mathrm{~g}) \\ \mathrm{As} & (2 \mathrm{~h}) \\ \mathrm{As} & (2 \mathrm{~h}) \\ \mathrm{Cs} & (1 \mathrm{a}) \\ \mathrm{Fe} & (4 \mathrm{i}) \\ \mathrm{Fe} & (4 \mathrm{i}) \\ \mathrm{Fe} & (4 \mathrm{i}) \\ \mathrm{Fe} & (4 \mathrm{i}) \\ \mathrm{Rb} & (1 \mathrm{~d})\end{array}$

$\mathrm{Nb}_{4}$ CoSi: AB4C_tP12_124_a_m_c - CIF

\# CIF file

data_findsym-output

audit_creation_method FINDSYM

_chemical_name_mineral ' $\mathrm{Nb} 4 \mathrm{CoSi}$,

chemical_formula_sum ' $\mathrm{Co} \mathrm{Nb} 4 \mathrm{Si}$

loop

_publ_author_name

E. I. Gladysherskii

'\{Yu\}. B. \{Kuz 'ma\}'

_journal_name_full_name

Journal of Structural Chemistry _journal_volume 6

journal_year 1965

_journal_page_first 60

journal_page_last 63

_publ_Section_title

The compounds $\mathrm{Nb} \$_{-}\{4\} \$ \mathrm{FeSi}, \mathrm{Nb} \$_{-}\{4\} \$ \mathrm{CoSi}, \mathrm{Nb} \$_{-}\{4\} \$ \mathrm{NiSi}$ and their $\hookrightarrow$ crystal structures

Found in Pearson's Crystal Data - Crystal Structure Database for $\hookrightarrow$ Inorganic Compounds, 2013

aflow_title'Nb\$_\{4\}\$CoSi Structure'

aflow_proto 'AB4C_tP12_124_a_m_c'

aflow_params 'a, c/a, x_\{3\}, _

_aflow_params_values 6.18848084

-aflow_Strukturbericht 'None'
-aflow_Pearson 'tP12,

_cell_length_a $\quad 6.1884808485$
_cell_length_b

cell_length_b $\quad 6.1884808485$

cell_length_c $\quad 5.0525761395$

cell_angle_alpha 90.0000000000

cell_angle_beta 90.0000000000

_cell_angle_gamma 90.0000000000

_symmetry_space_group_name_H-M "P $4 / \mathrm{m} \mathrm{2/c} 2 / \mathrm{c} "$

_symmetry_Int_Tables_number 124

loop

space_group_symop_id

space_group_symop_operation_xyz

$\overline{1} \mathrm{x}, \mathrm{y}, \mathrm{z}$

$2 \mathrm{x},-\mathrm{y},-\mathrm{z}+1 / 2$

$3-\mathrm{x}, \mathrm{y},-\mathrm{z}+1 / 2$

$4-\mathrm{x},-\mathrm{y}, \mathrm{z}$

$5-\mathrm{y},-\mathrm{x},-\mathrm{z}+1 / 2$

$6-\mathrm{y}, \mathrm{x}, \mathrm{z}$

$7 \mathrm{y},-\mathrm{x}, \mathrm{z}$

$8 \mathrm{y}, \mathrm{x},-\mathrm{z}+1 / 2$

$9-\mathrm{x},-\mathrm{y},-\mathrm{z}$

$10-\mathrm{x}, \mathrm{y}, \mathrm{z}+1 / 2$

$11 \mathrm{x},-\mathrm{y}, \mathrm{z}+1 / 2$

$12 \mathrm{x}, \mathrm{y},-\mathrm{z}$

$13 \mathrm{y}, \mathrm{x}, \mathrm{z}+1 / 2$

$14 \mathrm{y},-\mathrm{x},-\mathrm{z}$

$6-\mathrm{y},-\mathrm{x}, \mathrm{z}+1 / 2$

loop

atom_site_label

atom_site_type_symbol

atom_site_symmetry_multiplicity

atom_site_Wyckoff_label

atom_site_fract_x

atom_site_fract_y

atom_site_fract_z

atom_site_occupancy

$\begin{array}{lllllll}\mathrm{Co} 1 \mathrm{Co} & 2 & \mathrm{a} & 0.00000 & 0.00000 & 0.25000 & 1.00000\end{array}$

$\begin{array}{lllllllll}\mathrm{Si} & \mathrm{Si} & 2 & \mathrm{c} & 0.50000 & 0.50000 & 0.25000 & 1.00000\end{array}$

$\begin{array}{llllllllllll}\mathrm{Nb} 1 & \mathrm{Nb} & 8 & \mathrm{~m} & 0.16200 & 0.66200 & 0.00000 & 1.00000\end{array}$

$\mathrm{Nb}_{4}$ CoSi: AB4C_tP12_124_a_m_c - POSCAR

\begin{tabular}{|c|c|c|c|c|}
\hline \multicolumn{5}{|c|}{ 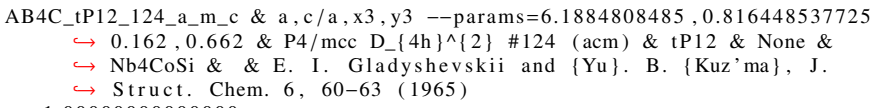 } \\
\hline \multicolumn{5}{|l|}{1.00000000000000} \\
\hline 6.18848084850000 & 0.00000000000000 & 0.00000000000000 & & \\
\hline 0.00000000000000 & 6.18848084850000 & 0.00000000000000 & & \\
\hline 0.00000000000000 & 0.00000000000000 & 5.05257613950000 & & \\
\hline Co & & & & \\
\hline 8 & & & & \\
\hline \multicolumn{5}{|l|}{ Direct } \\
\hline 0.00000000000000 & 0.00000000000000 & 0.25000000000000 & Co & $(2 \mathrm{a})$ \\
\hline 0.00000000000000 & 0.00000000000000 & 0.75000000000000 & Co & (2a) \\
\hline 0.16200000000000 & 0.66200000000000 & 0.00000000000000 & $\mathrm{Nb}$ & $(8 \mathrm{~m})$ \\
\hline-0.16200000000000 & -0.66200000000000 & 0.00000000000000 & $\mathrm{Nb}$ & $(8 \mathrm{~m})$ \\
\hline-0.66200000000000 & 0.16200000000000 & 0.00000000000000 & $\mathrm{Nb}$ & $(8 \mathrm{~m})$ \\
\hline 0.66200000000000 & -0.16200000000000 & 0.00000000000000 & $\mathrm{Nb}$ & $(8 \mathrm{~m})$ \\
\hline-0.16200000000000 & 0.66200000000000 & 0.50000000000000 & $\mathrm{Nb}$ & $(8 \mathrm{~m})$ \\
\hline 0.16200000000000 & -0.66200000000000 & 0.50000000000000 & $\mathrm{Nb}$ & $(8 \mathrm{~m})$ \\
\hline 0.66200000000000 & 0.16200000000000 & 0.50000000000000 & $\mathrm{Nb}$ & $(8 \mathrm{~m})$ \\
\hline-0.66200000000000 & -0.16200000000000 & 0.50000000000000 & $\mathrm{Nb}$ & $(8 \mathrm{~m})$ \\
\hline 0.50000000000000 & 0.50000000000000 & 0.25000000000000 & $\mathrm{Si}$ & $(2 \mathrm{c})$ \\
\hline 0.50000000000000 & 0.50000000000000 & 0.75000000000000 & $\mathrm{Si}$ & $(2 \mathrm{c})$ \\
\hline
\end{tabular}

$\mathrm{NbTe}_{4}$ : AB4_tP10_124_a_m - CIF

\# CIF file

data_findsym-output

_audit_creation_method FINDSYM

chemical name mineral 'NbTe4,

chemical formula sum ' $\mathrm{Nb} \mathrm{Te} 4$

loop

publ_author_name

K. Selte'

A. Kjekshus,

journal_name_full_name

Acta Chemica Scandinavica 
_journal_volume 18

_journal_year 1964

_journal_page_first 690

_journal_page_last 696

_publ_Section_title

On the crystal structure of $\mathrm{NbTe}_{-}\{4\} \$$

\# Found in Pearson's Crystal Data - Crystal Structure Database for $\hookrightarrow$ Inorganic Compounds, 2013

aflow_title 'NbTe\$_\{4\}\$ Structure

aflow_proto ,AB4_tP10_124_a_m,

-aflow_proto , AB4_tPlo_124_a_m,

-aflow params values $, 6.4989671731,1.05200800123,0.1425,0.3361$,

- aflow_Strukturbericht 'None'

-aflow_Pearson 'tP10,

_cell_length_a $\quad 6.4989671731$

_cell_length_b 6.4989671731

_cell_length_c 6.8369654658

_cell_angle_alpha 90.0000000000

_cell_angle_beta 90.0000000000

cell_angle_gamma 90.0000000000

_symmetry_space_group_name_H-M "P $4 / \mathrm{m} 2 / \mathrm{c} 2 / \mathrm{c} "$

_symmetry_Int_Tables_number 124

loop_

_space_group_symop_id

_space_group_symop_operation_xyz

$1 \mathrm{x}, \mathrm{y}, \mathrm{z}$

$2 \mathrm{x},-\mathrm{y},-\mathrm{z}+1 / 2$

$3-\mathrm{x}, \mathrm{y},-\mathrm{z}+1 / 2$

$4-\mathrm{x},-\mathrm{y}, \mathrm{z}$

$5-\mathrm{y},-\mathrm{x},-\mathrm{z}+1 / 2$

$6-\mathrm{y}, \mathrm{x}, \mathrm{z}$

$7 \mathrm{y},-\mathrm{x}, \mathrm{z}$

$8 \mathrm{y}, \mathrm{x},-\mathrm{z}+1 / 2$

$9-\mathrm{x},-\mathrm{y},-\mathrm{z}$

$11 \mathrm{x},-\mathrm{y}, \mathrm{z}+1 / 2$

$12 \mathrm{x}, \mathrm{y},-\mathrm{z}$

$13 \mathrm{y}, \mathrm{x}, \mathrm{z}+1 / 2$

$14 \mathrm{y},-\mathrm{x},-\mathrm{z}$

$16-y,-x, z+1 / 2$

loop_

atom_site_label

_atom_site_type_symbol

_atom_site_symmetry_multiplicity

_atom_site_Wyckoff_label

_atom_site_fract_x

-atom_site_fract_y

-atom_site_fract_z

atom_site_occupancy

$\begin{array}{lllllll}\overline{\mathrm{Nb}} 1 \mathrm{Nb} & 2 & \mathrm{a} & 0.00000 & 0.00000 & 0.25000 & 1.00000\end{array}$

$\begin{array}{llllllll}\mathrm{Te} 1 & \mathrm{Te} & 8 & \mathrm{~m} & 0.14250 & 0.33610 & 0.00000 & 1.00000\end{array}$

$\mathrm{NbTe}_{4}$ : AB4_tP10_124_a_m - POSCAR

\begin{tabular}{|c|c|c|c|c|}
\hline \multicolumn{5}{|c|}{$\begin{aligned} \text { AB4_tP10_124_a_m \& a, c } / \mathrm{a}, \mathrm{x} 2, \mathrm{y} 2--\mathrm{params}=6.4989671731,1.05200800123 & \\
& \hookrightarrow 0.1425,0.3361 \& \mathrm{P} 4 / \mathrm{mcc} \mathrm{D}_{-}\{4 \mathrm{~h}\}^{\wedge}\{2\} \# 124(\mathrm{am}) \& \mathrm{tP} 10 \text { \& None \& } \\
& \hookrightarrow \text { NbTe4\&\& \&. Selte and A. Kjekshus, Acta Chem. Scand. 18, } \\
& \hookrightarrow 690-696(1964)\end{aligned}$} \\
\hline \multicolumn{5}{|l|}{1.00000000000000} \\
\hline 6.49896717310000 & 0.00000000000000 & 0.00000000000000 & & \\
\hline 0.00000000000000 & 6.49896717310000 & 0.00000000000000 & & \\
\hline 0.00000000000000 & 0.00000000000000 & 6.83696546580000 & & \\
\hline $\mathrm{Nb}$ & & & & \\
\hline 2 & & & & \\
\hline \multicolumn{5}{|l|}{ Direct } \\
\hline 0.00000000000000 & 0.00000000000000 & 0.25000000000000 & $\mathrm{Nb}$ & $(2 a)$ \\
\hline 0.00000000000000 & 0.00000000000000 & 0.75000000000000 & $\mathrm{Nb}$ & (2a) \\
\hline 0.14250000000000 & 0.33610000000000 & 0.00000000000000 & $\mathrm{Te}$ & $(8 \mathrm{~m})$ \\
\hline-0.14250000000000 & -0.33610000000000 & 0.00000000000000 & $\mathrm{Te}$ & $(8 \mathrm{~m})$ \\
\hline-0.33610000000000 & 0.14250000000000 & 0.00000000000000 & $\mathrm{Te}$ & $(8 \mathrm{~m})$ \\
\hline 0.33610000000000 & -0.14250000000000 & 0.00000000000000 & $\mathrm{Te}$ & $(8 \mathrm{~m})$ \\
\hline-0.14250000000000 & 0.33610000000000 & 0.50000000000000 & $\mathrm{Te}$ & $(8 \mathrm{~m})$ \\
\hline 0.14250000000000 & -0.33610000000000 & 0.50000000000000 & $\mathrm{Te}$ & $(8 \mathrm{~m})$ \\
\hline 0.33610000000000 & 0.14250000000000 & 0.50000000000000 & $\mathrm{Te}$ & $(8 \mathrm{~m})$ \\
\hline-0.33610000000000 & -0.14250000000000 & 0.50000000000000 & $\mathrm{Te}$ & $(8 \mathrm{~m})$ \\
\hline
\end{tabular}

$\mathrm{PtPb}_{4}$ : A4B_tP10_125_m_a - CIF

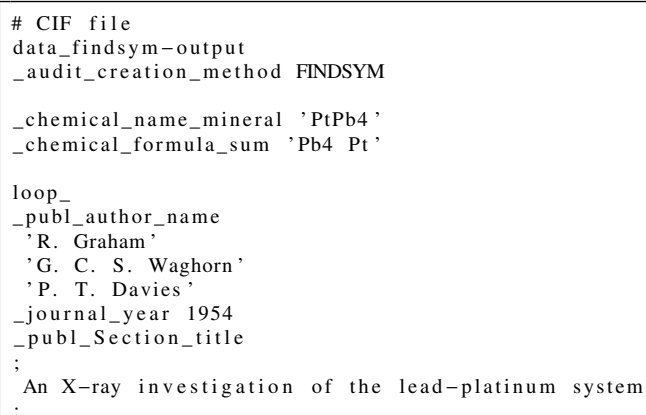

\# Found in Pearson's Crystal Data - Crystal Structure Database for $\hookrightarrow$ Inorganic Compounds, 2013

aflow_title $P$ PtPb\$_\{4\}\$ Structure

aflow_proto 'A4B_tP10_125_m_a'

aflow_params 'a, c/a, $x_{-}\{2\}, z_{-}\{2\}$ '

_aflow_params_values ' $6.6398746049,0.899096385539,0.425,0.255$,

aflow_Strukturbericht 'None'

aflow_Pearson 'tP10

cell_length_a 6.6398746049

cell 6.6398746049

cell_length_c 5.9698872577

cell lengle alpha 90.0000000000

cellangle 90.0000000000

_cell_angle_gamma 90.0000000000

symmetry_space_group_name_H-M "P 4/n 2/b 2/m (origin choice 2)" _symmetry_Int_Tables_number 125

loop

space_group_symop_id

_space_group_symop_operation_xyz

$\mathrm{x}, \mathrm{y}, \mathrm{z}$

$3-x+1 / 2, y,-z$

$4-x+1 / 2,-y+1 / 2, z$

$5-y+1 / 2,-x+1 / 2,-z$

$6-\mathrm{y}+1 / 2, \mathrm{x}, \mathrm{z}$

$7 \mathrm{y},-\mathrm{x}+1 / 2$,

$9-\mathrm{x}, \mathrm{z}$

$10-\mathrm{x}, \mathrm{y}+1 / 2, \mathrm{z}$

$11 \mathrm{x}+1 / 2,-\mathrm{y}, \mathrm{z}$

$12 \mathrm{x}+1 / 2, \mathrm{y}+1 / 2,-$

$13 \mathrm{y}+1 / 2, \mathrm{x}+1 / 2, \mathrm{z}$

$14 \mathrm{y}+1 / 2,-\mathrm{x},-\mathrm{z}$

$15-\mathrm{y}, \mathrm{x}+1 / 2,-\mathrm{z}$

$16-\mathrm{y},-\mathrm{x}, \mathrm{z}$

loop

atom site label

atom_site_type_symbol

atom_site_symmetry_multiplicity

atom_site_Wyckoff_label

atom_site_fract_x

atom_site_fract_y

atom_site_fract_z

atom_site_occupancy

$\begin{array}{lllllll} & & \end{array}$

$\begin{array}{lllllll}\mathrm{Pb} 1 \mathrm{~Pb} & 8 & \mathrm{~m} & 0.42500 & 0.57500 & 0.25500 & 1.00000\end{array}$

$\mathrm{PtPb}_{4}$ : A4B_tP10_125_m_a - POSCAR

A4B $\mathrm{tP} 10-125 \mathrm{~m}$ a \& a $\mathrm{c} / \mathrm{a}, \mathrm{x} 2, \mathrm{z2}-\mathrm{params}=6.6398746049,0.899096385539$ $\hookrightarrow 0.425,0.255 \& \mathrm{P} 4 / \mathrm{nbm} D_{-}\{4 \mathrm{~h}\}^{\wedge}\{3\} \# 125(\mathrm{am}) \& \mathrm{tP} 10 \&$ None \& PtPb4 $\hookrightarrow$ \& \& R. Graham and G. C. S. Waghorn and P. T. Davies, (1954)

1.00000000000000

$\begin{array}{lll}6.63987460490000 & 0.00000000000000 & 0.00000000000000\end{array}$

$\begin{array}{lll}0.00000000000000 & 6.63987460490000 & 0.00000000000000\end{array}$ $\begin{array}{lll}0.00000000000000 & 6.63987460490000 & 0.00000000000000 \\ 0.00000000000000 & 0.00000000000000 & 5.96988725770000\end{array}$

$\begin{array}{rr}\mathrm{Pb} & \mathrm{Pt} \\ 8 & 2\end{array}$

Direct

00000

0.92500000000000

$-0.42500000000000$

0.42500000000000

$-0.42500000000000$

0.9250000000000

0.92500000000000

0.25000000000000

0.42500000000000 0.92500000000000 0.42500000000000 0.07500000000000 0.42500000000000 0.92500000000000 0.42500000000000 0.07500000000000 0.25000000000000 0.7500000000000

0.25500000000000 0.25500000000000 0.25500000000000 0.25500000000000 $-0.25500000000000$ $-0.25500000000000$ $-0.25500000000000$ $-0.2550000000000$ 0.00000000000000

\begin{tabular}{ll}
$\mathrm{Pb}$ & $(8 \mathrm{~m})$ \\
$\mathrm{Pb}$ & $(8 \mathrm{~m})$ \\
$\mathrm{Pb}$ & $(8 \mathrm{~m})$ \\
$\mathrm{Pb}$ & $(8 \mathrm{~m})$ \\
$\mathrm{Pb}$ & $(8 \mathrm{~m})$ \\
$\mathrm{Pb}$ & $(8 \mathrm{~m})$ \\
$\mathrm{Pb}$ & $(8 \mathrm{~m})$ \\
$\mathrm{Pb}$ & $(8 \mathrm{~m})$ \\
$\mathrm{Pt}$ & $(2 \mathrm{a})$ \\
$\mathrm{Pt}$ & $(2 \mathrm{a})$ \\
\hline
\end{tabular}

$\mathrm{KCeSe}_{4}:$ ABC4_tP12_125_a_b_m - CIF

\# CIF file

data_findsym-output

audit_creation_method FINDSYM

chemical name mineral 'KCeSe4,

chemical formula sum ' $\mathrm{Ce} \mathrm{K} \mathrm{Se} 4$ '

loop

publ_author_nam

A. C. Sutorik

M. G. Kanatzidis

_journal_name_full_name

Angewandte Chemie (International ed.)

journal_volume 31

journal year 1992

journal_page first 1594

journal page last 1596

publ_section title

KCeSe\$_\{4 \$: A New Solid-State Lanthanide Polychalcogenide

\# Found in Pearson's Crystal Data - Crystal Structure Database for $\hookrightarrow$ Inorganic Compounds, 2013 


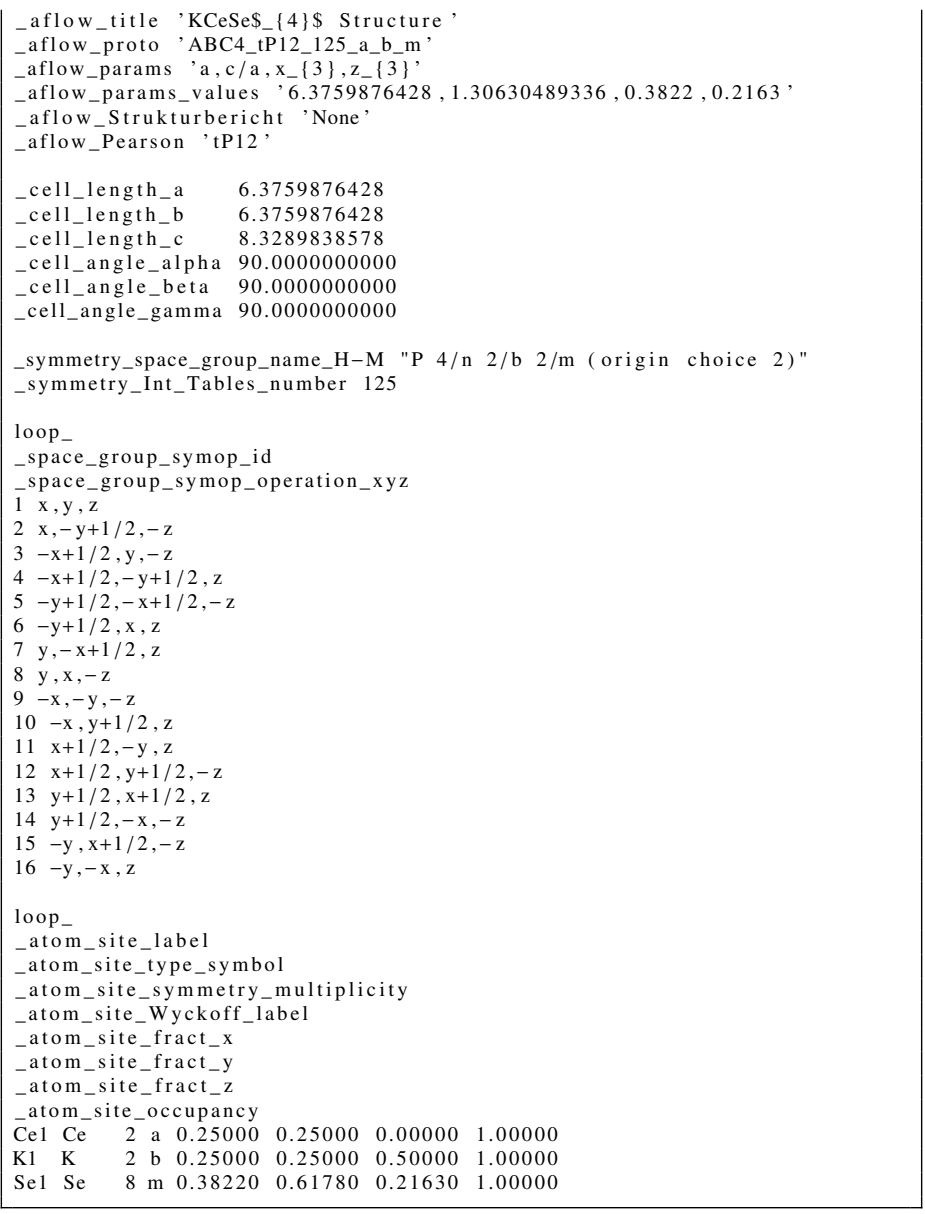

$\mathrm{KCeSe}_{4}$ : ABC4_tP12_125_a_b_m - POSCAR

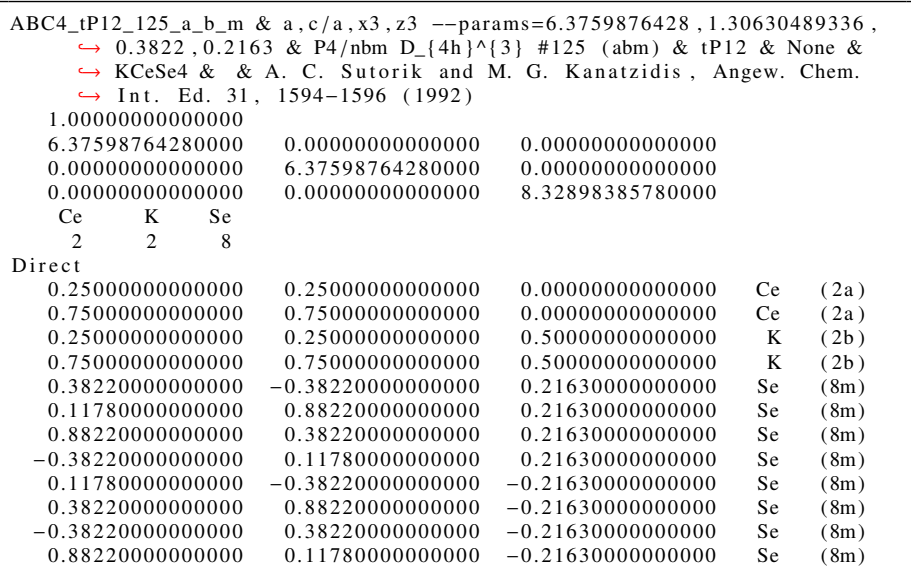

$\mathrm{BiAl}_{2} \mathrm{~S}_{4}$ : A2BC4_tP28_126_cd_e_k - CIF

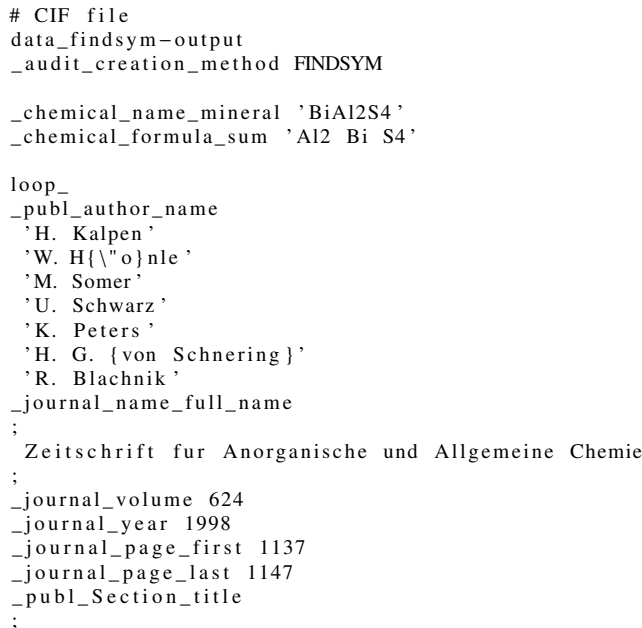

Bismut(II)-chalkogenometallate( III) Bi\$_\{2\}M $\{4\} \mathrm{X}\{8\} \$$, Verbindungen $\hookrightarrow$ mit $\mathrm{Bi}_{-}\{2\}^{\wedge}\{4+\} \$-$ Hanteln $(\$ M \$=\mathrm{Al}, \mathrm{Ga} ; \$ \mathrm{X} \$=\mathrm{S}, \mathrm{Se})$

\# Found in Pearson's Crystal Data - Crystal Structure Database for $\hookrightarrow$ Inorganic Compounds, 2013

aflow title BiAl\$ $\{2\} \$ S \$$ _ \{\} S Structure

aflow_proto 'A2BC4_tP28_126_cd_e_k

aflow_params 'a, c/a, z $\{3\}, x_{-}\{4\}, y_{-}\{4\}, z_{-}\{4\}$

aflow_params_values $\quad 7.4920241479,1.58609183128,0.11808,0.0865,0.5924$, $\hookrightarrow 0.1254$

aflow Strukturbericht ,None,

aflow_Pearson 'tP28,

cell_length_a $\quad 7.4920241479$

cell_length_b 7.4920241479

_cell_length_c 11.8830383007

_cell_angle_alpha 90.0000000000

_cell_angle_beta 90.0000000000

ccell_angle_gamma 90.0000000000

symmetry_space_group_name_H-M "P 4/n 2/n 2/c (origin choice 2)"

_symmetry_Int_Tables_number 126

loop

space_group_symop_id

space_group_symop_operation_xyz

$\mathrm{x}, \mathrm{y}, \mathrm{z}$

$\mathrm{x},-\mathrm{y}+1 / 2,-\mathrm{z}+1 / 2$

$-x+1 / 2, y,-z+1 / 2$

$4-\mathrm{x}+1 / 2,-\mathrm{y}+1 / 2, \mathrm{z}$
$5-\mathrm{y}+1 / 2,-\mathrm{x}+1 / 2,-\mathrm{z}+1 / 2$

$5-\mathrm{y}+1 / 2,-\mathrm{x}+1 / 2$
$6-\mathrm{y}+1 / 2, \mathrm{x}, \mathrm{z}$

$7 \mathrm{y},-\mathrm{x}+1 / 2, \mathrm{z}$

$8 \mathrm{y}, \mathrm{x},-\mathrm{z}+1 / 2$

$9-\mathrm{x},-\mathrm{y},-\mathrm{z}$

$10-\mathrm{x}, \mathrm{y}+1 / 2, \mathrm{z}+1 / 2$

$11 \mathrm{x}+1 / 2,-\mathrm{y}, \mathrm{z}+1 / 2$

$12 \mathrm{x}+1 / 2, \mathrm{y}+1 / 2,-\mathrm{z}$

$13 \mathrm{y}+1 / 2, \mathrm{x}+1 / 2, \mathrm{z}+1 / 2$

$14 \mathrm{y}+1 / 2,-\mathrm{x},-\mathrm{z}$

$15-\mathrm{y}, \mathrm{x}+1 / 2,-\mathrm{z}$

$16-\mathrm{y},-\mathrm{x}, \mathrm{z}+1 / 2$

loop

atom_site_label
atom

-atom_site_type_symbol

-atom_site_type_symbol
atom_site_symmetry_multiplicity

atom site Wyckoff label

atom_site_fract_x

atom_site_fract_y

atom_site_fract_z

-atom_site_occupancy

$\begin{array}{lllllllll}\text { Al1 } & \mathrm{Al} & 4 & \mathrm{c} & 0.25000 & 0.75000 & 0.75000 & 1.00000\end{array}$

$\begin{array}{llllllll}\mathrm{A} 12 & \mathrm{Al} & 4 & \mathrm{~d} & 0.25000 & 0.75000 & 0.00000 & 1.00000\end{array}$

$\begin{array}{llllllll}\mathrm{Bi} 1 \mathrm{Bi} & 4 & \mathrm{e} & 0.25000 & 0.25000 & 0.11808 & 1.00000\end{array}$

$\begin{array}{lllllllll}\mathrm{S} 1 & \mathrm{~S} & 16 & \mathrm{k} & 0.08650 & 0.59240 & 0.12540 & 1.00000\end{array}$

$\mathrm{BiAl}_{2} \mathrm{~S}_{4}$ : A2BC4_tP28_126_cd_e_k - POSCAR

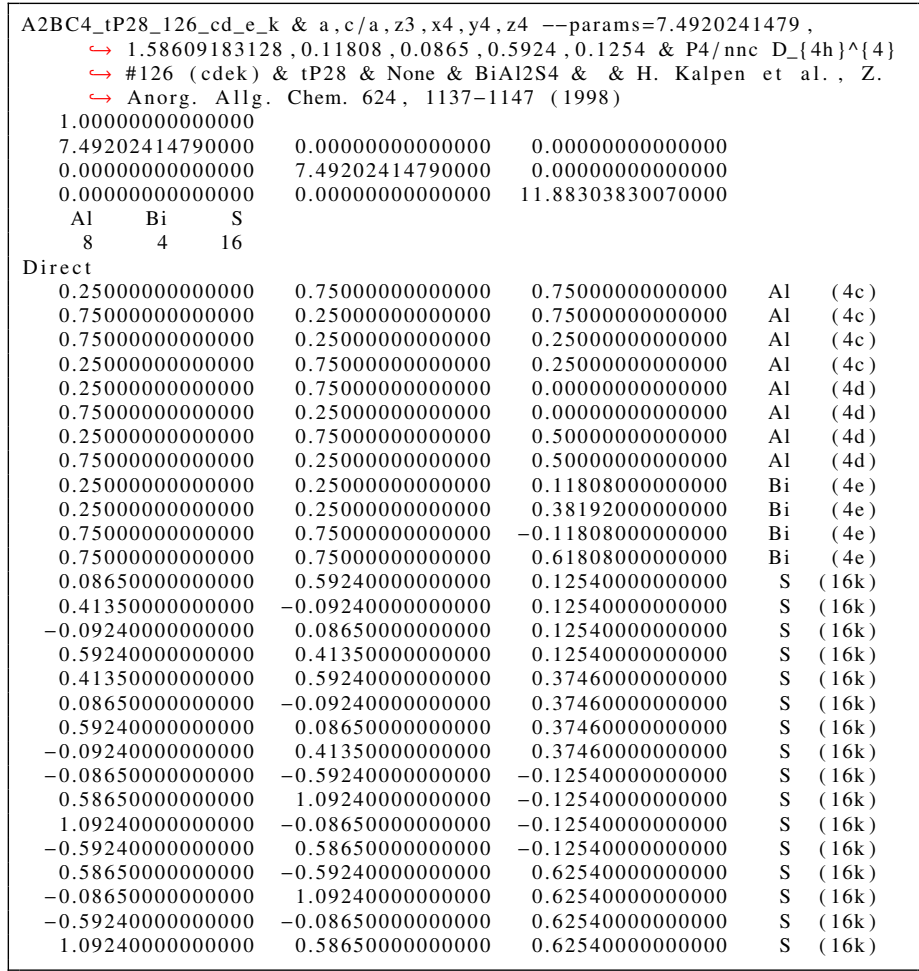

$\mathrm{ThB}_{4}\left(D 1_{e}\right):$ A4B_tP20_127_ehj_g - CIF

\# CIF file

data_findsym-output 
_audit_creation_method FINDSYM

_chemical_name_mineral 'ThB4,

_chemical_formula_sum 'B4 Th'

loop

publ_author_name

'A. Zalkin'

'D. H. Templeton'

_journal_name_full_name

Journal of Chemical Physics

_journal_volume 18

journal year 1950

journal_page_first 391

journal_page_last 391

publ_Section_title

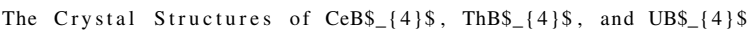

aflow_title $T h B \$ \_\{4\}$ ( $\$ D 1_{-}\{$e $\left.\} \$\right)$ Structure

aflow_proto 'A4B_tP20_127_ehj_g

aflow_params ,a,c/a, z $\{1\}, x_{-}\{2\}, x_{-}\{3\}, x_{-}\{4\}, y_{-}\{4\}$

aflow_params_values ' $7.256,0.56684123484,0.2,0.31,0.1,0.2,0.04$ '

aflow Strukturbericht, \$D1_\{e\}\$

aflow Pearson 'tP20'

symmetry_space_group_name_H-M "P 4/m 21/b 2/m"

symmetry_Int_Tables_number 127

cell_length_a $\quad 7.25600$

cell_length_b $\quad 7.25600$

cell_length_c 4.11300

cell_angle_alpha 90.00000

_cell_angle_beta 90.00000

_cell_angle_gamma 90.00000

loop

_space_group_symop_id

_space_group_symop_operation_xyz

$1 \mathrm{x}, \mathrm{y}, \mathrm{z}$

$2 \mathrm{x}+1 / 2,-\mathrm{y}+1 / 2,-\mathrm{z}$

$3-\mathrm{x}+1 / 2, \mathrm{y}+1 / 2,-\mathrm{z}$

$4-\mathrm{x},-\mathrm{y}, \mathrm{z}$

$5-\mathrm{y}+1 / 2,-\mathrm{x}+1 / 2,-\mathrm{z}$

$6-\mathrm{y}, \mathrm{x}, \mathrm{z}$

$8 \mathrm{y}+1 / 2, \mathrm{x}+1 / 2,-\mathrm{z}$

$9-x,-y,-z$

$10-x+1 / 2, y+1 / 2, z$

$11 \mathrm{x}+1 / 2,-\mathrm{y}+1 / 2, \mathrm{z}$

$12 \mathrm{x}, \mathrm{y},-\mathrm{z}$

$13 \mathrm{y}+1 / 2, \mathrm{x}+1 / 2, \mathrm{z}$

$14 \mathrm{y},-\mathrm{x},-\mathrm{z}$

$15-\mathrm{y}, \mathrm{x},-\mathrm{z}$

$16-y+1 / 2,-x+1 / 2, z$

loop

-atom_site type symbol

atom_site_symmetry_multiplicity

atom_site_Wyckoff_label

atom_site_fract_x

atom site fract

atom_site_fract_z

atom site occupancy

$\begin{array}{llllllll}\mathrm{B} 1 & \mathrm{~B} & 4 & \mathrm{e} & 0.00000 & 0.00000 & 0.20000 & 1.00000\end{array}$

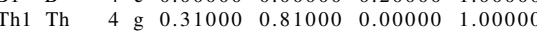

$\begin{array}{lllllllll}\mathrm{B} 2 & \mathrm{~B} & 4 & \mathrm{~h} & 0.10000 & 0.60000 & 0.50000 & 1.00000\end{array}$

$\begin{array}{lllllllll}\text { B } 3 & \text { B } & 8 & \text { j } & 0.20000 & 0.04000 & 0.50000 & 1.00000\end{array}$

$\mathrm{ThB}_{4}\left(D 1_{e}\right):$ A4B_tP20_127_ehj_g - POSCAR

A4B_tP20_127_ehj_g \& a,c/a, z1, x2, x3, x4,y4 --params $=7.256,0.56684123484$,

$\hookrightarrow 0.2,0.31,0.1,0.2,0.04 \&$ P4/mbm D_ $\{4 \mathrm{~h}\}^{\wedge}\{5\} \# 127$ (eghj) \& tP20\&

$\hookrightarrow$ \$D1_\{e\}\$\& ThB4\& ThB4 \& A. Zalkin and D. H. Templeton, J

$\hookrightarrow$ Chem. Phys. 18, 391(1950)

1.00000000000000

$\begin{array}{lll}7.25600000000000 & 0.00000000000000 & 0.00000000000000\end{array}$

$\begin{array}{lll}0.00000000000000 & 7.25600000000000 & 0.00000000000000\end{array}$

$0.00000000000000 \quad 0.00000000000000 \quad 4.11300000000000$

B Th

Direct

0.00000000000000

0.50000000000000

0.50000000000000

0.00000000000000

0.50000000000000

0.10000000000000

$-0.10000000000000$

0.40000000000000

0.60000000000000

0.20000000000000

$-0.20000000000000$

$-0.04000000000000$

0.04000000000000

0.30000000000000

0.70000000000000

0.54000000000000

0.46000000000000

0.31000000000000

$-0.31000000000000$

0.19000000000000

0.00000000000000 $0.50000000000000-0.20000000000000$ $0.00000000000000-0.20000000000000$ $\begin{array}{rr}0.50000000000000 & 0.20000000000000\end{array}$ $0.60000000000000 \quad 0.50000000000000$ $0.40000000000000 \quad 0.50000000000000$ $0.10000000000000 \quad 0.50000000000000$ $-0.10000000000000 \quad 0.50000000000000$ $0.04000000000000 \quad 0.50000000000000$ $\begin{array}{lll}0.04000000000000 & 0.50000000000000\end{array}$ $0.20000000000000 \quad 0.5000000000000$ 0.50000000000000 0.5000000000000 0.50000000000000 $0.70000000000000-0.50000000000000$ $0.30000000000000-0.50000000000000$ $0.81000000000000-0.00000000000000$ $0.19000000000000-0.00000000000000$ $\begin{array}{ll}0.19000000000000 & 0.00000000000000 \\ 0.31000000000000 & 0.00000000000000\end{array}$
$0.81000000000000 \quad-0.31000000000000$

$\mathrm{K}_{2} \mathrm{SnCl}_{6}$ (Low-temperature): A6B2C_tP18_128_eh_d_b - CIF

\# CIF fil

data findsym-output

audit_creation_method FINDSYM

chemical name mineral ' $\mathrm{K} 2 \mathrm{SnCl6}$ '

chemical_formula_sum, Cl6 K2 Sn,

loop

publ_author_nam

'H. Boysen'

'A. W. Hewat

journal_name_full_name

Acta Crystallographica Section B: Structural Science

journal_volume 34

journal_year 1978

journal_page first 1412

journal page last 1418

publ Section title

A neutron powder investigation of the structural changes in $K \$\{\{2\}$ $\hookrightarrow$ SSnCl\$_\{6\}\$

\# Found in Pearson's Crystal Data - Crystal Structure Database for $\hookrightarrow$ Inorganic Compounds, 2013

aflow_title $K \$ \_\{2\} \$ S n C l \$\{\{6\} \$$ (Low-temperature) Structure,

aflow_proto 'A6B2C_tP18_128_eh_d_b

aflow_params 'a, c/a, z $\{3\}, x_{-}\{4\}, y \_\{4\}$

aflow_params_values ' $7.057532571,1.41383170154,0.7523,0.7217,0.2489$

aflow_Strukturbericht , None'

aflow Pearson, tP18,

cell_length_a $\quad 7.0575325710$

cell_length_b 7.0575325710

cell_length_c $\quad 9.9781632835$

cell angle alpha 900000000000

cell_angle_beta 90.0000000000

_cell_angle_gamma 90.0000000000

symmetry_space_group_name_H-M "P $4 / \mathrm{m} \mathrm{21/n} \mathrm{2/c"}$

symmetry_Int_Tables_number 128

loop

space_group_symop_id

space group_symop_operation_xyz

$1 \mathrm{x}, \mathrm{y}, \mathrm{z}$

$\mathrm{x}+1 / 2,-\mathrm{y}+1 / 2,-\mathrm{z}+1 / 2$

$-x+1 / 2, y+1 / 2,-z+1 / 2$

$4-\mathrm{x},-\mathrm{y}, \mathrm{z}$

$5-\mathrm{y}+1 / 2,-\mathrm{x}+1 / 2,-\mathrm{z}+1 / 2$

$6-\mathrm{y}, \mathrm{x}, \mathrm{z}$

$7 \mathrm{y},-\mathrm{x}, \mathrm{z}$

$8 \mathrm{y}+1 / 2, \mathrm{x}+1 / 2,-\mathrm{z}+1 / 2$

-x, $-\mathrm{y},-$

$10-x+1 / 2, y+1 / 2, z+1 / 2$

$11 \mathrm{x}+1 / 2,-\mathrm{y}+1 / 2, \mathrm{z}+1 / 2$

$12 \mathrm{x}, \mathrm{y},-\mathrm{z}$

$13 \mathrm{y}+1 / 2, \mathrm{x}+1 / 2, \mathrm{z}+1 / 2$

$14 \mathrm{y},-\mathrm{x},-\mathrm{z}$

$15-\mathrm{y}, \mathrm{x},-\mathrm{z}$

$16-y+1 / 2,-x+1 / 2, z+1 / 2$

loop

atom_site label

atom_site_type_symbol

atom_site_symmetry_multiplicity

atom_site_Wyckoff_label

atom_site_fract_x

atom_site_fract_y

-atom_site_fract_z

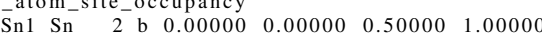

$\begin{array}{lllllllll}\mathrm{K} 1 & \mathrm{~K} & 4 & \mathrm{~d} & 0.00000 & 0.50000 & 0.25000 & 1.00000\end{array}$

$\begin{array}{llllllll}\mathrm{C} 11 & \mathrm{Cl} & 4 & \mathrm{e} & 0.00000 & 0.00000 & 0.75230 & 1.00000\end{array}$

$\begin{array}{lllllllll}\mathrm{Cl} 2 & \mathrm{Cl} & 8 & \mathrm{~h} & 0.72170 & 0.24890 & 0.00000 & 1.00000\end{array}$

$\mathrm{K}_{2} \mathrm{SnCl}_{6}$ (Low-temperature): A6B2C_tP18_128_eh_d_b - POSCAR

A6B2C_tP18_128_eh_d_b \& a , c/a , z3 , x4,y4 --params $=7.057532571$

$\hookrightarrow 1.41383170154,0.7523,0.7217,0.2489 \&$ P4/mnc D_ $\{4 \mathrm{~h}\} \wedge\{6\} \# 128$

$\hookrightarrow$ bdeh ) \& tP18 \& None \& K2SnCl6 \& \& H. Boysen and A. W. Hewat,

$\hookrightarrow$ Acta Crystallogr. Sect. B Struct. Sci. 34, 1412-1418 (1978)

1.00000000000000

$\begin{array}{lll}7.05753257100000 & 0.00000000000000 & 0.00000000000000\end{array}$

$\begin{array}{lll}0.00000000000000 & 7.05753257100000 & 0.00000000000000\end{array}$

$\begin{array}{lll}0.00000000000000 & 0.00000000000000 & 9.97816328350000\end{array}$

$\begin{array}{lll}\mathrm{Cl} & \mathrm{K} & \mathrm{Sn}\end{array}$

Direct

0.00000000000000

0.50000000000000

0.00000000000000

0.50000000000000

0.72170000000000

$-0.72170000000000$

$-0.24890000000000$

0.2489000000000

$-0.22170000000000$

0.00000000000000 0.50000000000000

0.00000000000000

0.50000000000000

0.24890000000000

$-0.24890000000000$

0.72170000000000

$-0.72170000000000$

0.74890000000000

0.75230000000000 $-0.25230000000000$ $-0.75230000000000$ 1. 25230000000000 0.0000000000000 0.00000000000000 0.00000000000000 0.00000000000000 0.50000000000000 


$\begin{array}{rrrrr}1.22170000000000 & 0.25110000000000 & 0.50000000000000 & \mathrm{Cl} & (8 \mathrm{~h}) \\ 0.74890000000000 & 1.22170000000000 & 0.50000000000000 & \mathrm{Cl} & (8 \mathrm{~h}) \\ 0.25110000000000 & -0.22170000000000 & 0.50000000000000 & \mathrm{Cl} & (8 \mathrm{~h}) \\ 0.00000000000000 & 0.50000000000000 & 0.25000000000000 & \mathrm{~K} & (4 \mathrm{~d}) \\ 0.50000000000000 & 0.00000000000000 & 0.25000000000000 & \mathrm{~K} & (4 \mathrm{~d}) \\ 0.00000000000000 & 0.50000000000000 & 0.75000000000000 & \mathrm{~K} & (4 \mathrm{~d}) \\ 0.50000000000000 & 0.00000000000000 & 0.75000000000000 & \mathrm{~K} & (4 \mathrm{~d}) \\ 0.00000000000000 & 0.00000000000000 & 0.50000000000000 & \mathrm{Sn} & (2 \mathrm{~b}) \\ 0.50000000000000 & 0.50000000000000 & 0.00000000000000 & \mathrm{Sn} & (2 \mathrm{~b})\end{array}$

$\mathrm{FeCu}_{2} \mathrm{Al}_{7}(E 9 a)$ : A7B2C_tP40_128_egi_h_e - CIF

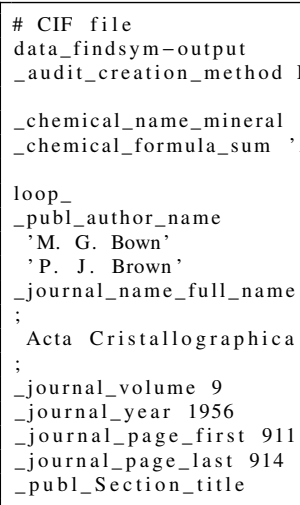

$\mathrm{FeCu}_{2} \mathrm{Al}_{7}\left(E 9_{a}\right)$ : A7B2C_tP40_128_egi_h_e - POSCAR

A7B2C_tP40_128_egi_h_e \& a , c/a, z1 , z2 , x3 $, \mathrm{x} 4, \mathrm{y} 4, \mathrm{x} 5, \mathrm{y} 5, \mathrm{z5}--$ params $=6.336$, $\hookrightarrow 2.34690656566,0.366,0.2008,0.165,0.278,0.088,0.198,0.42,0.1 \&$

$\hookrightarrow \mathrm{P} 4 / \mathrm{mnc} \mathrm{D}_{-}\{4 \mathrm{~h}\}^{\wedge}\{6\} \# 128\left(\mathrm{e}^{\wedge} 2 \mathrm{ghi}\right) \& \mathrm{tP} 40 \&$ \$E9_\{a\}\$ \& FeCu2Al7 \& $\hookrightarrow \mathrm{FeCu} 2 \mathrm{~A} 17$

1.00000000000000

6.3360000000000

$0.00000000000000 \quad 0.00000000000000$

$\begin{array}{lll}0.00000000000000 & 6.33600000000000 & 0.00000000000000\end{array}$

$\begin{array}{rrr}0.00000000000000 & 0.00000000000000 & 14.87000000000000\end{array}$

$\mathrm{Al} \quad \mathrm{Cu} \quad \mathrm{Fe}$

Direct

0.00000000000000

0.50000000000000

0.00000000000000 0.50000000000000
0.36600000000000 0.13400000000000
0.00000000000000 0.5000000000000 0.16500000000000 $-0.16500000000000$ 0.33500000000000 0.66500000000000 $-0.16500000000000$ 0.16500000000000 0.66500000000000 0.33500000000000 0.19800000000000 $-0.19800000000000$ $-0.42000000000000$ 0.4200000000000 0.4200000000000 0.30200000000000 0.69800000000000 0.92000000000000 0.08000000000000 $-0.19800000000000$ 0.19800000000000 0.42000000000000 $-0.42000000000000$ 0.69800000000000 0.30200000000000 0.08000000000000 0.92000000000000 0.27800000000000 $-0.27800000000000$ $-0.08800000000000$ 0.08800000000000 0.22200000000000 0.7780000000000 0.788000000000 0.5880000000000 0.4120000000000 0.00000000000000 0.50000000000000 0.0000000000000 0.50000000000000

.00000000000000 0.50000000000000 0.66500000000000 0.33500000000000 0.16500000000000 $-.16500000000000$ 0.33500000000000 0.66500000000000 $-0.16500000000000$ 0.16500000000000 0.42000000000000 $-0.42000000000000$ 0.19800000000000 0.19800000000000 0.92000000000000 0.08000000000000 0.69800000000000 0.6980000000000 0.3020000000000 $-0.42000000000000$ 0.42000000000000 0.19800000000000 0.19800000000000 0.08000000000000 0.92000000000000 0.30200000000000 0.69800000000000 0.08800000000000 $-0.08800000000000$ 0.27800000000000 $-0.27800000000000$ 0.58800000000000 0.41200000000000 0.7780000000000 0.77800000000 0.00000000000000 0.00000000000000 0.50000000000000 0.50000000000000

$\mathrm{CuBi}_{2} \mathrm{O}_{4}$ : A2BC4_tP28_130_f_c_g - CIF

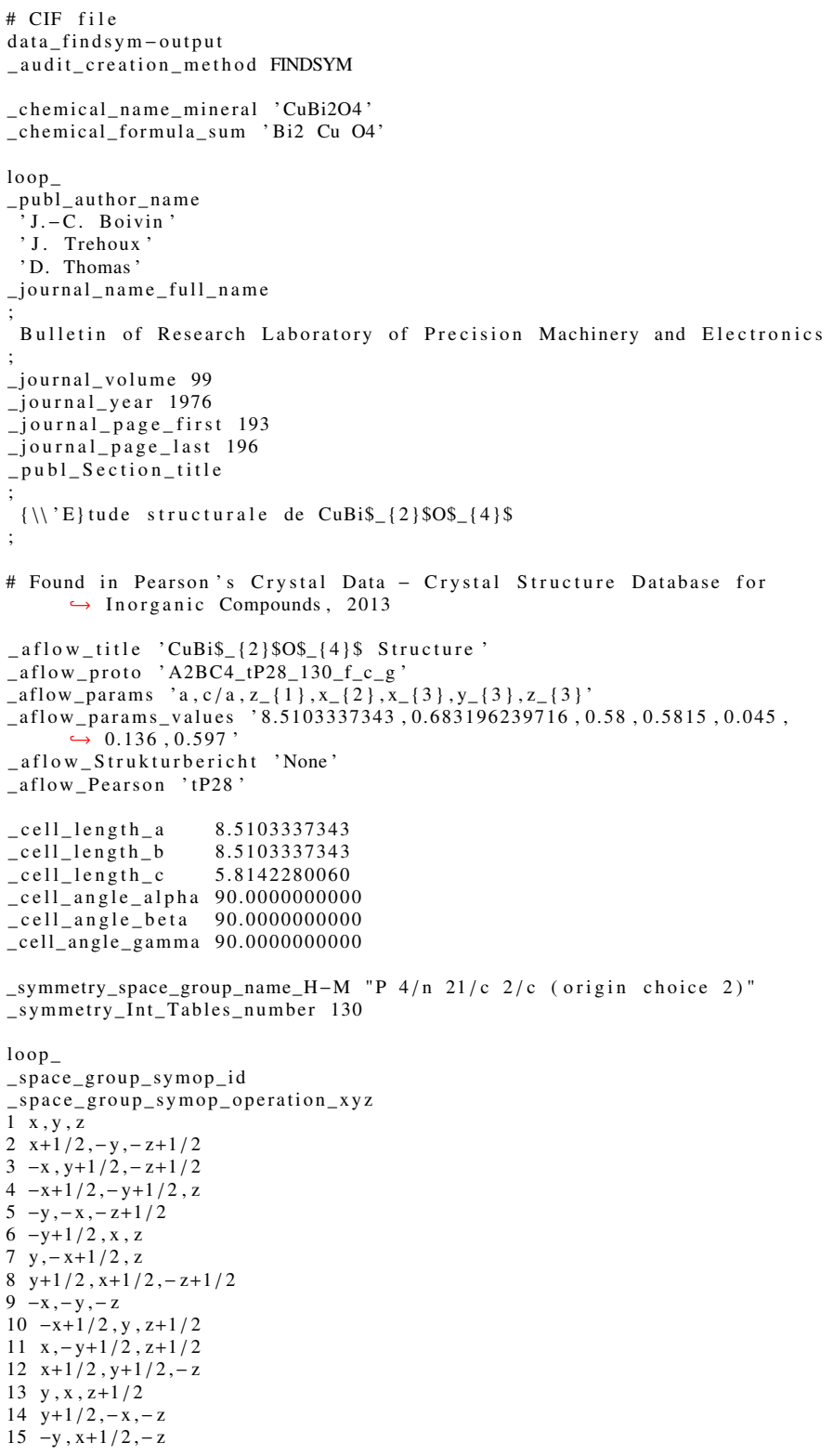

$-0.36600000000000$ 0.86600000000000 0.25000000000000 0.25000000000000 0.25000000000 0.25000000000000 0.75000000000000 0.75000000000000 0.75000000000000 0.75000000000000 0.10000000000000 0.10000000000000 0.10000000000000 0.10000000000000 0.40000000000000 0.40000000000000 0.40000000000000 0.40000000000000 0.40000000000 $-0.10000000000000$ 0.1000000000000 $-0.10000000000000$ . 1000000000000 0.60000000000000 $-100000000$ .60000000000000 0.60000000000000 0.00000000000000 0.00000000000000 0.00000000000000 0.00000000000000 0.50000000000000 0.50000000000000 .50000000000000 .50000000000000 0.20080000000000 0.29920000000000 $-0.20080000000000$ 0.70080000000000 


\begin{tabular}{|c|c|c|c|c|}
\hline \\
\hline \multirow{2}{*}{\multicolumn{5}{|c|}{$\begin{array}{l}\text { _atom_site_label } \\
\text { _atom_site_type_symbol }\end{array}$}} \\
\hline \multirow{2}{*}{\multicolumn{5}{|c|}{$\begin{array}{l}\text {-atom_site_type_symbol } \\
\text {-atom_site_symmetry_multiplicity }\end{array}$}} \\
\hline & & & & \\
\hline \multicolumn{5}{|c|}{ _atom_site_Wyckoff_label } \\
\hline \multicolumn{5}{|c|}{-atom_site_fract_x } \\
\hline \multicolumn{5}{|l|}{ _atom_site_fract_y } \\
\hline \multicolumn{5}{|l|}{$\begin{array}{l}\text {-atom_site_fract_z } \\
\text { atom_site_occupancy }\end{array}$} \\
\hline \multicolumn{5}{|c|}{ 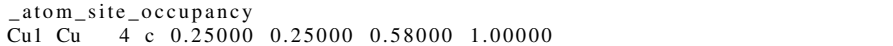 } \\
\hline $\mathrm{Bi} 1 \mathrm{Bi} \quad 8 \mathrm{f} \quad 0.58150$ & $\begin{array}{llll}0.41850 & 0.25000 & 1\end{array}$ & \\
\hline $\begin{array}{lllll}01 & \mathrm{O} & 16 & \mathrm{~g} & 0.04500\end{array}$ & $0.13600 \quad 0.59700 \quad 1$. & \multicolumn{3}{|c|}{$\begin{array}{l}1.00000 \\
1.00000\end{array}$} \\
\hline \multicolumn{5}{|c|}{$\mathrm{CuBi}_{2} \mathrm{O}_{4}:$ A2BC4_tP28_130_f_c_g - POSCAR } \\
\hline \multicolumn{5}{|c|}{ 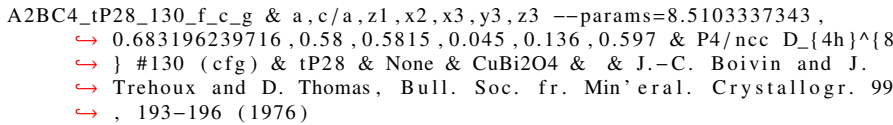 } \\
\hline \multicolumn{5}{|l|}{1.00000000000000} \\
\hline 8.51033373430000 & 0.00000000000000 & 0.00000000000000 & & \\
\hline \multirow{2}{*}{$\begin{array}{l}0.00000000000000 \\
0.00000000000000\end{array}$} & 8.51033373430000 & 0.00000000000000 & & \\
\hline & 0.00000000000000 & 5.81422800600000 & & \\
\hline $\begin{array}{cc}0.00000000000000 \\
\mathrm{Bi} & \mathrm{Cu} \\
\mathrm{O}\end{array}$ & & & & \\
\hline 16 & & & & \\
\hline \multicolumn{5}{|l|}{ Direct } \\
\hline 0.58150000000000 & -0.58150000000000 & 0.25000000000000 & $\mathrm{Bi}$ & $(8 \mathrm{f})$ \\
\hline-0.08150000000000 & -0.08150000000000 & 0.25000000000000 & $\mathrm{Bi}$ & $(8 \mathrm{f})$ \\
\hline 1.08150000000000 & 0.58150000000000 & 0.25000000000000 & $\mathrm{Bi}$ & $(8 \mathrm{f})$ \\
\hline-0.58150000000000 & -0.08150000000000 & 0.25000000000000 & $\mathrm{Bi}$ & ( $8 f$ f) \\
\hline-0.58150000000000 & 0.58150000000000 & 0.75000000000000 & $\mathrm{Bi}$ & ( $8 f$ f) \\
\hline 1.08150000000000 & -0.08150000000000 & 0.75000000000000 & $\mathrm{Bi}$ & ( $8 f$ f) \\
\hline-0.08150000000000 & -0.58150000000000 & 0.75000000000000 & $\mathrm{Bi}$ & $(8 \mathrm{f})$ \\
\hline 0.58150000000000 & 1.08150000000000 & 0.75000000000000 & $\mathrm{Bi}$ & $(8 \mathrm{f})$ \\
\hline 0.25000000000000 & 0.25000000000000 & 0.58000000000000 & $\mathrm{Cu}$ & $(4 c)$ \\
\hline 0.75000000000000 & 0.75000000000000 & -0.08000000000000 & $\mathrm{Cu}$ & $(4 c)$ \\
\hline 0.75000000000000 & 0.75000000000000 & -0.58000000000000 & $\mathrm{Cu}$ & $(4 c)$ \\
\hline 0.25000000000000 & 0.25000000000000 & 1.08000000000000 & $\mathrm{Cu}$ & $(4 c)$ \\
\hline 0.04500000000000 & 0.13600000000000 & 0.59700000000000 & $\mathrm{O}$ & $(16 \mathrm{~g})$ \\
\hline 0.45500000000000 & 0.36400000000000 & 0.59700000000000 & $\mathrm{O}$ & $(16 \mathrm{~g})$ \\
\hline 0.36400000000000 & 0.04500000000000 & 0.59700000000000 & $\mathrm{O}$ & $(16 \mathrm{~g})$ \\
\hline 0.13600000000000 & 0.45500000000000 & 0.59700000000000 & $\mathrm{O}$ & $(16 \mathrm{~g})$ \\
\hline-0.04500000000000 & 0.63600000000000 & -0.09700000000000 & $\mathrm{O}$ & $(16 \mathrm{~g})$ \\
\hline 0.54500000000000 & -0.13600000000000 & -0.09700000000000 & $\mathrm{O}$ & $(16 \mathrm{~g})$ \\
\hline 0.63600000000000 & 0.54500000000000 & -0.09700000000000 & $\mathrm{O}$ & $(16 \mathrm{~g})$ \\
\hline-0.13600000000000 & -0.04500000000000 & -0.09700000000000 & $\mathrm{O}$ & $(16 \mathrm{~g})$ \\
\hline-0.04500000000000 & -0.13600000000000 & -0.59700000000000 & $\mathrm{O}$ & $(16 \mathrm{~g})$ \\
\hline 0.54500000000000 & 0.63600000000000 & -0.59700000000000 & $\mathrm{O}$ & $(16 \mathrm{~g})$ \\
\hline 0.63600000000000 & -0.04500000000000 & -0.59700000000000 & $\mathrm{O}$ & $(16 \mathrm{~g})$ \\
\hline-0.13600000000000 & 0.54500000000000 & -0.59700000000000 & $\mathrm{O}$ & $(16 \mathrm{~g})$ \\
\hline 0.04500000000000 & 0.36400000000000 & 1.09700000000000 & $\mathrm{O}$ & $(16 \mathrm{~g})$ \\
\hline 0.45500000000000 & 0.13600000000000 & 1.09700000000000 & $\mathrm{O}$ & $(16 \mathrm{~g})$ \\
\hline 0.36400000000000 & 0.45500000000000 & 1.09700000000000 & $\mathrm{O}$ & $(16 \mathrm{~g})$ \\
\hline 0.13600000000000 & 0.04500000000000 & 1.09700000000000 & $\mathrm{O}$ & $(16 \mathrm{~g})$ \\
\hline
\end{tabular}

Ba $5 \mathrm{Si}_{3}$ : A5B3_tP32_130_cg_cf - CIF

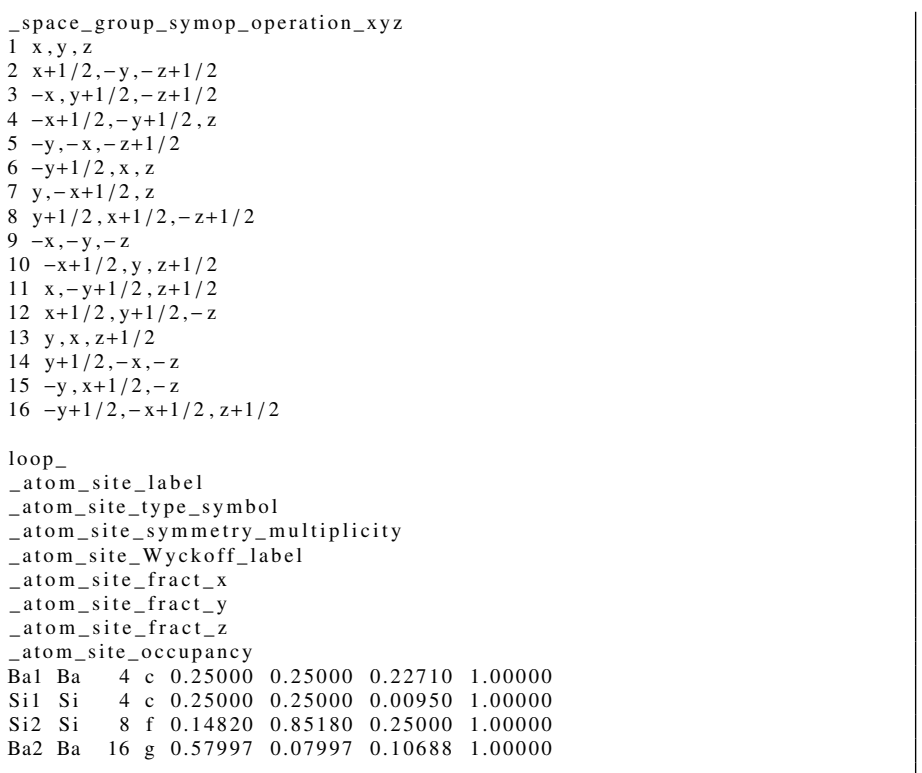

$\mathrm{Ba}_{5} \mathrm{Si}_{3}$ : A5B3_tP32_130_cg_cf - POSCAR

\begin{tabular}{|c|c|c|c|c|}
\hline & \multicolumn{3}{|c|}{ A5B3_tP32_130_cg_cf $\& \mathrm{a}, \mathrm{c} / \mathrm{a}, \mathrm{z} 1, \mathrm{z} 2, \mathrm{x} 3$} \\
\hline \multirow{2}{*}{\multicolumn{5}{|c|}{$\begin{array}{l}\hookrightarrow \text { 1.94329592439,0.2271, } 0.0095,0.1482,0.57997,0.07997,0.10688 \text { \& P4 } \\
\hookrightarrow / \text { ncc D_ }\{4 \mathrm{~h}\} \wedge\{8\} \# 130\left(\mathrm{c}^{\wedge} 2 \mathrm{fg}\right) \& \text { \& }\left\{\begin{array}{l}\text { \& } \\
\hookrightarrow\end{array} \text { None \& Ba5Si3 \& \& R. }\right. \\
\hookrightarrow \text { Nesper and F. Z }\{\backslash " \mathrm{u}\} \text { rcher, Z. Kristallogr. B } 214,20(1966)\end{array}$}} \\
\hline & & & & \\
\hline \multicolumn{5}{|c|}{1.00000000000000} \\
\hline 8.46500000000000 & 0.00000000000000 & 0.00000000000000 & & \\
\hline 0.00000000000000 & 8.46500000000000 & 0.00000000000000 & & \\
\hline 0.00000000000000 & 0.00000000000000 & 16.45000000000000 & & \\
\hline $\mathrm{Ba}$ & & & & \\
\hline 20 & & & & \\
\hline \multicolumn{5}{|l|}{ Direct } \\
\hline 0.25000000000000 & 0.25000000000000 & 0.22710000000000 & $\mathrm{Ba}$ & $(4 \mathrm{c})$ \\
\hline 0.75000000000000 & 0000 & 0.27290000000000 & $\mathrm{Ba}$ & \\
\hline 0.75000000000000 & 0.75000000000000 & -0.22710000000000 & $\mathrm{Ba}$ & $(4 \mathrm{c})$ \\
\hline 0.25000000000000 & 0.25000000000000 & 0.72710000000000 & $\mathrm{Ba}$ & \\
\hline 0.57997000 & 00 & 000 & $\mathrm{Ba}$ & $(16 \mathrm{~g})$ \\
\hline-0.07997000000000 & 0.42003000000000 & 0.10688000000000 & $\mathrm{Ba}$ & $(16 \mathrm{~g})$ \\
\hline 0.42003000000000 & 7000000000 & 0.10688000000000 & $\mathrm{Ba}$ & $(16 \mathrm{~g})$ \\
\hline 0.07997000000000 & -0.07997000000000 & 0.10688000000000 & $\mathrm{Ba}$ & $(16 \mathrm{~g})$ \\
\hline-0.57997000000000 & 0.579 & 0.39312000000000 & $\mathrm{Ba}$ & $(16 \mathrm{~g})$ \\
\hline 1.07997000000000 & -0.07997000000000 & 0.39312000000000 & $\mathrm{Ba}$ & $(16 \mathrm{~g})$ \\
\hline 0.57997000000000 & 1.07997000000000 & 0.39312000000000 & $\mathrm{Ba}$ & (16g) \\
\hline-0.07997000000000 & -0.57997000000000 & 0.39312000000000 & $\mathrm{Ba}$ & $(16 \mathrm{~g})$ \\
\hline-0.57997000000000 & -0.07997000000000 & -0.10688000000000 & $\mathrm{Ba}$ & $(16 \mathrm{~g})$ \\
\hline 1.07997000000000 & 0.57997000000000 & -0.10688000000000 & $\mathrm{Ba}$ & $(16 \mathrm{~g})$ \\
\hline 0.57997000000000 & -0.57997000000000 & -0.10688000000000 & $\mathrm{Ba}$ & $(16 \mathrm{~g})$ \\
\hline-0.07997000000000 & 1.07997000000000 & -0.10688000000000 & $\mathrm{Ba}$ & $(16 \mathrm{~g})$ \\
\hline 0.57997000000000 & 0.42003000000000 & 0.60688000000000 & $\mathrm{Ba}$ & $(16 \mathrm{~g})$ \\
\hline-0.07997000000000 & 0000000 & 0.60688000000000 & $\mathrm{Ba}$ & $(16 \mathrm{~g})$ \\
\hline 0.42003000000000 & -0.079970 & 0.60688000000000 & $\mathrm{Ba}$ & $(16 \mathrm{~g})$ \\
\hline 0.07997000000000 & 0.57997000000000 & 0.60688000000000 & $\mathrm{Ba}$ & $(16 \mathrm{~g})$ \\
\hline 0.25000000000000 & 0.25000000000000 & 0.00950000000000 & $\mathrm{Si}$ & $(4 c)$ \\
\hline 0.75000000000000 & 0.75000000000000 & 0.49050000000000 & $\mathrm{Si}$ & $(4 \mathrm{c})$ \\
\hline 0.75000000000000 & 0.75000000000000 & -0.00950000000000 & $\mathrm{Si}$ & $(4 c)$ \\
\hline 0.25000000000000 & 0.25000000000000 & 0.50950000000000 & $\mathrm{Si}$ & (4c) \\
\hline 0.14820000000000 & -0.14820000000000 & 0.25000000000000 & $\mathrm{Si}$ & (8f) \\
\hline 0.35180000000000 & 0.35180000000000 & 0.25000000000000 & $\mathrm{Si}$ & \\
\hline 0.64820000000000 & 0.14820000000000 & 0.25000000000000 & $\mathrm{Si}$ & $(8 \mathrm{f})$ \\
\hline-0.14820000000000 & 0.35180000000000 & 0.25000000000000 & $\mathrm{Si}$ & \\
\hline-0.14820000000000 & 0.14820000000000 & 0.75000000000000 & $\mathrm{Si}$ & $(8 \mathrm{f})$ \\
\hline 0.64820000000000 & 0.35180000000000 & 0.75000000000000 & $\mathrm{Si}$ & (8f) \\
\hline 0.35180000000000 & -0.14820000000000 & 0.75000000000000 & $\mathrm{Si}$ & (8f) \\
\hline 0.14820000000000 & 0.64820000000000 & 0.75000000000000 & $\mathrm{Si}$ & (8f) \\
\hline
\end{tabular}

$\mathrm{Rb}_{2} \mathrm{TiCu}_{2} \mathrm{~S}_{4}$ : A2B2C4D_tP18_132_e_i_o_d - CIF

\section{\# CIF file}

data_findsym-output

audit_creation_method FINDSYM

chemical name mineral 'Rb2TiCu2Se4,

chemical_formula_sum, Cu2 Rb2 S4 Ti,

loop

publ_author_name

'F. Q. Huang'

journal_name_full_name

Inorganic Chemistry

journal_volume 40

journal year 2001

journal_page_first 2602

journal_page_last 2607

publ_Section title

New Layered Materials: Syntheses, Structures, and Optical Properties of $\hookrightarrow \mathrm{K} \$_{-}\{2\} \$ \mathrm{TiCu} \$_{-}\{2\} \$ S \$_{-}\{4\} \$, \operatorname{Rb} \$_{-}\{2\} \$ \mathrm{TiCu} \$_{-}\{2\} \$ \mathrm{~S} \$_{-}\{4\} \$, \operatorname{Rb} \$_{-}\{2\}$

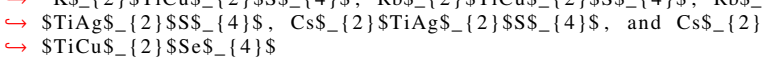




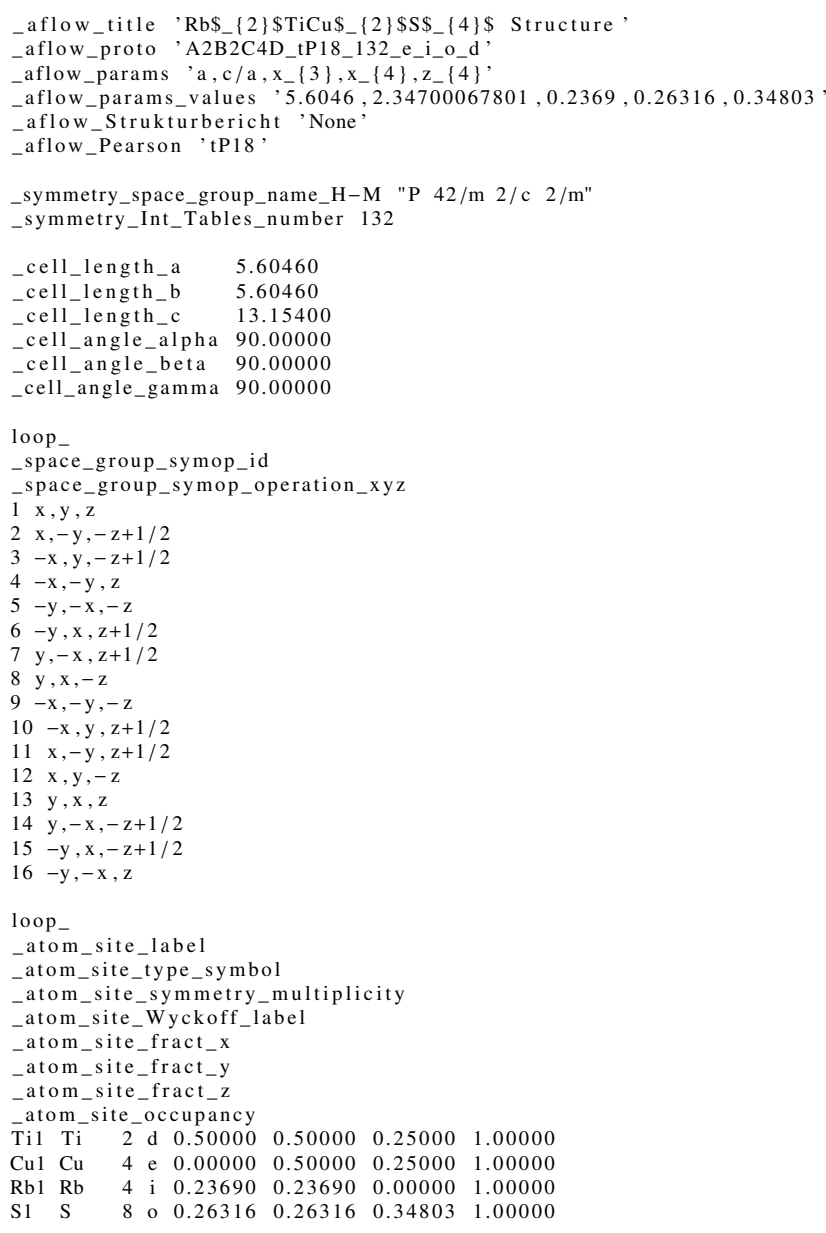

$\mathrm{Rb}_{2} \mathrm{TiCu}_{2} \mathrm{~S}_{4}$ : A2B2C4D_tP18_132_e_i_o_d - POSCAR

A2B2C4D_tP18 132 e i o d \& a c c/a, x3, x4, z4 -params $=5.6046,2.34700067801$ $\hookrightarrow 0.2369,0.26316,0.34803 \& \mathrm{P} 4 \_\{2\} / \mathrm{mcm} \mathrm{D}_{-}\{4 \mathrm{~h}\}^{\wedge}\{10\} \# 132$ (deio) \&

$\hookrightarrow \mathrm{tP} 18$ \& None \& Rb2TiCu2Se4 \& Rb2TiCu2Se4 \& F. Q. Huang and J. A. $\hookrightarrow$ Ibers, Inorg. Chem. 40, 2602-2607 (2001) 1.00000000000000 5.60460000000000 0.00000000000000 0.00000000000000 $\mathrm{Cu} \quad \mathrm{Rb} \quad \mathrm{S} \quad \mathrm{T}$

Direct

$\begin{array}{lll}0.00000000000000 & 0.50000000000000 & 0.25000000000000\end{array}$ $0.50000000000000 \quad 0.00000000000000$ $0.00000000000000 \quad 0.50000000000000$ $0.50000000000000 \quad 0.00000000000000$ $0.23690000000000 \quad 0.23690000000000$ $-0.23690000000000-0.23690000000000$ $-0.236900000000-0.2369000000000$ $0.23690000000000-0.2369000000000$ $0.26316000000000 \quad 0.2631600000000$ $-0.26316000000000-0.2631600000000$ $-0.26316000000000 \quad 0.2631600000000$ $0.26316000000000-0.26316000000000$ $-0.26316000000000 \quad 0.26316000000000$ $0.26316000000000-0.26316000000000$ $0.26316000000000 \quad 0.26316000000000$ $0.26316000000000-0.26316000000000$ $\begin{array}{ll}0.50000000000000 & 0.50000000000000 \\ 0.50000000000000 & 0.50000000000000\end{array}$ .75000000000000 0.25000000000000 0.00000000000000 0.00000000000000 0.50000000000000 0.500000000000 0.50000000000000 0.34803000000000 0.34803000000000 0.84803000000000 0.84803000000000 0.15197000000000 0.15197000000000 $-0.34803000000000$ $-0.34803000000000$ 0.25000000000000 0.75000000000000

$\mathrm{AgUF}_{6}: \mathrm{AB} 6 \mathrm{C}$ tP16 132 d io a - CIF

\section{\# CIF file}

data_findsym-output

audit_creation_method FINDSYM

chemical name mineral 'AgUF6'

chemical formula_sum ' $A g$ F6 U'

loop

_publ_author_name

'P. Charpin

journal_name_full_name

Comptes Rendus Hebdomadaires des S\{\'eances de l'Acad $\{\backslash$ 'e $\}$ mie de $\hookrightarrow$ Sciences

_journal_volume 260

_journal_year 196

_journal_page_first 1914 _journal_page_last 1916

-publ_Section_title

Structure cristalline des hexafluorures complexes $d \backslash$ 'uranium $V$ et $d \backslash$ ' $\hookrightarrow$ argent de potassium $d \backslash$ 'ammonium de rubidium ou de thallium

\# Found in Pearson's Crystal Data - Crystal Structure Database for $\hookrightarrow$ Inorganic Compounds, 2013

-aflow_title 'AgUF\$_\{6\}\$ Structure,

-aflow_proto 'AB6C_tP16_132_d_io_a',

aflow_params ,a,c/a, $x_{-}\{3\}, x_{-}\{4\}, z_{-}\{4\}$,

aflow_params_values ' $5.4229923801,1.46597824081,0.3,0.2,0.333$

aflow Strukturbericht 'None'

aflow_Pearson 'tP16'

cell_length_a $\quad 5.4229923801$

-cell_length_b $\quad 5.4229923801$

cell_length_c 7.9499888293

cell_angle_alpha 90.0000000000

ccell_angle_beta 90.0000000000

_cell_angle_gamma 90.0000000000

symmetry_space_group_name_H-M "P $42 / \mathrm{m} 2 / \mathrm{c} \quad 2 / \mathrm{m}$ "

symmetry_Int_Tables_number 132

loop

space_group_symop_id

space_group_symop_operation_xyz

$\mathrm{x}, \mathrm{y}, \mathrm{z}$

$\mathrm{x},-\mathrm{y},-\mathrm{z}+1 / 2$

$3-\mathrm{x}, \mathrm{y},-\mathrm{z}+1 / 2$
$4-\mathrm{x},-\mathrm{y}, \mathrm{z}$

$4-\mathrm{x},-\mathrm{y}, \mathrm{z}$

$-\mathrm{y},-\mathrm{x},-\mathrm{z}$
$6-\mathrm{y}, \mathrm{x}, \mathrm{z}+1 / 2$

$\begin{array}{ll}6 & -\mathrm{y}, \mathrm{x}, \mathrm{z}+1 / 2 \\ 7 & \mathrm{y},-\mathrm{x}, \mathrm{z}+1 / 2\end{array}$

$\mathrm{y}, \mathrm{x},-\mathrm{z}$

$9-\mathrm{x},-\mathrm{y},-\mathrm{z}$

$10-x, y, z+1 / 2$

$11 \mathrm{x},-\mathrm{y}, \mathrm{z}+1 / 2$

$12 \mathrm{x}, \mathrm{y},-\mathrm{z}$

$14 \mathrm{y},-\mathrm{x},-\mathrm{z}+1 / 2$

$15-\mathrm{y}, \mathrm{x},-\mathrm{z}+1 / 2$

$16-\mathrm{y},-\mathrm{x}, \mathrm{z}$
16

loop

atom_site_label

atom_site_type_symbol

atom_site_symmetry_multiplicity

atom_site_Wyckoff_label

atom_site_fract_x

atom_site_fract_y

-atom_site_fract_z

_atom_site_occupancy

$\begin{array}{lllllll}\mathrm{U} 1 & \mathrm{U} & 2 & 0.00000 & 0.00000 & 0.00000 & 1.00000\end{array}$

$\begin{array}{lllllllll}\mathrm{Ag} 1 & \mathrm{Ag} & 2 & \mathrm{~d} & 0.50000 & 0.50000 & 0.25000 & 1.00000\end{array}$

$\begin{array}{lllllllll}\text { F1 } & \text { F } & 4 & \text { i } & 0.30000 & 0.30000 & 0.00000 & 1.00000\end{array}$

$\begin{array}{llllllllll}\text { F2 } & \mathrm{F} & 8 & 0 & 0.20000 & 0.20000 & 0.33300 & 1.00000\end{array}$

AgUF 6 : AB6C_tP16_132_d_io_a - POSCAR

AB6C_tP16_132_d_io_a \& a,c/a, x3, x4, z4 --params $=5.4229923801$, $\rightarrow 1.46597824081,0.3,0.2,0.333 \&$ P $4 \_\{2\} / \mathrm{mcm}_{-}\{4 \mathrm{~h}\}^{\wedge}\{10\} \# 132$ (adio $\hookrightarrow)$ ) \& tP16 \& None \& AgUF6 \& \& P. Charpin, \{C. R. Hebd. S\{'e\} $\hookrightarrow$ ances Acad. Sci. 260, 1914-1916 (1965)

1.00000000000000

$\begin{array}{lll}5.42299238010000 & 0.00000000000000 & 0.00000000000000\end{array}$

$0.00000000000000 \quad 5.42299238010000 \quad 0.00000000000000$

$0.00000000000000 \quad 0.00000000000000-7.94998882930000$

$\mathrm{Ag} \quad \mathrm{F} \quad \mathrm{U}$

Direct

0.50000000000000

$0.50000000000000 \quad 0.50000000000000$

$\begin{array}{ll}0.30000000000000 & 0.30000000000000\end{array}$

$-0.30000000000000 \quad-0.30000000000000$

$\begin{array}{rr}-0.30000000000000 & -0.30000000000000\end{array}$

$\begin{array}{rr}0.30000000000000 & -0.30000000000000\end{array}$

$0.20000000000000 \quad 0.20000000000000$

$-0.20000000000000-0.20000000000000$

$-0.20000000000000 \quad 0.20000000000000$

$0.20000000000000-0.20000000000000$

$-0.20000000000000 \quad 0.20000000000000$

$0.20000000000000-0.20000000000000$

$0.20000000000000 \quad 0.20000000000000$

$-0.20000000000000-0.20000000000000$

$0.00000000000000 \quad 0.00000000000000$

$0.00000000000000 \quad 0.00000000000000$

0.25000000000000 0.75000000000000 0.00000000000000 0.00000000000000 0.50000000000000 0.50000000000000 0.33300000000000 0.33300000000000 0.83300000000000 0.83300000000000 0.16700000000000 0.16700000000000 $-0.33300000000000$ $-0.33300000000000$ 0.00000000000000 0.50000000000000

\# CIF file

data_findsym-output

audit_creation_method FINDSYM

chemical name mineral 'beta-V3S,

chemical formula sum 's V3,

loop

publ_author_name

'B. Pedersen

F. Gr $\{\backslash 0\}$ nvold

_journal_name_full_name 


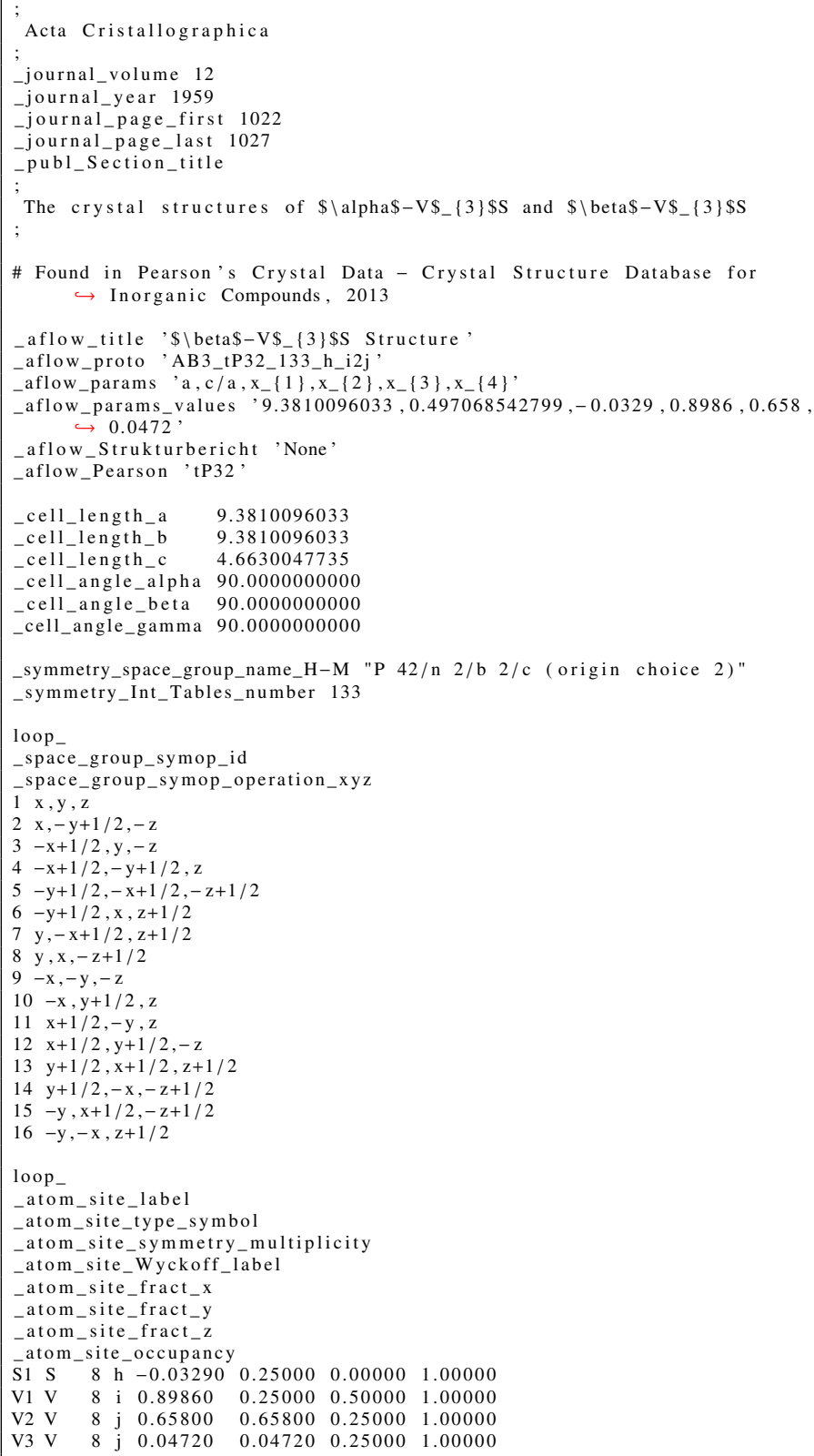

$\beta$ - $\mathrm{V}_{3} \mathrm{~S}$ : AB3_tP32_133_h_i2j - POSCAR

\begin{tabular}{|c|c|c|c|c|}
\hline \multicolumn{5}{|c|}{ AB3_tP32_133_h_i2j \& a,c/ } \\
\hline & & & & \\
\hline 1$\} \# 13$ & (biiin) \& $1 \mathrm{P} 32$ \& & V3S \& beta & & \\
\hline$\hookrightarrow$ and $\mathrm{F} . \mathrm{Gr}\{\backslash$ & old & $12,1022-1027$ & & \\
\hline 1.00000000000000 & & & & \\
\hline 9.38100960330000 & 0.00000000000000 & 0.00000000000000 & & \\
\hline 0.00000000000000 & 9.38100960330000 & 0.00000000000000 & & \\
\hline 0.00000000000000 & 0.00000000000000 & 4.66300477350000 & & \\
\hline $\mathrm{V}$ & & & & \\
\hline 24 & & & & \\
\hline$c t$ & & & & \\
\hline-0.03290000000000 & 0.25000000000000 & 0.00000000000000 & $\mathrm{~s}$ & \\
\hline 0.53290000000000 & 0.25000000000000 & 0.00000000000000 & $\mathrm{~s}$ & $(8 \mathrm{~h})$ \\
\hline 0.25000000000000 & -0.03290000000000 & 0.50000000000000 & S & \\
\hline 0.25000000000000 & 0.53290000000000 & 0.50000000000000 & S & $(8 \mathrm{~h})$ \\
\hline 0.03290000000000 & 0.75000000000000 & 0.0000000 & $\mathrm{~s}$ & \\
\hline 0.46710000000000 & 0.75000000000000 & 0.00000000000000 & $\mathrm{~s}$ & $(8 \mathrm{~h}$ \\
\hline 0.75000000000000 & 0.03290000000000 & 0.50000000000000 & $\mathrm{~s}$ & $(8 \mathrm{~h})$ \\
\hline 0.75000000000000 & 0.46710000000000 & 0.5000000 & $\mathrm{~s}$ & $(8 \mathrm{~h})$ \\
\hline 0.89860000000000 & 0.25000000000000 & 0.50000000000000 & $\mathrm{~V}$ & $(8 \mathrm{i})$ \\
\hline-0.39860000000000 & 0.25000000000000 & 0.50000000000000 & $\mathrm{~V}$ & \\
\hline 0.25000000000000 & 0.89860000000000 & 0.00000000000000 & $\mathrm{~V}$ & \\
\hline 0.25000000000000 & 000000000 & 0.0 & $\mathrm{~V}$ & \\
\hline-0.89860000000000 & 00000000 & 0.5000 & $\mathrm{~V}$ & \\
\hline 1.39860000000000 & 0.75000000000000 & 0.50000000000000 & $\mathrm{~V}$ & \\
\hline 0.75000000000000 & -0.898 & & $\mathrm{~V}$ & \\
\hline 0.75000000000000 & 1.39860000000000 & 0.00000000000000 & $\mathrm{~V}$ & \\
\hline 0.65800000000000 & 0.658 & & $\mathrm{~V}$ & \\
\hline-0.15800000000000 & -0.15800000000000 & 0.25000000000000 & $\mathrm{~V}$ & \\
\hline-0.15800000000000 & 0.65800000000000 & 0.75000000000000 & $\mathrm{~V}$ & \\
\hline 0.65800000000000 & -0.15800000000000 & 0.75000000 & $\mathrm{~V}$ & \\
\hline-0.65800000000000 & -0.65800000000000 & 0.75000000000000 & $\mathrm{~V}$ & \\
\hline 1.15800000000000 & 00000 & 0.75000000000000 & $\mathrm{~V}$ & \\
\hline 1.15800000000000 & -0.65800000000000 & 0.25000000000000 & $\mathrm{~V}$ & \\
\hline-0.65800000000000 & 1.15800000000000 & 0.25000000000000 & $\mathrm{~V}$ & \\
\hline 0.04720000000000 & 0.04720000000000 & 0.25000000000000 & $\mathrm{~V}$ & \\
\hline
\end{tabular}

$\begin{array}{rrrrr}0.45280000000000 & 0.45280000000000 & 0.25000000000000 & \mathrm{~V} & (8 \mathrm{j}) \\ 0.45280000000000 & 0.04720000000000 & 0.75000000000000 & \mathrm{~V} & (8 \mathrm{j}) \\ 0.04720000000000 & 0.45280000000000 & 0.75000000000000 & \mathrm{~V} & (8 \mathrm{j}) \\ -0.04720000000000 & -0.04720000000000 & 0.75000000000000 & \mathrm{~V} & (8 \mathrm{j}) \\ 0.54720000000000 & 0.54720000000000 & 0.75000000000000 & \mathrm{~V} & (8 \mathrm{j}) \\ 0.54720000000000 & -0.04720000000000 & 0.25000000000000 & \mathrm{~V} & (8 \mathrm{j}) \\ -0.04720000000000 & 0.54720000000000 & 0.25000000000000 & \mathrm{~V} & (8 \mathrm{j})\end{array}$

Downeyite $\left(\mathrm{SeO}_{2}, \mathrm{C} 47\right)$ : A2B_tP24_135_gh_h - CIF

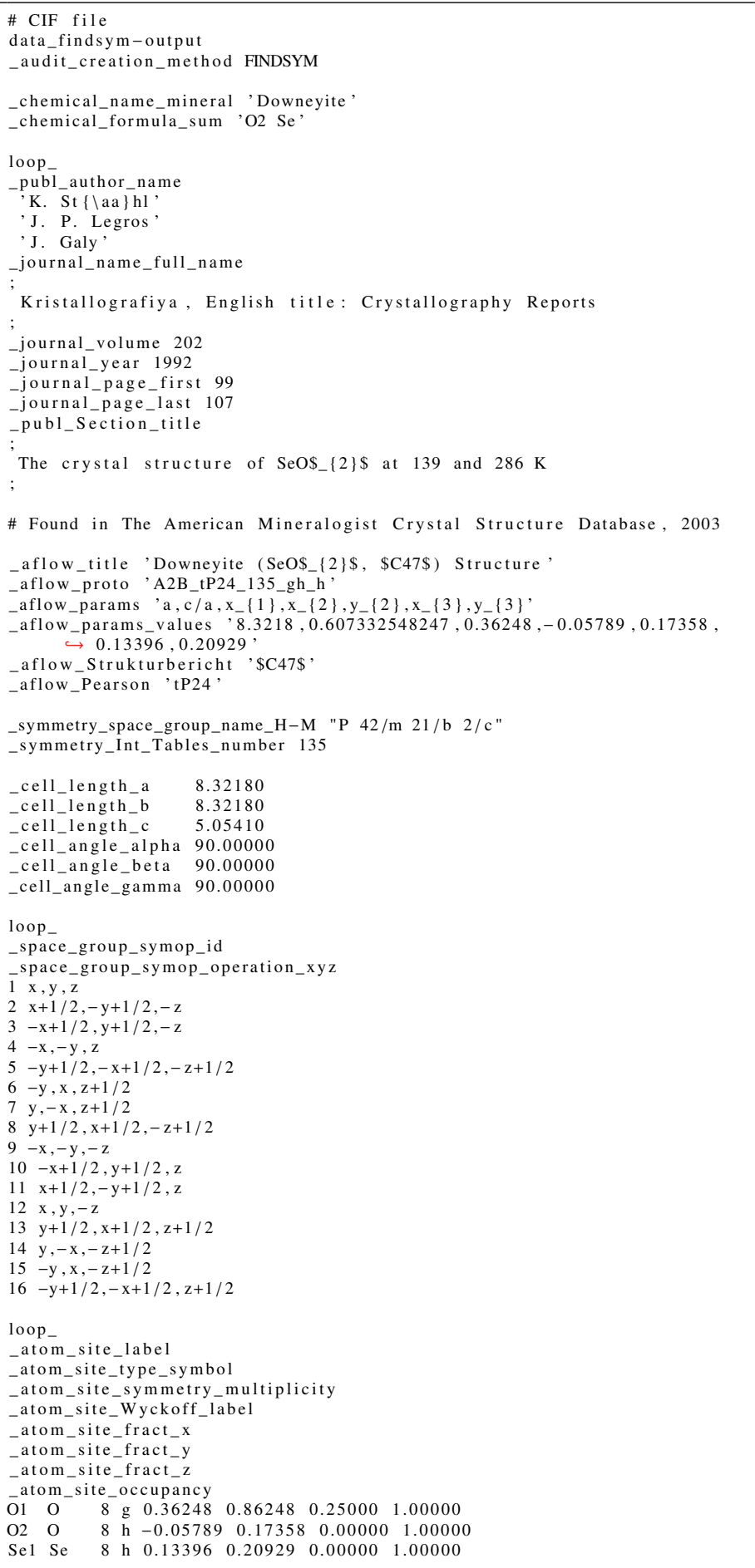

Downeyite $\left(\mathrm{SeO}_{2}, \mathrm{C} 47\right)$ : A2B_tP24_135_gh_h - POSCAR

A2B_tP24_135_gh_h \& a,c/a, x1, x2,y2, x3,y3 --params $=8.3218,0.607332548247$, $\hookrightarrow 0.36248,-0.05789,0.17358,0.13396,0.20929 \&$ P $4 \_\{2\} / m b c D_{-}\{4 h\}^{\wedge}\{$ $\hookrightarrow 13\} \# 135\left(\mathrm{gh}^{\wedge} 2\right) \& \mathrm{tP} 24$ \& $\$ \mathrm{C} 47 \$$ \& SeO2 \& Downeyite \& K. St $\{\backslash a \mathrm{a}\}$ $\hookrightarrow$ hl and J. P. Legros and J. Galy, Kristallografiya 202, 99-107 ( $\hookrightarrow 1992$ )

1.00000000000000

$8.32180000000000 \quad 0.00000000000000-0.00000000000000$

$8.3218000000000-8.32180000000000-0.00000000000000$ $\begin{array}{lll}0.00000000000000 & 8.32180000000000 & 0.00000000000000 \\ 0.00000000000000 & 0.00000000000000 & 5.05410000000000\end{array}$ $\begin{array}{rr}\mathrm{O} & \mathrm{Se} \\ 16 & 8\end{array}$

Direc

$\begin{array}{lll}0.36248000000000 & 0.86248000000000 & 0.25000000000000\end{array}$ $\begin{array}{lll}-0.36248000000000 & 0.13752000000000 & 0.25000000000000\end{array}$ $\begin{array}{lll}0.13752000000000 & 0.36248000000000 & 0.75000000000000\end{array}$ 
0.86248000000000 $-0.36248000000000$ 0.36248000000000 0.86248000000000 0.13752000000000 0.05789000000000 0.05789000000000 $-0.17358000000000$ 0.17358000000000 0.55789000000000 0.44211000000000 0.67358000000000 0.32642000000000 0.1339600000000 $-0.1339600000000$ $-0.1339600000000$ 0.2000000 0.20929000000000 0.36604000000000 0.63396000000000 0.29071000000000

$-0.36248000000000$ .1375200000000 0.8624800000000 $-0.36248000000000$ 0.36248000000000 0.17358000000000 $-0.17358000000000$ $-0.0578900000000$ 0.05789000000000 0.67358000000000 0.32642000000000 0.44211000000000 0.55789000000000 0.2092900000000 $-0.20929000000000$ 0.20200000 0.13396000000000 0.1339600000000 0.70929000000000 0.29071000000000 0.36604000000000

$\mathrm{ZnSb}_{2} \mathrm{O}_{4}$ : A4B2C_tP28_135_gh_h_d - CIF

CIF file

data_findsym-output

audit_creation_method FINDSYM

chemical_name_mineral ' $\mathrm{ZnSb2O4}$ '

chemical_formula_sum, $\mathrm{O} 4 \mathrm{Sb} 2 \mathrm{Zn}$

loop

publ_author_name

S. St $\{\backslash$ aa $\}$ hl,

_journal_name_full_name

Arkiv $f\{\backslash " o\} r$ Kemi, Mineralogi och Geologi

journal_volume 17B

journal year 1943

journal_page_first 1

journal_page_last

publ_Section_title

The crystal structure of $\mathrm{ZnSb} \$_{-}\{2\} \$ O \$_{-}\{4\} \$$ and isomorphous compound

\# Found in Pearson's Crystal Data - Crystal Structure Database for $\hookrightarrow$ Inorganic Compounds, 2013

aflow_title' ZnSb\$_\{2\}\$O\$_\{4\}\$ Structure

aflow_proto 'A4B2C_tP28_135_gh_h_d

aflow params , a, c/a, $x_{-}\{2\}, x_{-}\{3\}, y-\{3\}, x_{-}\{4\}, y-\{4\}$

aflow_params_values $, 8.4909023894,0.697208809336,0.169,0.114,0.386$, $\stackrel{-p a r a m s \_ \text {value }}{\hookrightarrow} 0.167,0.175$

aflow Strukturbericht 'None'

aflow_Pearson 'tP28,

cell_length_a $\quad 8.4909023894$

cell_length_b 8.4909023894

cell_length_c $\quad 5.9199319451$

cell_angle_alpha 90.0000000000

cell_angle_beta 90.0000000000

cell_angle_gamma 90.0000000000

symmetry_space_group_name_H-M "P $42 / \mathrm{m} \mathrm{21/b} 2 / \mathrm{c} "$

symmetry Int Tables_number 135

loop

space_group_symop_id

space_group_symop_operation_xyz

$1 \mathrm{x}, \mathrm{y}, \mathrm{z}$

$2 \mathrm{x}+1 / 2,-\mathrm{y}+1 / 2,-\mathrm{z}$

$3-x+1 / 2, y+1 / 2,-z$

$4-\mathrm{x},-\mathrm{y}, \mathrm{z}$

$5-\mathrm{y}+1 / 2,-\mathrm{x}+1 / 2,-\mathrm{z}+1 / 2$

$6-\mathrm{y}, \mathrm{x}, \mathrm{z}+1 / 2$

$7 \mathrm{y},-\mathrm{x}, \mathrm{z}+1 / 2$

$8 \mathrm{y}+1 / 2, \mathrm{x}+1 / 2,-\mathrm{z}+1 / 2$

$-\mathrm{x},-\mathrm{y},-\mathrm{z}$

$0-x+1 / 2, y+1 / 2, z$

$1 \mathrm{x}+1 / 2,-\mathrm{y}+1 / 2, \mathrm{z}$

$2 \mathrm{x}, \mathrm{y},-$

$3 \mathrm{y}+1 / 2, \mathrm{x}+1 / 2, \mathrm{z}+1 / 2$

$14 \mathrm{y},-\mathrm{x},-\mathrm{z}+1 / 2$
$15-\mathrm{y}, \mathrm{x},-\mathrm{z}+1 / 2$

$16-\mathrm{y}+1 / 2,-\mathrm{x}+1 / 2, \mathrm{z}+1 / 2$

loop

atom site label

atom_site_type_symbol

atom_site_symmetry_multiplicity

atom_site_Wyckoff_labe

atom_site_fract_x

atom_site_fract_y

atom_site_fract_z

_atom_site_occupancy

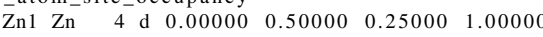

$\begin{array}{lllllllll}\mathrm{O} 1 & \mathrm{O} & 8 & \mathrm{~g} & 0.16900 & 0.66900 & 0.25000 & 1.00000\end{array}$

$\begin{array}{lllllllll}\mathrm{O} 2 & \mathrm{O} & 8 & \mathrm{~h} & 0.11400 & 0.38600 & 0.00000 & 1.00000\end{array}$

$\begin{array}{llllllll}\mathrm{Sb} 1 & \mathrm{Sb} & 8 & \mathrm{~h} & 0.16700 & 0.17500 & 0.00000 & 1.00000\end{array}$

$\mathrm{ZnSb}_{2} \mathrm{O}_{4}$ : A4B2C_tP28_135_gh_h_d - POSCAR
.75000000000000 0000000 0.75000000000000 . 0.25000000000000 0

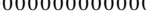
5000000000000 00000000000000 50000000000000 0000000000000 0000000000000 00000000000 00000000000 00000000000 000000000000 . 50000000000000

$\begin{array}{ll}\mathrm{O} & (8 \mathrm{~g}) \\ \mathrm{O} & (8 \mathrm{~g}) \\ \mathrm{O} & (8 \mathrm{~g}) \\ \mathrm{O} & (8 \mathrm{~g}) \\ \mathrm{O} & (8 \mathrm{~g}) \\ \mathrm{O} & (8 \mathrm{~h}) \\ \mathrm{O} & (8 \mathrm{~h}) \\ \mathrm{O} & (8 \mathrm{~h}) \\ \mathrm{O} & (8 \mathrm{~h}) \\ \mathrm{O} & (8 \mathrm{~h}) \\ \mathrm{O} & (8 \mathrm{~h}) \\ \mathrm{O} & (8 \mathrm{~h}) \\ \mathrm{O} & (8 \mathrm{~h}) \\ \mathrm{Se} & (8 \mathrm{~h}) \\ \mathrm{Se} & (8 \mathrm{~h}) \\ \mathrm{Se} & (8 \mathrm{~h}) \\ \mathrm{Se} & (8 \mathrm{~h}) \\ \mathrm{Se} & (8 \mathrm{~h}) \\ \mathrm{Se} & (8 \mathrm{~h}) \\ \mathrm{Se} & (8 \mathrm{~h}) \\ \mathrm{Se} & (8 \mathrm{~h})\end{array}$

A4B2C_tP28_135_gh_h_d \& a , c/a , x2 , x3 $, y 3, x 4, y 4--$ params $=8.4909023894$ , $0.169,0.114,0.386,0.167,0.175 \&$ P4_ $\{2\} / \mathrm{mbc} \mathrm{D}_{-}\{4 \mathrm{~h}$ $\leftrightarrow$ Ark Kem. Mineral Geol, 17B, 1-7 (1943)

$\hookrightarrow\{$ Ark. Kem.

$\begin{array}{llll}0.00000000000000 & 8.49090238940000 & 0.00000000000000\end{array}$

$\begin{array}{lll}0.00000000000000 & 0.00000000000000 & 5.91993194510000\end{array}$

Direct

$\begin{array}{rrr}\mathrm{O} & \mathrm{Sb} & \mathrm{Zn} \\ 16 & 8 & 4\end{array}$

0.16900000000000

$-0.16900000000000$

0.33100000000000

0.66900000000000

0.6690000000000

0.16900000000000

0.66900000000000

0.33100000000000

0.11400000000000

$-0.11400000000000$

$-0.38600000000000$

0.38600000000000

0.38600000000000

0.61400000000000

0.88600000000000

0.11400000000000

0.16700000000000

$-0.16700000000000$

$-0.17500000000000$

0.17500000000000

0.33300000000000

0.66700000000000

0.67500000000000

0.32500000000000

0.00000000000000

0.50000000000000

0.00000000000000

0.5000000000000

0.66900000000000 0.33100000000000

0.16900000000000

0.16900000000000

0.33100000000000

0.66900000000000

0.16900000000000

0.16900000000000

0.38600000000000

$-38600000000000$

0.11400000000000

0.11400000000000

0.88600000000000

-

600000000000

17500000000000

$-0.17500000000000$

.16700000000000

.16700000000000

0.67500000000000

0.66700000000000

0.33300000000000

0.50000000000000

0.00000000000000

0.50000000000000

0.00000000000000

0.25000000000000 0.25000000000000 0.75000000000000 0.75000000000000 0.75000000000000 0.75000000000000 0.25000000000000 0.25000000000000 0.00000000000000 0.00000000000000 0.50000000000000 0.5000000000000 0.00000000000000 0.00000000000000 .00000000000000 0.50000000000000 .50000000000000 0.00000000000000 0.50000000000000 0.50000000000000 0.0000000000000 0.000000000 0.50000000000000 0.50000000000 0.25000000000000 0.25000000000000 0.75000000000000 0.75000000000000 0.25000000000000

O $\quad(8 \mathrm{~g})$ O (8g) $\begin{array}{ll}\mathrm{O} & (8 \mathrm{~g}) \\ \mathrm{O} & (8 \mathrm{~g})\end{array}$ 0
0 O $(8 g)$ $0 \quad(8 g)$ $\mathrm{O} \quad(8 \mathrm{~g})$ $\mathrm{O} \quad(8 \mathrm{~h})$ $\mathrm{O} \quad(8 \mathrm{~h})$ $\mathrm{O} \quad(8 \mathrm{~h})$

$\mathrm{Zn}_{3} \mathrm{P}_{2}\left(D 5_{9}\right):$ A2B3_tP40_137_cdf_3g - CIF

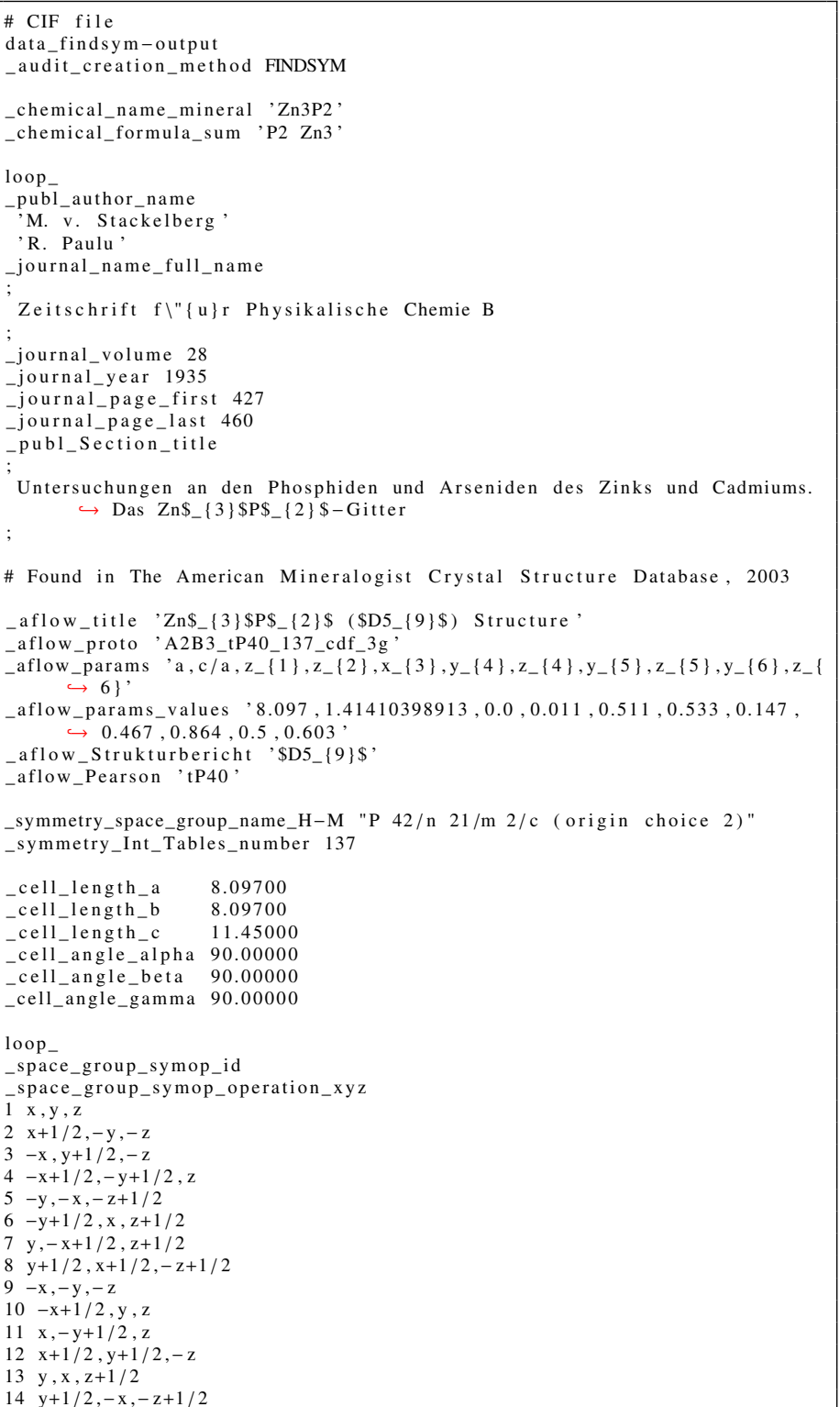




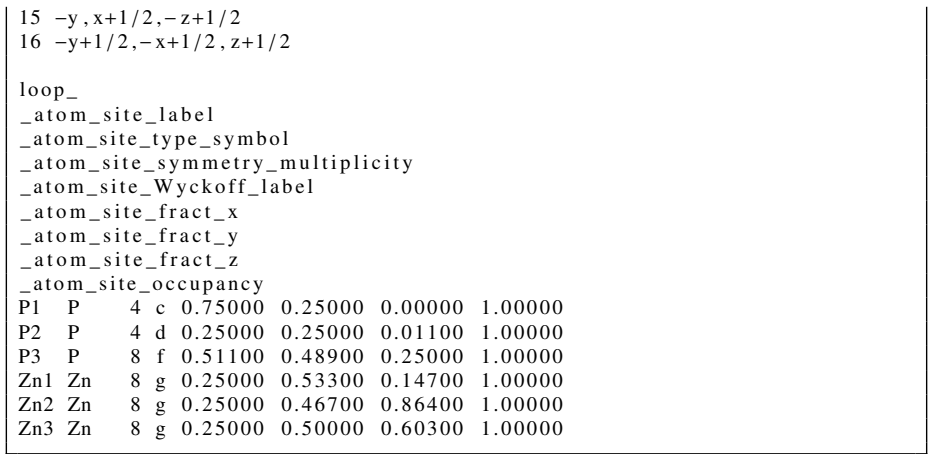

$\mathrm{Zn}_{3} \mathrm{P}_{2}\left(D 5_{9}\right)$ : A2B3_tP40_137_cdf_3g - POSCAR

A2B3_tP40_137_cdf_3g \& a, c/a, z1, z2, x3, y4, z4, y5 , z5, y6, z6 --params $=8.097$ $\hookrightarrow 1.41410398913,0.0,0.011,0.511,0.533,0.147,0.467,0.864,0.5,0.603$

$\hookrightarrow$ \& P4_\{2\}/nmc D_ $\{4 \mathrm{~h}\}^{\wedge}\{15\} \# 137(\operatorname{cdfg} \wedge 3) \& \operatorname{tP} 40 \&$ D 5_ $\{9\} \$ \&$

$\hookrightarrow$ Zn3P2 \& Zn3P2 \& M. v. Stackelberg and R. Paulu, Z. Phys. Chem. $\hookrightarrow$ B $28,427-460(1935)$

1.00000000000000

$8.09700000000000 \quad 0.00000000000000 \quad 0.00000000000000$

$\begin{array}{lll}0.00000000000000 & 8.09700000000000 & 0.00000000000000\end{array}$

$\begin{array}{lll}0.00000000000000 & 0.00000000000000 & 11.45000000000000\end{array}$

$$
\begin{array}{cc}
\mathrm{P} & \mathrm{Zn} \\
16 & 24
\end{array}
$$

Direct

0.75000000000000 0.25000000000000 0.25000000000000 0.75000000000000 0.25000000000000 0.25000000000000 0.750000000000 0.7500000000000 0.5110000000000 0.51100000000000 1.01100000000000 1.01100000000000 $-0.51100000000000$ $-0.51100000000000$ 1.01100000000000 $-0.01100000000000$ 0.51100000000000 0.25000000000000 0.25000000000000 $-0.03300000000000$ 0.53300000000000 0.75000000000000 0.75000000000000 1.033000000000 $-0.5330000000000$ 0.25000000000000 0.250000000000 0.0330000000000 0.46700000000000 0.75000000000000 0.75000000000000 0.96700000000000 $-0.46700000000000$ 0.25000000000000 0.25000000000000 0.00000000000000 0.50000000000000 0.75000000000000 0.75000000000000

1.00000000000000

$0.25000000000000 \quad 0.00000000000000$ 0.75000000000000 0.75000000000000 0.25000000000000 0.25000000000000 0.25000000000000 0.75000000000000 0.7500000000000 0.750000000000 1.01100000000000 0.5110000000000 $-0.01100000000000$ 0.51100000000000 $-0.01100000000000$ $-0.51100000000000$ 1.01100000000000 0.53300000000000 $-0.0330000000000$ 0.25000000000000 0.25000000000000 1.03300000000000 $-0.53300000000000$ 0.75000000000000 0.750000000000 0.75000000000000 0.4670000000000 0.0330000000000 0.2500000000000 0.25000000000000 0.96700000000000 $-0.46700000000000$ 0.75000000000000 0.75000000000000 0.50000000000000 0.00000000000000 0.25000000000000 0.25000000000000 1.00000000000000 $-0.50000000000000$ 0.75000000000000 0.75000000000000 .50000000000000 .00000000000000 0.50000000000000 0.01100000000000 0.51100000000000 $-0.01100000000000$ 0.48900000000000 0.25000000000000 0.25000000000000 0.75000000000000 0.75000000000000 0.75000000000000 0.75000000000000 0.7500000000000 0.25000000000000 0.25000000000000 . 0.64700000000000 0.64700000000000 $-0.14700000000000$ $-0.14700000000000$ 0.3530000000000 0.35300000000000 0.8640000000000 0.86400000000000 1.36400000000000 1.3640000000000 1.36400000000000 $-0.86400000000000$ $-0.86400000000000$ $-0.36400000000000$ $-0.36400000000000$ 0.6030000000000 0.6030000000000 1.10300000000000 1.10300000000000 $-0.60300000000000$ $-0.60300000000000$ $-0.10300000000000$ $-0.10300000000000$

$\begin{array}{ll}\mathrm{P} & (4 \mathrm{c}) \\ \mathrm{P} & (4 \mathrm{c}) \\ \mathrm{P} & (4 \mathrm{c}) \\ \mathrm{P} & (4 \mathrm{c}) \\ \mathrm{P} & (4 \mathrm{~d}) \\ \mathrm{P} & (4 \mathrm{~d}) \\ \mathrm{P} & (4 \mathrm{~d}) \\ \mathrm{P} & (4 \mathrm{~d}) \\ \mathrm{P} & (8 \mathrm{f}) \\ \mathrm{P} & (8 \mathrm{f}) \\ \mathrm{P} & (8 \mathrm{f}) \\ \mathrm{P} & (8 \mathrm{f}) \\ \mathrm{P} & (8 \mathrm{f}) \\ \mathrm{P} & (8 \mathrm{f}) \\ \mathrm{P} & (8 \mathrm{f}) \\ \mathrm{P} & (8 \mathrm{f}) \\ \mathrm{Zn} & (8 \mathrm{~g}) \\ \mathrm{Zn} & (8 \mathrm{~g}) \\ \mathrm{Zn} & (8 \mathrm{~g}) \\ \mathrm{Zn} & (8 \mathrm{~g}) \\ \mathrm{Zn} & (8 \mathrm{~g}) \\ \mathrm{Zn} & (8 \mathrm{~g}) \\ \mathrm{Zn} & (8 \mathrm{~g}) \\ \mathrm{Zn} & (8 \mathrm{~g}) \\ \mathrm{Zn} & (8 \mathrm{~g}) \\ \mathrm{Zn} & (8 \mathrm{~g}) \\ \mathrm{Zn} & (8 \mathrm{~g}) \\ \mathrm{Zn} & (8 \mathrm{~g}) \\ \mathrm{Zn} & (8 \mathrm{~g}) \\ \mathrm{Zn} & (8 \mathrm{~g}) \\ \mathrm{Zn} & (8 \mathrm{~g}) \\ \mathrm{Zn} & (8 \mathrm{~g}) \\ \mathrm{Zn} & (8 \mathrm{~g}) \\ \mathrm{Zn} & (8 \mathrm{~g}) \\ \mathrm{Zn} & (8 \mathrm{~g}) \\ \mathrm{Zn} & (8 \mathrm{~g}) \\ \mathrm{Zn} & (8 \mathrm{~g}) \\ \mathrm{Zn} & (8 \mathrm{~g}) \\ \mathrm{Zn} & (8 \mathrm{~g}) \\ \mathrm{Zn} & (8 \mathrm{~g}) \\ & \end{array}$

$\mathrm{ZrO}_{2}$ (High-temperature): A2B_tP6_137_d_a - CIF

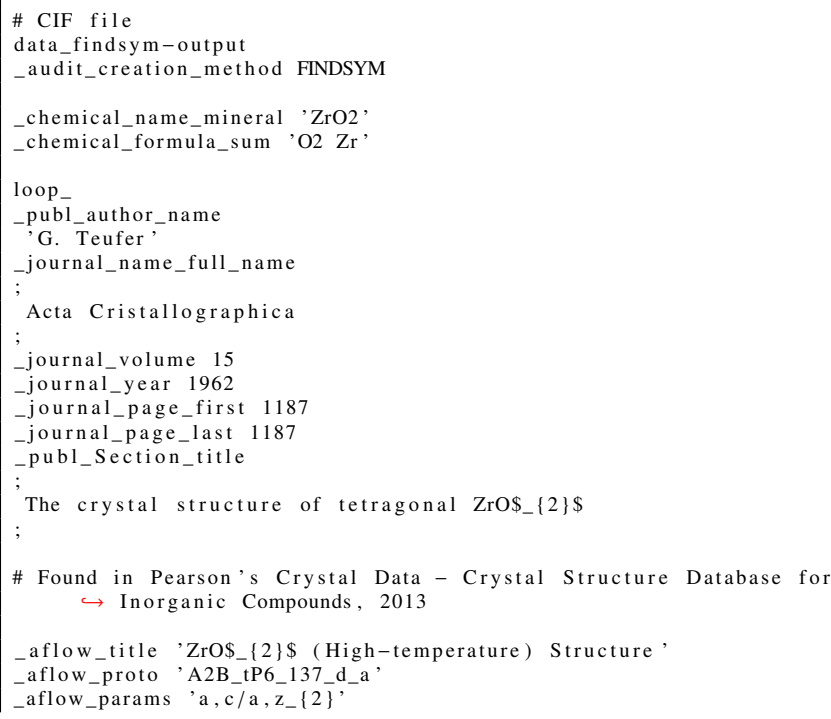

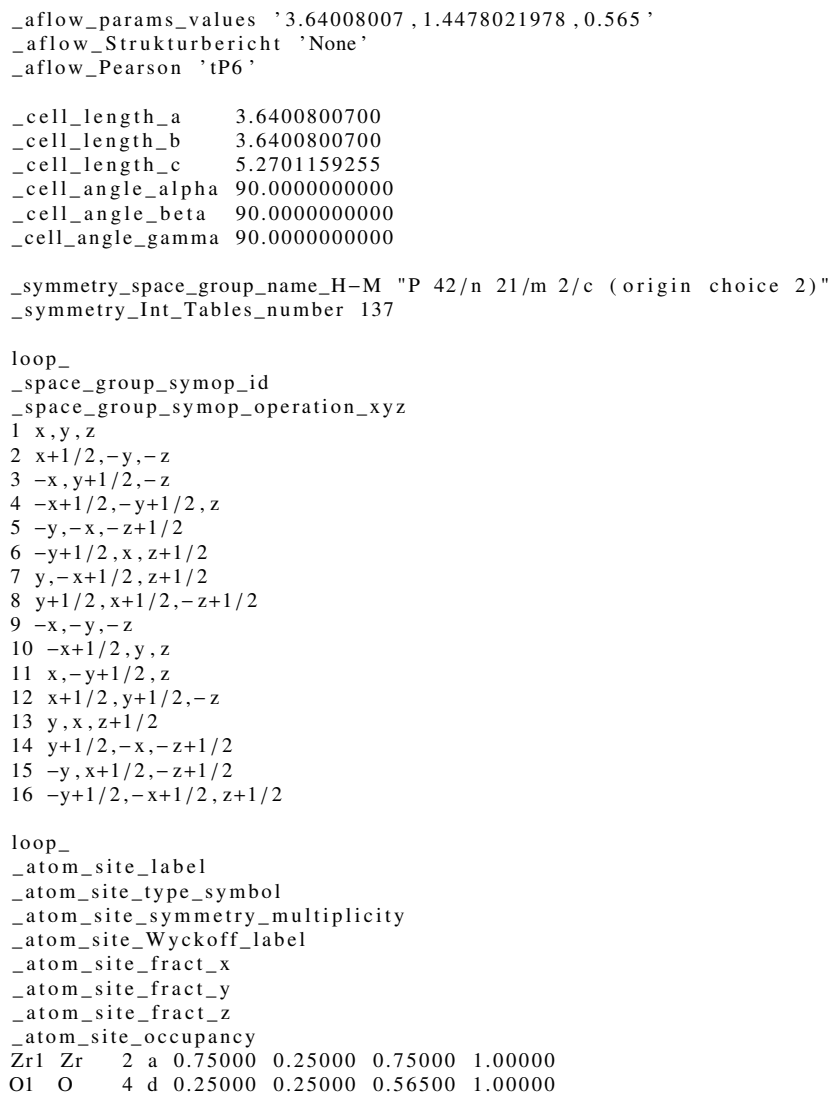

$\mathrm{ZrO}_{2}$ (High-temperature): A2B_tP6_137_d_a - POSCAR

\begin{tabular}{|c|c|c|c|c|}
\hline \multicolumn{5}{|c|}{ 2B_tP6_137_d_a \& a,c/a, z2 --params $=3.64008007,1.4478021978,0.565 \&$ \&4_\{ } \\
\hline$\hookrightarrow 2\} / \mathrm{nmc} D_{-}\{4 \mathrm{~h}$ & $\wedge\{15\} \# 137$ (ad) \& & tP6 \& None \& $\mathrm{ZrO} 2 \&$ & $\& \mathrm{G}$ & Teufer \\
\hline Acta Cryst & 15. $1187-1187$ & & & \\
\hline \multicolumn{5}{|l|}{1.00000000000000} \\
\hline 3.64008007000000 & 0.00000000000000 & 0.00000000000000 & & \\
\hline 0.00000000000000 & 3.64008007000000 & 0.00000000000000 & & \\
\hline 0.00000000000000 & 0.00000000000000 & 5.27011592550000 & & \\
\hline $\mathrm{Zr}$ & & & & \\
\hline 4 & & & & \\
\hline \multicolumn{5}{|l|}{ Direct } \\
\hline 0.25000000000000 & 0.25000000000000 & 0.565 & $\mathrm{O}$ & $(4 d)$ \\
\hline 0.25000000000000 & 0.2500000 & 1.065 & $\mathrm{O}$ & $(4 \mathrm{~d})$ \\
\hline 0.75000000000000 & 0.75000000000000 & -0.56500000000000 & $\mathrm{O}$ & $(4 d)$ \\
\hline 0.75000000000000 & 0.75000000000000 & -0.06500000000000 & $\mathrm{O}$ & (4d) \\
\hline 0.75000000000000 & 0.25000000000000 & 0.75000000000000 & $\mathrm{Zr}$ & $(2 a)$ \\
\hline 0.25000000000000 & 0.75000000000000 & 0.25000000000000 & $\mathrm{Zr}$ & (2a) \\
\hline
\end{tabular}

$\mathrm{CeCo}_{4} \mathrm{~B}_{4}$ : A4BC4_tP18_137_g_b_g - CIF

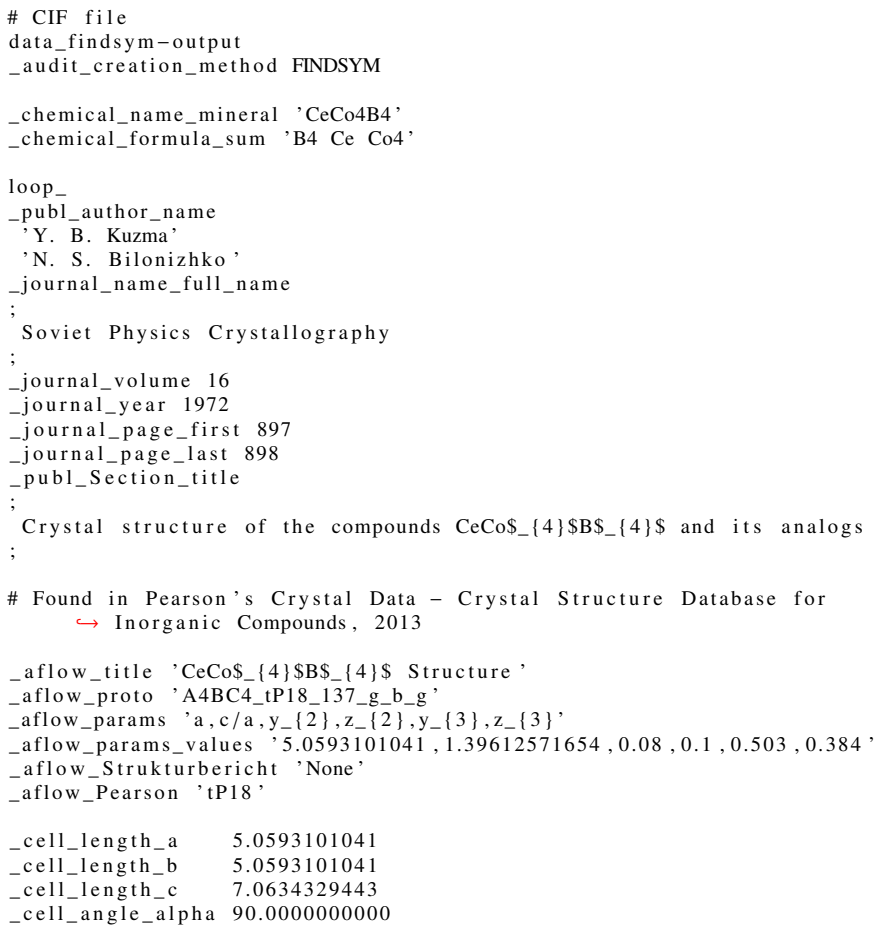




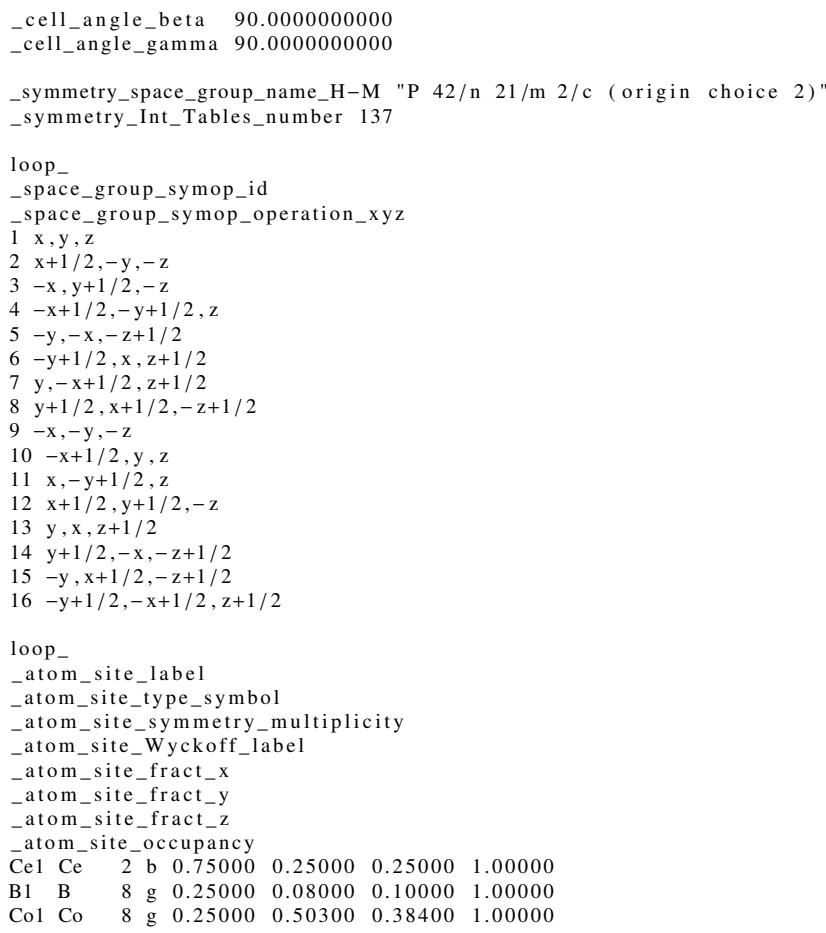

$\mathrm{CeCo}_{4} \mathrm{~B}_{4}$ : A4BC4_tP18_137_g_b_g - POSCAR

A4BC4_tP18_137_g_b_g \& a,c/a ,y2, z2,y3, z3 --params $=5.0593101041$,

$\hookrightarrow 1.39612571654,0.08,0.1,0.503,0.384 \& P_{4}\{2\} / \mathrm{nmc} \mathrm{D}_{-}\{4 \mathrm{~h}\}^{\wedge}\{15\}$

$\hookrightarrow 137\left(b^{\wedge} 2\right) \&$ tP18 \& None \& CeCo4B4 \& \& Y. B. Kuzma and N. S.

$\hookrightarrow$ Bilonizhko, Sov. Phys. Crystallogr. 16, 897-898 (1972)

1.00000000000000

$\begin{array}{lll}5.05931010410000 & 0.00000000000000 & 0.00000000000000\end{array}$

$\begin{array}{lll}0.00000000000000 & 5.05931010410000 & 0.00000000000000\end{array}$

$\begin{array}{lll}0.00000000000000 & 0.00000000000000 & 7.06343294430000\end{array}$

Direct

$\begin{array}{rrr}\mathrm{B} & \mathrm{Ce} & \text { Co } \\ 8 & 2 & 8\end{array}$

0.25000000000000

0.25000000000000

0.42000000000000

0.42000000000000

0.08000000000000

0.75000000000000

0.58000000000000

$-0.08000000000000$

0.75000000000000

0.25000000000000

0.25000000000000

0.25000000000000

$-0.00300000000000$

0.50300000000000

0.75000000000000

0.75000000000000

$-0.50300000000000$

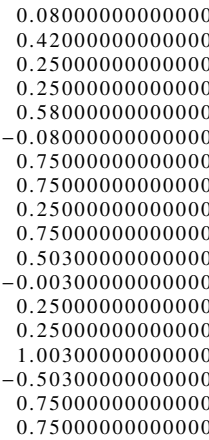

0.10000000000000

0.10000000000000

0.60000000000000

0.60000000000000

$-0.10000000000000$

$-0.1000000000000$

$-0.10000000000000$

0.40000000000000

0.40000000000000

0.25000000000000

0.75000000000000

0.38400000000000

0.38400000000000

0.88400000000000

0.88400000000000

$-0.3840000000000$

$-0.38400000000000$

0.11600000000000

0.11600000000000

$\mathrm{HgI}_{2}(C 13)$ : AB2_tP6_137_a_d - CIF

\section{\# CIF file}

data_findsym-output

-audit_creation_method FINDSYM

_chemical_name_mineral,

chemical formula sum ' $\mathrm{Hg}$ I2

loop

publ_author_name

D. Schwarzenbach,

'H. Birkedal'

M. Hostettler

'P. Fischer

_journal_name_full_name

Acta Crystallographica Section B: Structural Science

_journal_volume 63

_journal_year 2007

-journal_page_first 826

page_last

Neutron diffraction investigation of the temperature dependence of

$\hookrightarrow$ crystal structure and thermal motions of red $\mathrm{HgI \$}\{2\} \$$

\# Found in The American Mineralogist Crystal Structure Database, 2003

aflow_title 'HgI\$_ $\{2\} \$(\$ C 13 \$)$ Structure

_aflow_proto 'AB2_tP6_137_a_d,

-aflow_params a, c/a, z z $_{-}\{2\}$,

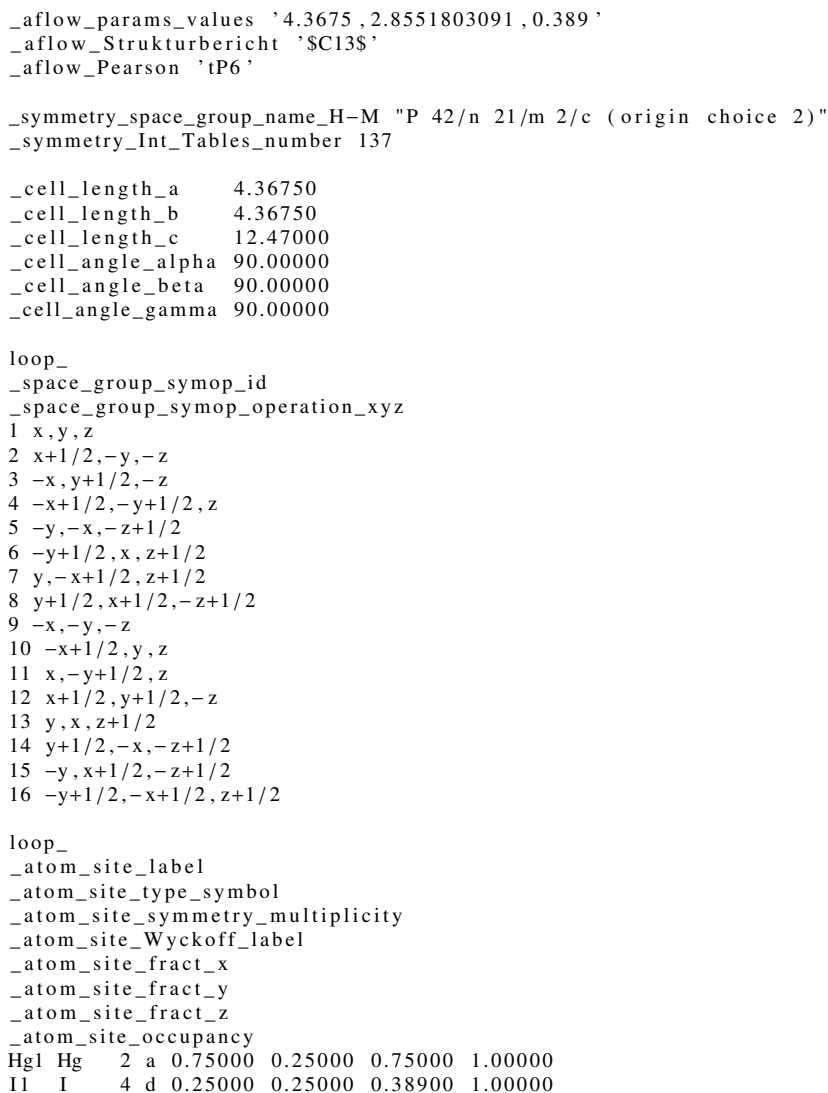

$\mathrm{HgI}_{2}$ (C13): AB2_tP6_137_a_d - POSCAR

\begin{tabular}{|c|c|c|c|c|}
\hline $\begin{aligned} \text { AB2_tP6_137_a_d \& a } & \\
& \hookrightarrow \text { nmc D_ }\{4 \mathrm{~h}\}^{\wedge} \\
& \hookrightarrow \text { Schwarzenba } \\
& \hookrightarrow, 826-835\end{aligned}$ & $\begin{array}{l}\text { /a, z2 --params }=4 . \\
15\} \# 137(\text { ad }) \& \text { tP } \\
\text { et al., Acta Cr } \\
07)\end{array}$ & $\begin{array}{l}575,2.8551803091,0 \\
\& \text { C } 13 \$ \text { HgI2 \& } \\
\text { stallogr. Sect. B }\end{array}$ & $\begin{array}{cc}9 \& & \& \\
D . \\
\text { uct }\end{array}$ & Sci. 63 \\
\hline 1.00000000000000 & & & & \\
\hline 4.36750000000000 & 0.00000000000000 & 0.00000000000000 & & \\
\hline 0.00000000000000 & 4.36750000000000 & 0.00000000000000 & & \\
\hline 0.00000000000000 & 0.00000000000000 & 12.47000000000000 & & \\
\hline $\mathrm{Hg}$ & & & & \\
\hline 2 & & & & \\
\hline irect & & & & \\
\hline 0.75000000000000 & 0.25000000000000 & 0.75000000000000 & $\mathrm{Hg}$ & (2a) \\
\hline 0.25000000000000 & 0.75000000000000 & 0.25000000000000 & $\mathrm{Hg}$ & (2a) \\
\hline 0.25000000000000 & 0.25000000000000 & 0.38900000000000 & I & $(4 d)$ \\
\hline 0.25000000000000 & 0.25000000000000 & 0.88900000000000 & I & $(4 d)$ \\
\hline 0.75000000000000 & 0.75000000000000 & -0.38900000000000 & I & $(4 d)$ \\
\hline 0.75000000000000 & 0.75000000000000 & 0.11100000000000 & I & $(4 d)$ \\
\hline
\end{tabular}

C (T12 Group IV): A_tP12_138_bi - CIF

\# CIF file

data_findsym-outpu

audit_creation_method FINDSYM

chemical_name_mineral 'T12

chemical_formula_sum ' $\mathrm{C}$ '

loop

publ_author_name

'Z. Zhao'

'F. Tian,

'X. Dong'

' $\mathrm{X}$. Li '

'Q. Wang'

Q. Wang,

'X. Wang',

'B. $\mathrm{Xu}$ ',

D. $\mathrm{Yu}$

'J. $\mathrm{He}$

H.-T. Wang

Y. Ma'

'Y. Tian

journal_name_full_name

Journal of the American Chemical Society

journal_volume 134

journal year 2012

journal_page_first 12362

journal page last 12365

publ_Section_title

Tetragonal Allotrope of Group 14 Elements

aflow_title 'C (T12 Group IV) Structure'

_aflow_proto A_tP12_138_bi 
_aflow_params 'a, c/a, $x_{-}\{2\}, z_{-}\{2\}$

_ flow_params_values ' $3.388,1.77420306966,0.086,0.107$,

_aflow_Strukturbericht 'None'

-aflow_Pearson 'tP12,

_symmetry_space_group_name_H-M "P 42/n 21/c 2/m (origin choice 2)"

_symmetry_Int_Tables_number 138

\begin{tabular}{ll} 
_cell_length_a & 3.38800 \\
\hline &
\end{tabular}

cell_length_c 6.01100

_cell_angle_alpha 90.00000

_cell_angle_beta 90.00000

cell_angle_gamma 90.00000

loop

_space_group_symop_id

-space_group_symop_operation_xyz

$1 \mathrm{x}, \mathrm{y}, \mathrm{z}$

$2 \mathrm{x}+1 / 2,-\mathrm{y},-\mathrm{z}+1 / 2$

$3-x, y+1 / 2,-z+1 / 2$

$4-\mathrm{x}+1 / 2,-\mathrm{y}+1 / 2, \mathrm{z}$

$5-\mathrm{y},-\mathrm{x},-\mathrm{z}$

$6-\mathrm{y}+1 / 2, \mathrm{x}, \mathrm{z}+1 / 2$

$7 \mathrm{y},-\mathrm{x}+1 / 2, \mathrm{z}+1 / 2$

$8 \mathrm{y}+1 / 2, \mathrm{x}+1 / 2,-\mathrm{z}$

$9-\mathrm{x},-\mathrm{y},-\mathrm{z}$

$10-\mathrm{x}+1 / 2, \mathrm{y}, \mathrm{z}+1 / 2$

$12 x+1 / 2, y+1 / 2,-z$

$13 \mathrm{y}, \mathrm{x}, \mathrm{z}$

$14 \mathrm{y}+1 / 2,-\mathrm{x},-\mathrm{z}+1 / 2$

$15-\mathrm{y}, \mathrm{x}+1 / 2,-\mathrm{z}+1 / 2$

$16-y+1 / 2,-x+1 / 2, z$

loop

_atom_site_label

_atom_site_type_symbo

_atom_site_symmetry_multiplicity

_atom_site_Wyckoff_label

_atom_site_fract_x

atom_site_fract_y

_atom_site_fract_z

$\begin{array}{lllllllll}\mathrm{C} 1 \mathrm{C} & 4 & \mathrm{~b} & 0.75000 & 0.25000 & 0.75000 & 1.00000\end{array}$

$\begin{array}{lllllllll}\text { C2 } & \text { C } & 8 & \text { i } & 0.08600 & 0.08600 & 0.10700 & 1.00000\end{array}$

C (T12 Group IV): A_tP12_138_bi - POSCAR

\begin{tabular}{|c|c|c|c|c|}
\hline \multicolumn{5}{|c|}{ 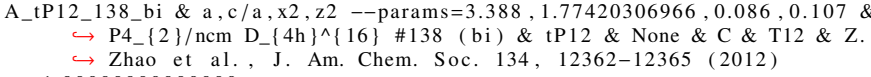 } \\
\hline \multicolumn{5}{|c|}{1.00000000000000} \\
\hline 3.38800000000000 & 0.00000000000000 & 0.00000000000000 & & \\
\hline 0.00000000000000 & 3.38800000000000 & 0.00000000000000 & & \\
\hline 0.00000000000000 & 0.00000000000000 & 6.01100000000000 & & \\
\hline \\
\hline \multicolumn{5}{|l|}{12} \\
\hline \multicolumn{5}{|l|}{$\begin{array}{l}\text { Direct } \\
075000000000000\end{array}$} \\
\hline 0.75000000000000 & 0.25000000000000 & 0.75000000000000 & $\mathrm{C}$ & (4b) \\
\hline 0.25000000000000 & 0.75000000000000 & 0.25000000000000 & $\mathrm{C}$ & (4b) \\
\hline 0.25000000000000 & 0.75000000000000 & 0.75000000000000 & $\mathrm{C}$ & (4b) \\
\hline 0.75000000000000 & 0.25000000000000 & 0.25000000000000 & $\mathrm{C}$ & (4b) \\
\hline 0.08600000000000 & 0.08600000000000 & 0.10700000000000 & $\mathrm{C}$ & ( $8 \mathrm{i})$ \\
\hline 0.41400000000000 & 0.41400000000000 & 0.10700000000000 & $\mathrm{C}$ & ( $8 \mathrm{i})$ \\
\hline 0.41400000000000 & 0.08600000000000 & 0.60700000000000 & $\mathrm{C}$ & ( $8 \mathrm{i})$ \\
\hline 0.08600000000000 & 0.41400000000000 & 0.60700000000000 & $\mathrm{C}$ & ( $8 \mathrm{i})$ \\
\hline-0.08600000000000 & 0.58600000000000 & 0.39300000000000 & $\mathrm{C}$ & ( $8 \mathrm{i})$ \\
\hline 0.58600000000000 & -0.08600000000000 & 0.39300000000000 & $\mathrm{C}$ & ( $8 \mathrm{i})$ \\
\hline 0.58600000000000 & 0.58600000000000 & -0.10700000000000 & $\mathrm{C}$ & ( $8 \mathrm{i})$ \\
\hline-0.08600000000000 & -0.08600000000000 & -0.10700000000000 & $\mathrm{C}$ & ( $8 \mathrm{i})$ \\
\hline
\end{tabular}

Calomel $\left(\mathrm{Hg}_{2} \mathrm{Cl}_{2}, D 3_{1}\right)$ : AB_tt8_139_e_e - CIF

\section{\# CIF file}

data_findsym-output

audit_creation_method FINDSYM

_chemical_name_mineral 'Calomel'

chemical_formula_sum ' $\mathrm{Cl} \mathrm{Hg}$ '

loop

publ author name

, N. J. Calos,

'C. H. L. Kennard,

'R. L. Davis

_journal_name_full_name

Zeitschrift $f\{\backslash " u\} r$ Kristallografiya

_journal_volume 187

_journal_year 1989

_journal_page_first 305

journal_page_last 307

_publ_Section_title

The structure of calomel, $\mathrm{Hg} \$_{-}\{2\} \$ C \mathrm{Cl} \$_{-}\{2\} \$$, derived from neutron powder $\hookrightarrow$ data

\# Found in The American Mineralogist Crystal Structure Database, 2003

_aflow_title 'Calomel $\left(\mathrm{Hg} \$_{-}\{2\} \$ \mathrm{Cl} \$_{-}\{2\} \$, \$ D 3_{-}\{1\} \$\right)$ Structure,

_aflow_proto 'AB_tI8_139_e_e

-aflow_params a, a/a, z- $\{1\}, z_{-}\{2\}$ _aflow_params_values ' $4.4795,2.43451278044,0.3356,0.119$ '

aflow_Strukturbericht ' $\$ D 3_{-}\{1\} \$$

_aflow_Pearson 'tI8,

symmetry_space_group_name_H-M "I $4 / \mathrm{m} 2 / \mathrm{m} 2 / \mathrm{m}^{\prime \prime}$

_symmetry_Int_Tables_number 139

_cell_length_a 4.47950

_cell_length_b 4.47950

_cell_length_c $\quad 10.90540$

_cell_angle_alpha 90.00000

cell_angle_gamma 90.00000

loop

_space_group_symop_id

space_group_symop_operation_xyz

$1 \mathrm{x}, \mathrm{y}, \mathrm{z}$

$2 \mathrm{x},-\mathrm{y},-\mathrm{z}$

$3-\mathrm{x}, \mathrm{y},-\mathrm{z}$

$4-\mathrm{x},-\mathrm{y}, \mathrm{z}$

$5-\mathrm{y},-\mathrm{x},-\mathrm{z}$

$6-\mathrm{y}, \mathrm{x}, \mathrm{z}$

$7 \mathrm{y},-\mathrm{x}, \mathrm{z}$

$8 \mathrm{y}, \mathrm{x},-\mathrm{z}$

$9-x,-y,-z$

$10-x, y, z$

$11 \mathrm{x},-\mathrm{y}, \mathrm{z}$

$12 \mathrm{x}, \mathrm{y},-\mathrm{z}$

$13 \mathrm{y}, \mathrm{x}, \mathrm{z}$

$14 \mathrm{y},-\mathrm{x},-\mathrm{z}$

$15-\mathrm{y}, \mathrm{x},-\mathrm{z}$

$\mathrm{x}+1 / 2, \mathrm{y}+1 / 2, \mathrm{z}+1 / 2$

$18 \mathrm{x}+1 / 2,-\mathrm{y}+1 / 2,-\mathrm{z}+1 / 2$

$19-\mathrm{x}+1 / 2, \mathrm{y}+1 / 2,-\mathrm{z}+1 / 2$

$20-\mathrm{x}+1 / 2,-\mathrm{y}+1 / 2, \mathrm{z}+1 / 2$

$21-\mathrm{y}+1 / 2,-\mathrm{x}+1 / 2,-\mathrm{z}+1 / 2$
$22-\mathrm{y}+1 / 2, \mathrm{x}+1 / 2, \mathrm{z}+1 / 2$

$22-\mathrm{y}+1 / 2, \mathrm{x}+1 / 2, \mathrm{z}+1 / 2$

$23 \mathrm{y}+1 / 2,-\mathrm{x}+1 / 2, \mathrm{z}+1 / 2$

$24 \mathrm{y}+1 / 2, \mathrm{x}+1 / 2,-\mathrm{z}+1 / 2$
$25-\mathrm{x}+1 / 2,-\mathrm{y}+1 / 2,-\mathrm{z}+1 / 2$
$26-\mathrm{x}+1 / 2, \mathrm{y}+1 / 2, z+1 / 2$

$26-x+1 / 2, y+1 / 2, z+1 / 2$

$27 \mathrm{x}+1 / 2,-\mathrm{y}+1 / 2, \mathrm{z}+1 / 2$

$28 \mathrm{x}+1 / 2, \mathrm{y}+1 / 2,-\mathrm{z}+1 / 2$

$29 \mathrm{y}+1 / 2, x+1 / 2, z+1 / 2$

$30 \mathrm{y}+1 / 2, \mathrm{x}+1 / 2, \mathrm{x}+1 / 2, \mathrm{z}$

$30 \mathrm{y}+1 / 2,-\mathrm{x}+1 / 2,-\mathrm{z}+1 / 2$

$31-\mathrm{y}+1 / 2, \mathrm{x}+1 / 2,-\mathrm{z}+1 / 2$

loop

atom_site_label

atom_site_type_symbol

atom_site_symmetry_multiplicity

atom_site_Wyckoff_label

atom_site_fract_x

-atom_site_fract_y

atom_site_fract_z

atom_site_occupancy

$\begin{array}{llllllll}\mathrm{C} 11 & \mathrm{Cl} & 4 & \mathrm{e} & 0.00000 & 0.00000 & 0.33560 & 1.00000\end{array}$

$\begin{array}{lllllllll}\mathrm{Hg} 1 \mathrm{Hg} & 4 & \mathrm{e} & 0.00000 & 0.00000 & 0.11900 & 1.00000\end{array}$

Calomel $\left(\mathrm{Hg}_{2} \mathrm{Cl}_{2}, D 3_{1}\right)$ : AB_tI8_139_e_e - POSCAR

AB_tI8_139_e_e \& a,c/a,z1,z2 --params $=4.4795,2.43451278044,0.3356,0.119$ $\hookrightarrow \& \mathrm{I} 4 / \mathrm{mmm} \mathrm{D}_{-}\{4 \mathrm{~h}\}^{\wedge}\{17\} \# 139\left(\mathrm{e}^{\wedge} 2\right) \& \mathrm{tI} 8 \&$ \& $3 \_\{1\} \$ \& \mathrm{Hg} 2 \mathrm{Cl} 2 \&$

$\hookrightarrow$ Calomel \& N. J. Calos and C. H. L. Kennard and R. L. Davis, Z.

$\hookrightarrow$ Kristallogr. 187, 305-307 (1989)

1.00000000000000

$\begin{array}{lll}-2.23975000000000 & 2.23975000000000 & 5.45270000000000\end{array}$

$\begin{array}{rrr}2.23975000000000 & -2.23975000000000 & 5.45270000000000\end{array}$

$\begin{array}{lll}2.23975000000000 & 2.23975000000000 & -5.45270000000000\end{array}$

$\mathrm{Cl} \quad \mathrm{Hg}$

Direc

0.33560000000000

$-0.33560000000000-0.33560000000000$

$-0.11900000000000$

0.11900000000000

0.00000000000000 0.00000000000000 0.00000000000000

$\mathrm{W}_{5} \mathrm{Si}_{3}\left(D 8_{m}\right):$ A3B5_tI32_140_ah_bk - CIF

\section{\# CIF file}

data_findsym-output

audit_creation_method FINDSYM

chemical_name_mineral 'W5Si3

_chemical_formula_sum ' $\mathrm{Si} 3$ W5

loop

publ_author_name

'B. Aronsson'

_journal_name_full_name

Acta Chemica Scandinavica

journal_volume 9

journal_year 1955

journal_page_first 1107

journal_page_last 1110

publ Section title

The Crystal Structure of $\operatorname{Mo}_{-}\{5\} \$ \operatorname{Si} \$_{-}\{3\} \$$ and $W \$_{-}\{5\} \$ \operatorname{Si} \$_{-}\{3\} \$$ 


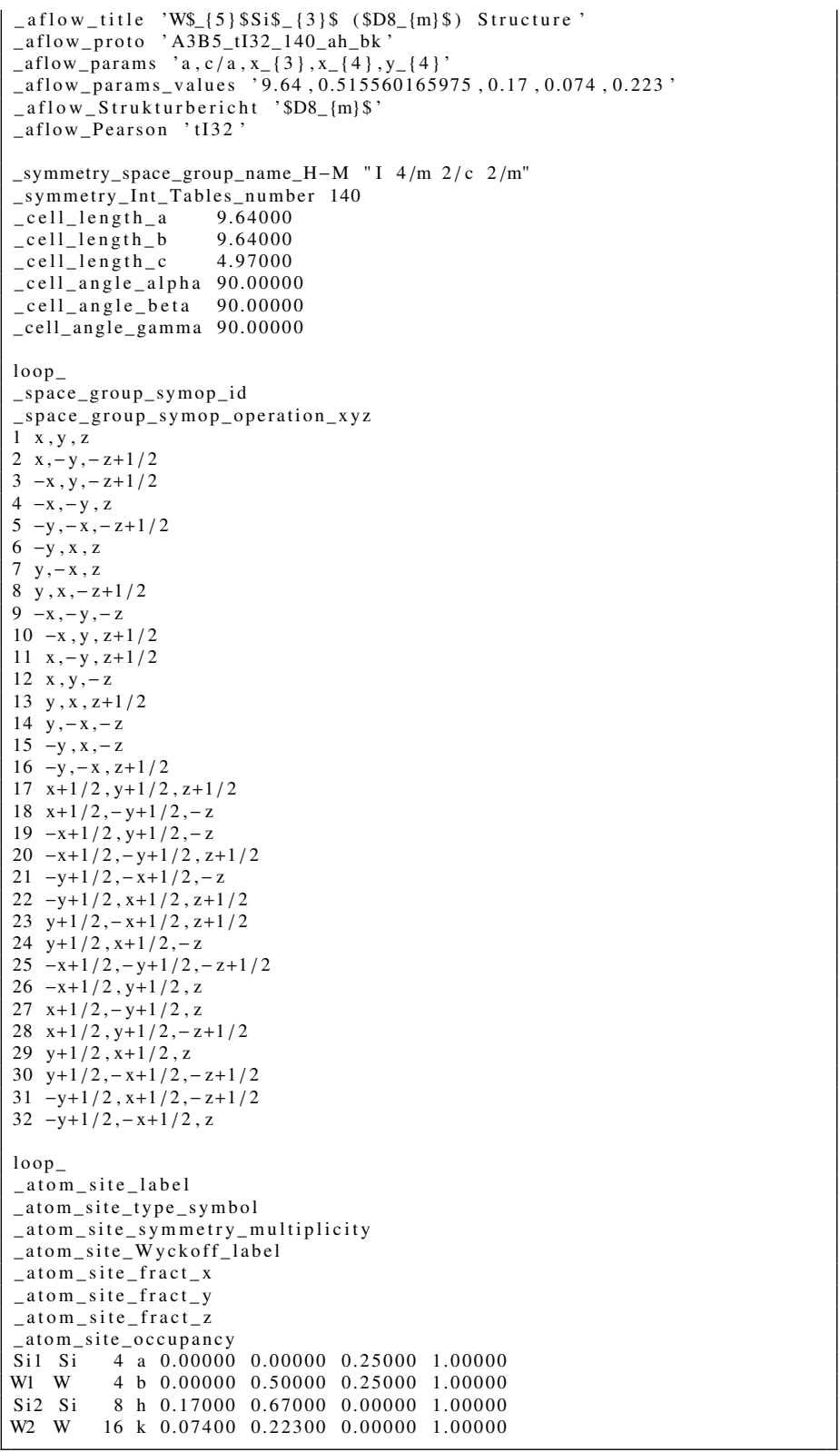

$\mathrm{W}_{5} \mathrm{Si}_{3}\left(D 8_{m}\right)$ : A3B5_tI32_140_ah_bk - POSCAR

\begin{tabular}{|c|c|c|c|c|}
\hline 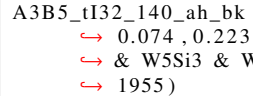 & $\begin{array}{l}\& \mathrm{a}, \mathrm{c} / \mathrm{a}, \mathrm{x} 3, \mathrm{x} 4, \mathrm{y} 4- \\
\& \mathrm{I} / \mathrm{mcm} \mathrm{D}_{-}\{4 \mathrm{~h}\}^{\wedge}\{ \\
5 \mathrm{Si} 3 \text { \& B. Aronsson }\end{array}$ & $\begin{array}{l}\text { params }=9.64,0.51556 \\
\text { A \#140 (abhk) \& t } \\
\text { Acta Chem. Scand. }\end{array}$ & & $\begin{array}{l}\text {, } 0.17, \\
8-\{\mathrm{m}\} \$ \\
-1110\end{array}$ \\
\hline 1.00000000000000 & & & & \\
\hline-4.82000000000000 & 4.82000000000000 & 2.48500000000000 & & \\
\hline 4.82000000000000 & -4.82000000000000 & 2.48500000000000 & & \\
\hline 4.82000000000000 & 4.82000000000000 & -2.48500000000000 & & \\
\hline $\mathrm{Si} \quad \mathrm{W}$ & & & & \\
\hline 10 & & & & \\
\hline Direct & & & & \\
\hline 0.25000000000000 & 0.25000000000000 & 0.00000000000000 & $\mathrm{Si}$ & (4a) \\
\hline 0.75000000000000 & 0.75000000000000 & 0.00000000000000 & $\mathrm{Si}$ & (4a) \\
\hline 0.67000000000000 & 0.17000000000000 & 0.84000000000000 & $\mathrm{Si}$ & $(8 \mathrm{~h})$ \\
\hline 0.33000000000000 & -0.17000000000000 & 0.16000000000000 & $\mathrm{Si}$ & $(8 \mathrm{~h})$ \\
\hline 0.17000000000000 & 0.33000000000000 & 0.50000000000000 & $\mathrm{Si}$ & $(8 \mathrm{~h})$ \\
\hline-0.17000000000000 & 0.67000000000000 & 0.50000000000000 & $\mathrm{Si}$ & $(8 \mathrm{~h})$ \\
\hline 0.75000000000000 & 0.25000000000000 & 0.50000000000000 & W & (4b) \\
\hline 0.25000000000000 & 0.75000000000000 & 0.50000000000000 & W & (4b) \\
\hline 0.22300000000000 & 0.07400000000000 & 0.29700000000000 & W & $(16 \mathrm{k})$ \\
\hline-0.22300000000000 & -0.07400000000000 & -0.29700000000000 & W & $(16 \mathrm{k})$ \\
\hline 0.07400000000000 & -0.22300000000000 & -0.14900000000000 & $\mathrm{~W}$ & $(16 \mathrm{k})$ \\
\hline-0.07400000000000 & 0.22300000000000 & 0.14900000000000 & W & $(16 \mathrm{k})$ \\
\hline 0.72300000000000 & 0.42600000000000 & 0.14900000000000 & W & $(16 \mathrm{k})$ \\
\hline 0.27700000000000 & 0.57400000000000 & -0.14900000000000 & W & $(16 \mathrm{k})$ \\
\hline 0.57400000000000 & 0.72300000000000 & 0.29700000000000 & W & $(16 \mathrm{k})$ \\
\hline 0.42600000000000 & 0.27700000000000 & -0.29700000000000 & W & $(16 \mathrm{k})$ \\
\hline
\end{tabular}

$\mathrm{Cr}_{5} \mathrm{~B} 3\left(D 8_{l}\right):$ A3B5_tI32_140_ah_cl - CIF

\section{\# CIF file}

data_findsym-output

_audit_creation_method FINDSYM

chemical_name_mineral 'Cr5B3,

_chemical_formula_sum 'B3 Cr5, loop

publ a

F. Bertaut,

'P. Blum

_journal_name_full_name

Comptes Rendus Hebdomadaires des S $\left\{\backslash^{\prime}\right.$ eances de l'Acad $\{\backslash$ 'e $\}$ mie des $\hookrightarrow$ Sciences

journal_volume 236

journal year 1953

journal_page_first 1055

journal page last 1056

publ_Section_title

Etude des borures de chrome

\# Found in The American Mineralogist Crystal Structure Database, 2003

aflow_title, Cr\$_ $\{5\} \$ B \$_{-}\{3\} \$\left(\$ D 8_{-}\{1\} \$\right)$ Structure,

aflow_proto 'A3B5_tI32_140_ah_cl

aflow_params 'a,c/a, $x_{-}\{3\}, x_{-}\{4\}, z_{-}\{4\}$

aflow_params_values $5.46,1.91575091575,0.625,0.166,0.15$

aflow_Strukturbericht $\$ D 8_{-}\{1\} \$$,

aflow_Pearson 'tI32

symmetry space group name H-M "I $4 / \mathrm{m} 2 / \mathrm{c} 2 / \mathrm{m}$

symmetry_Int_Tables_number 140

cell_length_a $\quad 5.46000$

cell_length_b 5.46000

cell_length_c $\quad 10.46000$

cell_angle_alpha 90.00000

a 90.00000

cell_angle_gamma 90.00000

loop

space_group_symop_id

_space_group_symop_operation_xyz

$\mathrm{x}, \mathrm{y}, \mathrm{z}$

$\mathrm{x},-\mathrm{y},-\mathrm{z}+1 / 2$

$3-\mathrm{x}, \mathrm{y},-\mathrm{z}+1 /$
$4-\mathrm{x},-\mathrm{y}, \mathrm{z}$

$5-\mathrm{y},-\mathrm{x},-\mathrm{z}+1 / 2$

$6-\mathrm{y}, \mathrm{x}, \mathrm{z}$

$\begin{array}{ll}6 & -y, x, z \\ 7 & y,-x, z\end{array}$

$8 \mathrm{y}, \mathrm{x},-\mathrm{z}+1 / 2$

$8 \mathrm{y}, \mathrm{x},-\mathrm{z}+1 / 2$
$9-\mathrm{x},-\mathrm{y},-\mathrm{z}$

$10-\mathrm{x}, \mathrm{y}, \mathrm{z}+1 / 2$

$1 \mathrm{x},-\mathrm{y}, \mathrm{z}+1 / 2$

$2 \mathrm{x}, \mathrm{y},-\mathrm{z}$

$13 \mathrm{y}, \mathrm{x}, \mathrm{z}+1 / 2$

$14 \mathrm{y},-\mathrm{x},-\mathrm{z}$

$15-\mathrm{y}, \mathrm{x},-\mathrm{z}$

$16-\mathrm{y},-\mathrm{x}, \mathrm{z}+1 / 2$

$7 \mathrm{x}+1 / 2, \mathrm{y}+1 / 2, \mathrm{z}+1 / 2$

$18 \mathrm{x}+1 / 2,-\mathrm{y}+1 / 2,-\mathrm{z}$

$19-\mathrm{x}+1 / 2, \mathrm{y}+1 / 2,-\mathrm{z}$

$20-x+1 / 2,-y+1 / 2, z+1 / 2$

$21-\mathrm{y}+1 / 2,-\mathrm{x}+1 / 2,-\mathrm{z}$

$22-y+1 / 2, x+1 / 2, z+1 / 2$

$23 x+1 / 2,-x+1 / 2, z+1 / 2$

$24 \mathrm{y}+1 / 2, \mathrm{x}+1 / 2, \mathrm{z}$

$24 \mathrm{y}+1 / 2, \mathrm{x}+1 / 2,-\mathrm{z}$
$25-\mathrm{x}+1 / 2,-\mathrm{y}+1 / 2,-\mathrm{z}+1 / 2$

$26-x+1 / 2, y+1 / 2, z$

$27 \mathrm{x}+1 / 2,-\mathrm{y}+1 / 2, \mathrm{z}$

$28 \mathrm{x}+1 / 2, \mathrm{y}+1 / 2,-\mathrm{z}+1 / 2$

$29 \mathrm{y}+1 / 2, \mathrm{x}+1 / 2, \mathrm{z}$

$30 \mathrm{y}+1 / 2,-\mathrm{x}+1 / 2,-\mathrm{z}+1 / 2$

$31-y+1 / 2, x+1 / 2,-z+1 / 2$

$32-\mathrm{y}+1 / 2,-\mathrm{x}+1 / 2, \mathrm{z}$

loop

atom_site_label

atom_site_type_symbol

_atom_site_symmetry_multiplicity

atom_site_Wyckoff_label

atom_site_fract_x

atom_site_fract_y

atom_site_fract_z

atom site occupancy

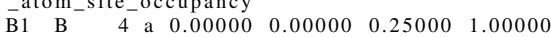

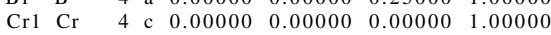

$\begin{array}{llllllll}\mathrm{B} 2 & \mathrm{~B} & 8 & \mathrm{~h} & 0.62500 & 0.12500 & 0.00000 & 1.00000\end{array}$

$\begin{array}{llrrrrrrr}\mathrm{Cr} 2 & \mathrm{Cr} & 16 & 1 & 0.16600 & 0.66600 & 0.15000 & 1.00000\end{array}$

$\mathrm{Cr}_{5} \mathrm{~B}_{3}\left(D 8_{l}\right):$ A3B5_tI32_140_ah_cl - POSCAR

A3B5_tI32_140_ah_cl \& a,c/a, x3, x4, z4 --params $=5.46,1.91575091575,0.625$

$\hookrightarrow 0.166,0.15 \& \mathrm{I} 4 / \mathrm{mcm} \mathrm{D}_{-}\{4 \mathrm{~h}\}^{\wedge}\{18\} \# 140$ (achl) \& $\mathrm{tI} 32 \&$ \&D8 $\{1\} \$$

$\hookrightarrow \mathrm{Cr} 5 \mathrm{~B} 3$ \& Cr5B3 \& F. Bertaut and P. Blum, \{C. R. Hebd. S $\{$ 'e\}

$\hookrightarrow$ ances Acad. Sci. 236, 1055-1056 (1953)

1.0000000000000

$\begin{array}{rrr}-2.73000000000000 & 2.73000000000000 & 5.23000000000000\end{array}$

$\begin{array}{rrr}2.73000000000000 & -2.73000000000000 & 5.23000000000000\end{array}$

$\begin{array}{lll}2.73000000000000 & 2.73000000000000 & -5.23000000000000\end{array}$

$\begin{array}{ll}\mathrm{B} & \mathrm{Cr} \\ 6 & 10\end{array}$

Direct

0.25000000000000

0.75000000000000

1.12500000000000

$-0.12500000000000$

0.25000000000000 0.75000000000000 0.62500000000000

$-0.62500000000000$

0.00000000000000 0.00000000000000 $-0.7500000000000$

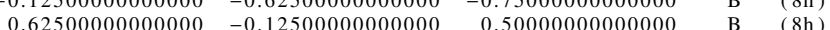




$\begin{array}{rrrrrr}-0.62500000000000 & 1.12500000000000 & 0.50000000000000 & \mathrm{~B} & (8 \mathrm{~h}) \\ 0.00000000000000 & 0.00000000000000 & 0.00000000000000 & \mathrm{Cr} & (4 \mathrm{c}) \\ 0.50000000000000 & 0.50000000000000 & 0.00000000000000 & \mathrm{Cr} & (4 \mathrm{c}) \\ 0.81600000000000 & 0.31600000000000 & 0.83200000000000 & \mathrm{Cr} & (161) \\ 0.48400000000000 & -0.01600000000000 & 0.16800000000000 & \mathrm{Cr} & (161) \\ 0.31600000000000 & 0.48400000000000 & 0.50000000000000 & \mathrm{Cr} & (161) \\ -0.01600000000000 & 0.81600000000000 & 0.50000000000000 & \mathrm{Cr} & (161) \\ 0.01600000000000 & 0.18400000000000 & 0.50000000000000 & \mathrm{Cr} & (161) \\ -0.31600000000000 & 0.51600000000000 & 0.50000000000000 & \mathrm{Cr} & (161) \\ 0.51600000000000 & 0.01600000000000 & 0.83200000000000 & \mathrm{Cr} & (161) \\ 0.18400000000000 & -0.31600000000000 & 0.16800000000000 & \mathrm{Cr} & (161)\end{array}$

$\alpha-\mathrm{ThSi}_{2}\left(C_{c}\right):$ A2B_tI12_141_e_a - CIF

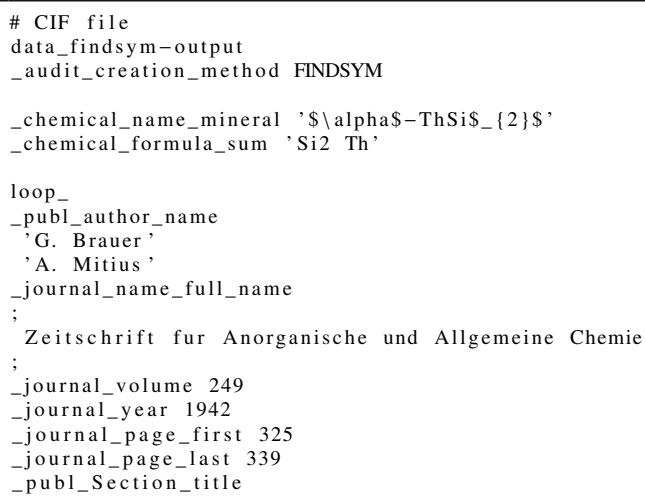

$\alpha-\mathrm{ThSi}_{2}\left(C_{c}\right):$ A2B_tI12_141_e_a - POSCAR

\begin{tabular}{|c|c|c|c|c|}
\hline \multicolumn{5}{|c|}{ 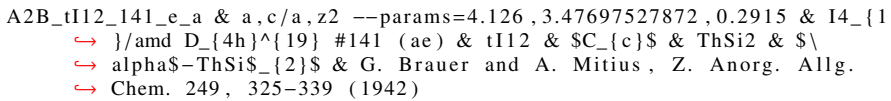 } \\
\hline \multicolumn{5}{|l|}{1.00000000000000} \\
\hline-2.06300000000000 & 2.06300000000000 & 7.17300000000000 & & \\
\hline 2.06300000000000 & -2.06300000000000 & 7.17300000000000 & & \\
\hline 2.06300000000000 & 2.06300000000000 & 7.17300000000000 & & \\
\hline Th & & & & \\
\hline 4 & & & & \\
\hline \multicolumn{5}{|l|}{ Direct } \\
\hline 0.54150000000000 & 0.29150000000000 & 0.25000000000000 & $\mathrm{Si}$ & (8e ) \\
\hline 0.29150000000000 & 0.54150000000000 & 0.75000000000000 & $\mathrm{Si}$ & $(8 \mathrm{e})$ \\
\hline 0.45850000000000 & -0.29150000000000 & 0.75000000000000 & $\mathrm{Si}$ & ( $8 \mathrm{e})$ \\
\hline-0.29150000000000 & 0.45850000000000 & 0.25000000000000 & $\mathrm{Si}$ & $(8 \mathrm{e})$ \\
\hline 0.87500000000000 & 0.12500000000000 & 0.75000000000000 & Th & (4a) \\
\hline 0.12500000000000 & 0.87500000000000 & 0.25000000000000 & Th & (4a) \\
\hline
\end{tabular}

S-III: A_tI16_142_f - CIF

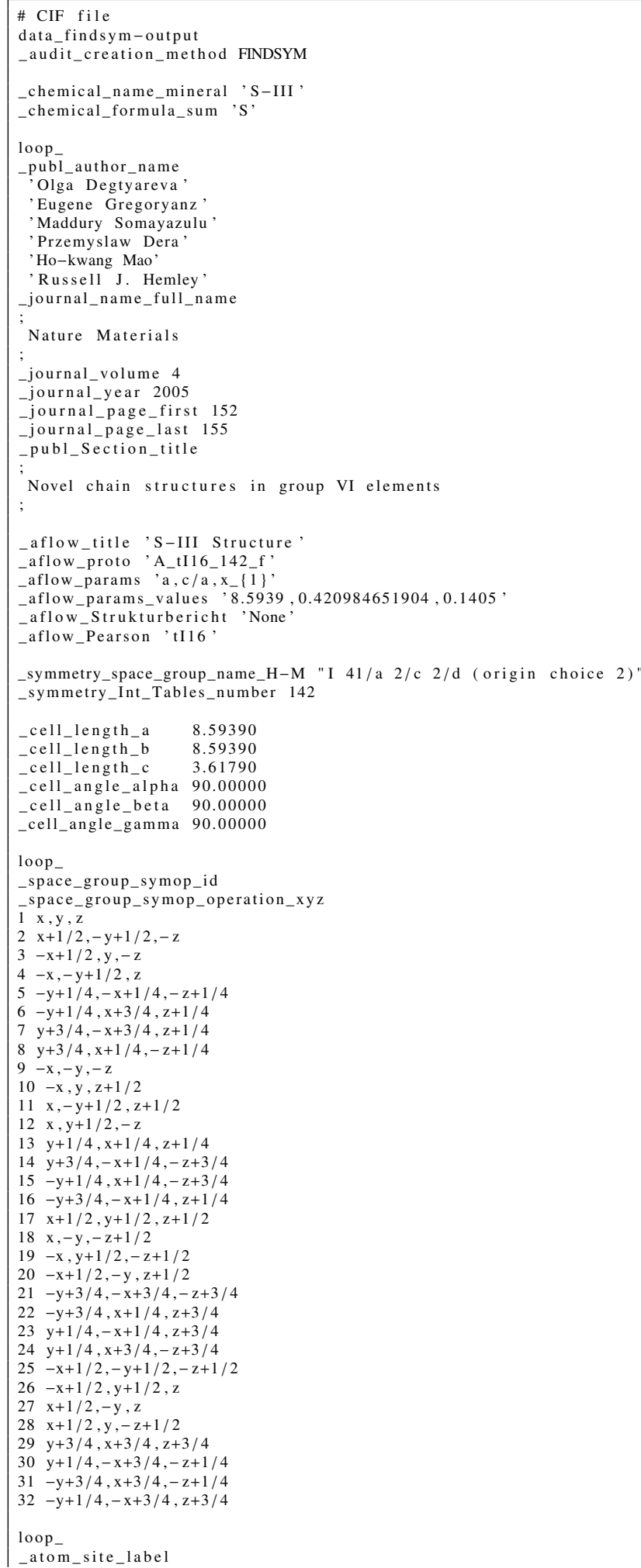


_atom_site_type_symbol

atom_site_symmetry_multiplicity

atom_site_Wyckoff_label

atom_site_fract_x

atom_site_fract_y

atom_site_fract_z

_atom_site_occupancy

$\begin{array}{llllllll}\text { S1 S } & 16 & \text { f } & 0.14050 & 0.39050 & 0.12500 & 1.00000\end{array}$

S-III: A_tI16_142_f - POSCAR

A_tI16_142_f \& a,c/a, x1 --params $=8.5939,0.420984651904,0.1405 \&$ I4_ $\{1\} /$

$\hookrightarrow$ acd D_ $\{4 \mathrm{~h}\}^{\wedge}\{20\} \# 142$ (f) \& tI16 \& None \& S \& S-III \& Olga

$\hookrightarrow$ Degtyareva et al., Nat. Mater. 4, 152-155 (2005)

1.00000000000000

$\begin{array}{lll}-4.29695000000000 & 4.29695000000000 & 1.80895000000000\end{array}$

$4.29695000000000 \quad-4.29695000000000 \quad 1.80895000000000$

$\begin{array}{lll}4.29695000000000 & 4.29695000000000 & -1.80895000000000\end{array}$

S

Direct

$\begin{array}{lll}0.51550000000000 & 0.26550000000000 & 0.53100000000000\end{array}$

0.23450000000000

0.2655000000000

$-0.0155000000000$

0.01550000000000

0.48450000000000

0.76550000000000

0.7345000000000

0.23450000000000

0.51550000000000

0.51550000000000

0.734500000000

0.03100000000000

0.75000000000000

0.75000000000000

0.46900000000000

0.46900000000000
1.0310000000000

0.76550000000000

1.03100000000000

0.2500000000000

S (16f)

S (16f)

(16f)

(16f)

$S \quad(16 f)$

$S$ (16f)

$S$ (16f)

Simpsonite $\left(\mathrm{Ta}_{3} \mathrm{Al}_{4} \mathrm{O}_{13}[\mathrm{OH}]\right)$ : A4B14C3_hP21_143_bd_ac4d_d - CIF

\section{\# CIF file}

data_findsym-output

_audit_creation_method_FINDSYM

_chemical_name_mineral 'Ta3Al4O13[OH]'

_chemical_formula_sum 'Al4 O14 Ta3

loop

publ_author_name

T. S. Ercit

F. C. Hawthorne

'P. Cerny'

journal_name_full_name

Canadian Mineralogist

journal_volume 30

journal year 1992

journal_page_first 653

journal_page_last 662

publ_Section_title

The crystal structure of alumotantite; its relation to the structure $\hookrightarrow$ of simpsonite and the $(\mathrm{Al}, \mathrm{Ga})(\mathrm{Ta}, \mathrm{Nb}) \mathrm{O} \$\{\{4\} \$$ compounds

\# Found in Pearson's Crystal Data - Crystal Structure Database for $\hookrightarrow$ Inorganic Compounds, 2013

aflow_title 'Simpsonite $\left(\mathrm{Ta} \$ \$_{-}\{3\} \$ \mathrm{Al} \$ \$_{-}\{4\} \$ \mathrm{O} \$ \$_{-}\{13\} \$[\mathrm{OH}]\right)$ Structure aflow_proto A4B14C3_hP21_143_bd_ac4d_dd

aflow_params a , c/a, z $\{1\}, z_{-}\{2\}, z_{-}\{3\}, x_{-}\{4\}, y_{1}\{4\}, z_{-}\{4\}, x_{-}\{5\}, y_{-}\{5\}, z_{-}\{$ $\hookrightarrow 5\}, x_{-}\{6\}, y_{-}\{6\}, z_{-}\{6\}, x_{-}\{7\}, y_{-}\{7\}, z_{-}\{7\}, x_{-}\{8\}, y_{-}\{8\}, z_{-}\{8\}, x_{-}\{9\}$ $\hookrightarrow \mathrm{y}_{-}\{9\}, \mathrm{z}_{-}\{9\}$,

aflow params_values $\quad 7.3813879247,0.611841213925,0.0,0.305,0.497,0.453$ $\hookrightarrow 0.09,0.302,0.087,0.605,0.536,0.253,0.059,0.583,0.141,0.429,0.08$ $\hookrightarrow, 0.444,0.296,0.071,0.2215,0.2757,0.806$

aflow Strukturbericht 'None'

aflow_Pearson 'hP21,

cell_length_a 7.3813879247

cell_length_b 7.3813879247

cell_length_c $\quad 4.5162373483$

cell_angle_alpha 90.0000000000

cell_angle_beta 90.0000000000

cell_angle_gamma 120.0000000000

ymmetry space group name $\mathrm{H}-\mathrm{M}$ "P 3 "

symmetry_Int_Tables_number 143

loop

_space_group_symop_id

_space_group_symop_operation_xyz

$1 \mathrm{x}, \mathrm{y}, \mathrm{z}$

$2-y, x-y, z$
$3-x+y,-x, z$

loop

atom_site_label

atom_site_type_symbol

atom_site_symmetry_multiplicity

atom_site_Wyckoff_label

atom_site_fract_x

atom_site_fract_y

atom_site_fract_z

atom_site_occupancy

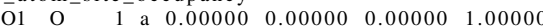

$\begin{array}{lllllllll}\mathrm{Al} 1 & \mathrm{Al} & 1 & \mathrm{~b} & 0.33333 & 0.66667 & 0.30500 & 1.00000\end{array}$

$\begin{array}{lllllllll}\mathrm{O} 2 & \mathrm{O} & 1 & \mathrm{c} & 0.66667 & 0.33333 & 0.49700 & 1.00000\end{array}$

$\begin{array}{llllllll}\mathrm{A} 12 & \mathrm{Al} & 3 & \mathrm{~d} & 0.45300 & 0.09000 & 0.30200 & 1.00000\end{array}$

$\begin{array}{lllllllll}03 & \mathrm{O} & 3 & \mathrm{~d} & 0.08700 & 0.60500 & 0.53600 & 1.00000\end{array}$

$\begin{array}{lllllllll}\mathrm{O} 4 & \mathrm{O} & & 3 & \mathrm{~d} & 0.25300 & 0.05900 & 0.58300 & 1.00000\end{array}$

$\begin{array}{lllllllll}\mathrm{O} 5 & \mathrm{O} & 3 & \mathrm{~d} & 0.14100 & 0.42900 & 0.08000 & 1.00000\end{array}$
O6 0 - 3 d $0.44400 \quad 0.29600 \quad 0.07100 \quad 1.00000$

$\begin{array}{lllllllll}\mathrm{Ta} 1 & \mathrm{Ta} & 3 & \mathrm{~d} & 0.22150 & 0.27570 & 0.80600 & 1.00000\end{array}$

Simpsonite $\left(\mathrm{Ta}_{3} \mathrm{Al}_{4} \mathrm{O}_{13}[\mathrm{OH}]\right)$ : A4B 14C3_hP21_143_bd_ac4d_d - POSCAR

\begin{tabular}{|c|c|c|c|c|}
\hline \\
\hline & \multicolumn{4}{|c|}{ 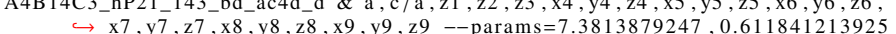 } \\
\hline \\
\hline \multicolumn{5}{|c|}{$\begin{array}{l}\hookrightarrow, 0.0,0.305,0.497,0.453,0.09,0.302,0.087,0.605,0.536,0.253,0.059 \\
\hookrightarrow, 0.583,0.141,0.429,0.08,0.444,0.296,0.071,0.2215,0.2757,0.806 \&\end{array}$} \\
\hline \multicolumn{5}{|c|}{$\hookrightarrow \mathrm{P}^{2} \mathrm{C}_{-}\{3\}^{\wedge}\{1\} \# 143\left(\mathrm{abcd}^{\wedge} 6\right) \& \mathrm{hP} 21 \&$ None \& Ta3Al4O13[OH] \& \& } \\
\hline \multirow{2}{*}{\multicolumn{5}{|c|}{$\begin{array}{l}\text { T. S. Ercit and F. C. Hawthorne and P. Cerny, Can. Mineral. 30, } \\
653-662(1992)\end{array}$}} \\
\hline \multicolumn{2}{|l|}{1.00000000000000} & & & \\
\hline 3.69069396235000 & -6.39246945797790 & 0.00000000000000 & & \\
\hline \multirow{2}{*}{$\begin{array}{l}3.69069396235000 \\
0.00000000000000\end{array}$} & 6.39246945797790 & 0.00000000000000 & & \\
\hline & 0.00000000000000 & 4.51623734830000 & & \\
\hline $\mathrm{Ta}$ & & & & \\
\hline 14 & & & & \\
\hline \multicolumn{5}{|l|}{ Direct } \\
\hline 0.33333333333333 & 0.666666666666667 & 0.30500000000000 & $\mathrm{Al}$ & (1b) \\
\hline 0.45300000000000 & 0.09000000000000 & 0.30200000000000 & $\mathrm{Al}$ & $(3 d)$ \\
\hline-0.09000000000000 & 0.36300000000000 & 0.30200000000000 & $\mathrm{Al}$ & $(3 d)$ \\
\hline-0.36300000000000 & -0.45300000000000 & 0.30200000000000 & $\mathrm{Al}$ & $(3 d)$ \\
\hline 0.00000000000000 & 0.00000000000000 & 0.00000000000000 & $\mathrm{O}$ & (1a) \\
\hline 0.666666666666667 & 0.33333333333333 & 0.49700000000000 & $\mathrm{O}$ & (1c) \\
\hline 0.08700000000000 & 0.60500000000000 & 0.53600000000000 & $\mathrm{O}$ & (3d) \\
\hline-0.60500000000000 & -0.51800000000000 & 0.53600000000000 & $\mathrm{O}$ & (3d) \\
\hline 0.51800000000000 & -0.08700000000000 & 0.53600000000000 & $\mathrm{O}$ & (3d) \\
\hline 0.25300000000000 & 0.05900000000000 & 0.58300000000000 & $\mathrm{O}$ & (3d) \\
\hline-0.05900000000000 & 0.19400000000000 & 0.58300000000000 & $\mathrm{O}$ & $(3 d)$ \\
\hline-0.19400000000000 & -0.25300000000000 & 0.58300000000000 & $\mathrm{O}$ & $(3 d)$ \\
\hline 0.14100000000000 & 0.42900000000000 & 0.08000000000000 & $\mathrm{O}$ & (3d) \\
\hline-0.42900000000000 & -0.28800000000000 & 0.08000000000000 & $\mathrm{O}$ & (3d) \\
\hline 0.28800000000000 & -0.14100000000000 & 0.08000000000000 & $\mathrm{O}$ & (3d) \\
\hline 0.44400000000000 & 0.29600000000000 & 0.07100000000000 & $\mathrm{O}$ & $(3 d)$ \\
\hline-0.29600000000000 & 0.14800000000000 & 0.07100000000000 & $\mathrm{O}$ & $(3 d)$ \\
\hline-0.14800000000000 & -0.44400000000000 & 0.07100000000000 & $\mathrm{O}$ & (3d) \\
\hline 0.22150000000000 & 0.27570000000000 & 0.80600000000000 & $\mathrm{Ta}$ & (3d) \\
\hline-0.27570000000000 & -0.05420000000000 & 0.80600000000000 & $\mathrm{Ta}$ & (3d) \\
\hline 0.05420000000000 & -0.22150000000000 & 0.80600000000000 & $\mathrm{Ta}$ & $(3 d)$ \\
\hline
\end{tabular}

$\mathrm{ScRh}_{6} \mathrm{P}_{4}$ : A4B6C_hP11_143_bd_2d_a - CIF

\# CIF file

data findsym-output

audit_creation method FINDSYM

chemical name mineral 'ScRh6P4'

chemical formula_sum 'P4 Rh6 Sc'

loop

publ_author_name

U. Pfannenschmidt,

U. C. Rodewald

R. $P\{\backslash$ o $\}$ ttgen

journal_name_full_name

Monatshefte $f\{\backslash " u\} r$ Chemie - Chemical Monthly

journal_volume 142

journal_year 2011

journal_page_first 219

journal page last 224

publ_section title

Bismuth flux crystal growth of \$RE\$Rh\$ \{6\}\$P\$ \{4\}\$ (\$RE\$ = Sc, Yb, Lu) : $\hookrightarrow$ new phosphides with a superstructure of the LiCo\$_\{6\}\$P\$_\{4\}\$ $\begin{array}{ll}\hookrightarrow & \text { new } \\ \hookrightarrow & \text { type }\end{array}$

\# Found in Pearson's Crystal Data - Crystal Structure Database for $\hookrightarrow$ Inorganic Compounds, 2013

aflow_title, ScRh\$_\{6\}\$P\$_ $\{4\} \$$ Structure

aflow_proto 'A4B6C_hP11_143_bd_2d_a

aflow_params 'a, c/a, $\mathrm{z}_{-}\{1\}, \mathrm{z}_{-}\{2\}, \mathrm{x}_{-}\{3\}, \mathrm{y}_{-}\{3\}, \mathrm{z}_{-}\{3\}, \mathrm{x}_{-}\{4\}, \mathrm{y}_{-}\{4\}, \mathrm{z}_{-}\{4\}, \mathrm{x}_{-}\{$ $\stackrel{\longrightarrow}{\hookrightarrow} 5\}, \mathrm{y}_{-}\{5\}, \mathrm{z}_{-}\{5\}$

flow_params_values $, 6.9672687469,0.526119402977,0.0004,-0.0007,0.8181$ $\hookrightarrow 0.1915,0.4998,0.5475,0.48,0.0003,0.1859,0.799,0.5002$

aflow_Strukturbericht 'None'

aflow_Pearson 'hP11'

cell_length_a $\quad 6.9672687469$

cell_length_b $\quad 6.9672687469$

cell_length_c 3.6656152735

_cell_angle_alpha 90.0000000000

_cell_angle_beta 90.0000000000

cell_angle_gamma 120.0000000000

symmetry_space_group_name_H-M "P 3"

_symmetry_Int_Tables_number 143

loop

space_group_symop_id

space_group_symop_operation_xyz

$\mathrm{x}, \mathrm{y}, \mathrm{z}$

$2-y, x-y, z$

loop

atom site label

atom_site_type_symbol

atom_site_symmetry_multiplicity

atom_site_Wyckoff_label

_atom_site_fract_x 
-atom_site_fract_y

atom_site_fract_z

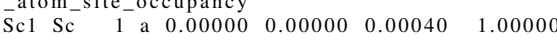

$\begin{array}{lllllllll}\mathrm{P} 1 & \mathrm{P} & 1 & \mathrm{~b} & 0.33333 & 0.66667 & -0.00070 & 1.00000\end{array}$

$\begin{array}{llllllll}\mathrm{P} 2 & \mathrm{P} & 3 & \mathrm{~d} & 0.81810 & 0.19150 & 0.49980 & 1.00000\end{array}$

$\begin{array}{lllllll}\mathrm{Rh} 1 \mathrm{Rh} & 3 & \mathrm{~d} & 0.54750 & 0.48000 & 0.00030 & 1.00000\end{array}$

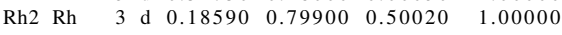

$\mathrm{ScRh}_{6} \mathrm{P}_{4}$ : A4B6C_hP11_143_bd_2d_a - POSCAR

A4B6C_hP11_143_bd_2d_a \& a , c/a, z1 , z2, x3 , y3 , z3 , x4 ,y4, z4, x5 , y5 , z5 --param $\hookrightarrow=6.9672687469,0.526119402977,0.0004,-0.0007,0.8181,0.1915$

$\hookrightarrow 0.4998,0.5475,0.48,0.0003,0.1859,0.799,0.5002 \& \mathrm{P} 3 \mathrm{C}_{-}\{3\}^{\wedge}\{1\} \#$

$\hookrightarrow 143\left(a^{\wedge} 3\right)$ \& hP11 \& None \& ScRh6P4 \& \& U. Pfannenschmidt and

$\hookrightarrow$ U. C. Rodewald and R. P $\{\backslash " \mathrm{o}\} \mathrm{ttgen,} \mathrm{Monatsh.} \mathrm{Chem.} \mathrm{142,} \mathrm{219-224}$ $\hookrightarrow(2011)$

1.00000000000000

$\begin{array}{lll}3.48363437345000 & -6.03383172980877 & 0.00000000000000\end{array}$

$\begin{array}{lll}3.48363437345000 & 6.03383172980877 & 0.00000000000000\end{array}$

0.00000000000000

$$
\begin{array}{rrr}
\mathrm{P} & \mathrm{Rh} & \mathrm{Sc} \\
4 & 6 & 1
\end{array}
$$

Direct

0.33333333333333

0.81810000000000

0.81810000000000

0.19150000000000

$-0.62660000000000$

0.54750000000000

$-0.48000000000000$

$-0.06750000000000$

0.18590000000000

$-0.79900000000000$

0.61310000000000

0.00000000000000

3.66561527350000

0.666666666666667 0.19150000000000 0.62660000000000 0.8181000000000 0.48000000000000 0.06750000000000 $-0.5475000000000$ 0.79900000000000 $-0.6131000000000$ $-0.18590000000000$ 0.00000000000000

$-0.00070000000000$ 0.49980000000000 0.49980000000000 0.49980000000000 0.00030000000000 0.00030000000000 0.00030000000000 0.00030000000000 0.50020000000000 0.50020000000000 0.50020000000000 0.00040000000000

$\mathrm{MoS}_{2}$ : AB2_hP12_143_cd_ab2d - CIF

\section{\# CIF file}

data_findsym-output

audit_creation_method FINDSYM

chemical_name_mineral 'MoS2'

chemical formula sum 'Mo S2'

loop

_publ_author_name

'K. E. Dungey'

J. E. \{Penner-Hahn\},

journal_name_full_name

Chemistry of Materials

journal_volume 10

journal_year 199

journal_page_first 2152

journal_page_last 2161

publ_Section_title

Structural characterization and thermal stability of MoS\$_\{2\}\$

$\hookrightarrow$ intercalation compounds

\# Found in Pearson's Crystal Data - Crystal Structure Database for

$\hookrightarrow$ Inorganic Compounds, 2013

aflow title 'MoS\$_\{2\}\$ Structure'

aflow proto, $\mathrm{AB} 2 \mathrm{hP12} 143 \mathrm{~cd}$ ab2d

aflow_params $, a, c / a, z_{-}\{1\}, z_{-}\{2\}, z_{-}\{3\}, x_{-}\{4\}, y_{-}\{4\}, z_{-}\{4\}, x_{-}\{5\}, y_{-}\{5\}, z_{-}\{$ $\hookrightarrow 5\}, x_{-}\{6\}, y_{-}\{6\}, z_{-}\{6\}$

aflow_params_values ' $6.5003859369,0.944615384626,0.0,0.5,0.25,0.0548$ $\hookrightarrow 0.2679,0.25,0.33333,0.16667,0.5,0.0,0.5,0.0$,

aflow_Strukturbericht 'None'

aflow_Pearson 'hP12,

cell_length_a $\quad 6.5003859369$

cell_length_b $\quad 6.5003859369$

cell_length_c 6.1403645620

cell_angle_alpha 90.0000000000

symmetry_space_group_name_H-M "P 3"

symmetry_Int_Tables_number 143

loop

space_group_symop_id

space_group_symop_operation_xyz

$1 \mathrm{x}, \mathrm{y}, \mathrm{z}$

$2-\mathrm{y}, \mathrm{x}-\mathrm{y}, \mathrm{z}$

loop

atom_site_type_symbol

atom_site_symmetry_multiplicity

atom_site_Wyckoff_label

atom_site_fract_x

atom_site_fract_y

atom_site_fract_z

-

S1 S $\quad 1$ a $0.00000 \quad 0.00000 \quad 0.00000 \quad 1.00000$

$\begin{array}{lllllllll}\mathrm{S} 2 & \mathrm{~S} & & 1 & \mathrm{~b} & 0.33333 & 0.66667 & 0.50000 & 1.00000\end{array}$

$\begin{array}{llllllll}\mathrm{Mo1} \text { Mo } & 1 & \mathrm{c} & 0.66667 & 0.33333 & 0.25000 & 1.00000\end{array}$ $\begin{array}{llllllll}\text { Mo2 } & \text { Mo } \quad 3 & d & 0.05480 & 0.26790 & 0.25000 & 1.00000\end{array}$

$\begin{array}{llllllllll} & \mathrm{S} & \mathrm{S} & 3 & \mathrm{~d} & 0.33333 & 0.16667 & 0.50000 & 1.00000\end{array}$

$\begin{array}{llllllll}\mathrm{S} 4 & \mathrm{~S} & 3 & \mathrm{~d} & 0.00000 & 0.50000 & 0.00000 & 1.00000\end{array}$

$\mathrm{MoS}_{2}$ : AB2_hP12_143_cd_ab2d - POSCAR

AB2_hP12_143_cd_ab2d \& a,c/a, z1, z2, z3, x4,y4,z4, x5, y5, z5 , x6, y6, z6 $\hookrightarrow$ params $=6.5003859369,0.944615384626,0.0,0.5,0.25,0.0548,0.2679$ $\hookrightarrow 0.25,0.33333,0.16667,0.5,0.0,0.5,0.0 \& \mathrm{P} 3 \mathrm{C}_{-}\{3\}^{\wedge}\{1\} \# 143\left(\mathrm{abcd}^{\wedge}\right.$ $\hookrightarrow 3)$ \& hP12 \& None \& MoS2 \& \& K. E. Dungey and M. D. Curtis and $\hookrightarrow$ J. E. \{Penner-Hahn\}, Chem. Mater. 10, 2152-2161 (1998)

1.0000000000000

$\begin{array}{lll}3.25019296845000 & -5.62949935575851 & 0.00000000000000\end{array}$

$\begin{array}{lll}3.25019296845000 & 5.62949935575851 & 0.00000000000000\end{array}$

$\begin{array}{lll}0.00000000000000 & 0.00000000000000 & 6.14036456200000\end{array}$

$\begin{array}{rr}\text { Mo } & \text { S } \\ 4 & 8\end{array}$

Direct

$\begin{array}{lll}0.66666666666667 & 0.333333333333333 & 0.25000000000000\end{array}$

$0.05480000000000 \quad 0.26790000000000-0.25000000000000$

$-0.26790000000000-0.21310000000000-0.25000000000000$

$0.21310000000000-0.05480000000000-0.25000000000000$

0.25000000000000

0.00000000000000

$0.33333333333333-0.66666666666667$

$0.33333000000000-0.16667000000000$

$-0.16667000000000 \quad 0.16666000000000$

$-0.16666000000000-0.33333000000000$

$0.00000000000000 \quad 0.50000000000000$

0.50000000000000

0.50000000000000

0.50000000000000

0.50000000000000

0.00000000000000

0.00000000000000

$\begin{aligned} \text { Mo } & (1 \mathrm{c}) \\ \text { Mo } & (3 d) \\ \text { Mo } & (3 d) \\ \text { Mo } & (3 d) \\ \text { S } & (1 \mathrm{a}) \\ \text { S } & (1 \mathrm{~b}) \\ \text { S } & (3 d) \\ \text { S } & (3 d) \\ \text { S } & (3 d) \\ \text { S } & (3 d) \\ \text { S } & (3 d) \\ \text { S } & (3 d)\end{aligned}$

$\mathrm{IrGe}_{4}: \mathrm{A} 4 \mathrm{~B} \_\mathrm{hP15} 144 \_4 \mathrm{a} \_$- $-\mathrm{CIF}$

\# CIF file

data_findsym-outpu

audit_creation_method FINDSYM

chemical_name_mineral 'TrGe4'

chemical_formula_sum ' Ge4 Ir

loop

publ author name

K. Schubert

S. Bhan'

T. K. Biswas

'T. Frank,

'P. K. Panday'

journal_name_full_name

Naturwissenschaften

journal_volume 55

journal_year 1968

_journal_page_first 542

journal_page_last 543

publ_Section_title

Einige Strukturdaten metallischer Phasen

Found in Pearson's Crystal Data - Crystal Structure Database for

$\hookrightarrow$ Inorganic Compounds, 2013

aflow title 'IrGe\$ $\{4\} \$$ Structure'

aflow_proto, A4B_hP15_144_4a_a,

aflow_params $, a, c / a, x_{-}\{1\}, y_{-}\{1\}, z_{-}\{1\}, x_{-}\{2\}, y_{-}\{2\}, z_{-}\{2\}, x_{-}\{3\}, y_{-}\{3\}, z_{-}\{$ $\longrightarrow 3\}, x_{-}\{4\}, y_{-}\{4\}, z_{-}\{4\}, x_{-}\{5\}, y_{-}\{5\}, z_{-}\{5\}$

aflow_param_values '6.2151204722, $1.25245374096,0.4904,0.2194,0.2268$, $\hookrightarrow 0.2226,0.4873,0.1142,0.0775,0.0012,0.0,0.6097,0.0014,0.0018$ $\hookrightarrow 0.3178,0.0008,0.5062$,

aflow_Pearson 'hP15'

cell_length_a $\quad 6.2151204722$

cell_length b $\quad 6.215120472$

_cell_length_c $\quad 7.7841508859$

cell angle alpha 90.0000000000

_cell_angle_alpha 90.0000000000

symmetry_space_group_name_H-M "P 31 "

symmetry_Int_Tables_number 144

loop

space_group_symop_ic

_space_group_symop_operation_xyz

$1 \mathrm{x}, \mathrm{y}, \mathrm{z}$

$2-y, x-y, z+1 / 3$

$-x+y,-x, z+2 / 3$

loop

atom_site_label

atom_site_type_symbol

atom_site_symmetry_multiplicity

atom_site_Wyckoff_label

atom_site_fract_x

atom_site_fract_y

atom_site_fract_z

atom site_occupancy

$\begin{array}{lllllll}\text { Ge1 Ge } & \text { G a } & 0.49040 & 0.21940 & 0.22680 & 1.00000\end{array}$

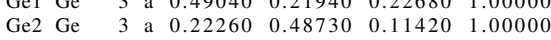

$\begin{array}{lllllll}\mathrm{Ge} 3 \mathrm{Ge} & 3 & \mathrm{a} & 0.07750 & 0.00120 & 0.00000 & 1.00000\end{array}$

$\begin{array}{llllllll}\mathrm{Ge} 4 \mathrm{Ge} & 3 & \text { a } & 0.60970 & 0.00140 & 0.00180 & 1.00000\end{array}$

$\begin{array}{lllllllll}\text { Ir } 1 & \text { Ir } & 3 & \text { a } & 0.31780 & 0.00080 & 0.50620 & 1.00000\end{array}$ 
IrGe 4 : A4B_hP15_144_4a_a - POSCAR

\begin{tabular}{|c|c|c|c|c|}
\hline \\
\hline \multicolumn{5}{|c|}{$\begin{array}{r}\text { A4B_hP15_144_4a_a \& a a c / a , x1 } 1, \mathrm{y} 1, \mathrm{z} 1, \mathrm{x} 2, \mathrm{y} 2, \mathrm{z2}, \mathrm{x} 3, \mathrm{y} 3, \mathrm{z} 3, \mathrm{x} 4, \mathrm{y} 4, \mathrm{z} 4, \mathrm{x} 5, \mathrm{y} 5, \mathrm{z} 5 \\
\hookrightarrow-\text { - params }=6.2151204722,1.25245374096,0.4904,0.2194,0.2268,0.2226\end{array}$} \\
\hline \multirow{2}{*}{\multicolumn{5}{|c|}{$\begin{array}{l}\hookrightarrow, 0.4873,0.1142,0.0775,0.0012,0.0,0.6097,0.0014,0.0018,0.3178, \\
\hookrightarrow 0.0008,0.5062 \& P_{-}\{1\} C_{-}\{3\}^{\wedge}\{2\} \# 144\left(a^{\wedge} 5\right) \& \text { hP15 \& None \& }\end{array}$}} \\
\hline & & & & \\
\hline \multirow{2}{*}{\multicolumn{5}{|c|}{ et al., \{Naturwissenschaften $55,542-543$}} \\
\hline & & & & \\
\hline \multicolumn{5}{|l|}{1.00000000000000} \\
\hline 3.10756023610000 & -5.38245221650594 & 0.00000000000000 & & \\
\hline \multirow{2}{*}{$\begin{array}{l}3.10756023610000 \\
0.00000000000000\end{array}$} & 5.38245221650594 & 0.00000000000000 & & \\
\hline & 0.00000000000000 & 7.78415088590000 & & \\
\hline $\mathrm{Ge}$ & & & & \\
\hline 12 & & & & \\
\hline \multicolumn{5}{|l|}{ Direct } \\
\hline 0.49040000000000 & 0.21940000000000 & 0.22680000000000 & $\mathrm{Ge}$ & (3a) \\
\hline-0.21940000000000 & 0.27100000000000 & 0.56013333333333 & $\mathrm{Ge}$ & (3a) \\
\hline-0.27100000000000 & -0.49040000000000 & 0.89346666666667 & $\mathrm{Ge}$ & (3a) \\
\hline 0.22260000000000 & 0.48730000000000 & 0.11420000000000 & $\mathrm{Ge}$ & (3a) \\
\hline-0.48730000000000 & -0.26470000000000 & 0.44753333333333 & $\mathrm{Ge}$ & (3a) \\
\hline 0.26470000000000 & -0.22260000000000 & 0.780866666666667 & $\mathrm{Ge}$ & (3a) \\
\hline 0.07750000000000 & 0.00120000000000 & 0.00000000000000 & $\mathrm{Ge}$ & (3a) \\
\hline-0.00120000000000 & 0.07630000000000 & 0.33333333333333 & $\mathrm{Ge}$ & (3a) \\
\hline-0.07630000000000 & -0.07750000000000 & 0.666666666666667 & $\mathrm{Ge}$ & (3a) \\
\hline 0.60970000000000 & 0.00140000000000 & 0.00180000000000 & $\mathrm{Ge}$ & (3a) \\
\hline-0.00140000000000 & 0.60830000000000 & 0.33513333333333 & $\mathrm{Ge}$ & (3a) \\
\hline-0.60830000000000 & -0.60970000000000 & 0.66846666666667 & $\mathrm{Ge}$ & (3a) \\
\hline 0.31780000000000 & 0.00080000000000 & 0.50620000000000 & $\mathrm{Ir}$ & (3a) \\
\hline-0.00080000000000 & 0.31700000000000 & 0.83953333333333 & $\mathrm{Ir}$ & (3a) \\
\hline-0.31700000000000 & -0.31780000000000 & 1.17286666666667 & $\mathrm{Ir}$ & (3a) \\
\hline
\end{tabular}

TeZn (High-pressure): AB_hP6_144_a_a - CIF

\# CIF file

data findsym-output

-audit_creation_method FINDSYM

chemical_name_mineral ' $Z n T e$ '

chemical_formula_sum ' $\mathrm{Te} \mathrm{Zn}$ ',

loop

publ_author_name

'K. Kusaba'

'D. J. Weidner'

journal_year 1994

Structure of high pressure phase $\mathrm{I}$ in $\mathrm{ZnTe}$

\# Found in Pearson's Crystal Data - Crystal Structure Database for

$\hookrightarrow$ Inorganic Compounds, 2013

aflow_title 'TeZn (High-pressure) Structure'

-aflow_proto 'AB_hP6_144_a_a'

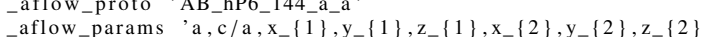

-aflow_params 'a,c/a, $x_{-}\{1\}, y_{-}\{1\}, z_{-}\{1\}, x_{-}\{2\}, y_{-}\{2\}, z_{-}\{2\}$
-aflow_params_values
- $4.0452497415,2.30951792334,0.33,0.16,0.197,0.13$, $\hookrightarrow 0.32,0.0$,

aflow_Strukturbericht 'None'

_aflow_Pearson 'hP6'

_cell_length_a $\quad 4.0452497415$

_cell_length_b 4.0452497415

_cell_length_c 9.3425767824

_cell_angle_alpha 90.0000000000

_cell_angle_beta 90.0000000000

cell_angle_gamma 120.0000000000

_symmetry_space_group_name_H-M "P 31 "

_symmetry_Int_Tables_number 144

loop

space_group_symop_id

_space_group_symop_operation_xyz

$1 \mathrm{x}, \mathrm{y}, \mathrm{z}$

$2-\mathrm{y}, \mathrm{x}-\mathrm{y}, \mathrm{z}+1 / 3$

loop

_atom_site_labe

_atom_site_type_symbol

atom_site_symmetry_multiplicity

atom_site_Wyckoff_label

-atom_site_fract_x

atom_site_fract_y

-atom_site_fract_z

$\begin{array}{llllll}\text { atom_site_occupancy } & & & & \\ \text { Te1 Te } 3 \text { a } & 0.33000 & 0.16000 & 0.19700 & 1.00000\end{array}$

$\begin{array}{llllllll}\mathrm{Zn} 1 & \mathrm{Zn} & 3 & \text { a } & 0.13000 & 0.32000 & 0.00000 & 1.00000\end{array}$

TeZn (High-pressure): AB_hP6_144_a_a - POSCAR

AB_hP6_144_a_a \& a , c/a, x1, y1 ,z1, x2, y2, z2 --params $=4.0452497415$,

$\hookrightarrow 2.30951792334,0.33,0.16,0.197,0.13,0.32,0.0 \& \mathrm{P} 3\{1\} \mathrm{C}_{-}\{3\}^{\wedge}\{2\}$

$\leftrightarrow \# 144\left(\mathrm{a}^{\wedge} 2\right)$ \& hP6 \& None \& ZnTe \& \& K. Kusaba and D. J. Weidner $(1994)$

1.00000000000000

$\begin{array}{lll}2.02262487075000 & -3.50328904079143 & 0.00000000000000\end{array}$

$\begin{array}{rrr}2.02262487075000 & -3.50328904079143 & 0.00000000000000 \\ 2.02262487075000 & 3.50328904079143 & 0.00000000000000\end{array}$

$\begin{array}{lll}2.02262487075000 & 3.50328904079143 & 0.00000000000000 \\ 0.00000000000000 & 0.00000000000000 & 9.34257678240000\end{array}$

$\mathrm{Te} \quad \mathrm{Zn}$

Direct

0.33000000000000

$-0.16000000000000$

0.16000000000000 0.17000000000000

0.19700000000000 $\begin{array}{lll}0.19700000000000 & \mathrm{Te} & (3 \mathrm{a}) \\ 0.53033333333333 & \mathrm{Te} & (3 \mathrm{a})\end{array}$
$-0.17000000000000-0.33000000000000$ $0.13000000000000 \quad 0.32000000000000$

-

0.19000000000000 -0.19000000000000
-0.13000000000000

Sheldrickite $\left(\mathrm{NaCa}_{3}\left[\mathrm{CO}_{3}\right]_{2} \mathrm{~F}_{3}\left[\mathrm{H}_{2} \mathrm{O}\right]\right):$ A2B3C3DE7_hP48_145_2a_3a_3a_a_7a - CIF

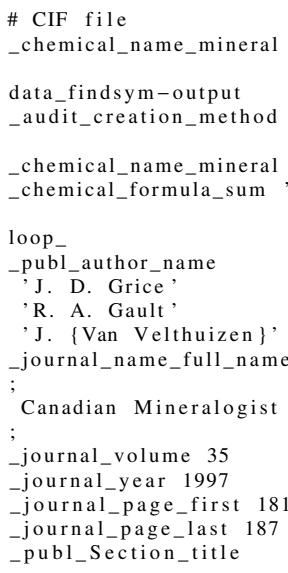

Sheldrickite, a new sodium-calcium-fluorocarbonate mineral species from $\hookrightarrow$ Mont Saint-Hilaire, Quebec

\# Found in Pearson's Crystal Data - Crystal Structure Database for $\hookrightarrow$ Inorganic Compounds, 2013

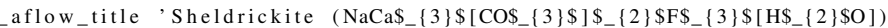
$\hookrightarrow$ Structure

aflow_proto 'A2B3C3DE7_hP48_145_2a_3a_3a_a_7a

aflow_params a, a/a, $x_{-}\{1\}, y_{-}\{1\}, z_{-}\{1\}, x_{-}\{2\}, y_{-}\{2\}, z_{-}\{2\}, x_{-}\{3\}, y_{-}\{3\}, z_{-}\{$ $\hookrightarrow 3\}, x_{-}\{4\}, y_{-}\{4\}, z_{-}\{4\}, x_{-}\{5\}, y_{-}\{5\}, z_{-}\{5\}, x_{-}\{6\}, y_{-}\{6\}, z_{-}\{6\}, x_{-}\{7\}$ $\hookrightarrow \mathrm{y}_{-}\{7\}, \mathrm{z}_{-}\{7\}, \mathrm{x}_{-}\{8\}, \mathrm{y}_{-}\{8\}, \mathrm{z}_{-}\{8\}, \mathrm{x}_{-}\{9\}, \mathrm{y}_{-}\{9\}, \mathrm{z}_{-}\{9\}, \mathrm{x}_{-}\{10\}, \mathrm{y}_{-}\{10\}$ $\hookrightarrow \mathrm{z}_{-}\{10\}, \mathrm{x}_{-}\{11\}, \mathrm{y}_{-}\{11\}, \mathrm{z}_{-}\{11\}, \mathrm{x}_{-}\{12\}, \mathrm{y}_{-}\{12\}, \mathrm{z}_{-}\{12\}, \mathrm{x}_{-}\{13\}, \mathrm{y}_{-}\{13\}$, $\hookrightarrow \mathrm{z}_{-}\{13\}, \mathrm{x}_{-}\{14\}, \mathrm{y}_{-}\{14\}, \mathrm{z}_{-}\{14\}, \mathrm{x}_{-}\{15\}, \mathrm{y}_{-}\{15\}, \mathrm{z}_{-}\{15\}, \mathrm{x}_{-}\{16\}, \mathrm{y}_{-}\{16\}$, $\rightarrow \mathrm{z}_{-}\{16\}$

(1) $6.7260126433,2.23669342849,0.567,0.317,0.168,0.432$ $\hookrightarrow, 0.752,0.169,0.6161,-0.0009,0.0,-0.0011,0.6284,0.0045,0.3706$ $\hookrightarrow 0.371,-0.0043,-0.003,0.266,0.01,0.271,0.002,-0.005,0.73,0.734,-$ $\hookrightarrow 0.02,0.001,0.297,0.165,0.664,0.348,0.09,0.668,0.333,0.238,0.353$ $\rightarrow, 0.273,0.167,0.321,0.654,-0.092,0.336,0.666,0.761,0.646,-0.079$, $\hookrightarrow 0.165,0.203,0.202,0.831$

aflow Strukturbericht 'None'

-aflow_Pearson 'hP48,

cell_length_a $\quad 6.7260126433$

cell_length_b 6.7260126433

cell_length_c 15.0440282792

_cell_angle_alpha 90.0000000000

cell_angle_beta 90.0000000000

_cell_angle_gamma 120.0000000000

_symmetry_space_group_name_H-M "P 32"

symmetry_Int_Tables_number 145

loop

space_group_symop_id

space

$2-y, x-y, z+2 / 3$

$3-x+y,-x, z+1 / 3$

loop

atom_site_label

atom_site_type_symbol

atom_site_symmetry_multiplicity

atom_site_Wyckoff_label

atom_site_fract_x

atom_site_fract_y

atom_site_fract_z

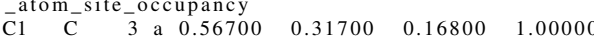

$\begin{array}{llllllll}\mathrm{C} 2 & \mathrm{C} & 3 & \mathrm{a} & 0.43200 & 0.75200 & 0.16900 & 1.00000\end{array}$

$\begin{array}{llllllll}\mathrm{Ca} 1 & \mathrm{Ca} & 3 & \mathrm{a} & 0.61610 & -0.00090 & 0.00000 & 1.00000\end{array}$

$\begin{array}{llllllll}\mathrm{Ca} 2 & \mathrm{Ca} & 3 & \mathrm{a} & -0.00110 & 0.62840 & 0.00450 & 1.00000\end{array}$

$\begin{array}{llllllll}\mathrm{Ca} 3 & \mathrm{Ca} & 3 & \mathrm{a} & 0.37060 & 0.37100 & -0.00430 & 1.00000\end{array}$

$\begin{array}{lllllllll}\mathrm{F} 1 & \mathrm{~F} & 3 & \mathrm{a} & -0.00300 & 0.26600 & 0.01000 & 1.00000\end{array}$

F2 $\quad \mathrm{F} \quad 3$ a $0.27100 \quad 0.00200-0.00500-1.00000$

$\begin{array}{llllllll}\text { F2 } & \mathrm{F} & 3 & \text { a } & 0.27100 & 0.00200 & -0.00500 & 1.00000\end{array}$

$\begin{array}{llllllll}\mathrm{F} 3 & \mathrm{~F} & 3 & \mathrm{a} & 0.73000 & 0.73400 & -0.02000 & 1.00000\end{array}$

$\begin{array}{llllllll}\mathrm{Na} 1 & \mathrm{Na} & 3 & \text { a } & 0.00100 & 0.29700 & 0.16500 & 1.00000 \\ \mathrm{O} 1 & \mathrm{O} & 3 & \mathrm{a} & 0.66400 & 0.34800 & 0.09000 & 1.00000\end{array}$

$\begin{array}{llllllll}\mathrm{O} 1 & \mathrm{O} & 3 & \mathrm{a} & 0.66400 & 0.34800 & 0.09000 & 1.00000 \\ \mathrm{O} 2 & \mathrm{O} & 3 & \mathrm{a} & 0.66800 & 0.33300 & 0.23800 & 1.00000\end{array}$

$\begin{array}{llllllll}\mathrm{O} 2 & \mathrm{O} & 3 & \mathrm{a} & 0.66800 & 0.33300 & 0.23800 & 1.00000 \\ \mathrm{O} 3 & \mathrm{O} & 3 & \mathrm{a} & 0.35300 & 0.27300 & 0.16700 & 1.00000 \\ \mathrm{O} 4 & \mathrm{O} & 3 & \mathrm{a} & 0.32100 & 0.65400 & -0.09200 & 1.00000\end{array}$

$\begin{array}{llllllll}\mathrm{O} 3 & \mathrm{O} & 3 & \mathrm{a} & 0.35300 & 0.27300 & 0.16700 & 1.00000 \\ \mathrm{O} 4 & \mathrm{O} & 3 & \mathrm{a} & 0.32100 & 0.65400 & -0.09200 & 1.00000\end{array}$

$\begin{array}{llllllll}\mathrm{O} 4 & \mathrm{O} & 3 & \mathrm{a} & 0.32100 & 0.65400 & -0.09200 & 1.00000 \\ \mathrm{O} 5 & \mathrm{O} & 3 & \text { a } & 0.33600 & 0.66600 & 0.76100 & 1.00000\end{array}$

$\begin{array}{llllllll}\mathrm{O} 6 & \mathrm{O} & 3 & \mathrm{a} & 0.64600 & -0.07900 & 0.16500 & 1.00000\end{array}$

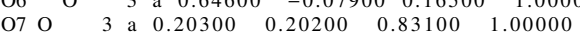

Sheldrickite $\left(\mathrm{NaCa}_{3}\left[\mathrm{CO}_{3}\right]_{2} \mathrm{~F}_{3}\left[\mathrm{H}_{2} \mathrm{O}\right]\right)$ : A2B3C3DE7_hP48_145_2a_3a_3a_a_7a - POSCAR

A2B3C3DE7_hP48_145_2a_3a_3a_a_7a \& a,c/a, x1,y1, z1, x2 ,y2, z2, x3, y3 , z3 , x4 $\hookrightarrow \mathrm{y} 4, \mathrm{z} 4, \mathrm{x} 5, \mathrm{y} 5, \mathrm{z} 5, \mathrm{x} 6, \mathrm{y} 6, \mathrm{z6}, \mathrm{x} 7, \mathrm{y} 7, \mathrm{z7}, \mathrm{x} 8, \mathrm{y} 8, \mathrm{z} 8, \mathrm{x} 9, \mathrm{y} 9, \mathrm{z} 9, \mathrm{x} 10, \mathrm{y} 10, \mathrm{z} 10$, $\hookrightarrow \mathrm{x} 11, \mathrm{y} 11, \mathrm{z} 11, \mathrm{x} 12, \mathrm{y} 12, \mathrm{z} 12, \mathrm{x} 13, \mathrm{y} 13, \mathrm{z} 13, \mathrm{x} 14, \mathrm{y} 14, \mathrm{z} 14, \mathrm{x} 15, \mathrm{y} 15, \mathrm{z} 15, \mathrm{x} 16$ $\hookrightarrow$, y16, z16 --params $=6.7260126433,2.23669342849,0.567,0.317,0.168$, 
$\longrightarrow 0.432,0.752,0.169,0.6161,-0.0009,0.0,-0.0011,0.6284,0.0045$

$\hookrightarrow 0.3706,0.371,-0.0043,-0.003,0.266,0.01,0.271,0.002,-0.005,0.73$

$\hookrightarrow 0.734,-0.02,0.001,0.297,0.165,0.664,0.348,0.09,0.668,0.333$,

$\hookrightarrow 0.238,0.353,0.273,0.167,0.321,0.654,-0.092,0.336,0.666,0.761$,

$\hookrightarrow 0.646,-0.079,0.165,0.203,0.202,0.831 \&$ \& $3 \_\{2\} \mathrm{C}_{-}\{3\}^{\wedge}\{3\} \# 145$ (a

$\left.\hookrightarrow \wedge^{\wedge} 16\right) \&$ hP48 \& None \& NaCa3 [CO3]2F3[H2O] \& Sheldrickite \& J. D.

$\hookrightarrow$ Grice and R. A. Gault and J. \{Van Velthuizen\}, Can. Mineral. 35 $\hookrightarrow$, 181-187 (1997)

1.00000000000000

3.36300632165000

3.36300632165000

0.00000000000000

$5.82489781527312-0.00000000000000$ $5.82489781527312-0.00000000000000$ $0.00000000000000-15.04402827920000$

$$
\begin{array}{rr}
\mathrm{C} & \mathrm{Ca} \\
6 & 9
\end{array}
$$

F

$\mathrm{Na} \quad \mathrm{O}$

irect
0.56700000000000

$-0.31700000000000$

$-0.25000000000000$ 0.43200000000000 $-0.75200000000000$ 0.32000000000000 0.61610000000000 0.00090000000000 $-0.61700000000000$ $-0.00110000000000$ $-0.62840000000000$ 0.62950000000000 0.37060000000000 $-0.37100000000000$ 0.00040000000000 $-0.00300000000000$ $-0.26600000000000$ .26600000000000 0.26900000000000 0.27100000000000 $-0.00200000000000$ $-0.26900000000000$ 0.73000000000000 $-0.73400000000000$ 0.00400000000000 0.00100000000000 $-0.29700000000000$ 0.29600000000000 0.66400000000000 $-0.34800000000000$ $-0.31600000000000$ 0.66800000000000 $-0.33300000000000$ $-0.33300000000000$ $-0.33500000000000$ $-0.2730000000000$ $-0.27300000000000$ $-0.08000000000000$ 0.32100000000000 0.65400000000000 0.33300000000000 0.33600000000000 $-0.66600000000000$ 0.33000000000000 0.64600000000000 0.07900000000000 $-0.72500000000000$ 0.20300000000000 $-0.20200000000000$

0.31700000000000 0.25000000000000 $-0.56700000000000$ 0.75200000000000 $-0.32000000000000$ $-0.43200000000000$ 0.00090000000000 0.6170000000000 0.61700000000000 0.6284000000000 284000000000 $-0.6295000000000$ 0.37100000000000 $-0.00040000000000$ $-0.3706000000000$ 0.2660000000000 . 0.003000000000 0.0020000000000 0.27100000000000 $-0.27100000000000$ 0.73400000000000 $-0.0040000000000$ $-0.7300000000000$ 0.29700000000000 $-0.2960000000000$ $-0.00100000000000$ 0.34800000000000 0.31600000000000 $-0.66400000000000$ 0.33300000000000 0.33500000000000 0.668000000000 0.6680000000000 0.2730000000000 0.0800000000000 0.65400000000000 0.65400000000000 $-0.3330000000000$ $-0.32100000000000$ 0.66600000000000 $-0.33000000000000$ $-0.33600000000000$ $-0.07900000000000$ 0.72500000000000 $-0.64600000000000$ 0.20200000000000 0.00100000000000 $-0.20300000000000$

0.16800000000000 0.83466666666667 0.50133333333333 0.16900000000000 0.83566666666667 0.50233333333333 0.00000000000000 0.06666666666667 0.666666666666667 .33333333333333 0.00450000000000 0.6711666666666 0.33783333333333 $-0.00430000000000$ 0.662366666666667 0.32903333333333 0.01000000000000 0.67666666666667 0.34333333333333 0.00500000000000 0.66166666666667 .32833333333333 $-0.02000000000000$ 0.64666666666667 0.31333333333333 0.16500000000000 0.83166666666667 0.4983333333333 0.09000000000000 0.75666666666667 0.4233333333333 0.23800000000000 0.90466666666667 0.57133333333333 0.16700000000000 0.83366666666667 0.0920000000000 0.57466666666667 0.57466666666667 0.24133333333333 0.76100000000000 1.42766666666666 1.0943333333333 0.16500000000000 0.8316666666666 0.4983333333333 0.83100000000000 1.497666666666667 1.16433333333333

C (3a)

C (3a)

C (3a)

(3a)

(3a)

$\begin{array}{ll}\mathrm{C} & (3 \mathrm{a}) \\ \mathrm{Ca} & (3 \mathrm{a})\end{array}$

$\mathrm{Ca}$ (3a)

$\mathrm{Ca} \quad(3 \mathrm{a})$

$\mathrm{Ca}$ (3a)

$\mathrm{Ca} \quad(3 \mathrm{a})$

$\mathrm{Ca}(3 \mathrm{a})$

$\mathrm{Ca} \quad(3 \mathrm{a})$

(3a)

(3a)

(3a)

$\gamma$-Ag 3 SI (Low-temperature): A3BC_hR5_146_b_a_a - CIF cell angle alpha 90.0000000000 cell_angle_beta 90.0000000000 cell_angle_gamma 120.0000000000

symmetry_space_group_name_H-M "R 3 (hexagonal axes)" _symmetry_Int_Tables_number 146

loop

space_group_symop_id

-space

$2-y, x-y, z$

$3-\mathrm{x}+\mathrm{y},-\mathrm{x}, \mathrm{z}$

$4 \mathrm{x}+1 / 3, \mathrm{y}+2 / 3, \mathrm{z}+2 / 3$

$5-\mathrm{y}+1 / 3, \mathrm{x}-\mathrm{y}+2 / 3, \mathrm{z}+2 / 3$

$6-x+y+1 / 3,-x+2 / 3, z+2 / 3$

$7 \mathrm{x}+2 / 3, \mathrm{y}+1 / 3, \mathrm{z}+1 / 3$

$8-y+2 / 3, x-y+1 / 3, z+1 / 3$

loop

-atom_site_label

atom_site_type_symbol

atom_site_symmetry_multiplicity

atom_site_Wyckoff_label

atom_site_fract_x

atom_site_fract_y

atom site

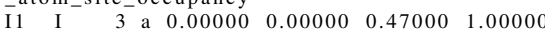

$\begin{array}{lllllllllll}\mathrm{S} 1 \mathrm{~S} & 3 & \mathrm{a} & 0.00000 & 0.00000 & 0.00000 & 1.00000\end{array}$

$\begin{array}{lllllllll}\mathrm{Ag} 1 & \mathrm{Ag} & 9 & \mathrm{~b} & 0.37533 & 0.11667 & 0.11467 & 1.00000\end{array}$

$\gamma-\mathrm{Ag}_{3} \mathrm{SI}$ (Low-temperature): A3BC_hR5_146_b_a_a - POSCAR

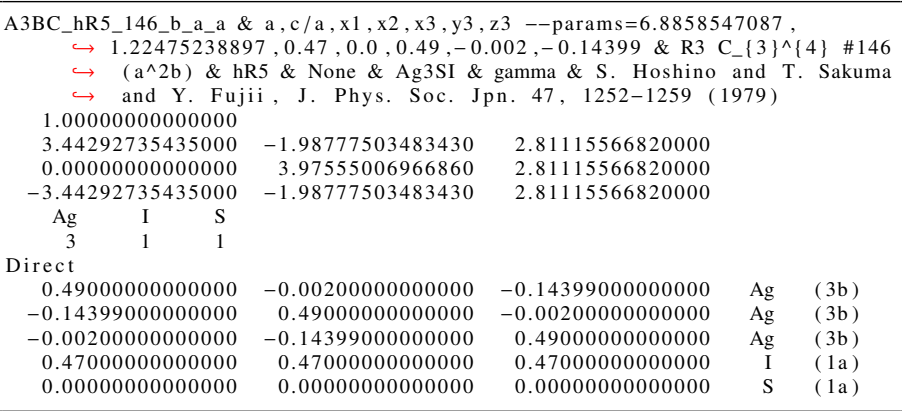

$\mathrm{FePSe}_{3}$ : ABC3_hR10_146_2a_2a_2b - CIF

\# CIF file

data_findsym-output

audit_creation_method FINDSYM

chemical_name_mineral 'FePSe3'

chemical_formula_sum ' $\mathrm{Fe}$ P $\mathrm{Se} 3$ '

loop

publ_author_name

W. Klingen'

G. Eulenberger,

'H. Hahn'

journal_name full_name

Zeitschrift fur Anorganische und Allgemeine Chemie

journal_volume 40

journal_year 1973

journal_page_first 97

journal_page_last 112

-publ_Section_title

Uber die Kristallstrukturen von Fe\$_\{2\}\$P\$_ $\{2\} \$ S e \$ \_\{6\} \$$ und Fe\$_ $\{2\} \$ P \$$ $\longrightarrow\{2\} \$ S \$+\{6\} \$$

\# Found in Pearson's Crystal Data - Crystal Structure Database for $\hookrightarrow$ Inorganic Compounds, 2013

aflow_title 'FePSe\$_\{3\}\$ Structure'

aflow proto, $\mathrm{ABC} 3 \mathrm{hR} 10$ 146 2a 2a $2 \mathrm{~b}$

aflow_params $, a, c / a, x_{-}\{1\}, x_{-}\{2\}, x_{-}\{3\}, x_{-}\{4\}, x_{-}\{5\}, y_{-}\{5\}, z_{-}\{5\}, x_{-}\{6\}, y_{-}\{$ $\hookrightarrow 6\}, z_{-}\{6\}$

aflow_params_values $\quad 6.2648898445,3.16041500397,0.3911,0.7256,0.1132$, $\hookrightarrow 0.0,0.1454,-0.1886,0.474,0.6151,-0.0128,0.3247$

aflow_Strukturbericht 'None'

-aflow_Pearson 'hR10'

cell_length_a $\quad 6.2648898445$

cell_length_b 6.2648898445

cell_length_c $\quad 19.7996518628$

cell_angle_alpha 90.0000000000

cell_angle_beta 90.0000000000

cell angle gamma 120.0000000000

symmetry_space_group_name_H-M "R 3 (hexagonal axes)"

symmetry_Int_Tables_number 146

loop

space_group_symop_i

_space_group_symop_operation_xy 


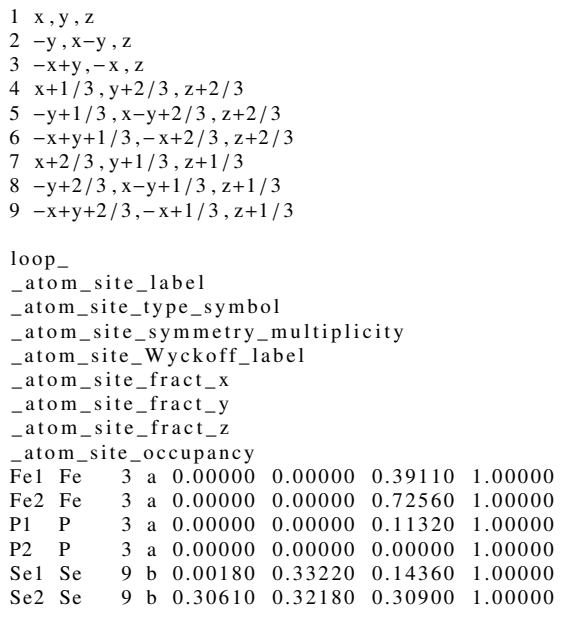

$\mathrm{FePSe}_{3}$ : ABC3_hR10_146_2a_2a_2b - POSCAR

ABC3_hR10_146_2a_2a_2b \& a , c/a, x1, x2 , x3, x4, x5,y5, z5, x6,y6, z6 --params $\hookrightarrow 6.2648898445,3.16041500397,0.3911,0.7256,0.1132,0.0,0.1454$, $\hookrightarrow 0.1886,0.474,0.6151,-0.0128,0.3247 \&$ \& $3 C_{-}\{3\}^{\wedge}\{4\} \# 146\left(a^{\wedge} 4 b^{\wedge} 2\right.$ $\hookrightarrow$ \& hR10 \& None \& FePSe3 \& \& W. Klingen and G. Eulenberger and $\hookrightarrow$ H. Hahn, Z. Anorg. Allg. Chem. 401, 97-112 (1973) 1.00000000000000

$\begin{array}{llll}3.13244492225000 & -1.80851791908271 & 6.59988395426667\end{array}$ $\begin{array}{rrr}0.00000000000000 & 3.61703583816543 & 6.59988395426667\end{array}$ $\begin{array}{rrr}-3.13244492225000 & -1.80851791908271 & 6.59988395426667\end{array}$

$$
\begin{array}{rrr}
-3.13244492225000 \\
\mathrm{Fe} & \mathrm{P} & \mathrm{Se} \\
2 & 2 & 6
\end{array}
$$

0.39110000000000

0.39110000000000 0.72560000000000 0.00000000000000 0.00000000000000 0.14540000000000 0.47400000000000 $-0.18860000000000$ 0.61510000000000 0.32470000000000 $-0.01280000000000$

0.39110000000000 0.72560000000000 0.11320000000000 0.00000000000000 $-0.18860000000000$ 0.14540000000000 0.4740000000000 $-0.01280000000000$ 0.61510000000000
0.32470000000000

Phenakite $\left(\mathrm{Be}_{2} \mathrm{SiO}_{4}, S 1_{3}\right)$ : A2B4C hR42_148_2f_4f_f - CIF

\section{\# CIF file}

data_findsym-output

audit_creation_method FINDSYM

chemical_name_mineral 'Phenakite,

chemical_formula_sum ' $\mathrm{Be} 2 \mathrm{O} 4 \mathrm{Si}$

loop

publ_author_name

R. M. Hazen,

'L. W. Finger'

journal_name_full_name

Physics and Chemistry of Minerals

_journal_volume 14

-journal_year 1987

_journal_page_first 426

journal_page_last 434

publ_Section_title

High-Temperature Crystal Chemistry of Phenakite (Be\$_ $2 \$ S i O \$ 4 \$$ ) and $\hookrightarrow$ Chrysoberyl $($ BeAl\$_ $2 \$ O \$$ \$ 4 )

aflow_title 'Phenakite (Be\$_ $\left.2 \$ S i O \$ 4 \$, \$ S 1_{-}\{3\} \$\right)$ Structure

aflow_proto, A2B4C_hR42_148_2f $4 \mathrm{f}_{-} \mathrm{f}$,

aflow_params $, a, c / a, x_{-}\{1\}, y_{-}\{1\}, z_{-}\{1\}, x_{-}\{2\}, y_{-}\{2\}, z_{-}\{2\}, x_{-}\{3\}, y_{-}\{3\}, z_{-}\{$ $\hookrightarrow 3\}, x_{-}\{4\}, y_{-}\{4\}, z_{-}\{4\}, x_{-}\{5\}, y_{-}\{5\}, z_{-}\{5\}, x_{-}\{6\}, y_{-}\{6\}, z_{-}\{6\}, x_{-}\{7\}$ $\hookrightarrow 3\}, x_{-}\{4\}, y_{-}\{4\}$

aflow_params_values $, 12.4401,0.661634552777,0.20546,-0.56835,0.60961$

$\hookrightarrow 0.87265,0.10211,-0.72078,0.66221,-0.37042,-0.03997,0.41696$

$\hookrightarrow 0.2507,0.0834,0.70548,0.00275,0.03736,0.37614,-0.32772,0.70785$

$\hookrightarrow 0.53815,-0.23404,-0.05466$

aflow_Strukturbericht $\$ \$ 1_{-}\{3\} \$$

_aflow_Pearson 'hR42,

symmetry_space_group_name_H-M "R -3 (hexagonal axes)"

symmetry_Int_Tables_number 148

\section{cell_length_a $\quad 12.44010$}

cell_length_c 8.23080

cell angle alpha 90.00000

cell_angle_alpha 90.00000

cell_angle gamma 120.00000

loop

space_group_symop_id

space_group_symop_operation_xyz

$1 \mathrm{x}, \mathrm{y}, \mathrm{z}$

$2-y, x-y, z$

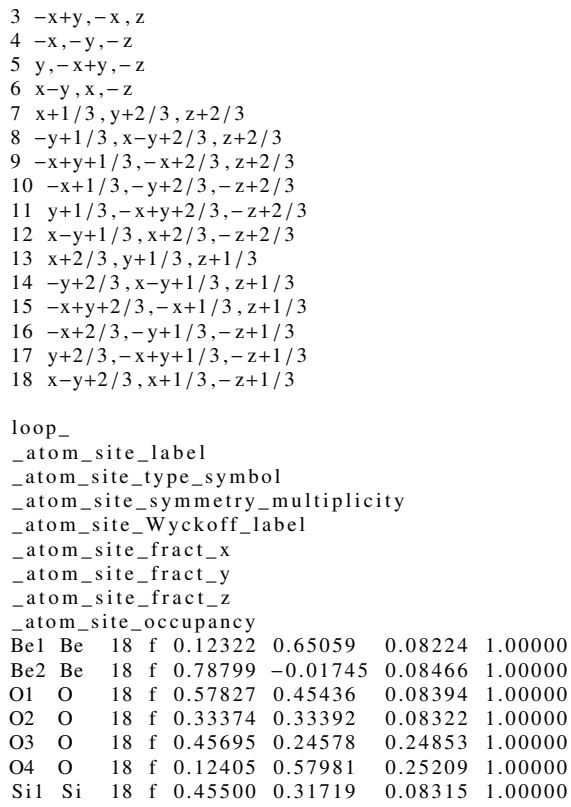

Phenakite $\left(\mathrm{Be}_{2} \mathrm{SiO}_{4}, S 1_{3}\right)$ : A2B4C_hR42_148_2f_4f_f - POSCAR

\begin{tabular}{|c|c|c|c|c|}
\hline & & & & \\
\hline $\begin{array}{r}\text { A2B4C_hR42_148_2f } \\
\hookrightarrow, \mathrm{z} 5, \mathrm{x} 6, \mathrm{y} 6,\end{array}$ & $\begin{array}{l}4 \mathrm{f} \text { ff \& \& a, c /a, x } 1, y 1, z 1 \\
66, x 7, y 7, z 7-\text { params }\end{array}$ & $\begin{array}{l}, \mathrm{x} 2, \mathrm{y} 2, \mathrm{z} 2, \mathrm{x} 3, \mathrm{y} 3, \mathrm{z3}, \\
=12.4401,0.661634552\end{array}$ & $77, y$ & $\begin{array}{l}\mathrm{z} 4, \mathrm{x} 5, \mathrm{y} 5 \\
20546,-\end{array}$ \\
\hline$\hookrightarrow 0.56835,0.6$ & $0961,0.87265,0.10211$ & $,-0.72078,0.66221$ & 0.370 & $2,-$ \\
\hline$\hookrightarrow 0.03997,0.4$ & $1696,-0.2507,0.0834$ & $0.70548,0.00275,0.0$ & 3736 & .37614 \\
\hline$\hookrightarrow,-0.32772,0$ & $0.70785,0.53815,-0.23$ & $404,-0.05466 \& R-3$ & $\mathrm{C}_{-}\{\}$ & $\wedge\{2\} \#$ \\
\hline$\hookrightarrow 148\left(\mathrm{f}^{\wedge} 7\right) \&$ & hR42 \& $\$ S 1_{-}\{3\} \$ \& 1$ & Be2SiO4 \& Phenakite & & \\
\hline$\hookrightarrow$ and L. W. & Finger, Phys. Chem. & Miner. 14, 426-434 & 698 & \\
\hline 1.00000000000000 & & & & \\
\hline 6.22005000000000 & -3.59114754187293 & 2.74360000000000 & & \\
\hline 0.00000000000000 & 7.18229508374586 & 2.74360000000000 & & \\
\hline-6.22005000000000 & -3.59114754187293 & 2.74360000000000 & & \\
\hline $\mathrm{Be}$ & & & & \\
\hline 12 & & & & \\
\hline Direct & & & & \\
\hline 0.20546000000000 & -0.56835000000000 & 0.60961000000000 & $\mathrm{Be}$ & $(6 \mathrm{f})$ \\
\hline 0.60961000000000 & 0.20546000000000 & -0.56835000000000 & $\mathrm{Be}$ & (6f) \\
\hline-0.56835000000000 & 0.60961000000000 & 0.20546000000000 & $\mathrm{Be}$ & (6f) \\
\hline-0.20546000000000 & 0.56835000000000 & -0.60961000000000 & $\mathrm{Be}$ & (6f) \\
\hline-0.60961000000000 & -0.20546000000000 & 0.56835000000000 & $\mathrm{Be}$ & (6f) \\
\hline 0.56835000000000 & -0.60961000000000 & -0.20546000000000 & $\mathrm{Be}$ & (6f) \\
\hline 0.87265000000000 & 0.10211000000000 & -0.72078000000000 & $\mathrm{Be}$ & (6f) \\
\hline-0.72078000000000 & 0.87265000000000 & 0.10211000000000 & $\mathrm{Be}$ & (6f) \\
\hline 0.10211000000000 & -0.72078000000000 & 0.87265000000000 & $\mathrm{Be}$ & (6f) \\
\hline-0.87265000000000 & -0.10211000000000 & 0.72078000000000 & $\mathrm{Be}$ & (6f) \\
\hline 0.72078000000000 & -0.87265000000000 & -0.10211000000000 & $\mathrm{Be}$ & (6f) \\
\hline-0.10211000000000 & 0.72078000000000 & -0.87265000000000 & $\mathrm{Be}$ & (6f) \\
\hline 0.66221000000000 & -0.37042000000000 & -0.03997000000000 & $\mathrm{O}$ & (6f) \\
\hline-0.03997000000000 & 0.66221000000000 & -0.37042000000000 & $\mathrm{O}$ & (6f) \\
\hline-0.37042000000000 & -0.03997000000000 & 0.66221000000000 & $\mathrm{O}$ & (6f) \\
\hline-0.66221000000000 & 0.37042000000000 & 0.03997000000000 & $\mathrm{O}$ & (6f) \\
\hline 0.03997000000000 & -0.66221000000000 & 0.37042000000000 & $\mathrm{O}$ & $(6 \mathrm{f})$ \\
\hline 0.37042000000000 & 0.03997000000000 & -0.66221000000000 & $\mathrm{O}$ & (6f) \\
\hline 0.41696000000000 & -0.25070000000000 & 0.08340000000000 & $\mathrm{O}$ & (6f) \\
\hline 0.08340000000000 & 0.41696000000000 & -0.25070000000000 & $\mathrm{O}$ & (6f) \\
\hline-0.25070000000000 & 0.08340000000000 & 0.41696000000000 & $\mathrm{O}$ & (6f) \\
\hline-0.41696000000000 & 0.25070000000000 & -0.08340000000000 & $\mathrm{O}$ & (6f) \\
\hline-0.08340000000000 & -0.41696000000000 & 0.25070000000000 & $\mathrm{O}$ & $(6 \mathrm{f})$ \\
\hline 0.25070000000000 & -0.08340000000000 & -0.41696000000000 & $\mathrm{O}$ & $(6 f)$ \\
\hline 0.70548000000000 & 0.00275000000000 & 0.03736000000000 & $\mathrm{O}$ & (6f) \\
\hline 0.03736000000000 & 0.70548000000000 & 0.00275000000000 & $\mathrm{O}$ & (6f) \\
\hline 0.00275000000000 & 0.03736000000000 & 0.70548000000000 & $\mathrm{O}$ & (6f) \\
\hline-0.70548000000000 & -0.00275000000000 & -0.03736000000000 & $\mathrm{O}$ & (6f) \\
\hline-0.03736000000000 & -0.70548000000000 & -0.00275000000000 & $\mathrm{O}$ & (6f) \\
\hline-0.00275000000000 & -0.03736000000000 & -0.70548000000000 & $\mathrm{O}$ & (6f) \\
\hline 0.37614000000000 & -0.32772000000000 & 0.70785000000000 & $\mathrm{O}$ & $(6 \mathrm{f})$ \\
\hline 0.70785000000000 & 0.37614000000000 & -0.32772000000000 & $\mathrm{O}$ & (6f) \\
\hline-0.32772000000000 & 0.70785000000000 & 0.37614000000000 & $\mathrm{O}$ & (6f) \\
\hline-0.37614000000000 & 0.32772000000000 & -0.70785000000000 & $\mathrm{O}$ & (6f) \\
\hline-0.70785000000000 & -0.37614000000000 & 0.32772000000000 & $\mathrm{O}$ & (6f) \\
\hline 0.32772000000000 & -0.70785000000000 & -0.37614000000000 & $\mathrm{O}$ & (6f) \\
\hline 0.53815000000000 & -0.23404000000000 & -0.05466000000000 & $\mathrm{Si}$ & (6f) \\
\hline-0.05466000000000 & 0.53815000000000 & -0.23404000000000 & $\mathrm{Si}$ & (6f) \\
\hline-0.23404000000000 & -0.05466000000000 & 0.53815000000000 & $\mathrm{Si}$ & (6f) \\
\hline-0.53815000000000 & 0.23404000000000 & 0.05466000000000 & $\mathrm{Si}$ & (6f) \\
\hline 0.05466000000000 & -0.53815000000000 & 0.23404000000000 & $\mathrm{Si}$ & (6f) \\
\hline 0.23404000000000 & 0.05466000000000 & -0.53815000000000 & $\mathrm{Si}$ & (6f) \\
\hline
\end{tabular}

$\beta-\mathrm{PdCl}_{2}:$ A2B_hR18_148_2f_f - CIF

\# CIF file

data findsym-output

audit_creation_method FINDSYM

chemical name mineral ' $\$ \backslash$ beta $\$-P d C l 2$,

chemical formula_sum ' $\mathrm{Cl} 2 \mathrm{Pd}$ '

loop

publ_author_nam

D. B. \{Dell \’Amico $\}$ ' 


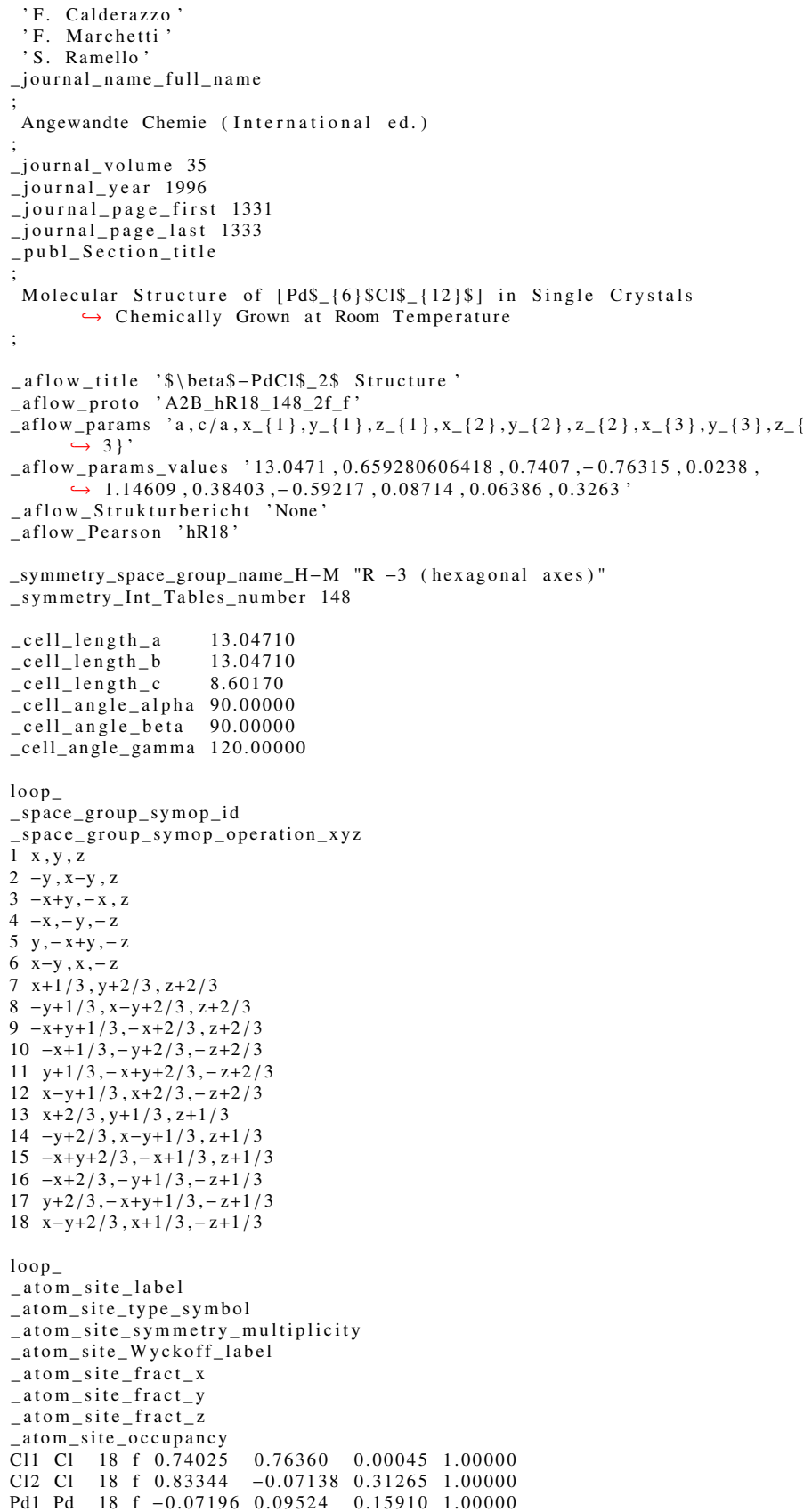

$\beta-\mathrm{PdCl}_{2}$ : A2B_hR18_148_2f_f - POSCAR

A2B_hR18_148_2f_f \& a,c/a, x1,y1,z1,x2,y2,z2, x3,y3,z3 --params $=13.0471$ $\hookrightarrow 0.659280606418$ $\hookrightarrow 0.08714,0.06386,0.3263 \& \mathrm{R}-3 \mathrm{C}_{-}\{3 \mathrm{i}\}^{\wedge}\{2\} \# 148\left(\mathrm{f}^{\wedge} 3\right) \& \mathrm{hR} 18 \&$

$\hookrightarrow$ None \& $\mathrm{PdCl} 2 \& \$ \backslash$ beta $\$-\mathrm{PdCl} 2 \&$ D. B. \{Dell 'Amico\} et al.,

$\hookrightarrow$ Angew. Chem. Int. Ed. 35, 1331-1333 (1996)

1.00000000000000

$\begin{array}{lll}6.52355000000000 & -3.76637334857198 & 2.867233333333333\end{array}$

$\begin{array}{lll}0.00000000000000 & 7.53274669714397 & 2.86723333333333\end{array}$

$\begin{array}{lll}-6.52355000000000 & -3.76637334857198 & 2.86723333333333\end{array}$

$$
\begin{array}{rr}
\mathrm{Cl} & \mathrm{Pd} \\
12 & 6
\end{array}
$$

Direct

0.74070000000000 0.74070000000000 $-0.7631500000000$ $-0.76315000000000$ $-0.7407000000000$ 0.72380000000000 0.76315000000000 1.14609000000000 0.59217000000000 0.38403000000000 $-1.14609000000000$ 0.59217000000000 0.38403000000000 0.08714000000000 0.32630000000000 0.06386000000000 $-0.08714000000000$ $-0.32630000000000$ $-0.06386000000000$

$-0.76315000000000$ $-0.76315000000000$ $0.76315000000000-0.02380000000000$ $-0.74070000000000-0.76315000000000$ $-0.74380000000000-0.74070000000000$ $0.38403000000000-0.59217000000000$ . 14609000000000 1.1460900000000 $\begin{array}{ll}.1 .14609000000000 & -0.38403000000000\end{array}$ $0.59217000000000-1.14609000000000$ 0.06386000000000 0.08714000000000 $0.32630000000000 \quad 0.08714000000000$ $-0.06386000000000-0.32630000000000$ $-0.08714000000000-0.06386000000000$ $-0.32630000000000-0.08714000000000$
\# CIF file

data findsym-output

audit creation method FINDSYM

chemical name mineral 'Ti3O'

chemical formula_sum 'O Ti3

loop

publ_author_nam

A. Jostsons

A. S. Malin

journal_name_full_name

Acta Crystallographica Section B: Structural Science

journal_volume 24

journal year 1968

_journal_page_first 21

journal page last 213

publ Section title

The ordered structure of $\mathrm{Ti} \$ \_\{3\} \$ O$

Found in Pearson's Crystal Data - Crystal Structure Database for $\hookrightarrow$ Inorganic Compounds, 2013

aflow_title Ti $\$_{-}\{3\} \$ O$ (Room-temperature) Structure

-aflow_proto 'AB3_hP24_149_acgi_31

aflow_params $, a, c / a, z_{-}\{3\}, z_{-}\{4\}, x_{-}\{5\}, y_{-}\{5\}, z_{-}\{5\}, x_{-}\{6\}, y_{-}\{6\}, z_{-}\{6\}, x_{-}\{$ $\hookrightarrow 7\}, \mathrm{y}_{-}\{7\}, \mathrm{z}_{-}\{7\}$

aflow_params_values $, 5.1418156677,2.78268310709,0.33333,0.33333,0.0$, $\hookrightarrow 0.33333,0.421,0.33333,0.33333,0.246,0.0,0.33333,0.088$

aflow Strukturbericht 'None' -aflow_Pearson 'hP24,

cell_length_a $\quad 5.1418156677$

cell_length_b $\quad 5.1418156677$

cell_length_c $\quad 14.3080435983$

_cell_angle_alpha 90.0000000000

_cell_angle_beta 90.0000000000

cell_angle_gamma 120.0000000000

symmetry_space_group_name_H-M "P $3 \quad 1 \quad 2$ "

symmetry_Int_Tables_number 149

loop

space_group_symop_id

space group symop operation $x y z$

$\mathrm{x}, \mathrm{y}, \mathrm{z}$

$-y, x-y, z$

$3-x+y,-x, z$
$4 x, x-y,-z$

$3-x, y-y,-z$
$5-x+y, y,-z$

$5-x+y, y,-z$
$6-y,-x,-z$

loop

atom_site_label

-atom_site_type_symbol

atom_site_symmetry_multiplicity

atom_site_Wyckoff_label

atom_site_fract_x

atom_site_fract_y

atom_site_occupancy

$\begin{array}{llllllll}\mathrm{O} 1 & \mathrm{O} & 1 & \mathrm{a} & 0.00000 & 0.00000 & 0.00000 & 1.00000\end{array}$

$\begin{array}{lllllllll}\mathrm{O} 2 & \mathrm{O} & 1 & \mathrm{c} & 0.33333 & 0.66667 & 0.00000 & 1.00000\end{array}$

$\begin{array}{llllllllll}\mathrm{O} 3 & \mathrm{O} & & 2 & \mathrm{~g} & 0.00000 & 0.00000 & 0.33333 & 1.00000\end{array}$

$\begin{array}{lllllllll}\mathrm{O} 3 & \mathrm{O} & & 2 & \mathrm{~g} & 0.00000 & 0.00000 & 0.33333 & 1.00000 \\ \mathrm{O} 4 & \mathrm{O} & & 2 & \mathrm{i} & 0.66667 & 0.33333 & 0.33333 & 1.00000\end{array}$

$\begin{array}{llllllll}\mathrm{O} 4 & \mathrm{O} & 2 & \mathrm{i} & 0.66667 & 0.33333 & 0.33333 & 1.00000 \\ \mathrm{Ti} 1 & \mathrm{Ti} & 6 & 1 & 0.00000 & 0.33333 & 0.42100 & 1.00000\end{array}$

$\begin{array}{lllllllll}\mathrm{Ti1} & \mathrm{Ti} & 6 & & 0.00000 & 0.33333 & 0.42100 & 1.00000 \\ \mathrm{Ti} 2 & \mathrm{Ti} & 6 & 1 & 0.33333 & 0.33333 & 0.24600 & 1.00000\end{array}$

$\begin{array}{lllllllll}\mathrm{Ti} 3 & \mathrm{Ti} & 6 & \mathrm{l} & 0.00000 & 0.33333 & 0.08800 & 1.00000\end{array}$

$\mathrm{Ti}_{3} \mathrm{O}$ (Room-temperature): AB3_hP24_149_acgi_31 - POSCAR

\begin{tabular}{|c|c|c|c|c|}
\hline \multicolumn{5}{|c|}{ AB3_hP24_149_acgi_31 \& a, c/a $, \mathrm{z} 3, \mathrm{z} 4, \mathrm{x} 5, \mathrm{y} 5, \mathrm{z} 5, \mathrm{x} 6, \mathrm{y} 6, \mathrm{z} 6, \mathrm{x} 7, \mathrm{y} 7, \mathrm{z} 7--$ params $=$} \\
\hline$\hookrightarrow 5.141815667$ & & & & \\
\hline$\hookrightarrow 0.33333,0.33$ & $333,0.246,0.0,0.3$ & $33,0.088 \&$ P $312 \quad D_{-}\{3$ & & \#149 \\
\hline$\hookrightarrow \operatorname{acgil} \wedge 3) \&$ & $24 \&$ None \& Ti3C & \& A. Jostsons and & & Malin \\
\hline$\hookrightarrow$ Acta Cryst & & & $(196$ & \\
\hline 1.00000000000000 & & & & \\
\hline 2.57090783385000 & -4.45294298980505 & 0.00000000000000 & & \\
\hline 2.57090783385000 & 4.45294298980505 & 0.00000000000000 & & \\
\hline 0.00000000000000 & 0.00000000000000 & 14.30804359830000 & & \\
\hline $\mathrm{Ti}$ & & & & \\
\hline 18 & & & & \\
\hline Direct & & & & \\
\hline 0.00000000000000 & 0.00000000000000 & 0000000 & $\mathrm{O}$ & (1a) \\
\hline 0.33333333333333 & 0.66666666666667 & 0.00000000000000 & $\mathrm{O}$ & (1c) \\
\hline 0.00000000000000 & 0.00000000000000 & 0.33333000000000 & $\mathrm{O}$ & $(2 \mathrm{~g})$ \\
\hline 0.00000000000000 & 0.00000000000000 & -0.33333000000000 & $\mathrm{O}$ & $(2 \mathrm{~g})$ \\
\hline 0.666666666666667 & 0.33333333333333 & 0.33333000000000 & $\mathrm{O}$ & ( $2 \mathrm{i})$ \\
\hline 0.666666666666667 & 0.33333333333333 & -0.33333000000000 & $\mathrm{O}$ & $(2 \mathrm{i})$ \\
\hline 0.00000000000000 & 0.33333000000000 & 0.42100000000000 & $\mathrm{Ti}$ & (61) \\
\hline-0.333333000000000 & -0.33333000000000 & 0.42100000000000 & $\mathrm{Ti}$ & (61) \\
\hline 0.33333000000000 & 0.00000000000000 & 0.42100000000000 & $\mathrm{Ti}$ & (61) \\
\hline-0.33333000000000 & 0.00000000000000 & -0.42100000000000 & $\mathrm{Ti}$ & (61) \\
\hline 0.33333000000000 & 0.33333000000000 & -0.42100000000000 & $\mathrm{Ti}$ & (61) \\
\hline 0.00000000000000 & -0.33333000000000 & -0.42100000000000 & $\mathrm{Ti}$ & (61) \\
\hline 0.33333000000000 & 0.33333000000000 & 0.24600000000000 & $\mathrm{Ti}$ & (61) \\
\hline-0.33333000000000 & 0.00000000000000 & 0.24600000000000 & $\mathrm{Ti}$ & (61) \\
\hline 0.00000000000000 & -0.33333000000000 & 0.24600000000000 & $\mathrm{Ti}$ & (61) \\
\hline-0.33333000000000 & -0.33333000000000 & -0.24600000000000 & $\mathrm{Ti}$ & (61) \\
\hline 0.00000000000000 & 0.33333000000000 & -0.24600000000000 & $\mathrm{Ti}$ & (61) \\
\hline 0.33333000000000 & 0.00000000000000 & -0.24600000000000 & $\mathrm{Ti}$ & (61) \\
\hline
\end{tabular}

$\begin{array}{ll}\mathrm{Cl} & (6 \mathrm{f}) \\ \mathrm{Cl} & (6 \mathrm{f}) \\ \mathrm{Cl} & (6 \mathrm{f}) \\ \mathrm{Cl} & (6 \mathrm{f}) \\ \mathrm{Cl} & (6 \mathrm{f}) \\ \mathrm{Cl} & (6 \mathrm{f}) \\ \mathrm{Cl} & (6 \mathrm{f}) \\ \mathrm{Cl} & (6 \mathrm{f}) \\ \mathrm{Cl} & (6 \mathrm{f}) \\ \mathrm{Cl} & (6 \mathrm{f}) \\ \mathrm{Cl} & (6 \mathrm{f}) \\ \mathrm{Cl} & (6 \mathrm{f}) \\ \mathrm{Pd} & (6 \mathrm{f}) \\ \mathrm{Pd} & (6 \mathrm{f}) \\ \mathrm{Pd} & (6 \mathrm{f}) \\ \mathrm{Pd} & (6 \mathrm{f}) \\ \mathrm{Pd} & (6 \mathrm{f}) \\ \mathrm{Pd} & (6 \mathrm{f}) \\ & \end{array}$


0.00000000000000 $-0.33333000000000$ 0.33333000000000 $-0.33333000000000$ 0.33333000000000 0.00000000000000

0.33333000000000 $-0.33333000000000$ 0.00000000000000 0.0000000000000 0.33333000000000

0.08800000000000 0.08800000000000 0.08800000000000 $-0.0880000000000$ $-0.08800000000000$ $-0.08800000000000$

$\begin{array}{ll}\mathrm{Ti} & (61) \\ \mathrm{Ti} & (61) \\ \mathrm{Ti} & (61) \\ \mathrm{Ti} & (61) \\ \mathrm{Ti} & (61) \\ \mathrm{Ti} & (61)\end{array}$

$\mathrm{CrCl}_{3}: \mathrm{A} 3 \mathrm{~B} \_$hP24_153 3c_2b - CIF

\section{\# CIF file}

data_findsym-output

audit_creation_method FINDSYM

chemical_name_mineral ' $\mathrm{CrCl} 3$,

chemical_formula_sum ' $\mathrm{Cl} 3 \mathrm{Cr}$ '

aflow_title $\mathrm{CrCl}_{-}\{3\} \$$ Structure

aflow_proto 'A3B hP24 153 3c_2b'

aflow_params $, a, c / a, x_{-}\{1\}, x_{-}\{2\}, x_{-}\{3\}, y_{-}\{3\}, z_{-}\{3\}, x_{-}\{4\}, y_{-}\{4\}, z_{-}\{4\}, x_{-}\{$ $\hookrightarrow 5\}, y_{-}\{5\}, z_{-}\{5\}$,

aflow_params_values $6.017,2.87518697025,0.1111,0.4444,0.1111,0.2222$ $\hookrightarrow 0.09357,0.4444,0.8888,0.09357,0.77778,0.55558,0.09357$,

aflow Strukturbericht 'None'

aflow_Pearson 'hP24,

cell_length_a $\quad 6.0170000000$

cell_length_b 6.0170000000

cell_length_c 17.3000000000

cell_angle_alpha 90.0000000000

cell_angle_beta 90.0000000000

cell_angle_gamma 120.0000000000

_symmetry_space_group_name_H-M "P $32 \quad 1 \quad 2 "$

symmetry Int Tables_number 153

loop_

space_group_symop_id

space_group_symop_operation_xyz

$1 \mathrm{x}, \mathrm{y}, \mathrm{z}$

$2-y, x-y, z+2 / 3$

$3-x+y,-x, z+1 / 3$

$4 \mathrm{x}, \mathrm{x}-\mathrm{y},-\mathrm{z}$

$5-x+y, y,-z+2 / 3$

$6-\mathrm{y},-\mathrm{x},-\mathrm{z}+1 / 3$

loop

atom_site_label

atom_site_type_symbol

atom_site_symmetry_multiplicity

atom_site_Wyckoff_label

atom_site_fract_x

atom_site_fract_y

atom_site_fract_z

- atom_site_occupancy

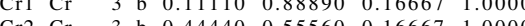

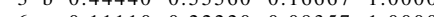

$\begin{array}{lllllllll}\mathrm{Cl} & \mathrm{Cl} & 6 & \mathrm{c} & 0.11110 & 0.22220 & 0.09357 & 1.00000\end{array}$

$\begin{array}{lllllllll}\mathrm{Cl} 2 & \mathrm{Cl} & 6 & \mathrm{c} & 0.44440 & 0.88880 & 0.09357 & 1.00000 \\ \mathrm{Cl} 3 & \mathrm{Cl} & 6 & \mathrm{c} & 0.77778 & 0.55558 & 0.09357 & 1.00000\end{array}$

$\mathrm{CrCl}_{3}$ : A3B_hP24_153_3c_2b - POSCAR

A3B_hP24_153_3c_2b \& a , c / a , x1 , x2 , x3 $, y 3, \mathrm{z} 3, \mathrm{x} 4, \mathrm{y} 4, \mathrm{z} 4, \mathrm{x} 5, \mathrm{y} 5, \mathrm{z} 5$--params $=$

$\hookrightarrow 0.8888,0.09357,0.77778,0.55558,0.09357 \& P_{-}\{2\} 12 D_{-}\{3\}^{\wedge}\{5\} \#$

$\hookrightarrow 153\left(\mathrm{~b}^{\wedge} 2 \mathrm{c}^{\wedge} 3\right) \& \mathrm{hP} 24$ \& None \& $\mathrm{CrCl} 3 \& \mathrm{CrCl} 3$ \&

1.00000000000000

$\begin{array}{lll}3.00850000000000 & -5.21087485457097 & 0.00000000000000\end{array}$

$\begin{array}{lll}3.00850000000000 & 5.21087485457097 & 0.00000000000000\end{array}$

$\begin{array}{lll}0.00000000000000 & 0.00000000000000 & 17.30000000000000\end{array}$

$\begin{array}{rr}\mathrm{Cl} & \mathrm{Cr} \\ 18 & 6\end{array}$

Direct

$0.11110000000000 \quad 0.22220000000000$

$-0.22220000000000-0.11110000000000$

$0.11110000000000-0.11110000000000$

$-0.22220000000000-0.11110000000000$

$0.11110000000000-0.111000000000$

$0.11110000000000-0.11110000000000$

$0.1111000000000-0.1111000000000$

$0.44440000000000-0.8888000000000$

$-0.88880000000000-0.44440000000000$

$0.44440000000000-0.44440000000000$

$-0.88880000000000-0.44440000000000$

$0.44440000000000 \quad 0.88880000000000$

$0.44440000000000-0.44440000000000$

$0.77778000000000 \quad 0.55558000000000$

$-0.55558000000000 \quad 0.22220000000000$

$-0.22220000000000 \quad-0.77778000000000$

$-0.55558000000000-0.77778000000000$

$-0.22220000000000 \quad 0.55558000000000$

$0.77778000000000 \quad 0.22220000000000$

$0.11110000000000-0.11110000000000$

$0.11110000000000 \quad 0.22220000000000$

$-0.22220000000000-0.11110000000000$

$0.44440000000000-0.44440000000000$

$0.444400000000-0.8888000000000$

S-II: A_hP9_154_bc - CIF

\section{\# CIF file}

data_findsym-output

_audit_creation_method FINDSYM

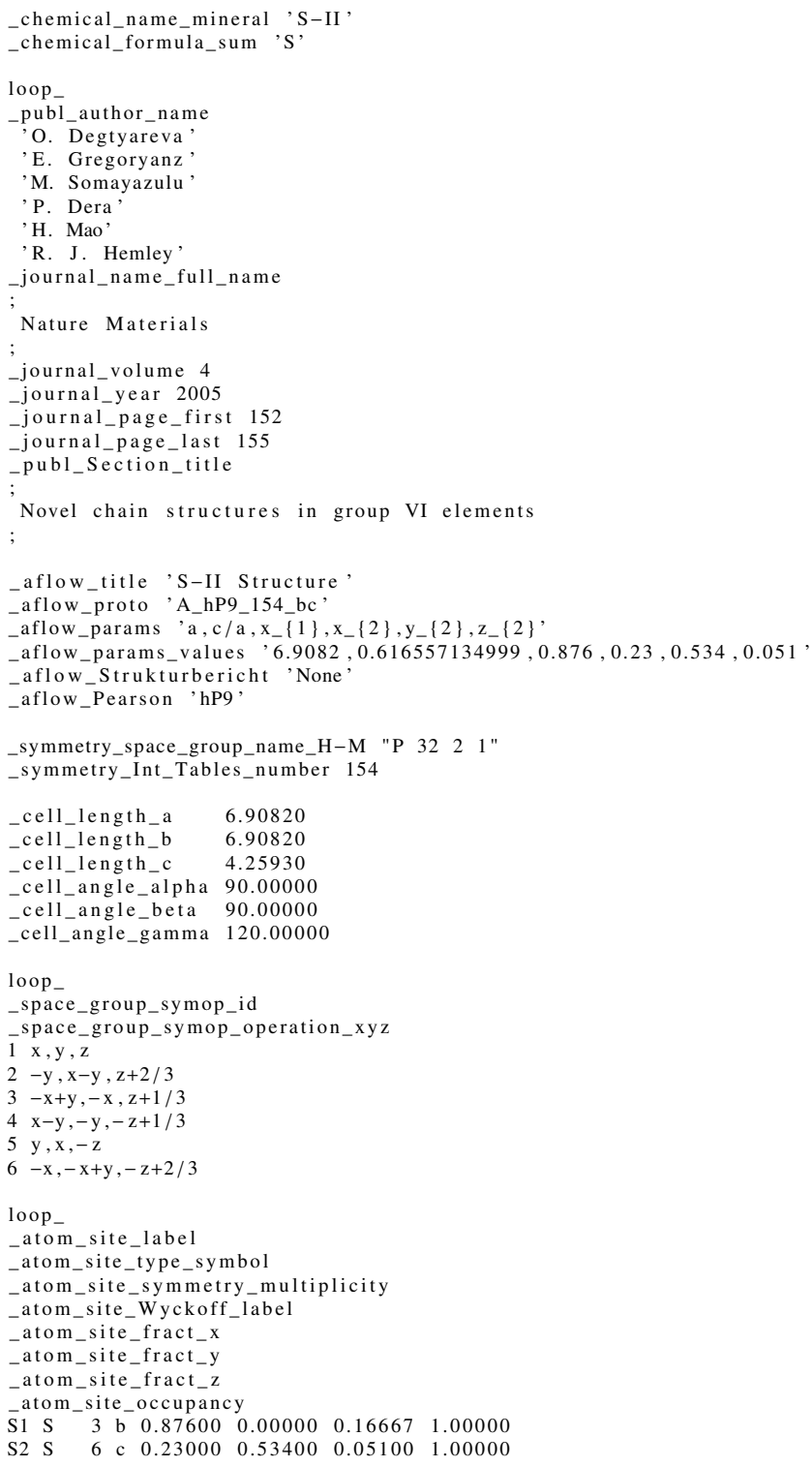

A_hP9_154_bc \& a , c/a, x1, x2,y2, z2 --params $=6.9082,0.616557134999,0.876$, $\rightarrow 0.23,0.534,0.051 \& \mathrm{P} 3-\{2\} 21 \mathrm{D}_{-}\{3\}^{\wedge}\{6\} \# 154$ (bc) \& hP9 \& None \& $\hookrightarrow$ S \& S-II \& O. Degtyareva et al., Nat. Mater. 4, 152-155 (2005) 1.00000000000000

3.45410000000000

$\begin{array}{lll}3.4541000000000 & -5.98267669442366 & 0.00000000000000\end{array}$

$\begin{array}{lll}5.98267669442366 & 0.00000000000000\end{array}$

(1.00000000000000-4.25930000000000 $\mathrm{S}$

Direct

$0.87600000000000-0.00000000000000-0.16666666666667$ $\begin{array}{rrr}0.00000000000000 & 0.87600000000000 & 0.83333333333333 \\ -0.87600000000000 & -0.87600000000000 & 0.50000000000000\end{array}$ $-0.87600000000000 \quad-0.87600000000000 \quad 0.50000000000000$ $\begin{array}{lll}0.23000000000000 & 0.53400000000000 & 0.05100000000000\end{array}$ $\begin{array}{lll}-0.53400000000000 & -0.30400000000000 & 0.71766666666667\end{array}$ $\begin{array}{lll}0.30400000000000 & -0.23000000000000 & 0.38433333333333\end{array}$ $\begin{array}{lll}0.53400000000000 & 0.23000000000000 & -0.05100000000000\end{array}$ $\begin{array}{rrr}-0.30400000000000 & -0.53400000000000 & 0.28233333333333 \\ -0.23000000000000 & 0.30400000000000 & 0.61566666666667\end{array}$ 0.61566666666667

$\mathrm{CdI}_{2}$ (Polytype $6 \mathrm{H}_{1}$ ): AB2_hP9_156_b2c_3a2bc - CIF

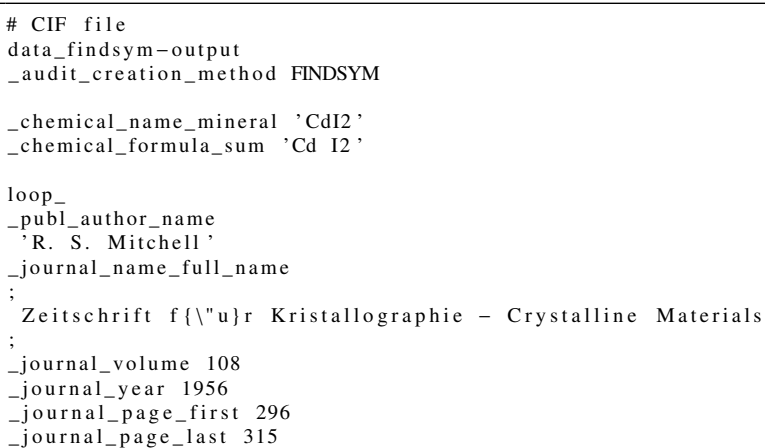


publ_Section_title

Polytypism of Cadmium Iodide and its Relationship to Screw Dislocations $\longrightarrow$ : I. Cadmium Iodide Polytypes

\# Found in Pearson's Crystal Data - Crystal Structure Database for $\hookrightarrow$ Inorganic Compounds, 2013

aflow title 'CdI\$_ $\{2\} \$$ (Polytype $6 \mathrm{H} \$\{1\} \$$ ) Structure

aflow_proto 'AB2_hP9_156_b2c_3a2bc'

aflow_params 'a, c/a, z- $\{1\}, z_{-}\{2\}, z_{-}\{3\}, z_{-}\{4\}, z_{-}\{5\}, z_{-}\{6\}, z_{-}\{7\}, z_{-}\{8\}, z_{-}\{$ $\hookrightarrow 9\}$,

aflow_params_values $, 4.239807232,4.83608490569,0.0,0.66667,0.33333,0.75$ $\hookrightarrow, 0.16667,0.5,0.08333,0.41667,0.83333$,

aflow Strukturbericht 'None'

aflow_Pearson 'hP9'

cell_length_a 4.2398072320

cell_length_b 4.2398072320

cell_length_c 20.5040677577

cell_angle_alpha 90.0000000000

cell_angle_beta 90.0000000000

_cell_angle_gamma 120.0000000000

symmetry_space_group_name_H-M "P $3 \mathrm{~m} 1$ "

symmetry Int Tables_number 156

loop

space_group_symop_id

space_group_symop_operation_xyz

$1 \mathrm{x}, \mathrm{y}, \mathrm{z}$

$2-\mathrm{y}, \mathrm{x}-\mathrm{y}, \mathrm{z}$

$3-x+y,-x$,
$4-x+y, y, z$

$4-\mathrm{x}+\mathrm{y}, \mathrm{y}, \mathrm{z}$

$5-y,-x, z$
$6 x, x-y, z$

loop_

atom_site_label

atom_site_type_symbol

atom_site_symmetry_multiplicity

atom_site_Wyckoff_label

atom_site_fract_x

atom_site_fract_y

atom_site_fract_z

I1 atom_site_occupancy

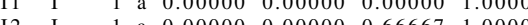

$\begin{array}{lllllllllll}12 & \mathrm{I} & \mathrm{I} & \mathrm{I} & 0.00000 & 0.00000 & 0.66667 & 1.00000\end{array}$

$\begin{array}{lllllllll}13 & 1 & 1 & \text { a } & 0.00000 & 0.00000 & 0.33333 & 1.00000\end{array}$

$\begin{array}{lllllllllllll}\mathrm{Cd} \mathrm{Cd} & \mathrm{l} & \mathrm{b} & 0.33333 & 0.66667 & 0.75000 & 1.00000\end{array}$

$\begin{array}{lllllllll}\mathrm{I} 4 & \mathrm{I} & \mathrm{l} & \mathrm{b} & 0.333333 & 0.66667 & 0.16667 & 1.00000\end{array}$

$\begin{array}{llllllll}15 & 1 & 1 & \mathrm{~b} & 0.333333 & 0.66667 & 0.16667 & 1.00000\end{array}$

$\begin{array}{lllllllll}\mathrm{Cd} 2 & \mathrm{Cd} & 1 & \mathrm{c} & 0.66667 & 0.33333 & 0.08333 & 1.00000\end{array}$

$\begin{array}{lllllllll}\mathrm{Cd} 3 & \mathrm{Cd} & 1 & \mathrm{c} & 0.66667 & 0.33333 & 0.41667 & 1.00000\end{array}$

$\begin{array}{llllllllll}\text { I6 } & \text { I } & 1 & \text { c } & 0.66667 & 0.33333 & 0.83333 & 1.00000\end{array}$

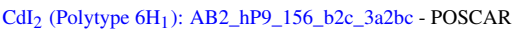

AB2_hP9_156_b2c_3a2bc \& a,c/a,z1, z2, z3, z4, z5, z6, z7, z8, z9 --params

$\hookrightarrow 4.239807232,4.83608490569,0.0,0.66667,0.33333,0.75,0.16667,0.5$

$\hookrightarrow 0.08333,0.41667,0.83333 \&$ \& $3 \mathrm{~m} 1 \mathrm{C}_{-}\{3 \mathrm{v}\}^{\wedge}\{1\} \# 156\left(\mathrm{a}^{\wedge} 3 \mathrm{~b}^{\wedge} 3 \mathrm{c}^{\wedge} 3\right)$

$\hookrightarrow$ hP9 \& None \& CdI2 \& \& R. S. Mitchell, Zeitschrift f" $\{u\} r$

$\hookrightarrow$ Kristallographie - Crystalline Materials 108, 296-315 (1956)

1.00000000000000

$\begin{array}{lll}2.11990361600000 & -3.67178077006098 & 0.00000000000000\end{array}$

$\begin{array}{lll}2.11990361600000 & 3.67178077006098 & 0.00000000000000\end{array}$

$\begin{array}{lll}0.00000000000000 & 0.00000000000000 & 20.50406775770000\end{array}$

$\begin{array}{cc}\mathrm{Cd} & \mathrm{I} \\ 3 & 6\end{array}$

Direct

0.33333333333333

0.66666666666667

0.66666666666667

0.00000000000000

0.0000000000000

0.00000000000000

0.33333333333333

0.33333333333333

0.66666666666667

0.666666666666667

0.75000000000000

0.08333000000000

0.41667000000000

0.00000000000000

(2)

0.66666666666667

.33333000000000

0.1666700000000

0.50000000000000
0.83333000000000
$\mathrm{Cd} \quad(1 \mathrm{~b})$

Cd (1c)

(1c)

(1a)

(1a)

(1a)

(1b)

(1c)
CuI: AB_hP12_156_2ab3c_2ab3c - CIF

\section{\# CIF file}

data_findsym-output

_audit_creation_method FINDSYM

_chemical_name_mineral 'CuI,

_chemical_formula_sum, Cu I,

loop

publ_author_name

R. N. Kurdyumova

'R. V. Baranova

_journal_name_full_name

Soviet Physics Crystallography

journal volume 6

journal year 1961

_journal_page_first 318

_journal_page_last 32

publ_Section_title

An electron diffraction study of thin films of cuprous iodide
\# Found in Pearson's Crystal Data - Crystal Structure Database for $\hookrightarrow$ Inorganic Compounds, 2013

aflow_title 'CuI Structure

aflow_proto 'AB_hP12_156_2ab3c_2ab3c,

aflow_params a,c/a, $z_{-}\{1\}, z_{-}\{2\}, z_{-}\{3\}, z_{-}\{4\}, z_{-}\{5\}, z_{-}\{6\}, z_{-}\{7\}, z_{-}\{8\}, z_{-}\{$ $\hookrightarrow 9\}, z_{-}\{10\}, z_{-}\{11\}, z_{-}\{12\}$

_aflow_params_values ' $4.2499813346,4.90823529409,0.375,0.70833,0.5$ $\hookrightarrow 0.83333,0.04167,0.16667,0.45833,0.79167,0.125,0.33333,0.66667$$$
\hookrightarrow 0.0
$$

aflow Strukturbericht 'None'

aflow_Pearson 'hP12,

_cell_length_a $\quad 4.2499813346$

cell_length_b 4.2499813346

_cell_length_c 20.8599083857

_cell_angle_alpha 90.0000000000

_cell_angle_beta 90.0000000000

_cell_angle_gamma 120.0000000000

_symmetry_space_group_name_H-M "P 3 m 1"

_symmetry_Int_Tables_number 156

loop

_space_group_symop_id

_space_group_symop_operation_xyz

$1 \mathrm{x}, \mathrm{y}, \mathrm{z}$

$2-\mathrm{y}, \mathrm{x}-\mathrm{y}, \mathrm{z}$

$-x+y,-x, z$

$4-x+y, y, z$

20 $\mathrm{x}, \mathrm{x}-\mathrm{x}, \mathrm{z}$

loop_

atom_site_label

atom_site_type_symbol

atom_site_symmetry_multiplicity

atom_site_Wyckoff_label

atom_site_fract_x

atom_site_fract_y

atom_site_fract_z

atom_site_occupancy

$\begin{array}{lllllll}\mathrm{Cu} 1 \mathrm{Cu} & 1 & \mathrm{a} & 0.00000 & 0.00000 & 0.37500 & 1.00000\end{array}$

$\begin{array}{llllllll}\mathrm{Cu} 2 \mathrm{Cu} & 1 & \mathrm{a} & 0.00000 & 0.00000 & 0.70833 & 1.00000\end{array}$

$\begin{array}{lllllll}\mathrm{Cu} 2 \mathrm{Cu} & 1 & \mathrm{a} & 0.00000 & 0.00000 & 0.70833 & 1.00000\end{array}$

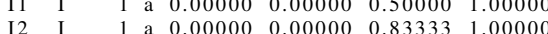

$\begin{array}{lllllllll}12 & \mathrm{~T} & 1 & \mathrm{a} & 0.00000 & 0.00000 & 0.83333 & 1.00000\end{array}$

$\begin{array}{llllllll}\mathrm{Cu} 3 \mathrm{Cu} & \mathrm{I} & \mathrm{b} & 0.33333 & 0.66667 & 0.04167 & 1.00000\end{array}$

\begin{tabular}{llllllll}
13 & $\mathrm{l}$ & $\mathrm{l}$ & $\mathrm{b}$ & 0.33333 & 0.66667 & 0.04167 & 1.00000 \\
\hline $\mathrm{C}$ & $\mathrm{C}$ & $\mathrm{l}$ & 0.16667 & 1.00000
\end{tabular}

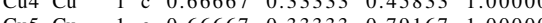

$\begin{array}{lllllllll}\mathrm{Cu} 5 \mathrm{Cu} & 1 & \mathrm{c} & 0.66667 & 0.33333 & 0.79167 & 1.000000\end{array}$

$\begin{array}{llllllll}\mathrm{Cu} 6 \mathrm{Cu} & 1 & \mathrm{c} & 0.66667 & 0.33333 & 0.12500 & 1.00000\end{array}$

$\begin{array}{lllllllll}\text { I4 } & \text { I } & 1 & c & 0.66667 & 0.33333 & 0.33333 & 1.00000 \\ \text { I5 } & \text { I } & 1 & c & 0.66667 & 0.33333 & 0.66667 & 1.00000\end{array}$

$\begin{array}{lllllllll}\text { I5 } & \text { I } & & 1 & \text { c } & 0.66667 & 0.33333 & 0.66667 & 1.00000 \\ \text { I6 } & \text { I } & & 1 & \text { c } & 0.66667 & 0.33333 & 0.00000 & 1.00000\end{array}$

CuI: AB_hP12_156_2ab3c_2ab3c - POSCAR

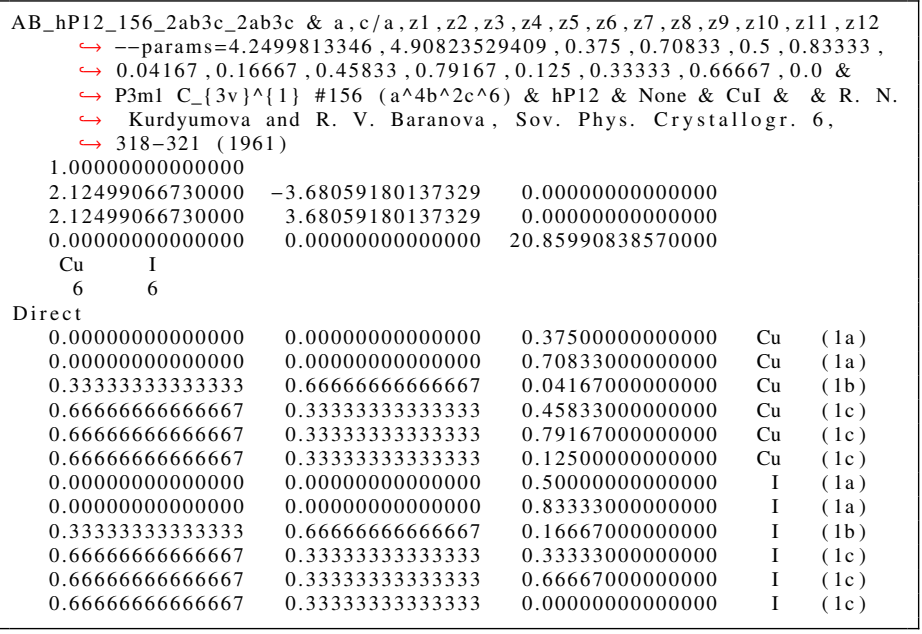

$\beta$-CuI: AB_hP4_156_ac_ac - CIF

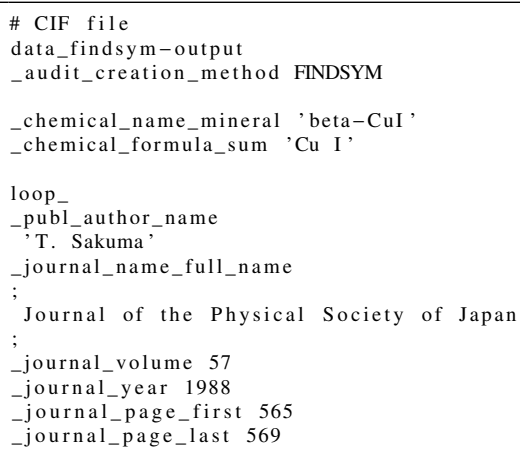




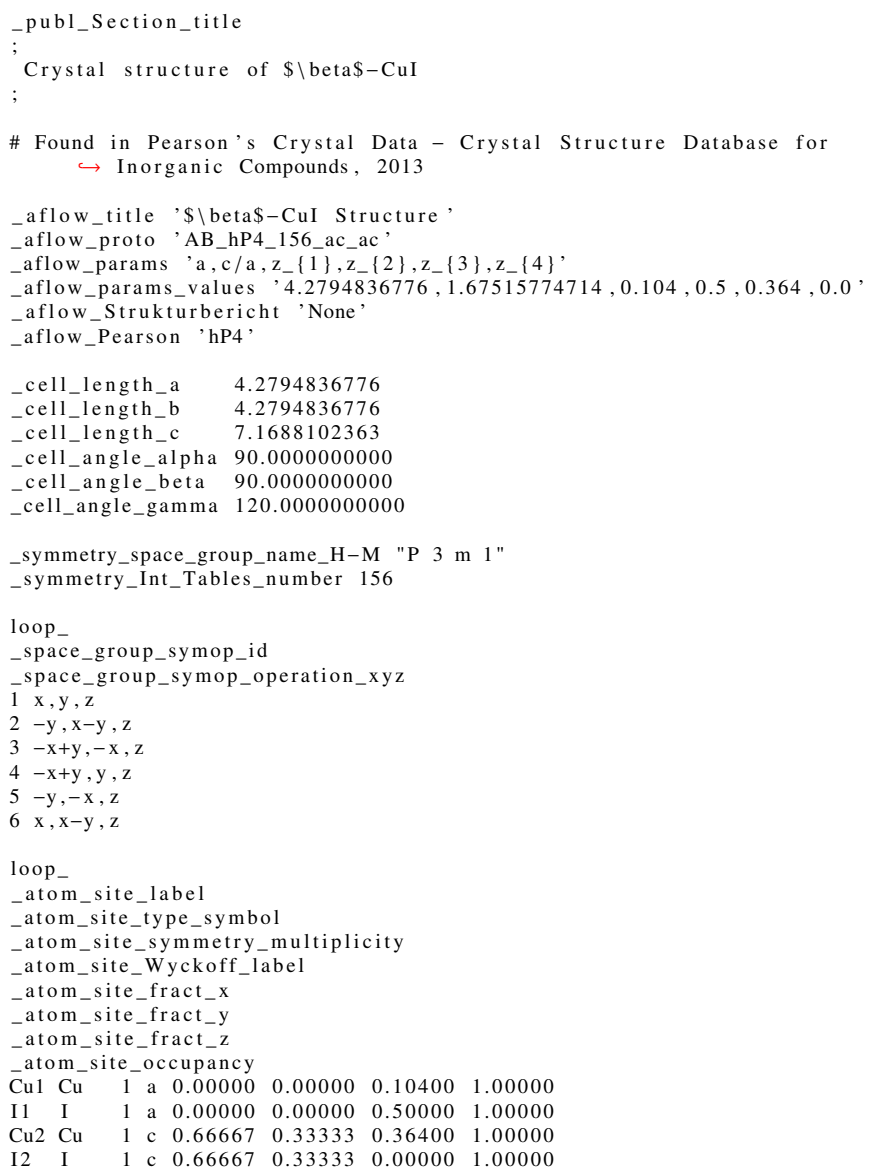

$\beta$-CuI: AB_hP4_156_ac_ac - POSCAR

\begin{tabular}{|c|c|c|c|c|}
\hline \multirow{2}{*}{\multicolumn{5}{|c|}{$\begin{array}{l}\hookrightarrow, 0.104,0.5,0.364,0.0 \& \text { P } 3 \mathrm{~m}_{1} \mathrm{C}_{-}\{3 \mathrm{v}\}^{\wedge}\{1\} \# 156\left(\mathrm{a}^{\wedge} 2 \mathrm{c}^{\wedge} 2\right) \& \mathrm{hP} 4 \& \\
\hookrightarrow \text { None \& CuI \& beta \& T. Sakuma, J. Phys. Soc. Jpn. 57, } 565-569 \\
\hookrightarrow 1988)\end{array}$}} \\
\hline & & & & \\
\hline \multicolumn{5}{|l|}{1.00000000000000} \\
\hline 2.13974183880000 & -3.70614157988245 & 0.00000000000000 & & \\
\hline \multirow{2}{*}{$\begin{array}{l}2.13974183880000 \\
0.00000000000000\end{array}$} & 3.70614157988245 & 0.00000000000000 & & \\
\hline & 0.00000000000000 & 7.16881023630000 & & \\
\hline $\mathrm{Cu}$ & & & & \\
\hline 2 & & & & \\
\hline \multicolumn{5}{|l|}{ Direct } \\
\hline 0.00000000000000 & 0.00000000000000 & 0.10400000000000 & $\mathrm{Cu}$ & (1a) \\
\hline 0.666666666666667 & 0.33333333333333 & 0.36400000000000 & $\mathrm{Cu}$ & (1c) \\
\hline 0.00000000000000 & 0.00000000000000 & 0.50000000000000 & I & (1a) \\
\hline 0.666666666666667 & 0.33333333333333 & 0.00000000000000 & I & (1c) \\
\hline
\end{tabular}

$\mathrm{Ag}_{5} \mathrm{~Pb}_{2} \mathrm{O}_{6}:$ A5B6C2_hP13_157_2ac_2c_b - CIF

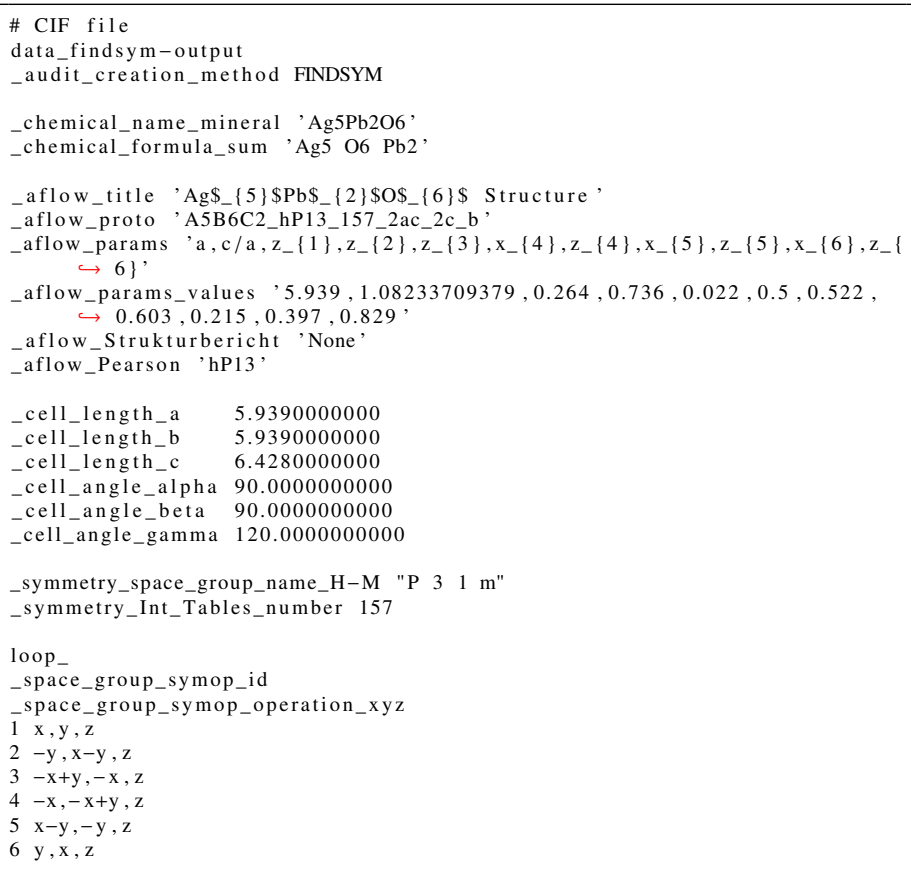

atom_site_label

atom_site_type_symbol

atom_site_symmetry_multiplicity

atom_site_Wyckoff_label

atom_site_fract_x

atom_site_fract_y

atom_site_fract_z

atom_site_occupancy

$\begin{array}{llllllll}\mathrm{Ag} 1 \mathrm{Ag} & 1 \mathrm{a} & 0.00000 & 0.00000 & 0.26400 & 1.00000\end{array}$

$\begin{array}{lllllllll}\mathrm{Ag} 2 & \mathrm{Ag} & 1 & \mathrm{a} & 0.00000 & 0.00000 & 0.73600 & 1.00000\end{array}$

$\begin{array}{lllllllll}\mathrm{Pb} 1 \mathrm{~Pb} & 2 & \mathrm{~b} & 0.33333 & 0.66667 & 0.02200 & 1.00000\end{array}$

$\begin{array}{llllllll}\mathrm{Ag} 3 & \mathrm{Ag} & 3 & \mathrm{c} & 0.50000 & 0.00000 & 0.52200 & 1.00000\end{array}$

$\begin{array}{llllllll}\mathrm{Ag} 3 & \mathrm{Ag} & 3 & \mathrm{c} & 0.50000 & 0.00000 & 0.52200 & 1.00000 \\ \mathrm{O} 1 & \mathrm{O} & 3 & \mathrm{c} & 0.60300 & 0.00000 & 0.21500 & 1.00000\end{array}$

$\begin{array}{lllllllll}\mathrm{O} 1 & \mathrm{O} & & 3 & \mathrm{c} & 0.60300 & 0.00000 & 0.21500 & 1.000000 \\ \mathrm{O} 2 & \mathrm{O} & 3 & \mathrm{c} & 0.39700 & 0.00000 & 0.82900 & 1.00000\end{array}$

$\mathrm{Ag}_{5} \mathrm{~Pb}_{2} \mathrm{O}_{6}:$ A5B6C2_hP13_157_2ac_2c_b - POSCAR

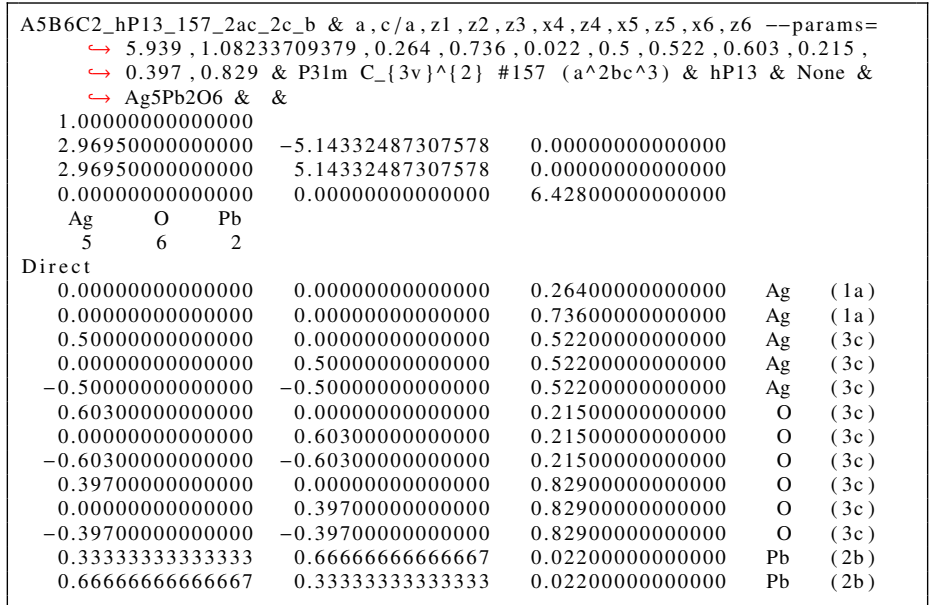

$\beta-\mathrm{RuCl}_{3}: \mathrm{A}$ 3B_hP8_158_d_a - CIF

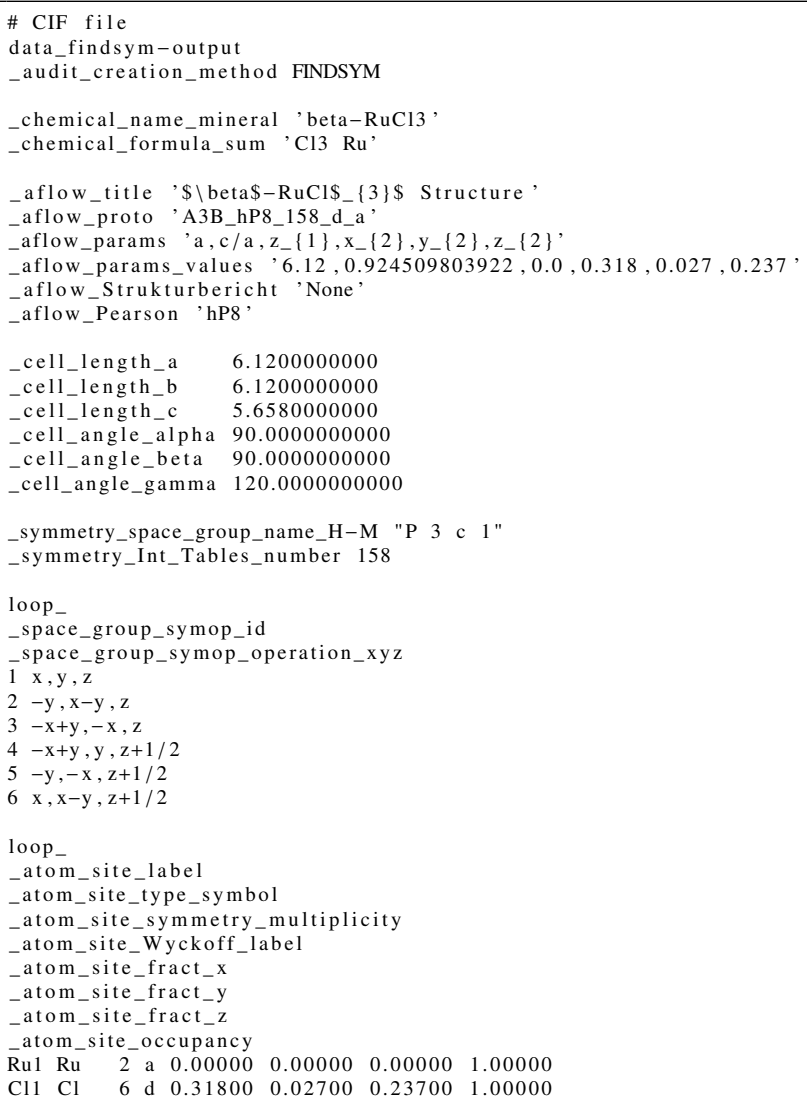

A3B_hP8_158_d_a \& a , c/a , z1 $, x 2, y 2, z 2--$ params $=6.12,0.924509803922,0.0$ $\hookrightarrow 0.318,0.027,0.237 \& \mathrm{P} 3 \mathrm{c} 1 \mathrm{C}_{-}\{3 \mathrm{v}\}^{\wedge}\{3\} \# 158(\mathrm{ad}) \& \mathrm{hP} 8 \&$ None \& $\hookrightarrow \mathrm{RuCl} 3$ \& beta \&

1.00000000000000

$\begin{array}{lll}3.06000000000000 & -5.30007547116076 & 0.00000000000000\end{array}$

$\begin{array}{lll}3.06000000000000 & 5.30007547116076 & 0.00000000000000\end{array}$

$0.00000000000000 \quad 0.00000000000000-5.65800000000000$

$\mathrm{Cl} \quad \mathrm{Ru}$

$\begin{array}{lll}0.31800000000000 & 0.02700000000000 & 0.23700000000000\end{array}$ 
$-0.02700000000000$ $-0.29100000000000$ -0.02700000000000
-0.29100000000000 $-0.29100000000000$ 0.31800000000000 0.00000000000000 0.00000000000000

0.29100000000000 $-0.31800000000000$ $-0.31800000000000$ 0.02700000000000 0.29100000000000 0.00000000000000 0.00000000000000

$\mathrm{Bi}_{2} \mathrm{O}_{3}$ (High-pressure): A2B3_hP20_159_bc_2c - CIF

\section{\# CIF file}

data_findsym-output

audit_creation_method FINDSYM

chemical_name_mineral 'Bi2O3,

chemical_formula_sum 'Bi2 O3,

loop

publ_author_name

'T. Locherer'

'D. L. V. K. Prasad,

'R. Dinnebier'

'U. Wedig ',

M. Jansen'

G. Garbarino

journal_name_full_name

Physical Review B

journal_volume 83

journal_year 201

journal_page_first 214102

journal_page_last 214102

publ_Section_title

High-pressure structural evolution of $\mathrm{HP}_{-\mathrm{Bi}} \$_{-}\{2\} \$ O{ }_{-}\{3\} \$$

Found in Pearson's Crystal Data - Crystal Structure Database fo $\hookrightarrow$ Inorganic Compounds, 2013

aflow_title $B i \$ \_\{2\} \$ O \$\{\{3\}$ (High-pressure) Structure,

aflow_proto 'A2B3_hP20_159_bc_2c,

aflow_params a,c/a, z $z_{-}\{1\}, x_{-}\{2\}, y_{-}\{2\}, z_{-}\{2\}, x_{-}\{3\}, y_{-}\{3\}, z_{-}\{3\}, x_{-}\{4\}, y_{-}\{$ $\hookrightarrow 4\}, z_{-}\{4\}$,

aflow_params_values $\quad 7.7488577892,0.813266227893,0.0,0.337,0.154,0.021$, $\hookrightarrow 0.451,0.061,0.287,0.149,0.282,0.083$

aflow_Strukturbericht 'None

aflow_Pearson 'hP20,

\section{cell_length_a $\quad 7.7488577892$}

cell_length_c 6.7488577892

cell angle alpha 90.0000000000

cell_angle_alpha 90.0000000000

symmetry_space_group_name_H-M "P 31 c"

symmetry_Int_Tables_number 159

loop

_space_group_symop_id

space_group_symop_operation_xyz

$1 \mathrm{x}, \mathrm{y}, \mathrm{z}$

$2-\mathrm{y}, \mathrm{x}-\mathrm{y}, \mathrm{z}$

$-x+y,-x, z$

$-\mathrm{x},-\mathrm{x}+\mathrm{y}, \mathrm{z}+1 / 2$

$\mathrm{x}-\mathrm{y},-\mathrm{y}, \mathrm{z}+1 / 2$

$6 \mathrm{y}, \mathrm{x}, \mathrm{z}+1 / 2$

loop

atom site label

atom_site_type_symbol

atom_site_symmetry_multiplicity

atom_site_Wyckoff_label

atom_site_fract_x

atom_site_fract_y
atom_site_fract_z

- atom_site_fract_Z

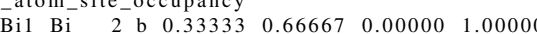

$\begin{array}{llllll}6 & \text { c } & 0.33700 & 0.15400 & 0.02100 & 1.0000\end{array}$

$\begin{array}{lllllllll}\mathrm{O} 1 & \mathrm{O} & 6 & \mathrm{c} & 0.45100 & 0.06100 & 0.28700 & 1.00000\end{array}$

$\begin{array}{lllllllll}\mathrm{O} 2 & \mathrm{O} & 6 & \mathrm{c} & 0.14900 & 0.28200 & 0.08300 & 1.00000\end{array}$

$\mathrm{Bi}_{2} \mathrm{O}_{3}$ (High-pressure): A2B3_hP20_159_bc_2c - POSCAR

A2B3_hP20_159_bc_2c \& a , c /a , z1 , x2 , y2 $, \mathrm{z} 2, \mathrm{x} 3, \mathrm{y} 3, \mathrm{z} 3, \mathrm{x} 4, \mathrm{y} 4, \mathrm{z} 4--$ params $=$

$\hookrightarrow 7.7488577892,0.813266227893,0.0,0.337,0.154,0.021,0.451,0.061$,

$\hookrightarrow 0.287,0.149,0.282,0.083 \& \mathrm{P} 31 \mathrm{c} \mathrm{C}_{-}\{3 \mathrm{v}\}^{\wedge}\{4\} \# 159\left(\mathrm{bc}^{\wedge} 3\right) \& \mathrm{hP} 20 \&$

$\hookrightarrow$ None \& Bi2O3\&\& \&. Locherer et al., Phys. Rev. B 83, 214102 ( $\hookrightarrow 2011$ )

1.00000000000000

$\begin{array}{lll}3.87442889460000 & -6.71070769576012 & 0.00000000000000\end{array}$

$3.87442889460000-6.71070769576012-0.00000000000000$

$$
\begin{array}{rr}
\mathrm{Bi} & \mathrm{O} \\
8 & 12
\end{array}
$$

Direct

0.33333333333333

0.33333333333333

0.33700000000000

$-0.15400000000000$

$-0.18300000000000$

0.15400000000000

0.666666666666667 0.33333333333333 0.15400000000000 0.18300000000000 $-0.33700000000000$ 0.33700000000000

\subsection{0}

0.00000000000000 0.50000000000000 0.02100000000000 0.02100000000000 0.52100000000000 0.02100000000000
0.18300000000000

$-0.3370000000000$

0.45100000000000
-0.06100000000000

$-0.06100000000000$

$-0.39000000000000$

0.06100000000000

0.39000000000000

$-0.45100000000000$

0.14900000000000

$-0.28200000000000$

0.28200000000000

$-0.13300000000000$

$-0.14900000000000$

$-15400000000000$ 0.18300000000000 0.06100000000000 0.39000000000000 0.45100000000000 0.45100000000000 $-0.06100000000000$ $-0.39000000000000$ 0.28200000000000 $-0.13300000000000$ $-0.14900000000000$ 0.14900000000000 $-0.28200000000000$ 0.13300000000000 0.52100000000000 0.28700000000000 0.28700000000000 0.28700000000000 0.78700000000000 0.78700000000000 0.78700000000000 0.08300000000000 0.08300000000000 0.08300000000000 0.58300000000000 0.58300000000000 0.58300000000000

$\mathrm{Bi} \quad(6 \mathrm{c})$

Nierite $\left(\alpha-\mathrm{Si}_{3} \mathrm{~N}_{4}\right)$ : A4B3_hP28_159_ab2c_2c - CIF

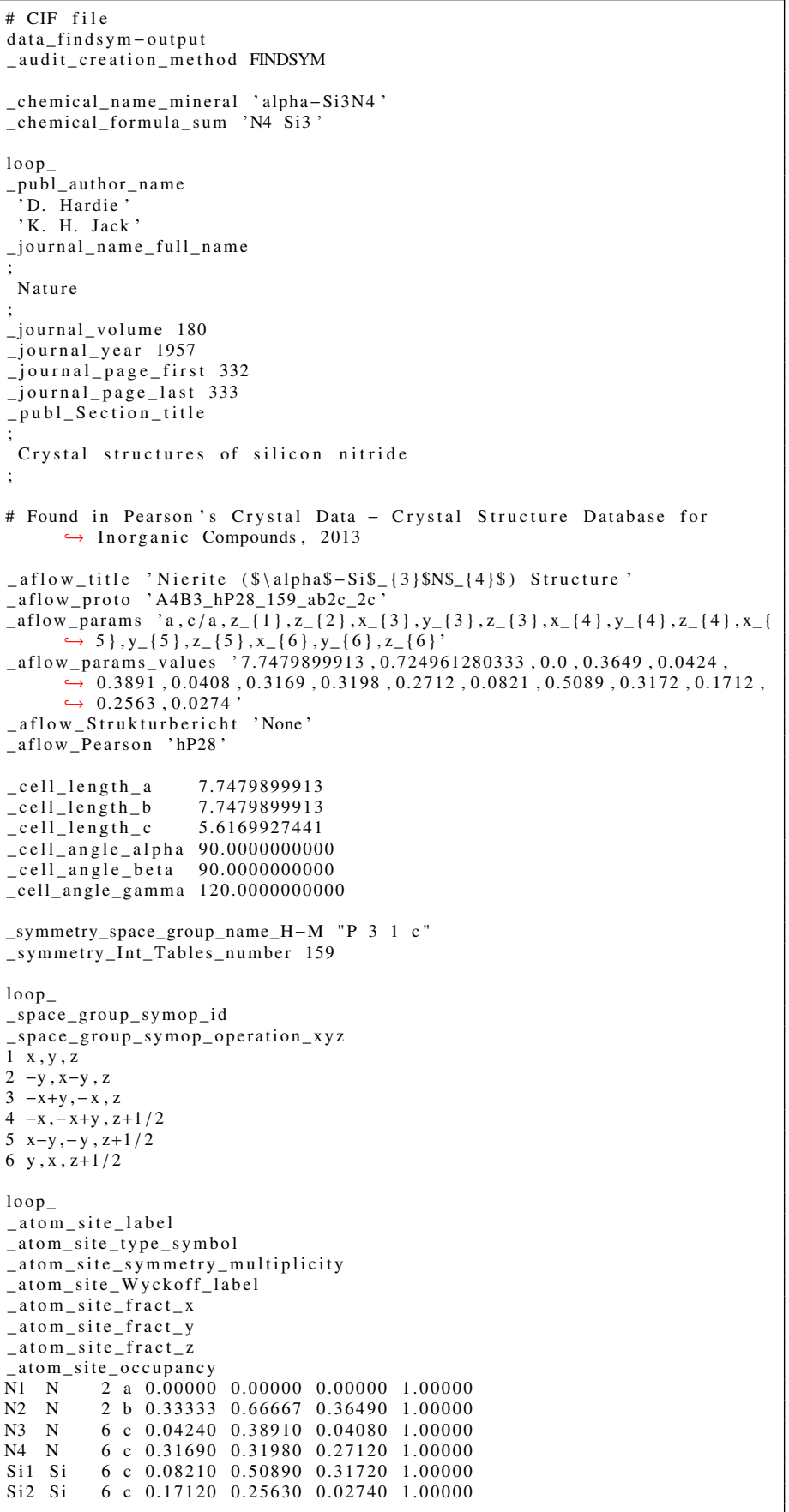

Nierite $\left(\alpha-\mathrm{Si}_{3} \mathrm{~N}_{4}\right)$ : A4B3_hP28_159_ab2c_2c - POSCAR

A4B3_hP28_159_ab2c_2c \& a , c/a , z1 , z2 , x3 $, y 33, \mathrm{z} 3, \mathrm{x} 4, \mathrm{y} 4, \mathrm{z} 4, \mathrm{x} 5, \mathrm{y} 5, \mathrm{z} 5, \mathrm{x} 6, \mathrm{y} 6, \mathrm{z} 6$ $\hookrightarrow-$-params $=7.7479899913,0.724961280333,0.0,0.3649,0.0424,0.3891$ $\leftrightarrow 0.0408,0.3169,0.3198,0.2712,0.0821,0.5089,0.3172,0.1712,0.2563$

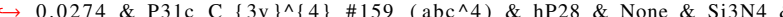
\& D. Hardie and K. H. Jack , Nature $180,332-333$ (1957) 1.00000000000000

$\begin{array}{llll}3.87399499565000 & -6.70995616073337 & 0.00000000000000\end{array}$

$\begin{array}{lll}3.87399499565000 & 6.70995616073337 & 0.00000000000000\end{array}$

$0.00000000000000 \quad 0.00000000000000 \quad 5.61699274410000$

$\mathrm{N} \quad \mathrm{Si}$

16
rect

$0.00000000000000 \quad 0.00000000000000 \quad 0.00000000000000$

$\mathrm{N} \quad(2 \mathrm{a})$ 


$\begin{array}{rrrrl}0.00000000000000 & 0.00000000000000 & 0.50000000000000 & \mathrm{~N} & (2 \mathrm{a}) \\ 0.33333333333333 & 0.66666666666667 & 0.36490000000000 & \mathrm{~N} & (2 \mathrm{~b}) \\ 0.66666666666667 & 0.33333333333333 & 0.86490000000000 & \mathrm{~N} & (2 \mathrm{~b}) \\ 0.04240000000000 & 0.38910000000000 & 0.04080000000000 & \mathrm{~N} & (6 \mathrm{c}) \\ -0.38910000000000 & -0.34670000000000 & 0.04080000000000 & \mathrm{~N} & (6 \mathrm{c}) \\ 0.34670000000000 & -0.04240000000000 & 0.04080000000000 & \mathrm{~N} & (6 \mathrm{c}) \\ 0.38910000000000 & 0.04240000000000 & 0.54080000000000 & \mathrm{~N} & (6 \mathrm{c}) \\ -0.34670000000000 & -0.38910000000000 & 0.54080000000000 & \mathrm{~N} & (6 \mathrm{c}) \\ -0.04240000000000 & 0.34670000000000 & 0.54080000000000 & \mathrm{~N} & (6 \mathrm{c}) \\ 0.31690000000000 & 0.31980000000000 & 0.27120000000000 & \mathrm{~N} & (6 \mathrm{c}) \\ -0.31980000000000 & -0.00290000000000 & 0.27120000000000 & \mathrm{~N} & (6 \mathrm{c}) \\ 0.00290000000000 & -0.31690000000000 & 0.27120000000000 & \mathrm{~N} & (6 \mathrm{c}) \\ 0.31980000000000 & 0.31690000000000 & 0.77120000000000 & \mathrm{~N} & (6 \mathrm{c}) \\ -0.00290000000000 & -0.31980000000000 & 0.77120000000000 & \mathrm{~N} & (6 \mathrm{c}) \\ -0.31690000000000 & 0.00290000000000 & 0.77120000000000 & \mathrm{~N} & (6 \mathrm{c}) \\ 0.08210000000000 & 0.50890000000000 & 0.31720000000000 & \mathrm{Si} & (6 \mathrm{c}) \\ -0.50890000000000 & -0.42680000000000 & 0.31720000000000 & \mathrm{Si} & (6 \mathrm{c}) \\ 0.42680000000000 & -0.08210000000000 & 0.31720000000000 & \mathrm{Si} & (6 \mathrm{c}) \\ 0.50890000000000 & 0.08210000000000 & 0.81720000000000 & \mathrm{Si} & (6 \mathrm{c}) \\ -0.42680000000000 & -0.50890000000000 & 0.81720000000000 & \mathrm{Si} & (6 \mathrm{c}) \\ -0.08210000000000 & 0.42680000000000 & 0.81720000000000 & \mathrm{Si} & (6 \mathrm{c}) \\ 0.17120000000000 & 0.25630000000000 & 0.02740000000000 & \mathrm{Si} & (6 \mathrm{c}) \\ -0.25630000000000 & -0.08510000000000 & 0.02740000000000 & \mathrm{Si} & (6 \mathrm{c}) \\ 0.08510000000000 & -0.17120000000000 & 0.02740000000000 & \mathrm{Si} & (6 \mathrm{c}) \\ 0.25630000000000 & 0.17120000000000 & 0.52740000000000 & \mathrm{Si} & (6 \mathrm{c}) \\ -0.08510000000000 & -0.25630000000000 & 0.52740000000000 & \mathrm{Si} & (6 \mathrm{c}) \\ -0.17120000000000 & 0.08510000000000 & 0.52740000000000 & \mathrm{Si} & (6 \mathrm{c})\end{array}$

$\mathrm{YbBaCo}_{4} \mathrm{O}_{7}$ : AB4C7D_hP26_159_b_ac_a2c_b - CIF

\section{\# CIF file}

data_findsym-output

audit_creation_method FINDSYM

_chemical_name_mineral 'YbBaCo4O7'

chemical_formula_sum 'Ba $\mathrm{Co} 4 \mathrm{O} 7 \mathrm{Y}$

loop

publ_author_name

'A. Huq'

J. F. Mitchell,

'H. Zheng'

L. C. Chapon'

P. G. Radaelli

K. S. Knight,

'P. W. Stephens

_journal_name_full_name

Journal of Solid State Chemistry

_journal_volume 179

journal year 2006

_journal_page_first 1136

_ournal_page_last 1145

publ_Section_title

Structural and magnetic properties of the Kagom $\{1)^{\prime}$ e $\}$ antiferromagnet $\hookrightarrow \mathrm{YbBaCo}_{-}\{4\} \$ O \mathrm{O}_{-}\{7\} \$$

Found in Pearson's Crystal Data - Crystal Structure Database for $\hookrightarrow$ Inorganic Compounds, 2013

aflow_title 'YbBaCo\$_ $\{4\} \$ O \$_{-}\{7\} \$$ Structure

aflow_proto, AB4C7D_hP26_159_b_ac_a2c_b

aflow_params ,a, c/a, $z_{-}\{1\}, z_{-}\{2\}, z_{-}\{3\}, z_{-}\{4\}, x_{-}\{5\}, y_{-}\{5\}, z_{-}\{5\}, x_{-}\{6\}, y_{-}\{$ $\hookrightarrow 6\}, z_{-}\{6\}, x_{-}\{7\}, y_{-}\{7\}, z_{-}\{7\}$,

aflow_params values $, 6.2653109789,1.6324735851,0.0,0.3057,0.0613,0.4379$ $\hookrightarrow, 0.3425,0.1575,0.2472,0.0015,0.5149,0.3102,0.3347,0.1157,0.0618$

aflow_Strukturbericht 'None'

-aflow_Pearson 'hP26,

cell_length_a $\quad 6.2653109789$

cell_length_b 6.2653109789

cell_length_c $\quad 10.2279546755$

cell_angle_alpha 90.0000000000

cell_angle_beta 90.0000000000

cell_angle_gamma 120.0000000000

symmetry_space_group_name_H-M "P $3 \quad 1 \quad$ c

symmetry_Int_Tables_number 159

loop

_space_group_symop_id

_space_group_symop_operation_xyz

$1 \mathrm{x}, \mathrm{y}, \mathrm{z}$

$2-y, x-y, z$

$3-\mathrm{x}+\mathrm{y},-\mathrm{x}, \mathrm{z}$
$4-\mathrm{x},-\mathrm{x}+\mathrm{y}, \mathrm{z}+1 / 2$

$5 \mathrm{x}-\mathrm{y},-\mathrm{y}, \mathrm{z}+1 / 2$

$6 \mathrm{y}, \mathrm{x}, \mathrm{z}+1 / 2$

loop

atom_site_label

atom_site_type_symbol

atom_site_symmetry_multiplicity

atom_site_Wyckoff_label

atom_site_fract_x

atom_site_fract_y

atom_site_fract_z

$\begin{array}{lllllll}\text { Co1 Co } & 2 & \text { a } & 0.00000 & 0.00000 & 0.00000 & 1.00000\end{array}$

$\begin{array}{lllllllll}\mathrm{O} 1 & \mathrm{O} & 2 & \text { a } & 0.00000 & 0.00000 & 0.30570 & 1.00000\end{array}$

$\begin{array}{llllllll}\mathrm{O} 1 & \mathrm{O} & 2 & \mathrm{a} & 0.00000 & 0.00000 & 0.30573 & 1.00000\end{array}$
$\begin{array}{llllllllll}\mathrm{Y} 1 & \mathrm{Y} & 2 & \mathrm{~b} & 0.33333 & 0.66667 & 0.43790 & 1.00000\end{array}$

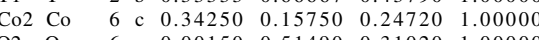

$\begin{array}{lllllllll}\mathrm{O} 2 & \mathrm{O} & 6 & \mathrm{c} & 0.00150 & 0.51490 & 0.31020 & 1.00000 \\ \mathrm{O} 3 & \mathrm{O} & 6 & \mathrm{c} & 0.33470 & 0.11570 & 0.06180 & 1.00000\end{array}$

$\begin{array}{llllllll}\mathrm{O} 3 & \mathrm{O} & 6 & \mathrm{c} & 0.33470 & 0.11570 & 0.06180 & 1.00000\end{array}$

$\mathrm{YbBaCo}_{4} \mathrm{O}_{7}$ : AB4C7D_hP26_159_b_ac_a2c_b - POSCAR

AB4C7D_hP26_159_b_ac_a2c_b \& a , c /a , z1 , z2 , z3 $, \mathrm{z} 4, \mathrm{x} 5, \mathrm{y} 5, \mathrm{z} 5, \mathrm{x} 6, \mathrm{y} 6, \mathrm{z} 6, \mathrm{x} 7, \mathrm{y} 7$,

$\hookrightarrow \mathrm{z} 7-$ params $=6.2653109789,1.6324735851,0.0,0.3057,0.0613,0.4379$

$\hookrightarrow 0.3425,0.1575,0.2472,0.0015,0.5149,0.3102,0.3347,0.1157,0.0618$

$\hookrightarrow \& \mathrm{P} 31 \mathrm{c} \mathrm{C}_{-}\{3 \mathrm{v}\}^{\wedge}\{4\} \# 159\left(\mathrm{a}^{\wedge} 2 \mathrm{~b}^{\wedge} 2 \mathrm{c}^{\wedge} 3\right) \& \mathrm{hP} 26 \&$ None \& YbBaCo4O7 \&

$\hookrightarrow$ \& A. Huq et al., J. Solid State Chem. 179, 1136-1145 (2006)

1.0000000000000

$\begin{array}{lll}3.13265548945000 & -5.42591847033695 & 0.00000000000000\end{array}$

$\begin{array}{lll}3.13265548945000 & 5.42591847033695 & 0.00000000000000\end{array}$

$\begin{array}{lll}0.00000000000000 & 0.00000000000000 & 10.22795467550000\end{array}$ $\begin{array}{rrrr}\mathrm{Ba} & \mathrm{Co} & \mathrm{O} & \mathrm{Y} \\ 2 & 8 & 14 & 2\end{array}$

Direct

0.33333333333333

0.66666666666667

0.00000000000000

0.34250000000000

$-0.1575000000000$

$-0.1575000000000$

0.15750000000000

0.18500000000000

0.18500000000000
-0.34250000000000

-0.34250000000000
0.00000000000000

0.00000000000000

0.00150000000000

$-0.51490000000000$

0.51340000000000

0.51490000000000

$-0.51340000000000$

$-0.00150000000000$

0.33470000000000

$-0.11570000000000$

$-0.21900000000000$

0.11570000000000

$-0.33470000000000$

0.33333333333333

0.666666666666667 0.33333333333333 0.00000000000000 0.00000000000000 0.15750000000000 0.18500000000000 0.34250000000000 0.34250000000000 0.15750000000000 0.18500000000000 0.00000000000000 0.00000000000000 0.51490000000000 $-0.51340000000000$ $-0.00150000000000$ 0.00150000000000 $-0.51490000000000$ 0.51340000000000 0.11570000000000 0.21900000000000 $-0.33470000000000$ 0.33470000000000 $-0.11570000000000$ $-0.21900000000000$ $-0.21900000000000$ 0.33333333333333

0.06130000000000 0.56130000000000 0.00000000000000 0.50000000000000 . 24720000000000 . 0.24720000000000
0.24720000000000 0.24720000000000 0.74720000000000 0.74720000000000 0.74720000000000 0.30570000000000 0.80570000000000 0.31020000000000 0.31020000000000 0.31020000000000 0.81020000000000 0.81020000000000 0.81020000000000 0.06180000000000 0.06180000000000 0.06180000000000 0.56180000000000 0.5618000000000 0.56180000000000 0.56180000000000 0.43790000000000 0.93790000000000

$\mathrm{H}_{3} \mathrm{~S}$ (130 GPa): A3B_hR4_160_b_a - CIF

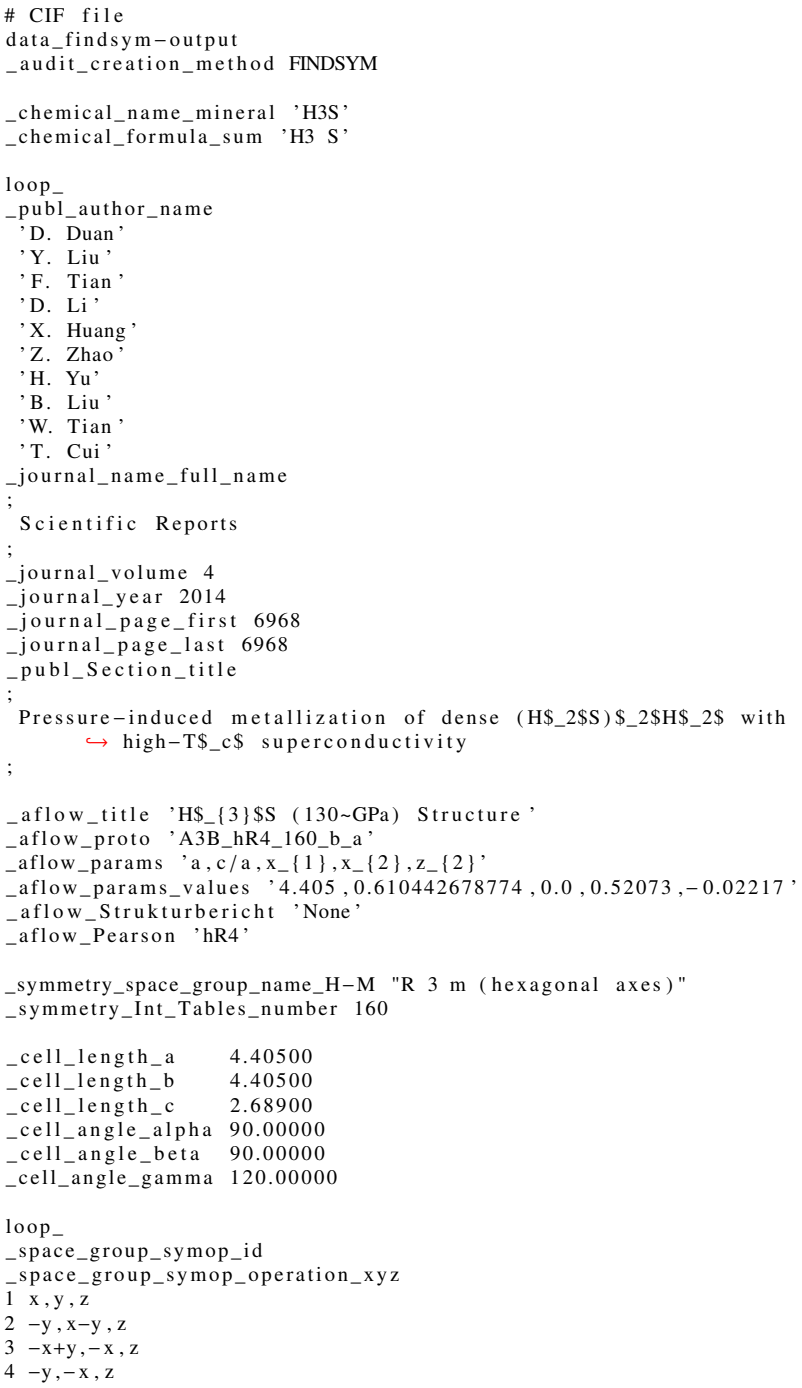




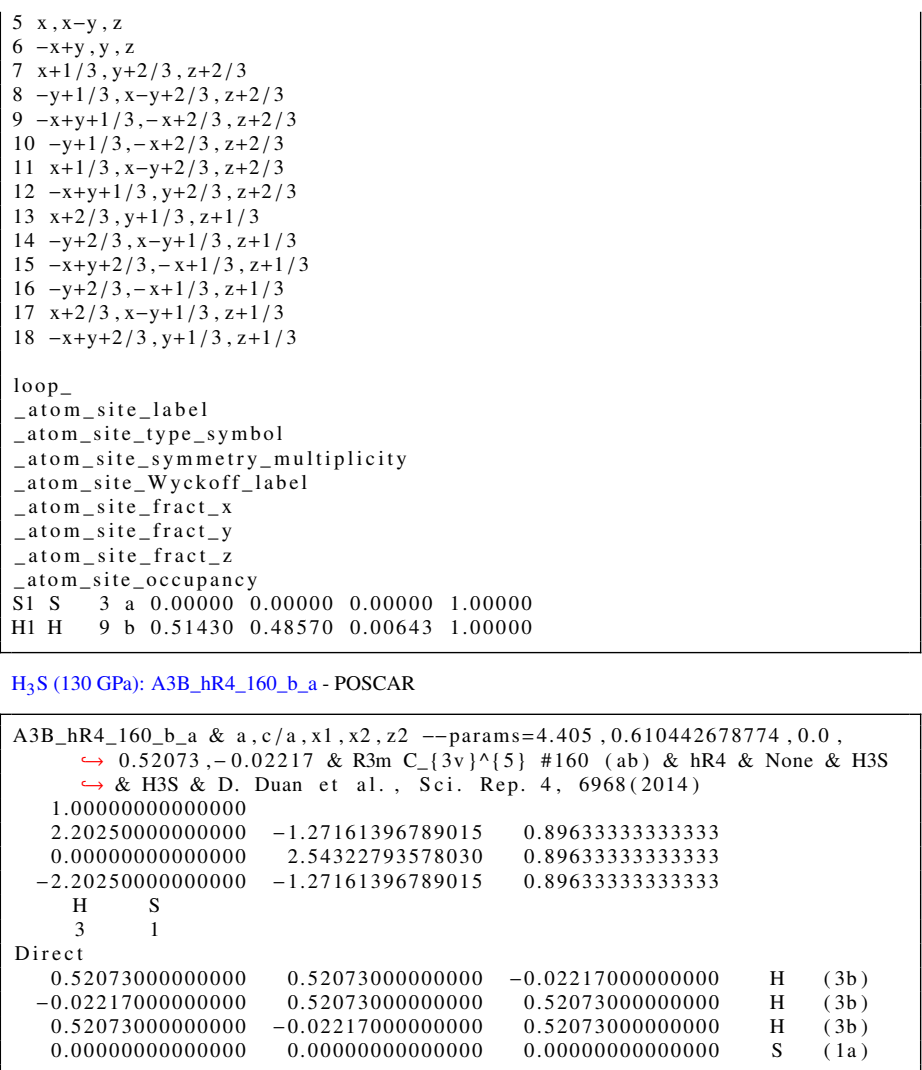

$\mathrm{Al}_{8} \mathrm{Cr}_{5}\left(D 8_{10}\right):$ A8B5_hR26_160_a3bc_a3b - CIF

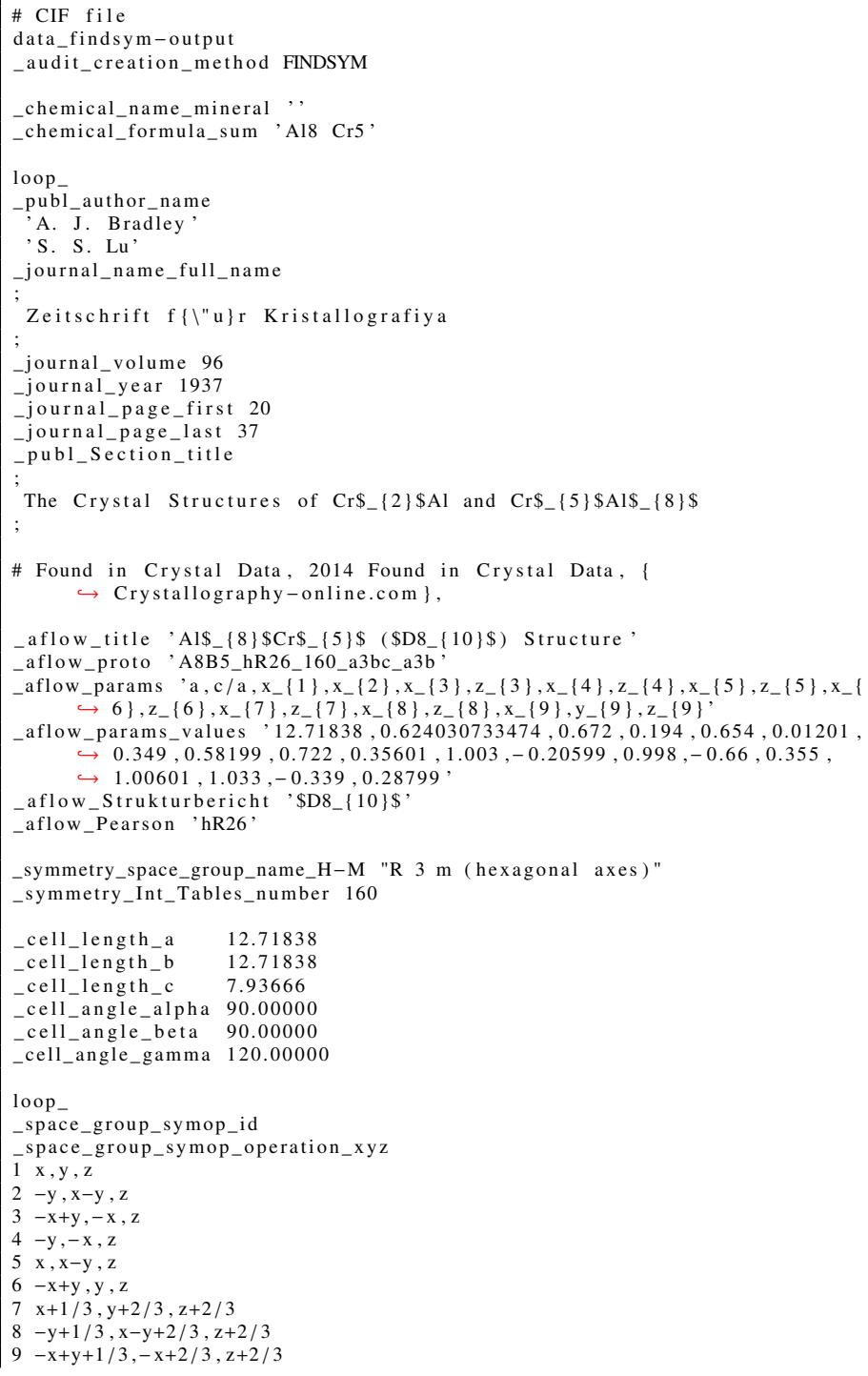

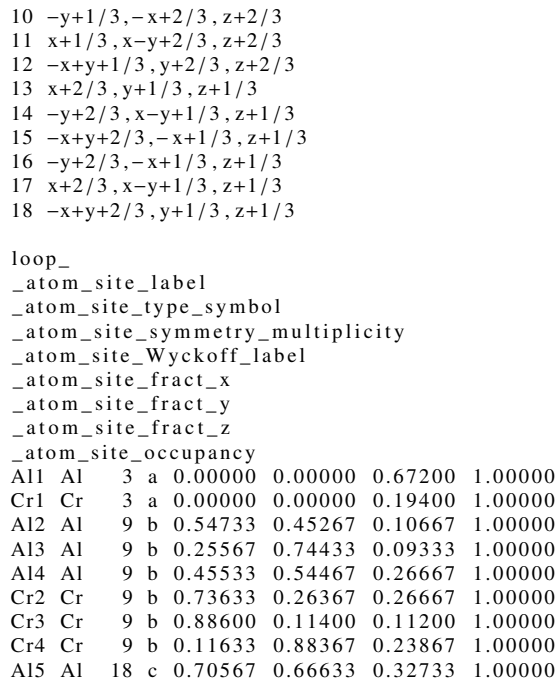

$\mathrm{Al}_{8} \mathrm{Cr}_{5}\left(D 8_{10}\right):$ A8B5_hR26_160_a3bc_a3b - POSCAR

\begin{tabular}{|c|c|c|c|c|}
\hline \multicolumn{5}{|c|}{ 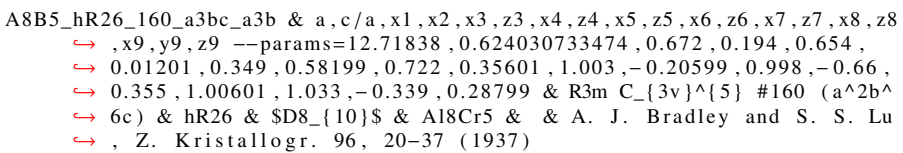 } \\
\hline \multicolumn{5}{|c|}{1.0000000000000} \\
\hline 6.35919000000000 & -3.67148005832798 & 2.64555333333333 & & \\
\hline \multirow{2}{*}{$\begin{array}{r}0.00000000000000 \\
-6.35919000000000\end{array}$} & 7.34296011665595 & 2.64555333333333 & & \\
\hline & -3.67148005832798 & 2.64555333333333 & & \\
\hline $\mathrm{Al}$ & & & & \\
\hline 16 & & & & \\
\hline \multicolumn{5}{|l|}{ Direct } \\
\hline 0.67200000000000 & 0.67200000000000 & 0.67200000000000 & $\mathrm{Al}$ & (1a) \\
\hline 0.65400000000000 & 0.65400000000000 & 0.01201000000000 & $\mathrm{Al}$ & (3b) \\
\hline 0.01201000000000 & 0.65400000000000 & 0.65400000000000 & $\mathrm{Al}$ & $(3 b)$ \\
\hline 0.65400000000000 & 0.01201000000000 & 0.65400000000000 & $\mathrm{Al}$ & $(3 b)$ \\
\hline 0.34900000000000 & 0.34900000000000 & 0.58199000000000 & $\mathrm{Al}$ & (3b) \\
\hline 0.58199000000000 & 0.34900000000000 & 0.34900000000000 & $\mathrm{Al}$ & $(3 b)$ \\
\hline 0.34900000000000 & 0.58199000000000 & 0.34900000000000 & $\mathrm{Al}$ & (3b) \\
\hline 0.72200000000000 & 0.72200000000000 & 0.35601000000000 & $\mathrm{Al}$ & $(3 b)$ \\
\hline 0.35601000000000 & 0.72200000000000 & 0.72200000000000 & $\mathrm{Al}$ & $(3 b)$ \\
\hline 0.72200000000000 & 0.35601000000000 & 0.72200000000000 & $\mathrm{Al}$ & (3b) \\
\hline 1.03300000000000 & -0.33900000000000 & 0.28799000000000 & $\mathrm{Al}$ & (6c) \\
\hline 0.28799000000000 & 1.03300000000000 & -0.33900000000000 & $\mathrm{Al}$ & $(6 c)$ \\
\hline-0.33900000000000 & 0.28799000000000 & 1.03300000000000 & $\mathrm{Al}$ & $(6 c)$ \\
\hline 0.28799000000000 & -0.33900000000000 & 1.03300000000000 & $\mathrm{Al}$ & $(6 c)$ \\
\hline-0.33900000000000 & 1.03300000000000 & 0.28799000000000 & $\mathrm{Al}$ & $(6 c)$ \\
\hline 1.03300000000000 & 0.28799000000000 & -0.33900000000000 & $\mathrm{Al}$ & $(6 c)$ \\
\hline 0.19400000000000 & 0.19400000000000 & 0.19400000000000 & $\mathrm{Cr}$ & (1a) \\
\hline 1.00300000000000 & 1.00300000000000 & -0.20599000000000 & $\mathrm{Cr}$ & (3b) \\
\hline-0.20599000000000 & 1.00300000000000 & 1.00300000000000 & $\mathrm{Cr}$ & $(3 b)$ \\
\hline 1.00300000000000 & -0.20599000000000 & 1.00300000000000 & $\mathrm{Cr}$ & $(3 b)$ \\
\hline 0.99800000000000 & 0.99800000000000 & -0.66000000000000 & $\mathrm{Cr}$ & \\
\hline-0.66000000000000 & 0.99800000000000 & 0.99800000000000 & $\mathrm{Cr}$ & (3b) \\
\hline 0.99800000000000 & -0.66000000000000 & 0.99800000000000 & $\mathrm{Cr}$ & (3b) \\
\hline 0.35500000000000 & 0.35500000000000 & 1.00601000000000 & $\mathrm{Cr}$ & (3b) \\
\hline 1.00601000000000 & 0.35500000000000 & 0.35500000000000 & $\mathrm{Cr}$ & (3b) \\
\hline 0.35500000000000 & 1.00601000000000 & 0.35500000000000 & $\mathrm{Cr}$ & (3b) \\
\hline
\end{tabular}

Carbonyl Sulphide $\left(\operatorname{COS}, F_{2}\right)$ : ABC_hR3_160_a_a_a - CIF

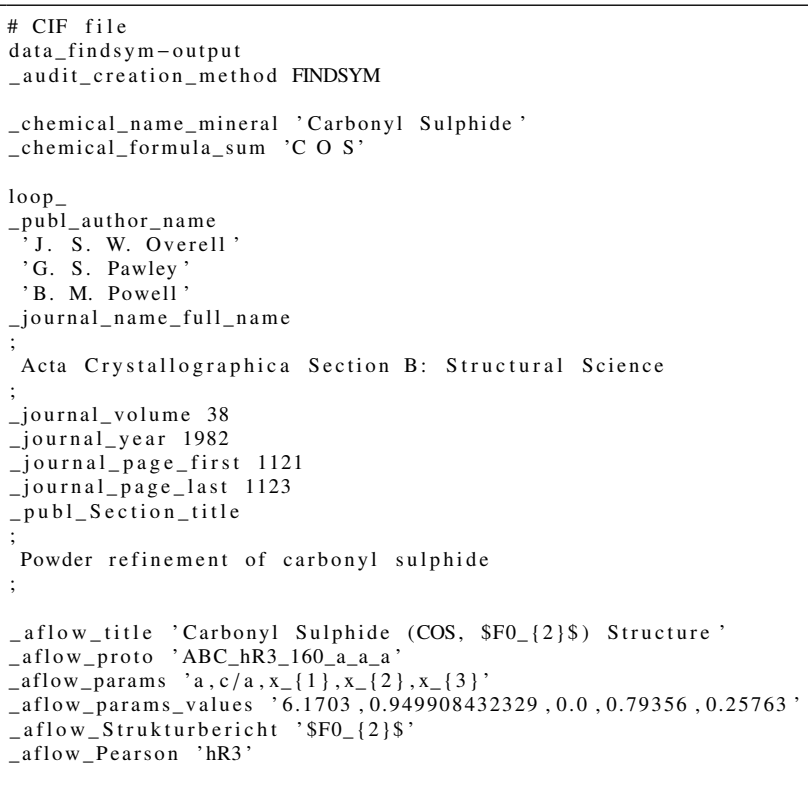




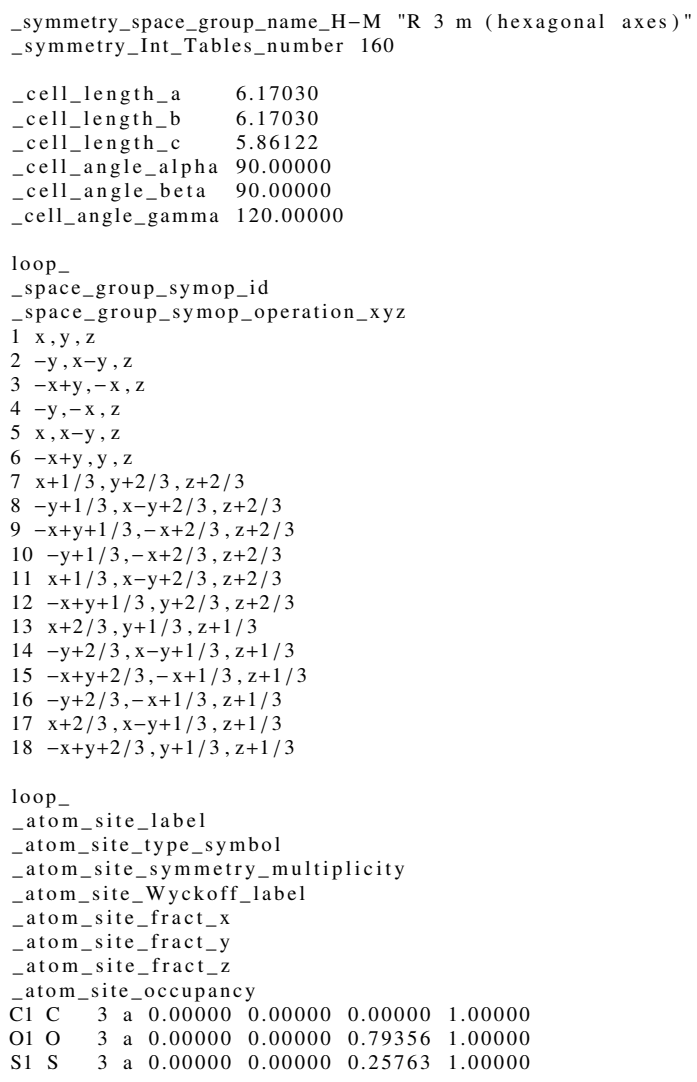

Carbonyl Sulphide $\left(\mathrm{COS}, \mathrm{FO}_{2}\right)$ : ABC_hR3_160_a_a_a - POSCAR

\begin{tabular}{|c|c|c|c|c|}
\hline \multicolumn{5}{|c|}{ 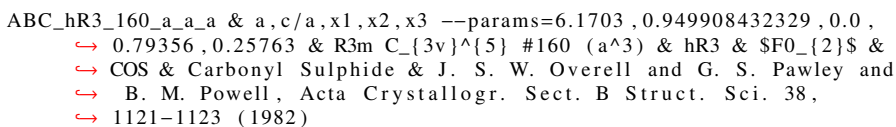 } \\
\hline \multicolumn{5}{|l|}{1.00000000000000} \\
\hline 3.08515000000000 & -1.78121218299037 & 1.95374000000000 & & \\
\hline \multirow{2}{*}{$\begin{array}{r}0.00000000000000 \\
-3.08515000000000\end{array}$} & 3.56242436598075 & 1.95374000000000 & & \\
\hline & -1.78121218299037 & 1.95374000000000 & & \\
\hline $\mathrm{O}$ & & & & \\
\hline 1 & & & & \\
\hline \multicolumn{5}{|l|}{ Direct } \\
\hline 0.00000000000000 & 0.00000000000000 & 0.00000000000000 & $\mathrm{C}$ & (1a) \\
\hline 0.79356000000000 & 0.79356000000000 & 0.79356000000000 & $\mathrm{O}$ & \\
\hline 0.25763000000000 & 0.25763000000000 & 0.25763000000000 & $\mathrm{~s}$ & (1a) \\
\hline
\end{tabular}

Moissanite-15R (SiC, B7): AB_hR10_160_5a_5a - CIF

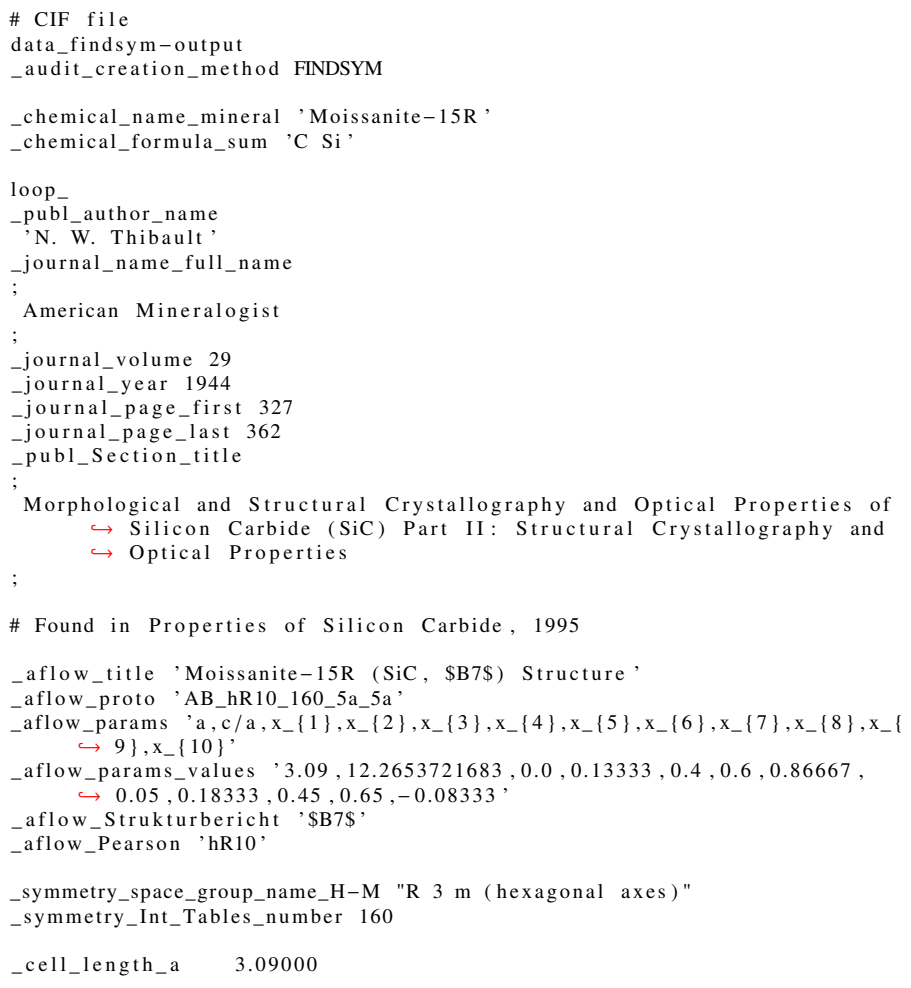

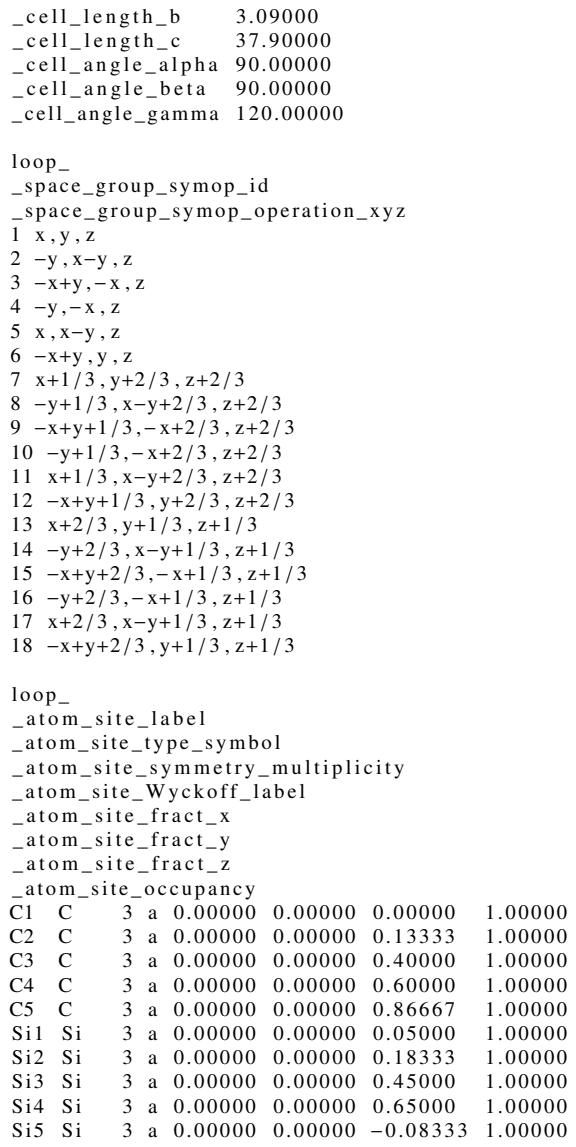

Moissanite-15R (SiC, B7): AB_hR10_160_5a_5a - POSCAR

\begin{tabular}{|c|c|c|c|c|}
\hline \multirow{2}{*}{\multicolumn{5}{|c|}{ 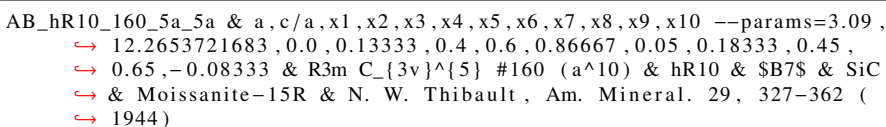 }} \\
\hline & & & & \\
\hline \multicolumn{5}{|c|}{1.00000000000000} \\
\hline 1.54500000000000 & -0.89200616589797 & 12.63333333333330 & & \\
\hline \multirow{2}{*}{$\begin{array}{r}0.00000000000000 \\
-1.54500000000000\end{array}$} & 1.78401233179594 & 12.63333333333330 & & \\
\hline & -0.89200616589797 & 12.63333333333330 & & \\
\hline $\mathrm{Si}$ & & & & \\
\hline 5 & & & & \\
\hline \multicolumn{5}{|l|}{ Direct } \\
\hline 0.00000000000000 & 0.00000000000000 & 0.00000000000000 & $\mathrm{C}$ & (1a) \\
\hline 0.13333000000000 & 0.13333000000000 & 0.13333000000000 & $\mathrm{C}$ & (1a) \\
\hline 0.40000000000000 & 0.40000000000000 & 0.40000000000000 & $\mathrm{C}$ & (1a) \\
\hline 0.60000000000000 & 0.60000000000000 & 0.60000000000000 & $\mathrm{C}$ & (1a) \\
\hline 0.86667000000000 & 0.86667000000000 & 0.86667000000000 & $\mathrm{C}$ & (1a) \\
\hline 0.05000000000000 & 0.05000000000000 & 0.05000000000000 & $\mathrm{Si}$ & (1a) \\
\hline 0.18333000000000 & 0.18333000000000 & 0.18333000000000 & $\mathrm{Si}$ & (1a) \\
\hline 0.45000000000000 & 0.45000000000000 & 0.45000000000000 & $\mathrm{Si}$ & (1a) \\
\hline 0.65000000000000 & 0.65000000000000 & 0.65000000000000 & $\mathrm{Si}$ & (1a) \\
\hline-0.08333000000000 & -0.08333000000000 & -0.08333000000000 & $\mathrm{Si}$ & (1a) \\
\hline
\end{tabular}

$\mathrm{La}_{2} \mathrm{O}_{3}\left(D 5_{2}\right)$ : A2B3_hP5_164_d_ad - CIF

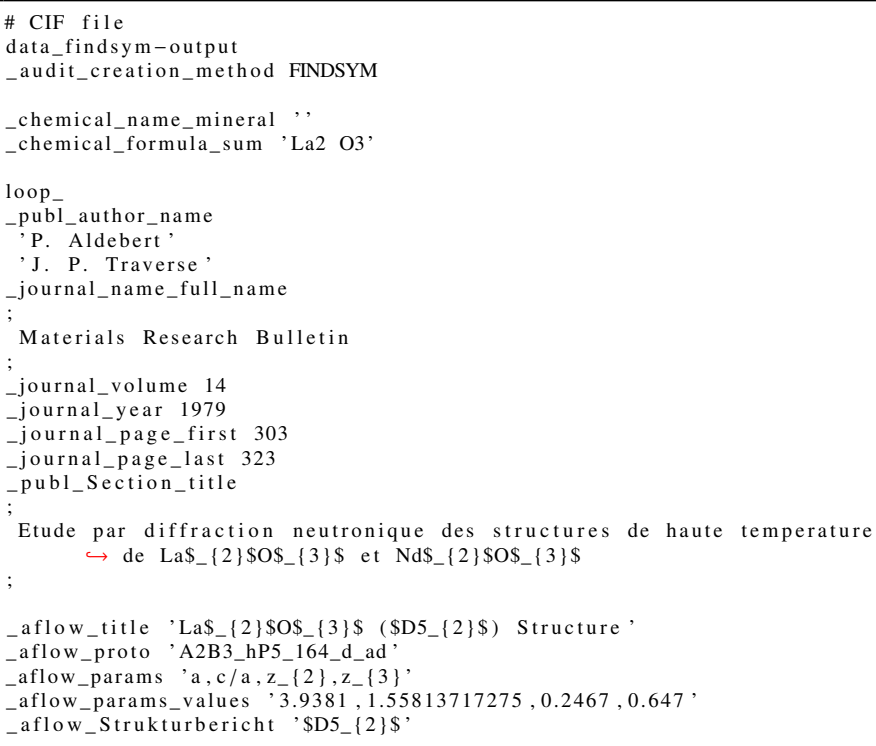




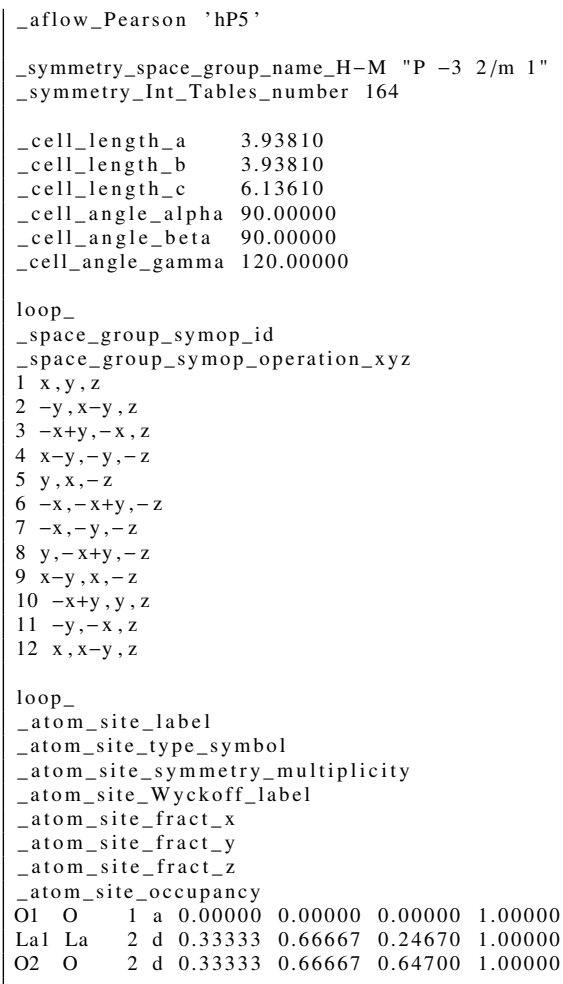

$\mathrm{La}_{2} \mathrm{O}_{3}\left(D 5_{2}\right)$ : A2B3_hP5_164_d_ad - POSCAR

A2B3_hP5_164_d_ad \& a , c /a , z2, z3 --params $=3.9381,1.55813717275,0.2467$ $\hookrightarrow 0.647 \&$ P-3m1 D_ $\{3 \mathrm{~d}\} \wedge\{3\} \# 164\left(\mathrm{ad}^{\wedge} 2\right) \& \mathrm{hP5} \&$ \$D5_ $\{2\} \$ \&$ La2O3 \& $\hookrightarrow \&$ P. Aldebert and J. P. Traverse, Mater. Res. Bull. 14 $\hookrightarrow 303-323(1979)$

1.00000000000000

$\begin{array}{lll}.96905000000000 & -3.41049464264350 & 0.00000000000000\end{array}$

$\begin{array}{lll}1.96905000000000 & 3.41049464264350 & 0.00000000000000\end{array}$

$\begin{array}{lll}0.00000000000000 & 0.00000000000000 & 6.13610000000000\end{array}$

La $\quad$ O

Direct

$\begin{array}{rrrrr}0.33333333333333 & 0.66666666666667 & 0.24670000000000 & \text { La } & (2 \mathrm{~d}) \\ 0.66666666666667 & 0.33333333333333 & -0.24670000000000 & \mathrm{La} & (2 \mathrm{~d}) \\ 0.00000000000000 & 0.00000000000000 & 0.00000000000000 & \mathrm{O} & (1 \mathrm{a}) \\ 0.33333333333333 & 0.66666666666667 & 0.64700000000000 & \mathrm{O} & (2 \mathrm{~d}) \\ 0.66666666666667 & 0.33333333333333 & -0.64700000000000 & \mathrm{O} & (2 \mathrm{~d})\end{array}$

$\begin{array}{llllll}0.66666666666667 & 0.33333333333333 & -0.64700000000000 & O & (2 \mathrm{~d})\end{array}$

$\delta_{H}^{I I}-\mathrm{NW}_{2}: \mathrm{AB} 2$ hP9_164_bd_c2d - CIF

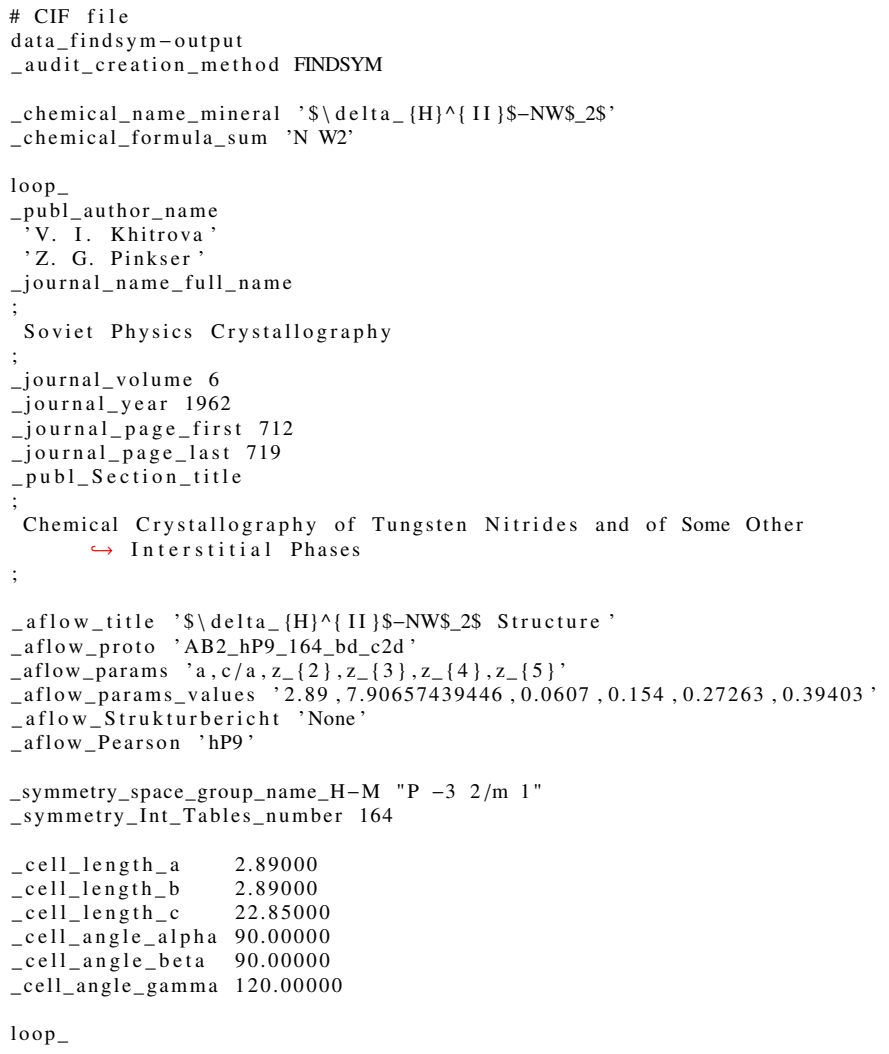

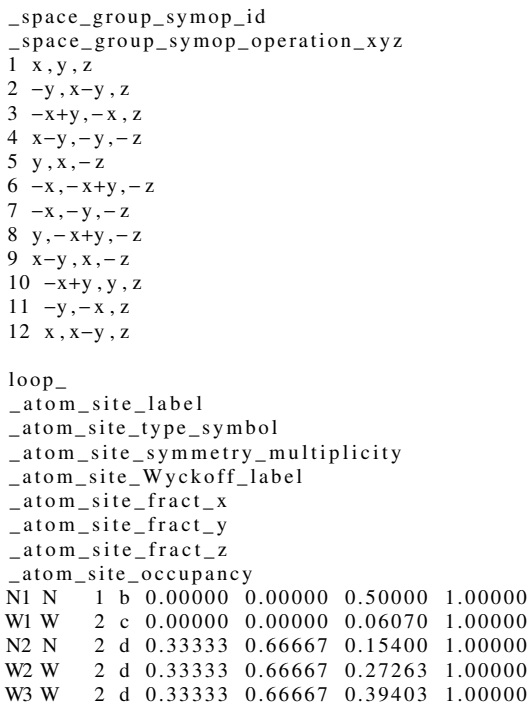

$\delta_{H}^{I I}-\mathrm{NW}_{2}$ : AB2_hP9_164_bd_c2d - POSCAR

\begin{tabular}{|c|c|c|c|c|}
\hline \\
\hline \\
\hline \multirow{2}{*}{\multicolumn{5}{|c|}{$\begin{array}{l}\hookrightarrow 0.0607,0.154,0.27263,0.39403 \& \text { P }-3 m 1 D_{-}\{3 d\} \wedge\{3\} \# 164\left(b^{\wedge} d^{\wedge} 3\right) \\
\hookrightarrow \text { hP9 \& None \& NW2 \& } \$ \backslash \text { delta- }\{\text { H }\}^{\wedge}\{\text { II }\} \$-N W \$ 2 \$ \& \text { V. Khitrova } \\
\hookrightarrow \text { and Z. G. Pinkser, Sov. Phys. Crystallogr. 6, 712-719 (1962) }\end{array}$}} \\
\hline & & & & \\
\hline \multicolumn{5}{|l|}{1.00000000000000} \\
\hline 1.44500000000000 & -2.50281341693703 & 0.00000000000000 & & \\
\hline \multirow{2}{*}{$\begin{array}{l}1.44500000000000 \\
0.00000000000000\end{array}$} & 2.50281341693703 & 0.00000000000000 & & \\
\hline & 0.00000000000000 & 22.85000000000000 & & \\
\hline W & & & & \\
\hline 3 & & & & \\
\hline \multicolumn{5}{|l|}{ Direct } \\
\hline 0.00000000000000 & 0.00000000000000 & 0.50000000000000 & $\mathrm{~N}$ & (1b) \\
\hline 0.33333333333333 & 0.666666666666667 & 0.15400000000000 & $\mathrm{~N}$ & (2d) \\
\hline 0.666666666666667 & 0.33333333333333 & -0.15400000000000 & $\mathrm{~N}$ & (2d) \\
\hline 0.00000000000000 & 0.00000000000000 & 0.06070000000000 & W & $(2 \mathrm{c})$ \\
\hline 0.00000000000000 & 0.00000000000000 & -0.06070000000000 & $\mathrm{~W}$ & $(2 \mathrm{c})$ \\
\hline 0.33333333333333 & 0.666666666666667 & 0.27263000000000 & $\mathrm{~W}$ & (2d) \\
\hline 0.666666666666667 & 0.33333333333333 & -0.27263000000000 & W & (2d) \\
\hline 0.33333333333333 & 0.666666666666667 & 0.39403000000000 & W & (2d) \\
\hline 0.666666666666667 & 0.33333333333333 & -0.39403000000000 & W & (2d) \\
\hline
\end{tabular}

$\mathrm{CuNiSb}_{2}$ : ABC2_hP4_164_a_b_d - CIF

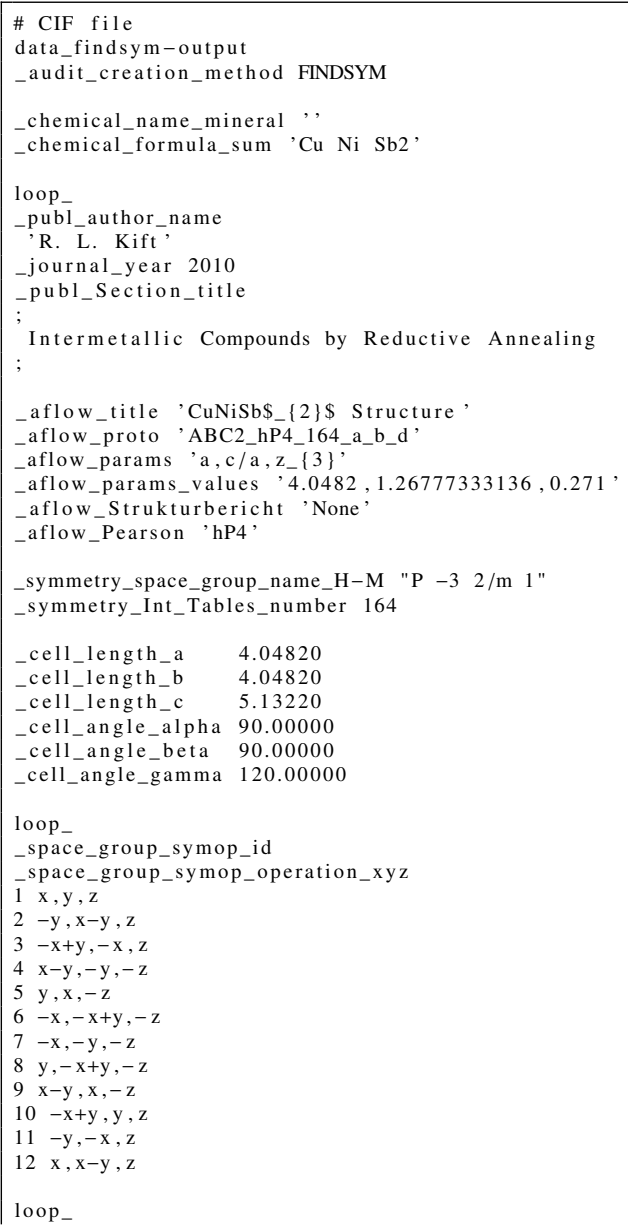


atom site label

_atom_site_type_symbol

_atom_site_symmetry_multiplicity

_atom_site_Wyckoff_label

atom_site_fract_x

atom_site_fract_y

atom_site_fract_z

atom_site_occupancy

$\begin{array}{lllllll}\mathrm{Cu} 1 \mathrm{Cu} & 1 & \mathrm{a} & 0.00000 & 0.00000 & 0.00000 & 1.00000\end{array}$

$\begin{array}{lllllllll}\mathrm{Ni} & \mathrm{Ni} & 1 & \mathrm{~b} & 0.00000 & 0.00000 & 0.50000 & 1.00000\end{array}$

$\begin{array}{lllllllll}\mathrm{Sb} 1 & \mathrm{Sb} & 2 & \mathrm{~d} & 0.33333 & 0.66667 & 0.27100 & 1.00000\end{array}$

$\mathrm{CuNiSb}_{2}$ : ABC2_hP4_164_a_b_d - POSCAR

ABC2_hP4_164_a_b_d \& a,c/a, z3 --params $=4.0482,1.26777333136,0.271 \&$ $\hookrightarrow \mathrm{P}-3 \mathrm{~m} 1 \mathrm{D}_{-}\{3 \mathrm{~d}\}^{\wedge}\{3\} \# 164$ (abd) \& hP4 \& None \& CuNiSb2 \& \& R. L. $\hookrightarrow$ Kift , $(2010)$

1.00000000000000

$2.02410000000000 \quad-3.50584403960016 \quad 0.00000000000000$

$\begin{array}{lll}2.02410000000000 & 3.50584403960016 & 0.00000000000000\end{array}$

$0.00000000000000 \quad 0.00000000000000 \quad 5.13220000000000$

$\mathrm{Cu} \quad \mathrm{Ni} \quad \mathrm{Sb}$

Direct

$\begin{array}{lll}0.00000000000000 & 0.00000000000000 & 0.00000000000000\end{array}$

$\begin{array}{llll}0.00000000000000 & 0.00000000000000 & 0.50000000000000\end{array}$

$\begin{array}{llll}0.33333333333333 & 0.66666666666667 & 0.27100000000000\end{array}$

0.666666666666667

$-0.27100000000000$

$\begin{array}{ll}\mathrm{Cu} & (1 \mathrm{a}) \\ \mathrm{Ni} & (1 \mathrm{~b}) \\ \mathrm{Sb} & (2 \mathrm{~d}) \\ \mathrm{Sb} & (2 \mathrm{~d})\end{array}$

$\mathrm{Cu}_{3} \mathrm{P}\left(D 0_{21}\right):$ A3B_hP24_165_bdg_f - CIF

\# CIF file

data findsym-output

-audit_creation_method FINDSYM

chemical name mineral,

chemical formula_sum ' $\mathrm{Cu} 3 \mathrm{P}$ '

loop

_publ_author_name

B. Steenberg

_journal_name_full_name

Arkiv $f\{\backslash " o\} r$ Kemi, Mineralogi och Geologi

_journal_volume A12

_journal_year 1938

_journal_page_first 1

journal page last 15

publ Section

The Crystal Structure of $\mathrm{Cu}_{-}\{3\} \$ \mathrm{As}$ and $\mathrm{Cu} \$_{-}\{3\} \$ P$

\# Found in A Handbook of Lattice Spacings and Structures of Metals and

$$
\hookrightarrow \text { Alloys , } 1958
$$

aflow_title 'Cu\$_\{3\}\$P (\$D0_\{21\}\$) Structure

_aflow_proto A3B_hP24_165_bdg_f

-aflow_params a,c/a, z $\{2\}, x_{-}\{3\}, x_{-}\{4\}, y_{-}\{4\}, z_{-}\{4\}$

_aflow_params_values ' $7.07,1.00919377652,0.17,0.38,0.69,0.07,0.08$

aflow_Strukturbericht '\$D0_\{21\}\$”

aflow_Pearson 'hP24'

_symmetry_space_group_name_H-M "P $-3 \quad 2 / \mathrm{c} 1$ "

symmetry Int Tables_number 165

\section{cell_length_a $\quad 7.07000$}

cell_length_b 7.07000

cell_length_c $\quad 7.13500$

cell_angle_alpha 90.00000

$\begin{array}{ll}\text { cell_angle_beta } & 90.00000 \\ \text { cell_angle_gamma } & 120.00000\end{array}$

loop

_space_group_symop_id

_space_group_symop_operation_xyz

$1 \mathrm{x}, \mathrm{y}, \mathrm{z}$

$2-\mathrm{y}, \mathrm{x}-\mathrm{y}, \mathrm{z}$

$3-x+y,-x, z$

$4 x-y,-y,-z+1 / 2$

$\mathrm{y}, \mathrm{x},-\mathrm{z}+1 / 2$

$6-x,-x+y,-z+1 / 2$

$\begin{array}{ll}7 & -\mathrm{x},-\mathrm{y}, \mathrm{y},-\mathrm{z} \\ 8 & \mathrm{y},-\mathrm{x}+\mathrm{y},-\mathrm{z} \\ 9 & \mathrm{x}-\mathrm{y}, \mathrm{x}, \mathrm{z}\end{array}$

$\begin{array}{ll}8 & y,-x+y,-z \\ 9 & x-y, x,-z\end{array}$

$10-\mathrm{x}+\mathrm{y}, \mathrm{y}, \mathrm{z}+1 / 2$

$11-\mathrm{y},-\mathrm{x}, \mathrm{z}+1 / 2$

$12 x, x-y, z+1 / 2$

loop

_atom_site_labe

_atom_site_type_symbo

_atom_site_symmetry_multiplicity

atom_site_Wyckoff_label

-atom_site_fract_x

atom_site_fract_y

atom_site_fract_z

\begin{tabular}{lllllll}
\hline $\mathrm{Cu} 1 \mathrm{Cu}$ & 2 & $\mathrm{~b}$ & 0.00000 & 0.00000 & 0.00000 & 1.00000
\end{tabular}

$\begin{array}{llllllll}\mathrm{Cu} 2 & \mathrm{Cu} & 4 & \mathrm{~d} & 0.33333 & 0.66667 & 0.17000 & 1.00000 \\ \mathrm{P} 1 & \mathrm{P} & 6 & \mathrm{f} & 0.38000 & 0.00000 & 0.25000 & 1.00000\end{array}$

$\begin{array}{llrlllll}\mathrm{P} 1 & \mathrm{P} & 6 & \mathrm{f} & 0.38000 & 0.00000 & 0.25000 & 1.00000 \\ \mathrm{Cu} 3 \mathrm{Cu} & 12 & \mathrm{~g} & 0.69000 & 0.07000 & 0.08000 & 1.00000\end{array}$

$\mathrm{Cu}_{3} \mathrm{P}\left(D 0_{21}\right)$ : A3B_hP24_165_bdg_f - POSCAR

\begin{tabular}{|c|c|c|c|c|}
\hline $\begin{aligned} \text { A3B_hP2 } 24 B_{-} 165 \_ \text {bdg_f } \& \\
\\
\hookrightarrow 0.17,0.38,0 \\
\hookrightarrow \& \$ D 0_{-}\{21\} \$ \\
\hookrightarrow \text { A } 12,1-15\end{aligned}$ & $\begin{array}{l}\mathrm{a}, \mathrm{c} / \mathrm{a}, \mathrm{z2}, \mathrm{x} 3, \mathrm{x} 4, \mathrm{y} 4, \\
69,0.07,0.08 \text { \& }-3 \\
\& \mathrm{Cu} 3 \mathrm{P} \& \& \text { B. Ste } \\
(1938)\end{array}$ & $\begin{array}{l}z 4-- \text { params }=7.07,1 . \\
1 D_{-}\{3 d\}^{\wedge}\{4\} \quad \# 165 \\
\text { nberg, }\{\text { Ark. Kem. }\end{array}$ & dig & $\begin{array}{c}77652 \\
\& \text { hP24 } \\
1 . \mathrm{Geol} .\end{array}$ \\
\hline 1.00000000000000 & & & & \\
\hline 3.53500000000000 & -6.12279960475598 & 0.00000000000000 & & \\
\hline 3.53500000000000 & 6.12279960475598 & 0.00000000000000 & & \\
\hline 0.00000000000000 & 0.00000000000000 & 7.13500000000000 & & \\
\hline $\mathrm{Cu}$ & & & & \\
\hline 18 & & & & \\
\hline irect & & & & \\
\hline 0.00000000000000 & 0.00000000000000 & 0.00000000000000 & $\mathrm{Cu}$ & $(2 b)$ \\
\hline 0.00000000000000 & 0.00000000000000 & 0.50000000000000 & $\mathrm{Cu}$ & $(2 b)$ \\
\hline 0.333333333333333 & 0.66666666666667 & 0.17000000000000 & $\mathrm{Cu}$ & (4d) \\
\hline 0.666666666666667 & 0.33333333333333 & 0.33000000000000 & $\mathrm{Cu}$ & (4d) \\
\hline 0.666666666666667 & 0.33333333333333 & -0.17000000000000 & $\mathrm{Cu}$ & (4d) \\
\hline 0.33333333333333 & 0.666666666666667 & 0.67000000000000 & $\mathrm{Cu}$ & (4d) \\
\hline 0.69000000000000 & 0.07000000000000 & 0.08000000000000 & $\mathrm{Cu}$ & $(12 \mathrm{~g})$ \\
\hline-0.07000000000000 & 0.62000000000000 & 0.08000000000000 & $\mathrm{Cu}$ & $(12 \mathrm{~g})$ \\
\hline-0.62000000000000 & -0.69000000000000 & 0.08000000000000 & $\mathrm{Cu}$ & $(12 \mathrm{~g})$ \\
\hline 0.07000000000000 & 0.69000000000000 & 0.42000000000000 & $\mathrm{Cu}$ & $(12 \mathrm{~g})$ \\
\hline 0.62000000000000 & -0.07000000000000 & 0.42000000000000 & $\mathrm{Cu}$ & $(12 \mathrm{~g})$ \\
\hline-0.69000000000000 & -0.62000000000000 & 0.42000000000000 & $\mathrm{Cu}$ & $(12 \mathrm{~g})$ \\
\hline-0.69000000000000 & -0.07000000000000 & -0.08000000000000 & $\mathrm{Cu}$ & $(12 \mathrm{~g})$ \\
\hline 0.07000000000000 & -0.62000000000000 & -0.08000000000000 & $\mathrm{Cu}$ & $(12 \mathrm{~g})$ \\
\hline 0.62000000000000 & 0.69000000000000 & -0.08000000000000 & $\mathrm{Cu}$ & $(12 \mathrm{~g})$ \\
\hline-0.07000000000000 & -0.69000000000000 & 0.58000000000000 & $\mathrm{Cu}$ & $(12 \mathrm{~g})$ \\
\hline-0.62000000000000 & 0.07000000000000 & 0.58000000000000 & $\mathrm{Cu}$ & $(12 \mathrm{~g})$ \\
\hline 0.69000000000000 & 0.62000000000000 & 0.58000000000000 & $\mathrm{Cu}$ & $(12 \mathrm{~g})$ \\
\hline 0.38000000000000 & 0.00000000000000 & 0.25000000000000 & $P$ & (6f) \\
\hline 0.00000000000000 & 0.38000000000000 & 0.25000000000000 & $\mathrm{P}$ & (6f) \\
\hline-0.38000000000000 & -0.38000000000000 & 0.25000000000000 & $\mathrm{P}$ & (6f) \\
\hline-0.38000000000000 & 0.00000000000000 & 0.75000000000000 & $\mathrm{P}$ & (6f) \\
\hline 0.00000000000000 & -0.38000000000000 & 0.75000000000000 & $\mathrm{P}$ & (6f) \\
\hline 0.38000000000000 & 0.38000000000000 & 0.75000000000000 & $\mathrm{P}$ & (6f) \\
\hline
\end{tabular}

$\mathrm{Al}_{4} \mathrm{C}_{3}\left(D 7_{1}\right):$ A4B3_hR7_166_2c_ac - CIF

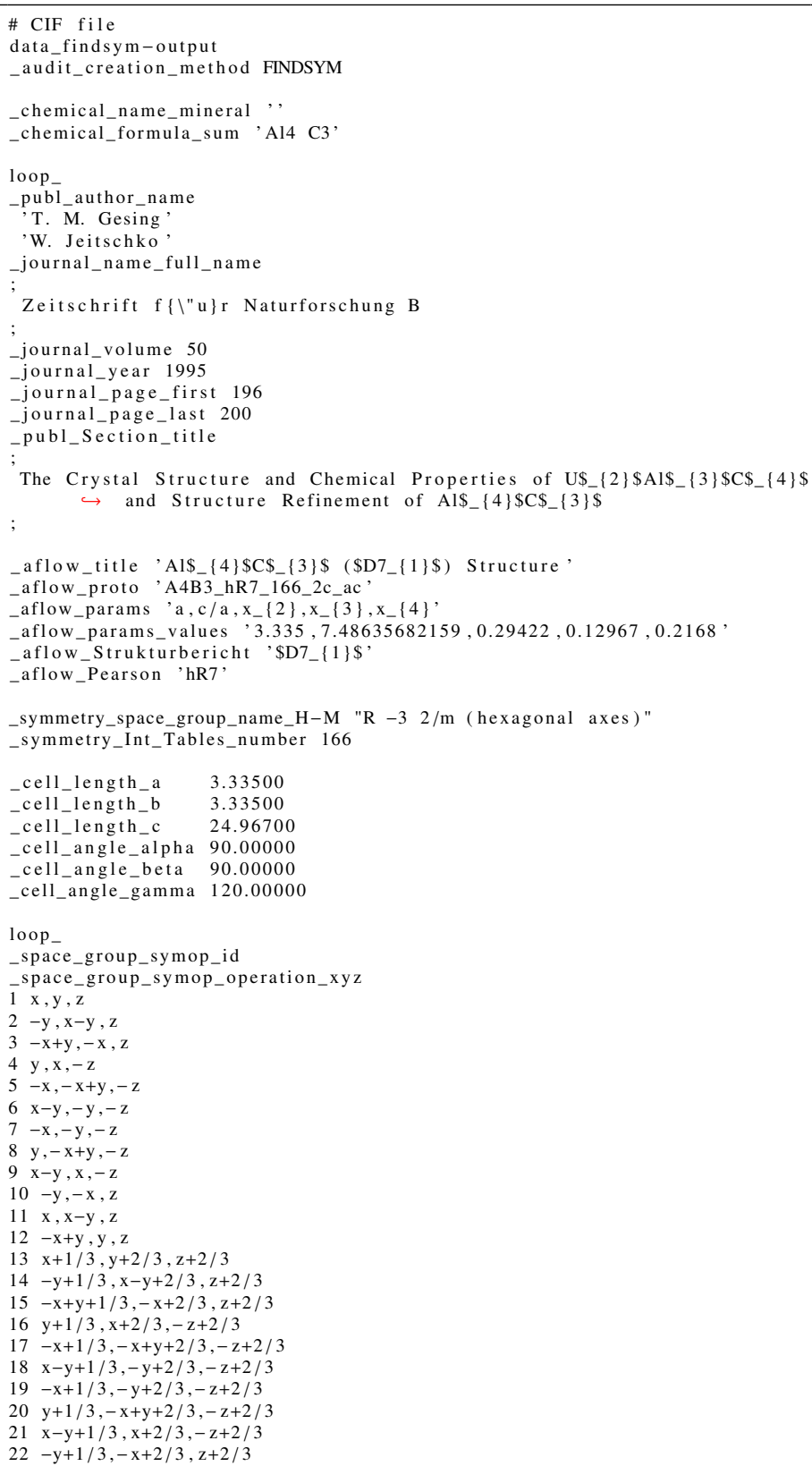


$23 \mathrm{x}+1 / 3, \mathrm{x}-\mathrm{y}+2 / 3, \mathrm{z}+2 / 3$

$24-x+y+1 / 3, y+2 / 3, z+2 / 3$

$25 \mathrm{x}+2 / 3, \mathrm{y}+1 / 3, \mathrm{z}+1 / 3$

$26-\mathrm{y}+2 / 3, \mathrm{x}-\mathrm{y}+1 / 3, \mathrm{z}+1 / 3$

$27-x+y+2 / 3,-x+1 / 3, z+1 / 3$

$28 \mathrm{y}+2 / 3, \mathrm{x}+1 / 3,-\mathrm{z}+1 / 3$

$29-\mathrm{x}+2 / 3,-\mathrm{x}+\mathrm{y}+1 / 3,-\mathrm{z}+1 / 3$

$30 x-y+2 / 3,-y+1 / 3,-z+1 / 3$

$31-x+2 / 3,-y+1 / 3,-z+1 / 3$

$32 \mathrm{y}+2 / 3,-\mathrm{x}+\mathrm{y}+1 / 3,-\mathrm{z}+1 / 3$

$33 \mathrm{x}-\mathrm{y}+2 / 3, \mathrm{x}+1 / 3,-\mathrm{z}+1 / 3$

$34-\mathrm{y}+2 / 3,-\mathrm{x}+1 / 3, \mathrm{z}+1 / 3$

$35 \mathrm{x}+2 / 3, \mathrm{x}-\mathrm{y}+1 / 3, \mathrm{z}+1 / 3$

$36-x+y+2 / 3, y+1 / 3, z+1 / 3$

loop

atom_site_label

-atom_site_type_symbol

_atom_site_symmetry_multiplicity

_atom_site_Wyckoff_label

atom_site_fract_x

atom_site_fract_y

atom_site_fract_z

$\begin{array}{lllllll}\text { C1 C } & \text { atom_site_occupancy } & & & & & \\ \text { a a } & 0.00000 & 0.00000 & 0.00000 & 1.00000\end{array}$

$\begin{array}{lllllllll}\text { Al1 } & \mathrm{Al} & 6 & \mathrm{c} & 0.00000 & 0.00000 & 0.29422 & 1.00000\end{array}$

$\begin{array}{lllllllll}\mathrm{Al} 2 & \mathrm{Al} & 6 & \mathrm{c} & 0.00000 & 0.00000 & 0.12967 & 1.00000\end{array}$

$\begin{array}{llllllllll}\mathrm{C} 2 & \mathrm{C} & 6 & \mathrm{c} & 0.00000 & 0.00000 & 0.21680 & 1.00000\end{array}$

$\mathrm{Al}_{4} \mathrm{C}_{3}\left(D 7_{1}\right)$ : A4B3_hR7_166_2c_ac - POSCAR

A4B3_hR7_166_2c_ac \& a , c/a , x2 , x3, x4 --params $=3.335,7.48635682159,0.29422$ $\hookrightarrow, 0.12967,0.2168 \& \mathrm{R}-3 \mathrm{~m} \mathrm{D} \mathrm{D}_{-}\{3 \mathrm{~d}\}^{\wedge}\{5\} \# 166\left(\mathrm{ac}^{\wedge} 3\right) \& \mathrm{hR} 7 \&$ \$D7_ $\{1\} \$$

$\hookrightarrow$ \& Al4C3 \& \& T. M. Gesing and W. Jeitschko, Z. Naturforsch. B $\hookrightarrow 50, \quad 196-200(1995)$

1.00000000000000

$\begin{array}{lll}1.66750000000000 & -0.96273157387370 & 8.322333333333333\end{array}$

$0.00000000000000 \quad 1.92546314774740-8.32233333333333$

(1.9625

$\begin{array}{rr}\mathrm{Al} & \mathrm{C} \\ 4 & 3\end{array}$

Direct

$\begin{array}{lll}0.29422000000000 & 0.29422000000000 & 0.29422000000000\end{array}$

$-0.29422000000000-0.29422000000000-0.29422000000000$

0.1296700000000

0.12967000000000

0.000700000000

0.21680000000000

$-0.29422000000000-0.29422000000000$

0.12967000000000

0.12967000000000
-0.12967000000000

-0.12967000000000
0.00000000000000

0.00000000000000

0.00000000000000

0.21680000000000

$-0.21680000000000$

$\mathrm{Al} \quad(2 \mathrm{c})$

$\mathrm{Al} \quad(2 \mathrm{c})$

$\mathrm{Al} \quad(2 \mathrm{c})$

(2c)

C (1a)

C $\quad(2 \mathrm{c})$

0.21680000000000

SmSI: ABC_hR6_166_c_c_c - CIF

\# CIF file

data_findsym-output

audit_creation_method FINDSYM

chemical_name_mineral 'SmSI'

chemical_formula_sum 'I S Sm'

loop

publ_author_name

'H. P. Beck'

journal_name_full_name

Zeitschrift fur Anorganische und Allgemeine Chemie

journal_volume 535

-journal_olume 535

_journal_page_first 222

_journal_page_last 239

-publ_Section_title

Zur Hochdruckpolymorphie der Seltenerdsulfidiodide LnSI

\# Found in Crystal structure of $\$ \backslash$ beta $\$-\$ M \$ N \$ X \$(\$ M \$=Z r, H f ; \quad X \$=C l$,

$$
\hookrightarrow \mathrm{Br}), 1998
$$

aflow_title 'SmSI Structure'

-aflow_proto 'ABC_hR6_166_c_c_c'

aflow_params 'a,c/a, $x_{-}\{1\}, x_{-}\{2\}, x_{-}\{3\}$ '

-aflow_params_values $3.8548,7.95397945419,0.1159,0.3017,0.3815$

- aflow Strukturbericht 'None'

aflow_Pearson 'hR6'

_symmetry_space_group_name_H-M "R $-3 \quad 2 / m: H "$

_symmetry_Int_Tables_number 166

_cell_length_a $\quad 3.85480$

_cell_length_b 3.85480

cell_length_c $\quad 30.66100$

cell_angle_alpha 90.00000

_cell_angle_beta 90.00000

cell angle gamma 120.00000

loop_

_space_group_symop_id

_space_group_symop_operation_xyz

$1 \mathrm{x}, \mathrm{y}, \mathrm{z}$

$\mathrm{x}, \mathrm{y}, \mathrm{z}$
$2-\mathrm{y}, \mathrm{x}-\mathrm{y}, \mathrm{z}$

$3-x+y,-x, z$

$4 \mathrm{y}, \mathrm{x},-\mathrm{z}$

$5-x,-x+y,-z$

$6 x-y,-y,-z$

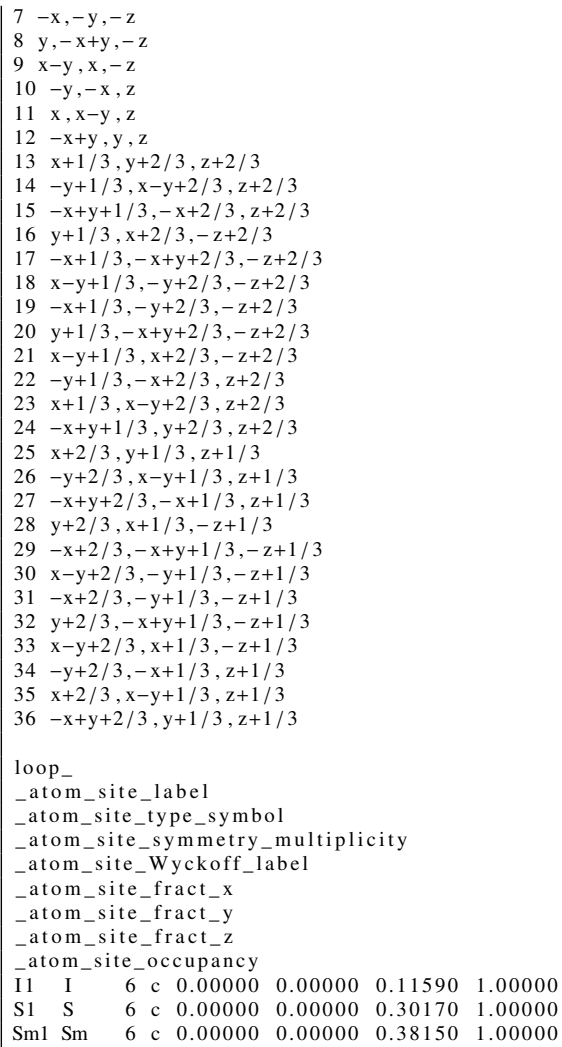

SmSI: ABC_hR6_166_c_c_c - POSCAR

\begin{tabular}{|c|c|c|c|c|}
\hline $\begin{aligned} \text { IBC_hR6_166_c_c_c \& } \\
$\[ 0.3017,0.38 \]$ \\
\quad \hookrightarrow \text { \& SmSI \& H. } \\
\quad \hookrightarrow 222-239 \quad(19\end{aligned}$ & $\begin{array}{l}\mathrm{a}, \mathrm{c} / \mathrm{a}, \mathrm{x} 1, \mathrm{x} 2, \mathrm{x} 3-- \\
5 \& \mathrm{R}-3 \mathrm{~m} \mathrm{D}_{-}\{3 \mathrm{~d}\}^{\wedge}\{ \\
\text { P. Beck and C. St } \\
6 \text { 6) }\end{array}$ & $\begin{array}{l}\mathrm{ams}=3.8548,7.9539 \\
\# 166\left(\mathrm{c}^{\wedge} 3\right) \& \mathrm{hR6} \\
\text { el }, \mathrm{Z} . \text { Anorg. Al }\end{array}$ & $\mathrm{Nor}$ & $\begin{array}{l}\text { \& SmSI } \\
\text { n. } 535 \text {, }\end{array}$ \\
\hline 1.00000000000000 & & & & \\
\hline 1.92740000000000 & -1.11278490883608 & 10.220333333333330 & & \\
\hline 0.00000000000000 & 2.22556981767217 & 10.220333333333330 & & \\
\hline-1.92740000000000 & -1.11278490883608 & 10.220333333333330 & & \\
\hline Sm & & & & \\
\hline 2 & & & & \\
\hline Direct & & & & \\
\hline 0.11590000000000 & 0.11590000000000 & 0.11590000000000 & I & $(2 c)$ \\
\hline-0.11590000000000 & -0.11590000000000 & -0.11590000000000 & I & $(2 \mathrm{c})$ \\
\hline 0.30170000000000 & 0.30170000000000 & 0.30170000000000 & $\mathrm{~S}$ & $(2 \mathrm{c})$ \\
\hline-0.30170000000000 & -0.30170000000000 & -0.30170000000000 & $\mathrm{~s}$ & $(2 \mathrm{c})$ \\
\hline 0.38150000000000 & 0.38150000000000 & 0.38150000000000 & $\mathrm{Sm}$ & $(2 \mathrm{c})$ \\
\hline-0.38150000000000 & -0.38150000000000 & -0.38150000000000 & $\mathrm{Sm}$ & $(2 c)$ \\
\hline
\end{tabular}

PrNiO $3:$ AB3C_hR10_167_b_e_a - CIF

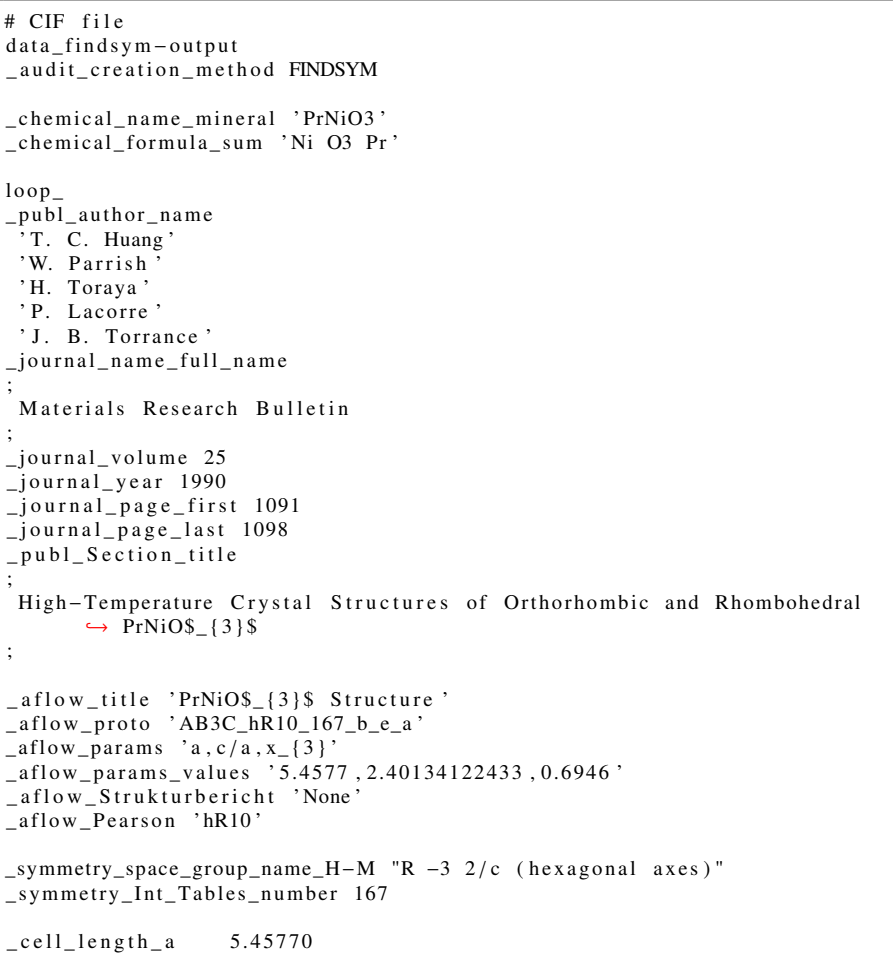


_cell_length_b $\quad 5.45770$

_cell_length_c $\quad 13.10580$

_cell_angle_alpha 90.00000

_cell_angle_beta 90.00000

_cell_angle_gamma 120.00000

loop

_space_group_symop_id

_space_group_symop_operation_xyz

$1 \mathrm{x}, \mathrm{y}, \mathrm{z}$

$2-y, x-y, z$

$3-\mathrm{x}+\mathrm{y},-\mathrm{x}, \mathrm{z}$

$4 \mathrm{y}, \mathrm{x},-\mathrm{z}+1 / 2$

$5-\mathrm{x},-\mathrm{x}+\mathrm{y},-\mathrm{z}+1 / 2$

$6 \mathrm{x}-\mathrm{y},-\mathrm{y},-\mathrm{z}+1 / 2$

$7-\mathrm{x},-\mathrm{y},-\mathrm{z}$

$8 \mathrm{y},-\mathrm{x}+\mathrm{y},-\mathrm{z}$

$9 \mathrm{x}-\mathrm{y}, \mathrm{x},-\mathrm{z}$

$10-y,-x, z+1 / 2$

$11 \mathrm{x}, \mathrm{x}-\mathrm{y}, \mathrm{z}+1 / 2$

$12-x+y, y, z+1 / 2$

$13 \mathrm{x}+1 / 3, \mathrm{y}+2 / 3, \mathrm{z}+2 / 3$

$14-y+1 / 3, x-y+2 / 3, z+2 / 3$

$5-\mathrm{x}+\mathrm{y}+1 / 3,-\mathrm{x}+2 / 3, \mathrm{z}+2 / 3$

$16 \mathrm{y}+1 / 3, \mathrm{x}+2 / 3,-\mathrm{z}+1 / 6$

$17-x+1 / 3,-x+y+2 / 3,-z+1 / 6$

$18 x-y+1 / 3,-y+2 / 3,-z+1 / 6$

$19-x+1 / 3,-y+2 / 3,-z+2 / 3$

$20 \mathrm{y}+1 / 3,-\mathrm{x}+\mathrm{y}+2 / 3,-\mathrm{z}+2 / 3$

$21 \mathrm{x}-\mathrm{y}+1 / 3, \mathrm{x}+2 / 3,-\mathrm{z}+2 / 3$

$22-y+1 / 3,-x+2 / 3, z+1 / 6$

$23 x+1 / 3, x-y+2 / 3, z+1 / 6$

$24-x+y+1 / 3, y+2 / 3, z+1 / 6$

$25 x+2 / 3, y+1 / 3, z+1 / 3$

$26-\mathrm{y}+2 / 3, \mathrm{x}-\mathrm{y}+1 / 3, \mathrm{z}+1 / 3$

$27-\mathrm{x}+\mathrm{y}+2 / 3,-\mathrm{x}+1 / 3, \mathrm{z}+1 / 3$

$28 \mathrm{y}+2 / 3, \mathrm{x}+1 / 3,-\mathrm{z}+5 / 6$

$29-\mathrm{x}+2 / 3,-\mathrm{x}+\mathrm{y}+1 / 3,-\mathrm{z}+5 / 6$

$30 x-y+2 / 3,-y+1 / 3,-z+5 / 6$

$31-x+2 / 3,-y+1 / 3,-z+1 / 3$

$32 \mathrm{y}+2 / 3,-\mathrm{x}+\mathrm{y}+1 / 3,-\mathrm{z}+1 / 3$

$33 x-y+2 / 3, x+1 / 3,-z+1 / 3$

$34-y+2 / 3,-x+1 / 3, z+5 / 6$

$35 x+2 / 3, x-y+1 / 3, z+5 / 6$

$36-x+y+2 / 3, y+1 / 3, z+5 / 6$

loop

atom site label

atom_site_type_symbol

atom_site_symmetry_multiplicity

atom_site_Wyckoff_labe

atom_site_fract_x

atom_site_fract_y

atom_site_fract_z

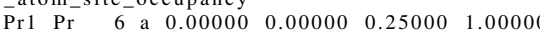

$\begin{array}{llllllll}\mathrm{Ni} 1 & \mathrm{Ni} & 6 & \mathrm{~b} & 0.00000 & 0.00000 & 0.00000 & 1.00000\end{array}$

$\begin{array}{llllllllll}\mathrm{O} 1 & \mathrm{O} & 18 & \text { e } & 0.44460 & 0.00000 & 0.25000 & 1.0000\end{array}$

$\mathrm{PrNiO}_{3}$ : AB3C_hR10_167_b_e_a - POSCAR

AB3C_hR10_167_b_e_a \& a,c/a, x3 --params $=5.4577,2.40134122433,0.6946 \&$

$\hookrightarrow \mathrm{R}-3 \mathrm{c} \mathrm{D}_{-}\{3 \mathrm{~d}\}^{\wedge}\{6\} \# 167$ (abe) \& hR10 \& None \& PrNiO3 \& PrNiO3 \& $\mathrm{T}$

$\hookrightarrow$ C. Huang et al., Mater. Res. Bull. 25, 1091-1098 (1990)

1.00000000000000

$\begin{array}{lll}2.72885000000000 & -1.57550228207811 & 4.36860000000000\end{array}$

$0.00000000000000 \quad 3.15100456415622 \quad 4.36860000000000$

$\begin{array}{lll}-2.72885000000000 & -1.57550228207811 & 4.36860000000000\end{array}$

$$
\begin{array}{rrr}
\mathrm{Ni} & \mathrm{O} & \mathrm{Pr} \\
2 & 6 & 2
\end{array}
$$

Direct

0.00000000000000

0.50000000000000

0.69460000000000

0.25000000000000

$-0.19460000000000$

$-0.69460000000000$

0.75000000000000

1.19460000000000

0.25000000000000

0.75000000000000

0.00000000000000

0.1946000000000

1.1946000000000

$-0.69460000000000$

0.75000000000000

0.2500000000000

0.75000000000000

0.00000000000000

0.50000000000000

0.25000000000000

0.19460000000000

0.69460000000000

0.75000000000000

1.19460000000000

$-0.69460000000000$

0.25000000000000

0.75000000000000

$\begin{array}{cc}\mathrm{Ni} & (2 \mathrm{~b}) \\ \mathrm{Ni} & (2 \mathrm{~b}) \\ \mathrm{O} & (6 \mathrm{e}) \\ \mathrm{O} & (6 \mathrm{e}) \\ \mathrm{O} & (6 \mathrm{e}) \\ \mathrm{O} & (6 \mathrm{e}) \\ \mathrm{O} & (6 \mathrm{e}) \\ \mathrm{O} & (6 \mathrm{e}) \\ \mathrm{Pr} & (2 \mathrm{a}) \\ \mathrm{Pr} & (2 \mathrm{a})\end{array}$

$\mathrm{KBO}_{2}\left(F 5_{13}\right):$ ABC2_hR24_167_e_e_2e - CIF

\section{\# CIF file}

data_findsym-output

_audit_creation_method FINDSYM

chemical_name_mineral 'KBO2'

_chemical_formula_sum 'B K O2,

loop

publ_author_name

'W. Schneider'

'G. B. Carpenter

_journal_name_full_name

Acta Crystallographica Section B: Structural Science

_journal_volume 26

journal year 1970

_journal_page_first 1189

_journal_page_last 1191

_publ_Section_title
Bond lengths and thermal parameters of potassium metaborate, K\$_\{3\}\$B\$ $\hookrightarrow\{3\} \$ O \$_{-}\{6\} \$$

\# Found in Landolt-B $\backslash$ " $\{0\}$ rnstein - Group III Condensed Matter (Numerica $\hookrightarrow$ Data and Functional Relationships in Science and Technology),
$\hookrightarrow 2007$

aflow_title 'KBO\$_ $\{2\} \$\left(\$ F 5 \_\{13\} \$\right)$ Structure

aflow_proto 'ABC2_hR24_167_e_e_2e

aflow_params 'a, c/a, $x_{-}\{1\}, x_{-}\{2\}, x_{-}\{3\}, x_{-}\{4\}$ '

aflow_params_values $, 12.76,0.575235109718,1.1389,0.8113,1.0343,0.3584$

aflow_Strukturbericht '\$F5_\{13\}\$'

aflow Pearson ',

symmetry space group name $H-M \quad R-32 / c$ (hexagonal axes)"

symmetry_Int Tables_number 167

cell_length_a $\quad 12.76000$

cell_length_b 12.76000

cell_length_c 7.34000

cell_angle_alpha 90.00000

_cell_angle_beta 90.00000

cell_angle_gamma 120.00000

loop

space_group_symop_id

space group symop operation xyz

$\mathrm{x}, \mathrm{y}, \mathrm{z}$

$2-\mathrm{y}, \mathrm{x}-\mathrm{y}, \mathrm{z}$

$3-\mathrm{x}+\mathrm{y},-\mathrm{x}, \mathrm{z}$
$4-\mathrm{y}, \mathrm{x},-\mathrm{z}+1 / 2$
5

$\mathrm{y}, \mathrm{x},-\mathrm{z}+1 / 2$
$-\mathrm{x},-\mathrm{x}+\mathrm{y},-\mathrm{z}+1 / 2$

$6 x-y,-y,-z+1 / 2$

$-x,-y,-z$

$8 \mathrm{y},-\mathrm{x}+\mathrm{y},-\mathrm{z}$

$9 \mathrm{x}-\mathrm{y}, \mathrm{x},-\mathrm{z}$

$10-y,-x, z+1 / 2$

$11 \mathrm{x}, \mathrm{x}-\mathrm{y}, \mathrm{z}+1 / 2$

$12-x+y, y, z+1 / 2$

$13 \mathrm{x}+1 / 3, \mathrm{y}+2 / 3, \mathrm{z}+2 / 3$

$4-y+1 / 3, x-y+2 / 3, z+2 / 3$

$15-x+y+1 / 3,-x+2 / 3, z+2 / 3$

$16 \mathrm{y}+1 / 3, \mathrm{x}+2 / 3,-\mathrm{z}+1 / 6$

$17-\mathrm{x}+1 / 3,-\mathrm{x}+\mathrm{y}+2 / 3,-\mathrm{z}+1 / 6$

$18 \mathrm{x}-\mathrm{y}+1 / 3,-\mathrm{y}+2 / 3,-\mathrm{z}+1 / 6$

$19-x+1 / 3,-y+2 / 3,-z+2 / 3$

$20 \mathrm{y}+1 / 3,-\mathrm{x}+\mathrm{y}+2 / 3,-\mathrm{z}+2 / 3$

$21 \mathrm{x}-\mathrm{y}+1 / 3, \mathrm{x}+2 / 3,-\mathrm{z}+2 / 3$

$22-y+1 / 3,-x+2 / 3, z+1 / 6$

$23 x+1 / 3, x-y+2 / 3, z+1 / 6$

$24-\mathrm{x}+\mathrm{y}+1 / 3, \mathrm{y}+2 / 3, \mathrm{z}+1 / 6$

$25 \mathrm{x}+2 / 3, \mathrm{y}+1 / 3, \mathrm{z}+1 / 3$

$26-\mathrm{y}+2 / 3, \mathrm{x}-\mathrm{y}+1 / 3, \mathrm{z}+1 / 3$

$27-\mathrm{x}+\mathrm{y}+2 / 3,-\mathrm{x}+1 / 3, \mathrm{z}+1 / 3$

$28 \mathrm{y}+2 / 3, \mathrm{x}+1 / 3,-\mathrm{z}+5 / 6$

$29-x+2 / 3,-x+y+1 / 3,-z+5 / 6$

$30 \mathrm{x}-\mathrm{y}+2 / 3,-\mathrm{y}+1 / 3,-\mathrm{z}+5 / 6$

$31-x+2 / 3,-y+1 / 3,-z+1 / 3$

$32 y+2 / 3,-x+y+1 / 3,-z+1 / 3$

$33 x-y+2 / 3, x+1 / 3,-z+1 / 3$

$34-\mathrm{y}+2 / 3,-x+1 / 3, z+5 / 6$

$35 x+2 / 3, x-y+1 / 3, z+5 / 6$

$36-x+y+2 / 3, y+1 / 3$

loop

atom site label

atom_site_type_symbol

atom_site_symmetry_multiplicity

atom_site_Wyckoff_labe

atom_site_fract_x

atom_site_fract_y

atom_site_fract_z

_atom_site_occupancy

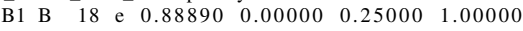

K1 K 18 e $0.56130 \quad 0.00000 \quad 0.25000 \quad 1.00000$

$\begin{array}{lllllllllll}\mathrm{O} 1 & \mathrm{O} & 18 & \mathrm{e} & 0.78430 & 0.00000 & 0.25000 & 1.00000\end{array}$

$\begin{array}{lllllllll}\mathrm{O} 2 & \mathrm{O} & 18 & \mathrm{e} & 0.10840 & 0.00000 & 0.25000 & 1.00000\end{array}$

$\mathrm{KBO}_{2}\left(F 5_{13}\right):$ ABC2_hR24_167_e_e_2e - POSCAR

ABC2_hR24_167_e_e_2e \& a , c /a, x1 , x2, x3, x4 --params $=12.76,0.575235109718$ $\hookrightarrow 1.1389,0.8113,1.0343,0.3584 \& \mathrm{R}-3 \mathrm{c} \mathrm{D}_{-}\{3 \mathrm{~d}\}^{\wedge}\{6\} \# 167\left(\mathrm{e}^{\wedge} 4\right) \& \mathrm{hR} 24$

$\hookrightarrow$ \& \$F5_\{13\}\$ \& $\mathrm{KBO} 2 \& \mathrm{KBO} 2 \& \mathrm{~W}$. Schneider and G. B. Carpenter-

$\hookrightarrow$ Acta Crystallogr. Sect, B Struct, Sci, 26, 1189-1191 (1970)

1.00000000000000

6.38000000000000

$-3.68349471742981$

7.36698943485963

$6.38000000000000-3.68349471742981$

B $\mathrm{K} \quad \mathrm{O}$

Direct

1.13890000000000

0.25000000000000

$-0.63890000000000$

$-1.13890000000000$

0.75000000000000

1.63890000000000

0.81130000000000

0.25000000000000

$-0.81130000000000$

0.75000000000000

1.31130000000000

1.03430000000000

-3.36698943485963
-3.68349471742981

446666666666667

2.446666666666667

2. 446666666666667

$-0.63890000000000$

1.13890000000000

0.25000000000000

1.63890000000000

$-1.13890000000000$

0.75000000000000

0.81130000000000

0.25000000000000

1.31130000000000

$-0.81130000000000$

0.75000000000000

$-0.53430000000000$

0.25000000000000

$-0.63890000000000$

1.13890000000000

0.75000000000000

1.63890000000000

$-1.13890000000000$

0.25000000000000

$-0.31130000000000$ 0.81130000000000 0.75000000000000

1.31130000000000

$-0.8113000000000$

0.25000000000000
B $\quad(6 e)$

$\begin{array}{ll}\mathrm{B} & (6 \mathrm{e}) \\ \mathrm{B} & (6 \mathrm{e})\end{array}$

B (6e) 
0.25000000000000 $-0.53430000000000$ $-1.03430000000000$ 0.75000000000000 1.53430000000000 0.35840000000000 0.25000000000000 0.14160000000000 $-0.35840000000000$ 0.75000000000000 0.85840000000000

1.03430000000000 0.25000000000000 1.53430000000000 $-1.03430000000000$ 0.75000000000000 0.14160000000000 0.35840000000000 0.25000000000000 0.85840000000000 $-0.35840000000000$
53430000000000 0.7500000000000 $-1.03430000000000$ 0.25000000000000 0.14160000000000 0.35840000000000 0.75000000000000 .85840000000000 $-0.35840000000000$ 1.03430000000000 1.53430000000000

$\begin{array}{ll}\mathrm{O} & (6 \mathrm{e}) \\ \mathrm{O} & (6 \mathrm{e}) \\ \mathrm{O} & (6 \mathrm{e}) \\ \mathrm{O} & (6 \mathrm{e}) \\ \mathrm{O} & (6 \mathrm{e}) \\ \mathrm{O} & (6 \mathrm{e}) \\ \mathrm{O} & (6 \mathrm{e}) \\ \mathrm{O} & (6 \mathrm{e}) \\ \mathrm{O} & (6 \mathrm{e}) \\ \mathrm{O} & (6 \mathrm{e}) \\ \mathrm{O} & (6 \mathrm{e})\end{array}$

$\mathrm{K}_{2} \mathrm{Ta}_{4} \mathrm{O}_{9} \mathrm{~F}_{4}$ : A2B13C4_hP57_168_d_c6d_2d - CIF

\section{\# CIF file}

data_findsym-output

audit_creation_method FINDSYM

chemical_name_mineral 'K2Ta4O9F4'

chemical formula_sum 'K2 $\mathrm{O} 13$ Ta4'

loop

publ author name

A. Boukhari

J. P. Chaminade

'M. Pouchard

journal_name_full_name

Acta Crystallographica Section B: Structural Science

_journal_volume 35

journal year 1979

_journal_page_first 1983

journal page last 1986

publ_Section_title

Structure cristalline de $1 \backslash$ oxyfluorure de tantale et de potassium, $\mathrm{K} \$$ $\hookrightarrow\{2\} \$ T a \$_{-}\{4\} \$ F \$_{-}\{4\} \$ O \$_{-}\{9\} \$$

\# Found in Pearson's Crystal Data - Crystal Structure Database for $\hookrightarrow$ Inorganic Compounds, 2013

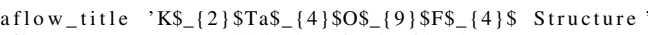

aflow_proto 'A2B13C4_hP57_168_d_c6d_2d

aflow_params $, a, c / a, z_{-}\{1\}, x_{-}\{2\}, y_{-}\{2\}, z_{-}\{2\}, x_{-}\{3\}, y_{-}\{3\}, z_{-}\{3\}, x_{-}\{4\}, y_{-}\{$ $\hookrightarrow 4\}, \mathrm{z}_{-}\{4\}, \mathrm{x}_{-}\{5\}, \mathrm{y}_{-}\{5\}, \mathrm{z}_{-}\{5\}, \mathrm{x}_{-}\{6\}, \mathrm{y}_{-}\{6\}, \mathrm{z}_{-}\{6\}, \mathrm{x}_{-}\{7\}, \mathrm{y}_{-}\{7\}, \mathrm{z}_{-}\{7\}$ $\hookrightarrow \mathrm{x}_{-}\{8\}, \mathrm{y}_{-}\{8\}, \mathrm{z}_{-}\{8\}, \mathrm{x}_{-}\{9\}, \mathrm{y}_{-}\{9\}, \mathrm{z}_{-}\{9\}, \mathrm{x}_{-}\{10\}, \mathrm{y}_{-}\{10\}, \mathrm{z}_{-}\{10\}$

_aflow_params_values ' $15.9361426085,0.244226907628,0.0,0.4965,0.179$,

$\hookrightarrow 0.555,0.035,0.187,0.007,0.349,0.034,0.018,0.168,0.388,0.01$,

$\hookrightarrow 0.195,0.569,0.02,0.093,0.454,0.539,0.273,0.095,0.555,0.2634$

$\hookrightarrow 0.09,0.086,0.0789,0.4368,0.071$,

aflow_Strukturbericht 'None'

aflow_Pearson 'hP57,

cell_length_a $\quad 15.9361426085$

cell_length_b 15.9361426085

cell_length_c 3.8920348288

cell_angle_alpha 90.0000000000

_cell_angle_beta 90.0000000000

cell_angle_gamma 120.0000000000

symmetry_space_group_name_H-M "P 6"

symmetry_Int_Tables_number 168

loop

space_group_symop_id

_space_group_symop_operation_xyz

$1 \mathrm{x}, \mathrm{y}, \mathrm{z}$

$2 \mathrm{x}-\mathrm{y}, \mathrm{x}, \mathrm{z}$

$3-y, x-y, z$

$4-\mathrm{x},-\mathrm{y}, \mathrm{z}$

$-\mathrm{x}$

$6,-x+y, z$

loop

atom_site_labe

atom_site_type_symbo

atom_site_symmetry_multiplicity

atom_site_Wyckoff_labe

atom_site_fract_x

atom_site_fract_y

atom site foccupancy

$\begin{array}{llllllll}\mathrm{O} 1 & \mathrm{O} & 3 & \mathrm{c} & 0.50000 & 0.00000 & 0.00000 & 1.00000\end{array}$

$\begin{array}{lllllllllll} & \mathrm{K} 1 & \mathrm{~K} & 6 & \mathrm{~d} & 0.49650 & 0.17900 & 0.55500 & 1.00000\end{array}$

$\begin{array}{lllllllllll}\mathrm{K} 1 & \mathrm{~K} & & -6 & \mathrm{~d} & 0.49650 & 0.1780 & 0.55700 & 1.00000\end{array}$

$\begin{array}{llllllll}\mathrm{O} 2 & \mathrm{O} & 6 & \mathrm{~d} & 0.03500 & 0.18700 & 0.00700 & 1.00000\end{array}$

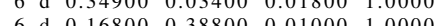

$\begin{array}{lllllllll}04 & 0 & -6 & \mathrm{~d} & 0.16800 & 0.38800 & 0.01000 & 1.00000 \\ 05 & 0 & 6 & \mathrm{~d} & 0.19500 & 0.56900 & 0.02000 & 1.00000\end{array}$

$\begin{array}{lllllllll}05 & 0 & -6 & \mathrm{~d} & 0.19500 & 0.56900 & 0.02000 & 1.00000\end{array}$

$\begin{array}{llllllllll}06 & 0 & 6 & d & 0.09300 & 0.45400 & 0.53900 & 1.00000\end{array}$

$\begin{array}{lllllllll}07 & 0 & 6 & \mathrm{~d} & 0.27300 & 0.05500 & 0.55500 & 1.00000\end{array}$

$\begin{array}{lllllll}6 & \mathrm{~d} & 0.26340 & 0.09000 & 0.08600 & 1.0000\end{array}$

T.

A2B13C4_hP57_168_d_c6d_2d \& a , c/a, z1 , x2,y2, z2, x3, y3 , z3 , x4,y4, z4, x5,y5, z5 $\hookrightarrow, \mathrm{x} 6, \mathrm{y} 6, \mathrm{z} 6, \mathrm{x} 7, \mathrm{y} 7, \mathrm{z} 7, \mathrm{x} 8, \mathrm{y} 8, \mathrm{z8}, \mathrm{x} 9, \mathrm{y} 9, \mathrm{z} 9, \mathrm{x} 10, \mathrm{y} 10, \mathrm{z} 10-$ params $=$

$\hookrightarrow 15.9361426085,0.244226907628,0.0,0.4965,0.179,0.555,0.035,0.187$

$\hookrightarrow, 0.007,0.349,0.034,0.018,0.168,0.388,0.01,0.195,0.569,0.02$

$\hookrightarrow 0.093,0.454,0.539,0.273,0.095,0.555,0.2634,0.09,0.086,0.0789$

$\hookrightarrow 0.4368,0.071 \&$ P6 $C_{-}\{6\}^{\wedge}\{1\} \# 168\left(\mathrm{~cd}^{\wedge} 9\right) \&$ hP57 \& None \&

$\hookrightarrow$ K2Ta4O9F4 \& \& A Boukhari et al., Acta Crystallogr. Sect. B $\hookrightarrow$ Struct. Sci. 35, 1983-1986 (1979)

1.00000000000000

$\begin{array}{lll}7.96807130425000 & -13.80110433729260 & 0.00000000000000\end{array}$

$\begin{array}{rrr}7.96807130425000 & 13.80110433729260 & 0.00000000000000\end{array}$

$\begin{array}{rrr}0.00000000000000 & 0.00000000000000 & 3.89203482880000\end{array}$

$\begin{array}{rrr}\mathrm{K} & \mathrm{O} & \mathrm{Ta} \\ 6 & 39 & 12\end{array}$

Direct

0.49650000000000

$-0.17900000000000$

$-0.31750000000000$

$-0.49650000000000$

0.17900000000000

0.31750000000000

0.50000000000000

0.00000000000000

0.50000000000000

0.03500000000000

-0.18700000000000
0.15200000000000

0.15200000000000

-0.03500000000000
0.18700000000000

$-0.1520000000000$

0.34900000000000

$-0.03400000000000$

$-0.31500000000000$

$-0.34900000000000$

0.03400000000000

0.31500000000000

0.16800000000000

$-0.38800000000000$

$-0.16800000000000$

0.38800000000000

$-0.22000000000000$

0.19500000000000

$-0.56900000000000$

0.37400000000000

$-0.19500000000000$

0.56900000000000

$-0.37400000000000$

0.09300000000000

0.36100000000000

$-0.09300000000000$

0.45400000000000

$-0.3610000000000$

0.27300000000000

$-0.0950000000000$

$-0.17800000000000$

$-0.27300000000000$

0.09500000000000
0.17800000000000

0.17800000000000
0.26340000000000

0.26340000000000
-0.09000000000000

$-0.17340000000000$

$-0.26340000000000$

0.09000000000000

0.17340000000000

0.07890000000000

$-0.43680000000000$

0.35790000000000

$-0.07890000000000$

0.43680000000000

$-0.35790000000000$

0.17900000000000 .31750000000000 .49650000000000 17900000000000 .31750000000000 0.49650000000000 0.00000000000000 0.50000000000000 8700000000000 0.18700000000000 . .03500000000000 .18700000000000 200000000000 (2) 400000000000 0000000000 34900000000000 0.03400000000000 .31500000000000 .34900000000000 .38800000000000 . 38800000000000 .32000000000000 0.16800000000000 .56800000000 0.37400000000000 0.19500000000000 . . 00000000000 .36100000000000 $-0.09300000000000$ 0.45400000000000 .36100000000000 0.09300000000000 0.09500000000000 0.17800000000000 . . . .27300000000000 0.09000000000000 0.17340000000000 0.26340000000000 0.09000000000000 0.17340000000000 .26340000000000 0.43680000000000 $-0.35790000000000$ $-0.43680000000000$ 0.35790000000000 0.07890000000000

0.55500000000000 0.55500000000000 0.55500000000000 0.55500000000000 0.55500000000000 0.55500000000000 0.00000000000000 0.00000000000000 0.00000000000000 0.00700004 0.0070000000000 0.00700000000000 0.007000000000 0.00700000000000 0.00700000000000 0.00700000000000 0.01800000000000 0.01800000000000 0.01800000000000 0.01800000000000 0.01800000000000 0.01800000000000 0.01000000000000 0.01000000000000 0.01000000000 0.010000000000 0.0100000000000 0.01000000000000 0.02000000000000 0.02000000000000 0.02000000000000 0.02000000000000 0.02000000000000 0.02000000000000 0.53900000000000 0.53900000000000 0.53900000000000 0.53900000000000 0.53900000000000 0.53900000000000 0.55500000000000 0.5500000000000 0.55500000000 0.55500000000000 0.55500000000000 0.55500000000000 0.55500000000000 0.08600000000000 0.08600000000000 0.08600000000000 0.08600000000000 0.08600000000000 0.08600000000000 0.07100000000000 0.07100000000000 0.07100000000000 0.07100000000000 0.07100000000000 0.0710000000000 
$0.1278,0.466,0.1246,0.4154,0.2053,0.0456,0.2066,0.4189,0.5576$ 年 $\hookrightarrow 0.0083,0.3667,0.4879,0.5693,0.1616,0.0357,0.1505,0.5625,0.5943$ $\hookrightarrow 0.4451,0.1173,0.0,0.1291,0.4589,0.4993$ _aflow_Strukturbericht 'None' _aflow_Pearson 'hP72,

\section{_cell_length_a $\quad 13.7599395580$}

_cell_length_c 8.3899631462

_cell_angle_alpha 90.0000000000

cell_angle beta 120.0000000000

symmetry space group name $\mathrm{H}-\mathrm{M}$ "P 6"

symmetry_Int_Tables_number 168

loop

_space_group_symop_id

_space_group_symop_operation_xyz

$1 \mathrm{x}, \mathrm{y}, \mathrm{z}$

$2 x-y, x, z$

$3-y, x-y, z$
$4-x,-y, z$

$4-x,-y, z$
$5-x+y,-x$,

$6 y,-x+y, z$

loop_

_atom_site_type_symbol

_atom_site_symmetry_multiplicity

atom_site_Wyckoff_label

atom_site_fract_x

atom_site_fract_y

atom_site_occupancy

$\begin{array}{llllllll}\text { Al1 } & \text { Al } & 6 & \text { d } & 0.44980 & 0.11300 & 0.62530 & 1.00000\end{array}$

$\begin{array}{lllllllll}\mathrm{Al} 2 & \mathrm{Al} & 6 & \mathrm{~d} & 0.12780 & 0.46600 & 0.12460 & 1.00000\end{array}$

$\begin{array}{llllllllll}\mathrm{O} 1 & \mathrm{O} & 6 & \mathrm{~d} & 0.41540 & 0.20530 & 0.04560 & 1.00000\end{array}$

$\begin{array}{lllllllll}\mathrm{O} 2 & \mathrm{O} & 6 & \mathrm{~d} & 0.20660 & 0.41890 & 0.55760 & 1.00000\end{array}$

$\begin{array}{lllllllll}\mathrm{O} 3 & \mathrm{O} & 6 & \mathrm{~d} & 0.42180 & 0.08940 & 0.82480 & 1.00000\end{array}$

$\begin{array}{lllllllll}\mathrm{O} 4 & \mathrm{O} & 6 & \mathrm{~d} & 0.15140 & 0.49070 & 0.32490 & 1.00000\end{array}$

$\begin{array}{lllllllll}\mathrm{O} 5 & \mathrm{O} & 6 & \mathrm{~d} & 0.37460 & 0.01060 & 0.09480 & 1.00000\end{array}$

$\begin{array}{lllllllll}\mathrm{O} 6 & \mathrm{O} & 6 & \mathrm{~d} & 0.00830 & 0.36670 & 0.48790 & 1.00000\end{array}$

$\begin{array}{lllllllll}\mathrm{O} 7 & \mathrm{O} & 6 & \mathrm{~d} & 0.56930 & 0.16160 & 0.03570 & 1.00000\end{array}$

$\begin{array}{lllllllll}\mathrm{O} 8 & \mathrm{O} & 6 & \mathrm{~d} & 0.15050 & 0.56250 & 0.59430 & 1.00000\end{array}$

$\begin{array}{lllllllllll}\mathrm{P} 1 & \mathrm{P} & & 6 & \mathrm{~d} & 0.44510 & 0.11730 & 0.00000 & 1.00000\end{array}$

$\begin{array}{llllllll}\mathrm{P} 1 & \mathrm{P} & 6 & \mathrm{~d} & 0.44510 & 0.11730 & 0.00000 & 1.00000 \\ \mathrm{P} 2 & \mathrm{P} & 6 & \mathrm{~d} & 0.12910 & 0.45890 & 0.49930 & 1.00000\end{array}$

$\mathrm{Al}\left[\mathrm{PO}_{4}\right]$ : AB4C_hP72_168_2d_8d_2d - POSCAR

AB4C_hP72_168_2d_8d_2d \& a,c/a, x1, y1 $\mathrm{z} 1, \mathrm{x} 2, \mathrm{y} 2, \mathrm{z} 2, \mathrm{x} 3, \mathrm{y} 3, \mathrm{z} 3, \mathrm{x} 4, \mathrm{y} 4, \mathrm{z} 4, \mathrm{x} 5, \mathrm{y} 5$ $\hookrightarrow, \mathrm{z} 5, \mathrm{x} 6, \mathrm{y} 6, \mathrm{z} 6, \mathrm{x} 7, \mathrm{y} 7, \mathrm{z} 7, \mathrm{x} 8, \mathrm{y} 8, \mathrm{z} 8, \mathrm{x} 9, \mathrm{y} 9, \mathrm{z} 9, \mathrm{x} 10, \mathrm{y} 10, \mathrm{z} 10, \mathrm{x} 11, \mathrm{y} 11, \mathrm{z} 11$ $\hookrightarrow, \mathrm{z} 5, \mathrm{x} 6, \mathrm{y6}, \mathrm{z6}, \mathrm{x} 7, \mathrm{y} 7, \mathrm{z} 7, \mathrm{x} 8, \mathrm{y} 8, \mathrm{z8}, \mathrm{x} 9, \mathrm{y} 9, \mathrm{z} 9, \mathrm{x} 10, \mathrm{y} 10, \mathrm{z} 10, \mathrm{x} 11, \mathrm{y} 11, \mathrm{z} 11$ $\hookrightarrow, \mathrm{x} 12, \mathrm{y} 12, \mathrm{z} 12-\mathrm{params}=13.759939558,0.609738372094,0.4498,0.113$,

$\hookrightarrow 0.6253,0.1278,0.466,0.1246,0.4154,0.2053,0.0456,0.2066,0.4189$,

$\hookrightarrow 0.5576,0.4218,0.0894,0.8248,0.1514,0.4907,0.3249,0.3746,0.0106$,

$\hookrightarrow 0.0948,0.0083,0.3667,0.4879,0.5693,0.1616,0.0357,0.1505,0.5625$,

$\hookrightarrow 168\left(\mathrm{~d}^{\wedge} 12\right) \& \mathrm{hP72} \&$ None \& Al[PO4] \& \& J. W. \{Richardson Jnr

$\hookrightarrow 168\left(\mathrm{~d}^{\wedge} 12\right) \&$ hP72 \& None \& Al[PO4] \& \& J. W. \{Richardson
$\hookrightarrow$ and J. J. Pluth and J. V. Smith, Acta Crystallogr. C 43 ,

$\hookrightarrow 1469-1472$ (1987)

1.00000000000000

$\begin{array}{lll}6.87996977900000 & -11.91645721176640 & 0.00000000000000\end{array}$

$\begin{array}{llll}6.87996977900000 & 11.91645721176640 & 0.00000000000000\end{array}$

$0.00000000000000 \quad 0.00000000000000 \quad 8.38996314620000$

$\begin{array}{rrr}\mathrm{Al} & \mathrm{O} & \mathrm{P} \\ 12 & 48 & 12\end{array}$

Direct

0.44980000000000

0.44980000000000
-0.11300000000000

$-0.11300000000000$

$-0.44980000000000$

0.11300000000000

0.33680000000000

0.12780000000000

$-0.46600000000000$

0.33820000000000

$-0.12780000000000$

0.46600000000000

$-0.33820000000000$

0.41540000000000

$-0.20530000000000$

$-0.21010000000000$

0.20530000000000

0.21010000000000

0.20660000000000

$-0.20660000000000$

$-.41890000000000$

0.41890000000000

$-0.21230000000000$

0.42180000000000

$-0.08940000000000$

$-0.33240000000000$

$-0.42180000000000$

0.08940000000000

0.33240000000000

0.15140000000000

$-0.49070000000000$

0.33930000000000

$-0.15140000000000$

0.49070000000000

$-0.33930000000000$

0.37460000000000
0.11300000000000 $-0.44980000000000$ 0.11300000000000 0.33680000000000 .44980000000000 0.46600000000000 ( $-0.127800$ 0.12780000000000 .46600000000000 0.33820000000000 0.12780000000000 0.20530000000000 . 21010000000000 0.20530000000000 $-0.21010000000000$ 0.41890000000000 $-0.20660000000000$ $-0.41890000000000$ 0.21230000000000 0.21230000000 0.08940000000000 0.08940000000000 0.33240000000000 $-0.42180000000000$ $-0.08940000000000$ 0.42180000000000 .49070000000000 $-0.33930000000000$ $-0.15140000000000$ $-0.49070000000000$ 0.15140000000000 0.01060000000000 0.33680000000000

0.62530000000000 0.62530000000000 0.62530000000000 0.62530000000000 0.62530000000000 0.62530000000000 0.12460000000000 0.12460000000000 0.12460000000000 0.12460000000000 0.12460000000000 0.12460000000000 0.04560000000000 0.04560000000000 0.04560000000000 0.04560000000000 0.04560000000000
$-0.01060000000000$ $-0.36400000000000$ $-0.37460000000000$ 0.01060000000000 0.36400000000000 0.00830000000000 $-0.36670000000000$ 0.35840000000000 $-0.00830000000000$ 0.36670000000000 $-0.35840000000000$ 0.56930000000000 $-0.16160000000000$ $-0.40770000000000$ $-0.56930000000000$ 0.16160000000000 0.15050000000000 $-0.56250000000000$ $-0.56250000000000$ $-0.41200000000000$ 0.44510000000000 $-0.32780000000000$ 0.11730000000000 0.12910000000000 $-0.45890000000000$ 0.32980000000000

$-0.12910000000000$

0.45890000000000 $-0.32980000000000$

0.36400000000000 $-0.37460000000000$ $-0.01060000000000$ $-0.36400000000000$ .37460000000000 0.36670000000000 $-0.35840000000000$ $-0.00830000000000$ $-0.36670000000000$ 0.35840000000000 0.00830000000000 0.16160000000000 0.40770000000000 $-0.56930000000000$ $-0.16160000000000$ $-0.40770000000000$ 0.56930000000000 0.56250000000000 0.41200000000000 0.42000000000 $-0.15050000000000$ . 0.41200000000000 0.15050000000000 .32780000000000 0.44510000000000 0.11730000000000 0.32780000000000 0.44510000000000 0.45890000000000 $-0.32980000000000$ $-0.12910000000000$ $-0.45890000000000$ 0.32980000000000 0.12910000000000

0.09480000000000 0.09480000000000 0.09480000000000 0.09480000000000 . 0.48790000000000 0.48790000000000 0.48790000000000 0.48790000000000 0.48790000000000 0.48790000000000 0.03570000000000 0.03570000000000 0.03570000000000 0.03570000000000 0.03570000000000 0.03570000000000 0.03570000000000 0.59430000000000 0.59430000000000 0.59430000000000 0.59430000000000 0.59430000000000 0.59430000000000 0.00000000000000 0.00000000000000 0.00000000000000 0.00000000000000 0.00000000000000 0.00000000000000 0.49930000000000 0.49930000000000 0.493000000 0.49930000000000 0.493000000 0.49930000000000

$\alpha-\mathrm{Al}_{2} \mathrm{~S}_{3}$ : A2B3_hP30_169_2a_3a-CIF

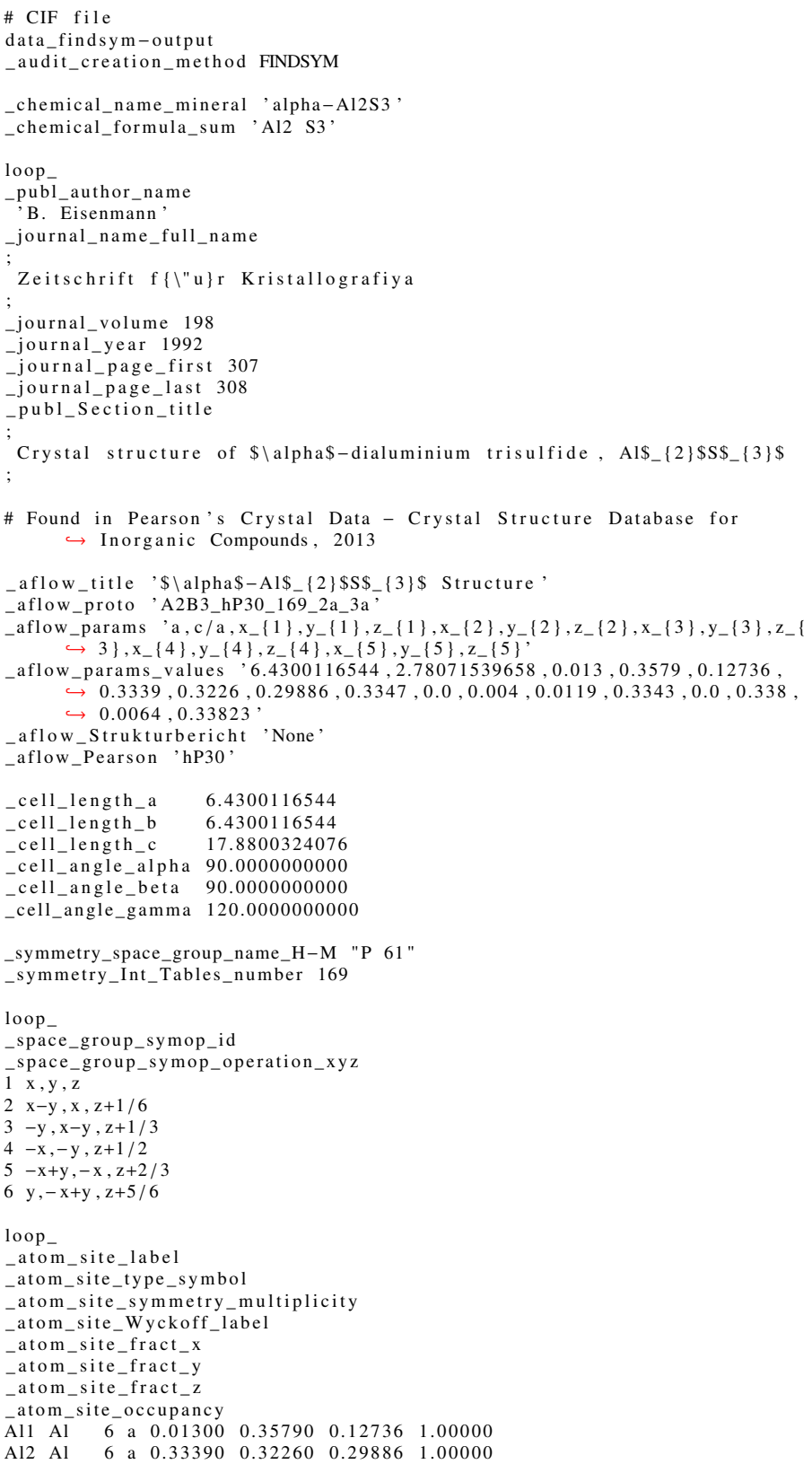


$\begin{array}{llllllll}\mathrm{S} 1 & \mathrm{~S} & 6 & \mathrm{a} & 0.33470 & 0.00000 & 0.00400 & 1.00000\end{array}$

6 a $0.01190 \quad 0.33430$

$\alpha-\mathrm{Al}_{2} \mathrm{~S}_{3}$ : A2B3 hP30_169_2a_3a - POSCAR

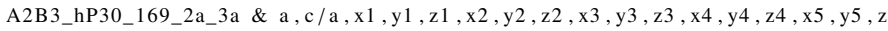
$\hookrightarrow \quad-$ params $=6.4300116544,2.78071539658,0.013,0.3579,0.12736$

年

$\hookrightarrow 0.0064,0.33823 \&$ \& $\left.6 \_11\right\} C_{-}\{6\}^{\wedge}\{2\} \# 169\left(a^{\wedge} 5\right) \&$ hP30 \& None \&

$\hookrightarrow$ Al2S3 \& alpha \& B. Eisenmann, Z. Kristallogr. 198, 307-308 $\hookrightarrow 1992$ )

1.00000000000000

3.21500582720000

3.21500582720000

0.00000000000000

$-5.5685534393404$

5.5685534393404

0.00000000000000

17.88003240760000

$$
\begin{array}{rr}
\mathrm{Al} & \mathrm{S} \\
12 & 18
\end{array}
$$

Direct

$0.01300000000000 \quad 0.35790000000000$

$-0.35790000000000-0.34490000000000$

0.34490000000000

$-0.01300000000000$

0.35790000000000

$-0.3449000000000$

$-0.34490000000000$

$-0.32260000000000$

$-0.01130000000000$

$-0.33390000000000$

0.32260000000000

0.01130000000000

0.33470000000000

0.00000000000000

$-0.33470000000000$

$-0.33470000000000$

0.00000000000000

0.33470000000000

0.01190000000000

$-0.33430000000000$

0.32240000000000

$-0.01190000000000$

0.33430000000000

$-0.32240000000000$

0.33800000000000

$-0.00640000000000$

$-0.33160000000000$

$-0.33800000000000$

0.00640000000000
0.33160000000000

$-0.01300000000000$

$-0.35790000000000$

0.34490000000000

0.0130000000000

0.32260000000000

0.322600000000

$-0.33390000000000$ $-0.32260000000000$ $-0.32260000000000$ 0.01130000000000 0.33390000000000 0.00000000000000 0.33470000000000 $-0.33470000000000$ 0.00000000000000 $-0.33470000000000$ 0.33470000000000 0.33430000000000 $-0.32240000000000$ $-0.01190000000000$ $-0.33430000000000$ 0.3224000000000 0.0119000000000 0.00640000000000 0.33160000000000 $-0.3380000000000$ $-0.33800000000000$ $-0.00640000000000$ 0.33800000000000

0.12736000000000 .4606933333333 0.79402666666667 0.62736000000000 0.96069333333333 0.29402666666667 0.29886000000000 0.63219333333333 0.63219333333333 0.96552666666667 . 1.13219333333333 0.00400000000000 0.33733333333333 0.6706666666666 0.50400000000000 0.8373333333333 0.17066666666667 0.00000000000000 0.33333333333333 0.66666666666667 0.5000000006067 0.83333333333333 0.16666666666667 0.33823000000000 1.00489666666667 1.0048966666666 0.83823000000000 1.17156333333333 0.5048966666666

Al (6a)

$\mathrm{Al} \quad(6 \mathrm{a})$

$(6 a)$

(6a)

(6a)

(6a)

(6a)

(6a)

(6a)

(6a)

$(6 a)$

\begin{tabular}{l}
$(6 a)$ \\
\hline$\quad(6 a)$
\end{tabular}

(6a)

(6a)

(6a)

(6a)

(6a)

(6a)

(6a)

(6a)

(6a)

(6a)

(6a)

(6a)

(6a)

(6a)

(6a)

(6a)

$(6 a)$
$(6 a)$

$\mathrm{Al}_{2} \mathrm{~S}_{3}$ : A2B3_hP30_170_2a_3a - CIF

\section{\# CIF file}

data_findsym-output

audit_creation_method FINDSYM

_chemical_name_mineral 'Al2S3,

_chemical_formula_sum, A12 S3,

aflow_title, Al\$_\{2\}\$S\$_\{3\}\$ Structure

aflow_proto 'A2B3_hP30_170_2a_3a

aflow_params $, a, c / a, x_{-}\{1\}, y_{-}\{1\}, z_{-}\{1\}, x_{-}\{2\}, y_{-}\{2\}, z_{-}\{2\}, x_{-}\{3\}, y_{-}\{3\}, z_{-}\{$ $\hookrightarrow 3\}, x_{-}\{4\}, y_{-}\{4\}, z_{-}\{4\}, x_{-}\{5\}, y_{-}\{5\}, z_{-}\{5\}$

aflow_params values $6.4300116544,2.78071539658,0.013,0.3579,0.87264$

$\hookrightarrow 0.3339,0.3226,0.70114,0.3347,0.0,-0.004,0.0119,0.3343,0.0,0.338$ $\hookrightarrow, 0.0064,0.66177$

_aflow_Strukturbericht 'None

-aflow_Pearson 'hP30'

_cell_length_a $\quad 6.4300116544$

cell length_b 6.4300116544

cell_length_c 17.8800324076

cell_angle_alpha 90.0000000000

_cell_angle_beta 90.0000000000

_cell_angle_gamma 120.0000000000

_symmetry_space_group_name_H-M "P 65 "

_symmetry_Int_Tables_number 170

loop

_space_group_symop_id

- space
1
$\mathrm{x}, \mathrm{y}, \mathrm{z}$

$2 \mathrm{x}-\mathrm{y}, \mathrm{x}, \mathrm{z}+5 / 6$

$3-y, x-y, z+2 / 3$

$4-x,-y, z+1 / 2$

$5-x+y,-x, z+1 / 3$

$6 \mathrm{y},-\mathrm{x}+\mathrm{y}, \mathrm{z}+1 / 6$

loop

_atom_site_label

atom_site_type_symbol

atom_site_symmetry_multiplicity

atom_site_Wyckoff_label

atom_site_fract_x

atom_site_fract_y

atom_site_fract_z

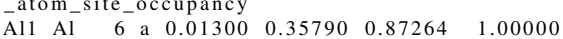

$\begin{array}{lllllllll}\mathrm{A} 12 & \mathrm{Al} & 6 & \mathrm{a} & 0.33390 & 0.32260 & 0.70114 & 1.00000\end{array}$

S1 S $\quad 6$ a $0.33470 \quad 0.00000-0.00400 \quad 1.00000$

$\begin{array}{llllllll}\mathrm{S} 1 & \mathrm{~S} & 6 & \mathrm{a} & 0.33470 & 0.00000 & -0.00400 & 1.00000 \\ \mathrm{~S} 2 & \mathrm{~S} & 6 & \mathrm{a} & 0.01190 & 0.33430 & 0.00000 & 1.00000\end{array}$

$\begin{array}{lllllllll}\text { S2 } & \text { S } & & 6 & \text { a } & 0.01190 & 0.33430 & 0.00000 & 1.00000 \\ \text { S3 } & \text { S } & 6 & \text { a } & 0.33800 & 0.00640 & 0.66177 & 1.00000\end{array}$

$\mathrm{Al}_{2} \mathrm{~S}_{3}$ : A2B3_hP30_170_2a_3a-POSCAR

A2B3_hP30_170_2a_3a \& a, c/a , x1,y1,z1, x2,y2, z2, x3 ,y3 $, \mathrm{z} 3, \mathrm{x} 4, \mathrm{y} 4, \mathrm{z} 4, \mathrm{x} 5, \mathrm{y} 5, \mathrm{z} 5$ $\hookrightarrow \quad-$ params $=6.4300116544,2.78071539658,0.013,0.3579,0.87264$ $\hookrightarrow 0.3339,0.3226,0.70114,0.3347,0.0,-0.004,0.0119,0.3343,0.0,0.338$ $\hookrightarrow, 0.0064,0.66177 \&$ P6 $\{5\} C_{-}\{6\}^{\wedge}\{3\} \# 170\left(a^{\wedge} 5\right) \&$ hP30 \& None \& $\hookrightarrow \mathrm{A} 12 \mathrm{~S} 3$ \& A12S3 \&

1.00000000000000

$3.21500582720000-5.56855343934041-0.00000000000000$

$\begin{array}{rrr}3.21500582720000 & 5.56855343934041 & 0.00000000000000\end{array}$

$\begin{array}{lll}0.00000000000000 & 0.00000000000000 & 17.88003240760000\end{array}$

$\begin{array}{rr}\mathrm{Al} & \mathrm{S} \\ 12 & 18\end{array}$

$0.01300000000000 \quad 0.35790000000000$

$-0.35790000000000-0.34490000000000$

$0.34490000000000-0.01300000000000$

$-0.01300000000000 \quad-0.35790000000000$

$0.35790000000000 \quad 0.34490000000000$

$-0.34490000000000 \quad 0.01300000000000$

$0.33390000000000 \quad 0.32260000000000$

$-0.3226000000000-0.01130000000000$

$-0.3226000000000-0.01130000000000$

$-0.0113000000000-0.33390000000000$

$0.32260000000000-0.01130000000000$

$\begin{array}{rr}0.01130000000000 & 0.33390000000000\end{array}$

$0.33470000000000 \quad 0.00000000000000$

$0.00000000000000 \quad 0.33470000000000$

$-0.33470000000000-0.33470000000000$

$-0.33470000000000 \quad 0.00000000000000$

$0.00000000000000-0.33470000000000$

$0.33470000000000 \quad 0.33470000000000$

$0.01190000000000 \quad 0.33430000000000$

$-0.33430000000000 \quad-0.32240000000000$

$0.32240000000000-0.01190000000000$

$-0.01190000000000-0.33430000000000$

$0.33430000000000 \quad 0.32240000000000$

$-0.32240000000000 \quad 0.01190000000000$

$0.33800000000000 \quad 0.00640000000000$

$-0.00640000000000 \quad 0.33160000000000$

$-0.33160000000000-0.33800000000000$

$-0.33800000000000-0.00640000000000$

$0.00640000000000 \quad-0.33160000000000$

0.33160000000000

0.33800000000000

0.87264000000000 1.53930666666667 1.20597333333333

1.37264000000000

1.03930666666667

1.70597333333333

0.70114000000000

1.36780666666667

1.03447333333333

1.20114000000000

0.86780666666667

1.53447333333333

$-0.00400000000000$

0.66266666666667

0.32933333333333

0.49600000000000

0.16266666666667

0.82933333333333

0.00000000000000

0.33333333333333

0.50000000000000

0.16666666666667

0.83333333333333

0.66177000000000

1.32843666666667

0.99510333333333

1.16177000000000

0.82843666666667

0.82843666666667
1.49510333333333

$\begin{array}{ll}\mathrm{Al} & (6 \mathrm{a}) \\ \mathrm{Al} & (6 \mathrm{a}) \\ \mathrm{Al} & (6 \mathrm{a}) \\ \mathrm{Al} & (6 \mathrm{a}) \\ \mathrm{Al} & (6 \mathrm{a}) \\ \mathrm{Al} & (6 \mathrm{a}) \\ \mathrm{Al} & (6 \mathrm{a}) \\ \mathrm{Al} & (6 \mathrm{a}) \\ \mathrm{Al} & (6 \mathrm{a}) \\ \mathrm{Al} & (6 \mathrm{a}) \\ \mathrm{Al} & (6 \mathrm{a}) \\ \mathrm{Al} & (6 \mathrm{a}) \\ \mathrm{S} & (6 \mathrm{a}) \\ \mathrm{S} & (6 \mathrm{a}) \\ \mathrm{S} & (6 \mathrm{a}) \\ \mathrm{S} & (6 \mathrm{a}) \\ \mathrm{S} & (6 \mathrm{a}) \\ \mathrm{S} & (6 \mathrm{a}) \\ \mathrm{S} & (6 \mathrm{a}) \\ \mathrm{S} & (6 \mathrm{a}) \\ \mathrm{S} & (6 \mathrm{a}) \\ \mathrm{S} & (6 \mathrm{a}) \\ \mathrm{S} & (6 \mathrm{a}) \\ \mathrm{S} & (6 \mathrm{a}) \\ \mathrm{S} & (6 \mathrm{a}) \\ \mathrm{S} & (6 \mathrm{a}) \\ \mathrm{S} & (6 \mathrm{a}) \\ \mathrm{S} & (6 \mathrm{a}) \\ \mathrm{S} & (6 \mathrm{a}) \\ \mathrm{S} & (6 \mathrm{a}) \\ & \end{array}$

$\mathrm{Sr}\left[\mathrm{S}_{2} \mathrm{O}_{6}\right]\left[\mathrm{H}_{2} \mathrm{O}\right]_{4}$ : A10B2C_hP39_171_5c_c_a - CIF

\# CIF file

data findsym-output

audit_creation_method FINDSYM

chemical name mineral ' $\mathrm{Sr}$ [S2O6][H2O]4

chemical formula_sum ' 010 S2 Sr,

loop

publ_author_nam

R. N. Hargreaves

E. Stanley,

journal_name_full_name

Zeitschrift f\{\"u\}r Kristallographie - Crystalline Materials

journal_volume 135

journal year 1972

journal_page first 399

journal page last 407

publ-Section title

The structure of strontium dithionate tetrahydrate

\# Found in Pearson's Crystal Data - Crystal Structure Database for $\hookrightarrow$ Inorganic Compounds, 2013

aflow_title $\operatorname{Sr}\left[S \$_{-}\{2\} \$ O \$_{-}\{6\} \$\right]\left[\mathrm{H} \$_{-}\{2\} \$ O\right] \$_{-}\{4\} \$$ Structure

aflow_proto 'A10B2C_hP39_171_5c_c_a

aflow_params 'a,c/a, z $\{1\}, x_{-}\{2\}, y_{-}\{2\}, z_{-}\{2\}, x_{-}\{3\}, y_{-}\{3\}, z_{-}\{3\}, x_{-}\{4\}, y_{-}\{$ $\hookrightarrow 4\}, \mathrm{z}_{-}\{4\}, \mathrm{x}_{-}\{5\}, \mathrm{y}_{-}\{5\}, \mathrm{z}_{-}\{5\}, \mathrm{x}_{-}\{6\}, \mathrm{y}_{-}\{6\}, \mathrm{z}_{-}\{6\}, \mathrm{x}_{-}\{7\}, \mathrm{y}_{-}\{7\}, \mathrm{z}_{-}\{7\}$

aflow_params_values ' $6.3199906634,3.05221518986,0.0,-0.004,0.253$,

$\hookrightarrow 0.23633,0.015,0.248,0.11034,0.681,0.192,0.16733,0.448,0.188$

$\hookrightarrow 0.29533,0.449,0.25,0.048,0.373,0.065,0.50467$

aflow_Strukturbericht 'None'

aflow_Pearson 'hP39'

cell_length_a $\quad 6.3199906634$

cell_length_b 6.3199906634

cell_length_c 19.2899715026

cell_angle_alpha 90.0000000000

$\begin{array}{ll} & \\ \text { cell_angle_gamma } & 120.0000000000\end{array}$

symmetry_space_group_name_H-M "P 62"

symmetry_Int_Tables_number 171

loop 


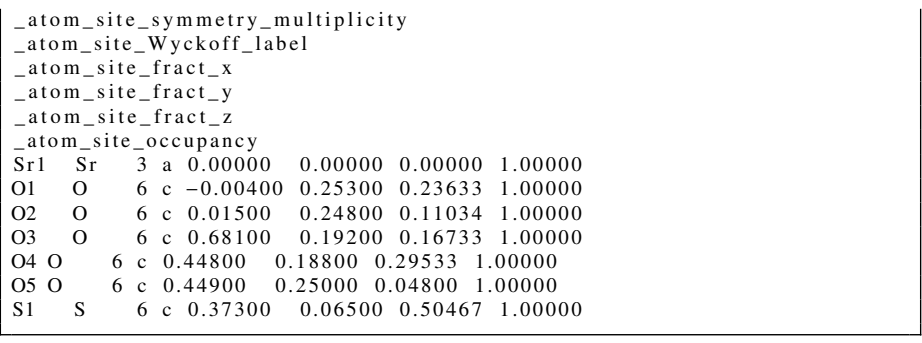

$\mathrm{Sr}\left[\mathrm{S}_{2} \mathrm{O}_{6}\right]\left[\mathrm{H}_{2} \mathrm{O}\right]_{4}$ : A10B2C_hP39_171_5c_c_a - POSCAR

A10B2C_hP39_171_5c_c_a \& a , c/a, z1 , x2 , y2 , z2 , x3, y3 , z3 $, \mathrm{x} 4, \mathrm{y} 4, \mathrm{z} 4, \mathrm{x} 5, \mathrm{y} 5, \mathrm{z} 5, \mathrm{x} 6$ $\hookrightarrow, \mathrm{y} 6, \mathrm{z6}, \mathrm{x} 7, \mathrm{y} 7, \mathrm{z} 7-$-params $=6.3199906634,3.05221518986,0.0,-0.004$ $\hookrightarrow 0.253,0.23633,0.015,0.248,0.11034,0.681,0.192,0.16733,0.448$,

$\hookrightarrow 0.188,0.29533,0.449,0.25,0.048,0.373,0.065,0.50467 \& \mathrm{P}_{-}\{2\} \mathrm{C}_{-}\{$

$\hookrightarrow 6\}^{\wedge}\{4\} \# 171\left(\mathrm{ac}^{\wedge} 6\right) \& \mathrm{hP} 39 \&$ None \& $\mathrm{Sr}[\mathrm{S} 2 \mathrm{O} 6][\mathrm{H} 2 \mathrm{O}] 4$ \& \& R. N.

$\hookrightarrow$ Hargreaves and E. Stanley, Zeitschrift f" $\{$ u $\}$ r Kristallographie

$\hookrightarrow$ - Crystalline Materials 135, 399-407 (1972)

1.00000000000000

$\begin{array}{lll}3.15999533170000 & -5.47327246618487 & 0.00000000000000\end{array}$

$\begin{array}{rrr}3.15999533170000 & 5.47327246618487 & 0.00000000000000\end{array}$

$\begin{array}{rrr}0.00000000000000 & 0.00000000000000 & 19.28997150260000\end{array}$

$$
\begin{array}{rrr}
\mathrm{O} & \mathrm{S} & \mathrm{Sr} \\
30 & 6 & 3
\end{array}
$$

Direct

$-0.00400000000000$

-0.25300000000000
0.25700000000000

0.00400000000000

0.25300000000000

$-0.25700000000000$

0.01500000000000

$-0.24800000000000$

0.23300000000000

0.2330000000000

0.0150000000000

$-0.23300000000000$

0.68100000000000

$-0.19200000000000$

$-0.48900000000000$

$-0.68100000000000$

0.19200000000000

0.48900000000000

0.44800000000000

$-0.18800000000000$

$-0.26000000000000$

$-0.44800000000000$

0.18800000000000

0.26000000000000

0.44900000000000

$-0.25000000000000$

$-0.44900000000000$

0.25000000000000

0.19900000000000

0.37300000000000

$-0.06500000000000$

$-0.30800000000000$

$-0.37300000000000$

0.06500000000000

0.30800000000000

0.00000000000000

0.00000000000000

0.00000000000000

0.25300000000000 $-0.2570000000000$ 0.00400000000000 $-0.25300000000000$ 0.2570000000000 $-0.00400000000000$ 0.24800000000000 $-0.23300000000000$ $-0.01500000000000$ 0.0150000000 0.23300000000000 0.01500000000000 0.0150000000000 0.48900000000000 $-0.68100000000000$ $-0.19200000000000$ 0.48900000000000 0.68100000000000 0.18800000000000 0.26000000000000 $-0.4480000000000$ $-0.18800000000000$ $-0.26000000000000$ 0.44800000000000 0.25000000000000 0.1990000000000 $-0.44900000000000$ $-0.25000000000000$ $-0.19900000000000$ 0.44900000000000 0.06500000000000 0.06500000000000 0.30800000000000 $-0.06500000000000$ $-0.3080000000000$ 0.3730000000000 0.00000000000000 0.00000000000000

0.23633000000000 0.9029966666666 0.5696633333333 0.23633000000000 0.90299666666667 0.56966333333333 0.11034000000000 0.77700666666667 0.4436733333333 0.11034000000000 0.77700666666667 0.77700666666667 0.4436733333333 0.5006633333333 0.16733000000000 0.83399666666667 0.50066333333333 0.2953300000000 0.96199666666667 0.62866333333333 0.29533000000000 0.96199666666667 0.62866333333333 0.04800000000000 0.71466666666667 0.38133333333663 0.38133333333333
0.04800000000000 0.0480000000000 0.71466666666667 0.50467000000000 0.50467000000000 1.17133666666667 0.83800333333333 0.50467000000000 1.1713366666666 0.83800333333333 0.00000000000000 0.6666666666666 0.33333333333333

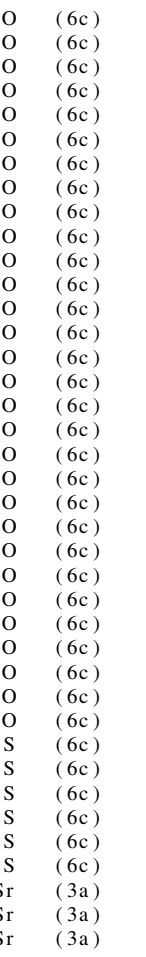

$\mathrm{Sr}\left[\mathrm{S}_{2} \mathrm{O}_{6}\right]\left[\mathrm{H}_{2} \mathrm{O}\right]_{4}:$ A10B2C_hP39_172_5c_c_a - CIF

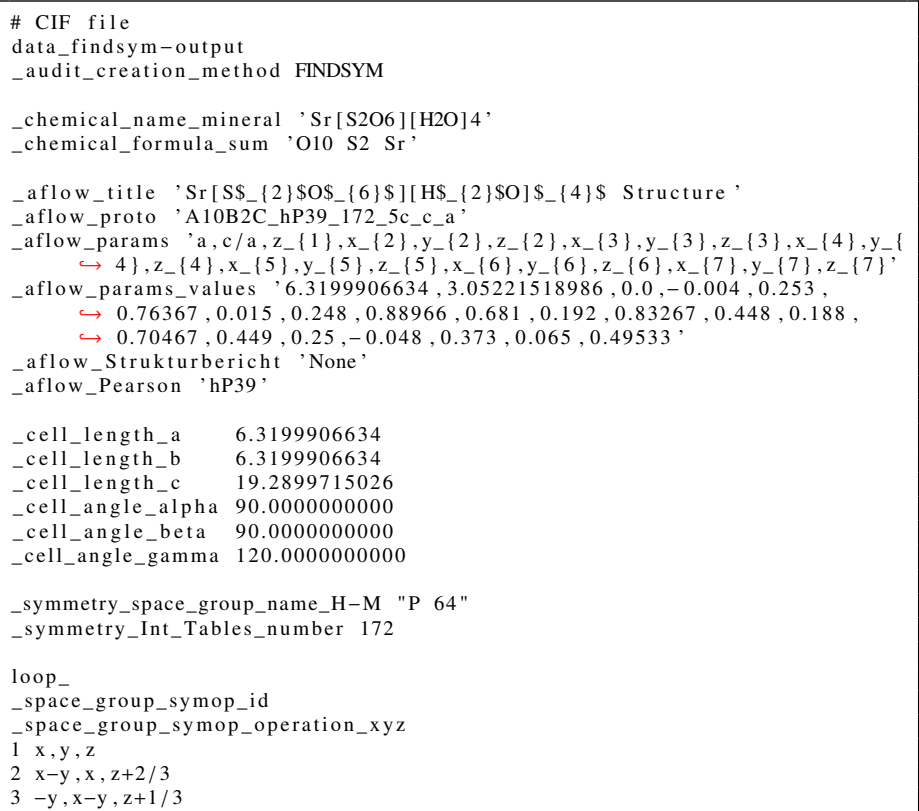

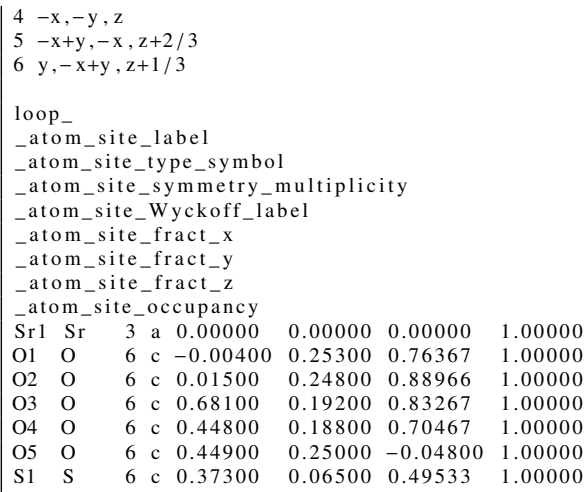

$\mathrm{Sr}\left[\mathrm{S}_{2} \mathrm{O}_{6}\right]\left[\mathrm{H}_{2} \mathrm{O}\right]_{4}$ : A10B2C_hP39_172_5c_c_a - POSCAR

A10B2C_hP39_172_5c_c_a \& a ,c/a, z1, x2,y2, z2, x3,y3,z3, x4,y4,z4, x5,y5, z5 , x6 $\hookrightarrow, \mathrm{y} 6, \mathrm{z} 6, \mathrm{x} 7, \mathrm{y} 7, \mathrm{z} 7-$-params $=6.3199906634,3.05221518986,0.0,-0.004$ $\Longrightarrow 0.253,0.76367,0.015,0.248,0.88966,0.681,0.192,0.83267,0.448$ $\left.\hookrightarrow \begin{array}{l}\hookrightarrow \\ \hookrightarrow\end{array} 6\right\}^{\wedge}\{5\} \# 172\left(\mathrm{ac}^{\wedge} 6\right) \& \mathrm{hP} 39 \&$ None \& Sr[S2O6][H2O $] 4 \& \mathrm{Sr}[\mathrm{S} 2 \mathrm{O} 6]\left[{ }_{-}\right.$

1.00000000000000

$\begin{array}{lll}3.15999533170000 & -5.47327246618487 & 0.00000000000000\end{array}$

$\begin{array}{llll}3.15999533170000 & 5.47327246618487 & 0.00000000000000\end{array}$

$\begin{array}{lll}0.00000000000000 & 0.00000000000000 & 19.28997150260000\end{array}$

$\begin{array}{rrr}\mathrm{O} & \mathrm{S} & \mathrm{Sr} \\ 30 & 6 & 3\end{array}$

Direc

$\begin{array}{lll}-0.00400000000000 & 0.25300000000000 & 0.76367000000000\end{array}$

$-0.25300000000000-0.25700000000000$

$0.25700000000000-0.00400000000000$

$0.2570000000000-0.0530000000000$

$0.0040000000000--0.25300000000000$

$0.2530000000000-0.25700000000000$

$0.01500000000000 \quad 0.24800000000000$

$-0.24800000000000-0.23300000000000$

$0.23300000000000-0.01500000000000$

$-0.01500000000000-0.24800000000000$

$0.24800000000000 \quad 0.23300000000000$

$-0.23300000000000 \quad 0.01500000000000$

$0.68100000000000 \quad 0.19200000000000$

$-0.19200000000000 \quad 0.48900000000000$

$-0.48900000000000-0.68100000000000$

$\begin{array}{ll}-0.68100000000000 & -0.19200000000000 \\ 0.19200000000000 & -0.48900000000000\end{array}$

$0.48900000000000-0.68100000000000$

$0.44800000000000 \quad 0.18800000000000$

$-0.18800000000000-0.26000000000000$

$-0.26000000000000-0.44800000000000$

$-0.44800000000000-0.1880000000000$

$0.18800000000000-0.26000000000000$

$0.26000000000000 \quad 0.44800000000000$

$0.44900000000000 \quad 0.25000000000000$

$-0.25000000000000 \quad 0.19900000000000$

$-0.19900000000000-0.44900000000000$

$-0.44900000000000-0.25000000000000$

$0.25000000000000-0.19900000000000$

$0.19900000000000 \quad 0.44900000000000$

$0.37300000000000 \quad 0.06500000000000$

$-0.06500000000000 \quad 0.30800000000000$

$-0.30800000000000-0.37300000000000$

$-0.37300000000000-0.06500000000000$

$0.06500000000000-0.30800000000000$

$0.30800000000000 \quad 0.37300000000000$

$0.00000000000000 \quad 0.0000000000000$

$0.000000000000-0.000000000000$

$\begin{array}{ll}0.00000000000000 & 0.00000000000000 \\ 0.00000000000000 & 0.00000000000000\end{array}$

1.43033666666667

0.76367000000000

1.09700333333333

1.43033666666667

0.88966000000000

1.22299333333333

1.55632666666667

0.88966000000000

1.22299333333333

1.55632666666667

0.83267000000000

1.16600333333333

1.49933666666667

0.83267000000000

1.16600333333333

1.49933666666667

0.70467000000000

1.03800333333333

1.37133666666667

0.70467000000000

1.03800333333333

1.37133666666667

0.04800000000000

0.28533333333333

0.61866666666667

$-0.04800000000000$

0.285333333333333

0.61866666666667

0.49533000000000

1. 16199666666667

0.49533000000000

0.82866333333333

1.16199666666667

0.00000000000000

0.33333333333333

0.66666666666667

$\mathrm{O} \quad(6 \mathrm{c})$

O $\quad(6 c)$

$\begin{array}{ll}\mathrm{O} & (6 \mathrm{c}) \\ \mathrm{O} & (6 \mathrm{c})\end{array}$

$\begin{array}{ll}\mathrm{O} & (6 \mathrm{c}) \\ \mathrm{O} & (6 \mathrm{c})\end{array}$

O $(6 c)$

O (6c)

$\mathrm{O} \quad(6 \mathrm{c})$

$\mathrm{O} \quad(6 \mathrm{c})$

$\mathrm{O} \quad(6 \mathrm{c})$

$\mathrm{O} \quad(6 \mathrm{c})$

$\mathrm{O} \quad(6 \mathrm{c})$

O (6c)

$\mathrm{O} \quad(6 \mathrm{c})$

$\mathrm{O} \quad(6 \mathrm{c})$

O $\quad(6 c)$

$\begin{array}{ll}\mathrm{O} & (6 \mathrm{c}) \\ \mathrm{O} & (6 \mathrm{c})\end{array}$

$\begin{array}{ll}0 \\ \mathrm{O} & (6 \mathrm{c})\end{array}$

O (6c)

O $(6 c)$

O $(6 c)$

$\mathrm{O} \quad(6 \mathrm{c})$

$\mathrm{O} \quad(6 \mathrm{c})$

$\mathrm{O} \quad(6 \mathrm{c})$

O (6c)

O (6c)

$\mathrm{O} \quad(6 \mathrm{c})$

$\mathrm{O} \quad(6 \mathrm{c})$

$\mathrm{S} \quad(6 \mathrm{c})$

$\mathrm{PI}_{3}$ : A3B_hP8_173_c_b - CIF

\# CIF file

data findsym-output

audit_creation_method FINDSYM

chemical name mineral 'PI3,

chemical formula sum , I3 P

loop

publ_author_nam

E. T. Lance

J. M. Haschke

D. R. Peacor

e_full_name

Inorganic Chemistry

journal_volume 15

journal_year 1976

journal_page_first 780

Journal page last 781

publ-Section title

Crystal and molecular structure of phosphorus triiodide

\# Found in Pearson's Crystal Data - Crystal Structure Database for 


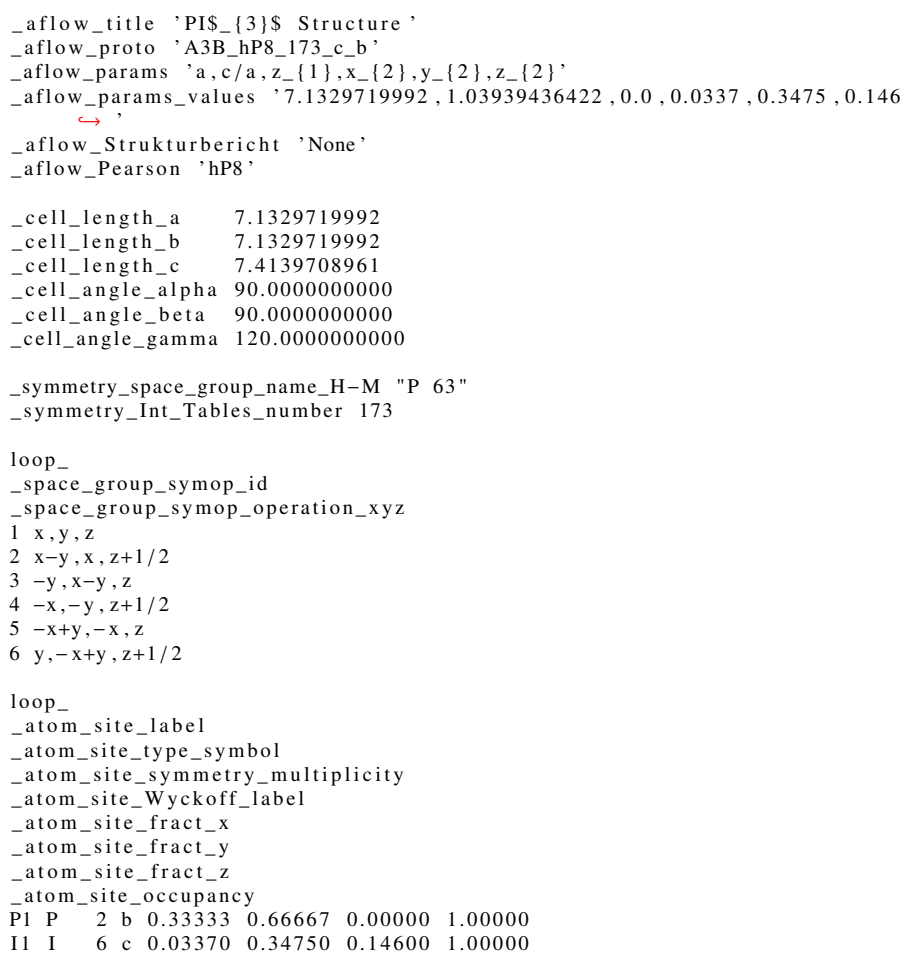

PI 3 : A3B_hP8_173_c_b - POSCAR

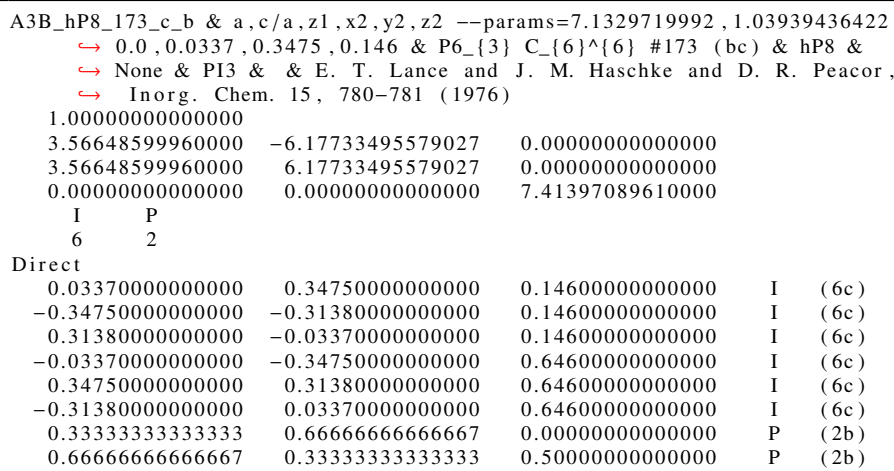

$\beta-\mathrm{Si}_{3} \mathrm{~N}_{4}$ : A4B3_hP14_173_bc_c - CIF

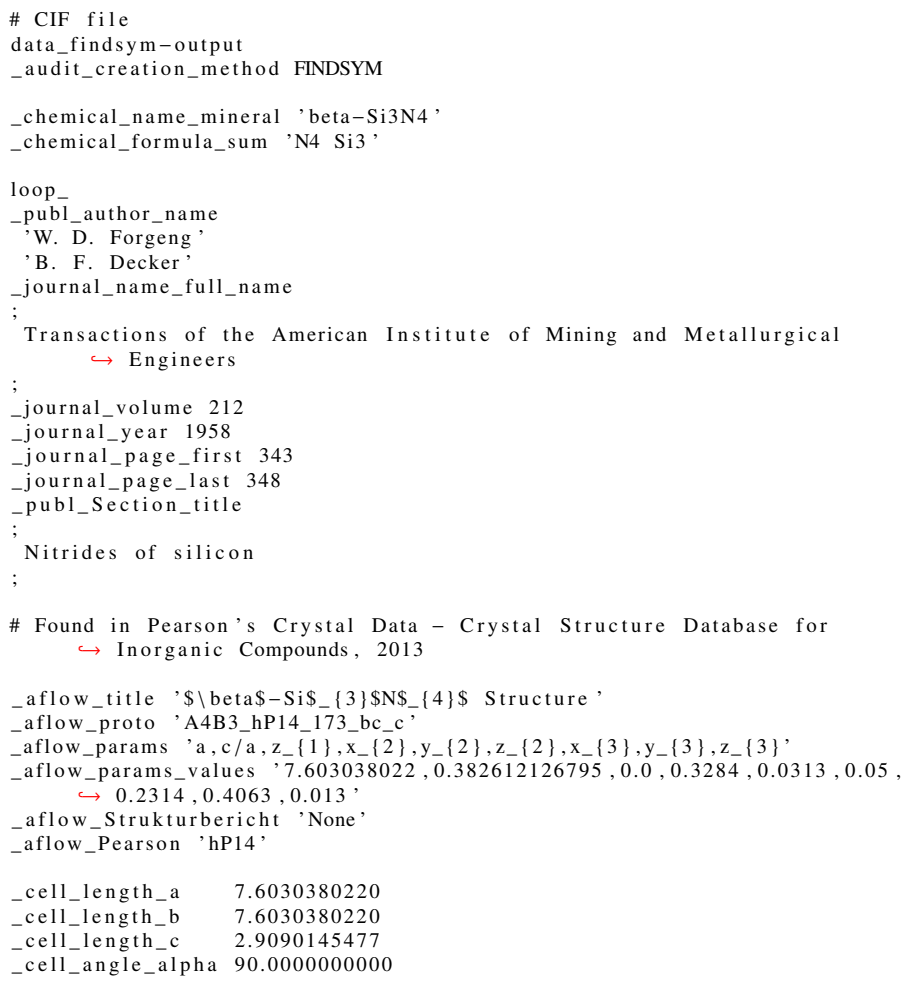

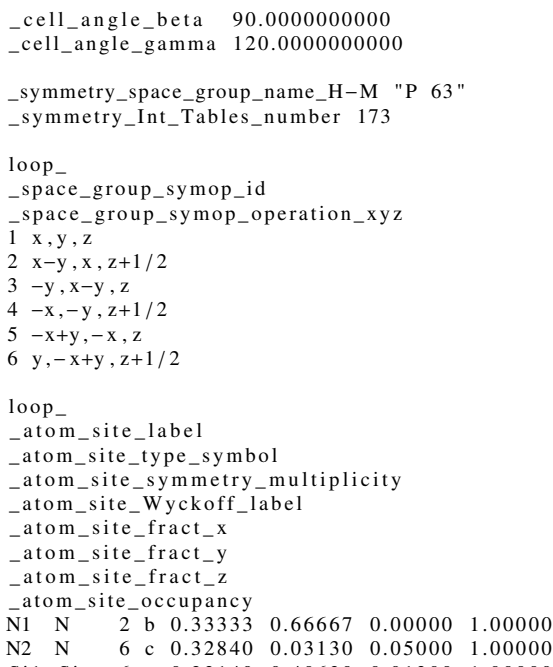

0.333333333333333 0.66666666666667 0.32840000000000 $-0.0313000000000$ $-0.29710000000000$ $-0.32840000000000$ 0.03130000000000 0.29710000000000 0.23140000000000 $-0.40630000000000$ 0.17490000000000 $-0.23140000000000$ 0.40630000000000 $-0.17490000000000$

$0.66666666666667-0.00000000000000$ 0.33333333333333 0.03130000000000 0.29710000000000 $-0.32840000000000$ $-0.03130000000000$ $-0.29710000000000$ 0.32840000000000 0.40630000000000 $-0.17490000000000$ $-0.23140000000000$ $-0.40630000000000$ $-0.174900000000$ 0.23140000000000 0.50000000000000 0.05000000000000 0.05000000000000 0.05000000000000 0.05000000000000 0.55000000000000 0.55000000000000 0.55000000000000 0.01300000000000 0.01300000000000 0.51300000000000 0.513000000000 0.51300000000000

$\mathrm{Fe}_{12} \mathrm{Zr}_{2} \mathrm{P}_{7}$ : A12B7C2_hP21_174_2j2k_ajk_cf - CIF

\# CIF file

data_findsym-output audit_creation_method FINDSYM

chemical name mineral ' $\mathrm{Fe} 12 \mathrm{Zr} 2 \mathrm{P} 7$, chemical formula sum 'Fe12 P7 Zr2'

loop

publ_author_nam

,E. Ganglberger

_journal_name_full_nam

Monatshefte $f\{\backslash " u\} r$ Chemie - Chemical Monthly

journal_volume 99

journal_year 1968

journal_page_first 557

journal page last 565

publ_Section_title

Die Kristallstruktur von Fe\$ $\{12\}$ ZZT $\{2\} \$ P \$\{\{7\}$

Found in Pearson's Crystal Data - Crystal Structure Database for $\hookrightarrow$ Inorganic Compounds, 2013

aflow_title ${ }_{-} \mathrm{Fe}_{-}\{12\} \$ \mathrm{Zr} \$_{-}\{2\} \$ P \$_{-}\{7\} \$$ Structure,

aflow_proto 'A12B7C2_hP21_174_2j2k_ajk_cf

aflow_params 'a, c/a, $x_{-}\{4\}, y_{-}\{4\}, x_{-}\{5\}, y_{-}\{5\}, x_{-}\{6\}, y_{-}\{6\}, x_{-}\{7\}, y_{-}\{7\}, x_{-}\{$ $\hookrightarrow 8\}, \mathrm{y}_{-}\{8\}, \mathrm{x}_{-}\{9\}, \mathrm{y}_{-}\{9\}$

aflow_params_values '9.0004021308, $0.399102242177,0.4309,0.3719,0.1189$, $\hookrightarrow 0.2772,0.4163,0.1204,0.0495,0.4359,0.2232,0.124,0.2889,0.4096$, aflow_Strukturbericht 'None' aflow_Pearson 'hP21

cell_length_a 9.0004021308 Ccell_length_b 9.0004021308 cell length c 3.5920806709

cellengle alpha 90.0000000000

_cell_angle_alpha 90.000000000

$\begin{array}{ll} & \\ \text { cell_angle_gamma } & 120.0000000000\end{array}$

symmetry_space_group_name_H-M "P -6"

symmetry_Int_Tables_number 174 
loop

_space_group_symop_id

_space_group_symop_operation_xyz

$1 \mathrm{x}, \mathrm{y}, \mathrm{z}$

$2-y, x-y, z$

$3-x+y,-x, z$
$4-x+y,-x,-z$

$5 \mathrm{x}, \mathrm{y},-\mathrm{z}$

$6-\mathrm{y}, \mathrm{x}-\mathrm{y},-$

loop

_atom_site_type_symbol

atom_site_symmetry_multiplicity

atom_site_Wyckoff_label

atom_site_fract_x

atom_site_fract_y

atom_site_fract_z

- atom_site_occupancy

\begin{tabular}{lllll}
\hline & 0.00000 & 0.00000 & 0.00000 & 1.00000
\end{tabular}

$\begin{array}{llllllllll}\mathrm{Zr} 1 & \mathrm{Zr} & 1 & \mathrm{c} & 0.33333 & 0.66667 & 0.00000 & 1.00000\end{array}$

$\begin{array}{lllllllll}\mathrm{Zr} 2 & \mathrm{Zr} & 1 & \mathrm{f} & 0.66667 & 0.33333 & 0.50000 & 1.00000\end{array}$

$\begin{array}{lllllllll}\mathrm{Fe} 1 & \mathrm{Fe} & 3 & \mathrm{j} & 0.43090 & 0.37190 & 0.00000 & 1.00000\end{array}$

$\begin{array}{llllllll}\mathrm{Fe} 2 & \mathrm{Fe} & 3 & \mathrm{j} & 0.11890 & 0.27720 & 0.00000 & 1.00000\end{array}$

$\begin{array}{llllllll}\mathrm{P} 2 & \mathrm{P} & 3 & \mathrm{j} & 0.41630 & 0.12040 & 0.00000 & 1.00000\end{array}$

$\begin{array}{llllllll}\mathrm{Fe} 3 & \mathrm{Fe} & 3 \mathrm{k} & 0.04950 & 0.43590 & 0.50000 & 1.00000\end{array}$

$\begin{array}{llllllll}\mathrm{Fe} 4 & \mathrm{Fe} & 3 & \mathrm{k} & 0.22320 & 0.12400 & 0.50000 & 1.00000\end{array}$

$\begin{array}{llllllllll}\mathrm{P} 3 & \mathrm{P} & 3 & \mathrm{k} & 0.28890 & 0.40960 & 0.50000 & 1.00000\end{array}$

$\mathrm{Fe}_{12} \mathrm{Zr}_{2} \mathrm{P}_{7}$ : A12B7C2_hP21_174_2j2k_ajk_cf - POSCAR

A12B7C2_hP21_174_2j2k_ajk_cf \& a,c/a, x4,y4, x5,y5, x6, y6, x7,y7, x8,y8, x9, y9 $\hookrightarrow-$ params $=9.0004021308,0.399102242177,0.4309,0.3719,0.1189$

$\hookrightarrow 0.2772,0.4163,0.1204,0.0495,0.4359,0.2232,0.124,0.2889,0.4096 \&$

$\hookrightarrow \mathrm{P}-6 \mathrm{C}_{-}\{3 \mathrm{~h}\}^{\wedge}\{1\} \# 174\left(\mathrm{acfj}^{\wedge} 3 \mathrm{k}^{\wedge} 3\right) \& \mathrm{hP} 21$ \& None \& Fe12Zr2P7 \& \&

$\hookrightarrow$ E. Ganglberger, Monatsh. Chem. 99, 557-565 (1968)

1.00000000000000

$\begin{array}{lll}4.50020106540000 & -7.79457688954839 & 0.00000000000000\end{array}$

$\begin{array}{lll}4.50020106540000 & 7.79457688954839 & 0.00000000000000\end{array}$

$\begin{array}{lll}0.00000000000000 & 0.00000000000000 & 3.59208067090000\end{array}$

$\mathrm{Fe} \quad \mathrm{P} \quad \mathrm{Zr}$

Direct

0.43090000000000

$-0.37190000000000$

$-0.05900000000000$

0.11890000000000

$-0.27720000000000$

0.15830000000000

0.04950000000000

$-0.43590000000000$

0.38640000000000

0.22320000000000

$-0.12400000000000$

$-0.09920000000000$

0.00000000000000

0.41630000000000

0.12040000000000

$-0.12040000000000$

0.2959000000000

0.28890000000000

$-0.40960000000000$

0.12070000000000

0.33333333333333
0.66666666666667

0.37190000000000 0.0590000000000 $-0.43090000000000$ 0.27720000000000 $-0.15830000000000$ $-0.11890000000000$ 0.4359000000000 $-0.38640000000000$ $-0.04950000000000$ 0.12400000000000 0.09920000000000 $-0.22320000000000$ 0.0000000000000 0.1204000000000 0.12059000000000 0.295900000000 $-0.4163000000000$ 0.4096000000000 $-0.1207000000000$ $-0.28890000000000$ 0.6666666666666 0.33333333333333

0.00000000000000 0.0000000000000 0.00000000000000 0.0000000000000 0.00000000000000 0.0000000000000 0.50000000000000 0.50000000000000 0.50000000000000 0.50000000000000 0.50000000000000 0.50000000000000 0.000000000000 0.000000000000 0.00000000000000 . 0.0000000000000 0.50000000000000 0.50000000000000 0.5000000000000 0.00000000000000 0.50000000000000

$\begin{aligned} \mathrm{Fe} & (3 \mathrm{j}) \\ \mathrm{Fe} & (3 \mathrm{j}) \\ \mathrm{Fe} & (3 \mathrm{j}) \\ \mathrm{Fe} & (3 \mathrm{j}) \\ \mathrm{Fe} & (3 \mathrm{j}) \\ \mathrm{Fe} & (3 \mathrm{j}) \\ \mathrm{Fe} & (3 \mathrm{k}) \\ \mathrm{Fe} & (3 \mathrm{k}) \\ \mathrm{Fe} & (3 \mathrm{k}) \\ \mathrm{Fe} & (3 \mathrm{k}) \\ \mathrm{Fe} & (3 \mathrm{k}) \\ \mathrm{Fe} & (3 \mathrm{k}) \\ \mathrm{P} & (1 \mathrm{a}) \\ \mathrm{P} & (3 \mathrm{j}) \\ \mathrm{P} & (3 \mathrm{j}) \\ \mathrm{P} & (3 \mathrm{j}) \\ \mathrm{P} & (3 \mathrm{k}) \\ \mathrm{P} & (3 \mathrm{k}) \\ \mathrm{P} & (3 \mathrm{k}) \\ \mathrm{Zr} & (1 \mathrm{c}) \\ \mathrm{Zr} & (1 \mathrm{f})\end{aligned}$

GdSI: ABC_hP12_174_cj_fk_aj - CIF

\section{\# CIF file}

data_findsym-output

_audit_creation_method FINDSYM

chemical_name_mineral 'GdSI'

chemical_formula_sum 'Gd I S,

loop

publ_author_name

'C. Dagron',

journal_name_full_name

Comptes Rendus Hebdomadaires des $\mathbf{S}\{\backslash$ ' eances de l'Acad $\{\backslash$ 'e $\}$ mie des $\hookrightarrow$ Sciences $\mathrm{S}\{\backslash$ ’e\}rie C - Sciences chimiques

journal_volume 268

-journal year 1969

_journal_page_first 1867

_journal_page_last 1869

-publ_Section_title

$R\{\backslash \backslash$ 'e $\}$ partition des types cristallins dans la s $\{\backslash \backslash$ 'e $\}$ rie des $\hookrightarrow$ iodosulfures et fluorosulfures des $\{\backslash \backslash$ 'e $\} 1\{\backslash \backslash$ 'e\}ments des $\hookrightarrow$ terres rares et d`yttrium

Found in Pearson's Crystal Data - Crystal Structure Database for $\hookrightarrow$ Inorganic Compounds, 2013

aflow title 'GdSI Structure'

aflow_proto 'ABC_hP12_174_cj_fk_aj,

aflow params a, c/a, $x_{-}\{4\}, y_{-}\{4\}, x_{-}\{5\}, y_{-}\{5\}, x_{-}\{6\}, y_{-}\{6\}$

aflow params values $, 10.7303215747,0.395153774459,0.30167,0.15433$ -P $0.03467,0.51733,0.14967,0.31433$

aflow_Strukturbericht 'None'

aflow_Pearson 'hP12,

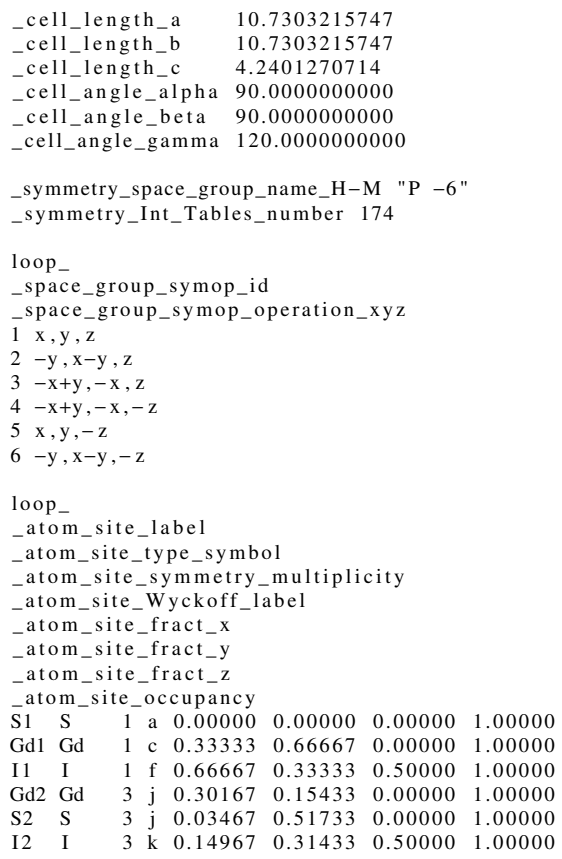

$\begin{array}{llllllll}\text { S1 S } & 1 \text { a } & 0.00000 & 0.00000 & 0.00000 & 1.00000\end{array}$ $\begin{array}{llllllll} & 1 & \text { a } & 0.00003 & 0.00000 & 0.00000 & 1.00000\end{array}$ $\begin{array}{lllllllll} & \mathrm{Gd} 1 \mathrm{Gd} & \mathrm{I} & \mathrm{c} & 0.33333 & 0.66667 & 0.00000 & 1.00000\end{array}$ $\begin{array}{lllllll}11 & 1 & 1 & 0.66667 & 0.33333 & 0.50000 & 1.00000\end{array}$ $\begin{array}{llllllll}\mathrm{Gd} 2 \mathrm{Gd} & 3 & \mathrm{j} & 0.30167 & 0.15433 & 0.00000 & 1.00000\end{array}$

$\begin{array}{lllllllll}\mathrm{S} 2 & \mathrm{~S} & & 3 & \mathrm{j} & 0.03467 & 0.51733 & 0.00000 & 1.00000 \\ \mathrm{I} 2 & \mathrm{I} & & 3 & \mathrm{k} & 0.14967 & 0.31433 & 0.50000 & 1.00000\end{array}$

GdSI: ABC_hP12_174_cj_fk_aj - POSCAR

\begin{tabular}{|c|c|c|c|c|}
\hline \multicolumn{5}{|c|}{$\begin{array}{l}\hookrightarrow \& \mathrm{P}-6 \mathrm{C}_{-}\{3 \mathrm{~h}\}^{\wedge}\{1\} \# 174\left(\mathrm{acfj}^{\wedge} 2 \mathrm{k}\right) \& \mathrm{hP} 12 \text { \& None \& GdSI \& \& } \mathrm{C} . \\
\hookrightarrow \text { Dagron and F. Thevet, }\{\mathrm{C} \text {. R. Hebd. S }\{\text { e }\} \text { ances Acad. Sci. C } 268\end{array}$} \\
\hline$\hookrightarrow \quad 1867-1869$ & (1969) & & & \\
\hline \multicolumn{5}{|l|}{1.00000000000000} \\
\hline 5.36516078735000 & -9.29273107446644 & 0.00000000000000 & & \\
\hline 5.36516078735000 & 9.29273107446644 & 0.00000000000000 & & \\
\hline \multicolumn{5}{|l|}{0.00000000000000} \\
\hline $\mathrm{Gd}$ & & & & \\
\hline 4 & & & & \\
\hline \multicolumn{5}{|l|}{ Direct } \\
\hline 0.33333333333333 & 0.6666666 & 0.00000000000000 & $\mathrm{Gd}$ & (1c) \\
\hline 0.30167000000000 & 0.15433000000000 & 0.00000000000000 & $\mathrm{Gd}$ & $(3 \mathrm{j})$ \\
\hline-0.15433000000000 & 0.14734000000000 & 0.00000000000000 & $\mathrm{Gd}$ & $(3 \mathrm{j})$ \\
\hline-0.14734000000000 & -0.30167000000000 & 0.00000000000000 & $\mathrm{Gd}$ & $(3 \mathrm{j})$ \\
\hline 0.666666666666667 & 0.33333333333333 & 0.50000000000000 & I & (1f) \\
\hline 0.14967000000000 & 0.31433000000000 & 0.50000000000000 & I & $(3 \mathrm{k})$ \\
\hline-0.31433000000000 & -0.16466000000000 & 0.50000000000000 & I & $(3 \mathrm{k})$ \\
\hline 0.16466000000000 & -0.14967000000000 & 0.50000000000000 & I & $(3 \mathrm{k})$ \\
\hline 0.00000000000000 & 0.00000000000000 & 0.00000000000000 & $\mathrm{~s}$ & (1a) \\
\hline 0.03467000000000 & 0.51733000000000 & 0.00000000000000 & $\mathrm{~s}$ & $(3 \mathrm{j})$ \\
\hline-0.51733000000000 & -0.48266000000000 & 0.00000000000000 & $\mathrm{~s}$ & $(3 \mathrm{j})$ \\
\hline 0.48266000000000 & -0.03467000000000 & 0.00000000000000 & $\mathrm{~s}$ & $(3 \mathrm{j})$ \\
\hline
\end{tabular}

$\mathrm{Nb}_{7} \mathrm{Ru}_{6} \mathrm{~B}_{8}$ : A8B7C6_hP21_175_ck_aj_k - CIF

\section{\# CIF file}

data_findsym-output

audit_creation_method FINDSYM

chemical_name_mineral 'Nb7Ru6B8,

chemical_formula_sum 'B8 Nb7 Ru6

loop

publ_author_name

'Q. Zheng',

M. Kohout,

'R. Gumeniuk,

'N. Abramchuk'

'H. Borrmann'

'Y. Prots,

U. Burkhardt

'W. Schnelle'

'L. Akselrud'

'H. Gu'

A. \{Leithe-Jasper

_journal_name_full_name

Inorganic Chemistry

journal_volume 51

journal year 2012

journal_page_first 7472

journal_page_last 7483

publ_Section_title

$\mathrm{TM} \$\{7\} \$ \mathrm{TM} \backslash$ '\$ $\left.\{6\} \$ B \$\{8\} \$(\mathrm{TM}=\mathrm{Ta}, \mathrm{Nb} ; \mathrm{TM}\rangle^{\prime}=\mathrm{Ru}, \mathrm{Rh}, \mathrm{Ir}\right):$ New $\hookrightarrow$ Compounds with [B\$_\{6\}\$] Ring Polyanions

Found in Pearson's Crystal Data - Crystal Structure Database for $\leftrightarrow$ Inorganic Compounds, 2013

aflow_title 'Nb\$_\{7\}\$Ru\$_ $\{6\} \$ B \$ \_\{8\} \$$ Structure

_aflow_proto 'A8B7C6_hP21_175_ck_aj_k 


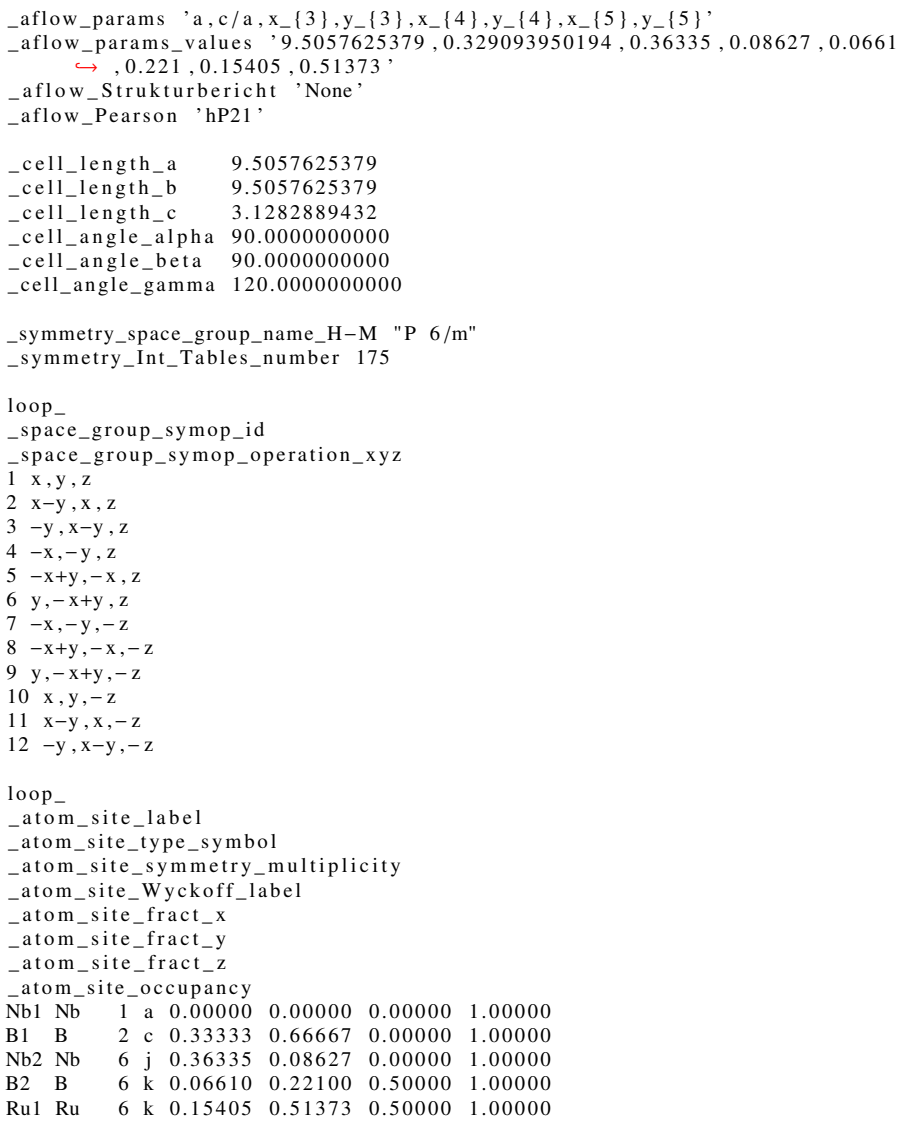

$\mathrm{Nb}_{7} \mathrm{Ru}_{6} \mathrm{~B}_{8}$ : A8B7C6_hP21_175_ck_aj_k - POSCAR

A8B7C6_hP21_175_ck_aj_k \& a , c/a, x3 ,y3 , x4,y4, x5,y5 --params $=9.5057625379$, $\hookrightarrow 0.329093950194,0.36335,0.08627,0.0661,0.221,0.15405,0.51373 \&$

$\hookrightarrow \mathrm{P} 6 / \mathrm{m} \mathrm{C} \_\{6 \mathrm{~h}\}^{\wedge}\{1\} \# 175\left(\mathrm{acjk}^{\wedge} 2\right) \& \mathrm{hP} 21 \&$ None \& Nb7Ru6B8 \& \&

$\hookrightarrow$ Zheng et al., Inorg. Chem. 51, 7472-7483 (2012) 1.00000000000000

4.75288126895000

$\begin{array}{llll}4.75288126895000 & -8.23223184016384 & 0.00000000000000\end{array}$

$\begin{array}{lll}4.75288126895000 & 8.23223184016384 & 0.00000000000000\end{array}$

$\begin{array}{lll}4.75288126895000 & 8.23223184016384 & 0.00000000000000 \\ 0.00000000000000 & 0.00000000000000 & 3.12828894320000\end{array}$

B $\mathrm{Nb} \quad \mathrm{Ru}$

Direct

0.33333333333333

0.6666666663333

0.66666666666667

0.22100000000000

0.15490000000000

0.06610000000000

0.22100000000000

$-0.15490000000000$

0.00000000000000

0.36335000000000

$-0.08627000000000$

$-0.27708000000000$

$-0.3633500000000$

0.08627000000000

0.0862700000000

0.15405000000000

$-0.51373000000000$

0.35968000000000

$-0.15405000000000$

0.51373000000000

0.666666666666667

0.33333333333333

0.22100000000000

$-0.15490000000000$

$-0.06610000000000$

$-0.22100000000000$

0.15490000000000

0.06610000000000

00000000000000

.08627000000000

0.27708000000000

$-0.3633500000000$

$-0.08627000000000$

$-0.27708000000000$

0.36335000000000

0.51373000000000

$-0.35968000000000$

$-0.15405000000000$

$-0.51373000000000$

0.3596800000000

0.15405000000000

0.00000000000000

0.0000000000000

0.50000000000000

0.50000000000000

0.50000000000000

0.50000000000000

0.50000000000000

0.50000000000000

0.00000000000000

0.00000000000000

0.00000000000000

0.0000000000000

0.00000000000000

.

(0.000000000000

0.50000000000000

0.50000000000000

0.50000000000000

0.50000000000000

0.50000000000000

0.50000000000000

0.50000000000000

journal_volume 50

journal_year 2010

journal_page_first 1116

journal_page_last 1122

publ_Section_title

Magnesium imide: synthesis and structure determination of an

$\hookrightarrow$ unconventional alkaline earth imide from decomposition of

$\hookrightarrow$ magnesium amid

Found in Pearson's Crystal Data - Crystal Structure Database for $\hookrightarrow$ Inorganic Compounds, 2013

aflow title 'Mg[NH] Structure,

aflow_proto 'ABC_hP36_175_jk_jk_jk,

aflow params $, a, c / a, x_{-}\{1\}, y_{-}\{1\}, x_{-}\{2\}, y_{-}\{2\}, x_{-}\{3\}, y_{-}\{3\}, x_{-}\{4\}, y_{-}\{4\}, x_{-} \mid$ $\hookrightarrow 5\}, \mathrm{y}_{-}\{5\}, \mathrm{x}_{-}\{6\}, \mathrm{y}_{-}\{6\}$

(1) $\leftrightarrow 0.416,0.3483,0.0762,0.2334,0.5727,0.4279,0.0715,0.1367,0.5046$; aflow_Strukturbericht 'None

-aflow_Pearson 'hP36'

cell_length_a $\quad 11.5799622371$

cell_length_b $\quad 11.5799622371$

cell_length_c $\quad 3.6812151535$

cell_angle_alpha 90.0000000000

20.0000000000

symmetry space group name_H-M "P $6 / \mathrm{m}$ "

symmetry_Int_Tables_number 175

loop

space_group_symop_i

_operation_xyz

$\mathrm{x}, \mathrm{y}, \mathrm{z}$

$\mathrm{x}-\mathrm{y}, \mathrm{x}, \mathrm{z}$

$3-y, x-y, z$

$4-x,-y, z$

$5-x+y,-x, z$

$6,-x+y, z$
$7-x,-y,-z$

$8-\mathrm{x}+\mathrm{y},-\mathrm{x},-\mathrm{z}$

9 y, $-x+y,-z$

$10 \mathrm{x}, \mathrm{y},-\mathrm{z}$

$1 \mathrm{x}-\mathrm{y}, \mathrm{x},-\mathrm{z}$

$12-y, x-y,-z$

loop

atom_site_label

atom_site_type_symbol

atom_site_symmetry_multiplicity

atom_site_Wyckoff_label

atom_site_fract_x

atom_site_fract_y

atom_site_fract_z

atom_site_occupancy

$\begin{array}{lllllllll}\mathrm{H} 1 & \mathrm{H} & 6 & \mathrm{j} & 0.25500 & 0.05980 & 0.00000 & 1.00000\end{array}$

$\begin{array}{llllllll}\mathrm{Mg} 1 \mathrm{Mg} & 6 & \mathrm{j} & 0.13230 & 0.41600 & 0.00000 & 1.00000\end{array}$

$\begin{array}{llllllllll}\mathrm{N} 1 & \mathrm{~N} & 6 & \mathrm{j} & 0.34830 & 0.07620 & 0.00000 & 1.00000\end{array}$

$\begin{array}{lllllllllll}\mathrm{H} 2 & \mathrm{H} & 6 & \mathrm{k} & 0.23340 & 0.57270 & 0.50000 & 1.00000\end{array}$

$\begin{array}{lllllllll}\mathrm{Mg} 2 & \mathrm{Mg} & 6 & \mathrm{k} & 0.42790 & 0.07150 & 0.50000 & 1.00000\end{array}$

$\begin{array}{lllllllll}\mathrm{Mg} 2 & \mathrm{Mg} & 6 & \mathrm{k} & 0.42790 & 0.07150 & 0.50000 & 1.00000 \\ \mathrm{~N} 2 & \mathrm{~N} & 6 & \mathrm{k} & 0.13670 & 0.50460 & 0.50000 & 1.00000\end{array}$

$\mathrm{Mg}[\mathrm{NH}]: \mathrm{ABC} \_\mathrm{hP} 36 \_175 \_j \mathrm{k} \_\mathrm{jk} \_\mathrm{jk}$ - POSCAR

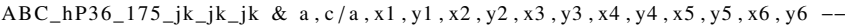
$\hookrightarrow$ params $=11.5799622371,0.317895264089,0.255,0.0598,0.1323,0.416$ $\hookrightarrow 0.3483,0.07620 .2334,0.5727,0.4279,0.0715,0.1367,0.5046 \& \mathrm{P} 6 / \mathrm{m}$ $\hookrightarrow \mathrm{C}\{6 \mathrm{~h}\}^{\wedge}\{1\} \# 175\left(\mathrm{j}^{\wedge} 3 \mathrm{k}^{\wedge} 3\right) \& \mathrm{hP} 36$ \& None \& $\mathrm{Mg}[\mathrm{NH}] \&$ \& F. Dolci $\hookrightarrow C_{-}\{6 \mathrm{~h}\} \wedge\{1\}$ \#

1.00000000000000

$5.78998111855000-10.02854147219310 \quad 0.00000000000000$

$\begin{array}{rrr}5.78998111855000 & 10.02854147219310 & 0.00000000000000\end{array}$

$\begin{array}{rrr}0.00000000000000 & 0.00000000000000 & 3.68121515350000\end{array}$

$\begin{array}{rrr}\mathrm{H} & \mathrm{Mg} & \mathrm{N} \\ 12 & 12 & 12\end{array}$

0.25500000000000 $-0.05980000000000$ $-0.19520000000000$ $-0.25500000000000$ 0.05980000000000 0.19520000000000 0.2334000000000 0.23340000000000 $-0.57270000000000$ 0.33930000000000 $-0.23340000000000$ 0.5727000000000 0.33930000000000 0.13230000000000 $-0.41600000000000$ 0.28370000000000 $-0.13230000000000$ 0.41600000000000 $-0.28370000000000$ 0.42790000000000 $-0.07150000000000$ $-0.35640000000000$ $-0.42790000000000$ 0.07150000000000

0.35640000000000

0.34830000000000

0.05980000000000 $-0.25500000000000$ $-0.05980000000000$ 0.19520000000000 .25500000000000 0.57270000000000 $-0.23340000000000$ 0.57270000000000 0.33930000000000 0.3330000000 0.23300000000 0.41600000000000 $-0.28370000000000$ $-0.13230000000000$ 0.41600000000000 0.28370000000000 0.07150000000000 0.35640000000000 $-0.42790000000000$ $-0.35640000000000$ 0.42790000000000 0.07620000000000
0.00000000000000 0.00000000000000 0.00000000000000 0.00000000000000 0.00000000000000 0.00000000000000 0.50000000000000 0.50000000000000 0.50000000000000 0.5000000000000 0.5000000000000 0.50000000000 0.00000000000000 0.00000000000000 0.00000000000000 0.00000000000000 0.00000000000000 0.00000000000000 0.50000000000000 0.50000000000000 0.50000000000000 0.50000000000000 0.50000000000000 0.50000000000000 0.00000000000000 


$\begin{array}{rrrrr}-0.07620000000000 & 0.27210000000000 & 0.00000000000000 & \mathrm{~N} & (6 \mathrm{j}) \\ -0.27210000000000 & -0.34830000000000 & 0.00000000000000 & \mathrm{~N} & (6 \mathrm{j}) \\ -0.34830000000000 & -0.07620000000000 & 0.00000000000000 & \mathrm{~N} & (6 \mathrm{j}) \\ 0.07620000000000 & -0.27210000000000 & 0.00000000000000 & \mathrm{~N} & (6 \mathrm{j}) \\ 0.27210000000000 & 0.34830000000000 & 0.00000000000000 & \mathrm{~N} & (6 \mathrm{j}) \\ 0.13670000000000 & 0.50460000000000 & 0.50000000000000 & \mathrm{~N} & (6 \mathrm{k}) \\ -0.50460000000000 & -0.36790000000000 & 0.50000000000000 & \mathrm{~N} & (6 \mathrm{k}) \\ 0.36790000000000 & -0.13670000000000 & 0.50000000000000 & \mathrm{~N} & (6 \mathrm{k}) \\ -0.13670000000000 & -0.50460000000000 & 0.50000000000000 & \mathrm{~N} & (6 \mathrm{k}) \\ 0.50460000000000 & 0.36790000000000 & 0.50000000000000 & \mathrm{~N} & (6 \mathrm{k}) \\ -0.36790000000000 & 0.13670000000000 & 0.50000000000000 & \mathrm{~N} & (6 \mathrm{k})\end{array}$

$\mathrm{Er}_{3} \mathrm{Ru}_{2}$ : A3B2_hP10_176_h_bd - CIF

\section{\# CIF file}

data_findsym-output

-audit_creation_method FINDSYM

_chemical_name_mineral 'Er3Ru2

_chemical_formula_sum 'Er3 Ru2'

loop

publ_author_name

'A. Palenzona'

_journal_name_full_name

Journal of the Less-Common Metals

_journal_volume 159

_journal_year 1990

_journal_page_first L21

_journal_page_last L23

publ_Section_title

The phase diagram of the Er-Ru system

\# Found in Pearson's Crystal Data - Crystal Structure Database for $\hookrightarrow$ Inorganic Compounds, 2013

aflow title 'Er\$_\{3\}\$Ru\$_\{2\}\$ Structure,

_aflow_proto 'A3B2_hP10_176_h_bd,

-aflow params, a, c/a, $x_{-}\{3\}, y-\{3\}$

_aflow_params_values ' $7.8700439404,0.50025412961,0.0915,0.7068$,

_aflow_Strukturbericht 'None'

_aflow_Pearson 'hP10,

\section{_cell_length_a $\quad 7.8700439404$}

_cell_length_b 7.8700439404

_cell_length_c 3.9370219814

_cell_angle_alpha 90.0000000000

_cell_angle_beta 90.0000000000

cell angle gamma 120.0000000000

symmetry_space_group_name_H-M "P 63/m"

_symmetry_Int_Tables_number 176

loop

space_group_symop_id

_space_group_symop_operation_xyz

$1 \mathrm{x}, \mathrm{y}, \mathrm{z}$

$2 \mathrm{x}-\mathrm{y}, \mathrm{x}, \mathrm{z}+1 / 2$

$3-\mathrm{y}, \mathrm{x}-\mathrm{y}, \mathrm{z}$

$4-\mathrm{x},-\mathrm{y}, \mathrm{z}+1 / 2$

$5-\mathrm{x}+\mathrm{y},-\mathrm{x}, \mathrm{z}$

$6 \mathrm{y},-\mathrm{x}+\mathrm{y}, \mathrm{z}+1 / 2$

$7-x,-y,-z$

$8-\mathrm{x}+\mathrm{y},-\mathrm{x},-\mathrm{z}+1 / 2$

$9 \mathrm{y},-\mathrm{x}+\mathrm{y},-\mathrm{z}$

$10 \mathrm{x}, \mathrm{y},-\mathrm{z}+1 / 2$

$11 \mathrm{x}-\mathrm{y}, \mathrm{x},-\mathrm{z}$
$12-\mathrm{y}, \mathrm{x}-\mathrm{y},-\mathrm{z}+1 / 2$

loop_

atom_site_label

-atom_site_type_symbol

_atom_site_symmetry_multiplicity

_atom_site_Wyckoff_label

-atom_site_fract_x

atom_site_fract_y

atom_site_fract_z

_atom_site_occupancy

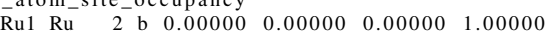

$\begin{array}{llllllll}\mathrm{Ru} 2 & \mathrm{Ru} & 2 & \mathrm{~d} & 0.66667 & 0.33333 & 0.25000 & 1.00000\end{array}$

$\begin{array}{lllllllll}\text { Er1 } & \text { Er } & 6 & \text { h } & 0.09150 & 0.70680 & 0.25000 & 1.00000\end{array}$

$\mathrm{Er}_{3} \mathrm{Ru}_{2}$ : A3B2_hP10_176_h_bd - POSCAR

\begin{tabular}{|c|c|c|c|c|}
\hline \multicolumn{5}{|c|}{ 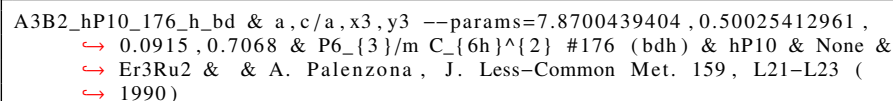 } \\
\hline \multicolumn{5}{|l|}{1.00000000000000} \\
\hline 3.93502197020000 & 128618 & \multicolumn{3}{|l|}{0.00000000000000} \\
\hline 3.93502197020000 & 8618 & \multicolumn{3}{|l|}{0.00000000000000} \\
\hline 0.00000000000000 & 0.00000000000000 & \multicolumn{3}{|l|}{3.93702198140000} \\
\hline Er & & & & \\
\hline 6 & & & & \\
\hline \multicolumn{5}{|l|}{ Direct } \\
\hline 0.091500000 & 000000 & 0.25000000 & $\mathrm{Er}$ & $(6 \mathrm{~h})$ \\
\hline-0.706800000 & -0.6 & 0.25 & $\mathrm{Er}$ & \\
\hline 0.61530000000000 & -0.09150000000000 & 0.25000000000000 & $\mathrm{Er}$ & $(6 \mathrm{~h})$ \\
\hline-0.09150000000000 & -0.70680000000000 & 0.75000000000000 & $\mathrm{Er}$ & $(6 \mathrm{~h})$ \\
\hline 0.70680000000000 & 0.61 & 0.750000000 & $\mathrm{Er}$ & $(6 \mathrm{~h})$ \\
\hline-0.61530000000000 & 0.09150000000000 & 0.75000000000000 & $\mathrm{Er}$ & $(6 \mathrm{~h})$ \\
\hline
\end{tabular}

$\begin{array}{lllll}0.00000000000000 & 0.00000000000000 & 0.00000000000000 & \mathrm{Ru} & (2 \mathrm{~b}) \\ 0.00000000000000 & 0.00000000000000 & 0.50000000000000 & \mathrm{Ru} & (2 \mathrm{~b}) \\ 0.66666666666667 & 0.33333333333333 & 0.25000000000000 & \mathrm{Ru} & (2 \mathrm{~d}) \\ 0.33333333333333 & 0.66666666666667 & 0.75000000000000 & \mathrm{Ru} & (2 \mathrm{~d})\end{array}$

$\mathrm{Fe}_{3} \mathrm{Te}_{3} \mathrm{Tl}$ : A3B3C_hP14_176_h_h_d - CIF

\# CIF_file
data_findsym-output
-audit_creation_method FINDSYM

chemical_name_mineral ' Fe3Te3Tl,

_chemical_formula_sum ' $\mathrm{Fe} 3 \mathrm{Te} 3 \mathrm{Tl}$

loop

publ_author_name

'K. Klepp',

journal_name_full_name

Acta Crystallographica Section A: Foundations and Advances

journal_volume 34

journal year 1978

journal_page_first $S 160$

journal_page_last S161

publ_Section_title

Crystal Structures of Thallium-Iron Chalcogenides

\# Found in Pearson's Crystal Data - Crystal Structure Database for $\hookrightarrow$ Inorganic Compounds, 2013

aflow_title ${ }^{\prime}$ Fe\$_ $\{3\} \$ T$ Te $\{3\} \$ T$ T Structure

aflow proto 'A3B3C hP14_176 h_h_d

aflow_params 'a, c/a, $x_{-}\{2\}, y_{-}\{2\}, x_{-}\{3\}, y\{3\}$

aflow_params_values $, 9.3500327107,0.451336898402,0.1701,0.0208,0.0462$, $\hookrightarrow 0.6892$,

aflow Strukturbericht 'None'

aflow_Pearson 'hP14,

cell_length_a 9.3500327107

cell_length_b 9.3500327107

cell_length_c $\quad 4.2200147636$

_cell_angle_alpha 90.0000000000

cell_angle_beta 90.0000000000

cell_angle_gamma 120.0000000000

symmetry_space_group_name_H-M "P $63 / \mathrm{m}^{\prime \prime}$

symmetry_Int_Tables_number 176

loop

space_group_symop_id

space_group_symop_operation_xyz

$\mathrm{x}, \mathrm{y}, \mathrm{z}$

$\mathrm{x}-\mathrm{y}, \mathrm{x}, \mathrm{z}+1 / 2$

$3-\mathrm{y}, \mathrm{x}-\mathrm{y}, \mathrm{z}$

$-\mathrm{x},-\mathrm{y}, \mathrm{z}+1 / 2$

$\begin{array}{ll}5 & -x+y,-x, z \\ 6 & y,-x+y, z+1 / 2\end{array}$

$7-x,-y,-z$

$8-\mathrm{x}+\mathrm{y},-\mathrm{x},-\mathrm{z}+1 / 2$

$9 \mathrm{y},-\mathrm{x}+\mathrm{y},-\mathrm{z}$

$10 \mathrm{x}, \mathrm{y},-\mathrm{z}+1 / 2$

$11 \mathrm{x}-\mathrm{y}, \mathrm{x},-\mathrm{z}$

$12-y, x-y,-z+1 / 2$

loop

atom_site_label

atom_site_type_symbol

atom_site_symmetry_multiplicity

atom_site_Wyckoff_label

atom_site_fract_x

atom_site_fract_y

atom_site_fract-z

atom_site_occupancy

$\begin{array}{llllllll}\text { T11 Tl } & 2 & \text { d } & 0.66667 & 0.33333 & 0.25000 & 1.00000\end{array}$

$\begin{array}{llllllll}\mathrm{Fe} 1 & \mathrm{Fe} & 6 & \mathrm{~h} & 0.17010 & 0.02080 & 0.25000 & 1.00000\end{array}$

$\begin{array}{llllllll}\mathrm{Te} 1 & \mathrm{Te} & 6 & \mathrm{~h} & 0.04620 & 0.68920 & 0.25000 & 1.00000\end{array}$

$\mathrm{Fe}_{3} \mathrm{Te}_{3} \mathrm{Tl}: \mathrm{A}$ 3B3C_hP14_176_h_h_d - POSCAR

A3B3C_hP14_176_h_h_d \& a , c/a, x2 ,y2, x3,y3 --params $=9.3500327107$,

$\hookrightarrow 0.451336898402,0.1701,0.0208,0.0462,0.6892 \& \mathrm{P}_{-}\{3\} / \mathrm{m} \mathrm{C}_{-}\{6 \mathrm{~h}\}^{\wedge}\{2$

$\hookrightarrow$ \} \#176 (dh^2) \& hP14 \& None \& Fe3Te3Tl \& \& K. Klepp and H.

$\hookrightarrow$ Boller, Acta Crystallogr. Sect. A 34, S160-S161 (1978)

1.00000000000000

$\begin{array}{lll}4.67501635535000 & -8.09736585368168 & 0.00000000000000\end{array}$

$\begin{array}{lll}4.67501635535000 & 8.09736585368168 & 0.00000000000000\end{array}$

$0.00000000000000 \quad 0.00000000000000 \quad 4.22001476360000$

$\begin{array}{rrr}\mathrm{Fe} & \mathrm{Te} & \mathrm{Tl} \\ 6 & 6 & 2\end{array}$

Direct

0.17010000000000

$-0.02080000000000$

$-0.14930000000000$

$-0.17010000000000$

0.02080000000000

0.14930000000000

0.04620000000000

-0.68920000000000
0.64300000000000

0.64300000000000
-0.04620000000000

-0.04620000000000
0.68920000000000

0.02080000000000 0.14930000000000

$-0.17010000000000$

$-0.02080000000000$

$-0.14930000000000$

0.17010000000000

0.68920000000000

$-0.64300000000000$

$-0.04620000000000$

$-0.68920000000000$

0.64300000000000

0.25000000000000 0.25000000000000 0.75000000000000 0.75000000000000 0.75000000000000 0.250000000000 0.250000000000 0.75000000000000 0.75000000000000 


$\begin{array}{rlllll}-0.64300000000000 & 0.04620000000000 & 0.75000000000000 & \mathrm{Te} & (6 \mathrm{~h}) \\ 0.66666666666667 & 0.33333333333333 & 0.25000000000000 & \mathrm{Tl} & (2 \mathrm{~d}) \\ 0.33333333333333 & 0.66666666666667 & 0.75000000000000 & \mathrm{Tl} & (2 \mathrm{~d})\end{array}$

$\mathrm{UCl}_{3}$ : A3B_hP8_176_h_d - CIF

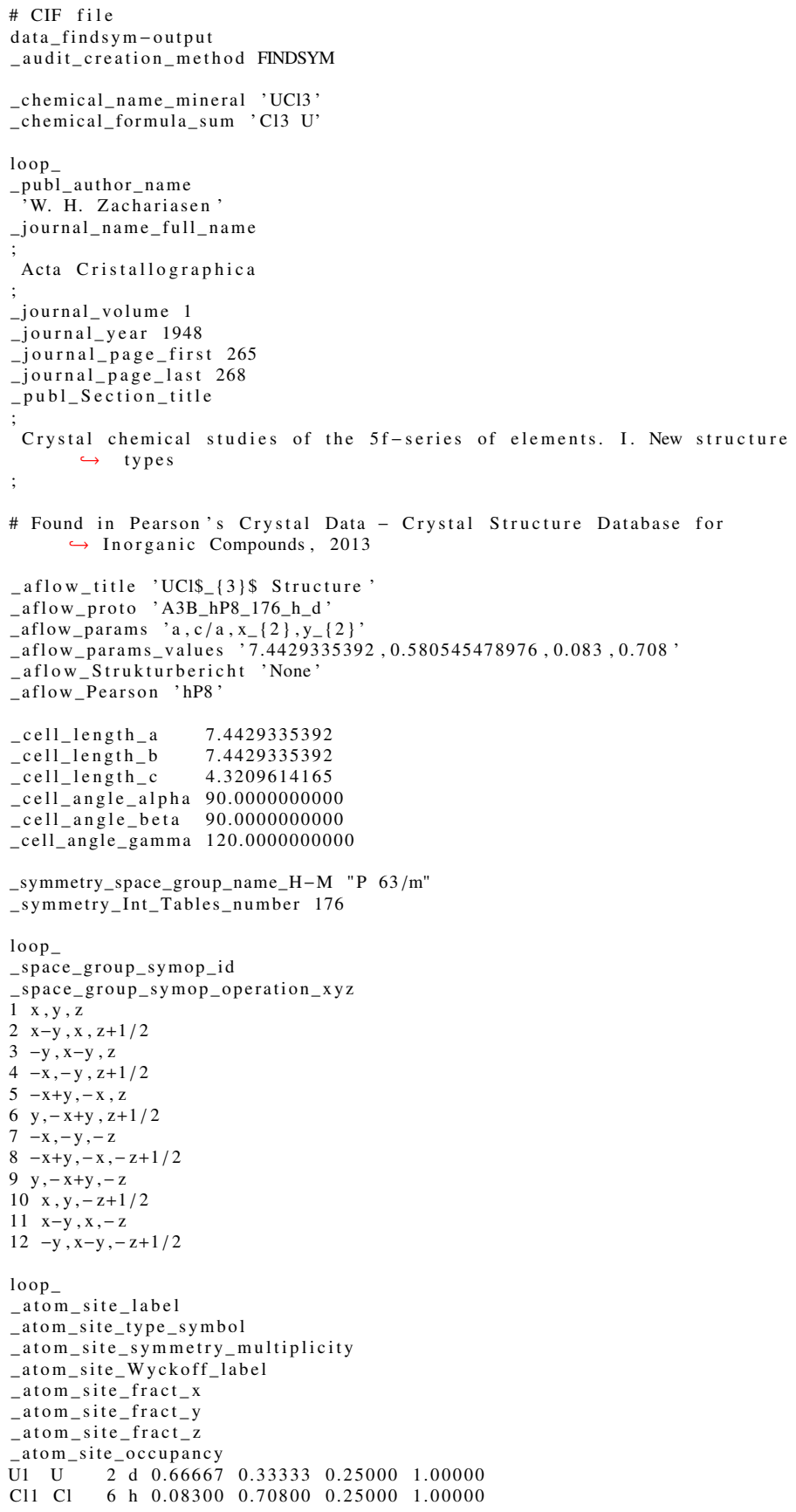

UCl 3 : A3B_hP8_176_h_d - POSCAR

A3B_hP8_176_h_d \& a,c/a, x2,y2 --params $=7.4429335392,0.580545478976,0.083$ $\hookrightarrow, 0.708 \& \mathrm{P} 6\{3\} / \mathrm{m} \mathrm{C}\{6 \mathrm{~h}\}^{\wedge}\{2\} \# 176(\mathrm{dh}) \& \mathrm{hP} 8 \&$ None \& UCl3 \& \& $\hookrightarrow$ W. H. Zachariasen, Acta Cryst. 1, 265-268 (1948) 1.00000000000000

$\begin{array}{lll}3.72146676960000 & -6.44576952362642 & 0.00000000000000\end{array}$ $\begin{array}{lll}3.72146676960000 & 6.44576952362642 & 0.00000000000000\end{array}$ $\begin{array}{lll}0.00000000000000 & 0.00000000000000 & 4.32096141650000\end{array}$

$$
\begin{array}{rr}
\mathrm{Cl} & \mathrm{U} \\
6 & 2
\end{array}
$$

Direct

$0.08300000000000 \quad 0.70800000000000$ $-0.70800000000000-0.62500000000000$ $0.62500000000000-0.08300000000000$ $-0.08300000000000-0.70800000000000$ $0.70800000000000-0.62500000000000$ 0.6250000000000 $-0.62500000000000 \quad 0.08300000000000$ $\begin{array}{ll}0.66666666666667 & 0.33333333333333 \\ 0.33333333333333 & 0.66666666666667\end{array}$

0.25000000000000 0.25000000000000 0.25000000000000 0.75000000000000 0.75000000000000 0.75000000000000 0.75000000000000 $0.66666666666667 \quad 0.75000000000000$

$\begin{aligned} \mathrm{Cl} & (6 \mathrm{~h}) \\ \mathrm{Cl} & (6 \mathrm{~h}) \\ \mathrm{Cl} & (6 \mathrm{~h}) \\ \mathrm{Cl} & (6 \mathrm{~h}) \\ \mathrm{Cl} & (6 \mathrm{~h}) \\ \mathrm{Cl} & (6 \mathrm{~h}) \\ \mathrm{U} & (2 \mathrm{~d}) \\ \mathrm{U} & (2 \mathrm{~d})\end{aligned}$

$\mathrm{SiO}_{2}$ : A2B_hP36_177_j2Im_n - CIF

\# CIF file

data_findsym-output

_audit_creation_method FINDSYM _chemical_name_mineral 'SiO2,

_chemical_formula_sum 'O2 $\mathrm{Si}$,

aflow_title 'SiO\$_ $\{2\} \$$ Structure

aflow_proto 'A2B_hP36_177_j2lm_n

aflow_params 'a,c/a, $x_{-}\{1\}, x_{-}\{2\}, x_{-}\{3\}, x_{-}\{4\}, x_{-}\{5\}, y_{-}\{5\}, z_{-}\{5\}$

aflow_params_values ' $12.7835,0.291064262526,0.61855,0.39242,0.79257$, $\hookrightarrow 0.44445,0.52169,0.86952,0.16458$,

aflow_Strukturbericht 'None'

aflow_Pearson 'hP36'

_cell_length_a $\quad 12.7835000000$

_cell_length_b $\quad 12.7835000000$

$\begin{array}{ll}\text { _cell_length_b } & 12.7835000000 \\ \text { _cell_length_c } & 3.7208200000\end{array}$

cell angle alpha 90.0000000000

cell_angle_beta 90.0000000000

cell_angle_gamma 120.0000000000

symmetry_space_group_name_H-M "P $6 \begin{array}{lllll} & 6 & 2\end{array}$

_symmetry_Int_Tables_number 177

loop

space_group_symop_id

space_group_symop_operation_xyz

$1 \mathrm{x}, \mathrm{y}, \mathrm{z}$

$2 \mathrm{x}-\mathrm{y}, \mathrm{x}, \mathrm{z}$

$3-y, x-y, z$

$3-\mathrm{x}, \mathrm{x}-\mathrm{y}, \mathrm{z}$
$4-\mathrm{x},-\mathrm{y}, \mathrm{z}$

$-\mathrm{x},-\mathrm{y}, \mathrm{z}$
$-\mathrm{x}+\mathrm{y},-\mathrm{x}, \mathrm{z}$

$6 \mathrm{y},-\mathrm{x}+\mathrm{y}, \mathrm{z}$

$7 \mathrm{x}-\mathrm{y},-\mathrm{y},-\mathrm{z}$

$8 \mathrm{x}, \mathrm{x}-\mathrm{y},-\mathrm{z}$

$10-\mathrm{x}+\mathrm{y}, \mathrm{y},-\mathrm{z}$

$11-\mathrm{x},-\mathrm{x}+\mathrm{y},-\mathrm{z}$

$12-\mathrm{y},-\mathrm{x},-\mathrm{z}$

loop

atom_site_label

atom_site_type_symbol

atom_site_symmetry_multiplicity

atom_site_Wyckoff_label

atom_site_fract_x

-atom_site_fract_y

atom_site_fract_z

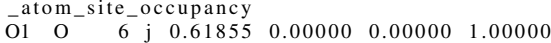

$\begin{array}{lllllllll}\mathrm{O} 2 & \mathrm{O} & 6 & 6 & 0.39242 & 0.60758 & 0.00000 & 1.00000\end{array}$

$\begin{array}{lllllllll}\mathrm{O} 2 & \mathrm{O} & 6 & 1 & 0.39242 & 0.60758 & 0.00000 & 1.00000 \\ \mathrm{O} 3 & \mathrm{O} & 6 & 1 & 0.79257 & 0.20743 & 0.00000 & 1.00000\end{array}$

$\begin{array}{lllllllll}\mathrm{O} 3 & \mathrm{O} & & 6 & 1 & 0.79257 & 0.20743 & 0.00000 & 1.00000 \\ \mathrm{O} 4 & \mathrm{O} & 6 & \mathrm{~m} & 0.44445 & 0.55555 & 0.50000 & 1.00000\end{array}$

$\begin{array}{llllllll}\mathrm{Si} 1 & \mathrm{Si} & 12 & \mathrm{n} & 0.52169 & 0.86952 & 0.16458 & 1.00000\end{array}$

$\mathrm{SiO}_{2}$ : A2B_hP36_177_j2Im_n - POSCAR

\begin{tabular}{|c|c|c|c|c|}
\hline \multicolumn{5}{|c|}{$\begin{aligned} \text { A2B_hP36_177_j2lm_n \& a, c/a }, \mathrm{x} 1, \mathrm{x} 2, \mathrm{x} 3, \mathrm{x} 4, \mathrm{x} 5, \mathrm{y} 5, \mathrm{z} 5-\mathrm{params}=12.7835, & \\
& \hookrightarrow 0.291064262526,0.61855,0.39242,0.79257,0.44445,0.52169,0.86952, \\
& \hookrightarrow 0.16458 \& \mathrm{P} 622 \mathrm{D}_{-}\{6\}^{\wedge}\{1\} \# 177\left(\mathrm{j} 1^{\wedge} 2 \mathrm{mn}\right) \& \mathrm{hP} 36 \& \text { None \& SiO2\& } \\
& \hookrightarrow \&\end{aligned}$} \\
\hline \multicolumn{5}{|l|}{1.00000000000000} \\
\hline 6.39175000000000 & -11.07083574927840 & 0.00000000000000 & & \\
\hline 6.39175000000000 & 11.07083574927840 & 0.00000000000000 & & \\
\hline 0.00000000000000 & 0.00000000000000 & 3.72082000000000 & & \\
\hline $\mathrm{Si}$ & & & & \\
\hline 24 & & & & \\
\hline \multicolumn{5}{|l|}{ Direct } \\
\hline 0.61855000000000 & 0.00000000000000 & 0.00000000000000 & $\mathrm{O}$ & $(6 \mathrm{j})$ \\
\hline 0.00000000000000 & 0.61855000000000 & 0.00000000000000 & $\mathrm{O}$ & $(6 j)$ \\
\hline-0.61855000000000 & -0.61855000000000 & 0.00000000000000 & $\mathrm{O}$ & $(6 j)$ \\
\hline-0.61855000000000 & 0.00000000000000 & 0.00000000000000 & $\mathrm{O}$ & $(6 \mathrm{j})$ \\
\hline 0.00000000000000 & -0.61855000000000 & 0.00000000000000 & $\mathrm{O}$ & $(6 \mathrm{j})$ \\
\hline 0.61855000000000 & 0.61855000000000 & 0.00000000000000 & $\mathrm{O}$ & $(6 \mathrm{j})$ \\
\hline 0.39242000000000 & -0.39242000000000 & 0.00000000000000 & $\mathrm{O}$ & (61) \\
\hline 0.39242000000000 & 0.78484000000000 & 0.00000000000000 & $\mathrm{O}$ & (61) \\
\hline-0.78484000000000 & -0.39242000000000 & 0.00000000000000 & $\mathrm{O}$ & (61) \\
\hline-0.39242000000000 & 0.39242000000000 & 0.00000000000000 & $\mathrm{O}$ & (61) \\
\hline-0.39242000000000 & -0.78484000000000 & 0.00000000000000 & $\mathrm{O}$ & (61) \\
\hline 0.78484000000000 & 0.39242000000000 & 0.00000000000000 & $\mathrm{O}$ & (61) \\
\hline 0.79257000000000 & -0.79257000000000 & 0.00000000000000 & $\mathrm{O}$ & (61) \\
\hline 0.79257000000000 & 1.58514000000000 & 0.00000000000 & $\mathrm{O}$ & (61) \\
\hline-1.58514000000000 & -0.79257000000000 & 0.0000000000 & $\mathrm{O}$ & (61) \\
\hline-0.79257000000000 & 0.79257000000000 & 0.0000000000 & $\mathrm{O}$ & (61) \\
\hline-0.79257000000000 & -1.58514000000000 & 0.00000000000000 & $\mathrm{O}$ & (61) \\
\hline 1.58514000000000 & 0.792570 & 0.0 & $\mathrm{O}$ & (61) \\
\hline 0.44445000000000 & -0.44445000000000 & 0.50000000000000 & $\mathrm{O}$ & $(6 \mathrm{~m})$ \\
\hline 0.44445000000000 & 0000000 & 0.5000000000 & $\mathrm{O}$ & $(6 \mathrm{~m})$ \\
\hline-0.88890000000000 & -0.44445000000000 & 0.50000000000 & & $(6 \mathrm{~m})$ \\
\hline-0.44445000000000 & 0.44445000000000 & 0.50000000000000 & $\mathrm{O}$ & $(6 \mathrm{~m})$ \\
\hline-0.44445000000000 & -0.88890000000000 & 0.50000000000000 & $\mathrm{O}$ & $(6 \mathrm{~m})$ \\
\hline 0.88890000000000 & 0.44445000000000 & 0.50000000000000 & $\mathrm{O}$ & $(6 \mathrm{~m})$ \\
\hline 0.52169000000000 & 0.86952000000000 & 0.16458000000000 & $\mathrm{Si}$ & $(12 n)$ \\
\hline-0.86952000000000 & -0.34783000000000 & 0.16458000000000 & $\mathrm{Si}$ & $(12 n)$ \\
\hline 0.34783000000000 & -0.52169000000000 & 0.16458000000000 & $\mathrm{Si}$ & $(12 n)$ \\
\hline-0.52169000000000 & -0.86952000000000 & 0.16458000000000 & $\mathrm{Si}$ & $(12 n)$ \\
\hline 0.86952000000000 & 0.34783000000000 & 0.16458000000000 & $\mathrm{Si}$ & $(12 n)$ \\
\hline-0.34783000000000 & 0.52169000000000 & 0.16458000000000 & $\mathrm{Si}$ & $(12 n)$ \\
\hline 0.86952000000000 & 0.52169000000000 & -0.16458000000000 & $\mathrm{Si}$ & $(12 n)$ \\
\hline-0.34783000000000 & -0.86952000000000 & -0.16458000000000 & $\mathrm{Si}$ & $(12 n)$ \\
\hline-0.52169000000000 & 0.34783000000000 & -0.16458000000000 & $\mathrm{Si}$ & $(12 n)$ \\
\hline-0.86952000000000 & -0.52169000000000 & -0.16458000000000 & $\mathrm{Si}$ & $(12 n)$ \\
\hline 0.34783000000000 & 0.86952000000000 & -0.16458000000000 & $\mathrm{Si}$ & $(12 n)$ \\
\hline 0.52169000000000 & -0.34783000000000 & -0.16458000000000 & $\mathrm{Si}$ & $(12 n)$ \\
\hline
\end{tabular}

AuF 3 : AB3_hP24_178_b_ac - CIF 
\# CIF file

data_findsym-output

_audit_creation_method FINDSYM

_chemical_name_mineral 'AuF3,

_chemical_formula_sum 'Au F3,

loop

_publ_author_name

L. B. Asprey

'F. H. Kruse'

K. H. Jack

K. H. Jack'

journal_name_full_name

Inorganic Chemistry

_journal_volume 3

-journal_year 1964

_journal_page_first 602

journal_page_last 604

-publ_Section_title

Preparation and properties of crystalline gold trifluoride

\# Found in Pearson's Crystal Data - Crystal Structure Database for $\hookrightarrow$ Inorganic Compounds, 2013

_aflow_title,$A u F \$ \_\{3\} \$$ Structure,

-aflow_proto 'AB3_hP24_178_b_ac

-aflow_params 'a,c/a, $x_{-}\{1\}, x_{-}\{2\}, x_{-}\{3\}, y_{-}\{3\}, z_{-}\{3\}$ '

aflow_params_values , $5.14898393,3.15789473684,0.8361,0.7601,0.5338$ $\stackrel{-\mathrm{P}}{\hookrightarrow} 0.3099,-0.0053$,

aflow Strukturbericht 'None'

-aflow_Pearson 'hP24,

_cell_length_a $\quad 5.1489839300$

_cell_length_b 5.1489839300

_cell_length_c $\quad 16.2599492526$

_cell_angle_alpha 90.0000000000

_cell_angle_beta 90.0000000000

cell_angle_gamma 120.0000000000

symmetry_space_group_name_H-M "P 61 2 2 2"

_symmetry_Int_Tables_number 178

loop

_space_group_symop_id

_space_group_symop_operation_xyz

$1 \mathrm{x}, \mathrm{y}, \mathrm{z}$

$2 \mathrm{x}-\mathrm{y}, \mathrm{x}, \mathrm{z}+1 / 6$

$3-\mathrm{y}, \mathrm{x}-\mathrm{y}, \mathrm{z}+1 / 3$

$4-x,-y, z+1 / 2$
$5-x+y,-x, z+2 / 3$

$\begin{array}{ll}5 & \mathrm{y},-\mathrm{x}+\mathrm{y}, \mathrm{z}+5 / 6\end{array}$

$7 \mathrm{x}-\mathrm{y},-\mathrm{y},-\mathrm{z}$

$8 \mathrm{x}, \mathrm{x}-\mathrm{y},-\mathrm{z}+1 / 6$

$9 \mathrm{y}, \mathrm{x},-\mathrm{z}+1 / 3$

$10-x+y, y,-z+1 / 2$

$11-\mathrm{x},-\mathrm{x}+\mathrm{y},-\mathrm{z}+2 / 3$

$12-y,-x,-z+5 / 6$

loop

atom site label

-atom_site_type_symbol

-atom_site_symmetry_multiplicity

_atom_site_Wyckoff_labe

-atom_site_fract_x

-atom_site_fract_y

- atom_site_fract_z

\begin{tabular}{llllllll}
\hline F1 & F & 6 & a & 0.83610 & 0.00000 & 0.00000 & 1.00000
\end{tabular}

$\begin{array}{llllllll}\mathrm{Au} 1 \mathrm{Au} & 6 & \mathrm{~b} & 0.76010 & 0.52020 & 0.25000 & 1.00000\end{array}$

$\begin{array}{llllllllll}\text { F2 } & \text { F } & 12 & \text { c } & 0.53380 & 0.30990 & -0.00530 & 1.00000\end{array}$

$\mathrm{AuF}_{3}$ : AB3_hP24_178_b_ac - POSCAR

AB3_hP24_178_b_ac \& a,c/a, x1, x2, x3,y3,z3 --params $=5.14898393$

$\hookrightarrow 3.15789473684,0.8361,0.7601,0.5338,0.3099,-0.0053 \&$ P6_ $\{1\} 22$ D

$\hookrightarrow\{6\}^{\wedge}\{2\} \# 178(\mathrm{abc}) \& \mathrm{hP} 24 \&$ None \& AuF3 \& \& L. B. Asprey et

$\hookrightarrow$ al., Inorg. Chem. 3, 602-604 (1964)

1.00000000000000

$\begin{array}{lll}2.57449196500000 & -4.45915088705784 & 0.00000000000000\end{array}$

$\begin{array}{llll}2.57449196500000 & 4.45915088705784 & 0.00000000000000\end{array}$

$\begin{array}{rrr}0.00000000000000 & 0.00000000000000 & 16.25994925260000\end{array}$

$\mathrm{Au} \quad \mathrm{F}$

Direct

0.76010000000000

$-1.52020000000000$

0.76010000000000

$-0.76010000000000$

1.52020000000000

$-0.76010000000000$

0.83610000000000

0.00000000000000

$-0.83610000000000$

$-0.83610000000000$ 0.00000000000000 0.83610000000000 0.53380000000000 $-0.30990000000000$

$-0.22390000000000$

$-0.53380000000000$

1.52020000000000 $-0.76010000000000$ $-0.76010000000000$ $-1.52020000000000$ 0.76010000000000 0.76010000000000 0.0000000000000 0.83610000000000 $-0.83610000000000$ 0.00000000000000 $-0.83610000000000$ 0.83610000000000 0.30990000000000 0.22390000000000 $-0.5338000000000$ $-0.30990000000000$
0.30990000000000 .22390000000000 0.30990000000000

0.22390000000000 $-0.53380000000000$ $-0.3099000000000$ $-0.22390000000000$

0.53380000000000

$-0.22390000000000$ 0.53380000000000 0.53380000000000 0.30990000000000 $-0.22390000000000$ $-0.53380000000000$ 0.30990000000000 0.22390000000000 0.338633333333333 0.00530000000000 0.67196666666667 0.83863333333333 0.50530000000000 0.171966666666667

Sc-V (High-pressure): A_hP6_178_a - CIF

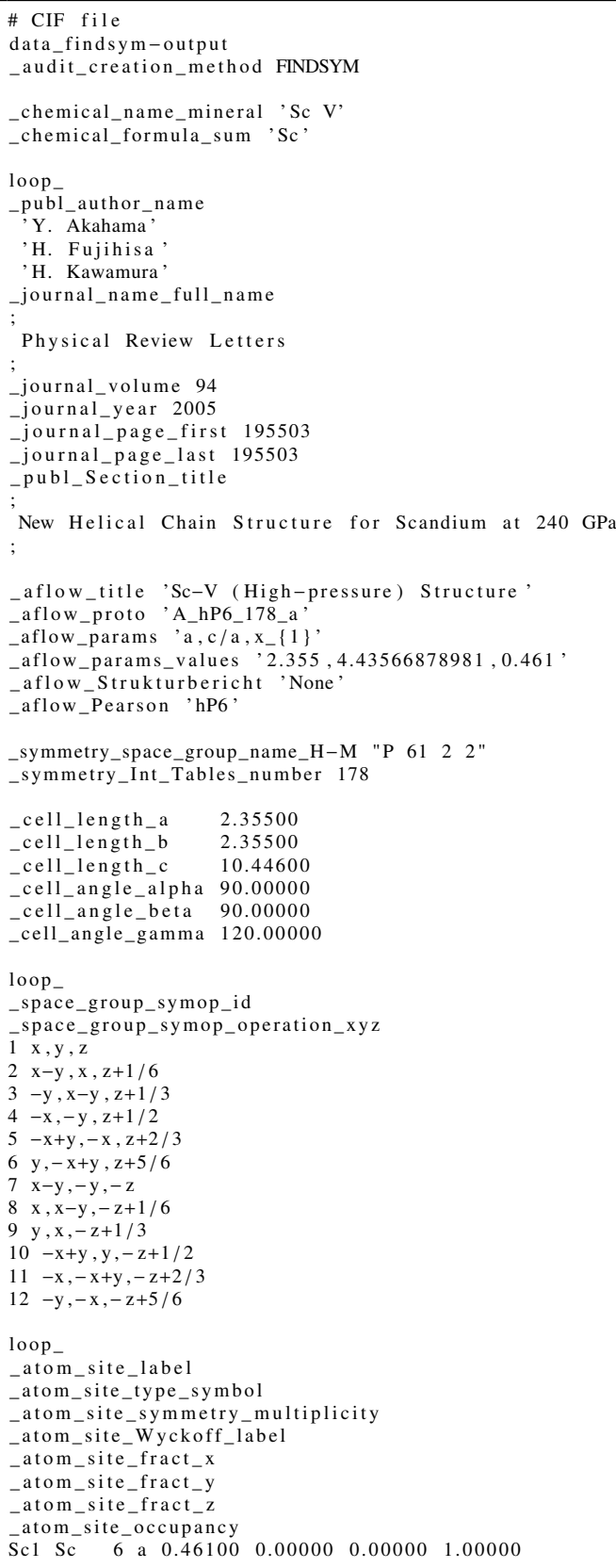

Sc-V (High-pressure): A_hP6_178_a - POSCAR

\begin{tabular}{|c|c|c|c|c|}
\hline \multirow{4}{*}{\multicolumn{5}{|c|}{$\begin{aligned} \text { A_hP6_178_a \& a,c/a, x } 1-\text { params }=2.355,4.43566878981,0.461 \& \text { P6_\{1 } 22 \\
\hookrightarrow 6\}^{\wedge}\{2\} \# 178 \text { (a) \& hP6 \& None \& Sc \& Sc V \& Y. Akahama and H. } \\
\hookrightarrow \text { Fujihisa and H. Kawamura, Phys. Rev. Lett. 94, 195503(2005) }\end{aligned}$}} \\
\hline & & & & \\
\hline & & & & \\
\hline & & & & \\
\hline 1.17750000000000 & -2.03948982591235 & \multicolumn{3}{|l|}{0.00000000000000} \\
\hline 1.17750000000000 & 2.03948982591235 & \multicolumn{3}{|l|}{0.00000000000000} \\
\hline 0.00000000000000 & 0.00000000000000 & \multirow{2}{*}{\multicolumn{3}{|c|}{10.44600000000000}} \\
\hline \multirow{2}{*}{\multicolumn{3}{|c|}{$\begin{array}{r}\text { Sc } \\
6\end{array}$}} & & \\
\hline \multirow{2}{*}{\multicolumn{5}{|c|}{$\begin{array}{r}6 \\
\mathrm{ct}\end{array}$}} \\
\hline & & & & \\
\hline 0.4610000 & 0.00000000000000 & 0.00000000000000 & $\mathrm{Sc}$ & (6a) \\
\hline 0.00000000000000 & 0.4610 & 0.33333333333333 & $\mathrm{Sc}$ & \\
\hline-0.46100000000000 & -0.46100000000000 & 0.66666666666667 & $\mathrm{Sc}$ & (6a) \\
\hline-0.46100000000000 & 0.00000000000000 & 0.50000000000000 & Sc & (6a) \\
\hline 0.00000000000000 & -0.46100000000000 & 0.83333333333333 & $\mathrm{Sc}$ & (6a) \\
\hline 0.46100000000000 & 0.46100000000000 & 0.166666666666667 & $\mathrm{Sc}$ & (6a) \\
\hline
\end{tabular}

AuF 3 : AB3_hP24_179_b_ac - CIF 
chemical_name_mineral 'AuF3,

_chemical_formula_sum 'Au F3

aflow_title, $\mathrm{AuF}_{-}\{3\} \$$ Structure

aflow_proto 'AB3_hP24_179_b_ac'

aflow_params 'a,c/a, $x_{-}\{1\}, x_{-}\{2\}, x_{-}\{3\}, y_{-}\{3\}, z_{-}\{3\}$,

aflow_params_values ' $5.14898393,3.15789473684,0.8361,0.7601,0.5338$. $\hookrightarrow 0.3099,0.0053$

aflow_Strukturbericht 'None

aflow_Pearson 'hP24,

\section{cell_length_a $\quad 5.1489839300$}

cell_length_b 5.1489839300

cell_length_c $\quad 16.2599492526$

cell_angle_alpha 90.000000000

cell_angle beta 90.0000000000

_symmetry_space_group_name_H-M "P $65 \quad 2 \quad 2$ "

_symmetry_Int_Tables_number 179

loop_

_space_group_symop_id

_space_group_symop_operation_xyz

$1 \mathrm{x}, \mathrm{y}, \mathrm{z}$

$2 \mathrm{x}-\mathrm{y}, \mathrm{x}, \mathrm{z}+5 / 6$

$3-y, x-y, z+2 / 3$

$4-x,-y, z+1 / 2$

$5-\mathrm{x}+\mathrm{y},-\mathrm{x}, \mathrm{z}+1 / 3$

$6 \mathrm{y},-\mathrm{x}+\mathrm{y}, \mathrm{z}+1 / 6$

$7 \mathrm{x}-\mathrm{y},-\mathrm{y},-\mathrm{z}$

$8 \mathrm{x}, \mathrm{x}-\mathrm{y},-\mathrm{z}+5 / 6$

$9 \mathrm{y}, \mathrm{x},-\mathrm{z}+2 / 3$

$10-\mathrm{x}+\mathrm{y}, \mathrm{y},-\mathrm{z}+1 / 2$

$11-x,-x+y,-z+1 / 3$

loop

atom_site_labe

atom_site_type_symbol

atom_site_symmetry_multiplicity

atom_site_Wyckoff_label

atom_site_fract_x

atom_site_fract_y

atom_site_fract_z

$\begin{array}{llllll}\text { atom_site_occupancy } & & & & & \\ \text { F1 F } & 6 & 0.83610 & 0.00000 & 0.00000 & 1.00000\end{array}$

$\begin{array}{lllllllll} & 6 & \text { a } & 0.83610 & 0.00000 & 0.00000 & 1.00000\end{array}$

$\begin{array}{llrllllll}\mathrm{F} 2 & \mathrm{~F} & 12 & \mathrm{c} & 0.53380 & 0.30990 & 0.00530 & 1.00000\end{array}$

$\mathrm{AuF}_{3}$ : AB3_hP24_179_b_ac - POSCAR

AB3_hP24_179_b_ac \& a,c/a, x1, x2, x3, y3, z3 --params $=5.14898393$

$\hookrightarrow 3.15789473684,0.8361,0.7601,0.5338,0.3099,0.0053 \&$ P6_\{5\}22 D_t $\hookrightarrow 6\}^{\wedge}\{3\} \# 179(\mathrm{abc}) \& \mathrm{hP} 24$ \& None \& AuF3 \& AuF3 \& $\hookrightarrow 6\}^{\wedge}\{3\} \# 179$ 1.00000000000000

$\begin{array}{lll}2.57449196500000 & -4.45915088705784 & 0.00000000000000\end{array}$ $\begin{array}{lll}2.57449196500000 & 4.45915088705784 & 0.00000000000000\end{array}$ $\begin{array}{rrr}0.00000000000000 & 0.00000000000000 & 16.25994925260000\end{array}$

$$
\begin{array}{rr}
\mathrm{Au} & \mathrm{F} \\
6 & 18
\end{array}
$$

Direct

0.76010000000000

$-1.52020000000000$

0.76010000000000

$-0.76010000000000$

1.52020000000000

$-0.76010000000000$

0.83610000000000

0.00000000000000

$-0.83610000000000$

$-0.83610000000000$

0.000000000000

0.000000000000

0.83610000000000

0.53380000000000

$-0.30990000000000$

$-0.22390000000000$

$-0.53380000000000$

0.30990000000000

0.22390000000000

0.30990000000000

0.22390000000000

$-0.53380000000000$

$-0.30990000000000$

$-0.22390000000000$

0.53380000000000

1.52020000000000

$-0.76010000000000$

$-0.76010000000000$

$-1.52020000000000$

0.76010000000000

0.76010000000000 0.00000000000000 0.83610000000000

$-0.83610000000000$

0.0000000000000

0.83610000000000

.8361000000000

0.8361000000000

0.22390000000000

$-0.53380000000000$

$-0.30990000000000$

$-0.22390000000000$

0.53380000000000

0.53380000000000

$-0.30990000000000$

$-0.22390000000000$

$-0.53380000000000$

0.30990000000000

0.75000000000000 0.41666666666667 0.08333333333333 0.25000000000000 0.91666666666667 0.58333333333333 0.00000000000000 0.66666666666667 0.3333333333333 0.50000000000000 0.16666666000007 0.83333333333333 0.00530000000000 0.67196666666667 0.67196666666667 0.33863333333333 0.50530000000000 0.1719666666666 0.83863333333333 0.6613666666666 0.00530000000000 0.32803333333333 0.16136666666667 0.49470000000000 0.82803333333333

$\begin{array}{rr}\mathrm{Au} & (6 \mathrm{~b}) \\ \mathrm{Au} & (6 \mathrm{~b}) \\ \mathrm{Au} & (6 \mathrm{~b}) \\ \mathrm{Au} & (6 \mathrm{~b}) \\ \mathrm{Au} & (6 \mathrm{~b}) \\ \mathrm{Au} & (6 \mathrm{~b}) \\ \mathrm{F} & (6 \mathrm{a}) \\ \mathrm{F} & (6 \mathrm{a}) \\ \mathrm{F} & (6 \mathrm{a}) \\ \mathrm{F} & (6 \mathrm{a}) \\ \mathrm{F} & (6 \mathrm{a}) \\ \mathrm{F} & (6 \mathrm{a}) \\ \mathrm{F} & (12 \mathrm{c}) \\ \mathrm{F} & (12 \mathrm{c}) \\ \mathrm{F} & (12 \mathrm{c}) \\ \mathrm{F} & (12 \mathrm{c}) \\ \mathrm{F} & (12 \mathrm{c}) \\ \mathrm{F} & (12 \mathrm{c}) \\ \mathrm{F} & (12 \mathrm{c}) \\ \mathrm{F} & (12 \mathrm{c}) \\ \mathrm{F} & (12 \mathrm{c}) \\ \mathrm{F} & (12 \mathrm{c}) \\ \mathrm{F} & (12 \mathrm{c}) \\ \mathrm{F} & (12 \mathrm{c})\end{array}$

$\beta-\mathrm{SiO}_{2}$ : A2B_hP9_181_j_c - CIF

\section{\# CIF file}

data_findsym-output

audit_creation_method FINDSYM

chemical_name_mineral 'beta-SiO2,

chemical_formula_sum ' $\mathrm{O} 2 \mathrm{Si}$,

aflow_title $\$ \backslash$ beta $\$-S i O \$\{\{2\} \$$ Structure,

aflow_proto 'A2B_hP9_181_j_c

aflow_params 'a, c/a, $x_{-}\{2\}$ '

aflow_params_values , $4.9977,1.09252256038,0.2072$,

aflow Strukturbericht "None'

aflow_Pearson 'hP9

_cell_length_a $\quad 4.9977000000$

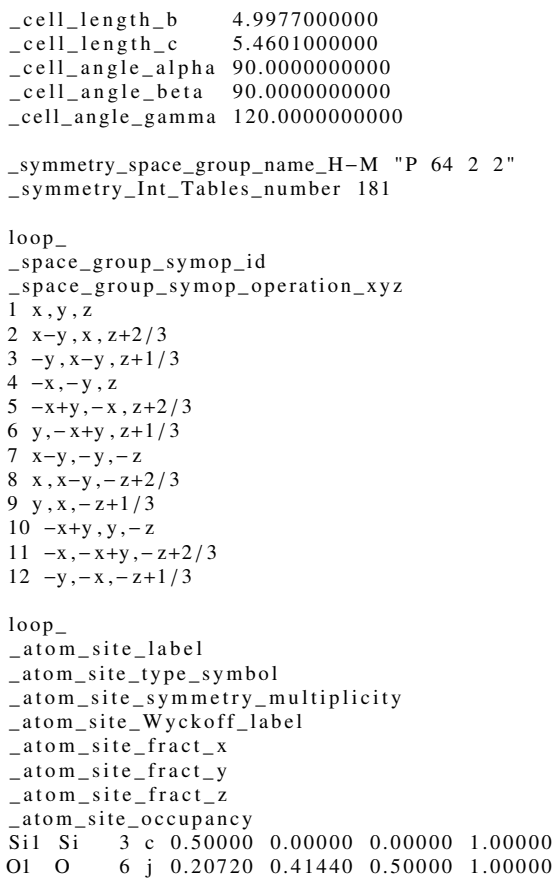

$\beta-\mathrm{SiO}_{2}$ : A2B_hP9_181_j_c - POSCAR

\begin{tabular}{|c|c|c|c|c|}
\hline \multicolumn{5}{|c|}{$\begin{array}{c}\text { A2B_hP9_181_j_c \& a, c/a, x2 --params }=4.9977,1.09252256038,0.2072 \& \text { P6_\{4 }\} \\
\hookrightarrow 22 \mathrm{D}_{-}\{6\}^{\wedge}\{5\} \# 181 \text { (cj) \& hP9 \& None \& SiO2 \& beta \& }\end{array}$} \\
\hline 2.49885000000000 & -4.32813516049349 & 0.00000000000000 & \\
\hline 2.49885000000000 & 4.32813516049349 & 0.00000000000000 & & \\
\hline 0.00000000000000 & 0.00000000000000 & 5.46010000000000 & & \\
\hline $\begin{array}{lr}\mathrm{O} & \mathrm{Si} \\
6 & 3\end{array}$ & & & & \\
\hline \multicolumn{5}{|l|}{ Direct } \\
\hline 0.20720000000000 & 0.41440000000000 & 0.50000000000000 & $\mathrm{O}$ & $(6 \mathrm{j})$ \\
\hline-0.41440000000000 & -0.20720000000000 & 0.83333333333333 & $\mathrm{O}$ & $(6 \mathrm{j})$ \\
\hline 0.20720000000000 & -0.20720000000000 & 0.166666666666667 & $\mathrm{O}$ & $(6 \mathrm{j})$ \\
\hline-0.20720000000000 & -0.41440000000000 & 0.50000000000000 & $\mathrm{O}$ & $(6 \mathrm{j})$ \\
\hline 0.41440000000000 & 0.20720000000000 & 0.83333333333333 & $\mathrm{O}$ & $(6 \mathrm{j})$ \\
\hline-0.20720000000000 & 0.20720000000000 & 0.166666666666667 & $\mathrm{O}$ & $(6 \mathrm{j})$ \\
\hline 0.50000000000000 & 0.00000000000000 & 0.00000000000000 & $\mathrm{Si}$ & (3c) \\
\hline 0.00000000000000 & 0.50000000000000 & 0.33333333333333 & $\mathrm{Si}$ & $(3 c)$ \\
\hline 0.50000000000000 & 0.50000000000000 & 0.666666666666667 & $\mathrm{Si}$ & $(3 c)$ \\
\hline
\end{tabular}

AuCN: ABC_hP3_183_a_a_a - CIF

\# CIF file

data_findsym-output

-audit_creation_method FINDSYM

chemical name mineral 'AuCN'

chemical formula sum ' $\mathrm{Au} \mathrm{C} \mathrm{N}$ '

loop

publ_author_nam

S. J. Hibble

A. C. Hannon
S. M. Cheyne

journal_name_full_name

Inorganic Chemistry

journal_volume 42

_journal_year 2003

_journal_page_first 4724

journal page last 4730

publ_Section_title

Structure of AuCN determined from total neutron diffraction

\# Found in Pearson's Crystal Data - Crystal Structure Database for $\hookrightarrow$ Inorganic Compounds, 2013

aflow_title 'AuCN Structure,

aflow_proto 'ABC_hP3_183_a_a_a

aflow_params 'a,c/a, $z_{-}\{1\}, z_{-}\{2\}, z_{-}\{3\}$

aflow_params_values ' $3.3908401495,1.49568037743,0.608,0.0,0.226$

aflow_Strukturbericht 'None'

aflow_Pearson 'hP3,

cell_length_a 3.3908401495

cell_length_b 3.3908401495

cell_length_c $\quad 5.0716130746$

cell_angle_alpha 90.0000000000

cell angle beta 90.0000000000

cell angle gamma 120.0000000000

symmetry_space_group_name_H-M "P $6 \mathrm{~m} \mathrm{~m}$ "

_symmetry_Int_Tables_number 183 
loop

_space_group_symop_id

_space_group_symop_operation_xyz

$1 \mathrm{x}, \mathrm{y}, \mathrm{z}$

$2 \mathrm{x}-\mathrm{y}, \mathrm{x}, \mathrm{z}$

$3-y, x-y, z$

$4-x,-y, z$

$5-x+y,-x, z$

$6 \mathrm{y},-\mathrm{x}+\mathrm{y}, \mathrm{z}$

$7-x+y, y, z$

$8-x,-x+y, z$

$9-\mathrm{y},-\mathrm{x}, \mathrm{z}$

$10 x-y,-y, z$

$11 \mathrm{x}, \mathrm{x}-\mathrm{y}, \mathrm{z}$

$12 \mathrm{y}, \mathrm{x}, \mathrm{z}$

loop

atom_site_label

_atom_site_type_symbol

_atom_site_symmetry_multiplicity

_atom_site_Wyckoff_label

atom_site_fract_x

atom_site_fract_y

atom_site_fract_z

atom_site_occupancy

$\begin{array}{lllllll}\mathrm{Au} 1 \mathrm{Au} & 1 & \mathrm{a} & 0.00000 & 0.00000 & 0.60800 & 1.00000\end{array}$

$\begin{array}{llllllllll}\mathrm{C} 1 & \mathrm{C} & 1 & \mathrm{a} & 0.00000 & 0.00000 & 0.00000 & 1.00000\end{array}$

$\begin{array}{llllllllll}\mathrm{N} 1 & \mathrm{~N} & 1 & \mathrm{a} & 0.00000 & 0.00000 & 0.22600 & 1.00000\end{array}$

AuCN: ABC_hP3_183_a_a_a - POSCAR

ABC_hP3_183_a_a_a \& a , c /a, z1 , z2, z3 --params $=3.3908401495,1.49568037743$, $\hookrightarrow 0.608,0.0,0.226 \& \mathrm{P}_{\mathrm{mm}} \mathrm{C}_{-}\{6 \mathrm{v}\}^{\wedge}\{1\} \# 183\left(\mathrm{a}^{\wedge} 3\right) \& \mathrm{hP} 3 \&$ None $\&$

$\hookrightarrow$ AuCN \& \& S. J. Hibble and A. C. Hannon and S. M. Cheyne,

$\hookrightarrow$ Inorg. Chem. 42, 4724-4730 (2003)

1.00000000000000

$\begin{array}{lll}1.69542007475000 & -2.93655370963922 & 0.00000000000000\end{array}$

$\begin{array}{rrr}1.69542007475000 & 2.93655370963922 & 0.00000000000000\end{array}$

$\begin{array}{lll}1.69542007475000 & 2.93655370963922 & 0.00000000000000 \\ 0.00000000000000 & 0.00000000000000 & 5.07161307460000\end{array}$

0.00000000000000

\section{(1)}

$\begin{array}{lll}0.00000000000000 & 0.00000000000000 & 0.60800000000000\end{array}$

$0.00000000000000 \quad 0.0000000000000$

0.00000000000000

0.00000000000000

0.00000000000000 0.22600000000000

(1a)

$\mathrm{CrFe}_{3} \mathrm{NiSn}_{5}: \mathrm{AB} \_$hP6_183_c_ab-CIF

\section{\# CIF file}

data_findsym-output

audit_creation_method FINDSYM

chemical_name_mineral 'CrFe3NiSn5,

_chemical_formula_sum 'M Sn

loop

_publ_author_name

'J. Huang '

'L. Zeng

journal_name_full_name

Powder Diffraction

journal volume 19

journal year 2004

journal_page_first 372

journal_page_last 374

publ_Section_titl

$\mathrm{X}$-ray powder diffraction data and Rietveld refinement of $\mathrm{CrFe}_{-}\{3\}$ $\longrightarrow \$ N i S n \$-\{5\} \$$

\# Found in Pearson's Crystal Data - Crystal Structure Database for $\hookrightarrow$ Inorganic Compounds, 2013

aflow_title 'CrFe\$_ $\{3\} \$ N i S n \$_{-}\{5\} \$$ Structure

-aflow_proto 'AB_hP6_183_c_ab

aflow_params ,a,c/a, z_\{1\},z_\{2\},z $\{3\}$,

aflow_params_values ' $5.3175214551,0.83247442072,0.0,0.513,0.01$,

aflow Strukturbericht 'None'

aflow_Pearson 'hP6'

_cell_length_a $\quad 5.3175214551$

_cell_length_b 5.3175214551

_cell_length_c $\quad 4.4267005930$

cell_angle_alpha 90.0000000000

cell_angle_beta 90.0000000000

cell_angle_gamma 120.0000000000

_symmetry_space_group_name_H-M "P $6 \mathrm{~m} \mathrm{~m}$

symmetry Int Tables number 183

loop

_space_group_symop_id

_space_group_symop_operation_xyz

$1 \mathrm{x}, \mathrm{y}, \mathrm{z}$

$2 \mathrm{x}-\mathrm{y}, \mathrm{x}, \mathrm{z}$

$-y, x-y, z$

$-\mathrm{x},-\mathrm{y}, \mathrm{z}$

$-\mathrm{x}+\mathrm{y},-\mathrm{x}, \mathrm{z}$

$6 \quad y,-x+y, z$

$7-x+y, y, z$
$8-x,-x+y, z$ $9-y,-x, z$

$10 x-y,-y, z$

$11 \mathrm{x}, \mathrm{x}-\mathrm{y}, \mathrm{z}$

$12 \mathrm{y}, \mathrm{x}, \mathrm{z}$

loop

atom_site_label

atom_site_type_symbol

atom_site_symmetry_multiplicity

_atom_site_Wyckoff_label

atom_site_fract_x

atom_site_fract_y

-atom_site_fract_z

_atom_site_occupancy

Sn 1 Sn 1 a 0.033330 .006

$\mathrm{CrFe}_{3} \mathrm{NiSn}_{5}$ : AB_hP6_183_c_ab - POSCAR

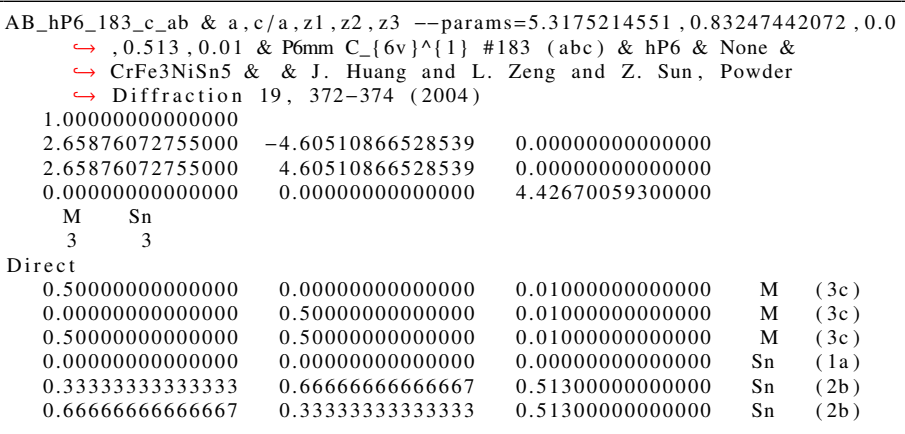

$\mathrm{Al}\left[\mathrm{PO}_{4}\right]$ (Framework type AFI): AB4C_hP72_184_d_4d_d - CIF

\section{\# CIF file}

data_findsym-output

audit_creation_method FINDSYM

_chemical_name_mineral 'Al[PO4]

chemical_formula_sum, Al O4 P,

loop

publ_author_name

- Gublathor Klap'

'H. \{van Koningsveld \}'

H. Traafsma

A. M. M. Schreurs,

_journal_name_full_name

Microporous and Mesoporous Materials

journal_volume 38

journal_year 2000

journal_page_first 403

journal_page_last 412

publ_Section_title

Absolute configuration and domain structure of AIPO $\{4\} \$-5$ studied by $\hookrightarrow$ single crystal X-ray diffraction

\# Found in Pearson's Crystal Data - Crystal Structure Database for $\hookrightarrow$ Inorganic Compounds, 2013

-aflow_title ${ }^{\prime}$ Al[PO\$_\{4\}\$] (Framework type AFI) Structure

aflow_proto 'AB4C_hP72_184_d_4d_d '

aflow_params $, a, c / a, x_{-}\{1\}, y_{-}\{1\}, z_{-}\{1\}, x_{-}\{2\}, y_{-}\{2\}, z_{-}\{2\}, x_{-}\{3\}, y_{-}\{3\}, z_{-}\{$ $\hookrightarrow 3\}, \mathrm{x}_{-}\{4\}, \mathrm{y}_{-}\{4\}, \mathrm{z}_{-}\{4\}, \mathrm{x}_{-}\{5\}, \mathrm{y}_{-}\{5\}, \mathrm{z}_{-}\{5\}, \mathrm{x}_{-}\{6\}, \mathrm{y}_{-}\{6\}, \mathrm{z}_{-}\{6\}$

aflow_params_values ' $13.7178848276,0.616168537688,0.45652,0.12053,0.0$, $\hookrightarrow 0.42,0.2069,0.071,0.1224,0.4519,0.2982,0.3629,0.0019,0.0649$,

$\hookrightarrow 0.1538,0.5737,0.0602,0.12298,0.4525,0.12746$

aflow_Strukturbericht 'None'

aflow Pearson 'hP72'

cell_length_a $\quad 13.7178848276$

cell_length_b $\quad 13.7178848276$

cell_length_c $\quad 8.4525290344$

cell angle alpha 90.0000000000

cell_angle_beta 90.0000000000

cell_angle_gamma 120.0000000000

symmetry_space_group_name_H-M "P 6 c c"

_symmetry_Int_Tables_number 184

loop

space_group_symop_id

space_group_symop_operation_xyz

$\mathrm{x}, \mathrm{y}, \mathrm{z}$

$2 x-y, x, z$

$3-y, x-y, z$

$3-x,-y, z$
$5-x+y,-x, z$

$4-x+y,-x$,
$6-y,-x+y, z$

6
$7-x,-x+y, z$
$8-x+y, y+1 / 2$

$-x+y, y, z+1 / 2$

$8-x,-x+y, z+1 / 2$

$-y,-x, z+1 / 2$

$10 \mathrm{x}-\mathrm{y},-\mathrm{y}, \mathrm{z}+1 / 2$

$11 \mathrm{x}, \mathrm{x}-\mathrm{y}, \mathrm{z}+1 / 2$
$12 \mathrm{y}, \mathrm{x}, \mathrm{z}+1 / 2$ 
loop

-atom_site_label

atom_site_type_symbo

atom_site_symmetry_multiplicity

atom_site_Wyckoff_label

atom_site_fract_x

atom_site_fract ${ }_{-}$

atom_site_fract_z

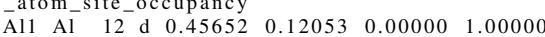

$\begin{array}{llllllllllll}\mathrm{O} 1 & \mathrm{O} & 12 & \mathrm{~d} & 0.42000 & 0.20690 & 0.07100 & 1.00000\end{array}$

$\begin{array}{llllllllllllll}02 & \mathrm{O} & 12 & \mathrm{~d} & 0.12240 & 0.45190 & 0.29820 & 1.00000\end{array}$

$\begin{array}{lllllllll}\mathrm{O} 3 & \mathrm{O} & 12 & \mathrm{~d} & 0.36290 & 0.00190 & 0.06490 & 1.00000\end{array}$

$\begin{array}{lllllllll}\mathrm{O} 4 & \mathrm{O} & 12 & \mathrm{~d} & 0.36290 & 0.00190 & 0.06490 & 1.00000\end{array}$

$\begin{array}{lllllllll}\mathrm{P} 1 & \mathrm{P} & 12 & \mathrm{~d} & 0.12298 & 0.45250 & 0.12746 & 1.00000\end{array}$

$\mathrm{Al}\left[\mathrm{PO}_{4}\right]$ (Framework type AFI): AB4C_hP72_184_d_4d_d - POSCAR

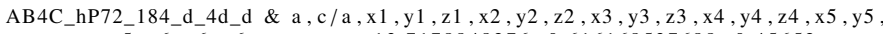
$\hookrightarrow \mathrm{z} 5, \mathrm{x} 6, \mathrm{y} 6, \mathrm{z} 6--$ params $=13.7178848276,0.616168537688,0.45652$,

$\hookrightarrow 0.12053,0.0,0.42,0.2069,0.071,0.1224,0.4519,0.2982,0.3629$,

$\hookrightarrow 0.0019,0.0649,0.1538,0.5737,0.0602,0.12298,0.4525,0.12746 \&$

$\hookrightarrow$ Klap et al., Microporous Mesoporous Mater. 38, 403-412 (2000) 1.00000000000000

$6.85894241380000-11.88003674689070 \quad 0.00000000000000$

$\begin{array}{rrr}6.85894241380000 & 11.88003674689070 & 0.00000000000000\end{array}$

$\begin{array}{rrr}6.8589424138000000000000000000000000 & 8.45252903440000\end{array}$

$$
\begin{array}{rrr}
\text { Al } & \text { O } & \text { P } \\
12 & 48 & 12
\end{array}
$$$$
\text { Direct }
$$

ect

$-0.12053000000000$

$-0.45652000000000$

0.12053000000000

0.33599000000000

$-0.12053000000000$

$-0.33599000000000$

0.45652000000000

0.12053000000000

0.33599000000000

$-0.45652000000000$

0.42000000000000

$-0.20690000000000$

$-0.21310000000000$

$-0.42000000000000$

0.20690000000000

0.21310000000000

$-0.20690000000000$

$-0.21310000000000$

0.42000000000000

0.20690000000000

0.21310000000000

$-0.42000000000000$

0.12240000000000

$-0.45190000000000$

0.32950000000000

$-0.12240000000000$

0.45190000000000

$-0.32950000000000$

$-0.45190000000000$

0.32950000000000

0.12240000000000

0.45190000000000

$-0.32950000000000$

$-0.12240000000000$

0.36290000000000

$-0.00190000000000$

$-0.36100000000000$

0.00190000000000

0.36100000000000

0.3610000000000

$-0.00190000000000$

0.36100000000000

0.00190000000000

0.36100000000000

$-0.36290000000000$

0.15380000000000

$-0.57370000000000$

0.41990000000000

$-0.15380000000000$

0.57370000000000

$-0.41990000000000$

$-0.57370000000000$

0.41990000000000

0.15380000000000

0.57370000000000

$-0.41990000000000$

$-0.4159000000000$

0.12298000000000

$-0.45250000000000$

0.32952000000000

$-0.12298000000000$

0.45250000000000

$-0.32952000000000$

$-0.45250000000000$

0.32952000000000

0.12298000000000

0.45250000000000

$-0.12298000000000$

0.12053000000000 0.33599000000000 .45652000000000 $-0.1205300000000$ $-0.33599000000000$ 0.45652000000000 0.45652000000000 .12053000000000 . 33599000000000 . .33599000000000 . . 0.21310000000000 0.42000000000000 0.20690000000000 42000000000000 0.20690000000000 0.21310000000000 0.42000000000000 $-0.20690000000000$ $-0.21310000000000$ 0.45190000000000 0.32950000000000 $-0.12240000000000$ $-0.45190000000000$ . 0.12240000000000 0.122400000000 .1224000000000 . . 32950000000000 0.12240000000000 $-0.45190000000000$ 0.3295000000000 0.00190000000000 0.36100000000000 $-0.36290000000000$ $-0.00190000000000$ $-0.36100000000000$ . 36290000000000 .36290000000000 0.00190000000000 0.36100000000000 0.3629000000000 0.3629000000 $-0.00190000000000$ 0.36100000000000 0.57370000000000 $-0.41990000000000$ . 57370000000000 $-1$ .15380000000000 $-0.15380000000000$ 0.57370000000000 0.41990000000000 0.15380000000000 $-0.57370000000000$ 0.41990000000000 0.45250000000000 0.32552000000000 $-0.12298000000000$ $-0.45250000000000$ 0.45250000000000 0.32952000000000 0.45250000000000 $-0.3295200000000$ 0.12298000000000 0.4525000000000
0.00000000000000 0.00000000000000 0.00000000000000 0.00000000000000 0.00000000000000 0.00000000000000 0.50000000000000 0.50000000000000 0.5000000000000 0.5000000000000 0.5000000000000 0.500000000 . .50700000000000 0.0710000000000 0.07100000000000 0.07100000000000 0.07100000000000 0.07100000000000 0.07100000000000 0.5710000000000 0.57100000000000 0.57100000000000 0.57100000000000 0.57100000000000 0.57100000000000 0.29820000000000 0.2982000000000 0.2982000000000 0.2982000000000 0.29820000000000 0.298200000000 0.29820000000000 0.79820000000000 0.79820000000000 0.7982000000000 0.7982000000000 0.79820000000000 0.7982000000000 0.0649000000000 0.06490000000000 0.0649000000000 0.06490000000000 0.06490000000000 0.06490000000000 0.56490000000000 0.5649000000000 0.564900000000 0.5649000000000 0.56490000000000 0.56490000000000 0.56490000000000 0.06020000000000 0.06020000000000 0.06020000000000 0.06020000000000 0.0602000000000 0.0602000000000 0.56020000000000 0.56020000000000 0.56020000000000 0.56020000000000 0.56020000000000 0.5602000000000 0.5602740000000 0.12746000000000 0.12746000000000 . 0.12746000000000 0.12746000000000 0.12746000000000 0.62746000000000 0.6274600000000 0.62746000000000 0.62746000000000 0.62746000000000 0.62746000000000

Al (12d)

Al $(12 \mathrm{~d})$

Al (12d)

$\mathrm{Al} \quad(12 \mathrm{~d})$

$\mathrm{Al} \quad(12 \mathrm{~d})$

$\mathrm{Al} \quad(12 \mathrm{~d})$

$\mathrm{Al}$ (12d)

$\mathrm{Al}$ (12d)

Al (12d)

Al (12d)

O (12d)

$\mathrm{O} \quad(12 \mathrm{~d})$

$\mathrm{O} \quad(12 \mathrm{~d})$

$\mathrm{O} \quad(12 \mathrm{~d})$

$\mathrm{O} \quad(12 \mathrm{~d})$

$\mathrm{O}(12 \mathrm{~d})$

O (12d)

(12d)

$\mathrm{O} \quad(12 \mathrm{~d})$

$\mathrm{O}(12 \mathrm{~d})$

$\mathrm{O} \quad(12 \mathrm{~d})$

$\mathrm{O} \quad(12 \mathrm{~d})$

$\mathrm{O} \quad(12 \mathrm{~d})$

$\mathrm{O}(12 \mathrm{~d})$

O (12d)

O $(12 \mathrm{~d})$

$\mathrm{O} \quad(12 \mathrm{~d})$

$\mathrm{O} \quad(12 \mathrm{~d})$

O (12d)

O (12d)

O (12d)

$\mathrm{O}(12 \mathrm{~d})$

$\mathrm{O}$ (12d)

$\mathrm{O} \quad(12 \mathrm{~d})$

$\mathrm{O} \quad(12 \mathrm{~d})$

O (12d)

O (12d)

$\mathrm{O}$ (12d)

O (12d)

$\mathrm{O} \quad(12 \mathrm{~d})$

$\mathrm{O} \quad(12 \mathrm{~d})$

$\mathrm{O} \quad(12 \mathrm{~d})$

$\mathrm{O} \quad(12 \mathrm{~d})$ 

$-0.66470000000000$ 0.33333333333333
0.50560000000000 $-0.33240000000000$ $-0.50560000000000$ 0.33240000000000 0.17320000000000 0.50560000000000 $-0.33240000000000$ $-0.17320000000000$ 0.66470000000000 0.00000000000000 $-0.66470000000000$ 0.00000000000000 0.66470000000000 0.00000000000000 0.00000000000000 0.00000000000000 0.33333333333333 0.66666666666667 0.33333333333333
0.66666666666667
0.33240000000000 0.17320000000000 $-0.17320000000000$ 0.5056000000000 0.5056000000000 0.3320000000 $-0.5056000000000$ $-0.50560000000000$ 0.3324000000000 0.00000000000000 0.66470000000000 $-0.66470000000000$ 0.00000000000000 $-0.66470000000000$ 0.66470000000000 0.00000000000000 0.00000000000000 0.66666666666667 0.66666666666667
0.61480000000000 0.61480000000000 0.61480000000000 0.61480000000000 0.61480000000000 0.6148000000 0.11480000000000 0.1148000000000 0.11480000000000 0.17060000000000 0.17060000000000 0.17060000000000 0.67060000000000 0.67060000000000 0.67060000000000 0.00000000000000 0.50000000000000 0.37700000000000 0.87700000000000 0.87700000000000 0.87700000000 0000000000

$\begin{aligned} \mathrm{Cl} & (12 \mathrm{~d}) \\ \mathrm{Cl} & (12 \mathrm{~d}) \\ \mathrm{Cl} & (12 \mathrm{~d}) \\ \mathrm{Cl} & (12 \mathrm{~d}) \\ \mathrm{Cl} & (12 \mathrm{~d}) \\ \mathrm{Cl} & (12 \mathrm{~d}) \\ \mathrm{Cl} & (12 \mathrm{~d}) \\ \mathrm{Cl} & (12 \mathrm{~d}) \\ \mathrm{K} & (6 \mathrm{c}) \\ \mathrm{K} & (6 \mathrm{c}) \\ \mathrm{K} & (6 \mathrm{c}) \\ \mathrm{K} & (6 \mathrm{c}) \\ \mathrm{K} & (6 \mathrm{c}) \\ \mathrm{K} & (6 \mathrm{c}) \\ \mathrm{Ni} & (2 \mathrm{a}) \\ \mathrm{Ni} & (2 \mathrm{a}) \\ \mathrm{Ni} & (4 \mathrm{~b}) \\ \mathrm{Ni} & (4 \mathrm{~b}) \\ \mathrm{Ni} & (4 \mathrm{~b}) \\ \mathrm{Ni} & (4 \mathrm{~b})\end{aligned}$

Cu 3 P: A3B_hP24_185_ab2c_c - CIF

\section{CIF file}

data_findsym-output

audit_creation_method FINDSYM

chemical_name_mineral,

chemical_formula_sum ' $\mathrm{Cu} 3 \mathrm{P}$,

oop

publ_author_name

'O. Olofsson'

journal_name_full_name

Acta Chemica Scandinavica

journal_volume 26

journal year 1972

journal_page_first 2777

journal page last 2787

publ Section title

The Crystal Structure of $\mathrm{Cu}_{-}\{3\} \$ \mathrm{P}$

flow title

aflow_proto 'A3B_hP24_185_ab2c_c

aflow_params 'a, c/a, z_ $\{1\}, \mathrm{z}_{-}\{2\}, \mathrm{x}_{-}\{3\}, \mathrm{z}_{-}\{3\}, \mathrm{x}_{-}\{4\}, \mathrm{z}_{-}\{4\}, \mathrm{x}_{-}\{5\}, \mathrm{z}_{-}\{5\}$, _params_values '6.9593,1.026396,

$\longrightarrow 0.3761,0.4246,0.3322,0$.

aflow Pearson, 'hP24,

ymmetry space group_name_H-M "P 63 c m"

symmetry_Int_Tables_number 185

cell_length_a $\quad 6.95930$

cell_length_b 6.95930

cell_length_c 7.14300

cell_angle_alpha 90.00000

cell_angle_beta 90.00000

ang_gamma 120.00000

oop

space_group_symop_id

space

$x-y, x, z+1 / 2$

$3-y, x-y, z$

$-x,-y, z+1 / 2$

$-\mathrm{x}+\mathrm{y},-\mathrm{x}, \mathrm{z}$

$\mathrm{y},-\mathrm{x}+\mathrm{y}, \mathrm{z}+1 / 2$

$-\mathrm{x}+\mathrm{y}, \mathrm{y}, \mathrm{z}+1 / 2$

$-\mathrm{x},-\mathrm{x}+\mathrm{y}, \mathrm{z}$

$0 \mathrm{x}-\mathrm{y},-\mathrm{y}, \mathrm{z}$

$\mathrm{x}, \mathrm{x}-\mathrm{y}, \mathrm{z}+1 / 2$

$12 \mathrm{y}, \mathrm{x}, \mathrm{z}$

oop

atom_site_type_symbol

atom_site_symmetry_multiplicity

atom_site_Wyckoff_label

atom_site_fract_x

atom_site_fract_y

atom_site_fract_z

atom_site_occupancy

$\begin{array}{llllll}2 & \text { a } & 0.00000 & 0.00000 & 0.32130 & 1.00000\end{array}$

$\begin{array}{lllllll}4 & \mathrm{~b} & 0.33333 & 0.66667 & 0.19980 & 1.00000\end{array}$

$\begin{array}{llllllllll}\mathrm{Cu} 4 & \mathrm{Cu} & 6 & \mathrm{c} & 0.37610 & 0.00000 & 0.42460 & 1.00000\end{array}$

$\begin{array}{lllllllll}\mathrm{P} 1 & \mathrm{P} & 6 & \mathrm{c} & 0.33220 & 0.00000 & 0.75000 & 1.00000\end{array}$

Cu3 P: A3B_hP24_185_ab2c_c - POSCAR

3B hP24 185 ab2c_c \& a , c/a , z1 $, \mathrm{z} 2, \mathrm{x} 3, \mathrm{z} 3, \mathrm{x} 4, \mathrm{z4}, \mathrm{x} 5, \mathrm{z} 5-$ - params $=6.9593$

$\hookrightarrow 1.02639633296,0.3213,0.1998,0.2806,0.0765,0.3761,0.4246,0.3322$

$\hookrightarrow 0.75 \& \mathrm{P} 6\{3\} \mathrm{cm} \mathrm{C}\{6 \mathrm{v}\}^{\wedge}\{3\} \# 185\left(\mathrm{abc}^{\wedge} 3\right) \& \mathrm{hP} 24$ \& None \& $\mathrm{Cu} 3 \mathrm{P}$ \&

$\hookrightarrow$ \& O. Olofsson, Acta Chem. Scand. 26, 2777-2787 (1972)

1.00000000000000

$\begin{array}{lll}3.47965000000000 & -6.02693059255704 & 0.00000000000000\end{array}$

$\begin{array}{llll}3.47965000000000 & 6.02693059255704 & 0.00000000000000\end{array}$

$\begin{array}{lll}0.00000000000000 & 0.00000000000000 & 7.14300000000000\end{array}$

\begin{tabular}{rrrrrr|}
$\begin{aligned} \mathrm{Cu} \\
18\end{aligned} \mathrm{P}$ & $\mathrm{P}$ & & & \\
Direct & & & & \\
0.00000000000000 & 0.00000000000000 & 0.32130000000000 & $\mathrm{Cu}$ & $(2 \mathrm{a})$ \\
0.00000000000000 & 0.00000000000000 & 0.82130000000000 & $\mathrm{Cu}$ & $(2 \mathrm{a})$ \\
0.33333333333333 & 0.66666666666667 & 0.19980000000000 & $\mathrm{Cu}$ & $(4 \mathrm{~b})$ \\
0.66666666666667 & 0.33333333333333 & 0.69980000000000 & $\mathrm{Cu}$ & $(4 \mathrm{~b})$ \\
0.3333333333333 & 0.66666666666667 & 0.69980000000000 & $\mathrm{Cu}$ & $(4 \mathrm{~b})$ \\
0.66666666666667 & 0.33333333333333 & 0.19980000000000 & $\mathrm{Cu}$ & $(4 \mathrm{~b})$ \\
0.28060000000000 & 0.00000000000000 & 0.07650000000000 & $\mathrm{Cu}$ & $(6 \mathrm{c})$ \\
0.00000000000000 & 0.28060000000000 & 0.07650000000000 & $\mathrm{Cu}$ & $(6 \mathrm{c})$ \\
-0.28060000000000 & -0.28060000000000 & 0.07650000000000 & $\mathrm{Cu}$ & $(6 \mathrm{c})$ \\
-0.28060000000000 & 0.00000000000000 & 0.57650000000000 & $\mathrm{Cu}$ & $(6 \mathrm{c})$ \\
0.00000000000000 & -0.28060000000000 & 0.57650000000000 & $\mathrm{Cu}$ & $(6 \mathrm{c})$ \\
0.28060000000000 & 0.28060000000000 & 0.57650000000000 & $\mathrm{Cu}$ & $(6 \mathrm{c})$ \\
0.37610000000000 & 0.00000000000000 & 0.42460000000000 & $\mathrm{Cu}$ & $(6 \mathrm{c})$ \\
0.00000000000000 & 0.37610000000000 & 0.42460000000000 & $\mathrm{Cu}$ & $(6 \mathrm{c})$ \\
-0.37610000000000 & -0.37610000000000 & 0.42460000000000 & $\mathrm{Cu}$ & $(6 \mathrm{c})$ \\
-0.37610000000000 & 0.00000000000000 & 0.92460000000000 & $\mathrm{Cu}$ & $(6 \mathrm{c})$ \\
0.00000000000000 & -0.37610000000000 & 0.92460000000000 & $\mathrm{Cu}$ & $(6 \mathrm{c})$ \\
0.37610000000000 & 0.37610000000000 & 0.92460000000000 & $\mathrm{Cu}$ & $(6 \mathrm{c})$ \\
0.33220000000000 & 0.00000000000000 & 0.75000000000000 & $\mathrm{P}$ & $(6 \mathrm{c})$ \\
0.00000000000000 & 0.33220000000000 & 0.75000000000000 & $\mathrm{P}$ & $(6 \mathrm{c})$ \\
-0.33220000000000 & -0.33220000000000 & 0.75000000000000 & $\mathrm{P}$ & $(6 \mathrm{c})$ \\
-0.33220000000000 & 0.00000000000000 & 1.25000000000000 & $\mathrm{P}$ & $(6 \mathrm{c})$ \\
0.00000000000000 & -0.33220000000000 & 1.25000000000000 & $\mathrm{P}$ & $(6 \mathrm{c})$ \\
0.33220000000000 & 0.33220000000000 & 1.25000000000000 & $\mathrm{P}$ & $(6 \mathrm{c})$ \\
\hline
\end{tabular}

$\beta$-RuCl 3 : A3B_hP8_185_c_a - CIF

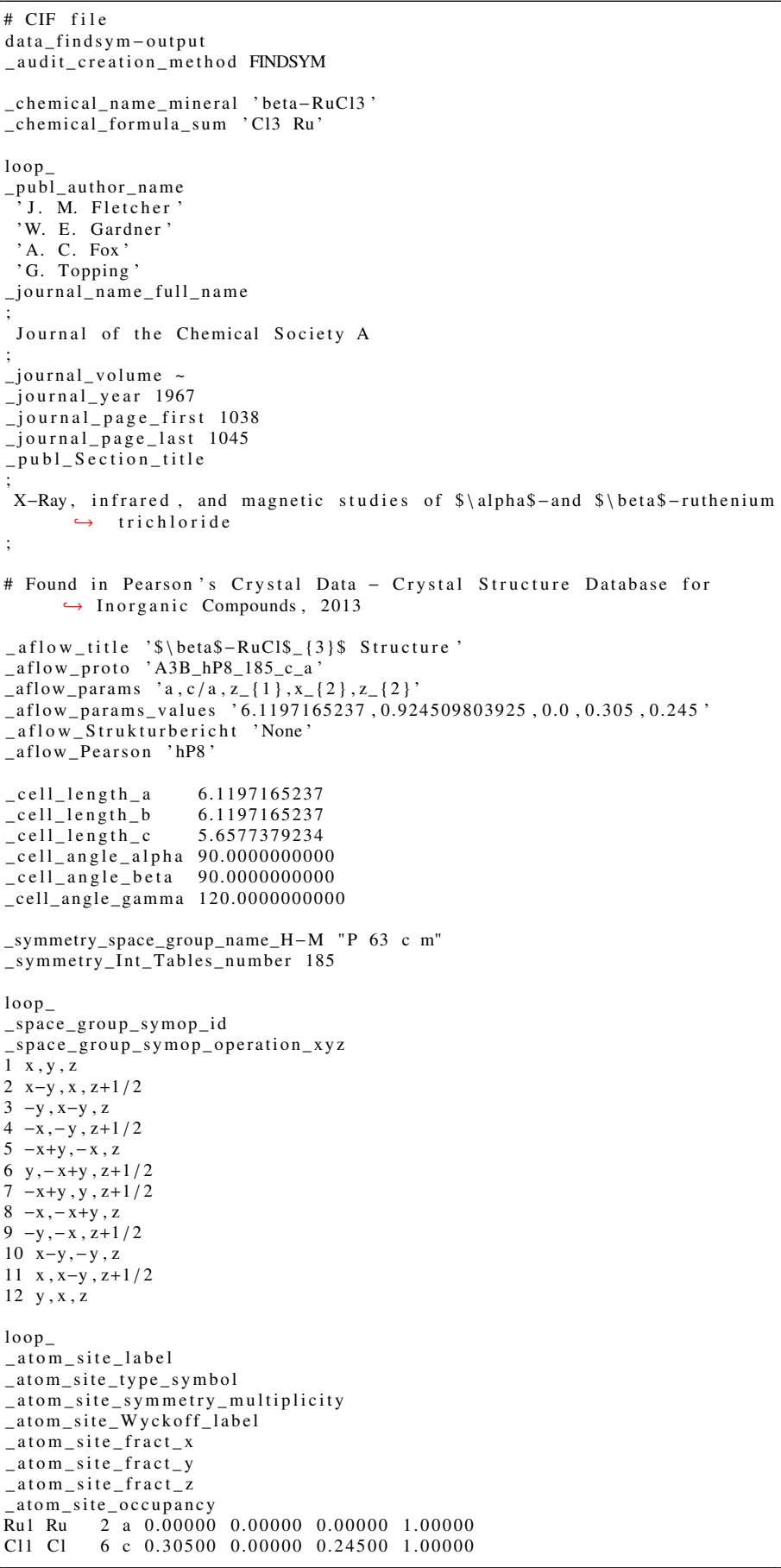


$\beta$-RuCl 3 : A3B_hP8_185_c_a - POSCAR

\begin{tabular}{|c|c|c|c|c|}
\hline \multicolumn{5}{|c|}{ 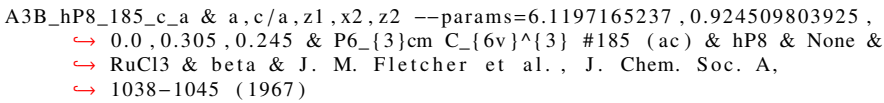 } \\
\hline \multicolumn{5}{|c|}{1.0000000000000} \\
\hline 3.05985826185000 & -5.29982997348359 & 0.00000000000000 & & \\
\hline 3.05985826185000 & 5.29982997348359 & 0.00000000000000 & & \\
\hline 0.00000000000000 & 0.00000000000000 & 5.65773792340000 & & \\
\hline $\mathrm{Cl}$ & & & & \\
\hline 6 & & & & \\
\hline \multicolumn{5}{|l|}{ Direct } \\
\hline 0.30500000000000 & 0.00000000000000 & 0.24500000000000 & $\mathrm{Cl}$ & $(6 c)$ \\
\hline 0.00000000000000 & 0.30500000000000 & 0.24500000000000 & $\mathrm{Cl}$ & $(6 c)$ \\
\hline-0.30500000000000 & -0.30500000000000 & 0.24500000000000 & $\mathrm{Cl}$ & (6c) \\
\hline-0.30500000000000 & 0.00000000000000 & 0.74500000000000 & $\mathrm{Cl}$ & (6c) \\
\hline 0.00000000000000 & -0.30500000000000 & 0.74500000000000 & $\mathrm{Cl}$ & (6c) \\
\hline 0.30500000000000 & 0.30500000000000 & 0.74500000000000 & $\mathrm{Cl}$ & $(6 c)$ \\
\hline 0.00000000000000 & 0.00000000000000 & 0.00000000000000 & $\mathrm{Ru}$ & (2a) \\
\hline 0.00000000000000 & 0.00000000000000 & 0.50000000000000 & $\mathrm{Ru}$ & $(2 a)$ \\
\hline
\end{tabular}

Na 3 As: AB3_hP24_185_c_ab2c - CIF

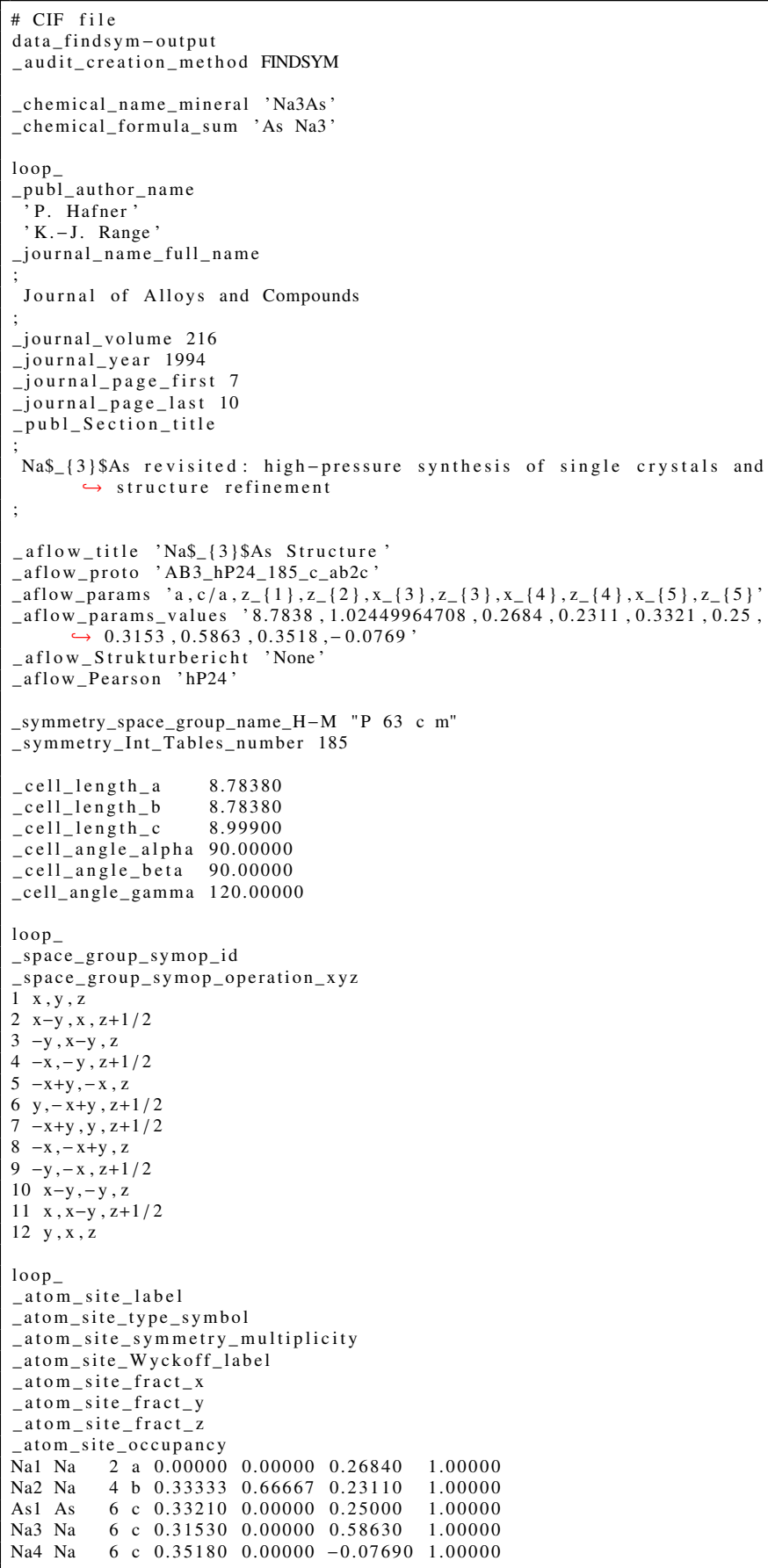

Na 3 As: AB3_hP24_185_c_ab2c - POSCAR

AB3_hP24_185_c_ab2c \& a , c /a , z1 , z2 , x3 , z3 , x4, z4, x5, z5 --params $=8.7838$ $\hookrightarrow \begin{array}{ll}\hookrightarrow \\ \hookrightarrow\end{array} 0.0769 \&$ P $6_{-}\{3\} \mathrm{cm} \mathrm{C} C_{-}\{6 \mathrm{v}\}^{\wedge}\{3\} \# 185\left(\mathrm{abc}^{\wedge} 3\right) \& \mathrm{hP} 24 \&$ None \& Na3As

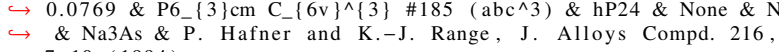
$\hookrightarrow 7-10(1994)$
1.00000000000000

4.39190000000000

4.39190000000000

0.00000000000000

$\begin{array}{rr}\text { As } & \mathrm{Na} \\ 6 & 18\end{array}$

Direct

0.33210000000000 0.00000000000000 $-0.33210000000000$ $-0.33210000000000$ 0.00000000000000 0.33210000000000 0.33210000000000 0.0000000000000

0.0000000000000

0.3333333333333
0.66666666666667

0.66666666666667

0.33333333333333

0.66666666666667

0.31530000000000

0.00000000000000

$-0.31530000000000$

$-0.31530000000000$

0.00000000000000

0.31530000000000

0.35180000000000

0.00000000000000

$-0.35180000000000$

$-0.35180000000000$

0.00000000000000

60699394176175 7.60699394176175 0.00000000000000

0.00000000000000 0.00000000000000 8.99900000000000

0.00000000000000 0.33210000000000 $-0.33210000000000$ 0.00000000000000 $-0.33210000000000$ 0.33210000000000 0.0000000000000 0.00000000000000 0.66666666666667 0.66666666666667 0.66666666666667 0.66666666666667 0.33333333333333 0.00000000000000 0.31530000000000 $-0.31530000000000$ 0.00000000000000 $-0.31530000000000$ 0.31530000000000 0.00000000000000 0.35180000000000 $-0.35180000000000$ 0.00000000000000 $-0.35180000000000$ 0.35180000000000

0.25000000000000 0.25000000000000 0.25000000000000 0.75000000000000 0.75000000000000 0.75000000000000 0.26840000000000 0.76840000000000 0.73110000000000 0.73110000000000 0.7311000000000 0.7311000000000 0.23110000000000 0.58630000000000 0.58630000000000 0.58630000000000 1.08630000000000 1.08630000000000 1.08630000000000 $-0.07690000000000$ $-0.07690000000000$ $-0.07690000000000$ 0.42310000000000 0.42310000000000 0.42310000000000

$\mathrm{T}_{3} \mathrm{Th}_{7}\left(D 10_{2}\right):$ A3B7_hP20_186_c_b2c - CIF

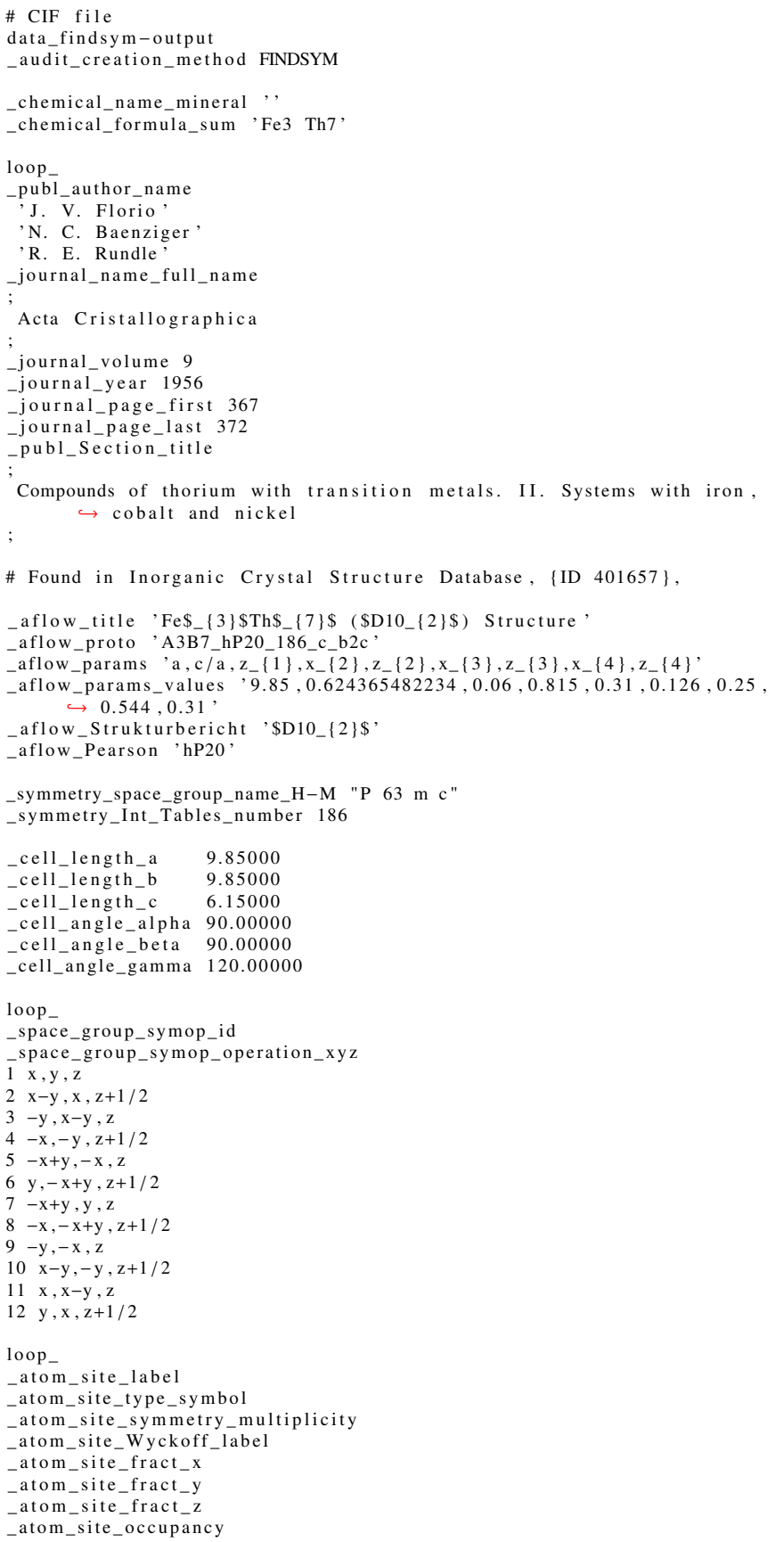


$\mathrm{Fe}_{3} \mathrm{Th}_{7}\left(D 10_{2}\right):$ A3B7_hP20_186_c_b2c - POSCAR

_atom_tite_Wyckoff_labe

atom_site_fract_x

atom_site_fract_y

atom_site_fract_z

A3B7_hP20_186_c_b2c \& a,c /a , z1, x2, z2, x3, z3, x4, z4 --params $=9.85$ $\hookrightarrow 0.624365482234,0.06,0.815,0.31,0.126,0.25,0.544,0.31 \&$ P6 $\{3\} \mathrm{m}$

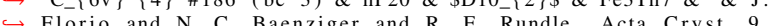

$\hookrightarrow$ Florio and N. C. Baenziger and R. E. Rundle, Acta Cryst. 9,

$\hookrightarrow 367-372(1956)$

1.00000000000000

$\begin{array}{lll}4.92500000000000 & -8.53035022727672 & 0.00000000000000\end{array}$

$\begin{array}{lrr}4.92500000000000 & 8.53035022727672 & 0.00000000000000\end{array}$

$\begin{array}{lll}0.00000000000000 & 0.00000000000000 & 6.15000000000000\end{array}$

$\begin{array}{rr}\mathrm{Fe} & \mathrm{Th} \\ 6 & 14\end{array}$

Direct

rect

$0.81500000000000 \quad-0.81500000000000$

$0.81500000000000 \quad 1.63000000000000$

$-1.63000000000000-0.81500000000000$

$-0.81500000000000-0.81500000000000$

$-0.81500000000000-1.63000000000000$

$\begin{array}{lll}1.63000000000000 & 0.81500000000000\end{array}$

$\begin{array}{ll}0.333333333333333 & 0.66666666666667\end{array}$

$0.66666666666667 \quad 0.33333333333333$

$0.12600000000000-0.1260000000000$

$0.12600000000000 \quad 0.25200000000000$

$-0.25200000000000-0.12600000000000$

$-0.12600000000000 \quad 0.12600000000000$

$-0.12600000000000-0.25200000000000$

$0.25200000000000 \quad 0.12600000000000$

$0.54400000000000 \quad-0.54400000000000$

$0.54400000000000 \quad 1.08800000000000$

$-1.08800000000000-0.54400000000000$

$-0.54400000000000 \quad 0.54400000000000$

$-0.54400000000000-1.08800000000000$

$\begin{array}{rr}1.08800000000000 & 0.54400000000000\end{array}$

0.31000000000000 0.31000000000000 0.31000000000000 0.81000000000000 0.81000000000000 0.81000000000000 0.06000000000000 0.56000000000000 0.2500000000000 .2500000000000 0.25000000000000 0.75000000000000 0.75000000000000 0.75000000000000 0.31000000000000 0.31000000000000 0.31000000000000 0.81000000000000 0.81000000000000 0.81000000000000

$\begin{array}{ll}\mathrm{Fe} & (6 \mathrm{c}) \\ \mathrm{Fe} & (6 \mathrm{c}) \\ \mathrm{Fe} & (6 \mathrm{c}) \\ \mathrm{Fe} & (6 \mathrm{c}) \\ \mathrm{Fe} & (6 \mathrm{c}) \\ \mathrm{Fe} & (6 \mathrm{c}) \\ \mathrm{Th} & (2 \mathrm{~b}) \\ \mathrm{Th} & (2 \mathrm{~b}) \\ \mathrm{Th} & (6 \mathrm{c}) \\ \mathrm{Th} & (6 \mathrm{c}) \\ \mathrm{Th} & (6 \mathrm{c}) \\ \mathrm{Th} & (6 \mathrm{c}) \\ \mathrm{Th} & (6 \mathrm{c}) \\ \mathrm{Th} & (6 \mathrm{c}) \\ \mathrm{Th} & (6 \mathrm{c}) \\ \mathrm{Th} & (6 \mathrm{c}) \\ \mathrm{Th} & (6 \mathrm{c}) \\ \mathrm{Th} & (6 \mathrm{c}) \\ \mathrm{Th} & (6 \mathrm{c}) \\ \mathrm{Th} & (6 \mathrm{c})\end{array}$

$\mathrm{Re}_{3} \mathrm{~N}$ : AB3_hP4_187_e_fh - CIF

\section{\# CIF file}

data_findsym-output

audit_creation method FINDSYM

chemical name mineral ' Re3N'

chemical_formula_sum ' $\mathrm{N} \mathrm{Re} 3$ ',

loop

publ_author_name

A. Friedrich

B. Winkler,

'L. Bayarjargal,

E. A. Juarez-Arellano

'V. Milman'

K. Refson,

'M. Kunz'

'K. Chen'

_journal_name_full_name

Physical Review Letters

journal volume 105

journal year 2010

journal_page_first 08550

journal_page_last 085504

publ_Section_title

Novel Rhenium Nitrides

aflow_title'Re\$_\{3\}\$N Structure

aflow_proto 'AB3_hP4_187_e fh '

aflow_params 'a, c/a, z $\{3\}$ '

aflow_params_values ' $2.8065,2.53411722786,0.198$ '

aflow Strukturbericht 'None'

aflow_Pearson 'hP4

ymmetry space_group_name_H-M "P $-6 \mathrm{~m} \mathrm{2"}$

symmetry_Int_Tables_number 187

cell_length_a 2.80650

cell_length_b 2.80650

cell_length_c $\quad 7.11200$

cell_angle_alpha 90.00000

_cell_angle_beta 90.00000

cell_angle_gamma 120.00000

loop

space_group_symop_id

space_group_symop_operation_xyz

$1 \mathrm{x}, \mathrm{y}, \mathrm{z}$

$2-y, x-y, z$

$-x+y,-x, z$

$\mathrm{x}, \mathrm{x}-\mathrm{y},-\mathrm{z}$

$-\mathrm{x}+\mathrm{y}, \mathrm{y},-\mathrm{z}$

$\begin{array}{ll}6 & -y,-x,-z \\ 7 & -x+y,-x,-z\end{array}$

$\begin{array}{ll}6 & -\mathrm{x}+\mathrm{y},-\mathrm{x} \\ 8 & \mathrm{x}, \mathrm{y},-\mathrm{z}\end{array}$

$-y, x-y,-z$

$-\mathrm{x}+\mathrm{y}, \mathrm{y}, \mathrm{z}$

$11-\mathrm{y},-\mathrm{x}, \mathrm{z}$

$12 \mathrm{x}, \mathrm{x}-\mathrm{y}, \mathrm{z}$

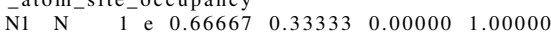

$\begin{array}{llllllll}\text { Re1 Re } 1 \text { f } & 0.66667 & 0.33333 & 0.50000 & 1.00000\end{array}$

$\begin{array}{lllllll}\mathrm{Re} 2 \mathrm{Re} & 2 & \mathrm{~h} & 0.33333 & 0.66667 & 0.19800 & 1.00000\end{array}$

$\mathrm{Re}_{3} \mathrm{~N}$ : AB3_hP4_187_e_fh - POSCAR

\begin{tabular}{|c|c|c|c|c|}
\hline \multicolumn{5}{|c|}{$\begin{aligned} \text { AB3_hP4_187_e_fh \& a, c/a, z3 }- \text { params }=2.8065,2.53411722786,0.198 \& \text { \& }-6 m 2 \\
\\
\hookrightarrow \text { D_ }\{3 \text { h }\} \wedge\{1\} \# 187 \text { (efh) \& hP4 \& None \& Re3N \& Re3N \& A. Friedrich } \\
\hookrightarrow \text { et al., Phys. Rev. Lett. 105, 085504(2010) }\end{aligned}$} \\
\hline 1.40325000000000 & -2.43050029572103 & 0.00000000000000 & & \\
\hline 1.40325000000000 & 2.43050029572103 & 0.00000000000000 & & \\
\hline 0.00000000000000 & 0.00000000000000 & 7.11200000000000 & & \\
\hline $\operatorname{Re}$ & & & & \\
\hline 1 & & & & \\
\hline \multicolumn{5}{|l|}{ Direct } \\
\hline 0.666666666666667 & 0.33333333333333 & 0.000000 & $\mathrm{~N}$ & (1e) \\
\hline 0.666666666666667 & 0.33333333333333 & 0.50000000000000 & $\operatorname{Re}$ & (1f) \\
\hline 0.33333333333333 & 0.666666666666667 & 0.19800000000000 & $\operatorname{Re}$ & ( $2 \mathrm{~h})$ \\
\hline 0.33333333333333 & 0.666666666666667 & -0.19800000000000 & $\operatorname{Re}$ & $(2 \mathrm{~h})$ \\
\hline
\end{tabular}

LiScI 3 : A3BC_hP10_188_k_a_e - CIF

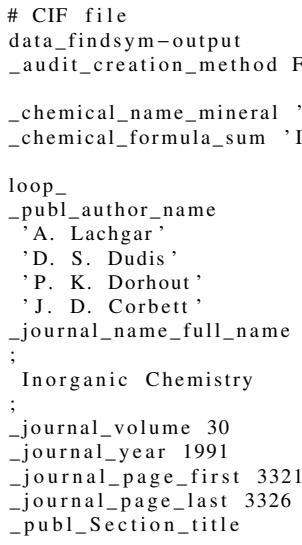

\# Found in Pearson's Crystal Data - Crystal Structure Database for $\hookrightarrow$ Inorganic Compounds, 2013

aflow_title, LiScI\$_ $\{3\} \$$ Structure

aflow_proto 'A3BC_hP10_188_k_a_e'

aflow_params 'a,c/a, $x_{-}\{3\}, y_{-}\{3\}$,

aflow params values $, \overline{7} .2864263258,0.928891999832,0.67077,0.01058$,

aflow_Strukturbericht 'None'

-aflow_Pearson 'hP10'

_cell_length_a $\quad 7.2864263258$

cell_length_b $\quad 7.2864263258$

cell_length_c $\quad 6.7683031214$

cell_angle_alpha 90.0000000000

cell_angle_beta 90.0000000000

_cell_angle_gamma 120.0000000000

symmetry_space_group_name_H-M "P -6 c 2 ("

symmetry_Int_Tables_number 188

loop

space_group_symop_id

space_group_symop_operation_xyz

$1 \mathrm{x}, \mathrm{y}, \mathrm{z}$

$2-\mathrm{y}, \mathrm{x}-\mathrm{y}, \mathrm{z}$

$3-\mathrm{x}+\mathrm{y},-\mathrm{x}, \mathrm{z}$

$4 \mathrm{x}, \mathrm{x}-\mathrm{y},-\mathrm{z}$

$5-x+y, y,-z$

$6-\mathrm{y},-\mathrm{x},-\mathrm{z}$

$7-\mathrm{x}+\mathrm{y},-\mathrm{x},-\mathrm{z}+1 / 2$

$8 \mathrm{x}, \mathrm{y},-\mathrm{z}+1 / 2$

$9-y, x-y,-z+1 / 2$

$10-\mathrm{x}+\mathrm{y}, \mathrm{y}, \mathrm{z}+1 / 2$

$11-\mathrm{y},-\mathrm{x}, \mathrm{z}+1 / 2$

$12 \mathrm{x}, \mathrm{x}-\mathrm{y}, \mathrm{z}+1 / 2$

loop

atom_site_label

atom_site_type_symbol

atom_site_symmetry_multiplicity

atom_site_Wyckoff_label

atom_site_fract_x

atom_site_fract_y

_atom_site_fract_z 
-atom_site_occupancy

$\begin{array}{lllll}0.00000 & 0.00000 & 0.00000 & 1.00000\end{array}$

$\begin{array}{lllllllll}\mathrm{Sc} 1 & \mathrm{Sc} & 2 & \mathrm{e} & 0.66667 & 0.33333 & 0.00000 & 1.00000\end{array}$

LiScI $I_{3}$ : A3BC_hP10_188_k_a_e - POSCAR

A3BC_hP10_188_k_a_e \& a , c /a , x3,y3 --params $=7.2864263258,0.928891999832$, $\hookrightarrow 0.67077,0.01058 \&$ P-6c2 D_ $\{3 \mathrm{~h}\}^{\wedge}\{2\} \# 188$ (aek) \& hP10 \& None \&

$\hookrightarrow$ LiScI3 \& \& A. Lachgar et al., Inorg. Chem. 30, 3321-3326 (1991

1.00000000000000

3.6432131629

$3.64321316290000-6.31023030094651$

$\begin{array}{lll}0.00000000000000 & 0.00000000000000 & 6.76830312140000\end{array}$

Direct

$\begin{array}{rrr}\mathrm{I} & \mathrm{Li} & \mathrm{Sc} \\ 6 & 2 & 2\end{array}$

0.67077000000000

$-0.01058000000000$

$-0.66019000000000$

$-0.01058000000000$

0.66019000000000

0.00000000000000

0.00000000000

0.00000000000000

0.66666666666667

0.01058000000000 0.66019000000000 $-0.67077000000000$ $-0.67077000000000$ 0.01058000000000 0.66019000000000 0.00000000000000 0.0000000000000 0.33333333333333 0.33333333333333

$\begin{array}{lrl}0.25000000000000 & \mathrm{I} & (6 \mathrm{k}) \\ 0.25000000000000 & \mathrm{I} & (6 \mathrm{k}) \\ 0.25000000000000 & \mathrm{I} & (6 \mathrm{k}) \\ 0.75000000000000 & \mathrm{I} & (6 \mathrm{k}) \\ 0.75000000000000 & \mathrm{I} & (6 \mathrm{k}) \\ 0.75000000000000 & \mathrm{I} & (6 \mathrm{k}) \\ 0.00000000000000 & \mathrm{Li} & (2 \mathrm{a}) \\ 0.50000000000000 & \mathrm{Li} & (2 \mathrm{a}) \\ 0.00000000000000 & \mathrm{Sc} & (2 \mathrm{e}) \\ 0.50000000000000 & \mathrm{Sc} & (2 \mathrm{e})\end{array}$

$\mathrm{BaSi}_{4} \mathrm{O}_{9}$ : AB9C4_hP28_188_e_kl_ak - CIF

\section{\# CIF file}

data_findsym-output

audit_creation_method FINDSYM

chemical_name_mineral 'BaSi409,

chemical_formula_sum 'Ba O9 $\mathrm{Si} 4$

loop

publ_author_name

L. W. Finger

R. M. Hazen

B. A. Fursenko,

_journal_name_full_name

Journal of Physics and Chemistry of Solids

journal_volume 56

journal year 1995

journal_page_first 1389

journal_page_last 1393

-publ_Section_title

Refinement of the crystal structure of $\mathrm{BaSi}_{-}\{4\} \$ \mathrm{O}_{-}\{9\} \$$ in the $\hookrightarrow$ benitoite form

\# Found in Pearson's Crystal Data - Crystal Structure Database for $\hookrightarrow$ Inorganic Compounds, 2013

aflow_title 'BaSi\$_\{4\}\$O\$ $\{9\} \$$ Structure

aflow_proto 'AB9C4 hP28 188 e kl_ak'

aflow_params 'a,c/a, $x_{-}\{3\}, y_{-}\{3\}, x_{-}\{4\}, y_{-}\{4\}, x_{-}\{5\}, y_{-}\{5\}, z_{-}\{5\}$,

aflow_params_values $6.4953629976,1.43896355826,0.07103,0.48306,0.12023$ $\hookrightarrow, 0.75436,0.22923,0.00127,0.6032$

aflow Strukturbericht 'None'

aflow_Pearson 'hP28,

cell_length_a 6.4953629976

cell_length_b 6.4953629976

cell_length_c 9.3465906512

cell_angle_alpha 90.0000000000

cell_angle_beta 90.0000000000

cell_angle_gamma 120.0000000000

symmetry_space_group_name_H-M "P -6 c 2 "

symmetry_Int_Tables_number 188

loop

space_group_symop_id

space_group_symop_operation_xyz

$\overline{1} \mathrm{x}, \mathrm{y}, \mathrm{z}$

$2-\mathrm{y}, \mathrm{x}-\mathrm{y}, \mathrm{z}$

$3-\mathrm{x}+\mathrm{y},-\mathrm{x}$,

$4 \mathrm{x}, \mathrm{x}-\mathrm{y},-\mathrm{z}$

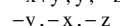

$-\mathrm{x}+\mathrm{y},-\mathrm{x},-\mathrm{z}+1 / 2$

$\mathrm{x}, \mathrm{y},-\mathrm{z}+1 / 2$

$-y, x-y,-z+1 / 2$
$0-x+y, y, z+1 / 2$

$1-\mathrm{y},-\mathrm{x}, \mathrm{z}+1 / 2$

$12 \mathrm{x}, \mathrm{x}-\mathrm{y}, \mathrm{z}+1 / 2$

loop

atom_site label

atom_site_type_symbol

atom_site_symmetry_multiplicity

atom_site_Wyckoff_label

atom_site_fract_x

atom_site_fract_y

atom_site_fract_z

\begin{tabular}{lllllll}
\hline Silom_site_occupancy & & & & & \\
Si & 2 & a & 0.00000 & 0.00000 & 0.00000 & 1.0000
\end{tabular}

$\begin{array}{lllllll}\mathrm{Ba} 1 \mathrm{Ba} & 2 & \mathrm{e} & 0.66667 & 0.33333 & 0.00000 & 1.00000\end{array}$ $\begin{array}{llllllllll}01 & \mathrm{O} & 6 & \mathrm{k} & 0.07103 & 0.48306 & 0.25000 & 1.00000\end{array}$

$\begin{array}{lllllllll}\mathrm{Si} 2 & \mathrm{Si} & 6 & \mathrm{k} & 0.12023 & 0.75436 & 0.25000 & 1.00000\end{array}$

$\begin{array}{lllllllll}\mathrm{O} 2 & \mathrm{O} & 12 & 1 & 0.22923 & 0.00127 & 0.60320 & 1.00000\end{array}$

$\mathrm{BaSi}_{4} \mathrm{O}_{9}$ : AB9C4_hP28_188_e_kl_ak - POSCAR

\begin{tabular}{|c|c|c|c|c|}
\hline \multirow{6}{*}{\multicolumn{5}{|c|}{ 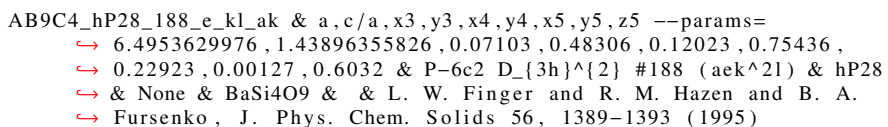 }} \\
\hline & & & & \\
\hline & & & & \\
\hline & & & & \\
\hline & & & & \\
\hline & & & & \\
\hline \multicolumn{5}{|l|}{1.00000000000000} \\
\hline 3.24768149880000 & -5.62514936272304 & 0.00000000000000 & & \\
\hline & 5.62514936272304 & 0.00000000000000 & & \\
\hline 0.00000000000000 & 0.00000000000000 & 9.34659065120000 & & \\
\hline $\mathrm{Ba}$ & & & & \\
\hline 18 & & & & \\
\hline \multicolumn{5}{|l|}{ Direct } \\
\hline 66666666667 & 0.33333333333333 & 0.00000000000000 & $\mathrm{Ba}$ & $(2 \mathrm{e})$ \\
\hline 0.666666666666667 & 0.33333333333333 & 0.50000000000000 & $\mathrm{Ba}$ & \\
\hline 0.07103000000000 & 0.483060 & 000 & $\mathrm{O}$ & $(6 \mathrm{k})$ \\
\hline-0.48306000000000 & -0.41203000000000 & 0.25000000000000 & $\mathrm{O}$ & $(6 \mathrm{k})$ \\
\hline 0.41203000000000 & -0.07103000000000 & 0.25000000000000 & $\mathrm{O}$ & $(6 \mathrm{k})$ \\
\hline-0.48306000000000 & -0.07103000000000 & 0.75000000000000 & $\mathrm{O}$ & $(6 \mathrm{k})$ \\
\hline 0.41203000000000 & 0.48306000000000 & 0.75000000000000 & $\mathrm{O}$ & $(6 \mathrm{k})$ \\
\hline 0.07103000000000 & -0.41203000000000 & 0.75000000000000 & $\mathrm{O}$ & $(6 \mathrm{k})$ \\
\hline 0.22923000000000 & 0.00127000000000 & 0.60320000000000 & $\mathrm{O}$ & (121) \\
\hline-0.00127000000000 & 0.22796000000000 & 0.60320000000000 & $\mathrm{O}$ & (121) \\
\hline-0.22796000000000 & -0.22923000000000 & 0.60320000000000 & $\mathrm{O}$ & (121) \\
\hline 0.22923000000000 & 0.00127000000000 & -0.10320000000000 & $\mathrm{O}$ & (121) \\
\hline-0.00127000000000 & 0.22796000000000 & -0.10320000000000 & $\mathrm{O}$ & $(121)$ \\
\hline-0.22796000000000 & -0.22923000000000 & -0.10320000000000 & $\mathrm{O}$ & (121) \\
\hline \multirow{2}{*}{$\begin{array}{l}-0.00127000000000 \\
-0.22796000000000\end{array}$} & -0.22923000000000 & 1.10320000000000 & $\mathrm{O}$ & (121) \\
\hline & 0.00127000000000 & 1.10320000000000 & $\mathrm{O}$ & (121) \\
\hline 0.22923000000000 & 0.22796000000000 & 1.10320000000000 & $\mathrm{O}$ & (121) \\
\hline-0.00127000000000 & -0.22923000000000 & -0.60320000000000 & $\mathrm{O}$ & (121) \\
\hline-0.22796000000000 & 0.00127000000000 & -0.60320000000000 & $\mathrm{O}$ & (121) \\
\hline 0.22923000000000 & 0.22796000000000 & -0.60320000000000 & $\mathrm{O}$ & (121) \\
\hline 0.00000000000000 & 0.00000000000000 & 0.00000000000000 & $\mathrm{Si}$ & $(2 a)$ \\
\hline 0.00000000000000 & 0.00000000000000 & 0.50000000000000 & $\mathrm{Si}$ & \\
\hline 0.12023000000000 & 0.75436000000000 & 0.25000000000000 & $\mathrm{Si}$ & $(6 \mathrm{k})$ \\
\hline-0.75436000000000 & -0.63413000000000 & 0.25000000000000 & $\mathrm{Si}$ & $(6 \mathrm{k}$ \\
\hline 0.63413000000000 & -0.12023000000000 & 0.25000000000000 & $\mathrm{Si}$ & $(6 \mathrm{k})$ \\
\hline-0.75436000000000 & -0.12023000000000 & 0.75000000000000 & $\mathrm{Si}$ & \\
\hline 0.63413000000000 & 0.7543600 & 0.75000000000000 & $\mathrm{Si}$ & $(6 \mathrm{k})$ \\
\hline 0.12023000000000 & -0.63413000000000 & 0.75000000000000 & $\mathrm{Si}$ & $(6 \mathrm{k})$ \\
\hline
\end{tabular}

$\pi-\mathrm{FeMg}_{3} \mathrm{Al}_{8} \mathrm{Si}_{6}\left(E 9_{b}\right):$ A8BC3D6_hP18_189_bfh_a_g_i - CIF

\section{\# CIF file}

data_findsym-outpu

audit_creation_method FINDSYM

chemical_name_mineral ' $\$ \backslash p i \$-F e M g \$ \_\{3\} \$ A I \$ \$_{-}\{8\} \$ S i \$ \_\{6\} \$$,

chemical_formula_sum, A18 Fe Mg3 $\mathrm{Si}$

loop

publ_author_name

'H. Perlitz',

A. Westgren

journal_name full_name

Arkiv $\mathrm{f}\{\backslash$ o $\} \mathrm{r}$ Kemi, Mineralogi och Geologi

journal_volume $15 \mathrm{~B}$

journal year 1942

journal_page_first

-journal_page_last 8

The Crystal Structure of $\mathrm{Al} \$_{-}\{8\} \$ \mathrm{Si} \$_{-}\{6\} \$ M g \$ \$_{-}\{3\} \$ \mathrm{Fe}$

\# Found in Determination of the crystal structure of the $\$ \backslash p i \$-A l F e M g S i$ $\hookrightarrow$ phase using symmetry- and site-sensitive electron microscope

$\hookrightarrow$ techniques, 2003

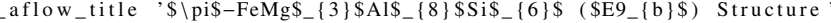
-aflow_proto 'A8BC3D6_hP18_189_bfh_a_g_i

aflow_params 'a, c/a, $x_{-}\{3\}, x_{-}\{4\}, z_{-}\{5\}, x_{-}\{6\}, z_{-}\{6\}$,

aflow_params_values $, 6.62,1.20241691843,0.403,0.444,0.231,0.75,0.222$,

aflow Strukturbericht '\$E9 $\{$ b $\}$,

aflow_Pearson 'hP18'

symmetry_space_group_name_H-M "P -62 m"

symmetry_Int_Tables_number 189

cell_length_a $\quad 6.62000$

cell_length_b $\quad 6.62000$

cell_length_c $\quad 7.96000$

cell_angle_alpha 90.00000

cell_angle_beta 90.00000

cell_angle_gamma 120.00000

loop

space_group_symop_id

space_group_symop_operation_xyz

$\mathrm{x}, \mathrm{y}, \mathrm{z}$

$2-\mathrm{y}, \mathrm{x}-\mathrm{y}, \mathrm{z}$

$3-\mathrm{x}+\mathrm{y},-\mathrm{x}, \mathrm{z}$

$\mathrm{x}-\mathrm{y},-\mathrm{y},-\mathrm{z}$
5
$\mathrm{6}, \mathrm{x},-\mathrm{z}$

2
$\mathrm{y}, \mathrm{x},-\mathrm{z}$

$6-\mathrm{x},-\mathrm{x}+\mathrm{y},-\mathrm{z}$

$7-x+y,-x,-z$
$8 x, y,-z$ 


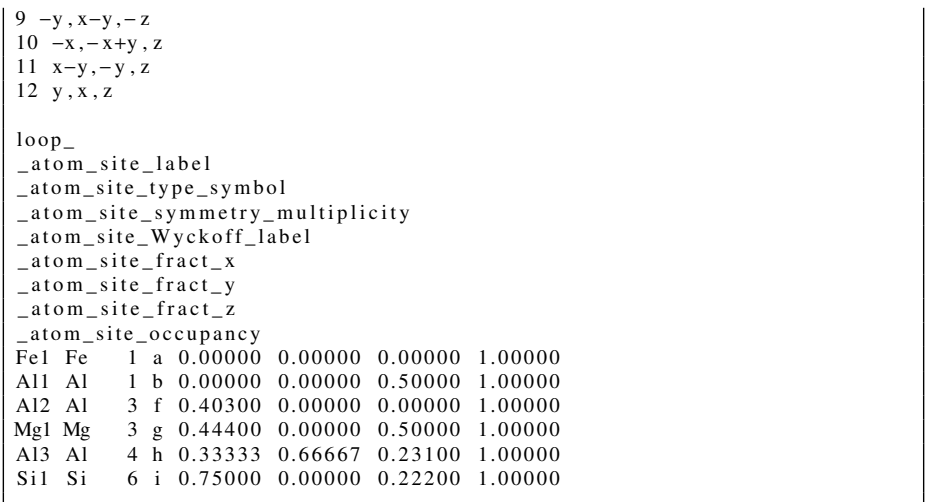

$\pi-\mathrm{FeMg}_{3} \mathrm{Al}_{8} \mathrm{Si}_{6}\left(E 9_{b}\right):$ A8BC3D6_hP18_189_bfh_a_g_i - POSCAR

A8BC3D6_hP18_189_bfh_a_g_i \& a, c/a , x3, x4, z5, x6, z6 --params $=6.62$

$\hookrightarrow 1.20241691843,0.403,0.444,0.231,0.75,0.222 \& \mathrm{P}-62 \mathrm{mD}_{-}\{3 \mathrm{~h}\}^{\wedge}\{3\}$

$\hookrightarrow 189$ (abfghi) \& hPl \&

$\hookrightarrow \$ \mathrm{Al}_{-}\{8\} \$ \mathrm{Si}_{-}\{6\} \$ \& \mathrm{H}$. Perlitz and A. Westgren, \{Ark. Kem.

$\hookrightarrow$ Mineral. Geol. 15B, $1-8(1942)$

1.00000000000000

$\begin{array}{lll}3.31000000000000 & -5.73308817305298 & 0.00000000000000\end{array}$

$\begin{array}{rrr}3.31000000000000 & 5.73308817305298 & 0.00000000000000\end{array}$

$\begin{array}{lll}0.00000000000000 & 0.00000000000000 & 7.96000000000000\end{array}$

$\begin{array}{rrrr}\mathrm{Al} & \mathrm{Fe} & \mathrm{Mg} & \mathrm{Si} \\ 8 & 1 & 3 & 6\end{array}$

Direct

$0.40300000000000 \quad 0.00000000000000$

$0.00000000000000-0.40300000000000$

$-0.4030000000000--0.40300000000000$

$0.33333333333333-0.666666666000067$

0.33333333333333

0.66666666666667

0.66666666666667

0.66666666666667

0.00000000000000

0.44400000000000

0.00000000000000

0.44400000000000

0.75000000000000

0.00000000000000

$-0.75000000000000$

0.75000000000000

0.00000000000000

0.333333333333333

0.33333333333333

0.00000000000000

0.00000000000000

0.44400000000000

$-0.44400000000000$

0.00000000000000

0.75000000000000

$-0.75000000000000$

0.00000000000000

$-0.75000000000000$

$-0.75000000000000$

0.50000000000000 0.00000000000000 0.00000000000000 0.00000000000000 0.23100000000000 $-0.23100000000000$ $-0.23100000000000$ 0.2310000000000 0.00000000000000 0.00000000000000 0.50000000000000 0.50000000000000 0.5000000000000 0.2220000000000 0.22200000000000 0.22200000000000 $-0.22200000000000$ $-0.22200000000000$ $-0.22200000000000$

$\begin{array}{ll}\mathrm{Al} & (1 \mathrm{~b}) \\ \mathrm{Al} & (3 \mathrm{f}) \\ \mathrm{Al} & (3 \mathrm{f}) \\ \mathrm{Al} & (3 \mathrm{f}) \\ \mathrm{Al} & (4 \mathrm{~h}) \\ \mathrm{Al} & (4 \mathrm{~h}) \\ \mathrm{Al} & (4 \mathrm{~h}) \\ \mathrm{Al} & (4 \mathrm{~h}) \\ \mathrm{Fe} & (1 \mathrm{a}) \\ \mathrm{Mg} & (3 \mathrm{~g}) \\ \mathrm{Mg} & (3 \mathrm{~g}) \\ \mathrm{Mg} & (3 \mathrm{~g}) \\ \mathrm{Si} & (6 \mathrm{i}) \\ \mathrm{Si} & (6 \mathrm{i}) \\ \mathrm{Si} & (6 \mathrm{i}) \\ \mathrm{Si} & (6 \mathrm{i}) \\ \mathrm{Si} & (6 \mathrm{i}) \\ \mathrm{Si} & (6 \mathrm{i})\end{array}$

$\pi$-FeMg $\mathrm{Al}_{9} \mathrm{Si}_{5}$ : A9BC3D5_hP18_189_fi_a_g_bh - CIF

\section{\# CIF file}

data_findsym-output

audit_creation_method FINDSYM

chemical_name_mineral $\$ \backslash$ pi $\$-F e M g \$ \_\{3\} \$ A I \$ \_\{9\} \$ S i \$\{\{5\} \$$

chemical_formula_sum ' $\mathrm{Al} 9 \mathrm{Fe} \mathrm{Mg} 3 \mathrm{Si} 5$

loop

publ_author_name

'S. Foss'

A. Olsen

C. J. Simensen

J. Taft $\{\backslash 0\}$

_journal_name_full_name

Acta Crystallographica Section B: Structural Science

journal_volume 59

journal year 2003

journal_page_first 36

journal_page_last 42

publ_Section_title

Determination of the crystal structure of the $\$ \backslash p i \$-A l F e M g S i$ phase $\hookrightarrow$ using symmetry- and site-sensitive electron microscope $\hookrightarrow$ using sym

\# Found in The Materials Project, $\operatorname{Mg} \$_{-}\{3\} \$ \mathrm{Al}_{-}\{9\} \$ \mathrm{FeSi}_{-}\{5\} \$$, IID $\hookrightarrow \mathrm{mp}-7062\}$,

aflow_title $\$ \backslash \mathrm{pi} \$-\mathrm{FeMg} \$_{-}\{3\} \$ \mathrm{~A} \mid \$_{-}\{9\} \$ \mathrm{Si}_{-}\{5\} \$$ Structure

aflow_proto 'A9BC3D5_hP18_189_fi_a_g_bh,

aflow_params $, a, c / a, x_{-}\{3\}, x_{-}\{4\}, z_{-}\{5\}, x_{-}\{6\}, z_{-}\{6\}$

aflow_params_values , $6.6,1.19696969697,0.378,0.43,0.266,0.755,0.236$ aflow_Strukturbericht 'None'

aflow_Pearson 'hP18,

symmetry space group_name_H-M "P $-622 \mathrm{m"}$

symmetry_Int_Tables_number 189

cell_length_a $\quad 6.60000$

cell_length_b 6.60000

cell_length_c 7.90000

cell_angle_alpha 90.00000

cell_angle beta 90.00000

_cell_angle_gamma 120.00000

loop_ _space_group_symop_id

-space_group_symop_operation_xy

$1 \mathrm{x}, \mathrm{y}, \mathrm{z}$

$2-\mathrm{y}, \mathrm{x}-\mathrm{y}, \mathrm{z}$

$3-x+y,-x, z$

$4 \mathrm{x}-\mathrm{y},-\mathrm{y},-\mathrm{z}$

$5 \mathrm{y}, \mathrm{x},-\mathrm{z}$

$6-\mathrm{x},-\mathrm{x}+\mathrm{y},-\mathrm{z}$
$7-\mathrm{x}+\mathrm{y},-\mathrm{x},-\mathrm{z}$

$8 \mathrm{x}, \mathrm{y},-\mathrm{z}$

$9-y, x-y,-z$

$10-x,-x+y, z$

$11 \mathrm{x}-\mathrm{y},-\mathrm{y}, \mathrm{z}$

$12 \mathrm{y}, \mathrm{x}, \mathrm{z}$

loop

atom site label

atom_site_type_symbol

atom_site_symmetry_multiplicity

_atom_site_Wyckoff_label

atom_site_fract_x

atom_site_fract_y

atom_site_fract_z

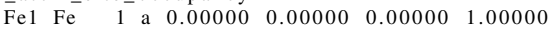

$\begin{array}{llllllll}\mathrm{Si} 1 & \mathrm{Si} & 1 & \mathrm{~b} & 0.00000 & 0.00000 & 0.50000 & 1.00000\end{array}$

$\begin{array}{lllllllll}\text { All } & \mathrm{Al} & 3 & \mathrm{f} & 0.37800 & 0.00000 & 0.00000 & 1.00000\end{array}$

$\begin{array}{llllllll}\mathrm{Mg} 1 \mathrm{Mg} & 3 & \mathrm{~g} & 0.43000 & 0.00000 & 0.50000 & 1.00000\end{array}$

$\begin{array}{lllllllll}\mathrm{Si} 2 & \mathrm{Si} & 4 & \mathrm{~h} & 0.33333 & 0.66667 & 0.26600 & 1.00000\end{array}$

$\begin{array}{lllllll}\mathrm{A} 12 & \mathrm{Al} & 6 \mathrm{i} & 0.75500 & 0.00000 & 0.23600 & 1.00000\end{array}$

$\pi$-FeMg $3 \mathrm{Al}_{9} \mathrm{Si}_{5}$ : A9BC3D5_hP18_189_fi_a_g_bh - POSCAR

\begin{tabular}{|c|c|c|c|c|}
\hline \multirow{5}{*}{\multicolumn{5}{|c|}{ 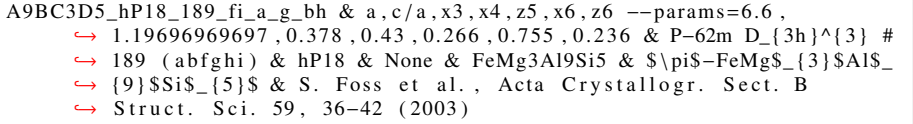 }} \\
\hline & & & & \\
\hline & & & & \\
\hline & & & & \\
\hline & & & & \\
\hline \multicolumn{5}{|l|}{1.00000000000000} \\
\hline 3.30000000000000 & -5.71576766497729 & 0.00000000000 & & \\
\hline & 5.71576766497729 & 0.00000000000000 & & \\
\hline $\begin{array}{l}3.30000000000000 \\
0.00000000000000\end{array}$ & 0.00000000000000 & 00000000 & & \\
\hline $\mathrm{Mg}$ & $\mathrm{Si}$ & & & \\
\hline 9 & 5 & & & \\
\hline \multicolumn{5}{|l|}{ Direct } \\
\hline 0.37800000000000 & 0.0 & 0000 & $\mathrm{Al}$ & (3f) \\
\hline 0.00000000000000 & & 00 & A & \\
\hline-0.37800000000000 & -0.3 & 0.0 & 1 & \\
\hline 0.75500000000000 & & 0.2 & 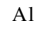 & \\
\hline 0.00000000000000 & 0.7 & 00000 & $\mathrm{Al}$ & ) \\
\hline-0.75500000000000 & -0.75 & & & \\
\hline 0.75500000000000 & 0.00000000000000 & -0.23600000000000 & $\mathrm{Al}$ & $(6 \mathrm{i})$ \\
\hline 0.00000000000000 & & -0.236 & & \\
\hline-0.75500000000000 & 00000000 & 0000000 & $\mathrm{Al}$ & \\
\hline 0.00000000000000 & & 0.000 & Ге & \\
\hline 0.43000000000000 & & 0.5 & $\mathrm{Mg}$ & \\
\hline 0.00000000000000 & 0.430 & 0.50000 & $\mathrm{Mg}$ & $(3 g)$ \\
\hline-0.43000000000000 & -0.43000000000000 & 0.50000000000000 & & \\
\hline 0.00000000000000 & 0.00000000000000 & 0.50000000000000 & $\mathrm{Si}$ & \\
\hline 0.33333333333333 & 0.66 & 0.26600000000 & $\mathrm{Si}$ & \\
\hline 0.33333333333333 & 0.666666666666667 & -0.26600000000000 & $\mathrm{Si}$ & $(4 \mathrm{~h})$ \\
\hline 0.666666666666667 & & & & \\
\hline 0.666666666666667 & 0.33333333333333 & 0.26600000000000 & $\mathrm{Si}$ & $(4 \mathrm{~h})$ \\
\hline
\end{tabular}

Li 2 Sb: A2B_hP18_190_gh_bf - CIF

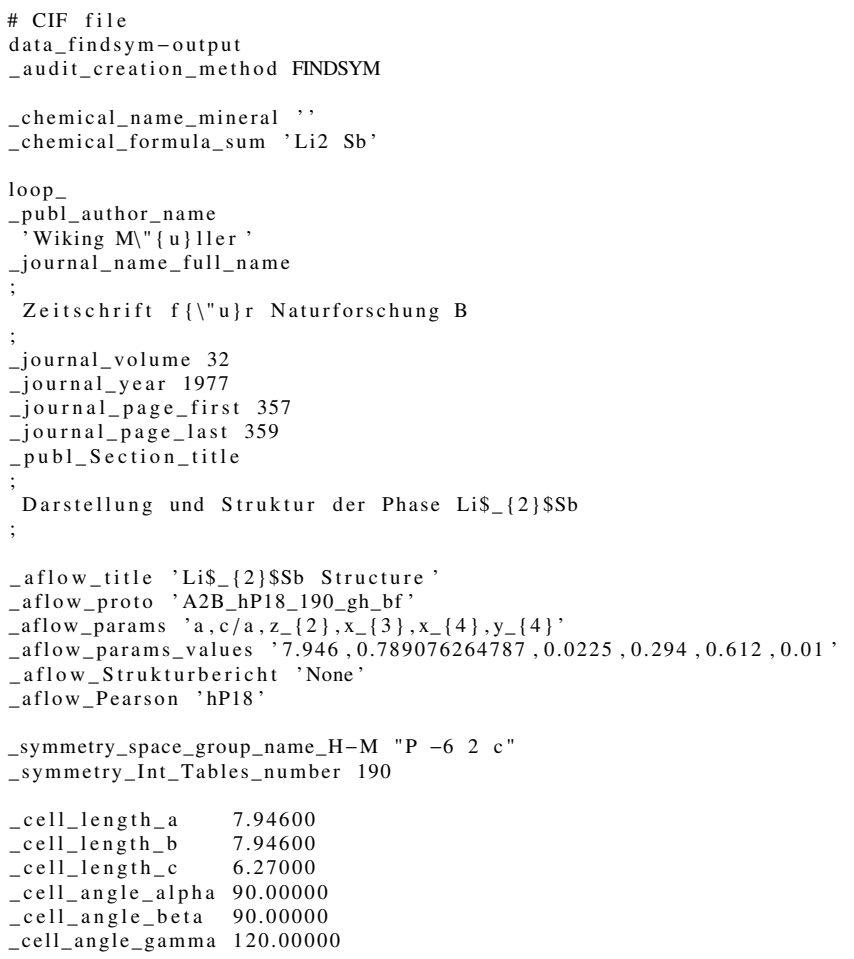


loop

_space_group_symop_id

_space_group_symop_operation_xyz

$1 \mathrm{x}, \mathrm{y}, \mathrm{z}$

$2-\mathrm{y}, \mathrm{x}-\mathrm{y}, \mathrm{z}$

$3-x+y,-x, z$

$4 x-y,-y,-z$

$5 \mathrm{y}, \mathrm{x},-\mathrm{z}$

$6-\mathrm{x},-\mathrm{x}+\mathrm{y},-\mathrm{z}$

$7-\mathrm{x}+\mathrm{y},-\mathrm{x},-\mathrm{z}+1 / 2$

$8 \mathrm{x}, \mathrm{y},-\mathrm{z}+1 / 2$

$9-y, x-y,-z+1 / 2$

$10-\mathrm{x},-\mathrm{x}+\mathrm{y}, \mathrm{z}+1 / 2$

$11 \mathrm{x}-\mathrm{y},-\mathrm{y}, \mathrm{z}+1 / 2$
$12 \mathrm{y}, \mathrm{x}, \mathrm{z}+1 / 2$

loop

atom_site_label

_atom_site_type_symbol

_atom_site_symmetry_multiplicity

_atom_site_Wyckoff_label

atom_site_fract_x

atom_site_fract_y

atom_site_fract_z

atom_site_occupancy

\begin{tabular}{llllllll}
\hline Sb1 & Sb & 2 & b & 0.00000 & 0.00000 & 0.25000 & 1.00000
\end{tabular}

$\begin{array}{lllllllll} & \mathrm{Sb} 2 & \mathrm{Sb} & 4 & \mathrm{f} & 0.33333 & 0.66667 & 0.02250 & 1.00000\end{array}$

$\begin{array}{llllllll}\mathrm{Li} 1 & \mathrm{Li} & 6 & \mathrm{~g} & 0.29400 & 0.00000 & 0.00000 & 1.00000\end{array}$

$\begin{array}{llllllll}\mathrm{L} i 2 & \mathrm{Li} & 6 & \mathrm{~h} & 0.61200 & 0.01000 & 0.25000 & 1.00000\end{array}$

Li 2 Sb: A2B_hP18_190_gh_bf - POSCAR

A2B_hP18_190_gh_bf \& a , c/a , z2, x3, x4,y4 --params $=7.946,0.789076264787$, $\hookrightarrow 0.0225,0.294,0.612,0.01 \&$ P-62c D_ $\{3 \mathrm{~h}\}^{\wedge}\{4\} \# 190(\mathrm{bfgh}) \& \mathrm{hP} 18$

$\hookrightarrow$ None \& Li2Sb \& \& Wiking $M \backslash "\{u\} 11$ er, Z. Naturforsch. B 32, $\hookrightarrow 357-359(1977)$

1.00000000000000

$\begin{array}{lll}3.97300000000000 & -6.88143785847115 & 0.00000000000000\end{array}$

$\begin{array}{rrr}3.97300000000000 & -6.88143785847115 & 0.00000000000000 \\ 3.97300000000000 & 6.88143785847115 & 0.00000000000000 \\ 0.0000000000000 & 0.00000000000 & 6.270000000000\end{array}$

$\begin{array}{lll}3.97300000000000 & 6.88143785847115 & 0.00000000000000 \\ 0.00000000000000 & 0.00000000000000 & 6.27000000000000\end{array}$

$\mathrm{Li} \quad \mathrm{Sb}$

Direct

0.29400000000000

0.00000000000000

$-0.29400000000000$

0.29400000000000

0.00000000000000

$-0.29400000000000$

0.61200000000000

$-0.01000000000000$

$-0.60200000000000$

0.01000000000000

0.60200000000000

$-0.6120000000000$

0.61200000000000

0.00000000000000

0.00000000000000

0.33333333333333

0.33333333333333
0.66666666666667

0.666666666666667

0.00000000000000

0.29400000000000

$-0.29400000000000$

0.00000000000000

0.29400000000000

$-0.29400000000000$

0.01000000000000

$-0.6120000000000$

0.61200000000000

$-0.01000000000000$

$-0.6020000000000$

0.0000000000000

0.000000000000

0.00000000000000

0.66666666666667

0.3333333333333

0.33333333333333

0.00000000000000

0.00000000000000

0.0000000000000

0.50000000000000

0.50000000000000

0.50000000000000

0.25000000000000

0.25000000000000

0.25000000000000

0.75000000000000

0.75000000000000

0.75000000000000

0.25000000000000

0.75000000000000

0.750000000000

0.02250000000000

0.02250000000000

0.52250000000000

$\begin{array}{ll}\mathrm{Li} & (6 \mathrm{~g}) \\ \mathrm{Li} & (6 \mathrm{~g})\end{array}$

(6g)

$(6 \mathrm{~g})$

$(6 \mathrm{~g})$

$(6 \mathrm{~g})$

(6g)

(6h)

i (6h)

i (6h)

(6h)

(6h)

(6h)

(2b)

$\alpha$-Sm $3 \mathrm{Ge}_{5}$ (High-temperature): A5B3_hP16_190_bdh_g - CIF

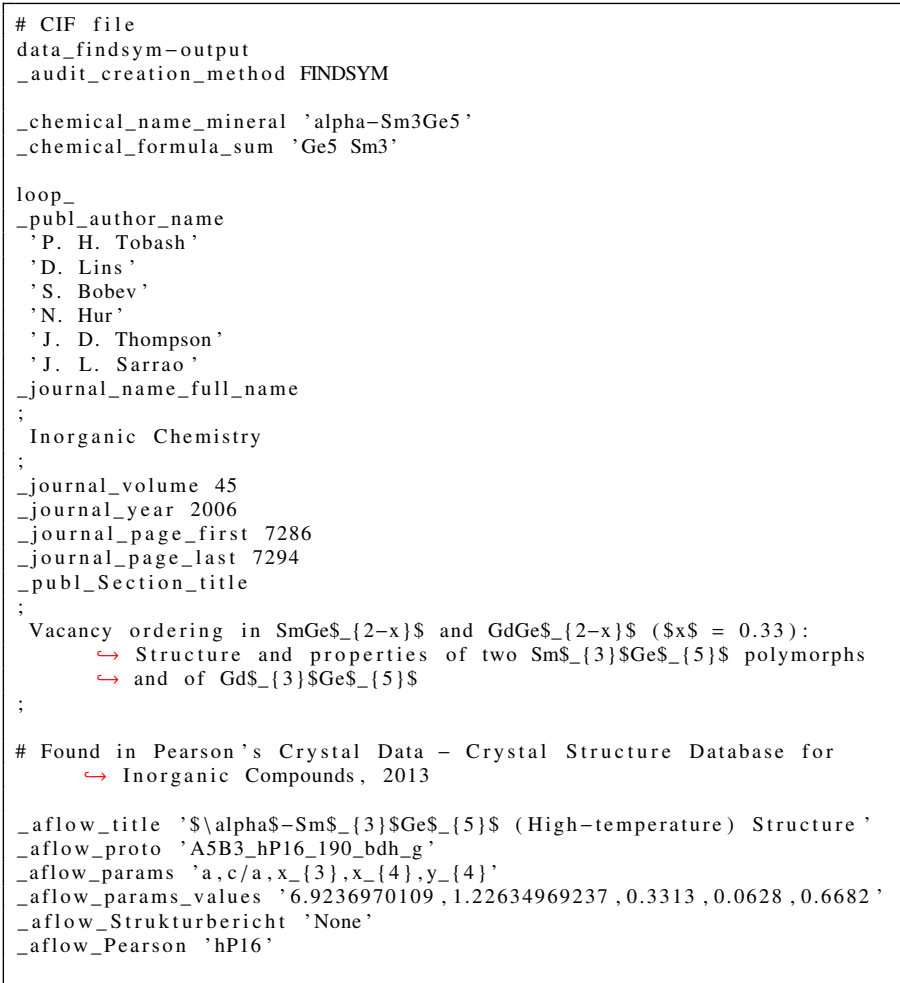

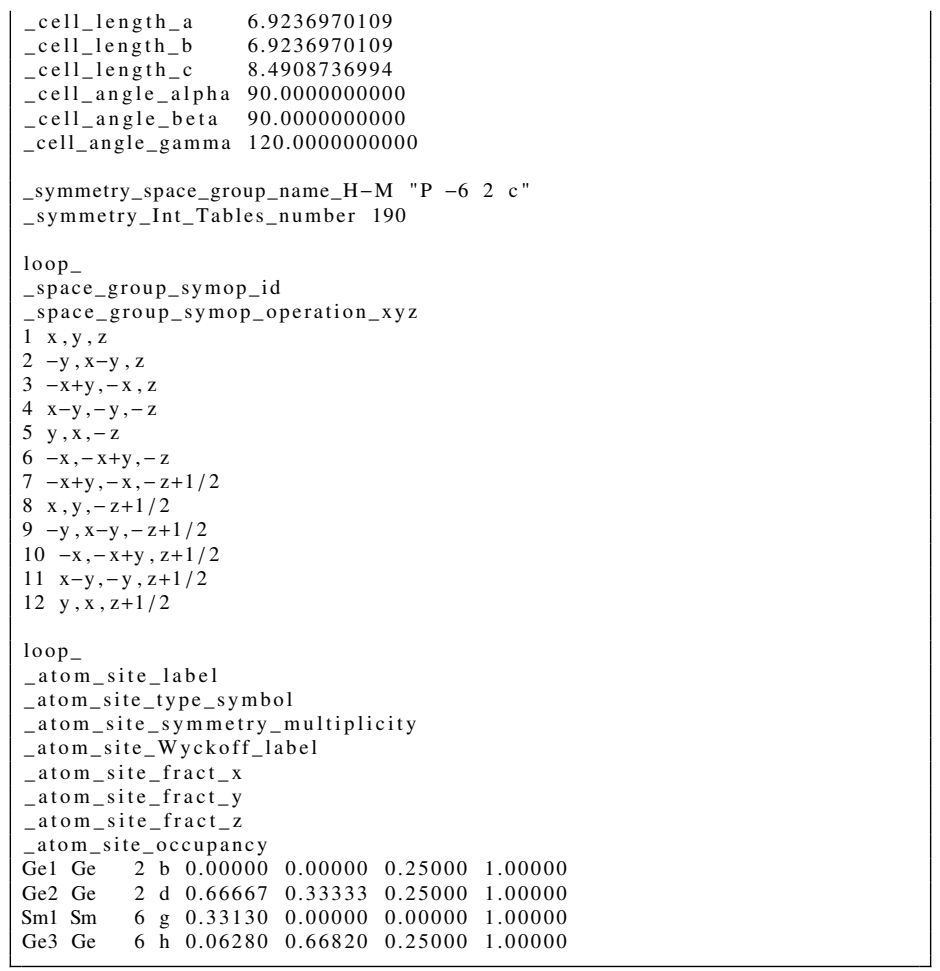

$\alpha$-Sm $3 \mathrm{Ge}_{5}$ (High-temperature): A5B3_hP16_190_bdh_g - POSCAR

\begin{tabular}{|c|c|c|c|c|}
\hline \\
\hline \multicolumn{5}{|c|}{$\begin{array}{l}\hookrightarrow \text { None \& Sm3Ge5 \& alpha \& P. H. Tobash et al. , Inorg. Chem. 45, } \\
\hookrightarrow \text { None } \\
\hookrightarrow 7286-7294(2006)\end{array}$} \\
\hline \multicolumn{5}{|c|}{1.00000000000000} \\
\hline \multirow{2}{*}{3.46184850545000} & -5.99609749954578 & 0.00000000000000 & & \\
\hline & 5.99609749954578 & 0.00000000000000 & & \\
\hline $\begin{array}{l}3.46184850545000 \\
0.00000000000000\end{array}$ & 0.00000000000000 & 8.49087369940000 & & \\
\hline $\mathrm{Sm}$ & & & & \\
\hline 10 & & & & \\
\hline \multicolumn{5}{|l|}{ Direct } \\
\hline 0.00000000000000 & 0.00000000000000 & 0.25000000000000 & $\mathrm{Ge}$ & (2b) \\
\hline 0.00000000000000 & 0.00000000000000 & 0.75000000000000 & $\mathrm{Ge}$ & (2b) \\
\hline 0.666666666666667 & 0.33333333333333 & 0.25000000000000 & $\mathrm{Ge}$ & (2d) \\
\hline 0.33333333333333 & 0.666666666666667 & 0.75000000000000 & $\mathrm{Ge}$ & (2d) \\
\hline 0.06280000000000 & 0.66820000000000 & 0.25000000000000 & $\mathrm{Ge}$ & $(6 \mathrm{~h})$ \\
\hline-0.66820000000000 & -0.60540000000000 & 0.25000000000000 & $\mathrm{Ge}$ & $(6 \mathrm{~h})$ \\
\hline 0.60540000000000 & -0.06280000000000 & 0.25000000000000 & $\mathrm{Ge}$ & (6h) \\
\hline 0.66820000000000 & 0.06280000000000 & 0.75000000000000 & $\mathrm{Ge}$ & (6h) \\
\hline-0.60540000000000 & -0.66820000000000 & 0.75000000000000 & $\mathrm{Ge}$ & $(6 \mathrm{~h})$ \\
\hline-0.06280000000000 & 0.60540000000000 & 0.75000000000000 & $\mathrm{Ge}$ & $(6 \mathrm{~h})$ \\
\hline 0.33130000000000 & 0.00000000000000 & 0.00000000000000 & $\mathrm{Sm}$ & $(6 \mathrm{~g})$ \\
\hline 0.00000000000000 & 0.33130000000000 & 0.00000000000000 & $\mathrm{Sm}$ & $(6 \mathrm{~g})$ \\
\hline-0.33130000000000 & -0.33130000000000 & 0.00000000000000 & $\mathrm{Sm}$ & $(6 \mathrm{~g})$ \\
\hline 0.33130000000000 & 0.00000000000000 & 0.50000000000000 & $\mathrm{Sm}$ & (6g) \\
\hline 0.00000000000000 & 0.33130000000000 & 0.50000000000000 & Sm & $(6 \mathrm{~g})$ \\
\hline-0.33130000000000 & -0.33130000000000 & 0.50000000000000 & $\mathrm{Sm}$ & $(6 \mathrm{~g})$ \\
\hline
\end{tabular}

Troilite (FeS): AB_hP24_190_i_afh - CIF

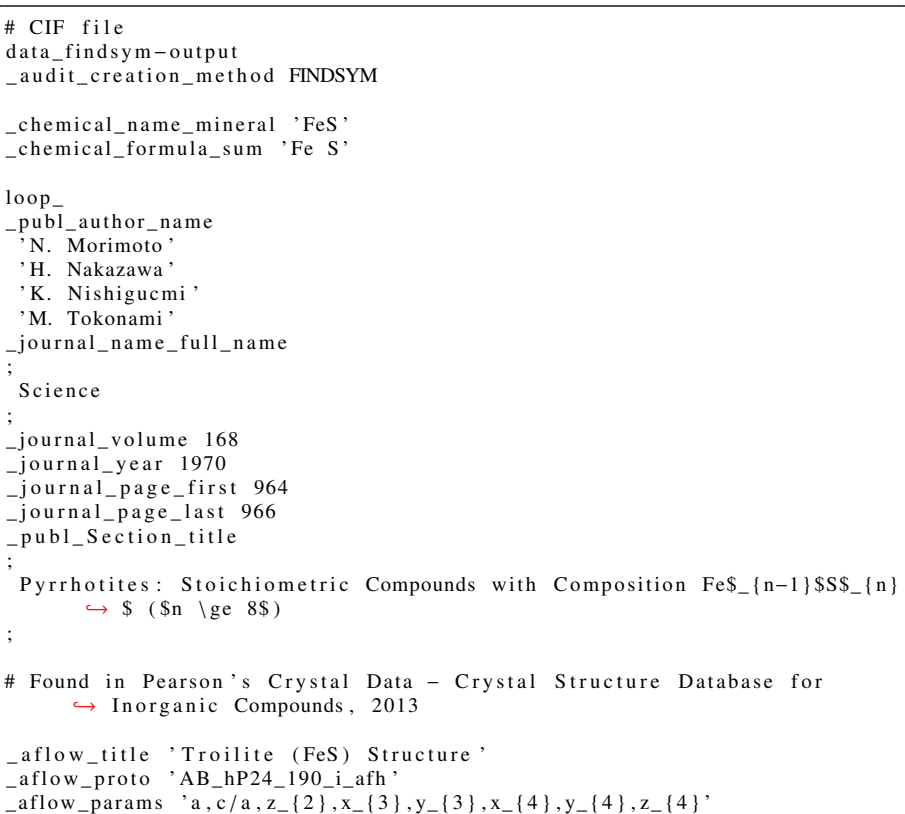




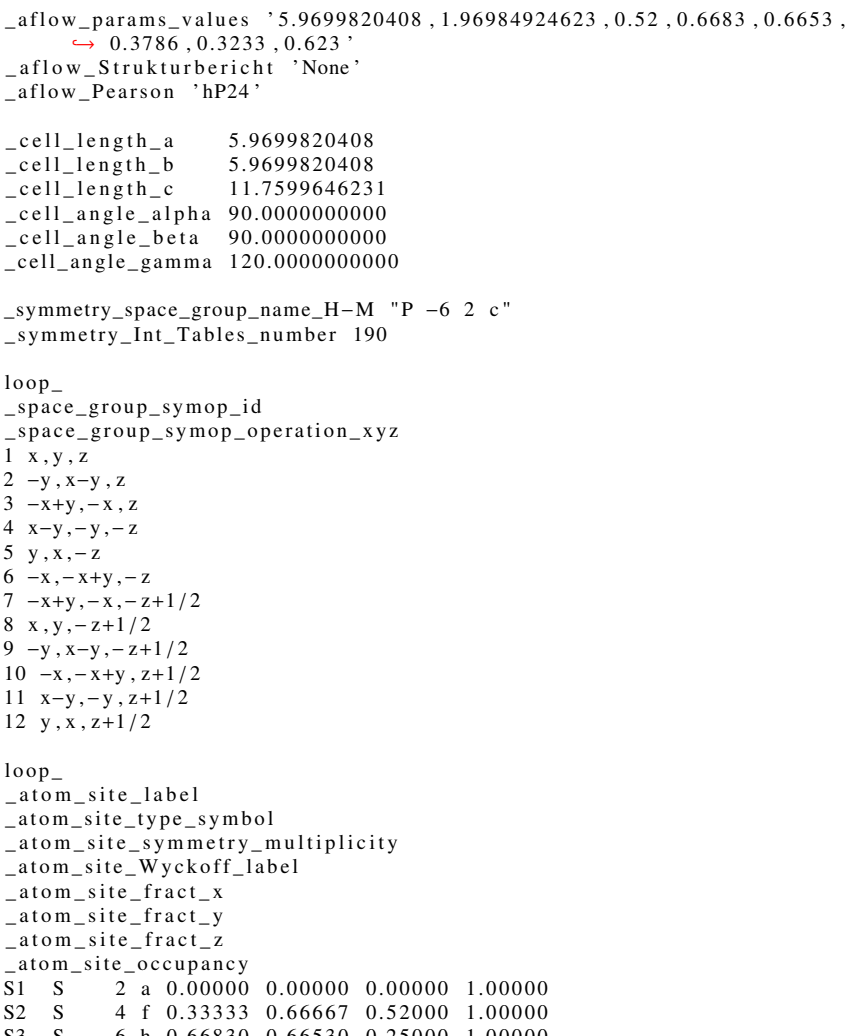

Troilite (FeS): AB_hP24_190_i_afh - POSCAR

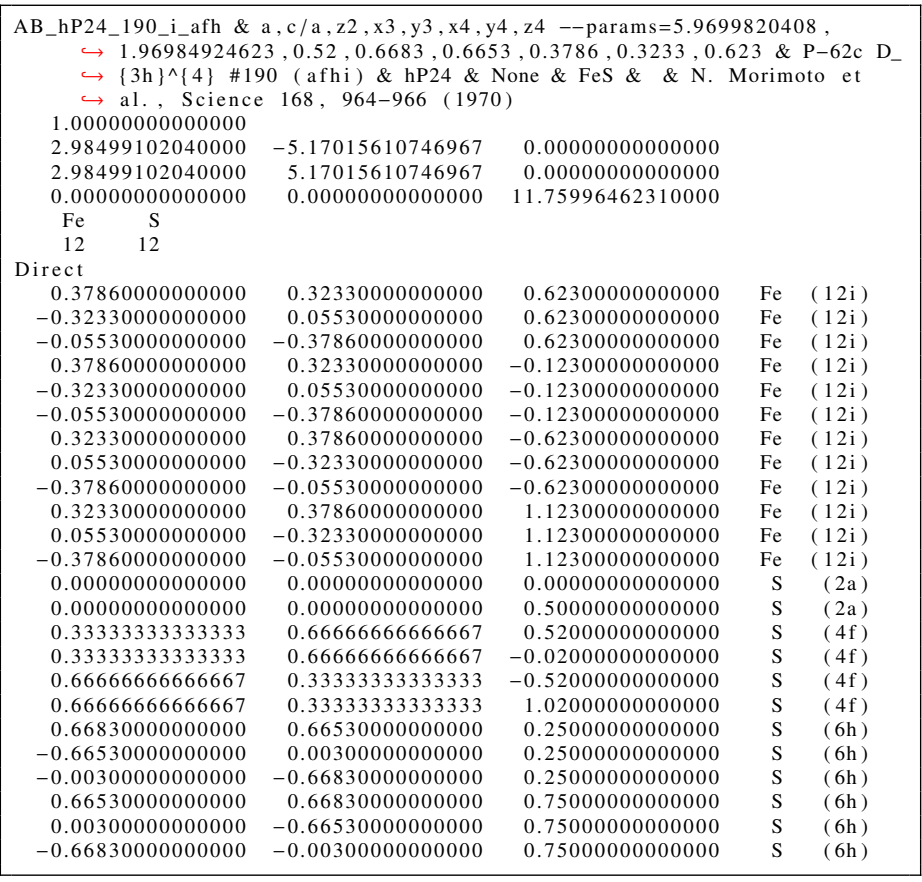

$\operatorname{Beryl}\left(\mathrm{Be}_{3} \mathrm{Al}_{2} \mathrm{Si}_{6} \mathrm{O}_{18}, G 3_{1}\right)$ : A2B3C18D6_hP58_192_c_f_Im_l - CIF

$$
\begin{aligned}
& \text { \# CIF file } \\
& \text { data_findsym-output } \\
& \text { _audit_creation_method FINDSYM } \\
& \text { _chemical name mineral 'Beryl' } \\
& \text { _chemical_formula_sum, A12 Be3 O18 Si6, } \\
& \text { loop } \\
& \text { publ_author_name } \\
& \text { R. M. Hazen, } \\
& \text { A. Y. Au' } \\
& \text { 'L. W. Finger, } \\
& \text { journal_name_full_name } \\
& \text { American Mineralogist } \\
& \text { _journal_volume } 71 \\
& \text { _journal_year } 1986 \\
& \text { _journal_page_first } 977 \\
& \text { _ournal_page_last } 984
\end{aligned}
$$


0.31030000000000 0.49850000000000 $-0.14560000000000$ $-0.35290000000000$ $-0.49850000000000$ 0.14560000000000 0.35290000000000 0.14560000000000 0.35290000000000 $-0.49850000000000$ $-0.14560000000000$ $-0.35290000000000$ 0.49850000000000 $-0.49850000000000$ 0.14560000000000 0.35290000000000 0.49850000000000 0.4985000000000 $-0.35290000000000$ $-0.1456000000000$ 0.35290000000000 0.49850000000000 0.14560000000000 0.35290000000000 $-0.49850000000000$ 0.38760000000000 $-0.11590000000000$ $-0.38760000000000$ 0.11590000000000 0.27170000000000 0.11590000000000 0.27170000000000 $-0.38760000000000$ $-0.11590000000000$ 0.27170000000000 0.38760000000000

0.07340000000000 0.1456000000000 0.3529000000000 $-0.49850000000000$ $-0.14560000000000$ $-0.3529000000000$ 0.49850000000000 0.49850000000000 $-0.14560000000000$ $-0.35290000000000$ $-0.49850000000000$ 0.14560000000000 0.35290000000000 $-0.1456000000000$ 0.35290000000000 0.49850000000000 0.14560000000000 0.35290000000 $-0.49850000000000$ 0.14560000000000 0.3529000000000 0.49850000000000 $-0.14560000000000$ $-0.35290000000000$ 0.11590000000000 0.27170000000000 $-0.38760000000000$ $-0.27170000000000$ 0.38760000000000 0.38760000000000 $-0.11590000000000$ $-0.27170000000000$ $-0.38760000000000$ 0.11590000000000 0.27170000000000

$\mathrm{AlPO}_{4}: \mathrm{AB} 2 \_\mathrm{hP7} 2192 \_\mathrm{m} \_\mathrm{j} 2 \mathrm{kl}-\mathrm{CIF}$

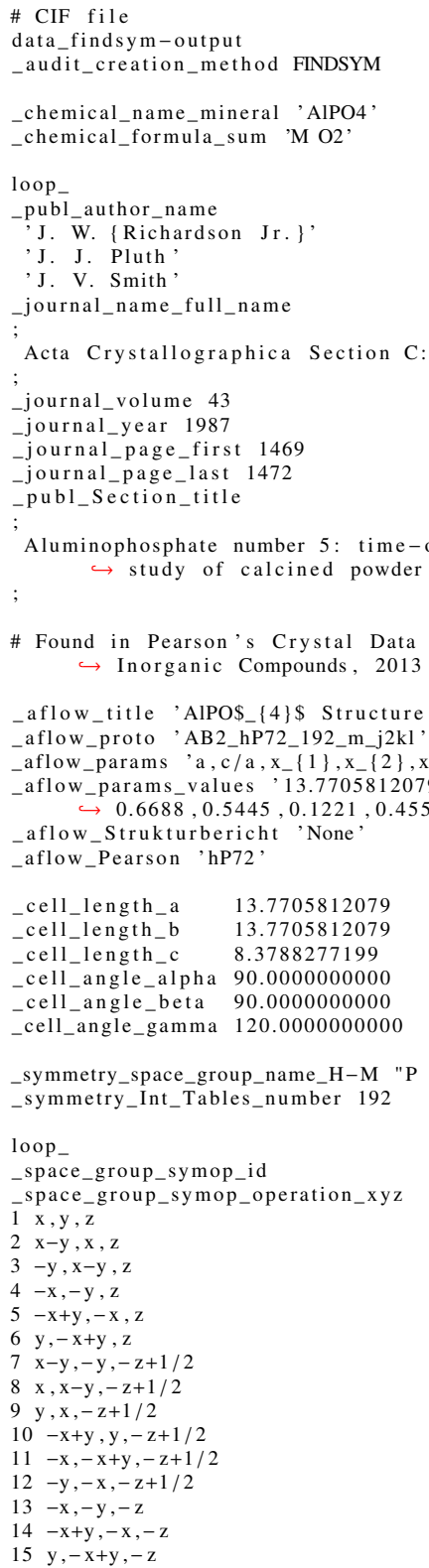

50000000000000 .1453000000000 0.1453000000000 0.14530000000000 0.14530000000000 0.1453000000000 0.14530000000000 0.35470000000000 .35470000000000 .35470000000000 0.35470000000000 0.35470000000000 $-0.14530000000000$ $-0.14530000000000$ $-0.14530000000000$ $-0.14530000000000$ $-0.14530000000000$ 0.64530000000000 0.64530000000000 0.64530000000000 0.64530000000000 .6453000000000 0.0000000000000 .00000000000000 0.00000000000000 0.00000000000000 0.00000000000000 0.50000000000000 0.50000000000000 0.50000000000000 0.50000000000000 .50000000000000 0.50000000000000
O (121)

O $(24 \mathrm{~m})$

$\begin{array}{ll}\mathrm{O} & (24 \mathrm{~m}) \\ \mathrm{O} & (24 \mathrm{~m})\end{array}$

O $(24 \mathrm{~m})$

$(24 \mathrm{~m})$

$(24 \mathrm{~m})$

$(24 \mathrm{~m})$
$(24 \mathrm{~m})$

O $(24 \mathrm{~m})$

(24m)

O $(24 \mathrm{~m})$

$(24 \mathrm{~m})$

$(24 \mathrm{~m})$
$(24 \mathrm{~m})$

$(24 \mathrm{~m})$

$\begin{array}{ll}\mathrm{O} & (24 \mathrm{~m}) \\ \mathrm{O} & (24 \mathrm{~m})\end{array}$

$(24 \mathrm{~m})$
$(24 \mathrm{~m})$

O $(24 \mathrm{~m})$

O $(24 \mathrm{~m})$

$\begin{array}{ll}\mathrm{Si} & (121) \\ \mathrm{Si} & (121)\end{array}$

$\mathrm{Si} \quad(121)$

$\begin{array}{ll}\mathrm{Si} & (121) \\ \mathrm{Si} & (121)\end{array}$

$\mathrm{Si} \quad(121)$

$\mathrm{Si}(121)$

Si (121)

Si (121)

$\begin{array}{ll}\mathrm{Si} & (121) \\ \mathrm{Si} & (121)\end{array}$

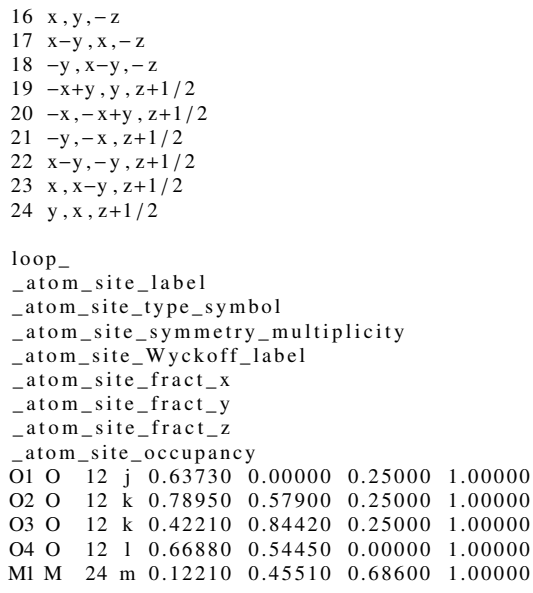

$\mathrm{AlPO}_{4}:$ AB2_hP72_192_m_j2kl - POSCAR

AB2_hP72_192_m_j2k1 \& a,c/a, x1, x2, x3, x4,y4, x5, y5 , z5 --params $=$

$\hookrightarrow 13.7705812079,0.608458538779,0.6373,0.7895,0.4221,0.6688,0.5445$ $\hookrightarrow, 0.1221,0.4551,0.686 \&$ P6/mcc D_ $\{6 \mathrm{~h}\}^{\wedge}\{2\} \# 192(\mathrm{jk} \wedge 21 \mathrm{~m}) \& \mathrm{hP72} \&$ $\hookrightarrow$ None \& AlPO4 \& \& J. W. \{Richardson Jr.\} and J. J. Pluth and $\hookrightarrow$ J. V. Smith, Acta Crystallogr. C 43, 1469-1472 (1987) 1.00000000000000

$\begin{array}{llll}6.88529060395000 & -11.92567315091800 & 0.00000000000000\end{array}$ $\begin{array}{rrr}6.88529060395000 & 11.92567315091800 & 0.00000000000000\end{array}$ $\begin{array}{rrr}0.00000000000000 & 0.00000000000000 & 8.37882771990000\end{array}$

$\begin{array}{cr}\mathrm{M} & \mathrm{O} \\ 24 & 48\end{array}$

Direct

0.12210000000000 0.33300000000000 $-0.12210000000000$ 0.45510000000000 $-0.33300000000000$ 0.45510000000000 $-0.33300000000000$ $-0.12210000000000$ 0.33300000000000 0.12210000000000 $-0.12210000000000$ 0.45510000000000 $-0.3330000000000$ $-0.33300000000000$ 0.12210000000000 $-0.33300000000000$ $-0.45510000000000$ 0.33300000000000 0.12210000000000 0.45510000000000 $-0.33300000000000$ $-0.12210000000000$ 0.63730000000000 $-0.63730000000000$ $-0.63730000000000$ 0.00000000000000 0.63730000000000 $-0.63730000000000$

0.0000000000000 0.63730000000000 0.00000000000000 $-0.63730000000000$ 0.78950000000000 $-1.57900000000000$ 0.78950000000000 $-0.7895000000000$ 1.57900000000000 $-0.78950000000000$ $-0.78950000000000$ 1.57900000000000 $-0.78950000000000$ 0.78950000000000 $-1.57900000000000$ 0.78950000000000 0.4221000000000 0.42210000000000 -0.84420000000000
0.42210000000000 $-0.42210000000000$ 0.84420000000000 $-0.42210000000000$ $-0.42210000000000$ 0.84420000000000 $-0.42210000000000$ 0.42210000000000 $-0.84420000000000$ 0.42210000000000 0.66880000000000 $-0.12430000000000$ $-0.66880000000000$

0.54450000000000 0.12430000000000

0.45510000000000 $-0.33300000000000$ $-0.12210000000000$ 0.45510000000000 0.33300000000000 0.12210000000000 0.12210000000000 $-0.45510000000000$ 


$\begin{array}{rrrrr}0.54450000000000 & 0.66880000000000 & 0.50000000000000 & \mathrm{O} & (121) \\ 0.12430000000000 & -0.54450000000000 & 0.50000000000000 & \mathrm{O} & (121) \\ -0.66880000000000 & -0.12430000000000 & 0.50000000000000 & \mathrm{O} & (121) \\ -0.54450000000000 & -0.66880000000000 & 0.50000000000000 & \mathrm{O} & (121) \\ -0.12430000000000 & 0.54450000000000 & 0.50000000000000 & \mathrm{O} & (121) \\ 0.66880000000000 & 0.12430000000000 & 0.50000000000000 & \mathrm{O} & (121)\end{array}$

Mavlyanovite $\left(\mathrm{Mn}_{5} \mathrm{Si}_{3}\right)$ : A5B3_hP16_193_dg_g - CIF

\section{\# CIF file}

data_findsym-output

_audit_creation_method FINDSYM

_chemical_name_mineral 'Mn5Si3,

_chemical_formula_sum 'Mn5 Si3

loop

_publ_author_name

B. Aronsson,

_journal_name_full_name

Acta Chemica Scandinavica

journal_volume 14

journal year 1960

_journal_page_first 1414

_journal_page_last 1418

_publ_Section_title

A note on the compositions and crystal structures of $\operatorname{MnB} \$_{-}\{2\} \$, \operatorname{Mn} \$_{-}\{3\}$ $\hookrightarrow \$ \mathrm{Si}, \mathrm{Mn}_{-}\{5\} \$ \mathrm{Si}_{-}\{3\} \$$, and $\mathrm{FeSi}_{-}\{2\} \$$

\# Found in Pearson's Crystal Data - Crystal Structure Database for $\hookrightarrow$ Inorganic Compounds, 2013

_aflow_title 'Mavlyanovite (Mn\$_\{5\}\$Si\$_\{3\}\$) Structure'

_aflow_proto 'A5B3_hP16_193_dg_g',

_aflow_params 'a, c/a, $x_{-}\{2\}, x_{-}\{3\}$ ', $0.696671490596,0.2358,0.5992$,

-aflow_params_values ' 6.910416

-aflow_Strukturbericht,
-aflow_Pearson 'hP16,

_cell_length_a $\quad 6.9104160691$

_cell_length_b 6.9104160691

_cell_length_c $\quad 4.8142898635$

_cell_angle_alpha 90.0000000000

_cell_angle_beta 90.0000000000

_cell_angle_gamma 120.0000000000

_symmetry_space_group_name_H-M "P $63 / \mathrm{m} \mathrm{2/c} 2 / \mathrm{m}^{\prime \prime}$

_symmetry_Int_Tables_number 193

loop

_space_group_symop_id

_space_group_symop_operation_xyz

$1 \mathrm{x}, \mathrm{y}, \mathrm{z}$

$2 \mathrm{x}-\mathrm{y}, \mathrm{x}, \mathrm{z}+1 / 2$

$3-\mathrm{y}, \mathrm{x}-\mathrm{y}, \mathrm{z}$

$4-x,-y, z+1 /$
$5-x+y,-x, z$

$6 \mathrm{y},-\mathrm{x}+\mathrm{y}, \mathrm{z}+1 / 2$

$7 \mathrm{x}-\mathrm{y},-\mathrm{y},-\mathrm{z}+1 / 2$

$8 \mathrm{x}, \mathrm{x}-\mathrm{y},-\mathrm{z}$

$9 \mathrm{y}, \mathrm{x},-\mathrm{z}+1 / 2$

$10-x+y, y,-z$

$11-x,-x+y,-z+1 / 2$

$12-y,-x,-z$

$14-\mathrm{x}+\mathrm{y},-\mathrm{x},-\mathrm{z}+1 / 2$

$15 \mathrm{y},-\mathrm{x}+\mathrm{y},-\mathrm{z}$

$16 \mathrm{x}, \mathrm{y},-\mathrm{z}+1 / 2$

$17 \mathrm{x}-\mathrm{y}, \mathrm{x},-\mathrm{z}$

$18-\mathrm{y}, \mathrm{x}-\mathrm{y},-\mathrm{z}+1 / 2$

$19-x+y, y, z+1 / 2$

$20-x,-x+y, z$

$21-\mathrm{y},-\mathrm{x}, \mathrm{z}+1$

$22 \mathrm{x}-\mathrm{y},-\mathrm{y}, \mathrm{z}$

$23 \mathrm{x}, \mathrm{x}-\mathrm{y}, \mathrm{z}+1 / 2$

$24 \mathrm{y}, \mathrm{x}, \mathrm{z}$

loop_

_atom_site_label

_atom_site_type_symbol

_atom_site_symmetry_multiplicity

_atom_site_Wyckoff_label

_atom_site_fract_x

_atom_site_fract_y

_atom_site_fract_z

atom site occupancy

\begin{tabular}{lllllll}
\hline $\mathrm{Mn} 1 \mathrm{Mn}$ & 4 & $\mathrm{~d}$ & 0.33333 & 0.66667 & 0.00000 & 1.00000
\end{tabular}

$\begin{array}{llllllll}\mathrm{Mn} 2 & \mathrm{Mn} & 6 & \mathrm{~g} & 0.23580 & 0.00000 & 0.25000 & 1.00000\end{array}$

$\begin{array}{llllllll}\mathrm{Si} 1 & \mathrm{Si} & 6 & \mathrm{~g} & 0.59920 & 0.00000 & 0.25000 & 1.00000\end{array}$

Mavlyanovite $\left(\mathrm{Mn}_{5} \mathrm{Si}_{3}\right)$ : A5B3_hP16_193_dg_g - POSCAR

A5B3_hP16_193_dg_g \& a , c/a, x2, x3 --params $=6.9104160691,0.696671490596$,

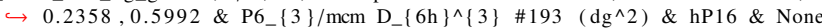

$\hookrightarrow$ \& Mn5Si3 \& \& B. Aronsson, Acta Chem. Scand. 14, 1414-1418

1.00000000000000

$\begin{array}{rrr}3.45520803455000 & -5.98459586656080 & 0.00000000000000 \\ 3.45520803455000 & 5.98459586656080 & 0.00000000000000\end{array}$

$\begin{array}{lll}0.00000000000000 & 0.00000000000000 & 4.81428986350000\end{array}$
Direct

0.33333333333333

0.6666666666666

0.6666666666666

0.33333333333333

0.23580000000000

0.00000000000000

$-0.23580000000000$

$-0.23580000000000$

0.00000000000000

0.23580000000000

0.59920000000000

0.00000000000000

$-0.59920000000000$

$-0.59920000000000$

0.00000000000000

0.59920000000000

0.666666666666667

0.33333333333333

0.33333333333333

0.666666666666667

0.00000000000000

0.23580000000000

$-0.23580000000000$

0.00000000000000

$-0.23580000000000$

0.23580000000000

0.00000000000000

0.59920000000000

$-0.59920000000000$

0.00000000000000

0.5000000000

0.59920000000000

0.00000000000000

0.50000000000000

0.00000000000000

0.50000000000000

0.25000000000000

0.25000000000000

0.25000000000000

0.75000000000000

0.75000000000000

0.75000000000000

0.25000000000000

.25000000000000

0.25000000000000

0.75000000000000

0.75000000000000

0.75000000000000

$\mathrm{Ni}_{3} \mathrm{Ti}\left(D 0_{24}\right)$ : A3B_hP16_194_gh_ac - CIF

\section{\# CIF file}

data_findsym-output

audit_creation_method FINDSYM

chemical_name_mineral,

chemical_formula_sum ' $\mathrm{Ni} 3 \mathrm{Ti}$

loop

publ_author_name

F. Laves'

H. J. Wallbaum

journal_name_full_name

Zeitschrift $f \backslash \backslash " u\} r$ Kristallografiya

journal_volume 10

journal year 1939

journal_page_first 78

journal page last 93

publ_Section title

Die Kristallstruktur von Ni\$_\{3\}\$Ti und $\mathrm{Si}_{-}\{2\} \$ \mathrm{Ti}$ (Zwei neue Typen.)

aflow_title, Ni\$_\{3\}\$Ti (\$D0_\{24\}\$) Structure

aflow_proto 'A3B_hP16_194_gh_ac

aflow_params 'a, c/a, x_\{4\}'

aflow_params_values ' $5.096,1.6295133438,-0.16667$ '

aflow_Strukturbericht '\$D0_\{24\}\$

aflow_Pearson 'hP16'

symmetry_space group_name_H-M "P $63 / \mathrm{m} 2 / \mathrm{m} \mathrm{2/c"}$

symmetry_Int_Tables_number 194

cell_length_a $\quad 5.09600$

cell_length_b $\quad 5.09600$

cell_length_c 8.30400

_cell_angle_alpha 90.00000

_cell_angle_beta 90.00000

cell_angle_gamma 120.00000

loop

space_group_symop_id

space_group_symop_operation_xyz

$\mathrm{x}, \mathrm{y}, \mathrm{z}$

$2 \mathrm{x}-\mathrm{y}, \mathrm{x}, \mathrm{z}+1 / 2$

$3-\mathrm{y}, \mathrm{x}-\mathrm{y}, \mathrm{z}$

$4-\mathrm{x},-\mathrm{y}, \mathrm{z}+1 / 2$

$5-\mathrm{x}+\mathrm{y},-\mathrm{x}, \mathrm{z}$
$6 \mathrm{y},-\mathrm{x}+\mathrm{y}, \mathrm{z}+1 / 2$

$6 \mathrm{y},-\mathrm{x}+\mathrm{y}, \mathrm{z}+1 / 2$

$7 \mathrm{x}-\mathrm{y},-\mathrm{y},-\mathrm{z}$

$8 \mathrm{x}, \mathrm{x}-\mathrm{y},-\mathrm{z}+1 / 2$

$\mathrm{y}, \mathrm{x},-\mathrm{z}$

$10-x+y, y,-z+1 / 2$

$11-\mathrm{x},-\mathrm{x}+\mathrm{y},-\mathrm{z}$

$12-\mathrm{y},-\mathrm{x},-\mathrm{z}+1 / 2$

$13-x,-y,-z$

$14-\mathrm{x}+\mathrm{y},-\mathrm{x},-\mathrm{z}+1 / 2$

$15 \mathrm{y},-\mathrm{x}+\mathrm{y},-\mathrm{z}$

$16 \mathrm{x}, \mathrm{y},-\mathrm{z}+1 / 2$

$17 \mathrm{x}-\mathrm{y}, \mathrm{x},-\mathrm{z}$

$8-\mathrm{y}, \mathrm{x}-\mathrm{y},-\mathrm{z}+1 / 2$

$19-x+y, y, z$

$20-x,-x+y, z+1 / 2$

$21-\mathrm{y},-\mathrm{x}, \mathrm{z}$

$22 \mathrm{x}-\mathrm{y},-\mathrm{y}, \mathrm{z}+1 / 2$

$23 \mathrm{x}, \mathrm{x}-\mathrm{y}, \mathrm{z}$

$24 \mathrm{y}, \mathrm{x}, \mathrm{z}+1 / 2$

loop

atom_site_label

_atom_site_type_symbol

_atom_site_symmetry_multiplicity

_atom_site_Wyckoff_label

atom_site_fract_x

atom_site_fract_y

atom_site_fract_z

atom_site_occupancy

$\begin{array}{llllllll}\mathrm{Ti} 1 & \mathrm{Ti} & 2 & \mathrm{a} & 0.00000 & 0.00000 & 0.00000 & 1.00000\end{array}$

$\begin{array}{lllllllll}\mathrm{Ti} 2 & \mathrm{Ti} & 2 & \text { c } & 0.33333 & 0.66667 & 0.25000 & 1.00000\end{array}$

$\begin{array}{lllllllll}\mathrm{Ni} 1 & \mathrm{Ni} & 6 & \mathrm{~g} & 0.50000 & 0.00000 & 0.00000 & 1.00000\end{array}$

$\begin{array}{llllllll}\mathrm{Ni} 2 & \mathrm{Ni} & 6 & \mathrm{~h} & -0.16667 & -0.33333 & 0.25000 & 1.00000\end{array}$

$\mathrm{Ni}_{3} \mathrm{Ti}\left(D 0_{24}\right)$ : A3B_hP16_194_gh_ac - POSCAR 


\begin{tabular}{|c|c|c|c|c|}
\hline \multirow{2}{*}{\multicolumn{5}{|c|}{ 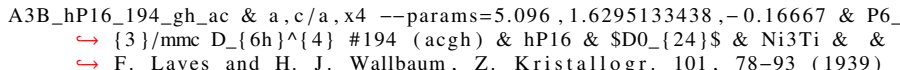 }} \\
\hline & & & & \\
\hline 2.54800000000000 & -4.41326545768550 & 0.00000000000000 & & \\
\hline 2.54800000000000 & 4.41326545768550 & 0.00000000000000 & & \\
\hline 0.00000000000000 & 0.00000000000000 & 8.30400000000000 & & \\
\hline $\mathrm{Ni}$ & & & & \\
\hline 12 & & & & \\
\hline \multicolumn{5}{|l|}{ Direct } \\
\hline 0.50000000000000 & 0.00000000000000 & 0.00000000000000 & $\mathrm{Ni}$ & $(6 \mathrm{~g})$ \\
\hline 0.00000000000000 & 0.50000000000000 & 0.00000000000000 & $\mathrm{Ni}$ & $(6 \mathrm{~g})$ \\
\hline 0.50000000000000 & 0.50000000000000 & 0.00000000000000 & $\mathrm{Ni}$ & $(6 \mathrm{~g})$ \\
\hline 0.50000000000000 & 0.00000000000000 & 0.50000000000000 & $\mathrm{Ni}$ & $(6 \mathrm{~g})$ \\
\hline 0.00000000000000 & 0.50000000000000 & 0.50000000000000 & $\mathrm{Ni}$ & $(6 \mathrm{~g})$ \\
\hline 0.50000000000000 & 0.50000000000000 & 0.50000000000000 & $\mathrm{Ni}$ & (6g) \\
\hline-0.16667000000000 & -0.33334000000000 & 0.25000000000000 & $\mathrm{Ni}$ & $(6 \mathrm{~h})$ \\
\hline 0.33334000000000 & 0.16667000000000 & 0.25000000000000 & $\mathrm{Ni}$ & $(6 \mathrm{~h})$ \\
\hline-0.16667000000000 & 0.16667000000000 & 0.25000000000000 & $\mathrm{Ni}$ & $(6 \mathrm{~h})$ \\
\hline 0.16667000000000 & 0.33334000000000 & 0.75000000000000 & $\mathrm{Ni}$ & $(6 \mathrm{~h})$ \\
\hline-0.33334000000000 & -0.16667000000000 & 0.75000000000000 & $\mathrm{Ni}$ & $(6 \mathrm{~h})$ \\
\hline 0.16667000000000 & -0.16667000000000 & 0.75000000000000 & $\mathrm{Ni}$ & (6h) \\
\hline 0.00000000000000 & 0.00000000000000 & 0.00000000000000 & $\mathrm{Ti}$ & (2a) \\
\hline 0.00000000000000 & 0.00000000000000 & 0.50000000000000 & $\mathrm{Ti}$ & (2a) \\
\hline 0.33333333333333 & 0.66666666666667 & 0.25000000000000 & $\mathrm{Ti}$ & $(2 \mathrm{c})$ \\
\hline 0.666666666666667 & 0.33333333333333 & 0.75000000000000 & $\mathrm{Ti}$ & $(2 \mathrm{c})$ \\
\hline
\end{tabular}

$\mathrm{Co}_{2} \mathrm{Al}_{5}\left(D 8_{11}\right)$ : A5B2_hP28_194_ahk_ch - CIF

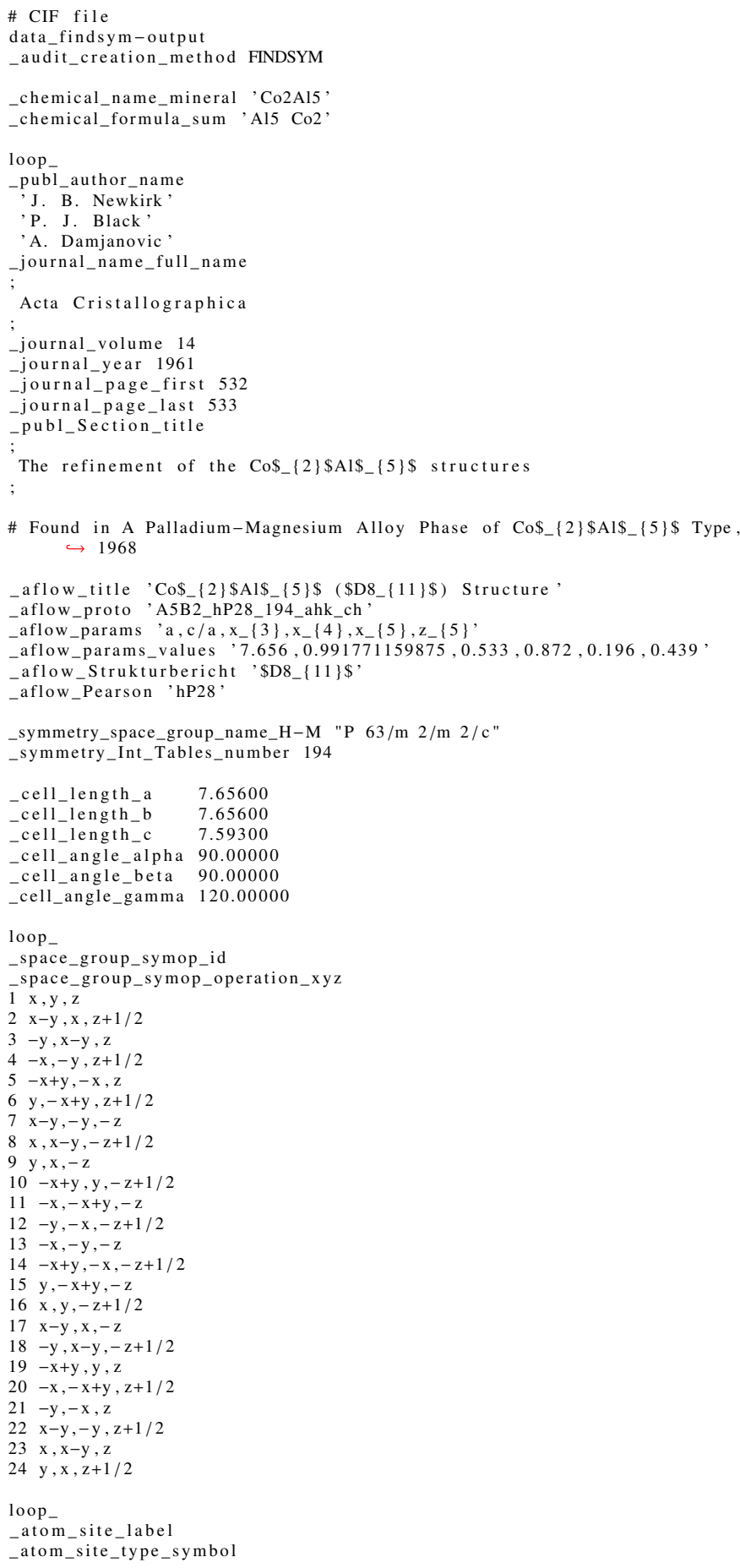

$\mathrm{Co}_{2} \mathrm{Al}_{5}\left(D 8_{11}\right)$ : A5B2_hP28_194_ahk_ch - POSCAR

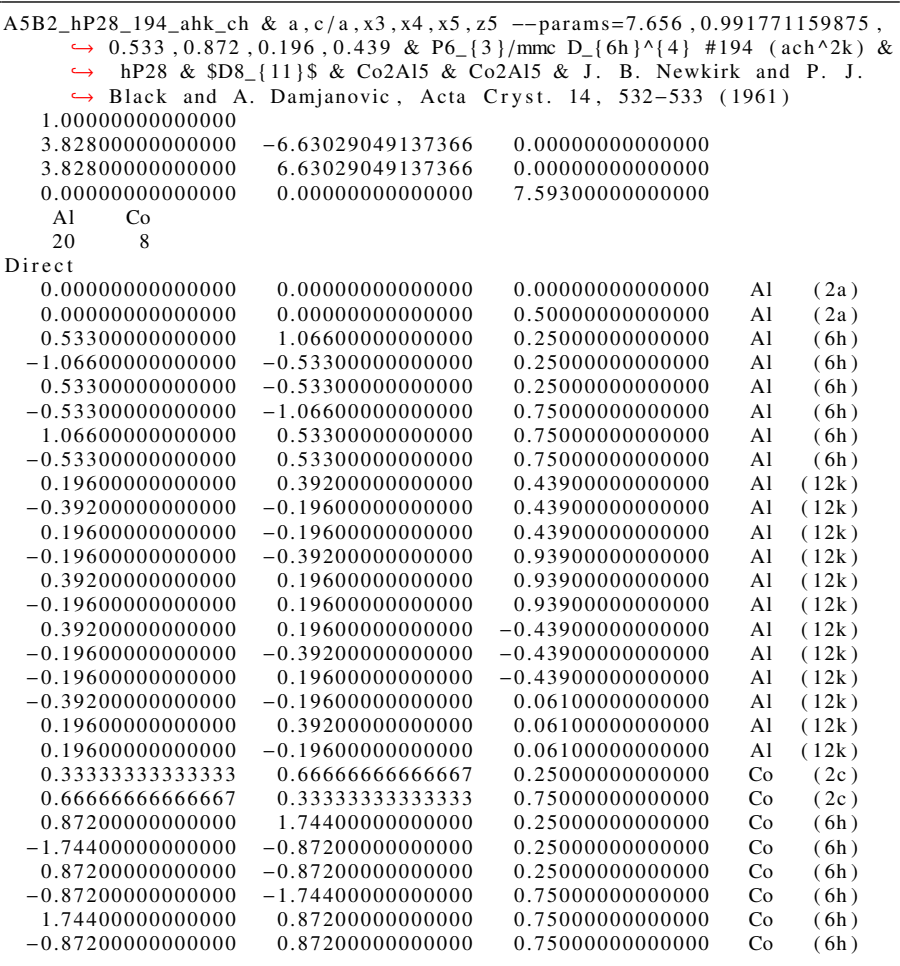

$\mathrm{Al}_{9} \mathrm{Mn}_{3} \mathrm{Si}\left(E 9_{c}\right):$ A9B3C_hP26_194_hk_h_a - CIF

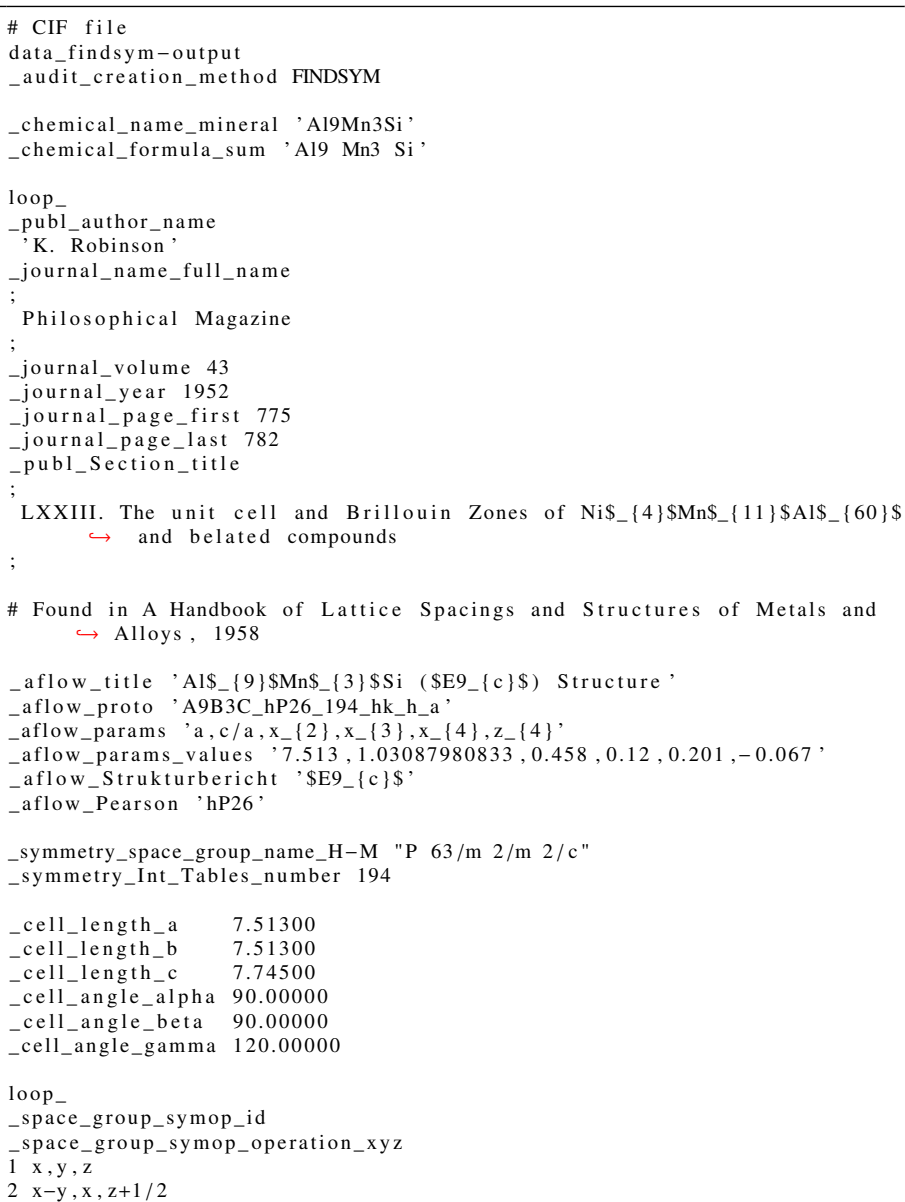




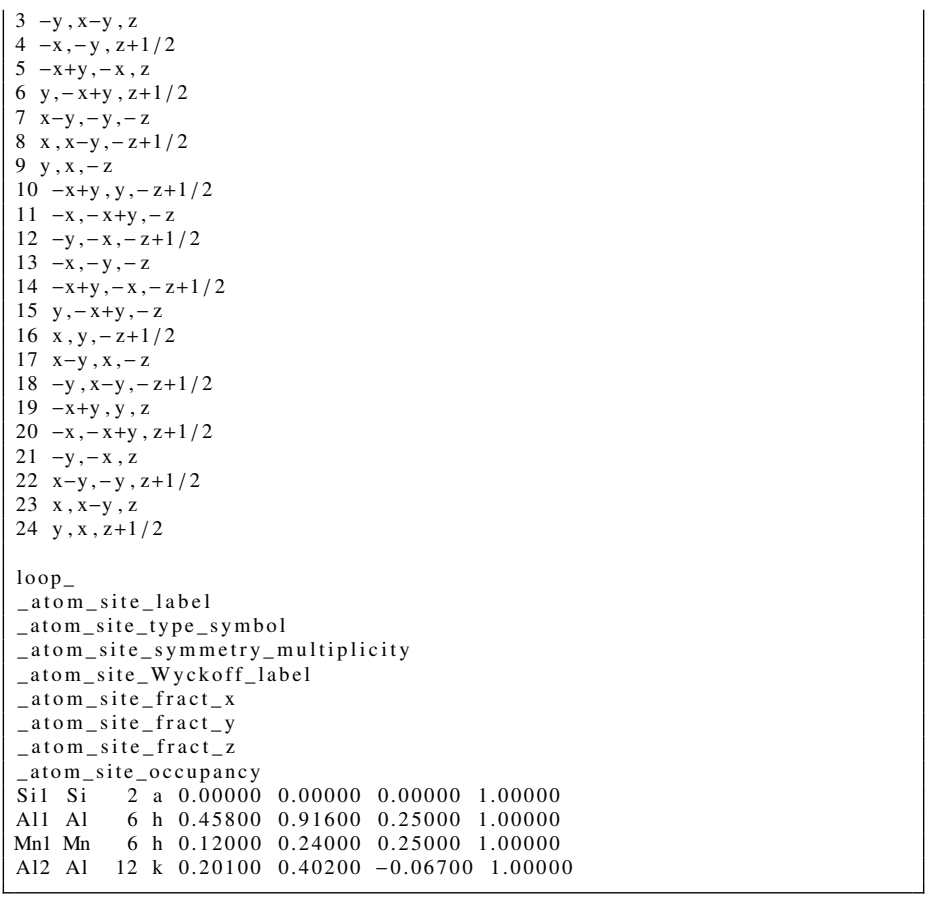

$\mathrm{Al}_{9} \mathrm{Mn}_{3} \mathrm{Si}\left(E 9_{c}\right):$ A9B3C_hP26_194_hk_h_a - POSCAR

A9B3C_hP26_194_hk_h_a \& a , c /a , x2 , x3, x4, z4 --params $=7.513,1.03087980833$,

$\hookrightarrow 0.458,0.12,0.201,-0.067 \& \mathrm{P}_{-}\{3\} / \mathrm{mmc} \mathrm{D}_{-}\{6 \mathrm{~h}\}^{\wedge}\{4\} \# 194 \quad\left(\mathrm{ah}^{\wedge} 2 \mathrm{k}\right)$

$\hookrightarrow$ Mag. 43, 775-782 (1952)

1.00000000000000

$\begin{array}{lll}3.75650000000000 & -6.50644885863249 & 0.00000000000000\end{array}$

$\begin{array}{lll}3.75650000000000 & 6.50644885863249 & 0.00000000000000\end{array}$

$\begin{array}{lll}0.00000000000000 & 0.00000000000000 & 7.74500000000000\end{array}$

$\begin{array}{rrr}\mathrm{Al} & \mathrm{Mn} & \mathrm{Si} \\ 18 & 6 & 2\end{array}$

Direct

$0.45800000000000 \quad 0.91600000000000$

$-0.91600000000000$

0.45800000000000

$-0.4580000000000$

0.4580000000000

0.9160000000000

0.20100000000000

$-0.40200000000000$

0.20100000000000

$-0.20100000000000$

0.40200000000000

$-0.20100000000000$

0.40200000000000

$-0.20100000000000$

$-0.20100000000000$

$-0.40200000000000$

0.20100000000000

0.20100000000000

0.12000000000000

$-0.24000000000000$

0.12000000000000

0.12000000000000

0.24000000000000

$-0.12000000000000$

0.00000000000000

0.00000000000000

0.91600000000000

$-0.45800000000000$

$-0.9160000000000$

0.4580000000000

0.45800000000000

.

$-0.20100000000000$

$-0.20100000000000$

$-0.40200000000000$

0.20100000000000

0.2010000000000

0.20100000000000

$-0.40200000000000$

0.2010000000000

$-0.20100000000000$

0.40200000000000

0.20100000000000

$-0.12000000000000$

$-0.1200000000000$

$-0.24000000000000$

0.12000000000000

0.12000000000000

0.00000000000000

0.00000000000000

0.25000000000000 0.25000000000000 0.25000000000000 0.75000000000000 0.75000000000000 0.75000000000000 $-0.06700000000000$ $-0.06700000000000$ $-0.06700000000000$ 0.43300000000000 0.43300000000000 0.43300000000000 0.06700000000000 0.06700000000000 0.06700000000000 0.5670000000000 0.56700000000000 0.56700000000000 0.25000000000000 0.25000000000000 0.25000000000000 0.7500000000000 0.7500000000000 0.750000000000 0.00000000000000 0.50000000000000

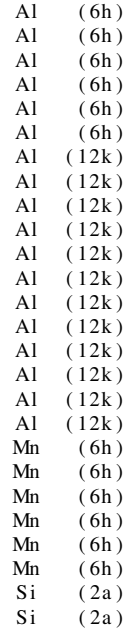

$\mathrm{PrRu}_{4} \mathrm{P}_{12}$ : A12BC4_cP34_195_2j_ab_2e - CIF

\section{CIF file}

data_findsym-output

_audit_creation_method FINDSYM

chemical_name_mineral 'PrRu4P12'

_chemical_formula_sum 'P12 Pr Ru4,

loop

publ_author_name

'C. H. Lee'

'H. Matsuhata'

'H. Yamaguchi'

'K. Kihou'

I. Shirotani

journal_name_full_name

Journal of Magnetism and Magnetic Materials

_journal_volume 272

-journal year 2004

_journal_page_first 426

journal_page_last 427

publ_Section_title

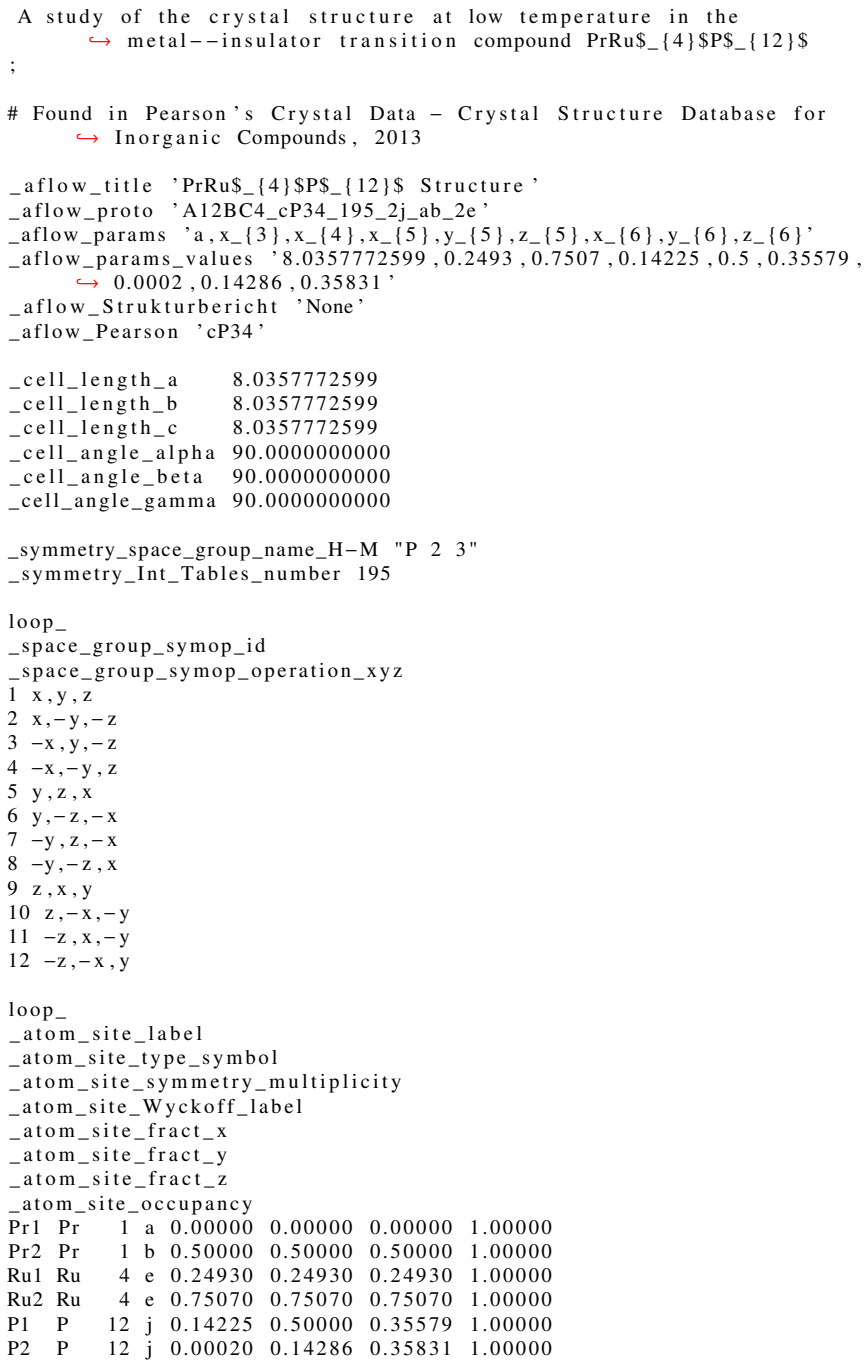

$\mathrm{PrRu}_{4} \mathrm{P}_{12}$ : A12BC4_cP34_195_2j_ab_2e - POSCAR

A12BC4_cP34_195_2j_ab_2e \& a , x3 , x4, x5 , y5 , z5 , x6, y6, z6 --params=

$\hookrightarrow 8.0357772599,0.2493,0.7507,0.14225,0.5,0.35579,0.0002,0.14286$

$\rightarrow 0.35831 \& \mathrm{P} 23 \mathrm{~T}^{\wedge}\{1\} \# 195\left(\mathrm{abe}^{\wedge} 2 \mathrm{j}^{\wedge} 2\right) \& \mathrm{cP} 34$ \& None \& PrRu4P12 \&

$\leftrightarrow$ \& C. H. Lee et al., J. Magn. Magn. Mater. 272, 426-427 (2004)

1.00000000000000

$\begin{array}{lll}8.03577725990000 & 0.00000000000000 & 0.00000000000000\end{array}$

$\begin{array}{lll}0.00000000000000 & 8.03577725990000 & 0.00000000000000\end{array}$

$\begin{array}{lll}0.00000000000000 & 0.00000000000000 & 8.03577725990000\end{array}$

$\begin{array}{rrr}\mathrm{P} & \mathrm{Pr} & \mathrm{Ru} \\ 24 & 2 & 8\end{array}$

Direc

0.14225000000000

$-0.14225000000000$

$-0.14225000000000$

0.1422500000000

0.14225000000000

0.35579000000000

$-0.35579000000000$

$-0.35579000000000$

0.50000000000000

$-0.5000000000000$

0.5000000000000

$-0.50000000000000$

0.00020000000000

$-0.00020000000000$

$-0.00020000000000$

0.00020000000000

0.35831000000000

0.35831000000000

$-0.35831000000000$

$-0.35831000000000$

0.1428600000000

0.14286000000000

0.14286000000000

$-0.14286000000000$

0.0000000000000

0.50000000000000

0.24930000000000

$-0.24930000000000$

$-0.24930000000000$

0.24930000000000

0.75070000000000

$-0.75070000000000$

0.75070000000000

0.50000000000000 0.50000000000000 0.50000000000000 0.50000000000000 0.14225000000000 $-0.14225000000000$ 0.14225000000000 0.14225000000000 0.35579000000000 0.35579000000000 $-0.35579000000000$ 0.35579000000000 0.14286000000000 0.14286000000000 0.14286000000000 0.14286000000000 0.00020000000000 $-0.00020000000000$ $-0.00020000000000$ 0.00020000000000 0.35831000000000 0.35831000000000 $-0.35831000000000$ 0.35831000000000 0.0000000000000 . 0.5000000000 0.24930000000000 0.24930000000000 0.24930000000000 .24930000000000 0.75070000000000 0.75070000000000 0.75070000000000

0.35579000000000 0.35579000000000 $-0.35579000000000$ $-0.35579000000000$ 0.5000000000000 $-0.5000004$ 0.50000000 $-0.500000$ 0.1425000 $-0.14225000000000$ .1422500000000 0.14225000000000 0.35831000000000 0.35831000000000 $-0.35831000000000$ $-0.35831000000000$ 0.14286000000000 $-0.14286000000000$ 0.14286000000000 $-0.14286000000000$ 0.0002000000000 $-0.00020000000000$ 0.0002000000000 . 0.00020000000000 0.00000000000000 0.50000000000000 0.24930000000000 0.24930000000000 $-0.24930000000000$ $-0.24930000000000$ 0.75070000000000 0.75070000000000 $-0.75070000000000$

\begin{tabular}{cl}
$P$ & $(12 \mathrm{j})$ \\
$P$ & $(12 \mathrm{j})$ \\
$P$ & $(12 \mathrm{j})$ \\
$P$ & $(12 \mathrm{j})$ \\
$P$ & $(12 \mathrm{j})$ \\
$P$ & $(12 \mathrm{j})$ \\
$P$ & $(12 \mathrm{j})$ \\
$P$ & $(12 \mathrm{j})$ \\
$P$ & $(12 \mathrm{j})$ \\
$P$ & $(12 \mathrm{j})$ \\
$P$ & $(12 \mathrm{j})$ \\
$P$ & $(12 \mathrm{j})$ \\
$P$ & $(12 \mathrm{j})$ \\
$P$ & $(12 \mathrm{j})$ \\
$P$ & $(12 \mathrm{j})$ \\
$P$ & $(12 \mathrm{j})$ \\
$P$ & $(12 \mathrm{j})$ \\
$P$ & $(12 \mathrm{j})$ \\
$P$ & $(12 \mathrm{j})$ \\
$P$ & $(12 \mathrm{j})$ \\
$P$ & $(12 \mathrm{j})$ \\
$P$ & $(12 \mathrm{j})$ \\
$P$ & $(12 \mathrm{j})$ \\
$P$ & $(12 \mathrm{j})$ \\
$P r$ & $(1 \mathrm{a})$ \\
$P r$ & $(1 \mathrm{~b})$ \\
$R u$ & $(4 \mathrm{e})$ \\
$R u$ & $(4 \mathrm{e})$ \\
$R u$ & $(4 \mathrm{e})$ \\
$R u$ & $(4 \mathrm{e})$ \\
$R u$ & $(4 \mathrm{e})$ \\
$R u$ & $(4 \mathrm{e})$ \\
$R u$ & $(4 \mathrm{e})$ \\
$R u$ & $(4 \mathrm{e})$ \\
\hline
\end{tabular}


$\mathrm{Cu}_{2} \mathrm{Fe}[\mathrm{CN}]_{6}$ : A12B2C_cF60_196_h_bc_a - CIF

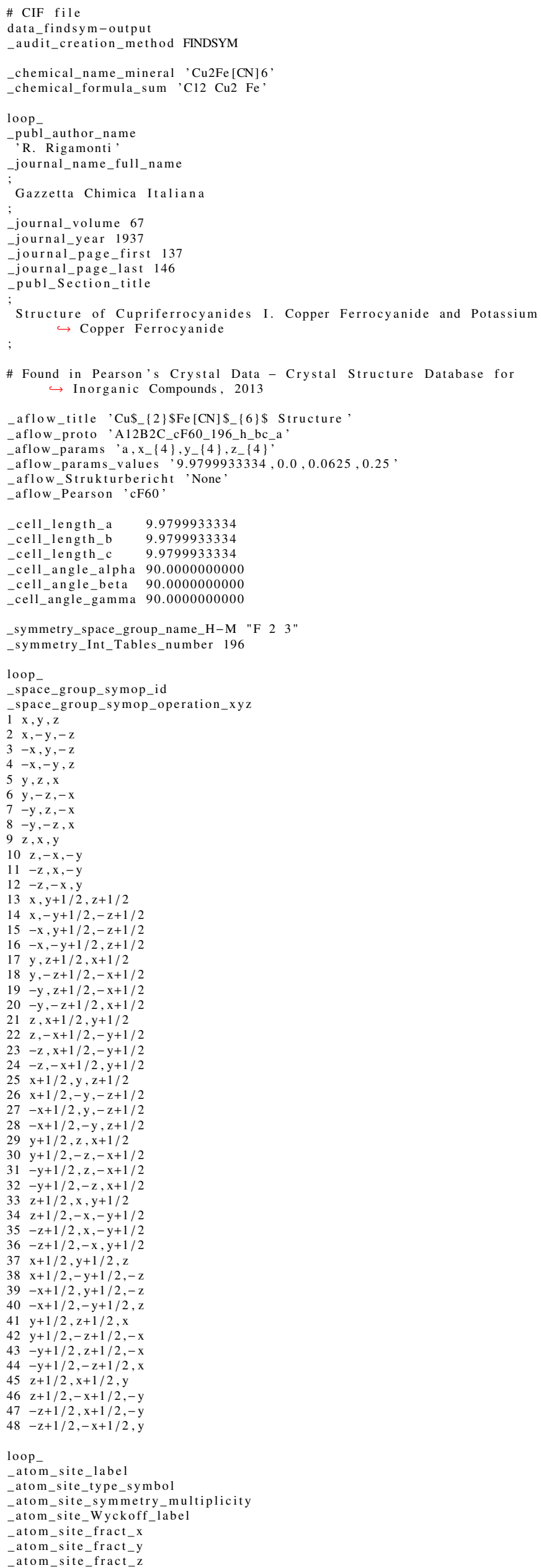

atom_site_occupancy

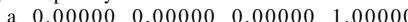

$\begin{array}{llllllll}\mathrm{Cu} 1 \mathrm{Cu} & 4 & \mathrm{~b} & 0.50000 & 0.50000 & 0.50000 & 1.00000\end{array}$ $\begin{array}{llllllll}\mathrm{Cu} 2 \mathrm{Cu} & 4 & \text { c } & 0.25000 & 0.25000 & 0.25000 & 1.00000\end{array}$ $\begin{array}{llllllll}\mathrm{C} 1 \mathrm{C} & 48 & \mathrm{~h} & 0.00000 & 0.06250 & 0.25000 & 1.00000\end{array}$

$\mathrm{Cu}_{2} \mathrm{Fe}[\mathrm{CN}]_{6}:$ A12B2C_cF60_196_h_bc_a - POSCAR

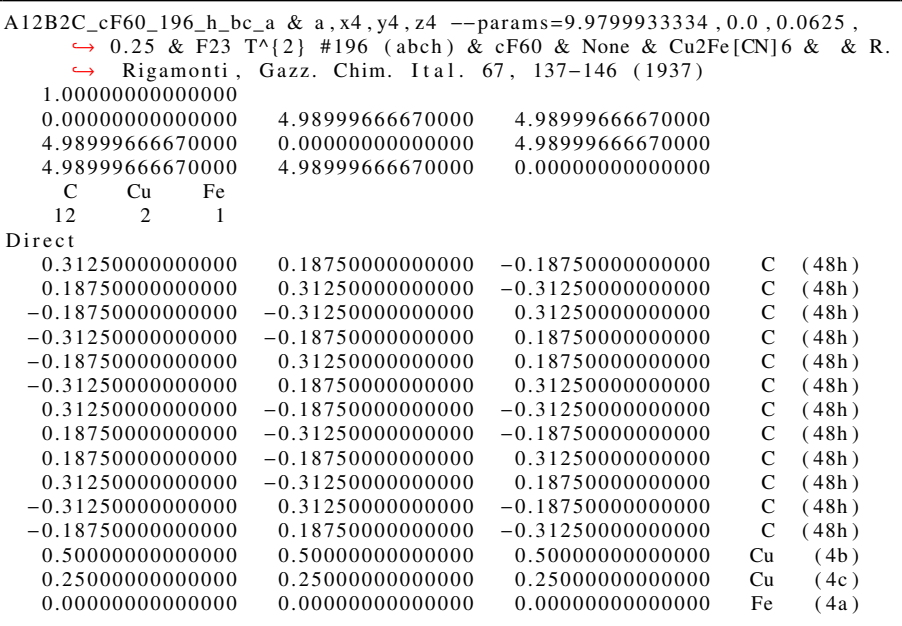

$\mathrm{MgB}_{12} \mathrm{H}_{12}\left[\mathrm{H}_{2} \mathrm{O}\right]_{12}$ : A12B36CD12_cF488_196_2h_6h_ac_fgh - CIF

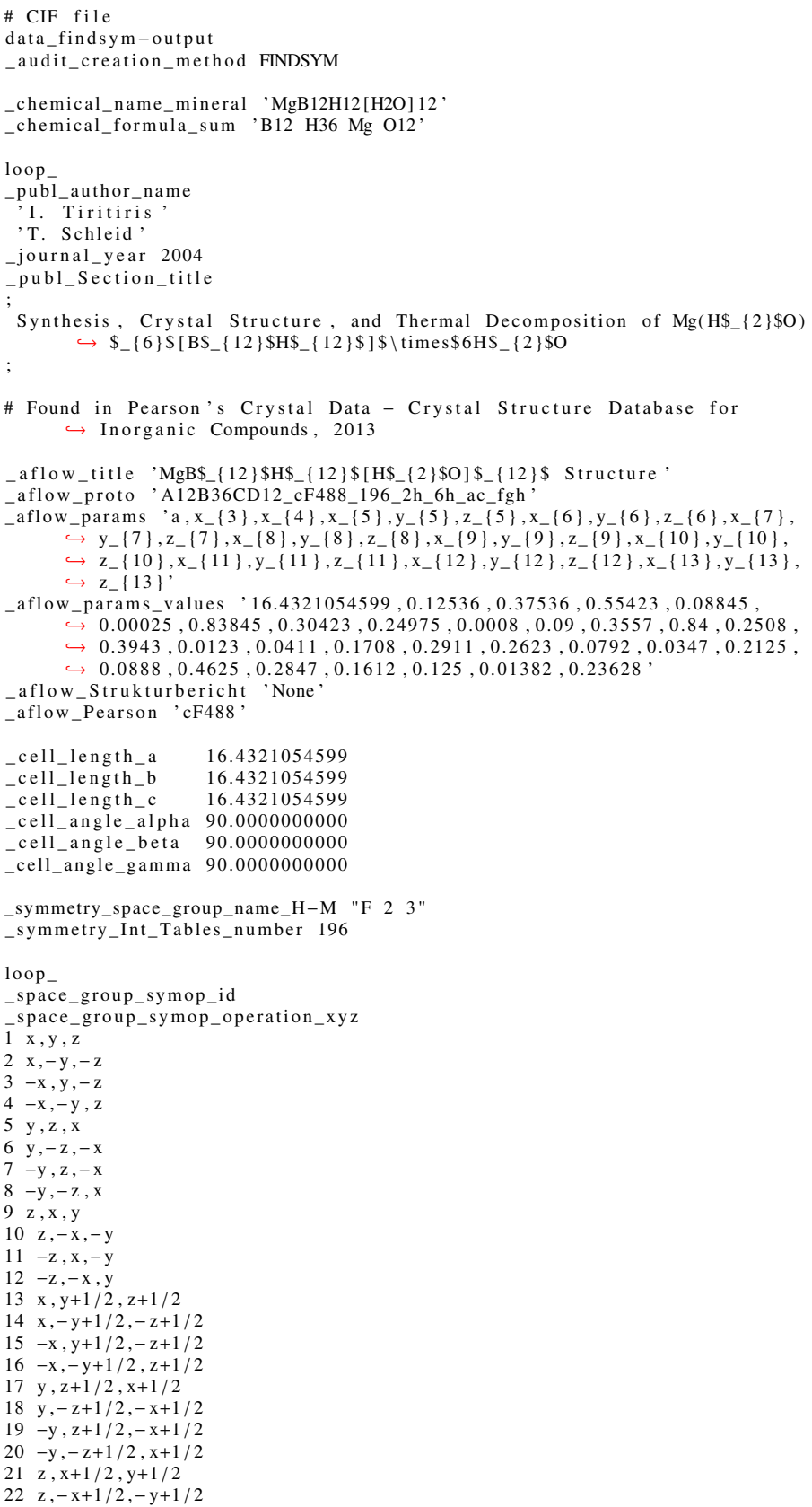




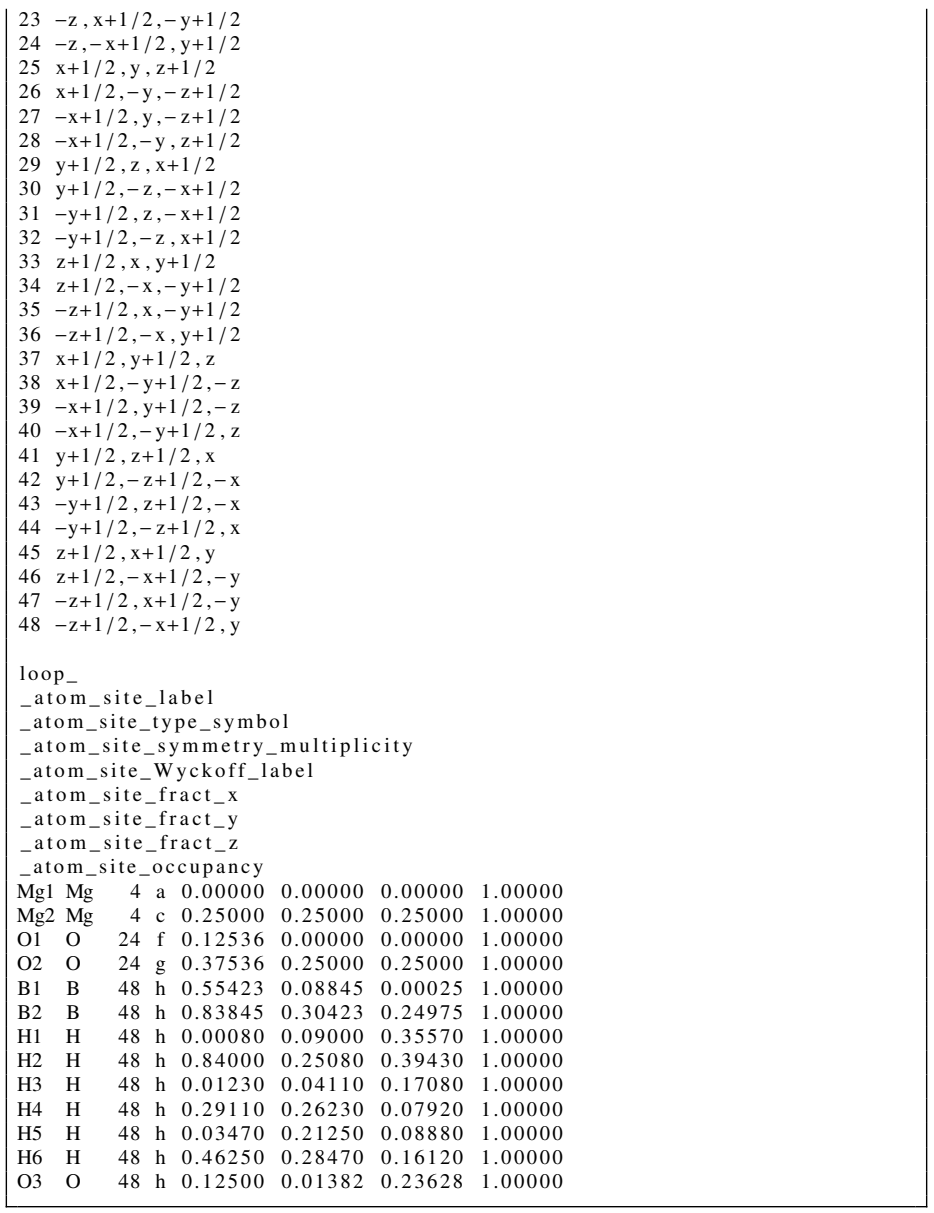

$\mathrm{MgB}_{12} \mathrm{H}_{12}\left[\mathrm{H}_{2} \mathrm{O}\right]_{12}$ : A12B36CD12_cF488_196_2h_6h_ac_fgh - POSCAR

A12B36CD12_cF488_196_2h_6h_ac_fgh \& a , x3, x4, x5 ,y5, z5 , x6, y6, z6, x7 ,y $7, \mathrm{z} 7$, $\hookrightarrow \mathrm{x} 8, \mathrm{y} 8, \mathrm{z} 8, \mathrm{x} 9, \mathrm{y} 9, \mathrm{z} 9, \mathrm{x} 10, \mathrm{y} 10, \mathrm{z} 10, \mathrm{x} 11, \mathrm{y} 11, \mathrm{z} 11, \mathrm{x} 12, \mathrm{y} 12, \mathrm{z} 12, \mathrm{x} 13, \mathrm{y}$

$\hookrightarrow$ z13 - - params $=16.4321054599,0.12536,0.37536,0.55423,0.08845$,

$\hookrightarrow 0.3943,0.0123,0.0411,0.1708,0.2911,0.2623,0.0792,0.0347,0.2125$

$\hookrightarrow 0.0888,0.4625,0.2847,0.1612,0.125,0.01382,0.23628 \& \mathrm{~F} 23 \mathrm{~T}^{\wedge}\{2\}$

$\hookrightarrow 196($ acfgh^9) \& cF488 \& None \& MgB12H12[H2O]12 \& \& I.

$\hookrightarrow$ Tiritiris and T. Schleid, (2004)

1.00000000000000

0.00000000000000

$\begin{array}{lll}8.21605272995000 & 0.00000000000000 & 8.21605272995000 \\ 8.21605272995000 & 8.21605272995000 & 0.00000000000000\end{array}$

8.21605272995000

$\begin{array}{lll}8.21605272995000 & 0.00000000000000 & 8.21605272995000 \\ 8.21605272995000 & 8.21605272995000 & 0.00000000000000\end{array}$

8.21605272995000

$\begin{array}{rrrr}\text { B } & \mathrm{H} & \mathrm{Mg} & \mathrm{O} \\ 24 & 72 & 2 & 24\end{array}$

Direct

$-0.46553000000000$

0.46603000000000

0.64243000000000

$-0.64293000000000$

0.64243000000000

$-0.64293000000000$

0.46553000000000

0.46603000000000

0.46603000000000

$-0.46553000000000$

$-0.64293000000000$

0.64243000000000

$-0.28447000000000$

0.78397000000000

0.89293000000000

0.89293000000000

$-1.39243000000000$

$-0.28447000000000$

0.78397000000000

0.78397000000000

$-0.28447000000000$

$-1.39243000000000$ 0.89293000000000 0.44490000000000 0.26650000000000 $-0.26490000000000$

$-0.44650000000000$

$-0.26490000000000$

$-0.44650000000000$

0.44490000000000

0.26650000000000

0.44490000000000

$-0.44650000000000$

$-0.26490000000000$

$-0.19490000000000$

0.98350000000000

0.69650000000000 $-1.48510000000000$ 0.69650000000000 $-1.48510000000000$ $-0.19490000000000$ 0.98350000000000 0.98350000000000 $-0.19490000000000$ $-1.48510000000000$ 0.69650000000000 0.19960000000000 0.14200000000000 $-0.22420000000000$

0.19960000000000 0.14200000000000 0.14200000000000 0.19960000000000 $-0.22420000000000$ $-0.11740000000000$ 0.05040000000000 0.10800000000000 0.47420000000000 $-0.63260000000000$ 0.47420000000000 $-0.63260000000000$ 0.05040000000000

0.10800000000000 $-0.63260000000000$ 0.47420000000000 0.26660000000000 $-0.08900000000000$ 0.15840000000000 $-0.33600000000000$ 0.15840000000000 $-0.33600000000000$ 0.26660000000000 $-0.08900000000000$ $-0.08900000000000$ 0.26660000000000 $-0.33600000000000$ 0.15840000000000 $-0.0166000000000$ 0.3390000000000 0.58600000000000 $-0.90840000000000$ 0.5860000000000 $-0.90840000000000$ $-0.01660000000000$ 0.33900000000000 0.33900000000000 $-0.01660000000000$ $-0.90840000000000$ 0.58600000000000 0.00000000000000 0.25000000000000 $-0.12536000000000$ 0.12536000000000 0.12536000000000 $-0.12536000000000$ 0.1253600000000 0.1253600000000 0.1246400000000 0.124640000000 0.37536000000000 0.37536000000000 0.12464000000000 0.37536000000000 0.1246400000000 0.12510000000000 0.34746000000000 $-0.09746000000000$ $-0.37510000000000$ $-0.09746000000000$ $-0.37510000000000$ 0.12510000000000 0.34746000000000 0.34746000000000

0.34746000000000
0.12510000000000

0.12510000000000
-0.37510000000000 $-0.09746000000000$

48510000000000 0.69650000000000 $-0.19490000000000$ 0.98350000000000 0.69650000000000 $-1.48510000000000$ 0.69650000000000 $-1.48510000000000$ $-0.19490000000000$ 0.98350000000000 0.14200000000000 0.19960000000000 $-0.11740000000000$ 0.19960000000000 0.14200000000000 ( 0.11740000000000 0.22420000000000 0.19960000000000 0.14200000000000 0.10800000000000 0.05040000000000 $-0.63260000000000$ 0.47420000000000 0.05040000000000 0.10800000000000 0.47420000000000 $-0.63260000000000$ 0.47420000000000 0.63260000000000 0.05040000000000 (2) 0.26660000000000 $-0.33600000000000$ 0.15840000000000 0.26660000000000 $-0.08900000000000$ 0.15840000000000 $-0.33600000000000$ 0.15840000000000 $-0.33600000000000$ 0.26660000000000 $-0.08900000000000$ 0.33900000000000 $-0.01660000000000$ $-0.01660000000000$ 0.58600000000000 0.58600000000000 0.01660000000000 0.33900000000000 0.58600000000000 $-0.90840000000000$ 0.58600000000000 $-0.90840000000000$ $-0.01660000000000$ 0.33900000000000 0.00000000000000 0.25000000000000 0.12536000000000 $-0.12536000000000$ $-0.12536000000000$ 0.12536000000000 0.12536000000000 0.37536000000000 0.12464000000000 0.12464000000000 0.37536000000000 0.37536000000000 0.12464000000000 0.34746000000000 0.12510000000000 $-0.37510000000000$ $-0.09746000000000$ 0.12510000000000 0.34746000000000 $-0.09746000000000$ $-0.37510000000000$ $-0.09746000000000$ $-0.37510000000000$ 0.37510000000000 0.34746000000000

$-0.19490000000000$ 0.98350000000000 0.98350000000000 $-0.19490000000000$ $-1.4851000000000$ 0.69650000000000 $-0.19490000000000$ 0.98350000000000 0.69650000000000 $-1.48510000000000$ $-0.11740000000000$ $-0.22420000000000$ 0.19960000000000 0.14200000000000 0.14200000000000 60000000000 0.14200000000000 $-0.11740000000000$ $-0.22420000000000$ 0.47420000000000 0.63260000000000 0.05040000000000 0.10800000000000 0.10800000000000 0.05040000000000 $-0.63260000000000$ 0.47420000000000 0.05040000000000 0.10800000000000 0.47420000000000 0.63260000000000 0.15840000000000 $-0.33600000000000$ 0.26660000000000 $-0.08900000000000$ 0.08900000000000 . 0.26660000000000 5840000000000 0.15840000000000 0.26660000000000 $-0.08900000000000$ 0.15840000000000 0.58600000000000 $-0.90840000000000$ $-0.90840000000000$ (0.3390000 0.908400 0.33900000000000 0.339000000000 0.58600000000000 0.90840000000000 0.00000000000000 0.25000000000000 $-0.12536000000000$ 0.12536000000000 $-0.12536000000000$ 0.375360 


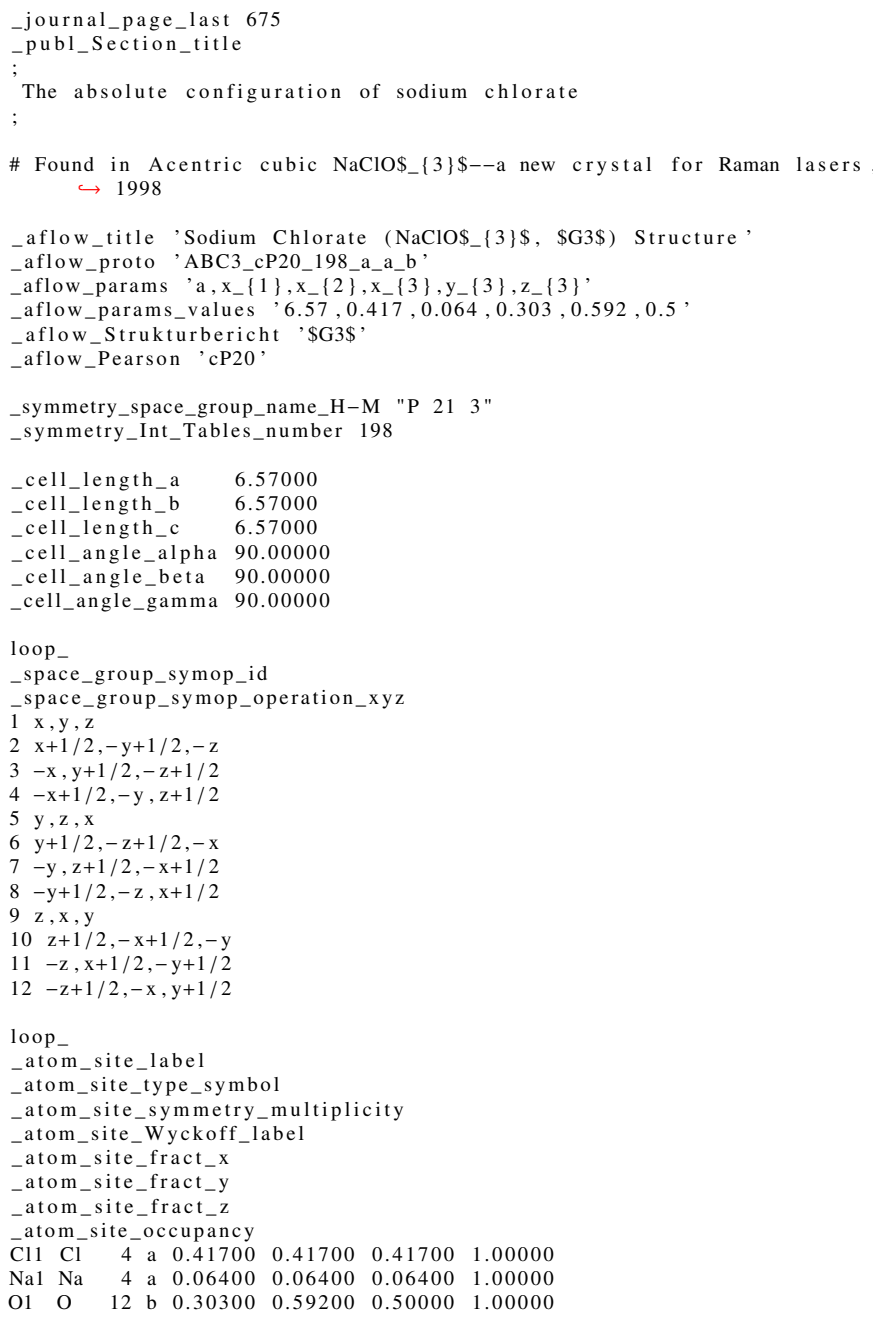

Sodium Chlorate $\left(\mathrm{NaClO}_{3}, \mathrm{G3}\right)$ : ABC3_cP20_198_a_a_b - POSCAR

ABC3_cP20_198_a_a_b \& a , x1 , x2 $, \mathrm{x} 3, \mathrm{y} 3, \mathrm{z3}--$ params $=6.57,0.417,0.064,0.303$ $\hookrightarrow 0.592,0.5 \& \mathrm{P} 2 \_\{1\} 3 \mathrm{~T}^{\wedge}\{4\} \# 198 \quad\left(\mathrm{a}^{\wedge} 2 \mathrm{~b}\right) \& \mathrm{cP} 20 \& \$ \mathrm{G} 3 \$ \& \mathrm{NaClO} 3 \&$ $\hookrightarrow$ Sodium chlorate \& G. N. Ramachandran and K. S. Chandrasekaran, $\hookrightarrow$ Acta Cryst. 10, 671-675 (1957) 1.00000000000000

$\begin{array}{lll}6.57000000000000 & 0.00000000000000 & 0.00000000000000 \\ 0.00000000000000 & 6.57000000000000 & 0.00000000000000\end{array}$

$\begin{array}{lll}0.00000000000000 & 0.00000000000000 & 6.57000000000000\end{array}$

$\mathrm{Cl} \mathrm{Na} \quad \mathrm{O}$

Direct

0.41700000000000 0.08300000000000 $-0.41700000000000$ 0.91700000000000 0.0640000000000 0.06400000000000 0.43600000000000 0.06400000000000 0.56400000000000 0.30300000000000 0.19700000000000 $-0.30300000000000$ 0.80300000000000 0.50000000000000 1.00000000000000 0.00000000000000 $-0.50000000000000$ 0.59200000000000 $-0.59200000000000$ 1.09200000000000 $-0.09200000000000$

$\begin{array}{rrrr}0.41700000000000 & 0.41700000000000 & \mathrm{Cl} & (4 \mathrm{a}) \\ -0.41700000000000 & 0.91700000000000 & \mathrm{Cl} & (4 \mathrm{a}) \\ 0.91700000000000 & 0.08300000000000 & \mathrm{Cl} & (4 \mathrm{a}) \\ 0.08300000000000 & -0.41700000000000 & \mathrm{Cl} & (4 \mathrm{a}) \\ 0.06400000000000 & 0.06400000000000 & \mathrm{Na} & (4 \mathrm{a}) \\ -0.06400000000000 & 0.56400000000000 & \mathrm{Na} & (4 \mathrm{a}) \\ 0.56400000000000 & 0.43600000000000 & \mathrm{Na} & (4 \mathrm{a}) \\ 0.43600000000000 & -0.06400000000000 & \mathrm{Na} & (4 \mathrm{a}) \\ 0.59200000000000 & 0.50000000000000 & \mathrm{O} & (12 \mathrm{~b}) \\ -0.59200000000000 & 1.00000000000000 & \mathrm{O} & (12 \mathrm{~b}) \\ 1.09200000000000 & 0.00000000000000 & \mathrm{O} & (12 \mathrm{~b}) \\ -0.09200000000000 & -0.50000000000000 & \mathrm{O} & (12 \mathrm{~b}) \\ 0.30300000000000 & 0.59200000000000 & \mathrm{O} & (12 \mathrm{~b}) \\ 0.19700000000000 & -0.59200000000000 & \mathrm{O} & (12 \mathrm{~b}) \\ -0.30300000000000 & 1.09200000000000 & \mathrm{O} & (12 \mathrm{~b}) \\ 0.80300000000000 & -0.09200000000000 & \mathrm{O} & (12 \mathrm{~b}) \\ 0.50000000000000 & 0.30300000000000 & \mathrm{O} & (12 \mathrm{~b}) \\ 1.00000000000000 & 0.19700000000000 & \mathrm{O} & (12 \mathrm{~b}) \\ 0.00000000000000 & -0.30300000000000 & \mathrm{O} & (12 \mathrm{~b}) \\ -0.50000000000000 & 0.80300000000000 & \mathrm{O} & (12 \mathrm{~b})\end{array}$

$\mathrm{Mg}_{2} \mathrm{Zn}_{11}$ : A2B11_cP39_200_f_aghij - CIF

\section{\# CIF file}

data_findsym-output

audit_creation method FINDSYM

_chemical_name_mineral 'Mg2Zn11'

chemical formula sum 'Mg2 $\mathrm{Zn} 11$,

loop

publ_author_name

S. Samson'

journal_name_full_name

Acta Chemica Scandinavica _journal_volume 3

journal_year 1949

_journal_page_first 835

_journal_page_last 843

_publ_Section_title

Die Kristallstruktur von $\operatorname{Mg} \$_{-}\{2\} \$ Z Z n \$_{-}\{11\} \$$ Isomorphie zwischen $\operatorname{Mg} \$_{-}\{2\}$ $\hookrightarrow \$ \mathrm{Zn} \$_{-}\{11\} \$$ und $\mathrm{Mg} \$_{-}\{2\} \$ \mathrm{Cu} \$ \$_{-}\{6\} \$ \mathrm{Al}_{-}\{5\} \$$

\# Found in Pearson's Crystal Data - Crystal Structure Database for $\hookrightarrow$ Inorganic Compounds, 2013

aflow_title 'Mg\$_\{2\}\$Zn\$_\{11\}\$ Structure

aflow_proto 'A2B11_cP39_200_f_aghij

aflow params , a, $x_{-}\{2\}, x_{-}\{3\}, x_{-}\{4\}, x_{-}\{5\}, y_{-}\{6\}, z_{-}\{6\}$,

aflow params values $8.5520223662,0.18,0.34,0.265,0.278,0.157,0.257$

aflow_Strukturbericht 'None'

aflow_Pearson 'cP39'

cell_length_a $\quad 8.5520223662$

-cell_length_b 8.5520223662

-cell_length_c 8.5520223662

cell_angle_alpha 90.0000000000

cell_angle_beta 90.0000000000

_cell_angle_gamma 90.0000000000

_symmetry_space_group_name_H-M "P $2 / \mathrm{m} \mathrm{-3"}$

_symmetry_Int_Tables_number 200

loop

space_group_symop_id

space_group_symop_operation_xyz

$1 \mathrm{x}, \mathrm{y}, \mathrm{z}$

$2 \mathrm{x},-\mathrm{y},-\mathrm{z}$

$3-\mathrm{x}, \mathrm{y},-\mathrm{z}$

$4-x,-y, z$

$5 \mathrm{y}, \mathrm{z}, \mathrm{x}$

$6 \mathrm{y},-\mathrm{z},-\mathrm{x}$

$7-\mathrm{y}, \mathrm{z},-\mathrm{x}$

$8-\mathrm{y},-\mathrm{z}, \mathrm{x}$

$9 \mathrm{z}, \mathrm{x}, \mathrm{y}$

$10 \mathrm{z},-\mathrm{x},-\mathrm{y}$

$11-\mathrm{z}, \mathrm{x},-\mathrm{y}$
$12-\mathrm{z},-\mathrm{x}, \mathrm{y}$

$12-z,-x, y$
$13-x,-y,-z$

$14-\mathrm{x}, \mathrm{y}, \mathrm{z}$

$15 \mathrm{x},-\mathrm{y}, \mathrm{z}$

$16 \mathrm{x}, \mathrm{y},-\mathrm{z}$

$17-y,-z,-x$

$18-\mathrm{y}, \mathrm{z}, \mathrm{x}$

$19 \mathrm{y},-\mathrm{z}, \mathrm{x}$

$20 \mathrm{y}, \mathrm{z},-\mathrm{x}$

$21-\mathrm{z},-\mathrm{x},-\mathrm{y}$

$22-z, x, y$

$23 \mathrm{z},-\mathrm{x}, \mathrm{y}$

$24 \mathrm{z}, \mathrm{x},-\mathrm{y}$

loop

atom_site_label

atom_site_type_symbol

_atom_site_symmetry_multiplicity

atom_site_Wyckoff_label

atom_site_fract_x

atom_site_fract_y

atom_site_fract_z

atom site occupancy

$\begin{array}{llllllll}\mathrm{Zn} 1 \mathrm{Zn} & 1 & 0 & 0 & 00000 & 0.00000 & 0.00000 & 1.00000\end{array}$

$\begin{array}{lllllll}\mathrm{Mg} 1 \mathrm{Mg} & 6 & \mathrm{f} & 0.18000 & 0.00000 & 0.50000 & 1.00000\end{array}$

$\begin{array}{llllllll}\mathrm{Zn} 2 & \mathrm{Zn} & 6 & \mathrm{~g} & 0.34000 & 0.50000 & 0.00000 & 1.00000\end{array}$

$\begin{array}{llllllll}\mathrm{Zn} 3 & \mathrm{Zn} & 6 & \mathrm{~h} & 0.26500 & 0.50000 & 0.50000 & 1.00000\end{array}$

$\begin{array}{lllllllll}\mathrm{Zn} 4 & \mathrm{Zn} & 8 & \mathrm{i} & 0.27800 & 0.27800 & 0.27800 & 1.00000\end{array}$

$\begin{array}{llllllll}\mathrm{Zn} 5 & \mathrm{Zn} & 12 & \mathrm{j} & 0.00000 & 0.15700 & 0.25700 & 1.00000\end{array}$

$\mathrm{Mg}_{2} \mathrm{Zn}_{11}$ : A2B11_cP39_200_f_aghij - POSCAR

A2B11_cP39_200_f_aghij \& a , x2 , x3 , x4, x5 , y6, z6 --params $=8.5520223662,0.18$ $\hookrightarrow 0.34,0.265,0.278,0.157,0.257 \& \mathrm{Pm}-3 \mathrm{~T}_{-}\{\mathrm{h}\}^{\wedge}\{1\} \# 200(\mathrm{afghij}) \&$ $\hookrightarrow$ cP39 \& None \& Mg2Zn11 \& \& S. Samson, Acta Chem. Scand. 3, $\hookrightarrow 835-843(1949)$

1.00000000000000

$\begin{array}{lll}8.55202236620000 & 0.00000000000000 & 0.00000000000000\end{array}$ $\begin{array}{lll}0.00000000000000 & 8.55202236620000 & 0.00000000000000\end{array}$ $\begin{array}{llll}0.00000000000000 & 0.00000000000000 & 8.55202236620000\end{array}$

$$
\begin{array}{rr}
\mathrm{Mg} & \mathrm{Zn} \\
6 & 33
\end{array}
$$

Direct

0.18000000000000 $-0.18000000000000$ 0.50000000000000 0.50000000000000 0.00000000000000 0.00000000000000 0.00000000000000

0.34000000000000

$-0.34000000000000$

0.00000000000000

0.00000000000000

0.50000000000000

0.50000000000000

0.26500000000000

$-0.26500000000000$

0.50000000000000

0.50000000000000

0.50000000000000

0.00000000000000 0.00000000000000 0.18000000000000 0.18000000000000 0.50000000000000 0.50000000000000 0.00000000000000 0.50000000000000 0.50000000000000 0.34000000000000 $-0.34000000000000$ 0.00000000000000 0.00000000000000 0.50000000000000 0.50000000000000 0.26500000000000 $-0.26500000000000$ 0.50000000000000

0.50000000000000 0.50000000000000 0.00000000000000 0.00000000000000 0.18000000000000 $-0.18000000000000$ 0.00000000000000 0.00000000000000 .00000000 0.50000000000 0.50000000000000 0.50000000000000 $-0.34000000000000$ 0.50000000000000 0.50000000000000 0.50000000000000 0.5000000000000 0.26500000000000
$\mathrm{Mg}$ (6f)

$\mathrm{Mg}$ (6f)

$\mathrm{Mg} \quad$ (6f) 


$\begin{array}{rrrrr}0.50000000000000 & 0.50000000000000 & -0.26500000000000 & \mathrm{Zn} & (6 \mathrm{~h}) \\ 0.27800000000000 & 0.27800000000000 & 0.27800000000000 & \mathrm{Zn} & (8 \mathrm{i}) \\ -0.27800000000000 & -0.27800000000000 & 0.27800000000000 & \mathrm{Zn} & (8 \mathrm{i}) \\ -0.27800000000000 & 0.27800000000000 & -0.27800000000000 & \mathrm{Zn} & (8 \mathrm{i}) \\ 0.27800000000000 & -0.27800000000000 & -0.27800000000000 & \mathrm{Zn} & (8 \mathrm{i}) \\ -0.27800000000000 & -0.27800000000000 & -0.27800000000000 & \mathrm{Zn} & (8 \mathrm{i}) \\ 0.27800000000000 & 0.27800000000000 & -0.27800000000000 & \mathrm{Zn} & (8 \mathrm{i}) \\ 0.27800000000000 & -0.27800000000000 & 0.27800000000000 & \mathrm{Zn} & (8 \mathrm{i}) \\ -0.27800000000000 & 0.27800000000000 & 0.27800000000000 & \mathrm{Zn} & (8 \mathrm{i}) \\ 0.00000000000000 & 0.15700000000000 & 0.25700000000000 & \mathrm{Zn} & (12 \mathrm{j}) \\ 0.00000000000000 & -0.15700000000000 & 0.25700000000000 & \mathrm{Zn} & (12 \mathrm{j}) \\ 0.00000000000000 & 0.15700000000000 & -0.25700000000000 & \mathrm{Zn} & (12 \mathrm{j}) \\ 0.00000000000000 & -0.15700000000000 & -0.25700000000000 & \mathrm{Zn} & (12 \mathrm{j}) \\ 0.25700000000000 & 0.00000000000000 & 0.15700000000000 & \mathrm{Zn} & (12 \mathrm{j}) \\ 0.25700000000000 & 0.00000000000000 & -0.15700000000000 & \mathrm{Zn} & (12 \mathrm{j}) \\ -0.25700000000000 & 0.00000000000000 & 0.15700000000000 & \mathrm{Zn} & (12 \mathrm{j}) \\ -0.25700000000000 & 0.00000000000000 & -0.15700000000000 & \mathrm{Zn} & (12 \mathrm{j}) \\ 0.15700000000000 & 0.25700000000000 & 0.00000000000000 & \mathrm{Zn} & (12 \mathrm{j}) \\ -0.15700000000000 & 0.25700000000000 & 0.00000000000000 & \mathrm{Zn} & (12 \mathrm{j}) \\ 0.15700000000000 & -0.25700000000000 & 0.00000000000000 & \mathrm{Zn} & (12 \mathrm{j}) \\ -0.15700000000000 & -0.25700000000000 & 0.00000000000000 & \mathrm{Zn} & (12 \mathrm{j})\end{array}$

$\mathrm{KSbO}_{3}$ (High-temperature): AB3C_cP60_201_ce_fh_g - CIF

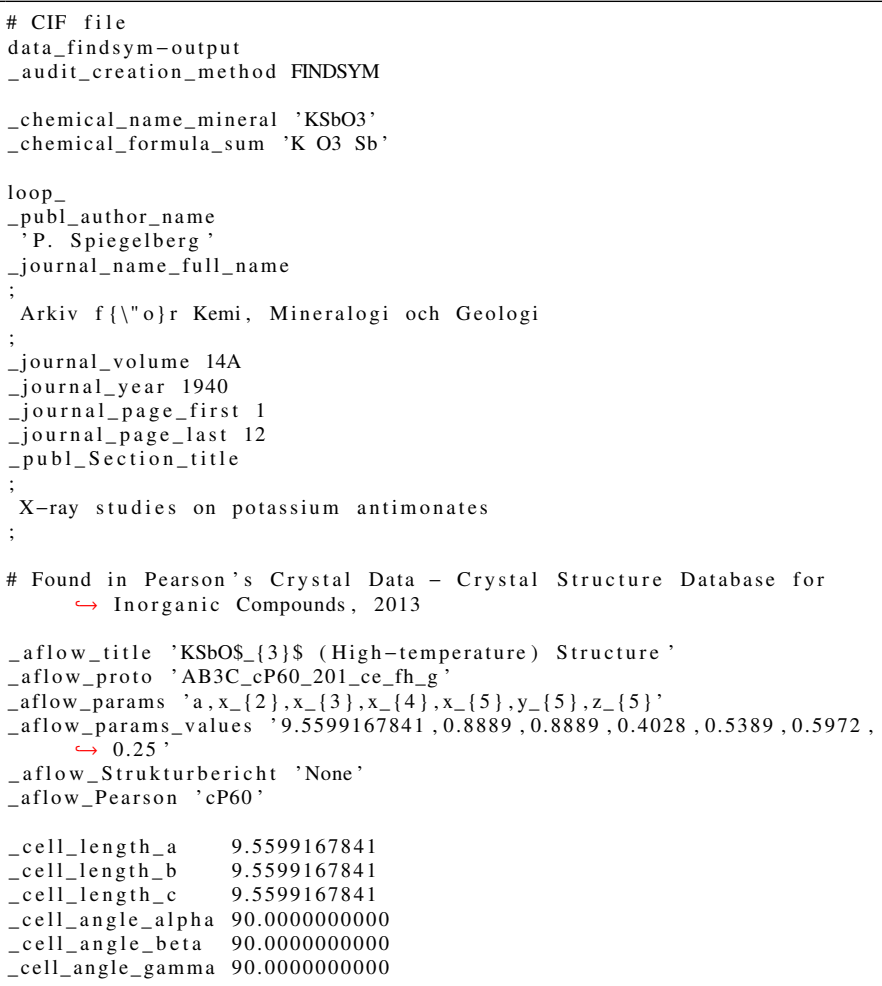

$\begin{array}{lllllllll}\mathrm{K} 1 & \mathrm{~K} & 4 & \mathrm{c} & 0.50000 & 0.50000 & 0.50000 & 1.00000 \\ \mathrm{~K} 2 & \mathrm{~K} & 8 & \mathrm{e} & 0.888800 & 0.88890 & 0.88890 & 1.00000\end{array}$

$\begin{array}{llrllllll}\mathrm{K} 2 & \mathrm{~K} & 8 & \mathrm{e} & 0.88890 & 0.88890 & 0.88890 & 1.00000 \\ \mathrm{O} 1 & \mathrm{O} & 12 & \mathrm{f} & 0.88880 & 0.25000 & 0.25000 & 1.00000\end{array}$

$\begin{array}{lllllllll}\mathrm{O} 1 & \mathrm{O} & 12 & \mathrm{f} & 0.88890 & 0.25000 & 0.25000 & 1.00000\end{array}$

$\begin{array}{llllllll}\mathrm{Sb} 1 & \mathrm{Sb} & 12 & \mathrm{~g} & 0.40280 & 0.75000 & 0.25000 & 1.00000 \\ \mathrm{O} 2 & \mathrm{O} & 24 & \mathrm{~h} & 0.53890 & 0.59720 & 0.25000 & 1.00000\end{array}$

$\mathrm{KSbO}_{3}$ (High-temperature): AB3C_cP60_201_ce_fh_g - POSCAR $\leftrightarrow, 0.8889,0.4028,0.5389,0.5972,0.25 \& \mathrm{Pn}-3 \mathrm{~T}_{-}\{\mathrm{h}\} \wedge\{2\} \# 201$ (cefgh) $\hookrightarrow$ \& cP60 \& None \& KSbO3 \& \& P. Spiegelberg, \{Ark. Kem. Mineral. Geol. 14A, 1-12 (1940)

.00000000000000

$\begin{array}{lll}9.55991678410000 & 0.00000000000000 & 0.00000000000000\end{array}$

$\begin{array}{lll}0.00000000000000 & 9.55991678410000 & 0.00000000000000\end{array}$

$\begin{array}{lll}0.00000000000000 & 0.00000000000000 & 9.55991678410000\end{array}$

$\begin{array}{rrr}\mathrm{K} & \mathrm{O} & \mathrm{Sb} \\ 12 & 36 & 12\end{array}$

Direct

0.50000000000000

0.00000000000000

0.00000000000000

0.00000000000000

0.88890000000000

$-0.38890000000000$

$-0.38890000000000$

0.88890000000000

-0.88890000000000
1.38890000000000

1.38890000000000

1.38890000000000
-0.88890000000000

0.88890000000000

$-0.38890000000000$

0.25000000000000

0.25000000000000

0.25000000000000

0.25000000000000

$-0.88890000000000$

1.38890000000000

0.75000000000000
0.75000000000000

0.75000000000000

0.75000000000000

0.53890000000000

$-0.03890000000000$

$-0.03890000000000$

0.5389000000000

0.25000000000000

0.25000000000000

0.25000000000000

0.5500000000000

$-0.09720000000000$

0.59720000000000

$-0.09720000000000$

$-0.5389000000000$

1.03890000000000

1.0389000000000

1.03890000000000

$-0.53890000000000$

$-0.2500000000000$

$-0.25000000000000$

0.75000000000000

0.75000000000000

$-0.59720000000000$

1.09720000000000

$-0.59720000000000$

1.09720000000000

0.40280000000000

0.09720000000000

0.25000000000000

0.25000000000000

0.75000000000000

0.7500000000000

0.7500000000000

$-0.4028000000000$

0.7500000000000

0.7500000000000

0.75000000000000

0.25000000000000

0.50000000000000 .00000000000000 0.50000000000000 0.00000000000000 0.8889000000000 . . .3889000000000 . . .88890000000000 1.38890000000000 $-0.88890000000000$ 1.38890000000000 0.25000000000000 0.25000000000000 0.88890000000000 $-0.38890000000000$ 0.25000000000000 0.25000000000000 0.75000000000000 0.75000000000000 0.8889000000000 1.3889000000000 0.75000000000000 0.750000000000 0.7500000000000 0.59720000000000 0.09720000000000 0.59720000000000 $-0.09720000000000$ 0.53890000000000 $-0.03890000000000$ $-0.03890000000000$ 0.53890000000000 0.25000000000000 0.25000000000000 0.25000000000000 0.25000000000000 $-0.59720000000000$ 1.0972000000000 1.59720000000000 $-0.59720000000000$ 1.0972000000000 0.53890000000000 1.03890000000000 1.03890000000000 $-0.53890000000000$ 0.25000000000000 0.25000000000000 0.75000000000000 0.75000000000000 0.75000000000000 0.75000000000000 0.40280000000000 0.09720000000000 0.25000000000000 0.25000000000000 0.25000000000000 0.250000000000 0.40280000000000 . .00280000000000 0.90280000000000 0.7500000000000

0.50000000000000 0.50000000000000 0.00000000000000 0.00000000000000 0.88890000000000 0.88890000000000 $-0.38890000000000$ $-0.38890000000000$ $-0.88890000000000$ 0.88890000000000 1.38890000000000 1.38890000000000 0.25000000000000 0.25000000000000 0.25000000000000 0.25000000000000 0.88890000000000 $-0.38890000000000$ 0.75000000000000 0.75000000000000 0.75000000000000 0.75000000000 0.75000000000 1.38890000000000 0.25000000000000 0.25000000000000 0.25000000000000 0.25000000000000 0.59720000000000 0.09720000000000 0.59720000000000 0.09720000000000 0.53890000000000 $-0.03890000000000$ 0.53890000000000 $-0.25000000000000$ $-0.25000000000000$ 0.75000000000000 0.75000000000000 0.750000000000 $-0.59720000000000$ 1.09720000000000 $-0.59720000000000$ 1.09720000000000 $-0.53890000000000$ 1.03890000000000 1.03890000000000 $-0.53890000000000$ 0.25000000000000 0.25000000000000 0.75000000000000 0.75000000000000 0.40280000000000 0.09720000000000 0.75000000000000 0.7500000000000 0.7500000000 0.2500000000 0.25000000000000 -0.40280000000000
0.90280000000000

K (4c)

$\mathrm{K} \quad(4 \mathrm{c})$

$\mathrm{K} \quad(4 \mathrm{c})$

$\mathrm{K} \quad(4 \mathrm{c})$

$\mathrm{K} \quad(8 \mathrm{e})$

$\mathrm{K} \quad(8 \mathrm{e})$

$\mathrm{K} \quad(8 \mathrm{e})$

$\mathrm{K} \quad(8 \mathrm{e})$

$\mathrm{K} \quad(8 \mathrm{e}$

$\mathrm{K} \quad(8 \mathrm{e})$

$\mathrm{K} \quad(8 \mathrm{e})$

O (12f)

$\mathrm{O} \quad(12 \mathrm{f})$

O (12f)

O (12f)

$\mathrm{O} \quad(12 \mathrm{f})$

$\mathrm{O} \quad(12 \mathrm{f})$

$\mathrm{O} \quad(12 \mathrm{f})$

$\mathrm{O} \quad(12 \mathrm{f})$

$\begin{array}{ll}\mathrm{O} & (12 \mathrm{f}) \\ \mathrm{O} & (12 \mathrm{f})\end{array}$

0 (12f)

$\mathrm{O} \quad(12 \mathrm{f})$

$\mathrm{O} \quad(12 \mathrm{f})$

O (12f)

$\mathrm{O} \quad(24 \mathrm{~h})$

$\mathrm{O} \quad(24 \mathrm{~h})$

$\mathrm{O} \quad(24 \mathrm{~h})$

O (24h) 
Found in Pearson's Crystal Data - Crystal Structure Database for $\hookrightarrow$ Inorganic Compounds, 2013

aflow_title ${ }^{\prime} \mathrm{KB} \$ \$_{-}\{6\} \$ \mathrm{H}_{-}\{6\} \$$ Structure

aflow_proto 'A6B6C_cF104_202_h_h_c

aflow_params , a, $y_{-}\{2\}, z_{-}\{2\}, y_{-}\{3\}, z_{-}\{3\}$,

aflow_params_values $, 10.6100296668,0.5827,0.6359,0.638,0.72$,

aflow_Strukturbericht 'None

aflow_Pearson 'cF104'

_cell_length_a $\quad 10.6100296668$

cell_length_b $\quad 10.6100296668$

cell_length_c $\quad 10.6100296668$

cell_angle_alpha 90.0000000000

cell angle beta 90.0000000000

cell_angle gamma 90.0000000000

_symmetry_space_group_name_H-M "F $2 / \mathrm{m} \mathrm{-3"}$

_symmetry_Int_Tables_number 202

loop_

space_group_symop_id

_space_group_symop_operation_xyz

$1 \mathrm{x}, \mathrm{y}, \mathrm{z}$

$2 \mathrm{x},-\mathrm{y},-\mathrm{z}$

$3-\mathrm{x}, \mathrm{y},-\mathrm{z}$

$3-\mathrm{x},-\mathrm{y}, \mathrm{z}$
$5 \mathrm{y}, \mathrm{z}, \mathrm{x}$

6 y, $-\mathrm{z},-\mathrm{x}$

$6 \mathrm{y},-\mathrm{z},-\mathrm{x}$
$7-\mathrm{y}, \mathrm{z},-\mathrm{x}$

$7-\mathrm{y}, \mathrm{z},-\mathrm{x}$
$8-\mathrm{y},-\mathrm{z}, \mathrm{x}$

$8-y,-z, x$
$9 z, x, y$

$10 \mathrm{z},-\mathrm{x},-\mathrm{y}$

$11-\mathrm{z}, \mathrm{x},-\mathrm{y}$

$12-z,-x, y$
$3-x,-y,-z$

$14-\mathrm{x}, \mathrm{y}, \mathrm{z}$

$15 \mathrm{x},-\mathrm{y}, \mathrm{z}$

$16 \mathrm{x}, \mathrm{y},-\mathrm{z}$

$17-y,-z,-x$

$18-\mathrm{y}, \mathrm{z}, \mathrm{x}$

$19 \mathrm{y},-\mathrm{z}, \mathrm{x}$

$21-z,-x,-y$

$22-\mathrm{z}, \mathrm{x}, \mathrm{y}$

$23-\mathrm{z}, \mathrm{x}, \mathrm{y}$

$24 \mathrm{z}, \mathrm{x},-\mathrm{y}$

$25 \mathrm{x}, \mathrm{y}+1 / 2, \mathrm{z}+1 / 2$

$26 \mathrm{x},-\mathrm{y}+1 / 2,-\mathrm{z}+1 / 2$

$27-\mathrm{x}, \mathrm{y}+1 / 2,-\mathrm{z}+1 / 2$

$28-x,-y+1 / 2, z+1 / 2$

$29 \mathrm{y}, \mathrm{z}+1 / 2, \mathrm{x}+1 / 2$

$30 \mathrm{y},-\mathrm{z}+1 / 2,-\mathrm{x}+1 / 2$

$31-y, z+1 / 2,-x+1 / 2$

$32-\mathrm{y},-\mathrm{z}+1 / 2, \mathrm{x}+1 / 2$

$33 \mathrm{z}, \mathrm{x}+1 / 2, \mathrm{y}+1 / 2$

$34 \mathrm{z},-\mathrm{x}+1 / 2,-\mathrm{y}+1 / 2$

$35-\mathrm{z}, \mathrm{x}+1 / 2,-\mathrm{y}+1 / 2$

$36-\mathrm{z},-\mathrm{x}+1 / 2, \mathrm{y}+1 / 2$

$37-x,-y+1 / 2,-z+1 / 2$

$38-\mathrm{x}, \mathrm{y}+1 / 2, \mathrm{z}+1 / 2$

$39 \mathrm{x},-\mathrm{y}+1 / 2, \mathrm{z}+1 / 2$

$40 x, y+1 / 2,-z+1 / 2$

$41-\mathrm{y},-\mathrm{z}+1 / 2,-\mathrm{x}+1 / 2$

$42-y, z+1 / 2, x+1 / 2$

$44 \mathrm{y}, \mathrm{z}+1 / 2, \mathrm{x}+1 / 2$

$44 \mathrm{y}, \mathrm{z}+1 / 2,-\mathrm{x}+1 / 2$
$45-\mathrm{z},-\mathrm{x}+1 / 2,-\mathrm{y}+1 / 2$

$46-\mathrm{z}, \mathrm{x}+1 / 2, \mathrm{y}+1 / 2$

$47 \mathrm{z},-\mathrm{x}+1 / 2, \mathrm{y}+1 / 2$

$48 \mathrm{z}, \mathrm{x}+1 / 2,-\mathrm{y}+1 / 2$

$49 \mathrm{x}+1 / 2, \mathrm{y}, \mathrm{z}+1 / 2$

$50 \mathrm{x}+1 / 2,-\mathrm{y},-\mathrm{z}+1 / 2$

$51-\mathrm{x}+1 / 2, \mathrm{y},-\mathrm{z}+1 / 2$

$52-x+1 / 2,-y, z+1 / 2$

$53 \mathrm{y}+1 / 2, \mathrm{z}, \mathrm{x}+1 / 2$

$55-y+1 / 2, z,-x+1 / 2$

$56-y+1 / 2,-x+1 / 2$

$56-y+1 / 2,-z, x+1 / 2$

$58 \mathrm{z}+1 / 2,-\mathrm{x},-\mathrm{y}+1 / 2$

$5 \mathrm{z}+1 / 2,-\mathrm{x},-\mathrm{y}+1 / 2$
$59-\mathrm{z}+1 / 2, \mathrm{x},-\mathrm{y}+1 / 2$

$60-z+1 / 2,-x, y+1 / 2$

$61-\mathrm{x}+1 / 2,-\mathrm{y},-\mathrm{z}+1 / 2$

$62-x+1 / 2, y, z+1 / 2$

$63 \mathrm{x}+1 / 2,-\mathrm{y}, \mathrm{z}+1 / 2$

$64 \mathrm{x}+1 / 2, \mathrm{y},-\mathrm{z}+1 / 2$
$65-\mathrm{y}+1 / 2,-\mathrm{z},-\mathrm{x}+1 / 2$

$65-\mathrm{y}+1 / 2,-\mathrm{z},-\mathrm{x}+1 / 2$
$66-\mathrm{y}+1 / 2, \mathrm{z}, \mathrm{x}+1 / 2$

$67 \mathrm{y}+1 / 2,-\mathrm{z}, \mathrm{x}+1 / 2$

$68 \mathrm{y}+1 / 2, \mathrm{z},-\mathrm{x}+1 / 2$

$69-\mathrm{z}+1 / 2,-\mathrm{x},-\mathrm{y}+1 / 2$

$70-\mathrm{z}+1 / 2, \mathrm{x}, \mathrm{y}+1 / 2$

$71 \mathrm{z}+1 / 2,-\mathrm{x}, \mathrm{y}+1 / 2$

$72 \mathrm{z}+1 / 2, \mathrm{x},-\mathrm{y}+1 / 2$

$73 \mathrm{x}+1 / 2, \mathrm{y}+1 / 2, \mathrm{z}$

$74 x+1 / 2,-y+1 / 2,-$

$75-x+1 / 2, y+1 / 2,-z$

$77-x+1 / 2,-y+1 / 2$,

$77 \mathrm{y}+1 / 2, \mathrm{z}+1 / 2, \mathrm{x}$

$78 \mathrm{y}+1 / 2,-\mathrm{z}+1 / 2,-\mathrm{x}$

$79-y+1 / 2, z+1 / 2,-x$

$80-y+1 / 2,-z+1 / 2$,
$81-z+1 / 2, x+1 / 2, y$

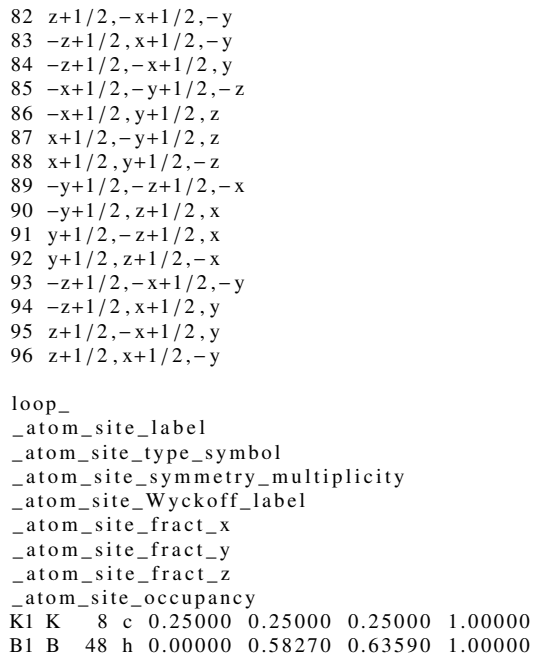

$\mathrm{KB}_{6} \mathrm{H}_{6}$ : A6B6C_cF104_202_h_h_c - POSCAR

A6B6C_cF104_202_h_h_c \& a ,y2, z2,y3, z3 --params $=10.6100296668,0.5827$, $\rightarrow 0.6359,0.638,0.72 \& \mathrm{Fm}-3 \mathrm{~T}_{-}\{\mathrm{h}\}^{\wedge}\{3\} \# 202\left(\mathrm{ch}^{\wedge} 2\right) \& \mathrm{cF} 104 \&$ None \& $\hookrightarrow$ KB6H6 \& \& J. A. Wunderlich and W. N. Lipscomb, J. Am. Ceram. $\hookrightarrow$ Soc. 82, 4427-4428 (1960)

1.00000000000000

$\begin{array}{lll}0.00000000000000 & 5.30501483340000 & 5.30501483340000\end{array}$

$5.30501483340000 \quad 0.00000000000000 \quad 5.30501483340000$

$30501483340000 \quad 5.30501483340000 \quad 0.00000000000000$

B $\quad \mathrm{H} \quad \mathrm{K}$

Direc

1.21860000000000 0.05320000 $-0.05320000000000$ $-1.21860000000000$ $-0.0532000000000$

$-1.21860000000000$

1.21860000000000

0.05320000000000

0.05320000000000

1.21860000000000

$-1.21860000000000$

$-0.05320000000000$

1.35800000000000

1.358000000000

$-0.08200000000000$

$-1.35800000000000$

$-1.358000000000$

$-0.08200000000000$

$-1.35800000000000$

1.35800000000000
0.08200000000000

0.08200000000000
0.08200000000000

0.08200000000000

1.35800000000000
-1.35800000000000

$-1.35800000000000$

$-0.08200000000000$

0.25000000000000

0.05320000000000

1.21860000000000

$-1.21860000000000$

$-0.05320000000000$

1.21860000000000

0.05320000000000

$-0.05320000000000$

$-1.21860000000000$

$-0.05320000000000$

1.21860000000000

1.21860000000000

0.05320000000000

. .08200000000000
-1.35800000000000

$-1.3580000000000$

$-1.35800000000000$

1.35800000000000

1.3580000000

$-0.08200000000000$

$-1.35800000000000$

$-0.08200000000000$

$-1.35800000000000$

1.35800000000000

0.08200000000000

0.25000000000000

0.75000000000000

0.05320000000000

1.21860000000000

1.21860000000000

0.05320000000000

0.05320000000000

1.21860000000000 $-1.21860000000000$ .21860000000000 0.05320000000000 $-0.05320000000000$ $-1.21860000000000$ $-0.08200000000000$ $-1.35800000000000$ 1.35800000000000 0.0820000 0.08200000 1.35800000000000 1.35800000 $-1.35800000000000$ .0820000000000 1.35800000000000 $-0.08200000000000$ $-1.35800000000000$ 0.25000000000000

B $(48 \mathrm{~h})$

B $(48 \mathrm{~h})$

B $(48 \mathrm{~h})$

B $(48 \mathrm{~h})$

B $(48 \mathrm{~h})$

B $(48 \mathrm{~h})$

B (48h)

B (48h)

B $(48 \mathrm{~h})$

B $(48 \mathrm{~h})$

B $(48 \mathrm{~h})$

B $(48 \mathrm{~h})$

$\mathrm{H} \quad(48 \mathrm{~h})$

$\mathrm{H} \quad(48 \mathrm{~h})$

$\begin{array}{ll}\mathrm{H} & (48 \mathrm{~h}) \\ \mathrm{H} & (48 \mathrm{~h})\end{array}$

$\mathrm{H} \quad(48 \mathrm{~h})$

$\mathrm{H} \quad(48 \mathrm{~h})$

$\mathrm{H} \quad(48 \mathrm{~h})$

$\mathrm{H} \quad(48 \mathrm{~h})$

$\mathrm{H} \quad(48 \mathrm{~h})$

$\mathrm{H}$ (48h)

$\mathrm{H} \quad(48 \mathrm{~h})$

$\mathrm{H}$ (48h)

$\mathrm{H} \quad(48 \mathrm{~h})$

$\mathrm{H} \quad(48 \mathrm{~h})$

$\mathrm{K} \quad(8 \mathrm{c}$

$\mathrm{K} \quad(8 \mathrm{c})$

FCC C 60 Buckminsterfullerine: A_cF240 202 h2i - CIF

\# CIF file

data_findsym-output

audit_creation_method FINDSYM

chemical_name mineral 'Buckminsterfullerene

chemical_formula_sum 'C'

loop

publ_author_nam

D. L. Dorset'

M. P. Mecourto

Acta Crystallographica Section A: Foundations and Advances

journal_volume 50

journal_year 1994

journal_page_first 344

journal_page_last 351

publ_Section_title

Disorder and the molecular packing of $\mathrm{C}_{-}\{60\} \$$

buckminsterfullerene: a direct

electron-crystallographic analysis

aflow title 'FCC C\$ $\{60\} \$$ Buckminsterfullerine Structure

aflow_proto, A_cF240_202_h2i

aflow_params a, a $\{1\}, z_{-}\{1\}, x_{-}\{2\}, y_{-}\{2\}, z_{-}\{2\}, x_{-}\{3\}, y_{-}\{3\}, z_{-}\{3\}$,

aflow params values $14.26,0.249,0.052,0.105,0.085,0.22,0.185,0.052$, $\stackrel{-}{\hookrightarrow} 0.165$

aflow_Strukturbericht 'None'

-aflow_Pearson 'cF240, 
_symmetry_space_group_name_H-M "F 2/m -3" symmetry_Int_Tables_number 202

_cell_length_a $\quad 14.26000$

_cell_length_b $\quad 14.26000$

_cell_length_c $\quad 14.26000$

_cell_angle_alpha 90.00000

_cell_angle_beta 90.00000

loop

space_group_symop_id

_space_group_symop_operation_xyz

$1 \mathrm{x}, \mathrm{y}, \mathrm{z}$

$2 \mathrm{x},-\mathrm{y},-\mathrm{z}$

$3-\mathrm{x}, \mathrm{y},-\mathrm{z}$

$4-x,-y, z$

$5 \mathrm{y}, \mathrm{z}, \mathrm{x}$

$6 \mathrm{y},-\mathrm{z},-\mathrm{x}$

$-y, z,-x$
$-y,-z, x$

$\mathrm{z}, \mathrm{x}, \mathrm{x}$

$0 \mathrm{z},-\mathrm{x},-\mathrm{y}$

$11-\mathrm{z}, \mathrm{x},-\mathrm{y}$

$2-\mathrm{z},-\mathrm{x}, \mathrm{y}$

$14-\mathrm{x}, \mathrm{y}, \mathrm{z}$

$15 x,-y, z$

$16 \mathrm{x}, \mathrm{y},-\mathrm{z}$

$17-y,-z,-x$

$18-\mathrm{y}, \mathrm{z}, \mathrm{x}$

19 y, $\mathrm{z}, \mathrm{x}$

$21-\mathrm{z},-\mathrm{x},-\mathrm{y}$
$22-\mathrm{z}, \mathrm{x}, \mathrm{y}$

$22-\mathrm{z}, \mathrm{x}, \mathrm{y}$

$23 \mathrm{z},-\mathrm{x}$,

$24 \mathrm{z}, \mathrm{x},-\mathrm{y}$

$25 \mathrm{x}, \mathrm{y}+1 / 2, \mathrm{z}+1 / 2$

$26 \mathrm{x},-\mathrm{y}+1 / 2,-\mathrm{z}+1 / 2$

$27-\mathrm{x}, \mathrm{y}+1 / 2,-\mathrm{z}+1 / 2$

$28-\mathrm{x},-\mathrm{y}+1 / 2, \mathrm{z}+1 / 2$

$29 \mathrm{y}, \mathrm{z}+1 / 2, \mathrm{x}+1 / 2$

$30 \mathrm{y},-\mathrm{z}+1 / 2,-\mathrm{x}+1 / 2$

$31-y, z+1 / 2,-x+1 / 2$

$33 z, x+1 / 2, y+1 / 2$

$34 z-x+1 / 2,-y+1 / 2$

$35-z, x+1 / 2,-y+1 / 2$

$-z, x+1 / 2,-y+1 / 2$

$37-\mathrm{x},-\mathrm{y}+1 / 2,-\mathrm{z}+1 / 2$

$-x, y+1 / 2, z+1 / 2$

$x,-y+1 / 2, z+1 / 2$

$\begin{array}{ll}40 & \mathrm{x}, \mathrm{y}+1 / 2,-\mathrm{z}+1 / 2 \\ 41 & -\mathrm{y},-\mathrm{z}+1 / 2,-\mathrm{x}+1 / 2\end{array}$

$42-\mathrm{y}, \mathrm{z}+1 / 2, \mathrm{x}+1 / 2$

$43 \mathrm{y},-\mathrm{z}+1 / 2, \mathrm{x}+1 / 2$

$44 \mathrm{y}, \mathrm{z}+1 / 2,-\mathrm{x}+1 / 2$
$45-\mathrm{z},-\mathrm{x}+1 / 2,-\mathrm{y}+1 / 2$

$46-\mathrm{z}, \mathrm{x}+1 / 2, \mathrm{y}+1 / 2$

$47 \mathrm{z},-\mathrm{x}+1 / 2, \mathrm{y}+1 / 2$

$48 \mathrm{z}, \mathrm{x}+1 / 2,-\mathrm{y}+1 / 2$

$49 \mathrm{x}+1 / 2, \mathrm{y}, \mathrm{z}+1 / 2$

$50 \mathrm{x}+1 / 2,-\mathrm{y},-\mathrm{z}+1 / 2$

$51-x+1 / 2, y,-z+1 / 2$

$52-x+1 / 2,-y, z+1 / 2$

$53 \mathrm{y}+1 / 2, \mathrm{z}, \mathrm{x}+1 / 2$

$54 \mathrm{y}+1 / 2,-z,-x+1 / 2$

$55-\mathrm{y}+1 / 2, \mathrm{z},-\mathrm{x}+1 / 2$

$56-y+1 / 2,-z, x+1 / 2$

$57 \mathrm{z}+1 / 2, \mathrm{x}, \mathrm{y}+1 / 2$

$58 \mathrm{z}+1 / 2,-\mathrm{x},-\mathrm{y}+1 / 2$

$59-\mathrm{z}+1 / 2, \mathrm{x},-\mathrm{y}+1 / 2$

$60-\mathrm{z}+1 / 2,-\mathrm{x}, \mathrm{y}+1 / 2$

$61-x+1 / 2,-y,-z+1 / 2$

$62-x+1 / 2, y, z+1 / 2$

$63 x+1 / 2,-y, z+1 / 2$

$64 \mathrm{x}+1 / 2, \mathrm{y},-\mathrm{z}+1 / 2$

$65-\mathrm{y}+1 / 2,-\mathrm{z},-\mathrm{x}+1 / 2$

$66-\mathrm{y}+1 / 2, \mathrm{z}, \mathrm{x}+1 / 2$

$67 \mathrm{y}+1 / 2,-\mathrm{z}, \mathrm{x}+1 / 2$

$68 \mathrm{y}+1 / 2, \mathrm{z},-\mathrm{x}+1 / 2$

$69-\mathrm{z}+1 / 2,-\mathrm{x},-\mathrm{y}+1 / 2$

$70-z+1 / 2, x, y+1 / 2$

$71 \quad z+1 / 2,-x, y+1 / 2$

$73 \mathrm{x}+1 / 2, \mathrm{x}+1 / 2, \mathrm{z}$

$73 \mathrm{x}+1 / 2, \mathrm{y}+1 / 2, \mathrm{z}$

$74 x+1 / 2,-y+1 / 2,-z$

$75-x+1 / 2, y+1 / 2,-$

$76-x+1 / 2,-y+1 / 2$,

$77 \mathrm{y}+1 / 2, \mathrm{z}+1 / 2, \mathrm{x}$

$78 \mathrm{y}+1 / 2,-\mathrm{z}+1 / 2,-\mathrm{x}$

$79-\mathrm{y}+1 / 2, \mathrm{z}+1 / 2,-\mathrm{x}$

$80-\mathrm{y}+1 / 2,-\mathrm{z}+1 / 2, \mathrm{x}$

$81 \mathrm{z}+1 / 2, \mathrm{x}+1 / 2, \mathrm{y}$

$82 \mathrm{z}+1 / 2,-\mathrm{x}+1 / 2,-\mathrm{y}$

$83-z+1 / 2, x+1 / 2,-y$

$84-\mathrm{z}+1 / 2,-\mathrm{x}+1 / 2, \mathrm{y}$

$85-x+1 / 2,-y+1 / 2,-2$

$86-x+1 / 2, y+1 / 2, z$

$88 \mathrm{x}+1 / 2,-\mathrm{y}+1 / 2, \mathrm{z}$

$88 x+1 / 2, y+1 / 2,-z$
$89-y+1 / 2,-z+1 / 2,-x$

$89-y+1 / 2,-z+1 / 2,-x$
$90-y+1 / 2, z+1 / 2, x$

$90-y+1 / 2, z+1 / 2, x$
$91 y+1 / 2,-z+1 / 2, x$

$92 \mathrm{y}+1 / 2, \mathrm{z}+1 / 2,-\mathrm{x}$

$93-z+1 / 2,-x+1 / 2,-y$

$94-z+1 / 2, x+1 / 2, y$

$95 \mathrm{z}+1 / 2,-\mathrm{x}+1 / 2$,

$\mathrm{z}+1 / 2, \mathrm{x}+1 / 2,-\mathrm{y}$

loop

atom_site_label

atom_site_type_symbol

atom_site_symmetry_multiplicity

atom_site_Wyckoff_label

atom_site_fract_x

atom_site_fract_y

- atom_site_fract_z

$\begin{array}{llllllll}\mathrm{C} 1 & \mathrm{C} & 48 & \mathrm{~h} & 0.00000 & 0.24900 & 0.05200 & 1.00000\end{array}$

$\begin{array}{lllllllll}\mathrm{C} 2 & \mathrm{C} & 96 & \mathrm{i} & 0.10500 & 0.08500 & 0.22000 & 1.00000\end{array}$

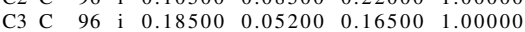

FCC C 60 Buckminsterfullerine: A_cF240_202_h2i - POSCAR

A_cF240_202_h2i \& a , y1, z1, x2, y2, z2, x3,y3, z3 --params $=14.26,0.249,0.052$ $\hookrightarrow 0.105,0.085,0.22,0.185,0.052,0.165 \&$ Fm-3 $T_{-}\{h\}^{\wedge}\{3\} \# 202\left(h i^{\wedge} 2\right)$ $\hookrightarrow$ \& cF240 \& None \& C \& Buckminsterfullerene \& D. L. Dorset and $\hookrightarrow$ M. P. McCourt, Acta Crystallogr. Sect. A 50,344-351 (1994)

1.00000000000000

0.00000000000000

7.13000000000000

7.13000000000000

7.13000000000000

0.00000000000000

7.13000000000000

7.13000000000000

0.00000000000000 c

Direct

0.19700000000000

$-0.30100000000000$

0.19700000000000

$-0.30100000000000$

0.30100000000000

$-0.19700000000000$

$-0.19700000000000$

0.30100000000000

$-0.30100000000000$

0.19700000000000

0.20000000000000

0.24000000000000

$-0.03000000000000$

$-0.41000000000000$

$-0.03000000000000$

$-0.41000000000000$

0.20000000000000

0.24000000000000

0.24000000000000

0.20000000000000

$-0.41000000000000$

$-0.03000000000000$

$-0.20000000000000$

$-0.24000000000000$

0.03000000000000

0.41000000000000

0.03000000000000

0.41000000000000

$-0.20000000000000$

$-0.24000000000000$

$-0.24000000000000$

$-0.2000000000000$

0.41000000000000

0.03000000000000

0.03200000000000

0.29800000000000

0.07200000000000

$-0.40200000000000$

0.07200000000000

$-0.40200000000000$

0.03200000000000

0.29800000000000

0.29800000000000

0.03200000000000

$-0.40200000000000$

0.07200000000000

$-0.03200000000000$

$-0.29800000000000$

$-0.07200000000000$

0.40200000000000

$-0.07200000000000$

0.40200000000000

$-0.03200000000000$

$-0.29800000000000$

$-0.29800000000000$

$-0.03200000000000$

0.40200000000000

$-0.07200000000000$

$-0.19700000000000$ 0.30100000000000 0.30100000000000

0.19700000000000 0.30100000000000 0.19700000000000 0.19700000000000 0.30100000000000 0.1970000000000 0.3010000000000 0.3010000000000 0.30100000000000 0.1970000000000 0.24000000000000 0.20000000000000 $-.41000000000000$ 0.03000000000000 0.20000000000000 0.24000000000000 0.03000000000000 $-0.41000000000000$ $-0.03000000000000$ 0.41000000000000 0.20000000000000 0.24000000000000 $-0.24000000000000$ $-0.200000000000$ 0.41000000000000 .03000000000000 . 20000000000000 0.24000000000000 0.03000000000000 0.41000000000000 0.03000000000000 0.41000000000000 $-0.20000000000000$ $-0.24000000000000$ 0.29800000000000 0.03200000000000 $-0.40200000000000$ 0.07200000000000 0.03200000000000 0.2980000000000 0.07200000000000 0.07200000000000 0.072000000000 . 0.40200000000000 0.03200000000000 0.29800000000000 $-0.29800000000000$ $-0.03200000000000$ 0.40200000000000 $-0.07200000000000$ $-0.03200000000000$ $-0.29800000000000$ 0.07200000000000 0.40200000000000 0.40200000000000 $-0.03200000000000$ $-0.29800000000000$

0.19700000000000 $-0.30100000000000$ 0.30100000000000 $-0.19700000000000$ $-0.19700000000000$ 0.30100000000000 $-0.30100000000000$ 0.19700000000000 0.30100000000000 $-0.1970000$ 0.19700004 $-0.301000$ (2) 0.41000000000000 0.2000000000000 0.24000000000000 0.24000000000000 0.20000000000000 $-0.41000000000000$ 0.20000000000000 0.24000000000000 $-0.03000000000000$ $-0.41000000000000$ .03000000000000 0.41000000000000 $-0.2000004$ 0.2400000000000 $-0.20000000000000$ 0.41000000000000 . 0.03000000000000 $-0.20000000000000$ $-10000000$ $-0.40200000000000$ 0.03200000000000 0.29800000000000 0.29800000000000 0.03200000000000 0.072000 0.03200000000000 0.29800000000000 0.07200000000000 
'Y. Xiao'

A. Senyshyn,

G. G. Simeoni

Y. Su'

, U. $R \backslash "\{$ u $\}$ cker
, P. K\"\{o\}gerler

'Thomas $\mathrm{Br} \backslash "\{\mathrm{u}\} \mathrm{ckel}$,

journal_name_full_name

\section{Physical Review B}

journal volume 87

journal year 2013

journal_page_first 214406

journal page last 214406

publ_Section_title

Coexistence of magnetic order and spin-glass-like phase in the $\hookrightarrow$ pyrochlore antiferromagnet $\mathrm{Na}_{\_} 3 \$ \mathrm{Co}\left(\mathrm{CO} \$ \_3 \$\right) \$ \_2 \$ \mathrm{Cl}$

aflow_title 'Pyrochlore (Na_3\$Co $\left.\left(\mathrm{CO} \$ \_3 \$\right) \$ \_2 \$ \mathrm{Cl}\right)$ Structure

aflow_proto 'A2BCD3E6_cF208_203_e_c_d_f_g'

aflow_params $, a, x_{-}\{3\}, x_{-}\{4\}, x_{-}\{5\}, y_{-}\{5\}, z_{-}\{5\}$,

aflow_params_values ' $13.9898,0.2826,-0.099,0.2257,0.2665,0.3531$,

aflow_Strukturbericht 'None'

aflow Pearson 'cF208,

symmetry_space group_name_H-M "F 2/d -3 (origin choice 2)"

symmetry_Int_Tables_number 203

cell_length_a $\quad 13.98980$

cell_length_b 13.98980

$\begin{array}{ll}\text { cell_length_c } & 13.98980 \\ \text { cell_angle_alpha } & 90.00000\end{array}$

cell_angle_beta 90.00000

cell_angle_gamma 90.00000

loop_

space_group_symop_id

space_group_symop_operation_xyz

$1 \mathrm{x}, \mathrm{y}, \mathrm{z}$

$2 \mathrm{x},-\mathrm{y}+3 / 4,-\mathrm{z}+3 / 4$

$-\mathrm{x}+3 / 4, \mathrm{y},-\mathrm{z}+3 / 4$

$-x+3 / 4,-y+3 / 4, z$

$\mathrm{y}, \mathrm{z}, \mathrm{x}$

$y,-z+3 / 4,-x+3 / 4$

$-y+3 / 4, z,-x+3 / 4$

$-y+3 / 4,-z+3 / 4, x$

$\mathrm{z}, \mathrm{x}, \mathrm{y}$

$\mathrm{z},-\mathrm{x}+3 / 4,-\mathrm{y}+3 / 4$

$11-\mathrm{z}+3 / 4, \mathrm{x},-\mathrm{y}+3 / 4$

$12-z+3 / 4,-x+3 / 4, y$

$13-\mathrm{x},-\mathrm{y},-\mathrm{z}$

$14-\mathrm{x}, \mathrm{y}+1 / 4, \mathrm{z}+1 / 4$

$15 \mathrm{x}+1 / 4,-\mathrm{y}, \mathrm{z}+1 / 4$

$6 \mathrm{x}+1 / 4, \mathrm{y}+1 / 4,-\mathrm{z}$

$17-y,-z,-x$

$18-\mathrm{y}, \mathrm{z}+1 / 4, \mathrm{x}+1 / 4$

$20 y+1 / 4, z+1 / 4,-x$

$21-\mathrm{z},-\mathrm{x},-\mathrm{y}$

$22-\mathrm{z}, \mathrm{x}+1 / 4, \mathrm{y}+1 / 4$

$23 \mathrm{z}+1 / 4,-\mathrm{x}, \mathrm{y}+1 / 4$

$24 \mathrm{z}+1 / 4, \mathrm{x}+1 / 4,-\mathrm{y}$

$25 \mathrm{x}, \mathrm{y}+1 / 2, \mathrm{z}+1 / 2$

$26 \mathrm{x},-\mathrm{y}+1 / 4,-\mathrm{z}+1 / 4$
$27-\mathrm{x}+3 / 4, \mathrm{y}+1 / 2,-\mathrm{z}+1 / 4$

$27-\mathrm{x}+3 / 4, \mathrm{y}+1 / 2,-\mathrm{z}+1 / 4$
$28-\mathrm{x}+3 / 4,-\mathrm{y}+1 / 4, \mathrm{z}+1 / 2$

$29 \mathrm{y}, \mathrm{z}+1 / 2, \mathrm{x}+1 / 2$

$30 \mathrm{y},-\mathrm{z}+1 / 4,-\mathrm{x}+1 / 4$

$31-y+3 / 4, z+1 / 2,-x+1 / 4$
$32-y+3 / 4,-z+1 / 4, x+1 / 2$

$33 \mathrm{z}, \mathrm{x}+1 / 2, \mathrm{y}+1 / 2$

$34 \mathrm{z},-\mathrm{x}+1 / 4,-\mathrm{y}+1 / 4$

$35-\mathrm{z}+3 / 4, \mathrm{x}+1 / 2,-\mathrm{y}+1 / 4$

$36-\mathrm{z}+3 / 4,-\mathrm{x}+1 / 4, \mathrm{y}+1 / 2$

$37-x,-y+1 / 2,-z+1 / 2$

$38-x, y+3 / 4, z+3 / 4$

$39 \mathrm{x}+1 / 4,-\mathrm{y}+1 / 2, \mathrm{z}+3 / 4$

$40 \mathrm{x}+1 / 4, \mathrm{y}+3 / 4,-\mathrm{z}+1 / 2$

$41-\mathrm{y},-\mathrm{z}+1 / 2,-\mathrm{x}+1 / 2$

$42-y, z+3 / 4, x+3 / 4$

$43 \mathrm{y}+1 / 4,-\mathrm{z}+1 / 2, \mathrm{x}+3 / 4$

$44 \mathrm{y}+1 / 4, z+3 / 4,-x+1 / 2$

$45-\mathrm{z},-\mathrm{x}+1 / 2,-\mathrm{y}+1 / 2$

$46-\mathrm{z}, \mathrm{x}+3 / 4, \mathrm{y}+3 / 4$

$47 \mathrm{z}+1 / 4,-\mathrm{x}+1 / 2, \mathrm{y}+3 / 4$

$48 \mathrm{z}+1 / 4, \mathrm{x}+3 / 4,-\mathrm{y}+1 / 2$

$49 \mathrm{x}+1 / 2, \mathrm{y}, \mathrm{z}+1 / 2$

$50 \mathrm{x}+1 / 2,-\mathrm{y}+3 / 4,-\mathrm{z}+1 / 4$

$51-\mathrm{x}+1 / 4, \mathrm{y},-\mathrm{z}+1 / 4$

$52-x+1 / 4,-y+3 / 4, z+1 / 2$

$53 \mathrm{y}+1 / 2, \mathrm{z}, \mathrm{x}+1 / 2$

$54 \mathrm{y}+1 / 2,-\mathrm{z}+3 / 4,-\mathrm{x}+1 / 4$

$55-\mathrm{y}+1 / 4, \mathrm{z},-\mathrm{x}+1 / 4$

$56-\mathrm{y}+1 / 4,-\mathrm{z}+3 / 4, \mathrm{x}+1 / 2$

$\begin{array}{ll}56 & -y+1 / 4,-z+3 / 4 \\ 57 & z+1 / 2, x, y+1 / 2\end{array}$

$\begin{array}{ll}57 & \mathrm{z}+1 / 2, \mathrm{x}, \mathrm{y}+1 / 2 \\ 58 & \mathrm{z}+1 / 2,-\mathrm{x}+3 / 4,-\mathrm{y}+1 / 4\end{array}$

$58 \mathrm{z}+1 / 2,-\mathrm{x}+3 / 4,-\mathrm{y}+1$
$59-\mathrm{z}+1 / 4, \mathrm{x},-\mathrm{y}+1 / 4$

$59-\mathrm{z}+1 / 4, \mathrm{x},-\mathrm{y}+1 / 4$
$60-\mathrm{z}+1 / 4,-\mathrm{x}+3 / 4, \mathrm{y}+1 / 2$

$60-z+1 / 4,-x+3 / 4, y+1$
$61-x+1 / 2,-y,-z+1 / 2$

$62-x+1 / 2, y+1 / 4, z+3 / 4$

$63 \mathrm{x}+3 / 4,-\mathrm{y}, \mathrm{z}+3 / 4$

$64 \mathrm{x}+3 / 4, \mathrm{y}+1 / 4,-\mathrm{z}+1 / 2$

$65-\mathrm{y}+1 / 2,-\mathrm{z},-\mathrm{x}+1 / 2$

$66-y+1 / 2, z+1 / 4, x+3 / 4$

$67 \mathrm{y}+3 / 4,-\mathrm{z}, \mathrm{x}+3 / 4$

$68 \mathrm{y}+3 / 4, \mathrm{z}+1 / 4,-\mathrm{x}+1 / 2$

$69-\mathrm{z}+1 / 2,-\mathrm{x},-\mathrm{y}+1 / 2$

$70-z+1 / 2, x+1 / 4, y+3 / 4$

$71 \mathrm{z}+3 / 4,-x, y+3 / 4$

$72 \mathrm{z}+3 / 4, x+1 / 4,-y+1 / 2$

$74 x+1 / 2,-y+1 / 4,-z+3 / 4$

$75-\mathrm{x}+1 / 4, \mathrm{y}+1 / 2,-\mathrm{z}+3 / 4$

$76-x+1 / 4,-y+1 / 4$,

$78 \mathrm{y}+1 / 2,-\mathrm{z}+1 / 4,-\mathrm{x}+3 / 4$

$78 \mathrm{y}+1 / 2,-\mathrm{z}+1 / 4,-\mathrm{x}+3 / 4$
$79-\mathrm{y}+1 / 4, \mathrm{z}+1 / 2,-\mathrm{x}+3 / 4$

$80-\mathrm{y}+1 / 4,-\mathrm{z}+1 / 4, \mathrm{x}$

$81 \mathrm{z}+1 / 2, \mathrm{x}+1 / 2, \mathrm{y}$

$82 \mathrm{z}+1 / 2,-\mathrm{x}+1 / 4,-\mathrm{y}+3 / 4$

$83-z+1 / 4, x+1 / 2,-y+3 / 4$

$84-\mathrm{z}+1 / 4,-\mathrm{x}+1 / 4, \mathrm{y}$

$85-x+1 / 2,-y+1 / 2,-z$

$86-x+1 / 2, y+3 / 4, z+1 / 4$
$87 x+3 / 4,-y+1 / 2, z+1 / 4$

$\begin{array}{ll}87 & \mathrm{x}+3 / 4,-\mathrm{y}+1 / 2, \mathrm{z}+1 / 4 \\ 88 \mathrm{x}+3 / 4, \mathrm{y}+3 / 4,-\mathrm{z}\end{array}$

$89-\mathrm{y}+1 / 2,-\mathrm{z}+1 / 2,-\mathrm{x}$

$90-\mathrm{y}+1 / 2, \mathrm{z}+3 / 4, \mathrm{x}+1 / 4$

$91 \mathrm{y}+3 / 4,-\mathrm{z}+1 / 2, \mathrm{x}+1 / 4$

$92 \mathrm{y}+3 / 4, \mathrm{z}+3 / 4,-\mathrm{x}$

$93-\mathrm{z}+1 / 2,-\mathrm{x}+1 / 2,-\mathrm{y}$

$93-z+1 / 2,-x+1 / 2,-y$
$94-z+1 / 2, x+3 / 4, y+1 / 4$

$95 z+3 / 4, x+1 / 2, y+1 / 4$

$96 \mathrm{z}+3 / 4, \mathrm{x}+3 / 4,-\mathrm{y}$

loop

atom_site_labe

atom_site_type_symbol

atom_site_symmetry_multiplicity

atom_site_Wyckoff_label

-atom_site_fract_x

atom_site_fract_y

atom_site_fract_z

atom_site_occupancy

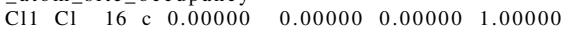

$\begin{array}{llllllll}\text { Co1 Co } & 16 & \text { d } & 0.50000 & 0.50000 & 0.50000 & 1.00000\end{array}$

$\begin{array}{llllllllll}\text { C1 } & \text { C } & 32 & \text { e } & 0.28260 & 0.28260 & 0.28260 & 1.00000\end{array}$

$\begin{array}{lllllllll}\mathrm{Na} 1 & \mathrm{Na} & 48 & \mathrm{f} & -0.09900 & 0.12500 & 0.12500 & 1.00000\end{array}$

$\begin{array}{llllllll}\mathrm{Na} & \mathrm{Na} & 48 & \mathrm{f} & -0.09900 & 0.12500 & 0.12500 & 1.00000\end{array}$

Pyrochlore $\left(\mathrm{Na}_{3} \mathrm{Co}\left(\mathrm{CO}_{3}\right)_{2} \mathrm{Cl}\right)$ : A2BCD3E6_cF208_203_e_c_d_f_g - POSCAR

A2BCD3E6_cF208_203_e_c_d_f_g \& a , x3, x4, x5, y5, z5 --params $=13.9898,0.2826$ $\hookrightarrow,-0.099,0.2257,0.2665,0.3531 \& \mathrm{Fd}_{-3} \mathrm{~T}_{-}\{\mathrm{h}\}^{\wedge}\{4\} \# 203(\mathrm{cdefg}) \&$

$\hookrightarrow \mathrm{cF} 208$ \& None \& $\mathrm{Na} 3 \mathrm{Co}(\mathrm{CO} 3) 2 \mathrm{Cl} \& \mathrm{Na} 3 \mathrm{Co}(\mathrm{CO} 3) 2 \mathrm{Cl} \& \mathrm{Z}$. Fu et al.

$\hookrightarrow$ Phys. Rev. B 87, $214406(2013)$

1.00000000000000

0.00000000000000

C 0.00000000000000

6.99490000000000 6.99490000000000 0.00000000000000

Direct

0.28260000000000 $\mathrm{Na}$

$\mathrm{Na} \quad 24$

$0.28260000000000 \quad 0.28260000000000$

$-0.34780000000000$

$-0.28260000000000$

$-0.28260000000000$

1.34780000000000

1.34780000000000

0.00000000000000

0.0000000000000

0.00000000000000

0.50000000000000

0.50000000000000

0.5000000000000

0.00000000000000

0.3490000000000

$-0.09900000000000$

$-0.09900000000000$

0.34900000000000

$-0.09900000000000$

0.34900000000000

0.65100000000000

0.09900000000000

0.0990000000000

0.6510000000000

0.65100000000000

0.6510000000000

0.65100000000000

0.3939000000000

0.31230000000000

0.13910000000000

$-0.34530000000000$

0.13910000000000

$-0.34530000000000$

0.39390000000000

0.31230000000000

0.31230000000000

0.39390000000000

$-0.34530000000000$

0.13910000000000

$-0.39390000000000$

$-0.31230000000000$

$-0.13910000000000$ 0.28260000000000 0.34780000000000 0.28260000000000 0.28260000000000 .28260000000000 34780000000000 0.28260000000000 0.00000000000000 0.5000000000000 0.50000000000 0.50000000000000 .50000000000000 . .50000000000000 0.00000000000000 0.50000000000000 $-0.09900000000000$ 0.34900000000000 0.34900000000000 $-0.09900000000000$ $-0.09900000000000$ 0.34900000000000 0.09900000000000 0.65100000000000 0.6510000000000 0.09900000000000 0.09900000000 0.65100000000000 0.65100000000000 0.312300000000 0.39390000000000 0.34530000000000 0.13910000000000 0.39390000000000 0.13910000000000 .34530000000000 0.13910000000000 0.34530000000000 0.39390000000000 $-0.39390000000000$ 1.34530000000000

0.28260000000000 $-0.34780000000000$ 0.28260000000000 0.28260000000000 $-0.28260000000000$ 1.34780000000000 $-0.28260000000000$ $-0.28260000000000$ 0.00000000000000 0.50000000000000 0.00000000000000 0.00000000000000 0.50000000000000 0.00000000000000 0.50000000000000 0.50000000000000 $-0.09900000000000$ 0.34900000000000 $-0.09900000000000$ 0.34900000000000 0.34900000000000 $-0.09900000000000$ 0.09900000000000 0.65100000000000 0.09900000000000 0.65100000000000 0.65100000000000 0.65100000000000 0.09900000000000 0.13910000000000 0.34530000000000 0.39390000000000 0.31230000000000 0.31230000000000 0.39390000000000 $-0.34530000000000$ 0.13910000000000 0.39390000000000 0.31230000000000 0.13910000000000 $-0.34530000000000$ $-0.13910000000000$ 1.34530000000000 $-0.39390000000000$ 
1.34530000000000 $-0.13910000000000$ 1.34530000000000
-0.39390000000000 $-0.39390000000000$ $-0.31230000000000$ $-0.31230000000000$ $-0.39390000000000$ 1.34530000000000 $-0.13910000000000$

$-0.13910000000000$ $-0.39390000000000$ $-0.31230000000000$ $-0.13910000000000$ 1.34530000000000 $-0.13910000000000$ 1.34530000000000 $-0.39390000000000$

$-0.31230000000000$ $-0.31230000000000$ $-0.39390000000000$ 1.34530000000000 $-0.13910000000000$ $-0.39390000000000$ $-0.31230000000000$ $-0.13910000000000$ 1.34530000000000

O (96g)

O $(96 \mathrm{~g})$

O (96g)

O $\quad(96 \mathrm{~g})$

$\mathrm{O} \quad(96 \mathrm{~g})$

O $\quad(96 \mathrm{~g})$

O $\quad(96 \mathrm{~g})$

O $(96 \mathrm{~g})$

Tychite $\left(\mathrm{Na}_{6} \mathrm{Mg}_{2}\left(\mathrm{SO}_{4}\right)\left(\mathrm{CO}_{3}\right)_{4}\right):$ A4B2C6D16E_cF232_203_e_d_f_eg_a - CIF

\section{\# CIF file}

data_findsym-output

audit_creation_method FINDSYM

chemical_name_mineral 'Tychite'

chemical_formula_sum 'C4 Mg2 Na6 $016 \mathrm{~S}$

loop

publ_author_name

G. R. Schmidt'

'R. Jacqueline'

'H. Yang'

R. T. Downs'

journal_name_full_name

Acta Crystallographica Section E: Crystallographic Communication

journal_volume 62

journal_year 2006

journal_page_first i207

journal_page_last i209

publ_Section_title

Tychite, $N a \$_{-}\{6\} \$ M g \$_{-}\{2\} \$\left(\operatorname{SO} \$_{-}\{4\} \$\right)\left(\operatorname{CO} \$_{-}\{3\} \$\right) \$ \$_{-}\{4\} \$$ : Structure analysis $\rightarrow$ and Raman spectroscopic data

Found in The American Mineralogist Crystal Structure Database, 2003

aflow_title, Tychite $\left(\mathrm{Na} \$_{-}\{6\} \$ M g \$_{-}\{2\} \$\left(\operatorname{SO} \$_{-}\{4\} \$\right)\left(\operatorname{CO}_{-}\{3\} \$\right) \$ \_\{4\} \$\right)$ $\hookrightarrow$ Structure

aflow_proto 'A4B2C6D16E_cF232_203_e_d_f_eg_a'

-aflow_params ,a, $x_{-}\{3\}, x_{-}\{4\}, x_{-}\{5\}, x_{-}\{6\}, y_{-}\{6\}, z_{-}\{6\}$

aflow_params_values , $13.9038,0.28207,0.06362,0.34379,0.26626,0.22529$, $\hookrightarrow 0.35333$

aflow Strukturbericht 'None'

aflow_Pearson 'cF232'

symmetry_space_group_name_H-M "F 2/d -3 (origin choice 2)"

symmetry_Int Tables number 203

cell_length_a $\quad 13.90380$

cell_length_b 13.90380

cell_length_c 13.90380

cell_angle_alpha 90.00000

cell-angle beta 90.00

cell_angle_gamma 90.00000

loop

space_group_symop_id

space_group_symop_operation_xyz

$1 \mathrm{x}, \mathrm{y}, \mathrm{z}$

$\mathrm{x},-\mathrm{y}+3 / 4,-\mathrm{z}+3 / 4$

$-\mathrm{x}+3 / 4, \mathrm{y},-\mathrm{z}+3 / 4$

$-x+3 / 4,-y+3 / 4, z$

$\mathrm{y}, \mathrm{z}, \mathrm{x}$

$6 \mathrm{y},-\mathrm{z}+3 / 4,-\mathrm{x}+3 / 4$

$7-y+3 / 4, z,-x+3 / 4$

$8-\mathrm{y}+3 / 4,-\mathrm{z}+3 / 4, \mathrm{x}$

$\mathrm{z}, \mathrm{x}, \mathrm{y}$

$0 \mathrm{z},-\mathrm{x}+3 / 4,-\mathrm{y}+3 / 4$

$-z+3 / 4, x,-y+3 / 4$

$2-z+3 / 4,-x+3 / 4, y$

$13-\mathrm{x},-\mathrm{y},-\mathrm{z}$

$14-x, y+1 / 4, z+1 / 4$

$15 \mathrm{x}+1 / 4,-\mathrm{y}, \mathrm{z}+1 / 4$

$16 \mathrm{x}+1 / 4, \mathrm{y}+1 / 4,-\mathrm{z}$

$17-\mathrm{y},-\mathrm{z},-\mathrm{x}$

$8-\mathrm{y}, \mathrm{z}+1 / 4, \mathrm{x}+1 / 4$

$19 \mathrm{y}+1 / 4,-\mathrm{z}, \mathrm{x}+1 / 4$

$20 \mathrm{y}+1 / 4, \mathrm{z}+1 / 4,-\mathrm{x}$

$21-\mathrm{z},-\mathrm{x},-\mathrm{y}$

$22-\mathrm{z}, \mathrm{x}+1 / 4, \mathrm{y}+1 / 4$

$23 \mathrm{z}+1 / 4,-\mathrm{x}, \mathrm{y}+1 / 4$

$24 \mathrm{z}+1 / 4, \mathrm{x}+1 / 4,-\mathrm{y}$

$25 \mathrm{x}, \mathrm{y}+1 / 2, \mathrm{z}+1 / 2$

$26 \mathrm{x},-\mathrm{y}+1 / 4,-\mathrm{z}+1 / 4$
$27-\mathrm{x}+3 / 4, \mathrm{y}+1 / 2,-\mathrm{z}+1 / 4$

$27-x+3 / 4, y+1 / 2,-z+1 / 4$
$28-x+3 / 4,-y+1 / 4, z+1 / 2$

$29 \mathrm{y}, \mathrm{z}+1 / 2, \mathrm{x}+1 / 2$

$-\mathrm{z}+1 / 4,-\mathrm{x}+1 / 4$

$31-\mathrm{y}+3 / 4, \mathrm{z}+1 / 2,-\mathrm{x}+1 / 4$

$32-\mathrm{y}+3 / 4,-\mathrm{z}+1 / 4, \mathrm{x}+1 / 2$

$33 \mathrm{z}, \mathrm{x}+1 / 2, \mathrm{y}+1 / 2$

$4 \mathrm{z},-\mathrm{x}+1 / 4,-\mathrm{y}+1 / 4$

$35-z+3 / 4, x+1 / 2,-y+1 / 4$

$36-z+3 / 4,-x+1 / 4, y+1 / 2$

$37-\mathrm{x},-\mathrm{y}+1 / 2,-\mathrm{z}+1 / 2$

$38-\mathrm{x}, \mathrm{y}+3 / 4, \mathrm{z}+3 / 4$

$39 \mathrm{x}+1 / 4,-\mathrm{y}+1 / 2, \mathrm{z}+3 / 4$

$40 \mathrm{x}+1 / 4, \mathrm{y}+3 / 4,-\mathrm{z}+1 / 2$

$41-\mathrm{y},-\mathrm{z}+1 / 2,-\mathrm{x}+1 / 2$

$43 \mathrm{y}+1 / 4,-\mathrm{z}+1 / 2, \mathrm{x}+3 / 4$

44 $y+1 / 4,-z+3 / 4,-x+1 / 2$

$45-\mathrm{z},-\mathrm{x}+1 / 2,-\mathrm{y}+1 / 2$

$47 \mathrm{z}+1 / 4,-\mathrm{x}+1 / 2, \mathrm{y}+3 / 4$

$48 \mathrm{z}+1 / 4, \mathrm{x}+3 / 4,-\mathrm{y}+1 / 2$

$49 \mathrm{x}+1 / 2, \mathrm{y}, \mathrm{z}+1 / 2$

$50 \mathrm{x}+1 / 2,-\mathrm{y}+3 / 4,-\mathrm{z}+1 / 4$

$51-\mathrm{x}+1 / 4, \mathrm{y},-\mathrm{z}+1 / 4$

$52-x+1 / 4,-y+3 / 4, z+1 / 2$

$53 \mathrm{y}+1 / 2, \mathrm{z}, \mathrm{x}+1 / 2$

$54 \mathrm{y}+1 / 2,-\mathrm{z}+3 / 4,-\mathrm{x}+1 / 4$

$55-\mathrm{y}+1 / 4, \mathrm{z},-\mathrm{x}+1 / 4$

$56-\mathrm{y}+1 / 4,-\mathrm{z}+3 / 4, \mathrm{x}+1 / 2$

$57 \mathrm{z}+1 / 2, \mathrm{x}, \mathrm{y}+1 / 2$

$58 \mathrm{z}+1 / 2,-\mathrm{x}+3 / 4,-\mathrm{y}+1 / 4$

$59-\mathrm{z}+1 / 4, \mathrm{x},-\mathrm{y}+1 / 4$

$60-z+1 / 4,-x+3 / 4, y+1 / 2$

$61-x+1 / 2,-y,-z+1 / 2$

$62-x+1 / 2, y+1 / 4, z+3 / 4$

$63 \mathrm{x}+3 / 4,-\mathrm{y}, \mathrm{z}+3 / 4$

$64 \mathrm{x}+3 / 4, \mathrm{y}+1 / 4,-\mathrm{z}+1 / 2$

$65-\mathrm{y}+1 / 2,-\mathrm{z},-\mathrm{x}+1 / 2$

$66-\mathrm{y}+1 / 2, \mathrm{z}+1 / 4, \mathrm{x}+3 / 4$

$67 \mathrm{y}+3 / 4,-\mathrm{z}, \mathrm{x}+3 / 4$

$68 \mathrm{y}+3 / 4, \mathrm{z}+1 / 4,-\mathrm{x}+1 / 2$

$69-z+1 / 2,-x,-y+1 / 2$

$70-z+1 / 2, x+1 / 4, y+3 / 4$

$72 z+3 / 4, x+1 / 4,-y+1 / 2$

$73 \mathrm{x}+1 / 2, \mathrm{y}+1 / 2, \mathrm{z}$

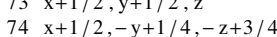

$74 \mathrm{x}+1 / 2,-\mathrm{y}+1 / 4,-\mathrm{z}+3 / 4$
$75-\mathrm{x}+1 / 4, \mathrm{y}+1 / 2,-\mathrm{z}+3 / 4$

$76-x+1 / 4,-y+1 / 4, z$

$77 \mathrm{y}+1 / 2, \mathrm{z}+1 / 2, \mathrm{x}$

$78 \mathrm{y}+1 / 2,-\mathrm{z}+1 / 4,-\mathrm{x}+3 / 4$

$79-y+1 / 4, z+1 / 2,-x+3 / 4$

$80-\mathrm{y}+1 / 4,-\mathrm{z}+1 / 4, \mathrm{x}$

$81 \mathrm{z}+1 / 2, \mathrm{x}+1 / 2, \mathrm{y}$

$82 \mathrm{z}+1 / 2,-\mathrm{x}+1 / 4,-\mathrm{y}+3 / 4$

$83-z+1 / 4, x+1 / 2,-y+3 / 4$

$84-\mathrm{z}+1 / 4,-\mathrm{x}+1 / 4, \mathrm{y}$

$85-x+1 / 2,-y+1 / 2,-z$

$86-x+1 / 2, y+3 / 4, z+1 / 4$

$87 x+3 / 4,-y+1 / 2, z+1 / 4$

$88 \mathrm{x}+3 / 4, \mathrm{y}+3 / 4,-\mathrm{z}$

$\begin{array}{ll}88 & x+3 / 4, y+3 / 4,-z \\ 89 & -y+1 / 2,-z+1 / 2,-x\end{array}$

$89-\mathrm{y}+1 / 2,-\mathrm{z}+1 / 2,-\mathrm{x}$

$90-y+1 / 2, z+3 / 4, x+1 / 4$

$91 \mathrm{y}+3 / 4,-\mathrm{z}+1 / 2, \mathrm{x}+1 / 4$
$92 \mathrm{y}+3 / 4, \mathrm{z}+3 / 4,-\mathrm{x}$

$92 \mathrm{y}+3 / 4, \mathrm{z}+3 / 4,-\mathrm{x}$
$93-\mathrm{z}+1 / 2,-\mathrm{x}+1 / 2,-\mathrm{y}$

$93-\mathrm{z}+1 / 2,-\mathrm{x}+1 / 2,-\mathrm{y}$
$94-\mathrm{z}+1 / 2, \mathrm{x}+3 / 4, \mathrm{y}+1 / 4$

$95 \mathrm{z}+3 / 4,-\mathrm{x}+1 / 2, \mathrm{y}+1 / 4$

$96 \mathrm{z}+3 / 4, \mathrm{x}+3 / 4,-\mathrm{y}$

loop

atom_site_label

atom_site_type_symbol

atom_site_symmetry_multiplicity

atom_site_Wyckoff_label

atom_site_fract_x

atom_site_fract_y

atom_site_fract_z

tom_site_occupancy

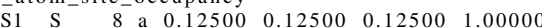

$\begin{array}{llllllll}\mathrm{Mg} 1 \mathrm{Mg} & 16 & \mathrm{~d} & 0.50000 & 0.50000 & 0.50000 & 1.00000\end{array}$

$\begin{array}{lllllllllll}\mathrm{C} 1 & \mathrm{C} & 32 & \mathrm{e} & 0.28207 & 0.28207 & 0.28207 & 1.00000\end{array}$

$\begin{array}{llllllllll}\mathrm{O} 1 & \mathrm{O} & 32 & \text { e } & 0.06362 & 0.06362 & 0.06362 & 1.00000\end{array}$

$\begin{array}{llllllll}\mathrm{Na} 1 & \mathrm{Na} & 48 & \mathrm{f} & 0.34379 & 0.12500 & 0.12500 & 1.00000\end{array}$

$\begin{array}{llllllll}\mathrm{O} 2 & \mathrm{O} & 96 & \mathrm{~g} & 0.26626 & 0.22529 & 0.35333 & 1.00000\end{array}$

Tychite $\left(\mathrm{Na}_{6} \mathrm{Mg}_{2}\left(\mathrm{SO}_{4}\right)\left(\mathrm{CO}_{3}\right)_{4}\right)$ : A4B2C6D16E_cF232_203_e_d_f_eg_a - POSCAR

A4B2C6D16E_cF232_203_e_d_f_eg_a \& a , x3, x4, x5, x6,y6, z6 --params $=13.9038$ $\hookrightarrow 0.28207,0.06362,0.34379,0.26626,0.22529,0.35333 \&$ \&d $-3 T_{-}\{h\}^{\wedge}\{4$ $\rightarrow\} \# 203\left(\operatorname{ade}^{\wedge} 2 \mathrm{fg}\right) \& \mathrm{cF} 232$ \& None \& Na6Mg2 (SO4) (CO3)4 \& Tychite \& $\hookrightarrow$ \} \#203 (ade^^2fg) \& cF232 \& None \& Na6Mg2(SO4)(CO3)4 \& Tychite \&
$\hookrightarrow$ G. R. Schmidt et al. Acta Crystallogr. E 62, i $207-\mathrm{i} 209$ (2006) 1.00000000000000

0.00000000000000

$6.95190000000000 \quad 6.95190000000000$

$\begin{array}{lll}6.95190000000000 & 0.00000000000000 & 6.95190000000000\end{array}$

$\begin{array}{lll}6.95190000000000 & 6.95190000000000 & 0.00000000000000\end{array}$

$\begin{array}{rrrrr}\mathrm{C} & \mathrm{Mg} & \mathrm{Na} & \mathrm{O} & \mathrm{S} \\ 8 & 4 & 12 & 32 & 2\end{array}$

Direct

0.28207000000000 0.28207000000000
0.28207000000000 $-0.34621000000000$ $-0.2820700000000$ $-0.2820700000000$ $-0.2820700000000$ 0.28207000000000 1.34621000000000 0.50000000000000 0.5000000000000

0.50000000000000

0.00000000000000

$-0.09379000000000$

0.34379000000000

0.34379000000000 0.34379000000000 $-0.09379000000000$ 1.09379000000000

$-0.34379000000000$

$-0.34379000000000$

0.28207000000000 0.28207000000000 $-0.34621000000000$ 0.2820700000000 $-0.28207000000000$ $-0.28207000000000$ 1.34621000000000 1.346210000000 0.500000000000 0.500000000000 0.50000000000000 0.00000000000000 0.50000000000000 0.34379000000000 $-0.09379000000000$ $-0.09379000000000$ 0.34379000000000 0.34379000000000 $-0.09379000000000$ $-0.34379000000000$ 1.09379000000000 1.09379000000000
0.28207000000000 $-0.34621000000000$ 0.28207000000000 0.28207000000000 $-0.28207000000000$ 1.34621000000000 $-0.28207000000000$ $-0.28207000000000$ 0.50000000000000 0.00000000000000 0.50000000000000 0.50000000000000 0.34379000000000 $-0.09379000000000$ 0.34379000000000 $-0.09379000000000$ $-0.09379000000000$ 0.34379000000000 $-0.34379000000000$ 1.09379000000000 $-0.34379000000000$
C $(32 \mathrm{e})$

C $(32 \mathrm{e})$

C $(32 \mathrm{e})$

C $(32 \mathrm{e})$

C $(32 \mathrm{e})$

C (32e)

C (32e)

C $(32 \mathrm{e})$

C (16d)

M (16d)

$\mathrm{Mg} \quad(16 \mathrm{~d})$

$\mathrm{Mg}$ (16d)

$\mathrm{Na}(48 \mathrm{f})$

$\mathrm{Na}(48 \mathrm{f})$

$\mathrm{Na}$ (48f)

$\mathrm{Na}$ (48f)

$\mathrm{Na}$ (48f)

$\mathrm{Na} \quad(48 \mathrm{f})$

$\mathrm{Na}(48 \mathrm{f})$

$\mathrm{Na}(48 \mathrm{f})$

$\mathrm{Na} \quad(48 \mathrm{f})$ 
1.09379000000000 $-0.34379000000000$ 1.09379000000000 0.06362000000000 0.06362000000000 0.06362000000000 0.30914000000000 $-0.06362000000000$ $-0.06362000000000$ $-0.06362000000000$ 0.69086000000000 0.31236000000000 0.39430000000000 0.13822000000000 $-0.34488000000000$ 0.13822000000000 0.3123600 0.312360000000 0.39430000000000 0.31236000000000 $-0.34488000000000$ 0.13822000000000 $-0.31236000000000$ $-0.39430000000000$ $-0.13822000000000$ 1.34488000000000 $-0.13822000000000$ 1.34488000000000 $-0.31236000000000$ $-0.39430000000000$ $-0.39430000000000$ $-0.3123600000000$ 1.34488000000000 $-0.13822000000000$ 0.12500000000000 0.87500000000000
$-0.34379000000000$ .34379000000000 1.09379000000000 0.06362000000000 0.06362000000000 0.30914000000000 0.06362000000000 $-0.06362000000000$ $-0.06362000000000$ 0.69086000000000 $-0.06362000000000$ 0.39430000000000 0.31236000000000 0.31236000000000 0.138800000000 0.1382200000000 0.3123600000000 0.3943000000000 0.13822000000000 0.13822000000000 $-0.34488000000000$ 0.31236000000000 0.39430000000000 $-0.39430000000000$ 1.34488000000000 $-0.13822000000000$ $-0.31236000000000$ $-0.39430000000000$ $-0.13822000000000$ 1.34488000000000 $-0.13822000000000$ 1.3448800000000 - .31236000000000 $-0.3123600000000$ $-0.3943000000000$ 0.12500000000000
0.87500000000000 $-0.34488000000000$ $-0.31236000000000$

.09379000000000 . $-0.34379000000000$ 0.06362000000000 0.30914000000000 0.06362000000000 0.06362000000000 0.06362000000000 0.6908600000000 $-0.0636200000000$ $-0.06362000000000$ 0.13822000000000 $-0.34488000000000$ 0.3123600000000 0.39430000000000 0.3943000000000 0.31236000000000 0.312360000000 0.34488000000000 0.1382200000000 0.31236000000000 0.39430000000000 0.13822000000000 $-0.34488000000000$ $-0.13822000000000$ 1.34488000000000 $-0.31236000000000$ $-0.39430000000000$ $-0.39430000000000$ $-0.31236000000000$ 1.34488000000000 $-0.13822000000000$ $-0.31236000000000$ $-0.31236000000000$ $-0.3943000000000$ 1. 3448800000000 .34488000000000 0.12500000000000 0.87500000000000

$\mathrm{Na} \quad(48 \mathrm{f})$

$\mathrm{Na} \quad(48 \mathrm{f})$

$\mathrm{Na} \quad(48 \mathrm{f})$

O $\quad(32 \mathrm{e})$

$\mathrm{O} \quad(32 \mathrm{e})$

O $(32 \mathrm{e})$

(32e)

(32e)

(32e)

(32e)

$(96 \mathrm{~g})$

$(96 \mathrm{~g})$

$(96 \mathrm{~g})$

$(96 \mathrm{~g})$

(96g)

$(96 \mathrm{~g})$

(96g)

$(96 \mathrm{~g})$

$(96 \mathrm{~g})$

$(96 \mathrm{~g})$

$(96 \mathrm{~g})$

$(96 \mathrm{~g})$

$(96 \mathrm{~g})$

O $(96 \mathrm{~g})$

O $(96 \mathrm{~g})$

$(96 \mathrm{~g})$

$(96 \mathrm{~g})$

$(96 \mathrm{~g})$

$(96 \mathrm{~g})$

O $(96 \mathrm{~g})$

O $(96 \mathrm{~g})$

$(96 \mathrm{~g})$

S $(8 a$

$\mathrm{S} \quad(8 \mathrm{a})$

$\mathrm{Rb}_{3} \mathrm{AsSe}_{16}: \mathrm{AB} 3 \mathrm{C} 16 \_\mathrm{cF} 160 \_203$ b_ad_eg - CIF

\section{CIF file}

data_findsym-output

_audit_creation_method FINDSYM

chemical_name_mineral 'Rb3AsSe16'

_chemical_formula_sum As Rb3 Se16

loop

publ_author_name

'M. Wachhold'

W. S. Sheldrick'

_journal_name_full_name

Zeitschrift f $\{\backslash " u\}$ r Naturforschung B

journal_volume 52

journal year 1997

journal_page_first 169

_journal_page_last 175

-publ_Section_title

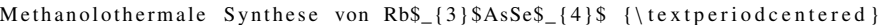

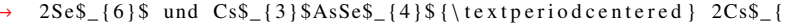
$\rightarrow\} \$ \mathrm{As}_{-}\{2\} \$ \mathrm{Se} \$_{-}\{4\} \$\{\backslash$ textperiodcentered $\} 6 \mathrm{Te} \$_{-}\{4\} \$ \mathrm{Se} \$ \_\{2\} \$$

$\hookrightarrow$ zwei Selenidoarsenate mit sechsgliedrigen Chalkogenringen/

$\rightarrow$ Methanolothermal Synthesis of $\operatorname{Rb\$ }\{3\} \$ A s S e \$ \_\{4\} \$\{$

$\rightarrow$ textperiodcentered $\} 2 \mathrm{Se} \$ \_\{6\} \$$ and $\mathrm{Cs}_{-}\{3\} \$ \mathrm{AsSe}_{-}\{4\} \$\{\backslash$

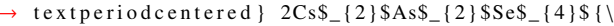

$\hookrightarrow$ textperiodcentered $\} 6 \mathrm{Te} \$\{4\} \$ S \mathrm{Se} \$\{2\} \$$. Two Selenidoarsenates

$\hookrightarrow$ with Six-Membered Chalcogen Rings

\# Found in Pearson's Crystal Data - Crystal Structure Database for

$\hookrightarrow$ Inorganic Compounds, 2013

-aflow_title 'Rb\$_ $\{3\} \$ A s S e \$_{-}\{16\} \$$ Structure

aflow_proto 'AB3C16_cF160_203_b_ad_eg'

aflow_params 'a, $x_{-}\{4\}, x_{-}\{5\}, y_{-}\{5\}, z_{-}\{5\}$ '

aflow_params_values $16.6600284675,0.70516,0.51201,0.111,0.42978$

aflow_Strukturbericht 'None'

aflow_Pearson 'cF160'

cell_length_a $\quad 16.6600284675$

cell_length_b $\quad 16.6600284675$

cell_length_c $\quad 16.6600284675$

cell angle alpha 90.0000000000

cell angle beta 90.0000000000

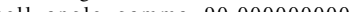

symmetry_space_group_name_H-M "F 2/d -3 (origin choice 2)"

symmetry_Int_Tables_number 203

loop

space_group_symop_id

space_group_symop_operation_xyz

$1 \mathrm{x}, \mathrm{y}, \mathrm{z}$

$\mathrm{x},-\mathrm{y}+3 / 4,-\mathrm{z}+3 / 4$

$-x+3 / 4, y,-z+3 / 4$

$-x+3 / 4,-y+3 / 4, z$

$5 \mathrm{y}, \mathrm{z}, \mathrm{x}$

$6 \mathrm{y},-\mathrm{z}+3 / 4,-\mathrm{x}+3 / 4$

$-y+3 / 4, z,-x+3 / 4$

$-y+3 / 4,-z+3 / 4, x$

$9 \mathrm{z}, \mathrm{x}, \mathrm{y}$

$10 \mathrm{z},-\mathrm{x}+3 / 4,-\mathrm{y}+3 / 4$

$11-\mathrm{z}+3 / 4, \mathrm{x},-\mathrm{y}+3 / 4$

$12-z+3 / 4,-x+3 / 4, y$

$13-x,-y,-z$

$14-x, y+1 / 4, z+1 / 4$

$15 \mathrm{x}+1 / 4,-\mathrm{y}, \mathrm{z}+1 / 4$

$16 \mathrm{x}+1 / 4, \mathrm{y}+1 / 4,-\mathrm{z}$

$17-\mathrm{y},-\mathrm{z},-\mathrm{x}$

$18-\mathrm{y}, \mathrm{z}+1 / 4, \mathrm{x}+1 / 4$

$19 \mathrm{y}+1 / 4,-\mathrm{z}, \mathrm{x}+1 / 4$

$20 \mathrm{y}+1 / 4, \mathrm{z}+1 / 4,-\mathrm{x}$

$21-\mathrm{z},-\mathrm{x},-\mathrm{y}$

$22-\mathrm{z}, \mathrm{x}+1 / 4, \mathrm{y}+1 / 4$

$23 \mathrm{z}+1 / 4,-\mathrm{x}, \mathrm{y}+1 / 4$

$24 \mathrm{z}+1 / 4, \mathrm{x}+1 / 4,-\mathrm{y}$

$25 \mathrm{x}, \mathrm{y}+1 / 2, \mathrm{z}+1 / 2$

$26 x,-y+1 / 4,-z+1 / 4$
$27-x+3 / 4, y+1 / 2,-z+1 / 4$

$\begin{array}{ll}27 & -x+3 / 4, y+1 / 2,-z+1 / 4 \\ 28 & -x+3 / 4,-y+1 / 4, z+1 / 2\end{array}$

$29 \mathrm{y}, \mathrm{z}+1 / 2, \mathrm{x}+1 / 2$

$30 \mathrm{y},-\mathrm{z}+1 / 4,-\mathrm{x}+1 / 4$

$31-\mathrm{y}+3 / 4, \mathrm{z}+1 / 2,-\mathrm{x}+1 / 4$

$32-\mathrm{y}+3 / 4,-\mathrm{z}+1 / 4, \mathrm{x}+1 / 2$

$33 \mathrm{z}, \mathrm{x}+1 / 2, \mathrm{y}+1 / 2$

$34 \mathrm{z},-\mathrm{x}+1 / 4,-\mathrm{y}+1 / 4$
$35-\mathrm{z}+3 / 4, \mathrm{x}+1 / 2,-\mathrm{y}+1 / 4$

$36-\mathrm{z}+3 / 4,-\mathrm{x}+1 / 4, \mathrm{y}+1 / 2$

$37-x,-y+1 / 2,-z+1 / 2$

$38-x, y+3 / 4, z+3 / 4$

$39 \mathrm{x}+1 / 4,-\mathrm{y}+1 / 2, \mathrm{z}+3 / 4$

$41-y,-z+1 / 2,-x+1 / 2$

$42-y, z+3 / 4, x+3 / 4$
$43 y+1 / 4,-z+1 / 2, x+3 / 4$

$44 \mathrm{y}+1 / 4, \mathrm{z}+3 / 4,-\mathrm{x}+1 / 2$

$45-\mathrm{z},-\mathrm{x}+1 / 2,-\mathrm{y}+1 / 2$

$46-\mathrm{z}, \mathrm{x}+3 / 4, \mathrm{y}+3 / 4$

$47 \mathrm{z}+1 / 4,-x+1 / 2, y+3 / 4$

$48 \mathrm{z}+1 / 4, \mathrm{x}+3 / 4,-\mathrm{y}+1 / 2$

$49 \mathrm{x}+1 / 2, \mathrm{y}, \mathrm{z}+1 / 2$

$50 \mathrm{x}+1 / 2,-\mathrm{y}+3 / 4,-\mathrm{z}+1 / 4$

$51-x+1 / 4, y,-z+1 / 4$

$52-x+1 / 4,-y+3 / 4, z+1 / 2$

$53 \mathrm{y}+1 / 2, \mathrm{z}, \mathrm{x}+1 / 2$

$54 \mathrm{y}+1 / 2,-\mathrm{z}+3 / 4,-\mathrm{x}+1 / 4$

$55-\mathrm{y}+1 / 4, z,-x+1 / 4$

$56-\mathrm{y}+1 / 4,-\mathrm{z}+3 / 4, \mathrm{x}+1 / 2$

$57 \mathrm{z}+1 / 2, \mathrm{x}, \mathrm{y}+1 / 2$

$58 \mathrm{z}+1 / 2,-\mathrm{x}+3 / 4,-\mathrm{y}+1 / 4$

$59-\mathrm{z}+1 / 4, \mathrm{x},-\mathrm{y}+1 / 4$

$60-z+1 / 4,-x+3 / 4, y+1 / 2$

$61-x+1 / 2,-y,-z+1 / 2$

$62-x+1 / 2, y+1 / 4, z+3 / 4$

$63 \mathrm{x}+3 / 4,-\mathrm{y}, \mathrm{z}+3 / 4$

$64 \mathrm{x}+3 / 4, \mathrm{y}+1 / 4,-\mathrm{z}+1 / 2$

$65-\mathrm{y}+1 / 2,-\mathrm{z},-\mathrm{x}+1 / 2$

$66-y+1 / 2, z+1 / 4, x+3 / 4$

$67 \mathrm{y}+3 / 4,-\mathrm{z}, \mathrm{x}+3 / 4$

$68 \mathrm{y}+3 / 4, \mathrm{z}+1 / 4,-\mathrm{x}+1 / 2$

$69-\mathrm{z}+1 / 2,-\mathrm{x},-\mathrm{y}+1 / 2$

$70-\mathrm{z}+1 / 2, \mathrm{x}+1 / 4, \mathrm{y}+3 / 4$

$71 \mathrm{z}+3 / 4,-\mathrm{x}, \mathrm{y}+3 / 4$

$72 \mathrm{z}+3 / 4, \mathrm{x}+1 / 4,-\mathrm{y}+1 / 2$

$73 \mathrm{x}+1 / 2, \mathrm{y}+1 / 2, \mathrm{z}$

$74 x+1 / 2,-y+1 / 4,-z+3 / 4$

$75-\mathrm{x}+1 / 4, \mathrm{y}+1 / 2,-\mathrm{z}+3 / 4$

$76-x+1 / 4,-y+1 / 4, z$

$77 \mathrm{y}+1 / 2, \mathrm{z}+1 / 2, \mathrm{x}$

$78 \mathrm{y}+1 / 2,-\mathrm{z}+1 / 4,-\mathrm{x}+3 / 4$

$79-y+1 / 4, z+1 / 2,-x+3 / 4$

$80-\mathrm{y}+1 / 4,-\mathrm{z}+1 / 4, \mathrm{x}$

$81 \mathrm{z}+1 / 2, \mathrm{x}+1 / 2, \mathrm{y}$

$82 \mathrm{z}+1 / 2,-\mathrm{x}+1 / 4,-\mathrm{y}+3 / 4$

$83-z+1 / 4, x+1 / 2,-y+3 / 4$

$84-\mathrm{z}+1 / 4,-\mathrm{x}+1 / 4, \mathrm{y}$

$85-x+1 / 2,-y+1 / 2,-z$

$86-x+1 / 2, y+3 / 4, z+1 / 4$

$87 x+3 / 4,-y+1 / 2, z+1 / 4$

$88 \mathrm{x}+3 / 4, \mathrm{y}+3 / 4,-\mathrm{z}$

$89-\mathrm{y}+1 / 2,-\mathrm{z}+1 / 2,-\mathrm{x}$

$90-y+1 / 2, z+3 / 4, x+1 / 4$

$90-\mathrm{y}+1 / 2, \mathrm{z}+3 / 4, \mathrm{x}+1 / 4$

$91 \mathrm{y}+3 / 4,-\mathrm{z}+1 / 2, \mathrm{x}+1 /$

$92 \mathrm{y}+3 / 4, \mathrm{z}+3 / 4,-\mathrm{x}$

$93-z+1 / 2,-x+1 / 2,-y$
$94-z+1 / 2, x+3 / 4, y+1 / 4$

$95 \mathrm{z}+3 / 4,-\mathrm{x}+1 / 2, \mathrm{y}+1 / 4$

$96 \mathrm{z}+3 / 4, \mathrm{x}+3 / 4,-\mathrm{y}$

loop

atom_site_label

atom_site_type_symbol

atom_site_symmetry_multiplicity 
$\hookrightarrow 0.51201,0.111,0.42978 \& \mathrm{Fd}-3 \mathrm{~T}\{\mathrm{~h}\}^{\wedge}\{4\}$ \#203 (abdeg) \& $\mathrm{cF} 160 \&$

$\hookrightarrow$ None \& Rb3AsSe16 \& \& M. Wachhold and W. S. Sheldrick, Z.

$\hookrightarrow$ Naturforsch. B 52, 169-175 (1997)

1.00000000000000

0.00000000000000

8.33001423375000

8.33001423375000

$\begin{array}{rrr}\text { As } & \mathrm{Rb} & \mathrm{Se} \\ 2 & 6 & 32\end{array}$

Direct

$8.33001423375000 \quad 8.33001423375000$

$0.00000000000000 \quad 8.33001423375000$

0.00000000000000

0.62500000000000

0.37500000000000

0.12500000000000

0.8750000000000

0.87500000000000

0.50000000000000

0.50000000000000

0.00000000000000

0.70516000000000

0.70516000000000

0.70516000000000

$-1.61548000000000$

$-0.70516000000000$

$-0.70516000000000$

0.70516000000000

2.61548000000000

0.02877000000000

0.19323000000000

$-0.55279000000000$

0.19323000000000

$-0.55279000000000$

0.02877000000000

0.83079000000000

0.83079000000000

0.02877000000000

$-0.55279000000000$

0.19323000000000

$-0.02877000000000$

$-0.83079000000000$

$-0.19323000000000$

1.55279000000000

$-0.19323000000000$

1.55279000000000

$-0.02877000000000$

$-0.83079000000000$

$-0.830790000000$

1.55279000000000

$-0.19323000000000$

0.62500000000000

.37500000000000

0.12500000000000

0.8750000000000

0.5000000000000

50000000000000

0.00000000000000

0.50000000000000

0.70516000000000

0.70516000000000

$-1.61548000000000$

0.70516000000000

0.7051600000000

$-0.70516000000000$

2.61548000000000

$-0.70516000000000$

0.83079000000000

0.02877000000000

$-0.55279000000000$

0.02877000000000

0.83079000000000

.19323000000000

0.19323000000000

0.19323000000000

$-0.55279000000000$

0.02877000000000

0.83079000000000

$-0.83079000000000$

0.02877000000000

279000000000

$-0.19323000000000$

$-0.02877000000000$

$-0.19323000000000$

1.55279000000000

$-0.19323000000000$

1.55279000000000

1.5527900000000

$-0.83079000000000$

0.62500000000000

0.37500000000000

0.12500000000000

0.87500000000000

0.50000000000000

0.00000000000000

0.50000000000000

0.50000000000000

.70516000000000

$-1.61548000000000$

.70516000000000

0.70516000000000

$-0.70516000000000$

2.61548000000000

0.70516000000000 0.70516000000000 0.19323000000000 .55279000000000 0.02877000000000 0.83079000000000

0.83079000000000

0.02877000000000

. . . . . 0.19323000000000

$-0.55279000000000$

.02877000000000

$-0.83079000000000$

$-0.83079000000000$

.02877000000000

$-0.19323000000000$

$-0.02877000000000$

$-0.83079000000000$

1.55279000000000

As (8b)

$\mathrm{Rb} \quad(8 \mathrm{a})$

$\begin{array}{ll}\mathrm{Rb} & (8 \mathrm{a}) \\ \mathrm{Rb} & (16 \mathrm{~d})\end{array}$

$\mathrm{Rb} \quad(16 \mathrm{~d})$

$\mathrm{Rb}$ (16d)

$\mathrm{Rb}$ (16d)

$\mathrm{Se} \quad(32 \mathrm{e})$

$\mathrm{Se} \quad(32 \mathrm{e})$

$\begin{array}{ll}\mathrm{Se} & (32 \mathrm{e}) \\ \mathrm{Se} & (32 \mathrm{e})\end{array}$

$\mathrm{Se} \quad(32 \mathrm{e})$

$\mathrm{Se} \quad(32 \mathrm{e})$

$\mathrm{Se} \quad(96 \mathrm{~g})$

Se $(96 \mathrm{~g})$

$\mathrm{Se}(96 \mathrm{~g})$

Se $(96 \mathrm{~g})$

$\mathrm{Se}(96 \mathrm{~g})$

Se $(96 \mathrm{~g})$

Se $(96 \mathrm{~g})$

Se $(96 \mathrm{~g})$

Se $(96 \mathrm{~g})$

$\mathrm{Se}(96 \mathrm{~g})$

Se $(96 \mathrm{~g})$

Se $(96 g)$

Se $(96 \mathrm{~g})$

Se $(96 \mathrm{~g})$

Se $(96 \mathrm{~g})$

Se $(96 g)$

Se $(96 \mathrm{~g})$

Se $(96 \mathrm{~g})$

Se $(96 \mathrm{~g})$

$\mathrm{Se}(96 \mathrm{~g})$

$\mathrm{Ca}_{3} \mathrm{Al}_{2} \mathrm{O}_{6}$ : A2B3C6_cP264_205_2d_ab2c2d_6d - CIF

\section{\# CIF file}

data_findsym-output

audit creation_method FINDSYM

chemical_name mineral 'Ca3Al2O6'

chemical_formula_sum, A12 Ca3 O6,

loop

publ_author_nam

P. Mondal

J. W. Jeffery

ournal_name full_name

Acta Crystallographica Section B: Structural Science

_journal_volume 31

journal_year 1975

journal_page_first 689

Journal_page

The crystal structure of tricalcium aluminate, $\operatorname{Ca} \$_{-}\{3\} \$ A 1 \$_{-}\{2\} \$ O \$_{-}\{6\} \$$

aflow_title 'Ca\$_ $\{3\} \$ \mathrm{Al}_{-}\{2\} \$ \mathrm{O}_{-}\{6\} \$$ Structure

aflow_proto , A2B 3 C6 cP264 205 2d ab2c2d $6 d$

aflow_params $, a, x_{-}\{3\}, x_{-}\{4\}, x_{-}\{5\}, y_{-}\{5\}, z_{-}\{5\}, x_{-}\{6\}, y_{-}\{6\}, z_{-}\{6\}, x_{-}\{7\}$

$\hookrightarrow \mathrm{y}_{-}\{7\}, \mathrm{z}_{-}\{7\}, \mathrm{x}_{-}\{8\}, \mathrm{y}_{-}\{8\}, \mathrm{z}_{-}\{8\}, \mathrm{x}_{-}\{9\}, \mathrm{y}_{-}\{9\}, \mathrm{z}_{-}\{9\}, \mathrm{x}_{-}\{10\}, \mathrm{y}_{-}\{10\}$,

$\hookrightarrow \mathrm{z}_{-}\{10\}, \mathrm{x}_{-}\{11\}, \mathrm{y}_{-}\{11\}, \mathrm{z}_{-}\{11\}, \mathrm{x}_{-}$

flow $\mathrm{z}_{-}\{13\}, \mathrm{x}_{-}\{14\}, \mathrm{y}_{-}\{14\}, \mathrm{z}_{-}\{14\}, 0.375,0.2526,0.0133,0.0197,0.2444$,

$\hookrightarrow 0.2335,0.0046,0.1386,0.3763,0.1272,0.38,0.3838,0.1209,0.2777$,

$\hookrightarrow 0.1241,0.0103,0.4835,0.1315,0.2536,0.2664,0.2841,0.1049,0.235$

$\hookrightarrow 0.4047,0.2921,0.3491,-0.0385,-0.1074,0.1509,-0.0104,-0.0242$,

aflow Strukturbericht 'None'

aflow_Pearson 'cP264

symmetry_space group_name_H-M "P $21 /$ a -3 "

symmetry_Int_Tables_number 205

cell_length_a $\quad 15.26300$

cell_length_b 15.26300

cell_length_c $\quad 15.26300$

cell_angle_alpha 90.00000

_ella 90.00000

loop

space_group_symop_id

space_group_symop_operation_xyz

$1 \mathrm{x}, \mathrm{y}, \mathrm{z}$

$2 x+1 / 2,-y+1 / 2,-z$

$3-\mathrm{x}, \mathrm{y}+1 / 2,-\mathrm{z}+1 / 2$

$4-x+1 / 2,-y, z+1 / 2$

$5 \mathrm{y}, \mathrm{z}, \mathrm{x}$

$6 \mathrm{y}+1 / 2,-\mathrm{z}+1 / 2,-\mathrm{x}$

$7-\mathrm{y}, \mathrm{z}+1 / 2,-\mathrm{x}+1 / 2$
$8-\mathrm{y}+1 / 2,-\mathrm{z}, \mathrm{x}+1 / 2$

$9 \mathrm{z}, \mathrm{x}, \mathrm{y}$

$10 \mathrm{z}+1 / 2,-\mathrm{x}+1 / 2,-\mathrm{y}$

$11-z, x+1 / 2,-y+1 / 2$

$13-\mathrm{x},-\mathrm{y},-\mathrm{z}$

$14-\mathrm{x}+1 / 2, \mathrm{y}+1 / 2, \mathrm{z}$

$16 \mathrm{x},-\mathrm{y}+1 / 2, \mathrm{z}+1 / 2$

$16 \mathrm{x}+1 / 2, y,-z$

$18-\mathrm{y}+1 / 2, \mathrm{z}+1 / 2, \mathrm{x}$

$19 \mathrm{y},-\mathrm{z}+1 / 2, \mathrm{x}+1 / 2$

$20 \mathrm{y}+1 / 2, \mathrm{z},-\mathrm{x}+1 / 2$

$21-\mathrm{z},-\mathrm{x},-\mathrm{y}$

$22-\mathrm{z}+1 / 2, \mathrm{x}+1 / 2, \mathrm{y}$

$24 \mathrm{z}+1 / 2, \mathrm{x},-\mathrm{y}+1 / 2$

loop

atom_site_label

atom_site_type_symbol

atom_site_symmetry_multiplicity

atom_site_Wyckoff_label

atom_site_fract_x

-atom_site_fract_y

- atom_site_fract_z

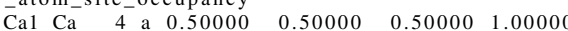

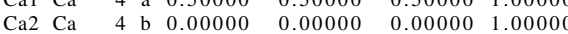

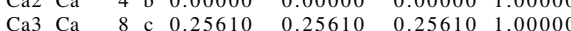

$\begin{array}{llllllll}\mathrm{Ca} 4 & \mathrm{Ca} & 8 & \mathrm{c} & 0.37500 & 0.37500 & 0.37500 & 1.00000\end{array}$

$\begin{array}{llllllll}\mathrm{Al} 1 & \mathrm{Al} & 24 & \mathrm{~d} & 0.25260 & 0.01330 & 0.01970 & 1.00000\end{array}$

$\begin{array}{llllllll}\mathrm{A} 12 & \mathrm{Al} & 24 & \mathrm{~d} & 0.24440 & 0.23350 & 0.00460 & 1.00000\end{array}$

$\begin{array}{llllllll}\mathrm{Ca} 5 & \mathrm{Ca} & 24 & \mathrm{~d} & 0.13860 & 0.37630 & 0.12720 & 1.00000\end{array}$

$\begin{array}{llllllll}\mathrm{Ca} 6 & \mathrm{Ca} & 24 & \mathrm{~d} & 0.38000 & 0.38380 & 0.12090 & 1.00000\end{array}$

$\begin{array}{lllllllll}\mathrm{O} 1 & \mathrm{O} & 24 & \mathrm{~d} & 0.27770 & 0.12410 & 0.01030 & 1.00000\end{array}$

$\begin{array}{lllllllll}\mathrm{O} 2 & \mathrm{O} & 24 & \mathrm{~d} & 0.48350 & 0.13150 & 0.25360 & 1.00000\end{array}$

$\begin{array}{lllllllll}\mathrm{O} 3 & \mathrm{O} & 24 & \mathrm{~d} & 0.26640 & 0.28410 & 0.10490 & 1.00000\end{array}$

$\begin{array}{lllllllll}\mathrm{O} 4 & \mathrm{O} & 24 & \mathrm{~d} & 0.23500 & 0.40470 & 0.29210 & 1.00000\end{array}$

$\begin{array}{lllllllll}\mathrm{O} 5 & \mathrm{O} & 24 & \mathrm{~d} & 0.34910 & -0.03850 & -0.10740 & 1.00000\end{array}$

O6 $\mathrm{O}-24 \mathrm{~d} 0.15090-0.01040-0.02420-1.00000$

$\mathrm{Ca}_{3} \mathrm{Al}_{2} \mathrm{O}_{6}$ : A2B3C6_cP264_205_2d_ab2c2d_6d - POSCAR

A2B3C6_cP264_205_2d_ab2c2d_6d \& a, x3, x4, x5,y5, z5, x6, y6, z6 , x7,y7,z7, x8,y8 $\hookrightarrow, \mathrm{z} 8, \mathrm{x} 9, \mathrm{y} 9, \mathrm{z} 9, \mathrm{x} 10, \mathrm{y} 10, \mathrm{z} 10, \mathrm{x} 11, \mathrm{y} 11, \mathrm{z} 11, \mathrm{x} 12, \mathrm{y} 12, \mathrm{z} 12, \mathrm{x} 13, \mathrm{y} 13, \mathrm{z} 13$

$\hookrightarrow \mathrm{x} 14, \mathrm{y} 14, \mathrm{z} 14$-params $=15.263,0.2561,0.375,0.2526,0.0133,0.0197$

$\hookrightarrow 0.2444,0.2335,0.0046,0.1386,0.3763,0.1272,0.38,0.3838,0.1209$,

$\hookrightarrow 0.2777,0.1241,0.0103,0.4835,0.1315,0.2536,0.2664,0.2841,0.1049$

$\hookrightarrow 0.235,0.4047,0.2921,0.3491,-0.0385,-0.1074,0.1509,-0.0104,-$

$\hookrightarrow 0.0242 \& \mathrm{~Pa}-3 \mathrm{~T}_{-}\{\mathrm{h}\}^{\wedge}\{6\} \# 205\left(\mathrm{abc}^{\wedge} 2 \mathrm{~d}^{\wedge} 10\right) \& \mathrm{cP} 264 \&$ None \&

$\hookrightarrow \mathrm{Ca} 3 \mathrm{Al} 2 \mathrm{O} 6$ \& Ca3Al2O6 \& P. Mondal and J. W. Jeffery, Acta

$\hookrightarrow$ Crystallogr. Sect. B Struct. Sci. 31, 689-697 (1975)

1.00000000000000

15.26300000000000

0.00000000000000

0.00000000000000

15.26300000000000

0.00000000000000

0.00000000000000

$\begin{array}{rrr}\mathrm{Al} & \mathrm{Ca} & \mathrm{O} \\ 48 & 72 & 144\end{array}$

Direct

0.25260000000000

0.24740000000000

0.75260000000000

0.01970000000000

0.51970000000000

0.48030000000000

$-0.0197000000000$

$-0.01970000000000$

0.01330000000000

0.51330000000000

0.48670000000000

$-0.25260000000000$

0.7526000000000

0.25260000000000

0.24740000000000

$-0.01970000000000$

0.01970000000000

0.48670000000000

0.24440000000000

0.25560000000000

0.74440000000000

0.00460000000000

0.50460000000000

0.49540000000000

$-0.00460000000000$

0.23350000000000 
$-0.00460000000000$ 0.49540000000000 0.00460000000000 $-0.23350000000000$ 0.23350000000000 0.26650000000000 0.73350000000000 0.00000000000000 0.00000000000000 0.50000000000000 0.50000000000000 0.50000000000000 0.00000000000000 0.25610000000000 0.24390000000000 $-0.25610000000000$ 0.75610000000000 $-0.25610000000000$ 0.75610000000000 0.25610000000000 0.24390000000000 0.37500000000000 0.12500000000000 0.87500000000000 $-0.37500000000000$ 0.87500000000000 0.37500000000000 0.12500000000000 0.13860000000000 0.36140000000000 0.13860000000000 0.63860000000000 0.12720000000000 0.62720000000000 0.3728000000000 0.37630000000000 $-0.37630000000000$ 0.87630000000000 0.12370000000000 0.63860000000000 0.13860000000000 0.36140000000000 $-0.12720000000000$ 0.37280000000000 0.62720000000000 0.12720000000000 0.37630000000000 0.37630000000000 0.87630000000000 0.38000000000000 0.12000000000000 $-0.38000000000000$ 0.88000000000000 0.62090000000000 0.37910000000000 $-0.12090000000000$ 0.38380000000000 0.38380000000000 0.88380000000000 0.11620000000000 $-0.38000000000000$ 0.88000000000000
0.38000000000000 0.12000000000000 $-0.12090000000000$ 0.37910000000000 0.62090000000000 $-0.38380000000000$ 0.38380000000000 0.11620000000000 0.88380000000000 0.27770000000000 0.22230000000000 $-0.27770000000000$ 0.77770000000000 0.01030000000000 0.48970000000000 0.01030000000000 0.12410000000000
-0.12410000000000 $-0.12410000000000$ 0.62410000000000 0.37590000000000 0.77770000000000 0.27770000000000 0.22230000000000 0.01030000000000 0.48970000000000 0.51030000000000 0.01030000000000 0.12410000000000 0.12410000000000 0.37590000000000 0.48350000000000
$-0.24440000000000$ . 0.2444000000000 0.25560000000000 0.0046000000000 0.49540000000000 0.00460000000000 0.00000000000000 0.5000000000000 0.50000000000000 0.50000000000000 0.500000000000 0.000000000000 0.25610000000000 $-0.25610000000000$ 0.75610000000000 0.24390000000000 $-0.25610000000000$ 0.25610000000000 0.2439000000000 0.75610000000000 0.3750000000000 $-0.37500000000000$ 0.87500000000000 $-0.37500000000000$ 0.37500000000000 0.12500000000000 0.87500000000000 0.37630000000000 0.37630000000000 0.87630000000000 0.12370000000000 0.13860000000000 0.36140000000000 $-0.13860000000000$ 0.63860000000000 0.12720000000000 0.62720000000000 0.372800000000 $-0.37630000000000$ 0.37630000000000 0.12370000000000 0.87630000000000 $-0.13860000000000$ 0.63860000000000 0.13860000000000 0.36140000000000 $-0.1272000000000$ 0.37280000000000 0.62720000000000 0.12720000000000 0.38380000000000 0.38380000000000 0.11620000000000 0.12000000000000 $-0.38000000000000$ 0.88000000000000 0.12090000000000 0.62090000000000 0.37910000000000 $-0.12090000000000$ $-0.38380000000000$ 0.3838000000000 0.11620000000000 0.8838000000000 $-0.3800000000000$ 0.88000000000000 0.1200000000000 $-0.12090000000000$ 0.37910000000000 0.62090000000000 0.12090000000000 0.1241000000000 $-0.12410000000000$ 0.62410000000000 0.37590000000000 0.27770000000000 0.22230000000000 $-0.27770000000000$ 0.7777000000000 0.5103000000000 $-0.01030000000000$ $-0.12410000000000$ 0.12410000000000 0.62410000000000 $-0.2777000000000$ 0.77770000000000 0.27770000000000 0.22230000000000 0.01030000000000 0.48970000000000 0.5103000000000 0.01030000000000

$-0.23350000000000$ 0.23350000000000 0.26650000000000 0.73350000000000 .24440000000000 0.7444000000000 0.2444000000000 0.25560000000000 0.0000000000000 0.50000000000000 0.50000000000000 0.00000000000000 0.50000000000000 0.00000000000000 0.00000000000000 0.50000000000000 0.25610000000000 .75610000000000 0.24390000000000 .25610000000000 .25610000000000 .24390000000000 0.75610000000000 0.25610000000000 0.37500000000000 0.87500000000000 0.12500000000000 .37500000000000 0.12500000000000 0.87500000000000 0.3750000000000 0.12720000000000 0.6272000000000 0.372800000000 0.372800000000 0.12720000000000 0.37630000000000 0.37630000000000 0.87630000000000 0.12370000000000 0.13860000000000 0.36140000000000 $-0.13860000000000$ 0.63860000000000 $-0.12720000000000$ 0.37280000000000 0.62720000000000 0.12720000000000 0.37630000000000 0.37630000000000 0.87630000000000 0.13860000000000 .63860000000000 0.13860000000000 0.3614000000000 0.12090000 0.62090000000000 0.37910000000000 $-0.12090000000000$ 0.38380000000000 $-0.38380000000000$ 0.88380000000000 0.11620000000000 0.38000000000000 0.12000000000000 38000000000000 .12090000000000 .37910000000000 .62090000000000 0.12090000000000 $-0.38380000000000$ 0.38380000000000 0.11620000000000 0.88380000000000 $-0.38000000000000$ 0.88000000000000 0.38000000000000 0.12000000000000 0.0103000000000 0.51030000000000 0.48970000000000 (2) .12410000000000 .12410000000000 0.62410000000000 0.37590000000000 0.27770000000000 . . 0.77770000000000 0.48970000000000 0.51030000000000 0.01030000000000 .12410000000000 0.1241000000000 .27770000000000 0.77770000000000 0.27770000000000 0.22230000000000
0.25360000000000 
0.48960000000000 0.51040000000000 $-0.15090000000000$ 0.65090000000000 0.15090000000000 0.34910000000000 0.02420000000000 0.52420000000000 0.47580000000000 $-0.02420000000000$ 0.01040000000000 $-0.01040000000000$ 0.51040000000000 0.48960000000000
0.52420000000000 0.02420000000000 0.0104000000000 0.51040000000000 0.48960000000000 $-0.15090000000000$ 0.65090000000000 0.15090000000000 0.34910000000000 0.02420000000000 0.52420000000000 0.47580000000000 $-0.02420000000000$ $-0.01040000000000$
.15090000000000 .6509000000000 0.52420000000000 0.47580000000000 0.02420000000000 0.0104000000000 $-0.01040000000000$ 0.5104000000000 0.48960000000000 $-0.15090000000000$ 0.65090000000000 0.15090000000000 0.02420000000000
O $(24 \mathrm{~d})$

O $(24 \mathrm{~d})$

$\mathrm{O} \quad(24 \mathrm{~d})$

$O \quad(24 d)$

$(24 d)$
$(24 d)$

$(24 d)$

$(24 d)$

$(24 d)$

0 (24d)

O $(24 \mathrm{~d})$

O $(24 \mathrm{~d})$

$\begin{array}{ll}\mathrm{O} & (24 \mathrm{~d}) \\ \mathrm{O} & (24 \mathrm{~d})\end{array}$
Simple Cubic $\mathrm{C}_{60}$ Buckminsterfullerine: A_cP240_205_10d - CIF

\# CIF file
data_findsym-output
_audit_creation_method FINDSYM
_chemical_name_mineral 'Buckminsterfullerene,
_chemical_formula_sum 'C'
loop_-
-publ_author_name
'W. I. F. David,
'R. M. Ibberson,
'J. C. Matthewman,
'K. Prassides,
'T. J. S. Dennis,
'J. P. Hare',
'H. W. Kroto'
'R. Taylor,
'D. R. M. Walton,
_journal_name_full_name
;
Nature
;
_journal_volume 353
-journal_year 1991
-journal_page_first 147
_journal_page_last 149
-publ_Section_title
;

Crystal structure and bonding of ordered $C \$ \_\{60\} \$$

aflow_title 'Simple Cubic C\$_ $\{60\} \$$ Buckminsterfullerine Structure' aflow_proto, A_cP240_205 $10 \mathrm{~d}$ '

aflow_params $, a, x_{-}\{1\}, y_{-}\{1\}, z_{-}\{1\}, x_{-}\{2\}, y_{-}\{2\}, z_{-}\{2\}, x_{-}\{3\}, y_{-}\{3\}, z_{-}\{3\}$, $\hookrightarrow \mathrm{x}_{-}\{4\}, \mathrm{y}_{-}\{4\}, \mathrm{z}_{-}\{4\}, \mathrm{x}_{-}\{5\}, \mathrm{y}_{-}\{5\}, \mathrm{z}_{-}\{5\}, \mathrm{x}_{-}\{6\}, \mathrm{y}_{-}\{6\}, \mathrm{z}_{-}\{6\}, \mathrm{x}_{-}\{7\}, \mathrm{y}_{-}$ $\hookrightarrow 7\}, z_{-}\{7\}, x_{-}\{8\}, y_{-}\{8\}, z_{-}\{8\}, x_{-}\{9\}, y_{-}\{9\}, z_{-}\{9\}, x_{-}\{10\}, y_{-}\{10\}, z_{-}\{$

aflow_params_values $\quad 14.04078,0.2294,-0.0325,0.101,0.2467,-0.054,0.006$ $\hookrightarrow \quad 0.2081,0.0646,0.1289,0.2066,0.8599,-0.036,0.171,-0.0963,0.159$

$\hookrightarrow 0.2236,0.1122,-0.0371,0.2439,0.0192,-0.0636,0.2053,0.1349$,

$\hookrightarrow 0.0616,0.1503,0.7983,0.0202,0.1323,0.8207,0.1186$,

aflow_Strukturbericht 'None,

_aflow_Pearson 'cP240,

symmetry_space_group_name_H-M "P $21 / \mathrm{a}-3$

_symmetry_Int_Tables_number 205

cell_length_a $\quad 14.04078$

cell_length_b 14.04078

cell_length_c 14.04078

$\begin{array}{ll}\text { _cell_angle_alpha } & 90.00000 \\ \text { cell_angle_beta } & 90.00000\end{array}$

cell_angle_gamma 90.00000

loop

space group_symop id

space group_symop_operation_xyz

$1 \mathrm{x}, \mathrm{y}, \mathrm{z}$

$2 \mathrm{x}+1 / 2,-\mathrm{y}+1 / 2,-\mathrm{z}$

$3-\mathrm{x}, \mathrm{y}+1 / 2,-\mathrm{z}+1 / 2$

$4-x+1 / 2,-y, z+1 / 2$

$5 \mathrm{y}, \mathrm{z}, \mathrm{x}$

$6 \mathrm{y}+1 / 2,-\mathrm{z}+1 / 2,-\mathrm{x}$

$7-y, z+1 / 2,-x+1 / 2$
$8-y+1 / 2,-z, x+1 / 2$

$9 \mathrm{z}, \mathrm{x}, \mathrm{y}$

$10 \mathrm{z}+1 / 2,-\mathrm{x}+1 / 2,-\mathrm{y}$

$11-\mathrm{z}, \mathrm{x}+1 / 2,-\mathrm{y}+1 / 2$

$12-\mathrm{z}+1 / 2,-\mathrm{x}, \mathrm{y}+1 / 2$

$13-\mathrm{x},-\mathrm{y},-\mathrm{z}$

$14-\mathrm{x}+1 / 2, \mathrm{y}+1 / 2, \mathrm{z}$

$15 \mathrm{x},-\mathrm{y}+1 / 2, \mathrm{z}+1 / 2$

$16 \mathrm{x}+1 / 2, \mathrm{y},-\mathrm{z}+1$
$17-\mathrm{y},-\mathrm{z},-\mathrm{x}$

$17-\mathrm{y},-\mathrm{z},-\mathrm{x}$
$18-\mathrm{y}+1 / 2, \mathrm{z}+1 / 2, \mathrm{x}$

$19 \mathrm{y},-\mathrm{z}+1 / 2, \mathrm{x}+1 / 2$

$20 \mathrm{y}+1 / 2, \mathrm{z},-\mathrm{x}+1 / 2$

$21-\mathrm{z},-\mathrm{x},-\mathrm{y}$

$22-\mathrm{z}+1 / 2, \mathrm{x}+1 / 2, \mathrm{y}$

$\begin{array}{ll}23 & \mathrm{z},-\mathrm{x}+1 / 2, \mathrm{y}+1 / 2 \\ 24 & \mathrm{z}+1 / 2, \mathrm{x},-\mathrm{y}+1 / 2\end{array}$

loop_

atom_site_label

atom_site_type_symbol

atom_site_symmetry_multiplicity

atom_site_Wyckoff_label

atom_site_fract_x

_atom_site_fract_y

-atom_site_fract_z
atom_site_occupancy

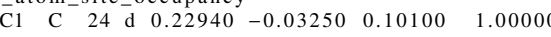

$\begin{array}{llllllll}\mathrm{C} 2 & \mathrm{C} & 24 & \mathrm{~d} & 0.24670 & -0.05400 & 0.00610 & 1.00000\end{array}$

$\begin{array}{llllllll}\mathrm{C} 3 & \mathrm{C} & 24 & \mathrm{~d} & 0.20810 & 0.06460 & 0.12890 & 1.00000\end{array}$

$\begin{array}{llllllll}\text { C4 } & \text { C } & 24 & \text { d } & 0.20660 & 0.85990 & -0.03600 & 1.00000\end{array}$

$\begin{array}{llllllll}\text { C5 } & \mathrm{C} & 24 & \mathrm{~d} & 0.17100 & -0.09630 & 0.15900 & 1.00000\end{array}$

$\begin{array}{lllllllll}\mathrm{C} 6 & \mathrm{C} & 24 & \mathrm{~d} & 0.22360 & 0.11220 & -0.03710 & 1.00000\end{array}$

$\begin{array}{llllllll}\text { C7 } & \text { C } & 24 & \text { d } & 0.24390 & 0.01920 & -0.06360 & 1.00000\end{array}$

$\begin{array}{llllllll}\mathrm{C} 8 & \mathrm{C} & 24 & \mathrm{~d} & 0.20530 & 0.13490 & 0.06160 & 1.00000\end{array}$

$\begin{array}{llllllll}\text { C9 } & \mathrm{C} & 24 & \mathrm{~d} & 0.15030 & 0.79830 & 0.02020 & 1.00000\end{array}$

$\begin{array}{lllllll}\mathrm{C} 10 \mathrm{C} & 24 & \mathrm{~d} & 0.15030 & 0.79830 & 0.02020 & 1.00000\end{array}$

Simple Cubic $\mathrm{C}_{60}$ Buckminsterfullerine: A_cP240_205_10d - POSCAR

cP240_205_10d \& a , x1, y1, z1, x2 , y2, z2, x3,y3, z3 , x4,y4, z4, x5 , y5 , z5 , x6, y6

$\hookrightarrow \mathrm{z} 6, \mathrm{x} 7, \mathrm{y} 7, \mathrm{z} 7, \mathrm{x} 8, \mathrm{y} 8, \mathrm{z} 8, \mathrm{x} 9, \mathrm{y} 9, \mathrm{z} 9, \mathrm{x} 10, \mathrm{y} 10, \mathrm{z} 10--\mathrm{param}=14.04078$

$\hookrightarrow 0.2066,0.8599,-0.036,0.171,-0.0963,0.159,0.2236,0.1122,-0.0371$

$\hookrightarrow 0.2439,0.0192,-0.0636,0.2053,0.1349,0.0616,0.1503,0.7983,0.0202$

$\hookrightarrow \quad 0.1323,0.8207,0.1186 \& \mathrm{~Pa}-3 \mathrm{~T}_{-}\{\mathrm{h}\}^{\wedge}\{6\} \# 205\left(\mathrm{~d}^{\wedge} 10\right) \& \mathrm{cP} 240 \&$

$\hookrightarrow$ None \& C \& Buckminsterfullerene \& W. I. F. David et al., Nature

$\hookrightarrow \quad 353,147-149$ (1991)

1.00000000000000

$1.000000000000-0.00000000000000$

$\begin{array}{lll}0.00000000000000 & 14.04078000000000 & 0.00000000000000\end{array}$

$\begin{array}{lll}0.00000000000000 & 0.00000000000000 & 14.04078000000000\end{array}$

240

Direc

$0.22940000000000-0.03250000000000$

0.27060000000000

$-0.22940000000000$

0.72940000000000

0.60100000000000

0.3990000000000

0.1000000000000

$-0.03250000000000$

0.03250000000000

0.46750000000000

0.53250000000000

$-0.22940000000000$

0.72940000000000

0.22940000000000
0.27060000000000

10100000000000

0.39900000000000

0.60100000000000

0.10100000000000

0.03250000000000

$-0.03250000000000$

0.53250000000000

0.4675000000000

0.4675000000000

0.25330000000000

$-0.24670000000000$

0.74670000000000

0.00610000000000

0.50610000000000

0.49390000000000

$-0.00610000000000$

$-0.05400000000000$

0.4460000000000

0.55400000000000

$-0.24670000000000$

0.74670000000000

0.24670000000000

0.24670000000000

0.2533000000000

$-0.00610000000000$

0.50610000000000

0.00610000000000

0.05400000000000

$-0.05400000000000$

0.55400000000000

0.44600000000000

0.20810000000000

0.29190000000000

$-0.20810000000000$

0.70810000000000

0.62890000000000

0.37110000000000

$-0.1289000000000$

$-0.12890000000000$

0.0646000000000

$-0.06460000000000$

0.43540000000000

$-0.20810000000000$

0.70810000000000

0.2081000000000

0.29190000000000

$-0.12890000000000$

0.37110000000000

0.6289000000000

0.12890000000000

$-0.06460000000000$

0.06460000000000

0.43540000000000

0.56460000000000

0.20660000000000

0.29340000000000

0.10100000000000 0.60100000000000 0.39900000000000 $-0.10100000000000$ $-0.03250000000000$ 0.03250000000000 0.46750000000000 0.46750000000000 0.22940000000000 0.2294000000000 0.27060000 0.22940000000000 0.72940000000000 $-0.10100000000000$ 0.39900000000000 0.60100000000000 0.10100000000000 0.03250000000000 $-0.03250000000000$ 0.53250000000000 0.46750000000000 $-0.22940000000000$ 0.72940000000000 0.22940000000000 0.27060000000000 0.27060000000000 0.0061000000000 0.50610000000000 0.49390000000000 $-0.00610000000000$ $-0.05400000000000$ 0.05400000000000 0.44600000000000 0.55400000000000 0.24670000000000 0.25330000000000 $-0.24670000000000$ 0.74670000000000 $-0.00610000000000$ 0.49390000000000 0.50610000000000 0.00610000000000 0.0540000000000 $-0.05400000000000$ 0.55400000000000 0.44600000000000 $-0.24670000000000$ 0.74670000000000 0.24670000000000 0.25330000000000 0.12890000000000 0.62890000000000 0.37110000000000 $-0.12890000000000$ 0.06460000000000 $-0.06460000000000$ 0.56460000000000 0.43540000000000 0.20810000000000 0.208100000000 $-0.20810000000$ 0.70810000000000 0.7081000000000 $-0.12890000000000$ 0.37110000000000 0.62890000000000 0.12890000000000 $-0.06460000000000$ 0.06460000000000 0.43540000000000 0.56460000000000 $-0.20810000000000$ 0.70810000000000 0.20810000000000 0.29190000000000 $-0.03600000000000$ 0.46400000000000

C (24d)

C (24d)

C (24d)

C (24d)

C $(24 d)$

C (24d) 
$-0.20660000000000$ 0.70660000000000 $-0.03600000000000$ 0.46400000000000 0.53600000000000 0.03600000000000 0.85990000000000 $-0.85990000000000$ 1.35990000000000 $-0.35990000000000$ $-0.20660000000000$ 0.70660000000000 0.20660000000000 0.29340000000000 0.03600000000000 0.53600000000000 0.46400000000000 $-0.03600000000000$ 0.85990000000000 0.85990000000000 $-0.35990000000000$ 1.35990000000000 0.17100000000000 0.32900000000000 $-0.17100000000000$ 0.67100000000000 0.15900000000000 0.34100000000000 $-0.15900000000000$ $-0.09630000000000$ 0.09630000000000 0.40370000000000 0.59630000000000 0.17100000000000 0.67100000000000 0.17100000000000 0.32900000000000 $-0.15900000000000$ 0.34100000000000 0.65900000000000 0.15900000000000 0.09630000000000 $-0.09630000000000$ 0.59630000000000 0.40370000000000 0.22360000000000 0.27640000000000 $-0.22360000000000$ 0.72360000000000 0.03710000000000 0.46290000000000 0.53710000000000 0.03710000000000 0.11220000000000
-0.11220000000000 $-0.11220000000000$ 0.61220000000000 0.38780000000000 0.7236000000000 0.22360000000000 0.27640000000000 0.03710000000000 0.53710000000000 0.46290000000000 $-0.03710000000000$ 0.11220000000000 0.11220000000000 0.38780000000000 0.61220000000000 0.24390000000000 0.25610000000000 0.24390000000000 0.74390000000000 $-0.06360000000000$ 0.43640000000000 0.56360000000000 0.01920000000000 $-0.01920000000000$ 0.51920000000000 0.48080000000000 $-0.24390000000000$ 0.74390000000000 0.24390000000000 0.25610000000000 0.06360000000000 0.56360000000000 0.43640000000000 $-0.06360000000000$ $-0.01920000000000$ 0.01920000000000 0.51920000000000 0.20530000000000 0.29470000000000 $-0.20530000000000$ 0.70530000000000 0.06160000000000 0.56160000000000 0.43840000000000 $-0.06160000000000$ 0.13490000000000 $-0.13490000000000$
1.35990000000000 0.3596000000000 0.20660000000000 0.29340000000000 0.7066000000000 $-0.0360000000000$ 0.46400000000000 0.53600000000000 0.03600000000000 0.85990000000000 $-0.35990000000000$ 1.3599000000000 $-0.20660000000000$ 0.70660000000000 0.20660000000000 0.29340000000000 0.03600000000000 0.53600000000000 0.46400000000000 $-0.03600000000000$ $-0.0963000000000$ 6300000000 0.40370000000000 0.59630000000000 0.17100000000000 0.32900000000000 $-0.17100000000000$ 0.67100000000000 0.15900000000000 0.65900000000000 0.34100000000000 $-0.15900000000000$ 0.09630000000000 $-0.09630000000000$ 0.5963000000000 0.40370000000000 $-0.1710000000000$ 0.67100000000000 0.1710000000000 0.32900000000000 $-0.15900000000000$ 0.34100000000000 0.65900000000000 0.15900000000000 0.11220000000000 $-0.1122000000000$ 0.6122000000000 0.38780000000000 0.22360000000000 0.27640000000000 $-0.22360000000000$ 0.72360000000000 0.03710000000000 0.46290000000000 0.53710000000000 0.0371000000000 $-0.1122000000000$ 0.11220000000000 0.38780000000000 0.61220000000000 $-0.22360000000000$ 0.72360000000000 0.22360000000000 0.27640000000000 0.03710000000000 0.53710000000000 0.4629000000000 $-0.0371000000000$ 0.01920000000000 0.0192000000000 0.51920000000000 0.48080000000000 0.24390000000000 0.25610000000000 $-0.24390000000000$ 0.74390000000000 0.43640000000000 0.56360000000000 0.06360000000000 $-0.01920000000000$ 0.01920000000000 0.48080000000000 0.51920000000000 $-0.24390000000000$ 0.74390000000000 0.2439000000000 0.25610000000000 0.06360000000000 0.56360000000000 0.43640000000000 $-0.06360000000000$ 0.13490000000000 $-0.13490000000000$ 0.63490000000000 0.36510000000000 0.20530000000000 0.29470000000000 $-0.20530000000000$ 0.70530000000000 0.06160000000000 0.5616000000000 0.43840000000000

0.53600000000000 0.0360000000000 0.85990000000000 0.85990000000000 1.35990000000000 0.35990000000000 0.20660000000000 0.29340000000000 $-0.20660000000000$ 0.70660000000000 0.03600000000000 0.53600000000000 0.46400000000000 $-0.03600000000000$ $-0.85990000000000$ 0.85990000000000 .35990000000000 35990000000000 20660000000000 0.70660000000000 0.20660000000000 0.29340000000000 0.15900000000000 0.65900000000000 0.34100000000000 $-0.15900000000000$ 0.09630000000000 0.09630000000000 0.40370000000000 0.59630000000000 0.17100000000000 0.32900000000000 17100000000000 0.67100000000000 0.15900000000000 .34100000000000 0.65900000000000 0.1590000000000 0.09630000000000 $-0.09630000000000$ 0.59630000000000 0.40370000000000 $-0.1710000000000$ 0.67100000000000 0.17100000000000 0.32900000000000 $-0.03710000000000$ 0.46290000000000 0.53710000000000 0.03710000000000 0.11220000000000 0.11220000000000 0.6122000000000 0.61220000000000 0.38780000000000 0.22360000000000 0.27640000000000 $-0.22360000000000$ 0.72360000000000 0.03710000000000 0.53710000000000 0.46290000000000 $-0.03710000000000$ 0.11220000000000 0.11220000000000 0.38780000000000 0.61220000000000 0.22360000000000 0.72360000000000 0.22360000000000 .27640000000000 0.06360000000000 0.43640000000000 0.56360000000000 0.0636000000000 0.01920000000000 $-0.01920000000000$ 0.51920000000000 0.48080000000000 0.24390000000000 0.25610000000000 $-0.24390000000000$ 0.74390000000000 .06360000000000 56360000000000 .43640000000000 $-0.06360000000000$ 0.01920000000000 0.01920000000000 0.48080000000000 0.51920000000000 
_cell_angle_alpha 90.00000

_cell_angle_beta 90.00000

loop_

_space_group_symop_id

_space_group_symop_operation_xyz

$1 \mathrm{x}, \mathrm{y}, \mathrm{z}$

$2 \mathrm{x},-\mathrm{y},-\mathrm{z}+1 / 2$

$3-x+1 / 2, y,-z$

$5 \mathrm{y}, \mathrm{z}, \mathrm{x}$

$6 \mathrm{y},-\mathrm{z},-\mathrm{x}+1 / 2$

$7-y+1 / 2, z,-x$

$9 \mathrm{z}, \mathrm{x}, \mathrm{y}$

$10 \mathrm{z},-\mathrm{x},-\mathrm{y}+1 / 2$

$11-z+1 / 2, x,-y$

$12-\mathrm{z},-\mathrm{x}+1 / 2, \mathrm{y}$

$13-\mathrm{x},-\mathrm{y},-\mathrm{z}$

$14-x, y, z+1 / 2$
$15-x+1 / 2,-y, z$

$15 \mathrm{x}+1 / 2,-\mathrm{y}, \mathrm{z}$
$16 \mathrm{x}, \mathrm{y}+1 / 2,-\mathrm{z}$

$17-\mathrm{y},-\mathrm{z},-\mathrm{x}$

$18-\mathrm{y}, \mathrm{z}, \mathrm{x}+1 / 2$

$19 \mathrm{y}+1 / 2,-\mathrm{z}, \mathrm{x}$

$21-\mathrm{z},-\mathrm{x},-\mathrm{y}$

$22-\mathrm{z}, \mathrm{x}, \mathrm{y}+1 / 2$

$23 \mathrm{z}+1 / 2,-\mathrm{x}, \mathrm{y}$

$24 \mathrm{z}, \mathrm{x}+1 / 2,-\mathrm{y}$

$25 \mathrm{x}+1 / 2, \mathrm{y}+1 / 2, \mathrm{z}+1 / 2$

$26 x+1 / 2,-y+1 / 2,-z$

$27-\mathrm{x}, \mathrm{y}+1 / 2,-\mathrm{z}+1 / 2$

$29 \mathrm{y}+1 / 2, \mathrm{z}+1 / 2, \mathrm{x}+1 / 2$

$30 \mathrm{y}+1 / 2,-\mathrm{z}+1 / 2,-\mathrm{x}$

$31-\mathrm{y}, \mathrm{z}+1 / 2,-\mathrm{x}+1 / 2$

$32-\mathrm{y}+1 / 2,-\mathrm{z}, \mathrm{x}+1 / 2$

$33 \mathrm{z}+1 / 2, \mathrm{x}+1 / 2, \mathrm{y}+1 / 2$

$34 \mathrm{z}+1 / 2,-\mathrm{x}+1 / 2,-\mathrm{y}$

$35-\mathrm{z}, \mathrm{x}+1 / 2,-\mathrm{y}+1 / 2$

$36-\mathrm{z}+1 / 2,-\mathrm{x}, \mathrm{y}+1 / 2$

$37-x+1 / 2,-y+1 / 2,-z+1 / 2$

$38-x+1 / 2, y+1 / 2, z$

$39 \mathrm{x},-\mathrm{y}+1 / 2, \mathrm{z}+1 / 2$

$40 \mathrm{x}+1 / 2, \mathrm{y},-\mathrm{z}+1 / 2$

$41-\mathrm{y}+1 / 2,-\mathrm{z}+1 / 2,-\mathrm{x}+1 / 2$

$42-\mathrm{y}+1 / 2, \mathrm{z}+1 / 2, \mathrm{x}$

$43 \mathrm{y},-\mathrm{z}+1 / 2, \mathrm{x}+1 / 2$

$44 \mathrm{y}+1 / 2, \mathrm{z},-\mathrm{x}+1 / 2$

$45-\mathrm{z}+1 / 2,-\mathrm{x}+1 / 2,-\mathrm{y}+1 / 2$

$46-\mathrm{z}+1 / 2, \mathrm{x}+1 / 2, \mathrm{y}$

$47 \mathrm{z},-\mathrm{x}+1 / 2, \mathrm{y}+1 / 2$

$48 \mathrm{z}+1 / 2, x,-y+1 / 2$

loop

atom_site_label

atom_site_type_symbol

atom_site_symmetry_multiplicity

atom_site_Wyckoff_label

atom_site_fract_x

atom_site_fract_y

atom_site_fract_z

$\begin{array}{llllllll}\mathrm{N} 1 & \mathrm{~N} & 8 & \text { a } & 0.00000 & 0.00000 & 0.00000 & 1.00000\end{array}$

$\begin{array}{lllllllll}\mathrm{Al} 1 & \mathrm{Al} & 16 & \mathrm{c} & 0.11500 & 0.11500 & 0.11500 & 1.00000\end{array}$

$\begin{array}{llllllllll}\mathrm{N} 2 & \mathrm{~N} & 24 & \mathrm{~d} & 0.20500 & 0.00000 & 0.25000 & 1.00000\end{array}$

$\begin{array}{lllllllll}\mathrm{Li} 1 & \mathrm{Li} & 48 & \mathrm{e} & 0.16000 & 0.38200 & 0.11000 & 1.00000\end{array}$

$\mathrm{AlLi}_{3} \mathrm{~N}_{2}\left(E 9_{d}\right):$ AB3C2_cI96_206_c_e_ad - POSCAR

$\mathrm{AB} 3 \mathrm{C} 2 \_\mathrm{cI} 96206$ c e ad \& a $\mathrm{x} 2, \mathrm{x} 3, \mathrm{x} 4, \mathrm{y} 4, \mathrm{z} 4-$-params $=9.46,0.115,0.205,0.16$

$\hookrightarrow 0.382,0.11 \&$ Ia $-3 \mathrm{~T}_{-}\{\mathrm{h}\} \wedge\{7\} \# 206$ (acde) \& cI96\& $\$$ E9_\{d $\} \$ \&$

$\hookrightarrow$ AlLi3N2 \& \& R. Juza and F. Hund, Z. Anorg. Allg. Chem. 257, $\hookrightarrow 13-25(1948)$

1.00000000000000

$-4.73000000000000 \quad 4.73000000000000 \quad 4.73000000000000$

$\begin{array}{rrr}4.73000000000000 & -4.73000000000000 & 4.73000000000000\end{array}$

$\begin{array}{lll}4.73000000000000 & 4.73000000000000 & -4.73000000000000\end{array}$

$\begin{array}{rrr}\mathrm{Al} & \mathrm{Li} & \mathrm{N} \\ 8 & 24 & 16\end{array}$

Direct

0.23000000000000

0.50000000000000

0.00000000000000

0.2700000000000

0.27000000000000

$-0.23000000000000$

0.50000000000000

0.00000000000000

0.73000000000000

0.49200000000000

0.22800000000000

0.27200000000000

0.00800000000000

0.54200000000000

$-0.04200000000000$

0.72200000000000

$-0.22200000000000$

0.27000000000000

$-0.05000000000000$

0.23000000000000

0.55000000000000

$-0.49200000000000$

0.77200000000000

0.23000000000000 0.50000000000000 0.23000000000000 0.0000000000000 0.73000000000000 0.50000000000000 0.57000000000000 0.2700000000 0.23000000000000 0.55000000000000 0.49200000000000 0.22800000000000 0.27200000000000 0.00800000000000 0.54200000000000 $-0.04200000000000$ $-0.22200000000000$ $-0.27000000000000$ 0.05000000000000
$-0.27200000000000$

0.99200000000000

$-0.54200000000000$

1.04200000000000

0.27800000000000

0.22200000000000

$-0.2700000000000$

0.05000000000000

0.77000000000000

0.45000000000000

0.00000000000000

0.50000000000000

0.5000000000000

0.25000000000000

0.75000000000000

0.20500000000000

0.205000000000

0.29500000000000

0.45500000000000

0.04500000000000

0.75000000000000

0.25000000000000

$-0.20500000000000$

0.70500000000000

0.54500000000000

0.95500000000000

.77000000000000 .45000000000000 $-0.49200000000000$ 0.77200000000000 0.27200000000000 0.99200000000000 $-0.54200000000000$ 1.04200000000000 0.27800000000000 0.22200000000000 0.00000000000000 0.00000000000000 0.50000000000000 0.50000000000000 0.45500000000000 0.04500000000000 .25000000000000 0.2500000000000 0.75000000000000 0.20500000000000 0.29500000000000 0.54500000000000 0.95500000000000 0.75000000000000 0.25000000000000 $-0.20500000000000$ 0.70500000000000

0.27800000000000 0.22200000000000 $-0.27000000000000$ 0.05000000000000 0.77000000000000 0.45000000000000 0.450000000000 0.77200000000000 0.77200000000000 0.27200000000000 0.99200000000000 0.00000000000000 0.50000000000000 0.00000000000000 0.20500000000000 0.29500000000000 0.45500000000000 0.04500000000000 0.0450000000000 0.25000000000000 0.75000000000000 $-0.20500000000000$ 0.70500000000000 0.54500000000000 0.95500000000000 0.75000000000000 0.25000000000000

Li $(48 \mathrm{e})$ $\mathrm{Li} \quad(48 \mathrm{e})$ $\mathrm{Li}(48 \mathrm{e})$ Li $(48 \mathrm{e}$ $\mathrm{Li} \quad(48 \mathrm{e})$ $\mathrm{Li} \quad(48 \mathrm{e})$ $\mathrm{Li} \quad(48 \mathrm{e}$ $\mathrm{Li} \quad(48 \mathrm{e})$ $\mathrm{Li} \quad(48 \mathrm{e})$ $\mathrm{Li} \quad(48 \mathrm{e})$ $\mathrm{N} \quad(8 \mathrm{a})$ $\mathrm{N} \quad(8 \mathrm{a})$ $\mathrm{N} \quad(8 \mathrm{a})$

N $(24 d)$

$\mathrm{Pd}_{17} \mathrm{Se}_{15}$ : A17B15_cP64_207_acfk_eij - CIF

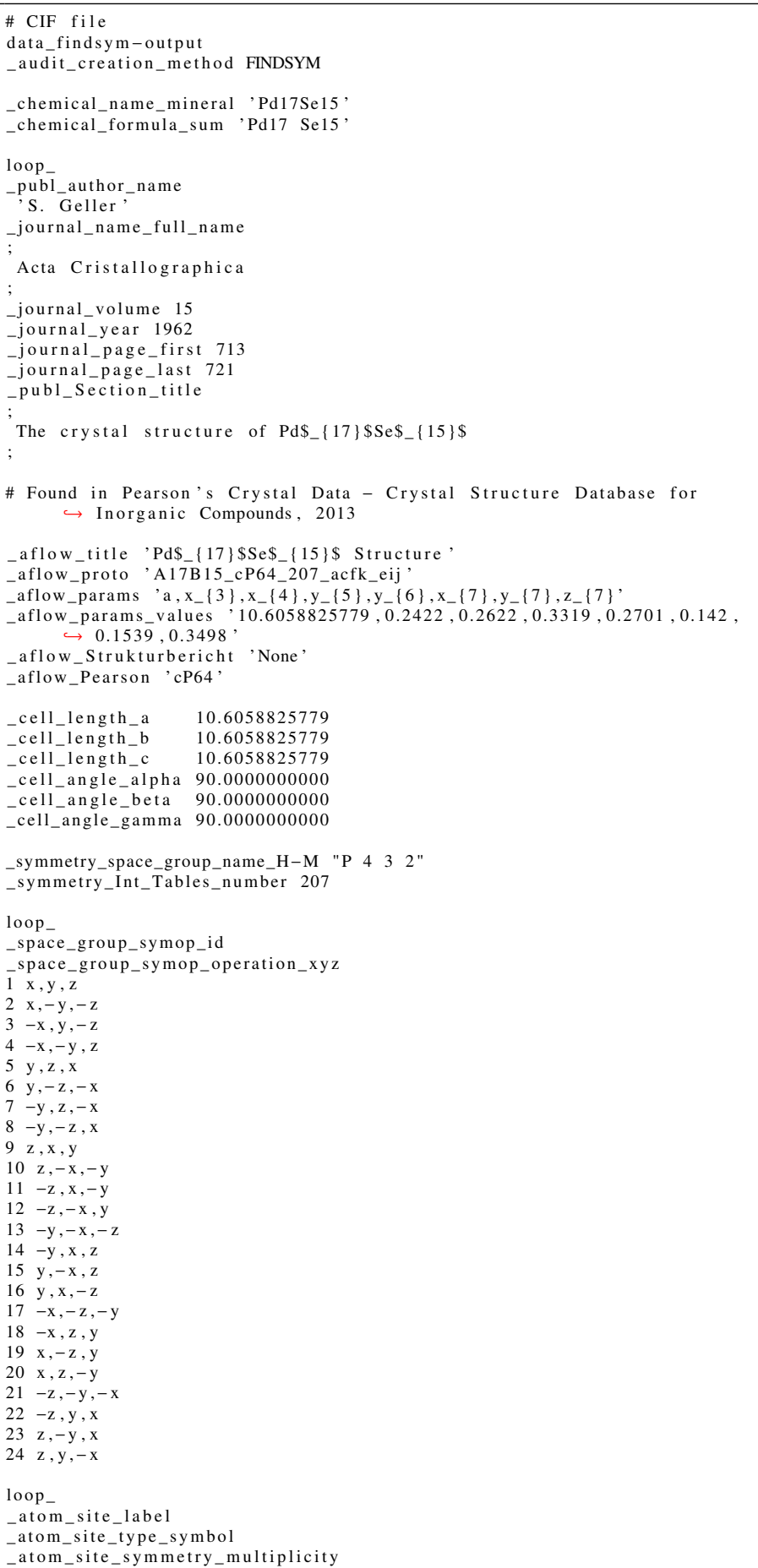

atom_site_symmetry_multiplicity $0.27000000000000 \quad \mathrm{Al} \quad(16 \mathrm{c})$ $0.50000000000000 \quad \mathrm{Al} \quad(16 \mathrm{c})$ $0.00000000000000 \quad \mathrm{Al} \quad(16 \mathrm{c})$ $-0.23000000000000 \quad \mathrm{Al} \quad(16 \mathrm{c})$ $0.73000000000000 \quad \mathrm{Al} \quad(16 \mathrm{c})$ 0.50000000000000 Al $(16 \mathrm{c})$ $0.00000000000000 \quad \mathrm{Al} \quad(16 \mathrm{c})$ 0.54200000000000 Li $(48 \mathrm{e})$ .04200000000000 Li $(48 \mathrm{e})$ $\begin{array}{lll}0.72200000000000 & \mathrm{Li} & (48 \mathrm{e})\end{array}$ $-0.72200000000000 \quad \mathrm{Li} \quad(48 \mathrm{e})$

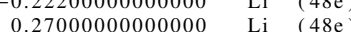
$-0.05000000000000 \quad \mathrm{Li} \quad(48 \mathrm{e})$ $0.23000000000000 \quad \mathrm{Li} \quad(48 \mathrm{e})$ $0.55000000000000 \quad \mathrm{Li} \quad(48 \mathrm{e})$ $0.49200000000000 \quad \mathrm{Li} \quad(48 \mathrm{e})$ $0.22800000000000 \quad \mathrm{Li} \quad(48 \mathrm{e})$ $.27200000000000 \quad \mathrm{Li} \quad(48 \mathrm{e})$ $-0.54200000000000 \quad \mathrm{Li} \quad(48 \mathrm{e}$ $1.04200000000000 \mathrm{Li} \quad(48 \mathrm{e})$ 
_atom_site_Wyckoff_label

atom_site_fract_x

atom_site_fract_y
atom_site_fract_z

\begin{tabular}{lllllll}
\hline Pd1 Pd & 1 a & 0.00000 & 0.00000 & 0.00000 & 1.00000
\end{tabular}

$\begin{array}{llllllll}\mathrm{Pd} 2 & \mathrm{Pd} & 3 & \mathrm{c} & 0.00000 & 0.50000 & 0.50000 & 1.0000\end{array}$

$\begin{array}{lllllllll}\mathrm{Se} 1 & \mathrm{Se} & 6 & \mathrm{e} & 0.24220 & 0.00000 & 0.00000 & 1.00000\end{array}$

$\begin{array}{llllllll}\mathrm{Pd} 3 & \mathrm{Pd} & 6 & \mathrm{f} & 0.26220 & 0.50000 & 0.50000 & 1.00000\end{array}$

$\begin{array}{llllllllll}\mathrm{Se} 2 & \mathrm{Se} & 12 & \mathrm{i} & 0.00000 & 0.33190 & 0.33190 & 1.00000\end{array}$

$\begin{array}{lllllllll}\mathrm{Se} 3 & \mathrm{Se} & 12 & \mathrm{j} & 0.50000 & 0.27010 & 0.27010 & 1.00000\end{array}$

$\begin{array}{lllllllll}\mathrm{Pd} 4 & \mathrm{Pd} & 24 & \mathrm{k} & 0.14200 & 0.15390 & 0.34980 & 1.00000\end{array}$

$\mathrm{Pd}_{17} \mathrm{Se}_{15}$ : A17B15_cP64_207_acfk_eij - POSCAR

A17B15_cP64_207_acfk_eij \& a , x3, x4,y5,y6, x7,y7,z7 --params $=10.6058825779$ $\hookrightarrow, 0.2422,0.2622,0.3319,0.2701,0.142,0.1539,0.3498 \& \mathrm{P} 432 \mathrm{O}^{\wedge}\{1\}$

$\hookrightarrow 207$ (acefijk) \& cP64 \& None \& Pd17Se15 \& \& S. Geller, Acta

$\hookrightarrow$ Cryst. $15,713-721(1962)$

1.00000000000000

10.60588257790000

0.00000000000000

10.60588257790000

0.00000000000000

$0.00000000000000 \quad 0.00000000000000$

$34-30$

Direct

0.00000000000000

0.00000000000000

0.50000000000000

0.50000000000000

0.26220000000000

0.26220000000000

0.50000000000000

0.50000000000000

0.50000000000000

0.50000000000000

0.14200000000000

$-0.14200000000000$

$-0.14200000000000$

0.14200000000000

0.34980000000000

0.34980000000000

$-0.34980000000000$

$-0.34980000000000$

0.15390000000000

$-0.15390000000000$

0.15390000000000

0.15390000000000

0.15390000000000

$-0.15390000000000$

0.15390000000000

$-0.15390000000000$

0.14200000000000

$-0.14200000000000$

$-0.14200000000000$

0.1420000000000

0.349800000000

0.34980000000000

0.349800000000

0.34900000000

0.34980000000000

0.24220000000000

$-0.24220000000000$

0.00000000000000

0.00000000000000

0.00000000000000

0.00000000000000

0.00000000000000

0.00000000000000

0.00000000000000

0.00000000000000

0.33190000000000

0.33190000000000

$-0.3319000000000$

$-0.33190000000000$

0.33190000000

0.33190000000

$-0.3319000000000$

0.33190000000000

$-0.33190000000000$

0.50000000000000

0.50000000000000

0.50000000000000

.50000000000000

0.27010000000000

0.27010000000000

$-0.27010000000000$

$-0.27010000000000$

0.27010000000000

$-0.27010000000000$

0.27010000000000

$-0.27010000000000$

00000000000000

.5000000000000

0.0000000000000

.50000000000000

.5000000000000

50000000000000

0.2622000000000

$-0.26220000000000$

0.50000000000000

0.50000000000000

0.15390000000000

$-0.15390000000000$

0.15390000000000

.15390000000000

0.14200000000000

(14.142000000000 . 14200000000000 . .14200000000000 .34980000000000 0.34980000000000 $-0.34980000000000$ $-0.34980000000000$ 0.14200000000000 .14200000000000 0.14200000000000 0.34980000000000 0.34980000000000 $-0.3498000000000$ $-0.34980000000000$ 0.15390000000000 0.15390000000000 0.15390000000000 . . 0.00000000000000 0.00000000000000 0.24220000000000 0.24220000000000 0.00000000000000 0.00000000000000 .33190000000000 .33190000000000 0.33190000000000 $-0.33190000000000$ 0.00000000000000 0.0000000000000 0.00000000000000 . .33190000000000 0.3319000000000 $-0.33190000000000$ $-0.33190000000000$ 0.27010000000000 0.27010000000000 . 27010000000000 0.50000000000000 0.50000000000000 0.27010000000000 0.27010000000000 $-0.27010000000000$ $-0.27010000000000$

0.00000000000000 .50000000000000 0.50000000000000 0.00000000000000 0.50000000000000 0.50000000000000 0.5000000000000 0.50000000000000 0.26220000000000 $-0.26220000000000$ 0.34980000000000 0.34980000000000 $-0.34980000000000$ $-0.34980000000000$ 0.1539000000000 $-0.15390000000000$ 0.15390000000000 $-0.15390000000000$ $-0.1539000000000$ 0.14200000000000 $-0.14200000000000$ 0.14200000000000 $-0.3498000000000$ $-0.34980000000000$ 0.3498000000000 0.34980000000000 $-0.15390000000000$ 0.15390000000000 $-0.15390000000000$ 0.1539000000000 $-0.14200000000000$ 0.14200000000000 0.14200000000000 0.142000000000 $-0.1420000000000$ 0.00000000000000 0.0000000000000 0.00000000000000 0.0000000000000 0.2422000000000 $-0.24220000000000$ 0.33190000000000 0.3319000000000 $-0.33190000000000$ $-0.33190000000000$ 0.33190000000000 $-0.33190000000000$ 0.33190000000000 $-0.3319000000000$ $-0.33190000000$ 0.000000000000 0.00000000000000 0.00000000000000 0.0000000000000 0.27010000000000 0.27010000000000 $-0.27010000000000$ $-0.27010000000000$ 0.27010000000000 $-0.2701000000000$ 0.27010000000000 $-0.27010000000000$ 0.50000000000000 0.50000000000000 0.5000000000000 0.50000000000000
$\mathrm{Pd} \quad(1 \mathrm{a})$

$\mathrm{Pd} \quad(3 \mathrm{c})$

$\mathrm{Pd} \quad(3 \mathrm{c})$

$\begin{array}{ll}\mathrm{Pd} & (3 \mathrm{c}) \\ \mathrm{Pd} & (6 \mathrm{f})\end{array}$

(6f)
(6f)

(6f)

(6f)

(6f)

(24k)

$\begin{array}{ll} & \text { Pd } \\ \mathrm{Pd} & (24 \mathrm{k})\end{array}$

$\mathrm{Pd} \quad(24 \mathrm{k})$

$\mathrm{Pd} \quad(24 \mathrm{k})$

Pd $(24 \mathrm{k})$

Pd $(24 \mathrm{k})$

Pd $(24 k)$

Pd (24k)

Pd $\quad(24 \mathrm{k})$

$\mathrm{Pd}(24 \mathrm{k})$

Pd (24k)

Pd (24k)

Pd (24k)

Pd $(24 k)$

$\mathrm{Pd} \quad(24 \mathrm{k})$

$\mathrm{Pd}(24 \mathrm{k})$

$\mathrm{Pd} \quad(24 \mathrm{k})$

$\mathrm{Pd}(24 \mathrm{k})$

$\mathrm{Pd}(24 \mathrm{k})$

Pd (24k)

Pd (24k

$\mathrm{Pd} \quad(24 \mathrm{k})$

$\mathrm{Se} \quad(6 \mathrm{e})$

$\mathrm{Se} \quad(6 \mathrm{e})$

$\begin{array}{ll}\mathrm{Se} & (6 \mathrm{e}) \\ \mathrm{Se} & (6 \mathrm{e})\end{array}$

$\mathrm{Se} \quad(6 \mathrm{e})$

$\mathrm{Se} \quad(6 \mathrm{e})$

$\mathrm{Se}(12 \mathrm{i})$

$\mathrm{Se} \quad(12 \mathrm{i})$

$\mathrm{Se} \quad(12 \mathrm{i})$

$\mathrm{Se} \quad(12 \mathrm{i})$

$\mathrm{Se} \quad(12 \mathrm{i})$

Se $(12 i)$

Se $(12 \mathrm{i})$

Se $(12 \mathrm{i})$

Se $(12 \mathrm{i})$

Se $(12 i)$

$\mathrm{Se} \quad(12 \mathrm{j})$

$\mathrm{Se}(12 \mathrm{j})$

Se $(12 \mathrm{j})$

$\begin{array}{ll}\mathrm{Se} & (12 \mathrm{j}) \\ \mathrm{Se} & (12 \mathrm{j})\end{array}$

Se $(12 \mathrm{j})$

Se $(12 \mathrm{j})$

$\mathrm{Se} \quad(12 \mathrm{j})$

Se $(12 \mathrm{j})$

Se $(12 \mathrm{j})$

$\mathrm{Se} \quad(12 \mathrm{j})$
$\mathrm{PH}_{3}$ : A3B_cP16_208_j_b - CIF

\# CIF file

data_findsym-output

audit_creation_method FINDSYM

chemical_name_mineral ' $\mathrm{PH} 3$,

chemical formula sum ' $\mathrm{H3}$ P'

loop

publ_author_name

'G. Natta'
E. Casazza

journal_name_full_name 


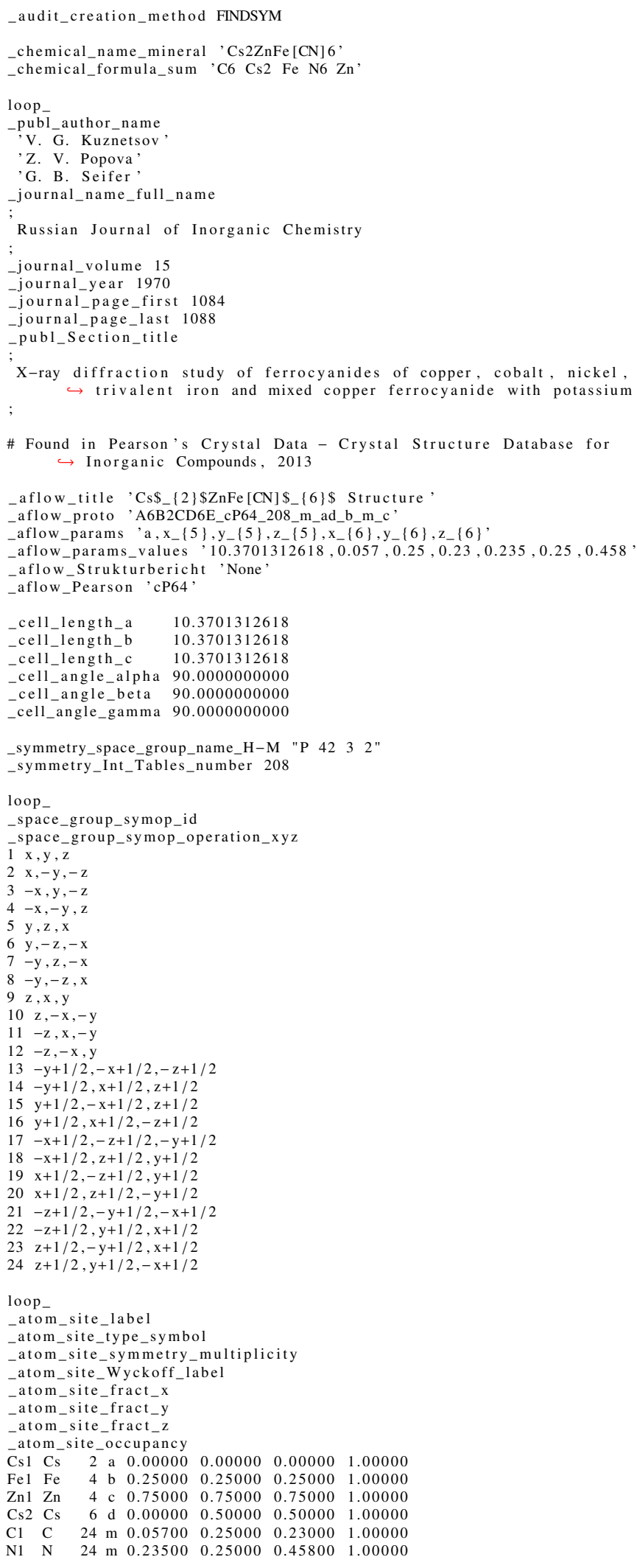

$\mathrm{Cs}_{2} \mathrm{ZnFe}[\mathrm{CN}]_{6}$ : A6B2CD6E_cP64_208_m_ad_b_m_c - POSCAR

A6B2CD6E_cP64_208_m_ad_b_m_c \& a , x $5, \mathrm{y} 5, \mathrm{z} 5, \mathrm{x} 6, \mathrm{y} 6, \mathrm{z} 6--$ params $=$ $\hookrightarrow 10.3701312618,0.057,0.25,0.23,0.235,0.25,0.458 \&$ \& $4 \_\{2\} 32 \mathrm{O}^{\wedge}\{2\}$ $\hookrightarrow$ \#208 (abcdm^2) \&

$\hookrightarrow$ Kuznetsov and Z. V. Popova and G. B. Seifer, Russ. J. Inorg. $\hookrightarrow$ Chem. 15, 1084-1088 (1970)

1.00000000000000

$\begin{array}{lll}1.0 .37013126180000 & 0.00000000000000 & 0.00000000000000\end{array}$ $\begin{array}{lll}0.00000000000000 & 10.37013126180000 & 0.00000000000000\end{array}$ $\begin{array}{lll}0.00000000000000 & 0.00000000000000 & 10.37013126180000\end{array}$ $\begin{array}{rrrrr}\mathrm{C} & \mathrm{Cs} & \mathrm{Fe} & \mathrm{N} & \mathrm{Zn} \\ 24 & 8 & 4 & 24 & 4\end{array}$

Direct

0.05700000000000

0.25000000000000

$-0.05700000000000$ 0.25000000000000
0.23000000000000 0.23000000000000 0.23000000000000
-0.23000000000000

0.05700000000000 23000000000000 0.23000000000000 $-0.23000000000000$ $-0.2300000000000$ 0.25000000000000 $-0.25000000000000$ 0.25000000000000 $-0.25000000000000$ 0.75000000000000 0.25000000000000 0.75000000000000 0.25000000000000 0.5570000000000 0.55700000000000 0.4430000000000 0.55700000000000 0.73000000000000 0.73000000000000 0.27000000000000 0.27000000000000

0.00000000000000

0.50000000000000

0.00000000000000

0.50000000000000

0.50000000000000

0.00000000000000

0.50000000000000

0.00000000000000

0.25000000000000

0.7500000000000

0.7500000000000

0.25000000000000

0.23500000000000 $-0.23500000000000$ $-0.23500000000000$ 0.2350000000000 0.4580000000000 0.45800000000000 $-0.45800000000000$ $-0.45800000000000$ 0.25000000000000 $-0.25000000000000$ 0.25000000000000 $-0.25000000000000$ 0.75000000000000 0.2500000000000 0.25000000000000 0.7500000000000 0.25000000000000 0.73500000000000 0.26500000000000 0.26500000000000 0.73500000000000 0.95800000000000 0.95800000000000 0.04200000000000 0.0420000000000 0.75000000000000 0.25000000000000 0.25000000000000 0.75000000000000

.25000000000000 0.05700000000000 0.05700000000000 $-0.05700000000000$ 0.05700000000000 0.23000000000000 0.23000000000000 $-0.23000000000000$ $-0.23000000000000$ 0.55700000000000 0.44300000000000 0.44300000000000 0.55700000000000 0.73000000000000 0.73000000000000 0.2700000000000 .27000000000000 0.270000000000 0.75000000000000 0.25000000000000 0.75000000000000 0.25000000000000 0.00000000000000 0.50000000000000 0.50000000000000 0.00000000000000 0.50000000000000 0.50000000000000 0.00000000000000 0.00000000000000 0.25000000000000 0.75000000000000 0.2500000000000 0.75000000000000 0.25000000000000 0.250000000000 0.25000000000000 $-0.25000000000000$ 0.23500000000000 $-0.23500000000000$ $-0.23500000000000$ 0.23500000000000 0.45800000000000 0.45800000000000 $-0.45800000000000$ $-0.45800000000000$ 0.73500000000000 0.26500000000000 0.26500000000000 0.73500000000000 0.7580000000000 0.95800000000000 0.9580000000000 0.04200000000000 0.04200000000000 0.75000000000000 0.25000000000000 0.75000000000000 0.25000000000000 0.75000000000000 0.25000000000000 0.75000000000000 0.25000000000000

0.23000000000000 0.25000000000000 $-0.25000000000000$ 0.25000000000000 0.250000000000 0.0570000000000 $-1$ $-0.05700000000000$ 0.05700000000000 0.05700000000000 0.27000000000000 0.27000000000000 0.73000000000000 0.73000000000000 0.25000000000000 0.75000000000000 0.7500000 0.55700000000000 0.5570000000000 0.5570000000000 0.44300000000000 0.00000000000000 0.50000000000000 0.50000000000000 0.50000000000000 0.00000000000000 0.00000000000000 0.00000000000000 0.50000000000000 0.25000000000000 0.25000000000000 0.75000000000000 0.75000000000000 0.45800000000000 0.45800000000000 $-0.45800000000000$ $-0.45800000000000$ 0.25000000000000 0.25000000000000 0.25000000000000 0.25000000000000 0.25000000000000 0.23500000000000 $-0.23500000000000$ 0.23500000000000 0.23500000000000 0.042000000000 0.9580000000000 0.95800000000000 0.25000000000000 0.250000000000 0.750000000000 0.25000000000000 0.75000000000000 0.26500000000000 0.73500000000000 0.73500000000000 0.26500000000000 0.75000000000000 0.75000000000000 0.25000000000000 0.25000000000000 
symmetry_space_group_name_H-M "F $4 \quad 3$ 2" _symmetry_Int_Tables_number 209

loop

space_group_symop_id

space_group_symop_operation_xyz

$1 \mathrm{x}, \mathrm{y}, \mathrm{z}$

$2 \mathrm{x},-\mathrm{y},-\mathrm{z}$

$3-\mathrm{x}, \mathrm{y},-\mathrm{z}$
$4-\mathrm{x},-\mathrm{y}, \mathrm{z}$

$5 \mathrm{y}, \mathrm{z}, \mathrm{x}$

6 y, $-\mathrm{z},-\mathrm{x}$

$\mathrm{y},-\mathrm{z},-\mathrm{x}$
$7-\mathrm{y}, \mathrm{z},-\mathrm{x}$

$-\mathrm{y}, \mathrm{z},-\mathrm{x}$
$8-\mathrm{y},-\mathrm{z}, \mathrm{x}$

$\mathrm{z}, \mathrm{x}, \mathrm{y}$

$0 \mathrm{z},-\mathrm{x},-\mathrm{y}$

$1-\mathrm{z}, \mathrm{x},-\mathrm{y}$

$11-z,-x, y$
$3-y,-x,-z$

$4-y, x, z$
$4-y,-x$

$5 \mathrm{y},-\mathrm{x}, \mathrm{z}$

$6 \mathrm{y}, \mathrm{x},-\mathrm{z}$

$17-x,-z,-y$

$18-\mathrm{x}, \mathrm{z}, \mathrm{y}$

$19 \mathrm{x},-\mathrm{z}, \mathrm{y}$

$20 \mathrm{x}, \mathrm{z},-\mathrm{y}$

$21-\mathrm{z},-\mathrm{y},-\mathrm{x}$

$23 \mathrm{z}, \mathrm{y}, \mathrm{x}$

$24 \mathrm{z}, \mathrm{y}, \mathrm{x}$

$25 \mathrm{x}, \mathrm{y}+1 / 2, \mathrm{z}+1 / 2$

$26 \mathrm{x},-\mathrm{y}+1 / 2,-\mathrm{z}+1 / 2$

$27-\mathrm{x}, \mathrm{y}+1 / 2,-\mathrm{z}+1 / 2$

$29 \mathrm{y}, \mathrm{z}+1 / 2, \mathrm{x}+1 / 2$

$30 \mathrm{y},-\mathrm{z}+1 / 2,-\mathrm{x}+1 / 2$

$31-\mathrm{y}, \mathrm{z}+1 / 2,-\mathrm{x}+1 / 2$

$32-\mathrm{y},-\mathrm{z}+1 / 2, \mathrm{x}+1 / 2$

$33 \mathrm{z}, \mathrm{x}+1 / 2, \mathrm{y}+1 / 2$

$34 \mathrm{z},-\mathrm{x}+1 / 2,-\mathrm{y}+1 / 2$

$35-\mathrm{z}, \mathrm{x}+1 / 2,-\mathrm{y}+1 / 2$

$36-\mathrm{z},-\mathrm{x}+1 / 2, \mathrm{y}+1 / 2$

$37-\mathrm{y},-\mathrm{x}+1 / 2,-\mathrm{z}+1 / 2$

$38-\mathrm{y}, \mathrm{x}+1 / 2, \mathrm{z}+1 / 2$

$39 \mathrm{y},-\mathrm{x}+1 / 2, \mathrm{z}+1 / 2$

$40 \mathrm{y}, \mathrm{x}+1 / 2,-\mathrm{z}+1 / 2$

$41-\mathrm{x},-\mathrm{z}+1 / 2,-\mathrm{y}+1 / 2$

$42-\mathrm{x}, \mathrm{z}+1 / 2, \mathrm{y}+1 / 2$

$43 \mathrm{x},-\mathrm{z}+1 / 2, \mathrm{y}+1 / 2$

$44 \mathrm{x}, \mathrm{z}+1 / 2,-\mathrm{y}+1 / 2$
$45-\mathrm{z},-\mathrm{y}+1 / 2,-\mathrm{x}+1 / 2$

$46-\mathrm{z}, \mathrm{y}+1 / 2, \mathrm{x}+1 / 2$

$47 \mathrm{z},-\mathrm{y}+1 / 2, \mathrm{x}+1 / 2$

$48 \mathrm{z}, \mathrm{y}+1 / 2,-\mathrm{x}+1 / 2$

$49 \mathrm{x}+1 / 2, \mathrm{y}, \mathrm{z}+1 / 2$

$50 \mathrm{x}+1 / 2,-\mathrm{y},-\mathrm{z}+1 / 2$

$52-x+1 / 2,-y, z+1 / 2$

$53 \mathrm{y}+1 / 2, \mathrm{z}, \mathrm{x}+1 / 2$

$54 \mathrm{y}+1 / 2,-\mathrm{z},-\mathrm{x}+1 / 2$

$55-\mathrm{y}+1 / 2, \mathrm{z},-\mathrm{x}+1 / 2$

$56-\mathrm{y}+1 / 2,-\mathrm{z}, \mathrm{x}+1 / 2$

$57 \mathrm{z}+1 / 2, \mathrm{x}, \mathrm{y}+1 / 2$

$58 \mathrm{z}+1 / 2,-\mathrm{x},-\mathrm{y}+1 / 2$

$59-z+1 / 2, x,-y+1 / 2$

$60-z+1 / 2,-x, y+1 / 2$

$61-\mathrm{y}+1 / 2,-\mathrm{x},-\mathrm{z}+1 / 2$

$62-\mathrm{y}+1 / 2, \mathrm{x}, \mathrm{z}+1 / 2$

$63 \mathrm{y}+1 / 2,-\mathrm{x}, \mathrm{z}+1 / 2$

$64 \mathrm{y}+1 / 2, \mathrm{x},-\mathrm{z}+1 / 2$
$65-\mathrm{x}+1 / 2,-\mathrm{z},-\mathrm{y}+1 / 2$

$66-\mathrm{x}+1 / 2, \mathrm{z}, \mathrm{y}+1 / 2$

$67 \mathrm{x}+1 / 2,-\mathrm{z}, \mathrm{y}+1 / 2$

$68 \mathrm{x}+1 / 2, \mathrm{z},-\mathrm{y}+1 / 2$

$69-z+1 / 2,-y,-x+1 / 2$

$70-\mathrm{z}+1 / 2, \mathrm{y}, \mathrm{x}+1 / 2$

$71 \mathrm{z}+1 / 2,-\mathrm{y}, \mathrm{x}+1 / 2$

$73 \mathrm{z}+1 / 2, \mathrm{y},-\mathrm{x}+1 / 2$

$73 x+1 / 2, y+1 / 2, z$

$74 x+1 / 2,-y+1 / 2,-z$

$75-x+1 / 2, y+1 / 2,-z$

$77-x+1 / 2,-y+1 / 2, z$

$77 \mathrm{y}+1 / 2, \mathrm{z}+1 / 2, \mathrm{x}$

$78 \mathrm{y}+1 / 2,-\mathrm{z}+1 / 2,-\mathrm{x}$

$80-y+1 / 2,-z+1 / 2, x$

$81 \mathrm{z}+1 / 2, \mathrm{x}+1 / 2, \mathrm{y}$

$82 \mathrm{z}+1 / 2,-\mathrm{x}+1 / 2,-\mathrm{y}$

$83-z+1 / 2, x+1 / 2,-y$

$84-\mathrm{z}+1 / 2,-\mathrm{x}+1 / 2, \mathrm{y}$

$85-y+1 / 2,-x+1 / 2,-z$

$86-y+1 / 2, x+1 / 2$,

$88 \mathrm{y}+1 / 2, x+1 / 2,-\mathrm{z}$

$89-\mathrm{x}+1 / 2,-\mathrm{z}+1 / 2,-\mathrm{y}$

$90-x+1 / 2, z+1 / 2, y$

$91-x+1 / 2, z+1 / 2, y$

$92 x+1 / 2, z+1 / 2,-y$

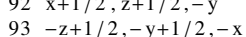

$94-\mathrm{z}+1 / 2, \mathrm{y}+1 / 2, \mathrm{x}$

$95 \mathrm{z}+1 / 2,-\mathrm{y}+1 / 2, \mathrm{x}$

$96 \mathrm{z}+1 / 2, \mathrm{y}+1 / 2,-\mathrm{x}$

loop_ atom site label

atom_site_type_symbol

atom_site_symmetry_multiplicity

atom_site_Wyckoff_label

atom_site_fract_x

atom_site_fract_y

atom_site_fract_z

atom site occupancy

$\begin{array}{llllllll}\mathrm{K} 1 \mathrm{~K} & 4 & \mathrm{a} & 0.00000 & 0.00000 & 0.00000 & 1.00000\end{array}$

$\begin{array}{lllllllll}\mathrm{P} 1 & \mathrm{P} & 4 & \mathrm{~b} & 0.50000 & 0.50000 & 0.50000 & 1.00000\end{array}$

$\begin{array}{llllllllll}\text { F1 } & F & 96 & j & 0.04300 & 0.10900 & 0.16500 & 1.00000\end{array}$

F6 KP: A24BC_cF104_209_j_a_b - POSCAR

\begin{tabular}{|c|c|c|c|c|}
\hline \multicolumn{4}{|c|}{ A24BC_cF104_209_j_a_b \& a , x3,y3,z3 --params $=7.7099775082,0.043,0.109}$, & $\hookrightarrow 0.165 \& \mathrm{~F} 432 \mathrm{O}^{\wedge}\{3\} \# 209(\mathrm{abj}) \& \mathrm{cF} 104 \&$ None \& F6KP \& \& Y. P. \\
\hline \multicolumn{5}{|l|}{ renhas } \\
\hline \multicolumn{5}{|l|}{1.00000000000000} \\
\hline 0.00000000000000 & 3.85498875410000 & 3.85498875410000 & & \\
\hline \multirow{2}{*}{$\begin{array}{l}3.85498875410000 \\
3.85498875410000\end{array}$} & 0.00000000000000 & 3.85498875410000 & & \\
\hline & 3.85498875410000 & 0.00000000000000 & & \\
\hline $\mathrm{P}$ & & & & \\
\hline 24 & & & & \\
\hline \multicolumn{5}{|l|}{ Direct } \\
\hline 0.23100000000000 & 0.09900000000000 & -0.01300000000000 & $\mathrm{~F}$ & $(96 \mathrm{j})$ \\
\hline 0.09900000000000 & 0.23100000000000 & -0.31700000000000 & $\mathrm{~F}$ & $(96 \mathrm{j})$ \\
\hline-0.01300000000000 & -0.31700000000000 & 0.23100000000000 & $\mathrm{~F}$ & $(96 \mathrm{j})$ \\
\hline-0.31700000000000 & -0.01300000000000 & 0.09900000000000 & $\mathrm{~F}$ & $(96 \mathrm{j})$ \\
\hline-0.01300000000000 & 0.23100000000000 & 0.09900000000000 & $\mathrm{~F}$ & $(96 \mathrm{j})$ \\
\hline-0.31700000000000 & 0.09900000000000 & 0.23100000000000 & $\mathrm{~F}$ & $(96 \mathrm{j})$ \\
\hline 0.23100000000000 & -0.01300000000000 & -0.31700000000000 & $\mathrm{~F}$ & $(96 \mathrm{j})$ \\
\hline 0.09900000000000 & -0.31700000000000 & -0.01300000000000 & $\mathrm{~F}$ & $(96 \mathrm{j})$ \\
\hline 0.09900000000000 & -0.01300000000000 & 0.23100000000000 & $\mathrm{~F}$ & $(96 \mathrm{j})$ \\
\hline 0.23100000000000 & -0.31700000000000 & 0.09900000000000 & $\mathrm{~F}$ & $(96 \mathrm{j})$ \\
\hline-0.31700000000000 & 0.23100000000000 & -0.01300000000000 & $\mathrm{~F}$ & $(96 \mathrm{j})$ \\
\hline-0.01300000000000 & 0.09900000000000 & -0.31700000000000 & $\mathrm{~F}$ & $(96 \mathrm{j})$ \\
\hline-0.23100000000000 & -0.09900000000000 & 0.31700000000000 & $\mathrm{~F}$ & $(96 \mathrm{j})$ \\
\hline-0.09900000000000 & -0.23100000000000 & 0.01300000000000 & $\mathrm{~F}$ & $(96 \mathrm{j})$ \\
\hline 0.01300000000000 & 0.31700000000000 & -0.09900000000000 & $\mathrm{~F}$ & $(96 \mathrm{j})$ \\
\hline 0.31700000000000 & 0.01300000000000 & -0.23100000000000 & $\mathrm{~F}$ & $(96 \mathrm{j})$ \\
\hline 0.01300000000000 & -0.23100000000000 & 0.31700000000000 & $\mathrm{~F}$ & $(96 \mathrm{j})$ \\
\hline 0.31700000000000 & -0.09900000000000 & 0.01300000000000 & $\mathrm{~F}$ & $(96 \mathrm{j})$ \\
\hline-0.23100000000000 & 0.01300000000000 & -0.09900000000000 & $\mathrm{~F}$ & $(96 \mathrm{j})$ \\
\hline-0.09900000000000 & 0.31700000000000 & -0.23100000000000 & $\mathrm{~F}$ & $(96 \mathrm{j})$ \\
\hline-0.09900000000000 & 0.01300000000000 & 0.31700000000000 & $\mathrm{~F}$ & $(96 \mathrm{j})$ \\
\hline-0.23100000000000 & 0.31700000000000 & 0.01300000000000 & $\mathrm{~F}$ & $(96 \mathrm{j})$ \\
\hline 0.31700000000000 & -0.23100000000000 & -0.09900000000000 & $\mathrm{~F}$ & $(96 \mathrm{j})$ \\
\hline 0.01300000000000 & -0.09900000000000 & -0.23100000000000 & $\mathrm{~F}$ & $(96 \mathrm{j})$ \\
\hline 0.00000000000000 & 0.00000000000000 & 0.00000000000000 & $\mathrm{~K}$ & (4a) \\
\hline 0.50000000000000 & 0.50000000000000 & 0.50000000000000 & $\mathrm{P}$ & (4b) \\
\hline
\end{tabular}

$\mathrm{Te}[\mathrm{OH}]_{6}$ : A12B6C_cF608_210_4h_2h_e - CIF

\# CIF file

data_findsym-outpu

audit_creation_method FINDSYM

chemical_name_mineral ' $\mathrm{Te}[\mathrm{OH}] 6$ '

chemical_formula_sum 'H12 O6 Te'

loop

publ_author_name

D. F. Mullica

W. O. Milligan

G. W. Beall

'G. W. Beall,

journal_name_full_name

Acta Crystallographica Section B: Structural Science

journal_volume 36

journal_year 1980

journal_page_first 2565

journal_page last 2570

publ_Section_title

Neutron structural refinement of cubic orthotelluric acid

Found in Pearson's Crystal Data - Crystal Structure Database for

$\leftrightarrow$ Inorganic Compounds, 2013

aflow_title 'Te $[\mathrm{OH}] \$ \_\{6\} \$$ Structure,

aflow_proto 'A12B6C_cB608_210_4

aflow_params $, a, x_{-}\{1\}, x_{-}\{2\}, y_{-}\{2\}, z_{-}\{2\}, x_{-}\{3\}, y_{-}\{3\}, z_{-}\{3\}, x_{-}\{4\}, y_{-}\{4\}$, $\hookrightarrow \mathrm{z}_{-}\{4\}, \mathrm{x}_{-}\{5\}, \mathrm{y}_{-}\{5\}, \mathrm{z}_{-}\{5\}, \mathrm{x}_{-}\{6\}, \mathrm{y}_{-}\{6\}, \mathrm{z}_{-}\{6\}, \mathrm{x}_{-}\{7\}, \mathrm{y}_{-}\{7\}, \mathrm{z}_{-}\{7\}$

_aflow_params_values ' $16.4321054599,0.3771,0.0157,0.2009,0.1224,0.5287$, $\hookrightarrow 0.1425,0.0068,0.0949,0.2596,0.2225,0.6117,0.1785,0.0224,0.0928$ $\hookrightarrow 0.2607,0.1616,0.0194,0.0793,0.3503$,

aflow_Strukturbericht 'None'

aflow_Pearson 'cF608,

cell_length_a $\quad 16.4321054599$

cell_length_b 16.4321054599

16.4321054599
$-c e l l$

_cell_angle_alpha 90.0000000000

symmetry space group name $\mathrm{H}-\mathrm{M}$ "F 4132 (3)

symmetry_Int_Tables_number 210

loop_ 
_space_group_symop_id

_space_group_symop_operation_xyz

$1 \mathrm{x}, \mathrm{y}, \mathrm{z}$

$2 \mathrm{x},-\mathrm{y},-\mathrm{z}$
$3-\mathrm{x}, \mathrm{y},-\mathrm{z}$

$4-x,-y, z$

$5 \mathrm{y}, \mathrm{z}, \mathrm{x}$

$6 \mathrm{y},-\mathrm{z},-\mathrm{x}$

$-y, z,-x$
$-y,-z, x$

$9 \mathrm{z}, \mathrm{x}, \mathrm{y}$

$10 \mathrm{z},-\mathrm{x},-\mathrm{y}$

$11-\mathrm{z}, \mathrm{x},-\mathrm{y}$
$12-\mathrm{z},-\mathrm{x}, \mathrm{y}$

$3-\mathrm{y}+1 / 4,-\mathrm{x}+1 / 4,-\mathrm{z}+1 / 4$

$4-y+1 / 4, x+1 / 4, z+1 / 4$

$15 \mathrm{y}+1 / 4,-x+1 / 4, z+1 / 4$

$6 \mathrm{y}+1 / 4, \mathrm{x}+1 / 4,-\mathrm{z}+1 / 4$

$17-x+1 / 4,-z+1 / 4,-y+1 / 4$

$18-\mathrm{x}+1 / 4, \mathrm{z}+1 / 4, \mathrm{y}+1 / 4$
$19 \mathrm{x}+1 / 4,-\mathrm{z}+1 / 4, \mathrm{y}+1 / 4$

$20 \mathrm{x}+1 / 4, \mathrm{z}+1 / 4,-\mathrm{y}+1 / 4$

$21-z+1 / 4,-y+1 / 4,-x+1 / 4$

$22-\mathrm{z}+1 / 4, \mathrm{y}+1 / 4, \mathrm{x}+1 / 4$

$23 \mathrm{z}+1 / 4,-\mathrm{y}+1 / 4, \mathrm{x}+1 / 4$

$25 \mathrm{x}, \mathrm{y}+1 / 2, \mathrm{z}+1 / 2$

$26 \mathrm{x},-\mathrm{y}+1 / 2,-\mathrm{z}+1 / 2$

$27-\mathrm{x}, \mathrm{y}+1 / 2,-\mathrm{z}+1 / 2$

$29 \mathrm{y}, \mathrm{z}+1 / 2, \mathrm{x}+1 / 2$

$30 \mathrm{y},-\mathrm{z}+1 / 2,-\mathrm{x}+1 / 2$

$31-\mathrm{y}, \mathrm{z}+1 / 2,-\mathrm{x}+1 / 2$

$32-y,-z+1 / 2, x+1 / 2$

$34 \mathrm{z},-\mathrm{x}+1 / 2,-\mathrm{y}+1 / 2$

$35-\mathrm{z}, \mathrm{x}+1 / 2,-\mathrm{y}+1 / 2$

$\begin{array}{ll}36 & -\mathrm{z},-\mathrm{x}+1 / 2, \mathrm{y}+1 / 2 \\ 37 & -\mathrm{y}+1 / 4,-\mathrm{x}+3 / 4,-\mathrm{z}+3 / 4\end{array}$

$38-\mathrm{y}+1 / 4, \mathrm{x}+3 / 4, \mathrm{z}+3 / 4$

$39 \mathrm{y}+1 / 4,-x+3 / 4, z+3 / 4$

$40 \mathrm{y}+1 / 4, x+3 / 4,-\mathrm{z}+3 / 4$

$41-x+1 / 4,-z+3 / 4,-y+3 / 4$
$42-x+1 / 4, z+3 / 4, y+3 / 4$

$43 \mathrm{x}+1 / 4,-\mathrm{z}+3 / 4, \mathrm{y}+3 / 4$

$44 \mathrm{x}+1 / 4, \mathrm{z}+3 / 4,-\mathrm{y}+3 / 4$

$45-\mathrm{z}+1 / 4,-\mathrm{y}+3 / 4,-\mathrm{x}+3 / 4$

$46-z+1 / 4, y+3 / 4, x+3 / 4$

$47 z+1 / 4,-y+3 / 4, x+3 / 4$

$49 \mathrm{x}+1 / 2, \mathrm{y}, \mathrm{z}+1 / 2$

$50 \mathrm{x}+1 / 2,-\mathrm{y},-\mathrm{z}+1 / 2$

$51-x+1 / 2, y,-z+1 / 2$

$52-\mathrm{x}+1 / 2,-\mathrm{y}, \mathrm{z}+1 / 2$

$53 \mathrm{y}+1 / 2, \mathrm{z}, \mathrm{x}+1 / 2$

$54 \mathrm{y}+1 / 2,-\mathrm{z},-\mathrm{x}+1 / 2$

$55-\mathrm{y}+1 / 2, \mathrm{z},-\mathrm{x}+1 / 2$

$56-\mathrm{y}+1 / 2,-\mathrm{z}, \mathrm{x}+1 / 2$

$57 \mathrm{z}+1 / 2, \mathrm{x}, \mathrm{y}+1 / 2$

$58 \mathrm{z}+1 / 2,-\mathrm{x},-\mathrm{y}+1 / 2$

$59-\mathrm{z}+1 / 2, \mathrm{x},-\mathrm{y}+1 / 2$
$60-\mathrm{z}+1 / 2,-\mathrm{x}, \mathrm{y}+1 / 2$

$61-\mathrm{y}+3 / 4,-\mathrm{x}+1 / 4,-\mathrm{z}+3 / 4$

$62-y+3 / 4, x+1 / 4, z+3 / 4$

$63 \mathrm{y}+3 / 4,-\mathrm{x}+1 / 4, \mathrm{z}+3 / 4$

$64 \mathrm{y}+3 / 4, \mathrm{x}+1 / 4,-\mathrm{z}+3 / 4$
$65-\mathrm{x}+3 / 4,-\mathrm{z}+1 / 4,-\mathrm{y}+3 / 4$

$66-x+3 / 4, z+1 / 4, y+3 / 4$

$67 \mathrm{x}+3 / 4,-\mathrm{z}+1 / 4, \mathrm{y}+3 / 4$

$68 \mathrm{x}+3 / 4, \mathrm{z}+1 / 4,-\mathrm{y}+3 / 4$
$69-\mathrm{z}+3 / 4,-\mathrm{y}+1 / 4,-\mathrm{x}+3 / 4$

$70-z+3 / 4, y+1 / 4, x+3 / 4$

$71 \mathrm{z}+3 / 4,-y+1 / 4, x+3 / 4$

$72 \mathrm{z}+3 / 4, \mathrm{y}+1 / 4,-\mathrm{x}+3 / 4$

$73 \mathrm{x}+1 / 2, \mathrm{y}+1 / 2, \mathrm{z}$

$74 x+1 / 2,-y+1 / 2,-z$

$75-x+1 / 2, y+1 / 2,-z$

$77 \mathrm{y}+1 / 2, \mathrm{z}+1 / 2, \mathrm{x}$

$78 \mathrm{y}+1 / 2, \mathrm{z}+1 / 2, \mathrm{x}$

$78 \mathrm{y}+1 / 2,-\mathrm{z}+1 / 2,-\mathrm{x}$
$79-\mathrm{y}+1 / 2, \mathrm{z}+1 / 2,-\mathrm{x}$

$80-\mathrm{y}+1 / 2,-\mathrm{z}+1 / 2, \mathrm{x}$

$81 \mathrm{z}+1 / 2, \mathrm{x}+1 / 2, \mathrm{y}$

$82 \mathrm{z}+1 / 2,-\mathrm{x}+1 / 2,-\mathrm{y}$

$83-z+1 / 2, x+1 / 2,-y$

$84-\mathrm{z}+1 / 2,-\mathrm{x}+1 / 2, \mathrm{y}$

$85-\mathrm{y}+3 / 4,-\mathrm{x}+3 / 4,-\mathrm{z}+1 / 4$

$86-\mathrm{y}+3 / 4, \mathrm{x}+3 / 4, \mathrm{z}+1 / 4$

$87 \mathrm{y}+3 / 4,-x+3 / 4, z+1 / 4$

$88 \mathrm{y}+3 / 4, \mathrm{x}+3 / 4,-\mathrm{z}+1 / 4$

$89-x+3 / 4,-z+3 / 4,-y+1 / 4$

$90-\mathrm{x}+3 / 4, \mathrm{z}+3 / 4, \mathrm{y}+1 / 4$

$\begin{array}{ll}91 & x+3 / 4,-z+3 / 4, y+1 / 4 \\ 92 & x+3 / 4, z+3 / 4,-y+1 / 4\end{array}$

$93-\mathrm{z}+3 / 4,-\mathrm{y}+3 / 4,-\mathrm{x}+1 / 4$

$94-z+3 / 4, y+3 / 4, x+1 / 4$

$95 \mathrm{z}+3 / 4,-\mathrm{y}+3 / 4, \mathrm{x}+1 / 4$

$96 \mathrm{z}+3 / 4, \mathrm{y}+3 / 4,-\mathrm{x}+1 / 4$

loop_

tom_site label

atom_site_type_symbol

atom_site_symmetry_multiplicity

atom_site_Wyckoff_label

_atom_site_fract_x

atom_site_fract_y

atom_site_fract_z

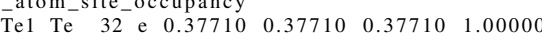

$\begin{array}{lllllllll}\mathrm{H} 1 & \mathrm{H} & 96 & \mathrm{~h} & 0.01570 & 0.20090 & 0.12240 & 1.00000\end{array}$

$\begin{array}{lllllllll}\mathrm{H} 2 & \mathrm{H} & 96 & \mathrm{~h} & 0.52870 & 0.14250 & 0.00680 & 1.00000\end{array}$

$\begin{array}{lllllllll}\mathrm{H} 3 & \mathrm{H} & 96 & \mathrm{~h} & 0.09490 & 0.25960 & 0.22250 & 1.00000\end{array}$

$\begin{array}{lllllllll}\mathrm{H} 4 & \mathrm{H} & 96 & \mathrm{~h} & 0.61170 & 0.17850 & 0.02240 & 1.00000\end{array}$

$\begin{array}{lllllllll}\mathrm{O} 1 & \mathrm{O} & 96 & \mathrm{~h} & 0.09280 & 0.26070 & 0.16160 & 1.00000\end{array}$

$\begin{array}{llllllllll}\mathrm{O} 2 & \mathrm{O} & 96 & \mathrm{~h} & 0.01940 & 0.07930 & 0.35030 & 1.00000\end{array}$

$\mathrm{Te}[\mathrm{OH}]_{6}$ : A12B6C_cF608_210_4h_2h_e - POSCAR

A12B6C_cF608_210_4h_2h_e \& a , x1, x2 , y2, z2, x3 ,y3 $, \mathrm{z} 3, \mathrm{x} 4, \mathrm{y} 4, \mathrm{z} 4, \mathrm{x} 5, \mathrm{y} 5, \mathrm{z} 5, \mathrm{x} 6$, $\hookrightarrow \mathrm{y} 6, \mathrm{z6}, \mathrm{x} 7, \mathrm{y} 7, \mathrm{z} 7--$ params $=16.4321054599,0.3771,0.0157,0.2009$

$\hookrightarrow 0.1224,0.5287,0.1425,0.0068,0.0949,0.2596,0.2225,0.6117,0.1785$,

$\hookrightarrow 0.0224,0.0928,0.2607,0.1616,0.0194,0.0793,0.3503 \& \mathrm{~F}_{-}\{1\} 32 \mathrm{O}^{\wedge}$

$\hookrightarrow$ al., Acta Crystallogr. Sect. B Struct. Sci, 36, 2565-2570 (1980

1.0000000000000

$\begin{array}{lll}0.00000000000000 & 8.21605272995000 & 8.21605272995000\end{array}$

$\begin{array}{lll}\mathrm{H} & \mathrm{O} & \mathrm{Te}\end{array}$

Direct

0.30760000000000

$-0.06280000000000$

0.09420000000000

$-0.33900000000000$

0.09420000000000

$-0.33900000000000$

$-0.06280000000000$

$-0.06280000000000$

0.30760000000000

$-0.3390000000000$

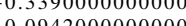

$-0.05760000000000$

0.31280000000000

0.15580000000000

0.58900000000000

0.15580000000000

0.58900000000000

$-0.05760000000000$

0.31280000000000

0.31280000000000

$-0.05760000000000$

0.15580000000000

$-0.37940000000000$

0.39300000000000

$-0.6740000000000$

$-0.67800000000000$

$-0.67800000000000$

$-0.37940000000000$

0.39300000000000

0.39300000000000

$-0.37940000000000$

$-0.6780000000000$

0.66440000000000

0.62940000000000

$-0.14300000000000$

$-0.41440000000000$

0.92800000000000

0.92800000000000

0.62940000000000

$-0.1430000000000$

$-0.14300000000000$

0.62940000000000

0.92800000000000

$-0.41440000000000$

0.38720000000000

0.05780000000000

0.13200000000000

$-0.57700000000000$

0.13200000000000

$-0.57700000000000$

0.38720000000000

0.05780000000000

0.38720000000000

$-0.57700000000000$

0.13200000000000

$-0.13720000000000$

0.1922000000000

0.11800000000000

0.82700000000000

0.11800000000000

0.82700000000000

$-0.13720000000000$

0.19220000000000

0.19220000000000

$-0.13720000000000$

0.82700000000000

0.11800000000000

$-0.41080000000000$

0.45560000000000

0.76780000000000

$-0.81260000000000$

0.76780000000000

$-0.81260000000000$

1605272995000 000000000000

8.21605272995000

$8.21605272995000 \quad 0.00000000000000$

0.06280000000000

0.30760000000000

0.33900000000000

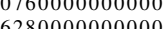

0.09420000000000

0.33900000000000

0.09420000000000

.33900000000000

0.30760000000000

.

0.062800000000

0.05760000000000

58900000000000

0.15580000000000

0.05760000000000

0.31280000000000

0.15580000000000

0.58900000000000

0.15580000000000

00000000000

.05760000000000

0.39300000000000

0.37940000000000

$-0.67800000000000$

0.66440000000000

. 37940000000000

0.39300000000000

0.66440000000000

.67800000000000

0.66440000000000

0.67800000000000

0.37940000000000

0.39300000000000

0.14300000000000

.92800000000000

.41440000000000

$-0.14300000000000$

$-0.41440000000000$

.92800000000000

.

0.92800000000000

.

0.14300000000000

0.05780000000000

0.38720000000000

$-0.57700000000000$

0.13200000000000

0.38720000000000

0.05780000000000

0.13200000000000

00000000000

.13200000000000

0.57700000000000

0.05780000000000

0.13720000000000

0.82700000000000

0.11800000000000

0.1180000000 0.192200000000 0.19220000000000 0.1180000000000 0.82700000000000 0.11800000000000 0.82700000000000 0.19220000000000 .45560000000000 0.41080000000000 0.76780000000000

0.41080000000000

0.45560000000000

0.09420000000000 0.33900000000000 0.30760000000000 0.06280000000000 $-0.06280000000000$ 30760000000000 .33900000000000 09420000000000 0.30760000000000 0.06280000000000 .09420000000000 (2) 0.05760000000000 0.58900000000000 0.15580000000000 0.31280000000000 $-0.05760000000000$ 0.58900000000000 0.31280000000000 05760000000000 66440000000000 $-0.67800000000000$ 0.37940000000000 0.39300000000000 0.39300000000000 


\begin{tabular}{|c|c|c|c|c|}
\hline 0.41080000000000 & 000000 & -0.81260000000000 & $\mathrm{H}$ & $(96 \mathrm{~h})$ \\
\hline 0.45560000000000 & -0.81260000000000 & 0.76780000000000 & $\mathrm{H}$ & (96h) \\
\hline 0.45560000000000 & 0.76780000000000 & -0.41080000000000 & $\mathrm{H}$ & $(96 \mathrm{~h})$ \\
\hline-0.41080000000000 & -0.81260000000000 & 0.45560000000000 & $\mathrm{H}$ & $(96 \mathrm{~h})$ \\
\hline-0.81260000000000 & -0.41080000000000 & 0.76780000000000 & $\mathrm{H}$ & (96h) \\
\hline 0.76780000000000 & 0.45560000000000 & -0.81260000000000 & $\mathrm{H}$ & (96h) \\
\hline 0.66080000000000 & -0.20560000000000 & 1.06260000000000 & $\mathrm{H}$ & (96h) \\
\hline-0.20560000000000 & 0.66080000000000 & -0.51780000000000 & $\mathrm{H}$ & $(96 \mathrm{~h})$ \\
\hline-0.51780000000000 & 1.06260000000000 & -0.20560000000000 & $\mathrm{H}$ & $(96 \mathrm{~h})$ \\
\hline 1.06260000000000 & -0.51780000000000 & 0.66080000000000 & $\mathrm{H}$ & $(96 \mathrm{~h})$ \\
\hline-0.51780000000000 & 0.66080000000000 & 1.06260000000000 & $\mathrm{H}$ & (96h) \\
\hline 1.06260000000000 & -0.20560000000000 & -0.51780000000000 & $\mathrm{H}$ & (96h) \\
\hline 0.66080000000000 & -0.51780000000000 & -0.20560000000000 & $\mathrm{H}$ & (96h) \\
\hline-0.20560000000000 & 1.06260000000000 & 0.66080000000000 & $\mathrm{H}$ & (96h) \\
\hline-0.20560000000000 & -0.51780000000000 & 1.06260000000000 & $\mathrm{H}$ & (96h) \\
\hline 0.66080000000000 & 1.06260000000000 & -0.51780000000000 & $\mathrm{H}$ & (96h) \\
\hline 1.06260000000000 & 0.66080000000000 & -0.20560000000000 & $\mathrm{H}$ & (96h) \\
\hline-0.51780000000000 & -0.20560000000000 & 0.66080000000000 & $\mathrm{H}$ & (96h) \\
\hline 0.32950000000000 & -0.00630000000000 & 0.19190000000000 & $\mathrm{O}$ & (96h) \\
\hline-0.00630000000000 & 0.32950000000000 & -0.51510000000000 & $\mathrm{O}$ & $(96 \mathrm{~h})$ \\
\hline 0.19190000000000 & -0.51510000000000 & 0.32950000000000 & $\mathrm{O}$ & (96h) \\
\hline-0.51510000000000 & 0.19190000000000 & -0.00630000000000 & $\mathrm{O}$ & $(96 \mathrm{~h})$ \\
\hline 0.19190000000000 & 0.32950000000000 & -0.00630000000000 & $\mathrm{O}$ & (96h) \\
\hline-0.51510000000000 & -0.00630000000000 & 0.32950000000000 & $\mathrm{O}$ & (96h) \\
\hline 0.32950000000000 & 0.19190000000000 & -0.51510000000000 & $\mathrm{O}$ & (96h) \\
\hline-0.00630000000000 & -0.51510000000000 & 0.19190000000000 & $\mathrm{O}$ & $(96 \mathrm{~h})$ \\
\hline-0.00630000000000 & 0.19190000000000 & 0.32950000000000 & $\mathrm{O}$ & (96h) \\
\hline 0.32950000000000 & -0.51510000000000 & -0.00630000000000 & $\mathrm{O}$ & (96h) \\
\hline-0.51510000000000 & 0.32950000000000 & 0.19190000000000 & $\mathrm{O}$ & (96h) \\
\hline 0.19190000000000 & -0.00630000000000 & -0.51510000000000 & $\mathrm{O}$ & (96h) \\
\hline-0.07950000000000 & 0.25630000000000 & 0.76510000000000 & $\mathrm{O}$ & $(96 \mathrm{~h})$ \\
\hline 0.25630000000000 & -0.07950000000000 & 0.05810000000000 & $\mathrm{O}$ & $(96 \mathrm{~h})$ \\
\hline 0.05810000000000 & 0.76510000000000 & 0.25630000000000 & $\mathrm{O}$ & (96h) \\
\hline 0.76510000000000 & 0.05810000000000 & -0.07950000000000 & $\mathrm{O}$ & (96h) \\
\hline 0.05810000000000 & -0.07950000000000 & 0.76510000000000 & $\mathrm{O}$ & (96h) \\
\hline 0.76510000000000 & 0.25630000000000 & 0.05810000000000 & $\mathrm{O}$ & (96h) \\
\hline-0.07950000000000 & 0.05810000000000 & 0.25630000000000 & $\mathrm{O}$ & (96h) \\
\hline 0.25630000000000 & 0.76510000000000 & -0.07950000000000 & $\mathrm{O}$ & (96h) \\
\hline 0.25630000000000 & 0.05810000000000 & 0.76510000000000 & $\mathrm{O}$ & $(96 \mathrm{~h})$ \\
\hline-0.07950000000000 & 0.76510000000000 & 0.05810000000000 & $\mathrm{O}$ & (96h) \\
\hline 0.76510000000000 & -0.07950000000000 & 0.25630000000000 & $\mathrm{O}$ & (96h) \\
\hline 0.05810000000000 & 0.25630000000000 & -0.07950000000000 & $\mathrm{O}$ & $(96 \mathrm{~h})$ \\
\hline 0.41020000000000 & 0.29040000000000 & -0.25160000000000 & $\mathrm{O}$ & (96h) \\
\hline 0.29040000000000 & 0.41020000000000 & -0.44900000000000 & $\mathrm{O}$ & $(96 \mathrm{~h})$ \\
\hline-0.25160000000000 & -0.44900000000000 & 0.41020000000000 & $\mathrm{O}$ & (96h) \\
\hline-0.44900000000000 & -0.25160000000000 & 0.29040000000000 & $\mathrm{O}$ & (96h) \\
\hline-0.25160000000000 & 0.41020000000000 & 0.29040000000000 & $\mathrm{O}$ & (96h) \\
\hline-0.44900000000000 & 0.29040000000000 & 0.41020000000000 & $\mathrm{O}$ & (96h) \\
\hline 0.41020000000000 & -0.25160000000000 & -0.44900000000000 & $\mathrm{O}$ & (96h) \\
\hline 0.29040000000000 & -0.44900000000000 & -0.25160000000000 & $\mathrm{O}$ & $(96 \mathrm{~h})$ \\
\hline 0.29040000000000 & -0.25160000000000 & 0.41020000000000 & $\mathrm{O}$ & (96h) \\
\hline 0.41020000000000 & -0.44900000000000 & 0.29040000000000 & $\mathrm{O}$ & (96h) \\
\hline-0.44900000000000 & 0.41020000000000 & -0.25160000000000 & $\mathrm{O}$ & $(96 \mathrm{~h})$ \\
\hline-0.25160000000000 & 0.29040000000000 & -0.44900000000000 & $\mathrm{O}$ & $(96 \mathrm{~h})$ \\
\hline-0.16020000000000 & -0.04040000000000 & 0.69900000000000 & $\mathrm{O}$ & $(96 \mathrm{~h})$ \\
\hline-0.04040000000000 & -0.16020000000000 & 0.50160000000000 & $\mathrm{O}$ & (96h) \\
\hline 0.50160000000000 & 0.69900000000000 & -0.04040000000000 & $\mathrm{O}$ & (96h) \\
\hline 0.69900000000000 & 0.50160000000000 & -0.16020000000000 & $\mathrm{O}$ & (96h) \\
\hline 0.50160000000000 & -0.16020000000000 & 0.69900000000000 & $\mathrm{O}$ & (96h) \\
\hline 0.69900000000000 & -0.04040000000000 & 0.50160000000000 & $\mathrm{O}$ & (96h) \\
\hline-0.16020000000000 & 0.50160000000000 & -0.04040000000000 & $\mathrm{O}$ & $(96 \mathrm{~h})$ \\
\hline-0.04040000000000 & 0.69900000000000 & -0.16020000000000 & $\mathrm{O}$ & (96h) \\
\hline-0.04040000000000 & 0.50160000000000 & 0.69900000000000 & $\mathrm{O}$ & (96h) \\
\hline-0.16020000000000 & 0.69900000000000 & 0.50160000000000 & $\mathrm{O}$ & (96h) \\
\hline 0.69900000000000 & -0.16020000000000 & -0.04040000000000 & $\mathrm{O}$ & (96h) \\
\hline 0.50160000000000 & -0.04040000000000 & -0.16020000000000 & $\mathrm{O}$ & (96h) \\
\hline 0.37710000000000 & 0.37710000000000 & 0.37710000000000 & $\mathrm{Te}$ & $(32 \mathrm{e})$ \\
\hline 0.37710000000000 & 0.37710000000000 & -1.13130000000000 & $\mathrm{Te}$ & $(32 \mathrm{e})$ \\
\hline 0.37710000000000 & -1.13130000000000 & 0.37710000000000 & $\mathrm{Te}$ & $(32 \mathrm{e})$ \\
\hline-1.13130000000000 & 0.37710000000000 & 0.37710000000000 & $\mathrm{Te}$ & $(32 \mathrm{e})$ \\
\hline-0.12710000000000 & -0.12710000000000 & 1.38130000000000 & $\mathrm{Te}$ & $(32 \mathrm{e})$ \\
\hline-0.12710000000000 & -0.12710000000000 & -0.12710000000000 & $\mathrm{Te}$ & $(32 \mathrm{e})$ \\
\hline-0.12710000000000 & 1.38130000000000 & -0.12710000000000 & $\mathrm{Te}$ & $(32 \mathrm{e})$ \\
\hline 1.38130000000000 & -0.12710000000000 & -0.12710000000000 & $\mathrm{Te}$ & $(32 \mathrm{e})$ \\
\hline
\end{tabular}

$\mathrm{SiO}_{2}$ : A2B_cl72_211_hi_i - CIF

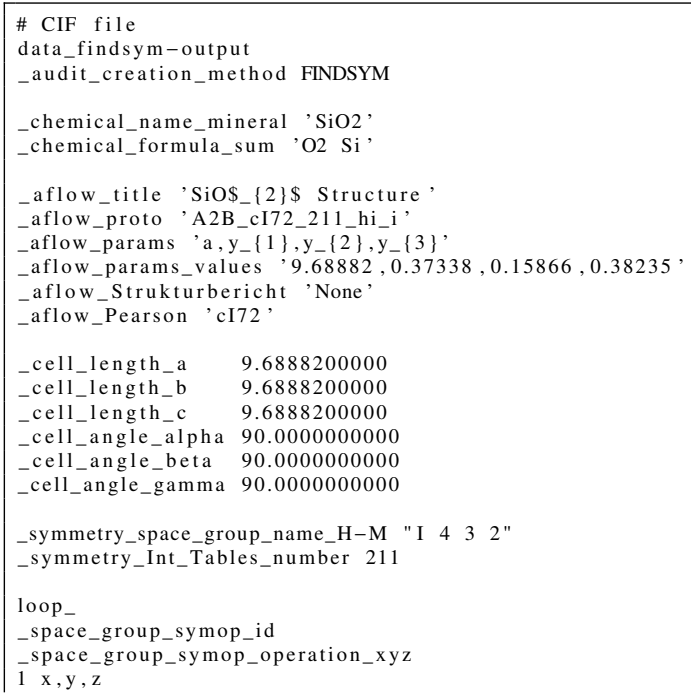

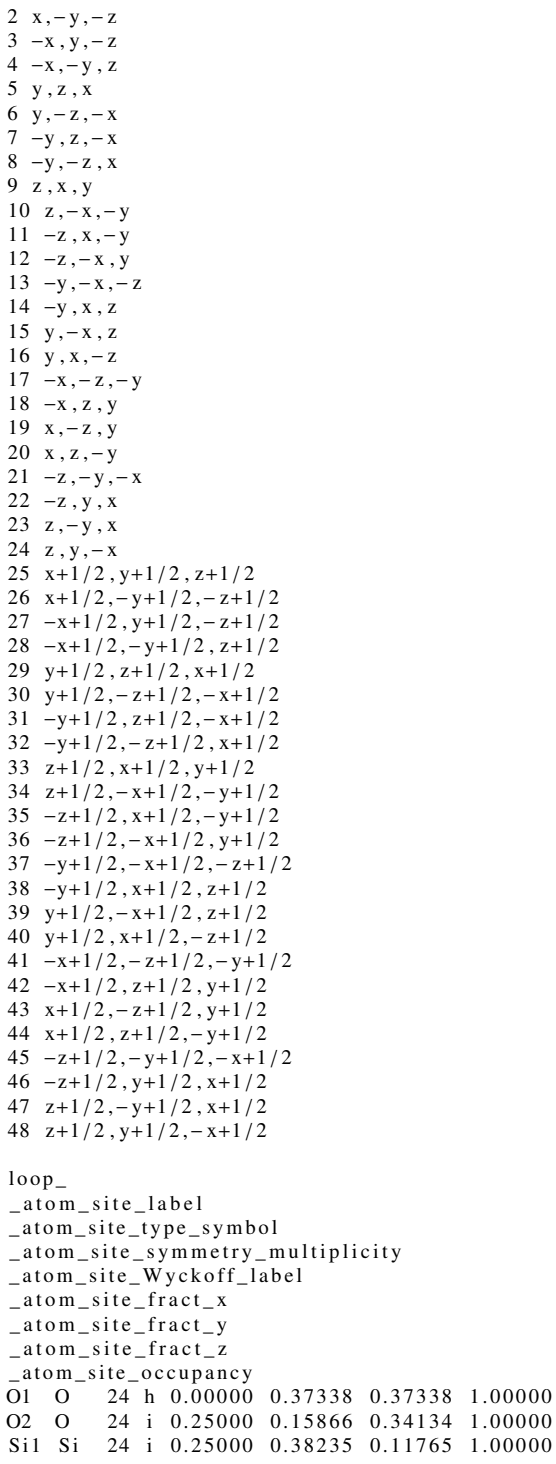

4.84441000000000 4.84441000000000

4.84441000000000 $-4.84441000000000$

0.37338000000000 0.37338000000000 . 37338000000000 0.37338000000000 0.74676000000000 0.00000000000000 0.00000000000000 $-0.74676000000000$ 0.37338000000000 $-0.37338000000000$ $-0.37338000000000$ 0.59134000000000 0.09134000000000 0.40866000000000 0.50000000000000 0.18268000000000 0.51732000000 0.40866000000000 0.59134000000000 0.90866000000000 0.09134000000000 0.36765000000000 0.13235000000000 0.63235000000000 1.13235000000000 0.50000000000000 1.26470000000000 0.50000000000000 0.63235000000000
0.37338000000000 $-0.37338000000000$ 0.37338000000000 $-0.37338000000000$ 0.37338000000000 0.37338000000000 0.37338000000000 $-0.37338000000000$ 0.7467600000000 00000000000000 0.0000000000000 0.00000000000000 $-.74676000000000$ 0.40866000000000 0.90866000000000 0.09134000000000 0.09134000000000 0.09134000 0.18268000000000 0.81732000000000 0.63235000000000 .13235000000000 $-100000000$ 0.36765000000000 $-0.13235000000000$ 1.13235000000000 0.50000000000000
O (24h)

O (24h)

O (24h)

O (24h)

$\mathrm{O} \quad(24 \mathrm{~h})$

O (24h)

O (24h)

O (24h)

O (24h)

$\mathrm{O}(24 \mathrm{~h})$

O (24i)

O (24i)

O (24i)

O (24i)

O (24i)

$O$ (24i)

O (24i)

O (24i)

O (24i)

(

O (24i)

O (24i)

O (24i)

Si (24i)

Si (24i)

Si (24i)

Si (24i)

Si (24i)

Si (24i)

Si (24i)

$\mathrm{Si} \quad(24 \mathrm{i})$ 


$\begin{array}{|rrrrr|}-0.13235000000000 & 0.36765000000000 & -0.26470000000000 & \mathrm{Si} & (24 \mathrm{i}) \\ 0.63235000000000 & 1.13235000000000 & 1.26470000000000 & \mathrm{Si} & (24 \mathrm{i}) \\ 1.13235000000000 & -0.13235000000000 & 0.50000000000000 & \mathrm{Si} & (24 \mathrm{i})\end{array}$

$\mathrm{SrSi}_{2}$ : A2B_cP12_212_c_a - CIF

\section{CIF file}

data_findsym-output

_audit_creation_method FINDSYM

_chemical_name_mineral 'SrSi2,

_chemical_formula_sum 'Si2 Sr,

_aflow_title 'SrSi\$_\{2\}\$ Structure

-aflow_proto 'A2B_cP12_212_c_a

_aflow_params 'a, $x_{-}\{2\}$

_aflow_params_values $6.54,0.428$,

-aflow_Strukturbericht 'None'

_aflow_Pearson 'cP12,

_cell_length_b 6.5400000000

cell_ength_c 6.5400000000

cell_angle_alpha 90.0000000000

_cell_angle_beta 90.0000000000

_cell_angle_gamma 90.0000000000

_symmetry_space_group_name_H-M "P $43 \quad 3 \quad 2$ "

_symmetry_Int_Tables_number 212

loop

_space_group_symop_id

_space_group_symop_operation_xyz

$1 \mathrm{x}, \mathrm{y}, \mathrm{z}$

$2 \mathrm{x}+1 / 2,-\mathrm{y}+1 / 2,-\mathrm{z}$

$3-x, y+1 / 2,-z+1 / 2$

$4-x+1 / 2,-y, z+1 / 2$

$5 \mathrm{y}, \mathrm{z}, \mathrm{x}$

$6 \mathrm{y}+1 / 2,-\mathrm{z}+1 / 2,-\mathrm{x}$

$7-y, z+1 / 2,-x+1 / 2$

$8-\mathrm{y}+1 / 2,-\mathrm{z}, \mathrm{x}+1 / 2$

$9 \mathrm{z}, \mathrm{x}, \mathrm{y}$

$10 \mathrm{z}+1 / 2,-\mathrm{x}+1 / 2,-\mathrm{y}$

$11-\mathrm{z}, \mathrm{x}+1 / 2,-\mathrm{y}+1 / 2$

$12-\mathrm{z}+1 / 2,-\mathrm{x}, \mathrm{y}+1 / 2$

$13-\mathrm{y}+1 / 4,-\mathrm{x}+1 / 4,-\mathrm{z}+1 / 4$

$14-\mathrm{y}+3 / 4, \mathrm{x}+1 / 4, \mathrm{z}+3 / 4$

$15 \mathrm{y}+3 / 4,-\mathrm{x}+3 / 4, \mathrm{z}+1 / 4$

$16 \mathrm{y}+1 / 4, \mathrm{x}+3 / 4,-\mathrm{z}+3 / 4$

$17-x+1 / 4,-z+1 / 4,-y+1 / 4$

$18-x+3 / 4, z+1 / 4, y+3 / 4$

$19 \mathrm{x}+3 / 4,-\mathrm{z}+3 / 4, \mathrm{y}+1 / 4$

$20 \mathrm{x}+1 / 4, \mathrm{z}+3 / 4,-\mathrm{y}+3 / 4$

$21-\mathrm{z}+1 / 4,-\mathrm{y}+1 / 4,-\mathrm{x}+1 / 4$

$22-\mathrm{z}+3 / 4, \mathrm{y}+1 / 4, \mathrm{x}+3 / 4$

$23 \mathrm{z}+3 / 4,-\mathrm{y}+3 / 4, \mathrm{x}+1 / 4$

$24 \mathrm{z}+1 / 4, \mathrm{y}+3 / 4,-\mathrm{x}+3 / 4$

loop_

_atom_site_label

_atom_site_type_symbol

_atom_site_symmetry_multiplicity

_atom_site_Wyckoff_label

_atom_site_fract_x

-atom_site fract_y

atom_site_fract_z

atom_site_occupancy

\begin{tabular}{llllllll}
\hline $\mathrm{Sr} 1$ & $\mathrm{Sr}$ & 4 & 0.12500 & 0.12500 & 0.12500 & 1.00000
\end{tabular}

$\begin{array}{lllllllll}\mathrm{Si} & \mathrm{Si} & 8 & \mathrm{c} & 0.42800 & 0.42800 & 0.42800 & 1.00000\end{array}$

$\mathrm{SrSi}_{2}$ : A2B_cP12_212_c_a - POSCAR

\begin{tabular}{|c|c|c|c|c|}
\hline \multicolumn{3}{|c|}{$\begin{array}{l}\text { A2B_cP12_212_c_a \& a , x } 2-- \text { params }=6.54,0.428 \& \text { P } 4 \_\{3\} 32 \mathrm{O}^{\wedge}\{6 \\
\hookrightarrow \text { cP12 \& None \& SrSi2 \& \& } \\
1.00000000000000\end{array}$} & \multicolumn{2}{|c|}{ \} \#212 (ac) \& } \\
\hline 6.54000000000000 & 0.00000000000000 & 0.00000000000000 & & \\
\hline 0.00000000000000 & 6.54000000000000 & 0.00000000000000 & & \\
\hline 0.00000000000000 & 0.00000000000000 & 6.54000000000000 & & \\
\hline $\mathrm{Si}$ & & & & \\
\hline 4 & & & & \\
\hline \multicolumn{5}{|l|}{ Direct } \\
\hline 0.42800000000000 & 0.42800000000000 & 0.42800000000000 & $\mathrm{Si}$ & $(8 c)$ \\
\hline 0.07200000000000 & -0.42800000000000 & 0.92800000000000 & $\mathrm{Si}$ & $(8 c)$ \\
\hline-0.42800000000000 & 0.92800000000000 & 0.07200000000000 & $\mathrm{Si}$ & $(8 c)$ \\
\hline 0.92800000000000 & 0.07200000000000 & -0.42800000000000 & $\mathrm{Si}$ & $(8 \mathrm{c})$ \\
\hline 0.67800000000000 & 1.17800000000000 & 0.32200000000000 & $\mathrm{Si}$ & $(8 \mathrm{c})$ \\
\hline-0.17800000000000 & -0.17800000000000 & -0.17800000000000 & $\mathrm{Si}$ & $(8 \mathrm{c})$ \\
\hline 1.17800000000000 & 0.32200000000000 & 0.67800000000000 & $\mathrm{Si}$ & $(8 c)$ \\
\hline 0.32200000000000 & 0.67800000000000 & 1.17800000000000 & $\mathrm{Si}$ & $(8 \mathrm{c})$ \\
\hline 0.12500000000000 & 0.12500000000000 & 0.12500000000000 & $\mathrm{Sr}$ & (4a) \\
\hline 0.37500000000000 & 0.87500000000000 & 0.62500000000000 & $\mathrm{Sr}$ & (4a) \\
\hline 0.87500000000000 & 0.62500000000000 & 0.37500000000000 & $\mathrm{Sr}$ & (4a) \\
\hline 0.62500000000000 & 0.37500000000000 & 0.87500000000000 & $\mathrm{Sr}$ & (4a) \\
\hline
\end{tabular}

$\mathrm{Ca}_{3} \mathrm{PI}_{3}$ : A3B3C_cI56_214_g_h_a - CIF

\# CIF file\# This file was generated by FINDSYM

\# Harold T. Stokes, Branton J. Campbell, Dorian M. Hatch

\# Brigham Young University, Provo, Utah, USA

data_findsym-output

audit_creation_method FINDSYM _chemical_formula_sum 'Ca3 I3 P'

loop

publ_author_name

'C. Hamon'

'R. Marchand

'Y. Laurent'

' J. Lang

journal_name_full_name

Bulletin of Research Laboratory of Precision Machinery and Electronics

journal_volume 97

journal__olume 97

journal

_journal_page_first

(1)

Etude $d \backslash$ 'halogenopictures. m. Structures de Ca\$ $\{2\} \$ P I$ et $C a \$ \_\{3\} \$ P I \$ \$_{-}$ $\hookrightarrow 3\} \$$. Sur structures de type $\mathrm{NaCl}$

\# Found in Pearson's Crystal Data - Crystal Structure Database for $\hookrightarrow$ Inorganic Compounds, 2013

aflow title ' $\mathrm{Ca} \$\{3\}$ PPI\$ $\{3\} \$$ Structure

aflow_proto 'A3B3C cI56_214_g_h_a'

_aflow_params 'a, $y_{-}\{2\}, y_{-}\{3\}$ '

aflow params values , $12.31504,0.108,0.384$

aflow_Strukturbericht "None,

aflow Pearson, cI56,

symmetry_space_group_name_H-M "I $4 \begin{array}{lllll}4 & 3 & 2 \text { " }\end{array}$

symmetry_Int_Tables_number 214

cell_length_a 12.31504

cell_length_b 12.31504

cell_length_c 12.31504

cell_angle_alpha 90.00000

cell_angle_beta 90.00000

cell_angle_gamma 90.00000

loop

space_group_symop_id

space_group_symop_operation_xyz

$\mathrm{x}, \mathrm{y}, \mathrm{z}$

$\mathrm{x},-\mathrm{y},-\mathrm{z}+1 / 2$

$3-x+1 / 2, y,-z$

$4-x,-y+1 /$

$\mathrm{y}, \mathrm{z}, \mathrm{x}$

$\mathrm{y},-\mathrm{z},-\mathrm{x}+1 / 2$

$7-y+1 / 2, z,-x$

$-\mathrm{y},-\mathrm{z}+1 / 2, \mathrm{x}$

$9 \mathrm{z}, \mathrm{x}, \mathrm{y}$

$10 \mathrm{z},-\mathrm{x},-\mathrm{y}+1 / 2$

$11-\mathrm{z}+1 / 2, \mathrm{x},-\mathrm{y}$

$12-\mathrm{z},-\mathrm{x}+1 / 2$,

$13-y+1 / 4,-x+1 / 4,-z+1 / 4$

$4-\mathrm{y}+1 / 4, \mathrm{x}+3 / 4, \mathrm{z}+1 / 4$

$15 \mathrm{y}+1 / 4,-\mathrm{x}+1 / 4, \mathrm{z}+3 / 4$

$16 \mathrm{y}+3 / 4, \mathrm{x}+1 / 4,-\mathrm{z}+1 / 4$

$17-\mathrm{x}+1 / 4,-\mathrm{z}+1 / 4,-\mathrm{y}+1 / 4$

$18-\mathrm{x}+1 / 4, \mathrm{z}+3 / 4, \mathrm{y}+1 / 4$

$19 x+1 / 4,-z+1 / 4, y+3 / 4$

$20 \mathrm{x}+3 / 4, \mathrm{z}+1 / 4,-\mathrm{y}+1 / 4$

$21-\mathrm{z}+1 / 4,-\mathrm{y}+1 / 4,-\mathrm{x}+1 / 4$

$22-z+1 / 4, y+3 / 4, x+1 / 4$

$23 \mathrm{z}+1 / 4,-\mathrm{y}+1 / 4, \mathrm{x}+3 / 4$

$24 \mathrm{z}+3 / 4, \mathrm{y}+1 / 4,-\mathrm{x}+1 / 4$

$25 \mathrm{x}+1 / 2, \mathrm{y}+1 / 2, \mathrm{z}+1 / 2$

$26 \mathrm{x}+1 / 2,-\mathrm{y}+1 / 2,-\mathrm{z}$

$27-\mathrm{x}, \mathrm{y}+1 / 2,-\mathrm{z}+1 / 2$

$28-\mathrm{x}+1 / 2,-\mathrm{y}, \mathrm{z}+1 / 2$

$29 \mathrm{y}+1 / 2, \mathrm{z}+1 / 2, \mathrm{x}+1 / 2$

$30 \mathrm{y}+1 / 2,-\mathrm{z}+1 / 2,-\mathrm{x}$

$31-\mathrm{y}, \mathrm{z}+1 / 2,-\mathrm{x}+1 / 2$

$32-y+1 / 2,-z, x+1 / 2$

$33 \mathrm{z}+1 / 2, \mathrm{x}+1 / 2, \mathrm{y}+1 / 2$

$34 \mathrm{z}+1 / 2,-\mathrm{x}+1 / 2,-\mathrm{y}$

$34 \mathrm{z}+1 / 2,-\mathrm{x}+1 / 2,-\mathrm{y}$

$35-\mathrm{z}, \mathrm{x}+1 / 2,-\mathrm{y}+1 / 2$
$36-\mathrm{z}+1 / 2,-\mathrm{x}, \mathrm{y}+1 / 2$

$36-z+1 / 2,-x, y+1 / 2$
$37-y+3 / 4,-x+3 / 4,-z+3 / 4$

$37-y+3 / 4,-x+3 / 4,-z+3 / 4$
$38-y+3 / 4, x+1 / 4, z+3 / 4$

$38-y+3 / 4, x+1 / 4, z+3 / 4$
$39-y+3 / 4,-x+3 / 4, z+1 / 4$

$40 \mathrm{y}+1 / 4, x+3 / 4,-\mathrm{z}+3 / 4$

$\begin{array}{ll}40 & y+1 / 4, x+3 / 4,-z+3 / 4 \\ 41-x+3 / 4,-z+3 / 4,-y+3 / 4\end{array}$

$42-\mathrm{x}+3 / 4, \mathrm{z}+1 / 4, \mathrm{y}+3 / 4$

$43 \mathrm{x}+3 / 4,-\mathrm{z}+3 / 4, \mathrm{y}+1 / 4$

$44 \mathrm{x}+1 / 4, \mathrm{z}+3 / 4,-\mathrm{y}+3 / 4$

$45-\mathrm{z}+3 / 4,-\mathrm{y}+3 / 4,-\mathrm{x}+3 / 4$

$46-\mathrm{z}+3 / 4, \mathrm{y}+1 / 4, \mathrm{x}+3 / 4$

$47 \mathrm{z}+3 / 4,-\mathrm{y}+3 / 4, \mathrm{x}+1 / 4$

$48 \mathrm{z}+1 / 4, \mathrm{y}+3 / 4,-\mathrm{x}+3 / 4$

loop_

-atom_site_label

atom_site_type_symbol

atom_site_symmetry_multiplicity

atom_site_Wyckoff_label

atom_site_fract $x$

-atom_site_fract_y

atom_site_fract_z

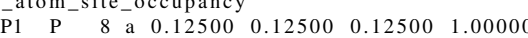

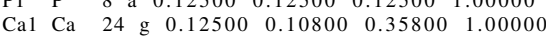

_chemical_name_mineral 'Ca3PI3, 
I1 I $\quad 24 \mathrm{~h} \quad 0.12500 \quad 0.38400 \quad 0.86600 \quad 1.00000$

$\mathrm{Ca}_{3} \mathrm{PI}_{3}$ : A3B3C_cI56_214_g_h_a - POSCAR

\begin{tabular}{|c|c|c|c|c|}
\hline \multicolumn{5}{|c|}{ A3B3C_cI56_214_g_h_a \& a,y2,y3 --params $=12.31504,0.108,0.384 \& I 4 \_\{1\} 3$} \\
\hline & & & & \\
\hline & 10 & $97,6-12$ & & \\
\hline 1.00000000000000 & & & & \\
\hline-6.15752000000000 & 6.15752000000000 & 6.15752000000000 & & \\
\hline 6.15752000000000 & -6.15752000000000 & 6.15752000000000 & & \\
\hline 6.15752000000000 & 6.15752000000000 & -6.15752000000000 & & \\
\hline $\mathrm{Ca}$ & & & & \\
\hline 12 & & & & \\
\hline Direct & & & & \\
\hline 0.46600000000000 & 0.48300000000000 & 0.23300000000000 & $\mathrm{Ca}$ & $(24 \mathrm{~g})$ \\
\hline 0.75000000000000 & 0.23300000000000 & 0.26700000000000 & $\mathrm{Ca}$ & $(24 \mathrm{~g})$ \\
\hline 0.75000000000000 & 0.01700000000000 & 0.48300000000000 & $\mathrm{Ca}$ & $(24 \mathrm{~g})$ \\
\hline 0.03400000000000 & 0.26700000000000 & 0.01700000000000 & $\mathrm{Ca}$ & $(24 \mathrm{~g})$ \\
\hline 0.23300000000000 & 0.46600000000000 & 0.48300000000000 & $\mathrm{Ca}$ & $(24 \mathrm{~g})$ \\
\hline 0.26700000000000 & 0.75000000000000 & 0.23300000000000 & $\mathrm{Ca}$ & $(24 \mathrm{~g})$ \\
\hline 0.48300000000000 & 0.75000000000000 & 0.01700000000000 & $\mathrm{Ca}$ & $(24 \mathrm{~g})$ \\
\hline 0.01700000000000 & 0.03400000000000 & 0.26700000000000 & $\mathrm{Ca}$ & (24g) \\
\hline 0.48300000000000 & 0.23300000000000 & 0.46600000000000 & $\mathrm{Ca}$ & $(24 \mathrm{~g})$ \\
\hline 0.23300000000000 & 0.26700000000000 & 0.75000000000000 & $\mathrm{Ca}$ & $(24 \mathrm{~g})$ \\
\hline 0.01700000000000 & 0.48300000000000 & 0.75000000000000 & $\mathrm{Ca}$ & $(24 \mathrm{~g})$ \\
\hline 0.26700000000000 & 0.01700000000000 & 0.03400000000000 & $\mathrm{Ca}$ & $(24 \mathrm{~g})$ \\
\hline 0.25000000000000 & -0.00900000000000 & 0.50900000000000 & I & $(24 \mathrm{~h})$ \\
\hline-0.01800000000000 & -0.25900000000000 & -0.00900000000000 & I & $(24 \mathrm{~h})$ \\
\hline 1.51800000000000 & 0.50900000000000 & 0.75900000000000 & I & $(24 \mathrm{~h})$ \\
\hline 0.25000000000000 & 0.75900000000000 & -0.25900000000000 & I & $(24 h)$ \\
\hline 0.50900000000000 & 0.25000000000000 & -0.00900000000000 & I & $(24 \mathrm{~h})$ \\
\hline-0.00900000000000 & -0.01800000000000 & -0.25900000000000 & I & $(24 h)$ \\
\hline 0.75900000000000 & 1.51800000000000 & 0.50900000000000 & I & $(24 \mathrm{~h})$ \\
\hline-0.25900000000000 & 0.25000000000000 & 0.75900000000000 & I & $(24 h)$ \\
\hline-0.00900000000000 & 0.50900000000000 & 0.25000000000000 & I & $(24 h)$ \\
\hline-0.25900000000000 & -0.00900000000000 & -0.01800000000000 & I & $(24 h)$ \\
\hline 0.50900000000000 & 0.75900000000000 & 1.51800000000000 & I & $(24 h)$ \\
\hline 0.75900000000000 & -0.25900000000000 & 0.25000000000000 & I & $(24 h)$ \\
\hline 0.25000000000000 & 0.25000000000000 & 0.25000000000000 & $\mathrm{P}$ & $(8 a)$ \\
\hline 0.50000000000000 & 0.00000000000000 & 0.25000000000000 & $\mathrm{P}$ & (8a) \\
\hline 0.00000000000000 & 0.25000000000000 & 0.50000000000000 & $\mathrm{P}$ & (8a) \\
\hline 0.25000000000000 & 0.50000000000000 & 0.00000000000000 & $\mathrm{P}$ & (8a) \\
\hline
\end{tabular}

Petzite $\left(\mathrm{Ag}_{3} \mathrm{AuTe}_{2}\right)$ : A3BC2_cI48_214_f_a_e - CIF

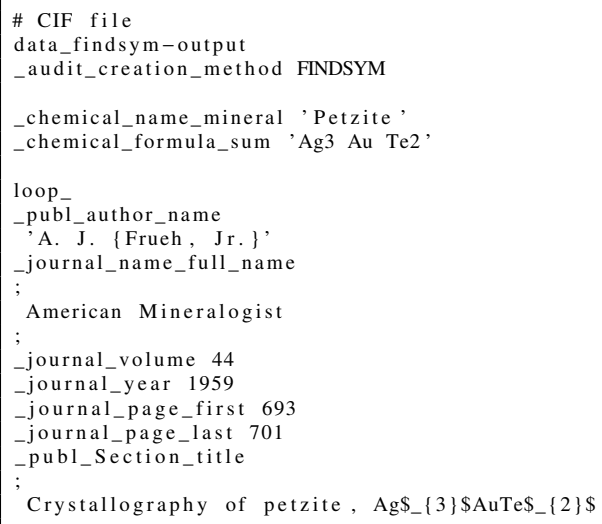

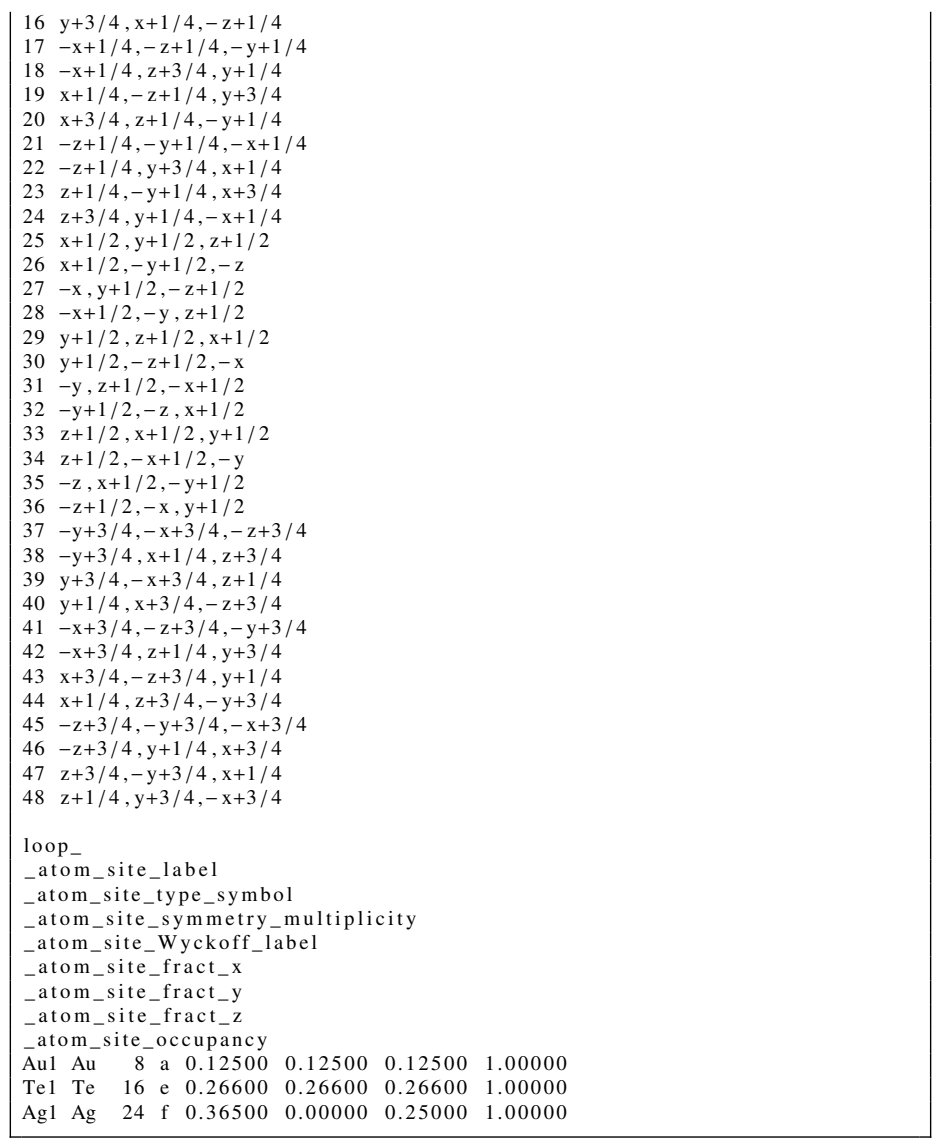

$\begin{array}{lllllllll}\mathrm{Ag} 1 & \mathrm{Ag} & 24 & \mathrm{f} & 0.36500 & 0.00000 & 0.25000 & 1.0000\end{array}$

Petzite $\left(\mathrm{Ag}_{3} \mathrm{AuTe}_{2}\right)$ : A3BC2_cI48_214_f_a_e - POSCAR

\begin{tabular}{|c|c|c|c|c|}
\hline \multirow{2}{*}{\multicolumn{5}{|c|}{ 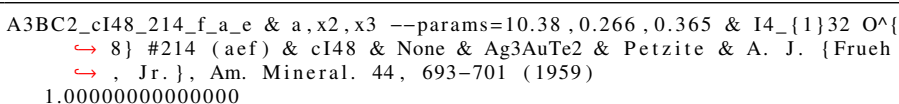 }} \\
\hline & & & & \\
\hline-5.19000000000000 & 5.19000000000000 & 5.19000000000000 & & \\
\hline 5.19000000000000 & -5.19000000000000 & 5.19000000000000 & & \\
\hline 5.19000000000000 & 5.19000000000000 & -5.19000000000000 & & \\
\hline $\mathrm{Ag}$ & & & & \\
\hline 12 & & & & \\
\hline \multicolumn{5}{|l|}{ Direct } \\
\hline 0.25000000000000 & 0.61500000000000 & 0.36500000000000 & $\mathrm{Ag}$ & $(24 f)$ \\
\hline 0.75000000000000 & -0.11500000000000 & 0.13500000000000 & $\mathrm{Ag}$ & $(24 f)$ \\
\hline 0.36500000000000 & 0.25000000000000 & 0.61500000000000 & $\mathrm{Ag}$ & $(24 \mathrm{f})$ \\
\hline 0.13500000000000 & 0.75000000000000 & -0.11500000000000 & $\mathrm{Ag}$ & $(24 f)$ \\
\hline 0.61500000000000 & 0.36500000000000 & 0.25000000000000 & $\mathrm{Ag}$ & $(24 f)$ \\
\hline-0.11500000000000 & 0.13500000000000 & 0.75000000000000 & $\mathrm{Ag}$ & $(24 f)$ \\
\hline 0.61500000000000 & 0.75000000000000 & 0.36500000000000 & $\mathrm{Ag}$ & $(24 f)$ \\
\hline-0.11500000000000 & 0.25000000000000 & 0.13500000000000 & $\mathrm{Ag}$ & $(24 f)$ \\
\hline 0.75000000000000 & 0.36500000000000 & 0.61500000000000 & $\mathrm{Ag}$ & $(24 f)$ \\
\hline 0.25000000000000 & 0.13500000000000 & -0.11500000000000 & $\mathrm{Ag}$ & $(24 f)$ \\
\hline 0.13500000000000 & -0.11500000000000 & 0.25000000000000 & $\mathrm{Ag}$ & $(24 f)$ \\
\hline 0.36500000000000 & 0.61500000000000 & 0.75000000000000 & $\mathrm{Ag}$ & $(24 f)$ \\
\hline 0.25000000000000 & 0.25000000000000 & 0.25000000000000 & $\mathrm{Au}$ & $(8 a)$ \\
\hline 0.50000000000000 & 0.00000000000000 & 0.25000000000000 & $\mathrm{Au}$ & ( $8 \mathrm{a})$ \\
\hline 0.00000000000000 & 0.25000000000000 & 0.50000000000000 & $\mathrm{Au}$ & (8a) \\
\hline 0.25000000000000 & 0.50000000000000 & 0.00000000000000 & $\mathrm{Au}$ & (8a) \\
\hline 0.53200000000000 & 0.53200000000000 & 0.53200000000000 & $\mathrm{Te}$ & $(16 \mathrm{e})$ \\
\hline 0.50000000000000 & 0.00000000000000 & -0.03200000000000 & $\mathrm{Te}$ & $(16 \mathrm{e})$ \\
\hline 0.00000000000000 & -0.03200000000000 & 0.50000000000000 & $\mathrm{Te}$ & $(16 \mathrm{e})$ \\
\hline-0.03200000000000 & 0.50000000000000 & 0.00000000000000 & $\mathrm{Te}$ & $(16 \mathrm{e})$ \\
\hline 0.50000000000000 & 0.00000000000000 & 0.53200000000000 & $\mathrm{Te}$ & $(16 \mathrm{e})$ \\
\hline-0.03200000000000 & -0.03200000000000 & -0.03200000000000 & $\mathrm{Te}$ & $(16 \mathrm{e})$ \\
\hline 0.00000000000000 & 0.53200000000000 & 0.50000000000000 & $\mathrm{Te}$ & $(16 \mathrm{e})$ \\
\hline 0.53200000000000 & 0.50000000000000 & 0.00000000000000 & $\mathrm{Te}$ & $(16 \mathrm{e})$ \\
\hline
\end{tabular}

$\gamma$-brass $\left(\mathrm{Cu}_{9} \mathrm{Al}_{4}, D 8_{3}\right)$ : A4B9_cP52_215_ei_3efgi - CIF 


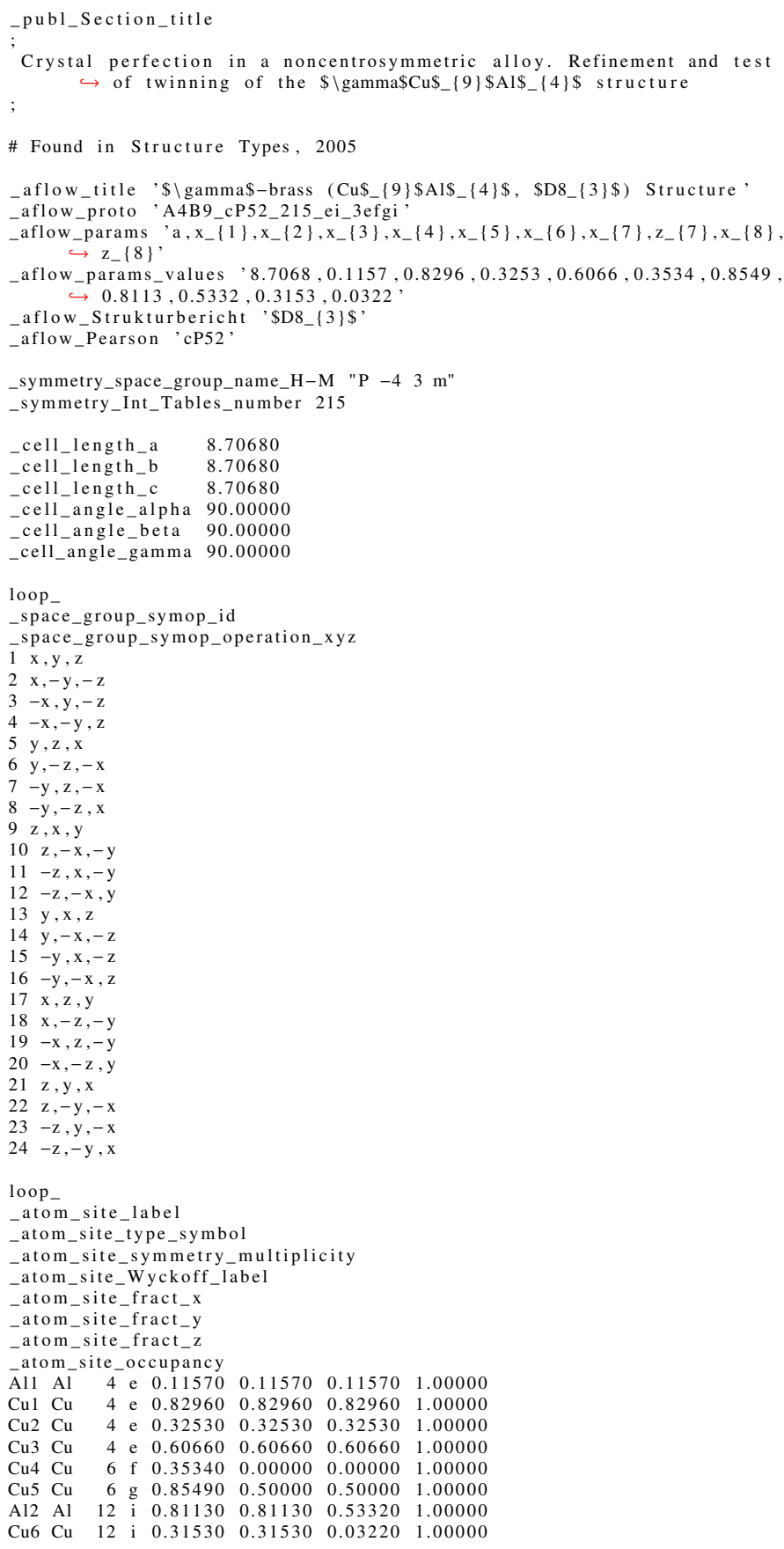

$\gamma$-brass $\left(\mathrm{Cu}_{9} \mathrm{Al}_{4}, D 8_{3}\right)$ : A4B9_cP52_215_ei_3efgi - POSCAR

A4B9_cP52_215_ei_3efgi \& a , x1, x2, x3, x4, x5, x6, x7, z7, x8, z8 --params $=8.7068$ $\hookrightarrow, 0.1157,0.8296,0.3253,0.6066,0.3534,0.8549,0.8113,0.5332,0.315$ $\hookrightarrow, 0.0322 \& \mathrm{P}-43 \mathrm{~m} \mathrm{~T} \mathrm{~T}_{-}\{\mathrm{d}\}^{\wedge}\{1\} \# 215\left(\mathrm{e}^{\wedge} 4 \mathrm{fgi} i^{\wedge} 2\right) \& \mathrm{cP} 52 \& \$ \mathrm{D} 8 \_\{3\} \$ \&$

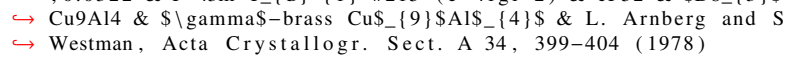
1.00000000000000

$\begin{array}{lll}8.70680000000000 & 0.00000000000000 & 0.00000000000000\end{array}$ $\begin{array}{lll}0.00000000000000 & 8.70680000000000 & 0.00000000000000\end{array}$ $\begin{array}{lll}0.00000000000000 & 0.00000000000000 & 8.70680000000000\end{array}$

$$
\begin{array}{ll}
\mathrm{Al} & \mathrm{Cu} \\
16 & 36
\end{array}
$$

Direct

0.11570000000000 $-0.11570000000000$ $-0.11570000000000$ $-0.11570000000000$ 0.81130000000000 $-0.81130000000000$ $-0.81130000000000$ 0.81130000000000 0.53320000000000 0.53320000000000 $-0.53320000000000$ $-0.53320000000000$ 0.81130000000000 $-0.81130000000000$ 0.81130000000000 $-0.81130000000000$ 0.82960000000000 0.82960000000000

0.11570000000000 $-0.11570000$ 0.81130000000000 0.81130000000000 0.81130000000000 0.81130000000000 0.81130000000000 $-0.81130000000000$ $-0.81130000000000$ 0.81130000000000 0.53320000000000 0.53320000000000 $-0.53320000000000$ $-0.53320000000000$ 0.82960000000000 $-0.82960000000000$

$-0.82960000000000$ 0.82960000000000 0.32530000000000 $-0.32530000000000$ $-0.32530000000000$ 0.32530000000000 0.60660000000000 $-0.60660000000000$ $-0.60660000000000$ 0.60660000000000 0.35340000000000 $-0.35340000000000$ 0.00000000000000 0.0000000000000 0.0000000000000 0.000000000000 0.8549000000000 0.854900000000 $-0.85490000000000$ 0.50000000000000 0.50000000000000 0.50000000000000 0.50000000000000 0.31530000000000 $-0.31530000000000$ $-0.31530000000000$ 0.31530000000000 0.03220000000000 0.03220000000000 $-0.03220000000000$ $-0.03220000000000$

$-0.31530000000000$ $-0.31530000000000$

$-0.31530000000000$ $-0.31530000000000$

0.82960000000000 0.82960000000000 0.32530000000000 0.32530000000000 0.32530000000000 $-0.32530000000000$ 0.60660000000000 $-0.60660000000000$ 0.60660000000000 $-0.60660000000000$ 0.00000000000000 0.00000000000000 0.35340000000000 0.35340000000000 0.00000000000000 0.00000000000000 0.50000000000000 0.500000000000 0.5000000000000 0.85490000000000 $-0.85490000000000$ 0.50000000000000 0.50000000000000 0.31530000000000 $-0.31530000000000$ 0.31530000000000 $-0.31530000000000$ 0.31530000000000 $-0.31530000000000$ $-0.31530000000000$ 0.31530000000000 0.03220000000000 0.03220000000000 $-0.0322000000000$ $-0.03220000000000$

$-0.82960000000000$ $-0.82960000000000$ 0.32530000000000 0.32530000000000 0.325300000000 $-0.3253000000000$ 0.60660000000000 . 0.60660000000000 0.60660000000000 0.60660000000000 0.00000000000000 0.00000000000000 0.00000000000000 0.3534000000000 0.3534000000000 0.50000000000000 0.500000000000 0.50000000000 0.500000000000 0.85490000000000 $-0.85490000000000$ 0.03220000000000 0.03220000000000 $-0.03220000000000$ 0.03220000000000 .31530000000000 0.31530000000000 0.31530000000000 $-0.31530000000000$ 0.31530000000000 $-0.31530000000000$ 0.31530000000000

$\mathrm{Cu} \quad(4 \mathrm{e})$ $\mathrm{Cu} \quad(4 \mathrm{e}$ $\mathrm{Cu} \quad(4 \mathrm{e}$ $\mathrm{Cu} \quad(4 \mathrm{e}$ $\mathrm{Cu} \quad(4 \mathrm{e})$ $\mathrm{Cu} \quad(4 \mathrm{e})$ $\mathrm{Cu} \quad(4 \mathrm{e})$ $\mathrm{Cu} \quad(4 \mathrm{e}$ $\mathrm{Cu} \quad(4 \mathrm{e})$ $\mathrm{Cu} \quad(4 \mathrm{e})$ $\mathrm{Cu}$ (6f)

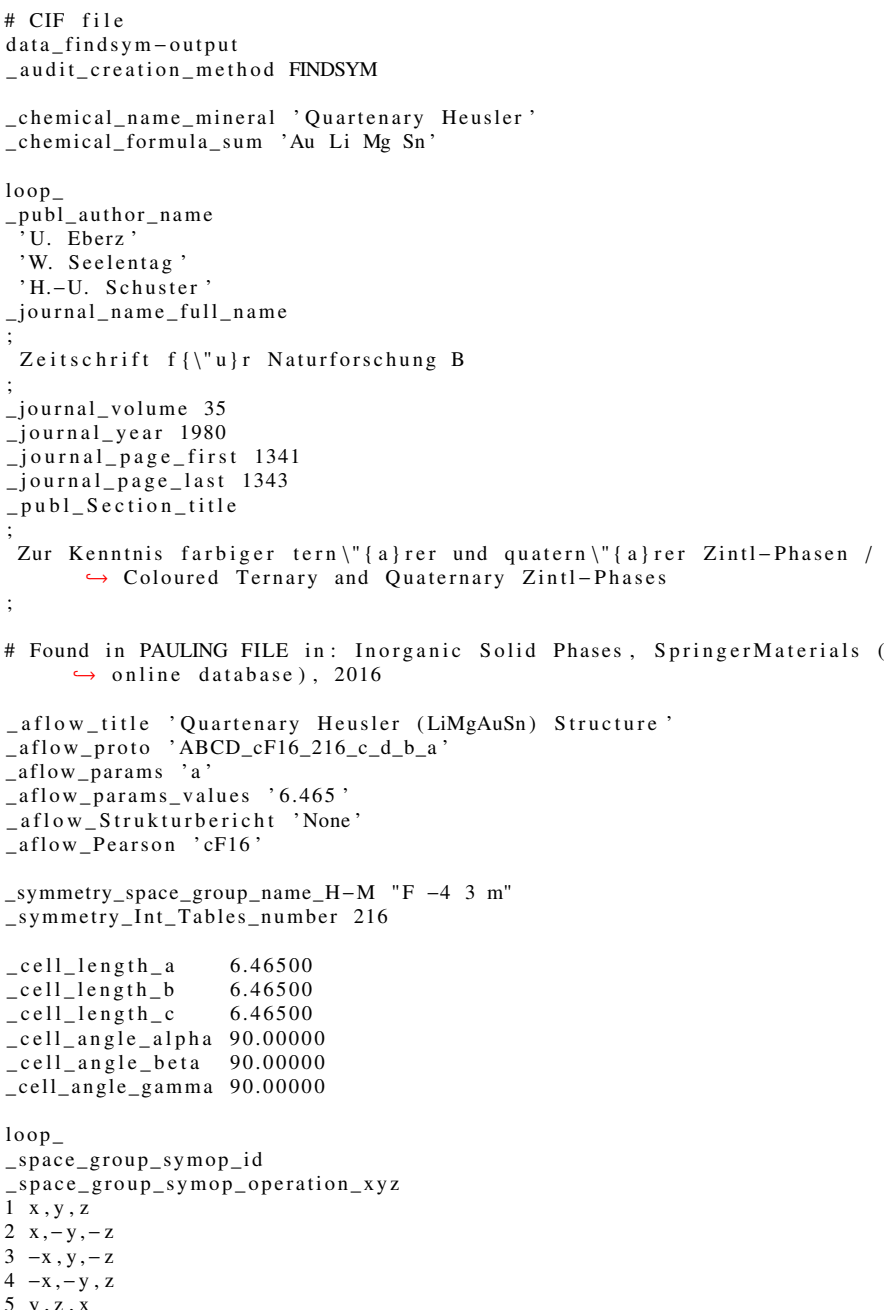


$20-\mathrm{x},-\mathrm{z}, \mathrm{y}$

$21 \mathrm{z}, \mathrm{y}, \mathrm{x}$

$22 \mathrm{z},-\mathrm{y},-\mathrm{x}$

$23-\mathrm{z}, \mathrm{y},-\mathrm{x}$

$25 \mathrm{x}, \mathrm{y}+1 / 2, \mathrm{z}+1 / 2$

$26 \mathrm{x},-\mathrm{y}+1 / 2,-\mathrm{z}+1 / 2$

$27-\mathrm{x}, \mathrm{y}+1 / 2,-\mathrm{z}+1 / 2$

$28-\mathrm{x},-\mathrm{y}+1 / 2, \mathrm{z}+1 / 2$

$29 \mathrm{y}, \mathrm{z}+1 / 2, \mathrm{x}+1 / 2$

$30 \mathrm{y},-\mathrm{z}+1 / 2,-\mathrm{x}+1 / 2$

$31-y, z+1 / 2,-x+1 / 2$

$32-y, z+1 / 2,-x+1 / 2$

$32-y,-z+1 / 2, x+1 / 2$

$33 z, x+1 / 2, y+1 / 2$

$34 \mathrm{z},-\mathrm{x}+1 / 2,-\mathrm{y}+1 / 2$
$35-\mathrm{z}, \mathrm{x}+1 / 2,-\mathrm{y}+1 / 2$

$35-z, x+1 / 2,-y+1 / 2$
$36-z,-x+1 / 2, y+1 / 2$

$36-\mathrm{z},-\mathrm{x}+1 / 2, \mathrm{y}+1 / 2$
$37 \mathrm{y}, \mathrm{x}+1 / 2, \mathrm{z}+1 / 2$

$38 \mathrm{y},-\mathrm{x}+1 / 2,-\mathrm{z}+1 / 2$

$39-\mathrm{y}, \mathrm{x}+1 / 2,-\mathrm{z}+1 / 2$

$40-y,-x+1 / 2, z+1 / 2$

$41 \mathrm{x}, \mathrm{z}+1 / 2, \mathrm{y}+1 / 2$

$42 \mathrm{x},-\mathrm{z}+1 / 2,-\mathrm{y}+1 / 2$

$43-\mathrm{x}, \mathrm{z}+1 / 2,-\mathrm{y}+1 / 2$

$44-\mathrm{x},-\mathrm{z}+1 / 2, \mathrm{y}+1 / 2$

$45 \mathrm{z}, \mathrm{y}+1 / 2, \mathrm{x}+1 / 2$

$46 \mathrm{z},-\mathrm{y}+1 / 2,-\mathrm{x}+1 / 2$

$47-\mathrm{z}, \mathrm{y}+1 / 2,-\mathrm{x}+1 / 2$

$48-\mathrm{z},-\mathrm{y}+1 / 2, \mathrm{x}+1 / 2$

$49 \mathrm{x}+1 / 2, \mathrm{y}, \mathrm{z}+1 / 2$

$50 \mathrm{x}+1 / 2,-\mathrm{y},-\mathrm{z}+1 / 2$

$51-\mathrm{x}+1 / 2, \mathrm{y},-\mathrm{z}+1 / 2$

$52-x+1 / 2,-y, z+1 / 2$

$53 \mathrm{y}+1 / 2, \mathrm{z}, \mathrm{x}+1 / 2$

$54 \mathrm{y}+1 / 2,-\mathrm{z},-\mathrm{x}+1 / 2$

$55-\mathrm{y}+1 / 2, \mathrm{z},-\mathrm{x}+1 / 2$

$\begin{array}{ll}56 & -y+1 / 2,-z, x+1 / 2 \\ 57 & z+1 / 2, x, y+1 / 2\end{array}$

$58 \mathrm{z}+1 / 2,-\mathrm{x},-\mathrm{y}+1 / 2$

$59-\mathrm{z}+1 / 2, \mathrm{x},-\mathrm{y}+1 / 2$

$60-\mathrm{z}+1 / 2,-\mathrm{x}, \mathrm{y}+1 / 2$

$61 \mathrm{y}+1 / 2, \mathrm{x}, \mathrm{z}+1 / 2$

$62 \mathrm{y}+1 / 2,-\mathrm{x},-\mathrm{z}+1 / 2$

$63-y+1 / 2, x,-z+1 / 2$

$64-y+1 / 2,-x, z+1 / 2$

$65 \mathrm{x}+1 / 2, \mathrm{z}, \mathrm{y}+1 / 2$

$66 x+1 / 2,-z,-y+1 / 2$

$67-x+1 / 2, z,-y+1 / 2$

$68-\mathrm{x}+1 / 2,-\mathrm{z}, \mathrm{y}+1 / 2$

$69 \mathrm{z}+1 / 2, y, x+1 / 2$

$70 \mathrm{z}+1 / 2,-\mathrm{y},-\mathrm{x}+1 / 2$

$71-z+1 / 2, y,-x+1 / 2$

$72-\mathrm{z}+1 / 2,-\mathrm{y}, \mathrm{x}+1 / 2$

$73 x+1 / 2, y+1 / 2, z$

$74 x+1 / 2,-y+1 / 2,-z$
$75-x+1 / 2, y+1 / 2,-z$

$76-\mathrm{x}+1 / 2,-\mathrm{y}+1 / 2, \mathrm{z}$

$77 \mathrm{y}+1 / 2, \mathrm{z}+1 / 2, \mathrm{x}$

$78 \mathrm{y}+1 / 2,-\mathrm{z}+1 / 2,-\mathrm{x}$

$79-y+1 / 2, z+1 / 2,-x$

$80-\mathrm{y}+1 / 2,-\mathrm{z}+1 / 2, \mathrm{x}$

$81 \mathrm{z}+1 / 2, \mathrm{x}+1 / 2, \mathrm{y}$

$82 \mathrm{z}+1 / 2,-\mathrm{x}+1 / 2,-\mathrm{y}$

$83-\mathrm{z}+1 / 2, \mathrm{x}+1 / 2,-\mathrm{y}$

$84-z+1 / 2,-x+1 / 2, y$

$86 \mathrm{y}+1 / 2, x+1 / 2, \mathrm{z}$

$86 \mathrm{y}+1 / 2,-x+1 / 2,-$

$87-y+1 / 2, x+1 / 2,-z$

$88-y+1 / 2,-x+1 / 2$,

$89 \mathrm{x}+1 / 2, \mathrm{z}+1 / 2, \mathrm{y}$

$90 \mathrm{x}+1 / 2,-\mathrm{z}+1 / 2,-\mathrm{y}$

$91-x+1 / 2, z+1 / 2,-y$

$92-\mathrm{x}+1 / 2,-\mathrm{z}+1 / 2, \mathrm{y}$

$93 \mathrm{z}+1 / 2, \mathrm{y}+1 / 2, \mathrm{x}$

$94 \mathrm{z}+1 / 2,-\mathrm{y}+1 / 2,-\mathrm{x}$

$95-\mathrm{z}+1 / 2, \mathrm{y}+1 / 2,-\mathrm{x}$

$96-\mathrm{z}+1 / 2,-\mathrm{y}+1 / 2, \mathrm{x}$

loop

_atom_site_type_symbol

_atom_site_symmetry_multiplicity

atom_site_Wyckoff_label

_atom_site_fract_x

atom_site_fract_y

atom_site_fract_z

_atom_site_occupancy

$\begin{array}{lllllll}\mathrm{S} n 1 \mathrm{Sn} & 4 & \text { a } & 0.00000 & 0.00000 & 0.00000 & 1.00000\end{array}$

$\begin{array}{llllllll}\mathrm{Mg} 1 \mathrm{Mg} & 4 & \mathrm{~b} & 0.50000 & 0.50000 & 0.50000 & 1.00000\end{array}$

$\begin{array}{llllllll}\mathrm{Au} 1 \mathrm{Au} & 4 & \mathrm{c} & 0.25000 & 0.25000 & 0.25000 & 1.00000\end{array}$

$\begin{array}{llllllll}\mathrm{Li} 1 & \mathrm{Li} & 4 & \mathrm{~d} & 0.75000 & 0.75000 & 0.75000 & 1.00000\end{array}$

Quartenary Heusler (LiMgAuSn): ABCD_cF16_216_c_d_b_a - POSCAR

ABCD_cF16_216_c_d_b_a \& a --params $=6.465 \&$ F-43m $T_{-}\{d\}^{\wedge}\{2\} \# 216$ (abcd) \& $\hookrightarrow$ cF16 \& None \& LiMgAuSn \& Quartenary Heusler \& U. Eberz and W.

$\hookrightarrow$ Seelentag and H.-U. Schuster, Z. Naturforsch. B 35, 1341-1343 $\hookrightarrow 1980$ )

1.00000000000000

$0.00000000000000 \quad 3.23250000000000 \quad 3.23250000000000$

$3.23250000000000 \quad 0.00000000000000 \quad 3.23250000000000$

$3.23250000000000-3.23250000000000 \quad 0.00000000000000$

$\mathrm{Au} \quad \mathrm{Li} \quad \mathrm{Mg} \quad \mathrm{Sn}$

Direct
.25000000000000

0.75000000000000

.50000000000000

0.00000000000000

$\mathrm{Ag}_{3}\left[\mathrm{PO}_{4}\right]: \mathrm{A} 3 \mathrm{~B} 4 \mathrm{C} \_\mathrm{cP} 16 \_218$ c_e e a - CIF

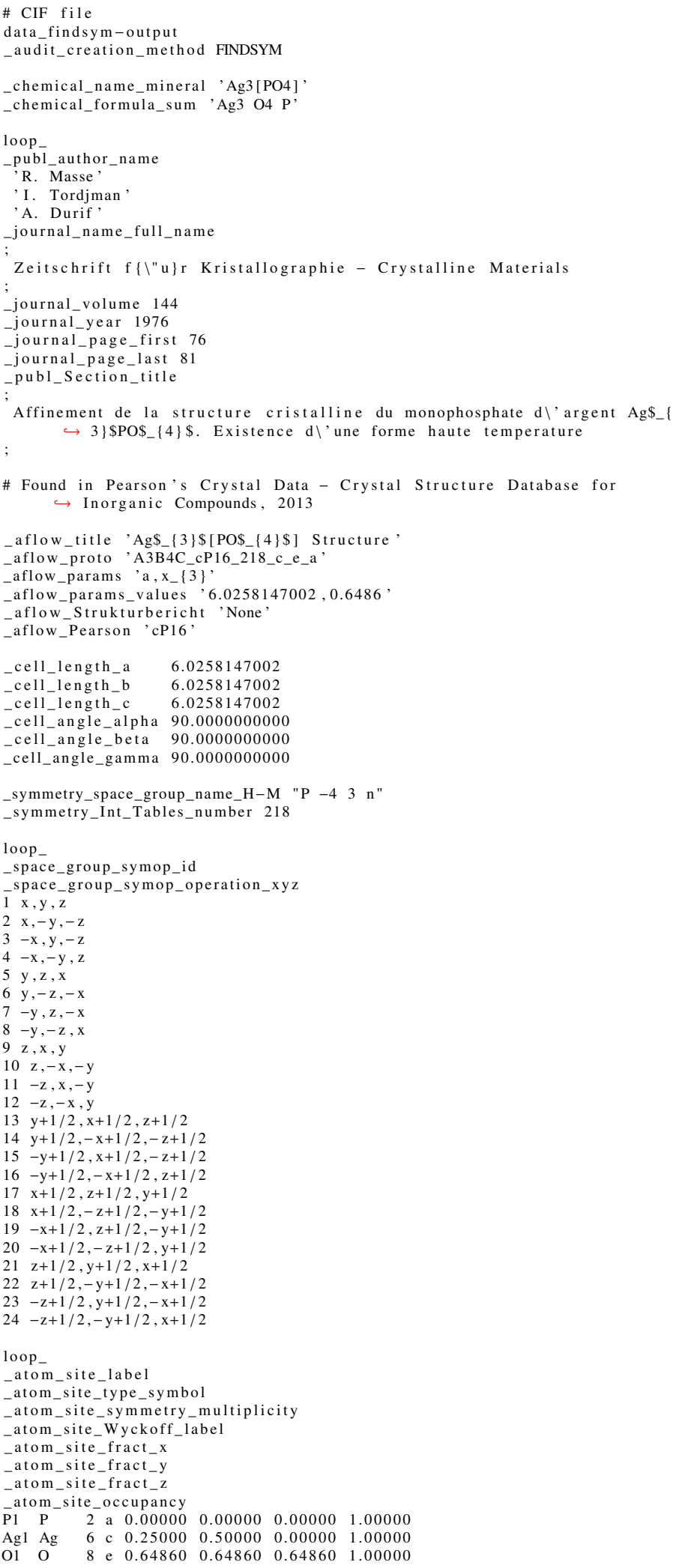

$\mathrm{Ag}_{3}\left[\mathrm{PO}_{4}\right]:$ A3B4C_cP16_218_c_e_a - POSCAR

A3B4C cP16 218 c e a \& a, x3 --params $=6.0258147002,0.6486 \&$ \& $-43 n$ T $\{\text { d }\}^{\wedge}$

$\hookrightarrow 4\} \# 218$ (ace) \& cP16 \& None \& Ag3[PO4] \& \& R. Masse and I.
$\hookrightarrow$ Tordjman and A. Durif, Zeitschrift f" $\{\mathrm{u}\} \mathrm{r}$ Kristallographie -

$\rightarrow$ Tordjman and A. Durif, Zeitschrift $\mathrm{f}$ \{u\}r

1.00000000000000

6.02581470020000 $\begin{array}{llll}0.0000000000000 & 6.02581470020000 & 0.00000000000000\end{array}$

0.0000000000000 $\mathrm{Ag}$ (
6.02581470020000 


\begin{tabular}{|crrrrr|}
6 & 8 & & & & \\
Direct & & & & & \\
0.25000000000000 & 0.50000000000000 & 0.00000000000000 & $\mathrm{Ag}$ & $(6 \mathrm{c})$ \\
0.75000000000000 & 0.50000000000000 & 0.00000000000000 & $\mathrm{Ag}$ & $(6 \mathrm{c})$ \\
0.00000000000000 & 0.25000000000000 & 0.50000000000000 & $\mathrm{Ag}$ & $(6 \mathrm{c})$ \\
0.00000000000000 & 0.75000000000000 & 0.50000000000000 & $\mathrm{Ag}$ & $(6 \mathrm{c})$ \\
0.50000000000000 & 0.00000000000000 & 0.25000000000000 & $\mathrm{Ag}$ & $(6 \mathrm{c})$ \\
0.50000000000000 & 0.00000000000000 & 0.75000000000000 & $\mathrm{Ag}$ & $(6 \mathrm{c})$ \\
0.64860000000000 & 0.64860000000000 & 0.64860000000000 & $\mathrm{O}$ & $(8 \mathrm{e})$ \\
-0.64860000000000 & -0.64860000000000 & 0.64860000000000 & $\mathrm{O}$ & $(8 \mathrm{e})$ \\
-0.64860000000000 & 0.64860000000000 & -0.64860000000000 & $\mathrm{O}$ & $(8 \mathrm{e})$ \\
0.64860000000000 & -0.64860000000000 & -0.64860000000000 & $\mathrm{O}$ & $(8 \mathrm{e})$ \\
1.14860000000000 & 1.14860000000000 & 1.14860000000000 & $\mathrm{O}$ & $(8 \mathrm{e})$ \\
-0.14860000000000 & -0.14860000000000 & 1.14860000000000 & $\mathrm{O}$ & $(8 \mathrm{e})$ \\
1.14860000000000 & -0.14860000000000 & -0.14860000000000 & $\mathrm{O}$ & $(8 \mathrm{e})$ \\
-0.14860000000000 & 1.14860000000000 & -0.14860000000000 & $\mathrm{O}$ & $(8 \mathrm{e})$ \\
0.00000000000000 & 0.00000000000000 & 0.00000000000000 & $\mathrm{P}$ & $(2 \mathrm{a})$ \\
0.50000000000000 & 0.50000000000000 & 0.50000000000000 & $\mathrm{P}$ & $(2 \mathrm{a})$ \\
\hline
\end{tabular}

Boracite $\left(\mathrm{Mg}_{3} \mathrm{~B}_{7} \mathrm{ClO}_{13}\right)$ : A7BC3D13_cF192_219_de_b_c_ah - CIF

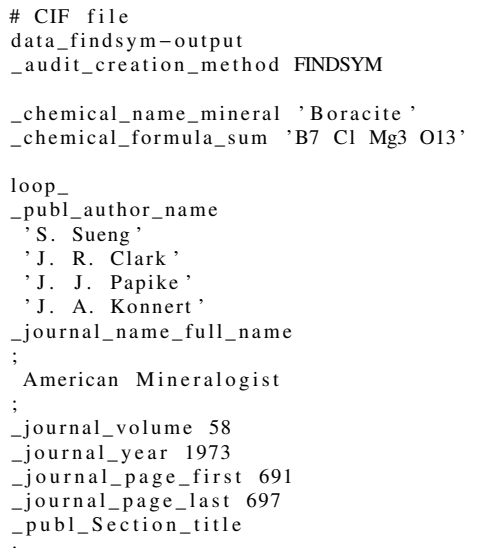

$38 \mathrm{y}+1 / 2,-\mathrm{x},-\mathrm{z}$

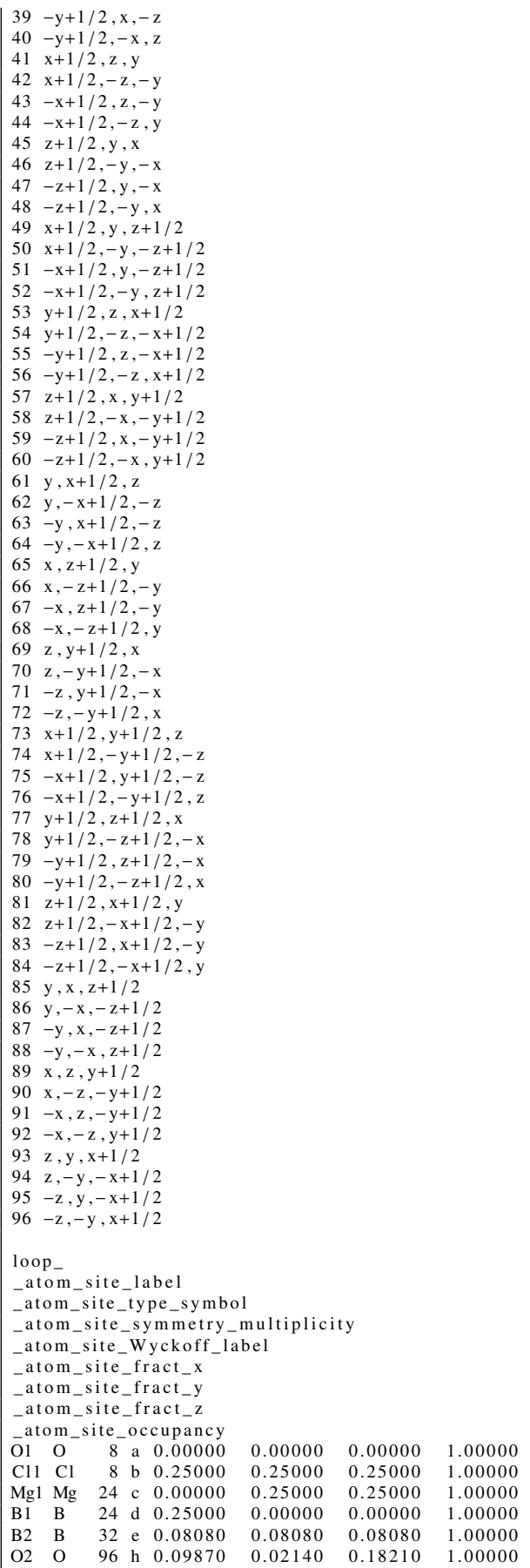

Boracite $\left(\mathrm{Mg}_{3} \mathrm{~B}_{7} \mathrm{ClO}_{13}\right)$ : A7BC3D13_cF192_219_de_b_c_ah - POSCAR

\begin{tabular}{|c|c|c|c|c|}
\hline \multicolumn{5}{|c|}{$\begin{aligned} \text { A7BC3D13_cF192_219_de_b_c } & \text { _ } \\
& \hookrightarrow 0.0987,0.0214,0.1 \\
& \hookrightarrow \text { None \& Mg3B7ClO13 } \\
& \hookrightarrow, 691-697(1973)\end{aligned}$} \\
\hline \\
\hline 0.00000000000000 & 6.04930000000000 & 6.04930000000000 & & \\
\hline \multirow{2}{*}{$\begin{array}{l}6.04930000000000 \\
6.04930000000000\end{array}$} & 0.00000000000000 & 6.04930000000000 & & \\
\hline & 6.04930000000000 & 0.00000000000000 & & \\
\hline $\mathrm{Mg}$ & $\mathrm{O}$ & & & \\
\hline 14 & 26 & & & \\
\hline \multicolumn{5}{|l|}{ Direct } \\
\hline 0.75000000000000 & 0.25000000000000 & 0.25000000000000 & B & $(24 d)$ \\
\hline 0.25000000000000 & 0.75000000000000 & 0.75000000000000 & B & $(24 d)$ \\
\hline 0.25000000000000 & 0.75000000000000 & 0.25000000000000 & B & $(24 d)$ \\
\hline 0.75000000000000 & 0.25000000000000 & 0.75000000000000 & B & $(24 d)$ \\
\hline 0.25000000000000 & 0.25000000000000 & 0.75000000000000 & B & $(24 d)$ \\
\hline 0.75000000000000 & 0.75000000000000 & 0.25000000000000 & B & $(24 d)$ \\
\hline 0.08080000000000 & 0.08080000000000 & 0.08080000000000 & B & $(32 \mathrm{e})$ \\
\hline 0.08080000000000 & 0.08080000000000 & -0.24240000000000 & B & $(32 \mathrm{e})$ \\
\hline 0.08080000000000 & -0.24240000000000 & 0.08080000000000 & B & $(32 \mathrm{e})$ \\
\hline-0.24240000000000 & 0.08080000000000 & 0.08080000000000 & B & $(32 \mathrm{e})$ \\
\hline 0.58080000000000 & 0.58080000000000 & 0.58080000000000 & B & $(32 \mathrm{e})$ \\
\hline 0.58080000000000 & 0.58080000000000 & 0.25760000000000 & B & $(32 \mathrm{e})$ \\
\hline 0.25760000000000 & 0.58080000000000 & 0.58080000000000 & B & $(32 \mathrm{e})$ \\
\hline 0.58080000000000 & 0.25760000000000 & 0.58080000000000 & B & $(32 \mathrm{e})$ \\
\hline 0.25000000000000 & 0.25000000000000 & 0.25000000000000 & $\mathrm{Cl}$ & $(8 \mathrm{~b})$ \\
\hline 0.75000000000000 & 0.75000000000000 & 0.75000000000000 & $\mathrm{Cl}$ & $(8 b)$ \\
\hline 0.50000000000000 & 0.00000000000000 & 0.00000000000000 & $\mathrm{Mg}$ & $(24 c)$ \\
\hline
\end{tabular}


0.00000000000000 0.00000000000000 0.50000000000000 0.00000000000000 0.50000000000000 0.00000000000000 0.50000000000000 0.10480000000000 0.25940000000000 $-0.06200000000000$ $-0.30220000000000$ $-0.30220000000000$ 0.10480000000000 0.25940000000000 0.25940000000000 0.10480000000000 $-0.30220000000000$ 0.06200000000 0.75940000000000 0.6048000000000 0.60480000000000 0.19780000000000 0.43800000000000 0.60480000000000 0.75940000000000 0.43800000000000 0.19780000000000 0.43800000000000 0.19780000000000 0.60480000000000 0.75940000000000
0.50000000000000 0.50000000000000 0.00000000000000 0.00000000000000 0.5000000000000 0.0000000000000 0.2594000000000 0.1048000000000 $-0.30220000000000$ $-0.06200000000000$ 0.10480000000000 0.25940000000000 $-0.06200000000000$ $-0.3022000000000$ $-0.06200000000000$ $-0.30220000000000$ $-0.3022000000000$ 0.104800000000 0.25940000000000 0.60480000000000 0.75940000000000 0.4380000000000 0.19780000000000 0.4380000000000 0.19780000000000 0.60480000000000 0.75940000000000 0.75940000000000 0.60480000000000 0.19780000000000 0.500000000000 0.43800000000000
.50000000000000 . 0.5000000000000 0.50000000000000 0.00000000000000 0.0000000000000 0.50000000000000 $-0.06200000000000$ $-0.30220000000000$ 0.1048000000000 0.25940000000000 0.25940000000000 0.10480000000000 $-0.3022000000000$ $-0.0620000000000$ 0.10480000000000 0.25940000000000 0.062000000000 $-0.062000000000$ 0.30220000000000 0.43800000000000 0.19780000000000 0.60480000000000 0.7594000000000 0.75940000000000 0.6048000000000 0.19780000000000 0.43800000000000 0.60480000000000 0.75940000000000 0.43800000000000 0.19780000000000

$\begin{array}{rr}\mathrm{Mg} & (24 \mathrm{c}) \\ \mathrm{Mg} & (24 \mathrm{c}) \\ \mathrm{Mg} & (24 \mathrm{c}) \\ \mathrm{Mg} & (24 \mathrm{c}) \\ \mathrm{Mg} & (24 \mathrm{c}) \\ \mathrm{O} & (8 \mathrm{a}) \\ \mathrm{O} & (8 \mathrm{a}) \\ \mathrm{O} & (96 \mathrm{~h}) \\ \mathrm{O} & (96 \mathrm{~h}) \\ \mathrm{O} & (96 \mathrm{~h}) \\ \mathrm{O} & (96 \mathrm{~h}) \\ \mathrm{O} & (96 \mathrm{~h}) \\ \mathrm{O} & (96 \mathrm{~h}) \\ \mathrm{O} & (96 \mathrm{~h}) \\ \mathrm{O} & (96 \mathrm{~h}) \\ \mathrm{O} & (96 \mathrm{~h}) \\ \mathrm{O} & (96 \mathrm{~h}) \\ \mathrm{O} & (96 \mathrm{~h}) \\ \mathrm{O} & (96 \mathrm{~h}) \\ \mathrm{O} & (96 \mathrm{~h}) \\ \mathrm{O} & (96 \mathrm{~h}) \\ \mathrm{O} & (96 \mathrm{~h}) \\ \mathrm{O} & (96 \mathrm{~h}) \\ \mathrm{O} & (96 \mathrm{~h}) \\ \mathrm{O} & (96 \mathrm{~h}) \\ \mathrm{O} & (96 \mathrm{~h}) \\ \mathrm{O} & (96 \mathrm{~h}) \\ \mathrm{O} & (96 \mathrm{~h}) \\ \mathrm{O} & (96 \mathrm{~h}) \\ \mathrm{O} & (96 \mathrm{~h}) \\ \mathrm{O} & (96 \mathrm{~h}) \\ & \end{array}$

$\mathrm{Cu}_{15} \mathrm{Si}_{4}\left(D 8_{6}\right):$ A15B4_cI76_220_ae_c - CIF

\# CIF file

data findsym-output

audit_creation_method FINDSYM

chemical name mineral,

chemical_formula_sum 'Cu15 Si4,

loop

publ_author_name

M. Mattern

R. Seyrich

L. Wilde'

C. Baehtz,

'M. Knapp',

journal_name_full_name

Journal of Alloys and Compounds

journal_volume 429

journal year 2007

journal_page_first 211

journal_page_last 215

publ_section title

Phase formation of rapidly quenched $\mathrm{Cu}-\mathrm{Si}$ alloys

Found in Experimental investigation of the $\mathrm{Cu}-\mathrm{Si}$ phase diagram at $\mathrm{x}(\mathrm{Cu}$ $\hookrightarrow) \$>0.72 \$, 201$

aflow_title 'Cu\$_ $\{15\} \$ S i \$ \_\{4\} \$\left(\$ D 8 \_\{6\} \$\right)$ Structure

aflow_proto 'A15B4_cI76_220_ae_c'

aflow_params 'a, $x_{-}\{2\}, x_{-}\{3\}, y_{-}\{3\}, z_{-}\{3\}$,

aflow_params_values $9.718,-0.042,0.12,0.16,-0.04$,

aflow Strukturbericht '\$D8_ $\{6\} \$$

aflow_Pearson 'cI76,

symmetry_space_group_name_H-M "I $-4 \quad 3$ d"

symmetry_Int_Tables_number 220

cell_length_a $\quad 9.71800$

cell_length_b $\quad 9.71800$

cell_length_c $\quad 9.71800$

cell_angle_alpha 90.00000

cell_angle_beta 90.00000

cell_angle_gamma 90.00000

loop

space_group_symop_id

space_group_symop_operation_xyz

$\mathrm{x}, \mathrm{y}, \mathrm{z}$

$\mathrm{x},-\mathrm{y},-\mathrm{z}+1 / 2$

$-x+1 / 2, y,-z$

$-\mathrm{x},-\mathrm{y}+1 /$
$\mathrm{y}, \mathrm{z}, \mathrm{x}$

$y,-z,-x+1 / 2$

$-\mathrm{y}+1 / 2, \mathrm{z},-\mathrm{x}$

$-\mathrm{y},-\mathrm{z}+1 / 2, \mathrm{x}$

$\mathrm{z}, \mathrm{x}, \mathrm{y}$

$10 \mathrm{z},-\mathrm{x},-\mathrm{y}+1 / 2$

$11-z+1 / 2, x,-y$

$2-z,-x+1 / 2, y$

$3 \mathrm{y}+1 / 4, \mathrm{x}+1 / 4, \mathrm{z}+1 / 4$

$14 \mathrm{y}+1 / 4,-\mathrm{x}+3 / 4,-\mathrm{z}+1 / 4$

$15-\mathrm{y}+1 / 4, \mathrm{x}+1 / 4,-\mathrm{z}+3 / 4$

$16-\mathrm{y}+3 / 4,-\mathrm{x}+1 / 4, \mathrm{z}+1 / 4$

$17 \mathrm{x}+1 / 4, \mathrm{z}+1 / 4, \mathrm{y}+1 / 4$

$18 \mathrm{x}+1 / 4,-\mathrm{z}+3 / 4,-\mathrm{y}+1 / 4$

$19-\mathrm{x}+1 / 4, \mathrm{z}+1 / 4,-\mathrm{y}+3 / 4$

$20-x+3 / 4,-z+1 / 4, y+1 / 4$

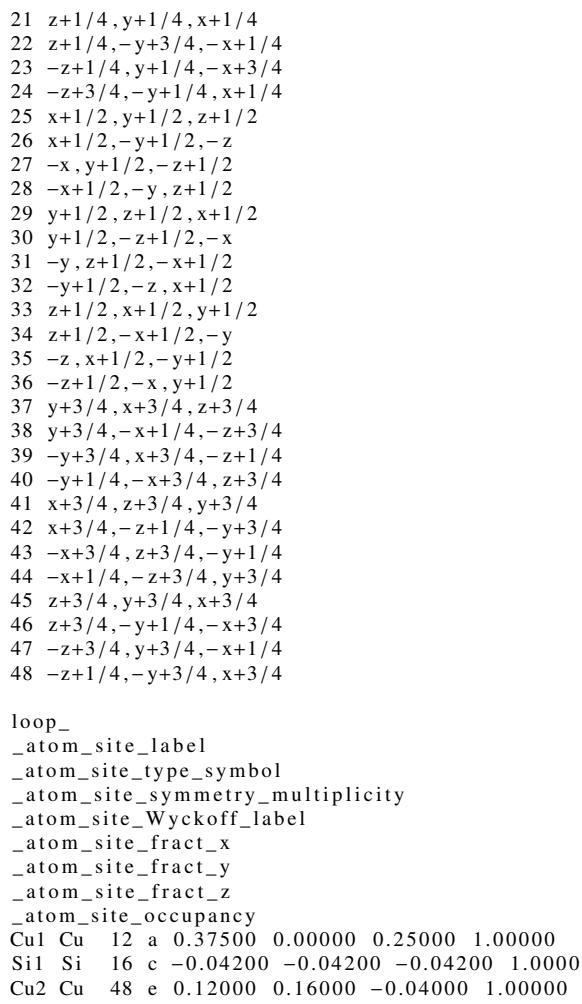

$\mathrm{Cu}_{15} \mathrm{Si}_{4}\left(D 8_{6}\right): \mathrm{A} 15 \mathrm{~B} 4 \_\mathrm{cI} 76 \_220 \_\mathrm{ae} \_\mathrm{c}-\mathrm{POSCAR}$

\begin{tabular}{|c|c|c|c|c|}
\hline \multirow{2}{*}{\multicolumn{5}{|c|}{ 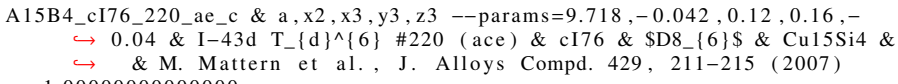 }} \\
\hline & & & & \\
\hline-4.85900000000000 & 4.85900000000000 & 4.85900000000000 & & \\
\hline \multirow{2}{*}{$\begin{array}{l}4.85900000000000 \\
4.85900000000000\end{array}$} & -4.85900000000000 & 4.85900000000000 & & \\
\hline & 4.85900000000000 & -4.85900000000000 & & \\
\hline \multicolumn{5}{|l|}{$\mathrm{Cu} \quad \mathrm{Si}$} \\
\hline 30 & & & & \\
\hline \multicolumn{5}{|l|}{ Direct } \\
\hline 0.25000000000000 & 0.62500000000000 & 0.37500000000000 & $\mathrm{Cu}$ & $(12 \mathrm{a})$ \\
\hline 0.75000000000000 & 0.87500000000000 & 0.12500000000000 & $\mathrm{Cu}$ & $(12 \mathrm{a})$ \\
\hline 0.37500000000000 & 0.25000000000000 & 0.62500000000000 & $\mathrm{Cu}$ & $(12 \mathrm{a})$ \\
\hline 0.12500000000000 & 0.75000000000000 & 0.87500000000000 & $\mathrm{Cu}$ & $(12 \mathrm{a})$ \\
\hline 0.62500000000000 & 0.37500000000000 & 0.25000000000000 & $\mathrm{Cu}$ & $(12 \mathrm{a})$ \\
\hline 0.87500000000000 & 0.12500000000000 & 0.75000000000000 & $\mathrm{Cu}$ & $(12 a)$ \\
\hline 0.12000000000000 & 0.08000000000000 & 0.28000000000000 & $\mathrm{Cu}$ & $(48 \mathrm{e})$ \\
\hline 0.30000000000000 & -0.16000000000000 & 0.22000000000000 & $\mathrm{Cu}$ & $(48 \mathrm{e})$ \\
\hline 0.20000000000000 & 0.42000000000000 & 0.54000000000000 & $\mathrm{Cu}$ & $(48 \mathrm{e})$ \\
\hline 0.38000000000000 & 0.66000000000000 & -0.04000000000000 & $\mathrm{Cu}$ & $(48 \mathrm{e})$ \\
\hline 0.28000000000000 & 0.12000000000000 & 0.08000000000000 & $\mathrm{Cu}$ & $(48 \mathrm{e})$ \\
\hline 0.22000000000000 & 0.30000000000000 & -0.16000000000000 & $\mathrm{Cu}$ & $(48 \mathrm{e})$ \\
\hline 0.54000000000000 & 0.20000000000000 & 0.42000000000000 & $\mathrm{Cu}$ & $(48 \mathrm{e})$ \\
\hline-0.04000000000000 & 0.38000000000000 & 0.66000000000000 & $\mathrm{Cu}$ & $(48 \mathrm{e})$ \\
\hline 0.08000000000000 & 0.28000000000000 & 0.12000000000000 & $\mathrm{Cu}$ & $(48 \mathrm{e})$ \\
\hline-0.16000000000000 & 0.22000000000000 & 0.30000000000000 & $\mathrm{Cu}$ & $(48 \mathrm{e})$ \\
\hline 0.42000000000000 & 0.54000000000000 & 0.20000000000000 & $\mathrm{Cu}$ & $(48 \mathrm{e})$ \\
\hline 0.66000000000000 & -0.04000000000000 & 0.38000000000000 & $\mathrm{Cu}$ & $(48 \mathrm{e})$ \\
\hline 0.58000000000000 & 0.62000000000000 & 0.78000000000000 & $\mathrm{Cu}$ & $(48 \mathrm{e})$ \\
\hline 0.34000000000000 & -0.20000000000000 & -0.28000000000000 & $\mathrm{Cu}$ & $(48 \mathrm{e})$ \\
\hline-0.08000000000000 & 0.70000000000000 & 0.04000000000000 & $\mathrm{Cu}$ & $(48 \mathrm{e})$ \\
\hline 0.16000000000000 & -0.12000000000000 & 0.46000000000000 & $\mathrm{Cu}$ & $(48 \mathrm{e})$ \\
\hline 0.62000000000000 & 0.78000000000000 & 0.58000000000000 & $\mathrm{Cu}$ & $(48 \mathrm{e})$ \\
\hline-0.20000000000000 & -0.28000000000000 & 0.34000000000000 & $\mathrm{Cu}$ & $(48 \mathrm{e})$ \\
\hline 0.70000000000000 & 0.04000000000000 & -0.08000000000000 & $\mathrm{Cu}$ & $(48 \mathrm{e})$ \\
\hline-0.12000000000000 & 0.46000000000000 & 0.16000000000000 & $\mathrm{Cu}$ & $(48 \mathrm{e})$ \\
\hline 0.78000000000000 & 0.58000000000000 & 0.62000000000000 & $\mathrm{Cu}$ & $(48 \mathrm{e})$ \\
\hline-0.28000000000000 & 0.34000000000000 & -0.20000000000000 & $\mathrm{Cu}$ & $(48 \mathrm{e})$ \\
\hline 0.04000000000000 & -0.08000000000000 & 0.70000000000000 & $\mathrm{Cu}$ & $(48 \mathrm{e})$ \\
\hline 0.46000000000000 & 0.16000000000000 & -0.12000000000000 & $\mathrm{Cu}$ & $(48 \mathrm{e})$ \\
\hline-0.08400000000000 & -0.08400000000000 & -0.08400000000000 & $\mathrm{Si}$ & $(16 \mathrm{c})$ \\
\hline 0.50000000000000 & 0.00000000000000 & 0.58400000000000 & $\mathrm{Si}$ & $(16 c)$ \\
\hline 0.00000000000000 & 0.58400000000000 & 0.50000000000000 & $\mathrm{Si}$ & $(16 \mathrm{c})$ \\
\hline 0.58400000000000 & 0.50000000000000 & 0.00000000000000 & $\mathrm{Si}$ & $(16 \mathrm{c})$ \\
\hline 0.41600000000000 & 0.41600000000000 & 0.41600000000000 & $\mathrm{Si}$ & $(16 \mathrm{c})$ \\
\hline 0.50000000000000 & 0.00000000000000 & 0.08400000000000 & $\mathrm{Si}$ & $(16 \mathrm{c})$ \\
\hline 0.08400000000000 & 0.50000000000000 & 0.00000000000000 & $\mathrm{Si}$ & \\
\hline 0.00000000000000 & 0.08400000000000 & 0.50000000000000 & $\mathrm{Si}$ & $(16 \mathrm{c})$ \\
\hline
\end{tabular}

$\mathrm{Th}_{3} \mathrm{P}_{4}\left(D 7_{3}\right):$ A4B3_cl28_220_c_a - CIF

\# CIF file

audit_creation_method FINDSYM

chemical name mineral 'Th3P4',

, P4 Th3'

oop

_publ_author_name

'K. Meisel, 
_journal_name_full_name

Zeitschrift fur Anorganische und Allgemeine Chemie

journal_volume 240

journal_year 1939

journal_page_first 300

journal_page_last 312

publ_Section_title

Kristallstrukturen von Thoriumphosphiden

aflow_title $T h \$\left\{\{3\} \$ P \$ \_\{4\} \$\left(\$ D 7 \_\{3\} \$\right)\right.$ Structure

aflow proto A4B 3 cI 28 _220_c a

aflow params , a, x $\{2\}$,

aflow_params_values 8.6, 0.08333 ,

aflow_Strukturbericht '\$D7_\{3\}\$'

aflow_Pearson 'cI28,

symmetry_space_group_name_H-M "I $-4 \quad 3$ d"

_symmetry_Int_Tables_number 220

\section{cell_length_a $\quad 8.60000$ \\ cell_length_b 8.60000}

cell_length_c 8.60000

cell_angle_alpha 90.00000

_cell_angle_beta 90.00000

_cell_angle_gamma 90.00000

loop

_space_group_symop_id

_space_group_symop_operation_xyz

$1 \mathrm{x}, \mathrm{y}, \mathrm{z}$

$2 \mathrm{x},-\mathrm{y},-\mathrm{z}+1 / 2$

$3-x+1 / 2, y,-z$

$4-\mathrm{x},-\mathrm{y}+1 / 2, \mathrm{z}$

$5 \mathrm{y}, \mathrm{z}, \mathrm{x}$

$6 \mathrm{y},-\mathrm{z},-\mathrm{x}+1 / 2$

$7-y+1 / 2, z,-x$

$-\mathrm{y},-\mathrm{z}+1 / 2, \mathrm{x}$

$\mathrm{z}, \mathrm{x}, \mathrm{y}$

$10 \mathrm{z},-\mathrm{x},-\mathrm{y}+1 / 2$

$12-\mathrm{z},-\mathrm{x}+1 / 2, \mathrm{y}$

$13 \mathrm{y}+1 / 4, \mathrm{x}+1 / 4, \mathrm{z}+1 / 4$

$14 \mathrm{y}+1 / 4,-\mathrm{x}+3 / 4,-\mathrm{z}+1 / 4$

$16-y+3 / 4, x+1 / 4,-z+3 / 4$

$17-y+3 / 4,-x+1 / 4, z+1 / 4$

$7 \mathrm{x}+1 / 4, \mathrm{z}+1 / 4, \mathrm{y}+1 / 4$

$18 \mathrm{x}+1 / 4,-\mathrm{z}+3 / 4,-\mathrm{y}+1 / 4$

$19-x+1 / 4, z+1 / 4,-y+3 / 4$

$20-x+3 / 4,-z+1 / 4, y+1 / 4$

$21 \mathrm{z}+1 / 4, \mathrm{y}+1 / 4, \mathrm{x}+1 / 4$

$22 \mathrm{z}+1 / 4,-\mathrm{y}+3 / 4,-\mathrm{x}+1 / 4$

$23-z+1 / 4, y+1 / 4,-x+3 / 4$

$24-\mathrm{z}+3 / 4,-\mathrm{y}+1 / 4, \mathrm{x}+1 / 4$

$25 \mathrm{x}+1 / 2, \mathrm{y}+1 / 2, \mathrm{z}+1 / 2$

$26 \mathrm{x}+1 / 2,-\mathrm{y}+1 / 2,-\mathrm{z}$

$27-\mathrm{x}, \mathrm{y}+1 / 2,-\mathrm{z}+1 / 2$

$29 \mathrm{y}+1 / 2, \mathrm{z}+1 / 2, \mathrm{x}+1 / 2$

$30 \mathrm{y}+1 / 2,-\mathrm{z}+1 / 2,-\mathrm{x}$

$31-\mathrm{y}, \mathrm{z}+1 / 2,-\mathrm{x}+1 / 2$

$32-\mathrm{y}+1 / 2,-\mathrm{z}, \mathrm{x}+1 / 2$

$33 \mathrm{z}+1 / 2, \mathrm{x}+1 / 2, \mathrm{y}+1 / 2$

$34 \mathrm{z}+1 / 2,-\mathrm{x}+1 / 2,-\mathrm{y}$

$35-\mathrm{z}, \mathrm{x}+1 / 2,-\mathrm{y}+1 / 2$

$36-\mathrm{z}+1 / 2,-\mathrm{x}, \mathrm{y}+1 / 2$

$37 \mathrm{y}+3 / 4, \mathrm{x}+3 / 4, \mathrm{z}+3 / 4$

$38 \mathrm{y}+3 / 4,-\mathrm{x}+1 / 4,-\mathrm{z}+3 / 4$

$39-\mathrm{y}+3 / 4, \mathrm{x}+3 / 4,-\mathrm{z}+1 / 4$

$40-y+1 / 4,-x+3 / 4, z+3 / 4$

$41 \mathrm{x}+3 / 4, \mathrm{z}+3 / 4, \mathrm{y}+3 / 4$

$42 \mathrm{x}+3 / 4,-\mathrm{z}+1 / 4,-\mathrm{y}+3 / 4$

$43-x+3 / 4, z+3 / 4,-y+1 / 4$

$44-x+1 / 4,-z+3 / 4, y+3 / 4$

$45 \mathrm{z}+3 / 4, y+3 / 4, \mathrm{x}+3 / 4$

$46 \mathrm{z}+3 / 4,-y+1 / 4,-x+3 / 4$

$47-\mathrm{z}+3 / 4, \mathrm{y}+3 / 4,-\mathrm{x}+1 / 4$

$48-\mathrm{z}+1 / 4,-\mathrm{y}+3 / 4, \mathrm{x}+3 / 4$

loop

-atom site label

-atom_site_type_symbol

-atom_site_symmetry_multiplicity

_atom_site_Wyckoff_label

atom_site_fract_x

atom_site_fract_y

atom_site_fract_z

atom_site_occupancy

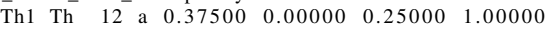

$\begin{array}{llllllllll}\text { P1 } & \text { P } & 16 & \text { c } & 0.08333 & 0.08333 & 0.08333 & 1.0000\end{array}$

$\mathrm{Th}_{3} \mathrm{P}_{4}\left(D 7_{3}\right):$ A4B3_cI28_220_c_a - POSCAR

A4B3_cI28_220_c_a \& a, x2 --params $=8.6,0.08333 \&$ \& I-43d T $\{\text { d }\}^{\wedge}\{6\} \# 220$ (ac $\hookrightarrow)$ ～\& cI28 \& \$D7_\{3\}\$\& Th3P4 \& Th3P4 \& K. Meisel, Z. Anorg.

$\hookrightarrow$ Allg. Chem. 240, 300-312 (1939)

1.00000000000000

$\begin{array}{rrr}-4.30000000000000 & 4.30000000000000 & 4.30000000000000\end{array}$

$\begin{array}{lll}4.30000000000000 & -4.30000000000000 & 4.30000000000000\end{array}$

$\begin{array}{lll}4.30000000000000 & 4.30000000000000 & -4.30000000000000\end{array}$

$\mathrm{P} \quad \mathrm{Th}$

Direct

0.16666000000000

0.50000000000000

0.00000000000000

0.33334000000000

0.66666000000000

0.50000000000000

$-0.16666000000000$

0.00000000000000

0.25000000000000

0.75000000000000

0.37500000000000

0.12500000000000

0.62500000000000

0.87500000000000

.16666000000000 .00000000000000 0.3333400000000 .50000000000000 .66666000000000 0.00000000000000 0.50000000000000 .16666000000000 0.62500000000000 0.87500000000000 0.25000000000000 0.75000000000000 0.37500000000000 0.12500000000000

0.16666000000000 0.33334000000000 0.50000000000000 0.00000000000000 0.66666000000000 0.6666600000000 . 0.00000000000000 0.50000000000000 0.37500000000000 0.62500000000000 0.87500000000000 0.25000000000000 0.75000000000000

P $(16 c)$

$\mathrm{P} \quad(16 \mathrm{c})$

P $\quad(16 \mathrm{c})$

P (16c)

P (16c)

$P-(16 c)$

$P \quad(16 c)$

(16c)

(12a)

Th (12a)

(12a)

Th (12a)

Th (12a)

$\mathrm{Ca}_{3} \mathrm{Al}_{2} \mathrm{O}_{6}\left(E 9_{1}\right): \mathrm{A} 2 \mathrm{~B} 3 \mathrm{C} 6 \_\mathrm{cP} 33 \_221 \_\mathrm{cd} \_a g \_f h-\mathrm{CIF}$

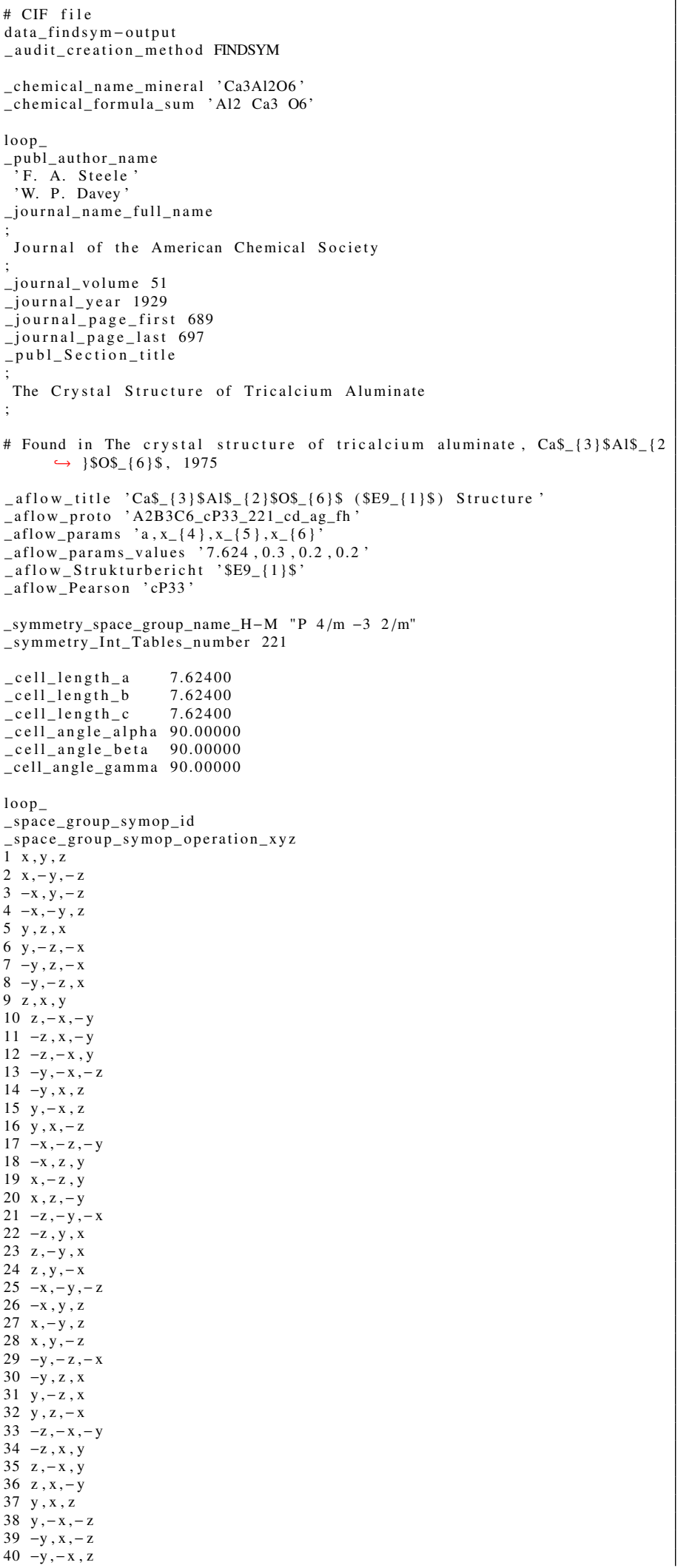


$41 \mathrm{x}, \mathrm{z}, \mathrm{y}$

$42 x,-z,-y$

$43-\mathrm{x}, \mathrm{z},-\mathrm{y}$

$44-\mathrm{x},-\mathrm{z}, \mathrm{y}$

$45 \mathrm{z}, \mathrm{y}, \mathrm{x}$

$\begin{array}{ll}46 & \mathrm{z},-\mathrm{y},-\mathrm{x} \\ 47-\mathrm{z}, \mathrm{y},-\mathrm{x}\end{array}$

$48-\mathrm{z},-\mathrm{y}, \mathrm{x}$

loop

atom_site label

atom_site type symbol

atom_site_symmetry_multiplicity

atom_site_Wyckoff_label

atom_site_fract_x

atom_site_fract_y

atom_site_fract_z

-atom_site_occupancy

$\begin{array}{lllllll}\mathrm{Ca} & 1 & \mathrm{a} & 0.00000 & 0.00000 & 0.00000 & 1.00000\end{array}$

$\begin{array}{lllllllll}\mathrm{Al} 1 & \mathrm{Al} & 3 & \mathrm{c} & 0.00000 & 0.50000 & 0.50000 & 1.0000\end{array}$

$\begin{array}{lllllllll}\mathrm{A} 12 & \mathrm{Al} & 3 & \mathrm{~d} & 0.50000 & 0.00000 & 0.00000 & 1.00000\end{array}$

$\begin{array}{lllllllll}\mathrm{O} 1 & \mathrm{O} & 6 & \mathrm{f} & 0.30000 & 0.50000 & 0.50000 & 1.00000\end{array}$

$\begin{array}{lllllllll}\mathrm{Ca} 2 & \mathrm{Ca} & 8 & \mathrm{~g} & 0.20000 & 0.20000 & 0.20000 & 1.00000\end{array}$

$\begin{array}{llllllllll}\mathrm{O} 2 & \mathrm{O} & 12 & \mathrm{~h} & 0.20000 & 0.50000 & 0.00000 & 1.00000\end{array}$

$\mathrm{Ca}_{3} \mathrm{Al}_{2} \mathrm{O}_{6}\left(E 9_{1}\right)$ : A2B3C6_cP33_221_cd_ag_fh - POSCAR

A2B3C6_cP33_221_cd_ag_fh \& a, x4, x5, x6 --params $=7.624,0.3,0.2,0.2 \&$ Pm-3m

$\hookrightarrow \mathrm{O}_{-}\{\mathrm{h}\}^{\wedge}\{1\} \# 221$ (acdfgh) \& cP33 \& \$E9_\{1\}\$\& Ca3A12O6 \&

$\hookrightarrow \mathrm{Ca} 3 \mathrm{~A} 12 \mathrm{O} 6$ \& F. A. Steele and W. P. Davey, J. Am. Chem. Soc. 51,

$\hookrightarrow 689-697(1929)$

1.00000000000000

$\begin{array}{lll}7.62400000000000 & 0.00000000000000 & 0.00000000000000\end{array}$

$\begin{array}{lll}0.00000000000000 & 7.62400000000000 & 0.00000000000000\end{array}$

$\begin{array}{lll}0.00000000000000 & 0.00000000000000 & 7.62400000000000\end{array}$

$$
\begin{array}{rrr}
\mathrm{Al} & \mathrm{Ca} & \mathrm{O} \\
6 & 9 & 18
\end{array}
$$

Direct

0.00000000000000 0.50000000000000 0.50000000000000 0.50000000000000 0.00000000000000 0.00000000000000 0.00000000000000 0.20000000000000

$-0.20000000000000$

$-0.20000000000000$

0.20000000000000

0.20000000000000

$-0.20000000000000$

0.20000000000000

$-0.20000000000000$

0.30000000000000

$-0.30000000000000$

0.5000000000000

0.50000000000000

0.50000000000000

0.500000000000

0.200000000000

$-0.20000000000000$

0.00000000000000

0.00000000000000

0.50000000000000

0.50000000000000

0.50000000000000

0.50000000000000

0.20000000000000

$-0.20000000000000$

0.00000000000000

0.00000000000000

0.50000000000000 0.5000000000000 . 0.00000000000000 0.2000000000000 0.20000000000000 0.20000000000000 0.20000000000000 0.20000000000000 $-0.2000000000000$ $-0.20000000000000$ 0.20000000000000 0.50000000000000 0.50000000000000 0.30000000000000 $-0.30000000000000$ 0.50000000000000 0.50000000000000 0.5000000000000 0.50000000000 0.50000000000000 0.20000000000000 $-0.20000000000000$ 0.00000000000000 000000000 00000000 0.20000000000000 0.00000000000000 0.50000000000000

0.50000000000000 0.50000000000000 0.0000000000000 0.00000000000000 0.0000000000000 0.5000000000000 0.50000000000000 0.00000000000000 0.20000000000000 0.20000000000000 $-0.20000000000000$ $-0.20000000000000$ $-0.2000000000000$ $-0.2000000000000$ 0.20000000000000 0.20000000000000 0.50000000000000 0.50000000000000 0.5000000000000 0.5000000000000 0.50000000000000 0.3000000000000 $-0.30000000000000$ 0.0000000000000 0.00000000000000 0.50000000000000 0.50000000000000 0.20000000000000 $-0.20000000000000$ 0.00000000000000 0.00000000000000 0.5000000000000 0.50000000000000 $-0.20000000000000$
$\mathrm{Al}(3 \mathrm{c})$

$\mathrm{Al} \quad(3 \mathrm{c})$

$\mathrm{Al} \quad(3 \mathrm{~d})$

$\mathrm{Al} \quad(3 \mathrm{~d})$

$\mathrm{Al} \quad(3 \mathrm{~d})$

$\mathrm{Ca} \quad(1 \mathrm{a})$

$\mathrm{Ca} \quad(8 \mathrm{~g})$

$\mathrm{Ca} \quad(8 \mathrm{~g})$

$\mathrm{Ca} \quad(8 \mathrm{~g})$

$\mathrm{Ca} \quad(8 \mathrm{~g})$

$\begin{array}{ll}\mathrm{Ca} & (8 \mathrm{~g}) \\ \mathrm{Ca} & (8 \mathrm{~g})\end{array}$

$\mathrm{Ca} \quad(8 \mathrm{~g})$

$\mathrm{O} \quad(6 \mathrm{f})$

$\mathrm{O} \quad(6 \mathrm{f})$

O (6f)

$\mathrm{O} \quad(6 \mathrm{f})$

(6f)

$(12 \mathrm{~h}$

$\mathrm{O} \quad(12 \mathrm{~h})$

$\mathrm{O} \quad(12 \mathrm{~h})$

$\mathrm{O} \quad(12 \mathrm{~h})$

$\mathrm{O} \quad(12 \mathrm{~h})$

$\mathrm{O} \quad(12 \mathrm{~h})$

$\mathrm{O} \quad(12 \mathrm{~h})$

$\mathrm{O} \quad(12 \mathrm{~h})$

$\mathrm{O} \quad(12 \mathrm{~h})$

O (12h)

O (12h)
$\mathrm{Ce}_{5} \mathrm{Mo}_{3} \mathrm{O}_{16}$ : A5B3C16_cP96_222_ce_d_fi - CIF

\section{\# CIF file}

data_findsym-output

audit_creation_method FINDSYM

chemical_name_mineral 'Ce5Mo3016',

chemical formula_sum ' $\mathrm{Ce} 5 \mathrm{Mo} 3 \mathrm{O}^{\prime} 6^{\prime}$

loop

-publ_author_name

P. H. Hubert'

journal_year 1974

publ_Section_title

Contribution $\{\backslash$ a $\} 1 \backslash$ ' $\{1\}^{\prime}$ e $\}$ tude des molybdites des terres rares: 1 $\hookrightarrow$ Bimolybdites Ln\$_ $\{2\} \$ M o \$_{-}\{2\} \$ O \$_{-}\{7\}$

\# Found in Pearson's Crystal Data - Crystal Structure Database for $\hookrightarrow$ Inorganic Compounds, 2013

aflow_title 'Ce\$_ $\{5\} \$ M O \$_{-}\{3\} \$ O \$_{-}\{16\} \$$ Structure

aflow_proto $A 5 B 3 \mathrm{C} 16$ _cP96_222_ce_d_fi

aflow_params , a, $x_{-}\{3\}, x_{-}\{4\}, x_{-}\{5\}, y_{-}\{5\}, z_{-}\{5\}$,

aflow_params_values $11.1199004528,0.0,0.125,0.084,0.166,0.625$

flow Strukturbericht 'None'

aflow_Pearson 'cP96,

cell_length_a 11.1199004528

cell_length_b $\quad 11.1199004528$

$\begin{array}{ll}\text { ccell_length_c } & 11.1199004528 \\ \text { cell_angle_alpha } & 90.0000000000\end{array}$

cell_angle_beta 90.0000000000

_cell_angle_gamma 90.0000000000

-symmetry_space_group_name_H-M "P $4 / \mathrm{n}-32 / \mathrm{n}$ (origin choice 2 )"

_symmetry_Int_Tables_number 222

loop

space_group_symop_id

$1 \mathrm{x}, \mathrm{y}, \mathrm{z}$

$2 \mathrm{x},-\mathrm{y}+1 / 2,-\mathrm{z}+1 / 2$

$3-x+1 / 2, y,-z+1 / 2$

$5 \mathrm{y}, \mathrm{z}, \mathrm{x}$

$\mathrm{y},-\mathrm{z}+1 / 2,-\mathrm{x}+1 / 2$

$7-\mathrm{y}+1 / 2, \mathrm{z},-\mathrm{x}+1 / 2$

$\mathrm{z}, \mathrm{x}, \mathrm{y}$

$10 \mathrm{z},-\mathrm{x}+1 / 2,-\mathrm{y}+1 / 2$

$11-z+1 / 2, x,-y+1 / 2$

$12-\mathrm{z}+1 / 2,-\mathrm{x}+1 / 2, \mathrm{y}$

$13-\mathrm{y}+1 / 2,-\mathrm{x}+1 / 2,-\mathrm{z}+1 / 2$

$14-\mathrm{y}+1 / 2, \mathrm{x}, \mathrm{z}$

$15 \mathrm{y},-\mathrm{x}+1 / 2, \mathrm{z}$
$16 \mathrm{y}, \mathrm{x},-\mathrm{z}+1 / 2$

$7-\mathrm{x}+1 / 2,-\mathrm{z}+1 / 2,-\mathrm{y}+1 / 2$

$18-\mathrm{x}+1 / 2, \mathrm{z}, \mathrm{y}$

$19 \mathrm{x},-\mathrm{z}+1 / 2, \mathrm{y}$
$20 \mathrm{x}, \mathrm{z},-\mathrm{y}+1 / 2$
$21 \mathrm{z}, \mathrm{z}+1 / 2,-\mathrm{y}+1 /$

$21-\mathrm{z}+1 / 2,-\mathrm{y}+1 / 2,-\mathrm{x}+1 / 2$

$22-\mathrm{z}+1 / 2, \mathrm{y}, \mathrm{x}$

$3 \mathrm{z},-\mathrm{y}+1 / 2, \mathrm{x}$

$24 \mathrm{z}, \mathrm{y},-\mathrm{x}+1$

$25-\mathrm{x},-\mathrm{y},-\mathrm{z}$
$26-\mathrm{x}, \mathrm{y}+1 / 2, \mathrm{z}+1 / 2$
$27 \mathrm{x}+1 / 2,-\mathrm{y}, \mathrm{z}+1 / 2$

$27 \mathrm{x}+1 / 2,-\mathrm{y}, \mathrm{z}+1 / 2$

$28 \mathrm{x}+1 / 2, \mathrm{y}+1 / 2,-\mathrm{z}$

$29-\mathrm{y},-\mathrm{z},-\mathrm{x}$

$30-y, z+1 / 2, x+1 / 2$

$31 \mathrm{y}+1 / 2,-\mathrm{z}, \mathrm{x}+1 / 2$

$32 \mathrm{y}+1 / 2, \mathrm{z}+1 / 2,-\mathrm{x}$

$33-\mathrm{z},-\mathrm{x},-\mathrm{y}$

$34-\mathrm{z}, \mathrm{x}+1 / 2, \mathrm{y}+1 / 2$

$36 \mathrm{z}+1 / 2, \mathrm{x}+1 / 2,-\mathrm{y}$

$37 \mathrm{y}+1 / 2, \mathrm{x}+1 / 2, \mathrm{z}+1 / 2$

$38 \mathrm{y}+1 / 2,-\mathrm{x},-\mathrm{z}$

$39-\mathrm{y}, \mathrm{x}+1 / 2,-\mathrm{z}$

$41 \mathrm{x}+1 / 2, \mathrm{z}+1 / 2, \mathrm{y}+1 / 2$

$42 \mathrm{x}+1 / 2,-\mathrm{z},-\mathrm{y}$

$43-\mathrm{x}, \mathrm{z}+1 / 2,-\mathrm{y}$

$44-\mathrm{x},-\mathrm{z}, \mathrm{y}+1 / 2$

$45 \mathrm{z}+1 / 2, \mathrm{y}+1 / 2, \mathrm{x}+1 / 2$

$46 \mathrm{z}+1 / 2,-\mathrm{y},-\mathrm{x}$

$47-\mathrm{z}, \mathrm{y}+1 / 2,-\mathrm{x}$

$48-\mathrm{z},-\mathrm{y}, \mathrm{x}+1 / 2$

loop

atom_site_label

atom_site_type_symbol

atom_site_symmetry_multiplicity

atom_site_Wyckoff_label

atom_site_fract_x

atom_site_fract_y

atom_site_fract_z

Celom_site_occupancy

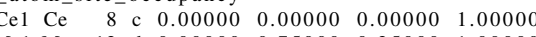

$\begin{array}{lllllll}\text { Mol Mo } 12 & \text { d } & 0.00000 & 0.75000 & 0.25000 & 1.00000\end{array}$

$\begin{array}{llllllll}\mathrm{Ce} 2 \mathrm{Ce} & 12 & \text { e } & 0.00000 & 0.25000 & 0.25000 & 1.00000\end{array}$

$\begin{array}{llllllll}\mathrm{O} 1 & \mathrm{O} & 16 & \mathrm{f} & 0.12500 & 0.12500 & 0.12500 & 1.00000 \\ \mathrm{O} 2 & \mathrm{O} & 48 & \mathrm{i} & 0.08400 & 0.16600 & 0.62500 & 1.00000\end{array}$

$\mathrm{Ce}_{5} \mathrm{Mo}_{3} \mathrm{O}_{16}$ : A5B3C16_cP96_222_ce_d_fi - POSCAR

A5B3C16_cP96_222_ce_d_fi \& a , x3, x4, x5,y5, z5 --params $=11.1199004528,0.0$ $\hookrightarrow 0.125,0.084,0.166,0.625 \&$ Pn-3n O_ $\{\text { h }\}^{\wedge}\{2\} \# 222$ (cdefi) \& cP96 \& $\hookrightarrow$ None \& Ce5Mo3O16 \& \& P. H. Hubert, (1974)

1.00000000000000

$\begin{array}{lll}11.11990045280000 & 0.00000000000000 & 0.00000000000000\end{array}$

$\begin{array}{lll}0.00000000000000 & 11.11990045280000 & 0.00000000000000\end{array}$

$\begin{array}{lrr}0.00000000000000 & 0.00000000000000 & 11.11990045280000\end{array}$

$\begin{array}{rrr}\mathrm{Ce} & \mathrm{Mo} & \mathrm{O} \\ 20 & 12 & 64\end{array}$

Direct

0.00000000000000

0.50000000000000

0.50000000000000

0.00000000000000

0.00000000000000

0.50000000000000

0.00000000000000

0.50000000000000

0.00000000000000

0.50000000000000

0.25000000000000

0.25000000000000

0.25000000000000

0.25000000000000

0.00000000000000

0.75000000000000

0.75000000000000

0.75000000000000

0.75000000000000

0.00000000000000 0.50000000000000 0.00000000000000 0.50000000000000 0.00000000000000 0.50000000000000 .50000000000000 0.00000000000000 0.25000000000000 0.25000000000000 0.00000000000000 0.50000000000000 
0.00000000000000 0.50000000000000 0.25000000000000 0.25000000000000 0.75000000000000 0.75000000000000 0.75000000000000 0.75000000000000 0.00000000000000 0.50000000000000 0.25000000000000 0.25000000000000 0.12500000000000 0.3750000000000 0.37500000000000 0.3750000000000 0.1250000000000 0.3750000000000 0.12500000000000 0.37500000000000 0.12500000000000 0.62500000000000 0.62500000000000 $-0.12500000000000$ $-0.12500000000000$ 0.62500000000000 $-0.12500000000000$ 0.62500000000000 0.08400000000000 0.41600000000000 0.41600000000000 0.41600000000000 0.08400000000000 0.6250000000000 0.62500000000000 $-0.12500000000000$ $-0.12500000000000$ 0.16600000000000 0.33400000000000 0.16600000000000 0.33400000000000 0.16600000000000 0.33400000000000 0.16600000000000 0.33400000000000 0.08400000000000 0.41600000000000 0.4160000000000 0.41600000000000 0.08400000000000 0.62500000000 0.62500000000000 $-0.12500000000000$ $-0.12500000000000$ $-0.08400000000000$ 0.58400000000000 0.58400000000000 $-0.08400000000000$ $-0.62500000000000$ $-0.62500000000000$ 1.12500000000000 1.12500000000000 $-0.16600000000000$ 0.66600000000000 $-0.16600000000000$ 0.66600000000000 $-0.16600000000000$ 0.16600000000000 $-0.6660000000000$ 0.16600000000000 0.66600000000000 $-0.08400000000000$ 0.58400000000000 0.58400000000000 0.08400000000000 $-0.62500000000000$ $-0.62500000000000$ $\begin{array}{rr}1.12500000000000 & -0.16600000000000 \\ 1.12500000000000 & 0.66600000000000\end{array}$

0.75000000000000 0.75000000000000 0.00000000000000 0.5000000000000 0.2500000000000 0.2500000000000 0.000000000000 0.0000000000000 0.50000000000000 0.2500000000000 0.25000000000000 0.75000000000000 0.12500000000000 0.3750000000000 0.3750000000000 0.1250000000000 0.3750000000000 0.375000000000 0.375000000000 0.12500000000000 0.12500000000000 0.62500000000000 0.12500000000000 0.62500000000000 $-0.1250000000000$ 0.62500000000000 0.62500000000000 0.12500000000000 0.33400000000000 0.1660000000000 0.3340000000000 0.08400000000000 0.41600000000000 0.4160000000000 0.08400000000000 0.084000000000 0.62500000000000 $-0.1250000000000$ $-0.12500000000000$ 0.0840000000000 0.41600000000000 0.41600000000000 0.08400000000000 0.62500000000000 0.62500000000000 $-0.12500000000000$ $-0.12500000000000$ 0.16600000000000 0.33400000000000 0.16600000000000 0.1660000000 $-0.16600000000000$ 0.66600000000000 0.66600000000000 0.66600000000000 0.66600000000000 0.58400000000000 0.58400000000000 $-0.08400000000000$ $-0.62500000000000$ $-0.62500000000000$ 1.12500000000000 1.12500000000000 $-0.08400000000000$ 0.58400000000000 $-0.08400000000000$ $-0.62500000000000$ $-0.6250000000000$ 1.12500000000000 1.12500000000000 $-0.1660000000000$ 0.66600000000000 0.66600000000000

$\mathrm{Th}_{6} \mathrm{Mn}_{23}\left(D 8_{a}\right):$ A23B6_cF116_225_bd2f_e - CIF

\section{CIF file}

ata_findsym-output

audit_creation_method FINDSYM

chemical_name_mineral 'Th6Mn23,

chemical formula sum 'Mn23 Th6'

loop

publ_author_nam

J. V. Florio

R. E. Rundle

journal_name_full_name

Acta Cristallographica

journal_volume 5

journal_year 1952

journal_page_first 445

journal_page last 457

publ_Section_title

Compounds of thorium with transition metals. I. The thorium-manganese $\hookrightarrow$ system

0.25000000000000 .25000000000000 0.75000000000000 0.75000000000000 0.00000000000000 0.50000000000000 0.25000000000000 0.25000000000000 0.75000000000000 0.75000000000000 0.50000000000000 0.00000000000000 .12500000000000 0.12500000000000 0.37500000000000 0.3750000000000 0.37500000000000 (0.3750000 0.375000000 0.125000000000 0.12500000000000 $-0.12500000000000$ $-0.12500000000000$ .62500000000000 0.62500000000000 $-0.12500000000000$ $-0.12500000000000$ 0.62500000000000 0.62500000000000 $-0.12500000000000$ $-0.12500000000000$ 0.16600000000000 0.33400000000000 0.16600000000000 0.33400000000000 0.0840000000000 0.41600000000000 0.41600000000000 0.08400000000000 $-0.12500000000000$ $-0.12500000000000$ 0.62500000000000 .62500000000000 0.33400000000000 .16600000000000 0.33400000000000 0.16600000000000 0.41600000000000 0.08400000000000 . .4160000000000 $-0.62500000000000$ $-0.62500000000000$ 1.12500000000000 1.12500000000000 $-0.16600000000000$ 0.6660000000000 $-0.16600000000000$ 0.66600000000000 $-0.08400000000000$ 0.58400000000000 0.58400000000000 $-0.08400000000000$ 1.12500000000000 . 12500000000000 $-0.62500000000000$ $-0.62500000000000$ 0.66600000000000 $-0.16600000000000$ 0.66600000000000 $-0.1660000000000$ 0.58400000000000 $-0.0840000000000$ 0.58400000000000

Mo $\quad(12 \mathrm{~d})$

Mo (12d)

Mo $(12 \mathrm{~d})$

Mo $(12 \mathrm{~d})$

Mo (12d)

Mo (12d)

Mo (12d)

Mo (12d)

Mo (12d)

Mo (12d)

$\begin{array}{ll}\mathrm{O} & (16 \mathrm{f}) \\ \mathrm{O} & (16 \mathrm{f})\end{array}$

O (16f)

O (16f)

O (16f)

$O \quad(16 f)$

$\mathrm{O}$ (16f)

$\mathrm{O}$ (16f)

$\mathrm{O}$ (16f)

$\mathrm{O} \quad(16 \mathrm{f})$

$\mathrm{O}$ (16f)

O (16f)

(16f)

O $(48 \mathrm{i})$

$\mathrm{O} \quad(48 \mathrm{i})$

O (48i)

(48i)

$(48 \mathrm{i})$

(48i)

O (48i)

$\mathrm{O} \quad(48 \mathrm{i})$

$\mathrm{O} \quad(48 \mathrm{i})$

O (48i)

O (48i)

O (48i)

O (48i)

O (48i)

O (48i)

(48i)

O $(48 \mathrm{i})$

O (48i)

O (48i) 
$83 \mathrm{z},-\mathrm{x}+1 / 2, \mathrm{y}+1 / 2$ $84 z, x+1 / 2,-y+1 / 2$ $85 \mathrm{y}, \mathrm{x}+1 / 2, \mathrm{z}+1 / 2$ $86 \mathrm{y},-\mathrm{x}+1 / 2,-\mathrm{z}+1 / 2$ $87-y, x+1 / 2,-z+1 / 2$ $88-\mathrm{y},-\mathrm{x}+1 / 2, \mathrm{z}+1 / 2$ $89 \mathrm{x}, \mathrm{z}+1 / 2, \mathrm{y}+1 / 2$ $90 \mathrm{x},-\mathrm{z}+1 / 2,-\mathrm{y}+1 / 2$ $91-\mathrm{x}, \mathrm{z}+1 / 2,-\mathrm{y}+1 / 2$ $92-\mathrm{x},-\mathrm{z}+1 / 2, \mathrm{y}+1 / 2$ $93 \mathrm{z}, \mathrm{y}+1 / 2, \mathrm{x}+1 / 2$ $94 \mathrm{z},-\mathrm{y}+1 / 2,-\mathrm{x}+1 / 2$ $95-\mathrm{z}, \mathrm{y}+1 / 2,-\mathrm{x}+1 / 2$ $96-z,-y+1 / 2, x+1 / 2$ $97 x+1 / 2, y, z+1 / 2$ $98 x+1 / 2, y, y+z+1 / 2$ $98 x+1 / 2,-y,-z+1 / 2$ $99-\mathrm{x}+1 / 2, \mathrm{y},-\mathrm{z}+1 / 2$ $100-x+1 / 2,-y, z+1 / 2$ $101 \mathrm{y}+1 / 2, z, x+1 / 2$ $102 \mathrm{y}+1 / 2,-\mathrm{z},-\mathrm{x}+1 / 2$ $103-y+1 / 2, z,-x+1 / 2$ $104-y+1 / 2,-z, x+1 / 2$ $105 \mathrm{z}+1 / 2, \mathrm{x}, \mathrm{y}+1 / 2$ $106 \mathrm{z}+1 / 2,-\mathrm{x},-\mathrm{y}+1 / 2$ $107-\mathrm{z}+1 / 2, \mathrm{x},-\mathrm{y}+1 / 2$ $108-\mathrm{z}+1 / 2,-\mathrm{x}, \mathrm{y}+1 / 2$ $109-\mathrm{y}+1 / 2,-x,-z+1 / 2$ $110-\mathrm{y}+1 / 2, \mathrm{x}, \mathrm{z}+1 / 2$ $111 \mathrm{y}+1 / 2,-\mathrm{x}, \mathrm{z}+1 / 2$ $112 \mathrm{y}+1 / 2, \mathrm{x},-\mathrm{z}+1 / 2$ $113-\mathrm{x}+1 / 2,-\mathrm{z},-\mathrm{y}+1 / 2$ $114-\mathrm{x}+1 / 2, \mathrm{z}, \mathrm{y}+1 / 2$ $115 \mathrm{x}+1 / 2,-\mathrm{z}, \mathrm{y}+1 / 2$ $116 x+1 / 2, z,-y+1 / 2$ $117-z+1 / 2,-y,-x+1 / 2$ $118-\mathrm{z}+1 / 2, \mathrm{y}, \mathrm{x}+1 / 2$ $119 \mathrm{z}+1 / 2,-\mathrm{y}, \mathrm{x}+1 / 2$ $120 \mathrm{z}+1 / 2, \mathrm{y},-\mathrm{x}+1 / 2$ $121-\mathrm{x}+1 / 2,-\mathrm{y},-\mathrm{z}+1 / 2$ $122-x+1 / 2, y, z+1 / 2$ $123 \mathrm{x}+1 / 2,-\mathrm{y}, \mathrm{z}+1 / 2$ $124 \mathrm{x}+1 / 2, \mathrm{y},-\mathrm{z}+1 / 2$ $125-\mathrm{y}+1 / 2,-\mathrm{z},-\mathrm{x}+1 / 2$ $126-\mathrm{y}+1 / 2, \mathrm{z}, \mathrm{x}+1 / 2$ $127 \mathrm{y}+1 / 2,-\mathrm{z}, \mathrm{x}+1 / 2$ $128 \mathrm{y}+1 / 2, \mathrm{z},-\mathrm{x}+1 / 2$ $129-\mathrm{z}+1 / 2,-\mathrm{x},-\mathrm{y}+1 / 2$ $130-\mathrm{z}+1 / 2, \mathrm{x}, \mathrm{y}+1 / 2$ $131 \mathrm{z}+1 / 2,-\mathrm{x}, \mathrm{y}+1 / 2$ $132 z+1 / 2, x,-y+1 / 2$ $133 y+1 / 2, x, z+1 / 2$ $134 \mathrm{y}+1 / 2,-\mathrm{x},-\mathrm{z}+1 / 2$ $135-\mathrm{y}+1 / 2, \mathrm{x},-\mathrm{z}+1 / 2$ $136-\mathrm{y}+1 / 2,-\mathrm{x}, \mathrm{z}+1 / 2$ $137 \mathrm{x}+1 / 2, \mathrm{z}, \mathrm{y}+1 / 2$ $138 \mathrm{x}+1 / 2,-\mathrm{z},-\mathrm{y}+1 / 2$ $139-x+1 / 2, z,-y+1 / 2$ $140-\mathrm{x}+1 / 2,-\mathrm{z}, \mathrm{y}+1 / 2$ $41 \mathrm{z}+1 / 2, \mathrm{y}, \mathrm{x}+1 / 2$ $42 \mathrm{z}+1 / 2,-\mathrm{y},-\mathrm{x}+1 / 2$ $143-\mathrm{z}+1 / 2, \mathrm{y},-\mathrm{x}+1 / 2$ $144-z+1 / 2,-y, x+1 / 2$ $145 \mathrm{x}+1 / 2, \mathrm{y}+1 / 2, \mathrm{z}$ $145 x+1 / 2, y+1 / 2, z$ $146 x+1 / 2,-y+1 / 2,-z$ $147-x+1 / 2, y+1 / 2,-z$ $148-x+1 / 2,-y+1 / 2, z$ $149 \mathrm{y}+1 / 2, z+1 / 2, \mathrm{x}$ $150 \mathrm{y}+1 / 2,-\mathrm{z}+1 / 2,-\mathrm{x}$ $151-\mathrm{y}+1 / 2, \mathrm{z}+1 / 2,-\mathrm{x}$ $152-y+1 / 2,-z+1 / 2, x$ $153 \mathrm{z}+1 / 2, \mathrm{x}+1 / 2, \mathrm{y}$ $154 \mathrm{z}+1 / 2,-\mathrm{x}+1 / 2,-\mathrm{y}$ $155-\mathrm{z}+1 / 2, \mathrm{x}+1 / 2,-\mathrm{y}$ $156-\mathrm{z}+1 / 2,-\mathrm{x}+1 / 2, \mathrm{y}$ $157-y+1 / 2,-x+1 / 2,-z$ $158-\mathrm{y}+1 / 2, \mathrm{x}+1 / 2, \mathrm{z}$ $159 y+1 / 2,-x+1 / 2, z$ $160 \mathrm{y}+1 / 2, \mathrm{x}+1 / 2,-\mathrm{z}$ $161-x+1 / 2,-z+1 / 2,-y$ $162-x+1 / 2, z+1 / 2, y$ $162-x+1 / 2, z+1 / 2, y$ $163 \mathrm{x}+1 / 2,-\mathrm{z}+1 / 2, \mathrm{y}$
$164 \mathrm{x}+1 / 2, \mathrm{z}+1 / 2,-\mathrm{y}$ $164 \mathrm{x}+1 / 2, \mathrm{z}+1 / 2,-\mathrm{y}$
$165-\mathrm{z}+1 / 2,-\mathrm{y}+1 / 2,-\mathrm{x}$ $166-\mathrm{z}+1 / 2, \mathrm{y}+1 / 2, \mathrm{x}$ $167 \mathrm{z}+1 / 2,-\mathrm{y}+1 / 2, \mathrm{x}$ $167 \mathrm{z}+1 / 2,-\mathrm{y}+1 / 2, \mathrm{x}$
$168 \mathrm{z}+1 / 2, \mathrm{y}+1 / 2,-\mathrm{x}$ $169-\mathrm{x}+1 / 2,-\mathrm{y}+1 / 2,-$ $170-x+1 / 2, y+1 / 2, z$ $171 x+1 / 2,-y+1 / 2, z$ $172 \mathrm{x}+1 / 2, \mathrm{y}+1 / 2,-\mathrm{z}$ $173-\mathrm{y}+1 / 2,-\mathrm{z}+1 / 2,-\mathrm{x}$ $174-\mathrm{y}+1 / 2, \mathrm{z}+1 / 2, \mathrm{x}$ $175 \mathrm{y}+1 / 2,-\mathrm{z}+1 / 2, \mathrm{x}$ $176 \mathrm{y}+1 / 2, \mathrm{z}+1 / 2,-\mathrm{x}$ $177-\mathrm{z}+1 / 2,-\mathrm{x}+1 / 2,-\mathrm{y}$ $178-\mathrm{z}+1 / 2, \mathrm{x}+1 / 2, \mathrm{y}$ $179 \mathrm{z}+1 / 2,-\mathrm{x}+1 / 2, \mathrm{y}$ $180 \mathrm{z}+1 / 2, \mathrm{x}+1 / 2,-\mathrm{y}$ $181 \mathrm{y}+1 / 2, x+1 / 2, z$ $182 \mathrm{y}+1 / 2,-\mathrm{x}+1 / 2,-\mathrm{z}$ $183-y+1 / 2, x+1 / 2,-z$ $183-y+1 / 2, x+1 / 2,-z$ $184-y+1 / 2,-x+1 / 2, z$ $185 \mathrm{x}+1 / 2, \mathrm{z}+1 / 2, \mathrm{y}$
$186 \mathrm{x}+1 / 2,-\mathrm{z}+1 / 2,-\mathrm{y}$ $187-\mathrm{x}+1 / 2, \mathrm{z}+1 / 2,-\mathrm{y}$ $188-\mathrm{x}+1 / 2,-\mathrm{z}+1 / 2, \mathrm{y}$

$189 \mathrm{z}+1 / 2, \mathrm{y}+1 / 2, \mathrm{x}$

$190 \mathrm{z}+1 / 2,-\mathrm{y}+1 / 2,-\mathrm{x}$

$191-z+1 / 2, y+1 / 2,-x$

$192-\mathrm{z}+1 / 2,-\mathrm{y}+1 / 2, \mathrm{x}$

loop

atom_site_label

atom_site_type_symbol

_atom_site_symmetry_multiplicity

_atom_site_Wyckoff_label

atom_site_fract_x

atom_site_fract_y

atom_site_fract_z

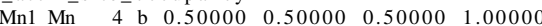

$\begin{array}{lllllll}\mathrm{Mn} 2 \mathrm{Mn} & 24 & \mathrm{~d} & 0.00000 & 0.25000 & 0.25000 & 1.00000\end{array}$

$\begin{array}{llllllll}\mathrm{Mn} 2 & \mathrm{Mn} & 24 & \mathrm{~d} & 0.00000 & 0.25000 & 0.25000 & 1.00000 \\ \mathrm{Th} 1 \mathrm{Th} & 24 & \mathrm{e} & 0.20300 & 0.00000 & 0.00000 & 1.00000\end{array}$

$\begin{array}{llllllll}\mathrm{Mn} 3 \mathrm{Mn} & 32 & \mathrm{f} & 0.17800 & 0.17800 & 0.17800 & 1.00000\end{array}$

$\begin{array}{lllllll}\mathrm{Mn} 4 \mathrm{Mn} & 32 & \mathrm{f} & 0.37800 & 0.37800 & 0.37800 & 1.00000\end{array}$

$\mathrm{Th}_{6} \mathrm{Mn}_{23}\left(D 8_{a}\right):$ A23B6_cF116_225_bd2f_e - POSCAR

A23B6_cF116 225_bd2f_e \& a , x 3, x4, x5 --params $=12.523,0.203,0.178,0.378 \&$ $\hookrightarrow$ Fm-3m O_ $\{\mathrm{h}\}^{\wedge}\{5\} \# 225\left(\mathrm{bdef}^{\wedge} 2\right) \& \mathrm{cF} 116 \&$ \&D8_\{a\}\$ \& Th6Mn23\& $\rightarrow$ Th6Mn23 \& J.V. Florio and R. E. Rundle and A. I. Snow, Acta $\hookrightarrow$ Cryst. 5, 445-457 (1952)

1.00000000000000

$\begin{array}{lll}0.00000000000000 & 6.26150000000000 & 6.26150000000000\end{array}$

$\begin{array}{lll}6.26150000000000 & 0.00000000000000 & 6.26150000000000\end{array}$

$\begin{array}{lll}6.26150000000000 & 6.26150000000000 & 0.00000000000000\end{array}$

$\begin{array}{lr}\mathrm{Mn} & \mathrm{Th} \\ 23 & 6\end{array}$

Direct

0.50000000000000

0.50000000000000

0.00000000000000

0.00000000000000

0.50000000000000

0.00000000000000

0.50000000000000

0.17800000000000

0.17800000000000

0.17800000000000

$-0.53400000000000$

$-0.17800000000000$

$-0.17800000000000$

$-0.17800000000000$

0.53400000000000

0.37800000000000

0.37800000000000

0.37800000000000

$-1.13400000000000$

$-0.37800000000000$

$-0.37800000000000$

$-0.37800000000000$

1.13400000000000

$-0.20300000000000$

0.20300000000000

0.20300000000000

$-0.20300000000000$

$-0.20300000000000$

0.50000000000000 .00000000000000

0.50000000000000

0.50000000000000

0.00000000000000

0.00000000000000

0.50000000000000

0.17800000000000

0.17800000000000

0.53400000000000

0.17800000000000

$-0.17800000000000$

0.17800000000000

0.53400000000000

$-0.17800000000000$

0.37800000000000

0.37800000000000

0.3780000000000

$-0.37800000000000$

$-0.37800000000000$

1.1340000000000

$-0.37800000000000$

0.20300000000000

$-0.20300000000000$

$-0.20300000000000$

0.20300000000000

0.20300000000000

0.50000000000000 .

.50000000000000

0.00000000000000

0.5000000000000

0.00000000000000

0.17800000000000 $-0.53400000000000$ 0.17800000000000 0.17800000000000 0.53400000000000 $-0.17800000000000$ $-0.17800000000000$ $-0.17800000000000$ 0.37800000000000 1.13400000000000 0.37800000000000 0.37800000000000 1.13400000000000 $-0.37800000000000$ 0.37800000000000 $-0.37800000000000$ 0.20300000000000 0.20300000000000 0.20300000000 0.2030000000 $-0.20300000000000$ 0.20300000000000 0.20300000000000

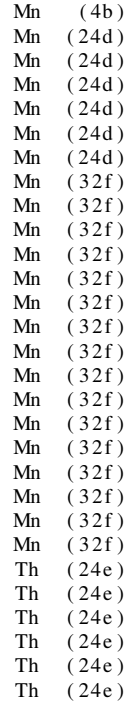

$\mathrm{K}_{2} \mathrm{PtCl}_{6}\left(J 1_{1}\right):$ A6B2C_cF36_225_e_c_a - CIF

\# CIF file

data findsym-outpu

audit_creation_method FINDSYM

chemical_name_mineral 'K2PtCl6'

chemical_formula_sum, Cl6 K2 Pt

loop

-publ_author_name

'G. Engel'

journal_name_full_name

Zeitschrift $f \backslash \backslash " u\}$ r Kristallografiya

journal_volume 90

journal year 1935

_journal_page_first 341

journal page last 373

publ_Section_title

Die Kristallstrukturen einiger Hexachlorokomplexsalze

\# Found in The American Mineralogist Crystal Structure Database, 2003

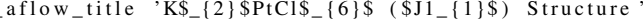

aflow_proto 'A6B2C_cF36_225_e_c_a

aflow_params 'a, $x_{-}\{3\}$

_aflow_params_values '9.725, 0.24 ,

aflow_Strukturbericht $\$ \mathrm{~J} 1$

aflow_Pearson 'cF36'

symmetry space group_name_H-M "F $4 / \mathrm{m}-3 \quad 2 / \mathrm{m} "$

symmetry_Int Tables_number 225

cell_length_a $\quad 9.72500$

cell_length_b 9.72500

_cell_length_c $\quad 9.72500$

_cell_angle_alpha 90.00000 
$100-\mathrm{x}+1 / 2,-\mathrm{y}, \mathrm{z}+1 / 2$

$101 \mathrm{y}+1 / 2, \mathrm{z}, \mathrm{x}+1 / 2$

$102 \mathrm{y}+1 / 2,-\mathrm{z},-\mathrm{x}+1 / 2$

$103-\mathrm{y}+1 / 2, \mathrm{z},-\mathrm{x}+1 / 2$

$104-\mathrm{y}+1 / 2,-\mathrm{z}, \mathrm{x}+1 / 2$

$105 \mathrm{z}+1 / 2, \mathrm{x}, \mathrm{y}+1 / 2$

$106 \mathrm{z}+1 / 2,-\mathrm{x},-\mathrm{y}+1 / 2$

$107-\mathrm{z}+1 / 2, \mathrm{x},-\mathrm{y}+1 / 2$

$108-\mathrm{z}+1 / 2,-\mathrm{x}, \mathrm{y}+1 / 2$

$109-\mathrm{y}+1 / 2,-\mathrm{x},-\mathrm{z}+1 / 2$

$110-y+1 / 2, x, z+1 / 2$

$111 \mathrm{y}+1 / 2,-\mathrm{x}, \mathrm{z}+1 / 2$

$112 y+1 / 2, x,-z+1 / 2$

$113-x+1 / 2,-z,-y+1 / 2$

$114-x+1 / 2,-z,-y+1 / 2$

$114-\mathrm{x}+1 / 2, \mathrm{z}, \mathrm{y}+1 / 2$

$115 \mathrm{x}+1 / 2,-\mathrm{z}, \mathrm{y}+1 / 2$

$116 \mathrm{x}+1 / 2, \mathrm{z},-\mathrm{y}+1 / 2$

$117-z+1 / 2,-y,-x+1 / 2$

$118-\mathrm{z}+1 / 2, \mathrm{y}, \mathrm{x}+1 / 2$

$119 \mathrm{z}+1 / 2,-\mathrm{y}, \mathrm{x}+1 / 2$

$120 \mathrm{z}+1 / 2, \mathrm{y},-\mathrm{x}+1 / 2$

$121-\mathrm{x}+1 / 2,-\mathrm{y},-\mathrm{z}+1 / 2$

$122-x+1 / 2, y, z+1 / 2$

$123 \mathrm{x}+1 / 2,-\mathrm{y}, \mathrm{z}+1 / 2$

$124 \mathrm{x}+1 / 2, \mathrm{y},-\mathrm{z}+1 / 2$

$125-\mathrm{y}+1 / 2,-\mathrm{z},-\mathrm{x}+1 / 2$

$126-\mathrm{y}+1 / 2, \mathrm{z}, \mathrm{x}+1 / 2$

$127 \mathrm{y}+1 / 2,-\mathrm{z}, \mathrm{x}+1 / 2$

$128 \mathrm{y}+1 / 2, \mathrm{z},-\mathrm{x}+1 / 2$

$129-\mathrm{z}+1 / 2,-\mathrm{x},-\mathrm{y}+1 / 2$

$130-\mathrm{z}+1 / 2, \mathrm{x}, \mathrm{y}+1 / 2$

$131 \mathrm{z}+1 / 2,-\mathrm{x}, \mathrm{y}+1 / 2$

$32 z+1 / 2, x,-y+1 / 2$

$133 \mathrm{y}+1 / 2, \mathrm{x}, \mathrm{z}+1 / 2$

$134 \mathrm{y}+1 / 2,-\mathrm{x},-\mathrm{z}+1 / 2$

$135-\mathrm{y}+1 / 2, \mathrm{x},-\mathrm{z}+1 / 2$

$136-\mathrm{y}+1 / 2,-\mathrm{x}, \mathrm{z}+1 /$

$137 \mathrm{x}+1 / 2, \mathrm{z}, \mathrm{y}+1 / 2$

$138 \mathrm{x}+1 / 2,-\mathrm{z},-\mathrm{y}+1 / 2$

$139-\mathrm{x}+1 / 2, \mathrm{z},-\mathrm{y}+1 / 2$

$140-\mathrm{x}+1 / 2,-\mathrm{z}, \mathrm{y}+1 / 2$

$141 \mathrm{z}+1 / 2, \mathrm{y}, \mathrm{x}+1 / 2$

$142 \mathrm{z}+1 / 2,-\mathrm{y},-\mathrm{x}+1 / 2$

$143-\mathrm{z}+1 / 2, \mathrm{y},-\mathrm{x}+1 / 2$

$144-z+1 / 2,-y, x+1 / 2$

$145 \mathrm{x}+1 / 2, \mathrm{y}+1 / 2, \mathrm{z}$

$146 x+1 / 2,-y+1 / 2, z$

$146 x+1 / 2,-y+1 / 2,-z$

$147-x+1 / 2, y+1 / 2,-z$

$148-x+1 / 2,-y+1 / 2, z$

$149 \mathrm{y}+1 / 2, \mathrm{z}+1 / 2, \mathrm{x}$

$150 \mathrm{y}+1 / 2,-\mathrm{z}+1 / 2,-\mathrm{x}$

$151-\mathrm{y}+1 / 2, \mathrm{z}+1 / 2,-\mathrm{x}$

$152-\mathrm{y}+1 / 2,-\mathrm{z}+1 / 2$,

$153 \mathrm{z}+1 / 2, \mathrm{x}+1 / 2, \mathrm{y}$

$154 \mathrm{z}+1 / 2,-\mathrm{x}+1 / 2,-\mathrm{y}$

$155-\mathrm{z}+1 / 2, \mathrm{x}+1 / 2,-\mathrm{y}$

$156-\mathrm{z}+1 / 2,-\mathrm{x}+1 / 2, \mathrm{y}$

$157-\mathrm{y}+1 / 2,-\mathrm{x}+1 / 2,-\mathrm{z}$

$158-y+1 / 2, x+1 / 2, z$

$159 \mathrm{y}+1 / 2,-x+1 / 2, \mathrm{z}$

$160 \mathrm{y}+1 / 2, \mathrm{x}+1 / 2,-\mathrm{z}$

$161-\mathrm{x}+1 / 2,-\mathrm{z}+1 / 2,-\mathrm{y}$

$162-\mathrm{x}+1 / 2, \mathrm{z}+1 / 2, \mathrm{y}$

$163 \mathrm{x}+1 / 2,-\mathrm{z}+1 / 2, \mathrm{y}$

$164 \mathrm{x}+1 / 2, \mathrm{z}+1 / 2,-\mathrm{y}$

$165-\mathrm{z}+1 / 2,-\mathrm{y}+1 / 2,-\mathrm{x}$

$166-\mathrm{z}+1 / 2, \mathrm{y}+1 / 2, \mathrm{x}$

$167 \mathrm{z}+1 / 2,-\mathrm{y}+1 / 2, \mathrm{x}$

$168 \mathrm{z}+1 / 2, \mathrm{y}+1 / 2,-\mathrm{x}$

$169-x+1 / 2,-y+1 / 2,-z$

$170-\mathrm{x}+1 / 2, \mathrm{y}+1 / 2, \mathrm{z}$

$171 \mathrm{x}+1 / 2,-\mathrm{y}+1 / 2, \mathrm{z}$

$172 \mathrm{x}+1 / 2, \mathrm{y}+1 / 2,-\mathrm{z}$

$173-\mathrm{y}+1 / 2,-\mathrm{z}+1 / 2,-\mathrm{x}$

$174-\mathrm{y}+1 / 2, \mathrm{z}+1 / 2, \mathrm{x}$

$175 \mathrm{y}+1 / 2,-\mathrm{z}+1 / 2, \mathrm{x}$

$176 \mathrm{y}+1 / 2, \mathrm{z}+1 / 2,-\mathrm{x}$

$177-\mathrm{z}+1 / 2,-\mathrm{x}+1 / 2,-\mathrm{y}$

$178-\mathrm{z}+1 / 2, \mathrm{x}+1 / 2, \mathrm{y}$

$179 z+1 / 2, x+1 / 2, y$

$180 z+1 / 2, x+1 / 2, y$

$181 \mathrm{z}+1 / 2, x+1 / 2,-y$

$181 \mathrm{y}+1 / 2, \mathrm{x}+1 / 2, \mathrm{z}$

$182 \mathrm{y}+1 / 2,-\mathrm{x}+1 / 2,-\mathrm{z}$

$183-\mathrm{y}+1 / 2, \mathrm{x}+1 / 2,-\mathrm{z}$

$184-\mathrm{y}+1 / 2,-\mathrm{x}+1 / 2$,

$185 \mathrm{x}+1 / 2, \mathrm{z}+1 / 2, \mathrm{y}$

$186 \mathrm{x}+1 / 2,-\mathrm{z}+1 / 2,-\mathrm{y}$

$187-\mathrm{x}+1 / 2, \mathrm{z}+1 / 2,-\mathrm{y}$

$188-\mathrm{x}+1 / 2,-\mathrm{z}+1 / 2, \mathrm{y}$

$189 \mathrm{z}+1 / 2, \mathrm{y}+1 / 2, \mathrm{x}$

$190 \mathrm{z}+1 / 2,-\mathrm{y}+1 / 2,-\mathrm{x}$

$191-z+1 / 2, y+1 / 2,-x$

$192-z+1 / 2,-y+1 / 2, x$

loop

atom_site_label

_atom_site_type_symbol

-atom_site_type_symbol

_atom_site_fract_x

-atom_site_fract_y

_atom_site_fract_z

$\begin{array}{llllllll}\text { Pt1 } & \mathrm{Pt} & 4 & 4 & 0.00000 & 0.00000 & 0.00000 & 1.00000\end{array}$

$\begin{array}{llllllll}\mathrm{K} 1 & \mathrm{~K} & 8 & \mathrm{c} & 0.25000 & 0.25000 & 0.25000 & 1.00000\end{array}$ 


\begin{tabular}{|c|c|c|c|c|}
\hline \multicolumn{5}{|c|}{$\mathrm{K}_{2} \mathrm{PtCl}_{6}\left(J 1_{1}\right):$ A6B2C_cF36_225_e_c_a - POSCAR } \\
\hline \multirow{2}{*}{\multicolumn{5}{|c|}{ 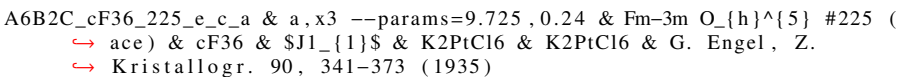 }} \\
\hline & & & & \\
\hline 0.00000000000000 & 1.00000000000000 & 4.86250000000000 & & \\
\hline 4.86250000000000 & 0.00000000000000 & 4.86250000000000 & & \\
\hline 4.86250000000000 & 4.86250000000000 & 0.00000000000000 & & \\
\hline $\mathrm{Cl}$ & & & & \\
\hline 6 & & & & \\
\hline \multicolumn{5}{|l|}{ Direct } \\
\hline-0.24000000000000 & 0.24000000000000 & 0.24000000000000 & $\mathrm{Cl}$ & $(24 \mathrm{e})$ \\
\hline \multirow{2}{*}{0.24000000000000} & -0.24000000000000 & -0.24000000000000 & $\mathrm{Cl}$ & $(24 \mathrm{e})$ \\
\hline & -0.24000000000000 & 0.24000000000000 & $\mathrm{Cl}$ & $(24 \mathrm{e})$ \\
\hline-0.24000000000000 & 0.24000000000000 & -0.24000000000000 & $\mathrm{Cl}$ & $(24 \mathrm{e})$ \\
\hline 0.24000000000000 & 0.24000000000000 & -0.24000000000000 & $\mathrm{Cl}$ & $(24 \mathrm{e})$ \\
\hline-0.24000000000000 & -0.24000000000000 & 0.24000000000000 & $\mathrm{Cl}$ & $(24 \mathrm{e})$ \\
\hline \multirow{2}{*}{0.25000000000000} & 0.25000000000000 & 0.25000000000000 & $\mathrm{~K}$ & $(8 \mathrm{c})$ \\
\hline & 0.75000000000000 & 0.75000000000000 & $\mathrm{~K}$ & $(8 \mathrm{c})$ \\
\hline 0.00000000000000 & 0.00000000000000 & 0.00000000000000 & $\mathrm{Pt}$ & (4a) \\
\hline
\end{tabular}

$\mathrm{NaZn}_{13}\left(D 2_{3}\right): \mathrm{AB} 13 \_c F 112 \_226 \_a$ bi - CIF

\section{\# CIF file}

data_findsym-output

-audit_creation_method FINDSYM

chemical_name_mineral,

chemical_formula_sum ' $\mathrm{Na} \mathrm{Zn13}$,

loop

-publ_author_name

D. P. Shoemaker

'R. E. Marsh'

'F. J. Ewing,
'L. Pauling,

journal_name_full_name

Acta Cristallographica

_journal_volume 5

journal year 1952

_journal_page_first 637

publ_Section_title

Interatomic distances and atomic valences in NaZn\$_\{13\}\$

_aflow_title 'NaZn\$_\{13\}\$(\$D2_\{3\}\$) Structure,

-aflow_proto 'AB 13 _cF112_226_a_bi,

_aflow_params 'a, __ $_{-}\{3\}, z_{-}\{3\}$

_aflow_params_values $12.2836,0.1806,0.1192$,

_aflow_Strukturbericht '\$D2_\{3\}\$,

_aflow_Pearson 'cF112,

_symmetry_space_group_name_H-M "F $4 / \mathrm{m}-3 \quad 2 / \mathrm{c} "$

_symmetry_Int_Tables_number 226

_cell_length_a $\quad 12.28360$

_cell_length_b $\quad 12.28360$

$\begin{array}{ll}\text { _cell_length_c } & 12.28360 \\ \text { _cell_angle_alpha } & 90.00000\end{array}$

$\begin{array}{ll}\text { _cell_angle_alpha } & 90.00000 \\ \text { cell_angle_beta } & 90.00000\end{array}$

_cell_angle_gamma 90.00000

loop

_space_group_symop_id

_space_group_symop_operation_xyz

$1 \mathrm{x}, \mathrm{y}, \mathrm{z}$

$2 \mathrm{x},-\mathrm{y},-\mathrm{z}$

$3-x, y,-z$
$4-x,-y, z$

$\begin{array}{ll}4 & -\mathrm{x},-\mathrm{y}, \mathrm{z} \\ 5 & \mathrm{y}, \mathrm{z}, \mathrm{x}\end{array}$

$6 \mathrm{y},-\mathrm{z},-\mathrm{x}$

$7-y, z,-x$

$8-\mathrm{y},-\mathrm{z}, \mathrm{x}$

$9 \mathrm{z}, \mathrm{x}, \mathrm{y}$

$10 \mathrm{z},-\mathrm{x},-\mathrm{y}$
$11-\mathrm{z}, \mathrm{x},-\mathrm{y}$

$11-\mathrm{z}, \mathrm{x},-\mathrm{y}$
$12-\mathrm{z},-\mathrm{x}, \mathrm{y}$

$13-\mathrm{y}+1 / 2,-\mathrm{x}+1 / 2,-\mathrm{z}+1 / 2$

$14-\mathrm{y}+1 / 2, \mathrm{x}+1 / 2, \mathrm{z}+1 / 2$

$15 \mathrm{y}+1 / 2,-\mathrm{x}+1 / 2, \mathrm{z}+1 / 2$

$16 \mathrm{y}+1 / 2, \mathrm{x}+1 / 2,-\mathrm{z}+1 / 2$
$17-\mathrm{x}+1 / 2,-\mathrm{z}+1 / 2,-\mathrm{y}+1 / 2$

$18-x+1 / 2, z+1 / 2, y+1 / 2$

$19 x+1 / 2,-z+1 / 2, y+1 / 2$

$20 \mathrm{x}+1 / 2, \mathrm{z}+1 / 2,-\mathrm{y}+1 / 2$

$21-\mathrm{z}+1 / 2,-\mathrm{y}+1 / 2,-\mathrm{x}+1 / 2$

$22-\mathrm{z}+1 / 2, \mathrm{y}+1 / 2, \mathrm{x}+1 / 2$

$23 \mathrm{z}+1 / 2,-\mathrm{y}+1 / 2, \mathrm{x}+1 / 2$

$24 \mathrm{z}+1 / 2, \mathrm{y}+1 / 2,-\mathrm{x}+1 / 2$

$25-\mathrm{x},-\mathrm{y},-\mathrm{z}$

$26-x, y$,

$27 \mathrm{x},-\mathrm{y}, \mathrm{z}$
$28 \mathrm{x}, \mathrm{y},-\mathrm{z}$

$28 x, y,-z$
$29-y,-z,-x$

$29-y,-z,-x$
$30-y, z, x$

$31 \mathrm{y},-\mathrm{z}, \mathrm{x}$

$32 \mathrm{y}, \mathrm{z},-\mathrm{x}$
$33-\mathrm{z},-\mathrm{x},-$

$34-\mathrm{z}, \mathrm{x}, \mathrm{y}$

$35 \mathrm{z},-\mathrm{x}, \mathrm{y}$

$\begin{array}{ll}36 & \mathrm{z}, \mathrm{x},-\mathrm{y} \\ 37 & \mathrm{y}+1 / 2, \mathrm{x}+1 / 2, \mathrm{z}+1 / 2 \\ 38 & \mathrm{y}+1 / 2,-\mathrm{x}+1 / 2,-\mathrm{z}+1 / 2 \\ 39 & -\mathrm{y}+1 / 2, \mathrm{x}+1 / 2 /,-z+1 / 2\end{array}$

$38 \mathrm{y}+1 / 2,-\mathrm{x}+1 / 2,-\mathrm{z}+1 / 2$

$39-\mathrm{y}+1 / 2, \mathrm{x}+1 / 2,-\mathrm{z}+1 / 2$

$40-\mathrm{y}+1 / 2,-\mathrm{x}+1 / 2, \mathrm{z}+1 / 2$

$41 \mathrm{x}+1 / 2, \mathrm{z}+1 / 2, \mathrm{y}+1 / 2$

$42 \mathrm{x}+1 / 2,-\mathrm{z}+1 / 2,-\mathrm{y}+1 / 2$

$43-x+1 / 2, z+1 / 2,-y+1 / 2$

$44-\mathrm{x}+1 / 2,-\mathrm{z}+1 / 2, \mathrm{y}+1 / 2$

$45 \mathrm{z}+1 / 2, \mathrm{y}+1 / 2, \mathrm{x}+1 / 2$

$46 x+1 / 2,-y+1 / 2,-x+1 / 2$

$46 \mathrm{z}+1 / 2,-\mathrm{y}+1 / 2,-\mathrm{x}+1 / 2$
$47-\mathrm{z}+1 / 2, \mathrm{y}+1 / 2,-\mathrm{x}+1 / 2$

$47-\mathrm{z}+1 / 2, \mathrm{y}+1 / 2,-\mathrm{x}+1 / 2$
$48-\mathrm{z}+1 / 2,-\mathrm{y}+1 / 2, \mathrm{x}+1 / 2$

$48-\mathrm{z}+1 / 2,-\mathrm{y}+1 / 2, \mathrm{x}+\mathrm{x}$

$50 \mathrm{x},-\mathrm{y}+1 / 2,-\mathrm{z}+1 / 2$

$50 \mathrm{x},-\mathrm{y}+1 / 2,-\mathrm{z}+1 / 2$

$51-\mathrm{x}, \mathrm{y}+1 / 2,-\mathrm{z}+1 / 2$

$52-x,-y+1 / 2, z+1 / 2$

$53 \mathrm{y}, \mathrm{z}+1 / 2, \mathrm{x}+1 / 2$

$54 \mathrm{y},-\mathrm{z}+1 / 2,-\mathrm{x}+1 / 2$

$55-\mathrm{y}, \mathrm{z}+1 / 2,-\mathrm{x}+1 / 2$

$56-\mathrm{y},-\mathrm{z}+1 / 2, \mathrm{x}+1 / 2$

$57 \mathrm{z}, \mathrm{x}+1 / 2, \mathrm{y}+1 / 2$

$58 \mathrm{z},-\mathrm{x}+1 / 2,-\mathrm{y}+1 / 2$

$59-\mathrm{z}, \mathrm{x}+1 / 2,-\mathrm{y}+1 / 2$

$60-\mathrm{z},-\mathrm{x}+1 / 2, \mathrm{y}+1 / 2$

$61-\mathrm{y}+1 / 2,-\mathrm{x},-\mathrm{z}$

$62-y+1 / 2, x, z$

$62-y+1 / 2, x, z$

$63 \mathrm{y}+1 / 2,-\mathrm{x}, \mathrm{z}$

$64 \mathrm{y}+1 / 2, \mathrm{x},-\mathrm{z}$
$65-\mathrm{x}+1 / 2,-\mathrm{z},-\mathrm{y}$

$66-x+1 / 2, z, y$

$67 \mathrm{x}+1 / 2,-\mathrm{z}, \mathrm{y}$

$68 \mathrm{x}+1 / 2, \mathrm{z},-\mathrm{y}$

$69-z+1 / 2,-y,-x$

$70-z+1 / 2, y, x$

$71 \mathrm{z}+1 / 2,-\mathrm{y}, \mathrm{x}$

$72 \mathrm{z}+1 / 2, \mathrm{y},-\mathrm{x}$

$73-\mathrm{x},-\mathrm{y}+1 / 2,-\mathrm{z}+1 / 2$

$74-\mathrm{x}, \mathrm{y}+1 / 2, \mathrm{z}+1 / 2$

$75 \mathrm{x},-\mathrm{y}+1 / 2, \mathrm{z}+1 / 2$

$76 \mathrm{x}, \mathrm{y}+1 / 2,-\mathrm{z}+1 / 2$

$77-y,-z+1 / 2,-x+1 / 2$

$78-\mathrm{y}, \mathrm{z}+1 / 2, \mathrm{x}+1 / 2$

$79 \mathrm{y},-\mathrm{z}+1 / 2, \mathrm{x}+1 / 2$

$80 \mathrm{y}, \mathrm{z}+1 / 2, \mathrm{x}+1 / 2$

$80 \mathrm{y}, \mathrm{z}+1 / 2,-\mathrm{x}+1 / 2$
$81-\mathrm{z},-\mathrm{x}+1 / 2,-\mathrm{y}+1 / 2$

$81-z,-x+1 / 2,-y+1 / 2$

$82-\mathrm{z}, \mathrm{x}+1 / 2, \mathrm{y}+1 / 2$

$83 \mathrm{z},-\mathrm{x}+1 / 2, \mathrm{y}+1 / 2$

$84 \mathrm{z}, \mathrm{x}+1 / 2,-\mathrm{y}+1 / 2$

$85 \mathrm{y}+1 / 2, \mathrm{x}, \mathrm{z}$

$86 y+1 / 2,-x,-z$

$87-\mathrm{y}+1 / 2, \mathrm{x},-\mathrm{z}$

$88-\mathrm{y}+1 / 2,-\mathrm{x}, \mathrm{z}$

$89 x+1 / 2, z, y$

$90 \mathrm{x}+1 / 2,-\mathrm{z},-\mathrm{y}$

$91-x+1 / 2, z,-y$

$92-x+1 / 2,-z, y$

$93 \mathrm{z}+1 / 2, \mathrm{y}, \mathrm{x}$

$94 \mathrm{z}+1 / 2,-\mathrm{y},-\mathrm{x}$

$94 \mathrm{z}+1 / 2,-\mathrm{y},-\mathrm{x}$
$95-\mathrm{z}+1 / 2, \mathrm{y},-\mathrm{x}$

$96-z+1 / 2, y, x$

$96-z+1 / 2,-y, x$

$98 x+1 / 2, y, z+1 / 2$

$98 \mathrm{x}+1 / 2,-\mathrm{y},-\mathrm{z}+1 / 2$

$99-\mathrm{x}+1 / 2, \mathrm{y},-\mathrm{z}+1 / 2$
$100-\mathrm{x}+1 / 2,-\mathrm{y}, \mathrm{z}+1 / 2$

$100-\mathrm{x}+1 / 2,-\mathrm{y}, \mathrm{z}+1 / 2$
$101 \mathrm{y}+1 / 2, \mathrm{z}, \mathrm{x}+1 / 2$

$101 \mathrm{y}+1 / 2, \mathrm{z}, \mathrm{x}+1 / 2$
$102 \mathrm{y}+1 / 2,-\mathrm{z},-\mathrm{x}+1 / 2$

$102 \mathrm{y}+1 / 2,-\mathrm{z},-\mathrm{x}+1 / 2$
$103-\mathrm{y}+1 / 2, \mathrm{z},-\mathrm{x}+1 / 2$

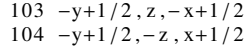

$105 \mathrm{z}+1 / 2, \mathrm{x}, \mathrm{y}+1 / 2$

$106 \mathrm{z}+1 / 2,-\mathrm{x},-\mathrm{y}+1 / 2$

$107-\mathrm{z}+1 / 2, \mathrm{x},-\mathrm{y}+1 / 2$

$108-\mathrm{z}+1 / 2,-\mathrm{x}, \mathrm{y}+1 / 2$

$109-y,-x+1 / 2,-z$

$110-y, x+1 / 2, z$

$111 \mathrm{y},-\mathrm{x}+1 / 2, \mathrm{z}$

$112 \mathrm{y}, \mathrm{x}+1 / 2,-\mathrm{z}$

$113-x,-z+1 / 2,-y$

$113-\mathrm{x},-\mathrm{z}+1 / 2,-\mathrm{y}$

$114-\mathrm{x}, \mathrm{z}+1 / 2, \mathrm{y}$

$15 \mathrm{x},-\mathrm{z}+1 / 2, \mathrm{y}$

$16 \mathrm{x}, \mathrm{z}+1 / 2,-\mathrm{y}$

$117-z,-y+1 / 2,-x$

$118-\mathrm{z}, \mathrm{y}+1 / 2, \mathrm{x}$

$119 \mathrm{z},-\mathrm{y}+1 / 2, \mathrm{x}$

$120 \mathrm{z}, \mathrm{y}+1 / 2,-\mathrm{x}$

$121-\mathrm{x}+1 / 2,-\mathrm{y},-\mathrm{z}+1 / 2$

$122-\mathrm{x}+1 / 2, \mathrm{y}, \mathrm{z}+1 / 2$

$123 \mathrm{x}+1 / 2,-\mathrm{y}, \mathrm{z}+1 / 2$

$124 \mathrm{x}+1 / 2, \mathrm{y},-\mathrm{z}+1 / 2$

$125-\mathrm{y}+1 / 2,-\mathrm{z},-\mathrm{x}+1 / 2$

$126-\mathrm{y}+1 / 2, \mathrm{z}, \mathrm{x}+1 / 2$

$127 \mathrm{y}+1 / 2,-\mathrm{z}, \mathrm{x}+1 / 2$

$128 \mathrm{y}+1 / 2, \mathrm{z},-\mathrm{x}+1 / 2$

$129-\mathrm{z}+1 / 2,-\mathrm{x},-\mathrm{y}+1 / 2$

$130-z+1 / 2, x, y+1 / 2$

$130-\mathrm{z}+1 / 2, \mathrm{x}, \mathrm{y}+1 / 2$

$131 \mathrm{z}+1 / 2,-x, y+1 / 2$

$132 \mathrm{z}+1 / 2, \mathrm{x},-\mathrm{y}+1 / 2$
$133 \mathrm{y}, \mathrm{x}+1 / 2, \mathrm{z}$

$133 \mathrm{y}, \mathrm{x}+1 / 2, \mathrm{z}$

$134 \mathrm{y},-\mathrm{x}+1 / 2,-\mathrm{z}$

$135-y, x+1 / 2,-z$
$136-y,-x+1 / 2, z$

$136-\mathrm{y},-\mathrm{x}+1 / 2$,
$137 \mathrm{x}, \mathrm{z}+1 / 2, \mathrm{y}$

$138 \mathrm{x},-\mathrm{z}+1 / 2,-\mathrm{y}$ 
$139-\mathrm{x}, \mathrm{z}+1 / 2,-\mathrm{y}$

$140-x,-z+1 / 2$,

$141 \mathrm{z}, \mathrm{y}+1 / 2, \mathrm{x}$

$142 \mathrm{z},-\mathrm{y}+1 / 2,-\mathrm{x}$
$143-\mathrm{z}, \mathrm{y}+1 / 2,-\mathrm{x}$

$144-z,-y+1 / 2, x$

$145 \mathrm{x}+1 / 2, \mathrm{y}+1 / 2$,

$146 x+1 / 2,-y+1 / 2,-z$

$147-x+1 / 2, y+1 / 2,-z$

$148-x+1 / 2,-y+1 / 2, z$

$149 \mathrm{y}+1 / 2, \mathrm{z}+1 / 2, \mathrm{x}$

$150 \mathrm{y}+1 / 2,-\mathrm{z}+1 / 2,-\mathrm{x}$

$151-\mathrm{y}+1 / 2, \mathrm{z}+1 / 2,-\mathrm{x}$

$152-\mathrm{y}+1 / 2,-\mathrm{z}+1 / 2, \mathrm{x}$

$153 \mathrm{z}+1 / 2, \mathrm{x}+1 / 2, \mathrm{y}$

$154 \mathrm{z}+1 / 2,-\mathrm{x}+1 / 2,-\mathrm{y}$

$155-\mathrm{z}+1 / 2, \mathrm{x}+1 / 2,-\mathrm{y}$

$156-\mathrm{z}+1 / 2,-\mathrm{x}+1 / 2, \mathrm{y}$

$157-\mathrm{y},-\mathrm{x},-\mathrm{z}+1 / 2$

$158-\mathrm{y}, \mathrm{x}, \mathrm{z}+1 / 2$
$159 \mathrm{y},-\mathrm{x}, \mathrm{z}+1 / 2$

$160 \mathrm{y}, \mathrm{x},-\mathrm{z}+1 / 2$

$161-x,-z,-y+1 / 2$

$162-x, z, y+1 / 2$

$163 \mathrm{x},-\mathrm{z}, \mathrm{y}+1 / 2$

$164 \mathrm{x}, \mathrm{z},-\mathrm{y}+1 / 2$

$166-\mathrm{z}, \mathrm{y}, \mathrm{x}+1 / 2$

$167 \mathrm{z},-\mathrm{y}, \mathrm{x}+1 / 2$

$168 \mathrm{z}, \mathrm{y},-\mathrm{x}+1 / 2$

$169-x+1 / 2,-y+1 / 2,-z$

$170-x+1 / 2, y+1 / 2$,

$171 x+1 / 2,-y+1 / 2, z$

$172 x+1 / 2, y+1 / 2,-z$
$173-y+1 / 2,-z+1 / 2,-x$

$174-\mathrm{y}+1 / 2, \mathrm{z}+1 / 2, \mathrm{x}$

$175 \mathrm{y}+1 / 2,-\mathrm{z}+1 / 2$,

$176 \mathrm{y}+1 / 2, \mathrm{z}+1 / 2,-\mathrm{x}$
$177-\mathrm{z}+1 / 2,-\mathrm{x}+1 / 2,-\mathrm{y}$

$178-\mathrm{z}+1 / 2, \mathrm{x}+1 / 2, \mathrm{y}$

$179 \mathrm{z}+1 / 2,-\mathrm{x}+1 / 2, \mathrm{y}$

$180 \mathrm{z}+1 / 2, \mathrm{x}+1 / 2,-\mathrm{y}$

$181 \mathrm{y}, \mathrm{x}, \mathrm{z}+1 / 2$

$182 \mathrm{y},-\mathrm{x},-\mathrm{z}+1 / 2$

$183-\mathrm{y}, \mathrm{x},-\mathrm{z}+1 / 2$

$184-\mathrm{y},-\mathrm{x}, \mathrm{z}+1 / 2$

$185 \mathrm{x}, \mathrm{z}, \mathrm{y}+1 / 2$

$186 x,-z,-y+1 / 2$

$187-\mathrm{x}, \mathrm{z},-\mathrm{y}+1 / 2$

$188-\mathrm{x},-\mathrm{z}, \mathrm{y}+1 / 2$

$89 \mathrm{z}, \mathrm{y}, \mathrm{x}+1 / 2$

$190 \mathrm{z},-\mathrm{y},-\mathrm{x}+1 / 2$
$191-\mathrm{z}, \mathrm{y},-\mathrm{x}+1 / 2$

$192-\mathrm{z},-\mathrm{y}, \mathrm{x}+1 / 2$

loop

atom_site_label

atom_site_type_symbol

atom_site_symmetry_multiplicity

atom_site_Wyckoff_label

atom_site_fract_x

atom_site_fract_y

atom_site_fract_z

atom_site_occupancy

$\begin{array}{lllllll}\mathrm{Na} 1 \mathrm{Na} & 8 & \text { a } & 0.25000 & 0.25000 & 0.25000 & 1.00000\end{array}$

$\begin{array}{lllllllll}\mathrm{Zn} 1 \mathrm{Zn} & 8 & \mathrm{~b} & 0.00000 & 0.00000 & 0.00000 & 1.00000\end{array}$

$\begin{array}{llllllll}\mathrm{Zn} 2 & \mathrm{Zn} & 96 & \mathrm{i} & 0.00000 & 0.18060 & 0.11920 & 1.00000\end{array}$

$\operatorname{NaZn}_{13}(D 23):$ AB13_cF112_226_a_bi - POSCAR

$\mathrm{AB} 13$ cF112 226 a bi \& a, y3, z3 - params $=12.2836,0.1806,0.1192 \&$ Fm-3c O

$\hookrightarrow \mathrm{h}\}^{\wedge}\{6\} \# 226$ (abi) \& cF112\& \$D2 $\{3\} \$ \&$ NaZn13\& \& D. P.

$\hookrightarrow$ Shoemaker et al. Acta Cryst 5, 637-644 (1952)

1.00000000000000

0.00000000000000

6.14180000000000

6.14180000000000

6.14180000000000

6.14180000000000

$\begin{array}{rr}\mathrm{Na} & \mathrm{Zn} \\ 2 & 26\end{array}$

Direct

0.25000000000000

0.75000000000000

0.00000000000000

0.50000000000000

0.29980000000000

$-0.06140000000000$

0.06140000000000

$-0.0614000000000$

0.06140000000000

0.06140000000000

$-0.29980000000000$

0.29980000000000

$-0.06140000000000$

$-0.06140000000000$

0.29980000000000
-0.29980000000000

0.06140000000000

0.20020000000000

0.56140000000000

0.43860000000000

0.79980000000000

0.43860000000000

0.79980000000000

0.20020000000000

0.56140000000000

0.56140000000000 0.00000000000000

6.14180000000000

0.00000000000000

25000000000000 0.0000000000000 0.50000000000000 $-0.06140000000000$ 0.29980000000000 $-0.2998000000000$ 0.06140000000000 0.299800000000 0.2998000000000 0.06140000000000 0.061400000000 0.06140000000000 0.0614000000000 0.29980000000000 0.29980000000000 $-0.06140000000000$ 0.56140000000000 0.20020000000000 0.79980000000000 0.43860000000000 0.20020000000000 0.56140000000000 0.43860000000000 0.43860000000000 0.79980000000000
.20020000000000

0.43860000000000

.79980000000000

20020000000000 0.56140000000000

Pyrochlore Iridate $\left(\mathrm{Eu}_{2} \mathrm{Ir}_{2} \mathrm{O}_{7}\right):$ A2B2C7_cF88_227_c_d_af - CIF

\# CIF fil

data findsym-output

audit_creation method FINDSYM

chemical_name_mineral 'Pyrochlore Iradate

chemical_formula_sum 'Eu2 Ir2 O7

loop

publ_author_name

'H. Sagayama'

'D. Uematsu

T. Arima'

'K. Sugimoto

'J. J. Ishikawa

E. O\'Farrell

S. Nakatsuji

journal_name_full_name

Physical Review B

journal_volume 87

journal_year 2013

journal_page_first 100403

journal_page_last 100403

-publ_Section_title

Determination of long-range all-in-all-out ordering of $\operatorname{Ir} \$ \wedge\{4+\} \$$ $\hookrightarrow$ moments in a pyrochlore iridate Eu\$_ $\{2\} \$ \operatorname{Ir} \$ \$_{-}\{2\} \$ O \$_{-}\{7\} \$$ by $\hookrightarrow$ resonant $x$-ray diffraction

\# Found in Magnetic Excitations across the Metal-Insulator Transition in $\hookrightarrow$ the Pyrochlore Iridate Eu\$ $\{2\} \$ I r \$\{2\} \$ O \$\{\} \$, 2018$

aflow title 'Pyrochlore Iridate (Eu\$ $\{2\} \$ I r \$\{2\} \$ 0 \$\{7\}$ ) Structure aflow_proto 'A2B2C7_cF88_227_c_d_af

aflow_params 'a, $x_{-}\{4\}$,

aflow_params_values $, 10.2663,0.4157$

aflow_Strukturbericht 'None'

aflow_Pearson 'cF88'

symmetry_space_group_name_H-M " $\mathrm{F}$ d $-3 \mathrm{~m}: 2$

symmetry_Int_Tables_number 227

cell_length_b $\quad 10.26630$

cell_length_c $\quad 10.26630$

cell_angle_alpha 90.00000

cell angle beta 90.00000

cell angle gamma 90.00000

loop

space_group_symop_i

-space_group_symop_operation_xy

$\mathrm{x}, \mathrm{y}, \mathrm{z}$

$\mathrm{x},-\mathrm{y}+1 / 4,-\mathrm{z}+1 / 4$

$3-x+1 / 4, y,-z+1 / 4$

$4-x+1 / 4,-y+1 / 4, z$

$5 \mathrm{y}, \mathrm{z}, \mathrm{x}$

$\mathrm{y},-\mathrm{z}+1 / 4,-\mathrm{x}+1 / 4$

$7-y+1 / 4, z,-x+1 / 4$

$8-y+1 / 4,-z+1 / 4, x$

$9 \mathrm{z}, \mathrm{x}, \mathrm{y}$

$10 \mathrm{z},-\mathrm{x}+1 / 4,-\mathrm{y}+1 / 4$

$11-z+1 / 4, x,-y+1 / 4$

$12-z+1 / 4,-x+1 / 4, y$

$13-y,-x,-z$

$14-\mathrm{y}, \mathrm{x}+1 / 4, \mathrm{z}+1 / 4$

$15 \mathrm{y}+1 / 4,-\mathrm{x}, \mathrm{z}+1 / 4$

$16 \mathrm{y}+1 / 4, \mathrm{x}+1 / 4,-\mathrm{z}$

$17-\mathrm{x},-\mathrm{z},-\mathrm{y}$

$18-\mathrm{x}, \mathrm{z}+1 / 4, \mathrm{y}+1 / 4$

$19 \mathrm{x}+1 / 4,-\mathrm{z}, \mathrm{y}+1 / 4$

$20 \mathrm{x}+1 / 4, \mathrm{z}+1 / 4,-\mathrm{y}$

$21-\mathrm{z},-\mathrm{y},-\mathrm{x}$

$22-\mathrm{z}, \mathrm{y}+1 / 4, \mathrm{x}+1 / 4$

$23 \mathrm{z}+1 / 4,-\mathrm{y}, \mathrm{x}+1 / 4$

$24 \mathrm{z}+1 / 4, \mathrm{y}+1 / 4,-\mathrm{x}$

$25-\mathrm{x},-\mathrm{y},-\mathrm{z}$

$26-\mathrm{x}, \mathrm{y}+1 / 4, \mathrm{z}+1 / 4$

$27 x+1 / 4,-y, z+1 / 4$

$28 x+1 / 4,-y, z+1 / 4$

$28 \mathrm{x}+1 / 4, \mathrm{y}+1 / 4$

$29-y,-z,-x$
$30-y, z+1 / 4, x+1 / 4$

$30-y, z+1 / 4, x+1 / 4$
$31 y+1 / 4,-z, x+1 / 4$

$32 \mathrm{y}+1 / 4, \mathrm{z}+1 / 4,-\mathrm{x}$

$33-\mathrm{z},-\mathrm{x},-\mathrm{y}$

$34-\mathrm{z}, \mathrm{x}+1 / 4, \mathrm{y}+1 / 4$

$35 \mathrm{z}+1 / 4,-\mathrm{x}, \mathrm{y}+1 / 4$

$36 \mathrm{z}+1 / 4, \mathrm{x}+1 / 4,-\mathrm{y}$

$37 \mathrm{y}, \mathrm{x}, \mathrm{z}$

$38 \mathrm{y},-\mathrm{x}+1 / 4,-\mathrm{z}+1 / 4$

$39-\mathrm{y}+1 / 4, \mathrm{x},-\mathrm{z}+1 / 4$

$40-y+1 / 4,-x+1 / 4, z$

$41 \mathrm{x}, \mathrm{z}, \mathrm{y}$

$42 \mathrm{x},-\mathrm{z}+1 / 4,-\mathrm{y}+1 / 4$

$43-x+1 / 4, z,-y+1 / 4$

$44-x+1 / 4,-z+1 / 4, y$

$44-\mathrm{x}+1 / 4,-\mathrm{z}+1 / 4, \mathrm{y}$
$45 \mathrm{z}, \mathrm{y}, \mathrm{x}$ 
$46 \mathrm{z},-\mathrm{y}+1 / 4,-\mathrm{x}+1 / 4$ $47-z+1 / 4, y,-x+1 / 4$ $48-\mathrm{z}+1 / 4,-\mathrm{y}+1 / 4, \mathrm{x}$ $49 \mathrm{x}, \mathrm{y}+1 / 2, \mathrm{z}+1 / 2$ $50 \mathrm{x},-\mathrm{y}+3 / 4,-\mathrm{z}+3 / 4$ $51-\mathrm{x}+1 / 4, \mathrm{y}+1 / 2,-\mathrm{z}+3 / 4$ $52-x+1 / 4,-y+3 / 4, z+1 / 2$ $53 \mathrm{y}, \mathrm{z}+1 / 2, \mathrm{x}+1 / 2$ $4 \mathrm{y},-\mathrm{z}+3 / 4,-\mathrm{x}+3 / 4$ $55-\mathrm{y}+1 / 4, \mathrm{z}+1 / 2,-\mathrm{x}+3 / 4$ $56-\mathrm{y}+1 / 4,-\mathrm{z}+3 / 4, \mathrm{x}+1 / 2$ $57 \mathrm{z}, \mathrm{x}+1 / 2, \mathrm{y}+1 / 2$ $58 \mathrm{z},-\mathrm{x}+3 / 4,-\mathrm{y}+3 / 4$ $59-\mathrm{z}+1 / 4, \mathrm{x}+1 / 2,-\mathrm{y}+3 / 4$ $60-z+1 / 4, x+1 / 2,-y+3 / 4, y+1 / 4$ $61-y,-x+1 / 2,-z+1 / 2$ $62-y, x+3 / 4, z+3 / 4$ $63 \mathrm{y}+1 / 4,-\mathrm{x}+1 / 2, \mathrm{z}+3 / 4$ $64 \mathrm{y}+1 / 4, \mathrm{x}+3 / 4,-\mathrm{z}+1 / 2$ $65-\mathrm{x},-\mathrm{z}+1 / 2,-\mathrm{y}+1 / 2$ $66-x, z+3 / 4, y+3 / 4$ $67 \mathrm{x}+1 / 4,-\mathrm{z}+1 / 2, \mathrm{y}+3 / 4$ $68 \mathrm{x}+1 / 4, \mathrm{z}+3 / 4,-\mathrm{y}+1 / 2$ $69-\mathrm{z},-\mathrm{y}+1 / 2,-\mathrm{x}+1 / 2$ $70-\mathrm{z}, \mathrm{y}+3 / 4, \mathrm{x}+3 / 4$ $71 \mathrm{z}+1 / 4,-\mathrm{y}+1 / 2, \mathrm{x}+3 / 4$ $72 \mathrm{z}+1 / 4, \mathrm{y}+3 / 4,-\mathrm{x}+1 / 2$ $73-x,-y+1 / 2,-z+1 / 2$ $75 \mathrm{x}+1 / 4,-\mathrm{y}+1 / 2, \mathrm{z}+3 / 4$ $76 \mathrm{x}+1 / 4, \mathrm{y}+3 / 4,-\mathrm{z}+1 / 2$ $76 \mathrm{x}+1 / 4, \mathrm{y}+3 / 4,-\mathrm{z}+1 / 2$
$77-\mathrm{y},-\mathrm{z}+1 / 2,-\mathrm{x}+1 / 2$ $77-\mathrm{x},-\mathrm{z}+1 / 2,-\mathrm{x}+1 / 1 / 2$
$78-\mathrm{y}, \mathrm{z}+3 / 4, \mathrm{x}+3 / 4$ $79 \mathrm{y}+1 / 4,-\mathrm{z}+1 / 2, \mathrm{x}+3 / 4$ $80 y+1 / 4, z+3 / 4,-x+1 / 2$ $81-z,-x+1 / 2,-y+1 / 2$ $82-\mathrm{z}, \mathrm{x}+3 / 4, \mathrm{y}+3 / 4$ $83 \mathrm{z}+1 / 4,-\mathrm{x}+1 / 2, \mathrm{y}+3 / 4$ $84 \mathrm{z}+1 / 4, \mathrm{x}+3 / 4,-\mathrm{y}+1 / 2$ $85 \mathrm{y}, \mathrm{x}+1 / 2, \mathrm{z}+1 / 2$ $86 \mathrm{y},-\mathrm{x}+3 / 4,-\mathrm{z}+3 / 4$ $87-y+1 / 4, x+1 / 2,-z+3 / 4$
$88-y+1 / 4,-x+3 / 4, z+1 / 2$ $89 x, z+1 / 2, y+1 / 2$ $90 x,-z+3 / 4,-y+3 / 4$ $91-x+1 / 4, z+1 / 2,-y+3 / 4$ $92-\mathrm{x}+1 / 4,-\mathrm{z}+3 / 4, \mathrm{y}+1 / 2$ $93 \mathrm{z}, \mathrm{y}+1 / 2, \mathrm{x}+1 / 2$ $93 \mathrm{z}, \mathrm{y}+1 / 2, \mathrm{x}+1 / 2$ $94 \mathrm{z},-\mathrm{y}+3 / 4,-\mathrm{x}+3 / 4$
$95-\mathrm{z}+1 / 4, \mathrm{y}+1 / 2,-\mathrm{x}+3 / 4$ $95-\mathrm{z}+1 / 4, \mathrm{y}+1 / 2,-\mathrm{x}+3 / 4$
$96-\mathrm{z}+1 / 4,-\mathrm{y}+3 / 4, \mathrm{x}+1 / 2$ $96-z+1 / 4,-y+3 / 4, x+$ $98 \mathrm{x}+1 / 2,-\mathrm{y}+1 / 4,-\mathrm{z}+3 / 4$ $98 \mathrm{x}+1 / 2,-\mathrm{y}+1 / 4,-\mathrm{z}+3 / 2$
$99-\mathrm{x}+3 / 4, \mathrm{y},-\mathrm{z}+3 / 4$ $100-x+3 / 4,-y+1 / 4, z+1 / 2$ $101 \mathrm{y}+1 / 2, \mathrm{z}, \mathrm{x}+1 / 2$ $102 \mathrm{y}+1 / 2,-\mathrm{z}+1 / 4,-\mathrm{x}+3 / 4$ $103-y+3 / 4, z,-x+3 / 4$ $104-\mathrm{y}+3 / 4,-\mathrm{z}+1 / 4, \mathrm{x}+1 / 2$ $105 \mathrm{z}+1 / 2, \mathrm{x}, \mathrm{y}+1 / 2$ $106 \mathrm{z}+1 / 2,-\mathrm{x}+1 / 4,-\mathrm{y}+3 / 4$ $107-z+3 / 4, x,-y+3 / 4$ $108-\mathrm{z}+3 / 4,-\mathrm{x}+1 / 4, \mathrm{y}+1 / 2$ $109-\mathrm{y}+1 / 2,-\mathrm{x},-\mathrm{z}+1 / 2$ $110-y+1 / 2, x+1 / 4, z+3 / 4$ $110-y+1 / 2, x+1 / 4, z+3$ $112 \mathrm{y}+3 / 4, \mathrm{x}+1 / 4,-\mathrm{z}+1 / 2$ $113-x+1 / 2,-z,-y+1 / 2$ $114-x+1 / 2, z+1 / 4, y+3 / 4$ $115 x+3 / 4,-z, y+3 / 4$ $116 \mathrm{x}+3 / 4, \mathrm{z}+1 / 4,-\mathrm{y}+1 / 2$ $117-\mathrm{z}+1 / 2,-\mathrm{y},-\mathrm{x}+1 / 2$ $118-\mathrm{z}+1 / 2, \mathrm{y}+1 / 4, \mathrm{x}+3 / 4$ $119 \mathrm{z}+3 / 4,-y, x+3 / 4$ $120 \mathrm{z}+3 / 4, \mathrm{y}+1 / 4,-\mathrm{x}+1 / 2$ $121-x+1 / 2,-y,-z+1 / 2$ $122-x+1 / 2, y+1 / 4, z+3 / 4$ $123 x+3 / 4,-y, z+3 / 4$ $124 \mathrm{x}+3 / 4, \mathrm{y}+1 / 4,-\mathrm{z}+1 / 2$ $125-\mathrm{y}+1 / 2,-\mathrm{z},-\mathrm{x}+1 / 2$ $126-\mathrm{y}+1 / 2, \mathrm{z}+1 / 4, \mathrm{x}+3 / 4$ $127 \mathrm{y}+3 / 4,-\mathrm{z}, \mathrm{x}+3 / 4$ $128 \mathrm{y}+3 / 4, \mathrm{z}+1 / 4,-\mathrm{x}+1 / 2$ $128 \mathrm{y}+3 / 4, \mathrm{z}+1 / 4,-\mathrm{x}+1 / 2$
$129-\mathrm{z}+1 / 2,-\mathrm{x},-\mathrm{y}+1 / 2$ $129-\mathrm{z}+1 / 2,-\mathrm{x},-\mathrm{y}+1 / 2$
$130-\mathrm{z}+1 / 2, \mathrm{x}+1 / 4, \mathrm{y}+3 / 4$ $130-\mathrm{z}+1 / 2, \mathrm{x}+1 / 4, \mathrm{y}+3 / 4$ $131 \mathrm{z}+3 / 4,-\mathrm{x}, \mathrm{y}+3 / 4$
$132 \mathrm{z}+3 / 4, \mathrm{x}+1 / 4,-\mathrm{y}+1 / 2$ $132 \mathrm{z}+3 / 4, \mathrm{x}+1 / 4,-\mathrm{y}+1$
$133 \mathrm{y}+1 / 2, \mathrm{x}, \mathrm{z}+1 / 2$ $134 \mathrm{y}+1 / 2,-\mathrm{x}+1 / 4,-\mathrm{z}+3 / 4$ $135-\mathrm{y}+3 / 4, \mathrm{x},-\mathrm{z}+3 / 4$ $136-\mathrm{y}+3 / 4,-\mathrm{x}+1 / 4, \mathrm{z}+1 / 2$ $137 \mathrm{x}+1 / 2, \mathrm{z}, \mathrm{y}+1 / 2$ $138 x+1 / 2,-z+1 / 4,-y+3 / 4$ $139-x+3 / 4, z,-y+3 / 4$ $140-\mathrm{x}+3 / 4,-\mathrm{z}+1 / 4, \mathrm{y}+1 / 2$ $141 \mathrm{z}+1 / 2, \mathrm{y}, \mathrm{x}+1 / 2$

$142 \mathrm{z}+1 / 2,-\mathrm{y}+1 / 4,-\mathrm{x}+3 / 4$ $142 \mathrm{z}+1 / 2,-\mathrm{y}+1 / 4,-\mathrm{x}+3 /$
$143-\mathrm{z}+3 / 4, \mathrm{y},-\mathrm{x}+3 / 4$ $144-z+3 / 4,-y+1 / 4, x+1 / 2$ $144-z+3 / 4,-y+1 / 4, x+$
$145 x+1 / 2, y+1 / 2, z$ $146 \mathrm{x}+1 / 2,-\mathrm{y}+3 / 4,-\mathrm{z}+1 / 4$ $146 x+1 / 2,-y+3 / 4,-z+1 / 4$
$147-x+3 / 4, y+1 / 2,-z+1 / 4$ $148-x+3 / 4,-y+3 / 4, z$ $149 \mathrm{y}+1 / 2, \mathrm{z}+1 / 2, \mathrm{x}$

$150 \mathrm{y}+1 / 2,-\mathrm{z}+3 / 4,-\mathrm{x}+1 / 4$

$151-\mathrm{y}+3 / 4, \mathrm{z}+1 / 2,-\mathrm{x}+1 / 4$

$152-y+3 / 4,-z+3 / 4, x$

$153 \mathrm{z}+1 / 2, \mathrm{x}+1 / 2, \mathrm{y}$

$154 \mathrm{z}+1 / 2,-x+3 / 4,-y+1 / 4$

$155-\mathrm{z}+3 / 4, \mathrm{x}+1 / 2,-\mathrm{y}+1 / 4$

$156-\mathrm{z}+3 / 4,-\mathrm{x}+3 / 4, \mathrm{y}$

$157-\mathrm{y}+1 / 2,-\mathrm{x}+1 / 2,-\mathrm{z}$

$158-y+1 / 2, x+3 / 4, z+1 / 4$

$159 \mathrm{y}+3 / 4,-x+1 / 2, z+1 / 4$

$161-x+1 / 2,-z+1 / 2,-y$

$162-x+1 / 2, z+3 / 4, y+1 / 4$

$163 \mathrm{x}+3 / 4,-\mathrm{z}+1 / 2, \mathrm{y}+1 / 4$

$164 \mathrm{x}+3 / 4, \mathrm{z}+3 / 4,-\mathrm{y}$

$165-z+1 / 2,-y+1 / 2,-x$

$166-\mathrm{z}+1 / 2, \mathrm{y}+3 / 4, \mathrm{x}+1 / 4$

$167 \mathrm{z}+3 / 4,-y+1 / 2, x+1 / 4$

$168 \mathrm{z}+3 / 4, y+3 / 4,-x$

$169-x+1 / 2,-y+1 / 2,-z$
$170-x+1 / 2, y+3 / 4, z+1 / 4$

$171 \mathrm{x}+3 / 4,-\mathrm{y}+1 / 2, \mathrm{z}+1 / 4$

$172 \mathrm{x}+3 / 4, \mathrm{y}+3 / 4,-\mathrm{z}$

$173-\mathrm{y}+1 / 2,-\mathrm{z}+1 / 2,-\mathrm{x}$

$174-\mathrm{y}+1 / 2, \mathrm{z}+3 / 4, \mathrm{x}+1 / 4$

$175 \mathrm{y}+3 / 4,-\mathrm{z}+1 / 2, \mathrm{x}+1 / 4$

$176 \mathrm{y}+3 / 4, \mathrm{z}+3 / 4,-\mathrm{x}$

$177-\mathrm{z}+1 / 2,-\mathrm{x}+1 / 2,-\mathrm{y}$

$178-\mathrm{z}+1 / 2, \mathrm{x}+3 / 4, \mathrm{y}+1 / 4$

$180 \mathrm{z}+3 / 4,-\mathrm{x}+1 / 2, \mathrm{y}+1 / 4$

$180 \mathrm{z}+3 / 4, \mathrm{x}+3 / 4,-\mathrm{y}$

$181 \mathrm{y}+1 / 2, \mathrm{x}+1 / 2, \mathrm{z}$
$182 \mathrm{y}+1 / 2,-\mathrm{x}+3 / 4,-\mathrm{z}+1 / 4$

$182 \mathrm{y}+1 / 2,-\mathrm{x}+3 / 4,-\mathrm{z}+1 / 4$
$183-\mathrm{y}+3 / 4, x+1 / 2,-\mathrm{z}+1 / 4$

$183-\mathrm{y}+3 / 4, \mathrm{x}+1 / 2,-\mathrm{z}+1 / 4$
$184-\mathrm{y}+3 / 4,-\mathrm{x}+3 / 4, \mathrm{z}$

$184-\mathrm{y}+3 / 4,-\mathrm{x}+3 / 4$,
$185 \mathrm{x}+1 / 2, \mathrm{z}+1 / 2, \mathrm{y}$

$186 \mathrm{x}+1 / 2,-\mathrm{z}+3 / 4,-\mathrm{y}+1 / 4$

$187-x+3 / 4, z+1 / 2,-y+1 / 4$

$188-\mathrm{x}+3 / 4,-\mathrm{z}+3 / 4, \mathrm{y}$

$189 \mathrm{z}+1 / 2, \mathrm{y}+1 / 2, \mathrm{x}$

$190 \mathrm{z}+1 / 2,-\mathrm{y}+3 / 4,-\mathrm{x}+1 / 4$

$191-\mathrm{z}+3 / 4, \mathrm{y}+1 / 2,-\mathrm{x}+1 / 4$

$192-\mathrm{z}+3 / 4,-\mathrm{y}+3 / 4, \mathrm{x}$

loop

atom site label

atom_site_type_symbol

atom_site_symmetry_multiplicity

atom_site_Wyckoff_label

atom_site_fract_x

atom_site_fract_y

atom_site_fract_z

-atom_site_occupancy

$\begin{array}{llll}2500 & 0.12500 & 0.12500 & 1.00000\end{array}$

$\begin{array}{llllllll}\text { Eu1 Eu } & 16 & \text { c } & 0.00000 & 0.00000 & 0.00000 & 1.00000\end{array}$

$\begin{array}{lllllllll}\mathrm{Ir} 1 & \mathrm{Ir} & 16 & \mathrm{~d} & 0.50000 & 0.50000 & 0.50000 & 1.00000\end{array}$

$\begin{array}{lllllllll}\mathrm{O} 2 & \mathrm{O} & 48 & \mathrm{f} & 0.41570 & 0.12500 & 0.12500 & 1.00000\end{array}$

Pyrochlore Iridate $\left(\mathrm{Eu}_{2} \mathrm{Ir}_{2} \mathrm{O}_{7}\right)$ : A2B2C7_cF88_227_c_d_af - POSCAR

A2B2C7_cF88_227_c_d_af \& a, x4 --params $=10.2663,0.4157 \&$ Fd-3m O_ $\{\mathrm{h}\}^{\wedge}\{7\}$ $\hookrightarrow$ \#227 (acdf) \& cF88 \& None \& Eu2Ir2O7 \& Pyrochlore Iradate \& H. $\hookrightarrow$ Sagayama et

1.00000000000000

$0.00000000000000 \quad 5.13315000000000 \quad 5.13315000000000$

$\begin{array}{lll}5.13315000000000 & 0.00000000000000 & 5.13315000000000\end{array}$

$\begin{array}{llll}5.13315000000000 & 5.13315000000000 & 0.00000000000000\end{array}$

$\begin{array}{rrr}\mathrm{Eu} & \mathrm{Ir} & \mathrm{O} \\ 4 & 4 & 14\end{array}$

Direct

0.00000000000000

0.00000000000000

0.5000000000000

0.50000000000000

0.50000000000000

0.500000000000

0.5000000000000

0.00000000000000

0.12500000000000

0.87500000000000

$-0.16570000000000$

0.41570000000000

0.41570000000000

$-0.16570000000000$

0.41570000000000

$-0.16570000000000$

1.16570000000000

$-0.41570000000000$

$-0.41570000000000$

1.16570000000000

-0.41570000000000
1.16570000000000

0.00000000000000 0.00000000000000 0.50000000000000 0.00000000000000 0.50000000000000 0.50000000000000 0.00000000000000 0.50000000000000 0.12500000000000 0.87500000000000 0.41570000000000 $-0.16570000000000$ 0.16570000000000 0.41570000000000 0.41570000000000 $-0.16570000000000$ $-0.41570000000000$ 1.16570000000000 1.16570000000000 $-0.41570000000000$ $-0.41570000000000$ 1.16570000000000

0.00000000000000 0.50000000000000 0.00000000000000 0.00000000000000 0.50000000000000 0.00000000000000 0.50000000000000 0.50000000000000 0.12500000000000 0.87500000000000 0.41570000000000 0.16570000000000 $-0.16570000000000$ $-0.16570000000000$ $-0.16570000000000$ 0.41570000000000 1.16570000000000 $-0.41570000000000$ 1.16570000000000 $-0.41570000000000$ 1.1657000000000 $-0.41570000000000$

$\mathrm{Eu} \quad(16 \mathrm{c})$ Eu $(16 c)$ Eu $(16 c)$ Eu $\quad(16 c)$ $\mathrm{Ir} \quad(16 \mathrm{~d})$ Ir $(16 d)$ Ir $(16 d)$ Ir (16d) O $(8 \mathrm{a})$ O $(8 \mathrm{a})$ $\mathrm{O} \quad(48 \mathrm{f})$ $\mathrm{O}(48 \mathrm{f})$ O (48f) $\mathrm{O} \quad(48 \mathrm{f})$ O (48f) O (48f) $\mathrm{O} \quad(48 \mathrm{f})$ O (48f) $\mathrm{O} \quad(48 \mathrm{f})$ $\begin{array}{ll}\mathrm{O} & (48 \mathrm{f}) \\ \mathrm{O} & (48 \mathrm{f})\end{array}$ $\mathrm{O} \quad(48 \mathrm{f})$ $\mathrm{O} \quad(48 \mathrm{f})$

Spinel $\left(\mathrm{Co}_{3} \mathrm{O}_{4}, D 7_{2}\right)$ : A3B4_cF56_227_ad_e - CIF

\# CIF file

data findsym-output

audit_creation_method FINDSYM

chemical name mineral 'Spinel,

chemical formula_sum ' $\mathrm{Co} 3 \mathrm{O} 4$ '

loop

publ_author_nam

O. Knop'

K. I. G. Reid' 
Chalkogenides of the transition elements. VI. X-Ray, neutron, an $\hookrightarrow$ magnetic investigation of the spinels $\operatorname{Co} \$\{3\}$ O $\$ 4\} \$, N i C o \$$ $\hookrightarrow 2\} \$ O \$_{-}\{4\} \$, \operatorname{Co} \$\{3\} \$ S \$_{-}\{4\} \$$, and NiCo\$_ $\{2\} \$ S \$ \$_{-}\{4\} \$$

aflow_title 'Spinel $\left(\operatorname{Co} \$_{-}\{3\} \$ O \$_{-}\{4\} \$, \$ D 7_{-}\{2\} \$\right)$ Structure,

aflow_proto 'A3B4_cF56_227_ad_e

aflow_params 'a, $x_{-}\{3\}$ '

aflow_params_values $8.0835,0.2642$

aflow_Strukturbericht '\$D7_\{2\}\$

aflow_Pearson 'cF56,

_symmetry_space_group_name_H-M "F d $-3 \mathrm{m:2"}$

_symmetry_Int_Tables_number 227

_cell_length_a $\quad 8.08350$

$\begin{array}{ll}\text { _cell_length_b } & 8.08350 \\ \text { _cell_length_c } & 8.08350\end{array}$

-cell_length_c 8.08350

cell_angle_alpha 90.00000

$\begin{array}{ll}\text { cell_angle_beta } & 90.00000 \\ \text { cell_angle_gamma } & 90.00000\end{array}$

loop

_space_group_symop_id

_space_group_symop_operation_xyz

$1 \mathrm{x}, \mathrm{y}, \mathrm{z}$

$2 \mathrm{x},-\mathrm{y}+1 / 4,-\mathrm{z}+1 / 4$

$3-x+1 / 4, y,-z+1 / 4$

$4-x+1 / 4,-y+1 / 4, z$

$5 \mathrm{y}, \mathrm{z}, \mathrm{x}$

$6 \mathrm{y},-\mathrm{z}+1 / 4,-\mathrm{x}+1 / 4$

$7-y+1 / 4, z,-x+1 / 4$

$8-y+1 / 4,-z+1 / 4, x$

$\begin{array}{ll}8 & -\mathrm{y}+1 / 4 \\ 9 & \mathrm{z}, \mathrm{x}, \mathrm{y}\end{array}$

$10 \mathrm{z},-\mathrm{x}+1 / 4,-\mathrm{y}+1 / 4$

$11-z+1 / 4, x,-y+1 / 4$

$11-z+1 / 4, x,-y+1 / 4$
$12-z+1 / 4,-x+1 / 4, y$

$13-\mathrm{y},-\mathrm{x},-\mathrm{z}$

$14-\mathrm{y}, \mathrm{x}+1 / 4, \mathrm{z}+1 / 4$

$15 \mathrm{y}+1 / 4,-\mathrm{x}, \mathrm{z}+1 / 4$

$16 \mathrm{y}+1 / 4, \mathrm{x}+1 / 4,-\mathrm{z}$

$17-x,-z,-y$

$18-\mathrm{x}, \mathrm{z}+1 / 4, \mathrm{y}+1 / 4$

$19 \mathrm{x}+1 / 4,-\mathrm{z}, \mathrm{y}+1 / 4$

$20 \mathrm{x}+1 / 4, \mathrm{z}+1 / 4,-\mathrm{y}$

$21-\mathrm{z},-\mathrm{y},-\mathrm{x}$

$22-\mathrm{z}, \mathrm{y}+1 / 4, \mathrm{x}+1 / 4$

$23 \mathrm{z}+1 / 4,-\mathrm{y}, \mathrm{x}+1 / 4$

$24 \mathrm{z}+1 / 4, \mathrm{y}+1 / 4,-\mathrm{x}$

$25-\mathrm{x},-\mathrm{y},-\mathrm{z}$

$26-\mathrm{x}, \mathrm{y}+1 / 4, \mathrm{z}+1 / 4$

$27 \mathrm{x}+1 / 4,-\mathrm{y}, \mathrm{z}+1 / 4$

$28 \mathrm{x}+1 / 4, \mathrm{y}+1 / 4,-\mathrm{z}$

$29-y,-z,-x$

$30-y, z+1 / 4, x+1 / 4$

$31 \mathrm{y}+1 / 4,-\mathrm{z}, \mathrm{x}+1 / 4$

$32 \mathrm{y}+1 / 4, \mathrm{z}+1 / 4,-\mathrm{x}$

$33-z,-x,-y$

$34-\mathrm{z}, \mathrm{x}+1 / 4, \mathrm{y}+1 / 4$

$35 \mathrm{z}+1 / 4,-\mathrm{x}, \mathrm{y}+1 / 4$

$36 \mathrm{z}+1 / 4, \mathrm{x}+1 / 4,-\mathrm{y}$

$37 \mathrm{y}, \mathrm{x}, \mathrm{z}$

$38 \mathrm{y},-\mathrm{x}+1 / 4,-\mathrm{z}+1 / 4$

$39-\mathrm{y}+1 / 4, \mathrm{x},-\mathrm{z}+1 / 4$

$40-y+1 / 4,-x+1 / 4, z$

$41 \mathrm{x}, \mathrm{z}, \mathrm{y}$

$42 \mathrm{x},-\mathrm{z}+1 / 4,-\mathrm{y}+1 / 4$

$43-x+1 / 4, z,-y+1 / 4$

$44-\mathrm{x}+1 / 4,-\mathrm{z}+1 / 4, \mathrm{y}$

$45 \mathrm{z}, \mathrm{y}, \mathrm{x}$

$46 \mathrm{z},-\mathrm{y}+1 / 4,-\mathrm{x}+1 / 4$

$47-\mathrm{z}+1 / 4, \mathrm{y},-\mathrm{x}+1 / 4$

$48-z+1 / 4,-y+1 / 4, x$

$49 x, y+1 / 2, z+1 / 2$

$50 x,-y+3 / 4,-z+3 / 4$

$51-\mathrm{x}+1 / 4, \mathrm{y}+1 / 2,-\mathrm{z}+3 / 4$

$52-\mathrm{x}+1 / 4,-\mathrm{y}+3 / 4, \mathrm{z}+1 / 2$

$53 \mathrm{y}, \mathrm{z}+1 / 2, \mathrm{x}+1 / 2$

$54 \mathrm{y},-\mathrm{z}+3 / 4,-\mathrm{x}+3 / 4$

$55-\mathrm{y}+1 / 4, \mathrm{z}+1 / 2,-\mathrm{x}+3 / 4$

$56-\mathrm{y}+1 / 4,-\mathrm{z}+3 / 4, \mathrm{x}+1 / 2$

$57 \mathrm{z}, \mathrm{x}+1 / 2, \mathrm{y}+1 / 2$

$58 \mathrm{z},-\mathrm{x}+3 / 4,-\mathrm{y}+3 / 4$

$59-\mathrm{z}+1 / 4, \mathrm{x}+1 / 2,-\mathrm{y}+3 / 4$

$60-\mathrm{z}+1 / 4,-\mathrm{x}+3 / 4, \mathrm{y}+1 / 2$

$61-\mathrm{y},-\mathrm{x}+1 / 2,-\mathrm{z}+1 / 2$

$61-y,-x+1 / 2,-z+1 / 2$

$63 \mathrm{y}+1 / 4,-x+1 / 2, z+3 / 4$

$63 \mathrm{y}+1 / 4,-\mathrm{x}+1 / 2, \mathrm{z}+3 / 4$

$64 \mathrm{y}+1 / 4, \mathrm{x}+3 / 4,-\mathrm{z}+1 / 2$
$65-\mathrm{x},-\mathrm{z}+1 / 2,-\mathrm{y}+1 / 2$

$65-\mathrm{x},-\mathrm{z}+1 / 2,-\mathrm{y}+1 / 2$
$66-\mathrm{x}, \mathrm{z}+3 / 4, \mathrm{y}+3 / 4$

$66-x, z+3 / 4, y+3 / 4$
$67 x+1 / 4,-z+1 / 2, y+3 / 4$
$68 x+1 / 4, z+3 / 4,-y+1 / 2$

$67 \mathrm{x}+1 / 4,-\mathrm{z}+1 / 2, \mathrm{y}+3 / 4$
$68 \mathrm{x}+1 / 4, \mathrm{z}+3 / 4,-\mathrm{y}+1 / 2$ $69-\mathrm{z},-\mathrm{y}+1 / 2,-\mathrm{x}+1 / 2$

$70-\mathrm{z}, \mathrm{y}+3 / 4, \mathrm{x}+3 /$

$71 \mathrm{z}+1 / 4,-\mathrm{y}+1 / 2, \mathrm{x}+3 / 4$

$72 \mathrm{z}+1 / 4, \mathrm{y}+3 / 4,-\mathrm{x}+1 / 2$

$73-x,-y+1 / 2,-z+1 / 2$

$74-x, y+3 / 4, z+3 / 4$

$75 \mathrm{x}+1 / 4,-\mathrm{y}+1 / 2, \mathrm{z}+3 / 4$

$76 \mathrm{x}+1 / 4, \mathrm{y}+3 / 4,-\mathrm{z}+1 / 2$

$77-\mathrm{y},-\mathrm{z}+1 / 2,-\mathrm{x}+1 / 2$

$78-\mathrm{y}, \mathrm{z}+3 / 4, \mathrm{x}+3 / 4$

$79 \mathrm{y}+1 / 4,-\mathrm{z}+1 / 2, \mathrm{x}+3 / 4$

$80 \mathrm{y}+1 / 4, \mathrm{z}+3 / 4,-\mathrm{x}+1 / 2$

$81-z,-x+1 / 2,-y+1 / 2$

$81-\mathrm{z},-\mathrm{x}+1 / 2,-\mathrm{y}+1 / 2$
$82-\mathrm{z}, \mathrm{x}+3 / 4, \mathrm{y}+3 / 4$

$82-\mathrm{z}, \mathrm{x}+3 / 4, \mathrm{y}+3 / 4$
$83 \mathrm{z}+1 / 4,-\mathrm{x}+1 / 2, \mathrm{y}+3 / 4$

$83 \mathrm{z}+1 / 4,-\mathrm{x}+1 / 2, \mathrm{y}+3 / 4$
$84 \mathrm{z}+1 / 4, \mathrm{x}+3 / 4,-\mathrm{y}+1 / 2$

$84 \mathrm{z}+1 / 4, \mathrm{x}+3 / 4,-\mathrm{y}+1 /$
$85 \mathrm{y}, \mathrm{x}+1 / 2, \mathrm{z}+1 / 2$

$85 \mathrm{y}, \mathrm{x}+1 / 2, \mathrm{z}+1 / 2$
$86 \mathrm{y},-\mathrm{x}+3 / 4,-\mathrm{z}+3 / 4$

$86 \mathrm{y},-\mathrm{x}+3 / 4,-\mathrm{z}+3 / 4$
$87-\mathrm{y}+1 / 4, \mathrm{x}+1 / 2,-\mathrm{z}+3 / 4$

$87-\mathrm{y}+1 / 4, \mathrm{x}+1 / 2,-\mathrm{z}+3 / 4$
$88-\mathrm{y}+1 / 4,-\mathrm{x}+3 / 4, \mathrm{z}+1 / 2$

$89 \mathrm{x}, \mathrm{z}+1 / 2, \mathrm{y}+1 / 2$

$90 \mathrm{x},-\mathrm{z}+3 / 4,-\mathrm{y}+3 / 4$

$91-x+1 / 4, z+1 / 2,-y+3 / 4$

$92-\mathrm{x}+1 / 4,-\mathrm{z}+3 / 4, \mathrm{y}+1 / 2$

$93 \mathrm{z}, \mathrm{y}+1 / 2, \mathrm{x}+1 / 2$

$94 \mathrm{z},-\mathrm{y}+3 / 4,-\mathrm{x}+3 / 4$

$95-\mathrm{z}+1 / 4, \mathrm{y}+1 / 2,-\mathrm{x}+3 / 4$

$96-\mathrm{z}+1 / 4,-\mathrm{y}+3 / 4, \mathrm{x}+1 / 2$

$97 \mathrm{x}+1 / 2, \mathrm{y}, \mathrm{z}+1 / 2$

$98 \mathrm{x}+1 / 2,-\mathrm{y}+1 / 4,-\mathrm{z}+3 / 4$

$98 \mathrm{x}+1 / 2,-\mathrm{y}+1 / 4,-\mathrm{z}+3 /$
$99-\mathrm{x}+3 / 4, \mathrm{y},-\mathrm{z}+3 / 4$

$99-\mathrm{x}+3 / 4, \mathrm{y},-\mathrm{z}+3 / 4$
$100-\mathrm{x}+3 / 4,-\mathrm{y}+1 / 4, \mathrm{z}+1 / 2$

$100-x+3 / 4,-y+1 / 4, z$

$102 \mathrm{y}+1 / 2,-\mathrm{z}+1 / 4,-\mathrm{x}+3 / 4$

$102 \mathrm{y}+1 / 2,-\mathrm{z}+1 / 4,-\mathrm{x}+3 / 4$
$103-\mathrm{y}+3 / 4, \mathrm{z},-\mathrm{x}+3 / 4$

$103-\mathrm{y}+3 / 4, \mathrm{z},-\mathrm{x}+3 / 4$
$104-\mathrm{y}+3 / 4,-\mathrm{z}+1 / 4, \mathrm{x}+1 / 2$

$104-\mathrm{y}+3 / 4,-\mathrm{z}+1 / 4$,
$105 \mathrm{z}+1 / 2, \mathrm{x}, \mathrm{y}+1 / 2$

$105 \mathrm{z}+1 / 2, \mathrm{x}, \mathrm{y}+1 / 2$
$106 \mathrm{z}+1 / 2,-\mathrm{x}+1 / 4,-\mathrm{y}+3 / 4$

$106 \mathrm{z}+1 / 2,-\mathrm{x}+1 / 4,-\mathrm{y}+3 / 4$
$107-\mathrm{z}+3 / 4, \mathrm{x},-\mathrm{y}+3 / 4$

$108-\mathrm{z}+3 / 4,-\mathrm{x}+1 / 4, \mathrm{y}+1 / 2$

$109-\mathrm{y}+1 / 2,-\mathrm{x},-\mathrm{z}+1 / 2$

$110-y+1 / 2, x+1 / 4, z+3 / 4$

$111 \mathrm{y}+3 / 4,-x, z+3 / 4$

$112 \mathrm{y}+3 / 4, \mathrm{x}+1 / 4,-\mathrm{z}+1 / 2$

$113-x+1 / 2,-z,-y+1 / 2$

$114-\mathrm{x}+1 / 2, \mathrm{z}+1 / 4, \mathrm{y}+3 / 4$

$114-\mathrm{x}+1 / 2, \mathrm{z}+1 / 4, \mathrm{y}+3 /$
$115 \mathrm{x}+3 / 4,-\mathrm{z}, \mathrm{y}+3 / 4$

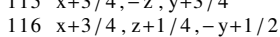

$116 x+3 / 4, z+1 / 4,-y+1 / 2$

$117-z+1 / 2,-y,-x+1 / 2$

$118-z+1 / 2, y+1 / 4, x+3 / 4$

$19 \mathrm{z}+3 / 4,-y, x+3 / 4$

$120 \mathrm{z}+3 / 4, \mathrm{y}+1 / 4,-\mathrm{x}+1 / 2$

$121-x+1 / 2,-y,-z+1 / 2$

$122-x+1 / 2, y+1 / 4, z+3 / 4$

$123 \mathrm{x}+3 / 4,-\mathrm{y}, \mathrm{z}+3 / 4$

$124 \mathrm{x}+3 / 4, y+1 / 4,-\mathrm{z}+1 / 2$

$125-\mathrm{y}+1 / 2,-\mathrm{z},-\mathrm{x}+1 / 2$

$126-\mathrm{y}+1 / 2, \mathrm{z}+1 / 4, \mathrm{x}+3 / 4$

$127 \mathrm{y}+3 / 4,-\mathrm{z}, \mathrm{x}+3 / 4$

$128 \mathrm{y}+3 / 4, \mathrm{z}+1 / 4,-\mathrm{x}+1 / 2$

$129-\mathrm{z}+1 / 2,-\mathrm{x},-\mathrm{y}+1 / 2$

$130-\mathrm{z}+1 / 2, \mathrm{x}+1 / 4, \mathrm{y}+3 / 4$

$131 z+3 / 4,-x, y+3 / 4$

$132 \mathrm{z}+3 / 4, \mathrm{x}+1 / 4,-\mathrm{y}+1 / 2$

$133 \mathrm{z}+1 / 2, x+1 / 4,-y+1$

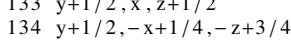

$134 \mathrm{y}+1 / 2,-\mathrm{x}+1 / 4,-\mathrm{z}+3 / 4$

$136-\mathrm{y}+3 / 4,-\mathrm{x}+1 / 4, \mathrm{z}+1 / 2$

$137 x+1 / 2, z, y+1 / 2$
$138 x+1 / 2,-z+1 / 4,-y+3 / 4$

$138 \mathrm{x}+1 / 2,-\mathrm{z}+1 / 4,-\mathrm{y}+3 / 4$
$139-\mathrm{x}+3 / 4, \mathrm{z},-\mathrm{y}+3 / 4$

$139-\mathrm{x}+3 / 4, \mathrm{z},-\mathrm{y}+3 / 4$
$140-\mathrm{x}+3 / 4,-\mathrm{z}+1 / 4, \mathrm{y}+1 / 2$

$141 \mathrm{z}+1 / 2, \mathrm{y}, \mathrm{x}+1 / 2$

$142 \mathrm{z}+1 / 2,-\mathrm{y}+1 / 4,-\mathrm{x}+3 / 4$

$143-z+3 / 4, y,-x+3 / 4$

$144-\mathrm{z}+3 / 4,-\mathrm{y}+1 / 4, \mathrm{x}+1 / 2$

$145 \mathrm{x}+1 / 2, \mathrm{y}+1 / 2, \mathrm{z}$

$146 \mathrm{x}+1 / 2,-\mathrm{y}+3 / 4,-\mathrm{z}+1 / 4$

$147-\mathrm{x}+3 / 4, \mathrm{y}+1 / 2,-\mathrm{z}+1 / 4$

$148-x+3 / 4,-y+3 / 4, z$

$149 \mathrm{y}+1 / 2, \mathrm{z}+1 / 2, \mathrm{x}$

$150 \mathrm{y}+1 / 2,-\mathrm{z}+3 / 4,-\mathrm{x}+1 / 4$

$151-y+3 / 4, z+1 / 2,-x+1 / 4$

$152-\mathrm{y}+3 / 4,-\mathrm{z}+3 / 4, \mathrm{x}$

$153 \mathrm{z}+1 / 2, x+1 / 2, y$

$154 \mathrm{z}+1 / 2,-\mathrm{x}+3 / 4,-\mathrm{y}+1 / 4$

$155-\mathrm{z}+3 / 4, \mathrm{x}+1 / 2,-\mathrm{y}+1 / 4$

$156-\mathrm{z}+3 / 4,-\mathrm{x}+3 / 4, \mathrm{y}$

$157-\mathrm{y}+1 / 2,-\mathrm{x}+1 / 2,-\mathrm{z}$

$158-\mathrm{y}+1 / 2, \mathrm{x}+3 / 4, \mathrm{z}+1 / 4$

$159 \mathrm{y}+3 / 4,-x+1 / 2, z+1 / 4$

$160 \mathrm{y}+3 / 4, \mathrm{x}+3 / 4,-\mathrm{z}$

$161-x+1 / 2,-z+1 / 2,-y$

$162-x+1 / 2, z+3 / 4, y+1 / 4$

$163 \mathrm{x}+3 / 4,-\mathrm{z}+1 / 2, \mathrm{y}+1 / 4$

$164 x+3 / 4, z+3 / 4,-y$

$165 x+z+1 / 2, y+1 / 2, x$

$165-z+1 / 2,-y+1 / 2,-x$

$166-z+1 / 2, y+3 / 4, x+1 / 4$

$167 z+3 / 4,-y+1 / 2, x+1 / 4$

$168 \mathrm{z}+3 / 4, y+3 / 4,-\mathrm{x}$

$169-\mathrm{x}+1 / 2,-\mathrm{y}+1 / 2,-\mathrm{z}$

$170-x+1 / 2, y+3 / 4, z+1 / 4$

$171 \mathrm{x}+3 / 4,-\mathrm{y}+1 / 2, \mathrm{z}+1 / 4$

$172 \mathrm{x}+3 / 4, \mathrm{y}+3 / 4,-\mathrm{z}$

$173-y+1 / 2,-z+1 / 2,-x$ 
$187-x+3 / 4, z+1 / 2,-y+1 / 4$
$188-x+3 / 4,-z+3 / 4, y$

$188-\mathrm{x}+3 / 4,-\mathrm{z}+3 / 4, \mathrm{y}$
$189 \mathrm{z}+1 / 2, \mathrm{y}+1 / 2, \mathrm{x}$

$190 \mathrm{z}+1 / 2,-\mathrm{y}+3 / 4,-\mathrm{x}+1 / 4$

$191-\mathrm{z}+3 / 4, \mathrm{y}+1 / 2,-\mathrm{x}+1 / 4$

$192-z+3 / 4,-y+3 / 4, x$

loop

_atom_site_label

_atom_site_type_symbol

atom_site_symmetry_multiplicity

_atom_site_Wyckoff_label

atom_site_fract_x

_atom_site_fract_y

_atom_site_fract_z

$\begin{array}{llllll}\text { Co1 Co } & \text { _site_occupancy } & & & & \\ \text { Co2 Co } & 0.12500 & 0.12500 & 0.12500 & 1.00000\end{array}$

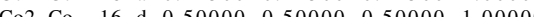

$\begin{array}{lllllllllll}\mathrm{Co} 2 \mathrm{Co} & 16 & \mathrm{~d} & 0.525000 & 0.500120 & 0.50000 & 1.00000\end{array}$

Spinel $\left(\mathrm{Co}_{3} \mathrm{O}_{4}, D 7_{2}\right)$ : A3B4_cF56_227_ad_e - POSCAR

\begin{tabular}{|c|c|c|c|c|}
\hline \\
\hline $\begin{array}{l}\hookrightarrow \text { (ade) \& cF5 } \\
\hookrightarrow \text { J. Chem. } 4\end{array}$ & $3463-3476$ & & & \\
\hline $\begin{array}{l}\hookrightarrow \text { J. Chem. } \\
1.00000000000000\end{array}$ & $3463-3476$ & & & \\
\hline 0.00000000000000 & 4.04175000000000 & 4.04175000000000 & & \\
\hline 4.04175000000000 & 0.00000000000000 & 4.04175000000000 & & \\
\hline 4.04175000000000 & 4.04175000000000 & 0.00000000000000 & & \\
\hline Co & & & & \\
\hline 6 & & & & \\
\hline $\mathrm{ct}$ & & & & \\
\hline 0.12500000000000 & 0.12500000000000 & 00000 & Co & (8a) \\
\hline 0.87500000000000 & 0.87500000000000 & 0.8 & Co & (8a) \\
\hline 0.50000000000000 & 0.50000000000000 & 00000 & Co & (16d) \\
\hline 0.50000000000000 & 0.50000000000000 & 0.00 & Co & $(16 d)$ \\
\hline 0.50000000000000 & 0.00000000000000 & 0.50000000000000 & Co & $(16 \mathrm{~d})$ \\
\hline 0.00000000000000 & 0.5000000 & 0.50 & Co & $(16 \mathrm{~d})$ \\
\hline 0.26420000000000 & 0.26420000000000 & 0.26420000000000 & $\mathrm{O}$ & $(32 \mathrm{e})$ \\
\hline 0.26420000000000 & 0.26420000000000 & -0.292600000000 & $\mathrm{O}$ & $(32 \mathrm{e})$ \\
\hline 0.26420000000000 & -0.29260000000000 & 0.26420 & $\mathrm{O}$ & $(32 \mathrm{e})$ \\
\hline 0.29260000000000 & 0.26420000000000 & 0.26420 & $\mathrm{O}$ & $(32 \mathrm{e})$ \\
\hline-0.26420000000000 & -0.26420000000000 & 1.29260000000000 & $\mathrm{O}$ & $(32 \mathrm{e})$ \\
\hline-0.26420000000000 & -0.26420000000000 & -0.26420000000000 & $\mathrm{O}$ & $(32 \mathrm{e})$ \\
\hline-0.26420000000000 & 1.29260000000000 & -0.26420000000000 & $\mathrm{O}$ & $(32 \mathrm{e})$ \\
\hline 1.29260000000000 & -0.26420000000000 & -0.26420000000000 & $\mathrm{O}$ & $(32 \mathrm{e})$ \\
\hline
\end{tabular}

$\mathrm{CuCrCl}_{5}\left[\mathrm{NH}_{3}\right]_{6}$ : A5BCD6_cF416_228_eg_c_b_h - CIF

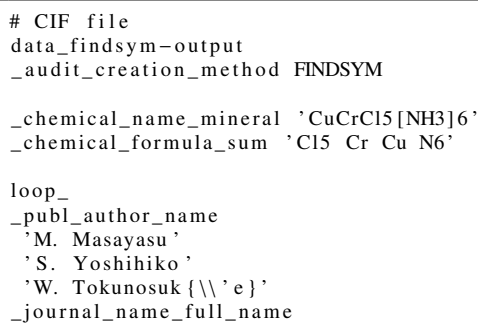


$101 \mathrm{y}+1 / 2, \mathrm{z}, \mathrm{x}+1 / 2$

$102 \mathrm{y}+1 / 2,-\mathrm{z}+1 / 4,-\mathrm{x}+3$

$103-y+3 / 4, z,-x+3 / 4$

$104-\mathrm{y}+3 / 4,-\mathrm{z}+1 / 4, \mathrm{x}+1 / 2$

$105 \mathrm{z}+1 / 2, \mathrm{x}, \mathrm{y}+1 / 2$

$106 \mathrm{z}+1 / 2,-\mathrm{x}+1 / 4,-\mathrm{y}+3 / 4$
$107-\mathrm{z}+3 / 4, \mathrm{x},-\mathrm{y}+3 / 4$

$108-\mathrm{z}+3 / 4,-\mathrm{x}+1 / 4, \mathrm{y}+1 / 2$

$109-\mathrm{y},-\mathrm{x}+1 / 2,-\mathrm{z}$

$110-y, x+1 / 4, z+3 / 4$

$111 \mathrm{y}+3 / 4,-x+1 / 2, z+3 / 4$

$112 y+3 / 4, x+1 / 4,-z$

$113-\mathrm{x},-\mathrm{z}+1 / 2,-\mathrm{y}$
$114-\mathrm{x}, \mathrm{z}+1 / 4, \mathrm{y}+3 / 4$

$114-\mathrm{x}, \mathrm{z}+1 / 4, \mathrm{y}+3 / 4$
$115 \mathrm{x}+3 / 4,-\mathrm{z}+1 / 2, \mathrm{y}+3 / 4$

$116 \mathrm{x}+3 / 4, \mathrm{z}+1 / 4,-\mathrm{y}$

$117-\mathrm{z},-\mathrm{y}+1 / 2,-\mathrm{x}$

$118-\mathrm{z}, \mathrm{y}+1 / 4, \mathrm{x}+3 / 4$
$119 \mathrm{z}+3 / 4,-\mathrm{y}+1 / 2, \mathrm{x}+3 / 4$

$120 \mathrm{z}+3 / 4, \mathrm{y}+1 / 4,-\mathrm{x}$

$121-x+1 / 2,-y,-z+1 / 2$
$122-x+1 / 2, y+1 / 4, z+3 / 4$

$123 \mathrm{x}+3 / 4,-\mathrm{y}, \mathrm{z}+3 / 4$

$124 \mathrm{x}+3 / 4, \mathrm{y}+1 / 4,-\mathrm{z}+1 / 2$

$125-\mathrm{y}+1 / 2,-\mathrm{z},-\mathrm{x}+1 / 2$

$126-\mathrm{y}+1 / 2, \mathrm{z}+1 / 4, \mathrm{x}+3 /$

$128 \mathrm{y}+3 / 4, \mathrm{z}+1 / 4,-\mathrm{x}+1 / 2$

$129-\mathrm{z}+1 / 2,-\mathrm{x},-\mathrm{y}+1 / 2$

$130-z+1 / 2, x+1 / 4, y+3$
$131 z+3 / 4,-x, y+3 / 4$

$\begin{array}{ll}131 & \mathrm{z}+3 / 4,-\mathrm{x}, \mathrm{y}+3 / 4 \\ 132 \mathrm{z}+3 / 4, \mathrm{x}+1 / 4,-\mathrm{y}+1 / 2\end{array}$

$133 \mathrm{y}, \mathrm{x}+1 / 2, \mathrm{z}$

$134 \mathrm{y},-\mathrm{x}+1 / 4,-\mathrm{z}+3 / 4$

$135-\mathrm{y}+3 / 4, \mathrm{x}+1 / 2,-\mathrm{z}+3 / 4$

$136-\mathrm{y}+3 / 4,-\mathrm{x}+1 / 4, \mathrm{z}$

$137 \mathrm{x}, \mathrm{z}+1 / 2, \mathrm{y}$

$138 \mathrm{x},-\mathrm{z}+1 / 4,-\mathrm{y}+3 / 4$

$139-x+3 / 4, z+1 / 2,-y+3 / 4$

$140-x+3 / 4,-z+1 / 4, y$

$141 \mathrm{z}, \mathrm{y}+1 / 2, \mathrm{x}$

$143-\mathrm{z}+3 / 4, \mathrm{y}+1 / 2,-\mathrm{x}+3 / 4$

$144-\mathrm{z}+3 / 4,-\mathrm{y}+1 / 4, \mathrm{x}$

$145 \mathrm{x}+1 / 2, \mathrm{y}+1 / 2, \mathrm{z}$

$146 \mathrm{x}+1 / 2,-\mathrm{y}+3 / 4,-\mathrm{z}+1 / 4$

$147-\mathrm{x}+3 / 4, \mathrm{y}+1 / 2,-\mathrm{z}+1 / 4$

$148-x+3 / 4,-y+3 / 4$

$149 \mathrm{y}+1 / 2, \mathrm{z}+1 / 2, \mathrm{x}$

$150-\mathrm{y}+1 / 2,-\mathrm{z}+3 / 4,-\mathrm{x}+1 / 4$

$151-\mathrm{y}+3 / 4, \mathrm{z}+1 / 2,-\mathrm{x}+1 / 4$

$152-\mathrm{y}+3 / 4,-\mathrm{z}+3 / 4, \mathrm{x}$

$153 \mathrm{z}+1 / 2, \mathrm{x}+1 / 2, \mathrm{y}$

$154 \mathrm{z}+1 / 2,-\mathrm{x}+3 / 4,-\mathrm{y}+1 / 4$

$155-\mathrm{z}+3 / 4, \mathrm{x}+1 / 2,-\mathrm{y}+1 / 4$
$156-\mathrm{z}+3 / 4,-\mathrm{x}+3 / 4, \mathrm{y}$

$157-\mathrm{y},-\mathrm{x},-\mathrm{z}+1 / 2$

$158-\mathrm{y}, \mathrm{x}+3 / 4, \mathrm{z}+1 / 4$

$159 \mathrm{y}+3 / 4,-\mathrm{x}, \mathrm{z}+1 / 4$

$160 \mathrm{y}+3 / 4, \mathrm{x}+3 / 4,-\mathrm{z}+1 / 2$

$161-x,-z,-y+1 / 2$

$162-x, z+3 / 4, y+1 / 4$

$163 \mathrm{x}+3 / 4,-\mathrm{z}, \mathrm{y}+1 / 4$
$164 \mathrm{x}+3 / 4, \mathrm{z}+3 / 4,-\mathrm{y}+1 / 2$

$165-z,-y,-x+1 / 2$

$166-\mathrm{z}, \mathrm{y}+3 / 4, \mathrm{x}+1 / 4$

$167 \mathrm{z}+3 / 4,-y, x+1 / 4$

$168 \mathrm{z}+3 / 4, \mathrm{y}+3 / 4,-\mathrm{x}+1 / 2$

$169-x+1 / 2,-y+1 / 2,-z$

$\begin{array}{ll}170 & -x+1 / 2, y+3 / 4, z+1 / 4 \\ 171 & x+3 / 4,-y+1 / 2, z+1 / 4\end{array}$

$172 \mathrm{x}+3 / 4, y+3 / 4,-$

$173-\mathrm{y}+1 / 2,-\mathrm{z}+1 / 2,-\mathrm{x}$

$174-y+1 / 2, z+3 / 4, x+1 / 4$
$175 y+3 / 4,-z+1 / 2, x+1 / 4$

$176 \mathrm{y}+3 / 4, \mathrm{z}+3 / 4,-\mathrm{x}$

$177-z+1 / 2,-x+1 / 2,-y$

$178-\mathrm{z}+1 / 2, \mathrm{x}+3 / 4, \mathrm{y}+1 / 4$

$179 \mathrm{z}+3 / 4,-x+1 / 2, y+1 / 4$

$180 \mathrm{z}+3 / 4, \mathrm{x}+3 / 4,-\mathrm{y}$

$181 \mathrm{y}, \mathrm{x}, \mathrm{z}+1 / 2$

$182 \mathrm{y},-\mathrm{x}+3 / 4,-\mathrm{z}+1 / 4$

$183-\mathrm{y}+3 / 4, \mathrm{x},-\mathrm{z}+1 / 4$
$184-\mathrm{y}+3 / 4,-\mathrm{x}+3 / 4, \mathrm{z}+1 / 2$

$\begin{array}{ll}184 & -\mathrm{y}+3 / 4,-\mathrm{x}+3 / 2 \\ 185 & \mathrm{x}, \mathrm{z}, \mathrm{y}+1 / 2\end{array}$

$186 \mathrm{x},-\mathrm{z}+3 / 4,-\mathrm{y}+1 / 4$

$187-x+3 / 4, z,-y+1 / 4$

$188-\mathrm{x}+3 / 4,-\mathrm{z}+3 / 4, \mathrm{y}+1 / 2$

$189 \mathrm{z}, \mathrm{y}, \mathrm{x}+1 / 2$

$190 \mathrm{z},-\mathrm{y}+3 / 4,-\mathrm{x}+1 / 4$

$191-\mathrm{z}+3 / 4, \mathrm{y},-\mathrm{x}+1 / 4$

$192-\mathrm{z}+3 / 4,-\mathrm{y}+3 / 4, \mathrm{x}+1 / 2$

loop_

atom site label

_atom_site_type_symbol

_atom_site_symmetry_multiplicity

_atom_site_Wyckoff_label

atom_site_fract_x

atom_site_fract_y

atom_site_fract_z

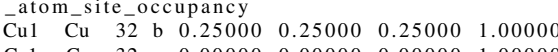

$\begin{array}{llllllllll}\mathrm{Cr} 1 & \mathrm{Cr} & 32 & \mathrm{c} & 0.00000 & 0.00000 & 0.00000 & 1.00000\end{array}$

$\begin{array}{llllllll}\mathrm{Cl} 1 & \mathrm{Cl} & 64 & \mathrm{e} & 0.19000 & 0.19000 & 0.19000 & 1.00000\end{array}$
$\begin{array}{llllllllll}\mathrm{C} 12 & \mathrm{Cl} & 96 & \mathrm{~g} & 0.25000 & 0.42500 & 0.57500 & 1.00000\end{array}$

$\mathrm{N} 1 \quad \mathrm{~N} \quad \begin{array}{llllllll}192 & \mathrm{~h} & 0.05000 & 0.18000 & 0.28000 & 1.00000\end{array}$

$\mathrm{CuCrCl}_{5}\left[\mathrm{NH}_{3}\right]_{6}$ : A5BCD6_cF416_228_eg_c_b_h - POSCAR

A5BCD6_cF416_228_eg_c_b_h \& a , x3 ,y4, x5 , y5 , z5 --params $=22.2649775014,0.19$ $\rightarrow, 0.425,0.05,0.18,0.28 \& \mathrm{Fd}-3 \mathrm{c}$ O_ $\{\mathrm{h}\}^{\wedge}\{8\} \# 228$ (bcegh) \& cF416 $\rightarrow$ None \& $\mathrm{CuCrCl} 5[\mathrm{NH} 3] 6$ \& \& M. Masayasu and S. Yoshihiko and $\stackrel{\hookrightarrow}{\hookrightarrow}$ Tokunosuk $\{\backslash>$

$\begin{array}{llll}0.00000000000000 & 11.13248875070000 & 11.13248875070000\end{array}$

$\begin{array}{rrr}11.13248875070000 & 0.00000000000000 & 11.13248875070000\end{array}$

$\begin{array}{lrr}11.13248875070000 & 11.13248875070000 & 0.00000000000000\end{array}$

Direct

$\begin{array}{rrrr}\mathrm{Cl} & \mathrm{Cr} & \mathrm{Cu} & \mathrm{N} \\ 40 & 8 & 8 & 48\end{array}$

0.19000000000000

0.19000000000000

$-0.0700000000000$

0.31000000000000

0.31000000000000

0.31000000000000

0.57000000000000

$-0.19000000000000$

$-0.19000000000000$

$-0.19000000000000$

1.0700000000000

0.69000000000000

0.69000000000000

0.69000000000000

$-0.57000000000000$

0.00000000000000

1.10000000000000

0.25000000000000

1.10000000000000

0.25000000000000

0.75000000000000

$-0.60000000000000$

$-0.60000000000000$

0.75000000000000

0.25000000000000

1.10000000000000

0.25000000000000

1.6000000000000

$-0.10000000000000$

0.75000000000000

0.75000000000000

0.25000000000000

1.60000000000000

.25000000000000

0.7500000000000

0.75000000000000

0.00000000000000

0.00000000000000

0.00000000000000

0.5000000000000

0.50000000000000

0.50000000000000

0.50000000000000

0.00000000000000

0.25000000000000

0.25000000000000

0.25000000000000

0.75000000000000

0.75000000000000

0.75000000000000

0.75000000000000

0.75000000000000

0.4100000000000

0.15000000000000

$-0.05000000000000$

$-0.01000000000000$

$-0.05000000000000$

$-0.01000000000000$

0.4100000000000

0.15000000000000

0.15000000000000

0.41000000000000

$-0.01000000000000$

$-0.05000000000000$

0.09000000000000

0.35000000000000

0.55000000000000

0.55000000000000

0.51000000000000

0.51000000000000

0.09000000000000

0.35000000000000

0.35000000000000

0.0900000000000

0.51000000000000

0.55000000000000

$-0.41000000000000$

$-0.15000000000000$

0.05000000000000

1.01000000000000

0.05000000000000

1.01000000000000

$-0.41000000000000$

$-0.15000000000000$

$-0.15000000000000$
.19000000000000 . .07000000000000 0.19000000000000 . 31000000000000 .5700000000000 57000000000000 19000000000000 19000000000000 07000000000000 0.19000000000000 0.69000000000000 0.69000000000000 .57000000000000 .69000000000000 0.35000000000000 0.75000000000000 0.25000000000000 1.10000000000000 0.75000000000000 0.60000000000000

10000000000000 25000000000000 10000000000000 .25000000000000 0.75000000000000 .60000000000000 1.60000000000000 0.25000000000000 0.75000000000000 $-0.10000000000000$ 0.25000000000000 60000000000000 0.10000000000000 0.75000000000000 $-0.10000000000000$ 0.75000000000000 .250000000000 1.6000000000000 1.60000000000000 . . 0.50000000000000 0.00000000000000 0.50000000000000 0.50000000000000 0.00000000000000 0.50000000000000 0.25000000000000 0.25000000000000 0.75000000000000 0.25000000000000 0.75000000000000 0.75000000000000 0.7500000000000 0.7500000000000 0.4100000000000 0.0500000000000 .05000000000000 . 1500000000000 0.15000000000000 0.05000000000000 $-0.01000000000000$ 0.05000000000000 0.01000000000000 0.41000000000000 0.15000000000000 0.35000000000000 0.51000000000000 0.55000000000000 0.09000000000000 0.35000000000000 0.51000000000000 0.51000000000 0.55000000000000 0.51000000000000 .09000000000000 0.35000000000000 0.41000000000000 1.01000000000000 $-0.41000000000000$ 0.05000000000000 0.15000000000000 0.05000000000000

0.19000000000000 $-0.07000000000000$ 0.19000000000000 0.57000000000000 0.3100000000000 0.310000000000 0.31000000000000 $-0.19000000000000$ 1.07000000000000 $-0.19000000000000$ $-0.19000000000000$ $-0.57000000000000$ 


$\begin{array}{rrrl}-0.41000000000000 & 1.01000000000000 & -0.15000000000000 & \text { N }(192 \mathrm{~h}) \\ 1.01000000000000 & -0.41000000000000 & 0.05000000000000 & \text { N }(192 \mathrm{~h}) \\ 0.05000000000000 & -0.15000000000000 & 1.01000000000000 & \text { N }(192 \mathrm{~h}) \\ 0.91000000000000 & 0.65000000000000 & -0.51000000000000 & \text { N }(192 \mathrm{~h}) \\ 0.65000000000000 & 0.91000000000000 & 0.45000000000000 & \mathrm{~N}(192 \mathrm{~h}) \\ 0.45000000000000 & -0.51000000000000 & 0.65000000000000 & \mathrm{~N}(192 \mathrm{~h}) \\ -0.51000000000000 & 0.45000000000000 & 0.91000000000000 & \mathrm{~N}(192 \mathrm{~h}) \\ 0.45000000000000 & 0.91000000000000 & -0.51000000000000 & \mathrm{~N}(192 \mathrm{~h}) \\ -0.51000000000000 & 0.65000000000000 & 0.45000000000000 & \mathrm{~N}(192 \mathrm{~h}) \\ 0.91000000000000 & 0.45000000000000 & 0.65000000000000 & \mathrm{~N}(192 \mathrm{~h}) \\ 0.65000000000000 & -0.51000000000000 & 0.91000000000000 & \mathrm{~N}(192 \mathrm{~h}) \\ 0.65000000000000 & 0.45000000000000 & -0.51000000000000 & \mathrm{~N}(192 \mathrm{~h}) \\ 0.91000000000000 & -0.51000000000000 & 0.45000000000000 & \mathrm{~N}(192 \mathrm{~h}) \\ -0.51000000000000 & 0.91000000000000 & 0.65000000000000 & \mathrm{~N}(192 \mathrm{~h}) \\ 0.45000000000000 & 0.65000000000000 & 0.91000000000000 & \mathrm{~N}(192 \mathrm{~h})\end{array}$

$\mathrm{TeO}_{6} \mathrm{H}_{6}$ : A6B_cF224_228_h_c - CIF

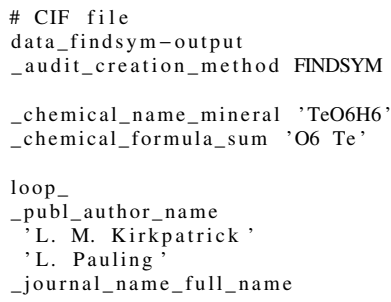

$41 \mathrm{x}+1 / 2, \mathrm{z}+1 / 2, \mathrm{y}+1 / 2$

$42 x+1 / 2,-z+1 / 4,-y+1 / 4$

$43-x+1 / 4, z+1 / 2,-y+1 / 4$

$44-\mathrm{x}+1 / 4,-\mathrm{z}+1 / 4, \mathrm{y}+1 / 2$

$45 \mathrm{z}+1 / 2, \mathrm{y}+1 / 2, \mathrm{x}+1 / 2$

$46 \mathrm{z}+1 / 2,-\mathrm{y}+1 / 4,-\mathrm{x}+1 / 4$

$47-\mathrm{z}+1 / 4, \mathrm{y}+1 / 2,-\mathrm{x}+1 / 4$

$48-\mathrm{z}+1 / 4,-\mathrm{y}+1 / 4, \mathrm{x}+1 / 2$

$49 \mathrm{x}, \mathrm{y}+1 / 2, \mathrm{z}+1 / 2$

$50 \mathrm{x},-\mathrm{y}+3 / 4,-\mathrm{z}+3 / 4$

$51-x+1 / 4, y+1 / 2,-z+3 / 4$

$52-x+1 / 4,-y+3 / 4, z+1 / 2$

$53 \mathrm{y}, \mathrm{z}+1 / 2, \mathrm{x}+1 / 2$

$54 \mathrm{y},-\mathrm{z}+3 / 4,-\mathrm{x}+3 / 4$

$54 \mathrm{y},-\mathrm{z}+3 / 4,-\mathrm{x}+3 / 4$
$55-\mathrm{y}+1 / 4, \mathrm{z}+1 / 2,-\mathrm{x}+3 / 4$

$55-\mathrm{y}+1 / 4, \mathrm{z}+1 / 2,-\mathrm{x}+3 / 4$
$56-\mathrm{y}+1 / 4,-\mathrm{z}+3 / 4, \mathrm{x}+1 / 2$

$56-\mathrm{y}+1 / 4,-\mathrm{z}+3 / 4, \mathrm{x}+1$
$57 \mathrm{z}, \mathrm{x}+1 / 2, \mathrm{y}+1 / 2$

$58 \mathrm{z},-\mathrm{x}+3 / 4,-\mathrm{y}+3 / 4$

$59-\mathrm{z}+1 / 4, \mathrm{x}+1 / 2,-\mathrm{y}+3 / 4$

$60-z+1 / 4,-x+3 / 4, y+1 / 2$

$61-\mathrm{y}+1 / 2,-\mathrm{x},-\mathrm{z}$

$62-y+1 / 2, x+3 / 4, z+3 / 4$

$63 \mathrm{y}+1 / 4,-\mathrm{x}, \mathrm{z}+3 / 4$

$64 \mathrm{y}+1 / 4, \mathrm{x}+3 / 4,-\mathrm{z}$

$65-\mathrm{x}+1 / 2,-\mathrm{z},-\mathrm{y}$

$66-x+1 / 2, z+3 / 4, y+3 / 4$

$67 \mathrm{x}+1 / 4,-\mathrm{z}, \mathrm{y}+3 / 4$

$68 \mathrm{x}+1 / 4, \mathrm{z}+3 / 4,-\mathrm{x}$

$69-z+1 / 2,-y,-x$

$69-z+1 / 2,-y,-x$
$70-z+1 / 2, y+3 / 4, x+3 / 4$

$71 \mathrm{z}+1 / 4,-\mathrm{y}, \mathrm{x}+3 / 4$

$71 \mathrm{z}+1 / 4,-\mathrm{y}, \mathrm{x}+3 / 4$
$72 \mathrm{z}+1 / 4, \mathrm{y}+3 / 4,-\mathrm{x}$

$72 \mathrm{z}+1 / 4, \mathrm{y}+3 / 4,-\mathrm{x}$
$73-\mathrm{x},-\mathrm{y}+1 / 2,-\mathrm{z}+1 / 2$

$73-\mathrm{x},-\mathrm{y}+1 / 2,-\mathrm{z}+1 / 2$
$74-\mathrm{x}, \mathrm{y}+3 / 4, \mathrm{z}+3 / 4$

$75 \mathrm{x}+1 / 4,-\mathrm{y}+1 / 2, \mathrm{z}+3 / 4$

$76 \mathrm{x}+1 / 4, \mathrm{y}+3 / 4,-\mathrm{z}+1 / 2$

$77-\mathrm{y},-\mathrm{z}+1 / 2,-\mathrm{x}+1 / 2$

$78-\mathrm{y}, \mathrm{z}+3 / 4, \mathrm{x}+3 / 4$

$79 \mathrm{y}+1 / 4,-\mathrm{z}+1 / 2, \mathrm{x}+3 / 4$

$80 \mathrm{y}+1 / 4, \mathrm{z}+3 / 4,-\mathrm{x}+1 / 2$

$81-z,-x+1 / 2,-y+1 / 2$

$82-\mathrm{z}, \mathrm{x}+3 / 4, \mathrm{y}+3 / 4$

$83 \mathrm{z}+1 / 4,-\mathrm{x}+1 / 2, \mathrm{y}+3 / 4$

$84 \mathrm{z}+1 / 4, \mathrm{x}+3 / 4,-\mathrm{y}+1 / 2$

$85 \mathrm{y}+1 / 2, \mathrm{x}, \mathrm{z}$

$86 \mathrm{y}+1 / 2,-\mathrm{x}+3 / 4,-\mathrm{z}+3 / 4$

$86-\mathrm{y}+1 / 2,-\mathrm{x}+3 / 4,-\mathrm{z}+3 / 4$
$87-\mathrm{y}+1 / 4, \mathrm{x},-\mathrm{z}+3 / 4$
$88-\mathrm{y}+1 / 4,-\mathrm{x}+3 / 4, \mathrm{~s}$

$88-\mathrm{y}+1 / 4,-\mathrm{x}+3 /$
$89 \mathrm{x}+1 / 2, \mathrm{z}, \mathrm{y}$

$89 x+1 / 2, z, y$
$90 x+1 / 2,-z+3 / 4,-y+3 / 4$

$90 \mathrm{x}+1 / 2,-\mathrm{z}+3 / 4,-\mathrm{y}+3 / 4$
$91-\mathrm{x}+1 / 4, \mathrm{z},-\mathrm{y}+3 / 4$

$91-\mathrm{x}+1 / 4, \mathrm{z},-\mathrm{y}+3 / 4$
$92-\mathrm{x}+1 / 4,-\mathrm{z}+3 / 4, \mathrm{y}$

$92-\mathrm{x}+1 / 4,-\mathrm{z}+3 / 2$

$94 \mathrm{z}+1 / 2,-\mathrm{y}+3 / 4,-\mathrm{x}+3 / 4$

$95-\mathrm{z}+1 / 4, \mathrm{y},-\mathrm{x}+3 / 4$

$96-z+1 / 4,-y+3 / 4, x$

$97 \mathrm{x}+1 / 2, \mathrm{y}, \mathrm{z}+1 / 2$

$98 \mathrm{x}+1 / 2,-\mathrm{y}+1 / 4,-\mathrm{z}+3 / 4$

$99-x+3 / 4, y,-z+3 / 4$

$100-x+3 / 4,-y+1 / 4, z+1 / 2$

$101 \mathrm{y}+1 / 2, \mathrm{z}, \mathrm{x}+1 / 2$

$102 \mathrm{y}+1 / 2,-\mathrm{z}+1 / 4,-\mathrm{x}+3 / 4$

$103-\mathrm{y}+3 / 4, \mathrm{z},-\mathrm{x}+3 / 4$

$104-\mathrm{y}+3 / 4,-\mathrm{z}+1 / 4, \mathrm{x}+1 / 2$

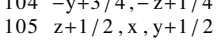

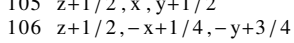

$106 \mathrm{z}+1 / 2,-\mathrm{x}+1 / 4,-\mathrm{y}+3$
$107-\mathrm{z}+3 / 4, \mathrm{x},-\mathrm{y}+3 / 4$

$107-z+3 / 4, x,-y+3 / 4$
$108-z+3 / 4,-x+1 / 4, y+1 / 2$

$109-\mathrm{y},-\mathrm{x}+1 / 2,-\mathrm{z}$

$110-y, x+1 / 4, z+3 / 4$

$111 \mathrm{y}+3 / 4,-x+1 / 2, z+3 / 4$

$112 \mathrm{y}+3 / 4, \mathrm{x}+1 / 4,-\mathrm{z}$

$113-\mathrm{x},-\mathrm{z}+1 / 2,-\mathrm{y}$

$114-\mathrm{x}, \mathrm{z}+1 / 4, \mathrm{y}+3 / 4$

$115 x+3 / 4,-z+1 / 2, y+3 / 4$

$116 \mathrm{x}+3 / 4, \mathrm{z}+1 / 4,-\mathrm{y}$

$117-\mathrm{z},-\mathrm{y}+1 / 2,-\mathrm{x}$

$118-\mathrm{z}, \mathrm{y}+1 / 4, \mathrm{x}+3 / 4$

$119 \mathrm{z}+3 / 4,-\mathrm{y}+1 / 2, \mathrm{x}+3 / 4$

$120 z+3 / 4, y+1 / 4,-x$

$120 z+3 / 4, y+1 / 4,-x$

$122-\mathrm{x}+1 / 2, \mathrm{y}+1 / 4, \mathrm{z}+3 / 4$

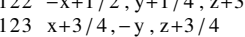

$124 x+3 / 4, y+1 / 4,-z+1 / 2$

$\mathrm{x}+3 / 4, \mathrm{y}+1 / 4,-\mathrm{z}+1 / 2$
$125-\mathrm{y}+1 / 2,-\mathrm{z},-\mathrm{x}+1 / 2$

$125-\mathrm{y}+1 / 2,-\mathrm{z},-\mathrm{x}+1 / 2$
$126-\mathrm{y}+1 / 2, \mathrm{z}+1 / 4, \mathrm{x}+3 / 4$

$127 \mathrm{y}+3 / 4,-\mathrm{z}, \mathrm{x}+3 / 4$

$128 \mathrm{y}+3 / 4, \mathrm{z}+1 / 4,-\mathrm{x}+1 / 2$

$129-\mathrm{z}+1 / 2,-\mathrm{x},-\mathrm{y}+1 / 2$

$130-z+1 / 2, x+1 / 4, y+3 / 4$

$131 \mathrm{z}+3 / 4,-x, y+3 / 4$

$132 \mathrm{z}+3 / 4, \mathrm{x}+1 / 4,-\mathrm{y}+1 / 2$

$133 \mathrm{y}, \mathrm{x}+1 / 2, \mathrm{z}$

$134 \mathrm{y},-\mathrm{x}+1 / 4,-\mathrm{z}+3 / 4$

$135-\mathrm{y}+3 / 4, \mathrm{x}+1 / 2,-\mathrm{z}+3 / 4$

$136-\mathrm{y}+3 / 4,-\mathrm{x}+1 / 4, \mathrm{z}$

$137 \mathrm{x}, \mathrm{z}+1 / 2, \mathrm{y}$

$138 \mathrm{x},-\mathrm{z}+1 / 4,-\mathrm{y}+3 / 4$

$139-x+3 / 4, z+1 / 2,-y+3 / 4$

$140-\mathrm{x}+3 / 4,-\mathrm{z}+1 / 4, \mathrm{y}$

$141 \mathrm{z}, \mathrm{y}+1 / 2, \mathrm{x}$

$142 \mathrm{z},-\mathrm{y}+1 / 4,-\mathrm{x}+3 / 4$

$143-z+3 / 4, y+1 / 2,-x+3 / 4$

$144-\mathrm{z}+3 / 4,-\mathrm{y}+1 / 4$,

$145 \mathrm{x}+1 / 2, \mathrm{y}+1 / 2, \mathrm{z}$ 
$148-x+3 / 4, y+1 / 2,-z+1 / 4$

$149-\mathrm{y}+1 / 2,-\mathrm{y}+3 / 4, \mathrm{z}$

$150 \mathrm{y}+1 / 2,-\mathrm{z}+3 / 4,-\mathrm{x}+1 / 4$

$151-\mathrm{y}+3 / 4, \mathrm{z}+1 / 2,-\mathrm{x}+1 / 4$

$152-\mathrm{y}+3 / 4,-\mathrm{z}+3 / 4, \mathrm{x}$

$153 \mathrm{z}+1 / 2, \mathrm{x}+1 / 2, \mathrm{y}$

$154 \mathrm{z}+1 / 2,-\mathrm{x}+3 / 4,-\mathrm{y}+1 / 4$

$155-\mathrm{z}+3 / 4, \mathrm{x}+1 / 2,-\mathrm{y}+1 / 4$

$156-\mathrm{z}+3 / 4,-\mathrm{x}+3 / 4, \mathrm{y}$

$157-\mathrm{y},-\mathrm{x},-\mathrm{z}+1 / 2$

$158-\mathrm{y}, \mathrm{x}+3 / 4, \mathrm{z}+1 / 4$

$159 \mathrm{y}+3 / 4,-\mathrm{x}, \mathrm{z}+1 / 4$
$160 \mathrm{y}+3 / 4, \mathrm{x}+3 / 4,-\mathrm{z}+1 / 2$

$161-\mathrm{x},-\mathrm{z},-\mathrm{y}+1 / 2$

$162-x, z+3 / 4, y+1 / 4$

$163 x+3 / 4,-z, y+1 / 4$

$164 x+3 / 4, z+3 / 4,-y+1 / 2$

$165-\mathrm{z},-\mathrm{y},-\mathrm{x}+1 / 2$

$166-\mathrm{z}, \mathrm{y}+3 / 4, \mathrm{x}+1 / 4$

$167 \mathrm{z}+3 / 4,-\mathrm{y}, \mathrm{x}+1 / 4$

$168 \mathrm{z}+3 / 4, \mathrm{y}+3 / 4,-\mathrm{x}+1 / 2$

$169-x+1 / 2,-y+1 / 2,-z$

$170-x+1 / 2, y+3 / 4, z+1 / 4$

$171 x+3 / 4,-y+1 / 2, z+1 / 4$

$172 x+3 / 4, y+3 / 4,-z$

$174-y+1 / 2, z+3 / 4, x+1 / 4$

$175 y+3 / 4,-z+1 / 2, x+1 / 4$

$176 \mathrm{y}+3 / 4, \mathrm{z}+3 / 4,-\mathrm{x}$

$177-z+1 / 2,-x+1 / 2,-y$

$178-z+1 / 2, x+3 / 4, y+1 / 4$

$179 \mathrm{z}+3 / 4,-x+1 / 2, y+1 / 4$

$180 \mathrm{z}+3 / 4, \mathrm{x}+3 / 4,-\mathrm{y}$

$181 \mathrm{y}, \mathrm{x}, \mathrm{z}+1 / 2$

$182 \mathrm{y},-\mathrm{x}+3 / 4,-\mathrm{z}+1 / 4$

$183-\mathrm{y}+3 / 4, \mathrm{x},-\mathrm{z}+1 / 4$

$184-\mathrm{y}+3 / 4,-\mathrm{x}+3 / 4, \mathrm{z}+1 / 2$

$185 \mathrm{x}, \mathrm{z}, \mathrm{y}+1 / 2$

$186 \mathrm{x},-\mathrm{z}+3 / 4,-\mathrm{y}+1 / 4$

$187-x+3 / 4, z,-y+1 / 4$

$188-x+3 / 4,-z+3 / 4, y+1 / 2$

$189 \mathrm{z}, \mathrm{y}, \mathrm{x}+1 / 2$

$190 \mathrm{z},-\mathrm{y}+3 / 4,-\mathrm{x}+1 / 4$

$192-\mathrm{z}+3 / 4,-\mathrm{y}+3 / 4, \mathrm{x}+1 / 2$

loop

atom site label

-atom_site_type_symbol

_atom_site_symmetry_multiplicity

_atom_site_Wyckoff_labe

_atom_site_fract_x

-atom_site_fract_y

atom_site_fract_z

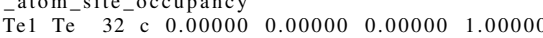

$\begin{array}{llllllllll}\mathrm{O} 1 & \mathrm{O} & 192 & \mathrm{~h} & 0.04300 & 0.13800 & 0.27800 & 1.00000\end{array}$

$\mathrm{TeO}_{6} \mathrm{H}_{6}$ : A6B_cF224_228_h_c - POSCAR

A6B_cF224_228_h_c \& a , x2,y2, z2 --params $=15.4800297791,0.043,0.138,0.278$ $\hookrightarrow$ \& Fd-3c O_ $\{\mathrm{h}\}^{\wedge}\{8\} \# 228$ (ch) \& $\mathrm{cF} 224$ \& None \& TeO6H6 \& \& L. M.

$\hookrightarrow$ Kirkpatrick and L. Pauling, Zeitschrift f" $\{u\}$ r Kristallographie

$\hookrightarrow$ - Crystalline Materials 63, 502-506 (1926)

1.00000000000000

0.00000000000000

7.74001488955000

7.74001488955000 0.00000000000000

7.74001488955000

7.74001488955000

7.74001488955000

7.74001488955000

$\begin{array}{rr}\mathrm{O} & \mathrm{Te} \\ 48 & 8\end{array}$

Direct

0.37300000000000

0.18300000000000

$-0.09700000000000$

0.04100000000000

$-0.09700000000000$

0.04100000000000

0.37300000000000

0.18300000000000

0.18300000000000

0.37300000000000

0.04100000000000

$-0.09700000000000$

0.12700000000000

0.31700000000000

0.31700000000000

0.59700000000000

0.45900000000000

0.59700000000000

0.45900000000000

0.12700000000000

0.31700000000000

0.31700000000000

0.12700000000000

0.45900000000000

0.59700000000000

$-0.37300000000000$

$-0.18300000000000$

0.09700000000000

0.95900000000000

0.09700000000000

0.95900000000000

$-0.37300000000000$

$-0.18300000000000$

0.18300000000000 0.37300000000000 0.04100000000000 $-0.09700000000000$ 0.37300000000000 0.1830000000000 $-0.09700000000000$ 0.04100000000000 $-0.09700000000000$ 0.04100000000000 0.37300000000000 0.18300000000000 0.31700000000000 0.1270000000000 0.4590000000000 0.5970000000000 0.5970000000000 0.12700000000000 0.31700000000000 0.5970000000000 0.45900000000000 0.59700000000000 0.45900000000000 0.12700000000000 0.31700000000000 $-0.1830000000000$ $-0.37300000000000$ 0.95900000000000 0.09700000000000 $-0.37300000000000$ $-0.18300000000000$ 0.09700000000000 0.95900000000000
$-0.18300000000000$ $-0.37300000000000$ 0.95900000000000 0.09700000000000 0.8730000000000 0.68300000000000 0.40300000000000 $-0.45900000000000$ 0.40300000000000 $-0.45900000000000$ 0.87300000000000 0.68300000000000 0.68300000000000 0.873000000000 0.87300000000000 $-0.45900000000000$ 0.4030000000000 0.00000000000000 0.00000000000000 0.00000000000000 0.50000000000000

0.50000000000000

0.50000000000000

0.50000000000000

0.00000000000000

0.09700000000000 0.95900000000000 $-0.37300000000000$ $-0.18300000000000$ 0.68300000000000 0.87300000000000 $-0.45900000000000$ 0.40300000000000 0.87300000000000 0.68300000000000 0.40300000000000 $-0.45900000000000$ 0.40300000000000 0.45900000000000 0.87300000000000 0.68300000000000 0.00000000000000 0.000000000000 0.50000000000 0.50000000000000 0.00000000000000 0.50000000000000 0.50000000000000 0.00000000000000 0.50000000000000

$-0.37300000000000$ $-0.18300000000000$ 0.09700000000000 0.95900000000000 $-0.45900000000000$ 0.40300000000000 0.68300000000000 0.87300000000000 $-0.45900000000000$ 0.40300000000000 0.68300000000000 0.87300000000000 $-0.45900000000000$ 0.40300000000000 0.68300000000000 0.87300000000000 0.87300000000000 0.000000000000 0.0000000000000 0.00000000000000 0.00000000000000 0.00000000000000 0.50000000000000 0.50000000000000 0.50000000000000

$\gamma$-brass $\left(\mathrm{Fe}_{3} \mathrm{Zn}_{10}, D 8_{1}\right):$ A3B10_cI52_229_e_fh - CIF

\# CIF file

data_findsym-outpu

audit_creation_method FINDSYM

chemical_name_mineral ' $\$ \backslash$ gamma $\$-$ brass Fe $\$$ _ $\{3\} \$ Z n \$$ \$ $\{10\} \$$

_chemical_formula_sum ' $\mathrm{Fe} 3 \mathrm{Zn} 10$

loop

publ_author_name

'J. Schramm'

journal_name_full_name

Zeitschrift fur Metallkunde

journal_volume 30

journal_year 1938

journal_page_first 122

journal_page_last 130

-publ_Section_title

X-Ray Investigation of Phases and Phase Limits of the Zn Alloy

Systems with $\mathrm{Fe}$, $\mathrm{Co}$ and $\mathrm{Ni}$

Found in A Handbook of Lattice Spacings and Structures of Metals and $\hookrightarrow$ Alloys , 1958

aflow title '\$ $\$$ gamma\$-brass (Fe\$_\{3\}\$Zn_\{10\}\$, \$D8_\{1\}\$) Structure aflow_proto 'A3B10_cI52_229_e_fh,

aflow_params, a, $x_{-}\{1\}, x_{-}\{2\}, y_{-}\{3\}$,

aflow params values $8.9822,0.3538,0.13505,0.3045$,

aflow_Strukturbericht $\$ D 8 \_\{1\} \$$,

aflow_Pearson 'cI52,

symmetry_space_group_name_H-M "I $4 / \mathrm{m}-3 \quad 2 / \mathrm{m}$

_symmetry_Int_Tables_number 229

cell_length_a $\quad 8.98220$

cell_length_b $\quad 8.98220$

cell_length_c $\quad 8.98220$

cell_angle_alpha 90.00000

cell_angle beta 90.00000

cell angle_gamm

loop

space_group_symop_id

space group symop_operation_xyz

$1 \mathrm{x}, \mathrm{y}, \mathrm{z}$

$\mathrm{x},-\mathrm{y},-\mathrm{z}$

$3-\mathrm{x}, \mathrm{y},-\mathrm{z}$

$4-\mathrm{x},-\mathrm{y}, \mathrm{z}$

$5 \mathrm{y}, \mathrm{z}, \mathrm{x}$

$6 \mathrm{y},-\mathrm{z},-\mathrm{x}$

$7-y, z,-x$

$8-\mathrm{y},-\mathrm{z}, \mathrm{x}$

$9 \mathrm{z}, \mathrm{x}, \mathrm{y}$

$10 \mathrm{z},-\mathrm{x},-\mathrm{y}$

$11-\mathrm{z}, \mathrm{x},-\mathrm{y}$

$12-\mathrm{z},-\mathrm{x}, \mathrm{y}$
$13-\mathrm{y},-\mathrm{x},-\mathrm{z}$

$14-y, x, z$

$13-y, x, z$
$15 y,-x, z$

$6 y, x, z$

$16-\mathrm{y}, \mathrm{x},-\mathrm{z}$
$-\mathrm{x},-\mathrm{z},-\mathrm{y}$

$18-x, z, y$

$19 \mathrm{x},-\mathrm{z}, \mathrm{y}$

$20 \mathrm{x}, \mathrm{z},-\mathrm{y}$

$21-\mathrm{z},-\mathrm{y},-\mathrm{x}$

$22-\mathrm{z}, \mathrm{y}, \mathrm{x}$

$23 \mathrm{z},-\mathrm{y}, \mathrm{x}$

$24 \mathrm{z}, \mathrm{y},-\mathrm{x}$

$25-x,-y,-z$

$26-x, y, z$

$27 \mathrm{x},-\mathrm{y}, \mathrm{z}$

$28 \mathrm{x}, \mathrm{y},-\mathrm{z}$

$29-y,-z,-x$

$30-y, z, x$

$31 \mathrm{y},-\mathrm{z}, \mathrm{x}$

O (192h

O (192h)

$\mathrm{O}(192 \mathrm{~h})$

O (192h)

$\mathrm{O}(192 \mathrm{~h})$

O (192h)

$O(192 \mathrm{~h})$

$\mathrm{O}(192 \mathrm{~h})$

O (192h)

$\mathrm{O}(192 \mathrm{~h})$

O (192h)

O (192h)

$\mathrm{Te} \quad(32 \mathrm{c})$

e $(32 \mathrm{c})$

Te $(32 \mathrm{c})$ 


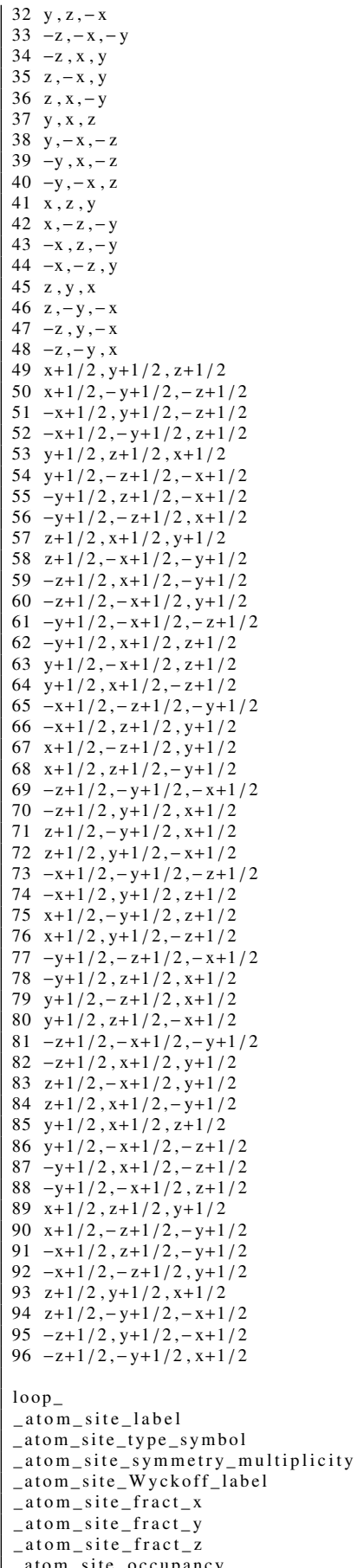

A3B10_cI52_229_e_fh \& a , x1 , x 2,y3 --params $=8.9822,0.3538,0.13505,0.3045 \&$ $\hookrightarrow \mathrm{Im}-3 \mathrm{~m} \mathrm{O}_{-}\{\mathrm{h}\}^{\wedge}\{9\}$ \#229 (efh) \& cI52 \& \$D8_\{1\}\$\& Fe3Zn10 \& \$ $\hookrightarrow$ gamma\$-brass $\mathrm{Fe}_{-}\{3\} \$ \mathrm{Zn} \$_{-}\{10\} \$ \&$ J. Schramm, Z. Metallkd. 30 $\hookrightarrow 122-130(1938)$

1.00000000000000

$-4.49110000000000 \quad 4.49110000000000 \quad 4.49110000000000$

$4.49110000000000 \quad-4.49110000000000 \quad 4.49110000000000$

$\begin{array}{rrr}4.49110000000000 & 4.49110000000000 & -4.49110000000000\end{array}$

$\mathrm{Fe} \quad \mathrm{Zn}$

Direct

0.00000000000000 0.00000000000000 0.35380000000000 $-0.35380000000000$ 0.35380000000000 $-0.35380000000000$ 0.27010000000000 0.00000000000000 0.00000000000000 $-0.27010000000000$ 0.00000000000000 $-0.27010000000000$ 0.00000000000000

0.35380000000000 $-0.35380000000000-0.35380000000000$ $0.00000000000000 \quad 0.35380000000000$ $\begin{array}{ll}0.00000000000000 & -0.35380000000000\end{array}$ $\begin{array}{lll}0.35380000000000 & 0.00000000000000\end{array}$ $-0.35380000000000 \quad 0.00000000000000$ $0.27010000000000 \quad 0.2701000000000$ $0.00000000000000-0.27010000000000$ $-0.27010000000000 \quad 0.00000000000000$ $0.00000000000000 \quad 0.00000000000000$ $0.00000000000000 \quad 0.27010000000000$ $-0.27010000000000-0.27010000000000$ $-0.270100000000-0.27010000000000$
0.27010000000000 0.60900000000000 0.00000000000000 0.00000000000000

$-0.60900000000000$

0.30450000000000

$-0.30450000000000$

0.30450000000000

$-0.30450000000000$

0.30450000000000

0.30450000000000

$-0.30450000000000$

$-0.30450000000000$

0.00000000000000 0.30450000000000 .30450000000000 $-0.30450000000000$ $-0.30450000000000$ 0.60900000000000 0.0000000000000 0.00000000000000 $-0.60900000000000$ 0.30450000000000 $-0.30450000000000$ 0.30450000000000 $-0.30450000000000$ 0.30450000000000 0.30450000000000 $-0.30450000000000$ 0.30450000000000 0.30450000000000 $-0.30450000000000$ $-0.30450000000000$ 0.60900000000000 0.00000000000000 0.00000000000000 0.60900000000000

$\beta$-Hg 4 Pt: A4B_cI10_229_c_a - CIF

\# CIF file

data_findsym-output

audit_creation method FINDSYM

chemical name mineral ' $\$ \backslash$ beta $\$-\mathrm{Hg} \$\{4\} \$ \mathrm{Pt}$ '

chemical_formula_sum ' $\mathrm{Hg} 4 \mathrm{Pt}$ ',

loop

publ_author_name

E. Bauer,

H. Nowotny
A. Stempfl,

journal_name_full_name

Monatshefte $f\{\backslash u\} r$ Chemie - Chemical Monthly

journal_volume 84

journal year 1953

journal_page_first 211

journal page last 212

publ_section title

$R \backslash "\{0\}$ ntgenographische Untersuchungen im System: Platin-Quecksilber

aflow title '\$ $\$$ beta $\$-\mathrm{Hg} \$\{4\} \$$ Pt Structure

_aflow_proto A4B_cI10_229_c_a,

aflow_params

aflow_params_values ' 6.186 '

aflow_Strukturbericht 'None'

aflow_Pearson 'cI10

symmetry_space_group_name_H-M

symmetry_Int_Tables_number 229

_cell_length_a $\quad 6.18600$

_cell_length_b 6.18600

6.18600

_cell_angle_alpha 90.00000

cell_angle_gamma 90.00000

loop

space_group_symop_id

_space_group_symop_operation_xy

$\mathrm{x}, \mathrm{y}, \mathrm{z}$

$2 \mathrm{x},-\mathrm{y},-\mathrm{z}$

$3-\mathrm{x}, \mathrm{y},-\mathrm{z}$

$4-x,-y, z$

$5 \mathrm{y}, \mathrm{z}, \mathrm{x}$

$6 \mathrm{y},-\mathrm{z},-\mathrm{x}$

$-y, z,-x$
$-y,-z, x$

$-y,-z, x$
$z, x, y$

$0, x, y$

$10 \mathrm{z},-\mathrm{x},-\mathrm{y}$
$11-\mathrm{z}, \mathrm{x},-\mathrm{y}$

$11-\mathrm{z}, \mathrm{x},-\mathrm{y}$

$12-\mathrm{z},-\mathrm{x}, \mathrm{y}$

$13-y,-x,-z$
$14-y, x, z$

$14-y, x, z$

$15 \mathrm{y},-\mathrm{x}, \mathrm{z}$

$16 \mathrm{y}, \mathrm{x},-\mathrm{z}$

$17-x,-z,-y$

$18-x, z, y$

$19 \mathrm{x},-\mathrm{z}, \mathrm{y}$

$20 \mathrm{x}, \mathrm{z},-\mathrm{y}$

$21-\mathrm{z},-\mathrm{y},-\mathrm{x}$

$22-\mathrm{z}, \mathrm{y}, \mathrm{x}$

$23 \mathrm{z},-\mathrm{y}, \mathrm{x}$

$24 \mathrm{z}, \mathrm{y},-\mathrm{x}$

$25-x,-y,-z$

$25-x,-y,-z$
$26-x, y, z$

$26-x, y, z$
$27 x,-y, z$

$27 \mathrm{x},-\mathrm{y}, \mathrm{z}$

$28 \mathrm{x}, \mathrm{y},-\mathrm{z}$

$29-y,-z,-x$
$30-y, z, x$

$30-\mathrm{y}, \mathrm{z}, \mathrm{x}$
$31 \mathrm{y},-\mathrm{z}, \mathrm{x}$

$31 \mathrm{y},-\mathrm{z}, \mathrm{x}$
$32 \mathrm{y}, \mathrm{z},-\mathrm{x}$

$32 \mathrm{y}, \mathrm{z},-\mathrm{x}$
$33-\mathrm{z},-\mathrm{x},-\mathrm{y}$

$34-\mathrm{z}, \mathrm{x}, \mathrm{y}$

$35 \mathrm{z},-\mathrm{x}, \mathrm{y}$

$36 \mathrm{z}, \mathrm{x},-\mathrm{y}$

$37 \mathrm{y}, \mathrm{x}, \mathrm{z}$

$38 \mathrm{y},-\mathrm{x},-\mathrm{z}$

$40-y,-x, z$

$41 \mathrm{x}, \mathrm{z}, \mathrm{y}$

$42 \mathrm{x},-\mathrm{z},-\mathrm{y}$

$42 x,-z,-y$

$43-x, z,-y$
$44-x,-z, y$ 


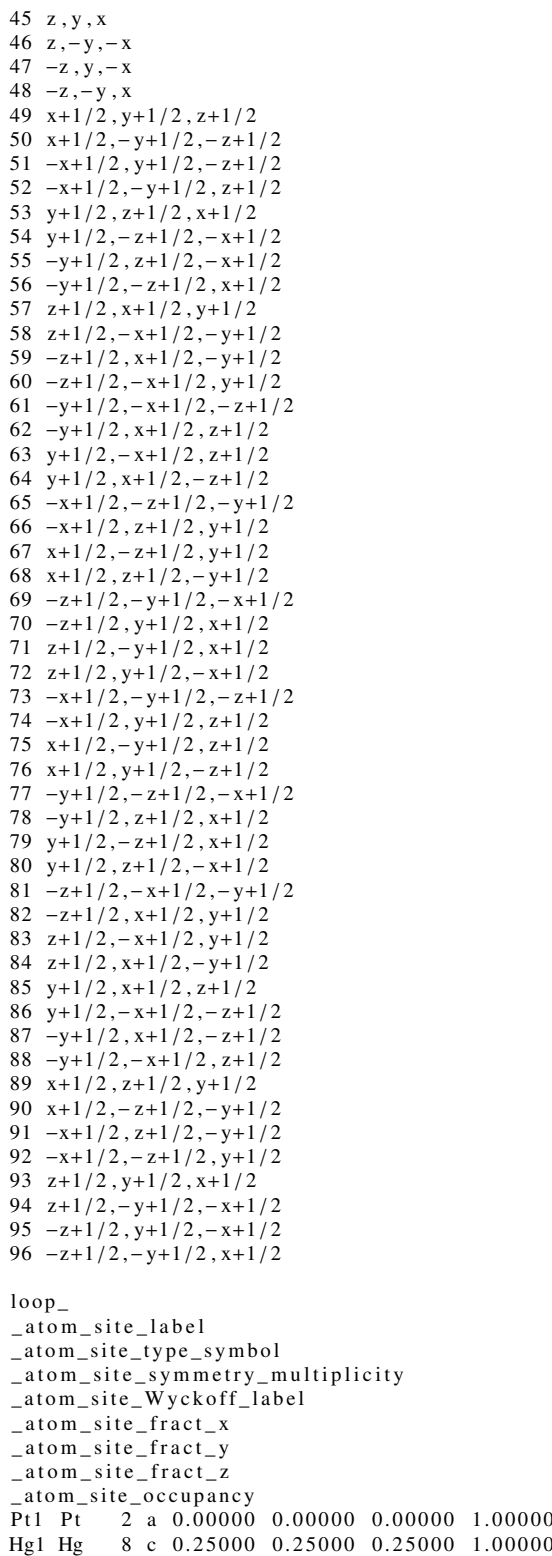

$\beta$-Hg ${ }_{4}$ Pt: A4B_cI10_229_c_a - POSCAR

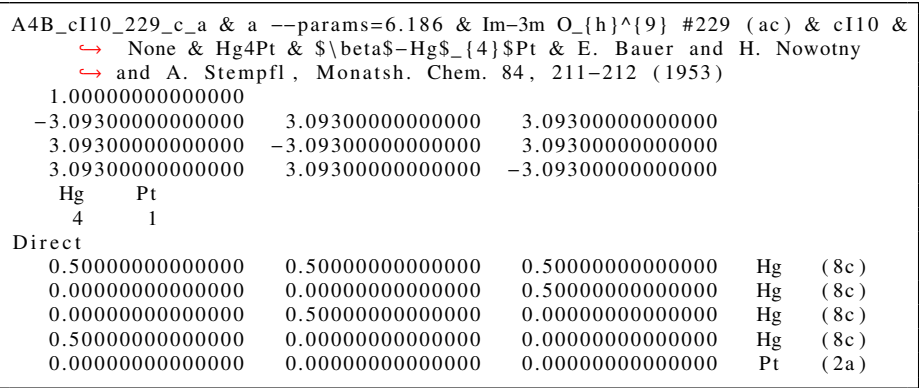

$\mathrm{Ir}_{3} \mathrm{Ge}_{7}\left(D 8_{f}\right)$ : A7B3_cI40_229_df_e - CIF

\section{\# CIF file}

data_findsym-output

_audit_creation_method FINDSYM

chemical_name_mineral ' $\mathrm{Ir} 3 \mathrm{Ge} 7$

_chemical_formula_sum 'Ge7 Ir3

loop

_publ_author_name

'U. $\mathrm{H} \backslash$ " $\{$ a $\}$ ussermann,

'M. \{ Elding-Pont $\backslash \backslash,\{e\} n\}$,

,C. Svensson

journal_name_full_name

Chemistry - A European Journal

_journal_volume

_ournal_year 1998

_ournal_page_first 1007 _journal_page_last 1015

-publ_Section_title

Compounds with the $\operatorname{Ir} \$ \_\{3\} \$ G e \$_{-}\{7\} \$$ Structure Type: Interpenetrating $\hookrightarrow$ Frameworks with Flexible Bonding Properties

\# Found in Diffusion of $\$ \wedge\{111\} \$ C d$ probes in $\mathrm{Ga}_{-}\{7\} \$ \mathrm{Pt} \$ \$_{-}\{3\} \$$ studied $\hookrightarrow$ via nuclear quadrupole relaxation, 2007

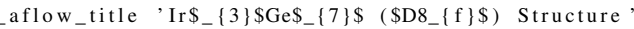

_aflow_proto 'A7B3_cI40_229_df_e

- aflow_params 'a, $x_{-}\{2\}, x_{-}\{3\}$,

-aflow_params_values $8.735,0.342,0$.

- aflow_Strukturberich,

symmetry_space_group_name_H-M "I $4 / \mathrm{m}-3 \quad 2 / \mathrm{m}^{\prime \prime}$

symmetry_Int_Tables_number 229

_cell_length_a 8.73500

cell_length_b 8.73500

cell_length_c $\quad 8.73500$

cell_angle_alpha 90.00000

cell_angle_beta 90.00000

_cell_angle_gamma 90.00000

loop

space_group_symop_id

space_group_symop_operation_xyz

$\mathrm{x}, \mathrm{y}, \mathrm{z}$

$2 \mathrm{x},-\mathrm{y},-\mathrm{z}$

$3-x, y,-z$
$4-x,-y, z$

$4-x,-y, z$

$\mathrm{y}, \mathrm{z}, \mathrm{x}$

$6 \mathrm{y},-\mathrm{z},-\mathrm{x}$

$7-y, z,-x$

$8-\mathrm{y},-\mathrm{z}, \mathrm{x}$

$\mathrm{z}, \mathrm{x}, \mathrm{y}$

$10 \mathrm{z},-\mathrm{x},-\mathrm{y}$

$2-z,-x, y$

$13-y,-x,-z$

$14-\mathrm{y}, \mathrm{x}, \mathrm{z}$

$15 \mathrm{y},-\mathrm{x}, \mathrm{z}$

$16 \mathrm{y}, \mathrm{x},-\mathrm{z}$

$17-x,-z,-y$

$18-\mathrm{x}, \mathrm{z}, \mathrm{y}$

$19 \mathrm{x},-\mathrm{z}, \mathrm{y}$

$20 \mathrm{x}, \mathrm{z},-\mathrm{y}$

$21-\mathrm{z},-\mathrm{y},-\mathrm{x}$

$22-\mathrm{z}, \mathrm{y}, \mathrm{x}$

$23 \mathrm{z},-\mathrm{y}, \mathrm{x}$

$24 \mathrm{z}, \mathrm{y},-\mathrm{x}$
$25-\mathrm{x},-\mathrm{y},-\mathrm{z}$

$26-\mathrm{x}, \mathrm{y}, \mathrm{z}$

$27 \mathrm{x},-\mathrm{y}, \mathrm{z}$

$28 \mathrm{x}, \mathrm{y},-\mathrm{z}$

$29-y,-z,-x$

$31 \mathrm{y},-\mathrm{z}, \mathrm{x}$

$31 \mathrm{y},-\mathrm{z}, \mathrm{x}$
$32 \mathrm{y}, \mathrm{z},-\mathrm{x}$

$33-z,-x,-y$

$33-z,-x,-y$
$34-z, x, y$

$35 \mathrm{z},-\mathrm{x}, \mathrm{y}$

$36 \mathrm{z}, \mathrm{x},-\mathrm{y}$

37, $\mathrm{y}, \mathrm{z}$

$\begin{array}{ll}38 & y,-x,-z \\ 39 & -y, x,-z\end{array}$

$39-\mathrm{y}, \mathrm{x},-\mathrm{z}$
$40-\mathrm{y},-\mathrm{x}, \mathrm{z}$

$40-\mathrm{y},-\mathrm{x}, \mathrm{z}$
$41 \mathrm{x}, \mathrm{z}, \mathrm{y}$

$42 \mathrm{x},-\mathrm{z},-\mathrm{y}$

$43-x, z,-y$

$44-x,-z, y$

$45 \mathrm{z}, \mathrm{y}, \mathrm{x}$

$46 \mathrm{z},-\mathrm{y},-\mathrm{x}$

$47-\mathrm{z}, \mathrm{y},-\mathrm{x}$

$48-\mathrm{z},-\mathrm{y}, \mathrm{x}$

$49 \mathrm{x}+1 / 2, \mathrm{y}+1 / 2, \mathrm{z}+1 / 2$

$50 \mathrm{x}+1 / 2,-\mathrm{y}+1 / 2,-\mathrm{z}+1 / 2$

$51-x+1 / 2, y+1 / 2,-z+1 / 2$

$52-\mathrm{x}+1 / 2,-\mathrm{y}+1 / 2, \mathrm{z}+1 / 2$

$52-\mathrm{x}+1 / 2,-\mathrm{y}+1 / 2, \mathrm{z}+1 / 2$
$53 \mathrm{y}+1 / 2, \mathrm{z}+1 / 2, \mathrm{x}+1 / 2$

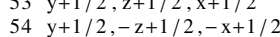

$54 \mathrm{y}+1 / 2,-\mathrm{z}+1 / 2,-\mathrm{x}+1 / 2$
$55-\mathrm{y}+1 / 2, \mathrm{z}+1 / 2,-\mathrm{x}+1 / 2$

$55-\mathrm{y}+1 / 2, \mathrm{z}+1 / 2,-\mathrm{x}+1 / 2$
$56-\mathrm{y}+1 / 2,-\mathrm{z}+1 / 2, \mathrm{x}+1 / 2$

$56-\mathrm{y}+1 / 2,-\mathrm{z}+1 / 2, \mathrm{x}+1 / 2$
$57 \mathrm{z}+1 / 2, \mathrm{x}+1 / 2, \mathrm{y}+1 / 2$

$58 \mathrm{z}+1 / 2,-\mathrm{x}+1 / 2,-\mathrm{y}+1 / 2$

$59-z+1 / 2, x+1 / 2,-y+1 / 2$

$60-z+1 / 2,-x+1 / 2, y+1 / 2$

$61-y+1 / 2,-x+1 / 2,-z+1 / 2$

$62-y+1 / 2, x+1 / 2, z+1 / 2$

$63 \mathrm{y}+1 / 2,-\mathrm{x}+1 / 2, \mathrm{z}+1 / 2$

$64 \mathrm{y}+1 / 2, \mathrm{x}+1 / 2,-\mathrm{z}+1 / 2$

$65-x+1 / 2,-z+1 / 2,-y+1 / 2$

$66-x+1 / 2, z+1 / 2, y+1 / 2$

$67 x+1 / 2,-z+1 / 2, y+1 / 2$

$68 \mathrm{x}+1 / 2, \mathrm{z}+1 / 2,-\mathrm{y}+1 / 2$

$69-\mathrm{z}+1 / 2,-\mathrm{y}+1 / 2,-\mathrm{x}+1 / 2$
$70-\mathrm{z}+1 / 2, \mathrm{y}+1 / 2, \mathrm{x}+1 / 2$

$70-\mathrm{z}+1 / 2, \mathrm{y}+1 / 2, \mathrm{x}+1 / 2$
$71 \mathrm{z}+1 / 2,-\mathrm{y}+1 / 2, \mathrm{x}+1 / 2$

$71 \mathrm{z}+1 / 2,-y+1 / 2, x+1 / 2$

$72 \mathrm{z}+1 / 2, \mathrm{y}+1 / 2,-\mathrm{x}+1 / 2$
$73-\mathrm{x}+1 / 2,-\mathrm{y}+1 / 2,-\mathrm{z}+1 / 2$

$73-\mathrm{x}+1 / 2,-\mathrm{y}+1 / 2,-\mathrm{z}+1 / 2$

$\begin{array}{ll}74 & -x+1 / 2, y+1 / 2, z+1 / 2 \\ 75 & x+1 / 2,-y+1 / 2, z+1 / 2\end{array}$ 


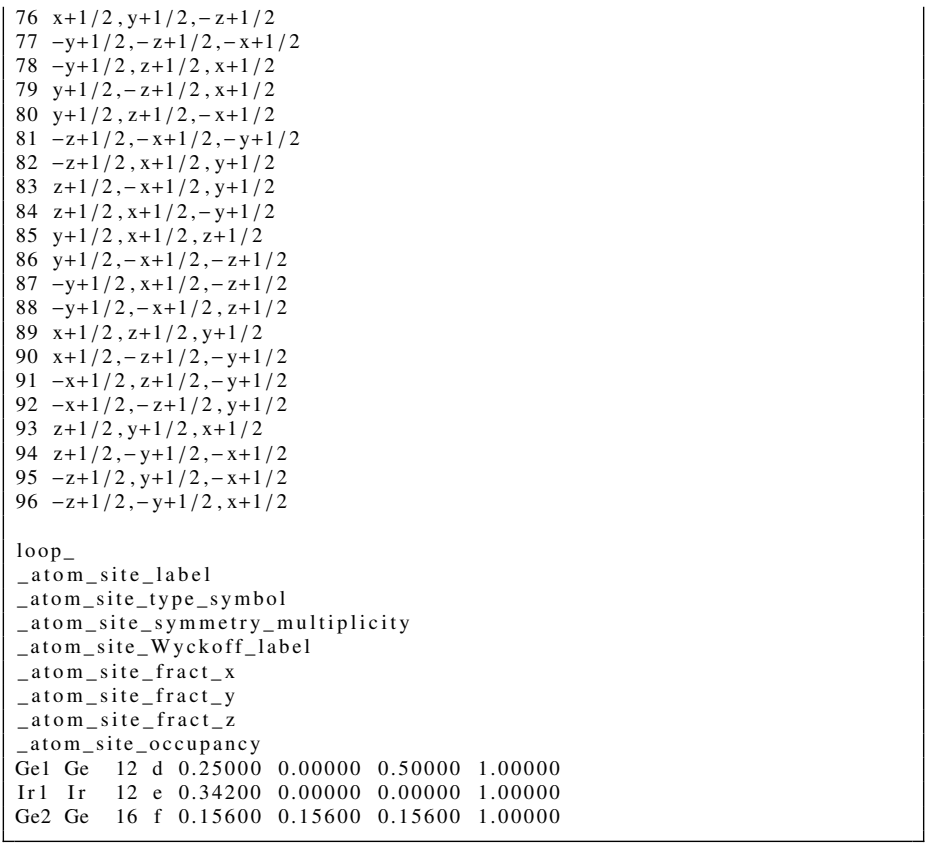

$\mathrm{Ir}_{3} \mathrm{Ge}_{7}\left(D 8_{f}\right)$ : A7B3_cI40_229_df_e - POSCAR

\begin{tabular}{|c|c|c|c|c|}
\hline \multicolumn{5}{|c|}{ A7B3_cI40_229_df_e $\&$ a , x2, x3 --params $=8.735,0.342,0.156 \&$ Im-3m O_ $\{\mathrm{h}\}^{\wedge}\{9$} \\
\hline \multicolumn{5}{|c|}{ 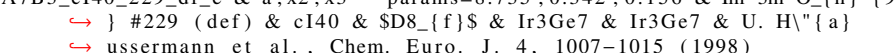 } \\
\hline \multicolumn{5}{|c|}{$\begin{array}{l}\hookrightarrow \text { ussermann et al., Chem. Euro. J. 4, 1007-1015 (1998) } \\
1.0000000000000\end{array}$} \\
\hline-4.36750000000000 & 4.36750000000000 & 4.36750000000000 & & \\
\hline 4.36750000000000 & -4.36750000000000 & 4.36750000000000 & & \\
\hline 4.36750000000000 & 4.36750000000000 & -4.36750000000000 & & \\
\hline $\mathrm{Ge}$ & & & & \\
\hline 14 & & & & \\
\hline \multicolumn{5}{|l|}{ Direct } \\
\hline 0.5000000 & 0.75000000000000 & 0.25000000000000 & $\mathrm{Ge}$ & $(12 d)$ \\
\hline 0.50000000 & 0.25000000000000 & 0.75000000000000 & $\mathrm{Ge}$ & \\
\hline 0.25000000 & 0. & 000 & $\mathrm{Ge}$ & \\
\hline 0.75000000 & 0000 & 0.25000000000000 & $\mathrm{Ge}$ & ( \\
\hline 0.75000000000000 & 0000000000 & 0.50000000000000 & G & $(12 \mathrm{~d})$ \\
\hline 0.25000000000000 & 000000000 & 0.50000000000000 & $\mathrm{Ge}$ & $(12 \mathrm{~d})$ \\
\hline 0.31200000000000 & 000000000 & 0.31200000000000 & $\mathrm{Ge}$ & $(16 \mathrm{f})$ \\
\hline 0.00000000000000 & 000000000 & -0.31200000000000 & $\mathrm{Ge}$ & ( 1 \\
\hline 0.00000000000000 & -0.31200000000000 & 0.00000000000000 & $\mathrm{Ge}$ & $(16 \mathrm{f})$ \\
\hline-0.31200000000000 & 0.00000000000000 & 0.00000000000000 & $\mathrm{Ge}$ & $(16 f)$ \\
\hline 0.00000000000000 & 0.00000000000000 & 0.31200000000000 & $\mathrm{Ge}$ & $(16 \mathrm{f}$ \\
\hline-0.31200000000000 & -0.31200000000000 & -0.31200000000000 & $\mathrm{Ge}$ & $(16 f)$ \\
\hline 0.00000000000000 & 0.31200000000000 & 0.00000000000000 & $\mathrm{Ge}$ & \\
\hline 0.31200000000000 & 0.00 & 0.00000000 & $\mathrm{Ge}$ & $(16 \mathrm{f})$ \\
\hline 0.00000000000000 & 0.3420 & 0.34200000 & Ir & $(12 \mathrm{e})$ \\
\hline 0.00000000000000 & -0.34200000000000 & -0.34200000000000 & $\mathrm{Ir}$ & $(12 \mathrm{e})$ \\
\hline 0.34200000000000 & 0.00000000000000 & 0.34200000000000 & Ir & \\
\hline-0.34200000000000 & 0.00000000000000 & -0.34200000000000 & Ir & $(12 \mathrm{e})$ \\
\hline 0.34200000000000 & 0.34200000000000 & 0.00000000000000 & Ir & $(12 \mathrm{e})$ \\
\hline-0.34200000000000 & -0.34200000000000 & 0.00000000000000 & Ir & $(12 \mathrm{e})$ \\
\hline
\end{tabular}

Garnet $\left(\mathrm{Co}_{3} \mathrm{Al}_{2} \mathrm{Si}_{3} \mathrm{O}_{12}, S 1_{4}\right)$ : A2B3C12D3_cI160_230_a_c_h_d - CIF

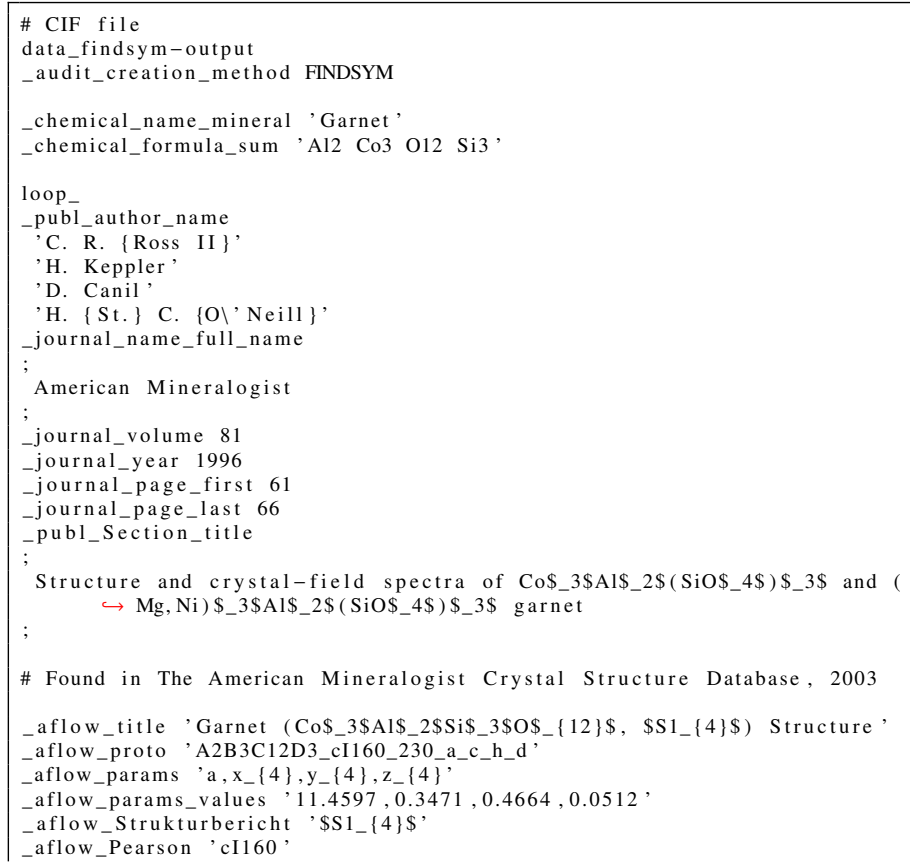

symmetry_space_group_name_H-M "I $41 /$ a $-3 \quad 2 / d "$

_symmetry_Int_Tables_number 230

_cell_length_a $\quad 11.45970$

_cell_length_b 11.45970

cell_length_c $\quad 11.45970$

cell_angle_alpha 90.00000

cell_angle_beta 90.00000

cell_angle_gamma 90.00000

loop

space_group_symop_id

space_group_symop_operation_xyz

$\mathrm{x}, \mathrm{y}, \mathrm{z}$

$\mathrm{x},-\mathrm{y},-\mathrm{z}+1 / 2$

$3-x+1 / 2, y,-z$

$4-\mathrm{x},-\mathrm{y}+1 / 2$
$5 \mathrm{y}, \mathrm{z}, \mathrm{x}$

5 y, z,

$6 \mathrm{y},-\mathrm{z},-\mathrm{x}+1 / 2$

$7-y+1 / 2, z,-x$

$8-\mathrm{y},-\mathrm{z}+1 / 2, \mathrm{x}$

$9 \mathrm{z}, \mathrm{x}, \mathrm{y}$

$10 \mathrm{z},-\mathrm{x},-\mathrm{y}+1 / 2$

$1-\mathrm{z}+1 / 2, \mathrm{x},-\mathrm{y}$

$12-\mathrm{z},-\mathrm{x}+1 / 2, \mathrm{y}$

$13-\mathrm{y}+1 / 4,-\mathrm{x}+1 / 4,-\mathrm{z}+1 / 4$

$14-y+1 / 4, x+3 / 4, z+1 / 4$

$15 \mathrm{y}+1 / 4,-\mathrm{x}+1 / 4, \mathrm{z}+3 / 4$

$16 \mathrm{y}+3 / 4, x+1 / 4,-z+1 / 4$

$16 \mathrm{y}+3 / 4, x+1 / 4,-z+1 / 4$
$17-\mathrm{x}+1 / 4,-\mathrm{z}+1 / 4,-y+1 / 4$

$17-x+1 / 4,-z+1 / 4,-y+1 / 4$

$18-x+1 / 4, z+3 / 4, y+1 / 4$

$19 x+1 / 4,-z+1 / 4, y+3 / 4$

$\begin{array}{ll}20 & x+3 / 4, z+1 / 4,-y+1 / 4 \\ 21 & -z+1 / 4,-y+1 / 4,-x+1 / 4\end{array}$

$22-\mathrm{z}+1 / 4, \mathrm{y}+3 / 4, \mathrm{x}+1 / 4$

$23 \mathrm{z}+1 / 4,-\mathrm{y}+1 / 4, \mathrm{x}+3 / 4$

$24 \mathrm{z}+3 / 4, \mathrm{y}+1 / 4,-\mathrm{x}+1 / 4$

$25-\mathrm{x},-\mathrm{y},-\mathrm{z}$

$26-\mathrm{x}, \mathrm{y}, \mathrm{z}+1 / 2$

$27 \mathrm{x}+1 / 2,-\mathrm{y}, \mathrm{z}$

$28 \mathrm{x}, \mathrm{y}+1 / 2,-\mathrm{z}$

$29-\mathrm{y},-\mathrm{z},-\mathrm{x}$

$30-\mathrm{y}, \mathrm{z}, \mathrm{x}+1 / 2$

$31 \mathrm{y}+1 / 2,-\mathrm{z}, \mathrm{x}$

$32 \mathrm{y}, \mathrm{z}+1 / 2,-\mathrm{x}$

$33-z,-x,-y$

$33-z,-x,-y$
$34-z, x, y+1 / 2$

$34-\mathrm{z}, \mathrm{x}, \mathrm{y}+1 / 2$
$35 \mathrm{z}+1 / 2,-\mathrm{x}, \mathrm{y}$

$35 \mathrm{z}+1 / 2,-\mathrm{x}, \mathrm{y}$
$36 \mathrm{z}, \mathrm{x}+1 / 2,-\mathrm{y}$
$37 \mathrm{y}+1 / 4, \mathrm{x}+1 / 4$

$36 \mathrm{z}, \mathrm{x}+1 / 2,-\mathrm{y}$
$37 \mathrm{y}+1 / 4, \mathrm{x}+1 / 4, \mathrm{z}+1 / 4$

$38 \mathrm{y}+1 / 4,-\mathrm{x}+3 / 4,-\mathrm{z}+1 / 4$

$38 \mathrm{y}+1 / 4,-\mathrm{x}+3 / 4,-\mathrm{z}+1 / 4$
$39-\mathrm{y}+1 / 4, \mathrm{x}+1 / 4,-\mathrm{z}+3 / 4$

$39-y+1 / 4, x+1 / 4,-z+3 / 4$
$40-y+3 / 4,-x+1 / 4, z+1 / 4$

$41 \mathrm{x}+1 / 4, \mathrm{z}+1 / 4, \mathrm{y}+1 / 4$

$42 \mathrm{x}+1 / 4,-\mathrm{z}+3 / 4,-\mathrm{y}+1 / 4$

$43-x+1 / 4, z+1 / 4,-y+3 / 4$

$44-\mathrm{x}+3 / 4,-\mathrm{z}+1 / 4, \mathrm{y}+1 / 4$

$45 \mathrm{z}+1 / 4, \mathrm{y}+1 / 4, \mathrm{x}+1 / 4$

$46 \mathrm{z}+1 / 4,-\mathrm{y}+3 / 4,-\mathrm{x}+1 / 4$

$47-\mathrm{z}+1 / 4, \mathrm{y}+1 / 4,-\mathrm{x}+3 / 4$

$48-\mathrm{z}+3 / 4,-\mathrm{y}+1 / 4, \mathrm{x}+1 / 4$

$49 x+1 / 2, y+1 / 2, z+1 / 2$

$50 x+1 / 2, y+1 / 2, z$

$51-x, y+1 / 2,-z+1 / 2$

$51-\mathrm{x}, \mathrm{y}+1 / 2,-\mathrm{z}+1 / 2$

$\begin{array}{ll}52 & -x+1 / 2,-y, z+1 / 2 \\ 53 & y+1 / 2, z+1 / 2, x+1 / 2\end{array}$

$54 \mathrm{y}+1 / 2,-\mathrm{z}+1 / 2,-\mathrm{x}$

$55-\mathrm{y}, \mathrm{z}+1 / 2,-\mathrm{x}+1 / 2$

$56-\mathrm{y}+1 / 2,-\mathrm{z}, \mathrm{x}+1 / 2$

$57 \mathrm{z}+1 / 2, \mathrm{x}+1 / 2, \mathrm{y}+1 / 2$

$58 \mathrm{z}+1 / 2,-\mathrm{x}+1 / 2,-\mathrm{y}$

$59-z, x+1 / 2,-y+1 / 2$

$60-\mathrm{z}+1 / 2,-\mathrm{x}, \mathrm{y}+1 / 2$

$61-y+3 / 4,-x+3 / 4,-z+3 / 4$

$62-y+3 / 4, x+1 / 4, z+3 / 4$

$63 y+3 / 4,-x+3 / 4, z+1 / 4$

$64 \mathrm{y}+1 / 4, \mathrm{x}+3 / 4,-\mathrm{z}+3 / 4$

$65-x+3 / 4,-z+3 / 4,-y+3 / 4$

$65-x+3 / 4,-z+3 / 4,-y+3 / 4$

$66-x+3 / 4, z+1 / 4, y+3 / 4$
$67 x+3 / 4,-z+3 / 4, y+1 / 4$

$67 x+3 / 4,-z+3 / 4, y+1 / 4$

$68 \mathrm{x}+1 / 4, \mathrm{z}+3 / 4,-\mathrm{y}+3 / 4$

$69-z+3 / 4,-y+3 / 4,-x+3 / 4$

$-\mathrm{z}+3 / 4, \mathrm{y}+1 / 4, \mathrm{x}+3 / 4$

$71 \mathrm{z}+3 / 4,-y+3 / 4, x+1 / 4$

$72 \mathrm{z}+1 / 4, \mathrm{y}+3 / 4,-\mathrm{x}+3 / 4$

$73-x+1 / 2,-y+1 / 2,-z+1 / 2$

$74-x+1 / 2, y+1 / 2, z$

$75 \mathrm{x},-\mathrm{y}+1 / 2, \mathrm{z}+1 / 2$

$76 \mathrm{x}+1 / 2, \mathrm{y},-\mathrm{z}+1 / 2$

$77-y+1 / 2,-z+1 / 2,-x+1 / 2$

$78-\mathrm{y}+1 / 2, \mathrm{z}+1 / 2, \mathrm{x}$

$79 \mathrm{y},-\mathrm{z}+1 / 2, \mathrm{x}+1 / 2$

$80 \mathrm{y}+1 / 2, \mathrm{z},-\mathrm{x}+1 / 2$

$81-z+1 / 2,-x+1 / 2,-y+1 / 2$

$82-z+1 / 2, x+1 / 2, y$

$83 \mathrm{z},-\mathrm{x}+1 / 2, \mathrm{y}+1 / 2$

$84 \mathrm{z}+1 / 2, x,-y+1 / 2$

$85 \mathrm{y}+3 / 4, x+3 / 4, z+3 / 4$

$86 \mathrm{y}+3 / 4,-x+1 / 4,-z+3 / 4$

$87-y+3 / 4, x+3 / 4,-z+1 / 4$

$88-\mathrm{y}+1 / 4,-\mathrm{x}+3 / 4, \mathrm{z}+3 / 4$

$89 x+3 / 4, z+3 / 4, y+3 / 4$

$90 \mathrm{x}+3 / 4,-\mathrm{z}+1 / 4,-\mathrm{y}+3 / 4$

$91-x+3 / 4, z+3 / 4,-y+1 / 4$ 


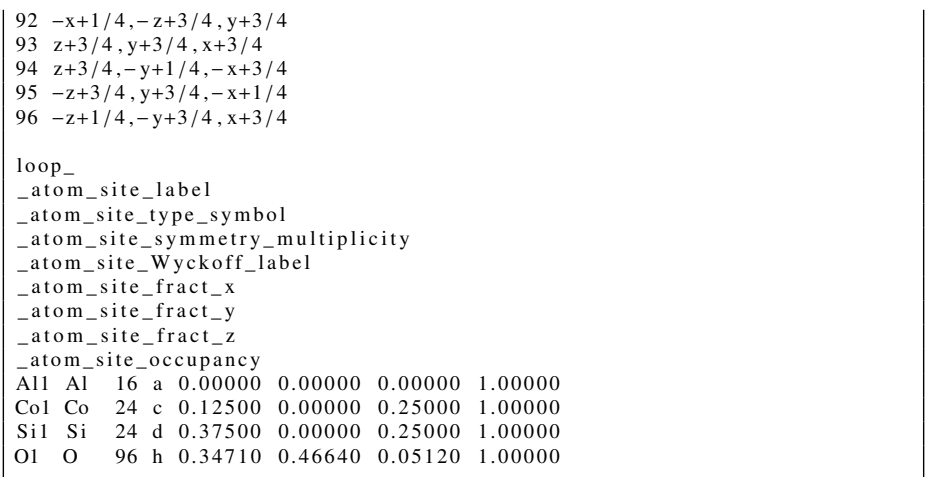

Garnet $\left(\mathrm{Co}_{3} \mathrm{Al}_{2} \mathrm{Si}_{3} \mathrm{O}_{12}, S 1_{4}\right)$ : A2B3C12D3_cI160_230_a_c_h_d - POSCAR

A2B3C12D3_cI160_230_a_c_h_d \& a, x4,y4,z4 --params $=11.4597,0.3471,0.4664$ $\hookrightarrow 0.0512 \& \mathrm{Ia}-3 \mathrm{~d} \mathrm{O}_{-}\{\mathrm{h}\} \wedge\{10\} \# 230(\mathrm{acdh}) \& \mathrm{cI} 160 \& \$ \mathrm{~S} 1_{-}\{4\} \$ \&$ $\hookrightarrow \mathrm{Co} 3 \mathrm{Al} 2 \mathrm{Si} 3 \mathrm{O} 12$ \& Garnet \& C. R. \{Ross II\} et al., Am. Mineral. 81 , 61-66 (1996)

1.00000000000000

$\begin{array}{rrr}-5.72985000000000 & 5.72985000000000 & 5.72985000000000\end{array}$

$\begin{array}{rrr}5.72985000000000 & -5.72985000000000 & 5.72985000000000\end{array}$

$\begin{array}{lrr}5.72985000000000 & 5.72985000000000 & -5.72985000000000\end{array}$

$\begin{array}{rrrr}\mathrm{Al} & \mathrm{Co} & \mathrm{O} & \mathrm{Si} \\ 8 & 12 & 48 & 12\end{array}$

Direct

0.00000000000000

0.50000000000000

0.00000000000000

0.50000000000000

0.50000000000000

0.50000000000000

0.50000000000000

0.00000000000000

0.00000000000000

0.25000000000000

0.75000000000000

0.12500000000000

0.37500000000000

0.37500000000000

0.12500000000000

0.75000000000000

0.25000000000000

0.87500000000000

0.62500000000000

0.62500000000000

0.87500000000000

0.5176000000000

0.51760000000000

0.08480000000000

0.41520000000000

$-0.01760000000000$

0.81350000000000

0.31350000000000
0.61930000000000

0.61930000000000
0.11930000000000

$-0.11930000000000$

0.39830000000000

$-0.29590000000000$

0.10170000000000

0.79590000000000

0.79590000000000

0.10170000000000

$-0.29590000000000$

0.39830000000000

0.08480000000000

0.51760000000000

0.5176000000000

$-0.01760000000000$

0.41520000000000

0.61930000000000

0.11930000000000

0.81350000000000

$-0.31350000000000$

$-0.51760000000000$

0.91520000000000

$-0.41520000000000$

1.01760000000000

$-0.81350000000000$

1.31350000000000 0.38070000000000

0.11930000000000

$-0.39830000000000$

0.2959000000000

0.29590000000000

0.89830000000000

0.20410000000000

0.20410000000000

0.89830000000000

0.29590000000000

0.39830000000000

0.91520000000000

$-0.51760000000000$

1.01760000000000

0.41520000000000

0.38070000000000

0.11930000000000

$-0.81350000000000$

1.31350000000000 0.25000000000000 0.75000000000000

0.37500000000000

0.12500000000000

0.00000000000000 00000000000000 0.50000000000000 0.50000000000000 0.0000000000000 0.0000000000000 . . .37500000000000 .12500000000000 0.25000000000000 0.75000000000000 0.12500000000000 0.37500000000000 0.62500000000000 0.87500000000000 0.75000000000000 0.25000000000000 0.87500000000000 0.62500000000000 0.3983000000000 0.395900000000 29590000000000 51760000000000 ( .08480000000000 0.41520000000000 0.81350000000000 . 930000000000 41520000000000 0.01760000000000 .51760000000000 0.08480000000000 $-0.11930000000000$ 0.61930000000000 $-0.3135000000000$ 0.81350000000000 $-0.29590000000000$ 0.39830000000000 .785000000 0.795000000000 0.10170000000000 0.3530000000000 0.29590000000000 0.51760000000000 0.91520000000000 0.41520000000000 1.01760000000000 1.31350000000000 0.38070000000000 0.11930000000000 $-0.4152000$ 1.01760000000000 $-0.51760000000000$ 0.91520000000000 0.38070000000000 0.38070000000000 1.31350000000000 $-0.81350000000000$ 0.29590000000000 $-0.39830000000000$ 0.20410000000000 0.89830000000000 0.25000000000000 0.75000000000000

0.00000000000000 $0.50000000000000 \quad \mathrm{Al} \quad(16 \mathrm{a})$ $0.00000000000000 \quad \mathrm{Al} \quad(16 \mathrm{a})$ $0.00000000000000 \quad \mathrm{Al}$ (16a) $0.50000000000000 \quad \mathrm{Al} \quad(16 \mathrm{a})$ $0.00000000000000 \quad \mathrm{Al} \quad(16 \mathrm{a})$ $0.12500000000000 \quad \mathrm{Co} \quad(24 \mathrm{c})$ $37500000000000-\mathrm{Co}(24 \mathrm{c})$ (co $(24 \mathrm{c})$ Co $(24 \mathrm{c})$ Co $(24 \mathrm{c})$ 0.25000000000000 Co $(24 \mathrm{c})$ .87500000000000 Co $(24 \mathrm{c})$ 0.62500000000000 Co $(24 \mathrm{c})$ 0.62500000000000 Co $(24 \mathrm{c})$ 0.87500000000000 Co $(24 \mathrm{c})$ $0.75000000000000 \quad$ Co $\quad(24 \mathrm{c})$ $0.25000000000000 \quad \mathrm{Co}(24 \mathrm{c})$ $0.81350000000000 \quad \mathrm{O}(96 \mathrm{~h})$ $-0.31350000000000 \quad 0 \quad(96 \mathrm{~h})$ $0.61930000000000 \quad 0 \quad(96 \mathrm{~h})$ $-0.11930000000000 \quad 0 \quad(96 \mathrm{~h})$ $0.39830000000000 \quad 0 \quad(96 \mathrm{~h})$ $-0.29590000000000-0$ (96h) $0.10170000000000 \quad$ O (96h) $0.795900000000 \quad$ O (96h) (96h) $0.51760000000000 \quad \mathrm{O} \quad(96 \mathrm{~h})$ $0.08480000000000 \quad$ O $\quad(96 \mathrm{~h})$ $-0.01760000000000 \quad \mathrm{O} \quad(96 \mathrm{~h})$ $0.81350000000000 \quad \mathrm{O} \quad(96 \mathrm{~h})$ $-0.31350000000000 \quad \mathrm{O} \quad(96 \mathrm{~h})$ $0.61930000000000 \quad$ O $(96 \mathrm{~h})$ $-0.11930000000000 \quad$ O $\quad(96 \mathrm{~h})$ $0.39830000000000 \quad$ O $(96 \mathrm{~h})$ $-0.29590000000000 \quad 0$ (96h) $0.1017000000000 \quad$ O $\quad(96 \mathrm{~h})$ $0.75900000000 \quad$ O $\quad(96 \mathrm{~h})$ $0.5176000000000 \quad \mathrm{O}(96 \mathrm{~h})$ $0.0848000000000 \quad 0$ (96h) $0.41520000000000 \quad$ O $(96 \mathrm{~h})$ $-0.01760000000000 \quad$ O $\quad(96 \mathrm{~h})$ $0.38070000000000 \quad$ O $\quad$ (96h) $0.11930000000000 \quad$ O $\quad(96 \mathrm{~h})$ $-0.39830000000000 \quad$ O (96h) $0.29590000000000 \quad \mathrm{O} \quad(96 \mathrm{~h})$ $0.89830000000000 \quad \mathrm{O} \quad(96 \mathrm{~h})$ $0.20410000000000 \quad \mathrm{O} \quad(96 \mathrm{~h})$ $-0.51760000000000 \quad \mathrm{O} \quad(96 \mathrm{~h})$ $0.91520000000000 \quad 0 \quad(96 \mathrm{~h})$ $-0.41520000000000 \quad 0 \quad(96 \mathrm{~h})$ $1.01760000000000 \quad$ O $\quad(96 \mathrm{~h})$ $-0.81350000000000 \quad$ O $(96 \mathrm{~h})$ $-0.81350000000000 \quad$ O $(96 \mathrm{~h})$ $0.38070000000000 \quad \mathrm{O} \quad(96 \mathrm{~h})$ $0.11930000000000 \quad \mathrm{O} \quad(96 \mathrm{~h})$ $-0.39830000000000 \quad \mathrm{O} \quad(96 \mathrm{~h})$ $-0.51760000000000 \quad$ O $\quad(96 \mathrm{~h})$ $0.91520000000000 \quad \mathrm{O} \quad(96 \mathrm{~h})$ $-0.41520000000000 \quad$ O $\quad$ (96h) $1.01760000000000 \quad$ O $\quad(96 \mathrm{~h})$ $0.37500000000000 \quad \mathrm{Si} \quad(24 \mathrm{~d})$ $0.12500000000000 \quad \mathrm{Si} \quad(24 \mathrm{~d})$ $0.62500000000000 \mathrm{Si}(24 \mathrm{~d})$ $\begin{array}{lll}0.62500000000000 & \mathrm{~S} & (24 \mathrm{~d}) \\ 0.87500000000000 & \mathrm{Si} & (24 \mathrm{~d})\end{array}$ $0.50000000000000-\mathrm{Al} \quad(16 \mathrm{a})$ 0.75000000000000 Co (24c) $0.41520000000000 \quad$ O $\quad(96 \mathrm{~h})$ $0.7959000000000 \quad$ O $(96 \mathrm{~h})$ $-0.81350000000000 \quad$ O $\quad(96 \mathrm{~h})$ $1.31350000000000 \quad \mathrm{O} \quad(96 \mathrm{~h})$ $0.29590000000000 \quad$ O $\quad$ (96h) $0.89830000000000 \quad$ O $(96 \mathrm{~h})$ $0.20410000000000 \quad$ O $\quad$ (96h) 


\section{Prototype Index}

1. $\alpha$-Al $\mathrm{A}_{2} \mathrm{~S}_{3}$ : A2B3_hP30_169_2a_3a $\ldots \ldots \ldots \ldots \ldots 547$

2. $\alpha-\mathrm{CuAlCl}_{4}: \mathrm{AB} 4 \mathrm{C} \_\mathrm{tP} 12 \_112 \_\mathrm{b} \_\mathrm{n} \_\mathrm{e} \ldots \ldots \ldots . . . . .378$

3. $\alpha-\mathrm{FeSe}^{\dagger}$ : AB_oC8_67_a_g ................. 240

4. $\alpha$-Naumannite: A2B_oP12_17_abe_e $\ldots \ldots \ldots \ldots 75$

5. $\alpha-\mathrm{NbO}_{2}$ : AB2_tI96_88_2f_4f ............... 296

6. $\alpha-\mathrm{P}_{3} \mathrm{~N}_{5}$ : A5B3_mC32_9_5a_3a $\ldots \ldots \ldots \ldots \ldots .42$

7. $\alpha-\mathrm{PbO}^{\dagger}$ : AB_oC8_67_a_g .................242

8. $\alpha-\mathrm{PdCl}_{2}$ : A2B_oP6_58_g_a $\ldots \ldots \ldots \ldots \ldots \ldots \ldots$

9. $\alpha-\mathrm{RbPr}\left[\mathrm{MoO}_{4}\right]_{2}$ :

A2B8CD_oP24_48_k_2m_d_b .................149

10. $\alpha-\mathrm{Sm}_{3} \mathrm{Ge}_{5}$ : A5B3_hP16_190_bdh_g ..........6 626

11. $\alpha$-ThSi 2 : A2B_tI12_141_e_a ................457

12. $\alpha-\mathrm{Tl}_{2} \mathrm{TeO}_{3}$ : A3BC2_oP48_50_3m_m_2m $\ldots \ldots \ldots 157$

13. $\alpha$-Toluene: A7B8_mP120_14_14e_16e .........64 64

14. $\beta-\mathrm{Bi}_{2} \mathrm{O}_{3}$ : A2B3_tP20_117_i_adgh.......... .393

15. $\beta$-CuI: AB_hP4_156_ac_ac ................. 497

16. $\beta-\mathrm{Hg}_{4} \mathrm{Pt}$ : A4B_cI10_229_c_a $\ldots \ldots \ldots \ldots \ldots . \ldots 793$

17. $\beta-\mathrm{NbO}_{2}$ : AB2_tI48_80_2b_4b ...............279

18. $\beta$-PdCl $\mathrm{PdCl}_{2}$ : A2B_hR18_148_2f_f $\ldots \ldots \ldots \ldots \ldots . \ldots 48$

19. $\beta$-RuCl $\mathrm{Ru}_{3}$ : A3B_hP8_158_d_a ...............501

20. $\beta-\mathrm{RuCl}_{3}$ : A3B_hP8_185_c_a $\ldots . . \ldots \ldots \ldots \ldots . \ldots 65$

21. $\beta-\mathrm{SeO}_{2}{ }^{*}$ : A2B_oP12_26_abc_ab $\ldots \ldots \ldots \ldots \ldots \ldots 97$

22. $\beta-\mathrm{Si}_{3} \mathrm{~N}_{4}$ : A4B3_hP14_173_bc_c $\ldots . . . \ldots \ldots \ldots . \ldots 56$

23. $\beta$ - $\mathrm{SiO}_{2}$ : A2B_hP9_181_j_c ................ 588

24. $\beta$ - $\mathrm{Ta}_{2} \mathrm{O}_{5}$ : A5B2_oP14_49_dehq_ab $\ldots \ldots \ldots \ldots \ldots 151$

25. $\beta$-ThI ${ }_{3}$ : A3B_oC64_66_kl2m_bdl $\ldots \ldots \ldots \ldots \ldots .233$

26. $\beta$-Toluene: A7B8_oP120_60_7d_8d ............185

27. $\beta$-V $\mathrm{V}_{3} \mathrm{~S}: \mathrm{AB} 3 \_\mathrm{tP} 32 \_133 \_\mathrm{h} \_\mathrm{i} 2 \mathrm{j} . . . . . . . . . . . . .433$

28. $\delta$-PdCl ${ }_{2}$ : A2B_mP6_10_mn_bg $\ldots \ldots \ldots \ldots \ldots \ldots 46$

29. $\delta_{H}^{I I}-\mathrm{NW}_{2}: \mathrm{AB} 2 \_\mathrm{hP9}{ }_{-164} \_\mathrm{bd} \_\mathrm{c} 2 \mathrm{~d} \ldots \ldots \ldots \ldots . \ldots 523$

30. $\epsilon-\mathrm{NiAl}_{3}$ : A3B_oP16_62_cd_c $\ldots . . . \ldots \ldots \ldots . .200$

31. $\epsilon-\mathrm{WO}_{3}:$ A3B_mP16_7_6a_2a $. . . \ldots \ldots \ldots . \ldots 38$

32. $\gamma-\mathrm{Ag}_{3}$ SI: A3BC_hR5_146_b_a_a $\ldots \ldots \ldots \ldots \ldots .475$

33. $\gamma$-MgNiSn: A7B7C2_tP32_101_bde_ade_d ...... 340

34. $\gamma-\mathrm{PdCl}_{2}$ : A2B_mP6_14_e_a $\ldots \ldots \ldots \ldots \ldots \ldots 62$

35. $\gamma$-brass: A4B9_cP52_215_ei_3efgi .............741

36. $\gamma$-brass: A3B10_cI52_229_e_fh .............. 790

37. $\kappa$-alumina: A2B3_oP40_33_4a_6a ............ 120

38. $\pi-\mathrm{FeMg}_{3} \mathrm{Al}_{8} \mathrm{Si}_{6}$ :

A8BC3D6_hP18_189_bfh_a_g_i .............6 619

39. $\pi-\mathrm{FeMg}_{3} \mathrm{Al}_{9} \mathrm{Si}_{5}$ :

A9BC3D5_hP18_189_fi_a_g_bh .............6 622

40. $\mathrm{Ag}_{3}\left[\mathrm{PO}_{4}\right]:$ A3B4C_cP16_218_c_e_a $\ldots \ldots \ldots \ldots 747$

41. $\mathrm{Ag}_{5} \mathrm{~Pb}_{2} \mathrm{O}_{6}:$ A5B6C2_hP13_157_2ac_2c_b ....... 499

42. AgUF 6 : AB6C_tP16_132_d_io_a ..............4 431

${ }^{\dagger} \alpha$-FeSe and $\alpha$-PbO have the same AFLOW prototype label. They are generated by the same symmetry operations with different sets of parameters.

${ }^{*} \mathrm{H}_{2} \mathrm{~S}$ and $\beta-\mathrm{SeO}_{2}$ have the same AFLOW prototype label. They are generated by the same symmetry operations with different sets of parameters.
43. Akermanite: A2BC7D2_tP24_113_e_a_cef_e ..... 380

44. $\mathrm{Al}_{2} \mathrm{CuIr}{ }^{\S}$ : A2BC_oC16_67_ag_b_g ............ 236

45. $\mathrm{Al}_{2} \mathrm{~S}_{3}:$ A2B3_hP30_170_2a_3a .............. 550

46. $\mathrm{Al}_{4} \mathrm{C}_{3}$ : A4B3_hR7_166_2c_ac ............... 530

47. $\mathrm{Al}_{4} \mathrm{U}$ : A4B_oI20_74_beh_e $. . . . \ldots \ldots \ldots \ldots . . .256$

48. $\mathrm{Al}_{8} \mathrm{Cr}_{5}$ : A8B5_hR26_160_a3bc_a3b ........... 514

49. $\mathrm{Al}_{9} \mathrm{Mn}_{3} \mathrm{Si}:$ A9B3C_hP26_194_hk_h_a ......... 646

50. $\mathrm{AlLi}_{3} \mathrm{~N}_{2}$ : AB3C2_cI96_206_c_e_ad .............704

51. $\mathrm{AlPO}_{4}: \mathrm{AB} 2 \_\mathrm{hP72} 1192 \_\mathrm{m} \_\mathrm{j} 2 \mathrm{kl} \ldots \ldots \ldots \ldots \ldots 635$

52. $\mathrm{Al}\left[\mathrm{PO}_{4}\right]: \mathrm{AB} 4 \mathrm{C} \_\mathrm{hP72} \_168 \_2 \mathrm{~d} \_8 \mathrm{~d} \_2 \mathrm{~d} \ldots . . . . . .543$

53. $\mathrm{Al}\left[\mathrm{PO}_{4}\right]$ : AB4C_hP72_184_d_4d_d .............594

54. Anhydrite: AB4C_oC24_63_c_fg_c ............2224

55. $\mathrm{As}_{2} \mathrm{Ba}$ : A2B_mP18_7_6a_3a ................. 36

56. $\mathrm{AsPh}_{4} \mathrm{CeS}_{8} \mathrm{P}_{4} \mathrm{Me}_{8}$ :

AB32CD4E8_tP184_93_i_16p_af_2p_4p ..........315

57. AuCN: ABC_hP3_183_a_a_a ................ 590

58. $\mathrm{AuF}_{3}: \mathrm{AB} 3 \_$hP24_178_b_ac ................. 582

59. $\mathrm{AuF}_{3}$ : AB3_hP24_179_b_ac ................ 586

60. BN: AB_oF8_42_a_a ...................... 143

61. $\mathrm{BPS}_{4}$ : ABC4_oI12_23_a_b_k ...............91

62. $\mathrm{Ba}_{5} \mathrm{In}_{4} \mathrm{Bi}_{5}$ : A5B5C4_tP28_104_ac_ac_c ......... 347

63. $\mathrm{Ba}_{5} \mathrm{Si}_{3}:$ A5B3_tP32_130_cg_cf ...............427

64. $\mathrm{BaCr}_{2} \mathrm{Ru}_{4} \mathrm{O}_{12}$ :

AB2C12D4_tP76_75_2a2b_2d_12d_4d ...........258

65. $\mathrm{BaCu}_{4}[\mathrm{VO}]\left[\mathrm{PO}_{4}\right]_{4}$ :

AB4C17D4E_tP54_90_a_g_c4g_g_c .............310

66. $\mathrm{BaGe}_{2} \mathrm{As}_{2}$ : A2BC2_tP20_105_f_ac_2e ........... 352

67. $\mathrm{BaSi}_{4} \mathrm{O}_{9}: \mathrm{AB} 9 \mathrm{C} 4 \_\mathrm{hP2} 8 \_188 \_\mathrm{e} \_\mathrm{kl} \_\mathrm{ak} \ldots \ldots . .666$

68. Barite: AB4C_oP24_62_c_2cd_c ............... 206

69. $\mathrm{Be}\left[\mathrm{BH}_{4}\right]_{2}:$ A2BC8_tI176_110_2b_b_8b ........ 368

70. Benzene: AB_oP48_61_3c_3c ............... 191

71. Beryl: A2B3C18D6_hP58_192_c_f_lm_1 ........631

72. $\mathrm{Bi}_{2} \mathrm{O}_{3}:$ A2B3_hP20_159_bc_2c $\ldots . . . . . . . .503$

73. $\mathrm{Bi}_{5} \mathrm{Nb}_{3} \mathrm{O}_{15}$ : A5B3C15_oP46_30_a2c_bc_a7c .....112

74. $\mathrm{BiAl}_{2} \mathrm{~S}_{4}$ : A2BC4_tP28_126_cd_e_k ............416

75. $\mathrm{BiGaO}_{3}$ : ABC3_oP20_54_e_d_cf ................. 168

76. Boracite: A7BC3D13_cF192_219_de_b_c_ah ......749

77. C: A_tP12_138_bi .......................449

78. $\mathrm{C}_{17} \mathrm{FeO}_{4} \mathrm{Pt}$ : A17BC4D_tP184_89_17p_p_4p_io ....300

79. $\mathrm{Ca}_{3} \mathrm{Al}_{2} \mathrm{O}_{6}$ : A2B3C6_cP264_205_2d_ab2c2d_6d ... 687

80. $\mathrm{Ca}_{3} \mathrm{Al}_{2} \mathrm{O}_{6}$ : A2B3C6_cP33_221_cd_ag_fh ........758

81. $\mathrm{Ca}_{3} \mathrm{PI}_{3}$ : A3B3C_cI56_214_g_h_a .............736

82. $\mathrm{Ca}_{4} \mathrm{Al}_{6} \mathrm{O}_{16} \mathrm{~S}$ :

A6B4C16D_oP108_27_abcd4e_4e_16e_e ..........101

83. $\mathrm{CaRbFe}_{4} \mathrm{As}_{4}$ : A4BC4D_tP10_123_gh_a_i_d ...... 406

84. Calomel: AB_tI8_139_e_e ...................441

85. Carbonyl Sulphide: ABC_hR3_160_a_a_a .......517

\footnotetext{
${ }^{\S} \mathrm{Al}_{2} \mathrm{CuIr}$ and $\mathrm{HoCuP}_{2}$ have similar AFLOW prototype labels (i.e., same symmetry and set of Wyckoff positions with different stoichiometry labels due to alphabetic ordering of atomic species). They are generated by the same symmetry operations with different sets of parameters.
} 
86. $\mathrm{CdAs}_{2}$ : A2B_tI12_98_f_a $\ldots \ldots \ldots \ldots \ldots \ldots \ldots \ldots 33$

87. $\mathrm{CdI}_{2}$ : AB2_hP9_156_b2c_3a2bc ..............493

88. $\mathrm{Ce}_{3} \mathrm{Si}_{6} \mathrm{~N}_{11}$ : A3B11C6_tP40_100_ac_bc2d_cd ..... 337

89. $\mathrm{Ce}_{5} \mathrm{Mo}_{3} \mathrm{O}_{16}$ : A5B3C16_cP96_222_ce_d_fi ........761

90. $\mathrm{CeCo}_{4} \mathrm{~B}_{4}$ : A4BC4_tP18_137_g_b_g . ........... 445

91. $\mathrm{CeRu}_{2} \mathrm{~B}_{2}$ : A2BC2_oF40_22_fi_ad_gh ........... 81

92. $\mathrm{CeTe}_{3}$ : AB3_oC16_40_b_3b ................ 139

93. $\mathrm{Co}_{2} \mathrm{Al}_{5}$ : A5B2_hP28_194_ahk_ch .............643

94. $\mathrm{Co}_{5} \mathrm{Ge}_{7}$ : A5B7_tI24_107_ac_abd ................. 358

95. Cobaltite: ABC_oP12_29_a_a_a ................110

96. $\mathrm{Cr}_{5} \mathrm{~B}_{3}$ : A3B5_tI32_140_ah_cl ................455

97. $\mathrm{CrCl}_{3}$ : A3B_hP24_153_3c_2b ...............488

98. $\mathrm{CrFe}_{3} \mathrm{NiSn}_{5}$ : AB_hP6_183_c_ab .............. 592

99. $\mathrm{Cs}_{2} \mathrm{ZnFe}[\mathrm{CN}]_{6}$ :

A6B2CD6E_cP64_208_m_ad_b_m_c ...........712

100. $\mathrm{Cs}_{3} \mathrm{P}_{7}$ : A3B7_tP40_76_3a_7a ............... 264

101. $\mathrm{CsPr}\left[\mathrm{MoO}_{4}\right]_{2}$ : AB2C8D_oP24_49_g_q_2qr_e .... 153

102. $\mathrm{Cu}_{15} \mathrm{Si}_{4}$ : A15B4_cI76_220_ae_c ...............753

103. $\mathrm{Cu}_{2} \mathrm{Fe}[\mathrm{CN}]_{6}$ : A12B2C_cF60_196_h_bc_a .......6 652

104. $\mathrm{Cu}_{3} \mathrm{P}: \mathrm{A} 3 \mathrm{~B} \_$hP24_165_bdg_f ................ 527

105. $\mathrm{Cu}_{3} \mathrm{P}^{\|}:$: A3B_hP24_185_ab2c_c ..............602

106. $\mathrm{CuBi}_{2} \mathrm{O}_{4}$ : A2BC4_tP28_130_f_c_g ............ 425

107. $\mathrm{CuBrSe}_{3}$ : ABC3_oP20_30_2a_c_3c .............115

108. $\mathrm{CuBrSe}_{3}$ : ABC3_oP20_53_e_g_hi ............. 166

109. $\mathrm{CuCrCl}_{5}\left[\mathrm{NH}_{3}\right]_{6}$ : A5BCD6_cF416_228_eg_c_b_h ..778

110. CuI: AB_hP12_156_2ab3c_2ab3c .............495

111. $\mathrm{CuNiSb}_{2}$ : ABC2_hP4_164_a_b_d .............5 525

112. Cubanite: AB2C3_oP24_62_c_d_cd .............202

113. Downeyite: A2B_tP24_135_gh_h .............436

114. $\mathrm{Er}_{3} \mathrm{Ru}_{2}$ : A3B2_hP10_176_h_bd .............. 573

115. F F $_{6}$ KP: A24BC_cF104_209_j_a_b .............. 716

116. FCC C $_{60}$ Buckminsterfullerine:

A_cF240_202_h2i ....................6 671

117. $\mathrm{Fe}_{12} \mathrm{Zr}_{2} \mathrm{P}_{7}$ : A12B7C2_hP21_174_2j2k_ajk_cf .... 564

118. $\mathrm{Fe}_{3} \mathrm{Te}_{3} \mathrm{Tl}: \mathrm{A} 3 \mathrm{~B} 3 \mathrm{C} \_\mathrm{hP14} \_176 \_\mathrm{h} \_\mathrm{h} \_\mathrm{d} \ldots \ldots \ldots \ldots 575$

119. $\mathrm{Fe}_{3} \mathrm{Th}_{7}$ : A3B7_hP20_186_c_b2c ...........6610

120. $\mathrm{FeCu}_{2} \mathrm{Al}_{7}$ : A7B2C_tP40_128_egi_h_e ...........422

121. FeNi: AB_mP4_6_2b_2a ................... 32

122. FeOCl: ABC_oP6_59_a_b_a ................. 178

123. $\mathrm{FePSe}_{3}$ : ABC3_hR10_146_2a_2a_2b ...........477

124. FeS: AB_oF8_22_a_c ..................... 83

125. $\mathrm{FeSb}_{2}$ : AB2_oP6_34_a_c ..................... 125

126. Forsterite: A2B4C_oP28_62_ac_2cd_c .......... 196

127. Fresnoite: A2B8C2D_tP26_100_c_abcd_c_a ..... 335

128. $\mathrm{GaCl}_{2}$ : A2B_oP24_52_2e_cd ................. 160

129. GaSb: AB_tI4_119_c_a ................... 402

130. Garnet: A2B3C12D3_cI160_230_a_c_h_d ....... 797

\footnotetext{
${ }^{\|} \mathrm{Cu}_{3} \mathrm{P}$ and $\mathrm{Na}_{3} \mathrm{As}$ have similar AFLOW prototype labels (i.e., same symmetry and set of Wyckoff positions with different stoichiometry labels due to alphabetic ordering of atomic species). They are generated by the same symmetry operations with different sets of parameters.
}

131. $\mathrm{Gd}_{3} \mathrm{Al}_{2}$ : A2B3_tP20_102_2c_b2c ..............343

132. GdSI: ABC_hP12_174_cj_fk_aj .............. 566

133. GeAs 2 : A2B_oP24_55_2g2h_gh .............. 170

134. GeP: AB_tI4_107_a_a ..................... 360

135. GeSe 2 : AB2_tP12_81_adg_2h ................282

136. $\mathrm{H}_{2} \mathrm{~S}$ : A2B_aP6_2_aei_i ......................28

137. $\mathrm{H}_{2} \mathrm{~S}$ : A2B_mP12_13_2g_ef $\ldots \ldots \ldots \ldots \ldots \ldots \ldots 60$

138. $\mathrm{H}_{2} \mathrm{~S}^{*}$ : A2B_oP12_26_abc_ab ................ 95

139. $\mathrm{H}_{2} \mathrm{~S}$ : A2B_oC24_64_2f_f ..................226

140. $\mathrm{H}_{2}$ S III: A2B_tP48_77_8d_4d .................2270

141. $\mathrm{H}_{2} \mathrm{~S}$ IV: A2B_mP12_7_4a_2a ................. 34

142. $\mathrm{H}_{3} \mathrm{Cl}$ : AB3_mC16_9_a_3a ...................44

143. $\mathrm{H}_{3} \mathrm{Cl}$ : AB3_mP16_10_mn_3m3n ..............48

144. $\mathrm{H}_{3} \mathrm{Cl}$ : AB3_mC16_15_e_cf .................. 71

145. $\mathrm{H}_{3} \mathrm{Cl}$ : AB3_oP16_19_a_3a ....................77

146. $\mathrm{H}_{3} \mathrm{~S}$ : A3B_oI32_23_ij2k_k .................. 85

147. $\mathrm{H}_{3} \mathrm{~S}$ : A3B_oC64_66_gi2lm_21 ...............230

148. $\mathrm{H}_{3} \mathrm{~S}$ : A3B_hR4_160_b_a .................. 512

149. H-III: A_mC24_15_2e2f .................... 73

150. HCl: AB_oC8_36_a_a ................... 129

151. $\mathrm{HgI}_{2}$ : AB2_tP12_115_j_egi ..................... 389

152. $\mathrm{HgI}_{2}{ }^{\mathrm{T}}$ : AB2_tP6_137_a_d ................... 447

153. $\mathrm{HoCuP}_{2}{ }^{\S}$ : ABC2_oC16_67_b_g_ag ............. 238

154. $\mathrm{Ir}_{3} \mathrm{Ga}_{5}$ : A5B3_tP32_118_g2i_aceh ............. 397

155. $\mathrm{Ir}_{3} \mathrm{Ge}_{7}$ : A7B3_cI40_229_df_e ................795

156. $\mathrm{IrGe}_{4}$ : A4B_hP15_144_4a_a ................ 467

157. $\mathrm{K}_{2} \mathrm{CdPb}$ : AB2C_oC16_40_a_2b_b ............. 137

158. $\mathrm{K}_{2} \mathrm{PtCl}_{6}$ : A6B2C_cF36_225_e_c_a ............ 769

159. $\mathrm{K}_{2} \mathrm{SnCl}_{6}$ : A6B2C_tP18_128_eh_d_b ...........420

160. $\mathrm{K}_{2} \mathrm{Ta}_{4} \mathrm{O}_{9} \mathrm{~F}_{4}$ : A2B13C4_hP57_168_d_c6d_2d ......539

161. $\mathrm{KAg}\left[\mathrm{CO}_{3}\right]$ : ABCD3_oI48_73_d_e_e_ef ......... 252

162. $\mathrm{KAu}_{4} \mathrm{Sn}_{2}$ : A4BC2_tI28_120_i_d_e ............ 404

163. $\mathrm{KB}_{6} \mathrm{H}_{6}$ : A6B6C_cF104_202_h_h_c ............669 669

164. $\mathrm{KBO}_{2}$ : ABC2_hR24_167_e_e_2e ..............536

165. $\mathrm{KCeSe}_{4}$ : ABC4_tP12_125_a_b_m ..............414

166. $\mathrm{KHg}_{2}$ : A2B_OI12_74_h_e .................. 254

167. $\mathrm{KNiCl}_{3}$ : A3BC_hP30_185_cd_c_ab .............599

168. $\mathrm{KSbO}_{3}$ : AB3C_cP60_201_ce_fh_g ............666

169. $\mathrm{La}_{2} \mathrm{NiO}_{4}$ : A2BC4_oP28_50_ij_ac_ijm ........... 155

170. $\mathrm{La}_{2} \mathrm{O}_{3}$ : A2B3_hP5_164_d_ad ................ 521

171. $\mathrm{La}_{43} \mathrm{Ni}_{17} \mathrm{Mg}_{5}$ :

A43B5C17_oC260_63_c8fg6h_cfg_ce3f2h ........212

172. LaPtSi: ABC_tI12_109_a_a_a ...................364

173. LaRhC $_{2}$ : A2BC_tP16_76_2a_a_a ..............262

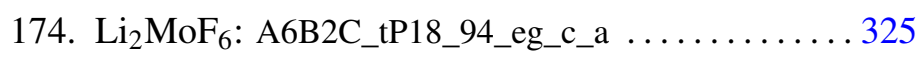

175. Li $_{2} \mathrm{Sb}$ : A2B_hP18_190_gh_bf ..............6624

176. $\mathrm{Li}_{2} \mathrm{Si}_{2} \mathrm{O}_{5}$ : A2B5C2_oC36_37_d_c2d_d ..........131

\footnotetext{
${ }^{\mathbb{I}} \mathrm{ZrO}_{2}$ and $\mathrm{HgI}_{2}$ have similar AFLOW prototype labels (i.e., same symmetry and set of Wyckoff positions with different stoichiometry labels due to alphabetic ordering of atomic species). They are generated by the same symmetry operations with different sets of parameters.
} 
177. LiScI $_{3}:$ A3BC_hP10_188_k_a_e ............614

178. LiSn: AB_mP6_10_en_am ............... 52

179. M-carbon: A_mC16_12_4i ............... 58

180. Mavlyanovite: A5B3_hP16_193_dg_g ........6 639

181. $\mathrm{Mg}_{2} \mathrm{Zn}_{11}$ : A2B11_cP39_200_f_aghij .........663

182. $\mathrm{MgB}_{12} \mathrm{H}_{12}\left[\mathrm{H}_{2} \mathrm{O}\right]_{12}$ :

A12B36CD12_cF488_196_2h_6h_ac_fgh .........654

183. $\mathrm{MgSO}_{4}:$ AB4C_oC24_63_a_fg_c ............. 222

184. $\mathrm{Mg}[\mathrm{NH}]$ : ABC_hP36_175_jk_jk_jk ..........5570

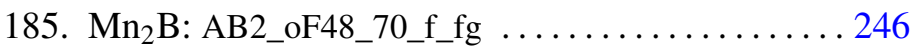

186. $\mathrm{MnAl}_{6}:$ A6B_oC28_63_efg_c ............... 218

187. $\mathrm{MnF}_{2}$ : A2B_tP12_111_2n_adf ............. 374

188. $\mathrm{MnGa}_{2} \mathrm{Sb}_{2}$ : A2BC2_oI20_45_c_b_c ........... 145

189. $\mathrm{Mo}_{8} \mathrm{P}_{5}$ : A8B5_mP13_6_a7b_3a2b ............ 30

190. $\mathrm{MoS}_{2}$ : AB2_hP12_143_cd_ab2d .............465

191. Moissanite-15R: AB_hR10_160_5a_5a ..........519

192. Molybdite: AB3_oP16_62_c_3c . . . . . . . . . . . 204

193. Muthmannite: ABC2_mP8_10_ac_eh_mn .........50

194. NV: AB_tP8_111_n_n ........................ 376

195. $\mathrm{Na}_{3} \mathrm{As}{ }^{\|}:$AB3_hP24_185_c_ab2c ............6 607

196. $\mathrm{Na}_{4} \mathrm{Ti}_{2} \mathrm{Si}_{8} \mathrm{O}_{22}\left[\mathrm{H}_{2} \mathrm{O}\right]_{4}$ :

A4B2C13D_tP40_90_g_d_cef2g_c ............. 307

197. $\mathrm{Na}_{5} \mathrm{Fe}_{3} \mathrm{~F}_{14}$ : A14B3C5_tP44_94_c3g_ad_bg . ..... 322

198. $\mathrm{NaFeS}_{2}$ : ABC2_oI16_23_ab_i_k .............89

199. $\mathrm{NaGdCu}_{2} \mathrm{~F}_{8}$ : A2B8CD_tI24_97_d_k_a_b ........ 329

200. $\mathrm{NaZn}_{13}:$ AB13_cF112_226_a_bi .............771

201. $\mathrm{NaZn}[\mathrm{OH}]_{3}$ : A3BC3D_tP64_106_3c_c_3c_c ...... 354

202. $\mathrm{Nb}_{4} \mathrm{CoSi}$ : AB4C_tP12_124_a_m_c . . . . . . . . . 408

203. $\mathrm{Nb}_{7} \mathrm{Ru}_{6} \mathrm{~B}_{8}:$ A8B7C6_hP21_175_ck_aj_k ....... 568

204. NbAs: AB_tI8_109_a_a ... . . . . . . . . . . . . . 366

205. NbPS: ABC_oI12_71_h_j_g ............. 250

206. $\mathrm{NbTe}_{4}:$ AB4_tP10_103_a_d ....................... 345

207. $\mathrm{NbTe}_{4}:$ AB4_tP10_124_a_m ................4 410

208. $\mathrm{Ni}_{3} \mathrm{P}:$ A3B_tI32_82_3g_g ................ 284

209. $\mathrm{Ni}_{3}$ Ti: A3B_hP16_194_gh_ac .............6. 641

210. Nierite: A4B3_hP28_159_ab2c_2c ...........506

211. $\mathrm{PH}_{3}$ : A3B_cP16_208_j_b ...............710

212. $\mathrm{PI}_{3}$ : A3B_hP8_173_c_b ................ 560

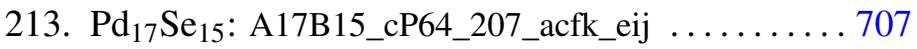

214. $\mathrm{Pd}_{4} \mathrm{Se}:$ A4B_tP10_114_e_a .................... 385

215. PdSn 4 : AB4_oC20_68_a_i .................. 244

216. Petzite: A3BC2_cI48_214_f_a_e ............. 739

217. Phenakite: A2B4C_hR42_148_2f_4f_f .........479

218. Pinnoite: A2B6CD7_tP64_77_2d_6d_d_ab6d ......267

219. Post-perovskite: AB3C_oC20_63_a_cf_c .........220

220. $\mathrm{PrNiO}_{3}$ : AB3C_hR10_167_b_e_a .............554

221. $\mathrm{PrRu}_{4} \mathrm{P}_{12}$ : A12BC4_cP34_195_2j_ab_2e .........649

222. $\mathrm{PtPb}_{4}:$ A4B_tP10_125_m_a ................412
223. Pyrite ${ }^{\dagger}:$ AB2_oP12_29_a_2a ............... 108

224. Pyrochlore: A2BCD3E6_cF208_203_e_c_d_f_g . . . 675

225. Pyrochlore Iridate: A2B2C7_cF88_227_c_d_af ................ 774

226. Quartenary Heusler:

ABCD_cF16_216_c_d_b_a .................. 745

227. R-carbon: A_oP16_55_2g2h ............... 174

228. Rasvumite: A2BC3_oC24_63_e_c_cg ........... 210

229. $\mathrm{Rb}_{2} \mathrm{TiCu}_{2} \mathrm{~S}_{4}:$ A2B2C4D_tP18_132_e_i_o_d .......4429

230. $\mathrm{Rb}_{3} \mathrm{AsSe}_{16}$ : AB3C16_cF160_203_b_ad_eg .......663

231. RbGa $:$ A3B_tI24_119_b2i_af .............. 400

232. $\mathrm{Re}_{2} \mathrm{O}_{5}\left[\mathrm{SO}_{4}\right]_{2}: \mathrm{A} 13 \mathrm{~B} 2 \mathrm{C} 2 \_$oP34_32_a6c_c_c ...... 117

233. $\mathrm{Re}_{3} \mathrm{~N}:$ AB3_hP4_187_e_fh ................6 612

234. $\mathrm{Rh}_{2} \mathrm{Ga}$ : A9B2_mP22_7_9a_2a ............40

235. $\mathrm{Rh}_{2} \mathrm{~S}_{3}$ : A2B3_oP20_60_d_cd ............. 180

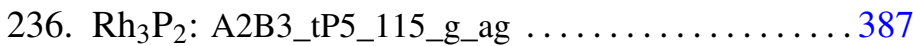

237. $\mathrm{Rh}_{5} \mathrm{Ge}_{3}$ : A3B5_oP16_55_ch_agh ............ 172

238. $\mathrm{Ru}_{2} \mathrm{Sn}_{3}:$ A2B3_tP20_116_bci_fj ............. 391

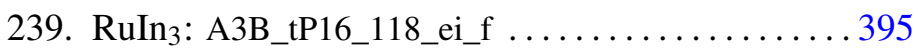

240. S-II: A_hP9_154_bc ..................491

241. S-III: A_tI16_142_f ......................459

242. S-carbon: A_mP8_10_2m2n ............... 54

243. Sc-V: A_hP6_178_a .................... 584

244. $\mathrm{ScRh}_{6} \mathrm{P}_{4}:$ A4B6C_hP11_143_bd_2d_a ......... 463

245. $\mathrm{SeO}_{3}$ : A3B_tP32_114_3e_e .................... 382

246. Sheldrickite:

A2B3C3DE7_hP48_145_2a_3a_3a_a_7a ........441

247. $\mathrm{SiO}_{2}$ : A2B_hP36_177_j2lm_n ...........579

248. $\mathrm{SiO}_{2}$ : A2B_cI72_211_hi_i .............. 731

249. Simple Cubic $\mathrm{C}_{60}$ Buckminsterfullerine:

A_cP240_205_10d .....................696

250. Simpsonite: A4B14C3_hP21_143_bd_ac4d_d .....461

251. SmSI: ABC_hR6_166_c_c_c ................552

252. Sodium Chlorate: ABC3_cP20_198_a_a_b ...... 661

253. Spinel: A3B4_cF56_227_ad_e .............776

254. $\mathrm{Sr}_{2} \mathrm{As}_{2} \mathrm{O}_{7}$ : A2B7C2_tP88_78_4a_14a_4a ........ 273

255. $\mathrm{Sr}_{2} \mathrm{Bi}_{3}$ : A3B2_oP20_52_de_cd ............. 162

256. $\mathrm{Sr}_{5} \mathrm{Si}_{3}$ : A3B5_tI32_108_ac_a2c .......... 362

257. $\mathrm{SrAl}_{2} \mathrm{Se}_{4}$ : A2B4C_oC28_66_1_kl_a ........... 228

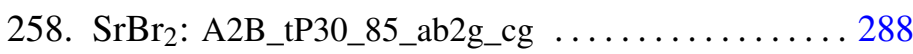

259. $\mathrm{SrH}_{2}$ : A2B_oP12_62_2c_c ............... 198

260. $\mathrm{SrSi}_{2}$ : A2B_cP12_212_c_a ................ 734

261. $\mathrm{Sr}\left[\mathrm{S}_{2} \mathrm{O}_{6}\right]\left[\mathrm{H}_{2} \mathrm{O}\right]_{4}: \mathrm{A} 10 \mathrm{~B} 2 \mathrm{C} \_$hP39_171_5c_c_a .... 553

262. $\mathrm{Sr}\left[\mathrm{S}_{2} \mathrm{O}_{6}\right]\left[\mathrm{H}_{2} \mathrm{O}\right]_{4}: \mathrm{A} 10 \mathrm{~B} 2 \mathrm{C} \_$hP39_172_5c_c_a .....557

263. Stannoidite:

A8B2C12D2E_oI50_23_bcfk_i_3k_j_a .........887

264. Ta 2 H: AB2_oC6_21_a_k ................. 79

${ }^{\ddagger} \mathrm{ZrO}_{2}$ and Pyrite have similar AFLOW prototype labels (i.e., same symmetry and set of Wyckoff positions with different stoichiometry labels due to alphabetic ordering of atomic species). They are generated by the same symmetry operations with different sets of parameters. 
265. $\mathrm{Ta}_{2} \mathrm{Se}_{8} \mathrm{I}:$ AB8C2_tI44_97_e_2k_cd ...............331

266. Ta $\mathrm{B}_{4}$ : A4B3_oI14_71_gh_cg ................248

267. $\mathrm{Ta}_{3} \mathrm{~S}_{2}$ : A2B3_oC40_39_2d_2c2d ............. 133

268. TaNiTe 2 : ABC2_oP16_53_h_e_gh .............. 164

269. $\mathrm{TeO}_{6} \mathrm{H}_{6}$ : A6B_cF224_228_h_c ...............785

270. TeZn: AB_hP6_144_a_a ...................469

271. Te[OH] $]_{6}$ : A12B6C_cF608_210_4h_2h_e .........719

272. $\mathrm{Th}_{3} \mathrm{P}_{4}$ : A4B3_cI28_220_c_a $\ldots . . . \ldots \ldots \ldots . . . .756$

273. $\mathrm{Th}_{6} \mathrm{Mn}_{23}$ : A23B6_cF116_225_bd2f_e ........... 766

274. ThB 4 : A4B_tP20_127_ehj_g .................418

275. ThBC: ABC_tP24_91_d_d_d ...................313

276. ThBC: ABC_tP24_95_d_d_d ...................327

277. $\mathrm{ThCl}_{4}$ : A4B_tI20_88_f_a ....................294

278. Thortveitite: A7B2C2_mC22_12_aij_h_i .........56

279. $\mathrm{Ti}_{2} \mathrm{Ge}_{3}$ : A3B2_tP10_83_adk_j ..............286

280. Ti 3 O: AB3_hP24_149_acgi_31 ................486

281. Ti 3 P: AB3_tP32_86_g_3g $\ldots . . . . . \ldots \ldots \ldots . . .291$

282. $\mathrm{TiAl}_{2} \mathrm{Br}_{8}$ : A2B8C_oP22_34_c_4c_a $\ldots \ldots \ldots \ldots . .123$

283. TiFeSi: ABC_ol36_46_ac_bc_3b .............. 147

284. $\mathrm{Tl}_{4} \mathrm{HgI}_{6}$ : AB6C4_tP22_104_a_2ac_c ............350

285. TIP 5 : A5B_oP24_26_3a3b2c_ab .............. 99

286. $\mathrm{TlZn}_{2} \mathrm{Sb}_{2}$ : A2BC2_tI20_79_c_2a_c ........... 277

287. Tongbaite: A2B3_oP20_62_2c_3c ............. 194

288. Troilite: AB_hP24_190_i_afh ...............6 628

289. Tychite: A4B2C6D16E_cF232_203_e_d_f_eg_a . . . 679

290. $\mathrm{UCl}_{3}$ : A3B_hP8_176_h_d ................. 577

291. $\mathrm{V}_{2} \mathrm{MoO}_{8}$ : AB8C2_oC22_35_a_ab3e_e .......... 127

292. VPCl 9 : A9BC_oC44_39_3c3d_a_c ..............135

293. $\mathrm{W}_{3} \mathrm{O}_{10}$ : A10B3_oF52_42_2abce_ab ............. 141

294. $\mathrm{W}_{5} \mathrm{Si}_{3}$ : A3B5_tI32_140_ah_bk .............. 453

295. $\mathrm{WO}_{3}$ : A3B_oP32_60_3d_d ................. 182

296. Weberite: AB7CD2_oI44_24_a_b3d_c_ac .........93

297. Westerveldite: AB_oP8_62_c_c .............. 208

298. $\mathrm{YbBaCo}_{4} \mathrm{O}_{7}$ : AB4C7D_hP26_159_b_ac_a2c_b ....509

299. $\mathrm{Zn}_{3} \mathrm{P}_{2}$ : A2B3_tP40_137_cdf_3g .............. 440

300. $\mathrm{ZnSb}_{2} \mathrm{O}_{4}$ : A4B2C_tP28_135_gh_h_d .......... 438

301. $\mathrm{ZrO}_{2}^{+}$: A2B_oP12_29_2a_a .................. 106

302. $\mathrm{ZrO}_{2}{ }^{\mathrm{II}}$ : A2B_tP6_137_d_a $\ldots . . . . . . . . . . . .443$

\section{Pearson Symbol Index}

aP

1. $\mathbf{a P 6}$

1.1. $\mathrm{H}_{2} \mathrm{~S}$ : A2B_aP6_2_aei_i $\ldots \ldots \ldots \ldots \ldots \ldots .28$

cF $\ldots \ldots$

1.1. Quartenary Heusler:

ABCD_cF16_216_c_d_b_a ................745

2. $\mathbf{c F 3 6}$

2.1. $\mathrm{K}_{2} \mathrm{PtCl}_{6}$ : A6B2C_cF36_225_e_c_a.... .769

3. $\mathbf{c F 5 6}$

3.1. Spinel: A3B4_cF56_227_ad_e ........... 776

4. $\mathbf{c F 6 0}$

4.1. $\mathrm{Cu}_{2} \mathrm{Fe}[\mathrm{CN}]_{6}$ : A12B2C_cF60_196_h_bc_a ...652

5. $\mathbf{c F 8 8}$

5.1. Pyrochlore Iridate:

A2B2C7_cF88_227_c_d_af ............... 774

6. $\mathbf{c F 1 0 4}$

6.1. $\mathrm{KB}_{6} \mathrm{H}_{6}$ : A6B6C_cF104_202_h_h_c . ........669

6.2. $\mathrm{F}_{6} \mathrm{KP}:$ A24BC_cF104_209_j_a_b .......... 716

7. $\mathbf{c F 1 1 2}$

7.1. $\mathrm{NaZn}_{13}$ : AB13_cF112_226_a_bi ...........771

8. $\mathbf{c F 1 1 6}$

8.1. $\mathrm{Th}_{6} \mathrm{Mn}_{23}$ : A23B6_cF116_225_bd2f_e ...... 766

9. $\mathbf{c F 1 6 0}$

9.1. $\mathrm{Rb}_{3} \mathrm{AsSe}_{16}$ : AB3C16_cF160_203_b_ad_eg ...683

10. $\mathbf{c F 1 9 2}$

10.1. Boracite:

A7BC3D13_cF192_219_de b _c $\_$ah ..........749

11. $\mathbf{c F 2 0 8}$

11.1. Pyrochlore:

A2BCD3E6_cF208_203_e_c_d_f_g .........675

12. $\mathbf{C F 2 2 4}$

12.1. $\mathrm{TeO}_{6} \mathrm{H}_{6}:$ A6B_cF224_228_h_c ........... 785

13. $\mathbf{c F 2 3 2}$

13.1. Tychite:

A4B2C6D16E_cF232_203_e_d_f_eg_a .......679

14. $\mathbf{c F 2 4 0}$

14.1. FCC C 60 Buckminsterfullerine:

A_cF240_202_h2i

15. $\mathbf{c F 4 1 6}$

15.1. $\mathrm{CuCrCl}_{5}\left[\mathrm{NH}_{3}\right]_{6}$ :

A5BCD6_cF416_228_eg_c_b_h .............778

16. $\mathbf{c F 4 8 8}$

16.1. $\mathrm{MgB}_{12} \mathrm{H}_{12}\left[\mathrm{H}_{2} \mathrm{O}\right]_{12}$ :

A12B36CD12_cF488_196_2h_6h_ac_fgh .....665

17. $\mathbf{c F 6 0 8}$

17.1. Te $[\mathrm{OH}]_{6}$ : A12B6C_cF608_210_4h_2h_e ....719

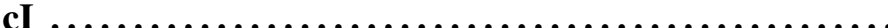

1. cI10

1.1. $\beta$-Hg $\mathrm{Hg}_{4} \mathrm{Pt}: \mathrm{A} 4 \mathrm{~B} \_\mathrm{cI} 10 \_229 \_\mathrm{c} \_\mathrm{a} \ldots \ldots . \ldots . . .793$

2. $\mathbf{c I 2 8}$

2.1. $\mathrm{Th}_{3} \mathrm{P}_{4}$ : A4B3_cI28_220_c_a $\ldots . . . . . . . .756$ 
3. $\mathbf{c I 4 0}$

3.1. $\mathrm{Ir}_{3} \mathrm{Ge}_{7}$ : A7B3_cI40_229_df_e .............795

4. cI48

4.1. Petzite: A3BC2_cI48_214_f_a_e .......... 739

5. $\mathbf{c I 5 2}$

5.1. $\gamma$-brass: A3B10_cI52_229_e_fh ..........790

6. $\mathbf{c I 5 6}$

6.1. $\mathrm{Ca}_{3} \mathrm{PI}_{3}$ : A3B3C_cI56_214_g_h_a $\ldots . . . . .736$

7. cI72

7.1. $\mathrm{SiO}_{2}$ : A2B_cI72_211_hi_i ..............731

8. $\mathbf{c I 7 6}$

8.1. $\mathrm{Cu}_{15} \mathrm{Si}_{4}:$ A15B4_cI76_220_ae_c $\ldots . . . \ldots . .753$

9. cI96

9.1. $\mathrm{AlLi}_{3} \mathrm{~N}_{2}$ : AB3C2_cI96_206_c_e_ad .........704

10. $\mathbf{c I 1 6 0}$

10.1. Garnet: A2B3C12D3_cI160_230_a_c_h_d ... 797

cP

1. $\mathbf{C P 1 2}$

1.1. $\mathrm{SrSi}_{2}$ : A2B_cP12_212_c_a ............. 734

2. $\mathbf{C P 1 6}$

2.1. $\mathrm{PH}_{3}: \mathrm{A} 3 \mathrm{~B} \_\mathrm{cP} 16 \_208 \_\mathrm{j} \_\mathrm{b} \ldots \ldots \ldots \ldots \ldots \ldots 710$

2.2. $\mathrm{Ag}_{3}\left[\mathrm{PO}_{4}\right]$ : A3B4C_cP16_218_c_e_a $\ldots . . .747$

3. $\mathbf{c P 2 0}$

3.1. Sodium Chlorate: ABC3_cP20_198_a_a_b . . 661

4. cP33

4.1. $\mathrm{Ca}_{3} \mathrm{Al}_{2} \mathrm{O}_{6}$ : A2B3C6_cP33_221_cd_ag_fh ....758

5. $\mathbf{c P 3 4}$

5.1. $\operatorname{PrRu}_{4} \mathrm{P}_{12}$ : A12BC4_cP34_195_2j_ab_2e ....649

6. cP39

6.1. $\mathrm{Mg}_{2} \mathrm{Zn}_{11}$ : A2B11_cP39_200_f_aghij ........663

7. $\mathbf{c P 5 2}$

7.1. $\gamma$-brass: A4B9_cP52_215_ei_3efgi

8. $\mathbf{c P 6 0}$

8.1. $\mathrm{KSbO}_{3}:$ AB3C_cP60_201_ce_fh_g .........666

9. cP64

9.1. $\mathrm{Pd}_{17} \mathrm{Se}_{15}$ : A17B15_cP64_207_acfk_eij ..... 707

9.2. $\mathrm{Cs}_{2} \mathrm{ZnFe}[\mathrm{CN}]_{6}$ :

A6B2CD6E_cP64_208_m_ad_b_m_c ....... 712

10. $\mathbf{c P 9 6}$

10.1. $\mathrm{Ce}_{5} \mathrm{Mo}_{3} \mathrm{O}_{16}$ : A5B3C16_cP96_222_ce_d_fi ...761

11. $\mathbf{c P 2 4 0}$

11.1. Simple Cubic $\mathrm{C}_{60}$ Buckminsterfullerine:

A_cP240_205_10d

.696

12. $\mathbf{c P 2 6 4}$

12.1. $\mathrm{Ca}_{3} \mathrm{Al}_{2} \mathrm{O}_{6}$ :

A2B3C6_cP264_205_2d_ab2c2d_6d

hP

1. hP3

1.1. AuCN: ABC_hP3_183_a_a_a

2. hP4

2.1. $\beta$-CuI: AB_hP4_156_ac_ac .............. 497

2.2. $\mathrm{CuNiSb}_{2}$ : ABC2_hP4_164_a_b_d .........5525

2.3. $\mathrm{Re}_{3} \mathrm{~N}$ : AB3_hP4_187_e_fh .............. 612
3. hP5

3.1. $\mathrm{La}_{2} \mathrm{O}_{3}$ : A2B3_hP5_164_d_ad ............521

4. hP6

4.1. TeZn: AB_hP6_144_a_a .................469

4.2. Sc-V: A_hP6_178_a .................. 584

4.3. $\mathrm{CrFe}_{3} \mathrm{NiSn}_{5}:$ AB_hP6_183_c_ab .......... 592

5. hP8

5.1. $\beta$-RuCl 3 : A3B_hP8_158_d_a .............501

5.2. $\mathrm{PI}_{3}$ : A3B_hP8_173_c_b ............... 560

5.3. $\mathrm{UCl}_{3}$ : A3B_hP8_176_h_d $\ldots . . . \ldots \ldots \ldots . \ldots 577$

5.4. $\beta-\mathrm{RuCl}_{3}$ : A3B_hP8_185_c_a ..............605

6. hP9

6.1. S-II: A_hP9_154_bc ..................491

6.2. $\mathrm{CdI}_{2}$ : AB2_hP9_156_b2c_3a2bc ..........493

6.3. $\delta_{H}^{I I}-\mathrm{NW}_{2}$ : AB2_hP9_164_bd_c2d $. . . \ldots \ldots . .523$

6.4. $\beta-\mathrm{SiO}_{2}$ : A2B_hP9_181_j_c $\ldots \ldots \ldots \ldots \ldots 58$

7. hP10

7.1. $\mathrm{Er}_{3} \mathrm{Ru}_{2}: \mathrm{A} 3 \mathrm{~B} 2 \_\mathrm{hP10} 176$ _h_bd $\ldots \ldots \ldots \ldots 573$

7.2. LiScI $_{3}$ : A3BC_hP10_188_k_a_e ..........6614

8. hP11

8.1. $\mathrm{ScRh}_{6} \mathrm{P}_{4}$ : A4B6C_hP11_143_bd_2d_a $\ldots . . .463$

9. hP12

9.1. $\mathrm{MoS}_{2}$ : AB2_hP12_143_cd_ab2d ..........465

9.2. CuI: AB_hP12_156_2ab3c_2ab3c .........4495

9.3. GdSI: ABC_hP12_174_cj_fk_aj ............ 566

10. hP13

10.1. $\mathrm{Ag}_{5} \mathrm{~Pb}_{2} \mathrm{O}_{6}$ : A5B6C2_hP13_157_2ac_2c_b ...499

11. hP14

11.1. $\beta$ - $\mathrm{Si}_{3} \mathrm{~N}_{4}$ : A4B3_hP14_173_bc_c $\ldots . . . . . .562$

11.2. $\mathrm{Fe}_{3} \mathrm{Te}_{3} \mathrm{Tl}$ : A3B3C_hP14_176_h_h_d ........575

12. hP15

12.1. IrGe 4 : A4B_hP15_144_4a_a .............467

13. $\mathbf{h P 1 6}$

13.1. $\alpha-\mathrm{Sm}_{3} \mathrm{Ge}_{5}$ : A5B3_hP16_190_bdh_g . . . . . 626

13.2. Mavlyanovite: A5B3_hP16_193_dg_g . . ... 639

13.3. Ni3 Ti: A3B_hP16_194_gh_ac ............641

14. hP18

14.1. $\pi-\mathrm{FeMg}_{3} \mathrm{Al}_{8} \mathrm{Si}_{6}$ :

A8BC3D6_hP18_189_bfh_a_g_i . ..........619

14.2. $\pi-\mathrm{FeMg}_{3} \mathrm{Al}_{9} \mathrm{Si}_{5}$ :

A9BC3D5_hP18_189_fi_a_g_bh ..........622

14.3. Li $_{2}$ Sb: A2B_hP18_190_gh_bf ............6624

15. hP20

15.1. $\mathrm{Bi}_{2} \mathrm{O}_{3}$ : A2B3_hP20_159_bc_2c ......... 503

15.2. $\mathrm{Fe}_{3} \mathrm{Th}_{7}$ : A3B7_hP20_186_c_b2c $\ldots . . . \ldots 610$

16. hP21

16.1. Simpsonite:

A4B14C3_hP21_143_bd_ac4d_d ............461

16.2. $\mathrm{Fe}_{12} \mathrm{Zr}_{2} \mathrm{P}_{7}$ :

A12B7C2_hP21_174_2j2k_ajk_cf ..........5564

16.3. $\mathrm{Nb}_{7} \mathrm{Ru}_{6} \mathrm{~B}_{8}$ : A8B7C6_hP21_175_ck_aj_k ... 568

17. hP24

17.1. Ti 3 O: AB3_hP24_149_acgi_31 .............486

17.2. $\mathrm{CrCl}_{3}$ : A3B_hP24_153_3c_2b ..............488

17.3. $\mathrm{Cu}_{3} \mathrm{P}:$ A3B_hP24_165_bdg_f $\ldots . . . \ldots \ldots . .527$ 
17.4. AuF 3 : AB3_hP24_178_b_ac ........... 582

17.5. AuF $:$ AB3_hP24_179_b_ac ........... 586

17.6. $\mathrm{Cu}_{3} \mathrm{P}^{\|}:$A3B_hP24_185_ab2c_c ..........606

17.7. $\mathrm{Na}_{3} \mathrm{As}{ }^{\|}:$AB3_hP24_185_c_ab2c ........ 607

17.8. Troilite: AB_hP24_190_i_afh ..........6 628

18. hP26

18.1. $\mathrm{YbBaCo}_{4} \mathrm{O}_{7}: \quad$ AB4C7D_hP26_159_b_ac_a2c_b 509

18.2. $\mathrm{Al}_{9} \mathrm{Mn}_{3} \mathrm{Si}$ : A9B3C_hP26_194_hk_h_a ..... 646

19. hP28

19.1. Nierite: A4B3_hP28_159_ab2c_2c .......506

19.2. $\mathrm{BaSi}_{4} \mathrm{O}_{9}: \mathrm{AB} 9 \mathrm{C} 4 \_\mathrm{hP} 28 \_188 \_\mathrm{e} \_\mathrm{kl}$ ak ......6 616

19.3. $\mathrm{Co}_{2} \mathrm{Al}_{5}$ : A5B2_hP28_194_ahk_ch ........6 643

\section{0. hP30}

20.1. $\alpha-\mathrm{Al}_{2} \mathrm{~S}_{3}$ : A2B3_hP30_169_2a_3a $\ldots \ldots \ldots .547$

20.2. $\mathrm{Al}_{2} \mathrm{~S}_{3}: \mathrm{A} 2 \mathrm{~B} 3 \_\mathrm{hP} 30 \_170 \_2 \mathrm{a} \_3 \mathrm{a} \ldots \ldots . . .550$

20.3. $\mathrm{KNiCl}_{3}$ : A3BC_hP30_185_cd_c_ab ........599

21. hP36

21.1. $\mathrm{Mg}[\mathrm{NH}]:$ ABC_hP36_175_jk_jk_jk .......5570

21.2. $\mathrm{SiO}_{2}$ : A2B_hP36_177_j21m_n ..........579

22. hP39

22.1. $\mathrm{Sr}\left[\mathrm{S}_{2} \mathrm{O}_{6}\right]\left[\mathrm{H}_{2} \mathrm{O}\right]_{4}$ :

A10B2C_hP39_171_5c_c_a .............553

22.2. $\mathrm{Sr}\left[\mathrm{S}_{2} \mathrm{O}_{6}\right]\left[\mathrm{H}_{2} \mathrm{O}\right]_{4}$ :

A10B2C_hP39_172_5c_c_a ..............557

23. hP48

23.1. Sheldrickite:

A2B3C3DE7_hP48_145_2a_3a_3a_a_7a .....471

24. hP57

24.1. $\mathrm{K}_{2} \mathrm{Ta}_{4} \mathrm{O}_{9} \mathrm{~F}_{4}:$ A2B13C4_hP57_168_d_c6d_2d .539

25. hP58

25.1. Beryl: A2B3C18D6_hP58_192_c_f_lm_1 ....631

26. hP72

26.1. $\mathrm{Al}\left[\mathrm{PO}_{4}\right]: \mathrm{AB} 4 \mathrm{C} \_$hP72_168_2d_8d_2d ......543

26.2. $\mathrm{Al}\left[\mathrm{PO}_{4}\right]: \mathrm{AB} 4 \mathrm{C} \_$hP72_184_d_4d_d ........ . 594

26.3. $\mathrm{AlPO}_{4}: \mathrm{AB} 2 \_\mathrm{hP} 72 \_192 \_\mathrm{m} \_\mathrm{j} 2 \mathrm{kl} \ldots \ldots \ldots \ldots 635$ hR

1. hR3

1.1. Carbonyl Sulphide: ABC_hR3_160_a_a_a ...517

2. hR4

2.1. $\mathrm{H}_{3} \mathrm{~S}:$ A3B_hR4_160_b_a ............ 512

3. hR5

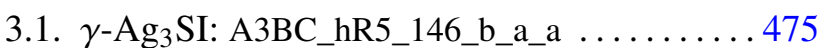

4. hR6

4.1. SmSI: ABC_hR6_166_c_c_c ...........552

5. hR7

5.1. $\mathrm{Al}_{4} \mathrm{C}_{3}: \mathrm{A} 4 \mathrm{~B} 3 \_\mathrm{hR} 7 \_166 \_2 \mathrm{c} \_\mathrm{ac} \ldots \ldots \ldots \ldots 530$

6. hR10

6.1. $\mathrm{FePSe}_{3}$ : ABC3_hR10_146_2a_2a_2b 477

${ }^{\|} \mathrm{Cu}_{3} \mathrm{P}$ and $\mathrm{Na}_{3} \mathrm{As}$ have similar AFLOW prototype labels (i.e., same symmetry and set of Wyckoff positions with different stoichiometry labels due to alphabetic ordering of atomic species). They are generated by the same symmetry operations with different sets of parameters.
6.2. Moissanite-15R: AB_hR10_160_5a_5a ......519

6.3. $\mathrm{PrNiO}_{3}$ : AB3C_hR10_167_b_e_a .........534

7. hR18

7.1. $\beta-\mathrm{PdCl}_{2}$ : A2B_hR18_148_2f_f $\ldots \ldots \ldots \ldots 483$

8. hR24

8.1. $\mathrm{KBO}_{2}$ : ABC2_hR24_167_e_e_2e ........536

9. hR26

9.1. $\mathrm{Al}_{8} \mathrm{Cr}_{5}$ : A8B5_hR26_160_a3bc_a3b ...... 514

10. hR42

10.1. Phenakite: A2B4C_hR42_148_2f_4f_f ..... 479

$\mathbf{m C}$

1. $\mathrm{mC16}$

1.1. $\mathrm{H}_{3} \mathrm{Cl}$ : AB3_mC16_9_a_3a ............44

1.2. M-carbon: A_mC16_12_4i ...........58

1.3. $\mathrm{H}_{3} \mathrm{Cl}$ : AB3_mC16_15_e_cf ............ 71

2. $\mathbf{m C 2 2}$

2.1. Thortveitite: A7B2C2_mC22_12_aij_h_i .....56

3. $\mathrm{mC24}$

3.1. H-III: A_mC24_15_2e2f ..............73

4. $\mathrm{mC32}$

4.1. $\alpha-\mathrm{P}_{3} \mathrm{~N}_{5}:$ A5B3_mC32_9_5a_3a $\ldots \ldots \ldots \ldots 42$

mP

1. $\mathbf{m P 4}$

1.1. FeNi: AB_mP4_6_2b_2a $\ldots \ldots \ldots \ldots \ldots \ldots 32$

2. $\mathbf{m P 6}$

2.1. $\delta-\mathrm{PdCl}_{2}$ : A2B_mP6_10_mn_bg $\ldots \ldots \ldots \ldots .46$

2.2. LiSn: AB_mP6_10_en_am ............52

2.3. $\gamma-\mathrm{PdCl}_{2}$ : A2B_mP6_14_e_a ..........6. 62

3. $\mathbf{m P 8}$

3.1. Muthmannite: ABC2_mP8_10_ac_eh_mn .....50

3.2. S-carbon: A_mP8_10_2m2n ............54

4. $\mathbf{m P 1 2}$

4.1. $\mathrm{H}_{2} \mathrm{~S}$ IV: A2B_mP12_7_4a_2a .......... 34

4.2. $\mathrm{H}_{2} \mathrm{~S}$ : A2B_mP12_13_2g_ef ...........6 60

5. $\mathbf{m P 1 3}$

5.1. Mos $\mathrm{P}_{5}$ : A8B5_mP13_6_a7b_3a2b ........ 30

6. $\mathrm{mP16}$

6.1. $\epsilon-\mathrm{WO}_{3}$ : A3B_mP16_7_6a_2a $\ldots \ldots \ldots \ldots . . . .68$

6.2. $\mathrm{H}_{3} \mathrm{Cl}$ : AB3_mP16_10_mn_3m3n .........48

7. $\mathbf{m P 1 8}$

7.1. As 2 Ba: A2B_mP18_7_6a_3a ............36

8. $\mathbf{m P 2 2}$

8.1. $\mathrm{Rh}_{2} \mathrm{Ga} 9$ : A9B2_mP22_7_9a_2a .........40

9. $\mathbf{m P 1 2 0}$

9.1. $\alpha$-Toluene:

A7B8_mP120_14_14e_16e 64

oC

1. $\mathbf{0 C 6}$

1.1. Ta $2 \mathrm{H}$ : AB2_oC6_21_a_k 79

2. $\mathbf{o C 8}$

2.1. $\mathrm{HCl}: \mathrm{AB} \_0 \mathrm{C} 8 \_36 \_a \_a$ 


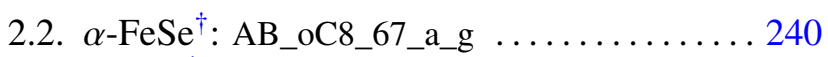

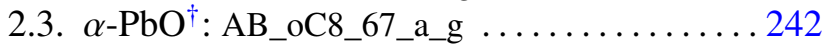

3. $\mathrm{oC16}$

3.1. $\mathrm{K}_{2} \mathrm{CdPb}:$ AB2C_oC16_40_a_2b_b ......... 137

3.2. $\mathrm{CeTe}_{3}$ : AB3_oC16_40_b_3b ............. 139

3.3. $\mathrm{Al}_{2} \mathrm{CuIr}^{\S}$ : A2BC_oC16_67_ag_b_g ........ 236

3.4. $\mathrm{HoCuP}_{2}{ }^{\S}$ : ABC2_oC16_67_b_g_ag ........ 238

4. $\mathbf{0 C 2 0}$

4.1. Post-perovskite: AB3C_oC20_63_a_cf_c ....220

4.2. $\mathrm{PdSn}_{4}: \mathrm{AB} 4 \_\mathrm{oC} 20 \_68 \_\mathrm{a} \_\mathrm{i} . . . . . \ldots \ldots . . .244$

5. $\mathrm{oC22}$

5.1. $\mathrm{V}_{2} \mathrm{MoO}_{8}: \mathrm{AB} 8 \mathrm{C} 2 \_$oC22_35_a_ab3e_e ..... 127

6. оC24

6.1. Rasvumite: A2BC3_oC24_63_e_c_cg ......210

6.2. $\mathrm{MgSO}_{4}$ : AB4C_oC24_63_a_fg_c .........222

6.3. Anhydrite: AB4C_oC24_63_c_fg_c .........224

6.4. $\mathrm{H}_{2} \mathrm{~S}$ : A2B_oC24_64_2f_f ..............226

7. $\mathbf{0} \mathbf{C 2 8}$

7.1. $\mathrm{MnAl}_{6}:$ A6B_oC28_63_efg_c .......... 218

7.2. $\mathrm{SrAl}_{2} \mathrm{Se}_{4}:$ A2B4C_oC28_66_1_kl_a ....... 228

8. $\mathbf{0 C 3 6}$

8.1. $\mathrm{Li}_{2} \mathrm{Si}_{2} \mathrm{O}_{5}$ : A2B5C2_oC36_37_d_c2d_d ..... 131

9. оC40

9.1. $\mathrm{Ta}_{3} \mathrm{~S}_{2}$ : A2B3_oC40_39_2d_2c2d ....... 133

10. оC44

10.1. VPCl : A9BC_oC44_39_3c3d_a_c ..........135

11. оC64

11.1. $\mathrm{H}_{3} \mathrm{~S}$ : A3B_oC64_66_gi2lm_21 .......... 230

11.2. $\beta-\mathrm{ThI}_{3}$ : A3B_oC64_66_k12m_bdl ......... 233

12. $\mathbf{o C 2 6 0}$

12.1. $\mathrm{La}_{43} \mathrm{Ni}_{17} \mathrm{Mg}_{5}$ :

A43B5C17_oC260_63_c8fg6h_cfg_ce3f2h ....212

$\mathbf{o F}$

\section{1. $\mathbf{0 F 8}$}

1.1. FeS: AB_oF8_22_a_c ............. 83

1.2. BN: AB_oF8_42_a_a ............... 143

2. $\mathbf{o F 4 0}$

2.1. $\mathrm{CeRu}_{2} \mathrm{~B}_{2}$ : A2BC2_oF40_22_fi_ad_gh ......81

3. $\mathbf{o F 4 8}$

3.1. $\mathrm{Mn}_{2} \mathrm{~B}$ : AB2_oF48_70_f_fg .......... 246

4. oF52

4.1. $\mathrm{W}_{3} \mathrm{O}_{10}: \mathrm{A} 10 \mathrm{~B} 3 \_\mathrm{oF} 52 \_42 \_2 \mathrm{abce} \_\mathrm{ab} \ldots . . . .141$

oI

1. $\mathbf{0 I 1 2}$

1.1. $\mathrm{BPS}_{4}$ : ABC4_oI12_23_a_b_k ..........91

1.2. NbPS: ABC_oI12_71_h_j_g . . . . . . . . . 250

1.3. $\mathrm{KHg}_{2}$ : A2B_oI12_74_h_e ........... 254

${ }^{\dagger} \alpha$-FeSe and $\alpha$-PbO have the same AFLOW prototype label. They are generated by the same symmetry operations with different sets of parameters.

${ }^{\S} \mathrm{Al}_{2} \mathrm{CuIr}$ and $\mathrm{HoCuP}_{2}$ have similar AFLOW prototype labels (i.e., same symmetry and set of Wyckoff positions with different stoichiometry labels due to alphabetic ordering of atomic species). They are generated by the same symmetry operations with different sets of parameters.
2. $\mathbf{0 I 1 4}$

2.1. $\mathrm{Ta}_{3} \mathrm{~B}_{4}$ : A4B3_oI14_71_gh_cg ........... 248

3. $\mathbf{0 I 1 6}$

3.1. $\mathrm{NaFeS}_{2}$ : ABC2_oI16_23_ab_i_k .........89

4. $\mathbf{0 I 2 0}$

4.1. $\mathrm{MnGa}_{2} \mathrm{Sb}_{2}$ : A2BC2_oI20_45_c_b_c ....... 145

4.2. $\mathrm{Al}_{4} \mathrm{U}$ : A4B_oI20_74_beh_e .............256

5. $\mathbf{0 I 3 2}$

5.1. $\mathrm{H}_{3} \mathrm{~S}$ : A3B_oI32_23_ij2k_k ...........85

6. $\mathbf{o I 3 6}$

6.1. TiFeSi: ABC_oI36_46_ac_bc_3b

7. $\mathbf{0 I 4 4}$

7.1. Weberite: AB7CD2_oI44_24_a_b3d_c_ac .....93

8. $\mathbf{0 I 4 8}$

8.1. $\mathrm{KAg}\left[\mathrm{CO}_{3}\right]$ : ABCD3_oI48_73_d_e_e_ef ..... 252

9. $\mathbf{0 I 5 0}$

9.1. Stannoidite:

A8B2C12D2E_oI50_23_bcfk_i_3k_j_a .......87

oP

1. $\mathbf{o P 6}$

1.1. $\mathrm{FeSb}_{2}$ : AB2_oP6_34_a_c ............. 125

1.2. $\alpha-\mathrm{PdCl}_{2}$ : A2B_oP6_58_g_a ...........176

1.3. FeOCl: ABC_oP6 59_a_b_a .......... 178

2. $\mathbf{0 P 8}$

2.1. Westerveldite: AB_oP8_62_c_c ........... 208

3. $\mathbf{0 P 1 2}$

3.1. $\alpha$-Naumannite: A2B_oP12_17_abe_e .......75

3.2. $\mathrm{H}_{2} \mathrm{~S}^{*}$ : A2B_oP12_26_abc_ab ...........95

3.3. $\beta-\mathrm{SeO}_{2}{ }^{*}$ : A2B_oP12_26_abc_ab ..........97

3.4. $\mathrm{ZrO}_{2}^{\dagger}$ : A2B_oP12_29_2a_a ............. 106

3.5. Pyrite ${ }^{\dagger}$ AB2_oP12_29_a_2a ............. 108

3.6. Cobaltite: ABC_oP12_29_a_a_a ........... 110

3.7. $\mathrm{SrH}_{2}$ : A2B_oP12_62_2c_c ............. 198

4. oP14

4.1. $\beta-\mathrm{Ta}_{2} \mathrm{O}_{5}$ : A5B2_oP14_49_dehq_ab ....... 151

5. $\mathbf{0 P 1 6}$

5.1. $\mathrm{H}_{3} \mathrm{Cl}$ : AB3_oP16_19_a_3a .............77

5.2. TaNiTe 2 : ABC2_oP16_53_h_e_gh ......... 164

5.3. $\mathrm{Rh}_{5} \mathrm{Ge}_{3}$ : A3B5_oP16_55_ch_agh .......... 172

5.4. R-carbon: A_oP16_55_2g2h ............ 174

5.5. $\epsilon-\mathrm{NiAl}_{3}: \mathrm{A} 3 \mathrm{~B} \_\mathrm{oP} 16 \_62 \_c d \_\mathrm{c} . . . \ldots \ldots \ldots .200$

5.6. Molybdite: AB3_oP16_62_c_3c . . . . . . . . . 204

6. $\mathbf{0 P 2 0}$

6.1. $\mathrm{CuBrSe}_{3}: \mathrm{ABC} 3 \_$oP20_30_2a_c_3c ....... 115

6.2. $\mathrm{Sr}_{2} \mathrm{Bi}_{3}$ : A3B2_oP20_52_de_cd ........... 162

6.3. $\mathrm{CuBrSe}_{3}: \mathrm{ABC} 3 \_$oP20_53_e_g_hi ......... 166

6.4. $\mathrm{BiGaO}_{3}:$ ABC3_oP20_54_e_d_cf ........... 168

6.5. $\mathrm{Rh}_{2} \mathrm{~S}_{3}$ : A2B3_oP20_60_d_cd .......... 180

${ }^{*} \mathrm{H}_{2} \mathrm{~S}$ and $\beta-\mathrm{SeO}_{2}$ have the same AFLOW prototype label. They are generated by the same symmetry operations with different sets of parameters.

$\mathrm{ZrO}_{2}$ and Pyrite have similar AFLOW prototype labels (i.e., same symmetry and set of Wyckoff positions with different stoichiometry labels due to alphabetic ordering of atomic species). They are generated by the same symmetry operations with different sets of parameters. 
6.6. Tongbaite: A2B3_oP20_62_2c_3c ........ 194

7. $\mathbf{0 P 2 2}$

7.1. $\mathrm{TiAl}_{2} \mathrm{Br}_{8}$ : A2B8C_oP22_34_c_4c_a $\ldots . . .123$

8. $\mathbf{~} \mathbf{P 2 4}$

8.1. TlP 5 : A5B_oP24_26_3a3b2c_ab ...........99

8.2. $\alpha-\operatorname{RbPr}\left[\mathrm{MoO}_{4}\right]_{2}$ :

A2B8CD_oP24_48_k_2m_d_b ..............149

8.3. $\mathrm{CsPr}\left[\mathrm{MoO}_{4}\right]_{2}$ : AB2C8D_oP24_49_g_q_2qr_e 153

8.4. $\mathrm{GaCl}_{2}$ : A2B_oP24_52_2e_cd ............. 160

8.5. $\mathrm{GeAs}_{2}$ : A2B_oP24_55_2g2h_gh ...........170

8.6. Cubanite: AB2C3_oP24_62_c_d_cd ........202

8.7. Barite: AB4C_oP24_62_c_2cd_c ........... 206

9. $\mathbf{0 P 2 8}$

9.1. $\mathrm{La}_{2} \mathrm{NiO}_{4}$ : A2BC4_oP28_50_ij_ac_ijm ....... 155

9.2. Forsterite: A2B4C_oP28_62_ac_2cd_c ...... 196

10. $\mathbf{o P 3 2}$

10.1. $\mathrm{WO}_{3}$ : A3B_oP32_60_3d_d .............. 182

11. oP34

11.1. $\mathrm{Re}_{2} \mathrm{O}_{5}\left[\mathrm{SO}_{4}\right]_{2}: \mathrm{A} 13 \mathrm{~B} 2 \mathrm{C} 2 \_\mathrm{OP} 34 \_32 \_a 6 c \_c \_c . .117$

12. $\mathbf{o P 4 0}$

12.1. $\kappa$-alumina: A2B3_oP40_33_4a_6a ......... 120

13. oP46

13.1. $\mathrm{Bi}_{5} \mathrm{Nb}_{3} \mathrm{O}_{15}$ : A5B3C15_oP46_30_a2c_bc_a7c .112

14. $\mathbf{o P 4 8}$

14.1. $\alpha-\mathrm{Tl}_{2} \mathrm{TeO}_{3}$ :

A3BC2_oP48_50_3m_m_2m ............ 157

14.2. Benzene: AB_oP48_61_3c_3c . ........... 191

15. $\mathbf{0 P 1 0 8}$

15.1. $\mathrm{Ca}_{4} \mathrm{Al}_{6} \mathrm{O}_{16} \mathrm{~S}$ :

A6B4C16D_oP108_27_abcd4e_4e_16e_e .....101

16. $\mathbf{o P 1 2 0}$

16.1. $\beta$-Toluene: A7B8_oP120_60_7d_8d ........ 185

tI

\section{1. tI4}

1.1. GeP: AB_tI4_107_a_a . ................ 360

1.2. GaSb: AB_tI4_119_c_a ................ 402

2. $\mathrm{tI8}$

2.1. NbAs: AB_tI8_109_a_a ................. 366

2.2. Calomel: AB_tI8_139_e_e ................451

3. $\mathbf{t I 1 2}$

3.1. $\mathrm{CdAs}_{2}$ : A2B_tI12_98_f_a $\ldots \ldots \ldots \ldots \ldots . \ldots 333$

3.2. LaPtSi: ABC_tI12_109_a_a_a ..............364

3.3. $\alpha$-ThSi 2 : A2B_tI12_141_e_a $\ldots . . . . . . .457$

4. $\mathbf{t I 1 6}$

4.1. S-III: A_tI16_142_f . .

5. $\mathbf{t I 2 0}$

5.1. $\mathrm{TlZn}_{2} \mathrm{Sb}_{2}$ : A2BC2_tI20_79_c_2a_c ........277

5.2. $\mathrm{ThCl}_{4}$ : A4B_tI20_88_f_a ................294

6. $\mathrm{tI24}$

6.1. $\mathrm{NaGdCu}_{2} \mathrm{~F}_{8}$ : A2B8CD_tI24_97_d_k_a_b ....329

6.2. $\mathrm{Co}_{5} \mathrm{Ge}_{7}$ : A5B7_tI24_107_ac_abd ...........358

6.3. RbGa $:$ A3B_tI24_119_b2i_af ........... 400

7. $\mathbf{t I 2 8}$

7.1. $\mathrm{KAu}_{4} \mathrm{Sn}_{2}$ : A4BC2_tI28_120_i_d_e ........ 404
8. $\mathbf{t I 3 2}$

8.1. $\mathrm{Ni}_{3} \mathrm{P}:$ A3B_tI32_82_3g_g $\ldots \ldots \ldots \ldots \ldots \ldots 284$

8.2. $\mathrm{Sr}_{5} \mathrm{Si}_{3}$ : A3B5_tI32_108_ac_a2c $\ldots \ldots \ldots \ldots . \ldots 2$

8.3. $\mathrm{W}_{5} \mathrm{Si}_{3}$ : A3B5_tI32_140_ah_bk ........... 453

8.4. $\mathrm{Cr}_{5} \mathrm{~B}_{3}$ : A3B5_tI32_140_ah_cl ............455

9. $\mathbf{t I 4 4}$

9.1. $\mathrm{Ta}_{2} \mathrm{Se}_{8} \mathrm{I}: \mathrm{AB} 8 \mathrm{C} 2$ _tI44_97_e_2k_cd ..........331

10. $\mathbf{t I 4 8}$

10.1. $\beta-\mathrm{NbO}_{2}:$ AB2_tI $48 \_80 \_2 \mathrm{~b} \_4 \mathrm{~b} \ldots . . . . . .279$

11. $\mathbf{t I 9 6}$

11.1. $\alpha-\mathrm{NbO}_{2}$ : AB2_tt96_88_2f_4f $\ldots \ldots \ldots \ldots .296$

12. $\mathbf{t I 1 7 6}$

12.1. $\mathrm{Be}\left[\mathrm{BH}_{4}\right]_{2}$ : A2BC8_tI176_110_2b_b_8b .... 368

tP ....

1. tP5

1.1. $\mathrm{Rh}_{3} \mathrm{P}_{2}$ : A2B3_tP5_115_g_ag ..............387

2. $\mathbf{t P 6}$

2.1. $\mathrm{ZrO}_{2}$ II: A2B_tP6_137_d_a $\ldots \ldots \ldots \ldots \ldots . . . .443$

2.2. $\mathrm{HgI}_{2}{ }^{\mathrm{TI}}:$ AB2_tP6_137_a_d .............. 447

3. $\mathbf{t P 8}$

3.1. NV: AB_tP8_111_n_n ..................376

4. tP10

4.1. $\mathrm{Ti}_{2} \mathrm{Ge}_{3}$ : A3B2_tP10_83_adk $\mathrm{j} \ldots \ldots \ldots \ldots .286$

4.2. $\mathrm{NbTe}_{4}$ : AB4_tP10_103_a_d ................345

4.3. $\mathrm{Pd}_{4} \mathrm{Se}$ : A4B_tP10_114_e_a .............. 385

4.4. $\mathrm{CaRbFe}_{4} \mathrm{As}_{4}$ : A4BC4D_tP10_123_gh_a_i_d . 406

4.5. $\mathrm{NbTe}_{4}$ : AB4_tP10_124_a_m ...............410

4.6. $\mathrm{PtPb}_{4}$ : A4B_tP10_125_m_a ..............412

5. $\mathbf{t P 1 2}$

5.1. $\mathrm{GeSe}_{2}$ : AB2_tP12_81_adg_2h ...........282

5.2. $\mathrm{MnF}_{2}$ : A2B_tP12_111_2n_adf ........... 374

5.3. $\alpha-\mathrm{CuAlCl}_{4}$ : AB4C_tP12_112_b_n_e ....... 378

5.4. $\mathrm{HgI}_{2}: \mathrm{AB} 2 \_t \mathrm{P} 12 \_115 \_\mathrm{j}_{-}$egi $\ldots . . . . . . . . . .389$

5.5. $\mathrm{Nb}_{4} \mathrm{CoSi}$ : AB4C_tP12_124_a_m_c . ........ 408

5.6. $\mathrm{KCeSe}_{4}$ : ABC4_tP12_125_a_b_m ..........4 414

5.7. C: A_tP12_138_bi .................. 449

6. $\mathrm{tP16}$

6.1. LaRhC $_{2}$ : A2BC_tP16_76_2a_a_a ..........262

6.2. RuIn 3 : A3B_tP16_118_ei_f . ............... 395

6.3. AgUF 6 : AB6C_tP16_132_d_io_a ...........431

7. $\mathbf{t P 1 8}$

7.1. $\mathrm{Li}_{2} \mathrm{MoF}_{6}$ : A6B2C_tP18_94_eg_c_a $\ldots \ldots \ldots 325$

7.2. $\mathrm{K}_{2} \mathrm{SnCl}_{6}:$ A6B2C_tP18_128_eh_d_b . . . . . . 420

7.3. $\mathrm{Rb}_{2} \mathrm{TiCu}_{2} \mathrm{~S}_{4}$ : A2B2C4D_tP18_132_e_i_o_d ..429

7.4. $\mathrm{CeCo}_{4} \mathrm{~B}_{4}$ : A4BC4_tP18_137_g_b_g . ....... 445

8. $\mathbf{t P 2 0}$

8.1. $\mathrm{Gd}_{3} \mathrm{Al}_{2}$ : A2B3_tP20_102_2c_b2c ..........343

8.2. $\mathrm{BaGe}_{2} \mathrm{As}_{2}$ : A2BC2_tP20_105_f_ac_2e ...... 352

8.3. $\mathrm{Ru}_{2} \mathrm{Sn}_{3}$ : A2B3_tP20_116_bci_fj ............391

8.4. $\beta-\mathrm{Bi}_{2} \mathrm{O}_{3}$ : A2B3_tP20_117_i_adgh $\ldots . . . \ldots 393$

8.5. ThB 4 : A4B_tP20_127_ehj_g ............. 418

${ }^{I} \mathrm{ZrO}_{2}$ and $\mathrm{HgI}_{2}$ have similar AFLOW prototype labels (i.e., same symmetry and set of Wyckoff positions with different stoichiometry labels due to alphabetic ordering of atomic species). They are generated by the same symmetry operations with different sets of parameters. 


\section{9. $\mathbf{t P 2 2}$}

9.1. $\mathrm{Tl}_{4} \mathrm{HgI}_{6}$ : AB6C4_tP22_104_a_2ac_c ........350

10. $\mathbf{t P 2 4}$

10.1. ThBC: ABC_tP24_91_d_d_d ...............313

10.2. ThBC: ABC_tP24_95_d_d_d ...............327

10.3. Akermanite:

A2BC7D2_tP24_113_e_a_cef_e ............380

10.4. Downeyite: A2B_tP24_135_gh_h ..........436

11. $\mathbf{t P 2 6}$

11.1. Fresnoite:

A2B8C2D_tP26_100_c_abcd_c_a ........... 335

\section{2. $\mathbf{t P 2 8}$}

12.1. $\mathrm{Ba}_{5} \mathrm{In}_{4} \mathrm{Bi}_{5}: \mathrm{A} 5 \mathrm{~B} 5 \mathrm{C} 4 \_\mathrm{tP} 28 \_104 \_\mathrm{ac} \_\mathrm{ac} \_\mathrm{c} \ldots . .347$

12.2. $\mathrm{BiAl}_{2} \mathrm{~S}_{4}$ : A2BC4_tP28_126_cd_e_k .........416

12.3. $\mathrm{CuBi}_{2} \mathrm{O}_{4}$ : A2BC4_tP28_130_f_c_g . ........425

12.4. $\mathrm{ZnSb}_{2} \mathrm{O}_{4}$ : A4B2C_tP28_135_gh_h_d ...... 438

13. tP30

13.1. $\mathrm{SrBr}_{2}$ : A2B_tP30_85_ab2g_cg $\ldots . . . \ldots . .288$

14. $\mathbf{t P 3 2}$

14.1. $\mathrm{Ti}_{3} \mathrm{P}:$ AB3_tP32_86_g_3g ............. 291

14.2. $\gamma$-MgNiSn:

A7B7C2_tP32_101_bde_ade_d . . .......... 340

14.3. $\mathrm{SeO}_{3}$ : A3B_tP32_114_3e_e ............... 382

14.4. $\mathrm{Ir}_{3} \mathrm{Ga}_{5}$ : A5B3_tP32_118_g2i_aceh .......... 397

14.5. $\mathrm{Ba}_{5} \mathrm{Si}_{3}$ : A5B3_tP32_130_cg_cf ..........427

14.6. $\beta$-V ${ }_{3} \mathrm{~S}: \mathrm{AB} 3 \_\mathrm{tP} 32 \_133 \_\mathrm{h} \_\mathrm{i} 2 \mathrm{j} . . . \ldots \ldots . . .433$

\section{5. $\mathbf{t P 4 0}$}

15.1. $\mathrm{Cs}_{3} \mathrm{P}_{7}$ : A3B7_tP40_76_3a_7a ........... 264

15.2. $\mathrm{Na}_{4} \mathrm{Ti}_{2} \mathrm{Si}_{8} \mathrm{O}_{22}\left[\mathrm{H}_{2} \mathrm{O}\right]_{4}$ :

A4B2C13D_tP40_90_g_d_cef2g_c ......... 307

15.3. $\mathrm{Ce}_{3} \mathrm{Si}_{6} \mathrm{~N}_{11}$ :

A3B11C6_tP40_100_ac_bc2d_cd ............337

15.4. $\mathrm{FeCu}_{2} \mathrm{Al}_{7}$ : A7B2C_tP40_128_egi_h_e ........422

15.5. $\mathrm{Zn}_{3} \mathrm{P}_{2}$ : A2B3_tP40_137_cdf_3g ........... 440

16. $\mathbf{t P 4 4}$

16.1. $\mathrm{Na}_{5} \mathrm{Fe}_{3} \mathrm{~F}_{14}$ : A14B3C5_tP44_94_c3g_ad_bg .. 322

\section{7. $\mathbf{t P 4 8}$}

17.1. $\mathrm{H}_{2} \mathrm{~S}$ III: A2B_tP48_77_8d_4d

\section{8. tP54}

18.1. $\mathrm{BaCu}_{4}[\mathrm{VO}]\left[\mathrm{PO}_{4}\right]_{4}$ :

AB4C17D4E_tP54_90_a_g_c4g_g_c .........310

19. tP64

19.1. Pinnoite:

A2B6CD7_tP64_77_2d_6d_d_ab6d .........267

19.2. $\mathrm{NaZn}[\mathrm{OH}]_{3}$ : A3BC3D_tP64_106_3c_c_3c_c .354

20. $\mathbf{t P 7 6}$

20.1. $\mathrm{BaCr}_{2} \mathrm{Ru}_{4} \mathrm{O}_{12}$ :

AB2C12D4_tP76_75_2a2b_2d_12d_4d ......258

\section{1. $\mathbf{t P 8 8}$}

21.1. $\mathrm{Sr}_{2} \mathrm{As}_{2} \mathrm{O}_{7}$ : A2B7C2_tP88_78_4a_14a_4a $\ldots .273$

22. $\mathbf{t P 1 8 4}$

22.1. $\mathrm{C}_{17} \mathrm{FeO}_{4} \mathrm{Pt}$ :

A17BC4D_tP184_89_17p_p_4p_io .......... 300

22.2. $\mathrm{AsPh}_{4} \mathrm{CeS}_{8} \mathrm{P}_{4} \mathrm{Me}_{8}$ :

AB32CD4E8_tP184_93_i_16p_af_2p_4p ..... 315

\section{Strukturbericht Designation Index}

B7

1. Moissanite-15R: AB_hR10_160_5a_5a .........519

\section{B14}

1. Westerveldite: AB_oP8_62_c_c ..............208

\section{C13}

1. $\mathrm{HgI}_{2}{ }^{\mathrm{II}}:$ AB2_tP6_137_a_d

C29

1. $\mathrm{SrH}_{2}:$ A2B_oP12_62_2c_c ................... 198

C47 ......................................

1. Downeyite: A2B_tP24_135_gh_h .............436

C50

1. $\alpha$-PdCl 2 : A2B_oP6_58_g_a $\ldots \ldots \ldots \ldots \ldots \ldots 176$

$C_{c}$

1. $\alpha$-ThSi 2 : A2B tII12_141_e a .................457

$\mathrm{DO}_{8}$

1. Molybdite: AB3_oP16_62_c_3c ...............204

D0 $0_{20}$.......................................

1. $\epsilon-\mathrm{NiAl}_{3}$ : A3B_oP16_62_cd_c $\ldots . . . \ldots \ldots \ldots . . .200$

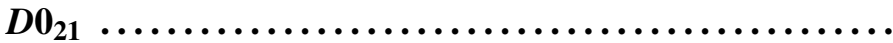

1. $\mathrm{Cu}_{3} \mathrm{P}: \mathrm{A} 3 \mathrm{~B} \_$hP24_165_bdg_f $\ldots . . . \ldots \ldots \ldots . . .527$

$D_{24}$...................................

1. Ni ${ }_{3} \mathrm{Ti}:$ A3B_hP16_194_gh_ac ................661 641

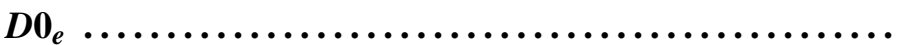

1. $\mathrm{Ni}_{3} \mathrm{P}:$ A3B_tI32_82_3g_g $\ldots . \ldots \ldots \ldots \ldots \ldots . . .284$

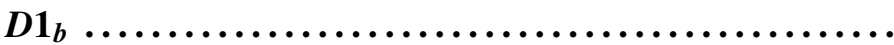

1. $\mathrm{Al}_{4} \mathrm{U}$ : A4B_ol20_74_beh_e $\ldots \ldots \ldots \ldots \ldots \ldots . . .256$

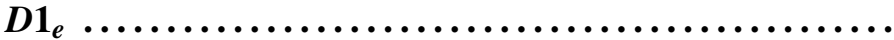

1. ThB 4 : A4B_tP20_127_ehj_g ................4 418

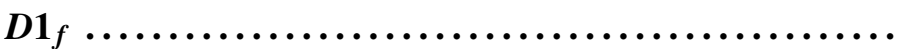

1. $\mathrm{Mn}_{2} \mathrm{~B}: \mathrm{AB} 2 \_\mathrm{oF} 48 \_70 \_f \_f g \ldots \ldots \ldots \ldots \ldots . \ldots 246$

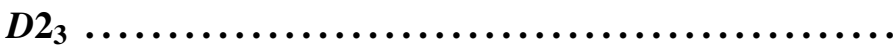

1. $\mathrm{NaZn}_{13}: \mathrm{AB} 13 \_c F 112 \_226 \_a \_b i \ldots . . . \ldots \ldots . . .771$

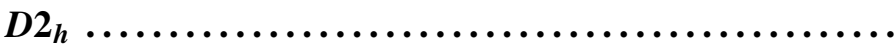

1. $\mathrm{MnAl}_{6}:$ A6B_oC28_63_efg_c $\ldots \ldots \ldots \ldots \ldots . . .218$

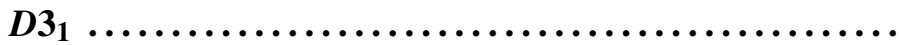

1. Calomel: AB_tI8_139_e_e ...................451

$\mathrm{DS}_{2}$

1. $\mathrm{La}_{2} \mathrm{O}_{3}$ : A2B3_hP5_164_d_ad ...............5 521

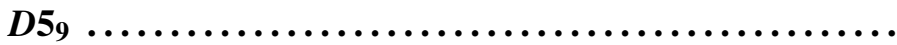

1. $\mathrm{Zn}_{3} \mathrm{P}_{2}:$ A2B3_tP40_137_cdf_3g ..............440

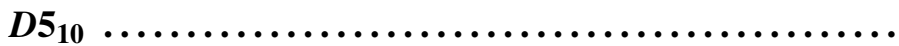

1. Tongbaite: A2B3_oP20_62_2c_3c ............ 194

D7 1 ..................................

1. $\mathrm{Al}_{4} \mathrm{C}_{3}$ : A4B3_hR7_166_2c_ac $\ldots \ldots \ldots \ldots \ldots \ldots 530$

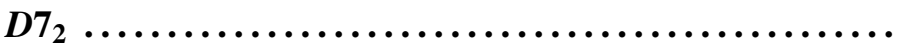

1. Spinel: A3B4_cF56_227_ad_e ............... 776 $D 7_{3}$

\footnotetext{
${ }^{\mathrm{I}} \mathrm{ZrO}_{2}$ and $\mathrm{HgI}_{2}$ have similar AFLOW prototype labels (i.e., same symmetry and set of Wyckoff positions with different stoichiometry labels due to alphabetic ordering of atomic species). They are generated by the same symmetry operations with different sets of parameters.
} 
1. $\mathrm{Th}_{3} \mathrm{P}_{4}:$ A4B3_cI28_220_c_a $\ldots \ldots \ldots \ldots \ldots \ldots .756$

$D_{b}$

1. $\mathrm{Ta}_{3} \mathrm{~B}_{4}$ : A4B3_oI14_71_gh_cg .............. 248

$D 8_{1}$

1. $\gamma$-brass: A3B10_cI52_229_e_fh ........... 790

$D 8_{3}$

1. $\gamma$-brass: A4B9_cP52_215_ei_3efgi ...........741

$D_{6}$

1. $\mathrm{Cu}_{15} \mathrm{Si}_{4}: \mathrm{A} 15 \mathrm{~B} 4 \_\mathrm{cI76} 2220 \_\mathrm{ae} \_\mathrm{c} \ldots \ldots \ldots \ldots \ldots . \ldots 753$

$D 8_{10}$

1. $\mathrm{Al}_{8} \mathrm{Cr}_{5}: \mathrm{A} 8 \mathrm{~B} 5 \_$hR26_160_a3bc_a3b ......... 514

D811

1. $\mathrm{Co}_{2} \mathrm{Al}_{5}$ : A5B2_hP28_194_ahk_ch ..........643

$D 8_{a}$

1. $\mathrm{Th}_{6} \mathrm{Mn}_{23}:$ A23B6_cF116_225_bd2f_e ..........766

$D 8_{f}$

1. $\mathrm{Ir}_{3} \mathrm{Ge}_{7}$ : A7B3_cI40_229_df_e ............... 795

$D 8_{l}$

1. $\mathrm{Cr}_{5} \mathrm{~B}_{3}$ : A3B5_tI32_140_ah_cl .............455

$D 8_{m}$

1. $\mathrm{W}_{5} \mathrm{Si}_{3}:$ A3B5_tI32_140_ah_bk

453

$\mathrm{D10}_{2}$

1. $\mathrm{Fe}_{3} \mathrm{Th}_{7}: \mathrm{A} 3 \mathrm{~B} 7 \_$hP20_186_c_b2c

610

E91

1. $\mathrm{Ca}_{3} \mathrm{Al}_{2} \mathrm{O}_{6}$ : A2B3C6_cP33_221_cd_ag_fh ........758

E9a

1. $\mathrm{FeCu}_{2} \mathrm{Al}_{7}$ : A7B2C_tP40_128_egi_h_e .........422

$E 9_{b}$

1. $\pi-\mathrm{FeMg}_{3} \mathrm{Al}_{8} \mathrm{Si}_{6}$ :

A8BC3D6_hP18_189_bfh_a_g_i ............619

E9.

1. $\mathrm{Al}_{9} \mathrm{Mn}_{3} \mathrm{Si}:$ A9B3C_hP26_194_hk_h_a ........6 646

E9

1. $\mathrm{AlLi}_{3} \mathrm{~N}_{2}$ : AB3C2_cI96_206_c_e_ad ............704

E9.

1. Cubanite: AB2C3_oP24_62_c_d_cd ...........202

$\mathrm{FO}_{2}$

1. Carbonyl Sulphide: ABC_hR3_160_a_a_a .......517

$F 5_{13}$

1. $\mathrm{KBO}_{2}$ : ABC2_hR24_167_e_e_2e ...........536 G3

1. Sodium Chlorate: ABC3_cP20_198_a_a_b .......661 661 G31

1. Beryl: A2B3C18D6_hP58_192_c_f_lm_l

.631

$\mathrm{HO}_{1}$

1. Anhydrite: AB4C_oC24_63_c_fg_c ...........224

$\mathrm{HO}_{2}$

1. Barite: AB4C_oP24_62_c_2cd_c ............. 206

$J 1_{1}$

1. $\mathrm{K}_{2} \mathrm{PtCl}_{6}$ : A6B2C_cF36_225_e_c_a ........... 769

$\mathrm{S1}_{2}$

1. Forsterite: A2B4C_oP28_62_ac_2cd_c ......... 196

$\mathrm{S1}_{3}$
1. Phenakite: A2B4C_hR42_148_2f_4f_f 479

$\mathrm{S1}_{4}$

1. Garnet: A2B3C12D3_cI160_230_a_c_h_d

797

$S 2_{1}$

1. Thortveitite: A7B2C2_mC22_12_aij_h_i .........56

$\mathrm{S5}_{3}$

1. Akermanite: A2BC7D2_tP24_113_e_a_cef_e ..... 380

None

1. $\mathrm{H}_{2} \mathrm{~S}$ : A2B_aP6_2_aei_i .................. 28

2. $\mathrm{Mo}_{8} \mathrm{P}_{5}$ : A8B5_mP13_6_a7b_3a2b ......... 30

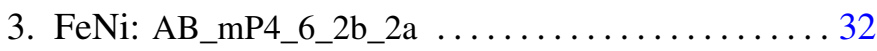

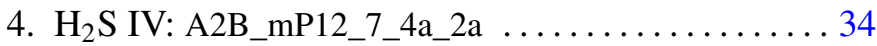

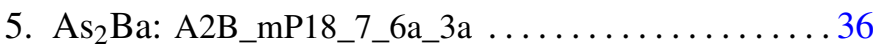

6. $\epsilon-\mathrm{WO}_{3}:$ A3B_mP16_7_6a_2a ............... 38

7. $\mathrm{Rh}_{2} \mathrm{Ga}_{9}:$ A9B2_mP22_7_9a_2a ...........40

8. $\alpha-\mathrm{P}_{3} \mathrm{~N}_{5}$ : A5B3_mC32_9_5a_3a .............42

9. $\mathrm{H}_{3} \mathrm{Cl}$ : AB3_mC16_9_a_3a ................44

10. $\delta$ - $\mathrm{PdCl}_{2}$ : A2B_mP6_10_mn_bg $\ldots \ldots \ldots \ldots \ldots \ldots 46$

11. $\mathrm{H}_{3} \mathrm{Cl}$ : AB3_mP16_10_mn_3m3n ...........48

12. Muthmannite: ABC2_mP8_10_ac_eh_mn ........50

13. LiSn: AB_mP6_10_en_am ............... 52

14. S-carbon: A_mP8_10_2m2n ............. 54

15. M-carbon: A_mC16_12_4i ............. 58

16. $\mathrm{H}_{2} \mathrm{~S}$ : A2B_mP12_13_2g_ef $\ldots \ldots \ldots \ldots \ldots \ldots \ldots 60$

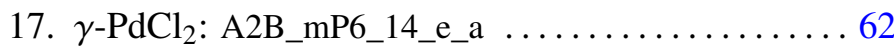

18. $\alpha$-Toluene: A7B8_mP120_14_14e_16e .........64 64

19. $\mathrm{H}_{3} \mathrm{Cl}$ : AB3_mC16_15_e_cf ............... 71

20. H-III: A_mC24_15_2e2f ................73

21. $\alpha$-Naumannite: A2B_oP12_17_abe_e .........75

22. $\mathrm{H}_{3} \mathrm{Cl}$ : AB3_oP16_19_a_3a ............... 77

23. $\mathrm{Ta}_{2} \mathrm{H}$ : AB2_oC6_21_a_k ................. 79

24. $\mathrm{CeRu}_{2} \mathrm{~B}_{2}$ : A2BC2_oF40_22_fi_ad_gh .........81

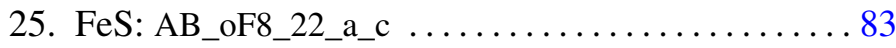

26. $\mathrm{H}_{3} \mathrm{~S}$ : A3B_oI32_23_ij2k_k ............. 85

27. Stannoidite:

A8B2C12D2E_oI50_23_bcfk_i_3k_j_a .........887

28. $\mathrm{NaFeS}_{2}$ : ABC2_oI16_23_ab_i_k .............89

29. $\mathrm{BPS}_{4}$ : ABC4_oI12_23_a_b_k ............. 91

30. Weberite: AB7CD2_oI44_24_a_b3d_c_ac ........99

31. $\mathrm{H}_{2} \mathrm{~S}^{*}$ : A2B_oP12_26_abc_ab ............. 95

32. $\beta-\mathrm{SeO}_{2}{ }^{*}$ : A2B_oP12_26_abc_ab ........... 97

33. T1P $:$ A5B_oP24_26_3a3b2c_ab ............ 99

34. $\mathrm{Ca}_{4} \mathrm{Al}_{6} \mathrm{O}_{16} \mathrm{~S}$ :

A6B4C16D_oP108_27_abcd4e_4e_16e_e ..........101

35. $\mathrm{ZrO}_{2}^{\dagger}$ : A2B_oP12_29_2a_a ............. 106

${ }^{*} \mathrm{H}_{2} \mathrm{~S}$ and $\beta-\mathrm{SeO}_{2}$ have the same AFLOW prototype label. They are generated by the same symmetry operations with different sets of parameters.

${ }^{\ddagger} \mathrm{ZrO}_{2}$ and Pyrite have similar AFLOW prototype labels (i.e., same symmetry and set of Wyckoff positions with different stoichiometry labels due to alphabetic ordering of atomic species). They are generated by the same symmetry operations with different sets of parameters. 
36. Pyrite ${ }^{\ddagger}$ AB2_oP12_29_a_2a ................ 108

37. Cobaltite: ABC_oP12_29_a_a_a ...............110

38. $\mathrm{Bi}_{5} \mathrm{Nb}_{3} \mathrm{O}_{15}$ : A5B3C15_oP46_30_a2c_bc_a7c . .... 112

39. $\mathrm{CuBrSe}_{3}$ : ABC3_oP20_30_2a_c_3c ............115

40. $\mathrm{Re}_{2} \mathrm{O}_{5}\left[\mathrm{SO}_{4}\right]_{2}$ : A13B2C2_oP34_32_a6c_c_c .......117

41. $\kappa$-alumina: A2B3_oP40_33_4a_6a $\ldots \ldots \ldots \ldots \ldots 120$

42. $\mathrm{TiAl}_{2} \mathrm{Br}_{8}$ : A2B8C_oP22_34_c_4c_a $\ldots \ldots \ldots \ldots . .123$

43. $\mathrm{FeSb}_{2}$ : AB2_oP6_34_a_c .................. 125

44. $\mathrm{V}_{2} \mathrm{MoO}_{8}$ : AB8C2_oC22_35_a_ab3e_e $\ldots \ldots \ldots \ldots 127$

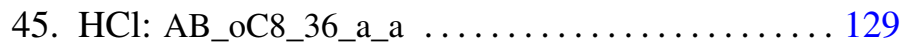

46. $\mathrm{Li}_{2} \mathrm{Si}_{2} \mathrm{O}_{5}$ : A2B5C2_oC36_37_d_c2d_d .......... 131

47. $\mathrm{Ta}_{3} \mathrm{~S}_{2}$ : A2B3_oC40_39_2d_2c2d $\ldots \ldots \ldots \ldots \ldots \ldots .133$

48. VPCl 9 : A9BC_oC44_39_3c3d_a_c ..............135

49. $\mathrm{K}_{2} \mathrm{CdPb}$ : AB2C_oC16_40_a_2b_b ............. 137

50. $\mathrm{CeTe}_{3}: \mathrm{AB} 3 \_0 \mathrm{C} 16 \_40 \_b \_3 b \quad \ldots . . . . . . . . . . .139$

51. $\mathrm{W}_{3} \mathrm{O}_{10}$ : A10B3_oF52_42_2abce_ab ............ 141

52. BN: AB_oF8_42_a_a ...................... 143

53. $\mathrm{MnGa}_{2} \mathrm{Sb}_{2}$ : A2BC2_oI20_45_c_b_c $\ldots \ldots \ldots \ldots . .145$

54. TiFeSi: ABC_ol36_46_ac_bc_3b ............... 147

55. $\alpha-\operatorname{RbPr}\left[\mathrm{MoO}_{4}\right]_{2}$ :

A2B8CD_oP24_48_k_2m_d_b ................. 149

56. $\beta-\mathrm{Ta}_{2} \mathrm{O}_{5}$ : A5B2_oP14_49_dehq_ab ............ 151

57. $\mathrm{CsPr}\left[\mathrm{MoO}_{4}\right]_{2}$ : AB2C8D_oP24_49_g_q_2qr_e .....153

58. $\mathrm{La}_{2} \mathrm{NiO}_{4}$ : A2BC4_oP28_50_ij_ac_ijm ............ 155

59. $\alpha-\mathrm{Tl}_{2} \mathrm{TeO}_{3}$ : A3BC2_oP48_50_3m_m_2m $\ldots \ldots \ldots 157$

60. $\mathrm{GaCl}_{2}$ : A2B_oP24_52_2e_cd ................ 160

61. $\mathrm{Sr}_{2} \mathrm{Bi}_{3}: \mathrm{A} 3 \mathrm{~B} 2 \_\mathrm{OP} 20 \_52 \_\mathrm{de} \_\mathrm{cd} \ldots \ldots \ldots \ldots \ldots \ldots 2$

62. TaNiTe 2 : ABC2_oP16_53_h_e_gh .............. 164

63. $\mathrm{CuBrSe}_{3}$ : ABC3_oP20_53_e_g_hi .............. 166

64. $\mathrm{BiGaO}_{3}$ : ABC3_oP20_54_e_d_cf ................ 168

65. GeAs 2 : A2B_oP24_55_2g2h_gh .............. 170

66. $\mathrm{Rh}_{5} \mathrm{Ge}_{3}$ : A3B5_oP16_55_ch_agh .............. 172

67. R-carbon: A_oP16_55_2g2h .................. 174

68. FeOCl: ABC_oP6_59_a_b_a .................. 178

69. $\mathrm{Rh}_{2} \mathrm{~S}_{3}$ : A2B3_oP20_60_d_cd ............... 180

70. $\mathrm{WO}_{3}$ : A3B_oP32_60_3d_d $\ldots . . . \ldots \ldots \ldots \ldots . \ldots 182$

71. $\beta$-Toluene: A7B8_oP120_60_7d_8d ............ 185

72. Benzene: AB_oP48_61_3c_3c .............. 191

73. Rasvumite: A2BC3_oC24_63_e_c_cg ..........210

74. $\mathrm{La}_{43} \mathrm{Ni}_{17} \mathrm{Mg}_{5}$ :

A43B5C17_oC260_63_c8fg6h_cfg_ce3f2h ........212

75. Post-perovskite: AB3C_oC20_63_a_cf_c ........220

76. $\mathrm{MgSO}_{4}$ : AB4C_oC24_63_a_fg_c .............222

77. $\mathrm{H}_{2} \mathrm{~S}$ : A2B_oC24_64_2f_f $\ldots \ldots \ldots \ldots \ldots \ldots . . .226$

78. $\mathrm{SrAl}_{2} \mathrm{Se}_{4}$ : A2B4C_oC28_66_1_kl_a ...........228

79. $\mathrm{H}_{3} \mathrm{~S}$ : A3B_oC64_66_gi2lm_21 ..............230

80. $\beta$-ThI ${ }_{3}$ : A3B_oC64_66_k12m_bdl .............233

81. $\mathrm{Al}_{2} \mathrm{CuIr}{ }^{\S}$ : A2BC_oC16_67_ag_b_g ............236

\footnotetext{
${ }^{\S} \mathrm{Al}_{2} \mathrm{CuIr}$ and $\mathrm{HoCuP}_{2}$ have similar AFLOW prototype labels (i.e., same symmetry and set of Wyckoff positions with different stoichiometry labels due to alphabetic ordering of atomic species). They are generated by the same symmetry operations with different sets of parameters.
}

82. $\mathrm{HoCuP}_{2}^{\S}$ : ABC2_oC16_67_b_g_ag ............2238

83. $\alpha$-FeSe ${ }^{\dagger}:$ AB_oC8_67_a_g .................. 240

84. $\alpha-\mathrm{PbO}^{\dagger}$ : AB_oC8_67_a_g .................. 242

85. PdSn 4 : AB4_oC20_68_a_i ...................244

86. NbPS: ABC_oI12_71_h_j_g ............... 250

87. $\mathrm{KAg}\left[\mathrm{CO}_{3}\right]$ : ABCD3_oI48_73_d_e_e_ef ......... 252

88. $\mathrm{KHg}_{2}$ : A2B_oI12_74_h_e ................. 254

89. $\mathrm{BaCr}_{2} \mathrm{Ru}_{4} \mathrm{O}_{12}$ : AB2C12D4_tP76_75_2a2b_2d_12d_4d ..........258

90. LaRhC $_{2}$ : A2BC_tP16_76_2a_a_a ..............262

91. $\mathrm{Cs}_{3} \mathrm{P}_{7}$ : A3B7_tP40_76_3a_7a ................. 264

92. Pinnoite: A2B6CD7_tP64_77_2d_6d_d_ab6d ......267

93. $\mathrm{H}_{2}$ S III: A2B_tP48_77_8d_4d ............... 270

94. $\mathrm{Sr}_{2} \mathrm{As}_{2} \mathrm{O}_{7}$ : A2B7C2_tP88_78_4a_14a_4a $\ldots \ldots \ldots 273$

95. $\mathrm{TlZn}_{2} \mathrm{Sb}_{2}$ : A2BC2_tI20_79_c_2a_c ............277

96. $\beta-\mathrm{NbO}_{2}$ : AB2_tI48_80_2b_4b ...............279

97. $\mathrm{GeSe}_{2}$ : AB2_tP12_81_adg_2h ...............282

98. $\mathrm{Ti}_{2} \mathrm{Ge}_{3}:$ A3B2_tP10_83_adk_j ...............286

99. $\mathrm{SrBr}_{2}$ : A2B_tP30_85_ab2g_cg $\ldots \ldots \ldots \ldots \ldots . \ldots 288$

100. Ti ${ }_{3} \mathrm{P}:$ AB3_tP32_86_g_3g ................. 291

101. $\mathrm{ThCl}_{4}$ : A4B_tI20_88_f_a .................. 294

102. $\alpha-\mathrm{NbO}_{2}$ : AB2_tI96_88_2f_4f . .............. 296

103. $\mathrm{C}_{17} \mathrm{FeO}_{4} \mathrm{Pt}$ : A17BC4D_tP184_89_17p_p_4p_io ....300

104. $\mathrm{Na}_{4} \mathrm{Ti}_{2} \mathrm{Si}_{8} \mathrm{O}_{22}\left[\mathrm{H}_{2} \mathrm{O}\right]_{4}$ : A4B2C13D_tP40_90_g_d_cef2g_c ............. 307

105. $\mathrm{BaCu}_{4}[\mathrm{VO}]\left[\mathrm{PO}_{4}\right]_{4}$ : AB4C17D4E_tP54_90_a_g_c4g_g_c ............310

106. ThBC: ABC_tP24_91_d_d_d ...................313

107. $\mathrm{AsPh}_{4} \mathrm{CeS}_{8} \mathrm{P}_{4} \mathrm{Me}_{8}$ :

AB32CD4E8_tP184_93_i_16p_af_2p_4p ......... 315

108. $\mathrm{Na}_{5} \mathrm{Fe}_{3} \mathrm{~F}_{14}$ : A14B3C5_tP44_94_c3g_ad_bg .......322

109. $\mathrm{Li}_{2} \mathrm{MoF}_{6}$ : A6B2C_tP18_94_eg_c_a ............ 325

110. ThBC: ABC_tP24_95_d_d_d ....................327

111. $\mathrm{NaGdCu}_{2} \mathrm{~F}_{8}$ : A2B8CD_tI24_97_d_k_a_b .........329

112. $\mathrm{Ta}_{2} \mathrm{Se}_{8} \mathrm{I}: \mathrm{AB} 8 \mathrm{C} 2$ _tI 44 _97_e_2k_cd ................331

113. CdAs 2 : A2B_tI12_98_f_a .................... 333

114. Fresnoite: A2B8C2D_tP26_100_c_abcd_c_a . . ... 335

115. $\mathrm{Ce}_{3} \mathrm{Si}_{6} \mathrm{~N}_{11}$ : A3B11C6_tP40_100_ac_bc2d_cd ..... 337

116. $\gamma$-MgNiSn: A7B7C2_tP32_101_bde_ade_d ...... 340

117. $\mathrm{Gd}_{3} \mathrm{Al}_{2}$ : A2B3_tP20_102_2c_b2c ...............343

118. $\mathrm{NbTe}_{4}$ : AB4_tP10_103_a_d ....................345

119. $\mathrm{Ba}_{5} \mathrm{In}_{4} \mathrm{Bi}_{5}$ : A5B5C4_tP28_104_ac_ac_c ..........347

120. $\mathrm{Tl}_{4} \mathrm{HgI}_{6}$ : AB6C4_tP22_104_a_2ac_c ............ 350

121. $\mathrm{BaGe}_{2} \mathrm{As}_{2}$ : A2BC2_tP20_105_f_ac_2e ...........352

122. $\mathrm{NaZn}[\mathrm{OH}]_{3}:$ A3BC3D_tP64_106_3c_c_3c_c ...... 354

123. $\mathrm{Co}_{5} \mathrm{Ge}_{7}$ : A5B7_tI24_107_ac_abd ................358

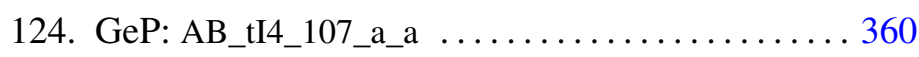

125. $\mathrm{Sr}_{5} \mathrm{Si}_{3}$ : A3B5_tI32_108_ac_a2c $\ldots \ldots \ldots \ldots \ldots . \ldots 362$

\footnotetext{
${ }^{\dagger} \alpha$-FeSe and $\alpha$-PbO have the same AFLOW prototype label. They are generated by the same symmetry operations with different sets of parameters.
} 
126. LaPtSi: ABC_tI12_109_a_a_a .................364

127. NbAs: AB_tI8_109_a_a ...................... 366

128. $\mathrm{Be}\left[\mathrm{BH}_{4}\right]_{2}$ : A2BC8_tI176_110_2b_b_8b ........ 368

129. $\mathrm{MnF}_{2}$ : A2B_tP12_111_2n_adf .............. 374

130. NV: AB_tP8_111_n_n .......................376

131. $\alpha$-CuAlCl 4 : AB4C_tP12_112_b_n_e ............378

132. $\mathrm{SeO}_{3}$ : A3B_tP32_114_3e_e ...................382

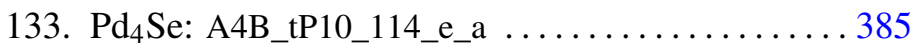

134. $\mathrm{Rh}_{3} \mathrm{P}_{2}$ : A2B3_tP5_115_g_ag ................... 387

135. $\mathrm{HgI}_{2}:$ AB2_tP12_115_j_egi .................... 389

136. $\mathrm{Ru}_{2} \mathrm{Sn}_{3}$ : A2B3_tP20_116_bci_fj ................391

137. $\beta-\mathrm{Bi}_{2} \mathrm{O}_{3}$ : A2B3_tP20_117_i_adgh $\ldots \ldots \ldots \ldots \ldots 393$

138. $\mathrm{RuIn}_{3}$ : A3B_tP16_118_ei_f .................. 395

139. $\mathrm{Ir}_{3} \mathrm{Ga}_{5}$ : A5B3_tP32_118_g2i_aceh ............. 397

140. RbGa $:$ A3B_tI24_119_b2i_af ............... 400

141. GaSb: AB_tI4_119_c_a .................... 402

142. $\mathrm{KAu}_{4} \mathrm{Sn}_{2}$ : A4BC2_tI28_120_i_d_e ............ 404

143. $\mathrm{CaRbFe}_{4} \mathrm{As}_{4}$ : A4BC4D_tP10_123_gh_a_i_d ...... 406

144. $\mathrm{Nb}_{4} \mathrm{CoSi}$ : AB4C_tP12_124_a_m_c ............ 408

145. $\mathrm{NbTe}_{4}$ : AB4_tP10_124_a_m .................410

146. $\mathrm{PtPb}_{4}$ : A4B_tP10_125_m_a $\ldots \ldots \ldots \ldots \ldots \ldots . \ldots 12$

147. $\mathrm{KCeSe}_{4}$ : ABC4_tP12_125_a_b_m ............. 414

148. $\mathrm{BiAl}_{2} \mathrm{~S}_{4}$ : A2BC4_tP28_126_cd_e_k ............416

149. $\mathrm{K}_{2} \mathrm{SnCl}_{6}$ : A6B2C_tP18_128_eh_d_b ...........420

150. $\mathrm{CuBi}_{2} \mathrm{O}_{4}$ : A2BC4_tP28_130_f_c_g $\ldots \ldots \ldots \ldots . . .425$

151. $\mathrm{Ba}_{5} \mathrm{Si}_{3}:$ A5B3_tP32_130_cg_cf ..............427

152. $\mathrm{Rb}_{2} \mathrm{TiCu}_{2} \mathrm{~S}_{4}$ : A2B2C4D_tP18_132_e_i_o_d ......4429

153. AgUF 6 : AB6C_tP16_132_d_io_a .............441

154. $\beta-\mathrm{V}_{3} \mathrm{~S}: \mathrm{AB} 3 \_\mathrm{tP} 32 \_133 \_\mathrm{h} \_\mathrm{i} 2 \mathrm{j} . . . \ldots \ldots \ldots \ldots . . \ldots 433$

155. $\mathrm{ZnSb}_{2} \mathrm{O}_{4}$ : A4B2C_tP28_135_gh_h_d .......... 438

156. $\mathrm{ZrO}_{2}$ II: A2B_tP6_137_d_a ...................443

157. $\mathrm{CeCo}_{4} \mathrm{~B}_{4}$ : A4BC4_tP18_137_g_b_g ........... 445

158. C: A_tP12_138_bi ........................449

159. S-III: A_tI16_142_f ......................459

160. Simpsonite: A4B14C3_hP21_143_bd_ac4d_d ..... 461

161. $\mathrm{ScRh}_{6} \mathrm{P}_{4}$ : A4B6C_hP11_143_bd_2d_a ......... 463

162. $\mathrm{MoS}_{2}$ : AB2_hP12_143_cd_ab2d ..............465

163. $\mathrm{IrGe}_{4}$ : A4B_hP15_144_4a_a ................ 467

164. TeZn: AB_hP6_144_a_a .....................469

165. Sheldrickite:

A2B3C3DE7_hP48_145_2a_3a_3a_a_7a ........ 471

166. $\gamma$-Agg 3 SI: A3BC_hR5_146_b_a_a .............. 475

167. $\mathrm{FePSe}_{3}$ : ABC3_hR10_146_2a_2a_2b ............477

168. $\beta$-PdCl $\mathrm{Pd}_{2}$ : A2B_hR18_148_2f_f $\ldots \ldots \ldots \ldots \ldots . . . . .483$

169. $\mathrm{Ti}_{3} \mathrm{O}: \mathrm{AB} 3$ hP24_149_acgi_31 ...............486

170. $\mathrm{CrCl}_{3}$ : A3B_hP24_153_3c_2b ................488

171. S-II: A_hP9_154_bc ..................... 491

172. CdI $_{2}$ : AB2_hP9_156_b2c_3a2bc .............493

173. CuI: AB_hP12_156_2ab3c_2ab3c ..............495

174. $\beta$-CuI: AB_hP4_156_ac_ac .................. 497

175. $\mathrm{Ag}_{5} \mathrm{~Pb}_{2} \mathrm{O}_{6}$ : A5B6C2_hP13_157_2ac_2c_b .......499

176. $\beta$-RuCl ${ }_{3}$ : A3B_hP8_158_d_a ................501
177. $\mathrm{Bi}_{2} \mathrm{O}_{3}:$ A2B3_hP20_159_bc_2c $\ldots \ldots \ldots \ldots \ldots . \ldots 503$

178. Nierite: A4B3_hP28_159_ab2c_2c ............. 506

179. $\mathrm{YbBaCo}_{4} \mathrm{O}_{7}$ : AB4C7D_hP26_159_b_ac_a2c_b ....509

180. $\mathrm{H}_{3} \mathrm{~S}$ : A3B_hR4_160_b_a .................. 512

181. $\delta_{H}^{I I}-\mathrm{NW}_{2}$ : AB2_hP9_164_bd_c2d .............5 523

182. CuNiSb 2 : ABC2_hP4_164_a_b_d ............. 525

183. SmSI: ABC_hR6_166_c_c_c ................. 532

184. $\mathrm{PrNiO}_{3}$ : AB3C_hR10_167_b_e_a ..............554

185. $\mathrm{K}_{2} \mathrm{Ta}_{4} \mathrm{O}_{9} \mathrm{~F}_{4}$ : A2B13C4_hP57_168_d_c6d_2d ......539

186. $\mathrm{Al}\left[\mathrm{PO}_{4}\right]$ : AB4C_hP72_168_2d_8d_2d ...........5543

187. $\alpha-\mathrm{Al}_{2} \mathrm{~S}_{3}$ : A2B3_hP30_169_2a_3a ............554

188. $\mathrm{Al}_{2} \mathrm{~S}_{3}$ : A2B3_hP30_170_2a_3a ..............550

189. $\mathrm{Sr}\left[\mathrm{S}_{2} \mathrm{O}_{6}\right]\left[\mathrm{H}_{2} \mathrm{O}\right]_{4}$ : A10B2C_hP39_171_5c_c_a .....553

190. $\mathrm{Sr}\left[\mathrm{S}_{2} \mathrm{O}_{6}\right]\left[\mathrm{H}_{2} \mathrm{O}\right]_{4}$ : A10B2C_hP39_172_5c_c_a . ....557

191. $\mathrm{PI}_{3}$ : A3B_hP8_173_c_b ................... 560

192. $\beta-\mathrm{Si}_{3} \mathrm{~N}_{4}$ : A4B3_hP14_173_bc_c ..............562

193. $\mathrm{Fe}_{12} \mathrm{Zr}_{2} \mathrm{P}_{7}$ : A12B7C2_hP21_174_2j2k_ajk_cf ..... 564

194. GdSI: ABC_hP12_174_cj_fk_aj ............... 566

195. $\mathrm{Nb}_{7} \mathrm{Ru}_{6} \mathrm{~B}_{8}$ : A8B7C6_hP21_175_ck_aj_k ........ 568

196. $\mathrm{Mg}[\mathrm{NH}]$ : ABC_hP36_175_jk_jk_jk ...........5570

197. $\mathrm{Er}_{3} \mathrm{Ru}_{2}$ : A3B2_hP10_176_h_bd .............. 573

198. $\mathrm{Fe}_{3} \mathrm{Te}_{3} \mathrm{Tl}$ : A3B3C_hP14_176_h_h_d ............5 575

199. UCl U $_{3}$ : A3B_hP8_176_h_d .................. 577

200. $\mathrm{SiO}_{2}$ : A2B_hP36_177_j2lm_n ................5579

201. $A u F_{3}:$ AB3_hP24_178_b_ac ................ 582

202. Sc-V: A_hP6_178_a ..................... 584

203. $\mathrm{AuF}_{3}$ : AB3_hP24_179_b_ac ................ 586

204. $\beta-\mathrm{SiO}_{2}$ : A2B_hP9_181_j_c $\ldots . . . \ldots \ldots \ldots . \ldots 58$

205. AuCN: ABC_hP3_183_a_a_a ................ 590

206. $\mathrm{CrFe}_{3} \mathrm{NiSn}_{5}$ : AB_hP6_183_c_ab .............. 592

207. Al[PO 4 ]: AB4C_hP72_184_d_4d_d ............5594

208. $\mathrm{KNiCl}_{3}$ : A3BC_hP30_185_cd_c_ab ............599

209. $\mathrm{Cu}_{3} \mathrm{P}^{\|}:$A3B_hP24_185_ab2c_c ...............602 602

210. $\beta-\mathrm{RuCl}_{3}$ : A3B_hP8_185_c_a ...............605

211. Na 3 As ${ }^{l l}:$ AB3_hP24_185_c_ab2c .............. 607

212. $\mathrm{Re}_{3} \mathrm{~N}$ : AB3_hP4_187_e_fh ................6 612

213. LiScI 3 : A3BC_hP10_188_k_a_e ..............6614

214. $\mathrm{BaSi}_{4} \mathrm{O}_{9}$ : AB9C4_hP28_188_e_kl_ak .........66 616

215. $\pi-\mathrm{FeMg}_{3} \mathrm{Al}_{9} \mathrm{Si}_{5}$ :

A9BC3D5_hP18_189_fi_a_g_bh .............6622

216. Li $2 \mathrm{Sb}$ : A2B_hP18_190_gh_bf ...............6624

217. $\alpha-\mathrm{Sm}_{3} \mathrm{Ge}_{5}$ : A5B3_hP16_190_bdh_g ..........66 626

218. Troilite: AB_hP24_190_i_afh .................6628

219. $\mathrm{AlPO}_{4}:$ AB2_hP72_192_m_j2kl ..............66 635

220. Mavlyanovite: A5B3_hP16_193_dg_g .........66 639

221. $\mathrm{PrRu}_{4} \mathrm{P}_{12}$ : A12BC4_cP34_195_2j_ab_2e ........649

222. $\mathrm{Cu}_{2} \mathrm{Fe}[\mathrm{CN}]_{6}$ : A12B2C_cF60_196_h_bc_a .......662

${ }^{\|} \mathrm{Cu}_{3} \mathrm{P}$ and $\mathrm{Na}_{3} \mathrm{As}$ have similar AFLOW prototype labels (i.e., same symmetry and set of Wyckoff positions with different stoichiometry labels due to alphabetic ordering of atomic species). They are generated by the same symmetry operations with different sets of parameters. 
223. $\mathrm{MgB}_{12} \mathrm{H}_{12}\left[\mathrm{H}_{2} \mathrm{O}\right]_{12}$ :

A12B36CD12_cF488_196_2h_6h_ac_fgh .........665

224. $\mathrm{Mg}_{2} \mathrm{Zn}_{11}$ : A2B11_cP39_200_f_aghij ............663

225. $\mathrm{KSbO}_{3}$ : AB3C_cP60_201_ce_fh_g ..............666

226. $\mathrm{KB}_{6} \mathrm{H}_{6}$ : A6B6C_cF104_202_h_h_c ............669

227. FCC $\mathrm{C}_{60}$ Buckminsterfullerine:

A_cF240_202_h2i ....................6671

228. Pyrochlore: A2BCD3E6_cF208_203_e_c_d_f_g . . 675

229. Tychite: A4B2C6D16E_cF232_203_e_d_f_eg_a . . . 679

230. $\mathrm{Rb}_{3} \mathrm{AsSe}_{16}$ : AB3C16_cF160_203_b_ad_eg .......683

231. $\mathrm{Ca}_{3} \mathrm{Al}_{2} \mathrm{O}_{6}$ : A2B3C6_cP264_205_2d_ab2c2d_6d ... 687

232. Simple Cubic $\mathrm{C}_{60}$ Buckminsterfullerine:

A_cP240_205_10d ....................666

233. $\mathrm{Pd}_{17} \mathrm{Se}_{15}$ : A17B15_cP64_207_acfk_eij .......... 707

234. $\mathrm{PH}_{3}$ : A3B_cP16_208_j_b ..................710

235. $\mathrm{Cs}_{2} \mathrm{ZnFe}[\mathrm{CN}]_{6}$ :

A6B2CD6E_cP64 $208 \_m \_a d \_b \_c \ldots . . . . .712$

236. $\mathrm{F}_{6} \mathrm{KP}:$ A24BC_cF104_209_j_a_b .............716

237. $\mathrm{Te}[\mathrm{OH}]_{6}$ : A12B6C_cF608_210_4h_2h_e ........719

238. $\mathrm{SiO}_{2}$ : A2B_cI72_211_hi_i ................731

239. $\mathrm{SrSi}_{2}$ : A2B_cP12_212_c_a ................ 734

240. $\mathrm{Ca}_{3} \mathrm{PI}_{3}$ : A3B3C_cI56_214_g_h_a ............736

241. Petzite: A3BC2_cI48_214_f_a_e .............. 739

242. Quartenary Heusler:

ABCD_cF16_216_c_d_b_a ...................745

243. $\mathrm{Ag}_{3}\left[\mathrm{PO}_{4}\right]:$ A3B4C_cP16_218_c_e_a $\ldots . . . \ldots \ldots . .747$

244. Boracite: A7BC3D13_cF192_219_de_b_c_ah ......749

245. $\mathrm{Ce}_{5} \mathrm{Mo}_{3} \mathrm{O}_{16}$ : A5B3C16_cP96_222_ce_d_fi ........761

246. Pyrochlore Iridate:

A2B2C7_cF88_227_c_d_af ................. 774

247. $\mathrm{CuCrCl}_{5}\left[\mathrm{NH}_{3}\right]_{6}:$ A5BCD6_cF416_228_eg_c_b_h ..778

248. $\mathrm{TeO}_{6} \mathrm{H}_{6}: \mathrm{A} 6 \mathrm{~B} \_\mathrm{cF} 224 \_228 \_\mathrm{h} \_\mathrm{c} \ldots \ldots \ldots \ldots \ldots . \ldots 785$

249. $\beta$-Hg $\mathrm{Hg}_{4} \mathrm{Pt}$ : A4B_cI10_229_c_a .............. 793

\section{Duplicate AFLOW Label Index}

\section{A2B_oP12_26_abc_ab}

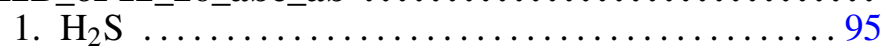

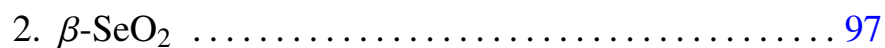

AB_oC8_67_a_g ................................

1. $\alpha$-FeSe ........................... 240

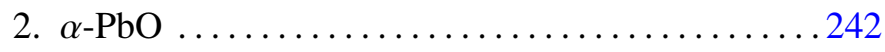

\section{Similar AFLOW Label Index}

A2B_oP12_29_2a_a

1. $\mathrm{ZrO}_{2}$ : A2B_oP12_29_2a_a ................... 106

2. Pyrite: AB2_oP12_29_a_2a .................. 108

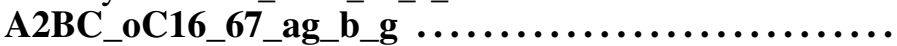

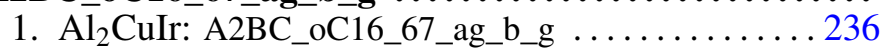

2. $\mathrm{HoCuP}_{2}$ : ABC2_oC16_67_b_g_ag ..............238

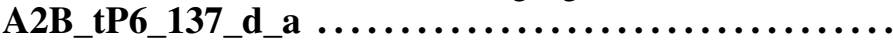

1. $\mathrm{ZrO}_{2}$ : A2B_tP6_137_d_a $\ldots . . . \ldots \ldots \ldots \ldots \ldots . . . . . .443$

2. $\mathrm{HgI}_{2}$ : AB2_tP6_137_a_d .................. 447

A3B_hP24_185_ab2c_c ........................

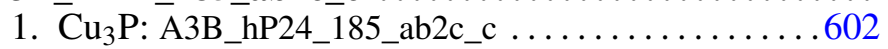

2. $\mathrm{Na}_{3}$ As: AB3_hP24_185_c_ab2c .............607

\section{CIF Index}

1. $\alpha-\mathrm{Al}_{2} \mathrm{~S}_{3}$ : A2B3_hP30_169_2a_3a $\ldots \ldots \ldots \ldots . .923$

2. $\alpha-\mathrm{CuAlCl}_{4}: \mathrm{AB} 4 \mathrm{C} \_\mathrm{tP} 12 \_112 \_\mathrm{b} \_\mathrm{n} \_\mathrm{e} . . . . . . . . .884$

3. $\alpha-\mathrm{FeSe}^{\dagger}$ : AB_oC8_67_a_g ................. 852

4. $\alpha$-Naumannite: A2B_oP12_17_abe_e .......... 812

5. $\alpha-\mathrm{NbO}_{2}:$ AB2_tI96_88_2f_4f $\ldots \ldots \ldots \ldots \ldots . . .666$

6. $\alpha-\mathrm{P}_{3} \mathrm{~N}_{5}:$ A5B3_mC32_9_5a_3a .............. 805

7. $\alpha-\mathrm{PbO}^{\dagger}$ : AB_oC8_67_a_g ................. 853

8. $\alpha-\mathrm{PdCl}_{2}$ : A2B_oP6_58_g_a $\ldots \ldots \ldots \ldots \ldots \ldots . \ldots 37$

9. $\alpha-\operatorname{RbPr}\left[\mathrm{MoO}_{4}\right]_{2}$ :

A2B8CD_oP24_48_k_2m_d_b ................8 830

10. $\alpha-\mathrm{Sm}_{3} \mathrm{Ge}_{5}$ : A5B3_hP16_190_bdh_g .......... 940

11. $\alpha$-ThSi $i_{2}$ : A2B_tI12_141_e_a ................ 904

12. $\alpha-\mathrm{Tl}_{2} \mathrm{TeO}_{3}$ : A3BC2_oP48_50_3m_m_2m $\ldots \ldots . .833$

13. $\alpha$-Toluene: A7B8_mP120_14_14e_16e $\ldots \ldots \ldots . .810$

14. $\beta-\mathrm{Bi}_{2} \mathrm{O}_{3}: \mathrm{A} 2 \mathrm{~B} 3 \_t \mathrm{P} 20 \_117 \_\mathrm{i} \_$adgh $\ldots \ldots \ldots \ldots . . . .687$

15. $\beta$-CuI: AB_hP4_156_ac_ac $\ldots . . . \ldots \ldots \ldots \ldots . \ldots 12$

16. $\beta$-Hg H $_{4} \mathrm{Pt}: \mathrm{A} 4 \mathrm{~B} \_\mathrm{cI} 10 \_229 \_\mathrm{c} \_\mathrm{a} \ldots \ldots \ldots \ldots \ldots . . . . . . .984$

17. $\beta-\mathrm{NbO}_{2}$ : AB2_tI48_80_2b_4b ............... 862

18. $\beta$-PdCl ${ }_{2}$ : A2B_hR18_148_2f_f $\ldots \ldots \ldots \ldots \ldots . . . .909$

19. $\beta-\mathrm{RuCl}_{3}$ : A3B_hP8_158_d_a $\ldots \ldots \ldots \ldots \ldots \ldots 913$

20. $\beta$-RuCl $\mathrm{Ru}_{3}$ : A3B_hP8_185_c_a $\ldots . . . \ldots \ldots \ldots . . . .935$

21. $\beta-\mathrm{SeO}_{2}{ }^{*}:$ A2B_oP12_26_abc_ab $\ldots \ldots \ldots \ldots \ldots . . .618$

22. $\beta-\mathrm{Si}_{3} \mathrm{~N}_{4}$ : A4B3_hP14_173_bc_c ..............9926

23. $\beta-\mathrm{SiO}_{2}$ : A2B_hP9_181_j_c $\ldots \ldots \ldots \ldots \ldots \ldots 932$

24. $\beta-\mathrm{Ta}_{2} \mathrm{O}_{5}$ : A5B2_oP14_49_dehq_ab $\ldots \ldots \ldots \ldots . . .631$

25. $\beta-\mathrm{ThI}_{3}$ : A3B_oC64_66_kl2m_bdl $\ldots \ldots \ldots \ldots \ldots . \ldots 51$

26. $\beta$-Toluene: A7B8_oP120_60_7d_8d ...........8839

27. $\beta-\mathrm{V}_{3} \mathrm{~S}$ : $\mathrm{AB} 3 \_\mathrm{tP} 32 \_133 \_\mathrm{h} \_\mathrm{i} 2 \mathrm{j} \ldots . . . . . . . . . . . .897$

28. $\delta$-PdCl ${ }_{2}$ : A2B_mP6_10_mn_bg $\ldots \ldots \ldots \ldots \ldots . . .606$

29. $\delta_{H}^{I I}-\mathrm{NW}_{2}$ : AB2_hP9_164_bd_c2d $\ldots \ldots \ldots \ldots \ldots . \ldots 18$

30. $\epsilon-\mathrm{NiAl}_{3}$ : A3B_oP16_62_cd_c ............... 843

31. $\epsilon-\mathrm{WO}_{3}:$ A3B_mP16_7_6a_2a ............... 804

32. $\gamma$-Ag S $_{3}$ SI: A3BC_hR5_146_b_a_a .............908

33. $\gamma$-MgNiSn: A7B7C2_tP32_101_bde_ade_d ...... 875

34. $\gamma-\mathrm{PdCl}_{2}$ : A2B_mP6_14_e_a $\ldots \ldots \ldots \ldots \ldots . . . . .610$

35. $\gamma$-brass: A4B9_cP52_215_ei_3efgi .............966

36. $\gamma$-brass: A3B10_cI52_229_e_fh $\ldots \ldots \ldots \ldots \ldots . . .983$

37. $\kappa$-alumina: A2B3_oP40_33_4a_6a $\ldots \ldots \ldots \ldots . . .823$

38. $\pi-\mathrm{FeMg}_{3} \mathrm{Al}_{8} \mathrm{Si}_{6}$ :

A8BC3D6_hP18_189_bfh_a_g_i .............. 938

39. $\pi-\mathrm{FeMg}_{3} \mathrm{Al}_{9} \mathrm{Si}_{5}$ :

A9BC3D5_hP18_189_fi_a_g_bh .............. 939

40. $\mathrm{Ag}_{3}\left[\mathrm{PO}_{4}\right]$ : A3B4C_cP16_218_c_e_a $\ldots \ldots \ldots \ldots 96$

41. $\mathrm{Ag}_{5} \mathrm{~Pb}_{2} \mathrm{O}_{6}$ : A5B6C2_hP13_157_2ac_2c_b ....... 913

42. AgUF 6 : AB6C_tP16_132_d_io_a ............... 897

${ }^{\dagger} \alpha$-FeSe and $\alpha$-PbO have the same AFLOW prototype label. They are generated by the same symmetry operations with different sets of parameters.

${ }^{*} \mathrm{H}_{2} \mathrm{~S}$ and $\beta-\mathrm{SeO}_{2}$ have the same AFLOW prototype label. They are generated by the same symmetry operations with different sets of parameters. 
43. Akermanite: A2BC7D2_tP24_113_e_a_cef_e .....884

44. $\mathrm{Al}_{2} \mathrm{CuIr}{ }^{\S}$ : A2BC_oC16_67_ag_b_g ............ 851

45. $\mathrm{Al}_{2} \mathrm{~S}_{3}$ : A2B3_hP30_170_2a_3a .............. 924

46. $\mathrm{Al}_{4} \mathrm{C}_{3}$ : A4B3_hR7_166_2c_ac .............. 919

47. $\mathrm{Al}_{4} \mathrm{U}$ : A4B_ol20_74_beh_e ................. 856

48. $\mathrm{Al}_{8} \mathrm{Cr}_{5}$ : A8B5_hR26_160_a3bc_a3b ........... 916

49. $\mathrm{Al}_{9} \mathrm{Mn}_{3} \mathrm{Si}$ : A9B3C_hP26_194_hk_h_a ......... 944

50. $\mathrm{AlLi}_{3} \mathrm{~N}_{2}$ : AB3C2_cI96_206_c_e_ad ............958

51. $\mathrm{AlPO}_{4}: \mathrm{AB} 2 \_\mathrm{hP72} 192 \_\mathrm{m} \_\mathrm{j} 2 \mathrm{kl} \ldots \ldots \ldots \ldots \ldots 942$

52. $\mathrm{Al}\left[\mathrm{PO}_{4}\right]:$ AB4C_hP72_168_2d_8d_2d ..........9922

53. $\mathrm{Al}\left[\mathrm{PO}_{4}\right]: \mathrm{AB} 4 \mathrm{C} \_\mathrm{hP72} \_184 \_\mathrm{d} \_4 \mathrm{~d} \_\mathrm{d} \ldots \ldots . . . . .933$

54. Anhydrite: AB4C_oC24_63_c_fg_c .............849

55. $\mathrm{As}_{2} \mathrm{Ba}$ : A2B_mP18_7_6a_3a ................ 803

56. $\mathrm{AsPh}_{4} \mathrm{CeS}_{8} \mathrm{P}_{4} \mathrm{Me}_{8}$ :

AB32CD4E8_tP184_93_i_16p_af_2p_4p .........870

57. AuCN: ABC_hP3_183_a_a_a ................ 932

58. $\mathrm{AuF}_{3}$ : AB3_hP24_178_b_ac ...............9930

59. $\mathrm{AuF}_{3}$ : AB3_hP24_179_b_ac ............... 931

60. BN: AB_oF8_42_a_a .................... 829

61. BPS 4 : ABC4_oI12_23_a_b_k ................ 816

62. $\mathrm{Ba}_{5} \mathrm{In}_{4} \mathrm{Bi}_{5}$ : A5B5C4_tP28_104_ac_ac_c ........ 877

63. $\mathrm{Ba}_{5} \mathrm{Si}_{3}$ : A5B3_tP32_130_cg_cf .............. 896

64. $\mathrm{BaCr}_{2} \mathrm{Ru}_{4} \mathrm{O}_{12}$ :

AB2C12D4_tP76_75_2a2b_2d_12d_4d ..........857

65. $\mathrm{BaCu}_{4}[\mathrm{VO}]\left[\mathrm{PO}_{4}\right]_{4}$ :

AB4C17D4E_tP54_90_a_g_c4g_g_c ............868

66. $\mathrm{BaGe}_{2} \mathrm{As}_{2}$ : A2BC2_tP20_105_f_ac_2e ..........878

67. $\mathrm{BaSi}_{4} \mathrm{O}_{9}$ : AB9C4_hP28_188_e_kl_ak .......... 938

68. Barite: AB4C_oP24_62_c_2cd_c .............. 844

69. $\mathrm{Be}\left[\mathrm{BH}_{4}\right]_{2}:$ A2BC8_tI176_110_2b_b_8b $\ldots \ldots \ldots . . .882$

70. Benzene: AB_oP48_61_3c_3c .............. 841

71. Beryl: A2B3C18D6_hP58_192_c_f_lm_1 .......941

72. $\mathrm{Bi}_{2} \mathrm{O}_{3}$ : A2B3_hP20_159_bc_2c ............. 914

73. $\mathrm{Bi}_{5} \mathrm{Nb}_{3} \mathrm{O}_{15}$ : A5B3C15_oP46_30_a2c_bc_a7c .....821

74. $\mathrm{BiAl}_{2} \mathrm{~S}_{4}$ : A2BC4_tP28_126_cd_e_k ............893

75. $\mathrm{BiGaO}_{3}$ : ABC3_oP20_54_e_d_cf ..............8. 835

76. Boracite: A7BC3D13_cF192_219_de_b_c_ah .....999

77. C: A_tP12_138_bi .....................901

78. $\mathrm{C}_{17} \mathrm{FeO}_{4} \mathrm{Pt}$ : A17BC4D_tP184_89_17p_p_4p_io ....866

79. $\mathrm{Ca}_{3} \mathrm{Al}_{2} \mathrm{O}_{6}:$ A2B3C6_cP264_205_2d_ab2c2d_6d ... 955

80. $\mathrm{Ca}_{3} \mathrm{Al}_{2} \mathrm{O}_{6}$ : A2B3C6_cP33_221_cd_ag_fh .......971

81. $\mathrm{Ca}_{3} \mathrm{PI}_{3}$ : A3B3C_cI56_214_g_h_a ............965

82. $\mathrm{Ca}_{4} \mathrm{Al}_{6} \mathrm{O}_{16} \mathrm{~S}$ :

A6B4C16D_oP108_27_abcd4e_4e_16e_e .........819

83. $\mathrm{CaRbFe}_{4} \mathrm{As}_{4}$ : A4BC4D_tP10_123_gh_a_i_d ..... 890

84. Calomel: AB_tI8_139_e_e ...................902

85. Carbonyl Sulphide: ABC_hR3_160_a_a_a .......916

\footnotetext{
${ }^{\S} \mathrm{Al}_{2} \mathrm{CuIr}$ and $\mathrm{HoCuP}_{2}$ have similar AFLOW prototype labels (i.e., same symmetry and set of Wyckoff positions with different stoichiometry labels due to alphabetic ordering of atomic species). They are generated by the same symmetry operations with different sets of parameters.
}

86. $\mathrm{CdAs}_{2}$ : A2B_tI12_98_f_a $\ldots \ldots \ldots \ldots \ldots \ldots . \ldots . \ldots . \ldots . \ldots 14$

87. CdI 2 : AB2_hP9_156_b2c_3a2bc .............911

88. $\mathrm{Ce}_{3} \mathrm{Si}_{6} \mathrm{~N}_{11}$ : A3B11C6_tP40_100_ac_bc2d_cd .... 875

89. $\mathrm{Ce}_{5} \mathrm{Mo}_{3} \mathrm{O}_{16}$ : A5B3C16_cP96_222_ce_d_fi .......972

90. $\mathrm{CeCo}_{4} \mathrm{~B}_{4}$ : A4BC4_tP18_137_g_b_g . ........... 900

91. $\mathrm{CeRu}_{2} \mathrm{~B}_{2}$ : A2BC2_oF40_22_fi_ad_gh ...........814

92. $\mathrm{CeTe}_{3}$ : AB3_oC16_40_b_3b ................ 828

93. $\mathrm{Co}_{2} \mathrm{Al}_{5}$ : A5B2_hP28_194_ahk_ch .............944

94. $\mathrm{Co}_{5} \mathrm{Ge}_{7}$ : A5B7_tI24_107_ac_abd ...............8879

95. Cobaltite: ABC_oP12_29_a_a_a ................88

96. $\mathrm{Cr}_{5} \mathrm{~B}_{3}$ : A3B5_tt32_140_ah_cl ............... 903

97. $\mathrm{CrCl}_{3}$ : A3B_hP24_153_3c_2b ...............911

98. $\mathrm{CrFe}_{3} \mathrm{NiSn}_{5}$ : AB_hP6_183_c_ab .............933

99. $\mathrm{Cs}_{2} \mathrm{ZnFe}[\mathrm{CN}]_{6}$ :

A6B2CD6E_cP64_208_m_ad_b_m_c ............990

100. $\mathrm{Cs}_{3} \mathrm{P}_{7}$ : A3B7_tP40_76_3a_7a ................. 858

101. CsPr[ $\left[\mathrm{MoO}_{4}\right]_{2}$ : AB2C8D_oP24_49_g_q_2qr_e ....8832

102. $\mathrm{Cu}_{15} \mathrm{Si}_{4}$ : A15B4_cI76_220_ae_c ..............970

103. $\mathrm{Cu}_{2} \mathrm{Fe}[\mathrm{CN}]_{6}$ : A12B2C_cF60_196_h_bc_a ...... 945

104. $\mathrm{Cu}_{3}$ P: A3B_hP24_165_bdg_f .................919

105. $\mathrm{Cu}_{3} \mathrm{P}^{\|}:$A3B_hP24_185_ab2c_c ............... 935

106. $\mathrm{CuBi}_{2} \mathrm{O}_{4}$ : A2BC4_tP28_130_f_c_g . ............ 895

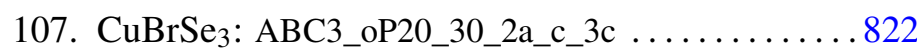

108. $\mathrm{CuBrSe}_{3}$ : ABC3_oP20_53_e_g_hi .............885

109. $\mathrm{CuCrCl}_{5}\left[\mathrm{NH}_{3}\right]_{6}:$ A5BCD6_cF416_228_eg_c_b_h ..980

110. CuI: AB_hP12_156_2ab3c_2ab3c ............9912

111. $\mathrm{CuNiSb}_{2}$ : ABC2_hP4_164_a_b_d ............ 918

112. Cubanite: AB2C3_oP24_62_c_d_cd .............843

113. Downeyite: A2B_tP24_135_gh_h ..............898

114. $\mathrm{Er}_{3} \mathrm{Ru}_{2}:$ A3B2_hP10_176_h_bd .............. 929

115. F 6 KP: A24BC_cF104_209_j_a_b ............. 961

116. $\mathrm{FCC} \mathrm{C}_{60}$ Buckminsterfullerine:

A_cF240_202_h2i .................... 950

117. $\mathrm{Fe}_{12} \mathrm{Zr}_{2} \mathrm{P}_{7}$ : A12B7C2_hP21_174_2j2k_ajk_cf ....9926

118. $\mathrm{Fe}_{3} \mathrm{Te}_{3} \mathrm{Tl}: \mathrm{A} 3 \mathrm{~B} 3 \mathrm{C} \_$hP14_176_h_h_d ........... 929

119. $\mathrm{Fe}_{3} \mathrm{Th}_{7}$ : A3B7_hP20_186_c_b2c ............. 936

120. $\mathrm{FeCu}_{2} \mathrm{Al}_{7}$ : A7B2C_tP40_128_egi_h_e ...........895

121. FeNi: AB_mP4_6_2b_2a .................. 802

122. FeOCl: ABC_oP6_59_a_b_a ................. 838

123. $\mathrm{FePSe}_{3}$ : ABC3_hR10_146_2a_2a_2b ........... 908

124. FeS: AB_oF8_22_a_c ....................... 814

125. FeSb 2 : AB2_oP6_34_a_c .................... 825

126. Forsterite: A2B4C_oP28_62_ac_2cd_c ...........8842

127. Fresnoite: A2B8C2D_tP26_100_c_abcd_c_a . . ...8874

128. $\mathrm{GaCl}_{2}$ : A2B_oP24_52_2e_cd ................ 833

129. GaSb: AB_tI4_119_c_a .................... 889

130. Garnet: A2B3C12D3_cI160_230_a_c_h_d .......9986

\footnotetext{
${ }^{\|} \mathrm{Cu}_{3} \mathrm{P}$ and $\mathrm{Na}_{3} \mathrm{As}$ have similar AFLOW prototype labels (i.e., same symmetry and set of Wyckoff positions with different stoichiometry labels due to alphabetic ordering of atomic species). They are generated by the same symmetry operations with different sets of parameters.
} 
131. $\mathrm{Gd}_{3} \mathrm{Al}_{2}$ : A2B3_tP20_102_2c_b2c $\ldots \ldots \ldots \ldots \ldots . \ldots 76$

132. GdSI: ABC_hP12_174_cj_fk_aj .............. 927

133. GeAs 2 : A2B_oP24_55_2g2h_gh ............. 836

134. GeP: AB_tI4_107_a_a ..................... 880

135. GeSe 2 : AB2_tP12_81_adg_2h .................862

136. $\mathrm{H}_{2} \mathrm{~S}$ : A2B_aP6_2_aei_i .................... 802

137. $\mathrm{H}_{2} \mathrm{~S}$ : A2B_mP12_13_2g_ef ................. 809

138. $\mathrm{H}_{2} \mathrm{~S}^{*}$ : A2B_oP12_26_abc_ab ............... 817

139. $\mathrm{H}_{2} \mathrm{~S}$ : A2B_oC24_64_2f_f .................... 849

140. $\mathrm{H}_{2}$ S III: A2B_tP48_77_8d_4d ................ 860

141. $\mathrm{H}_{2} \mathrm{~S}$ IV: A2B_mP12_7_4a_2a ............... 803

142. $\mathrm{H}_{3} \mathrm{Cl}: \mathrm{AB} 3 \_m C 16 \_9 \_a \_3 a \ldots . . . . . . . . . . .806$

143. $\mathrm{H}_{3} \mathrm{Cl}$ : AB3_mP16_10_mn_3m3n ............ 806

144. $\mathrm{H}_{3} \mathrm{Cl}$ : AB3_mC16_15_e_cf ................. 811

145. $\mathrm{H}_{3} \mathrm{Cl}$ : AB3_oP16_19_a_3a ................. 813

146. $\mathrm{H}_{3} \mathrm{~S}$ : A3B_ol32_23_ij2k_k .................. 815

147. $\mathrm{H}_{3} \mathrm{~S}$ : A3B_oC64_66_gi21m_21 ...............850

148. $\mathrm{H}_{3} \mathrm{~S}$ : A3B_hR4_160_b_a $\ldots \ldots \ldots \ldots \ldots \ldots \ldots . . \ldots 15$

149. H-III: A_mC24_15_2e2f ..................... 812

150. HCl: AB_oC8_36_a_a ................... 825

151. $\mathrm{HgI}_{2}:$ AB2_tP12_115_j_egi $\ldots \ldots \ldots \ldots \ldots \ldots . \ldots 86$

152. $\mathrm{HgI}_{2}{ }^{\mathrm{T}}$ : AB2_tP6_137_a_d .................. 901

153. $\mathrm{HoCuP}_{2}{ }^{\S}$ : ABC2_oC16_67_b_g_ag ............ 852

154. $\mathrm{Ir}_{3} \mathrm{Ga}_{5}$ : A5B3_tP32_118_g2i_aceh ............ 888

155. $\mathrm{Ir}_{3} \mathrm{Ge}_{7}$ : A7B3_cI40_229_df_e ............... 985

156. $\mathrm{IrGe}_{4}$ : A4B_hP15_144_4a_a ................906

157. $\mathrm{K}_{2} \mathrm{CdPb}$ : AB2C_oC16_40_a_2b_b .............8 828

158. $\mathrm{K}_{2} \mathrm{PtCl}_{6}$ : A6B2C_cF36_225_e_c_a ........... 974

159. $\mathrm{K}_{2} \mathrm{SnCl}_{6}$ : A6B2C_tP18_128_eh_d_b ........... 894

160. $\mathrm{K}_{2} \mathrm{Ta}_{4} \mathrm{O}_{9} \mathrm{~F}_{4}$ : A2B13C4_hP57_168_d_c6d_2d ..... 922

161. $\mathrm{KAg}\left[\mathrm{CO}_{3}\right]$ : ABCD3_oI48_73_d_e_e_ef .........855

162. $\mathrm{KAu}_{4} \mathrm{Sn}_{2}$ : A4BC2_tI28_120_i_d_e ........... 890

163. $\mathrm{KB}_{6} \mathrm{H}_{6}$ : A6B6C_cF104_202_h_h_c ........... 949

164. $\mathrm{KBO}_{2}$ : ABC2_hR24_167_e_e_2e .............9921

165. $\mathrm{KCeSe}_{4}$ : ABC4_tP12_125_a_b_m ............. 892

166. $\mathrm{KHg}_{2}$ : A2B_oI12_74_h_e ................. 856

167. $\mathrm{KNiCl}_{3}$ : A3BC_hP30_185_cd_c_ab ............9934

168. $\mathrm{KSbO}_{3}$ : AB3C_cP60_201_ce_fh_g ............. 949

169. $\mathrm{La}_{2} \mathrm{NiO}_{4}$ : A2BC4_oP28_50_ij_ac_ijm ...........8832

170. $\mathrm{La}_{2} \mathrm{O}_{3}$ : A2B3_hP5_164_d_ad ................ 917

171. $\mathrm{La}_{43} \mathrm{Ni}_{17} \mathrm{Mg}_{5}$ :

A43B5C17_oC260_63_c8fg6h_cfg_ce3f2h ........846

172. LaPtSi: ABC_tI12_109_a_a_a .................881

173. LaRhC $_{2}$ : A2BC_tP16_76_2a_a_a ............. 858

174. Li $_{2} \mathrm{MoF}_{6}$ : A6B2C_tP18_94_eg_c_a ........... 872

175. $\mathrm{Li}_{2} \mathrm{Sb}$ : A2B_hP18_190_gh_bf ................999

176. $\mathrm{Li}_{2} \mathrm{Si}_{2} \mathrm{O}_{5}$ : A2B5C2_oC36_37_d_c2d_d ..........8 826

\footnotetext{
${ }^{\mathrm{I}} \mathrm{ZrO}_{2}$ and $\mathrm{HgI}_{2}$ have similar AFLOW prototype labels (i.e., same symmetry and set of Wyckoff positions with different stoichiometry labels due to alphabetic ordering of atomic species). They are generated by the same symmetry operations with different sets of parameters.
}

177. LiScI $_{3}:$ A3BC_hP10_188_k_a_e ............937

178. LiSn: AB_mP6_10_en_am .................887

179. M-carbon: A_mC16_12_4i ................ 809

180. Mavlyanovite: A5B3_hP16_193_dg_g ........9943

181. $\mathrm{Mg}_{2} \mathrm{Zn}_{11}$ : A2B11_cP39_200_f_aghij .........9948

182. $\mathrm{MgB}_{12} \mathrm{H}_{12}\left[\mathrm{H}_{2} \mathrm{O}\right]_{12}$ :

A12B36CD12_cF488_196_2h_6h_ac_fgh .........9946

183. $\mathrm{MgSO}_{4}:$ AB4C_oC24_63_a_fg_c ............. 848

184. $\mathrm{Mg}[\mathrm{NH}]:$ ABC_hP36_175_jk_jk_jk ......... 928

185. $\mathrm{Mn}_{2} \mathrm{~B}:$ AB2_oF48_70_f_fg .............. 854

186. $\mathrm{MnAl}_{6}:$ A6B_oC28_63_efg_c . . . . . . . . . . . 847

187. $\mathrm{MnF}_{2}$ : A2B_tP12_111_2n_adf ............ 883

188. $\mathrm{MnGa}_{2} \mathrm{Sb}_{2}:$ A2BC2_oI20_45_c_b_c .......... 829

189. $\mathrm{Mo}_{8} \mathrm{P}_{5}$ : A8B5_mP13_6_a7b_3a2b ........... 802

190. $\mathrm{MoS}_{2}$ : AB2_hP12_143_cd_ab2d ............906

191. Moissanite-15R: AB_hR10_160_5a_5a ..........917

192. Molybdite: AB3_oP16_62_c_3c .............. 844

193. Muthmannite: ABC2_mP8_10_ac_eh_mn .......887

194. NV: AB_tP8_111_n_n .....................883

195. $\mathrm{Na}_{3} \mathrm{As}{ }^{\|}$: AB3_hP24_185_c_ab2c ............9936

196. $\mathrm{Na}_{4} \mathrm{Ti}_{2} \mathrm{Si}_{8} \mathrm{O}_{22}\left[\mathrm{H}_{2} \mathrm{O}\right]_{4}$ :

A4B2C13D_tP40_90_g_d_cef2g_c ............ 868

197. $\mathrm{Na}_{5} \mathrm{Fe}_{3} \mathrm{~F}_{14}: \mathrm{A} 14 \mathrm{~B} 3 \mathrm{C} 5 \_t \mathrm{tP} 44 \_94 \_c 3 g \_a d \_b g \ldots . .871$

198. $\mathrm{NaFeS}_{2}$ : ABC2_oI16_23_ab_i_k ............8 816

199. $\mathrm{NaGdCu} \mathrm{F}_{8}$ : A2B8CD_tI24_97_d_k_a_b ........ 873

200. $\mathrm{NaZn}_{13}: \mathrm{AB} 13 \_c F 112 \_226 \_a \_b i \ldots \ldots \ldots \ldots \ldots .976$

201. $\mathrm{NaZn}[\mathrm{OH}]_{3}:$ A3BC3D_tP64_106_3c_c_3c_c .....889

202. $\mathrm{Nb}_{4} \mathrm{CoSi}:$ AB4C_tP12_124_a_m_c ........... 891

203. $\mathrm{Nb}_{7} \mathrm{Ru}_{6} \mathrm{~B}_{8}:$ A8B7C6_hP21_175_ck_aj_k .......999

204. NbAs: AB_tI8_109_a_a .................. 881

205. NbPS: ABC_oI12_71_h_j_g ............. 855

206. $\mathrm{NbTe}_{4}$ : AB4_tP10_103_a_d .................877

207. $\mathrm{NbTe}_{4}:$ AB4_tP10_124_a_m ................ 891

208. $\mathrm{Ni}_{3} \mathrm{P}:$ A3B_tI32_82_3g_g ............... 863

209. Ni 3 Ti: A3B_hP16_194_gh_ac .............. 943

210. Nierite: A4B3_hP28_159_ab2c_2c ...........914

211. $\mathrm{PH}_{3}$ : A3B_cP16_208_j_b .................. 960

212. $\mathrm{PI}_{3}$ : A3B_hP8_173_c_b ................ 925

213. $\mathrm{Pd}_{17} \mathrm{Se}_{15}$ : A17B15_cP64_207_acfk_eij .........959

214. $\mathrm{Pd}_{4} \mathrm{Se}:$ A4B_tP10_114_e_a ................ 885

215. $\mathrm{PdSn}_{4}:$ AB4_oC20_68_a_i ................. 853

216. Petzite: A3BC2_cI48_214_f_a_e ............ 966

217. Phenakite: A2B4C_hR42_148_2f_4f_f ......... 909

218. Pinnoite: A2B6CD7_tP64_77_2d_6d_d_ab6d ......859

219. Post-perovskite: AB3C_oC20_63_a_cf_c .........8848

220. $\mathrm{PrNiO}_{3}$ : AB3C_hR10_167_b_e_a ............9920

221. $\operatorname{PrRu}_{4} \mathrm{P}_{12}$ : A12BC4_cP34_195_2j_ab_2e ........945

222. PtPb 4 : A4B_tP10_125_m_a .............. 892 
223. Pyrite ${ }^{\ddagger}:$ AB2_oP12_29_a_2a .................8 820

224. Pyrochlore: A2BCD3E6_cF208_203_e_c_d_f_g . . . 951

225. Pyrochlore Iridate:

A2B2C7_cF88_227_c_d_af ................. 977

226. Quartenary Heusler:

ABCD_cF16_216_c_d_b_a ..................967

227. R-carbon: A_oP16_55_2g2h . ............... 837

228. Rasvumite: A2BC3_oC24_63_e_c_cg ...........8845

229. $\mathrm{Rb}_{2} \mathrm{TiCu}_{2} \mathrm{~S}_{4}$ : A2B2C4D_tP18_132_e_i_o_d .......896

230. $\mathrm{Rb}_{3} \mathrm{AsSe}_{16}$ : AB3C16_cF160_203_b_ad_eg .......9954

231. RbGa3: A3B_tt24_119_b2i_af .............. 889

232. $\mathrm{Re}_{2} \mathrm{O}_{5}\left[\mathrm{SO}_{4}\right]_{2}:$ A13B2C2_oP34_32_a6c_c_c .......823

233. $\operatorname{Re}_{3} \mathrm{~N}$ : AB3_hP4_187_e_fh ................ 937

234. $\mathrm{Rh}_{2} \mathrm{Ga}$ : A9B2_mP22_7_9a_2a $\ldots \ldots \ldots \ldots \ldots . \ldots 04$

235. $\mathrm{Rh}_{2} \mathrm{~S}_{3}$ : A2B3_oP20_60_d_cd ............... 838

236. $\mathrm{Rh}_{3} \mathrm{P}_{2}$ : A2B3_tP5_115_g_ag . ............... 886

237. $\mathrm{Rh}_{5} \mathrm{Ge}_{3}$ : A3B5_oP16_55_ch_agh .............886

238. $\mathrm{Ru}_{2} \mathrm{Sn}_{3}$ : A2B3_tP20_116_bci_fj .............. 887

239. RuIn $:$ A3B_tP16_118_ei_f ................. 888

240. S-II: A_hP9_154_bc ..................... 911

241. S-III: A_tI16_142_f ......................... 904

242. S-carbon: A_mP8_10_2m2n ................ 808

243. Sc-V: A_hP6_178_a .................... 931

244. $\mathrm{ScRh}_{6} \mathrm{P}_{4}$ : A4B6C_hP11_143_bd_2d_a ......... 905

245. $\mathrm{SeO}_{3}$ : A3B_tP32_114_3e_e ................. 885

246. Sheldrickite:

A2B3C3DE7_hP48_145_2a_3a_3a_a_7a ........ 907

247. $\mathrm{SiO}_{2}$ : A2B_hP36_177_j2lm_n ..............9930

248. $\mathrm{SiO}_{2}$ : A2B_cI72_211_hi_i ................. 964

249. Simple Cubic $\mathrm{C}_{60}$ Buckminsterfullerine:

A_cP240_205_10d ......................957

250. Simpsonite: A4B14C3_hP21_143_bd_ac4d_d .....9905

251. SmSI: ABC_hR6_166_c_c_c ................9920

252. Sodium Chlorate: ABC3_cP20_198_a_a_b . . .... 947

253. Spinel: A3B4_cF56_227_ad_e .............. 978

254. $\mathrm{Sr}_{2} \mathrm{As}_{2} \mathrm{O}_{7}$ : A2B7C2_tP88_78_4a_14a_4a ........860

255. $\mathrm{Sr}_{2} \mathrm{Bi}_{3}$ : A3B2_oP20_52_de_cd .............. 834

256. $\mathrm{Sr}_{5} \mathrm{Si}_{3}$ : A3B5_tI32_108_ac_a2c $\ldots \ldots \ldots \ldots \ldots 80$

257. $\mathrm{SrAl}_{2} \mathrm{Se}_{4}$ : A2B4C_oC28_66_1_kl_a ............880

258. $\mathrm{SrBr}_{2}$ : A2B_tP30_85_ab2g_cg .............. 864

259. $\mathrm{SrH}_{2}$ : A2B_oP12_62_2c_c ................. 842

260. $\mathrm{SrSi}_{2}:$ A2B_cP12_212_c_a $\ldots \ldots \ldots \ldots \ldots \ldots . . \ldots 65$

261. $\mathrm{Sr}\left[\mathrm{S}_{2} \mathrm{O}_{6}\right]\left[\mathrm{H}_{2} \mathrm{O}\right]_{4}$ : A10B2C_hP39_171_5c_c_a ....924

262. $\mathrm{Sr}\left[\mathrm{S}_{2} \mathrm{O}_{6}\right]\left[\mathrm{H}_{2} \mathrm{O}\right]_{4}$ : A10B2C_hP39_172_5c_c_a ....9925

263. Stannoidite:

A8B2C12D2E_ol50_23_bcfk_i_3k_j_a ..........815

264. $\mathrm{Ta}_{2} \mathrm{H}$ : AB2_oC6_21_a_k .................. 813

\footnotetext{
${ }^{\ddagger} \mathrm{ZrO}_{2}$ and Pyrite have similar AFLOW prototype labels (i.e., same symmetry and set of Wyckoff positions with different stoichiometry labels due to alphabetic ordering of atomic species). They are generated by the same symmetry operations with different sets of parameters.
}

265. $\mathrm{Ta}_{2} \mathrm{Se}_{8} \mathrm{I}:$ AB8C2_tI44_97_e_2k_cd ...........873

266. Ta $3 \mathrm{~B}_{4}:$ A4B3_oI14_71_gh_cg ..............884

267. $\mathrm{Ta}_{3} \mathrm{~S}_{2}$ : A2B3_oC40_39_2d_2c2d .......... 826

268. TaNiTe 2 : ABC2_oP16_53_h_e_gh ........... 834

269. $\mathrm{TeO}_{6} \mathrm{H}_{6}:$ A6B_cF224_228_h_c ............. 982

270. TeZn: AB_hP6_144_a_a ..................907

271. Te[OH $]_{6}:$ A12B6C_cF608_210_4h_2h_e .........962

272. $\mathrm{Th}_{3} \mathrm{P}_{4}:$ A4B3_cI28_220_c_a .............. 970

273. $\mathrm{Th}_{6} \mathrm{Mn}_{23}$ : A23B6_cF116_225_bd2f_e ..........973

274. ThB ${ }_{4}$ : A4B_tP20_127_ehj_g ...............893

275. ThBC: ABC_tP24_91_d_d_d ..................869

276. ThBC: ABC_tP24_95_d_d_d .................872

277. $\mathrm{ThCl}_{4}$ : A4B_tI20_88_f_a ................. 865

278. Thortveitite: A7B2C2_mC22_12_aij_h_i ....... 808

279. $\mathrm{Ti}_{2} \mathrm{Ge}_{3}$ : A3B2_tP10_83_adk_j ............ 863

280. $\mathrm{Ti}_{3} \mathrm{O}:$ AB3_hP24_149_acgi_31 .............910

281. Ti 3 P: AB3_tP32_86_g_3g .............. 865

282. $\mathrm{TiAl}_{2} \mathrm{Br}_{8}:$ A2B8C_oP22_34_c_4c_a ......... 824

283. TiFeSi: ABC_oI36_46_ac_bc_3b ........... 830

284. $\mathrm{Tl}_{4} \mathrm{HgI}_{6}$ : AB6C4_tP22_104_a_2ac_c .......... 878

285. T1P 5 : A5B_oP24_26_3a3b2c_ab ........... 818

286. $\mathrm{TlZn}_{2} \mathrm{Sb}_{2}:$ A2BC2_tI20_79_c_2a_c .......... 861

287. Tongbaite: A2B3_oP20_62_2c_3c ........... 841

288. Troilite: AB_hP24_190_i_afh ...............940

289. Tychite: A4B2C6D16E_cF232_203_e_d_f_eg_a ...993

290. $\mathrm{UCl}_{3}$ : A3B_hP8_176_h_d .............. 930

291. $\mathrm{V}_{2} \mathrm{MoO}_{8}$ : AB8C2_oC22_35_a_ab3e_e ........ 825

292. VPCl9: A9BC_oC44_39_3c3d_a_c ............8827

293. $\mathrm{W}_{3} \mathrm{O}_{10}$ : A10B3_oF52_42_2abce_ab ........... 828

294. $\mathrm{W}_{5} \mathrm{Si}_{3}$ : A3B5_tI32_140_ah_bk ............. 902

295. $\mathrm{WO}_{3}$ : A3B_oP32_60_3d_d ............... 839

296. Weberite: AB7CD2_oI44_24_a_b3d_c_ac .........817

297. Westerveldite: AB_oP8_62_c_c ............. 845

298. $\mathrm{YbBaCo}_{4} \mathrm{O}_{7}$ : AB4C7D_hP26_159_b_ac_a2c_b ....915

299. $\mathrm{Zn}_{3} \mathrm{P}_{2}$ : A2B3_tP40_137_cdf_3g ............ 899

300. $\mathrm{ZnSb}_{2} \mathrm{O}_{4}$ : A4B2C_tP28_135_gh_h_d ......... 899

301. $\mathrm{ZrO}_{2}{ }^{\ddagger}$ : A2B_oP12_29_2a_a .............. 820

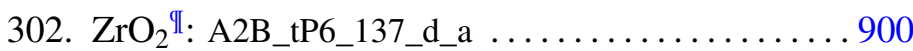




\section{POSCAR Index}

1. $\alpha-\mathrm{Al}_{2} \mathrm{~S}_{3}$ : A2B3_hP30_169_2a_3a ............924

2. $\alpha-\mathrm{CuAlCl}_{4}: \mathrm{AB} 4 \mathrm{C} \_\mathrm{tP} 12 \_112 \_\mathrm{b} \_\mathrm{n} \_\mathrm{e} \ldots \ldots \ldots . . .684$

3. $\alpha$-FeSe ${ }^{\dagger}:$ AB_oC8_67_a_g ................. 853

4. $\alpha$-Naumannite: A2B_oP12_17_abe_e .......... 813

5. $\alpha-\mathrm{NbO}_{2}$ : AB2_tI96_88_2f_4f $\ldots \ldots \ldots \ldots \ldots \ldots . \ldots 66$

6. $\alpha-\mathrm{P}_{3} \mathrm{~N}_{5}$ : A5B3_mC32_9_5a_3a ............... 805

7. $\alpha-\mathrm{PbO}^{\dagger}$ : AB_oC8_67_a_g ................ 853

8. $\alpha$-PdCl 2 : A2B_oP6_58_g_a $\ldots \ldots \ldots \ldots \ldots \ldots . \ldots 38$

9. $\alpha-\mathrm{RbPr}\left[\mathrm{MoO}_{4}\right]_{2}$ :

A2B8CD_oP24_48_k_2m_d_b ................8831

10. $\alpha-\mathrm{Sm}_{3} \mathrm{Ge}_{5}$ : A5B3_hP16_190_bdh_g ......... 940

11. $\alpha$-ThSi 2 : A2B_tI12_141_e_a .................904

12. $\alpha-\mathrm{Tl}_{2} \mathrm{TeO}_{3}$ : A3BC2_oP48_50_3m_m_2m $\ldots \ldots \ldots 833$

13. $\alpha$-Toluene: A7B8_mP120_14_14e_16e ......... 811

14. $\beta-\mathrm{Bi}_{2} \mathrm{O}_{3}$ : A2B3_tP20_117_i_adgh $\ldots . . \ldots \ldots \ldots . . . .888$

15. $\beta$-CuI: AB_hP4_156_ac_ac ................. 913

16. $\beta-\mathrm{Hg}_{4} \mathrm{Pt}$ : A4B_cI10_229_c_a $\ldots \ldots \ldots \ldots \ldots \ldots 98$

17. $\beta-\mathrm{NbO}_{2}$ : AB2_tI48_80_2b_4b $\ldots \ldots \ldots \ldots \ldots . . . .662$

18. $\beta$-PdCl $\mathrm{PdCl}_{2}$ : A2B_hR18_148_2f_f $\ldots \ldots \ldots \ldots \ldots \ldots 10$

19. $\beta$-RuCl $\mathrm{Ru}_{3}$ : A3B_hP8_158_d_a $\ldots \ldots \ldots \ldots \ldots \ldots 13$

20. $\beta-\mathrm{RuCl}_{3}$ : A3B_hP8_185_c_a $\ldots \ldots \ldots \ldots \ldots \ldots 935$

21. $\beta-\mathrm{SeO}_{2}{ }^{*}:$ A2B_oP12_26_abc_ab $\ldots \ldots \ldots \ldots \ldots . .618$

22. $\beta$-Si $\mathrm{Si}_{3} \mathrm{~N}_{4}$ : A4B3_hP14_173_bc_c ............. 926

23. $\beta-\mathrm{SiO}_{2}$ : A2B_hP9_181_j_c $\ldots \ldots \ldots \ldots \ldots \ldots . \ldots 32$

24. $\beta$ - $\mathrm{Ta}_{2} \mathrm{O}_{5}$ : A5B2_oP14_49_dehq_ab $\ldots \ldots \ldots \ldots . .631$

25. $\beta$-ThI ${ }_{3}$ : A3B_oC64_66_kl2m_bdl $\ldots \ldots \ldots \ldots \ldots . \ldots 51$

26. $\beta$-Toluene: A7B8_oP120_60_7d_8d ...........8840

27. $\beta$-V $\mathrm{V}_{3} \mathrm{~S}$ : $\mathrm{AB} 3 \_\mathrm{tP} 32 \_133 \_\mathrm{h} \_\mathrm{i} 2 \mathrm{j} \ldots \ldots . . . . . . . . .898$

28. $\delta$-PdCl 2 : A2B_mP6_10_mn_bg ..............806

29. $\delta_{H}^{I I}-\mathrm{NW}_{2}$ : AB2_hP9_164_bd_c2d $\ldots \ldots \ldots \ldots . . . .918$

30. $\epsilon-\mathrm{NiAl}_{3}$ : A3B_oP16_62_cd_c $\ldots \ldots \ldots \ldots \ldots . . .643$

31. $\epsilon-\mathrm{WO}_{3}:$ A3B_mP16_7_6a_2a ............... 804

32. $\gamma$-Ag g $_{3}$ SI: A3BC_hR5_146_b_a_a ............. 908

33. $\gamma$-MgNiSn: A7B7C2_tP32_101_bde_ade_d ...... 876

34. $\gamma-\mathrm{PdCl}_{2}$ : A2B_mP6_14_e_a $\ldots \ldots \ldots \ldots \ldots . \ldots 10$

35. $\gamma$-brass: A4B9_cP52_215_ei_3efgi ............. 967

36. $\gamma$-brass: A3B10_cI52_229_e_fh .............. 984

37. $\kappa$-alumina: A2B3_oP40_33_4a_6a ........... 824

38. $\pi-\mathrm{FeMg}_{3} \mathrm{Al}_{8} \mathrm{Si}_{6}$ :

A8BC3D6_hP18_189_bfh_a_g_i ..............939

39. $\pi-\mathrm{FeMg}_{3} \mathrm{Al}_{9} \mathrm{Si}_{5}$ :

A9BC3D5_hP18_189_fi_a_g_bh ............. 939

40. $\mathrm{Ag}_{3}\left[\mathrm{PO}_{4}\right]:$ A3B4C_cP16_218_c_e_a $\ldots \ldots \ldots \ldots 96$

41. $\mathrm{Ag}_{5} \mathrm{~Pb}_{2} \mathrm{O}_{6}$ : A5B6C2_hP13_157_2ac_2c_b ....... 913

42. AgUF 6 : AB6C_tP16_132_d_io_a .............. 897

${ }^{\dagger} \alpha$-FeSe and $\alpha$-PbO have the same AFLOW prototype label. They are generated by the same symmetry operations with different sets of parameters.

${ }^{*} \mathrm{H}_{2} \mathrm{~S}$ and $\beta-\mathrm{SeO}_{2}$ have the same AFLOW prototype label. They are generated by the same symmetry operations with different sets of parameters.
43. Akermanite: A2BC7D2_tP24_113_e_a_cef_e .....885

44. $\mathrm{Al}_{2} \mathrm{CuIr}^{\S}$ : A2BC_oC16_67_ag_b_g ............ 852

45. $\mathrm{Al}_{2} \mathrm{~S}_{3}:$ A2B3_hP30_170_2a_3a .............. 924

46. $\mathrm{Al}_{4} \mathrm{C}_{3}$ : A4B3_hR7_166_2c_ac .............. 920

47. $\mathrm{Al}_{4} \mathrm{U}$ : A4B_ol20_74_beh_e .................. 857

48. $\mathrm{Al}_{8} \mathrm{Cr}_{5}$ : A8B5_hR26_160_a3bc_a3b ........... 916

49. $\mathrm{Al}_{9} \mathrm{Mn}_{3} \mathrm{Si}$ : A9B3C_hP26_194_hk_h_a ......... 945

50. $\mathrm{AlLi}_{3} \mathrm{~N}_{2}$ : AB3C2_cI96_206_c_e_ad ............959

51. $\mathrm{AlPO}_{4}: \mathrm{AB} 2 \_\mathrm{hP72} 1192 \_\mathrm{m} \_\mathrm{j} 2 \mathrm{kl} \ldots \ldots \ldots \ldots \ldots 942$

52. $\mathrm{Al}\left[\mathrm{PO}_{4}\right]: \mathrm{AB} 4 \mathrm{C} \_\mathrm{hP7} 2168 \_2 \mathrm{~d} \_8 \mathrm{~d} \_2 \mathrm{~d} \ldots . . . . . .9923$

53. $\mathrm{Al}\left[\mathrm{PO}_{4}\right]$ : AB4C_hP72_184_d_4d_d .............994

54. Anhydrite: AB4C_oC24_63_c_fg_c .............8849

55. $\mathrm{As}_{2} \mathrm{Ba}$ : A2B_mP18_7_6a_3a ................. 804

56. $\mathrm{AsPh}_{4} \mathrm{CeS}_{8} \mathrm{P}_{4} \mathrm{Me}_{8}$ :

AB32CD4E8_tP184_93_i_16p_af_2p_4p .........8870

57. AuCN: ABC_hP3_183_a_a_a ............... 933

58. $\mathrm{AuF}_{3}:$ AB3_hP24_178_b_ac ................ 931

59. $\mathrm{AuF}_{3}$ : AB3_hP24_179_b_ac ................ 932

60. BN: AB_oF8_42_a_a .................... 829

61. BPS 4 : ABC4_oI12_23_a_b_k ............... 817

62. $\mathrm{Ba}_{5} \mathrm{In}_{4} \mathrm{Bi}_{5}$ : A5B5C4_tP28_104_ac_ac_c ........ 877

63. Ba5 $\mathrm{Si}_{3}$ : A5B3_tP32_130_cg_cf ...............896

64. $\mathrm{BaCr}_{2} \mathrm{Ru}_{4} \mathrm{O}_{12}$ :

AB2C12D4_tP76_75_2a2b_2d_12d_4d ..........857

65. $\mathrm{BaCu}_{4}[\mathrm{VO}]\left[\mathrm{PO}_{4}\right]_{4}$ :

AB4C17D4E_tP54_90_a_g_c4g_g_c .............869

66. $\mathrm{BaGe}_{2} \mathrm{As}_{2}$ : A2BC2_tP20_105_f_ac_2e ..........8878

67. $\mathrm{BaSi}_{4} \mathrm{O}_{9}$ : AB9C4_hP28_188_e_kl_ak .......... 938

68. Barite: AB4C_oP24_62_c_2cd_c .............. 845

69. $\mathrm{Be}\left[\mathrm{BH}_{4}\right]_{2}:$ A2BC8_tI176_110_2b_b_8b ........ 882

70. Benzene: AB_oP48_61_3c_3c .............. 841

71. Beryl: A2B3C18D6_hP58_192_c_f_lm_1 ........941

72. $\mathrm{Bi}_{2} \mathrm{O}_{3}:$ A2B3_hP20_159_bc_2c $\ldots \ldots \ldots \ldots . . .914$

73. $\mathrm{Bi}_{5} \mathrm{Nb}_{3} \mathrm{O}_{15}$ : A5B3C15_oP46_30_a2c_bc_a7c .....822

74. $\mathrm{BiAl}_{2} \mathrm{~S}_{4}$ : A2BC4_tP28_126_cd_e_k ............893

75. $\mathrm{BiGaO}_{3}$ : ABC3_oP20_54_e_d_cf .............. 836

76. Boracite: A7BC3D13_cF192_219_de_b_c_ah .....9969

77. C: A_tP12_138_bi .......................902

78. $\mathrm{C}_{17} \mathrm{FeO}_{4} \mathrm{Pt}$ : A17BC4D_tP184_89_17p_p_4p_io ....867

79. $\mathrm{Ca}_{3} \mathrm{Al}_{2} \mathrm{O}_{6}:$ A2B3C6_cP264_205_2d_ab2c2d_6d ...995

80. $\mathrm{Ca}_{3} \mathrm{Al}_{2} \mathrm{O}_{6}$ : A2B3C6_cP33_221_cd_ag_fh ........972

81. $\mathrm{Ca}_{3} \mathrm{PI}_{3}:$ A3B3C_cI56_214_g_h_a .............966

82. $\mathrm{Ca}_{4} \mathrm{Al}_{6} \mathrm{O}_{16} \mathrm{~S}$ :

A6B4C16D_oP108_27_abcd4e_4e_16e_e .........819

83. $\mathrm{CaRbFe}_{4} \mathrm{As}_{4}$ : A4BC4D_tP10_123_gh_a_i_d ......891

84. Calomel: AB_tI8_139_e_e ....................902

85. Carbonyl Sulphide: ABC_hR3_160_a_a_a .......917

\footnotetext{
${ }^{\S} \mathrm{Al}_{2} \mathrm{CuIr}$ and $\mathrm{HoCuP}_{2}$ have similar AFLOW prototype labels (i.e., same symmetry and set of Wyckoff positions with different stoichiometry labels due to alphabetic ordering of atomic species). They are generated by the same symmetry operations with different sets of parameters.
} 
86. $\mathrm{CdAs}_{2}$ : A2B_tI12_98_f_a .............. 874

87. $\mathrm{CdI}_{2}$ : AB2_hP9_156_b2c_3a2bc ........... 912

88. $\mathrm{Ce}_{3} \mathrm{Si}_{6} \mathrm{~N}_{11}$ : A3B11C6_tP40_100_ac_bc2d_cd ....8875

89. $\mathrm{Ce}_{5} \mathrm{Mo}_{3} \mathrm{O}_{16}$ : A5B3C16_cP96_222_ce_d_fi .......9972

90. $\mathrm{CeCo}_{4} \mathrm{~B}_{4}$ : A4BC4_tP18_137_g_b_g ......... 901

91. $\mathrm{CeRu}_{2} \mathrm{~B}_{2}$ : A2BC2_oF40_22_fi_ad_gh .........814

92. $\mathrm{CeTe}_{3}$ : AB3_oC16_40_b_3b .............88

93. $\mathrm{Co}_{2} \mathrm{Al}_{5}$ : A5B2_hP28_194_ahk_ch .......... 944

94. $\mathrm{Co}_{5} \mathrm{Ge}_{7}$ : A5B7_tI24_107_ac_abd ........... 880

95. Cobaltite: ABC_oP12_29_a_a_a .............8. 821

96. $\mathrm{Cr}_{5} \mathrm{~B}_{3}$ : A3B5_tI32_140_ah_cl ............. 903

97. $\mathrm{CrCl}_{3}$ : A3B_hP24_153_3c_2b ............ 911

98. $\mathrm{CrFe}_{3} \mathrm{NiSn}_{5}: \mathrm{AB} \_$hP6_183_c_ab ...........933

99. $\mathrm{Cs}_{2} \mathrm{ZnFe}[\mathrm{CN}]_{6}$ :

A6B2CD6E_cP64_208_m_ad_b_m_c ..........961

100. $\mathrm{Cs}_{3} \mathrm{P}_{7}: \mathrm{A} 3 \mathrm{~B} 7 \_t \mathrm{P} 40 \_76 \_3 \mathrm{a} \_7 \mathrm{a} \ldots \ldots \ldots \ldots . \ldots . . . .659$

101. $\mathrm{CsPr}\left[\mathrm{MoO}_{4}\right]_{2}:$ AB2C8D_oP24_49_g_q_2qr_e ....8832

102. $\mathrm{Cu}_{15} \mathrm{Si}_{4}:$ A15B4_cI76_220_ae_c ............970

103. $\mathrm{Cu}_{2} \mathrm{Fe}[\mathrm{CN}]_{6}:$ A12B2C_cF60_196_h_bc_a ...... 946

104. $\mathrm{Cu}_{3} \mathrm{P}:$ A3B_hP24_165_bdg_f .............. 919

105. $\mathrm{Cu}_{3} \mathrm{P}^{\|}:$A3B_hP24_185_ab2c_c ..............935

106. $\mathrm{CuBi}_{2} \mathrm{O}_{4}$ : A2BC4_tP28_130_f_c_g ...........886

107. $\mathrm{CuBrSe}_{3}: \mathrm{ABC} 3 \_$oP20_30_2a_c_3c ........... 822

108. $\mathrm{CuBrSe}_{3}$ : ABC3_oP20_53_e_g_hi ........... 835

109. $\mathrm{CuCrCl}_{5}\left[\mathrm{NH}_{3}\right]_{6}: \mathrm{A} 5 \mathrm{BCD} 6 \_\mathrm{cF} 416 \_228 \_$gg_c_b_h . .981

110. CuI: AB_hP12_156_2ab3c_2ab3c ..........912

111. $\mathrm{CuNiSb}_{2}$ : ABC2_hP4_164_a_b_d ........... 919

112. Cubanite: AB2C3_oP24_62_c_d_cd .............8844

113. Downeyite: A2B_tP24_135_gh_h .............8898

114. $\mathrm{Er}_{3} \mathrm{Ru}_{2}$ : A3B2_hP10_176_h_bd ........... 929

115. F6 KP: A24BC_cF104_209_j_a_b ........... 962

116. FCC $\mathrm{C}_{60}$ Buckminsterfullerine:

A_cF240_202_h2i ................... 951

117. $\mathrm{Fe}_{12} \mathrm{Zr}_{2} \mathrm{P}_{7}: \mathrm{A} 12 \mathrm{~B} 7 \mathrm{C} 2 \_\mathrm{hP} 21$ 174_2j2k_ajk_cf ....9927

118. $\mathrm{Fe}_{3} \mathrm{Te}_{3} \mathrm{Tl}: \mathrm{A} 3 \mathrm{~B} 3 \mathrm{C} \_\mathrm{hP1} 14 \_176 \_$h_h_d ..........999

119. $\mathrm{Fe}_{3} \mathrm{Th}_{7}$ : A3B7_hP20_186_c_b2c .......... 937

120. $\mathrm{FeCu}_{2} \mathrm{Al}_{7}$ : A7B2C_tP40_128_egi_h_e ..........895

121. FeNi: AB_mP4_6_2b_2a .................883

122. FeOCl: ABC_oP6_59_a_b_a .............8838

123. $\mathrm{FePSe}_{3}: \mathrm{ABC} 3 \_$hR10_146_2a_2a_2b ...........909

124. FeS: AB_oF8_22_a_c .................. 815

125. FeSb 2 : AB2_oP6_34_a_c .................825

126. Forsterite: A2B4C_oP28_62_ac_2cd_c ......... 842

127. Fresnoite: A2B8C2D_tP26_100_c_abcd_c_a .....8875

128. $\mathrm{GaCl}_{2}$ : A2B_oP24_52_2e_cd ............. 834

129. GaSb: AB_tI4_119_c_a ................... 890

130. Garnet: A2B3C12D3_cI160_230_a_c_h_d ........997

\footnotetext{
${ }^{\|} \mathrm{Cu}_{3} \mathrm{P}$ and $\mathrm{Na}_{3}$ As have similar AFLOW prototype labels (i.e., same symmetry and set of Wyckoff positions with different stoichiometry labels due to alphabetic ordering of atomic species). They are generated by the same symmetry operations with different sets of parameters.
}

131. $\mathrm{Gd}_{3} \mathrm{Al}_{2}$ : A2B3_tP20_102_2c_b2c ..........876

132. GdSI: ABC_hP12_174_cj_fk_aj .............9927

133. GeAs 2 : A2B_oP24_55_2g2h_gh ...........836

134. GeP: AB_tI4_107_a_a .................. 880

135. $\mathrm{GeSe}_{2}$ : AB2_tP12_81_adg_2h .............863

136. $\mathrm{H}_{2}$ S: A2B_aP6_2_aei_i ................. 802

137. $\mathrm{H}_{2} \mathrm{~S}$ : A2B_mP12_13_2g_ef $\ldots \ldots \ldots \ldots \ldots \ldots \ldots 810$

138. $\mathrm{H}_{2} \mathrm{~S}^{*}$ : A2B_oP12_26_abc_ab ............. 818

139. $\mathrm{H}_{2} \mathrm{~S}$ : A2B_oC24_64_2f_f .................. 849

140. $\mathrm{H}_{2}$ S III: A2B_tP48_77_8d_4d ............ 860

141. $\mathrm{H}_{2}$ S IV: A2B_mP12_7_4a_2a ............ 803

142. $\mathrm{H}_{3} \mathrm{Cl}$ : AB3_mC16_9_a_3a ................806

143. $\mathrm{H}_{3} \mathrm{Cl}: \mathrm{AB} 3 \_\mathrm{mP16} 10 \_\mathrm{mn} \_3 \mathrm{~m} 3 \mathrm{n} \ldots \ldots \ldots \ldots . . .607$

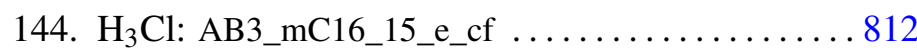

145. $\mathrm{H}_{3} \mathrm{Cl}$ : AB3_oP16_19_a_3a ................ 813

146. $\mathrm{H}_{3} \mathrm{~S}$ : A3B_oI32_23_ij2k_k ............... 815

147. $\mathrm{H}_{3} \mathrm{~S}$ : A3B_oC64_66_gi2lm_21 ............ 850

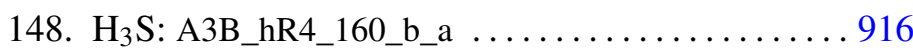

149. H-III: A_mC24_15_2e2f ..................812

150. HCl: AB_oC8_36_a_a ............... 826

151. $\mathrm{HgI}_{2}$ : AB2_tP12_115_j_egi .............. 887

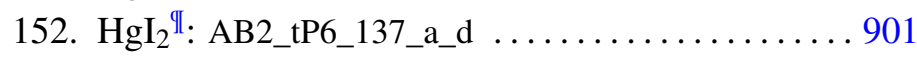

153. $\mathrm{HoCuP}_{2}{ }^{\S}: \mathrm{ABC} 2 \_\mathrm{oC} 16 \_67 \_b \_g \_a g \ldots . . . \ldots . . .852$

154. $\mathrm{Ir}_{3} \mathrm{Ga}_{5}:$ A5B3_tP32_118_g2i_aceh .......... 889

155. $\mathrm{Ir}_{3} \mathrm{Ge}_{7}$ : A7B3_cI40_229_df_e ............ 986

156. $\mathrm{IrGe}_{4}$ : A4B_hP15_144_4a_a ............... 907

157. $\mathrm{K}_{2} \mathrm{CdPb}:$ AB2C_oC16_40_a_2b_b ........... 828

158. $\mathrm{K}_{2} \mathrm{PtCl}_{6}$ : A6B2C_cF36_225_e_c_a .......... 976

159. $\mathrm{K}_{2} \mathrm{SnCl}_{6}$ : A6B2C_tP18_128_eh_d_b ........... 894

160. $\mathrm{K}_{2} \mathrm{Ta}_{4} \mathrm{O}_{9} \mathrm{~F}_{4}: \mathrm{A} 2 \mathrm{~B} 13 \mathrm{C} 4 \_$hP57_168_d_c6d_2d .....9922

161. $\mathrm{KAg}\left[\mathrm{CO}_{3}\right]: \mathrm{ABCD} 3 \_\mathrm{I} 48 \_73 \_\mathrm{d}$ e_e_ef ........ 856

162. $\mathrm{KAu}_{4} \mathrm{Sn}_{2}$ : A4BC2_tI28_120_i_d_e ...........890

163. $\mathrm{KB}_{6} \mathrm{H}_{6}:$ A6B6C_cF104_202_h_h_c ...........950

164. $\mathrm{KBO}_{2}$ : ABC2_hR24_167_e_e_2e ...........9921

165. $\mathrm{KCeSe}_{4}$ : ABC4_tP12_125_a_b_m ............883

166. $\mathrm{KHg}_{2}$ : A2B_oI12_74_h_e ............. 856

167. $\mathrm{KNiCl}_{3}$ : A3BC_hP30_185_cd_c_ab ...........934

168. $\mathrm{KSbO}_{3}:$ AB3C_cP60_201_ce_fh_g .............949

169. $\mathrm{La}_{2} \mathrm{NiO}_{4}$ : A2BC4_oP28_50_ij_ac_ijm .......... 832

170. $\mathrm{La}_{2} \mathrm{O}_{3}$ : A2B3_hP5_164_d_ad ............. 918

171. $\mathrm{La}_{43} \mathrm{Ni}_{17} \mathrm{Mg}_{5}$ :

A43B5C17_oC260_63_c8fg6h_cfg_ce3f2h ........846

172. LaPtSi: ABC_tI12_109_a_a_a ..............881

173. LaRhC $_{2}$ : A2BC_tP16_76_2a_a_a ............ 858

174. Li $_{2} \mathrm{MoF}_{6}:$ A6B2C_tP18_94_eg_c_a . . . . . . . . 872

175. Li $_{2} \mathrm{Sb}$ : A2B_hP18_190_gh_bf .............940

176. $\mathrm{Li}_{2} \mathrm{Si}_{2} \mathrm{O}_{5}:$ A2B5C2_oC36_37_d_c2d_d .........88 826

\footnotetext{
${ }^{I} \mathrm{ZrO}_{2}$ and $\mathrm{HgI}_{2}$ have similar AFLOW prototype labels (i.e., same symmetry and set of Wyckoff positions with different stoichiometry labels due to alphabetic ordering of atomic species). They are generated by the same symmetry operations with different sets of parameters.
} 
177. LiScI $_{3}:$ A3BC_hP10_188_k_a_e ............938

178. LiSn: AB_mP6_10_en_am ................. 808

179. M-carbon: A_mC16_12_4i ................. 809

180. Mavlyanovite: A5B3_hP16_193_dg_g .........9943

181. $\mathrm{Mg}_{2} \mathrm{Zn}_{11}$ : A2B11_cP39_200_f_aghij ..........9948

182. $\mathrm{MgB}_{12} \mathrm{H}_{12}\left[\mathrm{H}_{2} \mathrm{O}\right]_{12}$ :

A12B36CD12_cF488_196_2h_6h_ac_fgh .........9947

183. $\mathrm{MgSO}_{4}:$ AB4C_oC24_63_a_fg_c .............. 848

184. $\mathrm{Mg}[\mathrm{NH}]$ : ABC_hP36_175_jk_jk_jk .......... 928

185. $\mathrm{Mn}_{2} \mathrm{~B}: \mathrm{AB} 2 \_\mathrm{oF} 48 \_70 \_f \_f g \ldots \ldots \ldots \ldots . \ldots . \ldots . \ldots 54$

186. $\mathrm{MnAl}_{6}:$ A6B_oC28_63_efg_c ............... 847

187. $\mathrm{MnF}_{2}$ : A2B_tP12_111_2n_adf ........... 883

188. $\mathrm{MnGa}_{2} \mathrm{Sb}_{2}$ : A2BC2_oI20_45_c_b_c ..........8830

189. $\mathrm{Mo}_{8} \mathrm{P}_{5}:$ A8B5_mP13_6_a7b_3a2b ........... 802

190. $\mathrm{MoS}_{2}$ : AB2_hP12_143_cd_ab2d .............906

191. Moissanite-15R: AB_hR10_160_5a_5a ..........917

192. Molybdite: AB3_oP16_62_c_3c . . . . . . . . . . . 844

193. Muthmannite: ABC2_mP8_10_ac_eh_mn .......88 807

194. NV: AB_tP8_111_n_n ..................... 884

195. $\mathrm{Na}_{3} \mathrm{As}{ }^{\|}:$AB3_hP24_185_c_ab2c ........... 936

196. $\mathrm{Na}_{4} \mathrm{Ti}_{2} \mathrm{Si}_{8} \mathrm{O}_{22}\left[\mathrm{H}_{2} \mathrm{O}\right]_{4}$ :

A4B2C13D_tP40_90_g_d_cef2g_c ............. 868

197. $\mathrm{Na}_{5} \mathrm{Fe}_{3} \mathrm{~F}_{14}$ : A14B3C5_tP44_94_c3g_ad_bg . . . . . 871

198. $\mathrm{NaFeS}_{2}$ : ABC2_oI16_23_ab_i_k ........... 816

199. $\mathrm{NaGdCu}_{2} \mathrm{~F}_{8}$ : A2B8CD_tI24_97_d_k_a_b ........873

200. $\mathrm{NaZn}_{13}$ : AB13_cF112_226_a_bi ..............977

201. $\mathrm{NaZn}[\mathrm{OH}]_{3}$ : A3BC3D_tP64_106_3c_c_3c_c . . . . 879

202. $\mathrm{Nb}_{4} \mathrm{CoSi}:$ AB4C_tP12_124_a_m_c . . . . . . . . . 891

203. $\mathrm{Nb}_{7} \mathrm{Ru}_{6} \mathrm{~B}_{8}: \mathrm{A} 8 \mathrm{~B} 7 \mathrm{C} 6 \_\mathrm{hP} 21 \_175 \_\mathrm{ck} \_\mathrm{aj} \_\mathrm{k} \ldots \ldots . .928$

204. NbAs: AB_tI8_109_a_a ... . . . . . . . . . . . . . 882

205. NbPS: ABC_oI12_71_h_j_g ............. 855

206. $\mathrm{NbTe}_{4}:$ AB4_tP10_103_a_d ..................877

207. $\mathrm{NbTe}_{4}$ : AB4_tP10_124_a_m ................. 892

208. $\mathrm{Ni}_{3} \mathrm{P}:$ A3B_tI32_82_3g_g ................ 863

209. $\mathrm{Ni}_{3}$ Ti: A3B_hP16_194_gh_ac ............ 943

210. Nierite: A4B3_hP28_159_ab2c_2c ...........914

211. $\mathrm{PH}_{3}$ : A3B_cP16_208_j_b ................960

212. $\mathrm{PI}_{3}$ : A3B_hP8_173_c_b ................ 926

213. $\mathrm{Pd}_{17} \mathrm{Se}_{15}$ : A17B15_cP64_207_acfk_eij .........960

214. $\mathrm{Pd}_{4} \mathrm{Se}$ : A4B_tP10_114_e_a ............... 886

215. PdSn 4 : AB4_oC20_68_a_i ................. 854

216. Petzite: A3BC2_cI48_214_f_a_e ............ 966

217. Phenakite: A2B4C_hR42_148_2f_4f_f ........ 909

218. Pinnoite: A2B6CD7_tP64_77_2d_6d_d_ab6d ......859

219. Post-perovskite: AB3C_oC20_63_a_cf_c ......... 848

220. $\mathrm{PrNiO}_{3}$ : AB3C_hR10_167_b_e_a ............9921

221. $\mathrm{PrRu}_{4} \mathrm{P}_{12}$ : A12BC4_cP34_195_2j_ab_2e ........945

222. $\mathrm{PtPb}_{4}$ : A4B_tP10_125_m_a ................ 892
223. Pyrite ${ }^{\ddagger}:$ AB2_oP12_29_a_2a ..............8 821

224. Pyrochlore: A2BCD3E6_cF208_203_e_c_d_f_g ...952

225. Pyrochlore Iridate:

A2B2C7_cF88_227_c_d_af ................9978

226. Quartenary Heusler:

ABCD_cF16_216_c_d_b_a ...................9968

227. R-carbon: A_oP16_55_2g2h .............8837

228. Rasvumite: A2BC3_oC24_63_e_c_cg ...........8846

229. $\mathrm{Rb}_{2} \mathrm{TiCu}_{2} \mathrm{~S}_{4}:$ A2B2C4D_tP18_132_e_i_o_d .......897

230. $\mathrm{Rb}_{3} \mathrm{AsSe}_{16}$ : AB3C16_cF160_203_b_ad_eg .......9954

231. RbGa $:$ A3B_tI24_119_b2i_af ............. 889

232. $\mathrm{Re}_{2} \mathrm{O}_{5}\left[\mathrm{SO}_{4}\right]_{2}: \mathrm{A} 13 \mathrm{~B} 2 \mathrm{C} 2 \_$oP34_32_a6c_c_c ......8 823

233. $\mathrm{Re}_{3} \mathrm{~N}$ : AB3_hP4_187_e_fh ............... 937

234. $\mathrm{Rh}_{2} \mathrm{Ga9}$ : A9B2_mP22_7_9a_2a ........... 805

235. $\mathrm{Rh}_{2} \mathrm{~S}_{3}$ : A2B3_oP20_60_d_cd ............ 839

236. $\mathrm{Rh}_{3} \mathrm{P}_{2}$ : A2B3_tP5_115_g_ag ............ 886

237. $\mathrm{Rh}_{5} \mathrm{Ge}_{3}$ : A3B5_oP16_55_ch_agh ...........887

238. $\mathrm{Ru}_{2} \mathrm{Sn}_{3}:$ A2B3_tP20_116_bci_fj ............887

239. RuIn 3 : A3B_tP16_118_ei_f ............... 888

240. S-II: A_hP9_154_bc ................. 911

241. S-III: A_tI16_142_f ...................... 905

242. S-carbon: A_mP8_10_2m2n .............. 808

243. Sc-V: A_hP6_178_a .................. 931

244. $\mathrm{ScRh}_{6} \mathrm{P}_{4}:$ A4B6C_hP11_143_bd_2d_a ......... 906

245. $\mathrm{SeO}_{3}$ : A3B_tP32_114_3e_e ............... 885

246. Sheldrickite:

A2B3C3DE7_hP48_145_2a_3a_3a_a_7a ........ 907

247. $\mathrm{SiO}_{2}$ : A2B_hP36_177_j2lm_n ...........930

248. $\mathrm{SiO}_{2}$ : A2B_cI72_211_hi_i ...............964

249. Simple Cubic $\mathrm{C}_{60}$ Buckminsterfullerine:

A_cP240_205_10d ......................957

250. Simpsonite: A4B14C3_hP21_143_bd_ac4d_d .....905

251. SmSI: ABC_hR6_166_c_c_c ............... 920

252. Sodium Chlorate: ABC3_cP20_198_a_a_b . . . . . . 948

253. Spinel: A3B4_cF56_227_ad_e ..............9980

254. $\mathrm{Sr}_{2} \mathrm{As}_{2} \mathrm{O}_{7}$ : A2B7C2_tP88_78_4a_14a_4a ....... 861

255. $\mathrm{Sr}_{2} \mathrm{Bi}_{3}$ : A3B2_oP20_52_de_cd ............ 834

256. $\mathrm{Sr}_{5} \mathrm{Si}_{3}:$ A3B5_tI32_108_ac_a2c .......... 881

257. $\mathrm{SrAl}_{2} \mathrm{Se}_{4}$ : A2B4C_oC28_66_1_kl_a ...........8850

258. $\mathrm{SrBr}_{2}$ : A2B_tP30_85_ab2g_cg ............ 864

259. $\mathrm{SrH}_{2}$ : A2B_oP12_62_2c_c ............... 843

260. $\mathrm{SrSi}_{2}$ : A2B_cP12_212_c_a .............. 965

261. $\mathrm{Sr}\left[\mathrm{S}_{2} \mathrm{O}_{6}\right]\left[\mathrm{H}_{2} \mathrm{O}\right]_{4}: \mathrm{A} 10 \mathrm{~B} 2 \mathrm{C} \_$hP39_171_5c_c_a ....99

262. $\mathrm{Sr}\left[\mathrm{S}_{2} \mathrm{O}_{6}\right]\left[\mathrm{H}_{2} \mathrm{O}\right]_{4}:$ A10B2C_hP39_172_5c_c_a ....99

263. Stannoidite:

A8B2C12D2E_oI50_23_bcfk_i_3k_j_a .........816

264. Ta 2 H: AB2_oC6_21_a_k ................. 814

${ }^{\ddagger} \mathrm{ZrO}_{2}$ and Pyrite have similar AFLOW prototype labels (i.e., same symmetry and set of Wyckoff positions with different stoichiometry labels due to alphabetic ordering of atomic species). They are generated by the same symmetry operations with different sets of parameters. 


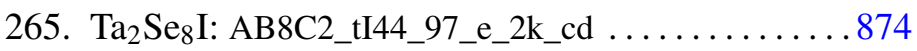

266. $\mathrm{Ta}_{3} \mathrm{~B}_{4}$ : A4B3_oI14_71_gh_cg ................ 855

267. $\mathrm{Ta}_{3} \mathrm{~S}_{2}$ : A2B3_oC40_39_2d_2c2d $\ldots \ldots \ldots \ldots \ldots . \ldots 27$

268. TaNiTe 2 : ABC2_oP16_53_h_e_gh ............. 835

269. $\mathrm{TeO}_{6} \mathrm{H}_{6}$ : A6B_cF224_228_h_c ..............998

270. TeZn: AB_hP6_144_a_a ...................907

271. Te[OH] $]_{6}$ : A12B6C_cF608_210_4h_2h_e ........963

272. $\mathrm{Th}_{3} \mathrm{P}_{4}:$ A4B3_cI28_220_c_a ................9971

273. $\mathrm{Th}_{6} \mathrm{Mn}_{23}$ : A23B6_cF116_225_bd2f_e .......... 974

274. ThB 4 : A4B_tP20_127_ehj_g . ................. 894

275. ThBC: ABC_tP24_91_d_d_d .................. 869

276. ThBC: ABC_tP24_95_d_d_d ................. 872

277. $\mathrm{ThCl}_{4}$ : A4B_tI20_88_f_a $\ldots . . . . . . . . . . . . .865$

278. Thortveitite: A7B2C2_mC22_12_aij_h_i . .......8 809

279. $\mathrm{Ti}_{2} \mathrm{Ge}_{3}$ : A3B2_tP10_83_adk_j .............. 864

280. $\mathrm{Ti}_{3} \mathrm{O}:$ AB3_hP24_149_acgi_31 ................910

281. Ti 3 P: AB3_tP32_86_g_3g $\ldots \ldots \ldots \ldots \ldots \ldots \ldots . \ldots 65$

282. $\mathrm{TiAl}_{2} \mathrm{Br}_{8}$ : A2B8C_oP22_34_c_4c_a $\ldots \ldots \ldots \ldots .624$

283. TiFeSi: ABC_ol36_46_ac_bc_3b .............. 830

284. $\mathrm{Tl}_{4} \mathrm{HgI}_{6}$ : AB6C4_tP22_104_a_2ac_c ........... 878

285. TlP : A5B_oP24_26_3a3b2c_ab .............. 819

286. $\mathrm{TlZn}_{2} \mathrm{Sb}_{2}$ : A2BC2_tI20_79_c_2a_c $\ldots \ldots \ldots \ldots . . . .662$

287. Tongbaite: A2B3_oP20_62_2c_3c ............ 842

288. Troilite: AB_hP24_190_i_afh ............... 941

289. Tychite: A4B2C6D16E_cF232_203_e_d_f_eg_a ...995

290. $\mathrm{UCl}_{3}$ : A3B_hP8_176_h_d ................ 930

291. $\mathrm{V}_{2} \mathrm{MoO}_{8}$ : AB8C2_oC22_35_a_ab3e_e ......... 825

292. $\mathrm{VPCl}_{9}$ : A9BC_oC44_39_3c3d_a_c ............8827

293. $\mathrm{W}_{3} \mathrm{O}_{10}$ : A10B3_oF52_42_2abce_ab ............ 829

294. $\mathrm{W}_{5} \mathrm{Si}_{3}$ : A3B5_tI32_140_ah_bk .............. 903

295. $\mathrm{WO}_{3}$ : A3B_oP32_60_3d_d ................. 839

296. Weberite: AB7CD2_oI44_24_a_b3d_c_ac ........8817

297. Westerveldite: AB_oP8_62_c_c .............. 845

298. $\mathrm{YbBaCo}_{4} \mathrm{O}_{7}$ : AB4C7D_hP26_159_b_ac_a2c_b ....915

299. $\mathrm{Zn}_{3} \mathrm{P}_{2}$ : A2B3_tP40_137_cdf_3g ..............900

300. $\mathrm{ZnSb}_{2} \mathrm{O}_{4}$ : A4B2C_tP28_135_gh_h_d .......... 899

301. $\mathrm{ZrO}_{2}{ }^{\ddagger}:$ A2B_oP12_29_2a_a .................88

302. $\mathrm{ZrO}_{2}{ }^{\mathrm{TI}}:$ A2B_tP6_137_d_a $\ldots . . . . . . . . . . . .900$ 